\title{
MISCELLANEOUS PUBLICATIONS
}

\author{
OP TH
}

NATIONAL BUREAU OF STANDARDS

NOs. $120 \cdot 127$ 







U. S. DEPARTMENT OF COMMERCE

DANIEL C. ROPER, Secretary

BUREAU OF STANDARDS

LYMAN J. BRIGGS, Acting Director

\title{
STANDARDS AND SPECIFICATIONS FOR METALS AND METAL PRODUCTS
}

\begin{abstract}
NATIONALLY RECOGNIZED STANDARDS AND SPECIFICATIONS FOR ORES, METALS, AND MANUFACTURES

EXCEPT MACHINERY, VEHICLES, AND ELECTRICAL SUPPLIES
\end{abstract}

MISCELLANEOUS PUBLICATION No. 120

[Issued March 11, 1933]

PREPARED BY GEORGE A. WARDLAW, UNDER THE DIRECTION OF A. S. MCALLISTER, CHIEF OF THE DIVISION OF SPECIFICATIONS

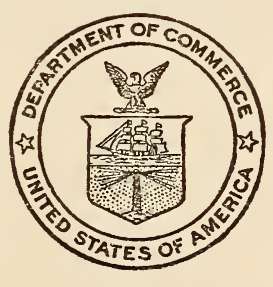

UNITED STATES

GOVERNMENT PRINTING OFFICE

WASHINGTON : 1933 
This volume represents an attempt on the part of the Department of Commerce to collect and publish the substance of the standards and specifications relating to ores, metals, and manufactures (except machinery, vehicles, and electrical supplies) formulated by the national technical societies, the trade associations having national recognition, or other organizations which speak for industry or with the authority of the Federal Government as a whole.

Criticisms and suggestions are desired from all interested persons, so that the presentation of the information concerning the nationallyrecognized specifications may be made most nearly universally satisfactory and that the collection of existing specifications and classification thereof may be kept as complete, accurate, and up to date as possible. All recommendations for improvement received will be given careful consideration when future editions are issued. 


\section{LETTER OF SUBMITTAL}

\section{Department of Commerce, Bureau of Standards, Washington, March 9, 1933.}

Sir: I have the honor to submit herewith for publication the manuscript for a compilation relating to "Standards and Specifications for Metals and Metal Products."

In conformity to plans outlined by the Secretary of Commerce in 1923, there has been made under the joint auspices of the Bureau of Standards and the Bureau of Foreign and Domestic Commerce a review of the nationally-recognized standards, specifications, simplifications, and testing methods in use in this country. The material obtained as a result of this review, following recommendations of an advisory board composed of official representatives of 14 national organizations interested in the utilization of specifications, was divided into two distinct parts. One part, in the nature of an index, was first issued in 1925, and revised in 1932, as the National Directory of Commodity Specifications. The second part, under the recommendations, was to contain copies of actual specifications (or abstracts thereof) instead of descriptive titles.

The first step in the accomplishment of this second part of the work was achieved in 1927, with the publication of the volume, Standards and Specifications in the Wood-Using Industries. In 1930 there appeared a second volume, on Standards and Specifications for Nonmetallic Minerals and their Products.

The present compilation represents a further step in the fulfillment of the second part of the foregoing program, being the third of the series of publications dealing with standards and specifications in various industries, to be issued as rapidly as conditions permit.

The arrangement and distribution of standards and specifications presented in this compilation conforms with the decimal system of indexing made use of in the National Directory of Commodity Specifications. In certain cases, due to the expansion of standardization, it has been necessary to enlarge upon the system laid out in the volume referred to.

Respectfully,

Hon. Daniel C. Roper,

LyMAN J. Briggs, Acting Director, Bureau of Standards.

Secretary of Commerce. 



\section{CONTENTS}

Page

Introduction

600-609

Class No.

600.

600. 0

600. 1

600. 2

600. 3

600. 4

600. 5

600. 6

600. 7

601.

601. 1

601. 2

601. 20

601. 21

601. 22

601. 23

601. 24

601.3

602 .

602. 1

602. 2

603.

603. 0

603. 1

603. 2

603. 21

603. 22

603. 23

603. 24

603. 25

603. 26

603. 27

603. 3

603. 31

603. 32

603. 33

603. 4

603. 40

603. 41

603. 42

603. 43

603. 5

603. 50

603.51

603.52

603.53

603. 54

603.55

603. 56

\section{IRON AND STEEL}

General items relating to both ferrous and nonferrous material .

Common basic requirements ....-

Testing of metals _............

Classification of metals . ........

Coating of metals .............

Definitions of metals ...........

Heat treatment of metals......

Plumbing .

Weights and tolerances of iron and steel

Ores, pig iron, and scrap

Iron ore

Pig iron.....................

General items_.............

Charcoal pig iron

Coke pig iron

Foundry pig iron............

Malleable pig iron _.........

Iron and steel scrap........

Finished and semifinished iron and steel .......................

Iron billets.

Steel bars and billets ........

Iron and steel bars, rods, and wire.

General items ..............

Wrought iron bars and rods....

Carbon steel bars and rods.....Steel stock for bolts, nuts, rivets, and screws........... Steel for case hardening ...... Commercial grade steel bars and rods ...............

Steel for shafts, axles, and forgings

Steel bars and rods for welding -

Steel bars and rods for automotive and railway use

Spring and tool steel bars and rods Drill tool steel bars and rods _.-

Hand, high-speed, and machine tool steels

Spring steel bars and rods...-

Iron and steel wire...........

General items

Bare and coated iron and steel

Bare and coated wire rope....

Manufactures of iron and steel wire.

Chains and attachments......

General items ....

Long-link chains

Short-link, straight-link chains _

Stud-link chains _..._._._.

Twisted-link chains

Straight and twisted link vehicle chains

Weldless chains, various link patterns.

\begin{tabular}{r|ll} 
Page & \multicolumn{2}{|c}{ Class No. } \\
1 & & \\
1 & & \\
7 & 603.57 \\
51 & 603.59 & \\
60 & 604. & I \\
71 & 604.0 & \\
72 & 604.1 & \\
113 & 604.10 \\
116 & 604.11 \\
119 & 604.12 \\
119 & 604.13 \\
119 & 604.14 \\
119 & 604.19 \\
129 & \\
129 & 604.2
\end{tabular}

604. 2

130 604. 20

131 604. 21

131

131

131

131

134

134

135

141

141

146

146

149

149

149

150

150

150

150

152

153

153

155

158

191

198

198

201

201

204
211

211

211
604. 22

604. 23

604. 24

604. 25

604. 29

604. 3

604. 30

604. 31

604. 32

605.

605. 0

605. 1

605. 11

605. 12

605. 13

605. 14

605. 15

605. 19

605. 2

605. 20

605. 21

605. 22

605. 23

605. 24

605. 25

606.

606. 0

606. 1 Continued.

Chains and attachments-Contd. Chain attachments......... 213

Miscellaneous chains

Iron and steel plates and sheets..-- 224

General items.............. 224

Iron and steel plates........ 226

General items_............. 226

Steel plates for pressure vessels_ $\quad 227$

Steel plates for nonpressure tanks and stacks

Steel plates for ship hulls .....

Wrought iron plates.......

Miscellaneous iron and steel plates...................

Iron and steel sheets........

General items......

Iron and steel sheets for magneto electric apparatus...--

Iron and steel strips, bands, and hoops _. . _............

Iron and steel sheets for drawing and forming ........

Spring and tool steel sheets.---

Iron and steel sheets for automotive and railway use

Iron and steel sheets for miscellaneous purposes.......-.--

Tin-coated and zinc-coated sheets

General items terneplate) ...............

Zinc-coated (galvanized) steel sheets _....................

Structural iron and steel.......

General items..............

Structural shapes and plates, not fabricated.

Structural steel for bridges...-

Structural steel for buildings.-

Structural steel for cars and locomotives

Structural steel for machinery.

Structural iron and steel for ships _.................

Miscellaneous structural steel.Structural shapes and plates, fabricated.

General items

Steel bridges and railway turntables

Steel buildings and metal parts of buildings . . . . . . . .

Steel tanks, towers, and flumes.

Metal lath_...........

Steel for concrete reinforcement_..................

Railway track material_.........

General items.............

Steel rails, tee, and girder......
234

234

234

235

236

236

236

236

239

239

239

241

241

241

241

243

246

246

249

249

252

254

267

268

272

273

273

273

275

283

300

304

310

310

311 
Class No.

Subject

Railway track material-Contd.

606. 2

606. 3

606. 4

606.5

606. 6

607.

607. 0

607. 1

607. 10

607.11

607. 12

607. 13

607. 14

607. 2

607. 3

607. 4

607.5

610-619

611.

611. 0

611. 1

611. 11

611. 12

611. 13

611. 14

611. 15

611. 16

611. 17

611. 18

611. 19

611. 2

611. 21

611. 22

611. 23

611. 29

611. 3

611. 4

611. 40

611. 41

611. 42

611. 43

611. 44

611. 49

611.5

611. 50

611. 51

611. 52

611. 53

611. 54

611. 55

611. 56

611. 59

612.

612. 1

612. 11

612. 12
Rail joint plates and tie plates... Switches and special track work

Track bolts, nuts, and washers

Track spikes

Track tie rods

Tubular products and fittings, cocks, and valves...............

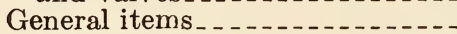

Cast-iron pipe, fittings, and connections..................

General items...............

Cast-iron pipe culverts, drains, and sewers

Cast-iron gas and water pipe -
Cast-iron pipe for railings and poles.

Cast-iron pipe fittings and connections . . . . . . . . . . .

Malleable iron pipe, fittings, and connections _...........

Wrought-iron pipe, fittings, and connections _._.

Steel pipe, fittings, and connec-

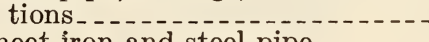

Sheet iron and steel pipe

\begin{tabular}{l|l} 
Page & \multicolumn{1}{|l}{ Class No } \\
326 & \\
334 & 607.6 \\
352 & \\
357 & 607.7 \\
360 & \\
& 608. \\
361 & 608.0 \\
361 & 608.1 \\
& 608.11 \\
369 & 608.12 \\
369 & 608.13 \\
& 608.14 \\
369 & 608.15 \\
374 & 608.2 \\
& 608.3 \\
385 & \\
& 608.31 \\
385 & 608.32 \\
& 608.4 \\
416 & 608.5 \\
& 608.51 \\
430 & \\
435 & 608.52 \\
488 & 608.6 \\
& 608.7
\end{tabular}

Subject

Tubular products and fittings, cocks, and valves-Continued.

Valves, cocks, and hydrants, ferrous and nonferrous........

Metallic hose, ferrous and nonferrous.-1-_-

Nails, screws, bolts, nuts, and rivets Neneral items Nails, brads, staples, and tacks.Nails

Brads_...

Staples_. - . . .

Tacks.

Spikes_-_._.

Screws.

Bolts and nuts, except railway track bolts.......... Bolts

Nuts

Rivets, iron or steel

Pins and shaft keys Pins, cotter, dowel, rod end pins, etc . Shaft keys

Washers Turnbuckles.

IRON AND STEEL MANUFACTURES

Castings and forgings.

635

General items

Gray iron castings

Gray cast iron

Sluice gates and mooring bitts

Floor drains and traps......-

Grates and grate bars.

Pulleys . Heating and cooking vessels ..-

Cast-iron wheels...........

Miscellaneous manufactures of gray cast iron

Malleable iron castings _.......

Malleable cast iron.

Journal boxes............

Rowlocks _... malleable cast iron ........

Wrought iron forgings

Steel castings _..._._._._._.

General items

Railway brake beams

Railway brake shoes, cast steel and cast iron forged steel.............

Miscellaneous manufactures of cast steel . . . . . . . . . .

Steel forgings

General items

Forged steel.

Axles, shafts, and similar forgings...-...-.

Wheels and tires, steel

Springs, helical and elliptical, and parts..............

Pinions and gears
Turbine bucket wheels.

Miscellaneous manufactures of steel forgings

Cutlery, hollow ware, and other household utensils..........

Cutlery, except tools......

Pocket knives....

Razors
612. 13

612. 14

612. 2

612. 20

612. 21

612. 22

612. 23

612. 29

613.

613. 1

613. 2

613. 3

613. 4

613. 5

613. 6

613. 7

613. 9

614.

614. 0

614. 1

614. 2

614. 3

614. 4

614. 9

615.

615. 0

615. 1

615. 11

615. 12

615. 13

615. 14

615. 15

615. 2

615. 20

615. 21

615. 22

Cutlery, hollow ware, and other household utensils-Contd.

Cutlery, except tools-Contd.

Scissors and shears

Table and kitchen cutlery....

Hollow and stamped ware, iron and steel.

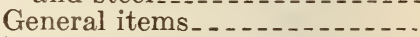

Bath tubs............

Hotel and household hollow and stamped ware.........

Water-closets and sinks....--

Miscellaneous hollow and stamped ware

Metal furniture and fixtures.....

Beds, bed springs, cots $\ldots \ldots$

Book cases.......

Chairs and stools.......

Desks.....

Lockers and safes...........

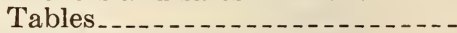

Filing furniture ..............

Miscellaneous metal furniture and

fixtures.
Stoves and furnaces, except electric.

General items

Gas stoves, ranges, and water heaters.

Oil and gasoline stoves, ranges, and water heaters.......

House heating boilers, radiators, and hot-water tanks...--

Miscellaneous heating and cooking equipment... Hand tools, metal working

General items taps, dies . . Chisels_........ Drills Punches. Reamers Taps and dies.... 
Hand tools, metal working-Contd

615. 3

615. 31

615. 32

615. 33

615. 39

615. 4

615. 41

615. 42

615. 43

615. 5

615. 51

615. 52

615. 53

615. 54

615. 55

615. 6

615. 61

615. 62

615. 63

615. 64

615. 7

615. 71

615. 72

615. 73

615. 8

615. 81

615. 82

615. 9

616.

616. 1

616. 11

616. 12

616. 13

616. 14

616. 15

616. 16

616. 17

616. 18

616. 2

616. 21

616. 22

616. 23

616. 3

616. 31

616. 32

616. 33

616. 34

616. 35

616. 4

616. 41

616. 42

616. 5

616. 6

616. 60

616. 61

616. 62

616. 63

616. 64
Pincers, pliers, plier cutters, and shears . .

Pincers. - -

Pliers and plier cutters ......

Shears.....................

Miscellaneous cutting tools...

Vises, wrenches, and vise clamps Vises

Wrenches

Vise clamps

Anvils, bellows, hardies, and forges . . . . . . . . . . . . .

Anvils

Hardies

Miscellaneous blacksmith tools

Files, rasps, hack saws, and scribers_......................

Files.

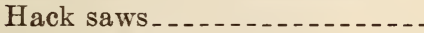

Rasps_...................

Tinners' and coppersmiths' tools .

Soldering coppers _...........

Blowtorches and soldering pots.

Bench plates and stakes.....

Measuring tools Calipers and dividers

Gages, dimension

Miscellaneous metal-working tools.

Hand tools other than metal-working tools......

Edge tools.................. Adzes, axes, and hatchets...Augers and carpenters' braces _ Chisels _. . . . . . . . . . . . . . . . .

Drills.......

Knives.......................

Planes and scrapers

Saws_.................... Glass cutters

Awls, punches, and screw drivers Awls_............... Punohes..... Screw drivers..............

Leveling and measuring tools...Bevels and miter boxes...... Levels and plumbs......... Rules

Squares

Tapes.

Hooks for manual handling of commodities

Nail pullers_

Tools for line construction men.-

Soil working tools............

General items _...........

Bars, digging and tamping

Hoes, rakes, and forks...

Scaops, shovels, and spades...

Picks and mattocks.......
Hand tools other than metal-working tools-Continued.

Harvesting, pruning, and trimming tools . - .

Sickles and scythes........

Shears, grass and hedge cutting .....................

Lawn mowers.............

Track tools than metal working .......

Jacks, levers, and leverage bars_

Mallets

Trowels

Hand tools not elsewhere classified

Furniture hardware, builders' hardware, and plumbing........

General items ples_...................

Sash-pole hangers, hooks, and sash-pole plates..._.....-

Hinges . . . . . .

Hasps and staples..........

Locks, latches, and lock parts..Locks._. . - . Latches.....................

Lock parts._._._.

Knobs, handles, and pulls..... Door knobs_...... Door and drawer pulls........ Push and kick plates and bars. Sash lifts_................. Checks, catches, and fasteners.-Door closers and checks..... Catches..................... Door, casement, and sash fasteners._._._.......

Door holders and stops.....

Transom and sash devices.-...--

Bolt locks, brackets, and hooks.Bolt locks _..._-_. - - - - - Brackets, handrail and shelf.-Hooks.

Plumbing fixtures

Slate sous and porcelain ware -.glass plumbing . . . Plumbing trimmings and fittings._................. Plumbing stopcocks and valves_ Pipe, plumbing - - Miscellaneous plumbing fix-

Miscellaneous builders' and furniture hardware
Miscellaneous iron and steel manufactures....

Firearms and ordnance.

Manufactures of sheet iron and sheet steel.

Screw posts, metal fence posts_--

Buoys of iron or steel........

Water filters.................

Manufactures of iron or steel not covered in above classification.
968

968

968

968

968

970

970

975

975

977

977

977

980

980

981

1004

1004

1004

1034

1035

1037

1037

1037

1038

1040

1041

1042

1042

1042

1043

1045

1046

1052

1052

1060

1060

1061

1061

1061

1062

1077

1077

1078

1080

1081

1081

1084

1085

1086

1086

1086

\section{0-629 FERRO-ALLOYING ORES AND METALS; ALLOY STEELS AND ALLOY-STEEL MANUFACTURES}

621.

621. 1

621. 11

621. 12

621. 13
Ferro-alloying ores and metals...--

Ferro-alloying ores........... Chromium ores..........-.

Manganese ores.........

Silicon ores
1088

1088

1088

1091

1092
621. 2

621. 20

621. 21

621. 22
Ferro-alloying ores and metals-Con.

Ferro-alloys................

General items

Ferrochromium

1092

1092

1102

1103 
Class No.

Ferro-alloying ores and metals-Con. Ferro-alloys-Continued.

621. 23

621. 24

621. 25

621. 26

621. 27

621. 3

621. 30

621. 31

621. 32

621. 33

630-639

630. 0

631.

631. 0

631. 1

631. 11

631. 12

631. 13

631. 2

631. 21

631. 22

631. 23

631. 24

631. 3

631. 31

631. 32

631. 33

640-649

640.

640. 1

641.

641. 0

641. 1

641. 11

641. 12

641. 2

641. 21

641. 22

641. 23

642.

642. 1

642. 11

642. 12

642. 13

642. 2

642. 21

642. 22

642. 23

642. 24

642.3

642. 4

642.5

642. 6

642. 61

642. 62

642. 9

643.

643. 1

643. 2

Ferromolybdenum . . . . . . .

Ferrotungsten

Ferrotitanium, ferrouranium
ferrovanadium ferrovanadium

Finished and semifinished alloy steel. rods strips _..._._._._._._._. steels_._._._._._._._._.

Rivets, aluminum
General items

Thickness tolerances for nonfer-
rous sheets and strips......Copper

General items.

Copper ingots and bars.......

Ingots and cast bars, copper.--

Rolled bars, copper.........

Copper plates, sheets, shapes, and strips.

Plates and sheets, copper....

Shapes, copper

Strips, copper

Manufactures of copper.

Copper rods, wires, and sleeves _Rods, copper.

Wires, copper

Sleeves, copper

Copper castings, ferrules, pipes, and tubes.........

Castings, copper

Ferrules, copper

Pipes, copper

Tubes, copper

Gravity battery coppers.....

Copper wire cloth

Hollow ware, copper....

Brush copper and mats...... Brush copper

Miscellaneous manufactures of copper

Manganese copper alloys

Phosphor copper.
Page

Class No.

Surject

Page

Ferrosilicon_..............

1104

1105621.34

1105 621. 35

621. 36

1106622.

1107622.1

622. 2

General items

Alloy-steel billets, bars, and Structural shapes of alloy

ALUMINUM, ANTIMONY

General items. . ...............

Aluminum

Aluminum and aluminum-alloys, ingots, and bars.......

Alloys, aluminum .........

Ingots, aluminum

Bars, aluminum aluminum-alloy

Aluminum and aluminum-alloy
plates, sheets, shapes, and plates, sheets, shapes, and
strips.

Plates, aluminum

Shapes, aluminum

Sheets, aluminum

Strips, aluminum__._.

Aluminum and aluminum-alloy
rods, wires, and rivets

Rods, aluminum

Wires, aluminum

1160

1141

1141

1146

1146

1150

1151

1151

1151

1151

1154

1157

1157

1157

1158

622. 3

622.4

622. 5

622. 6

622. 7

622. 9

Ferro-alloying ores and metals-Con.

Finished and semifinished alloy steel-Continued.

Alloy steel for springs _....... 1128

Alloy steel for tools ......... 1128

Alloy steel for magnets_...... 1129

Manufactures of alloy steels_...... 1130

Bolts, alloy steel

Railway rails, alloy steel__._._._. 1132

Tubular products and fittings, alloy steel.

Alloy-steel wire and manufactures.

Alloy-steel castings

Alloy-steel forgings.

Alloy-steel springs _..........

Miscellaneous manufactures of alloy steel

1132

1133

1133

1136

1138

1138

1160

1160

631. 41

631.42

631.5

631. 9

632.

632. 1

632.2

632. 3

633.

634.

635.

Aluminum-Continued.

Aluminum and aluminum-alloy castings and tubes........

Castings, aluminum

Tubes, aluminum _-..-_.-.--

Aluminum and aluminum-alloy hollow ware.

Miscellaneous aluminum articles_

Antimony

General specifications for antimony -..................

Antimony ingot metal

Antimony alloys

Bismuth

Cadmium

Cobalt

1163

1163

1163

1164

1164

1164

1164

1164

1164

1164
1165

1165

1170

1170

1173

1173

1176

1176

1176

1179

1179

1179

1179

1179

1181

1181

1181

1181

1182

1182

1184

1189

1190

1190

1190

1190

1190

1190

1191

1191

1191
643. 3

643. 4

643. 5

643. 6

643. 7

643. 9

644.

644. 0

644. 1

644. 11

644. 12

644. 2

644. 21

644. 22

644. 23

645.

645. 1

645. 11

645. 12

645. 2

645. 21

645. 22

645. 23

645. 24

645. 25

645. 3

645. 31

645. 32

645. 39

Copper alloys-Continued.

Silicon copper..............

Copper-tin-zinc alloy _..........

Copper-nickel alloy _............

Copper-nickel-zinc alloy _....... 
Class No

Manufactures of brass-Continued. Brass fittings and plumbing fixtures _...................

Brass oilers_................

Brass builders hardware ........

Miscellaneous manufactures of brass_......................

Bronze.......

General items...............

Bronze ingots and bars......... Ingots, bronze.............

Bars, bronze..............

Bronze plates, sheets, shapes, and strips.................. Plates and sheets, bronze...Shapes, bronze......... Strips, bronze.............

Bronze rods and wires........... Rods, bronze...... Wires, bronze.............

Bronze castings and tubes...... Castings, bronze............ Tubes, bronze.............

Manufactures of bronze......... Fittings, bronze........... Bronze-wire mänufactures..... Miscellaneous manufactures of bronze.................

Special bronzes (bronze alloys) ...

Aluminum bronze.............

Ingots, aluminum bronze....

Bars, aluminum bronze.......

\begin{tabular}{l|l} 
Page & \multicolumn{1}{|l}{ Class No. } \\
& \\
1230 & \\
1239 & 647.13 \\
1239 & 647.14 \\
& \\
1239 & 647.15 \\
1243 & 647.16 \\
1243 & 647.17 \\
1248 & 647.2 \\
1248 & 647.20 \\
1249 & 647.21 \\
1249 & 647.22 \\
1249 & 647.23 \\
1249 & 647.24 \\
1249 & \\
1249 & 647.25 \\
1249 & 647.26 \\
1249 & 647.27 \\
1250 & 647.3 \\
1250 & 647.31 \\
1255 & \\
1255 & 647.32 \\
1255 & 647.33 \\
1256 & \\
1257 & 647.34 \\
1258 & 647.35 \\
1258 & 647.37 \\
1258 & 647.38 \\
1258 & \\
&
\end{tabular}

Subject

Page

Special bronzes (bronze alloys)Continued.

Aluminum bronze-Continued.

Rods, aluminum bronze.......

Plates and sheets, aluminum bronze.

1259

Castings, aluminum bronze...-

Shapes, aluminum bronze....

Strips, aluminum bronze......

Manganese bronze...........

General items .............

Ingots, manganese bronze....

Bars, manganese bronze......

Rods, manganese bronze.....

Plates and sheets, manganese bronze.................

Shapes, manganese bronze... -

Castings, manganese bronze..-

Wires, manganese bronze.....

Phosphor bronze ............... Ingots and bearing metals, phosphor bronze...........

Bars, phosphor bronze

Plates and sheets, phosphor bronze................

Castings, phosphor bronze...Shapes, phosphor bronze.....

Strips, phosphor bronze.......

Tubing, phosphor bronze.....

Wires and rods, phosphor bronze....................
650.

651.

651.0

651. 1

651. 2

651. 3

651. 4

651. 5

651. 6

651.8

651. 9

652.

653.

653. 0

653. 1

653. 2

653. 3

653. 31

653. 32

654.

654.0

654. 1

654. 2

654.3

654. 4

660-669

661.

662 .

663.
General items _

Lead .............

General items .................

Lead ingots..................

Bar lead.

Castings, lead

Strips and sleeves, lead......

Sheet lead . . . . . .

Lead alloys.....

Lead pipe....................

Miscellaneous manufactures of lead.

Mercury

Nickel

General items

Nickel metal, ingots, and shot.--

Nickel bars . . . . . . . . . . . .

Nickel manufactures . . . Nickel sheets

Nickel wire

Nickel-copper alloys (Monel metal). General items . . . . . . . . ...... Ingots and shot, nickel-copper.-Bars, nickel-copper............

Strips, nickel-copper...........

Shapes, nickel-copper
1272

1272

1275

1277

1277

1277

1277

1278

1278

1279

1279

1279

1279

1281

1282

1282

1282

1282

1282

1282

1284

1284656

1284 656. 0

1284 656. 1
Manufactures, nickel-copper.

Castings and forgings, nickelcopper

Sheets and plates, nickel-copper.-

Rods, nickel-copper._._._...

Wires and cables, nickel-copper.-

Miscellaneous manufactures of nickel-copper

Nickel-copper-zinc alloys (nickel silver and German silver) _.....

General items................

Bars, nickel-copper-zinc alloy -..-

Sheets and plates, nickel-copperzinc alloy _..............

Shapes and castings, nickelcopper-zinc alloy _............

Strips, nickel-copper-zinc alloy -.-

Wires and rods, nickel-copperzinc alloy

Hollow and stamped ware, nickelcopper-zinc alloy --------

Miscellaneous specifications for nickel-copper-zinc alloy _.....- -

Nickel-chromium alloy . . . . .

General items . . . . . . .

Heating elements, nickel-chromium_
1284

1284

1285

1285

1286

1286

1286

1286

1287

1287

1287

1288

1288

1288

1288

1289

1289

1296
Platinum and platinum jewelry -..Gold and dental gold alloys......Silver
$1298 \mid 663.0$. 1298 663. 1

\begin{tabular}{l|l}
1299 & 663.2
\end{tabular}
General items

Silver metal and alloys

Silverware........................
1299 1299 1300

Dials 
680-689

Class No.

680.

681.

681.0

681. 1

681. 2

681. 3

681. 4

681. 41

681. 42

681. 43

681. 49

682.

\section{0-699}

691.

692.

692. 0

692. 1

692. 2

692. 3
General items........

Tin .........

General items

Ingots and pig metal, tin

Sheets, tin

Castings, tin

Manufactures of tin

Holloware, tin

Coatings, tin

Alloys, tin ...............

Miscellaneous manufactures of tin........

Phosphor tin

\section{TIN AND ZINC}

\begin{tabular}{l|l} 
Page & \multicolumn{2}{|c}{ Class No. } \\
1306 & 683. \\
1306 & 683.0 \\
1306 & 683.1 \\
1306 & 683.2 \\
1307 & 683.3 \\
1307 & 683.4 \\
1307 & 683.41 \\
1307 & 683.42 \\
1307 & 683.42 \\
1307 & 683.49 \\
1307 & 683.5
\end{tabular}

Subject

Zinc

1308

General items_............... 1308

Ingot metals, zinc or spelter

Sheets and plates, zinc........ 1311

Strips, zinc................... 1314

Manufactures of zinc _.......... 1315

Die castings, zinc-base _....... 1315

Gravity battery zincs......... 1316

Miscellaneous manufactures of zinc_................ 1316

Zine alloys_........... 1316

1307 684. Zinc coatings (galvanizing) ...- 1316

\section{MISCELLANEOUS ORES, METALS, ALLOYS, AND METAL MANUFACTURES}

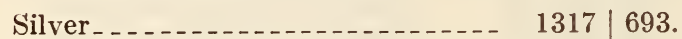

Babbitt and other bearing metals _-

1317 693. 1

Solders.

1330

General items...............

1317693.2

Babbitt metals $\ldots . . . . . . . . . .$.

1319

693. 3

Bearing metals, other than Bab-

bitt_................... 1324696.

695.

1327

Solder, tin-lead base

1330

Solders, copper-zinc base....... 1333

Silver solder...._............. 1335

Solders for aluminum ........ 1340

Magnesium and magnesium alloys 1340

Ores, metals, and alloys not else-

where classified.

1340

\section{SOCIETIES AND ASSOCIATIONS}

Technical societies, trade associations, and other organizations issuing standards and specifications dealing with metals and metal products

\section{INDEX}

Alphabetical list of commodities covered by standards and specifications 


\section{INTRODUCTION}

The preceding table of contents discloses the nature and extent of the material contained in this book. The subdivisions show that some commodity designations carry a number of specifications and standards, while other commodity designations carry only a few. Some commodity designations carry no specifications at all. In the latter case either there has not been sufficient demand to create a supply or else the requirements of these particular commodities are embodied in the specifications and standards of a related commodity.

Unlike the specifications and standards for commodities employed in the wood-using and nonmetallic-using industries, the specifications and standards of which have already appeared in the two preceding volumes of this series, the nationally recognized specifications and standards of the metal group do not possess the quality of detachment but appear to be interdependent. They lend themselves readily to comparison because of their more or less close relationship. One finds certain basic requirements to be inherent in a number of specifications and standards which treat of commodities of the same name though made of different material-for example, iron, steel, aluminum, brass, copper rods and bars, shapes and sheets. Again, commodities which seem to have nothing else in common, often approach uniformity in respect to chemical analysis. And regardless of the commodity or the organization producing the specification, it will be found that requirements covering finish, inspection, rejection, and rehearing are in a measure all-embracing.

These intimate relationships among the specifications and standards attaching themselves to the metals are reflected in this book in the unusual number of cross references. In a word, this spells interchangeability. Common ground, too, is covered by several different organizations producing similar or even identical specifications on the same commodity, or on metallic commodity attributes, such as screw threads, gages, tests, test specimens. In respect to screw threads, identical data take on a thin disguise of originality by the device of applied permutations and combinations in the arrangement of headings and tabular columns. Ferrous and nonferrous pipe fittings and hardware have much in common in the way of specification phrasing. Numerous cross references also emphasize these facts, facts which are apparent to anyone who takes the trouble to examine this book in some detail.

These many similarities may, perhaps, prompt the suggestion from the metal-using industries that the cause of economy and efficiency might be well served by setting up a specification-sifting agency along the lines of the Simplified Practice Division of the National Bureau of Standards.

In preparing the manuscript for this volume use has been made of the latest available issue of current nationally recognized standards and specifications. Information concerning the exact wording employed, and such changes as may have been made in these standards and specifications at some later date, can be obtained from the headquarters of the technical societies, trade associations, and other organizations sponsoring the standards and specifications reproduced herein. The names and addresses of these organizations are set forth on page 1341 . 



\section{STANDARDS AND SPECIFICATIONS FOR METALS AND METAL PRODUCTS}

600-609

IRON AND STEEL

\section{GENERAL ITEMS RELATING TO BOTH FERROUS AND NONFERROUS MATERIAL}

600.0. COMMON BASIC REQUIREMENTS.

\section{AMERICAN BUREAU OF SHIPPING RULES FOR BUILDING AND CLASSING STEEL VESSELS; MATERIALS FOR MACHINERY, BOILERS, AND PIPING, 1930}

(This organization has prepared specifications relating to steel ship construction, including ship parts from keel to masts, boilers, propelling and ther machinery, and electrical equipment. Specifications contained in the publication relating to ferrous and nonferrous metals have been placed in this compilation under their proper commodity headings, as follows):

\begin{tabular}{|c|c|c|}
\hline $\begin{array}{l}\text { Commodity } \\
\text { designation }\end{array}$ & Material & Page \\
\hline 600.0 & General & \\
\hline 600.1 & Testing requirements & 7 \\
\hline 603.1 & Iron stays for boilers & 135 \\
\hline $\begin{array}{l}603.21 \\
604.11\end{array}$ & $\begin{array}{l}\text { Steel bars and stays for boilers } \\
\text { Steel plates for boilers }\end{array}$ & $\begin{array}{l}141 \\
227\end{array}$ \\
\hline 607. & Steel pipes.. & 436 \\
\hline 608.4 & Steel rivets for boilers. & 612 \\
\hline 611.11 & Iron castings . . . . & 635 \\
\hline 611.3 & Iron forgings . & 705 \\
\hline 611.41 & 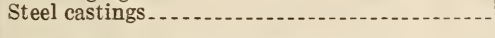 & 706 \\
\hline 611.51 & Steel forgings for machinery & 750 \\
\hline 641.2 & Copper plates and shapes... & 1176 \\
\hline 642.23 & Copper pipes and tubes & 1182 \\
\hline 644.21 & Brass plates and shapes & 1198 \\
\hline 645.21 & Brass castings & 1250 \\
\hline 645.23 & Brass pipes and tubes. & 1216 \\
\hline 645.24 & Condenser tubes and ferrule stock & 1219 \\
\hline & Bronze castings & 1250 \\
\hline
\end{tabular}

\section{GENERAL}

(See also 600.1, p. - -)

1. Where the process of manufacture is prescribed by the rules, the manufacturer must adopt a system of marking ingots, billets, slabs, finished plates, and forgings which will enable the material to be traced to its tests and original heat, and the surveyor must be given every facility for so tracing the material. 2. Three copies of the mill sheets or shipping invoices of all materials accepted by the surveyor should be furnished for his signature-one to be forwarded by the manufacturer to the purchaser, two to be retained for the use of the bureau. Before the mill sheets are signed the manufacturer must furnish the surveyor with a certificate guaranteeing that the material has been made by an approved process and that it had withstood satisfactorily the prescribed tests. The following form of certificate will be accepted if printed on each mill sheet with the name of the firm and initialed by the authorized representative of the manufacturer:

3. "We hereby certify that the material described herein has been made by the (describe process) process, and is that which has been satisfactorily tested in the presence of the surveyors in accordance with the tests of the American Bureau of Shipping."

4. Where steel is not produced in the works at which it is rolled, a certificate shall be supplied to the surveyor stating the process by which it was manufactured, the name of the manufacturer who supplied it, and the number of the heat from which it was made, for reference to the books of the manufacturer. The number of the heat shall be marked on each plate or bar for the purpose of identification.

\section{BOILER MATERIAL}

5. Physical requirements. - The structural steel used in the construction of boilers must be made by the open-hearth process. The finished material shall be free from defects and shall have a workmanlike finish. All boiler plates are to be surface inspected by the surveyor at the mills. The rolled surface should be retained on two opposite sides of the test specimens. For quench bend tests the specimens are to be heated to a light cherry red and quenched at once in water of a temperature of about $80^{\circ} \mathrm{F}$. The physical characteristics of the material must be in accordance with the requirements specified under their respective commodity designations as given above.

6. All material which has satisfactorily passed the requirements must be clearly stamped by the manufacturers, as indicated in the following table, in at least two places on each finished plate or bar. 


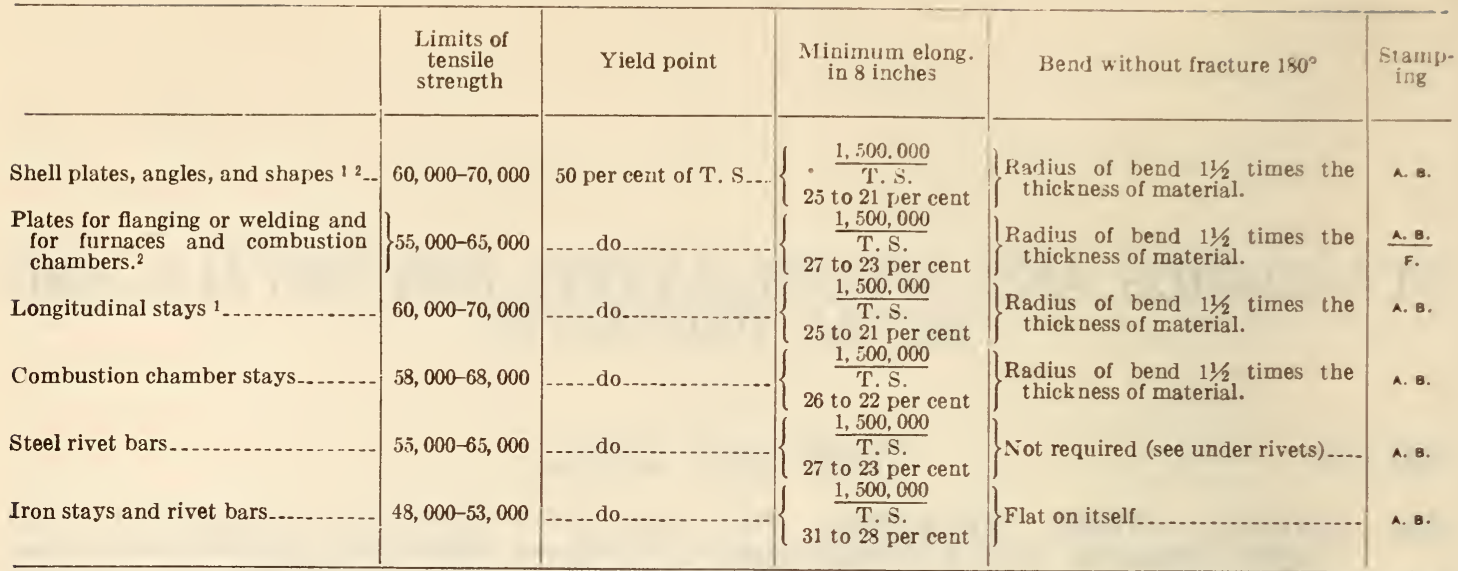

1 Materials other than those specified, within the limits of 58,000 to 78,000 pounds T. S. will be apyroved, provided the range of tensile strength of the materials used in any one construction does not exceed 10,000 pounds per square inch.

2 For plates over three-fourths inch in thickness, a deduction from the specified elongation of 0.125 per cent may be made for each in. crease of one thirty-second inch of the specified thickness above three-fourths incb, to a minimum of 20 per cent.

\section{AMERICAN BUREAU OF SHIPPING RULES FOR BUILDING AND CLASSING STEEL VESSELS; QUALITY AND TESTING OF MATERIALS-SHIP CONSTRUCTION, 1930}

(This organization has prepared specifications relating to steel ship construction, including ship parts from keel to masts, boilers, propelling and other machinery, and electrical equipment. Specifications contained in the publication relating to ferrous and nonferrous metals have been placed in this compilation under their proper commodity headings, as follows):

\begin{tabular}{|c|c|c|}
\hline $\begin{array}{l}\text { Commodity } \\
\text { designation }\end{array}$ & Material & Page \\
\hline 600.0 & General... & \\
\hline 603.1 & Round and flat iron bars & 135 \\
\hline 603.21 & Steel rivet bars. & 141 \\
\hline 603.42 & Steel wire ropes.......... & 159 \\
\hline 603.52 & Iron and steel chains ....... & 201 \\
\hline 603.53 & Cast steel stud-link chain. & 204 \\
\hline 603 & Shackles and shackle pins. & 213 \\
\hline & Iron plates and angles. & 234 \\
\hline 605 & Steel plates, angles, bulb angles, channels, etc.. & 268 \\
\hline 608 & Steel and iron rivets & 612 \\
\hline 611.3 & Iron forgings ... & 704 \\
\hline 611.3 & Forged wrought iron anchors....... & 704 \\
\hline 611.41 . & Steel castings & 706 \\
\hline 611.49 & Cast steel anchors & 742 \\
\hline $\begin{array}{l}611.51 \\
611.59\end{array}$ & $\begin{array}{l}\text { Ingot steel and welded steel forgings } \\
\text { Ingot steel anchors }\end{array}$ & $\begin{array}{l}750 \\
814\end{array}$ \\
\hline & & \\
\hline
\end{tabular}

\section{GENERAL}

1. The committee is prepared to consider proposals from owners or builders in cases where it may be desired to use materials of other tenacity than is provided for in the following rules:

2. Where materials are not required to be in accordance with the rules of the American Bureau of Shipping, the committee will approve of their surveyors testing any such materials to suitable specifications other than those stated in the rules, provided these are mutually agreed upon by the manufacturers and purchasers. Materials tested under these conditions must not be stamped A. B., as this mark is reserved exclusively to signify that the materials have satisfactorily complied with the tests required by the rules of the American Bureau of Shipping, and have been tested in the presence of the surveyor.
3. The whole of the steel to be used in the construction of steel vessels shall be made by the openhearth process, and the finished materials shall be free from injurious defects and shall have a workmanlike finish. The name or brand of the manufacturer and the melt number shall be legibly rolled or stamped on all finished material. The melt number shall be legibly stamped on each test specimen. Specimens are to be selected in such numbers as the surveyor may consider necessary, and tested in his presence at the place of manufacture prior to dispatch.

\section{SOCIETY OF AUTOMOTIVE ENGINEERS, IRON AND STEEL SPECIFICATIONS, S. A. E. STANDARD, 1928}

\section{INTRODUCTION}

1. The steel compositions included in this standard are considered adequate for pracically all parts made of ferrous metals that are necessary for the production of automotive apparatus, and include grades that have been found commercially available and technically adequate for the service required of such parts. In cases where very special requirements or exceptional quality make it necessary, grades containing less phosphorus and sulphur an d narrower ranges of carbon and manganese are avail. able, but such steels should be obtained by specia 1 arrangement between the mill and the purchaser.

\section{SPECIFICATION NOMBERS}

2. A numerical index system is used for the specifications contained in this report, which makes it pussible to use specification numerals on shop drawings and blue prints that are partially descriptive of the quality of material covered by such numbers. The first figure indicates the class to which the steel belongs; thus " $1-$ " indicates a carbon steel; "2-" a nickel steel; and "3-" a nickel-chromium steel. In the case of alloy steels, the second figure generally indicates the approximate percentage of the predominant alloying element. Usually the last two or three figures indicate the average carbon content in "points," or hundredths of 1 per cent. Thus 2340 indicates a nickel steel of approximately 3 per cent nickel (3.25 to 3.75 ) and 0.40 per cent carbon $(0.35$ to 0.45$)$. and 71360 indicates a tungsten steel of about 13 per cent tungsten (12 to 15$)$ and 0.60 per cen 
carbon $(0.50$ to 0.70$)$. The basic numerals for the various qualities of steel specified are:

Carbon steels
Nickel steels
Nickel-chromium steels.
Molybdenum steels.
Chromium steels.
Chromium-vanadium steels
Tungsten steels

3. Steels should be purchased on the basis of requirements as to chemical composition, and the specifications provided herein should be used in the purchase of the grades and types of steel covered by each. (For chemical composition of the various classes of steel enumerated above see the respective commodity designations of the several classes as follows:

\begin{tabular}{|c|c|}
\hline Carbon steels.... & 27 (p. \\
\hline Scr & $603.21(\mathrm{p}$. \\
\hline casting & $611.41(\mathrm{p}$. \\
\hline Nickel steels_. & 621.31 (p. 1124 \\
\hline Nickel-chromium steels _- & $621.31(\mathrm{p}$ \\
\hline Molybdenum steels . . . & $621.31($ p. 1124$)$ \\
\hline Chromium steels & 621.31 (p. 112 \\
\hline Chromium - vanadium & \\
\hline steels & \\
\hline ese steel & \\
\hline
\end{tabular}

4. Requirements as to physical properties have been omitted for all steels except steel castings, because the majority of steels for automotive purposes are either worked or given special heat treatments by the purchaser.

(Comments not included in S. A. E. standard specifications, covering the characteristics and uses of a large variety of automotive steels, will also be found in the handbook.)

UNITED STATES GOVERNMENT, FEDERAL SPECIFICATIONS BOARD, FEDERAL SPECIFICATION FOR RODS, WELDING, NONFERROUS, FOR GAS WELDING, QQR-571, MAY 3, 1932 .

\section{NONFERROUS WELDING RODS FOR GAS WELDING GENERAL SPECIFICATIONS}

1. United States Government General Specification for Metals, Federal Specifications Board specification 339a (current designation: QQ-M-151) or latest revision thereof, in effect on date of invitation for bids, shall form part of this specification. (See 339 a, below.)

\section{TYPES}

2. Nonferrous welding rods shall be of the following types as specified under their respective commodity designations:

Type A, manganese

bronze........- (See 647.23, p. 1266)

Type B, naval brass.-(See 645.11, p. 1205)

Type C, copper ...... (See 642.11; p. 1180)

Type D, aluminum --(See 631.31, p. 1157)

Type E, aluminum

alloy _........ (See 631.31, p. 1157)

Type $\mathrm{F}$, copper-

nickel alloy _._... (See 654.53, p. 1285)

\section{MATERIAL}

3. The material for the various grades shall be as specified under their respective commodity designations as given above.

\section{GENERAL REQUIREMENTS}

4. All rods shall be of homogeneous composition throughout, true to form, clean, smooth, of uniform color, quality, size, and free from all injurious defects. Rods furnished in bundles shall be straight, and rods furnished in coils shall be free from kinks. Cast rods shall be pickled or rattled if necessary to obtain a clean surface.

\section{DETAIL REQUIREMENTS}

(For chemical requirements and diameter in inches of the several types of nonferrous welding rods covered by this specification, see under their respective commodity designations as given above.)

5. Drawn welding rods shall be furnished in straight lengths or coils as may be ordered. When furnished in straight lengths, drawn rods shall be in 36 -inch lengths, and cast rods in 12 to 20 inch lengths.

\section{METHOD OF INSPECTION, TESTS, AND CHEMICAL ANALYSIS}

6. Welding rods shall demonstrate good weldability and shall flow smoothly and evenly without any unusual characteristics in flat and overhead positions. Sample rods may be selected by the inspector and forwarded to any Government laboratory for this test.

7. Check chemical analyses may be made by the inspector or through him by any Government laboratory or other designated representative and without cost to the contractor. The chemical composition thus determined shall conform to the requirements specified in the tables for the types ordered.

8. If check chemical analyses are made, the inspector shall obtain a composite sample of not less than 4 ounces from each 1,000 pounds or less of the same type. At least one wire from every size in each 1,000 pounds or less shall be selected. Where the 1,000 pounds or less consists of but one size, one wire from each of five bundles shall be selected. Samples shall consist of fine particles obtained by clipping, sawing, or drilling, depending on the size of the wire.

\section{PACKING AND MARKING OF SHIPMENTS}

9. When welding rods are ordered in lengths or coils they shall be securely wired and wrapped in heavy waterproof paper and burlap, or wrapped in heavy waterproof paper and boxed.

\section{NOTES}

10. Requisitions for welding rods should state the type, diameter, whether drawn or cast, and whether rods are desired in straight lengths or coils.

\section{UNITED STATES GOVERNMENT, FEDERAL SPECIFICATIONS BOARD, FEDERAL SPECIFICATIONS FOR METALS NO. 339a, JANUARY 25, 1929. (CURRENT DESIG- NATION : QQ-M-151)}

\section{GENERAL SPECIFICATION FOR METALS}

\section{PART I}

NoTE-A revision of this specification which appeared too late for (o incorporation in this publication includes additional provisions to those given below. New requirements are given concerning the use destroyed are to be in addition to the quantities called for in the contract; new requirements on the forms and dimensions of test specimens for tubing and sheet material; form and dimensions of bend test mens for tubing and and impact test specimentic limit, proof stress, and yield strength by use of extensometer. 
2. Subtechnical requirements.-This specification covers certain requirements which are common to all detail specifications for metals unless specifically excepted in the detail specifications. Strictly technical requirements which describe the material or articles are given in the detail specifications. The requirements of this specification do not describe the material or articles, but provide means for determining whether the technical requirements are being met.

3. Technical requirements.-While it is mandatory that the technical requirements of the specifications of any particular department of the Federal Government conform in detail to the technical requirements of Federal specifications, there is no compulsion requiring conformity with other than the technical requirements. However, the adoption of the provisions of this specification is strongly urged on all departments so that there may be greater uniformity among the various departments in their procurements.

4. Extent of application.-It is to be presumed that it will be desirable to make exceptions in certain detail specifications to certain of the requirements stated herein. The use of this specification avoids the repetition of most of its provisions in each detail specification.

\section{Part II}

\section{GENERAL}

5. Detail specifications.-This specification shall form a part of each detail specification for metals issued by the Federal Specifications Board.

6. Precedence.- Should any conflicts exist between the requirements of this specification, the detail specifications, the drawings or the contract; the requirements of the contract, the drawings, the detail specifications, and this specification shall prevail in the order named unless a different order of precedence be specifically indicated.

\section{MANUFACTURE}

7. Responsibility for subcontracts.-Contractors will be held responsible for the compliance by their subcontractors with all the requirements of the contract, drawings, and specifications.

8. Manufacturing methods.-When not specified in the contract, drawings, or detail specifications, the means and methods for executing the work will be determined by the contractor, but the obligation is on the contractor to satisfy the inspector as to full compliance with the requirements of the specifications, drawings, and contract.

\section{HEAT TREATMENT}

9. Equipment.-Materials that are subjected to heat treatment as a process of manufacture, or as a final operation, shall be heat treated by methods, and in equipment, suited to the purpose.

\section{INFORMATION AND FACILITIES}

10. Information furnished by contractor.-As soon as practicable after receiving an order under this specification, the contractor shall, if requested by the inspector, inform the latter when work will be commenced, and of the general plans and methods that he intends to follow. During the progress of the work, the contractor shall, if requested, furnish the inspector or such of his assistants as he may designate, the following:

(a) A copy of the results of chemical analyses for all material for which chemical requirements are stated in the detail specifications.

(b) Notification of the time when each operation which the specifications require the inspector to witness is to take place. (c) Such other notifications as may be required to insure that the inspector has the opportunity to witness any particular part of any operation which he may specify.

11. Facilities furnished by contractor. - The contractor shall furnish the inspector, free of cost, such facilities, including suitable office space and office furniture, as may be necessary for the performance of his work.

12. Inspector's access to plant.-The inspector and his assistants shall have free access at all times to all parts of any manufacturing plant which directly concern the manufacture of the material ordered.

13. Contractor's expense in connection with tests.All handling of material, sampling, preparation of test specimens, and testing thereof, except inspection analyses, shall be performed or furnished by the contractor without expense to the Government. Where the manufacturer has no facilities for making tests or analyses required by specifica cions, they will be made at some suitable place in the vicinity at the contractor's expense. Physical tests shall be made in the presence of the inspector.

14. Identity. - When material is inspected by melto or luts, the contractor shall so arrange his working, handling, and marking of the material that the inspector, at all times throughout its manufacture and inspection, may identify with certainty any or all portions of each heat, melt, or lot. In case the inspector can not satisfy himself as to the identity of the heat, melt, or lot from which a piece is made, each such piece may be tested singly, at the expense of the contractor.

15. Samples for Government inspection analysis. (a) Samples of material for inspection analysis shall be so taken as to represent the quality of material offered for inspection.

(b) All drillings, turnings, or cuttings of material shall be fine, clean, dry, and free from foreign substance. They shall be taken at slow speed and shall not be oxidized. Where surfaces are contaminated by fureign material or are otherwise not representative of the quality of the articles or material, surface chips shall be discarded.

(c) In addition to such analyses as are required to be made by the contractor, the inspector may have an analysis made from finished material representing each melt in cases where he may deem it necessary. The inspector may have the samples taken from the test coupons, test specimens, or from any part or parts of the material concerned, provided the serviceability of the part or parts is not destroyed. The sample taken shall be of sufficient size to permit five analyses to be made.

16. Check analysis.-(a) In case of dissatisfaction with results of the inspection analysis, the contractor may make claim for a check analysis, in which case the chemical composition shall be determined by a Government laboratory.

(b) Part of any sample taken for the above analysis shall be furnished the contractor if he so requests the inspector at the time the sample is taken.

17. Physical test specimens.-(a) The inspector shall select test coupons or test pieces and apply his stamp or other marks to them for future identification. Test coupons or pieces from which test specimens are to be prepared are to be subjected to the same treatment and identical processes which are applied to the material which they represent. Coupons or test pieces shall not be cut off or otherwise removed from the individual pieces or material until these pieces have received their final annealing or other treatment. Any marks applied by the inspector or his assistants shall remain on the coupons or test specimens until the pieces are tested and necessary record made. Before the inspector's stamp or other identification marks are effaced or I removed from one place, they shall be transferred to 
another place by the inspector. The Government may waive any or all of the requirements of this paragraph in cases where they are impracticable of application, wholly or in part.

(b) Test coupons or pieces, or the test specimens taken therefrom, shall receive no further or special working or treatment other than necessary machining after their removal from the individual pieces of material represented.

18. Tension specimens.-(a) Standard specimens, types 1, 2, and 3, as shown in Figures 1, 2, and 3, respectively, indicate standard tension test specimens which shall be used when practicable, unless otherwise provided in detail specifications. Type 1 is circular in cross section. Type 2 is rectangular in cross section and may be used for tests requiring either a 2 or an 8 inch gage length. Type 3 , which is also rectangular in cross section, shall be used only where it is impracticable to use type 2 . A variation of 5 per cent above or below the standard area of cross section shall be allowed in test specimens of tvpe 1 .

(b) All rods, bars, and shapes shall be pulled in full size when practicable.

(c) Subsize specimens.-When it is not possible to obtain from forgings or other shapes tension-test

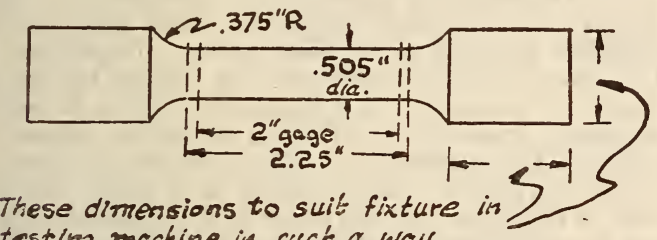

testing machine in such a way

that the load sholl be axia\%.

Figure 1.-Standard test specimen, type 1

specimens that conform to type 1 or to such other dimensions as may be provided in the respective detail specifications, round subsize tension-test specimens as shown in type 4 (see fig. 4) shall be used. A variation of 5 per cent above or below the standard area of cross section shall be allowed in test specimens of type 4 .

19. Bend-test specimens.--Whenever machined bend-test specimens are required by detail specifications, unless otherwise specified the specimen shall be from 6 to 8 inches long, about 1 inch wide or the full width of the material if it be less, and about onehalf inch thick or the full thickness of the material if it be less. The long edges will be rounded to about one-sixteenth inch radius.

20. Retests.-(a) If any test specimen shows defective machining or obvious lack of continuity of metal it may be discarded and replaced by another specimen selected by the inspector.

(b) If the percentage of elongation of any tensiontest specimen is less than that specified, and any part of the fracture is outside of the gage length, or within the gage length and less than 25 per cent of the gage length from either datum point, as indicated by scribe marks made on the specimen before testing, the inspector shall select another specimen in its stead and a retest shall be allowed.

(c) If any of the test specimens selected fail to meet the requirements specified and the Government permits a retest, at least twice the number of specimens that failed shall be selected by the inspector for replacement, all of which shall meet requirements.

21. Test methods.-(a) All tension and compression tests shall be made with a testing machine of standard make, maintained in good condition. All knife-edges shall be sharp and free from oil or dirt.
Such a machine shall be sensitive to a variation of one two-hundred-and-fiftieth of any registered load.

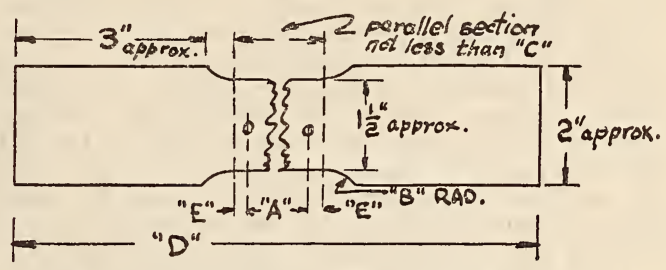

Frgure 2.-Standard test specimen, type 2

When $A=2$ inches, $B=0.25$ inches, $C=2.25$ inches, $D=9$ inches, $E=0.125$ inches.

When $A=8$ inches, $B=1$ to 3 inches, $C=9$ inches, $D=18$ inches, $E=0.5$ inches.

They shall be accurate within their loading ranges to $1 \frac{1}{2}$ per cent plus or minus. Machines shall be calibrated at least once every 12 months, using approved standard methods.

(b) During the conduct of the tests, the speed of the testing machine shall be such that the beam of

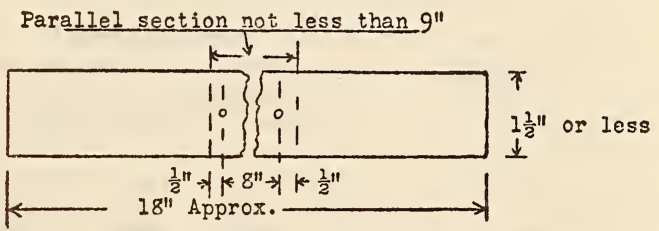

Figure 3.-Standard test specimen, type 3

NoтE.-In types 2 and 3 , the thickness shall not exceed the width of the parallel section.

the machine can be kept balanced, but in no case shall the values given in the following table be exceeded.

\begin{tabular}{|c|c|c|c|}
\hline \multirow{2}{*}{$\begin{array}{l}\text { Specified minimum tensile strength of } \\
\text { material (in pounds per square inch) }\end{array}$} & \multirow{2}{*}{$\begin{array}{l}\text { Gage } \\
\text { length }\end{array}$} & \multicolumn{2}{|c|}{$\begin{array}{l}\text { Maximum cross- } \\
\text { head speed (in } \\
\text { inches per } \\
\text { minute) }\end{array}$} \\
\hline & & $\begin{array}{l}\text { Up to } \\
\text { yield } \\
\text { point }\end{array}$ & $\begin{array}{l}\text { Above } \\
\text { yield } \\
\text { point }\end{array}$ \\
\hline 80,000 or under-_- & $\begin{array}{r}\text { Inches } \\
2\end{array}$ & 0.50 & 2.0 \\
\hline Over 80,000 & & $\begin{array}{r}.25 \\
.50\end{array}$ & $\begin{array}{l}1.0 \\
2.0\end{array}$ \\
\hline
\end{tabular}

(c) In determining the proportional limit or proof stress, the cross-head speed shall not exceed 0.025 inch per inch of gage length per minute.

\section{CORRESPONDENCE AND REPORTS}

22. Channels.-All communications between the contractor and the department involved shall be through the inspector, and shall be forwarded in triplicate. All important communications between the contractor and the inspector shall be in writing, and shall be forwarded in duplicate. All correspondence shall refer to the contract or purchase order applicable.

23. Report forms. - All reports covering details of manufacture, heat treatment, tests, etc., shall be submitted on forms approved by the department concerned. 


\section{PACKING AND MARKING OF SHIPMENTS}

24. Normal packing.-Unless otherwise specified in the contract, purchase order, or detail specifications, the material or articles shall be packed, boxed, or crated, and marked in accordance with standard commercial practice for similar material or articles, the cost of such special or commercial packing, boxing, crating, or marking being included in the bid.

25. Packing for ocean shipment.-In all cases where material is purchased to be packed for ocean shipment, such packing shall be witnessed by the inspector, and the quantity, weight, etc., checked, and such other inspection conducted as will permit

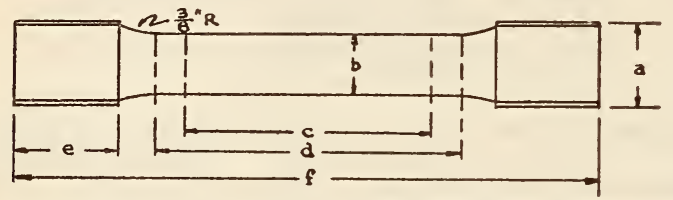

Figure 4.-Standard test specimen, type 4

Proportional size in round tension test specimen

\begin{tabular}{|c|c|c|c|}
\hline \multirow{2}{*}{ Dimension } & \multicolumn{3}{|c|}{ Diameter of material } \\
\hline & $\begin{array}{l}1 / 2 \text { to } 3 / 4 \\
\text { inch }\end{array}$ & $\begin{array}{l}36 \text { to } 1 / 6 \\
\text { inch }\end{array}$ & $\begin{array}{l}1 / 4 \text { to } \\
\text { inch }\end{array}$ \\
\hline 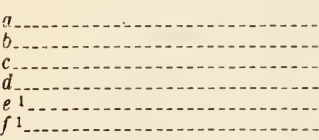 & $\begin{array}{r}0.500 \\
.375 \\
1.500 \\
1.750 \\
.750 \\
3.625\end{array}$ & $\begin{array}{r}0.375 \\
.250 \\
1.000 \\
1.250 \\
.500 \\
2.625\end{array}$ & $\begin{array}{r}0.250 \\
.125 \\
.500 \\
.750 \\
.375 \\
1.875\end{array}$ \\
\hline
\end{tabular}

1 Approximately.

it to be final; and proper notation thereof shall appear on the shipment report.

\section{Part III}

\section{INSPECTION AND TESTS}

26. Inspector.-The term "Inspector" refers to the representative of the Government assigned to the duty of inspecting the materials or articles involved.

27. Commercial quality.-Material specified as "Commercial quality," will not ordinarily be subject to tests or analyses; however, the right is reserved to make such tests as the inspector deems necessary.

28. Contractor's design.-Where approval of a manufacturer's plans and specifications by the Government is required, inspection will not be completed nor shipment authorized, except at the contractor's risk, in advance of the receipt of copies of such approved plans and specifications by the inspector.

29. Inspection at place of manufacture.-All material shall, as far as practicable, be inspected, tested, and accepted at the place of manufacture. Procedure as to inspection at place of manufacture divides itself into three general groups or conditions, as follows:

Condition 1.-When it is possible to conduct all required inspection and tests prior to shipment, acceptance at destination will be based on certification of inspection. Inspection will be conducted at destination for the purpose or checking the quantity, ascertaining damage in transit, and properly identifying the material. Rejections will not be made at destination unless substitutions have oc- curred, fraud exists, or material can not be properly identified as that covered by the shipping report.

Condition 2.- When for any reason material, furnished under contracts or orders requiring delivery f. o. b. carrier at works or point of shipment, is not given final inspection before shipment, it is agreed that title will pass to the Government at point of shipment with the understanding that final inspection will be made at destination and that the contractor will furnish the inspector with a written guarantee that the material is in accordance with the specifications. If the material is rejected at the point of delivery, title will revert to the contractor at the point of delivery, and he will be required to replace same with acceptable material, the expense of removing rejected material and the cost of transportation of replacement material being borne by the contractor. If the contractor declines to furnish the guarantee, shipment will not be authorized and the inspector will refer the matter to the department concerned, for instructions.

Condition 3.- When material which has been inspected, tested, and accepted contains inherent defects subsequently exposed, the contractor may be required to replace the defective material without expense to the Government.

30. Inspection at destination.-Inspection at place of manufacture shall be waived when the contract specifies inspection at destination. Inspection at the place of manufacture may be waived by the department concerned and the right to do so is hereby expressly reserved in the case of orders where the value of the material is small, or where the distance to be traveled by the inspector is so great that the cost of inspection at the place of manufacture would not be warranted, or where for other reasons inspection at the point of delivery or at destination can be more satisfactorily made. In such cases, the manufacturer shall ship the material at his own risk, subject to final acceptance or rejection after delivery at destination, however, waivers of any part of inspection or test of material purchased f. o. b. contractor's works without first having obtained the consent of the department involved are not authorized.

31. Inspection at subcontractor's plant.-The inspection of material purchased by the contractor may, at the option of the department involved, be at the works of the subcontractor, to whom all of the provisions of this specification, not in their nature inapplicable, shall apply.

Nothing contained herein shall operate to relieve the contractor of his responsibility for furnishing material conforming strictly with the requirements of the prescribed contract, drawings, and specifications.

32. Provisional acceptance.-The inspector may decide on the provisional acceptability of all manufactured material to be used by the contractor for the work covered by his order, but such acceptance shall not operate to transfer title to the United States, and shall not preclude subsequent rejection for discovered or developed defects. The inspector shall promptly notify the contractor or subcontractor of the existence of any defect which, in his opinion, will probably make the material unsuitable.

33. Inspector's stamps.-(a) In the case of small articles packed in bulk, or in the case of material which would be injured by stamping, the necessary stamp marks shall be applied by the inspector to the boxing or packing material of the article, or to tags securely wired or attached thereto.

(b) The removal of any inspector's official stamped markings or any other Government markings from material prior to its final acceptance, without the authority or permission of the inspector, shall be a sufficient cause for the rejection of such material. 


\section{DEFINITIONS}

34. Definition of tension test terms.-The following definitions and explanations of terms used in tension tests shall apply:

(a) The "elastic limit" is the greatest load per unit of original cross section which does not produce a permanent set.

(b) The "proportional limit" is the load per unit of original cross section at which the deformation ceases to be directly proportional to the loads.

(c) The "vield point" is the load per unit of original cross section at which a marked increase in the deformation of the specimen occurs without increase of load. It is usually determined by the drop of the beam of the testing machine or by the use of dividers.

(d) The "tensile strength" is the maximum load per square inch of original cross section.

(e) The "elongation" is the percentage difference between lengths laid off between gage marks before being subjected to any stress, and after rupture.

(f) The "reduction of area" is the percentage difference between the area of the bar before being subjected to stress and the area of the bar after rupture. The latter will be determined by measurements taken at the point of rupture.

35. Definition of heat-treating terms.-In general, heat treatment, when applied to steel, will consist of one or more of the following operations or combinations thereof:

(a) Normalizing.-Material ordered normalized shall be uniformly heated to and held at a temperature sufficiently above the critical range for a time sufficient to produce refining of the grain, after which it shall be allowed to cool freely in the air.

(b) Annealing.-Material ordered annealed shall, unless otherwise approved, be uniformly heated to and held at a temperature sufficiently above the critical range for a time sufficient to insure that the section has been soaked through and the grain refined, then allowed to cool slowly as desired.

If the contract or purchase order specifies a Brinell hardness or other physical requirement that necessitates a spheroidizing anneal, the contractor may perform such anneal at a temperature below the lower critical temperature, but such low temperature shall not be used for the usual annealing treatment to refine the grain.

(c) Heating and quenching.-Material ordered to meet physical requirements that necessitate heating and quenching shall be uniformly heated to and held at a temperature sufficiently above the critical range for a time sufficient to insure that the section has been thoroughly soaked through and the grain refined, then rapidly cooled in a suitable quenching medium. The heating and quenching operations may be preceded by a normalizing or annealing operation, or both, depending upon the character and condition of the steel, and the particular properties required.

(d) Tempering.-All hardened or quenched parts shall receive $\&$ tempering heat, which shall consist of uniformly reheating to some temperature below the lower critical temperature, holding at heat for a time sufficient to insure that the section has been soaked through, and then cooling as may be desired. Quenching from the tempering heat may be necessary with certain alloy steels in order to avoid socalled "temper brittleness."

\subsection{TESTING OF METALS.}

\section{METHODS OF TEST}

(In the ferrous and nonferrous metals group a test method that covers several items will be found under the commodity designation 600.1. Reference to this test method is then inserted under the commodity designation of each item. For example, tentative methods of tension testing - and also of compression testing-of metallic materials appear below. References to these test methods will be found under commodity designations $621.30,631.0,640.1,650.1$, 680.1, and 692.0. But when a test method is confined to a specific item, the test method is inserted under the commodity designation attached to that item. For example, chemical analysis of alloy steels will be found under 621.30; under 693.3 will be found chemical analysis of silver solders.)

(Federal Specification Board specification No. 339 a (current designation, $Q Q-M-151$ ) includes general requirements for tests which apply to a number of the Federal specifications. These test requirements will be found under $600.0, \mathrm{p} .3$.)

\section{AMERICAN BUREAU OF SHIPPING, RULES FOR BUILDING AND CLASSING STEEL VESSELS ; MATERIALS FOR MACHINERY, BOILERS, AND PIPING, 1930}

\section{TESTING REQUIREMENTS}

1. All materials subject to tests and inspection are to be tested and inspected by the bureau's surveyors in accordance with the following requirements: Materials of different characteristics than prescribed herein require the special approval of the committee. Such materials satisfactorily tested and inspected shall be stamped A. B., signifying that the material has satisfactorily passed the required test and inspection.

2 . Where materials are not required to be in accordance with the rules, the same may be tested by the surveyors to the bureau, upon request, to specifications agreed upon by the purchaser and manufacturer. Such materials are not to be stamped A. B.

3. All materials subject to test and inspection are to be free from defects. Welding or dressing for the purpose of remedying defects is not permitted without the previous sanction of the surveyor. Where inaterials have been so treated the surveyor may prescribe additional tests, and the part treated must be stamped with the surveyor's identification mark and surrounded with a ring of paint.

4. All test specimens shall be selected by the surveyor and must not be detached until stamped with his identification mark and until the material has received its final treatment. The yield point is to be determined by the drop of the beam. In the event of any test specimen failing to meet the requirements, unless two further specimens prove satisfactory, the particular material from which such test specimens have been taken shall be rejected. Further tests of specimens taken from the same heat shall then be made, and if these fail the whole of the material produced from that heat is to be rejected. Should a tension test piece break outside the middle half, the test piece may, at the manufacturer's option, be discarded and a new specimen substituted. All tests are to be conducted in the presence of the surveyor at the place of manufacture prior to shipping.

5. Any material proving unsatisfactory in the process of being worked shall be rejected notwithstanding any previous certificate of satisfactory testing.

\section{STANDARD TEST PIECES}

6. The test pieces should be made to the following dimension, as shown in Figure 5, on page 8 . 


\section{AMERICAN HIGHWAY \\ ASSOCIATION OFFICIALS, STANDARD METHODS OF SAMPLING AND TESTING HIGHWAY MATERIALS. UNITED STATES DEPARTMENT OF AG- RICULTURE, BULLETIN NO. 1216, 1928}

\section{SAMPIING AND TESTING HIGHWAY MATERIALS}

(This publication includes reprints of American Society for Testing Materials specifications Nos. E4-27, E8-27T, E9-27T, and E10-27, all of which, with certain cross references, are given below.)

\section{AMERICAN ELECTRIC RAILWAY ENGI- NEERING ASSOCIATION, PROOF TEST- ING OF FORGINGS TO DETERMINE THEIR SOUNDNESS AFTER QUENCHING AND TEMPERING, SERIAL NO. E108-25, 1925}

\section{PROOF TESTING OF FORGINGS}

Up to the present time proof testing has been mostly confined to locomotive driving axles and the

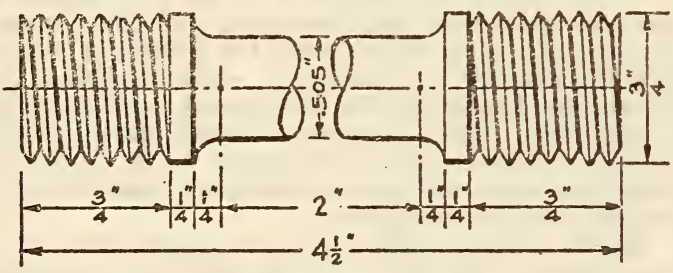

Tension Test Piece
The accompanying table has been prepared to give a suitable height of drop uniform for both ends of each size of axle, but increasing with the size, and includes heights of drop for both 1,640 and 2,000 pound tups, for reasons given later in this report. This table is based on the formula above given, with compensation made for the varying cross section of axles, and was calculated for the rough turned sizes of association's standard axles, allowing one-fourth inch on the diameter for finishing.

It is recommended that wherever practicable the proof tests be made after the rough turning of forging.

Due to the many variables governing a test of this kind, some of which can not be ascertained with any degree of accuracy, it is impossible to calculate the proper height of drop for a definite fiber stress. The ideal proof test is one that subjects all parts of the forging to a stress slightly under its elastic limit, and under no circumstances should the elastic limit of the material be exceeded; therefore when it is considered that the stress due to a drop test is dependent on not only the weight of tup, height of drop, and distance between supports, but also on the hardness of the material, the relation between the mass of the forging tested and the anvils upon which it rests, the capacity and flexibility of springs

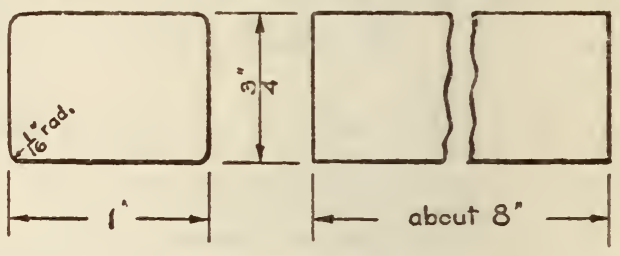

Bend Test Piece

TENSION AND BEND TEST PIECES FOR CASTINGS AND FORGINGS

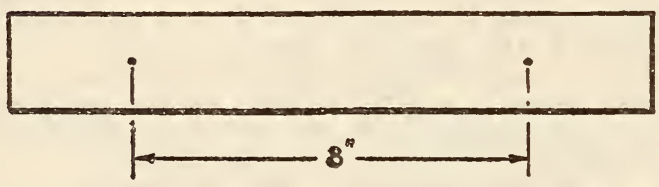

TENSION AND BEND TEST PIECES FOR

ROLLED BARS

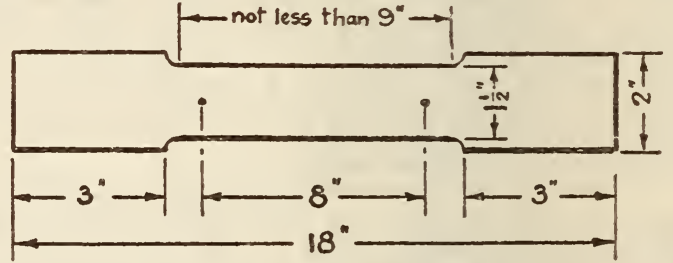

TENSION TEST PIECE

FOR PLATES AND SHAPES

Note:-Bend Test Piese may have parallel edges

FiguRe 5.-Standard test pieces

(See specification on p. 7)

distance between supports usually placed at 3 feet, with height of drop generally based on the following formula:

$$
H=\frac{d^{3}}{100}
$$

Wherein and

$$
H=\text { Height of drop in feet }
$$

$d=$ Diameter of axle in inches.

Testing machine.-Standard M. C. B. with spring supported anvils.

Weight of tup.-1,640 pounds.

So far as can be learned, these tests were established through experiments conducted to determine the blow required to produce permanent set, and the theory developed for the M. C. B. car axle drop test with the modifications necessary to change from a destructive to a proof test. which support the anvils, and other details of the testing apparatus, it can be readily seen that the ideal test can only be approximated, and the table given under Figure 6 should be taken, therefore, as a guide in indicating what has been found from actual use to fulfill as nearly as possible the requirements.

While a 1,640-pound tup has been generally used in the past in the standard proof testing machine, we are informed that the M. C. B. Association is considering adopting a tup weighing 2,000 pounds on account of the lighter weight not being sufficient for a destructive drop test with the present height of testing machine and the largest standard car axles, and in order that it shall not be necessary to change weight between the different tests, it has been suggested that the newly proposed standard weight of tup of 2,000 pounds be used for proof testing, with a corresponding reduction in height of drop. 
It is the usual practice to turn the forging after each test so as to provide drop test on two opposite sides. Any drop test of this sort should not cause permanent set in sound forging, and provision should be made in the agreement with the manufacturer that permanent set in forging shall be cause for rejection. A suggestion for gage to determine the set in forgings is shown in Figure 7.
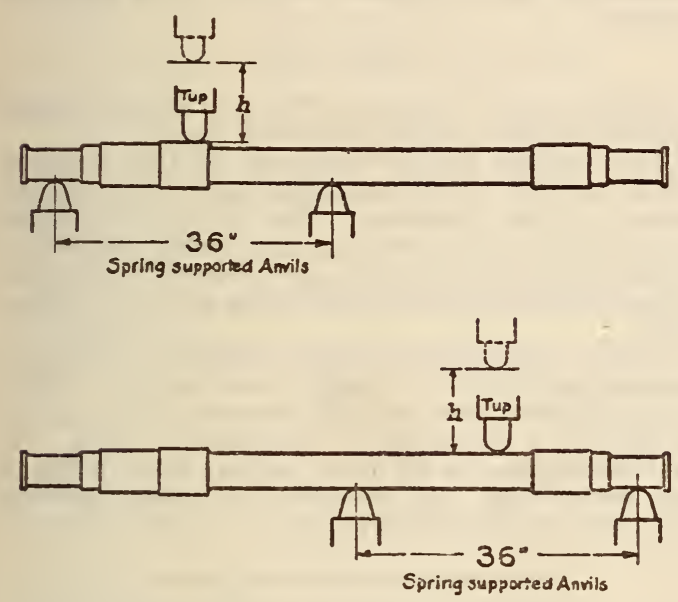

Table showing height of drop " $h$ "

\begin{tabular}{|c|c|c|c|}
\hline \multicolumn{2}{|c|}{ Axle } & Drop & Drop \\
\hline Size & $\begin{array}{l}\text { Designa- } \\
\text { tion }\end{array}$ & $\begin{array}{l}\text { pound } \\
\text { lup }\end{array}$ & $\begin{array}{l}\text { pound } \\
\text { tup }\end{array}$ \\
\hline $\begin{array}{r}\text { Inches } \\
33 / 4 \text { by } 7 \\
41 / 4 \text { by } 8 \\
41 / 4 \text { by } 8 \\
5 \text { by } 9 \\
5 \text { by } 9 \\
51 / 2 \text { by } 10\end{array}$ & $\begin{array}{ll} & \begin{array}{ll}\mathrm{E} \\
\mathrm{E}\end{array} \\
\mathrm{E} & \mathrm{B} \\
\mathrm{E} & \mathrm{B}-1 \\
\mathrm{E} & \mathrm{C} \\
\mathrm{E} & \mathrm{C} \\
\mathrm{E} & \mathrm{D}\end{array}$ & $\begin{array}{c}\text { Inches } \\
15 \\
19 \\
24 \\
30 \\
36 \\
45\end{array}$ & $\begin{array}{c}\text { Inches } \\
12 \\
15 \\
21 \\
24 \\
30 \\
39\end{array}$ \\
\hline
\end{tabular}

FIGURE 6.-Method of supporting axles with table giving suggested heights of drop

\section{AMERICAN SOCIETY OF MECHANICAL EN- GINEERS, GENERAL RULES FOR INSPEC- TION AND TESTS, BOILER CONSTRUC- TION CODE, 1927}

\section{INSPECTION AND TESTS}

(In respect to inspection, rejections, and rehearing, this specification is identical wilh pars. 7,8 , and 9 of the standard specifications for steel of the American Society for Testing Materials (see 605.0, p. 247.) Additional provisions are as follows:)

Ladle analyses.-The manufacturer shall make an analysis of each melt of steel, from a test ingot taken during the pouring of the melts, to determine the percentage of carbon, manganese, phosphorus, and sulphur. The results of analysis shall conform to requirements, and shall be reported to the purchaser or his representative.

Check analyses.-The purchaser may make an analysis from finished material representing each melt. The chemical composition thus determined shall conform to the requirements.

Physical tests. - The vield point shall be determined by the drop of the beam of the testing machine. For determination of the yield point the speed of the crosshead of the machine shall not exceed one-half inch per minute when the elongation is taken in 2 inches, nor exceed 2 inches per minute when the elongation is taken in 8 inches. After the yield point has been passed, the speed of the machine may be increased to not more than 2 inches per minute when the elongation is taken in 2 inches, nor more than 6 inches per minute when the elongation is taken in 8 inches.

\section{AMERICAN SOCIETY OF MECHANICAL EN- GINEERS, AMERICAN STANDARD SYM- BOLS FOR MECHANICS, STRUCTURAI ENGINEERING, AND TESTING MATE- RIALS, Z10A-1932, JANUARY, 1932}

[Approved by the American Standards Association, January, 1932]

\section{ENGINEERING SYMBOIS}

(This pamphlec contains the approved symbols for 69 different items which appear with varying frequency in publications related to the practice of engineering. Seven of these items-angular distance, angular velocity, angular acceleration, radius of curvature, deflection of a panel point of a truss, density, and the unit of elongation-are represented
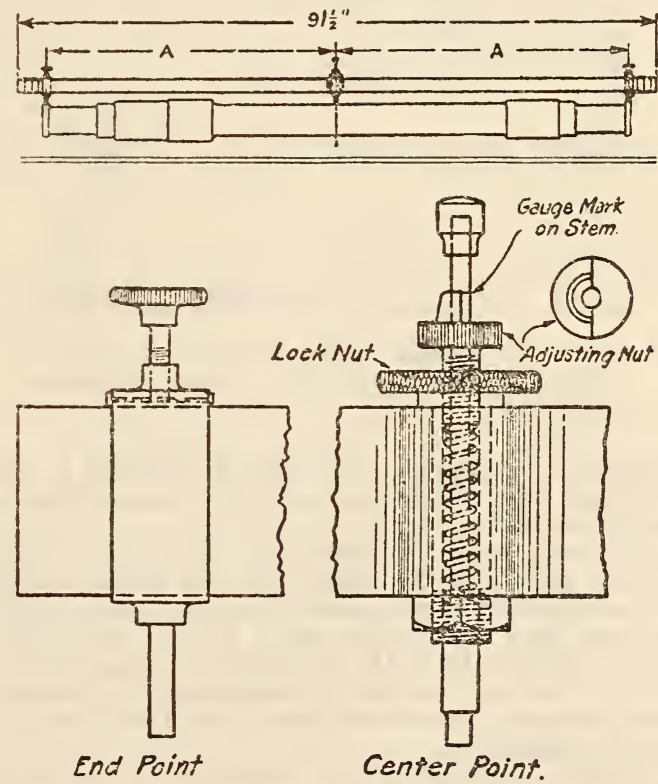

\begin{tabular}{|c|c|}
\hline Axle & $\mathrm{A}$ \\
\cline { 1 - 1 } Inches & Inches \\
$33 / 4$ by 7 & $411 / 4$ \\
$41 / 4$ by 8 & 4134 \\
5 by 9 & $423 / 4$ \\
$1 \frac{3}{2}$ by 10 & $433 / 4$ \\
\hline
\end{tabular}

Figure 7.-Suggested design of gage for determining if axles take set in proof testing

by Greek letters. English capitals and lower case letters represent the others. Capital $T$ covers four different items, including harmonic motion period, trrque, and absolute temperature.)

Note.-Sponsor organizations: American Association for the Ad vancement of Science, American Institute of Electrical Engineers, American Society of Civil Engineers, American Society of Mechanical Engineers, and Societ y for the Promotion of Engin 3 ering Education. 


\section{AMERICAN SOCIETY FOR TESTING MA- RIALS, STANDARD METHODS OF CHEMICAL ANALYSIS OF PLAIN CAR- BON STEEL, SERIAL DESIGNATION A33-24, 1924}

\section{CHEMICAL ANALYSIS OF PLAIN CARBON STEEL}

\section{DETERMINATION OF CARBON BY THE DIRECT COMBOS- TION METHOD}

(Substantially the same as in A64 (see 601.20, p.119), except for the alternative method of absorbing the carbon dioxide by means of barium hydroxide solution, given below, and that nickel boats should not be made of sheet nickel containing more than 0.3 per cent of carbon instead of 0.15 per cent as in A64.)

\section{DETERMINATION OF CARBON (CARBON DIOXIDE ABSORBED} IN BARIOM HYDROXIDE SOLUTION)

\section{Special Apparatus}

Purifying train.-This method eliminates the necessity of a purifying train following the furnace, inasmuch as no precautions are necessary to prevent access of water vapor or oxides of sulphur from the

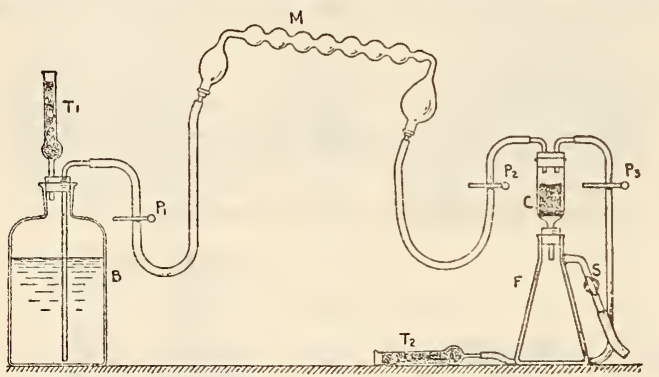

Figure 8.-Apparatus for filtration in determination of carbon by the direct-combustion method

absorbing apparatus. All that is needed is the purifying train before the combustion apparatus as mentioned above.

The train after the combustion apparatus.-This consists merely of the Meyer tube for absorption of the carbon dioxide, protected by a soda-lime or sodaasbestos tube at the far end. Meyer tubes with 7 to 10 bulbs of 10 to $15 \mathrm{c}$ c capacity each, and large bulbs at the ends, having volumes equal to the combined capacity of the small bulbs, have been used and found satisfactory.

Filtering apparatus. - In filtration for accurate work, care should be taken to protect the solution from access of extraneous carbon dioxide. This is accomplished in the apparatus shown in Figure 8. For work requiring less accuracy, the barium carbonate may be filtered off on a filter made by fitting a carbon funnel with a perforated porcelain disk and filtering by suction. The precipitate is then washed with distilled water from which the carbon dioxide has been removed by boiling.

\section{Reagents}

Tenth-normal hydrochloric acid.-This may be standardized by any of the accepted methods, or as follows: Measure out $20 \mathrm{c}$ c of the approximately $0.1 \mathrm{~N}$ acid (free from chlorides) with a pipette, and precipitate the silver chloride by an excess of silver-nitrate solution in a volume of 50 to $66 \mathrm{c} \mathrm{c}$. After digesting at $70^{\circ}$ to $80^{\circ} \mathrm{C}$., until the supernatant liquid is clear, filter off the chloride on a tared Gooch filter and wash with water con- taining $2 \mathrm{c}$ c of $\mathrm{HNO}_{3}$ (specific gravity 1.42) per $100 \mathrm{c} \mathrm{c}$ of water until free from silver nitrate. After drying to constant weight at $130^{\circ} \mathrm{C}$., note the increase of weight over the original tare and from this weight, corresponding to the silver chloride, calculate the strength of the hydrochloric acid, after which adjust it to the strength prescribed. The standardization should be based upon several concordant determinations using varying ainounts of acid.

\section{1cc $0.1 N \mathrm{HCl}=0.0006 \mathrm{~g}$ carbon}

Methyl orange. - Dissolve $0.02 \mathrm{~g}$ of methyl orange in $100 \mathrm{c}$ c of hot distilled water and filter.

Tenth-normal sodium hydroxide solution.-Standardize against the hydrochloric acid. Use methyl orange as the indicator. The sodium hydroxide solution should be stored in a large bottle from which it may be driven out by air pressure, protecting against carbon dioxide by soda-lime or soda-asbestos tubes.

Barium hydroxide solution.-Filter a saturated solution and store in a large reservoir from which it is delivered by air pressure, protecting from carbon dioxide by a soda-lime or soda-asbestos tube. In each determination fill three or four small bulbs of the Meyer tube, and add $\mathrm{CO}_{2}$-free water until the remaining small bulbs are filled.

\section{A pparatus and Procedure for Filtration}

The apparatus is shown to approximately onetenth size in Figure 8, which is self-explanatory. The stopcock is a 3 -way cock connected to the suction pipe. The rubber tubing connected to the Meyer tube should be of best-grade black rubber, and the lengths used should be so chosen as to permit of easy manipulation of the tube. The Meyer tube is connected or disconnected by the rubber stopper's which are left always attached to the rubber tubes. The carbon tube $C$ is fitted with a perforated porcelain plate sliding easily.

The funnel is prepared for filtration by making on the porcelain disk a felt of asbestos about onesixteenth to one-eighth inch in thickness, using amphibole (not serpentine) asbestos which has been carefully digested with strong hydrochloric acid for several hours and washed with water until it gives no acid reaction. On top of the asbestos pad is placed a layer of similarly treated quartz, mixed with asbestos, of the height shown. A mixture of quartz grains of various sizes (approximately 50 per cent passing a 20 -mesh sieve and 50 per cent passing a 10 -mesh and remaining on a 20 -mesh sieve) is suitable. The mixture of quartz and asbestos may be obtained by filling the funnel from a beaker (directing against it a stream from a wash bottle) while maintaining a gentle suction. In this way the asbestos is properly mixed with the quartz. A little experience and attention to these details will enable one to prepare the quartz bed in a manner that will greatly expedite filtration. The stopper is now inserted in the funnel, the Meyer tube connected as shown, and the liquid and precipitate sucked into the funnel. Only a gentle suction should be used. When necessary $P_{3}$ is opened to admit air back of the column of liquid in the Meyer tube. When the contents of the Meyer tube have been transferred, the large bulb nearest $B$ is half filled with water by opening $P_{1}$; the stopcock $S$ is operated during this and subsequent operations so as to maintain a gentle suction all the time. $M$ is now manipulated so as to bring the wash water in contact with all parts of the interior, after which the water is sucked through $C ; P_{2}$ is left open during this and subsequent washings. After eight washings as directed, allowing the wash water to drain off thoroughly each time before adding more, $M$ may 
be detached, the stopper removed from the funnel, and the washings completed by filling $C$ to the top with $\mathrm{CO}_{2}$-free water, draining completely and repeating the operation once. With care the washing may be done with $150 \mathrm{c}$ c of water. Air is now admitted through the side opening of $S, C$ is removed, and the porceiain disk carrying the asbestos, quartz, and barium carbonate is thrust, by means of a long glass rod, into a flask, removing any adhering varticles from the sides of $C$, by a stream of water from a wash bottle. An excess of the standard acid is now added from a burette or pipette, using a portion to wash out $M$, and after the contents of the flask have been thoroughly agitated by shaking, the excess of acid is titrated against the standard alkali, using three drops of the methyl-orange indicator.

Notes.-The operation of filtering can be carried out very rapidly after a little practice.

Glass wool should on no account be used as a substitute for the quartz, on account of the probability of errors arising from its attack by the alkali or acid.

It is well to wash out the rubber tubes connected to the Meyer tube with a little water each day before beginning work.

\section{Method}

After having properly set up and tested the apparatus, place $2 \mathrm{~g}$ of steel (note 1 ) in the form recommended above, in a moderately packed condition on the bed material and introduce the boat into the combustion apparatus, already heated to the proper temperature. After one-half to one minute, admit oxygen somewhat more rapidly than it is consumed, as shown by the rate of bubbling in the Meyer tube (note 2). A rate of $200 \mathrm{c}$ c per minute is satisfactory. The sample burns completely in one or two minutes, and all that is now necessary is to sweep all the carbon dioxide into the absorption apparatus. This is accomplished in 5 to 10 minutes by passing about 1 or 2 liters of oxygen. Detach the Meyer tube (note 2) and filter and wash the barium carbonate, using the special filtering apparatus shown. After solution in a measured excess of standard hydrochloric acid (the Meyer tube being washed out with a portion of the acid to remove adhering barium carbonate), titrate the excess of acid against alkali and from the data thus obtained calculate the percentage of carbon.

Notes. - 1. When working with steels high in carbon (above 1 per cent) it is advisable not to use more than $1 \mathrm{~g}$ in order that filtration may be sufficiently rapid.

2. As a precaution against error resulting from too rapid passage of the gases, it is well to attach a second barium-hydroxide tube to retain any carbon dioxide that may pass the first.

3 . For the most accurate work the Meyer tubes should be washed with dilute acid before beginning work each day. After a determination is finished, the tube should be completely filled two or three times with tap water, then rinsed with distilled water, in order to remove the carbon dioxide liberated when dissolving the carbonate from the previous determination.

4. The flask containing the carbonate should be thoroughly agi4. The lask containing the carbonate should be thoroughly agirather slowly if this is not done; this is particularly the case if it has packed much during filtration.

\section{DETERMINATION OF MANGANESE BY THE BISMTTHATE} METHOD (ABSENCE OF COBALT)

\section{Solutions Required}

(Dilute nitric acid $(1: 3)$, dilute nitric acid $(3: 97)$, ferrous ammonium sulphate solution, standard potassium permanganate solution, and standard sodium arsenite solution-all conform to like solutions in the determination of manganese by the bismuthate method in A64 (see 601.20, p. 119).)

\section{Method}

In a $300 \mathrm{c}$ c Erlenmeyer flask dissolve $1 \mathrm{~g}$ of steel in $50 \mathrm{c}$ c of dilute $\mathrm{HNO}_{3}(1: 3)$, and boil to expel the oxides of nitrogen. Cool, and add about $0.5 \mathrm{~g}$ of sodium bismuthate and heat for a few minutes, or until the pink color has disappeared, with or without precipitation of manganese dioxide. Add small portions of ferrous sulphate (or any suitable reducing agent) in sufficient quantity to clear the solution, and boil to expel the oxides of nitrogen. Cool to $15^{\circ} \mathrm{C}$., add an excess of sodium bismuthate and agitate for a few minutes. Add $50 \mathrm{c}$ c of dilute $\mathrm{HNO}_{3}(3: 97)$, filter through an alundum filter or asbestos pad, and wash with the same acid. Titrate by method $(a)$ or $(b)$ below.

(a) Ferrous sulphate-permanganate titration (less than 0.05 per cent chromium). (Same as in A64 (see 601.20, p. 119).)

(b) Arsenite titration (more than 0.05 per cent chromium).

Titrate immediately with the standard sodium arsenite solution to the clear green color used as an end point in the standardization of the solution.

Allowable error.-Percentages of manganese determined by the above method should be accurate to $\pm[0.01+(0.02 \times$ the percentage of manganese found)]

Notes.-In the method, the preliminary treatment with sodium bismuthate has been found by a number of investigators to be apparently unnecessary; however, the available data to confirm this position are not considered sufficient to warrant its omission.

In mared and as much surface as possible. This insures rapid filtration, and the filter may be used until it becomes clogged with bismuthate.

The filtrate must be perfectly clear, since the least particle of bis. muthate carried through the filter will vitiate the results.

This method can not be used in the presence of cobalt.

In the arsenite titration, permanganic acid does not react in accordance with the ratio $\mathrm{Mn}_{2} \mathrm{O}_{7}: 2 \mathrm{MnO}$, but more nearly as indicated by the ratio $\mathrm{Mn}_{2} \mathrm{O}_{7}: \mathrm{Mn}_{2} \mathrm{O}_{3}$. Consequently the theoretical titre of the arsenite can not be used and the manganese titre must be obthe arsenite can not be used and the manganese titre must be obthe conditions that obtain in the method.

\section{DETERMINATION OF MANGANESE BY THE PERSULPEATE} METHOD (ROUTINE AND IN THE PRESENCE OF COBALT)

Solutions Required

Conform to like solutions in the determination of manganese by the persulphate method in A64 (see 601.20, p. 119).)

\section{Method}

In a small Erlenmeyer flask or large test tube ( 8 by 1 inch) dissolve 0.1 to $0.3 \mathrm{~g}$ of steel, depending on the manganese content of the sample, in 15 c c of dilute $\mathrm{HNO}_{3}$ (specific gravity 1.20) with the aid of heat from a hot-plate or water bath. The solution of carbon may be hastened by the addition of $1 \mathrm{c}$ c of the ammonium persulphate solution (10 per cent). When solution is complete and the liquid is clear, add $15 \mathrm{c}$ c of $\mathrm{AgNO}_{3}$ solution (0.133 per cent). (Then proceed as in the determination of manganese by the persulphate method in A64 (see 601.20. p. 119).)

\section{DETERMINATION OF PHOSPHOROS BY THE MOLYBDATE-}

\section{Solutions Required}

Dilute nitric acid (specific gravity 1.20).-Mix 380 c c of $\mathrm{HNO}_{3}$ (specific gravity 1.42) and $620 \mathrm{c}$ c of distilled water.

Dilute nitric acid (2 : 100).-Mix 20 c c of $\mathrm{HNO}_{3}$ (specific gravity 1.42) and 1,000 c c of distilled water. Potassium permanganate (2.5 per cent).-Dissolve $25 \mathrm{~g}$ of $\mathrm{KMnO}_{4}$ in $1,000 \mathrm{c}$ c of distilled water.

Dilute ammonium hydroxide (1:2).-Mix $300 \mathrm{c} \mathrm{c}$ of $\mathrm{NH}_{4} \mathrm{OH}$ (specific gravity 0.90 ) and $600 \mathrm{c}$ c of distilled water.

Dilute ammonium hydroxide (1:19).-Mix 100 c c of $\mathrm{NH}_{4} \mathrm{OH}$ (specific gravity 0.90 ) and $1,900 \mathrm{c} \mathrm{c}$ of distilled water.

Ammonium bisulphite ( 3 per cent).-Dissolve 30 $\mathrm{g}$ of ammonium bisulphite in $1,000 \mathrm{c} \mathrm{c}$ of distilled water.

Ammonium molybdate.-(Same as B28 (see 646.0, p. 1243).) 
Magnesia mixture.-Dissolve $50 \mathrm{~g}$ of $\mathrm{MgCl}_{2} \cdot 6 \mathrm{H}_{2} \mathrm{O}$ and $125 \mathrm{~g}$ of $\mathrm{NH}_{4} \mathrm{Cl}$ in $750 \mathrm{c}$ c of distilled water, and then add $150 \mathrm{c} c$ of $\mathrm{NH}_{4} \mathrm{OH}$ (specific gravity 0.90 ).

\section{Method}

In a 300 c c Erlenmeyer flask dissolve $5 \mathrm{~g}$ of steel in $75 \mathrm{c}$ c of dilute $\mathrm{HNO}_{3}$ (specific gravity 1.20). Heat to boiling; while boiling add about $12 \mathrm{c} \mathrm{c}$ of the potassium permanganate solution, and continue boiling until manganese dioxide precipitates. Dissolve this precipitate by additions of ammonium bisulphite or ferreas sulphate solution, boil until clear and free from brown fumes, and cool to $80^{\circ} \mathrm{C}$. Add $100 \mathrm{c} \mathrm{c}$ of the ammonium molybdate solution at room temperature, shake or agitate for five minutes, allow to settle, filter on a $9 \mathrm{~cm}$ paper, and wash the flask and precipitate at least three times with the dilute $\mathrm{HNO}_{3}(2: 100)$, to free from iron.

Dissolve the precipitate on the filter with dilute $\mathrm{NH}_{4} \mathrm{OH}(1: 2)$, letting the solution run into the original flask and avoiding the use of more than 10 to $20 \mathrm{c}$ c of the reagent. Pour a solution of $0.5 \mathrm{~g}$ of citric acid in $15 \mathrm{c}$ c of water and $10 \mathrm{e}$ e of $\mathrm{HCl}$ (specific gravity 1.19) through the filter in order to dissolve any ferric phosphate on the paper and render acid the ammoniacal solution of the phosphate. Transfer the solution to a $100 \mathrm{c}$ c beaker, add $10 \mathrm{c} \mathrm{c}$ of the magnesia mixture and precipitate the phosphate by adding $\mathrm{NH}_{4} \mathrm{OH}$ (specific gravity 0.90 ) slowly and with constant stirring, until a crystalline precipitate is formed or the solution is alkaline. Finally add 10 per cent by volume excess and set aside in a cool place for two to six hours. Filter, wash with dilute $\mathrm{NH}_{4} \mathrm{OH}(1: 19)$, ignite, and weigh. Dissolve the precipitate of magnesium pyrophosphate in $5 \mathrm{c}$ c of dilute $\mathrm{HNO}_{3}(5: 6)$, and $20 \mathrm{c} \mathrm{c}$ of distilled water, filter, and wash with hot water. Ignite and weigh. The difference in weights represents pure magnesium pyrophosphate containing 27.87 per cent of phosphorus.

Allowable error.-Percentages of phosphorus determined by the above method should be accurate to $\pm[0.002+(0.02 \times$ the percentage of phosphorus found)].

NOTES.-The ammonium molybdate solution should be kept in a cool place and should always be absolutely clear before using.

If arsenic is present in the steel, it will come down in part with the phosphomolybdate and contaminate the pyrophosphate. In very accurate work, or where arsenic is present in appreciable amount steps must be taken for its removal before precipitation with magnesia mixture.

\section{DETERMINATION OF PHOSPHORUS BY THE ALBALIMETRIC}

Solutions Required

(Dilute nitric acid (specific gravity 1.20), dilute nitric acid $(2: 100)$, potassium permanganate, and ammonium bisulphite-all identical with molybdate-magnesia method given above. Ammonium molybdate, potassium nitrate (1 per cent), phenolphthalein indicator, standard sodium hydroxide, and standard nitric acid-all identical with B28 (see 646.0, p. 1243).)

Method

In a $300 \mathrm{c}$ c Erlenmever flask dissolve $2 \mathrm{~g}$ of steel in $50 \mathrm{c}$ c of dilute $\mathrm{HNO}_{3}$ (specific gravity 1.20). Heat the solution to boiling and while boiling add about $6 \mathrm{c}$ c of the $\mathrm{KMnO}_{4}$ solution and continue boiling until manganese dioxide precipitates. Dissolve this precipitate by additions of ammonium bisulphite or ferrous sulphate solution, boil until clear and free from brown fumes, cool to $80^{\circ} \mathrm{C}$., add $50 \mathrm{c} \mathrm{c}$ of the ammonium molybdate solution at room temperature, shake or agitate for five minutes, allow to settle, and filter on a $9 \mathrm{~cm}$ paper. Wash the flask and precipitate three times with dilute
$\mathrm{HNO}_{3}(2: 100)$ to free it from iron, and continue the washing with the 1 per cent potassium nitrate solution until the paper, precipitate, and flask are free from acid.

Transfer the paper and precipitate to the flask, add $20 \mathrm{c}$ c of distilled water, 5 drops of phenolphthalein solution as indicator, and an excess of standard sodium hydroxide solution. Insert a rubbes stopper and shake vigorously until solution of the precipitate is complete. Wash off the stopper with distilled water and determine the excess of sodium hydroxide solution by titrating with standard nitric acid solution. Each cubis centimeter of standard sodium hydroxide solution represents 0.01 percent of phosphorus.

NOTES.-The ammonium molybdate solution should be kept in a cool place and should always be absolutely clear before using.

All distilled water used in titration should be freed from carbon dioxide by boiling or otherwise.

A ppropriate Bureau of Stand.rds standard steels are recommended for the standardization of the sodium hydroxide solution.

The composition of phosphomolybdate varies with changes in conditions such as acidity, temperature, and concentration of the molybdate reagent; consequently care should be taken to keep conditions alike in standardization and in analysis. The ratio 23 molecules of sodium hydroxide to 1 atom of phosphorus represents the reaction very closely if the phosphomolybdate is precipitated at $35^{\circ}$ to $45^{\circ} \mathrm{C}$.

DETERMINATION OF SULPHOR BY THE OXIDATION METHOD

Solutions Required

Dilute hydrochloric acid (2:98).-Mix 20 c c of $\mathrm{HCl}$ (specific gravity 1.19) and $980 \mathrm{c}$ c of distilled water.

Barium-chloride solution (10 per cent).-Dissolve $100 \mathrm{~g}$ of $\mathrm{BaCl}_{2} \cdot 2 \mathrm{H}_{2} \mathrm{O}$ in $1,000 \mathrm{c}$ c of distilled water.

Barium chloride-hydrochloric acid washing solution.-Mix $10 \mathrm{c} \mathrm{c}$ of the above solution, $10 \mathrm{c} \mathrm{c}$ of $\mathrm{HCl}$ (specific gravity 1.19), and $1,000 \mathrm{c}$ of distilled water.

\section{Method}

Dissolve $4.57 \mathrm{~g}$ of the sample in $50 \mathrm{c}$ c of $\mathrm{HNO}_{3}$ (specific gravity 1.42) in a covered beaker or flask. In case solution is slow or difficult, $\mathrm{HCl}$ (specific gravity 1.19) mav be added dropwise at intervals. When solution is complete, add $0.5 \mathrm{~g}$ of $\mathrm{Na}_{2} \mathrm{CO}_{3}$, evaporate to dryness and bake for 1 hour on the hot plate. Add $30 \mathrm{c} \mathrm{c}$ of $\mathrm{HCl}$ (specific gravity 1.19), and repeat the evaporation and baking. Add another $30 \mathrm{c} \mathrm{c}$ of $\mathrm{HCl}$ (specific gravity 1.19), and evaporate to sirupy consistency. Add $5 \mathrm{c}$ c of $\mathrm{HCl}$ (specific gravity 1.19), $20 \mathrm{c}$ c of water, $5 \mathrm{~g}$ of 20 to 30 mesh zinc (free from sulphur) and warm on the steam bath until the iron is reduced to the ferrous state and the evolution of hydrogen has nearly ceased. Filter, by decantation on a small filter, from the silica and undissolved zinc, wash with small portions of approximately $75 \mathrm{c}$ c of dilute $\mathrm{HCl}(2: 98)$, warm to $60^{\circ}$ to $70^{\circ} \mathrm{C}$., and add $10 \mathrm{c} \mathrm{c}$ of $\mathrm{BaCl}_{2}$ solution (10 per cent). Let stand for 18 to 24 hours, filter on a paper of close texture, and discard the filtrate. Wash with hot $\mathrm{BaCl}_{2}-\mathrm{HCl}$ washing solution until free from iron and then with hot water until free from chlorides. Reserve the paper, and precipitate and evaporate the washings to dryness. Dissolve the slight residue in $50 \mathrm{c} \mathrm{c}$ of hot dilute $\mathrm{HCl}(2: 98)$, add $1 \mathrm{c}$ c of $\mathrm{BaCl}_{2}$ solution (10 per cent), and digest at $70^{\circ}$ to $80^{\circ} \mathrm{C}$. for an hour or so, avoiding any undue evaporation. Filter on a small paper of close texture, wash with hot $\mathrm{BaCl}_{2}-\mathrm{HCl}$ washing solution until free from iron and then with hot water until free from chlorides. Ignite both papers in a tared platinum crucible, and treat with one drop of dilute $\mathrm{H}_{2} \mathrm{SO}_{4}(1: 1)$ and $1 \mathrm{c} \mathrm{c}$ of hydrofluoric acid. Evaporate to dryness, ignite, and weigh. A blank should be carried through all steps of the determination and any $\mathrm{BaSO}_{4}$ found deducted. The corrected weight 
of the ignited $\mathrm{BaSO}_{4}$ multiplied by 3 represents the percentage of sulphur.

Allowable errer.-Percentages of sulphur determined by the above method should be accurate to $\pm[0.002+(0.02 \times$ the percentage of sulphur found)].

Notes.-The precipitation of $\mathrm{BaSO}_{4}$ must not be carried out in a solution containing more than $2 \mathrm{c}$ c of $\mathrm{HC}^{\prime} \mathrm{l}$ (specific gravit y 1.19! per $100 \mathrm{cc}$.

7 he recovery of $\mathrm{BaSO}_{4}$ which is obtained ordinarily represents approximately 0.001 per cent of sulphur.

Precinitation of $\mathrm{BaSO}_{4}$ in unreduced iron solutions of carefully regulated acidity gives low values of the order of 0.001 to 0.002 per cent of sulphur.

\section{DETERMIN ATION OF SULPHUR BY THE EVOLUTION-
TITRATION METHOD (ROUTINE)}

(In respect to diagram illustrating apparatus, solutions required, and method, this specification is identical with A64 (see 601.20, p. 119), except that in A64 the potassium iodate solution includes the chemical reactions, the starch solution in $\mathrm{A} 64$ is replaced by the starch indicator given below, and for the notes in A64 are substituted the following notes:)

Starch indicator.-(1) To 1,000 c c of boiling distilled water add a cold suspension of $6 \mathrm{~g}$ of starch in $100 \mathrm{c}$ c of water, and boil vigorously for five minutes. Cool the solution, add $6 \mathrm{~g}$ of $\mathrm{ZnCl}_{2}$ dissolved in 50 c c of cold water, thoroughly mix, and set aside for 24 hours. Decant the clear supernatant liquid into a suitable container, add $3 \mathrm{~g}$ of $\mathrm{KI}$, and mix thoroughly. (2) (Optional.) Prepare an emulsion of 6 $\mathrm{g}$ of soluble starch in $25 \mathrm{c}$ c of water, add a solution of $1 \mathrm{~g}$ of $\mathrm{NaOH}$ in $10 \mathrm{c} \mathrm{c}$ of water, and stir the solution until it gelatinizes. Dilute to $1,000 \mathrm{c} \mathrm{c}$, add $3 \mathrm{~g}$ of $\mathrm{KI}$, and mix thoroughly.

Notes.-This method succeeds best when the erolution of gas is rapid and the conditions are kept constant

Some analysts prefer to add the dilute $\mathrm{HCl}$ at a temperature of $80^{\circ}$ to $90^{\circ} \mathrm{C}$. and to heat the flask on an electric heater instead of over gas. The use of concentrated $\mathrm{HCl}$ necessitates a condensing arrangement and does not offer any general advantages over the arrangement a

diluted acid.

with most plain carbon steel the results for sulphur which are obtained by the evolution method and the use of the theoretical sulphur titre check the gravimetric results within \pm 0.002 per cent Some steels dissolve too slowly, and some, as, for example, certain steels containing high sulphur and carbon, do not yield all of their sulphur. In such cases the samples must be annealed as follows:

Transter $5 \mathrm{~g}$ of drillings to a $12.5 \mathrm{~cm}$ filter paper, and wrap the sample securely in the paper so that at least three thicknesses of paper cover the steel. Place the package in a $25 \mathrm{c}$ c porcelain crucible, cover the crucinle tightly so that no air can enter, and heat for 20 minutes at a bright-red neat over a burner or preferably in a muffie. The filter paper should be charred but not burned. Cool, and proceed as in the regular method.

\section{DETERMINATION OF SILICON BY TEE NITROSULPHURIC}

\section{METIOD}

Solutions Required

Nitrosulphuric acid.-Mix 1,000 c c of $\mathrm{H}_{2} \mathrm{SO}_{4}$ (specific gravity 1.84), 1,500 c c $\mathrm{HNO}_{3}$ (specific gravity 1.42), and $5,500 \mathrm{c} c$ of distilled water.

Dilute hydrochloric acid (5:95).-(Same as in A64 (see 601.20, p. 119).)

\section{Method}

(Identical with A64 (see 601.20,p. 119), except that $80 \mathrm{c}$ c of the nitrosulphuric acid is added cautiously to $4.676 \mathrm{~g}$ of steel, and that the difference in weights in milligrams divided by 100 equals the percentage of silicon instead of being divided by 50 as in A64.)

Allon able error. - Percentages of silicon determined by the above method should be accurate to $\pm[0.005+(0.02 \times$ the percentage of silicon found)].

Note. - A blank determination on all reagents used should be made and the results corrected accordingly.

\section{DETERMINATION OF SILICON BY THE SULPHURIC-ACID}

(Identical with A64 (see 601.20, p. 119), except that here $2.338 \mathrm{~g}$ of steel is substituted for the $2.336 \mathrm{~g}$ of iron specified in A64.)

\section{DETERMINATION OF COPPER}

(Identical with A64 (see 601.20, p. 119) except for the addition of the following clause in the method of precipitation by hydrogen sulphide:)

Dissolve the cooled melt in the crucible in 1 or 2 c c of $\mathrm{HCl}$ (specific gravity 1.19) and a few cubic centimeters of water, transfer to a $200 \mathrm{c}$ c beaker, dilute to $100 \mathrm{c}$ c, and add $\mathrm{NaOH}$ (5 per cent) solution in slight excess.

\section{DETERMINATION OF COPPER (ROUTINE)}

(Substantially the same as in A64 (see 601.20, p. 119).)

\section{DETERMINATION OF NICKEL}

See the determination of nickel in nickel steel by the gravimetric dimethylglyoxime method in A55 $(621.30$, p. 1107$)$; also the determination of nickel in nickel steel by the cyanide titration method (routine) in A55 (621.30, p. 1107).

\section{DETERMINATION OF CHROMIOM}

(Same as the determination of chromium in chrome-nickel steel by the persulphate oxidation method in A55 (see 621.30, p. 1107) except in solutions required, where dilute nitric acid (specific gravity 1.20 ) is the same as in the determination of manganese by the persulphate method in A64 (see 601.20 p. 119), and standard ferrous-sulphate and standard permanganate solution are the same as in the determination of manganese by the bismuthate method in $\mathrm{A} 64$ (see 601.20, p. 119).)

AMERICAN SOCIETY FOR TESTING MATERIALS, STANDARD METHOD OF TEST FOR MAGNETIC PROPERTIES OF IRON AND STEEL, DESIGNATION A34-28, 1928

\section{TESTING MAGNETIC PROPERTIES OF IRON AND STEEL}

\section{A. NORMAL INDUCTION AND HYSTERESIS}

\section{Definitions}

1. (a) Normal induction.-The induction, produced under a given magnetizing force, in a substance which has been previously demagnetized and then subjected to a sufficient number of reversals of the given magnetizing force to bring it to a cyclic condition.

Both the induction and magnetizing force shall be expressed in terms of the cgs electromagnetic units (gauss and gilbert per centimeter respectively).

(b) Magnetic permeability.-The ratio of the normal induction to the magnetizing force which produces it. Thus:

$$
\text { Permeability }=\frac{\text { Gausses }}{\text { Gilberts per centimeter }}
$$

(c) Residual induction.-The magnetic induction which exists in a substance after the magnetizing force has been reduced to zero.

Note-If the induction has been reduced from a point on the normal induction curve it is called the normal residual induction.

(d) Coercive force.-The negative value of magnetizing force required to reduce the induction in a substance to zero.

Note.-If the induction has been reduced to zero from a point on the normal induction curve the coercive force is called the normal coercive force.

\section{Test Specimens}

2. (a) Test specimens may consist of rings, straight bars, or strips of uniform cross section.

(b) Ring specimens shall be continuous, having no joints or welds. The ratio of mean diameter to radial width shall be not less than 10 . 
(c) Sharp shears or dies shall be used in the preparation of specimens of sheet material. If the radial width of ring specimens or the width of straight strips of sheet material is less than $3 \mathrm{~cm}$ ( $13 / 16$ inch), the punching or cutting strains shall be removed before testing by an annealing process acceptable both to manufacturer and to purchaser.

(d) Test specimens of sheet material consisting of straight strips shall be cut half parallel and half at right angles to the direction of rolling, and in types of test requiring companion samples each half shall contain an equal number of each kind of strips.

3. (a) Test specimens of sheet material shall have a cross-sectional area of not less than $2 \mathrm{~cm}^{2}(0.31$ square inch) nor more than $4 \mathrm{~cm}^{2}$ (0.62 square inch) and shall be so selected from not less than two sheets that their properties shall represent as nearly as practicable the average properties of the sheets. (See figs. 9 and 10.)

(b) The cross section of specimens of sheet material shall be calculated from measured values of length and mass and measured or assumed values of density.

(c) For silicon steels, a density of $7.7 \mathrm{~g}$ per cubic centimeter shall be assumed if the silicon content is 2 per cent or less and a density of $7.5 \mathrm{~g}$ per cubic centimeter shall be assumed if the silicon content is over 2 per cent.

(d) For nickel-iron alloys the density shall be assumed from the nickel content as given by the straight lines joining the points defined as follows. ${ }^{2}$

\begin{tabular}{|c|c|}
\hline Nickel & Density \\
\cline { 1 - 2 } Per cent & $\mathrm{g} / \mathrm{cm}^{3}$ \\
0 & 7.85 \\
30 & 8.00 \\
100 & 8.90 \\
\hline
\end{tabular}

(e) For other alloys, a density corresponding to the actual measured or estimated value shall be used

4. Specimens of magnet steel shall be hardened previous to testing in accordance with a procedure acceptable to both manufacturer and purchaser.

\section{Standard data}

5. (a) The standard normal-induction data for al magnetic materials shall consist of corresponding values of normal induction and magnetizing force at suitable intervals within the range of values agreed upon by the manufacturer and purchaser.

(b) Residual-induction and coercive-force values for transformer-core material and material of the type used for electromagnet cores shall be determined from a maximum normal induction of 10,000 gausses.

(c) For permanent magnet steel, the residual induction and coercive force shall be determined from the maximum normal induction corresponding to a magnetizing force of 200 gilberts per cen timeter unless the maximum permeability is less than 100 , in which case the maximum magnetizing force shall be not less than two and one-half times the value of the magnetizing force at which this maximum permeability occurs and shall be some multiple of 200.

\section{Methods of test}

6. The tests may be made by means of one of the following methods:

The Rowland ring method.-This method may be used for any material and any magnetizing forces.

The Burrows compensated double yoke.-This type of apparatus is suitable for tests a magnetizing forces up to 300 gilberts per centimeter. For higher magnetizing forces, serious difficulty due to heating

${ }_{1}^{1}$ For silicon steel having a 2 per cent silicon content the electrical resistance is 2.6 ohms per meter gram.

${ }_{2}^{2}$ For 50 per cent nickel content the density is $8.26 \mathrm{~g} / \mathrm{cm}^{3}$. For 80 per cent nickel content the density is $8.64 \mathrm{~g} / \mathrm{cm}^{3}$. may be encountered unless special temperature control is provided.

The magnetizing coils shall be not less than 20 centimeters ( $7 \% / 8$ inches) in length and the ratio of the area of the test coil to that of the specimen shall be such that the air-eorrection factor shall be not greater than 5. This air correction may be applied by calculation or eliminated by means of an adjustable mutual inductance having its primary in series with the main permeameter magnetizing winding and its secondary in series with the main test coil. This mutual inductance shall be set to such a value that the flux in the space between the sample and the test coil shall be automatically compensated for. If desired, the two main magnetizing coils may be connected in series and the average values for the whole sample ${ }^{3}$ determined by connecting the two middle test coils in series and using them as one. In this arrangement, four auxiliary test coils near the ends of the specimens are connected in series and used as one coil for adjusting the current in the compensating magnetizing coils.

The Fahy simplex permeameter.-This type of apparatus is suitable for tests at magnetizing forces up to 300 gilberts per centimeter without difficulties due to heating. For higher values of magnetizing force, the high magnetization adapter may be used.

Isthmus method.-This method, as described in Bureau of Standards Scientific Papers Nos. 361 and 383 , is suitable for tests at magnetizing forces exceeding 200 gilberts per centimeter.

\section{Accuracy}

7. (a) The accuracy of the results of magnetie tests depends not only upon the method or apparatus used but also upon the nacure of the specimen. The most common sources of error are (1) lack of uniformity in permeability along the length of the specimen, (2) mechanical strain, and (3) temperature variations. Errors due to these causes are indeterminate and may be large.

(b) For specimens having a satisfactory degree of uniformity, clamped so as to be free from mechanical strain and kept at a constant temperature within $5^{\circ} \mathrm{C}$., the methods specified above may be expected to give the following accuracy: For normal induction, the values of magnetiring force corresponding to inductions up to an induction equal to two-thirds of that corresponding to the maximum magnetizing force specified in section 5 (c) should be accurate within 3 per cent, provided the permeability does not exceed 10,000 . This permeability limitation does noi apply to the ring method. Values of induction greater than the above should be accurate within 1 per cent. For residual induction, values should be accurate within 2 per cent and for coercive force within 5 per cent.

\section{B. CORE LOSS}

Befinitions

8. (a) Core loss.-The power consumption in electrical sheet steel when subjected to an alternating magnetization.

(b) Standard core loss. - The total power in watts consumed in each kilogram of material at a temperature of $25^{\circ} \mathrm{C}$. when subjected to a harmonically varying induction having a maximum of 10,000 gausses and a frequency of 60 cycles per second, when determined in accordance with definite prescribed methods. It is represented by the symbol $W^{10} \%$.

Note.-If the loss corresponding to a higher induction is desired, the sample should be subjected to a harmonically varying induction having a maximum of 14,000 gausses. The corresponding symbol will be $W^{14} 60$

3 The limits of cross-sectional area given in soction 3 (a) shall apply to each half of the whole sample. 
(c) Aging coefficient.-The percentage change in in which

the standard core loss after continued heating at $100^{\circ}$ C. for 600 hours.

\section{Procedure}

9. The standard core loss shall be measured under the following conditions:

(a) The magnetic circuit shall consist of $10 \mathrm{~kg}$ (22 pounds) of the test material, cut with a sharp shear into strips $50 \mathrm{~cm}$ (1911/16 inches) in length and

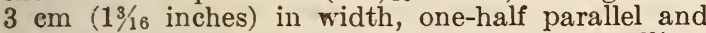
one-half at right-angles to the direction of rolling, made up into 4 equal bundles, 2 containing material parallel and 2 containing material at right angles to the direction of rolling, and finally built into the

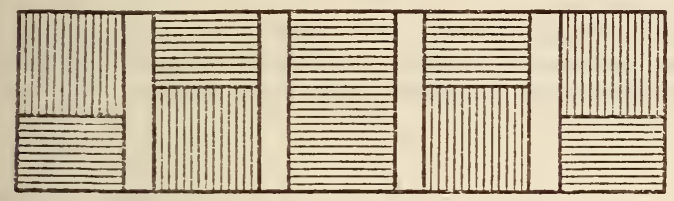

Figure 9

4 sides of a square with butt joints and opposite sides consisting of material cut in the same manner. No insulation other than the natural scale of the material (except in the case of scale-free material) shall be used between laminations, but the corner joints may be separated by tough paper $0.1 \mathrm{~mm}$ (0.004 inch) in thickness.

(b) The magnetizing winding shall consist of four solenoids surrounding the four sides of the magnetic circuit and joined in series. A secondary coil shall be used for energizing the voltmeter and the potential coil of the wattmeter.

These solenoids shall be wound on a form of any nonmagnetic nonconducting material of the following dimensions:

Inside cross-section.................... 4 by $4 \mathrm{~cm}$

Thickness of wall.

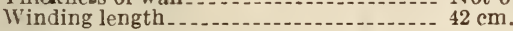

The primary winding on each solenoid shall consist of 150 turns of copper wire uniformly wound over

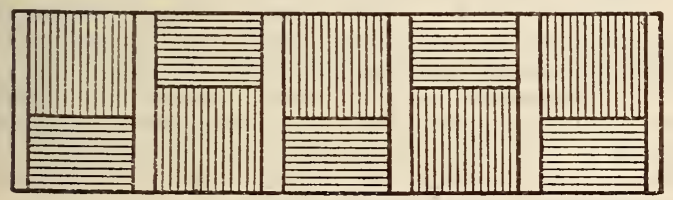

Figure 10

the $42 \mathrm{~cm}$ length. The total resistance of the magnetizing winding shall be between 0.3 and 0.5 onm. The secondary winding of 150 turns of copper wire on each solenoid shall be similarly wound beneath the primary winding. Its resistance shall not exceed $1 \mathrm{ohm}$.

(c) A voltmeter and the voltage coil of a wattmeter shall be connected in parallel to the terminals of the secondary winding of the apparatus. The current coil of the wattmeter shall be connected in series with the primary winding.

(d) An electromotive force having a wave form approximating a sine curve shall be applied to the primary winding and adjusted until the voltage of the secondary circuit is given by the equation:

$$
E=\frac{4 f N n B M}{4 l D 10^{3}}
$$

$f=$ form factor of primary emf

$N=$ number of secondary turns..._.

$n=$ number of cycles per second............. $=60$

$B=$ maximum induction, gausses............ $=10,000$.

$M=$ total mass in grams $\ldots=10,000$

$l=$ length of strips in centimeters _

7.5 for high-resistance steel. 7.7 for low-resistance steel.

$$
E=\left\{\begin{array}{c}
106.6 \text { volts for high-resistance steel for sine } \\
\text { voltage. } \\
103.8 \text { volts for low-resistance steel for sine } \\
\text { voltage. }
\end{array}\right.
$$

NotE.-For the 14,000-gauss test the voltages will be as follows:

$E=\{149.2$ volts for high-resistance steel for sine voltage

$E=\left\{\begin{array}{l}145.2 \text { volts for low-resistance steel for sine voltage. } \\ \text {. }\end{array}\right.$

The form factor of the applied electromotive force shall not depart more than 1 per cent from the value of 1.11. As an alternative to the use of a sine wave, the correct value of the voltage may be determined by applying it to an Epstein apparatus containing a sample which has been standardized by means of a sine. wave electromotive force. The wattmeter is brought to the correct reading for the standard sample. The corresponding voltage is the correct one to use.

10. (a) For silicon steels, a density of $7.7 \mathrm{~g} / \mathrm{cm}^{3}$ shall be assumed if the silicon content is 2 per cent or less and a density of $7.5 \mathrm{~g} / \mathrm{cm}^{3}$ shall be assumed if the silicon content is over 2 per cent.

(b) For nickel-iron alloys the density shall be assumed from the nickel content as given by the straight lines joining the points defined as follows: ${ }^{5}$

\begin{tabular}{|c|c|}
\hline Nickel & Density \\
\cline { 2 - 2 } Per cent & g/cm ${ }^{8}$ \\
0 & 7.85 \\
30 & 8.00 \\
100 & 8.90 \\
\hline
\end{tabular}

(c) For other alloys a density corresponding to the actual measured or estimated value shall be used.

11. The wattmeter gives the power consumed in the iron and the secondary circuit. The loss in the secondary circuit is given in terms of the total resistance and voltage. Subtracting this correction term from the total power gives the net power consumed in the steel as hysteresis and eddy-eurrent loss. Dividing this value by 10 gives the core loss in watts per kilogram.

12. The core-loss material shall be cut from two or more sheets taken at random from shipment. The strips should be distributed symmetrically over the sheet, as nearly as may be practicable. (See figs. 9 and 10.)

It is recommended that a test sample shall represent not more than $5,000 \mathrm{~kg}$ (11,000 pounds.)

13. (a) Cut the test material into strips 3 by 50 $\mathrm{cm}$ as indicated in section 9 (a).

(b) Place on the balance a pile of strips weighing $2.5 \mathrm{~kg}$. Add a second pile of the same kind, bringing the weight up to $5 \mathrm{~kg}$. In each case the weight shall be taken to the nearest strip. Add in suc-

4 For silicon steel having a 2 per cent silicon content the electrical resistance is 2.6 ohms per meter gram.

8 For 50 per cent nickel content the density is $8.26 \mathrm{~g} / \mathrm{cm}^{3}$ For 80 per cent nickel content the density is $8.64 \mathrm{~g} / \mathrm{cm}^{3}$ 
cession two piles of $2.5 \mathrm{~kg}$ each, of the other kind of strips, bringing the weight up to $7.5 \mathrm{~kg}$ and $10 \mathrm{~kg}$, respectively.

(c) Insert in the apparatus as indicated.

Note.-If desired, for convenience, the bundles may be loosely secured by tape or string (not wire).

(d) Apply the alternating voltage to the primary coil and tap the joints together until the current has a minimum value, as shown by an ammeter in

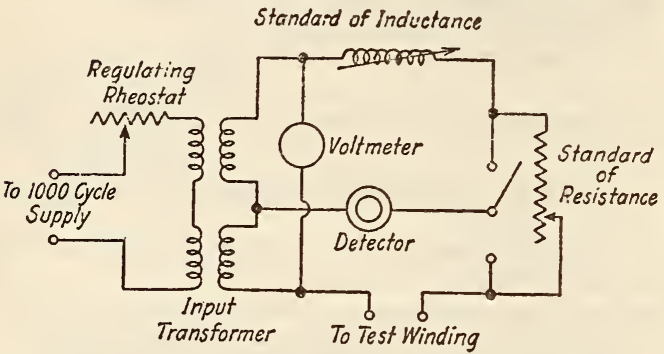

Figure 11.-Connections for balanced transformer method for magnetic properties at low inductions

series. Then clamp the corners firmly by some suitable device.

(e) Shunt the ammeter and adjust the primary current until the voltmeter indicates the proper value. This adjustment may be made by an autotransformer, by varying the field of the alternator, or by both, but not by the insertion of resistance or inductance in the primary circuit. Simultaneously the frequency must be adjusted to 60 cycles.

(f) Read the wattmeter.

(g) Calculations.- Subtract from the wattmeter reading the instrument losses, which will be con-

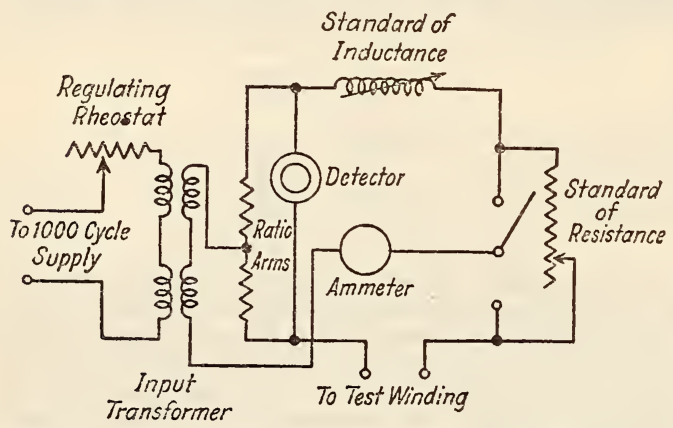

Figure 12.-Connections for ratio arm method for magnetic properties at low inductions

stant for any set of instruments and voltage, and divide by 10 . The result is the standard core loss.

Note.-If desired, a $5 \mathrm{~kg}$ sample may be used for routine acceptance tests, provided it is cut from at least two sheets, except in case of a dispute between the manufacturer and purchaser when the 10 $\mathrm{kg}$ sample shall be used.

\section{PROPERTIES AT LOW INDUCTIONS AND AUDIO- FREQUENCIES}

14. This method provides for the determination of the alternating-current permeability and core loss of magnetic materials at low inductions and audio-frequencies. These properties are indicative of the effect of the material on the impedance and loss characteristics of audio-frequency apparatus. Standard values are those for the standard low induction of 10 gausses and the standard audiofrequency of 1,000 cycles.

\section{Test Specimens}

15. (a) Test specimens shall preferably be of the ring form. Ring specimens shall conform to the requirements specified in sections 2 and 3 .

(b) If mutually agreed upon by the manufacturer and purchaser, the rings may be sheared at one point to facilitate insertion of the specimen into formwound coils. If this method of preparation is used, the slits shall be staggered so as to come at different points in the magnetic circuit.

(c) As an alternative, test specimens of sheet material not exceeding $0.05 \mathrm{~cm}(0.0197$ inch) in thickness may be of the form described in section 9 (a), except that the four bundles of strips shall be assembled so that at each corner consecutive layers mutually overlap the butt joints occurring in each single layer.

16. When testing for core loss, the separate laminations comprising each form of specimen shall be effectively insulated from each other.

\section{Apparatus}

17. (a) The apparatus shown in Figures 11 and 12 shall conform to the requirements specified in paragraphs $(b)$ to $(j)$, inclusive. The apparatus shall be connected for the balanced-transformer method as shown in Figure 11 or for the ratio-arm method as shown in Figure 12.

(b) Test winding.-Continuous ring specimens shall be wound with a toroidal test winding distributed uniformly around the circumference of the ring. If the sheared ring specimen is used, the windings may be applied in sections, provided the sections are of equal numbers of turns and uniformly spaced to cover at least 75 per cent of the mean circumference of the ring. The number of turns should be such that an inductance not less than 0.03 henry nor more than 0.15 henry is obtained. The winding conductor shall not be smaller than No. 28 nor larger than No. $20 \mathrm{~A}$. W. G. Care shall be taken to prevent any electrical contact between turns or to the core. It is recommended that double-cotton-insulated wire be used. The test winding for the strip form of specimen shall be the secondary winding of the solenoids described in section $9(b)$, care being taken that the primary winding is open circuited during the test.

(c) Power supply.-The supply voltage shall be of approximately a sine-wave form, having not more than 10 per cent of total harmonics present.

(d) Transformer. - The input transformer shall have a core formed from magnetic sheet material which is not more than $0.038 \mathrm{~cm}(0.015$ inch) in thickness and which has a standard low induction permeability of not less than 400 .

Note.-This corresponds to good quality 4 per cent silicon steel.

The primary and secondary windings shall have self and mutual inductance characteristics suited to the efficient transmission of power under the test conditions from the source to the test circuit. For the balanced-transformer method (fig. 11) the secondary winding shall have a tap at its electrical mid-point. The two half-windings so formed shall be closely balanced with respect to each other and with respect to mutual effects to the primary. The degree of balance will be satisfactory if, upon reversal of the complete secondary with respect to the test coils and standards, the readings of the standards are not changed for the new condition of balance by more than $0.02 \mathrm{ohm}$ in resistance or 1 per cent in inductance. For the ratio-arm method (fig. 12) a balanced secondary winding is not required.

Note.-A satisfactory degree of balance can be obtained by winding two conductors together on the core and taking each of the coils thus formed as one-half of the double secondary. 
(e) Ratio arms.-The ratio arms used in the ratio arm circuit (fig. 12) shall be balanced nonreactive resistors, the resistance of each of which may be of any value from 500 to 1,000 ohms. The degree of balance shall be equal to that specified for the secondary winding of the transformer used in the balanced-transformer method as specified in paragraph $(d)$.

(f) Standard of resistance.-The adjustable standard of resistance shall have a range of from 0 to at least $100 \mathrm{ohms}$, the smallest step being not more than $0.1 \mathrm{ohm}$. The resistance values for each decade shall be correct to within $\pm(0.1$ per cent +0.005 $\mathrm{ohm})$. The effective inductance for any setting shall not exceed 0.0001 henry.

(g) Standard of inductance.-The adjustable standard of inductance ${ }^{6}$ shall have a range of from 0.03 to 0.15 henry and shall be capable of being read to at least 0.0005 henry. It shall be wound with a stranded conductor, each strand of which shall not be larger than No. 35 A. W. G. and insulated from all other strands. The direct-current resistance shall not exceed 50 ohms. The inductance values shall be accurate, to within $\pm(0.5$ per cent +0.00025 henry).

(h) Detector.-Any zero-current detector having the desired sensitivity may be used. A telephone receiver having an impedence between 300 and 500 ohms at a frequency of 1,000 cycles is recommended.

(i) Voltmeter. - Any voltmeter capable of reading the output voltage at the test frequency with an accuracy of at least \pm 2 per cent may be used. A vacuum-thermocouple type having a full scale of 10 volts is recommended.

(j) Ammeter.-Any ammeter capable of reading the bridge current at the test frequency with an accuracy of at least \pm 2 per cent may be used. A vacuum-thermocouple type having a full scale of 0.020 ampere is recommended.

\section{Procedure}

18. (a) The test specimen shall be thoroughly demagnetized before testing.

(b) The apparatus having been connected in circuit as shown in Figure 11 or Figure 12, the standard of resistance shall be set at zero and the standard of inductance at approximately mid-scale. The output of the transformer shall then be adjusted by means of the regulating rheostat to give approximately the standard induction in the test specimen.

Note. -In this determination the following relations between the bridge voltage or current and the induction in the test specimen shall bridge volta

$E=8.88 f N A B \times 10^{-8}$ for the balanced-transformer method. (Fig. 11.)

$I=\frac{1.414 N A B \times 10^{-8}}{L}$ for the ratio-arm method. (Fig. 12.) where

$E=$ the secondary voltage (r.m.s. value);

$I=$ the bridge current (r.m.s. value);

$f=$ the test frequency in cycles per second;

$N=$ number of turns in the test winding;

$A=$ cross-sectional area of the test specimen in square centimeters;

$B=$ maximum induction in gausses; and

$L=$ inductance of the test coil in henrys.

The standards of resistance and inductance shall then be alternately adjusted and, if necessary, the regulating rheostat readjusted until, with the meter reading the voltage or current corresponding to the standard induction, the detector indicates zero difference of potential between its terminals. The readings of the standards shall be noted and the

- A general form of construction suitable for this instrument is described in Bureau of Standards Scientific Paper No. 290, Oct. 12, 1926. transformer secondary or ratio arms reversed and a second balance obtained. The mean of the two inductance readings shall be taken as the inductance of the test coil. The resistance readings shall be added to or subtracted from the resistance of the standard inductance, as indicated by the connection, and the mean of these resultants taken as the effective resistance of the test coil. Subtracting from this value the direct current resistance of the test winding gives the effective resistance due to the core loss in the test specimen.

\section{Calculations}

19. (a) Permeability.-The standard low induction permeability shall be calculated from the inductance value of the test coil determined as described in section $18(b)$ by means of the following formulas:

(1) For ring specimens:

$$
\mu_{x / 1,000}=\frac{L \times 10^{9}}{2 N^{2} h \log \frac{D}{d}}
$$

or

$$
\mu_{x / 1,000}=\frac{L \times 10^{9}(D+d)}{8 N^{2} A}
$$

(2) For straight-strip specimens:

$$
\mu_{x} / 1,000=\frac{41,600 L}{A}
$$

where

$\mu_{x / 1,000}=$ standard low induction permeability (permeability at 10 gausses and 1,000 cycles);

$L=$ inductance of the test coil in henrys;

$N=$ number of turns in the test winding;

$h=$ net axial height of the core in centimeters (see note);

$D=$ outer diameter of the core in centimeters ;

$d=$ inner diameter of the core in centimeters; and

$A=$ cross-sectional area of the test specimen in square centimeters.

Note. - The net axial height and cross-sectional area of the core shall be determined trom measured values of the diameters and weight and standard values of density.

(b) Core loss.-The standard low induction core loss shall be calculated from the measured effective resistance due to the core loss in the test specimen determined in section $18(b)$ by means of the following formula:

$W_{x / 1,000}=\frac{R_{1} E^{2} \times 10^{3}}{16 \pi^{2} 2^{2} L^{2} M}$ for the balanced-transformer method. (Fig. 11.)

$W_{x / 1,000}=\frac{R_{1} I^{2} \times 10^{3}}{4 M}$ for the ratio-arm method. (Fig. 12.)

where

$W_{x / 1,000}=$ the standard low induction core loss in watts per kilogram;

$R_{1}=$ the effective resistance due to the core loss in the test specimen;

$M=$ weight of the test specimen in grams;

$E=$ the bridge voltage (r. m. s. value);

$f=$ the test frequency in cycles per second;

$L=$ the inductance of the test coil in henrys; and

$I=$ the bridge current (r. m. s. value) in amperes.

AMERICAN SOCIETY FOR TESTING MATERIALS, STANDARD METHODS OF CHEMICAL ANALYSIS OF ALLOY STEELS, SERIAL DESIGNATION A55-24, 1924

\section{CHEMICAL ANALYSIS OF ALLOY STEELS}

(See 621.30, p. 1107.) 
AMERICAN SOCIETY FOR TESTING MATERIALS, STANDARD SPECIFICATIONS FOR THE ARBITRATION TEST BAR AND TENSION-TEST SPECIMEN FOR CAST IRON, DESIGNATION A124-29, 1929

\section{CAST-IRON TEST SPECIMENS}

1. These specifications cover the arbitration test bar for cast iron, 1.20 inches in diameter, intended

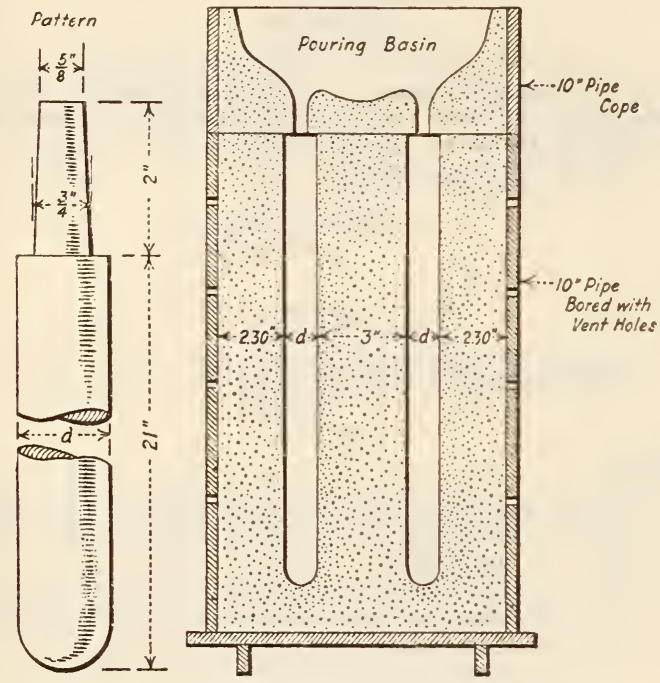

NOTE: The dimension "d"shall be made such that the casting shall measure approximately $1.20 \mathrm{in}$. in diameter at the mid point.

FIGURE 13.-Mold for arbitration test bar

for transverse testing placed horizontally upon supports 18 inches apart with a centrally applied load; and the tension-test specimen for determining the tensile strength of cast iron. The arbitration test bar represents the metal in the ladle and not in the casting.

\section{ARBITRATION TEST BAR}

2. The form and dimensions of the pattern and mold for the arbitration test bar shall conform to

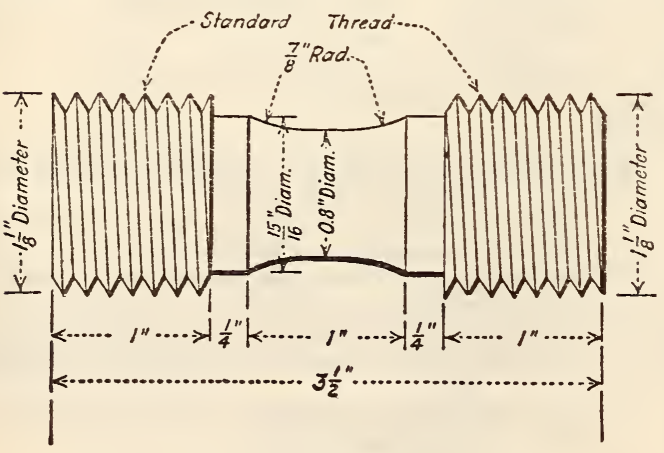

Figure 14.-Tension test specimen

those shown in Figure 13. The mold may be made in cores or in dry sand. The bottom of the bar shall be 0.05 inch smaller in diameter than the top, to allow for draft and for the strain of pouring. The pattern shall not be rapped before withdrawing. The flask shall be rammed-up with green molding sand, a little damper than usual, well mixed and put through a No. 8 sieve, with a mixture of 1 to 12 bituminous facing. The mold shall be rammed evenly and fairly hard, thoroughly dried, and not cast until it is cold. The test bar shall not be removed from the mold until cold enough to be handled. It shall not be rumbled or otherwise treated, being simply brushed off before testing.

3. The patterns shall be made of such size that the arbitration test bar will have a diameter of 1.20 inches at the center.

4. (a) In case the transverse test bar varies from the specified diameter of 1.20 inches, a correction factor conforming to that shown in the table shall be applied.

\begin{tabular}{|c|c||c|c|}
\hline $\begin{array}{c}\text { Diameter } \\
\text { of arbitra- } \\
\text { tion test bar } \\
\text { (in inches) }\end{array}$ & $\begin{array}{c}\text { Correction } \\
\text { factor }\end{array}$ & $\begin{array}{c}\text { Diameter } \\
\text { of arbitra- } \\
\text { tion test bar } \\
\text { (in inches) }\end{array}$ & $\begin{array}{c}\text { Correction } \\
\text { factor }\end{array}$ \\
1.10 & 0.770 & 1.21 & 1.025 \\
1.11 & .791 & 1.22 & 1.051 \\
1.12 & .813 & 1.22 & 1.077 \\
1.13 & .835 & 1.24 & 1.103 \\
1.14 & .857 & 1.25 & 1.130 \\
1.15 & .880 & 1.26 & 1.158 \\
1.16. & .903 & 1.27 & 1.185 \\
1.17 & .927 & 1.25 & 1.214 \\
1.18 & .951 & 1.29 & 1.242 \\
1.19 & .975 & 1.30 & 1.271 \\
1.20 & 1.000 & & \\
\hline
\end{tabular}

If the test bar is not round the correction factor can not be obtained from the preceding table and shall be calculated as follows: Square the depth of the bar measured at point of application of load, multiply by the width, and divide the product by 1.728 .

(b) In order to correct to a diameter of 1.20 inches the breaking load, obtained in testing the bar, shall be divided by the correction factor.

\section{TENSION-TEST SPECIMEN}

5. The tension-test specimen shall be machined from one of the broken pieces of the arbitration test bar, and shall conform to the dimensions shown in Figure 14.

\section{AMERICAN SOCIETY FOR TESTING MATE-} RIALS, TENTATIVE DEFINITIONS OF TERMS, WITH UNITS AND SYMBOLS, RELATING TO MAGNETIC TESTING, DESIGNATION A127-31T, 1931

\section{MAGNETIC TESTING DEFINITIONS}

In the following list of definitions the term defined is given first followed in parenthesis by any optional alternative terms which may be used when the context clearly indicates the intended conception. The symbol for the quantity is then given wherever such symbol is in use, followed by the definition of the standard term.

The name of the unit employed is included, together with the definition of the unit.

\section{BASIC CONCEPTIONS}

Magnetic field (field).-The region in space in which a magnetic state exists.

UNIT.-None.

Magnetic circuit.-A closed path of magnetic flux.

Magnetic flux (flux), Ф.-The magnetic quantity which by its variation is capable of producing in an 
associated electric circuit an electromotive force proportional to its time rate of variation.

UNIT: Maxuell.-The maximum value of the magnetic flux, the uniform variation of which between zero and its full value in one second induces one cgs. unit of electromotive force in a single turn electric circuit enclosing the flux.

Magnetomotive force, F.-The total magnetizing influence acting on any part of a magnetic circuit.

UxiT: Gilbert.-The magnetomotive force resulting from a current in an electric circuit of any number of turns linked with the magnetic circuit when the product of the current in amperes by the number of turns is $\frac{1}{0.4 \pi}$.

Note.-When a current of $I$ amperes is flowing in a circuit of $N$ turns, $\mathcal{F}=0.4 \pi N I$.

\section{DERIVED CONCEPTIONS}

Magnetic force (magnetizing force, field intensity), $H$. - The space rate of variation of magnetomotive force at any point in the magnetic circuit.

Uvir: Oersted.-The magnetic force of one Gilbert per centimeter.

Note. - At the center of an infinitely long solenoid having $n$ turns per centimeter in which a current of $I$ amperes is flowing, $H=0.4 \pi n I$.

Magnetic potential difference.-The magnetomotive force between two points.

Induction ( $f$ lux density), B.-The flux per unit area normal to the direction of the flux.

Uviт: Gauss.-An induction of one Maxwell per square centimeter.

Normal induction, B.-The induction in a magnetic material corresponding to a given magnetic force when the material is in a symmetrically cyclic magnetic condition with respect to the given magnetic force.

UnıT: Same as for Induction.

Intrinsic induction (ferric induction), $B_{i}$.- The excess of the normal induction over that corresponding to the same magnetic force in empty space.

UNiT: Same as for Induction.

Saturation induction, $B_{8}$.- The maximum intrinsic induction possible in a material.

UNIT: Same as for Induction.

Permeability, $\mu$.- When used without a qualifying adjective, understood to be the normal permeability as defined below.

UNIT: None.

Normal permeability, $\mu$.- The ratio of the normal induction to the corresponding magnetic force.

Unir: None.

Initial permeability, $\mu_{0}$.- The normal permeability when both the magnetic force and the induction are vanishingly small.

Uxit: None.

Differential permeability, $\mu_{d}$. The ratio of the positive increase of normal induction to the positive increase of magnetic force when these increases are vanishingly small.

Unir: None.

Incremental permeability, $\mu_{\Delta}$. - The ratio of the cyclic change in induction to the corresponding cyclic change in magnetic force when the mean induction differs from zero.

UNıT: None.

Reversible permeability, $\mu_{7}$ - The incremental permeability when the change in induction is vanishingly small.

UNIT: None.
Intrinsic permeability, $\mu_{j}$. - The ratio of the intrinsic induction to the corresponding magnetic force.

UNIT: None.

Permeance, $\odot$.- The property of any part of a magnetic circuit which determines the flux corresponding to a given magnetomotive force.

Note.-Permeance $=\frac{\Phi}{F}$ equivalent to $\frac{\mu A}{l}$ for uniform $\mu$.

where

$\mu=$ permeability;

$A=$ area in square centimeters; and

$l=$ length in centimeters.

UNIT: None.

Reluctivity. - The reciprocal of permeability.

Unit: None.

Reluctance, R.-The reciprocal of permeance.

NoTE. - Reluctance $=\frac{F}{\Phi}=\frac{l}{\mu A}$ for uniform $\mu$.

UNIT: The reluctance of a portion of an empty space 1 centimeter in length and having a cross section of 1 square centimeter.

Remanence.-The magnelic induction which remains in a magnetic circuit after the removal of an applied magnetic force.

NotE.-If there is an air gap in the magnetic circuit, the remanence will be less than the residual induction.

UNIT: Same as for Induction.

Residual induction, $B_{r}$. - The magnetic induction which remains in a magnetic material when the effective magnetic force has been reduced to zero.

Note.-When the material is in a symmetrically cyclic magnetic condition, the residual induction is called the normal residual induction.

UNIT: Same as for Induction.

Retentivity. - The property of a material measured by the normal residual induction remaining after the removal of an applied magnetic force corresponding to the saturation induction for the material.

UNiT: Same as for Induction.

Coercive force, $H_{c}$.-The reversed magnetic force that is just sufficient to reduce the residual induction in a material to zero.

Note.-When the residual induction is the normal residual induction, the coercive force required is called the normal coercive force.

UNIT: Same as for Magnetic force.

Coercivity. - The property of a material measured by the coercive force required to reduce to zero the induction remaining after the removal of an applied magnetic force corresponding to the saturation induction for the material.

UNIT: Same as for Magnetic force.

Core loss (iron loss), W.-The power expended in a magnetic material subjected to a varying induction.

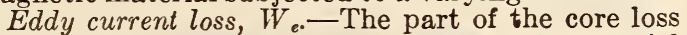
due to currents circulating in the magnetic material as a result of electromotive forces induced by the varying induction.

Hysteresis loss, $W_{h}$.-That part of the core loss due to so-called hysteresis effects.

Standard core loss, $W_{B / f}$-The total power in watts per kilogram expended in the magnetic material which is subjected to a harmonically varying induction of a specified maximum value $B$ and of $a$ specified frequency $f$.

Aging (of magnetic materials). - The normal or accelerated change in magnetic properties of a 
magnetic material under continued normal or specified artificial aging conditions. When used in reference to core loss this term, unless otherwise modified, implies an increase in loss. When used in reference to permeability or remanence the term, in a positive sense, indicates a decrease in these quantities.

Dia-magnetic.-Having a magnetic permeability less than unity.

Para-magnetic.-Having a magnetic permeability slightly greater than unity and approximately independent of the magnetic induction.

Ferromagnetic.-Having a magnetic permeability considerablygreater than unity.

Linkage. - The product of the flux in maxwells by the number of turns in a coil or winding linked therewith.

\section{AMERICAN SOCIETY FOR TESTING MATE- RIALS, STANDARD METHODS OF SAM- PLING ROLLED AND FORGED STEEL PRODUCTS FOR CHECK ANALYSIS, DES- IGNATION A130-30, 1930}

\section{SAMPLING ROLLED AND FORGED STEEL PRODUCTS}

SAMPLING

Different parts of a piece of steel vary in composition. For this reason, a sample from a single piece

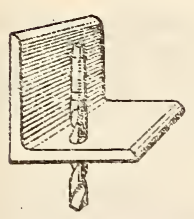

(a)

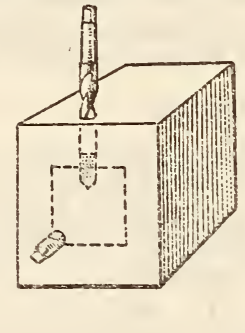

(b)

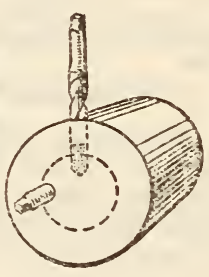

(c)
FIGURE 15.-Location of samples for check analysis

must be carefully selected if it is to be representative of that piece. If the analysis is to be representative of the melt, a number of representative pieces should be sampled and analyzed separately. In any case, the sample should be so selected as to be thoroughly representative, and large enough to suffice for all of the required determinations.

It should be remembered that the composition of the steel is changed by certain operations and that samples should be taken from the steel in its original condition.

\section{PREPARATION OF SAMPLES}

Samples shall consist of drillings or chips cut by some machine tool without the application of water, oil, or other lubricant, and shall be free from scale, surface metal, grease, dirt, or other foreign substances. If the samples are taken by drilling, a drill not less than one-half nor more than threefourths inch in diameter shall be used. Samples shall be uniform, thoroughly mixed, and free from dust. Chips too coarse to pass a No. 20 sieve are not recommended, nor shall long curly drillings which will not pack closely for the carbon determination be used.

In referring samples to other analysts for check analyses, pieces of the original full-size section, when possible, should be submitted rather than cuttings, unless the latter are specifically requested.

\section{LOCATION OF SAMPLES}

Large sections.-For large sections, including blooms, billets, slabs, rounds, squares, shapes, etc., samples shall be taken at any point midway between the outside and the center of the piece by drilling parallel to the axis. In cases where this is not practicable, the piece shall be drilled on the side (see figs. $15(b)$ and $(c)$ ), but the drillings shall not be collected until they represent the portion midway between the outside and the center. The tensiontest specimen may be used for sampling if it conforms to the above conditions.

Bored forgings.-For bored forgings, samples shall be taken midway between the inner and outer surface of the wall.

Thin material.-For thin material or material of small cross section, such as plates, shapes, bars, etc., if the method described above for large sections is not applicable, the sample shall be taken by machining off the entire cross section or, if this is not possible, by drilling entirely through the material at a point midway between the outside and the center. (See fig. $15(a)$.)

Sheets rolled longitudinally.-For sheets rolled from slabs or bars longitudinally, the specimen for sampling shall be cut 2 inches in width and across the full width of the sheet as rolled. The specimen shall be cleaned by pickling or grinding and then folded once or more by bringing the ends together and closing the bend. The sample for analysis shall be taken in the middle of this length by milling the inside sheared edges or drilling entirely through from the flat surface. Sampling by milling is preferable. For sheets of a light gage more than one specimen may be taken and stacked together before folding.

Sheets rolled transversely.-For sheets rolled from slabs or bars transversely, the specimen shall be cut from the side of the sheet, half way between the middle and end as rolled, 2 inches in width and 18 inches in length. If the sheet is No. 20 gage (24 ounces per square foot or 0.037 inch in thickness) or lighter, the specimen shall be cut from the full length of the sheet as rolled. The specimen selected shall be cleaned by pickling or grinding and then folded once or more by bringing the ends together and closing the bend. The sample for analysis shall be taken in the middle of this length by milling the inside sheared edges or drilling entirely through from the flat surface. Sampling by milling is preferable.

Sheets not of the full size rolled.-Sheets cut from larger sheets and not of the full size rolled shall be sampled by milling or drilling the sheet in a sufficient number of places so that the sample is representative of the entire sheet. The sampling may be facilitated by folding the sheet both ways.

AMERICAN SOCIETY FOR TESTING MATERIALS, STANDARD RULES GOVERNING THE PREPARATION OF MICROGRAPHS OF METALS AND ALLOYS, DESIGNATION E2-30; 1930

\section{MICROGRAPH WORK \\ STANDARD MAGNIFICATIONS}

1. The standard magnifications for general use in making micrographs, expressed in diameters, shall be as follows:

$$
10,25,50,75,100,200,500,1,000
$$




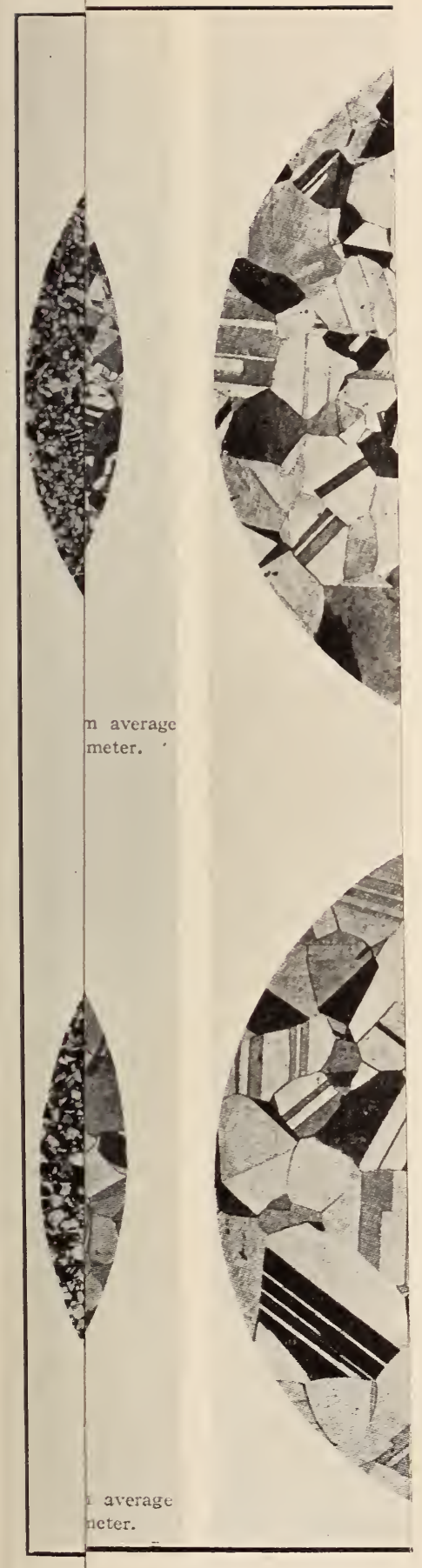

ularly nonferrous alloys, suc roxide. $(\times 75$.) 

sli

pe

co

th

th

A 
2. For general use in society reports, and for showing grain size, the following magnifications are recommended:

\begin{tabular}{|c|c|c|}
\hline Mnterial & Cast & Wrought \\
\hline $\begin{array}{l}\text { Steel, and ferrous materials } \\
\text { Copper } \\
\text { Copper-zinc alloys } \\
\text { Copper-nickel alloys } \\
\text { Copper-nickel-zinc alloys } \\
\text { Lead-tin-antimony alloys }{ }^{1}\end{array}$ & $\begin{array}{l}50 \text { and } 100 \\
50 \text { and } 100 \\
50 \text { and } 100 \\
50 \text { and } 100 \\
50 \text { and } 200\end{array}$ & $\begin{array}{r}100 \\
75 \\
75 \\
200 \\
200\end{array}$ \\
\hline
\end{tabular}

1 Including those containing small percentages of copper.

3. (a) Reproductions of micrographs in publications shall be made of exact standard magnifications except in those special cases where the details of structure are to be illustrated.

(b) If micrographs submitted for publication are not of standard magnification, they shall be enlarged or reduced to the nearest standard size. The actual magnification appearing in print shall be stated under the micrograph. (See par. $(d)$. )

(c) To facilitate the reproduction, authors should be advised that their micrographs should be of proper size to fit the printed page, and in order to conserve space that inicrographs be printed in the form of squares.

(d) With each micrograph shall be printed an explanatory title, together with actual magnification, etching medium, treatment, etc., indicating what the author intends to show, so that it will not be necessary to search the text for general information. With micrographs at magnifications of approximately 500 diameters and above, the numerical aperture of the objective and the type of illuminator shall be given.

(e) Photographs in which the magnification is less than 10 diameters are usually made with photo. graphic lenses or single magnifiers. These will be considered as "macrographs."

With each macrograph should be printed an explanatory title, together with the actual magnifications, method of illumination, and general treatment indicating what the author desires to illustrate, so that it will not be necessary to refer to the text for general information.

\section{LENSES}

4. For obtaining the magnifications recommended, the following types of lenses are suggested as suitable for general commercial work:

(a) For magnifications from 20 to 30: A photographic lens of approximately $35 \mathrm{~mm}$ focal length, to be used without ocular.

(b) For magnifications from 30 to 75: A $32 \mathrm{~mm}$ objective with Huyghens ocular (approximately $\times 5)$

(c) For magnifications from 75 to 150: A $16 \mathrm{~mm}$ objective with Huyghens ocular (approximately $\times 5)$.

(d) For magnifications from 250 to 500: A $4 \mathrm{~mm}$ objective (preferably apochromatic) with projection ocular.

5. (a) All objectives should be corrected for use without cover glasses.

(b) In using one of the lens combinations given above, the length of the camera bellows may be adjusted to give the exact magnification desired.

It should be borne in mind, however, that increasing the magnification by increasing the length of the camera bellows adds nothing to the detail of the micrograph. This depends mainly upon the resolving power of the objective used.

(c) The magnification shall be determined by accurately measuring the image of a stage microm- eter scale, and not by estimation from lens combinations, or microscope or camera adjustments.

\section{GRAIN SIZE}

6. (a) Alloys consisting of but one type of crystals (for example, copper, alpha brass, etc.).- In counting individual grains the original crystal, including the twinned layers, shall be called one grain.

(b) Alloys consisting of two metallographic components (for example, steels, Muntz metal, etc.).The original grain which has given rise to the aggregation of the two components shall be taken as the unit when determinable and the individual component in other cases. ${ }^{a}$ When grain size is included in actual specifications, the term should always be defined in such specifications.

7. For measuring grain size, two methods (see note 1) are recommended depending upon the condition of the material:

(a) For material in which the grains are equi-axed (for example, most metals in the cast and in the annealed state). - The planimetric method as modified by Jeffries (see note 2) is recommended for use as being very accurate and rapid. It is recommended that the circular area used should always include at least 50 grains.

(b) When the grains are not equi-axed (for example, in strained materials).-Heyn's intercept method should be used, the average grain size being determined by counting the number of grains at a given magnification along a line of known length on two axes at right angles to each other, one axis being parallel to the direction of rolling. In some cases a third count along a line perpendicular to the other two may be desirable. This will necessitate the preparation of another polished surface.

8. The grain size as determined by the planimetric method, section $7(a)$, should be expressed as the number of grains per unit area (square inch or square centimeter). This may also, but less preferably so, be expressed as the average grain area; or the average linear dimension of the grain may be given. The grain size as determined by the intercept method, section $7(b)$, should be expressed by giving the average number of grains per linear unit in the two directions; or the average number per unit area together with the ratio of length to breadth of grain $(L / B)$ may be given.

9. If grain size is to be included in specifications, it should be expressed as the maximum or minimum allowable as determined above. It is recommended that only in extreme cases should grain size be made the sole basis of rejection of material.

Note 1.-Standard micrographs for comparison of grain sizes.-The direct comparison of a micrograph or projected image with the standdirection of nonard micrographs shown in Figure 16, will facilitate inspection of nona sufficiently precise method of obtaining the grain count of a

specimen.

NOTE 2.-Jeffries' method for grain-size measurements.7-A circle $79.8 \mathrm{~mm}$ in diameter is drawn on the rough side of a ground-glass screen, the center of the circle being near the center of the rectangula section of the screen. This ground glass is mounted in a frame which fection ofthe fits a metallographic camera, the smooth side of the glass being on the outside. When the image of the specimen for grain-size determination is focused on the screen, the circle will be plair

and its circumference should be well within the image.
When the image is properly focused the grains intersected by the circumference of the circle are checked and counted. Since the check marks must be made on the smooth side of the glass, a soft red pencil such as is used in laboratories for marking beakers and flasks, wil such as is used in labor The marks used to indicate the boundary rou circumference grains are usually short, straight lines inter of the circle and perpendicular to it. The completely included grain are next checked and counted, after whicn the red marks are erased from the glass with a dry cloth. The specind
and other measurements made as desired.

$a$ See U. S. Navy specifications, 49-S-2-c., p. 7.

7 This method has been condensed from the paper by Zay Jeffiries appearing in Chemical and Metallurgical Engineering, Vol. XVIII p. 185,1918 . 
One-half the number of grains intersected by the circumference of the circle, added to the number of completely included grains, gives the number of equivalent whole grains within the circle. If the equivalent number of whole grains within the circle measured at a certain magnification be multiplied by the number in the third certann in the following table opposite the magnification used, the product will be the number of grains per square millimeter.

\begin{tabular}{|c|c|c|}
\hline Magnification used $(=m)$ & $\begin{array}{l}\text { Diameter } \\
\text { of circle }\end{array}$ & $\begin{array}{l}\text { Multiplier } \\
\text { to obtain } \\
\text { grains per } \\
\text { square } \\
\text { millimeter } \\
\qquad(=f)\end{array}$ \\
\hline $\begin{array}{r}\text { Full size. } \\
10 \\
250 \\
55\end{array}$ & $\begin{array}{r}m m \\
79.8 \\
79.8 \\
79.8 \\
79.8 \\
79.8\end{array}$ & $\begin{array}{l}0.0002 \\
.02 \\
.125 \\
.5 \\
1.125\end{array}$ \\
\hline $\begin{array}{l}100 \\
150 \\
200 \\
300\end{array}$ & $\begin{array}{l}79.8 \\
79.8 \\
79.8 \\
79.8 \\
79.8\end{array}$ & $\begin{array}{r}2.0 \\
4.5 \\
8.0 \\
12.5 \\
18.0\end{array}$ \\
\hline $\begin{array}{r}500 \\
1,000 \\
1,500 \\
2,000\end{array}$ & $\begin{array}{l}79.8 \\
79.8 \\
79.8 \\
79.8 \\
79.8\end{array}$ & $\begin{array}{r}50.0 \\
112.5 \\
200.0 \\
450.0 \\
800.0\end{array}$ \\
\hline
\end{tabular}

1 At 75 diameters, if a circle $84.5 \mathrm{~mm}$ in diameter, or a rectangle having an area of $5,625 \mathrm{~mm}^{2}$ is used, the factor $f$ becomes unity.

A circle $79.8 \mathrm{~mm}$ in diameter has an area of 5,000 $\mathrm{mm}^{2}$. If it is desired to use a rectangle of the same area in place of a circle for the determination, convenient sizes are given as follows:

$\begin{array}{lll}70.7 & \text { by } 70.7 \mathrm{~mm} \\ 65 & \text { by } 77 & \mathrm{~mm} \\ 60 & \text { by } 83.3 \mathrm{~mm} \\ 55 & \text { by } 91 & \mathrm{~mm} \\ 50 & \text { by } 100 & \mathrm{~mm}\end{array}$

Since each of these rectangles has an area of about $5,000 \mathrm{~mm}^{2}$, the multipliers given in the table can be used for any one of them, or any other rectangle having an area of $5,000 \mathrm{~mm}^{2}$. To make a grain-size determination with a rectangular in place of a circular area, the grains intersected by the periphery of the rectangle should be counted, one-half the number added to the completely included grains and the result will be the number of equivalent whole grains within the rectangle. If the determination is made at one of the magnifications given in the table the number of grains per square millimeter can be obtained by multiplying the equivalent number of whole grains within the area by the multiplier $(f)$ opposite the magnification used. The rectangle could be used on a ground glass as described above for the circle, or it could be used on a micrograph.

Some metallographists prefer to refer to the grain size in terms of the diameter of the average grain in millimeters, or the area of the average grain in $\mu^{2}$. The following simple formulas will be found helpful in these cases:

$$
\begin{aligned}
& x=1 / 2 w+z \\
& f=\frac{m^{2}}{5,000} \\
& n=f x \\
& d=\frac{1}{\sqrt{n}} \\
& a=\frac{1,000,000}{n}
\end{aligned}
$$

where

$w=$ number of boundary grains;

$z=$ number of completely included grains;

$x=$ equivalent number of whole grains in 5,000 $\mathrm{mm}^{2}$ (circle $79.8 \mathrm{~mm}$ in diameter or rectangle having area of $5,000 \mathrm{~mm}^{2}$ );

$m=$ magnification used;

$f=$ multiplier to obtain number of grains per square millimeter;

$n=$ number of grains per square millimeter;

$d=$ diameter of average grain in millimeters; and

$a=$ area of average grain in $\mu^{2}$.

After considering various criticisms, owing to the fact that 75 diameters has become an established practice for use in illustrating the structure and grain size of wrought copper and the copper-zinc alloys, and that a large volume of work has been done using this magnification, it seems advisable to retain a magnification of 75 diameters for this class of material.

(Recommended practice for photography as applied to metallography is added to this specification. It is considered under the following sections: Magnification, lenses, illumination of specimens, and photographic materials. An appendix treats of the care of the eyes when using a metallographic microscope.)

AMERICAN SOCIETY FOR TESTING MATERIALS, STANDARD METHODS OF METALLOGRAPHIC TESTING OF IRON AND STEEL, DESIGNATION E3-24, 1924

\section{METALLOGRAPHIC TESTING \\ MACROSCOPIC EXAMINATION \\ Preparation of Surface}

In order to show up both chemical and crystalline heterogeneity, sections of iron and steel are more or less deeply etched in order to bring out their macrostructure. In this way the persistence of the dendritic structure of castings, even after much heat treatment and mechanical working, physical unsoundness such as blowholes, gas cavities, porosity; fine internal cracks and flakes; discontinuities of structure such as found in welding; flow of metal as in riveting, punching, hot pressing and forging; etc., are brought out.

Suitable sections are cut, smoothed, and then ground on various grades of emery paper in order to obtain a smooth flat surface. In some cases it is necessary to continue polishing through Nos. 0 and 00 French emery papers. The sample ought to have a surface free from grease and dirt; if necessary it is then freed from all grease by cleaning with gasoline and then alcohol when it is ready for immersion in the solution. ${ }^{8}$

\section{Solutions Used in Etching}

Macroscopic etching of iron and steel was used as early as 1783 by Bergman. Many different solutions have been used and many of them are given by Campbell ${ }^{9}$ and more recently by Rawdon. ${ }^{10}$

Of the following solutions 1 (a) and 2 are recommended.

8 For large sections various methods have been devised depending on the material. Surface grinding when carefully done gives excellent results.

Proc., Am. Soc. Testing Mats., Vol. XV, Pt. II, p. 96, 1915

10 "Structure and Related Properties of Metals," Bureau of Standards Circular No. 113. 
1. To show up segregation and the like:

(a) Hot concentrated hydrochloric acid used at about $100^{\circ} \mathrm{C}$. A deep-etched specimen may be printed with printer's ink.

(b) Etching for 4 to 5 hour's with 5 per cent picric acid in alcohol.

2 . To show up variations in crystalline structure as well as segregation:

One to two grams of ammonium persulphate in $10 \mathrm{c}$ c of water. This is probably the best reagent for developing this phase of the structure in iron and steel.11

3. To show up the distribution of phosphorus:

Various reagents containing copper chloride are used. The copper coats those portions which are low in phosphorus and leaves the high phosphorus areas bright. It has recently been shown that nickel and other alloys give similar effects, also that oxide of iron acts in the same way. The accompanying list shows the various solutions which have been recommended:

Heyn. - Copper-ammonium chloride -g-- 10

A somewhat weaker solution is better, the specimen being etched two or three times in fresh solution if necessary. The liquid is gently agitated. The coating of spongy copper forming on the face of the specimen is easily removed with a swab of wet cotton. Portions high in carbon, sulphur and phosphorus will have darkened. If the copper film adheres, a 0.5 per cent solution of ammonium persulphate will facilitate its removal.

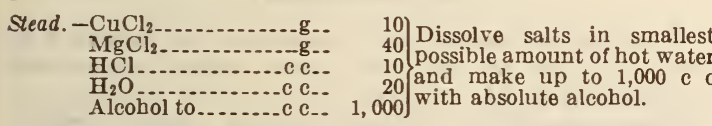

For small specimens the reagent is applied drop by drop until structure is developed. The specimen is then washed with boiling water and then alcohol.

Another method is to wash off the deposited copper with ammonia and so increase the contrast. Le Chatelier and Lemoine. $-\mathrm{CuCl}_{2}$

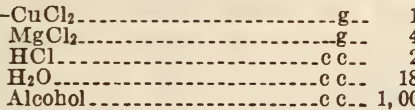

Applied like Stead's reagent. Contrast can be increased by electrolysis, using a single battery cell, keeping the current below 50 milliamperes.

Rosenhain and Haughton.-

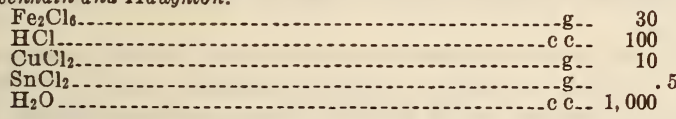

The copper adheres firmly. Pearlite areas remain bright. Ferrite darkened by deposited copper, the purest parts darkening the fastest. Pitting can be avoided by diluting the reagen $i$.

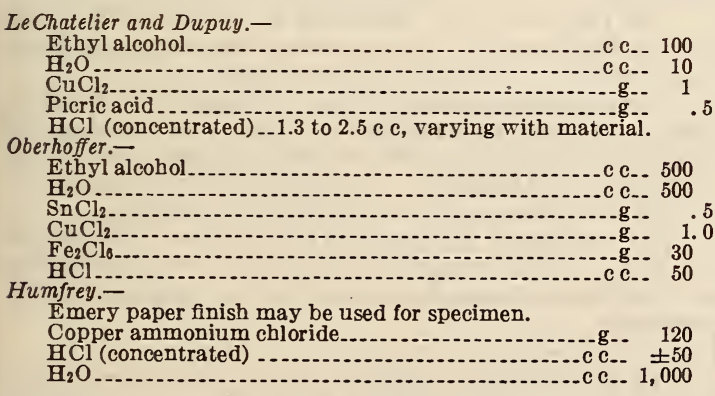

11 Rawdon, "Macro-etching with Ammonium Persulphate," Scientific Paper No. 402, Bureau of Standards, 1920.
The addition of $\mathrm{HCl}$ causes the copper deposit to adhere. Etching is therefore siarted with the neutral solution and continued until all traces of the scratches have disappeared. Then in successive applications the acidity of this solution is gradually increased up to the maximum. Alloy steels etch better with solutions of weaker acidity. After wiping away the deposited copper and drying the surface is lightly rubbed with fine emery paper and the relief portions brought out in strong contrast. The section may be printed with ordinary printer's ink applied to the surface by means of a roller.

\section{Dickenson. -}

First etch with 10 per cent $\mathrm{HNO}_{3}$. Then reetch with -

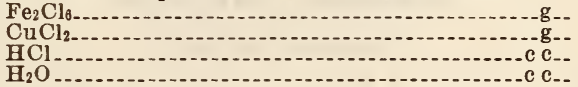

4. For etch tests to distinguish between wrought iron and steel, to develop the structure of welds, etc., the following rapidly acting reagents are recommended, the percentages given being by volume:

(a) Hydrochloric acid (specific gravity 1.19) Sulphuric acid (specific gravity 1.84) Water-. 60

(b) Nitric acid (specific gravity 1.42)

Water

\section{Methods for Indicating Distribution of Sulphur}

The distribution of sulphur is usually shown by the sulphur-print method. A solution of 2 to $3 \mathrm{c} \mathrm{c}$ of concentrated $\mathrm{H}_{2} \mathrm{SO}_{4}$ in $100 \mathrm{c}$ c of water is used to moisten a sheet of matte-finish photographic paper. The specimen, with its surface smoothed either with a fine file or grinding wheel, is pressed firmly on the paper on which a brown mark will indicate the sulphur-rich areas. About 60 seconds is usually sufficient to give a good print. The paper is then washed and fixed in hyposulphite solution.

With larger sections the paper is placed on their polished surface with care so that no air pockets occur. If a very dilute solution is used and a longer "etch," several prinús can be obtained. Fresh solution can be added with absorbent cotton should the paper tend to become dry.

\section{Methods Used for the Location of Minute Cracks Before Deep Etching}

Considerable difference of opinion has arisen as to the meaning of the results obtained by deep etching in transversely fissured rails, etc., because the presence of cracks in the material previous to etching had not been demonstrated. The following method, however, can be used to locate them. The sample, after careful polishing, is magnetized and then immersed in kerosene or a similar liquid containing very fine iron dust in suspension. The particles of iron dust collect upon the face of the specimen and orient themselves to correspond to the shape of the cracks because of discontinuity in the metal which causes a change in the density of the magneric flux. The samples are then washed in clean kerosene to remove as much as possible of the excess iron dust which clings to the surface. ${ }^{12}$

According to several authorities, their existence has been proven by annealing the specimens prior to etching, because a pronounced decarburized area then occurs around these cracks.

\section{Method of Photographing}

The camera should be arranged so that the specimen is illuminated by vertical light. After certain

12 Rawdon and Epstein, Technologic Paper No. 165, Bureau of Standards, 1920. 
kinds of etching, for example, ammonium persulphate, the surface should be immersed in water, alcohol, or light oil. For large specimens the surface may be covered with oil or glycerin.

\section{Printing the Surface}

The surface obtained by deep etching may be printed with printer's ink applied with a roller. The printing can be done on a glossy paper in a letter press. For small samples of wrought iron deeply etched a reproduction can be made using an ordinary ink pad and applying the specimen as in block printing.

\section{MICROSCOPIC EXAMINATION}

\section{Location of Test Pieces}

The location of the test piece for microscopic examination is of prime importance. In the case of a casting of steel or cast iron, the section should be cut perpendicular to the surface so as to show the variation of structure from the outside to the interior. In the case of forged and rolled metal both cross and longitudinal sections should be examined, their location being determined by the nature of the forging, etc. For crankshafts, definite locations are given with reference to the tension-test specimens. For guns, tangential specimens are required and for microstructure these should be cut parallel to the axis, etc. No one specimen is, of necessity, representative. The usual method of taking a cross section from rods and bars while showing segregation of the constituents fails to reveal the usual lamination in such material for which a longitudinal section is necessary. The location of test specimens may be determined by a preliminary macroscopic examination.

In defective material, the sample should be cut adjacent to the fracture or defect. When necessary the fracture can be protected during grinding and polishing by coating it with a heavy deposit of electrolytic copper, molten lead-tin solder or a mixture of litharge and glycerin. For hardened steel and other material liable to be tempered, fusible metals such as Rose's or Wood's alloys may be used.

\section{Method of Polishing}

The method used for polishing specimens will depend primarily on the material to be examined and on the polishing equipment. In general, samples should not be over 1 inch square and their thickness should be less than the smallest dimension of the polished surface; otherwise there is a tendency to round off the edges during the process.

The softer material can be cut with a saw while harder metal may be cut with an emery disk or broken off with a hammer. A flat surface is at first obtained either by rubbing on a file held in a rise or by grinding on an emery wheel. In both cases it is necessary to avoid tearing the surface of the metal or causing it to flow. Perfectly smooth surfaces can be obtained by commercial surface-grinding machines.

Having obtained a flat surface in this way, the sample is next rubbed down on a series of emery papers, usually starting with No. 00 commercial followed by Nos. 0 and 00 French emery. These papers may conveniently be mounted on revolving disks. Rubbing on one paper is stopped as soon as the scratches from the last operation have been taken out. From the No. 00 French emery the specimen may be taken to a revolving disk covered with broadcloth, preferably running in a horizontal position at such a speed that the polishing powders are not unnecessarily thrown off. For ordinary work, two such disks will be sufficient, the first armed with well-washed carborundum, 65 or a similar grade, while the second may be armed with well-washed alumina. This is preferable to rouge because it is much cleaner.

To obtain a fine polish it is a mistake to try to save time by hurrying through the papers because if this is done, when the specimen is etched, innumerable fine lines will be revealed in the ferrite. In some cases the appearance of the ferrite is actually blurred because the metal distorted in the grinding and by the coarse grades of emery has not been subsequently removed. Under no conditions should buffing be tried, because this causes an actual flow of the metal and the structure is thereby obliterated.

No general rules can be laid down as to the method to be pursued, but common sense will tell what is right and what is wrong. For example, in grinding on the emery wheel a copious flow of water must be maintained, otherwise the surface of the sample will heat up and the structure of the metal will be modified thereby.

For material of small size, such as sheet and wire, numerous methods of mounting have been devised. For wire, various mounting media such as wax, a mixture of litharge and glycerin, fusible metals and the like can be used in a small brass container. For sheet metal, a pack can be built up of pieces of the sample with alternate layers of red fiber board, bakelite, or other resistant material, the whole being held together by two brass end pieces and two screws. The whole can then be polished down.

\section{Methods of Etching}

The sample ought to be examined as polished in order to show up the nonmetallic inclusions as well as cracks and other defects. In order to show up the structure of the metallic matrix various etching reagents have been used depending on the material being examined. For general work, a 4 per cent solution of picric acid in ethyl alcohol is recoinmended. This darkens the pearlite in steel and leaves the ferrite or cementite bright, as the case may be. Long etching with picric acid in alcohol will develop the grain structure in very low carbon steel and in wrought iron. This may be done more rapidly with a 2 to 4 per cent solution of nitric acid in ethyl alcohol (95 per cent). For showing up pearlite alone, picric acid in absolute alcohol is best.

To distinguish between ferrite and cementite in high-carbon steels, the usual method is to boil in the sodium picrate solution, which darkens the cementite.

For hardened and tempered steel, picric acid may be used, but after etching, washing under the tap, and flooding with alcohol, the specimen should be dried by an air blast or if this is not available by shaking it in air. If the usual method of mopping on a soft towel be tried, the etching film will be spoiled. ${ }^{13}$

For the study of hardened and tempered steels under high magnifications other reagents have been devised such as those of Kourbatoff.

For distinguishing between cementite and phosphide of iron in cast iron, either heat tinting, or boiling with sodium picrate may be used, as recommended by Matweiff. This attacks the iron phosphide, but leaves the cementite unattacked.

For revealing the segregation of the phosphorus in steel, various reagents have been used, depending on the deposition of copper from a copper chloride solution.

${ }_{13}$ To dry specimens without the use of alcohol: After etching wash in very hot water and dry in an air-blast. With this method the results obtained are as good as with the older method of using alcohol after washing in water. After etching with dilute nitric acid solutions, steels in the unhardened condition do not show the stains which generally appear after drying with alcohol. 
Certain alloy steels, especially those hign in the alloying elements, are very resistant to etching. For these, special solutions have been devised such as ferric chloride for high-nickel material, and caustic soda solution of hydrogen peroxide, etc., for high-speed steels.

In many cases after etching, the ferrite or cementite may be found to be more or less stained or colored. This can often be remedied by a light repolish on a chamois-skin pad. Wnile this brightens up the ferrite or cementite, as the case may be, it markedly alters the appearance of the pearlite, giving it an abnormal structure, and therefore when this method is used, photographs should be so marked.

(Supplementing these methods are numerous references to the art of metallography; and a list of reagents for etching, including solutions for iron and plain-carbon steels, solutions for hardened steels, solutions for carbides, and solutions for alloy steels. Information on methods of identifying nonmetallic inclusions is added, also a bibliography on etching.)

\section{AMERICAN SOCIETY FOR TESTING MATE- RIALS, STANDARD METHOD OF VERIFI- CATION OF TESTING MACHINES, DES. IGNATION E4-27, 1927}

\section{VERIFYING TESTING MACHINES}

\section{DEFINITIONS}

1. (a) Testing machine (load applying).-A mechanical device for applying a load (force) to a specimen.

Nоте.-Usually the magnitude of the load can be changed at the will of the operator. Many testing machines are arranged to measure the load, but this is not always the case, especially with impact machines and machines for testing ductility.

(b) Error.-In the case of a testing machine, the value obtained by subtracting the correct value of the quantity measured (usually load) from the indicated value as given by the testing machine.

Note.-The error may be positive or negative.

(c) Percentage of error.-In the case of a testing machine, the ratio, expressed in per cent, of the error to the correct value of the quantity measured.

(d) Correction.14-In the case of a testing machine, the value obtained by subtracting the indicated value from the correct value of the quantity measured.

Note. - The correction has the same magnitude as the error but the opposite sign.

(e) Tolerance.-In the case of a testing machine, the maximum allowable error in the value of the quantity indicated.

NotE.-It is convenient to express tolerance in terms of percentage of error. The numerical value of the tolerance for a testing machine is so stated hereafter in these methods.

The word "accurate" applied to a testing machine shall be used without numerical values. The word "error" shall be used with numerical values. For example: "An accurate testing machine was used for the investigation"; but "the error of the testing machine at a load of 30,000 pounds was 15 pounds." A testing machine is said to be accurate if the indicated load is within the specified tolerance of the actual load.

The accuracy of a testing machine should not be confused with sensitiveness. For example, a testing machine might be very sensitive--that is, it might indicate quickly and definitely small changes in

14 It is recommended that, except for special cases, no corrections be used on machines tested and found to have errors within the tolerances given in these methods. the load-but, nevertheless, be very inaccurate. On the other hand, the accuracy of the results is, in general, limited by the sensitivity.

(f) Loading range.- In the case of a testing machine, the range of indicated loads for wnicn the testing machine gives results within the specified tolerances. The loading range shall not include loads either greater than the largest load or less than the smallest load applied in verifying the testing machine.

\section{METHODS FOR VERIFYING TESTING MACHINES THAT MEASORE}

2. Wherever practicable, testing machines shall be verified by applying standard weights directly to the weighing mechanism of the testing machine, or by the use of standardized proving levers in connection with standard weights. The test loads selected shall be applied in both ascending and descending order, and the indications for both the ascending series and the descending series of loads shall be considered in the application of tolerances.

Note.-For machines in which the load-indicating device is actvated by a Bourdon pressure tube, a hydraulic (or steam-engine) indicator spring, or other device depending on the elastic properties of a material, the tolerances for the descending load shall be applied to differences in the indication for the corresponding ascending and descending readings. In such testing machines the test for ascending load shall be applied as rapidly as practicable after the testin machine has remained unloaded for at least six hours, and the tim of applying the ascending series of loads shall not exceed thirty mintest mum test load has remained on the machine for one hour, and the readings for descending loads shall be taken as rapidly as possible, and in any event the descending load test shall be completed within a period of twenty minutes.

3. For any loading range, the testing machine shall be verified by at least four test loads. It is recommended that the test loads used be $10,20,50$, and 100 per cent of the maximum capacity at which the testing machine is to be used.

Note.-Where a large amount of testing of small specimens is to be done on a large-capacity machine, it is recommended that the verification outlined above be supplemented by a series of smaller test loads selected in reference to the light work to be done on the testing machine.

\section{(A) Method of Verification by Standard Weights}

4. Standard metal weights of suitable design, finish, and adjustment shall be placed on the weighing platform of the testing machine or upon trays or other supports suspended from the load-measuring mechanism in place of the specimen. The weights shall be applied in increments and removed in the reverse order. They shall be arranged symmetrically with respect to the weighing platform, so that the center of gravity of the load lies in the vertical line through the center of the platform. The applied load and the in dicated load shall be recorded for each test load applied, and the error computed from these data.

Note. - The method of verification by standard weights can be NoTE. The method of verifcation by total load is limited by the size of the platform and the number of weights available. Often 20 weights of 50 pounds each are used, a total of 1,000 pounds.

This method of verification is seldom used for any but small testing machines, as the loading range does not include the loads for which large machines are used.

\section{(B) Method of Verification by Standardized Proving Levers}

5. The arrangement of proving levers for verifying testing machines is shown in Figure 17. The two levers rest on supports on the weighing platf orm of the testing machine. These supports shall move easily in a horizontal direction, which insures that the forces at each of the knife-edges shall be very nearly vertical. The inner knife-edges in each lever bear against a suitable block in the movable head of the testing machine. Weight trays or hangers are suspended from each of the outer knife-edges 
and these trays or hangers are loaded with staniard weights. The increment of load put on the testing machine by the standard weights is the amount of standard weights multiplied by the lever ratio $m / n$, Figure 17 .

6. The knife-edges, as well as their supports, shall be of hardened tool steel. The knife-edges shall be

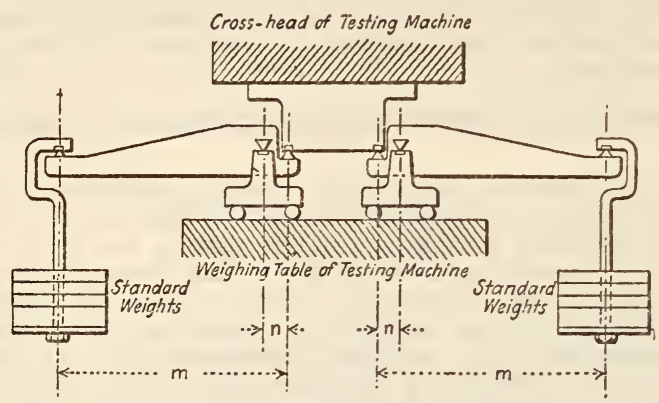

Figure 17.-Proving levers

ground sharp with an angle of $90^{\circ}$. The load on any knife-edge shall not exceed 7,000 pounds per linear inch. The three knife edges in each lever shall be parallel, and their edges shall lie in a plane. Each lever shall have machined surfaces in this or in

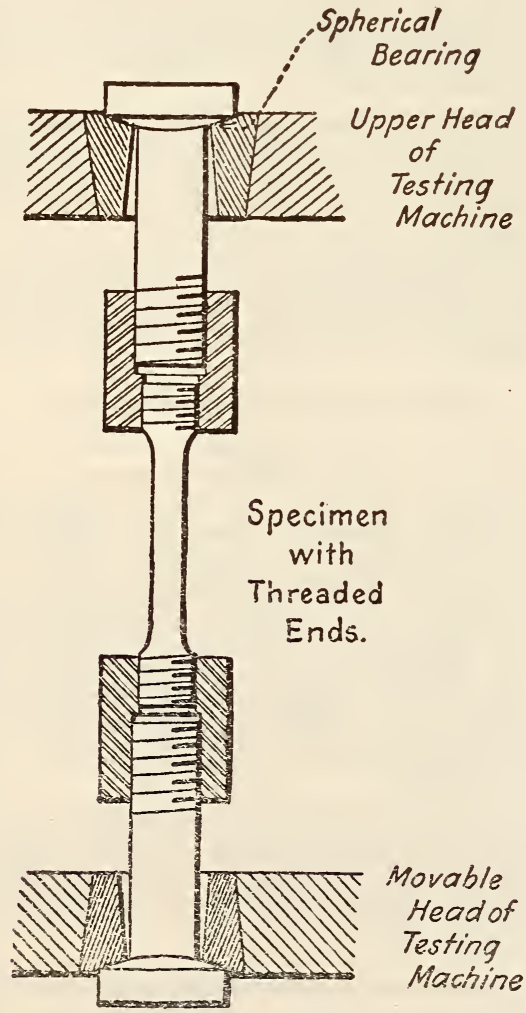

Figure 18

some parallel plane, upon which a spirit level can be placed.

7. The lever ratio of a proving lever shall be determined by the use of at least three test loads; the amount of weights used shall not be less than the maximum load applied upon one of the arms of a lever in using the levers to verify testing machines. The proving lever shall be balanced over its center knife edge with suitable weight trays suspended from the end knife-edges. Standard weights shall then be applied to the trays in three steps, corresponding approximately to 50,75 , and 100 per cent of the weights available, and the proving lever shall then be brought to a balance by the use of small weights and by observations of the freely swinging proving lever. From the weights in the two weight trays the lever ratio shall be computed.

8. The proving levers shall be placed symmetrically in the testing machine to be verified and both levers brought as near to a horizontal position as is feasible, after applying each increment of load, by means of the movable head of the testing machine. The testing machine shall be balanced with the levers in place and the weight trays empty. Standard weights shall be applied in increments, half an increment in each tray, and they shall be removed in the reverse order. The weights shall

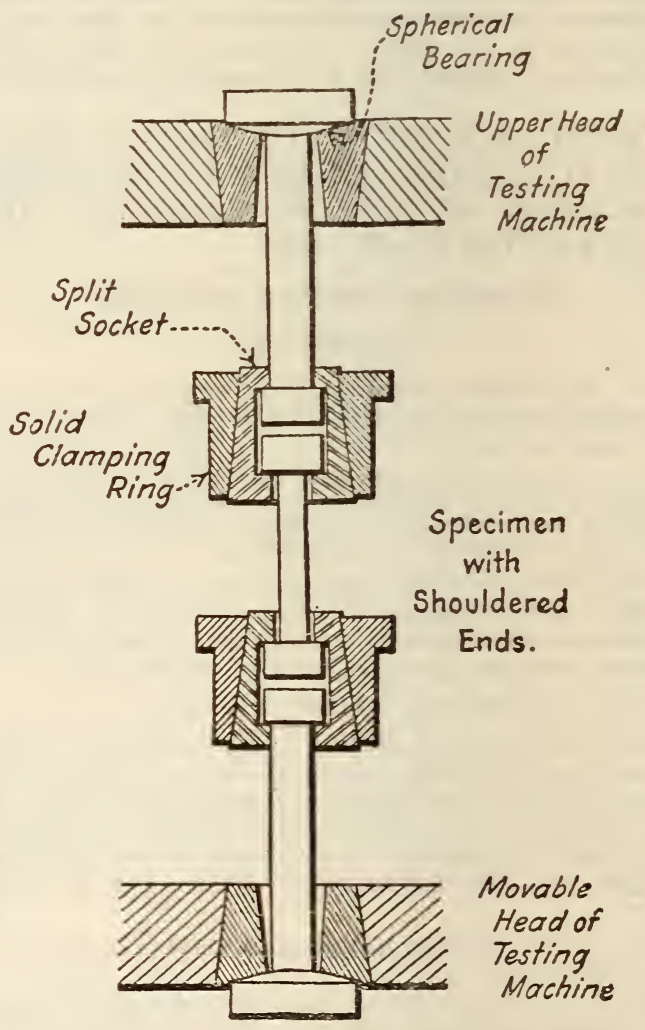

Figure 19

be placed symmetrically on the weight trays, with the center of gravity of the weights over the center of the tray. The applied load and the indicated load shall be recorded for each test load applied, and the error computed from these data.

Nот:.-The method of verification by standardized proving levers $\mathrm{s}$ used, at the present time, only on rertical testing machines. It is often used up to loads of 10,000 pounds.

(C) Method of Verification by Means of an Mlastic Calibration Device

9. In this method the testing machine to be verified is tested by comparing its load indications with the amount of elastic deformation of a calibration device, the deformation being measured with a delicate strainometer.

10. The calibration device may be a bar tested in tension or in compression, or an elastic loop or other shape whose elastic deformation can be measured, and if a tension or compression bar is used it shall be fitted with spherical-seated shackles or with 
a spherical-seated bearing block to insure as nearly axial loading as is possible. The arrangement shown in Figure 18 or Figure 19 is suggested for tension bars and the arrangement shown in Figure 20 is suggested for compression bars.

11. (a) The calibration device shall be of such size that under the greatest load for which the device is to be used (called its full load) the stress on any cross section will not be more than 80 per cent of the elastic limit of the material, as determined in accordance with Method II for the Determination of Elastic Limit as described under the definition of the term "Elastic Limit" of the Tentative Definitions of Terms Relating to Methods of Testing (A. S. T. M. Designation: E6-30T) of the American Society for Testing Materials on a test specimen cut from the bar, forging, or casting for the device after its final heat treatment. (See below.)

(b) Before the calibration device is first used for verification purposes, it shall be loaded to its full load at least six times, and between successive loadings it shall be heated to a temperature of $100^{\circ} \mathrm{C}$. $\left(212^{\circ}\right.$ F.) for a period of 30 minutes or shall be allowed to rest for a period of 24 hours. The object of this treatment is to adjust the device to its bearings and to relieve internal strain.

12. The loading range of a calibration device is the range of indicated loads for which the device gives results within specified tolerances. Obviously the loading range may not include loads either greater than the largest load or less than the smallest load applied in verifying the calibration device.

13. (a) The loading range of a calibration device shall be determined by verifying its indications by the use of standard weights or by standard weights and proving levers. In verifying the indications of the device, an initial load of 1 per cent of its full load shall first be applied. All changes of strainometer reading and of applied load shall be reckoned from the readings at this initial load. Successive loads shall be applied, a recommended series of loads being $10,20,50$, and 100 per cent of the full load of the device. Such a series shall be applied to the bar at least five times to determine the relation of applied load to strainometer reading, the shackles or bearing blocks being readjusted before each application of the series.

(b) The average of the five (or more) strainometer readings obtained in this way shall be taken for each load applied. The average of the differences (taken without regard to algebraic sign) between the individual strainometer readings for any load and the mean reading for that load constitutes the average deviation for that load. The lowest value of load for which the average deviation is not greater than 0.5 per cent of the strain for that load is the lower limit of the loading range of the calibration device.

NoTE. - If in taking five or more strainometer readings for any load the deviation of any one reading is more than 1 per cent from the mean reading, then this series of five or more readings shall be repeated.

14. The strainometer for elastic calibration devices, which employ elastic bars in axial tension or axial compression, shall be so designed as to indicate the average elongation or compression of the bar. For any elastic calibration device, it shall be sufficiently sensitive to indicate a change of 0.25 per cent of the minimum load in the loading range of the device. If the strainometer is not permanently attached to the calibration device, the strainometre shall be removed and replaced for each successive reading taken during the calibration of the device itself. This, of course, does not mean that the strainometer must be removed for each reading in the regular use of the instrument.

15. In using the calibration device it is important that just previous to taking any reading including, the initial reading of a series, there shall be no reversal in the direction of motion of the indicating mechanism of the strainometer, because such reversal may introduce error due to backlash in the instrument. That is, when taking readings for a series of increasing loads the load should be brought up to each predetermined test load, and when taking readings for a series of decreasing loads the load should be brought down to each predetermined test load.

16. In using a calibration device made of steel, correction for variation in mean temperature during any calibrations made on different occasions should be made by allowing a diminution of 0.015 per cent ( $15 / 1000$ of 1 per cent) in the modulus of elasticity of steel for each $1^{\circ} \mathrm{F}$. increase in temperature. During any calibration with an elastic calibration device, temperature conditions should be kept as uniform as is possible.

(D) Verification by Comparison Method

17. When the direct method of verification of a testing machine can not be carried out (as is the case at present with horizontal testing machines, for example) or when an elastic calibration device is not available, a comparison method of verification may

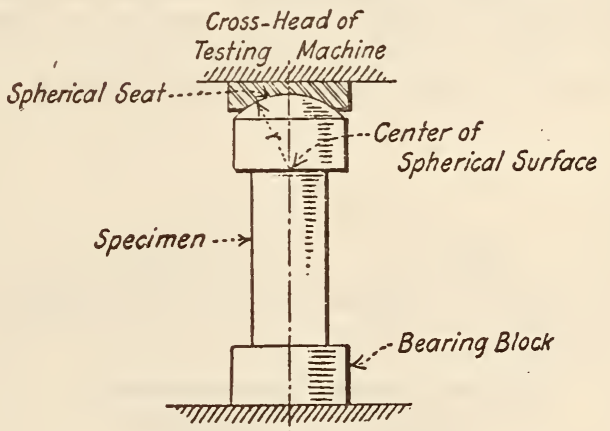

Weighing Table of

Figure 20

be used. In this method the machine to be verified is tested by comparing its indications with corresponding readings obtained under the same conditions by the use of another testing machine, especially tested and verified, as specified in section 18 . The method of verification by comparison shall be carried out by the use of a series of companion specimens, half of which are to be tested in tension in the machine to be verified and half of which are to be tested in tension in the especially verified testing machine which serves as a standard machine. The general provisions respecting the application of the test loads and the loading range which are outlined in the direct method of verifying testing machines shall apply to the verification of testing machines by the comparison method, so far as is practicable.

Nore.-The method of verification of a testing machine by standard weights (sec. 4) and the method of verification by standardized proving levers (secs. 5 to 8 ) are both regarded as superior to the proving levers (secs. 5 to 8 ) are both regarded as superior to the method of verification by the use of companion specimens. If the results of a verification test of a testing machine by the comparison method fail to agree with the results of a verification by either the standard-weight method or the proving-lever method, the resu

18. (a) A testing machine which is to be used as the standard for verifying other testing machines shall itself be verified by a special test, in which the verification is carried out twice. The loading range shall be determined by the tolerance for new testing machines, namely, \pm 1 per cent (sec. 22). 
For the loading range established, the difierences in indications found in the two tests of the standard machine shall not exceed 0.25 per cent.

(b) In verifying testing machines by the comparison method, corrections shall be applied to the indications of the standard machine and these corrections shall be based on the average correction obtained in the two tests used in its verification.

19. (a) For each test load, eight or more tensiontest specimens shall be cut from soft rolled or drawn steel and numbered consecutively, as in Figure $21(a)$.

(b) The tensile strength of the steel shall be determined by a preliminary test and the sets of specimens for the comparison test shall have such nominal cross-sectional areas as will give, approximately, the loads required. The form of the specimen shall be as shown in Figure 21 (b). The ends may be threaded, or otherwise machined, to fit holders, but it is recommended that those specimens shipped to another laboratory be left with cylindrical ends. The actual value of " $d$ " (fig. 21) for each specimen shall be determined by means of a micrometer.

Note.-It should be noted that the standard tension-test specimen, 18 0.5-inch in diameter and having a 2-inch gage length, satisfies the requirements for a specimen for the verification of a testing machine by the comparison method.

20. (a) The specimens having odd numbers shall be tested in the machine to be verified and those

(a)
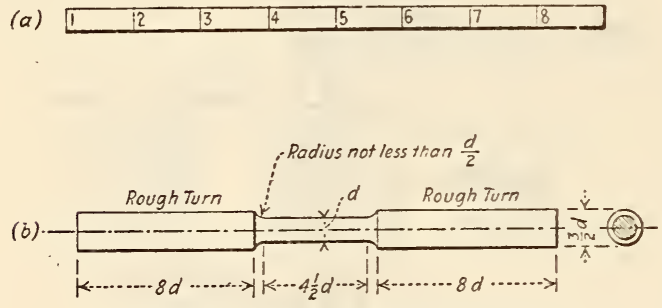

Figure 21.-Comparison specimen

(a) method of numbering specimens (b), form of test specimen

having even numbers in the standard testing machine. The tensile strength only shall be determined.

(b) The speed of the testing machine, by which is meant the speed of the pulling head when the machine is running idle with no specimen in the machine, when testing verification specimens shall be approximately the same for both the standard testing machine and the machine being verified. The speed shall be the slowest speed that can be obtained on both machines; in any case the speed shall not exceed 1 inch per minute per inch of diameter of comparison specimen.

(c) When testing verification specimens, especial care shall be taken to maintain the weighing beam of the testing machine in equilibrium as the tensile strength of the specimen is approached. It is recommended that a mark be placed at the middle of the trig loop and that a pointer be placed on the beam for verification tests, and that the pointer be kept as nearly on the mark as possible.

21. (a) The average tensile strength (in pounds per square inch) of the odd-numbered specimens, multiplied by the nominal area of cross section, shall be considered a measure of the indicated load, and the average tensile strength of the even-numbered specimens, multiplied by the nominal area of cross section, shall be considered a measure of the applied load. The error shall be computed from these data.

13 See Standard Specifications for Steel (605.0, p. 247). (b) If the tensile strength (in pounds per square inch) of any verification specimen varies more than 1 per cent from the mean of the group with which it is tested, its tensile strength shall not be used in computing the error of the testing machine. If more than one specimen in the odd-numbered group or one in the even-numbered group exceeds the allowable variation in tensile strength, all the specimens shall be discarded and another set prepared.

\section{TOLERANCES FOR TESTING MACHINES THAT MEASORE LOAD}

22. All testing machines that measure load shall conform to the following tolerances: 10

(a) New machines. - The error for loads in the loading range shall not exceed \pm 1.0 per cent for new machines or for those which have had any of the knife-edges in the weighing mechanism repaired or renewed.

(b) Used machines. - The error for loads in the loading range shall not exceed \pm 1.5 per cent.

23. The indicated load of a testing machine shall not be corrected either by computation or by the use of a calibration diagram to obtain values within the required tolerance.

\section{TIME INTERVAL BETWEEN VERIFICATIONS}

24. It is recommended that testing machines, when in constant use, be verified at intervals of six months and, when used intermittently, at intervals of two or three years. Testing machines shall, however, be verified immediately after making repairs or adjustments of the weighing mechanism, and whenever there is reason to doubt the accuracy of the results, without regard to the time interval since the last verification.

\section{REPORTS AND CERTIFICATES}

25. A clear and complete report shall be prepared of each verification of a testing machine. This report shall contain all data necessary to verify the accuracy of the report, and shall be filed for future reference. This report shall state clearly the method of verification used.

26. A certificate giving the maker's serial number and a brief description of the testing machine, the maker's name, the date of verification, and the loading range shall be signed by the person responsible for the maintenance of the testing machine, and this certificate shall be posted in plain view of a person operating the testing machine.

\section{AMERICAN SOCIETY FOR TESTING MATE- RIALS, STANDARD METHODS OF METAL. LOGRAPHIC TESTING OF NONFERROUS METALS AND ALLOYS, DESIGNATION E 5-27, 1927}

\section{METALLOGRAPHIC TESTING OF NONFERROUS METALS}

\section{MACROSCOPIC EXANINATION}

In order to show up both chemical and crystalline heterogeneity, sections of metals and alloy's should be more deeply etched than for microscopic examination. The dendritic structure in castings, dendritic shrinkage cavities, blowholes and other porosity, the flow of metal due to cold working, etc., are brought out.

${ }^{16}$ It is believed that the tolerances given in this section may be made much smaller when more perfect methods of rerification become generally available; and that the tolerances might well be materially reduced if there is available equipment for verifying testing machines by the use of standard weights (sec. 4) or by the use of standardized proving levers (secs. 5 to 8 ). 
After cutting the section, it is filed or ground smooth and flat, then rubbed on various grades of emery paper down to No. 00 French, so that all effects of surface cold working due to cutting are eliminated. After cleaning the surface of all grease it is ready for etching.

\section{Etching Solutions}

For macroscopic etching, most of the reagents used for microscopic examinations may be used, but the time of etching is usually much longer. Those solutions which give maximum grain contrast are usually to be preferred.

For copper and its alloys, a 50 per cent aqueous solution of nitric acid is recommended. Also an ammoniacal solution of copper ammonium chloride is very satisfactory.

The specimen is either immersed in the solution or flooded with it until the structure shows up strongly. It is then well washed with running water, flooded with alcohol, and dried. If the structure is to be recorded, it should be photographed immediately. The surface may be preserved for reference by covering with a thin coat of brass lacquer (a solution of gun cotton in amyl acetate) or similar preparation.

For aluminum and its alloys, a solution of $\mathrm{HF}-\mathrm{HCl}$ (solution No. 2) is recommended. A 10 per cent aqueous solution of $\mathrm{NaOH}$ and a 10 per cent $\mathrm{HF}$ also give good results. In copper-bearing alloys the latter solution should be followed by a cleaning in cold concentrated $\mathrm{HNO}_{3}$

For lead and its alloys, 10 per cent of nitric acid in water is recommended. For lead ingots, acetic acid gives excellent results.

\section{MICROSCOPIC EXAMINATION}

\section{Location of Test Pieces}

In the case of castings, the sections should be cut perpendicular to the surface and show the variation of structure from outside to center. For rods, sheet, etc., transverse, longitudinal, and frequently oblique sections should be examined. For large forgings, the threaded ends of the tension test pieces can usually be used by cutting a longitudinal section about one inch in length. No one specimen is of necessity representative. As in iron and steel, the usual method of taking a cross section from rods and bars while showing segregation, fails to reveal lamination. Surface sections of sheet metal are usually taken, but for cold-worked metal, transverse, longitudinal, and frequently oblique sections are also necessary.

For material of small size, the methods of mounting recommended for iron and steel can be used as described in the standard methods of metallographic testing of iron and steel in A. S. T. M. E3-24, given above.

\section{Method of Polishing}

The methods of polishing recommended for iron and steel as described in the standard methods of metallographic testing of iron and steel in A. S. T. M. E3-24, given above, are applicable for the harder alloys. For soft metals and alloys, emery wheels can not be used. The sample is rubbed on a file held in a vise, care being taken that the surface is cut and not torn. The file must be kept free from embedded particles of metals or the surface is bound to be torn.

In using the emery papers, only very little pressure may be used or the surface is apt to flow. For soft metals, rubbing by hand is recommended. If revolving disks are used, low speed must be maintained to prevent spoiling the surface. From the No. 00 French paper the specimen may be taken to a revolving disk covered with broadcloth and armed with well-washed alumina. Again, for soft metals, if the pressure is too great the surface is apt to be spoiled. Buffing can not be used. For very soft metals, such as lead and some of its alloys, even the most careful polishing leaves a film of "flowed" metal. Such samples can be etched fairly deeply with 10 per cent of nitric acid in water and then given a very light repolish with alumina, the surface of the disk being kept verv moist. It is then washed free from polishing powder and dried with alcohol.

In the preparation of specimens of aluminum and its alloys, the same precautions must be followed which are recommended for other soft metals. It is further recommended that following the cut on the file, two coarse emery papers, No. 2 and No. 1, be used, preceding the use of the finer metallographic polishing papers. The papers should be prepared by applying a solution of paraffin in kerosene and working well into the surface. The polishing must be done by hand. The papers may be used either as so prepared or moistened with additional solution. It is not always necessary to use this solution on the coarse papers, No. 2 and No. 1. The hand polishing may be stopped at either No. 000 or No. 0000 paper and the polishing continued on a wheel revolving at approximately $200 \mathrm{r}$. p. m. For the first cut on the wheel, a coarse grade of broadcloth is used with a grade of abrasive similar to $65 F$ alundum. The next cut is made using a softer broadcloth and "heavy magnesium oxide," applied as follows: The pad is moistened with distilled water and approximately one teaspoonful of powder applied evenly to the surface and thoroughly rubbed in, all hard and coarse particles being brushed off with the hand. After the scratches left by the $65 F$ material have been removed the pad is washed clean and a smaller quantity of powder worked in as before. This time the cut is made at right angles to the first treatment. This procedure may be repeated several times until the specimen shows a brilliant, reflecting surface, and under the microscope the aluminum solid-solution matrix appears clear white and the constituents stand out in even colors, free from tarnish. Only distilled water should be used during the polishing with the magnesia powder, otherwise there is danger of forming particles of carbonate, which are harsh and scratch the specimen. It is good practice frequently to raise the specimen from the pad and revolve it $180^{\circ}$ before replacing. The pressure and quantity of water to be used can only be determined after considerable practice and varies with the nature of the alloy.

\section{Methods of Etching}

The sample is first examined for nonmetallic inclusions, cavities, and the like. The structure is then developed by etching with a suitable reagent, a list of which is given below. No one reagent can be said to be the best for any alloy or group of alloys. Cast metal behaves quite differently from both hot and cold worked or annealed material.

For copper and its alloys a 50 per cent aqueous nitric acid solution is recommended. The sample is immersed until the structure shows up, then washed with water and dried with alcohol. ${ }^{17}$ This reagent, however, is liable to cause pitting.

For wrought brass, bronze, German silver, etc., the best reagent is ammonia and hydrogen peroxide. About $10 \mathrm{c} \mathrm{c}$ of strong ammonia are placed in a watch glass, 1 or 2 c c of peroxide ( 3.6 per cent) added and applied to the surface of the specimen with a small wad of cotton, rubbing gently till the grain structure appears.

17 To dry specimens without the use of alcohol: After etching, wash in very hoter and dry in an air-blast. With this method th in very obtained are as good as with the older method of using alcohol results obtained in water. After etching with dilute nitric acid soluaiter washing in water. Antened condition do not show the stain which generally appear after drying with alcohol. 
For cast gun metal, this method shows up the coring of the dendrites, but the eutectoid has little contrast with the saturated parts of the alpha. If the sample is then flooded with Grard's solution No. 1 until the alpha darkens slightly, the eutectoid still remains bright blue-white and shows up in marked contrast.

Nickel and its alloys frequently show a peculiar pitting. If a 50 per cent aqueous nitric acid solution be used, small pits appear with colored rings or halos. Merica's nitric-acetic acid gives much better results.

For wrought nickel alloys, concentrated hydrochloric acid is recommended. The action is very slow but the contrast is excellent.
The etching characteristics of copper and of nickel alloys are thoroughly dealt with by Rawdon and Lorentz.

Etching reagents for the identification of constituents in aluminum and its alloy's are generally unsatisfactory. Reagents intended differentially to color specific constituents are uncertain and their action varies in different alloys and especially under different conditions of polishing. For this reason it is reommended that the specimens be carefully polished and examined in the unetched condition. If a light filter is used to give a source of light approximating daylight, the constituents will appear as described below:

\section{Unetched characteristics}

\begin{tabular}{|c|c|c|c|}
\hline Constituent & Color & Usual shapes & Characteristics \\
\hline & Slate... & Irregular plat & Easily recognized. \\
\hline $\mathrm{FeAl} 3$ & Purplish gra & Needles..... & Tendency to purple tinge with relief polishing. \\
\hline $\begin{array}{l}\text { Other iron constit- } \\
\text { uents. }\end{array}$ & 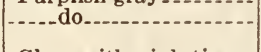 & $\begin{array}{l}\text { "Chinese script" and } \\
\text { needles. }\end{array}$ & $\begin{array}{l}\text { Less tendency to purplish tinge. Occurrence due to other elements, } \\
\text { notably Si. Composition uncertain. }\end{array}$ \\
\hline $\mathrm{CuAl}_{2 \ldots} \ldots$ & Clear with pink tinge. & Gobs, stringers........ & $\begin{array}{l}\text { Tendency to polish in relief and tarnish. A ppears bright on pass- } \\
\text { ing out of focus. }\end{array}$ \\
\hline $\mathrm{MnAl}_{3}$ & Flat gray to watery... & $\begin{array}{l}\text { Angular plates, often } \\
\text { hollow. A r row } \\
\text { heads. }\end{array}$ & $\begin{array}{l}\text { Does not polish in relief, but prolonged polishing gives watery ap- } \\
\text { pearance. In high-manganese alloys shapes are very character- } \\
\text { istic. }\end{array}$ \\
\hline $\mathrm{Mg}_{2} \mathrm{Al}_{8-}$ & Very faint watery. & Gobs, stringers....... & Same form as $\mathrm{CuAl}_{2}$ but much softer. Sometimes difficult to dis- \\
\hline 70 & Light gra & Needle & Difficult to distinguish from $\mathrm{FeAl}$. \\
\hline $\mathrm{Mg}{ }_{2} \mathrm{Si}$ & $\begin{array}{l}\text { Slate gray to iridescent } \\
\text { blue. }\end{array}$ & $\begin{array}{l}\text { Skeletons and rounded } \\
\text { particles. Angular } \\
\text { primary particles. }\end{array}$ & $\begin{array}{l}\text { Very careful polish gives even slate-gray particles which are easily } \\
\text { tarnished and are turned black or eaten out by too much water } \\
\text { in polishing. }\end{array}$ \\
\hline
\end{tabular}

With a little experience in the examination of specially prepared known standards, the identification of these constituents when they occur in sufficiently large particles is relatively simple, and etching reagents for distinguishing them are generally unnecessary. When the particles are smaller the identification becomes extremely difficult, as etching reagents which satisfactorily color large particles fail to identify the smaller particles.

In studying a specimen containing very small particles, such as finely precipitated $\mathrm{CuAl}_{2}$, there may be sufficient surface flow completely to obscure the particles. To guard against this condition and to reveal grain boundaries and other structural features, it is desirable to swab the specimen lightly with a soft cotton swab saturated with a dilute solution of HF ( 0.5 to 1 per cent preferred).

The white metals are usually easy to etch if properly polished. For the lead-tin alloys, however, the usual methods do not work so well. It is better to etch rather deeply with nitric acid and then very lightly to repolish on a chamois-skin pad until the structure shows up in marked contrast.

\section{Reagents for Etching}

(a) Solutions for Copper and Copper Alioys.

Nitric acid. -50 c c nitric acid (concentrated). $50 \mathrm{c}$ c water.

Ammonia.-10 c c ammonia (concentrated). 1 c c hydrogen peroxide (Marchand).
Grard No. 1.-5 g ferric chloride.

50 c c hydrochloric acid.

$100 \mathrm{c}$ c water.

Grard No. 2.-19 g ferric chloride.

6 c c hydrochloric acid.

100 c c water.

Ammonium persulphate - 10 per cent aqueous solution made just before use. This solution is improved materially by the addition of a few drops of ammonia.

Heyn's solution. $-5 \mathrm{~g}$ copper ammonium chloride. 120 c c water.

Add ammonia until precipitate which forms redissolves.

(b) Solutions for Nickel, Monel Metal, Cupro-Nickel.

Merica's solution. $-50 \mathrm{c}$ c of 70 per cent nitric acid. $50 \mathrm{c}$ c of 50 per cent acetic acid.

International Nickel Co. $-20-40$ c c nitric acid. 40-30 c c 75 per cent acetic acid.

40-30 c c acetone.

Hydrochloric acid.-(Concentrated). 
(c) Solutions for Alumindm and Aluminum Alloys

\begin{tabular}{|c|c|c|c|c|}
\hline $\begin{array}{l}\text { Solution } \\
\text { No. }\end{array}$ & Reagent & Concentration & Specific use & Authority \\
\hline & $\mathrm{HF}$ and $\mathrm{HCl}^{a} \ldots . . . . .$. & $\begin{array}{l}10 \text { to } 20 \text { per cent alcoholic } \mathrm{HF} \text { and concentrated } \\
\mathrm{HCl} \text {. }\end{array}$ & Macroscopic... & Czochralski. \\
\hline & $\mathrm{HF}$ and $\mathrm{HCl}{ }^{\circ}$ & $\begin{array}{l}10 \text { parts } \mathrm{HF}, 15 \text { parts } \mathrm{HCl}, 90 \text { parts } \mathrm{H}_{2} \mathrm{O} \\
0.1 \text { to } 10 \text { per cent }\end{array}$ & General............... & $\begin{array}{l}\text { Flick. } \\
\text { Various. }\end{array}$ \\
\hline 6-1 & $\begin{array}{l}\mathrm{NaOH} \\
\mathrm{NaOH} \mathrm{NO}_{3} \\
\mathrm{H} \mathrm{NO}_{3}\end{array}$ & $\begin{array}{l}0.1 \text { per cent in } 10 \text { to } 50 \text { per cent of alcohol } \\
1 \text { per cent in alcohol } \\
10 \text { per cent in alcohol }\end{array}$ & $\mid \mathrm{Mg}_{2} \mathrm{Al}_{3}-\mathrm{d}^{-2}$ & $\begin{array}{l}\text { Do. } \\
\text { Merica. } \\
\text { Hanson. } \\
\text { Do. }\end{array}$ \\
\hline 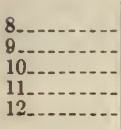 & $\begin{array}{l}\mathrm{H} \mathrm{NO}_{3} \mathrm{~b} \\
\mathrm{H} \mathrm{NO}_{3} \mathrm{~b} \\
\text { Ficric sulphate acid } \\
\mathrm{H}_{2} \mathrm{SO} \mathrm{O}_{4}^{6}\end{array}$ & $\begin{array}{l}20 \text { per cent quench } \\
25 \text { per cent quench } \\
4 \text { per cent in alcohol } \\
10 \text { per cent at } 60^{\circ} \text { to } 70^{\circ} \mathrm{C}\end{array}$ & $\begin{array}{l}\mathrm{CuAl}{ }_{2} \\
\mathrm{CuAl} \mathrm{A}_{2} \text { from } \mathrm{FeAl} \mathrm{F}_{3} \\
\mathrm{CuAl} \mathrm{I}_{2} \\
\mathrm{FeAl} \mathrm{I}_{3} \ldots\end{array}$ & $\begin{array}{l}\text { Do. } \\
\text { Dix. } \\
\text { R. J. Anderson. } \\
\text { A. Meyer. } \\
\text { Do. }\end{array}$ \\
\hline
\end{tabular}

- Czochralski originally etched for grain by using from 10 to 20 per cent of concentrated H F in $100 \mathrm{c} \mathrm{cabsolute} \mathrm{alcohol,} \mathrm{followed} \mathrm{by} \mathrm{dipping}$ in concentrated $\mathrm{HCl}$ solution. Flick has very satisfactorily modified this etch by using a single solution prepared as follows: $10 \mathrm{c} c$ of commercial $\mathrm{HF}$ and $90 \mathrm{c} \mathrm{c}$ of distilled water and $15 \mathrm{c} \mathrm{c}$ of concentrated $\mathrm{HCl}$.

By the 20 and 25 per cent nitric acid quench is meant the procedure of heating the solution to exactly $70^{\circ} \mathrm{C}$. and immersing the specimen for a period of from 2 or 3 seconds to 30 seconds and then immediately quenching the specimen in cold water. This same procedure may be used with the sulphuric acid etch, although the quench is not essential.

(d) Solutions for White Metals (Lead, Tin, or ZINC BASE).

Nitric acid.-2 per cent alcoholic solution. For tin-rich alloys.

5 per cent aqueous solution. For lead-rich alloys.

Hydrochloric acid. -5 per cent aqueous solution. For zinc-rich alloys.

The above list gives the principal reagent used for etching the chief commercial alloys. Numerous other methods have appeared in the literature, each worker apparently preferring to derelop his own method. The papers by Hudson, Czochralski, and Guertler covering etching reagents enumerate most of the ones which have had any vogue. Among these less-used reagents the following may be mentioned:

Gold, platinum, etc.-Aqua regia.

Zinc (Timofeeff). - A few drops added to 94 per cent nitric acid $\quad 100 \mathrm{c}$ c of water be6 per cent chromic acid fore use.

Tin. -5 per cent alcoholic solution of hydrochloric acid.

Magnesium and its alloys. -2 per cent nitric acid in water, 10 seconds.

Tungsten.-(1) Boil in ammonia and hydrogen peroxide or hydrogen peroxide alone.

(2) Electrolytic etch. 15 per cent $\mathrm{NaOH}$. 20 milliamperes per square centimeter. About 5 minutes.

(3) 2.5 per cent water solution of potassium ferrocyanide to which a few drops of ammonia are added just before use. Broadcloth or rubber is moistened with the solution and the specimen gently polished on the surface so prepared.

\section{Electrolytic Etching}

Electrolytic etching has been advocated by many workers, notably Le Chatelier, for brass and other alloys. The newer methods, as, for example, ammonia with hydrogen peroxide, give better results. For certain materials, however, electrolytic etching gives excellent results, especially where the samples are very resistant to ordinary etching reagents: For example, tungsten, certain of the nickel chromium alloys, white gold, etc. A simple method is to connect two short platinum wires to the electric-light circuit of 110 volt (direct current) through a 4candlepower lamp. Place the specimen on the anode wire so that its upper surface is perfectly level. Drop on enough dilute sodium hydroxide to cover the whole surface and then dip the cathode wire into the electrolyte. In order to get a uniform etch, the end of the wire should be bent at right angles and moved back and forth above the surface of the specimen to be etched. As soon as the structure is visible to the eye, the sample may be washed and dried in alcohol in the usual way. This gives a quick although somewhat rough-and-ready method, but one which usually works very well.

The specimen is held face downwards by a flexible clip attached to the positive connection. "The electrolyte is placed in a suitable dish in which the negative pole is immersed. The electrolyte surface is raised until it wets the surface of the specimen and then lowered slightly so as to produce a surface tension contact between the specimen and the solution. The results are very much superior to the old method of complete immersion of the specimen.

(Supplementing these methods are numerous references to the art of metallographic etching of nonferrous metals, including copper and copper alloys, nickel and nickel alloys, aluminum and aluminum alloys, brass, and bronze. There is also a bibliography of etching.)

\section{AMERICAN SOCIETY FOR TESTING MATE- RIALS, STANDARD DEFINITIONS OF TERMS RELATING TO METHODS OF TESTING, DESIGNATION E6-30, 1930}

\section{TESTING TERMS}

Stress.-The intensity (measured per unit area) of the internal distributed forces or components of force which resist a change in the form of a body. Stress is measured in force per unit area (pounds per square inch, kilograms per square millimeter, etc.).

Note.-In examining the definitions given in various authoritaNote.-In examining the cefinitions given "stress" were found. tive textbooks two definitions of the term "stress" were found. Textbooks in physics and some European textbooks in mechanics
of materials gave a definition substantially as above. American textbooks in engineering mechanics define stress as a "force," measured in "pounds." They call the intensity of force (pounds per square inch) "unit stress," "intensity of stress," or "fiber stress." This use of the term "stress" is illustrated by the "stress sheet" of the bridge engineer which gives forces, measured in pounds.

In view of the fact that even in engineering textbooks the term "stress" is often used to denote intensity of force per unit of area, and in view of the fact that the physicists' definitions involve somewhat simpler terms, the physicists' definitions have been followed what simpler terms,
in these standards.

While it is important to have a clear definition for the term "stress" it is even more important to keep clearly in mind the units used in computations and test data for materials of construction. In fact, if the units are always given, there can be no misunderstanding as to the sense in which the term "stress" is used. 
There are threc kinds of stress: Tensilc, compressive, and shearing. Flexure involves the combination of tensile stress and compressive stress. Torsion involves shearing stress.

It is customary to compute stress on the basis of the original dimensions of the cross section of the body.

Strain.-The change per unit of length in a linear dimension of a body, which change accompanies a stress. Strain is measured in inches per inch of length (millimeters per millimeter).

NoTE.-In American engineering textbooks the term "strain" is used in the sense of total deformation and is mcasured in inches. Change of dimension per unit length is called "unit strain," or "unit deformation." As in the consideration of the term "stress," the definitions given in textbooks in physics have been followed.

Under tensile stress or compressive strcss, strain is measured along the dimension under consideration. Shearing strain is mcasured at right angles to the dimension under consideration. In torsion tests.

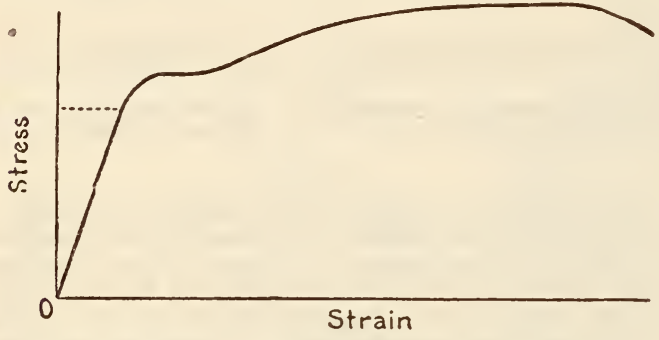

Figure 22.-A typical stress-strain diagram

which involve shearing stress, it is customary to measure the angle of twist, which may be translated into terms of strain.

Stress-strain diagram.-A diagram plotted with values of stress as ordinates and values of strain as abscissas.

NoTE.-The use of the term "stress-strain diagram" is frequently extended to cover diagrams plottcd with values of applied load or applied moment as ordinates, and with values of stretch, compression, deflection, or twist as abscissas.

Figure 22 is an example of a stress-strain diagram.

Stress-strain diagrams are in some cases drawn directly by an autographic attachment to the testing machine. A more usual method of procedure consists in taking a scries of load readings (from the balanced scale beam of the testing machine) with corresponding readings of the strain-indicating apparatus (extensometer, compressometer, deflectometer or torsion indicator) The term "strainometer reading" will be used to denote the reading of any strain-

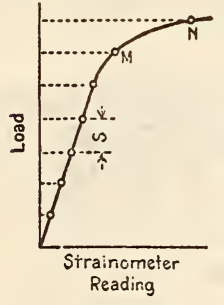

$a$

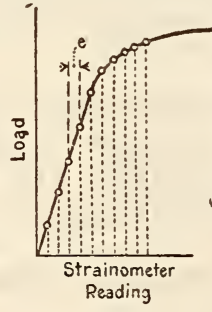

$b$

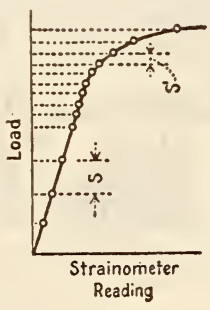

c
FIGURE 23.-Three methods of plotting load-deformation curves

$(a)$, with equal increments of stress; $(b)$, with equal increments

of strain; (c) with two values of increments of stress.

measuring instrument. From these readings, or from values computed from them, there is plotted a diagram with stress-indicating values (load, flexural moment, twisting moment, or stress) as ordinates, and strain-indicating values (elongation, shortening, deflection, twist, or strain) as abscissas. In planning such a test it is necessary to decide on the increment of load or the increment of reading of strainometer to be used between successive readings.

In Figure 23 are shown three typical stress-strain diagrams. The material for all three tests is the same, and the test specimens are all of the same size, so that the diagrams should be the same. The diagram shown in Figure $23(a)$ is plotted from points determined by taking increments of load $S$. In this diagram it is seen that owing to the shape of the curve, data for locating points between $M$ and $N$ are lacking. That particular portion of the curve (the "knee" of the curve) is the part for which it is especially desirable to locate several points on the diagram.

In Figure $23(b)$ is shown a diagram plotted from points determined by taking increments of strainometer reading $(e)$. It will be noted tirat for this diagram there are located sevcral points near the knee of the curve, and the shape of the diagram in this important region is much more definitely determined than for the curve shown in Figure $23(a)$

The custom of choosing incremcnts of load rather than increments of strainometer reading is quite common in tests of materials and is followed because, in gencral, it is easier to compute increments of load than it is to compute increments of strainometer read. ing. An estimate of the load necessary to stress the specimen ur to the knee of the curve is made and some fraction (frequently one. tenth) of this value is taken as an increment. The computation of the corresponding increment of strainometer rcading is more com. plicated.

Sometimes the practice is followed of applying a few increments of load as determined above, and then applying load in much smaller incremcnts $S^{\prime \prime}$ until the knee of the curve is jassed. Figure $23(\mathrm{c})$ shows a diagram obtained in this manner. This method involves a marked increase in the number of readings necessary for a test and with unknown material there is always some danger that the knee of the curve will be reached before the use of small increments of load is begun.

The following method of choosing increments for a test is sug gested: Estimate the load corresponding to the knee of the stressstrain diagram and choose a value for increment of load about one. tenth of this value. Apply this increment of load $S$ once and note the corresponding change of reading for the strainometer $e$. Then the corresponding change of reading for the strainometer $e$. Then
for the remainder of the test, use for the increment of strainometcr reading a value which corresponds to some convenient interval on the scale of the strainometer, and which is approximately equal to $e$.

Tensile strength. - The maximum tensile stress which a material is capable of developing.

NotE.-In practice, it is considered to be the maximum stress developed by a specimen representing the material in a tension test carried to rupture, under definite prescribed conditions. Tensile strength is computed from the maximum load carried during a tension test and the original cross-sectional area of the specimen.

Compressive strength. - The maximum compressive stress which a material is capable of developing.

NotE.-In the case of a material which fails in compression by a shattering fracture the compressive strength has a very definite value. In the case of materials which do not fail in compression by a shattering fracture the value obtained for compressive strength is an arbi. trary value depending upon the degree of distortion which is regarded as indicating complete failure of the metal.

Modulus of elasticity.-The ratio, within the elastic limit of a material, of stress to corresponding strain.

Note.-As there are three kinds of stress, so are there three moduli of elasticity for any material: The modulus in tension, the modulus in compression, and the modulus in shear. The value of the modulus of elasticity in tension is nearly the same, for most metals, as the ralue of the modulus of elasticity in compression. The value of the modulus of elasticity in shear is smaller than the value of the modulus of elasticity in tension. The modulus of elasticity is expressed in pounds per square inch (kilograms per square millimeter).

\section{AMERICAN SOCIETY FOR TESTING MATE- RIALS, TENTATIVE DEFINITIONS OF TERMS RELATING TO METHODS OF TESTING, DESIGNATION E6-30T, 1930}

\section{TENTATIVE TESTING TERMS}

Elastic limit.-The greatest stress which a material is capable of developing without a permanent deformation remaining upon complete release of the stress.

NoтE.-In certain specifications of the society the term "elastic limit" is used to designate a value obtained by a test method which does not involve the release of stress during a test but which does involve the determination of a limiting load at which there occurs an appreciable change in rate of strain with respect to stress. This limit is frequently given the name "proportional limit" (or "proportional elastic limit"), and the following definition may be given: Proportional limit. - The greatest stress which a material is capable of developing without a deviation from the law of proportionality of stress to strain (Hooke's law).

It is a matter of experience with many materials, especially with many metallic materials, that, using ordinary methods of testing, the values found for elastic limit by means of observations of permanent deformation (set) after release of stress do not differ widely from the values found for proportional limit. Since the determination of proportional limit is much more readily made than in the determination of elastic limit it is customary to accept the proportional limit as equivalent to the elastic limit for such materials.

Using any known method, the value determined either for proportional limit or for elastic limit is somewhat arbitrary; the value depends on various test conditions, among them precision of apparatus, the scales to $w$ hich stress-strain diagrams may be plotted, and the tolerance allowed before it is judged that there exists an appreciable deviation from the law of proportionality of stress to strain or an appreciable permanent deformation (set) upon release of stress. 
In connection with this note on elastic limit and proportional limit, it seems proper to consider the action of a material under steadily increasing stress, and to discuss briefly five stages which may be distinguished between the first application of stress and fracture of the metal. Not all materials show all fire stages, and for a material the end of one stage and the beginning of the next stage are not always marked.

In the first stage (OA, fig. $24(a)$ ), with the use of measuring instruments of ordinary sensitiveness, deriations from the law of proportionality of stress to strain (Hooke's law) will not be detected, nor will there be detected set after release of stress.

In the second stage ( $A B$, fig. $24(a)$ ) as stress increases there is detected an appreciable and growing deviation from the law of proportionality of stress to strain. This is usually accompanied by an appreciable and growing permanent deformation (set) after release of stress, although for some materials (for example, rubber) measurable permanent deformation may not occur after release of stress exceeding the proportional limit. Values for elastic limit and for proportional limit are found in this second stage.

The third stage ( $B C$, fig. $24(a))$ is characteristic of ductile metals. In this stage, strain increases without any increase of stress and in some cases increase of strain is accompanied by an actual decrease of stress. The yield point is found at the beginning of this stage (at B, fig. $24(a)$ ).

In the fourth stage ( $C D$, fig. $24(a))$ stress and strain both increase up to the maximum stress carried ( $D$, fig. $24(a)$, which gives the tensile strength in a tension test of a material).

In the fifth stage ( $D E$, fig. $24(a)$ ) strain increases while stress diminishes from the maximum value $(D$, fig. $24(a))$ to fracture
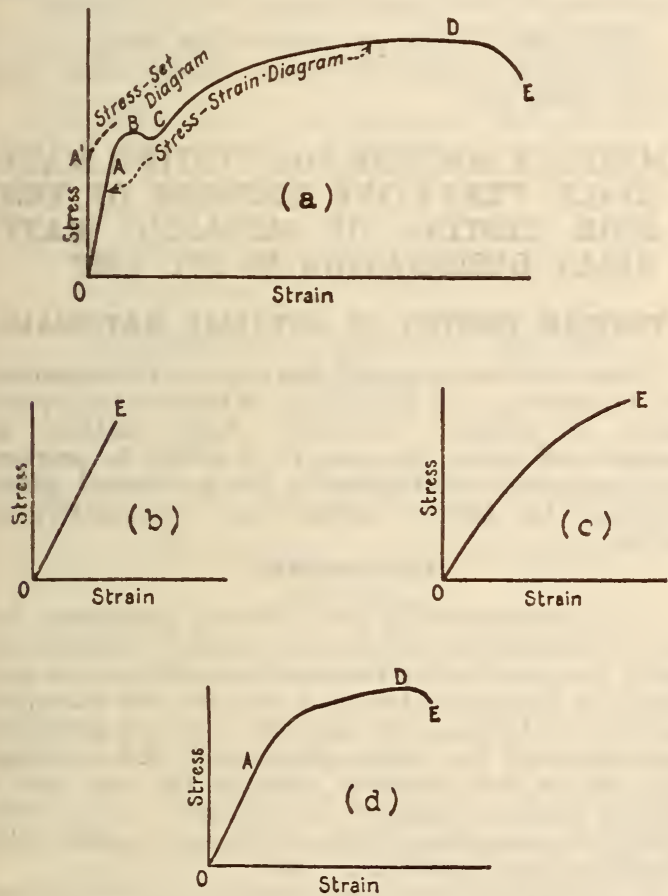

FIGURE 24.- Stress strain diagrams

$E$, fig. $24(a))$. In considering each of these stages it is important to note that stress is computed on the basis of original cross section of specimen.

Many materials, especially brittle materials, do not exhibit all the above-mentioned stages. Some (for example, glass and fused quartz) show only the first stage. Figure $24(b)$ gires a representative stressstrain diagram for such materials. Some materials (for example, cast iron) show only the second stage, and Figure 24 (c) shows a a typical stress-strain diagram for such materials. Some materials (for example, medium-hard steel) do not show the third stage, and the second stage merges into the fourth stage. Figure $24(d)$ shows a typical stress-strain diagram for such materials. The fifth stage is absent in many materials; it is, in fact, characteristic of ductile metals only.

The following methods of test are, strictly speaking, methods of determining proportional limit with various degrees of precision. However, the values determined by these methods may for certain materials, be regarded as satisfactory and reliable values for the elastic limit, and they are therefore so used in certain specifications of the society.

Method I. (For tension tests only, proposed by Committee A-1 on steel, and embodied in a number of specifications for steel.) The elastic limit shall be determined by an extensometer reading to 0.0002 inch. The extensometer shall be attached to the specimen at the gage marks and not to the shoulders of the specimen nor to any part of the testing machine. When the specimen is in place and the extensometer attached, the testing machine shall be operated so as to increase the load on the specimen at a uniform rate. The observer sholl watch the elongation of the specimen as shown extensometer and shall note, for this determination, the which the which the rate of elongation shows a sudden increase. The extensometer may then be removed from the specimen and the test coninued to determine the tensile strength.

Method II (proposed by the late J. B. Johnson) is a somewhat more delicate method for locating the elastic limit than is Method I In Method II, the elastic limit shall be taken as that stress at which the rate of deformation is 50 per cent greater than the initial rate of deformation. A stress-strain diagram is necessary for determining the elastic limit by Method II. Figure 25 illustrates the use of Method II The initial rate of for $m n: O m$. $n q=0.5 \mathrm{mn}, m q=1.5 \mathrm{mn}$, and the slope of $O q$ represents $m n: O m ., n q=0.5 \quad m n, m q=1.5 m n$, and the slope of $O q$ represents
a rate of deformation 50 per cent greater than the initial rate. $O^{\prime} q^{\prime}$ is drawn parallel to $O q$ and tangent to the stress-strain diagram The point of tangency $J$ locates the elastic limit. In using Method II the same precautions should be observed in attaching the strainometer as for the determination of the elastic limit by Method I. Method II, if used for tension tests of metals, gives lower values for the elastic limit than does Method I. It is recommended that in using Method II for tension tests the strainometer be of such in using Method II for tension tests the strainometer be of such sensitiveness that it will indicate a change of strain of 0.0001 inch
per inch of gage length $(0.0001 \mathrm{~mm}$ per millimeter of gage length) and that a corresponding degree of sensitiveness be required for strainometers used for compression tests, for flexure tests, and for torsion tests.

When it is desired to determine the elastic limit with a high degree of precision, it is suggested that the experimenter devise his own procedure, and in reporting his results describe the procedure in deratus used and of the procedure used in plotting the stress-strain diagram from which the determination of elastic limit is made It is to be remembered that the accurate determination of the elastic

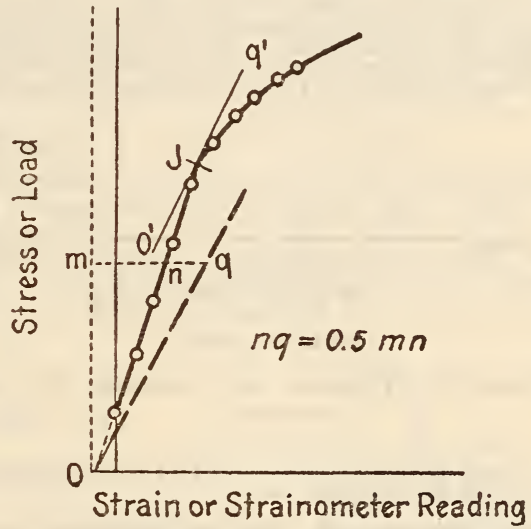

Figure 25.-Illustrating Method II for determining elastic limit

limit requires the use of accurate and sensitive instruments and the use of accurate methods of plotting test data.

Yield point.-The stress in a material at which there occurs a marked increase in strain without an increase in stress.

Note.-Some metals do not show a clearly defined yield point. Two methods are in use for determining the yield point: (I) The "drop of the beam" method, and (II) the method by use of dividers. In Method I, load is applied to the specimen at a steady rate of increase and the operator keeps the beam in balance by running out the poise the the poise at a steady rate. At the yleld point the increase of load tops, but the operator, running out the poise at a steady rate, runs it a trifle beyond the balance position, and the beam of the machine drops for a brief but appreciable interval of time. In a machine fitted with a self-indicating load-measuring device there is a sudden halt of the load-indicating pointer, corresponding to the drop of the beam. The load at the "halt" or the "drop" is recorded, and the corresponding stress is taken as the yield point. This method of determining the yield point requires only one man to conduct a test.

In Method II, an observer with a pair of dividers watches for risiIn Method II, an observer with a pair of dividers watches for risivisible stretch is observed, the load at that instant is noted, and the stress corresponding to the load is taken as the yield point. When a strainometer 18 is used in place of dividers a deformation of 0.01 inch over a gage length of 2 inches shall be regarded as indicating the yield point.

18 The determination of yield point with a strainometer is sometimes made in tests on nonferrous metals. 


\section{AMERICAN SOCIETY FOR TESTING MATE- RIALS, STANDARD DEFINITIONS OF TERMS RELATING TO METALLOGRAPHY, DESIGNATION E7-27, 1927}

\section{METALLOGRAPHIC TERMS}

Alloy.-A substance, having metallic properties, consisting of two or more metallic elements, or of metallic and nonmetallic elements, which are miscible with each other when molten, and have not separated into distinct layers when solid.

Note.-Alloys when solid may be composed of eutectics, eutectoids, solid solutions, chemical compounds, or of aggregates of these components with each other or with pure metals. In the commercial sense, the term "alloy" would also include the case where some separation into distinct layers had occurred.

Alpha brass.-A copper-zinc alloy with a copper content greater than approximately 64 per cent. It is composed of but one type of crystals all of which are of the same composition.

Beta brass.-A copper-zinc alloy with a copper content of approximately 54 per cent, composed of but one type of crystals all of which are of the same composition.

Note.-At ordinary temperatures, the normal range of beta brass is from 51 to 55 per cent copper. Quenching from higher temperatures considerably broadens this range. Between 64 and 55 per cent copper, the copper-zinc alloys consist of mixed crystals of alpha and beta, the relative proportion of which depends upon the composition and heat treatment. Alloys of this type can be me chanically worked while hot and are frequently referred to as munt metal. When rapidly cooled from high temperatures (that is,

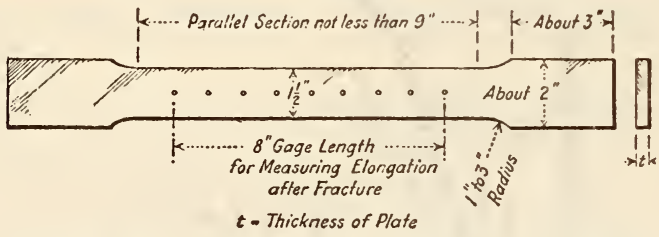

FIgURE 26.-Tension test specimen for plate material

from at or near the melting point) mixed alpha and beta may occur with copper as high as 70 per cent.

Etching reagent.-A reactive substance used to reveal the structure of a metal or alloy through the development of differences in the appearance of different constituent parts or different grains.

NoTE.-This substance is usually a solution of the reagent in water acid, or alkali, but etching may in some cases be brought out by a differential oxidation produced by "heat tinting."

Equi-axed grain.-A grain which has approximately equal dimensions in all directions.

NorE.-This term is practically restricted to unstrained metals.

Grain.-A term used for an allotrimorphic crystal present in metals and in one-component alloys.

NoTe.-Although a crystal may show division by "twinning," etc., it is regarded as the grain rather than smaller subdivisions. In the case of alloys of more than one component, the crystal which, by its transformation, gave rise to these constituents is taken as th grain when its limits are still discernible; if the limits are not discernible the individual constituents are considered as grains.

Grain size.-This is preferably expressed as the number of grains per unit area of cross section. The average cross-sectional area of the grain may also be given or the average dimensions. Grain size of strained material is expressed by the average number per linear unit in two directions, or by the average number per unit cross-sectional area, together with the ratio of length to breadth $(L / B)$.

Nore.-By the intercept method for grain count, the number of grains and fractions of grains along a line of known length on two axes at right angles to each other are counted. By the planimetric method for grain count, the number of grains and fractions of grains within a definite area are counted.
Intercept method for grain count.-See note under Grain size.

Macrograph.-A graphic reproduction of any object which has not been magnified more than 10 diameters.

Note.-When it is desired to indicate that it is a photographic reporoduction, the term "photomacrograph" may be employed.

Magnifica.ion.-The ratio of the size of the image to that of the object.

Note.-Magnification is generally expressed in diameters, thus $\times 100$ or 100 diameters.

Metal.-Any of the metallic elements, either of very high purity or of ordinary commercial grades.

Note.-Brass and many other alloys are metals in the commercial sense, but alloys in the scientific sense.

Metallography.-That branch of science which relates to the constitution and structure, and their relation to the properties, of metals and alloys.

Micrograph.-A graphic reproduction of any object magnified more than 10 diameters.

Note.-When it is desired to indicate that it is a photographic reproduction, the term "photomicrograph" may be employed.

Muntz metal.-See note under Beta brass.

Planimetric method for grain count.-See note under Grain size.

\section{AMERICAN SOCIETY FOR TESTING MATE- RIALS, TENTATIVE METHODS OF TEN- SION TESTING OF METALLIC MATE- RIALS, DESIGNATION E8-27T, 1927}

\section{TENSION TESTING OF METALLIC MATERIALS}

These methods deal with the form and dimensions, the machining, and the testing of tension-test specimens of metallic materials. Such matters as methods of taking samples from which to prepare test specimens are treated in the individual specifications for ferrous metals and for nonferrous metals.

\section{TEST SPECIMENS}

1. In recommending the following specimens for use in tension tests it is not intended to exclude entirely the use of other test specimens for special materials or for special forms of material; for example, in tests of tubing and pipe the form of specimen recommended for plate, shape, and flat material (fig. 26) is not generally suitable for use, and a special form of specimen is specified. It is, however, recommended that wherever feasible the specimens here specified be used.

2. Tension-test specimens of wire are of the full-size diameter as drawn, and tension-test specimens of rods of ductile metal are often of the full-size diameter as rolled. Tension-test specimens of pipe and of tubing are frequently of full size as manufactured, in which case the ends should be plugged with metal plugs which do not extend within the gage marks.

3. The tension-test specimen shown in Figure 26 is recommended for plate, shape, and flat material having a thickness of one-fourth inch or over. ${ }^{10}$

Note.-When it is desired to use a specimen with a gage length of less than 8 inches, the general proportions of Figure 26 should be followed. Specimens with a gage length of 2 inches are occasionally used. When it is not convenient to machine specimens to the standard shape shown in Figure 26, specimens may be used with edges machined parallel for the entire length of the specimen. If such specimens are machined with a tool cutting across the edges of the specimen it is important that the transverse tool marks be removed before men it is important that

For thick plate material, it is usually preferable to use a machined specimen rather than to use a specimen the full thickness of the plate.

10 Attention is called to the fact that two types of specimen are applicable for material one-fourth inch in thickness, the plate specimen shown in fig. 26 and the thin sheet specimen shown in fig. 28 . 
The thickness of the specimen is that of the material tested.

4. For tension tests of wrought ferrous and nonferrous metal in the form of plate, sheet, flat wire, ${ }^{20}$ strip, ${ }^{20}$ band, ${ }^{20}$ and hoop ${ }^{20}$ without respect to width, ${ }^{20}$ length, grade, or method of manufacture, and having a thickness of from 0.01 to 0.250 inch, the test specimen described in sections 5 and 6 is recommended.

Note. - This specimen may also be used in tests of material cut from tubing.

5. The specimen blank shall be cut from the material to be tested, having its length in such direction relative to the material as may be specified.

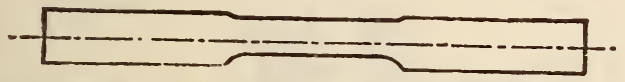

(a) Ends not Symmetrical with $\mathrm{Re}$ duced Section.

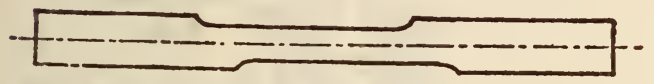

(b) Two Sides of Reduced Section not Symmetrical.

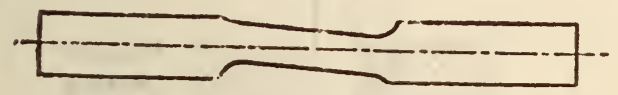
(c) Ends not Parallel to Reduced
Section.

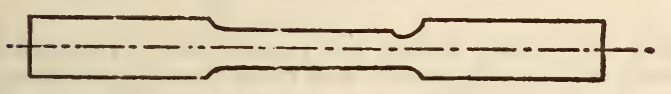

\section{(d) Narrow Width Due to "Hogging In" of Cutter.}

FIGURE 27.-Common errors in preparation of plate or sheet metal test specimens

The specimen blank shall conform to the dimensions shown in the following table:

\begin{tabular}{|c|c|c|}
\hline $\begin{array}{l}\text { Method of cutting long } \\
\text { edges of blank }\end{array}$ & $\begin{array}{l}\text { Nominal thickness of material } \\
\text { (in inches) }\end{array}$ & $\begin{array}{l}\text { Minimum } \\
\text { width of } \\
\text { blank }\end{array}$ \\
\hline $\begin{array}{l}\text { Sheared... } \\
\text { Sawed } 1 . .\end{array}$ & $\left\{\begin{array}{l}\text { Up to } 1 / 8, \text { inclusive } \\
\text { Over } 1 / 8 \text { to } 1 / 4 \text {, inclusive...... } \\
\text { Up to } 1 / 4, \text { inclusive......... }\end{array}\right.$ & Inch ${ }^{3 / 4}$ \\
\hline
\end{tabular}

1 Or otherwise machined without severely straining the metal.

Blanks cut with a torch shall not be used unless all metal affected by such cutting is removed by machining.

6 . Figure 28 shows the form of the finished specimen recommended for thin sheet metals. The reduced section of the specimen shall be machined in such a manner as to avoid leaving severe machining strains in the material. It is essential that the

${ }^{20} \mathrm{It}$ is to be noted that the recommended specimen can not be used for flat wire, strip, band, or hoop material whose width is less used for flat wire, strip, band, or hoop mate reduced section and the ends of the specimen be symmetrical with respect to the longitudinal axis of the specimen. ${ }^{21}$

In Figure 28, the 2-inch gage length should be laid off with a tolerance in length of \pm 0.005 inch.

7. The tension-test specimen is identical with Figure 129 in A. S. T. M. standard specifications for steel (see 605.0, p. 247). The gage length for measuring elongation after fracture is 2 inches.

8. When it is necessary to cut specimens from material (other than plate, shape, and flat material) which is of such size that the standard test specimen

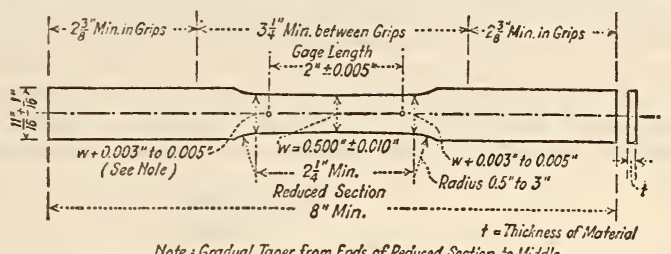

Note : Gradual Toper from Ends of Reauced Section to Mitdle.
All Mactining Dimensions are Shown below and Testing Dimensions above Specimen. FIGURE 28.- Standard tension test specimen for
thin sheet metals

referred to in the preceding paragraph can not be used, it is recommended that a specimen with dimensions proportional to those shown in Figure 129 be used, and that the specimen be made as large as feasible. In any such small-size specimen it is especially important that the gage length for measuring elongation be four times the diameter of the specimen.

9 . In all tension tests of metals the actual dimensions of cross section of the test specimens shall be measured with a micrometer reading to $1 / 200$ of the dimension measured, and the stresses shall be computed on the basis of the measured cross section; it should never be assumed that the dimensions of the

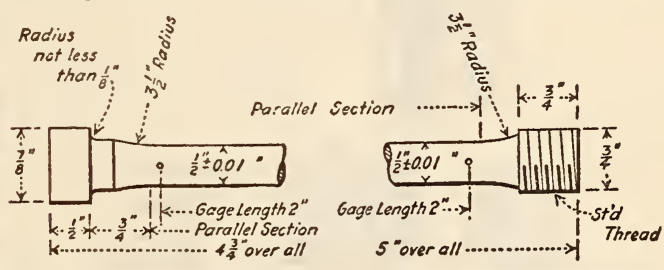

(u) Specimen with shouldered ends.

(b) Spccimen with threader ends

Figure 29

measured cross section are identical with the nominal dimensions.

NoTE.-It will be noted that the tolerance for diameter of specimens shown as Figure 129 in standard specifications for steel (see 605.0 , p. 247) permits the use of specimens 0.505 inch in diameter.

10. The shape of the ends of the specimen shown in Figure 129 are not specified above. For specimens of brittle metal it is desirable that the diameter of the specimen be changed gradually along its length from the minimum section at the gage length to the diameter at the ends. Figure 29 shows two forms of end of specimen which have given satisfactory results in tests of brittle metals.

11. Specimens shall be finished so that the surfaces are smooth and free from nicks and tool marks. All ragged edges shall be smoothed.

21 For all specimens for flat metallic materials, both for thin sheet and for thick plate, it is important so to machine the specimen as to avoid the conditions shown in fig. 27. 


\section{GRIPPING DEVICES FOR TENSION-TEST SPECIMENS}

12. For long bars of ductile metal and for the flat plate test specimen shown in Figure 26, wedge grips, bearing directly on the end of the test specimen, generally furnished a satisfactory means of gripping the specimens.

13. For short specimens and for specimens of brittle material, wedge grips are not a satisfactory means of holding test specimens; it is necessary to use machined specimens and to use special means for insuring that the specimen, when under load, shall be as nearly as possible in pure axial tension. Figure 30 shows a device for gripping tension specimens with threaded ends and Figure 31 shows a device for gripping specimens with shouldered ends. Both these gripping devices are attached to the heads of the testing machine through sphericalseated bearings. The distance between spherical

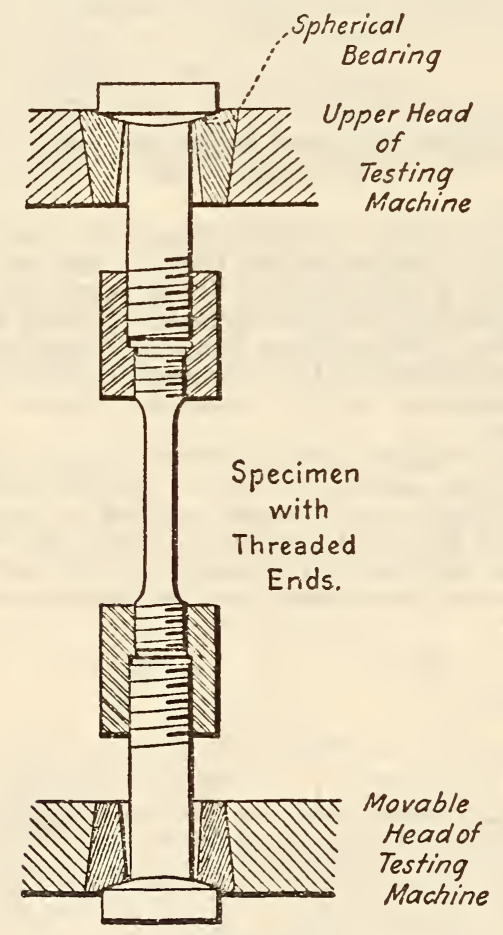

Figure 30

bearings should be as great as is feasible. For brittle materials, even the spherical-seated bearings shown in Figures 30 and 31 are not always effective in avoiding bending stress on the specimen. The gripping device shown in Figure 32 has proved satisfactory for testing such specimens, and is an excellent gripping device for any machined tensiontest specimen.

14. For specimens cut from thin plate material and from sheet material, ordinary wedge grips are liable to cut the specimens near the edges, giving a tearing stress rather than an axial tension. The selfadjusting grips shown in Figure 33 have proved satisfactory for tests of thin sheet copper and aluminum.

15. For tests of specimens of wire, ordinary wedge grips are liable to cut the wire at the edges of the grips. This cutting has been found to be largely eliminated by the use of grips of the form shown in Figure 34.

16. In making tension tests, care shall be taken to see that the axis of the test specimen is located in the center line of the heads of the testing machine and that the liners used behind wedge grips are of proper thickness. From time to time the openings in the heads of the testing machine shall be lined up

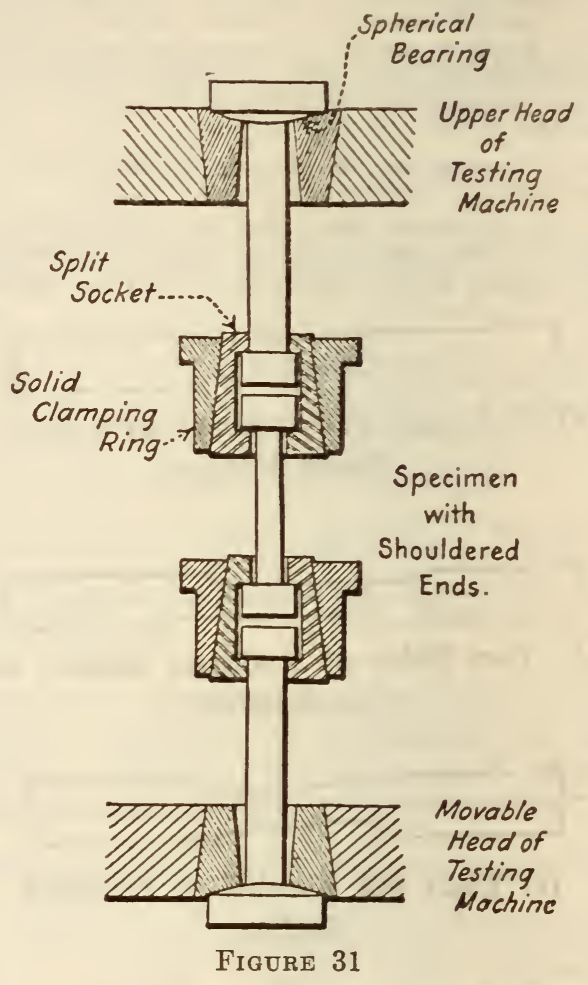

and the heads tested for parallelism. Deviations from correct alignment and from parallelism should not exceed 0.01 -inch for any position of the movable

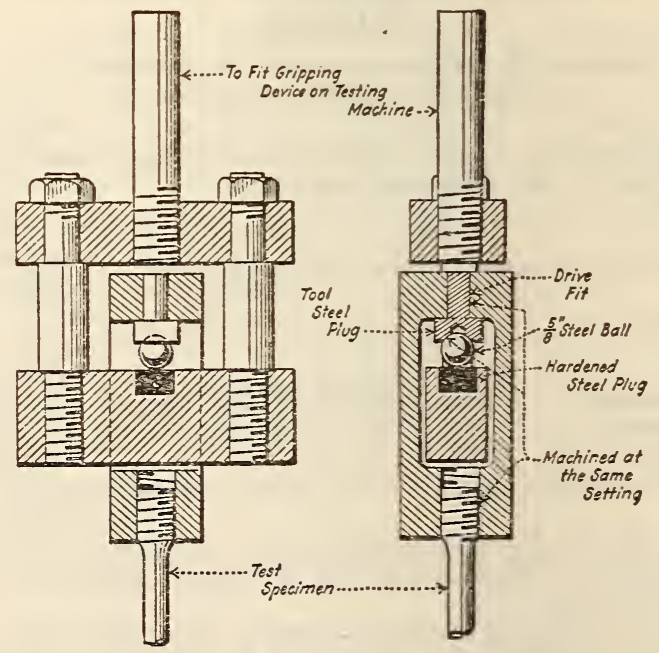

Figure 32

The gripping device is shown as used with threaded end specimens. A similar device fitted with split sockets would be used with shouldered specimens

head within the range of movement during test. The heads of the machine should be free from sidewise motion that is visible to the unaided eye as the machine is run at its fastest speed. 
SPEED OF OPERATION OF TESTING MACHINE

17. The pulling speed has a marked influence on the tensile properties shown by materials tested,n a increase in speed increasing the values found for yield point and tensile strength. In testing specimens of metallic materials in gage lengths of 2 and $\delta$ inches, the speed of the machine, by which is meant the speed of the crosshead when the machine

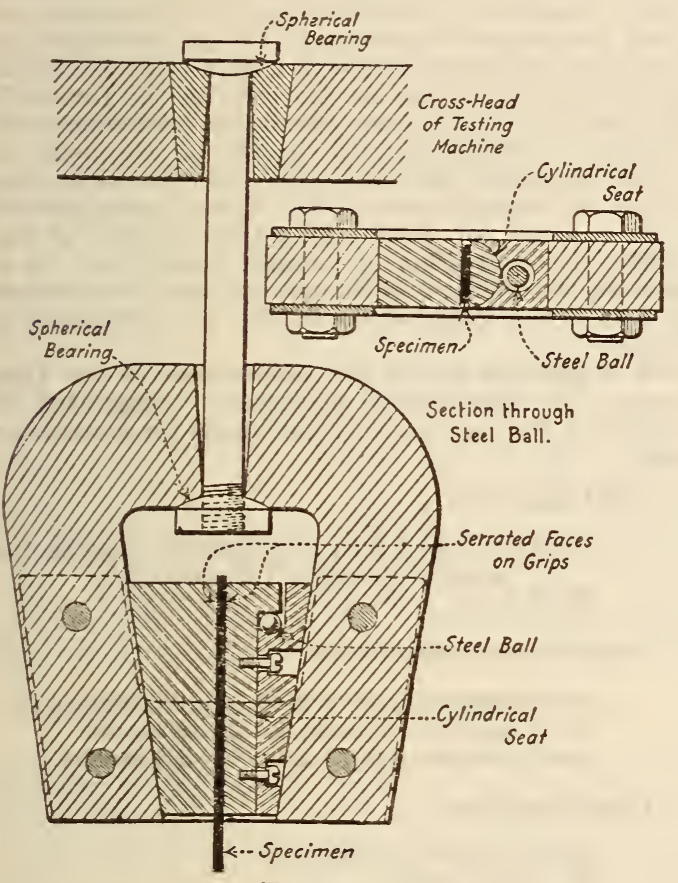

Figure 33

is running idle, shall conform to the following requirements:

The speed of head of the testing machine shall be such that the load can be accurately weighed, but in no case shall the speed be greater than the values given in the following table, which values represent the extreme of good laboratory practice for steel: ${ }^{22}$

\begin{tabular}{|c|c|c|c|}
\hline \multirow{2}{*}{$\begin{array}{l}\text { Specified minimum tensile strength of } \\
\text { material (in pounds per square inch) }\end{array}$} & \multirow{2}{*}{$\begin{array}{l}\text { Gage } \\
\text { length }\end{array}$} & \multicolumn{2}{|c|}{$\begin{array}{l}\text { Maximum cross- } \\
\text { head speed }\end{array}$} \\
\hline & & $\begin{array}{l}\text { Yield } \\
\text { point }\end{array}$ & $\begin{array}{l}\text { Tensile } \\
\text { strength }\end{array}$ \\
\hline 80,000 or under & $\left\{\begin{array}{r}\text { Inches } \\
2 \\
8\end{array}\right.$ & $\begin{array}{r}\text { In./min. } \\
0.50 \\
2.00\end{array}$ & $\begin{array}{r}\text { In./min. } \\
2.0 \\
6.0\end{array}$ \\
\hline О ver $80,000 \ldots$ & & $\begin{array}{l}0.25 \\
0.50\end{array}$ & $\begin{array}{l}1.0 \\
2.0\end{array}$ \\
\hline
\end{tabular}

18. In determining the elastic limit in accordance with Method I, section 20 (see A. S. T. M. E6-30T, above) the crosshead speed for the 2-inch gage length shall not exceed 0.125 inch per minute.

19. In determining the elastic limit by Method II, section 20 (see A. S. T. M. E6-30T, above) the crosshead speed shall not exceed 0.025 inch per inch of gage length per minute.

\section{DETERMINATION OF ELASTIC LIMIT, YIELD POINT, AND}

20. The term "elastic limit" is defined as follows: Elastic limit. - The greatest stress which a material

22 The speeds given here are probably too high for satisfactory use in testing nonferrous metals. is capable of developing without a permanent deformation remaining upon complete release of the stress.

Note. - The determination of the elastic limit as thus defined would logically involve the application and release of a succession of increasing loads on a test specimen until there was observed deformation after release of a load. This procedure is very slow and since for many materials experience does not indicate any appreciable difference between the elastic limit and the limit of proportionality of ence between the elastic limit and the limit of proportionality of
stress to strain (sometimes called the proportional limit), the determination of the stress at the limit of proportionality of stress to strain is regarded as an accurate determination of the elastic limit.

It is obvious that the values obtained in tests for determining the elastic limit will depend on the delicacy of methods and instruments used. It becomes necessary, therefore, that in any test the method used in obtaining the elastic limit be clearly stated. The following methods are in common use for determining a value designated as the elastic limit:

(Method I, Method II, and diagram, and methods of high precision for determining elastic limit are the same as A. S. T. M. E6-30T, given above. This is also true of Method I and Method II for determining the yield point.)

21. Some metals do not have a well-defined yield point. ${ }^{23}$

(Definition of tensile strength is the same as in A. S. T. M. E6-30, given above.)

22. The conditions that must be prescribed in a tension test of metallic materials are the form of specimen, the method of gripping the specimen, and the rate of application of load. These are covered in foregoing sections. No further detailed discussion of methods of determining tensile strength is necessary, as there is involved merely the observing

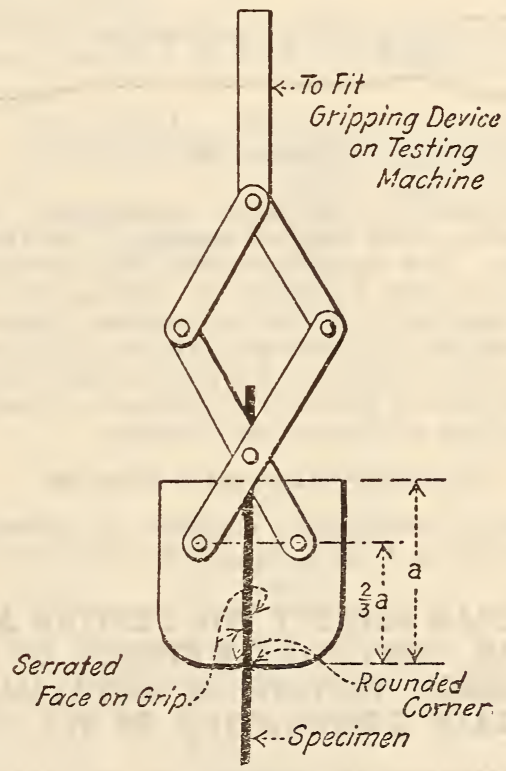

Figure 34

and the recording of the maximum load carried by the specimen during the test.

\section{THE MEASUREMENT, AFTER FRACTORE, OF TRE ELONGATION AND THE REDUCTION OF CROSS SECTION OF TENSION-TEST SPECIMENS}

23. The measurement of the elongation after fracture of tension-test specimens can be made with sufficient accuracy by means of a pair of dividers and a scale. The elongation should not be reported for any tension-test specimen which breaks outside the middle third of the gage length.

23 The determination of yield point with a strainometer is sometimes made in tests on nonferrous metals. 
Note.-If only a few specimens are furnished for testing, it is recommended that in marking the gage length for measuring elongation after fracture it be divided into eight parts, and that if a specimen breaks inside the gage length but outside the middle third of the gage length the requirement in the foregoing paragraph be waived, and the elongation be measured and reported as follows:

the elongation be measured and reported as follows: tween $C$ and $G$ ) (figs. $35(a)$ and $(b))$ the elongation is measured directly over the stretched gage length (fig. $35(b)$ ).

2. If the specimen break between $A$ and $C$ (or between $G$ and $I$ ), but nearer $B$ than $A$ (or nearer $H$ than $I$ ), as shown in Figure $35(c)$ the elongation from $A$ to $C$ (or from $G$ to $I$ ) is measured, and to this is added twice the measured elongation from $C$ to $F$ (or from $D$ to $G$ ).

3 . If the specimen break within one-half a division of an end-gage mark (see fig. $35(d)$ ) the elongation from A to $\mathrm{E}$ (or from $E$ to $I$ ) is measured and multiplied by 2 .

The above method can be applied to specimens with any gage length, and to turned specimens as well as flat specimens.

This method is not applicable to brass specimens.

24 . The measurement of reduction of the dimensions of the cross section of a tension-test specimen

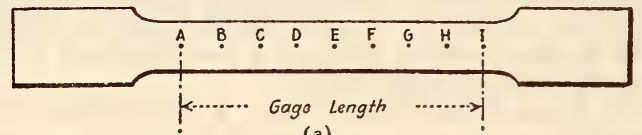

(a)

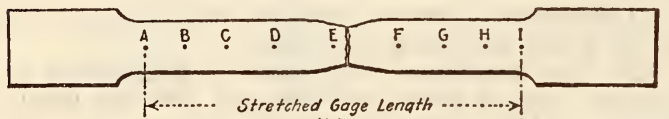

(b)

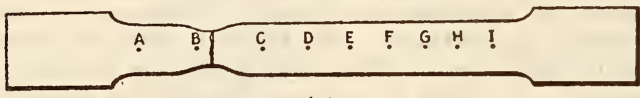

(c)

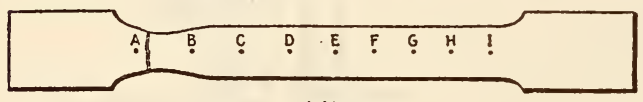

(d)

Figure 35

may be made by the direct measurement with a micrometer of the smallest section of the fractured specimen. For round specimens this measurement can usually best be made by holding the broken pieces together in a vise or between centers and then measuring the average diam eter of the smallest cross section by means of a micrometer fitted with points so shaped that they will come in contact with the specimen at its smallest diameter.

\section{PLOTTING STRESS-STRAIN DIAGRAMS}

(Plotting stress-strain diagrams is substantially the same as in A. S. T. M. E6-30, given above.)

\section{AMERICAN SOCIETY FOR TESTING MATE- RIALS, TENTATIVE METHODS OF COM- PRESSION TESTING OF METALIIC MA- TERIALS，DESIGNATION E9-27T, 1927}

\section{COMPRESSION TESTING OF METALLIC MATERIALS}

These methods deal with the form and dimensions, the machining, and the testing of compression-test specimens of metallic materials. Such matters as methods of taking samples from which to prepare test specimens are treated in the individual specifications for ferrous metals and for nonferrous metals.

\section{TEST SPECIMENS}

1. In recommending the foliowing specimens for use in compression tests it is not intended to exclude entirely the use of other test specimens for special materials or for special forms of material. It is, however, recommended that wherever it is feasible the specimens here recommended be used.
2. It is recommended that standard compression specimens be in the form of circular cylinders. The ends of a specimen should be plane, as nearly as can be determined by the use of a straight-edge and the unaided eye. In most cases this requirement necessitates the turning or the grinding of the ends of the specimen.

3. There are recognized three classes of compression specimens for metallic materials, designated as short specimens, medium-length specimens, and long specimens, respectively. Short specimens are used for compression tests of such metals as bearing metals, which in service are used in the form of a thin plate or shell to carry load perpendicular to the surface. Medium-length specimens ale used for determining the general compressive strength properties of metallic materials. Long specimens are best adapted for determining the modulus of elasticity in compression of metallic materials. In reporting the results of a compression test, it is important that the dimensions of the test specimen be given.

4. Figure 36 shows the proportions of the three classes of compression specimens. Suggested dimensions for compression specimens for general use are:

\section{Short specimens:}

$d=1 \frac{1}{8} 8$ inches \pm 0.01 inch (area of cross section $h=1$ inch; s appı oximately 1 square inch)

\section{Medium-length specimens}

$d=0.798$ inch \pm 0.01 inch, $h=23 / 8$ inches, or $d=1$ inch \pm 0.01 inch, $h=3$ inches, or $d=1 \frac{1}{8}$ inches \pm 0.01 inch, $h=3 \frac{3}{8}$ inches.

Long specimens:

$d=1 \frac{1}{4}$ inches \pm 0.01 inch

$h=12 \frac{1}{2}$ inches.

5. In all compression tests of metallic materials, the actual dimensions of cross section of the test

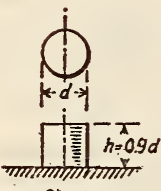

Short Specimen.

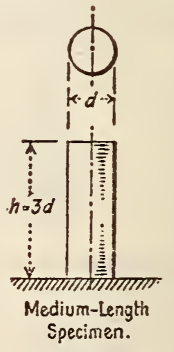

Figure 36

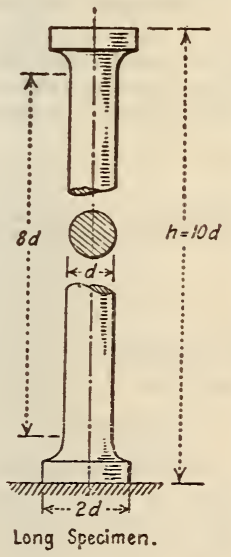

Long Specimen. specimens shall be measured with a micrometer reading to $1 / 200$ of the dimension measured, and stresses shall be computed on the basis of the measured cross section. It should never be assumed that the dimensions of the cross section of the specimen are identical with the nominal dimensions.

6. Specimens shall be finished so that the surfaces are smooth and free from nicks and tool marks. All ragged edges shall be smoothed. 


\section{BEARING BLOCKS FOR COMPRESSION-TEST SPECIMENS}

7. Both ends of a compression-test specimen shall bear on a carefully machined plane surface. The bearing blocks either shall be made of hardened steel or shall be faced with hardened steel.

8 . One end of a compression-test specimen shall bear on a spherical-seated compression block. ${ }^{24}$ Figure 37 shows a satisfactory arrangement of compression specimens and bearing blocks. It is desirable that the spherical-seated bearing block be at the upper end of the test specimen (for specimens tested with the axis vertical). It is important that the center of the spherical surface of the sphericalseated bearing block be in the flat face which beais on the specimen, otherwise lack of parallelism between faces of the specimen sets up a bending moment in the specimen. ${ }^{25}$

9. It should be borne in mind that the object of a spherical-seated bearing block is to give the specimen as even a distribution of initial load as is possible. Owing to friction, the spherical-seated bearing can not be relied on to adjust itself to bending action which may occur during the test.

10. In making compression tests, care should be taken to preserve the alignment of the heads of the testing machine, and to center the specimen in the testing machine. From time to time the heads of the testing machine shall be tested for parallelism. Deviations from parallelism should not exceed 0.01 inch. The movable head of the machine should be free from sidewise motion that is visible to the unaided eye as the machine is run at its fastest speed.

\section{SPEED OF OPERATION OF TESTING MACHINE}

11. In making compression tests of specimens of metallic materials the speed of the machine, by which is meant the speed of the crosshead when the machine is running idle, shall be such that the load can be accurately weighed, but in no case, shall the speed be greater than the values given in the following table:

\begin{tabular}{|c|c|}
\hline $\begin{array}{c}\text { Length of specimen } \\
\text { (in inches) }\end{array}$ & $\begin{array}{c}\text { Maximum } \\
\text { crosshead } \\
\text { speed (in } \\
\text { inches per } \\
\text { minute) }\end{array}$ \\
\hline $1.00 \ldots$ or greater......... & 0.05 \\
3.00 \\
\hline
\end{tabular}

12. In making tests to determine the elastic limit in compression of metallic materials, the crosshead speed of the testing machine shall in no case exceed the allowable speed of head for the corresponding tension test of the material, as given in sections 18 and 19 of A.S.T. M. E8-27T given above.

\section{THE DETERMIN ATION OF ELASTIC LIMIT, YIELD POINT, AND COMPRESSIVE STRENGTH}

13. (a) The term "elastic limit" in compression is defined the same as in tension, as follows:

Elastic limit. - The greatest stress which a material is capable of developing without a permanent deformation remaining upon complete release of the stress.

24 The device shown in Fig. 38 has been successfully used in compression tests of metallic specimens whose ends were machined with extreme care. It will be noted that this device does not involve a spherical seated compression block bearing directly on the test specimen. This device or one similar has been successfully used in the British National Physical Laboratory and in the laboratories of the U. S. Air Service at McCook Field, Dayton, Ohio.

${ }^{23}$ See Mont Schuyler, "Spherical Bearings," Procs. Am. Soc. Testing Mats., Vol. XIII, p. 1004, 1913. (b) The same general methods for determining elastic limit as given above in A. S. T. M. E8-27T (including the references in E8-27T to A. S. T. M. E6-30T) shall be used. For compression tests,

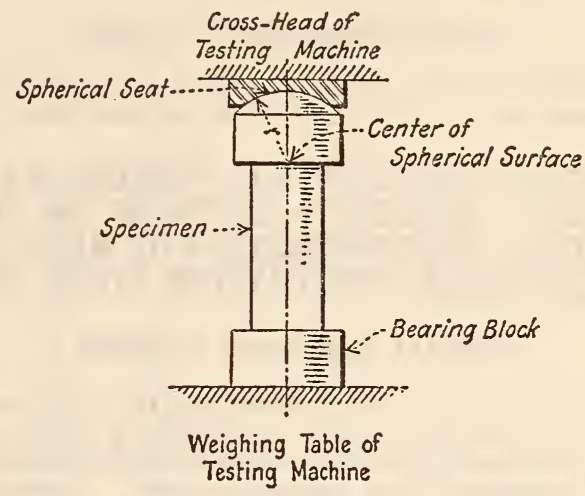

Figure 37

Method II or a still more precise method seems generally suitable.

14. (a) The term "yield point" in compression is defined the same as in tension, as follows:

Yield point. - The stress in a material at which there occurs a marked increase in strain without an increase in stress.

(b) The same methods for determining yield point as given above in A. S. T. M. E8-27T (including the

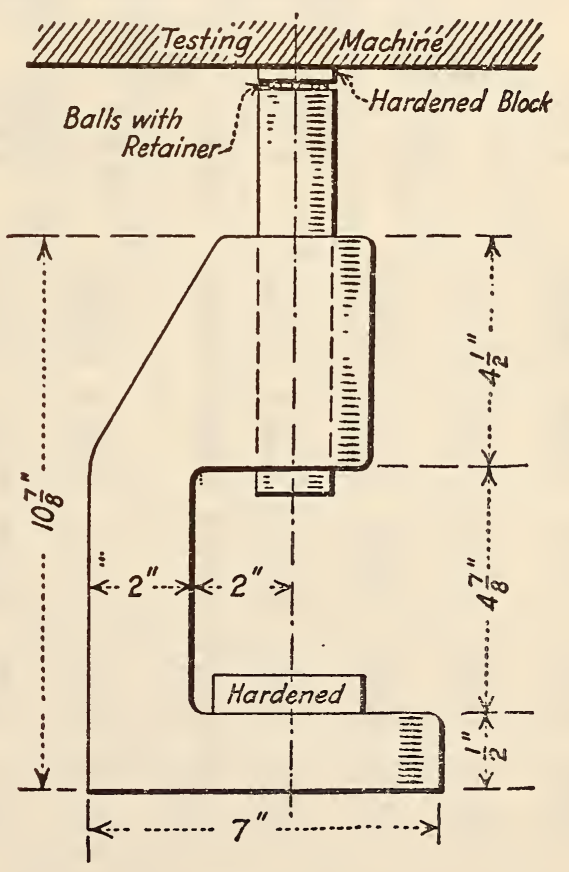

FIGURE 38

references in E8-27T to A. S. T. M. E6-30T) shall be used. The method by the use of dividers or by the use of a strainometer seems generally suitable for compression testing.

15. The term "compressive strength" is defined as follows:

Compressive strength.-The maximum compressive stress which a material is capable of developing. 
NotE.-In the case of a material which fails in compression by a shattering fracture the compressive strength has a very definite value. In the case of materials which do not fail in compression by a shattering fracture the value obtained for compressive strength is an arbitrary value depending upon the degree of distortion which is regarded as indicating complete failure of the metal.

\section{PLOTTING STRESS-STRAIN DIAGRAMS}

(Plotting stress-strain diagrams is substantially the same as in A. S. T. M. E6-30, given above.)

\section{AMERICAN SOCIETY FOR TESTING MATE- RIALS, STANDARD METHODS OF BRI- NELL HARDNESS TESTING OF METALLIC MATERIALS, DESIGNATION E10-27, 1927}

\section{BRINELL HARDNESS TESTING}

The Brinell test for determining the hardness of metallic materials consists in applying a known load to the surface of the material to be tested through a hardened steel ball of known diameter. The diameter (or depth) of the resulting permanent impression in the metal is measured. The Brinell hardness number is taken as the quotient of the applied load divided by the area of the surface of the impression, which is assumed to be spherical. If $P$ is the applied load (measured in kilograms), $D$ is the diam- eter of the steel ball (measured in millimeters), and $d$ is the diameter of the impression (measured in millimeters), then:

$$
\text { B. H. } N=\frac{P}{\frac{\pi D}{2}\left(D-\sqrt{D^{2}-d^{2}}\right)}
$$

in which $B . H . N$. is the Brinell hardness number in kilograms per square millimeter.

If the steel ball were not deformed under the applied load and if the impression were truly spherical, then the above formula would be a genera. one, and any combination of applied load and size of ball could be used. As the impression is not quite a spherical surface (since there must always be some deformation of the steel ball and some recovery of form of the metal in the impression), for a standard Brinell test the size and characteristics of the ball and the magnitude of the applied load must be standardized. (A standard ball $10 \mathrm{~mm}$ in diameter and a load of $3,000 \mathrm{~kg}$ for hard metals and $500 \mathrm{~kg}$ for soft metals is standard practice. See secs. 3 and 6.$)$

In Table 1 is given the Brinell hardness number corresponding to various diameters of impression for both 500 and $3,000 \mathrm{~kg} \mathrm{load}$, making it unnecessary to compute for each test the value of the Brinell hardness number by the above formula.

TABLE 1.-Brinell hardness numbers

[Steel ball, $10 \mathrm{~mm}$ in diameter, pressures of 500 and 3,000 kg. Prepared by the Engineering Mechanics Section, Bureau of Standards]

The values given in this table for hardness numbers are merely solutions of the equation given in the introductory paragraphs. They do not imply that Brinell tests are feasible on materials of a hardness indicated by the highest ralues in the table

\begin{tabular}{|c|c|c|c|c|c|c|c|c|c|c|c|c|c|c|}
\hline \multirow{2}{*}{$\begin{array}{l}\text { Diam- } \\
\text { eter of } \\
\text { inden- } \\
\text { tation }\end{array}$} & \multicolumn{2}{|c|}{$\begin{array}{l}\text { Brinell hard- } \\
\text { ness No. }\end{array}$} & \multirow{2}{*}{$\begin{array}{l}\text { Diam- } \\
\text { eter of } \\
\text { inden- } \\
\text { tation }\end{array}$} & \multicolumn{2}{|c|}{$\begin{array}{l}\text { Brivell hard- } \\
\text { ness No. }\end{array}$} & \multirow{2}{*}{$\begin{array}{l}\text { Diam- } \\
\text { eter of } \\
\text { inden- } \\
\text { tation }\end{array}$} & \multicolumn{2}{|c|}{$\begin{array}{l}\text { Brinell hard- } \\
\text { ness No. }\end{array}$} & \multirow{2}{*}{$\begin{array}{l}\text { Diam- } \\
\text { eter of } \\
\text { inden- } \\
\text { tation }\end{array}$} & \multicolumn{2}{|c|}{$\begin{array}{c}\text { Brinell hard- } \\
\text { ness No. }\end{array}$} & \multirow{2}{*}{$\begin{array}{l}\text { Diam- } \\
\text { eter of } \\
\text { inden- } \\
\text { tation }\end{array}$} & \multicolumn{2}{|c|}{$\begin{array}{l}\text { Brinell hard- } \\
\text { ness No. }\end{array}$} \\
\hline & $\begin{array}{l}500- \\
\mathrm{kg} \\
\text { load }\end{array}$ & $\begin{array}{c}3,000- \\
\mathrm{kg} \\
\text { load }\end{array}$ & & $\begin{array}{l}500- \\
\mathrm{kg} \\
\text { load }\end{array}$ & $\begin{array}{c}3,000- \\
\mathrm{kg} \\
\text { load }\end{array}$ & & $\begin{array}{c}500- \\
\mathrm{kg} \\
\mathrm{load}\end{array}$ & $\begin{array}{l}3,000- \\
\mathrm{kg} \\
\text { load }\end{array}$ & & $\begin{array}{l}500- \\
\mathrm{kg} \\
\text { load }\end{array}$ & $\begin{array}{l}3,000- \\
\mathrm{kg} \\
\text { load }\end{array}$ & & $\begin{array}{l}500- \\
\mathrm{kg} \\
\text { load }\end{array}$ & $\begin{array}{c}3,000- \\
\mathrm{kg} \\
\text { load }\end{array}$ \\
\hline $\begin{array}{l}m m \\
2.00 \\
2.01 \\
2.02 \\
2.03 \\
2.04\end{array}$ & $\begin{array}{l}158 \\
156 \\
154 \\
153 \\
151\end{array}$ & $\begin{array}{l}945 \\
963 \\
926 \\
917 \\
908\end{array}$ & $\begin{array}{l}m m \\
2.35 \\
2.36 \\
2.37 \\
2.38 \\
2.39\end{array}$ & $\begin{array}{l}114 \\
113 \\
112 \\
111 \\
110\end{array}$ & $\begin{array}{l}682 \\
676 \\
670 \\
665 \\
659\end{array}$ & $\begin{array}{r}m m \\
2.70 \\
2.71 \\
2.72 \\
2.73 \\
2.74\end{array}$ & $\begin{array}{l}85.7 \\
85.1 \\
84.4 \\
83.8 \\
83.2\end{array}$ & $\begin{array}{l}514 \\
510 \\
507 \\
503 \\
499\end{array}$ & $\begin{array}{l}m m \\
3.05 \\
3.06 \\
3.07 \\
3.08 \\
3.09\end{array}$ & $\begin{array}{l}66.8 \\
66.4 \\
65.9 \\
65.5 \\
65.0\end{array}$ & $\begin{array}{l}401 \\
398 \\
395 \\
393 \\
390\end{array}$ & $\begin{array}{l}m m \\
3.40 \\
3.41 \\
3.42 \\
3.43 \\
3.44\end{array}$ & $\begin{array}{l}53.4 \\
53.1 \\
52.8 \\
52.5 \\
52.2\end{array}$ & $\begin{array}{l}321 \\
319 \\
317 \\
315 \\
313\end{array}$ \\
\hline $\begin{array}{l}2.05 \\
2.06 \\
2.07 \\
2.08 \\
2.09\end{array}$ & $\begin{array}{l}150 \\
148 \\
147 \\
146 \\
144\end{array}$ & $\begin{array}{l}899 \\
890 \\
882 \\
873 \\
865\end{array}$ & $\begin{array}{l}2.40 \\
\text { 2. } 41 \\
\text { 2. } 42 \\
\text { 2. } 43 \\
\text { 2. } 44\end{array}$ & $\begin{array}{l}109 \\
108 \\
107 \\
106 \\
105\end{array}$ & $\begin{array}{l}653 \\
648 \\
643 \\
637 \\
632\end{array}$ & $\begin{array}{l}\text { 2. } 75 \\
\text { 2. } 76 \\
\text { 2. } 77 \\
\text { 2. } 78 \\
\text { 2. } 79\end{array}$ & $\begin{array}{l}82.6 \\
81.9 \\
81.3 \\
80.8 \\
80.2\end{array}$ & $\begin{array}{l}495 \\
492 \\
488 \\
485 \\
481\end{array}$ & $\begin{array}{l}\text { 3. } 10 \\
\text { 3. } 11 \\
\text { 3. } 12 \\
\text { 3. } 13 \\
\text { 3. } 14\end{array}$ & $\begin{array}{l}64.6 \\
64.2 \\
63.8 \\
63.3 \\
62.9\end{array}$ & $\begin{array}{l}388 \\
385 \\
383 \\
380 \\
378\end{array}$ & $\begin{array}{l}\text { 3. } 45 \\
\text { 3. } 46 \\
\text { 3. } 47 \\
\text { 3. } 48 \\
\text { 3. } 49\end{array}$ & $\begin{array}{l}51.8 \\
51.5 \\
51.2 \\
50.9 \\
50.6\end{array}$ & $\begin{array}{l}311 \\
309 \\
307 \\
306 \\
304\end{array}$ \\
\hline $\begin{array}{l}\text { 2. } 15 \\
\text { 2. } 16 \\
\text { 2. } 17 \\
\text { 2. } 18 \\
\text { 2. } 19\end{array}$ & $\begin{array}{l}136 \\
135 \\
134 \\
132 \\
131\end{array}$ & $\begin{array}{l}817 \\
809 \\
802 \\
794 \\
-87\end{array}$ & $\begin{array}{l}2.50 \\
\text { 2. } 51 \\
\text { 2. } 52 \\
\text { 2. } 53 \\
\text { 2. } 54\end{array}$ & $\begin{array}{r}100 \\
99.4 \\
98.6 \\
97.8 \\
97.1\end{array}$ & $\begin{array}{l}601 \\
597 \\
592 \\
587 \\
582\end{array}$ & $\begin{array}{l}2.85 \\
2.86 \\
2.87 \\
2.88 \\
2.89\end{array}$ & $\begin{array}{l}76.8 \\
76.2 \\
75.7 \\
75.1 \\
74.6\end{array}$ & $\begin{array}{l}461 \\
457 \\
454 \\
451 \\
448\end{array}$ & $\begin{array}{l}\text { 3. } 20 \\
\text { 3. } 21 \\
\text { 3. } 22 \\
\text { 3. } 23 \\
\text { 3. } 24\end{array}$ & $\begin{array}{l}60.5 \\
60.1 \\
59.8 \\
59.4 \\
59.0\end{array}$ & $\begin{array}{l}363 \\
361 \\
359 \\
356 \\
354\end{array}$ & $\begin{array}{l}3.55 \\
3.56 \\
3.57 \\
3.58 \\
3.59\end{array}$ & $\begin{array}{l}48.9 \\
48.6 \\
48.3 \\
48.0 \\
47.7\end{array}$ & $\begin{array}{l}293 \\
292 \\
290 \\
288 \\
256\end{array}$ \\
\hline $\begin{array}{l}2.20 \\
2.21 \\
2.22 \\
2.23 \\
2.24\end{array}$ & $\begin{array}{l}130 \\
129 \\
129 \\
126 \\
125\end{array}$ & $\begin{array}{l}780 \\
772 \\
765 \\
758 \\
752\end{array}$ & $\begin{array}{l}2.55 \\
2.56 \\
2.57 \\
2.58 \\
2.59\end{array}$ & $\begin{array}{l}96.3 \\
95.5 \\
94.8 \\
94.0 \\
93.3\end{array}$ & $\begin{array}{l}578 \\
573 \\
569 \\
564 \\
560\end{array}$ & $\begin{array}{l}\text { 2. } 90 \\
\text { 2. } 91 \\
\text { 2. } 92 \\
\text { 2. } 93 \\
\text { 2. } 94\end{array}$ & $\begin{array}{l}74.1 \\
73.6 \\
73.0 \\
72.5 \\
72.0\end{array}$ & $\begin{array}{l}444 \\
441 \\
438 \\
435 \\
432\end{array}$ & $\begin{array}{l}\text { 3. } 25 \\
\text { 3. } 26 \\
\text { 3. } 27 \\
\text { 3. } 25 \\
\text { 3. } 29\end{array}$ & $\begin{array}{l}58.6 \\
58.3 \\
57.9 \\
57.5 \\
57.2\end{array}$ & $\begin{array}{l}352 \\
350 \\
347 \\
345 \\
343\end{array}$ & $\begin{array}{l}3.60 \\
3.61 \\
3.62 \\
3.63 \\
3.61\end{array}$ & $\begin{array}{l}47.5 \\
47.2 \\
46.9 \\
46.7 \\
46.4\end{array}$ & $\begin{array}{l}255 \\
283 \\
282 \\
250 \\
278\end{array}$ \\
\hline $\begin{array}{l}2.25 \\
2.26 \\
2.27 \\
2.28 \\
2.29\end{array}$ & $\begin{array}{l}124 \\
123 \\
122 \\
121 \\
120\end{array}$ & $\begin{array}{l}745 \\
738 \\
732 \\
725 \\
719\end{array}$ & $\begin{array}{l}\text { 2. } 60 \\
\text { 2. } 61 \\
\text { 2. } 62 \\
\text { 2. } 63 \\
\text { 2. } 64\end{array}$ & $\begin{array}{l}92.6 \\
91.8 \\
91.1 \\
90.4 \\
89.7\end{array}$ & $\begin{array}{l}555 \\
551 \\
547 \\
543 \\
538\end{array}$ & $\begin{array}{l}\text { 2. } 95 \\
\text { 2. } 96 \\
\text { 2. } 97 \\
\text { 2. } 98 \\
\text { 2. } 99\end{array}$ & $\begin{array}{l}71.5 \\
71.0 \\
70.5 \\
70.1 \\
69.6\end{array}$ & $\begin{array}{l}429 \\
426 \\
423 \\
420 \\
417\end{array}$ & $\begin{array}{l}\text { 3. } 30 \\
\text { 3. } 31 \\
\text { 3. } 32 \\
\text { 3. } 33 \\
\text { 3. } 34\end{array}$ & $\begin{array}{l}56.8 \\
56.5 \\
56.1 \\
55.8 \\
55.4\end{array}$ & $\begin{array}{l}341 \\
339 \\
337 \\
335 \\
333\end{array}$ & $\begin{array}{l}3.65 \\
3.66 \\
3.67 \\
3.68 \\
3.69\end{array}$ & $\begin{array}{l}46.1 \\
45.9 \\
45.6 \\
45.4 \\
45.1\end{array}$ & $\begin{array}{l}277 \\
275 \\
274 \\
272 \\
271\end{array}$ \\
\hline
\end{tabular}


TABLE 1.-Brinell hardness numbers-Continued

\begin{tabular}{|c|c|c|c|c|c|c|c|c|c|c|c|c|c|c|}
\hline $\begin{array}{l}\text { Diam- } \\
\text { eter of } \\
\text { inden- } \\
\text { tation }\end{array}$ & \multicolumn{2}{|c|}{$\begin{array}{l}\text { Brinell hard- } \\
\text { ress No. }\end{array}$} & $\begin{array}{l}\text { Diam- } \\
\text { eter of } \\
\text { inden- } \\
\text { tation }\end{array}$ & \multicolumn{2}{|c|}{$\begin{array}{l}\text { Brinell hard- } \\
\text { ness No. }\end{array}$} & $\begin{array}{l}\text { Diam- } \\
\text { eter of } \\
\text { inden- } \\
\text { tation }\end{array}$ & \multicolumn{2}{|c|}{$\begin{array}{l}\text { Brinell hard- } \\
\text { ness No. }\end{array}$} & $\begin{array}{l}\text { Diam- } \\
\text { eter of } \\
\text { inden- } \\
\text { tation }\end{array}$ & \multicolumn{2}{|c|}{$\begin{array}{c}\text { Brinell hard- } \\
\text { ness No. }\end{array}$} & $\begin{array}{l}\text { Diam- } \\
\text { eter of } \\
\text { inden- } \\
\text { tation }\end{array}$ & \multicolumn{2}{|c|}{$\begin{array}{l}\text { Brinell hard- } \\
\text { ness No. }\end{array}$} \\
\hline $\begin{array}{l}m m \\
3.75 \\
3.76 \\
3.77 \\
3.78 \\
3.79\end{array}$ & $\begin{array}{l}43.6 \\
43.4 \\
43.1 \\
42.9 \\
42.7\end{array}$ & $\begin{array}{l}262 \\
260 \\
259 \\
257 \\
256\end{array}$ & $\begin{array}{l}m m \\
\text { 4. } 40 \\
\text { 4. } 41 \\
\text { 4. } 42 \\
\text { 4. } 43 \\
\text { 4. } 44\end{array}$ & $\begin{array}{l}31.2 \\
31.1 \\
30.9 \\
30.8 \\
30.6\end{array}$ & $\begin{array}{l}187 \\
186 \\
185 \\
185 \\
184\end{array}$ & $\begin{array}{l}m m \\
5.05 \\
5.06 \\
5.07 \\
5.08 \\
5.09\end{array}$ & $\begin{array}{l}23.3 \\
23.2 \\
23.1 \\
23.0 \\
22.9\end{array}$ & $\begin{array}{l}140 \\
139 \\
138 \\
138 \\
137\end{array}$ & $\begin{array}{l}m m \\
5.70 \\
5.71 \\
5.72 \\
5.73 \\
5.74\end{array}$ & $\begin{array}{l}17.8 \\
17.8 \\
17.7 \\
17.6 \\
17.6\end{array}$ & $\begin{array}{l}107 \\
107 \\
106 \\
106 \\
105\end{array}$ & $\begin{array}{l}m m \\
6.35 \\
6.36 \\
6.37 \\
6.38 \\
6.39\end{array}$ & $\begin{array}{l}14.0 \\
13.9 \\
13.9 \\
13.8 \\
13.8\end{array}$ & $\begin{array}{l}84.0 \\
83.7 \\
83.4 \\
83.1 \\
82.8\end{array}$ \\
\hline $\begin{array}{l}3.80 \\
3.81 \\
3.82 \\
3.83 \\
3.81\end{array}$ & $\begin{array}{l}42.4 \\
42.2 \\
42.0 \\
41.7 \\
11.5\end{array}$ & $\begin{array}{l}255 \\
253 \\
252 \\
250 \\
249\end{array}$ & $\begin{array}{l}4.45 \\
4.46 \\
4.47 \\
4.48 \\
4.49\end{array}$ & $\begin{array}{l}30.5 \\
30.3 \\
30.2 \\
30.0 \\
29.9\end{array}$ & $\begin{array}{l}183 \\
182 \\
181 \\
180 \\
179\end{array}$ & $\begin{array}{l}5.10 \\
5.11 \\
5.12 \\
5.13 \\
5.14\end{array}$ & $\begin{array}{l}22.8 \\
22.7 \\
22.6 \\
22.5 \\
22.4\end{array}$ & $\begin{array}{l}137 \\
136 \\
135 \\
135 \\
134\end{array}$ & $\begin{array}{l}\text { 5. } 75 \\
\text { 5. } 76 \\
\text { 5. } 77 \\
\text { 5. } 78 \\
5.79\end{array}$ & $\begin{array}{l}17.5 \\
17.4 \\
17.4 \\
17.3 \\
17.2\end{array}$ & $\begin{array}{l}105 \\
105 \\
104 \\
104 \\
103\end{array}$ & $\begin{array}{l}6.40 \\
6.41 \\
6.42 \\
6.43 \\
6.44\end{array}$ & $\begin{array}{l}13.7 \\
13.7 \\
13.6 \\
13.6 \\
13.5\end{array}$ & $\begin{array}{l}82.5 \\
82.2 \\
81.9 \\
81.6 \\
81.3\end{array}$ \\
\hline $\begin{array}{l}3.85 \\
3.86 \\
3.87 \\
3.88 \\
3.89\end{array}$ & $\begin{array}{l}41.3 \\
41.1 \\
40.9 \\
40.6 \\
40.4\end{array}$ & $\begin{array}{l}248 \\
246 \\
245 \\
244 \\
242\end{array}$ & $\begin{array}{l}4.50 \\
\text { 4. } 51 \\
\text { 4. } 52 \\
\text { 4. } 53 \\
4.54\end{array}$ & $\begin{array}{l}29.8 \\
29.6 \\
29.5 \\
29.3 \\
29.2\end{array}$ & $\begin{array}{l}179 \\
178 \\
177 \\
176 \\
175\end{array}$ & $\begin{array}{l}5.15 \\
5.16 \\
5.17 \\
5.18 \\
5.19\end{array}$ & $\begin{array}{l}22.3 \\
22.2 \\
22.1 \\
22.0 \\
21.9\end{array}$ & $\begin{array}{l}134 \\
133 \\
133 \\
132 \\
132\end{array}$ & $\begin{array}{l}5.80 \\
5.81 \\
5.82 \\
5.83 \\
5.84\end{array}$ & $\begin{array}{l}17.2 \\
17.1 \\
17.0 \\
17.0 \\
16.9\end{array}$ & $\begin{array}{l}103 \\
103 \\
102 \\
102 \\
101\end{array}$ & $\begin{array}{l}6.45 \\
6.46 \\
6.47 \\
6.48 \\
6.49\end{array}$ & $\begin{array}{l}13.5 \\
13.4 \\
13.4 \\
13.4 \\
13.3\end{array}$ & $\begin{array}{l}81.0 \\
80.7 \\
80.4 \\
80.1 \\
79.8\end{array}$ \\
\hline $\begin{array}{l}3.90 \\
3.91 \\
3.92 \\
3.93 \\
3.94\end{array}$ & $\begin{array}{l}40.2 \\
40.0 \\
39.8 \\
39.6 \\
39.4\end{array}$ & $\begin{array}{l}241 \\
240 \\
239 \\
237 \\
236\end{array}$ & $\begin{array}{l}\text { 4. } 55 \\
\text { 4. } 56 \\
\text { 4. } 57 \\
\text { 4. } 58 \\
\text { 4. } 59\end{array}$ & $\begin{array}{l}29.1 \\
28.9 \\
28.8 \\
28.7 \\
28.5\end{array}$ & $\begin{array}{l}174 \\
174 \\
173 \\
172 \\
171\end{array}$ & $\begin{array}{l}5.20 \\
5.21 \\
5.22 \\
5.23 \\
5.24\end{array}$ & $\begin{array}{l}21.8 \\
21.7 \\
21.6 \\
21.6 \\
21.5\end{array}$ & $\begin{array}{l}131 \\
130 \\
130 \\
129 \\
129\end{array}$ & $\begin{array}{l}5.85 \\
5.86 \\
5.87 \\
5.88 \\
5.89\end{array}$ & $\begin{array}{l}16.8 \\
16.8 \\
16.7 \\
16.7 \\
16.5\end{array}$ & $\begin{array}{c}101 \\
101 \\
100 \\
99.9 \\
99.5\end{array}$ & $\begin{array}{l}6.50 \\
6.51 \\
6.52 \\
6.53 \\
6.54\end{array}$ & $\begin{array}{l}13.3 \\
13.2 \\
13.2 \\
13.1 \\
13.1\end{array}$ & $\begin{array}{l}79.6 \\
79.3 \\
79.0 \\
78.7 \\
78.4\end{array}$ \\
\hline $\begin{array}{l}3.95 \\
3.96 \\
3.97 \\
3.98 \\
3.99\end{array}$ & $\begin{array}{l}39.1 \\
38.9 \\
38.7 \\
38.5 \\
38.3\end{array}$ & $\begin{array}{l}235 \\
234 \\
232 \\
231 \\
230\end{array}$ & $\begin{array}{l}\text { 4. } 60 \\
\text { 4. } 61 \\
\text { 4. } 62 \\
\text { 4. } 63 \\
\text { 4. } 64\end{array}$ & $\begin{array}{r}28.4 \\
28.3 \\
28.1 \\
28.0 \\
27.9\end{array}$ & $\begin{array}{l}170 \\
170 \\
169 \\
168 \\
167\end{array}$ & $\begin{array}{l}\text { 5. } 25 \\
\text { 5. } 26 \\
5.27 \\
5.28 \\
5.29\end{array}$ & $\begin{array}{l}21.4 \\
21.3 \\
21.2 \\
21.1 \\
21.0\end{array}$ & $\begin{array}{l}128 \\
128 \\
127 \\
127 \\
126\end{array}$ & $\begin{array}{l}5.90 \\
5.91 \\
5.92 \\
5.93 \\
5.94\end{array}$ & $\begin{array}{l}16.5 \\
16.5 \\
16.4 \\
16.3 \\
16.3\end{array}$ & $\begin{array}{l}99.2 \\
98.8 \\
98.4 \\
98.0 \\
97.7\end{array}$ & $\begin{array}{l}6.55 \\
6.56 \\
6.57 \\
6.58 \\
6.59\end{array}$ & $\begin{array}{l}13.0 \\
13.0 \\
12.9 \\
12.9 \\
12.8\end{array}$ & $\begin{array}{l}78.2 \\
77.9 \\
77.6 \\
77.3 \\
77.1\end{array}$ \\
\hline $\begin{array}{l}4.00 \\
4.01 \\
\text { 4. } 02 \\
4.03 \\
4.04\end{array}$ & $\begin{array}{l}38.1 \\
37.9 \\
37.7 \\
37.5 \\
37.3\end{array}$ & $\begin{array}{l}229 \\
223 \\
226 \\
225 \\
224\end{array}$ & $\begin{array}{l}4.65 \\
4.66 \\
4.67 \\
4.68 \\
4.69\end{array}$ & $\begin{array}{l}27.8 \\
27.6 \\
27.5 \\
27.4 \\
27.3\end{array}$ & $\begin{array}{l}167 \\
166 \\
165 \\
164 \\
164\end{array}$ & $\begin{array}{l}5.30 \\
5.31 \\
5.32 \\
5.33 \\
5.34\end{array}$ & $\begin{array}{l}20.9 \\
20.9 \\
20.8 \\
20.7 \\
20.6\end{array}$ & $\begin{array}{l}126 \\
125 \\
125 \\
124 \\
124\end{array}$ & $\begin{array}{l}5.95 \\
5.96 \\
5.97 \\
5.98 \\
5.99\end{array}$ & $\begin{array}{l}16.2 \\
16.2 \\
16.1 \\
16.0 \\
16.0\end{array}$ & $\begin{array}{l}97.3 \\
96.9 \\
96.6 \\
96.2 \\
95.9\end{array}$ & $\begin{array}{l}6.60 \\
6.61 \\
6.62 \\
6.63 \\
6.64\end{array}$ & $\begin{array}{l}12.8 \\
12.8 \\
12.7 \\
12.7 \\
12.6\end{array}$ & $\begin{array}{l}76.8 \\
76.5 \\
76.2 \\
76.0 \\
75.7\end{array}$ \\
\hline $\begin{array}{l}4.05 \\
\text { 4. } 06 \\
4.07 \\
4.08 \\
4.09\end{array}$ & $\begin{array}{l}37.1 \\
37.0 \\
36.8 \\
36.6 \\
36.4\end{array}$ & $\begin{array}{l}223 \\
222 \\
221 \\
219 \\
218\end{array}$ & $\begin{array}{l}\text { 4. } 70 \\
\text { 4. } 71 \\
\text { 4. } 72 \\
\text { 4. } 73 \\
\text { 4. } 74\end{array}$ & $\begin{array}{l}27.1 \\
27.0 \\
26.9 \\
26.8 \\
26.6\end{array}$ & $\begin{array}{l}163 \\
162 \\
161 \\
161 \\
160\end{array}$ & $\begin{array}{l}5.35 \\
5.36 \\
5.37 \\
5.38 \\
5.39\end{array}$ & $\begin{array}{l}20.5 \\
20.4 \\
20.3 \\
20.3 \\
20.2\end{array}$ & $\begin{array}{l}123 \\
123 \\
122 \\
122 \\
121\end{array}$ & $\begin{array}{l}6.00 \\
6.01 \\
6.02 \\
6.03 \\
6.04\end{array}$ & $\begin{array}{l}15.9 \\
15.9 \\
15.8 \\
15.7 \\
15.7\end{array}$ & $\begin{array}{l}95.5 \\
95.1 \\
94.8 \\
94.4 \\
94.1\end{array}$ & $\begin{array}{l}6.65 \\
6.66 \\
6.67 \\
6.68 \\
6.69\end{array}$ & $\begin{array}{l}12.6 \\
12.5 \\
12.5 \\
12.4 \\
12.4\end{array}$ & $\begin{array}{l}75.4 \\
75.2 \\
74.9 \\
74.7 \\
74.4\end{array}$ \\
\hline $\begin{array}{l}\text { 4. } 10 \\
\text { 4. } 11 \\
\text { 4. } 12 \\
\text { 4. } 13 \\
\text { 4. } 14\end{array}$ & $\begin{array}{l}36.2 \\
36.0 \\
35.8 \\
35.7 \\
35.5\end{array}$ & $\begin{array}{l}217 \\
216 \\
215 \\
214 \\
213\end{array}$ & $\begin{array}{l}\text { 4. } 75 \\
\text { 4. } 76 \\
\text { 4. } 77 \\
\text { 4. } 78 \\
\text { 4. } 79\end{array}$ & $\begin{array}{l}26.5 \\
26.4 \\
26.3 \\
26.2 \\
26.1\end{array}$ & $\begin{array}{l}159 \\
158 \\
158 \\
157 \\
156\end{array}$ & $\begin{array}{l}5.40 \\
5.41 \\
5.42 \\
5.43 \\
5.44\end{array}$ & $\begin{array}{l}20.1 \\
20.0 \\
19.9 \\
19.9 \\
19.8\end{array}$ & $\begin{array}{l}121 \\
120 \\
120 \\
119 \\
119\end{array}$ & $\begin{array}{l}6.05 \\
6.06 \\
6.07 \\
6.08 \\
6.09\end{array}$ & $\begin{array}{l}15.6 \\
15.6 \\
15.5 \\
15.4 \\
15.4\end{array}$ & $\begin{array}{l}93.7 \\
93.4 \\
93.0 \\
92.7 \\
92.3\end{array}$ & $\begin{array}{l}6.70 \\
6.71 \\
6.72 \\
6.73 \\
6.74\end{array}$ & $\begin{array}{l}12.4 \\
12.3 \\
12.3 \\
12.2 \\
12.2\end{array}$ & $\begin{array}{l}74.1 \\
73.9 \\
73.6 \\
73.4 \\
73.1\end{array}$ \\
\hline $\begin{array}{l}4.15 \\
4.16 \\
4.17 \\
4.18 \\
4.19\end{array}$ & $\begin{array}{l}35.3 \\
35.1 \\
34.9 \\
34.8 \\
34.6\end{array}$ & $\begin{array}{l}212 \\
211 \\
210 \\
209 \\
203\end{array}$ & $\begin{array}{l}4.80 \\
4.81 \\
4.82 \\
4.83 \\
4.84\end{array}$ & $\begin{array}{l}25.9 \\
25.8 \\
25.7 \\
25.6 \\
25.5\end{array}$ & $\begin{array}{l}156 \\
155 \\
154 \\
154 \\
153\end{array}$ & $\begin{array}{l}5.45 \\
5.46 \\
5.47 \\
5.48 \\
5.49\end{array}$ & $\begin{array}{l}19.7 \\
19.6 \\
19.5 \\
19.5 \\
19.4\end{array}$ & $\begin{array}{l}118 \\
118 \\
117 \\
117 \\
116\end{array}$ & $\begin{array}{l}6.10 \\
6.11 \\
6.12 \\
6.13 \\
6.14\end{array}$ & $\begin{array}{l}15.3 \\
15.3 \\
15.2 \\
15.2 \\
15.1\end{array}$ & $\begin{array}{l}92.0 \\
91.7 \\
91.3 \\
91.0 \\
90.6\end{array}$ & $\begin{array}{l}6.75 \\
6.76 \\
6.77 \\
6.78 \\
6.79\end{array}$ & $\begin{array}{l}12.1 \\
12.1 \\
12.1 \\
12.0 \\
12.0\end{array}$ & $\begin{array}{l}72.8 \\
72.6 \\
72.3 \\
72.1 \\
71.8\end{array}$ \\
\hline $\begin{array}{l}\text { 4. } 20 \\
\text { 4. } 21 \\
\text { 4. } 22 \\
\text { 4. } 23 \\
\text { 4. } 24\end{array}$ & $\begin{array}{l}34.4 \\
34.2 \\
34.1 \\
33.9 \\
33.7\end{array}$ & $\begin{array}{l}207 \\
205 \\
204 \\
203 \\
202\end{array}$ & $\begin{array}{l}4.85 \\
4.86 \\
4.87 \\
4.88 \\
4.89\end{array}$ & $\begin{array}{l}25.4 \\
25.3 \\
25.1 \\
25.0 \\
24.9\end{array}$ & $\begin{array}{l}152 \\
152 \\
151 \\
150 \\
150\end{array}$ & $\begin{array}{l}5.50 \\
5.51 \\
5.52 \\
5.53 \\
5.54\end{array}$ & $\begin{array}{l}19.3 \\
19.2 \\
19.2 \\
19.1 \\
19.0\end{array}$ & $\begin{array}{l}116 \\
115 \\
115 \\
114 \\
114\end{array}$ & $\begin{array}{l}6.15 \\
6.16 \\
6.17 \\
6.18 \\
6.19\end{array}$ & $\begin{array}{l}15.1 \\
15.0 \\
14.9 \\
14.9 \\
14.8\end{array}$ & $\begin{array}{l}90.3 \\
90.0 \\
89.6 \\
89.3 \\
89.0\end{array}$ & $\begin{array}{l}6.80 \\
6.81 \\
6.82 \\
6.83 \\
6.84\end{array}$ & $\begin{array}{l}11.9 \\
11.9 \\
11.8 \\
11.8 \\
11.8\end{array}$ & $\begin{array}{l}71.6 \\
71.3 \\
71.1 \\
70.8 \\
70.6\end{array}$ \\
\hline $\begin{array}{l}\text { 4. } 25 \\
\text { 4. } 26 \\
\text { 4. } 27 \\
\text { 4. } 28 \\
\text { 4. } 29\end{array}$ & $\begin{array}{l}33.6 \\
33.4 \\
33.2 \\
33.1 \\
32.9\end{array}$ & $\begin{array}{l}201 \\
200 \\
199 \\
198 \\
198\end{array}$ & $\begin{array}{l}\text { 4. } 90 \\
\text { 4. } 91 \\
\text { 4. } 92 \\
\text { 4. } 93 \\
\text { 4. } 94\end{array}$ & $\begin{array}{l}24.8 \\
24.7 \\
24.6 \\
24.5 \\
24.4\end{array}$ & $\begin{array}{l}149 \\
148 \\
148 \\
147 \\
146\end{array}$ & $\begin{array}{l}5.55 \\
5.56 \\
5.57 \\
5.58 \\
5.59\end{array}$ & $\begin{array}{l}18.9 \\
18.9 \\
18.8 \\
18.7 \\
18.6\end{array}$ & $\begin{array}{l}114 \\
113 \\
113 \\
112 \\
112\end{array}$ & $\begin{array}{l}6.20 \\
6.21 \\
6.22 \\
6.23 \\
6.24\end{array}$ & $\begin{array}{l}14.8 \\
14.7 \\
14.7 \\
14.6 \\
14.6\end{array}$ & $\begin{array}{l}88.7 \\
88.3 \\
88.0 \\
87.7 \\
87.4\end{array}$ & $\begin{array}{l}6.85 \\
6.86 \\
6.87 \\
6.88 \\
6.89\end{array}$ & $\begin{array}{l}11.7 \\
11.7 \\
11.6 \\
11.6 \\
11.6\end{array}$ & $\begin{array}{l}70.4 \\
70.1 \\
69.9 \\
69.6 \\
69.4\end{array}$ \\
\hline $\begin{array}{l}\text { 4. } 30 \\
\text { 4. } 31 \\
\text { 4. } 32 \\
\text { 4. } 33 \\
\text { 4. } 34\end{array}$ & $\begin{array}{l}32.8 \\
32.6 \\
32.4 \\
32.3 \\
32.1\end{array}$ & $\begin{array}{l}197 \\
196 \\
195 \\
194 \\
193\end{array}$ & $\begin{array}{l}4.95 \\
4.96 \\
4.97 \\
4.98 \\
4.99\end{array}$ & $\begin{array}{l}24.3 \\
24.2 \\
24.1 \\
24.0 \\
23.9\end{array}$ & $\begin{array}{l}146 \\
145 \\
144 \\
144 \\
143\end{array}$ & $\begin{array}{l}\text { 5. } 60 \\
\text { 5. } 61 \\
\text { 5. } 62 \\
\text { 5. } 63 \\
\text { 5. } 64\end{array}$ & $\begin{array}{l}18.6 \\
18.5 \\
18.4 \\
18.3 \\
18.3\end{array}$ & $\begin{array}{l}111 \\
111 \\
110 \\
110 \\
110\end{array}$ & $\begin{array}{l}6.25 \\
6.26 \\
6.27 \\
6.28 \\
6.29\end{array}$ & $\begin{array}{l}14.5 \\
14.5 \\
14.4 \\
14.4 \\
14.3\end{array}$ & $\begin{array}{l}87.1 \\
86.7 \\
86.4 \\
86.1 \\
85.8\end{array}$ & $\begin{array}{l}6.90 \\
6.91 \\
6.92 \\
6.93 \\
6.94\end{array}$ & $\begin{array}{l}11.5 \\
11.5 \\
11.4 \\
11.4 \\
11.4\end{array}$ & $\begin{array}{l}69.2 \\
68.9 \\
68.7 \\
68.4 \\
68.2\end{array}$ \\
\hline $\begin{array}{l}\text { 4. } 35 \\
\text { 4. } 36 \\
\text { 4. } 37 \\
\text { 4. } 38 \\
\text { 4. } 39\end{array}$ & $\begin{array}{l}32.0 \\
31.8 \\
31.7 \\
31.5 \\
31.4\end{array}$ & $\begin{array}{l}192 \\
191 \\
190 \\
189 \\
188\end{array}$ & $\begin{array}{l}5.00 \\
5.01 \\
5.02 \\
5.03 \\
5.04\end{array}$ & $\begin{array}{l}23.8 \\
23.7 \\
23.6 \\
23.5 \\
23.4\end{array}$ & $\begin{array}{l}143 \\
142 \\
141 \\
141 \\
140\end{array}$ & $\begin{array}{l}5.65 \\
5.66 \\
5.67 \\
5.68 \\
5.69\end{array}$ & $\begin{array}{l}18.2 \\
18.1 \\
18.1 \\
18.0 \\
17.9\end{array}$ & $\begin{array}{l}109 \\
109 \\
108 \\
108 \\
107\end{array}$ & $\begin{array}{l}6.30 \\
6.31 \\
6.32 \\
6.33 \\
6.34\end{array}$ & $\begin{array}{l}\text { 14. } 2 \\
14.2 \\
14.1 \\
14.1 \\
14.0\end{array}$ & $\begin{array}{l}85.5 \\
85.2 \\
84.9 \\
84.6 \\
84.3\end{array}$ & $\begin{array}{l}6.95 \\
6.96 \\
6.97 \\
6.98 \\
6.99\end{array}$ & $\begin{array}{l}11.3 \\
11.3 \\
11.3 \\
11.2 \\
11.2\end{array}$ & $\begin{array}{l}68.0 \\
67.7 \\
67.5 \\
67.3 \\
67.0\end{array}$ \\
\hline
\end{tabular}

\section{APPARATUS}

1. The divisions on the micrometer scale of the microscope or other measuring device used for the measurement of the diameter of impression shall be such as to permit the direct measuring of the diameter to $0.1 \mathrm{~mm}$ and the estimation of the diameter to $0.01 \mathrm{~mm}$.

2. (a) The balls used in Brinell hardness testing shall be of hardened steel or other equally hard material.

(b) A ball to be suitable for use shall not show a permanent change in diameter greater than 0.0025 
$\mathrm{mm}(0.0001$ inch $)$ when pressed with a force of $3,000 \mathrm{~kg}$ against a piece of steel having a Brinell hardness number of 500 or greater. ${ }^{26}$

(c) If a ball is used in a test of a specimen which shows a Brinell hardness number greater than 500 , the ball shall be remeasured after the test. Should the ball show a permanent change in diameter greater than that permitted in paragraph $(b)$, the ball shall be unsuitable for further use in a standard test. ${ }^{26}$

3. The standard ball for Brinell hardness testing shall be $10 \mathrm{~mm}(0.3937 \mathrm{inch})$ in diameter with a deviation from this value of not more than $0.01 \mathrm{~mm}$ (0.0004 inch) in any diameter, but the diameter of any ball measured at various points shall be constant within a tolerance of $\pm 0.0025 \mathrm{~mm}(0.0001$ inch).

For testing very small specimens or very thin specimens it is sometimes necessary to make Brinell hardness tests with a ball less than $10 \mathrm{~mm}$ in diameter. For such tests (which are not to be regarded as standard Brinell tests) the relation between applied load, $P$, measstandard in kilograms, and diameter of ball, $D$, measured in millimeters, should be: and

$$
P=3,000 \frac{D^{2}}{10^{2}}=30 D^{2} \text { for iron and steel; }
$$

$$
P=500 \frac{D^{2}}{10^{2}}=5 D^{2} \text { for brass, bronze, and soft metals in general. }
$$

For extremely soft metals the relation $P=D^{2}$ may be used.

Balls differing in size from the standard $10 \mathrm{~mm}$ ball shall conform to the requirements for the material and the tolerance in size of diameter as specified for the standard ball.

\section{TEST SPECIMENS}

Specimens used in Brinell hardness testing vary greatly in form since it is frequently desirable to make the impression upon a part to be used in the finished product rather than upon a sample test specimen.

4. The thickness of the piece tested shall be such that no bulge or other marking showing the effect of the load appears on the side of the piece opposite the impression. In any event the thickness of the specimen shall be at least ten times the depth of impression.

5 . The surface on which the impression is to be made shall be filed, ground, machined, or polished with emery paper so that the edge of the impression shall be clearly enough defined to permit of the measurement of the diameter within $0.01 \mathrm{~mm}$ (0.0004 inch).

\section{APPLICATION AND MAGNITUDE OF LOAD}

6. The load in the standard Brinell test shall be $3,000 \mathrm{~kg}$ for iron and steel and $500 \mathrm{~kg}$ for brass, bronze, and soft metals generally.

Note.-For extremely soft metals a load of $100 \mathrm{~kg}$ is sometimes used. If for any reason it is necessary to use loads differing from those specified above, the load used should be specifically stated in the test report, conveniently as a subscript of the hardness numeral.

7. (a) The load shall be applied steadily to the specimen without jerk.

(b) The load shall be applied for at least $10 \mathrm{sec}-$ onds in the case of iron and steel and for at least 30 seconds in the case of other metals. ${ }^{27}$

(c) The center line of the applied load shall be far enough from any edge of the specimen so that when a straight-edge is applied to the edge after the load has been applied, the eye can detect no bulge in the edge due to the load and the resulting impression.

26 In testing softer metals, it is not, of course, necessary to have balls of this extreme hardness. However, a ball to be suitable for use should withstand pressure against the hardest material to be tested without showing a permanent change in diameter greater than $0.0025 \mathrm{~mm}$.

\section{MEASUREMENT OF IMPRESSION}

8. In the standard Brinell hardness test the diameter of the impression shall be measured.

9. When a micrometer microscope is used to measure the diameter of the impression the sharpness of definition of the edge of the impression can be increased by the use of a movable lamp for illuminating the specimen, placing the lamp so that the contrast of light and shade will bring first one edge of the impression, then the other into sharp definition. In testing very hard material, the sharpness of definition of the impression can be somewhat increased by the use of a ball lightly etched with nitric acid, or by the use of some pigment, such as Prussian blue, on the ball. In testing material in which there is considerable recovery of shape, the material may first be coated with a dull-black pigment, such as drawing ink or a mixture of graphite and alcohol. The edge of the impression is rendered clear on a surface so coated.

\section{BRINELL HARDNESS TESTS IN WHICH DEPTH OF IMPRESSION IS MEASURED IN PLACE OF DIAMETER}

When it is necessary to make Brinell hardness tests very rapidly, as is sometimes the case when the Brinell test is used as a control test for the output of a heat-treating furnace, the depth of impression under load or after the release of load can be measured more expeditiously than the diameter of impression. The depth of impression is usually determined from the relative motion of the plunger carrying the ball and the specimen. This method of determining Brinell hardness is not to be regarded as a standard method, and when requirements of speed necessitate its use the Brinell hardness number should not be determined from the geometrical relation of depth of impression to diameter, based on the assumption that the impression has a spherical surface. In such tests the relation between the Brinell hardness number and depth of impression may be determined with a fair degree of accuracy from tests on specimens of material of different degrees of hardness, several impressions being made on each specimen, and the depth under load, or the depth on release of load, and the diameter of impression after release of load being measured for each impression. By the use of a diagram or by interpolation, a table giving the Brinell hardness number for a given depth of impression can be prepared.

\section{CALIBRATION OF BRINELL HARDNESS-TESTING APPARATUS}

10. A load-measuring device which is in common use for Brinell hardness-testing machines involves the measurement of fiuid pressure on a piston of known diameter working in a cylinder with so perfect a fit that packing is not necessary. To this piston is attached the ball. Other load-measuring devices use combinations of weights and levers. The use of dead weights acting on a small piston is to be preferred to the ordinary Bourdon gage for measuring the intensity of fluid pressure.

11. The load-measuring device of a Brinell hardness-testing machine may be calibrated by the use of dead weights, or by the use of dead weights and proving levers, or by an elastic calibration device or spring, in the manner described in the standard

27 It was suggested in discussion that for magnesium and mag. nesium alloys the minimum time of application be two minutes. It wesium alloys the minimum time of application be two minutes. It was also suggested that for any given soft metal a definite time for
holding the load be specified rather than a minimum time. See holding the load be specified rather than a minimum time. See
discussion of the Report of Committee E-1, Proc. Am. Soc. Testing Mats. vol. 24, Pt. I, pp. 729 and $730,1924$. 
methods of verification of testing machines (serial designation E4) of the American Society for Testing Materials. ${ }^{28}$ (See above.)

12. The load-measuring device of a Brinell hardness-testing machine may be calibrated by making a series of impressions on specimens of different degrees of hardness, measuring the diameters of the impressions, making a second series of impressions by the use of any standardized testing machine and standard steel ball, and, using the same measuring device as for the first series of impressions, measuring the diameters of this second series of impressions. The error of the machine under calibration can be determined by the relative average values of the hardness numbers determined by its use and by the use of the standardized testing machine.

13. When the above companion-impression method of calibrating a Brinell harndess-testing machine is used, it is recommended that the specimens used be approximately 1 by 1 by 12 inches in size and that specimens with as wide a range of hardness as is feasible be used. It is recommended that impressions made by the machine under calibration be spaced about 2.5 inches apart along the specimen, and that the impressions made by the standardized testing machine be spaced alternately along the length of the specimen with the impressions made by the machine under calibration.

14. If any determination of hardness, either for the machine under calibration or for the standard machine, differ by more than 2 per cent from the mean of the four determinations made with that machine, it is recommended that such determination be discarded. If two determinations differ more than 2 per cent from the mean, the whole series should be discarded.

15. A Brinell hardness-testing machine is acceptable for use over a loading range within which its load-measuring device is correct within 3 per cent.

16. The micrometer microscope or other device for measuring diameter of impression may be calibrated by using it to measure lengths on a standard steel or glass scale. The eyepiece of a micrometer microscope should be adjusted so that throughout the range covered the error of reading does not exceed $0.01 \mathrm{~mm}$.

\section{AMERICAN SOCIETY FOR TESTING MATE- RIALS, STANDARD DEFINITIONS OF TERMS RELATING TO SPECIFIC GRAV- ITY, DESIGNATION E12-27, 1927}

\section{SPECIFIC GRAVITY TERMS}

1. Absolute specific gravity (of solids and liquids).The ratio of the weight referred to vacuum uf a given volume of the material at a stated temperature to the weight referred to vacuum of an equal volume of gas-free distilled water ${ }^{20}$ at a stated temperature. It shall be stated thus:

(a) When the temperatures of the material and of the water are the same:

Absolute specific gravity $x^{\circ} \mathrm{C}$.,

where $x$ is the temperature of the material and the water.

(b) When the temperatures of the material and of the water are not the same:

Absolute specific gravity $\frac{x^{\circ} \mathrm{C} . \text {, }}{y^{\circ} \mathrm{C} \text {. }}$

where $x$ is the temperature of the material and $y$ the temperature of the water.

28 See J. L. Jones and C. H. Marshall, “A New Method for Calibrating Brinell Hardness Testing Machines," Proc. Am. Soc. Testing Mats., vol. 20, Pt. II, p. 392, 1920.

${ }_{20}$ Distilled water boiled vigorously in racuum.
NoTE.- In the interest of staridardization and simplification, the first form of statement of specific gravity $(a)$ should be employed wherever practicable.

2. Specific gravity (of solids and liquids).-The ratio of the weight in air of a given volume of the material at a stated temperature to the weight in air of an equal volume of distilled water at a stated temperature. It shall be stated thus:

(a) When the temperatures of the material and of the water are the same:

Specific gravity $x^{\circ} \mathrm{C}$,

where $x$ is the temperature of the material and the water.

(b) When the temperatures of the material and of the water are not the same:

Specific gravity $x^{\circ} / y^{\circ} \mathrm{C}$,

where $x$ is the temperature of the material and $y$ is the temperature of the water.

NotE.-In the interest of standardization and simplification, the first form of statement of specific gravity $(a)$ should be employed wherever practicable.

3. Apparent specific gravity (of solids).-The ratio of the weight in air of a given volume of the impermeable portion of a permeable material (that is, the solid matter including its impermeable pores or voids) at a stated temperature to the weight in air of an equal volume of distilled water at a stated temperature. It shall be stated thus:

(a) When the temperatures of the material and of the water are the same:

A pparent specific gravity $x^{\circ} / x^{\circ} \mathrm{C}$,

where $x$ is the temperature of the material and the water.

(b) When the temperatures of the material and of the water are not the same:

Apparent specific gravity $x^{\circ} / y^{\circ} \mathrm{C} .$, ..........,

where $x$ is the temperature of the material and $y$ is the temperature of the water.

Note 1.-In scientific circles, specific gravity and density determinations made in air (that is, uncorrected to vacuum) are frequently distinguished by the arljective apparent. Thus, the specific gravity defined by definition No. 2 would be designated as apparent specific gravity and that defined by definition No. 1 as specific gravity. But in industry, the terminology is more generally in accordance But in industry, the terminology is

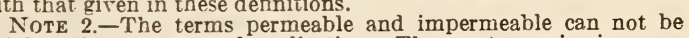
rigidly defined for general application. The exact meaning in a particular application is the conventional one inferred by the procedure specified for determining the specific gravity of the material in question.

NOTE 3 -In the interest of standardization and simplification, the first form of statement of specific gravity $(a)$ should be employed wherever practicable.

4. Bulk specific gravity (of solids).-The ratio of the weight in air of a given volume of a permeable material (including both permeable and impermeable voids normal to the material) at a stated temperature to the weight in air of an equal volume of distilled water at a stated temperature. It shall be stated thus:

(a) When the temperatures of the material and of the water are the same:

Bulk specific gravity $x^{\circ} \mathrm{C}$.

where $x$ is the temperature of the material and the water.

(b) When the temperatures of the material and of the water are not the same:

Bulk specific gravity $\frac{x^{\circ} \mathrm{C} \text {., }}{y^{\circ} \mathrm{C} \text {. }}$

where $x$ is the temperature of the material and $y$ is the temperature of the water.

Note 1.-See note 2 under Apparent specific gravity.

NoTE 2.-In the interest of standardization and simplification, the first form of statement of specific gravity $(a)$ should be employed wherever practicable. 
AMERICAN SOCIETY FOR TESTING MATERIALS, TENTATIVE RECOMMENDED PRACTICE FOR THERMAL ANALYSIS OF STEEL, DESIGNATION E14-30T, 1930

\section{THERMAL ANALYSIS OF STEEL}

1. Thermal analysis is the process of determining the temperature at which physical changes accompanied by an abrupt change in heat content occur in metals by observing the aberrations which the heat content changes impose on the rate of temperature change of a specimen, situated in an environment whose temperature is changing at a constant rate. The essential units required for thermal analysis are: (a) A furnace which may be heated and cooled at a substantially constant rate, (b) a temperature measuring device, and (c) a means for measuring time. The specific apparatus chosen depends largely on which of the several possible methods of thermal analysis is adopted with the exception that electrical resistance furnaces and potentiometric measurement of thermocouple electromotive force are in general use for the type of furnace and a temperature measuring device, respectively, and are recommended when applicable.

\section{METHODS}

2. (a) The transformation data concerning steels usually required can be obtained with either the inverse-rate or differential method of thermal analysis. With the inverse-rate method, the time intervals required for the temperature to change through successive equal increments are observed and plotted against the mean temperatures of the temperature increments. With the differential method, the temperature difference between the specimen and a neutral body, that is, one undergoing no abrupt change in heat content over the temperature range concerned, is plotted against the temperature of observation. With both methods it is permissible and much more convenient to plot electromotive force readings of a thermocouple instead of actual temperatures.

(b) Both of the methods are recommended for steel. Choice between them depends on the purposes for which they are employed. The inverserate method using the Rosenhain type of furnace is superior for the detection of transformation at low temperatures $\left(100^{\circ}\right.$ to $500^{\circ}$ C.) and for the study of effects of rate of temperature change. The differential method has the advantage of being easily adaptable to autographic recording and high sensitivity is possible with it. A machined-test specimen is required in the differential method whereas with the inverse-rate method small test specimens of irregular shape may be used.

3. Inverse-rate method.-The modified type of Rosenhain furnace is recommended for obtaining inverse-rate thermal curves on steel. Details of construction can be obtained from the literature.

A specimen in the form of a disk about one-eighth inch in thickness and three-eighths inch in diameter mounted with the hot junction of a thermocouple at its center and in firm metallic contact with it is recommended. The axis of the specimen should coincide with that of the tube in which it is mounted.

Time intervals may be measured most convenently by the double stop-watch method of Merica. For maximum precision, chronograph measurement of time intervals is required. Curves may be plotted autographically by the recording chronograph described by French.

4. Differential method. - An electric-resistance furnace having flexible control of the rate of temperature change is recommended. The thermal insula- tion and heat capacity of the furnace must be reduced to a minimum to achieve this control.

The temperature of the specimen should be measured by a thermocouple, the hot junction of which is embedded in the specimen. For the measurement of temperature differences a differential thermocouple is required. The two lead-in wires should be of the same metal and their hot terminals should be connected by a wire of different composition. One of the hot junctions should be placed in the specimen and the other in the neutral body. The electromotive force generated by temperature difference between specimen and neutral body may be measured by galvanometer deflection. Temperature differences of $0.04^{\circ} \mathrm{C}$. should be detectable.

The specimen and neutral body should be thermally and geometrically similar and symmetrically placed in the furnace. The preferred form of specimen is a cylinder. The thermocouple hot junctions need not come in metallic contact with the specimen or neutral body, but should be symmetrically placed with reference to the specimen and neutral body. Time measurements are necessary only to establish rates of temperature change for which purpose a watch or clock with second hand suffices.

\section{PRECAUTIONS}

5. The use of a high-vacuum environment (less than $0.01 \mathrm{~mm}$ mercury) for the specimen is desirable, but when not feasible it is recommended that precautions be taken to minimize decarbonization and convection currents. Thermocouples may be either of platinum-base or nickel-base alloys, and should be of moderate size $(0.020$ to 0.040 inch in diameter). The cold junctions should be kept at $0^{\circ} \mathrm{C}$.; that is, in melting ice or a suitable cold-junction correction applied.

Thermocouples should be checked frequently to insure their accuracy, particularly nickel-base thermocouples used above $900^{\circ} \mathrm{C}$. This may be done either by comparison with standardized couples or by the transformations in standard steel specimens. For the latter purpose the $A_{2}$ point of ingot iron is recommended when the inverse-rate method is used and the $A c_{1}$ point of unalloyed carbon tool steel when the differential method is used. The temperatures of these changes, taken when the rate of temperature change of the specimen is a minimum, may be taken as $768^{\circ}$ and $733^{\circ}$ C., respectively. The temperature of $A_{2}$ is practically independent of rate of temperature change, but that of $A c_{1}$ corresponds to a rate of heating of $0.10^{\circ} \mathrm{C}$. per second only.

The interpretation of thermal curves requires recognition of the fact that a peak on an inverserate curve does not represent the same stage of progress of the transformation as does one on a differential curve. The time ordinates of an inverse-rate curve are in effect the reciprocals of the slopes of the differential curve at corresponding temperatures and, therefore, the temperature of a minimum rate of change of heat content on a differential curve is that at which the curve has minimum slope with respect to the differential ordinate; that is, where its slope is most nearly perpendicular to the temperature ordinate. It is recommended that the interpretation and comparison of inverse-rate and differential curves be conducted on the basis outlined above.

In referring to temperatures of transformations, it is recommended that the stage of the transformation to which the temperature applies; for example, beginning, maximum, or end, be always stated.

\section{RECORDS}

6. It is recommended that the following data be recorded for every thermal curve taken:

(a) Source and composition of steel; 
(b) Method of thermal analysis;

(c) Size and mounting of specimen;

(d) Maximum temperature to which specimen is heated and time at that temperature;

(e) Previous treatment of specimen, including number of times thermal curves have been taken previously on the same specimen;

(f) The temperature of minimum rate of temperature change of the specimen at recognized and identified inflections, and the direction of temperature change;

(g) The estimated rate of change of temperature of the furnace at the time of minimum rate of temperature change of the specimen for each inflection observed;

(h) The temperatures at which the beginnings and ends of inflections can be definitely recognized;

(i) The maximum and minimum temperatures of decalescene and recalescene in case either phenomenon is observed;

(j) Any departures from the foregoing recommendations.

(Attached to this recommendation is a list of references in which will be found details of construction and operation of apparatus for obtaining thermal curves.)

\section{AMERICAN SOCIETY FOR TESTING MATE- RIALS, RECOMMENDED PRACTICE FOR RADIOGRAPHIC TESTING OF METAL CASTINGS, DESIGNATION E15-29, 1929}

\section{RADIOGRAPHIC TESTING}

\section{GENERA L CONSIDERATIONS}

On account of their very short wave lengths, $\mathrm{X}$ rays penetrate materials opaque to ordinary light. They are absorbed rapidly by heavy elements much as light is absorbed by fog. If $\mathrm{X}$ radiation is passed through metal that contains cracks, cavities, sand, or slag inclusions, the radiation that passes through the defects is absorbed to a less extent than that which passes through the adjacent sound metal. A photographic film placed to intercept the emergent beam will show, on development, regions of greater photographic density that may be definite images of the internal defects. Radiographic tests are concrerned with the obtaining of such photographic indications of internal defects. They may be extended to the examination of various other hidden details of gross structure.

There are at present two important fields for application of metal radiography. One of these concerns the development of manufacturing technique in the foundry, the other the final inspection of the manufactured product.

In foundry development work pilot castings are examined, defects are studied, and changes made in casting technique to correct the defects. This study leads to a developed technique that may be relied on to produce dependably sound castings, the extent of the elimination of defects depending, to a certain extent, upon the service for which the casting is intended. An important by-product of such application is the education of foundry personnel. Interior defects are present in castings partly because there has been no easy method of detecting their presence and no particular effort has been made to overcome them because of this fact. Such application to manufacturing metbods is probably the most economical use of radiographic tests. But on account of the fact that even with the most fully developed technique accidents or mistakes occur, there is still a pcssibility for defective castings.

For this reason, and also because very few castings are manufactured at present according to a carefully developed technique, it is important to have a method of testing in final inspection that will reveal internal gross defects and that will not injure the casting. Radiography seems to offer the best solution to this problem. The method is expensive, but in cases where the possible failure of a casting would cause damage far in excess of the cost of the tests, radiographic examination becomes a matter of insurance and the question to be answered is often not whether such inspection can be afforded but rather whether it can be afforded not to make it. The rapid development of high-pressure steam installations, high-head hydraulic power, high-pressure oil-cracking systems, higher-power locomotives, aerial transportation, and various other lines of industrial activity presents increasingly numerous cases where such tests may be applied and where sometimes they will be found absolutely necessary.

Standards of soundness that must be met by castings in order to pass X-ray inspection can not be set up at the present time with any degree of fairness to consumer or manufacturer. This fact is due partly to the newness of this method of testing, partly to a lack of sufficient information as to tne effects on strength or other properties of various types of defects, but mostly to the fact that each casting is a study in itself. Defects that would be injurious in some castings may not be serious in others and may be even advantageous in peculiar circumstances. It seems that castings must be regarded in this sense as structural units rather than as materials of construction. That they, in fact, have been so regarded, is illustrated by the way in which radiographic inspection tests have been handled. That is, revealed defects are usually considered with regard to known stress distribution or other conditions of service and rejections are made where the defects render the casting in question unfit for its particular service. It is probable that in the future foundries will be able to eliminate the more important casting defects. This will come about through intensive studies of casting technique. When this is accomplished the problem of final inspection will be simplified, but for many important cases will still remain a problem of individual study of individual castings.

\section{DEFECTS THAT MAY BE REVEALED BY X RAYS}

The more common defects found in castings by radiographic tests may be enumerated as follows:

1. Gas cavities:

(a) Due to gases liberated from the hot metal;

(b) Due to gases from the mold.

2. Sand inclusions.

3. Slag inclusions.

4. Pipe cavities.

5. Porosity:

(a) Due to small gas cavities;

(b) Due to small shrinkage cavities.

\section{Cracks.}

7. Metal segregations.

Most of these may be eliminated by improved manufacturing methods. Cracks, pipes, and porous sections are sometimes traceable to faulty design.

\section{RADIOGRAPHIC EQUIPMENT}

The apparatus needed for radiographic work depends to a certain extent on the nature of the materials to be investigated. For the study of steel castings whose sections do not exceed $3 \frac{11}{2}$ inches in thickness, the air-cooled Coolidge X-ray tube rated at $200 \mathrm{kv}$ and 8 milliamperes has been found satisfactory. A finer-focus tube rated at $100 \mathrm{kv}$ and 10 milliamperes is available for steel sections that do not exceed $1 \frac{1}{2}$ inches in thickness. The higherpowered tube will in general be more satisfactory. The accessory electrical equipment should include 
a high-tension transformer that will deliver 10 milliamperes at $300 \mathrm{kv}$ from the secondary, a rectifier for rectifying the output of the transformer, a milliameter for measuring the current across the tube, a standard sphere gap for determining potentials across the tube, and a remote-control stand containing the operating controls.

The X-ray tube mounted in a flexible support to facilitate manipulation is preferably contained in a lead-lined operating chamber and the control stand in a separate chamber. Intensifying screens are required for metal sections greater than three-fourths of an inch. Mounted screens are used where possible, but a supply of unmounted screens of various sizes is desirable. A well-equipped photographic dark room completes the necessary equipment for making radiographs. In addition to the $\mathrm{X}$-ray equipment, the laboratory should be supplied with a good 8 by 10 inch photographic camera and with facilities for handling heavy castings.

\section{RADIOGRAPHIC TECHNIQUE}

The procedure in the laboratory is somewhat as follows: A casting to be radiographed is given a superficial examination, and areas through which pictures are to be taken are charted. These areas are selected with a view to showing general conditions in the metal, and particular conditions in regions that, in service, are subjected to greater stresses or that for other reasons are of particular importance. The selected areas are numbered with white chalk or paint, after which the piece is photographed. The casting is now moved into the operating chamber and the X-ray tube arranged above one of the numbered areas. Lead numerals are placed over the painted ones. These show in the developed picture and serve to identify the areas and to permit exact orientation of the finished negative with the casting so that revealed defects may be charted on the surface of the metal. The special X-ray photograph film is placed in a paper holder or in a metal cassette between the intensifying screens and adjusted in as close contact with the metal as possible, to intercept the radiation that is to be passed through the selected area. Sheet lead is used to protect the film from all $\mathrm{X}$ radiation except that passing through the area under investigation. This protection is important. It is a pecularity of $\mathrm{X}$ radiation that, when it encounters matter, secondary radiation is sent out from the material and this secondary radiation, coming from points remote from the target, may fog the X-ray film. Secondary radiation is given off by the atoms of the metal under test, but this radiation, being weaker than that of the primary beam, is absorbed by the metal and does not affect the film except that the radiation from the underside of the metal adjacent to the film is not absorbed, and does produce a certain amount of fog in the negative.

In many cases, pieces to be radiographed vary in thickness of section to such an extent that a proper exposure for the thicker portions would overexpose the portion of the photographic film under the thinner part. This difficulty may be overcome with large castings by first exposing for the thin section after which the thin portion is covered with lead sheet and the exposure continued for the thicker section. This difficulty may be overcome for small objects by immersing them in a liquid whose absorbing power for $\mathrm{X}$ radiation is nearly equal to that of the metal.30 The exposure is then made through the liquid containing the pieces under investigation.

30 Suggested by Ancel St. John.
The method is excellent where it can be used Metal radiography owes very much of its success to intensifying screens. As stated above, matter exposed to $\mathrm{X}$ radiation itself begins to radiate. This secondary radiation may be partly reflected primary radiation and partly radiation of a longer wave-length characteristic of the material itself. Also, the greater the intensity of the primary radiation, the greater is the intensity of the secondary. These facts are taken advantage of in the intensifying screen. The special X-ray photographic film, sensitized on both sides, is placed between two thin sheets of material, usually calcium tungstate, that reacts strongly to $\mathrm{X}$ rays. The resultant photographic density is due partly to the direct action of the primary X-ray beam, but mostly to the secondary radiation from the calcium tungstate. On account of the very intimate contact between film and screens there is no tendency for the radiation from the screen material to fog the film, although there is some spreading of the image with resultant loss in definition.

The same effects may be obtained with metal used in the place of the calcium tungstate except that the intensifying factor is much less. The use of lead foil for intensifying screens has some advantage over calcium tungstate. While the intensifying factor is less, the lead absorbs part of the secondary radiation from the underside of the metal being investigated, which, as stated above, tends to cause fog in the negative, and in cutting down fog it increases the contrast in the negative. Lead screens also give finer-grained images and consequently sharper definition. These screens have been found particularly useful in examining castings for fine cracks. Unfortunately, their intensifying factor is too low for them to be very useful with sections above 2 inches in thickness. A combination of lead and calcium tungstate has been found advantageous for sections up to $23 / 4$ inches. In this case the lead screen is placed on the side of the film next to the metal to be investigated and the calcium tungstate on the remote side. Inasmuch as one of the present limitations in radiographing thick metal sections is the fogging of the negative by secondary radiation from the under side of the metal, it seems that metal screens may play an increasingly important part as tube power is increased and greater metal penetration obtained. Possibly the combination of screens, described above, with increase in tube power to offset the lower intensifying factor, will be found most advantageous.

\section{FLUOROSCOPIC EXAMINATION}

With present available tubes, X-ray visual examination for structural defects may be made where the thickness of section does not exceed about $11 / 2$ inches of steel, and where the contour of the metal is favorable. For this work the radiation after passing through the metal is caught on a screen that gives a visible fluorescence under the influence of $\mathrm{X}$ rays. Cavities or other defects stand out as brighter spots on the apple-green background of the fluorescing screen. The method is not so sensitive as the radiographic method, can not be used for as thick sections, does not give a permanent record, and is applicable only to metal pieces of relatively simple contours. The method is also open to the objection that it is more difficult to protect the operator from the injurious effects of the rays. There is an advantage in the method in that it is more rapid than radiography and consequently less expensive. 
ASSOCIATION OF AMERICAN STEEL MANUFACTURERS, STANDARD METHODS OF SAMPLING AND PERMISSIBLE VARIATIONS FOR CHECK ANALYSIS OF ROLLED AND FORGED STEEL PRODUCTS, 1928

\section{SAMPLING AND CHECK ANALYSIS OF ROLIED AND FORGED STEEL PRODUCTS}

(In respect to sampling, preparation of samples, and location of samples, this specification conforms to A. S. T. M. A130-30, given above. In addition, it provides that:)

\section{METHODS OF ANALYSIS}

Only. well-known accurate methods of analysis shall be employed. Carbon shall be determined by the combustion method.

\section{NOMBER OF CHECK ANALYSES}

(a) Material shall not be subject to rejection for any element or elements unless determinations have been made on the following minimum number of samples from each melt, except as provided in paragraph $(b)$ : 3 for 5 tons or less;

4 for over 5 tons to 10 tons;

5 for over 10 tons to 15 tons; and

6 for over 15 tons.

(b) If the number of pieces of a melt is less than the number of samples specified in paragraph $(a)$, one sample shall be taken from each piece.

\section{STANDARD PERMISSIBLE VARIATIONS FROM ORDERED LIMITS}

Ordinary Commercial Quality Material Without Special Discard

When check analyses are made of the material as furnished, the composition, based on the average of all the separate determinations made, may vary from that ordered to the extent permitted in the following table:

Permissible variations from ordered limits-average composition

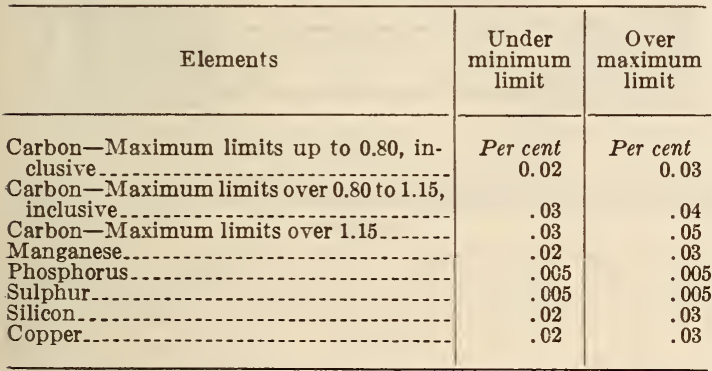

Nore.-Variations both under and over apply only when a range is specified.

\section{Forging Quality and Special Discard Material}

When check analyses are made of the material as furnished, the composition, based on the average of all the separate determinations made, may vary from that ordered to the extent permitted in the following table:
Permissible variations from ordered limits-average composition

\begin{tabular}{|c|c|c|}
\hline Elements & $\underset{\substack{\text { Under } \\
\text { limimit }}}{\text { Unimum }}$ & $\underset{\substack{\text { Over } \\
\text { limit }}}{\text { maximum }}$ \\
\hline $\begin{array}{l}\text { Carbon } \\
\text { Manganese-1 } \\
\text { Phosphorus } \\
\text { Sulphur } \\
\text { Silicon } \\
\text { Copper }\end{array}$ & $\begin{array}{c}\text { Per cent } \\
0.02 \\
.02 \\
.005 \\
.005 \\
.02 \\
.02\end{array}$ & $\begin{array}{c}\text { Percent } \\
0.02 \\
.02 \\
.005 \\
.005 \\
.03 \\
.03\end{array}$ \\
\hline
\end{tabular}

Note.-Variations both under and orer apply only when a range is specified.

The composition of individual samples may vary from that ordered to the extent permitted in the following table, except that the carbon, the manganese, and the silicon in the same melt may not vary both above and below the range ordered.

Permissible variations from ordered limits-individual samples

\begin{tabular}{|c|c|c|}
\hline Elements & $\underset{\substack{\text { Under } \\
\text { limimit }}}{\text { Unum }}$ & $\begin{array}{c}\text { Over } \\
\text { maximum } \\
\text { limit }\end{array}$ \\
\hline $\begin{array}{l}\text { Carbon-Maximum limits up to } 0.25 \text { in- } \\
\text { clusive } \\
\text { Carbon-Maximum limits over } 0.25 \text { to } 0.60 \\
\text { inclusive } \\
\text { Carbon-Maximum limits over } 0.60 \text { to } 1.15 \\
\text { inclusive } \\
\text { Carbon-Maximum limits over } 1.15 \\
\text { Manganese } \\
\text { Phosphorus } \\
\text { Sulphur } \\
\text { Silicon } \\
\text { Copper }\end{array}$ & $\begin{array}{r}\text { Per cent } \\
0.02 \\
.03 \\
.03 \\
.03 \\
.05 \\
.005 \\
.005 \\
.02 \\
.02\end{array}$ & $\begin{array}{r}\text { Per cent } \\
0.03 \\
.04\end{array}$ \\
\hline
\end{tabular}

\section{SPECIAL PERMISSIBLE VARIATIONS FROM ORDERED LIMITS}

Forging Quality and Special Discard Material Subject to Special Restrictions on Check Analyses

When check analyses are made of the material as furnished, the composition, based on the average of all the separate determinations made, shall be within the limits ordered.

The composition of individual samples may vary from that ordered to the extent permitted in the following table, except that the carbon, the manganese, and the silicon in the same melt may not vary both above and below the range ordered.

Permissible variations from ordered limits-individual samples

\begin{tabular}{|c|c|c|}
\hline Elements & $\begin{array}{c}\text { Under } \\
\text { minimum }\end{array}$ & $\begin{array}{c}\text { Over } \\
\text { maximum } \\
\text { limit }\end{array}$ \\
\hline $\begin{array}{l}\text { Carbon } \\
\text { Manganese } \\
\text { Phosphorus. } \\
\text { Sulphur } \\
\text { Silicon } \\
\text { Copper }\end{array}$ & $\begin{array}{c}\text { Per cent } \\
0.02 \\
.03 \\
.005 \\
.005 \\
.02 \\
.02\end{array}$ & $\begin{array}{c}\text { Per cent } \\
0.02 \\
.03 \\
.005 \\
.005 \\
.02 \\
.02\end{array}$ \\
\hline
\end{tabular}


NATIONAL ENGINEERING INSPECTION ASSOCIATION, TENTATIVE METHODS OF PROCEDURE FOR INSPECTION OF MATERIALS, 1930

\section{INSPECTION OF MATERIALS}

(Covers general procedure, such as checking of order, witnessing of tests, surface inspection, marking, and reports for the following: Mill, shop, and field inspection of structural steel for buildings and bridges; inspection of $T$ and girder rails, steel plate pipe, rolled or drawn steel and iron pipe, boiler and firebox steel, and cast-iron pipe.)

\section{SOCIETY OF AUTOMOTIVE ENGINEERS, IRON AND STEEL SPECIFICATIONS, TEST SPECIMENS, MARCH, 1922}

\section{TEST SPECIMENS}

1. S. A. E. steels shall be purchased on the basis of requirements as to chemical composition, and the

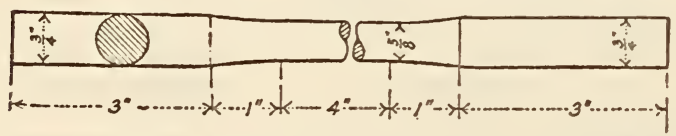

FIGURE 39. - Malleable iron tension test specimen

specifications provided herein should be used in the purchase of the grades and types of steel covered by each. When the steel user desires physical tests on forged parts, bars, or billets, the test specimens shall be selected and prepared in accordance with the following standards.

\section{CONSTRUCTION}

2. Tension and bend test specimens shall be taken from the rolled or forged material, except that in the case of irregularly shaped forgings they may be taken from a full-size prolongation. Test specimens shall not be annealed or otherwise treated, except as specified in the following paragraph. Bend tests shall be made cold.

3. Tension and bend test specimens for material that is to be annealed or otherwise treated befora use shall, for rolled material, be cut from properly annealed or similarly treated short lengths of the full section of the piece and for forged material from the treated forgings.

4. The axis of tension and bend test specimens for rolled bars and forgings of uniform cross section of over $1 \frac{11}{2}$ inches thickness of diameter and for forgings of irregular sections shall, when practicable, be located at any point midway between the center and the outer surface when solid, and at any point midway between the inner and outer surfaces of the wall when bored, and shall be parallel to the axis of the piece in the direction in which the metal is most drawn out. Round tension test specimens shall be machined to the form and dimensions shown in Figure 129, A. S. T. M. 605.0, p. 247. The gage length and fillets shall be ground or lap polished free from scratches. The ends shall be of a form to fit the holders of the testing machine in such a way that the load shall be axial. Threaded ends or self-aligning ball ends are recommended.

5. The bend-test specimens shall be one-half inch square in section and not over 6 inches in length with corners rounded to a radius of not over onesixteenth inch.

6 . The axis of tension-test specimens for rolled bars and forgings of uniform cross-section $1 \frac{1}{2}$ inches or under in thickness or diameter shall be machined concentrically from the specimen. Round tensiontest specimens may be machined to the form and dimensions shown in Figure 129, A. S. T. M. 605.0, p. 247, or may be of larger dimensions provided the length of the pull section conforms to the formula

\section{gage length, $d=4.5 \sqrt{\text { area of cross section. }}$}

The gage length and fillets shall be ground or lap polished free from scratches. The ends shall be of a form to fit the holders of the testing machine in such a way that the load shall be axial. Threaded ends or self-aligning ball ends are recommended.

7. Bend test specimens shall be one-half inch square in section and not over 6 inches in length with corners rounded to a radius of not over onesixteenth inch.

8. Round tension-test specimens for rolled bars or forgings, of dimensions too small to permit the use of the standard full-size test specimen, may be machined to the dimensions of the proportional-size round test specimens shown in Figure 40 and the accompanying table. The gage length and fillets shall be ground or lap polished free from scratches. The ends shall be of a form to fit the holders of a testing machine in such a way that the load will be axial. Threaded or self-aligning ball ends are recommended.

9. Flat tension and bend test specimens for plates and shapes shall be of the full thickness of material as rolled and tensile-test specimens shall be machined to the form and dimensions shown in Figure 128 in A. S. T. M. 605.0 , p. 247 . The gage width of test specimens one-fourth inch or less in thickness should be equal to five times the thickness, except that in no case shall the width be less than three-fourths inch. The gage length should equal trenty-four times the thickness of the test specimen, except that in no case shall it be less than 2 inches. The gage length on test specimens shall be finished on all sides smooth and free from scratches.

10. Tension-test specimens for malleable castings as specified in that specification (see 611.21, p. 666).

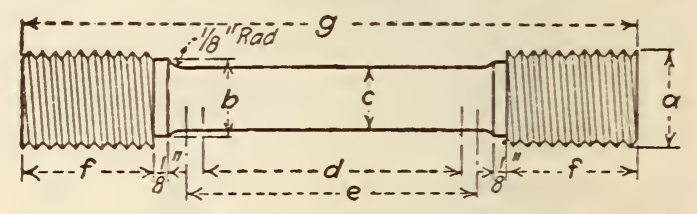

Figdre 40.-Proportional-size round tension testspecimen

\begin{tabular}{|c|c|c|c|}
\hline & \multicolumn{3}{|c|}{$\begin{array}{c}\text { Diameter of stock } \\
\text { (inches) }\end{array}$} \\
\hline & $1 / 2$ to $3 / 4$ & 36 to 36 & $1 / 4$ to $3 / 8$ \\
\hline $\begin{array}{l}\text { (a) } \\
\text { (b) } \\
\text { Game length }(d) \\
\text { (e) } \\
\text { (f) (approximately) } \\
\text { (g) (approximately) }\end{array}$ & $\begin{array}{r}\text { Inches } \\
0.500 \\
.391 \\
.375 \\
1.500 \\
1.750 \\
.750 \\
3.625\end{array}$ & $\begin{array}{r}\text { Inches } \\
0.375 \\
.281 \\
.250 \\
1.000 \\
1.250 \\
.500 \\
2.625\end{array}$ & $\begin{array}{r}\text { Inches } \\
0.250 \\
.172 \\
.125 \\
.500 \\
.750 \\
.375 \\
1.875\end{array}$ \\
\hline
\end{tabular}

Gage length, $d=4.5 \sqrt{\text { area of cross section. }}$

shall conform to the shape and dimensions shown in Figure 39 and shall not be machined.

\section{NUMBER OF TESTS}

11. Unless otherwise specified by the purchaser, one tension and one bend test shall be made from each melt; except that if material rolled from one 
melt differs three-eighths inch or more in thickness, one tension and one bend test shall be made from both the thickest and the thinnest material.

12. If any test specimen shows defective machining or develops flaws, it may be discarded; in which case the manufacturer and the purchaser or his representative shall agree upon the selection of another test specimen in its stead.

13. If the percentage of elongation of any tensiontest specimen is less than that specified and any part of the fracture is more than three-fourths inch from the center of the gage length of a 2-inch test specimen or is outside the middle third of the gage length of an 8-inch test specimen before testing, a retest shall be allowed.

\section{SOCIETY OF AUTOMOTIVE ENGINEERS,} IRON AND STEEL SPECIFICATIONS; SPECIFICATIONS FOR AUTOMOTIVE STEELS, S. A. E. STANDARD, AUGUST, 1922

\section{AUTOMOTIVE STEELS}

1. Process. - The steel shall be made by the Bessemer, openhearth, crucible, electric, or any other process approved by the purchaser.

2. Discard.-A sufficient discard shall be made from each ingot to secure freedom from injurious piping and undue segregation.

\section{CHEMICAL PROPERTIES}

3. The steel shall conform to the specified requirements as to chemical composition.

4. Ladle analysis.-An analysis of each melt of steel shall be made by the manufacturer to determine the percentages of the elements specified. This analysis shall be made from drillings taken at least one-fourth inch beneath the surface of a test ingot obtained during the pouring of the melt, and in as sound metal as possible. The chemical composition thus determined shall be reported to the purchaser or his representative, and shall conform to the requirements specified.

5. Check analysis. - Analsyes may be made by the purchaser. The chemical composition thus determined shall conform to the requirements specified.

6. Drillings for analyses of bars, billets, or other regular shapes shall be taken parallel to the axis, at any point midway between the center and the surface.

7. Drillings or cuttings for analyses of irregularly shaped pieces shall be taken from both the thickest and the thinnest sections. Surface drillings shall be discarded.

8. Wire, tubing, sheets, and rods less than $11 / 4$ inches thick shall be sampled through or across the entire section.

9. Defects.-The material shall be free from injurious defects and shall have a workmanlike finish.

\section{INSPECTION AND REJECTION}

10. Inspection.-The inspector representing the purchaser shall have free entry at all times to all parts of the manufacturer's works that concern the manufacture of the material ordered while work on the contract of the purchaser is being performed. The manufacturer shall afford the inspector free of cost all reasonable facilities to satisfy him that the material is being furnished in accordance with these specifications. Tests and inspection at the place of manufacture shall be made prior to shipment.

11. The purchaser may make the tests to govern the acceptance or rejection of the material in his own laboratory or elsewhere. Such tests, however, shall be made at the expense of the purchaser.

12. All tests and inspection shall be so conducted as not to interfere unnecessarily with the operation of the works.

13. Rejections.-Unless otherwise specified, any rejection based on tests made by the purchaser in its own laboratory shall be reported within 10 working days from the receipt of samples.

14. Material which shows injurious defects while being finished by the purchaser will be rejected, and the manufacturer shall be notified.

15. Rejection tests.- Samples tested by the purchaser in its own laboratory that represent rejected material shall be preserved for one month from the date of the test report. In case of dissatisfaction with the results of the tests, the manufacturer may make claim for a rehearing within that time.

\section{SOCIETY OF AUTOMOTIVE ENGINEERS, IRON AND STEEL SPECIFICATIONS, S. A. E. RECOMMENDED PRACTICE, 1924}

\section{HARDNESS TESTS}

\section{BRINELI HARDNESS TEST}

(The S. A. E. recommended practice for Brinell hardness testing is substantially the same as that of the American Society for Testing Materials in E-10-27, given above. Here and there in the table of hardness numbers appears a slight difference, a few tenths one way or the other, due to the omission from the S. A. E. table of all decimals.)

\section{ROCKWELL HARDNESS TESTS}

1. Principle of test.-The Rockwell tester measures hardness by determining the depth of penetration of a steel ball or diamond cone (Brale) in the material being tested, under conditions of load application. In elevating the work to testing position, the machine first applies a preliminary, or minor, load of $10 \mathrm{~kg}$ (22.046 pounds), which clamps and seats the piece being tested and allows the penetrator to break through the light scale and come in contact with the true material beneath. The direct-reading dial is set to zero penetration, directly after due application of the minor load. With the minor load applied and a zero penetration reading known, a final or major load is applied. This forces the penetrator into the metal being tested. The indication at this time on the direct-reading dial comprises not alone the depth of penetration but also the deformation of the testing-machine frame and components, and the work undergoing test. The true Rockwell hardness is read directly from the dial, after removing the major load. This automatically reinstates the $10 \mathrm{~kg}$ (22.046 pounds) minor load and removes the deformation of the machine and part under test. The result is the permanent deformation of the tested metal due to the penetrator and its major load. The Rockwell hardness readings are based on the depth to which the major load forces the penetrator below the point it was forced previously by the minor load.

2. Preparation of surfaces.-Concordant results are dependent on surface roughness being much less than the size of the impression. Surfaces that are ridged perceptibly to the eye by rough grinding or machining offer unequal resistance to the penetrator. The degree of surface preparation then, depends to some extent on the requirements of testing, whether they be production or research.

3. Thickness of specimens. - Practically any thickness in excess of 0.020 inch can be tested for comparative hardness. For true hardness, the piece must be of such thickness that the undersurface of 
the specimen, after testing, does not show a point of compression. The minimum possible thickness of any specimen varies according to the hardness, the load applied, and the kind of test point or penetrator used. The hardest steels give true hardness readings if over 0.027 inch in thickness.

4. Curved surfaces. - The true hardness of parts having curved surfaces can be found if the radius of curvature of the surface at the point tested is threesixteenths inch or more. If the radius is less than three-sixteenths inch only comparative results can be had. Data for hardness tests on a highly curved surface should be accompanied by a statement of the radius of curvature. In testing small rounds the effect of curvature can be eliminated by making a small flat spot on the specimen.

5. Rockwell scales.- Since 2 loads and 2 penetrators are used, 2 scales of hardness are placed on the dial of the machine. The black divisions and the letter $C$ apply when the $120^{\circ}$ diamond-cone penetrator or Brale and the $150-\mathrm{kg}$ (330.693-pound) load are employed. The red divisions and the letter $B$ apply when the $100-\mathrm{kg}$ (220.462-pound) load and the one-sixteenth inch steel-ball penetrator are employed. All data should be prefixed by a letter showing whether the values are on the $C$ or the $B$ scale. Other loads and penetrators can be used, but only comparative results can be obtained. In recording such results all factors relating to them should be clearly stated-the load; the penetrator; the scale used; the thickness of the piece, if very thin; and the curvature of the surface.

6. Penetrators and loads. - The Rockwell scale being based on depth measurements, the Brale penetrator gives the more accurate readings on hard metals and the steel ball on soft metals. Hard metals require the $150-\mathrm{kg}$ (330.693-pound) load. The diamond-cone penetrator of $120^{\circ}$ angle with the point ground spherically to a definite radius is the standard Rockwell diamond cone or Brale.

7. If the readings are below $\mathrm{C}-20$, it is preferable to test the material with the $100-\mathrm{kg}$ (220.462-pound) load and the one-sixteenth inch steel ball (replaceable) and use the $B$ scale. Readings on the $B$ scale are not generally taken higher than B-100, when it is advisable to change to the $C$ scale with the 150$\mathrm{kg}$ (330.693-pound) load and the Brale penetrator.

8. Measuring hardness greater than $\mathrm{B}-100$ is apt to deform the one-sixteenth inch steel ball, thus destroying its usefulness for testing softer materials.

9. On truly homogeneous material sublow readings are due to burrs on the undersurface of the material tested, causing a movement of the material on its chuck during application of the major load. Assuming that the machine checks correctly on its test blocks, too high readings can be secured only by testing on a spot previously tested, by testing too thin sections or by the penetrator being supported by two ridges caused by poor surface preparation, the last-named being a minor consideration in production testing.

10. In testing tubing or any shape that will deform under test, a blunt nose should be used in place of the penetrator, and the test conducted in the regular way to determine if the specimen takes a permanent set, that would invalidate the hardness test and necessitate consideration of another manner of chucking.

11. Homogeneity of metals.-The Rockwell tester measures the hardness of the specimen at the point of penetration, but the reading is also influenced by the hardness of the material under the impression. For example, in testing the hardness of carbon steels, when using the Brale penetrator and the $150-\mathrm{kg}$ (330.693-pound) load, the actual depth of penetration is akout 0.0027 inch while the effects of pene- tration extend to about 0.027 inch, and if any softer layer is located within 0.027 inch of the impression, the impression will be deeper and the apparent hardness less. Therefore, due regard for this condition must be given when testing material with a superficial hardness, such as cyanided or nitrogenized surfaces.

12. When testing bar stock and forgings, it will be found advisable to remove enough of the surface metal by machining or grinding that the penetrator will test the true metal underneath and the hardness reading will not be affected by the heary scale and decarburization usually present.

13. Materials, such as cast iron with graphite particles and some nonferrous materials whose crystalline aggregates are greater than the area of the penetrator, must be tested, if possible, with a penetrator of sufficient size to overcome local or grain hardness in order to secure mass hardness.

14. Conversion to other methods of hardness testing.-Care should be used in converting readings from one method of hardness testing to another. The factors that cause erroneous conversions are: (a) Lack of true homogeneity in metals, (b) different depths from the surface at which various hardness tests operate, $(c)$ different physical characteristics of varous testing devices and the properties measured, and $(d)$ different properties that are assumed by different metals under cold working.

15. No one conversion table is practical for close conversion on all metals. Tables and formulas have been compiled for some materials that can be used as guides. When conversion is necessary, it is advisable to make such conversion for each particular type of metal and each condition of heat treatment or mechanical work.

\section{SHORE HARDNESS TEST}

\section{General Information}

1. The Shore hardness number is the reading obtained on an arbitrary scale ranging from 0 to 120 , by the rebound of a small diamond-pointed hammer dropped from a fixed height. Two types of instrument are in common use; one in which the rebound is read directly on a vertical scale, and the other on which the reading is registered by the instrument on a recording dial. It is suggested that, unless special means are used to compensate for the variations in mass, form, surface, composition, and physical condition of different specimens being tested, the user determine for himself the characteristic readings obtained on his particular product.

(The S. A. E. handbook for 1930 contains a number of charts on which are shown the scleroscope values obtained by the Shore tests.)

\section{UNITED STATES GOVERNMENT, DEPART- MENT OF COMMERCE, BUREAU OF STANDARDS, STANDARD SAMPLES, C25 AND SUPPLEMENT, 1927}

\section{STANDARD SAMPLES OF STEELS}

\section{ACID OPEN-HEARTH STEELS}

(Standard samples No. 19c of $0.2 \mathrm{C}$, No. 20c of $0.4 \mathrm{C}$, No. $21 \mathrm{c}$ of $0.6 \mathrm{C}$, No. $34 \mathrm{a}$ of $0.8 \mathrm{C}$, and No. $35 \mathrm{a}$ of $1.0 \mathrm{C}$ are prepared and sold by the Bureau of Standards.)

\section{BASIC OPEN-HEARTH STEELS}

(Standard samples No. 15b of $0.1 \mathrm{C}$, No. $11 \mathrm{~d}$ of $0.2 \mathrm{C}$, No. $12 \mathrm{~d}$ of $0.4 \mathrm{C}$, No. $13 \mathrm{c}$ of $0.6 \mathrm{C}$, No. $14 \mathrm{~b}$ of $0.8 \mathrm{C}$, and No. $16 \mathrm{~b}$ of $1.0 \mathrm{C}$ are prepared and sold by the Bureau of Standards.) 
BESSEMER STEEIS

(Standard samples No. $8 \mathrm{~d}$ of $0.1 \mathrm{C}$, No. $9 \mathrm{c}$ of $0.2 \mathrm{C}$, No. $10 \mathrm{~d}$ of $0.4 \mathrm{C}$, and No. $22 \mathrm{~b}$ of $0.6 \mathrm{C}$ are prepared and sold by the Bureau of Standards.)

\section{ELECTRIC STEELS}

(Standard samples No. 51 of $1.2 \mathrm{C}$ and No. $65 \mathrm{a}$ of acid electric steel are prepared and sold by the Bureau of Standards.)

(The above samples are sold to and used by industrial organizations and others for checking analyses of similar products. Each sample is accompanied by a certificate giving its analysis as regards the important constituents.)

\subsection{CLASSIFICATION OF METALS.}

\section{AMERICAN ELECTRIC RAILWAY ENGI-} NEERING ASSOCIATION, RECOMMENDED CLASSIFICATION OF SCRAP MATERIALS, PS100-29, 1929

\section{CLASSIFICATION OF SCRAP MATERIALS}

In order that a uniform selling method may be resorted to, the following conditions should be embodied in the bid or acknowledgment:

All ferrous scrap should be sold on a net-ton (2,000-pound) basis f. o. b. cars point of shipment, consignee to accept weights as invoiced which are based on railroad scale weights at shipping point. The question of cars being light weighed before loading and the cost of light weighing should be left to the judgment of each road.

Nonferrous scrap if shipped in carload lots should be sold f. o. b. cars point of shipment. If taken away by trucks it should be sold f. o. b. seller's plant.

Inspection of all scrap materials is to be made by bidder prior to making bid. It is understood that bid is made on materials as classified by seller and constitutes acceptance by purchaser. All bids should be opened at a specified date and time of day. No verbal bids should be accepted and no attention should be paid to bids unless they are addressed in the desired way.

To do away with the possibility of opening bids before the specified time it is suggested that special envelopes be provided with the bids.

Upon notification of award, purchaser is to send shipping instructions accompanied by certified check for the approximate amount of the purchase to the official designated.

If the sale involves a small amount it should be sold on "cash on delivery" basis.

Adjustment between the actual deposit and the actual amount shipped on each contract should be adjusted with the last billing.

In case of embargoes purchaser should arrange for permits for movement of cars.

If shipping instructions are not received by specified time the sale may be canceled at the discretion of the seller.

\section{CLASSIFICATION OF FERROUS SCRAP}

1. Axles-Steel car axles.-May include armature shafts.

2. Brake shoes.-To include steel-back brake shoes. Not to include wheel-trueing shoes.

3. Cast iron No. 1.-To include pieces weighing more than 3 pounds but not over 150 pounds. Castiron parts of cars, automobiles, machinery, and miscellaneous castings; clean soil and water pipes. Must be cupola size, not over 24 by 30 inches in dimensions.
4. Cast iron No. 2.-Pieces weighing over 150 pounds. Otherwise same as No. 1 cast iron.

5. Cast iron No. 4, burnt.-To include grate bars, stoker castings, sand drying and other stove parts, rheostat grids, and also any other cast-iron pieces less than 3 pounds in weight.

6. Galvanized scrap.-To include all kinds of galvanized castings, pipe, wire, shapes, irrespective of size or weight.

7. Malleable iron No. 1.-All sizes. To include malleable parts of automobiles, cars, and miscellaneous track, and other malleable castings including malleable pipe fittings, reasonably clean. To be free from cast iron, steel, or galvanized pieces.

8. Melting steel, No. 1, heavy.-Steel scrap onefourth inch and over in thickness, not over 18 inches in width and not over 5 feet long. No pieces to weigh less than 5 pounds. To include structural shapes, bars, rods and plates, angle bars, rail joints, rail braces, tie plates, short pieces of rail, couplers, drawbars, forgings, steel castings, heavy chain, steel gears and pinions, leaf and coil springs (all coil springs to be three-eighths inch or larger diameter), carbon tool steel, also cut boiler plates if practically clean and free from attachments. Individual pieces must be cut into such shape that they will lie reasonably flat in a charging box. All to be free from dirt, excessive rust, scale, or foreign material.

9. Melting steel, No. 2.-Plate scrap, such as car sides, automobile frame stock, tank scrap, steel parts from scrapped automobiles, including automobile springs (cut apart), bars, rods, shafts, links, brake drums and bands, wheel rims and wheel disks, etc., one-eighth inch and over in thickness. Maximum size 15 inches wide by 3 feet long excepting car sides and all light plates to be 15 by 15 inches or under and all light rods to be 12 inches and under in length. Any curved or twisted pieces to be sheared into such shape that they will lie flat in a charging box. All to be free from cast or malleable iron attachments.

10. Pipe.-To include all kinds of iron and steel pipe, tubing, conduit, trolley poles. To be free from galvanized material.

11. Rail, steel girder.-To include girder and guard rail 9 inches and under in height; also slot rail and contact rail from underground-conduit track construction. To contain no manganese steel scrap, cast welds, or attachments. Free from excessive rust, dirt, or concrete.

12. Rail, steel T.-Standard section, 40 pounds and heavier per yard, not less than 36 inches long, to be free from frog and switch rails not cut apart. To contain no manganese steel scrap, cast welds, or attachments and to be free from excessive rust, dirt, or concrete.

13. Special track work, broken.-Broken or cut to charging-box size. To include pieces not over 18 inches in width and not over 5 feet long. To contain no manganese steel scrap, cast welds, babbitt metal, or spelter and to be free from excessive dirt or concrete. (May be included as heavy melting steel by mutual agreement between buyer and seller.)

14. Special track work unbroken.-To include switches, frogs, and mates not cut apart. To be free from excessive dirt or concrete.

15. Tool steel.-To include all high-carbon-content tool steel irrespective of size.

16. Turnings.-Mixed steel or iron. To include drillings, borings, cuttings, turnings. To be free from excessive corroded material, oil, or foreign material.

17. Wheels, steel.-Solid rolled, forged, or caststeel wheels 36 inches and under in diameter. Steel tires. 
18. Wheels, cast-iron.-To include solid and spoked car wheels.

19. Wrought No. 1.-Wrought iron and soft steel over 6 inches in length. Flats to be one-fourth inch and over in thickness, rounds to be threeeighths inch and over diameter. To include bars, bolts, chain, tie rods, and similar material. To contain no riveted material and to be free from excessive rust or dirt.

20. Wrought No. 2.-Wrought iron and soft steel under 6 inches in length, any thickness. To include bolts, pins, rivets, nuts, washers, spikes, and similar material.

21. Miscellaneous mixed iron and steel.-To include sheets, car shapes, or any ferrous scrap unsorted, irrespective of size, shape, or kind, which can not be considered in any of the accepted ferrous scrap classifications. To be free from galvanized material.

\section{CLASSIFICATION OF NONFERROUS SCRAP}

1. Heavy copper.-To imclude copper not less than one-sixteenth-inch thick, including field-coil wire, armature wire, bus bars, and copper clippings untinned. All to be free from insulation.

2. Light copper.-Less than one-sixteenth-inch in thickness. To include hair wire or any copper wire free from insulation, or sheets which are too light for heavy classification.

3. Copper trolley wire. -0000 and less in size, to be cut into suitable lengths as desired.

4. Copper segments and fingers. - Free from attachments. (This may be included in heavy copper.)

5. Copper bonds. - (This may be included in heavy copper.)

6. Copper wire, insulated.-To consist of armature wire, field wire, and other kinds of copper wire and cable from which the insulation has not been burned off.

7. Brittle copper.-To consist of miscellaneous copper wire which became brittle in burning off the insulation.

8. Copper borings and turnings.-To be free from all ferrous contents or babbitt.

9. Bronze journal bearings. - To be free from babbitt or lead.

10. Bronze armature and axle bearings.-To be free from babbitt or lead.

11. Trolley wheels. - To be free from bushings or screws.

12. Heavy red brass or bronze.-To include onesixteenth-inch and over in thickness.

13. Heavy yellow brass. - To include one-sixteenth inch and over in thickness.

14. Light brass. - To include red and yellow brass which is too light for heavy classification.

15. Brass or bronze borings and turnings.-To include red and yellow brass or bronze.

16. Aluminum.-To include aluminum sheet wire, castings, and pipe.

17. Monel metal.-To include sheet, clippings, and cast bars or rods free from iron.

18. Babbitt dross, tin base.-When the volume warrants it, such accumulation should be sold on the basis of random chemical analysis for metallic contents.

19. Babbitt dross, lead base.-When the volume warrants it, such accumulation should be sold on the basis of random chemical analysis for metallic contents.

20. Heavy lead.-To include sheet, pipe, etc. May be put in pig shape.

21. Battery lead.-To include dry battery plates, etc. To be free from wood, paper, and rubber.

22. Zinc.-To include sheet and castings.

\section{AMERICAN NEERING ELECTRIC RAILWAY ENGI- ASSOCIATION, CLASSIFICA TION OF MATERIALS AND SUPPLIES FOR ELECTRIC RAILWAYS, PS200-26, 1926}

\section{CLASSIFICATION OF MATERIALS AND SUPPLIES}

This classification contemplates uniformity in the physical grouping of stock for more economical operation and provides a basis for statistics for control of investment and simplified accounting.

All requisitions for the purchase or delivery of material should show the class number. Separate requisitions are required for material in each class to facilitate shipment and accounting.

NotE.-Fach class shall embrace all parts and the appurtenances of the articles described therein unless they are otherwise specifically provided for.

\section{(A) WAY AND STRUCTJRES}

3. Rails.-(a) Straight line, (b), special trackwork, $(c)$, relaying, $(d)$, third rail.

4. Rail fastenings.-Includes splice bars, continuous joints, compromise joints, welded joints, braces, shims, tie plates, tie rods, tie plugs, track bolts, track spikes, boat spikes, clips, plate washers, etc.

5. Special track work.-Includes steam and street railroad crossings, crossovers, curves, switches, mates, tongues, frogs, run-offs, track switch boxes, electric switch boxes, etc., and their repair parts.

6. Track tools, power and hand.-Includes all kinds of track bars, adzes, mauls, tool handles, chisels, shovels, picks, electric track-drills, track wrenches, spike drivers, tamping machines, air compressors, welding outfits, and their repair parts, etc.

7. Building and paving materials. -Includes castiron pipe and fittings, gutter and down spouts, etc.

11. Castings and forgings, rough and finished, not otherwise specified, for way and structures.-Includes manhole covers, sewer drain boxes, escalator and turnstile parts, etc.

12. Foundry supplies.-Includes pig iron, crucibles, etc.

\section{(C) EQUIPMENT}

27. Car wheels.-All kinds.

28. Car axles.-All kinds.

29. Brake shoes.-All kinds.

30. Drawheads, automatic couplers, and journal boxes complete.-All kinds, complete units only. (Repair parts under castings and forgings.)

32. Iron and steel bars, sheets, and shapes.-Includes spring steel, tool steel, shaped steel, sheet iron and steel, chain, channels, I beams, etc.

33. Pipe, conduits, and tubing.-Iron and steel only, including trolley poles. (Copper and other metal tubing under class 34 .)

34. Metals. - Includes brass, bronze, and copper in ingots, bars, sheets, tubes, etc.; Babbitt metals, lead, spelter, zinc, solder, aluminum, thermit, German silver, welding metals, etc.

35. Castings, brass and other nonferrous metals.Includes rough and finished castings (rough castings which finish in a given class to appear there and not hereunder).

36. Castings, malleable iron.-Includes rough and finished castings (rough castings which finish in a given class to appear there and not hereunder).

37. Castings, gray iron and steel.-Includes rough and finished castings (rough castings which finish in a given class to appear there and not hereunder).

38. Forgings. - Includes forged side frames, brake levers, turnbuckles, and all forgings not otherwise specified. 
39. S'prings.-Includes all elliptical, helical, coil, body, truck, motor suspension, and other springs not otherwise specified. (Springs peculiar to air brakes, controllers, motors, or other equipment will be classed with that equipment.)

40. Bolts, nuts, nails, etc.-Includes flat washers, lock washers, rivets, pins, studs, lag screws, common nails, etc. (Flat-head nails, brads, cotters, tinners' rivets, and other package goods and screws under hardware, class 65. .)

41. Pipe and conduit fittings.-All kinds for steam, air, water, etc., including condulets, valves, and cocks for same, etc. (Cast-iron sewer pipe and fittings under class 7.)

42. Gears, pinions, and gear cases for railyay-type motors.-(Repair parts under class 45.)

43. Bearings.-For railway-type motors, armature, axle, and truck journal bearings, machined and finished, as well as rough castings to be used for these purposes.

\section{(D) GENERAL}

65. Hardware and tinware- - Includes screws, cotters, brads, stove bolts, buckets (water, coal, and sand-whether of tin, iron, or fiber), wire cloth, wire netting, wire fencing, barbed wire, metal drinking cups, oil cans, sprinkling cans, lanterns, torches, gasoline furnaces, fire extinguishers, sprocket chains, weather strip, stepladders, steel-wire rope, etc.

66. Tools, all kinds, except machine and track tools.- Includes hammers, wrenches (except only track wrenches), wire cutters, bolt cutters, pliers, soldering irons, files, saws, hatchets, axes, chisels, screw drivers, bits, glass cutters, ladles, knives, blacksmith tools, twist drills, emery and buffing wheels, wheel dressers, stocks and dies, jacks and parts, putty knives, scrapers, etc.

71. Scrap, nonferrous metals.-Includes brass, copper, Babbitt, tin, Gernian silver, lead, zinc, lead ashes, etc.

72. Scrap, ferrous metals.-Includes iron and steel scrap of all kinds, scrap rails, scrap wheels, scrap axles, etc.

(Other classification numbers, omitted here, include materials which have no place under ores, metals, and manufactures (except machinery and vehicles).)

\section{AMERICAN RAILWAY ASSOCIATION, PUR. CHASES AND STORES DIVISION, STAND. ARD MATERIAL CLASEIFICATION, 1922}

\section{MATERIAI CLASSIFICATION}

The object of this classification is to secure uniformity in all departments in the ordering of and accounting for materials, also in the preparation of inventories and the rendering of reports of material received, disbursed, and on hand.

1-A. Frogs, switches, and crossings, and parts of same.

1-B. Track fastenings, track bolts, spikes, etc.

1-C. Track and roadway tools, all kinds, miscellaneous track material and wire fencing.

6. Iron bridges, turntables, and structural steel, all kinds.

8-A. Rail, new.

8-B. Rail, secondhand, except scrap.

11. Bolts, nuts, washers, rivets, lag screws, pins, and studs.

12. Springs, helical and elliptical, all kinds, for locomotives and cars.

14. Brass, copper, and steel tubing; copper ferrules; soft metals in bars, pigs, or sheets.

15. Bar iron and steel, spring steel, tool steel, unfabricated rolled shapes, wire netting and cnain, except light coil.
16. Boiler, fire box, tank and sheet iron and steel, all kinds.

17. Forgings and pressed steel parts for locomotives, such as crank pins, piston rods, quadrants and levers, motion links, valve yokes, pressed steel grate side bars, front ends, cylinder and steam chest casing, etc.

18. Car forgings, iron and steel, and fabricated or shaped steel, for passenger and freight cars.

19. Locomotive and miscellaneous shop castings, gray iron, malleable and steel. Including cylinders and wheel centers, sand dryers, roundhouse stoves, etc., rough and finished.

20. Car castings, including gray iron, malleable and steel, all kinds, brake beams and parts, couplers and parts, metal bolsters, side frames, and metal car roofs.

21. Rough and finished brass castings and journal bearings.

29. Wheels, cast iron, steel tired and rolled steel, tires and axles, for locomotives and cars

42. Pipe, iron and steel, and fittings, all kinds, for steam, air and water, valves and cocks for same.

45-A. Hardware, all kinds, including nails.

45-B. Hand and small machine tools, such as drills, taps, reamers, dies, chssers, including air tools and parts.

50. Scrap, all kinds, including scrap rail.

(Other classification numbers, omitted here, include materials which have no place under ores, metals, and manufactures (except machinery and vehicles).)

\section{AMERICAN RAILWAY ASSOCIATION, PUR. CHASES AND STORES DIVISION, CLAS- SIFICATION OF SCRAP IRON AND STREL, 1927}

\section{CLASSIFICATION OF SCRAP IRON AND STEEI}

1. Arch bars, iron.-Railroad arch, transom and tie bars, and coupler yokes.

2. Axles, steel.- - Solid car and locomotive, $53 / 4$ inches diameter and over at center. (Free of axles with keyways between wheel seats.)

3. Axles, steel.-Solid car and locomotive under $5 \% \frac{1}{4}$ inches diameter at center.

4. Axles, steel.-Hollow bored.

5. Axles, iron.-Railroad car and locomotive axles, A. R. A. and M. C. B. sizes. Free from defective or imperfect forgings.

6. Angle and splice bars.-Fish plates and patented joints, iron or steel.

7. Boilers, fire boxes and tanks, uncut.-All kinds, attached or separate. Specify whether with or without flues.

8. Boilers, fire boxes, and tanks, cut up.--Iron or steel boiler or tank plate cut into sheets and rings (with or without stay bolts).

9. Bolsters and side frames.-Cast steel.

10. Busheling, No. 1.-Clean iron and soft steel pipes and flues, tank and bands No. 12 gage and heavier, steel-plate punchings and clippings, soft steel and iron forgings and flashings. No dimensions over 8 inches. To be free from burnt material, hard steel, cast, malleable and metal-coated material of any kind.

11. Busheling, No. 2.-Netting, sheet and similar light material lighter than No. 12 gage. No dimension over 8 inches. To be free from hard steel, cast and metal-coated material of any kind.

12. Cast, No. 1.-Cast iron scrap, such as columns, pipes, plates, and castings of miscellaneous nature, but free from stove plate, brake shoes, and burnt scrap. Must be cupola size, not over 24 by 30 inches in dimensions, and no piece ts weigh over 150 pounds. Must be free from foreign material. 
13. Cast, No. 2.-Pieces weighing over 150 pounds, but not more than 500 pounds. Free from burnt cast.

14. Cast, No. 3.-Pieces weighing over 500 pounds; includes cylinders, driving wheel centers, and all other castings.

15. Cast, No. 4.-Burnt cast iron scrap, such as grate bars, stove parts, and any miscellaneous burnt scrap.

16. Cast-iron brake shoes.-Driving and car brake shoes of all types except composition filled shoes.

17. Couplers and knuckles.-Railroad car and locomotive steel couplers, knuckles and locks stripped clean of all other attachments.

18. Frogs and switches, uncut.-Steel and iron frogs and switches that have not been cut apart, exclusive of manganese.

19. Flues, wrought-iron and steel.-Two inches and $2 \frac{1}{4}$ inches in diameter. 8 feet long and over, rattled.

20. Flues, wrought-iron and steel.-Five inches and over in diameter 8 feet long and over, rattled.

21. Flues, tubes, and pipe-Wrought-iron and soft steel. Must be free from dirt, excessive corrosion or lime, and riveted seams. Fitting attached permitted.

22. Limed iron and steel.-All kinds of material from interior of boilers (except flues), which are encrusted with lime, such as crown bars, crown bar bolts, stay bolts, etc.

23. Malleable.-Malleable parts of automobiles, railroad cars, locomotives, and miscellaneous malleable castings. Must be free from steel and cast iron parts.

24. Melting steel, No. 1.- Steel scrap one-fourth inch and over in thickness, not over 18 inches in width, and not over 5 feet Jons. Individual pieces must be cut into such shape that they will be free from attachments and will lie reasonably straight in a charging box. Cut boiler plate must be practically cleaned of lime, free from stay bolts and not over 3 feet long. Smaller dimensions of scrap may be furnished upon mutual agreement between buver and seller. May include structural shapes, plates, rods and bars one-half inch and heavier, stee] castings, heavy chain, carbon tool steel, heavy forgings, forged butts, and similar heavy material. This grade may also include new mashed pipe ends (original diameter 4 inches and over, thoroughly flattened), sheet bars, billets, blooms, rail bends, railroad steel and wrought scrap such as angle and splice bars, couplers, knuckles, drawbars, cut cast-steel bolsters, and coil and leaf springs (all coil springs to be three-eighths inch or larger diameter). No needle or skeleton plate scrap agricultural shapes, annealing pots, boiler tubes, grate bars, cast iron, malleable iron, or curly or unwieldy pieces will be accepted. Must be free from dirt, excessive rust or scale, or foreign material of any kind.

25. Melting steel, No. 2.-Plate scrap, such as car sides one-eighth inch to one-fourth inch in thickness. Punchings one-fourth inch and over in thickness, heavy clippings, new unmashed pipe ends under 4 inches in diameter, and similar material. Car sides and all light plate to be sheared 15 by 30 inches or under and all light rods to be 12 inches and under in length. Any curved or twisted pieces must be sheared into such shape that they will lie reasonably flat in a charging box and not tangle in handling with a magnet; all to be free from cast iron, malleable iron, burnt scrap, dirt, or foreign material of any kind. Maximum size 15 inches wide by 3 feet long.

26. Rail, iron.-Standard section $T$ rails, original weight 40 pounds per yard or heavier; free from frog, guard, and switch rails.

27. Rail, sieel No. 1.- Standard section T rails, original weight 50 pounds per yard or heavier, 5 feet long and over. Suitable for rerolling. Free from bent and twisted rails; frog, switch, and guard rails, or rails with split heads and broken flanges.

28. Rail, steel No. 2.-Cropped rail ends under 3 feet long, 50 pounds and over standard section.

29. Rail, steel No. 3.-Standard section $T$ and guard rails, not less than 36 inches long, to be free from frog and switch rails not cut apart, and to contain no manganese, cast welds, or attachments of any kind except angle bars. Free from concrete, dirt, or foreign material of any kind:

30. Sheet scrap No. 1.-Under one-fourth inch thick, consisting of cut stacks and stack netting hoops, band iron and steel, pressed steel, hand-car wheels, scoops and shovels (free of wood), and wire rope. Must be free from burnt or metal-coated material, cushion and other similar springs, and lime-encrusted pipe and flues from boilers.

31. Sheet scrap No. 2.-Includes netting (other than stack wire), galvanized or tinned material, composition brake shoes, gas retorts, and any other iron or steel material not otherwise classified.

32. Steel, high-speed.-Specify kind in offering.

33. Steel, manganese.-All kinds of manganese, rails, guard rails, frogs, and switch points, cut or uncut.

34. Steel, spring-Coil and elliptic, made of material not less than three-eighths inch diameter or one-fourth inch thickness, not over 18 inches wide. May be assembled or apart.

35. Structural wrought iron and steel, uncut.All steel or steel mixed with iron from bridges, structures, and equipment that has not been cut apart. May include uncut bolsters, brake beams, steel trucks, underframes, channel bars, steel bridge plates, frog and crossing plates, and other steel of similar character.

36. Tires, No. 1.-All locomotive or car tires 36 inches and over inside diameter, smooth inside, not grooved for retaining rings or lipped. Cut or uncut.

37. Tires, No. 2.-All tires not included in class 36 , cut or uncut.

38. Turnings, No. 1.- Heavy turnings from wrought-iron and steel railroad axles or heavy forgings and rail chips, to weigh not less than 75 pounds per cubic foot. Free from dirt or other foreign material of any kind. Alloy steel scrap may be excluded from these specifications by mutual agreement between buyer and seller.

39. Turnings, drillings, and borings No. 2.-Cast, wrought, steel, and malleable-iron borings, turnings, and drillings mixed with other metals.

40. Wheels, No. 1.-Cast-iron car and locomotive wheels.

41. Wheels, No. 2.-Includes all kinds of built-up or steel-tired wheels 36 inches and under. (Specify kind in offering.)

42. Wheels, No. 3.- Solid cast steel, forged, pressed or rolled steel car and locomotive wheels, not over 36 inches diameter. (Specify kind in offering.)

43. Wrought iron and soft steel, No. 1.-Heavy wrought iron and soft steel from railroad shops and cars, 6 inches and longer, including iron links and pins. Flats one-fourth inch thick and over; rounds and squares three-eighths inch thick and over. Free from any riveted materials.

44. Wrought iron and soft steel No. 2.-Wroughtiron and soft-steel scrap from railroad shops and cars, under 6 inches long. May include tie plates, track bolts, spikes, and nuts. All to be free from steel shapes and plates.

45. Destroyed steel cars and locomotive tenders.Includes underframes and bodies of steel cars cut apart sufficiently to load; excludes trucks and caststeel underframes. (Specify kind in offering.)

46. Aluminum. 
47. Brass No. 1.-Locomotive bearing metals, such as driving crown and rod brass. Free from white metals; excluding car and tender bearings.

48. Brass No. 2.- Steam-metal brass, including valves and fittings, injector and lubricating bodies and parts, and check valves.

49. Brass No. 3.- Journal bearings free from babbitt.

50. Brass No. 4.-Brass or bronze borings, drillings, and turnings.

51. Brass No. 5.-Yellow-brass castings, including coach trimmings, light brass, hose couplings, pipe, tubes, etc.

52. Copper, cable, insulated.-Specify kind.

53. Copper No. 1.-Wire, free from insulation; flue ferrules, pipe, and tubes.

54. Copper No. 2.-Sheet, sheathing, and roofing, free from paint and nails.

55. Copper No. 3.- Sheathing and roofing with paint and nails.

56. Copper No. 4.-Battery.

57. Copper No. 5.-Dross and oxide. (Report separately.)

58. Lead.-Sheet, pipe, etc.

59. Lead.-Battery.

60. Lead.-Battery mud or sediment. (Specify wet or dry.)

61. White metal No. 1.- Including various mixtures of clean bearing or lining metals, such as babbitt, metallic packing, etc.

62. White metal No. 2.-All nonbearing white metals, such as lavatory basins, exclusive of aluminum.

63. Zinc, battery or sheet.-(Specify kind.)

Classifications as outlined above are for general use. Not necessary that all items specified in a particular classification be included. If, by separating, a higher price may be obtained, it is recommended that sale be made under the classification specifying as consisting of single item.

NATIONAL ASSOCIATION OF WASTE MATERIAL DEALERS (INC.), STANDARD CLASSIFICATION FOR OLD METALS, CIKCULAR L, 1930

\section{CLASSIFICATION OF OLD METALS}

1. Delivery.-(a) Delivery of more or less on the specified quantity up to $11 / 4$ per cent is permissible. (b) If the term "about" is used, it is understood that 5 per cent more or less of the quantity may be delivered.

Upon request of the shipper, rejections shall be returnable to the seller on domestic shipments within one week and on foreign shipments within 30 days from the time notice of rejection is received by them and upon payment by them of 1 cent a pound on material rejected to cover cost of sorting and packing; the seller to be responsible for freight both ways.

(Additional requirements under "delivery" include damages for failure to make deliveries as specified, arbitration of claims, definitions of carload and ton, shipment for export, and adjustments of claims under differences in weight.)

2. No. 1 copper wire.-To consist of clean untinned copper wire not smaller than No. 16 B. \& S. wire gage to be free from burnt copper wire which is brittle and all foreign substances.

3. No. 2 copper wire.-To consist of miscellaneous clean copper wire which may contain a percentage of tinned wire and soldered ends, but to be free of hair wire and burnt wire which is brittle; the tinned wire not to be over 15 per cent of the total weight.

4. No. 1 heavy copper. - This shall consist of tinned copper not less than one-sixteenth inch thick, and may include trolley wire, heavy field wire, heavy armature wire, that is not tangled, and also new, untinned, and clean copper clippings and punchings, and copper segments that are clean.

5. Mixed heavy copper.-May consist of tinned and untinned copper, consisting of copper clippings, clean copper pipe and tubing, and copper wire free of hair wire and burnt and brittle wire; free from nickel-plated material.

6. Light copper.-May consist of the bottoms of kettles and boilers, bathtub linings, hair wire, burnt copper wire which is brittle, roofing copper, and similar copper; free from radiators, brass, lead and solder connections, readily removable iron, old electrotype shells, and free of excessive paint, tar, and scale.

7. Composition or red brass.-May consist of red scrap brass, valves, machinery bearings and other parts of machinery, including miscellaneous castings made of copper, tin, zinc and/or lead, no piece to measure more than 12 inches over any one part or to weigh over 60 pounds; to be free of railroad boxes, and other similarly excessively leaded material, cocks and faucets, gates, pot pieces, ingots and burned brass, aluminum composition, manganese, and iron.

8. Railroad bearing.-Shall consist of railroad boxes or car journal bearings, must be old standard used scrap, free of yellow boxes, also iron-backed boxes, and must be free of babbitt, also free of excessive grease and dirt.

9. Cocks and faucets. - To be mixed red and yellow brass, free of gas cocks and beer faucets.

10. Heavy yellow brass.-May consist of heavy brass castings, rolled brass, rod brass ends, chandelier brass, tubing, not to contain over 15 per cent of tinned and/or nickel-plated material; no piece to measure more than 12 inches over any one part and must be in pieces not too large for crucibles. Must be free of manganese mixture, condenser tubes, iron, dirt, and excessively corroded tubing. Must be free of aluminum brass containing over 0.20 per cent aluminum.

11. Yellow brass casings.-Shall consist of brass castings in crucible shape, that is, no piece to measure more than 12 inches over any one part; must be free of manganese mixtures, tinned and nickel-plated material, and must be free of visible aluminum brass.

12. Light brass.-May consist of miscellaneous brass, tinned or nickel plated that is too light for heavy brass; to be free of gun shells containing paper, ashes, or iron; loaded lamp bases; clock works; and automobile gaskets. Free of visible iron unless otherwise specified.

13. Old rolled brass. - May consist exclusively of old pieces of sheet brass and pipe free from solder, tinned and nickel plated material, iron, paint and corrosion, ship sheathing, rod brass, condenser tubes and Muntz metal material.

14. New brass clippings.- Shall consist of the cuttings of new sheet brass to be absolutely clean and free from any foreign substances and not to contain more than 10 per cent of clean brass punchings to be not smaller than one-fourth inch in diameter.

15. Brass pipe.-Shall consist of brass pipe, free of nickel-plated, tinned, soldered pipes or pipes with cast-brass connections. To be sound clean pipes free of sediment, and condenser tubes.

16. No. 1 red composition turnings.-To be free of railroad car-box turnings and similarly excessively leaded material; aluminum, manganese, and yellowbrass turnings; not to contain over 2 per cent free iron; to be free of grindings and foreign material, especially babbitt. Turnings not according to this specification to be sold subject to sample. 
17. No. 1 yellow rod-brass turnings.-Shall consist of strictly rod turnings, free of aluminum, manganese, composition, and Tobin and Muntz metal turnings; not to contain over 3 per cent free iron, oil or other moisture; to be free of grindings and babbitts; to contain not more than 0.30 per cent tin and not more than 0.15 per cent combined iron.

18. No. 1 yellow-brass turnings.-Shall consist of yellow-brass turnings, free of aluminum, manganese, and composition turnings; not to contain over 3 per cent of free iron, oil or other moisture; to be free of grindings and babbitts. To avoid dispute, to be sold subject to sample.

19. Auto radiators (unsweated).-All radiators to be subject to deduction of actual iron. The tonnage specification should cover the gross weight of the radiators unless otherwise specified.

20. No. 1 pewter. - Shall consist of tableware and soda-fountain boxes, but in any case must test 84 per cent tin. Siphon tops to be treated for separately.

21. Zinc.- - Iiust consist of clean sheet and cast zinc, also cast batteries; to be free of loose oxide and dross, sal ammoniac cans and other foreign materials.

22. Zinc dross. - Must be unsweated in slabs and must contain a minimum of 92 per cent of zinc.

23. Tin foil.- Shall consist of pure foil free of lead compositions and other foreign ingredients and matters.

24. Electrotype shells.-Must be hand picked and free of loose dross and chunks of dross.

25. Scrap lead.-Should be clean, soft scrap lead. 26. Baitery lead plates.-(a) Shall consist of dry battery lead plates, moisture not to exceed 1 per cent, allowance to be made for wood, rubber and paper and excess moisture, or (b) lead plus antimony content, dry basis, less a treatment charge.

NoTE.-Contracts covering this item should specify which method is to be used as a basis of settlement.

27. New sheet aluminum clippings and new aluminum wire.-Shall consist of new sheet aluminum, cuttings, and new aluminum wire. Must be free from oil, grease, and any other foreign substances. Must be guaranteed not less than 98 per cent pure aluminum and not to contain more than 10 per cent of clean aluminum punchings to be not smaller than one-quarter inch in diameter.

28. Old aluminum wire.-Shall consist of old aluminum wire guaranteed 98 to 99 per cent pure aluminum and must be free from iron cores, insulation, and any other foreign substance.

29. Alloy sheet aluminum.-To be sold on specification and sample.

30. Painted sheet aluminum.-Shall consist of painted sheet aluminum and must be absolutely free from iron, dirt, or any other foreign substance.

31. Old sheet aluminum.- Shall consist of old and manufactured sheet aluminum. Must be free from painted sheet aluminum, iron, dirt, and any other foreign substance.

32. Aluminum castings.-Shall consist of aluminum castings, free from iron, babbitt, brass, and any other foreign substance. Must not exceed 2 per cent of oil and grease. Free of pistons.

33. Aluminum borings.-To avoid dispute, should be sold subject to sample.

34. Aluminum foil. - Shall consist of pure aluminum foil, free from paper and any foreign ingredients.

35. Babbitt metal. - Shall contain bearing metal of all kinds. Shall not contain scrap hard metal, Allen metal (which is copper and lead alloy), die cast, ornamental metal, casket metal, zinc boxes, or type metal.

36. Packages. - Shall be good strong packages suitable for shipment, and each package shall be plainly marked with separate shipping marks and numbers and with the gross and tare weights so that the packages may reach their destination and their weights can be easily checked.

\section{SOCIETY OF AUTOMOTIVE ENGINEERS IRON AND STEEL SPECIFICATIONS, FEBRUARY, 1928}

\section{SPECIFICATION NUMBERS}

(A scheme for classifying alloy stecls so that at a glance one can tell the percentage of all alloying elements is detailed in 600.0, page 2. Bv these means partially descriptive specification numbers can be inserted on shop drawings and blue prints.)

\section{URTTED STATES GOVERNMENT, DEPART. IKENT OF COMMERCE, BUREAU OF STANDARDS, SIMPLIFIED PRACTICE RECOMMENDATION R58-28, JANUARY, 1928}

\section{CLASSIFICATION OF IRON AND STEEL SCRAP}

(Simplified practice recommended and accepted by industry establishing and defining various standard classes and grades of iron and steel scrap.)

\section{SPECIFICATIONS FOR CLASSES OF SCRAP FOR USE IN BLAST} FURNACES

1. Pipe busheling scrap.-Iron and steel pipe and flues (clean), bedstead tubing, and similar material cut 8 inches and under in length, free from galvanized material and foreign metals.

2. Cast-iron borings. - Clean cast-iron borings free from badly corroded material, lumps, scale, other metals, dirt, or foreign material of any kind.

3. Shoveling turnings.-Clean, short, steel and wrought-iron turnings, drillings, or screw cuttings; free from stringy, bushy, or tangled material, corroded or rusty lumps, excessive oil, scale, other metals, dirt, or foreign material of any kind. Alloysteel scrap may be excluded from these specifications by mutual agreement between buver and seller.

4. Mixed borings and turnings.-Clean, short, steel and wrought-iron turnings, drillings, screw cuttings, and cast or malleable iron borings and drillings; free from stringy, bushy, tangled, corroded material, lumps, excessive oil, scale, other metals, dirt, or foreign material of any kind. Alloy-steel scrap may be excluded from these specifications by mutual agreement between buyer and seller.

5. Corroded borings and turnings.-Corroded and lump borings, turnings, and similar stock; free from scale, other metals, dirt, or foreign material of any kind. No dimensions to exceed 9 inches. Alloysteel scrap may be excluded from these specifications by mutual agreement between buyer and seller.

6. Burnt iron scrap.-Old annealing boxes, stools and pots, grate bars, and similar burnt iron. No dimension to exceed 9 inches, except brake shoes which may be included.

7. Mill scale.-Iron oxide produced in rolling-mill practice, from drop-forge hammers or from a busheling-mill squeezer. Should contain not less than 65 per cent metallic iron, and must be reasonably free from dirt, grease, and other foreign material. Scale from alloy steel may be excluded from these specifications by mutual agreement between buyer and seller.

8. Mill cinder.-Cinder from puddling furnaces, heating furnaces, and soaking pits. Should contain not less than 50 per cent iron, and be reasonably free from dirt and other foreign material. Cinder from alloy steel may be excluded from these specifica. tions by mutual agreement between buyer and seller.

\section{SPECIFICATIONS FOR CLASSES OF SCRAP FOR USE IN BASIC} OPEN-HEARTH FURNACES

9. No. 1 heavy melting steel scrap.-Steel scrap one-fourth inch and over in thickness, not over 18 
inches in width, and not over 5 feet long. Individual pieces must be cut into such shape that they will be free from attachments and will lie flat in a charging box. Cut boiler plate must be practically clean and free from stay bolts and not over 3 feet long and lie reasonably flat in charging box. Smaller dimensions of plate scrap may be required upon mutual agreement between buyer and seller. No piece to weigh less than 5 pounds.

May include structural shapes, angle bars and plates, steel castings, heavy ohain, carbon tool steel, heavy forgings, forge butts, and similar heavy material.

This grade may also include new mashed pipe ends, original diameter 4 inches and over, thoroughly flattened, sheet bars, billets, blooms, rail ends, railroad steel, and wrought scrap, such as angles, splices, couplers, knuckles, short rails, drawbars, cut caststeel bolsters, coil and leaf springs (all coil springs to be three-eighths inch or larger diameter).

No needle or skeleton plate scrap, agricultural shapes, annealing pots, boiler tubes, grate bars, cast iron, malleable iron, or curly or unwieldy pieces will be accepted.

Must be free from dirt, excessive rust or scale, or foreign material of any kind. Alloy-steel scrap may be excluded from these specifications by mutual agreement between buyer and seller.

10. No. 2 melting steel.-Plate scrap, such as car sides, automobile frame stock, tank and skelp crops, one-eighth inch and heavier; steel parts of agricultural implements, wagons, buggies, and scrapped automobiles; auto and buggy springs cut apart; rods and bars, one-half inch and heavier; punchings, onefourth inch and over in thickness; heavy clippings; new unmashed pipe ends, under 4 inches in diameter; horseshoes; and sinilar material. Car sides and all light plates to be sheared 15 by 15 inches or under and all tires and light rods to be 12 inches and under in length. Any curved or twisted pieces must be sheared into such shape that they will lie flat in a charging box and not tangle in handling with a magnet, all to be free from cast iron, malleable iron, burnt scrap, dirt, or foreign material of any kind.

Maxirnum size 15 inches wide by 3 feet long, excepting car sides. Alloy steel scrap may be excluded from these specifications by mutual agreement between buyer and seller.

11. Heavy shoveling steel scrap.-Heavy, clean, wrought-iron and steel scrap, one-fourth inch and over in thickness, not exceeding 8 inches in breadth or length, and no piece to be less than the equivalent of one-half inch square by 3 inches long. May include clean horseshoes, railroad spikes, bolts, nuts, tie-plates, etc.; boiler, bridge, and structural punching and clippings; small bar and shafting crop ends; and other similar material.

To contain no burnt material, cast or malleable scrap, cut pipe and tank, skeleton stock, badly corroded material, or any twisted or tangled scrap. Must be free from foreign metals of all kinds, from galvanized, painted, enameled, or other coated stock, and from dirt and rubbish of all kinds.

Must be loaded in separate cars from other grades of scrap.

Alloy steel scrap may be excluded from these specifications by mutual agreement between the buyer and seller.

12. Short shoveling flashings.-Flashings or trimmings from iron or steel forgings to be 10 inches or under in length, suitable for hand shoveling, and to include no tangled or twisted material. Alloy-steel scrap may be excluded from these specifications by mutual agreement between the buyer and seller.

13. Long flashings.-Flashings or trimmings from iron or steel forgings, which have been strictly separated to a minimum length of 20 inches and not to exceed a maximum of 36 inches. There must be a thickness in some section of each of these flashings of not less than three-sixteenths inch. Alloy-steel scrap may be excluded from these specifications by mutual agreement between the buyer and seller.

14. Mixed flashings.-Flashings or trimmings from iron or steel forgings, not over 36 inches in length. Alloy-steel scrap may be excluded from these specifications by mutual agreement between the buyer and seller.

15. No. 1 busheling.-Clean iron and soft-steel pipes and flues, tank, cut hoops and bands No. 12 gage and heavier, steel-plate punchings and clippings, soft-steel and iron forgings, and flashings; no dimension over 3 inches. To be free from burnt material, hard steel (cast, malleable), and galvanized, or metal-coated stock of any kind.

16. No. 2 busheling. - Cut hoops, necting, cotton ties, sheet, and similar material lighter than No. 12 gage, no dimension over 8 inches. To be free from hard steel (cast, malleable), and galvanized or metalcoated material of any kind.

17. No. 1 selected rail scrap. - Standard section $T$ and guard rails, 40 pounds and heavier per yard, not less than 36 inches long, to be free from frog and switch rails not cut apart; and contain no manganese steel scrap or cast welds or attachments of any kind; or concrete, dirt, or foreign material of any kind.

18. No. 1 selected shearing scrap.-Angles from 2 inches up to 8 inches, structural shapes in single members from 4 inches up to 9 inches, bars one-half inch and over up to 4 inches diameter, and flats one-half inch to 2 inches thick and from 1 to 10 inches wide. To be in straight lengths and free from dirt and foreign material of any kind. Long-length girder rails free from cast welds and attachments may be included by mutual agreement between the buyer and seller.

19. Axle turnings.-Heavy, short first-cut turnings from wrought iron and steel railroad axles or heavy forgings, and rail chips, to weigh not less than 75 pounds per cubic foot, free from dirt or other foreign material of any kind. Alloy-steel scrap may be excluded from these specifications by mutual agreement between the buyer and seller.

20. No. 1 machine-shop turnings.-New, clean steel or wrought-iron turnings; free from lumps, badly tangled or matted material, cast-iron borings, other metals, excessive oil, dirt, or foreign material of any kind. Badly rusted or corroded stock will not be accepted.

21. No. 2 machine-shop turnings.-New, clean steel or wrought-iron turnings, curly, bushy stock; may contain tangled materisl, but must be free from badly rusted, lumpy, and corroded stock. To concain no cast-iron borings, other merals, excessive oil, dirt, or foreign material of any kind.

22. Cast-iron borings. - New, clean cast-iron borings and drillings; free from steel turnings and from badly corroded or rusty material, lumps, oil, scale, other mecals, dirt, or foreign material of any kind.

23. Hydraulically compressed sheet scrap.--New, black steel sheet clippings, shearings, skeleton stamping scrap, side and end sheet and tin-mill scrap, hydraulically compressed into compact, rectangular packages not to exceed 20 inches longest dimension; weighing not less than 75 pounds per cubic foot; must be clean and free from excessive rust, paint, or proteccive coating of any kind. No detinned scrap, elecurical sheets, or material over 0.50 per cent silicon will be accepted. Further limitation of silicon content may be made by mutual agreement between buyer and seller.

24. Baied sheet scrap.-New, black steel sheet clippings, shearings, skeleton stamping scrap, side and end sheet and tin-mill scrap; machine baled into angular or rectangular packages; ried with wire or 
bands, or sufficiently compact not to corne apart in handling with a magner; not over 24 inches longest dimension; weighing not less than 45 pounds per cubic foot; must be clean and free from excessive rust, paint, or protective coating of any kind. No detinned scrap, electrical sheets, or material over 0.50 per cent silicon will be accepted. Further limitation of silicon content may be made by mutual agreement between buyer and seller.

25. Hand-bundled sheet scrap.-New, black steel slippings, shearings, skeleton stamping scrap, side and end sheet and tin-mill scrap; securely tied with not less than two wires or bands into packages not over 18 by 18 inches by 3 feet; weighing not over 125 pounds; to be bundled in such a manner that packages will not come apart in handling with a magnet; must be clean and free from excessive rust, paint, or protective coating of any kind. No detinned scrap, electrical sheets, or material over 0.50 per cent silicon will be accepted. Further limitation of silicon content may be made by mutual agreement between buyer and seller.

26. Loose sheet clippings.-New, black steel sheet clippings, shearings, and stampings; three-sixteenths inch and lighter; free from excessive rust, paint, or protective coating of any kind; to be not over 18 inches wide or long; or if edge trimmings or shearings, to be not over 12 inches by 5 feet long. No detinned scrap, elecirical sheets, or material over 0.50 per cent silicon will be accepted. Further limitation of silicon content may be made by mutual agreement between buyer and seller.

27. Galvanized or coated sheet scrap.-New, clean steel sheet scrap, composed in whole or in part of material having a coating of zinc, paint, or protective material. Must be clearly specified as "galvanized or coated scrap" and be classified as stated above under $15,16,17$, or 18 , according to the manner in which it is prepared for shipment. No material over 0.50 per cent silicon will be accepied. Further limitation of silicon content may be made by mutual agreement between buyer and seller.

28. Detinned sheet scrap.- Sheet steel clippings and shearings, originally covered wiuh a protective coating of tin, or lead and tin, but from which such foreign metals have been removed. Must be clean, concain no material over 0.50 per cent silicon and be free from badly rusted and corroded stock. Must be clearly specified as "detinned scrap" and classified as stated above under $15,16,17$, or 18, according to the manner in which is is prepared for shipment. Further limitation of silicon content may be made by mutual agreement between buyer and seller.

29. Electrical sheet scrap.-New, clean steel sheet scrap, composed in whole or in part of material from 0.50 per cent to 1 per cent silicon. Must be clearly specified as "electrical sheet scrap" and be classified as stated above under $15,16,17$, or 18, according to the manner in which it is prepared for shipment.

30. High silicon sheet scrap. - New, clean steel sheet scrap, composed in whole or in pari of material over 1 per cent silicon. Must be clearly specified as "high silicon sheet scrap" and be classified as stated above under $15,16,17$, or 18 , according to the manner in which it is prepared.

31. No. 1 cast-iron scrap. - To contain all kinds of machinery and similar cast-iron scrap, nothing under 10 pounds or over 500 pounds in weight, nor over 48 inches long or 18 inches wide. To contain no brake shoes, cast-iron soil pipe or water pipe, stove scrap, or burnt iron of any description, and to be free from steel parts.

32. No. 2 cast-iron scrap.-To contain all kinds of agricultural implements of cast iron, free from steel parts, nothing under 10 pounds or over 500 pounds in weight, nor over 48 inches long or 18 inches wide. To contain no stove scrap or burnt iron of any description.
33. No. 3 cast-iron scrap. - To consist of cast-iron scrap with steel parts attached, nothing under 10 pounds or over 500 pounds in weight, nor over 48 inches long or 18 inches wide.

34. Heavy breakable cast scrap.-Heavy cast iron suitable for breaking under and with buyer's drop. Pieces not to exceed 10 tons in weight. Free from anvil blocks, hammer bases, and like material, over charging box size and containing no burnt iron.

\section{SPECIFICATIONS FOR CLASSES OF LOW PHOSPHOROS AND HEARTH FURNACES}

35. Billet and bar crops.-Billet, bloom, axle, heavy forge, or bar crops not over 0.04 per cent phosphorus or sulphur, and not over 0.50 per cent silicon; free from alloys. Not less than 2 inches square or diameter, not over 18 inches wide and sheared to lengths not over 36 inches. No piece to weigh less than 10 pounds nor more than 500 pounds. Must be new material and free from excessive rust. Longer lengths may be allowed by mutual agreement between buyer and seller.

36. Plate crops.-Plate crops, or sheet-bar crops, not over 0.04 per cent phosphorus or sulphur, and not over 0.50 per cent silicon; free from alloys. Not less than one-fourth inch thick, not over 18 inches wide and sheared to lengths not over 36 inches. No piece to weigh less than 10 pounds or more than 350 pounds. To be free from needle and sliver stock. Must be new material and free from excessive rust. Longer lengths may be allowed by mutual agreement between buyer and seller.

37. Structural and miscellaneous scrap.-Bar ends, forging crops, structural crops, and plate shearings smaller and lighter than grades $\mathrm{A}$ and $\mathrm{B}$, not over 0.50 per cent silicon, 0.04 per cent phosphorus, and 0.05 per cent sulphur; free from alloys. Not less than one-fourth inch thick, not over 18 inches wide or 36 inches long. To be fully described at time of sale and purchase. Must be clean and free from excessive rust. Longer lengths may be allowed by mutual agreement between buyer and seller.

38. Tubescrap.-Tube scrap, seamless or welded, not over 0.04 per cent phosphorus or sulphur, free from alloys. Not less than No. 12 gage thickness of wall; if over 6 inches inside diameter they must be mashed flat and sheared to lengths not over 36 inches. Must be only new material that has not been in use for any purpose, and free from excessive rust.

39. Punchings.-Punchings from plate and structural-steel scrap, not less than one-fourth inch thick and not less than one-half inch diameter, not over 0.04 per cent phosphorus or 0.05 per cent sulphur, not over 0.50 per cent silicon, free from alloys. Must be free frum dirt and excessive rust or corrosion.

40. Car wheels.-Solid cast steel, forged, pressed, or rolled steel car and locomotive wheels, not over 36 inches diameter. (Specify kind when offering.)

41. Spring-steel scrap.-Coil and elliptic steel springs, made of material not less than three-eighths inch diameter or one-fourth inch thickness, not over 18 inches wide, and sheared to lengths not over 48 inches. May be assembled or cut apart.

42. Couplers and knuckles.-Railroad car and locomotive steel couplers, knuckles, and locks, stripped clean of all other attachments. Gates, heads, and risers may be included by mutual agreement between buyer and seller.

43. Scrap tires. - Steel tires from car and locomotive wheels, cut to not over 24 inches long.

44. Car bolsters. - Railroad car steel bolsters and side frames stripped clean of all iron attachments. Not over 24 inches wide and cut to lengths not over 48 inches.

45. Medium silicon scrap.-High silicon billet, bloom, bar crops, plate crops, or sheet-bar crops, not over 0.04 per cent phosphorus or sulphur, not less 
than 0.05 per cent to 2 per cent silicon, free from other alloys, not less than one-fourth inch thick, not over 18 inches wide and 36 inches long, and no piece to weigh less than 10 pounds or more than 500 pounds. To be free from needle and sliver stock. If ust be new material and free from excessive rust. 46. High-silicon scrap.-High-silicon billet, bloom, bar erops, plate crops, or sheet-bar crops, not over 0.04 per cent phosphorus or sulphur, not less than 2 per cent or over 4.5 per cent silicon, free from other alloys, not less than one-fourth inch thick, not over 18 inches wide and 36 inches long, and no piece to weigh less than 10 pounds or more than 500 pounds. To be free from needle and sliver stock. Must be new material and free from excessive rust.

47. High-nickel scrap.-Nickel-steel billet, bloom, bar crops, or other shapes of similar section and equal grade, not over 0.04 per cent phosphorus or sulphur, between 3 per cent and 3.5 per cent nickel (lower or higher in nickel content to be duly considered), free from other alloys. Not less than 2 inches square or diameter, not over 18 inches wide, and cut to lengths suitable for charging boxes of buyers, and no piece to weigh less than 25 pounds. Must be clean material.

48. No. 1 chrome-nickel scrap.-Chrome-nickel steel billet, bloom, bar crop, or other shapes of similar section and equal grade, not over 0.04 per cent phosphorus or sulphur, between 3 and 3.5 per cent nickel (lower or higher nickel content to be duly considered), not over 0.5 per cent chrome, free from other alloys. Not less than 2 inches square or diameter, not over 18 inches wide, and cut to lengths suitable for buyers' charging boxes, and no piece to weigh less than 25 pounds. Must be clean material.

49. No. 2 chrome-nickel scrap.-Chrome-nickel steel billet, bloom, bar crops, or other shapes of similar section and equal grade, not over 0.04 per cent phosphorus or sulphur, between 1.5 and 2.5 per cent nickel (lower or higher nickel content to be duly considered), between 0.5 and 1 per cent chrome, free from other alloys. Not less than 2 inches square or diameter, not over 18 inches wide, and cut to lengths suitable for buyers' charging boxes, and no piece to weigh less than 25 pounds. Must be clean material.

50. Light chrome-nickel scrap.-Nickel or chromenickel steel of the analyses and descriptions, as shown for grades 47,48 , and 49 , except it may be lighter and of miscellaneous shapes. To be fully described at time of sale and purchase.

51. Special chrome-nickel scrap.-Nickel or cliromenickel steel of the analyses and descriptions, as shown for grades 47,48 , and 49 , except the phosphorus and sulphur may go up to but not over 0.05 per cent. To be fully described at time of sale and purchase.

52. Nicleel-steel turnings.-Heavy, short first-cut turnings from nickel-steel forgings. Nickel and chrome content to be specified on each individual sale. To weigh not less than 75 pounds per cubic foot. To be free from dirt and other foreign materials.

\section{SPECIFICATIONS FOR CLASSES OF SCRAP FOR USE IN} ELECTRIC FORNACES

Alloy steel scrap may be excluded from these specifications by mutual agreement between buyer and seller.

53. Punchings and clippings.-Open-hearth steel punchings and clippings, one-fourth inch and heavier, 4 inches and under in length. Not over 0.04 per cent in phosphorus or sulphur and suitable for shovel charging. Must be clean and free from galvanized or coated stock, dirt, and excessive rust or corrosion.

54. Guaranteed heavy scrap.-Open-hearth steel plates, structural shapes, crop ends, shearings, broken steel tires, knuckles, tool steel, and spring steel. To be not less than one-fourth inch thick, other dimensions subject to agreement. Not over 0.04 per cent in phosphorus or sulphur, clean, free from excessive rust and corrosion, and contain no foreign material.

55. Unguaranteed scrap.-Steel plate, shearings, nut and bolt punchings, short rails, and other similar material, not less than one-fourth inch in thickness and not over 12 inches in width or length. Not guaranteed under 0.04 per cent phosphorus or sulphur. To be suitable for hand charging. Must be free from galvanized and coated stock, foreign material, and excessive rust or corrosion.

56. Guaranteed axle turnings.-Heavy steel or iron axle or forge turnings, guaranteed not over 0.04 per cent in phosphorus or sulphur. To contain no foreign material and must be clean and free from excessive rust and corrosion. To weigh not less than 75 pounds per cubic foot.

57. Unguaranieed turnings.- Short, heavy shoveling steel or wrought-iron turnings or rail chips, not guaranteed under 0.04 per cent in phosphorus or sulphur. To contain no foreign material and must be free from excessive rust or corrosion. To weigh not less than 75 pounds per cubic foot.

\section{SPECIFICATIONS FOR CIASSES OF SCRAP FOR USE IN GRAY IRON FOUNDRY PRACTICE}

58. No. 1 machinery cupola scrap.-Clean, machinery cast-iron scrap. Must be cupola size, not over 24 by 30 inches in dimensions, and no piece to weigh over 150 pounds.

59. No. 1 machinery breakable scrap.-Clean, machinery cast-iron scrap, weighing over 150 pounds, which can be easily broken, by an ordinary drop, into cupola size.

60. No. 1 standard cupola scrap.-Clean castiron scrap, such as columns, pipes, plates, and castings of miscellaneous nature, but free from stoveplate and agricultural scrap. Must be cupola size, not over 24 by 30 inches in dimensions, and no piece to weigh over 150 pounds. Must be free from foreign material.

61. No. 1 standard breakable scrap.-Clean castiron scrap, such as columns, pipes, plates, and castings of miscellaneous nature, weighing over 150 pounds, which can be broken, by an ordinary drop, into cupola size.

62. Burnt cast scrap.-Burnt cast-iron scrap, such as grate bars, stove parts, and any miscellaneous burnt scrap.

63. Stove plate scrap.-Clean cast-iron stove plate. Must be free from malleable and steel parts, window weights, plow points, grates, burnt iron, etc.

64. Agricultural scrap.-Cast-iron parts of agricultural machinery including plow points. Must be free from steel, and malleable, and full-chilled iron.

65. Cast-iron car wheels.-Cast-iron car and locomotive wheels.

66. Brake shoes.-Driving and car brake shoes of all types, except composition-filled shoes.

67. No. 1 radiator scrap.-Broken radiator castings, cupola size, with all steel parts removed. Must be free from excessive scale, rust, and corrosion.

68 . No. 2 radiator scrap.-Unbroken radiator castings. Must be free from excessive scale, rust, and corrosion.

69. No. 1 malleable scrap.-Malleable parts of automobiles, railroad cars, and miscellaneous malleable castings. Must be free from steel and cast-iron parts.

70. No. 2 malleable scrap.-Malleable parts of agricultural implements and other miscellaneous malleable castings. Must be free from steel and cast-iron parts. May include No. 2 rail steel, cropped rail ends under 3 feet long, 50-pound and over, standard section. 
SPECIFTCATIONS FOR CLASSES OF SCRAP FOR USE IN BESEEMER CONVERTERS

71. Mild steel converter scrap.-Mild open-hearth steel scrap, such as structural shapes, rolling mill crop ends, forgings, and forge-crop ends. No piece to weigh over 150 pounds or less than 10 pounds, not under three-eighths incl section, and must not exceed 24 inches in any one dimension. Must be free from excessive rust or corrosion.

72. High-carbon steel converter scrap.-High-carbon or hard-steel scrap, such as steel rails and materials of similar analysis. No piece to weigh over 150 pounds or less than 10 pounds, and must not exceed 24 inches in any one dimension. Must be free from excessive rust or corrosion.

73. Steel-spring scrap.-Coil and leaf springs, made from stock three-eighths inch in thickness or heavier. Elliptical springs must be cut apart and must not exceed 24 inches in length. Must be free from plates and from excessive rust or corrosion.

SPECIFICATIONS FOR CLASSES OF MISCELLANEOUS SCRAP

74. Crucible-steel scrap.-Boiler steel punchings, under 0.04 per cent in phosphorus or sulphur. This is a basic classification for crucible-steel scrap. In the event any other material is specified, no piece shall exceed 5 inches in width or 8 inches in length. In the event that small, soft-steel castings \&re specified as crucible scrap, the weight shall not exceed 10 pounds per piece. Must be clean and free from dirt and excessive rust or corrosion.

75. Chemical borings.-Clean, fine, drv, cast-iron borings free from cil and grease. Shall contain no lumpy or rusty stock and be free from other inetals.

\subsection{COATING OF INETALS.}

AMERICAN ASSOCIATION OF STATE HIGHWAY OFFICIALS, METHODS OF DETERMIINING WEIGIT OF COATING ON ZINCCOATED ARTICLES, TENTATIVE METHOD T-65, 1930

\section{WEIGHT OF ZINC COATING}

(Same as A. S. T. M. A90-30, given below.)

\section{AMERICAN ASSOCIATION OF STATE HIGHWAY OFFICIALS, METHOD OF TEST FOR UNIFORMITY OF GALVANIZING OR SPELTER COATING ON WIRE, TENTA- TIVE METHOD T-66, 1930}

TESTING SPELTER COATING ON WIRE

(Same as A. R. A. signal section specification 2912, given below.)

\section{AIPEICAN ELECTRIC RAILWAY ENGT- NEERING ASSOCIATION, STANDARD SPECIFICATION FOR GALVANIZING OR SHERARDIZING ON IRON AND STEEL, DQ-14, 1914}

\section{GALVANIZING OR SHERARDIZING ON IRON OR STEEL}

(This specification is similar to that of the American Railway Association, signal section, specification 2912 , given below, with the following additions and modifications:)

\section{GTNERAI DESCRIPTION}

This specification shall apply to all galvanized or sherardized materia].

\section{COATING REQUIREMENTS}

The coating shall consist of a continuous coating of pure zinc, zinc-iron alloy, or a combination of the two.

\section{METZIOD OF TEST}

In the case of sherardized material the specimens must be vigorously brushed in running water after each dip.

In testing wire, the No. 12 B. W. G. and smaller sizes shall be required to stand three 1 -minute and one $1 / 2$-minute immersions.

In case the article has a cut screw, the thread shall be recut after galvanizing and shall stand at least one 1-minute immersion in the test solution. The rest of the article shall withstand the specified four immersions.

The threads of nuts shall be cut after the nut has been galvanized, and the threads shall not be required to stand the test.

\section{INSPECTIO N}

All material shall be inspected on receipt; samples shall be taken at random and submitted to tests hereinafter specified. The shipment will be accepted or rejected upon these samples.

\section{R.EJECTION}

Any material which fails to meet the requirements hereinabove specified may be rejected and returned at the expense of the contractor.

\section{METHOD OF SHIPMENT}

All material furnished under this specification shall be so packed for shipment that it will be suitably protected from injury. Each package shall be plainly marked, giving the kind of material and the number of the company's order on which the shipment was made.

\section{AMERICAN MARINE STANDARDS COM- MITTEE, STANDARD WIRE ROPES FOR MARINE USES, O NO. 25-1930}

\section{WIRE ROPES FOR BIARINE USES}

(For galvanizing see 603.42 , p. 163.)

AMERICAN RAICWAY ASSOCIATION, SIGNAI SECTION, SPECIFICATION 2211, GALVANIZED E. B. B. BONDING WIRES, 1911

\section{GAIVANIZING E. B. B. BONDING WIRES}

(In respect to coating, cleaning, solution, samples, test, and acceptance, this specification conforms to A. R. A. signal section, specification 2912, given below. Otherwise it provides as follows:)

\section{GENWRAL}

(a) The intent of this specification is to provide for the furnishing of No. 8 B. W. G. galvanized E. B. B. wires for bonding rail joints of steam railroads where electric track circuits are to be used.

\section{CONDJCTOR}

(a) The wire must be cylindrical in form, free from scales, flaws, inequalities, splits, and all imperfections. The wires shall be cut and straightened to the length specified on order. The ends shall be sheared, cut, and free from burrs. 


\section{PROPERTIES}

(a) The mechanical and electrical properties of the finished wire must be in accordance with the following requirements:

\begin{tabular}{l|r|r|r|r}
\hline 33. W. G. & Diameter & $\begin{array}{c}\text { Breaking } \\
\text { resistance }\end{array}$ & $\begin{array}{c}\text { Per cent } \\
\text { eiongation } \\
\text { in 10 inches }\end{array}$ & $\begin{array}{c}\text { Resistance } \\
\text { in ohims } \\
\text { per mile at } \\
68^{\circ} \mathrm{F} .\end{array}$ \\
\hline & $\begin{array}{r}\text { Mils } \\
165\end{array}$ & $\begin{array}{r}\text { Pounds } \\
975\end{array}$ & 15 & 12.05 \\
\hline
\end{tabular}

The wire shall not vary more than 3 mils from the normal diameter.

\section{GALVANIZITKG TEST}

(a) A sufficient number of samples shall be taken from the wire submitted for inspection and shall be tested, these pieces being not less than 8 inches long and not more than seven pieces of wire shall be immersed in the specified quantity of solution.

\section{INSPECTION AND TESTS}

(a) The purchaser is to have the riglit to make such inspection and tests as he may desire of the wire at any stage of manufacture.

(b) The manufacturer must provide, at the mill, all apparatus and labor for making the required tests under the supervision of the purchaser.

(c) Tests shall be made at the mill on the wire before it is cut into lengths, or on samples submitted by the manufacturer, and may also be made on the wire upon its arrival at destination.

(d) If, upon arrival at destination, the wires do not meet the requirements of this specification they will be rejected and returned to the manufacturer, who shall pay all freight charges.

\section{PACKING FOR SHIPIENT}

(a) The wire shall be put up in bundles of 100 or 300 , as ordered, well burlapped at ends and securely fastened in not less than three places.

\section{TAGGING}

(a) A tag shall be securely fastened to each bundle, having plainly and indelibly marked thereon the number of and length of the wires, the purchaser's order and inspection number, and the proper shipping address.

\section{AMERICAN RAILWAY ASSOCIATION, SIG- NAL SECTION, SPECIFICATION 2912, GALVANIZING FOR IRON OR STEEL, 1912}

\section{GALVANIZING FOR IRON OR STEEL}

\section{PURPOSE}

(a) This specification gives in detail the tests to be applied to galvanized material. All specimens shall be capable of withstanding these tests.

\section{COATING}

(a) The galvanizing shall consist of a continuous coating of pure zinc of uniform thickness, and so applied that it adheres firmly to the surface of the iron or steel. The finished product shall be smooth.

\section{CLEANING}

(a) The samples shall be cleaned before testing' first with carbon tetrachloride, benzine, or turpentine and cotton waste (not with a brush), and then thoroughly rinsed in clean water and wiped dry with c ean cotton waste.

(b) The sample shall be clean and dry before each immersion in the solution.

\section{SOLUTION}

(a) The standard solution of copper sulphate shall consist of commercial copper sulphate crystals dissolved in cold water, about in the proportion of 36 parts, by weight, of crystals to 100 parts, by weight, of water. The solution shall be neutralized by the addition of an excess of chemically pure cupric oxide (CuO). The presence of an excess of cupric oxide will be shown by the sediment of this reagent at the bottom of the containing vessel.

(b) The neutralized solution shall be filtered before using by passing through filter paper. The filtered solution shall have a specific gravity of 1.186 at $65^{\circ}$ F. (reading the scale at the level of the solution) at the beginning of each test. In case the filtered solution is high in specific gravity, clean water shall be added to reduce the specific gravity to 1.186 at $65^{\circ} \mathrm{F}$ In case the filtered solution is low in specific gravity, filtered solution of a higher specific gravity shall be added to make the specific gravity 1.186 at $65^{\circ} \mathrm{F}$.

(c) As soon as the stronger solution is taken from the vessel containing the unfiltered neutralized stock solution, additional crystals and water must be added to the stock solution. An excess of cupric oxide shall always be kept in the unfiltered stock solution.

\section{QUANTITY OF SOLUTION}

(a) Wire samples shall be tested in a glass jar of at least 2 inches inside diameter. The jar without the wire samples shall be filled with standard solution to a depth of at least 4 inches. Hardware samples shall be tested in a glass or earthenware jar containing at least one-half pint of standard solution for each hardware sample.

(b) Solution shall not be used for more than one series of four immersions.

\section{SAMILISS}

(a) Not more than seven wires shall be simultaneously immersed, and not more than one sample of galvanized material other than wire shall be immersed in the specified quantity of solution.

(b) The samples shall not be grouped or twisted together, but shall be well separated so as to permit the action of the solution to be uniform upon all immersed portions of the samples.

\section{TEST}

(a) Clean and dry samples shall be immersed in the required quantity of standard solution in accordance with the following cycle of immersions.

(b) The temperature of the solution shall be maintained between $62^{\circ}$ and $68^{\circ} \mathrm{F}$. at all times during the following test: (1) Immerse for one minute, wash, and wipe dry; (2) immerse for one minute, wash, and wipe dry; (3) immerse for one minute, wash, and wipe dry; and (4) immerse for one minute, wash, and wipe dry.

(c) After each immersion the samples shall be immediately washed in clean water having a temperature between $62^{\circ}$ and $68^{\circ} \mathrm{F}$., and wiped dry with cotton waste.

$(d)$ In the case of number 14 B. W. G. galvanized iron or steel wire, the time of the fourth immersion shall be reduced to one-half minute.

\section{ACCEPTANCE}

(a) If, aiter the test described in section 7 (a) there should be a bright metallic copper deposit upon the samples, the lot represented by the samples shall be rejected. 
(b) Copper deposits on zinc or within 1 inch of the cut end shall not be considered causes for rejection.

(c) In the case of a failure of only 1 wire in a group of 7 wires immersed together, of if there is a reasonable doubt as to the copper deposit, 2 check tests shall be made on these 7 wires and the lot reported in accordance with the majority of the sets of tests.

(A note appended to this specification enumerates the equipment necessary for conducting the tests outlined above.)

\section{AMERICAN RAILWAY ASSOCIATION, TELE- GRAPII AND TELEPHONE SECTION, SPECIFICATIOHS FOR GELVANIZING OR SHERARDIZING ON IRON AND STEEL, S-1, 1923}

\section{GALVANIZING OR SHERARDIZING ON IRON AND STEEL}

(Substantially the same as AEREA, D6-14, given above, including the references in D6 to A. R. A. signal section specification 2912.)

\section{AMERICAN RAILWAY ASSOCIATION, TELE- GRAPH AND TELEPHONE SECTION, SPECIIICATION FOR GALVANIZING FOR IRON AND STEEL, 1-D-5, 1930}

\section{GALVANIZING FOR IRON AND STEEL}

(Identical with A. R. A. signal section specification 2912 , given above.)

\section{AMERICAN SOCIETY FOR TESTING MATE- RIALS, STANDARD METHOD OF DETER- MTNING WIGHT OF COATING ON ZINC- COATED ARTICIES, DESIGNATION A90- 30,1930}

\section{WEIGHT OF ZINC COATING}

\section{SHOP WEIGHIYG TESTS}

Select a pickled and washed sheet. Stand the sheet on edge and allow to drain for two minutes. The water remaining on the sheet will then amount to approximately 0.01 pound per square foot.

Weigh this sheet with an accuracy of 0.05 pound, and subtract the weight of the water to obtain the real weight of the uncoated sheet. After galvanizing, reweigh the sheet. The increase in weight represents the coating.

Where greater accuracy is desired, wash, dry, and weigh 10 sheets; then coat and reweigh.

To determine the weight of coating in ounces per square foot, divide the weight of coating in ounces by the area in square feet in the sheet or sheets.

\section{SARIPLING}

(a) Sheets.-The laboratory samples for determining the average weight of coating upon a galvanized sheet shall be taken as follows:

A $2 \frac{1}{2}$-inch strip shall be cut transversely or diagonally across the middle of the sheet with its ends approximately 1 inch from each edge. Three samples, $2^{1} y_{4}^{\prime}$ by $2^{1} y_{4}^{\prime}$ inches, shall be accurately cut from the middle and the two ends of this strip. These samples shall be cleaned with gasoline or benzol, then with alcohol and dried thoroughly before proceeding with the test.
The weight of coating in grams on a $2^{\frac{1}{4}}$ by $2 \frac{1}{4}$ inch piece (5.08 square inches) is numerically equal to the coating in oinces per square foot.

(b) Wire.-For determining the weight of coating on zinc-coated wire, the use of a sample of a spccific length is not necessary, since the density of the steel ( 0.283 pound per cubic inch) is known, it is only required to determine the diameter of the stripped wire and the ratio of the weight of zinc to the weight of the stripped wire. The sample of galvanized wire may be of any length over 12 inches, but preferably about 24 inches.

\section{HYDROCHLORIC $\triangle$ CID-ANTIMONY CHLORIDE IIETHOD (Standard methoa) \\ Solutions Required}

Antimony chloride solution.-Dissolve $20 \mathrm{~g}$ of antimony trioxide or $32 \mathrm{~g}$ of $\mathrm{SbCl}_{3}$ in $1,000 \mathrm{c} \mathrm{c}$ of $\mathrm{HCl}$ (specific gravity 1.19).

Hydrochloric acid.-Concentrated $\mathrm{HCl}$ (specific gravity 1.19$)$.

Biethod

(a) Sheets. - Cut three $23_{4}^{\prime}$ by $2^{1 / 4}$ inch laboratory samples, as described under Sampling, and weigh together ${ }^{31}$ after cleaning and drying. They should be immersed singly in $100 \mathrm{c}$ c of hydrochloric acid (specific gravity 1.19) to which has been added $5 \mathrm{c} \mathrm{c}$ of antimony chloride solution, and allowed to remain therein until the evolution of hydrogen has ccased or until only a few bubbles are being evolved. This requires only about 15 to 30 seconds, except in the case of sherardized coatings which require a somewhat longer time. The same $100 \mathrm{c} c$ of hydrcchloric acid can be used for at least five samples, but $5 \mathrm{c} \mathrm{c}$ of the antimony chloride solution should be added before the immersion of each sample. After stripping, the samples should be washed and scrubbed under running water, dried with a towel and laid in a warm place for a short time. The samples should again be weighed together 31 and the number of grams lost divided by the number of samples taken. Each gram then corresponds numerically to 1 ounce of coating per square foot.

(b) Wire.-

Solutions Required

Antimony chloride solution.-Dissolve $20 \mathrm{~g}$ of antimony trioxide or $32 \mathrm{~g}$ of $\mathrm{SbCl}_{3}$ in $1,000 \mathrm{c} \mathrm{c}$ of $\mathrm{HCl}$ (specific gravity 1.19).

Hyarochloric acid.-Concentrated $\mathrm{HCl}$ (specific gravity 1.19).

\section{Procedure}

The sample shall be cleaned with gasoline or benzine and dried thoroughly and then carefully weighed to $0.01 \mathrm{~g}$.

The sample shall then be stripped of the zinc coating by immersing in a solution made by adding $1 \mathrm{c} \mathrm{c}$ of the antimony chloride solution to $100 \mathrm{c} \mathrm{c}$ of the concentrated $\mathrm{HCl}$

As soon as the violent chemical action on the wire ceases, the wire shall be removed from the acid, washed thoroughly, and wiped dry.

The diameter of the wire shall then be determined to 0.001 inch by taking the mean of two measurements at right angles to each other. The stripped sample shall then be weighed to $0.01 \mathrm{~g}$.

Calculation.-The original weight minus the weight of the stripped sample, divided by the weight of the stripped sample, gives the ratio of zinc to iron for the sample under test. The weight of coating in ounces per square foot of stripped wire surface is

31 If it is of interest to know the individual weight of coating on each piece, each must be analyzed individually instead of collectively as described. 
determined by multiplying the constant 163 by the diameter in inches of the stripped wire by the above ratio. This calculation may be expressed by the following formula:

Ounces of zinc per square foot of stripped wire surface $=163 d r$ where $d=$ the diameter in inches of stripped wire; and

$$
\begin{gathered}
r=\frac{\text { original weight - stripped weight }}{\text { stripped weight }} \\
\text { BASIC LEAD ACETATE METHOD } \\
\text { (Alternate method) } \\
\text { Solutions Required }
\end{gathered}
$$

Lead acetate solution.-Dissolve $400 \mathrm{~g}$ of crystallized lead acetate in 1 liter of water. When dissolved, add $4 \mathrm{~g}$ of finely powdered litharge and after thorough agitation, allow the solution to settle and decant the clear portion for use.

\section{Method}

Cut three $21 / 4$ by $21 / 4$ inch laboratory samples, clean and weigh as described in the Hydrochloric acid-antimony chloride method. ${ }^{32}$ The samples should then be submerged separately in a tumbler containing sufficient lead acetate solution to cover the samples. The samples should be maintained in an upright position in order to enable the lead acetate to attack all portions.

After being immersed about three minutes, the sample should be taken out and the adherent lead carefully removed under running water in order that no burnishing action will cause the lead to plate onto the sample. Four immersions of three minutes each are usually sufficient to remove all zinc from the sample, when the bright surface of the iron will be exposed. The test pieces should then be washed in water, dried carefully, and weighed. The loss in grams divided by the number of samples represents the weight of coating in grams, which is numerically equal to the weight of coating in ounces per square foot.

\section{SULPHORIC ACID PERMANGANATE METHOD}

(Alternate method)

\section{Solutions Required}

Distilled water should be 1 sed in all cases where water is mentioned as a reagent.

Standard potassium permanganate, 0.1 N.-Dissolve pure, crystallized potassium permanganate in water in the proportion of $3.2 \mathrm{~g}$ per liter. Allow to stand at least two weeks and filter through asbestos. Standardize the solution in the following manner:

Weigh accurately 0.45 to $0.5 \mathrm{~g}$ of U. S. Bureau of Standards' sodium oxalate. Place in a 400 c c beaker and dissolve in $250 \mathrm{c}$ c of water at $80^{\circ}$ to $90^{\circ}$ C. Add $10 \mathrm{c} \mathrm{c}$ of sulphuric acid composed of $5 \mathrm{c} \mathrm{c}$ of water and $5 \mathrm{c} \mathrm{c}$ of c. p. $\mathrm{H}_{2} \mathrm{SO}_{4}$ (specific gravity 1.84) and titrate the solution at once with the potassium permanganate solution, stirring the liquid vigorously and continuously. The permanganate must not be added more rapidly than 10 to $15 \mathrm{c} \mathrm{c}$ per minute and the last 0.5 to $1 \mathrm{c} c$ must be added dropwise with particular care to allow each drop to be completely decolorized before the next is introduced. The solution should not be below $60^{\circ} \mathrm{C}$. by the time the end point is reached. More rapid cooling may be prevented by allowing the beaker to stand on a small asbestos-covered electric hot plate during the titration. The use of a small thermometer as a stirring rod is most convenient.

${ }^{22}$ If it is of interest to know the individual weight of coating on each pieco, each must be analyzed individually instead of collectively as described.
Calculate the iron equivalent of the permanganate solution using the iron value furnished by the Bureau of Standards for the sodium oxalate used.

Sulphuric acid.-Mix 100 c c c. p. $\mathrm{H}_{2} \mathrm{SO}_{4}$ (specific gravity 1.84), with $900 \mathrm{c}$ c of water.

Method

Carefully weigh the $2 \frac{1}{4}$ by $2 \frac{1}{4}$ inch piece, containing exactly 5.08 square inches.

After determining the weight of the plate, place the piece in a $250 \mathrm{c}$ c beaker (Griffin form). Allow the piece to rest on or come in contact with a small piece of platinum foil. Add $125 \mathrm{c}$ c or a sufficient amount of the sulphuric acid solution to cover the inclined piece. Cover the beaker with a watch glass and allow the zinc coating to dissolve. In from 10 to 20 minutes the zinc will be dissolved, as will be indicated by the cessation of the rapid evolution of gas, disregarding the small amount of gas coming mainly from the edges of the plate, due to the action of the acid on the base plate. Wash the plate with a jet of water from a wash bottle, allowing the washings to run into the beaker. Wipe the plate dry, and immediately titrate the solution in the beaker with the standard potassium permanganate solution and calculate the weight of iron dissolved with the zinc coating. Weigh the plate, and subtract this weight from the weight of the plate before removal of the coating. From the difference deduct the weight of iron found to have been dissolved with the coating, which will give the weight in grams of zinc coating on 5.08 square inches of the sample. The weight of the zinc coating in grams on the $2 \frac{1}{4}$ by $2 \frac{1}{4}$-inch piece wili be numerically equal to the weight of zinc coating in ounces per square foot.

\section{APPENDIX}

Field Tests for Determination of Zinc-Coated Articles

\section{PREECE OR COPPER SULPHATE DIP TEST}

The samples for the Preece test may be of any size, but should be free from as much cut surface as possible, and should be carefully cleaned before starting the test. The samples are then dipped into not less than $100 \mathrm{c}$ c of copper sulphate solution maintained at a temperature of $18^{\circ} \mathrm{C}$. The samples are allowed to remain exactly one minute in the solution. They are then washed in running water, and the copper deposit lightly rubbed off. Successive immersions of one minute each are continued, with the washing and cleaning of the sample between each one, until the appearance of bright adherent copper indicates that the iron beneath the coating has been exposed. Should a small amount of copper be coated adherently to the zinc coating, it must not be mistaken for the end point in which the copper plates-out onto the iron. The appearance of copper within 1 inch of a cut surface is likewise not considered to be the end point of the test.

The copper sulphate solution is prepared by dissolving 36 parts of commercial copper sulphate crystals in 100 parts of water, then adding enough cupric oxide to neutralize any free acid. The solution is filtered or allowed to settle and decanted, then diluted with water until its specific gravity is 1.186 at $18^{\circ} \mathrm{C}$. It should always be used at this temperature.

When material is tested by the Preece or copper sulphate method, the number of 1-minute immersions which the sample shall withstand is specified.

The amount of coating removed by a 1-minute immersion in the copper sulphate solution is roughly between 0.20 and 0.25 ounce per square foot of actual surface, or double this per square foot of sheets.

This test is not as accurate in determining the average weight of coating upon a sample as is the 
hydrochloric acid-antimony chloride method, but is useful, however, in determining which are the thinnest portions of the coating.

\section{THERMAL METHOD 33}

This method makes possible the determination of the zinc coating on iron and steel sheets and other articles without necessitating the use or an analytical balance. An accurately measured galvanized sample is immersed in a measured amount of acid, the maximum temperature rise being recorded. Each $0.1^{\circ} \mathrm{C}$. rise in temperature is equivalent to a definite weight of zinc.

The apparatus necessary for conducting this test is as follows:

1. Testing jar, about $2 \frac{1}{2}$ inches in inside diameter, with a capacity of about $400 \mathrm{c}$ c, flaring at the top if possible.

2. Thermometer, graduated in $0.1^{\circ} \mathrm{C}$, with a range of about $50^{\circ} \mathrm{C}$.

3. 300 c c graduated cylinder.

4. Glass-stoppered bottle containing concentrated $\mathrm{HCl}$ (specific gravity 1.19).

5. Rule for measuring.

The sample is cleaned with gasoline, dried and accurately measured. For testing zinc-coated sheet, the usual $2 \frac{1}{4}$ by $2 \frac{1}{4}$ inch specimen may be used, and for wire, suitable lengths may be used as given in the table under Sampling of wire.

A measured amount of hydrochloric acid (usually $200 \mathrm{c} \mathrm{c}$ ) is poured into the testing jar. The temperature of the acid is recorded after stirring, and the sample is immersed in the acid, care being taken that the acid does not overflow the jar. During this time, and until the coating has been stripped from the piece, the temperature is watched until the highest point is reached and the temperature starts to decline. The rise in temperature is then recorded, and from a table of factors prepared for various quantities of acid, the amount of zinc may be calculated. From the number of grams of zinc and the area of the piece in square inches, the weight of coating may be calculated as follows:

Coating, ounces per square foot, actual surface $=$ Weight of zinc in grams $\times \frac{5.08 \text { square inches }}{\text { area, square inches }}$

In this formula, the total area; that is, both sides of the sheet, is measured. To place this on the ordinary sheet basis, as expressed on zinc-coated sheet, the above figure should be doubled. For very light coatings (about 1 ounce per square foot) $100 \mathrm{c} \mathrm{c}$ of acid are sufficient. With coatings of $2 \frac{1}{2}$ ounces per square foot or heavier, $300 \mathrm{c} \mathrm{c}$ of acid should be used. To standardize the equipment, 5 or 6 zinc-coated specimens of any convenient gage or size are weighed, the rise in temperature determined for the apparatus, thermometer and volume of acid. The samples are washed, dried, and reweighed, and the difference in weight noted. The weight loss in grams is divided by the corresponding rise in temperature in degrees. When carefully determined, the average of the above five or six determinations will give a conversion factor which is reliable for the equipment used and for the volume of acid employed. Factors may be determined for any volume of acid in like manner.

\section{EVOLUTION OF HYDROGEN METHOD}

This method consists in measuring the hydrogen evolved from the action of hydrochloric acid containing antimony chloride upon a definite area of galvanized surface. By this method, it is not

33 Described in paper by D. M. Strickland, "A Method for Determining the Spelter Coating on Iron and Steel Sheets by Measuring the Rise in Temperature of the Acid Employed," Proc. A. m. Soc. Testing Mats., vol. 22, Pt. II, p. 227, 1922. required to cut any portion of the article to be tested, as with the Cushman coating tester, a $2 \frac{1}{2}$-inch ring is sealed to a portion of the surface. Acid is then added which attacks the coating within the area of the ring, and the evolved hydrogen is measured by a suitable gas burette. After removal of the spent acid, the stripped spot is waslied, dried, and may either be painted or coated with a suitable metallic coating. The article is, therefore, not mutilated. A full description of the test is found in the 1920 Proceedings of the American Society for Testing Materials. ${ }^{34}$

The results obtained by this method check very closely with those obtained by the regular hydrochloric acid-antimony chloride method. The inain objection to this method is the fact that the coating upon only one side of a sheet is obtained, and as the coating upon galvanized sheecs is not always exactly the same on each side; doubling the figures obtained on one side of the sheet will not always give the real amount of coating on both sides of the sheet.

This tester finds use, however, when it is uecessary to test sheets or culverts already installed, and where it is desired that the articles shall not be mutilated as would be necessary in taking a regular sample.

\section{AHERICAN SOCIETY FOR TESTING MATE- RIALS, STAYDARD METHODS OF DE- TRRMINING WEIGHT OF COATING ON TIN, TERNE, AND LEAD-COATED SHEETS, DESIGNATION A91-24, 1924}

\section{COATING ON TIN AND TERNE PLATE}

\section{SAMPLING}

From each grade or shipment take one sheet for analysis. Cut four 2 by 2 inch pieces; one from each of the four sides of the sheet, parallel with and about one-half inch from the sides, and near the middle of each side-or cut similar samples from corner, middle, and opposite corner of the sheet, keeping in at least 1 inch from the edge in each case.

Note.-For more accurate work, 9 samples are cut, 3 from across the top, 3 across the middle, and 3 across the bottom of each sheet.

\section{DETERMINATION OF WEIGHT OF COATING}

\section{Method}

Clean the laboratory samples with chloroform, carbon tetrachloride, benzol, or gasoline and finally with alcohol, and dry thoroughly; then weigh accurately the four pieces together.

Wrap a stiff platinum or nickel wire about the 2 by 2 inch piece in such a manner that it may be held in the acid in a horizontal position. Using a $400 \mathrm{c} \mathrm{c}$ beaker, heat from 60 to $150 \mathrm{c}$ c of sulphuric acid (specific gravity 1.84 ) to $250^{\circ} \mathrm{C}$. Immerse the sample for about one minute in the hot acid, then remove and momentarily immerse in $50 \mathrm{c}$ c of distilled water contained in a 600 c c beaker. Rub the surface with a "policeman," while washing with about $50 \mathrm{c}$ c of distilled water from a wash bottle. If the coating has not been completely remored, again immerse in the acid and repeat the procedure. Treat all four samples in the same manner, using the same acid. Thoroughly dry and reweigh the samples. If desired, the samples may be retained for the analysis of the base metal.

The loss in weight represents the coating together with some iron.

Cool the sulphuric acid in which the samples were stripped and combine with the washings in a 600

34 Allerton S. Cushman, "A New Method of Testing Galvanized Coatings," Proc., Am. Soc. Testing Mats., rol. 20, Pt. II, p. 411, 1920. 
c c beaker. Pour the solution into a 1,000 c c volumetric flask, and rinse the beaker with hydrochloric acid (specific gravity 1.12). Add rinsings to the flask and fill to the mark with hydrochloric acid of the same strength, again adjusting to the mark if necessary after cooling:

If it is desired to make a direct determination of the lead, the lead chloride should all be in solution. For heavy lead-coated sheets it may, therefore, be necessary to dilute the solution to $2,000 \mathrm{c}$ c with hydrochloric acid (specific gravity 1.12), in order to have all the lead chloride in solution.

The solution may now be analyzed for iron, tin, lead, or any other elements.

\section{DETERMINATION OF IRON}

\section{Method}

Remove an aliquot portion of $200 \mathrm{c}$ c from the $1,000 \mathrm{c}$ c volumetric flask to a $600 \mathrm{c}$ c beaker. Add 1 or $2 \mathrm{c}$ c of a saturated solution of potassium permanganate to oxidize the iron and tin, and heat the solution to boiling. Cool and add a slight excess of ammonia. Filter the solution and wash the precipitate with hot water. Dissolve the precipitate in the original beaker with hydrochloric acid (specific gravity 1.12), and hot water. Evaporate with liydrochloric acid solution to a volume of about $10 \mathrm{c} \mathrm{c}$ and reduce with stannous chloride.

To the cooled solution add a mixture of $25 \mathrm{c} \mathrm{c}$ of a saturated mercuric chloride solution, $10 \mathrm{c} c$ of phosphoric acid manganese sulphate solution and $400 \mathrm{c}$ c of boiled distilled water. Titrate immediately with $0.1 \mathrm{~N}$ potassium permanganate solution. Calculate the amount of iron stripped from the four pieces, from the number of cubic centimeters of permanganate used.

\section{Calculation of the Weight of Coating}

From the loss in weight of the four 2 by 2 inch pieces, subtract the amount of iron found by the above test. This figure represents the weight of coating in grams on the four pieces.

\section{Calculation Factors}

The weight of coating in grams on the four 2 by 2 inch pieces

multiplied by $4.3210=$ weight of coating in pounds per box of 112 sheets, 14 by 20 inches

multiplied by $8.6421=$ weight of coating in pounds per case of 112 sheets, 20 by 28 inches

multiplied by $0.3175=$ weight of coating in ounces per square foot.

Pounds of coating per case of 112 sheets, 20 by 28 inches, multiplied by $0.03673=$ ounces per square foot.

Ounces per square foot multiplied by $27.22=$ pounds per case of 112 sheets, 20 by 28 inches.

\section{DETERMINATION OE TIN ON TERNE PLATE}

Method

Remove an aliquot portion of $200 \mathrm{c}$ c from the volumetric flask. Place in a $300 \mathrm{c}$ c Erlenmey er flask, and add $1 \mathrm{~g}$ of powdered antimony. Fit the flask with a 1-hole rubber stopper containing a glass tube bent twice at right angles, with the end of the short bend projecting through the stopper, the other end being long enough to reach almost to the bottom of a beaker placed on a level with the flask. This beaker contains about $300 \mathrm{c} c$ of dilute sodium bicarbonate solution made up with boiled distilled water.

Place the flask on the hot plate, with the glass tube extending into the beaker of sodium bicarbonate solution. After boiling for about five minutes, substitute the beaker of dilute sodium bicarbonate solution with another containing about $50 \mathrm{c} \mathrm{c}$ of a saturated solution of sodium bicarbonate dissolved in boiled distilled water, and remove both the beaker and flask to a cool place. This will cause a small amount of sodium bicarbonate to enter the fiask and exclude the air. Finally bring the solution to a low temperature. Add several cubic centimeters of starch solution and titrate with $0.05 \mathrm{~N}$ iodine solution. It is desirable to run a duplicate analysis for tin, adding the iodine quickly to a point slightly less than the end point found in the previous determination, then finishing the titration.

From these determinations, calculate the percentage of tin in the coating. In the case of tin plate, the amount of tin can be calculated to pounds per box or pounds per case by factors given under Calculation of weight of coating.

\section{DETERMINATION OF IEAD ON TERNE PLATE}

\section{Method}

After ascertaining that all the lead chloride is in solution, remove an aliquot portion of $100 \mathrm{c} \mathrm{c}$ from the volumetric flask and place in a $400 \mathrm{c}$ c beaker. Add $10 \mathrm{c}$ c of sulphuric acid (specific gravity 1.84), cover, and evaporate to fumes of sulphuric anhydride. Cool and dilute to $300 \mathrm{c}$ c with water. Allow to settle and then filter on a weighed Gooch crucible, washing with 5 per cent sulphuric acid solution. Dry and ignite at a dull red heat. Cool and reweigh. The gain in weight multiplied by 0.6831 equals the weight of lead, from which calculate the percentage of lead in the sample.

DETERMINATION OF TIN ON TIN-COATED SHEQTS

\section{Method}

Bend or cut up each sample and place each in a separate Erlenmeyer flask and cover with about 40 c c of strong hydrochloric acid. Connect each flask through a rubber stopper with a source of pure oxygen-free carbon dioxide. Dissolve on a hot plate with carbon dioxide passing through to exclude air. Cool the flask in running water and titrate immediately with standard iodine solution, using starch indicator. The iodine solution is made up so that $1 \mathrm{c} \mathrm{c}$ is equivalent to $0.005786 \mathrm{~g}$ of tin, and is standardized against pure tin. The number of cubic centimeters of iodine used divided by 10 reads directly in pounds of tin per base box of plate.

Nots.-A base box contains 112 sheets, 14 by 20 inches, and weighs 100 pounds.

When it is desired to analyze only a small number of samples, satisfactory results may be obtained by adding to the flask, with the sample, a small pinch of pure powdered antimony. Cover with a watch glass and dissolve at boiling temperature. As soon as solution is complete, insert a rubber stopper with glass tube extending just through the stopper, and bent twice at right angles, the long end extending approximately level with the bottom of the flask. Insert the long end of the tube into a dilute solution of sodium bicarbonate. Place the flask in cold running water, and as the flask cools, the bicarbonate solution is sucked into the flask, the carbon dioxide set free driving out the air. As soon as cool, titrate as above.

ANIERCAN SOCTETY FOR TESTING MATERIAIS, STANDAPD SPECIFICATIONS FOR ZINC-COATED (GALVANIZED) SHEETS, DESIGNATION A93-2\%, $192 \%$

$$
\text { ZINC-COATED (GALVANIZED) SHEETS }
$$

(See 604.32, p. 244.) 
AMIERICAN SOCIETY FOR TESTING MATE. RIALS, STANDARD METHODS OF TESTING ZINC-COATED (GALVAIIZED) IRON AND STEEL WIRE AND WIRE PRODUCTS, DESIGNATION A110-30, 1930

\section{TESTING GALVANIZED WIRE}

\section{SCOPE}

1. These methods cover the testing of zinc coatings applied by the hot-dip and electrodeposition methods to iron and steel wire.

\section{WEIGHT OF COATING}

2. Weights of coating shall be determined by the hydrochloric acid-antimony chloride method as described in the standard methods of determining weight of coating on zinc-coated articles, A. S. T. M., A90, given above.

3. The classes of weights of coating per unit area on zinc-coated products shall be designated in accordance with the following illustrative scheme:

$\begin{array}{ll}\begin{array}{c}\text { Class } \\ \text { designation }\end{array} & \begin{array}{c}\text { Weight of coating, } \\ \text { ounces per square foot }\end{array} \\ \text { Weight 15 } & \text { Minimum of } 1.5 \\ \text { Weight 10 } & \text { Weight } 6 \ldots \\ \text { Weight } 1 \ldots & \end{array}$

This classification may be extended or interpolated to provide a selection of classes as needed in preparing specifications for zinc-coated wire and wire products.

4. The weight of coating of any class of wire being found on inspection to be heavier than that defined for the class designated shall not constitute cause for rejection unless so specified on the purchase order.

\section{UNIFORMITY OF COATING}

5. The uniformity of zinc coatings shall be determined by the Preece test as described in the appendix to the standard methods of determining weight of coating on zinc-coated articles, A. S. T. M., A90, given above.

6 . The requirements of zinc-coated wire in withstanding the Preece test shall be designated as 1 dip, 2 dip, 3 dip, 4 dip, etc., depending on the number of one-minute immersions in the standard Preece test solution that the coated wire is required to withstand. Fractional dip classes may be used, as $3 \frac{1}{2}$ dip, meaning 3 one-minute dips plus 1 half-minute dip.

7. There is a general relation between the weight of zinc coating and the number of dips in copper sulphate which the coating will withstand. Ordinarily a coating of weight 8 or heavier will withstand at least four immersions; one of weight 6 , at least three immersions; one of weight 4, at least two immersions.

\section{PHYSICAL PROPERTIES OF COATINGS}

8. (a) Wrap tests of zinc-coated wire, performed to measure adherence of the coating, may be required in some specifications.

(b) When a wrap test is specified, the coating shall be tested by wrapping two turns around a cylindrical mandrel of the diameter specified at a rate of about 15 turns per minute, and a coating shall be considered as meeting the test requirements if, after wrapping, it does not flake and none of the coating can be removed from the wire by rubbing with the bare fingers.

9. The diameters of the test mandrels used shall be approximately even multiples of the diameter of the wire tested. Mandrel sizes shall be designated as mandrel 1, mandrel 5, mandrel 12 , etc., depending upon whether the mandrel diameters are $1,5,12$, etc. times the diameter of the wire to be tested.

Note.-In general, wires carrying coatings of the higher weights and meeting higher dip-test requirements are used in the more severe conditions of exposure.

AMERICAN SOCIETY FOR TESTIIYG MATERIALS, STANDARD SPECIFICATIONS FOR ZINC-COATED (GALVANIZED) STEEL WIRE STRAND, DESIGNATION A122-30, 1930

GALVANIZED STEEL WIRE STRAND

(See 603.42, p. 181.)

AMERICAN SOCIETY FOR TESTING MATERIALS, STANDARD SPECIFICATIONS FOR ZINC (HOT-GALVANIZED) COATINGS ON STRUCTURAL-STEEL SHAPES, PLATES, AND BARS, AND THEIR PRODUCTS, DESIGNATION A123-30, 1930

[Approved as tentative American Standard by the American Standards Association, 1930]

\section{ZINC COA'TINGS ON STEEL SHAPES}

SCOPZ

1. (a) These specifications cover the protective zinc coatings, applied on structural-steel shapes, plates, and bars, and their products (note 1) by dipping the articles in a molten bath of zinc (note 2 ).

(b) These specifications presuppose the use of steel of recognized manufacture conforming to standard specifications.

\section{CHEMICAI PROPERTIES}

2. The slab zinc used shall conform to the requirements of the standard specifications for slab zinc (spelter), A. S. T. M., B6 (see 683.1, p. 1310.), and shall at least be equal to the grade designated as "prime western."

3. The amount of lead or iron in the bath during actual galvanizing operations shall not exceed the limits allowed for "prime western" slab zinc (spelter) namely, lead 1.60 per cent and iron 0.08 per cent. The aluminum content shall not exceed 0.01 per cent.

4. (a) The analyses for impurities in the bath shall be made on specimens which are combinations of borings from at least three test ingots taken during actual operations. Samples for test ingots shall be taken on the center line of the pot, one at the middle and the other two at opposite ends of the pot, but not nearer the ends than one-half the width of the pot. The samples shall be taken from a depth of at least 2 inches below the surface of the zinc but not greater than 25 per cent of the depth of the zinc.

(b) The implements and molds used shall be of materials which will not change the proportions of lead, iron, or aluminum in the test ingot.

(c) The cost of analyzing samples of the zinc bath shall be paid by the purchaser.

\section{PHYSICAL PROPERTIES AND TESTS}

5. (a) The finished material shall not be embrittled. (Note 3.)

(b) The purchaser shall bear the expense of making all embrittlement tests and the responsibility for delays in shipment incidental thereto. 
6. The weight of the zinc coating per square foot of astual surface shall not average less than 2 ounces, and no individual specimen shall show less than 1.8 ounces.

7. (a) Test specimens shall be selected from the material being galvanized and shall be galvanized at the same time, in the same manner, and in the same pot as the material whose coating characteristics they are intended to indicate. If the lot of material is of inconvenient lengths, pieces of the same shape but of convenient lengths for handling (at least 3 feet long) may be substituted as test specimens. (Note 4.)

(b) Three specimens may be required from each shift for carrying out the determination of the weight of coating as described in section 8 .

(c) No test specimen shall be used for the determination of the weight of coating which would fail to conform to other requirements of these specifications.

8. (a) The weight of the zinc coating shall be determined by weighing one or more specimens after pickling and drying and again after coating.

(b) In the case of material manufactured without inspection, the weight of coating may be determined by stripping an entire piece in accordance with the standard methods of determining weight of coating on zinc-coated articles, A. S. T. M., A90, given above.

\section{WORKMANSHIP AND FINISH}

9. The zinc coating shall be adherent, smooth, continuous, and thorough. It shall be free from such imperfections as lumps, blisters, gritty areas, uncoated spots, acid and black spots, dross (note 5), and flux. The coating shall not be so loosely adherent as to be removable by any reasonable process of handling and erection. Light blows with a one-half-pound hammer shall not cause peeling of the coating adjacent to the area deformed by the haminer blow. The coating shall not interfere with the intended use of the material.

10. After immersion in the molten zinc, the structural shapes, plates, or bars shall not be subjected to any process of scraping or wiping which will reduce the uniformity or the specified weight of the zinc coating.

11. The uniformity of the zinc coating shall be determined by visual inspection. If, in the opinion of the inspector, visual examination is not conclusive, he may use the Preece test as described in the appendix to the standard methods for determining weight of coating on zinc-coated articles, A. S. T. M., A90, given above. This test shall not be used to determine the weight of coating, and shall only be applied after the coating has been found to conform to the requirements of section 6 . (Note 6.)

\section{INSPECTION AND REJECTION}

12. (a) (Inspection conforms to the requirements of A. S. T. M. standard specifications for steel, par. 7.) (See 605.0, p. 247.)

(b) The material shall be inspected at the manufacturer's plant during the process of manufacture. However, by agreement the purchaser may make the tests which govern the acceptance or rejection of the materials in his own laboratory or elsewhere; such tests shall be made at the expense of the purchaser.

13. (a) Visual inspection of materials ready for shipment shall be made to determine conformity with the requirements of section 9; when partial inspection warrants rejection of a lot, the manufacturer may re-sort the lot and submit it once again for inspection.

(b) If one specimen should fail to conform to the requirements specified in section 6 for the weight of coating, a second and a third specimen shall be tested. Failure of either the second or third specimen to conform to the requirements shall be cause for rejection of the lot which the samples represent.

(c) Materials that have been rejected may be stripped and regalvanized and again submitted for test and inspection when they shall conform to the requirements of these specifications; otherwise the entire lot shall be rejected.

Note 1.-The use of the word "products" is not meant to imply an unqualified indorsement of the indiscriminate practice of apply. ing zinc coatings on assembled parts.

Note 2.-The zinc coating on the materials covered by these specifications is too heavy to permit bending and other severe fabrication of the galvanized article without seriously damaging the coating.

NOTE 3.- Steels are encountered which are susceptible to embrittlement by the normal hot-dip galvanizing processes. Such em. brittlement may depend on properties inherent in the steel, or on a method of cold working the material, or on the galvanizing processes. The present state of the art being such that embrittlement can not be controlled and it being the belief that the causes and remedies can only be ascertained through cooperative research by the parties interested, these specifications do not unqualifiedly reject embrittled materials. Therefore, if, despite precautions taken by the manufacturers in the galvanizing and in the selection of the steel, embrittlement results, rejections are not to be enforced unless the liability is specifically provided for by contractual agreement between the purchaser and the manufacturer.

Note 4.-The use of convenient lengths of material for test specimens should not be interpreted to mean that small pieces of flat plate or odd shapes can be substituted for test specimens. Practice has shown that coupon specimens do not give true indications of the weight of coating on the shapes.

Note 5. - It is highly desirable to keep the materials being coated from coming in contact with the dross, and in all cases where practi. cable such contact should be avoided. Every practice tending to agitate the dross should be avoided.

Note 6.-Accepted experience indicates that the minimum weight of coating to be applied under these specifications will be removed in from 7 to 9 one-minute dips, and the average weight of coating in from 8 to 10 one-minute dips.

\section{ASPHALT SHINGLE AND ROOFING INSTI- TUTE, NAILS, ACCELERATED CORRO- SION TEST, 1923}

(For A. S. R. I. specifications on zinc-coated nails see 608.11, p. 558.)

\section{CORROSION TEST}

The salt-spray test shall be used for this purpose as described in circular No. 80, Bureau of Standards, Department of Commerce, Washington, D. C., issued October 4, 1919, page 24, which is as follows:

The test is made in a box with a glass cover, the construction of which is indicated in Figure 41.

The box is inclined so that drops of solution collecting on the cover will run down to the edge instead of dripping on the samples.

A 20 per cent solution (by weight) of commercial sodium chloride $\left(20 \mathrm{~g}\right.$ salt and $80 \mathrm{~cm}^{3}$ water, or 2 pounds salt and 1 gallon water) filtered if necessary, is used, and with an air pressure of about 6 or 7 pounds per square inch, a very fine mist is produced.

The compressed air is passed through a glass-wool or cotton plug and then through water to remove oil and dust, and to saturate the air with water vapor which prevents concentration of the salt solution and crystallization of the salt on the tips of the atomizer. The baffle plate prevents the salt spray from blowing directly against the test pieces.

The samples, after being washed with gasoline and ether to remove all grease, are placed in the spray box in a vertical position on the glass rods or strips. They are removed from the bath every 24 hours, washed with water, using a moderately stiff bristle brush, and after drying are carefully examined for the presence of red or yellow iron rust. The first appearance of rust indicates the conclusion of the test, but valuable information may be obtained by continuing the test and observing the ex tent of the corrosion produced by longer exposure. 
ASPHAL' SHINGLE AND ROOFING INSTITUTE, MAILS, RINC IN PROTECTIVE COATING, 1921

(For A. S. R. I. specifications on zinc-coated nails see 608.11, p. 558.)

\section{ZINC IN PROTECTIVE COATING}

The hydrochloric acid-antimony chloride method is recommended for this purpose. Select 100 nails at random from the shipment. Weigh these in aggregate and calculate the average weight per nail. Select ten nails from the hundred taken, of which the weight per nail will not vary more than 2

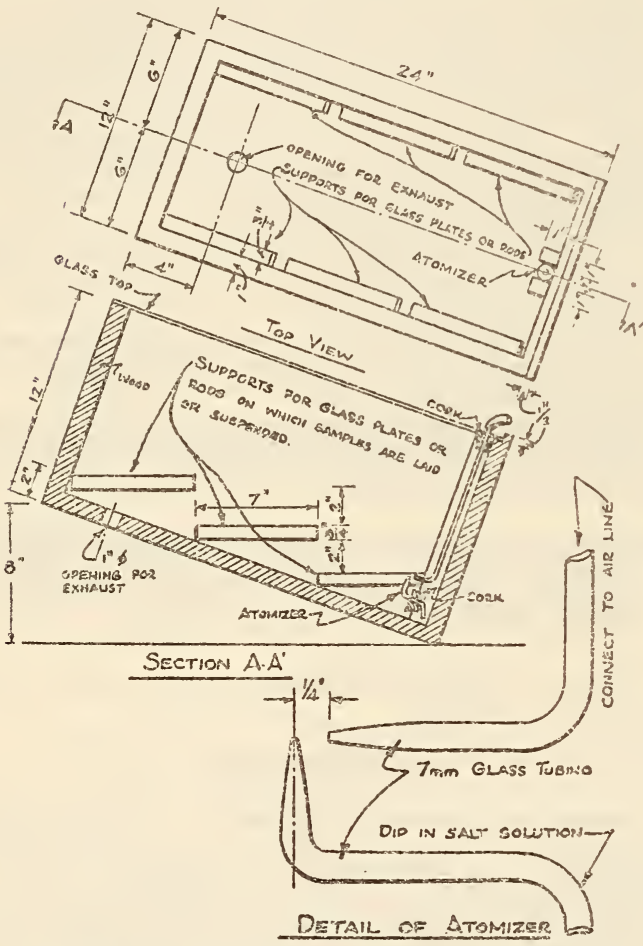

FigURE 41.-Apparatus for salt spray test

per cent from the average calculated. Test these nails individually as follows:

Calculate the area of the nail's surface in square inches in the following manner:

If $a$ represents the diameter of the nail head measured in inches; $b$ the average length of the shank measured in inches; $c$ the diameter of the shank measured in inches, then:

Area of nail's surface in square inches

$$
=\frac{11}{7} a^{2}+\frac{22}{7} b c
$$

The apparatus as shown in Figure 42 consists of a separatory funnel $(A)$ connected with the open end of a burette $(B)$ by means of a stout rubber tubing $(C)$ and rubber stopper $(D)$ carrying a glass tube $(E)$. The funnel should have a mouth sufficiently large to allow the nail to be introduced. This opening should be closed with a rubber stopper $(F)$ through which passes a thistle tube $(G)$ proviced with a glass stopcock $(H)$. The apparatus is connected up as follows:

Introduce the nail into the separatory funnel $(A)$ and fill the apparatus with water to displace all the air in the funnel $(A)$, the rubber tubing $(C)$ and the burette $(B)$. Place in the thistle tube $\left(G^{\prime}\right)$ a rrixture of $30 \mathrm{c}$ c of conceritrated hydrochloric acid (specific gravity 1.20) and $3 \mathrm{c} \mathrm{c}$ of a 5 per cent solution of antimony chloride and allow albout $10 \mathrm{c} \mathrm{c}$ to enter the funnel $(A)$. As soon as hydrogen is generated, admit the rest of the mixture to the funnel. When the evolution of hydrogen ceases, or only a bubble of gas comes every few seconds, invert the funnel to displace the hydrogen into the burette. The end-point is fairly sharp so that it is easy to distinguish the moment the zinc is all in solution and the iron or steel base begins to be slowly attacked by the acid. Return the funnel to its original position and allow the contents to cool to $20^{\circ} \mathrm{C}$. Open the stopcock $(H)$, bring the liquid in the funnel to the same level as the liquid in the burette, and read off the volume of hydrogen in the burette $(B)$. Calculate the ounces of zinc per square font nail surface from the following formula:

Ounces zinc per square foot $=$

$$
\frac{\mathrm{H} \text { (in c c at } 20^{\circ} \mathrm{C} \text {. and } 760 \mathrm{~mm} \text { ) } \times 0.0138176}{\text { Area of nail's surface (square inches) }}
$$

The method is claimed to be accurate to within 0.05 ounces per square font. The average weight of zinc on the 10 nails exanined should be reported as the average weight of zinc on the shipinent under examination.

The committee is unconvinced of the value of lead in the protective coating and regards its pres-

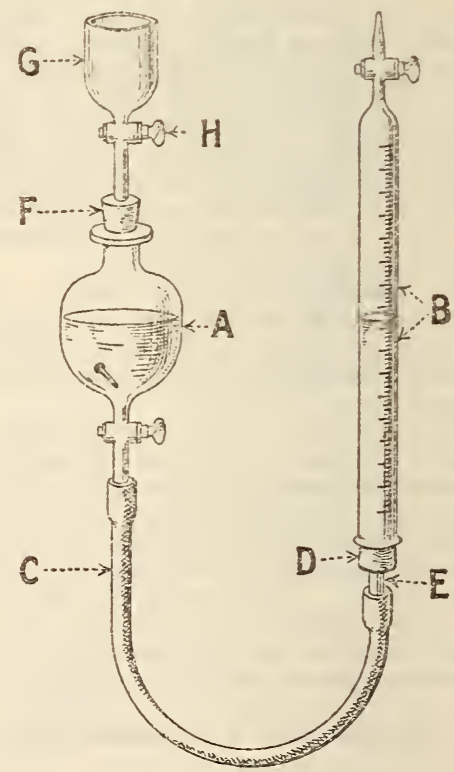

Figure 42.-Apparatus for determining zinc

ence with skepticism. The hydrochloric acidantimony chloride method does not take into consideration any lead carried in the zine coating and is favored for this reason:

\section{SOCIETY OF AUTOROTIVE ENGINEERS, RLECTRICAI EQUIPMENT; FLEXIBLE- CONDUIT FERRULES, S. A. E. STAND. ARD, AUGUST, 1928}

\section{FLEXIBLE-CONDUIT FERRULES}

(See 645.22, p. 1215.) 
UNDER WRITERS' IABORATORIES, STANDARD FOR RIGID STEEL CONDUIT, APRIL, 1931

\section{PROTECTIVE COATINGS}

1. Both inside and outside surfaces of rigid steel conduit shall be protected against corrosion, and the character and appearance of the coating on the inside surface shall be such that conduit can be readily distinguished from ordinary commercial pipe commonly used for other than electrical purposes. Coatings other than enamel or zinc as described below shall be made the subject of special investigation before being used.

2. Zinc coating.-The zinc coating on either the interior or exterior surface of conduit shall be of standard weight and quality to afford protection against corrosion. If the interior surface does not have a standard zinc coating it shall have a standard enamel coating.

3 . The protective zinc coating on rigid steel conduit is generally applied by the electroplating, by the sherardizing, or by the hot-dip process. The zinc is always applied to the outside surface, but the coating on the interior surface may be either zinc or enamel.

4. An additional coating of enamel, varnish, or other substance mav be used in order to distinguish conduit from ordinary commercial pipe, as required in paragraph 1 , when the interior surface has a zinc coating. When used for this purpose or for other reasons, the additional coating or coatings will not need to meet the requirements for standard enamel and shall not be considered as furnishing corrosion protection for the steel tube.

5. At any time up to one year from the time of manufacture the zinc coating on either the inside or outside of finished conduit shall be of uniform quality throughout, shall have a smooth and even appearance, and shall not soften at ordinary temperatures.

6. Compliance of conduit with the requirements of the preceding paragraph shall be determined by visual inspection.

7. Elasticity.-The zinc coating shall be sufficiently elastic to prevent its cracking or flaking at any time up to one year from the time of manufacture when a sample of finished $1 / 2$-inch conduit is bent in a semicircle, the inner edge of which has a radius of $3 \frac{1}{2}$ inches.

8. Compliance of conduit with the requirement of the preceding paragraph shall be determined by means of a suitable form as shown in Figure 43, and when so tested the zinc shall not crack or fly off at any point on either the inside or outside surface of the conduit.

9. The requirement of paragraph 7 does not apply to enamel or other substance applied to an interior zinc coating for the purpose of distinguishing the conduit from ordinary commercial pipe, nor to additional coatings of enamel or other substance applied to either interior or exterior zinc coatings.

10. Adequacy of zinc coating.-The zinc coating shall be such that a sample of finished conduit will not show a fixed deposit of copper after the prescribed number of immersions or dips in a standard copper sulphate solution in accordance with the following table:

\begin{tabular}{|c|c|}
\hline Kind of coating & $\begin{array}{l}\text { Number } \\
\text { of dips } \\
\text { after } \\
\text { which } \\
\text { fixed } \\
\text { copper } \\
\text { deposit } \\
\text { shall not } \\
\text { show }\end{array}$ \\
\hline $\begin{array}{l}\text { Electroplated, with or without copper subcoat. } \\
\text { Sherardized } \\
\text { Hot-dipped and wiped. }\end{array}$ & $\begin{array}{l}2 \\
2 \\
4\end{array}$ \\
\hline
\end{tabular}

11. Preparation of sample.-A piece of finished conduit approximately 4 inches in length and free from abrasions is to be used. The end to be immersed is closed with a rubber stopper as shown in Figure 44. All grease and paraffin are removed by washing the sample in carbon tetrachloride or chloroform, and, when the zinc coating is covered with paint or varnish, this also is removed with a suitable solvent. Finally the sample is rinsed in water and dried with clean cheesecloth. The zinc surface is to be perfectly clean before the sample is immersed in the copper sulphate solution, due care being exercised to avoid contact of the cleaned surface with the hands or any foreign materials.

12. Standard copper sulphate soluiion.-The copper sulphate solution shall have a specific gravity of $1.186 \pm 0.003$ at $65^{\circ} \mathrm{F}$. (approximately $18^{\circ} \mathrm{C}$.), and shall be prepared according to the following directions:

13. Approximately $350 \mathrm{~g}$ of C. P. copper sulphate $\left(\mathrm{CuSO}_{4} \cdot 5 \mathrm{H}_{2} \mathrm{O}\right)$ are weighed out for each liter of standard solution to be prepared. The required volume of distilled water is measured into a copper vessel and heated nearly to boiling. The weighed crystals of copper sulphate are then added slowly

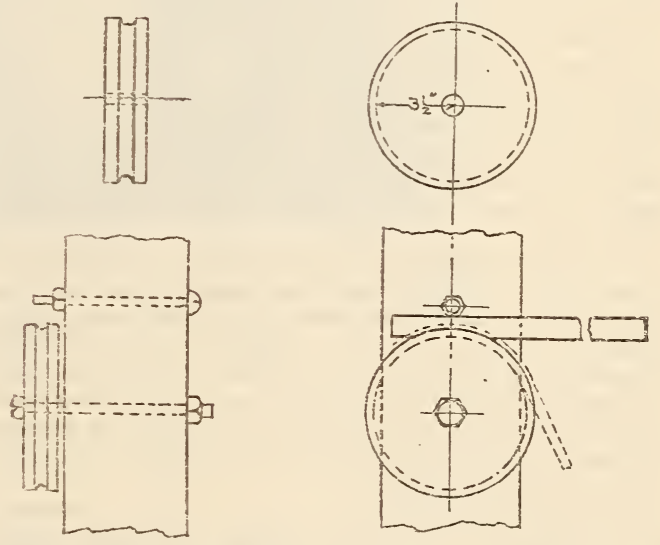

Figure 43.-Form for bending one-half inch conduit

and the solution stirred with a wooden rod. Approximately $1 \mathrm{~g}$ of cupric oxide $(\mathrm{CuO})$ per liter of water used shall be added to neutralize any free acid. The solution is stirred until the crystals are dissolved and then allowed to cool for a few minutes. About $200 \mathrm{c}$ c of the solution is filtered into a hydrometer cylinder, the temperature of the solution noted, and the specific gravity determined by means of a hydrometer. In case the temperature of the trial solution is somewhat above $65^{\circ} \mathrm{F}$. when the hydrometer reading is taken, the specific gravity of the solution at $65^{\circ} \mathrm{F}$. (approximately $18^{\circ} \mathrm{C}$.) can be computed by reference to a specific gravitytemperature chart. From the specific gravity of the trial solution, the required amount of water to be added in order to obtain a solution of standard strength can be calculated according to the following formula:

$$
X=1.15 \frac{(A-1.186) V}{0.186}
$$

where

$X=$ number of cubic centimeters of water to be added;

$A=$ specific gravity of the trial solution at $65^{\circ} \mathrm{F}$. (18 C.);

$V=$ number of cubic centimeters of water used in making up solution. 
In case $V=35,000$ c c, a chart may be employed in calculating the volume of water to be added to obtain a solution of standard strength.

14. The calculated amount of water and the trial solution are added to the original solution, which is then thoroughly stirred, filtered, and tested again as described above. Not less than 24 hours should elapse between the addition of the cupric oxide and the final filtration of the solution. The solution is considered standard when its specific gravity is $1.186 \pm 0.003$ at $65^{\circ} \mathrm{F} .\left(18^{\circ} \mathrm{C}.\right)$. The standard solution is to be stored in glass-stoppered bottles labeled to show the specific gravity of the solution and the date of its preparation.

15. Method of immersion.-A glass vessel having a diameter approximately twice as great as the outside diameter of the conduit is filled with the standard solution to a depth sufficient to immese $2 \frac{1}{2}$ inches of the length of the sample, the temperature of the solution being approximately $65^{\circ} \mathrm{F}$. The sample is then placed in a vertical position with the closed end at the bottom in the center of the vessel containing the solution, so that not less than 2 inches of the length is immersed. The sample is allowed to remain in the copper sulphate for a period of 60 seconds, during which time it is not to be removed, nor the solution stirred. At the end of the 60-second period the sample is removed from the vessel, immediately washed in a stream of running water, and rubbed with cheesecloth until any loosely adhering deposits of copper are removed The sample is then dried with clean, dry cheesecloth, care being taken to avoid contact of the cleaned surface with foreign objects, particularly the hands.

16. The operation just described, including the immersion or dipping and the washing and wiping is to be successively repeated, using the same copper sulphate solution, until a bright deposit of firmly adheling reguline copper remains on the sample. The sample is to be subjected to at least one more dip than the minimum number required for acceptable performance for the kind of coating in question as given in paragraph 10 .

17. The fixed deposit of reguline coppes generally occurs first at the weakest points in the zinc coating and increases in area upon successive dips, until the entire zinc coating disappears. After the dips have been completed on any one sample, the used copper sulphate solution is to be discarded and a fresh portion of the standard solution employed for any succeeding immersions.

18. Test results.-The results are to be expressed as an estimate of the percentage of the immersed surface which shows a fixed copper deposit after each dip-after the sample has been washed, rubbed, and dried. Failure is to be recorded for any sample on which the fixed copper deposit appears as the result of a number of dips equal to or less than the number given in paragraph 10 for the kind of coating in question.

UNITED STATES GOVERNMEIT, FEDERAI SPECIFICATIONS BOARD, FEDERAL SPECIFICATION FOR ROPE, WIRE, NO. 29\%, 1925. (CURRENT DESIGNATION: $R R-R-5 \% 1)$

\section{WIRE ROPE}

(See 603.42 , p. 183 , for galvanized wire rope.)
UNITED STATES GOVERNMENT, FEDERAL SPECIFICATIONS BOARD, FEDERAL SPECIFICATION FOR PLUIIBING FIXTURES (FOR LAND USE), NO. $\triangle 48$, NO. VEMBER 22, 1926 . CURRENT DESIGNANATION : FF-P-451)

(See also 617.73, p. 1062.)

\section{PLUMBING FIXTURES (FOR IAND USE)}

(i) Nickel plating.-The average thickness of nickel on articles subject to abrasion, such as faucet handles, toilet-door latches, coat hooks, etc., shall be not less than 0.0002 inch; and on articles not subject to abrasion, such as pipe, escutcheons, etc., shall be not less than 0.0001 inch. A tolerance of 10 per cent shall be permitted in these values to allow for uncertainties in measurement. These thicknesses are equivalent, respectively, to 0.144 ounce and 0.072 ounce per square foot, plating about one hour and one-half hour, respectively.

(j) Test of nickel plating on brass. - The area of the piece should be measured as closely as possible. It is then cleaned by immersion in an alkaline solution, rinsed, and hung in a solution containing about 10 per cent by volume of chemically pure concentrated hydrochloric acid. The article is connected as the anode and a strip of lead is made the cathode. A current is applied at 4 to 6 volts, until all the nickel is dissolved, usually in from one to two minutes. The resultant solution is diluted to a measured volume (for example, $250 \mathrm{c} \mathrm{c}$ ) and a definite portion (for example, $10 \mathrm{c}$ c) is used for the determination of the nickel content by the dimethylglyoxime method. If the area has been measured in square centimeters, the results can best be calculated to grams per 100 square centimeters or to thickness in millimeters, which may then be converted to ordinary units-

$$
\frac{\text { Weight of nickel }(\mathrm{g})}{\text { Area of sample }\left(\mathrm{cm}^{2}\right)} \times 100=\mathrm{g} \text { per } 100 \mathrm{~cm}^{2}\left(\mathrm{~g} / \mathrm{dm}^{2}\right)
$$

$\mathrm{g} / \mathrm{dm}^{2} \times 0.0115=$ thickness in millimeters.

Thickness $(\mathrm{mm}) \times 0.039=$ thickness in inches.

$\mathrm{g} / \mathrm{dm}^{2} \times 0.328=$ ounce per square foot.

\section{UIITED STATES GOVERNMENT, FEDERAL} SPECIFICATIONS BOARD, FEDERAI SPECIFICATION FOR BUILDERS' HARD. WARE (NONTEMPLATE), FF-H-101, AUGUST 19, 1930

\section{BUILDERS' HARDVARE (NONTEMFLATE)}

(For applicable Federal specifications; types, grades, and classes; material and workmanship; general requirements; detail requirements; packaging, packing, and marking; notes; and table of standard finishes; see 617.0 , p. 978.)

(Specific commodities, such as locks, latches, hinges, door pulls, etc., will be found under their respective commodity designations. For a list of these commodities see under detail requirements in 617.0, p. 979.)

$D-6$. Chromium plating.-

D-6a. Chromium plating on nonferrous metals.Chromium plating on nonferrous metals shall be applied over a coating of nickel. The specification for this procedure is as follows: Chromium plating 
Miscellaneous Publications, Bureau of Standards, No. 120

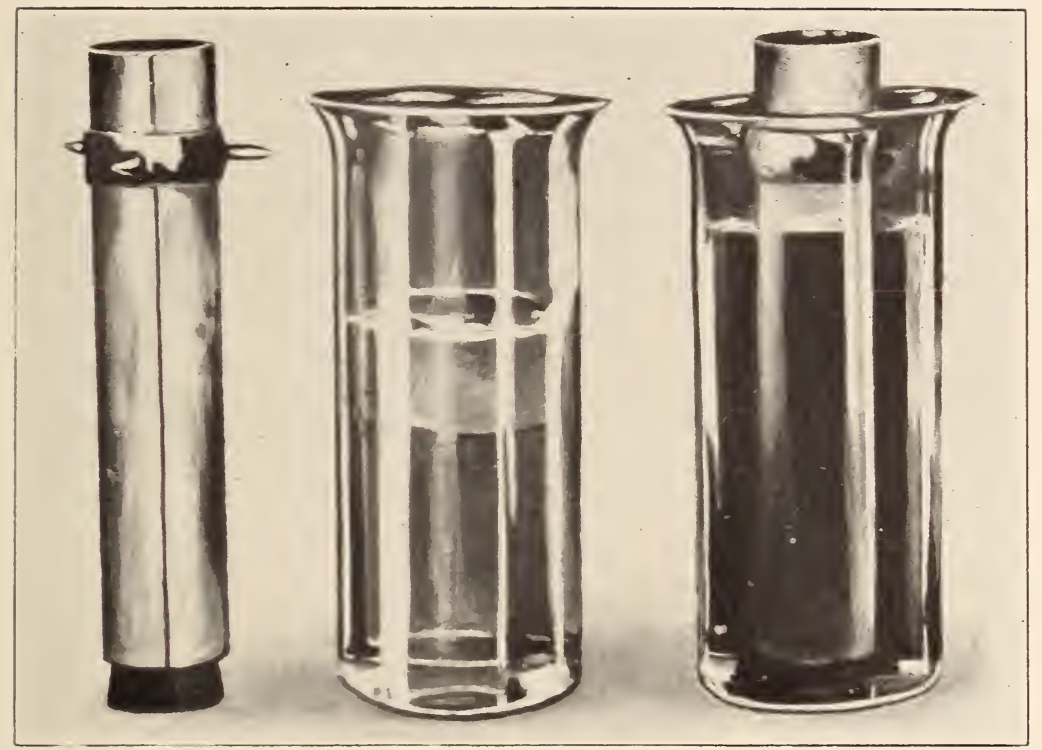

Figtre 44.-Test sections of conduit before and during immersion in copper sulphate solution

(For specification see p. 69.)

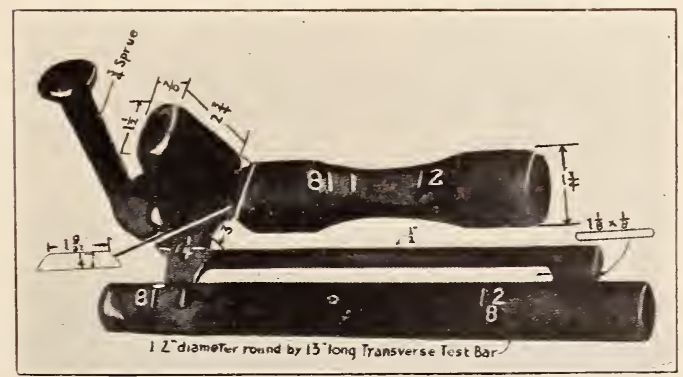

FIGURE 392.-Mold of tension and transverse test specimens

(For specification see A.S. T. M. 1126, p. 637.) 

over nickel plating on nonferrous metals. The nickel coating shall have an average tnickness of at least 0.0001 inch ( 0.0025 millimeter) and the chromium coating of at least 0.00002 inch $(0.0005$ millimeter). These are equivalent, respectively, to 0.074 ounce per square foot $\left(0.22 \mathrm{~g} / \mathrm{din}^{2}\right)$ of nickel and 0.011 ounce per square foot $\left(0.034 \mathrm{~g} / \mathrm{dm}^{2}\right)$ of chromium. A tolerance of 20 per cent shall be permitted on individual pieces, and of 10 pes cent on the average of thiee or more samples from the same lot or shipment.

D-6b. Chromium plating on polished steel.-Chromium plating on polished steel shall be applied over a coating of nickel which has been applied over base plate of cadmium. The chromium and nickel shall be applied as required for plating on nonferrous metals. (See paragraphs D-6a and D-7a.) The requirement for cadmium plating is as follows: Cadmium plating on polished steel. The average thickness of cadmium shall be not less than 0.00013 inch. This thickness is equivalent to 0.11 ounce per square foot $\left(0.34 \mathrm{~g} / \mathrm{dm}^{2}\right)$ of cadmium. A tolerance of 20 per cent shall be permitted on individual pieces and of 10 per cent on the average of three or more samples from the same lot or shipment.

D-\%. Nickel plating.-

D-7a. Nickel plating on nonferrous metals.-The average thickness of nickel when plated on nonferrous metals shall be not less than 0.0001 inch. This thickness is equivalent to 0.074 ounce per square foot $\left(0.22 \mathrm{~g} / \mathrm{dm}^{2}\right)$ of nickel. A tolerance of 20 per cent shall be permitted on individual pieces, and of 10 per cent on the average of three or more samples from the same lot or shipment.

D-7b. Nickel plating on polished steel.-Nickel plating on polished steel shall be applied over a suitable base plate, such as cadmium or copper. The combined thickness of nickel and the base plate shall be not less than 0.00015 inch. This combined thickness is equivalent to 0.107 ounce per square foot $\left(0.33 \mathrm{~g} / \mathrm{dm}^{2}\right)$ of nickel. A tolerance of 20 per cent sliall be permitted on individual pieces, and of 10 per cent on the average of three or more samples from the same lot or shipment.

$D-8$. Zinc coatings.-

D-8a. Zinc coatings on iron or steel-galvanized or sherardized.-The word "galvanized" where used in the detail specifications is intended to include the application of zinc by hot dipping, sherardizing, or zinc plating (electro galvanizing), provided that hot dipping and sherardizing shall not be applied on such alloy or heat-treated steels as will be injuriously affected at the temperatures employed. For such steels the zinc-plating process shall be used.

$D-8 b$. The average thickness of zinc coating shall be not less than 0.001 inch or 0.60 ounce per square foot. On accurately dimensioned parts such as screw threads the average thickness of zinc coating shall not exceed 0.002 inch or 1.20 ounces per square foot.

D-9. Illustrations. - Illustrations in the specifications show only the type of the various devices. However, a construction other than that illustrated or described shall constitute a full commercial equivalent of the one shown in order to be acceptable. Illustrations precede the descriptions in every instance.

\section{E. DETAIL REQUIREMENTS}

(Sec. 617.0, p. 978.)

\section{F. METHOD OF INSPECTION AND TESTS}

\section{F-2. Chromium plating.-}

F-2a.-Test of chromium plating on brass or nickel-plated brass. The area of the piece should be measured as closely as possible. It is then cleaned by immersion in an alkaline solution, rinsed, thoroughly dried and weighed to the nearest milli- gram for each square decimeter (about 16 square inches) of surface. It is then completely immersed in a solution containing about 10 per cent by volume of chemically pure concentrated liydrochloric acid, which has been warmed to about $50^{\circ} \mathrm{C}$. $\left(120^{\circ} \mathrm{F}\right.$.) When all the chromium has dissolved, as indicated by the ceasing of effervescence (usually in five minutes or less) the articles are removed, thoroughly rinsed, dried, and reweighed. The loss in weight is equal to the weight of chromium originally present.

Weight of chromium (g)

Area of sample $\left(\mathrm{cm}^{2}\right)=100=\mathrm{g}$ per square decimeter $\left(\mathrm{g} / \mathrm{dm}^{2}\right)$

$\mathrm{g} / \mathrm{dm}^{2} \times 0.0147=$ thickness of chromium in millimeters.

Thickness $(\mathrm{mm}) \times 0.039=$ thickness in inches.

$\mathrm{g} / \mathrm{dm}^{2} \times 0.328=$ ounces per square foot.

F-2b.-After the chromium plating has been removed from nickel-plated brass, the nickel may be determined on the same sample by the method described below.

F-3.-Nickel plating.-

$F-3 a$ - - (Identical with test of nickel plating on brass in FF-P-451, given above.)

$F$-4.- Solid nickel-bronze trimmings and fittings will be acceptable in lieu of nickel-plated brass.

F-5. Zinc co atings.-Hydrochloric acid-antimony chloride method of determining thickness. The solution is prepared by adding 100 cubic centimeters concentrated hydrochloric acid (specific gravity 1.20 ) to 5 cubic centimeters of a solution inade by dissolving 20 grams of antimony trioxide in 1,000 cubic centimeters of concentrated hydrochloric acid. A sufficient number of specimens should be used in each test to have an area not less than 25 square centimeters ( 4 square inches). The specimens are weighed carefully and then dipped in sufficient of the above solution to cover them completely. The samples are kept in the solution for one minute, or longer if necessary, to remove all of coating. They are then washed and scrubbed in running water to remove the deposited antimony. They are then dried and reweighed. The loss in weight represents the weight of the zinc coating, which is calculated directly to grams per square decimeter or to ounces per square foot (g/dm² multiplied by 0.328 ). On irregular-shaped parts the zinc may be expressed in grams per piece.

\subsection{DEFINITIONS OF METALS.}

\section{AMERICAN SOCIETY FOR TESTING MATE-} RIALS, STANDARD DEFINITIONS OF TERMS RELATING TO WROUGHT-IRON SPECIFICATIONS, DESIGNATION A8130,1930

\section{WROUGHT-IRON SPECIFICATION DEFINITIONS}

Wrought iron.-A ferrous material, aggregated from a solidifying mass of pasty particles of highly refined metallic iron with which, without subsequent fusion, is incorporated a minutely and uniformly distributed quantity of slag.

Busheling.-The process of heating to a welding heat in a reverberatory furnace, miscellaneous iron, steel, or a mixture of iron and steel scrap cut into small pieces.

Fagoting.-The making of a "fagot" or "box," the bottom and sides of which are formed of muck or scrap bars and the interior of miscellaneous iron scrap or a mixture of iron and steel scrap.

Muck bar or puddled bar.-Bar rolled from puddled balls, made wholly from puddled pig iron.

Common iron.- - Iron made from rerolled scrap iron or a mixture of iron and steel scrap, no attempt being made to separate the iron and steel scrap. 
Slab pile.-A pile built up wholly of flat bars of iron, all bars running the full length of the pile.

Box pile.-In manufacturing wrought-iron bars, a pile, the outside of which is formed of flat bars and the interior of a number of small bars, all bars running the full length of the pile.

Refined bar iron or refined wrought-iron bars.-Iron bars rolled from a muck bar pile or from a box pile, of muck bars and wrought iron scrap bars free from steel, all bars running the full length of the pile.

Double-refined iron.-Iron rolled from a slab pile or box pile of refined iron bars, all bars running the full length of the pile.

AMEMICAN SOCTETY TOR TESTING INATERIALS, STANDARD DEFINTTIONS OF TERMS RELATING TO METALIOGRAPHY, DESIGNATION E\%-2\%, 1927

\section{METALIOGRAPHY DEFINITIONS}

(See 600.1 , p. 34.)

\section{SOCIETY OF AUTOMOTIVE ENGINEERS IRON AND STEEL SPECIEICATIONS, GENERAI DEFINITIONS, JUNE, 1927}

\section{GENERAL DEFINITIONS}

1. Cold working. - The operation of forming the metal, without the application of heat, by rolling, hammering, drawing, pressing, or other means, to obtain accurate size, fine finish, or increased strength.

2. Hot working. - The operation of rolling, hammering, pressing, or extruding metal which has been made plastic by heating.

3. Yield point.- The load per unit of original cross section at which a marked increase in the deformation of the specimen occurs without increase of load. It is usually calculated from the load determined by the drop of the beam of the testing machine or by use of dividers.

4. Tensile strength.- The maximum load per unit of original cross-sectional area obtained before rupture.

5. Percentage elongation.-The percentage of increase in length of a tension-test specimen after rupture.

6. Percentage reduction of area.-The percentage of decrease of cross-sectional area of a tension-test specimen after rupture.

7. Shore hardness. - The reading on a conventional scale determined by the rebound of the hammer of the Shore scleroscope on striking the surface of the specimen.

8. Brinell hardness.-The number obtained from the ratio between the load applied on and the spherical area of the impression made by a steel ball forced into the surface of the material tested.

\subsection{HEAT TREATMENT OF MIETIS}

AJERICAN SOCIETY OF MECHANICAI ENGINEERS, A BIBIIOGRAPHY ON EFECT OT TEMPERATURE ON THE PROPERTIES OF METALS, 1928

\section{TEMPERATURT AND METAIS}

(A pamphlet containing 307 separate items on the effect of temperature both on ferrous and on nonferrous metals. In a subject index the effect of temperature is divided into two groups. In one group the time factor controls, but has no part in the other group. Thus divided, both ferrous and nonferrous metals are considered in terms of tensile creep, elasticity, apparatus and methods, service tests and requirements, chemical action-theoretical and general. In addition, ferrous metals connote compression and shear, hardness, shock, expansion, and heat conduction.)

AMERICAN SOCIETY FOR STEEL TREATING, DETINITIONS OF TERITS PELATING ING TO IEAT-TREATMENT OPERATIONS, 1929

\section{FEAT-TEEATMENT DEFINITIONS}

(Same as A. S. T. M., A119-30, given below.)

AMERICAN SOCIETY FOR STEEL TREATING, TENTATIVE GENERAL RECOMMENDATIONS FOR THE HEAT TREATOF TOOL STEELS, 1929

\section{HEAT TREATMENT OF TOOL STEELS}

Purpose.-Much of the general procedure for the heat treatment of one type of tool steel is applicable to others. These general instructions are to avoid repetitions in the Recommended practices.

General.-Often when correct temperatures are used, many hardening failures result from improper manipulation during heat treatment. The heat treatments given in the Recommended practices apply specifically to certain types of steels, the basic compositions of which are given. This does not imply that such steels are the only suitable ones. These practices are primarily for the purpose of recommending heat treatments.

If more specific information is desired on any type of steel, it should be obtained from the supplier of the material.

Rate of heating. - The rate of heating for carbon tool steels is given in the Recommended practices for the heat treatment of plain carbon tool steels.

The rate of heating for alloy tool steels is proportionally slower than for plain carbon steels as the alloying content increases.

Charging furnace.-Cold tools should be charged at comparatively low furnace temperature. Sudden changes in temperature should be avoided, except where steels, such as high-speed, are changed from preheating furnaces to high-heating furnaces to obtain rapid heating. It is especially important to avoid sudden changes when reheating hardened steel, as breakage may result.

The steel should be placed in the furnace so as to expose the maximum surface. It is often advisable to place supports under the tool to raise it from the furnace bottom, thus allowing better heat circulation. An intricate tool should be supported in such a. manner as to avoid warping.

Large sizes. - A range of temperature is usually given in recommendations for hardening heats. This does not imply that satisfactory results can be obtained on any tool if held anywhere within that range. Generally the larger pieces are heated to the high side of the range while small pieces are heated to the low side.

Uniform heating of tools. - Cylindrical tools or intricate parts that have a tendency to heat nonuniformly should be frequently rotated.

Holding at heat.-Care should be taken to hold the tool at temperature long enough to insure uniform heating throughout the section of the piece. 
Usually steel of higher alloy content will require more time, as noted in specific Recommended practices. This gives a better solution of the constituents, but may cause some grain growth and surface decarburization, particularly at temperatures well above the critical range.

For the time of holding high-speed steel at quenching heat, see the Recommended practice covering this stecl.

Furnace atmosphere.-Proper control of furnace gases is important. As far as possible a reducing atmosphere should be maintained at all times. Flames should not strike the steel.

Effect of scale.-If tool steel is heated above a low red heat while in contact with scale, even though in a neutral or reducing atmosphere, it will be decarburized. Therefore, care should be taken to keep steel, furnace hearth, and containers as free from scale as possible.

Thermocouples. - The thermocouple should be so placed that the flames do not strike it and so located as to indicate as nearly as possible the temperature of the tool.

It is generally better to insert the thermocouple through the end or side of the furnace rather than in the top. For specific instructions on pyrometry see the A. S. S. T. Handbook.

\section{COOLING}

Quenching medium.-The quenching medium should vary with the kind of steel treated as well as the speed of cooling necessary for various types of tools. Sufficient bath rolume should be provided to maintain the quenching medium at proper temperature for uniform results. The use of air as a means of agitation and cooling for liquid baths is not recommended.

Oils.-Oils generally give best results when used between the temperatures of $75^{\circ}$ and $125^{\circ} \mathrm{F}$. Some oils crack or otherwise deteriorate faster than others; consequently, should be replaced as found necessary.

Water. - Water should be held at a fairly constant temperature, usually between $70^{\circ}$ to $110^{\circ} \mathrm{F}$. Fresh tap water is often aerated and may produce soft spots, so that water which has been previously boiled, or has been used frequently, is recommended. A strong stream of water will produce better results and help to throw the scale, while a spray sometimes gives more uniform results.

Brine.-Tools quenched in brine will more readily throw their scale, producing a cleaner surface of more uniform hardness.

Air.-Certain types of tool steels require air cooling. When using an air blast, it should be applied through a properly designed device and care should be taken to avoid the presence of water in the air line. If tools are cooled naturally (without blast) they should be supported so as to allow uniform cooling.

Quenching.-Tools should be quenched in such a manner that warping is at a minimum. When immersing tools avoid the formation of gas pockets by vigorous agitation or by jets. It is important that the cooling medium come in contact with all parts of the work to be hardened. Tools should usually be removed before reaching the temperature of the quenching bath. For specific instances see the Tool Steel Recommended Practice, below.

\section{TEMPERING}

Strains.-Tools, after removal from the quenching bath and before tempering, are always in a highly and nonuniformly strained condition and danger of cracking is most pronounced. Tools should not become cold before tempering.

Uniform temperimg.- Satisfactory tempering results are obtained by heating slowly and uniformly in a freely circulating medium. If a liquid is used, the tools should be placed in a metal basket for immersion which prevents contact with the sides or bottom of the container.

Charging the tempering furnace.-When charging tools for tempering, the heating medium should be at a low temperature and the tools still warm.

Rate of heating tools.-Tools should be brought to heat slowly to prevent rapid release of hardening strains and warpage. Better results are obtained by holding at a lower temperature for a longer time. Generally the length of time should not be less than one hour at temperature, but for specific steels reference should be made to the Tool steel recommended practices.

Cooling from tempering.-Slow cooling from the tempering operation is recommended, since rapid cooling may induce strains in the steel.

Tempering by color.-Temper colors are the result of slight oxidation of the surface of steel when heated at low temperatures, and are dependent upon time as well as temperature. They are only indicative of the temperature of the surface and do not indicate that the steel has been heated uniformly throughout. Tempering by color is not conducive to uniformity. When necessity requires that tools be tempered by color they should be heated slowly as in other operations and the heat raised progressively to the point or color after which further tempering is usually stopped by quenching or withdrawing from the heating medium. The quenching should be done in the least drastic manner, preferably in oil.

\section{NORMALIZING}

Cementite segregates.-Normalizing implies heating steel far enough above the critical range to carry all cementite into solution and when this is done, it is followed by air cooling; cementite segregates may thus be broken up and a more uniform distribution obtained. This operation is more important on pieces that have been forged and should not be necessary on any material that has been annealed.

Rate of heating.-Heating tools for normalizing should be done in a manner similar to that of hardening.

Correct soaking time is proportional to the size of the piece. Prolonged soaking will coarsen the grain, rendering subsequent refinement difficult.

Rate of cooling. - The material is cooled from the normalizing temperatures in still air.

\section{ANNEALING}

Purpose.-Tool steel is annealed to soften it for machining, forming, and grain refinement.

Packing. - When the pieces are small it is advisable to pack them in charcoal to prevent scaling, decarburization, and too rapid changes of temperature. Care must be taken to keep the steel from contact with the container, and to avoid the presence of scale.

Heating.-Heat slowly and uniformly to the temperature desired. (See also specific Recommended practices.)

Holding at heat.-Hold at the annealing temperature long enough for complete penetration of heat and readjustment of grain. The time will increase with the weight of the charge and the alloy content of the steel.

Cooling.-Cool slowly, preferably in the furnace, or in mica or other good heat insulating material. In general, the slower the cooling, particularly through the critical range, the softer the steel. As the alloy content of the steel increases the slower the steel must be cooled.

Strain removal.- Heating is sometimes carried out at a temperature below the critical range for pur- 
poses of strain removal. This operation is beneficial as a preparatory treatment for hardening tools on which considerable machining has been done. This treatment should be performed after the rough machining operations, but before heating for quenching.

Warping.-The amount of warping on hardening is dependent on the shape of the tool, the kind of steel, the conditions of heating, and the severity of the quench. In general, in water hardening, steels warp more than in oil hardening, and long, thin tools more than short, heavy ones.

Some causes of undue warpage are improper tool design, the use of the wrong type of steel, nonuniform or too rapid heating, improper support of the steel while heating, heavy machining, cold-working strains, insufficient removal of surface, improper manner of quenching, nonuniform quenching, and nonuniform or too rapid tempering.

Soft spols.-Common causes of soft spots are decarburization, improper heating, quenching or bath agitation, gas pockets, tong contact, adhering scale, insufficient removal of surface, or defective furnace bottoms.

Straightening.-When necessary to straighten, the operation should be performed when quenching on the descending heat. The tool may change somewhat in the tempering operation, but the peening required will not be nearly as much as if the tools are straightened on the quenching heat. A continuous press should be available with an adjustable control and knife-edge rests, so that if one load does not satisfactorily straighten the tool another load can immediately be applied. For straightening on the descending heat, the tools should be withdrawn from the bath at a temperature from $500^{\circ}$ to $600^{\circ}$ F., which is just below the point where the oil ceases to flash, or in the case of water-quenched tools, just where the water steams or dries off the tools immediately.

AMERICAN SOCIETY FOR STEEL TREATING, RECOMMENDED PRACTICE FOR THE HEAT TREATMENT OF PLAIN CARBON TOOL STEEL, 1925

\section{HEAT TREATMENT OF PIAIN CARBON TOOL STEEL}

General.-This recommended practice covers the process to be followed in the heat treating of plain carbon tool steel.

\section{NORMALIZING OF TOOL STEEL BEFORE HARDENING}

\section{Operations.-Heating and cooling.}

Heating. - Place steel in furnace so as to expose maximum surface area. Heat uniformly to a temperature above the upper critical point, as given in Table 1, and hold at this temperature for sufficient time to obtain complete penetration of heat and for complete refinement of grain.

Cooling.-Remove from furnace and cool freely in air.

TABLE 1.-Normalizing temperature and carbon range

\begin{tabular}{|c|c|}
\hline Carbon & $\circ \mathrm{F}$. \\
\hline 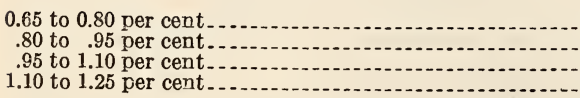 & $\begin{array}{l}1,475-1,525 \\
1,475-1,500 \\
1,500-1,575 \\
1,575-1,650\end{array}$ \\
\hline
\end{tabular}

CROSS SECTIONS, WEIGHT, AND TIME

\begin{tabular}{|c|c|c|c|}
\hline $\begin{array}{l}\text { Thickness of largest sec- } \\
\text { tion of unit }\end{array}$ & $\begin{array}{l}\text { Weight of unit in } \\
\text { pounds (aprrox- } \\
\text { imate) }\end{array}$ & $\begin{array}{c}\text { Timie of } \\
\text { heating, } \\
\text { in hours } \\
\text { (approxi- } \\
\text { mate) }\end{array}$ & $\begin{array}{l}\text { Timse of } \\
\text { hoi ling, } \\
\text { in hours } \\
\text { (approsi- } \\
\text { mate) }\end{array}$ \\
\hline $\begin{array}{l}\text { Up to and including } 1 \text { inch- } \\
\text { Over } 1 \text { inch and including } \\
2 \text { inches. } \\
\text { over } 2 \text { inches and includ- } \\
\text { ing } 3 \text { inches. } \\
\text { Over } 3 \text { inches and includ- } \\
\text { ing } 4 \text { inches. } \\
\text { Over } 4 \text { inches and includ- } \\
\text { ing } 5 \text { inches. } \\
\text { Over } 5 \text { inches and includ- } \\
\text { ing } 8 \text { inches. }\end{array}$ & $\begin{array}{l}\text { Up to } 100 . \\
\text { Over } 100 \text { and in- } \\
\text { cluding } 300 . \\
\text { Over } 300 \text { and in- } \\
\text { cluding } 500 \text {. } \\
\text { Over } 500 \text { and in- } \\
\text { cluding } 1,000 \text {. } \\
\text { Over } 1,000 \text { and in- } \\
\text { cluding } 1,500 \text {. } \\
\text { Over } 1,500 \text { and in- } \\
\text { cluding } 2,000 \text {. }\end{array}$ & $\begin{array}{r}8 / 4 \\
11 / 4 \\
18 / 4 \\
21 / 4 \\
28 / 4 \\
31 / 2\end{array}$ & $\begin{array}{l}1 / 2 \\
1 / 2 \\
2 / 4 \\
1 \\
1 \\
11 / 2\end{array}$ \\
\hline
\end{tabular}

HEAT TREATING OF PLAIN CAREON TOOL STEEL

Operations.-Heating for quenching, quenching, and temsering.

Heating.- Heat the steel uniformly to the temperature as given in Table 2 .

Quenching.-Quench from this temperature in water, but do not cool below temperature of boiling water $\left(212^{\circ} \mathrm{F}\right.$.).

\section{TABLE 2.-Quenching temperatures}

[Quenching temperature, water at $70^{\circ} \mathrm{F}$.]

\begin{tabular}{|c|c|c|c|c|}
\hline Carbon range, & $0.65-0.80$ & $0.81-0.95$ & $0.96-1.10$ & 1. $11-1.25$ \\
\hline $\begin{array}{l}\text { Hardening tempera- } \\
\text { ture. }\end{array}$ & $1,550-1,450$ & $1,460-1,410$ & $1,300-1,430$ & $1,3 \& 0-1,420$ \\
\hline
\end{tabular}

Tempering.-Reheat immediately in oil, salt $\left(\mathrm{NaNO}^{3}\right)$, or furnace for the time and at the ten perature specified in Table 3 and conl.

TABLE 3.-Tempering temperatures

\begin{tabular}{|c|c|c|}
\hline Results desired & $\begin{array}{l}\text { Tempering } \\
\text { medium }\end{array}$ & $\begin{array}{l}\text { Temper- } \\
\text { ature }\end{array}$ \\
\hline $\begin{array}{l}\text { Relieving strains } \\
\text { Relieving strain and reducing brittleness... } \\
\text { To relieve strains and to toughen........... }\end{array}$ & 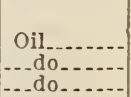 & $\begin{array}{l}\circ F . \\
350-375 \\
400-500 \\
500-600\end{array}$ \\
\hline
\end{tabular}

\section{NOTES ABOUT THE PROCESS}

General.-The recommended practice for the heat treatment of tool steel applies to the highest quality performance of tools for general purposes only. For specific applications, where special structural requirements seem to be necessary, deviation from th:e recommended practice must be left to the judgment of the individual.

Normalizing.-A normalizing treatment for all tool steels is recommended to obtain a uniform and refined grain structure, which enables the operator to predict the behavior and perforrriance of the tool steel during heating and quenching for hardening.

The variation in temperature for the different carbon ranges becomes evident upon a careful examination of the critical-range diagram, as shown in Figure 45. A temperature very much in excess of that required to produce solid solution (austenite) is conducive to austonitic grain growth and intergranular weakness.

A low normalizing temperature for high-carbon steels ( 1.25 to 1.50 per cent carbon) which fails to 
break up the massive cementite results in a brittle structure, since the rate of diffusion of the excess cementite depends upon the temperature. The solubility of carbide in iron being greater, or increasing as the temperature rises, the rate of this solubility is equivalent to the slope of the Acm. line in the diagram. (Figure 45.)

The higher normalizing temperatures given in Table 1 apply to the lower carbon ranges and the lower temperatures to the higher carbon ranges for steel of the hypoeutectoid comiposition, or, in other words, "the normalizing temperature for hypoeutectoid steels varies indirectly as the carbon percentage rises or falls, and the normalizing temperature of hypereutectoid steels varies directly with the jercentage of carbon."

Heating and quenching.-The wide range of temperature in the 0.65 to 0.80 per cent carbon steel is needed, as a number of tools, such as shear blades, arbors, mandrels, and others, are all around the low point, and because of mass and forms require somewhat higher temperatures for quenching.

The quenching temperatures given are at the lowest temperature range consistent with highest

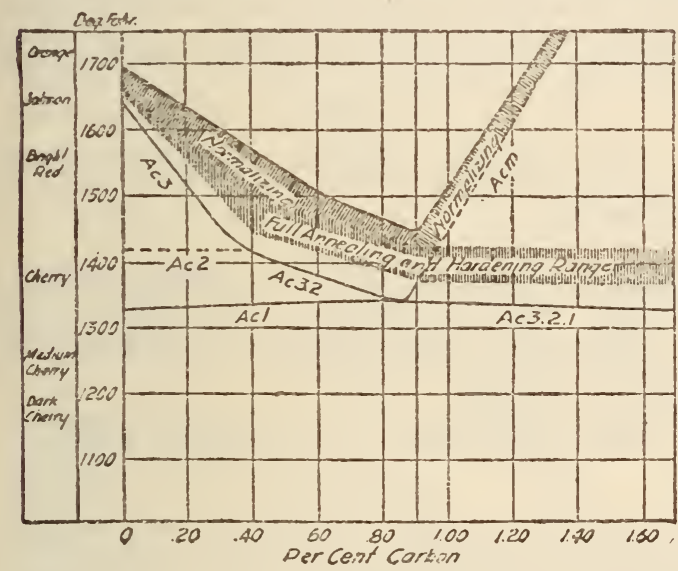

FIgURe 45.-Critical range diagram for ironcarbon alloys, showing normalizing, annealing, and hardening ranges

quality tools; deviations from it are not recommended, but may be practical for diverse reasons.

Quenching.-Water is the universal quenching medium and by varying its temperature and manner of application for the abstraction of heat, almost any degree of variation of structural conditions of the tool steel can be obtained. There are, however, special cases where oil may be a more suitable quenching medium.

\section{CARBON STEELS SUITABLE FOR VARIOUS USES}

\section{Carbon content 0.65 to 0.85 per cent}

Shear blades, boiler snaps and cups, hammers, stamping and pressing dies, mining drills.

\section{Carbon content 0.81 to 0.95 per cent}

Hot and cold sets, chisels, dies, shear blades, mining drills, smiths' tools, set hammers, swages, flatteners.

Carbon content 0.96 to 1.10 per cent

Small cold chisels, hot sets, small shear blades, large pinchers, large taps, granite drills, trimming dies, turning tonls, planer tools, drills, cutters, slotting and milling tools, mill picks, circular cutters, threading dies.
Carbon content 1.11 to 1.25 per cent

Small cutters, small taps, drills, slotting and planing tools, wood-cutting tools, turning tools, razors, etc.

\section{SUITABLE TEMPERIIG HEATS FOR VARIOUS TOOLS}

$$
\text { Temperature } 350^{\circ} \text { to } 390^{\circ} \mathrm{F} \text {. }
$$

Lathe tools for brass and copper alloys. Milling cutters for brass and copper alloys. Scraper and cutting tools for soft metals and micarta. Drawing mandrels, drawing dies, bone-cutting tools, hammer faces, steel engraving tools, wood-carving tools, cutting tools for iron and steel, hand tools, threading dies for brass.

$$
\text { Temperature } 400^{\circ} \text { to } 500^{\circ} \mathrm{F} \text {. }
$$

Hand taps and dies, hand reamers, drills, bits, cutting dies, penknives, milling cutters, chasers, inserted sawteeth, press dies for sheet steel, rock drills, taps and dies, wire-drawing dies, dental and surgical instruments, twist drills.

\section{Temperature $500^{\circ}$ to $6 \mathrm{CO}^{\circ} \mathrm{F}$.}

Bending and forming dies, shear blades, chuck jaws, forging dies, drifts, gages, press dies, flat drills, reamers, chisels and tools for woodcutting, hammers and drop dies, axes, lathe tools for copper augers, cold chisels, coppersmiths' tools, grinders, screw drivers, molding and planing tools, hack saws, needles, butcher knives, saws.

\section{AMERICAN SOCIETY FOR STEEL TREAT- ING, RECOMIENDED PRACTICE FOR THE HEAT TREATMENT OF 18 PER CENT TUNGSTEN HIGH-SPEED STEEL, 1925}

\section{HEAT TREATIENT OF HIGH-SPEED STEEL}

\section{GENERAL}

This recommended practice covers the process to be followed in the heat treating of high-speed steel.

\section{ANISEALING}

Heat slowly and uniformly to a temperature of $1,600^{\circ} \mathrm{F}$. and hold for complete adjustment and for complete uniformity of grain. Cool in furnace, infusorial earth, mica, lime or any medium that will permit of uniform slow cooling.

\section{HARDENITG}

(a) Preheating.-Heat slowly and uniformly to $1,500^{\circ} \mathrm{F}$. in a furnace of sufficient size.

(b) Heating for quenching.-Transfer preheated steel to a high-temperature furnace that is maintained at a temperature of $2,250^{\circ}$ to $2,400^{\circ} \mathrm{F}$., depending on the type of tools being hardened.

In order to obtain the most satisfactory "red hardness" conditions, the steel should be brought rapidly to the higher temperature; but in many cases the character of the cutting edges of certain form tools, such as milling cutters, threading tools, etc., makes it inadvisable to use the higher temperatures owing to destruction of the delicate edges through blistering, pitting, etc. It is, therefore, usual to use the higher temperatures for tools such as rough lathe tools, while the finer class of tools is hardened at the lower temperatures.

High-speed-steel tools should not be held at the high heat longer than necessary, since holding at the high hardening temperatures causes excessive grain growth, with subsequent brittleness of the hardened tools. 
Tools that can not be ground after hardening are often heated in a barium chloride or some such suitable salt bath.

Quench the steel in oil or air from the hardening temperature. It is advisable to maintain the oil quenching bath at a temperature of $150^{\circ}$ to $200^{\circ} \mathrm{F}$. to eliminate possibility of breakage with intricately shaped tools.

Tempering for secondary hardness.-Reheat uniformly in an open furnace, lead bath, or preferably in a sodium nitrate $\left(\mathrm{NaNO}_{3}\right)$ bath, or any other suitable salt bath, to a temperature of $1,050^{\circ}$ to $1,150^{\circ} \mathrm{F}$. for a sufficient length of time, and cool in air.

\section{NOT2S ABOUT TEE PROCESS}

Cooling from annealing.-Cooling in air should not be permitted, since air cooling from the annealing temperature is likely to result in partial hardening of the tool.

Heating for hardening. - It is the customary practice always to preheat for hardening in an open furnace, since preheating in a salt bath causes the salt to adhere to the tool, and the subsequent high temperature treatment causes unusual corroding from the adhering salt. Even preheating in lead is objectionable from small quantities of adhering lead. There is not much advantage to be gained in using a molten bath for the preheating, since the preheating temperature may vary over the comparatively wide range of $1,400^{\circ}$ to $1,600^{\circ} \mathrm{F}$., and an open furnace is invariably used in practice.

Quenching from tempering temperalures.-Quenching from the tempering temperatures is not advisable with high-speed steel.

\section{AMERICAN SOCIETY FOR STEEL TREAT. ING, RECOMMENDED PRACTICE FOR THE HEAT TREATIIENT OF NONSHRINK- ING, NONDEFORMING, OIL HARDENING TOOL STEELS, 1928}

\section{HEAT TREATMENT OF TOOL STEELS}

\section{GENERAL}

This recommended practice covers the process to be followed for the heat treatment of so-called nondeforming, oil-hardening steels. Because there are steels of other analyses used for nondeforming steels which require different heat treating temperatures, this recommended practice applies only to the steels given in Table 1.

TABLE 1.-Approximate chemical composition

\begin{tabular}{|c|c|c|c|c|}
\hline Steel No. & $\mathrm{C}$ & Mn & $\mathrm{Cr}$ & $w$ \\
\hline & $\begin{array}{r}0.90-0.95 \\
0.90\end{array}$ & $\begin{array}{r}1.00-1.25 \\
1.50\end{array}$ & 0.50 & \\
\hline
\end{tabular}

\section{ANNEALING OF STEEL AFTER FORGING AND BEFOR MACHINING}

Place steel in furnace so as to expose maximum surface area. For steel No. 1 as listed in Table 1, heat slowly and uniformly to a temperature of approximately $1,475^{\circ} \mathrm{F}$. and to $1,400^{\circ} \mathrm{F}$. for steel No. 2. Hold the steels at these temperatures for sufficient time to obtain complete penetration of heat and to obtain complete refinement of grain.

Cool in furnace, infusorial earth, mica, lime, or any medium that will permit uniform, slow cooling.

\section{HERDENING}

Heat the steel very slowly but uniformly to a dark red heat of approximately $1,200^{\circ} \mathrm{F}$., then raise temperature inore rapidly but uniformly to the quenching heat of $1,450^{\circ}$ to $1,500^{\circ} \mathrm{F}$. for steel No. 1 and to $1,400^{\circ}$ to $1,475^{\circ} \mathrm{F}$. for steel No. 2 . Hold the steels at these temperatures for complete penetration of heat.

Quench from these temperatures in light oil, but do not cool below temperature of boiling water, $212^{\circ} \mathrm{F}$.

Tempering.-For steels Nos. 1 and 2 reheat immediately in oil or other heating medium at a temperature of $325^{\circ}$ to $400^{\circ} \mathrm{F}$., hold for about one-half hour and cool from the tempering temperature in still air.

\section{NOTES ABOUT THE PROCISS}

Nonshrinking steels are essentially oil-hardening steels. The ratio of bath volume to mass of steel is a very important factor, and the heating up of the oil during prolonged and continuous quenching operation should be avoided.

Heating should be done very gradually and uniformly, perferably in a muffie furnace, the steel being out of contact with flame and furnace combustion gases.

The absorption of heat or its diffusion through these steels seems to be much slower than in ordinary carbon or alloy steels. Therefore, these steels should be heated more slowly and quenched less drastically.

Taps; reamers; broaches; blanking-trimmingpunching-forming and threading-dies; master-tools and gages.

AMERICAN SOCIETY FOR STEEL TREATING, RECOMMENDED PRACTICE FOR THE HEAT TREATMENT OF FINISHING STEEL, 1927

\section{HEAT TREATMENT OF FINISHING STEEL}

\section{GENERAL}

This recommended practice covers the process to be followed in the heat treatment of so-called fastfinishing steel, sometimes also referred to as semihigh-speed steel. This steel is fast finishing in comparison to carbon tool steel, but is not to be confused with high speed steel.

\section{CHEMICAI CONPOSITION}

There are various types of fast-finishing steels manufactured and it is not recommended that this is the most suitable steel to use, but simply points out that the heat treatments recommended refer to steels of the following approximate chemical composition:

\section{Approximaie Chemical Analysis}

Per cent

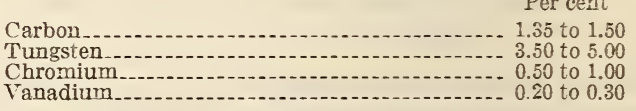

\section{ANNEALING OF STEEI, AFTER FORGING AND BEFORE}

Place steel in furnace so as to expose maximum surface area. Heat slowly and uniformly to a temperature of approximately $1,450^{\circ}$ to $1,525^{\circ} \mathrm{F}$. and hold at this temperature for a sufficient time to obtain complete penetration of heat and complete refinement of grain.

It is recommended that the steel be heated in a closed pipe or box containing a small amount of carbonaceous material, similar to regular tool steel practice.

Cool the steel in the furnace. 
BARDENING

Heat the steel uniformly to $1,475^{\circ}$ to $1,525^{\circ} \mathrm{F}$. and hold for complete penetration of heat. Quench from this temperature in water.

\section{TEMPERING}

Reheat immediately in oil or other heating medium at a temperature of $300^{\circ}$ to $500^{\circ} \mathrm{F}$. according to the use to which the steel is to be put. For intricate or small sections quench in oil or air.

\section{AMERICAN SOCIETY FOR STEEL TREAT- ING, RECOMMENDED PRACTICE FOR THE HEAT TREATMENT OF TAPS AND MILIING CUTTERS, 1927}

\section{HEAT TREATMENT OF TAPS AND MILLING CUTTERS}

\section{GENERAI}

This recommended practice covers the process to be followed in the heat treatment of taps and nilling cutters made from plain carbon tool steel, 18 per cent tungsten high-speed tool steel, and 1.50 to 2 per cent tungsten tool steel, and must not be applied to taps and inilling cutters having a chemical composition other than given below.

\section{Approximate chemical composition}

\begin{tabular}{|c|c|c|c|}
\hline & $\begin{array}{l}\text { Plain car- } \\
\text { bon tool } \\
\text { steel }\end{array}$ & $\begin{array}{c}18 \text { per cent } \\
\text { tungsten } \\
\text { high-speed } \\
\text { steel }\end{array}$ & $\begin{array}{l}1.50 \text { to } 2 \\
\text { per cent } \\
\text { tungsten } \\
\text { tool steel }\end{array}$ \\
\hline Carbon. & $\begin{array}{r}\text { Per cent } \\
1.00-1.30\end{array}$ & $\begin{array}{c}\text { Per cent } \\
0.65-0.75\end{array}$ & $\begin{array}{l}\text { Per cent } \\
1.00-1.30\end{array}$ \\
\hline Tungsten & 19-2- & $17.50-18.50$ & $1.50-2.00$ \\
\hline Chromium & $---n-1$ & $3.25-4.25$ & 1.50 \\
\hline Vanadium & & $.75-1.25$ & 1. 25 \\
\hline Manganese _. . maximum & .35 & .35 & .25 \\
\hline Phosphorus . . . . . . & .030 & .030 & .030 \\
\hline Sulphur _............ do & .030 & .030 & .030 \\
\hline Silicon_..._- & & & \\
\hline
\end{tabular}

1 Optional.

\section{ANKTAIING OF STEEL AFTER FORGING AND BEFORE MACHINING}

Place the steel in the furnace on a charcoal-covered bottom (except high-speed steel) and heat slowly and uniformly to the temperature given in Table 1 , and hold at this temperature for sufficient time to obtain complete penetration of heat and complete refinement of grain. It is recommended that the steel, especially the high-speed steel, be heated in a closed pipe or box containing some carbonaceous material.

Cool the steel in the furnace, infusorial earth, mica, lime, or any medium that will permit uniform, slow cooling and prevent excessive oxidation.

\section{ANNEALING FOR RELIEVING MACHINING STRAINS}

Place the taps and milling cutters in a box containing some carbonaceous material (except in case of high-speed steel). Place the box and contents in the furnace and heat to the temperature given in Table 2. Cool in furnace.

\section{TABLE 1.-Annealing after forging and before machining}

\begin{tabular}{|c|c|c|c|c|c|}
\hline Type of steel & Method & $\begin{array}{l}\text { Tem- } \\
\text { pera- } \\
\text { ture }\end{array}$ & $\begin{array}{l}\text { Time of } \\
\text { heating to } \\
\text { uniform } \\
\text { tempera- } \\
\text { ture }\end{array}$ & $\begin{array}{l}\text { Time } \\
\text { of } \\
\text { hold- } \\
\text { ing at } \\
\text { tem- } \\
\text { pera- } \\
\text { ture }\end{array}$ & Cool in- \\
\hline Plain carbon. & $\begin{array}{l}\text { Packannealor } \\
\text { on charcoal } \\
\text { bottom. }\end{array}$ & $\stackrel{\circ}{F}$ & $\begin{array}{l}1 \text { hour per } \\
\text { inch of } \\
\text { d i a m - } \\
\text { eter or } \\
\text { thick- } \\
\text { ness. }{ }^{1}\end{array}$ & $\begin{array}{r}\text { Hour } \\
1 / 2\end{array}$ & Furnace. \\
\hline $\begin{array}{l}18 \text { per cent } \\
\text { tungsten } \\
\text { high speed. }\end{array}$ & $\begin{array}{l}\text { Nonoxidizing } \\
\text { atmosphere. }\end{array}$ & $\begin{array}{l}1,600 \\
1,650\end{array}$ & -..._do_.... & $1 / 2$ & Do. \\
\hline $\begin{array}{l}.5 \text { i to } 2 \text { per } \\
\text { cent tung- } \\
\text { sten. }\end{array}$ & $\begin{array}{l}\text { Packanneal or } \\
\text { on charcoal } \\
\text { bottom. }\end{array}$ & 1,475 & . do do.... & $1 / 2$ & Do. \\
\hline
\end{tabular}

1 When pack annealing, consider the container and contents as unit.

2 If lead bath or salt bath is used for heating, cool in lime, mica, or infusorial earth.

\section{IIRDENING}

Heat the taps or milling cutters uniformly to the teinperature given in Table 3 and hold for complete penetration of heat.

Quench from this temperature into water, brine, or oil as directed in Table 3, but do not cool below the temperature of boiling water, $212^{\circ} \mathrm{F}$.

\section{TEMPERING}

After quenching, reheat immediately in oil, salt, or furnace for the time and temperature specified in Table 4.

TABLE 2.-Annealing for relieving machining strains

\begin{tabular}{|c|c|c|c|c|}
\hline Type of steel & $\begin{array}{l}\text { Tem- } \\
\text { pera- } \\
\text { ture }\end{array}$ & $\begin{array}{l}\text { Time of heating to } \\
\text { uniform temper- } \\
\text { ature }\end{array}$ & $\begin{array}{l}\text { Time } \\
\text { of } \\
\text { hold- } \\
\text { ing at } \\
\text { tem- } \\
\text { pera- } \\
\text { ture }\end{array}$ & Cool in- \\
\hline Plain carbon. & $\begin{array}{l}\circ F \\
1,400\end{array}$ & $\begin{array}{l}1 \text { hour per inch of } \\
\text { diameter or thick- }\end{array}$ & $\begin{array}{r}\text { Hour } \\
1 / 4\end{array}$ & Furnace. \\
\hline 18 per cent tung- & 1,500 & $\begin{array}{l}\text { ness. } \\
\text { ndo...... }\end{array}$ & $1 / 4$ & Do. \\
\hline $\begin{array}{l}\text { sten high speed. } \\
1.50 \text { to } 2 \text { per cent } \\
\text { tungsten. }\end{array}$ & 1,450 & ......do_. & $1 / 4$ & Do. \\
\hline
\end{tabular}

TABLE 3.-Heating and quenching of taps and milling cutters

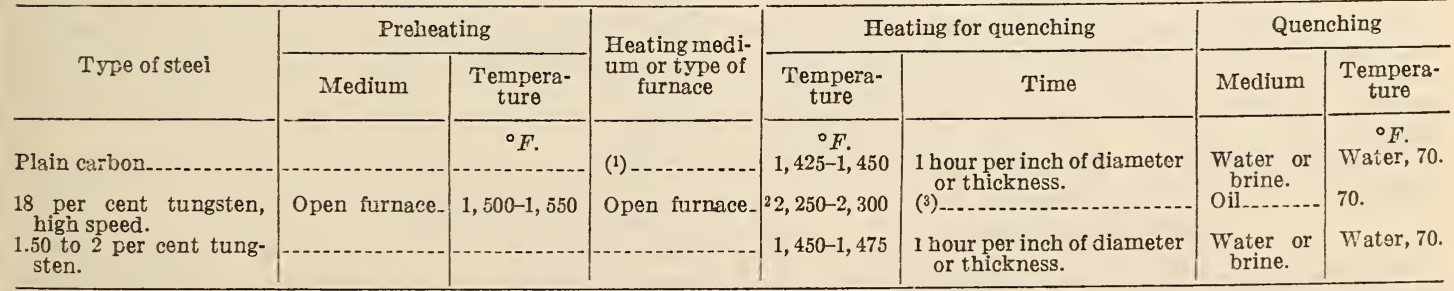

1 If a lead bath is used for heating, a higher temperature and shorter time should be used. When heating the steel in an open furnace either an indirect fired furnace (muffe) should be used or a smoky flame should be maintained.

${ }^{2} \mathrm{~A}$ temperature as high as $2,350^{\circ} \mathrm{F}$., may be used provided the furnace design is correct and the atmosphere is suitable.

3 Eold shortest time possible to thoroughly heat through. 
TABLE 4.-Tempering of taps and milling cutters

\begin{tabular}{|c|c|c|c|c|}
\hline Type of steel & $\begin{array}{l}\text { Pesults } \\
\text { desired }\end{array}$ & $\begin{array}{l}\text { Tempering } \\
\text { medium }\end{array}$ & $\begin{array}{l}\text { Tempering } \\
\text { temperature }\end{array}$ & Time \\
\hline $\begin{array}{l}\text { Plain carbon } \\
18 \text { per cent tung- } \\
\text { ten high speed. } \\
1.50 \text { to } 2 \text { per cent } \\
\text { tungsten. }\end{array}$ & $\begin{array}{l}\text { (1) } \\
(2) \\
(1)\end{array}$ & $\begin{array}{l}\text { Oil......... } \\
\text { Furnace... } \\
\text { Oil_......... }\end{array}$ & $\begin{array}{c}{ }^{\circ} F . \\
325-500 \\
1,050-1,150 \\
325-500\end{array}$ & $\begin{array}{l}\text { Hour } \\
1 / 2 \text { to } 1 \text {. } \\
1 / 2 \text {. } \\
1 / 2 \text { to } 1 .\end{array}$ \\
\hline
\end{tabular}

1 Depending on degree of hardness or toughness desired.

2 Development of secondary hardness and toughness.

\section{NOTES ABOUT THE PROCESS}

In the above practice it is assumed that the tap shanks are below the hardening temperature and are not fully hardened. If the shanks have been fully hardened they must be tempered in order to toughen them, especially the smaller sizes.

\section{AMERICAN SOCIETY FOR STEEL TREAT- ING, RECOMMENDED PRACTICE FOR THE HEAT TREATMENT OF DIES FOR DIE CASTINGS, 1930}

\section{HEAT TREATMENT OF DIE CASTINGS}

\section{INTRODUCTION}

A die casting is a finished, or practically finished, casting made by forcing a liquid metal or alloy under pressure into a metallic mold or die. Iittle or no machining other than drilling for screws, bolts, and the removal of fins by dressing is required to put the casting in condition for use.

The essential feature in the die-casting process, which distinguishes it from other processes involving metallic molds, is that the alloy is forced into the die cavity by pressure; such pressure may be mechanical as a plunger working in a cylinder, or air pressure.

Die casting, as is the case with other processes involving metallic molds, is essentially a quantity production process. This is necessarily so because of the heavy expense involved in designing and preparing the dies. In small lots, the heary die expense will be proportionately reflected in the cost of tne castings.

The die-casting process is practical for tin, lead, zinc, and aluminum base alloys. Alloys of higher melting points, like the brasses and bronzes, can be die cast, but such alloys have so strong an erosive effect on ordinary die steels that the process is not practical for these alloys. The life of a die when casting brass is exceedingly short and at the present time the die-casting process is confined to alloys of relatively low melting points.

The die-casting process is especially applicable to the production of small interchangeable parts which are required to be well finished and with accurate dimensions. Die castings up to 8 and 10 pounds are being commercially produced to-day.

\section{GENERAL}

This recommended practice covers the process for the heat treatment of dies for die castings. There are other steels used for this purpose, but this recommended practice applies only to the steels listed in Table 1.

Die-casting dies for aluminum-alloy work are generally made of alloy steel.

\section{CHEMICAI COMPOSITION}

The approximate chemical compositions of the steels generally used for die casting dies are given in Table 1.
TABLE 1.-Approximale chemical composition of steels for dies for die casting

\begin{tabular}{|c|c|c|c|c|c|c|}
\hline $\begin{array}{l}\text { Steel } \\
\text { No. }\end{array}$ & C & $\mathrm{Mn}$ & $\begin{array}{c}\text { Si } \\
\text { (maxi- } \\
\text { mum) }\end{array}$ & $\mathrm{Cr}$ & $w$ & \\
\hline $\begin{array}{lll} & 1 & \ldots \\
2 & 1 & \ldots \\
2 & & \ldots\end{array}$ & $\begin{array}{r}0.20-0.50 \\
.25-.35\end{array}$ & $\begin{array}{c}\text { Other el } \\
\text { rar } \\
0.60-0.70\end{array}$ & $\begin{array}{ll}\text { ements } \\
y & 0.30\end{array}$ & $20.65-0.75$ & & \\
\hline $44 \ldots$ & $\begin{array}{l}.20-.35 \\
.40-.55\end{array}$ & $\begin{array}{l}.50-.80 \\
.40-.80\end{array}$ & $\begin{array}{r}.30 \\
.30\end{array}$ & $\begin{array}{l}\text { With or } \\
\text { without } \\
\text { about } \\
1.00 \\
2.00-2.50\end{array}$ & & $\begin{array}{l}\text { Al } \\
0-1.50\end{array}$ \\
\hline $5 \ldots$ & $.30-.45$ & $.20-.35$ & $.50-1.00$ & 4. $50-6.00$ & 4. $00-5.00$ & $\begin{array}{l}\text { Co, with } \\
\text { or with- } \\
\text { out } \\
\text { 8bout } \\
0.50\end{array}$ \\
\hline 6 & $.25-.40$ & $.20-.35$ & .35 & 2.7 & $8.50-10.50$ & \\
\hline
\end{tabular}

1 Die steel for zinc, tin, and lead base alloys. The other steels are used for aluminum.

Nickel range for this steel is 1 to 1.50 per cent.

3 Molrbdenum range for this steel is 0.20 -1 per cent. Steels containing low percentages of molvbdenum, ordinsrils contain chromium. When the chromium is not present the molybdenum is on the high side of the range.

Vanadium content for this steel is $0.20-0.30$ per cent.

5 This steel may or mas not contain ranadium. The range is 0.30-0.60 per cent ranadium.

\section{PEAT TREATNENT}

Steels Nos. 1 and 2, as listed in Table 1, do not require a heat treatment, but are used in the soft condition as received from the mill.

Unless otherwise stated, the heat treatments as outlined in this recommended practice refer to treatments as performed in a muffle or semimuffle type furnace.

\section{IITRIDING STEEIS}

One of the more recent developments in die steels for die castings is the use of nitrided steels. This steel is finished machined and the impression polislied before subjecting it to the nitriding process. Steel No. 3 in Table 1 shows the approximate composition of nitriding steels. These steels can be softened after nitriding for necessary alterations by a short anneal in a double chloride bath (50 per cent sodium chloride and potassium chloride) at a temperature of $1,400^{\circ}$ to $1,500^{\circ} \mathrm{F}$.

\section{HEAT TREATMENT OF THE CEROMIOM-TATADIUM DIR CASTING STEEI}

(Steel No. 4 in Table 1)

Normalizing after forging.-The chromium-vanadium steel (steel No. 4 as listed in Table 1) is usually normalized after forging by couling from about $1,750^{\circ}$ to $1,800^{\circ} \mathrm{F}$. The steel is heated at the rate of about one hour per inch of diameter or thickness ${ }^{35}$ and held at the normalizing temperature for onefifth of the total heating time. The steel is cooled from this temperature in air.

Annealing after normalizing.-After the steel has been normalized it should be annealed by heating approximately one hour per inch of diameter or thickness to a temperature from $1,425^{\circ}$ to $1,475^{\circ} \mathrm{F}$. After holding the steel at the annealing temperature for one-fifth of the total heating time, it is cooled with the furnace. After this annealing the steel should have a maximum Brinell hardness number of 200 .

Packing.-Instructions for paching are given at the end of this practice.

Hardening. - The dies are heated to the hardening temperature of $1,600^{\circ} \mathrm{F}$. at a rate of approximately one and one-half hours per inch of greatest thickness. The dies are held at this hardening temperature for about one hour for every inch of diameter or thich- 
ness. After holding the heat for the required time the dies are quenched into oil which should be at a temperature of $80^{\circ}$ to $120^{\circ} \mathrm{F}$. The dies should be removed from the oil when cooled just far enough so that the cil does not flash and catch fire on the die block after it comes out of the quenching bath.

For intricate dies, hold the hardening temperature at $1,600^{\circ} \mathrm{F}$. for the full specified time, then drop the furnace temperature with the work to $1,500^{\circ} \mathrm{F}$. and hold until this temperature is uniform throughout, then quench as above.

Tempering.36-After quenching, the dies should be charged into the tempering furnace without being allowed to cool to room temperature. When the dies are charged into the tempering furnace, the furnace temperature should not be higher than $450^{\circ} \mathrm{F}$. The temperature is then raised slowly and uniformly to $800^{\circ}$ to $900^{\circ} \mathrm{F}$. and held at this temperature for about one hour for every inch of diameter or thickness. A Brinell hardness number of about 444 is desirable.

\section{HEAT TREATMENT OF THE TUNGSTEN-CEROMIUM DIE CASTING STEEL}

(Steel No. 5 in Table 1)

Normalizing after forging.-The tungsten-chronium die steel (steel No. 5 as listed in Table 1) is usually normalized after forging by cooling from about $1,750^{\circ}$ to $1,800^{\circ} \mathrm{F}$. The steel is heated at the rate of about one hour per inch of diameter or thickness and held at the normalizing temperature for one-fifth of the total heating time. The steel is cooled from this temperature in air.

Annealing.-After the steel has been normalized it should be annealed by heating approximately one hour per inch of diameter or thickness to a temperature of $1,550^{\circ}$ to $1,600^{\circ} \mathrm{F}$. After holding the steel at the annealing temperature for one-fifth of the total heating time, it is cooled slowly with the furnace.

Packing.- Instructions for packing are given at the end of this practice.

Hardening. - The dies should be heated at the rate of approximately one and one-half hours for each inch of maximum thickness to a hardening temperature of about $1,800^{\circ} \mathrm{F}$. The dies should be held at the hardening temperature approximately one-fifth of the heating time and then quenched into oil. For hardening large or intricate dies, quench into air from a temperature of about $1,875^{\circ} \mathrm{F}$.

Tempering.-After quenching in either oil or air the dies should be reheated immediately to a temperature not exceeding $1,075^{\circ} \mathrm{F}$. The dies should be held at the tempering heat five hours with an additional one hour for each inch of diameter or thickness. A Brinell hardness number from 400 to 460 has been found satisfactory.

\section{EEAT TREATMENT OF THE TUNGSTEN DIE CASTING STEEL}

(Steel Ko. 6 in Table i)

Annealing.-This steel should be annealed after forging and before machining. For this annealing treatment the steel is heated to a temperature of $1,600^{\circ}$ to $1,650^{\circ} \mathrm{F}$. at the rate of approximately one hour per inch of diameter or thickness and held at this temperature for a minimum time of one hour. From this temperature the steel is cooled with the furnace. The cooling is retarded at $1,450^{\circ}$ to $1,400^{\circ}$

F. for sufficient time (about one hour) to bring about a properly annealed structure.

Packing.-Instructions for packing are given at the end of this practice.

${ }^{35}$ This dimension is taken for all heat treatments at the largest section of the die.

${ }_{36}$ Then a die must be machined after heat treatment to exact size, it may, be desirable to temper this steel at $1,100^{\circ} \mathrm{F}$. or higher in orde to finish machining.
Preheating for hardening.-Before hardening, this steel is first preheated to a temperature of $1,500^{\circ}$ to $1,550^{\circ} \mathrm{F}$. and held at this temperature for about one hour for every inch of diameter or thickness.

Hardening.- After the dies have been preheated, they should be heated to a hardening temperature of approximately $1,800^{\circ}$ to $1,900^{\circ} \mathrm{F}$. and removed for cooling from the furnace as soon as this hardening temperature has been uniformly attained. The dies may either be cooled in still air or quenched into oil.

Oil quenching protects the surface but produces slightly greater movement than air cooling. Air cooling is usually resorted to when the permissible movement is at a minimum, and when the dies are air cooled the impression should be protected against excessive scaling.

When quenched into oil the dies should be withdrawn while still warm and immediately tempered.

Tempering.-After hardening, the dies should be reheated immediately to a temperature of $1,050^{\circ}$ to $1,250^{\circ} \mathrm{F}$. and held at this temperature for five hours with an additional one hour for each inch of diameter or thickness. A Brinell hardness number from 401 to 444 is desirable.

\section{PACKING}

Because of the high temperatures used in the heat treatment of steels Nos. 4, 5, and 6, it is necessary to protect the finished surface from scaling. The dies may be packed in cast-iron chips, spent charcoal, or other materials, which have proven satisfactory. However, when the dies are packed in these materials, excessive carburization may occur. It has been found that sealing the die in a box in which a mixture of charcoal with approximately 3 per cent by weight of soda ash surrounding the die, but not in contact with the die face, will produce a surface freer from carburization or decarburization than with the other methods and still prevent scale formation. Soda ash must not come in contact with the working face of the die.

\section{NOTES ABOUT THE PKOCESS}

When die casting aluminum base alloy castings, first heat the dies uniformly to a temperature of approximately $350^{\circ} \mathrm{F}$.

Dies for die casting are usually given a protective coating before going into service. This coating depends upon the alloy being cast.

\section{AMERICAN SOCIETY FOR STEEL TREAT- ING, TENTATIVE RECONMENDED PRACTICES FOR THE HEAT TREAT- MENT OF PIAIN CARBON AND ALLOY. STEEI DIE BIOCKS, 1931}

\section{HEAT TREATMENT OF DIE BIOCRS}

General.-This recommended practice covers the process to be followed in the heat treatment of die blocks made from plain carbon and alloy steels, but must not be applied to die blocks having chemical compositions other than those given in Tables 1 and 3.

It is not recommended that these steels are the most suitable for die blocks, because there are other steels used that are equally as satisfactory, but, of course, the heat treatments given in this recommended practice can not be applied.

Many die blocks are purchased from the manufacturer in the heat-treated condition. These blocks usually have a scleroscope hardness range of 40 to 57 , according to the depth of impression. Shallow impressions usually require the higher hardness 
Prior to any heat treatment, die blocks should have received hot forging on the six sides, in order to refine the grain and lessen the tendency toward directional structures.

When quenching die blocks, it is important that the quenching bath be sufficiently agitated to avoid the formation of gas pockets. When hardening with air, the blast should be dry and so directed as to strike the face of the block uniformly. The pressure of the air blast should be at least 12 ounces, and the volume should be adequate.

\section{PLAIN CARBON STEEL DIE BLOCKS}

(Cold and Hot Forming)

Chemical composition.-There are three classes of carbon steels generally used for die blocks. The chemical compositions of these steels are given in Table 1.

TABLE 1.-Approximate chemical composition for carbon-steel die blocks

\begin{tabular}{|c|c|c|c|c|}
\hline $\begin{array}{c}\text { Die } \\
\text { block } \\
\text { No. }\end{array}$ & Application or type & $\mathrm{C}$ & Mn & $\mathrm{Si}$ \\
\hline & Carbon-steel die blcck & $\begin{array}{r}0.50-0.65 \\
.60-.75\end{array}$ & $\begin{array}{r}0.60-0.80 \\
.20-.40\end{array}$ & $\begin{array}{r}0.15-0.30 \\
.15-.30\end{array}$ \\
\hline & $\begin{array}{l}\text { Cold-forming dies; ordi- } \\
\text { nary hammer die block }\end{array}$ & $.75-.90$ & $.20-.40$ & $.15-.30$ \\
\hline
\end{tabular}

1 This steel may or may not contain vanadium up to 0.30 per cent.

Normalizing after forging.-The normalizing temperatures and directions for heating and cooling are given in Table 2.

TABLE 2.-Heat treatments for carbon-steel die blocks-Cold and hot forming die blocks

\begin{tabular}{|c|c|c|c|c|}
\hline \multirow{2}{*}{$\begin{array}{l}\text { No. as listed in } \\
\text { Table } 1\end{array}$} & \multirow{2}{*}{$\frac{\begin{array}{c}\text { Normaliz- } \\
\text { ing }{ }^{1}\end{array}}{\begin{array}{c}\text { Tempera- } \\
\text { ture }\end{array}}$} & \multicolumn{2}{|c|}{$\begin{array}{l}\text { Annealing and sphe- } \\
\text { roidizing } 2\end{array}$} & \multirow{2}{*}{$\begin{array}{c}\text { Hardening } \\
\\
\begin{array}{c}\text { Tempera- } \\
\text { ture }\end{array}\end{array}$} \\
\hline & & $\begin{array}{l}\text { Tempera- } \\
\text { ture }\end{array}$ & Cool in- 1 & \\
\hline 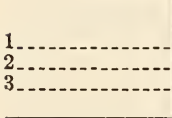 & $\begin{array}{c}\circ F . \\
1,450-1,500 \\
1,475-1,550 \\
1,550-1,575\end{array}$ & $\begin{array}{c}\circ F . \\
1,300-1,350 \\
1,350-1,375 \\
1,300-1,350\end{array}$ & $\mid \begin{array}{c}\text { Furnace.. } \\
-d 0_{2}\end{array}$ & $\begin{array}{c}\circ F . \\
1,400-1,425 \\
1,450-1,500 \\
1,425-1,475\end{array}$ \\
\hline
\end{tabular}

1 The time required to bring the blocks to normalizing heat should be 1 hour per inch of diameter or thickness. The time at the normalizing heat should be one-fifth of the total heating time. With large blocks, the time of holding at heat should be increased in order to refine the center of the block.

2 It is assumed for this heat treatment that the die block was first normalized. Time heating is 1 hour for every inch of diameter or thickness plus 1 hour. Time at heat is 1 hour per inch of diameter or thickness pl

${ }_{3}^{3}$ Quench into water. After quenching, temper to desired hardness. Time heating 1 hour per inch of diameter or thickness. Time at heat one-fifth of heating time.

${ }_{4}$ After this heat treatment, the scleroscope hardnẻss should not be over 30 .

sion

TABLE 3.-Approximate chemical composition for alloy-steel die blocks

(x)

\begin{tabular}{|c|c|}
\hline $\begin{array}{c}\text { Die block } \\
\text { No.1 }\end{array}$ & Application or type \\
\hline & Drop hammer die block. \\
\hline & Drop hammer die blocks \\
\hline $3-$ & Oil or air hardening die block ${ }^{3}$ \\
\hline $4-$ & Hot-forming die block \\
\hline $\begin{array}{l}5-- \\
6--\end{array}$ & $\begin{array}{l}\text { Special hot-working die steel } \\
\text { Chromium hot-working die-steel }\end{array}$ \\
\hline & Header die steel \\
\hline & Hot-working die steel \\
\hline
\end{tabular}

\begin{tabular}{|c|}
$\mathrm{C}$ \\
\hline $0.50-0.70$ \\
$.50-.70$ \\
$.50-.70$ \\
$.60-.70$ \\
$.45-.65$ \\
$.80-1.05$ \\
$.40-.55$ \\
$.30-.45$
\end{tabular}

The temperature of the normalizing furnace should not be over $500^{\circ} \mathrm{F}$. when the die blocks are charged into it. After normalizing, cool the blocks in air until they are below the lower critical point.

Annealing and spheroidizing.-The annealing and spheroidizing temperatures and directions for heating and cooling are given in Table 2.

From the annealing and spheroidizing treatment, cool the blocks slowly in the furnace until they become black. ${ }^{37}$

Hardening. - The quenching temperature, the time required to bring the die blocks to heat, the time at heat, and the quenching medium used for these die blocks are given in Table 2 .

The block is immersed from 1 to 2 inches below the deepest part of the impression 38 and held in the water until the block is cooled down to a temperature where reheating from the inside is not likely to occur to a sufficient degree to cause softening. This is determined by examining, with the hand, the deepest part of the impression. After quenching, the block is immediately placed in the tempering furnace, hot oil or salt bath, or transferred to a hot plate for tempering.

\section{TEMPERING}

1. Tempering hot-forming die blocks.-After quencling, the blocks are tempered immediately at a temperature from $450^{\circ}$ to $1,000^{\circ} \mathrm{I}$., depending upon the hardness desired.

A scleroscope hardness of 50 to 70 is suggested; the approximate Brinell hardness number is 331 to 495 .

2. Tempering cold-forming die blocks.-After quenching, the blocks are tempered immediately at a temperature of $350^{\circ}$ to $600^{\circ} \mathrm{F}$., depending upon the hardness desired.

A scleroscope hardness of 70 to 80 is suggested; the approximate Brinell hardness number is 495 to 578 .

\section{ALLOY STEEL DIE BLOCKS}

(See General Remarks)

Chemical compositions.-The chemical compositions of the alloy steel die blocks are given in Table 3 .

Normalizing.-Directions for the normalizing of the die blocks (except No. 8, which is not normalized) are given in Table 4.

When the normalized die blocks become black or approximately $900^{\circ} \mathrm{F}$., they should be charged back into the furnace for annealing or spheroidizing. Cool the blocks from the normalizing temperature in still air until they are below the lower critical point.

37 To test the soundness of the steel (structural condition and im perfections) the block mar be rehested to the hardening temperature and quenched completely after the normolizing and spheroidizin operations. The block is then annealed as directed in Table 2.

38 Dions. The block is then annealed as directed in Table 2 . quenched entirely in water and cooled to about $700^{\circ} \mathrm{F}$. (slightly below a red heat). They are then withd rawn and cooled in oil to a temperature of approximately $200^{\circ} \mathrm{F}$. and immediately tempered to desired hardness. When quenching the block, a strong geyser of wate should be directed into the impression of the block. To prevent warping during quenching, the shank may be cooled to a black heat by pouring water on it.
1 The silicon content for these steels is 0.15 to 0.30 per cent 2 These steels may or may not contain vanadium.
3 When this steel is use for upsetters' tools, it is usually air hardened. 4 This steel is mainly used for inserts and forging-machine dies. 
The temperature of the normalizing furnace should not be over $500^{\circ} \mathrm{F}$. when the die blocks are charged into it.

Annealing and spheroidizing.-For annealing and and spheroidizing of the alloy blocks, heat to the temperatures given in Table 4 and hold at these temperatures for the time specified.

Hardening.-Unless otherwise stated, heat die blocks to the hardening temperatures given in Table 4 and hold at these temperatures for the time specified before quenching.

Preheating for hardening (die block No. 8).-Die blocks made from this steel should be preheated before hardening. They are heated to $1,500^{\circ}$ to $1,550^{\circ} \mathrm{F}$. and held at this temperature for 1 hour for every inch of diameter or thickness. The blocks are then heated quickly to the hardening temperature.
Hardening (die block No. 8).-When the die blocks have been thoroughly preheated, they are heated quickly to the hardening temperature shown in Table 4 and cooled in air or quenched into oil.

Hardening (die block No. 3).-For hardening, the die blocks should be heated to the temperature shown in Table 4 and held for the time specified. With intricate impressions, the blocks should be pack hardened and cooled with a blast of dry air. Cool to about $400^{\circ} \mathrm{F}$. and temper at $1,000^{\circ} \mathrm{F}$.

Tempering.-After quenching, the die blocks are tempered immediately at the proper temperature, depending upon the hardness desired. The object of inost users of die blocks is to secure a hardness above the machinable range to increase production.

Before using a die of analysis No. 8, it should first be heated to approximately $200^{\circ}$ Fi.

TABLE 4.-Heat treatments for alloy-steel die blocks

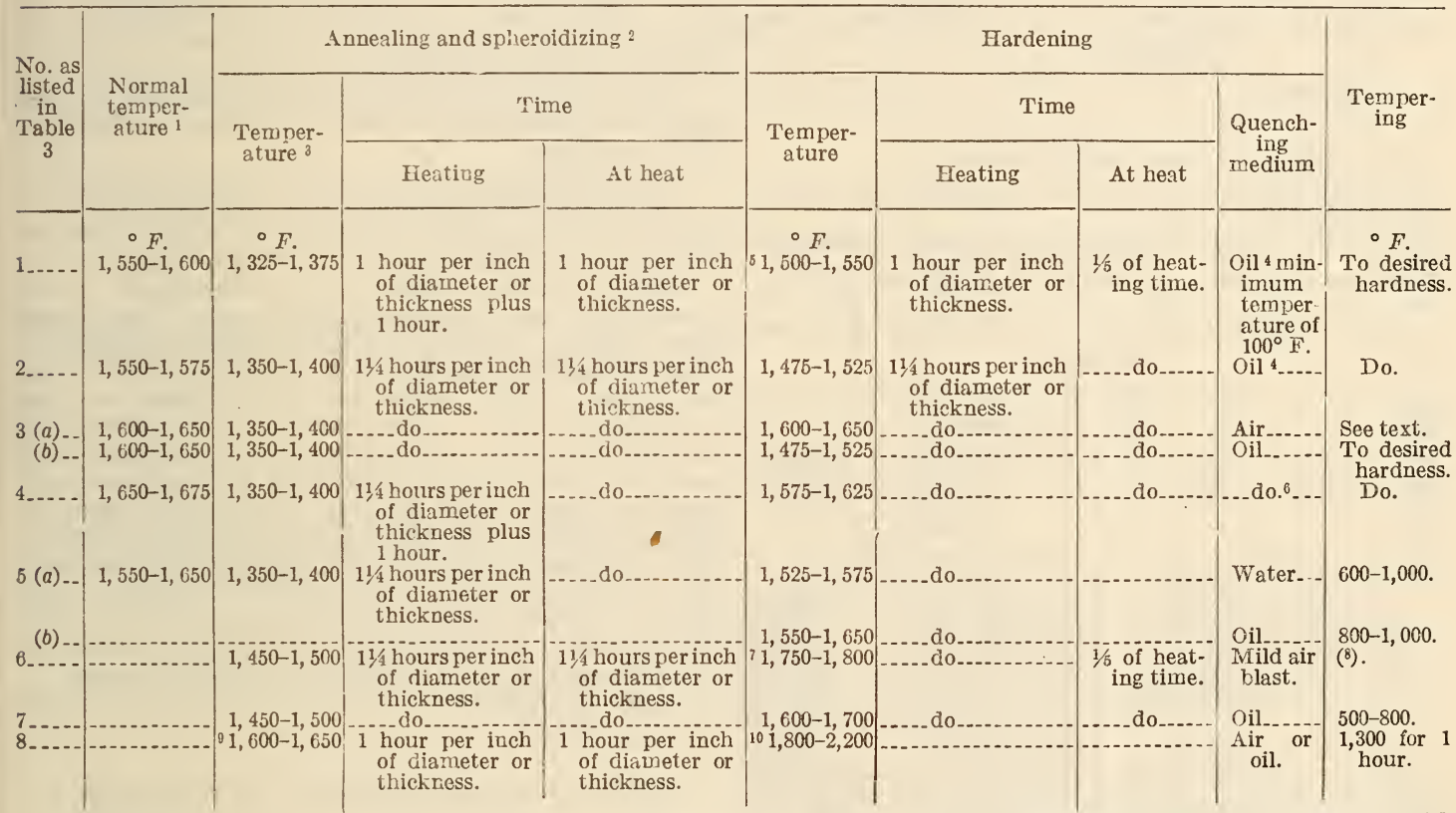

1 For normalizing, heat at the rate of 1 hour per inch of diameter or thickness, and hold at temperature one-fifth of the heating time. Cool in air.

2 It is assumed for this heat treatment that the die block was first normalized. After this treatment the scleroscope hardness should not exceed 35 .

1 From these temperatures, cool slowly to a black heat in the furnace. remove it while still hot enough, so that the oil smokes freely on the surface of the block, but does not flash. While smoking hot, the block is then put at once into the tempering furnace or hot plate. This is to prevent breakage of blocks.

$\checkmark$ For air quenching, heat to $1,550^{\circ}$ to $1,600^{\circ} \mathrm{F}$. The same rules for heating and holding at heat apply.

- Small blocks of approximately a 6 -inch cube or under should be quenched into oil which has first been heated to $150^{\circ} \mathrm{F}$.

7 At high temperatures this steel is susceptible to grain growth. It is best to hold for a longer time at the lower temperature. If air cooling is employed, preheat at $1,500^{\circ} \mathrm{F}$., then heat quickly to $1,700^{\circ} \mathrm{F}$.

8 When air hardened, this die block may be tempered as high as $1,000^{\circ} \mathrm{F}$

o This annealing operation of the blocks should be pack annealed Retard the cooling at $1,350^{\circ}$ to $1,400^{\circ} \mathrm{F}$. for sufficient time to bring about proper annealing structure; this applies particularly to the higher carbon ranges.

10 See direction for prebeating this die block in the text, under preheating for hardening.

ANERICAN SOCIETY FOR STEEL TREATING, TENTATIVE RECOMMERDED PRACTICE FOR THE HEAT TREATHENT OF BLANKING DIES AND PUNCHES, 1928

\section{HEAT TREATHENT OF BLANKING DIES AND PUNCEES}

General.-This is a tentative recommended practice for the heat treatment of blanking dies and punches, and must not be applied to blanking dies and punches with a composition other than given in Table 1. The steels considered in this practice are in common use for blanking dies and punches, but are not the only steels used for this purpose. The heat treatments, therefore, apply only to those steels considered in this practice.

In this practice a blanking die is defined as consisting of two main parts - the stationary "die" and the movable "punch."

Approximate chemical compositions.-The approximate chemical compositions of the steels generally used for blanking dies are given in Table 1 .

Heat treatments. - The heat treatments for blanking dies and punches are given in Table 2.

Notes and general information.-Usually, but not always, both the punch and die are made from the 
same steel. For dies of intricate design an oil hardening steel should be used, but for a more simple design a water-hardening steel may be used.

TABLE 1.-Approximate chemical composition

\begin{tabular}{|c|c|c|c|c|c|c|c|}
\hline $\begin{array}{l}\text { Steel } \\
\text { No. }\end{array}$ & $\begin{array}{l}\text { Type of } \\
\text { steel }\end{array}$ & Purpose & $\mathrm{C}$ & $\begin{array}{l}\min \\
\max .\end{array}$ & $\mathrm{W}$ & $\mathrm{Cr}$ & V \\
\hline 1 & Plain carbon & $\begin{array}{l}\text { Cold-work dies } \\
\text { and punches. }\end{array}$ & $\left\{\begin{array}{r}0.90 \\
1.10\end{array}\right.$ & 0.35 & & & \\
\hline 2 & $\mathrm{C}-\mathrm{V} \ldots$ & ..... do & & .35 & & & 0.25 \\
\hline 3 & $\mathrm{Cr}-\mathrm{V} \ldots \ldots$ & 0 & .6 & .35 & & 0.90 & .20 \\
\hline 41 & $\begin{array}{l}\text { Oil harden- } \\
\text { ing. }\end{array}$ & $\ldots \mathrm{do}^{\circ}$ & & 21.15 & 0.50 & .50 & - \\
\hline 51 & _.... Do & . d & & ${ }^{2} 1.50$ & & & 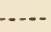 \\
\hline 6 & $\ldots D c$ & 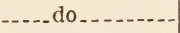 & $\left\{\begin{array}{r}1.10 \\
1.30\end{array}\right.$ & \}. .55 & 1.50 & .50 & .20 \\
\hline 7 & $\mathrm{~W}-\mathrm{Cr}-\mathrm{V}$ & $\begin{array}{l}\mathrm{C} \text { o l d - w or } \mathrm{k} \\
\text { punches. }\end{array}$ & & & 2. 00 & 1.25 & .25 \\
\hline 8 & & $\begin{array}{l}\text { Hot-work dies } \\
\text { and punches. }\end{array}$ & .85 & .35 & & 3.50 & \\
\hline 9 & W.... & - do do - & .40 & .35 & 10.00 & 3.50 & .40 \\
\hline 10 & High speed.- & $\begin{array}{l}\text { Hot and cold } \\
\text { work dies and } \\
\text { punches. }\end{array}$ & 4. 65 & .35 & 18.00 & 4.00 & 1. 00 \\
\hline $11^{5}$ & $\begin{array}{c}\text { High carbon } \\
\text { high } \mathrm{Cr} .\end{array}$ & $\ldots d$ & 2. 25 & & \%. & $|12.0 C|$ & $\ldots$ \\
\hline
\end{tabular}

1 These steels may or may not contain vanadium.

2 Approximately.

3 This steel may or may not contain vanadium to the approximate amount given.

1 The carbon content for this steel may be lower for special purposes.

5 This steel may or may not contain vanadium or cobalt.

TABLE 2.-Heat treatments for blanking dies and punches

\begin{tabular}{|c|c|c|c|c|c|}
\hline \multirow{2}{*}{$\begin{array}{c}\text { Steel } \\
\text { Nos. } \\
\text { as } \\
\text { listed } \\
\text { in } \\
\text { Table } \\
1\end{array}$} & \multirow[b]{2}{*}{$\begin{array}{l}\text { Normal- } \\
\text { izing }\end{array}$} & \multirow[b]{2}{*}{$\begin{array}{c}\text { Anneal- } \\
\text { ing }{ }^{1}\end{array}$} & \multicolumn{2}{|c|}{ Hardening } & \multirow{2}{*}{$\begin{array}{l}\text { Temper- } \\
\text { ing tem- } \\
\text { perature }\end{array}$} \\
\hline & & & ${ }^{\circ} \mathrm{F}$. & $\begin{array}{l}\text { Quench- } \\
\text { ing } \\
\text { medium }\end{array}$ & \\
\hline $\begin{array}{l}1 \\
2 \\
3 \\
4 \\
5 \\
6 \\
7 \\
8 \\
9\end{array}$ & $\begin{array}{c}{ }^{\circ} F . \\
1,500-1,575 \\
1,500-1,575 \\
\\
\end{array}$ & \begin{tabular}{r}
${ }^{\circ} F$ \\
$1,375-1,425$ \\
$1,375-1,425$ \\
$1,400-1,450$ \\
$31,425-1,475$ \\
$31,375-1,425$ \\
$1,450-1,500$ \\
\hdashline $1,425-1,475$ \\
$1,500-1,550$ \\
$1,575-1,625$ \\
$1,600-1,650$ \\
$1,600-1,650$
\end{tabular} & $\begin{array}{r}1,400-1,450 \\
1,400-1,475 \\
1,425-1,475 \\
1,450-1,500 \\
1,400-1,475 \\
1,425-1,500 \\
1,550-1,625 \\
1,600-1,675 \\
1,700-1,800 \\
51,850-2,150 \\
562,200-2,325 \\
71,950-2,200 \\
81,850-2,100 \\
0101,725-1,800\end{array}$ & 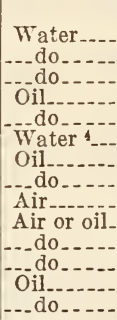 & $\begin{array}{r}{ }^{\circ} F \\
300-550 \\
300-550 \\
350-600 \\
300-550 \\
300-550 \\
325-600 \\
325-600 \\
400-600 \\
900-1,100 \\
1,000-1,250 \\
1,050-1,200 \\
1,050-1,200 \\
1,000-1,100 \\
400-1,000\end{array}$ \\
\hline
\end{tabular}

1 The degree of softness depends upon the rate of cooling from the annealing temperature. Up to a certain degree of softness, the the annealing temperature. Up to a cer

2 After the steel has reached the desired tempering temperature,
(n) the tempering should be continued from 30 minutes to 1 hour. The tempering temperatures given must necessarily cover a wide range; however, for the major portion of blanking dies and punches, the tempering temperature is towards the lower side of the range given.

${ }^{3}$ Steels Nos. 4 and 5 should be heated very slowly, but uniformly, to a dark-red heat of approximately $1,200^{\circ} \mathrm{F}$. then raised more rapidly but uniformly to the hardening temperatures giren in rapidly

${ }_{4}$ Oil quenching is considered preferable, but water quenching is sometimes used.

5 These steels should be preheated at $1,500^{\circ}$ to $1,550^{\circ} \mathrm{F}$.

6 Heat treatment for tools for cold work.

7 Heat treatment for tools for hot work.

8 Feat treatment for pack hardening. Charred sawdust or charred excelsior has been found best adapted for the packing material Carburizing compounds should not be used. Care must be used in the use of this process that the steel does not become too highly carburized. Too high a carbon concentration on the surface will cause spalling or even an actual fusion of the surface of the die during

cause spalli

Do not hold longer than is necessary to assure that the die has reached the desired temperature.

This steel should be preheated at a temperature of $1,300^{\circ}$ to $1,400^{\circ} \mathrm{F}$.

10 This steel absorbs heat slowly, so, consequently, should be heated much more slowly than the other steels listed.

This steel is often pack-hardened to prevent a soft surface resulting from decarburization. For dies which do not admit of grinding after hardening, pack-hardening is recommended.
AMERICAN SOCIETY FOR STEEL TREATING, TENTATIVE RECOMMENDED PRACTICE FOR THE HEAT TREATMENT OF SHEAR BLADES, 1928

\section{HEAT TREATMENT OF SHEAR BLADES}

General.-This is a tenfative recommended practice for the heat treatment of shear blades, and covers the steels that are generally used for cold and hot shearing. When other steels than those listed in Table 1 are used for shear blades, this recommended practice does not apply.

Chemical composition.-The approximate chemical compositions of the steels generally used for shear blades are given in Table 1.

Notes and general information.-The hardness of laid shear blades (steel No. 2) may have a scleroscope hardness from 70 to 85 , but the majority of such blades have a scleroscope hardness over 75 . For hot work, the scleroscope hardness should not be over 70 , and preferably under 60 .

When snearing very thin material, as well as hard steel, it is recommended that tre blades be placed in close contact.

When quenching heavy and long shear blades, it is recommendea that the bath be agitated to insure uniform cooling, otherwise the ends will cool before the center, resulting in nonuniform hardening.

Shear blades should be straightened, wien quenching, on the descending heat. The blades may change somewhat in the tempering operation, but the peening required will not be anywhere near as much if the blades are straightened on the quenching heat. A continuous press should be available with an adjustable control and knife-edge rests, so that if one load does not satisfactorily straighten the blade another load can be immediately applied. For straightening on the descending heat, the blades should be withdrawn from the bath at a temperature from $500^{\circ}$ to $600^{\circ} \mathrm{F}$., which is just below the point where the oil ceases to flash, or in the case of water-quenched blades, just where the water steams or dries off the blades immediately.

Heat treatments for the shear-blade steels are given in Table 2 .

\section{TABLE 1.-Approximate chemical composition ${ }^{1}$}

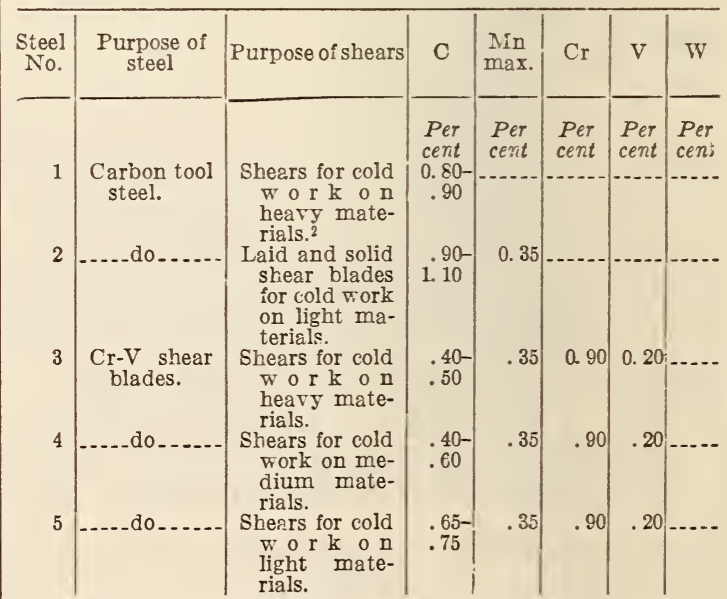

1 A steel that is finding some favor for hot shearing work is a chromium-nickel-molybdenum steel. Various compositions, of this steel may be used. The usual range is as follows: $\mathrm{C}, 0.35-0.60 ; \mathrm{Mn}$,
$0.60-1.50$; $\mathrm{Si}, 0.30-1.50 ; \mathrm{Ni}, 1.00-2.75 ; \mathrm{Mo}, 0.30-1.00 ; \mathrm{Cr}, 0.60-2.75$.

$2 \mathrm{By}$, diameter or thickness; light material one-fourth inch and under: and medium material is thin stock up to three-fourths inch in thickness or diameter. 
TABLE 1.-Approximate chemical composition-Con.

\begin{tabular}{|c|c|c|c|c|c|c|c|}
\hline $\begin{array}{l}\text { Steel } \\
\text { No. }\end{array}$ & $\begin{array}{l}\text { Purpose of } \\
\text { steel }\end{array}$ & Purpose of shears & C & $\underset{\max }{\operatorname{Mn}}$ & $\mathrm{Cr}$ & $\mathrm{V}$ & W \\
\hline & & & $\begin{array}{c}\text { Per } \\
\text { cent }\end{array}$ & $\begin{array}{l}\text { Per } \\
\text { cent } \\
\end{array}$ & $\begin{array}{l}\text { Per } \\
\text { cent }\end{array}$ & Per & Per \\
\hline 6 & $\begin{array}{c}\mathrm{W}-\mathrm{Cr}-\mathrm{V} \\
\mathrm{s} \mathrm{h} \text { e a r } \\
\text { blades. }\end{array}$ & $\begin{array}{l}\text { Shears for cold } \\
\text { work; prin- } \\
\text { cipally for } \\
\text { medium and } \\
\text { heary mate- } \\
\text { rials. }\end{array}$ & & 0.35 & 1.25 & 0.25 & 2.00 \\
\hline 7 & $\begin{array}{l}\text { Cr-steel for } \\
\text { hot shear- } \\
\text { ing. }\end{array}$ & $\begin{array}{l}\text { Shears for hot } \\
\text { rough shear- } \\
\text { ing. }\end{array}$ & .85 & .35 & 3.50 & & \\
\hline 8 & $\begin{array}{l}\text { Tungsten } \\
\text { hot work- } \\
\text { ing steel. }\end{array}$ & Hot shearing... & .40 & .35 & 3.50 & .40 & 10.00 \\
\hline 9 & High speed. & $\begin{array}{l}\text { Cold shearing }{ }^{34} \\
\text { for light ma- } \\
\text { terials. }\end{array}$ & .65 & .35 & 4. 00 & 1.00 & 18.00 \\
\hline 10 & $\begin{array}{l}\text { High carbon } \\
\text { high chro- } \\
\text { mium. }\end{array}$ & $\begin{array}{l}\text { Cold shearing }{ }^{3} \\
\text { for light ma- } \\
\text { terials. }\end{array}$ & 2.25 & & 12. 00 & & \\
\hline
\end{tabular}

- For certain classes of work, these steels may be used for both light and heary hot shearing.

- With a lower carbon content, steel No. 9 is generally used for hot shearing.

\section{TABLE 2.-Heat treatments for shear blades}

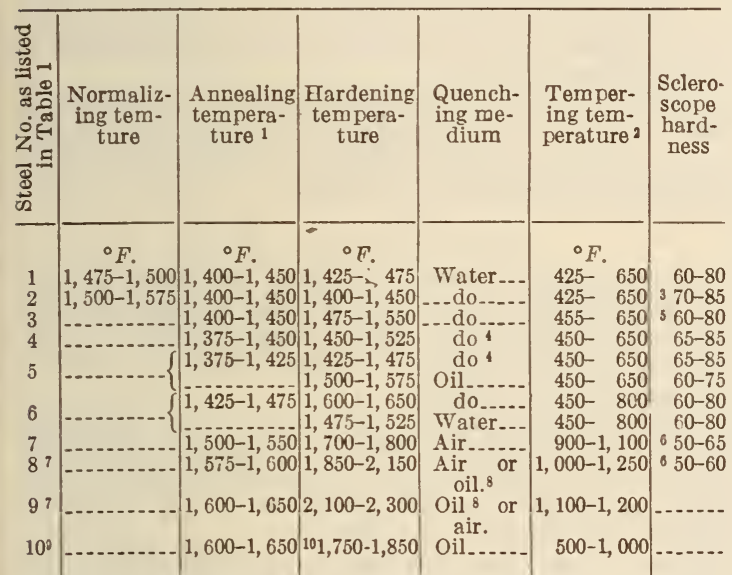

${ }^{1}$ After forging and for easy machining the steels are annealed at the temperatures given and cooled slowly.

2 The tempering temperature depends upon the character of the work to which the shears are to be subjected. For carbon-steel blades, either solid or laid, a range of $425^{\circ}$ to $650^{\circ} \mathrm{F}$. covers the tempering range.

${ }^{3}$ To shear very thin material the scleroscope hardness should be on the higher side of the range given.

${ }^{4}$ With all chromium-vanadium steels, the shear blades should be remored from the water at about $500^{\circ} \mathrm{F}$. and cooling continued in oil.

o The hardness of chromium-vanadium shear blades is about the same as for plain carbon blades, but as chromium-vanadium blades are used because of their greater toughness, a somewhat lower hardness is often used.

- For extreme hot work, the scleroscope hardness should not be over 60 .

7 Before hardening these steels should be preheated at a temperature of $1,500^{\circ}$ to $1,550^{\circ} \mathrm{F}$.

8 For toughness, the blades are usually quenched in air. When oil-quenched, they should be removed from the bath at a temperature of approximately $400^{\circ} \mathrm{F}$., and immediately tempered. If, howerer, the blades are to be straightened, they should be removed from the bath at approximately $900^{\circ} \mathrm{F}$., straightened, allowed to ccol in the air to a temperature of about $400^{\circ} \mathrm{F}$, and then tempered.

$\checkmark$ Before hardening, this steel should be preheated at a temperature of $1,300^{\circ}$ to $1,400^{\circ} \mathrm{F}$.

${ }_{10}$ This steel absorbs heat slowly, so time must be allowed for solution of the carbides. It should be held at temperature a much longer time than other steels. To prevent decarburization, it is often necessary to pack this steel in a suitable carbonaceous material.
AMERICAN SOCIETY FOR STEEL TREAT-

ING, TENTATIVE RECOMMENDED PRACTICE FOR THE HEAT TREATMENT OF CHISELS, 1928

\section{HEAT TREATMENT OF CHISELS}

GENERAL.-Chisels must meet severe fatigue stresses and retain good cutting edges. Various steels as well as heat treatments are employed to meet these conditions. Only the principal steels and simpler treatments are given here.

TABLE 1.-Approximate chemical composition

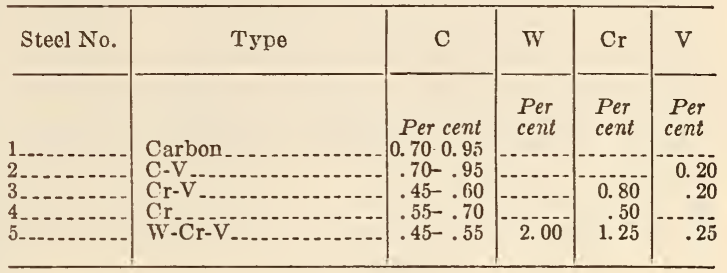

'TABLE 2.-Heat treatment for chisels

\begin{tabular}{|c|c|c|c|c|}
\hline $\begin{array}{l}\text { Steel Nos. } \\
\text { as listed } \\
\text { in Table } 1\end{array}$ & $\begin{array}{l}\text { Forging } \\
\text { tempers- } \\
\text { tures }^{1}\end{array}$ & $\begin{array}{l}\text { Hardening } \\
\text { tempera- } \\
\text { tures }\end{array}$ & $\begin{array}{l}\text { Quenching } \\
\text { media }\end{array}$ & $\begin{array}{l}\text { Temper- } \\
\text { ing } \\
\text { tempera- } \\
\text { tures }\end{array}$ \\
\hline $\begin{array}{l}4 \\
5\end{array}$ & $\begin{array}{c}{ }^{\circ} F . \\
1,6001,400 \\
1,600-1,400 \\
1,650-1,400 \\
1,650-1,400 \\
1,700-1,400 \\
1,700-1,400\end{array}$ & $\begin{array}{c}\circ F . \\
1,400-1,475 \\
1,400-1,475 \\
1,450-1,500 \\
1,450-1,500 \\
1,650-1,700 \\
1,550-1,600\end{array}$ & $\begin{array}{l}\text { Water.... } \\
\text { Oil do } \\
\text { Water...... } \\
\end{array}$ & $\begin{array}{l}{ }^{\circ} F . \\
350-550 \\
350-550 \\
350-550 \\
350-550 \\
450-550 \\
450-550\end{array}$ \\
\hline
\end{tabular}

1 The initial forging temperature depends upon the amount of, work to be done upon the steel and in no case should the finishing temperature be below $1,400^{\circ} \mathrm{F}$.

Nore. - With pneumatic chisels, various hardening methods are followed to put the shank in proper condition. Sometimes the chisel is treated as above and sometimes the shank is given a preliminary quench in oil from the temperatures given in Table 2 after which the cutting edge is hardened as given in Table 2 , and the whole chisel tempered at the temperatures given.

\section{AMERICAN SOCIETY FOR STEEL TREAT- ING, RECOMMENDED PRACTICE FOR THE HEAT TREATMENT OF CARBON STEELS, S. A. E. SERIES, 1929}

\section{HEAT TREATMENT OF CARBON STEELS GENERAI}

This recommended practice covers the process for the heat treatment of carbon steels of the S. A. E. series. The heat treatments prescribed for the steels considered are applicable for bar stock or for articles fabricated from the particular steel as listed under "uses." The heat treatments, of course, may be applied to other articles fabricated from the same steel, but consideration should be given to the design and heat-treatment methods, because it is often necessary to alter the treatments to cope with designs, and local conditions.

\section{CHEMICAL COMPOSITION}

The chemical compositions of the steels considered in this recommended practice are given in Table 1. 
TABLE 1.-Chemical compositions

\begin{tabular}{|c|c|c|c|c|}
\hline S. A. E. steels & $\mathrm{C}$ & $\mathrm{Mn}$ & $\begin{array}{l}\text { Phos. } \\
\text { (maxi- } \\
\text { mim) }\end{array}$ & $\begin{array}{l}\text { Sulphur } \\
\text { (maxi- } \\
\text { mum) }\end{array}$ \\
\hline $\begin{array}{l}1010 \\
1020 \\
1025 \\
1035 \\
1040 \\
1045\end{array}$ & $\begin{array}{l}\text { Per cent } \\
0.05-0.15 \\
.15-.25 \\
.20-.30 \\
.25-.35 \\
.30-.40 \\
.35-.45 \\
.40-.50\end{array}$ & $\begin{array}{l}\text { Per cent } \\
0.30-0.60 \\
.30-.60 \\
.50-.80 \\
.50-.80 \\
.50-.80 \\
.50-.80 \\
.50-.80\end{array}$ & $\begin{array}{r}\text { Percent } \\
0.015 \\
.045 \\
.045 \\
.045 \\
.045 \\
.045 \\
.045\end{array}$ & $\begin{array}{r}\text { Per cent } \\
0.055 \\
.055 \\
.055 \\
.055 \\
.055 \\
.055 \\
.055\end{array}$ \\
\hline
\end{tabular}

\section{HEAT TREATNRENT}

The heat treatments are given in Table 2 and are prescribed to develop grain refinements, better phyical properties, and better machining qualities. Carburizing and cyaniding treatments are purposely omitted from this recommended practice.

In all of these heat-treating operations, it is good practice to heat the steel 1 hour for every inch of diameter or thickness and to hold at temperature for at least one-fifth of the time required for heating.

\section{STEEL FO. 1010}

S. A. E. steel No. 1010 has low tensile strength in the annealed condition, but the strength can be greatly increased by heat treatment, especially in bolts, nuts, cap screws, and similar articles of diameters not greater than three-fourths inch. This treatment consists of heating the articles at or above the critical temperature range of the material and drastically quenching by means of suitable fixtures. ${ }^{39}$ The proper result may require a quenching medium of water or soluble oil. The tensile strength of the smaller diameters, one-half inch and less, may be as high as 90,000 pounds per square inch with other desirable physical properties retained. The temperature generally used is $1,650^{\circ}$ to $1,700^{\circ} \mathrm{F}$. Quenching by this method in a 5 per cent soluble oil solution gives approximately 70,000 to 80,000 pounds per square inch tensile strength.

TABLE 2-Heat treatments for carbon steels-S. A. E. series

\begin{tabular}{|c|c|c|c|c|c|c|}
\hline 这 & Normalize 1 & Anneal & Quench & $\begin{array}{l}\text { Quench- } \\
\text { ing me- } \\
\text { dium }\end{array}$ & $\begin{array}{l}\text { Tem- } \\
\text { perature } \\
\text { of me- } \\
\text { dium }\end{array}$ & 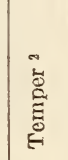 \\
\hline 10 . & $\stackrel{\circ F}{{ }^{\circ} \mathrm{F}}$ & $\stackrel{\circ F}{{ }_{1,600-1,700}}$ & ${ }^{\circ} F$ & & $\stackrel{\circ}{\circ}$. & ${ }^{\circ} F$ \\
\hline 20. & $1,600-$ & $1,575-1,625$ & ${ }^{3} 1,600-1,675$ & _.._do.4 - & $\begin{array}{lr}\text { Oil, } & 125 \\
\mathrm{H}_{2} \mathrm{O} & 60\end{array}$ & $0=700$ \\
\hline 25. & $1,600-1,750$ & $1,525-1,575$ & ${ }^{3} 1,575-1,625$ & Water... & & $700-$ \\
\hline 30 & $1,600-1,700$ & $1,525-1,575$ & $31,550-1,600$ & $\ldots d$ & & 0,70 \\
\hline 35. & $1,575-\mathbf{h}, 675$ & $1,500-1,550$ & $1,525-1$, ö75 & ...do. ${ }^{5}-\ldots$ & & 0 \\
\hline 35 & $1,575-1,675$ & $1,500-1,550$ & $1,550-1,600$ & Oil_. & & \\
\hline 0. & $1,575-1,650$ & $1,475-1,525$ & $\left\{\begin{array}{l}1,500-1,550 \\
1,525-1,575\end{array}\right.$ & Water... & & 1,1 \\
\hline 45 & ${ }^{7} 1,575-1,650$ & $1,450-1,500$ & $\left\{\begin{array}{l}1,500-1,550 \\
1,475-1,525\end{array}\right.$ & & & $\begin{array}{r}750- \\
1,200\end{array}$ \\
\hline
\end{tabular}

1 From these temperatures cool in air.

2 The tempering temperature depends upon the properties desired. See also the text for steels 1035 and 1040 .

3 For a heat treatment to improve machining qualities, see text.

4 The oil for this quench is a 5 per cent soluble oil solution.

5 Many parts may be quenched into a 6 per cent sodium hydroxide solution.

0 With complicated sections, this steel should be quenched into oil.

7 See text.

39 "Some Physical Properties of Low Carbon Steel," R. H. Smith, Trans., Am. Soc. Steel Treating, vol. 7, p. 569.
In the annealed condition, steel 1010 does not machine freely, but tears badly when turued, threaded, and broached. The machining qualities are improved by quenching from $1,700^{\circ} \mathrm{F}$. into water; tempering is not required, as the hardness and strength are only slightly increased with this heat treatment.

Uses.-The heat treatments for S. A. E. steel 1010 , as covered in this practice, are especially suitable for bolts, pins, cap screws, and similar parts.

\section{STELI NO. 1020}

This steel is used where a trifle higher tensile strength is required. It responds more readily to heat treatment than steel 1010, especially if the carbon is around the upper limit.

High tensile strength bolts, pins, ete., are produced from this steel by drastically quenching from $1,450^{\circ}$ to $1,525^{\circ} \mathrm{F} .{ }^{49}$ into a 5 per ceut soluble oil solution, which also produces a rust-resisting coating.

When the carbon is near the middle of the range, the tensile strength is approximately 85,000 to 95,000 pounds per square inch, with an elongation of 25 per cent.

Steel 1020 machines better than 1010, but is improved by quenching into water from a temperature of $1,600^{\circ}$ to $1,675^{\circ} \mathrm{F}$. If the carbon is around 0.25 per cent, it is well to temper from $500^{\circ}$ to $800^{\circ}$ $\mathrm{F}$., in order to produce a Brinell hardness number from 143 to 179 , which is desirable for good machining qualities.

Uses.-The heat treatments for S. A. E. 1020 steel, as covered in this practice are suitable for the same articles as listed under 1010 steel, but where higher tensile strength is desired.

\section{STEEL NOS. 1025 AND 1030}

These steels are used to quite an extent in the untreated condition for unimportant forgings and for pressed and stamped parts. Heat treatment only slightly increases the physical properties, but greatly increases the toughness.

These steels machine fairly well in the annealed condition, but for best machining qualities the steel should be quenched, after normalizing, into water from a temperature of $1,575^{\circ}$ to $1,625^{\circ} \mathrm{F}$., then tempered from $600^{\circ}$ to $1,100^{\circ} \mathrm{F}$.

Uses.-The heat treatment for steels 1025 and 1030 , as covered in this practice, are suitable for the following and similar articles:

Forgings; pressed or stamped parts (queuched after normalizing and annealing - where toughness is desired); frames; washing-machine parts; unimportant bolts.

\section{STEEL NO. 1085}

This steel responds readily to heat treatment and develops fair physical properties. It also machines readily.

When the carbon and the manganese are on the high side of the range, the quenching temperature should not exceed $1,550^{\circ} \mathrm{F}$., and the quenching water or oil should be warm; otherwise quenching cracks are likely to be encountered. When the carbon and manganese are both low it is often necessary to quench from a temperature as high as $1,600^{\circ} \mathrm{F}$. Even with this temperature, it is difficult to meet physical property requirements. For the heat treatment of small forgings, the low side of the quenching range should be used.

The quenching medium may be either oil or water. Higher physical properties are, of course, obtained with the water quench.

10 When not drastically quenched, use temperature giren in Table 2 . 
Uses.-The heat treatments for steel 1035, as covered in this practice, are suitable for the following and similar articles:

\begin{tabular}{|c|c|c|}
\hline 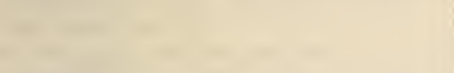 & $\begin{array}{l}\text { Temper } \\
\text { at- }\end{array}$ & $\begin{array}{c}\text { Arerage } \\
\text { Brinell } \\
\text { No. }\end{array}$ \\
\hline & ${ }^{\circ} F$ & \\
\hline $\begin{array}{l}\text { Front automobile axles. } \\
\text { Crankshafts. }\end{array}$ & $\begin{array}{l}900-1,100 \\
850-1,100\end{array}$ & $\begin{array}{l}207-241 \\
228-269\end{array}$ \\
\hline $\begin{array}{l}\text { Large and small forgings, bolts, pins, cap } \\
\text { screws }\end{array}$ & $800-1,100$ & $\begin{array}{l}1217-321 \\
2196-241\end{array}$ \\
\hline $\begin{array}{l}\text { Connecting rods } \\
\text { Wheel hubs }\end{array}$ & $1,100-1,150$ & $\begin{array}{l}196-235 \\
196-235\end{array}$ \\
\hline $\begin{array}{l}\text { Wrake-band anchors } \\
\text { Brabs }\end{array}$ & $\begin{array}{l}950-1,100 \\
850-1,000\end{array}$ & $\begin{array}{l}196-235 \\
241-286\end{array}$ \\
\hline
\end{tabular}

1 Water-quenched.

2 Oil-quenched.

STEEL NO. 1040

Steel 1040, like 1035, responds well to heat treatment, develops good physical properties, and machines readily.

Forgings that are made in such a manner that portions of the parts receive only little work and are, therefore, quite coarse, should be normalized; otherwise normalizing can be dispensed with.

It is advisable to time-quench all parts quenched in cold water. When removed from the water the temperature of the pieces should be from $300^{\circ} \mathrm{F}$. for heavy sections to $700^{\circ} \mathrm{F}$. for light sections.

As breakage may occur if quenched into water, irregularly shaped pieces, that is, parts with a heavy section adjacent to a thin section with only small fillets, are usually quenched into oil.

Uses. - The heat treatments covered in this practice for steel 1040 are suitable for the following and similar articles:

\begin{tabular}{|c|c|c|}
\hline & $\begin{array}{l}\text { Temper } \\
\text { at }-\end{array}$ & $\begin{array}{c}\text { A verage Brinell } \\
\text { No. }\end{array}$ \\
\hline & $\circ F$ & \\
\hline Axle shafts & $750-\quad 950$ & $241-321$ \\
\hline Front axle centers & $950-1,150$ & $196-241$ \\
\hline 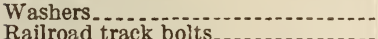 & $400-500$ & Rockwell C-45-50 \\
\hline $\begin{array}{l}\text { Railroad track bolts } \\
\text { Connecting rods. }\end{array}$ & $700-900$ & 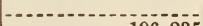 \\
\hline $\begin{array}{l}\text { Connecting rods } \\
\text { Torque-rod ends }\end{array}$ & 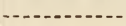 & $196-235$ \\
\hline Torque-rod ends. & $-\cdots$ & $187-223$ \\
\hline Brake levers.... & $-2-1-2-1-2$ & $187-223$ \\
\hline Tie-rod ends & - & $187-223$ \\
\hline Stocker crankshafts & $-\ldots$ & $187-217$ \\
\hline Propeller shafts (tubing) & $-\cdots$ & (-2.2. \\
\hline $\begin{array}{l}\text { Clutch plates } \\
\text { Large gears }\end{array}$ & & \\
\hline & & \\
\hline
\end{tabular}

STEEL NO. 1045

When steel 1045 is used for large forgings, it should be normalized and quenched. For this treatment the forgings are heated to $1,625^{\circ}$ to $1,650^{\circ}$ $\mathrm{F}$., cooled in air, and reheated to $1,500^{\circ}$ to $1,550^{\circ}$ F., and quenched into oil. The tempering depends upon the properties desired; generally $750^{\circ}$ to $900^{\circ}$ $\mathrm{F}$., is satisfactory.

When used for connecting rods, this steel is sometimes quenched into water from a temperature of $1,480^{\circ}$ to $1,510^{\circ} \mathrm{F}$. The temperature of the water is approximately $180^{\circ} \mathrm{F}$.

When quenching this steel into cold water there is a tendency to develop cracks. It is, therefore, advisable to quench into oil wherever possible, especially with small, irregularly shaped pieces.
AMERICAN SOCIETY FOR STEEL TREATING, RECOMMENDED PRACTICE FOR THE HEAT TREATMENT OF CARBONSTEEL CASTINGS, 1929

\section{HEAT TREATMENT OF CARBON-STEEL CASTINGS}

\section{GENERAI}

This recommended practice covers the process to be followed for the heat treatment of carbon-steel castings and applies generally to commercial castings for ordinary construction purposes and not for special use.

\section{CHEMICAL COMPOSITION}

The chemical ranges for the steel castings considered in this recommended practice are given in Table 1.

TABLE 1.-Chemical composition

\begin{tabular}{|c|c|c|}
\hline Carbon 1 & Manganese & Silicon \\
\hline $0.15-0.60$ & $0.50-0.90$ & $0.20-0.60$ \\
\hline
\end{tabular}

1 The carbon content is subdivided in the tables where heat treatments are prescribed.

\section{COOLING AND CLEANING CASTINGS AFTER POURING}

After pouring, all castings should be allowed to cool to a temperature below the critical range, preferably in the mold.

After shaking out and before heat treating, the castings should be cleaned sufficiently to insure thorough and complete penetration of heat during the heat-treating operations.

After the castings have been cooled and cleaned, they are subjected to one or more of the heat treatments considered below.

\section{SINGLE NORMALIZING OR SINGLE ANNEALING PRACTICES}

The castings are placed in the furnace and heated slcwly and uniformly to the temperature given in Table 2.

To obtain the correct structural condition throughout the castings, they should be held at temperature for approximately 60 minutes for each inch of thickness of the largest section of the castings.

To develop better ductility and relieve cooling strains, normalized castings may be reheated to a suitable temperature below the upper critical point. Usually reheating to about $1,200^{\circ} \mathrm{F}$. is sufficient.

\section{TABLE 2.-Single normalizing or single annealing}

\begin{tabular}{|c|c|c|c|}
\hline $\begin{array}{l}\text { Carbon } \\
\text { range }\end{array}$ & $\begin{array}{l}\text { Tempera- } \\
\text { ture }\end{array}$ & $\begin{array}{l}\text { For nor- } \\
\text { malizing } \\
\text { cool in- }\end{array}$ & For annealing cool in- \\
\hline $\begin{array}{l}0.15-0.40 \\
0.40-0.60\end{array}$ & $\begin{array}{l}\stackrel{\circ F}{1,600-1,700} \\
1,550-1,600\end{array}$ & Still air ..... & $\begin{array}{l}\text { Furnace or other medium for } \\
\text { cooling slowly.1 } \\
\text { Do.1 }\end{array}$ \\
\hline
\end{tabular}

1 The castings are cooled slowly until the temperature has dropped to $800^{\circ} \mathrm{F}$. or below. 


\section{DOUBLE TREATMENT-NORMALIZING AND ANNEALING}

Recent investigations indicate that where time and expense are minor considerations, but quality is of utmost importance, double annealing or normalizing treatments are often beneficial.

The first treatment is usually conducted at relatively high temperatures ranging from $1,600^{\circ}$ to $2,000^{\circ} \mathrm{F}$., while the second treatment is conducted at a temperature only slightly above the critical temperature range of the steel being treated. These treatments may be followed by a tempering or a spheroidizing treatment if so desired.

The mechanism of the above practice is based on the fact that, in the annealing of cast steel, two separate structural changes are involved. The first consists in breaking up or diffusing the dendritic segregation of metalloids as far as possible, so as to form a homogeneous, but not necessarily finegrained, structure. Since this is a process of diffusion of the metalloids, and not of critical transformation, the temperature employed is not a definite one, but rather depends on the original degree of segregation. Generally stated, segregation increases with the section of casting, so that the homogenizing temperature will also depend somewhat on the thickness of the casting.

As a result of the severe homogenizing treatment, the grain structure will be relatively coarse, and a second treatment at a temperature only slightly above the critical temperature range is given to refine the grain.

\section{OIL AND WATER QUENCHING STEEL CASTINGS}

When exceptional physical properties, high degree of structural refinement, and resistance to wear are required, heating and quenching will greatly assist in producing the desired results. When the carbon content of the castings is above 0.30 per cent, quenching and tempering are especially effective in producing these requirements.

Castings should not be quenched unless they have first been thoroughly annealed.

When water is used as the quenching medium, the temperature of the water should preferably be maintained at $125^{\circ}$ to $150^{\circ} \mathrm{F}$., especially for the higher carbon contents. With carbon under 0.25 per cent the quenching may be more drastic.

When the size and design of the castings are such that quenching might cause cracking if kept in the water or oil until cold, the castings should be removed from the quenching bath when they have become black, but still hot enough to dry the water quickly or, in case of oil, the castings should be hot enough to cause the oil to smoke freely on the surface of the casting.

\section{TEMPERING}

After quenching, the castings should be tempered immediately. When the load is charged into the tempering furnace, the temperature of the furnace should be approximately the same temperature as the castings (about $700^{\circ} \mathrm{F}$.). The tempering temperature ranges from $800^{\circ}$ to $1,350^{\circ} \mathrm{F}$., depending upon the physical properties desired. Where hardness is not a factor, it is recommended to temper in the neighborhood of $1,200^{\circ} \mathrm{F}$. for the average commercial casting.

The quenching and tempering temperatures are given in Table 3 .

\section{NOTES ABOUT THE PROCESS}

With some castings that have had considerable machine work performed on them, such as heavyduty gears, it is often advisable to remove the ma- chine strains by heating tne castings slowly and uniformly to a temperature of $400^{\circ}$ to $600^{\circ} \mathrm{F}$., then cool in air.

Castings should be charged into the furnace in such a manner that lighter castings will be protected from the heat of the furnace by the heavier castings. Should the castings be of similar section, care must be exercised so as not to overheat those on the outside of the charge before the inner ones have reached the annealing temperature.

TABLE 3.-Quenching and tempering of carbon-steel castings

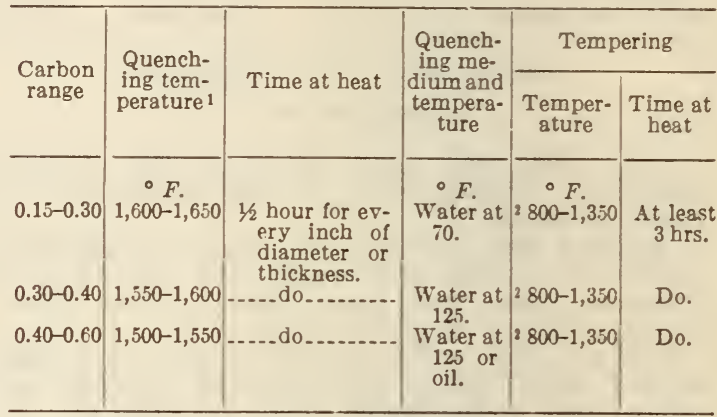

1 These temperatures are based on the assumption that the castings were first annealed.

'See text for tempering.

When charging the furnace, the castings should be piled loosely so that the hot gases will have free circulation throughout the charge. To further insure free circulation of the gases, it is advisable to pile the castings loosely on perforated racks or other suitable fixtures which elevate the castings a few inches above the floor of the furnace and so permit free circulation of the hot gases. When cooling from the normalizing temperature, this practice of piling the castings on racks also insures free circulation of the air.

Sufficient number of thermocouples should be used and so arranged in the furnace and, if possible, in the charge of castings, that the desired temperature can be maintained at the top, center, bottom, and sides of the charge.

Steel castings should be grouped for heat treatment, so that the carbon content of the entire charge falls within one of the carbon ranges given in the recommended practice.

Castings of intricate design, or where a heavy and a light section are adjacent, should not be quenched, except with utmost care.

As a basis for determining the physical properties of the metal in the casting, it is commercial practice to use test coupons cut from separately cast blocks or attached to the castings. When attached to the casting they should be so placed that they are properly fed and represent, as nearly as possible, the condition of the metal in the casting. Test coupons should be heat treated with the castings they represent.

To check the heating operation and to obtain closer control of the temperature, it is advisable to distribute several test specimens, for fracture inspection, throughout the charge of castings. The crosssectional dimensions of the specimens should represent the largest cross section of the castings, and the carbon content should be approximately the same as the castings being heat treated. After completion of the heat treatment, an examination of the fractures of the specimens for grain refinement indicates the uniformity of heat distribution without destroying a casting for fracture inspection. 
AMERICAN SOCIETY FOR STEEL TREATING, RECOMMENDED PRACTICE FOR THE HEAT TREATMENT OF ALLOYSTEEL CASTINGS, 1929

\section{HEAT TREATMENT OF ALLOY-STEEL CASTINGS}

\section{GENERAI}

This recommended practice covers the process to be followed for the heat treatment of alloy-steel castings and applies generally to commercial alloy castings for ordinary construction purposes and not for special use.

\section{CHEMICAL COMPOSITION}

The chemical compositions of the steel considered in this recommended practice are given in Table 1.

\section{COOLING AND CLEANING OF CASTINGS}

Alloy castings should be cooled in the molds to a temperature below the critical range, but as the brittleness increases with the decrease of temperature they should be shaken out, preferably as soon as they become black, and cleaned while they are still warm.

Cleaning should be performed rapidly so that the castings can be placed in the annealing furnace while still as hot as possible, but the cleaning must be sufficient to insure a thorough and complete penetration of heat during the heat-treating operations.

Flame cutting of gates and risers must be done before heat treating and is somewhat dangerous unless the castings are hot. If the risers or gates are broken instead of cut, the operation should be performed while the castings are warm, otherwise stresses are set up and minute cracks are developed which show up in the quenching operation. To avoid cracks when cutting with a torch at least one inch of metal should be left between the torch cut and the body of the castings. When castings are placed in the furnace, the temperature of the latter should preferably be the same as the castings.

\section{HEAT TREATMENT OF ALLOY CASTINGS}

Heat treatments may be classified as follows: Normalizing, annealing, quenching, and tempering.

The heat treatments to be used depend upon the chemical composition of the castings and the physical properties desired. Quenching, followed by a suitable tempering treatment, develops highest combinations of properties and permits a wide range of adjustment in the balancing of properties to suit individual requirements. Normalizing, followed by tempering, usually is a cheaper process than direct quenching, and while the results will not equal those obtained by quenching and tempering, nevertheless this treatment may be considered to yield maximum improvement in physical properties per unit of cost. It is one of the most.common of commercial treatments and is satisfactory for many applications. Tempering should follow rapid cooling as in normalizing and quenching, to relieve strains which otherwise might cause cracking. Full annealing increases the machinability of most steels, helps to break up the cast structure, and generally (though not always) induces maximum softness and ductility.
TABLE 1.-Approximate chemical compositions ${ }^{1}$

\begin{tabular}{|c|c|c|c|c|c|c|c|}
\hline क्ष & $\begin{array}{c}\text { Type of } \\
\text { steel }\end{array}$ & $\mathrm{C}$ & $\mathrm{Mn}$ & $\mathrm{Si}$ & $\mathrm{Cr}$ & $\mathrm{Ni}$ & $\mathrm{V}$ \\
\hline $\begin{array}{l}1 \\
2 \\
3\end{array}$ & $\begin{array}{l}\mathrm{C}-\mathrm{V}_{-} \\
\mathrm{C}-\mathrm{V}_{-} \\
\mathrm{C}-\mathrm{V}_{\ldots}\end{array}$ & $\mid \begin{array}{r}0.25-0.35 \\
.35-.45 \\
.45-.55\end{array}$ & $\begin{array}{r}0.60-0.90 \\
.60-.90 \\
.60-.90\end{array}$ & $\begin{array}{l}0.20-0.50 \\
2.20-.50 \\
2.20-.50\end{array}$ & $\mid \begin{array}{l}- \\
-\end{array}$ & - & $\begin{array}{l}.15-.20 \\
.15-20\end{array}$ \\
\hline $\begin{array}{l}4 \\
5 \\
6\end{array}$ & $\begin{array}{l}\mathrm{C}-\mathrm{Cr} . . \\
\mathrm{C}-\mathrm{Cr} . .- \\
\mathrm{C}-\mathrm{Cr} . .-\end{array}$ & $\begin{array}{l}.25-.35 \\
.35-.45 \\
.45-.55\end{array}$ & $\begin{array}{l}.60-.90 \\
.60-.90 \\
.60-.90\end{array}$ & $\begin{array}{l}.20-.60 \\
.20-.60 \\
.20-.60\end{array}$ & $\begin{array}{r}0.80-1.10 \\
.80-1.10 \\
.80-1.10\end{array}$ & $\cdots$ & \\
\hline $\begin{array}{l}7 \\
8 \\
9\end{array}$ & $\begin{array}{l}\mathrm{Cr}-\mathrm{V}_{-.-} \\
\mathrm{Cr}-\mathrm{V}_{-}-{ }^{-} \\
\mathrm{Cr}-\mathrm{V}_{-.-}\end{array}$ & $\begin{array}{l}.25-.35 \\
.35-.45 \\
.45-. .55\end{array}$ & $\begin{array}{l}.60-.90 \\
.60-.90 \\
.60-.90\end{array}$ & $\begin{array}{l}.20-.60 \\
.20-.60 \\
.20-.60\end{array}$ & $\begin{array}{l}.80-1.10 \\
.80-1.10 \\
.80-1.10\end{array}$ & & $\begin{array}{l}.15-.20 \\
.15-.20 \\
.15-.20\end{array}$ \\
\hline $\begin{array}{l}10 \\
11 \\
12\end{array}$ & $\begin{array}{l}\mathrm{Ni} \\
\mathrm{Ni} \\
\mathrm{Ni}\end{array}$ & $\begin{array}{l}.15-.25 \\
.25-.35 \\
.35-.45\end{array}$ & - & 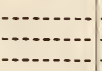 & & $\begin{array}{l}3.25-3.75 \\
3.25-3.75 \\
3.25-3.75\end{array}$ & \\
\hline $\begin{array}{l}13 \\
14 \\
15\end{array}$ & $\begin{array}{l}\mathrm{Ni}-\mathrm{Cr} .- \\
\mathrm{Ni}-\mathrm{Cr}-- \\
\mathrm{Ni}-\mathrm{Cr} .-\end{array}$ & $\begin{array}{l}.25-.35 \\
.35-.45 \\
.45-.55\end{array}$ & $\begin{array}{l}.60-.90 \\
.60-.90 \\
.60-.90\end{array}$ & $\begin{array}{l}.20-.60 \\
.20-.60 \\
.20-.60\end{array}$ & $\begin{array}{l}.50-.90 \\
.50-.90 \\
.50-.90\end{array}$ & $\begin{array}{l}\text { 1. } 25 \\
\text { 1. } 25 \\
1.25\end{array}$ & \\
\hline $\begin{array}{l}16 \\
17 \\
18\end{array}$ & $\begin{array}{l}\mathrm{Ni}-\mathrm{Cr}_{--} \\
\mathrm{Ni}-\mathrm{Cr}_{-} \\
\mathrm{Ni}-\mathrm{Cr}_{-}\end{array}$ & $\begin{array}{l}.25-.35 \\
.35-.45 \\
.45-.55\end{array}$ & & & $\begin{array}{l}1.25-1.75 \\
1.25-1.75 \\
1.25-1.75\end{array}$ & $\begin{array}{l}3.25-3.75 \\
3.25-3.75 \\
\text { 3. } 25-3.75\end{array}$ & \\
\hline $\begin{array}{l}19 \\
20 \\
21\end{array}$ & $\begin{array}{l}\mathrm{Cr}-\mathrm{Mo}^{3} \\
\mathrm{Cr}-\mathrm{Mo}^{3} \\
\mathrm{Cr}-\mathrm{Mo}^{3}\end{array}$ & $\begin{array}{l}.25-.35 \\
.35-.45 \\
.45-.55\end{array}$ & $\begin{array}{l}.60-.90 \\
.60-.90 \\
.60-.90\end{array}$ & $\begin{array}{l}.20-.60 \\
.20-.60 \\
.20-.60\end{array}$ & $\begin{array}{l}.70-1.00 \\
.70-1.00 \\
.70-1.00\end{array}$ & & \\
\hline $\begin{array}{l}22 \\
23 \\
24 \\
25\end{array}$ & $\begin{array}{l}\text { Mn.... } \\
M n \\
M n \\
M n \\
M n\end{array}$ & $\begin{array}{l}25-.35 \\
.25-.35 \\
35-.45 \\
.35-.45\end{array}$ & $\begin{array}{l}1.00-1.50 \\
1.50-1.75 \\
1.00-1.50 \\
1.50-1.75\end{array}$ & $\begin{array}{l}.20-.50 \\
.20-.50 \\
.20-.50 \\
.20-.50\end{array}$ & & & \\
\hline
\end{tabular}

1 Maximum sulphur, 0.060 per cent. Maximum phosphorus, 0.050 per cent.

20.20 to 0.30 per cent silicon preferred.

3 Molybdenum range, 0.25 to 0.35 per cent.

Normalizing. - The purpose of normalizing is to relieve cooling strains, refine the cast structure, and produce as high physical properties as are possible without liquid quenching. To accomplish this purpose, castings are placed in the furnace, preferably while they are still warm, as noted above, and heated slowly and uniformly to the normalizing temperatures as given in Table 2 , after which they should be allowed to cool uniformly and as rapidly as possible in still air. (Air blast is not usually considered acceptable.)

Castings should be held at temperature approximately one hour for each inch of thickest cross section. Manganese steels, numbers 22 to 25 , require a longer period of heating.

After normalizing, the castings should be further heat-treated by tempering, or by quenching and tempering as described later in this practice.

TABLE 2.-Normalizing treatments

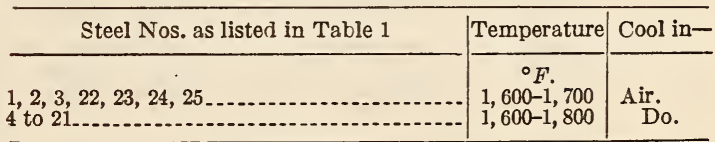

Full annealing.-Although full annealing of alloy steel castings is not generally recommended, it is essential for heavy sections and where machinability is desired. If full annealed, the castings should be further heat treated after machining to bring out the available physical qualities.

When the castings have had no preliminary treatment, they may be full annealed at the normalizing temperatures as given in Table 2 , followed by furnace cooling. 
If the steel has been previously normalized it may be annealed at lower temperatures as given in Table 3 , holding at temperature a sufficient time to insure thorough soaking. The rate of cooling should be regulated to give the desired machining qualities.

TABLE 3.-Annealing temperatures for alloy castings

\begin{tabular}{|c|c|c|}
\hline Steel Nos. as listed in Table 1 & Temperature & Cool in- \\
\hline $\begin{array}{l}1 \text { to } 3 ; 10 \text { to } 12 ; 16 \text { to } 18 ; 22 \text { to } 25 \\
4 \text { to } 9 ; 13 \text { to } 15 ; 19 \text { to } 21\end{array}$ & $\begin{array}{c}{ }^{\circ} \mathrm{F} . \\
1,500-1,600 \\
1,550-1,650\end{array}$ & $\begin{array}{l}\text { Furnace. } \\
\text { Do. }\end{array}$ \\
\hline
\end{tabular}

Double treatment-Normalizing and full-annealing.-For information on these treatments, refer to the practice for the heat treatment of carbon-steel castings.

Quenching.-Quenching should be employed where maximum physical properties are necessary.

After properly normalizing or annealing, the castings should be heated slowly and uniformly to the quenching temperature as given in Table 4. Before quenching, they should be held at temperature for at least 30 minutes for each inch of cross section.

TABLE 4.-Quenching and tempering temperatures for alloy castings

\begin{tabular}{|c|c|c|c|c|c|}
\hline $\begin{array}{c}\text { Steel Nos. } \\
\text { as listed } \\
\text { in Table } 1\end{array}$ & $\begin{array}{c}\text { Type of } \\
\text { steel }\end{array}$ & $\begin{array}{l}\text { Quenching } \\
\text { tempera- } \\
\text { tures }\end{array}$ & $\begin{array}{l}\text { Quenching } \\
\text { medium } 1\end{array}$ & $\begin{array}{l}\text { Maxi- } \\
\text { mum } \\
\text { tem- } \\
\text { pering } \\
\text { tem- } \\
\text { pera- } \\
\text { tures }\end{array}$ & Cool in- \\
\hline $1 .$. & C.V... & 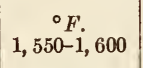 & Water or & ${ }_{1}^{\circ} \boldsymbol{F}$. & Air or fur- \\
\hline $\begin{array}{l}2 \\
3 \ldots \\
3-\cdots\end{array}$ & $\begin{array}{l}\mathrm{C} \cdot \mathrm{V} . . \\
\mathrm{C} \cdot \mathrm{V} . .\end{array}$ & $\begin{array}{l}1,500-1,550 \\
1,475-1,500\end{array}$ & _.do_... & $\begin{array}{l}1,300 \\
1,300\end{array}$ & $\begin{array}{l}\text { nace. } \\
\text { Do. } \\
\text { Furnace. }\end{array}$ \\
\hline $4 \ldots$ & $\mathrm{C} \cdot \mathrm{Cr}$ - & $1,575-1,650$ & -._do_... & 1,275 & Air or fur- \\
\hline $\begin{array}{l}5 \ldots \\
6 \ldots-. .\end{array}$ & $\begin{array}{l}\text { C.Cr-.- } \\
\text { C-Cr-.- }\end{array}$ & $\begin{array}{l}1,525-1,575 \\
1,500-1,525\end{array}$ & _._do.... & $\begin{array}{l}1,275 \\
1,275\end{array}$ & $\begin{array}{l}\text { Do. } \\
\text { Furnace. }\end{array}$ \\
\hline $7 \ldots$ & $\mathrm{Cr}-\mathrm{V}$ & $1,575-1,650$ & ...do... & 1,275 & Air or fur- \\
\hline $\begin{array}{l}8 \\
9 \ldots \\
9-. .-\end{array}$ & $\begin{array}{l}\mathrm{Cr}-\mathrm{V}-. \\
\mathrm{Cr} \cdot \mathrm{V}=\end{array}$ & $\begin{array}{l}1,525-1,575 \\
1,500-1,525\end{array}$ & $\begin{array}{l}\text {-..do-. } \\
\text {-..do-. }\end{array}$ & $\begin{array}{l}1,275 \\
1,275\end{array}$ & $\begin{array}{l}\text { Do. } \\
\text { Furnace. }\end{array}$ \\
\hline 10 & Ni.... & $1,475-1,525$ & .._do. & 1,200 & $\begin{array}{l}\text { Air or fur- } \\
\text { nace. }\end{array}$ \\
\hline $11 \ldots$ & $\begin{array}{l}\mathrm{Ni} . . . . \\
\mathrm{Ni} . . .\end{array}$ & $\begin{array}{l}1,450-1,500 \\
1,425-1,475\end{array}$ & -..do-..- & $\begin{array}{l}1,200 \\
1,200\end{array}$ & $\begin{array}{l}\text { Do. } \\
\text { Do. }\end{array}$ \\
\hline $\begin{array}{l}13 \\
14 \\
15 \\
-\end{array}$ & $\begin{array}{l}\mathrm{Ni}-\mathrm{Cr} . . \\
\text { Ni-Cr.-. } \\
\text { Ni-Cr.-. }\end{array}$ & $\begin{array}{l}1,515-1,550 \\
1,490-1,515 \\
1,475-1,490\end{array}$ & 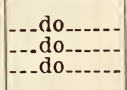 & $\begin{array}{l}1,250 \\
1,250 \\
1,250\end{array}$ & $\begin{array}{l}\text { Do. }{ }^{2} \\
\text { Do. } \\
\text { Furnace. }{ }^{2}\end{array}$ \\
\hline 16 & $\mathrm{Ni}-\mathrm{Cr}$ & $1,475-1,525$ & Oil.- & 1,200 & Air or fur- \\
\hline $17--$. & $\begin{array}{l}\mathrm{Ni}-\mathrm{Cr} \\
\mathrm{Ni}-\mathrm{Cr} . . .\end{array}$ & $\begin{array}{l}1,450-1,500 \\
1,425-1,475\end{array}$ & -.-do-...- & $\begin{array}{l}1,200 \\
1,200\end{array}$ & $\begin{array}{l}\text { nace. } \\
\text { Do. } \\
\text { Do. }\end{array}$ \\
\hline $\begin{array}{l}19 \ldots \\
20 \ldots \\
21 . .\end{array}$ & $\begin{array}{l}\text { Cr-Mo.- } \\
\text { Cr-Mo.- } \\
\text { Cr-Mo.. }\end{array}$ & $\begin{array}{l}1,575-1,650 \\
1,525-1,575 \\
1,500-1,525\end{array}$ & $\begin{array}{l}\text { Water-.- } \\
\text { Oil-..- }\end{array}$ & $\begin{array}{l}1,300 \\
1,300 \\
1,300\end{array}$ & $\begin{array}{l}\text { Do. }{ }^{3} \\
\text { Do. } \\
\text { Furnace. }{ }^{3}\end{array}$ \\
\hline 22 & Mn... & $1,600-1,650$ & Water... & 1,250 & Air or fur- \\
\hline $\begin{array}{l}23 \ldots \\
24-. \\
25 .-\end{array}$ & $\begin{array}{l}\mathrm{Mn} . \\
\mathrm{Mn} \\
\mathrm{Mn} .\end{array}$ & $\begin{array}{l}1,600-1,650 \\
1,550-1,600 \\
1,550-1,600\end{array}$ & \begin{tabular}{|l|}
$\ldots$ do \\
- do \\
- do $_{-}$
\end{tabular} & $\begin{array}{l}1,250 \\
1,250 \\
1,250\end{array}$ & $\begin{array}{l}\text { Do. } \\
\text { Do. } \\
\text { Do. }\end{array}$ \\
\hline
\end{tabular}

1 Air quenching is often made use of in the hardening of alloy steel castings of intricate design which would be likely to crack in the more drastic water or oil quench.

2 The shock resistance is usually improved by oil quenching from the tempering temperature.

i A long soak is required at the tempering temperature.

When water is used as the quenching medium, the temperature of the water should preferably be maintained at $125^{\circ}$ to $150^{\circ} \mathrm{F}$., especially for the higher carbon steels.
When the size and design of the castings are such that quenching might cause cracking, if kept in the water or oil until cold, the castings should be removed from the quenching bath while still hot enough to dry the water quickly, or in case of oil, the castings should be hot enough to cause the oil to smoke freely on the surface of the castings.

Tempering. - As soon as possible after normalizing or quenching, castings should be reheated for tempering. The temperature of the furnace receiving the charge should be approximately the same as that of the castings.

The purpose of tempering is to relieve the strains and brittleness caused by quenching or normalizing and to moderate the hardness; that is, to toughen the material. The temperature to be used depends upon the hardness and physical properties desired. Usually the higher the tempering temperature, up to the critical range, the softer and more ductile the steel. For maximum hardness and strength, a tempering temperature only sufficient to relieve strains is used. The maximum tempering temperatures recominended are given in Table 4 .

Except in special cases, the time the inaterial is held at the tempering temperatures should be at least sufficient to insure complete equalization of the heat throughout the heaviest section of the casting, and preferably an hour or more per inch of thickness.

\section{USES}

The alloy steels listed possess desirable combinations of hardness, strength, and toughness not obtainable with carbon cast steel. The castings are used for locomotive frames, gear blanks, dies, stamps, crusher jaws, pinions, car wheels, shovel-teeth racks, chain, valves and fittings, caterpillar treads, etc. The selection of the alloy depends upon the service required.

Due to their higher physical properties it is possible to obtain greater strength for a given cross section than with carbon steel castings. These alloys, therefore, find particular application where reduction of weight is important, and where high stresses are encountered.

\section{NOTES ABOUT THE PROCESS}

Castings should be charged into the furnace in such a manner that lighter castings will be protected from the heat of the furnace by the heavier castings. Should the castings be of similar section, care must be exercised so as not to overheat those on the outside of the charge before the inner ones have reached the desired temperature.

When charging the furnace, the castings should be piled loosely so that the hot gases will have free circulation throughout the charge. To further insure free circulation of the gases, it is advisable to pile the castings loosely on perforated racks or other suitable fixtures which elevate the castings a few inches above the floor of the furnace. When cooling from the normalizing temperature, this practice of piling the castings on racks also insures free circulation of the air, producing more rapid and uniform cooling.

Sufficient number of thermocouples should be used and so arranged in the furnace and, if possible, in the charge of castings that the desired temperature can be maintained at the top, center, bottom, and sides of the charge.

Steel castings should be grouped for heat treatment so that the carbon content of the entire charge falls within one of the carbon ranges in Table 1.

Castings of intricate design, or where a heavy and a light section are adjacent, should not be quenched. However, when quenching is necessary, utmost care should be exercised to avoid cracking.

Alloy-steel castings, in most cases, will harden appreciably if heated above the critical range and 
allowed to cool in the air. Therefore, they should always be tempered after normalizing.

When the suggested methods of annealing are not effective, alloy castings may sometimes be appreciably softened by heating within the critical range and holding for a considerable length of time.

The physical properties obtained by heat treating alloy castings are destroyed by the local application of heat, such as in welding or hot straightening. These operations produce dangerous local disturbances of grain structure, hardness, and excessive strains. Castings should, therefore, be retreated after such practice.

The rate of cooling in the normalizing practice is merely relative, depending upon the size of the charge. The smaller the load, the more drastic the cooling, and, therefore, the higher the physical strength and the lower the ductility.

It is commercial practice to use test specimens cut from a separately cast block or from test coupons attached to the casting as a basis for determining the physical properties of the castings. Test pieces or test coupons should be heat treated with the castings which they represent.

AMERICAN SOCIETY FOR STEEL TREATING. RECOMMENDED PRACTICE FOR THE HEAT TREATMENT AND CARE OF SLING AND CRANE CHAIN, 1929

HEAT TREATMENT OF SLING AND CRANE CHAIN

\section{INTRODUCTION}

The reliability of chain depends upon the quality of the welded links and the metal from which they are made. It is of paramount importance to ascertain if the chain is of ample size to sustain the safe working load. The quality of the metal should be carefully and thoroughly tested by the chain manufacturer, both for chemical and physical properties, also a metallographic examination is considered desirable.

Wrought iron for chain usually has the following approximate chemical composition:

Carbon, under. Per cent

Manganese, under

The A. S. T. M. specification A56-24 limits the manganese to 0.10 per cent for the best grade of wrought iron for chain.

The average physical properties of wrought iron for chain are as follows:

Tensile strength. Ibs./in. ${ }^{2}-$ 46, 000

Yield point

Elongation in 8 inches do _.. 23,000

Reduction of area

Other tests that are often performed on wrought iron for chain are: Cold-bend, quench-bend, and drift tests. In addition, a cold feathering test may be applied by flaring the end of the bar under a hammer. This test is an aid in judging the workmanship put into the welded fagot. The results of these tests should show a high degree of ductility, and a fibrous, silky fracture free from bright spots.

Because mild steel requires different welding temperatures and methods than wrought iron, a satisfactory weld may not be obtained if the two materials are mixed in rerolling or reheating processes. To determine if an admixture of wrought iron and steel has been used, metallographic examination should be resorted to.

Low-carbon steels are largely used for small chain, other than sling chain, but in large sizes there are welding difficulties which are not encountered with wrought iron. Most chain, therefore, above $1 \frac{1}{2}$ inch, is made of wrought iron.
NORMALIZING, TESTING, AND INSPECTING AFTER WELDING

Normalizing.--If the welding operation has been properly performed the welded portion may not need a normalizing treatment to refine the structure, but in the case of large chain, portions of the link adjacent to the welds have been heated to or near the welding temperature, and if not hot worked a weak, coarse, crystalline structure is developed which requires a normalizing treatment for refinement.

To normalize, the chain is placed in the annealing furnace, usually of the car-bottom type, and heated until the complete charge, top, center, and bottom is at a temperature of $1,750^{\circ} \mathrm{F}$. The time for heating is about three hours. The charge is held at this temperature for 10 to 15 minutes, or until the heat has completely penetrated the work. The car, if the car-bottom type has been used, is then drawn out and the chain allowed to cool in the air. In other furnace types, the furnace doors may be opened or the chain removed from the furnace and allowed to cool on the shop floor.

Proof test and inspection.-After cooling, the chain is proof tested. The test consists of fastening one end of the chain to the head of the testing machine and the other end to the anchor crosshead. The required proof load is 50 per cent of the breaking load. Each link is then carefully inspected for flaws

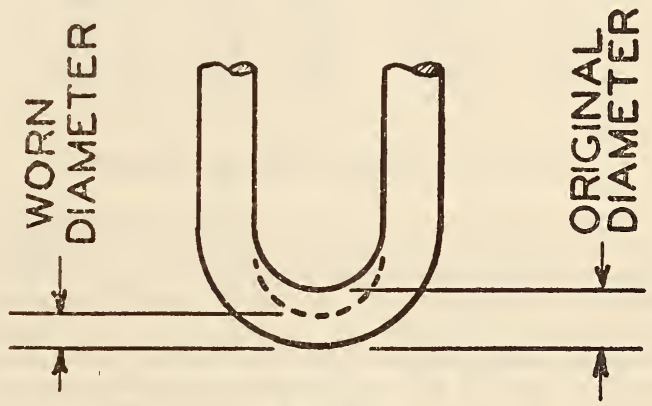

FIgURE 46

or other defects. I link measurements are made to determine if the chain has stretched beyond the specified dimensions. All chain suffers permanent elongation after the proof test.

After subjecting the chain to a proof test, it may be annealed and the yield point thereby lowered. This step makes overloading more evident and to that extent increases the factor of safety. Because of the increased cost, this operation is usually dispensed with, so the problem should be referred to the manufacturer before this particular annealing operation is specified.

\section{CARE AND USE OF CHAIN}

Overloading is probably the most serious service abuse to which chain is subjected. A new chain may be impaired by overloading the first few times it is used. The elasticity of the chain metal is sufficient to take care of the safe working load. If the chain nas not been overloaded, or stressed beyond the yield point, the chain will regain normal shape as soon as it is relieved of its load. When stressed beyond the yield point by an overload the metal does not go back to its normal condition or shape after the load is removed. When the yield point of the metal is once passed by an overload, the metal is never as strong under impact as before it had been stretched. If the chain is corefully measured at frequent intervals, any elongation that is not the result of wear is sure proof of overload. 
Improper application of the chain sets up stresses that are far in excess of those caused by the weight of the load lifted, and which are the cause of fatigue failure. These increased stresses may be caused by any of the following: (1) Sudden application of the load, shocks, and sudden jolts; (2) twisting of chain, kinks in chain, and crossing of chain; (3) bending of links around sharp corners; and (4) large angles between the branches or legs of a double-sling chain.

TABLE 1.-Safe loads in pounds for sling chains under different loading conditions

\begin{tabular}{|c|c|c|c|c|}
\hline \multirow{2}{*}{$\begin{array}{l}\text { Diameter } \\
\text { of chain } \\
\text { stock (in } \\
\text { inches) }\end{array}$} & \multirow{2}{*}{$\begin{array}{l}\text { Single } \\
\text { chain } \\
\text { when } \\
\text { used } \\
\text { straight }\end{array}$} & \multicolumn{3}{|c|}{$\begin{array}{l}\text { Double-sling chains when } \\
\text { used to form base angles of - }\end{array}$} \\
\hline & & $60^{\circ}$ & $45^{\circ}$ & $30^{\circ}$ \\
\hline $\begin{array}{r}1 / 4 \\
5 / 16 \\
3 / 8 \\
7 / 16 \\
1 / 2\end{array}$ & $\begin{array}{l}1,060 \\
1,655 \\
2,385 \\
3,250 \\
4,240\end{array}$ & $\begin{array}{l}1,850 \\
2,875 \\
4,150 \\
5,600 \\
7,350\end{array}$ & $\begin{array}{l}1,500 \\
2,350 \\
3,350 \\
4,600 \\
6,000\end{array}$ & $\begin{array}{l}1,060 \\
1,655 \\
2,385 \\
3,250 \\
4,240\end{array}$ \\
\hline $\begin{array}{l}9 / 16 \\
5 / 8 \\
3 / 4 \\
7 / 8 \\
1\end{array}$ & $\begin{array}{r}5,370 \\
6,630 \\
9,540 \\
12,960 \\
16,950\end{array}$ & $\begin{array}{r}9,300 \\
11,450 \\
16,500 \\
22,450 \\
29,350\end{array}$ & $\begin{array}{r}7,600 \\
9,350 \\
13,500 \\
18,300 \\
23,950\end{array}$ & $\begin{array}{r}5,370 \\
6,630 \\
9,540 \\
12,960 \\
16,950\end{array}$ \\
\hline $\begin{array}{l}11 / 8 \\
11 / 4 \\
13 / 8 \\
11 / 2\end{array}$ & $\begin{array}{l}20,040 \\
24,750 \\
29,910 \\
35,600\end{array}$ & $\begin{array}{l}34,700 \\
42,850 \\
51,800 \\
61,650\end{array}$ & $\begin{array}{l}23,300 \\
35,000 \\
42,350 \\
50,350\end{array}$ & $\begin{array}{l}20,040 \\
24,750 \\
29,910 \\
35,600\end{array}$ \\
\hline $\begin{array}{l}15 / 8 \\
13 / 4 \\
11 / 8 \\
2\end{array}$ & $\begin{array}{l}41,800 \\
48,450 \\
55,300 \\
63,300\end{array}$ & $\begin{array}{r}72,400 \\
83,800 \\
95,800 \\
119,600\end{array}$ & $\begin{array}{l}59,100 \\
68,500 \\
78,200 \\
89,500\end{array}$ & $\begin{array}{l}41,800 \\
48,450 \\
55,300 \\
63,300\end{array}$ \\
\hline
\end{tabular}

\section{FAILURE CAUSED BY REPEATED LOADING}

Chain may break without showing elongation if loaded below the yield point a sufficient number of times. Failure may take place by ordinary fatigue, but is much more likely to occur by the following mechanism: Through the repeated peening action of the chain links against each other and their being dropped and hit against stone or metal, the surface of the soft iron is given a work-hardened skin. Under impact loads, usually difficult to avoid, this skin is broken and the crack constitutes a notch which localizes stress and weakens the chain under conditions of further impact or repeated load. This hard skin may be removed and the impact strength of the chain maintained by periodic annealing. How often this is desirable depends on the particular service involved.

\section{ANGLE OF SLING CHAINS}

The angle between the legs of the branches of a double-sling chain should always be taken into consideration, especially when handling heavy loads. As this angle increases, the angle between the legs and the work decreases. This reduces greatly the safe working load of the chain, because as the angle between the two legs of the chain increases the tension in each leg is also increased. The safe load in pounds for double-sling chains, using different angles between the legs, is given in Table 1 .
TABLE 2.-Reduced diameler of link stock in chain slings

(Original safety factor $=7$ )

\begin{tabular}{|c|c|c|c|c|}
\hline \multirow{3}{*}{$\begin{array}{l}\text { Chain } \\
\text { size (in } \\
\text { inches) }\end{array}$} & \multicolumn{4}{|c|}{ Reduced safety factors } \\
\hline & 6 & 5 & 4 & 3 \\
\hline & \multicolumn{4}{|c|}{ Reduced diameters } \\
\hline $\begin{array}{l}1 / 4 \\
2 / 8 \\
1 / 2 \\
5 / 8 \\
3 / 4\end{array}$ & $\begin{array}{c}\text { Inches } \\
0.23 \\
.35 \\
.47 \\
.58 \\
.70\end{array}$ & $\begin{array}{c}\text { Inches } \\
0.21 \\
.32 \\
.42 \\
.52 \\
.63\end{array}$ & $\begin{array}{c}\text { Inches } \\
0.19 \\
.38 \\
.38 \\
.47 \\
.57\end{array}$ & $\begin{array}{c}\text { Inches } \\
0.16 \\
.25 \\
.33 \\
.42 \\
.49\end{array}$ \\
\hline $\begin{array}{l}1^{7 / 8} \\
11 / 8 \\
11 / 4 \\
12 / 8\end{array}$ & $\begin{array}{r}.81 \\
.93 \\
1.04 \\
1.17 \\
1.28\end{array}$ & $\begin{array}{r}.74 \\
.84 \\
.95 \\
1.05 \\
1.16\end{array}$ & $\begin{array}{r}.66 \\
.75 \\
.85 \\
.95 \\
1.04\end{array}$ & $\begin{array}{l}.57 \\
.66 \\
.73 \\
.82 \\
.90\end{array}$ \\
\hline $\begin{array}{l}11 / 2 \\
13 / 8 \\
13 / 4 \\
13 / 8 \\
2\end{array}$ & $\begin{array}{l}1.39 \\
1.50 \\
1.63 \\
1.73 \\
1.86\end{array}$ & $\begin{array}{l}1.26 \\
1.37 \\
1.47 \\
1.58 \\
1.68\end{array}$ & $\begin{array}{l}1.13 \\
1.22 \\
1.32 \\
1.42 \\
1.51\end{array}$ & $\begin{array}{l}.98 \\
1.06 \\
1.15 \\
1.23 \\
1.31\end{array}$ \\
\hline
\end{tabular}

Links to be measured at point of greatest wear.

Example.-A 1/2-inch chain is worn to about 0.40 inches in diameter. The remaining safety factor, full load, is (from table) about 4.5.

\section{FACTOR OF SAFETY}

Chain is always manufactured with a factor of safety sufficient to take care of a reasonable amount of abuse, but even this is of little avail with the careless handling which is given some chain.

The cold working caused from overloading will increase the breaking load, but decreases the ductility and resistance to shock, thereby reducing the factor of safety. Chain links are reduced in diameter, due to dirt, grit, and abrasives grinding between the links when the load is applied. As the diameter is reduced, the factor of safety is lowered. In Table 2 is given the factor of safety for reduced chain links caused by wear.

If properly carried out, annealing will automatically increase the factor of safety, except where the link is reduced in diameter by wear. Annealing should not be done periodically as a "cure all," but carried out in conjunction with careful inspection. Inspection and measurements of the chain will give notice of any abuse. Wear, however, will cause elongation of the chain, so it is necessary to distinguish between this effect and the elongation caused from overload.

Annealing can only restore ductility in the metal of the reduced cross section and does not restore the chain to its original condition because the effects of the permanent deformation to the links are unchanged. No chain should be used on heavy work after it has been badly overloaded and has, thereby, become deformed. The tensile strength may not be impaired but the deformation of the original links considerably reduces the reserve of shock resistance in the links. 
RECOMMENDED PRACTICE FOR THE HEAT TREATMENT OF SLING AND CRANE CHAIN

General. - This recommended practice covers the process to be followed for the annealing of sling and crane chain.

Process. - The process of annealing chain consists of loading the furnace, heating, and cooling the chain.

Loading the furnace.-Place the chain in uniform layers on the furnace bottom so as to expose maximum surface area. To prevent scale from forming, a hood should be placed over the chain and the edges of the hood sealed with sand. The openings of the hood are sealed with fire clay.

TABLE 3.-Annealing treatment for iron sling and crane chains

\begin{tabular}{|c|c|c|c|}
\hline $\begin{array}{l}\text { Diameter of chain link (in } \\
\text { inches) }\end{array}$ & $\begin{array}{l}\text { Annealing } \\
\text { tempera- } \\
\text { ture }^{1}\end{array}$ & $\begin{array}{l}\text { Time of } \\
\text { holding } \\
\text { at tem- } \\
\text { perature }\end{array}$ & $\begin{array}{l}\text { Cool } \\
\text { in }\end{array}$ \\
\hline $\begin{array}{l}1 / 4 \text { to } 1 / 2 \\
8 / 8 \text { to } 17 / 8 \\
17 / 8 \text { and above................... }\end{array}$ & $\begin{array}{c}\stackrel{\circ}{F} . \\
1,200-1,400 \\
1,200-1,400 \\
1,200-1,400\end{array}$ & $\begin{array}{r}\text { Hours } \\
1 / 4 \\
3 / 4 \\
2^{3 / 4}\end{array}$ & $\begin{array}{l}\text { Air. } \\
\text { Do. } \\
\text { Do. }\end{array}$ \\
\hline
\end{tabular}

1 In case there is doubt as to whether the chain to be treated is iron or steel, a temperature of $1,600^{\circ}$ to $1,650^{\circ}$ instead of $1,200^{\circ}$ to $1,400^{\circ} \mathrm{F}$. should be used. This higher temperature is not required for iron, but will not injure it. The lower temperature is undesirable for steel. Heating to this higher temperature and coming down again brings bout a recrystallization and decreases the danger of excessive grain growth such as may occur below the critical range, especially if the chain had been severely strained in service.

Heating.-Heat to a temperature of $1,200^{\circ}$ to $1,400^{\circ} \mathrm{F}$., and hold at this temperature for the time specified in Table 3 . The time required to bring the chain up to temperature, and whether the chain is charged into a hot or cold furnace have little influence on the result. The heat should thoroughly penetrate the work, and the top, center, and bottom of the charge should reach approximately the same temperature.

A sufficient number of thermocouples should be used to assure uniform temperature throughout the charge. A pyrometric record of the heating operation should be delivered with the annealed chain.

Cooling.-After the chain has been held at the annealing temperature for sufficient time, the charge is pulled on to the cooling platform, or onto the floor. The chain should be spread in single layers, with ample space between each chain, so as to allow it to cool rapidly and uniformly to room temperature.

\section{AMERICAN SOCIETY FOR STEEL TREAT- ING, RECOMMENDED PRACTICE FOR THE HEAT TREATMENT OF OIL-HARD- ENING ALLOY GEARS, 1929}

\section{HEAT TREATMENT OF ALLOY GEARS}

\section{GENERAL}

This recommended practice covers the process to be followed in the heat treatment of oil-hardening gears. It is recognized that the size, composition, and type of gears govern entirely the heat-treating process. This recommended practice, therefore, pertains only to gears of approximately the size of a passenger-car sliding gear.

All the temperatures recommended in this practice refer to the temperature of the stock and not to the furnace temperature.

\section{CHEMICAL COMPOSITION}

The alloy steels most commonly used for gears, together with their chemical compositions, are listed in Table 1.

All the alloy steels for gears should be selected as closely as is commercially possible from the middle of the carbon range, as given in Table 1 .

\section{TABLE 1.-Approximate chemical composition}

\begin{tabular}{|c|c|c|c|c|c|c|c|}
\hline $\begin{array}{l}\text { Steels, } \\
\text { S. A. E. } \\
\text { Nos. }\end{array}$ & C & $\mathrm{Mn}$ & $P$ & S & $\mathrm{Cr}$ & $\underset{\substack{\text { mini- } \\
\text { mum) }}}{\mathrm{V}}$ & $\mathrm{Ni}$ \\
\hline $\begin{array}{l}2345 \ldots . . \\
3250 \ldots \ldots \\
5150 \ldots \\
6150 \ldots \\
3150^{1} \ldots\end{array}$ & $\begin{array}{r}0.40-0.50 \\
.45-.55 \\
.45-.55 \\
.45-.55 \\
.45-.55\end{array}$ & $\begin{array}{l}0.50-0.80 \\
.30-.60 \\
.50-.80 \\
.50-.80 \\
.50-.80\end{array}$ & $\begin{array}{l}0.04 \\
.04 \\
.04 \\
.04 \\
.04\end{array}$ & $\begin{array}{l}0.05 \\
.045 \\
.05 \\
.045 \\
.045\end{array}$ & $\begin{array}{r}0.90-1.25 \\
.80-1.10 \\
.80-1.10 \\
.45-.75\end{array}$ & 0.15 & \begin{tabular}{l}
$3.25-3.75$ \\
$1.50-2.00$ \\
\hdashline $1.00-1.50$
\end{tabular} \\
\hline
\end{tabular}

1 This is not an S. A. E. steel, but follows the S. A. E. numbering system.

\section{HEAT TREATMENT AFTER FORGING AND BEFORE MACHINING}

To eliminate forging structure and to obtain desirable machining properties, either with bar stock or gear blanks, the steel should be normalized, annealed, or quenched and annealed. The preliminary heat treatments are given in Table 2 .

From the normalizing temperatures the steels are cooled in air. After the preliminary quenching, the steels are heated for at least one hour at the annealing temperature as indicated in Table 2 , then cooled in the furnace to about $800^{\circ} \mathrm{F}$. Below this temperature the steels may be cooled in air. After this heat treatment, the Brinell hardness number should be between 170 to 217 , which produces desirable machining properties.

To assure uniformity of the product, it is good practice to test, after the preliminary heat treatment, a sufficient number of the gears for hardness.

TABLE 2.-Normalizing, preliminary quenching, and preliminary annealing after forging

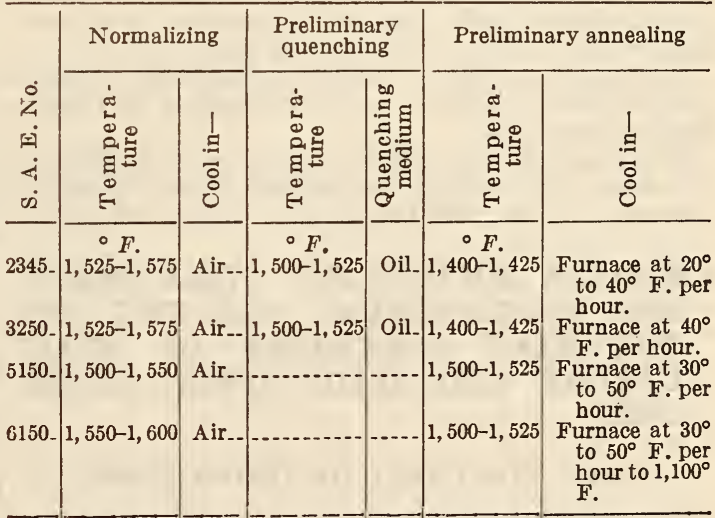

$3150^{1} 1,525-1,575$ Air_- $1,500-1,525$ Oil_ ${ }^{21,350-1,400}$ Furnace.

1 This is not an S. A. E. steel, but follows the S. A. E. numbering system.

2 When the quenching treatment is omitted, this steel should be annealed at $1,450^{\circ}$ to $1,500^{\circ} \mathrm{F}$

\section{HEAT TREATMENT OF FINISHED MACHINED GEARS}

After machining and cutting the teeth, the gears are hardened and tempered.

Heating for quenching.-The gears are heated slowly and uniformly to the quenching tempera- 
tures given in Table 3 , and held for sufficient time to assure complete and thorough penetration of heat. The heating medium should be so selected that scaling is held to a minimum.

Quenching.-The gears are quenched from the quenching temperature into oil as indicated in Table 3. The quenching oil should flow through the tank in large volumes, and the quenching should be so performed that the quenching oil circulates freely between the gear teeth.

TABLE 3.-Heating, quenching, and tempering of gears

\begin{tabular}{|c|c|c|c|}
\hline \multirow{2}{*}{ Steels, S. A. E. No. } & \multicolumn{2}{|c|}{ Heating for quenching } & \multirow{2}{*}{$\begin{array}{l}\text { Temper- } \\
\text { ing tem- } \\
\text { perature }\end{array}$} \\
\hline & $\begin{array}{c}\text { Tempera- } \\
\text { ture }\end{array}$ & $\begin{array}{l}\text { Quenching } \\
\text { medium }\end{array}$ & \\
\hline $\begin{array}{l}2345 \\
3250 \\
6150 \\
3150\end{array}$ & $\begin{array}{l}\circ F . \\
1,425-1,475 \\
1,425.1,475 \\
1,475-1,525 \\
1,500-1,550 \\
1,450-1,500\end{array}$ & 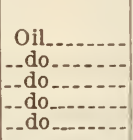 & $\begin{array}{l}\circ F . \\
400-450 \\
425-475 \\
425-475 \\
450-500 \\
425-475\end{array}$ \\
\hline
\end{tabular}

1 This is not an S. A. E. steel, but follows the S. A. E. numbering system.

Tempering.-After quenching, the sears are reheated to tne temperature indicated in Table 3 , and held for sufficient time to produce toughness. For tempering, either a liquid or circulating-air heating medium is satisfactory.

\section{NOTES ABOUT THE PROCESS}

Upset gear blanks produce teeth of uniform strength all tne way around, whereas pegged out flats when drop tested usually reveal strong teeth on two sides of the gear and weak teeth on two sides between the strong teeth.

Inspecting and testing.- At least three teeth on every gear should be polished and tested for hardness. After quenching and tempering, the scleroscope readings should be 70 to 76, Rockwell hardness C-52 to C-57. Special fixtures are of advantage when obtaining the hardness tests.

Specimens should be broken periodically under a drop hammer to check up on the forging and heattreating methods.

Cleaning.-Before grinding the holes and teeth of the gears, they should be subjected to a cleaning process, such as wire brushing or electric cleaning.

\section{AMERICAN SOCIETY FOR STEEL TREAT- ING, RECOMMENDED PRACTICE FOR THE HEAT TREATMENT OF PLAIN CARBON AND ALLOY SPRING STEEL, 1929}

\section{HEAT TREATMENT OF SPRING STEEL}

General.-This practice covers the recommended process to be followed in the manufacture and heat treatment of springs, and is divided into three sections: A. Flat springs (watch springs, etc., not included); B. Helical springs-wire diameter onehalf inch and over; and C. Helical springs-wire diameter under one-half inch.

\section{DIVISIONS A AND B}

Flat Springs and Helical Springs One-half Inch and Over

Hardness of steel as received from manufacturer.The maximum hardness limit for flat springs will depend to a considerable extent upon the nature of any cold work to be performed upon them. A maxi- mum Brinell hardness number of 321 is satisfactory for the thinner gages (less than five-sixteenth inch) where only the simple cold working operation, such as shearing, punching, and trimming are necessary. Heavier gages (five-sixteenths inch or over) should have a maximum Brinell hardness number of 302 . A minimum hardness limit is necessary only in special cases.

All coil-spring bars one-half inch or over should be soft enough to permit shearing without breaking.

Imperfections. - The maximum decarburization should not exceed 0.003 of an inch carbon free metal or a total depth of 0.008 of an inch of total and partial decarburization, as measured with the microscope.

Where springs are subjected to a large number of load applications per minute or to heavy stresses, surface imperfections such as seams with a depth of only 0.005 of an inch, generally cause early failure.

Quenching cracks, warping, and low-fatigue resistance are frequently encountered when considerable carbide is segregated.

Forging, forming, and coiling.-The lowest temperature at which the steel will work satisfactorily should be used. This temperature should not exceed $1,850^{\circ} \mathrm{F}$., and the steel should be held at this temperature for only sufficient time thoroughly to heat throughout. The method of cooling after forging or forming is usually not important. When severe coiling or other severe forming operations are performed, the steel should be allowed to cool in the air until it becomes black.

Annealing.-When annealing is necessary for machining to relieve machining strains, to relieve cold working strains, or to relieve strains from local heating, the steel may be heated to the annealing temperatures given in Table 2. The rate of cooling to vary with requirements.

Chemical composition. - The steelc listed in Table 1 are recommended for flat springs. The second carbon steel listed in this table is generally used for large helical springs, as alloy steels have proved successful only in special applications.

TABLE 1

\begin{tabular}{|c|c|c|c|c|c|c|c|}
\hline Steel & C & $\mathrm{Mn}$ & $\begin{array}{c}\mathrm{P} \\
\text { (maxi- } \\
\text { mum) }\end{array}$ & $\frac{\mathrm{S}}{\text { (maxj- }}$ & $\mathrm{Si}$ & $\mathrm{Cr}$ & $\left.\right|_{\text {mini- }} ^{\mathrm{V}}$ \\
\hline $\begin{array}{l}\mathrm{C} r \\
\mathrm{Cr}-\mathrm{V} \\
\mathrm{Si}-\mathrm{Mn} .\end{array}$ & $\begin{array}{r}10.70-0.80 \\
.90-1.05 \\
45-.55 \\
.45-.55 \\
.50-.60\end{array}$ & $\begin{array}{l}0.50-0.80 \\
.30-.50 \\
.80-1.00 \\
.70-.90 \\
.60-.90\end{array}$ & $\begin{array}{l}0.040 \\
.040 \\
.040 \\
.040 \\
.045\end{array}$ & $\begin{array}{l}0.040 \\
.050 \\
.045 \\
.040 \\
.045\end{array}$ & $1.80-2.20$ & $1.00-1.20$ & 0.15 \\
\hline
\end{tabular}

1 Used for flat springs one-eighth inch and under.

Heat treatment.-Specific heat treatments of steels for flat and large helical springs are given in Table 2. Variations in the heat-treatment operations are of ten due to the personnel, variations in the composition of the steel or manufacture, and changes in local conditions, such as the control and sensitiveness of heat-treating equipment.

\section{TABLE 2}

\begin{tabular}{|c|c|c|c|c|}
\hline $\begin{array}{r}\text { Steel (as } \\
\text { Tab }\end{array}$ & $\begin{array}{l}\text { Annealing } \\
\text { tempera- } \\
\text { ture, }\end{array}$ & $\begin{array}{c}\text { Quenching } \\
\text { tempera- } \\
\text { ture, }\end{array}$ & $\begin{array}{c}\text { Tempering } \\
\text { tempera- } \\
\text { ture, }\end{array}$ & $\begin{array}{c}\text { Brinell } \\
\text { hard- } \\
\text { ness }\end{array}$ \\
\hline$r_{-}-90-1.05$ & \begin{tabular}{c}
$\circ F$ \\
\hdashline $1,575-1,600$ \\
$1,600-1,625$ \\
$1,600-1,625$ \\
$1,600-1,625$
\end{tabular} & $\begin{array}{c}\circ F \\
1,450-1,475 \\
1,525-1,550 \\
1,550-1,575 \\
1,550-1,575 \\
1,575-1,600\end{array}$ & $\begin{array}{c}{ }^{\circ} F \\
2700- \\
850-1,050 \\
850-1,050 \\
850-1,050 \\
850-1,050\end{array}$ & 36 \\
\hline
\end{tabular}

1 The tempering temperatures are to be used only as a guide to obtain the Brinell hardness numbers given in the table.

2 Rockwell C40 to C50, depending upon use. 
Quenching.-The steel should be heated uniformly to the temperature shown in Table 2 and quenched in thin oil. The time, in minutes, of heating in the furnace may be determined approximately by multiplying the diameter or thickness of the steel in inches by 60 . More uniform results are obtained with good circulation of the quenching medium and with regulation of the temperature from $100^{\circ}$ to $140^{\circ} \mathrm{F}$.

Tempering.-The steel should be heated in a suitable tempering medium to the temperature shown in Table 2. It should be held at this temperature for sufficient time to permit complete penetration of the heat. ${ }^{41}$ The method of cooling after tempering is optional.

\section{DIVISION C}

\section{Helical Springs Under One-half Inch}

Hardness of steel as reccived from manufacturer.Wires made of steels listed in Table 3 are purchased either pretempered or annealed. The Rockwell Chardness of the pretempered wire is the same as shown in Table 4. For uniform coiling the annealed wire should have a Rockwell C-hardness of 15-25.

Imperfections.-The wire should be free from all evidence of decarburization. It is recommended that the alloy sieels should be tested for defects by macroetching.

Forging, forming, and cciling.-Springs of this type are seldom formed or coiled hot.

TABLE 3.-Steels for helical springs under one-half inch diameter

\begin{tabular}{|c|c|c|c|c|c|c|}
\hline Steels & C & Mn & $\begin{array}{l}\mathrm{P} \\
\text { (maxi- }\end{array}$ & $\begin{array}{c}\mathrm{S} \\
\text { (maxi- } \\
\text { mum) }\end{array}$ & $\mathrm{Cr}$ & $\begin{array}{l}\text { V } \\
\text { (mini- } \\
\text { mum) }\end{array}$ \\
\hline $\begin{array}{l}\mathrm{C} \\
\mathrm{C} \\
\mathrm{C}\end{array}$ & $\begin{array}{r}10.45-0.70 \\
.50-.70 \\
.60-.70 \\
.80-.90 \\
.90-1.05 \\
.45-.55\end{array}$ & $\begin{array}{r}0.80-1.20 \\
.60-.90 \\
.30-.60 \\
.30-.60 \\
2.30-.50 \\
.50-.80\end{array}$ & $\begin{array}{r}0.040 \\
.040 \\
.040 \\
.040 \\
.040 \\
.040\end{array}$ & $\begin{array}{l}0.045 \\
.045 \\
.025 \\
.040 \\
.040 \\
.040\end{array}$ & $0.80-1.10$ & י. \\
\hline
\end{tabular}

1 This steel is furnished as "hard drawn" wire, and in small sizes the carbon is often lower.

"In "music" wire the manganese is often lower.

Chemical composition.-Steels listed in Table 3 are recommended for helical springs under $1 / 2$-inch wire diameter.

Heat treatment.- Specific heat treatments of steels for helical springs under one-half inch are given in Table 4. Variations in the heat-treatment operations are often due to the personnel, variations in the composition of the steel or manufacture, and changes in local conditions, such as the control and sensitiveness of heat-treating equipment.

TABLE 4.- Heat treatments jor helical springs under one-halJ inch in diameter

\begin{tabular}{|c|c|c|c|}
\hline $\begin{array}{l}\text { C range from } \\
\text { Table } 3\end{array}$ & $\begin{array}{l}\text { Quenching tempera- } \\
\text { ture, }\end{array}$ & $\begin{array}{c}\text { Tempering } \\
\text { temperature, }\end{array}$ & $\begin{array}{c}\text { Rock- } \\
\text { well } \\
\text { hardness }\end{array}$ \\
\hline $\begin{array}{l}0.45-0.70 \\
.50-.70 \\
.50-.70 \\
.60-.70 \\
.80-.90 \\
.80-.90 \\
.90-1.05 \\
.90-1.05 \\
\text { Cr-V..... }\end{array}$ & 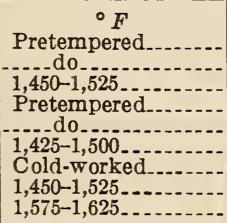 & $\begin{array}{c}\circ F \\
450-600 \\
450-600 \\
700-850 \\
450-600 \\
450-600 \\
800-850 \\
325-375 \\
800-900 \\
850-950\end{array}$ & $\begin{array}{r}140-45 \\
40-45 \\
40-45 \\
40-45 \\
40-45 \\
40-45 \\
(2) \\
40-45 \\
40-45\end{array}$ \\
\hline
\end{tabular}

1 On cushion springs this may be $35-45$.

2 Music wire is brought to tensile properties, not hardness.

41 Flash tempering should never be resorted to.
Quenching.-The steel should be heated uniformly to the temperature shown in Table 4 and quenched in thin oil. More uniform results are obtained with good circulation of the quenching medium and with regulation of the temperature from $100^{\circ}$ to $140^{\circ} \mathrm{F}$.

Tempering.-The steel should be heated in a suitable tempering medium to the temperature shown in Table 4. It should be held at this temperature for sufficient time to permit complete penetration of the heat. The method of cooling after tempering is optional.

AMERICAN SOCIETY FOR STEEL TREATING, TENTATIVE RECOMMENDED PRACTICE FOR THE HEATING, FORGING, AND HEAT TREATING OF IOCOMOTIVE FORGINGS, 1931

\section{LOCOMOTIVE FORGINGS}

\section{INTRODUCTION}

This recommended practice is to be used as a guide in the manufacture and heat treatment of locomotive forgings, such as locomotive driving and trailer axles, crank pins, main and side rods, piston rods, and similar forgings.

\section{HEATING BILLETS FOR FORGING}

As rapid heating may produce internal ruptures, care must be taken to avoid rapid heating, particularly from the low temperatures. Ample time should also be given for the heat to penetrate uniformly and thoroughly into the billet before forging. The actual heating time required will depend on the type of steel and the equipment and methods used for heating.

Cold billets should not be charged into a hot furnace. Billets that have been stored out of doors, particularly during winter months, should be brought into the shop for a warming period of 10 to 12 hours before charging into the furnace for heating.

\section{PREHEATING FORGING BILLETS}

It is good practice to preheat the billets. This preheating is best done in a separate furnace or one with a preheating chamber. If such a furnace is not available, the billets can be charged into a forging furnace after the operations for the day are completed, and the furnace has been cooled down to a dull red. The billets will absorb the furnace heat and by the next morning will be preheated to a temperature of several hundred degrees Fahrenheit. The billets are then ready for direct firing, which should proceed slowly. When a separate furnace is available for preheating, the billets are charged into the cold furnace and brought up slowly and uniformly to a temperature of $1,100^{\circ}$ to $1,200^{\circ} \mathrm{F}$. The time required to heat to this temperature should be approximately three-quarters to 1 hour per inch of least thickness.

To avoid loss of heat the billets should be transferred quickly from the preheating furnace to the forge-heating furnace. As the danger of rupturing the billets has been practically eliminated by preheating, the forging furnace can be at considerably higher temperature than the preheated billets. Raising the preheated billets to the forging temperature is carried out with the usual precautions to insure uniform heating and thorough penetration of the heat. The minimum time required to bring preheated billets from a temperature of about $1,100^{\circ}$ $\mathrm{F}$. to the forging heat $\left(2,100^{\circ}\right.$ to $2,200^{\circ} \mathrm{F}$.) is approximately one-half to 1 hour per inch of least thick- 
ness. For billets of various thicknesses, the following schedule is typical:

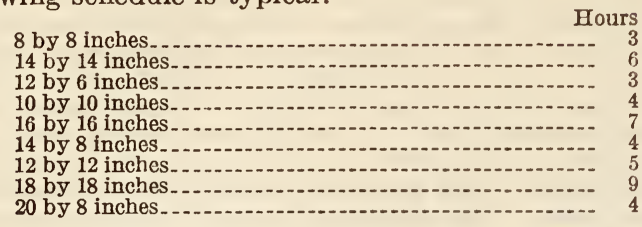

FORGING TEMPERATURES FOR BILLETS

The proper forging temperature for both carbon and alloy steel billets, of the types generally used for locomotive forgings, is from $2,000^{\circ}$ to $2,200^{\circ} \mathrm{F}$. which is very pale yellow, fading into white. Overheating and nonuniform heating must be avoided, as both are sources of forging ruptures. It is desirable to have the heating furnace under pyrometric control, preferably by thermocouples.

The temperature at which the forgings are finished will be determined by the initial forging temperature, and by the temperature lost during forging, which depend on the size of the forging and on the amount of work required to shape the forging from the billet. Generally they are finished at a temperature of $1,500^{\circ}$ to $1,800^{\circ} \mathrm{F}$. Lighter sections, such as connecting rods, are usually finished at the lower temperature, while heavy sections, such as driving axles, are finished near the higher temperature mentioned.

\section{HEAT TREATMENT OF LOCOMOTIVE FORGINGS}

After the forging operation is completed, the forgings should be allowed to cool to below the critical range under suitable conditions to prevent injury by too rapid cooling. To remove forging stresses and to refine the grain, an appropriate heat treatment should be given.

The heat treatment may consist of annealing, but it is usual practice for high-duty locomotive forgings, where better physical properties are required, to use a treatment consisting of normalizing followed by tempering.

When forgings are to be bored or rough machined, they may be annealed to improve the machineability, preliminary to normalizing and tempering.

\section{ANNEALING}

The purpose of annealing a forging is to refine the grain structure, to increase the ductility, or to soften it for ease of machining. Annealing consists of heating to a temperature above the critical range, holding a sufficient length of time at that temperature, and then cooling in the furnace or cooling under suitable conditions to secure uniform retarded cooling.

\section{NORMAIIZING AND TEMPERING LOCOMOTIVE FORGINGS}

The purpose of normalizing locomotive forgings is to refine the grain structure of a forging and to secure the highest combination of physical properties without resorting to quenching in a liquid medium. The normalizing temperature is therefore above the critical range. The temperature must be held a sufficient time to permit complete grain refinement in the forgings.

Normalizing may consist of a single heating to a temperature above the critical range followed by cooling in the air, or of a sequence of such heatings and coolings. If a sequence of heatings and coolings is used, the temperature may vary. A decrease in temperature is usually made for each successive heating, but the temperature for each heating should be above the critical range. Normalizing should be followed by tempering.
To eliminate the lack of uniformity in the grain structure of forgings, resulting from variations in reduction and finishing temperatures, and to produce a homogeneous grain structure throughout the forgings which will respond uniformly to the usual normalizing temperature, it is of ten desirable particularly with large forgings, first to normalize the forgings at a temperature much above the upper critical (approximately $1,750^{\circ}$ to $1,850^{\circ} \mathrm{F}$.) temperature.

\section{TEMPERING}

The purpose of tempering is to remove strains set up in the forgings during the cooling from the normalizing temperature. The ductility of the forgings is increased by tempering, while the tensile strength may be slightly reduced. Tempering is not intended to change the grain size; therefore, the temperature is below the critical range. After tempering, the forgings may be cooled in the air, slowly with the furnace, or under conditions to secure uniform retarded cooling. The forgings should be held at temperature a sufficient time to insure complete penetration of the heat.

In annealing, normalizing, and tempering, choice as to the temperatures used, the number of normalizing treatments, the length of time that the forgings are held at temperature, and the method of cooling in annealing and tempering must be made according to experience, taking into account the type of steel, the size and character of the forgings, and the condition of the furnace used.

\section{TEMPERATURES AND TIMES OF HEATING}

In view of the large number of variable conditions involved, it is impossible to lay down exact rules of procedure for annealing, normalizing, and tempering, with stated temperatures and times of heating which will be applicable under all conditions. The following sections give general information regarding temperatures and times of heating which may be expected to give satisfactory results under favorable conditions. The information is given only as a guide and not as standard practice for all cases. Experience based on the actual conditions involved will sometimes show that better results are obtained by the use of other temperatures.

The rate at which forgings are heated to the normalizing and tempering temperatures must not be too rapid or the forgings may be injured. The proper rate will depend to a considerable extent on the design of the furnace. In general, the time allowed for each inch of diameter or thickness of the forging should be 45 minutes for raising to the normalizing temperature and 30 minutes for raising to the tempering temperature.

After the temperatures desired for normalizing have been reached, the forgings should be held at these temperatures a sufficient time to permit the desired changes in structure to be completed. In general, a holding time of one hour per inch of diameter or maximum thickness will usually be satisfactory.

Table 1 gives, for general guidance, the time for heating and holding a medium furnace charge of forgings.

TABLE 1

\begin{tabular}{|c|c|c|}
\hline Thickness of largest section of forging & $\begin{array}{l}\text { Time of } \\
\text { heating } \\
\text { (approxi- } \\
\text { mate) } 1\end{array}$ & $\begin{array}{l}\text { Time of } \\
\text { holding } \\
\text { (approxi- } \\
\text { mate) } 1\end{array}$ \\
\hline $\begin{array}{l}\text { Up to and including } 5 \text { inches } \\
\text { Over } 5 \text { inches and including } 8 \text { inches. } \\
\text { Over } 8 \text { inches and including } 10 \text { inches.- } \\
\text { Over } 10 \text { inches and including } 12 \text { inches... }\end{array}$ & $\begin{array}{r}\text { Hours } \\
8 \\
8 \\
10 \\
12\end{array}$ & $\begin{array}{r}\text { Hours } \\
5 \\
8 \\
12 \\
16\end{array}$ \\
\hline
\end{tabular}

1 Medium charge. 
NORMALIZING AND TEMPERING TEMPERATURES

Table 2 gives, for general guidance, temperatures which may be used in normalizing and tempering forgings of the various compositions indicated. The figures are based on a single normalizing treatment with slow cooling in the furnace from the tempering temperature. As previously stated, the tempera- tures may require modification to meet individual requirements.

Annealing temperatures will usually be lower than the normalizing temperatures. The difference is approximately $50^{\circ} \mathrm{F}$. lower for annealing the plain carbon steel, $25^{\circ} \mathrm{F}$. for the nickel steels, and $100^{\circ} \mathrm{F}$. for the vanadium steels.

TABLE 2.-Normalizing and tempering locomotive forgings

\begin{tabular}{|c|c|c|c|c|c|c|c|}
\hline C & Mn & $\mathrm{Si}$ & V & $\mathrm{Ni}$ & $\begin{array}{l}\text { Normalizing } \\
\text { temperature }\end{array}$ & $\begin{array}{l}\text { Tempering } \\
\text { temperature }\end{array}$ & $\begin{array}{l}\text { Approxi- } \\
\text { mate } \\
\text { tensile } \\
\text { strength }\end{array}$ \\
\hline $\begin{array}{l}0.40-0.45 \\
.45-.50 \\
.50-.55 \\
.45-.50 \\
.50-.55 \\
.35-.40 \\
.40-.45 \\
.20-.30\end{array}$ & $\begin{array}{l}0.50-0.85 \\
.50-.85 \\
.59-.85 \\
.70-.95 \\
.70-.95 \\
.50-.80 \\
.50-.80 \\
.80-1.00\end{array}$ & $\begin{array}{l}0.18-0.30 \\
.18-.30 \\
.18-.30 \\
.18-.30 \\
.18-.30 \\
.18-.35 \\
.18-.35 \\
.18-.35\end{array}$ & $\begin{array}{r}0.15-0.25 \\
.15-.25\end{array}$ & $\begin{array}{l}3.25-3.75 \\
3.25-3.75 \\
2.50-3.00\end{array}$ & $\begin{array}{c}\circ F . \\
1,500-1,550 \\
1,475-1,525 \\
1,450-1,500 \\
1,600-1,650 \\
1,575-1,625 \\
1,425-1,475 \\
1,425-1,475 \\
1,425-1,475\end{array}$ & $\begin{array}{c}\circ F . \\
1,000-1,100 \\
1,100-1,250 \\
1,200-1,350 \\
1,050-1,200 \\
1,150-1,300 \\
1,000-1,150 \\
1,050-1,200 \\
1,000-1,200\end{array}$ & $\begin{array}{r}\text { Lbs./in. }{ }^{2} \\
80,000 \\
80,000 \\
80,000 \\
95,000 \\
95,000 \\
90,000 \\
90,000 \\
80,000\end{array}$ \\
\hline
\end{tabular}

AMERICAN SOCIETY FOR STEEL TREATING, TENTATIVE RECOMMENDED PRACTICE FOR THE HEAT TREATMENT OF SPLINE SHAFTS, 1931

\section{SPLINE SHAFTS}

\section{GENERAL}

This recommended practice covers the processes to be followed for the heat treatment of spline shafts. A spline shaft is so constructed that it interlocks, either rigidly or sliding, with a part or parts, such as gears. Thus a spline shaft may have one or more keyways, or one or more keys, but it is not customary to have both keys and keyways on the same shaft. Spline shafts are used in two general classes of work-automotive parts and machine-tool parts. Short shafts are widely used in automotive parts, whereas long spline shafts are more common in machine tools. No arbitrary dimensions are set in distinguishing between long and short shafts but, in general, shafts not over 12 inches in length or over $1 \frac{1}{2}$ inches in diameter may be called "short" spline shafts.

\section{DESIGN}

As spline shafts in operation are subjected to alternating stresses, their endurance limit or resistance to fatigue is of importance. The shaft should be designed to eliminate unnecessary necks and sharp corners where stresses might be concentrated. Fillets and machined grooves in splines should always be gradually rounded up.

Stiffness is one of the necessary properties of some types of spline shafts, particularly long shafts. Shafts should be designed of sufficient section so that harmful deflection will not occur under the applied load. Where the design is incorrect, the shaft may twist, or bend, thus causing undesirable effects due to alternating stress, vibration, and the like, which will be felt in the gears or other moving parts as well as in the shaft. Heat treatment may raise the elastic limit of the steel, but it will not change the modulus of elasticity. For this reason, heat-treated shafts may not be any stiffer than untreated shafts when operating below the elastic limit.

Distortion is the trouble most commonly encountered in the heat treatment of spline shafts. To avoid distortion care must be exercised in all operations. The steel must be carefully selected and inspected. If much machining is performed on the shafts, the machining strains should be relieved by normalizing or annealing before subjecting the shafts to the final heat treatments.

The carburizing and heat-treating processes should all be carried out with extreme care. It may be necessary with some carburized spline shafts to omit the double treatment and to carburize at a lower temperature to avoid distortion.

There are many spline shafts made of bar stock which is either black hot-rolled finish, or bright colddrawn finish. In either case this material is more prone to distortion than a properly normalized forging, and, therefore, where minimum straightening is essential, spline shafts made of bar stock should be normalized the same as forgings unless the straightening operation is considered absolutely necessary on the finished spline shaft. If hot-rolled or cold-drawn bar stock is used, it is essential that one sixty-fourth to one thirty-second of an inch should first be machined from the outer surface to insure a clean surface for hardening.

Keyways in a spline shaft may cause distortion in quenching. Unless designed with ample section, a shaft with a keyway has less stiffness than one carrying keys integral with the shaft. To minimize distortion in quenching, it is advantageous to have more than one integral key. These keys are equally spaced about the shaft to facilitate equal cooling and balance the section.

\section{CHEMICAL COMPOSITION OF SPLINE SHAFT STEELS}

Three types of steel are commonly used for spline shafts. These are the carburizing, water-hardening, and oil-hardening types. In Table 1 typical compositions are given, but no attempt has been made to include every steel that might be used for some specific purpose. 
TABLE 1.-Chemical composition of spline shaft steels

CARBURIZING STEELS

\begin{tabular}{|c|c|c|c|c|c|c|c|c|}
\hline Steels, S. A. E. No. & $\mathrm{C}$ & Mn & $\underset{\text { mum })}{P(\operatorname{maxi}-}$ & $\begin{array}{l}\text { S (maxi- } \\
\text { mum) }\end{array}$ & $\mathrm{Ni}$ & $\mathrm{Cr}$ & Mo & V \\
\hline 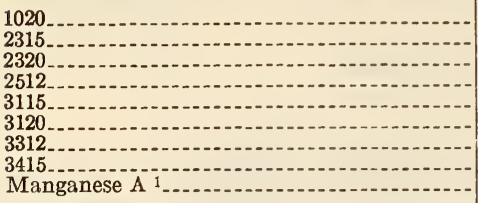 & $\begin{array}{r}0.15-0.20 \\
.10-.20 \\
15-.25 \\
\mathrm{Max} .17 \\
.10-.20 \\
15-.25 \\
\mathrm{Max} .17 \\
.10-.20 \\
.15-.25\end{array}$ & $\begin{array}{l}0.30-0.60 \\
.30-.60 \\
.30-.60 \\
.30-.60 \\
.30-.60 \\
.30-.60 \\
.30-.60 \\
.30-.60 \\
.90-1.10\end{array}$ & $\begin{array}{r}0.045 \\
.040 \\
.040 \\
.040 \\
.040 \\
.040 \\
.040 \\
.040 \\
.04-.06\end{array}$ & $\begin{array}{r}0.055 \\
.050 \\
.050 \\
.050 \\
.050 \\
.050 \\
.045 \\
.045 \\
.04-.06\end{array}$ & $\begin{array}{r}3.25-3.75 \\
3.25-3.75 \\
4.75-5.25 \\
1.00-1.50 \\
1.00-1.50 \\
3.25-3.75 \\
2.75-3.25\end{array}$ & $\begin{array}{r}0.45-0.75 \\
.45-.75 \\
1.25-1.75 \\
.60-.95 \\
.15-.25\end{array}$ & \begin{tabular}{|l} 
\\
\\
\end{tabular} & $\mid \begin{array}{l}-1 \\
-1 \\
-1\end{array}$ \\
\hline
\end{tabular}

WATER-HARDENING STEELS

\begin{tabular}{|c|c|c|c|c|c|c|c|c|}
\hline 1045 & $\begin{array}{r}0.40-0.50 \\
.15-.25 \\
.25-.35\end{array}$ & $\begin{array}{r}0.50-0.80 \\
.50-.60 \\
.50-.80\end{array}$ & $\begin{array}{l}0.045 \\
.04 \\
.04\end{array}$ & $\begin{array}{r}0.055 \\
.050 \\
.050\end{array}$ & $3.25-3.75$ & $0.50-0.80$ & $0.15-0.25$ & \\
\hline
\end{tabular}

OIL-HARDENING STEELS

\begin{tabular}{|c|c|c|c|c|c|c|c|c|}
\hline 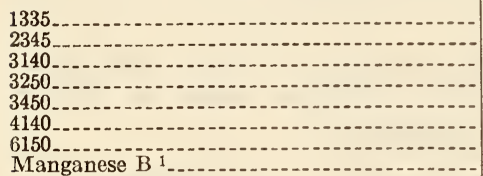 & $\begin{array}{l}0.30-0.40 \\
.40-.50 \\
.35-.45 \\
.45-.55 \\
.45-.55 \\
.35-.45 \\
.45-.55 \\
.45-.55\end{array}$ & $\begin{array}{l}1.60-1.90 \\
.50-.80 \\
.50-.80 \\
.30-.60 \\
.30-.60 \\
.50-.80 \\
.50-.80 \\
.90-1.10\end{array}$ & $\begin{array}{l}0.04 \\
.04 \\
.04 \\
.04 \\
.04 \\
.04 \\
.04 \\
.045\end{array}$ & $\begin{array}{l}0.050 \\
.05 \\
.05 \\
.045 \\
.045 \\
.05 \\
.045 \\
.050\end{array}$ & $\begin{array}{l}3.25-3.75 \\
1.00-1.50 \\
1.50-2.00 \\
2.75-3.25 \\
\end{array}$ & $\begin{array}{l}0.45-0.75 \\
.90-1.25 \\
.60-.95 \\
.80-1.10 \\
.80-1.10 \\
.45-.60\end{array}$ & $0.15-0.25$ & (2) \\
\hline
\end{tabular}

1 These are not S. A. E. steels.

\section{SELECTION OF STEELS FOR SPLINE SHAFTS}

Spline shafts which are not to be highly stressed can be satisfactorily made from steel No. 1020. To make the shaft sufficiently stiff, it is sometimes designed large enough so that this steel may be used instead of an alloy steel.

For small shafts which are to be carburized, steels Nos. $3115,2512,3312$, and 2315 are satisfactory. If the diameter is over $1 \frac{1}{2}$ inches, the carbon can be increased slightly without encountering brittleness on quenching. For example, for large shaft steels Nos. 3415,3120 , or 2320 are satisfactory.

While the water-hardening steels can be used, it should be pointed out that water quenching may result in cracking, particularly if the design is such that the shaft will be susceptible to cracking. Steel No. 1045, although used for spline shafts, is not entirely satisfactory. It does not always respond uniformly to heat treatment.

The steels given in Table 1 designated as manganese A, B, and 1335 are representative of the manganese structural steels. Steels of this general type are used successfully in spline shafts. They machine freely, have excellent physical properties, and harden without difficulty.

In addition to these two manganese steels, steels Nos. 1340 and 1345 are used to some extent for spline shafts. These steels contain carbon 0.35 $0.45,0.40-0.50$ per cent respectively with $1.60-1.90$ per cent manganese. These steels do not machine so readily as the manganese steels listed, but have excellent physical properties and are oil quenched.

The oil-hardening steels listed are in general quite satisfactory.

\section{HEAT TREATMENT OF CARBURIZING STEELS}

Normalizing and Annealing of Carburizing Steels

To normalize or anneal the carburizing steels as given in Table 1, heat openly, exposing maximum surface area, to a temperature of $1,650^{\circ}$ to $1,750^{\circ} \mathrm{F}$. Hold at heat only long enough for complete refinement of grain and complete penetration of the heat. Cool in air or at a rate to give the desired machining qualities.

\section{Carburizing}

Carburize to a depth to suit individual requirements. The conventional methods of carburizing, as given in the National Metals Handbook, may be followed. The carburizing temperatures and the heat treatments for carburized spline shafts are given in Table 2 .

TABLE 2.-Carburizing and heat treating of carburized spline shafts

\begin{tabular}{|c|c|c|c|c|c|c|c|}
\hline Steel No. as listed in Table 1 & $\begin{array}{l}\text { Carburizing } \\
\text { temperature }\end{array}$ & Cool in - & Reheat & $\underset{\text { in- }}{\text { Quench }}$ & Reheat & $\begin{array}{l}\text { Quench } \\
\text { in- }\end{array}$ & $\begin{array}{l}\text { Tempering } \\
\text { temperature }\end{array}$ \\
\hline $\begin{array}{l}1020 \\
231520 \\
2312 \\
31200 \\
3312 \\
3415 \\
\text { Manganese } \mathrm{A}\end{array}$ & $\begin{array}{l}\circ F .700 \\
1,600-1,700 \\
1,600-1,700 \\
1,600-1,700 \\
1,600-1,700 \\
1,600-1,700 \\
1,600-1,700 \\
1,600-1,700 \\
1,600-1,700 \\
1,600-1,650\end{array}$ & 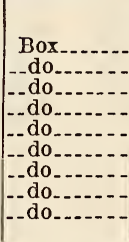 & $\begin{array}{l}{ }^{F} . \\
1,550 \\
1,540 \\
1,540 \\
1,525 \\
1,550 \\
1,550 \\
1,525 \\
1,525 \\
1,575\end{array}$ & 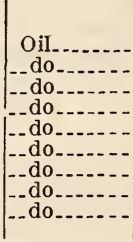 & $\begin{array}{c}\circ F . \\
1,400-1,450 \\
1,375-1,400 \\
1,375-1,400 \\
1,375-1,400 \\
1,400-1,425 \\
1,400-1,425 \\
1,375-1,400 \\
1,375-1,400 \\
1,400-1,425\end{array}$ & 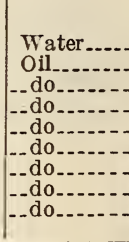 & $\begin{array}{r}{ }^{\circ} F \\
350-425 \\
350-425 \\
350-500 \\
350-500 \\
350-425 \\
350-500 \\
350-500 \\
350-500 \\
400\end{array}$ \\
\hline
\end{tabular}

1 Carburizing at the lower temperature causes less distortion, while at the higher temperature more rapid penetration is secured. 
Single-Quenching Treatments for Carburizing Spline Shafts

A single quench, or single treatment, may be given where resistance to impact or shock is not important and where it is important to hold distortion to a minimum. This treatment consists essentially in cooling the material in the box, reheating sufficiently high to refine the case and partially refine the core in one operation, followed by a suitable quench. This treatment is suited particularly to S. A. E. Nos. 2315 and 2512.

It is advisable, when the single-quenching treatment is employed, to use the lower carburizing temperature, and after carburizing if the structure is coarse it is advisable to use the higher reheating temperature, as given in Table 3 .

TABLE 3.-Single-quenching treatment for carburized spline shaft

\begin{tabular}{|c|c|c|c|c|c|}
\hline $\begin{array}{l}\text { Steel No. as } \\
\text { listed in } \\
\text { Table } 1\end{array}$ & Carburize & $\begin{array}{l}\text { Cool } \\
\text { in- }\end{array}$ & $\underset{\text { to- }}{\text { Reheat }}$ & $\underset{\text { in- }}{\text { Quench }}$ & $\begin{array}{l}\text { Tem- } \\
\text { pering } \\
\text { tem- } \\
\text { pera- } \\
\text { tures }\end{array}$ \\
\hline $\begin{array}{l}1020 \\
2315 \\
2320 \\
3115 \\
3120 \\
3312 \\
3415 \\
\text { Manganese A }\end{array}$ & $\begin{array}{c}\circ F . \\
1,600-1,700 \\
1,600-1,700 \\
1,600-1,700 \\
1,600-1,700 \\
1,600-1,700 \\
1,600-1,700 \\
1,600-1,700 \\
1,600-1,700 \\
1,600-1,650\end{array}$ & 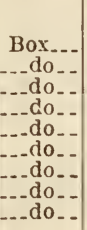 & $\begin{array}{c}\circ F . \\
1,4001,450 \\
1,325-1,375 \\
1,325-1,375 \\
1,300-1,350 \\
1,375-1,425 \\
1,375-1,425 \\
1,375-1,425 \\
1,350-1,400 \\
1,400-1,425\end{array}$ & 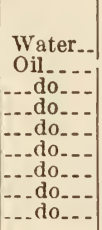 & $\begin{array}{c}\circ F \text {. } \\
350-425 \\
350-425 \\
350-500 \\
350-500 \\
350-425 \\
350,500 \\
350-500 \\
350-500 \\
300-400\end{array}$ \\
\hline
\end{tabular}

Double-Quenching Treatment for Carburized Spline Shafts

A double quench as given in Table 2 is recommended for obtaining case and core refinement, maximum ductility, and resistance to shock and impact. The conventional carburizing process (packing the material in a carburizing compound in a box) is satisfactory. Long and short shafts should stand vertically in the box, but uniform heating is essential which, in some furnaces, may require horizontal heating at a sacrifice of straightness.

It is suggested for hardening that the spline shafts may be hung vertically from eyebolts in the furnace, and also quenched vertically from the same eyebolts on both single and double quenched shafts. Spline shafts are always out in relation to the keyway. Shafts can be quenched in a fixture or on a machine on the final heat, so that they will come straight enough to clean up on grinding when an ample amount of grinding stock is allowed. By this method, in many cases, the straightening operation may be unnecessary.

Spline shafts can also be heated in a horizontal position in a continuous furnace provided the supports are properly spaced. The shafts come out fairly straight although they usually require some straightening.

\section{Tempering Carburized Spline Shafts}

In tempering, the highest temperature should be used that will give the desired hardness.

\section{HEAT TREATMENT OF WATER-HARDENING STEELS}

\section{Normalizing and Annealing}

To normalize or anneal the water-hardening steels for spline shafts as given in Table 1 , heat openly, exposing maximum surface area, to a temperature of $1,600^{\circ}$ to $1,700^{\circ} \mathrm{F}$. for steel No. 1045 , and to $1,650^{\circ}$ to $1,750^{\circ} \mathrm{F}$. for steels Nos. 2320 and 4130. For this treatment the steels should only be held at temperature long enough to insure complete penetration of heat. From these temperatures the steels may be cooled in the air or cooled at a rate to produce the desired machining qualities.

\section{Hardening and Tempering}

The quenching and tempering temperatures for the water-hardening steels are given in Table 4. Before quenching in water, it may be advisable first to quench these steels for a few seconds in oil. The shafts should always be removed from the water before they become cold.

\section{TABLE 4.-Hardening and tempering of water- hardening spline shafts}

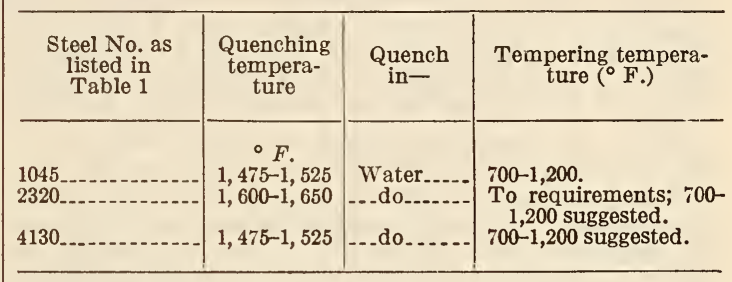

\section{HEAT TREATMENT OF OIL-HARDENING STERLS}

\section{Normalizing and Annealing}

After forging, these steels should be normalized or annealed. The following is a form of normalizing suitable for steels Nos. 1335, 2345, 3250, 3450, 6150, and manganese B. This treatment produces good machining qualities.

Heat the steels slowly, while exposing maximum surface area, to temperature of $1,650^{\circ}$ to $1,700^{\circ} \mathrm{F}$. Hold at heat only long enough to insure thorough and uniform heating. Cool rapidly by opening furnace doors to $1,280^{\circ} \mathrm{F}$. Cool slowly from $1,280^{\circ}$ to $1,000^{\circ} \mathrm{F}$. over a period from 6 to 10 hours for good machining.

Steel No. 3140 may be normalized by heating slowly and uniformly while exposing maximum surface area to a temperature of $1,575^{\circ}$ to $1,625^{\circ} \mathrm{F}$. and cooling from this temperature slowly in air.

Steel No. 4140 may be normalized by heating slowly and uniformly while exposing maximum surface area to a temperature of $1,650^{\circ}$ to $1,700^{\circ} \mathrm{F}$. and cooling slowly in air.

\section{Hardening and Tempering}

The quenching and tempering temperature for the spline shafts made from oil-hardening steels are given in Table 5 .

\section{TABLE 5.-Hardening and tempering oil-hardening} spline shafts

\begin{tabular}{|c|c|c|c|c|}
\hline $\begin{array}{c}\text { Steel No. as listed in } \\
\text { Table } 1\end{array}$ & $\begin{array}{l}\text { Quenching } \\
\text { temperature }\end{array}$ & $\begin{array}{l}\text { Quench } \\
\text { in- }\end{array}$ & $\begin{array}{c}\text { Tempering } \\
\text { tempera- } \\
\text { tures }\end{array}$ & $\begin{array}{c}\text { Approx- } \\
\text { imate } \\
\text { Brinell } \\
\text { hardness }\end{array}$ \\
\hline 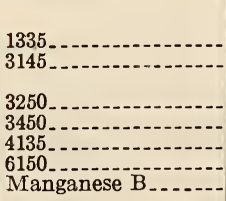 & $\begin{array}{c}{ }^{\circ} F . \\
1,500-1,525 \\
1,425-1,450 \\
1,525-1,550 \\
1,475-1,500 \\
1,475-1,500 \\
1,525-1,550 \\
1,575-1,600 \\
1,475-1,525\end{array}$ & 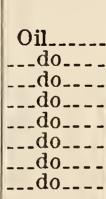 & 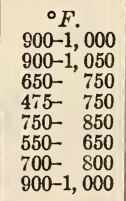 & $\begin{array}{l}217-255 \\
351-300 \\
430-375 \\
529-430 \\
460-387 \\
400-387 \\
444-351 \\
400-321\end{array}$ \\
\hline
\end{tabular}

Hardening steel No. 3145 may be accomplished in two different ways, as indicated in Table 5. The method giving a high-tempering temperature con- 
sists in hardening the shaft vertically, with the spline or keyway not cut, and leaving ample stock for grinding. The keyway is then milled after the heat treatment. If the steel has been properly normalized and carefully handled, straightening is rarely needed, but it can be done at the tempering heat if necessary. After tempering, the shafts are rough ground to 0.010 inch large, then the spline is milled, and the spline shafts are then finish ground.

This same sequence of operations may be followed for any of the steels listed in Table 5. The tempering temperature would then necessarily be higher than indicated in the table in order to make machining possible. By using this treatment on steels Nos. 3250 and 3450 , more ductility may be obtained than is possible with steel No. 3145 .

Spline shafts may be difficult to straighten after quenching. For example, shafts made with ample grinding stock from steel No. 3450 may be designed either to have two splines equally spaced or three integral keys which eliminate the unbalanced distortion. If steels Nos. 3250 and 3450 have to be straightened, it is done at the tempering temperature.

When steel No. 3145 is tempered at the lower temperature, the same method as outlined for steel No. 3450 is used. However, it may be possible to jig or machine quench this steel with a single spline, so that it will come within a reasonable grinding allowance.

Steel No. 6150 distorts in heat treating more than the steels previously discussed. However, it withstands abuse, has high strength, and high fatigueresistance values.

\section{AMERICAN SOCIETY FOR STEEL TREAT- ING, TENTATIVE RECOMMENDED PRAC- TICE FOR THE HEAT TREATMENT OF RIVET SETS FOR HOT WORK, 1931}

\section{RIVET SETS FOR HOT WORK}

\section{GENERAL}

This tentative recommended practice deals with two types of rivet dies; first, pneumatic rivet sets used in air guns, and, second, compression rivet dies which head by pressure rather than blows.

The approximate chemical compositions of the steels generally used for both types of rivet dies are given in Tables 1 and 3 .

TABLE 1.-Approximate chemical composition for
pneumatic rivet sets

\begin{tabular}{|c|c|c|c|c|c|c|c|}
\hline $\begin{array}{l}\text { Steel } \\
\text { No. }\end{array}$ & Type & C & $\mathrm{Si}$ & $\operatorname{Mn}$ & W & $\mathrm{Cr}$ & $V$ \\
\hline$\ldots$ & $\begin{array}{l}\text { Carbon } \\
\mathrm{C}-\mathrm{V}= \\
\mathrm{Cr}-\mathrm{V} \\
\mathrm{C} r-\mathrm{V} \\
\mathrm{Cr} \\
\mathrm{W}-\mathrm{Cr}-\mathrm{V} \\
\mathrm{Si}-\mathrm{Mn} \mathrm{1}\end{array}$ & $\begin{array}{l}0.70-0.90 \\
.70-.90 \\
.45-.60 \\
.45-.60 \\
.55-.70 \\
.40-.55 \\
.45-.60\end{array}$ & 2.00 & $\begin{array}{l}0.30 \\
.30 \\
.35 \\
.55 \\
.30 \\
.30 \\
.75\end{array}$ & . & $\begin{array}{r}0.80 \\
.80 \\
.50 \\
1.25 \\
\end{array}$ & $\begin{array}{r}0.20 \\
.20 \\
.20 \\
.25 \\
. .2\end{array}$ \\
\hline
\end{tabular}

1 This steel may or may not contain chromium up to 0.50 per cent; vanadium up to 0.25 ; or molybdenum up to 0.50 per cent.

TABLE 2.-Heat treatments for pneumatic rivet sets

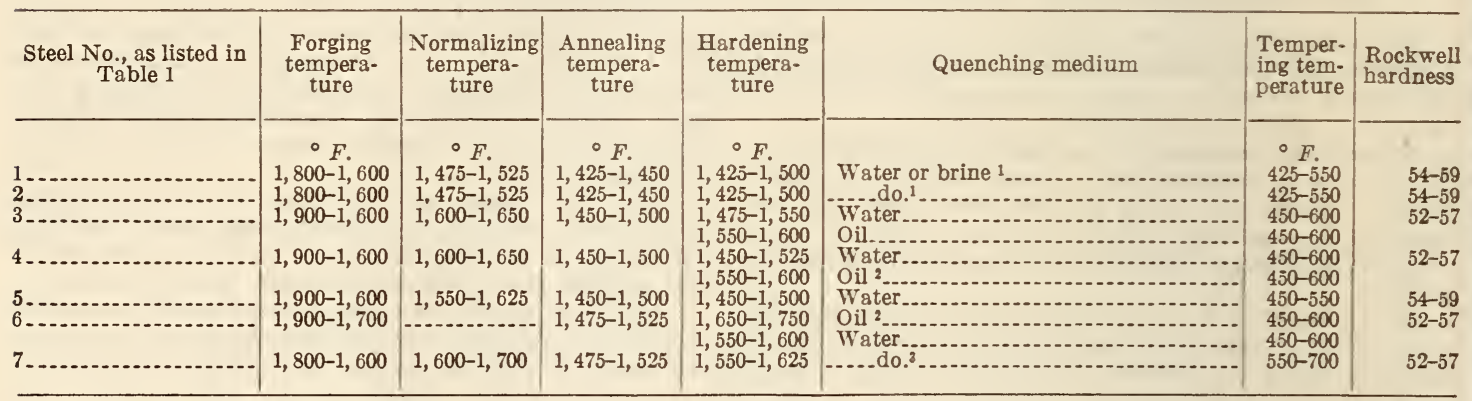

1 A 10 per cent brine solution is used.

2 For this treatment, oil is the preferred quenching medium.

${ }^{3}$ If this steel contains around 0.50 per cent molybdenum it may be quenched in oil by using a temperature approximately $25^{\circ}$ to $50^{\circ} \mathrm{F}$. higher than the temperature for water quenching.

\section{QUENCHING AND TEMPERING}

It is recommended that the rivet sets should be completely immersed while a stream of the quenching medium is being forced into the cup. When quenching, it is good practice to hold the rivet set with a special pair of tongs which keeps the water away from the ring, but at the same time permits uniform cooling of the cup and shank.

The sets should be tempered all over at the temperatures given in Tables 2 and 4 .
TABLE 3.-Approximate chemical composition of steel for rivet dies for hot pressing

\begin{tabular}{|c|c|c|c|c|c|}
\hline Steel No. & Type of steel & C & $\mathrm{Cr}$ & $\mathrm{v}$ & W \\
\hline 1 & $\mathrm{C}-\mathrm{Cr}-\mathrm{W}-\mathrm{V}^{-}$ & $\begin{array}{r}0.85-1.00 \\
.30-.45\end{array}$ & $\begin{array}{l}\text { 3. } 75 \\
3.00\end{array}$ & 0.50 & 10.00 \\
\hline
\end{tabular}

TABLE 4.-Heat treatment for rivet dies for hot pressing

\begin{tabular}{|c|c|c|c|c|c|c|c|}
\hline $\begin{array}{l}\text { Steel No. as listed in } \\
\text { Table } 3\end{array}$ & $\begin{array}{c}\text { Forging } \\
\text { temperature }\end{array}$ & $\begin{array}{c}\text { Annealing } \\
\text { temperature }\end{array}$ & $\begin{array}{l}\text { Preheating } \\
\text { temperature }\end{array}$ & $\begin{array}{l}\text { Quenching } \\
\text { temperature }\end{array}$ & Quenching medium & $\begin{array}{l}\text { Tempering } \\
\text { temperature }\end{array}$ & $\begin{array}{l}\text { Rockwell } \\
\text { hardness }\end{array}$ \\
\hline 12 & $\begin{array}{c}{ }^{\circ} \mathrm{F} \\
1,900-\mathrm{i}, 650 \\
2,050-1,800\end{array}$ & $\begin{array}{c}{ }^{\circ} F \\
1,475-1,525 \\
1,600-1,650\end{array}$ & $\begin{array}{c}{ }^{\circ} \mathrm{F} . \\
1,500-1,550\end{array}$ & $\begin{array}{c}{ }^{\circ} F \text {. } \\
1,750-1,850 \\
1,850-2,150\end{array}$ & $\begin{array}{l}\text { Air } 1 \\
\text { Oil or air..... }\end{array}$ & $\begin{array}{l}{ }^{\circ} \mathrm{F} \\
2950-\mathrm{i}, 100 \\
1,050-1,250\end{array}$ & $\begin{array}{l}43-50 \\
45-50\end{array}$ \\
\hline
\end{tabular}

1 Quench in low-pressure air blast or still air.

2 This tempering range is for dies quenched in air blast. 
AMERICAN SOCIETY FOR STEEL TREATING, RECOMMENDED PRACTICE FOR THE CARBURIZING AND HEAT TREATING OF CAMSHAFTS, 1929

\section{HEAT TREATMENT OF CAMSHAFTS}

\section{GENERAL}

This recommended practice covers the process to be followed in the carburizing and heat treating of camshafts.

\section{CHEMICAL COMPOSITION}

The steels listed in Table 1 may be used for camshafts and this recommended practice is not applicable to steels having any other chemical composition.

TABLE 1.-Chemical composition

\begin{tabular}{|c|c|c|c|c|c|}
\hline Steels & C & $\mathrm{Mn}$ & $\begin{array}{l}\mathrm{P} \text { (max- } \\
\text { imum) }\end{array}$ & $\begin{array}{l}\text { S(max- } \\
\text { imum) }\end{array}$ & $\mathrm{Ni}$ \\
\hline $\begin{array}{l}\text { S. A. E. } 1015 \\
\text { S. A. E. } 10201= \\
\text { S.A. E. } 2320 \\
0.50 \text { per cent Ni. } \\
\text { S. A. E. } 2512 \\
\text { S.A. E. } 3120^{2} \\
\text { Ni-M } \\
\text { S. A. E. } 6120^{4} \\
\text { Cr.V }\end{array}$ & $\begin{array}{l}0.10-0.20 \\
.15-.25 \\
.15-.25 \\
10-.20 \\
\text { Max. } 17 \\
.15-.25 \\
.10-.20 \\
.15-.25 \\
10-.15\end{array}$ & $\begin{array}{l}0.30-0.60 \\
.30-.60 \\
.30-.60 \\
.30-.60 \\
.30-.60 \\
.30-.60 \\
.30-.50 \\
.30-.60 \\
.30-.50\end{array}$ & $\begin{array}{l}0.045 \\
.045 \\
.040 \\
.045 \\
.040 \\
.040 \\
.040 \\
.040 \\
.040\end{array}$ & $\begin{array}{l}0.055 \\
.055 \\
.05 \\
.050 \\
.05 \\
.05 \\
.045 \\
.045 \\
.040\end{array}$ & $\begin{array}{r}3.25-3.75 \\
.50-.75 \\
4.75-5.25 \\
1.00-1.50 \\
1.25-1.75 \\
-\end{array}$ \\
\hline
\end{tabular}

1 This steel is the one most commonly used for camshafts. 2 The chromium content for this steel is 0.45 to 0.75 per cent. The molybdenum content of this steel is 0.20 to 0.30 per cent

- The chromium content for this steel is 0.80 to 1.10 per cent and the ranadium content is 0.15 to 0.20 per cent.

The chromium content for this steel is 0.25 to 0.40 per cent and the vanadium content is 0.15 to 0.20 per cent.

\section{ANNEALING AFTER FORGING AND BEFORE MACHINING}

For annealing the S. A. E. steels Nos. 1015, 1020, $2320,3120,6120$, the 0.50 per cent nickel, nickelmolybdenum, and the chromium-vanadium steels, place the steels in the furnace so as to expose maximum surface area. Heat slowly and uniformly to a temperature of $1,600^{\circ}$ to $1,650^{\circ} \mathrm{F}$., and cool at a rate to give the desired machinability.

For annealing the 5 per cent nickel steel (S. A. E. No. 2512), place in furnace so as to expose maximum surface area. Heat slowly and uniformly to $1,600^{\circ}$ F., and hold at this temperature for about 1 hour. Cool slowly in furnace to $800^{\circ} \mathrm{F}$., and then cool in air.

Before machining hollow camshafts, quenching and tempering operations, at the proper temperatures, are advisable to give the desired machinability.

\section{PREPARATION OF CAMSEAFTS FOR CARBURIZING}

Two methods are used for machining a camshaft that will straighten without breaking after hardening.

One method consists of copper plating the entire shaft prior to carburizing and removing the copper by grinding or turning from those areas which are to be carburized and hardened.

The second method consists of forging or machining the camshafts somewhat larger with an excess of metal between the cams and bearings. This excess metal is machined off after carburizing and before hardening.

Many companies are now finish-machining the camshafts before carburizing, and when hardening quench directly into a straightening machine.

\section{CARBURIZING CAMSHAFTS}

The carburizing operation should be carried out in containers and furnaces of suitable dimensions and design to permit uniform heating throughout the charge. The carburizing temperatures for the various steels are given in Table 2 .

The camshafts should be so packed that the areas to be hardened do not come in contact with each other or with the walls of the containers.

Sufficient compound should be used so that the camshafts are not exposed at any time during the carburizing and cooling operations; after carburizing, the camshafts are allowed to cool in the containers.

\section{CASE DEPTH}

The time required to attain the depth of case depends upon a number of factors, such as temperature of the furnace at time of loading, rate of heating, type of furnace, thickness of the containers, amount of stock left for grinding, etc. However, the carburizing time and the amount of stock left before grinding should be so regulated that the depth of case after grinding is at least 0.045 of an inch.

\section{HEAT TREATING OF CAMSHAFTS AFTER CARBURIZING}

Hardening. - The camshafts should be heated uniformly to the temperature given in Table 2, and held for complete penetration of heat. The heating medium should be of such a character that no decarburization will take place.

To prevent warpage, the shafts should be arranged properly in the heating medium and quenched either vertically or rolled horizontally into the quenching bath.

Tempering.-After quenching, reheat the cam shafts in a suitable tempering medium to the temperature given in Table 2 and hold at this temperature for at least 1 hour to insure complete penetration of heat. The highest temperature that will not affect the hardness should be used. The hardness of the camshaft should not be below a scleroscope reading of 75 .

TABLE 2.-Carburizing and heat treating camshafts

\begin{tabular}{|c|c|c|c|c|c|}
\hline Steels & $\begin{array}{c}\text { Carbu- } \\
\text { rizing } \\
\text { tempera- } \\
\text { ture }\end{array}$ & $\underset{\text { in } \rightarrow}{\text { Cool }}$ & $\begin{array}{c}\text { Reheat } \\
\text { to- }\end{array}$ & Quench in- & $\begin{array}{l}\text { Tem } \\
\text { per- } \\
\text { ing } \\
\text { tem- } \\
\text { pera- } \\
\text { ture }\end{array}$ \\
\hline $\begin{array}{l}\text { S. A. E. } 1015 .- \\
\text { S. A. E. } 1020 . \\
0.50 \text { per cent } \\
\text { Ni. }\end{array}$ & $\begin{array}{c}\circ F \\
1,625-1,750 \\
1,625-1,750 \\
1,650-1,700\end{array}$ & $\begin{array}{l}\text { Box.. } \\
\text { Bor.: } \\
\text { Box.. }\end{array}$ & $\begin{array}{c}\circ F \\
11,425-1,450 \\
11,425-1,450 \\
11,375-1,425\end{array}$ & $\begin{array}{l}\text { Water or brine } \\
\text { Water or oil. }\end{array}$ & $\begin{array}{c}\circ F \\
350-400 \\
350-400 \\
250-450\end{array}$ \\
\hline $\begin{array}{l}\text { Ni. } \\
\text { S. A. E. } 2320 \\
\text { S. A. E. } 3120 \\
\text { S. A. E. } 2512 \\
\text { Ni-Mo } \\
\text { S. A. E. } 6120 . \\
\text { Cr-V........ }\end{array}$ & $\begin{array}{r}1,575-1,625 \\
1,625-1,675 \\
1,600-1,650 \\
1,650 \\
1,675-1,725 \\
1,675-1,725\end{array}$ & $\begin{array}{l}\text { Bor.- } \\
\text { Bor.- } \\
\text { Bor-. } \\
\text { Bor.- } \\
\text { Bor.- } \\
\text { Bor.- }\end{array}$ & $\begin{array}{r}11,325-1,375 \\
11,375-1,425 \\
11,350-1,400 \\
1,475-1,525 \\
11,425-1,475 \\
11,425-1,475\end{array}$ & $\begin{array}{l}\text { Oil } \\
\text { Water or oil. } \\
\text { Oil } \\
\text { Water or brine }\end{array}$ & $\begin{array}{r}250-450 \\
250-450 \\
250-450 \\
350 \\
400-450 \\
400-450\end{array}$ \\
\hline
\end{tabular}

1 Use lowest possible temperature to produce maximum hardness. 2 After rough machining anneal at $1,450^{\circ} \mathrm{F}$,, air cool, finish the machining, and then carburize.

\section{NOTES ABOUT THE PROCESS}

The mixture of the carburizing compound is left to the judgment of the individual. Some add only enough new compound to replace the amount used during carburizing, while others may vary the ratio from the above to a mixture of 50 per cent old and 50 per cent new.

There are several methods used for packing the camshafts. For example, a single shaft may be packed in a tube, or a number of shafts may be packed in a tube or a box of suitable size. The majority of the methods give good results if properly carried out. 
When using the plain carbon steel (S. A. E. No. 1020), satisfactory results may be obtained without annealing or otherwise heat treating before machining, although by following the above practice the steel will be in a better condition.

When using a salt bath as a heating medium for hardening, the shafts are sometimes preheated at approximately $600^{\circ} \mathrm{F}$., transferred to the salt bath in which they are suspended vertically to prevent warping, and maintained in the heating medium until they attain the proper hardening temperature. They are then quenched and tempered.

\section{AMERICAN SOCIETY FOR STEEL TREAT- ING, RECOMMENDED PRACTICE FOR THE CARBURIZING AND HEAT TREAT- ING OF GEARS, 1929}

\section{HEAT TREATMENT OF GEARS GENERAL}

This recommended practice covers the process to be followed in the carburizing and heat treating of gears. The gears may be carburized by either the pack method or in retort furnaces, using either gas or a solid compound.

\section{CHEMICAL COMPOSITION}

There are steels used for gears of other analyses than those listed below, but they require different heat treatments and, therefore, this recommended practice does not apply to them. The chemical compositions of the steels generally used for gears are given in Table 1.

TABLE 1.-Approximate chemical composition of gear steels for carburizing

\begin{tabular}{|c|c|c|c|c|}
\hline Steel No. & C & $M n$ & $\begin{array}{l}\mathrm{P} \\
\text { (maxi- }\end{array}$ & $\underset{\text { (maxi- }}{\mathrm{S}}$ \\
\hline $\begin{array}{l}4{ }^{1}-10 \\
5 \\
6 \\
7^{2}\end{array}$ & $\begin{array}{r}0.10-0.20 \\
.10-.20 \\
.10-.20 \\
\text { Max. } .17 \\
.10-.20 \\
.12-.20 \\
.10-.20 \\
.06-.16\end{array}$ & $\begin{array}{r}0.30-0.60 \\
.30-.60 \\
.30-.60 \\
.30-.60 \\
.30-.60 \\
.55-.75 \\
.30-.50 \\
.30-.60\end{array}$ & $\begin{array}{l}0.045 \\
.040 \\
.040 \\
.040 \\
.040 \\
.040 \\
.040 \\
.040\end{array}$ & $\begin{array}{l}0.055 \\
.05 \\
.05 \\
.05 \\
.05 \\
.05 \\
.05 \\
.045\end{array}$ \\
\hline
\end{tabular}

\begin{tabular}{|c|c|c|c|c|}
\hline Steel No. & $\mathrm{Ni}$ & $\mathrm{Cr}$ & $\mathrm{V}$ & Mo \\
\hline $\begin{array}{c}0 \\
0\end{array}$ & $\begin{array}{r}1.25-1.75 \\
3.25-3.75 \\
4.50-5.25 \\
1.00-1.50 \\
1.25-1.75 \\
3.75-4.25\end{array}$ & $\begin{array}{r}0.45-0.75 \\
.70-1.00 \\
1.25-1.75\end{array}$ & $0.15-0.20$ & י \\
\hline
\end{tabular}

1 For most uniform and satisfactory results the carbon content for these steels should be within a 5-point range.

\section{ANNEAIING AFTER FORGING AND BEFORE MACHINING}

For annealing, place the steel in the furnace to expose maximum surface area, then heat slowly and uniformly to the temperature given in Table 2.

The 5 per cent nickel steel (steel No. 4 in Table 1) is heated to the annealing temperature as given in Table 2 and usually cooled with the furnace, allowing from five to eight hours for the steel to become cold.

To obtain the best conditions for machining and grain size, it is sometimes desirable to quench the $3 \frac{1}{2}$ and 5 per cent nickel steels from the annealing tem- peratures into water or oil. Then anneal to give a Brinell hardness number from 163 to 179 . The 5 per cent nickel steel also gives satisfactory results when air cooled from the annealing temperature.

\section{CARBORIZING}

The carburizing temperatures for the gear steels considered in this recommended practice are given in Table 3.

\section{HEAT TREATMENT OF CAREURIZED GEARS}

The quenching temperatures for the carburized gears are given in Table 3.

TABLE 2.-Annealing temperatures for carburizing gear steels-after forging and before machining

\begin{tabular}{|c|c|c|c|c|}
\hline $\begin{array}{c}\text { Steel } \\
\text { No. as } \\
\text { listed } \\
\text { in } \\
\text { Table } \\
1\end{array}$ & $\begin{array}{l}\text { Heating } \\
\text { medium }\end{array}$ & Time beating & $\begin{array}{c}\text { Tempera- } \\
\text { ture }\end{array}$ & $\begin{array}{l}\text { Method of cool- } \\
\text { ing }\end{array}$ \\
\hline $\begin{array}{l}1 \ldots \\
2 \ldots \\
3 \ldots \\
4 \ldots \\
5 \ldots \\
6 \ldots \\
7 \ldots \ldots \\
8 \ldots\end{array}$ & 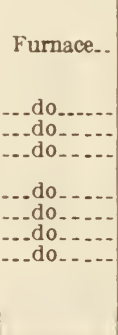 & $\begin{array}{l}1 \text { hourperinch } \\
\text { of diameter } \\
\text { or thickness. }\end{array}$ & $\begin{array}{r}\stackrel{\circ}{\circ} . \\
1,525-1,575 \\
1,500-1,550 \\
1,500-1,550 \\
1,600 \\
\\
1,500-1,550 \\
1,500-1,550 \\
1,500-1,550 \\
1,475-1,525\end{array}$ & $\begin{array}{l}\text { Air or furnace. } \\
\text { Furnace. } \\
\text { Do. } \\
\text { Air or furnace. } \\
\text { (See text). } \\
\text { Furnace. } \\
\text { Do. } \\
\text { Do. } \\
\text { Furnace. } \\
\text { (Do not remove } \\
\text { from furnace } \\
\text { over } 800 . \text { ) }\end{array}$ \\
\hline
\end{tabular}

When bevel gears are quenched, the last operation should be done on a quenching machine with a properly designed die.

For large gears (larger than automobile gears), where surface hardness is required in excess of that obtained from quenching in oil, water is sometimes used for the last quenching operation. This is especially true for steel No. 2 .

The heating medium considered in this recommended practice is the indirect-fired or muffle-type furnace.

\section{TEMPERING}

All gears should be tempered at $300^{\circ}$ to $350^{\circ} \mathrm{F}$. except clash gears, which are tempered at $350^{\circ}$ to $400^{\circ} \mathrm{F}$.

\section{CARBON CONTENT OF CASE}

The carburizing process should be so carried out that the carbon content of the outer portion of the case will be approximately 1.00 per cent. Care should, therefore, be exercised to avoid the use of badly spent carburizing compound.

\section{DEPTH OF CASE}

The depth of case on each side of the gear tooth should be about 15 per cent of the total thickness of the tooth, measured at the pitch line, until a case depth of 0.060 to 0.070 of an inch is reached. It is not considered practical to produce carburized cases of much greater depth, so this is about the limit of case depth even with gears having a greater total thickness of tooth.

\section{COPPER PLATING}

Where it is desired to keep a portion of the work soft after heat treatment, to permit machining or to assist in controlling distortion, copper plating to prevent the penetration of carbon is recommended. 
TABLE 3.-Carburizing and heat treating of carburized gears

[Purpose of heat treatments: $\mathrm{A}$, to be used when hardness and tough ness are both governing factors, and to hold the cementite in solu. tion. B, to refine case and core. C, to be used when distortion

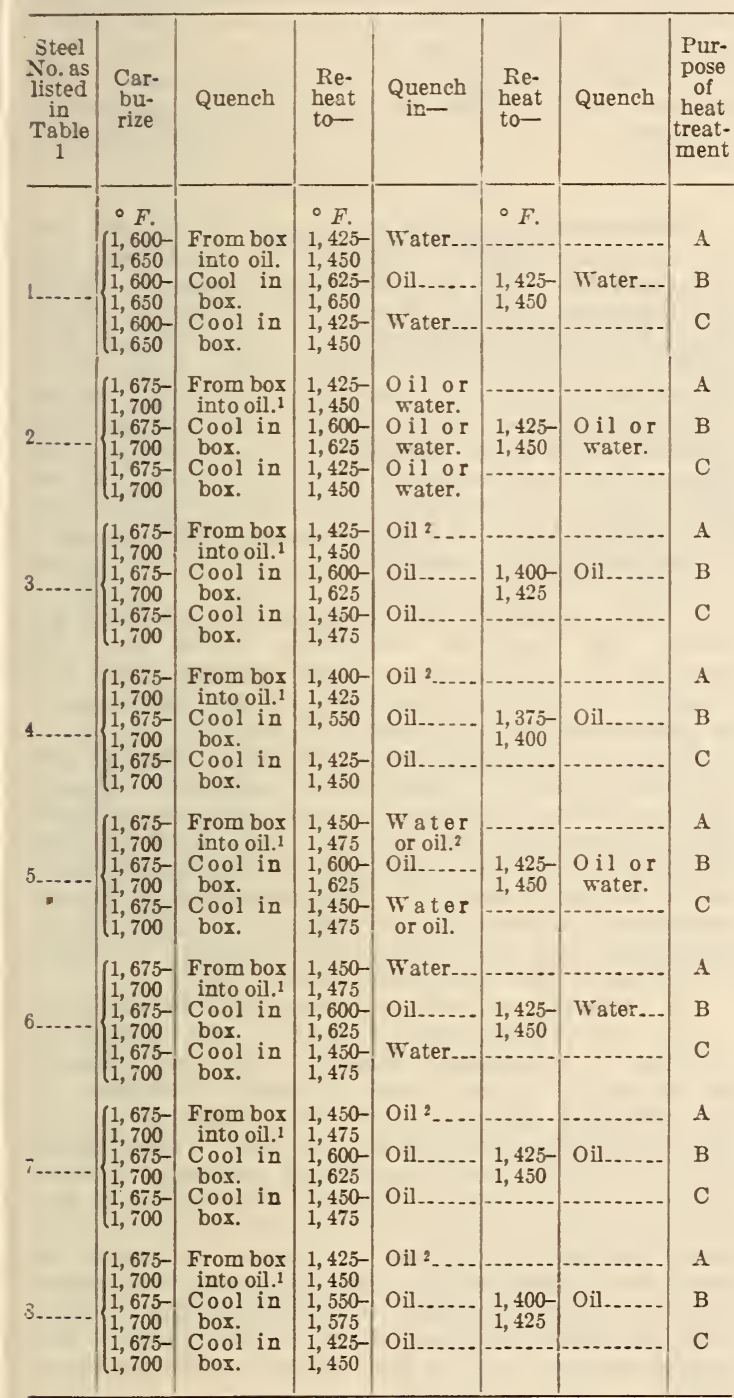

1 This quench is to hold the cementite in solution.

2 The oil for this quench should be flowing rapidly.

\section{AMERICAN SOCIETY FOR STEEL TREAT- ING, RECOMMENDED PRACTICE FOR THE CARBURIZING AND HEAT TREAT- ING OF PISTON PINS, 1929}

\section{HEAT TREATMENT OF PISTON PINS}

\section{GETERAL}

This recommended practice covers the processes to be followed in the carburizing and heat treating of piston pins made from plain carbon and alloy steels.

\section{CHEIICAL COMPOSITION}

The steels listed in Table 1 may be used for piston pins and this recommended practice is not applicable to steels having any other chemical composition.
TABLE 1.-Chemical composition of steels used for piston pins

\begin{tabular}{|c|c|c|c|c|}
\hline S. A. E. steel No. & C. 1 & Mn & $\begin{array}{c}\mathrm{P} \\
\text { (maxi- } \\
\text { mum) }\end{array}$ & $\begin{array}{l}\text { S } \\
\text { (maxi- } \\
\text { mum) }\end{array}$ \\
\hline $\begin{array}{l}1015 \\
2315 \\
25115 \\
6115\end{array}$ & \begin{tabular}{c|c}
$0.10-0$. \\
$.10-.2$ \\
Max. \\
$.10-.2$ \\
$.10-.2$ \\
.10
\end{tabular} & $\begin{array}{l}0.30-0.6 \\
.30-.6 \\
.30-.6 \\
.30-.6 \\
.30-.6 \\
.50-.8\end{array}$ & $\begin{array}{r}0.045 \\
.040 \\
.040 \\
.040 \\
.040 \\
.040\end{array}$ & $\begin{array}{l}0.055 \\
.05 \\
.05 \\
.05 \\
.05 \\
.045\end{array}$ \\
\hline S. A. E. steel No. & $\mathrm{Cr}$ & $\mathrm{Ni}$ & $\mathrm{V}$ & Mo \\
\hline $\begin{array}{l}1015 \\
2315 \\
2512 \\
4615 \\
6115\end{array}$ & $0.45-0.75$ & $\begin{array}{r}3.25-3.75 \\
\text { 4. } 50-5.25 \\
\text { 1. } 00-1.50 \\
\text { 1. } 50-2.00\end{array}$ & $0.15-0.20$ & $0.20-0.30$ \\
\hline
\end{tabular}

1 Some users specify the carbon for these steels to be 0.08 to 0.14 per cent and 0.15 to 0.25 .

\section{PREPARATION OF PISTON PINS FOR CARBURIZING}

Piston pins are generally made from either regular or special finish bar stock or tubing. If made from bar stock the hole may be drilled before carburizing. After drilling, the pins may be packed in the carburizing compound; but to prevent carburizing of the hole the pins may be strung on a rod, or they may be plugged. The pins may also be drilled after carburizing, which will leave the centers soft.

\section{CARBORIZING OF PISTON PINS}

The carburizing operation for piston pins may be carried out in containers and furnaces of suitable dimension and design to permit uniform heating throughout the charge, or in rotary retort furnaces using either gas or a solid compound. The carburizing temperatures for the various steels are given in Table 2 .

To keep the areas to be hardened from coming in contact with each other, or with the walls of the containers, and to prevent the piston pins from being exposed at any time during the carburizing and cooling operations, at least three-eighths of an inch of carburizing compound should separate the pins from each other and from the walls of the containers.

To determine the actual depth of case before the containers are allowed to cool, a test piece may be attached to a wire, one end of which is exposed. The test piece can then be conveniently removed while the container is still hot and the depth of case checked.

TABLE 2.-Carburizing and heat-treating of piston pins

\begin{tabular}{|c|c|c|c|c|c|}
\hline $\begin{array}{l}\text { S. A. E. } \\
\text { steel } \\
\text { No. }\end{array}$ & $\begin{array}{c}\text { Carburizing } \\
\text { tempera- } \\
\text { ture }\end{array}$ & $\begin{array}{l}\text { Cool } \\
\text { in- }\end{array}$ & Reheat to- & Quench in- & $\begin{array}{l}\text { Tem- } \\
\text { pering }\end{array}$ \\
\hline 15. & $\begin{array}{c}\circ F . \\
1,650-1,725 \\
1,575-1,625 \\
1,600-1,650 \\
1,650-1,700 \\
1,600-1,650 \\
1,650-1,700\end{array}$ & $\begin{array}{l}\text { Box... } \\
\text { Box... } \\
\text { Box... } \\
\text { Box... } \\
\text { Box... } \\
\text { Box... }\end{array}$ & $\begin{array}{c}\circ F . \\
1,425-1,450 \\
1,400-1,450 \\
1,350-1,400 \\
1,400-1,450 \\
1,475-1,525 \\
1,425-1,475\end{array}$ & $\begin{array}{l}\text { Water or brine.. } \\
\text { Oil or water... } \\
\text { Oil do or water. } \\
\text { Oil }\end{array}$ & $\begin{array}{c}\circ F . \\
275-375 \\
375 \\
250-450 \\
350-400 \\
350-400 \\
350-400\end{array}$ \\
\hline
\end{tabular}

CASE DEPTH

The carburizing time and the amount of stock left before grinding should be so regulated that the depth after grinding is at least 0.030 of an inch. From 0.008 to 0.010 inch is the amount of stock generally removed by grinding.

\section{HEAT TREATING OF PISTON PINS AFTER CARBURIZING}

Hardening.-For hardening, the piston pin should be heated uniformly to the temperature given in 
Table 2, and held for complete penetration of heat. The heating medium should be of such a character that no decarburization takes place.

Before hardening the case, the piston pins are sometimes given a core-refining treatment, the hardening temperature for the case then being lowered. However, a single heat treatment is usually satisfactory.

Tempering.-After quenching, piston pins may be tempered by reheating to the temperature given in Table 2.

The hardness of the piston pin should not be below a scleroscope reading of 75 , the approximate Rockwell being $\mathrm{C}-54$.

\section{AMERICAN SOCIETY FOR STEEL TREAT- ING, HEAT TREATMENT OF WROUGHT ALUMINUM ALLOYS OF HIGH STRENGTH, RECONMENDED PRACTICE, 1927}

\section{HEAT TREATMENT OF ALOMINUM ALLOYS}

\section{SUBJECT}

These data sheets cover the heat treatment of wrought aluminum alloys capable of being hardened by heat treatment. The word "hardened" as here used indicates an increase in Brinell hardness, yield point, tensile strength, and a decrease in plasticity.

\section{ALLOYS}

There are many possible aluminum-alloy compositions which may be worked and whose properties may be greatly improved by heat treatment. The same general principles and methods of heat treatment would apply to these various alloys, although the temperatures and other details depend on the specific compositions. These principles and methods will be illustrated with reference to the high-strength aluminum alloys commercially manufactured in the United States. Since these alloys are not yet recognized by general names or specifications, it will be necessary to refer to them by their trade symbols or designations. The alloys include duralumin "A" and "B" and 17S, 25S, and 51S. These are fundamentally wrought alloys, and the statements here made concerning their properties and heat treatment apply to material which has received sufficient working to destroy the cast structure.

\section{COMPOSITION}

The alloys 17S and duralumin are characterized by containing both copper and magnesium. Manganese is nearly always present and sometimes a small amount of chromium. Typical specifications for these alloys contain the following composition limits:

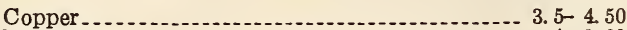

Manganese
Magnesium (minimum)
Aluminum

The composition of commercial alloys of the duralumin type may, however, vary somewhat from these values. The manufacturer's tentative operating limits for $25 \mathrm{~S}$ and $51 \mathrm{~S}$ are as follows:

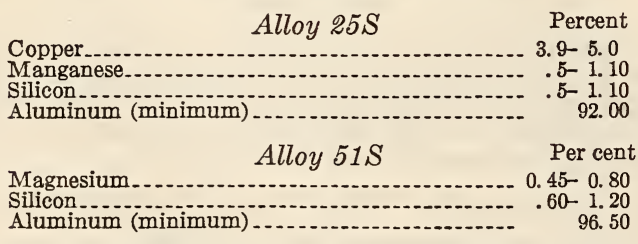

Iron and silicon occur as impurities in all commercial aluminum alloys. In $25 \mathrm{~S}, 51 \mathrm{~S}$, and some alloys of the duralumin type, the silicon content may be definitely controlled. The iron content is ordinarily less than 1 per cent in all these products.

\section{FORMS AVAILABLE}

Each of the following fabricated forms is commercially available in some or all of the alloys mentioned: Plate, sheet (both flat and coiled), bar, rod, wire, seamless tubing (round, square, and oval), shapes (both rolled and extruded), stampings, forgings, rivets, and automatic screw machine products.

\section{GRADES AND TEMPERS}

Duralumin is supplied in at least two grades and $17 \mathrm{~S}$ in four grades, produced by modifying the composition. The two standard grades of Baush duralumin are distinguished by the letters $A$ and $B$, while the various modifications of $17 \mathrm{~S}$ are designated $17 \mathrm{~S}, \mathrm{~A}-17 \mathrm{~S}, \mathrm{~B}-17 \mathrm{~S}$, and Special 17S. Each of the alloys is supplied in several tempers. The temper is determined by the condition as regards heat treatment or cold working. Like other metals, these alloys are hardened by cold working. If the alloy was in the hot rolled or annealed condition, as distinguished from the heat-treated condition, prior to cold rolling, the cold-rolled product is designated "hard" or "hard wrought."

In this condition the strength and hardness are fairly high and the elongation quite low. On heating the alloys to suitable temperatures and cooling, they are softened and the resulting temper is designated as the "annealed" temper.

The above statements concerning temper are applicable to all of the alloys mentioned. If any of these alloys be heated to a suitable temperature, then quenched, the strength and hardness are materially increased over the values characteristic of the annealed temper. The elongation is usually somewhat increased with respect to that of the annealed material, and is very markedly increased over that of the hard-wrought temper.

On standing at ordinary temperatures after the above quenching treatment, $17 \mathrm{~S}$ and duralumin undergo a spontaneous change, commonly referred to as aging, which involves further increases in strength and hardness, with little change in elongation, but with a decrease in plasticity. This change is at first rapid, but proceeds at a gradually decreasing rate. The change is largely effected in the first few hours and is generally considered to be substantially complete in about four days. Heating the alloy to the temperature of boiling water increases the rate of aging, but has little effect on the mechanical properties attained after several hours. The aging process may be illustrated by the following data:

$17 S$ sheet, 0.064 inch quenched from $940^{\circ}$ to $960^{\circ} \mathrm{F}$. AGED AT ROOM TEMPERATURE

\begin{tabular}{|c|c|c|c|}
\hline Aging time & $\begin{array}{c}\text { Tensile } \\
\text { strength }\end{array}$ & $\begin{array}{l}\text { Yield } \\
\text { point }\end{array}$ & $\begin{array}{l}\text { Elonga- } \\
\text { tion in ? } \\
\text { inches }\end{array}$ \\
\hline $\begin{array}{l}\text { hour } \\
\text { day } \\
\text { days } \\
\text { days } \\
\text { oys. days }\end{array}$ & $\begin{array}{r}\text { Lbs./in.2 } \\
44,830 \\
54,755 \\
56,115 \\
56,765 \\
57,175\end{array}$ & $\begin{array}{r}\text { Lbs./in.2 } \\
21,150 \\
32,350 \\
33,000 \\
34,850 \\
34,350\end{array}$ & $\begin{array}{r}\text { Per cent } \\
19.0 \\
18.7 \\
18.7 \\
19.7 \\
19.7\end{array}$ \\
\hline
\end{tabular}

AGED IN BOILING WATER

\begin{tabular}{|c|c|c|}
\hline $\begin{array}{l}1 \text { hour } \\
1 \text { day } \\
2 \text { days } \\
10 \text { days. }\end{array}$ & $\begin{array}{l}53,705 \\
55,505 \\
56,370 \\
56,040\end{array}$ & $\begin{array}{l}29,900 \\
32,350 \\
33,000 \\
33,650\end{array}$ \\
\hline
\end{tabular}

In order to discuss the properties of these alloys in various grades and tempers, it will be necessary to refer to them by their trade symbols, as follows: 


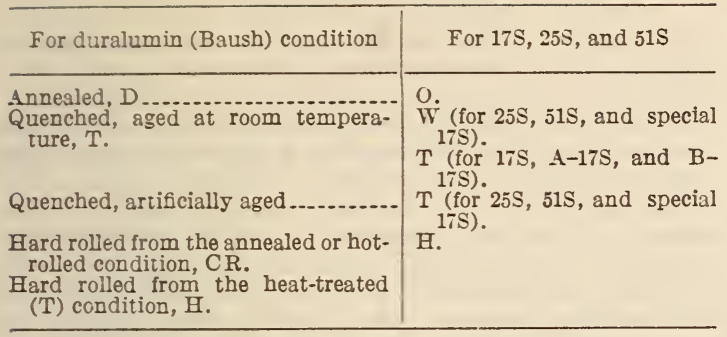

\section{MECHANICAL PROPERTIES}

The actual mechanical properties of these alloys in their various tempers depend somewhat on their fabrication history, especially on the total reduction by mechanical working; and in the case of hard material, on the amount of cold working. Lack of sufficiently thorough mechanical working results in low strength and elongation. This influence is apt to become apparent in sheets heavier than about one-fourth inch in thickness, in bars larger than three-fourths inch in diameter, and in heavy forgings. In very heavy material the strength may be affected by as much as 5,000 pounds per square inch and the elongation in 2 inches by 4 per cent or more.

Another factor which affects the tensile properties is the form of the test specimen. In testing very thin metal specimens the elongation values are usually less than when measured over the same gage length on thicker specimens of material possessing the same inherent physical properties. This is due to the proportions of the specimen (ratio of cross section area to gage length) and not to inferior properties of thin sheet.

With these qualifications, the following properties are given by the manufacturers as characteristic of the alloys in various tempers:

$17 \mathrm{~S}, 25 \mathrm{~S}, \mathrm{AND} 51 \mathrm{~S}$

\begin{tabular}{|c|c|c|c|c|c|}
\hline Material & Condition & $\begin{array}{l}\text { Yield } \\
\text { point }\end{array}$ & $\begin{array}{l}\text { Tensile } \\
\text { strength }\end{array}$ & $\begin{array}{l}\text { Elon- } \\
\text { gation } \\
\text { in } 2 \\
\text { inches }\end{array}$ & $\begin{array}{c}\text { Brinell } \\
\text { hard- } \\
\text { ness } \\
500 \mathrm{~kg} \\
10 \mathrm{~mm} \\
\text { ball }\end{array}$ \\
\hline $17 \mathrm{ST} . .$. & Quenched and aged at & Lbs, in $^{2}{ }^{2}$ & Lbs./in. ${ }^{2}$ & $\begin{array}{c}\text { Per } \\
\text { cent } \\
18-25\end{array}$ & $90-105$ \\
\hline $17 \mathrm{SO}$ & $\begin{array}{l}\text { room temperature. } \\
\text { Annealed................. }\end{array}$ & $\begin{array}{l}40,000 \\
7,000\end{array}$ & $\begin{array}{l}63,000 \\
25,000\end{array}$ & $14-22$ & $45-55$ \\
\hline $25 \mathrm{SC}$ & .....do. & $7,000-$ & $23,000-$ & $12-20$ & $45-55$ \\
\hline $25 \mathrm{~S} W \ldots$ & Quenched and aged at & 12,000 & $\begin{array}{l}35,000 \\
45,000\end{array}$ & $15-22$ & $68-85$ \\
\hline $25 \mathrm{ST} .$. & $\begin{array}{l}\text { Quenched and aged at } \\
\text { elevated tempera- } \\
\text { tures. }\end{array}$ & $\begin{array}{l}30,000- \\
40,000\end{array}$ & $\begin{array}{l}55,000- \\
63,000\end{array}$ & $16-25$ & $90-105$ \\
\hline $51 \mathrm{SO} \ldots$ & Annealed ................. & $4,000-$ & $\begin{array}{l}14,000- \\
19000\end{array}$ & $22-32$ & $25-32$ \\
\hline $51 \mathrm{SW}$ & $\begin{array}{l}\text { Quenched and aged at } \\
\text { room temperature. }\end{array}$ & $15,000-$ & $30,000-$ & $20-30$ & $55-70$ \\
\hline $51 \mathrm{ST}$ & $\begin{array}{l}\text { Quenched and aged at } \\
\text { elevated tempera- } \\
\text { tures. }\end{array}$ & $\begin{array}{l}30,000- \\
40,000\end{array}$ & $55,000-$ & $10-18$ & $90-100$ \\
\hline $\mathrm{A}-17 \mathrm{SO}$ & Annealed & & $20,000-$ & $20-28$ & $30-40$ \\
\hline A-17ST.-. & $\begin{array}{l}\text { Quenched and aged at } \\
\text { room temperature. }\end{array}$ & $\begin{array}{l}15,000- \\
20,000\end{array}$ & $\begin{array}{l}35,000- \\
45,000\end{array}$ & $20-28$ & $55-75$ \\
\hline B-17SO.. & Annealed ................... & & $20,000-$ & $20-28$ & $30-40$ \\
\hline B-17ST -- & $\begin{array}{l}\text { Quenched and aged at } \\
\text { room temperature. }\end{array}$ & $\begin{array}{l}20,000- \\
25,000\end{array}$ & $42,000-$ & $20-28$ & $65-85$ \\
\hline $\begin{array}{l}\text { Special } \\
17 \text { SO. }\end{array}$ & & $\begin{array}{r}7,000- \\
10,000\end{array}$ & $\begin{array}{l}25,000- \\
35,000\end{array}$ & $12-20$ & $42-55$ \\
\hline $\begin{array}{l}\text { Special } \\
17 \mathrm{SW} .\end{array}$ & $\begin{array}{l}\text { Quenched and aged at } \\
\text { room temperature. }\end{array}$ & $\begin{array}{l}30,000 \\
40,000\end{array}$ & $\begin{array}{l}55,000- \\
63,000\end{array}$ & $18-25$ & $90-105$ \\
\hline $\begin{array}{l}\text { Special } \\
\text { 17ST. }\end{array}$ & $\begin{array}{l}\text { Quenched and aged at } \\
\text { elevated tempera- } \\
\text { tures. }\end{array}$ & $\begin{array}{l}50,000 \\
55,000\end{array}$ & $\begin{array}{l}63,000- \\
70,000\end{array}$ & $8-14$ & $95-125$ \\
\hline
\end{tabular}

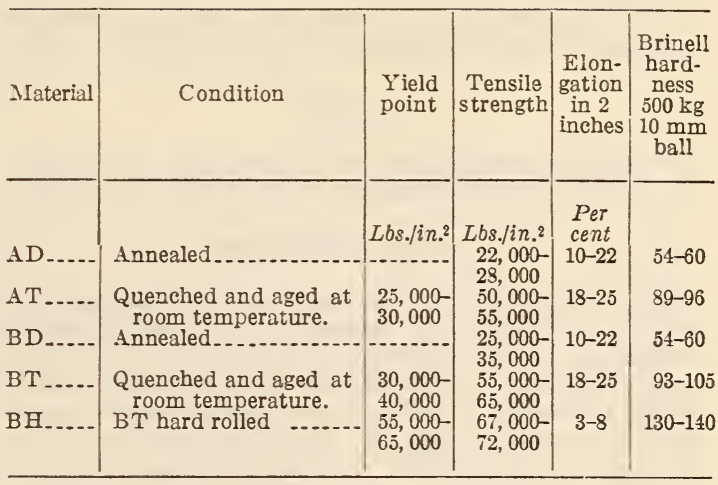

These alloys are seldom used in the cold-worked condition, being intended primarily to be hardened by heat treatment. The properties of work-hardened materials depend, of course, on the amount of cold work, which varies with the product. In general, a given percentage reduction by cold working gives roughly the same percentage increase in tensile strength. The yield point rises more rapidly, in per cent, than the tensile strength, and the elongation decreases rapidly. By way of example, it may be mentioned that certain samples of $17 \mathrm{SH}$ sheet (14 gage) which had been reduced in section 50 per cent by cold rolling after annealing had 37,625 pounds per square inch yield point, 39,400 pounds per square inch tensile strength, and 3 per cent elongation in 2 inches.

Material which has been quenched and aged is similarly hardened by cold work, but the reductions employed are relatively small. The figures for the material $\mathrm{BH}$ in the above table represent from 10 to 20 per cent reduction. While these properties are desirable for certain work, the field is limited as it is mainly in the case of sheet that any considerable cold work can be done on quenched and aged material without damage.

\section{ELASTIC MODULUS}

Young's modulus $(E)$ is about $10,000,000$ pounds per square inch for all of these alloys.

\section{SPECIFIC GRAVITY}

Approximate values are as follows:

\begin{tabular}{|c|c|c|c|c|c|}
\hline & \multicolumn{5}{|c|}{ Alloy } \\
\hline & $\begin{array}{c}\text { Dural- } \\
\text { umin, } \\
17 \mathrm{~S}\end{array}$ & $\begin{array}{c}25 \mathrm{~S}, \\
\text { Special } \\
17 \mathrm{~S}\end{array}$ & $51 \mathrm{~S}$ & A-17S & B-17S \\
\hline $\begin{array}{l}\text { Specific gravity } \\
\left.\text { Weight (lbs./in. }{ }^{3}\right)\end{array}$ & $\begin{array}{l}2.79 \\
.101\end{array}$ & $\begin{array}{l}2.79 \\
.101\end{array}$ & $\begin{array}{l}2.69 \\
.098\end{array}$ & $\begin{array}{l}2.74 \\
.099\end{array}$ & $\begin{array}{l}2.76 \\
.100\end{array}$ \\
\hline
\end{tabular}

\section{ILECTRICAL CONDUCTIVITY}

The conductivities of heat-treated duralumin, $17 \mathrm{ST}$ and $25 \mathrm{ST}$ are approximately 30 to 35 per cent of the annealed copper standard, while for 51ST the values are approximately 40 to 45 per cent.

\section{THERMAL CONDUCTIVITY}

The thermal conductivities in cgs units are numerically very close to the above values for electrical conductivity. 
FEAT-TREATMENT PROCESSES

The heat-treatment processes usually applied to the high-strength aluminum alloys are of three types:

1. Annealing

Normally applied to work-hardened metal with the object of softening and improving workability.

\section{Solution Heat Treatment}

A heat treatment at temperatures upward of $900^{\circ} \mathrm{F}$. followed by quenching. Metallurgically, the object of this treatment is to bring the soluble constituents of the alloys into solid solution, and to retain this solid solution in a supersaturated condition by rapid cooling to room temperature. This produces a susceptibility to hardening by natural or "artificial" aging. Immediately after quenching, the alloys are harder than in the annealed condition but softer than in the quenched and aged condition.

\section{Precipitation Heat Treatment}

The hardening which takes place on aging at room temperature or at elevated temperatures is believed to be due to the formation of a very fine precipitate of the soluble constituents from the supersaturated solid solutions. Hence, any heat treatment designed to accomplish this result may be referred to as a "precipitation heat treatment."

\section{ANNEALING}

Ordinarily the object of annealing is to remove the hardening effects of cold work. To accomplish this result, it is necessary to heat the metal above its recrystallization temperature, as in the annealing of copper and brass. In the case of high-strength aluminum alloys, however, if the metal is heated to higher temperatures and cooled at any other than a very slow rate, some hardening will take place, as in the regular solution-heat treatment. It is, therefore, necessary to avoid heating to temperatures above that required to remove the work-hardening. This temperature varies with the composition of the alloy and the amount of cold working, from about $575^{\circ}$ to $675^{\circ} \mathrm{F}$. In general, a temperature of $650^{\circ} \mathrm{F}$. is suitable for all of the alloys. The material should be left in the furnace for a sufficient length of time to make sure that all of the metal has reached the annealing temperature. Slow cooling to a temperature well below the annealing temperature is desirable, since this will tend to remove the hardening effect that would result if any part of the load had been overheated.

It is sometimes desired to anneal material which has been previously hardened by heat treatment ( $W$ or $T$ temper). Heating to the usual annealing temperature, as described above, will soften such material to some extent but not sufficiently to produce the workability characteristic of the normal annealed temper. A special annealing treatment which has been found effective in such cases consists in heating for an hour or more at $800^{\circ} \mathrm{F}$. and cooling very slowly to below $500^{\circ} \mathrm{F}$. The longer the time at $800^{\circ} \mathrm{F}$., and the slower the cooling, the more thoroughly will the material be annealed.

The condition of greatest softness in these alloys is attained when the work-hardening is removed by recrystallization and grain growth, and when the soluble constituents are precipitated from solid solution as completely as possible in the form of large particles. The drawing qualities can, however, be adversely affected by over-annealing, with the development of coarse grain.

\section{SOLUTION-HEAT TREATMENT}

Accurate temperature control is essential for the best results. The hardening effect increases gradually with the temperature of treatment until the maximum solution of soluble constituents is attained. If the heat-treating temperature is too low, the hardening will be incomplete, while if it is too high, partial melting of the alloy will take place with consequent loss of ductility and strength. This effect, known as "burning," should be carefully avoided. The damage done by burning increases gradually as the temperature of treatment and time of holding increase until finally the material is completely ruined. Badly burned material is characterized by a crystalline fracture, with or without discoloration; surface cracks are apt to appear on bending or stretching; the microstructure shows areas which have been fused. In its early stages, burning is fairly difficult to detect. Material once burned can not be completely restored by heat treatment.

The exact temperature at which burning will begin depends on the chemical composition, and perhaps also on the fabrication history of the alloy. It is apt, therefore, to vary a little from heat to heat of the same alloy. The temperature ranges recommended below are such that burning will not take place in any lot of material within the usual range of chemical composition.

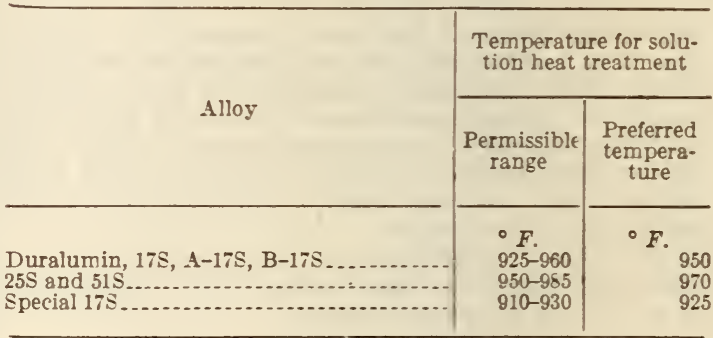

The time required at neat depends somewhat on the amount of mechanical working the material has received; for ordinary well worked sections 15 to 30 minutes, after due allowance for the thorough beating of heavy sections and large masses of material, will usually be sufficient. Long soaking after the material is at heat is ordinarily not beneficial, unless the material has not been thoroughly worked. Practically, the type of heating equipment and its method of operation as well as the sections, mass, and distribution of the charge, determine the total time taken. For example, a single piece of thin sheet might be heated and quenched in 15 minutes, while several hours might be required properly to heat and quench a large batch of heavy forgings. The same exercise of judgment required in heattreating varying sections and masses of steel will be sufficient in handling aluminum alloys.

After this treatment, the metal is cooled rapidly, as by quenching in water or oil. The time interval between the removal of the metal from the heating medium and its quenching should be as short as possible. The physical properties finally obtained are about the same whether the quenching medium is cold water, boiling water, or oil. Quenching from a nitrate bath into boiling water is apt to be accompanied by dangerous spattering, and operators should be protected by shields, goggles, etc. Good physical properties, with reduction of distortion, can also be obtained by cooling in a current of airespecially in the case of light sections. Another method of quenching may be described as diequenching. The act of hot-forming some of these materials, such as $17 \mathrm{~S}$ and duralumin, at substan- 
tially the same temperatures that are used for the solution-heat treatment, results in a sufficiently rapid quench to produce substantially fully heattreated material.

Although the quenching rate has very little effect upon the final mechanical properties of the material, the susceptibility to corrosion of quenched and aged material is affected to a much greater extent by this variable. It has been found that the resistance to salt-water corrosion, and to corrosion by chloride solutions in general, is better the more rapid the quenching. As a rule, cold-water quenching is recommended for materials which will be exposed to corrosive conditions, especially sheet material and structures fabricated from sheets or other thin sections. Since corrosion begins at the surface, its effect on the mechanical properties of the piece as a whole, is, of course, more serious the thinner the material. For articles of relatively large cross section, for example, forgings, the question of the corrodibility of the material, as affected by the rate of quenching is comparatively neslicible.

The properties produced by the solution heat treatment are substantially independent of the initial condition of the material; that is whether hard, annealed or heat treated, except that in the case of material which has received a relatively slight degree of cold work, either locally or generally, there is some possibility of excessive grain growth. In such cases rapid heating and short time at heat usually tend to reduce grain growth.

$$
\text { - }
$$

\section{AGING}

It should be noted that temperatures much below room temperature $\left(68^{\circ} \mathrm{F}\right.$.) will temporarily retard the normal age-hardening of duralumin and 17S, while extreme cold actually arrests aging. On return to normal room temperature, normal aging is resumed. In making tests, care should be taken that the aging has not been retarded by chilling. If there is any doubt as to rate of aging, hardness tests may be used as a check before pulling test bars.

\section{PRECIPITATION HEAT TREATMENT}

This type of heat treatment is ordinarily applied only to $25 \mathrm{~S}, 51 \mathrm{~S}$, and special $17 \mathrm{~S}$, of the alloys mentioned above. (Duralumin and 17S ordinarily receive their "precipitation heat treatment" by aging at room temperature.) It is to be preceded by the solution heat treatment.

The general effects of this treatment are increased hardness, yield point, and tensile strength, and decreased plasticity. The per cent elongation in the tensile test may increase slightly during the first stages of the treatment, but decreases by the time maximum hardening is attained.

Tne most favorable range of temperature for the treatment is usually from about $250^{\circ}$ to $320^{\circ} \mathrm{F}$. The time required is greater the lower the temperature of treatment, and the greater the hardening effect desired. As a rule, better combinations of tensile strength and elongation can be obtained by a long treatment at a low temperature than by a short treatment at a high temperature.

As the treatment progresses, the hardness rises to a maximum and then, after prolonged heating at $250^{\circ} \mathrm{F}$. or higher, begins to decrease slowly. In actual practice the precipitation treatment usually stops well short of this point.

To attain the physical properties given above for $25 \mathrm{ST}, 51 \mathrm{ST}$, and special 17ST, the following precipitation treatments have been found suitable: For $25 \mathrm{~S}, 8$ to 15 hours at $285^{\circ}$ to $295^{\circ} \mathrm{F}$.; 51S, 18 hours at $310^{\circ}$ to $320^{\circ} \mathrm{F}$.; special $17 \mathrm{~S}, 18$ hours at $310^{\circ}$ to $320^{\circ} \mathrm{F}$.
Some experimentation may be required to determine the best treatment for any particular class of product.

The manner of cooling after the precipitation treatment is immaterial.

\section{WELDING}

It is not intended to discuss here in detail the welding of the strong aluminum alloys nor the properties of welded joints, but it is felt that a few general principles should be pointed out. To make a weld it is necessary to melt some of the metal. This molten metal solidifies rather rapidly after welding, and acquires the structure and properties of a more or less sound chill casting, rather than those of a wrought metal. The adjacent metal which actually has not been melted has been heated to various temperatures including those used for the precipitation treatment, for annealing, and for the solution heat treatment as well as that required for burning. The properties of the metal, accordingly, will vary with the distance from the weld.

Both the mechanical properties and corrosion resistance of welded joints can be improved materially by heat treatment after welding.

\section{FORMING}

The cold-forming properties of the various alloys are best in the annealed condition. Articles formed from annealed material must be given the solution heat treatment before the material will develop its full physical properties either on natural or artificial aging. Because of the high temperatures used in the solution heat treatment there may be more or less difficulty, depending on the shape of the article, from warping or distortion. To avoid this difficulty it is often desirable to carry out forming operations after the solution heat treatment. This can best be done immediately after quenching, especially in the case of alloys which age-harden materially at room temperature. Even then the operations which can be carried out are usually more limited than in the case of annealed material. The capacity for cold forming decreases as the material is age-hardened at room temperature, and still further on aging at elevated temperatures. In the case of the alloys to which aging treatments at elevated temperatures are applied (special 17S, $25 \mathrm{~S}$, and $51 \mathrm{~S}$ ), the material may be obtained in the quenched condition, formed and then further hardened by a precipitation treatment. It is some times possible to combine the solution heat treatment with the forming operation. Articles have been successfully made from 17S and duralumin sheet, for example, by hot pressing at about $900^{\circ} \mathrm{F}$., the quenching effect of the dies being sufficient to produce nearly full development of physical properties in the finished article.

\section{TECHNICAL METHODS OF HEAT TREATHENT}

Uniformity of temperature distribution and accuracy of control are essential in the heat treatment of the strong aluminum alloys. These conditions can be realized satisfactorily in various types of heating equipment.

Annealing may be carried out in an air atmosphere, or in furnaces of either batch or continuous type, heated by electricity, gas, or oil. For the best results, close temperature control is desirable, but is not so vital as in the solution and precipitation treatments, especially if care is taken to cool very slowly from the annealing heat to $500^{\circ} \mathrm{F}$. or below.

The solution treatment of articles of light section, such as sheet, may readily be carried out in a bath 
of fused sodium nitrate heated with gas or oil so as to permit close regulation of temperature. It may be necessary to stir such a bath to avoid inequalities in temperature. This can be readily accomplished by alternately raising and lowering the load while it is being heated, making sure, however, that the metal is not raised above the surface of the bath. This will insure the circulation of the liquid between the different parts of the load.

For heavier sections, such as bars and forgings, or for large amounts of material, a furnace for treatment in an air atmosphere has certain advantages over nitrate baths and is preferred by some. Such a furnace must be so constructed and operated as to give proper uniformity and control of temperature. Standard gas and oil fired furnaces of several types, as well as electric furnaces with automatic temperature control, are in commercial use.

When metal is heated in a nitrate bath, it is necessary that the adhering nitrate be completely removed as, if left on, it may cause corrosion. Quenching in hot water facilitates the removal of nitrate. If the metal is allowed to cool slowly, the salt forms a sort of enamel that is very difficult to remove. For this reason the nitrate bath is not very suitable for the annealing treatment, from which the metal is preferably cooled slowly.

For the precipitation treatment, good temperature control and distribution can be obtained by the use of live steam, as in tanks employed for vulcanizing. This method insures rapid heating and the temperature can be controlled by a pressure-regulating device. The steam has some action on the surface of the aluminum, especially if the solution treatment has been carried out in niter. This surface action is more serious with thin than with heavy material. It is rather objectionable in the case of sheets, for example, and not of much consequence in the case of forgings.

The precipitation treatment may also be carried out in an oven heated by steam coils or electricity and provided with a fan for air circulation. When steam coils are used, the temperature can be varied by changing the pressure in the coils.

A circulated oil bath with automatic temperature control is, of course, ideal for the precipitation treatment from the standpoint of temperature distribution and freedom from chemical action on the aluminum, but may be uneconomical.

\section{NITRATE HAZARD}

Although fused nitrate baths are commonly used in the heat treatment of steel as well as aluminum, it is felt that attention may well be called here to certain dangers in their operation. Care should be taken to prevent contact of the fused salt with carbonaceous or organic material (wood, charcoal, coke, oil, cloth, etc.) or with red hot iron or steel. Articles to be heat treated, or sludge, should not be allowed to lie on the bottom of the tank, especially if the tank is underfired, as they are apt to cause the tank to become locally overheated and be burned through. The possibility of combustible gases accumulating around the tank should be guarded against, and it is desirable to have at some distance from the tank a gas or oil shut-off valve which can be operated in emergency. Care should be taken not to put anything wet into the nitrate bath. Extra sacks of nitrate should be stored in a dry place and at a distance from the tank. Care should be exercised in adding fresh salts to the bath if at all wet; this is best done when the bath is frozen. In melting a solid bath, the salt must melt freely at the sides of the tank before melting at the bottom, to avoid generation of pressure and possible explosion. In case of a nitrate fire, avoid the use of water or of any fire extinguisher containing water. Perhaps the best fire extinguisher is dry sand, a supply of which should be kept near the tank.
AMERICAN SOCIETY FOR TESTING MA. TERIALS, RECOMMENDED PRACTICE FOR ANNEALING OF MISCELLANEOUS ROLLED AND FORGED CARBON-STEEL OBJECTS, DESIGNATION A35-24, 1924

\section{ANNEALING CARBON-STEEL OBJECTS}

1. Purpose and general procedure.-The usual purpose in annealing miscellaneous forged or rolled carbon-steel objects is to remove existing coarseness of grain. This removal is brought about by heating the object to an annealing temperature, which varies with the carbon content of the metal. But the rate at which the object cools from this annealing temperature influences its properties very profoundly. Hence this rate of cooling should be suited to the duties which the object has to perform in service, and hence to the properties which we seek to give it.

Therefore these specifications first consider the annealing temperature to which the piece must be heated in order to remove existing coarse grain, and then the rate of cooling from that temperature.

Under certain special conditions an annealing is required in order to remove not coarseness of grain but the effects of rolling or otherwise working the metal at a temperature so low as to set up serious internal stress. Appropriate treatment for these conditions is given in section 14 .

As these specifications are intended to apply to a great variety of miscellaneous objects, the 5 are purposely made suggestive rather than mandatory in many respects, because no single set of rules can be applied rigorously to such widely varying classes of objects and purposes.

\section{ANNEALING FOR REMOVING EXISTING COARSE GRAIN}

2. Method of heating.-In the case of large objects, the heating of the interior of which lags behind that of the outside, the final approach to the annealing temperature aimed at should be slow, so that the interior may be brought fully up to it without carrying the exterior far beyond it, because in general any needlessly high temperature is injurious, and tends to recoarsen the grain. The temperature should be held long enough at the annealing point to insure that the whole of the interior reaches that point, and that the refining of the grain may become complete. An exposure of 45 minutes should be long enough for each inch of thickness.

3. Control of temperature-Pyrometers.-For all important work in careful hands the use of some trustworthy pyrometer is strongly recommended. But most pyrometers should be checked frequently against some standard. For those who are unwilling to take this trouble it is safer to rely on a trained eye than on an unchecked pyrometer. The operator should have clearly before him the fact that no pyrometer indicates the temperature of the interior of the object neated, and that the temperature which most pyrometers indicate is one between the temperature of the outside of the object heated and the temperature of the flame which is supplying the heat. Wherever practicable the part of the pyrometer which is supposed to reach the temperature of the object heated should be in immediate contact with that object, and should be protected from the flame or other source of heat by a suitable insulation, as for instance by covering it with sand. In important cases the gas or other source of heat should be shut off for at least ten minutes before taking the observations.

4. Control without the use of a pyrometer. - In working without a pyrometer and relying on the eve, the light surrounding the furnace should be duill, and should be kept as nearly constant as practicable, in order that the eye may not be misled by the chang- 
ing contrasts between the surrounding light and that of the object heated. In particular, direct sunlight should be excluded, and any arc or other strong lights should be so placed that neither they themselves nor any concentrated part of their light is in the field of the operator's sight when he is estimating by eye the temperature of the objects to be annealed. Allowance should be made for the brighter surroundings by day than at night, and on sunny than on dark days.

5. Magnetic indications.-The annealing temperature for steels containing between 0.50 and 0.90 per cent of carbon is that at which the metal suddenly ceases to be magnetic. This fact may sometimes be used for the purpose of fixing or verifying the annealing temperature.

6. Annealing temperature.-In general, the higher the carbon content the lower should be the annealing temperature. Hence different temperatures are given for different ranges of carbon content. Further, in order to bring the interior of large objects up to an effective annealing temperature, their outside may often be raised advantageously somewhat above that temperature. Hence for each range of carbon content a range of temperature is given. The upper limit of this range applied (1) to larger objects, and (2) to the lower part of the range of carbon content given.

The following ranges of temperature should be used for the several ranges of carbon content indicated. They refer to the usual moderate manganese content. For steels with a manganese content greater than 0.75 per cent, slightly lower temperatures suffice.

\begin{tabular}{|c|c|}
\hline Range of carbon content & Range of annealing temperature \\
\hline 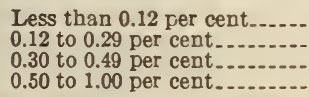 & $\begin{array}{l}875^{\circ} \text { to } 925^{\circ} \mathrm{C} \cdot\left(1,607-1,697^{\circ} \mathrm{F} .\right) \\
840^{\circ} \text { to } 870^{\circ} \mathrm{C}\left(\left(1,544-1,598^{\circ} \mathrm{F} .\right)\right. \\
815^{\circ} \text { to } 840^{\circ} \mathrm{C} .\left(1,499-1,544^{\circ} \mathrm{F} .\right) \\
790^{\circ} \text { to } 815^{\circ} \mathrm{C}\left(1,454-1,499^{\circ} \mathrm{F} .\right)\end{array}$ \\
\hline
\end{tabular}

7. Care in heating. - Care should be taken that all parts of the object reach the same temperature. To that end it may be necessary to mask from the heat, by means of bricks, the thinner parts of objects of varying thickness. When the heating is nearly finished, these bricks may be removed. In particular the flame should never be allowed to touch any part of the object under treatment.

8. Cooling.-After the object has been held at the annealing temperature long enougn to make its temperature nearly uniform throughout, and to complete the refining of the grain, it should be cooled in a way suited to its carbon content and to giving it the specific properties needed. The general principles are: First, the higher the carbon the slower should be the cooling; and second, the slower the cooling the softer and more ductile the metal will be, and the lower will be its tensile strength, elastic limit, and yield point. The greatest softness and ductility are obtained at a certain sacrifice of strength and elasticity, and the greatest strength and elasticity at a certain sacrifice of softness and ductility.

For most purposes neither of these extremes is desired, and it is not only sufficient as regards quality but also economical to remove the object from the furnace as soon as it has been thoroughly annealed, and to allow it to cool in air, always completely protected not only from rain and snow but from sharp drafts of air. Objects containing more than 0.50 per cent of carbon should cool more slowly till the color dies out, say, at $500^{\circ} \mathrm{C}$. $\left(932^{\circ} \mathrm{F}\right.$.), as for instance by leaving them in the furnace. They may then be removed and cooled in air. Further, thin objects containing between 0.25 and 0.50 per cent of carbon should be treated like those of 0.50 per cent of carbon, unless they can be so massed ogether that their collective bulk will retard their cooling, so that they will collectively cool even in air with moderate slowness, like single large objects.

\section{SPECIAL ANNEALING METHODS}

9. The foregoing methods are economical because they release the furnaces early for further use. In case special qualities are desired the following methods may be used:

10. To give the greatest softness and ductility of which the metal is capable, even at a certain sacrifice of strength and elastic limit, for instance for ease of machining or to resist a small number of severe distortions, the metal should be cooled slowly, either within the furnace or, in the case of large objects, under a cover of lime, clay, or other slow conductor of heat. The slower the cooling and the lower the temperature to which slow cooling is carried, the softer and weaker will be the steel. But for most cases for which even unusual softness and ductility are required, it suffices to remove the object from the furnace when it has become dead black, and to cool it thenceforth in air.

11. To give great tensile strength and high elastic limit even at a certain sacrifice of ductility, the cooling should be more rapid, the rapidity to be governed by the thickness and carbon content of the object. Thin objects and those with high-carbon content can not stand so rapid a cooling as thick and low-carbon ones, lest their ductility be too far sacrificed. For instance, thick objects with less than 0.50 per cent of carbon may be cooled completely in air, of course protected from rain or snow. Objects with 0.50 per cent of carbon or more, and thin objects with from 0.30 to 0.50 per cent of carbon, may be cooled in air if their cooling is somewhat retarded, as for instance by massing them together, as happens in the case of rails.

12. To give an unusually high combination of ductility with tensile strength and elastic limit. Water or oil quenching and annealing.-This process needs great care and intelligence, and should in general be used only by those familiar with high-grade steels. After holding at the annealing temperature suited to the particular steel, as indicated in section 6 , the object is quenched in oil, which should be kept in circulation. It may be quenched in water if its carbon content is so low and its shape so simple that it is not in danger of cracking or receiving permanently harmful stress. In any event the danger of such cracking and stress is lessened by removing the object from the oil or water before it has cooled completely, say when its temperature has fallen to $160^{\circ} \mathrm{C}$. $\left(320^{\circ} \mathrm{F}\right.$.). It should if possible not cool below $100^{\circ} \mathrm{C}$. $\left(212^{\circ} \mathrm{F}\right.$.) and certainly not below $20^{\circ}$ C. $\left(68^{\circ}\right.$ F.). The annealing should begin within a very few hours after the quenching and if possible without ever allowing the piece to cool below $100^{\circ}$ C. $\left(212^{\circ} \mathrm{F}\right.$.) and certainly not below $20^{\circ}$ C. $\left(68^{\circ} \mathrm{F}\right.$. $)$.

The steel thus hardened should next be annealed by heating to a temperature suited for giving the properties needed. In general the higher this annealing goes the more ductile will the steel become and the lower will be its strength and elastic limit.

For very high elastic limit and tensile strength, anneal at 500 to $650^{\circ} \mathrm{C}$. $\left(932\right.$ to $1,202^{\circ} \mathrm{F}$.). In this case the ductility will be low. Some steels, such as watch-springs and shafting, are annealed at $350^{\circ} \mathrm{C}$. Little commercial annealing is done below $500^{\circ} \mathrm{C}$.

For intermediate tensile strength, elastic limit, and ductility, best suited to the majority of cases, anneal at 600 to $650^{\circ} \mathrm{C} .\left(1,112\right.$ to $1,202^{\circ} \mathrm{F}$.).

For the greatest ductility, with good strength and elastic limit, anneal at from 725 to $750^{\circ} \mathrm{C}$. (1,337 to $1,382^{\circ} \mathrm{F}$.)

The object should be held at this second annealing temperature long enough not only to allow its interior to reach it but also to relieve the stress 
given in the water or oil quenching. For pieces of moderate thickness a 4-hour exposure should suffice.

13. To give a moderate increase of strength and elastic limit above that given by a simple slow cooling without the full sacrifice of ductility which a simple air cooling would cause.-After holding at the annealing temperature suited to the carbon content, as indicated in section 6 , hasten the cooling till the object is at a dull red, say $650^{\circ} \mathrm{C}$. $\left(1,202^{\circ} \mathrm{F}.\right)$, and thenceforth cool slowly. The hastening of the cooling may be brought about in the case of thin objects in relatively shallow furnaces by opening the furnace door till the temperature falls to dull redness, or by running the objects out in the air on a movable carbottom, and returning them to the furnace when they have cooled to dull redness. In cases in which such operations are to be performed frequently, special unfired chambers may be used for the final slow cooling, thus leaving the annealing furnaces properly a vailable for their regular work. The results obtained in this way are not as good as those obtained with the method described in section 11.

14. Special annealing to remove the effects of rolling or otherwise working the object in the cold or at any unduly low temperature.-The object should be heated to about $775^{\circ}$ C. $\left(1,427^{\circ}\right.$ F.) and cooled with a slowness which should increase with the thickness that is, the least dimension of the piece. In the case of thin plates a mere heating to $725^{\circ} \mathrm{C}$. $\left(1,337^{\circ} \mathrm{F}\right.$.), followed immediately by slow cooling, should suffice. In the case of thick forgings, in which much time may be needed to allow severe stress to relieve itself, the sojourn at $775^{\circ} \mathrm{C}$. $\left(1,427^{\circ} \mathrm{F}\right.$.) may be prolonged for several hours, though always at the cost of superficial decarburizing.

Such annealing for steel containing less than 0.15 per cent carbon should be at $900^{\circ} \mathrm{C}$. $\left(1,652^{\circ} \mathrm{F}\right.$.). Large grain size may be caused by annealing very low carbon steel in the neighborhood of $700^{\circ} \mathrm{C}$. $\left(1,292^{\circ}\right.$ F.), after cold working.

\section{AMERICAN SOCIETY FOR TESTING MATE- RIALS, RECOMMENDED PRACTICE FOR HEAT TREATMENT OF CARBON-STEEL CASTINGS, DESIGNATION A36-24, 1924}

\section{HEAT TREATMENT OF CARBON-STEEL CASTINGS}

1. This recommended practice is intended solely for guidance, and is not to be construed as entering in any manner into specifications of the American Society for Testing Materials.

2 . This recommended practice is intended to cover: (a) Annealing, (b) normalizing, (c) normalizing followed by a reheating, and $(d)$ quenching followed by a reheating.

3. After pouring, the castings should be allowed to cool in the sand to a temperature below the critical range.

4. The castings should be sufficiently cleaned of adhering sand before heat treatment to insure thorough and uniform heating.

5. The temperatures recommended when treatment above the upper critical point is desired should vary with the carbon content, approximately as follows:

\begin{tabular}{|c|c|}
\hline \multicolumn{1}{|c|}{ Carbon (per cent) } & Temperature \\
\hline $\begin{array}{l}\text { Up to } 0.40 \text {, inclusive } \\
\text { Over } 0.40 \text { to } 0.60 \text {, inclusive }\end{array}$ & $900^{\circ} \mathrm{C} .\left(1,650^{\circ} \mathrm{F}.\right)$ \\
Over 0.60 & $850^{\circ} \mathrm{C} .\left(1,560^{\circ} \mathrm{F}.\right)$ \\
\hline
\end{tabular}

6. The use of pyrometers (preferably recording pyrorneters) is recommended. Pyrometers should be checked frequently against a standard. Every precaution should be taken to procure due assurance that the castings throughout the furnace reach the required temperature.

7. After cooling as indicated in section 3 , the castings should be heated slowly and uniformly to the desired temperature. Sufficient time should be allowed for the heat to accomplish the desired structural transformations throughout the mass of the castings. For these reasons it is necessary that each furnace containing castings be held for a prolonged period at the desired temperature after the castings have attained that temperature. It is recommended that this prolonged period should be not less than 60 minutes for each inch of thickness of the thickest casting segment in the charge.

8. To anneal castings properly, they should be heated as described in section 7 to one of the teinperatures given in section 5 . They should then be slowly cooled in the furnace until they reach a faint-red color, after which the cooling mav be continued in air.

9. To secure finer structure (with probably less ductility than can be obtained by annealing as described in sec. 8) castings should be heated as described in section 7 to one of the temperatures given in section 5 , and cooled in still air.

10. To secure fine structure, accompanied by great ductility, normalizing as described in section 9 should be followed by a reheating and subsequent cooling. In this reheating, the castings may be kept for a short time just above the lower critical point or they may be kept for several hours at a suitable temperature below the critical range. After this reheating, the castings should be cooled slowly in the furnace until they reach a faint-red color (provided they reach this color in the reheating), after which the cooling may be continued in air.

11. To secure maximum refinement of the structure coupled with the greatest possible combination of strength and toughness, castings should be heated as described in section 7 to one of the temperatures given in section 5 , and quenched in water or oil. They should then be reheated. In this reheating, the castings may be kept for a short time just above the lower c.itical point or they may be kept for several hours at a suitable temperature below the critical range. After this reheating the castings should be cooled slowly in the furnace until they reach a faint-red color (provided that color was reached in the reheating operation), after wnich the cooling may be continued in air.

12. (a) Certain castings, which from their mass or design would crack on quenching if kept in the water or oil until completely cold, may be safely treated by removing them from the quenching bath when they have reached a black heat, immediately charging them into a furnace heated to approximately the same temperature as the castings, and reheating them as described in section 11 .

(b) The advisability of attempting to quench and reheat any given casting must be considered with judgment in the light of experience; and to attempt to apply this method of heat treatment without taking the precautions demanded by the shape, size, and sections of each individual casting can only result in the loss of many castings through the development of cracks either in quenching, in reheating, or at some later time. 
AMERICAN SOCIETY FOR TESTING MATERIALS, RECOMMENDED PRACTICE FOR CARBURIZING AND HEAT TREATMENT OF CARBURIZED OBJECTS, DESIGNATION A37-27, 1927

\section{CARBORIZING}

1. This recommended practice is intended solely for guidance, and is not to be construed as entering in any manner into specifications of the American Society for Testing Materials.

2. Carburizing is intended to increase the carbon content in the surface layers of low-carbon steel objects so they may be made hard and wear-resistant on the outside with a strong, tough, inner portion or core. This type of structure is suitable for parts which are required to stand considerable shock and friction. The process consists in heating the steel in contact with a carbonaceous material for a long time at temperatures above the critical temperature of the steel.

3 . The primary factors governing the process are: (a) Chemical composition of the steel, (b) the craburizing operation, and (c) the hardening operation.

4. The steel should be selected according to the service requirements. A well-made carbon steel will be satisfactory for most ordinary purposes. The carbon content of simple and alloy steels should preferably be below 0.20 per cent. High manganese above 0.60 per cent is sometimes specified to obtain increased hardness and increased core strength. High phosphorus and sulphur contents improve the machineability but generally indicate a steel of poor quality. These elements are, however, often added intentionally to steels of good quality to improve their machining qualities.

In alloy steels, nickel tends to retard the grain growth at carburizing temperatures, depresses the critical temperature, and allows the steel to be hardened at lower quenching temperatures. Chromum is used alone in percentages up to 1.25 per cent and gives a greater abrasive hardness to the case. S. A. E. steel No. 3115 , containing about 1.25 per cent nickel and 0.60 per cent chromium is recommended for general high-grade use. A molybdenum content of about 0.25 per cent or combined with about 1.75 per cent of nickel is used with good results. The use of molybdenum steel containing chromium is not recommended as this steel has an inherent tendency to distort in the carburizing operation.

5. Carburizing materials are divided into three general classes: Solids, liquids, and gases, the solid type being generally used. The recommendations given apply to this type only. Commercial compounds usually are better than those made by the user. One part of new solid compound to three parts of used compound is recommended as a shop mixture. The used compounds should be screened to remove the dust.

6. Packing pots are made from cast iron, cast and pressed steel, and special heat-resisting alloys. The latter are recommended. Comparisons are usually made on a heat-hour-cost basis, the life of the containers being determined by actual service and this figure divided into the cost of the pot which gives the cost per service-heat hour. The proportions of the pot in relation to the parts to be carburized is of primary importance as well as the proper distribution of metal in the walls, reinforcement ribs, legs, etc. A compromise in size is found between the very small containers which are expensive in handling practice and the large containers which have a tendency to heat unevenly. The size of the pot should be governed by the size of the parts.

7. The effective temperature is the temperature inside the pot. The carburizing time should be designated as the number of hours at the carburizing temperature. The heating through of the pots is judged in two ways: By estimating with the eye, and by placing thermocouples inside the pot as shown in Figure 47. The latter method is recommended except on pots which are so small that it would be impracticable to place a thermocouple inside them.

8. The use of recording pyrometers is recommended. Where the method shown in Figure 47 is used, an extra couple should be placed in the furnace so that the furnace temperature may be more readily controlled, as otherwise excessive furnace temperatures or extremely variable heats may be obtained.

9. Quick heating to the carburizing temperature will cause more distortion of the parts than slow heating. The temperature in any pot must be comparable with the temperature inside any other. To

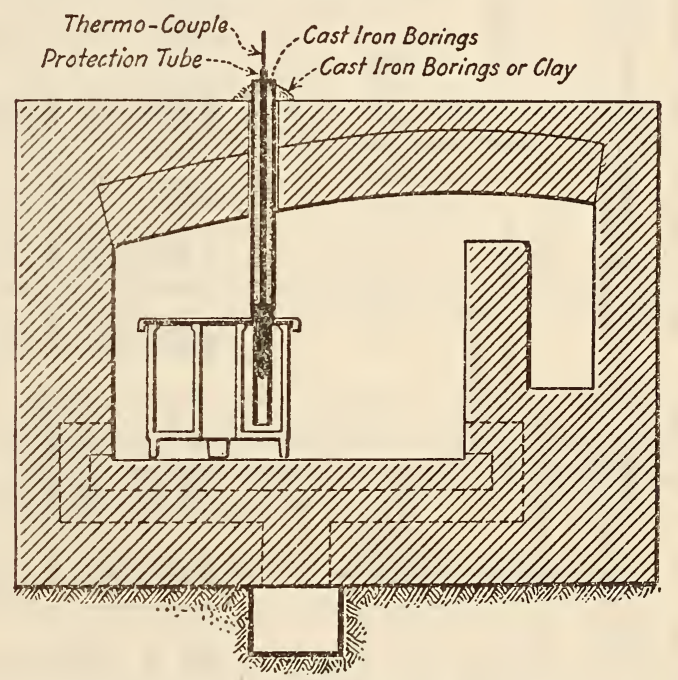

FIGURE 47

this end, the furnace must be designed to heat evenly and the pots must be spaced on the furnace hearth so that they will be heated evenly and uniformly.

10. The carbon content at the surface of the work increases with the carburizing temperature. The formation of free carbide at the grain boundaries is undesirable, as it leaves a very brittle surface exposed to the service stresses. The surface carbon should not exceed 1.15 per cent. This will be reduced by the finish grinding operation. The carbon content is governed by the carburizing compound and the temperature, while gradation depends upon the analysis of the steel and the rate of cooling. The allowable temperature depends upon the nature of the work. Temperatures of $941^{\circ}$ to $954^{\circ} \mathrm{C}$. $\left(1,725^{\circ}\right.$ to $1,750^{\circ} \mathrm{F}$.) may be used where speed and low expense are primary considerations. For general practice, $899^{\circ} \mathrm{C}$. $\left(1,650^{\circ} \mathrm{F}\right.$.) is recommended. The carburizing temperature should be lowered about $38^{\circ} \mathrm{C}$. $\left(100^{\circ} \mathrm{F}\right.$.) when the regenerative quench is not to be used. Thin cases, of 0.010 to 0.015 inch, are better obtained with a carburizing temperature of $843^{\circ} \mathrm{C}$. $\left(1,550^{\circ} \mathrm{F}.\right)$. 


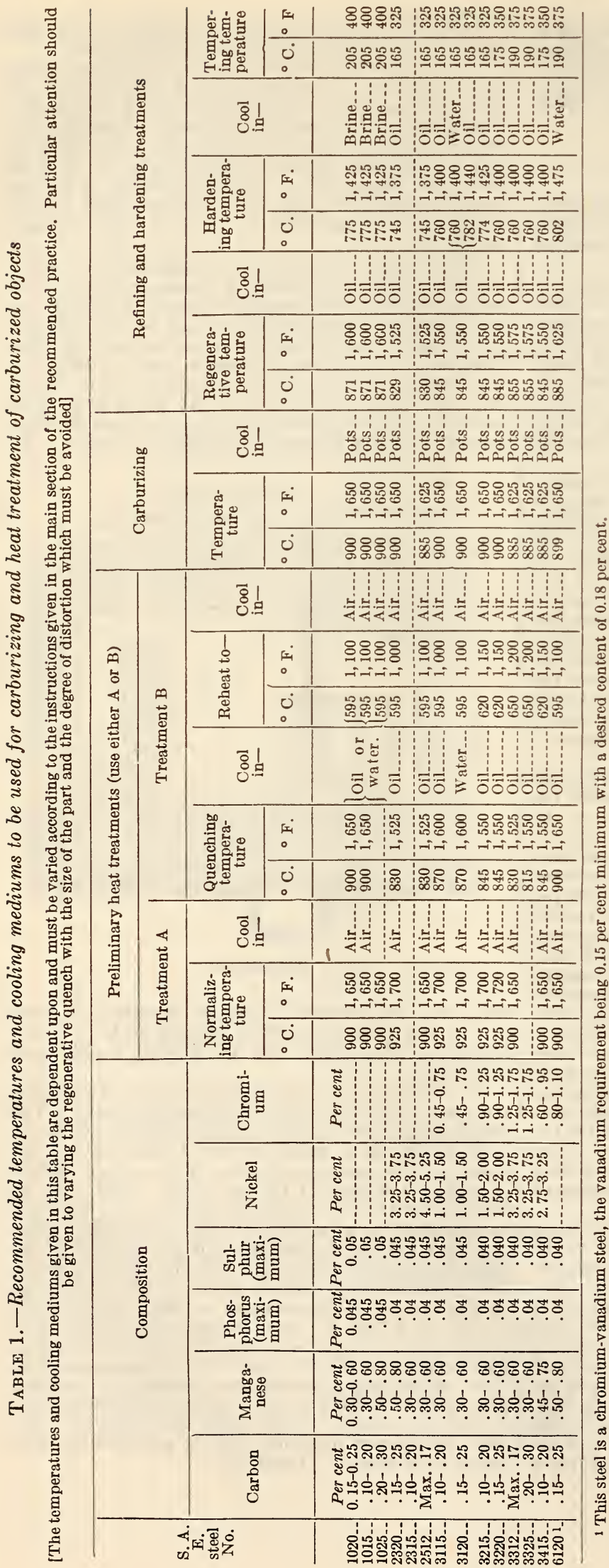


11. The "depth of case" shall be taken to be the depth of the refined case to be observed with the naked eye after carburizing, hardening at the temperature giving maximum case refinement, and breaking for fracture.

12. To obtain the best core condition, the part should be allowed to cool in the pots, outside the furnace, then reheated and quenched from a temperature which will refine the core and break up any undesirable structures of cementite. This is called a regenerative quench.

\section{TABLE 2.-Common defects in carburized objects}

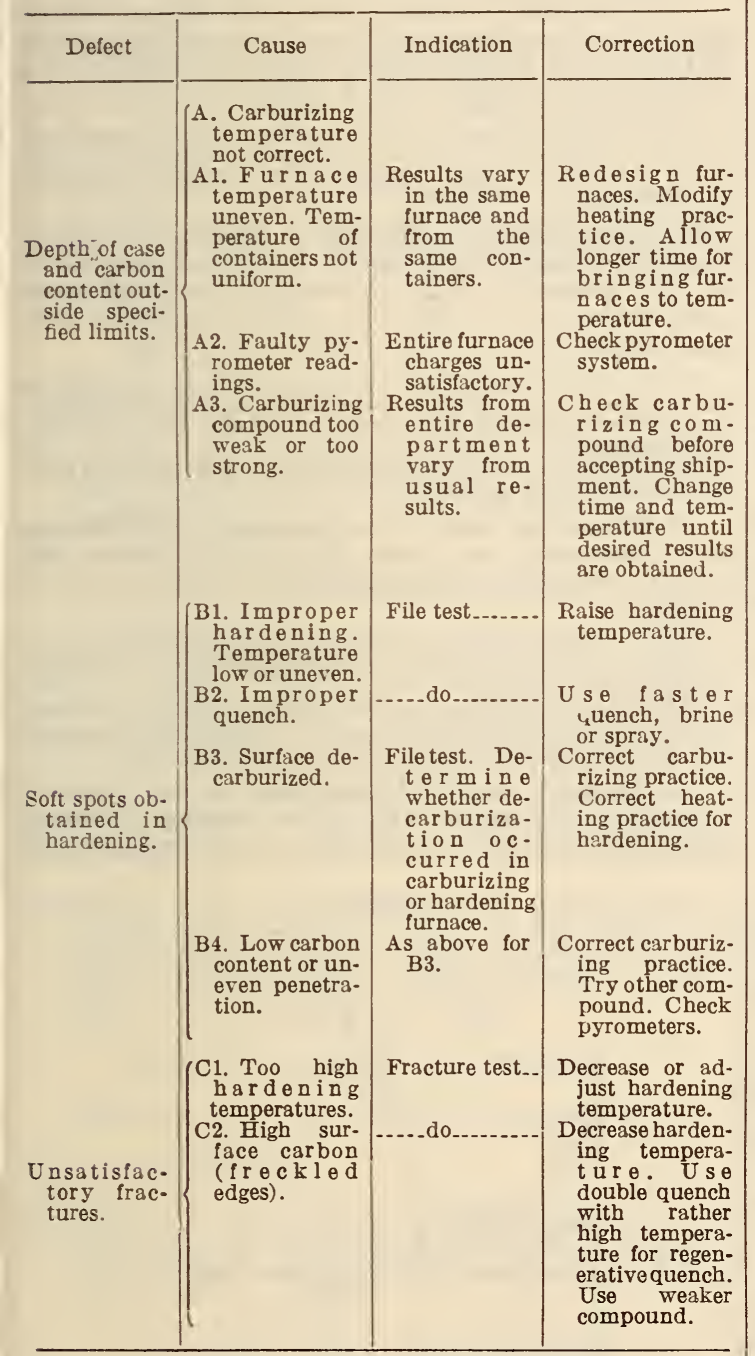

13. The cooling of the carburized parts should take place in the containers, outside of the furnace. Where cost of production is vital, the parts may be quenched directly from the pot in either oil or water.

14. In determining the depth of case desired, sufficient allowance must be made for the metal which will later be removed by grinding. A tem- pering operation will tend to eliminate the tendency to the formation of grinding cracks.

15. The hardening temperature of the case is below the critical temperature of the core and the grain size produced in the core, by the carburizing temperature or regenerative quench, is not affected. The hardening temperature should be chosen as the temperature which gives the best fracture on several dummies or experimental pieces hardened from various temperatures. The figures given in Table 1 are the middle of the probable range of hardening temperatures.

16. The tendency to distort may be avoided by a preliminary heat treatment, either before or after the rough machining operation. The method of carburizing must be studied and the subsequent hardening operations carried on with extreme care as to the heating and cooling cycles. In many cases, the regenerative quench must be omitted as the high temperature of this operation causes too mucn warping. When omitted, the carburizing temperature should be lowered about $38^{\circ} \mathrm{C}$. $\left(100^{\circ} \mathrm{F}\right.$.).

17. Tempering may be carried on at low temperatures, for the relief of strains; or at high temperatures, for the increase of toughness and uniformity without materially decreasing the hardness. An oil bath is preferable to an open furnace for temperatures up to $316^{\circ} \mathrm{C}$. $\left(600^{\circ}\right.$ F.). For simple carbon steels, a softening of the surface is found when the tempering temperature exceeds $190^{\circ}$ to $204^{\circ} \mathrm{C}$. $\left(375^{\circ}\right.$ to $400^{\circ}$ F.). For maximum hardness the temperature should be kept below this figure.

18. Table 2 shows in condensed form the most common defects in carburized work together with the possible causes and methods for their identification and correction.

\section{AMERICAN SOCIETY FOR STEEL TREAT- ING, RECOMMENDED PRACTICES FOR THE HEAT TREATMENT OF SPRINGS, MAY 25, 1932}

\section{HEAT TREATMENT OF SPRINGS}

(These recommended practices cover the heat treatment of small helical springs cold coiled from wire, large helical springs hot coiled from bars one-half inch or over in diameter, small flat springs cold formed from plates less than three-sixteenths inch in thickness, and large flat springs hot formed from plates three-sixteenths inch or over in thickness. It includes the chemical compositions of the various carbon and alloy steels usually used for these springs and the recommended physical characteristics. The recommended heat treatment for each of the steels is given, including the normalizing, quenching, and tempering temperature ranges, the quenching medium, and the approximate hardness of the resulting product.)

AMERICAN SOCIETY FOR TESTING MATERIALS, STANDARD SPECIFICATIONS FOR VALVES, FLANGES, AND FITTINGS FOR HIGH-TEMPERATURE SERVICE, DESIGNATION A95-29, 1929

\section{CASTINGS FOR HIGH TEMPERATURES}

For heat treatment (see 611.41, p. 709.) 
AMERICAN SOCIETY FOR TESTING MATERIALS, STANDARD DEFINITIONS OF TERMS RELATING TO HEAT-TREATMENT OPERATIONS (ESPECIALLY AS RELATED TO FERROUS ALLOYS), DESIGNATION A119-30, 1930

\section{HEAT-TREATMENT DEFINITIONS 42}

In preparing the definitions included herein, the terms have been so defined, as most in keeping with present-day usage, that they shall mean definite

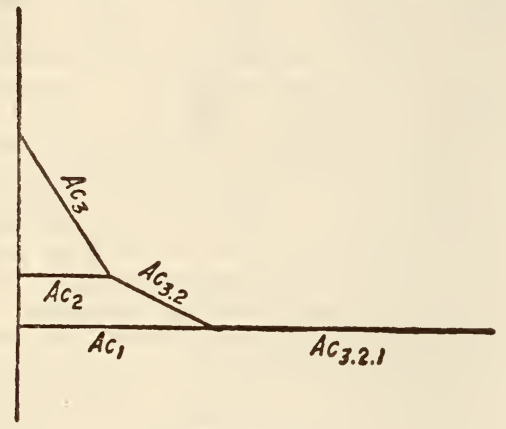

FigURe 48

operations and shall not be considered as referring to the resultant structures or general conditions.

By "critical temperature range," as used in the definitions, is meant that temperature range illustrated in Figure 48, taken from Howe.

Heat treatment.-An operation or combination of operations involving the heating and cooling of a metal or an alloy in the solid state.

Note.-This is for the purpose of obtaining certain desirable conditions or properties. Heating and coling for the sole purpose mechanical working are excluded from the meaning of this definition.

Quenching.-Rapid cooling by imersion.

Note.-Immersion may be in liquids, gases, or solids.

Hardening.-Heating and quenching certain ironbase alloys from a temperature either within or above the critical temperature range.

Annealing.-A heating and cooling operation of a material in the solid state.

Note 1.-Annealing usually iniplies a relatively slow cooling.

Note 2.-Annealing is a comprehensive term. The purpose of such a heat treatment may be: $(a)$ To remove stresses; $(b)$ to induce softness; $(c)$ to alter ductility, toughness, or electrical, magnetic, or other physical properties; $(d)$ to refine the crystaline structure, and (e) to remove gases.

In annealing, the temperature of the operation and the rate of cooling depend upon the material being heat treated and the purpose of the treatment.

Certain specific heat treatments coming under the comprehensive term "annealing" are:

(a) Full annealing.- - Heating iron base alloys above the critical temperature range, and holding above that range for a proper period of time followed by slow cooling through the range.

NoTE.-The annealing temperature is generally about $100^{\circ} \mathrm{F}$. $\left(55^{\circ} \mathrm{C}\right.$.) above the upper limit of the critical temperature range, and the time of holding is usually not less than one hour for each inch of section of the heaviest objects being treated. The objects being treated are ordinarily allowed to cool slowly in the furnace. They may, however, be removed from the furnace, and cooled in some may, however, be removed from the furnace, and cooled in some medium which will prolong

42 These definitions are those prepared by the Joint Committee on Definitions of Terms Relating to Heat Treatment and submitted by the joint committee to the society in 1927. The following organizations are represented on the joint committee: American Society for Steel Treating, Society of Automotive Engineers, and the American Society for Testing Materials. (b) Process annealing.- Heating iron base alloys to a temperature below or close to the lower limit of the critical temperature range followed by cooling as desired.

NoTe.-This heat treatment is common!y applied in the sheet and wire industries and the temperatures generally used are from $1,020^{\circ}$ to $1,200^{\circ} \mathrm{F}$. $\left(550^{\circ}\right.$ to $650^{\circ} \mathrm{C}$.).

(c) Normalizing.-Heating iron-base alloys above the critical temperature range followed by cooling to below that range in still air at ordinary temperature.

Note.-In the case of hypereutectoid steel, it is often desirable to heat above the $A c_{c m}$ line, as shown in Figure 49.

(d) Patenting.-Heating iron-base alloys above the critical temperature range followed by cooling to below that range in molten lead maintained at a temperature of about $700^{\circ} \mathrm{F}$. ( $365^{\circ} \mathrm{C}$.)

Note.-This treatment is usually applied in the wire industry either as a finishing treatment or especially in the case of eutectoi steel as a treatment previous to further wire drawing. Its plirpose is to produce a sorbitic structure.

(e) Spheroidizing.-Prolonged heating of ironbase alloys at a temperature in the neighborhood of, but generally slightly below, the critical temperature range, usually followed by relatively slow cooling.

Note 1.-In the case of small objects of high-carbon steels, the spheroidizing result is achieved more rapidly by prolonged heating to temperatures alternately within and slightly below the critical temperature range,

Note 2.-The object of this heat treatment is to produce a globular condition of the carbicie.

(f) Tempering (also termed drawing).-Reheating after hardening, to some temperature below the critical temperature range followed by any rate of cooling.

Note 1.-Although the terms "tempering" and "drawing" are practically synonymous as used in commercial practice, the term "tempering" is preferred.

NOTE 2.-Tempering meaning the operation of hardening followed by reheating is a usage which is illogical and confusing in the present state of the art of heat treating and should be discouraged.

(g) Malleablizing.-Malleablizing is a type of annealing operation with slow cooling whereby combined carbon in white cast iron is transformed

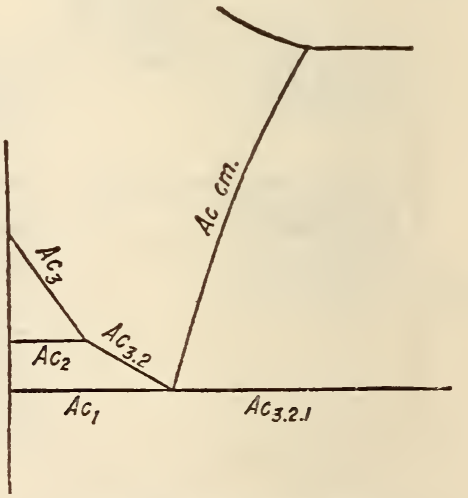

Figdre 49

to temper carbon and in some cases the carbon is entirely removed from the iron.

Note.-Temper carbon is free carbon in the form of rounded nodules made up of an aggregate of minute crystals.

(h) Graphitizing.-Graphitizing is a type of annealing of cast iron whereby some or all of the combined carbon is transformed to free or uncombined carbon.

Carburizing (cementation).-Adding carbon to iron-base alloys by heating the metal below its 
melting point in contact with carbonaceous material.

NotE.-The term "carbonizing" used in this sense is incorrect so its use should be discourage 1 .

Case hardening.-Carburizing and subsequently hardening by suitable heat treatment, all or part of the surface portions of a piece of iron-base alloy.

Case.-That portion of a carburized iron-base alloy article in which the carbon content has been substantially increased.

Core.-That portion of a carburized iron-base alloy article in which the carbon content has not been substantially increased.

Note.-The terms "case" and "core" apply both to carburizing and case hardening.

Cyaniding.-Surface hardening of an iron-base alloy article or portion of it by heating at a suitable temperature in contact with a cyanide salt, followed by quenching.

AMERICAN SOCIETY FOR TESTING MATERIALS, HEAT-TREATED, CARBONSTEEL HELICAL SPRINGS, DESIGNATION A125-31T, 1931

\section{HEAT-TREATED HELICAL SPRINGS}

(See 611.54, p. 805.)

SOCIETY OF AUTOMOTIVE ENGINEERS, IRON AND STEEL SPECIFICATIONS, S.A.E. STANDARD, 1931

\section{HEAT TREATMENT OF METALS}

(Voluminous notes on heat treatments of various kinds of steels are contained in the S. A. E. Handbook for 1931. These notes are not to be considered in any way a part of the standard specifications for S. A. E. steels. They are added solely for the information of users of the steels and the guidance of purchasers in the selection of proper materials for different purposes. They should not be incorporated in the customer's specifications when ordering steel.)

SOCIETY OF AUTOMOTIVE ENGINEERS, DEFINITIONS OF TERMS RELATING TO HEAT-TREATMENT OPERATIONS, GENERAL INFORMATION, 1930

\section{HEAT-TREATMENT DEFINITIONS}

(Same as A. S. T. M., A119-30, given above.)

600.6 PLUMBING.

AMERICAN RAILWAY ENGINEERING ASSOCIATION, PLUMBING FOR RAILWAY BUILDINGS, 1929

\section{CAST-IRON PIPE AND FITTINGS}

(See 607.11, p. 371.)

\section{NATIONAL ASSOCIATION OF MASTER PLUMBERS, PLUMBING SYMBOLS, 1927}

\section{PLOMBING SYMBOLS}

The standard symbols shown in the accompanying illustrations have been prepared by the standardization committee of the National Association of Master Plumbers for use by architects and engineers in designing plumbing and drainage systems.

Proper location of fixtures eliminates unnecessary cutting and notching of timbers and simplifies piping installation. Adequate pipe space must be provided in walls or partitions or beneath floors to eliminate the use of unnecessary fittings which add to the cost of labor and material without increasing the efficiency of the system.

UNITED STATES GOVERNMENT, DEPARTMENT OF COMMERCE, NATIONAI BU. REAU OF STANDARDS, RECOMMENDED MINIMUM REQUIREMENTS FOR PLUMBING, BH13, 1929

\section{PLUMBING REQUIREMENTS}

(This is an illustrated publication of 280 pages. It is divided into four parts: Part 1 covers general considerations; part 2 covers plumbing materials and their installation; experimental investigations and the physics of plumbing are included in part 3 ; and in part 4 will be found appendixes covering corrosion of metals in plumbing systems, air currents in a horizontal waste pipe, and the specifications of the American Society for Testing Materials that concern themselves with plumbing materials. In the article on quality and weights of materials, this publication provides as follows:)

Vitrified-clay pipe.-All vitrified clay pipe shall conform to the A. S. T. M. standard specifications for clay sewer pipe, C13-24. (See Standards and Specifications for Nonmetallic Minerals and their Products, p. 517.)

Cast-iron pipe.-(a) Quality.-All cast-iron pipe and fittings shall conform to the A. S. T. M. standard specifications for cast-iron soil pipe and fittings, A74-29. (See 607.11, p. 371.)

(b) Coating.-All cast-iron pipe and fittings for underground use shall be coated with asphaltum or coal-tar pitch.

Wrought-iron pipe.-All wrought-iron pipe shall conform to the A. S. T. M. standard specifications for welded wrought-iron pipe, A72-31. (See 607.3, p. 431 .)

Mild-steel pipe.-All steel pipe shall conform to the A. S. T. M. standard specifications for welded and seamless steel pipe, A53-30. (See 607.4, p. 476.)

Brass and copper pipe.-Brass and copper pipe shall conform, respectively, to the standard specifications of the A. S. T. M. for brass pipe, standard sizes, and for copper pipe, standard sizes, B43-24 (see 645.23, p. 1216) and B42-24 (see 642.23, p. 1182).

Lead pipe, diameter, weights.-All lead pipe shall be of best quality drawn pipe, of weight per linear foot not less than shown below.

(a) Lead soil, waste, vent, or flush pipes, including bends and traps (extra light):

\begin{tabular}{|c|c|c|c|}
\hline $\begin{array}{c}\text { Internal } \\
\text { diameter } \\
\text { (inches) }\end{array}$ & $\begin{array}{l}\text { Weight } \\
\text { per foot } \\
\text { (pounds } \\
\text { and } \\
\text { ounces) }\end{array}$ & $\begin{array}{l}\text { Internal } \\
\text { diameter } \\
\text { (inches) }\end{array}$ & $\begin{array}{l}\text { Weight } \\
\text { per foot } \\
\text { (pounds } \\
\text { and } \\
\text { ounces) }\end{array}$ \\
\hline $\begin{array}{l}1 \\
11 / 4 \\
11 / 2\end{array}$ & $\begin{array}{ll}2 & 0 \\
2 & 8 \\
3 & 8\end{array}$ & $\begin{array}{l}2 \\
3 \\
4\end{array}$ & $\begin{array}{rr}4 & 0 \\
4 & 12 \\
6 & 0\end{array}$ \\
\hline
\end{tabular}

(b) Lead water-supply pipe above ground (strong):

\begin{tabular}{|c|c|c|c|}
\hline $\begin{array}{c}\text { Internal } \\
\text { diameter } \\
\text { (inches) }\end{array}$ & $\begin{array}{l}\text { Weight } \\
\text { per foot } \\
\text { (pounds } \\
\text { and } \\
\text { ounces) }\end{array}$ & $\begin{array}{c}\text { Internal } \\
\text { diameter } \\
\text { (inches) }\end{array}$ & $\begin{array}{l}\text { Weight } \\
\text { per foot } \\
\text { (nounds } \\
\text { and } \\
\text { ounces) }\end{array}$ \\
\hline $\begin{array}{l}1 / 2 \\
5 / 8 \\
3 / 4 \\
1^{1 / 4}\end{array}$ & $\begin{array}{ll}2 & 0 \\
2 & 8 \\
3 & 0 \\
4 & 0\end{array}$ & $\begin{array}{l}11 / 4 \\
11 / 2 \\
13 / 4 \\
2\end{array}$ & $\begin{array}{rr}4 & 12 \\
6 & 0 \\
6 & 8 \\
7 & 0\end{array}$ \\
\hline
\end{tabular}




\section{SYMBOLS FOR EXPOSED PIPING}

CHARACTER

Sanitary seweragुe

soil stack

Waste stack

Vent stack

Combined Sewerage

storm sewerage

Roof Leader

Indirect Waste

Industrial Sewerage

Acid or Chemical Waste

cold City Water

Hot City Water

Cir. Hot City Water

Chilled Drinking Water

Fire Line

Cold Industrial Water

Hot Industrial Water

Cir. Hot Industrial Water

Air

Gas

Oi]

Vacuirm cleaner

Local or Surface VenI
PLAN

LINE

BAND

INITIAL, COLOR

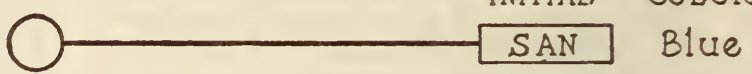

(24)

SS "

(17)

W S

(18) $---\cdots-\cdots---\cdots \mathrm{VS}$

(D) $+-+-+-+-+-+++C \operatorname{CS}$

(O) - - - - - STORM Gree:

(E) $-----1 R I$

A

IW

$\theta-1-1-1-1-$ IS

$\mathrm{B} \rightarrow \longrightarrow \rightarrow-\mathrm{AW}$

6

cw. White

(-

Hiv

D

$\mathrm{CR}$

$\theta$

DWV

(1)

(2)

FL

Red

Yellow

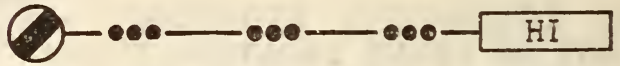

11

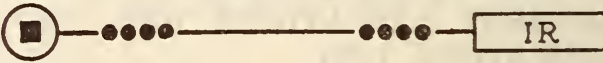

11

A-0-0 Gray

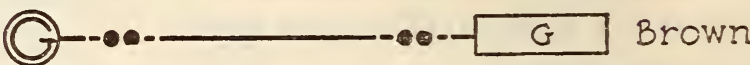

(3)-000-- Black

Q--000-1-0000-- $\mathrm{v}$ Cream

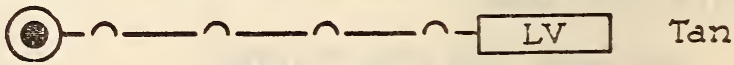

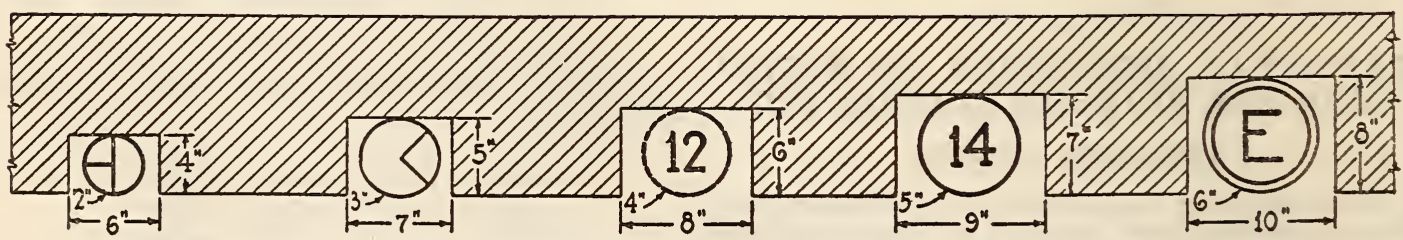

DIMENSIONS OF PIPE CHANNELS TO ACCOMODATE VARIOUS SIZE PIPES 
SYMBOLS FOR PLUMBING CHARACTER Bidet

Sitz Bath

(0)

Combination sink

\& Laundry Tray

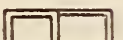

$\square \square$

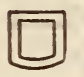

Slop Sink

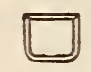

Water Heater

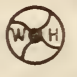

Flushing Slop Sink

Range Boiler

Dressing Table

Washing Machine

Kitchen Sink-Roll Rim R. E L.Hand Drain Birds.

Kitchen Sink-Roll Rim L.Hand Drain Board

Kitchen Sink Plain
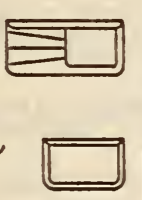

scale of Symbols on this - sheet $1 / 8$ in $=1$ ft

Bath Túb Roll Type
FIXTURES

CHARACTER L.H. Corner

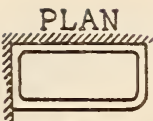

Bath Tub Built In

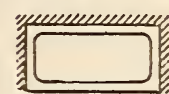

Shower Bath Stall

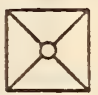

Drinking Fountain 00

Laundry Trays $[\mathbb{1 0}$

Lavatory Pedestal

\section{Lavatory} Wall Hung Type

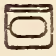

Stall Type-24" Size

Urinal

Wall Hung Type

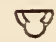

Urinals

Stall Type-18"Size

\section{DRAIN SYMBOLS}

CHARACTER

PLAN

Floor Drain

FIAARACTER

PLAN

BackwaterValve

(3)

CHARACTER

PLAN

Cleanout

(C)

Grease Separator

G

Shower Drain

Refrigerator Drain

(2)

Oil Separator 
(c) Lead water-supply pipe under ground (extra strong):

\begin{tabular}{|c|c|c|c|}
\hline $\begin{array}{c}\text { Internal } \\
\text { diameter } \\
\text { (inches) }\end{array}$ & $\begin{array}{l}\text { Weight } \\
\text { per foot } \\
\text { (pounds } \\
\text { and } \\
\text { ounces) }\end{array}$ & $\begin{array}{l}\text { Internal } \\
\text { diameter } \\
\text { (inches) }\end{array}$ & $\begin{array}{l}\text { Weight } \\
\text { pel foot } \\
\text { (pounds } \\
\text { and } \\
\text { ounces) }\end{array}$ \\
\hline $\begin{array}{l}1 / 2 \\
5 / 3 \\
3 / 4 \\
1^{3 / 4}\end{array}$ & $\begin{array}{rr}2 & 8 \\
3 & 0 \\
3 & 8 \\
4 & 12\end{array}$ & $\begin{array}{l}11 / 4 \\
11 / 2 \\
13 / 4 \\
2\end{array}$ & $\begin{array}{ll}6 & 0 \\
7 & 8 \\
8 & 0 \\
9 & 0\end{array}$ \\
\hline
\end{tabular}

Sheet lead.-Sheet lead shall weigh not less than 4 pounds per square foot.

Sheet copper or brass. - Sheet copper or brass shall be not lighter than No. 18 B. and S. gage, except that for local and interior ventilating pipe it shall be not lighter than No. 26 B. and S. gage.

Galvanizing sheet iron.-Galvanized sheet iron shall be not lighter than the following B. and S. gages:

No. 26 for 2 to 12 inch pipe.

No. 24 for 13 to 20 inch pipe.

No. 22 for 21 to 26 inch pipe.

Threaded fittings. - (a) Plain screwed fittings shall be of cast iron, malleable iron, or brass of standard weight and dimensions. (b) Drainage fittings shall be of cast iron, malleable iron, or brass with smooth interior waterway, with threads tapped out of solid metal. (c) All cast-iron fittings used for water-supply distribution shall be galvanized. (d) All malleable iron fittings shall be galvanized.

Calking ferrules.-Brass calking ferrules shall be of the best quality red cast brass, with weights and dimensions in accordance with the following table:

\begin{tabular}{r|r|r|r}
\hline Pipe size (inches) & $\begin{array}{r}\text { Actual } \\
\text { inside } \\
\text { diameter }\end{array}$ & Length & $\begin{array}{c}\text { Weight } \\
\text { (pounds } \\
\text { and } \\
\text { ounces) }\end{array}$ \\
\hline Inches & Inches \\
$41 / 2$ & \\
$41 / 2$ & \\
$411 / 2$ & \\
\hline
\end{tabular}

Soldering nipples and bushings.-(a) Soldering nipples shall be of brass pipe, iron-pipe size, or of heavy, cast red brass not less than the following weights:

\begin{tabular}{|c|c|c|c|}
\hline $\begin{array}{l}\text { Diameter } \\
\text { (inches) }\end{array}$ & $\begin{array}{l}\text { Weight } \\
\text { (ounces) }\end{array}$ & $\begin{array}{c}\text { Diameter } \\
\text { (inches) }\end{array}$ & $\begin{array}{l}\text { Weight } \\
\text { (pounds } \\
\text { and } \\
\text { ounces) }\end{array}$ \\
\hline $\begin{array}{l}11 / 4 \\
11 / 2 \\
2\end{array}$ & $\begin{array}{r}6 \\
8 \\
14\end{array}$ & $\begin{array}{l}21 / 2 \\
3 \\
4\end{array}$ & $\begin{array}{ll}1 & 6 \\
2 & 0 \\
3 & 8\end{array}$ \\
\hline
\end{tabular}

(b) Soldering bushings shall be of brass pipe, iron-pipe size, or of heavy, cast red brass.

Floor flanges for water-closets.-Floor flanges for water-closets shall be not less than three-sixteenths of an inch thick, and of brass or cast iron.
600.7. WEIGHTS AND TOLERANCES OF IRON AND STEEL.

AMERICAN SOCIETY FOR TESTING MA. TERIALS, STANDARD DEFINITIONS OF TERMS RELATING TO SPECIFIC GRAVITY, DESIGNATION E12-2\%, 1927

\section{SPECIFIC-GRAVITY DEFINITIONS}

(See 600.1 , p. 43.)

ASSOCIATION OF AMERICAN STEEL MANUFACTURERS, STANDARD PRACTICE GOVERNING THE ALLOWABLE VARIATIONS IN SIZE AND WEIGHT OF HOTROLLED BARS, 1910

\section{HOT-ROLLED BARS}

\section{STANDARD ALLOWABLE VARIATIONS IN WEIGHT OF BAR} SIZES OF ANGLES, TEES, ZEES, AND CHANNEIS

For bar sizes of angles, tees, zees, and channels the following average variations in weight will be permitted for sections of the various dimensions and thicknesses stated, viz:

\begin{tabular}{|c|c|c|}
\hline Dimensions & Thickness & Variation in weight \\
\hline $\begin{array}{l}\text { Any dimension over } 11 / 2 \\
\text { inches. } \\
\text { All dimensions } 11 / 2 \text { inches } \\
\text { and less. } \\
\text { Any dimension over } 112 \\
\text { inches. } \\
\text { All dimensions } 11 / 2 \text { inches } \\
\text { and less. }\end{array}$ & $\begin{array}{l}\text { Over } 3 / 16 \text { inch. } \\
3 / 16 \text { inch and } \\
\text { less. }\end{array}$ & $\begin{array}{l}4 \text { per cent over and } \\
\text { under. } \\
5 \text { per cent over and } \\
\text { under. } \\
6 \text { per cent over and } \\
\text { under. } \\
7 \text { per cent over and } \\
\text { under. }\end{array}$ \\
\hline
\end{tabular}

Note.-A channel is in "bar" size when its greatest dimension in less than 3 inches. An angle, T or $z$ is in "bar" size when its greatest dimension is less than 3 inches; or when it is 3 inches or more and at the same time the thickness is less than one-fourth inch.

STANDARD ALLOWABLE VARIATIONS IN THE SIZE OF HOTROLLED BARS

Rounds, squares, hexagons

Up to and including $1 / 2 \mathrm{inch}$

Over $1 / 2$ inch up to and including 1 inch

Over 1 inch up to and including 2 inches.

Over 2 inches up to and including 3 inches

Over 3 inches up to and including 5 inches............

Over 5 inches up to and including 8 inches........

\begin{tabular}{|c|r}
\multicolumn{2}{|c}{ Variation in size } \\
\hline Under & Over \\
& \\
\hline Inch & Inch \\
0.007 & 0.007 \\
.010 & .010 \\
164 & $1 / 32$ \\
$1 / 32$ & $3 / 64$ \\
$1 / 32$ & $3 / 32$ \\
$1 / 16$ & $1 / 8$ \\
&
\end{tabular}


Flats

\begin{tabular}{|c|c|c|c|c|c|c|}
\hline \multirow{3}{*}{ Width of flats } & \multirow{2}{*}{\multicolumn{2}{|c|}{$\begin{array}{l}\text { Variation } \\
\text { in width }\end{array}$}} & \multicolumn{4}{|c|}{$\begin{array}{l}\text { Variation in thickness, under } \\
\text { and over }\end{array}$} \\
\hline & & & \multicolumn{4}{|c|}{ Thickness of flats } \\
\hline & $\begin{array}{l}\text { Un- } \\
\text { der }\end{array}$ & Over & $\begin{array}{l}3 / 16 \text { inch } \\
\text { and } \\
\text { under }\end{array}$ & $\begin{array}{c}\text { Over } \\
3 / 16 \text { inch } \\
\text { up to } \\
36 \text { inch }\end{array}$ & $\begin{array}{c}\text { Over } \\
1 / 2 \text { inch } \\
\text { up to } \\
1 \text { inch }\end{array}$ & $\begin{array}{c}\text { Over } \\
1 \text { inch } \\
\text { up to } \\
2 \text { inches }\end{array}$ \\
\hline $\begin{array}{l}\text { Up to and including } \\
1 \text { inch }\end{array}$ & $\begin{array}{c}\text { Inch } \\
364\end{array}$ & $\begin{array}{r}\text { Inch } \\
1 / 32\end{array}$ & $\begin{array}{l}\text { Inch } \\
0.006\end{array}$ & $\begin{array}{l}\text { Inch } \\
0.008\end{array}$ & $\begin{array}{l}\text { Inch } \\
0.010\end{array}$ & Inch \\
\hline $\begin{array}{l}\text { Over } 1 \text { inch up to } \\
\text { and including } 2 \\
\text { inches.............. }\end{array}$ & $1 / 32$ & 364 & 008 & . & 0 & \\
\hline $\begin{array}{l}\text { Over } 2 \text { inches up to } \\
\text { and including } 4 \\
\text { inches............ }\end{array}$ & 364 & 116 & .010 & .015 & .020 & $1 / 32$ \\
\hline $\begin{array}{l}\text { Over } 4 \text { inches up to } \\
\text { and including } 6 \\
\text { inches. }\end{array}$ & 316 & $3 / 32$ & .010 & .015 & .020 & $1 / 32$ \\
\hline
\end{tabular}

ASSOCIATION OF AMERICAN STEEL MAN. UFACTURERS, STANDARD PERMISSIBLE VARIATIONS IN GAGE WEIGHT, GAGE THICKNESS, AND SIZE AND FLATNESS OF SHEETS AND LIGHT PLATES BLUE ANNEALED, 1929

\section{PERMISSIBLE VARIATIONS IN SHEETS AND PLATES}

\section{GAGE WEIGHT}

When ordered by weight per unit area, sheets shall conform to Table 1 . If ordered by gage number, the gage weights shall be those of the United States standard gage table for uncoated sheets and for long ternes, and of the galvanized sheet gage table for zinc-coated (galvanized) sheets.

TABLE 1

\begin{tabular}{|c|c|c|c|c|c|c|}
\hline \multicolumn{4}{|c|}{ Ordered gage weight } & \multicolumn{3}{|c|}{$\begin{array}{l}\text { Permissible variation } \\
\text { from ordered gage } \\
\text { weight, in percentage } \\
\text { of estimated weight }\end{array}$} \\
\hline \multicolumn{2}{|c|}{ Less than- } & \multicolumn{2}{|c|}{ Not less than- } & \multirow{2}{*}{$\begin{array}{l}\text { All of } \\
1 \text { gage, } \\
\text { and } \\
\text { size in } \\
\text { ship- } \\
\text { ment }\end{array}$} & \multirow[b]{2}{*}{$\begin{array}{c}\text { Single } \\
\text { package }\end{array}$} & \multirow[b]{2}{*}{$\begin{array}{l}\text { Single } \\
\text { sheet }\end{array}$} \\
\hline $\begin{array}{l}\text { Ounce } \\
\text { per } \\
\text { square } \\
\text { foot }\end{array}$ & $\begin{array}{l}\text { Pound } \\
\text { per } \\
\text { square } \\
\text { foot }\end{array}$ & $\begin{array}{l}\text { Ounce } \\
\text { per } \\
\text { square } \\
\text { foot }\end{array}$ & $\begin{array}{l}\text { Pound } \\
\text { per } \\
\text { square } \\
\text { foot }\end{array}$ & & & \\
\hline $\begin{array}{l}140 \\
220\end{array}$ & $\begin{array}{l}2.5 \\
1.25\end{array}$ & $\begin{array}{r}140 \\
220 \\
-\end{array}$ & \begin{tabular}{l}
2.5 \\
1.25 \\
\hdashline-2
\end{tabular} & $\begin{array}{l}5.0 \\
3.5 \\
2.5\end{array}$ & $\begin{array}{l}7.0 \\
5.5 \\
4.0\end{array}$ & $\begin{array}{l}10.0 \\
10.0 \\
10.0\end{array}$ \\
\hline
\end{tabular}

1 No. 16.

No.22.

"All of one gage and size in shipment" shall apply to lots of not less than 6,000 pounds.

References are to gross weights of bundled material and to net weights of crated and boxed material.

If minimum or maximum weight per unit area be ordered, the tolerance used shall be the one for the mean gage weight determined by that tolerance, and double that tolerance shall be allowed on the permissible side. Ordering by minimum or maximum weight per unit area is objectionable, as it is liable to cause error in manufacture and gage classification.

Sheets are not weighed singly at the mill and the 10 per cent limit can not be assured, but any sheets found by purchaser that are outside this limit may be rejected.

Sheets ordered by weight per unit area shall be subject to weight gage tolerances only, and not to thickness gage tolerances.

Should weight variations be considered more important, yet sheets be so used that uniform thickness is desirable, they should be ordered by weight gage, and order should stipulate "Make of as nearly uniform thickness as practicable." Sheets so ordered will be of less average thickness than those ordered by the equivalent thickness.

Both resquared and nonresquared sheets, by custom of long standing, are subject to these weight gage tolerances, based upon the ordered area; though, logically, nonresquared sheets should have smaller under and greater over tolerances.

\section{GAGE THICKNESS}

When ordered by thickness, sheets shall conform to Table 2.

TABLE 2

\begin{tabular}{|c|c|c|c|}
\hline $\begin{array}{l}\text { Ordered } \\
\text { thickness }\end{array}$ & $\begin{array}{l}\text { Permis- } \\
\text { sible } \\
\text { variation } \\
\text { from } \\
\text { ordered } \\
\text { thick- } \\
\text { ness }\end{array}$ & $\begin{array}{l}\text { Ordered } \\
\text { thickness }\end{array}$ & $\begin{array}{l}\text { Permis- } \\
\text { sible } \\
\text { variation } \\
\text { from } \\
\text { ordered } \\
\text { thick- } \\
\text { ness }\end{array}$ \\
\hline $\begin{array}{c}\text { Inch } \\
\text { Over } 0.249 \\
0.249-.220 \\
.219-.190 \\
189-.160 \\
.159-.140 \\
\\
.139-.120 \\
.119-.100\end{array}$ & $\begin{array}{l}\text { Inch } \\
0.016 \\
.015 \\
.014 \\
.013 \\
.012 \\
\\
.011 \\
.010\end{array}$ & $\begin{array}{c}\text { Inch } \\
0.099-0.080 \\
.079-.070 \\
.069-.060 \\
.059-.050 \\
.049-.040 \\
.039-.030 \\
.029\end{array}$ & $\begin{array}{l}\text { Inch } \\
0.009 \\
.008 \\
.007 \\
\\
.006 \\
.005 \\
.004 \\
.003\end{array}$ \\
\hline
\end{tabular}

The thickness variation range in any one sheet shall not exceed (to the nearest thousandth) one and one-fifth times the tabular limit for the ordered thickness.

If minimum or maximum thickness be ordered, the tolerance used shall be the one for the mean thickness determined by that tolerance, and double that tolerance shall be allowed on the permissible side. Ordering by minimum or maximum thickness is objectionable, as it is liable to cause error in manufacture and gage classification.

Sheets are not all gaged at the mill and the limits shown can not be assured, but any sheets found by purchaser that are outside the limits may be rejected except in extreme widths.

Sheets ordered by thickness shall be subject to thickness gage tolerances only, and not to weight gage tolerances.

Should weight variations be more important than thickness variations, but sheets be ordered by thickness by preference or custom, order should stipulate "Make of equivalent weight per unit area." In such case, sheets shall be subject to weight gage tolerances only, based on 40.80 pounds per square foot per inch thick.

\section{Gage Thickness-Expected Average and Limiting Weights}

While sheets ordered by thickness are not subject to weight gage tolerances, weights of such sheets are affected by the following considerations:

Weight of sheets ordered by thickness should be computed on the basis of the ordered size and ordered thickness at 40.80 pounds per square foot per inch thick, to which should be added 2 per cent 
for greater cross section of the sheet than is represented by the mean of the thickest and thinnest points.

When resquared, no adjustment should be made to the weight so computed (41.62 pounds per square foot per inch thick).

When neither resquared nor cold-rolled after annealing (blue-annealed, black, one pass, hot-rolled pickled), 1.5 per cent should be added to account for excess width and length within the standard tolerance limits (42.24 pounds per square foot per inch thick).

When not resquared but cold-rolled after annealing (full cold-rolled, pickled, and cold-rolled), 3 per cent should be added to account for excess width and length within the standard tolerance limits (42.86 pounds per square foot per inch thick).

Estimated weights on orders and in records and correspondence should be computed as shown above.

The weight computed on the proper basis with proper adjustments as explained, is, of course, affected by the usual permissible variation of 5 per cent for not lighter than No. 16 gage, of 3.5 per cent for lighter than No. 16 but not lighter than No. 22 gage, and of 2.5 per cent for lighter than No. 22 gage, as applied to all of one gage and size in a shipment.

These considerations are expressed in tabular form in Table 3.

\section{TABLE 3.-Gage thickness-Expected average and limiting weights}

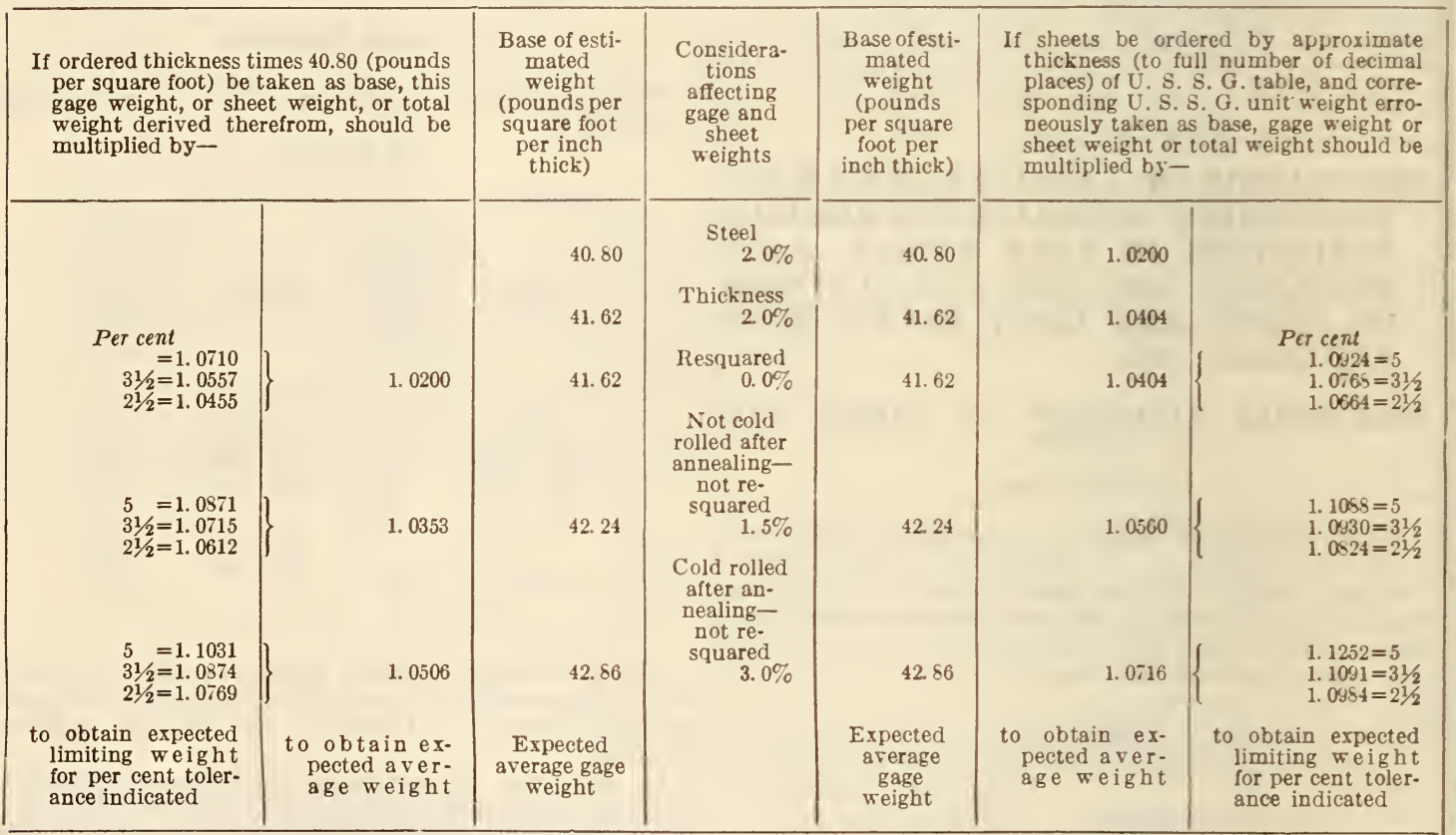

SIZE

Sheets shall be not less than widths and lengths ordered.

Width of sheets not cold rolled (blue-annealed, hot-rolled pickled) shall not exceed that ordered by more than-

One-fourth inch if ordered 48 inches wide and less and 144 inches long and less.

Five-sixteenth inch if ordered 48 inches wide and less and more han 144 inches long.

Three-eighths inch if ordered more than 48 inches wide and 144 inches long and less.

Seven-sixteenths inch if ordered more than 48 inches wide and more than 144 inches long.

Width of sheets cold rolled shall not exceed that ordered by more than-

One-fourth inch if ordered 36 inches wide and less and 120 inches long and less.

Five-sixteenth inch if ordered 36 inches wide and less and more than 120 inches long.

Three-eighths inch if ordered more than 36 inches wide and 120 inches long and less.

Seven-sixteenth inch if ordered more than 36 inches wide and more than 120 inches long.

Length of sheets not cold rolled (blue annealed, hot-rolled pickled) shall not exceed that ordered by more than-

One-half inch if ordered 120 inches long and less.

Three-fourths inch if ordered more than 120 inches long to 180 inches, inclusive. sire

One and one-fourth inches if ordered more than 240 inches long to 300 inches, inclusive.

One and one-half inches if ordered more than 300 inches long.

Length of sheets cold rolled before annealing (black, one pass, light gage pickled) shall not exceed that ordered by more than-

Three-fourths inch if ordered 96 inches long and less.

One inch if ordered more than 96 inches long to 120 inches, inclusire. One and one-fourth inches if ordered more than 120 inches long to 144 inches, inclusire.

One and one-half inches if ordered more than 144 inches long to 165 inches, inclusive.

One and three-fourths inches if ordered more than 168 inches long.

Length of sheets cold rolled after annealing (full cold rolled, pickled and cold rolled) shall not exceed that ordered by more than 2 per cent of ordered length.

Out-of-square of sheets not cold rolled shall not be more than one-sixteenth inch per 6 inches, or fraction thereof, of sheet width, along end edge.

Out-of-square of sheets cold rolled shall not be more than one-sixteenth inch per 4 inches, or fraction thereof, of sheet width, along end edge.

Total out-of-square is the greatest deviation of an end edge from a straight line at right angle to a side and touching one corner.

Camber shall not exceed the tolerances given in Table 4. 
TABLE 4

\begin{tabular}{|c|c|c|c|c|c|}
\hline \multicolumn{2}{|c|}{$\begin{array}{l}\text { Sheets not cold } \\
\text { rolled, ordered } \\
\text { length }\end{array}$} & \multirow{2}{*}{$\begin{array}{c}\text { Camber } \\
\text { Not } \\
\text { more } \\
\text { than- }\end{array}$} & \multicolumn{2}{|c|}{$\begin{array}{l}\text { Sheets cold } \\
\text { rolled, ordered } \\
\text { length }\end{array}$} & \multirow{2}{*}{ 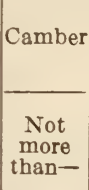 } \\
\hline $\begin{array}{l}\text { More } \\
\text { than- }\end{array}$ & $\begin{array}{l}\text { Not } \\
\text { more } \\
\text { than- }\end{array}$ & & $\begin{array}{l}\text { More } \\
\text { than- }\end{array}$ & $\begin{array}{c}\text { Not } \\
\text { more } \\
\text { than- }\end{array}$ & \\
\hline $\begin{array}{c}\text { Inches } \\
144 \\
180 \\
216 \\
240 \\
264 \\
288\end{array}$ & $\begin{array}{c}\text { Inches } \\
144 \\
180 \\
216 \\
240 \\
264 \\
288\end{array}$ & $\begin{array}{c}\text { Inch } \\
1 / 4 \\
3 / 8 \\
1 / 2 \\
5 / 8 \\
3 / 4 \\
7 / 8 \\
1\end{array}$ & $\begin{array}{c}\text { Inches } \\
72 \\
96 \\
120 \\
144 \\
156 \\
168\end{array}$ & $\begin{array}{c}\text { Inches } \\
72 \\
96 \\
120 \\
144 \\
156 \\
168\end{array}$ & $\begin{array}{c}\text { Inch } \\
1 / 4 \\
3 / 8 \\
1 / 2 \\
5 / 8 \\
3 / 4 \\
7 / 8 \\
1\end{array}$ \\
\hline
\end{tabular}

Camber is the greatest deviation of a side edge from a straight line touching both ends of the side.

Sheets required of accurate size shall be ordered "resquared." Such sheets shall not be in excess of ordered width and length by more than one-sixteenth inch and shall not be out of square or cambered by more than one-sixteenth inch, if not more than 48 inches wide and 120 inches long. For wider and longer sheets, the tolerance shall be one-eighth inch in each respect. For widths 12 inches and less and for all circle diameters, excess tolerance shall be one-eighth inch.

Resquaring excess allowance for sheets not stretcher-leveled (patent-leveled) shall be oneeighth for each intended cut, in addition to the foregoing tolerances.

Resquaring excess allowance for sheets stretcherleveled (patent-leveled) shall be in accordance with Table 5 .

TABLE 5

\begin{tabular}{|c|c|c|c|}
\hline \multicolumn{2}{|c|}{ Ordered length } & \multirow{2}{*}{$\begin{array}{l}\text { Width } \\
\text { excess } \\
\text { not } \\
\text { more } \\
\text { than- }\end{array}$} & \multirow[b]{2}{*}{ Length } \\
\hline $\begin{array}{c}\text { More } \\
\text { than- }\end{array}$ & $\begin{array}{c}\text { Not } \\
\text { more } \\
\text { than- }\end{array}$ & & \\
\hline $\begin{array}{l}\text { Inches } \\
120 \\
156 \\
204\end{array}$ & $\begin{array}{r}\text { Inches } \\
120 \\
156 \\
204 \\
-\end{array}$ & \begin{tabular}{c|} 
Inches \\
1 \\
$11 / 4$ \\
$11 / 4$ \\
$11 / 2$
\end{tabular} & $\left\{\begin{array}{l}\text { Not less than ordered between grip marks, } \\
\text { and not more than } 6 \text { inches in excess. } \\
\text { If "No allowance for grip marks" be } \\
\text { ordered, excess shall be not more than } \\
3 \text { inches for lengths not over } 120 \text { inches, } \\
\text { and } 4 \text { inches for longer sheets. }\end{array}\right.$ \\
\hline
\end{tabular}

In addition, one-eighth inch for each additional intended cut.

\section{FLATNESS}

Sheets not ordered stretcher-leveled (patentleveled) shall not have buckles exceeding the limits shown in Table 6.

TABLE 6

\begin{tabular}{|c|c|c|c|c|}
\hline \multicolumn{2}{|c|}{ Ordered gage } & \multicolumn{2}{|c|}{ Ordered width } & \multirow{2}{*}{$\begin{array}{l}\text { Maxi- } \\
\text { mum } \\
\text { height } \\
\text { of } \\
\text { buckles }\end{array}$} \\
\hline Less than- & Not less than- & $\begin{array}{l}\text { More } \\
\text { than- }\end{array}$ & $\begin{array}{c}\text { Not } \\
\text { more } \\
\text { than- }\end{array}$ & \\
\hline & $\begin{array}{l}80 \text { ounces } \\
5 \text { pounds.-. } \\
0.123 \text { inch } \\
\text { No. } 11 \text { U. S. S. G.- }\end{array}$ & $\begin{array}{r}\text { Inches } \\
48 \\
60 \\
72\end{array}$ & $\begin{array}{r}\text { Inches } \\
48 \\
60 \\
72\end{array}$ & $\begin{array}{r}\text { Inches } \\
5 / 8 \\
3 / 4 \\
1 \\
11 / 4\end{array}$ \\
\hline $\begin{array}{l}80 \text { ounces } \\
5 \text { pounds... } \\
0.123 \text { inch } \\
\text { No. } 11 \text { U. S. S. G. }\end{array}$ & $\begin{array}{l}40 \text { ounces } \\
21 / 2 \text { pounds } \\
0.061 \text { inch } \\
\text { No. } 16 \text { U. S. S. G.. }\end{array}$ & $\begin{array}{l}-48 \\
60 \\
72\end{array}$ & $\begin{array}{l}48 \\
60 \\
72\end{array}$ & $\begin{array}{l}3 / 4 \\
1^{3 / 4} \\
11 / 4 \\
11 / 2\end{array}$ \\
\hline $\begin{array}{l}40 \text { ounces... } \\
21 / 2 \text { pounds } \\
0.061 \text { inch } \\
\text { No. } 16 \text { U. S. S. G. }\end{array}$ & & $\begin{array}{l}36 \\
48 \\
60\end{array}$ & $\begin{array}{l}36 \\
48 \\
60\end{array}$ & $\begin{array}{l}1^{3 / 4} \\
11 / 4 \\
11 / 2\end{array}$ \\
\hline
\end{tabular}

Height of buckles is the maximum rise from a flat surface on which the sheet lies.

Sheets ordered stretcher-leveled (patent-leveled) shall not have buckles exceeding one-fourth inch in height. This standard of flatness can not be assured in sheets of less than 20 ounces per square foot (1.25 pounds per square foot, No. 22 U. S. S. G., 0.030 inch).

These height limits are for not more than two buckles in a side or in the middle. If more, the tolerance shall be proportionately less, but an approach to corrugation shall not be permitted.

\section{ORES, PIG IRON, AND SCRAP.}

\subsection{IRON ORE.}

\section{UNITED STATES GOVERNMENT, DEPART-}

MENT OF COMMERCE, BUREAU OF STANDARDS, STANDARD SAMPLES, CIRCULAR NO. 25 AND SUPPLEMENT, 1927

\section{IRON ORE SAMPLES}

(The Bureau of Standards prepares and sells standard samples of iron ores and other materials, for use by industrial organizations and others, as comparison standards for checking the accuracy of analysis of iron ores, etc. Each sample is accompanied by a certificate giving the composition of that particular sample as regards certain important constituents. Sample No. 25b is manganese ore, sample No. 26 is crescent iron ore, sample No. $27 \mathrm{a}$ is Sibley iron ore, sample No. 28 is Norrie iron ore, and sample No. 29 is magnetite iron ore (titaniferous).)

\section{UNITED STATES GOVERNMENT, DEPART- MENT OF COMMERCE, BUREAU OF STANDARDS, ANALYZED IRON AND MAN- GANESE ORES-METHODS OF ANALY- SIS, CIRCULAR N0. 26, APRIL 5, 1921}

\section{METHODS OF ANALYSIS OF IRON AND MAN- GANESE ORES}

(The methods of analysis described in Circular No. 26 are methods which have been found satisfactory at the Bureau of Standards and which may be used in the analysis of standard iron-ore samples described in Circular No. 25 above.)

601.2 PIG IRON.

601.20 GENERAL ITEMS.

AMERICAN SOCIETY FOR TESTING MATERIALS, STANDARD METHODS OF SAMPLING AND CHEMICAL ANALYSIS OF PIG AND CAST IRON, SERIAL DESIG. NATION A64-2\%, $192 \%$

\section{SAMPLING AND CHEMICAL ANALYSIS OF PIG AND CAST IRON}

\section{SAMPLING}

Pig iron.-One pig shall be selected at random from each 4 tons of iron and 10 such pigs (representing 40 tons of iron) shall constitute a unit for sampling. The surface of each pig shall be cleansed with a stiff wire brush or in any manner that will remove all loose sand without introducing deleterious matter.

The skin, down to clean metal, shall then be removed with an emery wheel at the center of the 
upper face of each pig and the surface carefully brushed off.

Drillings shall be taken with a 1/4-in. twist drill, from top to bottom of each pig, starting from the center of the cleared space and stopping when the point of the drill appears below. One hole only shall be bored in each pig.

Suitable precautions shall be taken to prevent the escape of fine particles during the drilling. To this end a disk of clean sheet metal shall be clamped upon the pig after the skin has been removed. This disk shall have a hole in its center just large enough to receive the drill. Most of the drillings should then accumulate on top of the disk and can be brushed off after the drill is withdrawn. The pig shall then be turned bottom side up over any suitable receptacle for collecting what may have remained in the drill hole.

Castings.- - In accordance with the standard specifications for gray-iron castings, A48 (see 611.11, p. 636), three test bars, 1.2 in. in diameter, shall be cast in sand from each heat at the beginning and again at the end of the pouring.

One bar from each set having been broken, one end of each next to the fracture shall be thoroughly cleaned and the outer skin removed for a sufficient distance from the fracture and down to clean metal. Chips shall then be taken by means of a lathe or milling machine across the whole face of the bar and uncil not less than $100 \mathrm{~g}$ has been collected. The same amount shall be taken from each bar. The bar shall be so clamped as to permit the attachment or use of any suitable device for collecting every part of the sample and the machine shall be run slowly enough to reduce to a minimum the danger of loss of fine particles.

Subsequent treatment of sample from both pig and castings. - In the determination of total and graphitic carbon, and in the case of check analyses on the other constituents, the following precautions shall be taken: The entire unit shall be weighed and then sifted on No. 80 (177 micron) (and if need be No. 120 (125 micron)) tight-fitting sieves (with cover).43 The finer sieve need be used only in case the particles passing the coarse sieve are not sufficiently uniform in size and shape to meet the requirements of the treatment that follows.

The two (or three) portions so obtained shall be separately weighed. Each one shall then be thoroughly mixed without any loss of material and divided by weight into two (or three) exactly equal portions, each of which shall be placed in a clean, glass-stoppered bottle or other suitable receptacle and appropriately labeled. Of the three sets of the subdivided samples, one shall be retained by the works, one sent to the purchaser's chemist, and the third reserved.

Before weighing out for analysis, the contents of each bottle shall be thoroughly mixed, and each portion used for analysis shall be made up of the two (or three) partial samples in the same proportion which they bear to the gross sample. Thus, if the gross sample, weighing, say, $500 \mathrm{~g}$, has been separated into portions weighing 400,80 , and $20 \mathrm{~g}$, the amounts of each that must be weighed to yield a $2 \mathrm{~g}$ portion for analysis are $1.6 \mathrm{~g}, 0.32 \mathrm{~g}$, and $0.08 \mathrm{~g}$, respectively.

As a general rule the very fine material is richest in carbon, the coarse material next, while the intermediate sizes are the poorest. For routine analyses, the aim should be to obtain the iron in as small particles as possible, as for example, by milling. In such case, the samples for graphite and carbon may be taken by forming a cone, flattening it out, and taking small dabs with a spatula from at least ten

${ }^{13}$ For detailed specifications for these sieves see E11 (645.31, p. 1226). representative positions for each weighed portion. Less care is needed in taking portions for the determinations of the other constituents, grab samples from the container after gentle tumbling is satisfactory.

\section{METHODS OF CHEMICAL ANALYSIS}

In all of the following methods it is suggested that the analyst check the methods and his technique against Bureau of Standards standard samples of cast iron, or irons that have been standardized in comparison with these, except that in the case of analyses on the iron of chilled car wheels, the method and technique shall be checked against standard analyzed samples of wheel iron prepared by the Association of Manufacturers of Chilled Car Wheels.

Premissible variations in analyses of cast iron.The following tabulation shows the variations that may occur between the results obtained by two good laboratories in the analysis of a proper sample of cast iron by the umpire method:

Total carbon Per cent (0.0. .

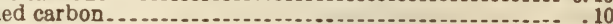
Manganese

Phosphorus, alkalimetric or gravimetric $0.003+(0.02 \times$ per cent phosphorus found)

Sulphur, gra rimetric...... 005 Sulphur, evolution. . . .

Determination of Total Carbon by the Direct-Combustion Method

The method of direct combustion of the metal in oxygen is recommended, the carbon dioxide obtained being absorbed in soda-asbestos with suitable purifying and protecting trains following the furnace. Owing to the diversity of apparatus by which correct results may be obtained in the determination of carbon, the recommendations are intended more to indicate what is acceptable than to prescribe definitely what shall be used.

\section{Apparatus and Reagents}

Material for lining boats.-Alundum, "RR alundum, alkali free, specially prepared for carbon determination," as supplied by dealers, is suitable and is recommended. The 90 -mesh or finer grades are used. Low-silica chrome ore, properly sized and freed from materials causing a blank, may also be employed. Freedom from alkali or alkaline earth metals, or carbon as carbonates or in other form, must be established before the above compounds are used as a lining material. Quartz sand owing to its liability to fuse or to slag with the oxides of iron, causing bubbles of gas to be inclosed, is objectionable. Aluminum oxide, made by calcining alum or otherwise, often contains sulphate not easily destroyed, or may contain objectionable substances of an alkaline nature.

Catalyzers. - Suitable catalyzers are asbestos, copper oxide, platinized quartz or asbestos, or platinum gauze. One of these should be used in the forward part of the combustion apparatus, as well as in the preheater preceding the combustion tube. (See below.) Platinized materials sometimes give off volatile substances on heating, and whatever material is used should not be subject to this defect.

Accelerators.-Millings of ingot iron or other iron with 0.01 to 0.02 per cent of carbon are the most satisfactory aids in the combustion of large drillings.

14 For example, with iron containing 3 per cent carbon, this represents 0.08 per cent.

45 This will vary, depending on whether or not the sample is annealed before test, or the manner in which the titer of the solution is obtained. 
Combustion apparatus.-Any apparatus heated by electricity or gas which will bring the sample to a temperature of $950^{\circ}$ to $1,100^{\circ} \mathrm{C}$. may be used. Combustion tubes may be porcelain, glazed on one or both sides, quartz, or platinum. Quarts is liable to devitrification when used continuously at temperatures above $1,000^{\circ}$ C., and may then become porous. Tubes 30 inches long and $1 \frac{1}{4}$ inches inside diameter drawn out at one end are convenient. This length permits at least 12 inches of the exit end to project from the furnace, the drawn-out end allows direct connection with the absorber and the large inner diameter accommodates larger boats and small Gooch crucibles as in the determination of graphite.

Boats or other containers of samples being burned.These may be porcelain, quartz, alundum, clay, platinum, or nickel, and should always receive a lining of granular alundum or any other material found to be suitable for the purpose. Nickel boats should not be made of sheet nickel containing more than 0.15 per cent of carbon. Before use, new boats should always be preheated in oxygen until no more carbon dioxide is given off. In order to prevent spattering and attack of the tube, a platinum or nickel cover, open at both ends and allowing free access of oxygen, is desirable.

Purifying train before combustion apparatus.-This consists of a tower filled with soda asbestos, soda lime, or granular sodium hydroxide preceded by a preheater when necessary.

Oxygen.-Oxygen of not less than 97 per cent purity is recommended. Endeavor should be made to obtain oxygen which gives no blank, since the correction for or elimination of this is troublesome and uncertain. For the most accurate work, particularly with low-carbon products, such as ingot iron, etc., the blank should be completely eliminated by the use of a preheater before the furnace, with a carbon dioxide absorbent interposed between furnace and preheater.

\section{Factors Influencing Rapid Combustion}

Size of particles of sample.-The finer the chips (short of dust, which causes low values on a hot boat) the better, except with samples which burn too vigorously. (See Rate of Admitting Oxygen.) Particles too coarse to pass a No. 20 ( 840 micron) sieve are not recommended. Oil, dust, and other foreign matter should be carefully excluded.

Manner of distributing sample in boat.-This is of considerable importance. With all samples, close packing in a small space is conducive to rapid combustion. In the case of samples which burn too vigorously, a satisfactory regulation may sometimes be attained by spreading the sample loosely over the lining in the boat.

Rate of admitting oxygen. - The rate at which oxygen is admitted is also a factor in the velocity of combustion. Assuming the combustion apparatus to be heated to the temperature range above recommended $\left(950^{\circ}\right.$ to $1,100^{\circ} \mathrm{C}$.), it is possible, if the material is closely packed and if oxygen is admitted at too rapid a rate, that the combustion may be so violent as to cause excessive spattering of fused oxides, and such fluidity of the molten slag that the boat or other container may be injured or destroyed; therefore, a moderate rate of burning is to be sought. Sufficient oxygen, however, must be run in to insure a current of gas through the absorber at all stages of the combustion. The factors, temperature of combustion apparatus, manner of distribution of sample, and rate of admission of oxygen, can be governed so as to burn successfully irons of a very wide range of compositions in either fine or coarse particles.
Determination of Total Carbon (Carbon Dioxide Absorbed in SodaAs bestos)

\section{Special Apparatus}

Purifying and protecting trains.-This method requires a purifying train before and after the combustion furnace.

The purifying train before the furnace, or between the furnace and preheater, if the latter is necessary for the purpose of oxidizing organic matter in the oxygen, may be a calcium chloride tower filled with soda asbestos, soda lime, or granular sodium hydroxide.

The purifying train after the furnace should be so designed that it will accomplish the following: (1) Remove impalpable oxide of iron which readily passes through loosely packed plugs of glass wool or asbestos or even solutions through which the oxygen is rapidly bubbled; (2) remove oxides of sulphur which are always formed; (3) dry the gases before entering the weighed carbon dioxide absorber; (4) dry the gases issuing from this absorber to the same extent as when they entered; in other words, the desiccant in the weighed absorber must be of the same kind as that which precedes it; and (5) protect the weighed absorber from outside effects.

Absorbing bulbs.-No special types are recommended, although the Fleming, Miller, and Midvale (sometimes called Stetzer and Norton) bulbs have proved satisfactory. When filled, the tubes should not weigh over $200 \mathrm{~g}$ and they should always be weighed filled with oxygen and against a like counterpoise. Open bulbs such as the Midvale lose oxygen by diffusion. They should be filled with oxygen before weighing when not in continuous use, and the same time interval shall be held between weighings.

Absorbents. - The most desirable absorbent is soda asbestos. The use of soda lime is not recommended unless the reagent has been carefully tested and found to be satisfactory. Pulverized sodium hydroxide may be used.

General arrangement of apparatus.-Figure 50 presents a typical arrangement in works' laboratories. Combustion tubes as large as 30 inches in length

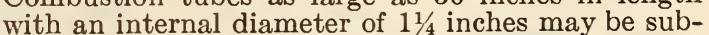
stituted if desired. For work of the highest accuracy the issuing gases in this train would be bubbled through concentrated sulphuric acid saturated with chromic acid before passing through the phosphorus pentoxide tube, and the upper chamber of the Fleming bulb would be filled with phosphorus pentoxide. "Dehydrite" (magnesium perchlorate trihydrate) may be substituted for phosphorus pentoxide and is more convenient to use.

\section{Method}

After having properly set up and tested the apparatus, moderately pack the desired weight of iron (in the form recommended above) on the bed material in the boat and introduce the boat into the combustion apparatus, which has already been heated to the proper temperature. Allow the sample to heat up for one to three minutes, depending upon the size of particles, and then admit the gas at such a rate that the gases leave the absorbents at the rate of 200 to $400 \mathrm{c}$ c per minute. The sample burns completely in one or two minutes, and all that is now necessary is to sweep all of the carbon dioxide into the absorption bulb. Regulate the rate of flow of the oxygen between 200 and 400 c c per minute and continue the flow for 10 minutes. Withdraw the absorption tube while filled with oxygen, place in the balance case for 10 minutes, open momentarily, and weigh against a like tube used as a counterpoise. The increase in weight represents 
carbon dioxide which when multiplied by 27.27 and divided by the weight of the sample represents the percentage of total carbon in the iron. Remove the boat from the tube and examine the fusion for evidences of incomplete combustion. If the drillings are not thoroughly fused in a solid pig the determination must be rejected.

Notes.-1. Oxides of sulphur cause positive errors when no provision is made for their removal. Sulphur trioxide can be almost entirely removed by interposing some baffle which condenses it, such as asbestos, phosphorus pentoxide, or sulphuric acid. Sulphur dioxide is not so easily removed; the most satisfactory medium is concentrated $\mathrm{H}_{2} \mathrm{SO}_{4}$ saturated with chromic acid.

2. The oxides of sulphur which are fixed and accumulate in the end of the combustion tube should be periodically removed by heating the end of the tube in the hot zone of the furnace.

3 . In accurate work it is desirable to include in the weighed system a dehydrating agent which will dry the escaping gas to the same degree as when it entered the system. The loss of water from a good grade of soda asbestos may cause losses higher than 0.01 per
Ignited asbestos.-Gently ignite prepared asbestos under oxidizing conditions until all carbonaceous matter has been consumed. Prepared asbestos is asbestos that has been digested with $\mathrm{HCl}$ and then thoroughly washed with water.

Potassium hydroxide solution (specific gravily approximately 1.10).-Dissolve $120 \mathrm{~g}$ of stick $\mathrm{KOH}$ in $1,000 \mathrm{c}$ c of water.

Dilute hydrochloric acid (1:20).-Mix 50 c c of $\mathrm{HCl}$ (specific gravity 1.19) and 1,000 c c of water.

\section{Method}

Dissolve 1 to $3 \mathrm{~g}$ of the sample in $50 \mathrm{c}$ c of $\mathrm{HNO}_{3}$ (specific gravity 1.20) and heat gently on the steam bath until action ceases. If silicic acid separates, add $5 \mathrm{c} \mathrm{c}$ of $\mathrm{HF}$ to facilitate filtration. Collect the residue conveniently on ignited asbestos by the use

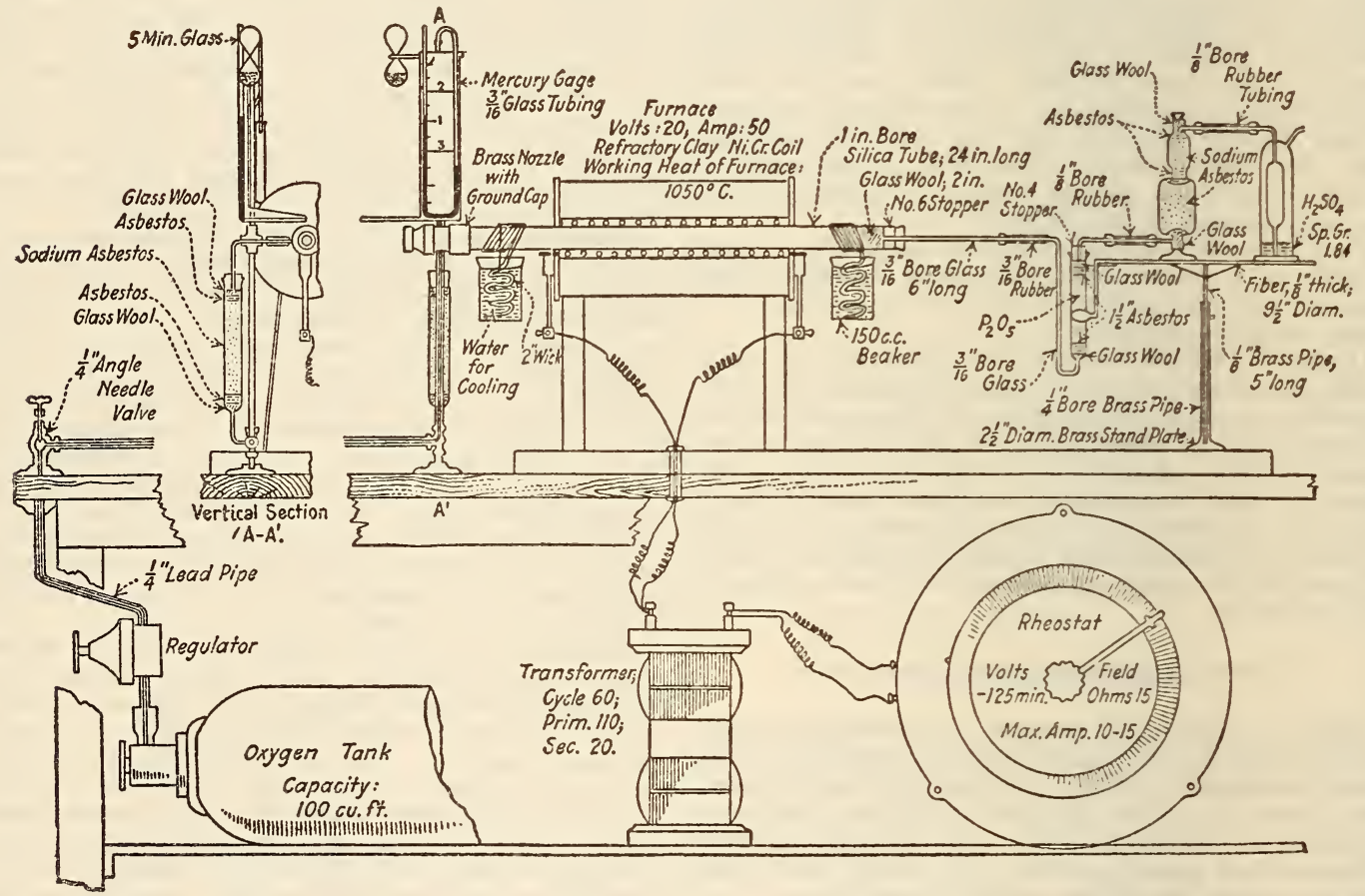

FIGURE 50.-Apparatus for determination of carbon by the direct-combustion method

cent carbon in ordinary combustions. In c $a$ reful work it is also desirable to protect the weighed system from moisture and carbon dioxide by means of suitable end tubes.

4. Soda asbestos is commercially known as "Ascarite," and directions for its preparation are to be found in J. Ind. Eng. Chem., vol. 8 , pp. 1038-9, 1916. For the use of granular sodium hydroxide see J. Ind. Eng. Chem., vol. 13, $p_{i} 1052,1921$.

5 . In some laboratories the absorbing bulbs are always kept in the balance case. In this case the balances are placed on the same table with the furnaces and each balance usually contains two absorbing bulbs which are connected by short lengths of rubber tubing to glass tubes extending out of the balance and connecting with a pair of tubes exten
furnaces.

6. The operator should occasionally test the apparatus and his technique by means of the irons noted in the introductory paragraph or irons which have been standardized in comparison with these.

(For the alternative method of absorbing carbon dioxide - carbon dioxide absorbed in barium hydroxide solution-see A33 (600.1, p. 10).)

\section{Determination of Graphite}

\section{Solutions Required}

Dilute nitric acid (specific gravity approximately 1.20).- Mix $380 \mathrm{c}$ c of $\mathrm{HNO}_{3}$ (specific gravity 1.42) and $620 \mathrm{c} \mathrm{c}$ of distilled water. of suction and fused-silica Gooch crucibles of such diameter as will go in the combustion tube. Wash the residue thoroughly with hot water, then with a hot solution of $\mathrm{KOH}$ (specific gravity 1.10 ), then with dilute $\mathrm{HCl}(1: 20)$ and finally with hot water. Dry at a temperature not to exceed $150^{\circ} \mathrm{C}$. and determine the graphite by direct combustion in the apparatus used for the determination of total carbon, taking care to close the combustion tube immediately after inserting the sample.

It is preferable to run the combustion at approximately $700^{\circ} \mathrm{C}$. An oxidant, such as oxidized copper gauze, should be provided in the hot exit end of the tube to insure complete combustion to carbon dioxide.

\section{Determination of Combined Carbon}

Direct determinations of combined carbon in cast iron are not satisfactory and, therefore, for the present it is recommended that the percentage of combined carbon be obtained by subtracting the percentage of graphite from the percentage of total carbon. 
Determination of Manganese by the Bismuthate Method

(REFEREE METHOD IN THE ABSENCE OF COBAIT)

Solutions and Reagents Required

Dilute nitric acid (1.3).-Mix 500 c c of $\mathrm{HNO}_{3}$ (specific gravity 1.42) and $1,500 \mathrm{c}$ c of distilled water.

Dilute nitric acid (3.97).-Mix 30 c c of $\mathrm{HNO}_{3}$ (specific gravity 1.42) (free from oxides of nitrogen) and $970 \mathrm{c} \mathrm{c}$ of distilled water.

Asbestos.-The asbestos for this work should be shredded, digested with $\mathrm{HCl}$, thoroughly washed with water, then digested on the steam bath with dilute $\mathrm{HNO}_{3}(1: 3)$, and finally thoroughly washed with water to remove chlorides and to oxidize any ferrous iron contained in the asbestos. In making the asbestos filter-pad it is advisable to have a thin bed and as much surface as possible. This insures rapid filtration, and the filter can be used until it becomes clogged with bismuthate.

Bismuthate. - The sodium bismuthate should contain at least 70 per cent of $\mathrm{NaBiO}_{3}$ or 4 per cent of active oxygen and not more than 0.005 per cent of manganese. Each bottle of bismuthate should be tested for strength and manganese contents. Its strength can be determined as follows: Weigh accurately about $3 \mathrm{~g}$ of the bismuthate, mix in a 100 c c graduated flask with a solution of $20 \mathrm{~g}$ of $\mathrm{KI}$ in $30 \mathrm{c}$ c of water and shake well. Add $20 \mathrm{c} \mathrm{c}$ of $\mathrm{HCl}$ (specific gravity 1.19), stopper the flask, allow to stand for one hour in the dark, and dilute to the mark. Mix and titrate $10 \mathrm{c}$ c of the solution with $0.1 N$ sodium thiosulphate, using starch as an indicator. One cubic centimeter of $0.1 N$ thiosulphate equals $0.0008 \mathrm{~g}$ of active oxygen. Determine the manganese content by boiling $3 \mathrm{~g}$ of bismuthate with $30 \mathrm{c}$ c of dilute $\mathrm{HNO}_{3}(1: 3)$ and matching the color by adding standard permanganate solution to a like amount of acid.

Ferrous ammonium sulphate solution.-Dissolve 12 $\mathrm{g}$ of ferrous ammonium sulphate in a cold mixture of $950 \mathrm{c}$ c of distilled water and $50 \mathrm{c}$ c of $\mathrm{H}_{2} \mathrm{SO}_{4}$ (specific gravity 1.84).

Standard potassium permanganate solution.-Dissolve $1 \mathrm{~g}$ of $\mathrm{KMnO}_{4}$ in $1,000 \mathrm{c}$ c of distilled water. Allow it to stand for at least one week and then filter through purified asbestos. Standardize against the Bureau of Standards standard sodium oxalate as follows: In a $200 \mathrm{c}$ c beaker, dissolve $0.1 \mathrm{~g}$ of sodium oxalate in 75 to $100 \mathrm{c} \mathrm{c}$ of hot water $\left(80^{\circ}\right.$ to $90^{\circ} \mathrm{C}$.) and add $5 \mathrm{c} \mathrm{c}$ of $\mathrm{H}_{2} \mathrm{SO}_{4}(1: 1)$. Titrate at once with the permanganate solution, stirring the liquid vigorously and continuously. The permanganate must not be added more quickly than 10 to $15 \mathrm{c}$ c per minute, and the last 0.5 to $1 \mathrm{c} \mathrm{c}$ must be added dropwise, with particular care to allow each drop to be fully decolorized before the next is introduced. The excess of permanganate used to cause an end-point color must be estimated by matching the color in another beaker containing the same bulk of acid and hot water. The temperature of the solution should not be below $60^{\circ} \mathrm{C}$. by the time the end point is reacned.

Standard sodium arsenite solution.-Dissolve $2 \mathrm{~g}$ of sodium arsenite in distilled water, filter if necessary, and dilute to 1 liter. Standardize against the standard permanganate solution by titrating $10 \mathrm{c} \mathrm{c}$ portions under the acidity and dilution conditions that obtain in the method. A clear green color free from brownish or purplish tints will be found a satisfactory and reproducible end point. The solution can also be standardized rgainst a standard cast iron or steel.

\section{Method}

Dissolve $1.000 \mathrm{~g}$ of the sample in $25 \mathrm{c} \mathrm{c}$ of dilute $\mathrm{HNO}_{3}(1: 3)$ and boil to expel oxides of nitrogen.
Cool, filter, and wash with 25 to $50 \mathrm{c}$ c of dilute $\mathrm{HNO}_{3}(1: 3)$. Add $0.5 \mathrm{~g}$ of sodium bismuthate to the filtrate, heat to boiling, and boil for at least three minutes. Clear the solution if permanganic acid or manganese dioxide is present by adding a few drops of a saturated solution of sulphur dioxide or other suitable reducing agent free from manganese and chlorides, and boil to expel oxides of nitrogen and sulphur. Cool to $15^{\circ}$ C., add an excess of sodium bismuthate, agitate, and let stand for a few minutes. Add $50 \mathrm{c}$ c of 3 per cent $\mathrm{HNO}_{3}$ (free from nitrous acid) and filter through asbestos. Wash with $\mathrm{HNO}_{3}$ (3 per cent) until the washings run through colorless. The filtrate must be clear and absolutely free from particles of bismuthate. Titrate by either method $(a)$ or $(b)$ as follows:

(a) Ferrous sulphate-permanganate titration.Add from a burette or pipette 10 to $50 \mathrm{c}$ c (depending on the amount of permanganic acid) of ferrous ammonium sulphate solution and then titrate with the standard $\mathrm{KMnO}_{4}$ solution. In exactly the same manner carry through a blank determination using the same amounts of acid and bismuthate as was done with the regular sample. Finally add the exact volume of ferrous ammonium sulphate solution which was employed and titrate with the standard $\mathrm{KMnO}_{4}$ solution. The difference between the volumes required in the two titrations represents the manganese in the sample.

(b) Arsenite titration.-Titrate immediately with the standard sodium arsenite solution to the clear green color used as an end point in the standardization of the solution. In this titration, permanganic acid does not react in accordance with the ratio $\mathrm{Mn}_{2} \mathrm{O}_{7}: 2 \mathrm{MnO}$. Consequently the theoretical titre of the arsenite solution can not be employed.

\section{Determination of Manganese by the Persulphate Method}

(ROUTINE AND IN THE PRESENCE OF COBALT)

\section{Solutions required}

Dilute nitric acid (specific gravity approximately 1.20).-Mix $380 \mathrm{c} \mathrm{c}$ of $\mathrm{HNO}_{3}$ (specific gravity 1.42) and $620 \mathrm{c} \mathrm{c}$ of distilled water.

Silver nitrate (0.133 per cent).-Dissolve $1.33 \mathrm{~g}$ of $\mathrm{AgNO}_{3}$ in 1,000 $\mathrm{c} \mathrm{c}$ of distilled water.

Ammonium persulphate solution (10 per cent).Prepare as needed by dissolving $10 \mathrm{~g}$ of the salt in $100 \mathrm{c} c$ of distilled water. The salt should show at least 80 per cent of persulphate by test. The pure salt ordinarily contains 95 per cent of persulphate. For method of test see solutions required in determination of small amounts of chromium, given below.

Sodium chloride (0.2 per cent).-Dissolve $2 \mathrm{~g}$ of $\mathrm{NaCl}$ in $1,000 \mathrm{c}$ c of distilled water.

Standard sodium arsenite.-Dissolve $0.8 \mathrm{~g}$ of sodium arsenite in distilled water, filter if necessary; dilute to 1 liter and standardize against measured portions of a standard permanganate solution which have been reduced by hydrogen peroxide or ferrous sulphate, that is free from manganese and then treated as in the method to be described, or against standard samples of iron.

\section{Method}

In a small Erlenmeyer flask or large test tube (8 by 1 inches) dissolve 0.1 to $0.3 \mathrm{~g}$ of iron, depending on the manganese content of the sample, in $15 \mathrm{c} \mathrm{c}$ of dilute $\mathrm{HNO}_{3}$ (specific gravity 1.20) and boil to expel oxides of nitrogen. Add $5 \mathrm{c}$ c of $\left(\mathrm{NH}_{4}\right)_{2} \mathrm{~S}_{2} \mathrm{O}_{8}$ solution (10 per cent) and heat several minutes to oxidize the combined carbon. Remove graphite by filtration through a small rapid paper, and wash the paper with $15 \mathrm{c}$ c of $\mathrm{AgNO}_{3}$ solution ( 0.133 per cent) and a small amount of water. If a tube 
is used, add $10 \mathrm{c}$ c of the $\left(\mathrm{NH}_{4}\right)_{2} \mathrm{~S}_{2} \mathrm{O}_{8}$ solution (10 per cent) and heat in the water bath until the color develops. If a flask is emoloyed, bring the solution to boiling, add $10 \mathrm{c}$ c of the persulphate solution and then set aside in a warm place until the color develops. When the full color has developed, cool the solution in running water, transfer to a $250 \mathrm{c} \mathrm{c}$ beaker and dilute to a volume of $75 \mathrm{c} \mathrm{c}$. Add $10 \mathrm{c} \mathrm{c}$ of $\mathrm{NaCl}$ solution ( 0.2 per cent) and titrate with standard arsenite solution.

Notes.-In the arsenite titration, permanganic acid does not react in accordance with the ratio $\mathrm{Mn}_{2} \mathrm{O}: 2 \mathrm{MnO}$, but more nearly as indicated by the ratio $\mathrm{Mn}_{2} \mathrm{O}_{7}: \mathrm{Mn}_{2} \mathrm{O}_{3}$. Consequently, the theoretical titre of the arsenite can not be used and the manganese titre must be obtained by titration of material of known manganese content and under the conditions that obtain in the method.

The persulphate oxidation requires careful attention; the solution must not be too acid and sufficient $\mathrm{AgNO}_{3}$ must be present (abou fiften times as manganese content the amount of $\mathrm{AgNO}_{3}$ and $\mathrm{NaCl}$ should be increased.

No $\mathrm{MnO}_{2}$ must be separate on heating after the first addition of persulphate, for this would be lost when the solution is filtered.

Determination of Phosphorus by Weighing as Magnesium Pyrophosphate

(REFEREE METHOD)

Solutions required

Dilute nitric acid $(1: 1)$ - Mix equal parts of $\mathrm{HNO}_{3}$ (specific gravity 1.42) and water.

Dilute hydrochloric acid (1:1).-Mix equal parts of $\mathrm{HCl}$ (specific gravity 1.19) and water.

Dilute hydrochloric acid (1:20).-Mix 50 c c of $\mathrm{HCl}$ (specific gravity 1.19) and $1,000 \mathrm{c} \mathrm{c}$ of water.

Dilute ammonium hydroxide (1:2).-Mix 300 c of $\mathrm{NH}_{4} \mathrm{OH}$ (specific gravity 0.90 ) and $600 \mathrm{c}$ c of distilled water.

Dilute ammonium hydroxide (1:20).-Mix $100 \mathrm{c} \mathrm{c}$ of $\mathrm{NH}_{4} \mathrm{OH}$ (specific gravity 0.90 ) and 2,000 c c of distilled water.

Dilute nitric acid (2:100).-Mix 20 c c $\mathrm{HNO}$ (specific gravity 1.42) and 1,000 $\mathrm{c} \mathrm{c}$ of distilled water.

(Ammonium molybdate solutions No. 1 and No. 2 and directions for handling are identical with the determination of phosphorus by the alkalimetric method in B28. See 646.0, p. 1243.)

Magnesia mixture.-Dissolve $50 \mathrm{~g}$ of $\mathrm{MgCl}_{2} \cdot 6 \mathrm{H}_{2} \mathrm{O}$ and $100 \mathrm{~g}$ of $\mathrm{NH}_{4} \mathrm{Cl}$ in $200 \mathrm{c} \mathrm{c}$ of distilled water, and then add $5 \mathrm{c}$ c of $\mathrm{NH}_{4} \mathrm{OH}$ (specific gravity 0.90 ). Let stand overnight, filter if a precipitate appears, make just acid with $\mathrm{HCl}$, and dilute to $1,000 \mathrm{c} \mathrm{c}$ with water.

\section{Method}

Dissolve 1 to $3 \mathrm{~g}$ of the sample in a covered casserole in $30 \mathrm{c}$ c of dilute $\mathrm{HNO}_{3}(1: 1)$. When solution is complete add $10 \mathrm{c} \mathrm{c}$ of dilute $\mathrm{HCl}(1: 1)$, evaporate to dryness, and bake for 15 to 20 minutes on the hot plate. Cool, drench the residue with concentrated $\mathrm{HCl}$, dilute to $50 \mathrm{c} \mathrm{c}$ with hot water, and warm until the salts are in solution. Filter without delay, wash with dilute $\mathrm{HCl}(1: 20)$, and evaporate the filtrate to sirupy consistency. In the meantime, place the paper containing graphite and silica in a platinum crucible, burn off the carbon, cool, and add 5 to 10 drops of concentrated $\mathrm{HCl}$ and 1 to $2 \mathrm{c} \mathrm{c}$ of $\mathrm{HF}$. Evaporate just to dryness, take up any residue in $5 \mathrm{c}$ c of concentrated $\mathrm{HCl}$ and add the solution to the main solution which is being evaporated. When the solution is of sirupy consistency, transfer it to a $300 \mathrm{c} \mathrm{c}$ Erlenmeyer flask by alternate washing with dilute $\mathrm{HNO}_{3}(1: 1)$ and hot water, using not more than $30 \mathrm{c} c$ of the former and $70 \mathrm{c} c$ of the latter. Heat to boiling, add $\mathrm{NH}_{4} \mathrm{OH}$ until a permanent precipitate has formed, dissolve in dilute $\mathrm{HNO}_{3}$ (1:1) and then add $100 \mathrm{c} \mathrm{c}$ of molybdate reagent. Shake for 10 minutes and allow to stand not less than six hours.

Filter on a paper of close texture, and wash the flask and the precipitate with not more than $50 \mathrm{c}$ c of cold dilute $\mathrm{HNO}_{3}(1: 99)$. Set the filtrate and washings aside after thorough mixing. If further separation of phosphomolybdate occurs in four hours recover the precipitate and determine its phosphorus content. Dissolve the precipitate on the filter and in the flask in $20 \mathrm{c}$ c of dilute $\mathrm{NH}_{4} \mathrm{OH}(1: 1)$ to which has been added $2 \mathrm{~g}$ of citric acid, catch the solution in a $250 \mathrm{c}$ c beaker, wash the filter several times with dilute $\mathrm{NH}_{4} \mathrm{OH}(1: 20)$, then with hot water, and finally several times with dilute $\mathrm{HCl}(1: 20)$. If the ammoniacal solution of the phosphomolybdate is not clear at this point, heat it to boiling, filter through the same paper, wash the paper with hot water, ignite in a small platinum crucible, fuse any residue with as little carbonate as possible (not over $0.5 \mathrm{~g}$ ), extract the cooled melt with hot water, filter, and add the water solution to the ammoniacal solution.

Render the ammoniacal solution acid with $\mathrm{HCl}$, add $20 \mathrm{c}$ c of magnesia mixture, then add $\mathrm{NH}_{4} \mathrm{OH}$ (specific gravity 0.90 ) cautiously until a crystalline precipitate appears. From this point proceed very slowly until the solution is alkaline and then add 5 cc of $\mathrm{NH}_{4} \mathrm{OH}$ in excess. The volume of the solution at this point should not exceed $100 \mathrm{c} \mathrm{c}$. Allow the solution to stand in a cool place for not less than six hours. Filter, and wash the precipitate moderately with dilute $\mathrm{NH}_{4} \mathrm{OH}(1: 19)$. Dissolve the precipitate on the filter in $25 \mathrm{c}$ c of dilute $\mathrm{HCl}$ $(1: 1)$, catching the solution in the original beaker. Wash the filter thoroughly with dilute $\mathrm{HCl}(1: 20$.) Add 0.5 to $1 \mathrm{~g}$ of ammonium bromide and boil the $\mathrm{HCl}$ solution to a volume of 5 to $10 \mathrm{c} \mathrm{c}$ (but not to dryness) to eliminate small amounts of arsenic. Precipitation with hydrogen sulphide can be substituted for the evaporation with hydrobromic acid and is desirable in case much arsenic is present. Dilute to a volume of 50 to $75 \mathrm{c} \mathrm{c}$, add 0.1 to $0.2 \mathrm{~g}$ of citric acid, 2 to $3 \mathrm{c}$ c of magnesia mixture, make ammoniacal as above, and allow to stand in a cool place for not less than four hours.

Filter, wash with dilute $\mathrm{NH}_{4} \mathrm{OH}(1: 20)$ until chlorides have been removed; ignite the precipitate carefully and at as low a temperature as possible, until the carbon has been destroyed and the residue is white. Finally, ignite at approximately $1,000^{\circ}$ C. to constant weight. Dissolve the ignited precipitate in $10 \mathrm{c}$ c of dilute $\mathrm{H}_{2} \mathrm{SO}_{4}(1: 4)$. Evaporate to fumes of sulphuric acid, dilute, filter, and wash with hot water. Ignite, weigh, treat with a few drops of $\mathrm{HF}$, evaporate to dryness, ignite, and reweigh. Subtract any loss of weight from the original weight of the pyrophosphate, and calculate on the basis of this purified magnesium pyrophosphate.

\section{Determination of phosphorus by the alkalimetric method}

(ROCTINe)

\section{Solutions required}

Dilute nitric acid (specific gravity approximately 1.135).- Mix 280 c c of $\mathrm{HNO}_{3}$ (specific gravity 1.42 ) and $720 \mathrm{c} \mathrm{c}$ of water.

Potassium permanganate (1.5 per cent).-Dissolve $15 \mathrm{~g}$ of $\mathrm{KMnO}_{4}$ in $1,000 \mathrm{c}$ c of water.

Ammonium bisulphite (3 per cent).-Dissolve 30 $\mathrm{g}$ of ammonium bisulphite in $1,000 \mathrm{c} \mathrm{c}$ of distilled water. This salt must be free from phosphorus.

Ammonium hydroxide (specific gravity 0.96).Mix $400 \mathrm{c} \mathrm{c}$ of $\mathrm{NH}_{4} \mathrm{OH}$ (Specific gravity 0.90 ) and $600 \mathrm{c} \mathrm{c}$ of water. The strength of this solution should be verified by hydrometer or titration.

(Ammonium molybdate solution, potassium nitrate (1 per cent), phenolphthalein indicator, standard sodium hydroxide, and standard nitric acidall are the same as in B28. (See 646.0, p. 1243.) 
Dissolve 0.5 to $2 \mathrm{~g}$ of the sample in $50 \mathrm{c} \mathrm{c}$ of $\mathrm{HNO}_{3}$ (specific gravity 1.135) and destroy organic matter by boiling with $10 \mathrm{c}$ c of a 1.5 per cent solution of $\mathrm{KMnO}_{4}$ until oxide of manganese is precipitated. Dissolve the oxide by addition of ammonium bisulphite, boil to expel the oxides of nitrogen, filter, and wash with 50 c c of $\mathrm{HNO}_{3}$ (specific gravity 1.135). Add to the cool solution 40 c c of $\mathrm{NH}_{4} \mathrm{OH}$ (specific gravity 0.96), 40 c c of ammonium molybdate solution, and shake for 5 minutes. Let settle and filter on a $9 \mathrm{~cm}$ No. 42 Whatman or equivalent paper which had been fitted tightly in the funnel. With irons high in vanadium or titanium the solution should be allowed to settle for two hours. Wash the flask, the precipitate, and the filter paper with $25 \mathrm{c}$ c portions of a 1 per cent solution of $\mathrm{KNO}_{3}$ until 10 c c of washings will not decolorize one drop of the standard alkali plus one drop of phenolphthalein. Return the paper and precipitate to the precipitating vessel, add a 2 to $5 \mathrm{c}$ c excess of standard $\mathrm{NaOH}$ solution and 25 cc of water, both free from carbon dioxide, and shake or stir until the precipitate is dissolved. Dilute to from 100 to $200 \mathrm{c}$ c witn water free from carbon dioxide, add three drops of 0.2 per cent phenolphthalein solution, and discharge the pink color with standard acid. Finish the titration by adding standard alkali until the reappearance of the pink color. In the analysis of low phosphorus iron this titration should be corrected by a blank carried through all steps of the method.

Note. -With irons containing ranadium in excess of 0.05 per cent, cool the solution to $10^{\circ}$ to $15^{\circ} \mathrm{C}$. and add $1 \mathrm{~g}$ of ferrous sulphate just before the addition of molybdate reagent.

Determination of Sulphur by the Gravimetric Method

(REFEREE METHOD)

Solutions required

Dilute hydrochloric acid (1:99).-Mix $10 \mathrm{c}$ c of $\mathrm{HCl}$ (specific gravity 1.19) and xxx c c of water. Barium chloride solution (10 per cent).-Dissolve $100 \mathrm{~g}$ of $\mathrm{BaCl}_{2} \cdot 2 \mathrm{H}_{2} \mathrm{O}$ in $1,000 \mathrm{c}$ c of distilled water.

Dilute sulphuric acid (1:1).-Carefully add while stirring $100 \mathrm{c} \mathrm{c}$ of $\mathrm{H}_{2} \mathrm{SO}_{4}$ (specific gravity 1.84 ) to 100 c c of water.

\section{Method}

Dissolve the sample $(4.57 \mathrm{~g})$ in $50 \mathrm{c}$ c of $\mathrm{HNO}_{3}$, (specific gravity 1.42 ) in a covered beaker or flask. Add $0.5 \mathrm{~g}$ of $\mathrm{Na}_{2} \mathrm{CO}_{3}$, evaporate to dryness and bake for one hour on the hot plate. Add $30 \mathrm{c}$ c of $\mathrm{HCl}$ (specific gravity 1.19) and repeat the evaporation and baking. Add another $30 \mathrm{c} \mathrm{c}$ of $\mathrm{HCl}$ (specific gravity 1.19) and evaporate to dryness. Add $25 \mathrm{c} \mathrm{c}$ of $\mathrm{HCl}$ (specific gravity 1.19), $25 \mathrm{c} \mathrm{c}$ of water, $5 \mathrm{~g}$ of 20 to 30 mesh zinc free from sulphur, and warm on the steam bath until the iron is reduced to ferrous chloride and the evolution of hydrogen has nearly ceased. Filter from the silica and undissolved zinc, wash with approximately $75 \mathrm{c} \mathrm{c}$ of $\mathrm{HCl}$ (1 per cent by volume), warm to $60^{\circ}$ to $80^{\circ} \mathrm{C}$., and add $10 \mathrm{c}$ c of 10 per cent barium chloride. Let stand 18 to 24 hours, filter on a paper of close texture and discard the filtrate. Wash once or twice with cold 1 per cent $\mathrm{HCl}$ and then with hot water until free from chlorides. Reserve the precipitate and evaporate the washings to dryness. Dissolve the slight residue in $1 \mathrm{c} \mathrm{c}$ of dilute $\mathrm{HCl}(1: 1)$, dilute to $25 \mathrm{c} \mathrm{c}$ with hot water, add $1 \mathrm{c} c$ of 10 per cent $\mathrm{BaCl}_{2}$ solution, and digest at the side of the steam bath for one hour. Filter on a small paper of close texture, and wash with hot water until free from chlorides. Ignite both papers in platinum and treat with one drop of $\mathrm{H}_{2} \mathrm{SO}_{4}(1: 1)$ and $1 \mathrm{c} \mathrm{c}$ of $\mathrm{HF}$. Evaporate to dryness, ignite, and weigh. A blank should be carried through all steps of the determination. The corrected weight of the ignited barium sulphate multiplied by 3 is equal to the percentage of sulphur.

Note.-The solution should preferebly contain not more than 2 per cent by volume of $\mathrm{HCl}$ at the time of the precipitation with $\mathrm{BaCl}_{2}$ Ordinarily, there will be no hydrolysis of iron during the filtration and washing of the undissolved zinc or the precipitation with $\mathrm{BaCl}_{2}$. Should this occur, the solution must be cleared by the addition of $\mathrm{HCl}$, having due regard to the final permissible acidity.

\section{Determination of Sulphur by the Gravimetric Method}

(OPTIONAL REFEREE METHOD)

\section{Solutions required}

Double chloride solution.-Dissolve $756 \mathrm{~g}$ of copper potassium chloride $\left(\mathrm{CuCl}_{2} \cdot 2 \mathrm{KCl} .2 \mathrm{H}_{2} \mathrm{O}\right)$ in $2,000 \mathrm{c} \mathrm{c}$ of water, and add $200 \mathrm{c}$ c of $\mathrm{HCl}$ (specific gravity 1.19).

\section{Method}

Transfer $4.57 \mathrm{~g}$ of the sample to an Erlenmeyer flask, treat with 250 c c of the double chloride solution, and stir gently by mechanical means until all of the iron is in solution. For more rapid solution of the iron, first place the double chloride solution in a flask provided with a stopper containing inlet and outlet tubes for carbon dioxide, warm to approximately $40^{\circ} \mathrm{C}$. as a stream of $\mathrm{CO}_{2}$ is bubbled through the solution, introduce the sample, replace the stopper, and continue the passage of the gas with occasional shaking of the flask to prevent clogging of the inlet tube.

When solution is complete, let settle for a moment, decant through a rapid filter, and wash quickly, by decantation as much as possible, with a little hot water. Place filter paper and contents in the original flask, add $25 \mathrm{c}$ c of water, then $5 \mathrm{c}$ c of bromine and finally $5 \mathrm{c}$ c of concentrated $\mathrm{HCl}$. Heat gently till free of bromine, dilute to $100 \mathrm{c} c$, filter, and wash the residue thoroughly with hot water. Precipitate with $\mathrm{BaCl}_{2}$ and finish the determination as described in the determination of sulphur by the gravimetric method (referee method).

The results must be corrected by blanks run under the same conditions as the determination.

Determination of Sulphur by the Evolution Method

(Routine)

\section{Apparatus}

Figure 51 shows a typical apparatus for this determination. A spray trap may be put in the outlet tube to prevent iron from passing over into the ammoniacal solution.

\section{Solutions required}

Dilute hydrochloric acid (1:1).-Mix 500 c c of $\mathrm{HCl}$ (specific gravity 1.19) and $500 \mathrm{c} \mathrm{c}$ of distilled water.

Ammoniacal cadmium chloride.-Dissolve $10 \mathrm{~g}$ of cadmium chloride in $400 \mathrm{c}$ c of distilled water and add $600 \mathrm{c}$ c of $\mathrm{NH}_{4} \mathrm{OH}$ (specific gravity 0.90 ).

Ammoniacal zinc sulphate (optional solution).Dissolve $200 \mathrm{~g}$ of zinc sulphate in $1,080 \mathrm{c}$ c of water, and add $920 \mathrm{c}$ c of $\mathrm{NH}_{4} \mathrm{OH}$ (specific gravity 0.90 ).

Starch solution.-To $500 \mathrm{c} \mathrm{c}$ of boiling distilled water, add a cold suspension of $5 \mathrm{~g}$ of soluble starch in $25 \mathrm{c}$ c of distilled water, cool, add a cool solution of $5 \mathrm{~g}$ of $\mathrm{NaOH}$ in $50 \mathrm{c}$ c of distilled water, then add $15 \mathrm{~g}$ of KI and mix thoroughly.

Potassium iodate.-Dissolve $1.12 \mathrm{~g}$ of $\mathrm{KIO}_{3}$ and $12 \mathrm{~g}$ of KI in $1,000 \mathrm{c} \mathrm{c}$ of distilled water. For general work the theoretical sulphur titre of this solution should be used; for specialized work on one kind of 
material, the solution may be standardized against like material. The theoretical titre is based on standard sodium oxalate and is obtained as follows: To $300 \mathrm{c}$ c of water in a $600 \mathrm{c}$ c flask, preferably glass stoppered, add $10 \mathrm{c} \mathrm{c}$ of $\mathrm{HCl}$ (specific gravity $1.19)$ and $1 \mathrm{~g}$ of KI. Cool and add $25 \mathrm{c}$ c of 0.03 $\mathrm{NKMnO}_{4}$ solution which has been standardized against sodium oxalate. Swirl gently, stopper, and let stand for five minutes. Titrate the liberated iodine with thiosulphate solution until the color nearly fades. Then add $10 \mathrm{c} \mathrm{c}$ of starch solution and continue the titration until the blue color is destroyed. In a new flask, repeat the experiment with the sole difference that $25 \mathrm{c}$ c of the iodate solution is substituted for the standard permanganate solution. Calculate the normality of the iodate solution, and dilute if desired so that its normality is 0.0312 , or $1 \mathrm{c} \mathrm{c}$ is equivalent to 0.01 per cent of sulphur on a $5 \mathrm{~g}$ sample. For standardization against like material, $5 \mathrm{~g}$ portions of the proper standard cast iron is carried through all steps of the method. The

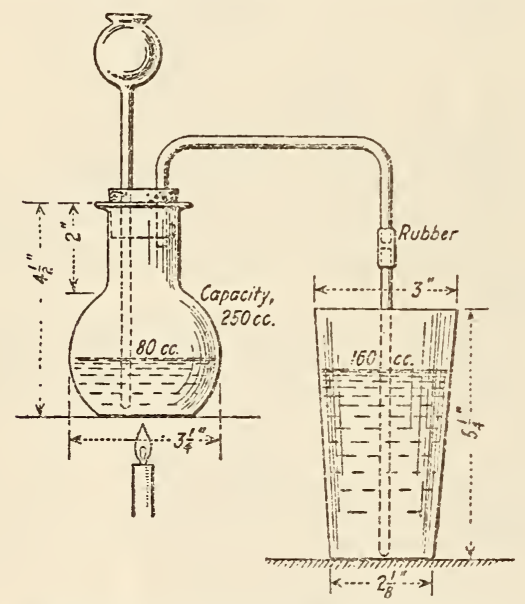

Figure 51.-Apparatus for determining sulphur by the evolution method

following equations represent the reactions that are involved:

$5 \mathrm{Na}_{2} \mathrm{C}_{2} \mathrm{O}_{4}+8 \mathrm{H}_{2} \mathrm{SO}_{4}+2 \mathrm{KMnO}_{4}=5 \mathrm{Na}_{2} \mathrm{SO}_{4}$ $+\mathrm{K}_{2} \mathrm{SO}_{4}+2 \mathrm{MnSO}_{4}+8 \mathrm{H}_{2} \mathrm{O}+10 \mathrm{CO}_{2}$

$10 \mathrm{KI}+16 \mathrm{HCl}+2 \mathrm{KMnO}_{4}=10 \mathrm{I}+12 \mathrm{KCl}$ $10 \mathrm{I}+10 \mathrm{Na}_{2}+2 \mathrm{MnCl}_{2}+8 \mathrm{H}_{2} \mathrm{O}$

$10 \mathrm{I}+10 \mathrm{Na}_{2} \mathrm{~S}_{2} \mathrm{O}_{3}=5 \mathrm{Na}_{2} \mathrm{~S}_{4} \mathrm{O}_{6}+10 \mathrm{NaI}$

$\mathrm{KIO}_{3}+5 \mathrm{KI}+6 \mathrm{HCl}=6 \mathrm{I}+6 \mathrm{KCl}+3 \mathrm{H}_{2} \mathrm{O}$

$\mathrm{KIO}_{3}+5 \mathrm{KI}+6 \mathrm{HCl}+3 \mathrm{H}_{2} \mathrm{~S}=3 \mathrm{~S}+6 \mathrm{KCl}$

The ratios are, therefore:

$$
+6 \mathrm{HI}+3 \mathrm{H}_{2} \mathrm{O}
$$

$5 \mathrm{Na}_{2} \mathrm{C}_{2} \mathrm{O}_{4}: 2 \mathrm{KMnO}_{4}=10 \mathrm{I}: 5 \mathrm{~S}$

\section{Method}

Place $5 \mathrm{~g}$ of the cast-iron sample in the flask (fig. 51) and connect the latter as shown in the sketch. Place $10 \mathrm{c}$ c of the ammoniacal solution of cadmium chloride or zinc sulphate solution and $150 \mathrm{c} c$ of distilled water in the tumbler. Add $80 \mathrm{c} c$ of the dilute $\mathrm{HCl}(1: 1)$ to the flask through the thistle tube, heat the flask and its contents gently until the solution of the iron is complete, then boil the solution for one-half minute. The heat must be so adjusted that there is a rapid, steady, evolution of gas. Disconnect the delivery tube leaving it in the solution and remove the tumbler. Add $5 \mathrm{c}$ c of starch solution, then 40 c c of dilute $\mathrm{HCl}(1: 1)$ and titrate immediately with the iodate solution to a permanent blue color.

Notes-If cast-iron samples are not annealed and the theoretical titre is used, the results obtained by the evolution method will be low by as much as 0.015 per cent. Annealed samples behave more nearly normal, but there is enough uncertainty so that the evolution method should never be used when high accuracy or settlement if disputes are in ruestion.

Samples are annealed as follows: Transfer $5 \mathrm{~g}$ of the drillings to a $12.5 \mathrm{~cm}$ filter paper, and wrap the sample securely in the parer so that at least three thicknesses of paper cover the iron. Place the packages in a 25 c c porcelain crucible, cover the crucible tightly so that air can not enter freely, and heat for 20 minutes at a bright red heat over a Meker birner or nreferably in a muffe. The filter paper should be charred but not burned.

Direct sunlight should be aroided if cadmium solutions are used, otherwise low results are obtained. Cast irons of high titanium content do not yield all of their sulphur even after snnealing.

\section{Determination of Silicon by the Nitro-Sulphuric Acid Method}

\section{Solutions required}

Nitro sulphuric acid.-Mix 160 c c of $\mathrm{H}_{2} \mathrm{SO}_{4}$ (specific gravity 1.84), $180 \mathrm{c}$ c of $\mathrm{HNO}_{3}$ (specific gravity $1.42)$, and $660 \mathrm{c} \mathrm{c}$ of water.

Dilute hydrochloric acid (5:95).-Mix 50 c c of $\mathrm{HCl}$ (specific gravity 1.19) and $950 \mathrm{c}$ c of distilled water.

\section{Method}

Cautiously add $100 \mathrm{c}$ c of the nitro-sulphuric acid to $2.336 \mathrm{~g}$ of iron in a platinum or porcelain dish of 300 c c capacity, cover with a watch glass, heat until the iron is dissolved, and evaporate until copious fumes of sulphuric acid are evolved. Cool, add 125 $\mathrm{c} \mathrm{c}$ of distilled water and $5 \mathrm{c}$ c of $\mathrm{HCl}$ (specific gravity 1.19), heat with frequent stirring until all salts are dissolved, and immediately filter on a $9 \mathrm{~cm}$ paper. Wash the precipitate with cold dilute $\mathrm{HCl}$ (5:95) and hot water alternately to complete the removal of iron salts, and finally with hot water, until free from acid. Transfer the filter to a platinum crucible, burn the paper without inflaming, and finally ignite with the crucible covered over a blast lamp or in a muffle furnace at $1,000^{\circ} \mathrm{C}$., for at least 10 minutes, cool in a desiccator, and weigh. Add sufficient dilute $\mathrm{H}_{2} \mathrm{SO}_{4}(1: 1)$ to moisten the silica, and then a small amount of HF. Evaporate to dryness, ignite, and weigh. The difference in weights in milligrams divided by 50 equals the percentage of silicon.

A blank determination on all reagents used should be made and the results corrected accordingly.

Determination of Silicon by the Hydrochloric-Sulphuric Acid Method

To $2.336 \mathrm{~g}$ of iron in a covered $600 \mathrm{c} \mathrm{c}$ beaker or a $250 \mathrm{c}$ c casserole add $40 \mathrm{c}$ c of dilute $\mathrm{HCl}(1: 1)$, heat gently until the solution is complete, and then wash and remove the cover glass. Evaporate to dryness, but do not bake. If basic salts are formed by accidental baking, moisten the hot mass with 1 to $3 \mathrm{c} \mathrm{c}$ of $\mathrm{HCl}$ (specific gravity 1.19). Cool slightly, add $15 \mathrm{c} \mathrm{c}$ of $\mathrm{H}_{2} \mathrm{SO}_{4}$ (specific gravity 1.84), and scrape down any salts on the sides of the vessel. Evaporate to fumes of sulphuric acid, cool, add $150 \mathrm{c} c$ of water, and heat with frequent stirring until salts are in solution. Immediately filter on a $9 \mathrm{~cm}$ paper, and proceed as in the determination of silicon by the nitro-sulphuric acid method.

NOTE.-This determination can be carried out in the $\mathrm{HCl}$ solution of the iron obtained in the determination of sulphur by the evolution method (routine).

Determination of Silicon by the Sulphuric Acid Method

(Optional)

\section{Solutions required}

Dilute hydrochloric acid (5:95).-(Same as in determination of silicon by the nitro-sulphuric acid methods given above.)

\section{Method}

To $2.336 \mathrm{~g}$ of iron in a $500 \mathrm{c}$ c beaker of low form add $60 \mathrm{c}$ c of distilled water, and then cautiously 15 
c c of $\mathrm{H}_{2} \mathrm{SO}_{4}$ (specific gravity 1.84). Cover with a watch glass, heat until the iron is dissolved, remove the cover, and evaporate until copious fumes of sulphuric acid are evolved. Cool, add $100 \mathrm{c} \mathrm{c}$ of distilled water, and heat with frequent stirring until the salts are in solution. Immediately filter on a $9 \mathrm{~cm}$ paper and proceed as in the determination of silic on by the nitro-sulphuric acid method.

Determination of Small Amounts of Chromium

\section{Solutions required}

Dilute sulphuric acid (1:9).-Slowly stir $100 \mathrm{c}$ c of $\mathrm{H}_{2} \mathrm{SO}_{4}$ (specific gravity 1.84) into cool water, cool, and then dilute to $1,000 \mathrm{c}$ c. It is important that this acid be of the indicated strength.

Sodium bicarbonate solution.-Dissolve $80 \mathrm{~g}$ of $\mathrm{NaHCO}_{3}$ in water and dilute to $1,000 \mathrm{c}$ c.

Dilute sulphuric acid $(1: 1)$. - Slowly stir $500 \mathrm{c}$ c of $\mathrm{H}_{2} \mathrm{SO}_{4}$ (specific gravity 1.84 ) into $500 \mathrm{c}$ c of water. Dilute nitric acid (specific gravity approximately 1.20).-Mix $380 \mathrm{c}$ c of $\mathrm{HNO}_{3}$ (specific gravity 1.42) and $620 \mathrm{c} \mathrm{c}$ of distilled water.

Silver nitrate solution (0.133 per cent).-Dissolve $1.33 \mathrm{~g}$ of $\mathrm{AgNO}_{3}$ in $1,000 \mathrm{c} \mathrm{c}$ of water.

Ammonium persulphate solution (10 per cent).Make up as needed by dissolving $10 \mathrm{~g}$ of the salt in $100 \mathrm{c} c$ of water. The salt should be at least 95 per cent pure and can be tested as follows: Dissolve $0.3 \mathrm{~g}$ of the salt, accurately weighed,in $25 \mathrm{c} c$ of a 10 per cent solution of potassium iodide and add $10 \mathrm{c} c$ of dilute sulphuric acid (1:9). Let it stand for one-half hour and titrate the liberated iodine with a $0.1 \mathrm{~N}$ solution of sodium thiosulphate. One cubic centimeter 0.1 $N \mathrm{Na}_{2} \mathrm{~S}_{2} \mathrm{O}_{3}$ equals $0.01141 \mathrm{~g}$ of $\left(\mathrm{NH}_{4}\right)_{2} \mathrm{~S}_{2} \mathrm{O}_{8}$. Or, the salt can be tested by trealing $0.3 \mathrm{~g}$ of the salt with 30 c c of $0.1 \mathrm{~N} \mathrm{FeSO}_{4}$ solution and then with $200 \mathrm{c} \mathrm{c}$ of hot water in a nonoxidizing atmosphere. After the salt has dissolved, cool, and determine the excess of $\mathrm{FeSO}_{4}$ by titration with $0.1 \mathrm{~N} \mathrm{KMnO}_{4}$ solution.

Sodium chloride solution (5 per cent).-Dissolve 5 $\mathrm{g}$ of $\mathrm{NaCl}$ in $100 \mathrm{c}$ c of water.

Standard ferrous sulphate solution $(0.03 N)$.- See the determination of manganese by the bismuthate method.

Standard potassium permanganate solution.-See the determination of manganese by the bismuthate method.

Sodium hydroxide solution (5 per cent).-Dissolve $50 \mathrm{~g}$ of stick $\mathrm{NaOH}$ in $1,000 \mathrm{c}$ c of water.

Standard potassium chromate.-Dissolve $0.2828 \mathrm{~g}$ of C. P. $\mathrm{K}_{2} \mathrm{Cr}_{2} \mathrm{O}_{7}$ in $1,000 \mathrm{c}$ c of water. One cubic centimeter of this solution contains $0.1 \mathrm{mg}$ of chromium. In tre colorimetric comparison the solution is added to a $\mathrm{NaOH}$ solution of the same strength as the solution which is to be tested.

\section{Method}

(a) Titration method (referee method).-Treat $10 \mathrm{~g}$ of the sample, contained in a $500 \mathrm{c}$ c Erlenmeyer flask, with $110 \mathrm{c}$ c of dilute $\mathrm{H}_{2} \mathrm{SO}_{4}$ (exactly 1:9). Cover, heat to boiling, and as soon as the action is complete dilute to $200 \mathrm{c}$ c with boiling water. (See note.) Immediately add $50 \mathrm{c} \mathrm{c}$ of sodium bicarbonate solution slowly and with stirring from a pipette, and then continue the addition from a burette until a permanent precipitate appears (10 to $30 \mathrm{c}$ c depending on the composition of the iron) and then add $4 \mathrm{c} \mathrm{c}$ more. Boil for one minute, let settle, filter on a rapid filter and quickly wash the flask and precipitate two or three times with hot water. If the proper portions of iron and $\mathrm{H}_{2} \mathrm{SO}_{4}$ were taken in the beginning and the precipitation is properly performed, there will be no more precipitate than can be conveniently handled on an $11 \mathrm{~cm}$ paper. The filtrate will become cloudy in the funnel stem and in the receiving vessel on account of oxidation and hydrolysis. Ignite the paper and precipitate in an iron or nickel crucible (free from chromium), mix the ash with 10 or 12 times its volume of sodium peroxide (free from chromium) and heat gently until the contents are fused. Dissolve the cooled melt by immersing it in $100 \mathrm{c}$ c of cold water, remove the crucible, and destroy any remaining peroxide by heating for at least one-half hour on the steam bath. Filter through paper or an asbestos pad, wash with hot water, and discard the residue.

If some iron passes into the filtrate, it will not interfere with the subsequent titration of chromium. Neutralize the filtrate with $\mathrm{H}_{2} \mathrm{SO}_{4}$ (1:1), using litmus paper as indicator, and add $20 \mathrm{c} \mathrm{c}$ in excess. Add $5 \mathrm{c}$ c of $\mathrm{HNO}_{3}$ (specific gravity 1.20), $10 \mathrm{c} \mathrm{c}$ of $\mathrm{AgNO}_{3}$ solution and $20 \mathrm{c}$ c of freshly prepared ammonium persulphate solution (10 per cent). Boil for 10 minutes. If small amounts of permanganic acid are now indicated, add $5 \mathrm{c}$ c of $\mathrm{NaCl}$ solution ( 5 per cent) and continue the boiling for five minutes after the pink color has been destroyed. If no permanganate acid is indicated, as is usually the case, the boiling with $\mathrm{NaCl}$ can be omitted. Cool the solution, dilute to $300 \mathrm{c} \mathrm{c}$ and titrate for chromium by adding a small measured excess of the standard ferrous sulphate solution and then titrating back with an approximately $0.03 \quad N$ standard potassium permanganate solution. The titration should be corrected by a "blank" run carried through the various steps of the method, not omitting the fusion which tests the crucible.

(b) Colorimetric method (routine).-A reasonably correct determination of small amounts of chromium requires such care that a rapid routine method is not possible. The titration method (referee method), above, can be shortened somewhat for less accurate work as follows:

When the hydrogen peroxide has been destroyed, filter the $\mathrm{NaOH}$ solution through an asbestos pad, preferably on a Büchner funnel, and wash with a cold 5 per cent solution of $\mathrm{NaOH}$. Dilute to a measured volume (preferably $100 \mathrm{c} \mathrm{c}$ ) and compare the color with a standard solution of $\mathrm{K}_{2} \mathrm{Cr}_{2} \mathrm{O}_{7}$. In this method peroxide need not be destroyed, but the solution is heated to give better separation of iron which occasionally goes through the filter and gives a false color.

In the colorimetric work asbestos should be used as the filtering medium as coloring matter may be extracted from filter paper by strongly alkaline solutions.

Vanadium is quantitatively precipitated by sodium bicarbonate in this method, but it does not interfere in the determination of chromium in either volumetric or colorimetric methods.

NотE.-As soon as attack is complete, the solution can be rapidly filtered by suction, the paper and contents washed with hot water and burned with the bircabonate precipitate. This aids a beginner to determine the bicarbonate end point.

\section{Determination of Nickel by the Dimethylglyoxime Method}

\section{Solutions required}

Dilute hydrochloric acid (1:1).-Mix 500 c c of $\mathrm{HCl}$ (specific gravity 1.19) and $500 \mathrm{c}$ c of water.

Dilute hydrochloric acid $(1: 20)$ - - Mix $50 \mathrm{c}$ c of $\mathrm{HCl}$ (specific gravity 1.19) and $1,000 \mathrm{c} \mathrm{c}$ of water.

Dimethylglyoxime solution (1 per cent).-Dissolve $20 \mathrm{~g}$ of dimethylglyoxime in $1,300 \mathrm{c} \mathrm{c}$ of $\mathrm{NH}_{4} \mathrm{OH}$ (specific gravity 0.90 ) and dilute to $2,000 \mathrm{c} \mathrm{c}$. Ten cubic centimeters of this solution allow sufficient excess completely to precipitate $0.015 \mathrm{~g}$ of nickel.

\section{Method}

Dissolve $5 \mathrm{~g}$ of the iron in $40 \mathrm{c}$ c of dilute $\mathrm{HCl}$ (1:1), add about $10 \mathrm{c}$ c of $\mathrm{HNO}_{3}$ (specific gravity 
1.42) to oxidize the iron, and evaporate to dryness on the steam bath. Drench the dried mass with $10 \mathrm{c} \mathrm{c}$ of $\mathrm{HCl}$ (specific gravity 1.19) and then dilute with $100 \mathrm{c}$ c of hot water. Filter, wash with dilute $\mathrm{HCl}(1: 20)$ and evaporate the filtrate to a sirupy consistency. Take up in $15 \mathrm{c} \mathrm{c}$ of $\mathrm{HCl}(1: 1)$ and transfer to a 150 or 250 c c separatory funnel, rinsing the beaker several times with small portions of dilute $\mathrm{HCl}(1: 1)$. Cool, add $50 \mathrm{c}$ c of ether, shake for one to two minutes in a stream of cold water, let settle for one minute, and then draw off the lower clear solution. Add $10 \mathrm{c} \mathrm{c}$ of cold dilute $\mathrm{HCl}(1: 1)$ to the solution in the separatory funnel, shake thoroughly, allow to settle for one minute, and again draw off the clear solution into the same beaker as was used for the first. Gently heat the solution in the beaker to expel the ether, add $0.2 \mathrm{~g}$ of potassium chlorate, boil until the chlorate is decomposed, dilute to $100 \mathrm{c}$ c and add $3 \mathrm{~g}$ of tartaric acid. Make the solution alkaline with ammonia and filter. If a precipitate of iron is present insufficient tartaric acid was added. In this case the precipitate must be dissolved in $\mathrm{HCl}$, the whole solution acidified, and treated with more tartaric acid. If no precipitate appear, render the solution slightly acid with $\mathrm{HCl}$, dilute to $200 \mathrm{c} \mathrm{c}$ and add $20 \mathrm{c}$ c of dimethylglyoxime solution. Heat to boiling, make slightly ammoniacal, and let stand on the steam bath for one hour. Filter on a weighed Gooch Pyrex-Alundum, or fritted-glass crucible, wash with hot water and dry for one hour at $110^{\circ}$ to $120^{\circ} \mathrm{C}$. Weigh as nickel-dimethylglyoxime and multiply the weight by 0.2031 to obtain the weight of nickel.

Note.-In routine analyses, the dimethylglyoxime precipitate can be caught on paper and ignited to $\mathrm{NiO}$ or decomposed and titrated with cyanide. In the former case, care must be taken to prevent sublimation of the undecomposed salt. This can be accomplished $\mathrm{y}$ enclosing the wet paper and precipitate in one or two wet ashless papers and slowly igniting so that the papers char before taking fire. $\mathrm{n}$; his way the compound is decomposed and ignition can then proceed at full red heat. In the latter case proceed as follows: Dissolve the nickel-dimethylglyoxime in $\mathrm{HNO}_{3}$, oxidize with ammonium persulphate or potassium chlorate and boil for five minutes. Cool, neutralize with $\mathrm{NaOH}$ and add $5 \mathrm{c} \mathrm{c}$ in excess. Rosolic acid is a convenient indicator. Dilute to $200 \mathrm{c} \mathrm{c}, \mathrm{cool}$ thoroughly, add $2 \mathrm{c} \mathrm{c}$ of a 10 per cent solution of KI and then from a burette $1 \mathrm{c} c$ of standard $\mathrm{AgNO}_{3}$ solution (5.7903 $\mathrm{g}$ per liter). Titrate with a standard solution of potassium cyanide ( $4.5 \mathrm{~g} \mathrm{KCN}$ per liter) until the AgI is dissolved. Finish the titration by adding $\mathrm{AgNO}_{3}$ to a reappearance of the opalesence. With exactly agreaing $\mathrm{AgNO}_{3}$ and $\mathrm{KCN}$ solutions, each cubic centimeter excess of standard cyanide solution over the $\mathrm{AgNO}_{3}$ olution will equal 0.1 per cent nickel on a $1 \mathrm{~g}$ sample.

\section{Determination of Copper}

\section{Solutions required}

Dilute sulphuric acid $(1: 5)$.- Slowly stir $200 \mathrm{c}$ c of $\mathrm{H}_{2} \mathrm{SO}_{4}$ (specific gravity 1.84) into $1,000 \mathrm{c}$ c of distilled water.

Dilute sulphuric acid (2:98).-Mix $20 \mathrm{c}$ c of $\mathrm{H}_{2} \mathrm{SO}_{4}$ (specific gravity 1.84) and $980 \mathrm{c} \mathrm{c}$ of distilled water. Acidulated hydrogen sulphide water.-Saturate 500 c c of dilute sulphuric acid $(2: 98)$ with hydrogen sulphide.

Sodium hydroxide (5 per cent).-Dissolve $50 \mathrm{~g}$ of stick $\mathrm{NaOH}$ in $1,000 \mathrm{c}$ c of distilled water and filter through asbestos if necessary.

Sodium thiosulphate (50 per ceni).-Dissolve $100 \mathrm{~g}$ of $\mathrm{Na}_{2} \mathrm{~S}_{2} \mathrm{O}_{3} \cdot 5 \mathrm{H}_{2} \mathrm{O}$ in $100 \mathrm{c}$ c of distilled water and filter if necessary.

Dilute nitric acid $(1: 1)$.-Mix 500 c c of $\mathrm{HNO}$ (specific gravity 1.42) and $500 \mathrm{c} \mathrm{c}$ of distilled water

\section{Method}

(a) Precipitation by hydrogen sulphide (referee method).-Dissolve $10 \mathrm{~g}$ of iron in $100 \mathrm{c} \mathrm{c}$ of dilute $\mathrm{H}_{2} \mathrm{SO}_{4}(1: 5)$, and when solution is complete evapo- rate to fumes of sulphuric acid, cool, dilute to 100 c c and filter. Wash the residue with hot water and dilute the filtrate to $500 \mathrm{c} \mathrm{c}$ with water. Heat to boiling and saturate with $\mathrm{H}_{2} \mathrm{~S}$. Digest for one to two hours, filter on paper or paper pulp and wash with acidulated hydrogen sulphide water. Ignite the precipitate and paper in a porcelain crucible and fuse with a small amount of alkali pyrosulphate. Dissolve the cooled melt in $50 \mathrm{c}$ c of dilute $\mathrm{H}_{2} \mathrm{SO}_{4}$ $(2: 98)$, transfer to a $200 \mathrm{c} \mathrm{c}$ beaker, dilute to $100 \mathrm{c} \mathrm{c}$ and add $\mathrm{NaOH}$ (5 per cent) solution in slight excess. Boil, digest, and filter, in order to separate such elements as vanadium, tungsten, and molybdenum. Dissolve the precipitate in hot dilute $\mathrm{HNO}_{3}(1: 1)$, add $5 \mathrm{c}$ c of $\mathrm{H}_{2} \mathrm{SO}_{4}$ (specific gravity 1.84 ), evaporate to the appearance of fumes of sulphuric acid, cool, dilute to $40 \mathrm{c}$ c, filter, and add sufficient $\mathrm{NH}_{4} \mathrm{OH}$ (specific gravity 0.90 ) to reduce the acidity to approximately $2 \mathrm{c}$ c of $\mathrm{H}_{2} \mathrm{SO}_{4}$ (specific gravity 1.84) per $100 \mathrm{c}$ c of solution. Heat the acid solution to boiling and saturate with $\mathrm{H}_{2} \mathrm{~S}$. Digest a while, filter, wash thoroughly with acidulated $\mathrm{H}_{2} \mathrm{~S}$ water, and proceed according to method (1) or (2) under (b) Precipitation by thiosulphate.

(b) Precipitation by thiosulphate.-Transfer $5 \mathrm{~g}$ of the sample to a $600 \mathrm{c}$ c beaker, add $100 \mathrm{c}$ c of $\mathrm{H}_{2} \mathrm{SO}_{4}$ $(1: 5)$ and heat until the sample is dissolved. Dilute to $300 \mathrm{c} \mathrm{c}$ heat to boiling, add $15 \mathrm{c} \mathrm{c}$ of thiosulphate solution ( 50 per cent), and continue the boiling for five minutes or until the precipitate of copper sulphide has coagulated. Collect the precipitate upon an $11 \mathrm{~cm}$ paper and wash slightly with dilute $\mathrm{H}_{2} \mathrm{SO}_{4}(2: 98)$. Ignite the residue and paper in a porcelain crucible and fuse with alkali pyrosulphate. Dissolve the cooled melt in the crucible in 1 to 2 c c of $\mathrm{HCl}$ (specific gravity 1.19) and a few cubic centimeters of water, transfer to a $200 \mathrm{c}$ c beaker, dilute to $100 \mathrm{c}$ c, and add $\mathrm{NaOH}$ (5 per cent) solution in slight excess. Boil, digest, and filter in order to separate such elements as vanadium, tungsten, and molybdenum. Dissolve the precipitate in hot dilute $\mathrm{HNO}_{4}(1: 1)$, carefully add $20 \mathrm{c} \mathrm{c}$ of $\mathrm{H}_{2} \mathrm{SO}_{4}$ (specific gravity 1.84), evaporate to the appearance of fumes of $\mathrm{H}_{2} \mathrm{SO}_{4}$, cool, dilute, and filter. Dilute the solution to a volume of $300 \mathrm{c} \mathrm{c}$ and precipitate the copper with thiosulphate as above. Filter, wash with dilute $\mathrm{H}_{2} \mathrm{SO}_{4}(2: 98)$ until sodium salts have been removed, and proceed according to method 1 or 2 below.

(1) Electrolytic method.-Dissolve the sulphides obtained as above in hot dilute $\mathrm{HNO}_{3}(1: 1)$, and electrolyze after the addition of $\mathrm{H}_{2} \mathrm{SO}_{4}$ according to usual procedures.

(2) Gravimetric method.-Ignite the sulphides obtained as above and weigh as copper oxide. As this compound is somewhat hygroscopic, care must be observed to prevent the absorption of moisture. It is difficult to convert large amounts of CuS to $\mathrm{CuO}$ by ignition and if the percentage of copper is in excess of 0.2 , the electrolytic method should be used or the size of sample reduced.

\section{Determination of Copper}

(Routine method)

\section{Solutions required}

Potassium ferrocyanide.-Dissolve $10 \mathrm{~g}$ of potassium ferrocyanide in $100 \mathrm{c} \mathrm{c}$ of distilled water.

Standard copper nitrate.-Dissolve $2 \mathrm{~g}$ of purest electrolytic copper in $20 \mathrm{c}$ c of $\mathrm{HNO}_{3}(1: 1)$, and dilute to 1,000 c c with distilled water. Each cubic centimeter is equivalent to 0.02 per cent of copper on the basis of a $10 \mathrm{~g}$ sample. 


\section{Method}

Proceed as in (a) precipitation by hydrogen sulphide or (b) precipitation by thiosulphate, under determination of copper; until the alkali pyrosulphate fusion has been made. Extract the cooled melt with hot water, filter, and complete the determination colorimetrically as follows:

Evaporate the filtrate to about $25 \mathrm{c}$ c, filter into a $100 \mathrm{c}$ c Nessler tube, wash with hot water, add $5 \mathrm{c} \mathrm{c}$ of $\mathrm{NH}_{4} \mathrm{OH}$ (specific gravity 0.90 ) and dilute to $50 \mathrm{c} \mathrm{c}$.

(a) If the solution is a strong blue.-To another 100 c c Nessler tube add 50 c c of distilled water, 5 c c of $\mathrm{NH}_{4} \mathrm{OH}$ (specific gravity 0.90 ) and from a burette the standard copper nitrate solution until the blue colors match.

(b) If the solution is a faint blue.-To the filtrate in a Nessler tube add dilute $\mathrm{H}_{2} \mathrm{SO}_{4}(1: 5)$ to faint acidity and then a few drops of the potassium ferrocyanide solution. To another $100 \mathrm{c}$ c Nessler tube add $50 \mathrm{c}$ c of distilled water, a few drops of the potassium ferrocyanide solution, and from a burette the standard copper nitrate solution until the reddish brown colors match.

\section{Determination of Titanium by the Colorimetric Method}

\section{Solutions required}

Dilute hydrochloric acid (1:2).-Mix 500 c c of $\mathrm{HCl}$ (specific gravity 1.19) with $1,000 \mathrm{c}$ c of water. Dilute sulphuric acid $(1: 9)$.-Carefully stir $100 \mathrm{c} \mathrm{c}$ of $\mathrm{H}_{2} \mathrm{SO}_{4}$ (specific gravity 1.84) into $900 \mathrm{c}$ c of water. Standard titanium solution.-Transfer $3 \mathrm{~g}$ of potassium titanium fluoride $\left(\mathrm{K}_{2} \mathrm{TiF}_{6}\right)$, which has been twice recrystallized from hot water, to a platinum dish, add $100 \mathrm{c}$ c of $\mathrm{H}_{2} \mathrm{SO}_{4}$ (specific gravity 1.84) and evaporate three times to fumes of the acid, with intervening washing down of the sides of the vessel. Cool and pour the solution rapidly and with stirring into $900 \mathrm{c}$ c of water. Cool to room temperature, and standardize the solution by taking $100 \mathrm{c} \mathrm{c}$ portions, precipitating with ammonia in boiling solution, filtering, igniting and weighing as $\mathrm{TiO}_{2}$.

Ferric sulphate solution.-Dissolve $10 \mathrm{~g}$ of ingot iron or plain carbon steel which is free from titanium in $\mathrm{HNO}_{3}$, add $20 \mathrm{c}$ c of $\mathrm{H}_{2} \mathrm{SO}_{4}$ (specific gravity 1.84), evaporate to fumes of sulphuric acid, cosl and dilute to $100 \mathrm{c} \mathrm{c}$.

\section{Method}

Dissolve $5 \mathrm{~g}$ of the sample in $100 \mathrm{c} \mathrm{c}$ of $\mathrm{HCl}(1: 2)$. When all action has ceased, filter, wash the insoluble matter with water, dry, and ignite in a platinum crucible under good oxidizing conditions. Treat with 1 to $2 \mathrm{c} c$ of $\mathrm{HF}$ and $1 \mathrm{c} c$ of dilute $\mathrm{H}_{2} \mathrm{SO}_{4}(1: 1)$ to eliminate silica, and evaporate to dryness. Fuse the residue with sodium carbonate, dissolve the fusion in water, digest for 15 minutes, filter and wash with water. Ignite the residue in platinum, fuse with a small quantity of alkali pryosulphate, cool, and take up in dilute $\mathrm{H}_{2} \mathrm{SO}_{4}(1: 9)$. Compare the amount of titanium in this solution with a standard titanium solution as follows: Treat the proper amount of a standard titanium solution with the same quantity of alkali pyrosulphate as was used in the above fusion and add sufficient ferric sulphate solution to give the same tint as the sample when both are at the same dilution. Add $2 \mathrm{c}$ c of 3 per cent hydrogen peroxide to the solution and standard respectively and compare in a colorimeter.

\section{Determination of Arsenic}

(Umpire method)

(As $\mathrm{As}_{2} \mathrm{~S}_{3}$ or by titration with $\mathrm{KSCN}$ )

Dissolve the sample $(10 \mathrm{~g})$ in $200 \mathrm{c}$ c of dilute $\mathrm{HNO}_{3}(1: 3)$. Retard action by cooling if it grows violent. After solution is complete, cool, add 50 c c of concentrated $\mathrm{H}_{2} \mathrm{SO}_{4}$ and evaporate until fumes of sulphuric acid begin to appear. Remove from the hot plate, cool, wash down the sides of the beaker, and fume again. (It is better to fume twice moderately than to fume once very strongly on account of the possibility of volatilizing arsenic at the higher temperature.) Cool, carefully add $50 \mathrm{c} \mathrm{c}$ of water and again cool to room temperature.

Transfer the solution by means of 150 to $175 \mathrm{c} c$ of concentrated $\mathrm{HCl}$ to a distillation flask fitted with a two-holed stopper, preferably of glass, carrying a separatory funnel and an outlet tube. The latter should be provided with a hole on the side about $1 \mathrm{~cm}$ from the end within the distillation flask. This allows for circulation in the outlet tube and prevents small particles of the material in the flask from passing over into the distillate. Add $20 \mathrm{~g}$ of cuprous chloride, stopper, and connect the outlet tube with a vertical condenser having its lower end dipping into a beaker containing $150 \mathrm{c} \mathrm{c}$ of distilled water and immersed in ice water. Distill until 100 c c of distillate have passed over.

After the distillation is done, add $15 \mathrm{c}$ c of strong $\mathrm{NH}_{4} \mathrm{OH}$ (specific gravity 0.90 ), to the distillate in order to neutralize some of the acid and pass a rapid stream of $\mathrm{H}_{2} \mathrm{~S}$ through the solution until saturated. Allow to stand at least one hour, preferably overnight.

(a) To weigh as $A s_{2} S_{3}$. - Filter on a weighed Gooch crucible, wash with alcohol, then with carbon disulphide, then with alcohol, and dry at $100^{\circ}$ to $105^{\circ} \mathrm{C}$. Weigh as $\mathrm{As}_{2} \mathrm{~S}_{3}$ and multiply by the factor 0.6092 to obtain the weight of arsenic.

(b) To Titrate with KSCN.-Titration with KSCN does away with uncertainty concerning the purity of the arsenic sulphide and is done as follows:

Filter through asbestos or paper and wash the precipitate moderately with dilute $\mathrm{H}_{2} \mathrm{SO}_{4}$ (1:99) that has been saturated with $\mathrm{H}_{2} \mathrm{~S}$. Dissolve the washed sulphide in 2 to $3 \mathrm{c}$ c of strong $\mathrm{NH}_{4} \mathrm{OH}$ or the same volume of a 10 per cent solution of $\mathrm{NaOH}$. Wash the pad or paper with water, evaporate nearly to dryness, add $10 \mathrm{c}$ c of concentrated $\mathrm{HNO}_{3}$ and again evaporate nearly to dryness. Take up with $50 \mathrm{c}$ c of water, add a few drops of phenolphthalein and then a 10 per cent solution of $\mathrm{NaOH}$ drop by drop until the solution is decidedly alkaline. Now add dilute acetic acid ( $1: 1)$ drop by drop until the solution just turns colorless, and precipitate the arsenic as silver arsenate by the addition of $10 \mathrm{c} \mathrm{c}$ of $\mathrm{AgNO}_{3}$ solution (34 $\mathrm{g}$ per liter). Heat just to boiling to coagulate the precipitate, cool, filter on an asbestos pad, and wash with cold water.

Dissolve the silver arsenate in dilute $\mathrm{HNO}_{3}(1: 1)$ and titrate the silver in the solution with $0.01 \mathrm{~N}$ potassium or ammonium sulphocyanate, using ferric alum (free from chlorides) as the indicator. The sulphocyanate solution may be standardized against either pure silver or pure silver nitrate and its arsenic titre calculated from the ratio 3 $\mathrm{Ag}: 1 \mathrm{As}$.

Notes.-A blank determination should be carried through all steps of the determination.

Distillation should be carried out so that no copper from the cuprous chloride is carried over into the distillate.

In routine analyses, the $\mathrm{HCl}$ distillate may be nearly neutralized with NaOH, the still acid solution treated with an exchs of sodium bicarbonate and the arsenic determined by titration with a standarc as indicator. The iodine solution is preferably standardized against a standard iron carried through the method.

601.21 CHARCOAL PIG IRON.

(See foundry pig iron.)

601.22 COKE PIG IRON.

(See foundry pig iron.) 
601.23 FOUNDRY PIG IRON.

AMERICAN RAILWAY ASSOCIATION, MECHANICAL DIVISION, IRON, FOUNDRY PIG, RECOMMENDED PRACTICE, 1916

\section{FOUNDKY PIG IRON}

1. Scope.-These specifications apply to all foundry pig iron which should be bought according to the grades shown by section 3 .

\section{MANUFACTURE}

2. Process. - The pig iron shall be made in accordance with the best modern practice and may be either sand or machine cast.

CHEMICAL PROPERTIES AND TESTS

3. Chemical composition. - The pig iron shall conform to the following requirements as to chemical composition:

\begin{tabular}{|c|c|c|c|}
\hline 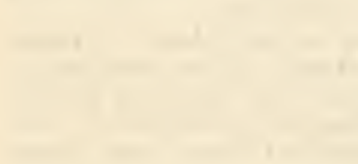 & $\begin{array}{l}\text { No.1 } \\
\text { Foundry } \\
\text { coke iron }\end{array}$ & $\begin{array}{l}\text { No. } 2 \\
\text { Low sili- } \\
\text { con coke } \\
\text { foundry } \\
\text { iron }\end{array}$ & $\begin{array}{l}\text { No. } 3 \\
\text { High } \\
\text { manga- } \\
\text { nese coke } \\
\text { foundry } \\
\text { iron }\end{array}$ \\
\hline $\begin{array}{l}\text { Silicon } \\
\text { Manganese } \\
\text { Phosphorus } \\
\text { Sulphur...... }\end{array}$ & $\begin{array}{r}\text { Per cent } \\
2.50-3.25 \\
.50-1.00 \\
.50-.90 \\
.05\end{array}$ & $\begin{array}{r}\text { Per cent } \\
1.00-1.50 \\
.50-1.00 \\
.20-.70 \\
.05\end{array}$ & $\begin{array}{r}\text { Per cent } \\
1.00-1.50 \\
1.75-3.00 \\
.20-.70 \\
.05\end{array}$ \\
\hline
\end{tabular}

Foundry charcoal pig iron will be bought in grades according to the following limits:

$\begin{array}{lr}\text { Silicon, maximum variation, any grade } & \text { Per cent } \\ \text { Manganese, maximum variation, any grade } & \\ \text { Phosphorus (not over) } & \end{array}$

4. Ladle analyses.-An analysis to determine the percentages of silicon, manganese, phosphorus, and sulphur shall be made by the manufacturer from a test ingot taken during the pouring of each melt, a copy of which shall be given to the purchaser or his representative. This analysis shall conform to the requirements specified in section 3 .

5. Check analyses. - Analyses may be made by the purchaser from the pigs in accordance with section 6 . This analysis shall conform to the requirements specified in section 3 .

6. Sampling.- Ten pigs shall be taken at random from the various parts of each carload or less. These pigs shall be broken in half, and from one-half of each pig the same amount of drillings shall be taken at or near the center by boring parallel to the axis of the pigs. These drillings shall be well mixed and a sample for chemical analysis shall be taken from this mixture.

\section{INSPECTION AND REMEARING}

(Inspection and rehearing are identical with the requirements for A. R. A. galvanized sheets. See 604.32 , p. 243.)

\section{AMERICAN SOCIETY FOR TESTING MA- TERIALS, STANDARD SPECIFICATIONS FOR FOUNDRY PIG IRON, SERIAL DES- IGNATION A43-24, 1924}

\section{FOUNDRY PIG IRON \\ MANUFACTURE}

1. The pig iron shall be clean foundry pig iron, as free as possible from an excessive amount of dross and sand, and may be either sand or machine cast.

\section{CHEMICAL PROPERTIES}

2. (a) The pig iron shall conform to the chemical requirements specified by the purchaser at the time of purchase, with the following permissible variations from the specified percentages in the case of pig iron intended for the manufacture of gray-iron castings:

\begin{tabular}{|c|c|}
\hline Element & $\begin{array}{l}\text { Variation from specified } \\
\text { percentage }\end{array}$ \\
\hline $\begin{array}{l}\text { Silicon } \\
\text { Manganese } \\
\text { Phosphorus. } \\
\text { Sulphur } \\
\text { Total carbon... }\end{array}$ & $\begin{array}{l}0.25 \text { per cent abore or below. } \\
0.20 \text { per cent above or below. } \\
0.15 \text { per cent above or below: } \\
\text { Not over specified percentage. } \\
\text { Not less than specifed percent. } \\
\text { age. }\end{array}$ \\
\hline
\end{tabular}

(b) In the case of pig iron for making steel and malleable-iron castings, a maximum percentage of phosphorus, and either a maximum or minimum percentage of manganese, may be specified.

\section{SAMPLING AND ANALYSIS}

3. (a) In sampling, each carload or its equivalent shall be considered as a unit.

(b) One pig shall be taken to every 4 tons in the car, and they shall be so chosen from different parts of the car as to secure, as nearly as possible, a sample representing the average quality of the iron.

(c) The pigs thus taken shall be sampled by drilling so as fairly to represent the composition of the pigs as cast.

(d) An equal weight of the drillings from each pig shall be thoroughly mixed to make up the sample for analysis.

4. It is recoinmended that analysis be made in accordance with the standard methods of sampling and chemical analysis of pig and cast iron. (See A. S. T. M. A64-27, 601.20, p. 119.)

\section{INSPECTION AND REJECTION}

5. In case of dispute, the sampling and chemical analysis shall be made in accordance with the standard methods of sampling and chemical analysis of pig and cast iron (see preceding paragraph) by an independent chemist, mutually agreed upon-if practicable at the time the contract is made-whose decision shall be final. The cost of such resampling and reanalysis shall be borne by the party in error.

6. All pig iron which fails to conform to these specifications shall be subject to rejection.

\section{UNITED STATES GOVERNMENT, FEDERAL SPECIFICATIO IFICATION FOR IRON; PIG, FOUNDRY, QQ-I-676, FEBRUARY 2, 1932}

\section{FOUNDRY PIG IRON}

(This specification is substantially the same as A. S. T. M. A43-24, given above, except that reference is made herein to four regular grades of foundry pig iron, and to special grades as required. In the matter of general and detail requirements this specification states that in addition to conforming to F. S. B. No. 339a (current designation QQ-M-151) (see 600.0, p. 3) it shall meet the following:)

\section{GENERAL REQUIREMENTS}

1. The pigs shall be free from excessive sand or slag.

2. Not more than 12 per cent of sow iron shall be of a size easily handled. 


\section{DETAIL REQUIREMENTS}

3. Grades A, B, C, and D pig iron shall conform to the respective requirements given in the accompanying table:

\begin{tabular}{|c|c|c|c|c|}
\hline Grade & $\begin{array}{c}\text { Silicon } \\
\text { (maximum) }\end{array}$ & Sulphur & Manganese & Phosphorus \\
\hline & $\begin{array}{l}\text { Per cent } \\
2.75-3.25 \\
2.25-2.75 \\
1.75-2.25 \\
1.25-1.75\end{array}$ & $\begin{array}{r}\text { Per cent } \\
0.04 \\
.05 \\
.05 \\
.05\end{array}$ & $\begin{array}{l}\text { Per cent } \\
0.50-1.00 \\
.50-1.00 \\
.50-1.00 \\
.50-1.00\end{array}$ & $\begin{array}{l}\text { Per cent } \\
0.50-0.80 \\
.50-.80 \\
.50-.80 \\
.50-.80\end{array}$ \\
\hline
\end{tabular}

4. Contracts and purchase orders for special grades of foundry pig iron should state the maximum or minimum total carbon desired, as well as the limits for the other elements.

5. Bidders should state whether the iron they propose to furnish is machine made or sand cast. Preference will be given to machine-cast foundry pig iron, other things being equal.

601.24 MALLEABLE PIG IRON.

(No nationally recognized specifications available.) 601.3 IRON AND STEEL SCRAP.

AMERICAN ELECTRIC RAILWAY ENGINEERING ASSOCIATION, CLASSIFICATION OF SCRAP MATERIALS, PS100-29, 1929

SCRAP MATERIALS

(See 600.2, p. 51.)

602. FINISHED AND SEMIFINISHED IRON AND STEEL

602.1 IRON BILLETS.

AMERICAN SOCIETY FOR TESTING MATERIALS, STANDARD SPECIFICATIONS FOR WROUGHT-IRON ROLLED OR FORGED BLOOMS AND FORGINGS FOR LOCOMOTIVES AND CARS, DESIGNATION A73-30, 1930

\section{WROUGHT-IRON FORGINGS}

(See 611.3, p. 705.)

602.2 STEEL BARS AND BILLETS.

AMERICAN RAILWAY ASSOCIATION, MECHANICAL DIVISION, SPECIFICATIONS FOR CARBON-STEEL BLOOMS, BILLETS, AND SLABS FOR FORGINGS, 1925

\section{CARBON STEEL BILLETS} SCOPE

These specifications cover five classes of billets, determined by their carbon ranges. (The carbon ranges are identical with those specified below in A. S. T. M., A17-29 as Grades Nos. 1, 2, 4, 7, and 8.)

The purposes for which these classes of billets are frequently used are as follows:

Class A (grade 1 in A. S. T. M., A17)-for welding and casehardening.

Class B (grade 2 in A. S. T. M., A17)-for casehardening when subsequently heat treated.

Class C (grade 4 in A. S. T. M., A17)-for special purposes.

Class D (grade 7 in A. S. T. M., A17)-for axles, shafts, connecting

rods, and similar forgings.
Class $\mathrm{E}$ (grade 8 in A. S. T. M., A 1 ) - for class $\mathrm{D}$ forgings when they are to be heat treated.
CHEMICAL COMPOSITION

The steel shall conform to the following requirements as to chemical composition:

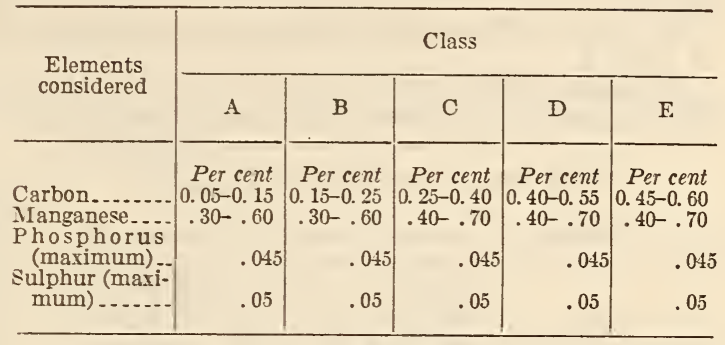

BASIS OF PURCHASE

Billets shall be purchased as semifinished rolled or forged material. In ordering, all dimensions shall be expressed in feet and inches.

\section{MANOFACTURE}

The steel shall be made by the open-hearth process.

(In respect to definition of terms, discard, ladle analyses, check analyses, workmanship and finish, marking, and inspection and rejection, this specification conforms with A. S. T. M., A17, given below, including reference therein to A. S. T. M. standard specifications for steel in 605.0, p. 247.)

\section{AMERICAN SOCIETY FOR TESTING MATE- RIALS, STANDARD SPECIFICATIONS FOR CARBON-STEEL AND ALLOY-STEEL BLOOMS, BILLETS, AND SLABS FOR FORGINGS, DESIGNATION A17-29, 1929}

\section{STEEL BILLETS}

1. The term "billet" is used in these specifications to include blooms, billets, and slabs.

\section{SCOPE}

2. (a) These specifications cover billets of carbon and alloy steel. Eight types of steel are covered, one type of carbon and seven types of alloy steel, classification by type being made according to the chemical composition other than carbon.

(b) Each type of steel is subdivided into grades according to the carbon content. There are eight grades of carbon steel and seven grades of each type of alloy steel.

(c) The billets are further divided into two classes, designated Classes I and II. Class I is the standard for all types and grades, and Class II is a special class applicable to grades Nos. 4 to 8 , inclusive, in type $\mathrm{A}$, and to grades Nos. 14 to 17 , inclusive, in all other types. Class II differs from Class I in the method of sampling for chemical analysis and in chemical requirements.

3. Billets shall be purchased as semifinished rolled or forged material.

\section{MAN UFACTURE}

4. The steel shall be made by either or both of the following processes: Open-hearth or electric furnace.

5. A sufficient discard shall be made from each ingot to secure freedom from injurious piping and undue segregation.

6. Unless otherwise specified, the billets shall be made from ingots of at least three times the crosssectional area of the billet. 


\section{CHEMICAL PROPERTIES AND TESTS}

7. The steel shall conform to the following requirements as to chemical composition for type and grade:

(a) Type.-The types of steel shall be as indicated in Table 1.

(b) Grade.-The carbon ranges for the various grades shall be as follows for carbon and alloy steel:

\begin{tabular}{|c|c|c|c|}
\hline \multicolumn{2}{|c|}{ Carbon steel-Type A } & \multicolumn{2}{|c|}{$\begin{array}{c}\text { Alloy steels-Types B to } \\
\mathrm{H} \text {, inclusive }\end{array}$} \\
\hline Grade & Carbon & Grade & Carbon \\
\hline $\begin{array}{l}1 . \\
2 . \\
3 . \\
4 . \\
5 . \\
6 . \\
7 . \\
8 .\end{array}$ & $\begin{array}{c}\text { Per cent } \\
0.05-0.15 \\
.15-.25 \\
.20-.30 \\
.25-.40 \\
.30-.45 \\
.35-.50 \\
.40-.55 \\
.45-.60\end{array}$ & $\begin{array}{l}11 \ldots \ldots \\
12 \ldots \ldots \\
13 \ldots \ldots \\
14 \ldots \ldots \\
15 \ldots \ldots \\
16 \ldots \ldots \\
17 \ldots \ldots\end{array}$ & $\begin{array}{c}\text { Per cent } \\
0.10-0.20 \\
.15-.25 \\
.20-.30 \\
.25-.38 \\
.30-.43 \\
.35-.50 \\
.45-.60\end{array}$ \\
\hline
\end{tabular}

Note 1.- When the steel is to be used for casehardening purposes, the manganese should be specified not to exceed 0.50 per cent. When the minimum carbon specified is 0.35 per cent or over, the manganese range may be specified $0.30-0.60$ per cent.

NOTE 2.-In selecting billets for making forgings of classes $K, L$, and $M$, as defined in the standard specifications for carbon-steel an alloy-steel forgings, A. S. T. M., A 18 (see 611.51, p. 754), it is suggested that the use of steel with a relatively high carbon content will permit the use of a high draw-back temperature and thus put the steel in it best physical condition for service, shock, and similar conditions.

8. An analysis of each melt of steel shall be made by the manufacturer to determine the percentages of the elements specified in section 7. This analysis shall be made from a test ingot taken during the pouring of the melt. The chemical composition thus determined shall be reported to the purchaser or his representative, and shall conform to the requirements specified in section 7 .

9. (a) Class I. - For billets of Class I, an analysis may be made by the purchaser from one billet representing each melt. The chemical composition thus determined shall conform to the requirements specified in section 7. Drillings for analysis shall be taken with a $5 / 8$-inch drill, parallel to the axis of the ingot as cast, at any point midway between the center and surface of the billet.

(b) Class II.-For billets of Class II, the purchaser or his representative may select one top-cut billet from which to make check analyses to represent each melt. Two sets of drillings shall be taken from the top face of this billet at points on the same diagonal of the billet. The drillings shall be taken with a $5 / 8$-inch drill parallel to the axis of the ingot as cast. The distance from the center of the billet to the drilling points shall be respectively 15 and 80 per cent of the length of the half diagonal of the billet.

(c) From the drillings taken at the 80 per cent point a complete analysis may be made. The chemical composition thus determined shall conform to the requirements specified in section 7 .

TABLE 1.-Types of steel

\begin{tabular}{|c|c|c|c|c|}
\hline \multirow{4}{*}{ Elements considered } & \multicolumn{4}{|c|}{ Type } \\
\hline & A & B & C & $\mathrm{D}$ \\
\hline & \multirow{2}{*}{$\begin{array}{c}\text { Carbon } \\
\text { steel }\end{array}$} & \multirow{2}{*}{$\begin{array}{l}\text { Nickel } \\
\text { steel }\end{array}$} & \multicolumn{2}{|c|}{ Chrome-nickel steel } \\
\hline & & & $\begin{array}{l}1-1.50 \mathrm{per} \\
\text { cent } \mathrm{Ni}\end{array}$ & $\begin{array}{l}1.50-2 \text { per } \\
\text { cent } \mathrm{Ni}\end{array}$ \\
\hline $\begin{array}{l}\text { Manganese } \\
\text { Phosphorus (maxi- }\end{array}$ & $\begin{array}{l}\text { Per cent } \\
0.50-0.80\end{array}$ & $\begin{array}{l}\text { Per cent } \\
0.50-0.80\end{array}$ & $\begin{array}{l}\text { Per cent } \\
0.50-0.80\end{array}$ & $\begin{array}{l}\text { Per cent } \\
0.30-0.60\end{array}$ \\
\hline $\begin{array}{l}\text { mum) } \\
\text { Sulphur (maximum) }\end{array}$ & $\begin{array}{l}.045 \\
.050\end{array}$ & .04 & .04 & .04 \\
\hline $\begin{array}{l}\text { Nickel } \\
\text { Chromium. }\end{array}$ & - - & 13.00 & 1. $00-1.50$ & 1. $50-2.040$ \\
\hline $\begin{array}{l}\text { Chromium } \\
\text { Vanadium (minimum) }\end{array}$ & $-\cdots$ & & $.45-.75$ & $.90-1.25$ \\
\hline
\end{tabular}

TABLE 1.-Types of steel-Continued

\begin{tabular}{|c|c|c|c|c|}
\hline \multirow{4}{*}{ Elements considered } & \multicolumn{4}{|c|}{ Type } \\
\hline & E & $\mathrm{F}$ & G & H \\
\hline & \multicolumn{2}{|c|}{ Chrome-nickel steel } & \multirow[b]{2}{*}{$\begin{array}{c}\text { Chrome } \\
\text { steel }\end{array}$} & \multirow{2}{*}{$\begin{array}{l}\text { Chrome- } \\
\text { vanad- } \\
\text { ium steel }\end{array}$} \\
\hline & $\begin{array}{l}2.75-3.25 \\
\text { per cent } \\
\mathrm{Ni}\end{array}$ & $\begin{array}{l}3 \text { per cent } \\
\text { Ni (min- } \\
\text { imum) }\end{array}$ & & \\
\hline Manganese.......... & \multirow{4}{*}{$\begin{array}{r}\text { Per cent } \\
0.45-0.75 \\
.04 \\
.045 \\
2.75-3.25 \\
.60-.95 \\
0 . . .6\end{array}$} & \multirow{4}{*}{$\begin{array}{c}\text { Per cent } \\
0.30-0.60 \\
\\
.04 \\
13.045 \\
11.00 \\
11.00\end{array}$} & $\begin{array}{c}\text { Per cent } \\
0.30-0.60\end{array}$ & $\begin{array}{l}\text { Per cent } \\
0.50-0.80\end{array}$ \\
\hline $\begin{array}{l}\text { Phosphorus (maxi- } \\
\text { mum) } \\
\text { Sulphur (maximum) } \\
\text { Nickel }\end{array}$ & & & $\begin{array}{l}.04 \\
.045\end{array}$ & $\begin{array}{l}.04 \\
.045\end{array}$ \\
\hline Chromium & & & $.60-.90$ & $.80-1.10$ \\
\hline Vanadium (minimum) & & & n. & .15 \\
\hline
\end{tabular}

1 Minimum.

(d) From the drillings taken at the 15 per cent point a carbon determination may be made. The difference between the carbon content of the drillings from the 15 per cent point and that of the drillings from the 80 per cent point, expressed as a percentage of the latter, shall not exceed the following values: (1) For billets 15 inches or under in thickness, 15 per cent; and (2) for billets over 15 inches in thickness, 20 per cent.

(e) If in any melt the drillings taken in accordance with paragraph (b) do not conform to the requirements of paragraph $(d)$, additional drillings may be taken in a similar manner after making a further top discard from the material from each ingot of at least 10 per cent of the original ingot weight. The results of this analysis shall conform to the requirements of paragraph $(d)$; otherwise, the melt represented shall be rejected.

\section{WORKMANSHIP AND FINISH}

10. (a) Billets may be chipped to remove surface defects, provided that the depth of chipping does not exceed one-sixteenth inch for each inch of dimension concerned, up to a maximum depth of threefourths inch, and provided that the width of the chipping is at least four times its greatest depth; except that in the case of slabs where the width is at least twice the thickness, the depth of chipping on the wide surfaces may exceed this allowance by 50 per cent, up to a maximum depth of three-fourths inch.

(b) In special cases-particularly, large alloy-steel billets - where it is necessary and is not injurious, greater depth of chipping may be permitted by special agreement between the manufacturer and the purchaser.

11. The billets shall be free from injurious defects and shall have a workmanlike finish.

\section{MARKING}

12. (a) The melt number shall be legibly stamped on each billet 6 inches or over in thickness, and on billets of smaller section when so specified.

(b) For billets of Class I, the top end of all top-cut billets 6 inches or over in thickness shall be legibly hot stamped with the letter " $T$ " and marked with paint.

(c) For billets of Class II, the top ends of the top, second, third, and following cut billets from each ingot shall be legibly hot stamped with the letters "A," "B," "C," etc., to show the position of the billet in the ingot, and marked with paint. 


\section{INSPECTION AND REJECTION}

(Requirements covering inspection, rejection, and rehearing conform with those specified in A. S. T. M. standard specifications for steel, paragraphs 7, 8, and 9 (see 605.0, p. 247), with the addition of the following:)

The purchaser may make the tests to govern the acceptance or rejection of billets in his own laboratory or elsewhere. Such tests, however, shall be made at the expense of the purchaser.

UNITED STATES GOVERNMENT, FEDERAL SPECIFICATIONS BOARD, FEDERAL SPECIFICATION FOR STEEL BLOOMS, BILLETS, SLABS, AND BARS FOR REFORGING, CARBON AND ALLOY, NO. 316, AUGUST 8, 1925. (CURRENT DESIGNATION : QQ-S-675.)

\section{STEEL BLOOMS FOR REFORGING}

\section{GENERAL SPECIFICATIONS}

(General specifications for metals, Federal Specifications Board specification No. 339a (current designation: $Q Q-M-151$ ), in effect on date of invitation for bids, shall form part of this specification. See 600.0, p. 3.)

\section{Types and Grades}

I. Steel blooms, billets, slabs, and bars for reforging, carbon and alloy (hereinafter referred to as billets), in a semifinished condition, shall be furnished in the several types and grades listed in Table 1 .

\section{Material}

II. Billets shall be reasonably straight and of uniform quality and condition, free from twists, pipes, seams, laps, cracks, sand, hard spots, porosity, excessive nonmetallic inclusions, segregations, or other defects that affect their suitability for the service intended.

\section{General Requirements}

III. 1. Billets for reforging purposes shall be reduced from ingots by hot rolling, pressing, or hammering.

2. The ingots from which the billets are made shall be poured from dead-melted steel manufactured by the open-hearth, crucible, or electricfurnace process and shall be cast in metal molds, unless a particular process and method of casting is specified in the contract or purchase order.

3. Sufficient discard shall be taken from each ingot to insure freedom from injurious piping and undue segregation. Such discard, in terms of the total ingot weight, shall be not less than 4 per cent from the bottom and 30 per cent from the top in the case of top-poured ingots, and not less than 4 per cent from the bottom and 20 per cent from the top in the case of bottom-poured ingots or fluid-compressed ingots not be to bored. When the billets are to be made from bored, fluid-compressed ingots, the discard shall be not less than 3 per cent from the bottom and 10 per cent from the top.

4. The percentage of top discard may be reduced by the inspector in specific instances where the contractor can submit evidence, based on fracture and metallographic examination of the crop ends of representative billets from the ingots under consideration, which indicates entirely sound and normal metal.

5. Unless otherwise specified in the contract or purchase order, material 18 inches or less in diameter, or 250 square inches or less in cross-sectional area, shall be rolled or forged from ingots with a cross-sectional area of at least four times that of the billet. Material over 18 inches in diameter or over 250 square inches in cross-sectional area shall be rolled or forged from ingots with a cross-sectional area of at least twice that of the billet.

6. Billets shall contain no welds.

(Paragraphs 7 and 8 are identical with paragraphs 10 (a) and 10 (b) of A. S. T. M., A17-29, given above.)

\section{Detail Requirements}

IV. 1. Chemical composition--(a) Material shall conform to the requirements as to chemical composition specified in Table 1.

(b) An analysis of each heat of steel shall be furnished by the contractor showing the percentages of the elements designated.

2. Metallographic requirements.-Billets may be subjected to macroscopic or microscopic examination to determine compliance with Section II. In the case of material intended for important forgings, definite metallographic requirements may be incorporated in the contract, requisition, or purchase order.

3. Dimensions and permissible variations.-Billets shall conform to the shapes and dimensions specified in the contract or purchase order with a permissible variation of plus or minus 3 per cent on transverse dimensions.

4. Physical condition.-Unless otherwise specified in the contract or purchase order, material shall be furnished mill or hotbed annealed.

\section{Methods of Inspection and Tests}

V. 1. Check analyses.-(a) Check analyses may be made by the inspector, or through him by any Government laboratory or other designated representative, and without cost to the contractor.

(b) Three sets of drillings for check analyses shall be taken from each heat or part of heat submitted for inspection, each from a different billet. The drillings for determination of the elements other than carbon shall be taken from the end of the material which was uppermost in the ingot mold, at a position halfway between the center or the inner surface and the outer surface of a solid or bored object, in a line parallel to the axis lying in the direction in which the material is most drawn out. Drillings for the determination of carbon shall be taken as specified above, except that they should be made to represent, by the location from which they are taken and by mixing, the average content for top and bottom of the ingot. The samples shall be fine, clean, and free from oil, dirt, grit, or other foreign matter, and shall consist of not less than 2 ounces.

2. Definition of lot.-For purposes of physical inspection, a lot shall consist of all material from the same heat or melt differing not more than 1 inch in any cross-sectional dimension.

3. Fracture test.-A cold-fracture test shall be made of the top portion of each ingot to insure that all piping, porosity, and other injurious defects have been removed. The fracture shall show a fresh surface not less than one-sixth of the sectional area of the ingot and shall be centrally located. In case the fracture test reveals piping or other defects, further discard may be made until the inspector is satisfied as to the soundness of the material.

4. Metallographic tests.-(a) When miscrosopic examinations are made, the specimens therefor shall be selected in the following manner, unless otherwise provided in the purchase order or drawing pertaining thereto: 
For material, the cross-sectional area of which is 50 square inches or more, at least 3 metallographic specimens shall be taken- 1 from the center, 1 from the outer edge, and 1 from a point halfway between the center and outer edge. If the end section is other than round, the specimen from the outer edge shall be taken from the middle of one of the sides.

For material, the cross-sectional area of which is less than 50 square inches, and which is made from the same heat, 2 billets shall be sclected; if possible, 1 from the first ingot poured in the heat and 1 from the last ingot poured in the heat. From each end of each billet so selected, 2 metallographic specimens shall be taken, 1 from the center and 1 from the outer edge. If the end section is other than round, the second specimen shall be taken from the middle of one of the sides.

(b) Specimens for microscopic examinations shall be at least one-fourth square inch in area and at least one-half inch in length.

\section{Packing and Marking}

VI. All material over 16 square inches in crosssectional area ordered under this specification shall be legibly hot stamped with the melt or heat number, and the words "For reforging" on each end of each billet, and the ends of the billet corresponding respectively to the top and bottom of the ingot from which it was rolled or forged shall be plainly marked "M" to indicate the top, or "B" to indicate the bottom. Material 16 square inches or less in cross-sectional area shall be stamped with the heat number only, unless otherwise required by the contract or purchase order.

\section{Additional Information}

VII. Table 2 is for the information of purchasing agencies and designers. It gives the minimum physical properties that may be expected of the material of the corresponding grade designation in Table 1 after normal working and heat treatment.

TABLE 1.-Chemical composition

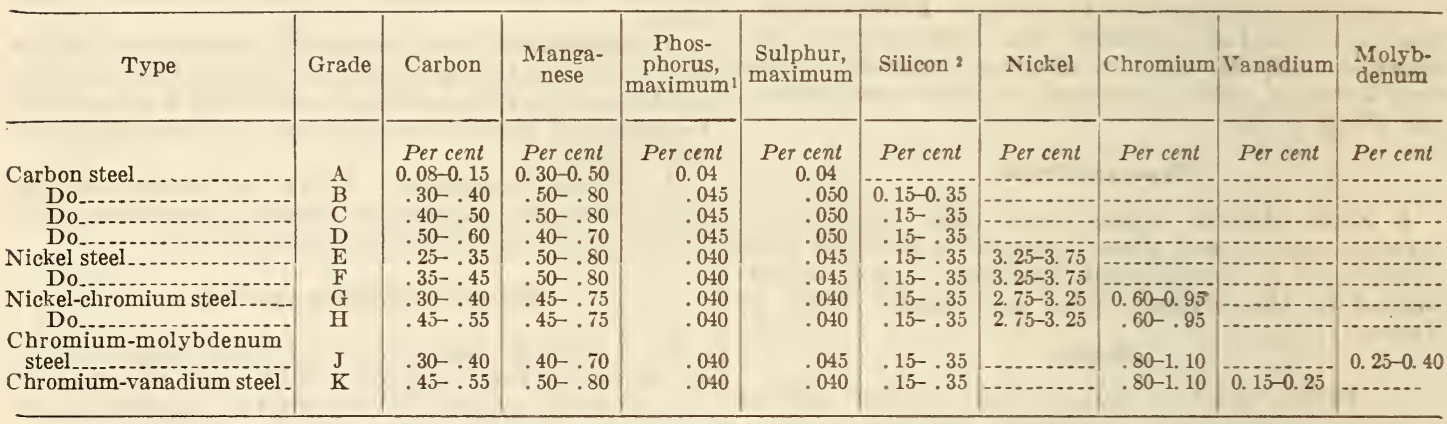

1 Maximum of 0.050 per cent permitted when acid steel is specified.

2 Waived except when acid steel is specified.

NotE.-The silicon requirements of Table 1 shall apply only when the contract or purchase order specifies an "acid steel"; that is, a steel made by either the acid open-hearth or acid electric process.

The grade letters assigned are not mandatory on the several departments. It is anticipated that these designations will be changed as soon as an authoritative numbering system for steel comes into general use.

The limits prescribed for phosphorus and sulphur are advisory only. Each department may insert such limits for phosphorus and sulphur in its specifications as are considered necessary for its particular use.

TABLE 2.-Physical properties

\begin{tabular}{|c|c|c|c|c|c|c|c|c|}
\hline \multirow{2}{*}{ Grade } & \multirow{2}{*}{$\begin{array}{c}\text { Tensile } \\
\text { strength, } \\
\text { mini- } \\
\text { mum }\end{array}$} & \multirow{2}{*}{$\begin{array}{l}\text { Yield } \\
\text { point, } \\
\text { mini- } \\
\text { mum }\end{array}$} & \multicolumn{2}{|c|}{$\begin{array}{l}\text { Elongation } \\
\text { in } 2 \text { inches, } \\
\text { minimum }\end{array}$} & \multicolumn{2}{|c|}{$\begin{array}{l}\text { Reduction } \\
\text { of area, } \\
\text { minimum }\end{array}$} & \multicolumn{2}{|c|}{$\begin{array}{l}\text { Cold-bend } \\
\text { test }\end{array}$} \\
\hline & & & $\begin{array}{l}\text { Longi- } \\
\text { tudi- } \\
\text { nal }\end{array}$ & $\begin{array}{l}\text { Trans- } \\
\text { verse }\end{array}$ & $\begin{array}{l}\text { Longi- } \\
\text { tudi- } \\
\text { nal }\end{array}$ & $\begin{array}{l}\text { Trans- } \\
\text { verse }\end{array}$ & $\begin{array}{l}\text { De- } \\
\text { grees }\end{array}$ & $\begin{array}{l}\text { Diam- } \\
\text { eter of } \\
\text { pin } \\
\text { equal } \\
\text { to- }\end{array}$ \\
\hline & $\begin{array}{r}\text { Lbs./in. }{ }^{2} \\
48,000\end{array}$ & $\begin{array}{r}\text { Lbs./in. }{ }^{2} \\
24,000\end{array}$ & $\begin{array}{l}\text { Per } \\
\text { cent } \\
32.0\end{array}$ & $\begin{array}{l}\text { Per } \\
\text { cent } \\
28.0\end{array}$ & $\begin{array}{l}\text { Per } \\
\text { cent }\end{array}$ & $\begin{array}{l}\text { Per } \\
\text { cent }\end{array}$ & 180 & $\begin{array}{c}\text { Inches } \\
\text { Flat. }\end{array}$ \\
\hline & 60,000 & 30, & 30. & 25 & 45. 0 & 40.0 & 180 & 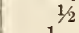 \\
\hline & & & & & & & & 1 \\
\hline & & & & & & & & \\
\hline & & 65 & & & & & & \\
\hline & 105,000 & 80,000 & 20.0 & 18.0 & 50.0 & 40.0 & 180 & 1 \\
\hline & 120,000 & 105,000 & 20 & 15 & 50.0 & 40 & 180 & 1 \\
\hline & 170,000 & 140,000 & 10.0 & 8.0 & 35.0 & 20.0 & & \\
\hline
\end{tabular}

603. IRON AND STEEL BARS, RODS, AND WIRE

\subsection{GENERAL ITEMS.}

AMERICAN RAILWAY ASSOCIATION, PURCHASES AND STORES DIVISION, STAND. ARDIZATION AND SIMPLIFICATION OF STORES STOCKS, PROCEEDINGS 1928, AND NO. D-V1-238, 1930

\section{SIMPLIFICATION OF STORES STOCKS}

(Recommendations contained in these publications relating to the standardization and simplification of stores stocks have been placed in this compilation under their proper commodity headings, as follows: 


\begin{tabular}{|c|c|c|}
\hline $\begin{array}{c}\text { Com- } \\
\text { modity } \\
\text { desig- } \\
\text { nation }\end{array}$ & Material & Page \\
\hline 603.0 & Iron and steel bars & 135 \\
\hline 603.32 & Carbon tool steel & 150 \\
\hline 604.10 & $\begin{array}{l}\text { Annealed steel sheets.- } \\
\text { Sheet steel and iron. }\end{array}$ & $\begin{array}{l}226 \\
236\end{array}$ \\
\hline $\begin{array}{l}604.20 \\
607.4\end{array}$ & Steel tubing & 463 \\
\hline 608.12 & Wire brads. & 572 \\
\hline 603.2 & Screws ...... & 574 \\
\hline $\begin{array}{l}608.31 \\
608.4\end{array}$ & $\begin{array}{l}\text { Bolts } \\
\text { Rivets.......... }\end{array}$ & $\begin{array}{l}588 \\
612\end{array}$ \\
\hline 608.51 & Cotter keys & 616 \\
\hline & Files_......-- & 834 \\
\hline $\begin{array}{l}621.35 \\
645.9\end{array}$ & $\begin{array}{l}\text { High-speed tool stee } \\
\text { Brass wood screws.. }\end{array}$ & 1128 \\
\hline & & \\
\hline
\end{tabular}

AMERICAN RAILWAY ASSOCIATION, PURCHASES AND STORES DIVISION, STANDARDIZATION AND SIMPLIFICATION OF STORES STOCK, NO. D-V1-135, 1926

STANDARD METHOD OF DESIGNATING CLASS, KIND, OR GRADE OF STEEL AND IRON BARS BY PAINTING THE ENDS

1. The coinmittee recommends the following color designations:

Engine bolt iron

Staybolt iron

2. Since there are so many different grades and rands of tool steel and welding rod being used, it has seemed impracticable to standardize color designation for this class of material.

AMERICAN RAILWAY ASSOCIATION, PURCHASES AND STORES DIVISION, STANDARDIZATION AND SIMPLIFICATION OF STORES STOCKS, PROCEEDINGS, 1929

\section{MILD STEEL OR OPEN HEARTH BARS}

1. One feature essential to the simplification of bar iron or steel is to bring about the adoption of one grade of steel to cover all (or at least 90 per cent) of the roads' general requirements. This has been done and is proving successful on many roads, and does away with carrying the same size bar in possibly three or four grades, such as merchant bar, mild steel, double refined, etc.

2. The committee recommends standard stocks of 24 rounds ranging in diameter from one-fourth inch to 4 inches; 11 squares ranging from one-half inch to $2 \frac{1}{2}$ inches; 4 half-rounds ranging from threefourths inch to $1 \frac{112}{2}$ inches; and 89 flat bars, bands, and plates ranging in size from $1 / 8$ by $1 / 2$ inch to 2 by 12 inches.

603.1 WROUGHT IRON BARS AND RODS.

AMERICAN BUREAU OF SHIPPING, RULES FOR BUILDING AND CLASSING STEEL VESSELS; QUALITY AND TESTING OF MATERIALS-SHIP CONSTRUCTION,1930

\section{ROUND AND FLAT IRON BARS}

(For general information and requirements, see 600.0 , p. 2.)

1. Round and flat iron bars are to have a tensile strength of not less than 45,000 pounds per square inch of prepared section, with an elongation of 20 per cent in 8 inches. For round bars under one- half inch in diameter and for flat bars under threeeighths inch thick, which are tested as rolled, and for round and flat bars, the test specimens for which are reduced by machining, an elongation of 16 per cent in 8 inches will be approved. On bars having a nominal area of 4 square inches or under, a test specimen must stand being bent cold through $180^{\circ}$ without fracture on the outside of the bent portion, the radius of the bend being twice the diameter or thickness of the specimen.

2. A test specimen when heated to a temperature between $1,700^{\circ}$ and $1,800^{\circ} \mathrm{F}$. must bend through $180^{\circ}$ without fracture on the outside of the bent portion as follows: For round bars under 2 square inches in section, flat on itself; for round bars 2 square inches or over in section; and for all flat bars, around a pin the diameter of which is equal to the diameter or thickness of the specimen.

3. Tension and bend test specimens are to be of the full section of the material as rolled, if possible; otherwise, the specimens are to be machined from the material as rolled. One bar of each size ordered shall be selected for testing from each 100 bars or fraction thereof. In the event of the above tests failing, one additional bar shall be selected and the tests repeated; and if these are satisfactory, the batch of bars represented shall be accepted. If the bars are not satisfactory, the batch of bars represented shall be rejected.

AMERICAN BUREAU OF SHIPPING, RULES FOR BUILDING AND CLASSING STEEL VESSELS; MATERIALS FOR MACHINERY, BOILERS, AND PIPING, 1930

\section{IRON STAYS FOR BOILERS}

(For general information and requirements, see 600.0, p. 1. For test requirements, see 600.1, p. 7.)

1. At least 4 per cent of the bars are to be selected by the surveyor from each diameter for tension and cold bend tests.

AMERICAN ELECTRIC RAILWAY ENGINEERING ASSOCIATION, STANDARD SPECIFICATION FOR REINED WROUGHTIRON BARS, E16-30, 1930

\section{WROUGHT-IRON BARS}

(Identical with A. S. T. M. A41-30, given below.)

AMERICAN ELECTRIC RAILWAY ENGINEERING ASSOCIATION, SPECIFICATION FOR MATERIALS FOR USE IN THE MANUFACTURE OF SPECIAL TRACKWORK, W104-28, 1928

(Approved as tentative American standard by the American Standards Association, January 23, 1929)

\section{SPECIAL TRACKWORK MATERIALS}

(See 606.3, p. 342.)

AMERICAN RAILWAY ASSOCIATION, MECHANICAL DIVISION, IRON, ENGINE BOLT, RECOMMENDED PRACTICE, 1926

\section{IRON, ENGINE BOLT}

\section{MANUFACTURE}

1. Process.-The iron shall be made wholly from all-pig puddled iron and shall be free from any admixture of iron scrap or steel. 
2. Definition of terms.-The term "iron scrap" applies only to foreign or bought scrap and does not include local mill products free from foreign or bought scrap.

\section{PHYSICAL PROPERTIES AND TESTS}

3. Tension tests.-(a) The iron shall conform to the following requirements as to tensile properties:

Tensile strength, pounds per square inch:

$11 / 4$ square inches and under in sectional area ..... 49,000-53,000 Over $11 / 4$ square inches in sectional area........ 47,000-53,000 Yield point, pounds per square inch:

$11 / 4$ square inch under in sectional area...... 0.6 tensile strength Over $1 \frac{1}{4}$ square inches to 4 square inches, inclusive, in seo-

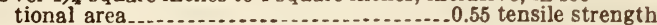
Over 4 square inches in sectional area........ 0.5 tensile strength Elongation in 8 inches, minimum . . . . . . . . . . . . . . . . . . per cent.. 25 Reduction in area, minimum................................. 40

(b) The yield point shall be determined by the drop of the beam of the testing machine. The speed of the crosshead of the machine shall not exceed three-fourths inch per minute. After passing the yield point the testing speed shall not exceed a maximum of 3 inches per minute.

4. Bend tests. - (a) Cold bend test.-The test specimen shall bend cold through $180^{\circ}$ around a pin the diameter of which is equal to the diameter of the specimen without fracture on the outside of the bent portion.

(b) Hot-bend test.-The test specimen, when heated to a temperature between $1,700^{\circ}$ and $1,800^{\circ}$ F., shall bend through $180^{\circ}$ flat on itself without fracture on the outside of the bent portion.

(c) Nick-bend test. - At the option of the purchaser, the following test shall be made: The specimen, when nicked 25 per cent around with a tool having a $60^{\circ}$ cutting edge, to a depth of not less than 8 nor more than 16 per cent of the diameter of the specimen, and broken, shall show a clean fiber, free from crystallization.

(d) Bend tests may be made by pressure or by blows.

5. Step test. - The test specimen, when reduced to 50 per cent of its original diameter by smoothturned steps, each 2 inches long and one-eighth inch less in diameter than the preceding, shall indicate freedom from injurious seams, laminations, and other internal defects.

6. Etch test. - The cross section of the test specimen shall be ground or polished and etched for a sufficient period to develop the structure. This test shall show the material to be free from steel.

7. Test specimens. - (a) Tension test specimens shall be the full section of material as rolled, if possible. Otherwise, the specimens shall be taken from the material as rolled; for bars $2 \frac{1}{2}$ inches or less in diameter, the axis of the specimen shall coincide with the axis of the bar; for bars over $2 \frac{1}{2}$ inches in diameter, the axis of the specimen shall be located at any point one-half the distance from the center to the surface and shall be parallel to the axis of the bar; and the specimens shall be turned to a diameter of 1 inch for a length of at least 9 inches, with enlarged ends.

(b) Bend and etch test specimens shall be of the full section of material as rolled, except that for bars over $1 \frac{1}{2}$ inches in diameter the cold-bend test specimen may be machined to not less than 1 square inch in section.

8. Number of tests. - (a) Bars of one size shall be sorted into lots of 100 each. Two bars shall be selected at random from each lot or fraction thereof, and tested as specified in sections 3 and 4 , but only one of these bars shall be tested as specified in section 6 .

(b) If any test specimen from either of the bars originally selected to represent a lot of material contains surface defects not visible before testing but visible after testing, or if a tension test specimen breaks outside the middle third of the gage length, one retest from a different bar will be allowed.

\section{PERMISSIBLE VARIATIONS IN GAGE}

9. Permissible variations.-(a) Round, square, and hexagon bars shall not vary more than the limits given in the following table:

\begin{tabular}{|c|c|c|}
\hline & \multicolumn{2}{|c|}{$\begin{array}{l}\text { Variations in } \\
\text { size }\end{array}$} \\
\hline & Under & Orer \\
\hline 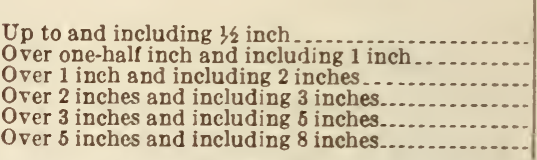 & $\begin{array}{c}\text { Inch } \\
0.007 \\
.010 \\
164 \\
363 \\
1 / 32 \\
14 \mathrm{e}\end{array}$ & $\begin{array}{r}\text { Inch } \\
0.007 \\
.010 \\
1 / 22 \\
364 \\
3 / 52 \\
\text { Is }\end{array}$ \\
\hline
\end{tabular}

(b) The widths or thickness of flat bars shall not vary more than 2 per cent from that specified.

\section{FINISH}

10. Finish. - The bars shall be smoothly finished and free from slivers, depressions, seams, crop ends, and evidences of being burned.

\section{MAREING}

11. Marking. - The bars shall be stamped or marked as designated by the purchaser.

\section{INSPECTION AND REJECTION}

(Requirements covering inspection, rejection, and rehearing conform to those specified in A. S. T. M. standard specifications for steel. See 605.0, p. 247.)

\section{AMERICAN RAILWAY ASSOCIATION, ME- CHANICAL DIVISION, STAY-BOLT IRON, HOLLOW, LOCOMOTIVE; RECOMMENDED PRACTICE, 1926}

\section{STAY-BOLT IRON, HOLLOW}

\section{MANUFACTURE}

1. Process. - The iron shall be rolled from a bloom, slab pile, or box pile, made wholly from reworked all-pig puddled iron or reworked knobbled charcoal iron. The puddle mixture and the component parts of the bloom, slab pile, or box pile shall be free from any admixture of iron scrap or steel.

2. Definition of terms.-(a) A bloom is a solid mass of iron that has been hammered into a convenient size for rolling.

(b) Slab pile. - A slab pile is built up wholly of flat bars of iron of the full length of the pile.

(c) Box pile. - A box pile is a pile the sides, top, and bottom of which are formed by four flat bars and the interior of which consists of a number of small bars the full length of the pile.

(d) Iron scrap. - This term applies only to foreign or bought scrap and does not include local mill products free from foreign or bought scrap.

\section{CHEMICAL PROPERTIES AND TESTS}

3. Chemical composition. - The iron shall conform to the following requirement as to chemical composition:

Manganese not over 0.05 per cent

4. Check analyses.-(a) An analysis may be made by the purchaser from a broken tension test specimen representing each lot as specified in section 10 (a). The chemical composition thus determined shall conform to the requirements specified in section 3. 
(b) Drillings for analysis shall be so taken as to represent the entire cross section of the specimen.

\section{PHYSICAL PROPERTIES AND TESTS}

5. Tension tests.-(a) The iron shall conform to the following requirements as to tensile properties:

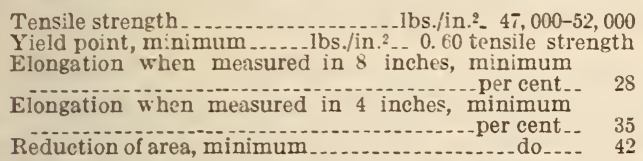

(b) The yield point shall be determined by the drop of the beam of the testing machine. The speed of the crosshead of the machine shall not exceed three-fourths inch per minute. For the tensile strength the speed shall not exceed 3 inches per minute.

6. Cold-bend tests.-(a) The test specimen shall bend cold through $180^{\circ}$ flat on itself in both directions without fracture on the outside of the bent portion.

(b) Bend tests may be made by pressure or by blows.

7. Splitting test.-For bars rolled or drawn hollow, a piece not less than 2 inches long shall be split open from end to end by driving a drift through the hole. The structure thus exhibited shall be free from signs of imperfect welding and the presence of slag or scale in the welds.

8. Etch tests.6-The cross section of the test specimen shall be ground or polished, and etched for a sufficient period to develop the structure. This test shall show the material to have been rolled from a bloom, slab pile, or box pile, and to be free from steel.

9. Test specimens. - All test specimens shall be of the full section of material as rolled.

10. Number of tests. - (a) Bars of each size shall be sorted into lots of 1,600 lineal feet. Two bars shall be selected at random from each lot or fraction thereof and tested as specified in sections 5, 6, and 7 , but only one of these bars shall be tested as specified in sections 3 and 8 .

(b) If any test specimen from either of the bars originally selected to represent a lot of material contains surface defects not visible before testing but visible after testing, or if a tension test specimen breaks outside the middle third of the gage length, the individual bar shall be rejected, and one retest from a different bar will be allowed.

\section{PERMISSIBLE VARIATIONS IN GAGE}

11. Permissible variations. - The bars shall be truly round within $0.01 \mathrm{inch}$. The diameter shall not be less than 0.005 inch under nor more than 0.020 inch over the specified diameter.

\section{FINISH}

12. Finish. - The bars shall be smoothly finished and free from slivers, depressions, seams, crop ends, and evidences of being burnt. The hole shall be as nearly axial and as nearly round as the best manufacturing practice permits, and shall have an area equivalent to that of a round hole three-sixteenths inch in diameter. The hole shall be free from slag and other obstructions.

\section{INSPECTION AND REJECTION}

(Requirements covering inspection and rejection, marking, and rehearing conform to those specified in A. S. T. M. standard specifications for steel (see 605.0 , p. 247 ), with the addition of the following item:)

\footnotetext{
${ }^{46}$ A solution of two parts water, one part concentrated hydrochloric acid, and one part concentrated sulphuric acid is recommended or the etch test.
}

Bars which will not take a clean, sharp thread, with dies in fair condition, or which develop defects in forging or machining, will be rejected, and the manufacturer shall be notified.

\section{AMERICAN RAILWAY ASSOCIATION, ME- CHANICAL DIVISION, STAY-BOIT IRON, SOLID, LOCOMOTIVE, RECOVIMENDED PRACTICE, 1926}

\section{STAY-BOLT IRON, SOLID}

(Identical with specification for stay-bolt iron, hollow, given above, except no splitting test is required, and that in tension tests the elongation in 8 inches, minimum is 30 per cent; and the reduction of area, minimum is 48 per cent; and the addition of the following items:)

In no case shall the variation in tensile strength between the maximum and minimum figures, for one offering of iron under these specifications, be more than 3,000 pounds.

Nick-bend test.-At the option of the purchaser, the following test shall be made: The specimen, when nicked 25 per cent around with a tool having a $60^{\circ}$ cutting edge, to a depth not less than 8 nor more than 16 per cent of the diameter of the specimen; and broken, shall show a clean fiber, free from crystallization.

\section{INSPECTION AND REJECTION}

(Requirements covering inspection and rejection, marking, and rehearing, conform to those specified in A. S. T. M. standard specifications for steel. See 605.0 , p. 247.)

AMERICAN RAILWAY ASSOCIATION, MECHANICAL DIVISION, WROUGHT-IRON BARS, REFINED, RECOMMENDED PRACTICE, 1914

\section{WROUGHT-IRON BARS}

\section{MANUFACTORE}

1. Process.-The finished product shall consist either of new muck-bar iron or a mixture of muckbar iron and scrap, but shall be free from any admixture of steel. Muck bars shall be made wholly from puddled iron.

\section{PHYSICAL PROPERTIES AND TESTS}

2. Tension tests.- Unless otherwise specified, the iron shall conform to the following requirements as to tensile properties:

Tensile strength................... lbs./in. ${ }^{2}{ }_{-} 47,000-53,000$

Elongation in 8 inches, minimum. .- per cent.-

3. Permissible variations in physical properties.(a) Tensile strength.-Large sections reduced or flats and rounds of one-half inch or under may show a tensile strength of $45,000-52,000$ pounds per square inch.

(b) Elongation. - Twenty per cent of the test specimens representing one size may show the following percentage of elongation in 8 inches:

Round bars:

Per cent $1 / 2$ inch or over, tested as rolled ................ 20

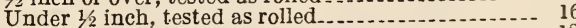
Reduced by machining

Flat bars: $3 / 8$ inch or over, tested as rolled . . . Under 8 inch, tested as rolled....................... 16

Reduced by machining .......................... 16

4. Bend tests. - (a) Cold-bend test.-Round, square, and hexagon bars under 2 square inches in section and flats less than three-fourths inch thick shall bend 
cold around a pin the diameter of which is equal to the diameter or thickness of the specimen, without fracture on the outside of the bent portion. Rounds, flats, and hexagon bars 2 square inches or over in section and all flat bars over three-fourths inch in thickness shall bend cold around a pin the diameter of which is equal to twice the diameter or thickness of the specimen.

(b) Hot-bend test.-The test specimen, when heated to a temperature between $1,700^{\circ}$ and $1,800^{\circ} \mathrm{F}$. (light cherry red), shall bend through $180^{\circ}$ without fracture on the outside of the bent portion, as follows: Round, flat, and hexagon bars under 2 square inches in section, flat on itself; round, flat, and hexagon bars 2 square inches and over in section, around a pin the diameter of which is equal to the diameter or thickness of the specimen.

(c) Nick-bend test.-The test specimen, when nicked 25 per cent around the round bar, and along one side for flat bars, with a tool having a $60^{\circ}$ cutting edge, to a depth of not less than 8 or more than 16 per cent of the diameter or thickness of the specimen, and broken, shall not show more than 10 per cent of the fractured surface to be crystalline.

5. Test specimen.-Tension and bend test specimens shall be of the full section of material as rolled if possible, otherwise the specimens shall be machined from the material as rolled; the axis of the machined specimen shall be located at any point one-half the distance from the center to the surface of round bars, or from the center to the edge of flat bars, and shall be parallel to the axis of the bar.

6. Number of tests.-(a) All bars of one size shall be piled separately. One bar from each 200 or less shall be selected at random and tested as specified.

(b) If any test specimen from the bar originally selected to represent a lot of material contains surface defects not visible before testing, but visible after testing, or if a tension test specimen breaks outside the middle third of the gage length, one retest from a different bar will be allowed.

\section{PER MISSIBLE VARIATIONS IN GAGE}

7. Permissible variations.-(a) Round bars shall conform to the standard A. R. A. limit gages (see 615.82 , p. 900 ).

(b) Flat bars.-The width and thickness of flat bars shall not vary more than 2 per cent from that specified.

(1) Sizes under 3 inches wide shall not be more than one thirty-second inch under or over size in width.

(2) Sizes 3 inches and over shall not be under size or more than one-sixteenth inch wider than ordered.

\section{INSPECTION AND REJECTION}

(Requirements covering inspection, rejection, and rehearing conform to those specified in A. S. T. M. standard specifications for steel. See 605.0, p. 247.)

\section{AMERICAN RAILWAY ASSOCIATION, SIG- NAL SECTION, SPECIFICATION FOR WROUGHT-IRON BARS, JULY, 1930}

\section{WROUGHT-IRON BARS}

(In respect to physical properties, determination of yield point, tensile tests, cold-bend tests, and etch tests, this specification is identical with A. S. T. M. A41-30, given below. In respect to permissible variations in elongation, hot-bend tests, nick-bend tests, tension and bend test specimens, number of tests, and permissible variations in gage (omitting references to sizes over and under 3 inches), it is identical with the specification of the American Railway Association for hollow stay-bolt iron, given above. In other respects it provides that:)

\section{PURPOSE}

The purpose of this specification is to provide for wrought-iron bars.

DRA WINGS

Contractor shall furnish with his tender, drawings forming an essential part thereof.

\section{TENDER}

The tender shall be for apparatus meeting the requirements of this specification. If the contractor wishes to vary from the specification, a tender may be submitted covering the apparatus he desires to furnish. This tender shall be accompanied by full information showing wherein variations from the requirements of the specification exists.

\section{MATERIAL AND WORKMANSHIP}

Material and workmanship shall be first class in every respect.

All sections must be true to size and shape ordered. Round iron must conform to the Master Car Builders' standard limit gages. (See 615.82, p. 900.)

Iron must be free from cracks, flaws, unwelded seams, and mechanical defects of all kinds, and must he free from steel scrap.

\section{INSPECTION}

Purchaser may inspect the material at all stages of manufacture.

Purchaser may inspect the completed product to determine that the requirements of this specification have been met.

If the material has not been accepted at point of production and if, upon arrival at destination, it does not meet the requirements of this specification, it may be rejected, and the contractor, upon request, shall advise the purchaser what disposition is to be made of the defective material. The contractor shall pay all freight charges.

If the purchaser is to make inspection at point of production it shall be so stated.

\section{TESTS}

Tests may be made at point of production, or on samples submitted, and may also be made at destination.

Contractor shall give the purchaser sufficient notice of time when material will be ready for testing.

Contractor shall provide, at point of production, apparatus and labor for making the required tests under supervision of the purchaser.

\section{PACKING}

Material shall be so prepared as to permit convenient handling and to protect against loss or damage during shipment.

\section{MARIING}

Purchaser's order, requisition, and package number; name of consignor; and name and address of consignee shall be plainly marked on outside of package.

Detail list of loose pieces, containers, and their contents shall be furnished for each shipment. Where carload shipments are made, routing and car identification shall be shown. Where carload shipments are made, name and address of consignee may be omitted.

\section{WARRANTY}

Contractor shall warrant the material covered by this specification to be free from defects in material 
and workmanship under ordinary use and service, his obligation under this warranty being limited to making, at point of production, any part or parts to replace those which shall be found defective after shipment to the purchaser. This warranty shall not apply to any apparatus which has been subjected to misuse, negligence, or accident.

\section{AMERICAN SOCIETY OF MECHANICAL ENGINEERS, SPECIFICATIONS FOR BOIL- ER-RIVET, STAY-BOLT, AND EXTRA- REFINED BAR IRON, S-16, 1930}

\section{WROUGHT-IRON BARS}

(In substantial agreement with A. S. T. M. A84-30, given below.)

\section{AMERICAN SOCIETY OF MUNICIPAL EN- GINEERS, STANDARD SPECIFICATIONS FOR SEWERS, A-27, 1927}

\section{WROUGHT IRON}

Quality.-All wrought iron to be used on the work shall be of the class known as merchant iron, grade $\mathrm{A}$, and must be free from cinder spots, injurious flaws, buckles, blisters, or cracks. Wrought iron shall be made by the puddling process, or rolled from fagots or piles made from wrought-iron scrap, alone or with muck bar added. All iron shall have a minimum tensile strength of 50,000 pounds per square inch and an elastic limit of at least 25,000 pounds per square inch. A specimen cut from the iron, when nicked on one side and bent back through an angle of $180^{\circ}$, shall show a long, clean, silky fiber; free from slag, dirt, or coarse crystalline spots.

Finishing painting-Wrought iron before leaving the factory shill be thoroughly cleaned and dipped in a preparation of asphalt or coal tar and oil.

\section{AMERICAN SOCIETY FOR TESTING MATE- RIALS, STANDARD SPECIFICATIONS FOR REFINED WROUGHT-IRON BARS, DESIGNATION A41-30, 1930}

\section{REFINED WROUGHT-IRON BARS}

(In respect to permissible variations in elongation, hot-bend tests, nick-bend tests, test specimens, number of tests, permissible variations in gage (omitting references to sizes under and over 3 inches), this specification conforms to the specification of the American Railway Association for wrought-iron bars, refined, given above. In respect to finish, inspection and rejection, and rehearing, it conforms to A. S. T. M. standard specifications for steel. See 605.0, p. 247 . In other respects it provides that:)

\section{MANUFACTURE}

1. The bars shall be made from wrought iron, and may consist either of all new iron or a mixture of new iron and scrap, but shall be free from any admixture of steel.

\section{PHYSICAL PROPERTIES AND TESTS}

2. (a) The iron shall confoim to the following minimum requirements as to tensile properties:

\footnotetext{
Tensile strength _.............. (See sections 3 and 4 .)

Yield point ...do_... 25,000

Elongation in 8 inches per cent.
}

(b) The yield point shall be determined by the drop of the beam of the testing machine. The speed of the crosshead of the machine shall not exceed three-fourths inch per minute.

3. For material over 4 square inches in sectional area, a reduction of 500 pounds per square inch from the tensile strength specified in section 2 will be permitted for each additional 2 square inches and a proportionate amount of reduction for fractional parts thereof; provided that the tensile strength shall not be less than 45,000 pounds per square inch.

4. Twenty per cent of the test specimens representing one size, may show tensile strengths 1,000 pounds per square inch under, or 5,000 pounds per square inch over that specified in sections 2 and 3 ; but no specimen shall show a tensile strength less than 45,000 pounds per square inch.

5. (a) Cold-bend tests. - Cold-bend tests will be made only on bars having a nominal sectional area of 4 square inches or under, in which case the test specimen shall stand being bent cold through $180^{\circ}$ without fracture on the outside of the bent portion, around a pin the diameter of which is equal to twice the diameter or thickness of the specimen.

(b) Bend tests may be made by pressure or by blows.

6. (a) The cross section of the test specimen shall be ground or polished, and etched for a sufficient period to develop the structure. This test shall show the material to be free from steel.

(b) Etch test specimens shall be of the full section of material as rolled.

\section{AMERICAN SOCIETY FOR TESTING MA- TERIALS, STANDARD SPECIFICATIONS FOR STAY BOLT, ENGINE-BOLT, AND. EXTRA REFINED WROUGHT IRON BARS, DESIGNATION A84-30, 1930}

\section{WROUGHT-IRON BARS}

(In respect to definitions of terms, check analysis, determination of yield point, number of tests, and permissible variations in gage of grade $A$, this specification conforms to the specification of the American Railway Association for stay-bolt iron, hollow, locomotive, given above. In respect to workmanship and finish, marking, inspection and rejection, and rehearing, it conforms to A. S. T. M. standard specifications for steel. See 605.0, p.247. In other respects it provides that:)

1. These specifications cover three grades of bar iron: Grade A, stay-bolt iron; grade B, engine-bolt iron; and grade $\mathrm{C}$, extra refined wrought iron bars.

\section{MANUFACTURE}

2. Grade A.-The bars shall be rolled from a bloom, slab pile, or box pile made from reworked wrought iron or reworked knobbled charcoal iron. The original muck or knobbled bar shall be twice piled and rerolled. All bars shall be the full length of the piles. The wrought iron and the component parts of the bloom, slab pile, or box pile shall be free from any admixture of iron scrap or steel.

Grades $B$ and $C$.- The bars shall be made from wrought iron and shall be free from any admixture of iron scrap or steel.

\section{CEEMICAL PROPERTIES AND TESTS}

4. Grade A.-At the option of the purchaser and when so specified, the iron shall conform to the following requirement as to chemical composition:

Manganese............................. Not over 0.10 per cent 
PHYSICAL PROPERTIES AND TESTS

6. (a) The iron shall conform to the following requirements as to tensile properties:

\begin{tabular}{|c|c|c|c|}
\hline & Grade A & Grade $\mathbf{B}$ & Grade C \\
\hline 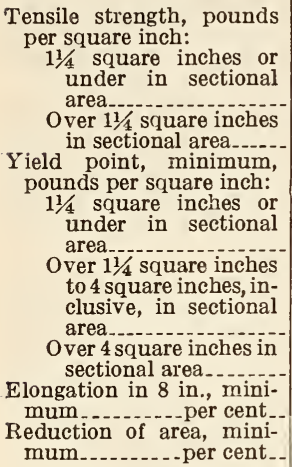 & $\begin{array}{c}48,000-52,000 \\
\text { (1) }\end{array}$ & $\begin{array}{r}0.6 \\
\text { (Ten.Str.) } \\
0.55 \\
\text { (Ten. Str.) } \\
0.5 \\
\text { (Ten. Str.) } \\
25 \\
40\end{array}$ & $\mid \begin{array}{c}0.6 \\
\text { (Ten.Str.) } \\
0.55 \\
\text { (Ten.Str.) } \\
0.5 \\
\text { (Ten.Str.) } \\
{ }^{3}{ }_{25} \\
{ }^{3} 37\end{array}$ \\
\hline
\end{tabular}

7. (a) Grade A.-For bars seven-eighths inch in diameter, a reduction in the minimum elongation in 8 inches specified in section $6(a)$ shall be permitted but in no case shall the elongation be less than 28 per cent. For bars three-fourths inch or under in diameter, the elongation shall be not less than 25 per cent.

(b) Grade A.-For bars over $1 \frac{1}{4}$ square inches in sectional area, the following deductions from the minimum requirements specified in section $6(a)$ shall be made for each square inch of nominal section above $1 \frac{1}{4}$ square inches:

Tensile strength _. 250 pounds, but not under 46,000 pounds per square inch.

Reduction of area.. 3 per cent, but not under 40 per cent.

(c) Grade A.-For flats of all sizes the minimum reduction of area shall be 40 per cent.

(d) Grade C.-For material over 4 square inches in sectional area, the following deductions from the minimum requirements specified in section $6(a)$ shall be made for each square inch of nominal section above 4 square inches:

Tensile strength _. 250 pounds, but not under 46,000 pounds per square inch.

Elongation -...-..- 0.5 per cent, but not under 22 per cent.

Reduction of area.- 1.0 per cent, but not under 32 per cent.

8. (a) Cold-bend tests-Grade A.-The test specimen shall withstand being bent cold through $180^{\circ}$ flat on itself in both directions, without fracture on the outside of the bent portions.

Grade B.-The test specimen shall withstand being bent cold through $180^{\circ}$ around a pin, the diameter of which is equal to the diameter of the specimen, without fracture on the outside of the bent portion.

Grade C.-Cold-bend tests will be made only on bars having a nominal section area of 4 square inches or under, in which case the test specimen shall withstand being bent cold through $180^{\circ}$ without fracture on the outside of the bent portion, around a pin the diameter of which is equal to the thickness of the specimen.

(b) Hot-bend tests.-

Grades $B$ and $C$. - The test specimen when heated to a temperature between $1,700^{\circ}$ and $1,800^{\circ} \mathrm{F}$. shall stand being bent through $180^{\circ}$ flat on itself without fracture on the outside of the bent portion.

(c) Nick-bend tests-All grades. - The test specimen when nicked 25 per cent around with a tool having a $60^{\circ}$ cutting edge, to a depth of not less than
8 nor more than 16 per cent of the diameter of the specimen, and broken, shall show a wholly fibrous fracture.

$(d)$ Bend tests may be made by pressure or by blows.

9. The cross section of the test specimen shall be ground or polished, and etched ${ }^{47}$ for a sufficient period to develop the structure. This test shall show the material as follows:

Grade A.-The material to have been rolled from a bloom, slab pile, or box pile and to be uniform and free from steel.

Grades $B$ and $C$.- The material to be uniform and free from steel.

10. (a) Tension and bend test specimens shall be of the full section of material as rolled, if possible. Otherwise the specimens shall be machined from the material as rolled; the axis of the specimen shall be located at any point midway between the center and surface of round bars, or midway between the center and edge of flat bars, and shall be parallel to the axis of the bar.

(The table of standard limit gages for engine-bolt iron is the same as that in the specification of the American Railway Association for limit gages for round iron. See 615.82 , p. 900 . Grade B bars shall conform to this table. Grade $\mathrm{C}$ bars shall not vary, in width or thickness, more than 2 per cent from that specified.)

(b) Etch test specimens shall be of the full section of material as rolled.

\section{AMERICAN SOCIETY FOR TESTING MA- TERIALS, STANDARD SPECIFICATIONS FOR COMMON IRON BARS, DESIGNA- TION A85-27, 1927}

\section{COMMON IRON BARS}

(In respect to physical properties, determination of yield point, permissible variations in tensile strength, cold-bend tests, etch tests, and etch-test specimens, this specification is identical with A. S. T. M., A41-30, given above. In respect to permissible variations in elongation, hot-bend tests, nick-bend tests, test specimens, number of tests, and permissible variations in gage (omitting references to sizes under and over 3 inches), it is identical with the specification of the American Railway Association for wrought-iron bars, refined given above. In respect to finish, inspection and rejection, and rehearing, it conforms to A. S. T. M. standard specifications for steel. See 605.0, p. 247. In other respects it provides that:)

\section{MANDFACTURE}

1. Common iron bars may be made from reworked iron scrap or a reworked mixture of iron and steel scrap, but shall be free from large areas of steel.

\section{CHEMICAL PROPERTIES AND TESTS}

2 . The iron shall conform to the following requirement as to chemical composition:

Manganese Not over 0.30 per cent

3. (a) An analysis may be made by the purchaser from a broken tension-test specimen representing each lot as specified. The chemical composition thus determined shall conform to the requirement specified in section 2 .

47 A solution of 10 per cent hydrochloric acid (specific gravity 1.19) 30 per solution of 10 per cent hydrochloric acid (specific gravity 1.19 ) 30 per cent sulphuric acid (specific gravity 1.84 ), and 60 per cent
water; or 25 per cent nitric acid (specific gravity 1.42 ), and 75 per cent water; or 25 per cent nitric acid (specific gra
water, is recommended for the etch test. 
(b) Drillings for analysis shall be so taken as to represent the entire cross section of the specimen.

4. Appearance of fracture. - The broken ends of the tension-test specimen shall show a uniform fibrous structure similar to that of wrought iron.

5. For material over 4 square inches in sectional area, a reduction of 250 pounds per square inch from the tensile strength specified will be permitted for each additional square inch, and a proprotionate amount of reduction for fractional parts thereof; provided that the tensile strength shall not be less than 45,000 pounds per square inch.

\section{AMERICAN SOCIETY FOR TESTING MA- TERIALS, STANDARD SPECIFICATIONS FOR HOLLOW ROLLED STAY-BOLT IRON, DESIGNATION A86-30, 1930}

\section{HOLLOW ROLLED STAY-BOLT IRON}

(Identical with specification of American Railway Association for hollow stay-bolt iron, given above, except that manganese shall not be over 0.10 per cent, the tensile strength ranges from 48,000 to 52,000 pounds per square inch, and the reduction of area, minimum, is 48 per cent.)

\section{INSPECTION AND REJECTION}

(Requirements covering inspection and rejection, marking, and rehearing conform to those specified in A. S. T. M. standard specifications for steel. See 605.0$, p. 247.$)$

\section{UNITED STATES GOVERNMENT, FEDERAI SPECIFICATIONS BOARD, FEDERAL SPECIFICATION FOR IRON BAR, WROUGHT, REFINED, NO. 391, MARCH 1. 1926. (CURRENT DESIGNATION : QQ-I-686)}

\section{IRON BARS}

(This specification is substantially the same as A. S. T. M., A41, given above, except that No. 391 includes F. S. B., No. 339 (current designation: QQ-M-151) general specifications for metals, for which see 600.0, p. 3.)

\subsection{CARBON-STEEL BARS AND RODS. \\ 603.21 STEEL STOCK FOR BOLTS, NUTS, RIVETS, AND SCREWS.}

\section{AMERICAN BUREAU OF SHIPPING, RULES FOR BUILDING AND CLASSING STEEL VESSELS; QUALITY AND TESTING OF MATERIALS, SHIP CONSTRUCTION, 1930}

\section{STEEL RIVET BARS}

(For general information and requirements, see 600.0, p. 2.)

For steel rivet bars at least one tension test shall be taken from each heat. Where the heat wieght exceeds 22,000 pounds, a second tension test will be required. For rivet bars no bend tests will be required. The tensile breaking strength of the bars shall be between the limits of 55,000 and 65,000 pounds per square inch, with an elongation of not less than 25 per cent on a gage length of eight times the diameter, or 31 per cent on three and one-half times the diameter of the test specimen. Intermediate proportions of length to diameter are to have intermediate percentages.
AMERICAN BUREAU OF SHIPPING, RULES FOR BUILDING AND CLASSING STEEL VESSELS ; MATERIALS FOR MACHINERY, BOILERS, AND PIPING, 1930

\section{STEEL BARS AND STAYS FOR BOILERS}

(For general information and requirements, see 600.0 , p. 1. For test requirements, see 600.1, p. 7.)

At least one tension, one cold-bend, and one quench-bend test shall be made for each 2,000 pounds or less of bars rolled from each heat.

AMERICAN RAILWAY ASSOCIATION, MECHANICAL DIVISION, RIVET STEEL AND RIVETS FOR LOCOMOTIVE TENDERS AND PASSENGER AND FREIGHT EQUIPMENT CARS, 1925

\section{RIVET STEEL AND CAR RIVETS}

SCOPE

1. These specifications cover steel bars for the manufacture of rivets, and finished steel rivets for locomotive tanks and underframes and passenger and freight equipment cars.

\section{MANUFACTURE}

2. The steel shall be made by the open-hearth process.

\section{CHEMICAL PROPERTIES AND TESTS}

3. The steel shall conform to the following requirements as to chemical composition:

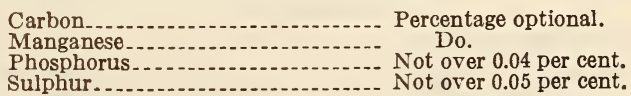

Sulphur.

\section{LADLE AND CHECK ANALYSES}

(Same as in American Railway Association specification for steel tank plates. See 621.32, p. 1124.)

\section{PHYSICAL PROPERTIES AND TESTS}

A. Requirements for Bars

4. Tension tests.-The bars shall conform to the following requirements as to tensile properties:

Tensile strength ............................ lbs./in. ${ }^{2}-4_{-1} \quad 45,000-60,000$ Elongation in 8 inches (minimum) (But need not exceed 30 per cent.)

5. Bend test.-The test specimen shall bend cold through $180^{\circ}$ flat on itself without cracking on the outside of the bent portion.

\section{B. Requirements for Rivets}

6. Bend tests.-The rivet shank shall bend cold through $180^{\circ}$ flat on itself, as shown in Figure 52 without cracking on the outside of the bent portion.

7. Flattening test.-Rivet heads shall be flattened sideways, when cold, to a thickness of one-third, and when at a driving heat to a thickness of one-fourth the original diameter of the shank, without splitting.

8. Test specimens-(a) Bars.-Tension and bend test specimens shall be of the full section of bars as rolled.

(b) Rivets.-Bend and flattening test specimens shall be of the full section of rivets as manufactured.

(c) When accurate account of the material has been kept and the melts can be identified, only one 
set of specimens for each diameter in each melt shall be taken from either bars or the finished rivets.

9. Number of tests-(a) Bars.-One tension and one bend test shall be made from each lot of 200 bars, or from each diameter in any one melt, each of which shall conform to the requirements specified.

(b) Rivets.- One bend and one flattening test shall be made from each lot of $50 \mathrm{kegs}$ of each diameter or

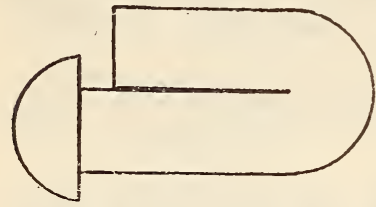

FIguRE 52.-Rivet shank bent cold flat on itself from each diameter in any one melt, each of which shall conform to the requirements specified.

(c) If any test specimen from the bar or rivets originally selected to represent a lot of bars or rivets contain surface defects not visible before testing but visible after testing, or if a tension-test specimen breaks outside the middle third of the gage length, one retest shall be allowed.

\section{PERMISSIBLE VARIATIONS IN GAGE}

(Identical with A. S. T. M., A31, given below.)

10. Dimensions of rivet heads. - Rivet heads shall conform to the dimensions shown on the purchaser's standard drawings when so specified, otherwise the heads shall conform, within 5 per cent above or below, to the dimensions shown in the accompanying table.
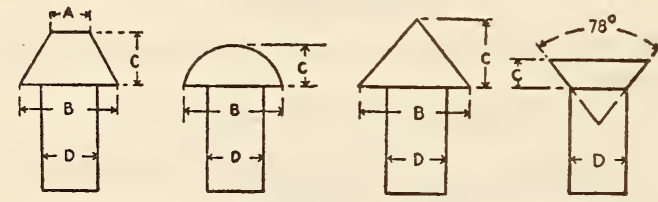

Figure 53

\begin{tabular}{|c|l|l|l|}
\hline Cone head & Button head & Steeple head & $\begin{array}{l}\text { Countersunk } \\
\text { head }\end{array}$ \\
\hline $\begin{array}{c}A=15 / 16 \text { by } D . \\
B=134 \text { by } D . \\
C=7 / 8 \text { by } D .\end{array}$ & $\begin{array}{l}B=134 \text { by } D . \\
C=3 / 4 \text { by } D .\end{array}$ & $\begin{array}{l}B=2 \text { by } D . \\
C=D .\end{array}$ & $\begin{array}{l}C=1 / 2 \text { by } D . \\
\text { Taper }=78^{\circ} .\end{array}$ \\
\hline
\end{tabular}

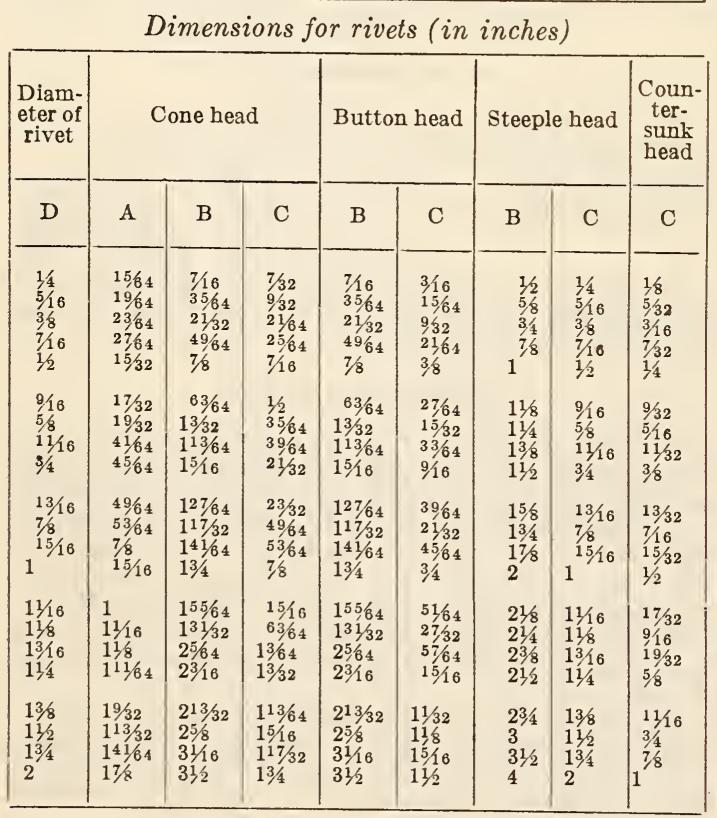

\section{WORKMANSHIP AND FINISH}

11. Workmanship.-The rivets shall be concentric, true to form, and shall be made in a workmanlike manner.

12. Finish.-The finished bars and rivets shall be free from injurious defects and shall have a workmanlike finish.

\section{MARIING}

13. Marking-(a) Bars.-Rivet bars shall, when ready for shipment, be properly separated and marked with the name or brand of the manufacturer and the melt or lot number for identification.

(b) Rivets. - Kegs of finished rivets shall, when ready for shipment, be properly marked with the name or brand of the manufacturer, diameter of rivets, and the melt or lot numbers for identification.

(c) Samples.-The melt or lot number shall be legibly stamped on each test specimen representing a lot of bars. Samples representing a lot of rivets shall be marked in a manner that will not impair their value for test purposes.

\section{INSPECTION AND REJECTION}

(Requirements covering inspection, rejection, and rehearing conform to A.S. T. M. standard specifications for steel, paragraphs 7, 8, and 9. See 605.0. p. 247.)

\section{AMERICAN RAILWAY ASSOCIATION, ME. CHANICAL DIVISION, RIVET STEEL AND RIVETS FOR STEAM BOILERS AND OTHER PRESSURE VESSELS, 1923}

\section{RIVET STEEL FOR BOILERS}

\section{SCOPE}

1. These specifications cover steel bars for the manufacture of rivets and finished steel rivets.

\section{MANOFACTORE}

2. The steel shall be made by the open-hearth process.

\section{CHEMICAL COMPOSITION}

The steel shall conform to the following requirements as to chemical composition:

\begin{tabular}{|c|c|c|}
\hline & Bars & Rivets \\
\hline $\begin{array}{l}\text { Carbon.......- } \\
\text { Manganese.... } \\
\text { Phosphorus...- } \\
\text { Sulphur.....-. }\end{array}$ & $\begin{array}{l}0.30 \text { to } 0.50 \text { per cent } \\
\text { Not orer } 0.04 \text { per cent.-- } \\
\text { Not orer } 0.045 \text { per cent.- }\end{array}$ & $\begin{array}{l}\text { Not orer } 0.18 \text { per cent. } \\
0.30 \text { to } 0.50 \text { per cent. } \\
\text { Not over } 0.04 \text { per cent. } \\
\text { Not orer } 0.045 \text { per cent. }\end{array}$ \\
\hline
\end{tabular}

\section{LADLE AND CHECK ANALYSES}

(Same as in American Railway Association specification for steel tank plates. See 621.32, p. 1124.)

\section{PHYSICAL PROPERTIES AND TESTS}

(Requirements covering tension tests for bars, determination of yield point, bend tests for bars and rivets, and quench-bend test all are the same as in A. S. T. M. A31, given below. Requirements covering flattening test for rivet heads, and test specimens for bars and rivets, are the same as in the A. R. A. specification for rivet steel and rivets for locomotive tenders and equipment cars, given below, with the following additional provision as to cold-worked test specimens:) 
(d) If rivet bars or rivets have been cold worked the test specimens shall be heated to a drawing heat and allowed to cool in air before testing.

3. Number of tests-(a) Bars.-Two tension, two cold-bend and two quench-bend tests shall be made from each lot of 200 bars, or from each diameter in any one melt, each of which shall conform to the requirements specified.

(b) Rivets.-One cold-bend, one quench-bend, and one flattening test shall be made from each lot of 50 kegs of each diameter or from each diameter in any one melt, each of which shall conform to the requirements specified.

(c) If any test specimen from the bar or rivets originally selected to represent a lot of bars or rivets contain surface defects not visible before testing, but visible after testing, or if the percentage of elongation of any tension test specimen be less than that specified, and any part of the fracture is outside the middle third of the gage length, as indicated by scribe scratches marked on the specimen before testing, a retest shall be allowed.

\section{PERMISSIBLE VARIATIONS}

(Same as A. S. T. M. A31, given below.)

\section{MARKING}

(Same as A. R. A. specification for rivet steel and rivets for locomotive tenders and equipment cars, given above.)

\section{INSPECTION AND REJECTION}

(Requirements covering workmanship and finish, inspection and rejection, and relhearing conform to A. S. T. M. standard specifications for steel, paragraphs $5,7,8$, and 9 . See 605.0 , p. 247.)

AMERICAN RAILWAY ENGINEERING ASSOCIATION, SPECIFICATIONS FOR STEEL RAILWAY BRIDGES, 1924

\section{STRUCTURAL AND RIVET STEEL}

(See 605.11, p. 249. See also 611.11, p. 636; 611.41 , p. 707 ; and 611.51 , p. 753 .)

\section{AMERICAN SOCIETY OF MECHANICAL ENGINEERS, BOILER-CONSTRUCTION CODE SPECIFICATIONS FOR BOILER RIVET STEEL, S-5, 1930}

\section{BOILER RIVET STEEL}

(Identical with A. S. T. M. A31-24, given below.)

\section{AMERICAN SOCIETY OF MECHANICAL ENGIN EERS, BOILER-CONSTRUCTION CODE SPECIFICATIONS FOR STEEL BARS FOR STAY BOLTS, S-6, 1930}

\section{STEEL BARS FOR STAY BOLTS}

\section{A. BARS}

1. Solid or hollow stay bolts.-Steel for solid or hollow bars for stay bolts shall conform to the requirements for boiler rivet steel, except as follows:

(a) Tension tests. - The bars shall conform to the following requirements as to tensile properties:

\begin{tabular}{|c|c|}
\hline 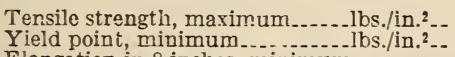 & $\begin{array}{l}60,000 \\
26,000\end{array}$ \\
\hline Elongation in 8 inches, minimum & $1,500,000$ \\
\hline
\end{tabular}

(b) Permissible variation in gage. - Solid or hollow bars for stay bolts, not exceeding $11 / 4$ inches in diameter, which are to be threaded as rolled, shall be truly round within 0.01 inch and shall not vary more than 0.005 inch under or more than 0.01 inch over. All other bars for stay bolts shall conform to the specified tolerances for steel bars.

\section{B. SEAMLESS STEEL TUBING FOR STAY BOLTS}

2. Scope.-These specifications cover seamless steel tubing for threaded stay bolts. They do not apply to stay tubes for locomotive boilers.

3. Process. - The steel shall be made by the openhearth process.

4. Chemical composition. - The steel shall conform to the following requirements as to chemical composition:

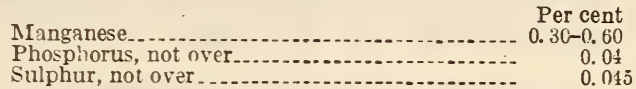

5. Check analyses.-(a) Analyses of two tubes in each lot of 250 tubes or less, may be made by the purchaser. The chemical composition thus determined shall conform to the requirements specified. Drillings for analyses shall be taken from several points around each tube selected for analysis.

(b) If the analysis of one tube does not conform to the requirements specified, analyses of two additional tubes from the same lot shall be made, each of which shall conform to the requirements specified.

6. Tension tests. - The tubes shall conform to the following requirements as to tensile properties:

$$
\begin{aligned}
& \text { Tensile strength, maximum.....-lbs./in. }{ }^{2}--\quad 60,000 \\
& \text { Y ield point, minimum _.._-_._-_lbs./in. }{ }^{2} \quad 25,000 \\
& \text { Elongation in } \mathrm{s} \text { inches, minimum } 1,500,000 \\
& \overline{\text { Tensile strength }}
\end{aligned}
$$

7. Test specimens.-Test specimens shall consist of either full-section specimens or of sections cut longitudinally from a tube.

8. Number of tests.-(a) Two tension tests from each 2,500 feet of tubing shall be made, or in case the tubing from separate melts can be identified, two tension tests shall be made from each melt.

(b) If the results of the physical tests of one specimen from any lot do not conform to the requirements specified, two additional specimens from the same lot may be tested, each of which shall conform to the requirements specified.

(c) If any test specimen develops flaws, it may be discarded and another specimen substituted.

(d) If the percentage of elongation of any tensiontest specimen is less than that specified, and any part of the fracture is outside the middle third of the gage length, as indicated by scribe scratches marked on the specimen before testing, a retest shall be allowed.

9. Fracture test.-A special fracture test shall be made from each 2,500 feet of tubing by cutting partly through the wall of the tube and breaking it off when removing the crop end. The fracture thus produced shall be fine grained.

10. Permissible variation in gage.-The finished tubing shall be circular within 0.015 inch, and the outside diameter shall not vary more than 0.01 inch over or under the size specified.

(Requirements covering finish, inspection and rejection, and rehearing conform to A. S. T. M. standard specifications for steel, paragraphs $5,7,8$, and 9 . See 605.0, p. 247.)

\section{AMERICAN SOCIETY OF MECHANICAL ENGINEERS, SMALL RIVETS, SEVEN- SIXTEENTHS INCH NOMINAL DIAME- TER AND UNDER, B-18a, $192 \%$}

[Approved as American Standard by the American Standards Association, July 1927]

SMALL RIVETS

(See 608.4, p. 612.) 
AMERICAN SOCIETY OF MECHANICAL ENGINEERS, TINNERS', COOPERS', AND BELT RIVETS, B-18g, 1928

Approved as tentative American Standard by the American Standards Association, May, 192\%

TINNERS', COOPERS', AND BELT RIVETS

(See 608.4, p. 614.)

AMERICAN SOCIETY FOR TESTING MATERIALS, STANDARD SPECIFICATIONS FOR STRUCTURAL STEEL FOR BRIDGES, DESIGNATION A\%-29, 1929

STRUCTURAL STEEL FOR BRIDGES

(See 605.11, p. 249.)

AMERICAN SOCIETY FOR TESTING MATERIALS, STANDARD SPECIFICATIONS FOR STRUCTURAL STEEL FOR BUILDINGS, DESIGNATION A9-29, 1929

\section{STRUCTURAL STEEL FOR BUILDINGS}

(See 60b.12, p. 252.)

\section{AMERICAN SOCIETY FOR TESTING MA- TERIALS, STANDARD SPECIFICATIONS FOR RIVET STEEL FOR SHIPS, DESIGNA- TION A13-24, 1924}

\section{RIVET STEEL FOR SHIPS}

(See 605.15, p. 269.)

\section{AMERICAN SOCIETY FOR TESTING MA- TERIALS, STANDARD SPECIFICATIONS FOR BOILER RIVET STEEL, DESIGNA- TION A31-24, 1924}

\section{BOILER RIVET STEEI}

(In respect to manufacture, determination of yield point, cold-bend tests, test specimens, flaws in test specimens, physical properties and tests of rivets, and workmanship and finish, this specification is identical with A. S. T. M. A13. See 605.15, p. 269. In respect to ladle and check analyses it is identical with A. S. T. M. A70. See 604.11, p. 227. In respect to marking, inspection and rejection, and rehearing it conforms to A. S. T. M. standard specifications for steel. See 605.0, p. 247. Its original provisions are as follows:)

\section{REQUIREMENTS FOR ROLIED BARS}

Chemical Composition

1. The steel shall conform to the following requirements as to chemical composition:

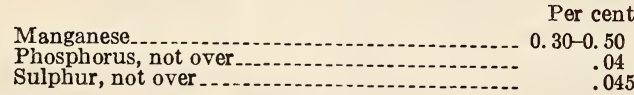

Physical Properties and Tests

2. The bars shall conform to the following requirements as to tensile properties:

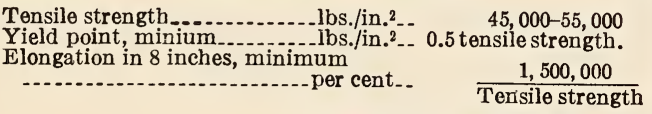

But need not exceed 30 per cent
3. Quench-bend tests. - The test specimen, when heated to a light cherry red as seen in the dark (not less than $1,200^{\circ}$ F.), and quenched at once in water the temperature of which is between $80^{\circ}$ and $90^{\circ}$ F., shall stand being bent through $180^{\circ}$ flat on itself without cracking on the outside of the bent portion.

4. Number of tests. - Two tension, two cold-bend, and two quench-bend tests shall be made from each melt, each of which shall conform to the requirements specified.

\section{Permissible Variations in Diameter}

5. The diameter of hot-finished rivet bars shall not vary from that specified by more than the amount given in the following table:

\begin{tabular}{|c|c|c|c|}
\hline \multirow{2}{*}{ Diameter (in inches) } & \multicolumn{2}{|c|}{$\begin{array}{l}\text { Variations in } \\
\text { diameter }\end{array}$} & \multirow{2}{*}{$\begin{array}{l}\text { Out of } \\
\text { round }\end{array}$} \\
\hline & Under & Over & \\
\hline $\begin{array}{l}\text { Up to } 1 / 2 \text {, inclusive } \\
\text { Over } 1 / 2 \text { to } 1 \text {, inclusive. } \\
\text { Over } 1 \text { to } 11 / 4 \text {, inclusive } \\
\text { Over } 11 / 4 \text { to } 11 / 2 \text {, inclusive. } \\
\text { Over } 11 / 2 \text { to } 2 \text {, inclusive.... }\end{array}$ & $\begin{array}{l}\text { Inch } \\
0.007 \\
.010 \\
.012 \\
.015 \\
.022\end{array}$ & $\begin{array}{l}\text { Inch } \\
0.007 \\
.010 \\
.012 \\
.015 \\
.022\end{array}$ & $\begin{array}{r}\text { Inch } \\
0.010 \\
.013 \\
.016 \\
.021 \\
.023\end{array}$ \\
\hline
\end{tabular}

\section{REQUIREMENTS FOR RIVETS}

Permissible Variations in Diameter

6. Finished rivets.-The diameter of finished rivets, measured cold, shall not vary from that specified more than the amounts given in the following table:

\begin{tabular}{|c|c|c|}
\hline \multirow[b]{2}{*}{ Diameter (in inches) } & \multicolumn{2}{|c|}{$\begin{array}{l}\text { Variations in } \\
\text { diameter }\end{array}$} \\
\hline & $\begin{array}{l}\text { Snap } \\
\text { gage } \\
\text { meas- } \\
\text { ure } \\
\text { ment } \\
\text { (under) }\end{array}$ & $\begin{array}{l}\text { Ring } \\
\text { gage } \\
\text { meas- } \\
\text { ure } \\
\text { ment } \\
\text { (over) }\end{array}$ \\
\hline $\begin{array}{l}\text { Up to } 1 / 2 \text {, inclusive } \\
\text { Over } 1 / 2 \text { to } 1 \text {, inclusive } \\
\text { Over } 1 \text { to } 11 / 4 \text {, inclusive } \\
\text { Over } 11 / 4 \text { to } 11 \frac{1}{2} \text {, inclusive } \\
\text { Over } 11 / 2 \text { to } 2 \text {, inclusive }\end{array}$ & $\begin{array}{l}\text { Inch } \\
0.022 \\
.025 \\
.027 \\
.030 \\
.037\end{array}$ & $\begin{array}{r}\text { Inch } \\
0.020 \\
.030 \\
.035 \\
.040 \\
.040\end{array}$ \\
\hline
\end{tabular}

Snap gage measurement shall be made at the point of minimum diameter, but it is not required that the rivet shall turn completely in the gage. Measurements of the maximum tolerance shall be made with a ring gage, all rivets to slip full to the head in the gage of the required size for the various diameters.

7. Rivet heads. - The dimensions of the rivet heads shall not vary from that specified more than the amounts given in the following table:

\begin{tabular}{|c|c|c|}
\hline \multirow{2}{*}{$\begin{array}{l}\text { Ordered } \\
\text { diame- } \\
\text { ter of } \\
\text { rivets }\end{array}$} & \multicolumn{2}{|c|}{ Head tolerances } \\
\hline & Width & Height \\
\hline $\begin{array}{r}\text { Inch } \\
1 / 2 \\
9 / 16 \\
5 / 8 \\
11 / 16 \\
34 \\
7 / 8 \\
1\end{array}$ & \begin{tabular}{cc}
\multicolumn{2}{c}{ Inch } \\
+116 & $-1 / 32$ \\
+116 & $-1 / 32$ \\
$+1 / 16$ & $-1 / 32$ \\
+116 & $-1 / 32$ \\
+564 & $-1 / 32$ \\
$+5 / 64$ & $-1 / 32$ \\
+564 & $-1 / 32$
\end{tabular} & $\begin{array}{l}\text { Inch } \\
+1 / 32 \\
+1 / 32 \\
+1 / 32 \\
+1 / 32 \\
+1 / 32 \\
+1 / 32 \\
+1 / 32\end{array}$ \\
\hline
\end{tabular}


AMERICAN SOCIETY FOR TESTING MATERIALS, STANDARD SPECIFICATIONS FOR COMMERCIAL QUALITY HOTROLLED BAR STEELS, DESIGNATION A107-30, 1930

\section{BAR STEELS}

(See 603.23, p. 147.)

AMERICAN SOCIETY FOR TESTING MATERIALS, STANDARD SPECIFICATIONS FOR COMMERCIAL COLD-FINISHED BAR STEELS AND COLD-FINISHED SHAFTING, DESIGNATION A108-30, 1930

BAR STEELS AND SHAFTING

(See 603.23, p. 147.)

AMERICAN SOCIETY FOR TESTING MATERIALS, STANDARD SPECIFICATIONS FOR STRUCTURAL STEEL FOR LOCOMOTIVES AND CARS, DESIGNATION A11329, 1929

\section{STEEL FOR LOCOMOTIVES AND CARS}

(See 605.13, p. 266.)

ASSOCIATION OF AMERICAN STEEL MANUFACTURERS, STANDARD SPECIFICATIONS FOR STRUCTURAL AND BOILER STEEL, 1922

\section{STRUCTURAL AND BOILER STEEL}

(See 604.11, p. 232, and 605.11, p. 250.)

SOCIETY OF AUTOMOTIVE ENGINEERS, AERONAUTIC; CASTLE-HEXAGON NUTS, S. A. E. STANDARD, AUGUST, 1928

STEEL FOR CASTLE-HEXAGON NUTS

(See 608.32, p. 608.)

SOCIETY OF AUTOMOTIVE ENGINEERS, AER ONAUTIC; PLAIN HEXAGONAL NUTS, S. A. E. STANDARD, AUGUST, 1928

STEEL FOR PLAIN HEXAGONAL NUTS

(See 608.32, p. 608.)

SOCIETY OF AUTOMOTIVE ENGINEERS, IRON AND STEEL SPECIFICATIONS, CHEMICAL COMPOSITIONS, JANUARY, 1930

\section{SCREW STOCK}

(For prefatory remarks, see 600.0, p. 2 ; for specification details, see 600.1, p. 49.)

Chemical Composition

\begin{tabular}{|c|c|c|c|c|}
\hline $\begin{array}{c}\text { S. A. E. } \\
\text { steel } \\
\text { No. }\end{array}$ & Carbon & Manganese & Phosphorus & Sulphur \\
\hline $\begin{array}{r}1112 \\
1120\end{array}$ & $\begin{array}{r}0.08-0.16 \\
.15-.25\end{array}$ & $\begin{array}{r}0.60-0.80 \\
.60-.90\end{array}$ & $\begin{array}{r}0.09-0.13 \\
1.06\end{array}$ & $\begin{array}{r}0.075-0.15 \\
.075-.15\end{array}$ \\
\hline
\end{tabular}

1 Maximum.
These two steels are intended for general automotive screw-machine products not requiring more particular properties.

UNITED STATES GOVERNMENT, FEDERAĬ SPECIFICATIONS BOARD, FEDERAL SPECIFICATION FOR STRUCTURAL STEEL FOR BRIDGES, NO. 351a, MARCH 25, 1929. (CURRENT DESIGNATION: QQ-S-711)

STRUCTURAL STEEL FOR BRIDGES

(See 605.11, p. 251.)

UNITED STATES GOVERNMENT, FEDERAI SPECIFICATIONS BOARD, FEDERAI SPECIFICATION FOR STRUCTURAI STEEL FOR BUILDINGS, NO. 352a, MARCH 25, 1929. (CURRENT DESIGNATION : QQ-S-721)

\section{STRUCTURAL STEEL FOR BUILDINGS}

(See 605.12, p. 254.)

UNITED STATES GOVERNMENT, FEDERAL SPECIFICATIONS BOARD, FEDERAI SPECIFICATION FOR STRUCTURAL STEEL FOR CARS, NO. 373a, MARCH 25, 1929. (CURRENT DESIGNATION : QQS-731)

STRUCTURAL STEEL FOR CARS

(See 605.13, p. 267.)

UNITED STATES GOVERNMENT, FEDERAL SPECIFICATIONS BOARD, FEDERAL SPECIFICATION FOR STRUCTURAL STEEL FOR SHIPS (OTHER THAN NAVAL VESSELS), QQ-S-751, JUNE 23, 1931

STRUCTURAL STEEL FOR SHIPS

(See 605.15, p. 270.)

UNITED STATES GOVERNMENT, FEDERAL SPECIFICATIONS BOARD, FEDERAL SPECIFICATION FOR STEEL, STAY BOLT, BOILER, QQ-S-701, MARCH 1, 1932

\section{STAY-BOLT STEEL, BOILER}

\section{GENERAL SPECIFICATIONS}

United States Government general specification for metals, Federal Specifications Board specification No. 339 (current designation: QQ-M-151), in effect on date of invitation for bids, shall form a part of this specification. (See 600.0, p. 3.)

\section{GRADES}

This specification covers a single grade of material for either solid or hollow stay bolts.

\section{MATERIAI AND WORKMANSHIP}

1. The steel shall be made by the open-hearth or electric-furnace process.

2. The workmanship shall be first class in every respect. 


\section{GENERAL REQUIREMENTS}

1. Surface defects.-The solid or hollow bars shall be straight, true to form, concentric, free from bends, blisters, or other injurious defects which might affect their strength or serviceability.

2. Permissible variation.-The diameter of each bar shall not vary from that specified more than the amount given in the following table:

\begin{tabular}{|c|c|c|c|}
\hline \multirow{2}{*}{ Diameter (in inches) } & \multicolumn{2}{|c|}{$\begin{array}{l}\text { Variation in } \\
\text { diameter }\end{array}$} & \multirow{2}{*}{$\begin{array}{l}\text { Out of } \\
\text { round }\end{array}$} \\
\hline & Under & Over & \\
\hline $\begin{array}{l}\text { Up to } 1 \text { inclusive } \\
\text { Over } 1 \text { to } 11 / 4 \text {, inclusive } \\
\text { Over } 11 / 4 \text { to } 11 / 2 \text {, inclusive } \\
\text { Over } 11 / 2 \text { to } 2 \text {, inclusive }\end{array}$ & $\begin{array}{l}\text { Inch } \\
0.01 \\
.012 \\
.015 \\
.022\end{array}$ & $\begin{array}{l}\text { Inch } \\
0.01 \\
.012 \\
.015 \\
.022\end{array}$ & $\begin{array}{c}\text { Inch } \\
0.013 \\
.016 \\
.021 \\
.023\end{array}$ \\
\hline
\end{tabular}

3. Ends of stay-bolt material upset for threading or flanged for riveting shall be thoroughly annealed.

\section{DETAIL REQUIREMENTS}

1. The stay-bolt steel shall conform to the chemical and physical requirements contained in the following table:

Tensile strength, maximum.......lbs./in. ${ }^{2}$.

Minimum yield point...............

Minimum elongation in 8 inches - per cent.-

Phosphorus (not more than) -.....-. do -..-

\begin{tabular}{r}
60.000 \\
26.000 \\
$1,500,000$ \\
\hline Tensile strength \\
0.04 \\
.045
\end{tabular}

2. The yield point shall be determined by the use of dividers or by the drop of the beam of the testing machine, as required by the inspector.

\section{METHODS OF INSPECTION AND TESTS}

1. All stay-bolt material shall be surface inspected.

2. Drillings or millings for chemical analysis shall be taken from several points from each bar selected for test and the chips combined and mixed. The resultant sample shall be free from dirt, grit, or other foreign matter and consist of at least one ounce.

3. Two tension, one bend and one fracture, test specimen shall be taken from each lot of 2,500 feet of material, or fraction thereof, or in case separate melts can be identified, the above-mentioned specimens shall be taken from each melt. Where upset or flanged stay bolts are required, the physical and chemical tests shall be made before upsetting or flanging.

4. Tension test specimens shall consist of full section specimens, or, if over $1 \frac{1}{2}$ inches diameter, of specimens machined to a diameter of at least three-fourths inch for a length of at least 9 inches,

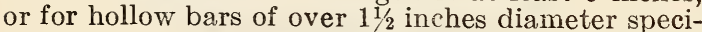
mens may be cut longitudinally and machined to shape. Flattening of hollow bar specimens shall not be permitted.

5. Bend test specimens cut from hollow bars or of full section if solid bars, shall withstand being bent cold through $180^{\circ}$ flat on itself without fracture on the outside of the bent portion for material 1 inch or under in diameter; for material over 1 inch to and including $1 \frac{1}{2}$ inches diameter around a pin the diameter of which is equal to the diameter of the specimen; and for material over $1 \frac{1}{2}$ inches in diameter around a pin the diameter of which is equal to twice the diameter of the specimen.

6. A fracture test shall be made by cutting partly through the bar or the wall of the hollow bar, and breaking it off when removing the crop end. The fracture thus produced shall be fine grained.

7 . If the results of the physical tests of one specimen from any lot do not conform to the requirements specified, two additional specimens, each from a different bar, of the same lot may be tested, each of which shall conform to the requirements specified.

8. If the percentage of elongation of any test specimen is less than that specified and any part of the fracture is outside the middle third of the gage length as indicated by scribe scratches marked on the specimen before testing, a retest shall be permitted.

\section{PACKING AND MARKING OF SHIPMENTS}

Shipments shall be packed and marked as required by the contract or order.

\section{NOTES}

1. Stay bolt material should be ordered by giving the type of material required, whether hollow or solid, outside and inside diameters, and length.

2. Material furnished in accordance with this specification is intended for staying surfaces or pressure boilers and may be applied by threading and riveting or by flanging the solid bar and connecting to the boiler plate with rivets.

3. The following specification was considered in the preparation of this specification.

A. S. M. E. Boiler Construction Code, 1930.

603.22. STEEL FOR CASE HARDENING.

SOCIETY OF AUTOMOTIVE ENGINEERS, IRON AND STEEL SPECIFICATIONS; SPECIFICATIONS FOR AUTOMOTIVE STEELS, S. A. E. STANDARD, FEBRUARY, 1929

\section{CARBON STEELS}

(See 603.27 , p. 150 , including certain references to 600.0 , p. 2 , and 600.1 , p. 49 ).

603.23. COMMERCIAL GRADE STEEL BARS AND RODS.

\section{AMERICAN SOCIETY OF MECHANICAL EN- GINEERS, BOILER CONSTRUCTION CODE, SPECIFICATIONS FOR STEEL BARS, S-7, 1930}

\section{STEEL BARS}

(This specification is based on and conforms to A. S. T. M. A7-29 (see 605.11 , p. 249), except that in tension tests the elongation in 2 inches, minimum, per cent is 26 instead of 22 as in A7-29, and the addition of the following data on gage variation:)

Permissible variation in gage. -The diameter of each bar shall not vary from that specified more than the amount given in the following table:

\begin{tabular}{|c|c|c|c|}
\hline \multirow{2}{*}{ Diameter (in inches) } & \multicolumn{2}{|c|}{$\begin{array}{l}\text { Variation in } \\
\text { diameter }\end{array}$} & \multirow{2}{*}{$\begin{array}{l}\text { Out of } \\
\text { round }\end{array}$} \\
\hline & Under & Over & \\
\hline $\begin{array}{l}\text { Up to } 1 / 2 \text {, inclusive } \\
\text { Over } 1 / 2 \text { to } 1 \text {, inclusive } \\
\text { Over } 1 \text { to } 11 / 4 \text {, inclusive } \\
\text { Over } 11 / 4 \text { to } 11 / 2 \text {, inclusive... } \\
\text { Over } 11 / 2 \text { to } 2 \text {, inclusive. } \\
\text { Over } 2 \text { to } 21 / 2 \text {, inclusive.... } \\
\text { Over } 21 / 2 \text { to } 3 \text {, inclusive...... }\end{array}$ & $\begin{array}{l}\text { Inch } \\
0.007 \\
.010 \\
.012 \\
.015 \\
.022 \\
1 / 64 \\
1 / 32\end{array}$ & $\begin{array}{l}\text { Inch } \\
0.007 \\
.010 \\
.012 \\
.015 \\
.022 \\
3646 \\
3,64\end{array}$ & $\begin{array}{r}\text { Inch } \\
0.010 \\
.013 \\
.016 \\
.021 \\
.023 \\
.023 \\
035\end{array}$ \\
\hline
\end{tabular}


AMERICAN SOCIETY FOR TESTING MATERIALS, STANDARD SPECIFICATIONS FOR COMMERCIAL QUALITY HOT-ROLLED BAR STEELS, DESIGNATION A107-30, 1930

\section{COMMERCIAL QUALITY BAR STEELS}

(In respect to scope, process, chemical composition, ladle analyses, check analyses, and finish this specification conforms with that of the Association of American Steel Manufacturers for commercial quality bar steel (see below), except for the addition in A107-30 of the following chemical compositions of screw steel grades:)

\begin{tabular}{|c|c|c|c|c|}
\hline Grades & Carbon & $\begin{array}{l}\text { Manga- } \\
\text { nese }\end{array}$ & $\begin{array}{l}\text { Phos- } \\
\text { phorus }\end{array}$ & Sulphur \\
\hline $\begin{array}{l}\text { Bessemer screw } \\
\text { Open-hearth screw..... }\end{array}$ & $\begin{array}{c}\text { Per cent } \\
0.08-0.16 \\
.15-.25\end{array}$ & $\begin{array}{l}\text { Per cent } \\
0.60-0.90 \\
.60-.90\end{array}$ & $\begin{array}{c}\text { Per cent } \\
0.09-0.13 \\
.06 \text { max. }\end{array}$ & $\begin{array}{l}\text { Per cent } \\
0.10-0.18 \\
.075-.15\end{array}$ \\
\hline
\end{tabular}

(In respect to permissible variations in sizes of bars, this specification is identical with that of the Association of American Steel Manufacturers for hot-rolled bars. (See 600.7, p. 116.)

(In respect to samples for analysis it is identical with the specification of the Association of American Steel Manufacturers for sampling rolled and forged steel products. See 600.1 , p. 47.)

(In respect to inspection, rejection, and rehearing it conforms with the A. S. T. M. standard specifications for steel in 605.0 , p. 247.)

\section{AMERICAN SOCIETY FOR TESTING MATE- RIALS, STANDARD SPECIFICATIONS FOR COMMERCIAL COLD-FINISHED BAR STEELS AND COLD-FINISHED SHAFT- ING, DESIGNATION A108-30, 1930}

\section{COMMERCIAL COLD-FINISHED BAR STEELS AND COLD-FINISHED SHAFTING}

\section{SCOPE}

1. (a) These specifications cover commercial cold-finished bar steels of grades considered to be in common use, including cold-finished Bessemer screw stock and cold-finished open-hearth screw stock, also cold-finished shafting and commercial key stock.

(b) The sections of bar steel covered are rounds, squares, and hexagons of all sizes, and flats not over 6 inches wide. The sections of shafting covered are rounds up to 6 inches, inclusive.

\section{BASIS OF PURCHASE}

2. (a) Bars and shafting may be cold-rolled, colddrawn, turned and polished, or turned and ground.

(b) The purchaser shall specify each grade of bar steel desired, by its grade designation or by its complete chemical limits.

(c) The steel in Bessemer and open-hearth screw stock shall be free-cutting and suitable for highspeed screw machine work, leaving a smooth finish after machining under proper conditions.

(d) Cold-finished shafting, when not specified by the purchaser in any particular grade, shall be in such grades as are in accordance with the manufacturer's practice for the respective sizes.

\section{MANUFACTURE}

3. (a) Bar steel shall be made by the openhearth or Bessemer process, as specified.

(b) Shafting shall be made by either or both the following processes: Bessemer or open-hearth.

\section{CHEMICAL PROPERTIES AND TESTS}

4. Bar steel shall conform to the requirements as to chemical composition specified in Tables 1 and 2 for the respective grades.

TABLE 1.-Grades and chemical compositions for commercial cold-finished bar steels

BESSEMER GRADES

\begin{tabular}{c|r|r|r}
\hline Grades & $\begin{array}{r}\text { Car- } \\
\text { bon }\end{array}$ & $\begin{array}{r}\text { Man- } \\
\text { ganese }\end{array}$ & $\begin{array}{r}\text { Phos- } \\
\text { phorus }\end{array}$ \\
\hline Bessemer soft, maximum_............. & $\begin{array}{r}\text { Per cent } \\
0.15\end{array}$ & $\begin{array}{r}\text { Per cent } \\
0.60\end{array}$ & $\begin{array}{r}\text { Per cent } \\
0.11\end{array}$ \\
\hline
\end{tabular}

(Bessemer screw and open-hearth screw grades are identical with like grades in A. S. T. M. A107-30, given above. Open-hearth grades ranging from $0.05-0.15$ to $0.40-0.50$ carbon per cent are the same as in A. A. S. M. commercial quality bar steel, given below, except for 0.005 per cent more phosphorus in A. S. T. M. A 108, and 0.005 per cent more sulphur in the A. A. S. M. specification.)

5. An analysis of each melt of open-hearth steel shall be furnished by the manufacturer when requested, showing the percentage of the elements specified, which shall conform to the requirements for the grade specified in Table 1 (including reference to the A. A. S. M. specification).

\section{CHECK ANALYSES}

6. (a) Analyses may be made by the purchaser from finished bars representing each melt of openhearth steel and each melt, or lot of 10 tons, of Bessemer steel. The chemical composition thus determined shall conform to the requirements in Table 1, with the permissible variations in ranges and in maximum limits specified in Table 2.

(b) Samples for analysis shall be taken by machining off the entire cross section of the bar, or by drilling parallel to the axis of the bar at any point midway between the center and surface.

\section{PERMISSIBLE VARIATIONS IN DIMENSIONS}

7. The variations from the specified diameters, or distances between parallel faces, of cold-finished bars specified in carbon up to and including 0.30 per cent shall not exceed the limits specified in Table 3 . For bars specified in carbon over 0.30 per cent, the variations shall be a matter of agreement between the purchaser and the manufacturer.

(b) The variations from the specified diameters of cold-finished shafting shall not exceed the limits specified in Table 4.

(In respect to finish, inspection, rejection, and rehearing, this specification conforms to the requirements specified in A. S. T. M. standard specifications for steel. See 605.0 , p. 247.) 
TABLE 2.-Variations in ranges and in maximum limits specified in Table 1 permitted on check analysis

(Open-hearth and Bessemer screw stock not included) OPEN-HEARTH GRADES

\begin{tabular}{|c|c|c|c|c|c|c|}
\hline \multirow{3}{*}{ Grades } & \multicolumn{6}{|c|}{$\begin{array}{l}\text { Permissible variations from } \\
\text { limits specified in Table } 1\end{array}$} \\
\hline & \multicolumn{2}{|c|}{ Carbon } & \multicolumn{2}{|c|}{$\begin{array}{l}\text { Manga- } \\
\text { nese }\end{array}$} & \multirow{2}{*}{ 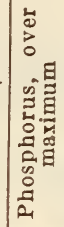 } & \multirow{2}{*}{ 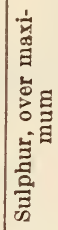 } \\
\hline & 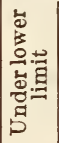 & 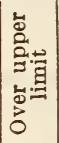 & 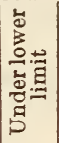 & 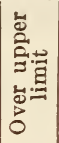 & & \\
\hline $\begin{array}{l}\text { Open-hearth } 05-15 \text { carbo } \\
\text { Open-hearth } 10-20 \text { and }\end{array}$ & \multirow{3}{*}{$\begin{array}{r}0.02 \\
.03 \\
.03 \\
.03\end{array}$} & \multirow{3}{*}{\begin{tabular}{|c|}
0.03 \\
.04 \\
.05 \\
.06 \\
.07
\end{tabular}} & \multirow{3}{*}{$\begin{array}{r}0.05 \\
.05 \\
.05 \\
.05 \\
.05\end{array}$} & \multirow{3}{*}{$\begin{array}{r}0.05 \\
.05 \\
.10 \\
.10 \\
.10\end{array}$} & \multirow{3}{*}{$\begin{array}{c}0.01 \\
.01 \\
.01 \\
.01 \\
.01\end{array}$} & \multirow{3}{*}{$\begin{array}{r}0.01 \\
.01 \\
.01 \\
.01 \\
.01\end{array}$} \\
\hline open-hearth $20-30$ and $15-35$ car- & & & & & & \\
\hline $\begin{array}{l}\text { Open-hearth } 30-40 \text { and } 35-45 \text { car- } \\
\text { bon } \\
\text { Open-hearth } 40-50 \text { carbon }\end{array}$ & & & & & & \\
\hline
\end{tabular}

BESSEMER GRADES

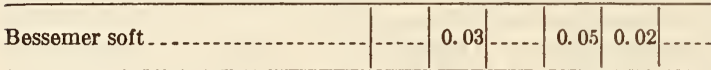

TABLE 3.-Permissible variations in the sizes of coldfinished bars

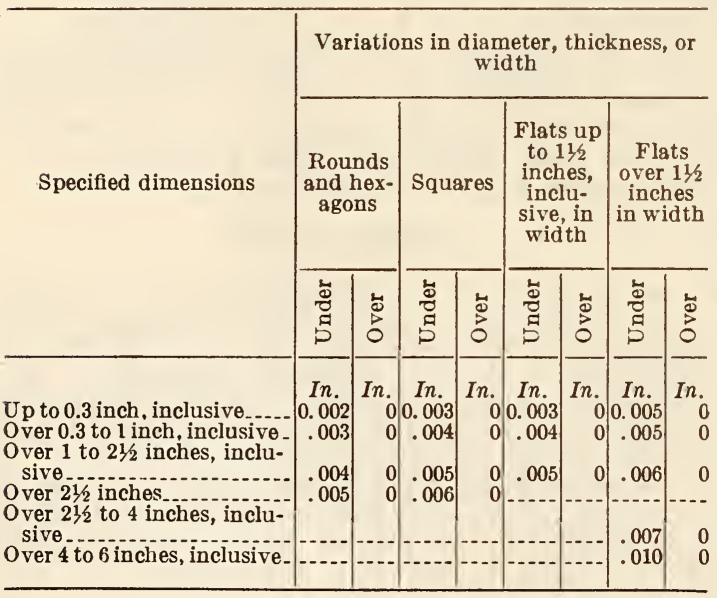

TABLE 4.-Permissible variations in the sizes of coldfinished shafting

ROUND SHAFTING

\begin{tabular}{|c|c|c|}
\hline \multirow{2}{*}{ Diameters specified } & \multicolumn{2}{|c|}{$\begin{array}{l}\text { Variations in } \\
\text { dimensions }\end{array}$} \\
\hline & Under & Over \\
\hline $\begin{array}{l}\text { Up to } 1 \text { inch, inclusive } \\
1116 \text { to } 2 \text { inches, inclusive } \\
2116 \text { to } 4 \text { inches, inclusive } \\
4116 \text { to } 6 \text { inches, inclusive }\end{array}$ & $\begin{array}{l}\text { Inch } \\
0.002 \\
.003 \\
.004 \\
.005\end{array}$ & Inch $\begin{array}{r}0 \\
0 \\
0 \\
0\end{array}$ \\
\hline
\end{tabular}

ASSOCIATION OF AMERICAN STEEL MANUFACTURERS, STANDARD SPECIFICATIONS FOR COMMERCIAL-QUALITY BAR STEEL, 1924

\section{COMMERCIAL-QUALITY BAR STEEL}

1. Scope.-These specifications cover hot-rolled carbon-steel bars, produced in accordance with good mill practice for general commercial purposes. Bars for drop-forging and heat-treatment purposes should be purchased to the Association of American Steel Manufacturers' standard specifications for special forging-quality bar steel. (See 603.24, p. 149.)

2. Process. - The steel shall be made by the openhearth process or the Bessemer process, as specified.

3. Chemical composition.-The steel shall conform to the requirements as to chemical composition specified in the following table:

\section{Chemical compositions}

\begin{tabular}{|c|c|c|c|c|}
\hline & Carbon & $\begin{array}{c}\text { Mang9- } \\
\text { nese }\end{array}$ & $\begin{array}{l}\text { Phos- } \\
\text { phorus } \\
\text { (mari- } \\
\text { mum) }\end{array}$ & $\begin{array}{l}\text { Sulphur } \\
\text { (maxi- } \\
\text { mum) }\end{array}$ \\
\hline \multirow{2}{*}{ Open-hearth steel } & $\begin{array}{c}\text { Per cent } \\
0.05-0.15 \\
.10-.20 \\
.15-.25 \\
.20-.30 \\
.25-.35 \\
.30-.40 \\
.35-.45\end{array}$ & $\begin{array}{l}\text { Pet cent } \\
0.30-0.60 \\
.30-.60 \\
.30-.60 \\
.50-.80 \\
.50-.80 \\
.50-.80 \\
.50-.80\end{array}$ & $\begin{array}{r}\text { Per cent } \\
0.04 \\
.01 \\
.04 \\
.04 \\
.04 \\
.04 \\
.04\end{array}$ & $\begin{array}{r}\text { Percent } \\
0.055 \\
.055 \\
.055 \\
.055 \\
.055 \\
.055 \\
.055\end{array}$ \\
\hline & $\mid \begin{array}{c}.40-.50 \\
.45-.55 \\
.50-.65 \\
.55-.70 \\
.60-.75 \\
.65-.80\end{array}$ & $\begin{array}{l}.50-.80 \\
.50-.80 \\
.50-.80 \\
.50-.80 \\
.50-.80 \\
.50-.80\end{array}$ & $\begin{array}{l}.04 \\
.04 \\
.04 \\
.04 \\
.04 \\
.04\end{array}$ & $\begin{array}{r}.055 \\
.055 \\
.055 \\
.055 \\
.055 \\
.055\end{array}$ \\
\hline Bessemer steel.. & $\left\{\begin{array}{c}.05-.10 \\
.08-.15 \\
.15-.25 \\
.25-.35 \\
.30-.40 \\
.40-.50 \\
.50-.60\end{array}\right.$ & $\begin{array}{l}.30-.60 \\
.40-.70 \\
.50-.90 \\
.50-.90 \\
.50-.90 \\
.50-.90 \\
.60-1.00\end{array}$ & $\begin{array}{l}11 \\
11 \\
.11 \\
.11 \\
.11 \\
11 \\
.11\end{array}$ & $\begin{array}{c}.08 \\
0.0 \\
\end{array}$ \\
\hline
\end{tabular}

4. Ladle analysis.-(a) For Bessemer steel a carbon determination shall be made of each melt, and determinations for manganese, phosphorus, and sulphur, representing the average of the melts made in each 12-hour period.

(b) For open-hearth steel an analysis of each melt shall be made for carbon, manganese, phosphorus, and sulphur.

(c) These analyses shall be made by the manufacturer from test ingots taken during the pouring of each melt. The chemical composition thus determined shall conform to the requirements specified, and shall be reported to the purchaser or his representative, if requested.

5. Check analyses.-Check analyses of the finished material may be made by the purchaser, using samples obtained in accordance with the manufacturers' standard methods of sampling for check analysis. (See 600.1, p. 47.) The average chemical composition thus determined shall not vary from the limits specified more than 0.03 over or 0.02 under in carbon, 0.03 over or 0.02 under in manganese, 0.005 over in phosphorus, and 0.005 over in sulphur.

6. Permissible variations in dimensions.-The dimensions of bars shall conform to the manufacturers' 
standard practice governing the allowable variations in size of hot-rolled bars. (See 600.7, p. 116.)

7. Finish.-T he bars shall have as good surface finish as is consistent with good hot-mill practice. They shall be free from injurious piping and undue segregation, and free from surface defects which would be injurious for general or ordinary purposes.

8. Rejection.-(a) Any rejection for chemical composition must be based on the manufacturers' standard methods of sampling for check analysis. (See 600.1, p. 47.)

(b) The manufacturer shall be notified, within six months from date of shipment, of any rejection at destination.

9. Rehearing.-Samples analyzed in accordance with sections 5 and 8 , which represent material rejected by the purchaser, shall be preserved for one month from the date of notice to the manufacturer. In case of dissatisfaction with the results of the tests, the manufacturer may make claim for a rehearing within that time.

603.24 STEEL FOR SHAFTS, AXLES, AND FORGINGS.

AMERICAN SOCIETY FOR TESTING MATERIALS, STANDARD SPECIFICATIONS FOR COMMERCIAL COLD-FINISHED BAR STEELS AND COLD-FINISHED SHAFTING, DESIGNATION A108-30, 1930

\section{BAR STEELS AND SHAFTING}

(See 603.23, p. 147.)

ASSOCIATION OF AMERICAN STEEL MANUFACTURERS, STANDARD SPECIFICATIONS FOR SPECIAL FORGING-QUALITY BAR STEEL, 1924

\section{FORGING-QUALITY BAR STEEL}

1. Scope.-These specifications cover hot-rolled carbon-steel bars, in the manufacture of which special precautions are taken with a view of securing material suitable for forging, heat treating, or similar purposes.

2. Process. - The steel shall be made by the openhearth process.

3. Discard.-A sufficient discard shall be made from each ingot to secure freedom from injurious piping and undue segregation.

\section{CHEMICAL COMPOSITION}

(Chemical composition is identical with that for commercial-quality bar steel for carbon percentages ranging from $0.05-0.15$ to $0.65-0.80$ except for the addition in commercial-quality bar steel of 0.005 per cent of sulphur in each grade of open-hearth steel. See 603.23 , p. 148.)

5. Ladle analysis.-(Conforms with commercialquality bar steel. See 603.23 , p. 148.)

6. Check analyses.-(a) Check analyses of the finished material may be made by the purchaser, using samples obtained in accordance with the manufacturers' standard methods of sampling for check analysis. (See 600.1, p. 47.) The average chemical composition thus determined shall not vary from the limits specified more than 0.02 over or under in carbon, 0.02 over or under in manganese, 0.005 over in phosphorus, and 0.005 over in sulphur.

(b) The chemical compositions of individual samples shall not exceed the permissible variations specified below, and no single melt shall vary both above and below the range specified.

Variations from limits of chemical compositions permitted in individual samples

\begin{tabular}{|c|c|c|}
\hline \multirow{2}{*}{ Elements } & \multicolumn{2}{|c|}{$\begin{array}{l}\text { Permissible } \\
\text { variations }\end{array}$} \\
\hline & $\begin{array}{l}\text { Under } \\
\text { lower } \\
\text { limit }\end{array}$ & $\begin{array}{l}\text { Over } \\
\text { upper } \\
\text { limit }\end{array}$ \\
\hline $\begin{array}{l}\text { Carbon-maximum limits up to } 0.25 \text {, inclusive } \\
\text { Carbon-maximum limits over } 0.25 \text { to } 0.30 \text {, in- } \\
\text { clusive }\end{array}$ & & \\
\hline $\begin{array}{l}\text { Carbon-maximum limits over } 0.30 \text { to } 0.80 \text {, in- } \\
\text { clusive } \\
\text { Manganese-all ranges } \\
\text { Phosphorus } \\
\text { Sulphur. }\end{array}$ & $\begin{array}{l}.03 \\
.03 \\
.05 \\
.03\end{array}$ & $\begin{array}{l}.04 \\
.05 \\
.05 \\
.01 \\
.01\end{array}$ \\
\hline
\end{tabular}

(Permissible variations in dimensions, and finish, rejection, and rehearing, conform with specifications for commercial-quality bar steel, including references therein to A. A. S. M. under 600.1 and 600.7. (See 603.23 , p. 148.)

AMERICAN SOCIETY OF AUTOMOTIVE ENGINEERS, IRON AND STEEL SPECIFICATIONS; SPECIFICATIONS FOR AUTOMOTIVE STEELS, S. A. E. STANDARD, FEBR UARY, 1929

\section{CARBON STEELS}

(See 603.27 , p. 150 including certain references to 600.0 , p. 2 , and 600.1 , p. 49.)

603.25 STEEL REINFORCING BARS AND RODS.

(See 605.25.)

603.26 STEEL BARS AND RODS FOR WELDING.

AMERICAN ELECTRIC RAILWAY ENGINEERING ASSOCIATION, STANDARD SPECIFICATION FOR WELDING RODS, W46-30, 1930

\section{WELDING RODS}

\section{SCOPE}

The specification is intended to cover the manufacture and general characteristics of plain uncoated steel arc-welding rods for purchase and use by the electric-railway industry. Coated, covered, fluxbearing welding rods, or those produced by patented processes, are not intended to be covered by this specification.

\section{MANUFACTURE}

\section{Process}

101. (a) The steel for the welding rod shall be either hot-rolled or cold-drawn from crucible, electric-furnace, or open-hearth steel, as specified by the purchaser.

(b) The welding rod shall be commercially straight, of uniform longitudinal structure, and free from irregularities in surface and hardness, segregation, pipes, and seams.

(c) The welding rod shall be unannealed, unless otherwise specified by the purchaser. 
Chemical Composition

102. (a) High-carbon welding rod (for metallic-arc welding) -

$\begin{array}{lr}\text { Carbon } & \\ \text { Manganese } & .95-1.10 \\ \text { Phosphorus, not more than } & .03 \\ \text { Sulphur, not more than } & .03 \\ \text { Silicon, not more than } & .20\end{array}$

(b) Medium low-carbon welding rod (for metallic and carbon arc welding)-

$\begin{array}{ll}\text { Carbon } & \\ \text { Manganese } & 0.13-0.18 \\ \text { Phosphorus, not more than } & \\ \text { Sulphur, not more than } & 03 \\ \text { Silicon, not more than } & \end{array}$

(c) Extra low carbon welding rod (for metallic and carbon arc welding)-

$\begin{array}{ll}\text { Carbon, not more than } & 0.05 \\ \text { Manganese, not more than } & .30 \\ \text { Phosphorus, not more than } & 025 \\ \text { Sulphur, not more than } & \\ \text { Silicon, }\end{array}$

Tolerances

103. (a) The diameter of the welding rod shall not vary more than 0.003 inch over or under that specified.

(b) The length of the welding rod shall not vary more than one-fourth inch under or over that specified.

$$
\text { II. SIZES }
$$

201. The following sizes shall be considered standard, as covered by this specification:

(a) High-carbon welding rod. $-3 / 16$ by 36 inches; $1 / 4$ by 36 inches.

(b) Medium low-carbon welding rod.-5/32 by 24 inches; $1 / 4$ by 24 inches; $3 / 16$ by 24 inches; $9 / 32$ by 24 inches.

(c) Extra low-carbon welding rod. $-5 / 32$ by 24 inches; $3 / 16$ by 24 inches; $9 / 32$ by 24 inches.

\section{PHYSICAL PROPERTIES AND TESTS}

301. The purchaser or his representatives shall select two welding rods from three separate bundles selected at random from each 1,000-pound lot, or less, and these welding rods in the hands of an experienced welder shall demonstrate gocd welding properties such as smooth even flowing, without excessive sputtering at the arc or other unusual characteristics when tested in positions encountered in actual welding practice. The deposited metal must be free from blow holes or gas pockets. If the test welding rods do not pass this test the shipment from which the welding rods were selected shall be rejected.

\section{WORKMANSHIP AND FINISH}

401. The surface of the welding rod shall be smooth and free from rust, oil, grease, or any other injurious substance.

\section{MARKING AND SHIPPING}

501. (a) The welding rods shall be securely tied in bundles weighing 50 pounds each, net weight, and fully wrapped in burlap or other suitable covering to protect them from moisture, etc.

(b) To each bundle shall be securely attached a tag bearing the size, specification number, and the name of the manufacturer.

\section{INSPECTION AND REJECTION}

601. (a) Unless otherwise agreed upon, the purchaser will make the tests to govern the acceptance or rejection of the welding rod. Such tests shall be made at the expense of the purchaser.

(b) Any rejection based on tests made in accordance with this specification shall be reported within 10 working days after the receipt of the welding rod.

\subsection{STEEL BARS AND RODS FOR AUTOMO- TIVE AND RAILWAY USE.}

AMERICAN ELECTRIC RAILWAY ENGINEERING ASSOCIATION, SPECIFICATION FOR MATERIALS FOR USE IN THE MANUFACTURE OF SPECIAL TRACKWORK, W104-28, 1928

[Approved as tentative American Standard by the American Standards Association, January 23, 1929]

\section{SPECIAL TRACKWORK MATERIALS}

(See 606.3, p. 342.)

\section{SOCIETY OF AUTOMOTIVE ENGINEERS,} IRON AND STEEL SPECIFICATIONS, CHEMICAL COMPOSITIONS, JUNE, 1929

\section{CARBON STEELS}

(For prefatory remarks, see 600.0, p. 2 ; for specification details, see 600.1 , p. 49.)

Chemical composition

\begin{tabular}{|c|c|c|c|c|}
\hline S. A. E. steel No. & $\begin{array}{l}\text { Carbon } \\
\text { range }\end{array}$ & $\begin{array}{l}\text { Man- } \\
\text { ganese } \\
\text { range }\end{array}$ & $\begin{array}{l}\text { Phos- } \\
\text { phorus } \\
\text { (maxi- } \\
\text { mum) }\end{array}$ & $\begin{array}{c}\text { Sulphur } \\
\text { (maximum) }\end{array}$ \\
\hline $\begin{array}{l}1010 \\
1015 \\
1020 \\
1030\end{array}$ & $\begin{array}{c}\text { Per cent } \\
0.05-0.15 \\
.10-.20 \\
.15-.25 \\
.20-.30 \\
.25-.35\end{array}$ & $\begin{array}{c}\text { Per cent } \\
0.30-0.60 \\
.30-.60 \\
.30-.60 \\
.50-.80 \\
.50-.80\end{array}$ & $\begin{array}{r}\text { Per cent } \\
0.045 \\
.045 \\
.045 \\
.045 \\
.045\end{array}$ & $\begin{array}{r}\text { Percent } \\
0.055 \\
.055 \\
.055 \\
.055 \\
.055\end{array}$ \\
\hline $\begin{array}{l}1035 \\
1040 \\
1045 \\
1050\end{array}$ & $\begin{array}{l}.30-.40 \\
.35-.45 \\
.40-.50 \\
.45-.55\end{array}$ & $\begin{array}{l}.50-.80 \\
.50-.80 \\
.50-.80 \\
.50-.80\end{array}$ & $\begin{array}{l}.045 \\
.045 \\
.045 \\
.045\end{array}$ & $\begin{array}{l}.055 \\
.055 \\
.055 \\
.055\end{array}$ \\
\hline $\begin{array}{l}1095 \\
1315 \\
1350 \\
1360\end{array}$ & $\begin{array}{l}.90-1.05 \\
.10-.20 \\
.45-.55 \\
.55-.70\end{array}$ & $\begin{array}{r}.25-.50 \\
1.25-1.55 \\
.90-1.20 \\
.90-1.20\end{array}$ & $\begin{array}{l}.040 \\
.050 \\
.040 \\
.040\end{array}$ & $\begin{array}{r}.050 \\
0.080-0.130 \\
.050 \\
.050\end{array}$ \\
\hline
\end{tabular}

1 The silicon content for steels Nos. 1350 and 1360 shall not exceed 0.30 per cent.

\subsection{SPRING AND TOOL STEEL BARS AND RODS.}

603.31 DRILL TOOL STEEL BARS AND RODS.

(No nationally recognized specifications available.)

603.32 HAND, HIGH-SPEED, AND MACHINE TOOL STEELS.

AMERICAN RAILWAY ASSOCIATION, PURCHASES AND STORES DIVISION, STAND. ARDIZATION AND SIMPLIFICATION OF STORES STOCKS, D-V1-238, 1930

\section{CARBON TOOL STEEL}

(Recommended practice in stocking certain specified sizes of octagon, square, and round carbon tool steel.) 


\section{AMERICAN RAILWAY ENGINEERING AS- SOCIATION, SPECIFICATIONS FOR MOV- ABLE RAILWAY BRIDGES, TOOL STEEL, 1929}

(See also 611.41, p. $707 ; 611.51$, p. $753 ; 647.31$, р. $1268 ; 692.1$, p. 1320.)

\section{TOOL STEEL}

Tool steel shall be made by the open-hearth or the crucible process. It shall conform to the following chemical requirements:

$\begin{array}{lr} & \begin{array}{c}\text { Per } \\ \text { cent }\end{array} \\ \text { Carbon, minimum } & 1.00 \\ \text { Shosphorus, maximum } & 04 \\ \text { Ianganese, maximum } & .04\end{array}$

\section{AMERICAN SOCIETY FOR TESTING MATE- RIALS, STANDARD SPECIFICATIONS FOR CARBON TOOL STEEL, DESIGNATION A71-26, 1926}

\section{CARBON TOOL STEEL}

\section{SCOPE}

1. (a) These specifications cover carbon tool steels, the composition of which shall be as agreed upon by the purchaser and the vendor, with the permissible variations specified in section 4 .

(b) These steels are suitable for lathe and planer tools, drills and milling cutters, dies and other miscellaneous purposes, etc.

\section{MANUFACTURE}

2. The steel shall be made either by the crucible or electric-furnace process.

3. The steel shall be furnished unannealed unless otherwise specified.

\section{CHEMICAL PROPERTIES AND TESTS}

4. The composition of the tool steels shall be as agreed upon by the purchaser and the vendor, with the following permissible variations in composition:

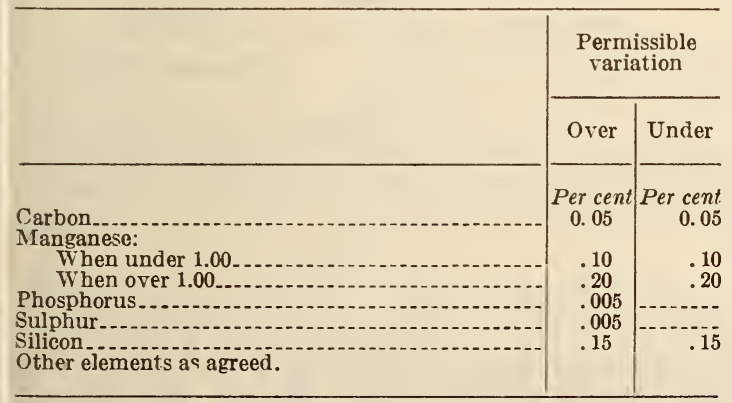

5. (a) Milled samples for analysis shall be taken from the sample bar to represent the full cross section, or drillings shall be taken by drilling a bar midway between the center and the surface, parallel to the axis of the bar. In either case, the decarburized material shall be rejected.

(b) In general, the minimum depth to which the surface shall be removed before a sample is taken shall be in accordance with the following requirements:

\begin{tabular}{l|c}
\hline \multicolumn{1}{c|}{ Thickness or diameter (in inches) } & $\begin{array}{c}\text { Materia } \\
\text { removed }\end{array}$ \\
\hline & Inch \\
$1 / 32$ \\
1 or under \\
$\begin{array}{l}1 / 8 \\
\text { Over } 1 \text { to } 215 .\end{array}$ \\
Over 213 to to 4 , inclusive \\
Larger sizes
\end{tabular}

(c) In the case of drill rod and other material of similar finish where freedom from decarburization is specified, samples shall be taken from the surface in addition to the methods described in paragraph $(a)$.

6. In case of dispute, the chemical analysis shall be made in accordance with the standard methods of chemical analysis of plain carbon steel. See A. S. T. M. A33 (600.1, p. 10).

\section{MANUFACTURING TOLERANCES IN DIMENSIONS}

7. Variations in the cross-sectional dimensions of the material shall not exceed the following amounts:

$$
\text { FORGED AND ROLLED MATERIAL }
$$

\begin{tabular}{|c|c|c|}
\hline & \multicolumn{2}{|c|}{ Permissible variation } \\
\hline & Over & Under \\
\hline $16 \mathrm{i}$ & Inch & Inch \\
\hline Over 32 inch to 1 inch, inclusive. & $.015(1 / 64)$ & $.015(1 / 64)$ \\
\hline Over 1 inch to 132 inches, inclusive & $.031(1 / 32)$ & $.031 \quad(1 / 32)$ \\
\hline Over 116 inches to 2 inches, in & $.046(3 \% 4)$ & $.031 \quad(132)$ \\
\hline Over 2 inches to 4 inches, inclusive & $.125 \quad(1 / 8)$ & $.031 \quad(1 / 32)$ \\
\hline Over 4 inches to 6 inches, inclusive. & $.156 \quad(5 / 32)$ & $.031 \quad(1 / 32)$ \\
\hline Over 6 inches to 8 inches, inclus & $.188 \quad(3 / 16)$ & $.063(1 \mathrm{Ko})$ \\
\hline Over 8 inches. & $250 \quad(1 / 4)$ & .0 กิว $(1 / 16)$ \\
\hline
\end{tabular}

FINISHED MATERIAL (DRILL ROD)

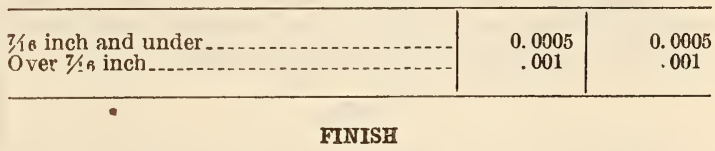

8. (a) The material shall be free from injurious defects and shall have a workmanlike finish.

(b) All drill rods shall be coated with a rust preventive.

9. (a) Indentification marks shall be stamped on the material as agreed by the purchaser and the vendor. All identification marks shall be about one-fourth inch in height when the size permits.

(b) In lieu of marking as specified in paragraph (a), the ends of bars and rods may be painted as agreed by the purchaser and the vendor.

10. All rods and bars of which the largest crosssectional dimension is three-eighths inch or less shall be boxed or bundled, each box or bundle to contain bars or rods of the same size, shape, and class of material. A metal tag shall be securely attached to the wrapping wire or box. Rods and bars of crosssectional dimensions exceeding three-eighths inch and not exceeding $1 \frac{1}{2}$ in., where convenient to purchaser and vendor, may be bundled and marked as specified above. Bundles shall be of suitable size to permit easy handling and shall be securely fastened.

\section{INSPECTION AND REJECTION}

11. The vendor shall afford the inspector, without charge, all reasonable facilities to satisfy him 
that the material is being furnished in accordance with these specifications.

12. The steel shall correspond in quality and characteristics to the samples submitted under the specifications and approved by the purchaser.

13. Material which does not conform to the chemical composition and specified dimensions, or which at any time shows injurious defects inherent in the steel, will be rejected and the vendor shall be notified.

603.33 SPRING STEEL BARS AND RODS.

AMERICAN ELECTRIC RAILWAY ENGINEERING ASSOCIATION, SPECIFICATION FOR MATERIALS FOR USE IN THE MANUFACTURE OF SPECIAL TRACK WORK, W104-28, 1928

[Approved as tentative American Standard by the American Standards Association, January 23, 1929]

\section{SPECIAL TRACK-WORK MATERIALS}

(See 606.3, p. 342.)

AMERICAN RAILWAY ASSOCIATION, MECHANICAL DIVISION, STEEL BARS, CARBON, FOR RAILWAY SPRINGS, 1917

\section{BARS FOR RAILWAY SPRINGS}

(Identical with A. S. T. M. A14, given below, including references in A14 to A. R. A. and A. S. T. M. specifications, except for permissible variations in gage, which are as follows:)

\section{PERMISSIBLE VARIATIONS IN GAGE}

Permissible variations. - The dimensions of the bars shall not vary from those ordered more than the amount shown in the following table:

\section{Flats}

\begin{tabular}{|c|c|c|c|c|c|c|}
\hline \multirow{3}{*}{ Size } & \multirow{2}{*}{\multicolumn{2}{|c|}{ Width }} & \multicolumn{4}{|c|}{ Thickness } \\
\hline & & & \multicolumn{2}{|c|}{$\begin{array}{c}\text { Up to } 3 / 8 \\
\text { inch, inclu- } \\
\text { sive }\end{array}$} & \multicolumn{2}{|c|}{$\begin{array}{l}\text { Over } 3 / 8 \text { to } \\
3 / 4 \text { inch, } \\
\text { inclusive }\end{array}$} \\
\hline & Over & $\begin{array}{l}\text { Un- } \\
\text { der }\end{array}$ & Over & $\begin{array}{l}\text { Un- } \\
\text { der }\end{array}$ & Over & $\begin{array}{l}\text { Un- } \\
\text { der }\end{array}$ \\
\hline & Inch & Inch & Inch & Inch & Inch & Inch \\
\hline Over 2 to 4 inches, inclusive. & $\begin{array}{r}.011 \\
.047 \\
0.04\end{array}$ & .015 & .015 & .005 & .015 & .010 \\
\hline $\begin{array}{l}\text { Over } 4 \text { to } 5 \text { inches, inclusive- } \\
\text { Over } 5 \text { to } 6 \text { inches, inclusive- }\end{array}$ & $\begin{array}{r}.047 \\
.062\end{array}$ & $\begin{array}{l}.032 \\
.032\end{array}$ & $\begin{array}{l}2 \\
2 \\
2 \\
.015 \\
.015\end{array}$ & $\begin{array}{l}.005 \\
.005\end{array}$ & $\begin{array}{r}.015 \\
.015\end{array}$ & .010 \\
\hline
\end{tabular}

Rounds and squares

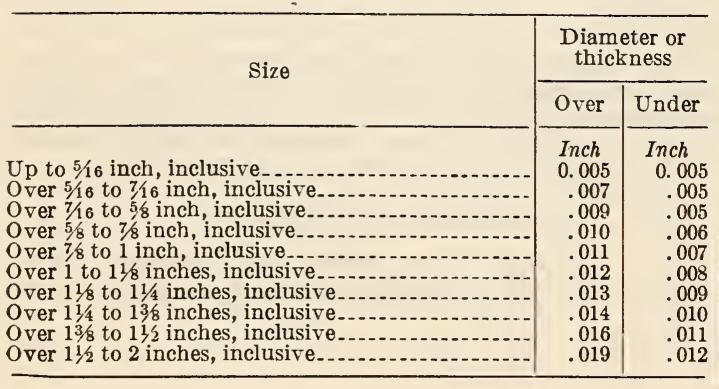

AMERICAN SOCIETY FOR TESTING MATERIALS, STANDARD SPECIFICATIONS FOR CARBON-STEEL BARS FOR RAILWAY SPRINGS, DESIGNATION A14-27, 1927

\section{BARS FOR RAILWAY SPRINGS \\ SCOPE}

1. These specifications cover two grades of carbonsteel bars to be used for the manufacture of railway springs, determined by the carbon ranges specified in section 3 . The choice of the grade of bar to be used for the manufacture of any spring will depend on the design of the spring and the stresses and service for which it is intended. The purposes for which these grades are frequently used are as follows: Grade A, for elliptical and helical springs; and grade B, for helical springs.

\section{MANUFACTURE}

2. The steel shall be made by one or more of the following processes: Open-hearth, crucible, or electric furnace.

\section{CHEMICAL PROPERTIES AND TESTS}

3 . The steel shall conform to the following requirements as to chemical composition:

\begin{tabular}{|c|c|c|}
\hline Elements considered & Grade A & Grade B \\
\hline $\begin{array}{l}\text { Marbon } \\
\text { Panganese, maximum } \\
\text { Sulphur, maximum }\end{array}$ & $\begin{array}{r}\text { Per cent } \\
0.90-1.10 \\
.50 \\
.05 \\
.05\end{array}$ & $\begin{array}{r}\text { Per cent } \\
0.95-1.15 \\
.50 \\
.05 \\
.05\end{array}$ \\
\hline
\end{tabular}

\section{LADLE AND CHECK ANALYSES}

(Same as in American Railway Association specification for steel tank plates. See 621.32, p. 1142.)

\section{PERMISSIBLE VARIATIONS IN DIMENSIONS}

6. The permissible variations in the width and thickness of the bars shall be:

\section{Flats}

\begin{tabular}{|c|c|c|c|c|c|c|}
\hline & \multirow{2}{*}{\multicolumn{2}{|c|}{$\begin{array}{l}\text { Variations } \\
\text { in width }\end{array}$}} & \multicolumn{4}{|c|}{$\begin{array}{l}\text { Variations in thick- } \\
\text { ness }\end{array}$} \\
\hline & & & \multicolumn{2}{|c|}{$\begin{array}{l}\text { Up to } 3 / 8 \\
\text { inch, inclu- } \\
\text { sive }\end{array}$} & \multicolumn{2}{|c|}{$\begin{array}{l}\text { Over } 3 / 8 \text { to } \\
5 / 8 \text { inch, in- } \\
\text { clusive }\end{array}$} \\
\hline & Over & $\begin{array}{l}\text { Un- } \\
\text { der }\end{array}$ & Over & $\begin{array}{l}\text { Un- } \\
\text { der }\end{array}$ & Over & $\begin{array}{l}\text { Un- } \\
\text { der }\end{array}$ \\
\hline $\begin{array}{l}1 \text { to } 2 \text { inches, inclusive } \\
\text { Over } 2 \text { to } 4 \text { inches, inclusire- } \\
\text { Over } 4 \text { to } 6 \text { inches, inclusive- }\end{array}$ & $\begin{array}{l}\text { Inch } \\
0.030 \\
.047 \\
.062\end{array}$ & $\begin{array}{l}\text { Inch } \\
0.015 \\
.015 \\
.032\end{array}$ & $\begin{array}{l}\text { Inch } \\
0.015 \\
.015 \\
.015\end{array}$ & $\begin{array}{l}\text { Inch } \\
0.005 \\
.005 \\
.005\end{array}$ & $\begin{array}{l}\text { Inch } \\
0.015 \\
.015 \\
.015\end{array}$ & $\begin{array}{l}\text { Inch } \\
0.010 \\
.010 \\
.010\end{array}$ \\
\hline
\end{tabular}

Rounds and squares

Up to $1 / 2$ inch, inclusive.

Over $1 / 2$ to 1 inch, inclusive.-
Over 1 to 2 inches, inclusive.

\begin{tabular}{c|c}
$\begin{array}{l}\text { Variations in } \\
\text { diameter or } \\
\text { thickness }\end{array}$ \\
\hline Over & Under \\
\hline Inch & $\begin{array}{r}\text { Inch } \\
0.007\end{array}$ \\
0.007 \\
.010 & .010 \\
.016 & .016 \\
\hline
\end{tabular}


(Workmanship and finish, marking, inspection, and rejection, and rehearing conform to paragraphs $5,6,7,8$, and 9 in A. S. T. M. standard specifications for steel. See 605.0 , p. 247.)

AMERICAN SOCIETY FOR TESTING MATERIALS, STANDARD SPECIFICATIONS FOR CARBON-STEEL BARS FOR VEHICLE AND GENERAL PURPOSE SPRINGS, DESIGNATION A58-27, 1927

\section{BARS FOR VEHICLE SPRINGS}

SCOPE

1. These specifications cover two grades of carbonsteel bars to be used for the manufacture of vehicle and general purpose springs, determined by the carbon ranges specified in section 3 . The choice of the grade of bar to be used for the manufacture of any spring will depend on the design of the spring and the stresses and service for which it is intended.

\section{MANUFACTURE}

2. The steel shall be made by one or more of the following processes: Open-hearth, crucible, or electric-furnace.

\section{CHEMICAL PROPERTIES AND TESTS}

3. The stee- shall conform to the following requirements as to chemical composition:

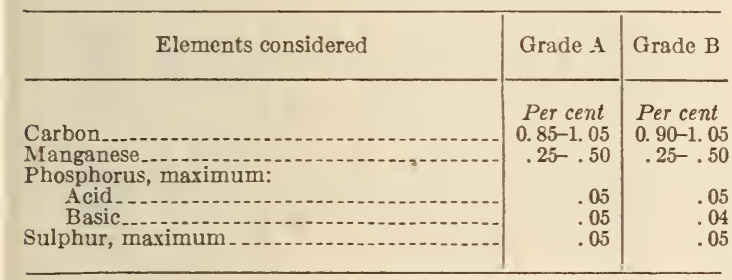

LADLE AND CHECK ANALYSES

(Same as in American Railway Association specification for steel tank plates. See 621.32, p. 1124.)

\section{PERMISSIBLE VARIATIONS IN DIMENSIONS}

4. The permissible variations in the width and thickness of the bars shall be agreed upon by the manufacturer and the purchaser.

(Workmanship and finish, marking, inspection and rejection, and rehearing conform to paragraphs $5,6,7,8$, and 9 in A. S. T. M. standard specifications for steel. See 605.0, p. 247.)

\section{AMERICAN SOCIETY FOR TESTING MATE. RIALS, STANDARD SPECIFICATIONS FOR CARBON-STEEL BARS FOR RAILWAY SPRINGS WITH SPECIAL SILICON RE- QUIREMENTS, DESIGNATION A68-27, 1927}

\section{BARS FOR RAILWAY SPRINGS}

(Identical with A. S. T. M. A14, given above, including references in $\mathrm{A} 14$ to $\mathrm{A}$. R. A. under 621.32, and A. S. T. M. standard specifications for steel under 605.0 , with the exception that the two grades of carbon steel in A68 have special silicon requirements. These requirements are specified under chemical composition as a silicon percentage in grades $\mathrm{A}$ and $\mathrm{B}$ of 0.25 to 0.50 , respectively.)
SOCIETY OF AUTOMOTIVE ENGINEERS, IRON AND STEEL SPECIFICATIONS; AU. TOMOTIVE STEEL SPECIFICATIONS, S. A. E. STANDARD, FEBRUARY, 1929

\section{CARBON STEELS}

(See 603.27 , p. 150 , including certain references to 600.0 , p. 2 , and 600.1, p. 49 .)

SOCIETY OF AUTOMOTIVE ENGINEERS, FRAMES AND SPRINGS; IEAF SPRINGS, S. A. E. RECOMMENDED PRACTICE, FEBRUARY, 1929

\section{LEAF SPRINGS}

(See 611.54, p. S08.)

603.4 IRON AND STEEL WIRE. 603.40 GENERAL ITEMS.

UNITED STATES GOVERNMENT, DEPARTMENT OF COMMERCE, BUREAU OF STANDARDS, WIRE GAGES, CIRCULAR NO. 67, JANUARY 17,1918

\section{WIRE GAGES}

Combined table of sizes in the principal wire gages 1

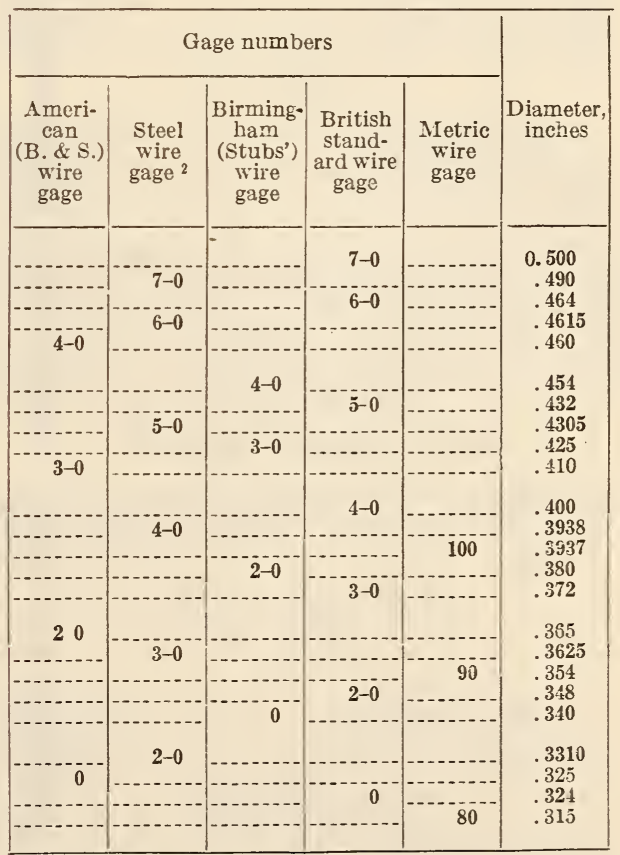

1 In this table the values printed in boldfaced type are exact as given values which are not exact have been rounded off to four significant values which are not exact have been rounded of to four significant figures, except that the diameters in inches of the American (B. \& S.) wire gage and of the metric wire gage in the column headed "Diameter, inches," have been given to 0.001 inch for the larger sizes and to 0.0001 inch for the smaller. This repre

2 The steel wire gage is the same gage which has been known by the various names: "Washburn and Moen," "Roebling," "American Steel and Wire Co.'s." Its abbreviation should be written "Stl. W. G." to distinguish it from "S. W. G.," the usual abbreviation for the (British standard) wire gage. 
Combined table of sizes in the principal wire gages ${ }^{1}$ - Continued

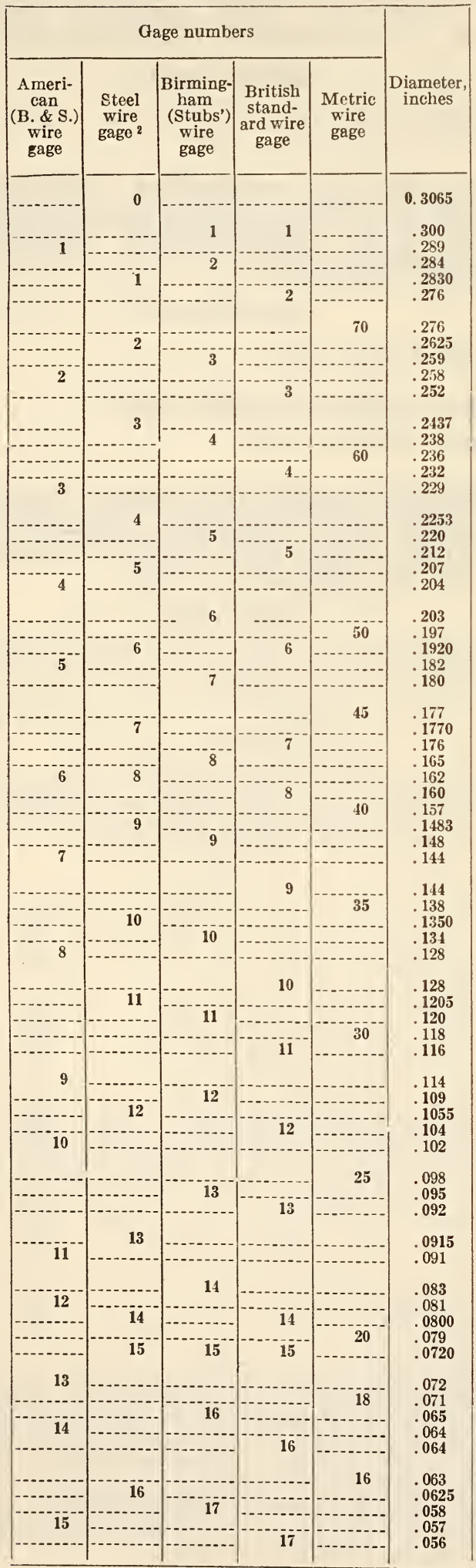

Combined table of sizes in the principal wire gages ${ }^{1-C o n t i n u e d}$

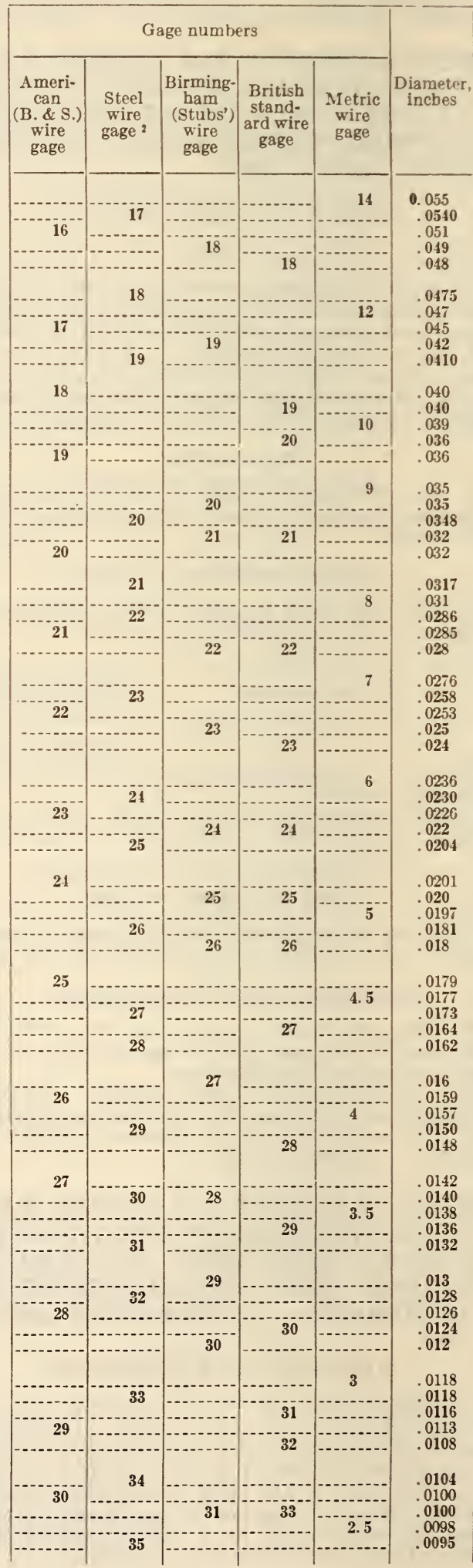


Combined table of sizes in the principal wire gages ${ }^{1-C o n t i n u e d}$

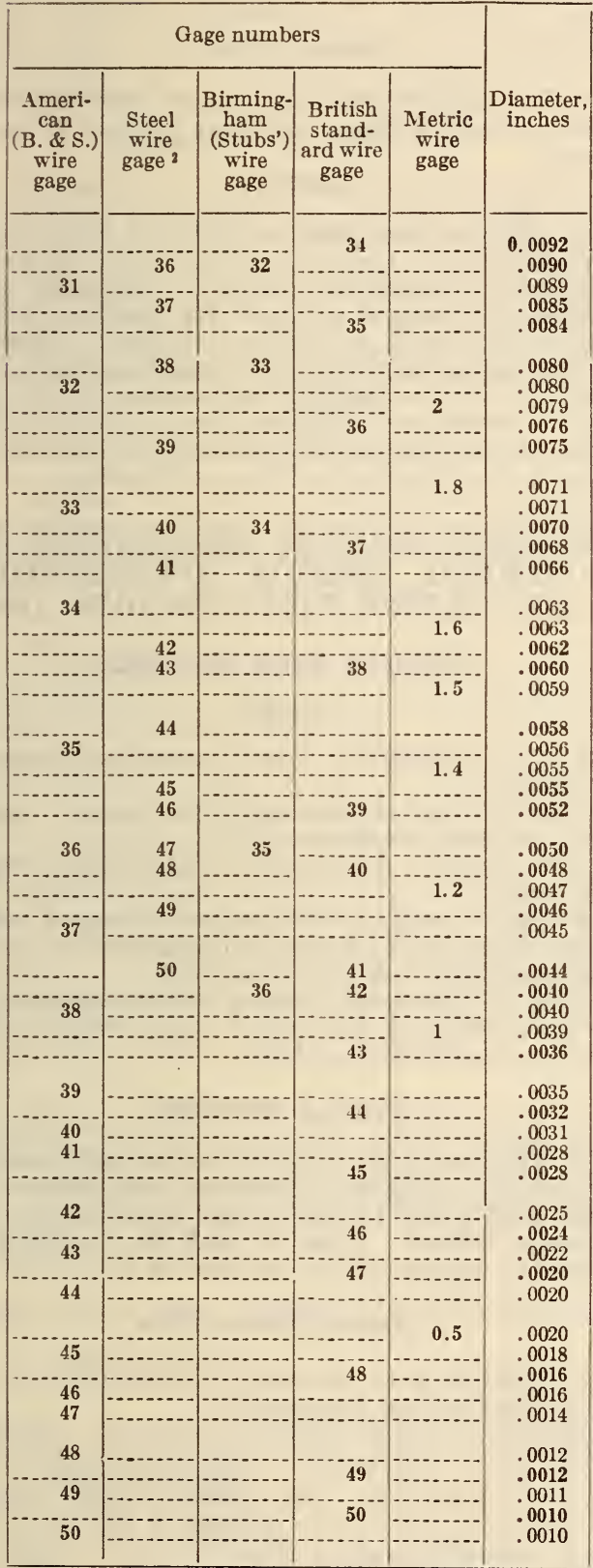

\subsection{BARE AND COATED IRON AND STEEL WIRE.}

\section{AMERICAN BUREAU OF WELDING, WELD- ING WIRE SPECIFICATIONS AND FOLIOS, BULLETIN NO. 2, 1921}

\section{BARE IRON AND STEEL ELECTRODES}

\section{GENERAL}

1. The following specifications prefixed by the letter $\mathrm{E}$ are recommended for the purchase of all bare iron and steel electrodes for use in arc welding.

The use of each particular type of electrode is specified below.

\section{SCOPE}

2. The electrodes herein specified are recommended as covering the usual railroad, shipyard, and industrial requirements as are allowed by authoritative regulating bodies, such as the American Bureau of Shipping, the Interstate Commerce Commission, etc.

\section{MATERIAL}

3. Material made by the puddling process is not permitted.

\section{PHYSICAL PROPERTIES}

4. Electrodes shall be made of commercially straight wire of uniform homogeneous structure, free from irregularities in surface hardness, segregation, oxides, pipe, seams, etc. Diameter shall not vary more than plus or minus 3 per cent from diameter specified.

\section{NOMENCLATURE}

5. The prefix letter $\mathrm{E}$ is to indicate that the material is intended for electric-arc welding.

\section{CHEMICAL COMPOSITION}

6. The chemical composition of electrodes shall be within the following limits for uses specified:

\section{LOW CARBON IRON OR STEEL}

E-No. 1A

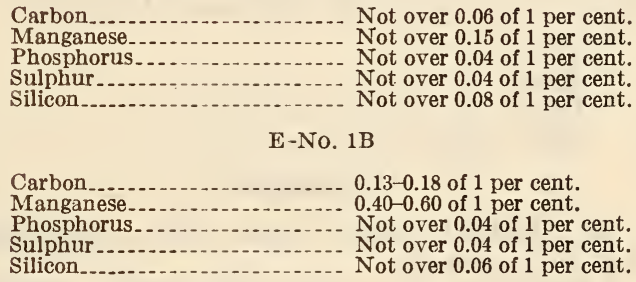

The difference between E-No. 1A and E-No. 1B electrodes is one of chemical composition incident to manufacture only. In order to assure uniformity of product, close limits are demanded. Either E-No. 1A or E-No. 1B may be supplied unless the purchaser, in ordering, specifically excludes either.

\section{MEDIUM CARBON STEEL}

The data at hand show that bare steel electrodes having a carbon content of 0.18 to 0.85 are of no particular value.

\section{HIGH CARBON STEEL}

E-No. $1 \mathrm{C}$

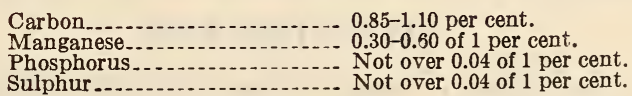
Not over 0.04 of 1 per cent.

7.

\section{RECOMMENDED SIZE}

Diameter (in inches)

E-No. 1A and E-No. 1B...... 1/16, 3/32, 1/8, 5/32,3/16, 1/4. E-No. 1C ................... $3 / 8,5 / 32,3 / 16,7 / 32,34,9 / 32,5 / 16$

\section{USES}

8. E-No. 1A and E-No. 1B for welding mild steel structural shapes, plates, bars, or low-carbon steel forgings and castings. E-No. $1 \mathrm{C}$ for welding highcarbon steel and worn surfaces where great resistance to abrasive wear is desired, and where machin- 
ing is not necessary, such as rails, frogs, switch points, bearing surfaces, etc.

\section{SURFACE FINISH}

9. The surface shall be smooth and free from rust, oil, or grease.

TESTS

10. In the hands of an experienced welder electrodes shall demonstrate good weldability in flat and overhead positions, and shall pass through the arc smoothly and evenly without any unusual characteristics.

\section{PACKING}

11. Electrodes shall be delivered in coils or in straight 14-inch lengths, packed and wrapped as follows:

(a) Bundles of 50 pounds net weight, securely wired and wrapped in heavy weatherproof paper.

(b) Bundles of 50 pounds net weight, securely wrapped in heavy burlap.

(c) Boxes or kegs of 100,200 , or 300 pounds net weight, and wrapped as per paragraph $(a)$.

(d) Boxes or kegs of 100,200 , or 300 pounds net weight, and wrapped as in paragrapn $(b)$.

(e) Coils of approximately 50 or 100 pound net weight, and wrapped as per paragraph $(a)$ or (b).

\section{MARKING}

12. All bundles, coils, boxes, or kegs shall be provided with a metal, linen, or strong fiber tag securely wired to, or in the case of boxes or kegs, nailed on the outside, bearing the following information:

Make.

Specif. No_.

Dia

Nom. Weight.

\section{ORDERING}

13. Material ordered under these specifications shall be known as:

"Electrodes, iron and steel, bare," American Welding Society Specifications Number 1, Revised May 1, 1921.

All orders should be specified in pounds. In addition, requisitions shall show the following:

\section{Specif. No. \\ Size \\ Packing}

As a guide in ordering, the following information is of use:

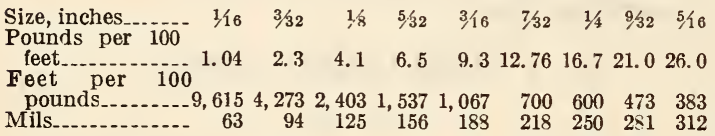

\section{GAS WELDING RODS}

(In respect to scope, material, chemical composition of No. 1A rods, uses, tests, packing, marking, the specifications for gas welding rods are identical with those for electrodes, given above, with the following exceptions:)

\section{PHYSICAL PROPERTIES}

4. Welding rods shall be made of annealed wire.

\section{NOMENCLATORE}

5. The use of the prefix letter $G$ is to indicate that the material is intended for gas welding.
7.

RECOMMENDED SIZES

Diameter (in inches) ........... 1/16, 3/32, 1/8, 5/32, 3/16, 1/4, 5/16, 3/6

\section{SURFACE FINISH}

9. The surface shall be smooth and free from scale, rust, oil or grease, and may be plain or copper coated.

\section{PACKING}

11. Welding rods shall be delivered in coils or in straight 36 -inch lengths (packed and wrapped as specified for electrodes above). (Information for ordering is the same as above for electrodes excent that there is no $7 / 32$-inch gas welding rod. Chemical analyses of coated or covered electrodes and of gas welding rods for welding high-carbon steels, nonferrous metals, and mild steel are appended to these specifications as a guide in selecting welding material. They do not form part of the specifications.)

\section{AMERICAN RAILWAY ASSOCIATION, ME- CHANICAL DIVISION, SPECIFICATIONS FOR WELDING WIRE AND RODS, 1924}

\section{WELDING WIRE AND RODS}

\section{SCOPE}

1. This specification covers six classes of material, as follows:

A or B. Wire for gas or electric welding of wrought iron, and cast or wrought steel.

C. Cast-iron welding rods for gas welding of gray cast iron.

D. Brass welding rods for gas welding of miscellaneous brass and gray cast-iron parts. (For chemical composition see 645.11 , p. 1203.)

$\mathrm{E}$ or $\mathrm{F}$. Aluminum welding rods for gas welding all parts made of aluminum. (For chemical composition see 631.31 , p. 1157.)

\section{PHYSICAL PROPERTIES}

2. All wire and rods shall be commercially straight, of uniform homogeneous structure, and shall be free from oxides, slivers, seams, and all other injurious physical defects. Class $A$ shall be furnished annealed or unannealed as specified on the order.

\section{CHEMICAL COMPOSITION}

3. Welding wire and rods shall conform to the following chemical composition:

\begin{tabular}{|c|c|c|c|}
\hline & $\begin{array}{l}\text { Class A } \\
\text { (maxi- } \\
\text { mum) }\end{array}$ & $\begin{array}{l}\text { Class B } \\
\text { (maxi- } \\
\text { mum) }\end{array}$ & Class C \\
\hline $\begin{array}{l}\text { Carbon } \\
\text { Manganese } \\
\text { Phosphorus } \\
\text { Sulphur } \\
\text { Silicon }\end{array}$ & $\begin{array}{r}\text { Per cent } \\
0.06 \\
.15 \\
.04 \\
.04 \\
.08\end{array}$ & $\begin{array}{r}\text { Per cent } \\
0.13-0.18 \\
.40-.60 \\
.04 \\
.04 \\
.06\end{array}$ & $\begin{array}{r}\text { Per cent } \\
13.00 \\
0.50-0.75 \\
.50-0.70 \\
2.10 \\
12.90\end{array}$ \\
\hline
\end{tabular}

1 Minimum.

2 Maximum.

\section{WELDING TEST}

4. Welding wire and rods shall render satisfactory welding properties and shall flow smoothly and evenly without unusual characteristics when used by a competent operator upon the class of work for which intended. 
SAMPLES FOR CHEMICAL ANALYSIS

5. From each class of material crdered upon every shipment, one sample for chemical analysis shall be taken, representative of each lot of 1,000 pieces or less, when cut to length, or of each five coils of uncut wire. This analysis snall in each case conform to the requirements of section 3 .

\section{DIMENSIONS AND TOLERANCES}

6. Welding wire and rods shall conform closely to the dimensions specified upon orders. Drawn wires shall not vary more than 3 per cent from the diameter specified. Cast rods shall not vary more than 10 per cent from the diameter specified. All electrodes shall be furnished in 14-inch lengths and drawn wire for gas welding in 36-inch lengths, unless otherwise specified. Cast rods shall be furnished in lengths of not less than 12 inches.

\section{WORKMANSHIP AND FINISH}

7. Welding wire and rods shall have a smooth clean surface, free from rust and scale, and shall not carry more than a slight trace of oil or grease.

\section{PACKING AND MARKING}

8. Rods, and welding wire cut to length, shall be securely tied at both ends with wire and wrapped in heavy weatherproof paper or burlap for shipment in bundles of 50 pounds net weight. If the manufacturer desires, the wire and rods may be packed in suitable boxes or kegs of 100 or 200 pounds net weight. Wire ordered in coils shall be furnished in coils of approximately 50 pounds net weight, unless otherwise specified. Each bundle of rods or wire cut to length, and each coil of wire, shall be provided with a linen or metal tag, securely wired on, showing the manufacturer, the letters A. R. A. and the class of wire, and the diameter and nominal weight. (Inspection, rejection, and rehearing are substantially the same as in A. S. T. M. standard specifications for steel. (See 605.0, p. 247.)

AMERICAN SOCIETY FOR TESTING MATERIALS, STANDARD SPECIFICATIONS FOR COLD-DRAWN STEEL WIRE FOR CONCRETE REINFORCEMENT, DESIGNATION A82-27, 1927

STEEL WIRE FOR CONCRETE REINFORCEMENT

(See 605.25, p. 305.)

\section{UNITED STATES GOVERNMENT, FEDERAL SPECIFICATIONS BOARD, FEDERAL SPECIFICATION FOR WELDING WIRE, IRON AND STEEL, NO. 174, JUNE 30, 1924. (CURRENT DESIGNATION: QQ- W-351)}

\section{WELDING WIRE, IRON AND STEEL}

\section{GRADES AND TYPES}

1. (a) This specification covers three types of welding wire, viz:

I. Electrodes, iron and steel, bare.

II. Gas welding, wire, iron, and steel, plain.

III. Cast-iron welding rod.

(b) The welding wire shall be of grades as given in Tables 1, 2, 3, paragraph $5(a)$.

\section{MATERIAL}

2. (a) Welding wire made by the puddling process will not be accepted.

\section{GENERAL REQUIREMENTS}

3. (a) Electrodes shall be made of straight wire of uniform homogeneous structure free from irregularities in surface hardness, segregation, oxides, other nonmetallic inclusions, pipes, seams, etc. The surface shall be free from scale and rust, and shall be free from oil or grease except that resulting from the last drawing operation.

(b) Gas welding rods shall be made of annealed, straight wire of uniform homogeneous structure free from irregularities in surface hardness, segregation, oxides, other nonmetallic inclusions, pipes, seams, etc. The surface shall be free from scale, rust, oil, or grease.

(c) Cast-iron welding rods shall be of uniform homogeneous structure, straight, cleaned, and free from rust, sand, dirt, scale, oil, or grease.

(d) The diameter of all wire except cast iron shall not vary more than plus or minus 3 per cent from the diameter specified.

\section{DETAIL REQUIREMENTS}

4. (a) The welding wire shall conform to the requirements shown in Tables 1,2 , and 3 below.

TABLE 1.-Chemical composition electrodes, iron and steel, bare

\begin{tabular}{|c|c|c|c|c|c|}
\hline Grades & $\mathrm{C}$ & Mn & $\begin{array}{c}\mathrm{P} \\
\text { (maxi- } \\
\text { mum) }\end{array}$ & $\begin{array}{l}\mathrm{S} \\
\text { (maxi- } \\
\text { mum) }\end{array}$ & $\begin{array}{l}\text { Si } \\
\text { (maxi- } \\
\text { mum) }\end{array}$ \\
\hline $\begin{array}{l}\mathrm{A} \\
\mathrm{B} \\
\mathrm{B}\end{array}$ & $\begin{array}{r}\text { Per cent } \\
10.06 \\
0.10-.18 \\
.35-.45 \\
.85-1.10\end{array}$ & $\begin{array}{r}\text { Per cent } \\
10.15 \\
0.40-.60 \\
.40-.60 \\
.30-.60\end{array}$ & $\begin{array}{c}\text { Per cent } \\
0.04 \\
.04 \\
.04 \\
.04\end{array}$ & $\begin{array}{c}\text { Per cent } \\
0.04 \\
.04 \\
.04 \\
.04\end{array}$ & $\begin{array}{r}\text { Per cent } \\
0.08 \\
.06 \\
.15 \\
.25\end{array}$ \\
\hline
\end{tabular}

1 Maximum.

TABLE 2.-Chemical composition gas-welding wire, iron and steel, plain

\begin{tabular}{|c|c|c|c|c|c|}
\hline Grade & C & $\mathrm{Mn}$ & $\underset{(\text { maxi- }}{\mathbf{P}}$ & $\begin{array}{c}\mathrm{S} \\
\text { (maxi- } \\
\text { mum) }\end{array}$ & $\mathrm{Si}$ \\
\hline $\mathrm{E}$ & $\begin{array}{r}\text { Per cent } \\
10.06 \\
0.10-.18 \\
.20-.30\end{array}$ & $\begin{array}{r}\text { Per cent } \\
10.15 \\
0.45-.60 \\
.40-.60\end{array}$ & $\left|\begin{array}{r}\text { Per cent } \\
0.04 \\
.04 \\
.04\end{array}\right|$ & $\mid \begin{array}{r}\text { Per cent } \\
0.04 \\
.04 \\
.04\end{array}$ & $\begin{array}{c}\text { Per cent } \\
10.08 \\
1.06 \\
(2)\end{array}$ \\
\hline
\end{tabular}

1 Maximum.

$2 \mathrm{Ni}, 2.00$ to 3.50 per cent; Cr (maximum), 0.20 .

TABLE 3.-Chemical composition cast-iron welding rod

\begin{tabular}{c|c|c|c|c|c}
\hline Grade & Total C & $\mathrm{Mn}$ & $\mathrm{P}$ & $\begin{array}{c}\mathrm{S} \\
(\text { maxi- } \\
\text { mum })\end{array}$ & Si \\
\hline & $\begin{array}{c}\text { Per cent } \\
\text { H.00-3.50 }\end{array}$ & $\begin{array}{c}\text { Per cent } \\
0.50-0.75\end{array}$ & $\begin{array}{c}\text { Per cent } \\
0.50-0.70\end{array}$ & $\begin{array}{c}\text { Per cent } \\
0.10\end{array}$ & $\begin{array}{c}\text { Per cent } \\
3.00-3.50\end{array}$ \\
\hline
\end{tabular}

\section{METHODS OF INSPECTION AND TESTS}

5. (a) Electrodes shall demonstrate good weldability in flat and overhead positions and shall pass through the arc smootrly and evenly without any unusual characteristics. Gas welding and cast-iron 
welding rods shall demonstrate good weldability and shall flow smoothly and evenly without any unusual characteristics.

(b) Check chemical analyses may be made by the inspector or through him by any Government laboratory or other designated representative and without cost to the contractor. The chemical composition thus determined shall conform to the requirements specified in the tables for the grade ordered.

(c) If a check analysis be made, a composite sample of not less than 4 ounces shall be taken for analysis from each 1,000 pounds or less. At least one wire from every size in each 1,000 pounds or less shall be selected. Where the 1,000 pounds or less consists of but one size, one wire from each of five bundles shal be selected. Samples shall consist of fine particles obtained by clipping, sawing, or drilling, depending on the size of the wire.

(d) Umpire method of analysis.-Suitable methods for umpire chemical analysis are being developed and will be added at later revision.

6. (a) Electrodes shall be delivered straight in 14inch lengths, gas-welding wire straight in 36-inch lengths, and cast-iron rods in 12 to 20 inch lengths, in bundles of 50 pounds net weight securely wired and wrapped in heavy waterproof paper and burlap. Each bundle shall be provided with a metal tag and indelibly marked with the manufacturer's name, grade, quantity, diameter of wire, and the contract or order number.

(b) Electrodes and gas-welding wire may be delivered in coils of 50 pounds net weight when so ordered. The coils to be securely wired, wrapped, and marked as specified above.

\section{ADDITIONAL INFORMATION}

7. (a) Coated or covered welding wire is not to be furnished under these specifications unless specifically required in the purchase order.

(b) Grades A, B, and E are used for welding mild steel structural shapes, plates, and bars, or lowcarbon steel forgings and castings.

(c) Grade $\mathrm{C}$ is used for building up worn surfaces that require a machine finish.

(d) Grade D is used on high-carbon steels and worn surfaces subject to resistance to abrasive wear and where machining is not necessary.

(e) Grade $\mathrm{F}$ is used for welding mild steel and in general for same purpose as Grade E.

$(f)$ Grade $\mathrm{G}$ is used for welding mild or nickel steels, producing a weld of greater strength than with Grades A, B, or C.

(g) Grade $\mathrm{H}$ is used for general welding of castiron parts.

(h) Grades covered in Tables 1 and 2 are furnished commercially in the following sizes: $1 / 16,3 / 32,1 / 8,5 / 32$, $3 / 16,1 / 4,5 / 16$, and $3 / 8$ inch. The grade covered in the Table 3 is furnished commercially in the following sizes: $3 / 16,1 / 4,3 / 8$, and $1 / 2$ inch.

\subsection{BARE AND COATED WIRE ROPE.}

AMERICAN ASSOCIATION OF STATE
HIGHWAY OFFICIALS, TENTATIVE
STANDARD SPECIFICATION FOR WIRE
ROPE AND FITTINGS FOR HIGHWAY
GUARD RAIL, MI-30, 1927

\section{WIRE ROPE AND FITTINGS}

\section{A. Material Covered}

1. This specification covers $3 / 4$-inch and 1 -inch wire rope and fittings for use in the construction of wire-rope railing supported by wood or other suitable posts erected as indicated.

\section{WIRE ROPE}

B. General Requirements

2. All rope shall be manufactured of doublegalvanized annealed steel having the properties hereinafter required. The wire shall be cylindrical in form and shall be free from scales, inequalities, flaws, and splits.

\section{Physical Properties}

3. Galvanizing.-Each wire from the rope shall be galvanized by the hot-dip method and shall have a continuous coating of zinc, of a uniform thickness, so appliea that it will adhere firmly to the surface of the wire. The weight of zinc coating for cach wire shall be 0.8 ounce minimum of zinc per square foot of surface after fabrication, and it shall be capable of withstanding four immersions in a standard testing solution of copper sulphate without showing any trace of metallic copper on the steel. The first three immersions shall be for a period of one minute each and the fourth immersion for a period of one-half minute.

4. Three-fourths inch rope.-The rope shall be composed of three strands, each strand having seven wires. The diameter of the finished rope shall not be less than three-fourths inch. The wire composing the rope shall be of such quality that the finished rope shall satisfy all the requirements hereinafter set forth. All the wires in the rope shall be of the same grade of steel and shall have approximately the same breaking strain.

5 . The lay of the finished rope shall be not more than $7 \frac{1}{2}$ inches. The lay of wires in the strand shall be not more than $4 \frac{1}{2}$ inches.

6. The diameter of the finished wires entering into the rope shall be not less than 0.117 inch and not more than 0.124 inch.

7. The minimum tensile strength of the rope shall be 13,000 pounds.

8. One-inch rope.-The rope shall be composed of six strands with wire-strand center, each strand having seven wires. The diameter of the finished rope shall be not less than 1 inch. The wires composing the rope shall be of such quality that the finished rope shall satisfy the requirements hereinafter set forth. All the wires in the rope shall be of the same grade of steel and shall have approximately the same breaking strain.

9. The lay of the finished rope shall be not more than 10 inches. The lay of the wires in the strand shall be not more than $4 \frac{1}{2}$ inches.

10. The diameter of the finished wires entering into the rope shall be not less than 0.105 inch and not more than 0.112 inch.

11. The minimum tensile strength of the rope shall be 30,000 pounds.

\section{Marking and Shipping}

12. All wire rope must be shipped upon substantial wooden reels. Each reel shall have the length and weight of the rope plainly and indelibly marked on a strong tag, firmly attached. The wooden reel shall be mounted so that it will revolve and the rope run off by pulling straight ahead.

\section{EYEBOLTS}

13. All eyebolts shall be drop-forged, 1 inch in diameter, with an eye having a minimum diameter of $13 / 8$ inches. They shall be double galvanized by the hot-dip method. Each eyebolt of 12-inch and 24inch lengths shall have double-galvanized wroughtiron washers and nuts attached, and these washers shall be at least one-eighth of an inch thick and 4 inches in diameter. The eyebolts of 48-inch lengths shall have attached washers consisting of steel plates, three-quarters of an inch thick and 6 inches in 
diameter, or 5 inches by 5 inches, having a center hole $11 / 4$ inches in diameter.

14. 'The eyebolts shall have a minimum tensile strength of 30,000 pounds.

15. The eyebolts shall be of the following lengths, the length specified being for the straight shaft only:

\begin{tabular}{|c|c|}
\hline Length & $\begin{array}{c}\text { Length, } \\
\text { threaded }\end{array}$ \\
Inches & Inches \\
48 & 12 \\
24 & 18 \\
12 & 6 \\
\hline
\end{tabular}

\section{CLIPS}

16. All clips shall be double galvanized and of approved design. Galvanizing shall be by the hotdip process.

\section{E. Sampling and Testing}

17. Sampling and testing of wire rope and fittings for highway guard rail shall be in accordance with A. A. S. H. O. T-39, given below.

\section{AMERICAN ASSOCIATION OF STATE HIGHWAY OFFICIALS, METHODS OF TEST FOR WIRE ROPE (GUARD RAIL), TENTATIVE METHOD T-39, 1931}

\section{TESTING WIRE ROPE}

1. The report of the physical test of wire rope shall include the following information: Diameter of the rope, diameter of the wire, number of strands and wires, length of lay of strands and wires, crosssectional area in square inches, breaking load in pounds, and a note as to whether the failure was in the body of the rope or at the socket.

\section{DEFINITIONS}

2. (a) The lay of the rope is the length expressed in inches for each complete turn of a strand around the axis of the rope measured along the axis.

(b) The lay of the strand is the length expressed in inches for each complete turn of a wire around the axis of the strand measured along the axis.

(c) The diameter of a wire rope is the diameter of a circle inclosing it.

3. The cross-sectional area of the rope shall be determined as follows: Measure the diameter of the component wires of a strand and obtain the mean diameter. The area calculated from the mean diameter of the wire multiplied by the number of wires in each strand and by the number of strands gives the aggregate area of the wires in the rope.

4. The test specimen for the tension test shall be free from bends and not less than 4 feet in length. Before cutting the test pieces from the coil of rope, the ends must be "served" or wound about with wire for a length of about 1 inch to prevent the strands from unlaying.

\section{PREPARATION OF SPECIMEN FOR TEST}

5. A socket shall be attached to each end of the rope for the tension test. In preparing the rope for socketing the ends are first served or wound around with fine soft wire for a length of about one-half inch at the ends and also at a distance from the ends equal to the lengths of the basket of the socket. The ends of the rope are then slipped through the socket and the serving wire removed from the end only. Unlay the strands, separate the individual wires, and straigthen them. Cut out the hemp center, if any. Cleanse the wire thoroughly with kerosene or gasoline and wipe dry. Tin the wires by dipping first in a mild zinc-chloride solution and then in molten zinc. When removed from the latter, they should be knocked with a stick or hammer to remove the excess zinc. Repeat the tinning until all the wires are thoroughly coated. Pull the frayed ends back into the basket of the socket and spread the wires evenly in the socket and even with the top of the basket. Put fire clay or asbestos fiber around the rope at the bottom of the socket, heat the socket for a short time with a blow torch, and pour in molten pure zinc (not babbitt or lead).

6. Put the sockets through the heads of the testing machine and place pins through the eyes of the sockets, using steel blocks on each side of the socket, if necessary, to secure a firm bearing (pins should be as large as possible). Apply the load slowly to give the strands and wires opportunity properly to bed upon one another during the application of the load. Continue loading until fracture of one or more strands of the rope occurs.

7. The uniformity of the galvanizing or spelter coating, if any, shall be determined in accordance with the method for uniformity of spelter coating.

\section{AMERICAN BUREAU OF SHIPPING, RULES FOR BUILDING AND CLASSING STEEL VESSELS, 1930}

\section{STEEL WIRE ROPES}

(For general information and requirements see 600.0 , p. 2.)

1. When these are adopted for stream cables, towlines, hawsers, and warps, they are to be subjected to the following breaking tests in the presence of the surveyors of the American Bureau of Shipping. (Those marked with an asterisk (*) comprise standard equipment adopted by the American Bureau of Shipping for steam vessels, sailing vessels and barges, and for seagoing tugs.) Substitution of steel wire ropes of equivalent strength to those prescribed as standard equipment will also be approved as indicated in the table following. 
[Diameter in inches]

\begin{tabular}{|c|c|c|c|c|c|c|c|c|c|c|c|c|}
\hline \multirow{4}{*}{ Break test (in pounds) } & \multicolumn{11}{|c|}{ Stranding } & \\
\hline & \multicolumn{4}{|c|}{6 by 12} & \multicolumn{4}{|c|}{6 by 24} & \multicolumn{4}{|c|}{6 by 37} \\
\hline & \multicolumn{4}{|c|}{ Grades } & \multicolumn{4}{|c|}{ Grades } & \multicolumn{4}{|c|}{ Grades } \\
\hline & 1 & 2 & 3 & 4 & 1 & 2 & 3 & 4 & 1 & 2 & 3 & 4 \\
\hline $\begin{array}{l}10,000 \\
13,000 \\
16,000 \\
23,000\end{array}$ & $\begin{array}{l}9 / 16 \\
3 / 4 \\
3 / 4 \\
7 / 8 \\
1\end{array}$ & $\begin{array}{l}9 / 16 \\
5 / 8 \\
3 / 4 \\
13 / 16 \\
7 / 8\end{array}$ & $\begin{array}{l}* 1 / 2 \\
* 5 / 8 \\
* 3 / 4 \\
* 13 / 6 \\
* 7 / 8\end{array}$ & $\begin{array}{l}1 / 2 \\
9 / 16 \\
5 / 8 \\
3 / 4 \\
13 / 16\end{array}$ & $\begin{array}{l}1 / 2 \\
5 / 8 \\
5 / 8 \\
3 / 4 \\
3 / 4\end{array}$ & $\begin{array}{l}1 / 2 \\
1 / 2 \\
5 / 8 \\
3 / 4 \\
3 / 4\end{array}$ & $\begin{array}{l}1 / 2 \\
1 / 2 \\
58 \\
5 / 8 \\
3 / 4\end{array}$ & $\begin{array}{l}3 / 8 \\
1 / 2 \\
1 / 2 \\
5 / 8 \\
3 / 4\end{array}$ & $\begin{array}{l}7 / 16 \\
1 / 2 \\
9 / 16 \\
5 / 8 \\
3 / 4\end{array}$ & $\begin{array}{l}7 / 16 \\
7 / 16 \\
1 / 2 \\
5 / 8 \\
3 / 4\end{array}$ & $\begin{array}{l}3 / 8 \\
7 / 16 \\
1 / 2 \\
5.8 \\
5 / 8\end{array}$ & $\begin{array}{l}3 / 8 \\
7,16 \\
7 / 16 \\
9 / 10 \\
5,8\end{array}$ \\
\hline $\begin{array}{l}31,000- \\
39,000 \\
44,000 \\
56,000\end{array}$ & $\begin{array}{l}1 \\
11 / 8 \\
13 / 16 \\
13 / 8 \\
13 / 8\end{array}$ & $\begin{array}{l}1 \\
1116 \\
11 / 8 \\
11 / 4 \\
13 / 8\end{array}$ & $\begin{array}{l}{ }^{*} 1 \\
{ }^{1} 1 \\
*_{11} 118 \\
*_{13} \\
*_{1} 1 / 4\end{array}$ & $\begin{array}{l}7 / 8 \\
1 \\
11 / 8 \\
11 / 8\end{array}$ & $\begin{array}{l}13 / 16 \\
1 \\
11 / 16 \\
118\end{array}$ & $\begin{array}{l}13 / 16 \\
178 \\
1 \\
11 / 16\end{array}$ & $\begin{array}{l}3 / 4 \\
7 / 8 \\
7 / 8 \\
1 \\
1\end{array}$ & $\begin{array}{l}3 / 4 \\
13 / 16 \\
7 / 8 \\
1 \\
1\end{array}$ & $\begin{array}{l}3 / 4 \\
7 / 8 \\
7 / 8 \\
1 \\
1\end{array}$ & $\begin{array}{l}3 / 4 \\
7 / 8 \\
7 / 8 \\
1 \\
1\end{array}$ & $\begin{array}{r}3 / 4 \\
3 / 4 \\
7 / 8 \\
7 / 8 \\
1\end{array}$ & $\begin{array}{l}5 / 8 \\
3 / 4 \\
3 / 4 \\
738 \\
7 / 8\end{array}$ \\
\hline $\begin{array}{l}62,000 \\
76,000 \\
82,000 \\
90,000\end{array}$ & $\begin{array}{l}17 / 16 \\
15 / 8 \\
15 / 8 \\
111 / 16 \\
115 / 16\end{array}$ & $\begin{array}{l}13 / 8 \\
11 / 2 \\
15 / 8 \\
158 \\
113 / 16\end{array}$ & $\begin{array}{l}* 13 / 8 \\
*^{*} 17 / 16 \\
*^{1} 11 / 2 \\
*^{15} 18 \\
{ }^{1} 11 / 16\end{array}$ & $\begin{array}{l}13 / 16 \\
13 / 8 \\
13 / 8 \\
17 / 16 \\
158\end{array}$ & $\begin{array}{l}13 / 16 \\
13 / 8 \\
138 \\
17 / 16 \\
158\end{array}$ & $\begin{array}{l}11 / 8 \\
11 / 4 \\
138 \\
138 \\
11 / 2\end{array}$ & $\begin{array}{l}11 / 16 \\
13 / 16 \\
11 / 4 \\
13 / 8 \\
17 / 16\end{array}$ & $\begin{array}{l}1 \\
11 / 8 \\
118 \\
131 / 16 \\
138\end{array}$ & $\begin{array}{l}138 \\
11 / 4 \\
11 / 4 \\
13 / 8 \\
11 / 2\end{array}$ & $\begin{array}{l}1 \\
1138 \\
11 / 4 \\
11 / 4 \\
13 / 8\end{array}$ & \begin{tabular}{l|l}
1 \\
118 \\
$11 / 8$ \\
$11 / 4$ \\
$13 / 8$
\end{tabular} & $\begin{array}{l}1 \\
1 \\
118 \\
118 \\
11 / 4\end{array}$ \\
\hline $\begin{array}{l}126,000 \\
148,000 \\
152,000 \\
164,000 \\
174,000\end{array}$ & $21 / 16$ & $115 / 16$ & $\begin{array}{c}{ }^{*} 1^{15} / 16 \\
2 \\
21 / 16\end{array}$ & $\begin{array}{l}13 / 4 \\
1^{15 / 16} \\
115 / 16 \\
2 \\
21 / 16\end{array}$ & $\begin{array}{l}111 / 16 \\
113 / 16 \\
113 / 16 \\
115 / 16 \\
2\end{array}$ & $\begin{array}{l}15,8 \\
13 / 4 \\
13 / 4 \\
113 / 16 \\
115 / 16\end{array}$ & $\begin{array}{l}* 11 / 2 \\
* 15 / 8 \\
* 111 / 16 \\
* 13 / 4 \\
* 113 / 16\end{array}$ & $\begin{array}{l}17 / 16 \\
15,8 \\
158 \\
1518 \\
111 / 16\end{array}$ & $\begin{array}{l}11 / 2 \\
15 / 8 \\
13 / 4 \\
13 / 4 \\
17,8\end{array}$ & $\begin{array}{l}13 / 2 \\
15 / 8 \\
158 \\
13 / 4 \\
13 / 4\end{array}$ & $\begin{array}{l}13 / 8 \\
* 11 / 2 \\
* 11 / 2 \\
* 15,8 \\
* 15 / 8\end{array}$ & $\begin{array}{l}138 \\
112 \\
112 \\
112 \\
158\end{array}$ \\
\hline $\begin{array}{l}194,000 \\
208,000 \\
224,000 \\
232,700\end{array}$ & & & & & $21 / 16$ & $\begin{array}{l}2 \\
21 / 16 \\
-\end{array}$ & $\begin{array}{l}* 115 / 16 \\
* 2 \\
* 21 / 16\end{array}$ & $\begin{array}{l}13 / 4 \\
113 / 16 \\
115 / 6 \\
115 / 16\end{array}$ & $\begin{array}{l}17 / 8 \\
2 \\
21 / 4 \\
21 / 4\end{array}$ & $\begin{array}{l}17 / 8 \\
17 / 8 \\
2 \\
2\end{array}$ & $\begin{array}{l}* 134 \\
* 178 \\
* 1178 \\
* 178\end{array}$ & $\begin{array}{l}15 / 8 \\
13 / 4 \\
13 / 4 \\
13 / 4\end{array}$ \\
\hline $\begin{array}{l}241,300 \\
249,800 \\
267,200 \\
285,300\end{array}$ & & & & & & & - & $\begin{array}{l}2 \\
2 \\
21 / 16\end{array}$ & $\begin{array}{l}21 / 4 \\
21 / 4 \\
214 \\
21 / 2\end{array}$ & $\begin{array}{l}2 \\
21 / 4 \\
21 / 4 \\
21 / 4\end{array}$ & $\begin{array}{l}* 2 \\
* 2 \\
* 21 / 4 \\
* 21 / 1\end{array}$ & $\begin{array}{l}17 / 8 \\
178 \\
17 / 8 \\
2\end{array}$ \\
\hline $\begin{array}{l}303,900 \ldots \ldots \\
327,000 \ldots \ldots \\
347,200 \ldots \\
367,300 \ldots\end{array}$ & & & & & & & & & $\begin{array}{l}21 / 2 \\
21 / 2 \\
23 / 4\end{array}$ & $\begin{array}{l}21 / 4 \\
21 / 2 \\
21 / 2 \\
21 / 2\end{array}$ & $\begin{array}{l}* 21 / 4 \\
* 21 / 4 \\
* 21 / 2 \\
* 21 / 2\end{array}$ & $\begin{array}{l}2 \\
21 / 4 \\
21 / 4 \\
214\end{array}$ \\
\hline
\end{tabular}

NOTE.-In above table, grade 1 refers to cast-steel wire; grade 2 refers to extra strong cast-steel wire; grade 3 refers to plow-steel wire and grade 4 refers to improved plow-steel wire.

2. Steel wire rigging is to be subjected to the following breaking tests:

[Diameter in inches]

\begin{tabular}{|c|c|c|c|c|c|c|c|c|c|c|c|c|}
\hline \multirow{4}{*}{ Break test (in pounds) } & \multicolumn{12}{|c|}{ Stranding } \\
\hline & \multicolumn{4}{|c|}{6 by 7} & \multicolumn{4}{|c|}{6 by 19} & \multicolumn{4}{|c|}{8 by 19} \\
\hline & \multicolumn{4}{|c|}{ Grades } & \multicolumn{4}{|c|}{ Grades } & \multicolumn{4}{|c|}{ Grades } \\
\hline & 1 & 2 & 3 & 4 & 1 & 2 & 3 & 4 & 1 & 2 & 3 & 4 \\
\hline $\begin{array}{l}26,600 \\
32,200 \\
38,500 \\
45,000 \\
52,400 \\
60,200\end{array}$ & $\begin{array}{l}* 11 / 16 \\
* 33 / 4 \\
* 7 / 8 \\
* 1 \\
* 1 \\
* 11 / 8\end{array}$ & $\begin{array}{l}11 / 16 \\
3 / 4 \\
7 / 8 \\
7 / 8 \\
7 / 8 \\
1\end{array}$ & $\begin{array}{l}5 / 8 \\
11 / 16 \\
3 / 4 \\
7 / 8 \\
178 \\
1\end{array}$ & \begin{tabular}{l|}
$5 / 8$ \\
$11 / 16$ \\
$3 / 4$ \\
344 \\
$7 / 8$ \\
$7 / 8$
\end{tabular} & $\begin{array}{r}3 / 4 \\
3 / 4 \\
7 / 8 \\
17 \\
11 / 8 \\
11 / 8\end{array}$ & $\begin{array}{l}34 \\
3 / 4 \\
778 \\
7 / 8 \\
1 \\
1\end{array}$ & $\begin{array}{r}5 / 8 \\
3.4 \\
34 \\
7 / 8 \\
1 / 8\end{array}$ & $\begin{array}{l}5 / 8 \\
5 / 8 \\
3 / 4 \\
3 / 4 \\
7 / 8 \\
7 / 8\end{array}$ & $\begin{array}{r}3 / 4 \\
7 / 8 \\
1 / 8 \\
11 / 8 \\
11 / 8\end{array}$ & $\begin{array}{l}3 / 4 \\
3 / 4 \\
7 / 8 \\
1 \\
11 / 8 \\
11 / 8\end{array}$ & \begin{tabular}{r|}
$3 / 4$ \\
$3 / 4$ \\
$7 / 8$ \\
$7 / 8$ \\
1 \\
1
\end{tabular} & $\begin{array}{r}5 / 8 \\
3 / 4 \\
3 / 4 \\
7 / 8 \\
1 / 8 \\
17\end{array}$ \\
\hline $\begin{array}{l}68,500 \\
86,300 \\
96,500\end{array}$ & $\begin{array}{l}{ }^{*} 11 / 8 \\
{ }^{*} 11 / 4 \\
{ }^{*} 11 / 4 \\
* 13 \\
* 11 / 2\end{array}$ & $\begin{array}{l}1 \\
11 / 8 \\
11 / 8 \\
11 / 4 \\
13 / 8\end{array}$ & $\begin{array}{l}1 \\
11 / 8 \\
11 / 8 \\
11 / 4 \\
13 / 8\end{array}$ & $\begin{array}{l}1 \\
1 \\
11 / 8 \\
11 / 8 \\
11 / 4\end{array}$ & $\begin{array}{l}11 / 8 \\
11 / 4 \\
11 / 4 \\
13 / 8 \\
11 / 2\end{array}$ & $\begin{array}{l}11 / 8 \\
11 / 8 \\
11 / 4 \\
114 \\
13 / 8\end{array}$ & \begin{tabular}{l|}
1 \\
$11 / 8$ \\
$11 / 8$ \\
114 \\
$13 / 8$
\end{tabular} & $\begin{array}{l}1 \\
1 \\
11 / 8 \\
11 / 8 \\
11 / 4\end{array}$ & \begin{tabular}{l|l|}
$11 / 4$ \\
$11 / 4$ \\
$13 / 8$ \\
$13 / 8$
\end{tabular} & $\begin{array}{l}11 / 8 \\
114 \\
11 / 4 \\
13 / 8 \\
11 / 2\end{array}$ & $\begin{array}{l}11 / 8 \\
11 / 8 \\
11 / 4 \\
11 / 4 \\
11 / 2\end{array}$ & $\begin{array}{l}1 \\
11 / 8 \\
11 / 3 \\
11 / 4 \\
13 / 8\end{array}$ \\
\hline
\end{tabular}

Manufacturers must furnish a certificate guaranteeing that the ropes will stand the above tests before breaking and that in addition the individual wires have been twisted around themselves at least eight times, then untwisted and straightened, without breaking.
This testing is to be open at any time to the inspection of the surveyors of the American Bureau of Shipping, who are also empowered to retest any wire rope, irrespective of any previous certificate in regard to same that may have been issued by a manufacturer. 
AMERICAN ELECTRIC RAILWAY ENGI- suit strength of rope used, can be procured if so

NEERING ASSOCIATION, OVERHEAD LINE MATERIAL FOR DIRECT AND CATENARY SUSPENSION, RECOMMENDED SPECIFICATION, D102-30, 1930

\section{OVERHEAD LINE MATERIAL} galvanized steel strand cable. For specification see 611.29, p. 682.)

AMERICAN MARINE STANDARDS COMMITTEE, SHIP FITTINGS FOR DECKS AND RIGGING, H NO. 9-1926

\section{WIRE ROPE SOCKETS}

1. Material.-Wrought steel, 55,000 to 65,000 pounds per square inch tensile strength.
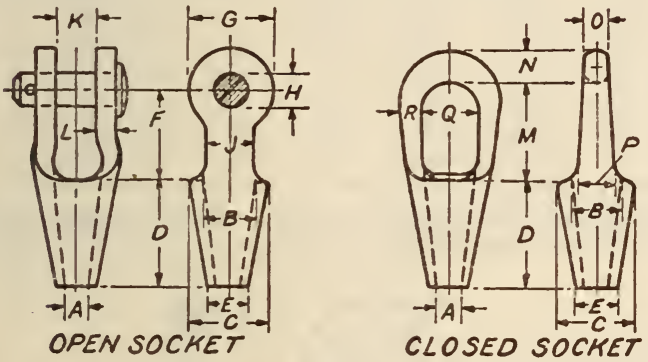

FrguRE 54.- Wire rope sockets

2. These sockets are commercially manufactured. columns X and Y. (See general notes accompany3. The sizes shown for pin $\mathrm{H}$ are commercial ing standard of rigging screws with spliced ropes, standards. Sockets with pins of different sizes, to $\mathrm{H}$ No. 6-1926 (608.7, p. 626).)

4. Instructions for attaching sockets to rope is a subject for a separate standard.

\section{AMERICAN MARINE STANDARDS COM- MITTEE, SHIP FITTINGS FOR DECKS AND RIGGING, H NO. 10-1926}

\section{SOLID THIMBLES FOR WIRE ROPE}

1. Material.-Cast iron, galvanized.

2. Thimbles for ropes to be wormed, parceled, or canvased and served, are to be of sizes to suit
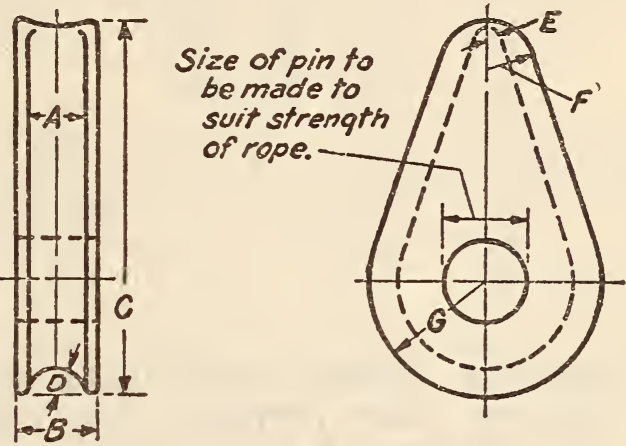

Frgure 55.-Solid thimble

(For dimensions of solid thimbles for wire rope, see table top of p. 162.)
(D102-30, par. 313, includes requirements for

Dimensions of wire rope sockets

[All dimensions in inches]

\begin{tabular}{|c|c|c|c|c|c|c|c|c|c|c|c|c|c|c|c|c|c|c|}
\hline \multicolumn{2}{|c|}{ Size } & \multicolumn{5}{|c|}{ Common dimensions } & \multicolumn{6}{|c|}{ Open sockets only } & \multicolumn{6}{|c|}{ Closed sockets only } \\
\hline $\begin{array}{c}\text { Diam- } \\
\text { eter } \\
\text { of } \\
\text { rope }\end{array}$ & $\begin{array}{c}\text { Cir- } \\
\text { cum- } \\
\text { fer- } \\
\text { ence } \\
\text { of } \\
\text { rope }\end{array}$ & A & B & $\mathrm{C}$ & $\mathrm{D}$ & $\mathrm{E}$ & F & G & $\mathrm{HI}$ & $\mathbf{J}$ & $\mathrm{K}$ & $\mathrm{L}$ & $\mathrm{M}$ & $\mathrm{N}$ & $\mathrm{O}$ & $\mathbf{P}$ & Q & $\mathrm{R}$ \\
\hline $\begin{array}{l}1 / 4 \\
5 / 16 \\
3 / 8 \\
7 / 16 \\
1 / 2\end{array}$ & $\begin{array}{l}3 / 4 \\
1^{3 / 1} \\
11 / 4 \\
11 / 2\end{array}$ & $\begin{array}{l}9 / 32 \\
11 / 32 \\
13 / 32 \\
15 / 32 \\
17 / 32\end{array}$ & $\begin{array}{l}3 / 4 \\
1^{3 / 4} \\
1 \\
13 / 16\end{array}$ & $\begin{array}{l}15 / 16 \\
15 / 16 \\
15 / 8 \\
158 \\
15 / 16\end{array}$ & $\begin{array}{l}15 / 8 \\
15 / 8 \\
21 / 2 \\
21 / 2 \\
23 \%\end{array}$ & $\begin{array}{l}17 / 32 \\
19 / 32 \\
3 / 4 \\
25 / 32 \\
15 / 16\end{array}$ & $\begin{array}{l}11 / 4 \\
11 / 4 \\
13 / 4 \\
13 / 4 \\
21 / 4\end{array}$ & $\begin{array}{l}1 \\
1 \\
13 / 8 \\
13 / 8 \\
17 / 8\end{array}$ & $\begin{array}{l}1 / 2 \\
1 / 2 \\
5 / 8 \\
5 / 8 \\
7 / 8\end{array}$ & $\begin{array}{r}58 \\
588 \\
7 / 8 \\
7 / 8 \\
1\end{array}$ & $\begin{array}{l}11 / 16 \\
11 / 16 \\
7 / 8 \\
7 / 8 \\
15 / 16\end{array}$ & $\begin{array}{l}5 / 16 \\
5 / 16 \\
3 / 8 \\
3 / 8 \\
1 / 2\end{array}$ & $\begin{array}{l}15 / 8 \\
15 / 8 \\
2 \\
2 \\
21 / 2\end{array}$ & $\begin{array}{l}1 / 2 \\
1 / 2 \\
11 / 16 \\
11 / 16 \\
3 / 4\end{array}$ & $\begin{array}{l}5 / 16 \\
5 / 16 \\
3 / 8 \\
3 / 8 \\
17 / 32\end{array}$ & $\begin{array}{l}5 / 8 \\
5 / 8 \\
3 / 4 \\
3 / 4 \\
15 / 16\end{array}$ & $\begin{array}{l}15 / 16 \\
15 / 16 \\
116 \\
11 / 8 \\
15 / 16\end{array}$ & $\begin{array}{l}3 / 8 \\
3 / 8 \\
1 / 2 \\
1 / 2 \\
17 / 32\end{array}$ \\
\hline $\begin{array}{c}9 / 16 \\
5 / 8 \\
34 \\
7 / 8 \\
1\end{array}$ & $\begin{array}{l}13 / 4 \\
2 \\
21 / 4 \\
23 / 4 \\
3\end{array}$ & $\begin{array}{c}19 / 32 \\
11 / 16 \\
13 / 16 \\
15 / 16 \\
11 / 16\end{array}$ & $\begin{array}{l}13 / 16 \\
13 / 8 \\
15 / 8 \\
17 / 8 \\
21 / 8\end{array}$ & $\begin{array}{l}115 / 16 \\
21 / 4 \\
25,8 \\
3,8 \\
31 / 2\end{array}$ & $\begin{array}{l}23 / 4 \\
3 \\
33 / 8 \\
4 \\
41 / 2\end{array}$ & $\begin{array}{l}1 \\
21 / 8 \\
15 / 16 \\
11 / 2 \\
1^{13} / 16\end{array}$ & $\begin{array}{l}21 / 4 \\
21 / 2 \\
23 / 4 \\
338 \\
33 / 4\end{array}$ & $\begin{array}{l}17 / 8 \\
238 \\
21 / 2 \\
3 \\
31 / 2\end{array}$ & $\begin{array}{l}1^{7 / 8} \\
11 / 8 \\
11 / 4 \\
15 / 8\end{array}$ & $\begin{array}{l}1 \\
13 / 8 \\
138 \\
13 / 4 \\
17 / 8\end{array}$ & $\begin{array}{l}15 / 16 \\
11 / 8 \\
15 / 16 \\
19 / 16 \\
17 / 8\end{array}$ & $\begin{array}{l}1 / 2 \\
9 / 16 \\
5 / 8 \\
11 / 16 \\
7 / 8\end{array}$ & $\begin{array}{l}21 / 2 \\
23 / 4 \\
31 / 2 \\
4 \\
41 / 2\end{array}$ & $\begin{array}{c}3 / 4 \\
7 / 8 \\
11 / 16 \\
17 / 32 \\
113 / 32\end{array}$ & $\begin{array}{l}17 / 32 \\
11 / 16 \\
3 / 4 \\
1^{7 / 8}\end{array}$ & $\begin{array}{l}15 / 16 \\
11 / 16 \\
11 / 4 \\
13 / 8 \\
11 / 16\end{array}$ & $\begin{array}{l}15 / 16 \\
11 / 2 \\
13 / 4 \\
2 \\
21 / 4\end{array}$ & $\begin{array}{l}17 / 32 \\
5 / 8 \\
334 \\
7 / 8 \\
1\end{array}$ \\
\hline $\begin{array}{l}11 / 8 \\
11 / 4 \\
13 / 8 \\
11 / 2\end{array}$ & $\begin{array}{l}31 / 2 \\
4 \\
414 \\
434 \\
\end{array}$ & $\begin{array}{l}13 / 16 \\
111 / 32 \\
115 / 32 \\
15 / 8\end{array}$ & $\begin{array}{l}21 / 8 \\
21 / 2 \\
21 / 2 \\
3\end{array}$ & $\begin{array}{l}31 / 2 \\
37 / 8 \\
37 / 8 \\
41 / 2\end{array}$ & $\begin{array}{l}41 / 2 \\
5 \\
5 \\
51 / 2\end{array}$ & $\begin{array}{l}115 / 16 \\
21 / 8 \\
21 / 4 \\
21 / 2\end{array}$ & $\begin{array}{l}33 / 4 \\
41 / 2 \\
41 / 2 \\
5\end{array}$ & $\begin{array}{l}31 / 2 \\
4 \\
4 \\
5\end{array}$ & $\begin{array}{l}15 / 8 \\
17 / 8 \\
17 / 8 \\
21 / 4\end{array}$ & $\begin{array}{l}17 / 8 \\
21 / 8 \\
21 / 8 \\
23 / 4\end{array}$ & $\begin{array}{l}17 / 8 \\
21 / 8 \\
21 / 8 \\
27 / 16\end{array}$ & $\begin{array}{c}7 / 8 \\
15 / 16 \\
15 / 16 \\
11 / 16\end{array}$ & $\begin{array}{l}41 / 2 \\
41 / 2 \\
41 / 2 \\
51 / 2\end{array}$ & $\begin{array}{l}13 / 32 \\
19 / 16 \\
19 / 16 \\
15 / 8\end{array}$ & $\begin{array}{l}1 \\
11 / 2 \\
11 / 2 \\
11 / 16\end{array}$ & $\begin{array}{l}1^{11 / 16} \\
2 \\
2 \\
21 / 4\end{array}$ & $\begin{array}{l}21 / 4 \\
23 / 4 \\
23 / 4 \\
23 / 4\end{array}$ & $\begin{array}{l}1 \\
11 / 4 \\
114 \\
138\end{array}$ \\
\hline $\begin{array}{l}15 / 8 \\
13 / 4 \\
2 \\
21 / 4\end{array}$ & $\begin{array}{l}5 \\
51 / 2 \\
61 / 4 \\
71 / 8\end{array}$ & $\begin{array}{l}111 / 16 \\
115 / 16 \\
21 / 4 \\
21 / 2\end{array}$ & $\begin{array}{l}31 / 8 \\
358 \\
41 / 8 \\
458\end{array}$ & $\begin{array}{l}41 / 2 \\
6 \\
61 / 2 \\
71 / 2\end{array}$ & $\begin{array}{l}61 / 8 \\
71 / 4 \\
814 \\
91 / 4\end{array}$ & $\begin{array}{l}21 / 2 \\
33 / 4 \\
41 / 4 \\
43 / 4\end{array}$ & \begin{tabular}{l|}
5 \\
6 \\
$73 / 4$ \\
$81 / 2$
\end{tabular} & \begin{tabular}{l|}
5 \\
$67 / 8$ \\
8 \\
$81 / 2$
\end{tabular} & $\begin{array}{l}21 / 4 \\
31 / 4 \\
334 \\
4\end{array}$ & $\begin{array}{l}23 / 4 \\
4 \\
41 / 2 \\
43 / 4\end{array}$ & $\begin{array}{l}27 / 16 \\
23 / 4 \\
31 / 2 \\
33 / 4\end{array}$ & $\begin{array}{l}11 / 16 \\
11 / 4 \\
11 / 2 \\
11 / 2\end{array}$ & $\begin{array}{l}6 \\
7 \\
7 \\
8\end{array}$ & $\begin{array}{l}13 / 4 \\
21 / 4 \\
21 / 2 \\
3\end{array}$ & $\begin{array}{l}13 / 4 \\
21 / 4 \\
21 / 2 \\
3\end{array}$ & $\begin{array}{l}21 / 4 \\
21 / 4 \\
21 / 2 \\
3\end{array}$ & $\begin{array}{l}3 \\
31 / 2 \\
4 \\
41 / 4\end{array}$ & $\begin{array}{l}138 \\
11 / 2 \\
13 / 4 \\
2\end{array}$ \\
\hline
\end{tabular}


Dimensions of solid thimbles

[All dimensions in inches]

\begin{tabular}{|c|c|c|c|c|c|c|c|c|c|c|c|c|}
\hline \multicolumn{6}{|c|}{ Size of rope-bare } & \multicolumn{7}{|c|}{ Dimension } \\
\hline \multirow{3}{*}{$\begin{array}{l}\text { Approxi- } \\
\text { mate } \\
\text { circum- } \\
\text { ference }\end{array}$} & \multirow{3}{*}{ Diameter } & \multirow{2}{*}{\multicolumn{2}{|c|}{$\frac{\begin{array}{c}\text { Before parceled } \\
\text { and served }\end{array}}{\mathrm{X}}$}} & \multirow{2}{*}{\multicolumn{2}{|c|}{$\begin{array}{c}\begin{array}{c}\text { Before wormed, par- } \\
\text { celed, and served }\end{array} \\
\mathrm{Y}\end{array}$}} & Score & \multirow{3}{*}{ B } & \multirow{3}{*}{$\mathrm{C}$} & \multirow{3}{*}{$\mathrm{D}$} & \multirow{3}{*}{ E } & \multirow{3}{*}{$\mathbf{F}$} & \multirow{3}{*}{ G } \\
\hline & & & & & & & & & & & & \\
\hline & & $\begin{array}{l}\text { Approx- } \\
\text { imate } \\
\text { circum- } \\
\text { ference }\end{array}$ & $\begin{array}{c}\text { Diam- } \\
\text { eter }\end{array}$ & $\begin{array}{l}\text { Approxi- } \\
\text { inate } \\
\text { circum- } \\
\text { ference }\end{array}$ & Diameter & A & & & & & & \\
\hline $\begin{array}{l}5 / 8 \\
3 / 4 \\
1 \\
11 / 8 \\
11 / 4\end{array}$ & $\begin{array}{l}3 / 16 \\
1 / 4 \\
5 / 16 \\
3 / 8 \\
7 / 16\end{array}$ & $1^{3 / 4}$ & $\begin{array}{l}3 / 16 \\
1 / 4 \\
5 / 16\end{array}$ & & & $\begin{array}{l}3 / 16 \\
1 / 4 \\
5 / 16 \\
3 / 8 \\
7 / 16\end{array}$ & $\begin{array}{l}7 / 32 \\
5 / 16 \\
7 / 16 \\
1 / 2 \\
9 / 16\end{array}$ & $\begin{array}{l}17 / 16 \\
13 / 4 \\
2 \\
21 / 4 \\
21 / 2\end{array}$ & $\begin{array}{l}3 / 32 \\
1 / 8 \\
5 / 32 \\
3 / 16 \\
7 / 32\end{array}$ & $\begin{array}{l}1 / 32 \\
1 / 32 \\
1 / 32 \\
1 / 16 \\
1 / 16\end{array}$ & $\begin{array}{l}1 / 8 \\
3 / 16 \\
1 / 4 \\
5 / 16 \\
3 / 8\end{array}$ & $\begin{array}{l}7 / 16 \\
1 / 2 \\
5 / 8 \\
11 / 16 \\
13 / 16\end{array}$ \\
\hline $\begin{array}{r}11 / 2 \\
13 / 4 \\
2 \\
21 / 4 \\
21 / 2-23 / 4\end{array}$ & $\begin{array}{r}1 / 2 \\
9 / 16 \\
5 / 8 \\
3 / 4 \\
13 / 16-7 / 8\end{array}$ & $\begin{array}{l}11 / 8 \\
11 / 4 \\
11 / 2 \\
13 / 4\end{array}$ & $\begin{array}{l}3 / 8 \\
7 / 16 \\
1 / 2 \\
9 / 16\end{array}$ & $2-21 / 4$ & $5 / 8-3 / 4$ & $\begin{array}{l}1 / 2 \\
9 / 16 \\
5 / 8 \\
3 / 4 \\
7 / 8\end{array}$ & $\begin{array}{l}5 / 8 \\
11 / 16 \\
7 / 8 \\
1 \\
118\end{array}$ & $\begin{array}{l}23 / 4 \\
3 \\
31 / 4 \\
33 / 4 \\
41 / 4\end{array}$ & $\begin{array}{l}1 / 4 \\
9 / 36 \\
5 / 16 \\
3 / 8 \\
7 / 16\end{array}$ & $\begin{array}{l}3,32 \\
1 / 8 \\
1 / 8 \\
1,8 \\
3 / 16\end{array}$ & $\begin{array}{l}1 / 2 \\
9 / 16 \\
5 / 8 \\
3 / 4 \\
7 / 8\end{array}$ & $\begin{array}{l}15 / 16 \\
11 / 16 \\
118 \\
15 / 16 \\
11 / 2\end{array}$ \\
\hline $\begin{array}{c}3 \\
31 / 4-31 / 2 \\
33 / 4-4 \\
41 / 4 \\
41 / 2-43 / 4\end{array}$ & $\begin{array}{r}1 \\
11 / 16-11 / 8 \\
13 / 16-11 / 4 \\
13 / 8 \\
17 / 16-11 / 2\end{array}$ & & & $\begin{array}{r}21 / 2 \\
23 / 4 \\
3 \\
31 / 4-31 / 2 \\
33 / 4-4\end{array}$ & $\begin{array}{c}13 / 16 \\
7 / 8 \\
11 / 16-11 / 8 \\
1316-11 / 4\end{array}$ & $\begin{array}{l}1 \\
11 / 8 \\
11 / 4 \\
13 / 8 \\
11 / 2\end{array}$ & $\begin{array}{l}11 / 4 \\
13 / 8 \\
11 / 2 \\
13 / 4 \\
17 / 8\end{array}$ & $\begin{array}{l}43 / 4 \\
51 / 4 \\
53 / 4 \\
61 / 4 \\
63 / 4\end{array}$ & $\begin{array}{l}1 / 2 \\
9 / 16 \\
5 / 8 \\
11 / 16 \\
3 / 4\end{array}$ & $\begin{array}{l}3 / 16 \\
1 / 4 \\
1 / 4 \\
1 / 4 \\
1 / 4\end{array}$ & $\begin{array}{l}1 \\
116 \\
13 / 16 \\
1516 \\
136\end{array}$ & $\begin{array}{l}1^{11} 1 / 16 \\
17 / 8 \\
21 / 16 \\
21 / 4 \\
27 / 16\end{array}$ \\
\hline $\begin{array}{l}5 \\
51 / 2 \\
53 / 4 \\
61 / 4\end{array}$ & $\begin{array}{l}15 / 8 \\
13 / 4 \\
17 / 8 \\
2\end{array}$ & & & $\begin{array}{r}41 / 4 \\
41 / 2-43 / 4 \\
5 \\
51 / 2\end{array}$ & $\begin{array}{r}13 / 8 \\
17 / 16-11 / 2 \\
15 \% \\
13 / 4\end{array}$ & $\begin{array}{l}15 / 8 \\
13 / 4 \\
17 / 8 \\
2\end{array}$ & $\begin{array}{l}2 \\
21 / 4 \\
23 / 8 \\
21 / 2\end{array}$ & $\begin{array}{l}71 / 4 \\
73 / 4 \\
81 / 4 \\
834\end{array}$ & $\begin{array}{l}13 / 16 \\
7 / 8 \\
15 / 16 \\
1\end{array}$ & $\begin{array}{l}1 / 4 \\
1 / 4 \\
1 / 4 \\
1 / 4\end{array}$ & $\begin{array}{l}13 / 2 \\
15 / 8 \\
13 / 4 \\
17 / 8\end{array}$ & $\begin{array}{l}25 / 8 \\
213 / 16 \\
3 \\
33 / 16\end{array}$ \\
\hline
\end{tabular}

AMERICAN MARINE STANDARDS COM- standard of rigging screws with spliced ropes MITTEE, SHIP FITTINGS FOR DECKS AND RIGGING, $\mathrm{H}$ NO. 11-1926

\section{OPEN THIMBLES FOR WIRE ROPE}

1. Material.-Wrought steel, galvanized.

2. Wire rope thimbles as listed are commercial standards, excepting those with $2 \frac{3}{4}$ and 3 inch scores, which are special sizes.

3. Thimbles for ropes to be wormed, parceled, or canvased and served, are to be of sizes to suit columns X and Y. (See general notes accompanying H No. 6-1926 (608.7, p. 626).)

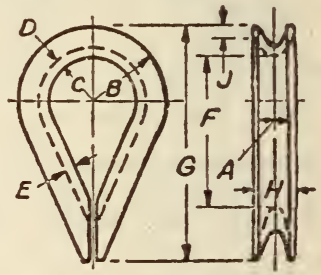

FiguRe 56.-Open thimble for wire rope

Dimensions of open thimbles

[All dimensions in inches]

\begin{tabular}{|c|c|c|c|c|c|c|c|c|c|c|c|c|c|c|}
\hline \multicolumn{6}{|c|}{ Size of rope-bare } & \multicolumn{9}{|c|}{ Dimension } \\
\hline \multirow{3}{*}{$\begin{array}{l}\text { Approxi- } \\
\text { mate cir- } \\
\text { cumfer- } \\
\text { ence }\end{array}$} & \multirow{3}{*}{ Diameter } & \multirow{2}{*}{\multicolumn{2}{|c|}{$\begin{array}{c}\begin{array}{c}\text { Before parceled } \\
\text { and served }\end{array} \\
\mathbf{X} \\
\end{array}$}} & \multirow{2}{*}{\multicolumn{2}{|c|}{$\frac{\begin{array}{c}\text { Before wormed, par- } \\
\text { celed, and served }\end{array}}{\mathrm{Y}}$}} & Score & \multirow{3}{*}{ B } & \multirow{3}{*}{$\mathrm{C}$} & \multirow{3}{*}{$\mathrm{D}$} & \multirow{3}{*}{$\mathrm{E}$} & \multirow{3}{*}{$F$} & \multirow{3}{*}{ G } & \multirow{3}{*}{ H } & \multirow{3}{*}{$\boldsymbol{J}$} \\
\hline & & & & & & & & & & & & & & \\
\hline & & $\begin{array}{l}\text { Approx- } \\
\text { imate } \\
\text { circum- } \\
\text { ference }\end{array}$ & $\begin{array}{l}\text { Diam- } \\
\text { eter }\end{array}$ & $\begin{array}{l}\text { Approxi- } \\
\text { mate cir- } \\
\text { cumfer- } \\
\text { ence }\end{array}$ & Diameter & A & & & & & & & & \\
\hline \begin{tabular}{l|}
$5 / 8$ \\
$3 / 4$ \\
$1^{11 / 8}$ \\
$11 / 4$
\end{tabular} & $\begin{array}{l}3 / 16 \\
1 / 4 \\
5 / 16 \\
38 \\
7 / 16\end{array}$ & $\begin{array}{r}5 / 8 \\
1^{3 / 4} \\
\end{array}$ & $\begin{array}{l}3 / 16 \\
114 \\
5 / 16\end{array}$ & & & $\begin{array}{l}3 / 16 \\
1 / 4 \\
5 / 16 \\
3 / 8 \\
7 / 16\end{array}$ & $\begin{array}{l}7 / 16 \\
1 / 2 \\
19 / 32 \\
11 / 16 \\
3 / 4\end{array}$ & $\begin{array}{l}1 / 4 \\
5 / 16 \\
3 / 8 \\
13 / 32 \\
7 / 16\end{array}$ & $\begin{array}{l}1 / 16 \\
1 / 8 \\
1 / 8 \\
1 / 8 \\
1 / 8\end{array}$ & $\begin{array}{l}1 / 16 \\
1 / 8 \\
1 / 8 \\
1 / 8 \\
1 / 8\end{array}$ & $\begin{array}{l}7 / 8 \\
11 / 8 \\
1516 \\
11 / 2 \\
111 / 16\end{array}$ & $\begin{array}{l}13 / 8 \\
13 / 4 \\
21 / 8 \\
21 / 4 \\
25 \%\end{array}$ & $\begin{array}{l}7 / 32 \\
5 / 16 \\
3 / 8 \\
7 / 16 \\
1 / 2\end{array}$ & $\begin{array}{l}1 / 16 \\
1 / 16 \\
3 / 32 \\
5 / 32 \\
3 / 16\end{array}$ \\
\hline $\begin{array}{r}11 / 2 \\
13 / 4 \\
2 \\
21 / 4 \\
21 / 2-23 / 4\end{array}$ & $\begin{array}{r}1 / 2 \\
9 / 16 \\
5 / 8 \\
3 / 4 \\
13 / 10-7 / 8\end{array}$ & $\begin{array}{l}11 / 8 \\
11 / 4 \\
11 / 2 \\
13 / 4\end{array}$ & $\begin{array}{l}3 / 8 \\
7 / 16 \\
1 / 2 \\
9116\end{array}$ & $21 / 4$ & $3 / 4$ & $\begin{array}{l}1 / 2 \\
9 / 16 \\
5,8 \\
3 / 4 \\
7 / 8\end{array}$ & $\begin{array}{l}7 / 8 \\
15 / 16 \\
11 / 32 \\
13 / 16 \\
13 / 8\end{array}$ & $\begin{array}{l}1 / 2 \\
17 / 32 \\
19 / 32 \\
11 / 16 \\
25 / 32\end{array}$ & $\begin{array}{l}1 / 8 \\
3 / 16 \\
3 / 16 \\
3 / 16 \\
3 / 16\end{array}$ & $\begin{array}{l}1 / 8 \\
3 / 16 \\
3 / 16 \\
3 / 16 \\
3 / 16\end{array}$ & $\begin{array}{l}17 / 8 \\
2 \\
21 / 8 \\
21 / 2 \\
23 / 4\end{array}$ & $\begin{array}{l}3 \\
3 \\
31 / 4 \\
33 / 4 \\
41 / 2\end{array}$ & $\begin{array}{l}19 / 32 \\
11 / 16 \\
3 / 4 \\
39 / 32 \\
11 / 16\end{array}$ & $\begin{array}{l}7 / 32 \\
7 / 32 \\
1 / 4 \\
5 / 16 \\
13 / 32\end{array}$ \\
\hline $\begin{array}{c}3 \\
31 / 4-31 / 2 \\
33 / 4 \\
4 \\
41 / 4\end{array}$ & $\begin{array}{c}1 \\
11 / 16-11 / 8 \\
1316 \\
11 / 4 \\
13 / 8\end{array}$ & & & $\begin{array}{r}21 / 2 \\
23 / 4 \\
3 \\
31 / 4-31 / 2\end{array}$ & $1_{11 / 16-11 / 8}^{\frac{13 / 16}{7 / 8}}$ & $\begin{array}{l}1 \\
11 / 8 \\
11 / 4 \\
15 / 16 \\
13 / 8\end{array}$ & $\begin{array}{l}117 / 32 \\
123 / 32 \\
17 / 8 \\
1^{31 / 32} \\
21 / 16\end{array}$ & $\begin{array}{c}7 / 8 \\
31 / 32 \\
11 / 16 \\
13 / 32 \\
15 / 32\end{array}$ & $\begin{array}{l}3 / 16 \\
14 \\
144 \\
144 \\
1 / 4\end{array}$ & $\begin{array}{l}3 / 16 \\
3 / 16 \\
3 / 16 \\
3 / 16 \\
3 / 16\end{array}$ & $\begin{array}{l}3 \\
35 / 16 \\
31 / 2 \\
35 / 8 \\
33 / 4\end{array}$ & $\begin{array}{l}43 / 4 \\
53 / 8 \\
53 / 4 \\
57 / 8 \\
6\end{array}$ & $\begin{array}{l}13 / 16 \\
13,8 \\
11 / 2 \\
155 \\
111 / 16\end{array}$ & $\begin{array}{l}15,32 \\
1,3 \\
916 \\
19,32 \\
5,8\end{array}$ \\
\hline $\begin{array}{c}41 / 2-43 / 4 \\
5 \\
51 / 2 \\
53 / 4 \\
61 / 4\end{array}$ & $\begin{array}{r}17 / 16-11 / 2 \\
15 / 8 \\
13 / 4 \\
17 / 8 \\
2\end{array}$ & & & $\begin{array}{c}33 / 4-4 \\
41 / 4 \\
41 / 2-43 / 4 \\
5 \\
51 / 2\end{array}$ & $\begin{array}{r}13 / 16-11 / 4 \\
13 / 8 \\
17 / 16-11 / 2 \\
15 \% \\
13 / 4\end{array}$ & $\begin{array}{l}11 / 2 \\
15 / 8 \\
13 / 4 \\
17 / 8 \\
2\end{array}$ & $\begin{array}{l}21 / 4 \\
23 / 8 \\
29 / 16 \\
23 / 4 \\
2^{15} / 16\end{array}$ & $\begin{array}{l}114 \\
15 / 16 \\
1716 \\
11 / 2 \\
158\end{array}$ & $\begin{array}{l}5 / 16 \\
5 / 16 \\
3 / 8 \\
3 / 8 \\
3 / 8\end{array}$ & $\begin{array}{l}3 / 16 \\
3 / 16 \\
3 / 16 \\
3 / 16 \\
3 / 16\end{array}$ & $\begin{array}{l}4 \\
43 / 16 \\
43 / 8 \\
41 / 2 \\
4 \% 16\end{array}$ & $\begin{array}{l}61 / 2 \\
71 / 4 \\
73 / 8 \\
71 / 2 \\
73 / 4\end{array}$ & $\begin{array}{l}1^{13 / 16} \\
2 \\
21 / 8 \\
25 / 16 \\
2^{7 / 16}\end{array}$ & $\begin{array}{l}11 / 16 \\
3 / 4 \\
3 / 4 \\
13 / 16 \\
7 / 8\end{array}$ \\
\hline $\begin{array}{l}71 / 8 \\
77 / 8 \\
85 / 8 \\
93 / 8\end{array}$ & $\begin{array}{l}21 / 4 \\
21 / 2 \\
23 / 4 \\
3\end{array}$ & & & $\begin{array}{r}53 / 4-61 / 4 \\
71 / 8 \\
77 / 8 \\
85 / 8\end{array}$ & $\begin{aligned} & 17 / 8- 2 \\
& 21 / 4 \\
& 21 / 2 \\
& 23 / 4\end{aligned}$ & $\begin{array}{l}21 / 4 \\
21 / 2 \\
23 / 4 \\
3\end{array}$ & $\begin{array}{l}33 / 16 \\
338 \\
37 / 8 \\
41 / 4\end{array}$ & $\begin{array}{l}17 / 8 \\
178 \\
2116 \\
21 / 4\end{array}$ & $\begin{array}{l}7 / 16 \\
1 / 2 \\
9 / 16 \\
5 / 8\end{array}$ & $\begin{array}{l}3 / 16 \\
3 / 16 \\
1 / 4 \\
5 / 16\end{array}$ & $\begin{array}{l}53 / 16 \\
534 \\
61 / 4 \\
7\end{array}$ & $\begin{array}{l}91 / 2 \\
93 / 4 \\
11 \\
12\end{array}$ & $\begin{array}{l}211 / 16 \\
3 \\
31 / 4 \\
31 / 2\end{array}$ & $\begin{array}{l}1 \\
11 / 8 \\
11 / 4 \\
138\end{array}$ \\
\hline
\end{tabular}




\section{AMERICAN MARINE STANDARDS COM- MITTEE, STANDARD WIRE ROPES FOR MARINE USES, 0 N0. 25-1930}

\section{WIRE ROPES FOR MARINE USES}

\section{Section I. BASIC INFORMATION}

\section{Scope of Specifications}

These specifications describe those types of wire ropes deemed most suitable for marine service. Uses are stated for the respective types to cover prevailing practices in different services, but only for general information and guidance of the users and not as part of the specifications.

\section{Standard Defined}

(a) Steel rope grades.-Steel ropes are made in a variety of grades and construction, some of wnich are marketed under various trade names. For the purpose of these specifications, uncoated cast-steel rope is made the basic standard, and this rope shall be supplied when another grade, or coated rope, is not specifically called for. This, however, shall not apply to the 6 by 24 and 6 by 12 ropes, types $\mathrm{E}$ and $\mathrm{F}$, hereinafter described in subdivision 4 , which are generally manufactured and marketed in galvanized form, and which shall be so furnished unless otherwise ordered. Four grades, which are deemed sufficient to cover all likely requirements of marine service, are classified as follows:

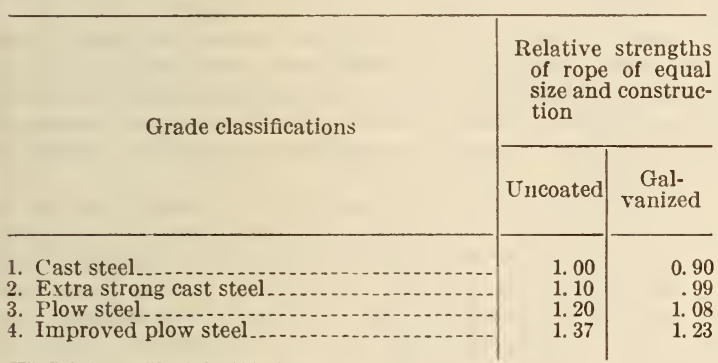

The size of rope, choice of grade, and whether or not the rope should be galvanized are matters which depend upon the requirements and judgment of the user. If any one of the grades other than cast steel is required, or the rope is to be different in any particular from its standard herein defined, such special requirements must be specifically stated in ship contract specifications, in asking for quotations and in ordering. (See tables in Sec. II for the relative physical properties of ropes of various types and sizes.)

(b) Diameter or size.-The nominal diameter of a wire strand or of wire rope is its greatest diameter, which coincides with that of a circumscribing circle inclosing all wires and strands.

(c) Lay and stranding.-The lay of a strand or rope is the distance parallel to their respective axes in which a wire of the strand or a strand of the rope makes one complete turn about the axis. This is generally more for galvanized ropes than for uncoated ropes. (See Sec. III, par. 3.) The ropes comprised in these specifications are of right regular lay. In this type of construction, the wires form a left-hand helix about the axis of the strand, and the strands form a right-hand helix about the axis of the rope. The outer wires in the finished rope are approximately parallel to the axis of the rope.

(d) Strand fabrication.-All of the wires in a strand shall, so far as practicable in the process of manufacture, be laid in a common helix, so that each wire is in continuous contact with the same adjacent wires. This may be accomplished in one or more operations of stranding, at the option of the manufacturer. This type of stranding is deemed best for marine work, especially for galvanized ropes; first, because the load is evenly distributed on, or carried by, all the wires; and, second, because the wires have the greatest possible surfaces in contact, and spot-wear or nicking across wires is avoided.

(e) Cores.-Hard fiber shall be the standard for all cores except the small sizes, which shall be cotton. Jute shall not be used. Cotton is used in the small sizes only because it can be spun into small diameters more satisfactorily than other fibers. Jute is unsatisfactory because, as compared with the harder fibers, it has less cellulose for its volume and is therefore weaker; it is also more hygroscopic and, therefore, subject to more rapid deterioration.

\section{Kinds and Uses in General}

(a) Uncoated rope.-This rope should be used for all purposes wherever it is protected from the elements, especially for hoisting. When used where it is exposed to the elements, a protective coating should be applied to it at regular intervals.

(b) Galvanized rope.-This rope is advisable only for standing rigging. It should not, in general, be used for hoisting or other services involving chafing or abrasion, because zinc coating is rapidly worn off by friction. Furthermore, galvanizing decreases the strength of the wires by about 10 per cent and, therefore, galvanized ropes must be correspondingly larger.

(c) Marline-clad rope.-(The covering of this rope has no relation to the marline covering or serving of wire ropes as applied on various parts of ships' standing rigging.) This type of rope is easily lubricated and coated for preservation. The marline covering protects the wires from wear and supplies them with lubricant. Compared with uncovered wire rope, it is appreciably larger for equivalent strength; it has a greater coefficient of friction on smooth drums, and the covering protects the wires against gases, grit, and moisture. It is not, however, advocated for heavy duty or services in which failure would endanger human life or cause material damage, because the wires are not visible for inspection.

(d) Phosphor-bronze rope-Compared to steel rope, phosphor-bronze rope has less strength and, therefore, larger sizes are required for equivalent safe working loads, and the sheaves or drums over which it is run must be comparatively larger. It is superior to steel rope from the standpoint of its noncorrodible and nonmagnetic properties.

\section{Standard Ropes and Their Uses}

The following are hereby designated as standard types of ropes for marine services. The statements made regarding their uses are to be understood only as indicating prevailing practice. They are intended for general information and guidance of users. The proper type of rope to order for any particular purpose depends upon the service requirements and the judgment of the user.

Type A. 6 by 7 rope.- Six strands of 7 wires each, total 42 wires single core of hard fiber. Lay not greater than $71 / 2$ times the nominal diameter of the rope if the wires are uncoated, or eight times the nominal diameter of the rope if the wires are galvanized.

Uses.-This is the stiffest of the ropes herein described, and its stretch under tension is less than any other type. Steel rope of this type for marine uses is generally galvanized, but may be used 
uncoated if properly protected. It is suitable for standing rigging, such as stays, shrouds, and guys; also for other purposes in which it is not subject to bending stresses, such as straight transmission leads, life lines, awning ridges, vang and topping lift pendants, davit slings, and guys, and, in general, where flexibility is not a requisite and comparatively large wires and a minimum of stretch are desirable. Phosphor-bronze rope of this type is not as strong as steel rope and is advocated only for uses where noncorrodible or nonmagnetic properties are specified requisites.

For physical properties, see Section II, Tables 1 and 2 for steel ropes, and Table 8 for phosphorbronze ropes.

Type B. 6 by 19 rope.-Six strands which may be of one or more diameters of wires. The numerical designation for this rope is only typical as the number of wires in ropes made by different manufacturers may vary if filler wires are used. Each strand shall contain 19 main wires, but when filler wires are used, the number of wires per strand may be up to 25 . The total wires in the rope may, therefore, be from 114 to 150 . The single core shall be of hard fiber.

Uses.-Uncoated steel rope of this type in sizes up to $13 / 4$ inches in diameter is suitable for general hoisting. When rope of diameter exceeding $13 / 4$ inches would be required for hoisting, it is advisable to use one of the more flexible ropes, such as 8 by 19 or 6 by 37 . Among uses for which this uncoated rope is suitable are cargo falls, topping lifts, slings, relieving tackles, and radio halyards; also standing rigging and guys if the rope can be properly protected. Galvanized rope of this type is suitable for standing rigging, guys, boat slings, topping lifts, boom vangs, anchor buoys, fire lines, shrouds, preventors, towlines, stream cables, warps, and hawsers. Phosphor-bronze rope of this type is not as strong as steel rope, and is advocated only for uses where noncorrodible or nonmagnetic properties are specfiied requisites.

For physical properties, see Section II, Tables 1 and 2 for steel ropes, and Table 8 for phosphorbronze ropes.

Type C. 8 by 19 rope.-Eight strands of one or more diameters of wire. The numerical designation for this rope is only typical as the number of wires in rope made by different manufacturers may vary if filler wires are used. Each strand shall have 19 main wires, but if filler wires are used, the number of wires per strand may be up to 25 and the total wires in the rope may, therefore, be from 152 to 200 . The single core shall be of hard fiber.

Uses.-This rope is more flexible, but not as strong for any given size as the 6 by 19 construction. It is suitable for similar uses, but is advisable in lieu thereof only in cases where rope of greater flexibility is required.

For physical properties, see Section II, Tables 1 and 2.

Type D. 6 by 37 rope--Six strands made up of main and filler wires of various diameters. The numerical designation of this rope is only typical as the number of wires in ropes made by different manufacturers may vary according to position and size of main and filler wires. Each strand shall have not less than 34 nor more than 46 wires. The total wires in the rope may, accordingly, be from 204 to 276. The number of outside wires of each strand shall be not less than 15 or more than 20 . The single core shall be of hard fiber.

Uses.-This rope is flexible by reason of its comparatively small wires. It is efficient because at least 50 per cent of the wires and, consequently, over 50 per cent of the strength are in the inner layers of the strand and thus protected from abrasion. Uncoated rope of this type is suitable for hoisting gears, steering gear transmission, boat-crane falls, relieving tackle, and running rigging where sheaves or drums are of necessity smaller than desirable for 6 by 19 rope; also for bridles, dip ropes, slings for general hoisting; and for hawsers, mooring lines, stream cables, towlines, and warps requiring great strength; provided that the rope can be properly protected. Galvanized rope is suitable for any of the purposes stated for the uncoated rope where the rope could not be adequately protected against corrosion, except hoisting and running rigging.

For physical properties, see Section II, Tables 1 and 2.

Type E. 6 by 24 rope.-This rope is generally made and marketed in galvanized form. It shall be so furnished on the basis of these specifications unless specifically ordered to be uncoated. It has 6 strands, each consisting of 24 wires laid in 2 lavers over a soft core. Total wires in the rope 144 . This rope has 7 soft cores, 1 in each strand and 1 main core, all of which shall be of hard fiber, except the strand cores for ropes of diameters under 1 inch which shall be of cotton.

Uses.-This type of rope is more flexible than any of the types hereinbefore described. It is suitable for hawsers, mooring lines, stream cables, towlines, warps, topping lift pendants, and similar uses where greater flexibility than that of previously described ropes is required.

For physical properties, sce Section II, Table 3,

Type F. 6 by 12 rope.-This rope is generally made and marketed in galvanized form. It shall be so furnished on the basis of these specifications unless specifically ordered to be uncoated. It has 6 strands, each of 12 wires laid over a soft core; total wires in the rope 72 . This rope has 7 soft cores, 1 il each strand and 1 main core, all of which shall be of hard fiber, except the strand cores for ropes of diameters under five-eighths inch, which shall be of cotton.

Uses.-This rope is more flexible than, but not as strong as, any of the types hereinbefore described. It is deemed suitable in preference to previously described ropes only for aerial hoists, life lines, awning ridge ropes, boat ladders, pilot ladders, and boom pendants; also for mooring lines, hawsers, stream cables, towlines, and warps of comparatively low strength, and for running rigging requiring great flexibility rather than strength.

For physical properties, see Section II, Table 3.

Type $G .6$ by 6 by 7 ( 6 by 42) tiller rope.-Six main strands of 42 wires, each of which is composed of 6 strands of 7 wires, being in effect 6 separate ropes twisted together. There are " cores, 1 in each strand and 1 main core, all of which shall be of hard fiber except the strand cores which may be of either hard fiber or cotton.

Uses.-This is the most flexible of all ropes herein specified and is capable of being used around small sheaves or drums. It is, however, unsuitable for hoisting or for heavy or continuous duty owing to the comparatively fine wires of which it is composed and its inability to withstand abrasion. Steel rope of this type is used for steering ropes and signal pulls which are required to wind over small diameters. Phosphor-bronze rope of this type is not as strong as steel rope, and its use is advocated only where noncorrodible and nonmagnetic properties are specified requisites.

For physical properties, see Section II, Tables 1 and 2 for steel ropes, and Table 8 for phosphorbronze ropes.

Type H. 5 by 19 marline-clad steel rope-This type of rope has each of its strands completely inclosed in a continuous marline or hemp covering. It is composed of 5 strands which may be of one or more diameters of wires. The numerical designa- 
tion for this type of rope is only typical as the number of wires in ropes made by different manufacturers may vary if filler wires are used. Each strand shall have 19 main wires. If filler wires are used each strand may have from 19 to 25 wires. The total wires in the rope may, therefore, be from 95 to 125 . Each strand has a hemp covering tightly wound or laid on, uniformly smooth, and free of knots or imperfections. The single soft core shall be of hard fiber. The wires may be either uncoated or galvanized, but the uncoated type of rope is most generally marketed as the wires are deemed to be adequately protected from corrosion by the covering if the latter is kept saturated with suitable lubricating compound.

Uses.-This type of rope is stronger than manila rope and suitable for similar uses, especially all kinds of running rigging, topping lifts, boat-handling gear, and comparatively light hoisting; also mooring lines.

For physical properties, see Section II, Table 4.

Type I. Strands.-(1) 1 by 7 stiff strand.-Six wires twisted around a central wire. Lay not more than twelve times the nominal diameter of the strand.

Uses.-This is the stiffest wire-strand construction. This strand is generally made of galvanized wire and shall be so furnished on the basis of these specifications unless specifically ordered to be uncoated. It is used on ships only in comparatively small sizes for guys and other purposes where stiffness is desired and comparatively great strength for size is required.

For physical properties and various grades obtainable, see Section II, Table 5.

(2) Seizing strands.- Sizes under three-sixteenthsinch diameter shall be of 7 -wire construction. Sizes three-sixteenths inch and more in diameter shall be of 19-wire construction, each of these numerical constructions comprising a single wire core. The wires may be of iron or low-carbon steel, annealed and galvanized. The lay shall be not greater than 12 times the nominal diameter of the strand. These strands are used mostly for seizing the ends of wire ropes.

For physical properties see Section II, Table 6 .

Type J. 6 by 7 sash and bell cord.-Six strands of 7 wires each. Single soft core of cotton. Lay to be not greater than eight times the nominal diameter of the rope. This may be required of annealed iron, tinned or galvanized, or of copper or phosphorbronze wire, according to the requirements of the work in which the cord is to be used.

Uses. - The principal uses for this cord are on sash weights, window shades, curtains, and for bell and whistle cords.

For physical properties, see Section II, Table 7.

Type $K$. Lead line with wire-cord center.-This is a cotton cord braided over a center of phosphorbronze wire cord composed of 6 strands, each containing 7 wires and a single cotton core. It is marketed in several sizes, the following of which are deemed adequate for all likely requirements on shipboard:

\begin{tabular}{|c|r|r|r}
\hline Trade No. & $\begin{array}{c}\text { Approxi- } \\
\text { mate } \\
\text { over-all } \\
\text { diameter }\end{array}$ & $\begin{array}{c}\text { Approxi- } \\
\text { mate } \\
\text { diameter } \\
\text { of wire- } \\
\text { cord } \\
\text { center }\end{array}$ & $\begin{array}{c}\text { Approxi- } \\
\text { mate } \\
\text { breaking } \\
\text { strength }\end{array}$ \\
\hline 8 & Inch & 114 & Inch \\
$1 / 16$ & Pounds \\
300 \\
500
\end{tabular}

The cotton yarn used in the braided covering shall be of good quality 3-ply warp twist, free from husks and other defects. It shall be dyed mahogany color while in the skein in order to insure a solid color in the finished article, and thoroughly waterproofed. The number of cotton strands and yarn ends per strand shall be such as to give a firmly braided covering over the wire cord to the over-all diameter specified and finished with a hard and smooth surface free from imperfections of braid or finish. The phosphor-bronze wires shall possess the characteristics of the phosphor-bron ze wire herein specified for phosphor-bronze ropes.

This type of cord does not stretch, and its use is advocated for lead lines in the interest of accurate soundings. It is serviceable as tiller rope on motor boats, also as bell cord, and is suitable for other purposes of similar character in ship operation.

\section{Section II. PHYSICAL PROPERTIES}

The approximate weights and minimum breaking strengths of the ropes, etc., hereinbefore specified are set forth in the annexed tables numbered 1 to 8 inclusive. The breaking strengths given in Table 2 are for uncoated cast-steel ropes. Factors are given in footnotes on this table from which the relative breaking strengths for other grades of steel ropes can be approximately determined. The properties of galvanized ropes, marline-clad ropes, galvanized-wire strands, sash and bell cords, and phosphor-bronze ropes are separately compiled in Tables 3 to 8, inclusive. (See Appendix II, notes on wire-rope strengths and working loads.)

\section{TABLE 1.-Approximate weights, in pounds per foot; uncoated steel wire ropes}

\begin{tabular}{|c|c|c|c|c|c|}
\hline \multirow{2}{*}{$\begin{array}{c}\text { Rope } \\
\text { diame- } \\
\text { ters }\end{array}$} & \multicolumn{5}{|c|}{ Types } \\
\hline & $\begin{array}{c}\text { A } \\
6 \text { by } 7\end{array}$ & $\begin{array}{c}\text { B } \\
6 \text { by } 19\end{array}$ & $\begin{array}{c}\mathrm{C} \\
8 \text { by } 19\end{array}$ & $\begin{array}{c}\text { D } \\
6 \text { by } 37\end{array}$ & $\begin{array}{c}\mathrm{G} \\
6 \text { by } 6 \text { by } \\
7 \text { tiller }\end{array}$ \\
\hline Inches & & 0.10 & 0.09 & 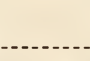 & 0.07 \\
\hline $\begin{array}{l}3 / 32 \\
5 / 16 \\
3 / 8 \\
7 / 16\end{array}$ & $\begin{array}{l}.12 \\
.15 \\
.21 \\
.29\end{array}$ & $\begin{array}{l}.16 \\
.23 \\
.31\end{array}$ & $\begin{array}{l}.14 \\
.20 \\
.28\end{array}$ & $\begin{array}{r}0.22 \\
.30\end{array}$ & $\begin{array}{l}.11 \\
.16 \\
.21\end{array}$ \\
\hline $\begin{array}{l}1 / 6 \\
916 \\
5,8 \\
11 / 6\end{array}$ & $\begin{array}{r}.38 \\
.48 \\
.59 \\
71\end{array}$ & $\begin{array}{l}.40 \\
.51 \\
.63\end{array}$ & $\begin{array}{l}.36 \\
.46 \\
.57\end{array}$ & $\begin{array}{l}.39 \\
.49 \\
.61\end{array}$ & $\begin{array}{l}.28 \\
.35 \\
.43\end{array}$ \\
\hline $\begin{array}{l}3 / 16 \\
3 / 4\end{array}$ & .84 & .90 & .82 & .87 & .62 \\
\hline $\begin{array}{l}1^{7 / 8} \\
118 \\
11 / 4\end{array}$ & $\begin{array}{l}1.15 \\
1.50 \\
1.90 \\
2.34\end{array}$ & $\begin{array}{l}1.23 \\
1.60 \\
2.03 \\
2.50\end{array}$ & $\begin{array}{l}1.11 \\
1.45 \\
1.84 \\
2.27\end{array}$ & $\begin{array}{l}1.19 \\
1.55 \\
1.96 \\
2.42\end{array}$ & $\begin{array}{r}.84 \\
1.10 \\
-.\end{array}$ \\
\hline $\begin{array}{l}13 / 8 \\
11 / 2 \\
158 \\
13 / 4\end{array}$ & $\begin{array}{r}2.84 \\
3.38 \\
\end{array}$ & $\begin{array}{l}3.03 \\
3.60 \\
4.23 \\
4.90\end{array}$ & $\begin{array}{l}2.74 \\
3.26 \\
-\end{array}$ & $\begin{array}{l}2.93 \\
3.49 \\
4.09 \\
4.75\end{array}$ & \\
\hline $\begin{array}{l}17 / 8 \\
2 \\
21 / 4 \\
21 / 2\end{array}$ & & $\begin{array}{r}5.63 \\
6.40 \\
8.10 \\
10.00\end{array}$ & & $\begin{array}{l}5.45 \\
6.20 \\
7.85 \\
9.69\end{array}$ & $\begin{array}{l}-- \\
-- \\
-- \\
--\end{array}$ \\
\hline $\begin{array}{l}23 / 4 \\
3 \\
31 / 4 \\
31 / 2\end{array}$ & & 12.10 & & $\begin{array}{l}11.72 \\
13.95 \\
16.37 \\
19.00\end{array}$ & $-\cdot-$ \\
\hline
\end{tabular}

For weights per fathom, multiply the above by 6 . 
TABLE 2.-Minimum breaking strengths, in pounds; uncoated cast-steel wire ropes

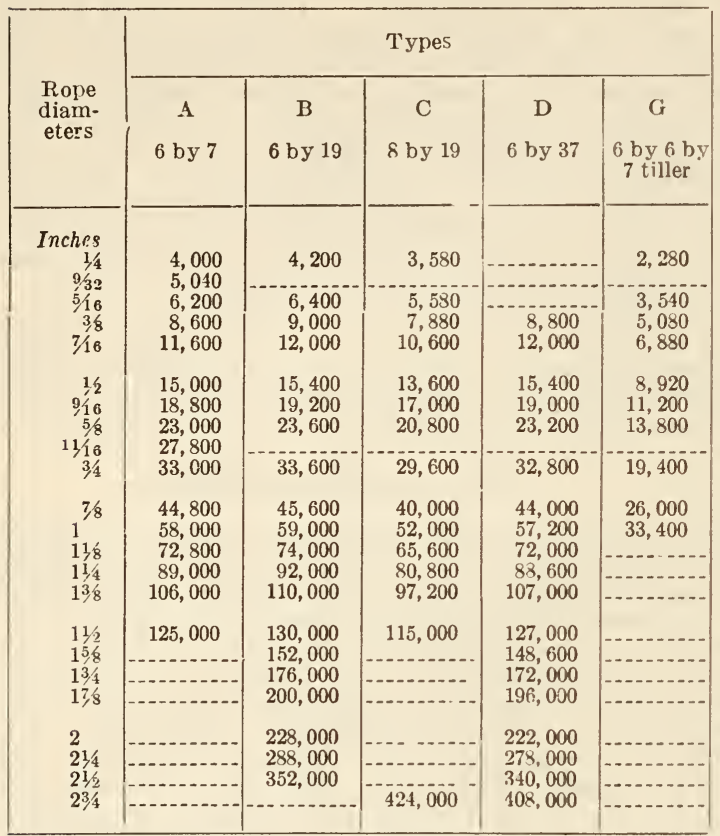

The above breaking strengths are for ropes of uncoated cast-steel wires. The minimum breaking strengths of ropes inade of other accuracy for the purpose of these specifications by multiplying the strengths above tabulated by factors as follows: Extra strong cast steel, 1.10; plow steel, 1.20: improved plow steel, 1.37.

If the wires are galvanized, the minimum breaking strengths are 10 per cent less than those tabilated or ascertained as above.

TABLE 3.-Properties of 6 by 24 and 6 by 12 galvanized cast-steel wire ropes, types $E$ and $F$

\begin{tabular}{|c|c|c|c|c|}
\hline \multirow{2}{*}{$\begin{array}{l}\text { Rope } \\
\text { diam- } \\
\text { sters }\end{array}$} & \multicolumn{2}{|c|}{$\begin{array}{l}\text { Approximate } \\
\text { weights per foot }\end{array}$} & \multicolumn{2}{|c|}{$\begin{array}{l}\text { Minimum breaking } \\
\text { strengths }\end{array}$} \\
\hline & $\frac{\mathrm{E}}{6 \mathrm{by} 24}$ & $6 \stackrel{\mathrm{F}}{6 \mathrm{by} 12}$ & $\begin{array}{c}\mathrm{E} \\
6 \mathrm{by} 24\end{array}$ & $\frac{\mathrm{F}}{6 \mathrm{by} 12}$ \\
\hline $\begin{array}{c}\text { Inches } \\
1 / 4 \\
5 / 16 \\
3 / 8 \\
7 / 16 \\
1 / 2\end{array}$ & $\begin{array}{c}\text { Pounds } \\
0.194 \\
35\end{array}$ & $\begin{array}{c}\text { Pounds } \\
0.07 \\
.10 \\
.15 \\
.20 \\
.26\end{array}$ & $\begin{array}{c}\text { Pounds } \\
7,340 \\
12,740\end{array}$ & $\begin{array}{r}\text { Pounds } \\
2,300 \\
3,520 \\
4,940 \\
6,580 \\
8,460\end{array}$ \\
\hline $\begin{array}{l}9 / 16 \\
5 / 8 \\
3 / 4 \\
13 / 16 \\
7 / 8\end{array}$ & $\begin{array}{r}.54 \\
.78 \\
.91 \\
1.06\end{array}$ & $\begin{array}{r}.33 \\
.41 \\
.59 \\
.69 \\
.80\end{array}$ & $\begin{array}{l}19,180 \\
27,200 \\
31,600 \\
36,600\end{array}$ & $\begin{array}{l}10,520 \\
12,920 \\
18,520 \\
21,600 \\
25,000\end{array}$ \\
\hline $\begin{array}{l}1 \\
11 / 16 \\
11 / 8 \\
13 / 16 \\
114\end{array}$ & $\begin{array}{l}1.38 \\
1.56 \\
1.75 \\
1.95 \\
2.16\end{array}$ & $\begin{array}{l}1.05 \\
1.19 \\
1.33 \\
1.48 \\
1.64\end{array}$ & $\begin{array}{l}47,400 \\
53,400 \\
59,800 \\
66,400 \\
73,400\end{array}$ & $\begin{array}{l}32,600 \\
36,600 \\
41,000 \\
45,600 \\
50,200\end{array}$ \\
\hline $\begin{array}{l}13 / 8 \\
17 / 16 \\
11 / 2 \\
15 / 8 \\
111 / 16\end{array}$ & $\begin{array}{l}2.61 \\
2.85 \\
3.11 \\
3.64 \\
3.93\end{array}$ & $\begin{array}{l}1.99 \\
2.17 \\
2.36 \\
2.77 \\
2.99\end{array}$ & $\begin{array}{r}88,800 \\
96,800 \\
105,200 \\
123,200 \\
132,600\end{array}$ & $\begin{array}{l}60,400 \\
66,000 \\
71,600 \\
83,600 \\
90,000\end{array}$ \\
\hline $\begin{array}{l}13 / 4 \\
113 / 16 \\
115 / 16 \\
2 \\
21 / 16\end{array}$ & $\begin{array}{l}4.23 \\
4.53 \\
5.18 \\
5.52 \\
5.87\end{array}$ & $\begin{array}{l}3.22 \\
3.45 \\
3.94 \\
4.20 \\
4.47\end{array}$ & $\begin{array}{l}142,400 \\
152,400 \\
173,200 \\
184,000 \\
196,000\end{array}$ & $\begin{array}{r}96,600 \\
103,400 \\
118,000 \\
125,400 \\
133,200\end{array}$ \\
\hline
\end{tabular}

For weights per fathom, multiply tabulated weights by 6 .

The tabulated breaking strengths are for ropes of galvanized cast. steel wires. The minimum breaking strengths of ropes made of other grades of galvanized steel wires may be ascertained with sufficient accuracy for the purpose of these specifications by multiplying the tabulated strengths by factors as follows: Extra strong cast steel, 1.10; plow steel, 1.20; improved plow steel, 1.37. (Should ropes of these types be made of uncoated steel wires, their minimum breaking strengths would be about 11 per cent more than those tabulated or ascertained as above.)
TABLE 4.-Properties of 5 by 19 marline-clad uncoated cast-steel wire ropes, type $H$

\begin{tabular}{|c|c|c|c|}
\hline \multicolumn{2}{|c|}{ Nominal diameters } & \multirow{2}{*}{$\begin{array}{l}\text { A pproximate } \\
\text { weights per } \\
\text { foot }\end{array}$} & \multirow{2}{*}{$\begin{array}{l}\text { Minimum } \\
\text { breaking } \\
\text { strengths }\end{array}$} \\
\hline $\begin{array}{c}\text { Equivalent } \\
\text { bare-wire } \\
\text { rope }\end{array}$ & $\begin{array}{l}\text { After serv- } \\
\text { ing }\end{array}$ & & \\
\hline $\begin{array}{c}\text { Inches } \\
1 / 4 \\
5 / 16 \\
3 / 8 \\
7 / 16 \\
1 / 2\end{array}$ & $\begin{array}{c}\text { Inches } \\
9 / 16 \\
5 / 8 \\
11 / 16 \\
3 / 4 \\
1316\end{array}$ & $\begin{array}{c}\text { Pounds } \\
0.21 \\
.28 \\
.36 \\
.42 \\
.51\end{array}$ & $\begin{array}{r}\text { Pounds } \\
3,800 \\
5,800 \\
8,200 \\
11,000 \\
14,200\end{array}$ \\
\hline $\begin{array}{l}9 / 16 \\
5 / 8 \\
3 / 4 \\
7 / 8 \\
1^{7 / 8}\end{array}$ & $\begin{array}{l}1^{7 / 8} \\
118 \\
114 \\
138\end{array}$ & $\begin{array}{r}.62 \\
.81 \\
1.10 \\
1.32 \\
1.70\end{array}$ & $\begin{array}{l}17,600 \\
21,600 \\
30,800 \\
42,000 \\
54,000\end{array}$ \\
\hline $\begin{array}{l}136 \\
11 / 4 \\
136 \\
116 \\
158\end{array}$ & $\begin{array}{l}11 / 2 \\
156 \\
134 \\
17,8 \\
2\end{array}$ & $\begin{array}{l}2.12 \\
2.55 \\
3.14 \\
3.69 \\
4.29\end{array}$ & $\begin{array}{r}68,000 \\
84,000 \\
101,000 \\
120,000 \\
140,000\end{array}$ \\
\hline $\begin{array}{l}13 / 4 \\
17,8 \\
2 \\
21 / 4\end{array}$ & $\begin{array}{l}21 / 8 \\
21 / 4 \\
23 / 8 \\
258\end{array}$ & $\begin{array}{l}5.00 \\
5.69 \\
6.38 \\
8.00\end{array}$ & $\begin{array}{l}162,000 \\
184,000 \\
210,000 \\
264,000\end{array}$ \\
\hline
\end{tabular}

For weights per fathom, multiply tabulated weights by 6 .

The tabulated breaking strengths are for ropes of uncoated cast. steel wires. The minimum breaking strengths of ropes made of other grades of uncoated steel wires may be ascertained with sufficient accuracy for the purpose of these specifications by multiplying the tabulated strengths by factors as follows: Extra strong cast steel, 1.10; plow steel, 1.20; improved plow steel, 1.37.

If the wires are galvanized, the minimum breaking strengths are 10 per cent less than those tabulated or ascertained as above.

TABLE 5.-Properties of galvanized stiff strands, type $I(1)$

\begin{tabular}{|c|c|c|c|c|}
\hline \multicolumn{2}{|c|}{$\begin{array}{l}\text { A pproximate } \\
\text { diameters }\end{array}$} & \multirow{2}{*}{$\begin{array}{l}\text { Number } \\
\text { nf wires }\end{array}$} & \multirow{2}{*}{$\begin{array}{l}\text { Approx- } \\
\text { imate } \\
\text { weights } \\
\text { per foot }\end{array}$} & \multirow{2}{*}{$\begin{array}{l}\text { Approx- } \\
\text { imate } \\
\text { breaking } \\
\text { strength }\end{array}$} \\
\hline Strand & Wire & & & \\
\hline $\begin{array}{r}\text { Inch } \\
1 / 4 \\
9 / 32 \\
5 / 16 \\
3 / 8 \\
7 / 16\end{array}$ & $\begin{array}{l}\text { Inch } \\
0.053 \\
.095 \\
.109 \\
.120 \\
.148\end{array}$ & $\begin{array}{l}7 \\
\frac{7}{7} \\
\frac{7}{7} \\
7\end{array}$ & $\begin{array}{c}\text { Pounds } \\
0.121 \\
.164 \\
.205 \\
.296 \\
.399\end{array}$ & $\begin{array}{c}\text { Pounds } \\
1,900 \\
2,570 \\
3,200 \\
4,250 \\
5,700\end{array}$ \\
\hline $\begin{array}{r}1 / 2 \\
9 / 16 \\
5 / 8 \\
3 / 4\end{array}$ & $\begin{array}{l}.165 \\
.203 \\
.220 \\
.259\end{array}$ & $\begin{array}{l}\frac{7}{7} \\
\frac{7}{7}\end{array}$ & $\begin{array}{r}.517 \\
.671 \\
.813 \\
1.200\end{array}$ & $\begin{array}{r}7,400 \\
9,600 \\
11,600 \\
16,700\end{array}$ \\
\hline
\end{tabular}

The tabulated breaking strengths are for strands of galvanized caststeel wires, the grade in which they are generally marketed. These strands are obtainable in extragalvanized wires, the latter being strands are obtainable in extragalter to meet requirements for protected by hearier coating of spelter to meet requirements for additional protection and wearing ralue. Strands made of higher
grades of wires may also be obtained, the relatively greater strengths of which may be ascertained with sufficient accuracy for the purpose of these specifications by multiplying the tabulated strengths by factors as follows: Siemens Martin steel, 1.60; high strength steel, 2.50; extra high strength steel, 3.50.

TABLE 6.-Properties of galvanized seizing strands, type $I$ (2)

\begin{tabular}{|c|c|c|c|c|c|c|}
\hline \multicolumn{2}{|c|}{$\begin{array}{c}\text { Approximate } \\
\text { diameters }\end{array}$} & $\begin{array}{c}\text { Number } \\
\text { of wires }\end{array}$ & $\begin{array}{c}\text { Approx- } \\
\text { imate } \\
\text { weights } \\
\text { per foot }\end{array}$ & $\begin{array}{c}\text { Approx- } \\
\text { imate } \\
\text { breaking } \\
\text { strengths }\end{array}$ & \multicolumn{2}{c|}{$\begin{array}{c}\text { Quantities on } \\
\text { reels }\end{array}$} \\
\hline Strand & Wire & Weights & Lengths \\
\hline Inch & Inch & & Pounds & Pounds & Pounds & Feet \\
$1 / 16$ & 0.022 & 7 & 0.010 & 130 & 50 & 5,000 \\
$3 / 32$ & .032 & 7 & .020 & 250 & 50 & 2,500 \\
138 & .042 & 7 & .0318 & 490 & 50 & 1,570 \\
$5 / 32$ & .058 & 7 & .0513 & 750 & 50 & 970 \\
$3 / 16$ & .042 & 19 & .0729 & 1,100 & 100 & 1,370 \\
$1 / 4$ & .052 & 19 & .121 & 1,900 & 100 & 820 \\
\hline
\end{tabular}

The tabulated breaking strengths are for iron or low-carbon-steel wires, annealed and galvanized, which is the grade in which these strands are generally marketed. 
TABLE 7.-Properties of 6 by 7 wire sash and bell cords, type $J$

\begin{tabular}{|c|c|c|c|c|}
\hline \multirow[b]{2}{*}{$\begin{array}{c}\text { Nominal } \\
\text { diameters }\end{array}$} & \multicolumn{2}{|c|}{$\begin{array}{c}\text { Approximate } \\
\text { weights per foot }\end{array}$} & \multicolumn{2}{|c|}{$\begin{array}{l}\text { Approximate } \\
\text { breaking strengths }\end{array}$} \\
\hline & Iron & $\begin{array}{c}\text { Copper } \\
\text { or phos- } \\
\text { phor- } \\
\text { bronze }\end{array}$ & $\begin{array}{l}\text { Annealed } \\
\text { iron } \\
\text { tinned } \\
\text { or galva- } \\
\text { nized or } \\
\text { bright } \\
\text { copper }\end{array}$ & $\begin{array}{l}\text { Phos- } \\
\text { phor- } \\
\text { bronze }\end{array}$ \\
\hline $\begin{array}{c}\text { Inch } \\
116 \\
3132 \\
118 \\
3 / 15 \\
7 / 32 \\
1 / 4\end{array}$ & $\begin{array}{r}\text { Pound } \\
0.006 \\
.014 \\
.025 \\
.056 \\
.076 \\
.100\end{array}$ & $\begin{array}{r}\text { Pound } \\
0.007 \\
.016 \\
.029 \\
.064 \\
.057 \\
.115\end{array}$ & $\begin{array}{r}\text { Pounds } \\
77 \\
172 \\
306 \\
688 \\
940 \\
1,225\end{array}$ & $\begin{array}{r}\text { Pounds } \\
242 \\
383 \\
715 \\
1,470 \\
2,100 \\
2.550\end{array}$ \\
\hline
\end{tabular}

TABLE 8.-Properties of phosphor-bronze wire ropes, types $A, B$, and $G$

\begin{tabular}{|c|c|c|c|c|c|c|}
\hline \multirow{2}{*}{$\begin{array}{l}\text { Rope } \\
\text { diam- } \\
\text { eters }\end{array}$} & \multicolumn{3}{|c|}{$\begin{array}{l}\text { A pproximate weights per } \\
\text { foot }\end{array}$} & \multicolumn{3}{|c|}{ Minimum breaking strengths } \\
\hline & $\underset{6 \text { by } 7}{\mathrm{~A}}$ & $\underset{6 \text { by } 19}{\text { B }}$ & $\begin{array}{c}G \\
6 \text { by } 6 \text { by } \\
7 \text { tiller }\end{array}$ & $\underset{6}{A}$ by 7 & $\begin{array}{l}\text { B } \\
6 \text { by } 19\end{array}$ & $\begin{array}{c}G \\
6 \text { by } 6 \text { by } \\
7 \text { tiller }\end{array}$ \\
\hline $\begin{array}{r}\text { Inch } \\
1 / 4 \\
5 / 16 \\
3,8 \\
7 / 16 \\
1 / 2\end{array}$ & $\begin{array}{c}\text { Pounds } \\
0.113 \\
.178 \\
.256 \\
.333 \\
.439\end{array}$ & $\begin{array}{c}\text { Pounds } \\
0.110 \\
.160 \\
.230 \\
.320 \\
.410\end{array}$ & \begin{tabular}{|c|} 
Pounds \\
0.076 \\
.120 \\
.174 \\
.229 \\
.305
\end{tabular} & $\begin{array}{c}\text { Pounds } \\
2,675 \\
3,645 \\
5,500 \\
6,500 \\
9,500\end{array}$ & $\begin{array}{c}\text { Pounds } \\
1,900 \\
3,000 \\
4,400 \\
5,900 \\
7,700\end{array}$ & $\begin{array}{c}\text { Pounds } \\
1,680 \\
2,600 \\
3,800 \\
4,750 \\
6,900\end{array}$ \\
\hline $\begin{array}{r}9 / 16 \\
5 \% 8 \\
34 \\
7,8\end{array}$ & $\begin{array}{r}.55 \\
.69 \\
1.01 \\
1.35\end{array}$ & $\begin{array}{r}.520 \\
.640 \\
.920 \\
1.260\end{array}$ & $\begin{array}{l}.382 \\
.469 \\
.676\end{array}$ & $\begin{array}{l}11,500 \\
14,850 \\
20,900 \\
30,000\end{array}$ & $\begin{array}{r}9,800 \\
12,100 \\
17,100 \\
23,400\end{array}$ & $\begin{array}{r}8,000 \\
10,360 \\
16,100\end{array}$ \\
\hline
\end{tabular}

For weights per fathom, multiply tabulated weights by 6 .

\section{Section III. MATERIALS AND WORKMANSHIP}

1. In general. - The rope shall be made from the best quality of the specified materials, shall be of good workmanship, and shall be free from defects which might affect its appearance or serviceability.

2. Diameter and tolerances.-No strand or rope, other than marline-clad rope, shall be accepted, the diameter of which is under the diameter ordered. The amount which the actual diameter of rope may be oversize from the diameter ordered shall not exceed the following:

Rope diameters:

Oversize

0 to $3 / 4$ inches...... tolerances

$13 / 16$ to $11 / 2$ inches

196 to 214 inches

$1 \% 6$ to 214 inch

Stiff strands all sizes

Stiff strands all sizes
Seizing strands all sizes.

The actual diameter of marline-clad rope, after serving, shall be not more than one-sixteenth inch larger or smaller than its specified nominal diameter.

3. Lay. - The ropes covered by these specifications shall be of right regular lay. Except as otherwise hereinbefore specified for ropes of types A, 6 by 7 and I, strands, the lay of the ropes included in these specifications shall be not greater than six and three-fourths times the nominal diameter of the rope if the wires are uncoated, or seven and onequarter times the diameter of the rope if the wires are galvanized. In the case of the marline-clad rope, type $\mathrm{H}, 5$ by 19 , the nominal diameter of the rope flom which the lay is determined is the diameter after serving.
4. Tensile strength of steel wires.-The minimum tensile strength of the steel wires of grades hereinbefore specified shall be as follows:

\begin{tabular}{c|r|r}
\hline Grade & Uncoated & Galvanized \\
\hline & $\begin{array}{r}\text { Pounds per } \\
\text { square inch } \\
170,000\end{array}$ & $\begin{array}{r}\text { Pounds per } \\
\text { square inch } \\
155,000 \\
190,000\end{array}$ \\
Cast steel & 210,000 & 190,000 \\
Extra strong cast steel & 230,000 & 210,000 \\
Plow steel & & \\
\hline
\end{tabular}

5. Phosphor-bronze wire.-The tensile strength of phosphor-bronze wire shall be not less than 100,000 pounds per square inch. The chemical composition shall be as follows: Copper (minimum), 94 per cent; tin (minimum), 3.5 per cent; phosphorus, 0.05 to 0.5 per cent; lead (maximum), 0.2 per cent; iron (maximum), 0.1 per cent; and zinc (maximum), trace.

6. Galvanizing wire.-Wires for galvanized rope shall be uniformly and continuously coated with zinc adhering firmly to the wire. The zinc shall be applied in a molten condition by what is known in the trade as "hot-process galvanizing."

7. Fiber cores.-These shall be of either hard fiber or cotton as specified in Section I. Jute fiber shall not be used. Hard fiber may be abaca (manila), sisal, or other suitable and generally recognized trade variety. A mixture of two or more kinds of the hard fibers mentioned may be used. All fiber shall be of the best quality, thoroughly cleaned, and free from waste. The cotton for the cores of the small ropes shall be of the best suitable quality. The cores shall be of uniform ply, evenly twisted, and of good workmanship.

8. Lubrication.-The fiber cores shall be thoroughly impregnated with suitable lubricant.

9. Marline covering.-The covering for wire rope of type H, Section I, 4 , shall be of good quality hard American or Russian hemp saturated with pine tar.

\section{Section IV. INSPECTION AND TESTS}

1. General.-Unless otherwise agreed upon, inspection of ropes purchased on the basis of these specifications shall be made after delivery. The physical tests hereinafter specified serve further to define the character of the ropes. The tests shall not be made, however, unless agreed upon in the conditions of purchase, as for small orders especially the cost of making such tests would be prohibitive. When tests are to be made, they shall, unless otherwise agreed upon, be made by the manufacturer, who shall furnish certified reports of the results thereof to the purchaser. The omission of test requirements in connection with any purchase shall not be construed to deprive the purchaser of the right to make tests as herein specified, at his own expense, to verify the grade and quality of the rope, or of the right to require replacement of any inferior rope supplied, provided that claim for replacement shall be presented within 30 days following delivery of the rope.

2. Guaranty in lieu of tests.-When rope purchased on the basis of these specifications is to be delivered without tests, the manufacturer or dealer shall, upon request, furnish to the purchaser a written guaranty that the rope delivered meets the requirements of these specifications.

3. Diameter. - The diameter of the rope (over-all diameter), shall be measured at three places at least 5 feet apart and at least 5 feet from the end. The average of the three measurements shall be taken as the diameter of the rope. (Measuring the diameter of a rope over the flats between strands is incorrect. 
See Sec. III, (2), for requirements as to diameter and tolerances.)

4. Lay. - The lay of wire rope shall be ascertained by measuring, parallel to the axis of the rope, the distance in which a strand makes five or more complete turns around the rope. This distance divided by the number of turns is the lay of the rope. When measuring the lay, there shall be no axial load on the rope, and the measured distance shall not be within 10 feet of the end of the rope. (See preceding sections for construction requirements.)

5. Strand fabrication.-To determine whether a strand consisting of two or more layers has been fabricated in accordance with these specifications, remove one of the strands from the rope and unlay one outside wire, holding the remaining wires in their proper pcsitions. The outside wire, which is unlayed, should be found in contact continuously with the same underlying wires. If it was in contact with different underlying wires, the stranding does not comply with the requirements. Strands having more than two layers are tested by applying this test to the adjacent layers successively.

6. Tensile test of wires.-For tensile tests of the wires, a section shall be cut from each length of rope. From eacn section at least six tensile tests shall be made, one or more wires being selected from each strand. Specimens for obtaining the tensile strength of the wires shall be not less than 15 inches long, and the distance between the grips of the testing machine shall be not less than 10 inches. The speed of the movable head of the testing machine, under no load, shall not exceed 1 inch per minute.

7. Torsional tests of wires.-(a) This test shall apply only to wires for uncoated steel ropes. Specimens for torsional tests of the wires, taken either before or after the rope is fabricated, shall be straight and at least 12 inches long. Not less than one specimen of each size of wire from each strand of the rope shall be selected, but the total number of specimens shall not exceed 25 per cent of the total number of wires in the rope. The distance between the clamps in which the wire is held before the test shall be 8 inches. One clamp shall be movable, parallel to the axis of the wire, and of adequate weight to cause sufficient tension on the specimen to keep it straight during the test. The wire shall be twisted by rotating one of the clamps at a uniform rate graded according to size of wire, but not exceeding 60 revolutions per minute for the smallest wire. If the temperature of the wire increases preceptibly during the test, the speed shall be decreased. Under this test the wires shall not break at less than the number of complete revolutions (of $360^{\circ}$ ) given in the following table:

\begin{tabular}{|c|c|}
\hline Material & Number of revolutions (in 8 inches) \\
\hline $\begin{array}{l}\text { Cast steel. } \\
\text { Extra strong cast steel. } \\
\text { Plow steel. } \\
\text { Improved plow steel...... }\end{array}$ & 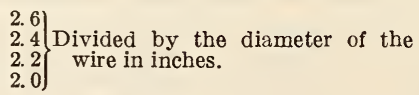 \\
\hline
\end{tabular}

(b) If the first torsional specimen fails, two more specimens shall be tested from the same wire. If both of these comply with the requirements, the test shall be deemed satisfactory.

8. Wrapping test for wires.-(a) When wrapped in a close helix, for six complete turns about a mandrel, and then unwrapped, the wire for galvanized steel wire rope shall not break. The diameter of the mandrel shall be twice the diameter of the wire.

(b) The specimens for the wrapping test, taken either before or after the rope is fabricated, shall be straight and of any convenient length. At least one specimen of each size of wire from each strand of the rope shall be selected, but the total number of specimens shall not exceed 25 per cent of the total number of wires in the rope.

(c) One end of the specimen shall be secured in any convenient way and the wire held by the free end shall be tightly wrapped around the mendrel, with the six coils of the helix practically in contact and then unwrapped.

9. Tensile test of rope.-(a) The breaking strength shall be determined as far as possible from the full section of the completed rope. The breaking strength thus ascertained shall conform to the requirements given for each size and type in the tables and notes comprised in Section II of these specifications. One tensile test specimen shall be cut from each type and size of rope.

(b) The distance between the sockets or grips may be from twenty-five to sixty times the nominal diameter of the rope. The ends of the specimen may be secured in tapered sockets by using molten zinc or held in any other suitable device. (See par. 7 on socketing in Appendix I.)

(c) Whenever the size to be tested is such that the tensile test can not be made on the completed rope, the strength shall be determined by obtaining the breaking strength of each individual wire. The breaking strength of ropes of 6 by 7 and 6 by 19 construction shall be assumed as 85 per cent, and that of the more flexible ropes as 80 per cent of the sum of the breaking strength of all of the wires in the rope section.

10. Galvanizing.-(a) Tests to determine the quality of the galvanizing will not be made unless specifically required in asking for quotations and in the purchase order, or unless the inspector has reason to believe that the galvanizing does not meet the requirements of these specifications.

(b) When this test is required, it shall be made by the Preece method, in which wire specimens are subjected to specified immersions in a standard solution of copper sulphate, washed thoroughly in water, and wiped dry. This produces a bright metallic copper deposit on the specimens wherever zinc coating is lacking. The procedure is as follows:

(1) Specimens.- Specimens at least 6 inches long shall be cut from the galvanized wire to be tested, before stranding. Not less than three specimens shall be cut from each size of wire to be used in the rope. The specimens shall be cleaned before testing, using cotton waste saturated with carbon tetrachloride, benzine, or turpentine. A brush shall not be used. The specimens shall then be rinsed thoroughly in clear water and wiped dry with clean cotton waste.

(2) Copper solution.-The standard solution of copper sulphate consists of 36 parts by weight of commercial copper-sulphate crystals dissolved in 100 parts by weight of cold water. To neutralize any free acid, chemically pure cupric oxide $(\mathrm{CuO})$ shall be added until excess thereof is indicated by the oxide appearing on the bottom of the container. The neutralized solution shall be filtered through filter paper before using. It shall have a specific gravity of 1.186 at $65^{\circ} \mathrm{F}$. at the beginning of each test. If the specific gravity is too high, clean water should be added; if too low, a filtered solution of a higher specific gravity should be added. The solution shall be contained in a glass vessel at least 2 inches inside diameter and filled to a depth of at least 4 inches before immersing the specimens. Its temperature shall be between $60^{\circ}$ and $65^{\circ} \mathrm{F}$.

(3) Test.-One immersion shall consist of dipping the wire into the solution for one minute, after which it shall be removed, washed thoroughly in water, and wiped dry. Not more than seven specimens shall be immersed simultaneously, and they shall be 
separated to allow the solution to act on all of their immersed surfaces. The specimens shall not show the appearance of bright adherent copper, indicating exposed iron, as a result of the number of immersions shown in the following table:

\begin{tabular}{|c|c|}
\hline Diameter of wire (in inches) & $\begin{array}{c}\text { Number } \\
\text { of immer- } \\
\text { sions }\end{array}$ \\
\hline 0.028 to 0.047 & 1 \\
0.048 to 0.054 & 2 \\
0.055 to 0.063 & $121 / 2$ \\
0.064 to 0.079 & 3 \\
0.080 to 0.092 & $131 / 2$ \\
0.093 to 0.114 & 4 \\
0.115 and larger........... & 5 \\
\hline
\end{tabular}

1 The duration of one half immersion in one-half minute.

If, after the last immersion, any specimen shows a bright metallic copper deposit, the galvanizing shall be regarded as defective, and the wire represented by that specimen shall be rejected: Provided, however, That no wire shall be rejected because a copper deposit appeared within 1 inch of the ends of its test specimen.

(4) Color samples.-Each day that lests of the galvanizing are made, standard color samples of the bright metallic copper deposit shall be made, with which the test specimens shall be compared. These color samples, cut from the wire to be tested, shall be immersed in concentrated hydrochloric acid until all zinc is removed. They shall be immediately washed and wiped dry and then immersed in the standard copper solution for one minute, removed, washed, and wiped dry.

\section{Section $\nabla$. PACKING AND MARKING OF SHIPMENTS}

(a) Rope furnished under these specifications shall be properly protected for shipment by the shipper. The ends of all ropes shall be suitably selzed to prevent untwisting. The manner of preparing the rope for shipment, whether in coils or on reels, shall be determined by the shipper unless specific instructions are given in the purchase order.

(b) The cost of preparing ropes for shipment shall be borne by the shipper, provided that if so agreed at the time of purchase, reels may be billed as extra cost and the purchaser may return them to the shipper in good condition, normal wear and tear excepted, within 12 months from date of shipment and receive a refund of the amount paid therefor.

(c) Each package of a shipment shall be marked with the name of the material, the size and leng ih of rope, the name of the manufacturer or shipper, and the purchaser's order number or date.

(d) In all cases where the purchase order calls for inspection and tests before shipment, a metal seal or tag stamped by the inspector for identification shall be securely fastened to each lenguh of rope inspected or tested, anc accepted.

\section{APPENDIX I}

Recommended Practice for the Storage, Handling, Use, Inspection, and Maintenance of Wire Ropes

1. Storage.-Rope should be stored in a dry and cool place. Heat would impair or destroy the core. 2. Handling.-Great care should be used in handling wire rope to avoid either twisting or untwisting and "kinks." The latter are caused by loops in the rope which are "pulled through" by tension on the rope until the diameter of the loop is only a few inches. The wires a i a kink are permanently bent and injured. Wire rope that has been kinked will not give satisfactory service.

3. Unreeling. $-(a)$ The best way to unreel a rope is to place an axle through the hole in the center of the reel and support it horizontally so that the reel can turn freely. The rope should then be pulled off in a straight line. The reel should be prevented from turning more rapidly than the rope is pulled away in order to prevent loops of the rope falling over the flange as this would probably result in kinks or other damage. This is easily accomplished by using a board or other form of brake against the flange of the reel.

(b) An alternative method which, however, is deemed to be inferior, is to mount the reel on a turntable with the axis vertical. The rope is pulled off in a straight line as by the other methoa, but it is likely to drop over the bottom flange of the reel and liable to be pulled around the axle with resultant damage. This can be avoided by keeping the loops on the reel. Under no circumstances should a loop be thrown over the upper flange of the reel and pulled out, as the rope would, when pulled straight, either be twisted or untwisted or even kinked, and the length of its service would be shortened.

4. Uncoiling. - If wire rope is in a coil, the outer end of the rope should be released and the coil rolled along the ground in a straight line, unwinding the rope as it proceeds. Under no circumstances should the coil be laid flat on the ground and the loops thrown off and pulled out, as the rope would thereby be twisted or untwisted and kinked as in the case of improper unreeling.

5. Seizing and seizing wire.-(a) The end of a wire rope should have at least three seizings to prevent unlaying. Unlaid rope would be useless. The length of each seizing should be at least equal to the diameter of the rope. Fittings which prevent unlaying of the rope may be substituted.

(b) Satisfactory seizing may be accomplished in either of two ways, viz:

Method $A$, in which both ends of the seizing wire are brought out at the same end of the seizing. This is done by laying the wire in the groove between two strands and wrapping the other end of the wire in a close helix around the rope back over this portion. A seizing iron (round bar, one-half to five-eighths inch in diameter by 18 inches long) should be used, especially if the rope is over 1 inch in diameter. To keep the necessary tension in the wire, the free end should be given one complete turn about the rope. The ends of the wire should be twisted tightly together.

Method B.- (1) Wind the seizing wire on the rope by hand, keeping the coils together and considerable tension on the wire. (2) Twist the ends of the wire together by hand, so that the twisted portion of the wires is near the middle of the seizing. (3) Using a proper tool, tighten the twist just enough to take up the slack, but do not try to tighten the seizing itself by twisting. (4) Tighten the seizing by prying the twist away from the rope and tighten the twist again as in (3). Repeat this operation as far as necessary to make the seizing tight. (5) Cut off the ends of the wires and pound the twist flat against the rope.

(c) Any annealed low-carbon steel wire may be used for temporary seizings. Proper sizes are as follows:

\begin{tabular}{|c|c|}
\hline $\begin{array}{c}\text { Wire rope diameters } \\
\text { (in inches) }\end{array}$ & $\begin{array}{c}\text { Seizing wire } \\
\text { diameters }\end{array}$ \\
\hline & $\begin{array}{l}\text { Inch } \\
1 / 4 \text { to } 15 / 16\end{array}$ \\
1 to $15 / 8$ & 054 \\
$13 / 4$ and over. & .105 \\
\hline
\end{tabular}

(d) Permanent seizings should be made with seizing strands described in the preceding specifications. 6. Cutting.-Before cutting a wire rope, three seizings of the character described in the preceding paragraphs should be placed on each side of the place to be cut, the first on each side to be close to the 
cut, and the others at short distances apart. These seizings are very important as they prevent unlaying or untwisting and ensure equal tension in the strands when load will be applied to the rope.

7. Socketing.- Standard forged steel sockets may be attached to a rope as follows:

(a) The rope should be securely seized before cutting as per preceding paragraph, but with at least an extra seizing placed at a distance from the end equal to the length of the basket of the socket. For large ropes these seizings should be several inches long and securely wrapped with a seizing iron.

(b) After cutting, the end seizing should be taken off, leaving the next or extra seizing at a distance from the end of the rope equal to the length of the socket basket. The fiber core should be cut back to this seizing, and the wires should all be untwisted and broomed out, but not necessarily straightened.

(c) The wires for the distance that they are to be inserted in the socket, should be washed with benzine, naphtha, or gasoline, and then dipped, for a distance not greater than three-fourths of the cleaned length, in commercial muriatic acid for fiom 30 seconds to one minute, or until the acid has thoroughly cleaned each wire. Care should be taken that the acid does not come in contact with any other portion of the rope, as if it does the wires will become brittle. The wires should then be dipped in boiling water containing a small amount of soda to neutralize the acid and be thoroughly dried. Do not handle the cleansed wires with greasy hands or tools.

(d) Insert the wires into the basket of the socket taking care that the socket is in line with the axis of the rope. The socket and wires must be dry, and the socket slightly heated before pouring the zinc. The base of the socket should be sealed with putty, clay, or any similar substance. Molten zinc should then be poured solwly and evenly into the socket until it is full, tapping its side with a light hammer to jar the zinc into the crevices between the wires. Do not use babbitt metal or lead for socketing wire ropes, as these metals would produce a fastening of much less strength than that of the rope. A safety mask should be worn by the man while he pours the molten zinc in order to guard against possible injury.

(e) The zinc must not be too hot or it will anneal the wires, particularly for small ropes. The temperature should not be above $830^{\circ} \mathrm{F}$. When the zinc has solidified sufficiently the socket may be plunged into cold water. If socketing is properly done, the strength of the connection will at least equal that of the rope. (A simple and practical method to determine the proper temperature of molten zinc is as follows: A dry soft pine stick dipped into the zinc and quickly withdrawn must have no zinc adhering to it, and should be only slightly discolored by scorching. If the zinc adheres to the stick, it is too cold for pouring. If the stick is charred, the zinc is too hot.)

$(f)$ Socketed connections used for services where failure would endanger human life should be tested to twice the maximum working load to which they are to be subjected.

(See American Marine Standards H No. 7-1928, Rigging Screws with Sockets, see 608.7, p. 626, and H No. 9-1926; Wire Rope Sockets, above.)

8. Clips and clamps. - (a) A wire rope may be attached to its load by looping its end around a thimble which is to sustain the load, with sufficient length in the short end of the rope above the thimble to be fastened to the long end by means of properly designed clips. The thimbles may be of pressed steel or cast, galvanized, and of adequate size. (See American Marine Standards H No. 10-1926, Solid Thimbles, and $\mathrm{H}$ No. 11-1926, Open Thimbles, above.)

(b) A wire rope clip consists of a forged steel "roddle," or base, having diagonal grooves which fit the strands of the rope and a steel $U$-bolt. A forged steel saddle for the $U$-bolt is advisable. The $U$-bolt is placed over its saddle and its ends pass through the roddle. The ropes are fastened by tightening the nuts on each end of the U-bolt. It is good practice to place the roddle in contact with the main rope and the $U$-bolt in contact with the loop end.

(c) Although fastening with clips requires little skill and can be readily inspected, rope so fastened is apt to slip, the clips frequently crush and bruise the rope, and the strength of the fastening is usually less than 80 per cent of that of the rope. Clip fastenings should, therefore, be limited to uses where failure would not endanger human life.

(d) Wire rope clamps consist of two similar pieces, each having two straight parallel grooves which fit the rope, one of which may have diagonal depressions which fit the strands of the rope. They have a comparatively low holding power and, therefore, they should not be used except where the stress in the rope is low in comparison to its strength.

(e) The distance between clips should not be less than six times the diameter of the rope. The minimum number of clips per fastening should be as follows:

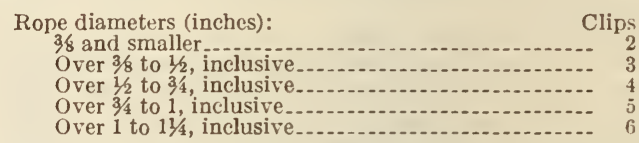

For ropes larger than $1 \frac{1 / 4}{4}$ inches, it is advisable to use sockets instead of clips.

9. Splicing. - (a) There are two types of splices, viz, long splices, which secure ends of rope together so as to form a longer or an endless rope; and short splices, wnich secure the loop end of a rope over an eye or thimble. Splicing should be done only by men experienced in such work.

(b) Generally speaking, the longer a splice is made, the greater is its strength. The minimum lengths recommended are as follows:

Long splices. - Four feet for every one-eighth inch of rope diameter. (Example: Minimum length of long splice for 1 inch diameter rope, $8 \times 4=32$ feet.)

Short splices.-Four inches for every one-eighth inch of rope diameter; provided that no such splice should be less than 12 inches long. This is additional to the length of rope passing around the eye or thimble. (Example: Minimum length of short splice for 1 inch diameter rope, $8 \times 4=32$ inches.)

(c) Splices, failure of which would endanger human life, should be tested to at least twice their minimum working load before being placed in service.

10. Sheaves and drums.-(a) The length of service of wire rope is greatly affected by the diameters of the sheaves or drums over which it is run. The length of service decreases as the diameter of sheaves or drums is made smaller. It is seldom possible on ships to have the sheaves or drums as large as they should be for utmost life of rope, but it is in the interest of safety and economy to make them as large as practicable.

(b) In the following data, given for general guidance, the tread diameters of sheaves or drums are expressed in times the diameters of the ropes.

\begin{tabular}{|c|c|c|c|}
\hline Typical rope constructions..................... & $\begin{array}{l}6 \text { by } 19 ; \\
5 \text { by } 19 \text { i }\end{array}$ & 8 by 19 & 6 by 37 \\
\hline $\begin{array}{l}\text { Tread diameters recommended for eco- } \\
\text { nomical results. } \\
\text { Allowable minimum tread diameters for } \\
\text { reasonable service and safety.......... }\end{array}$ & $\begin{array}{l}45 \\
30\end{array}$ & $\begin{array}{l}27 \\
18\end{array}$ & $\begin{array}{l}31.5 \\
21\end{array}$ \\
\hline
\end{tabular}

1 For 5 by 19 marline-clad rope compute tread diameter on the basis of the equivalent bare rope size. (See Table 4 , Sec. II of basis of the equifications.)
specificatis 
(c) If lesser tread diameters than the minimum specified in the preceding paragraph are unavoidable, comparatively rapid deterioration of the rope may be expected and it should be closely watched in operation for signs of failure. It is inadvisable in any case to use tread diameters less than 75 per cent of the allowable minimum. When sheaves or drums of lesser diameters must unavoidably be used, proportionately stronger rope should be provided for any given maximum load. This means higher grade rather than larger size rope as the bending stress of rope over a given sheave or drum increases rapidly as the rope diameter is increased and a condition is soon reached where increased strength due to increased size would be overbalanced $b$, the increased bending stress. (See Appendix II re safe loads.)

(d) Whenever possible the drums or sheaves for wire rope of any construction should be so arranged that the rope is not bent in service first in one direction then in the reverse direction. If reverse bending is unavoidable, the life of the rope will be prolonged by making the sheave about which the reverse bending occurs larger than the preceding one.

11. Lubrication.-(a) The wear upon a running rope is both external and internal and, it being impossible in manufacturing rope to provide lubricant for its entire life, it is necessary to lubricate it at frequent intervals in order to protect the wires from friction and corrosion. This applies especially to ropes in running rigging.

(b) The lubricant should be chemically neutral and should not dry out rapidly under service conditions. A heavy-bodied oil with graphite is satisfactory and comparatively inexpensive. A heavy viscous preparation may be desirable to cover the outside of the rope, but it does not give the requisite internal lubrication.

12. Deterioration and replacement.-(a) The principal causes of deterioration of wire rope are abrasion, overstrain, bending, and corrosion. Evidence of abrasion is shown by wear on the outside wires. If the wires are slightly worn, and break off squarely, sticking out all over the rope, there is evidence of an overload or severe working.

(b) No definite rules can be given which will enable an inexperienced person to determine when a worn rope should be discarded and replaced. The service for which the rope is used, and the possible consequences if it broke, are important factors in such determination. If breaking of the rope would cause little or no damage, it might theoretically be used until it fails, but this is not to be contemplated under any circumstances. The following suggestions are made for general guidance only:

(1) If material only is hoisted and no serious damage would result from failure of the rope, it may be used, if there are no broken wires, until the thickness of the outside wires is worn to about 35 per cent of their original diameter. It should, in any event, be renewed if it shows 10 per cent of brcken wires per lay, or when adjacent broken wires in one of the strands exceed 20 to 25 per cent of the wires in that strand, even if it does not show appreciable wear.

(2) If persons are hoisted, or if material damage would result from failure of the rope, it should be renewed when, in the judgment of the inspector, its strength is appreciably impaired by broken wires, or in any event when the outside wires are worn to a thickness of one-half of their original diameter, even if there are no broken wires.

13. Refastening. - When there is reason to believe that a socket or clip fastening is impaired, it is.advisable to renew it. It is recommended that in renewing fastenings, a section of 4 to 10 feet of the rope above the socket or the upper clip be cut off. This removes a portion of the rope which is likely to fail. The effective life of fastenings depends upon the service to which the rope is subjected. Under ordinary conditions it may be assumed to be but one-sixth or even less of the expected life of the rope.

14. Service inspection.-(a) All ropes should be inspected at intervals sufficiently frequent to detect indications of wear or corrosion. The interval between inspections depends upon the character and importance of the service and the prevailing conditions.

(b) Fastenings should be inspected at regular frequent intervals. Clips and clamps should be inspected daily and their bolts tested for tightness, and tightened when necessary to the proper degree.

(c) Hoisting rope should be carefully examined at least once a day by a competent and responsible inspector, a signed record of whose inspection should be kept in a suitable record book.

(d) It is advisable to keep general records of the life of hoisting ropes with the name of their manufacturer or dealer and their characteristics, the date when they were put in service, what was done in way of their maintenance, and the date and cause of their removal from service.

(e) It has been found that wires nearly always break on the outside or crown of the strand. Therefore, visual inspection along this line is likely to disclose the condition of the rope as regards broken wires. This, however, might not apply to ropes which are not sufficiently lubricated, and in which undetectable corrosion of internal wires might set in. Therefore, condition of lubrication should be regularly inspected; and upon discovering lack of it, the internal condition of the rope should be ascertained.

15. Available additional information. - (a) Trade publications issued by manufacturers of wire ropes and of elevators contain valuable data relative to the characteristics of wire ropes, and illustrated descriptions of the right and wrong way to perform various operations in handling and working them.

(b) Technical Paper No. 237, of the Bureau of Mines of the United States Department of Commerce, entitled "Safe Practice in Using Wire Ropes in Mines," by Kudlich and Hood, and Technologic Paper No. 229, of the Bureau of Standards, entitled "Some Tests of Steel-Wire Rope Sheaves" also contain much valuable information.

(c) Users of wire ropes are advised to study such publications for their full information and guidance, especially as to seizing, socketing, proper application of clips, and splicing.

(d) Wire rope for elevator installations should comply with the requirements of the Safety Code for Elevators and Escalators, published by the American Society of Mechanical Engineers, New York.

\section{APPENDIX II}

Notes on Wire Rope Strengths and Working Loads

1. Certificates of strength of wire ropes, required in foreign countries. - Some foreign authorities, including the British Board of Trade, require certificates. drawn up on their own forms, of the strength of rope rigging used in handling cargoes at ports of their respective countries. Operators of ships engaged in foreign trade should ascertain the requirements of those authorities, assure themselves that the rope rigging on ships intended to handle cargo in those countries is of adequate strength to comply with the requirements, instruct the officers of such ships accordingly, and provide them with the requisite certificates in proper form or with authenticated data for use abroad.

2. Working loads.-Determination of the proper working load for any rope is a matter best left open for decision by the user. It is deemed advisable to limit the working load of a wire rope for ordinary services, particularly running ropes, to one-fifth of 
the breaking strength. This means that the factor of safety should be not less than 5. To determine the proper working load, divide the breaking strength of the rope by the proper factor of safety; for example, a 1-inch diameter 6 by 19 uncoated caststeel rope has a breaking strength of 59,000 pounds and with a factor of safety of 5 the proper working load would be $\underline{5} \cdot 0 \frac{0}{5} 000=11,800$ pounds. (For breaking strengths, see tables in Sec. II of specifications.)

3. Factor of safety. - Factors of safety in excess of 5 , varying up to 8 and even more, are often required for safe and economical operation. The proper factor of safety for a wire rope should be determined by careful and thorough consideration of all pertinent data. Such data should include the probable maximum and average loads, acceleration, deceleration, rope speed, and character of rope attachments, with special consideration to the number, size, and arrangement of all sheaves and drums, existing conditions which may cause corrosion and abrasion, length of rope in service, economical rope life, and the degree of danger to life and property. No fixed arbitrary factor of safety can properly be set for any classification of service; but such factor can safely vary, within reasonable limits, with the conditions present on individual installations. Should any doubt arise as to the proper factor for any rope or service, it is suggested that rope manufacturers or other authorities be consulted.

4. Ship classification requirements.-The ship classification societies prescribe rules governing the sizes of wire ropes for stream cables, towlines, hawsers, and warps, also for rigging. The wire ropes for any ship or service subject to such rules should conform thereto. The breaking tests for wire ropes specified by the American Bureau of Shipping and the diameters of standard steel ropes to meet such tests are set forth in the American Bureau of Shipping, Rules for Building and Classing Steel Vessels, given above.

\section{AMERICAN MINING CONGRESS, WIRE ROPES FOR MINES, 1927}

[Approved as American Standard M11-1927, by the American Standards Association, February 24, 1927]

\section{Part 1. TYPES}

Section 100.-Rule 1,000.-The wire ropes covered by this specification are of the following types:

1 by 7 seizing strand.

1 by 7 steel strand.

6 by 7 cast steel.

6 by 7 extra strong cast steel (mild plow).

6 by 7 plow steel.

6 by 19 cast steel.

6 by 19 extra strong cast steel (mild plow).

6 by 19 plow steel.

6 by 37 cast steel.

6 by 37 extra strong cast steel (mild plow).

6 by 37 plow steel.

8 by 19 cast steel.

8 by 19 extra strong cast steel (mild plow).

8 by 19 plow steel.

Any other manufacturer's trade name of equivalent material which passes the tests complies with this specification.

(In respect to material and workmanship, general requirements, inspection and tests, and recommended practice in the use of wire rope, this specification conforms to the specification of the American Marine Standards Committee Specification O No. 25, wire rope for marine uses, given above. Detail requirements, including both text and illustrations, are identical for 1 by 7 seizing strand and 6 by 7 extra strong cast steel with those in Federal Specifi- cations Board Specification No. 297 (current designation: RR-R-571, given below.)

(Wire rope specified in Federal Specifications Board Specification No. 297 (current designation: $\mathrm{RR}-\mathrm{R}-571$ ), given below, but not included in this specification are as follows:)

\section{1 by 19 seizing strand.}

5 by 19 marline covered extra strong cast steel. 5 by 19 marline covered high-grade plow steel. 6 by 12 cast steel.

6 by 12 high-grade plow steel.

6 by 12 phosphor bronze.

6 by 19 high-grade plow steel.

6 by 19 phosphor bronze.

6 by 24 cast steel.

6 by 24 high-grade plow steel.

(Detail requirements in this specification covering original wire rope specifications are as follows:)

Section 401 . - 1 by 7 , steel strand.

Rule $4010 .-1$ by 7 steel strand shall be made as described here and have the properties given in Table 1. (Illustration same as in F. S. B. 297, for 1 by 7 seizing strand.)

\section{TABLe 1.- Properties of 1 by 7 steel strand}

\begin{tabular}{|c|c|c|c|}
\hline $\begin{array}{c}\text { Nominal } \\
\text { diameter }\end{array}$ & $\begin{array}{c}\text { A pproxi- } \\
\text { mate weight } \\
\text { per foot }\end{array}$ & $\begin{array}{c}\text { Minimum } \\
\text { breaking } \\
\text { strength }\end{array}$ & $\begin{array}{c}\text { Maximum } \\
\text { safe work- } \\
\text { ing load }\end{array}$ \\
\cline { 2 - 3 } Inch & Pound & Pounds & Pounds \\
$3 / 8$ & 0.295 & 4,500 & 1,000 \\
$1 / 2$ & .510 & 7,900 & 1.760 \\
$5 / 8$ & .800 & 12,400 & 2,750 \\
\hline
\end{tabular}

(Requirements covering construction, material, diameter, and lay are identical with those for 1 by 7 seizing strand in F. S. B. 297, given below, except that the diameter tolerance shall be one-sixteenth inch instead of one-sixty-fourth-inch.)

Section 402.-6 by 7 , cast steel.

Rule 4020.- Wire rope 6 by 7 , cast steel, shall be made as described here and shall have the properties shown in Table 8 in F. S. B. 297, given below, except for working and breaking strengths of both uncoated and galvanized rope, which are as follows:

\section{TABLE 2.-Properties of 6 by 7 cast steel wire rope}

\begin{tabular}{|r|r|r|r|r|}
\hline & \multicolumn{2}{|c|}{ Uncoated } & \multicolumn{2}{c|}{ Galvanized } \\
\cline { 2 - 3 } $\begin{array}{c}\text { Nominal } \\
\text { diameter }\end{array}$ & $\begin{array}{c}\text { Minimum } \\
\text { breaking } \\
\text { strength }\end{array}$ & $\begin{array}{c}\text { Maximum } \\
\text { safe work- } \\
\text { ing load }\end{array}$ & $\begin{array}{c}\text { Minimum } \\
\text { breaking } \\
\text { strength }\end{array}$ & $\begin{array}{c}\text { Maximum } \\
\text { safe work- } \\
\text { ing load }\end{array}$ \\
\cline { 5 - 5 } Inches & Pounds & Pounds & Pounds & Pounds \\
$3 / 8$ & 7,500 & 1,670 & 6,750 & 1,500 \\
$1 / 2$ & 11,700 & 2,600 & 10,500 & 2,330 \\
$5 / 8$ & 21,200 & 4,720 & 19,100 & 4,240 \\
$3 / 4$ & 30,600 & 6,800 & 27,600 & 6,130 \\
$7 / 8$ & 41,800 & 9,280 & 37,600 & 8,360 \\
1 & & 12,100 & 49,100 & 10,900 \\
$11 / 8$ & 64,500 & 15,400 & 62,400 & 13,900 \\
$11 / 4$ & 85,000 & 18,900 & 76,500 & 17,000 \\
$13 / 8$ & 102,000 & 22,700 & 91,800 & 20,400 \\
$11 / 2$ & 122,000 & 27,100 & 110,000 & 24,400 \\
\hline
\end{tabular}

Note.-The maximum safe working loads are the highest allowable under any conditions. Safety requires that most installations have a higher factor of safety, and therefore a lower working load.

Rule 4021-Construction.-One diameter of wire. one operation. Six strands of seven wires each. One main core. (Illustration same as for F. S. B. 6 by 7 extra strong cast steel) 
Rule 4022-Material.-Wires: Cast steel, either uncoated or galvanized. Main core, hard fiber.

Rule 4023-Diameter. - The difference between the nominal diameter and the actual diameter shall not be greater than the tolerances given in American Marine Standards Committee Specification O-25, given above.

Rule 4024-Lay. - The rope shall be regular lay, and the lay shall not be greater than seven and onehalf times the nominal diameter if the wires are uncoated steel, nor more than eight times the diameter if the wires are galvanized steel.

(The 6 by 7 extra strong cast steel rope in this specification requires that the lay shall not be greater than seven and one-half times the nominal diameter, instead of eight times as in the F. S. B. specification. Also this specification carries the breaking strengths and safe working loads of uncoated wire rope, instead of the galvanized rope requirements in F. S. B. Specification No. 297. These properties of the uncoated wire rope are as follows:)

TABLE 3.- Properties of 6 by 7 extra strong cast steel wire rope

\begin{tabular}{|c|r|r|}
\hline & \multicolumn{2}{|c|}{ Uncoated } \\
\cline { 2 - 3 } Nominal \\
\cline { 2 - 3 } & $\begin{array}{c}\text { Minimeter } \\
\text { breaking } \\
\text { strength }\end{array}$ & $\begin{array}{c}\text { Maximum } \\
\text { safe work- } \\
\text { ing load }\end{array}$ \\
\hline Inches & Pounds & Pounds \\
$1 / 2$ & 16,000 & 3,580 \\
$5 / 8$ & 25,000 & 5,560 \\
$3 / 4$ & 35,600 & 7,890 \\
$7 / 8$ & 47,800 & 10,600 \\
1 & 62,800 & 14,000 \\
$11 / 8$ & 78,900 & 17,600 \\
$11 / 4$ & 97,800 & 21,700 \\
$13 / 8$ & 119,000 & 26,400 \\
$11 / 2$ & 140,000 & 31,100 \\
\hline
\end{tabular}

NoTE.-The maximum safe working loads are the highest allowable under any conditions. Safety requires that most installations have a higher factor of safety, and therefore a lower working load.

Section 404.- 6 by 7 , plow-steel wire rope.

Rule 4040.-Wire rope, 6 by 7 , plow steel, shall be made as described here, and shall have the properties given in Table 4. (Illustration same as for F. S. B. No. 297,6 by 7 extra strong cast steel.)

TABLE 4.-Properties of 6 by 7 plow-steel wire rope

\begin{tabular}{|c|c|c|c|c|c|}
\hline \multirow{2}{*}{$\begin{array}{l}\text { Nominal } \\
\text { diameter }\end{array}$} & \multirow{2}{*}{$\begin{array}{l}\text { Approxi- } \\
\text { mate cir- } \\
\text { cumfer- } \\
\text { ence }\end{array}$} & \multirow{2}{*}{$\begin{array}{l}\text { Approxi- } \\
\text { mate } \\
\text { weight per } \\
\text { foot }\end{array}$} & \multicolumn{2}{|c|}{ Uncoated } & \multirow{2}{*}{$\begin{array}{l}\text { Recom- } \\
\text { mended } \\
\text { minimum } \\
\text { diameter } \\
\text { of sheave } \\
\text { or drum }\end{array}$} \\
\hline & & & $\begin{array}{l}\text { Minimum } \\
\text { breaking } \\
\text { strength }\end{array}$ & $\begin{array}{l}\text { Maximum } \\
\text { safe work- } \\
\text { ing load }\end{array}$ & \\
\hline $\begin{array}{r}\text { Inches } \\
1 / 2 \\
3 / 8 \\
3 / 4 \\
7 / 8 \\
1 \\
11 / 8 \\
11 / 4 \\
13 / 8 \\
11 / 2\end{array}$ & $\begin{array}{c}\text { Inches } \\
11 / 2 \\
2 \\
21 / 4 \\
23 / 4 \\
3 \\
31 / 2 \\
4 \\
41 / 4 \\
43 / 4\end{array}$ & $\begin{array}{r}\text { Pounds } \\
0.39 \\
.62 \\
.89 \\
1.20 \\
1.58 \\
2.03 \\
2.52 \\
3.05 \\
3.65\end{array}$ & $\begin{array}{r}\text { Pounds } \\
17,000 \\
27,200 \\
38,400 \\
51,800 \\
68,000 \\
86,500 \\
106,000 \\
128,000 \\
153,000\end{array}$ & $\begin{array}{r}\text { Pounds } \\
3,780 \\
6,020 \\
8,540 \\
11,500 \\
15,100 \\
19,300 \\
23,500 \\
28,400 \\
34,000\end{array}$ & $\begin{array}{r}\text { Feet } \\
3.8 \\
4.5 \\
5.5 \\
6.5 \\
7.2 \\
8.2 \\
9.0 \\
10.0 \\
11.0\end{array}$ \\
\hline
\end{tabular}

NотE.-The maximum safe working loads are the highest allowable under any conditions. Safety requires that most installations have a higher factor of safety, and therefore a lower working load.

Rule 4041-Construction.-One diameter of wire, one operation. Six strands of seven wires each. One main core.
Rule 4042-Material.-Wires: Plow steel, uncoated. Main core, hard fiber.

Rule 4043-Diameter.-(Same as in rule 4023, above.)

Rule 4044-Lay.-The rope shall be regular lay, and the lay shall not be greater than seven and onehalf times the nominal diameter.

Section $405 .-6$ by 19 , cast steel.

Rule 4050.-Wire rope, 6 by 19 , cast steel, shall be made as described here, and shall have the properties shown in Table 12 of F. S. B. No. 297, given below, with the following additional properties for galvanized rope:

TABLE 5.-Properties of 6 by 19 cast-steel wire rope

\begin{tabular}{|r|r|r|}
\hline & \multicolumn{2}{|c|}{ Gal vanized } \\
\cline { 2 - 3 } Nominal \\
diameter & $\begin{array}{c}\text { Minimum } \\
\text { breaking } \\
\text { strength }\end{array}$ & $\begin{array}{c}\text { Maximum } \\
\text { safe work- } \\
\text { ing load }\end{array}$ \\
& Pounds & Pounds \\
$3 / 8$ & 7,560 & 1,680 \\
$1 / 2$ & 13,500 & 3,000 \\
$3 / 8$ & 20,700 & 4,600 \\
$3 / 4$ & 28,400 & 6,310 \\
$1 / 8$ & 37,500 & 8,330 \\
1 & 48,600 & 10,800 \\
\hline
\end{tabular}

Note.-The maximum safe working loads are the highest allowable under any conditions. Safety requires that most installations have a higher factor of safety, and therefore a lower working load.

Rule 4051-Construction.-Each strand shall be fabricated in one operation of stranding. Six strands. Each strand shall have not less than 16 and not more than 31 wires. One main core.

Rule 4052-Material.-Wires: Cast steel, either uncoated or galvanized. Main core, hard fiber.

Rule 4053-Diameter.- (Same as in rule 4023, above.)

Rule 4054-Lay. - The rope shall be regular lay, and the lay shall not be greater than seven and onefourth times the nominal diameter, if the wires are uncoated steel, nor more than nine times the diameter if the wires are galvanized steel.

Section 406. - 6 by 19 extra strong cast steel.

Rule 4060.-Wire rope, 6 by 19 , extra strong cast steel shall be made as described here, and shall have the properties shown in Table 13 of F. S. B. No. 297 , given below, in respect to uncoated rope only.

Rule 4061-Construction.-Each strand shall be fabricated in one operation of stranding. Six strands. Each strand shall have not less than 16 and not more than 31 wires. One main core.

Rule 4062-Material.-Wires, extra strong cast steel, uncoated. Main core, hard fiber.

Rule 4063-Diameter--(Same as rule 4023, above.)

Rule 4064-Lay. - The rope shall be regular lay, and the lay shall not be greater than seven and onefourth times the nominal diameter.

Section $40 \%$-6 by 19 , plow steel.

(Properties are the same as for uncoated wire rope in Table 14 of F. S. B. No. 297, given below. Material, diameter, and lay are the same as for 6 by 19 extra strong cast stee', given above, except that plow steel takes the place of extra strong cast steel.)

Section $408 .-6$ by 37 , cast steel.

Rule 4080.-Wire rope, 6 by 37 , cast steel, shall be made as described here, and shall have the properties given in Table 6. (1llustration same as F. S. B. No. 297,6 by 37 extra strong cast steel, given bel $\mathrm{w}^{\mathrm{r}}$.) 
TABLE 6.-Properties of 6 by 37 cast-steel wire rope

\begin{tabular}{|c|c|c|c|c|c|}
\hline \multirow{2}{*}{$\begin{array}{l}\text { Nominal } \\
\text { diameter }\end{array}$} & \multirow{2}{*}{$\begin{array}{l}\text { Approxi- } \\
\text { mate cir- } \\
\text { cumfer- } \\
\text { ence }\end{array}$} & \multirow{2}{*}{$\begin{array}{l}\text { Approxi- } \\
\text { mate } \\
\text { weight per } \\
\text { foot }\end{array}$} & \multicolumn{2}{|c|}{ Uncoated } & \multirow{2}{*}{$\begin{array}{l}\text { Recom- } \\
\text { mended } \\
\text { minimum } \\
\text { diameter } \\
\text { of sheave } \\
\text { or drum }\end{array}$} \\
\hline & & & $\begin{array}{l}\text { Minimum } \\
\text { breaking } \\
\text { strength }\end{array}$ & $\begin{array}{c}\text { Maximum } \\
\text { safe work- } \\
\text { ing load }\end{array}$ & \\
\hline $\begin{array}{c}\text { Inches } \\
1 / 2 \\
5 / 8 \\
3 / 4 \\
1 / 8 \\
1\end{array}$ & $\begin{array}{c}\text { Inches } \\
11 / 2 \\
2 \\
21 / 4 \\
23 / 4 \\
3\end{array}$ & $\begin{array}{r}\text { Pounds } \\
0.39 \\
.62 \\
.89 \\
1.20 \\
1.58\end{array}$ & $\begin{array}{r}\text { Pounds } \\
12,400 \\
19,300 \\
28,000 \\
38,000 \\
49,500\end{array}$ & $\begin{array}{r}\text { Pounds } \\
2,750 \\
4,290 \\
6,220 \\
8,440 \\
11,000\end{array}$ & $\begin{array}{l}\text { Feet } \\
1.2 \\
1.5 \\
1.9 \\
2.1 \\
2.5\end{array}$ \\
\hline $\begin{array}{l}11 / 8 \\
11 / 4 \\
13 / 8 \\
11 / 2 \\
15 / 8\end{array}$ & $\begin{array}{l}31 / 2 \\
4 \\
41 / 4 \\
43 / 4 \\
5\end{array}$ & $\begin{array}{l}2.00 \\
2.45 \\
2.95 \\
3.55 \\
4.15\end{array}$ & $\begin{array}{r}62,800 \\
77,600 \\
94,000 \\
112,000 \\
132,000\end{array}$ & $\begin{array}{l}14,000 \\
17,200 \\
20,900 \\
26,600 \\
29,300\end{array}$ & $\begin{array}{l}2.7 \\
3.1 \\
3.3 \\
3.7 \\
3.9\end{array}$ \\
\hline
\end{tabular}

NotE.-The maximum safe working loads are the highest allow. able under any conditions. Safety requires that most installation have a higher factor of safety, and therefore a lower working load.

Rule 4081-Construction.-Each strand shall be fabricated in either one or two operations of stranding. Six strands. Each strand shall have not less than 29 and not more than 46 wires. One main core.

Rule 4082-Material.-Wires: Cast steel, uncoated. Main core, hard fiber.

Rule 4083-Diameter.-(Same as rule 4023, above.)

Rule 4084-Lay.-(Same as rule 4064, above.)

Section $409 .-6$ by 37 , extra strong, cast steel.

(Properties are the same as for uncoated wire rope in Table 19 of F. S. B. No. 297, given below. Illustration same as for F. S. B. 6 by 37 extra strong cast steel, given below. Diameter and lay are the same as in rule 4023 and rule 4064, respectively, given above. Construction same as rule 4081, above. Material of extra strong cast steel, uncoated. Main core, hard fiber.)

Section $410 .-6$ by 37 , plow steel.

Rule 4100.-Wire rope, 6 by 37 , plow steel, shall be made as described here, and shall have the properties given in Table 7 . (Illustration same as for F. S. B. 6 by 37 high-grade plow steel, given below.)

TABLE 7.-Properties of 6 by 37 plow-steel wire rope

\begin{tabular}{|c|c|c|c|c|c|}
\hline \multirow{2}{*}{$\begin{array}{l}\text { Nominal } \\
\text { diameter }\end{array}$} & \multirow{2}{*}{$\begin{array}{l}\text { Approxi- } \\
\text { mate cir- } \\
\text { cumfer- } \\
\text { ence }\end{array}$} & \multirow{2}{*}{$\begin{array}{l}\text { Approxi- } \\
\text { mate } \\
\text { weight per } \\
\text { foot }\end{array}$} & \multicolumn{2}{|c|}{ Uncoated } & \multirow{2}{*}{$\begin{array}{l}\text { Recom- } \\
\text { mended } \\
\text { minimum } \\
\text { diameter } \\
\text { of sheave } \\
\text { or drum }\end{array}$} \\
\hline & & & $\begin{array}{l}\text { Minimum } \\
\text { breaking } \\
\text { strength }\end{array}$ & $\begin{array}{l}\text { Maximum } \\
\text { safe work- } \\
\text { ing load }\end{array}$ & \\
\hline $\begin{array}{c}\text { Inches } \\
1 / 2 \\
5 / 8 \\
3 / 4 \\
7 / 8 \\
1 \\
11 / 8 \\
11 / 4 \\
13 / 8 \\
11 / 2 \\
15 / 8 \\
13 / 4 \\
17 / 8 \\
2\end{array}$ & $\begin{array}{c}\text { Inches } \\
11 / 2 \\
2 \\
21 / 4 \\
23 / 4 \\
3 \\
31 / 2 \\
4 \\
41 / 4 \\
43 / 4 \\
5 \\
51 / 2 \\
53 / 4 \\
61 / 4\end{array}$ & $\begin{array}{c}\text { Pounds } \\
0.39 \\
.62 \\
.89 \\
1.20 \\
1.58 \\
2.00 \\
2.45 \\
2.95 \\
3.55 \\
4.15 \\
4.85 \\
5.50 \\
6.30\end{array}$ & $\begin{array}{r}\text { Pounds } \\
15,600 \\
24,400 \\
35,400 \\
47,800 \\
63,000 \\
79,200 \\
98,300 \\
119,000 \\
142,000 \\
157,000 \\
194,000 \\
222,000 \\
252,000\end{array}$ & $\begin{array}{c}\text { Pounds } \\
3,470 \\
5,420 \\
7,860 \\
10,600 \\
14,000 \\
17,600 \\
21,900 \\
26,400 \\
31,500 \\
37,100 \\
43,200 \\
49,400 \\
56,000\end{array}$ & $\begin{array}{l}\text { Feet } \\
1.2 \\
1.5 \\
1.9 \\
2.1 \\
2.5 \\
2.7 \\
3.1 \\
3.3 \\
3.7 \\
3.9 \\
4.3 \\
4.5 \\
4.8\end{array}$ \\
\hline
\end{tabular}

NotE.-The maximum safe working loads are the highest allowable under any conditions. Safety requires that most installations have a higher factor of safety, and therefore a lower working load.

(Construction same as rule 4081, above. Diameter same as rule 4023 above. Lay same as rule 4064 above. Material of plow steel, uncoated. Main core, hard fiber.)

Section $411 .-8$ by 19 , cast steel.

Rule 4110.-Wire rope, 8 by 19 , cast steel.

(Properties and material are the same as for $8 \mathrm{by}$ 19 cast-steel rope in F. S. B. No. 297, given below. Diameter and lay are the same as in rule 4023 and rule 4064, respectively, given above. Construction is as follows:)

Rule 4111-Construction.-Each strand shall be fabricated in one operation of stranding. Eight strands. Each strand shall have not less than 16 and not more than 31 wires. One main core.

Section 412. - 8 by 19 extra strong cast steel.

(Properties and material are the same as for 8 by 19 extra strong cast steel rope in F. S. B. No. 297, given below. Construction is the same as in rule 4111 , above. Diameter and lay are the same as in rule 4023 and rule 4064, respectively, given above.

Section $413 .-8$ by 19 , plow steel.

Rule 4130.-Wire rope, 8 by 19 , plow steel, shall be made as described here and shall have the properties given in Table 8.

TABLE 8.-Properties of 8 by 19 plow-steel wire rope

\begin{tabular}{|c|c|c|c|c|c|}
\hline \multirow{2}{*}{$\begin{array}{l}\text { Nominal } \\
\text { diameter }\end{array}$} & \multirow{2}{*}{$\begin{array}{l}\text { Approxi- } \\
\text { mate cir- } \\
\text { cumfer- } \\
\text { ence }\end{array}$} & \multirow{2}{*}{$\begin{array}{l}\text { Approxi- } \\
\text { mate } \\
\text { weight per } \\
\text { foot }\end{array}$} & \multicolumn{2}{|c|}{ Uncoated } & \multirow{2}{*}{$\begin{array}{l}\text { Recom- } \\
\text { mended } \\
\text { minimum } \\
\text { diameter } \\
\text { of sheave } \\
\text { or drum }\end{array}$} \\
\hline & & & $\begin{array}{l}\text { Minimum } \\
\text { breaking } \\
\text { strength }\end{array}$ & $\begin{array}{l}\text { Maximum } \\
\text { safe work- } \\
\text { ing load }\end{array}$ & \\
\hline $\begin{array}{c}\text { Inches } \\
1 / 2 \\
5 / 8 \\
33 / 4 \\
7 / 8 \\
1 \\
11 / 8 \\
11 / 4 \\
13 / 8 \\
11 / 2\end{array}$ & $\begin{array}{l}\text { Inches } \\
11 / 2 \\
2 \\
21 / 4 \\
23 / 4 \\
3 \\
31 / 2 \\
41 / 4 \\
41 / 4 \\
43 / 4\end{array}$ & $\begin{array}{c}\text { Pounds } \\
0.35 \\
.55 \\
.80 \\
1.08 \\
1.42 \\
1.80 \\
2.20 \\
2.67 \\
3.19\end{array}$ & $\begin{array}{r}\text { Pounds } \\
14,800 \\
23,200 \\
33,800 \\
45,700 \\
60,000 \\
75,700 \\
93,700 \\
114,000 \\
135,000\end{array}$ & $\begin{array}{c}\text { Pounds } \\
3,290 \\
5,160 \\
7,520 \\
10,200 \\
13,400 \\
16,800 \\
20,800 \\
25,300 \\
30,000\end{array}$ & $\begin{array}{l}\text { Feet } \\
1.3 \\
1.6 \\
1.9 \\
2.2 \\
2.5 \\
2.8 \\
3.1 \\
3.5 \\
3.7\end{array}$ \\
\hline
\end{tabular}

NotE.-The maximum safe working loads are the highest allowable under any conditions. Safety requires that most installations have a higher factor of safety, and therefore a lower working load.

Rule 4132-Material.-Wires: Plow steel, uncoated. Main core, hard fiber.

(Construction is the same as rule 4111 above. Diameter and lay are the same as in rule 4023 and rule 4064, respectively, given above. Illustrations showing the correct and incorrect way of measuring the diameter of wire rope are the same as in the specification of the American Petroleum Institute, given below.)

\section{AMERICAN PETROLEUM INSTITUTE, SPECIFICATIONS FOR WIRE ROPE, STANDARD NO. 9-A (8TH ED.), JULY, 1931 \\ WIRE ROPE}

\section{SECTION I}

Material Covered

1. (a) These specifications apply to wire rope for oil country use, and include the following: Cable tool drilling lines, cable tool casing lines, sucker rod and tubing lines, sand lines, rotary drilling or casing lines, and rotary bailing lines.

(b) Specifications for the following lines are covered in Section VI.

Galvanized wire guy strands.-1. (c) Specifications to be added later for torpedo lines and wellmeasuring tapes. 
SECTION II

Grades of Material

2. (a) Grade $\mathbf{J}$ shall refer to a good grade acid or basic open-hearth steel of the physical properties hereinafter specified. (Otherwise called "plow steel.")

(b) Grade L shall refer to a good grade of acid or basic open-hearth steel of the physical properties hereinafter specified. (Otherwise called "extra strong cast steel.")

(c) Grade $\mathrm{N}$ shall refer to an acid or basic open-hearth steel of the physical properties hereinafter specified. (Otherwise called "cast steel.")

$(d)$ Lines composed of combination of two grades shall be permitted: The first letter referring to grade of outside wires, the second letter to grade of inside wires, each of which must pass the physical characteristics of the respective grades. For example: Grade NJ would mean that all outside wires are to be made of grade $\mathrm{N}$ and all inside wires to be of grade $J$.

\section{SECTION III}

\section{Properties and Tests for Wire}

3. Selection of wires to be tested.-(a) For tests of the wire in a rope a section shall be cut from a finished piece of unused and undamaged wire rope, samples to be taken within 30 days of actual delivery of the rope.

(b) From each strand there shall be selected and tested certain wires as follows: The total number to be tested shall be equal to the number of wires in any one strand. They shall be selected from all strands of the rope. The specimens shall be selected from all locations or positions so that they would constitute a complete composite strand exactly similar to a regular strand in the rope. The specimen for all like positioned wires to be selected so as to use as nearly as possible an equal number from each strand.

Note.-Whenever "like positioned" wires are mentioned it will be understood to mean wires symmetrically placed in a strand. For instance, in Warrington all the outside wires are not necessarily "like positioned," since in the outside layer arc placed alternately large and small wires. All large wires are "like positioned" with respect to each other and all small wires are "like positioned" with respect to each other.

(c) Any unsymmetrically placed wires or marker wires are to be disregarded entirely. Annealed heart wires not to be considered in averages, but must have a minimum tensile strength of 50,000 pounds per square inch; must stand a test of at least 60 twists as described under "Torsion test" and a Bending test as described under paragraph 7, "Bend test of wires."

(d) Each specimen except those for tension tests shall be carefully hand straightened.

(e) The tests shall be so run and records kept in such a manner that the results of each of the various tests on any one specimen are associated and may be studied separately from other specimens.

(f) In case of controversy the purchaser may at his expense test all of the wires.

(g) If the first specimen fails to pass the minimum requirement for individual wires, two more specimens shall be tested from the same wire, both of which must comply with the requirements for the wire. The average of the two tests showing either failure or acceptance of the three made shall be used as the value to represent the wire.

(h) In torsion tests the additional tests may be made at a reduced rate of speed, not exceeding 30 twists per minute.

(i) In bending tests the additional tests may be made at a reduced rate of speed, not exceeding 15 bends per minute.
4. Tensile strength of wire-(a) Specimens shall not be less than 18 inches long, and the distance between the grips of the testing machine shall not be less than 12 inches. The speed of the movable head of the testing machine, under no load, shall not exceed one inch per minute. Any specimen breaking within one-fourth inch from the jaws shall be disregarded and a retest made.

NotE.-It has been found that the diameter of wire can more easily and accurately be determined by placing the wire specimen in the test machine and applying a load not over 25 per cent of the breaking strength of the wire.)

(b) The tensile strength of the various grades shall be within the value shown in Table 1 .

TABLE 1.-Tensile strength

[Pounds per square inch]

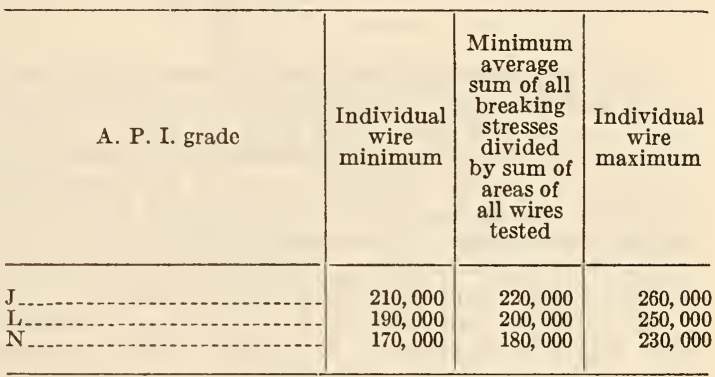

5. Elongation.-(a) The ultimate elongation shall be measured when making the tensile tests of wire. The ultimate elongation, measured on a 10-inch length of specimen, at instant of rupture, which must occur within the 10-inch length, shall not be less than the values given in Table 2 for grades $J$. L, and N. When determining elongation a stress shall be imposed upon the wire equal for grades $\mathrm{L}$ and $\mathrm{N}$, to 75,000 pounds per square inch, at which point the extensometer is applied. Direct to the reading of the extensometer shall be added 0.3 per cent to allow for the initial elongation occuring before application of the extensometer. For grade $J$ and stronger 100,000 pounds per square inch shall be applied; the additive elongation to be 0.4 per cent.

TABLE 2.-Elongation

\begin{tabular}{|c|c|}
\hline Size of wire & $\begin{array}{l}\text { Minimum } \\
\text { elonga- } \\
\text { tion }\end{array}$ \\
\hline $\begin{array}{l}\text { Wire } 0.07 \text { inch diameter and larger } \\
\text { Wire less than } 0.070 \text { to } 0.040 \text { inch diameter } \\
\text { Wire less than } 0.040 \text { to } 0.020 \text { inch diameter } \\
\text { Wire less than } 0.020 \text { inch diameter }\end{array}$ & $\begin{array}{c}\text { Per cent } \\
2 \\
13,4 \\
11 / 2 \\
1\end{array}$ \\
\hline
\end{tabular}

6. Torsion tests of wire--(a) The distance between the jaws of the testing machine shall be 8 inches, plus or minus one-sixteenth inch when the wire is straight. One end of the wire is to be rotated with respect to the other end of the wire at a continuous, uniform speed until breakage occurs. During the test the applied tension shall not exceed 5 per cent of its breaking strength as measured in the tensile test. The speed of rotation shall not exceed 60 twists per minute. A distance on the untwisted wire equal to one hundred times the diameter is to be taken as the unit of test length and the wire under test must withstand the number of twists given in Table 3 computed on this unit of length. One clamp of the torsion machine shall 
be movable in a direction parallel to the axis of the wire. The wire shall be twisted by rotating one or both clamps at a uniform rate not exceeding a sum of 60 twists per minute. Tests in which breakage occurs within one-eighth inch of jaw shall be discarded.

\section{TABLE 3.-Torsion test}

[Minimum number of twists of $360^{\circ}$ for individual wires in a length equal to one hundred times the wire diameter]

\begin{tabular}{|c|c|}
\hline A. P. I. grade & $\begin{array}{c}\text { Number of } \\
\text { twists for } \\
\text { individual } \\
\text { wires }\end{array}$ \\
\hline J & $271 / 2$ \\
\hline N. & 30 \\
N. & $321 / 2$ \\
\hline
\end{tabular}

(b) If when making the specified test of wires any wires fail to meet the torsion-test requirement, all the wires in the rope must then be tested and 95 per cent of all such wires must meet the requirement for torsion herein specified, none of the remaining 5 per cent to be less than 60 per cent of the torsion values herein given. (See par. $3(h)$.)

(c) The torsion-testing machine for rope wire testing shall have following characteristics:

(d) One jaw fixed axially, and the other jaw movable axially and arranged to permit tension weights to be applied to wire under test.

(e) The jaws to be capable of holding wire firmly without slippage. Machine must be equipped with automatic counter to record the number of twists. Control of speed so that it can be uniform can best be had with a power-operated machine, and this is recommended as standard.

$(f)$ Weights to be applied shall be as shown in Table 4.

TABLE 4.-Tension weights for torsion tests

\begin{tabular}{|c|c|}
\hline Size wire & $\begin{array}{c}\text { Applied } \\
\text { weights }\end{array}$ \\
\hline Inch & $\begin{array}{c}\text { Pounds } \\
0.011-0.016\end{array}$ \\
$.017-.020$ & 2 \\
$.021-.030$ & 4 \\
$.031-.040$ & 6 \\
$.041-.050$ & 8 \\
$.051-.060$ & 9 \\
$.061-.070$ & 11 \\
$.071-.080$ & 13 \\
$.081-.090$ & 16 \\
$.091-.100$ & 19 \\
$.101-.110$ & 21 \\
\hline
\end{tabular}

7. Bend test of wires. ${ }^{47}-(a)$ The machine shall be so designed that the mandrels touch the wire and maintain it in a symmetrical position with respect to the axis for all sizes of wire under test. The center of rocation for bend test shall fall on the center line of the axis of the wire and directly in line with the point of tangency of the mandrel with the wire. Bends shown in Table 5 shall be at a speed of between 30 and 40 bends per minute. At

47 Because of the difficulties experienced by operators in bending hand-straightened wire, the committee recommends that no rope be condemned by the bend test, pending further investigations. a greater speed values will be reduced. (See par 3 (i).) Each bend will be understood to mean bending the wire at right angles and returning to the central position. The machine should be arranged so that it can be driven with power to secure uniformity of speed.

(b) The distance between top of jaw or mandrel and lower edge of guide not to exceed 2 inches.

(c) The wire must stand the bend test shown in Table 5 before fracture.

\section{TABLE 5.-Bend test on rope wire}

\begin{tabular}{|c|c|c|}
\hline & $\begin{array}{c}\text { Number of bends } \\
\text { over radius equal } \\
\text { to six times } \\
\text { diameter of wire }\end{array}$ \\
\cline { 2 - 3 } & Grades L & Grade J \\
and N & \\
\hline Inch & & \\
$.031-0.050$ & 50 & 45 \\
$.071-.070$ & 47 & 43 \\
$.091-.090$ & 43 & 39 \\
\hline
\end{tabular}

(d) Mandrels for bend test shall be accurate to correspond to the values given in Table 6 .

\section{TABLE 6.-Mandrel sizes for bend test}

\begin{tabular}{|c|c|}
\hline Wire size & $\begin{array}{c}\text { Mandrel } \\
\text { radius }\end{array}$ \\
\hline Inch & Inch \\
$0.031-0.040$ & 0.210 \\
$.041-.050$ & .270 \\
$.051-.060$ & .330 \\
$.061-.070$ & .390 \\
$.071-.080$ & .450 \\
$.051-.090$ & .510 \\
$.091-.100$ & .570 \\
$.101-.110$ & .630 \\
\hline
\end{tabular}

(e) The bend test not to apply to wire 0.030 inch diameter and smaller.

8. Strength of ropes: Testing full-rope size specimens.-(a) Minimum ultimate strength of ropes shall be as specified in Tables 7 and 8 . When testing finished ropes to their ultimate strength, suitable sockets shall be attached by the method described under the beading "Directions for attaching sockets to wire rope," as contained in Appendix D.

(b) The length of test specimen shall not be less than 3 feet between sockets for ropes up to 1 -inch diameter, inclusive, and for ropes $11 / 8$ inches diameter and larger the minimum length between testing sockets shall be 5 feet.

(c) If the first specimen fails at a value below the specified ultimate strength, a second test shall be made, and if the second test meets the strength requirements the wire rope shall be accepted.

(d) The machine used for the finished rope test shall not have a capacity of more than twenty times the strength of the rope being tested. When testing, it is recommended that oversize wire rope sockets be used; that is a socket for a rope $1 / 4$-inch larger in diameter than the rope under test. The counterpoise used on such a machine shall not be in excess of five times the strength of the rope being tested. 
TABLE 7.-Ultimate strength of 6 by 19 ropes

\begin{tabular}{|c|c|c|c|c|}
\hline $\begin{array}{c}\text { Nominal } \\
\text { diameter } \\
\text { of rope }\end{array}$ & $\begin{array}{c}\text { Approx- } \\
\text { imate } \\
\text { weight }\end{array}$ & Grade N & Grade L & Grade J \\
\hline $\begin{array}{c}\text { Inches } \\
36 \\
7 / 16 \\
16 \\
916\end{array}$ & $\begin{array}{c}\text { Pounds } \\
\text { per foot } \\
0.23 \\
.31 \\
.40 \\
.51\end{array}$ & $\begin{array}{c}\text { Pounds } \\
9,000 \\
12,000 \\
15,400 \\
19,200\end{array}$ & $\begin{array}{c}\text { Pounds } \\
10,000 \\
13,200 \\
17,000 \\
21,200\end{array}$ & $\begin{array}{c}\text { Pounds } \\
11,000 \\
14,600 \\
18,800 \\
23,400\end{array}$ \\
\hline $\begin{array}{l}5 / 8 \\
3 / 4 \\
7 / 8 \\
1^{7 / 8}\end{array}$ & $\begin{array}{r}.63 \\
.90 \\
1.23 \\
1.60\end{array}$ & $\begin{array}{l}23,600 \\
33,600 \\
45,600 \\
59,000\end{array}$ & $\begin{array}{l}26,200 \\
37,400 \\
50,800 \\
66,000\end{array}$ & $\begin{array}{l}28,800 \\
41,200 \\
56,000 \\
73,000\end{array}$ \\
\hline $\begin{array}{l}11 / 8 \\
11 / 4 \\
138 \\
116\end{array}$ & $\begin{array}{l}2.03 \\
2.50 \\
3.03 \\
3.60\end{array}$ & $\begin{array}{r}74,000 \\
92,000 \\
110,000 \\
130,000\end{array}$ & $\begin{array}{r}83,000 \\
102,000 \\
123,000 \\
145,000\end{array}$ & $\begin{array}{r}92,000 \\
113,000 \\
136,000 \\
161,000\end{array}$ \\
\hline
\end{tabular}

TABLE 8.-Ultimate strength of 6 by 7 ropes

\begin{tabular}{|c|c|c|c|c|}
\hline $\begin{array}{c}\text { Nominal } \\
\text { diameter } \\
\text { of rope }\end{array}$ & $\begin{array}{l}\text { Approx- } \\
\text { imate } \\
\text { weight }\end{array}$ & Grade $N$ & Grade L & Grade J \\
\hline $\begin{array}{c}\text { Inch } \\
3 / 8 \\
7 / 16 \\
1 / 2 \\
9 / 16 \\
5 / 8 \\
3 / 4 \\
7 / 8 \\
1\end{array}$ & $\begin{array}{c}\text { Pounds } \\
\text { per foot } \\
0.21 \\
.29 \\
.38 \\
.48 \\
.59 \\
.84 \\
1.15 \\
1.50\end{array}$ & $\begin{array}{c}\text { Pounds } \\
8,600 \\
11,600 \\
15,000 \\
18,800 \\
23,000 \\
33,000 \\
44,800 \\
58,000\end{array}$ & $\begin{array}{c}\text { Pounds } \\
9,400 \\
12,600 \\
16,400 \\
20,600 \\
25,200 \\
36,200 \\
49,200 \\
63,800\end{array}$ & $\begin{array}{c}\text { Pounds } \\
10,300 \\
13,800 \\
18,000 \\
22,600 \\
27,600 \\
39,600 \\
53,600 \\
69,600\end{array}$ \\
\hline
\end{tabular}

\section{SECTION IV}

\section{Manufacture and Tolerances}

9. Strand construction.-(a) Strands for 6 by 19 construction ropes shall have not less than 16 wires and not more than 25 wires per strand. Individual strands shall be fabricated in one operation of stranding, as stipulated in Federal Specifications Board specification for wire rope No. 297 (current designation: RR-R-571) given below.

(b) Strands for 6 by 7 and 6 by 8 construction ropes shall contain the number of wires as specified by the trade name; namely, 7 wires per strand for the 6 by 7 construction, and 8 wires per strand for the 6 by 8 construction.

10. Joints in wire.-(a) In the manufacture of wire ropes, wire shall be made continuous by brazing or electric welding, and no two joints in any one strand shall be closer than 25 feet to each other. Joints shall be made so they will withstand a strength of at least 50 per cent of the original strength of the wire.

11. Direction of lay--(a) Standard right-lay rope will be furnished unless specified to the contrary by the purchaser.

12. Length of lay.-(a) For 6 by 7 and 6 by 8 drilling lines, also for 6 by 7 ropes used for sand lines, the lay of the finished rope shall not exceed eight times the diameter of the rope.

(b) For 6-strand ropes made of strands containing 16 to 25 wires, the lay shall not be more than seven and one-fourth times the nominal diameter.

13. Diameter of ropes-Tolerance limits.-(a) The diameter of a wire rope shall be measured as least 5 feet from end with a suitable measuring device, with at least 3 inches length of jaw. Figure 57 illustrates the correct and incorrect methods of measuring the diameter of wire rope when using a machinist's caliper.
MEASUREMENT OF DIAMETER

(b) Figure 58 illustrates an approved type of caliper for measuring the diameter of wire rope.
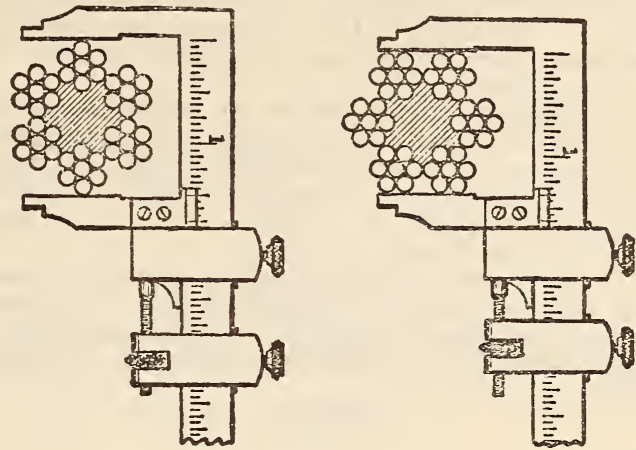

Figure 57.-Correct and incorrect way to measure diameter of wire rope

Use a machinist's caliper square.

(c) The diameter of a wire rope is the diameter of the circumscribed circle. The amount which the actual diameter of a rope differs from the nominal diameter shall not be greater than the values in Table 9 .

\section{TABLE 9.-Tolerance on rope diameters}

\begin{tabular}{|c|c|c|}
\hline $\begin{array}{c}\text { Nominal } \\
\text { diameter } \\
\text { of rope }\end{array}$ & $\begin{array}{c}\text { Under- } \\
\text { size }\end{array}$ & $\begin{array}{c}\text { Over- } \\
\text { size }\end{array}$ \\
\hline Inches & Inch & Inch \\
$0-3 / 4$ & 0 & $3 / 64$ \\
$13 / 16-11 / 8$ & 0 & $1 / 16$ \\
$13 / 16-11 / 2$ & 0 & 564 \\
\hline
\end{tabular}

14. Diameter of wire-Tolerance limits.-(a) In separating the wire rope for gaging of wire, care must be taken to separate the various sizes of wire

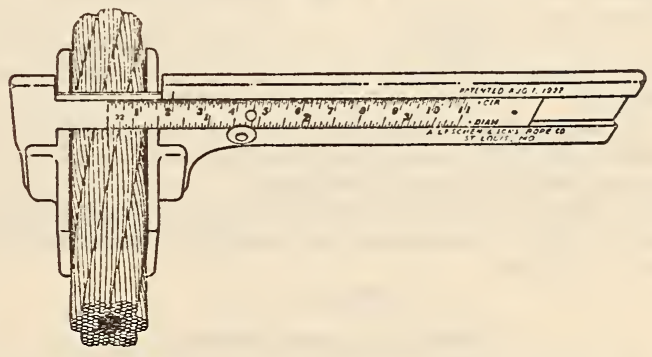

Figure 58.-Wide faced wire line calipers

composing the different layers of wires in strand. In like-positioned wires total variations of wire diameters shall not exceed:

\begin{tabular}{|c|c|}
\hline Wire size & $\begin{array}{c}\text { Total } \\
\text { variation }\end{array}$ \\
\hline S.064 and larger & $\begin{array}{r}\text { Inch } \\
0.004 \\
.003\end{array}$ \\
\hline
\end{tabular}


15. Fiber cores.-(a) For all wire ropes manufactured under this specification, all fiber cores will be of hard-twisted, best-quality Manila or Java hemp, or its equivalent. They shall be of uniform diameter and hardness, and effectively support tne strands. They snall be thorougnly impregnated with a suitable lubricating compound, free from acid. No jute cores shall be used.

16. Lubrication. - (a) All wire ropes, unless otherwise specified, shall be lubricated and impregnated in the manufacturing process with suitable compound thoroughly to protect the ropes both internally and externally, to minimize rust or ccrrosion, until the rope is put into service.

17. Workmanship.- (a) The rope shall be made from the best quality of specified grade of material, shall be of good workmanship, and shall be free from defects which might affect its appearance or serviceability.

\section{SECTION $\nabla$}

Type of Lines

18. (a) Cable-tool drilling lines shall be $3 / 4,7 / 8,1$, and $1 \frac{1}{1} 8$ inches in diameter, lengths to vary in multiples of 500 feet.

(b) Strand fabrication may be 6 by 7,6 by 8 , or 6 by 19 of either Seale, Warrington, or filler-wire construction. However, the arrangement of the different-size wires must be uniform and according to a regular symmetrical plan throughout.

(c) Warrington construction is to be made in one operation, consisting of 6 strands of 19 wires to the strand; the outside of each strand shall be of large and small diameter wires, laid alternately. There are three diameters of wire in the strand.

(d) Seale construction is to be made in one operation, consisting of 6 strands of 19 wires to the strand; the outside wires are to be 9 in number of one diameter around 9 smaller wires; the center wire to be of a larger diameter. There are three diameters of wire in the strand.

(e) Filler-wire construction is to be made in one operation consisting of 6 strands of 19 wires to the strand. All the wires except the center wire to be of approximately the same size; the center wire to be slightly larger. The filler wires are used to support the structure and are therefore not considered as component wires in the rope so far as strength is concerned.

$(f)$ Rotary drilling lines or casing lines shall be $3 / 4,7 / 8,1$, and $1 \frac{1}{8}$ inches, length to vary in multiples of 250 feet.

(g) Sand lines shall be 6 by 7 construction and shall be $3 / 8,1 / 2,9 / 16$, and $5 / 8$ inch in diameter. Lengths to vary in multiples of 500 feet.

(h) Rotary bailing lines shall be $9 / 16,5 / 8$, and $3 / 4$ inch, shall be 6 by 19 construction, lengths to vary in 500foot multiples.

\section{SECTION VI}

\section{Galvanized-Wire Guy Strands}

19. Single galvanized-wire guy strand.-(a) This shall consist of a strand made up of seven twisted galvanized wires of the following minimum breaking stresses per strand:

TABLE 10

\begin{tabular}{|c|c|}
\hline Size & $\begin{array}{c}\text { Minimum } \\
\text { breaking } \\
\text { stresses }\end{array}$ \\
\hline Inch & Lbs./in 2 \\
$1 / 4$ & 1,900 \\
$5 / 16$ & 3,200 \\
$3 / 8$ & 4,250 \\
$7 / 16$ & 5,700 \\
$1 / 2$ & 7,400 \\
\hline
\end{tabular}

(b) No test of galvanizing shall be applied to single guy wire strand.

20. Double or extra galvanized wire guy strand.(a) This shall consist of a strand made up of seven twisted galvanized wires of the following mininum breaking stresses per strand:

TABLE 11

\begin{tabular}{|c|c|}
\hline Size & $\begin{array}{c}\text { Minimum } \\
\text { breaking } \\
\text { stresses }\end{array}$ \\
\cline { 1 - 2 } Inch & Lbs./in. \\
$1 / 4$ & 1,900 \\
$5 / 16$ & 3,200 \\
$3 / 8$ & 4,250 \\
$7 / 16$ & 5,700 \\
$1 / 2$ & 7,400 \\
\hline
\end{tabular}

(b) Galvanized wire composing the above strand shall be uniformly and continuously coated with zinc which adheres firmly to the wire. The zinc shall be applied in a molten condition by what is designated commercially as "hot-process galvanizing." Using the Preece test, the wire shall not show the appearance of bright, adherent copper, indicating exposed iron, for the number of immersions shown in the following table:

Wires taken from 14 inch strand-

Wires taken from strands $5 / 16$ to $1 / 2$ inch diameter, inclusive. 4

21. Test of galvanizing.-(Conforms to specification of American Marine Standards Committee, given above.)

\section{SECTION VII \\ Packing and Marking}

22. Reels.-(a) Finished wire rope, when so required, shall be shipped in round head, substantial, wooden reels; reels shall preferably have a round arbor hole of 5 inches in diameter for drilling lines, casing lines and sand lines. When reel is full of rope, there shall be a clearance of not less than 2 inches between the full reel and the outside diameter of the flange.

(b) The manufacturer shall properly serve the outer end of rope so that the required 10 -foot sample can be cut out for preservation by the purchaser for test purposes, as described in paragraph $24(c)$.

23. Marking of reels.-(a) The following data shall be plainly marked on the face of the reel on which is wound wire rope meeting the A. P. I. specifications:

(1) Name of manufacturer.

(2) Reel number.

(3) A. P. I. monogram.

(Size of monogram to be in proportion to size of reel, with a minimum height of 2 inches and a maximum height of 4 inches.)

(4) Grade (J, L, N, or NJ, etc.)

(5) Diameter of line.

(6) Length of line.

(7) Type of construction (Warrington, Seale, or filler wire.)

(8) Type of core (fiber, wire).

(b) Wire rope which does not comply with these specifications shall not be wound on reels carrying the A. P. I. monogram.

(c) Authority to use the A. P. I. monogram will be granted in accordance with the rules and regulations contained in an appendix.

\section{SECTION VIII}

\section{Inspection and Rejection}

24. Inspection.-(a) Manufacturer will, on request of purchaser, conduct tests as called for in this specification on reasonable notice from the purchaser; 
during which tests the manufacturer will afford opportunity to the purchaser's representative to be present.

(b) A manufacturer delivering wire rope bearing the A. P. I. monogram and grade designation warrants that such material complies with these specifications. No rejections under these or any other specifications are to be wound on reels bearing the A. P. I. monogram, or sold as A. P. I. wire rope. When wire rope wound on reels bearing the A.P. I. monogram is rejected, it shall be removed from said reel; or the A. P. I. monogram on said reel shall be canceled by the painting or stenciling of two horizontal and two vertical lines through the body of the monogram.

(c) It is recommended that whenever possible the purchaser upon receipt shall test all new rope purchased in accordance with these specifications. It is realized after a rope is put in use and it fails to render satisfactory service that it is impracticable to retest such used rope. It is therefore required that the purchaser shall preserve at least one test specimen of all new rope purchased; length of specimen to be at least 10 feet, properly identified by reel number, etc. Care must be taken that no damage will result by storage of specimen.

$(d)$ If a purchaser receiving rope purchased on these specifications does not make physical test before putting rope into use, or does not preserve in proper manner the sample as outlined, recourse on the manufacturer shall be considered waived.

(Appendixes attached to this specification cover recommended practice for the use and preservation of wire rope, suggested improvements in design of oil-field equipment to lengthen life of wire rope, field troubles and their causes, and directions for attaching sockets to wire ropes.)

\section{AMERICAN RAILWAY ASSOCIATION, SIG- NAL SECTION, GALVANIZED MESSEN- GER WIRE, SPECIFICATION 4714, 1914}

\section{GALVANIZED MESSENGER WIRE}

\section{GENERAL}

(a) The intent of this specification is to provide for the furnishing of galvanized messenger wire in strand form.

(b) The strand shall be composed of seven galvanized wires twisted together to the specified lay.

(c) The wires composing the strand shall be cylindrical in form, free from scales, flaws, inequalities, splits, and all imperfections. Each length shall contain no weld joint or splice. All the wires of a strand shall be of the same grade of steel and shall have the same breaking strength.

$(d)$ The galvanizing shall consist of a continuous coating of pure zinc of uniform thickness so applied that it adheres firmly to the wire and presents a smooth surface.

\section{PROPERTIES}

(a) The mechanical properties of the finished wire shall be in accordance with the following requirements:

Wire

\begin{tabular}{|c|c|c|}
\hline $\begin{array}{c}\text { Diam- } \\
\text { eter } \\
\text { of } \\
\text { strand }\end{array}$ & Diameter & Area \\
\hline & & \\
\hline Inch & Inch & $\begin{array}{c}\text { Square } \\
\text { inch } \\
1 / 2\end{array}$ \\
$7 / 16$ & 0.162 & 0.0206 \\
$3 / 8$ & .144 & .0163 \\
.120 & .0113 \\
\hline
\end{tabular}

Strand

\begin{tabular}{|c|c|c|c|c|c|}
\hline $\begin{array}{l}\text { Diam- } \\
\text { eter } \\
\text { of } \\
\text { strand }\end{array}$ & Area & $\begin{array}{l}\text { Breaking } \\
\text { strength }\end{array}$ & $\begin{array}{l}\text { Elonga- } \\
\text { tion } 24 \\
\text { inches }\end{array}$ & Lay & W eight \\
\hline $\begin{array}{l}\text { Inch } \\
1,2 \\
7 / 16 \\
7 / 16 \\
3,8 \\
3 / 8\end{array}$ & $\begin{array}{c}\text { Square } \\
\text { inch } \\
0.144 \\
.114 \\
.114 \\
.079 \\
.079\end{array}$ & $\begin{array}{r}\text { Pounds } \\
11,000 \\
9,000 \\
15,000 \\
6,800 \\
11,500\end{array}$ & $\begin{array}{c}\text { Percent } \\
10 \\
10 \\
6 \\
10 \\
6\end{array}$ & $\begin{array}{c}\text { Inches } \\
5.5 \\
5.0 \\
5.0 \\
4.5 \\
4.5\end{array}$ & $\begin{array}{c}\text { Pound } \\
\text { per foot } \\
0.510 \\
.415 \\
.415 \\
.295 \\
.295\end{array}$ \\
\hline
\end{tabular}

\section{BENDING TEST}

(a) The wires shall withstand, without fracture, being wrapped five times around mandrels of certain sizes. This shall not be construed as a test of the galvanizing. Sizes as follows:

\begin{tabular}{|c|c|c|c|}
\hline $\begin{array}{c}\text { Diam- } \\
\text { eter of } \\
\text { strand }\end{array}$ & $\begin{array}{c}\text { Diam- } \\
\text { eter of } \\
\text { wire }\end{array}$ & $\begin{array}{c}\text { Strand } \\
\text { breaking } \\
\text { strength }\end{array}$ & $\begin{array}{c}\text { Diam- } \\
\text { eter of } \\
\text { mandrel }\end{array}$ \\
\cline { 1 - 2 } Inch & Inch & Pounds & Inch \\
$1 / 2$ & 0.162 & 11,000 & $1 / 2$ \\
$7 / 16$ & .144 & 9,000 & $1 / 2$ \\
$7 / 16$ & .144 & 15,000 & $3 / 4$ \\
$3 / 8$ & .120 & 6,800 & $1 / 2$ \\
$3 / 8$ & .120 & 11,500 & $5 / 8$ \\
\hline
\end{tabular}

(b) If the wire breaks, three additional tests shal be inade from the same wire and if any fail the strand shall be rejected.

\section{GALVANIZING TEST}

(a) Galvanizing shall comply with Railway Signa] Association specification for galvanizing for iron or steel.

\section{INSPECTION AND TESTS}

(a) The purchaser is to have the right to make such inspection and tests as he may desire of the materials and of the wire at any stage of manufacture.

(b) The manufacturer must provide, at the mill, all apparatus and labor for making the required tests under the supervision of the purchaser.

(c) Tests shall be made at the mill or on samples submitted by the manufacturer and may also be made on the wire upon its arrival at destination.

(d) If, upon arrival at destination, the wire does not meet the requirements of this specification, it will be rejected and returned to the manufacturer, who shall pay all freight charges.

\section{PACKING FOR SHIPMENT}

(a) The wire shall be furnished in $\left\{\begin{array}{l}\text { coils } \\ \text { reels }\end{array}\right\}$ of the following minimum lengths:

Feet.

$1 / 2$-inch strand.

7/16-inch strand 1. 500

(b) When furnished in reels, each reel shall have the weight, length of strand, the name of the manufacturer and purchaser's order or inspection number plainly and indelibly marked on a strong tag securely fastened to the strand and also stenciled on the outside of the reel.

(c) When furnished in coils, each coil shall have the weight, length and size of strand, the name of the maker, the purchaser's order and inspection number and the proper shipping address plainly and indelibly marked on a strong tag securely fastened to the coil. 
AMERICAN RAILWAY ASSOCIATION, TELEGRAPH AND TELEPHONE SECTION, SPECIFICATION FOR GALVANIZED STEEL GUY AND MESSENGER STRAND, $1-\mathrm{A}-17,1927$

\section{GALVANIZED STEEL GUY AND MESSENGER STRAND \\ PURPOSE}

A-1. The purpose of this specification is to guide the seller or contractor in the manufacture of galvanized steel guy and messenger strand for use in pole line construction and aerial cable installations in the telegraph and telephone plant.

\section{GENERAL}

C-1. The quality of the material used and the methods of manufacture shall be such as to insure for the completed wire the properties called for in this specification.

C-2. The seller or contractor must make sure that all material and work are in accordance with the specification before the wire is offered.

(Composition of strand, character, and quality of wires, and galvanizing conform to requirements for A. R. A. Signal Section specification for galvanized messenger wire, given above.)

D-4. All joints in wire composing a strand shall be well made, thoroughly brazed or welded and galvanized. No two joints in the wire of the strand shall be less than 150 feet apart. The outside of the completed strand shall be marked with paint or by other suitable means at each spliced wire.

\section{PHYSICAL AND MECHANICAL REQUIREMENTS}

E-1. Any section of the galvanized strand, free from factory joints, shall conform to the requirements of Table 1.

TABLE 1.-Galvanized steel guy and messenger strand

\begin{tabular}{|c|c|c|c|c|c|c|c|c|c|}
\hline \multirow{3}{*}{ 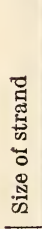 } & \multicolumn{5}{|c|}{ Wires } & \multicolumn{4}{|c|}{ Strand } \\
\hline & \multirow{2}{*}{ 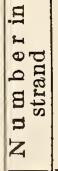 } & \multirow{2}{*}{$\begin{array}{l}\text { Gage each } \\
\text { wire }\end{array}$} & \multicolumn{3}{|c|}{ Diameter } & \multirow{2}{*}{ 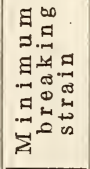 } & \multicolumn{2}{|c|}{$\begin{array}{l}\text { Elongation } \\
\text { in } 2 \text { feet }\end{array}$} & \multirow[b]{2}{*}{ त्ञ } \\
\hline & & & 富泉白 & 离胥 & 究品品 & & 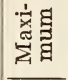 & 豈突 & \\
\hline $\begin{array}{c}\text { Inch } \\
1 / 4 \\
5 / 16 \\
38 \\
7 / 16\end{array}$ & $\begin{array}{l}7 \\
7 \\
7 \\
7\end{array}$ & $\begin{array}{r}13 \text { B.W.G. G. } \\
12 \text { B.W.G. } \\
11 \text { B.W.G. } \\
7 \text { A.W.G. }\end{array}$ & \begin{tabular}{|c|} 
Inch \\
0.093 \\
.107 \\
.116 \\
.138
\end{tabular} & $\begin{array}{l}\text { Inch } \\
0.095 \\
.109 \\
.120 \\
.144\end{array}$ & $\begin{array}{l}\text { Inch } \\
0.097 \\
.111 \\
.124 \\
.150\end{array}$ & $\begin{array}{r}\text { Pounds } \\
4,000 \\
6,000 \\
11,500 \\
218,000\end{array}$ & $\begin{array}{r}P . c t . \\
22 \\
17 \\
15\end{array}$ & $\begin{array}{l}\text { P.ct. } \\
11 \\
11 \\
41 / 2 \\
4\end{array}$ & $\begin{array}{r}\text { P.ct. } \\
3.5 \\
3.5 \\
4.5 \\
5.0\end{array}$ \\
\hline
\end{tabular}

1 Minimum breaking strain, with 1 wire cut, 10,000 pounds.

2 Minimum breaking strain, with 1 wire cut, 16,000 pounds.

\section{INSPECTION}

G-1. Place and facilities.-All testing and inspection shall be made at the place of manufacture. The seller or contractor shall afford the inspector representing the purchaser all reasonable facilities to enable him to satisfy himself that the material conforms to the requirements of this specification.

G-2. Time.-The seller or contractor shall notify the purchaser when the strand is ready for inspection, unless otherwise arranged by the purchaser, and shall agree upon a time convenient to the purchaser for inspection.

G-3. Rejection.- Strand failing to meet the requirements of this specification in any respect shall be rejected.
G-4. Factory inspection waived.-If factory inspection be waived by the purchaser, it shall be so stated on the order and detailed factory test sheets for the wire for which inspection is so waived shall be forwarded to the purchaser.

\section{TESTS}

H-1. Samples.-Samples for tests shall be selected by the inspector from each 2,500 feet of strand in the shipment or from each reel if more than 2,500 feet is contained thereon. If, upon testing a sample from any lot of strand, the results are found to not agree with the requirements of this specification, tests upon two additional samples shall be made and the average of the three tests shall determine acceptance or rejection of the lot represented by the samples.

H-2. Tensile strength and elongation.-Tensile tests shall be made upon the samples and the elongation shall be determined as the permanent increase in length, due to the breaking of the strand in tension, measured between the jaws of the testing machine. The zero length shall be the distance between the jaws when a load equal to 5 per cent of the required ultimate breaking strength shall have been applied, and the final length shall be the distance between the jaws at the time of rupture. The zero length shall be as near 2 feet as possible. The fracture shall be between the jaws and not closer than 1 inch to either jaw.

\section{PREPARATION FOR SHIPMENT}

J-1. Strand for shipment in lots of less than 500 feet shall be wound in coils with a diameter of eye of from 20 to 30 inches. Each coil shall be securely. bound in not less than four separate places with strong twine or soft galvanized iron wire.

$J-2$. Lots of 500 feet or more shall be wound on reels in the lengths specified on the order.

J-3. Strand ends shall be securely wrapped to prevent untwisting.

\section{MARKING}

K-1. Each coil or reel shall have the size, weight, and length of strand plainly and indelibly marked on a strong tag securely attached.

In less than carload lots, tags shall show in addition the purchaser's order and requisition numbers, name of consignor and name and address of consignee.

K-2. For carload shipments, routing and car identification shall be furnished the purchaser.

(Warranty conforms to that in A. R. A. Signal Section No. 1630. See 611.11, p. 635.)

$\mathrm{L}-2$. The seller or contractor shall covenant and agree to save harmless and indemnify the purchaser against all claims, suits, actions or proceedings, damages, costs, fees, and expenses by reason of alleged infringement of patents, or for patent royalties involved, in consequence of the purchase or the use of the material covered hereby.

\section{AMERICAN RAILWAY ENGINEERING AS- SOCIATION, SPECIFICATIONS FOR MOV- ABLE RAILWAY BRIDGES, WIRE ROPES AND ATTACHMENTS, 1922 \\ WIRE ROPES AND ATTACHMENTS MANUFACTURER}

501. Wire rope shall be made by a manufacturer whose facilities and reputation are approved by the engineer.

\section{LAYING}

502. Ropes shall be laid in accordance with the best practice, in an approved lubricant. 


\section{SPLICES}

503. Ropes shall not be spliced.

\section{WIRES-SIZE AND NUMBER}

504. The diameter of counterweight ropes shall be not less than 1 inch, not more than $2 \frac{1}{2}$ inches; of operating ropes not less than three-fourths of an inch.

The ropes shall be made of improved plow steel and shall consist of six strands of 19 wires each laid around a hemp center.

\section{SOCKETS}

505. Sockets shall be forged without welds from solid steel. The dimensions of sockets shall be such that no part under tension will be stressed higher than 65,000 pounds per square inch when the rope is stressed to its specified ultimate strength. The sockets shall be attached to the rope by a method which is reliable and which will not permit the rope to slip in its attachment to the socket.

\section{WIRE-PHYSICAL PROPERTIES}

506. The wire from which counterweight ropes are made shall be tested in the presence of an inspector designated by the engineer. It shall show the following physical properties:

(a) A tensile strength of not less than 225,000 pounds a square inch.

(b) A total elongation (measured on a piece 12 inches long) of not less than 2.4 per cent.

(c) The number of times a piece 6 inches long can be twisted around its longitudinal axis without rupture shall be not less than 1.4 divided by the diameter in inches.

(d) The number of times the wire can be bent $90^{\circ}$ alternately in opposite directions over a jaw the radius of which is equal to twice the diameter of the wire, without fracture, shall be not less than six. This test shall be made in a mechanical bender so constructed that the wire actually conforms to the radius of the jaws and is subjected to little tensile stress.

\section{ULTIMATE STRENGTH}

507. In order to test the strength of the rope and fastenings, a number of test pieces not more than 12 feet long, with sockets (selected at random from those which are to be used in filling the order) attached to each end, shall be stressed to destruction in a suilable testing machine. The number of test pieces shall be not more than 10 per cent of the total number of finished lengths of rope to be made, nor less than two from each original length. Under this test, the rope shall develop the ultimate strength given in the table below:

Ultimate strength of 6 by 19 improved plow-steel rope

\begin{tabular}{|c|c|c|c|}
\hline \multirow{2}{*}{$\begin{array}{l}\text { Diam- } \\
\text { eter o } 1 \\
\text { rope }\end{array}$} & \multirow{2}{*}{$\begin{array}{l}\text { Area of } \\
\text { section } \\
0.4 D^{2}\end{array}$} & \multicolumn{2}{|c|}{ Ultimate strength } \\
\hline & & $\begin{array}{c}\text { Per square } \\
\text { inch }\end{array}$ & $\begin{array}{l}\text { Entire } \\
\text { rope }\end{array}$ \\
\hline $\begin{array}{c}\text { Inches } \\
1 / 2 \\
5 / 8 \\
3 / 4 \\
7 / 8 \\
1\end{array}$ & $\begin{array}{r}\text { Sq. In. } \\
0.100 \\
.156 \\
.225 \\
.306 \\
.400\end{array}$ & $\begin{array}{l}\text { Pounds } \\
210,000 \\
208,000 \\
206,000 \\
204,000 \\
202,000\end{array}$ & $\begin{array}{r}\text { Pounds } \\
21,000 \\
32,000 \\
46,000 \\
63,000 \\
81,000\end{array}$ \\
\hline $\begin{array}{l}11 / 8 \\
11 / 4 \\
13 / 8 \\
11 / 2 \\
15 / 8\end{array}$ & $\begin{array}{r}.506 \\
.625 \\
.756 \\
.900 \\
1.056\end{array}$ & $\begin{array}{l}200,000 \\
198,000 \\
196,000 \\
194,000 \\
192,000\end{array}$ & $\begin{array}{l}101,000 \\
124,000 \\
148,000 \\
175,000 \\
202,000\end{array}$ \\
\hline $\begin{array}{l}13 / 4 \\
17 / 8 \\
2 \\
21 / 4 \\
21 / 2\end{array}$ & $\begin{array}{l}1.225 \\
1.406 \\
1.600 \\
2.025 \\
2.500\end{array}$ & $\begin{array}{l}190,000 \\
188,000 \\
186,000 \\
184,000 \\
182,000\end{array}$ & $\begin{array}{l}233,000 \\
264,000 \\
298,000 \\
373,000 \\
455,000\end{array}$ \\
\hline
\end{tabular}

\section{REJECTION}

508. If the physical qualities of the rope or of its individual wires fall below those specified, the entire length from which the test pieces were taken shall be replaced by the manufacturer with a new length, the physical qualities of which conform to those specified.

\section{TESTING SOCKETS}

509. If slipping in the sockets should occur during the test, the method of fastening the sockets shall be changed until slipping does not occur. The sockets shall be stronger than the rope to which they are attached. If a socket should break during the test, two others shall be selected and attached to another piece of rope and the test repeated. This process shall be continued until the inspector is satisfied as to the reliability of the sockets. If the inspector is satisfied, the lot shall be accepted. If, however, 10 per cent or more of the sockets tested break at a load less than the specified minimum ultimate strength of the rope, the lot shall be rejected.

\section{FACILITIES FOR TESTING}

510. The manufacturer shall provide proper facilities for making the tests, and shall make, at his own expense, the tests required. Tests shall be made in the presence of an inspector representing the engineer.

\section{LENGTH}

511. The length of each rope shall be measured while the rope is supported throughout its length in a straight line and under a tension of 2 per cent of its ultimate strength. A variation from the required length of not more than one-quarter of an inch in 100 feet, will be allowed.

\section{COILS}

512. Ropes shall be shipped on reels of a diameter not less than thirty times that of the rope.

\section{EQUALIZING LEVERS}

513. The equalizing levers, and their pins more than 7 inches in diameter, shall be of forged steel. Pins 7 inches or less in diameter shall be of either rolled steel or forged steel.

\section{AMERICAH SOCIETY FOR TESTING MATE- RIALS, STANDARD SPECIFICATIONS FOR ZINC-COATED (GAIVANIZED) STEEI WIRE STRAND, DESIGNATION A122-30, 1930}

\section{GALVANIZED STEEL WIRE STRAND SCOPE}

1. These specifications cover zinc-coated steel wire strand composed of a multiplicity of wires and suitable for use as guy wires, messenger wires, span wires, etc. The weight classification and all test procedure shall be in accordance with the standard methods of testing zinc-coated (galvanized) iron and steel wire and wire products A. S. T. M., A110. (See 600.3, p. 66.)

\section{MAN UFACTURE}

2. (Requirements covering zinc used for coating are identical with A. S. T. M., A116. See 603.43, p. 192.)

3. The base metal shall be iron or steel of such quality and purity that, when drawn and galvanized to the size of wire specified, the finished strand will have the properties and characteristics herein specified. 


\section{WEIGHT AND UNIFORMITY OF COATING}

4. The zinc coating shall be tested for quantity by a stripping test only and for uniformity by the Preece test. The weight and uniformity of the coating shall be defined and tested in accordance with the requirements of the standard methods of texting zinc-coated (galvanized) iron and steel wire products, A. S. T. M., A110. (See 600.3, p. 66.)

TABLE 1. - Commercially available zinc-coated steel wire strand

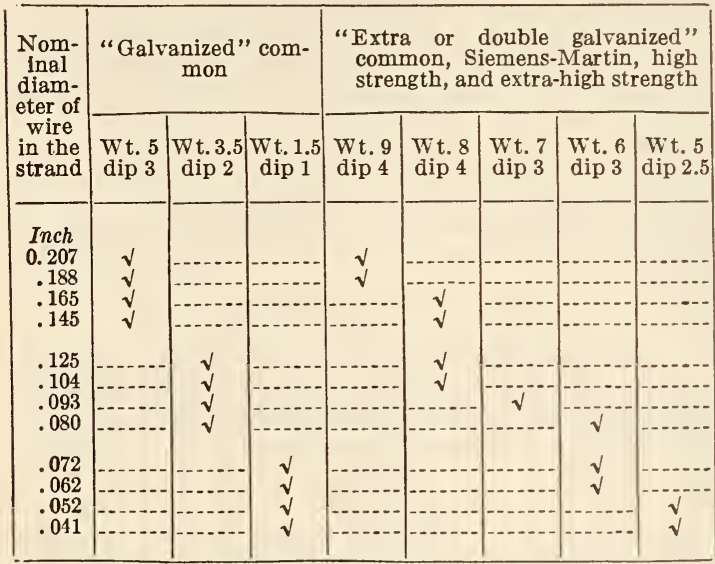

Nore.-Sizes in which any given weight of coating may be regularly obtained in the open market are indicated by a $\sqrt{ }$ opposite the nominal diameter in the above table.

For intermediate sizes of wire in the strand the weight and dip designations are the same as for the next finer size shown in above table.

TABLE 2.-Physical properties of zinc-coated steel wire strand

\begin{tabular}{|c|c|c|c|c|c|c|c|}
\hline \multirow{2}{*}{$\begin{array}{l}\text { Ap- } \\
\text { proxi- } \\
\text { mate } \\
\text { diam- } \\
\text { eter } \\
\text { of the } \\
\text { strand }\end{array}$} & \multirow{2}{*}{$\begin{array}{l}\text { Num- } \\
\text { ber of } \\
\text { wires } \\
\text { in } \\
\text { strand }\end{array}$} & \multirow{2}{*}{$\begin{array}{l}\text { Diam- } \\
\text { eter of } \\
\text { wires } \\
\text { in } \\
\text { strand }\end{array}$} & \multirow{2}{*}{$\begin{array}{c}\text { Weight } \\
\text { of } \\
\text { strand } \\
\text { per } \\
1,000 \\
\text { feet }\end{array}$} & \multicolumn{4}{|c|}{ Minimum breaking strength } \\
\hline & & & & $\begin{array}{l}\text { Com- } \\
\text { mon }\end{array}$ & $\begin{array}{c}\text { Sie- } \\
\text { mens- } \\
\text { Martin }\end{array}$ & $\begin{array}{c}\text { High } \\
\text { strength }\end{array}$ & $\begin{array}{l}\text { Extra- } \\
\text { high } \\
\text { strength }\end{array}$ \\
\hline $\begin{array}{c}\text { Inches } \\
1 / 8 \\
5 / 32 \\
3 / 16 \\
7 / 32 \\
1 / 4\end{array}$ & $\begin{array}{l}7 \\
7 \\
7 \\
7 \\
7\end{array}$ & $\begin{array}{l}\text { Inch } \\
0.041 \\
.052 \\
.062 \\
.072 \\
.080\end{array}$ & \begin{tabular}{|r|} 
Pounds \\
31.8 \\
51.3 \\
72.9 \\
98.3 \\
121
\end{tabular} & $\begin{array}{r}\text { Pounds } \\
540 \\
870 \\
1,150 \\
1,540 \\
1,900\end{array}$ & $\begin{array}{r}\text { Pounds } \\
910 \\
1,470 \\
1,900 \\
2,560 \\
3,150\end{array}$ & $\begin{array}{r}\text { Pounds } \\
1,330 \\
2,140 \\
2,850 \\
3,850 \\
4,750\end{array}$ & $\begin{array}{c}\text { Pounds } \\
1,830 \\
2,940 \\
3,990 \\
5,400 \\
6,650\end{array}$ \\
\hline $\begin{array}{l}9 / 32 \\
5 / 16 \\
3 / 8 \\
7 / 16\end{array}$ & $\begin{array}{l}7 \\
7 \\
7 \\
7\end{array}$ & $\begin{array}{l}.093 \\
.104 \\
.125 \\
.145\end{array}$ & $\begin{array}{l}164 \\
205 \\
296 \\
399\end{array}$ & $\begin{array}{l}2,570 \\
3,200 \\
4,250 \\
5,700\end{array}$ & $\begin{array}{l}4,250 \\
5,350 \\
6,950 \\
9,350\end{array}$ & $\begin{array}{r}6,400 \\
8,000 \\
10,800 \\
14,500\end{array}$ & $\begin{array}{r}8,950 \\
11,200 \\
15,400 \\
20,800\end{array}$ \\
\hline $\begin{array}{r}1 / 2 \\
1 / 2 \\
916 \\
916\end{array}$ & $\begin{array}{r}7 \\
19 \\
7 \\
19\end{array}$ & $\begin{array}{l}.165 \\
.100 \\
.188 \\
.113\end{array}$ & $\begin{array}{l}517 \\
504 \\
671 \\
637\end{array}$ & $\begin{array}{l}7,400 \\
7,620 \\
9,600 \\
9,640\end{array}$ & $\begin{array}{l}12,100 \\
12,700 \\
15,700 \\
16,100\end{array}$ & $\begin{array}{l}18,800 \\
19,100 \\
24,500 \\
24,100\end{array}$ & $\begin{array}{l}26,900 \\
26,700 \\
35,000 \\
33,700\end{array}$ \\
\hline $\begin{array}{l}5 / 8 \\
58 \\
3,4 \\
7 / 8\end{array}$ & $\begin{array}{r}7 \\
19 \\
19 \\
19\end{array}$ & $\begin{array}{l}.207 \\
.125 \\
.150 \\
.177\end{array}$ & $\begin{array}{r}813 \\
796 \\
1,155 \\
1,581\end{array}$ & $\begin{array}{l}11,600 \\
11,000 \\
16,000 \\
21,900\end{array}$ & $\begin{array}{l}19,100 \\
18,100 \\
26,200 \\
35,900\end{array}$ & $\begin{array}{l}29,600 \\
28,100 \\
40,800 \\
55,800\end{array}$ & $\begin{array}{l}42,400 \\
40,200 \\
58,300 \\
79,700\end{array}$ \\
\hline $\begin{array}{l}1 \\
1 \\
118 \\
114\end{array}$ & $\begin{array}{l}19 \\
37 \\
37 \\
37\end{array}$ & $\begin{array}{l}.200 \\
.143 \\
.161 \\
.179\end{array}$ & $\begin{array}{l}2,073 \\
2,057 \\
2,691 \\
3,248\end{array}$ & $\begin{array}{l}28,700 \\
28,300 \\
36,000 \\
44,600\end{array}$ & $\begin{array}{l}47,000 \\
46,200 \\
58,900 \\
73,000\end{array}$ & $\begin{array}{r}73,200 \\
71,900 \\
91,600 \\
113,600\end{array}$ & $\begin{array}{l}104,500 \\
102,700 \\
130,800 \\
162,200\end{array}$ \\
\hline
\end{tabular}

5. The weight and uniformity of the coating of the various sizes of wire composing the strand shall be as specified by the purchaser and in accordance with the weights and dips designated in Table 1 . Commercial strand designated as common strand is a vailable in two weights of coating known as "gal- vanized" and "extra or double galvanized." Strand designated as Siemens-Martin, high strength, and extra-high strength is furnished and used in one weight of coating only, namely, that which is ordinarily known as "extra galvanized" or "double galvanized."

6. All strand shall have a length of lay not to exceed sixteen times the nominal or approximate diameter of the strand, in inches.

\section{PHYSICAL PROPERTIES AND TESTS}

7. The number of wires in a strand, the size of the component wires, the weight per 1,000 feet of strand, and the minimum breaking strength shall conform to the values specified in Table 2 .

8. The elongation of the strand in 24 inches shall not be less than the following

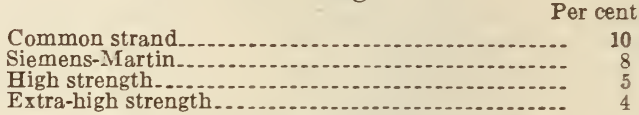

\section{SIZE AND PERMISSIBLE VARIATIONS}

9. (a) The size of the finished strand shall be expressed as the diameter of the wire in fractions of an inch.

(b) The diameter of the wire forming the strand shall be within the limits shown in Table 3 .

\section{WORKMANSHIP AND FINISH}

10. (a). The wire shall be free from splinters, scales, inequalities, flaws, and other imperfections not consistent with good commercial practice.

(b) The zinc coating shall be smooth and continuous.

\section{PACKING AND MARKING}

11. Strand wire shall be furnished in lengths as specified and in compact coils or on reels, and shall be well protected against mechanical injury in shipping as agreed upon at the time of placing the order.

\section{INSPECTION, SAMPLING, AND REJECTION}

12. (Inspection conforms to A. S. T. M. standard specifications for steel, par. 7 . See 605.0, p. 247.)

TABLE 3.-Permissible variations in diameter of zinccoated steel wire strand

\begin{tabular}{|c|c|c|c|c|}
\hline \multirow{2}{*}{$\begin{array}{l}\text { Nominal } \\
\text { or approx } \\
\text { imate } \\
\text { diameter } \\
\text { of strand }\end{array}$} & \multirow{2}{*}{$\begin{array}{l}\text { Number } \\
\text { of wires } \\
\text { in strand }\end{array}$} & \multicolumn{3}{|c|}{ Diameter of wire } \\
\hline & & Nominal & Maximum & Minimum \\
\hline $\begin{array}{r}\text { Inches } \\
1 / 8 \\
5 / 32 \\
3116 \\
7 / 32 \\
1 / 4\end{array}$ & $\begin{array}{l}7 \\
7 \\
7 \\
7 \\
7\end{array}$ & $\begin{array}{l}\text { Inch } \\
0.041 \\
.052 \\
.062 \\
.072 \\
.080\end{array}$ & $\begin{array}{c}\text { Inch } \\
0.043 \\
.054 \\
.065 \\
.075 \\
.083\end{array}$ & $\begin{array}{l}\text { Inch } \\
0.039 \\
.049 \\
.059 \\
.069 \\
.077\end{array}$ \\
\hline $\begin{array}{r}9 / 32 \\
5 / 16 \\
3 / 8 \\
7 / 16\end{array}$ & $\begin{array}{l}7 \\
7 \\
7 \\
7\end{array}$ & $\begin{array}{l}.093 \\
.104 \\
.120 \\
.145\end{array}$ & $\begin{array}{l}.097 \\
.108 \\
.125 \\
.151\end{array}$ & $\begin{array}{l}.089 \\
.100 \\
.115 \\
.139\end{array}$ \\
\hline $\begin{array}{r}1 / 2 \\
1 / 2 \\
916 \\
916\end{array}$ & $\begin{array}{r}7 \\
19 \\
7 \\
19\end{array}$ & $\begin{array}{l}.165 \\
.100 \\
.188 \\
.113\end{array}$ & $\begin{array}{r}.171 \\
.104 \\
.194 \\
.118\end{array}$ & $\begin{array}{l}.159 \\
.096 \\
.182 \\
.108\end{array}$ \\
\hline $\begin{array}{l}5 / 8 \\
5 / 8 \\
3 / 4 \\
7 / 6\end{array}$ & $\begin{array}{r}7 \\
19 \\
19 \\
19\end{array}$ & $\begin{array}{l}.207 \\
.125 \\
.150 \\
.177\end{array}$ & $\begin{array}{l}.213 \\
.130 \\
.156 \\
.183\end{array}$ & $\begin{array}{l}.201 \\
.120 \\
.144 \\
.171\end{array}$ \\
\hline $\begin{array}{l}1 \\
1 \\
11 / 8 \\
11 / 4\end{array}$ & $\begin{array}{l}19 \\
37 \\
37 \\
37\end{array}$ & $\begin{array}{l}.200 \\
.143 \\
.161 \\
.179\end{array}$ & $\begin{array}{l}.206 \\
.149 \\
.167 \\
.185\end{array}$ & $\begin{array}{l}.194 \\
.137 \\
.155 \\
.173\end{array}$ \\
\hline
\end{tabular}


13. In quantities of three or less coils, a sample for all tests shall be taken from each reel or coil.
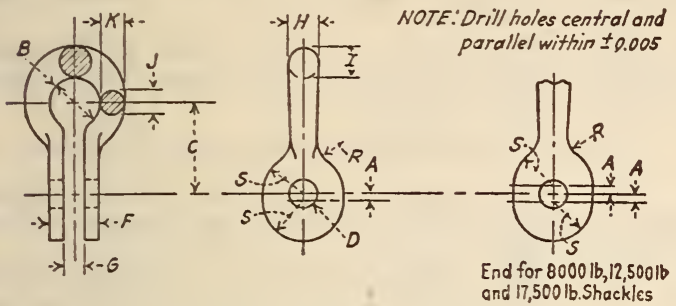

Figure 59.-Shackles

On larger quantities, samples shall be taken from 10 per cent of the lot. If over 3 per cent of the samples fail in any one test, the entire lot may be rejected. In case there is a reasonable doubt as to the failure of the wire or strand in the first trial, two additional tests shall be made, and if no failure occurs in either of these tests, the wire or strand shall be considered acceptable.

\section{SOCIETY OF AUTOMOTIVE ENGINEERS,} AERONAUTIC; SHACKLES, S. A. E. STANDARD, AUGUST, 1928

\section{SHACKLES}

\section{PHYSICAL PROPERTIES}

Shackles shall show the ultimate strength required when subjected to a tensile test. Shackles, when subjected to a bend test made by opening the shackle and bending flat, shall show no signs of cracking.

Shackel Dimensions (dimensions in inches)

\begin{tabular}{|c|c|c|c|c|c|c|c|c|c|c|c|c|c|}
\hline No. & $\begin{array}{c}\text { Shackle } \\
\text { and } \\
\text { cable } \\
\text { strength } \\
\text { (pounds) }\end{array}$ & $\begin{array}{c}\mathrm{D} \\
+0.010 \\
-0.000\end{array}$ & $\begin{array}{c}G \\
+0.010 \\
-0.000\end{array}$ & $F$ & $\mathrm{~S}$ & $\mathbf{J}$ & K & H & I & A & B & C & $\mathbf{R}$ \\
\hline $\begin{array}{l}8 \\
16 \\
32\end{array}$ & $\begin{array}{r}800 \\
1,600 \\
2,100 \\
3,200 \\
4,600\end{array}$ & $\begin{array}{l}0.188 \\
.188 \\
.188 \\
.250 \\
.313\end{array}$ & $\begin{array}{l}0.109 \\
.150 \\
.150 \\
.203 \\
.203\end{array}$ & $\begin{array}{l}0.250 \\
.313 \\
.313 \\
.438 \\
.500\end{array}$ & $\begin{array}{r}0.250 \\
.250 \\
.281 \\
.313 \\
.375\end{array}$ & $\begin{array}{r}0.172 \\
.172 \\
.172 \\
.219 \\
.219\end{array}$ & $\begin{array}{l}0.172 \\
.172 \\
.172 \\
.219 \\
.219\end{array}$ & $\begin{array}{r}0.172 \\
.172 \\
.172 \\
.250 \\
.281\end{array}$ & $\begin{array}{l}0.172 \\
.172 \\
.172 \\
.250 \\
.281\end{array}$ & $\begin{array}{l}0.031 \\
.031 \\
.031 \\
.031 \\
.063\end{array}$ & $\begin{array}{l}0.250 \\
.250 \\
.250 \\
.375 \\
.438\end{array}$ & $\begin{array}{l}0.563 \\
.563 \\
.563 \\
.750 \\
.813\end{array}$ & $\begin{array}{l}0.375 \\
.375 \\
.375 \\
.438 \\
.500\end{array}$ \\
\hline $\begin{array}{l}61 \\
80 \\
125 \\
175\end{array}$ & $\begin{array}{r}6,100 \\
8,000 \\
12,500 \\
17,500\end{array}$ & $\begin{array}{l}.375 \\
.375 \\
.438 \\
.500\end{array}$ & $\begin{array}{l}.203 \\
.266 \\
.360 \\
.406\end{array}$ & $\begin{array}{l}.563 \\
.563 \\
.719 \\
.813\end{array}$ & $\begin{array}{l}.375 \\
.406 \\
.531 \\
.625\end{array}$ & $\begin{array}{l}.281 \\
.375 \\
.469 \\
.563\end{array}$ & $\begin{array}{l}.281 \\
.375 \\
.469 \\
.563\end{array}$ & $\begin{array}{l}.313 \\
.438 \\
.594 \\
.688\end{array}$ & $\begin{array}{l}.313 \\
.375 \\
.469 \\
.563\end{array}$ & $\begin{array}{l}.063 \\
.063 \\
.094 \\
.125\end{array}$ & $\begin{array}{l}.500 \\
.438 \\
.625 \\
.625\end{array}$ & $\begin{array}{r}.875 \\
1.000 \\
1.125 \\
1.250\end{array}$ & $\begin{array}{l}.500 \\
.406 \\
.750 \\
.500\end{array}$ \\
\hline
\end{tabular}

Finished sizes include plating or protective coating.

Tolerances are \pm 0.010 except where otherwise indicated.

SOCIETY OF AUTOMOTIVE ENGINEERS, AERONAUTIC; STEEL THIMBLES, S. A. E. STANDARD, MARCH, 1918

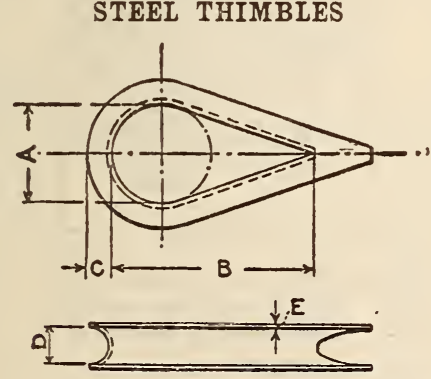

Figure 591/2.-Steel thimbles

[Dimensions in inches]

\begin{tabular}{|c|c|c|c|c|c|c|c|}
\hline \multicolumn{2}{|c|}{$\begin{array}{c}\text { Diameter of } \\
\text { cable }\end{array}$} & \multirow{2}{*}{ A } & \multirow{2}{*}{ B } & \multirow{2}{*}{0} & \multirow{2}{*}{$\mathrm{D}$} & \multirow{2}{*}{$\mathbf{E}$} & \multirow{2}{*}{$\begin{array}{l}\text { Approx } \\
\text { imate } \\
\text { weight, } \\
\text { pound } \\
\text { per } 1,000 \\
\text { pieces }\end{array}$} \\
\hline $\begin{array}{l}\text { Nom- } \\
\text { inal }\end{array}$ & $\begin{array}{c}\text { Deci- } \\
\text { mal }\end{array}$ & & & & & & \\
\hline $\begin{array}{l}1 / 16 \\
3 / 32 \\
18 \\
532 \\
3 / 16\end{array}$ & $\begin{array}{r}0.062 \\
.094 \\
.125 \\
.156 \\
.187\end{array}$ & $\begin{array}{r}0.35 \\
.35 \\
.35 \\
.40 \\
.50\end{array}$ & $\begin{array}{r}0.70 \\
.70 \\
.70 \\
.80 \\
1.00\end{array}$ & $\begin{array}{l}0.07 \\
.07 \\
.07 \\
.10 \\
.135\end{array}$ & $\begin{array}{r}0.09 \\
.09 \\
.13 \\
.17 \\
.21\end{array}$ & $\begin{array}{r}0.032 \\
.032 \\
.032 \\
.032 \\
.032\end{array}$ & $\begin{array}{l}3.00 \\
3.00 \\
4.34 \\
6.36 \\
9.00\end{array}$ \\
\hline $\begin{array}{l}7 / 32 \\
1 / 4 \\
9 / 32 \\
5 / 16 \\
3 / 8 \\
\end{array}$ & $\begin{array}{r}.219 \\
.250 \\
.281 \\
.312 \\
.375 \\
\end{array}$ & $\begin{array}{r}.60 \\
.70 \\
.80 \\
.90 \\
1.00 \\
\end{array}$ & $\begin{array}{l}1.20 \\
1.40 \\
1.60 \\
1.80 \\
2.00 \\
\end{array}$ & $\begin{array}{l}.15 \\
.17 \\
.198 \\
.21 \\
.26 \\
\end{array}$ & $\begin{array}{l}.24 \\
.25 \\
.30 \\
.33 \\
.39 \\
\end{array}$ & $\begin{array}{l}.032 \\
.032 \\
.040 \\
.040 \\
.060 \\
\end{array}$ & $\begin{array}{l}13.50 \\
16.63 \\
30.36 \\
33.00 \\
74.00 \\
\end{array}$ \\
\hline
\end{tabular}

The thimbles shall be galvanized by either the electrical or hot-dip process.

\section{UNITED STATES GOVERNMENT, DEPART- MENT OF AGRICULTURE, BUREAU OF PUBLIC ROADS, SPECIFICATIONS FOR FOREST ROAD CONSTRUCTION, 1929}

\section{WIRE-CABLE GUARD RAIL}

(This specification conforms to tentative specification $\mathrm{M}-30$ of the American Association of State Highway Officials, given above, except that here only $3 / 4$-inch cables are provided for, no mention is made of eyebolts, and requirements covering weight of zinc coating for each wire are omitted.)

\section{UNITED STATES GOVERNMENT, FEDERAI SPECIFICATIONS BOARD, FEDERAL SPECIFICATION FOR ROPE, WIRE, NO. 297, 1925. (CURRENT DESIGNATION : $R R-R-571)$}

\section{WIRE ROPE}

(In respect to material and workmanship, general requirements, inspection and tests, packing and marking, and additional information, this specification conforms to American Marine Standards Committee O No. 25-1930, given above. In respect to detail requirements, covering types and their physical properties, this specification makes the following provisions:) 


\section{DETAIL REQUIREMENTS}

\section{Type A, 1 by 7 Seizing Strand}

Seizing strand of type A shall be made as described and shown in Figure 60 and have the properties given in Table 4.

Construction.- One diameter of wire, one operation. One strand of seven wires, total seven wires. No core.

Material.-Wires: Iron or low carbon steel, annealed and galvanized.

Diameter.- The actual diameter of seizing strand, type A, shall not be smaller than the nominal

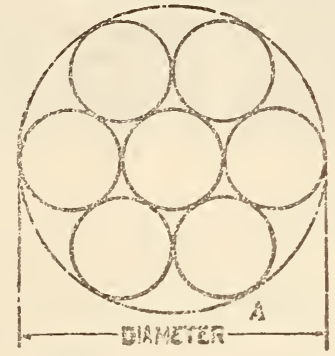

Figure 60.-Type $A$, 1 by 7 seizing strand

diameter nor larger than the nominal diameter plus one-sixty-fourth-inch.

Lay.-Seizing strand, type A, shall be standard lay, and the lay shall not be more than twelve times the nominal diameter.

TABLE 3.-Diameter tolerances for wire rope

\begin{tabular}{|c|c|c|}
\hline Nominal diameter of rope (in inches) & $\begin{array}{c}\text { Under } \\
\text { size }\end{array}$ & $\begin{array}{l}\text { Orer } \\
\text { size }\end{array}$ \\
\hline 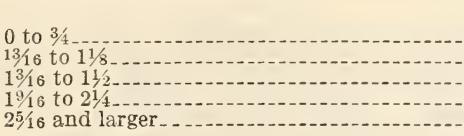 & $\begin{array}{r}\text { Inch } \\
0 \\
0 \\
0 \\
0 \\
0\end{array}$ & $\begin{array}{l}\text { Inch } \\
1 / 32 \\
3,64 \\
1 / 16 \\
3,32 \\
1 / 8\end{array}$ \\
\hline
\end{tabular}

Table 4.-Properties of seizing strand, type $A$

\begin{tabular}{|c|c|c|c|c|c|c|c|}
\hline $\begin{array}{l}\text { Nomi- } \\
\text { nal } \\
\text { diam- } \\
\text { eter }\end{array}$ & $\begin{array}{l}\text { Apprcx- } \\
\text { imate } \\
\text { diam- } \\
\text { eter } \\
\text { of wire }\end{array}$ & \begin{tabular}{|c} 
Approx- \\
imate \\
weight \\
per \\
foot
\end{tabular} & $\begin{array}{l}\text { Approx- } \\
\text { imate } \\
\text { weight } \\
\text { per } \\
\text { fathom }\end{array}$ & $\begin{array}{l}\text { Weight } \\
\text { of reel }\end{array}$ & \multicolumn{2}{|c|}{$\begin{array}{l}\text { Length of } \\
\text { strand } \\
\text { on reel }\end{array}$} & $\begin{array}{l}\text { Mini- } \\
\text { mum } \\
\text { break- } \\
\text { ing } \\
\text { strength }\end{array}$ \\
\hline $\begin{array}{c}\text { Inch } \\
1 / 16 \\
3 / 32 \\
1 / 8 \\
5 / 32\end{array}$ & $\begin{array}{r}\text { Inch } \\
0.022 \\
.032 \\
.042 \\
.052\end{array}$ & $\begin{array}{l}\text { Pound } \\
0.010 \\
.020 \\
.033 \\
.050\end{array}$ & $\begin{array}{c}\text { Pound } \\
0.06 \\
.12 \\
.20 \\
.30\end{array}$ & $\begin{array}{c}\text { Pounds } \\
50 \\
50 \\
50 \\
50\end{array}$ & $\begin{array}{r}\text { Feet } \\
5,000 \\
2,500 \\
1,500 \\
1,000\end{array}$ & $\begin{array}{c}\text { Fathoms } \\
834 \\
416 \\
250 \\
166\end{array}$ & $\begin{array}{c}\text { Pounds } \\
130 \\
280 \\
490 \\
750\end{array}$ \\
\hline
\end{tabular}

\section{Type $B, 1$ by 19 Seizing Strand}

Seizing strand of type $B$ shall be made as described and shown in Figure 61 and have the properties given in Table 5.

Construction.- One diameter of wire, one operation. One strand of 19 wires, total 19 wires. No core.

Material.-Wires: Iron or low carbon steel, annealed and galvanized.
Diameter.-The actual diameter of seizing strand, type $B$, shall not be smaller than the nominal diameter nor larger than the nominal diameter plus one-sixty-fourth inch.

Lay.- Seizing strand, type B, shall be standard lay, and the lay shall not be more than twelve times the nominal diameter.

\section{Type C, 5 by 19 Marline Covered Extra Strong Cast Steel}

Wire rope of type $\mathrm{C}$ shall be made as described and shown in Figure 62 and shall have the properties given in Table 6.

Construction.-Either $(a),(b),(c)$, or $(d)$ of IV, 9. Five strands of 19 wires eacn, total 95 wires.

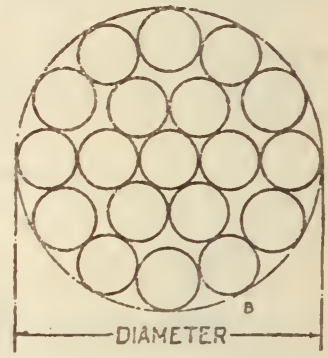

Figure 61.-Type B, 1 by 19 seizing strand

Each strand shall be served with marline wound tightly on the strand, so that it is firm, durahle, uniformly smooth, and free from knots and imperfections. One main core.

Material.-Wires: Extra strong cast steel, uncoated. Main core, hard fiber.

Diameter. - The actual diameter of wire rope, type $\mathrm{C}$, shall not be more than one-sixteenth inch larger or smaller than the nominal diameter.

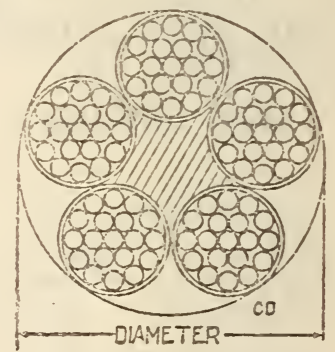

Figure 62.-Type $C, 5$ by 19 marline covered exira strong cast steel

Lay. - Wire rope, type $\mathrm{C}$, shall be regular lay, and the lay shall not be greater than six and three-fourths times the nominal diameter.

TABLE 5.-Properties of seizing strand, type $B$

\begin{tabular}{|c|c|c|c|c|c|c|c|}
\hline $\begin{array}{l}\text { Nomi- } \\
\text { nal } \\
\text { diam- } \\
\text { eter }\end{array}$ & $\begin{array}{l}\text { Approx- } \\
\text { imate } \\
\text { diam- } \\
\text { eter } \\
\text { of wire }\end{array}$ & $\begin{array}{l}\text { Approx- } \\
\text { imate } \\
\text { weight } \\
\text { per } \\
\text { foot }\end{array}$ & $\begin{array}{l}\text { Approx- } \\
\text { imate } \\
\text { weight } \\
\text { per } \\
\text { fathom }\end{array}$ & $\begin{array}{l}\text { Weight } \\
\text { of reel }\end{array}$ & \multicolumn{2}{|c|}{$\begin{array}{l}\text { Length of } \\
\text { strand } \\
\text { on reel }\end{array}$} & $\begin{array}{l}\text { Mini- } \\
\text { mum } \\
\text { break- } \\
\text { ing } \\
\text { strength }\end{array}$ \\
\hline $\begin{array}{l}\text { Inch } \\
9 / 16 \\
1 / 4\end{array}$ & $\begin{array}{r}\text { Inch } \\
0.042 \\
.052\end{array}$ & $\begin{array}{l}\text { Pound } \\
0.083 \\
.133\end{array}$ & $\begin{array}{c}\text { Pound } \\
0.50 \\
.80\end{array}$ & $\begin{array}{c}\text { Pounds } \\
100 \\
100\end{array}$ & $\begin{array}{r}\text { Feet } \\
1,200 \\
750\end{array}$ & $\begin{array}{c}\text { Fathoms } \\
200 \\
125\end{array}$ & $\begin{array}{l}\text { Pounds } \\
1,100 \\
1,900\end{array}$ \\
\hline
\end{tabular}


TABLE 6.-Properties of wire rope, type C

\begin{tabular}{|c|c|c|c|c|c|c|}
\hline \multirow[b]{2}{*}{$\begin{array}{l}\text { Diam- } \\
\text { eter } \\
\text { before } \\
\text { serving }\end{array}$} & \multirow{2}{*}{$\begin{array}{l}\text { Approx- } \\
\text { imate } \\
\text { diam- } \\
\text { eter } \\
\text { after } \\
\text { serving }\end{array}$} & \multirow[b]{2}{*}{$\begin{array}{c}\text { A pprox- } \\
\text { imate } \\
\text { weight } \\
\text { per } \\
\text { foot }\end{array}$} & \multirow{2}{*}{$\begin{array}{l}\text { Approx- } \\
\text { imate } \\
\text { weight } \\
\text { per } \\
\text { fathom } \\
\text { (6 feet) }\end{array}$} & \multicolumn{2}{|c|}{ Uncoated } & \multirow{2}{*}{$\begin{array}{l}\text { Recom- } \\
\text { mended } \\
\text { minimum } \\
\text { diameter } \\
\text { of sheave } \\
\text { or drum }\end{array}$} \\
\hline & & & & $\begin{array}{l}\text { Mini- } \\
\text { mum } \\
\text { breaking } \\
\text { strength }\end{array}$ & $\begin{array}{l}\text { Maxi- } \\
\text { mum } \\
\text { safe } \\
\text { working } \\
\text { load }^{1}\end{array}$ & \\
\hline $\begin{array}{c}\text { Inches } \\
1 / 4 \\
38 \\
18 \\
92 \\
916 \\
58\end{array}$ & $\begin{array}{c}\text { Inches } \\
1,2 \\
1116 \\
27 / 32 \\
29,32 \\
1\end{array}$ & $\begin{array}{c}\text { Pounds } \\
0.21 \\
.36 \\
.54 \\
.65 \\
.80\end{array}$ & $\begin{array}{c}\text { Pounds } \\
\text { 1. } 26 \\
2.16 \\
3.24 \\
3.90 \\
4.80\end{array}$ & $\begin{array}{r}\text { Pounds } \\
3,600 \\
8,000 \\
14,400 \\
18,000 \\
22,400\end{array}$ & $\begin{array}{c}\text { Pounds } \\
890 \\
1,910 \\
3,200 \\
4,000 \\
5,000\end{array}$ & $\begin{array}{l}\text { Feet } \\
1.0 \\
1.5 \\
2.0 \\
2.2 \\
2.5\end{array}$ \\
\hline $\begin{array}{l}3 / 4 \\
7 / 8 \\
1 \\
113 \\
1,4\end{array}$ & $\begin{array}{l}118 \\
114 \\
138 \\
112 \\
158\end{array}$ & $\begin{array}{l}1.05 \\
1.35 \\
1.70 \\
2.07 \\
2.52\end{array}$ & $\begin{array}{r}6.30 \\
8.10 \\
10.2 \\
12.4 \\
15.1\end{array}$ & $\begin{array}{l}32,000 \\
44,600\end{array}$ & $\begin{array}{r}7,100 \\
9,900 \\
12,700\end{array}$ & $\begin{array}{l}3.0 \\
3.5 \\
4.0 \\
4.5 \\
5.0\end{array}$ \\
\hline $\begin{array}{l}138 \\
116 \\
15,8 \\
134\end{array}$ & $\begin{array}{l}13 / 4 \\
17 / 8 \\
2 \\
218\end{array}$ & $\begin{array}{l}\text { 3. } 06 \\
\text { 3. } 64 \\
4.26 \\
4.90\end{array}$ & $\begin{array}{l}18.4 \\
21.8 \\
25.6 \\
29.4\end{array}$ & $\begin{array}{l}107,000 \\
128,000 \\
149,000 \\
174,000\end{array}$ & $\begin{array}{l}23,800 \\
29,400 \\
33,100 \\
38,700\end{array}$ & $\begin{array}{l}5.5 \\
6.0 \\
6.5 \\
7.0\end{array}$ \\
\hline
\end{tabular}

1 If the diameter of the drum or sheare is less than the recommended diameter, lower safe working loads should be used.

\section{Type D, 5 by 19 Marline Covered High-Grade Plow Steel}

Wire rope of type D shall be made as described and shown in Figure 63 and shall have the properties given in Table 7.

Construction.-Either $(a),(b),(c)$, or $(d)$ of IV, 9. Five strands of 19 wires each, total, 95 wires. Each strand shall be served with marline wound tightly on the strand, so that it is firm, durable, uniformly smooth, and free from knots and imperfections. One main core.

Material.-Wires: High-grade plow steel, uncoated. Main core, hard fiber.

Diameter.-The actual diameter of wire rope, type $D$, shall not be more than one-sixteenth inch larger or smaller than the nominal diameter.

Lay. - Wire rope, type D, shall be regular lay, and the lay shall not be greater than six and threefourths times the nominal diameter.

TABLE 7.-Properties of wire rope, type D

\begin{tabular}{|c|c|c|c|c|c|c|}
\hline \multirow[b]{2}{*}{$\begin{array}{l}\text { Diam- } \\
\text { eter } \\
\text { before } \\
\text { serving }\end{array}$} & \multirow[b]{2}{*}{$\begin{array}{l}\text { Approx } \\
\text { imate } \\
\text { diam- } \\
\text { eter } \\
\text { after } \\
\text { serving }\end{array}$} & \multirow[b]{2}{*}{$\begin{array}{c}\text { Approx- } \\
\text { imate } \\
\text { weight } \\
\text { per } \\
\text { foot }\end{array}$} & \multirow[b]{2}{*}{$\begin{array}{c}\text { Approx- } \\
\text { imate } \\
\text { weight } \\
\text { per } \\
\text { fathom } \\
\text { (6 feet) }\end{array}$} & \multicolumn{2}{|c|}{ Uncoated } & \multirow[b]{2}{*}{$\begin{array}{l}\text { Recom- } \\
\text { mended } \\
\text { minimum } \\
\text { diameter } \\
\text { of sheave } \\
\text { or drum }\end{array}$} \\
\hline & & & & $\begin{array}{l}\text { Mini- } \\
\text { mum } \\
\text { breaking } \\
\text { strength }\end{array}$ & $\begin{array}{l}\text { Maxi- } \\
\text { mum } \\
\text { safe } \\
\text { working } \\
\text { load }^{1}\end{array}$ & \\
\hline $\begin{array}{c}\text { Inches } \\
14 \\
3,8 \\
1,2 \\
9,16 \\
5,8\end{array}$ & $\begin{array}{l}\text { Inches } \\
132 \\
1116 \\
27 / 32 \\
29 / 32 \\
1\end{array}$ & $\begin{array}{c}\text { Pounds } \\
0.21 \\
.36 \\
.54 \\
.65 \\
.80\end{array}$ & $\begin{array}{c}\text { Pounds } \\
1.26 \\
2.16 \\
3.24 \\
3.90 \\
4.80\end{array}$ & $\begin{array}{r}\text { Pounds } \\
4,800 \\
10,500 \\
18,400 \\
23,200 \\
23,800\end{array}$ & $\begin{array}{c}\text { Pounds } \\
1,160 \\
2,330 \\
4,020 \\
4,980 \\
6,330\end{array}$ & $\begin{array}{l}\text { Feet } \\
1.0 \\
1.5 \\
2.0 \\
2.2 \\
2.5\end{array}$ \\
\hline $\begin{array}{l}3 / 4 \\
7 / 8 \\
11 \\
118 \\
114\end{array}$ & $\begin{array}{l}118 \\
11 / 4 \\
138 \\
11 / 2 \\
158\end{array}$ & $\begin{array}{l}1.05 \\
1.35 \\
1.70 \\
2.07 \\
2.52\end{array}$ & $\begin{array}{l}6.30 \\
8.10 \\
10.2 \\
12.4 \\
15.1\end{array}$ & $\begin{array}{r}40,000 \\
53,000 \\
70,000 \\
90,000 \\
110,000\end{array}$ & $\begin{array}{r}8,890 \\
11,800 \\
15,600 \\
20,000 \\
24,400\end{array}$ & $\begin{array}{l}3.0 \\
3.5 \\
4.0 \\
4.5 \\
5.0\end{array}$ \\
\hline $\begin{array}{l}138 \\
112 \\
158 \\
134\end{array}$ & $\begin{array}{l}13 / 4 \\
17,8 \\
2 \\
218\end{array}$ & $\begin{array}{l}3.06 \\
3.64 \\
4.26 \\
4.90\end{array}$ & $\begin{array}{l}18.4 \\
21.8 \\
25.6 \\
29.4\end{array}$ & $\begin{array}{l}134,000 \\
159,000 \\
187,000 \\
218,000\end{array}$ & $\begin{array}{l}29,800 \\
35,300 \\
41,600 \\
48,400\end{array}$ & $\begin{array}{l}5.5 \\
6.0 \\
6.5 \\
7.0\end{array}$ \\
\hline
\end{tabular}

1 If the diameter of the drum or sheave is less than the recommended diameter, lower safe working loads should be used.

\section{Type E, 6 by 7 Extra Strong Cast Steel}

Wire rope of type $\mathrm{E}$ shall be made as described and shown in Figure 64 and shall have the properties given in Table 8.
Construction.-One diameter of wire, one operation. Six strands of 7 wires each, total 42 wires. One main core.

Material.-Wires: Extra strong cast steel, galvanized. Main core, hard fiber.

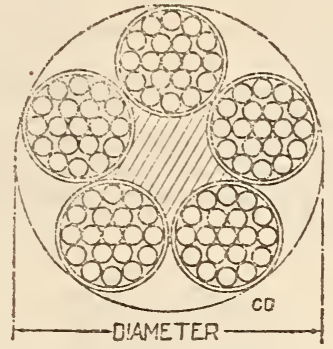

Figure 63.-Type D, 5 by 19 , marline covered high grade plow steel

Diameter.-The difference between the nominal diameter of wire rope, type $\mathrm{E}$, and the actual diameter shall not be greater than the tolerances given in American Marine Standards Committee Specification 0-25, given above.

Lay.-Wire rope, type E, shall be regular lay, and the lay shall not be greater than eight times the nominal diameter.

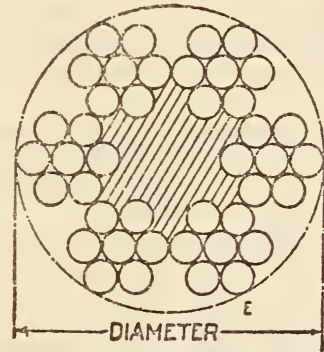

Figure 64.-Type E, 6 by $\%$, extra strong cast steel

TABLE 8.-Properties of wire rope, type $E$

\begin{tabular}{|c|c|c|c|c|c|c|}
\hline \multirow{2}{*}{$\begin{array}{l}\text { Nomi- } \\
\text { nal di- } \\
\text { ameter }\end{array}$} & \multirow{2}{*}{$\begin{array}{l}\text { Approx- } \\
\text { imate } \\
\text { circum- } \\
\text { ference }\end{array}$} & \multirow{2}{*}{$\begin{array}{l}\text { Approx- } \\
\text { imate } \\
\text { weight } \\
\text { per foot }\end{array}$} & \multirow{2}{*}{$\begin{array}{l}\text { Approx- } \\
\text { imate } \\
\text { weight } \\
\text { per } \\
\text { fathom } \\
\text { (6 feet) }\end{array}$} & \multicolumn{2}{|c|}{ Galvanized } & \multirow{2}{*}{$\begin{array}{l}\text { Recom- } \\
\text { mended } \\
\text { mini- } \\
\text { mum di- } \\
\text { ameter } \\
\text { of sheave } \\
\text { or drum }\end{array}$} \\
\hline & & & & $\begin{array}{c}\text { Minimum } \\
\text { breaking } \\
\text { strength }\end{array}$ & $\begin{array}{c}\text { Maxi- } \\
\text { mum safe } \\
\text { working } \\
\text { load }{ }^{1}\end{array}$ & \\
\hline $\begin{array}{c}\text { Inches } \\
9 / 32 \\
5116 \\
3 / 8 \\
716 \\
1 / 2\end{array}$ & $\begin{array}{c}\text { Inches } \\
7 / 8 \\
11 / 8 \\
118 \\
114 \\
11 / 2\end{array}$ & \begin{tabular}{|c|} 
Pounds \\
0.125 \\
.150 \\
.220 \\
.300 \\
.390
\end{tabular} & $\begin{array}{c}\text { Pounds } \\
0.75 \\
.90 \\
1.32 \\
1.80 \\
2.34\end{array}$ & $\begin{array}{r}\text { Pounds } \\
4,600 \\
5,800 \\
8,000 \\
11,000 \\
14,400\end{array}$ & $\begin{array}{c}\text { Pounds } \\
1,060 \\
1,420 \\
1,890 \\
2,440 \\
3,220\end{array}$ & $\begin{array}{l}\text { Feet } \\
2.0 \\
2.2 \\
2.8 \\
3.2 \\
3.8\end{array}$ \\
\hline $\begin{array}{r}9 / 16 \\
5 / 8 \\
11 / 16 \\
3 / 4 \\
7 / 8\end{array}$ & $\begin{array}{l}13 / 4 \\
2 \\
218 \\
21 / 4 \\
23 / 4\end{array}$ & $\begin{array}{r}.500 \\
.62 \\
.75 \\
.89 \\
1.20\end{array}$ & $\begin{array}{l}3.00 \\
3.72 \\
4.50 \\
5.34 \\
7.20\end{array}$ & $\begin{array}{l}18,200 \\
22,500 \\
27,000 \\
32,000 \\
43,000\end{array}$ & $\begin{array}{l}4,000 \\
5,000 \\
5,900 \\
7,100 \\
9,560\end{array}$ & $\begin{array}{l}4.0 \\
4.5 \\
5.0 \\
5.5 \\
6.5\end{array}$ \\
\hline $\begin{array}{l}1 \\
118 \\
11 / 4 \\
138 \\
11 / 2\end{array}$ & $\begin{array}{l}3 \\
31 / 2 \\
4 \\
41 / 4 \\
43 / 4\end{array}$ & $\begin{array}{l}1.58 \\
2.03 \\
2.52 \\
3.05 \\
3.65\end{array}$ & $\begin{array}{r}9.48 \\
12.20 \\
15.20 \\
18.30 \\
21.90\end{array}$ & $\begin{array}{r}56,500 \\
71,000 \\
88,000 \\
107,000 \\
126,000\end{array}$ & $\begin{array}{l}12,600 \\
15,800 \\
19,500 \\
23,800 \\
28,000\end{array}$ & $\begin{array}{r}7.2 \\
8.2 \\
9.0 \\
10.0 \\
11.0\end{array}$ \\
\hline
\end{tabular}

1 If the diameter of the drum or sheave is less than the recommended diameter, lower safe working loads should be used. 


\section{B. Type $F, 6$ by 12 Cast Steel}

Wire rope of type $F$ shall be made as described and shown in Figure 65 and shall have the properties given in Table 9.

Construction.-One diameter of wire, one operation. Six strands of 12 wires each, total 72 wires. Six strand cores and one main core, total seven cores.

Material.-Wires: Cast steel, galvanized. Cores. (See Table 9.)

Diameter.-The difference between the nominal diameter of wire rope, type $\mathrm{F}$, and the actual diameter shall not be greater than the tolerances given in Table 3 .

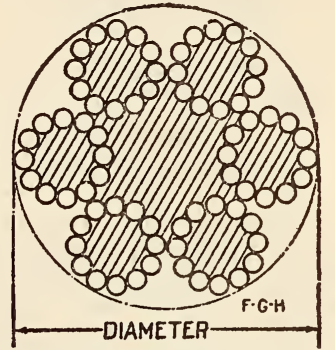

Figure 65.-Type $F^{\prime}, 6$

by 12 , cast steel

Lay.-Wire rope, type $\mathrm{F}$, shall be regular lay, and the lay shall not be greater than seven and onefourth times the nominal diameter.

TABLE 9.-Properties of wire rope, type $F$

\begin{tabular}{|c|c|c|c|c|c|c|c|}
\hline \multirow[b]{2}{*}{ 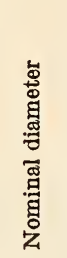 } & \multirow{2}{*}{ 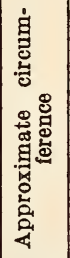 } & \multirow{2}{*}{ 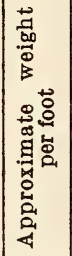 } & \multirow{2}{*}{ 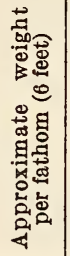 } & \multicolumn{2}{|c|}{ Galvanized } & \multicolumn{2}{|c|}{ Core } \\
\hline & & & & 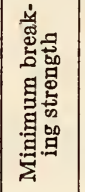 & 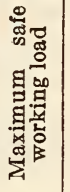 & Strand & Main \\
\hline $\begin{array}{l}7516 \\
3,6 \\
71,16 \\
1 / 2\end{array}$ & $\begin{array}{r}\text { Inches } \\
3 / 4 \\
1 \\
118 \\
11 / 4 \\
132 \\
\end{array}$ & $\begin{array}{c}L b s . \\
0.07 \\
.10 \\
.14 \\
.26 \\
.26\end{array}$ & \begin{tabular}{|c|}
$L b s$. \\
0.42 \\
.60 \\
.84 \\
1.20 \\
1.56
\end{tabular} & \begin{tabular}{|l|}
$L b s$. \\
2,000 \\
3,600 \\
5,200 \\
7,000 \\
9,300
\end{tabular} & $\begin{array}{r}c b s . \\
470 \\
790 \\
1,140 \\
1,560 \\
2,070\end{array}$ & 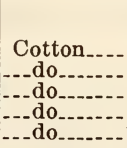 & $\begin{array}{l}\text { Hard fiber. } \\
\text { Do. } \\
\text { Do. } \\
\text { Do. } \\
\text { Do. }\end{array}$ \\
\hline $\begin{array}{c}9 / 16 \\
5 / 8 \\
3 / 4 \\
13 / 16 \\
7 / 8\end{array}$ & $\begin{array}{l}13 / 4 \\
2 \\
21 / 4 \\
21 / 2 \\
23 / 4\end{array}$ & $\begin{array}{l}.33 \\
.42 \\
.59 \\
.68 \\
.80\end{array}$ & $\begin{array}{l}1.98 \\
2.52 \\
3.54 \\
4.08 \\
4.80\end{array}$ & \begin{tabular}{|l|}
11,700 \\
14,400 \\
22,700 \\
24,300 \\
27,900 \\
\end{tabular} & $\begin{array}{l}2,600 \\
3,200 \\
4,600 \\
5,400 \\
6,200\end{array}$ & 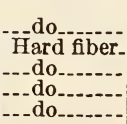 & $\begin{array}{l}\text { Do. } \\
\text { Do. } \\
\text { Do. } \\
\text { Do. } \\
\text { Do. }\end{array}$ \\
\hline $\begin{array}{l}1 \\
11 / 16 \\
118 \\
13 / 16 \\
114\end{array}$ & $\begin{array}{l}3 \\
314 \\
314 \\
313 \\
4\end{array}$ & \begin{tabular}{l|}
1.05 \\
1.18 \\
1.33 \\
1.47 \\
1.63
\end{tabular} & $\begin{array}{l}6.30 \\
7.08 \\
7.98 \\
8.82 \\
9.78\end{array}$ & $\mid \begin{array}{l}36,000 \\
40,500 \\
46,000 \\
50,400 \\
56,000\end{array}$ & $\begin{array}{r}8,000 \\
9,000 \\
11,200 \\
12,200\end{array}$ & 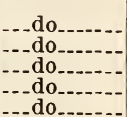 & $\begin{array}{l}\text { Do. } \\
\text { Do. } \\
\text { Do. } \\
\text { Do. } \\
\text { Do. }\end{array}$ \\
\hline $\begin{array}{l}13 / 8 \\
17 / 16 \\
112 \\
158 \\
11 / 16\end{array}$ & $\begin{array}{l}41 / 4 \\
41 / 2 \\
43 / 4 \\
5 \\
51 / 4\end{array}$ & $\begin{array}{l}2.00 \\
2.16 \\
2.36 \\
2.76 \\
2.94\end{array}$ & \begin{tabular}{|l|}
12.00 \\
13.00 \\
14.20 \\
16.60 \\
17.60
\end{tabular} & \begin{tabular}{|c|}
68,000 \\
74,000 \\
81,000 \\
94,00 \\
102,000 \\
\end{tabular} & $\begin{array}{l}15,100 \\
16,400 \\
118,000 \\
22,900 \\
22,700\end{array}$ & 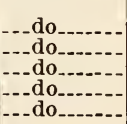 & $\begin{array}{l}\text { Do. } \\
\text { Do. } \\
\text { Do. } \\
\text { Do. } \\
\text { Do. }\end{array}$ \\
\hline $\begin{array}{l}13 / 4 \\
113116 \\
11516 \\
21 / 16 \\
21 / 16\end{array}$ & $\begin{array}{l}53 / 2 \\
53 / 4 \\
6 \\
63 / 4 \\
61 / 2\end{array}$ & $\begin{array}{l}3.23 \\
3.42 \\
3.89 \\
4.20 \\
4.43\end{array}$ & & 09,000 & & \begin{tabular}{l}
.- do.- \\
\hdashline- do-. \\
\hdashline- doo. \\
-- do.
\end{tabular} & $\begin{array}{l}\text { Do. } \\
\text { Do. } \\
\text { Do. } \\
\text { Do. } \\
\text { Do. }\end{array}$ \\
\hline
\end{tabular}

\section{Type G, 6 by 12 High-Grade Plow Steel}

Wire rope of type $\mathrm{G}$ shall be made as described and shown in Figure 66 and shall have the properties given in Table 10.
Construction.-One diameter of wire, one opera tion. Six strands of 12 wires each, total 72 wires. Six strand cores and one main core, total seven cores.

Material.-Wires: High-grade plow steel, galvanized. Cores. (See Table 10.)

Diameter.-The difference between the nominal diameter of wire rope, type $\mathrm{G}$, and the actual di-

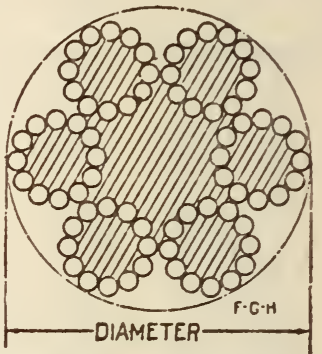

Frgure 66.-Type $G, 6$ by 12 , high-grade plow steel

ameter shall not be greater than the tolerances given in Table 3.

Lay.-Wire rope, type $\mathrm{G}$, shall be regular lay, and the lay shall not be greater than seven and onefourth times the nominal diameter.

TABLE 10.-Properties of wire rope, type $G$

\begin{tabular}{|c|c|c|c|c|c|c|c|}
\hline \multirow[b]{2}{*}{ 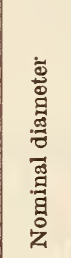 } & \multirow{2}{*}{ 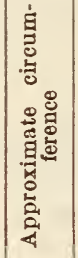 } & \multirow{2}{*}{ 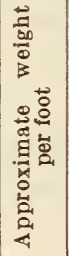 } & \multirow{2}{*}{ 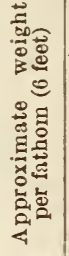 } & \multicolumn{2}{|c|}{ Galvanized } & \multicolumn{2}{|c|}{ Core } \\
\hline & & & & 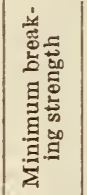 & 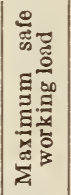 & Strand & Main \\
\hline $\begin{array}{c}\text { Inches } \\
1 / 4 \\
5 / 16 \\
3 / 8 \\
7 / 16 \\
1 / 2\end{array}$ & $\begin{array}{r}\text { Inches } \\
3 / 4 \\
1 \\
11 / 8 \\
11 / 4 \\
11 / 2\end{array}$ & $\begin{array}{c}\text { Lbs. } \\
0.07 \\
.10 \\
.14 \\
.20 \\
.26\end{array}$ & $\begin{array}{r}L b s . \\
0.42 \\
.60 \\
.84 \\
1.20 \\
1.56\end{array}$ & \begin{tabular}{r}
\multicolumn{1}{c}{ Lbs. } \\
2.400 \\
4,600 \\
6,600 \\
9,000 \\
11,800
\end{tabular} & $\begin{array}{c}\text { Lbs. } \\
534 \\
1,022 \\
1,470 \\
2,000 \\
2,620\end{array}$ & 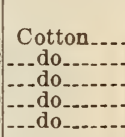 & $\begin{array}{c}\text { Hard fiber. } \\
\text { Do. } \\
\text { Do. } \\
\text { Do. } \\
\text { Do. }\end{array}$ \\
\hline $\begin{array}{l}9 / 16 \\
5 / 8 \\
3 / 4 \\
13 / 16 \\
7 / 8\end{array}$ & $\begin{array}{l}13 / 4 \\
2 \\
21 / 4 \\
21 / 2 \\
23 / 4\end{array}$ & $\begin{array}{l}.33 \\
.42 \\
.59 \\
.68 \\
.80\end{array}$ & $\begin{array}{l}1.98 \\
2.52 \\
3.54 \\
4.08 \\
4.80\end{array}$ & $\begin{array}{l}15,000 \\
18,500 \\
26,400 \\
31,100 \\
35,300\end{array}$ & $\begin{array}{l}3,330 \\
4,110 \\
5,870 \\
6,910 \\
7,840\end{array}$ & \begin{tabular}{l} 
Hard fiber \\
- do do \\
\hdashline do \\
- do
\end{tabular} & $\begin{array}{l}\text { Do. } \\
\text { Do. } \\
\text { Do. } \\
\text { Do. } \\
\text { Do. }\end{array}$ \\
\hline $\begin{array}{l}1 \\
11 / 16 \\
11 / 8 \\
13 / 16 \\
11 / 4\end{array}$ & $\begin{array}{l}3 \\
31 / 4 \\
31 / 2 \\
33 / 4 \\
4\end{array}$ & $\begin{array}{l}1.05 \\
1.18 \\
1.33 \\
1.47 \\
1.63\end{array}$ & $\begin{array}{l}6.30 \\
7.08 \\
7.98 \\
8.82 \\
9.78\end{array}$ & $\begin{array}{l}46,000 \\
51,000 \\
58,000 \\
64,500 \\
71,500\end{array}$ & $\begin{array}{l}10,200 \\
11,300 \\
12,900 \\
14,300 \\
15,900\end{array}$ & 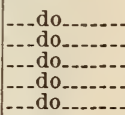 & $\begin{array}{l}\text { Do. } \\
\text { Do. } \\
\text { Do. } \\
\text { Do. } \\
\text { Do. }\end{array}$ \\
\hline $\begin{array}{l}13 / 8 \\
17 / 16 \\
11 / 2 \\
15 / 8 \\
111 / 16\end{array}$ & $\begin{array}{l}41 / 4 \\
416 \\
43 / 4 \\
5 \\
51 / 4\end{array}$ & $\begin{array}{l}2.00 \\
2.16 \\
2.36 \\
2.76 \\
2.94\end{array}$ & $\begin{array}{l}12.00 \\
13.00 \\
14.20 \\
16.60 \\
17.60\end{array}$ & $\begin{array}{r}85,000 \\
93,200 \\
101,000 \\
118,000 \\
127,000\end{array}$ & $\begin{array}{l}18,900 \\
20,700 \\
22,400 \\
26,200 \\
28,200\end{array}$ & $\begin{array}{l}- \text { - do } \\
- \text { do }\end{array}$ & $\begin{array}{l}\text { Do. } \\
\text { Do. } \\
\text { Do. } \\
\text { Do. } \\
\text { Do. }\end{array}$ \\
\hline $\begin{array}{l}13 / 4 \\
113 / 16 \\
115 / 16 \\
2 \\
21 / 16\end{array}$ & $\begin{array}{l}516 \\
53 / 4 \\
6 \\
61 / 4 \\
615\end{array}$ & \begin{tabular}{l|}
3.23 \\
3.42 \\
3.83 \\
4.20 \\
4.43
\end{tabular} & $\begin{array}{l}19.40 \\
20.50 \\
23.30 \\
25.20 \\
26.60\end{array}$ & $\begin{array}{l}136,000 \\
146,000 \\
167,000 \\
178,000 \\
189,000\end{array}$ & $\begin{array}{l}30,200 \\
32,400 \\
37,100 \\
39,600 \\
42,000\end{array}$ & 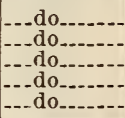 & $\begin{array}{l}\text { Do. } \\
\text { Do. } \\
\text { Do. } \\
\text { Do. } \\
\text { Do. }\end{array}$ \\
\hline
\end{tabular}

\section{Type H, 6 by 12 Phosphor Bronze}

Wire rope of type $H$ shall be made as described and shown in Figure 67, and shall have the properties given in Table 11.

Construction.-One diameter of wire, one operation. Six strands of 12 wires each, total 72 wires. Six strand cores and 1 main core, total 7 cores.

Material.-Wires: Phosphor bronze. Cores. (See Table 11.)

Diameter.-The difference between the nominal diameter of wire rope, type $H$, and the actual 
diameter shall not be greater than the tolerances given in Table 3.

Lay. - Wire rope, type $\mathrm{H}$, shall be regular lay, and the lay shall not be greater than six and three-fourths times the nominal diameter.

TABLE 11.-Properties of wire rope, type $H$

\begin{tabular}{|c|c|c|c|c|c|c|c|}
\hline \multirow[b]{2}{*}{ 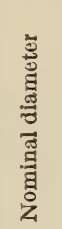 } & \multirow{2}{*}{ 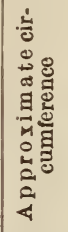 } & \multirow{2}{*}{ 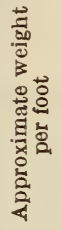 } & \multirow{2}{*}{ 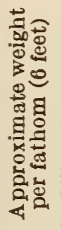 } & \multirow{2}{*}{ 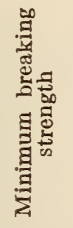 } & \multirow{2}{*}{ 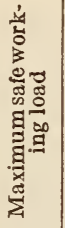 } & \multicolumn{2}{|c|}{ Core } \\
\hline & & & & & & Strand & Main \\
\hline $\begin{array}{r}\text { Inch } \\
1 / 4 \\
5116 \\
38 \\
7 / 16 \\
1 / 2\end{array}$ & $\begin{array}{c}\text { Inches } \\
3 / 4 \\
1 \\
118 \\
114 \\
116\end{array}$ & $\begin{array}{l}\text { Lbs. } \\
0.075 \\
.115 \\
.168 \\
.227 \\
.295\end{array}$ & $\begin{array}{r}\text { Lbs. } \\
0.45 \\
.69 \\
1.01 \\
1.36 \\
1.77\end{array}$ & $\begin{array}{l}\text { Lbs. } \\
1,400 \\
2,100 \\
3,000 \\
4,000 \\
5,200\end{array}$ & $\begin{array}{r}L b s . \\
310 \\
460 \\
670 \\
890 \\
1,160\end{array}$ & $\begin{array}{c}\text { Cotton } \\
\text { do do... }\end{array}$ & $\begin{array}{c}\text { Hard fiber. } \\
\text { Do. } \\
\text { Do. } \\
\text { Do. } \\
\text { Do. }\end{array}$ \\
\hline $\begin{array}{r}910 \\
58 \\
34 \\
1316 \\
7 / 8\end{array}$ & $\begin{array}{l}13 / 4 \\
2 \\
21 / 4 \\
21 / 2 \\
231\end{array}$ & $\begin{array}{l}.370 \\
.460 \\
.660 \\
.766 \\
.895\end{array}$ & \begin{tabular}{l|}
2.22 \\
2.76 \\
3.96 \\
4.60 \\
5.37
\end{tabular} & $\begin{array}{r}6,500 \\
8,100 \\
11,600 \\
13,500 \\
15,700\end{array}$ & $\begin{array}{l}1,450 \\
1,800 \\
2,580 \\
3,000 \\
3,490\end{array}$ & \begin{tabular}{l} 
Hard fiber \\
- \\
\hdashline do do
\end{tabular} & $\begin{array}{l}\text { Do. } \\
\text { Do. } \\
\text { Do. } \\
\text { Do. } \\
\text { Do. }\end{array}$ \\
\hline
\end{tabular}

9. Types J, K, L, M, and N, 6 by 19 General

The four constructions described and shown in Figure $68,(a),(b),(c)$, and $(d)$, may be used at the option of the seller or manufacturer for wire rope of

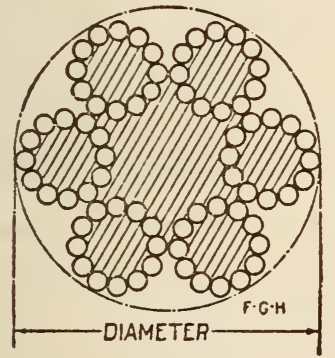

FigURe 67.-Type $H, 6$

by 12, phosphor bronze

types $J, K, L, M$, and $N$. If Seale construction is desired by the purchaser, this requirement shall be so stated in the order.

Construction.-(a) One diameter of wire, two operations. Six strands of 19 wires each, total 114 wires. All the wires in each strand shall be laid left hand. All the outside wires of each strand shall be of the same diameter. One main core.

(b) Three diameters of wire (Warrington), one operation. Six strands of 19 wires each, total 114 wires. The outside wires of each strand shall be of large and small diameter wires, laid alternately. One main core.

(c) Three diameters of wire (Seale) one operation. Six strands of 19 wires each total 114 wires. All the outside wires of each strand shall be of the same diameter. All the inside wires in each strand, except the central wire, shall be of the same diameter. One main core.

(d) Three diameters of wire and filler wires, one operation. Six strands of 25 wires each, total 150 wires. All the outside wires of each strand shall be of the same diameter. All the inside wires of each strand except the small filler wires and the central wire, shall be of the same diameter. All the small filler wires shall be the same diameter. One main core.

(e) Diameter.-The difference between the nominal diameter of wire rope, types $J, K, L, M$, and $N$, and the actual diameter shall not be greater than the tolerances given in Table 3. (f) Lay.-Wire rope, types J, K, L, M, and N, shall be regular lay, and the lay shall not be greater than six and three-fourths times the nominal diameter, if the wires are uncoated steel or phosphor

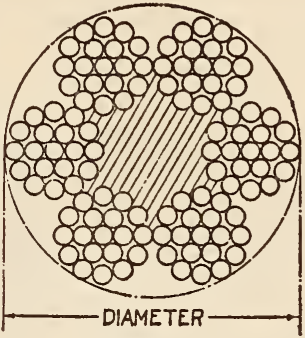

(a)

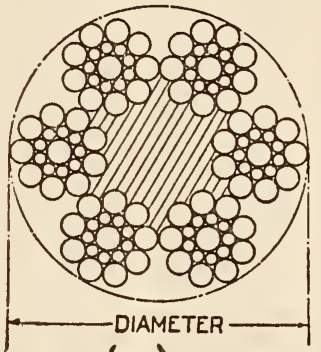

(c)

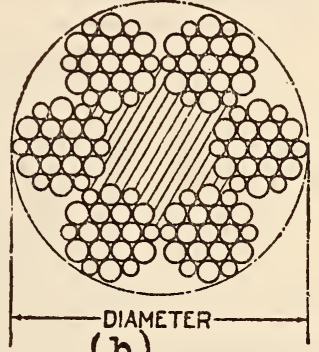

(b)

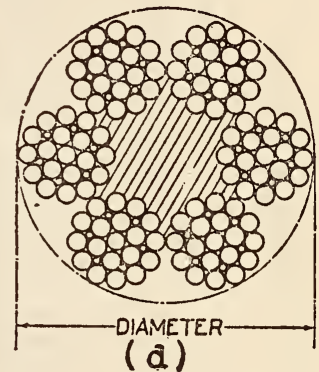

FigURe 68.-Types $J, K, L, M$, and $N ; 6$ by 19 cast steel; 6 by 19 extra strong cast steel; 6 by 19 plow steel; 6 by 19 high-made plow steel; 6 by 19 phosphor bronze.

bronze, nor more than seven and one-fourtn times the diameter, if the wires are galvanized steel.

\section{Type J, 6 by 19 Cast Steel}

Wire rope of type $J$ shall be made as described here and in IV, 9, and shall have the properties given in Table 12.

Material.-Wires: Cast steel, uncoated. Main core, hard fiber.

TABLE 12.-Properties of wire rope, type $J$

\begin{tabular}{|c|c|c|c|c|c|c|}
\hline \multirow[b]{2}{*}{$\begin{array}{l}\text { Nom- } \\
\text { inal } \\
\text { diam- } \\
\text { eter }\end{array}$} & \multirow[b]{2}{*}{$\begin{array}{c}\text { Approx- } \\
\text { imate } \\
\text { circum- } \\
\text { ference }\end{array}$} & \multirow[b]{2}{*}{$\begin{array}{c}\text { Approx- } \\
\text { imate } \\
\text { weight } \\
\text { per } \\
\text { foot }\end{array}$} & \multirow[b]{2}{*}{$\begin{array}{c}\text { Approx- } \\
\text { imate } \\
\text { weight } \\
\text { per } \\
\text { fathom } \\
\text { (6 feet) }\end{array}$} & \multicolumn{2}{|c|}{ Uncoated } & \multirow[b]{2}{*}{$\begin{array}{l}\text { Recom- } \\
\text { mended } \\
\text { minimum } \\
\text { diameter } \\
\text { of sheave } \\
\text { or drum }\end{array}$} \\
\hline & & & & $\begin{array}{c}\text { Mini- } \\
\text { mum } \\
\text { breaking } \\
\text { strength }\end{array}$ & $\begin{array}{l}\text { Maxi- } \\
\text { mum } \\
\text { safe } \\
\text { working } \\
\text { load }^{1}\end{array}$ & \\
\hline $\begin{array}{c}\text { Inches } \\
1 / 4 \\
5 / 16 \\
3 / 8 \\
7 / 16 \\
1 / 2 \\
9 / 16\end{array}$ & $\begin{array}{c}\text { Inches } \\
3 / 4 \\
1 \\
11 / 8 \\
11 / 4 \\
112 \\
13 / 4\end{array}$ & \begin{tabular}{|c} 
Pounds \\
0.10 \\
.15 \\
.22 \\
.30 \\
.39 \\
.50
\end{tabular} & $\begin{array}{c}\text { Pounds } \\
0.60 \\
.90 \\
1.32 \\
1.80 \\
2.34 \\
3.00\end{array}$ & $\begin{array}{r}\text { Pounds } \\
3,800 \\
6,000 \\
8,400 \\
11,600 \\
15,000 \\
19,000\end{array}$ & $\begin{array}{r}\text { Pounds } \\
880 \\
1,240 \\
1,920 \\
2,600 \\
3,360 \\
4,000\end{array}$ & $\begin{array}{l}\text { Feet } \\
1.0 \\
1.2 \\
1.5 \\
1.8 \\
2.0 \\
2.2\end{array}$ \\
\hline $\begin{array}{l}5 / 6 \\
3 / 4 \\
7 / 8 \\
1 \\
11 / 8\end{array}$ & $\begin{array}{l}2 \\
21 / 4 \\
23 / 4 \\
3 \\
31 / 2\end{array}$ & $\begin{array}{r}.62 \\
.89 \\
1.20 \\
1.58 \\
2.00\end{array}$ & $\begin{array}{r}3.72 \\
5.34 \\
7.20 \\
9.48 \\
12.00\end{array}$ & $\begin{array}{l}23,000 \\
31,600 \\
41,400 \\
54,000 \\
67,000\end{array}$ & $\begin{array}{r}5,000 \\
7,000 \\
9,200 \\
12,000 \\
14,900\end{array}$ & $\begin{array}{l}2.5 \\
3.0 \\
3.5 \\
4.0 \\
4.5\end{array}$ \\
\hline $\begin{array}{l}11 / 4 \\
13 / 8 \\
11 / 2 \\
15 \% \\
13 / 4\end{array}$ & $\begin{array}{l}4 \\
41 / 4 \\
43 / 4 \\
5 \\
51 / 2\end{array}$ & $\begin{array}{l}2.45 \\
3.00 \\
3.55 \\
4.15 \\
4.85\end{array}$ & $\begin{array}{l}14.70 \\
18.00 \\
21.30 \\
24.90 \\
29,10\end{array}$ & $\begin{array}{r}82,500 \\
98,000 \\
115,000 \\
133,000 \\
153,000\end{array}$ & $\begin{array}{l}18,300 \\
21,800 \\
25,500 \\
29,500 \\
34,000\end{array}$ & $\begin{array}{l}5.0 \\
5.5 \\
6.0 \\
6.5 \\
7.0\end{array}$ \\
\hline $\begin{array}{l}17 / 8 \\
2 \\
21 / 4 \\
21 / 2 \\
23 / 4\end{array}$ & $\begin{array}{l}53 / 4 \\
6114 \\
718 \\
77 \% 8 \\
85,8\end{array}$ & $\begin{array}{r}5.55 \\
6.30 \\
8.00 \\
9.85 \\
11.95\end{array}$ & $\begin{array}{l}33.30 \\
37.80 \\
48.00 \\
59.10 \\
71.70\end{array}$ & $\begin{array}{l}175,000 \\
200,000 \\
253,000 \\
313,000 \\
379,000\end{array}$ & $\begin{array}{l}38,900 \\
44,400 \\
56,200 \\
69,600 \\
84,200\end{array}$ & $\begin{array}{r}7.5 \\
8.0 \\
9.0 \\
10.0 \\
11.0\end{array}$ \\
\hline
\end{tabular}

1 If the diameter of the drum or sheave is less than the recommended diameter, lower safe working loads should be used. 
11. Type K, 6 by 19, Extra Strong Cast Steel

Wire rope of type $K$ shall be made as described here and in IV, 9 , and shall have the properties given in Table 13

Material.-Wires: Extra strong cast steel, either uncoated or galvanized. Main core, hard fiber.

TABLE 13.-Properties of wire rope, type $K$

\begin{tabular}{|c|c|c|c|c|c|c|c|c|}
\hline \multirow{2}{*}{ 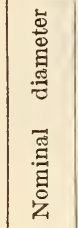 } & \multirow{2}{*}{ 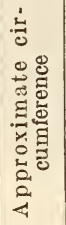 } & \multirow{2}{*}{ 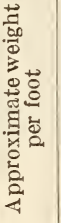 } & \multirow{2}{*}{ 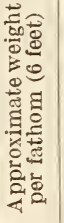 } & \multicolumn{2}{|c|}{ Uncosted } & \multicolumn{2}{|c|}{ Galvanized } & \multirow{2}{*}{ 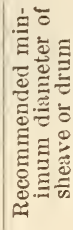 } \\
\hline & & & & 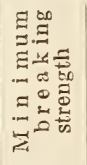 & 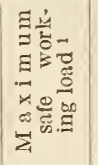 & 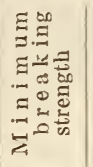 & 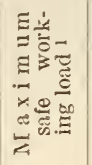 & \\
\hline 916 & $\begin{array}{r}\text { Inches } \\
3 / 4\end{array}$ & $\begin{array}{c}L b s . \\
0.10 \\
.15 \\
.22 \\
.30 \\
.39 \\
.50\end{array}$ & $\begin{array}{c}\text { Lbs. } \\
0.60 \\
.90 \\
1.32 \\
1.80 \\
2.34 \\
3.00\end{array}$ & $\begin{array}{r}\text { Pounds } \\
4,200 \\
6,500 \\
9,200 \\
12,600 \\
16,400 \\
20,600\end{array}$ & $\begin{array}{r}\text { Pounds } \\
930 \\
1,440 \\
2,040 \\
2,800 \\
3,640 \\
4,580\end{array}$ & $\begin{array}{r}\text { Pounds } \\
3,780 \\
5,840 \\
8,270 \\
11,300 \\
14,800 \\
18,500\end{array}$ & Pounds & \\
\hline 56 & $\begin{array}{l}2 \\
21 / 4 \\
23 / 4 \\
3 \\
31 / 2\end{array}$ & $\begin{array}{r}.62 \\
.89 \\
1.20 \\
1.58 \\
2.00\end{array}$ & $\begin{array}{r}3.72 \\
5,34 \\
7.20 \\
9.48 \\
12.00\end{array}$ & $\begin{array}{l}25,600 \\
36,400 \\
48,000 \\
62,000 \\
77,000\end{array}$ & $\begin{array}{r}5,680 \\
8,050 \\
10,700 \\
13,800 \\
17,100\end{array}$ & & 5,11 & \\
\hline & $\begin{array}{l}4 \\
41 / 4 \\
43 / 4 \\
5 \\
51 / 2\end{array}$ & $\begin{array}{l}2.45 \\
3.00 \\
3.55 \\
4.15 \\
4.85\end{array}$ & $\begin{array}{l}14.7 \\
18.0 \\
21.3 \\
24.9 \\
29.1\end{array}$ & & $\begin{array}{l}21,100 \\
25,100 \\
29,500 \\
34,200 \\
39,600\end{array}$ & & & \\
\hline $\begin{array}{l}17 \\
2 \\
21\end{array}$ & $\begin{array}{l}77 / 8 \\
85 / 8\end{array}$ & $\begin{array}{r}5.55 \\
6.30 \\
8.00 \\
9.85 \\
11.95\end{array}$ & $\begin{array}{l}33.3 \\
37.8 \\
48.0 \\
59.1 \\
71.7\end{array}$ & $\begin{array}{l}202,000 \\
231,000 \\
292,000 \\
362,000 \\
437,000\end{array}$ & $\begin{array}{r}44,900 \\
51,300 \\
64,800 \\
80,400 \\
97,100\end{array}$ & $\begin{array}{l}262,000 \\
326,000 \\
393,000\end{array}$ & $\begin{array}{l}40,400 \\
46,200 \\
58,200 \\
72,400 \\
87,200\end{array}$ & 11. \\
\hline
\end{tabular}

1 If the diameter of the drum or sheaves is less than the recommended diameter, lower safe working loads should be used

\section{Type L, 6 by 19 Plow Steel}

Wire rope of type $\mathrm{L}$ shall be made as described here and in IV, 9, and shall have the properties given in Table 14.

Material.-Wires: Plow steel either uncoated or galvanized. Main core, hard fiber.

TABLE 14.- Properties of wire rope, type $L$

\begin{tabular}{|c|c|c|c|c|c|c|c|c|}
\hline \multirow{2}{*}{ 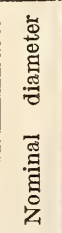 } & \multirow{2}{*}{ 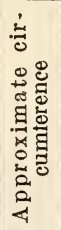 } & \multirow{2}{*}{ 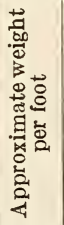 } & \multirow{2}{*}{ 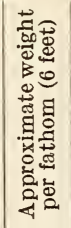 } & \multicolumn{2}{|c|}{ Uncoated } & \multicolumn{2}{|c|}{ Galvanized } & \multirow{2}{*}{ 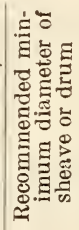 } \\
\hline & & & & 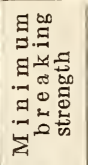 & 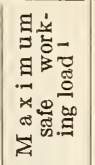 & 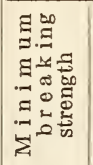 & 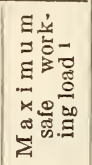 & \\
\hline 92 & $\begin{array}{r}\text { nches } \\
3 / 4 \\
1 \\
11 / 8 \\
114 \\
11 / 2 \\
13 / 4\end{array}$ & $\begin{array}{c}\text { Lbs. } \\
0.10 \\
.15 \\
.22 \\
.30 \\
.39 \\
.50\end{array}$ & $\begin{array}{c}\text { Lbs. } \\
0.60 \\
.90 \\
1.32 \\
1.80 \\
2.34 \\
3.00\end{array}$ & $\begin{array}{r}\text { Pounds } \\
4,500 \\
7,000 \\
10,100 \\
13,800 \\
17,900 \\
22,600\end{array}$ & \begin{tabular}{|r|} 
Pounds \\
1,000 \\
1,560 \\
2,240 \\
3,070 \\
3,980 \\
5,020
\end{tabular} & \begin{tabular}{|r|} 
Pounds \\
4,050 \\
6,300 \\
9,100 \\
12,400 \\
16,100 \\
20,400
\end{tabular} & $\begin{array}{r}\text { ourds } \\
900 \\
1,400\end{array}$ & $\begin{array}{l}2.0 \\
2.2\end{array}$ \\
\hline $\begin{array}{l}5 / 8 \\
334 \\
76\end{array}$ & $\begin{array}{l}2 \\
21 / 4 \\
23 / 4 \\
3 \\
31 / 2\end{array}$ & $\begin{array}{r}.62 \\
.89 \\
1.20 \\
1.58 \\
2.00\end{array}$ & $\begin{array}{r}3.72 \\
5.34 \\
7.20 \\
9.48 \\
12.00\end{array}$ & & $\begin{array}{r}6,200 \\
8,890 \\
11,800 \\
15,400 \\
19,100\end{array}$ & & 5 & 2 \\
\hline $11 / 4$ & $\begin{array}{l}5 \\
51 / 2\end{array}$ & & $\begin{array}{l}21.30 \\
24.90 \\
29.10\end{array}$ & & & & & \\
\hline $\begin{array}{l}17 / 8 \\
2 \\
21 / 4 \\
21 / 2 \\
23 / 4\end{array}$ & $53 / 4$ & 5.55 & & $\begin{array}{l}231,000 \\
263,000 \\
333,000 \\
412,000 \\
496,000\end{array}$ & & & & 10.0 \\
\hline
\end{tabular}

1 If the diameter of the drum or sheave is less than the recommended diameter, lower safe working loads should be used.

\section{Type $\mathbf{M}, 6$ by 19 High-Grade Plow Steel}

Wire rope of type $M$ shall be made as described here and in IV, 9, and shall have the properties given in Table 15 .

Material.-Wires: High-grade plow steel, either uncoated or galvanized. Main core, hard fiber.

TABLE 15.-Properties of wire rope, type $M$

\begin{tabular}{|c|c|c|c|c|c|c|c|c|}
\hline 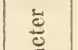 & $\stackrel{\check{0}}{0}$ & 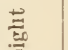 & $\overrightarrow{\mathrm{z}}$ & Unco & ated & Galra & nized & 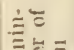 \\
\hline 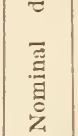 & 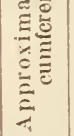 & 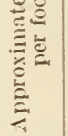 & 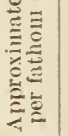 & 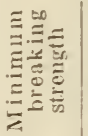 & 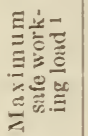 & 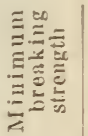 & 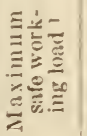 & 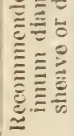 \\
\hline 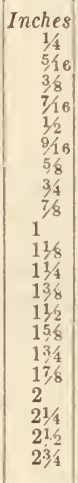 & $\begin{array}{r}\text { Inches } \\
3 / 4 \\
1 \\
11 / 8 \\
114 \\
11 / 2 \\
13 / 4 \\
2 \\
21 / 4 \\
23 / 4 \\
3 \\
31 / 2 \\
4 \\
414 \\
434 \\
5 \\
51 / 2 \\
534 \\
614 \\
71 / 8 \\
77 / 8 \\
555\end{array}$ & \begin{tabular}{r}
\multicolumn{1}{c}{$L$ bs. } \\
0.10 \\
.15 \\
.22 \\
.30 \\
.39 \\
.50 \\
.62 \\
.89 \\
1.20 \\
1.55 \\
2.00 \\
2.45 \\
3.00 \\
3.55 \\
4.15 \\
4.85 \\
5.55 \\
6.30 \\
8.00 \\
9.85 \\
11.95
\end{tabular} & $\begin{array}{c}L b s . \\
0.60 \\
.90 \\
1.32 \\
1.80 \\
2.34 \\
3.00 \\
3.72 \\
5.34 \\
7.20 \\
9.48 \\
12.00 \\
14.70 \\
18.00 \\
21.30\end{array}$ & \begin{tabular}{r}
\multicolumn{1}{c}{ Lbs. } \\
5,200 \\
7,800 \\
11,400 \\
15,600 \\
20,200 \\
25,600 \\
31,800 \\
46,000 \\
62,000 \\
80,000 \\
100,000 \\
123,000 \\
144,000 \\
175,000 \\
203,000 \\
237,000 \\
269,000 \\
302,000 \\
383,000 \\
472.000 \\
570,000
\end{tabular} & \begin{tabular}{r}
\multicolumn{1}{c}{$L b s}$. \\
1,160 \\
1,730 \\
2,530 \\
3,460 \\
4,490 \\
5,690 \\
7,060 \\
10,200 \\
13,800 \\
17,800 \\
22,200 \\
27,300 \\
32,900 \\
35,900 \\
45,100 \\
52,700 \\
59,800 \\
67,100 \\
85,100 \\
105,000 \\
127,000
\end{tabular} & 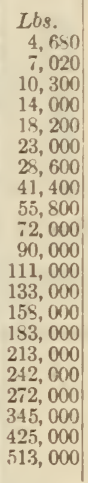 & $\begin{array}{r}\text { Lbs. } \\
1.040 \\
1,560 \\
2,290 \\
3,110 \\
4,040 \\
5,110 \\
6,360 \\
9,200 \\
12,400 \\
16,000 \\
20,000 \\
24,700 \\
29,500 \\
35,100 \\
40,600 \\
47,400 \\
53,500 \\
60,400 \\
76,600 \\
94,400 \\
114,000\end{array}$ & $\begin{array}{r}\text { Feet } \\
1.0 \\
1.2 \\
1.5 \\
1.8 \\
2.0 \\
2.2 \\
2.5 \\
3.0 \\
3.5 \\
4.0 \\
4.5 \\
5.0 \\
5.5 \\
6.0 \\
6.5 \\
7.0 \\
7.5 \\
8.0 \\
9.0 \\
10.0 \\
11.0\end{array}$ \\
\hline
\end{tabular}

${ }^{1}$ If the diameter of the drum or sheare is less than the recommended diameter, lower safe working loads should be used.

\section{Type $N, 6$ by 19 Phosphor Bronze}

Wire rope of type $\mathrm{N}$ shall be made as described here and shown in Figure 68, and shall have the properties given in Table 16 .

Material.-Wires: Phosphor bronze. Main core, hard fiber.

TABLE 16.-Properties of wire rope, type $N$

\begin{tabular}{|c|c|c|c|c|c|}
\hline $\begin{array}{l}\text { Nominal } \\
\text { diameter }\end{array}$ & $\begin{array}{l}\text { Approxi- } \\
\text { mate } \\
\text { circum- } \\
\text { ference }\end{array}$ & $\begin{array}{l}\text { Approxi- } \\
\text { mate } \\
\text { weight } \\
\text { per foot }\end{array}$ & $\begin{array}{l}\text { Approxi- } \\
\text { mate } \\
\text { weight } \\
\text { per } \\
\text { fathom } \\
\text { (6 feet) }\end{array}$ & $\begin{array}{l}\text { Mini- } \\
\text { mum } \\
\text { breaking } \\
\text { strength }\end{array}$ & $\begin{array}{l}\text { Maxi- } \\
\text { mum } \\
\text { safe } \\
\text { working } \\
\text { load }\end{array}$ \\
\hline $\begin{array}{c}\text { Inch } \\
1 / 4 \\
5116 \\
318 \\
716 \\
1 / 2\end{array}$ & $\begin{array}{r}\text { Inches } \\
3 / 4 \\
1 \\
118 \\
114 \\
11 / 2\end{array}$ & $\begin{array}{c}\text { Pounds } \\
0.110 \\
.160 \\
.230 \\
.320 \\
.410\end{array}$ & $\begin{array}{c}\text { Pounds } \\
0.660 \\
.960 \\
1.380 \\
1.920 \\
2.460\end{array}$ & $\begin{array}{c}\text { Pounds } \\
1,900 \\
3,000 \\
4,400 \\
5,900 \\
7,700\end{array}$ & $\begin{array}{c}\text { Pounds } \\
422 \\
666 \\
978 \\
1,310 \\
1,710\end{array}$ \\
\hline $\begin{array}{l}9 / 16 \\
5 / 8 \\
3 / 4 \\
13 / 16 \\
7 / 8\end{array}$ & $\begin{array}{l}13 / 4 \\
2 \\
21 / 4 \\
21 / 2 \\
23 / 4\end{array}$ & $\begin{array}{r}.520 \\
.640 \\
.920 \\
1.080 \\
1.260\end{array}$ & $\begin{array}{l}3.120 \\
3.840 \\
5.520 \\
6.480 \\
7.560\end{array}$ & $\begin{array}{r}9,800 \\
12,100 \\
17,100 \\
20,200 \\
23,400\end{array}$ & $\begin{array}{l}2,180 \\
2,690 \\
3,800 \\
4,480 \\
5,200\end{array}$ \\
\hline
\end{tabular}

\section{Type $P, 6$ by 24 Cast Steel}

Wire rope of type $\mathrm{P}$ shall be made as described and shown in Figure 69 and shall have the properties given in Table 17.

Construction. - Either 1 diameter of wire, 2 operations (9 inside, 15 outside wires), or 3 diameters of wire, 1 operation ( 8 inside, 16 outside wires). Six strands of 24 wires each, either 9 inside and 15 outside wires as shown, or 8 inside and 16 outside 
wires, total 144 wires. Six strand cores and 1 main core, total 7 cores.

Material.-Wires: Cast steel, galvanized. Cores (see Table 17).

Diameter. - The difference between the nominal diameter of wire rope, type $P$, and the actual diameter shall not be greater than the tolerances given in Table 3 .

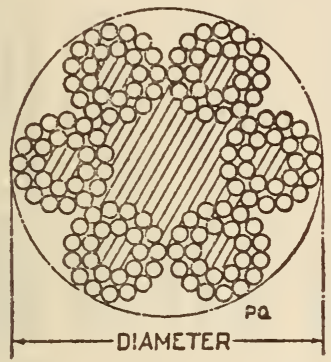

Figure 69.-Type $P$, 6 by 24 , cast steel

Lay.-Wire rope, type $\mathrm{P}$, shall be regular lay, and the lay shall not be greater than seven and onefourth times the nominal diameter.

TABLE 17.-Properties of wire rope, type $P$

\begin{tabular}{|c|c|c|c|c|c|c|c|}
\hline \multirow[b]{2}{*}{ 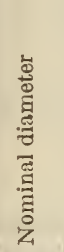 } & \multirow{2}{*}{ 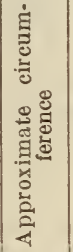 } & \multirow{2}{*}{ 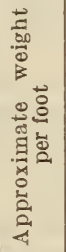 } & \multirow[b]{2}{*}{ 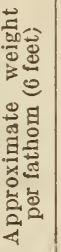 } & \multicolumn{2}{|c|}{ Galvanized } & \multicolumn{2}{|c|}{ Core } \\
\hline & & & & 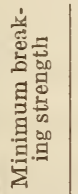 & 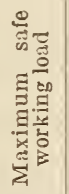 & Strand & Main \\
\hline $\begin{array}{c}\text { Inches } \\
3 / 4 \\
13 / 16 \\
7 / 8 \\
1 \\
11 / 16 \\
118\end{array}$ & $\begin{array}{r}\text { Inches } \\
21 / 4 \\
21 / 2 \\
23 / 4 \\
3 \\
31 / 4 \\
31 / 2\end{array}$ & $\begin{array}{c}L b s . \\
0.78 \\
.90 \\
1.05 \\
1.38 \\
1.56 \\
1.75\end{array}$ & $\begin{array}{r}\text { Lbs. } \\
4.68 \\
5.40 \\
6.30 \\
8.28 \\
9.36 \\
10.50\end{array}$ & $\begin{array}{c}\text { Lbs. } \\
26,000 \\
31,000 \\
36,000 \\
48,000 \\
54,000 \\
60,000\end{array}$ & \begin{tabular}{r}
\multicolumn{1}{c}{$L$ bs. } \\
5,780 \\
6,880 \\
8,000 \\
10,700 \\
12,000 \\
13,300
\end{tabular} & $\begin{array}{l}\text { Cotton } \\
\text { do } \\
\text { Hard fo fiber } \\
\text { Hado }\end{array}$ & $\begin{array}{c}\text { Hard fiber. } \\
\text { Do. } \\
\text { Do. } \\
\text { Do. } \\
\text { Do. } \\
\text { Do. }\end{array}$ \\
\hline $\begin{array}{l}13 / 16 \\
11 / 4 \\
13 / 8 \\
17 / 10\end{array}$ & $\begin{array}{l}33 / 4 \\
4 \\
41 / 4 \\
41 / 2\end{array}$ & $\begin{array}{l}1.96 \\
2.17 \\
2.65 \\
2.90\end{array}$ & $\begin{array}{l}11.80 \\
13.00 \\
15.90 \\
17.40\end{array}$ & $\begin{array}{l}67,000 \\
74,000 \\
90,000 \\
99,000\end{array}$ & $\begin{array}{l}14,900 \\
16,400 \\
20,000 \\
22,000\end{array}$ & 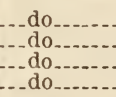 & $\begin{array}{l}\text { Do. } \\
\text { Do. } \\
\text { Do. } \\
\text { Do. }\end{array}$ \\
\hline $\begin{array}{l}11 / 2 \\
15 / 6 \\
11116 \\
134\end{array}$ & \begin{tabular}{l|}
$43 / 4$ \\
5 \\
514 \\
$51 / 2$
\end{tabular} & $\begin{array}{l}3.15 \\
3.68 \\
3.97 \\
4.27\end{array}$ & $\begin{array}{l}18.90 \\
22.10 \\
23.80 \\
25.60\end{array}$ & $\begin{array}{l}108,000 \\
126,000 \\
136,000 \\
146,000\end{array}$ & $\begin{array}{l}24,000 \\
28,000 \\
30,200 \\
32,400\end{array}$ & $\begin{array}{l}\text {. do } \\
\text { do } \\
\\
\end{array}$ & $\begin{array}{l}\text { Do. } \\
\text { Do. } \\
\text { Do. } \\
\text { Do. }\end{array}$ \\
\hline $\begin{array}{l}113 / 10 \\
115 / 16 \\
2 \\
21 / 16\end{array}$ & $\begin{array}{l}53 / 4 \\
6 \\
61 / 4 \\
612\end{array}$ & $\begin{array}{l}4.57 \\
5.22 \\
5.55 \\
5.77\end{array}$ & $\begin{array}{l}27.40 \\
31.30 \\
33.30 \\
34.60\end{array}$ & $\begin{array}{l}157,000 \\
178,000 \\
190,000 \\
202,000\end{array}$ & $\begin{array}{l}34,900 \\
39,600 \\
42,200 \\
44,900\end{array}$ & \begin{tabular}{l}
-.-do \\
\hdashline- do $_{-}$
\end{tabular} & $\begin{array}{l}\text { Do. } \\
\text { Do. } \\
\text { Do. } \\
\text { Do. }\end{array}$ \\
\hline
\end{tabular}

16. Type Q, 6 by 24 High-Grade Plow Steel

Wire rope of type $\mathrm{Q}$ shall be made as described and shown in Figure 70 and shall have the properties given in Table 18.

Construction.- Either 1 diameter of wire, 2 operations (9 inside, 15 outside wires) or 3 diameters of wire, 1 operation ( 8 inside, 16 outside wires). Six strands of 24 wires each, either 9 inside wires and 15 outside wires, as shown, or 8 inside and 16 outside wires, total 144 wires. Six strand cores and 1 main core, total 7 cores.

Material.-Wires: High-grade plow steel, galvanized. Cores (see Table 18).

Diameter. - The difference between the nominal diameter of wire rope, type $Q$, and the actual diameter shall not be greater than the tolerances given in Table 3.
Lay.-Wire rope, type $\mathrm{Q}$, shall be regular lay, and the lay shall not be greater than seven and one-fourth times the nominal diameter.

TABLE 18.-Properties of wire rope, type $Q$

\begin{tabular}{|c|c|c|c|c|c|c|c|}
\hline \multirow[b]{2}{*}{ 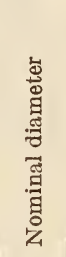 } & \multirow{2}{*}{ 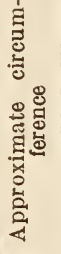 } & \multirow{2}{*}{ 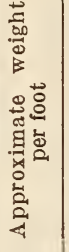 } & \multirow{2}{*}{ 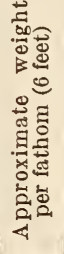 } & \multicolumn{2}{|c|}{ Galvanized } & \multicolumn{2}{|c|}{ Core } \\
\hline & & & & 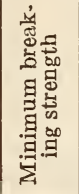 & 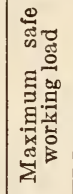 & Strand & Main \\
\hline $\begin{array}{c}\text { Inches } \\
3 / 4 \\
13 / 16 \\
1^{7 / 8} \\
1116\end{array}$ & $\begin{array}{r}\text { Inches } \\
21 / 4 \\
21 / 2 \\
23 / 4 \\
3 \\
31 / 4\end{array}$ & $\begin{array}{l}\text { Lbs. } \\
0.78 \\
.90 \\
1.05 \\
1.38 \\
1.56\end{array}$ & $\begin{array}{c}\text { Lbs. } \\
4.68 \\
5.40 \\
6.30 \\
8.28 \\
9.36\end{array}$ & $\begin{array}{l}\text { Lbs. } \\
34,000 \\
39,000 \\
45,000 \\
59,000 \\
67,000\end{array}$ & $\begin{array}{l}\text { Lbs. } \\
7,550 \\
8,660 \\
10,000 \\
13,100 \\
14,900\end{array}$ & \begin{tabular}{l} 
Cotton \\
\hdashline do \\
do \\
Hard fiber \\
do
\end{tabular} & $\begin{array}{c}\text { Hard fiber. } \\
\text { Do. } \\
\text { Do. } \\
\text { Do. } \\
\text { Do. }\end{array}$ \\
\hline $\begin{array}{l}11 / 8 \\
13 / 16 \\
11 / 4 \\
13 / 8 \\
17 / 16\end{array}$ & $\begin{array}{l}31 / 2 \\
33 / 4 \\
4 \\
41 / 4 \\
41 / 2\end{array}$ & $\begin{array}{l}1.75 \\
1.96 \\
2.17 \\
2.65 \\
2.90\end{array}$ & $\begin{array}{l}10.50 \\
11.80 \\
13.00 \\
15.90 \\
17.40\end{array}$ & $\begin{array}{r}75,000 \\
84,000 \\
93,000 \\
113,000 \\
124,000\end{array}$ & $\begin{array}{l}16,700 \\
18,700 \\
20,700 \\
25,100 \\
27,500\end{array}$ & 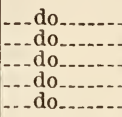 & $\begin{array}{l}\text { Do. } \\
\text { Do. } \\
\text { Do. } \\
\text { Do. } \\
\text { Do. }\end{array}$ \\
\hline $\begin{array}{l}11 / 2 \\
15 / 8 \\
111 / 16 \\
13 / 4\end{array}$ & $\begin{array}{l}43 / 4 \\
5 \\
51 / 4 \\
51 / 2\end{array}$ & \begin{tabular}{l|}
3.15 \\
3.68 \\
3.97 \\
4.27
\end{tabular} & $\begin{array}{l}18.90 \\
22.10 \\
23.80 \\
25.60\end{array}$ & $\begin{array}{l}135,000 \\
158,000 \\
170,000 \\
183,000\end{array}$ & $\begin{array}{l}30,000 \\
35,100 \\
37,800 \\
40,600\end{array}$ & 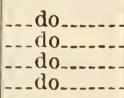 & $\begin{array}{l}\text { Do. } \\
\text { Do. } \\
\text { Do. } \\
\text { Do. }\end{array}$ \\
\hline $\begin{array}{l}113 / 16 \\
115 / 16 \\
2 \\
21 / 16\end{array}$ & $\begin{array}{l}53 / 4 \\
6 \\
61 / 4 \\
61 / 2\end{array}$ & $\begin{array}{l}4.57 \\
5.22 \\
5.55 \\
5.77\end{array}$ & \begin{tabular}{r|}
27.40 \\
31.30 \\
33.30 \\
34.60
\end{tabular} & $\begin{array}{l}196,000 \\
224,000 \\
238,000 \\
248,000\end{array}$ & $\begin{array}{l}43,600 \\
49,800 \\
52,900 \\
55,100\end{array}$ & $\begin{array}{l}\text { do } \\
\text { do } \\
\text { do } \\
\text { do do }\end{array}$ & $\begin{array}{l}\text { Do. } \\
\text { Do. } \\
\text { Do. } \\
\text { Do. }\end{array}$ \\
\hline
\end{tabular}

17. Type R, 6 by 37 Extra Strong Cast Steel

Wire rope of type $R$ shall be made as described and shown in Figure 71 and shall have the properties given in Table 19.

Construction.- Either 1 diameter of wire, 3 operations, or 4 or 5 diameters of wire, 2 operations.

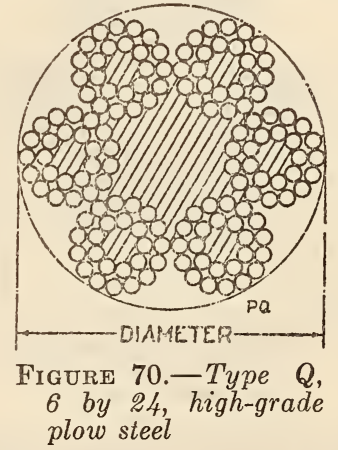

Six strands. Each strand shall have not less than 37 nor more than 43 wires. The number of outside wires of each strand shall be not less than 18 nor more than 20. Total 222 to 258 wires. One main core.

Material.-Wires: Extra strong cast steel, either uncoated or galvanized. Main core. Hard fiber.

Diameter.-The difference between the nominal diameter of wire rope, type $R$, and the actual diameter shall not be greater than the tolerances given in Table 3.

Lay.-Wire rope, type $\mathrm{R}$, shall be regular lay, and the lay shall not be greater than six and threefourths times the nominal diameter, if the wire is uncoated, nor greater than seven and one-fourth times the nominal diameter, if the wire is galvanized. 
TABLE 19.-Properties of wire rope, type $R$

\begin{tabular}{|c|c|c|c|c|c|c|c|c|}
\hline \multirow[b]{2}{*}{ 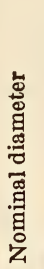 } & \multirow[b]{2}{*}{ 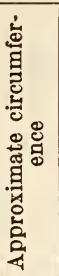 } & \multirow[b]{2}{*}{ 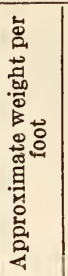 } & \multirow[b]{2}{*}{ 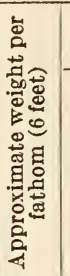 } & \multicolumn{2}{|c|}{ Uncoated } & \multicolumn{2}{|c|}{ Galvanized } & \multirow{2}{*}{ 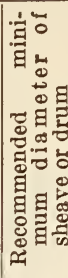 } \\
\hline & & & & 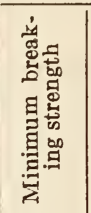 & 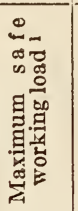 & 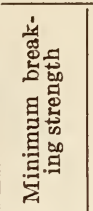 & 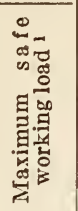 & \\
\hline $\begin{array}{l}\text { In. } \\
3.8 \\
7 / 16 \\
1 / 2 \\
9 / 16 \\
5 / 8\end{array}$ & $\begin{array}{l}\text { In. } \\
11 / 8 \\
11 / 4 \\
11 / 2 \\
13 / 4 \\
2\end{array}$ & $\begin{array}{c}\text { Lbs. } \\
0.22 \\
.30 \\
.39 \\
.50 \\
.62\end{array}$ & $\begin{array}{c}\text { Lbs. } \\
1.32 \\
1.80 \\
2.34 \\
3.00 \\
3.72\end{array}$ & $\begin{array}{r}L b s . \\
8,600 \\
11,600 \\
15,200 \\
19,000 \\
23,700\end{array}$ & $\begin{array}{c}L b s . \\
1,910 \\
2,580 \\
3,370 \\
4,220 \\
5,270\end{array}$ & \begin{tabular}{|c|}
$L b s$. \\
7,740 \\
10,400 \\
13,700 \\
17,100 \\
21,300
\end{tabular} & $\begin{array}{c}\text { Lbs. } \\
1,720 \\
2,310 \\
3,040 \\
3,800 \\
4,740\end{array}$ & \\
\hline $\begin{array}{l}3 / 4 \\
7 / 8 \\
1 \\
11 / 8 \\
11 / 4\end{array}$ & $\begin{array}{l}21 / 4 \\
23 / 4 \\
3 \\
31 / 2 \\
4\end{array}$ & $\begin{array}{r}.89 \\
1.20 \\
1.58 \\
2.00 \\
2.45\end{array}$ & $\begin{array}{r}5.34 \\
7.20 \\
9.48 \\
12.00 \\
14.70\end{array}$ & $\begin{array}{l}34,000 \\
47,000 \\
60,000 \\
74,000 \\
90,000\end{array}$ & $\begin{array}{r}7,560 \\
10,400 \\
13,300 \\
16,500 \\
20,000\end{array}$ & $\begin{array}{r}30,600 \\
42,300 \\
54,000 \\
66,600 \\
81,000\end{array}$ & $\begin{array}{r}6,800 \\
9,400 \\
12,000 \\
14,800 \\
18,000\end{array}$ & \\
\hline $\begin{array}{l}13 / 8 \\
112 \\
158 \\
13 / 4 \\
178\end{array}$ & $\begin{array}{l}41 / 4 \\
43 / 4 \\
5 \\
51 / 2 \\
53 / 4\end{array}$ & $\begin{array}{l}2.95 \\
\text { 3. } 55 \\
4.15 \\
4.85 \\
5.50\end{array}$ & $\begin{array}{l}17.70 \\
21.30 \\
24.90 \\
29.10 \\
33.00\end{array}$ & $\begin{array}{l}108,000 \\
128,000 \\
149,000 \\
170,000 \\
192,000\end{array}$ & $\begin{array}{l}24,000 \\
28,400 \\
33,100 \\
37,800 \\
42,700\end{array}$ & $\begin{array}{r}97,200 \\
115,000 \\
134,000 \\
153,000 \\
173,000\end{array}$ & $\begin{array}{l}34,000 \\
38,500\end{array}$ & \\
\hline $\begin{array}{l}2 \\
21 / 4 \\
21 / 2 \\
23 / 4\end{array}$ & $\begin{array}{l}61 / 4 \\
713 \\
77 / 8\end{array}$ & $\begin{array}{r}6.30 \\
7.95 \\
9.85 \\
11.90\end{array}$ & $\begin{array}{l}37.80 \\
47.70 \\
59.10 \\
71.40\end{array}$ & $\begin{array}{l}217,000 \\
274,000 \\
339,000 \\
410,000\end{array}$ & $\begin{array}{l}48,200 \\
60,900 \\
75,400 \\
91,100\end{array}$ & $\begin{array}{l}195,000 \\
246,000 \\
305,000 \\
369,000\end{array}$ & $\begin{array}{l}43,300 \\
54,600 \\
67,800 \\
82,000\end{array}$ & \\
\hline
\end{tabular}

1 If the diameter of the drum or sheave is less than the recommended diameter, lower safe working loads should be used.

\section{Type $S, 6$ by 37 High-Grade Plow Steel}

Wire rope of type $\mathrm{S}$ shall be made as described and shown in Figure 72 and shall have the properties given in Table 20 .

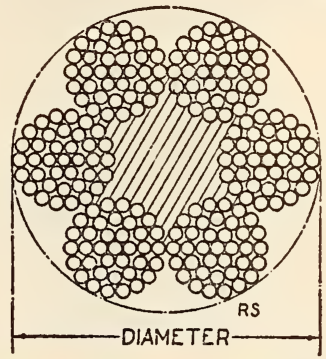

Figure 71.-Type $R$, 6 by 37 , extra strong cast steel

Construction.-Either 1 diameter of wire, 3 operations, or 4 or 5 diameters of wire, 2 operations. Six strands. Each strand shall have not less than 37 nor more than 43 wires. The number of outside wires of each strand shall be not less than 18 nor more than 20. Total, 222 to 258 wires. One main core.

Material.-Wires: High-grade plow steel, either uncoated or galvanized. Main core, hard fiber.

Diameter.-The difference between the nominal diameter of wire rope, type $\mathrm{S}$, and the actual diameter shall not be greater than the tolerances given in Table 3.

Lay.-Wire rope, type $\mathrm{S}$, shall be regular lay, and the lay shall not be greater than six and threefourths times the nominal diameter, if the wire is uncoated, nor greater than seven and one-fourth times the diameter, if the wire is galvanized.

TABLE 20.-Properties of wire rope, type $S$

\begin{tabular}{|c|c|c|c|c|c|c|c|c|}
\hline \multirow[b]{2}{*}{ 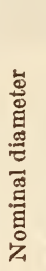 } & \multirow{2}{*}{ 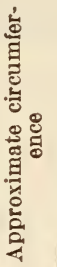 } & \multirow{2}{*}{ 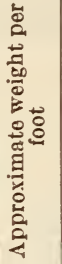 } & \multirow{2}{*}{ 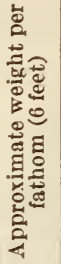 } & \multicolumn{2}{|c|}{ Uncoated } & \multicolumn{2}{|c|}{ Galvanized } & \multirow[b]{2}{*}{ 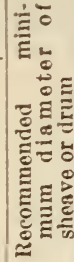 } \\
\hline & & & & 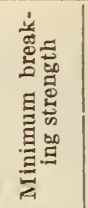 & 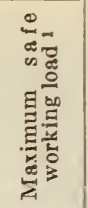 & 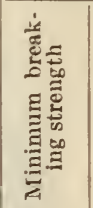 & 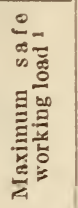 & \\
\hline $\begin{array}{c}\text { In. } \\
3 / 8 \\
7 / 16 \\
1 / 2 \\
9 / 10 \\
5 / 8\end{array}$ & $\begin{array}{c}I n . \\
11 / 8 \\
11 / 4 \\
11 / 2 \\
13 / 4 \\
2\end{array}$ & $\begin{array}{c}\text { Lbs. } \\
0.22 \\
.30 \\
.39 \\
.50 \\
.62\end{array}$ & $\begin{array}{c}L b 8 . \\
1.32 \\
1.80 \\
2.34 \\
3.00 \\
3.72\end{array}$ & $\begin{array}{l}L b 8 . \\
10,200 \\
13,800 \\
18,000 \\
22,500 \\
28,400\end{array}$ & $\begin{array}{c}\text { Lbs. } \\
2,270 \\
3,070 \\
4,000 \\
5,000 \\
6,310\end{array}$ & $\begin{array}{c}L b s . \\
9,160 \\
12,400 \\
16,200 \\
20,200 \\
25,500\end{array}$ & $\begin{array}{c}L b 8 . \\
2,040 \\
2,750 \\
3,600 \\
4,490 \\
5,660\end{array}$ & $\begin{array}{l}0.2 \\
0 \\
0\end{array}$ \\
\hline $\begin{array}{l}3 / 4 \\
7 / 8 \\
1 \\
11 / 8 \\
11 / 4\end{array}$ & $\begin{array}{l}21 / 4 \\
2^{3,4} \\
3 \\
31 / 2 \\
4\end{array}$ & $\begin{array}{r}.89 \\
1.20 \\
1.58 \\
2.00 \\
2.45\end{array}$ & $\begin{array}{r}5.34 \\
7.20 \\
9.48 \\
12.00 \\
14.70\end{array}$ & $\begin{array}{r}41,000 \\
54,000 \\
70,000 \\
87,000 \\
107,000\end{array}$ & $\begin{array}{r}9,110 \\
12,000 \\
15,600 \\
19,300 \\
23,800\end{array}$ & $\begin{array}{l}36,800 \\
48,600 \\
62,900 \\
78,200 \\
96,100\end{array}$ & $\begin{array}{r}8,180 \\
10,800 \\
14,000 \\
17,400 \\
21,400\end{array}$ & \\
\hline $\begin{array}{l}13 / 8 \\
11 / 2 \\
15 / 8 \\
13 / 4 \\
17 / 8\end{array}$ & $\begin{array}{l}41 / 4 \\
43 / 4 \\
5 \\
51 / 2 \\
53 / 4\end{array}$ & $\begin{array}{l}2.95 \\
3.55 \\
4.15 \\
4.85 \\
5.50\end{array}$ & $\begin{array}{l}17.70 \\
21.30 \\
24.90 \\
29.10 \\
33.00\end{array}$ & $\begin{array}{l}129,000 \\
153,000 \\
176,000 \\
202,000 \\
232,000\end{array}$ & $\begin{array}{l}28,700 \\
34,000 \\
39,100 \\
44,900 \\
51,600\end{array}$ & $\begin{array}{l}116,000 \\
138,000 \\
158,000 \\
182,000 \\
209,000\end{array}$ & $\begin{array}{l}35,100 \\
40,400 \\
46,400\end{array}$ & $\begin{array}{l}4.3 \\
4.5\end{array}$ \\
\hline $\begin{array}{l}2 \\
21 / 4 \\
213 \\
23 / 4\end{array}$ & $\begin{array}{l}61 / 4 \\
718 \\
77 / 8 \\
85 / 8\end{array}$ & $\begin{array}{r}6.30 \\
7.95 \\
9.85 \\
11.90\end{array}$ & $\begin{array}{l}37.80 \\
47.70 \\
59.10 \\
71.40\end{array}$ & $\begin{array}{l}264,000 \\
334,000 \\
413,000 \\
500,000\end{array}$ & $\begin{array}{r}58,600 \\
74,200 \\
91,800 \\
111,000\end{array}$ & $\begin{array}{l}237,000 \\
300,000 \\
372,000 \\
449,000\end{array}$ & $\begin{array}{l}52,600 \\
66,600 \\
82,600 \\
99,800\end{array}$ & $\begin{array}{l}4.8 \\
5.4 \\
6.1 \\
6.5\end{array}$ \\
\hline
\end{tabular}

1 If the diameter of the drum or sheare is less than the recom. mended diameter, lower safe working loads should be used.

\section{Type T, 8 by 18 Cast Steel}

Wire rope of type $T$ shall be made as described and shown in Figure 73 and shall have the properties given in Table 21.

Construction. - One diameter of wire, two operations. Eight strands of 19 wires each, total 152 wires. Strands for wire rope of type $\mathrm{T}$ may be of the Warrington, Seale, or of three diameters of wire and filler wire construction as shown for types $\mathrm{J}, \mathrm{K}, \mathrm{L}, \mathrm{M}$, and N. One main core.

Material.-Wires: Cast steel, uncoated. Main core, hard fiber.

Diameter. - The difference between the nominal diameter of wire rope, type $\mathrm{T}$, and the actual diam-

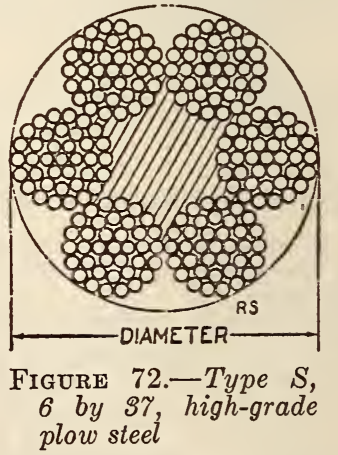

eter shall not be greater than the tolerances given in Table 3.

Lay. - Wire rope, type $\mathrm{T}$, shall be regular lay, and the lay shall not be greater than six and threefourths times the nominal diameter. 
TABLE 21. - Properties of wire rope, type $T$

\begin{tabular}{|c|c|c|c|c|c|c|}
\hline \multirow[b]{2}{*}{$\begin{array}{l}\text { Nomi- } \\
\text { nal } \\
\text { diam- } \\
\text { eter }\end{array}$} & \multirow[b]{2}{*}{$\begin{array}{l}\text { Ap- } \\
\text { proxi- } \\
\text { mate } \\
\text { cir- } \\
\text { cum- } \\
\text { ference }\end{array}$} & \multirow[b]{2}{*}{$\begin{array}{l}\text { Ap- } \\
\text { proxi- } \\
\text { mate } \\
\text { weight } \\
\text { per } \\
\text { foot }\end{array}$} & \multirow[b]{2}{*}{$\begin{array}{c}\text { A pproxi- } \\
\text { mate } \\
\text { weight } \\
\text { per } \\
\text { fathom } \\
(6 \text { feet) }\end{array}$} & \multicolumn{2}{|c|}{ Uncoated } & \multirow[b]{2}{*}{$\begin{array}{l}\text { Recom- } \\
\text { mended } \\
\text { minimum } \\
\text { diameter } \\
\text { of sheave } \\
\text { or drum }\end{array}$} \\
\hline & & & & $\begin{array}{l}\text { Mini- } \\
\text { mum } \\
\text { breaking } \\
\text { strength }\end{array}$ & $\begin{array}{l}\text { Maxi- } \\
\text { mum } \\
\text { safe } \\
\text { working } \\
\text { load } 1\end{array}$ & \\
\hline $\begin{array}{c}\text { Inches } \\
134 \\
516 \\
38 \\
716 \\
132\end{array}$ & $\begin{array}{c}\text { Inches } \\
3 / 4 \\
1 \\
118 \\
114 \\
11 / 2\end{array}$ & $\begin{array}{c}\text { Pounds } \\
0.09 \\
.14 \\
.20 \\
.27 \\
.35\end{array}$ & $\begin{array}{c}\text { Pounds } \\
0.54 \\
.84 \\
1.20 \\
1.62 \\
2.10\end{array}$ & $\begin{array}{r}\text { Pounds } \\
3,200 \\
5,000 \\
7,000 \\
9,600 \\
12,400\end{array}$ & $\begin{array}{r}\text { Pounds } \\
710 \\
1,110 \\
1,560 \\
2,140 \\
2,750\end{array}$ & $\begin{array}{r}\text { Feet } \\
0.6 \\
.8 \\
.9 \\
1.1 \\
1.3\end{array}$ \\
\hline $\begin{array}{l}916 \\
5 / 8 \\
3 / 4 \\
7 / 8\end{array}$ & $\begin{array}{l}13 / 4 \\
2 \\
21 / 4 \\
23 / 4 \\
3\end{array}$ & $\begin{array}{r}.45 \\
.55 \\
.80 \\
1.08 \\
1.42\end{array}$ & $\begin{array}{l}2.70 \\
3.30 \\
4.80 \\
6.48 \\
8.52\end{array}$ & $\begin{array}{l}15,700 \\
19,500 \\
27,800 \\
39,000 \\
49,000\end{array}$ & $\begin{array}{r}3,490 \\
4,340 \\
6,180 \\
8,670 \\
10,900\end{array}$ & $\begin{array}{l}1.4 \\
1.6 \\
1.9 \\
2.2 \\
2.5\end{array}$ \\
\hline $\begin{array}{l}118 \\
11 / 4 \\
138 \\
136\end{array}$ & $\begin{array}{l}31 / 2 \\
4 \\
41 / 4 \\
43 / 4\end{array}$ & $\begin{array}{l}1.80 \\
2.20 \\
2.67 \\
3.19\end{array}$ & $\begin{array}{l}10.80 \\
13.20 \\
16.00 \\
19.10\end{array}$ & $\begin{array}{r}61,000 \\
75,000 \\
90,000 \\
107,000\end{array}$ & $\begin{array}{l}13,600 \\
16,700 \\
20,000 \\
23,800\end{array}$ & $\begin{array}{l}2.8 \\
3.1 \\
3.5 \\
3.7\end{array}$ \\
\hline
\end{tabular}

1 If the diameter of the drum or sheave is less than the recommended diameter, lower safe working loads should be used.

\section{Type U, 8 by 19 Extra Strong Cast Steel}

Wire rope of type $U$ shall be made as described and shown in Figure 74 and shall have the properties given in Table 22 .

Construction. - One diameter of wire, two operations. Eight strands of 19 wires each, total 152

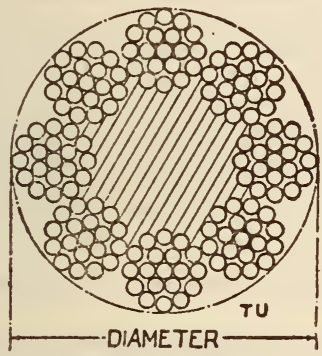

FIgdRE 73.-Type T, 8 by 19 , cast steel

wires. Strands for wire rope of this type may be of the Warrington, Seale, or of three diameters of wire and filler wire construction, as shown for types $\mathrm{J}, \mathrm{K}, \mathrm{L}, \mathrm{M}$, and $\mathrm{N}$. One main core.

Material.-Wires: Extra strong cast steel, uncoated. Main core, hard fiber.

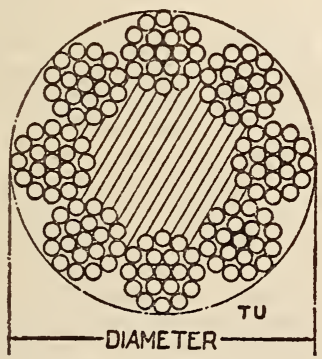

Figure 74.-Type $U$,

8 by 19 , extra strong cast steel

Diameter. - The difference between the nominal diameter of wire rope, type $U$, and the actual diameter shall not be greater than the tolerances given in Table 3.

Lay. - Wire rope, type $U$, shall be regular lay, and the lay shall not be greater than six and threefourths times the nominal diameter.
Table 22.-Properties of wire rope, type $U$

\begin{tabular}{|c|c|c|c|c|c|c|}
\hline \multirow[b]{2}{*}{$\begin{array}{l}\text { Nomi- } \\
\text { nal } \\
\text { diam- } \\
\text { eter }\end{array}$} & \multirow{2}{*}{$\begin{array}{c}\text { Ap- } \\
\text { proxi- } \\
\text { mate } \\
\text { cir- } \\
\text { cum- } \\
\text { ference }\end{array}$} & \multirow{2}{*}{\begin{tabular}{|c|} 
Ap- \\
proxi- \\
mate \\
weight \\
per \\
foot
\end{tabular}} & \multirow{2}{*}{$\begin{array}{c}\text { Approxi } \\
\text { mate } \\
\text { weight } \\
\text { per } \\
\text { fathom } \\
\text { (6 feet) }\end{array}$} & \multicolumn{2}{|c|}{ Uncoated } & \multirow{2}{*}{$\begin{array}{l}\text { Recom- } \\
\text { mended } \\
\text { minimum } \\
\text { diameter } \\
\text { of sheave } \\
\text { or drum }\end{array}$} \\
\hline & & & & $\begin{array}{l}\text { Mini- } \\
\text { mum } \\
\text { breaking } \\
\text { strength }\end{array}$ & $\begin{array}{c}\text { Maxi- } \\
\text { mum } \\
\text { safe } \\
\text { working } \\
\text { load } 1\end{array}$ & \\
\hline $\begin{array}{c}\text { Inches } \\
1 / 4 \\
516 \\
38 \\
7 / 16 \\
1 / 2\end{array}$ & $\begin{array}{c}\text { Inches } \\
3 / 4 \\
118 \\
118 \\
114 \\
11 / 2\end{array}$ & $\begin{array}{c}\text { Pounds } \\
0.09 \\
.14 \\
.20 \\
.27 \\
.35\end{array}$ & $\begin{array}{c}\text { Pounds } \\
0.54 \\
.84 \\
1.20 \\
1.62 \\
2.10\end{array}$ & $\begin{array}{r}\text { Pounds } \\
3,600 \\
5,600 \\
8,000 \\
10,900 \\
14,200\end{array}$ & $\begin{array}{c}\text { Pounds } \\
800 \\
1,240 \\
1,780 \\
2,420 \\
3,150\end{array}$ & $\begin{array}{r}\text { Feet } \\
0.6 \\
.8 \\
.9 \\
1.1 \\
1.3\end{array}$ \\
\hline $\begin{array}{l}9 / 16 \\
58 \\
3 / 4 \\
7 / 8 \\
1\end{array}$ & $\begin{array}{l}13 / 4 \\
2 \\
214 \\
234 \\
3\end{array}$ & $\begin{array}{r}.45 \\
.55 \\
.80 \\
1.08 \\
1.42\end{array}$ & $\begin{array}{l}2.70 \\
3.30 \\
4.80 \\
6.48 \\
8.52\end{array}$ & $\begin{array}{l}17,800 \\
22,200 \\
31,600 \\
42,000 \\
55,000\end{array}$ & $\begin{array}{r}3,960 \\
4,940 \\
7,020 \\
9,340 \\
12,200\end{array}$ & $\begin{array}{l}1.4 \\
1.6 \\
1.9 \\
2.2 \\
2.5\end{array}$ \\
\hline $\begin{array}{l}118 \\
11 / 4 \\
138 \\
112\end{array}$ & $\begin{array}{l}31 / 2 \\
4 \\
414 \\
434\end{array}$ & $\begin{array}{l}1.80 \\
2.20 \\
2.67 \\
3.19\end{array}$ & $\begin{array}{l}10.80 \\
13.20 \\
16.02 \\
19.14\end{array}$ & $\begin{array}{r}68,000 \\
84,000 \\
101,000 \\
119,000\end{array}$ & $\begin{array}{l}15,100 \\
18,700 \\
22,400 \\
26,400\end{array}$ & $\begin{array}{l}2.8 \\
3.1 \\
3.5 \\
3.7\end{array}$ \\
\hline
\end{tabular}

${ }^{1}$ If the diameter of the drum or sheave is less than the recommended diameter, lower safe working loads should be used.

603.43. MANUFACTURES OF IRON AND STEEL WIRE.

\section{AMERICAN RAILWAY ENGINEERING AS- SOCIATION, SPECIFICATIONS FOR STANDARD RIGHT-OF-WAY FENCES, 1926}

\section{RIGHT-OF-WAY FENCES}

\section{CLASSES}

1. Standard right-of-wav fences shall be divided into four classes, the height to conform to statutory requirements, generally about 4 feet 6 inches above the ground.

\section{Class A Fence}

2. Class A fence shall consist of nine longitudinal smooth galvanized steel wires; the top and bottom wires shall be No. 7 gage; the intermediate and stay wires shall be No. 9 gage.

The spacing of the longitudinal wires (commencing at the bottom) shall be $4,4 \frac{1}{2}, 5,5 \frac{1}{2}, 6,7,8$, and 9 inches. The bottom wires shall be 5 inches above the ground and the stay wires shall be spaced 12 inches apart.

When used as a hog-tight fence, a strand of barbed wire shall be added $2 \frac{1}{2}$ inches below the woven wire.

\section{Class B Fence}

3. Class B fence shall consist of seven longitudinal smooth galvanized steel wires; the longitudinal and stay wires shall be No. 9 gage.

The spacing of the longitudinal wires, commencing at the bottom, shall be $61 / 2,7,71 / 2,8,81 / 2$, and 9 inches. The bottom wire shall be 7 inches above the ground and stay wires shall be spaced 12 inches apart.

\section{Class C Fence}

4. Class $\mathrm{C}$ fence shall consist of woven wire fencing $251 / 2$ inches high with three strands of barbed wire above. The woven wire fencing shall consist of seven longitudinal, smooth galvanized steel wires. The longitudinal and stay wires shall be No. 9 gage and the stay wires shall be 12 inches apart. The spacing of the longitudinal wires, cor.mencing at the bottom, shall be $3,3 \frac{1}{2}, 4,4 \frac{1}{2}, 5$, and $5 \frac{1}{2}$ inches, and the bottom wire shall be 2 inches above the ground. The spacing of the barbed wires above the woven wire shall be $41 / 2,10$, and 12 inches. 


\section{Class D Fence}

5. Class D fence shall consist of five strands of galvanized steel ribbon, smooth, round, or barbed wire fencing.

The spacing of the wires, commencing at the bottom, shall be $10,10,12$, and 12 inches. The bottom wire shall be 10 inches above the ground.

The longitudinal wires of all woven wire fencing under classes $\mathrm{A}, \mathrm{B}$, and $\mathrm{C}$ shall be provided with tension curves to take up expansion and contraction

\section{AMERICAN SOCIETY FOR TESTING MATE- RIALS, STANDARD SPECIFICATIONS FOR ZINC-COATED (GALVANIZED) FARM- FIELD AND RAILROAD RIGHT-OF-WAY WIRE FENCING, DESIGNATION A116-30, 1930}

\section{GALVANIZED WIRE FENCING}

\section{SCOPE}

1. These specifications cover zinc-coated wire fencing of the following commercial types: Farmfield fencing and railroad right-of-way fencing. The weight classification and all test procedure shall be in accordance with the standard methods of testing zinc-coated (galvanized) iron and steel wire and wire products, A. S. T. M., A110. (See 600.3, p. 66.)

\section{MANUFACTURE}

2. It is recommended that styles and sizes of fence fabrics covered by these specifications be in accordance with those recommended by the United States Department of Commerce in its Simplified Practice Recommendation No. 9 or its subsequent recommendations. (See below.)

3. The zinc used for the coating shall be any grade of zinc conforming to the requirements of the standard specifications for slab zinc (spelter), A. S. T. M., B6. (See 683.1, p. 1310.)

4. The base metal of the fabric shall be of a good commercial quality of steel or iron wire. If copper is desired, the copper content shall conform to the following requirements:

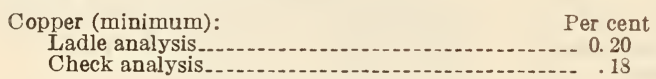

WEIGHT AND UNIFORMITY OF COATING

5. The weight and uniformity of the coating shall be defined and tested in accordance with the requirements of the standard methods of testing zinccoated (galvanized) iron and steel wire and wire products, A. S. T. M., A110. (See 600.3, p. 66.)

6 . The weight and uniformity of the coating for the various gages of wire composing the fencing shall be as specified by the purchaser and in accordance with the weights and dips designated in Tables 1 and 2.

TABLE 1.-Zinc-coated farm-field fence wire

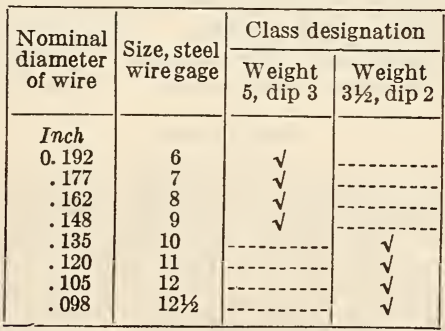

TABLE 2.-Zinc-coated railroad right-of-way fence wire

\begin{tabular}{|c|c|c|}
\hline $\begin{array}{c}\text { Nominal } \\
\text { diameter }\end{array}$ & $\begin{array}{c}\text { Size, steel } \\
\text { wire gage }\end{array}$ & $\begin{array}{c}\text { Class } \\
\text { designa- } \\
\text { tion- } \\
\text { weight 6, } \\
\text { dip 4 }\end{array}$ \\
\hline Inch & & \\
0.192 & 6 & $\sqrt{ }$ \\
.177 & 7 & $\sqrt{ }$ \\
.162 & 8 & $\sqrt{ }$ \\
.148 & 9 & $\sqrt{ }$ \\
\hline
\end{tabular}

Note.-Sizes in which any given weight of coating may be regularly obtained in the open market are indicated by $\sqrt{ }$ opposite the gage in the above tables.

In special cases wire may be obtained carrying weights 6 and 5 on smaller gages than indicated in the abore table.

\section{SIZE AND PERMISSIBLE VARIATIONS}

7. (a) The size of the finished wire shall be expressed as the diameter of the wire in decimal fractions of an inch.

(b) The permissible variations from the nominal diameter shall be:

For wire, 0.109 inch or over in diameter Inch

For wire, under 0.109 inch in diameter $\pm 0.004$

\section{PACKING AND MARKING}

8. Each length of fencing shall be tightly rolled and firmly tied. For ease of handling, consideration should be given to the weight of the roll, depending upon the length of fencing. Each roll shall carry a tag showing the class of coating, the gage of the wire, the length of fencing in the roll, type of fencing, and the manufacturer's name or mark.

\section{INSPECTION, SAMPLING, AND REJECTION}

9. (Inspection conforms to A. S. T. M. standard specifications for steel, paragraph 7. See 605.0, p. 247.)

10. All rolls of fencing accepted by the purchaser shall be billed to him on the basis of the original weight or footage of the rolls before sampling.

11. For purpose of tests, 1 roll from each 20 rolls of fencing, but not less than 3 rolls of each lot, shall be taken for a sample. Sufficient length of the fencing shall be cut from the end of the roll to include three stay wires. The test specimens for the line wire shall be cut between these three stays. The test specimens for the stays shall be cut from two of these three stays. All wire knots, wraps, or welds shall be excluded from the test. The resultant specimens of line and stay wire from each sample roll shall be put into separate groups. Three specimens from each of these groups shall be subjected to the test for the weight of coating in accordance with sections 5 and 6 , and three specimens from each of these groups shall be subjected to the test for uniformity of coating, in accordance with sections 5 and 6 . If 20 per cent of the specimens tested by the weight-of-coating test, or the uniformity test, fail to meet the requirements of these specifications, additional specimens shall be selected as described above, and a retest for the nonconforming properties shall be made; and if 20 per cent of these specimens fail, the entire lot thus inspected may be rejected. 
AMERICAN SOCIETY FOR TESTING MATERIALS, STANDARD SPECIFICATIONS FOR ZINC-COATED CHAIN-LINK FENCE FABRIC, GALVANIZED AFTER WEAVING, DESIGNATION A117-30, 1930

\section{GALVANIZED CHAIN-LINK FENCE FABRIC}

\section{SCOPE}

1. These specifications cover zinc-coated chainlink fence fabric in which the individual pickets are helically wound and interwoven in the form of a continuous link fabric without knots or ties. The weight classification and all test procedure shall be in accordance with the standard methods of testing zinc-coated (galvanized) iron and steel wire and wire products, A. S. T. M. A110. (See 600.3, p. 66.)

2. These specifications cover the usual sizes of wire and mesh as follows:

\begin{tabular}{|c|c|c|c|}
\hline $\begin{array}{l}\text { Nominal } \\
\text { diameter } \\
\text { of wire }\end{array}$ & $\begin{array}{l}\text { Size, } \\
\text { steel } \\
\text { wire } \\
\text { gage }\end{array}$ & $\begin{array}{l}\text { Size of } \\
\text { mesh }\end{array}$ & Height of fence fabric \\
\hline $\begin{array}{l}\text { Inch } \\
0.192 \\
.148 \\
.120 \\
.120\end{array}$ & $\begin{array}{r}6 \\
9 \\
11 \\
11\end{array}$ & $\begin{array}{c}\text { Inches } \\
2 \\
\frac{2}{2} \\
13 / 4\end{array}$ & $\begin{array}{c}\text { Inches } \\
36,42,48,60,72,84,96,108,120,144 \\
36,42,48,60,72,84,96,108,120,144 \\
36,42,48,60,72,84,-\overline{9},-\overline{108}, \overline{120}, 144\end{array}$ \\
\hline
\end{tabular}

\section{MANUFACTURE}

3. The wire shall be woven in the form of approximately uniform square mesh throughout, having parallel sides and horizontal and vertical diagonals of approximately uniform dimensions. The top and bottom of the fabric shall be knuckled or barbed as specified. The fencing shall be galvanized after weaving.

4. (Requirements covering zinc usecl for coating are identical with A. S. T. M. A116, given above.)

5. The base metal of the fabric shall be of good commercial quality of steel or iron wire. If copper is desired, the copper content shall not ',e less than 0.2 per cent.

\section{WEIGHT AND UNIFORNITY OF COATING}

6. (Requirements covering definition and testing are identical witn A. S. T. M. A116, given above.)

7. The weight and uniformity of the coating for the various gages of wire composing the fencing shall be as specified by the purchaser and in accordance with the weights and dips designated in Table 1.

TABLE 1.-Zinc-coated chain-link fence fabric galvanized after weaving

\begin{tabular}{|c|r|c|}
\hline $\begin{array}{c}\text { Nominal } \\
\text { diameter } \\
\text { of wire }\end{array}$ & $\begin{array}{c}\text { Size, } \\
\text { steel } \\
\text { wire } \\
\text { gage }\end{array}$ & $\begin{array}{c}\text { Class } \\
\text { tion } \\
\text { mini- } \\
\text { mum } \\
\text { weight } \\
12, \\
\text { dip } 4\end{array}$ \\
\hline Inch & & \\
0.192 & 6 & $\sqrt{ }$ \\
.148 & 9 & $\sqrt{ }$ \\
.120 & 11 & $\sqrt{ }$ \\
\hline
\end{tabular}

NoTE.-Sizes in which any given weight of coating may be regularly obtained in the open market are indicated by a $\sqrt{ }$ opposite the gage in the above table.

\section{PACKING AND MARKING}

8. The continuous lengths of fence fabric shall be 25, 50, or 100 feet, or as specified. Each length of fabric shall be tightly rolled and firmly tied. For ease of handling, consideration should be given to the weight of the roll, depending upon the length of fabric. Each roll shall carry a tag showing the class of coating, the gage of the wire, the length of fencing in the roll, and the manufacturer's name or mark.
INSPECTION, SAMPLING, AND REJECTION

9. (Inspection conforms to A. S. T. M. standard specifications for steel, paragraph 7. See 605.0 p. 247.)

10. All rolls of fencing accepted by the purchaser shall be billed to him on the basis of the original weight or footage of the rolls before sampling.

11. For purpose of tests, one roll for each 10 per cent of the rolls of fabric in shipments of less than 50 rolls shall be taken as a sample. One roll representing each 5 per cent of the rolls in shipments of over 50 rolls shall be taken as a sample. In no case shall there be less than three samples taken. The specimens for testing shall consist of 1 square foot of woven fencing selected at any point in the width of the fence fabric, exclusive of the twisted or knuckled portions, and taken from the end of the roll. A specimen from each sample shall be subjected to the test for the weight of coating in accordance with sections 6 and 7 , and a specimen from each sample shall be subjected to the test for uniformity of coating in accordance with sections 6 and 7 , including reference to A. S. T. M. A116.

By means of the following formulas, the wire surface area in square feet contained in 1 square foot of fabric can be calculated to facilitate the computation of the surface area of the specimens:

Square feet of wire surface of size 6 (steel wire gage) wire $=0.516 \mathrm{~W}$ Square feet of wire surface of size 9 (steel wire gage) wire $=0.674 \mathrm{~W}$ Square feet of wire surface of size 11 (steel wire gage) wire $=0.830 \mathrm{~W}$ where $W$ is the weight in pounds of wire in 1 square foot of fabric after being stripped of galvanizing.

If any specimen tested fails to meet the requirements of these specifications, two additional specimens shall be cut from the remainder of the sample and tested, both of which shall meet the requirements in every respect or the lot represented by the sample may be rejected.

\section{AMERICAN SOCIETY FOR TESTING MATE- RIAIS, TENTATIVE SPECIFICATIONS FOR ZINC-COATED CHAIN-LINK FENCE FABRIC, GALVANIZED BEFORE WEAV- ING, DESIGNATION A118-30T, 1930}

\section{GALVANIZED CHAIN-LINK FEINCE FABRIC}

(This specification is identical with A. S. T. M. A117, given above (including the references in A117 to A. S. T. M. A110, A116, and A. S. T. M. standard specifications for steel under 605.0) except for the weight and uniformity of the coating for the various gages of wire composing the fencing, and permissible variations. In A118-30T the weights and dips and permissible variations are as follows:)

TABLE 1.-Commercially available zinc-coated chainlink fence fabric galvanized before weaving

\begin{tabular}{|c|c|c|}
\hline $\begin{array}{c}\text { Nominal } \\
\text { diameter } \\
\text { of wire }\end{array}$ & $\begin{array}{c}\text { Size, steel } \\
\text { wire gage }\end{array}$ & $\begin{array}{c}\text { Class } \\
\text { designa- } \\
\text { tion, } \\
\text { weight 5, } \\
\text { dip 3 }\end{array}$ \\
\hline Inch & & \\
0.192 & 6 & $\sqrt{ }$ \\
.148 & 9 & $\sqrt{ }$ \\
.120 & 11 & $\sqrt{ }$ \\
\hline
\end{tabular}

Note.-Sizes in which any given weight of coating may be regularly obtained in the open market are indicated by a $\sqrt{ }$ opposite the gage in the above table.

\section{SIZE AND PERMISSIBLE VARIATIONS}

8. (a) The size of the finished wire shall be expressed as the diameter of the wire in decimal fractions of an inch.

(b) The permissible variations from the nominal diameter shall be plus or minus 0.004 inch. 


\section{AMERICAN SOCIETY FOR TESTING MATE- RIALS, STANDARD SPECIFICATIONS FOR ZINC-COATED (GALVANIZED) BARB WIRE, DESIGNATION A121-30, 1930}

\section{GALVANIZED BARB WIRE}

SCOPE

1. These specifications cover zinc-coated barb wire in which the wire is coated before or after fabrication, as herein noted. The weight classification and all test procedure shall be in accordance with the standard methods of testing zinc-coated (galvanized) iron and steel wire and wire products A. S. T. M. A110. (See 600.3, p. 66.)

2. These specifications cover the usual sizes and standard constructions of barb wire as follows:

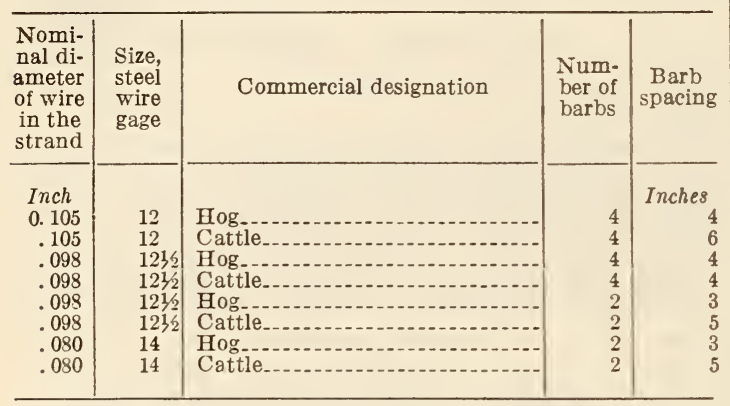

MANUFACTURE

3. (Requirements covering zinc used for coating are identical with A. S. T. M. A116, given above.)

4. The base metal of the barb wire shall be of a good commercial quality of steel or iron wire. If copper is desired, the copper content shall not be less than 0.2 per cent.

\section{WEIGHT AND ONIFORMITY OF COATING}

5. (Requirements covering definition and testing are jdentical with A. S. T. M., A116, given above.)

6. The weight and uniformity of the coating for the various gages of wire composing the barb wire shall be as specified by the purchaser and in accordance with the weights and dips designated in Table 1.

TABLE 1.-Zinc-coated barb wire

\begin{tabular}{|l|c|c|}
\hline $\begin{array}{l}\text { Size, } \\
\text { steel } \\
\text { wire } \\
\text { gage }\end{array}$ & $\begin{array}{c}\text { Nominal } \\
\text { diameter } \\
\text { of strand } \\
\text { wire }\end{array}$ & $\begin{array}{c}\text { Class des- } \\
\text { ignation, } \\
\text { weight } \\
31 / 2 \text {, dip 2 }\end{array}$ \\
\hline & $\begin{array}{c}\text { Inch } \\
0.105\end{array}$ \\
12 & .098 & $\sqrt{ }$ \\
$121 / 2$ & .080 & $\sqrt{ }$ \\
14 & & \\
\hline
\end{tabular}

Note.-Sizes in which any given weight of coating may be regularly obtained in the open market are indicated by a $\sqrt{ }$ opposite the gage in the above table.

For special purposes, barb wire galvanized before weaving may be obtained in $121 / 2$ and larger gages, carrying heavier coatings than indicated in the above table.

For special purposes, barb wire galvanized after weaving is available in the above and larger sizes with a coating of weight 10, dip 4

\section{SIZE AND PERMISSIBLE VARIATIONS}

7. (a) The size of the finished wire shall be expressed as the nominal diameter of the wire in decimal fractions of an inch. (b) The permissible variations from the nominal diameter in barb wire galvanized before weaving shall be \pm 0.005 inch.

\section{PACKING AND MARKING}

8. Each spool of barb wire shall be compactly wound and fastened and shall be legibly marked in accordance with the specifications of the purchaser.

\section{INSPECTION, SAMPLING, AND REJECTION}

9. (Inspection conforms to A. S. T. M. standard specifications for steel, paragraph 7. See 605.0, p. 247.)

10. All spools of barb wire accepted by the purchaser shall be billed to him on the basis of the original weight or footage of the spools before sampling.

11. (a) For purpose of tests, one spool shall be selected at random from every 10 spools or fraction thereof in a shipment. Sufficient length of barb wire shall be cut from the end of the reel to include three barbs.

(b) In the case of barb wire galvanized before weaving, the barbs shall be cut from the strand and discarded. The strand wires shall be tested separately.

(c) Barb wire galvanized after weaving shall be tested as woven with barbs and strand in place.

12. If 20 per cent of the samples tested fail to conform to the requirements of these specifications, then a check test of these same sample spools shall be made, and if in the check test 20 per cent of the samples fail to conform to the requirements, then the entire lot represented by the samples may be rejected.

AMERICAN SOCIETY FOR TESTING MATERIALS, STANDARD SPECIFICATIONS FOR SIEVES FOR TESTING PURPOSES, DESIGNATION E11-26, 1926

SIEVES FOR TESTING PURPOSES

(See 645.31, p. 1226.)

AMERICAN SOCIETY FOR TESTING MATERIALS, TENTATIVE DEFINITION OF THE TERM SCREEN (SIEVE), DESIGNATION E13-28T, 1928

\section{DEFINITION OF SIEVE}

(See 645.31, p. 1226.)

\section{SOCIETY OF AUTOMOTIVE ENGINEERS,} PARTS AND FITTINGS, WIRE CLOTH, S. A. E. STANDARD, JULY, 1924

$$
\text { WIRE CLOTH }
$$

(See 646.52, p. 1256.)

SOCIETY OF AUTOMOTIVE ENGINEERS, AERONAUTIC; WIRE LOOPS AND FERRULES, S. A. E. RECOMMENDED PRACTICE, AUGUST, 1917

\section{WIRE LOOPS AND FERRULES \\ AERONAOTIC}

The loops for round tinned steel wire are made by threading the tension wire through a ferrule, forming a loop of the specified size, slipping the ferrule 
up against the loop and bending the free end of the tension wire over the ferrule as shown in Figure 75. The ferrule is then thoroughly soldered in place without drawing the temper of the tension wire.
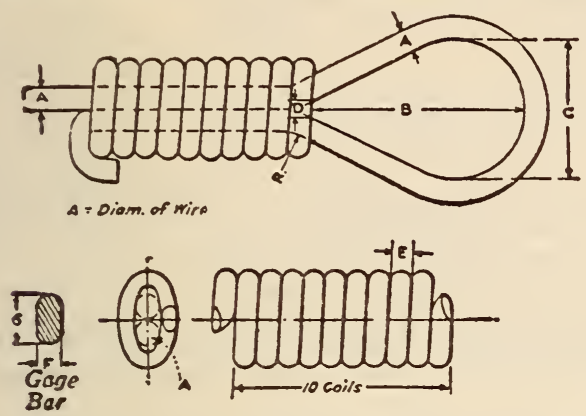

Figure 75

\begin{tabular}{|c|c|c|c|c|c|c|c|c|c|}
\hline \multicolumn{6}{|c|}{ Round wire loops } & \multicolumn{2}{|c|}{ Ferrule E } & \multicolumn{2}{|c|}{ Gage bar } \\
\hline \multicolumn{2}{|c|}{$\begin{array}{l}\text { Size of wire } \\
\text { (A) }\end{array}$} & \multirow{2}{*}{ B } & \multirow{2}{*}{$\mathrm{C}$} & \multirow{2}{*}{$\mathrm{D}$} & \multirow{2}{*}{$\mathrm{R}$} & \multirow{2}{*}{$\begin{array}{l}\text { B.\& S. } \\
\text { gage }\end{array}$} & \multirow{2}{*}{ Inch } & \multirow{2}{*}{$\mathrm{F}$} & \multirow{2}{*}{ G } \\
\hline $\begin{array}{c}\text { B. \& S. } \\
\text { gage }\end{array}$ & Inch & & & & & & & & \\
\hline $\begin{array}{r}8 \\
8 \\
10 \\
12 \\
14\end{array}$ & $\begin{array}{r}0.128 \\
.128 \\
.102 \\
.08 \\
.064\end{array}$ & $\begin{array}{r}9 / 16 \\
7 / 16 \\
15 / 32 \\
13 / 32 \\
13 / 32\end{array}$ & $\begin{array}{r}23664 \\
17 / 64 \\
9 / 32 \\
17 / 64 \\
17 / 64\end{array}$ & $\begin{array}{l}5 / 64 \\
1 / 16 \\
1 / 32 \\
1 / 32 \\
1 / 32\end{array}$ & $\begin{array}{r}13 / 64 \\
9 / 64 \\
964 \\
964 \\
964\end{array}$ & \begin{tabular}{r|r}
9 \\
9 \\
11 \\
13 \\
15
\end{tabular} & $\begin{array}{r}0.114 \\
.114 \\
.091 \\
.072 \\
.057\end{array}$ & $\begin{array}{r}0.130 \\
.130 \\
.104 \\
.083 \\
.066\end{array}$ & $\begin{array}{r}0.260 \\
.260 \\
.208 \\
.166 \\
.132\end{array}$ \\
\hline
\end{tabular}

Dimensions given in inches. Dimensions "A" and "E" are minima-tolerance, plus 0.002 inch. The gage bar has semicircular edges and shall pass through the ferrule with a clearance of approximately 0.003 inch.

\section{UNITED STATES GOVERNMENT, DEPART-} MENT OF COMMERCE, BUREAU OF STANDARDS, WOVEN-WIRE FENCING, SIMPLIFIED PRACTICE RECOMMENDATION R9-28, 1928

\section{WOVEN-WIRE FENCING}

In accordance with the unanimous action of the joint conference of representative manufacturers, distributors, and users of woven-wire fencing, the industry has adopted, and approved for promulgation by the United States Department of Commerce, a simplified practice recommendation establishing the following schedule of sizes and varieties of woven-wire fencing:

NotE.-The changes made by the rerision meeting of May, 1928, are indicated in the following tables by *:

\section{TABLE 1.-Field fence}

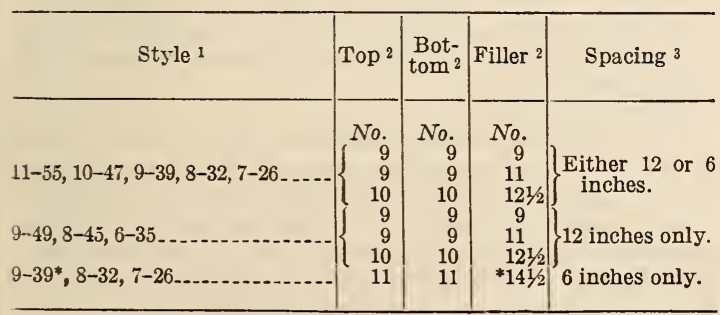

TABLE 2.-Close-mesh hog and cattle field fence

\begin{tabular}{|c|c|c|c|c|c|}
\hline \multicolumn{2}{|l|}{ Style 1} & Top ${ }^{2}$ & \multirow{2}{*}{$\begin{array}{c}\begin{array}{c}\text { Bot- } \\
\text { tom }{ }^{2}\end{array} \\
\begin{array}{c}\text { No. } \\
10\end{array}\end{array}$} & \multirow{2}{*}{$\mid \begin{array}{c}\text { Filler } 2 \\
\text { No. } \\
121 / 2\end{array}$} & Spacing ${ }^{3}$ \\
\hline $13-46,12-40,11-34$ & 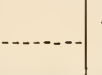 & $\begin{array}{r}\text { No. } \\
10\end{array}$ & & & $\begin{array}{l}\text { Either } 12 \text { or } 6 \\
\text { inches. }\end{array}$ \\
\hline Gage No. & $\begin{array}{l}\text { Wire } \\
\text { diameter }\end{array}$ & & $\mathrm{Ga}$ & ge No. & $\begin{array}{l}\text { Wire } \\
\text { diameter }\end{array}$ \\
\hline 90 & $\begin{array}{l}\text { Inch } \\
0.1483 \\
.1350 \\
.1205 \\
.1055 \\
.0985\end{array}$ & $\begin{array}{l}13 \\
14 \\
14 \\
15 \\
15\end{array}$ & $1 / 2 \ldots$ & & $\begin{array}{l}\text { Inch } \\
0.0915 \\
.0800 \\
.0760 \\
.0720 \\
.0672\end{array}$ \\
\hline
\end{tabular}

TABLE 3.-Wolf-proof fence

\begin{tabular}{|c|c|c|c|c|}
\hline Style 1 & Top 2 & $\begin{array}{l}\text { Bot- } \\
\text { tom² }\end{array}$ & Filler ${ }^{2}$ & Spacing ${ }^{3}$ \\
\hline $15-48,14-43,10-35,9-30,8-25 \ldots$ & $\begin{array}{l}\text { No. } \\
11\end{array}$ & $\begin{array}{r}\text { No. } \\
11\end{array}$ & $\begin{array}{l}\text { No. } \\
{ }^{*} 141 / 2\end{array}$ & $\begin{array}{l}\text { Either } 12 \text { or } 6 \\
\text { inches. }\end{array}$ \\
\hline
\end{tabular}

TABLE 4.-Poultry fence

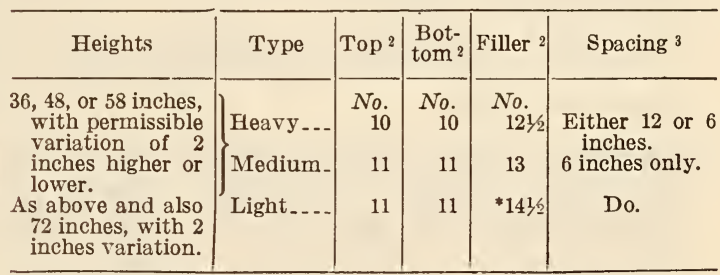

1 Style expressed in accordance with regular trade practice, the first number indicating the number of bars and the second the overall height in inches. Thus, 11-55, an 11-bar fence, 55 inches high. ${ }_{2}$ Steel wire gage (formerly Washburn \& Moen gage). The gage numbers referred to in this recommendation are given under numbers referred to in this recommendation are given under These gage numbers should not be confused with those of the American Wire Gage (formerly Brown \& Sharpe).

32 and 16 stays per rod considered equivalent, respectively, to 6 and 12 inch spacing.

This recommendation eliminates all fencing known as farm or field fence made out of Nos. 13, 14, 141/2, and lighter gage wire, except the types specifically noted above, and provides that no fencing known as poultry fence shall be made of lighter gage than No. 141/2. Fencing made as equivalent to poultry netting is to be so named and of gage not heavier than $151 / 2$.

It is further recommended that rolls of field fence be restricted to 10,20 , and $40 \mathrm{rod}$ lengths and rolls of poultry fence to 10 and 20 rod lengths.

\section{UNITED STATES GOVERNMENT, DEPART- MLENT OF COMIMERCE, BUREAU OF STANDARDS, WIRE INSECT-SCREEN CLOTH, SIMPLIFIED PRACTICE RECOM- MENDATION NO. 122, 1931}

\section{WIRE INSECT-SCREEN CLOTH}

(For copper and commercial bronze-wire insectscreen cloth, see 646.52 , p. 1256.)

In accordance with the unanimous action of a general conference of the industry held on June 18, 1931, the United States Department of Commerce submitted for the acceptance of the industry the following recommendation for the stock varieties of wire insect-screen cloth. The industry has since accepted this recommendation. 
TABLE 1. - Steel wire insect screen cloth

[Black painted and electrogalvanized, selvaged both edges]

\begin{tabular}{|c|c|c|c|c|c|c|}
\hline \multirow{2}{*}{ Description } & \multirow{2}{*}{$\begin{array}{c}\text { Mesh } \\
\text { openings } \\
\text { per linear } \\
\text { inch } 1\end{array}$} & \multicolumn{2}{|c|}{$\begin{array}{l}\text { Wire size before } \\
\text { weaving }{ }^{2}\end{array}$} & \multirow{2}{*}{ Width of roll ${ }^{3}$} & \multirow{2}{*}{$\begin{array}{l}\text { Length } \\
\text { of roll } \\
\text { (approx- } \\
\text { imate) }\end{array}$} & \multirow{2}{*}{ Packing } \\
\hline & & Warp & Filling & & & \\
\hline
\end{tabular}

1 Commercially exact for warp wires; one-half mesh variation plus or minus in filler wires in any one inch.

2 Two different sizes of warp and filler wire, approximately the same, having an average diameter of 0.0110 inch permissible.

3 Permissible variation in width plus or minus one-eighth inch.

The process of manufacture requires the inclusion of an occasional 2-piece roll; all 2-piece rolls have an extra footage of 2 to 4 linear feet

UNITED STATES GOVERNMENT, FEDERAL SPECIFICATIONS BOARD, FEDERAL SPECIFICATION FOR BUILDERS' HARDWARE (NONTEMPLATE), FF-H-101, AUGUST 19,1930

BUILDERS' HARDWARE (NONTEMPLATE)

(See 617.0, p. 978.)

UNITED STATES GOVERNMENT, FEDERAL SPECIFICATIONS BOARD, FEDERAL SPECIFICATION FOR CHAIN AND ATTACHMENTS; STANDARD, MISCELLANEOUS, RR-C-271, DECEMBER 8, 1931.

\section{WELDLESS CHAIN}

(See 603.56, p. 212.)

UNITED STATES GOVERNMENT, FEDERAL SPECIFICATIONS BOARD, FEDERAL SPECIFICATION FOR FENCING; WIRE (BARBED, NETTING, AND WOVEN), BLACK AND GALVANIZED, RR-F-221, JUNE 24, 1930

\section{WIRE FENCING}

\section{A. APPLICABLE FEDERAL SPECIFICATIONS}

The following Federal specifications, or parts thereof that are below indicated, of the issue in effect on date of invitation for bids, shall form a part of this specification:

\begin{tabular}{|c|c|c|c|}
\hline $\begin{array}{l}\text { Federal spec- } \\
\text { ification }\end{array}$ & $\begin{array}{l}\text { Former } \\
\text { number }\end{array}$ & Title & See \\
\hline QQ-M-151... & $339 a$ & General specifications for met- & 600.0, p. 3 \\
\hline QQ-Z-351.... & $91 \mathrm{a}$ & Slab zinc (spelter)... & 683.1, p. 1311. \\
\hline
\end{tabular}

\section{B. TYPES}

B-1. This specification covers three types of fence wire, as follows: Type A, wire, barbed, zine coated; type B, wire, woven, farm or field fences, black or zinc coated; and type $\mathrm{C}$, wire, netting, zinc coated.

\section{MATERIAL AND WORKMANSHIP, ETC.}

C-1. Material.-Unless otherwise specified, the base metal of any type of fence wire shall be a good commercial quality of steel. The zine used for coating shall be grade $\mathrm{E}$ conforming to the requirements of Federal specification QQ-Z-351 for slab zinc (spelter), in effect on date of invitation for bids. (See 683.1, p. 1311.)

C-2. Workmanship. - The manufacture of the types and sizes of fence covered by this specification and the zinc coating shall conform to the detail requirements under section $\mathrm{E}$. Welding in the manufacture of the woves fence will not be permitted. This does not prevent the welding of one coil of line wire to another during manufacture. On all type $\mathrm{C}$ hexagon wire netting the zinc coating shall be done after weaving.

\section{GENERAL REQUIREMENTS}

\section{D-1. See section E.}

\section{E. DETAIL REQUIREMENTS}

E-1. The type and sizes of wire fencing covered by this specification, and the weight and dip of the zinc coating, shall be as follows:

E-2. Type A.

E-2a. In all type $A$ fencing, the twist shall be uniform throughout and so twisted that the strain shall come equally on all main strands. The minimum zinc coating for type $\mathrm{A}$ wire shall be weight $3 \frac{1}{2}$, dip 2 .

E-2b. Four-point, 0.1055 wire, barbed, zinc coated. - This shall be zinc-coated barbed wire made of two twisted wires, each 0.1055 inch in diameter, complete with 4-point thick-set barbs sharp pointed and spaced not more than 4 inches apart. The diameter of wire for the barb shall be 0.098 inch, and the length of the barb shall be approximately one-half inch.

E-2c. Two-point, 0.098 wire, barbed, zinc coated.This shall be zine coated, barbed wire made of tro twisted wires, each 0.098 inch in diameter com. plete with 2-point thick-set barbs, sharp pointed and spaced approximately 5 inches apart. The diameter of wire for the barbs shall be 0.080 inch, and the length of the barb shall be approximately one-half inch or approximately seven-eighths inch as specified.

E-3. Type B.

E-3a. Wire, woven, farm or field fences, zinc coated.-This shall be fencing made of steel wire, and of the gage as specified in the invitation for bids. It is intended for fencing in corrals or yards. Unless otherwise specified, it shall be 55 inches wide, with meshes not over 6 inches square, and shall conform to style and sizes recommended by the United States Department of Commerce in simplified practice recommendation No. R9-28 (woven-wire fencing) or th ir subsequent recommendations. (See above.) For zinc-coated fences, the zinc coat- 
ing for the various gages of wire composing the fence shall be as specified by the purchaser and in accordance with the weights and dips designated in Table 1 .

$\mathrm{E}-3 \mathrm{~b}$. Sizes in which any giren weight of coating may be regularly obtained in the open marke' are indicated by an $\mathrm{X}$.

E-3c. In special cases wire may be obtaineo carrying heavier weight of coating on smaller sizes than indicated in Table 1.

E-4. Type C

E-4a. Wire, retting, zinc coated.-This shall be poultry netting of widths and meshes indicated below, or any commercial width of mesh that may be specified in the invitation for bids.

TABLE 1.-Commercially arailable zinc-coated fence wire

\begin{tabular}{|c|c|c|c|}
\hline Diameter & $\begin{array}{l}\text { Weight } 5 \text {, } \\
\text { dip } 3\end{array}$ & $\begin{array}{c}\text { Weight } 31 / 2, \\
\text { dip } 2\end{array}$ & $\begin{array}{l}\text { Weight } 2 \text {, } \\
\text { dip } 1\end{array}$ \\
\hline $\begin{array}{c}\text { Inch } \\
0.192 \\
.177 \\
.162 \\
.148 \\
.135 \\
.120 \\
.105 \\
.098 \\
.091 \\
.080 \\
.076 \\
.072 \\
.062 \\
.054\end{array}$ & $\begin{array}{c}{ }_{\mathrm{X}}^{\mathrm{X}} \\
\mathrm{X} \\
\mathrm{X}\end{array}$ & $\frac{\mathrm{X}}{\mathrm{X}}$ & $\begin{array}{l} \\
0\end{array}$ \\
\hline
\end{tabular}

\begin{tabular}{|c|c|}
\hline $\begin{array}{c}\text { Mesh in } \\
\text { inches }\end{array}$ & $\begin{array}{c}\text { Width of } \\
\text { netting }\end{array}$ \\
\hline & $\begin{array}{c}\text { Inches } \\
3 / 4\end{array}$ \\
10 \\
$1 / 2$ & 72 \\
2 & 36 \\
& 72 \\
\hline
\end{tabular}

(The first eight items in the upper table are the same as for farm field fence given in A. S. T. M. specification A116-30 above.)

E-4b. Unless otherwise specified, the netting shall be made of steel wire 0.041 inch in diameter. The weight and uniformity of the zinc coating shall be not less than weight 2, dip 1 .

\section{F. METHODS OF SAMPLING, INSPECTION, AND TESTS}

F-1. The tests for weight and uniformity of the zinc coating are optional with the purchaser.

F-2. Inspection.-The inspection, sampling, and rejection shall be as follows:

F-2a. The manufacturer shall afford the inspector representing the purchaser, without charge, all reasonable facilities to satisfy himself that the material is being furnished in accordance with this specification. All tests and inspection shall be made at the place of manufacture, prior to shipment, and shall be so conducted as not to interfere unnecessarily with the operation of the works.

F-3. For test purposes.-

F-3a. Wire, barbed, zinc coated; and wire, netting, zinc coated.- One roll for each 100 rolls or fraction thereof of each kind of wire shall be taker! at random for a sample for test. Sufficient length of both kinds of wire shall be cut from the ends of the roll and the specimen subjected to the tests for the weight of coating as described above.
F-3b. Wire, woven, farm or field fences, zinc coated.-(Method of sampling is the same as given in A. S. T. M. specification A116-30 for zinc-coated farm field fence given above.)

F-4. Tests. - The weight and uniformity of the zinc coating shall be as follows:

F-4a. Uniformity of coating.-The Preece, or copper sulphate, dip tests. (This method is the same as given in A. S. T. M. standard methods of determining weight of coating on zinc-coated articles, designation A90-30. See 600.3, p. 62.)

The requirements of zinc-coated wire withstanding the Preece test shall be designated as 1 dip, 2 dips 3 dips, 4 dips, etc., depending on the number of 1-minute immersions in the standard Preece test solution the coated wire is required to withstand. Fractional dip classes may be used as $3 \frac{1}{2}$ dips, meaning three 1-minute dips plus one 1/2-minute dip. There is a general relation between the weight of zinc coating and the number of dips in copper sulphate which the coating will withstand.

$\mathrm{F}-4 \mathrm{~d}$. Weight of coating. - The weight of coating shall be determined by the hydrochloric acid-antimony chloride method as follows: (This method is the same as given under the hydrochloric acidantimony chloride method of the standard methods of the A. S. T. M. for determining the weight of coating on zinc-coated articles, designation A90-30. See 600.3 , p. 62.)

The sample of galvanized wire may be of any length over 12 inches but preferably about 24 inches.

$\mathrm{F}-4 \mathrm{e}$. The classes of weights of coating per unit area on zinc-coated products shall be designated in accordance with the following illustrative scheme:

\begin{tabular}{|c|c|}
\hline Class designation & Weight of coating \\
\hline $\begin{array}{l}\text { Weight } 15 \\
\text { Weight } 10 \\
\text { Weight } 6 \\
\text { Weight } 1 \text {. } \\
\text { Etc- }\end{array}$ & $\begin{array}{l}\text { Minimum of } 1.5 \text { ounces per square foot. } \\
\text { Minimum of } 1.0 \text { ounce per square foot. } \\
\text { Minimum of } 0.6 \text { ounce per square foot. } \\
\text { Minimum of } 0.1 \text { ounce per square foot. } \\
\text { Etc. }\end{array}$ \\
\hline
\end{tabular}

(This classification is the same as given in A. S. T. M. standard methods of testing zinc-coated wire products, designation A110-30. See 600.3, p. 66.)

F-4f. This classification shall be extended or interpolated to provide a selection of classes as needed in writing specifications for zinc-coated wire and wire products.

$\mathrm{F}-4 \mathrm{~g}$. The weight of coating of any class of wire being found on inspection to be heavier than that defined for the class shall not constitute cause for rejection unless so specified on the purchase order.

F-5. See paragraph $\mathrm{A}-1$.

\section{G. PACKAGING, PACKING, AND MARKING}

\section{G-2. Packaging.-}

G-2a. Wire, barbed, zinc coated.-This shall be furnished in approximately 75-pound or approximately 100-pound coils, as ordered, wound on reels or spools and firmly secured thereto.

G-2b. Wire, woven, farm or field fences, black or zinc coated. - Each roll of fabric shall be compactly rolled and tied. Commercial lengths will be acceptable under this specification.

G-2c. Wire, netting, zinc coated.-This shall be furnished in rolls of 150 feet each, unless otherwise specified. Each roll shall be compactly tied.

G-3. Packing.-Unless otherwise specified, the subject commodity shall be delivered so packed as to insure acceptance by common or other carriers, for safe transportation, at the lowest rate, to the point of delivery.

G-4. Marking.-Unless otherwise specified, ship. ping containers shall be marked with the name of 
the material, the size, type, and the quantity contained therein, as defined by the contract or order under which the shipment is made, the name of the contractor, and the number of the contract or order.

G-4a. Wire, barbed, zinc coated.-Each coil shall carry a tag showing the net weight, manufacturer's name, and any other marking designated by the purchaser.

G-4b. Wire, woven, farm or field fences, black or zinc coated.-Each roll for identification shall carry a tag showing the nominal weight of coating, as ordered, corresponding to Table 1, the gage of the wire, the length of fencing in the roll, type of fence, and the manufacturer's name or mark.

$\mathrm{G}-4 \mathrm{c}$. Wire, netting, zinc coated.-Each roll shall carry a tag showing the length, net weight, manufacturer's name, and any other marking designated by the purchaser.

\section{NOTES}

I-1. All rolls of fencing accepted by the purchaser shall be billed to him on the basis of the original weight or footage of the rolls before sampling.

$\mathrm{I}-2$. It is believed that this specification adequately describes the characteristics necessary to secure the desired material, and that normally no samples will be necessary prior to award to determine compliance with this specification. If, for any particular purpose, samples with bids are necessary, they should be specifically asked for in the invitation for bids.

I-3. This specification governs all United States Government purchases of this commodity.

\subsection{CHAINS AND ATTACHMENTS.}

\subsection{GENERAL ITEMS.}

\section{UNITED STATES GOVERNIMENT, DEPART- MENT OF COMMERCE, BUREAU OF STANDARDS, SIMPLIFIED PRACTICE RECOMMENDATION FOR WELDED CHAIN, R100-29, FEBRUARY 1, 1929}

\section{WELDED CHAIN}

On November 15, 1928, a general conference of representative manufacturers, distributors, and users of welded chain drafted a simplified practice recommendation. The industry has since adopted, and approved for promulgation by the Department of Commerce, this recommendation, which eliminates from the list of stock items the following sizes and varieties:

Note.-Unless otherwise indicated, the dimensions shown below represent nominal sizes.

Straight link proof coil chain.-Eliminate sizes $11 / 16,13 / 16,15 / 16,11 / 16,13 / 16,15 / 16,17 / 16$, and $1 \% 16$ inches.

Straight link $B B$ chain.-Eliminate sizes 11/16, 13/16, $15 / 16,11 / 16,13 / 16,15 / 16,17 / 16$, and $1 \% / 16$ inches.

Straight link $B B B$ chain.-Eliminate sizes 11/16, $13 / 16,15 / 16,11 / 16,13 / 16,15 / 16,17 / 16$, and $1 \% 116$ inches.

Dredge chain.-Eliminate sizes 11/16, 13/16, 15/16, 11/16, $13 / 16,15 \% 16,1716$, and $1 \% 16$, inches.

Proof, $B B$, and $B B B$ coil chain (exact sizes).Eliminate sizes $3 / 16,1 / 4,5 / 16,3 / 8$, and $7 / 16$ inch.

Single breast chains. - Eliminate all sizes except 26-8-2, 28-10-2, and 30-10-1.

Double slack breast chains.-Eliminate all items except 26, 28, and 30 inch regular pattern.

Single tapered breast chains.-Eliminate all items except 27-inch, tapered $5 / 16$ to $1 / 4$ inch, and 32-inch, tapered $3 / 8$ to $5 / 16$ inch.

Bow breast chains.-Eliminate all items.

Slide breast chains.-Eliminate all items.

Single cart back chains.-Eliminate all sizes except \%/32-inch diameter.
Double cart back chains.-Eliminate.

Cart breeching chains.-Eliminate all sizes except $9 / 32$-inch diameter.

Cold shuts.-Elimiate all sizes of No. 2 pattern with shoulder.

Double chin chains.-Eliminate.

Butt chains.-Eliminate all items except 8 links to the foot, made from No. 2, No. 1, and No. 0 gages.

Hip strap chains.-Eliminate.

Jockey chains. - Eliminate.

Lazy chains.-Eliminate.

Lap rings.-Eliminate.

$C$ links.-Eliminate all items except $1 / 2$ by $21 / 2$ inches.

Trace chains.-Eliminate all items except 8 links per foot and 10 links per foot, made from No. 2 No. 1 , and No. 0 gages, and all to be made with 6 links measuring $5 / 8$ by 2 inches inside between ring and swivel.

Stage traces.-Eliminate all items except 31/2-10-1 buckeye pattern, $31 / 2-8-2$ regular pattern, $3 \frac{1}{2}-10-2$ Pennsylvania pattern, and $3 \frac{1}{2}-10-2$ South Jersey pattern.

Stake chains.-Eliminate all items except 61/2-8-2 and $6 \frac{1}{2}-8-1$

Tug chains.-Eliminate.

Fifth chains.-Eliminate.

Wagon chains.-Eliminate $1 / 4$-inch diameter.

Cow ties.-Eliminate all open-ring type; all closed-ring type; all sizes of Ohio pattern except $2 / 0-4 \frac{1}{2}, 3 / 0-4 \frac{1}{2}, 4 / 0-5,5 / 0-5,6 / 0-5 \frac{1}{2}$, and $7 / 0-6$ foot.

Chain dogs, ring dogs.-Eliminate all items except the following:

Style A chain dogs, plain point.-Blades, $3 / 8$ by $1 \frac{1}{8}$ by $6 \frac{1}{2}$ inches; center links, $11 / 32$ inch; and end links, $13 / 32$ inch.

Style B chain dogs, diamond point.-Blades, $1 / 2$ by $1 \frac{1}{2}$ by $6 \frac{1}{2}$ inches; center links, $13 / 32$ inch; and end links, $15 / 32$ inch.

Style $C$ plain ring dog.-Blades, $3 / 8$ by $1 \frac{1}{2}$ by $61 / 2$ inches; and ring, $1 / 2$ by 3 inches internal diameter.

Style $D$ diamond point ring dog.-Blades, $1 / 2$ by $1 \frac{1}{2}$ by $6 \frac{1}{2}$ inches; and ring, $1 / 2$ by 3 inches internal diameter.

Style $E$ Norfolk pattern ring dog.-Blades, $1 / 2$ by 2 by $6 \frac{1}{2}$ inches; and loop, $9 / 16$ by 3 by 4 inches internal diameter.

Heel chains.-

Nos. 5 and 8 eliminate.

Nos. 9, 10, and 11 eliminate No. 2 wire; make in No. 1 only.

Nos. 25, 35, 40, and 41 eliminate.

No. 45 eliminate No. 2 and No. 00, making only in No. 1 and No. 0 gages.

Nos. 46, 50, and 51 eliminate.

No. 52 eliminate No. 2 and No. 00 gages, making in No. 1 and No. 0 gages only.

Nos. 55 and 56 eliminate.

No. 57 eliminate No. 1 gage, making in No. 0 gage only.

No. 60 eliminate.

No. 61 eliminate No. 2 and No. 0 gages, making in No. 1 gage only.

Nos. 63, 64, and 65 eliminate.

No. 66 eliminate No. 1 and No. 2 gages, making in No. 0 gage only.

Nos. 80 and 81 eliminate.

Nos. 85 and 86 eliminate No. 2 gage, making in No. 0 and No. 1 gages only.

Nos. 88,90 , and 95 eliminate.

No. 99 eliminate No. 1 and No. 2 gages, making in No. 0 gage only. 
UNITED STATES GOVERNMENT, FEDERAI SPECIFICATIONS BOARD, FEDERAI SPECIFICATION RR-C-271, FOR STAND. ARD MISCELLANEOUS CHAIN AND ATTACHMENTS, DECEMBER 8, 1931

\section{CHAIN AND CHAIN ATTACHMENTS}

(Specifications contained in this publication relating to chain and chain attachments have been placed in this compilation under their proper commodity designations, as follows:)

\begin{tabular}{|c|c|c|}
\hline $\begin{array}{l}\text { Com- } \\
\text { modity } \\
\text { designa- } \\
\text { tion } \\
\end{array}$ & Material & Page \\
\hline 603.50 & General specifications & 199 \\
\hline 603.51 & Long straight link chair & 201 \\
\hline 603.52 & Close link coil chain... & 204 \\
\hline 603.54 & Twist link coil chain...... & 211 \\
\hline 603.56 & Weldless chain & 212 \\
\hline 603.57 & Chain attachments........ & 217 \\
\hline 617.5 & Sash attachments. & 1049 \\
\hline 631.9 & Aluminum safety chain & 1164 \\
\hline 642.9 & Plumbers, copper chain.- & 1191 \\
\hline 645.9 & Brass chain & 1242 \\
\hline 646.59 & Bronze sash chain... & 1258 \\
\hline
\end{tabular}

\section{GENERAL SPECIFICATIONS}

General specification for metals, Federal Specifications Board No. 339a (current designation: QQ-M-151), in effect on date of issuance of proposals, shall form part of this specification. (See 600.0, p. 3.)

\section{MATERIAL AND WORKMANSHIP}

1. Class 1.-Wrought-iron chain. The wrought iron shall be any wrought iron which has been rolled first into muck bars and these bars twice piled and rerolled. It shall be free from any admixture of iron scrap or steel.

NotE.-The term "iron scrap" applies only to foreign or bought scrap and does not include local mill products free from foreign or bought scrap.

2. Class 2.-Wrought-iron or open-hearth steel chain. The wrought iron may be made from a mixture of all pig-puddled iron and reworked scrap or from all reworked scrap. Unless otherwise specified, chain one-half inch and smaller may be made from open-hearth steel.

NotE.-The term "reworked scrap" applies to steel and iron scrap, or a misture of both.

4. Weldless chain shall be made from steel, copper, brass, or bronze wire; or stamped from sheets of steel, copper, brass, or bronze.

5. Chain attachments shall be of material as hereinafter specified. When a drop-forged steel piece is specified, the steel shall be of class 2 . If the chain is of class 1 wrought iron, the attachments shall be of class 2 steel.

6 . The chain and attachments shall be free from injurious defects and shall have a workmanlike finish.

\section{GENERAL REQUIREMENTS}

7. The nominal weights of 100 feet, nominal lengths of 100 links, and nominal dimensions of links for chain of various sizes shall be as given in Table 1 below.

8. The weights of 100 feet and lengths of 100 links of chain shall not vary more than 4 per cent over or under the nominal weights and lengths specified in Table 1 below. Excess weight over the permissible variations of 4 per cent may be cause for rejection.
9. Nominal weights and dimensions.-

TABLE 1

\begin{tabular}{|c|c|c|c|c|c|}
\hline \multirow{2}{*}{$\begin{array}{l}\text { Nominal } \\
\text { size of } \\
\text { chain } \\
\text { bar }\end{array}$} & \multirow{2}{*}{$\begin{array}{c}\text { Actual } \\
\text { size of } \\
\text { mate- } \\
\text { rial }\end{array}$} & \multicolumn{2}{|c|}{$\begin{array}{c}\text { Nominal length of } \\
100 \text { links }\end{array}$} & \multicolumn{2}{|c|}{$\begin{array}{l}\text { Nominal weight per } \\
100 \text { feet }\end{array}$} \\
\hline & & $\begin{array}{l}\text { Class } 1 \\
\text { crane }\end{array}$ & $\begin{array}{c}\text { Class 2 } \\
\text { proof coil }\end{array}$ & $\begin{array}{c}\text { Class } 1 \\
\text { crane }\end{array}$ & $\begin{array}{c}\text { Class 2 } \\
\text { proof coil }\end{array}$ \\
\hline $\begin{array}{r}\text { Inches } \\
1 / 4 \\
5 / 16 \\
3 / 8 \\
7 / 16\end{array}$ & $\begin{array}{r}\text { Inches } \\
9 / 32 \\
11 / 32 \\
1332 \\
15 / 32\end{array}$ & $\begin{array}{r}\text { Inches } \\
80 \\
100 \\
109 \\
122\end{array}$ & $\begin{array}{r}\text { Inches } \\
100 \\
111 \\
123 \\
138\end{array}$ & $\begin{array}{r}\text { Pounds } \\
78 \\
115 \\
166 \\
220\end{array}$ & $\begin{array}{c}\text { Pounds } \\
70 \\
105 \\
158 \\
210\end{array}$ \\
\hline $\begin{array}{r}1 / 2 \\
9 / 16 \\
5 / 8 \\
3 / 4\end{array}$ & $\begin{array}{l}17 / 32 \\
19,32 \\
21332 \\
25 / 32\end{array}$ & $\begin{array}{l}134 \\
156 \\
169 \\
188\end{array}$ & $\begin{array}{l}150 \\
175 \\
188 \\
213\end{array}$ & $\begin{array}{l}275 \\
350 \\
430 \\
615\end{array}$ & $\begin{array}{l}265 \\
335 \\
410 \\
580\end{array}$ \\
\hline $\begin{array}{l}1^{7 / 8} \\
118 \\
114 \\
138\end{array}$ & $\begin{array}{l}29 / 32 \\
11 / 32 \\
15 / 32 \\
19 / 32 \\
113 / 32\end{array}$ & $\begin{array}{l}225 \\
256 \\
288 \\
306 \\
363\end{array}$ & $\begin{array}{l}250 \\
275\end{array}$ & $\begin{array}{r}820 \\
1,045 \\
1,310 \\
1,600 \\
1,930\end{array}$ & $\begin{array}{r}780 \\
1,000 \\
\end{array}$ \\
\hline $\begin{array}{l}11 / 2 \\
15 / 8 \\
13 / 4 \\
17 / 8 \\
2\end{array}$ & $\begin{array}{l}117 / 32 \\
121 / 32 \\
125 / 32 \\
129 / 32 \\
21 / 32\end{array}$ & $\begin{array}{l}387 \\
425 \\
475 \\
525 \\
575\end{array}$ & & $\begin{array}{l}2,335 \\
2,740 \\
3,180 \\
3,650 \\
4,100\end{array}$ & \\
\hline
\end{tabular}

NOMINAL DIMENSIONS OF LINRS

\begin{tabular}{|c|c|c|c|c|c|c|c|c|}
\hline$\overleftarrow{\circ}$ & \multicolumn{4}{|c|}{ Class 1 crane } & \multicolumn{4}{|c|}{ Class 2 proof coil } \\
\hline & \multicolumn{2}{|c|}{ Outside } & \multicolumn{2}{|c|}{ Inside } & \multicolumn{2}{|c|}{ Outside } & \multicolumn{2}{|c|}{ Inside } \\
\hline 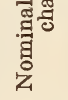 & 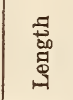 & 营 & 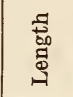 & 吾 & 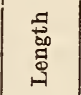 & 蔦 & 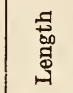 & 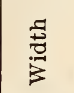 \\
\hline $\begin{array}{r}\text { Inches } \\
1 / 4 \\
5 / 16 \\
3 / 8 \\
7 / 16\end{array}$ & $\begin{array}{l}\text { Inches } \\
1^{27} / 64 \\
1^{11} 1 / 16 \\
129 / 32 \\
25 / 32\end{array}$ & $\begin{array}{l}\text { Inches } \\
1 \\
13 / 16 \\
17 / 16 \\
15 / 8\end{array}$ & $\begin{array}{c}\text { Inches } \\
{ }^{55 / 64} \\
1 \\
13 / 32 \\
17 / 32\end{array}$ & \begin{tabular}{|c} 
Inches \\
$7 / 16$ \\
$1 / 2$ \\
$5 / 8$ \\
$11 / 16$
\end{tabular} & \begin{tabular}{|l|} 
Inches \\
$19 / 16$ \\
$151 / 64$ \\
$23 \% 4$ \\
$25 / 16$
\end{tabular} & \begin{tabular}{|c} 
Inches \\
$11 / 16$ \\
$13 / 16$ \\
$17 / 16$ \\
11116
\end{tabular} & $\begin{array}{l}\text { Inches } \\
1 \\
17 / 64 \\
115 / 64 \\
13 / 8\end{array}$ & $\begin{array}{c}\text { Inches } \\
1 / 2 \\
1 / 2 \\
5 / 8 \\
3 / 4\end{array}$ \\
\hline $\begin{array}{r}1 / 2 \\
9 / 16 \\
5 / 8 \\
3 / 4\end{array}$ & $\begin{array}{l}213 / 32 \\
23 / 4 \\
3 \\
37 / 16\end{array}$ & $\begin{array}{l}113 / 16 \\
115 / 16 \\
23 / 16 \\
2 \% 16\end{array}$ & $\begin{array}{l}111 / 32 \\
19 / 16 \\
111 / 16 \\
17 / 8\end{array}$ & $\begin{array}{r}3 / 4 \\
3 / 4 \\
1^{7 / 8}\end{array}$ & $\begin{array}{l}29 / 16 \\
215 / 16 \\
33 / 16 \\
3^{11} 1 / 16\end{array}$ & \begin{tabular}{|l}
$17 / 8$ \\
$21 / 16$ \\
$25 / 16$ \\
$2^{11} 1 / 16$
\end{tabular} & $\begin{array}{l}11 / 2 \\
13 / 4 \\
17 / 8 \\
21 / 8\end{array}$ & $\begin{array}{l}13 / 16 \\
1^{7 / 8} \\
11 / 8\end{array}$ \\
\hline $\begin{array}{l}1^{7 / 8} \\
118 \\
114 \\
13 / 8\end{array}$ & $\begin{array}{l}41 / 16 \\
45 / 8 \\
53 / 16 \\
55 / 8 \\
67 / 16\end{array}$ & $\begin{array}{l}31 / 16 \\
37 / 16 \\
315 / 16 \\
45 / 16 \\
411 / 16\end{array}$ & $\begin{array}{l}21 / 4 \\
2916 \\
27 / 8 \\
31 / 16 \\
35 / 8\end{array}$ & $\begin{array}{l}134 \\
13 / 8 \\
15 / 8 \\
13 / 4 \\
17 / 8\end{array}$ & $\begin{array}{l}45 / 16 \\
4^{13 / 16}\end{array}$ & \begin{tabular}{|l|}
$33 / 16$ \\
$39 / 16$
\end{tabular} & $\begin{array}{l}21 / 2 \\
23 / 4\end{array}$ & $\begin{array}{l}13 / 8 \\
11 / 2\end{array}$ \\
\hline $\begin{array}{l}115 \\
158 \\
13 / 4 \\
17 / 8 \\
2\end{array}$ & $\begin{array}{l}615 / 16 \\
79 / 16 \\
85 / 16 \\
91 / 16 \\
9^{13} / 16\end{array}$ & $\begin{array}{l}51 / 16 \\
57 / 16 \\
5^{13} / 16 \\
6^{5} / 16 \\
6^{11} 1 / 16\end{array}$ & $\begin{array}{l}37 / 8 \\
41 / 4 \\
43 / 4 \\
514 \\
53 / 4\end{array}$ & $\begin{array}{l}2 \\
21 / 8 \\
21 / 4 \\
21 / 2 \\
25 \%\end{array}$ & & & & \\
\hline
\end{tabular}

Nоте.-Class 2. For ordinary chain, various lengths of links, dimensions, and weights are approximate only.

10. The diameter of the bar in all chain shall not be less than nominal size in the body and welds, and unless otherwise specified, the weld shall not exceed the diameter of the bar more than 25 per cent.

11. Class 1 chain shall be hot formed, hot scarfed and lap-fire-welded, unless otherwise specified. When handmade chain is specified, it shall be branded with the word "Handmade" every 6 feet. Class 2 may be made by either fire or electric welding processes.

12. Chain and attachments shall be self-colored, rough tumbled bright finish, black, hot or cold galvanized, electrocopperplated, brass, bronze, or nickel-plated finish, as specified. 


\section{DETAIL REQUIREMENTS}

13. The wrought iron and steel of the respective classes of chain shall conform to the following requirements as to chemical composition.

\begin{tabular}{|c|c|c|}
\hline & $\begin{array}{l}\text { Iron, } \\
\text { class } 1\end{array}$ & $\begin{array}{l}\text { Steel, } \\
\text { class } 2\end{array}$ \\
\hline Manganese, not over & $\begin{array}{r}\text { Per cent } \\
0.10\end{array}$ & Per cent \\
\hline Phosphorus, not over & -...-- & 0.05 \\
\hline Sulphur, not over & - n-n & \\
\hline
\end{tabular}

\section{PROOF AND BREAKING LOADS}

14. Class 1.-The proof and breaking loads shall be in accordance with the requirements of class 1 chain. (See comparative table facing p. 200.) The percentage of elongation shall be as specified for class 1 chain.

15. Class 2.-The proof and breaking loads shall be in accordance with the requirements for class 2 chain. (See comparative table facing p. 200.) The dimensions and weights shall be in accordance with Table 1. The percentage of elongation shall be as specified for this claiss of chain.

\section{METHOD OF INSPECTION AND TESTS}

17. All chain having breaking and proof requirements, shall first be subjected to the breaking tests and after satisfactorily passing that, to the proof test. These requirements are covered in the comparative table facing page 200 , except in those cases where individual tables are given or where tests are not required.

18. Samples from finished chain shall conform to the minimum requirements as to tensile strength specified in the comparative table facing page 200.

19. The inspector representing the purchaser shall have free entry at all times while the work on the contract of the purchaser is being performed, to all parts of the manufacturer's works which are concerned with the manufacture of the chain ordered. The manufacturer shall afford the inspector, free of charge, all reasonable facilities to satisfy him that the chain is being manufactured under this specification. Tests and inspection shall be conducted so as not to interfere unnecessarily with the operation of the works.

20. All handling of material necessary for purposes of inspection shall be performed, and all test specimens necessary for determining the qualities of the materials used, shall be prepared by and at the expense of the contractor.

21. All material when practicable shall be inspected and tested at the place of manufacture. Acceptance by the inspector shall be provisional only, subject to final acceptance upon delivery, except where delivery at the manufacturer's plant is specified.

22. Prior to testing and inspection the chain shall be free from paint or other coating which would tend to conceal defects. If chains are to be galvanized, surface inspection shall be made before and after galvanizing and proof vests and breaking tests shall be made before galvanizing.

23. When requested the manufacturer shall furnish a certificate of proof test. Unless break tests are made in the presence of the purchaser or his representative, the manufacturer shall furnish a certificate of break tests.

24. Proof tests of finished chain: After breaking tests, each lot which has satisfactorily passed that test shall be proof tested where required by subjecting it to the loads specified under each group. After proof test, the chain shall be thoroughly inspected and any links showing cold welding, surface rupture, deformation, inferior workmanship, or other defects shall be taken out and replaced and the chain again subjected to proof stress. If the cotal number of defective links exceeds 5 per cent, the lot shall be rejected.

25. Tension-test specimens shall consist of a length not less than 2 feet cut from the firished chain. One tension test shall be made to represent each lot of 200 feet of chain or fraction thereof ordered. If the tension test fails to cor form to the requirements specified, two additional tension tests may be made from the same lot, each of which shall meet the requirements.

26. The elongation, determined in a gage length of from 12 to 18 inches to the nearest link, shall conform to the following minimum requirements for the respective classes:

Class 1

Per cent

Class 2

27. Standard chain attachments shall be suifjected to the same proof-test load as the chain with which they are to be used. At least 1 out of every 50 or less to be furnished under a contract or order shall be selected for test on quantities of 100 or less and 1 for each additional 200 or less. If any of these pieces fails or shows defective welds the total number of test samples shall be doubled and a second failure will result in rejection of the lot.

28. Analysis of material for class 1 or class 2 may be made from bars or from finished chain. One sample of not less than 2 ounces of drillings or cuttings shall be taken from each class of chain delivered or from each ton or fraction thereof of wrought iron or each melt of steel. The cuttings or drillings shall be clean, fine, dry, free from all foreign substances and representative of the material and shall conform to the requirements specified.

29. Drillings for analysis shall be taken with a drill the diameter of which is approximately one-half the diameter of the bar from which drillings are taken, and the cutting shall be taken through the bar from surface to surface.

30 . The length shall be measured after the chain has been proof tested and shall be measured to the inside of the end links.

31. In determining the length of the chain, a load not exceeding 10 per cent of the proof load shall be applied to take up the slack.

\section{PACKIVG AND MARKING}

32. Chain and chain attachments purchased under this specification shall be packed and marked as required by the purchaser.

\section{NOTES}

33. Bidders shall in all cases state the kind of material they proposed to furnish under class 2 and may submit separate prices for wrought iron or steel or both.

34. Coil chain will be paid for on a basis of actual weight delivered up to and including 4 per cent excess. Excess weight above 4 per cent if accepted will not be paid for.

35. Class 1 is for slings, cranes, hoists, steam shovels, and marine uses, and where an all-iron chain is desired and is sold under the trade designation "crane chain."

36. Class 2 is for ordinary slings and hoists and general service where safety is not a factor and is sold under the trade designation "proof coil."

37. Brass chain for mechanical communication is required for use in pulleys having a $5 / 16$-inch groove used on a line of signal transmission of mechanical telegraphs.

38. Weldless close-link chain made from rolled steel or iron bar will be acceptable under this specification.

39. Weldless chain will be paid for by the foot. 


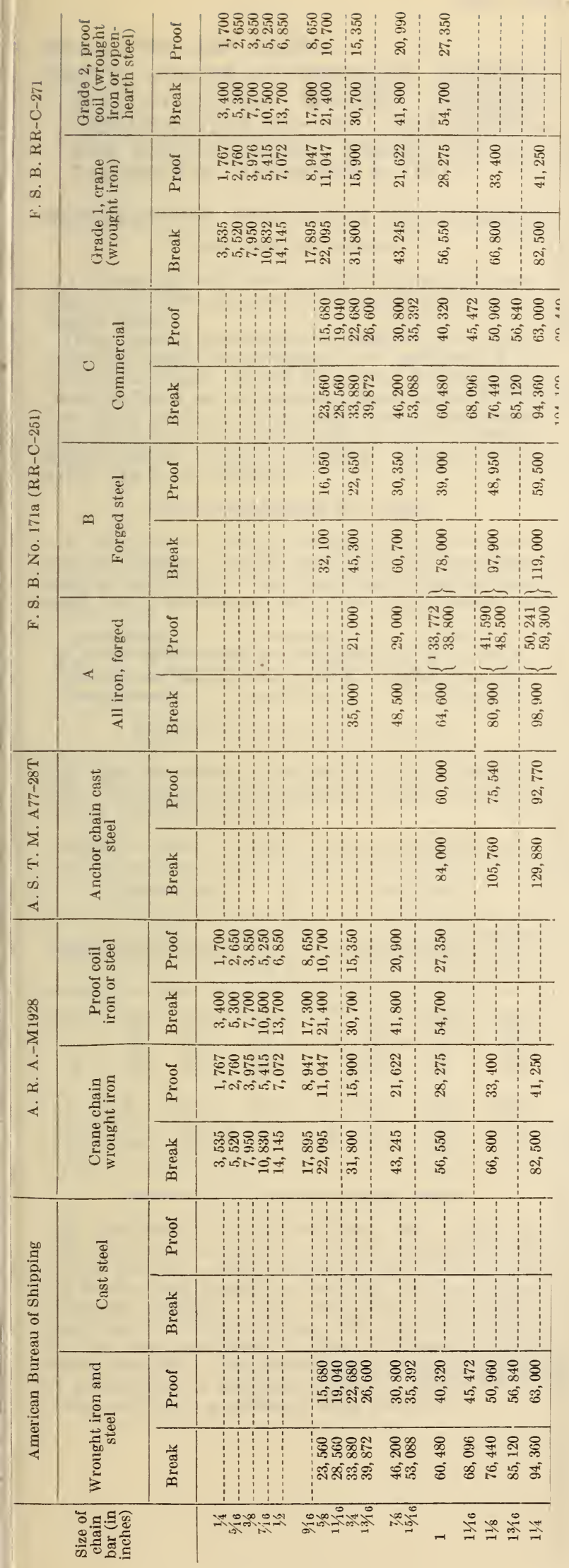




\begin{tabular}{|c|c|c|c|c|c|c|c|c|c|c|c|c|c|c|c|c|c|c|c|c|}
\hline \multirow{3}{*}{$\begin{array}{l}\text { Bize of } \\
\text { chain } \\
\text { bar (In } \\
\text { lnehess) }\end{array}$} & \multicolumn{4}{|c|}{ 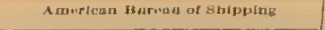 } & \multicolumn{4}{|c|}{ A H. A.-MIUDa } & \multirow{2}{*}{\multicolumn{2}{|c|}{ 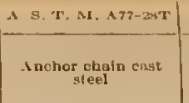 }} & \multicolumn{6}{|c|}{ 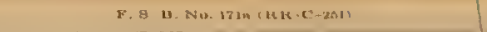 } & \multicolumn{4}{|c|}{$y$ \& $18,11182.271$} \\
\hline & \multicolumn{2}{|c|}{$\begin{array}{c}\text { Wrought Iron and } \\
\text { stenl }\end{array}$} & \multicolumn{2}{|c|}{ Cast steal } & \multicolumn{2}{|c|}{$\begin{array}{l}\text { Crane chain } \\
\text { wrought iron }\end{array}$} & \multicolumn{2}{|c|}{$\begin{array}{l}\text { Proor coil } \\
\text { iron or steel }\end{array}$} & & & \multicolumn{2}{|c|}{ All iroh, forged } & \multicolumn{2}{|c|}{ Iu } & \multicolumn{2}{|c|}{ Commercial } & \multicolumn{2}{|c|}{ 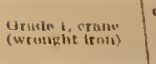 } & \multicolumn{2}{|c|}{ 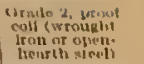 } \\
\hline & Areak & Proof & Break & Proot & Break & Proof & Bresk & Proot & Break & Proor & Break & Proo! & Break. & Proot & Break & l'root & Brouk & Frnol & Mreuk & Pront \\
\hline $\begin{array}{r}34 \\
510 \\
310 \\
310 \\
313\end{array}$ & & & & & $\begin{array}{r}3,533 \\
5,520 \\
7,950 \\
10,830 \\
14,145\end{array}$ & $\begin{array}{l}1,767 \\
2,760 \\
3,975 \\
5,415 \\
7,072\end{array}$ & $\begin{array}{r}3.400 \\
5,300 \\
7,700 \\
10,500 \\
13,700\end{array}$ & $\begin{array}{l}1,700 \\
2,650 \\
3,850 \\
5,250 \\
0,850\end{array}$ & & & & & & & & & $\begin{array}{r}3,635 \\
5,620 \\
7,050 \\
11,832 \\
11,114\end{array}$ & $\begin{array}{l}1,717 \\
2,760 \\
3,1070 \\
5,116 \\
7,072\end{array}$ & $\begin{array}{c}3,7100 \\
5,3(Y) \\
7,700 \\
10,800 \\
13,7000\end{array}$ & $\begin{array}{l}1,7400 \\
2,8301 \\
3,850 \\
5,2600 \\
0,850\end{array}$ \\
\hline $\begin{array}{c}9 / 10 \\
3,8 \\
11 f_{0}\end{array}$ & $\begin{array}{l}23,560 \\
23,560\end{array}$ & $\begin{array}{l}15,650 \\
10,040\end{array}$ & & & $\begin{array}{l}17,895 \\
22,095\end{array}$ & $\begin{array}{r}8,917 \\
11,017\end{array}$ & $\begin{array}{l}17,300 \\
21,400\end{array}$ & $\begin{array}{r}8,050 \\
10,700\end{array}$ & & & & & 32,100 & 10,050 & $\begin{array}{l}23,560 \\
2 \times, 560\end{array}$ & $15,0 \times 0$ & $\begin{array}{l}17, \sin \\
22,815\end{array}$ & $\begin{aligned} 5,4017 \\
11,017\end{aligned}$ & $\begin{array}{l}17,360) \\
21,400\end{array}$ & $\begin{array}{r}8,660 \\
10,700\end{array}$ \\
\hline $\begin{array}{c}33 \\
1310\end{array}$ & $\begin{array}{r}33,850 \\
39,572\end{array}$ & $\begin{array}{l}22,680 \\
26,600\end{array}$ & & & 31.800 & 15.900 & 00 & $150^{\circ}$ & & & 35,000 & 21,600 & 45,300 & 22.150 & $\begin{array}{l}33, \quad 30 \\
39,572\end{array}$ & $\begin{array}{l}22,1380 \\
26,1600\end{array}$ & 31,800 & is, $\{104$ & 30,700 & 15,350 \\
\hline $\begin{array}{c}78 \\
1810\end{array}$ & $\begin{array}{l}46,200 \\
53,088\end{array}$ & $\begin{array}{l}30,800 \\
35,302\end{array}$ & & & 43,245 & 21,622 & 100 & 9 & & & 48,500 & 39,000 & 60.700 & 30,350 & $\begin{array}{l}10,200 \\
53,056\end{array}$ & $\begin{array}{l}30,800 \\
35,302\end{array}$ & $43,21:$ & 21,022 & $\begin{array}{c}41,800 \\
. .\end{array}$ & $\begin{array}{l}30,000 \\
\cdots\end{array}$ \\
\hline 1 & 60,480 & 40,320 & & & 56,550 & 28,275 & 54,700 & 27,350 & 84,000 & 60,000 & $64,600\}$ & $\begin{array}{r}133.772 \\
38.800\end{array}$ & 78,000 & 39,000 & 60,480 & 40,320 & $50,5.50$ & $2 \times, 275$ & 51,760 & 27,350 \\
\hline $11_{0}$ & 6s, $0 \mathrm{~m})$ & 45,472 & & & & & & & & & & 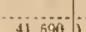 & & & is. 000 & 45,472 & & & & \\
\hline $11 \$$ & 76,440 & $\begin{array}{r}50,960 \\
50,840\end{array}$ & & & 66,800 & 33,400 & & & 105,700 & 75,510 & $80,900\{$ & $\begin{array}{l}41,590 \\
48,500\end{array}$ & 97.600 & 18,950 & 70.410 & 30,460 & $(4), x)(1)$ & 83,4113 & & \\
\hline 1310 & $\begin{array}{l}85,120 \\
94,360\end{array}$ & $\begin{array}{l}30,840 \\
63,000\end{array}$ & & & 82,500 & 41,250 & & & 129,850 & 92,770 & 08 & 60,241 & & ..... & 84,120 & $56, \times 10$ & & - & & \\
\hline 14, & $\begin{array}{r}94,360 \\
10-1,160\end{array}$ & 69,440 & & & & & & & & 92,770 & 98,900 & 59,300 & 119,000 & 54,500 & 94,360 & 13,000 & 32,500 & 11,220 & & 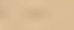 \\
\hline $\begin{array}{l}1350 \\
136\end{array}$ & $\begin{array}{l}104,1180 \\
114,240\end{array}$ & 76,1219 & 150,330 & 111,600 & $(w, \times(x)$ & 49,900 & & & 156,330 & iii, 610 & 118,600 & 71,200 & 142,500 & 71,250 & $\begin{array}{l}104,160 \\
114,240\end{array}$ & $\begin{array}{l}69.410 \\
76,120\end{array}$ & 99,8901 & $16, \operatorname{sic} 0$ & & \\
\hline $1^{*}$ io & 124,000 & 83,160 & 170,450 & 121.720 & & & & & & & & & & & $12-, 600$ & $\times 3,160$ & & & & \\
\hline $11 / 3$ & $131,4 \mathrm{ke}$ & 90,720 & $18,5,0 \times 0$ & 132,190 & $11 \times, 700$ & 59,350 & & & 185,0 in & 132,190 & 140,000 & $\begin{array}{l}70,0833 \\
81,000\end{array}$ & 168.000 & $4,0 \times 0$ & 131,120 & 00,720 & 118,700 & $50,3 m$ & & \\
\hline 19 is & 137,536 & p., 396 & $=00,270$ & 143,050 & & & & & & . & & & $\ldots$. & $\ldots$. & $13 \pi, 530$ & 98,330 & & & & 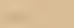 \\
\hline $11 i$ & 148,960 & 100,100 & 210,030 & 154,310 & $130,5(h)$ & 60,750 & & & 210,030 & 154,310 & 163,000 & $\begin{array}{l}x 1,380 \\
97,300\end{array}$ & 145,601 & $4=, 500$ & $11 \times, 960$ & $10 k, 10 \mathrm{NJ}$ & 130,5010 & (i), 750 & . & \\
\hline 113 in & 160,702 & 111,800 & 232,360 & 165,960 & & & & & $\ldots$ & $\ldots \ldots \ldots$ & (............ & 93,560 & & & 190,720 & 114,800 & & & & \\
\hline $1 x_{3}$ & 172,700 & $123,4 \leq 0$ & 219,2111 & 188,000 & $1 f i 1, a(x)$ & 80,800 & & & 249,210 & 178,000 & $1 N 7,800$ & 112,700 & 224,500 & 112,250 & 172,700 & $123.4 \times 0$ & $191, a n 0$ & $\mathrm{~S}(1, \mathrm{~K}(\mathrm{~N})$ & & . \\
\hline lisia & 185,300 & $132,4+0$ & 266,020 & 100,430 & & & & & & & & 93 & & & 185,360 & 132,110 & & & & .. \\
\hline 178 & $\begin{array}{l}198,240 \\
211, \text { fivo }\end{array}$ & $\begin{array}{l}141,680 \\
151,200\end{array}$ & $\begin{array}{l}24,540 \\
303,000\end{array}$ & $\begin{array}{l}203,250 \\
216,430\end{array}$ & 185,5001 & 92,750 & & & 254.540 & 203,250 & 214,300 & 128,500 & $25 \pi, 000$ & 128,5041 & $195,2: 10$ & 111,680 & $185, \sin 10$ & 122,750 & & .. \\
\hline 1340 & $\begin{array}{l}211, \text { fivo } \\
2245,702\end{array}$ & $\begin{array}{l}151,200 \\
161,250\end{array}$ & $\begin{array}{l}303,000 \\
322,000\end{array}$ & $\begin{array}{l}216,430 \\
230,000\end{array}$ & 311,100 & 105,500 & & & 322,000 & & 242,300 & & 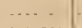 & 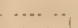 & 211.650 & 151,290 & $\ldots$ & $\therefore$ & & - \\
\hline 2 & $\begin{array}{l}245,702 \\
20,904\end{array}$ & $\begin{array}{l}161,280 \\
171,360\end{array}$ & $\begin{array}{l}322,000 \\
341,510\end{array}$ & $\begin{array}{l}230,000 \\
243,930\end{array}$ & & & & & 32,000 & 230,000 & 242,300 & $\begin{array}{l}120,7(3) \\
145, f(x)\end{array}$ & 291,000 & 115.500 & 225,792 & $161,2 \times 0$ & 211,100 & $105.5,50$ & .. & . \\
\hline $21 \% 6$ & 230,904 & 17.360 & 341,510 & $\begin{array}{l}243,930 \\
259,240\end{array}$ & & & & & 361.530 & $0=0=0$ & (2). & & ... & $\ldots \ldots$ & 239,004 & 171,300 & & & & 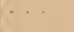 \\
\hline $\begin{array}{l}246 \\
2 \cdot t / 46\end{array}$ & $\begin{array}{l}25.1,309 \\
969,020\end{array}$ & $\begin{array}{l}182,000 \\
192,6200\end{array}$ & $\begin{array}{l}361,530 \\
3 \times 2,0 \times 00\end{array}$ & $\begin{array}{l}255,210 \\
272,910\end{array}$ & & & & & 361,530 & 255,210 & $2 \div 2,000$ & 163,200 & $32 \%$, sino & 163,250 & $\begin{array}{l}251,800 \\
239,923\end{array}$ & $\begin{array}{l}182,0007 \\
192,920\end{array}$ & & & & \\
\hline $\begin{array}{ll}21 \\
213 \\
13\end{array}$ & $\begin{array}{l}205,010 \\
301,810\end{array}$ & $\begin{array}{l}304,120 \\
215,800\end{array}$ & 403,100 & $\begin{array}{l}257.030 \\
303.320\end{array}$ & & & & & 403,100 & 257,930 & 300,300 & in 2,000 & 361,0006 & $18 \%, 6 \times 0$ & $2 \times, 1000$ & $3(4,121)$ & & & & \\
\hline$v^{10}$ & 315,304 & 225,360 & $\begin{array}{l}424,039 \\
413,860\end{array}$ & 319,050 & & & & & $116,660^{\circ}$ & 319,050 & $33 t, 200$ & 2001,700 & 302,000 & 20i, oin & 318,304 & $225,3(2)$ & & & & \\
\hline 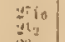 & $\begin{array}{l}3335,100 \\
352, \times 00\end{array}$ & $\begin{array}{l}239,454 \\
252,000\end{array}$ & $\begin{array}{l}180 \\
1100\end{array}$ & $\begin{array}{l}335,1 \\
351.5\end{array}$ & & & & & 192,190 & 351,500 & $370 \times 800$ & 222,500 & 43,000 & za1. $\operatorname{sinj}$ & $\begin{array}{l}335,160 \\
352,8100\end{array}$ & 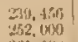 & & & .. & . \\
\hline 20 & $\begin{array}{l}3185,060 \\
374,120\end{array}$ & 23i1. this & 515, 1600 & 364,340 & 年 & & & & (1) & nos & (0) & & . & . & & 2851,114 & & & & \\
\hline 215 & $\begin{array}{l}374,120 \\
392,200\end{array}$ & $\begin{array}{l}270,816 \\
2 \times 0,224\end{array}$ & 534,0820 & $\begin{array}{l}3 \times 3,4 \pm 9 \\
402,890\end{array}$ & & & & & 339,620 & $3 \times 5,440$ & 407,000 & $241,2 \times 1)$ & $48(j, 000)$ & $24,8,0.00$ & $\begin{array}{l}373,120 \\
392,200\end{array}$ & $\begin{array}{l}270,816 \\
2 \times 0,224\end{array}$ & & & & \\
\hline 23, & $105,+10$ & $\begin{array}{l}284,63 \% \\
304,616\end{array}$ & $\begin{array}{l}545,930 \\
614,270\end{array}$ & $\begin{array}{l}+20,6601 \\
432, \pi t i n\end{array}$ & & & & & 585,930 & 427,6801 & $44,-000$ & 2666,800 & $530,00 \times 1$ & 205,000 & $405,4 \pm 0$ & 2009,8392 & & & & -1 \\
\hline 37 & 431. $4 \leq 0$ & $30 \mathrm{n}, 204$ & 140,071 & 190 & & & & & 640,070 & 457,190 & $14 i, 100$ & $290,50)$ & $59 \bar{s}, 0 \mathrm{OB}$ & 293,500 & $131,+40$ & $3 \mathrm{du}, 224$ & & & 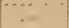 & - \\
\hline$\frac{213}{3}$ & $\begin{array}{l}44,360 \\
157,184\end{array}$ & $\begin{array}{l}317, \text { the } \\
32 t, 592\end{array}$ & 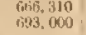 & $\begin{array}{l}175.940 \\
445,000\end{array}$ & ... & & & & 603,000 & $-145,000$ & 525,000 & 315, un & 625,000 & 312000 & $\begin{array}{l}414,350 \\
45+, 181\end{array}$ & $\begin{array}{l}317,4 \pi x \\
320,3132\end{array}$ & & & $\ldots$ & \\
\hline $\begin{array}{l}34, \\
348 \\
33_{10}\end{array}$ & $\begin{array}{l}460,-735 \\
4 \div, 160 \\
+24,400\end{array}$ & $\begin{array}{l}335,552 \\
344,400 \\
353,219\end{array}$ & 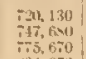 & $\begin{array}{l}514.349 \\
534,060 \\
554,050\end{array}$ & & & & & $-47,0 \times 0$ & 334,060 & 560.500 & 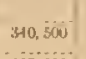 & 605,000 & $337, b(x)$ & 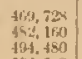 & $\begin{array}{l}335,552 \\
344,400 \\
353,218\end{array}$ & & & & |.. \\
\hline 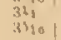 & 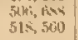 & $\begin{array}{l}391, \text { ve4 } \\
370,496\end{array}$ & $\begin{array}{l}804,060 \\
\times 32<<0\end{array}$ & $\begin{array}{l}5.4,340 \\
594,930\end{array}$ & $\therefore$ & & & & 804.070 & $55 \mathrm{i} .340$ & 612,000 & 367,000 & $72 x, 000$ & $3 F, 0 \times 10$ & $\begin{array}{l}8040,648 \\
51 \%, 560\end{array}$ & $\begin{array}{l}361, y=1 \\
370,404\end{array}$ & & & ..... & $\therefore$ \\
\hline $\begin{array}{l}3 R_{4} \\
3 i_{10}\end{array}$ & $\begin{array}{l}530,320 \\
51,132\end{array}$ & $\begin{array}{l}3 \mathrm{~s}, 540 \\
3 \times 6,9+20\end{array}$ & $\sin 1,130$ & & & & & & $\sin 2,130$ & 615,500 & 657,000 & 34,000 & $T s 0,(160$ & 300,000 & $\begin{array}{l}530,330 \\
5 \$ 1,632\end{array}$ & $\begin{array}{l}378,840 \\
360,8000\end{array}$ & .. & & & \\
\hline $\begin{array}{l}34 \\
3 \%\end{array}$ & 553,056 & $\begin{array}{c}395,130 \\
\cdots\end{array}$ & $021, \$ 10$ & $\begin{array}{l}65 s, 440 \\
\ldots . \ldots\end{array}$ & $\ldots$ & & & & $\begin{array}{r}921,010 \\
903,000\end{array}$ & $\begin{array}{l}65 ., 110 \\
702,200\end{array}$ & ....... & $\therefore \cdots$ & ......... & & $353,0=6$ & 365,133 & - & 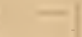 & --1 & $\therefore$ \\
\hline $\begin{array}{l}3 x_{4} \\
3^{2} \\
4 \\
4,8\end{array}$ & $\sin 3: 0$ & 425,370 & $1,015,900$ & $\div 5,070$ & & & & $\because$ & $\begin{array}{l}1,045,200 \\
1,110,210 \\
1,176.000 \\
1,213,200\end{array}$ & $\begin{array}{l}747.070 \\
73,010 \\
840,000 \\
548,000\end{array}$ & & & & & S.s. 330 & $\begin{array}{l}+25,370 \\
\cdots\end{array}$ & - & I & i & - \\
\hline
\end{tabular}

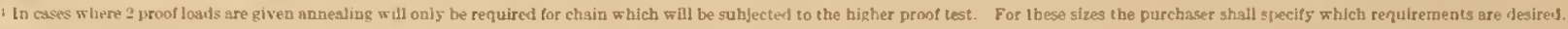
Sote,-1. S. T. M As6-30 hreut and proof lest loads ideatical with F \&. B. RR-C-2F1. 

603.วั1. LONG-LINK CHAINS.

UNITED STATES GOVERNMENT, FEDERAL SPECIFICATIONS BOARD, FEDERAL SPECIFICATION RR-C-271, FOR STAND. ARD MISCELLANEOUS CHAIN AND ATTACHMENTS, DECEMBER 8, 1931.

\section{MISCELLANEOUS CHAINS AND ATTACHMENTS}

\section{LONG LINK COIL CHAIN}

1. Long link coil chain shall be of the following classes: (1) Wrought iron, (2) wrought iron or openhearth steel.

(For general specifications, material and workmanship, detail requirements, and methods of inspecticn and tests, see 603.50, p. 199. For proof and breaking loads see comparative table facing p. 200. For other kinds of chain and chain attachments specified in F. S. B. No. 348 (RR-C-271) see 603.52, $603.54,603.56,603.57$, and 617.5. See also 631.9, $642.9,645.9$, and 646.59.)

\section{BUOY OR SUBMARINE NET CHAIN}

2. Buoy or submarine net chain shall be made of class 2 material wrought iron only, and in accordance with Table 1 . The proof and breaking loads shall be in accordance with the requirements for

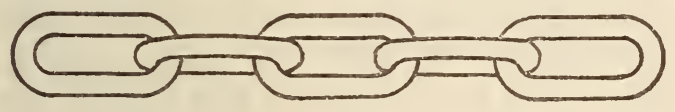

Figure 76.-Long link coil chain

this class as shown in comparative table facing $p$. 200. The percentage of elongation shall be as specified for this class of chain.

TABLE 1.-Buoy or submarine net chain (dimensions)

\begin{tabular}{|c|c|c|c|c|c|c|c|}
\hline \multicolumn{4}{|c|}{ Common links } & \multicolumn{4}{|c|}{ End links } \\
\hline $\begin{array}{l}\text { Size of } \\
\text { iron }\end{array}$ & $\begin{array}{l}\text { Outside } \\
\text { length }\end{array}$ & $\begin{array}{l}\text { Outside } \\
\text { width }\end{array}$ & $\begin{array}{l}\text { Length } \\
\text { of } 100 \\
\text { links }\end{array}$ & $\begin{array}{l}\text { Size of } \\
\text { iron }\end{array}$ & $\begin{array}{l}\text { Outside } \\
\text { length }\end{array}$ & Outside & $\begin{array}{l}\text { Weight } \\
\text { of } 100 \\
\text { feet }\end{array}$ \\
\hline $\begin{array}{c}\text { Inches } \\
1 / 2 \\
5 / 8 \\
3 / 4 \\
7 / 8\end{array}$ & $\begin{array}{c}\text { Inches } \\
21 / 2 \\
33 / 4 \\
41 / 2 \\
51 / 4\end{array}$ & $\begin{array}{c}\text { Inches } \\
17 / 8 \\
23 / 16 \\
25,8 \\
31 / 8\end{array}$ & $\begin{array}{c}\text { Inches } \\
138 \\
181 \\
206 \\
231\end{array}$ & $\begin{array}{c}\text { Inches } \\
3 / 4 \\
34 \\
7 / 8 \\
1^{7 / 8}\end{array}$ & $\begin{array}{l}\text { Inches } \\
37 / 8 \\
411 / 16 \\
55 / 8 \\
63 \% 8\end{array}$ & $\begin{array}{c}\text { Inches } \\
2^{11} 1 / 16 \\
2^{11 / 2} \\
3 \\
31 / 2\end{array}$ & $\begin{array}{c}\text { Pounds } \\
270 \\
430 \\
615 \\
820\end{array}$ \\
\hline $\begin{array}{l}1 \\
11 / 4 \\
11 / 2 \\
15 / 8\end{array}$ & $\begin{array}{l}6 \\
71 / 2 \\
9 \\
93 / 4\end{array}$ & $\begin{array}{l}31 / 2 \\
438 \\
51 / 4 \\
5^{11116}\end{array}$ & $\begin{array}{l}269 \\
331 \\
400\end{array}$ & $\begin{array}{l}11 / 8 \\
11 / 2 \\
13 / 4 \\
17 / 8\end{array}$ & $\begin{array}{r}71 / 4 \\
91 / 2 \\
111 / 4 \\
121 / 2 \\
\end{array}$ & $\begin{array}{l}37 / 8 \\
51 / 8 \\
57 / 8 \\
61 / 4\end{array}$ & $\begin{array}{l}1,030 \\
1,660 \\
2,450\end{array}$ \\
\hline
\end{tabular}

CONVEYOR OR SPROCKET-WHEEL CHAIN

3. Conveyor or sprocket-wheel chain shall be made of class 2 material. Chain from $1 / 2$ inch to $11 / 4$ inches, inclusive, shall be made of steel, chain from $13 / 8$ inches and larger to be made of iron unless otherwise specified. The proof and the breaking loads shall be in accordance with the requirements for this class as shown in the comparative table facing page 200 . The percentage of elongation shall be as specified for this class of chain. Otherwise the chain shall be in accordance with Table 2 .
TABLE 2.-Conveyor or sprocket-wheel chain (dimensions)

\begin{tabular}{|c|c|c|c|}
\hline Size & $\begin{array}{l}\text { Inside } \\
\text { width } \\
\text { of link }\end{array}$ & $\begin{array}{l}\text { Inside } \\
\text { length } \\
\text { of link }\end{array}$ & $\begin{array}{l}\text { Weight } \\
\text { per } 100 \\
\text { feet }\end{array}$ \\
\hline $\begin{array}{c}\text { Inches } \\
1 / 2 \\
1 / 2 \\
1 / 2 \\
5 / 8 \\
5 / 8\end{array}$ & $\begin{array}{c}\text { Inches } \\
3 / 4 \\
7 / 8 \\
1 \\
1 \\
1\end{array}$ & $\begin{array}{c}\text { Inches } \\
31 / 2 \\
31 / 2 \\
31 / 2 \\
41 / 2 \\
5\end{array}$ & $\begin{array}{c}\text { Pounds } \\
205 \\
210 \\
215 \\
305 \\
295\end{array}$ \\
\hline $\begin{array}{l}5 / 8 \\
3 / 4 \\
3 / 4 \\
3 / 4 \\
3 / 4\end{array}$ & $\begin{array}{l}118 \\
1 \\
118 \\
11 / 2 \\
118\end{array}$ & $\begin{array}{l}5 \\
5 \\
5 \\
5 \\
6\end{array}$ & $\begin{array}{l}300 \\
420 \\
430 \\
450 \\
400\end{array}$ \\
\hline $\begin{array}{l}3 / 4 \\
7 / 8 \\
7 / 8 \\
7 / 8 \\
7 / 8\end{array}$ & $\begin{array}{l}11 / 2 \\
11 / 8 \\
11 / 2 \\
11 / 4 \\
11 / 2\end{array}$ & $\begin{array}{l}6 \\
5 \\
5 \\
6 \\
6\end{array}$ & $\begin{array}{l}415 \\
595 \\
625 \\
575 \\
590\end{array}$ \\
\hline $\begin{array}{l}7 / 8 \\
1^{7 / 8} \\
1 \\
1\end{array}$ & $\begin{array}{l}11 / 4 \\
2 \\
11 / 2 \\
11 / 2 \\
13 / 4\end{array}$ & $\begin{array}{l}7 \\
8 \\
5 \\
6 \\
7\end{array}$ & $\begin{array}{l}535 \\
630 \\
825 \\
770 \\
745\end{array}$ \\
\hline $\begin{array}{l}1 \\
1 \\
118 \\
118 \\
118\end{array}$ & $\begin{array}{l}2 \\
2 \\
11 / 2 \\
13 / 4 \\
2\end{array}$ & $\begin{array}{r}7 \\
8 \\
-6 \\
6 \\
7\end{array}$ & $\begin{array}{r}765 \\
735 \\
1,000 \\
1,025 \\
985\end{array}$ \\
\hline $\begin{array}{l}118 \\
11 / 4 \\
11 / 4 \\
13 / 8 \\
11 / 2\end{array}$ & $\begin{array}{l}2 \\
21 / 4 \\
21 / 4 \\
21 / 4 \\
21 / 4\end{array}$ & $\begin{array}{l}8 \\
7 \\
8 \\
8 \\
8\end{array}$ & $\begin{array}{r}940 \\
1,260 \\
1,200 \\
1,450 \\
1,750\end{array}$ \\
\hline $\begin{array}{l}11 / 2 \\
15 / 8 \\
13 / 4 \\
13 / 4\end{array}$ & $\begin{array}{l}21 / 8 \\
23 / 8 \\
21 / 8 \\
21 / 4\end{array}$ & $\begin{array}{l}81 / 2 \\
8 \\
8 \\
8\end{array}$ & $\begin{array}{l}1,650 \\
2,120 \\
2,450 \\
2,485\end{array}$ \\
\hline $\begin{array}{l}13 / 4 \\
13 / 4 \\
17 / 8 \\
2\end{array}$ & $\begin{array}{l}21 / 2 \\
25,8 \\
21 / 2 \\
21 / 2\end{array}$ & $\begin{array}{l}10 \\
10 \\
10 \\
10\end{array}$ & $\begin{array}{l}2,300 \\
2,325 \\
2,700 \\
3,100\end{array}$ \\
\hline
\end{tabular}

603.52 SHORT LINK, STRAIGHT-LINK CHAINS.

\section{AMERICAN BUREAU OF SHIPPING, RULES FOR BUILDING AND CIASSING STEEI VESSELS; WROUGHT-IRON AND STEEL CHAINS, 1930}

\section{WROUGHT-IRON AND STEEL CHAINS}

(For general information and requirements see 600.0 , p. 2.)

1. No vessel can be classed with the letter $E$ unless the chain cables have been tested, and the weights and dimensions are in accordance with the requirements of the American Bureau of Shipping. (The dimensions and weights for like diameter of chain bar are the same for outside length and outside width as those for stud-link chain cable shown in American Bureau of Shipping, Table 1. (See 603.53, p. 206.).)

2. All chain cables, stream chains, and steering chains are to be tested under the inspection of a surveyor to the American Bureau of Shipping, and in a machine recognized for such purposes by the committee of the A. B. S. 


\section{MATERIAL AND WORKMANSHIP}

3. Chains are to be manufactured from a good quality of wrought iron, or from steel made by the open-hearth process, which shall be ductile and of satisfactory welding quality. They may be hand forged, machine or die made, or electrically welded. The finished chain is to be of good workmanship and free from injurious defects, and is to be carefully gaged to ascertain if the material is full to size and in accordance with the table.

4. There is to be an odd number of links to each shot to insure shackles leading over the windlass in the same direction. The size for descriptive and test purposes is to be the diameter of the common link of the chain with a plus tolerance which is not to exceed one-thirty-second-inch in the case of chains up to and including $1 \frac{1}{2}$ inches in diameter. In the case of chains above $1 \frac{1}{2}$ inches in diameter the tolerance is not to exceed one-sixteenth inch.

5. The sectional area of the material at the weld of the common link is not to be reduced below that of the prescribed minimum diameter of the common links of the chain. The links may be either end or side welded. Prior to testing and inspection, the chain shall be free from paint or any other coating which would tend to conceal defects.

6. All chain studs must be well centered and firmly held in place, and may be of cast iron, wrought iron, or steel. Each shot should be stamped with a distinctive mark in order to identify it through the several processes of gaging, testing, measuring, examining, repairing, and weighing. In the event of the surveyor being in attendance at the works while chains are being fabricated which are ultimately proposed to be submitted for testing, he will arrange to select the break-test specimens as far as possible during the process of fabrication.

\section{BREAKING AND PROOF TESTS}

7. Where the rods from which the chain is made have been tested by the bureau's surveyor in accordance with the requirements for bars (see 603.1, p. 135) a specimen of 2 links or more, as may be found necessary, is to be cut from 1 shot of 15 fathoms or less of each lot of 5 shots presented and subjected to the breaking test. If this test is satisfactory the shot from which the specimen has been taken shall be joined with a joining link and the shot subjected to the proof test, along with the four remaining shots presented. If the first test is not satisfactory, one more specimen is to be cut out and subjected to the breaking test; and if this fails the shots shall be rejected, and, in addition, a specimen of 2 links or more as may be found necessary is to be cut from each of the 4 remaining shots of 15 fathoms or less and subjected to the breaking test. If this test is satisfactory then each shot from which the specimen has been taken shall be joined with a joining link and the shots subjected to a proof test.

8. Where the rods from which the chain is made have not been tested by the bureau's surveyor, a specimen of two links or more from each shot of 15 fathoms or less is to be subjected to the breaking test described above. If the break-test specimen satisfactorily withstands the breaking test as prescribed by the rules, the specimen need not be tested to destruction, but must be discarded. Each 15 fathoms or less is to be subjected to the proof test.

9. After the proof test the length of the chain is to be ascertained. The chain shall then be carefully examined and any link showing surface rupture or deformation is to be taken out, the chain repaired, the proof test again applied, and the chain reexamined. If one link breaks under the proof test, a joining link shall be inserted and the proof test again applied. If a second link breaks under the second proof test, or if 5 per cent of the links are found defective, the shot shall be rejected.

10. The end shackles, joining shackles, spare shackles, and swivels required for a length of chain are to be tested to the same proof test as the chain, and at least 1 out of every 25 of the shackles is to be subjected to the breaking test, and must be discarded.

11. Where a chain which has already been in use is submitted for testing or retesting, the size for descriptive and testing purposes is to be the mean diameter of the part most worn. The certificate issued in regard to same will be marked in red: "This chain is not new but has been previously used."

12. When chains are supplied in continuous lengths without joining shackles it will be so noted on the certificate. In such cases a specimen of two links or more, as may be found necessary, is to be cut from each 30 fathoms or less and subjected to the breaking test. If this test is satisfactory the several shots are to be joined up and each 15 fathoms or less is to be subjected to the proof test.

13. Care must be taken that arrangements are made for each link to be tested once and only once during the test, and the gripping arrangements must be such that they do not put any stress on the end links of the portion under test, except such stress as is equally put on every link tested. Otherwise the requirements are to be as prescribed in this paragraph.

14. Where chains are not to be used for ships, and are not required to be in accordance with the rules of the American Bureau of Shipping, the committee will approve of their surveyors testing such chains to suitable specifications other than those stated in the rules, provided these are mutually agreed upon by the manufacturers and purchasers. A special form of certificate will be issued in such cases.

15. The breaking and proof tests, and minimum weights per 15 fathoms, of unstudded short-link chains are as shown in the table below.

\section{WEIGHING AND MARKING}

16. When chains have satisafctorily passed the above requirements they are to be weighed, together with the shackles forming the outfit, and this actual weight will be given on the certificate of test. The weight of the chain must not be more than $2 \frac{1}{2}$ per cent under the weight specified in the table, and it should not be more than 5 per cent over the weight specified in the table. After being weighed, the shackles and the end links of each shot, and every 15 fathoms in the case of chain which is in one continuous length without joining shackles, are to be clearly stamped by the manufacturer. (The stampings include identifying number, initials of surveyor, date of test, breaking test, proof test, and $A B$, the bureau's mark of approval.) 
Unstudded short link chain (dimensions, weights, tests)

\begin{tabular}{|c|c|c|c|}
\hline $\begin{array}{l}\text { Diameter } \\
\text { of com- } \\
\text { mon } \\
\text { links }\end{array}$ & $\begin{array}{c}\text { Breaking } \\
\text { test }\end{array}$ & $\begin{array}{l}\text { Proof } \\
\text { test }\end{array}$ & $\begin{array}{l}\text { Weight } \\
\text { per } 15 \\
\text { fathoms }\end{array}$ \\
\hline $\begin{array}{c}\text { Inches } \\
516 \\
616 \\
716 \\
816 \\
916\end{array}$ & $\begin{array}{r}\text { Pounds } \\
5,040 \\
7,280 \\
10,080 \\
13,440 \\
16,800\end{array}$ & $\begin{array}{c}\text { Pounds } \\
2,520 \\
3,640 \\
5,040 \\
6,720 \\
8,400\end{array}$ & $\begin{array}{c}\text { Pounds } \\
110 \\
150 \\
215 \\
270 \\
325\end{array}$ \\
\hline $\begin{array}{l}1 \% / 16 \\
11 / 16 \\
12 / 16 \\
13 / 16 \\
14 / 16\end{array}$ & $\begin{array}{l}20,720 \\
25,200 \\
30,240 \\
35,392 \\
40,880\end{array}$ & $\begin{array}{l}10,360 \\
12,600 \\
15,120 \\
17,696 \\
20,440\end{array}$ & $\begin{array}{l}390 \\
445 \\
525 \\
620 \\
710\end{array}$ \\
\hline $\begin{array}{l}15 / 16 \\
1316 \\
1316 \\
13 / 16\end{array}$ & $\begin{array}{l}47,040 \\
53,760 \\
60,480 \\
67,760 \\
75,712\end{array}$ & $\begin{array}{l}23,520 \\
26,880 \\
30,240 \\
33,880 \\
37,856\end{array}$ & $\begin{array}{r}815 \\
925 \\
1,035 \\
1,165 \\
1,300\end{array}$ \\
\hline $\begin{array}{l}1416 \\
15 / 16 \\
1916 \\
17 / 16 \\
1816\end{array}$ & $\begin{array}{r}84,000 \\
92,400 \\
101,360 \\
110,880 \\
120,960\end{array}$ & $\begin{array}{l}42,000 \\
46,200 \\
50,680 \\
55,440 \\
60,480\end{array}$ & $\begin{array}{l}1,435 \\
1,585 \\
1,735 \\
1,895 \\
2,050\end{array}$ \\
\hline
\end{tabular}

AMERICAN MARINE STANDARDS COMMITTEE, CARGO-HANDLING GEAR FOR SHIPS, AMERICAN MARINE STANDARDS H NO. 14-1926, AND H NO. 15-1926, JANUARY 25,1926

(The specifications for this gear conform to the requirements of the American Bureau of Shipping

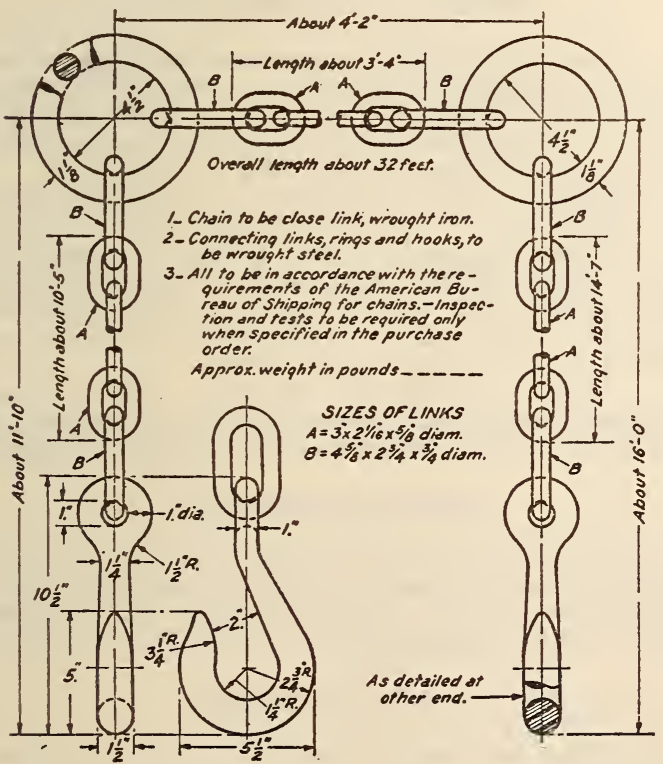

FIGURE 77.-Railroad iron sling; working load 2 tons

for wrought-iron and steel chains, given above. Inspection and tests are required only when specified in the purchase order.)
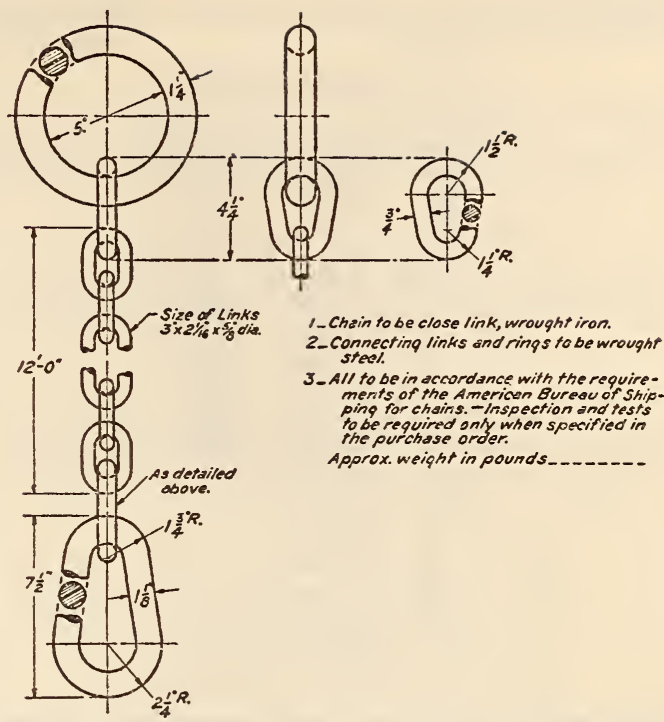

FIGURE 78.-Chain sling; working load 2 tons

\section{AMERICAN RAILWAY ASSOCIATION, ME- CHANICAL DIVISION, SPECIFICATION FOR CHAIN, 1930}

\section{MATERIAL AND MANUFACTURE}

1. SCOPE

These specifications cover two classes of chain generally used for the following purposes:

Crane chain, for slings, hoists, steam shovels, and marine uses, and where an all-iron chain is desired.

Proof coil, for railroad cars and construction and forestry work.

\section{MATERIAI}

The material shall be as follows:

Crane chain.-Wrought iron, which shall be made from all-pig puddled iron, free from any admixture of iron scrap or steel.

NoTE.-The term "iı on scrap" applies only to foreign or bought scrap, and does not include local mill products of crane chain iron quality.

Proof coil.-Wrought-iron or open-hearth steel. The wrought iron may be made from all reworked scrap.

Note.-The term "reworked scrap" applies to steel and iron scrap or to a mixture of both.

\section{WELDING}

Crane chain shall be lap fire welded. Unless otherwise specified, proof-coil chain may be made by either fire or electric welding process.

(In chemical composition and tests, physical properties and tests, standard weights and dimensions, workmanship and finish, inspection and rejection, this specification is in substantial agreement with F. S. B., RR-C-271. See 603.50, p. 199.)

(The standard weights and dimensions of crane chain in this specification are, for like sizes, the same as in F. S. B. RR-C-271. Weights and dimensions of corresponding proof coils are not specified. The A. R. A. specification also contains a table which 
shows safe working loads in pounds. This is reproduced as Table 1.)

TABLE 1.-Safe working load

\begin{tabular}{|c|c|c|}
\hline $\begin{array}{l}\text { Nominal } \\
\text { size of } \\
\text { chain } \\
\text { bar }\end{array}$ & $\begin{array}{l}\text { Crane } \\
\text { chain, } \\
\text { wrought } \\
\text { iron }\end{array}$ & $\begin{array}{l}\text { Proof } \\
\text { coil, } \\
\text { iron or } \\
\text { steel }\end{array}$ \\
\hline $\begin{array}{r}\text { Inches } \\
1 / 4 \\
5 / 16 \\
3 / 8 \\
7 / 16 \\
1 / 2\end{array}$ & $\begin{array}{c}\text { Pounds } \\
1,060 \\
1,655 \\
2,385 \\
3,250 \\
4,240\end{array}$ & $\begin{array}{c}\text { Pounds } \\
850 \\
1,325 \\
1,925 \\
2,625 \\
3,425\end{array}$ \\
\hline $\begin{array}{l}9 / 16 \\
5 / 8 \\
3 / 4 \\
7 / 8 \\
1^{7 / 8}\end{array}$ & $\begin{array}{r}5,370 \\
6,630 \\
9,540 \\
12,960 \\
16,950\end{array}$ & $\begin{array}{r}4,325 \\
5,350 \\
7,675 \\
10,450 \\
13,675\end{array}$ \\
\hline $\begin{array}{l}11 / 8 \\
11 / 4 \\
13 / 8 \\
11 / 2\end{array}$ & $\begin{array}{l}20,040 \\
24,750 \\
29,910 \\
35,600\end{array}$ & - \\
\hline $\begin{array}{l}15 / 8 \\
13 / 4 \\
17 / 8 \\
2\end{array}$ & $\begin{array}{l}41,800 \\
48,450 \\
55,300 \\
63,300\end{array}$ & 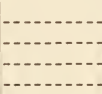 \\
\hline
\end{tabular}

AMERICAN SOCIETY FOR TESTING MATERIALS, STANDARD SPECIFICATIONS FOR IRON AND STEEL CHAIN, A56-30, 1930

\section{IRON AND STEEL CHAIN}

(Identical with A. R. A. M., given above, in respect to scope, material, welding, and safe working loads. In respect to physical properties and tests, standard weights and dimensions, workmanship and finish, inspection and rejection, this specification conforms to F. S. B. RR-C-271. See 603.50, p. 199.) It provides as follows for-

\section{CHEMICAL PROPERTIES AND TESTS}

1. The wrought iron and steel of the respective classes of chain shall conform to the following requirements as to chemical composition:

\begin{tabular}{l|r|r}
\hline & $\begin{array}{r}\text { Wrought- } \\
\text { iron crane } \\
\text { chain }\end{array}$ & $\begin{array}{r}\text { Steel } \\
\text { proof coil }\end{array}$ \\
\hline Manganese, maximum & Per cent \\
0.10 & Per cent \\
Phosphorus, maximum & 0.05 \\
Sulphur, maximum. & \\
\hline
\end{tabular}

\section{CHECK ANALYSIS}

2. (a) Analyses may be made by the purchaser from the bars or from the finished chain. The chemical composition thus determined shall conform to the requirements specified above.

(b) Drillings for analysis shall be taken from the entire cross section of the bar.

\section{UNITED STATES GOVERNMENT, FEDERAL SPECIFICATIONS BOARD, FEDERAL SPECIFICATION RR-C-2\%1 FOR STAND. ARD MISCELLANEOUS CHAIN AND AT- TACHMENTS, DECEMBER 8, 1931.}

\section{CLOSE-LINK COIL CHAIN}

1. Close-link coil chain shall be of the following classes: (1) Wrought iron, (2) wrought iron or openhearth steel.
(For general specifications, material and workmanship, weights and dimensions, detail requirements, and method of inspection and tests, see F. S. B. RR-C-271, (603.50, p. 199). For proof and breaking loads see comparative table facing page 200 . For other kinds of chain and for chain attachments specified in F. S. B. RR-C-271 see $603.51,603.54$,

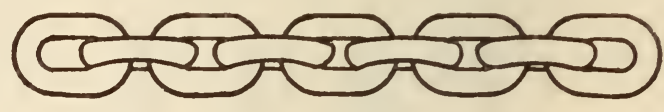

FIgURE 79.-Close link coil chain

$603.56,603.57$, and 617.5. See also 631.9, 642.9, 645.9 , and 646.59.)

603.53. STUD-LINK CHAINS.

AMERICAN BUREAU OF SHIPPING, RULES FOR BUILDING AND CLASSING STEEL VESSELS; QUALITY AND TESTING OF MATERIALS-SHIP CONSTRUCTION, 1930

\section{WROUGHT-IRON AND STEEL STUD-LINK CHAIN; CAST-STEEL STUD-LINK CHAIN}

(Specifications for wrought-iron and steel studlink chain are the same as those for wrought-iron and steel unstudded short-link chain. See 603.52, p. 201.)

\section{CAST-STEEL STUD-LINK CHAIN}

(For general information and requirements see 600.0 , p. 2.)

\section{MATERIAL AND WORKMANSHIP}

1. Cast steel made in accordance with the following specifications will be approved for use in all parts of stud-link chain, including stud links, enlarged links, end links, shackles, and shackle pins for chains $13 / 8$ inches and over in diameter.

2 . The steel shall be made by the electric furnace process.

3. All castings shall be true to form and free from shrinkage, porosity, projections, or other defects in such location or of such nature as to impair the strength of the chain; and all castings shall be produced in dry sand molds or cores.

4. Each complete shot of chain shall be heat treated in such manner that the treatment will be complete and uniform throughout its entire length.

\section{WEIGHTS AND DIMENSIONS}

5. The weight of each shot of chain shall not be less than the minimum amount specified in Table 1. The dimensions and tolerances shall be in accordance with tabular requirements. (See Tables 1 and 2.)

\section{PHYSICAL PROPERTIES}

6. From each heat of steel entering into the production of any shot of chain, shackles, shackle pins, etc., there shall be cast not less than one link in each of two test chains, said test chains to be poured from heats in such manner as to insure that not more than two 15-fathom shots of chains are represented by any one break test. The links entering into these test chains shall be of the same wire diameter and general dimensions as the common "A" links that form a part of the shot or shots of chain they represent. Each of these test chains shall contain not 
less than three links. These test chains shall be heat treated along with any shot or shots of chain containing links poured from the same heat or heats.

\section{BREAKING AND PROOF TESTS}

7. After heat treatment one of the test chains shall be subjected to the breaking stress, which shall consist of anchoring the ends of the test chain in an approved static testing machine, and pulling it to a breaking load in accordance with the comparative table facing page 200 . If the test chain satisfactorily withstands this breaking load, the heats of steel represented by the links in the test chains shall be accepted so far as this test is concerned.

8. If the first test chain fails to withstand the breaking load, then the second test chain representing the same heats of steel shall be subjected to the required breaking test; and if it satisfactorily withstands the pull, the heats of steel represented by the links in this second test chain shall be accepted so far as this test is concerned. If any link or links in this second test chain fail during the test, then all the links in any shots of chain cast from the corresponding heat or heats represented by the links that fail in both the first and second test chains shall be rejected. Such rejected links may be cut out and replaced by new links cast in the shot.

9. Retreatment will be permitted between first and second tests, but such treatment shall be applied simultaneously to shots and corresponding test links.

10. If the second test chain is not required to be used for this test, it may be subsequently incorporated in any shot of chain.

11. After satisfactorily withstanding the required breaking tests, the complete lot of chain shall then pass through the heat-treating process more than three times.

\section{WEIGHING AND MARKING}

13. Each shot of chain accepted shall be legibly marked in accordance with requirements. (The markings include identifying number, initials of
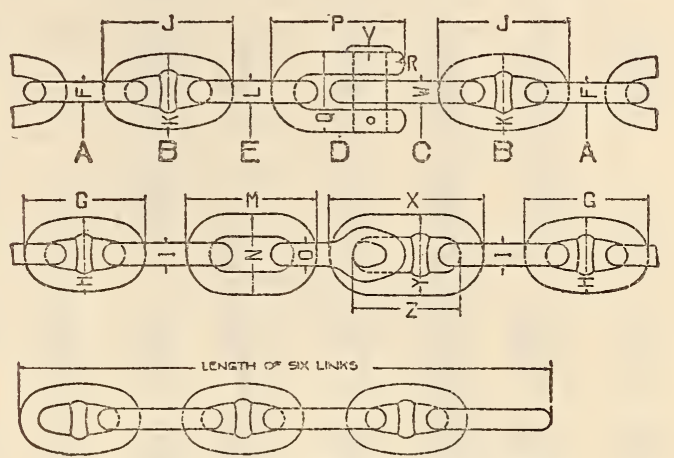

FIguRE 80.-Stud link chain cable

surveyor, date of test, breaking test, proof test, and $A B$, the bureau's mark of approval.)

All chain studs must be well centered and firmly held in place, and may be of cast iron, wrought iron, or steel. Each shot should be stamped with a distinctive mark in order to identify it through the several processes of gaging, testing, measuring, examining, repairing, and weighing. In the event of the surveyor being in attendance at the works while
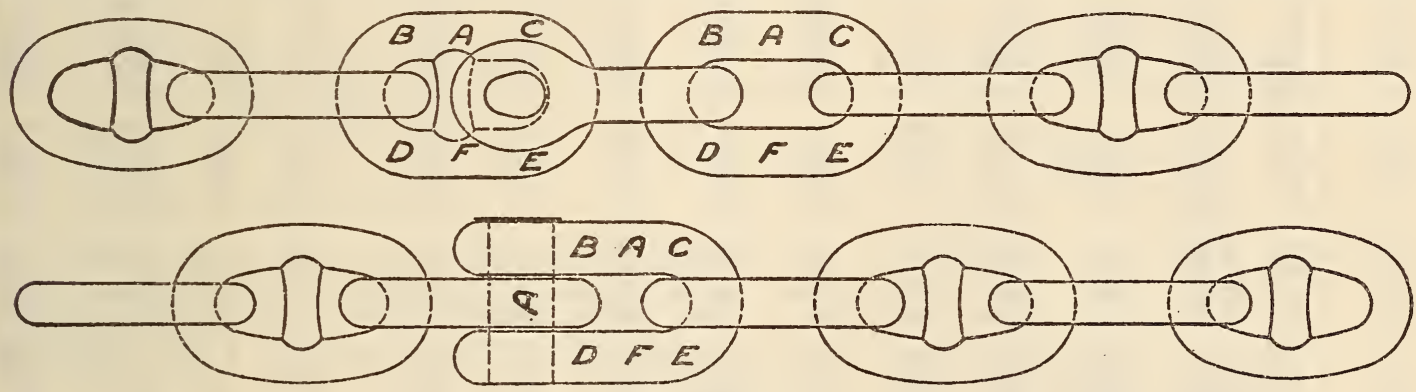

Figure 81

be subjected to the proof test given in table facing page 200, and if such shot of chain passes this test it shall be accepted.

12. If any link or links fail during the proof test they may be replaced by casting in new links from a heat which has satisfactorily passed the break test, and the shot may then be retreated and again subjected to the required proof test. If any link or links fail during the retest then all the links cast from the same heat or heats shall be rejected. Any links not so rejected may be used in making a new shot of chain, provided that in no case shall links chains are being fabricated which are ultimately proposed to be submitted for testing, he will arrange to select the break test specimens as far as possible during the process of fabrication.

\section{INSPECTION}

14. Each complete shot of chain shall be carefully examined to insure that there are no fractured links or other defects, that the chain has been properly cleaned to remove all sand and scale, that all fins and partings have been removed, and that it is finished in a workmanlike manner. 
TABLE 1.-Stud-link chain cable (dimensions)

\begin{tabular}{|c|c|c|c|c|c|c|c|c|c|c|c|c|c|c|}
\hline \multirow{2}{*}{$\begin{array}{c}\text { Size } \\
\text { of } \\
\text { chain }\end{array}$} & \multirow{2}{*}{$\begin{array}{c}\text { Length } \\
\text { of } 6 \\
\text { links }\end{array}$} & \multirow{2}{*}{$\begin{array}{l}\text { Weight } \\
\text { of } 15 \\
\text { fathoms }\end{array}$} & \multicolumn{3}{|c|}{ Common links A } & \multicolumn{3}{|c|}{ Enlarged links B } & \multicolumn{3}{|c|}{ End links $E$} & \multicolumn{3}{|c|}{ End links C } \\
\hline & & & $\begin{array}{l}\text { Size of } \\
\text { iron F }\end{array}$ & $\begin{array}{l}\text { Outside } \\
\text { length G }\end{array}$ & $\begin{array}{c}\text { Outside } \\
\text { width H }\end{array}$ & $\begin{array}{l}\text { Size of } \\
\text { iron I }\end{array}$ & $\begin{array}{r}\text { Outside } \\
\text { length J }\end{array}$ & $\begin{array}{r}\text { Outside } \\
\text { width K }\end{array}$ & $\begin{array}{l}\text { Size of } \\
\text { iron } L\end{array}$ & $\begin{array}{l}\text { Outside } \\
\text { length M }\end{array}$ & $\begin{array}{r}\text { Outside } \\
\text { width N }\end{array}$ & $\begin{array}{l}\text { Size of } \\
\text { iron W }\end{array}$ & $\begin{array}{l}\text { Outside } \\
\text { length X }\end{array}$ & $\begin{array}{l}\text { Outside } \\
\text { width Y }\end{array}$ \\
\hline $\begin{array}{c}\text { Inches } \\
5 / 8 \\
11 / 16 \\
3 / 4 \\
13 / 16 \\
7 / 8\end{array}$ & $\begin{array}{rr}F t . & \text { In. } \\
1 & 41 / 4 \\
1 & 57 / 8 \\
1 & 71 / 2 \\
1 & 91 / 8 \\
1 & 103 / 4\end{array}$ & \begin{tabular}{|r|} 
Pounds \\
365 \\
405 \\
480 \\
470 \\
655
\end{tabular} & $\begin{array}{c}\text { Inches } \\
5 / 8 \\
11 / 16 \\
3 / 4 \\
13 / 16 \\
7 / 8\end{array}$ & $\begin{array}{r}\text { Inches } \\
33 / 4 \\
41 / 8 \\
41 / 2 \\
47 / 8 \\
51 / 4\end{array}$ & \begin{tabular}{|c|} 
Inches \\
23116 \\
$27 / 16$ \\
$25 \% 8$ \\
278 \\
318
\end{tabular} & $\begin{array}{c}\text { Inches } \\
11 / 16 \\
3 / 4 \\
13 / 16 \\
7 / 8 \\
15 / 16\end{array}$ & $\begin{array}{c}\text { Inches } \\
4 \\
43 / 8 \\
43 / 4 \\
5316 \\
5 \% 16\end{array}$ & \begin{tabular}{|c|} 
Inches \\
$23 / 8$ \\
$25 / 8$ \\
21316 \\
$31 / 16$ \\
$.35 \% 10$
\end{tabular} & $\begin{array}{c}\text { Inches } \\
3 / 4 \\
13 / 16 \\
15 / 16 \\
1 \\
1116\end{array}$ & $\begin{array}{c}\text { Inches } \\
41 / 16 \\
47 / 16 \\
47 / 8 \\
51 / 4 \\
511 / 16\end{array}$ & \begin{tabular}{|c|} 
Inches \\
$21 / 2$ \\
$23 / 4$ \\
3 \\
$31 / 4$ \\
$31 / 2$
\end{tabular} & $\begin{array}{c}\text { Inches } \\
3 / 4 \\
13 / 16 \\
15 / 16 \\
1 \\
11 / 16\end{array}$ & $\begin{array}{c}\text { Inches } \\
411 / 16 \\
5316 \\
558 \\
618 \\
69 / 16\end{array}$ & $\begin{array}{c}\text { Inches } \\
21 / 2 \\
2314 \\
3 \\
31 / 4 \\
31 / 2\end{array}$ \\
\hline $\begin{array}{l}15 / 16 \\
1 \\
11 / 16 \\
11 / 8 \\
13 / 16\end{array}$ & $\begin{array}{rl}2 & 03 / 8 \\
2 & 2 \\
2 & 35 / 8 \\
2 & 51 / 4 \\
2 & 67 / 8\end{array}$ & $\begin{array}{r}755 \\
855 \\
970 \\
1,085 \\
1,215\end{array}$ & $\begin{array}{l}1^{15 / 16} \\
11 / 16 \\
138 \\
13 / 16\end{array}$ & $\begin{array}{l}55 / 8 \\
6 \\
63 / 8 \\
63 / 4 \\
71 / 8\end{array}$ & $\begin{array}{l}35 / 16 \\
39 / 16 \\
33 / 4 \\
4 \\
41 / 4\end{array}$ & $\begin{array}{l}1 \\
11 / 16 \\
11 / 8 \\
13 / 16 \\
11 / 4\end{array}$ & $\begin{array}{l}6 \\
63 / 8 \\
63 / 4 \\
73 / 16 \\
79 / 16\end{array}$ & $\begin{array}{l}3 \% / 16 \\
33 / 4 \\
4 \\
41 / 4 \\
41 / 2\end{array}$ & $\begin{array}{l}11 / 8 \\
13 / 16 \\
11 / 4 \\
15 / 16 \\
17 / 16\end{array}$ & $\begin{array}{l}61 / 8 \\
61 / 2 \\
615 / 16 \\
75 / 16 \\
73 / 4\end{array}$ & $\begin{array}{l}33 / 4 \\
4 \\
41 / 4 \\
415 \\
43 / 4\end{array}$ & $\begin{array}{l}11 / 8 \\
13 / 16 \\
11 / 4 \\
15 / 16 \\
17 / 16\end{array}$ & $\begin{array}{l}71 / 16 \\
7 \% 16 \\
8 \\
81 / 2 \\
815 / 16\end{array}$ & $\begin{array}{l}33 \\
4 \\
41 \\
4 \\
4\end{array}$ \\
\hline $\begin{array}{l}11 / 4 \\
15 / 16 \\
13 / 8 \\
17 / 16 \\
11 / 2\end{array}$ & $\begin{array}{rr}2 & 81 / 2 \\
2 & 101 / 8 \\
2 & 113 / 4 \\
3 & 13,8 \\
3 & 3\end{array}$ & $\begin{array}{l}1,345 \\
1,485 \\
1,625 \\
1,775 \\
1,935\end{array}$ & $\begin{array}{l}11 / 4 \\
15 / 16 \\
13 / 8 \\
17 / 16 \\
11 / 2\end{array}$ & $\begin{array}{l}71 / 2 \\
77 / 8 \\
81 / 4 \\
85 / 8 \\
9\end{array}$ & $\begin{array}{l}41 / 2 \\
43 / 4 \\
415 / 16 \\
53 / 16 \\
53 / 8\end{array}$ & $\begin{array}{l}15 / 16 \\
13 / 8 \\
17 / 16 \\
11 / 2 \\
19 / 16\end{array}$ & $\begin{array}{l}715 / 16 \\
83 / 8 \\
83 / 4 \\
93 / 16 \\
99 / 16\end{array}$ & $\begin{array}{l}43 / 4 \\
415 / 16 \\
53 / 16 \\
57 / 16 \\
511 / 16\end{array}$ & $\begin{array}{l}11 / 2 \\
19 / 16 \\
15 / 8 \\
111 / 16 \\
113 / 16\end{array}$ & $\begin{array}{l}81 / 8 \\
81 / 2 \\
815 / 16 \\
93 / 8 \\
913 / 16\end{array}$ & $\begin{array}{l}5 \\
51 / 4 \\
51 / 2 \\
53 / 4 \\
6\end{array}$ & $\begin{array}{l}11 / 2 \\
19 / 16 \\
15 / 8 \\
111 / 16 \\
113 / 16\end{array}$ & $\begin{array}{c}97 / 16 \\
97 / 8 \\
1038 \\
107 / 8 \\
115 / 16\end{array}$ & $\begin{array}{l}5 \\
51\end{array}$ \\
\hline $\begin{array}{l}1 \% / 6 \\
15 / 8 \\
111 / 16 \\
13 / 4 \\
113 / 16\end{array}$ & $\begin{array}{rr}3 & 45 / 8 \\
3 & 61 / 4 \\
3 & 77 / 8 \\
3 & 916 \\
3 & 111 / 8\end{array}$ & $\begin{array}{l}2,090 \\
2,235 \\
2,410 \\
2,590 \\
2,785\end{array}$ & $\begin{array}{l}19 / 16 \\
15 / 8 \\
111 / 16 \\
13 / 4 \\
113 / 16\end{array}$ & $\begin{array}{r}93 / 8 \\
93 / 4 \\
101 / 8 \\
101 / 2 \\
107 / 8\end{array}$ & $\begin{array}{l}55 / 8 \\
57 / 8 \\
61 / 16 \\
65116 \\
61 / 2\end{array}$ & $\begin{array}{l}111 / 16 \\
13 / 4 \\
113 / 16 \\
17 / 8 \\
115 / 16\end{array}$ & $\begin{array}{c}915 / 16 \\
103 / 8 \\
103 / 4 \\
1118 \\
119 / 16\end{array}$ & $\begin{array}{l}57 / 8 \\
61 / 8 \\
63 / 8 \\
65 / 8 \\
67 / 8\end{array}$ & $\begin{array}{l}17 / 8 \\
115 / 16 \\
2 \\
21 / 16 \\
23 / 16\end{array}$ & $\begin{array}{l}103 / 16 \\
10 \% / 16 \\
11 \\
1136 \\
11^{13} / 16\end{array}$ & $\begin{array}{l}61 / 4 \\
61 / 2 \\
63 / 4 \\
7 \\
71 / 4\end{array}$ & $\begin{array}{l}17 / 8 \\
1^{15 / 16} \\
2 \\
21 / 16 \\
23 / 16\end{array}$ & $\begin{array}{l}i 13 / 4 \\
121 / 4 \\
123 / 4 \\
133 / 16 \\
13^{11 / 16}\end{array}$ & $\begin{array}{l}63 \\
63 \\
63 \\
7 \\
73\end{array}$ \\
\hline $\begin{array}{l}17 / 8 \\
115 / 16 \\
2 \\
21 / 16 \\
23 / 8\end{array}$ & $\begin{array}{ll}4 & 03 / 4 \\
4 & 236 \\
4 & 4 \\
4 & 556 \\
4 & 71 / 4\end{array}$ & $\begin{array}{l}2,975 \\
3,175 \\
3,355 \\
3,570 \\
3,785\end{array}$ & $\begin{array}{l}i 7 / 8 \\
1^{15 / 16} \\
2 \\
21116 \\
21 / 8\end{array}$ & $\begin{array}{l}111 / 4 \\
115 / 8 \\
12 \\
1236 \\
123 / 4\end{array}$ & $\begin{array}{l}63 / 4 \\
7 \\
73 / 16 \\
77 / 16 \\
75 / 8\end{array}$ & $\begin{array}{l}2 \\
21166 \\
21,8 \\
2316 \\
2144\end{array}$ & $\begin{array}{l}1115 / 16 \\
123 / 8 \\
123 / 4 \\
131 / 8 \\
131 / 2\end{array}$ & $\begin{array}{l}71 / 16 \\
75 / 16 \\
79 / 16 \\
713 / 16 \\
8\end{array}$ & $\begin{array}{l}21 / 4 \\
25 / 16 \\
23 / 8 \\
27 / 16 \\
21 / 2\end{array}$ & $\begin{array}{l}1214 \\
125 / 8 \\
131 / 16 \\
137 / 16 \\
1378\end{array}$ & $\begin{array}{l}71 / 2 \\
73 / 4 \\
8 \\
81 / 4 \\
81 / 2\end{array}$ & $\begin{array}{l}21 / 4 \\
25 / 16 \\
23 / 8 \\
27 / 16 \\
216\end{array}$ & $\begin{array}{l}141 / 8 \\
145 / 8 \\
151 / 16 \\
15 \% / 16 \\
16\end{array}$ & \\
\hline $\begin{array}{l}23 / 16 \\
21 / 4 \\
25 / 16 \\
23 / 8 \\
27 / 16\end{array}$ & $\begin{array}{rr}4 & 87 / 8 \\
4 & 101 / 2 \\
5 & 01 / 8 \\
5 & 13 / 4 \\
5 & 33 / 8\end{array}$ & $\begin{array}{l}4,015 \\
4,245 \\
4,485 \\
4,725 \\
4,960\end{array}$ & $\begin{array}{l}23 / 16 \\
21 / 4 \\
25 / 16 \\
23 / 8 \\
27 / 16\end{array}$ & $\begin{array}{l}131 / 8 \\
131 / 2 \\
137 / 8 \\
141 / 4 \\
145 / 8\end{array}$ & $\begin{array}{l}77 / 8 \\
81 / 8 \\
85 / 16 \\
89 / 16 \\
83 / 4\end{array}$ & $\begin{array}{l}25 / 16 \\
23 / 8 \\
27 / 16 \\
21 / 2 \\
29 / 16\end{array}$ & $\begin{array}{l}1315 / 16 \\
143 / 8 \\
143 / 4 \\
153 / 16 \\
15 \% 16\end{array}$ & $\begin{array}{l}81 / 4 \\
81 / 2 \\
83 / 4 \\
9 \\
93 / 16\end{array}$ & $\begin{array}{l}29 / 16 \\
2^{1} 1 / 16 \\
23 / 4 \\
213 / 16 \\
27 / 8\end{array}$ & $\begin{array}{l}141 / 4 \\
1411 / 16 \\
151 / 16 \\
151 / 2 \\
157 / 8\end{array}$ & $\begin{array}{l}83 / 4 \\
9 \\
91 / 4 \\
91 / 2 \\
93 / 4\end{array}$ & $\begin{array}{l}29 / 16 \\
2^{1} 1 / 16 \\
23 / 4 \\
2^{13} 3 / 16 \\
2^{7} / 8\end{array}$ & $\begin{array}{l}161 / 2 \\
16^{15 / 16} \\
177 / 16 \\
177 / 8 \\
183 / 8\end{array}$ & 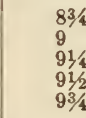 \\
\hline $\begin{array}{l}2^{13} / 16 \\
27 / 8 \\
2^{15} / 16 \\
3 \\
3116\end{array}$ & $\begin{array}{ll}6 & 11 / 8 \\
6 & 23 / 4 \\
6 & 43 / 8 \\
6 & 6 \\
6 & 75 / 8\end{array}$ & $\begin{array}{l}6,705 \\
7,015 \\
7,330 \\
7,650 \\
7,980\end{array}$ & $\begin{array}{l}213 / 16 \\
27 / 8 \\
2^{15} / 16 \\
3 \\
31 / 16\end{array}$ & \begin{tabular}{l|}
$167 / 8$ \\
$171 / 4$ \\
$175 \% 8$ \\
18 \\
1838
\end{tabular} & $\begin{array}{l}10^{1 / 8} \\
10^{3} / 8 \\
10^{9} / 16 \\
10^{13} / 16 \\
11\end{array}$ & $\begin{array}{l}3 \\
31 / 16 \\
31 / 8 \\
33 / 16 \\
31 / 4\end{array}$ & $\begin{array}{l}1715 / 16 \\
185 / 16 \\
183 / 4 \\
191 / 8 \\
191 / 2\end{array}$ & $\begin{array}{l}105 / 8 \\
107 / 8 \\
111 / 8 \\
113 / 8 \\
115 / 16\end{array}$ & $\begin{array}{l}33 / 8 \\
37 / 16 \\
31 / 2 \\
39 / 16 \\
35 / 8\end{array}$ & $\begin{array}{l}185 / 16 \\
183 / 4 \\
191 / 8 \\
199 / 16 \\
1915 / 16\end{array}$ & $\begin{array}{l}111 / 4 \\
111 / 2 \\
113 / 4 \\
12 \\
121 / 4\end{array}$ & $\begin{array}{l}33 / 8 \\
37 / 16 \\
31 / 2 \\
39 / 16 \\
35 / 8\end{array}$ & $\begin{array}{l}213 / 16 \\
211 / 16 \\
221 / 8 \\
225 / 8 \\
231 / 16\end{array}$ & $\begin{array}{l}113 \\
113 \\
12 \\
123\end{array}$ \\
\hline $\begin{array}{l}31 / 8 \\
33 / 16 \\
31 / 4 \\
35 / 16\end{array}$ & $\begin{array}{rr}6 & 91 / 4 \\
6 & 107 / 8 \\
7 & 01 / 2 \\
7 & 21 / 8\end{array}$ & $\begin{array}{l}8,320 \\
8,660 \\
9,010 \\
9,360\end{array}$ & $\begin{array}{l}31 / 8 \\
33 / 16 \\
31 / 4 \\
35 / 16\end{array}$ & $\begin{array}{l}183 / 4 \\
191 / 8 \\
191 / 2 \\
197 / 8\end{array}$ & $\begin{array}{l}111 / 4 \\
111 / 2 \\
11^{11 / 16} \\
11^{15} / 16\end{array}$ & $\begin{array}{l}35 / 16 \\
33 / 8 \\
37 / 16 \\
31 / 2\end{array}$ & $\begin{array}{l}1915 / 16 \\
205 / 16 \\
203 / 4 \\
211 / 8\end{array}$ & $\begin{array}{l}1113 / 16 \\
121 / 16 \\
121 / 4 \\
121 / 2\end{array}$ & $\begin{array}{l}3^{11} 1 / 6 \\
3^{13} / 16 \\
37 / 8 \\
3^{15} / 16\end{array}$ & $\begin{array}{l}207 / 16 \\
2013 / 16 \\
213 / 16 \\
215 / 8\end{array}$ & $\begin{array}{l}121 / 2 \\
123 / 4 \\
13 \\
131 / 4\end{array}$ & $\begin{array}{l}311 / 16 \\
313 / 16 \\
37 / 8 \\
315 / 16\end{array}$ & $\begin{array}{l}239 / 16 \\
24 \\
241 / 2 \\
2415 / 16\end{array}$ & $\begin{array}{l}123 \\
123 \\
13 \\
133\end{array}$ \\
\hline $\begin{array}{l}33 / 8 \\
37 / 16 \\
316 \\
334\end{array}$ & $\begin{array}{ll}7 & 33 / 4 \\
7 & 53 / 8 \\
7 & 7 \\
8 & 116\end{array}$ & $\begin{array}{r}9,725 \\
10,095 \\
10,475 \\
12,025\end{array}$ & $\begin{array}{l}33 / 8 \\
37 / 16 \\
31 / 2 \\
33 / 4\end{array}$ & $\begin{array}{l}201 / 4 \\
205 / 8 \\
21 \\
221 / 6\end{array}$ & $\begin{array}{l}121 / 8 \\
123 / 8 \\
125 / 8 \\
131 / 2\end{array}$ & $\begin{array}{l}39 / 16 \\
35 \% 8 \\
33 / 4 \\
4\end{array}$ & $\begin{array}{l}211 / 2 \\
2115 / 16 \\
225 / 16 \\
237 / 8\end{array}$ & $\begin{array}{l}123 / 4 \\
13 \\
131 / 4 \\
143 / 16\end{array}$ & $\begin{array}{l}4 \\
41 / 16 \\
43 / 16 \\
47 / 16\end{array}$ & $\begin{array}{l}22 \\
227 / 16 \\
2213 / 16 \\
241 / 2\end{array}$ & $\begin{array}{l}131 / 2 \\
133 / 4 \\
14 \\
15\end{array}$ & $\begin{array}{l}4 \\
41 / 16 \\
4316 \\
47 / 16\end{array}$ & $\begin{array}{l}257 / 16 \\
2515 / 16 \\
2638 \\
281 / 4\end{array}$ & $\begin{array}{l}131 \\
13 \\
14 \\
15\end{array}$ \\
\hline
\end{tabular}

TABLE 2.-Maximum tolerances in outside dimensions of stud link chain cables above and below the tabulated dimensions given in Table 1

\begin{tabular}{|c|c|c|c|c|}
\hline \multirow{3}{*}{$\begin{array}{l}\text { Size of } \\
\text { chain }\end{array}$} & \multirow{3}{*}{\begin{tabular}{|c|}
$\begin{array}{c}\text { Common } \\
\text { links }\end{array}$ \\
$\begin{array}{c}\text { Length } \\
\text { and } \\
\text { width, } \\
\text { above } \\
\text { and } \\
\text { below }\end{array}$
\end{tabular}} & \multicolumn{3}{|c|}{$\begin{array}{l}\text { Enlarged links, end links, } \\
\text { and shackles }\end{array}$} \\
\hline & & \multicolumn{2}{|c|}{ Length } & \multirow{2}{*}{$\begin{array}{l}\text { Width, } \\
\text { above } \\
\text { and } \\
\text { below }\end{array}$} \\
\hline & & Above & Below & \\
\hline $\begin{array}{c}\text { Inches } \\
7 / 16 \\
3 / 4 \\
1 \\
11 / 4 \\
11 / 2\end{array}$ & $\begin{array}{l}\text { Inch } \\
1 / 32 \\
1316 \\
3 / 32 \\
1 / 8 \\
5 / 32\end{array}$ & $\begin{array}{l}\text { Inch } \\
11 / 6 \\
118 \\
3 / 16 \\
1 / 4 \\
5 / 16\end{array}$ & $\begin{array}{l}\text { Inch } \\
1 / 32 \\
1316 \\
3 / 32 \\
1 / 8 \\
5 / 32\end{array}$ & $\begin{array}{l}\text { Inch } \\
1 / 32 \\
1 / 16 \\
3 / 32 \\
18 \\
5 / 32\end{array}$ \\
\hline $\begin{array}{l}13 / 4 \\
21 / 4 \\
21 / 4 \\
21 / 2\end{array}$ & $\begin{array}{l}3 / 16 \\
7 / 32 \\
1 / 4 \\
9 / 32\end{array}$ & $\begin{array}{l}3 / 8 \\
7 / 16 \\
1 / 2 \\
9 / 16\end{array}$ & $\begin{array}{l}3 / 16 \\
7 / 32 \\
1 / 4 \\
9 / 32\end{array}$ & $\begin{array}{l}3 / 16 \\
7 / 32 \\
1 / 4 \\
9 / 32\end{array}$ \\
\hline $\begin{array}{l}23 / 4 \\
3 \\
31 / 2 \\
4\end{array}$ & $\begin{array}{l}5 / 16 \\
1132 \\
3 / 8 \\
3 / 8\end{array}$ & $\begin{array}{l}5 / 8 \\
1 / 1 / 16 \\
3 / 4 \\
3 / 4\end{array}$ & $\begin{array}{l}5 / 16 \\
11 / 32 \\
3 / 8 \\
3 / 8\end{array}$ & $\begin{array}{l}5 / 16 \\
11 / 32 \\
3 / 8 \\
3 / 8\end{array}$ \\
\hline
\end{tabular}

\section{AMERICAN SOCIETY FOR TESTING MATE- RIALS, TENTATIVE SPECIFICATIONS FOR ELECTRIC CAST-STEEL STUD-LINK ANCHOR CHAIN, SERIAL DESIGNATION A77-28T, 1928}

\section{CAST-STEEL STUD-LINK ANCHOR CHAIN}

The specifications for manufacture, physical properties, and tests are substantially the same as those shown in the American Bureau of Shipping specifications, given above. For like sizes of chain the corresponding dimensions of common links A, enlarged links $\mathrm{B}$, end links $\mathrm{C}$, and end links $\mathrm{E}$ are identical with those shown in A. B. S. Table 1 . In that table the size of chain ranges from five-eighths of an inch to $3 \frac{3}{4}$ inches, with one-sixteenth inch gradations; while the A. S. T. M. range in A77-28T is from 1 inch to $4 \frac{1}{8} 8$ inches, with 1/8-inch gradations. Dimensions of cast-steel stud-link anchor chain in A77-28T which have no corresponding dimensions in the A. B. S. specification are as shown below in 
Table 2 and Figures 82, 83, and 84. For like sizes of chain the weight in pounds per 15 fathoms is identical in both A. S. T. M. A77-28T and A. B. S.

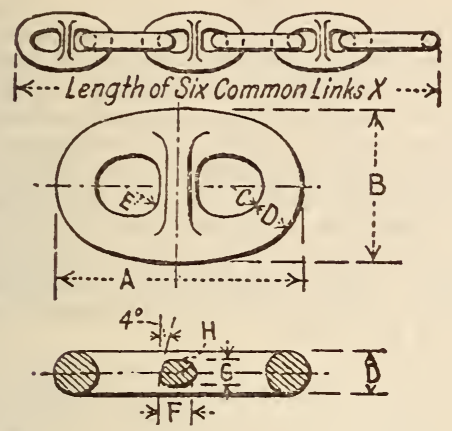

FIgURE 82.-Common links " $A$ "

specifications. For break and proof test loads see comparative table facing page 200. (See also 603.57.)

\section{PERMISSIBLE VARIATIONS}

1. Permissible variations in dimensions for common A links shall conform to the tolerances specified

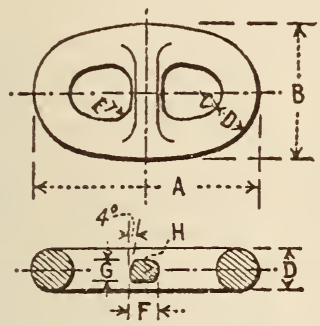
FrguRE $83 .-$ Enlarged
links "B"

in Table 1. Permissible variations for enlarged links and links, swivels, shackles, and shackle pins shall be permitted in accordance with the tolerances specified in Table 1 for common A links having the same size wire. In determining the permissible

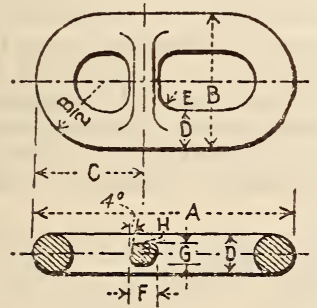

Figure $84 .-$ End links

variations for wire diameters, the diameter shall be measured at right angles, and one-half the sum of the two measurements shall be considered as representing the wire diameter.
TaBle 1.-Permissible variations of common A links, plus or minus

\begin{tabular}{|c|c|c|c|}
\hline $\begin{array}{l}\text { Size of chain } \\
\text { cable (in inches) }\end{array}$ & $\begin{array}{l}\text { Wire } \\
\text { diam- } \\
\text { eter }\end{array}$ & Width & Length \\
\hline $\begin{array}{l}1 \text { and } 11 / 8 \\
11 / 4-13 / 8 \\
11 / 2-15,8 \\
13 / 4-17 / 8 \\
2-21 / 8 \\
21 / 4-23 / 8\end{array}$ & $\begin{array}{r}\text { Inch } \\
1 / 32 \\
1 / 32 \\
1 / 32 \\
1 / 32 \\
3 / 64 \\
3 / 64\end{array}$ & $\begin{array}{c}\text { Inch } \\
1 / 16 \\
5 / 64 \\
3 / 32 \\
7 / 64 \\
1 \% 8 \\
9 / 64\end{array}$ & $\begin{array}{c}\text { Inch } \\
3 / 32 \\
7 / 64 \\
16 \\
18 \\
9 / 64 \\
5 / 32 \\
11 / 64\end{array}$ \\
\hline $\begin{array}{l}21 / 2-27 / 8 \ldots \\
3-336 \\
31 / 2-37 / 8 \\
4-43,8 \\
41 / 2-47 / 8\end{array}$ & $\begin{array}{l}3 / 64 \\
116 \\
1 / 16 \\
1 / 16 \\
1 / 16\end{array}$ & $\begin{array}{r}5 / 32 \\
1164 \\
3 / 16 \\
13 / 64 \\
7 / 32\end{array}$ & $\begin{array}{r}3 / 16 \\
13 / 64 \\
7 / 32 \\
15 / 64 \\
1 / 4\end{array}$ \\
\hline
\end{tabular}

Manufaciurer's Trademark or Initials and Size of Cable,

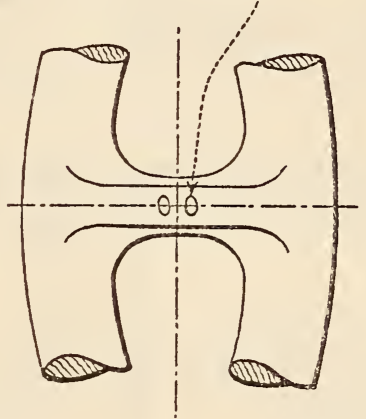

Obverse Side.

Same Marking on Reverse Side when not otherwise Specified.

Customer's Markings

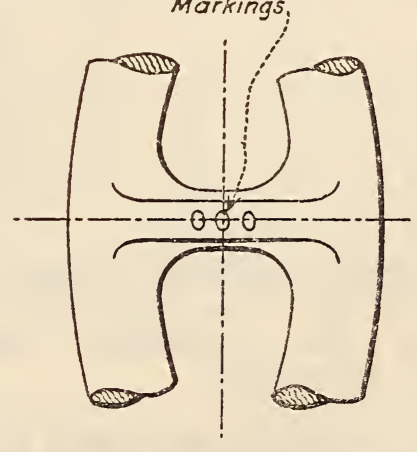

Reverse Side.

This Marking only when Specified. Otherwise Same as Obverse Side.

Frgure 85.-Location of markings on chain cable

\section{MARKING}

2. The manufacturer's trade-mark or initials and the size of cable, if used, shall be marked on the cable as shown in Figure 85 . These markings shall 
be required only on alternate common A links unless otherwise agreed upon with the manufacturer. Other markings, including shot numbers, etc., shall be in accordance with the customer's or inspector's requirements. All markings shall appear on the studs of precast links only and shall be raised letters or figures of the maximum height permissible by the size of the stud but not to exceed three-fourths inch in height by one-eighth inch in relief. A standard practice of marking shall be followed in so far as possible and shall be the same for all sizes of chain cable.

\section{INSPECTION AND REJECTION}

3. Following the proof tests, all shots of chain' swivels, shackles, shackle pins, and other appendages, shall be laid out for surface inspection. Any part showing defects that will be injurious to the strength and service of the chain shall be rejected and replaced subject to the above provisions of the specifications covering the part rejected.

4. The inspector representing the purchaser shall have free entry, at all times while work on the contract of the purchaser is being performed, to all parts of the manufacturer's works which concern the manufacture of the material ordered. The manufacturer shall afford the inspector, without charge, all reasonable facilities to satisfy him that the material is being furnished in accordance with these specifications. All tests and inspections shall be made at the place of manufacture prior to shipment, unless otherwise specified, and shall be so conducted as not to interfere unnecessarily with the operation of the works.

TABLE 2.-Supplementary dimensions of common, enlarged, and end linitis of electric cast-steel anchor chain.

\begin{tabular}{|c|c|c|c|c|c|c|c|c|c|c|c|c|c|c|c|c|}
\hline \multirow{2}{*}{$\begin{array}{c}\text { Size of } \\
\text { chain } \\
\text { cable }\end{array}$} & \multicolumn{6}{|c|}{ Common links $\mathrm{A}$} & \multicolumn{5}{|c|}{ Enlarged links B } & \multicolumn{5}{|c|}{ End links C } \\
\hline & $\mathrm{C}$ & E & $\mathrm{F}$ & G & $\mathrm{H}$ & $\mathrm{X}$ & $\mathrm{C}$ & $\mathrm{E}$ & $\mathrm{F}$ & G & II & $\mathrm{C}$ & $\mathrm{E}$ & $\mathrm{F}$ & G & $\Pi$ \\
\hline $\begin{array}{c}\text { Inches } \\
1 \\
11 / 8 \\
11 / 4 \\
13 / 8 \\
11 / 2\end{array}$ & $\begin{array}{c}\text { Inches } \\
21 / 32 \\
23 / 32 \\
25 / 32 \\
7 / 8 \\
15 / 16\end{array}$ & $\begin{array}{c}\text { Inches } \\
1 / 2 \\
9 / 16 \\
5 / 8 \\
11 / 16 \\
3 / 4\end{array}$ & $\begin{array}{c}\text { Inches } \\
11 / 16 \\
3 / 4 \\
7 / 8 \\
15 / 16 \\
1\end{array}$ & $\begin{array}{c}\text { Inches } \\
5 / 8 \\
11 / 16 \\
3 / 4 \\
1316 \\
7 / 8\end{array}$ & $\begin{array}{c}\text { Inch } \\
3 / 16 \\
3 / 16 \\
3 / 16 \\
1 / 4 \\
1 / 4\end{array}$ & $\begin{array}{c}\text { Inches } \\
26 \\
291 / 2 \\
321 / 2 \\
353 / 4 \\
39\end{array}$ & $\begin{array}{c}\text { Inches } \\
11 / 16 \\
3 / 4 \\
13 / 16 \\
15 / 16 \\
1\end{array}$ & $\begin{array}{c}\text { Inches } \\
1 / 2 \\
9 / 16 \\
5 / 8 \\
11 / 16 \\
3 / 4\end{array}$ & $\begin{array}{c}\text { Inches } \\
11 / 16 \\
3 / 4 \\
7 / 8 \\
1.516 \\
1\end{array}$ & $\begin{array}{c}\text { Inches } \\
5 / 8 \\
11 / 16 \\
3 / 4 \\
13 / 16 \\
7 / 8\end{array}$ & $\begin{array}{c}\text { Inch } \\
3 / 16 \\
3 / 16 \\
3 / 16 \\
1 / 4 \\
1 / 4\end{array}$ & $\begin{array}{c}\text { Inches } \\
33 / 16 \\
3916 \\
315 / 16 \\
438 \\
41116\end{array}$ & $\begin{array}{c}\text { Inches } \\
1 / 2 \\
916 \\
58 \\
1116 \\
34\end{array}$ & $\begin{array}{c}\text { Inches } \\
11 / 16 \\
34 \\
78 \\
15 / 16 \\
1\end{array}$ & $\begin{array}{c}\text { Inches } \\
5 / 8 \\
11 / 16 \\
3 / 4 \\
13 / 16 \\
7 / 8\end{array}$ & $\begin{array}{r}\text { Inch } \\
1 / 4 \\
1 / 4 \\
1 / 4 \\
1 / 4 \\
1 / 4\end{array}$ \\
\hline $\begin{array}{l}15 / 8 \\
13 / 4 \\
17 / 8 \\
2 \\
216\end{array}$ & $\begin{array}{l}1 \\
11 / 16 \\
11 / 8 \\
11 / 4 \\
15 / 16\end{array}$ & $\begin{array}{l}13 / 16 \\
7 / 8 \\
15 / 16 \\
1 \\
11 / 16\end{array}$ & $\begin{array}{l}11 / 8 \\
13 / 16 \\
11 / 4 \\
13 / 8 \\
17 / 16\end{array}$ & $\begin{array}{l}1^{15 / 16} \\
11 / 8 \\
13 / 16 \\
11 / 4\end{array}$ & $\begin{array}{l}1 / 4 \\
1 / 4 \\
1 / 4 \\
1 / 4 \\
1 / 4 \\
1 / 4\end{array}$ & $\begin{array}{l}421 / 4 \\
451 / 2 \\
483 / 4 \\
52 \\
551 / 4\end{array}$ & $\begin{array}{l}11 / 16 \\
11 / 8 \\
11 / 4 \\
15 / 16 \\
13 / 8\end{array}$ & $\begin{array}{l}13 / 16 \\
78 \\
15 / 16 \\
1 \\
11 / 16\end{array}$ & $\begin{array}{l}11 / 8 \\
13 / 16 \\
11 / 4 \\
13 / 8 \\
17 / 16\end{array}$ & $\begin{array}{l}1^{15 / 16} \\
11 / 8 \\
13 / 16 \\
11 / 4\end{array}$ & $\begin{array}{l}1 / 4 \\
144 \\
1 / 4 \\
1 / 4 \\
1 / 4\end{array}$ & $\begin{array}{l}51,5 \\
5916 \\
57,5 \\
65 / 16 \\
63 / 4\end{array}$ & $\begin{array}{l}13 / 10 \\
7,8 \\
15 / 16 \\
1 \\
11116\end{array}$ & $\begin{array}{l}116 \\
13 / 16 \\
11 / 4 \\
136 \\
17 / 16\end{array}$ & $\begin{array}{l}15 / 16 \\
1 \\
11 / 8 \\
13 / 16 \\
114\end{array}$ & $\begin{array}{l}1 / 4 \\
1 / 4 \\
2 / 4 \\
1 / 4 \\
1 / 4\end{array}$ \\
\hline $\begin{array}{l}21 / 4 \\
23 / 8 \\
21 / 2 \\
25 / 8\end{array}$ & $\begin{array}{l}13 / 8 \\
17 / 16 \\
11 / 2 \\
19 / 16\end{array}$ & $\begin{array}{l}11 / 8 \\
13 / 16 \\
11 / 4 \\
15 / 16\end{array}$ & $\begin{array}{l}11 / 2 \\
158 \\
111 / 16 \\
11316\end{array}$ & $\begin{array}{l}13 / 8 \\
17 / 16 \\
11 / 2 \\
19 / 16\end{array}$ & $\begin{array}{l}1 / 4 \\
1 / 4 \\
1 / 4 \\
1 / 4\end{array}$ & $\begin{array}{l}581 / 2 \\
613 / 4 \\
65 \\
681 / 4\end{array}$ & $\begin{array}{l}17 / 16 \\
11 / 2 \\
19 / 16 \\
15 \%\end{array}$ & $\begin{array}{l}11 / 8 \\
13 / 16 \\
11 / 4 \\
15 / 16\end{array}$ & $\begin{array}{l}11 / 2 \\
15 / 8 \\
111 / 16 \\
113 / 16\end{array}$ & $\begin{array}{l}13 / 8 \\
17 / 16 \\
11 / 2 \\
19 / 16\end{array}$ & $\begin{array}{l}1 / 4 \\
1 / 4 \\
1 / 4 \\
1 / 4\end{array}$ & $\begin{array}{l}71 / 16 \\
736 \\
778 \\
8516\end{array}$ & $\begin{array}{l}11 / 8 \\
1316 \\
11 / 4 \\
1516\end{array}$ & $\begin{array}{l}11 / 2 \\
15 / 8 \\
111 / 16 \\
113 / 16\end{array}$ & $\begin{array}{l}13 / 8 \\
17 / 16 \\
11,2 \\
1916\end{array}$ & $\begin{array}{l}1 / 4 \\
1 / 4 \\
1 / 4 \\
1 / 4\end{array}$ \\
\hline $\begin{array}{l}23 / 4 \\
27 / 8 \\
3 \\
31 / 8\end{array}$ & $\begin{array}{l}15 / 8 \\
111 / 16 \\
113 / 16 \\
178\end{array}$ & $\begin{array}{l}13 / 8 \\
17 / 16 \\
11 / 2 \\
19 / 16\end{array}$ & $\begin{array}{l}17 / 8 \\
115 / 16 \\
21 / 16 \\
21 / 8\end{array}$ & $\begin{array}{l}15 / 8 \\
13 / 4 \\
113 / 16 \\
17 / 8\end{array}$ & $\begin{array}{l}1 / 4 \\
1 / 4 \\
1 / 4 \\
1 / 4\end{array}$ & $\begin{array}{l}711 / 2 \\
743 / 4 \\
78 \\
811 / 4\end{array}$ & $\begin{array}{l}111 / 16 \\
113 / 16 \\
17 / 8 \\
115 / 16\end{array}$ & $\begin{array}{l}13 / 8 \\
17 / 16 \\
11 / 2 \\
19 / 16\end{array}$ & $\begin{array}{l}17 / 8 \\
11516 \\
21 / 6 \\
216\end{array}$ & $\begin{array}{l}15 / 8 \\
13 / 4 \\
113 / 16 \\
17 / 8\end{array}$ & $\begin{array}{l}1 / 4 \\
1 / 4 \\
1 / 4 \\
1 / 4\end{array}$ & $\begin{array}{l}81116 \\
91 / 16 \\
91 / 2 \\
97 / 8\end{array}$ & $\begin{array}{l}13 / 8 \\
17 / 16 \\
11 / 2 \\
19 / 16\end{array}$ & $\begin{array}{l}17 / 8 \\
11516 \\
21 / 16 \\
21 / 8\end{array}$ & $\begin{array}{l}15,8 \\
13 / 4 \\
113 / 16 \\
178\end{array}$ & $\begin{array}{l}1 / 4 \\
1 / 4 \\
1 / 4 \\
1 / 4\end{array}$ \\
\hline $\begin{array}{l}31 / 4 \\
33 / 8 \\
31 / 2 \\
35 \%\end{array}$ & $\begin{array}{l}1^{15} / 16 \\
2 \\
21 / 16 \\
21 / 8\end{array}$ & $\begin{array}{l}15 / 8 \\
111 / 16 \\
13 / 4 \\
1^{13} / 16\end{array}$ & $\begin{array}{l}23 / 16 \\
25 / 16 \\
23 / 8 \\
27 / 16\end{array}$ & $\begin{array}{l}1^{15 / 16} \\
2 \\
21 / 8 \\
23 / 16\end{array}$ & $\begin{array}{l}1 / 4 \\
1 / 4 \\
1 / 4 \\
1 / 4\end{array}$ & $\begin{array}{l}841 / 2 \\
873 / 4 \\
91 \\
941 / 4\end{array}$ & $\begin{array}{l}2 \\
21 / 16 \\
21 / 8 \\
23 / 16\end{array}$ & $\begin{array}{l}15 / 8 \\
111 / 16 \\
13 / 4 \\
113 / 16\end{array}$ & $\begin{array}{l}23 / 16 \\
25 / 16 \\
238 \\
27 / 16\end{array}$ & $\begin{array}{l}1^{15 / 16} \\
2 \\
21 / 8 \\
2^{33 / 16}\end{array}$ & $\begin{array}{l}1 / 4 \\
1 / 4 \\
1 / 4 \\
1 / 4\end{array}$ & $\begin{array}{l}101 / 4 \\
105 / 8 \\
11 \\
117 / 16\end{array}$ & $\begin{array}{l}158 \\
111 / 16 \\
13 / 4 \\
113 / 16\end{array}$ & $\begin{array}{l}23 / 16 \\
2516 \\
2368 \\
27 / 16\end{array}$ & $\begin{array}{l}1^{15} / 6 \\
2 \\
218 \\
23 / 16\end{array}$ & $\begin{array}{l}1 / 4 \\
1 / 4 \\
1 / 4 \\
1 / 4\end{array}$ \\
\hline $\begin{array}{l}33 / 4 \\
37 / 8 \\
4 \\
41 / 8\end{array}$ & $\begin{array}{l}23 / 16 \\
21 / 4 \\
25 / 16 \\
23 / 8\end{array}$ & $\begin{array}{l}17 / 8 \\
115 / 16 \\
2 \\
21 / 16\end{array}$ & $\begin{array}{l}29 / 16 \\
25 / 8 \\
23 / 4 \\
2^{13} / 16\end{array}$ & $\begin{array}{l}21 / 4 \\
25 / 16 \\
23 / 8 \\
21 / 2\end{array}$ & $\begin{array}{l}1 / 4 \\
1 / 4 \\
114 \\
1 / 4\end{array}$ & $\begin{array}{c}971 / 2 \\
100^{3 / 4} \\
104 \\
1071 / 4\end{array}$ & $\begin{array}{l}21 / 4 \\
25 / 16 \\
23 / 8 \\
27 / 16\end{array}$ & $\begin{array}{l}17 / 8 \\
115 / 16 \\
2 \\
21 / 16\end{array}$ & $\begin{array}{l}29 / 16 \\
25 / 8 \\
23 / 4 \\
213 / 16\end{array}$ & $\begin{array}{l}21 / 4 \\
25116 \\
23,8 \\
21 / 2\end{array}$ & $\begin{array}{l}1 / 4 \\
1 / 4 \\
1 / 4 \\
1 / 4\end{array}$ & $\begin{array}{l}117 / 8 \\
123 / 16 \\
125 / 8 \\
131 / 16\end{array}$ & $\begin{array}{l}178 \\
1^{11516} \\
2 \\
2116\end{array}$ & $\begin{array}{l}29 / 16 \\
25,8 \\
23 / 4 \\
213 / 16\end{array}$ & $\begin{array}{l}21 / 4 \\
2516 \\
23,8 \\
21 / 2\end{array}$ & $\begin{array}{l}1 / 4 \\
1 / 4 \\
1 / 4 \\
1 / 4\end{array}$ \\
\hline
\end{tabular}

FEDERAL SPECIFICATIONS BOARD, SPECIFICATION NO. 1\%1a, FOR SHIP CHAIN, JUNE $30,1924$. (CURRENT DESIGNANATION : RR-C-251)

(For general specifications, material and workmanship, and methods of inspection, see F. S. B. RR-C-271 603.50, p. 199) (See also 603.57.)

\section{CLASSES AND TYPE}

1. The classes of chain shall be as follows: A. This class shall be used where an all-iron forged chain is desired. B. This class shall be used where a forged-steel chain is desired. C. This class corresponds to the commercial chain acceptable to the classification bureaus and shall be used where that grade will satisfactorily meet service requirements.

2. The chain shall be of the stud-link type for use as anchor, mooring, buoy, and towing chain.

3. Finished chain may have the welds in either the sides or ends of the links.

4. Studs.-The studs shall be well centered and firmly held in place and shall be in accordance with the design and dimensions submitted or approved by the purchaser. For class A chain the studs shall be made of cast iron or drop-forged from common steel, as may be ordered. If drop-forged, the steel used shall conform to the following requirements:

(a) Material.-Open-hearth or Bessemer steel.

(b) Tensile strength, minimum. - 55,000 pounds per square inch.

(c) Elongation, minimum.--27 per cent in 2 inches, 22 per cent in 8 inches.

(d) Cold-bend test. $-180^{\circ}$ around a diameter of one thickness.

(e) For class B the studs shall be of forged steel or cast steel.

(f) For class $\mathrm{C}$ the studs shall be of forged iron, steel, or cast iron.

\section{GENERAL REQUIREMENTS}

5. Link dimensions.-Stud link chain shall be in accordance with the dimensions listed in A. B. S. Table 1, above.

6. Tolerances in dimensions and weights.-After proof test, and while the length of chain is still subjected to a load not in excess of 60 per cent of the 
proof load, each link shall be inspected for outside length and widtn. Also, the over-all length of six links, measuring from every third link, shall be tested. The manufacturing tolerances allowed are as follows:

(a) Tolerance in wire diameler of finished link.The size for descriptive and test purposes (nominal wire diameter) shall be the diameter of the common link of the chain with a plus tolerance which is not to exceed one thirty-second inch in the case of chains up to and including $1 \frac{1}{2}$ inches diameter. In the case of chains above $11 / 2$ inches diameter the tolerance shall not exceed one-sixteenth inch. The sectional area of the inaterial at the weld of the common link shall not be reduced below that of the prescribed minimum diameter of the common links of chain.

(b) Tolerances in lengths and widths of links.Maximum plus and minus tolerances in the designed widths of links, as listed in A. B. S. Table 1, above, will be permitted. Maximum plus and minus tolerances in the designed length of individual links as listed in A. B. S. Table 1 will be permitted provided the outside lengths of six consecutive links above or below that specified for chain of corresponding size do not exceed 60 per cent of the wire diameter.

(c) Tolerance in weight.- When chains have satisfactorily passed the above requirements, they shall be weighed, together with the shackles forming the outfit (if ordered), and this actual weight shall be given on the certificate of test. The weight of the chain shall be not more than $2 \frac{1}{2}$ per cent under nor more than 5 per cent above the weight specified in A. B. S. Table 1, above.

7. Arrangement of links and shackles.-Unless otherwise specified, chain shall be in 15-fathom lengths. There shall be an odd number of links in a shot or length of chain. The end links, also the swivels and shackles when ordered, and the shots of chain in complete cables shall be arranged in accordance with A. B. S. Figure 81, above. The enlarged end links may be omitted from the shots of chain at the option of the purchaser. (For end links, swivels, and shackles, see 603.57.)

8. Finish.-After inspection and before shipment, unless other finish is specified, the contractor shall coat the chain by hauling it through a bath of asphaltum diluted with turpentine substitute, in accordance with Federal specifications, in proportion of 25 gallons of turpentine substitute to 100 pounds of asphaltum, the bath to be maintained as near the boiling point as practicable.

\section{DETAIL REQUIREMENTS}

9. Chemical requirements. - The wrought iron and steel from which the respective classes of chain are to be manufactured shall conform to the following requirements as to chemical composition:

\begin{tabular}{l|r|r|r}
\hline Classes of chain & $\begin{array}{r}\text { Manga- } \\
\text { nese }\end{array}$ & $\begin{array}{r}\text { Phos- } \\
\text { phorus }\end{array}$ & Sulphur \\
\cline { 2 - 4 } & $\begin{array}{r}\text { Per cent } \\
10.10 \\
30-\end{array}$ & $\begin{array}{r}\text { Per cent } \\
10.10 \\
1.04\end{array}$ & $\begin{array}{r}\text { Per cent } \\
10.015 \\
1.040\end{array}$ \\
\hline
\end{tabular}

1 Maximum.

NoTE.-No chemical analysis of material for class $C$ chain is required. At the purchaser's option it may also be waived for class A chain.

10. Physical requirements.-The wrought iron and steel from which the respective classes of chain are to be manufactured shall conform to the following requirements as to physical properties:

\begin{tabular}{|c|c|c|c|c|}
\hline $\begin{array}{l}\text { Classes of } \\
\text { chain }\end{array}$ & $\begin{array}{l}\text { Tensile } \\
\text { strength }\end{array}$ & $\mid$\begin{tabular}{|} 
Yield point \\
(minimum)
\end{tabular} & $\begin{array}{l}\text { Elongation } \\
\text { (minimum) }\end{array}$ & $\begin{array}{l}\text { Reduction } \\
\text { of area } \\
\text { (minimum) }\end{array}$ \\
\hline A.. & $\begin{array}{c}\text { Lbs./in. }{ }^{2} \\
\text { (Min.) } 48,000\end{array}$ & $\begin{array}{c}\text { Per cent } \\
0.5 \text { tensile }\end{array}$ & $\begin{array}{c}\text { Per cent } \\
26 \text { in } 8 \text { inches.- }\end{array}$ & ${ }^{P e r}$ cent ${ }_{40}$ \\
\hline B.. & $45,000-55,000$ & $\begin{array}{l}0.5 \text { tensile } \\
\text { strength } \\
\text { obtained. }\end{array}$ & $\begin{array}{l}30 \text { in } 8 \text { inches; } \\
35 \text { in } 2 \text { in- } \\
\text { ches. }\end{array}$ & 60 \\
\hline
\end{tabular}

NotE. - No physical tests are required of material for class $\mathrm{C}$ chain. The elongation shall be measured and the physical requirements modified for certain sizes of bar as hereinafter specified.

11. Modification for physical requirements.-For wrought iron for class A chain over 4 square inches in sectional area, the following deductions from the minimum requirements specified above shall be allowed for each square inch of sectional area above 4 square inches:

Tensile strength _..._lbs./in. ${ }^{2}-250$, but not under 45,000 .
Elongation

Elongation
Reduction of area........

\section{MARKING}

12. All test specimens before being cut from the bars, forgings, etc., shall be stamped for identification by the inspector unless otherwise specified.

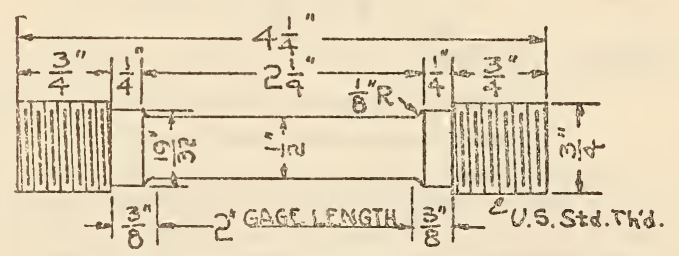

FIGURE 86.-Type I test specimen

NoTE.-The gage length, parallel portions, and fillets shall ends are approved by the chief inspector.

Each shot or length of chain shall be stamped with a distinctive identification number, the number to be supplied by the purchaser at his option and located as specified. The studs shall be marked at purchaser's option in accordance with plan or sketch submitted by the purchaser. Such other distinctive marks as are necessary for identification by the manufacturer may be placed on the links, shackles, swivels, shots, etc., provided they do not interfere with markings specified.

\section{TESTING}

13. Wrought iron for class A chain shall be tested in the sizes rolled, where practicable, and for each ton or less of each size the following tests shall be made; one tensile, one cold-bend, one hot-bend, one nick, one drift, and one etch test. Each test specimen shall be taken from a different bar. When material can not be tested in the sizes rolled, test bars shall be prepared of a sectional area as large as possible within the capacity of the testing machine.

14. Class B material shall be tested in the sizes rolled where practicable; otherwise test specimens as shown herein, shall be used. For each melt entering into each size, four tensile tests and one bend test shall be made. Each test specimen shall be taken from a different bar. When the material can not be tested in the sizes rolled, test bars of Type II or III as shown in Figures 87 and 88 shall be prepared if practicable; if not, a Type I test bar, as shown in Figure 86, shall be accepted. 
15. Measurement of elongation.-The elongation of test specimens of wrought iron for class A chain shall be measured in 8 inches except for material of less than three-fourths inch diameter, in which case the elongation shall be measured on a length equal to ten times the diameter of the material to be tested.

16. The elongation of test specimens of soft steel for class B chain shall be measured as follows:

\section{Nominal diameter}

Length in
which
measured

Over $1 / 4$ to $1 / 2$ inch, inclusive.

Inches

Over $1 / 2$ to $3 / 4$ inch, inclusive..-

ver $3 / 4$ to $11 / 2$ inches, inclusive

17. Tensile tests.-Tensile tests of specimens shall be made on a type of testing machine approved

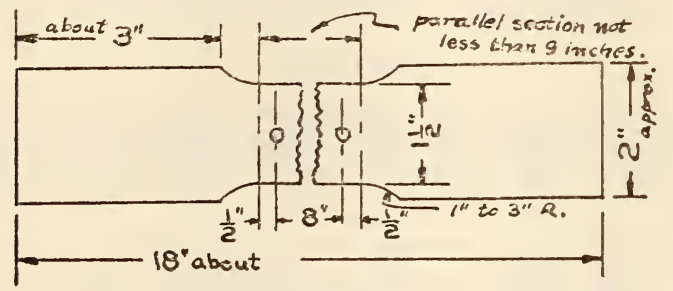

Figure 87.-Type II test specimen

[Parallel section not less than 9 inches]

by the purchaser. The pulling speeds shall be such as to permit of determination of the yield point by drop of the beam of the testing machine or by use of the dividers, but in no case should exceed $11 / 2$ inches per minute at the yield point nor 6 inches per minute after the yield point is passed.

18. Cold-bend tests. - Test specimens for class A material shall be bent cold through $180^{\circ}$ around a diameter of one thickness, without a fracture on the outside of the bent portion.

19. Test specimens for class $B$ material shall be bent cold through $180^{\circ}$ flat on themselves without sign of fracture on the outside of the bent portion. If one of these specimens fails, each bar from that melt entering into the chain of that size shall pass the cold-bend test before being cut to lengths.

20. Quench tests. - Test specimens for class A material, when heated to a temperature between

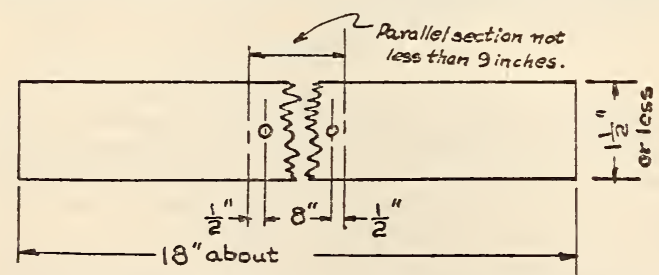

Figure 88.-Type III test specimen

[Parallel section not less than 9 inches]

$1,700^{\circ}$ and $1,800^{\circ} \mathrm{F}$. and quenched in water at $80^{\circ} \mathrm{F}$., shall bend back on themselves through $180^{\circ}$, without fracture.

21. Nick tests. - Those test specimens, when nicked to a depth of approximately 20 per cent of their thicknesses with a tool having a $60^{\circ}$ cutting edge and bent back at this point through an angle of $180^{\circ}$, shall show a long, clean, silky fiber, free from slag or dirt. A few crystalline spots may be tolerated, provided they do not, in the aggregate, exceed 10 per cent of the fractured area, and provided the etch test shows that such crystalline spots are not indicatire of the presence of steel.

22. Drift tests. - Test specimens for class A material shall be punched and expanded hot by pointed drifts until a round hole is formed, the diameter of which shall not be less than nine-tenths the diameter of the bar. Anv indication of fracture, cracks, or flaws, developed by this test shall be sufficient cause for rejection of the lot represented by the bar.

23. Etch tests.-The cross-section area of a test specimen for class A material shall be ground, polished, and etched in a solution of 2 parts water by weight, 1 part concentrated hydrochloric acid, and 1 part concentrated sulphuric acid by weight for a sufficient period to develop the structure. Under a magnification of 90 to 100 diameters, the material shall show no trace of steel.

24. Tests of finished chain.- The proof and breaking tests of finished chain shall be made on a chaintesting machine approved by the purchaser. The gripping arrangements shall be such that the stress applied to the end links of the portion under test shall be the same as equally put on every link tested; and the arrangement shall be such that each link is tested once and only once during the test.

25. Breaking tests of finished chain.-For class A or B, material, the tensile test specimen shall consist of two or, if the inspector considers it necessary, three links of finished chain taken at random from each length or shot. This specimen shall be tested for breaking strength as given in comparative table facing, page 200 , for that class of material. If the test specimen fails, two additional test specimens located adjacent to it in the shot shall be taken and the same stresses applied; and if either of these specimens fails the shot shall be rejected, and a defective weld, even though the link satisfactorily withstood the prescribed stress, shall be sufficient cause to require additional break tests. At the purchaser's option one test specimen selected at random shall be tested to destruction to determine the ultimate strength.

26. For class $\mathrm{C}$ chain the tensile-test specimen shall consist of two or, if considered necessary by the inspector, three links from one shot of 15 fathoms or less of each shot presented. This specimen shall then be subjected to the breaking test specified herein for this class of chain. If the test is satisfactory the shots represented shall be considered satisfactory as far as this test is concerned. If the first test is not satisfactory, one more specimen shall be selected and subjected to the breaking test, and if this fails the shot shall be rejected. If the break-test specimen satisfactorily withstands the prescribed stress, it need not be tested to destruction. but shall be discarded.

27. Proof test of finished chain.-After breaking test, each shot or length of chain which has satisfactorily passed that test, shall have the links which were cut out of it for breaking test replaced by new links and the complete shot or length subjected to proof test as given in comparative table facing page 200 for that class of material. Two proof loads are listed in comparative table facing page 200 for certain sizes of chain. When ordering chain of such sizes the purchaser shall stipulate the load to be applied. After proof test the chain shall be thoroughly inspected and any links showing cold welding, surface rupture, unusual deformation, inferior workmanship, or other defects shall be taken out and replaced and the chain again subjected to proof stress, provided the defective links do not exceed five per cent. If the defective links exceed five per cent, the shot shall be rejected. Shots of chain of class A or B material in which a link breaks under 
proof load shall be rejected. If under proof load, a joining link shall be inserted and the proof test again applied, and if a second link breaks under the second proof test, the shot shall be finally rejected.

603.54 TWISTED-IINK CHAINS.

UNITED STATES GOVERNMENT, FEDERAL SPECIFIC $\triangle T I O N S$ BOARD, FEDERAL SPECIFICATION RR-C-271, FOR STANDARD MISCELLANEOUS CHAIN AND ATTACHMENTS, DECEMBER 8, 1931.

\section{TWIST LINK COIL CHAIN}

(For general specifications, material and workmanship, detail requirements, and methods of inspection and tests, see F. S. B. RR-C-271, 603.50, p. 199. For proof and breaking loads see comparative table facing p. 200. For other kinds of chain and chain attachments specified in F. S. B. RR-C-271, see 603.51, $603.52,603.56,603.57$, and 617.5. See also 631.9, $642.9,645.9$, and 646.59.)

1. Twist link coil chain shall be of the following classes: (1) Wrought iron, (2) wrought iron or openhearth steel.

2. Twist link coil chain shall be made of class 2 material and in accordance with the following table. No proof test shall be required. The breaking load shall be in accordance with the requirements for this class as shown in comparative table facing page 200 . The percentage of elongation shall be as specified for this class of chain.

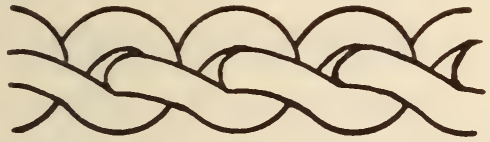

FIGURE 89.-Twist link coil chain

Twist link chain, plain pattern (weights and dimensions)

\begin{tabular}{|c|c|c|c|}
\hline Size & $\begin{array}{c}\text { Actual } \\
\text { diameter }\end{array}$ & $\begin{array}{c}\text { Length } \\
\text { of } 100 \\
\text { links }\end{array}$ & $\begin{array}{l}\text { Weight } \\
\text { per } 100 \\
\text { feet }\end{array}$ \\
\hline Inch & Inch & Inches & Pounds \\
$1 / 4$ & $8 / 32$ & 103 & 75 \\
$5 / 16$ & $11 / 32$ & 120 & 124 \\
$3 / 8$ & $133 / 32$ & 128 & 180 \\
$7 / 16$ & $15 / 32$ & 159 & 225 \\
$1 / 2$ & $17 / 32$ & 165 & 285 \\
$9 / 16$ & $19 / 32$ & 187 & 345 \\
$5 / 8$ & $21 / 32$ & 200 & 425 \\
\hline
\end{tabular}

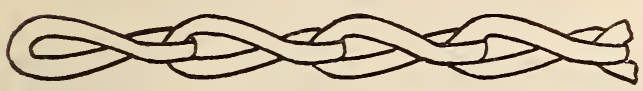

FIGURE 90.-Twist-link coil chain, long-link pattern

3. Twist link chain (long-link pattern) shall be made of class 2 material and in accordance with the following table:
Twist link chain, long-link pattern (weights and dimensions)

\begin{tabular}{|c|c|c|c|}
\hline Diameter & $\begin{array}{l}\text { Number } \\
\text { links }\end{array}$ & $\begin{array}{c}\text { Minimum } \\
\text { tensile } \\
\text { strength }\end{array}$ & $\begin{array}{l}\text { Weight } \\
\text { per } 100 \\
\text { feet }\end{array}$ \\
\hline $\begin{array}{c}\text { Inch } \\
0.092 \text { or } 3 / 32 \\
.105 \text { or } 7 / 64 \\
.120 \text { or } 18 \\
.135 \text { or } 9 / 64 \\
.148 \text { or } 10 \% 64\end{array}$ & $\begin{array}{c}\text { Per foot } \\
11.00 \\
11.00 \\
11.00 \\
10.50 \\
10.50\end{array}$ & $\begin{array}{r}\text { Pounds } \\
475 \\
590 \\
710 \\
830 \\
950\end{array}$ & $\begin{array}{r}\text { Pounds } \\
6.00 \\
8.00 \\
10.00 \\
13.00 \\
15.75\end{array}$ \\
\hline $\begin{array}{l}162 \text { or } 11 / 64 \\
177 \text { or } 11 / 64 \\
192 \text { or } 3 / 16 \\
207 \text { or } 13 / 64\end{array}$ & $\begin{array}{l}9.75 \\
9.25 \\
8.75 \\
8.75\end{array}$ & $\begin{array}{l}1,150 \\
1,240 \\
1,430 \\
1,670\end{array}$ & $\begin{array}{l}20.00 \\
23.00 \\
28.00 \\
33.00\end{array}$ \\
\hline $\begin{array}{l}.244 \text { or } 1 / 4 \\
.263 \text { or } 17 / 64 \\
283 \text { or } 9 / 32 \\
.307 \text { or } 5 / 16\end{array}$ & $\begin{array}{l}7.75 \\
7.75 \\
7.75 \\
8.00\end{array}$ & $\begin{array}{l}2,530 \\
3,000 \\
3,250 \\
4,050\end{array}$ & $\begin{array}{l}49.00 \\
48.50 \\
64.00 \\
84.00\end{array}$ \\
\hline
\end{tabular}

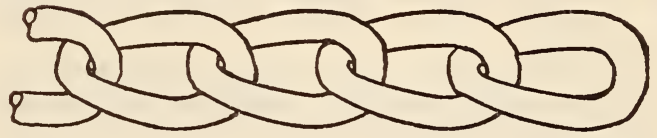

FIGURE 901/2. - Twist-link chain, short length pattern

4. Twist link chain (short-link pattern) shall be made of class 2 material. No proof test is required. Strength, dimensions, and weights as follows:

\begin{tabular}{|c|c|c|c|}
\hline Diameter & $\begin{array}{l}\text { Number } \\
\text { links }\end{array}$ & $\begin{array}{l}\text { Minimum } \\
\text { tensile } \\
\text { strength }\end{array}$ & $\begin{array}{l}\text { Weight } \\
\text { per } 100 \\
\text { feet }\end{array}$ \\
\hline $\begin{array}{c}\text { Inch } \\
0.105 \text { or } 7 / 64 \\
.120 \text { or } 1 / 8 \\
135 \text { or } 9 / 64 \\
.148 \text { or } 5 / 32\end{array}$ & $\begin{array}{c}\text { Per foot } \\
25.00 \\
23.50 \\
21.50 \\
21.00\end{array}$ & $\begin{array}{r}\text { Pounds } \\
620 \\
750 \\
875 \\
1,000\end{array}$ & $\begin{array}{r}\text { Pounds } \\
10 \\
13 \\
16 \\
21\end{array}$ \\
\hline $\begin{array}{l}162 \text { or } 5 / 32 \\
.177 \text { or } 11 / 64 \\
192 \text { or } 3 / 16 \\
207 \text { or } 13 / 64\end{array}$ & $\begin{array}{l}20.50 \\
17.50 \\
16.50 \\
15.00\end{array}$ & $\begin{array}{l}1,140 \\
1,310 \\
1,510 \\
1,760\end{array}$ & $\begin{array}{l}25 \\
29 \\
34 \\
40\end{array}$ \\
\hline $\begin{array}{l}.275 \text { or } 7 / 32 \\
.244 \text { or } 1 / 4 \\
.283 \text { or } 9 / 32 \\
.307 \text { or } 5 / 16\end{array}$ & $\begin{array}{l}14.75 \\
14.00 \\
13.00 \\
10.25\end{array}$ & $\begin{array}{l}1,990 \\
2,660 \\
3,420 \\
4,260\end{array}$ & $\begin{array}{l}50 \\
64 \\
78 \\
94\end{array}$ \\
\hline
\end{tabular}

603.55 STRAIGHT AND TWISTED LINK VEHICLE CHAINS.

(See 603.50, p. 198.)

603.56 WELDLESS CHAINS, VARIOUS IINK PATTERNS.

UNITED STATES GOVERNMENT, DEPARTMENT OF COMMERCE, BUREAU OF STANDARDS, COMMERCIAL STANDARDS FOR BUILDERS' HARDWARE (NONTEMPLATE), CS22-30, 1930

\section{TRANSOM CHAINS}

(See F. S. B. FF-H-101, 617.5, p. 1050.) 
UNITED STATES GOVERNMENT, FEDERAL SPECIFICATIONS BOARD, FEDERAL SPECIFICATION RR-C-271 FOR STANDARD MISCELLANEOUS CHAIN AND ATTACHMENTS, DECEMBER 8, 1931.

\section{WELDLESS CHAIN}

(For general specifications, material and workmanship, detail requirements, and methods of inspection and tests, see F. S. B. RR-C-271 (603.50, p. 199). For other kinds of chain and chain attachments specified in F. S. B. RR-C-271 see 603.51, $603.52,603.54,603.57,617.5,631.9,642.9,645.9$, and 646.59 .)

1. Weldless chain shall be automatic machinemade chain of the following classes: $(a)$ Single-loop pattern; $(b)$ double-loop pattern; $(c)$ sash and flat link, long pitch chain; $(d)$ plumber's, and flat link short pitch chain; (e) single jack chain; $(f)$ double jack chain; $(g)$ ladder chain; $(h)$ register chain; and (i) brass chain for mechanical communication. (See 645.9.)

2. Weldless chain shall be made from steel, copper, brass, or bronze wire, or stamped from sheets of steel, copper, brass, or bronze.

3 . Weldless close link chain made from rolled steel or iron bar will be acceptable under this specification.

4. Weldless chain will be paid for by the foot.

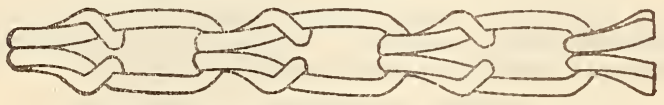

Figure 91.-Single-loop pattern chain

TABLE 1.-Single-loop pattern chain (dimensions, strength, weights)

\begin{tabular}{|c|c|c|c|c|}
\hline Material & $\begin{array}{c}\text { Diameter } \\
\text { of wire }\end{array}$ & $\begin{array}{l}\text { Approxi- } \\
\text { mate } \\
\text { number } \\
\text { links per } \\
\text { foot }\end{array}$ & $\begin{array}{c}\text { Minimum } \\
\text { tensile } \\
\text { strength }\end{array}$ & $\begin{array}{l}\text { Approxi- } \\
\text { mate } \\
\text { weight } \\
\text { per } 100 \\
\text { feet }\end{array}$ \\
\hline $\begin{array}{l}\text { Steel } \\
\text { Do } \\
\text { Do } \\
\text { Do } \\
\text { Do }\end{array}$ & $\begin{array}{l}\text { Inch } \\
0.047 \\
.054 \\
.062 \\
.072 \\
.080\end{array}$ & $\begin{array}{l}19 \\
15 \\
15 \\
13 \\
11\end{array}$ & $\begin{array}{r}\text { Pounds } \\
180 \\
205 \\
320 \\
405 \\
495\end{array}$ & $\begin{array}{r}\text { Pounds } \\
21 / 2 \\
3 \\
41 / 4 \\
6 \\
7\end{array}$ \\
\hline 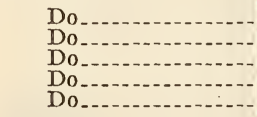 & $\begin{array}{l}.091 \\
.105 \\
.120 \\
.135 \\
.148\end{array}$ & $\begin{array}{r}11 \\
10 \\
10 \\
8 \\
8\end{array}$ & $\begin{array}{r}560 \\
940 \\
1,055 \\
1,545 \\
1,750\end{array}$ & $\begin{array}{l}10 \\
13 \\
17 \\
22 \\
26\end{array}$ \\
\hline $\begin{array}{l}\text { Do } \\
\text { Do } \\
\text { Do } \\
\text { Do }\end{array}$ & $\begin{array}{l}.162 \\
.177 \\
.192 \\
.207\end{array}$ & $\begin{array}{l}6 \\
5 \\
5 \\
5\end{array}$ & $\begin{array}{l}1,915 \\
2,440 \\
2,940 \\
2,325\end{array}$ & $\begin{array}{l}31 \\
35 \\
41 \\
50\end{array}$ \\
\hline
\end{tabular}

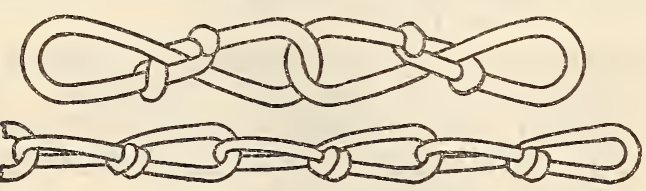

Figure 92.-Double-loop pattern chain

TABLE 2.-Double-loop pattern chain (dimensions, strength, weights)

STYLE 1

\begin{tabular}{c|r|r|r|r}
\hline Material & $\begin{array}{c}\text { Diameter } \\
\text { of wire }\end{array}$ & $\begin{array}{c}\text { Approxi- } \\
\text { mate } \\
\text { number } \\
\text { links per } \\
\text { foot }\end{array}$ & $\begin{array}{c}\text { Minimum } \\
\text { tensile } \\
\text { strength }\end{array}$ & $\begin{array}{c}\text { Approxi- } \\
\text { mate } \\
\text { weight } \\
\text { per 100 } \\
\text { feet }\end{array}$ \\
\hline & Inch & & Pounds & Pounds \\
Steel or brass.-.0unds & 13 & 260 & $31 / 2$ \\
Do & 0.062 & 12 & 320 & $41 / 2$ \\
Do & .072 & 11 & 375 & 5314 \\
Do & .080 & 9 & 430 & $71 / 2$
\end{tabular}

TABLE 2.-Double-loop pattern chain (dimensions, strength, weights)-Continued

STYLE 1-Continued

\begin{tabular}{|c|c|c|c|c|}
\hline Material & $\begin{array}{c}\text { Diameter } \\
\text { of wire }\end{array}$ & $\begin{array}{l}\text { Approxi- } \\
\text { mate } \\
\text { number } \\
\text { links per } \\
\text { foot }\end{array}$ & $\begin{array}{l}\text { Minimum } \\
\text { tensile } \\
\text { strength }\end{array}$ & $\begin{array}{l}\text { A pproxi- } \\
\text { mate } \\
\text { weight } \\
\text { per } 100 \\
\text { feet }\end{array}$ \\
\hline $\begin{array}{l}\text { Steel or brass } \\
\text { Do } \\
\text { Do } \\
\text { Do }\end{array}$ & $\begin{array}{c}\text { Inch } \\
0.105 \\
.120 \\
.135 \\
.148\end{array}$ & $\begin{array}{l}8 \\
7 \\
7 \\
51 / 2\end{array}$ & $\begin{array}{r}\text { Pounds } \\
785 \\
1,000 \\
1,205 \\
1,280\end{array}$ & $\begin{array}{r}\text { Pounds } \\
10 \\
13 \\
17 \\
20\end{array}$ \\
\hline $\begin{array}{l}\text { Do } \\
\text { Do } \\
\text { Do } 0.1 \\
\text { Do }\end{array}$ & $\begin{array}{l}.162 \\
.177 \\
.192 \\
.207\end{array}$ & $\begin{array}{l}51 / 2 \\
41 / 4 \\
4 \\
5\end{array}$ & $\begin{array}{l}1,500 \\
1,850 \\
2,025 \\
2,600\end{array}$ & $\begin{array}{l}25 \\
27 \\
32 \\
41\end{array}$ \\
\hline
\end{tabular}

STYLE 2

\begin{tabular}{|c|c|c|c|c|}
\hline $\begin{array}{l}\text { Steel or brass. } \\
\text { Do } \\
\text { Do } \\
\text { Do }\end{array}$ & $\begin{array}{l}\text { Inch } \\
0.062 \\
.072 \\
.080 \\
.091\end{array}$ & $\begin{array}{r}11.29 \\
9.66 \\
8.41 \\
7.35\end{array}$ & $\begin{array}{r}\text { Pounds } \\
285 \\
420 \\
505 \\
690\end{array}$ & $\begin{array}{r}\text { Pounds } \\
3.53 \\
4.71 \\
5.80 \\
7.50\end{array}$ \\
\hline $\begin{array}{l}\text { Do } \\
\text { Do } \\
\text { Do } \\
\text { Do }\end{array}$ & $\begin{array}{l}.105 \\
.120 \\
.135 \\
.148\end{array}$ & $\begin{array}{l}7.31 \\
6.62 \\
6.04 \\
5.41\end{array}$ & $\begin{array}{r}900 \\
1,130 \\
1,370 \\
1,675\end{array}$ & $\begin{array}{l}10.44 \\
13.44 \\
16.87 \\
20.25\end{array}$ \\
\hline $\begin{array}{l}\text { Do } \\
\text { Do } \\
\text { Do } \\
\text { Do } 0.1\end{array}$ & $\begin{array}{l}.162 \\
.177 \\
.192 \\
.207\end{array}$ & $\begin{array}{l}4.79 \\
4.52 \\
4.25 \\
3.66\end{array}$ & $\begin{array}{r}1,845 \\
2,220 \\
2,530 \\
3,100\end{array}$ & $\begin{array}{l}24.56 \\
29.37 \\
33.90 \\
40.00\end{array}$ \\
\hline
\end{tabular}

\section{एDDDDPDA}

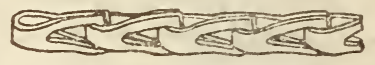

Figure 93.-Sash chain

$\mathrm{T}_{\mathrm{ABLE}}$ 3.-Sash chain (dimensions, strenglh, weights)

\begin{tabular}{|c|c|c|c|c|c|}
\hline Material & $\begin{array}{c}\text { Metal } \\
\text { thickness }\end{array}$ & $\begin{array}{l}\text { A pproxi- } \\
\text { mate } \\
\text { number } \\
\text { links per } \\
\text { foot }\end{array}$ & $\begin{array}{l}\text { Mini- } \\
\text { mum } \\
\text { tensile } \\
\text { strength }\end{array}$ & $\begin{array}{l}\text { A pproxi- } \\
\text { mate } \\
\text { weight } \\
\text { per } 100 \\
\text { feet }\end{array}$ & $\begin{array}{l}\text { Width } \\
\text { of links } \\
\text { (maxi- } \\
\text { mum) }\end{array}$ \\
\hline $\begin{array}{l}\text { Steel, sash. } \\
\text { Do.. } \\
\text { Do. } \\
\text { Do. } \\
\text { Bronze, sash. } \\
\text { Do } \\
\text { Do.... } \\
\text { Do... } \\
\text { Do... }\end{array}$ & $\begin{array}{c}\text { Inch } \\
0.035 \\
0.042-.045 \\
.051 \\
.054-.050 \\
.035 \\
.042-.045 \\
.049 \\
.054-.062 \\
.062\end{array}$ & $\begin{array}{l}21 \\
22 \\
21 \\
191 / 2 \\
21 \\
22 \\
22 \\
191 / 2 \\
17\end{array}$ & $\begin{array}{r}\text { Pounds } \\
475 \\
600 \\
725 \\
850 \\
425 \\
550 \\
650 \\
800 \\
800\end{array}$ & $\begin{array}{r}\text { Pounds } \\
51 / 2 \\
63 / 4 \\
83 / 4 \\
8-11 \\
53 / 4 \\
71 / 2 \\
81 / 2 \\
111 / 2 \\
12\end{array}$ & $\begin{array}{l}\text { Inch } \\
0.353 \\
.353 \\
.360 \\
.387 \\
.353 \\
.353 \\
.360 \\
.387 \\
.387\end{array}$ \\
\hline
\end{tabular}

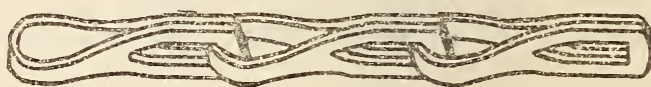

FigURe 94.-Flat-link long pitch chain

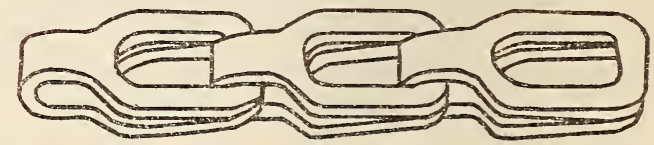

FIgURE 95.-Flat-link short pitch chain

TABLE 4.-Flat-link (long pitch) and flat-link (short pitch) chain

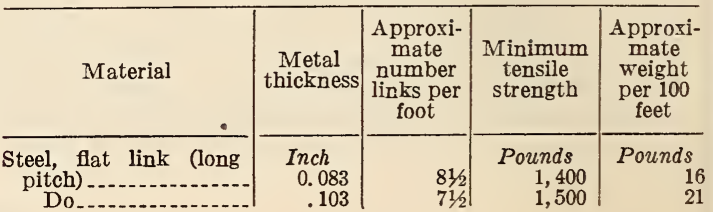


TABLE 4.-Flat-link (long pitch) and flat-link (short pitch) chain-Continued

\begin{tabular}{|c|c|c|c|c|}
\hline Material & $\begin{array}{c}\text { Metal } \\
\text { thickness }\end{array}$ & $\begin{array}{l}\text { A pproxi- } \\
\text { mate } \\
\text { number } \\
\text { links per } \\
\text { foot }\end{array}$ & $\begin{array}{c}\text { Minimum } \\
\text { tensile } \\
\text { strength }\end{array}$ & $\begin{array}{c}\text { A pproxi- } \\
\text { mate } \\
\text { weight } \\
\text { per } 100 \\
\text { feet }\end{array}$ \\
\hline $\begin{array}{l}\text { Steel or brass, flat link } \\
\text { (short pitch) } \\
\text { Do } \\
\text { Do } \\
\text { Do }\end{array}$ & $\begin{array}{l}\text { Inch } \\
0.065 \\
.083 \\
.103 \\
.125\end{array}$ & $\begin{array}{l}17 \\
834 \\
934 \\
9\end{array}$ & $\begin{array}{r}\text { Pounds } \\
900 \\
1,500 \\
2,100 \\
3,000\end{array}$ & $\begin{array}{c}\text { Pounds } \\
121 / 2 \\
25 \\
35 \\
40\end{array}$ \\
\hline
\end{tabular}

\section{बren}

FIGURE 96.-Plumber's chain

TABLE 5.-Plumber's chain

\begin{tabular}{|c|c|c|c|c|}
\hline Material & $\begin{array}{c}\text { Metal } \\
\text { thickness }\end{array}$ & $\begin{array}{l}\text { A pproxi- } \\
\text { mate } \\
\text { number } \\
\text { links per } \\
\text { foot }\end{array}$ & $\begin{array}{l}\text { Minimum } \\
\text { tensile } \\
\text { strength }\end{array}$ & $\begin{array}{c}\text { Approxi- } \\
\text { mate } \\
\text { weight } \\
\text { per } 100 \\
\text { feet }\end{array}$ \\
\hline $\begin{array}{l}\text { Brass or steel. } \\
\text { Do } \\
\text { Copper. }\end{array}$ & $\begin{array}{c}\text { Inch } \\
0.018 \\
.022 \\
.032\end{array}$ & $\begin{array}{l}23 \\
22 \\
21\end{array}$ & $\begin{array}{r}\text { Pounds } \\
150 \\
165 \\
150\end{array}$ & $\begin{array}{c}\text { Pounds } \\
13 / 4 \\
2 \\
31 / 2\end{array}$ \\
\hline
\end{tabular}

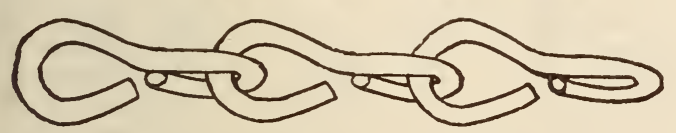

FigURe 97.--Single jack chain

TABLE 6.-Single jack chain

\begin{tabular}{|c|c|c|c|c|}
\hline Material & $\begin{array}{c}\text { Diameter } \\
\text { of wire }\end{array}$ & $\begin{array}{l}\text { Approxi- } \\
\text { mate } \\
\text { number } \\
\text { links per } \\
\text { foot }\end{array}$ & $\begin{array}{l}\text { Minimum } \\
\text { tensile } \\
\text { strength }\end{array}$ & $\begin{array}{l}\text { Approxi- } \\
\text { mate } \\
\text { weight } \\
\text { per } 100 \\
\text { feet }\end{array}$ \\
\hline $\begin{array}{l}\text { Steel or brass. } \\
\text { Do } \\
\text { Do Do } \\
\text { Do }\end{array}$ & $\begin{array}{c}\text { Inch } \\
0.207 \\
.192 \\
.162 \\
.135 \\
.105\end{array}$ & $\begin{array}{r}83 / 4 \\
91 / 2 \\
11 \\
13 \\
16\end{array}$ & $\begin{array}{r}\text { Pounds } \\
475 \\
350 \\
275 \\
220 \\
135\end{array}$ & $\begin{array}{c}\text { Pounds } \\
331 / 2 \\
30 \\
21 \\
14 \\
815\end{array}$ \\
\hline $\begin{array}{l}\text { Do } \\
\text { Do } \\
\text { Do } \\
\text { Do } 1 . .\end{array}$ & $\begin{array}{l}.080 \\
.062 \\
.048 \\
.036\end{array}$ & $\begin{array}{l}19 \\
23 \\
31 \\
40\end{array}$ & $\begin{array}{l}80 \\
45 \\
30 \\
20\end{array}$ & $\begin{array}{l}41 / 2 \\
3 \\
13 / 4 \\
1\end{array}$ \\
\hline
\end{tabular}

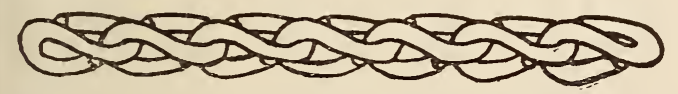

Figure 98.-Double jack chain

TABLE 7.-Double jack chain

\begin{tabular}{|c|c|c|c|c|}
\hline Material & $\begin{array}{l}\text { Diameter } \\
\text { of wire }\end{array}$ & $\begin{array}{l}\text { A pproxi- } \\
\text { mate } \\
\text { number } \\
\text { links per } \\
\text { foot }\end{array}$ & $\begin{array}{c}\text { Minimum } \\
\text { tensile } \\
\text { strength }\end{array}$ & $\begin{array}{l}\text { A pproxi- } \\
\text { mate } \\
\text { weight } \\
\text { per } 100 \\
\text { feet }\end{array}$ \\
\hline 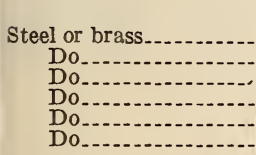 & $\begin{array}{c}\text { Inch } \\
0.135 \\
.105 \\
.080 \\
.062 \\
.048 \\
.036\end{array}$ & $\begin{array}{l}211 / 2 \\
263 / 4 \\
361 / 4 \\
451 / 4 \\
491 / 4 \\
60\end{array}$ & $\begin{array}{r}\text { Pounds } \\
190 \\
90 \\
40 \\
25 \\
17 \\
15\end{array}$ & $\begin{array}{c}\text { Pounds } \\
191 / 2 \\
12 \\
71 / 2 \\
41 / 4 \\
21 / 4 \\
11 / 4\end{array}$ \\
\hline
\end{tabular}

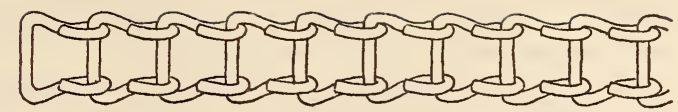

FigdRe 99.-Ladder chain

TABLE 8.-Ladder chain

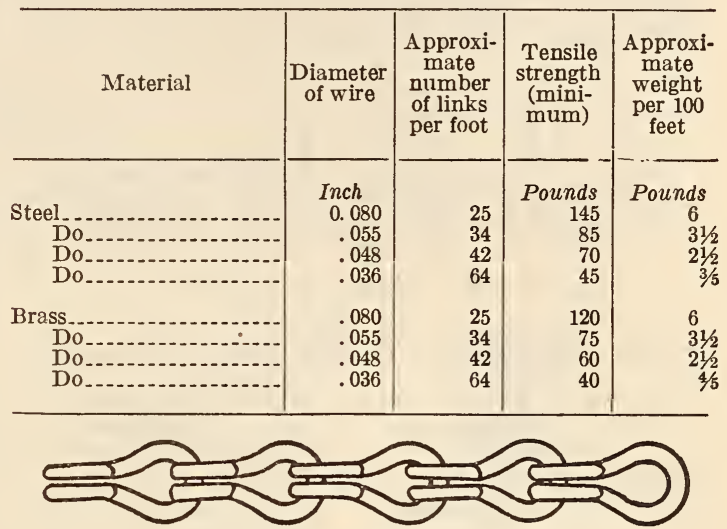

FIGURE 100.-Register chain

TABLE 9.-Register chain

\begin{tabular}{|c|c|c|c|c|}
\hline Material & $\begin{array}{c}\text { Diameter } \\
\text { of wire }\end{array}$ & $\begin{array}{l}\text { Approxi- } \\
\text { mate } \\
\text { number } \\
\text { of links } \\
\text { per foot }\end{array}$ & $\begin{array}{c}\text { Tensile } \\
\text { strength } \\
\text { (mini- } \\
\text { mum) }\end{array}$ & $\begin{array}{l}\text { Approxi- } \\
\text { mate } \\
\text { weight } \\
\text { per } 100 \\
\text { feet }\end{array}$ \\
\hline 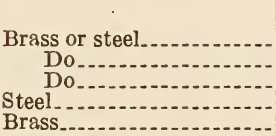 & $\begin{array}{c}\text { Inch } \\
0.148 \\
.091 \\
.062 \\
.036 \\
.036\end{array}$ & $\begin{array}{l}11 \\
143 / 4 \\
251 / 4 \\
35 \\
35\end{array}$ & $\begin{array}{r}\text { Pounds } \\
450 \\
165 \\
90 \\
30 \\
20\end{array}$ & $\begin{array}{r}\text { Pounds } \\
261 / 4 \\
101 / 4 \\
41 / 2 \\
11 / 2 \\
13 / 5\end{array}$ \\
\hline
\end{tabular}

\subsection{CHAIN ATTACHMENTS.}

\section{AMERICAN BUREAU OF SHIPPING, RULES FOR BUILDING AND CLASSING STEEL VESSELS; SHACKLES AND SHACKLE PINS, 1930}

(Specifications for wrought iron and steel shackles and pins, and cast-steel shackles and pins, are the same as for wrought iron and steel stud-link chains and cast-steel stud-link chains. (See 603.52, p. 201, and 603.53, p. 204.) This is also true in respect to tolerances. Maximum tolerances in shackles D above and below the dimensions given in Table 1 below, will be found in Table 2. (See 603.53, p. 206.) The dimensions of shackles $\mathrm{D}$ and their respective pins are as shown in Table 1 and Figure 101. The dimensions of shackles $\mathrm{F}$ and their respective pins are as shown in Table 2 and Figure 102.)

\section{SHACKLES AND SHACKLE PINS}

(For general information and requirements see 600.0 , p. 2.)

1. The end shackles, joining shackles, spare shackles, and swivels required for a length of chain are to be tested to the same proof test as the chain, and at least 1 out of every 25 of the shackles is to 
be subjected to the breaking test, and must be discarded. (For the respective breaking and proof tests see comparative table facing p. 200.)

2 . When shackles have satisfactorily passed the test requirements they are to be weighed, and this actual weight will be given on the certificate of test. After being weighed, the shackles and end links of each shot are to be clearly stamped by the manufacturer with identifying number, initials of surveyor, date of test, breaking test, proof test, and the bureau's mark of approval, A. B.

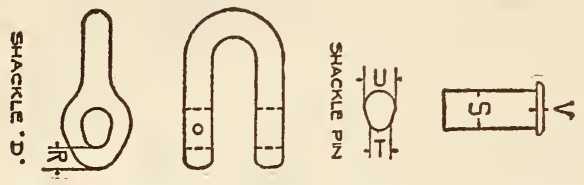

FiguRE 101.- Shackle $D$ and shackle pin

TABLE 1.-Shackles $D$ and pins (dimensions)-

\begin{tabular}{|c|c|c|c|c|c|c|c|c|}
\hline \multirow[b]{2}{*}{$\begin{array}{l}\text { Size of } \\
\text { chain }\end{array}$} & \multicolumn{4}{|c|}{ Shackles D } & \multicolumn{4}{|c|}{ Shackle pins } \\
\hline & $\begin{array}{c}\text { Size of } \\
\text { iron } \\
0\end{array}$ & $\begin{array}{c}\text { Out- } \\
\text { side } \\
\text { length } \\
\mathbf{P}\end{array}$ & $\mid \begin{array}{c}\text { Out- } \\
\text { side } \\
\text { width } \\
\text { Q }\end{array}$ & $\mathrm{R}$ & $\mathrm{S}$ & $\mathrm{T}$ & $\mathrm{U}$ & V \\
\hline $\begin{array}{c}\text { Inches } \\
5 / 8 \\
11 / 16 \\
3 / 4 \\
13 / 16 \\
7 / 8\end{array}$ & \begin{tabular}{|c|} 
Inches \\
$3 / 4$ \\
$13 / 16$ \\
$15 / 16$ \\
1 \\
$11 / 16$
\end{tabular} & $\begin{array}{c}\text { Inches } \\
41 / 16 \\
47 / 16 \\
47 / 8 \\
514 \\
511 / 16\end{array}$ & \begin{tabular}{|r|} 
Inches \\
$21 / 2$ \\
$23 / 4$ \\
3 \\
$31 / 4$ \\
$31 / 2$
\end{tabular} & $\begin{array}{c}\text { Inches } \\
1 / 2 \\
9 / 16 \\
5 / 8 \\
11 / 16 \\
11 / 16\end{array}$ & $\begin{array}{c}\text { Inches } \\
1 \\
11 / 8 \\
11 / 4 \\
15 / 16 \\
17 / 16\end{array}$ & $\begin{array}{c}\text { Inches } \\
5 / 8 \\
11 / 16 \\
3 / 4 \\
13 / 16 \\
7 / 8\end{array}$ & \begin{tabular}{|c|} 
Inches \\
$3 / 4$ \\
$13 / 16$ \\
$7 / 8$ \\
$15 / 16$ \\
$11 / 10$
\end{tabular} & $\begin{array}{l}\text { Inch } \\
1 / 8 \\
1 / 8 \\
1 / 8 \\
168 \\
3 / 10\end{array}$ \\
\hline $\begin{array}{l}15 / 16 \\
11 / 16 \\
11 / 8 \\
13 / 16\end{array}$ & $\begin{array}{l}11 / 8 \\
13 / 16 \\
11 / 4 \\
15 / 16 \\
17 / 16\end{array}$ & $\begin{array}{l}61 / 16 \\
61 / 2 \\
67 / 8 \\
75 / 16 \\
71116\end{array}$ & $\begin{array}{l}33 / 4 \\
4 \\
41 / 4 \\
41 / 2 \\
43 / 4\end{array}$ & $\begin{array}{l}3 / 4 \\
13 / 16 \\
7 / 8 \\
15 / 16 \\
15 / 16\end{array}$ & $\begin{array}{l}11 / 2 \\
15 / 8 \\
13 / 4 \\
113 / 16 \\
115 / 16\end{array}$ & $\begin{array}{l}15 / 16 \\
1 \\
11 / 16 \\
11 / 8 \\
13 / 16\end{array}$ & $\begin{array}{l}11 / 8 \\
13 / 16 \\
11 / 4 \\
15 / 16 \\
17 / 16\end{array}$ & $\begin{array}{l}3 / 16 \\
3 / 16 \\
3 / 16 \\
3 / 16 \\
1 / 4\end{array}$ \\
\hline $\begin{array}{l}134 \\
15 / 16 \\
13 / 8 \\
17 / 16 \\
11 / 2\end{array}$ & $\begin{array}{l}11 / 2 \\
19 / 16 \\
15 / 8 \\
111 / 16 \\
113 / 16\end{array}$ & $\begin{array}{l}818 \\
816 \\
815 / 16 \\
95 / 16 \\
911 / 16\end{array}$ & \begin{tabular}{l|}
5 \\
$51 / 4$ \\
$51 / 2$ \\
$53 / 4$ \\
6
\end{tabular} & $\begin{array}{l}1 \\
11 / 16 \\
11 / 8 \\
13 / 16 \\
11 / 4\end{array}$ & $\begin{array}{l}2 \\
21 / 8 \\
21 / 4 \\
25 / 16 \\
27 / 16\end{array}$ & $\begin{array}{l}11 / 4 \\
15 / 16 \\
136 \\
17 / 16 \\
11 / 2\end{array}$ & $\begin{array}{l}11 / 2 \\
19 / 16 \\
15 / 8 \\
111 / 16 \\
113 / 16\end{array}$ & $\begin{array}{l}1 / 4 \\
1 / 4 \\
1 / 4 \\
1 / 4 \\
5 / 10\end{array}$ \\
\hline $\begin{array}{l}1 \% / 16 \\
15 / 8 \\
111 / 16 \\
13 / 4 \\
113 / 16\end{array}$ & $\begin{array}{l}17 / 8 \\
1^{15 / 16} \\
2 \\
21 / 16 \\
23 / 16\end{array}$ & $\begin{array}{l}101 / 8 \\
101 / 2 \\
10^{15} / 16 \\
115 / 16 \\
113 / 4\end{array}$ & $\begin{array}{l}61 / 4 \\
61 / 2 \\
63 / 4 \\
7 \\
71 / 4\end{array}$ & $\begin{array}{l}134 \\
15 / 16 \\
138 \\
17 / 16 \\
11 / 2\end{array}$ & $\begin{array}{l}29 / 16 \\
25 / 8 \\
2^{3 / 4} \\
2^{7 / 8} \\
2^{15 / 16}\end{array}$ & $\begin{array}{l}19 / 16 \\
15 / 8 \\
111 / 16 \\
13 / 4 \\
113 / 16\end{array}$ & $\begin{array}{l}17 / 8 \\
115 / 16 \\
2 \\
21 / 16 \\
218\end{array}$ & $\begin{array}{l}5 / 10 \\
5 / 16 \\
5 / 16 \\
5 / 16 \\
5 / 16\end{array}$ \\
\hline $\begin{array}{l}17 / 8 \\
115 / 16 \\
2 \\
21 / 16 \\
21 / 8\end{array}$ & $\begin{array}{l}21 / 4 \\
25 / 16 \\
23 / 8 \\
27 / 16 \\
21 / 2\end{array}$ & $\begin{array}{l}1218 \\
129 / 16 \\
1215 / 16 \\
133 / 8 \\
133 / 4\end{array}$ & $\begin{array}{l}71 / 2 \\
73 / 4 \\
8 \\
81 / 4 \\
81 / 2\end{array}$ & $\begin{array}{l}11 / 2 \\
19 / 16 \\
15 / 8 \\
111 / 16 \\
13 / 4\end{array}$ & $\begin{array}{l}31 / 16 \\
318 \\
31 / 4 \\
33 / 8 \\
37 / 16\end{array}$ & $\begin{array}{l}17 / 8 \\
115 / 16 \\
2 \\
21 / 16 \\
218\end{array}$ & $\begin{array}{l}21 / 4 \\
25 / 16 \\
236 \\
27 / 16 \\
21 / 2\end{array}$ & $\begin{array}{l}3 / 8 \\
3 / 8 \\
3 / 8 \\
3 / 8 \\
3 / 8\end{array}$ \\
\hline $\begin{array}{l}23 / 16 \\
21 / 4 \\
25 / 16 \\
238 \\
27 / 16\end{array}$ & $\begin{array}{l}29 / 16 \\
2^{11} 1 / 16 \\
23 / 4 \\
2^{13} / 16 \\
2^{7 / 8}\end{array}$ & $\begin{array}{l}143 / 16 \\
149 / 16 \\
1415 / 16 \\
153 / 8 \\
153 / 4\end{array}$ & $\begin{array}{l}83 / 4 \\
9 \\
91 / 4 \\
91 / 2 \\
93 / 4\end{array}$ & $\begin{array}{l}13 / 4 \\
113 / 16 \\
17 / 8 \\
115 / 16 \\
2\end{array}$ & $\begin{array}{l}39 / 16 \\
311 / 16 \\
33 / 4 \\
37 / 8 \\
3^{15 / 16}\end{array}$ & $\begin{array}{l}23 / 16 \\
21 / 4 \\
25 / 16 \\
23 / 8 \\
27 / 16\end{array}$ & $\begin{array}{l}25 / 8 \\
2^{11} 1 / 16 \\
23 / 4 \\
2^{13} / 16 \\
2^{7} / 8\end{array}$ & $\begin{array}{l}7 / 16 \\
7 / 16 \\
7 / 16 \\
7 / 16 \\
7 / 16\end{array}$ \\
\hline $\begin{array}{l}21 / 2 \\
29 / 16 \\
25 / 8 \\
211 / 16 \\
23 / 4\end{array}$ & $\begin{array}{l}3 \\
31 / 16 \\
31 / 8 \\
33_{16} \\
314\end{array}$ & $\begin{array}{l}163 / 16 \\
169 / 16 \\
17 \\
173 / 8 \\
17^{13} / 16\end{array}$ & \begin{tabular}{l|}
10 \\
$101 / 4$ \\
$101 / 2$ \\
$103 / 4$ \\
11
\end{tabular} & $\begin{array}{l}2116 \\
2168 \\
21 / 8 \\
23116 \\
21 / 4\end{array}$ & $\begin{array}{l}41 / 16 \\
43 / 16 \\
41 / 4 \\
43 / 8 \\
41 / 2\end{array}$ & $\begin{array}{l}21 / 2 \\
29 / 16 \\
25 / 8 \\
211 / 16 \\
23 / 4\end{array}$ & $\begin{array}{l}3 \\
31 / 16 \\
31 / 8 \\
33 / 16 \\
31 / 4\end{array}$ & $\begin{array}{l}1 / 2 \\
1 / 2 \\
1 / 2 \\
1 / 2 \\
1 / 2\end{array}$ \\
\hline $\begin{array}{l}213 / 16 \\
27 / 8 \\
2^{15 / 16} \\
3 \\
31 / 16\end{array}$ & \begin{tabular}{|l|l|}
$33 / 8$ \\
$37 / 16$ \\
$31 / 2$ \\
$39 / 16$ \\
356
\end{tabular} & $\begin{array}{l}183 / 16 \\
185 / 8 \\
19 \\
197 / 8 \\
1913 / 16\end{array}$ & $\begin{array}{l}111 / 4 \\
111 / 2 \\
113 / 4 \\
12 \\
121 / 4\end{array}$ & $\begin{array}{l}25 / 16 \\
25 / 16 \\
236 \\
27 / 16 \\
21 / 2\end{array}$ & $\begin{array}{l}49 / 16 \\
4^{11} 1 / 16 \\
43 / 4 \\
47 / 8 \\
5\end{array}$ & $\begin{array}{l}2^{13 / 16} \\
2^{7 / 8} \\
2^{15 / 16} \\
3 \\
31 / 16\end{array}$ & $\begin{array}{l}35 / 16 \\
37 / 16 \\
31 / 2 \\
39 / 16 \\
35 / 8\end{array}$ & $\begin{array}{l}1 / 2 \\
9 / 16 \\
916 \\
9 / 16 \\
916\end{array}$ \\
\hline $\begin{array}{l}31 / 8 \\
33 / 16 \\
31 / 4 \\
35 / 16\end{array}$ & $\begin{array}{l}3^{11} 1 / 16 \\
3^{13} / 16 \\
3^{7} / 8 \\
3^{15} / 16\end{array}$ & $\begin{array}{l}201 / 2 \\
205 / 8 \\
21 \\
217 / 16\end{array}$ & $\begin{array}{l}121 / 2 \\
123 / 4 \\
13 \\
131 / 4\end{array}$ & $\begin{array}{l}29 / 16 \\
29 / 16 \\
258 \\
2^{11} 1 / 16\end{array}$ & $\begin{array}{l}51 / 16 \\
53 / 16 \\
55 / 16 \\
53 / 8\end{array}$ & $\begin{array}{l}31 / 8 \\
33 / 16 \\
31 / 4 \\
35 / 16\end{array}$ & $\begin{array}{l}311 / 16 \\
3^{13} / 16 \\
37 / 8 \\
3^{15} / 16\end{array}$ & $\begin{array}{l}9 / 16 \\
5 / 8 \\
5 / 8 \\
5 / 8\end{array}$ \\
\hline $\begin{array}{l}33 / 8 \\
37 / 16 \\
31 / 2 \\
33 / 4\end{array}$ & $\begin{array}{l}4 \\
41 / 16 \\
43 / 16 \\
47 / 16\end{array}$ & $\begin{array}{l}2113 / 16 \\
221 / 4 \\
225 / 8 \\
241 / 4\end{array}$ & $\begin{array}{l}131 / 2 \\
133 / 4 \\
14 \\
15\end{array}$ & $\begin{array}{l}23 / 4 \\
2^{13} / 16 \\
27 / 8 \\
31 / 16\end{array}$ & $\begin{array}{l}51 / 2 \\
59116 \\
511 / 16 \\
61 / 8\end{array}$ & $\begin{array}{l}33 / 8 \\
37 / 16 \\
31 / 2 \\
334\end{array}$ & $\begin{array}{l}4 \\
41 / 16 \\
43 / 16 \\
47 / 16\end{array}$ & $\begin{array}{c}5 / 8 \\
5 / 8 \\
1116 \\
1116\end{array}$ \\
\hline
\end{tabular}

SHACKLE "F"

SHACKLE PIN
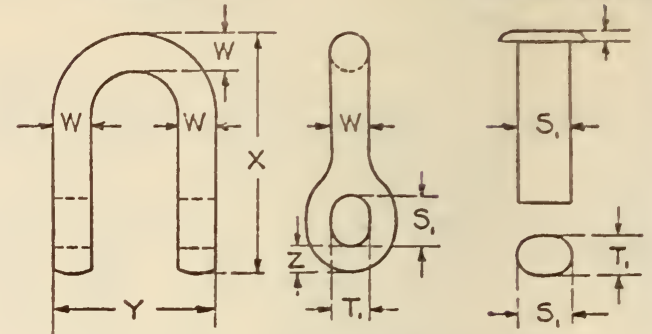

FIGURE 102.- Shackle $F$ and shackle pin

TABLE 2.-End or anchor shackles $F$ and pins (dimensions)

\begin{tabular}{|c|c|c|c|c|c|c|c|}
\hline \multirow[b]{2}{*}{$\begin{array}{l}\text { Size of } \\
\text { chain }\end{array}$} & \multicolumn{4}{|c|}{ Shackles F } & \multicolumn{3}{|c|}{ Shackle pins } \\
\hline & $\begin{array}{c}\text { Size of } \\
\text { iron } \\
\text { W }\end{array}$ & $\begin{array}{c}\text { Outside } \\
\text { length } \\
\mathrm{X}\end{array}$ & $\begin{array}{c}\text { Outside } \\
\text { width } \\
\text { Y }\end{array}$ & $\mathrm{Z}$ & $\mathrm{S}$ & $\mathrm{T}$ & V \\
\hline $\begin{array}{c}\text { Inches } \\
5 / 8 \\
11 / 16 \\
3 / 4 \\
13 / 16 \\
7 / 8\end{array}$ & $\begin{array}{c}\text { Inches } \\
7 / 8 \\
1 \\
11 / 16 \\
11 / 8 \\
13 / 16\end{array}$ & $\begin{array}{c}\text { Inches } \\
51 / 2 \\
6 \\
65 / 8 \\
71 / 8 \\
734\end{array}$ & $\begin{array}{r}\text { Inches } \\
33 / 4 \\
414 \\
41 / 2 \\
43 / 4 \\
51 / 8\end{array}$ & $\begin{array}{c}\text { Inches } \\
9 / 16 \\
5 / 8 \\
1116 \\
3 / 4 \\
13 / 16\end{array}$ & $\begin{array}{c}\text { Inches } \\
118 \\
11 / 4 \\
138 \\
11 / 2 \\
158\end{array}$ & $\begin{array}{c}\text { Inches } \\
7 / 8 \\
1 \\
1116 \\
118 \\
13 / 16\end{array}$ & $\begin{array}{c}\text { Inch } \\
1 / 8 \\
1 / 8 \\
3 / 8 \\
3 / 16 \\
3 / 16\end{array}$ \\
\hline $\begin{array}{l}15 / 16 \\
1 \\
11 / 16 \\
118 \\
13 / 16\end{array}$ & $\begin{array}{l}15 / 16 \\
17 / 16 \\
11 / 2 \\
19 / 16 \\
1568\end{array}$ & $\begin{array}{r}81 / 4 \\
83 / 4 \\
93 / 8 \\
97 / 8 \\
101 / 2\end{array}$ & $\begin{array}{l}55 / 8 \\
6 \\
63 / 8 \\
65 / 8 \\
67 / 8\end{array}$ & $\begin{array}{l}7 / 8 \\
15 / 16 \\
1 \\
1 \\
11 / 16\end{array}$ & $\begin{array}{l}111 / 16 \\
113 / 16 \\
17 / 8 \\
2 \\
21 / 8\end{array}$ & $\begin{array}{l}15 / 16 \\
17 / 16 \\
11 / 2 \\
1 \% / 16 \\
1568\end{array}$ & $\begin{array}{l}3 / 16 \\
3 / 10 \\
1 / 4 \\
1 / 4 \\
1 / 4\end{array}$ \\
\hline $\begin{array}{l}11 / 4 \\
15 / 16 \\
13 / 8 \\
17 / 16 \\
11 / 2\end{array}$ & $\begin{array}{l}13 / 4 \\
17 / 8 \\
115 / 16 \\
2 \\
216\end{array}$ & $\begin{array}{l}11 \\
111 / 2 \\
121 / 8 \\
125 / 8 \\
131 / 4\end{array}$ & $\begin{array}{l}71 / 4 \\
75 / 8 \\
77 / 8 \\
81 / 8 \\
81 / 2\end{array}$ & $\begin{array}{l}11 / 8 \\
13 / 16 \\
11 / 4 \\
15 / 16 \\
13 / 8\end{array}$ & $\begin{array}{l}21 / 4 \\
23 / 8 \\
21 / 2 \\
25 / 8 \\
2^{11 / 16}\end{array}$ & $\begin{array}{l}13 / 4 \\
178 \\
115 / 16 \\
2 \\
238\end{array}$ & $\begin{array}{l}1 / 4 \\
5 / 16 \\
5 / 16 \\
5 / 16 \\
5 / 16\end{array}$ \\
\hline $\begin{array}{l}19 / 16 \\
15 / 8 \\
111 / 16 \\
13 / 4 \\
113 / 16\end{array}$ & $\begin{array}{l}23 / 16 \\
21 / 4 \\
23 / 8 \\
27 / 16 \\
21 / 2\end{array}$ & $\begin{array}{l}133 / 4 \\
141 / 4 \\
147 / 8 \\
153 / 8 \\
16\end{array}$ & $\begin{array}{l}83 / 4 \\
9 \\
93 / 8 \\
95 / 8 \\
97 / 8\end{array}$ & $\begin{array}{l}17 / 16 \\
11 / 2 \\
1 \% / 16 \\
15 / 8 \\
15 / 8\end{array}$ & $\begin{array}{l}213 / 16 \\
2^{15} / 16 \\
3 \\
316 \\
31 / 4\end{array}$ & $\begin{array}{l}23 / 16 \\
21 / 4 \\
238 \\
27 / 16 \\
21 / 2\end{array}$ & $\begin{array}{l}5 / 16 \\
5 / 16 \\
3 / 8 \\
3 / 8 \\
3 / 8\end{array}$ \\
\hline $\begin{array}{l}17 / 8 \\
1^{15 / 16} \\
2 \\
21 / 16 \\
218\end{array}$ & $\begin{array}{l}258 \\
2^{33 / 4} \\
2^{13} / 16 \\
2^{7} / 8 \\
3\end{array}$ & $\begin{array}{l}161 / 2 \\
17 \\
175 / 8 \\
181 / 8 \\
183 / 4\end{array}$ & $\begin{array}{l}101 / 4 \\
103 / 4 \\
111 / 8 \\
111 / 2 \\
12\end{array}$ & $\begin{array}{l}111 / 16 \\
13 / 4 \\
113 / 16 \\
17 / 8 \\
115 / 16\end{array}$ & $\begin{array}{l}33 / 8 \\
31 / 2 \\
35 / 8 \\
33 / 4 \\
37 / 8\end{array}$ & $\begin{array}{l}25 / 8 \\
23 / 4 \\
2^{13} / 16 \\
27 / 8 \\
3\end{array}$ & $\begin{array}{l}3 / 8 \\
3 / 8 \\
7 / 16 \\
7 / 16 \\
7 / 16\end{array}$ \\
\hline $\begin{array}{l}23 / 16 \\
21 / 4 \\
25 / 16 \\
23 / 8 \\
27 / 16\end{array}$ & $\begin{array}{l}31 / 16 \\
31 / 8 \\
31 / 4 \\
336 \\
37 / 16\end{array}$ & $\begin{array}{l}191 / 4 \\
193 / 4 \\
203 / 8 \\
21 \\
211 / 2\end{array}$ & $\begin{array}{l}123 / 8 \\
123 / 4 \\
131 / 4 \\
133 / 4 \\
141 / 8\end{array}$ & $\begin{array}{l}2 \\
21 / 16 \\
216 \\
21 / 8 \\
23 / 16\end{array}$ & $\begin{array}{l}315 / 16 \\
41 / 16 \\
41 / 8 \\
41 / 4 \\
43 / 8\end{array}$ & $\begin{array}{l}31 / 16 \\
318 \\
31 / 4 \\
338 \\
37 / 16\end{array}$ & $\begin{array}{l}7 / 16 \\
1 / 2 \\
1 / 2 \\
1 / 2 \\
1 / 2\end{array}$ \\
\hline $\begin{array}{l}21 / 2 \\
29 / 16 \\
25,8 \\
211 / 16 \\
23 / 4\end{array}$ & $\begin{array}{l}31 / 2 \\
35 / 8 \\
311 / 16 \\
33 / 4 \\
37 / 8\end{array}$ & $\begin{array}{l}22 \\
221 / 2 \\
231 / 8 \\
235 / 8 \\
241 / 4\end{array}$ & $\begin{array}{l}141 / 2 \\
15 \\
153 / 8 \\
153 / 4 \\
161 / 4\end{array}$ & $\begin{array}{l}21 / 4 \\
25 / 16 \\
23 / 8 \\
27 / 16 \\
21 / 2\end{array}$ & $\begin{array}{l}41 / 2 \\
45 / 8 \\
43 / 4 \\
47 / 8 \\
4^{15} 516\end{array}$ & $\begin{array}{l}31 / 2 \\
35 / 8 \\
3^{11} 1 / 6 \\
33 / 4 \\
37 / 8\end{array}$ & $\begin{array}{l}1 / 2 \\
9 / 16 \\
9 / 16 \\
9 / 16 \\
9 / 16\end{array}$ \\
\hline $\begin{array}{l}2^{13} / 16 \\
2^{7 / 8} \\
2^{15} / 16 \\
3 \\
31 / 16\end{array}$ & $\begin{array}{l}315 / 16 \\
4 \\
41 / 8 \\
43 / 16 \\
41 / 4\end{array}$ & $\begin{array}{l}243 / 4 \\
251 / 4 \\
257 / 8 \\
263 / 8 \\
27\end{array}$ & $\begin{array}{l}165 / 8 \\
17 \\
171 / 2 \\
177 / 8 \\
181 / 4\end{array}$ & $\begin{array}{l}29 / 16 \\
25 / 8 \\
211 / 16 \\
2^{11} 1 / 16 \\
23 / 4\end{array}$ & $\begin{array}{l}51 / 16 \\
53 / 16 \\
51 / 4 \\
53 / 8 \\
51 / 2\end{array}$ & $\begin{array}{l}315 / 16 \\
4 \\
41 / 8 \\
43 / 16 \\
41 / 4\end{array}$ & $\begin{array}{l}9 / 16 \\
5 / 8 \\
5 / 8 \\
5 / 8 \\
5 / 8\end{array}$ \\
\hline $\begin{array}{l}31 / 8 \\
33 / 16 \\
31 / 4 \\
35 / 16\end{array}$ & $\begin{array}{l}43 / 8 \\
41 / 2 \\
49 / 16 \\
45 / 8\end{array}$ & $\begin{array}{l}271 / 2 \\
28 \\
285 / 8 \\
291 / 4\end{array}$ & $\begin{array}{l}185 / 8 \\
191 / 8 \\
191 / 2 \\
20\end{array}$ & $\begin{array}{l}2^{13 / 16} \\
2^{7 / 8} \\
2^{15 / 16} \\
3\end{array}$ & $\begin{array}{l}55 / 8 \\
53 / 4 \\
57 / 8 \\
5^{15 / 16}\end{array}$ & $\begin{array}{l}43 / 8 \\
416 \\
49 / 16 \\
45,8\end{array}$ & $\begin{array}{c}5 / 8 \\
11 / 16 \\
11 / 16 \\
11 / 16\end{array}$ \\
\hline $\begin{array}{l}33 / 8 \\
37 / 16 \\
31 / 2 \\
33 / 4\end{array}$ & $\begin{array}{l}4^{11} 1 / 16 \\
4^{13} / 16 \\
4^{15} / 16 \\
51 / 4\end{array}$ & $\begin{array}{l}293 / 4 \\
301 / 4 \\
303 / 4 \\
33\end{array}$ & $\begin{array}{l}203 / 8 \\
203 / 4 \\
211 / 4 \\
23\end{array}$ & $\begin{array}{l}31 / 16 \\
31 / 8 \\
33 / 16 \\
33 / 8\end{array}$ & $\begin{array}{l}61 / 16 \\
618 \\
61 / 4 \\
63 / 4\end{array}$ & $\begin{array}{l}411 / 16 \\
413 / 16 \\
415 / 16 \\
51 / 4\end{array}$ & $\begin{array}{c}11 / 16 \\
11 / 16 \\
3 / 4 \\
3 / 4\end{array}$ \\
\hline
\end{tabular}


AMERICAN SOCIETY FOR TESTING MATERIALS, TENTATIVE SPECIFICATIONS FOR ELECTRIC CAST-STEEL STUD-LINK ANCHOR CHAIN, DESIGNATION A7728T, 1928

\section{SHACKLES AND SHACKLE PINS}

(The specifications for materials for cast-steel shackles and shackle pins are the same as those for electric cast-steel stud-link anchor chain. (See A. S. T. M. A77-28T. 603.53, p. 206.)

\section{BREAKING AND PROOF TESTS}

1. Break tests. - In the event that shackles and shackle pins only are poured from any heat or heats of steel, one shackle and shackle pin shall be selected from each such heat of steel. If shackle and shackle pins of more than one size are poured from one heat of steel, the shackle and shackle pin selected for the break test shall be the average wire size. Shackles and shackle pins so selected shall be anchored in an approved static testing machine by connecting with one $\mathrm{E}$ and one $\mathrm{C}$ end link of the respective size chain and subjected to the break test load for the respective size chain. (Break-test loads for corresponding sizes of chain are as shown in comparative table facing p. 200.)

2 . If the test shackle or shackle pin fails in the break test as described in the preceding paragraph, then two additional shackles and shackle pins shall be selected and tested individually in accordance with the preceding paragraph. If either of these shackles or shackle pins fail during this retest, then the shackles and shackle pins represented shall be rejected.

3. Proof tests. - All shackles and shackle pins that have met the requirements of the break tests described in the previous paragraphs shall be subjected to the proof test loads for the respective size of chain. (Proof test loads are as shown in comparative table facing p. 200.)

4. Shackles and shackle pins shall be proof tested individually, and for anchoring in the static testing machine shall be connected on either end with one $\mathrm{E}$ and one $\mathrm{C}$ end link or chain cable of respective size or shackles and shackle pins of the same size in the form of a chain. If the test shackle or shackle pin fails during the test, then the shackles and shackle pins represented shall be rejected.

\section{DIMENSIONS OF SHACKLES, SHACKLE PINS, AND SWIVELS}

(The dimensions of shackles $\mathrm{D}$ and $\mathrm{F}$, and their pins, in the manual of the American Bureau of Shipping and in A. S. T. M. A77-28T are identical in some respects. In other respects the dimensions differ. Table 1, Figure 103, and Table 2, Figure 104, given below, show in what respect the A. S. T. M. dimensions differ from the dimensions in Table 1, Figure 101, and Table 2, Figure 102, given above.)

\section{SWIVELS}

(The specifications for cast-steel swivels are the same as those for electric cast-steel stud-link anchor chain. (See A. S. T. M. A77-28T, (603.53, p. 206.))
The dimensions of swivels are as shown in Table 3, Figure 105.)

\section{PERMISSIBLE VARIATIONS OF SHACKLES, SHACKLE PINS, AND SWIVELS}

5. Permissible variations for swivels, shackles, and shackle pins shall be permitted in accordance with the tolerances specified in Table 1 for common A links. (See A.S. T. M. A77-28T (603.53, p. 206).) In determining the permissible variations for wire diameters, the diameter shall be measured at right angles, and one-half the sum of the two measurements shall be considered as representing the wire diameter.

(The specifications for cast-steel shackles and shackle pins are the same as those for electric caststeel stud-link anchor chain. (See A. S. T. M. A7728T. 603.53, p. 206.)

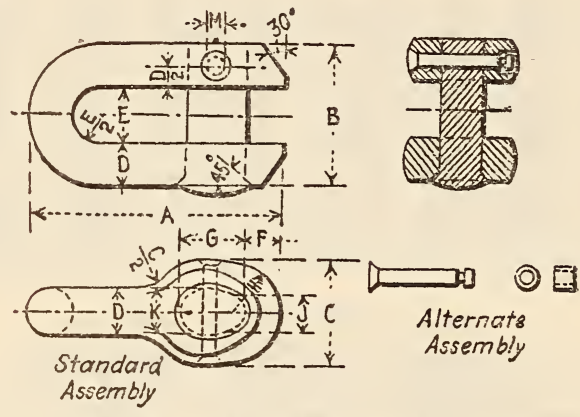

Figure 103.-Shackles $D$ and shackle pins

TABLE 1.-Shackles $D$ and shackle pins (dimensions)

\begin{tabular}{|c|c|c|c|c|c|c|c|}
\hline \multirow{2}{*}{$\begin{array}{l}\text { Size of } \\
\text { chain } \\
\text { cable }\end{array}$} & \multicolumn{7}{|c|}{ Shackles D and shackle pins } \\
\hline & A & B & $\mathrm{C}$ & $\mathbf{E}$ & $\mathrm{F}$ & H & M \\
\hline $\begin{array}{c}\text { Inches } \\
1 \\
11 / 8 \\
11 / 4 \\
138 \\
11 / 2\end{array}$ & $\begin{array}{c}\text { Inches } \\
611 / 16 \\
79 / 16 \\
87 / 16 \\
91 / 4 \\
101 / 16\end{array}$ & $\begin{array}{c}\text { Inches } \\
313 / 16 \\
43 / 16 \\
413 / 16 \\
53 / 16 \\
513 / 16\end{array}$ & $\begin{array}{c}\text { Inches } \\
2^{13} / 16 \\
33 / 16 \\
31 / 2 \\
37 / 8 \\
45 / 16\end{array}$ & $\begin{array}{c}\text { Inches } \\
17 / 16 \\
19 / 16 \\
113 / 16 \\
115 / 16 \\
23 / 16\end{array}$ & $\begin{array}{c}\text { Inches } \\
1 \\
13 / 16 \\
15 / 16 \\
17 / 16 \\
15 / 8\end{array}$ & $\begin{array}{c}\text { Inches } \\
15 / 16 \\
11 / 2 \\
19 / 16 \\
13 / 4 \\
115 / 16\end{array}$ & $\begin{array}{c}\text { Inches } \\
3 / 8 \\
3 / 8 \\
1 / 2 \\
1 / 2 \\
1 / 2\end{array}$ \\
\hline $\begin{array}{l}15 / 8 \\
13 / 4 \\
17 / 8 \\
2 \\
218\end{array}$ & $\begin{array}{l}107 / 8 \\
1111 / 16 \\
121 / 2 \\
135 / 16 \\
141 / 8\end{array}$ & $\begin{array}{l}63 / 16 \\
65 / 8 \\
73 / 16 \\
75 / 8 \\
8\end{array}$ & $\begin{array}{l}49 / 16 \\
415 / 16 \\
51 / 4 \\
55 / 8 \\
6\end{array}$ & $\begin{array}{l}25 / 16 \\
21 / 2 \\
2^{11} 1 / 16 \\
27 / 8 \\
3\end{array}$ & $\begin{array}{l}111 / 16 \\
113 / 16 \\
17 / 8 \\
2 \\
21 / 8\end{array}$ & $\begin{array}{l}21 / 16 \\
21 / 4 \\
23 / 8 \\
29 / 16 \\
23 / 4\end{array}$ & $\begin{array}{l}5 / 8 \\
5 / 8 \\
5 / 8 \\
3 / 4 \\
3 / 4\end{array}$ \\
\hline $\begin{array}{l}21 / 4 \\
23 / 8 \\
21 / 2 \\
25 / 8\end{array}$ & $\begin{array}{l}1415 / 16 \\
1513 / 16 \\
165 / 8 \\
171 / 2\end{array}$ & $\begin{array}{l}85 / 8 \\
9 \\
95 / 8 \\
10\end{array}$ & $\begin{array}{l}65 / 16 \\
6^{11 / 16} \\
71 / 8 \\
73 / 8\end{array}$ & $\begin{array}{l}31 / 4 \\
33 / 8 \\
35 / 8 \\
33 / 4\end{array}$ & $\begin{array}{l}23 / 16 \\
23 / 8 \\
21 / 2 \\
25 / 8\end{array}$ & $\begin{array}{l}27,8 \\
31 / 16 \\
31 / 4 \\
37 / 16\end{array}$ & $\begin{array}{l}3 / 4 \\
7 / 8 \\
7 / 8 \\
7 / 8\end{array}$ \\
\hline $\begin{array}{l}23 / 4 \\
27 / 8 \\
3 \\
31 / 8\end{array}$ & $\begin{array}{l}185 / 16 \\
191 / 8 \\
1915 / 16 \\
20^{13} / 16\end{array}$ & $\begin{array}{l}107 / 16 \\
11 \\
117 / 16 \\
11^{13} / 16\end{array}$ & $\begin{array}{l}73 / 4 \\
81 / 16 \\
87 / 16 \\
813 / 16\end{array}$ & $\begin{array}{l}315 / 16 \\
41 / 8 \\
45 / 16 \\
47 / 16\end{array}$ & $\begin{array}{l}2^{3} / 4 \\
2^{13} / 16 \\
2^{15} / 16 \\
31 / 8\end{array}$ & $\begin{array}{l}39 / 16 \\
33 / 4 \\
315 / 16 \\
41 / 16\end{array}$ & $\begin{array}{l}1 \\
1 \\
1 \\
11 / 8\end{array}$ \\
\hline $\begin{array}{l}31 / 4 \\
33 / 8 \\
31 / 2 \\
35 / 8\end{array}$ & $\begin{array}{l}219 / 16 \\
223 / 8 \\
231 / 4 \\
241 / 16\end{array}$ & $\begin{array}{l}127 / 16 \\
12^{13} / 16 \\
137 / 16 \\
13^{13} / 16\end{array}$ & $\begin{array}{c}91 / 8 \\
91 / 2 \\
915 / 16 \\
105 / 16\end{array}$ & $\begin{array}{l}4^{1} 1 / 16 \\
413 / 16 \\
51 / 16 \\
53 / 16\end{array}$ & $\begin{array}{l}33 / 16 \\
35 / 16 \\
31 / 2 \\
35 \% 8\end{array}$ & $\begin{array}{l}416 \\
45 / 16 \\
41 / 2 \\
411 / 16\end{array}$ & $\begin{array}{l}11 / 8 \\
11 / 8 \\
11 / 4 \\
11 / 4\end{array}$ \\
\hline $\begin{array}{l}33 / 4 \\
37 / 8 \\
4 \\
41 / 8\end{array}$ & $\begin{array}{l}247 / 8 \\
255 / 8 \\
267 / 16 \\
273 / 8\end{array}$ & $\begin{array}{l}141 / 4 \\
1411 / 16 \\
151 / 8 \\
15^{1} 1 / 16\end{array}$ & $\begin{array}{l}109 / 16 \\
1015 / 16 \\
115 / 16 \\
115 \% 8\end{array}$ & $\begin{array}{l}53 / 8 \\
59 / 16 \\
53 / 4 \\
515 / 16\end{array}$ & $\begin{array}{l}311 / 16 \\
313 / 16 \\
315 / 16 \\
41 / 16\end{array}$ & $\begin{array}{l}413 / 16 \\
5 \\
53 / 16 \\
55 / 16\end{array}$ & $\begin{array}{l}11 / 4 \\
11 / 4 \\
11 / 4 \\
11 / 4\end{array}$ \\
\hline
\end{tabular}


TABLE 2.-Bending shackles $F$ and shackle pins

\begin{tabular}{|c|c|c|c|c|c|c|c|}
\hline \multirow{2}{*}{$\begin{array}{l}\text { Size of } \\
\text { cable } \\
\text { chain }\end{array}$} & \multicolumn{7}{|c|}{ Bending shackles $\mathrm{F}$ and shackle pins } \\
\hline & $\mathrm{A}$ & $\mathrm{B}$ & $\mathrm{C}$ & $\mathrm{E}$ & $\mathbf{F}$ & $\mathrm{H}$ & $\mathrm{J}$ \\
\hline $\begin{array}{c}\text { Inches } \\
1 \\
118 \\
11 / 4 \\
13 / 8 \\
11 / 2\end{array}$ & $\begin{array}{c}\text { Inches } \\
815 / 16 \\
101 / 8 \\
111 / 4 \\
123 / 8 \\
131 / 2\end{array}$ & $\begin{array}{c}\text { Inches } \\
51 / 8 \\
55 / 8 \\
61 / 4 \\
67 / 8 \\
71 / 2\end{array}$ & $\begin{array}{c}\text { Inches } \\
35 / 16 \\
39 / 16 \\
4 \\
47 / 16 \\
47 / 8\end{array}$ & $\begin{array}{c}\text { Inches } \\
2 \\
21 / 4 \\
21 / 2 \\
23 / 4 \\
3\end{array}$ & $\begin{array}{c}\text { Inches } \\
11 / 8 \\
11 / 4 \\
13 / 8 \\
11 / 2 \\
15 / 8\end{array}$ & $\begin{array}{c}\text { Inches } \\
15 / 8 \\
13 / 4 \\
1^{15} / 16 \\
23 / 16 \\
23 / 8\end{array}$ & $\begin{array}{c}\text { Inches } \\
1 / 2 \\
1 / 2 \\
5 / 8 \\
5 / 8 \\
5 / 8\end{array}$ \\
\hline $\begin{array}{l}15 / 8 \\
13 / 4 \\
17 / 8 \\
2 \\
216\end{array}$ & $\begin{array}{l}141 / 2 \\
15^{5 / 8} \\
16^{13 / 16} \\
1715 / 16 \\
191 / 16\end{array}$ & $\begin{array}{l}8 \\
85 / 8 \\
91 / 4 \\
97 / 8 \\
101 / 2\end{array}$ & $\begin{array}{l}51 / 4 \\
5^{11 / 16} \\
6 \\
6^{7 / 116} \\
6^{7 / 8}\end{array}$ & $\begin{array}{l}31 / 4 \\
31 / 2 \\
33 / 4 \\
4 \\
41 / 4\end{array}$ & $\begin{array}{l}13 / 4 \\
17 / 8 \\
2 \\
21 / 8 \\
21 / 4\end{array}$ & $\begin{array}{l}2 \% / 16 \\
23 / 4 \\
215 / 16 \\
31 / 8 \\
31 / 2\end{array}$ & $\begin{array}{l}3 / 4 \\
3 / 4 \\
3 / 4 \\
7 / 8 \\
7 / 8\end{array}$ \\
\hline $\begin{array}{l}21 / 4 \\
23 / 3 \\
21 / 2 \\
25 / 8\end{array}$ & $\begin{array}{l}201 / 16 \\
213 / 8 \\
223 / 8 \\
231 / 2\end{array}$ & $\begin{array}{l}11 \\
113 / 4 \\
121 / 4 \\
127 / 8\end{array}$ & $\begin{array}{l}71 / 4 \\
75 / 8 \\
8 \\
87 / 16\end{array}$ & $\begin{array}{l}41 / 2 \\
43 / 4 \\
5 \\
51 / 4\end{array}$ & $\begin{array}{l}23 / 8 \\
21 / 2 \\
25 / 8 \\
23 / 4\end{array}$ & $\begin{array}{l}33 / 4 \\
4 \\
41 / 4 \\
41 / 2\end{array}$ & $\begin{array}{l}1^{7 / 8} \\
1\end{array}$ \\
\hline $\begin{array}{l}23 / 4 \\
27 / 8 \\
3 \\
31 / 8\end{array}$ & $\begin{array}{l}245 / 8 \\
255 / 8 \\
26^{13} / 16 \\
27^{15} / 16\end{array}$ & $\begin{array}{l}131 / 2 \\
14 \\
145 / 8 \\
151 / 4\end{array}$ & $\begin{array}{c}87 / 8 \\
91 / 4 \\
99 / 16 \\
10\end{array}$ & $\begin{array}{l}51 / 2 \\
53 / 4 \\
6 \\
61 / 4\end{array}$ & $\begin{array}{l}27 / 8 \\
3 \\
31 / 8 \\
31 / 4\end{array}$ & $\begin{array}{l}43 / 4 \\
5 \\
51 / 4 \\
51 / 2\end{array}$ & $\begin{array}{l}11 / 8 \\
11 / 8 \\
118 \\
11 / 4\end{array}$ \\
\hline $\begin{array}{l}31 / 4 \\
33 / 8 \\
31 / 2 \\
35 / 8\end{array}$ & $\begin{array}{l}291 / 16 \\
303 / 16 \\
313 / 16 \\
323 / 8\end{array}$ & $\begin{array}{l}157 / 8 \\
16^{3 / 8} \\
1718 \\
1711 / 16\end{array}$ & $\begin{array}{l}10^{7} / 16 \\
10^{13} / 16 \\
11^{5} / 16 \\
11^{1} 1 / 16\end{array}$ & $\begin{array}{l}61 / 2 \\
63 / 4 \\
7 \\
71 / 4\end{array}$ & $\begin{array}{l}33 / 8 \\
31 / 2 \\
35 \% 8 \\
33 / 4\end{array}$ & $\begin{array}{l}53 / 4 \\
61 / 8 \\
63 / 8 \\
69 / 16\end{array}$ & $\begin{array}{l}11 / 4 \\
11 / 4 \\
11 / 4 \\
11 / 4\end{array}$ \\
\hline $\begin{array}{l}33 / 4 \\
37 / 8 \\
4 \\
41 / 8\end{array}$ & $\begin{array}{l}331 / 2 \\
34^{11} 116 \\
35^{13} / 16 \\
36^{15} / 16\end{array}$ & $\begin{array}{l}181 / 4 \\
187 / 8 \\
197 / 16 \\
201 / 16\end{array}$ & $\begin{array}{l}12 \\
12^{7} / 16 \\
12^{13} / 16 \\
135 / 16\end{array}$ & $\begin{array}{l}71 / 2 \\
73 / 4 \\
8 \\
81 / 4\end{array}$ & $\begin{array}{l}37 / 8 \\
4 \\
41 / 8 \\
41 / 4\end{array}$ & $\begin{array}{l}63 / 4 \\
7 \\
71 / 4 \\
77 / 16\end{array}$ & $\begin{array}{l}11 / 4 \\
11 / 4 \\
11 / 4 \\
11 / 4\end{array}$ \\
\hline
\end{tabular}

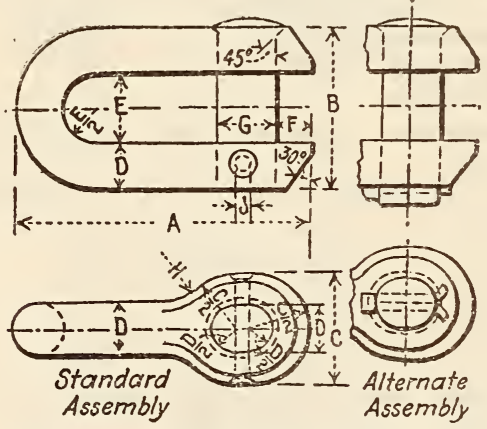

FIgURe 104.-Bending shackles $F$ and shackle pins

TABLE 3.-Swivels (dimensions)

\begin{tabular}{|c|c|c|c|c|c|c|}
\hline \multirow{2}{*}{$\begin{array}{l}\text { Size of } \\
\text { chain } \\
\text { cable }\end{array}$} & \multicolumn{6}{|c|}{ Swivels } \\
\hline & A & B & C & $\mathrm{D}$ & $\mathrm{E}$ & $\mathrm{F}$ \\
\hline $\begin{array}{c}\text { Inches } \\
1 \\
118 \\
11 / 4 \\
13 / 8 \\
11 / 2\end{array}$ & $\begin{array}{c}\text { Inches } \\
77 / 8 \\
77 / 8 \\
111 / 4 \\
111 / 4 \\
125 / 8\end{array}$ & $\begin{array}{c}\text { Inches } \\
45 / 8 \\
51 / 8 \\
55 / 8 \\
618 \\
65 \%\end{array}$ & $\begin{array}{c}\text { Inches } \\
23 / 32 \\
25 / 32 \\
27 / 32 \\
7 / 8 \\
15 / 16\end{array}$ & $\begin{array}{c}\text { Inches } \\
13 / 16 \\
15 / 16 \\
11 / 2 \\
15 \% \\
13 / 4\end{array}$ & $\begin{array}{c}\text { Inches } \\
13 / 4 \\
17 / 8 \\
2168 \\
238 \\
21 / 2\end{array}$ & $\begin{array}{c}\text { Inches } \\
3 / 8 \\
3 / 8 \\
3 / 8 \\
3 / 8 \\
3 / 8\end{array}$ \\
\hline $\begin{array}{l}15 / 8 \\
13 / 4 \\
17 / 8 \\
2 \\
21 / 8\end{array}$ & $\begin{array}{l}125 / 8 \\
15 \\
15 \\
15 \\
15\end{array}$ & $\begin{array}{l}71 / 8 \\
71 / 2 \\
8 \\
81 / 2 \\
9\end{array}$ & $\begin{array}{l}1 \\
11 / 16 \\
118 \\
11 / 4 \\
15 / 16\end{array}$ & $\begin{array}{l}17 / 8 \\
2 \\
21 / 8 \\
21 / 4 \\
23 / 8\end{array}$ & $\begin{array}{l}25 / 8 \\
23 / 4 \\
27 / 8 \\
318 \\
31 / 4\end{array}$ & $\begin{array}{l}1 / 2 \\
1 / 2 \\
1 / 2 \\
5 / 8 \\
5 / 8\end{array}$ \\
\hline $\begin{array}{l}21 / 4 \\
23 / 8 \\
21 / 2 \\
25 / 8\end{array}$ & $\begin{array}{l}181 / 4 \\
181 / 4 \\
181 / 4 \\
181 / 4\end{array}$ & $\begin{array}{l}91 / 2 \\
10 \\
101 / 2 \\
11\end{array}$ & $\begin{array}{l}13 / 8 \\
17 / 16 \\
11 / 2 \\
19 / 16\end{array}$ & $\begin{array}{l}21 / 2 \\
25 / 8 \\
23 / 4 \\
27 / 8\end{array}$ & $\begin{array}{l}33 / 8 \\
31 / 2 \\
35 / 8 \\
33 / 4\end{array}$ & $\begin{array}{l}5 / 8 \\
3 / 4 \\
3 / 4 \\
3 / 4\end{array}$ \\
\hline $\begin{array}{l}23 / 4 \\
27 / 8 \\
3 \\
31 / 8\end{array}$ & $\begin{array}{l}213 / 8 \\
213 / 8 \\
213 / 8 \\
213 / 8\end{array}$ & $\begin{array}{l}111 / 2 \\
12 \\
121 / 2 \\
13\end{array}$ & $\begin{array}{l}15 / 8 \\
111 / 16 \\
113 / 16 \\
17 / 8\end{array}$ & $\begin{array}{l}3 \\
31 / 8 \\
33 / 8 \\
31 / 2\end{array}$ & $\begin{array}{l}37 / 8 \\
4 \\
41 / 4 \\
43 / 8\end{array}$ & $\begin{array}{l}7 / 8 \\
7 / 8 \\
1^{7 / 8}\end{array}$ \\
\hline $\begin{array}{l}31 / 4 \\
33 / 8 \\
31 / 2 \\
35 / 8\end{array}$ & $\begin{array}{l}241 / 2 \\
241 / 2 \\
241 / 2 \\
241 / 2\end{array}$ & $\begin{array}{l}131 / 2 \\
14 \\
141 / 2 \\
15\end{array}$ & $\begin{array}{l}115 / 16 \\
2 \\
21 / 16 \\
21 / 8\end{array}$ & $\begin{array}{l}35 / 8 \\
33 / 4 \\
37 / 8 \\
4\end{array}$ & $\begin{array}{l}41 / 2 \\
45 / 8 \\
43 / 1 \\
47 / 8\end{array}$ & $\begin{array}{l}1 \\
1 \\
11 / 6 \\
11 / 8\end{array}$ \\
\hline $\begin{array}{l}33 / 4 \\
37 / 8 \\
4 \\
41 / 8\end{array}$ & $\begin{array}{l}273 / 4 \\
273 / 4 \\
273 / 4 \\
273 / 4\end{array}$ & $\begin{array}{l}151 / 2 \\
16 \\
161 / 2 \\
17\end{array}$ & $\begin{array}{l}23 / 16 \\
21 / 4 \\
23 / 8 \\
27 / 16\end{array}$ & $\begin{array}{l}41 / 8 \\
41 / 4 \\
43 / 8 \\
41 / 2\end{array}$ & $\begin{array}{l}5 \\
51 / 8 \\
53 / 8 \\
51 / 2\end{array}$ & $\begin{array}{l}11 / 8 \\
11 / 8 \\
11 / 8 \\
11 / 8\end{array}$ \\
\hline
\end{tabular}
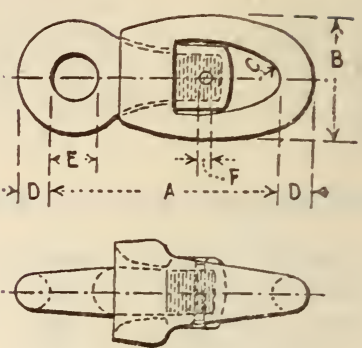

FiguRe 105.-Swivels

\section{UNITED STATES GOVERNMENT, FEDERAL SPECIFICATIONS BOARD, FEDERAL SPECIFICATION NO. 171A, FOR SHIP CHAIN, MAY 22, 1926. (CURRENT DES- IGNATION : RR-C-251)}

\section{SHACKLES AND SWIVELS}

(For general specifications, general requirements, material and workmanship, and methods of inspection see F. S. B. RR-C-271, (603.50, p. 199). For detail requirements and methods of test see F. S. B. No. $171 \mathrm{a}$ (RR-C-251), (603.53, p. 208).)

(The dimensions of shackles $\mathrm{D}$ and pins are the same as those of the American Bureau of Shipping, shown above in Table 1 and Figure 101. The breaking and proof tests of class $\mathrm{C}$ shackles D and pins are the same as those of the American Bureau of Shipping wrought iron and steel chain, as shown in comparative table facing p. 200. The breaking and proof tests of class A and class B shackles D and pins do not agree with the A. B. S. tests, but are as shown in comparative table facing p. 200.) (See also 603.53.)

1. No connection shackles, bending shackles, or swivels shall be furnished with chain ordered under this specification unless they are specifically called for. Shackles when ordered shall be in accordance with A. B. S. Table 1, above. When ordered, swivels shall be in accordance with design and dimensions to be submitted or approved by the purchaser.

2. Material.-When ordered, the shackles and swivels shall be drop-forged from the same grade of steel as is specified for use in the manufacture of class B chain, unless iron is specified, in which case the iron shall be of the same grade as the chain with which they are intended to be used.

3. Manufacture.-The wrought iron in all swivels and shackles shall be so worked that the strain, when they are in use, shall be across the fibers in shear and with the fibers in tension. All eves and loops shall be formed by wrapping and welding the iron. The use of punched eyes will not be permitted except in forged-steel shackles. Shackles or swivels which are cold bent shall be annealed after fabrication or forging.

4. Heat treatment.-All class A chain shall be annealed except as stated in footnote in comparative table facing page 200. Annealing shall be done by a method approved by the purchaser. It shall consist of heating the chain to $950^{\circ} \mathrm{C}$., holding at that temperature for 10 minutes, and air cooling.

5. Arrangement of links and shackles.-Unless otherwise specified, chain shall be in 15-fathom lengths. There shall be an odd number of links in a shot or length of chain. The end links, also the swivels and shackles when ordered, and the shots of chain in complete cables, shall be arranged in accordance with A. B. S. 603.53, (fig. 80). The enlarged 
end links may be omitted from the shots of chain at the option of the purchaser.

6. Test of shackles, swivels, etc.-Shackles, swivels, etc., shall be proof tested to the same proof test as the chain with which they are intended to be used, and not less than 1 out of every 25 or less shall be subjected to a tensile breaking test equal at least to specified breaking strength of the chain with which they are intended to be used. The shackles so tested for breaking strength shall, after test, be discarded.

7. Tolerances.-(The tolerances are identical with those shown in A. B. S. Table 2. See 603.53, p. 206.)

UNITED STATES GOVERNMENT, FEDERAL SPECIFICATIONS BOARD, FEDERAL SPECIFICATION RR-C-271, FOR STANDARD MISCELLANEOUS CHAIN AND ATTACHMENTS, DECEMBER 8, 1931.

\section{ATTACHMENTS}

(For general specifications, general requirements, material and workmanship, detail requirements, and methods of inspection and tests, see F. S. B., RR-C-271, (603.50, p. 199.) (See also 603.51, 603.52, $603.53,603.54,603.56,617.5,631.9,642.9,645.9$, 646.59 .

1. The attachments shall be of the following types:
A. Chain dogs.
B. Ring dogs.
C. Hooks.
D. Connecting links.
E. Repair or end lap links.
F. Oval side lap links.
G. Shackles.
H. Rings.
I. Toggles.
J. Swivels.

K. Sash.

(a) Chain dogs shall be of the following classes-

(1) Plain point.

(2) Diamond point.

(b) Ring dogs shall be of the following classes-

(1) Diamond point.

(2) Norfolk pattern.

(3) Plain.

(c) Hooks shall be of the following classes-

(1) Vehicle and harness hooks.

(2) Slip hooks.

(3) Grab hooks.

(4) Hoist or sling hooks.

(5) Bale hooks.

(6) Box hooks.

(7) Barrel hooks.

(8) Stone-hoist hooks.

(9) Hogshead hooks.

(10) Can hooks.

(d) Connecting links shall be of but one class.

(e) Repair or end lap links shall be of but one class.

(f) Oval-side lap links shall be as covered by table, page 220 .

(g) Shackles shall be of the following classes:

(1) Screw-pin anchor shackle.

(2) Screw-pin chain shackle.

(3) Oval-pin anchor shackle

(4) Oval-pin chain shackle.

(5) Round-pin anchor shackle.

(b) Round-pin chain shackle.
K. Sash-Continued.

(h) Rings shall be of but one class.

(i) Toggles shall be of the following classes:

(1) Sheared toggles.

(2) Forged toggles.

(j) Swivels shall be of but one class.

(k) Sash attachments shall be as covered by sketches. (See F. S. B., RR-C271, (figs. $981-983)(617.5$, p. 1049).)

2. Chain attachments shall be of material as hereinafter specified. When a drop-forged steel piece is specified, the steel shall be of class 2 , as ordered, depending on the chain with which the attachments are to be used. If the chain is class 1 wrought iron, the attachments shall be of class 2 steel.

3. The chain and attachments shall be free from injurious defects and shall have a workmanlike finish.

4. Standard chain attachments shall be subjected to the same proof-test load as the chain with which they are to be used. One attachment out of every 50 or less to be furnished under a contract or order shall be selected for test on quantities of 100 or less and 1 for each additional 200 or less. If any of these fails or shows defective welds the total number of test samples shall be doubled and a second failure will result in rejection of the lot. (For breaking and proof-test loads see comparative table facing p. 200.)

\section{CHAIN DOGS}

5. Chain dogs shall be of steel, grade 2, as specified. The types shall be in accordance with the following table. Forms of rings shall be in accodance with the sketches. When ordering chain dogs, the number of links between dogs shall be as specified.

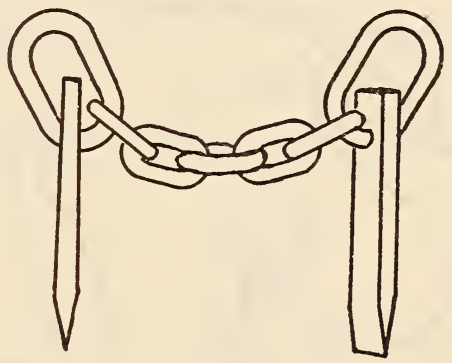

Class 1

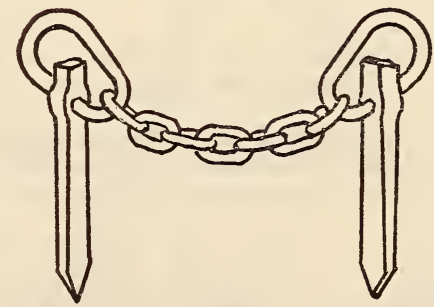

Class 2

Figdre 106.-Chain dogs

TABLE 1.-Chain dogs (dimensions)

\begin{tabular}{|c|c|c|c|c|}
\hline \multirow{2}{*}{ Class } & \multirow{2}{*}{ Style } & \multirow{2}{*}{ Dimensions } & \multicolumn{2}{|c|}{$\begin{array}{l}\text { Diameter of } \\
\text { chain }\end{array}$} \\
\hline & & & $\begin{array}{l}\text { Center } \\
\text { links }\end{array}$ & $\begin{array}{l}\text { End } \\
\text { links }\end{array}$ \\
\hline $\begin{array}{l}1 \\
2\end{array}$ & $\begin{array}{l}\text { Plain point } \\
\text { Diamond point... }\end{array}$ & $\begin{array}{c}\text { Inches } \\
3 / 8 \text { by } 11 / 8 \text { by } 61 / 2 \\
1 / 2 \cdot \text { by } 11 / 2 \text { by } 61 / 2\end{array}$ & $\begin{array}{l}\text { Inch } \\
11 / 32 \\
13 / 32\end{array}$ & $\begin{array}{l}\text { Inch } \\
13 / 32 \\
15 / 32\end{array}$ \\
\hline
\end{tabular}




\section{RING DOGS}

6. Ring dogs shall be of steel, grade 2, as specified. The types shall be in accordance with the following table. Forms of rings shall be in accordance with the sketches.

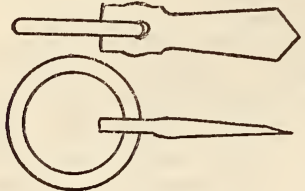

Class 1

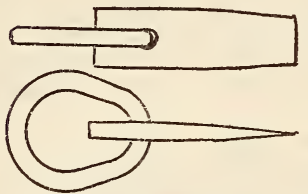

Class 2

Figure 107.-Ring dogs

TABLE 2.-Ring dogs (dimensions)

\begin{tabular}{|c|c|c|c|c|}
\hline Class & Pattern & Dimensions of dog & $\begin{array}{l}\text { Ring, } \\
\text { diam- } \\
\text { eter of } \\
\text { metal }\end{array}$ & $\begin{array}{l}\text { Ring, } \\
\text { inside } \\
\text { diam- } \\
\text { eter }\end{array}$ \\
\hline $\begin{array}{l}1 \\
2 \\
3\end{array}$ & $\begin{array}{l}\text { Diamond point } \\
\text { Norfolk pattern } \\
\text { Plain }\end{array}$ & $\begin{array}{l}\text { Inches } \\
1 / 2 \text { by } 11 / 2 \text { by } 61 / 2 \\
1 / 2 \text { by } 2 \text { by } 61 / 2 \\
3 / 8 \text { by } 11 / 2 \text { by } 61 / 2\end{array}$ & $\begin{array}{r}\text { Inch } \\
1 / 2 \\
9 / 16 \\
1 / 2\end{array}$ & $\begin{array}{r}\text { Inches } \\
3 \text { by } 4 \\
3\end{array}$ \\
\hline
\end{tabular}

\section{ноО KS}

7. Vehicle and harness hooks.

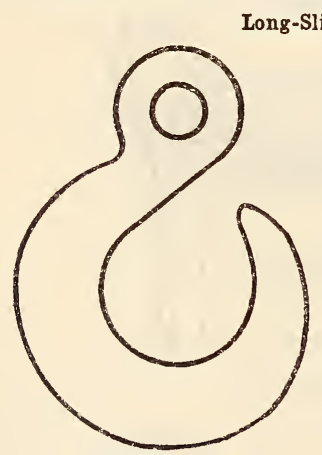

With direct eye

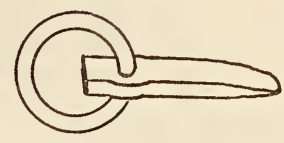

Class 3
With reverse eye

Figure 108.-Slip hooks

TABLE 3.-Drop-forged steel, long-slip pattern, for stay chains (dimensions and weights)

\begin{tabular}{|c|c|c|c|c|}
\hline & \multicolumn{4}{|c|}{ Size } \\
\hline & 1 & 2 & 3 & 4 \\
\hline $\begin{array}{l}\text { Approximate length over } \\
\text { all } \\
\text { Depth of throat (inside) } \\
\text { Opening of throat } \\
\text { Width of throat at bottom. } \\
\text { Size of metal at shank. } \\
\text { Size of metal at throat... } \\
\text { Size of chain. }\end{array}$ & \begin{tabular}{|r|} 
Inches \\
$31 / 8$ \\
$15 / 8$ \\
$1 / 2$ \\
$11 / 16$ \\
\\
$5 / 16$ by $7 / 16$ \\
$3 / 8$ by $1 / 2$ \\
$1 / 4$
\end{tabular} & $\begin{array}{l}\text { Inches } \\
31 / 2 \\
17 / 8 \\
9 / 16 \\
3 / 4 \\
3 / 8 \text { by } 1 / 2 \\
1 / 2 \text { by } 9 / 16 \\
5 / 16\end{array}$ & \begin{tabular}{|c|c|} 
Inches \\
$37 / 8$ \\
2 \\
$3 / 4$ \\
$7 / 8$ \\
$7 / 16$ by $9 / 16$ \\
$1 / 2$ by $5 / 8$ \\
$3 / 8$
\end{tabular} & \begin{tabular}{|r} 
Inches \\
$41 / 2$ \\
$23 / 4$ \\
$7 / 8$ \\
$13 / 16$ \\
$7 / 16$ by $9 / 16$ \\
$1 / 2$ by $5 / 8$ \\
$11 / 32$
\end{tabular} \\
\hline $\begin{array}{l}\text { Approximate weight per } \\
100 \text { hooks... }\end{array}$ & $\begin{array}{r}\text { Pounds } \\
20\end{array}$ & $\begin{array}{r}\text { Pounds } \\
28\end{array}$ & $\begin{array}{r}\text { Pounds } \\
35\end{array}$ & ${ }_{60}^{\text {Pounds }}$ \\
\hline
\end{tabular}

Slip Hooks

8. Slip hooks shall be of drop-forged steel. The eyes shall be parallel to hook, or shall be at angle to it, as specified.

TABLE 4.-Drop-forged steel slip hooks (dimensions and weights)

\begin{tabular}{|c|c||c|c|}
\hline $\begin{array}{c}\text { Size of } \\
\text { chain }\end{array}$ & $\begin{array}{c}\text { Approxi- } \\
\text { mate } \\
\text { weight } \\
\text { each }\end{array}$ & $\begin{array}{c}\text { Size of } \\
\text { chain }\end{array}$ & $\begin{array}{c}\text { Approxi- } \\
\text { mate } \\
\text { weight } \\
\text { each }\end{array}$ \\
\hline & & & \\
Inch & Pounds & Inch & Pounds \\
$1 / 4$ & 0.4 & $1 / 2$ & 1.8 \\
$5 / 16$ & .5 & $5 / 8$ & 2.8 \\
$3 / 8$ & .8 & $3 / 4$ & 3.5 \\
$7 / 16$ & 1.3 & & \\
\hline
\end{tabular}

Grab Hooks

9. Grab hooks shall be of drop-forged steel with eyes parallel to hooks or at an angle to them, as specified.
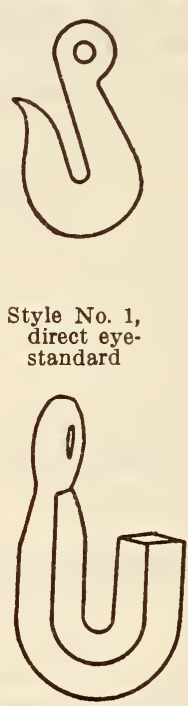

Style No. 4, reverse eyesquare

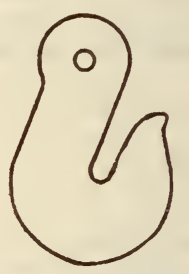

Style No. 2, direct eyeflat

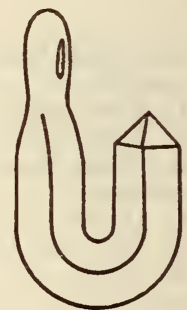

Style No. 5, reverse eyesquare

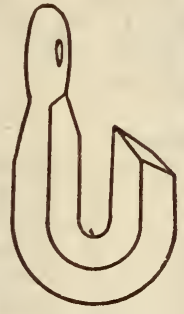

Style No. 3, reverse eye square

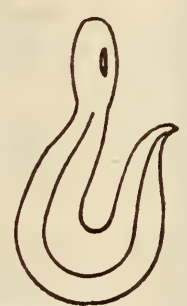

Style No. 6 reverse eye
FiguRe 109.-Grab hooks

TABLE 5.-Various styles of grab hooks (dimensions and weights)

\begin{tabular}{|c|c||c|c|}
\hline $\begin{array}{c}\text { Size of } \\
\text { chain }\end{array}$ & $\begin{array}{c}\text { Approxi- } \\
\text { mate } \\
\text { weight } \\
\text { each }\end{array}$ & $\begin{array}{c}\text { Size of } \\
\text { chain }\end{array}$ & $\begin{array}{c}\text { Approxi- } \\
\text { mate } \\
\text { weight } \\
\text { each }\end{array}$ \\
\hline Inch & $\begin{array}{c}\text { Pounds } \\
1 / 4\end{array}$ & $\begin{array}{c}\text { Inch } \\
5 / 16\end{array}$ & $\begin{array}{c}\text { Pounds } \\
1.8 \\
3 / 8\end{array}$ \\
$7 / 16$ & .5 & $5 / 2$ & 2.8 \\
& 1.3 & $3 / 4$ & 4.2 \\
\hline
\end{tabular}


Hoist or Sling Hooks

10. Hoist or sling hooks shall be of drop-forged steel with eyes parallel to hooks.

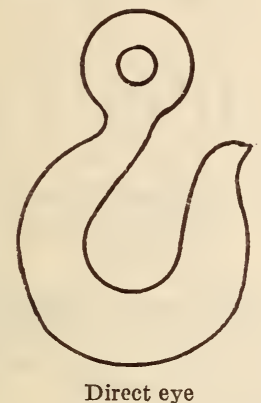

FIgURE 110.-Hoist or sling hook

TABLE 6.-Hoists or sling hooks (dimensions and weights)

\begin{tabular}{|c|c|c|c|}
\hline $\begin{array}{l}\text { Size of } \\
\text { chain }\end{array}$ & $\begin{array}{l}\text { Approxi- } \\
\text { mate } \\
\text { weight } \\
\text { each }\end{array}$ & $\begin{array}{l}\text { Size of } \\
\text { chain }\end{array}$ & $\begin{array}{l}\text { Approxi- } \\
\text { mate } \\
\text { weight } \\
\text { each }\end{array}$ \\
\hline $\begin{array}{c}\text { Inches } \\
1 / 4 \\
5116 \\
318 \\
7 / 16 \\
1 / 2 \\
5 / 8 \\
34\end{array}$ & $\begin{array}{r}\text { Pounds } \\
0.4 \\
.7 \\
1.5 \\
2.8 \\
4.5 \\
7.3 \\
15.0\end{array}$ & $\begin{array}{c}\text { Inches } \\
7 / 8 \\
1 \\
11 / 8 \\
11 / 4 \\
11 / 2 \\
15 / 8 \\
13 / 4\end{array}$ & $\begin{array}{c}\text { Pounds } \\
27.0 \\
35.0 \\
45.0 \\
58.0 \\
\end{array}$ \\
\hline
\end{tabular}

Bale and Box Hooks

11. Bale hooks and box hooks shall be of class 2 steel unless otherwise specified.
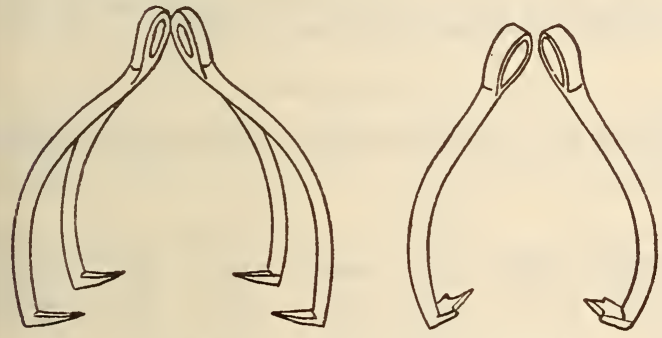

Figure 111.-Bale and box hooks

TABLE 7.-Bale and box hooks (dimensions)

\begin{tabular}{|c|c|c|c|}
\hline Bale hooks & Size & Box hooks & Size \\
\hline $\begin{array}{r}\text { Diameter of iror } \\
\text { Do } \\
\text { Do } \\
\text { Do }\end{array}$ & $\begin{array}{c}\text { Inch } \\
1 / 2 \\
5 / 8 \\
3 / 4 \\
7 / 8\end{array}$ & $\begin{array}{l}\text { Diameter of iron } \\
\text { do }\end{array}$ & $\begin{array}{c}\text { Inch } \\
1 / 2 \\
5 / 8 \\
3 / 4\end{array}$ \\
\hline
\end{tabular}

Barrel and Stone-Hoist Hooks

12. Barrel hooks and stone-hoist hooks shall be of class 2 steel unless otherwise specified.
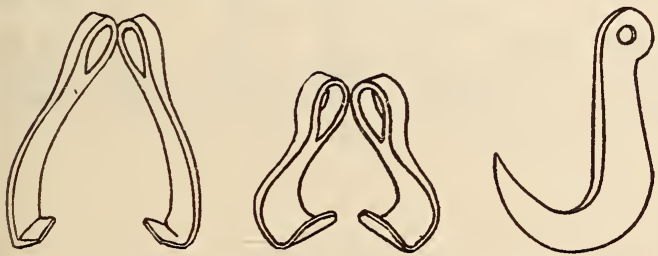

FIGURE 112.-Barrel and stone hoist hooks
TABLE 8.-Barrel and stone-hoist hooks (dimensions)

\begin{tabular}{|c|c|c|}
\hline $\begin{array}{l}\text { Barrel } \\
\text { hooks } \\
\text { (size) }\end{array}$ & $\begin{array}{l}\text { Barrel } \\
\text { hooks } \\
\text { (size) }\end{array}$ & $\begin{array}{l}\text { Stone-hoist } \\
\text { hooks (size) }\end{array}$ \\
\hline Large & Small & \\
\hline $\begin{array}{c}\text { Inches } \\
61 / 2 \\
101 / 4\end{array}$ & $\begin{array}{c}\text { Inches } \\
61 / 2 \\
101 / 4\end{array}$ & $\begin{array}{c}\text { Inches } \\
1 \text { by } 4 \\
1 / 2 \text { by } 21 / 2 \\
1 \text { by } 3 \\
2 \text { by } 4 \\
2 \text { by } 5\end{array}$ \\
\hline
\end{tabular}

Hogshead Hooks

13. Hogshead hooks shall be of class 2 steel.

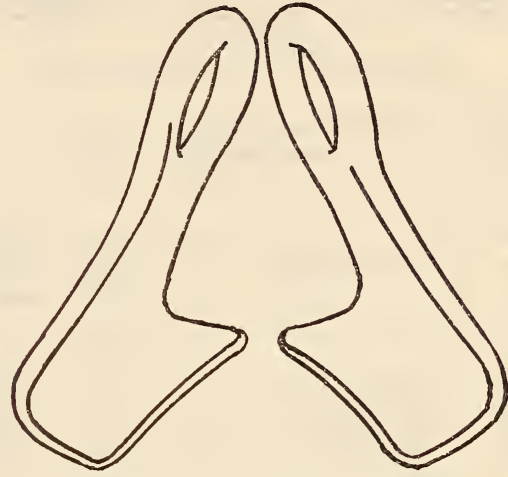

FIgURE 113.-Hogshead hooks

Hogshead hooks (dimensions)

Inches

Width at bend

$-91 / 2$

Can Hooks

14. Can hooks shall be made of class 2 steel.

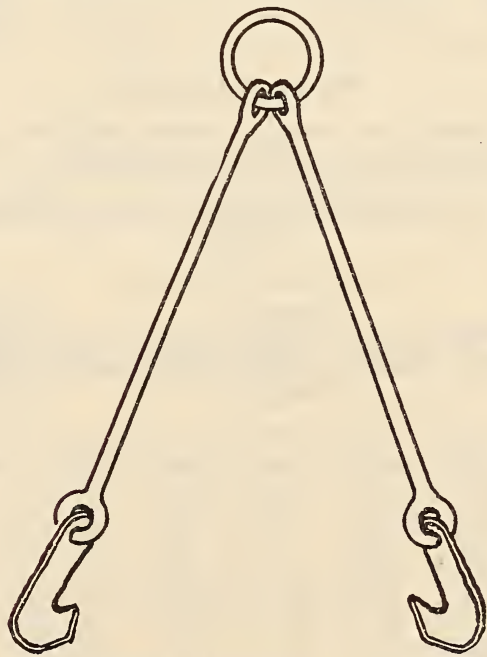

Figure 114.-Can hooks

TABLE 9.-Can hooks (dimensions)

\begin{tabular}{|c|c|c|c|}
\hline \multicolumn{2}{|l|}{ Can hooks } & \multicolumn{2}{|l|}{ Can hooks } \\
\hline $\begin{array}{r}\text { Diameter of iron.... } \\
\text { Do } \\
\text { Do } \\
\text { Do }\end{array}$ & $\begin{array}{l}\text { Inch } \\
3 / 8 \\
7 / 16 \\
1 / 2 \\
5 / 8\end{array}$ & $\begin{array}{r}\text { Diameter of iron.... } \\
\text { Do } \\
\text { Do }\end{array}$ & $\begin{array}{l}\text { Inch } \\
3 / 4 \\
1 / 8 \\
1\end{array}$ \\
\hline
\end{tabular}




\section{Connecting Links}

15. Drop-forged connecting links shall be made of class 2 steel.

16. Finish shall be plain or hot-galvanized as specified.

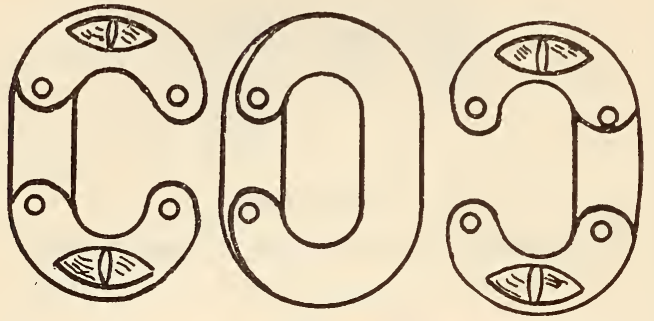

Figdre 115.-Connecting links

TABLE 10.-Connecting links (dimensions, strength, and weights)

\begin{tabular}{|c|c|c|c|}
\hline Size & $\begin{array}{l}\text { Outside dimension of links } \\
\text { (in inches) }\end{array}$ & $\begin{array}{l}\text { Weight } \\
\text { per } \\
\text { dozen }\end{array}$ & $\begin{array}{l}\text { Approxi- } \\
\text { mate } \\
\text { tensile } \\
\text { strength }\end{array}$ \\
\hline $\begin{array}{l}\text { Inches } \\
114 \\
5 / 16 \\
3 / 8 \\
7 / 16\end{array}$ & $\begin{array}{l}11 / 2 \text { by } 1 \\
111 / 16 \text { by } 13 / 16 \\
21 / 16 \text { by } 13 / 8 \\
23 / 8 \text { by } 13 / 2\end{array}$ & $\begin{array}{l}\text { Pounds } \\
\quad 3 / 4 \\
1 \\
2 \\
31 / 8\end{array}$ & $\begin{array}{r}\text { Pounds } \\
5,580 \\
8,300 \\
11,200 \\
13,980\end{array}$ \\
\hline $\begin{array}{l}1 / 2 \\
9 / 16 \\
5 / 8 \\
1116\end{array}$ & $\begin{array}{l}25 / 8 \text { by } 13 / 4 \ldots \\
3 \text { by } 115 / 16 \\
3516 \text { by } 23 / 16 \\
31 / 2 \text { by } 25 / 16\end{array}$ & $\begin{array}{c}41 / 2 \\
65 / 8 \\
9 \\
11\end{array}$ & $\begin{array}{l}15,900 \\
19,200 \\
23,900 \\
27,300\end{array}$ \\
\hline $\begin{array}{l}3 / 4 \\
7 / 8 \\
1^{718}\end{array}$ & $\begin{array}{l}37 / 8 \text { by } 21 / 2 \\
41 / 2 \text { by } 215 / 16 \\
5 \text { by } 31 / 4 \\
55 / 8 \text { by } 31 / 2\end{array}$ & $\begin{array}{l}141 / 2 \\
201 / 4 \\
29 \\
40\end{array}$ & $\begin{array}{l}34,000 \\
38,600 \\
59,900 \\
70,800\end{array}$ \\
\hline $\begin{array}{l}11 / 4 \\
138 \\
11 / 2 \\
158\end{array}$ & $\begin{array}{l}61 / 4 \text { by } 4 \\
63 / 4 \text { by } 43 / 44 \\
71 / 4 \text { by } 518 \\
8 \text { by } 512\end{array}$ & $\begin{array}{l}531 / 2 \\
75 \\
103 \\
131\end{array}$ & $\begin{array}{l}103,100 \\
112,800 \\
128,000 \\
200,000\end{array}$ \\
\hline
\end{tabular}

Repair or End Lap Links

17. Repair or end lap links shall be made of class 2 steel unless otherwise specified.

TABLE 11.-Repair or end lap links (dimensions and weights)

\begin{tabular}{|c|c|c|c|c|c|}
\hline $\begin{array}{l}\text { Diam- } \\
\text { eter of } \\
\text { metal }\end{array}$ & $\begin{array}{l}\text { Length } \\
\text { inside }\end{array}$ & $\begin{array}{c}\text { Approxi- } \\
\text { mate } \\
\text { weight } \\
\text { per } 100 \\
\text { links }\end{array}$ & $\begin{array}{l}\text { Diam- } \\
\text { eter of } \\
\text { metal }\end{array}$ & $\begin{array}{l}\text { Length } \\
\text { inside }\end{array}$ & $\begin{array}{l}\text { Approxi- } \\
\text { mate } \\
\text { weight } \\
\text { per } 100 \\
\text { links }\end{array}$ \\
\hline $\begin{array}{c}\text { Inch } \\
3 / 16 \\
7 / 32 \\
1 / 4 \\
9 / 32 \\
5 / 16 \\
38 \\
1 / 4 \\
1 / 4 \\
9 / 32 \\
9 / 32 \\
5 / 16 \\
5 / 16 \\
5 / 16 \\
3 / 8\end{array}$ & $\begin{array}{c}\text { Inches } \\
11 / 4 \\
11 / 4 \\
11 / 4 \\
11 / 4 \\
11 / 2 \\
13 / 4 \\
2 \\
3 \\
2 \\
21 / 2 \\
2 \\
21 / 2 \\
3 \\
2\end{array}$ & $\begin{array}{c}\text { Pounds } \\
5 \\
5.25 \\
6 \\
7.5 \\
11 \\
19 \\
9 \\
11 \\
11 \\
12.5 \\
13 \\
15 \\
17.5 \\
21.5\end{array}$ & $\begin{array}{c}\text { Inch } \\
3 / 8 \\
3 / 8 \\
7 / 16 \\
7 / 16 \\
7 / 16 \\
7 / 16 \\
1 / 2 \\
1 / 2 \\
1 / 2 \\
1 / 2 \\
9 / 16 \\
9 / 16 \\
5 / 8 \\
5 / 8\end{array}$ & $\begin{array}{c}\text { Inches } \\
21 / 2 \\
3 \\
13 / 4 \\
2 \\
21 / 2 \\
3 \\
2 \\
21 / 2 \\
3 \\
4 \\
21 / 2 \\
3 \\
21 / 2 \\
3\end{array}$ & $\begin{array}{c}\text { Pounds } \\
26 \\
31 \\
28 \\
31 \\
34 \\
40 \\
42 \\
50 \\
56 \\
65 \\
69 \\
76 \\
82 \\
90\end{array}$ \\
\hline
\end{tabular}

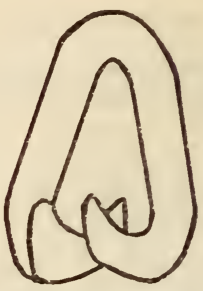

Figure 116 . Repair or end lap links

\section{OVAL SIDE LAP LINKS}

19. Oval side lap links shall be made of class 2 steel unless otherwise specified.

TABLE 13.-Oval side lap links (dimensions and weights)

\begin{tabular}{|c|c|c||c|c|c|}
\hline $\begin{array}{c}\text { Size of } \\
\text { metal }\end{array}$ & $\begin{array}{c}\text { Length } \\
\text { inside }\end{array}$ & $\begin{array}{c}\text { Approxi- } \\
\text { mate } \\
\text { weight } \\
\text { per 100 } \\
\text { rings }\end{array}$ & $\begin{array}{c}\text { Size of } \\
\text { metal }\end{array}$ & $\begin{array}{c}\text { Length } \\
\text { inside }\end{array}$ & $\begin{array}{c}\text { Approxi- } \\
\text { mate } \\
\text { weight } \\
\text { per 100 } \\
\text { rings }\end{array}$ \\
\hline Inch & Inches & Pounds & Inch & Inches & Pounds \\
$5 / 16$ & $11 / 2$ & 11 & $1 / 2$ & $21 / 2$ & 46 \\
$5 / 16$ & 2 & 12 & $1 / 2$ & 3 & 50 \\
$3 / 8$ & $13 / 4$ & 18 & $9 / 16$ & $21 / 2$ & 63 \\
$3 / 8$ & 2 & 19 & $9 / 16$ & 3 & 69 \\
318 & $21 / 2$ & 24 & $5 / 8$ & $21 / 2$ & 73 \\
$7 / 16$ & 2 & 28 & $5 / 8$ & 3 & 80 \\
$7 / 16$ & $21 / 2$ & 31 & $3 / 4$ & 3 & 130 \\
15 & 2 & 38 & & & \\
\hline
\end{tabular}

\section{Anchor Shackles}

20. Screw-pin anchor shackles shall be of dropforged steel unless otherwise specified.

\section{SHACKLES}

TABLE 14.-Anchor shackles (dimensions and weights)

\begin{tabular}{|c|c|c|c|c|c|c|}
\hline $\begin{array}{c}\text { Size (di- } \\
\text { ameter } \\
\text { (D)) } \\
\text { (mini- } \\
\text { mum) }\end{array}$ & $\begin{array}{l}\text { Length } \\
\text { inside } \\
\text { (L) }\end{array}$ & $\begin{array}{l}\text { Toler- } \\
\text { ance } \\
\text { (plus or } \\
\text { minus) } \\
\text { in inside } \\
\text { length } \\
\text { (L) }\end{array}$ & $\begin{array}{l}\text { Width } \\
\text { between } \\
\text { eyes } \\
\text { (W) }\end{array}$ & $\begin{array}{l}\text { Tolerance } \\
\text { (plus or } \\
\text { minus) in } \\
\text { width } \\
\text { between } \\
\text { eyes (W) }\end{array}$ & $\begin{array}{l}\text { Diam- } \\
\text { eter of } \\
\text { pin (P) } \\
\text { (mini- } \\
\text { mum) }\end{array}$ & $\begin{array}{c}\text { Approxi- } \\
\text { mate } \\
\text { weight } \\
\text { per } 100 \\
\text { shackles }\end{array}$ \\
\hline $\begin{array}{c}\text { Inches } \\
3 / 16 \\
1 / 4 \\
5 / 16 \\
38 \\
7 / 16\end{array}$ & $\begin{array}{c}\text { Inches } \\
7 / 8 \\
11 / 8 \\
11 / 4 \\
1716 \\
111 / 16\end{array}$ & $\begin{array}{c}\text { Inch } \\
1 / 32 \\
1 / 16 \\
1 / 16 \\
1 / 16 \\
1 / 16\end{array}$ & $\begin{array}{l}\text { Inches } \\
3 / 8 \\
15 / 32 \\
17 / 32 \\
2132 \\
23 / 32\end{array}$ & $\begin{array}{l}\text { Inch } \\
1 / 32 \\
1 / 32 \\
1 / 32 \\
1 / 32 \\
1 / 32\end{array}$ & $\begin{array}{c}\text { Inches } \\
144 \\
5116 \\
33 \\
7 / 16 \\
1 / 2\end{array}$ & $\begin{array}{c}\text { Pounds } \\
8 \\
12 \\
21 \\
32 \\
49\end{array}$ \\
\hline $\begin{array}{l}1 / 2 \\
9 / 16 \\
5 / 8 \\
24 \\
7 / 8\end{array}$ & $\begin{array}{l}115 / 6 \\
218 \\
21 / 2 \\
3 \\
31 / 4\end{array}$ & $\begin{array}{l}1 / 16 \\
1 / 16 \\
18 \\
1 / 4 \\
1 / 4\end{array}$ & $\begin{array}{l}13 / 16 \\
7 / 8 \\
11 / 16 \\
114 \\
1 \% 16\end{array}$ & $\begin{array}{l}1 / 16 \\
1 / 16 \\
1 / 16 \\
1 / 16 \\
1 / 16\end{array}$ & $\begin{array}{l}5 / 8 \\
558 \\
34 \\
7 / 8 \\
1\end{array}$ & $\begin{array}{r}73 \\
92 \\
151 \\
226 \\
340\end{array}$ \\
\hline $\begin{array}{l}1 \\
11 / 8 \\
114 \\
138\end{array}$ & $\begin{array}{l}33 / 4 \\
414 \\
41 / 2 \\
51 / 4\end{array}$ & $\begin{array}{l}1 / 4 \\
1\end{array}$ & $\begin{array}{l}111 / 16 \\
113 / 16 \\
2 \\
214\end{array}$ & $\begin{array}{l}1 / 16 \\
1 / 16 \\
1 / 16 \\
1 / 8\end{array}$ & $\begin{array}{l}11 / 8 \\
11 / 4 \\
13 / 8 \\
11 / 2\end{array}$ & $\begin{array}{r}495 \\
691 \\
899 \\
1,219\end{array}$ \\
\hline $\begin{array}{l}13 / 2 \\
158 \\
13 / 4 \\
2\end{array}$ & $\begin{array}{l}53 / 4 \\
634 \\
73 / 4\end{array}$ & $\begin{array}{l}1 / 4 \\
144 \\
1 / 4 \\
1 / 4\end{array}$ & $\begin{array}{l}23,8 \\
258 \\
27,8 \\
314\end{array}$ & $\begin{array}{l}1 / 8 \\
1 / 8 \\
1 / 8 \\
18\end{array}$ & $\begin{array}{l}15 / 8 \\
13 / 4 \\
22 \\
234\end{array}$ & $\begin{array}{l}1,595 \\
1,910 \\
2,750 \\
3,900\end{array}$ \\
\hline
\end{tabular}




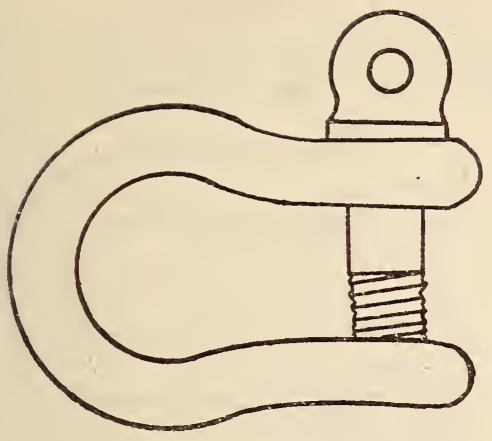

FigURe 117.-Anchor shackles

\section{Chain Shackles}

21. Screw-pin chain shackles shall be of dropforged steel unless otherwise specified.

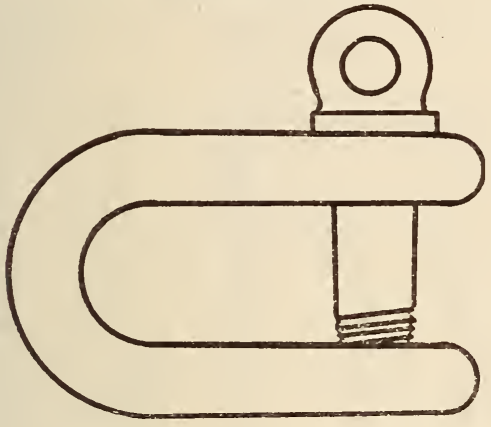

Figure 118.-Chain shackles

TABLE 15.-Chain shackles (dimensions and weights)

\begin{tabular}{|c|c|c|c|c|c|c|}
\hline $\begin{array}{l}\text { Size (di- } \\
\text { ameter } \\
\text { (D)) } \\
\text { (mini- } \\
\text { mum) }\end{array}$ & $\begin{array}{l}\text { Length } \\
\text { inside } \\
\text { (L) }\end{array}$ & $\begin{array}{l}\text { Toler- } \\
\text { ance } \\
\text { (plus or } \\
\text { minus) } \\
\text { in inside } \\
\text { length } \\
\text { (L) }\end{array}$ & $\begin{array}{c}\text { Width } \\
\text { between } \\
\text { eyes } \\
\text { (W) }\end{array}$ & $\begin{array}{c}\text { Tolerance } \\
\text { (plus or } \\
\text { minus) } \\
\text { in width } \\
\text { between } \\
\text { eyes } \\
\text { (W) }\end{array}$ & $\begin{array}{l}\text { Diam- } \\
\text { eter of } \\
\text { pin (P) } \\
\text { (mini- } \\
\text { mum) }\end{array}$ & $\begin{array}{l}\text { A pproxi } \\
\text { mate } \\
\text { weight } \\
\text { per } 100 \\
\text { shackles }\end{array}$ \\
\hline $\begin{array}{c}\text { Inches } \\
3116 \\
1 / 4 \\
5 / 16 \\
3 / 8 \\
7 / 16\end{array}$ & $\begin{array}{l}\text { Inches } \\
3 / 4 \\
7 / 8 \\
1 \\
11 / 4 \\
11 / 2\end{array}$ & $\begin{array}{c}\text { Inch } \\
1 / 32 \\
1 / 16 \\
1 / 16 \\
1 / 16 \\
1 / 16\end{array}$ & $\begin{array}{c}\text { Inches } \\
5 / 16 \\
15 / 32 \\
17 / 32 \\
21 / 32 \\
23 / 32\end{array}$ & $\begin{array}{l}\text { Inch } \\
1 / 32 \\
1 / 32 \\
1 / 32 \\
1 / 32 \\
1 / 32\end{array}$ & $\begin{array}{c}\text { Inches } \\
1 / 4 \\
5 / 16 \\
3 / 8 \\
7 / 16 \\
1 / 2\end{array}$ & $\begin{array}{c}\text { Pounds } \\
7 \\
12 \\
18 \\
28 \\
44\end{array}$ \\
\hline $\begin{array}{l}1 / 2 \\
9 / 16 \\
5 / 8 \\
3 / 4 \\
7 / 8\end{array}$ & $\begin{array}{l}111 / 16 \\
113 / 16 \\
25 / 16 \\
27 \%\end{array}$ & $\begin{array}{l}1 / 16 \\
1 / 16 \\
1 / 8 \\
1 / 4 \\
1 / 4\end{array}$ & $\begin{array}{l}13 / 16 \\
7 / 8 \\
11 / 16 \\
11 / 4 \\
17 / 16\end{array}$ & $\begin{array}{l}1 / 16 \\
1 / 16 \\
1 / 16 \\
1 / 16 \\
1 / 16\end{array}$ & $\begin{array}{r}5 / 8 \\
5 / 8 \\
3 / 4 \\
1^{7 / 8}\end{array}$ & $\begin{array}{r}68 \\
88 \\
130 \\
201 \\
288\end{array}$ \\
\hline $\begin{array}{l}1 \\
1 / 8 \\
11 / 4 \\
13 / 8 \\
11 / 2 \\
15 / 8 \\
13 / 4 \\
2\end{array}$ & $\begin{array}{l}31 / 4 \\
31 / 2 \\
311 / 16 \\
41 / 2 \\
5 \\
51 / 4 \\
57 / 8 \\
63 / 4\end{array}$ & $\begin{array}{l}1 / 4 \\
1 / 4 \\
134 \\
1 / 4 \\
1 / 4 \\
144 \\
14 \\
14\end{array}$ & $\begin{array}{l}111 / 16 \\
113 / 16 \\
2 \\
21 / 4 \\
23 / 8 \\
258 \\
27 / 8 \\
314\end{array}$ & $\begin{array}{l}1 / 16 \\
1 / 16 \\
116 \\
118 \\
1 / 8 \\
1 / 8 \\
1 / 8 \\
1 / 8\end{array}$ & $\begin{array}{l}11 / 8 \\
114 \\
13 / 8 \\
11 / 2 \\
158 \\
13 / 4 \\
2 \\
21 / 4\end{array}$ & $\begin{array}{r}441 \\
625 \\
800 \\
1,110 \\
1,372 \\
1,635 \\
2,600 \\
3,600\end{array}$ \\
\hline
\end{tabular}

Oval-Pin Anchor Shackles

22. Oval-pin anchor shackles shall be of dropforged steel unless otherwise specified.

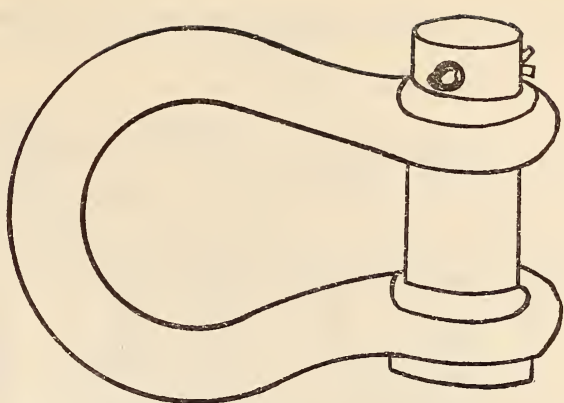

Figure 119.-Oval pin anchor shackles

TABLE 16.-Oval-pin anchor shackles (dimensions and weights)

\begin{tabular}{|c|c|c|c|c|c|}
\hline \multirow{2}{*}{ Size } & \multirow{2}{*}{$\begin{array}{l}\text { Length } \\
\text { inside }\end{array}$} & \multirow{2}{*}{$\begin{array}{l}\text { Width } \\
\text { between } \\
\text { eyes }\end{array}$} & \multicolumn{2}{|c|}{ Size of pin } & \multirow{2}{*}{$\begin{array}{c}\text { Esti- } \\
\text { mated } \\
\text { weight } \\
\text { per } \\
\text { hundred }\end{array}$} \\
\hline & & & Thick & Wide & \\
\hline $\begin{array}{c}\text { Inches } \\
1 / 4 \\
5 / 16 \\
3 / 8 \\
7 / 16 \\
1 / 2\end{array}$ & $\begin{array}{l}\text { Inches } \\
1 \\
11 / 8 \\
17 / 16 \\
15 / 8 \\
17 / 8\end{array}$ & $\begin{array}{c}\text { Inches } \\
7 / 16 \\
1 / 2 \\
5 / 8 \\
11 / 16 \\
3 / 4\end{array}$ & $\begin{array}{c}\text { Inches } \\
5 / 16 \\
11 / 32 \\
13 / 32 \\
7 / 16 \\
1 / 2\end{array}$ & $\begin{array}{c}\text { Inches } \\
3 / 8 \\
7 / 16 \\
1 / 2 \\
9 / 16 \\
11 / 16\end{array}$ & $\begin{array}{c}\text { Pounds } \\
13 \\
19 \\
29 \\
44 \\
85\end{array}$ \\
\hline $\begin{array}{l}9 / 16 \\
5 / 8 \\
3 / 4 \\
7 / 8 \\
1^{7 / 8}\end{array}$ & $\begin{array}{l}21 / 8 \\
23 / 8 \\
27 / 8 \\
31 / 4 \\
31 / 2\end{array}$ & $\begin{array}{l}7 / 8 \\
1 \\
11 / 4 \\
11 / 2 \\
15 / 8\end{array}$ & $\begin{array}{l}1 / 2 \\
11 / 16 \\
3 / 4 \\
13 / 16 \\
1^{13}\end{array}$ & $\begin{array}{l}11 / 16 \\
7 / 8 \\
1 \\
13 / 16 \\
13 / 8\end{array}$ & $\begin{array}{l}100 \\
178 \\
281 \\
380 \\
591\end{array}$ \\
\hline $\begin{array}{l}11 / 8 \\
11 / 4 \\
13 / 8 \\
11 / 2\end{array}$ & $\begin{array}{l}41 / 8 \\
43 / 16 \\
4^{13 / 16} \\
6\end{array}$ & $\begin{array}{l}17 / 8 \\
2 \\
25 / 16 \\
21 / 2\end{array}$ & $\begin{array}{l}11 / 16 \\
13 / 16 \\
13 / 8 \\
15 / 16\end{array}$ & $\begin{array}{l}11 / 2 \\
13 / 4 \\
21 / 8 \\
21 / 8\end{array}$ & $\begin{array}{r}690 \\
961 \\
1,353 \\
1,570\end{array}$ \\
\hline $\begin{array}{l}15 / 8 \\
13 / 4 \\
2 \\
21 / 4\end{array}$ & $\begin{array}{l}61 / 2 \\
7 \\
73 / 4 \\
83 / 4\end{array}$ & $\begin{array}{l}23 / 4 \\
3 \\
31 / 2 \\
33 / 4\end{array}$ & $\begin{array}{l}11 / 2 \\
11 / 2 \\
13 / 4 \\
17 / 8\end{array}$ & $\begin{array}{l}21 / 8 \\
21 / 8 \\
23 / 8 \\
21 / 2\end{array}$ & $\begin{array}{l}1,895 \\
2,400 \\
3,910 \\
4,400\end{array}$ \\
\hline $\begin{array}{l}23 / 8 \\
21 / 2 \\
25 / 8 \\
23 / 4\end{array}$ & & & & & \\
\hline
\end{tabular}

Note.-Tolerances (plus or minus) shall be $1 / 16$ inch on inside length for sizes up to $5 / 8$-inch, $1 / 8$ inch for $5 / 8$-inch size and $1 / 4$ inch for all other sizes. Tolerances (plus or minus) on width between eyes shall be $1 / 32$ inch for sizes up to and including $7 / 16$ inch, $1 / 16$ for sizes $1 / 2$ to $11 / 4$ inches, and $1 / 8$ for sizes $13 / 8$ inches and larger.

\section{Oval-Pin Chain Shackles}

23. Oval-pin chain shackles shall be of dropforged steel unless otherwise specified.

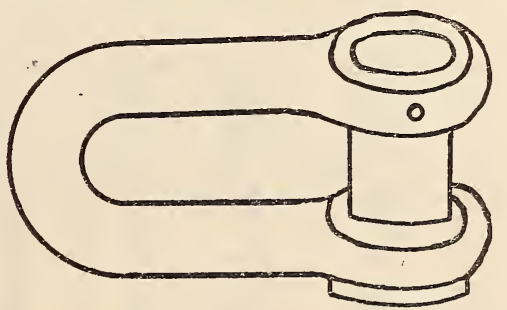

FIGURE 120.- Oval pin chain shackles 
TABLE 17.-Oval-pin chain shackles (dimensions and weights)

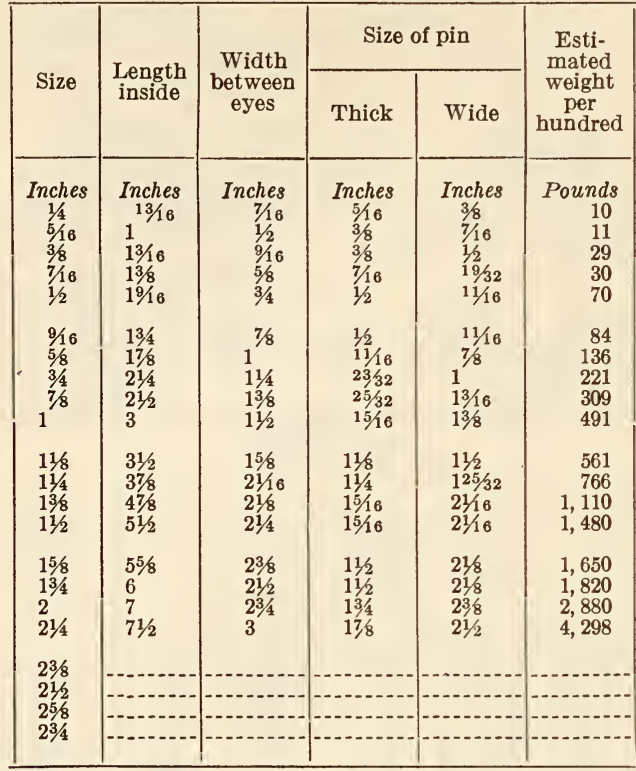

Note.-Tolerances same as given in note under Table 16.

24. Round-pin anchor shackles shall be of dropforged steel unless otherwise specified.

Round-Pin Anchor Shackles

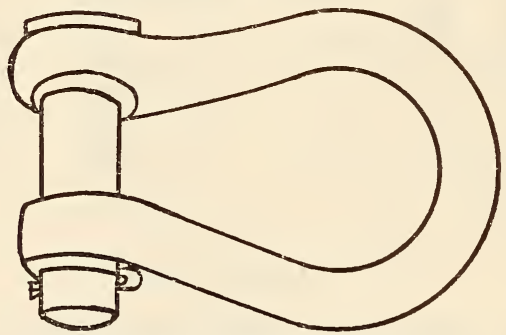

FIGURE 121.-Round-pin anchor shackles

TABLE 18.-Round-pin anchor shackles (dimensions and weights)

\begin{tabular}{|c|c|c|c|c|}
\hline Size & $\begin{array}{l}\text { Length } \\
\text { inside }\end{array}$ & $\begin{array}{l}\text { Width } \\
\text { between } \\
\text { eyes }\end{array}$ & $\begin{array}{l}\text { Diameter } \\
\text { of pin }\end{array}$ & $\begin{array}{l}\text { Estimated } \\
\text { weight } \\
\text { per } \\
\text { hundred }\end{array}$ \\
\hline $\begin{array}{c}\text { Inches } \\
1 / 4 \\
5 / 16 \\
3.8 \\
7 / 16 \\
1 / 2\end{array}$ & $\begin{array}{c}\text { Inches } \\
11 / 16 \\
11 / 4 \\
1716 \\
11116 \\
115 / 16\end{array}$ & $\begin{array}{r}\text { 1nches } \\
15 / 32 \\
17 / 32 \\
21 / 32 \\
23 / 32 \\
13 / 16\end{array}$ & $\begin{array}{c}\text { Inches } \\
5 / 16 \\
3 / 8 \\
7 / 16 \\
1 / 2 \\
5 / 8\end{array}$ & $\begin{array}{r}\text { Pounds } \\
12 \\
21 \\
32 \\
49 \\
73\end{array}$ \\
\hline $\begin{array}{l}9 / 16 \\
5 / 8 \\
3 / 4 \\
7 / 8\end{array}$ & $\begin{array}{l}21 / 8 \\
21 / 2\end{array}$ & $\begin{array}{l}7 / 8 \\
11 / 16 \\
11 / 4 \\
17 / 16\end{array}$ & $\begin{array}{r}5 / 8 \\
3 / 4 \\
7 / 8 \\
1^{7 / 8}\end{array}$ & $\begin{array}{r}92 \\
151 \\
226 \\
340\end{array}$ \\
\hline $\begin{array}{l}1 \\
11 / 8 \\
114 \\
138\end{array}$ & $\begin{array}{l}33 / 4 \\
41 / 4 \\
41 / 2 \\
51 / 4\end{array}$ & $\begin{array}{l}111 / 16 \\
113 / 16 \\
2 \\
21 / 4\end{array}$ & $\begin{array}{l}11 / 8 \\
11 / 4 \\
13 / 8 \\
11 / 2\end{array}$ & $\begin{array}{r}495 \\
691 \\
899 \\
1,219\end{array}$ \\
\hline $\begin{array}{l}11 / 2 \\
158 \\
13 / 4\end{array}$ & $\begin{array}{l}53 / 4 \\
61 / 4 \\
7 \\
73 / 4\end{array}$ & $\begin{array}{l}23 / 8 \\
25 / 8 \\
27 / 8 \\
31 / 4\end{array}$ & $\begin{array}{l}15 / 8 \\
13 / 4 \\
2 \\
21 / 4\end{array}$ & $\begin{array}{l}1,595 \\
1,910 \\
2,750 \\
3,900\end{array}$ \\
\hline
\end{tabular}

Note.-Tolerances are same as given in note under Table 16.
Round-Pin Chain Shackles

25. Round-pin chain shackles shall be of dropforged steel unless otherwise specified.

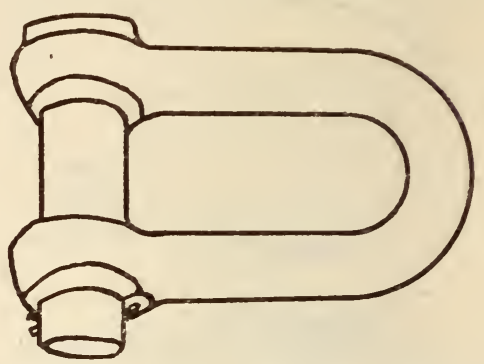

FIGURE 122.-Round-pin chain shackles

TABLE 19.-Round-pin chain shackles (dimensions and weights)

\begin{tabular}{|c|c|c|c|c|}
\hline Size & $\begin{array}{l}\text { Length } \\
\text { inside }\end{array}$ & $\begin{array}{l}\text { Width } \\
\text { between } \\
\text { eyes }\end{array}$ & $\begin{array}{c}\text { Diameter } \\
\text { of pin }\end{array}$ & $\begin{array}{l}\text { Estimated } \\
\text { weight } \\
\text { per } \\
\text { hundred }\end{array}$ \\
\hline $\begin{array}{c}\text { Inches } \\
1 / 4 \\
5 / 16 \\
3 / 8 \\
7 / 16 \\
1 / 2\end{array}$ & $\begin{array}{c}\text { Inches } \\
7 / 8 \\
1 \\
11 / 4 \\
11 / 2 \\
111 / 16\end{array}$ & $\begin{array}{r}\text { Inches } \\
15 / 32 \\
17 / 32 \\
21 / 32 \\
23 / 32 \\
13 / 16\end{array}$ & $\begin{array}{c}\text { Inches } \\
5 / 16 \\
3 / 8 \\
7 / 16 \\
1 / 2 \\
5 / 8\end{array}$ & $\begin{array}{r}\text { Pounds } \\
12 \\
18 \\
28 \\
44 \\
68\end{array}$ \\
\hline $\begin{array}{l}9 / 16 \\
5 / 8 \\
3 / 4 \\
7 / 8\end{array}$ & $\begin{array}{l}1^{13 / 16} \\
2 \\
25 / 16 \\
27 / 8\end{array}$ & $\begin{array}{l}7 / 8 \\
11 / 16 \\
11 / 4 \\
17 / 16\end{array}$ & $\begin{array}{l}5 / 8 \\
3 / 4 \\
7 / 8 \\
1^{7 / 8}\end{array}$ & $\begin{array}{r}88 \\
130 \\
201 \\
288\end{array}$ \\
\hline $\begin{array}{l}1 \\
11 / 8 \\
11 / 4 \\
13 / 8\end{array}$ & $\begin{array}{l}31 / 4 \\
31 / 2 \\
311 / 16 \\
41 / 2\end{array}$ & $\begin{array}{l}111 / 16 \\
113 / 16 \\
2 \\
21 / 4\end{array}$ & $\begin{array}{l}11 / 8 \\
11 / 4 \\
136 \\
11 / 2\end{array}$ & $\begin{array}{r}441 \\
625 \\
800 \\
1,110\end{array}$ \\
\hline $\begin{array}{l}11 / 2 \\
158 \\
13 / 4 \\
2\end{array}$ & $\begin{array}{l}5 \\
51 / 4 \\
57 / 8 \\
63 / 4\end{array}$ & $\begin{array}{l}23 / 8 \\
258 \\
27 / 8 \\
31 / 4\end{array}$ & $\begin{array}{l}15 / 8 \\
13 / 4 \\
2 \\
21 / 4\end{array}$ & $\begin{array}{l}1,372 \\
1,635 \\
2,600 \\
3,600\end{array}$ \\
\hline
\end{tabular}

Note.-Tolerances are same as given in note under Table 16.

\section{RINGS}

26. Rings shall be of class 2 steel. The types shall be in accordance with the following table:

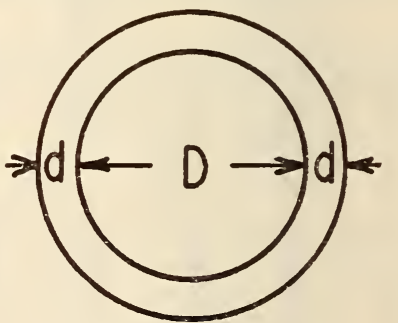

Figure 123.-Rings

TABLE 20.-Rings (dimensions and weights)

\begin{tabular}{|c|c|c|c|}
\hline Size d by D & $\begin{array}{l}\text { Approx- } \\
\text { imate } \\
\text { weight } \\
\text { per 100 } \\
\text { rings }\end{array}$ & Size d by D & $\begin{array}{l}\text { Approx- } \\
\text { imate } \\
\text { weight } \\
\text { per 100 } \\
\text { rings }\end{array}$ \\
\hline 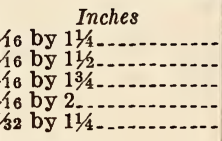 & $\begin{array}{r}\text { Pounds } \\
3.85 \\
4.45 \\
5.05 \\
5.75 \\
5.00\end{array}$ & 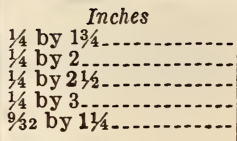 & $\begin{array}{r}\text { Pounds } \\
9.00 \\
10.00 \\
12.25 \\
14.35 \\
8.75\end{array}$ \\
\hline 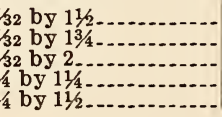 & $\begin{array}{l}5.75 \\
6.60 \\
7.50 \\
6.72 \\
7.80\end{array}$ & $\begin{array}{l}9 / 22 \text { by } 11 / 2 \\
9 / 22 \text { by } 13 / 4-\ldots \\
9,32 \text { by } 2 \\
9 / 32 \text { by } 215- \\
9 / 32 \text { by } 3\end{array}$ & $\begin{array}{l}10.02 \\
11.45 \\
12.75 \\
15.52 \\
18.33\end{array}$ \\
\hline
\end{tabular}


TABLE 20.-Rings (dimensions and weights)-Con.

\begin{tabular}{|c|c|c|c|}
\hline Size d by D & $\begin{array}{l}\text { Approx- } \\
\text { imate } \\
\text { weight } \\
\text { per } 100 \\
\text { rings }\end{array}$ & Size d by D & $\begin{array}{l}\text { Approx- } \\
\text { imate } \\
\text { weight } \\
\text { per } 100 \\
\text { rings }\end{array}$ \\
\hline 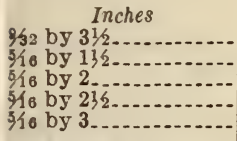 & $\begin{array}{r}\text { Pounds } \\
21.15 \\
12.56 \\
16.00 \\
19.42 \\
22.88\end{array}$ & $\begin{array}{l}\text { Inches } \\
5 / 8 \text { by } 31 / 2 \ldots \\
5 / 8 \text { by } 4 . \\
344 \text { by } 3 \\
3 / 4 \text { by } 31,2- \\
3 / 4 \text { by } 4\end{array}$ & $\begin{array}{r}\text { Pounds } \\
125 \\
140 \\
162 \\
183 \\
204\end{array}$ \\
\hline 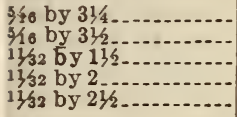 & $\begin{array}{l}24.50 \\
26.32 \\
15.50 \\
20 \\
24\end{array}$ & $\begin{array}{l}3 / 4 \text { by } 416 \\
3 / 4 \text { by } 6 \\
7 / 8 \text { by } 315,8 \\
7 / 8 \text { by } 4 \\
7 / 8 \text { by } 41,2 \ldots\end{array}$ & $\begin{array}{l}225 \\
275 \\
253 \\
282 \\
310\end{array}$ \\
\hline 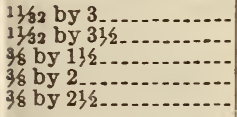 & $\begin{array}{l}28 \\
32 \\
19 \\
24 \\
29\end{array}$ & $\begin{array}{l}7,8 \text { by } 5 \\
78 \text { by } 6 \\
1 \text { by } 4 \text { by } 5 \\
1 \text { by } 512\end{array}$ & $\begin{array}{l}339 \\
375 \\
360 \\
440 \\
470\end{array}$ \\
\hline 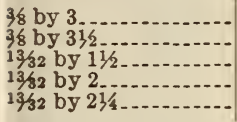 & $\begin{array}{l}34 \\
38 \\
23 \\
28 \\
31\end{array}$ & $\begin{array}{l}1 \text { by } 6 \\
118 \text { by } 5 \\
118 \text { by } 6 \\
13,8 \text { by } 6 \\
15,8 \text { by } 612\end{array}$ & $\begin{array}{r}500 \\
550 \\
650 \\
1,000 \\
1,600\end{array}$ \\
\hline 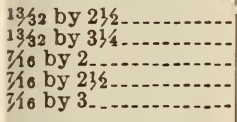 & $\begin{array}{l}34 \\
43 \\
33 \\
40 \\
46\end{array}$ & $\begin{array}{l}13 / 4 \text { by } 7 \\
17 / 8 \text { by } 8 \\
21 / 8 \text { by } 9 \\
21 / 4 \text { by } 9 \\
21 / 2 \text { by } 10\end{array}$ & $\begin{array}{l}2,000 \\
2,500 \\
3,600 \\
4,000 \\
5,500\end{array}$ \\
\hline 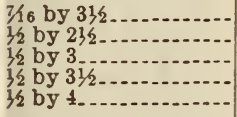 & $\begin{array}{l}53 \\
53 \\
61 \\
70 \\
79\end{array}$ & $\begin{array}{l}25,8 \text { by } 10 \\
23 / 4 \text { by } 10 \\
3 \text { by } 12 \\
318 \text { by } 14\end{array}$ & $\begin{array}{r}6,300 \\
7,000 \\
9,600 \\
12,000\end{array}$ \\
\hline $\begin{array}{l}\% 6 \text { by } 21 / 2 \ldots \\
916 \text { by } 3 \\
6 / 16 \text { by } 3132 \ldots \\
96 \text { by } 4 \\
5,8 \text { by } 3 \ldots\end{array}$ & $\begin{array}{r}77 \\
91 \\
110 \\
113 \\
110\end{array}$ & & \\
\hline
\end{tabular}

\section{TOGGLES}

27. Toggles shall be of class 2 steel. The types shall be in accordance with the following tables:

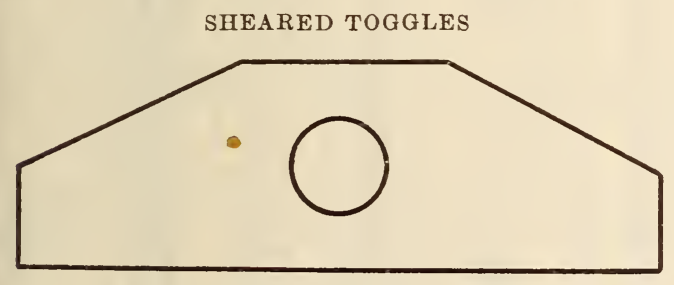

Figure 124.- Sheared toggles

TABLE 21.-Toggles (dimensions and weights)

\begin{tabular}{|c|c|c|c|}
\hline $\begin{array}{l}\text { Size } \\
\text { chain } \\
\text { used } \\
\text { on }\end{array}$ & Toggle dimensions & $\begin{array}{l}\text { Approxi- } \\
\text { mate } \\
\text { weight } \\
\text { each }\end{array}$ & $\begin{array}{l}\text { Ring, in- } \\
\text { side di- } \\
\text { ameter }\end{array}$ \\
\hline $\begin{array}{c}\text { Inch } \\
7 / 16 \\
1 / 2 \\
58 \\
334 \\
7 / 8\end{array}$ & $\begin{array}{l}\text { Inches } \\
6 \text { by } 21 / 2 \\
7 \text { by } 21 / 2 \\
8 \text { by } 21 / 4 \text { by } 5 / 8 \\
9 \text { by } 215 \text { by } 3 / 4 \\
10 \text { by } 23 / 4 \text { by } 3 / 4\end{array}$ & \begin{tabular}{|r} 
Pounds \\
r 114 \\
$11 / 2$ \\
214 \\
358 \\
$41 / 2$
\end{tabular} & $\begin{array}{l}\text { Inches } \\
5 / 8 \text { by } 3 \\
58 \text { by } 31 / 4 \\
3 / 4 \text { by } 4 \\
7 / 8 \text { by } 41 / 2 \\
1 \text { by } 5\end{array}$ \\
\hline
\end{tabular}

FORGED TOGGLES

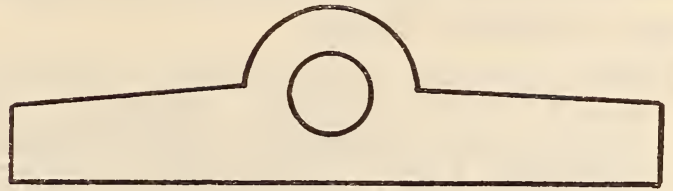

Figure 125.-Forged toggies

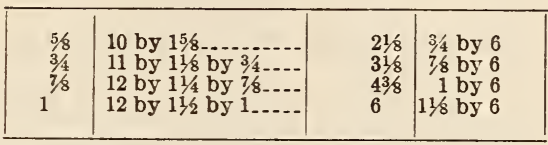

\section{SWIVELS}

28. Swivels shall be made of malleable iron. They shall be in accordance with the following table:

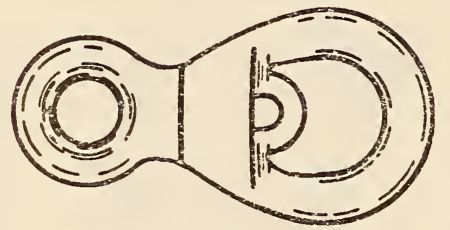

Figure 126.-Swivels

TABLE 22.-Swivels (dimensions and weights)

\begin{tabular}{|c|c|c|c|}
\hline $\begin{array}{c}\text { Size } \\
\text { chain }\end{array}$ & Reach & $\begin{array}{c}\text { Hole in } \\
\text { eye }\end{array}$ & $\begin{array}{c}\text { Approxi- } \\
\text { mate } \\
\text { weight } \\
\text { per 100 } \\
\text { swivels }\end{array}$ \\
\cline { 1 - 2 } Inch & Inches & Inches & Pounds \\
$1 / 4$ & $23 / 16$ & $7 / 16$ & 21 \\
$5 / 16$ & $21 / 2$ & $7 / 16$ & 32 \\
$3 / 8$ & $33 / 8$ & $7 / 16$ & 61 \\
$7 / 16$ & $31 / 2$ & $9 / 16$ & 78 \\
$1 / 2$ & 358 & $13 / 16$ & 93 \\
$5 / 8$ & $45 / 8$ & $15 / 16$ & 168 \\
\hline
\end{tabular}

603.59 MISCELLANEOUS CHAINS.

SOCIETY OF AUTOMOTIVE ENGINEERS, SILENT CHAINS, S. A. E. STANDARD, 1917

\section{SILENT CHAINS}

(This recommendation contains an illustrated tabulation which shows, for different widths and pitches of silent chain, the maximum width in inches. This maximum width is always equal to the chain width plus 0.6 times the pitch in inches. The width of chain tabulated ranges from $1 / 2$ to 6 inches, while the range in pitch is from $3 / 8$ to 2 inches.) 
604 IRON AND STEEL PLATES AND SHEETS 604.0 GENERAL ITEMS.

UNITED STATES GOVERNMENT, DEPARTMENT OF COMMERCE, BUREAU OF STANDARDS, STANDARD THICKNESSES, WEIGHTS, AND TOLERANCES OF SHEET METAL (CUSTOMARY PRACTICE), CIRCULAR NO. 391, MARCH 24, 1931

\section{IRON AND STEEL SHEET AND PLATE}

The United States standard gage for sheet and plate iron and steel is the legal standard formerly used in determining duties and taxes levied by the
United States, and is the recognized commercial standard for all uncoated sheet and plate iron and steel. It is a weight gage, having been based upon weights in ounces per square foot. The provisions of the act of Congress, approved March 3, 1893 (27 Stat. L. 746), establishing this gage are as follows:

\section{(a) AN ACT ESTABLISHING A STANDARD GAGE FOR SHEET} AND PLATE IRON AND STEEL

Be it enacted by the Senate and House of Representatives of the United States of America in Congress assembled, That for the purpose of securing uniformity, the following is established as the only standard gage for sheet and plate iron and steel in the United States of America, namely:

\begin{tabular}{|c|c|c|c|c|c|c|c|c|}
\hline Gage No. & $\begin{array}{c}\text { Approxi- } \\
\text { mate } \\
\text { thickness } \\
\text { in fractions } \\
\text { of an inch }\end{array}$ & $\begin{array}{l}\text { Approximate } \\
\text { thickness in } \\
\text { decimal parts } \\
\text { of an inch }\end{array}$ & $\begin{array}{l}\text { Approximate } \\
\text { thickness in } \\
\text { millimeters }\end{array}$ & $\begin{array}{l}\text { Weight } \\
\text { per } \\
\text { square } \\
\text { foot in } \\
\text { ounces } \\
\text { a voirdu- } \\
\text { pois }\end{array}$ & $\begin{array}{l}\text { Weight per } \\
\text { square foot } \\
\text { in pounds } \\
\text { avoirdupois }\end{array}$ & $\begin{array}{l}\text { Weight } \\
\text { per } \\
\text { square } \\
\text { foot in } \\
\text { kilo- } \\
\text { grams }\end{array}$ & $\begin{array}{l}\text { Weight } \\
\text { per } \\
\text { square } \\
\text { meter in } \\
\text { kilo- } \\
\text { grams }\end{array}$ & $\begin{array}{l}\text { Weight } \\
\text { per } \\
\text { square } \\
\text { meter in } \\
\text { pounds } \\
\text { avoirdu- } \\
\text { pois }\end{array}$ \\
\hline $\begin{array}{r}0000000 \\
000000 \\
00000 \\
0000 \\
000\end{array}$ & $\begin{array}{c}1-2 \\
15-32 \\
7-16 \\
13-32 \\
3-5\end{array}$ & $\begin{array}{l}0.5 \\
.46875 \\
.4375 \\
.40625 \\
.375\end{array}$ & $\begin{array}{l}12.7 \\
11.90625 \\
11.1125 \\
10.31875 \\
9.525\end{array}$ & $\begin{array}{l}320 \\
300 \\
280 \\
260 \\
210\end{array}$ & $\begin{array}{l}20.00 \\
18.75 \\
17.50 \\
16.25 \\
15\end{array}$ & $\begin{array}{l}9.072 \\
8.505 \\
7.983 \\
7.371 \\
6.804\end{array}$ & $\begin{array}{l}97.65 \\
91.55 \\
85.44 \\
79.33 \\
73.24\end{array}$ & $\begin{array}{l}215.28 \\
201.82 \\
188.37 \\
174.91 \\
161.46\end{array}$ \\
\hline $\begin{array}{r}00 \\
0 \\
1 \\
2 \\
3\end{array}$ & $\begin{array}{r}11-32 \\
5-16 \\
9-32 \\
17-64 \\
1-4\end{array}$ & $\begin{array}{l}.31375 \\
.3125 \\
.28125 \\
.265625 \\
.25\end{array}$ & $\begin{array}{l}8.73125 \\
7.9375 \\
7.14375 \\
6.746875 \\
6.35\end{array}$ & $\begin{array}{l}220 \\
200 \\
180 \\
170 \\
160\end{array}$ & $\begin{array}{l}13.75 \\
12.50 \\
11.25 \\
10.625 \\
10\end{array}$ & $\begin{array}{l}\text { 6. } 237 \\
5.67 \\
5.103 \\
4.819 \\
4.536\end{array}$ & $\begin{array}{l}67.13 \\
61.03 \\
54.93 \\
51.88 \\
48.82\end{array}$ & $\begin{array}{l}148.00 \\
134.55 \\
121.09 \\
114.37 \\
107.64\end{array}$ \\
\hline $\begin{array}{l}4 \\
5 \\
6 \\
7 \\
8\end{array}$ & $\begin{array}{r}15-64 \\
7-32 \\
13-64 \\
3-16 \\
11-64\end{array}$ & $\begin{array}{l}.234375 \\
21875 \\
.203125 \\
.1875 \\
.171875\end{array}$ & $\begin{array}{l}5.953125 \\
5.55625 \\
5.159375 \\
4.7625 \\
4.365625\end{array}$ & $\begin{array}{l}150 \\
140 \\
130 \\
120 \\
110\end{array}$ & $\begin{array}{l}9.375 \\
8.75 \\
8.125 \\
7.5 \\
6.875\end{array}$ & $\begin{array}{l}\text { 4. } 252 \\
\text { 3. } 969 \\
\text { 3. } 685 \\
\text { 3. } 402 \\
\text { 3. } 118\end{array}$ & $\begin{array}{l}45.77 \\
42.72 \\
39.67 \\
36.62 \\
33.57\end{array}$ & $\begin{array}{r}100.91 \\
94.18 \\
87.45 \\
80.72 \\
74.00\end{array}$ \\
\hline $\begin{array}{r}9 \\
10 \\
11 \\
12 \\
13\end{array}$ & $\begin{array}{l}5-32 \\
9-64 \\
1-8 \\
7-64 \\
3-32\end{array}$ & $\begin{array}{l}.15625 \\
.140625 \\
125 \\
.109375 \\
.09375\end{array}$ & $\begin{array}{l}\text { 3. } 96875 \\
\text { 3. } 571875 \\
\text { 3. } 175 \\
\text { 2. } 778125 \\
2.38125\end{array}$ & $\begin{array}{r}100 \\
90 \\
80 \\
70 \\
60\end{array}$ & $\begin{array}{l}6.25 \\
5.625 \\
5 \\
4.375 \\
3.75\end{array}$ & $\begin{array}{l}\text { 2. } 835 \\
\text { 2. } 552 \\
2.268 \\
\text { 1. } 984 \\
1.701\end{array}$ & $\begin{array}{l}30.52 \\
27.46 \\
24.41 \\
21.36 \\
18.31\end{array}$ & $\begin{array}{l}67.27 \\
60.55 \\
53.82 \\
47.09 \\
40.36\end{array}$ \\
\hline $\begin{array}{l}14 \\
15 \\
16 \\
17 \\
18\end{array}$ & $\begin{array}{l}5-61 \\
9-123 \\
1-16 \\
9-160 \\
1-20\end{array}$ & $\begin{array}{l}.078125 \\
.0703125 \\
.0625 \\
.05625 \\
.05\end{array}$ & $\begin{array}{l}1.984375 \\
1.7859375 \\
1.5875 \\
1.42875 \\
1.27\end{array}$ & $\begin{array}{l}50 \\
45 \\
40 \\
36 \\
32\end{array}$ & $\begin{array}{l}3.125 \\
2.8125 \\
2.5 \\
2.25 \\
2\end{array}$ & $\begin{array}{c}1.417 \\
1.276 \\
1.134 \\
1.021 \\
.9072\end{array}$ & $\begin{array}{l}15.26 \\
13.73 \\
12.21 \\
10.99 \\
9.765\end{array}$ & $\begin{array}{l}33.64 \\
30.27 \\
26.91 \\
24.22 \\
21.53\end{array}$ \\
\hline $\begin{array}{l}19 \\
20 \\
21 \\
22 \\
23\end{array}$ & $\begin{array}{c}7-160 \\
3-80 \\
11-320 \\
1-32 \\
9-320\end{array}$ & $\begin{array}{l}.04375 \\
.0375 \\
.034375 \\
.03125 \\
.028125\end{array}$ & $\begin{array}{l}1.11125 \\
.9525 \\
.873125 \\
.793750 \\
.714375\end{array}$ & $\begin{array}{l}28 \\
24 \\
22 \\
20 \\
18\end{array}$ & $\begin{array}{l}1.75 \\
1.50 \\
1.375 \\
1.25 \\
1.125\end{array}$ & $\begin{array}{l}.7988 \\
.6804 \\
.6237 \\
.567 \\
.5103\end{array}$ & $\begin{array}{l}8.544 \\
7.324 \\
6.713 \\
6.103 \\
5.493\end{array}$ & $\begin{array}{l}18.84 \\
16.15 \\
14.80 \\
13.46 \\
12.11\end{array}$ \\
\hline $\begin{array}{l}24 \\
25 \\
26 \\
27 \\
28\end{array}$ & $\begin{array}{c}1-40 \\
7-320 \\
3160 \\
11-640 \\
1-64\end{array}$ & $\begin{array}{l}.025 \\
.021875 \\
.01875 \\
.0171875 \\
.015625\end{array}$ & $\begin{array}{l}.635 \\
.555625 \\
.47625 \\
.4365625 \\
.396875\end{array}$ & $\begin{array}{l}16 \\
14 \\
12 \\
11 \\
10\end{array}$ & $\begin{array}{l}1 \\
.875 \\
.75 \\
.6975 \\
.625\end{array}$ & $\begin{array}{l}.4536 \\
.3969 \\
.3402 \\
.3119 \\
.2835\end{array}$ & $\begin{array}{l}4.882 \\
4.272 \\
3.662 \\
3.357 \\
3.052\end{array}$ & $\begin{array}{r}10.76 \\
9.42 \\
8.07 \\
7.40 \\
6.73\end{array}$ \\
\hline $\begin{array}{l}29 \\
30 \\
31 \\
32 \\
33\end{array}$ & $\begin{array}{c}9-640 \\
1-80 \\
7-640 \\
13-1280 \\
3-320\end{array}$ & $\begin{array}{l}.0140625 \\
.0125 \\
.0109375 \\
.01015625 \\
.009375\end{array}$ & $\begin{array}{l}.3571875 \\
.3175 \\
.2778125 \\
.25796875 \\
.238125\end{array}$ & $\begin{array}{l}9 \\
8 \\
7 \\
61 / 2 \\
6\end{array}$ & $\begin{array}{l}.5625 \\
.5 \\
.4375 \\
.40625 \\
.375\end{array}$ & $\begin{array}{l}.2551 \\
.2268 \\
.1984 \\
.1843 \\
.1701\end{array}$ & $\begin{array}{l}2.746 \\
2.441 \\
2.136 \\
1.983 \\
1.831\end{array}$ & $\begin{array}{l}6.05 \\
5.35 \\
4.71 \\
4.37 \\
4.04\end{array}$ \\
\hline $\begin{array}{l}34 \\
35 \\
36 \\
37 \\
38\end{array}$ & $\begin{array}{c}11-1280 \\
5-640 \\
9-1280 \\
17-2560 \\
1-160\end{array}$ & $\begin{array}{l}.00859375 \\
.0078125 \\
.00703125 \\
.006640625 \\
.00625\end{array}$ & $\begin{array}{l}.21828125 \\
.1984375 \\
.17959375 \\
.168671875 \\
.15875\end{array}$ & $\begin{array}{l}51 / 2 \\
5 \\
41 / 2 \\
41 / 4 \\
4\end{array}$ & $\begin{array}{l}.34375 \\
.3125 \\
.28125 \\
.265625 \\
.25\end{array}$ & $\begin{array}{l}.1559 \\
.1417 \\
.1276 \\
.1205 \\
.1134\end{array}$ & $\begin{array}{l}1.678 \\
1.526 \\
1.373 \\
1.297 \\
1.221\end{array}$ & $\begin{array}{l}3.70 \\
3.36 \\
3.03 \\
2.87 \\
2.69\end{array}$ \\
\hline
\end{tabular}

And on and after July 1, 1893, the same and no other shall be used in determining duties and taxes levied by the United States of America on sheet and plate iron and steel. But this act shall not be construed to increase duties upon any articles which may be imported.

SEC. 2. That the Secretary of the Treasury is authorized and required to prepare suitable standards in accordance herewith.

SEc. 3. That in the practical use and application of the standard gage hereby established a variation of $2 \frac{1}{2}$ per cent either way may be allowed.

Approved, March 3, 1893.

\section{(b) APPROXIMATE THICKNESSES OF STEEL PLATES AND} SHEETS

The thicknesses given in the law as approximate equivalents were based upon the density of wrought iron of 480 pounds per cubic foot, or 0.2778 pound per cubic inch. Since the United States standard gage was established, steel and commercially pure open-hearth iron have come into general use for plates and sheets. For most commercial purposes the density of steel is taken as 489.6 pounds per cubic foot, 0.2833 pound per cubic inch, or in terms more familiar to the sheet maker and user, steel weighs 40.80 pounds per square foot per inch thick, 
2 per cent more than wrought iron, which weighs 40 pounds per square foot per inch thick.

The density of steel varies, depending upon a number of factors, such as carbon content, presence of alloying elements, heat treatment, tempering temperature, amount and manner of cold working, etc. ${ }^{4}$ The total variation for carbon steel, due to these factors, may be of the order of 1 per cent. In the case of hot-rolled steel, it has been found that the mechanical working of the metal during hotrolling tends to decrease the density of the material, and of two sheets of different thicknesses rolled from the same material, the thicker sheet is always the denser. Cold-rolled steel sheets have been said to have a greater density than 0.2833 pound per cubic inch; however, two samples of full pickled, full cold-rolled sheets showed an average density of 0.2833 pound per cubic inch. Tests also have shown this value to be representative of commercially pure open-hearth iron.

In Table 1 the weights, and approximate thicknesses based upon 40 pounds per square foot per inch thick for wrought iron, and 40.80 for steel and open-hearth iron, are given for practical use. ${ }^{49}$ Also, the gage numbers above No. 38 are included, which have become standardized by custom, but were not included in the congressional enactment. The numbers of decimal places given for weights in pounds and for equivalent thicknesses have been limited, but the values have been carried out further than they should be used practically.

Attention is directed to United States Department of Commerce Simplified Practice Recommendations R28-29, sheet steel, which covers simplified sizes and weights of galvanized flat sheets, one pass coldrolled box annealed sheets, and blue annealed sheets (see 604.20, p. 236); and R78-28, iron and steel roofling (see 604.32, p. 246).

TABLE 1.-United States standard gage for sheet and plate iron and steel, and extension

\begin{tabular}{|c|c|c|c|c|c|c|c|}
\hline \multirow{2}{*}{ Gage No. } & \multirow{2}{*}{\multicolumn{2}{|c|}{$\begin{array}{l}\text { Weight per } \\
\text { square foot }\end{array}$}} & \multirow{2}{*}{$\begin{array}{l}\text { Weight } \\
\text { per } \\
\text { square } \\
\text { meter }\end{array}$} & \multicolumn{4}{|c|}{ Approximate thickness } \\
\hline & & & & \multicolumn{2}{|c|}{ Wrought iron } & \multicolumn{2}{|c|}{$\begin{array}{l}\text { Steel and open } \\
\text { hearth iron }\end{array}$} \\
\hline & Ounces & Pounds & $\mathrm{kg}$ & Inch & $m m$ & Inch & $m m$ \\
\hline 000 & 320 & 20.00 & 97.65 & 0.500 & 12. 70 & 0.490 & 12.45 \\
\hline 00 & $\begin{array}{l}300 \\
280\end{array}$ & $\begin{array}{l}18.75 \\
17.50\end{array}$ & $\begin{array}{l}91.55 \\
85.44\end{array}$ & $\begin{array}{l}.469 \\
.438\end{array}$ & $\begin{array}{l}11.91 \\
11.11\end{array}$ & $\begin{array}{l}.460 \\
.429\end{array}$ & $\begin{array}{l}11.67 \\
10.90\end{array}$ \\
\hline 000 & 260 & 16. 25 & 79. 34 & .406 & 10. 32 & .398 & 10. 12 \\
\hline 000 & 240 & 15.00 & 73. 24 & .375 & 9.52 & .368 & 9.34 \\
\hline 00 & 220 & 13. 75 & 67. 13 & .344 & 8. 73 & .337 & 8. 56 \\
\hline & 200 & 12.50 & 61.03 & .312 & 7. 94 & .306 & 7.78 \\
\hline & 180 & 11.25 & 54. 93 & .2812 & 7. 14 & .2757 & 7. 00 \\
\hline 2. & 170 & 10.6 & 51.88 & .2656 & & .2604 & 6.62 \\
\hline 3. & 160 & 10.00 & 48.82 & .2500 & 6.35 & .2451 & 6.23 \\
\hline & 150 & 9. 375 & 45.77 & .2344 & 5.95 & .2298 & 5. 84 \\
\hline & 140 & 8.750 & 42.72 & .2188 & 5. 56 & .2145 & 5.45 \\
\hline 6 & 130 & 8. 125 & 39.67 & . 2031 & 5. 16 & .1991 & 5.06 \\
\hline 8 & $\begin{array}{l}120 \\
110\end{array}$ & 7.5 & 36.62 & $\begin{array}{r}1875 \\
\cdot 1719\end{array}$ & & $\begin{array}{r}.1838 \\
1.85\end{array}$ & 4. 67 \\
\hline & & 0.810 & & & 4. $3 t$ & .1080 & \\
\hline
\end{tabular}

${ }^{4}$ See Density of Hot-Rolled and Heat-Treated Carbon Steels, by H. C. Cross and E. E. Hill, B. S. Sci. Paper No. 562.

49 According to an opinion rendered by the Attorney General of the United States, Apr. 22, 1929, the commercial use of the data presented in Table 1 , or of tolerances as presented in Secs. II $(c)$ and II (d) herein, does not violate the act of Mar. 3, 1893, establishing the United States standard gage. An excerpt of the opinion is as follows:

"Compliance with the standard gage established by the above act is not made mandatory either upon American manufacturers or upon the Government departments in preparing their specifications lor sheet and plate iron and steel. The only mandatory provision in determining duties and taxes levied by the United States of America on sheet and plate iron and steel.' 'There is no prohibition againśt the importation of sheet and plate iron or steel not complying with the standard gage, but it is provided merely that the standard shal be followed in determining the duties and taxes levied thereon."
TABLE 1.-United States standard gage for sheet and plate iron and steel, and extension-Continued

\begin{tabular}{|c|c|c|c|c|c|c|c|}
\hline \multirow{2}{*}{ Gage No. } & \multirow{2}{*}{\multicolumn{2}{|c|}{$\begin{array}{l}\text { Weight per } \\
\text { square foot }\end{array}$}} & \multirow{2}{*}{$\begin{array}{l}\text { Weight } \\
\text { per } \\
\text { square } \\
\text { meter }\end{array}$} & \multicolumn{4}{|c|}{ Approximate thickness } \\
\hline & & & & \multicolumn{2}{|c|}{ Wrought iron } & \multicolumn{2}{|c|}{$\begin{array}{l}\text { Steel and open } \\
\text { hearth iron }\end{array}$} \\
\hline 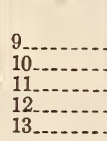 & $\begin{array}{c}\text { Ounces } \\
100 \\
90 \\
80 \\
70 \\
60\end{array}$ & $\begin{array}{c}\text { Pounds } \\
6.250 \\
5.625 \\
5.000 \\
4.375 \\
3.750\end{array}$ & $\begin{array}{c}k g \\
30.52 \\
27.46 \\
24.41 \\
21.36 \\
18.31\end{array}$ & $\begin{array}{l}\text { Inch } \\
0.1562 \\
.1406 \\
.1250 \\
.1094 \\
.0938\end{array}$ & $\begin{array}{l}m m \\
3.97 \\
3.57 \\
3.18 \\
2.778 \\
2.381\end{array}$ & $\begin{array}{l}\text { Inch } \\
0.1532 \\
.1379 \\
.1225 \\
.1072 \\
.0919\end{array}$ & $\begin{array}{l}m m \\
3.89 \\
3.50 \\
3.11 \\
2.724 \\
2.335\end{array}$ \\
\hline $\begin{array}{l}14-. . \\
15-. . \\
16-. . \\
17 .- \\
18 . .\end{array}$ & $\begin{array}{l}50 \\
45 \\
40 \\
36 \\
32\end{array}$ & $\begin{array}{l}3.125 \\
2.812 \\
2.500 \\
2.250 \\
2.000\end{array}$ & \begin{tabular}{|c|}
15.26 \\
13.73 \\
12.21 \\
10.99 \\
9.765
\end{tabular} & $\begin{array}{l}.0781 \\
.0703 \\
.0625 \\
.0562 \\
.0500\end{array}$ & $\begin{array}{l}1.984 \\
1.786 \\
1.588 \\
1.429 \\
1.270\end{array}$ & $\begin{array}{l}.0766 \\
.0689 \\
.0613 \\
.0551 \\
.0490\end{array}$ & $\begin{array}{l}1.946 \\
1.751 \\
1.557 \\
1.400 \\
1.245\end{array}$ \\
\hline $\begin{array}{l}19 \\
20 \ldots \\
21 \ldots \\
22 . \\
23 \ldots\end{array}$ & $\begin{array}{l}28 \\
24 \\
22 \\
20 \\
18\end{array}$ & $\begin{array}{l}\text { 1. } 750 \\
1.500 \\
1.375 \\
1.250 \\
1.125\end{array}$ & $\begin{array}{l}8.544 \\
7.324 \\
6.713 \\
6.103 \\
5.493\end{array}$ & $\begin{array}{l}.0438 \\
.0375 \\
.0344 \\
.0312 \\
.0281\end{array}$ & $\begin{array}{l}1.111 \\
.952 \\
.873 \\
.794 \\
.714\end{array}$ & $\begin{array}{l}.0429 \\
.0368 \\
.0337 \\
.0306 \\
.0276\end{array}$ & $\begin{array}{l}1.090 \\
.934 \\
.856 \\
.778 \\
.700\end{array}$ \\
\hline $\begin{array}{l}24 \ldots \\
25 \ldots \\
26 \\
27 . . \\
28 . \\
2 . .\end{array}$ & $\begin{array}{l}16 \\
14 \\
12 \\
11 \\
10\end{array}$ & $\begin{array}{l}1.000 \\
.8750 \\
.7500 \\
.6875 \\
.6250\end{array}$ & $\begin{array}{l}4.882 \\
4.272 \\
3.662 \\
3.357 \\
3.052\end{array}$ & $\begin{array}{l}.0250 \\
.0219 \\
.0188 \\
.0172 \\
.0156\end{array}$ & $\begin{array}{l}.635 \\
.556 \\
.476 \\
.437 \\
.397\end{array}$ & $\begin{array}{l}.0245 \\
.0214 \\
.0184 \\
.0169 \\
.0153\end{array}$ & $\begin{array}{l}.623 \\
.545 \\
.467 \\
.428 \\
.389\end{array}$ \\
\hline $\begin{array}{l}29 . . . \\
30 \ldots \\
31 . . \\
32 . .\end{array}$ & $\begin{array}{l}9 \\
8 \\
7 \\
61 / 2\end{array}$ & $\begin{array}{l}.5625 \\
.5000 \\
.4375 \\
.4062\end{array}$ & $\begin{array}{l}2.746 \\
2.441 \\
2.136 \\
1.983\end{array}$ & $\begin{array}{l}.0141 \\
.0125 \\
.0109 \\
.0102\end{array}$ & $\begin{array}{l}.357 \\
.318 \\
.278 \\
.258\end{array}$ & $\begin{array}{l}.0138 \\
.0123 \\
.0107 \\
.0100\end{array}$ & $\begin{array}{l}.350 \\
.311 \\
.272 \\
.253\end{array}$ \\
\hline & $\begin{array}{l}6 \\
5132 \\
5 \\
4132\end{array}$ & $\begin{array}{r}.3750 \\
.3438 \\
.3125 \\
.2812\end{array}$ & $\begin{array}{l}\text { 1. } 831 \\
\text { 1. } 678 \\
\text { 1. } 526 \\
1.373\end{array}$ & $\begin{array}{r}.0094 \\
.0086 \\
.0078 \\
.0070\end{array}$ & $\begin{array}{l}.238 \\
.218 \\
.198 \\
.179\end{array}$ & $\begin{array}{l}.0092 \\
.0084 \\
.0077 \\
.0069\end{array}$ & $\begin{array}{l}.233 \\
.214 \\
.195 \\
.175\end{array}$ \\
\hline $\begin{array}{l}37 \ldots-. \\
38 . . . \\
39 . .- \\
40 \ldots\end{array}$ & $\begin{array}{l}41 / 4 \\
4 \\
33 / 4 \\
31 / 2\end{array}$ & $\begin{array}{r}.2656 \\
.2500 \\
.2344 \\
.2188\end{array}$ & $\begin{array}{l}\text { 1. } 297 \\
\text { 1. } 221 \\
\text { 1. } 144 \\
\text { 1. } 068\end{array}$ & $\begin{array}{l}.0066 \\
.0062 \\
.0059 \\
.0055\end{array}$ & $\begin{array}{r}.169 \\
.159 \\
.149 \\
.139\end{array}$ & $\begin{array}{l}.0065 \\
.0061 \\
.0057 \\
.0054\end{array}$ & $\begin{array}{l}.165 \\
.156 \\
.146 \\
.136\end{array}$ \\
\hline $\begin{array}{l}41 \ldots \\
42 \ldots \\
43-\ldots \\
44 \ldots\end{array}$ & $\begin{array}{l}33 / 8 \\
31 / 4 \\
318 \\
3\end{array}$ & $\begin{array}{l}.2109 \\
.2031 \\
.1953 \\
.1875\end{array}$ & $\begin{array}{l}1.030 \\
.9917 \\
.9536 \\
.9155\end{array}$ & $\begin{array}{l}.0053 \\
.0051 \\
.0049 \\
.0047\end{array}$ & $\begin{array}{r}.134 \\
.129 \\
.124 \\
.119\end{array}$ & $\begin{array}{l}.0052 \\
.0050 \\
.0048 \\
.0046\end{array}$ & $\begin{array}{l}.131 \\
.126 \\
.122 \\
.117\end{array}$ \\
\hline
\end{tabular}

\section{c) PERMISSIBLE VARIATIONS IN WEIGHT AND THICKNESS
OF PLATES}

Manufacturers have had considerable difficulty in keeping within the tolerance of plus or minus $2 \frac{1}{2}$ per cent specified in the law establishing the United States standard gage for sheet and plate iron and steel. As the law does not make this tolerance mandatory for commercial purposes, the Association of American Steel Manufacturers has adopted specifications regarding permissible variations in weight and thickness, which have been applied to platemill products regularly, but not to sheet and tin mill products. (See A. A. S. M. standard specifications for structural and boiler steel in 605.0, p. 248.)

\section{(d) PERMISSIBLE VARIATIONS IN WEIGHT AND THICKNESS}

(Conforms to Association of American Steel Manufacturers, standard permissible variations in gage weight, gage thickness, size, and flatness of sheets and light plates blue annealed in $600.7, \mathrm{p} .117$.)

\section{(e) GALVANIZED SHEET GAGE}

The galvanized sheet gage given in Table 2 is based upon the United States standard gage for sheet and plate iron and steel; 2.5 ounces per square foot being added to the weight per square foot corresponding to a given gage number of the United States standard gage to determine the weight per square foot of the corresponding gage number of 
the galvanized sheet gage. This gage is a weight gage for finished sheet regardless of the weight of coating and has been established by custom in the United States. It appears, accordingly, that the sheet prior to coating must be rolled to such weight, depending upon the weight of coating to be applied, as to produce a sheet of the proper weight when coated. This is contrary to the principles of simplified practice, as it multiplies the number of gages to be rolled, a commercial practice which does not appear to be economically justified.

Tolerances for the weights per square foot of galvanized sheets, adopted March 28, 1929, by the Association of American Steel Manufacturers will be found in 600.7 , page 117. Those of the American Society for Testing Materials, as given in standard specifications A93-27, zinc-coated (galvanized) sheets, will be found in 604.32 , page 244 .

The resistance of galvanized sheet to corrosion depends upon the evenness and weight of coating. The weight of coating is also a factor in determining the degree of deformation which a sheet can undergo without breaking the coating. " For specifications covering this subject see A. S. T. M. A93-27. (604.32, p. 244.)

TABLE 2.-Galvanized sheet gage, weights for all classes

\begin{tabular}{|c|c|c|c|c|c|}
\hline \multirow{2}{*}{ Gage No. } & \multicolumn{2}{|c|}{$\begin{array}{l}\text { Weight per } \\
\text { square foot }\end{array}$} & \multirow{2}{*}{ Gage No. } & \multicolumn{2}{|c|}{$\begin{array}{l}\text { Weight per } \\
\text { square foot }\end{array}$} \\
\hline & Pounds & Ounces & & Pounds & Ounces \\
\hline $\begin{array}{l}8- \\
9-- \\
10- \\
11- \\
12 .\end{array}$ & $\begin{array}{l}7.031 \\
6.406 \\
5.781 \\
5.156 \\
4.531\end{array}$ & $\begin{array}{r}112.5 \\
102.5 \\
92.5 \\
82.5 \\
72.5\end{array}$ & $\begin{array}{l}22 \\
23 . \\
24 \\
25 \\
26 \\
\end{array}$ & $\begin{array}{l}1.406 \\
1.281 \\
1.156 \\
1.031 \\
0.906\end{array}$ & $\begin{array}{l}22.5 \\
20.5 \\
18.5 \\
16.5 \\
14.5\end{array}$ \\
\hline $\begin{array}{l}13 . \\
14 . \\
15 . \\
16 .\end{array}$ & $\begin{array}{l}3.906 \\
3.281 \\
2.969 \\
2.656\end{array}$ & $\begin{array}{l}62.5 \\
52.5 \\
47.5 \\
42.5\end{array}$ & $\begin{array}{l}27 . \\
28 \\
29- \\
30\end{array}$ & $\begin{array}{l}.844 \\
.781 \\
.719 \\
.656\end{array}$ & $\begin{array}{l}13.5 \\
12.5 \\
11.5 \\
10.5\end{array}$ \\
\hline $\begin{array}{l}17 \\
18 \\
19 \\
20 \\
21\end{array}$ & $\begin{array}{l}2.406 \\
2.156 \\
1.906 \\
1.656 \\
1.531\end{array}$ & $\begin{array}{l}38.5 \\
34.5 \\
30.5 \\
26.5 \\
24.5\end{array}$ & $\begin{array}{l}31 . . . \\
32 . \\
33 . \\
34-. .\end{array}$ & $\begin{array}{l}.594 \\
.562 \\
.531 \\
.500\end{array}$ & $\begin{array}{l}9.5 \\
9.0 \\
8.5 \\
8.0\end{array}$ \\
\hline
\end{tabular}

(f) TIN-PLATE GAGE

Tin plates, which consist of soft sheet steel coated with tin, and roofing terneplates in which the coating is approximately 25 per cent tin and 75 per cent lead, are measured in a unit of area known as the base box. This is an old British unit amounting to 31,360 square inches, or 217.78 square feet, is independent of weight, and corresponds to the area covered by 112 plates, each 14 by 20 inches.

The tin-plate gage is based on pounds per base box. In Table 10 are given the essential dimensions and trade symbols of the tin-plate gage as published in the Reference Book of the American Sheet \& Tin Plate Co. This gage is established by long custom and the symbols noted in the table are inherited from the British industry. It should be borne in mind that the corrosion-resisting qualities of terneplate depend on the thickness of the coating rather than on the total thickness of the plate. The term "coke tin plates" is used to designate tin plates of the lighter coating weights. "Charcoal tin plates" designates higher grades of finish, the amount of coating and the degree of finish being distinguished by letters $\mathrm{A}$ to $5 \mathrm{~A}$, the greater number of A's in the symbol, the heavier the coating. "AAA" tin plate, for example, has approximately 4 pounds of tin coating per base box. The designation "taggers tin" is usually applied to either "coke" or "charcoal plates," 65 pounds per base box or lighte:
Terneplate, used extensively as roofing, is manufactured in standard weights of coating of $8,15,20$, $25,30,32$, and 40 pounds per double base box. For roofing it is accepted practice to manufacture terneplates not lighter than IC weight (107 pounds per base box). (See U.S. Department of Commerce Simplified Practice Recommendation No. 30-28, roofing ternes 604.31, p. 241.) The United States standard gage is also used for long ternes.

Long terne, roofing terne, and tin plate are regarded by the industry as subject to the weight-gage tolerances given in A. A. S. M. (see 600.7, p. 117). Weights and permissible variations in weights, of resquared terneplates (roofing tin) are specified in Federal specification QQ-T-201 (see 604.31, p. 241).

TABLE 3.-Tin-plate gage

\begin{tabular}{|c|c|c|c|c|}
\hline \multirow[b]{2}{*}{ Trade symbol } & \multicolumn{3}{|c|}{ Weights } & \multirow[b]{2}{*}{$\begin{array}{l}\text { A pproxi- } \\
\text { mate } \\
\text { equiva- } \\
\text { lent } \\
\text { (inch) } \\
\text { thick- } \\
\text { ness } 1\end{array}$} \\
\hline & $\begin{array}{c}\text { Pounds } \\
\text { per } \\
\text { base } \\
\text { box }\end{array}$ & $\begin{array}{l}\text { Pounds } \\
\text { per } \\
\text { square } \\
\text { foot, } \\
\text { approxi- } \\
\text { mate }\end{array}$ & $\begin{array}{l}\text { Ounces } \\
\text { per } \\
\text { square } \\
\text { foot, } \\
\text { approxi- } \\
\text { mate }\end{array}$ & \\
\hline $\begin{array}{l}\text { 55-pound } \\
60 \text {-pound } \\
65 \text {-pound } \\
70 \text {-pound } \\
75 \text {-pound }\end{array}$ & $\begin{array}{l}55 \\
60 \\
65 \\
70 \\
75\end{array}$ & $\begin{array}{l}0.253 \\
.276 \\
.298 \\
.321 \\
.344\end{array}$ & $\begin{array}{l}\text { 4. } 04 \\
4.41 \\
\text { 4. } 78 \\
5.14 \\
5.51\end{array}$ & $\begin{array}{r}0.0062 \\
.0068 \\
.0073 \\
.0079 \\
.0084\end{array}$ \\
\hline $\begin{array}{l}\text { 80-pound } \\
\text { 85-pound } \\
90 \text {-pound } \\
\text { 95-pound } \\
\text { ICL }\end{array}$ & $\begin{array}{r}80 \\
85 \\
90 \\
95 \\
100\end{array}$ & $\begin{array}{l}.367 \\
.390 \\
.413 \\
.436 \\
.459\end{array}$ & $\begin{array}{l}5.88 \\
6.24 \\
6.61 \\
6.98 \\
7.35\end{array}$ & $\begin{array}{l}.0090 \\
.0096 \\
.0101 \\
.0107 \\
.0113\end{array}$ \\
\hline $\begin{array}{l}\text { IC } \\
108 \text {-pound } \\
110 \text {-pound } \\
112-\text { pound } \\
118 \text {-pound }\end{array}$ & $\begin{array}{l}107 \\
108 \\
110 \\
112 \\
118\end{array}$ & $\begin{array}{l}.491 \\
.496 \\
.505 \\
.514 \\
.542\end{array}$ & $\begin{array}{l}7.86 \\
7.93 \\
8.08 \\
8.23 \\
8.67\end{array}$ & $\begin{array}{l}.0120 \\
.0122 \\
.0124 \\
.0126 \\
.0133\end{array}$ \\
\hline $\begin{array}{l}\text { 123-pound } \\
\text { 125-pound } \\
\text { IXL } 1 \mathrm{X} \\
\text { DC }\end{array}$ & $\begin{array}{l}123 \\
125 \\
128 \\
135 \\
139\end{array}$ & $\begin{array}{l}.565 \\
.574 \\
.588 \\
.620 \\
.638\end{array}$ & $\begin{array}{r}9.04 \\
9.18 \\
9.40 \\
9.92 \\
10.21\end{array}$ & $\begin{array}{l}.0138 \\
.0141 \\
.0144 \\
.0152 \\
.0156\end{array}$ \\
\hline $\begin{array}{l}\text { 143-pound. } \\
2 \mathrm{XL} \\
2 \mathrm{X} \\
\text { 163-pound..... } \\
\text { 3XL...... }\end{array}$ & $\begin{array}{l}143 \\
148 \\
155 \\
163 \\
168\end{array}$ & $\begin{array}{l}.657 \\
.680 \\
.712 \\
.748 \\
.771\end{array}$ & $\begin{array}{l}10.51 \\
10.87 \\
11.39 \\
11.98 \\
12.34\end{array}$ & $\begin{array}{l}.0161 \\
.0167 \\
.0174 \\
.0183 \\
.0189\end{array}$ \\
\hline (2-20 & $\begin{array}{l}175 \\
180 \\
188 \\
195 \\
208\end{array}$ & $\begin{array}{l}.804 \\
.827 \\
.863 \\
.895 \\
.955\end{array}$ & $\begin{array}{l}12.86 \\
13.22 \\
13.81 \\
14.33 \\
15.28\end{array}$ & $\begin{array}{l}.0197 \\
.0203 \\
.0212 \\
.0219 \\
.0234\end{array}$ \\
\hline $\begin{array}{l}\mathrm{D} 2 \mathrm{X} \\
5 \mathrm{X} \\
6 \mathrm{X} \mathrm{L} \\
\mathrm{D} 3 \mathrm{X}\end{array}$ & $\begin{array}{l}210 \\
215 \\
228 \\
235 \\
240\end{array}$ & $\begin{array}{r}.964 \\
.987 \\
1.047 \\
1.079 \\
1.102\end{array}$ & $\begin{array}{l}15.43 \\
15.80 \\
16.75 \\
17.27 \\
17.63\end{array}$ & $\begin{array}{l}.0236 \\
.0242 \\
.0257 \\
.0264 \\
.0270\end{array}$ \\
\hline 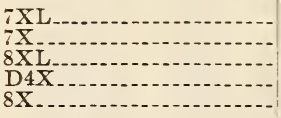 & $\begin{array}{l}248 \\
255 \\
268 \\
270 \\
275\end{array}$ & $\begin{array}{l}\text { 1. } 139 \\
1.171 \\
1.231 \\
1.240 \\
1.263\end{array}$ & $\begin{array}{l}18.22 \\
18.73 \\
19.69 \\
19.84 \\
20.20\end{array}$ & $\begin{array}{l}.0279 \\
.0287 \\
.0302 \\
.0304 \\
.0309\end{array}$ \\
\hline
\end{tabular}

1 Assuming that tin plate weighs 40.80 pounds per square foot per inch thick.

604.1 IRON AND STEEL PLATES.

604.10 GENERAL ITEMS.

AMERICAN RAILWAY ASSOCIATION, PURCHASES AND STORES DIVISION, STANDARDIZATION AND SIMPLIFICATION OF STORES STOCKS, PROCEEDINGS, 1928

\section{ANNEALED STEEL SHEETS}

(Recommended practice in stocking certain specified sizes of blue annealed steel sheets or tank steel.) 
ASSOCIATION OF AMERICAN STEEL MANUFACTURERS, STANDARD PERMISSIBLE VARIATIONS IN GAGE WEIGHT, GAGE THICKNESS, SIZE, AND FLATNESS OF SHEETS AND LIGHT PLATES BLUE ANNEALED, 1929

\section{PERMISSIBLE VARIATIONS IN SHEETS AND PLATES}

(See 600.7, p. 117.)

604.11 STEEL PLATES FOR PRESSURE VESSELS.

AMERICAN BUREAU OF SHIPPING, RULES FOR BUILDING AND CLASSING STEEL VESSELS ; MATERIAIS FOR MACHINERY, BOILERS, AND PIPING, 1930

\section{STEEL PLATES FOR BOILERS}

(For general information and requirements see 600.0, p. 1 . For test requirements see 600.1, p. 7.)

1. One tension and one quench or cold-bend test shall be taken from each plate as rolled. For plates exceeding 5,600 pounds in weight, one tension and one bend test shall be taken from each end. Quenchbend tests are required for all plates exposed to flame, or which require to be worked, hot or cold. Cold-bend tests are required for all other plates.

AMERICAN MARINE STANDARDS COMMITTEE, SPECIFICATION FOR MARINE BOILER STEEL PLATES, E NO. 27-1929

\section{MARINE BOILER STEEL PLATES}

(Identical with A. S. T. M. A114-29T, given below, including references in $114-29 \mathrm{~T}$ to A. S. T. M. A70-27, and to both A. S. T. M. and A. A. S. M. under commodity designation 605.0.)

AMERICAN RAILWAY ASSOCIATION, MECHANICAL DIVISION, STANDARD SPECIFICATIONS FOR STEEL, BOILER AND FIRE BOX FOR LOCOMOTIVE EQUIPMENT, 1925

BOILER AND FIRE-BOX STEEL FOR LOCOMOTIVES

(Identical with A. S. T. M. A30-24, given below, including the references in $\mathrm{A} 30-24$ to A. S. T. M. A70-27, and to both A. S. T. M. and A. A. S. M. under commodity designation 605.0.)

AMERICAN SOCIETY OF MECHANICAL ENGINEERS, BOILER CONSTRUCTION CODE, SPECIFICATIONS FOR STEEL BOILER PLATE, S-1, 1930

\section{STEEL BOILER PLATE}

(Identical with A. S. T. M. A70-27, given below, including the references in A70-27 to both A. S. T. M. and A. A. S. M. under commodity designation 605.0.)

AMERICAN SOCIETY OF MECHANICAL ENGINEERS, BOILER CONSTRUCTION CODE, SPECIFICATIONS FOR STEEL PLATE OF FLANGE QUALITY FOR FORGE WELDING, S-2, 1930

\section{STEEL PLATE FOR FORGE WELDING}

(Same as A. S. T. M. A89-31T, given below, including references in A89-31T to A. S. T. M. A70-27, and to both A. S. T. M. standard specifications for steel and A. A. S. M. standard specifications for structural and boiler steel under commodity designation 605.0 .)

AMERICAN SOCIETY FOR TESTING MATERIALS, STANDARD SPECIFICATIONS FOR BOILER AND FIRE-BOX STEEL FOR LOCOMOTIVES, DESIGNATION A30-24, 1924

\section{BOILER AND FIRE-BOX STEEL FOR LOCOMOTIVES}

(Identical with A. S. T. M. A70-27, given below, including references in A70-27 to both A. S. T. M. anc A. A. S. M. under commodity designation 605.0, except for the following:)

\section{SCOPE}

1. These specifications cover two classes of steel for boilers for locomotives, namely; flange and fire box.

\section{PHYSICAL PROPERTIES AND TESTS}

6. (a) The material shall conform to the following requirements as to tensile properties:

\begin{tabular}{|c|c|c|}
\hline & Flange & Fire box \\
\hline $\begin{array}{l}\text { Tensile strength } \\
\text { Yield point, minimum }\end{array}$ & $\begin{array}{l}55,000-65,000 \\
0.5 \text { tensile } \\
\text { strength. }\end{array}$ & $\begin{array}{l}52,000-62,000 \\
0.5 \text { tensile } \\
\text { strength. }\end{array}$ \\
\hline $\begin{array}{l}\text { Elongation in } 8 \text { inches, minimum } \\
\text { (See sec. } 7 \text { in } \mathrm{A} 70-27 .)\end{array}$ & $\frac{1,500,000}{\text { Tensile }}$ & 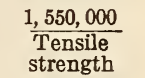 \\
\hline
\end{tabular}

(Omit paragraphs $6(b)$ and $(c)$ and $8(b)$ in A. S. T. M. A70-27 from this specification.)

3 . One tension and one bend test shall be made from each plate as rolled.

AMERICAN SOCIETY FOR TESTING MATERIALS, STANDARD SPECIFICATIONS FOR BOILER AND FIRE-BOX STEEL FOR STATIONARY SERVICE, DESIGNATION A70-27, 1927

BOILER AND FIRE-BOX STEEL FOR STATIONARY SERVICE

SCOPE

1. These specifications cover two classes of steel for boilers for stationary service, namely: Flange and fire box.

\section{MANUFACTURE}

2. The steel shall be made by the open-hearth process.

\section{CHEMICAL PROPERTIES AND TESTS}

3 . The steel shall conform to the following requirements as to chemical composition:

\begin{tabular}{|c|c|c|}
\hline & Flange & Firebox \\
\hline $\begin{array}{l}\text { Carbon: } \\
\text { For plates } 3 / 4 \text { inch or under in thickness.-.- } \\
\text { For plates over } 3 / 4 \text { inch in thickness.-.-.- }\end{array}$ & Per cent & $\begin{array}{r}\text { Per cent } \\
0.25 \\
1.30\end{array}$ \\
\hline $\begin{array}{l}\text { Manganese: } \\
\text { For plates } 3 / 4 \text { inch or under in thickness.- } \\
\text { For plates over } 3 / 4 \text { inch in thickness...- }\end{array}$ & $\begin{array}{r}0.30-0.60 \\
.30-.60\end{array}$ & $\begin{array}{r}0.30-.50 \\
.30-.60\end{array}$ \\
\hline $\begin{array}{l}\text { Phosphorus: } \\
\text { Acid. } \\
\text { Basic. } \\
\text { Sulphur. }\end{array}$ & $\begin{array}{l}1.05 \\
1.04 \\
1.05\end{array}$ & $\begin{array}{ll}1.04 \\
1.035 \\
1.04\end{array}$ \\
\hline
\end{tabular}

1 Maximum. 
4. An analysis of each melt of steel shall be made by the manufacturer to determine the percentages of the elements specified in section 3 . This analysis shall be made from a test ingot taken during the pouring of the melt. The chemical composition thus determined shall be reported to the purchaser or his representative, and shall conform to the requirements specified in section 3 .

5. An analysis may be made by the purchaser from a broken tension test specimen representing each plate as rolled. The chemical composition thus determined shall conform to the requirements specified in section 3 .

\section{PHYSICAL PROPERTIES AND TESTS}

6. (a) The material shall conform to the following requirements as to tensile properties:

\begin{tabular}{|c|c|c|}
\hline & Flange & Firebox \\
\hline Tensile strength_._._._._._. Ibs./in. ${ }^{2}$ & $55,000-65,000$ & $55,000-65,000$ \\
\hline Yield point, minimum....lbs./in. ${ }^{2}$ & $\begin{array}{l}0.5 \text { tensile } \\
\text { strength. }\end{array}$ & $\begin{array}{l}0.5 \text { tensile } \\
\text { strength. }\end{array}$ \\
\hline Elongation in 8 inches, minimum & $\begin{array}{l}\frac{1,500,000}{\text { Tensile }} \\
\text { strength }\end{array}$ & 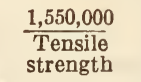 \\
\hline
\end{tabular}

(b) Tensile strength only need be determined on the test specimen taken longitudinally from the top of fire-box plates, and it shall not exceed 70,000 pounds per square inch.

(c) Should the above rule for minimum allowable elongation give a value of less than 25 per cent for fire-box steel, the minimum allowable elongation shall be 25 per cent, subject to the modifications of section 7 .

(d) The yield point shall be determined by the drop of the beam of the testing machine.

7. (a) For material over three-fourths inch in thickness, a deduction from the percentage of elongation specified in section $6(a)$ of 0.125 per cent shall be made for each increase of one-thirty-second inch of the specified thickness above three-fourths inch, to a minimum of 20 per cent.

(b) For material one-fourth inch or under in thickness, the elongation shall be measured on a gage length of twenty-four times the thickness of the specimen.

8. (a) The test specimen shall stand being bent cold through $180^{\circ}$ without cracking on the outside of the bent portion, as follows: For material 1 inch or under in thickness, around a pin the diameter of which is equal to the thickness of the specimen; and for material over 1 inch in thickness, around a pin the diameter of which is equal to twice the thickness of the specimen.

(b) Bend test for universal edge rolled plates when permitted for double-butt-strap construction.-The bend test specimen shall stand being bent cold through $180^{\circ}$ without cracking on the outside of the bent portion, as follows: For material 1 inch or under in thickness, around a pin the diameter of which is equal to one and one-half times the thickness of the specimen; and for material over 1 inch in thickness, around a pin the diameter of which is equal to three times the thickness of the specimen.

9. For fire-box steel, a sample taken from a broken tension-test specimen shall not show any single seam or cavity more than one-fourth inch in length, in either of the three fractures obtained in the test for homogeneity, which shall be made as follows:

The specimen shall be either nicked with a chisel or grooved on a machine, transversely, about onesixteenth inch deep, in three places about 2 inches apart. The first groove shall be made 2 inches from the square end; each succeeding groove shall be made on the opposite side from the preceding one. The specimen shall then be firmly held in a vise, with the first groove about one-fourth inch above the jaws, and the projecting end broken off by light blows of a hammer, the bending being away from the groove. The specimen shall be broken at the other two grooves in the same manner. The object of this test is to open and render visible to the eye any seams due to failure to weld up, or interposed foreign matter, or any cavities due to gas bubbles in the ingot. One side of each fracture shall be examined and the lengths of the seams and cavities determined, a pocket lens being used if necessary.

10. (a) Tension-test specimens shall be taken longitudinally from the top and bottom corners of the finished rolled fire-box material and from the bottom only of flange material. Bend-test specimens shall be taken transversely from the middle of the top of the finished rolled material. The longitudinal test specimen shall be taken in the direction of the longitudinal axis of the ingot, and the transverse test specimen at right angles to that axis.

(b) Tension and bend test specimens shall be of the full thickness of material as rolled, and shall be machined to the form and dimensions shown in Figure 128 in A. S. T. M. standard specifications for steel (see 605.0, p. 247); except that bend-test specimens may be machined with both edges parallel.

11. (a) Two tension and one bend test shall be made from each fire-box steel plate as rolled. One tension and one bend test shall be made from each flange steel plate as rolled.

(b) If any test specimen shows defective machining or develops flaws, it may be discarded and another specimen substituted.

(c) If the percentage of elongation of any tension test specimen is less than that specified in section $6(a)$ and any part of the fracture is outside the middle third of the gage length, as indicated by scribe scratches marked on the specimen before testing, a retest shall be allowed.

\section{PERMISSIBLE VARIATIONS IN WEIGHT AND THICKNESS}

12. The thickness of each plate shall not vary more than 0.01 inch under that ordered.

(For permissible overweights of plates ordered to thickness see Table 2 in Association of American Steel Manufacturers standard specification for structural and boiler steel (605.0, p. 247).)

13. The overweight of each lot (for definition of "lot" also see A. A. S. M. specification under 605.0) in each shipment shall not exceed the amount specified in the A. A. S. M. Table 2 referred to above. One cubic inch of rolled steel is assumed to weigh 0.2833 pound.

\section{MARKING}

(a) The name or brand of the manufacturer, the manufacturer's test identification number, class, and lowest tensile strength specified shall be legibly stamped on each finished plate in two places not less than 12 inches from the edges and on each butt strap near the center line not less than 12 inches from each end. The manufacturer's test identification number shall be legibly stamped on each test specimen.

(b) When specified on the order, plates shall be match marked as defined in paragraph (c) so that the test specimens representing them may be identified. When the plate rolled from a slab or ingot is cut into two or more smaller plates, each shall be match marked so that they may all be identified with the test specimens representing them.

(c) Each match mark shall consist of two overlapping circles, each not less than $1 \frac{1 / 2}{2}$ inches in 
diameter, placed upon the shear lines, and made by separate impressions of a single-circle steel die.

(d) Match-marked coupons shall match with the sheets represented and only those which match properly shall be accepted.

(Requirements covering finish, inspection and rejection, and rehearing conform to those specified in A. S. T. M. standard specifications for steel. See 605.0 , p. 247.)

AMERICAN SOCIETY FOR TESTING MATERIAIS, STANDARD SPECIFICATIONS FOR STEEL PLATES OF FLANGE QUALITY FOR FORGE WELDING, DESIGNATION A89-30, 1930

\section{STEEL PLATES OF FLANGE QUALITY FOR FORGE WELDING}

1. These specifications cover steel plates of flange quality suitable for forge welding, without the use of fluxes, where the weld is not reinforced.

\section{MANUFACTURE}

2. The steel shall be made by the open-hearth process.

\section{CHEMTCAL PROPERTIES AND TESTS}

3. (a) The steel shall conform to the following requirements as to chemical composition:

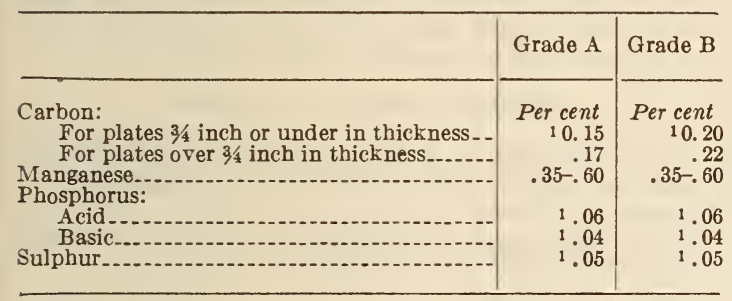

1 Maximum.

(b) The steel should preferably be free from nickel and chromium. When these elements are present the maximum quantity of either one shall not exceed 0.05 per cent. Unless otherwise specified, the silicon shall not exceed 0.05 per cent.

4. An analysis of each melt of steel shall be made by the manufacturer to determine the percentages of carbon, manganese, phosphorus and sulphur. This analysis shall be made from a test ingot taken during the pouring of the melt. The mechanical composition thus determined shall be reported to the purchaser or his representative, and shall conform to the requirements specified in section 3 .

5. An analysis may be made by the purchaser from a broken tension-test specimen representing each plate as rolled. The chemical composition thus determined shall conform to the requirements specified in section 3 .

\section{PHYSICAL PROPERTIES AND TESTS}

6. (a) The material shall conform to the following minimum requirements as to tensile properties:

\begin{tabular}{|c|c|c|}
\hline & Grade A & Grade B \\
\hline 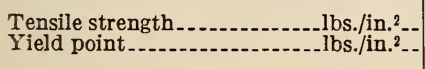 & $\begin{array}{l}45,000 \\
0.5 \text { tensile }\end{array}$ & $\begin{array}{l}50,000 \\
0.5 \text { tensile }\end{array}$ \\
\hline $\begin{array}{l}\text { But in no case less than } \\
\text { Elongation in } 8 \text { inches. }\end{array}$ & $\begin{array}{r}24,000 \\
30\end{array}$ & 27,090 \\
\hline
\end{tabular}

(b) The yield point shall be determined by the drop of the beam of the testing machine.

7. (a) For material over three-fourths inch in thickness, a deduction from the percentages of elongation specified in section $6(a)$ of 1.25 per cent shall be made for each increase of one-thirty-second inch of the specified thickness above three-fourths inch.

(b) For material under five-sixteenths inch in thickness, a deduction from the percentage of elongation in 8 inches specified in section $6(a)$ of 1.25 per cent shall be made for each decrease of one-thirty-second inch of the specified thickness below five-sixteenths inch.

8. The test specimen shall stand being bent cold through $180^{\circ}$ without cracking on the outside of the bent portion, as follows: For material 1 inch or under in thickness, around a pin the diameter of which is equal to the thickness of the specimen; and for material over 1 inch in thickness, around a pin the diameter of which is equal to twice the thickness of the specimen.

9. (a) Tension test specimen shall be taken longitudinally from the bottom of the finished rolled material, and bend test,specimens shall be taken transversely from the middle of the top of the finished rolled material. The longitudinal test specimens shall be taken in the direction of the longitudinal axis of the ingot, and the transverse test specimens at right angles to that axis.

(b) Tension and bend test specimens shall be of the full thickness of material as rolled, and shall be machined to the form and dimensions shown in Figure 128 in A. S. T. M. standard specifications for steel (see 605.0, p. 247), except that bend test specimens may be machined with both edges parallel.

(c) Test specimens for plates over $1 \frac{1}{2}$ inches in thickness may be machined to a thickness or diamameter of at least three-fourths inch for a length of at least 9 inches.

(d) The machined sides of rectangular bend test specimens may have the corners rounded to a radius not over one-sixteenth inch.

10. (a) One tension and one bend test shall be made from each plate as rolled.

(b) If any test specimen shows defective machining or develops flaws, it may be discarded and another specimen substituted.

(c) If the percentage of elongation of any tension test specimen is less than that specified in section 6 (a) and any part of the fracture is outside the middle third of the gage length, as indicated by scribe scratches marked on the specimen before testing, a retest shall be allowed.

\section{PERMISSIBLE VARIATIONS IN WEIGHT AND THICKNESS}

11. The thickness of each plate shall not vary more than 0.01 inch under that ordered.

The overweight of each lot in each shipment shall not exceed the amount given in Table 2 in Association of American Steel Manufacturers under 605.0, p. 248. One cubic inch of rolled steel is assumed to weigh $0.2833 \mathrm{lb}$.

\section{FINISH}

12. The finished material shall be free from injurious defects and shall have a workmanlike finish.

\section{MARKING}

13. (a) The name or brand of the manufacturer, melt or slab number, class, and tensile strength for the grade specified in section $6(a)$ shall be legibly stamped on each plate. The melt or slab number shall be legibly stamped on each test specimen.

(b) When specified on the order, plates shall be match marked as defined in paragraph (c) so that 
the test specimens representing them may be identified. When the plate rolled from a slab or ingot is cut into two or more smaller plates, each shall be match marked so that they may all be identified with the test specimens representing them.

(c) Each match mark shall consist of two overlapping circles each not less than $1 \frac{1}{2}$ inches in diameter, placed upon the shear lines, and made by separate impressions of a single-circle steel die.

(d) Match-marked coupons shall match with the sheets represented and only those which match properly shall be accepted.

(In respect to inspection and rejection, and rehearing, this specification conforms to A. S. T. M. standard specifications for steel. See 605.0 , p. 247. For table of permissible overweights of plates ordered to thickness, see Association of American Steel Manufacturers, standard specifications for structural and boiler steel, also under 605.0, p. 248.)

\section{AMERICAN SOCIETY FOR TESTING MATE- RIALS, TENTATIVE SPECIFICATIONS FOR STEEL PLATES OF FLANGE AND FIRE-BOX QUALITIES FOR FORGE WELD- ING, DESIGNATION A89-31T, 1931}

\section{STEEL PLATES FOR FORGE WELDING}

(In respect to ladle and check analyses, determination of yield point, modifications in elongation, bend tests, homogeneity tests, test specimens, number of tests, permissible variations, and marking, these specifications are substantially the same as A. S. T. M., A70-27, given above, including the references in A70-27 to both A. S. T. M. standard specifications for steel and A. A. S. M. standard specifications for structural and boiler steel under commodity designation 605.0. In addition these specifications provide for the following requirements:)

\section{$\mathrm{SCOPE}$}

1. These specifications cover two grades of steel plate of flange and fire-box qualities suitable for forge welding without the use of fluxes where the weld is not reinforced.

\section{MANUFACTURE}

2. The steel shall be made by either or both the following processes: Open-hearth or electric furnace.

\section{CHEMICAL COMPOSITION AND TESTS}

3. (a) The steel shall conform to the following requirements as to chemical composition:

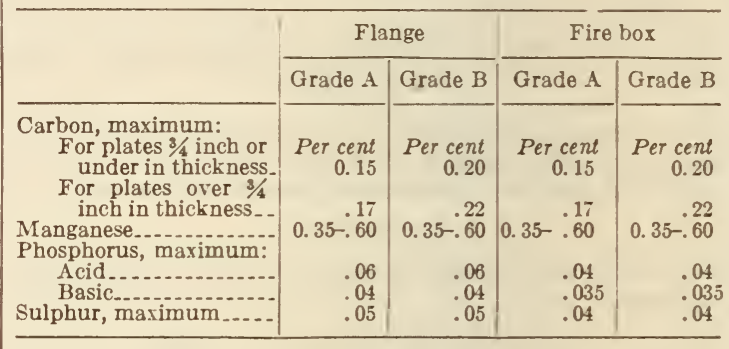

(b) The steel should preferably be free from nickel ${ }^{50}$ and chromium. When these elements are present the maximum quantity of either one shall not exceed 0.05 per cent. Unless otherwise specified, the silicon shall not exceed 0.05 per cent.

\section{PHYSICAL PROPERTIES AND TESTS}

4. (a) The material shall conform to the following minimum requirements as to tensile properties:

30 When these specifications are used for material to be fusion welded, the maximum limit for nickel shall not apply.

\begin{tabular}{|c|c|c|c|c|}
\hline & \multicolumn{2}{|c|}{ Flange } & \multicolumn{2}{|c|}{ Fire box } \\
\hline & Grade A & Grade B & Grade A & Grade B \\
\hline $\begin{array}{l}\text { Tensile strength } \\
\text { Yield point } \\
\text { But in no case less than } \\
\text { Elongation in } 8 \text { inches }{ }^{b} \\
\text { Elongation in } 2 \text { inches. }\end{array}$ & $\begin{array}{r}45,000 \\
0.5 \text { tensile strength. } \\
24,000 \\
\frac{1,500,000}{\text { tensile strength }} \\
\frac{1,600,000}{\text { tensile strength }}\end{array}$ & $\begin{array}{r}50,000 \\
0.5 \text { tensile strength. } \\
27,000 \\
\frac{1,500,000}{\text { tensile strength }} \\
\frac{1,600,000}{\text { tensile strength }}\end{array}$ & $\begin{array}{r}a 45,000 \\
0.5 \text { tensile strength. } \\
24,000 \\
\frac{1,550,000}{\text { tensile strength }} \\
\frac{1,650,000}{\text { tensile strength }}\end{array}$ & $\begin{array}{l}\quad b 50,000 \\
0.5 \text { tensile strength. } \\
27,000 \\
\frac{1,550,000}{\text { tensile strength }} \\
\frac{1,650,000}{\text { tensile strength }}\end{array}$ \\
\hline
\end{tabular}

a See par. (b).

$b$ See modifications in elongation in A. S. T. M., A70-27, above.

(b) Tensile strength only need be determined on the test specimen taken longitudinally from the top of fire-box plates, and it shall not exceed 60,000 pounds per square inch for grade $A$ and 65,000 pounds per square inch for grade B.

\section{TEST SPECIMENS}

5. Tension-test specimens for plates over $1 \frac{1 / 2}{2}$ inches in thickness may be machined to a thickness or diameter of at least three-fourth inch for a length of at least 9 inches, or they may conform to the dimensions shown in Figure 129 in A. S. T. M. standard specifications for steel. (See 605.0, p. 247.)

6 . Bend test specimens for plates over $11 / 2$ inches in thickness may be machined to a thickness or diameter of at least three-fourth inch, or to $1 \mathrm{by}$ $1 / 2$ inch in section.

7. The machined sides of rectangular bend test specimens may have the corners rounded to a radius not over one-sixteenth inch.

\section{MARKING}

8. The name or brand of the manufacturer, the manufacturer's test identification number, class, and lowest tensile strength for the grade specified above under physical properties and tests shall be legibly stamped on each plate. The manufacturer's test identification number shall be legibly stamped on each test specimen. 
AMERICAN SOCIETY FOR TESTING MATERIALS, TENTATIVE SPECIFICATIONS FOR MARINE-BOILER STEEL PLATES, DESIGNATION A114-29T, 1929

\section{MARINE-BOILER STEEL PLATES}

\section{SCOPE}

1. These specifications cover two grades of steel for marine boilers; namely, grade A and grade B.

\section{MANUFACTORE}

2. The steel shall be made by the open-hearth process.

\section{CHEMTCAL PROPERTIES AND TESTS}

3. The steel shall conform to the following requirements as to chemical composition:

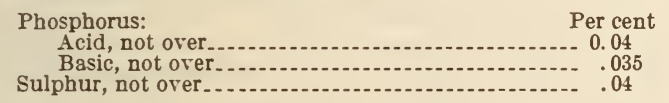

\section{LADLE AND CHECK ANALYSIS}

(Conform to A. S. T. M., A70-27, given above.)

\section{PHYSICAL PROPERTIES AND TESTS}

4. (a) The material shall conform to the following requirements as to tensile properties:

\begin{tabular}{|c|c|c|}
\hline & Grade A & Grade B \\
\hline $\begin{array}{l}\text { Tensile strength } \\
\text { Yield point, minimum }\end{array}$ & $\begin{array}{l}60,000-70,000 \\
0.5 \text { tensile }\end{array}$ & $\begin{array}{l}55,000-65,000 \\
0.5 \text { tensile }\end{array}$ \\
\hline Elongation in 8 inches, minimum & $\begin{array}{l}\text { strength. } \\
\frac{1,500,000}{\text { Tensile }} \\
\text { strength }\end{array}$ & $\begin{array}{l}\text { strength. } \\
\frac{1,550,000}{\text { Tensile }} \\
\text { strength }\end{array}$ \\
\hline
\end{tabular}

(b) The yield point shall be determined by the drop of the beam of the testing machine.

\section{MODIFICATION IN ELONGATION}

5. For material over three-fourth inch in thickness, a deduction from the percentages of elongation specified in section $6(a)$ of 0.125 per cent shall be made for each increase of one-thirty-second inch of the specified thickness above three-fourth inch to a minimum of 20 per cent for grade A steel and 22 per cent for grade B steel.

\section{BEND-TESTS}

6. (a) The test specimen for grade A steel shall stand being bent cold through $180^{\circ}$ without cracking on the outside of the bent portion, as follows: For material 1 inch or under in thickness, around a pin the diameter of which is equal to one and one-half times the thickness of the specimen; for material over 1 inch to and including $1 \frac{1}{2}$ inches in thickness, around a pin the diameter of which is equal to three times the thickness of the specimen; and for material over $1 \frac{1}{2}$ inches in thickness, around a pin the diameter of which is equal to four times the thickness of the specimen.

(b) The test specimen for grade B steel shall stand being bent cold through $180^{\circ}$ without cracking on the outside of the bent portion, as follows: For material 1 inch or under in thickness, around a pin the diameter of which is equal to the thickness of the specimen; for material over 1 inch to and including
$1 \frac{1}{2}$ inches in thickness, around a pin the diameter of which is equal to twice the thickness of the specimen; and for material over $1 \frac{1}{2}$ inches in thickness, around a pin the diameter of which is equal to three times the thickness of the specimen.

\section{TEST SPECIMENS}

7. (a) The tension-test specimens shall be taken longitudinally from the finished rolled material. In the case of plates rolled from ingots, these specimens shall be taken in the direction of the longitudinal axis of the ingot; one from the top and one from the bottom of the same side of the plates as rolled. In the case of plates rolled from slabs, the tension test specimens shall be taken from diagonal corners.

(b) In the case of plates rolled from ingots, the bend-test specimen shall be taken from the middle of the top of the finished rolled material at right angles to the longitudinal axis of the ingot. In the case of plates rolled from slabs, it shall be taken from the middle of one end at right angles to the longitudinal axis of the plate as rolled.

(c) Tension and bend test specimens shall be of the full thickness of the material as rolled, and shall be machined to the form and dimensions shown in Figure 128 in A. S. T. M. standard specifications for steel (see 605.0, p. 247.); except that bend-test specimens may be machined with both edges parallel.

(d) The machined sides of rectangular bend test specimens may have the corners rounded to a radius not over one-sixteenth inch.

8. (a) Two tension tests and one bend test shall be made from each plate as rolled.

(Requirements covering flaws in or defective machining of test specimens, also retests of tests specimens, are identical with paragraphs $11(b)$ and (c) in A. S. T. M., A70-27, given above.)

\section{PERMISSIBLE VARIATIONS IN WEIGHT AND THICKNESS}

(Identical with those specified in A. S. T. M. A70-27, given above, including references in A70-27 to Table 2 in Association of American Steel Manufacturers standard specification for structural and boiler steel (see 605.0. p. 248), and definition of term "lot".)

\section{MARKING}

9. (a) The name of the manufacturer, the place where manufactured, marine $\mathrm{A}$ or $\mathrm{B}$, the manufacturer's test identification number, and the tensile strength as specified in paragraphs (b) and (c) shall be legibly stamped in two places on each finished plate not less than 12 inches from the edges. Each butt strap shall be stamped near the middle of the plate. The manufacturer's identification number shall be legibly stamped on each test specimen.

(b) Plates not over one-half inch in thickness to be used in externally fired boilers for steamers navigated under the provisions of title $\mathrm{L} 11$. United States Revised Statutes, shall be stamped with the least tensile strength, expressed in thousands, as determined by the tests.

(c) Plates other than those described in paragraph (b) shall be stamped with the minimum tensile strength specified.

(d) The manufacturer shall furnish facilities to the representative of the United States Steamboat Inspection Service and, when required, to the representative of the ship classification society, to place their official monogram stamp adjacent to the stamping specified in paragraph $(a)$.

(Requirements covering finish, inspection and rejection, and rehearing conform to those specified in A. S. T. M. standard specifications for steel (see 605.0, p. 247).) 
AMERICAN SOCIETY FOR TESTING MATERIALS, TENTATIVE SPECIFICATIONS FOR OPEN-HEARTH IRON PLATES OF FLANGE QUALITY, DESIGNATION A129-30T, 1930

\section{IRON PLATES}

\section{SCOPE}

1. These specifications cover three types of openhearth iron plates of flange quality.

\section{MANUFACTURE}

2. The iron shall be made by the basic openhearth process.

\section{CHEMTCAL PROPERTIES AND TESTS}

3 . The iron shall conform to the following requirements as to chemical composition:

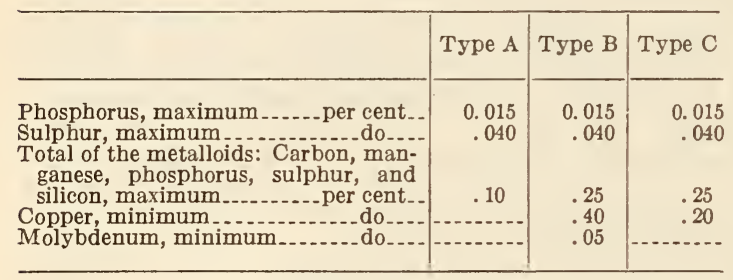

\section{IADLE AND CHECK ANALYSES}

4. Ladle analyses.-(Conform to A. S. T. M., A70-27, given above.)

5. Check analyses. - An analysis may be made by the purchaser from a broken tension-test specimen representing each plate as rolled, in which a variation of plus 0.01 per cent in sulphur, minus 0.02 per cent in copper, and plus 0.04 per cent in the total of the five metalloids will be permitted.

\section{PHYSICAL PROPERTIES AND TESTS}

6. (a) The material shall conform to the following minimum requirements as to tensile properties:

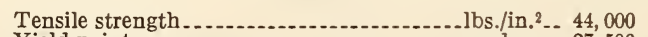

Yield point_..........

Elongation in 8 inches...................... 25

(b) The yield point shall be determined by the drop of the beam of the testing machine.

7. (a) For material over three-fourths inch in thickness, a deduction from the percentages of elongation specified in section $6(a)$ of 0.125 per cent shall be made for each increase of one-thirty-second inch of the specified thickness above three-fourth inch.

(b) For material under five-sixteenths inch in thickness, a deduction from the percentage of elongation in 8 inches specified in section $6(a)$ of 1.25 per cent shall be made for each decrease of one thirty-second inch of the specified thickness below five-sixteenths inch.

8. The test specimen shall stand being bent cold through $180^{\circ}$ without cracking on the outside of the bent portion, as follows: For material 1 inch or under in thickness, flat on itself; and for material over 1 inch in thickness, around a pin the diameter of which is equal to the thickness.

\section{TEST SPECIMENS}

9. (a) Tension test specimens shall be taken parallel to the longitudinal axis and from the bottom of the plate as rolled. Bend-test specimens shall be taken from the middle of the top and at right angles to the longitudinal axis of the plate as rolled. The longitudinal test specirnen shall be taken in the direction of rolling, and the transverse specimen at right angles to that direction.

(b) Tension and bend test specimens shall be of the full thickness of the material as rolled and shall be machined to the form and dimensions shown in Figure 128 in A. S. T. M. standard specifications for steel (see 605.0, p. 247); except that bend-test specimens may be machined with both edges parallel.

(c) Test specimens for plates over $1 \frac{1}{2}$ inches in thickness may be machined to a thickness or diameter of at least three-fourths inch for a length of at least 9 inches.

(d) The machined sides of rectangular bend-test specimens may have the corners rounded to a radius not over one-sixteenth inch.

\section{NOMBER OF TESTS}

10. (a) One tension and one bend test shall be made from each plate as rolled.

(b) If any test specimen shows defective machining or derelops flaws, it may be discarded and another specimen substituted.

(c) If the percentage of elongation of any tensiontest specimen is less than that specified in section 6 (a), and any part of the fracture is outside the middle third of the gage length, as indicated by scribe scratches marked on the specimen before testing, a retest shall be allowed.

\section{PERMISSIBLE VARIATIONS IN WEIGHT AND THICENESS}

11. The thickness of each plate shall not vary more than 0.01 inch under that ordered.

(For permissible overweights of plates ordered to thickness, see Table 2 in Association of American Steel Manufacturers standard specification for structural and boiler steel (605.0, p. 248).)

The overweight of each lot (for definition of "lot" also see A. A. S. M. specification under 605.0) in each shipment shall not exceed the amount specified in the A. A. S. M. table referred to above. One cubic inch of rolled open-hearth iron is assumed to weigh 0.2833 pound.

(Requirements covering marking are substantially the same as in A. S. T. M., A70-27, given above. Requirements covering finish, inspection, and rejection, and rehearing conform to those specified in A. S. T. M. standard specifications for steel (see 605.0, p. 247).)

\section{ASSOCIATION OF AMERICAN STEEL MAN- UFACTURERS, STANDARD SPECIFICA- TIONS FOR STRUCTURAL AND BOILER STEEL, NOVEMBER 24, 1922}

(See also 605.11, p. 250.)

\section{BOILER STEEL}

(This specification is, in certain particulars, substantially the same as specification A7 of the American Society for Testing Materials. For requirements relating to process, ladle analysis, and test specimens, see A7 (605.11, p. 249), paragraphs 2, 4, and $8(a),(c),(d),(e)$, and $(j)$, omitting reference to shapes and eyebar flats in paragraph $8(e)$. For requirements relating to yield point, number of tests, finish, inspection, rejection, and test specimen figures, see A. S. T. M. specifications (605.0, p. 247), paragraphs $2,3(c), 5,7$, and $8(b)$, and Figures 128 and 129 , omitting, in paragraph $3(c)$, reference to 2-inch test specimen.)

(For tables of permissible variations in weight and thickness of rectangular sheared plates when ordered 
to unit weight and when ordered to thickness, permissible variations in weight of bar sizes of angles, tees, zees, and channels, and permissible variations in size of hot-rolled rounds, squares, hexagons, and flats, see A. A. S. M., Tables $1,2,3,4$, and 5 (605.0, p. 24S).)

1. Classes. - There shall be three classes of steel for boilers, namely: Flange, fire box, and boiler rivet.

2. Chemical composition. - The steel shall conform to the following requirements as to chemical composition:

\begin{tabular}{|c|c|c|c|}
\hline Elements considered & $\begin{array}{l}\text { Flange } \\
\text { steel }\end{array}$ & $\begin{array}{l}\text { Fire-box } \\
\text { steel }\end{array}$ & $\begin{array}{l}\text { Boiler- } \\
\text { rivet } \\
\text { steel }\end{array}$ \\
\hline & Per cent & Per cent & Per cent \\
\hline Manganese & - & - & $0.30-0.50$ \\
\hline $\begin{array}{l}\text { For plates } \\
\text { thickness inch or under in }\end{array}$ & $0.30-0.60$ & $0.30-0.50$ & \\
\hline For plates over $3 / 4$ inch in & $.30-.60$ & $.30-.60$ & \\
\hline Phosphorus, maximum: & & & \\
\hline 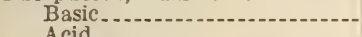 & .04 & .035 & .04 \\
\hline Sulphur, maximum & .05 & $\begin{array}{l}.04 \\
.04\end{array}$ & .04 \\
\hline
\end{tabular}

3. Check analyses.-An analysis may be made by the purchaser from a broken tension test specimen representing each plate as rolled, or from finished material representing each melt of rivet steel. The chemical composition thus determined shall conform to the requirements specified in the section on chemical composition.

4. Tension tests. - The steel shall conform to the following requirements as to tensile properties:

\begin{tabular}{|c|c|c|c|}
\hline Properties considered & Flange steel & Fire-box steel & $\begin{array}{l}\text { Boiler-rivet } \\
\text { steel }\end{array}$ \\
\hline $\begin{array}{l}\text { Tensile strength } \\
\text { Yield point, minimum } \\
\text { Elongation in } 8 \text { inches, } \\
\text { minimum. per cent }\end{array}$ & $\begin{array}{l}55,000-65,000 \\
0.5 \text { tensile } \\
\text { strength. } \\
\frac{11,450,000}{\text { Tensile }} \\
\text { strength }\end{array}$ & $\begin{array}{l}52,000-62,000 \\
\begin{array}{l}0.5 \text { tensile } \\
\text { strength. }\end{array} \\
\frac{11,500,000}{\text { Tensile }} \\
\text { strength }\end{array}$ & $\begin{array}{l}45,000-55,000 \\
\begin{array}{l}0.5 \text { tensile } \\
\text { strength. } \\
\frac{1,450,000}{\text { Tensile }} \\
\text { strength }\end{array}\end{array}$ \\
\hline
\end{tabular}

1 See section on modifications in elongation.

5. Modifications in elongation.-(a) For plates over three-fourths inch in thickness a deduction from the percentage of elongation specified in table of tensile properties shall be made in the proportion of 0.5 for each increase of one-eighth inch in thickness above three-fourths inch, to the minima of 20 per cent for flange steel and 22 per cent for fire-box steel.

(b) For plates under five-sixteenths inch in thickness a deduction from the percentage of elongation specified in table of tensile properties shall be made in the proportion of 2.5 for each decrease of onesixteenth inch in thickness below five-sixteenths inch.

6. Bend tests. - (a) Cold-bend tests shall be made on the material as rolled.

(b) Quench-bend test specimens, before bending, shall be heated to a light cherry red as seen in the dark (about $1,200^{\circ} \mathrm{F}$.), and quenched in water the temperature of which is about $80^{\circ} \mathrm{F}$.

(c) Specimens for cold-bend and quench-bend tests of flange and fire-box steel shall bend through $180^{\circ}$ without fracture on the outside of the bent portion, as follows: For plates 1 inch or under in thickness, flat on themselves; for plates over 1 inch to and including $11 / 2$ inches in thickness, around a pin the diameter of which is equal to the thickness of the specimen; and for plates over $11 / 2$ inches in thick- ness, around a pin the diameter of which is equal to one and one-half times the thickness of the specimen.

(d) Specimens for coal-bend and quench-bend tests of boiler rivet steel shall bend through $180^{\circ}$ flat on themselves without fracture on the outside of the bent portion.

7. Test specimens.-Test specimens for rivet bars shall be of the full section of the bars as rolled.

8. Number of tests.-(a) One tension, one coldbend, and one quench-bend test shall be made from each plate as rolled.

(b) Two tension, two cold-bend and two quenchbend tests shall be made from each melt of rivet steel.

(c) If any test specimen shows defective machining or develops flaws, it may be discarded and another specimen substituted.

9. Permissible variations.- $(a)$ The thickness or weight of sheared mill plates shall conform to the manufacturers' standard practice governing the permissible variations for sheared plates. (See A. A. S. M., 605.0, p. 248.)

(b) The dimensions of rivet bars shall conform to the manufacturers' standard practice governing the allowable variations in the size of hot-rolled bars. (See A. A. S. M., 605.0, p. 248.)

10. Marking.-(a) The name or brand of the manufacturer, the manufacturer's test identification number, class, and the minimum tensile strength for its class specified in table of tensile properties shall be legibly stamped on each plate. The test identification number shall be legibly stamped on each test specimen.

(b) Rivet steel shall be shipped in securely fastened bundles, with the manufacturer's name and melt number legibly stamped on attached metal tags.

\section{UNITED STATES GOVERNMENT, FEDERAL SPECIFICATIONS BOARD, FEDERAL SPECIFICATION FOR STEEL PLATES, MARINE BOILER, QQ-S-691, MARCH 1, 1932}

\section{MARINE BOILER STEEL PLATES}

(This specification conforms to A. S. T. M. A114-29T, given above, except that a third class of marine boiler steel is provided for here. This is known as class $\mathrm{C}$, and may be made by the Bessemer process. It has no specified physical and chemical properties. Class C plates are intended for smokestacks, boiler casings, uptakes, etc. Included in F. S. B. No. 549 (QQ-S-691) as part of the specification is F. S. B. $339 a$ (QQ-M-151), general specifications for metals, for which see 600.00, p. 3.)

\section{UNITED STATES GOVERNMENT, DEPART- MENT OF COMMERCE, STEAMBOAT IN- SPECTION SERVICE, GENERAL RULES AND REGULATIONS, MAY 4, 1929}

\section{BOILER PLATE}

\section{CHEMLICAL PROPERTIES, STEEL PLATES}

1. Steel plates shall be made by the open-hearth process, except that steel for plates to be used in the manufacture of boiler tubes may be made by the Bessemer process.

Open-hearth steel shall contain not more than 0.04 per cent of phosphorus nor more than 0.05 per cent of sulphur.

The manufacturer shall furnish the inspector, with each order tested, a certificate stating the process by which the steel was manufactured and a 
copy of the analysis of each melt. The analysis may, if deemed expedient by the supervising inspector general, be verified at the expense of the manufacturer. (Secs. 4405, 4430, R. S.)

\section{PHYSICAL PROPERTIES, STEEL PLATES}

2. All steel plates tested shall show an elongation of at least 20 per cent measured in a gage length or 8 inches. (Sec. 4430, R. S.)

\section{PHYSICAL PROPERTIES, IRON PLATES}

3. The tensile strength shall be not less than 45,000 pounds per square inch. The elongation shall be not less than 15 per cent. The reduction of area shall be not less than 15 per cent for 45,000 pounds tensile strength, and for each increase of 1,000 pounds tensile strength up to 55,000 pounds, an addition of 1 shall be made to the required percentage of reduction of area. The bend test specimen shall bend cold through $90^{\circ}$ around a curve the radius of which is not greater than one and one-half times the thickness of the specimen. (Sec.4430, R.S.)

4. All tension test specimens shall be milled to the form as shown in Figure 127, with the following dimensions: Length at least 16 inches, ends from $1 \frac{1}{2}$ to $31 / 2$ inches wide by about 3 inches in length, and parallel section at center 1 to $1 \frac{1}{2}$ inches wide by 9 inches in length. The percentage of elongation shall be measured in a gage length of 8 inches.

5. Where specimens are to be tested on the testing machines of the steamboat inspection service, they

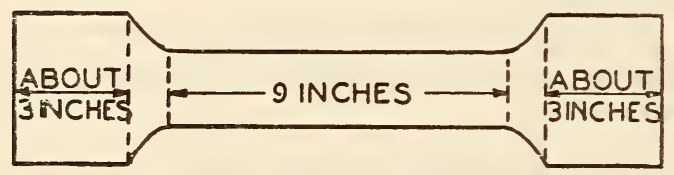

Figure 127

shall be 1 inch wide at parallel section in center, and shall not exceed 2 inches in width on the ends.

6. All bend test specimens shall be at least 12 inches in length and from 1 to $3 \frac{1}{2}$ inches in width, and the full thickness as rolled. The edges may be planed. The corners shall not be rounded, but the sharpness may be removed with a fine file. After bending, the specimens shall show no cracks or flaws on the outside of the bent portion.

\section{TEST SPECIMENS FOR STEEL PLATES}

7. Two tension tests and one cold-bend test shall be made from each plate as first rolled from a billet, slab, or ingot, the tensile test specimens to be taken from two diagonal corners of the plate, and a coldbend specimen to be taken transversely from the middle of the top of the plate.

8. The bend test specimen shall bend cold $180^{\circ}$ around a pin without showing any sign of cracking or other injurious defects on the outside of the bent portion, as follows: For plates under 1 inch in thickness the diameter of the pin shall be equal to one and one-half times the thickness of the plate; for plates 1 inch and over, up to and including 11/2 inches in thickness, the diameter of the pin shall be equal to three times the thickness of the plate, and for all plates over $1 \frac{1}{2}$ inches in thickness the diameter of the pin shall be equal to four times the thickness of the plate.

9. The finished material shall be free from all injurious defects, and shall have a good and workmanlike finish.

10. All measurements of test specimens and material shall be made by any standard American gage, and record of tests shall be submitted on Form 934. (Secs. 4405, 4430, R. S.)
604.12 STEEL PLATES FOR NONPRESSURE TANKS AND STACKS.

UNITED STATES GOVERNMENT, FEDERAL SPECIFICATIONS BOARD, FEDERAL SPECIFICATION FOR STEEL PLATES, MARINE BOILER, QQ-S-691) MARCH 1, 1932.

\section{SMOKESTACKS, UPTAKES}

(See 604.11. p. 233. with references to A. S. T. M. A114, and F. S. B. No. 339a [QQ-M-151].)

604.13 STEEL PLATES FOR SHIP HULIS.

AMERICAN BUREAU OF SHIPPING, RULES FOR BUILDING AND CLASSING STEEL VESSELS; QUALITY AND TESTING OF MATERIALS-SHIP CONSTRUCTION, 1930

\section{STEEL PLATES}

(See 605.15. p. 268.)

AMERICAN BUREAU OF SHIPPING, RULES FOR BUILDING AND CLASSING STEEL VESSELS; QUALITY AND TESTING OF MATERIALS-SHIP CONSTRUCTION, 1930

IRON PLATES AND ANGLES

(See 604.14. p. 234.)

AMERICAN SOCIETY FOR TESTING MATERIALS, STANDARD SPECIFICATIONS FOR STRUCTURAL STEEL FOR SHIPS, DESIGNATION A12-21, 1921

STRUCTURAL STEEL FOR SHIPS

(See 605.15. p. 269.)

ASSOCIATION OF AMERICAN STEEL MANUFACTURERS, STANDARD SPECIFICATIONS FOR STRUCTURAL AND BOILER STEEL, NOVEMBER 24, 1922

\author{
STRUCTURAL AND BOILER STEEL
}

(See 605.11. p. 250.)

UNITED STATES GOVERNMENT, FEDERAL SPECIFICATIONS BOARD, FEDERAL SPECIFICATION FOR STEEL; STRUC. TURAL (INCLUDING STEEL FOR COLD FLANGING), AND STEEL, RIVET FOR SHIPS OTHER THAN NAVAL VESSELS, QQ-S-751, JUNE 23, 1931

\section{STEEL SHIP PLATES}

(See 605.15. p. 270.)

604.14 WROUGHT-IRON PLATES.

AMERICAN BUREAU OF SHIPPING, RULES FOR BUILDING AND CLASSING STEEL VESSELS; QUALITY AND TESTING OF MATERIALS-SHIP CONSTRUCTION, 1930

\section{IRON PLATES AND ANGLES}

(For general information and requirements see 600.0 . p. 2.)

1. Iron plates and angles are to be of the best quality and free from surface pitting and lamination, 
and legibly stamped with the manufacturer's name or trade-mark. They are to have a tensile strength of $4 \mathrm{~S}, 000$ pounds per square inch of prepared section with the fiber and an elongation of 12 per cent in 8 inches. For plates under seven-sixteenths inch in thickness a deduction of one from the percentage of elongation shall be made for each decrease of onesixteenth inch in thickness below seven-sixteenths inch.

2. Tension and bend test specimens shall be taken from the finished plates and must be of the full thickness of the plates as rolled. A test specimen must stand being bent cold through $90^{\circ}$ without fracture on the outside of the bent portion, the radius of the bend being one and one-half times the thickness of the specimen. One tension and one coldbend test shall be made for each variation of thickness of one-eighth inch and not less than one test for each 10 plates as rolled.

3 . For angle bars pieces shall be heated red and so bent as to have their flanges shut until the outside edges touch and to have their flanges flattened out without fracture or cracks.

4. If any test specimen fails to conform to the above requirements through an apparent local defect, a retest shall be taken; and should the retest fail the plates or angle bars represented by such tests shall be rejected.

\section{AMERICAN SOCIETY FOR TESTING MATE- RIALS, STANDARD SPECIFICATIONS FOR WROUGHT-IRON PLATES, DESIG- NATION A42-30, $1930^{51}$}

\section{WROUGHT-IRON PLATES}

SCOPE

1. These specifications cover two classes of wrought-iron plates, as determined by the kind of material used in their manufacture, namely, class A, as defined in section $2(b)$, and class.B, as defined in section 2 (c).

\section{MANUFACTURE}

2. (a) All plates shall be rolled from piles entirely free from any admixture of steel.

(b) Piles for class A plates shall be made from wrought iron ${ }^{52}$ and such scrap as results from rolling the plates.

(c) Piles for class B plates shall be made from wrought iron 52 together with wrought-iron scrap.

\section{PHYSICAL PROPERTIES AND TESTS}

3. (a) The plates shall conform to the following minimum requirements as to tensile properties:

\begin{tabular}{|c|c|c|c|c|}
\hline \multirow[b]{2}{*}{ Properties considered . } & \multicolumn{2}{|c|}{ Class A } & \multicolumn{2}{|c|}{ Class B } \\
\hline & $\begin{array}{l}6 \text { to } 24 \\
\text { inches } \\
\text { inclu- } \\
\text { sire, in } \\
\text { width }\end{array}$ & $\begin{array}{l}\text { Orer } 24 \\
\text { inches } \\
\text { to } 90 \\
\text { inches, } \\
\text { inclu- } \\
\text { sive, in } \\
\text { width }\end{array}$ & $\begin{array}{l}6 \text { to } 24 \\
\text { inches } \\
\text { inclu- } \\
\text { sire, in } \\
\text { width }\end{array}$ & $\begin{array}{l}\text { Over } 24 \\
\text { inches } \\
\text { to } 90 \\
\text { inches, } \\
\text { inclu- } \\
\text { sive, in } \\
\text { width }\end{array}$ \\
\hline $\begin{array}{l}\text { Tensile strength } \\
\text { Yield point } \\
\text { Elongation in } 8 \text { inches.-. per cent.- } \\
\quad \text { (See sec. } 4 . \text {.) }\end{array}$ & $\begin{array}{r}49,000 \\
26,000 \\
16\end{array}$ & $\begin{array}{r}48,000 \\
26,000 \\
12\end{array}$ & $\begin{array}{r}48,000 \\
26,000 \\
14\end{array}$ & $\begin{array}{r}47,000 \\
26,000 \\
10\end{array}$ \\
\hline
\end{tabular}

$\$ 1$ These specifications prior to their revision in $1930 \mathrm{had}$ been ap proved as American Tentative Standard by the American Standards Association. The revised specifications hare been resubmitted for approral.

52 Wrought iron is defined as follows in the Standard Definitions of Terms Relating to Wrought-Iron Specifications (A. S. T. M Designation: A81) of the American Society for Testing Materials: Wrought iron.-A ferrous material, aggregated from a solidifying mass of pasty particles of highly refined metallic iron with whlch without subsequent fusion, is incorporated a minutely and uniforml distributed quantity of slag. (b) The yield point shall be determined by the drop of the beam of the testing machine. The speed of the crosshead of the machine shall not exceed three-fourths inch per minute.

(Modifications in elongation, bend tests, test specimens, and number of tests are the same as in American Bureau of Shipping, iron plates and angles, given above, except that in A42, class B plates shall withstand bending around a pin the diameter of which is equal to three times the thickness of the specimen; and in addition to one tension, and one cold-bend test, there shall be one nick-bend test.)

4. Nick-bend tests.-The test specimen, when nicked on one side and broken, shall show for class A plates a wholly fibrous fracture, and for class B plates, not more than 10 per cent of the fractured surface to be crystalline.

\section{FINISH}

5. The plates shall be straight, smooth, and free from cinder spots and holes, injurious flaws, buckles, blisters, seams, and laminations.

\section{MARKING}

6. The plates shall be stamped or otherwise marked as designated by the purchaser.

\section{INSPECTION AND REJECTION}

(Conform with A. S. T. M. standard specifications for steel. See 605.0, p. 247.)

604.19 MISCELLANEOUS IRON AND STEEL PLATES.

\section{AMERICAN SOCIETY OF MECHANICAL ENGINEERS, BOILER CONSTRUCTION CODE, SPECIFICATIONS FOR STEEL PLATE FOR BRAZING, S-3, 1930}

\section{STEEL PLATE FOR BRAZING}

1. The steel shall conform to the following re quirements as to chemical composition:

Per cent

Carbon, not over. 0.28

Manganese, not orer

Sulphur, not over..........

2. Ladle analyses.-(a) The manufacturer shall make an analysis of each melt of steel, from a test ingot taken during the pouring of the melts, to determine the percentage of carbon, manganese, phosphorus, and sulphur. The results of analysis shall conform to requirements, and shall be reported to the purchaser or his representative.

Check analyses.- (b) The purchaser may make an analysis from finished material representing each melt. The chemical composition thus determined shall conform to the requirements.

3. Tension tests.-The material shall conform to the following requirements as to tensile properties:

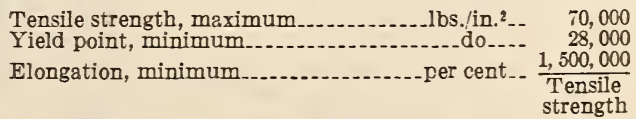

The elongation shall be measured on a gage length of twenty-four times the thickness of specimen, except that this may be reduced by $2 \frac{1}{2}$ per cent for each one-sixteenth inch under five-sixteenth inch.

4. Bend test.-The bend-test specimen shall bend cold through $180^{\circ}$ without cracking on the outside of the bent portion, around a pin the diameter of which is equal to the thickness of the specimen.

5. Number of tests. - Two tension tests and two bend tests shall be taken from each heat, but not both tension or both bend tests from the same slab. 
6. Sheets less than one-fourth inch in thickness shall not be required to be stamped at the mill on account of the small size and light weight of the sheets. The manufacturer must mark each vessel in some permanent manner which will enable him to identify the heat from which the sheet in each tank has been rolled.

AMERICAN SOCIETY FOR TESTING MATERIALS, STEEL PLATES OF STRUCTURAL QUALITY FOR FORGE WELDING, DESIGNATION A78-30, 1930

\section{STEEL PLATES FOR FORGE WELDING}

(See 605.19, p. 272.)

\subsection{IRON AND STEEL SHEETS.}

\author{
604.20 GENERAL ITEMS.
}

AMERICAN RAILWAY ASSOCIATION, PUR-
CHASES AND STORES DIVISION, STAND-
ARDIZATION AND SIMPLIFICATION OF
STORES STOCKS, PROCEEDINGS 1928

\section{LIGHT SHEET STEEL AND IRON}

(Recommended practice in stocking certain specified sizes of galvanized steel sheets, one pass cold-rolled box annealed sheets, and jacket steel.)

ASSOCIATION OF AMERICAN STEEL MAN-

UFACTURERS, STANDARD PERMISSIBLE VARIATIONS IN GAGE WEIGHT, GAGE THICKNESS, SIZE, AND FLATNESS OF SHEETS AND LIGHT PLATES BLUE ANNEALED, 1929

\section{PERMISSIBLE VARIATIONS IN SHEETS AND} (See 600.7, p. 117.)

UNITED STATES GOVERNMENT, DEPARTMENT OF COMMERCE, BUREAU OF STANDARDS, SHEET STEEL, SIMPLIFIED PRACTICE RECOMMENDATION R28-29, 1929

\section{SHEET STEEL}

This revision limits the stock sizes of sheet steel in various gages to those shown in the following schedule: All dimensions in inches.

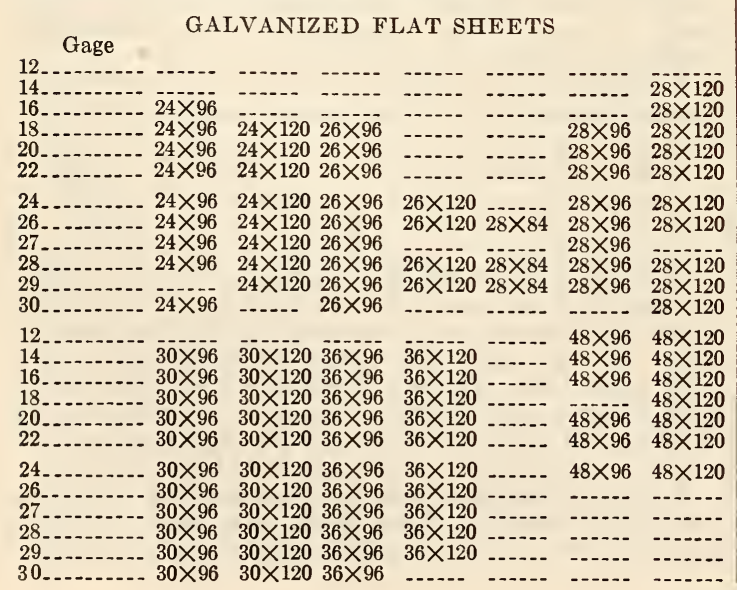

ONE PASS COLD-ROLLED BOX ANNEALED SHEETS

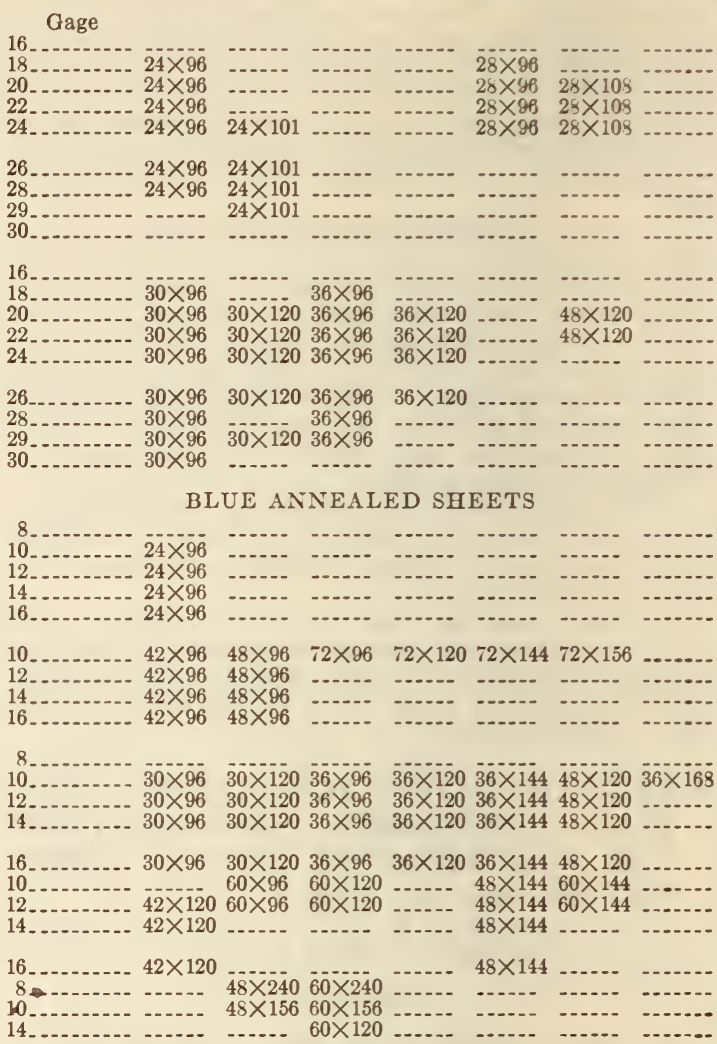

The United States standard gage for sheet and plate iron and steel, as adopted by Congress, as applied to steel sheets, and the galvanized sheet gage as applied to galvanized steel sheets, will be found in Circular of the Bureau of Standards No. 391. (See 604.0, p. 224.)

\subsection{IRON AND STEEL SHEETS FOR MAGNETO} ELECTRIC APPARATUS.

(No nationally recognized specifications available.)

604.22 IRON AND STEEL STRIPS, BANDS, AND HOOPS.

AMERICAN RAILWAY ENGINEERING ASSO. CIATION, SPECIFICATION FOR TANK HOOPS, 1920

\section{TANK HOOPS}

\section{MATERIAI}

1. The tank hoops shall preferably be wrought iron. They may be open-hearth steel. Such wrought iron or steel shall fulfill all of the requirements of quality, strength, inspection, and test for wrought iron and open-hearth steel given in the specifications for steel railway bridges of the American Railway Engineering Asssociation.

\section{SHAPE}

2. Hoops to be round in cross section, the same size throughout, and shall be bent to a true radius to fit the tank.

3. To be of such a size and so spaced that the stress shall not exceed 12,500 pounds per square inch, when computed from area at base of thread. No hoop less than three-fourths-inch diameter to be used. 


\section{SPACING}

4. Spacing of hoops to be figured by the following formula:

Spacing of hoops in inches $=\frac{\text { Safe load for giren hoop in pounds }}{\left.2.6 \text { diameter }(\text { feet }) \times \text { depth }, \text { s3 }^{\text {feet }}\right)}$

Top hoop to be placed within 2 inches of top of staves. No space between hoops to exceed 21 inches. Hoops to be so placed that lugs will not come in a vertical line.

On account of the swelling of the tank bottom, the hoops near the bottom may be subjected to a strain greater than that due to the water pressure alone, therefore additional hoops should be provided. Two hoops of the size used next above it should be placed around the bottom opposite the croze, one of which shall not be considered as withstanding any water pressure.

\section{THREADS}

5. The ends of each section of hoop shall be threaded with United States standard thread for length of $4 \frac{1}{2}$ inches.

\section{NOTS}

6. Each end of each section shall be provided with two hexagon nuts tapped to fit the thread on hoop.

\section{LUGS}

7. The lugs shall be of standard pattern, at least 8 inches long, and as strong as the hoop; they shall preferably be made of malleable iron, but cast iron may be used if approved by the engineer.

\section{GENERAI}

8. Each hoop shall be made in three sections for 16 by 24 foot tanks and in four sections for 20 by 30 foot tanks.

The several pieces constituting one hoop shall be tied together for shipment.

All pieces shall be furnished in full lengths, unwelded, and must not vary from the lengths given on order more than one-half inch.

NoтE.-The following table gives proper working strength for hoops of common sizes, based on the above allowable stress:

\begin{tabular}{|c|c|c|c|}
\hline $\begin{array}{c}\text { Diameter } \\
\text { of round } \\
\text { rod }\end{array}$ & $\begin{array}{c}\text { Area of } \\
\text { section } \\
\text { of rod }\end{array}$ & $\begin{array}{c}\text { Net area } \\
\text { of root of } \\
\text { thread }\end{array}$ & $\begin{array}{c}\text { Safe } \\
\text { working } \\
\text { load }\end{array}$ \\
\cline { 2 - 4 } $\begin{array}{c}\text { Inches } \\
3 / 4\end{array}$ & $\begin{array}{c}\text { Square } \\
\text { inch }\end{array}$ & $\begin{array}{c}\text { Square } \\
\text { inch }\end{array}$ & Pounds \\
$7 / 8$ & .44 & 0.30 & 3,750 \\
1 & .60 & .42 & 5,250 \\
118 & .79 & .55 & 6,875 \\
.99 & .69 & 8,625 \\
\hline
\end{tabular}

\section{AMERICAN SOCIETY FOR TESTING MATE- RIAIS, TENTATIVE SPECIFICATIONS FOR COLD-ROLLED STRIP STEEL, DESIG- NATION A109-27T, 1927}

\section{COLD-ROLLED STRIP STEEL}

1. These specifications cover five grades of commercial cold-rolled strip steel as follows:

Grade No. 1, or "Hard" temper;

Grade No. 2, or "Half hard" temper;

Grade No. 3, or "Quarter hard" temper;

Grade No. 4, or "Planished" temper;

Grade No. 5, or "Dead soft" temper.

2. (a) These five grades of cold-rolled strip steel are commonly used for blanking, bending, forming, spinning, and deep drawing.

(b) It should be understood, however, that the tempers and uses given are general. It is recomso Depth refers to distance from top of stave to point where hoop is
to be located. mended that the purchaser furnish the manufacturer with a sample or sketch of the article to be made.

\section{MANUFACTURE}

3. The steel shall be made by the open-hearth process for temper Nos. 3, 4, and 5.

\section{CHEMICAL REQUIREMENTS}

4. The steel shall conform to the following requirements as to chemical composition:

\section{Temper Nos. 1 and 2}

Per cent

Carbon, not over. 0.25

\section{Temper Nos. 3, 4, and 5}

Carbon...

Manganese 05 to 0.15

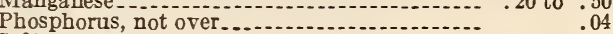

Sulfur, not over....................................

5. Samples for analysis shall be taken by machining the entire cross-section of the strip.

\section{PHYSICAL PROPERTIES AND TESTS}

6. The material shall conform to the following requirements as to tensile properties:

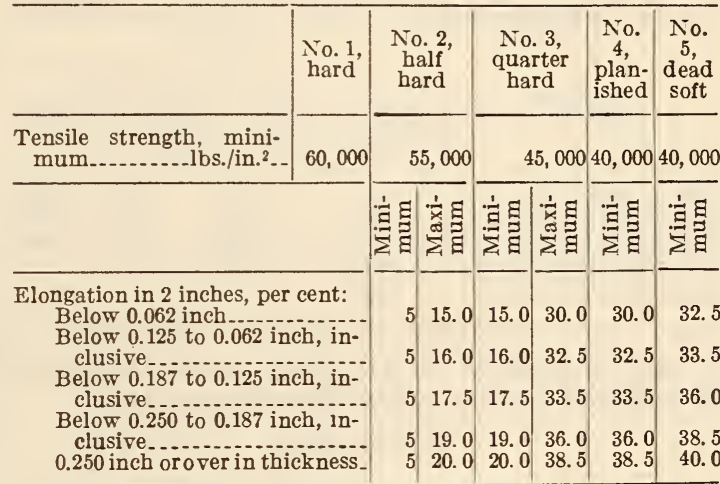

7. The bend test specimens shall stand being bent cold as specified below without cracking on the outside of the bent portion:

\begin{tabular}{c|c|c|c|c}
\hline No. 1, hard & $\begin{array}{c}\text { No. 2, half } \\
\text { hard }\end{array}$ & $\begin{array}{c}\text { No. 3, quarter } \\
\text { hard }\end{array}$ & $\begin{array}{c}\text { No. 4, plan- } \\
\text { ished }\end{array}$ & $\begin{array}{c}\text { No. 5, dead } \\
\text { soft }\end{array}$ \\
\cline { 2 - 3 } $\begin{array}{c}\text { Shall not be } \\
\text { required } \\
\text { to make } \\
\text { bends in } \\
\text { either di- } \\
\text { rection of } \\
\text { grain. }\end{array}$ & $\begin{array}{c}\text { Shall bend } \\
\text { to a sharp } \\
\text { right an- } \\
\text { gle across } \\
\text { the grain. }\end{array}$ & $\begin{array}{c}\text { Shall bend } \\
\text { flat on it- } \\
\text { self across } \\
\text { the grain } \\
\text { and to a } \\
\text { sharp right } \\
\text { angle along } \\
\text { the grain. }\end{array}$ & $\begin{array}{c}\text { Shall bend } \\
\text { flat upon } \\
\text { itself both } \\
\text { ways of } \\
\text { the grain. }\end{array}$ & $\begin{array}{c}\text { Shall bend } \\
\text { flat upon } \\
\text { itself both } \\
\text { ways of } \\
\text { the grain. }\end{array}$ \\
\end{tabular}

NOTE-To bend across the grain means that the axis of the ben shall be at right angles to the direction of rolling. To bend with the grain means that the axis of the bend shall be parallel to the direction of rolling.

8. (a) The tension test specimens shall be cut in both a longitudinal and transverse direction from the strip except in the case of widths narrower than 10 inches, in which case only longitudinal specimens shall be taken. The specimen shall be machined to the form and dimensions shown in Figure 127-A.

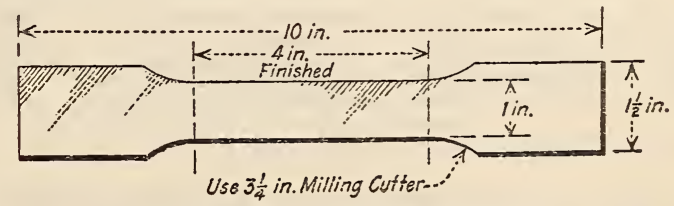

FIGURE 127-A.-Test specimen

(b) The test specimens shall be marked off for every 1-inch length within the 4 -inch gage length. The percentage of elongation in 2 inches shal be 
determined by averaging the increase in length of the two 2-inch lengths that include the break.

(c) Bend-test specimens may have the edges rounded.

\section{PERMISSIBLE VARIATIONS IN DIMENSIONS}

9. The thickness of the strip measured at a point one-half inch from the edge shall not vary from the thickness specified by more than the following plus or minus variations:

[All dimensions in inches]

\begin{tabular}{|c|c|c|c|c|}
\hline \multirow[b]{2}{*}{ Thickness } & \multicolumn{4}{|c|}{ Width } \\
\hline & $\begin{array}{l}\text { Up to } 2 \\
\text { inches, in- } \\
\text { clusive }\end{array}$ & $\begin{array}{c}\text { Over } 2 \\
\text { to } 4 \\
\text { inches, in- } \\
\text { clusive }\end{array}$ & $\begin{array}{c}\text { Over } 4 \\
\text { to } 6 \\
\text { inches, in- } \\
\text { clusive }\end{array}$ & $\begin{array}{c}\text { Over } 6 \\
\text { to } 8 \\
\text { inches, in- } \\
\text { clusive }\end{array}$ \\
\hline 0.200 or over & 0.003 & 0.004 & 0.005 & 0.005 \\
\hline Below 0.200 to 0.100 & .002 & .003 & .004 & .004 \\
\hline Below 0.100 to 0.050 . & .002 & .002 & .003 & .003 \\
\hline Below 0.050 to 0.035 .- & .002 & .002 & .002 & .002 \\
\hline Below 0.035 to 0.015 & .0015 & .002 & .002 & .002 \\
\hline Below 0.015 to 0.010 & .001 & .0015 & .0015 & .0015 \\
\hline Below 0.010 to 0.006 & .00075 & .001 & .001 & - n \\
\hline Below 0 & 0005 & 3005 & 0005 & 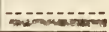 \\
\hline
\end{tabular}

\begin{tabular}{|c|c|c|c|c|}
\hline \multirow[b]{2}{*}{ Thickness } & \multicolumn{4}{|c|}{ Width } \\
\hline & $\begin{array}{c}\text { Over } 8 \\
\text { to } 12 \\
\text { inches, in- } \\
\text { clusive }\end{array}$ & $\begin{array}{c}\text { Over } 12 \\
\text { to } 16 \\
\text { inches, in- } \\
\text { clusive }\end{array}$ & $\begin{array}{c}\text { Over } 16 \\
\text { to } 24 \\
\text { inches, in- } \\
\text { clusive }\end{array}$ & $\begin{array}{l}\text { Over } 24 \\
\text { inches }\end{array}$ \\
\hline 0.200 or over & 0.006 & 0.006 & 0.007 & 0.008 \\
\hline Below 0.200 to 0.100 & .005 & .005 & .006 & .007 \\
\hline Below 0.100 to 0.050 & .004 & .004 & .005 & .006 \\
\hline Below 0.050 to 0.035 & .003 & .003 & .004 & $-\ldots . .$. \\
\hline Below 0.035 to 0.015 & .002 & - & -....- & - \\
\hline Below 0.015 to 0.010 .- & .0015 & & & \\
\hline
\end{tabular}

10. The width of the strip shall not vary from that specified by more than the following amounts:

Mill edge steel

\begin{tabular}{|c|c|c|}
\hline & Plus & Minus \\
\hline $\begin{array}{l}\text { Up to } 6 \text { inches, inclusive } \\
\text { Over } 6 \text { to } 10 \text { inches } \\
\text { Over } 10 \text { to } 16 \text { inches. } \\
\text { Over } 16 \text { inches. }\end{array}$ & $\begin{array}{c}\text { Inch } \\
1 / 16 \\
3 / 32 \\
5 / 32 \\
1 / 4\end{array}$ & $\begin{array}{c}\text { Inch } \\
1 / 16 \\
3 / 32 \\
5 / 32 \\
1 / 4\end{array}$ \\
\hline
\end{tabular}

Slit, sheared, or file edge steel

\begin{tabular}{|c|c|c|}
\hline & Plus & $\overline{\text { Minus }}$ \\
\hline $\begin{array}{l}\text { Up to } 0.100 \mathrm{inch} \\
0.100 \text { to } 0.200 \text { inch } \\
0.200 \text { to } 0.300 \text { inch } \\
0.300 \text { to } 0.400 \text { inch- } \\
0.400 \text { to } 0.500 \text { inch }\end{array}$ & $\begin{array}{l}\text { Inch } \\
0.015 \\
.025 \\
.035 \\
1 / 16 \\
18\end{array}$ & $\begin{array}{l}\text { Inch } \\
0.015 \\
.020 \\
.025 \\
1 / 16 \\
18\end{array}$ \\
\hline
\end{tabular}

Round or square-rolled edge steel

\begin{tabular}{|c|c|c|}
\hline Width & Thickness & $\begin{array}{l}\text { Toler- } \\
\text { ances in } \\
\text { width, } \\
\text { plus or } \\
\text { minus }\end{array}$ \\
\hline $\begin{array}{l}1 / 2 \text { inch and narrower } \\
\text { Over } 1 / 2 \text { to } 1 \text { inch, inclusive-- } \\
\text { Over } 1 \text { to } 2 \text { inches, inclusive- } \\
\text { Over } 2 \text { to } 4 \text { inches, inclusive-- } \\
\text { Over } 4 \text { to } 6 \text { inches, inclusive. }\end{array}$ & $\begin{array}{l}3 / 16 \text { inch and thinner- } \\
3 / 16 \text { to } 0.025 \text { inch, inclusive.- } \\
1 / 4 \text { to } 0.025 \text { inch, inclusive.- } \\
14 \text { to } 0.035 \text { inch, inclusive-- } \\
1 / 4 \text { to } 0.047 \text { inch, inclusive.- }\end{array}$ & $\begin{array}{r}\text { Inch } \\
1 / 64 \\
1 / 32 \\
1 / 32 \\
3 / 64 \\
3 / 64\end{array}$ \\
\hline
\end{tabular}

Strip wider than 6 inches is not furnished.

11. (a) Cold-rolled strip steel is usually furnished in coils, but may be furnished in cut lengths when ordered. Unless otherwise specified, the cut lengths will be 8 feet and ends. When so furnished the allowance shall be plus 3 inches with no minus allowance. (b) When a definite length in inches is specified, the permissible variation shall be plus 1 inch with no minus allowance. Cutting to definite lengths over 36 inches does not relieve the buyer from scrap loss and short lengths will be included, cut to multiples, if so desired.

12. The standard for straightness shall be one-half inch bow in 8 feet.

13. When flatness is an essential to the purchaser, it should be so specified. Flatness shall be determined by placing the strips in 8-foot lengths on a flat surface, when no part of the strip shall rise more than one-fourth inch from the surface.

(Requirements covering finish, inspection, rejection, and rehearing conform to those specified in A. S. T. M. standard specifications for steel (see 605.0 , p. 247) except that rejections shall be reported within 10 working days from receipt of samples, and that rejected material shall be preserved for one month from the date of the test report.)

ASSOCIATED COOPERAGE INDUSTRIES OF AMERICA, STANDARD SPECIFICATIONS OF STEEL HOOPS, WIDTH AND GAGE, FOR TIGHT BARRELS AND KEGS, OTHER THAN INTERSTATE COMMERCE COMMISSION BARRELS AND KEGS, 1930

\section{STEEL HOOPS}

\begin{tabular}{|c|c|c|c|}
\hline Size & $\begin{array}{l}\text { Head hoop } \\
\text { thickness } \\
\text { gage }\end{array}$ & $\begin{array}{c}\text { Quarter } \\
\text { hoop thick- } \\
\text { ness gage }\end{array}$ & $\begin{array}{l}\text { Bilge hoop } \\
\text { thickness } \\
\text { gage }\end{array}$ \\
\hline $\begin{array}{l}\text { 5-gallon } \\
10 \text {-gallon } \\
\text { 15-16-gallon } \\
20 \text {-25-gallon }\end{array}$ & $\begin{array}{l}\text { Inches } \\
11 / 8 \text { by } 19 \\
11 / 8 \text { by } 19 \\
11 / 4 \text { by } 19 \\
17 / 16 \text { by } 18\end{array}$ & $\begin{array}{c}\text { Inches } \\
1 \text { by } 20 \\
1 \text { by } 20 \\
1 \text { by } 20 \\
114 \text { by } 19\end{array}$ & $\begin{array}{l}\text { Inches } \\
1 \text { by } 20 \\
118 \text { by } 19 \\
118 \text { by } 19 \\
136 \text { by } 19\end{array}$ \\
\hline $\begin{array}{l}\text { 30-32-gallon } \\
40 \text {-gallon } \\
45 \text {-gallon } \\
50-57 \text {-gallon } \\
\text { (For commodities that con- } \\
\text { geal and other commod- } \\
\text { ities of low commercial } \\
\text { value.) } \\
\text { 50-57-gallon } \\
\text { (For commodities of high } \\
\text { commercial value.) }\end{array}$ & $\begin{array}{l}17 / 16 \text { by } 18 \\
111 / 16 \text { by } 18 \\
111 / 6 \text { by } 18 \\
111 / 16 \text { by } 18\end{array}$ & $\begin{array}{l}11 / 4 \text { by } 19 \\
17 / 16 \text { by } 19 \\
17 / 16 \text { by } 19 \\
17 / 16 \text { by } 19\end{array}$ & $\begin{array}{l}13 / 8 \text { by } 19 \\
111 / 16 \text { by } 19 \\
111 / 16 \text { by } 19 \\
111 / 16 \text { by } 19\end{array}$ \\
\hline
\end{tabular}

SOCIETY OF AUTOMOTIVE ENGINEERS,
IRON AND STEEL; STEEL BANDS AND
STRIPS, S. A. E. STANDARD, JUNE, 1911 STEEL BANDS AND STRIPS

\begin{tabular}{|c|c|c|c|}
\hline \multicolumn{2}{|c|}{$\begin{array}{l}\text { Width varying } \\
\text { by } 1 / 16 \text { inch }\end{array}$} & \multicolumn{2}{|c|}{ Thickness, B. W. G. No. } \\
\hline From- & To- & From- & To- \\
\hline $\begin{array}{c}\text { Inches } \\
\\
112 \\
1116 \\
21 / 16\end{array}$ & $\begin{array}{c}\text { Inches } \\
3,8 \\
7 / 16 \\
1 \\
2 \\
3\end{array}$ & $\begin{array}{cc}\text { Gage } & \text { Inch } \\
4 & (0.238) \\
4 & (.238) \\
4 & (.238) \\
4 & (.238) \\
1 & (.300)\end{array}$ & $\begin{array}{cc}\text { Gage } & \text { Inch } \\
18 & (0.049) \\
19 & (.042) \\
23 & (.025) \\
22 & (.028) \\
21 & (.032)\end{array}$ \\
\hline $\begin{array}{l}31 / 16 \\
41 / 16 \\
49116 \\
413 / 16 \\
5138\end{array}$ & $\begin{array}{l}4 \\
41 / 2 \\
43 / 4 \\
51 / 16 \\
63 / 4\end{array}$ & $\begin{array}{ll}1 & (.300) \\
1 & (.300) \\
00 & (.380) \\
00 & (.380) \\
00 & (.380)\end{array}$ & $\begin{array}{ll}20 & (.035) \\
19 & (.042) \\
18 & (.049) \\
17 & (.058) \\
16 & (.065)\end{array}$ \\
\hline & $\begin{array}{l}7 \\
71 / 2 \\
75,8 \\
73 / 4 \\
77 / 8\end{array}$ & $\begin{array}{ll}1 & (.300) \\
1 & (.300) \\
1 & (.300) \\
1 & (.300) \\
1 & (.300)\end{array}$ & $\begin{array}{ll}14 & (.083) \\
14 & (.083) \\
14 & (.083) \\
14 & (.083) \\
14 & (.083)\end{array}$ \\
\hline - & $\begin{array}{l}8 \\
814 \\
81 / 2 \\
85 / 8 \\
95 \%\end{array}$ & $\begin{array}{ll}1 & (.300) \\
1 & (.300) \\
1 & (.300) \\
1 & (.300) \\
1 & (.300)\end{array}$ & $\begin{array}{ll}14 & (.083) \\
14 & (.083) \\
14 & (.083) \\
14 & (.083) \\
12 & (.109)\end{array}$ \\
\hline
\end{tabular}


The Birmingham wire gage is used by the manufacturers of steel bands, hoops, and strips. The thickness desired, however, should be expressed in thousandths of an inch when writing specifications for bands, hoops, or strips.

UNITED STATES GOVERNMENT, INTERSTATE COMMERCE COMIMISSION, REGULATIONS FOR TRANSPORTATION BY RAIL OF EXPLOSIVES AND OTHER DANGEROUS ARTICLES. SHIPPING CONTAINER SPECIFICATIONS, 1930

\section{A-WOODEN BARRELS AND KEGS (TIGHT)}

8. (a) Hoops must be made from cooperage-grade hoop steel, and as follows:

Carbon content: At least 0.16 per cent.

Tensile strength: Maximum 90,000 pounds per square inch.

Elongation in 8 inches: At least 15 per cent.

(b) Hoops must be as follows with respect to number and size:

\begin{tabular}{|c|c|c|c|c|c|c|c|c|c|}
\hline \multirow{3}{*}{$\begin{array}{l}\text { Maximum capacity } \\
\text { of container } \\
\text { (gallons) }\end{array}$} & \multirow{3}{*}{ 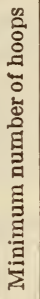 } & \multicolumn{8}{|c|}{$\begin{array}{c}\text { Minimum size of hoops (inches in width, } \\
\text { and Birmingham gage) }\end{array}$} \\
\hline & & \multicolumn{2}{|c|}{ Head } & \multicolumn{2}{|c|}{$\begin{array}{l}\text { First } \\
\text { quarter }\end{array}$} & \multicolumn{2}{|c|}{$\begin{array}{l}\text { Second } \\
\text { quarter }\end{array}$} & \multicolumn{2}{|c|}{ Bilge } \\
\hline & & 兽 & $\underset{\substack{\infty \\
ٍ}}{\infty}$ & 总 & 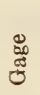 & 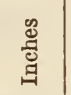 & 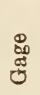 & 怤 & 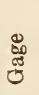 \\
\hline $\begin{array}{l}50 \\
30 \\
15 \\
10 \\
5\end{array}$ & $\begin{array}{l}8 \\
6 \\
6 \\
6 \\
6\end{array}$ & $\begin{array}{l}13 / 4 \\
1162 \\
11 / 4 \\
11 / 8 \\
1168\end{array}$ & $\begin{array}{l}16 \\
17 \\
18 \\
19 \\
19\end{array}$ & $\begin{array}{l}11 / 2 \\
114 \\
118 \\
1 \\
1\end{array}$ & $\begin{array}{l}18 \\
19 \\
19 \\
19 \\
19\end{array}$ & 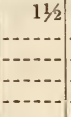 & 18 & $\begin{array}{l}13 / 4 \\
112 \\
11 / 4 \\
1138 \\
1\end{array}$ & $\begin{array}{l}17 \\
18 \\
19 \\
19 \\
19\end{array}$ \\
\hline
\end{tabular}

\section{B-WOODEN BARREIS AND KEGS (TIGHT)}

4. Head hoops with minimum width and thickness prescribed for bilge hoops in paragraph 8 (b) (see above) are authorized.

\section{C-WOODEN BARRELS AND KEGS (TIGHT)}

4. Head hoops with minimum width and thickness prescribed for bilge hoops in paragraph 8 (b) (see above) are authorized. Second-quarter hoops not required.

\section{5-WOODEN BARRELS WITH REMOVABLE HEADS}

4. Hoops must be at least six in number with minimum size as follows:
Head hoops. - 11116 inches by 17 gage.
Quarter hoops $17 / 16$ inches by 18 gage.
Bilge hoops....................... $111 / 16$ inches by 17 gage.

\section{A-WOODEN BARRELS AND KEGS (SLACK)}

5. (a) Hoops must be of cooperage-grade hoop steel-of beaded or rolled edge type-or of coiled elm, well finished and free from defects.

(b) Steel hoops must have one beaded or rolled edge, and with respect to number and size must be as follows:

\begin{tabular}{|c|c|c|c|}
\hline Maximum: & & & \\
\hline Net weight of article shipped..... pounds.. & 150 & 300 & 600 \\
\hline Head diameter of barrel or keg.--_inches.. & 16 & 17 & 20 \\
\hline Stave length of barrel or keg & 28 & 30 & 34 \\
\hline Minimum: & & & \\
\hline Steel hoops required. & 4 & 6 & 6 \\
\hline Head hoops, width & $13 / 8$ & $15 \%$ & $15 \%$ \\
\hline Quarter hoops, width....... & & 138 & $13 \%$ \\
\hline Bilge hoops, width_. & $13 / 8$ & $13 / 8$ & $13 / 8$ \\
\hline Head hoops (Birmingham gag & 23 & 22 & 21 \\
\hline Quarter hoops (Birmingham gage No. & & 23 & 23 \\
\hline Bilge hoops (Birmingham gage No. & 23 & 23 & 23 \\
\hline
\end{tabular}

\section{B-WOODEN BARRELS AND KEGS (SLACK)}

Steel hoops must conform to the following:

\begin{tabular}{|c|c|c|c|}
\hline Maximum: & & & \\
\hline Net weight of article shipped..._pounds.- & 150 & 300 & 600 \\
\hline Head diameter of barrel or keg.....inches.- & 16 & 17 & 20 \\
\hline Stave length of barrel or keg......... do.... & 28 & 30 & 34 \\
\hline Minimum: & & & \\
\hline Steel hoops required...... & 4 & 4 & 6 \\
\hline Head hoops, width & $13 / 8$ & $15 \%$ & $\begin{array}{l}15 \% 8 \\
13 / 8\end{array}$ \\
\hline Bilge hoops, width & $13 / 8$ & $13 / 8$ & 138 \\
\hline Head hoops (Birmingham gage No.). & 23 & 21 & 21 \\
\hline Quarter hoops (Birmingham & & & 23 \\
\hline Bilge hoops (Birmingham gage No & 23 & 23 & 23 \\
\hline
\end{tabular}

604.23 IRON AND STEEL SHEETS FOR DRAWING AND FORMING.

AMERICAN SOCIETY FOR TESTING MATERIALS, TENTATIVE SPECIFICATIONS FOR COLD-ROLLED STRIP STEEL, DESIGNATION A109-27T, 1927

COLD-ROLLED STRIP STEEL

(See 604.22, p. 237.)

604.24 SPRING AND TOOL STEEL SHEETS.

(No nationally recognized specifications available.)

604.25 IRON AND STEEL SHEETS FOR AUTOMOTIVE AND RAILWAY USE.

AMERICAN RAILWAY ASSOCIATION, MECHANICAL DIVISION, STEEL SHEETS AND THIN PLATES, RECOMMENDED PRACTICE, 1926

\section{STEEL SHEETS AND THIN PLATES}

1. Scope.-These specifications apply to all uncoated steel sheets and plates under three-sixteenth inch in thickness for locomotives, freight and passenger equipment cars. Plates are rolled on a plate mill and are usually rolled to a minimum thickness of 0.109 inch (12 USSG). Sheets are rolled on a jobbing or sheet mill and are usually rolled to a maximum thickness of 0.187 inch ( 7 USSG), though occasionally to 0.250 inch (3 USSG).

\section{MANUFACTURE}

2. Process.-The steel shall be made by the openhearth process.

\section{CHEMICAL PROPERTIES AND TESTS}

3. Chemical composition.-The steel shall conform to the following requirements as to chemical composition.

Phosphorus in material not less than 0.04 inch thick (1.6 pounds per square foot) not over 0.06 per cent.

Phosphorus in material less than 0.04 inch thick (1.6 pounds per square foot) not over 0.10 per cent. Sulphur, not over 0.06 per cent.

Copper (when so ordered), not under 0.20 per cent.

4. Ladle analysis. - An analysis shall be made by the maufacturer from a test ingot taken during the pouring of each melt to determine the percentage of carbon, manganese, phosphorus, and sulphur, also copper, when copper steel is ordered. Drillings for 
analysis shall be taken not less than one-fourth inch beneath the surface of the test ingot. Upon request, a copy of this analysis shall be given to the purchaser or his representative for all lots ordered not less than 20,000 pounds of one gage and size. This analysis shall conform to the requirements specified in section 3 .

5. Check analysis.-Check analysis may be made by the purchaser from the bend-test specimens. The chemical composition thus determined shall conform to the requirements specified in section 3 .

\section{PHYSICAL PROPERTIES AND TESTS}

6. Bend tests. - The test specimen shall bend cold through $180^{\circ}$ flat upon itself without cracking on the outside of the bent portion.

7. Test specimens.-(a) Bend-test specimens shall be taken from the finished product.

(b) Bend-test specimens shall be taken longitudinally as rolled and shall be cut from the edge of the sheet or plate midway between middle and end. They may be machined with both edges parallel.

8. Number of tests.- (a) One bend test shall be made from the thickest and thinnest material rolled from each melt, if sheets or plates are ordered in lots of not less than 20,000 pounds of one gage and size; otherwise, from each lot of 60,000 pounds, or fraction thereof, of each thickness (or weight per square foot).

(b) If the bend-test specimen shows defective machining or develops flaws, it may be discarded and another specimen substituted.

(c) If the bend-test specimen fails to meet the physical requirements of these specifications, two retests shall be taken from two other sheets or plates from the same lot, both of which shall meet all the bend-test requirements.

\section{PERMISSIBLE VARIATIONS IN WEIGHT, THICKNESS, AND}

9. When ordered to weight per square foot, the weight of sheets and plates shall be determined from draft lots. The theoretical weight of sheets and plates ordered by gage number shall be based upon the weights of the United States standard gage table. The maximum allowable variation from the theoretical weight shall not exceed the amounts given in Table 1.

TABLE 1.-Permissible variation in weight of sheets and plates

\begin{tabular}{|c|c|}
\hline Ordered weight (lbs. per sq. ft.) & $\begin{array}{r}\text { Permissi- } \\
\text { ble varia. } \\
\text { tion over } \\
\text { or under }\end{array}$ \\
\hline & Per cent \\
& 5.0 \\
Under 2.45 to and including 1.22.......... & 3.5 \\
Under 1.22. & 2.5 \\
\hline
\end{tabular}

10. When ordered to thickness, the thickness of each sheet or plate shall not vary more than 0.01 inch under that ordered. The overweight of each lot in each shipment shall not exceed the amount given in Table 2. The term "lot" means all of the sheets or plates of each group width and each group thickness. One cubic inch of rolled steel is assumed to weigh 0.2833 pound.
TABLE 2

\begin{tabular}{|c|c|c|c|c|}
\hline \multirow{2}{*}{ Ordered thickness } & \multicolumn{4}{|c|}{$\begin{array}{l}\text { Permissible excess in average weights } \\
\text { per square foot of sheets or plates } \\
\text { for widths given, expressed in per } \\
\text { centages of nominal weights }\end{array}$} \\
\hline & $\begin{array}{l}\text { Under } \\
48 \text { inches }\end{array}$ & $\begin{array}{l}48 \text { to } 60 \\
\text { inches, } \\
\text { exclusive }\end{array}$ & $\begin{array}{l}60 \text { to }: 2 \\
\text { inches, } \\
\text { exclusive }\end{array}$ & $\begin{array}{l}72 \text { to } 84 \\
\text { inches, } \\
\text { exclusive }\end{array}$ \\
\hline $\begin{array}{l}\text { Under } 1 / 8 \text { inch } \\
1 / 8 \text { to } 3_{1} 6 \text { inch, exclusire..... }\end{array}$ & $\begin{array}{l}9 \\
8\end{array}$ & $\begin{array}{r}10 \\
9\end{array}$ & $\begin{array}{l}12 \\
10\end{array}$ & $\begin{array}{l}14 \\
12\end{array}$ \\
\hline
\end{tabular}

11. Size.-(a) All sheets shall be sheared not less than the widths and lengths ordered.

(b) Sheets ordered 48 inches and less in width shall not exceed the ordered width by more than one-fourth inch, and for sheets ordered over 48 inches wide, the overwidth shall not exceed three-eighths inch.

(c) The length ordered for sheets 96 inches and less shall not be exceeded by more than three-fourths inch. For each 24 inches over 96 inches in length, an additional one-fourth inch shall be permitted to a maximum of $1 \frac{1}{2}$ inches.

(d) On full cold-rolled sheets, there shall be permitted excess length of not more than 2 inches.

(e) Sbeets required of accurate size shall be ordered "resquared," and such sheets shall not be in excess of ordered width and length by more than one-sixteenth inch, if not more than 48 inches wide and 120 inches long, and one-eighth inch for wider or longer sheets.

(f) All plates shall be sheared not more than onefourth inch less than the widths and lengths ordered.

12. Flatness.- (a) Sheets not ordered "patent leveled" and plates shall have the following tolerances for height of buckles:

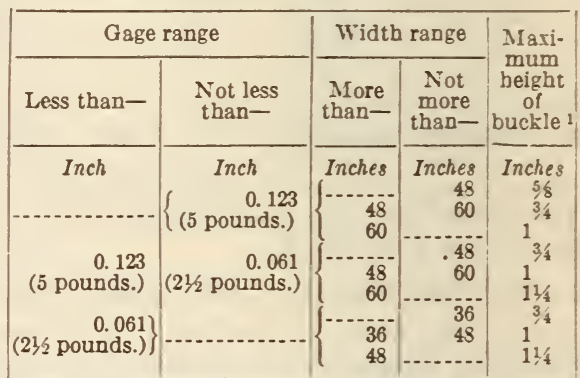

1 Height of buckle refers to maximum rise from a flat surface on which sheet or plate lies.

(b) Sheets ordered "patent leveled" shall not have buckles exceeding one-fourth inch in height. Sheets shall not be ordered patent leveled when under 0.03 inch thick (1.22 pounds per square foot).

\section{FINISH}

13. Finish.-The finished material shall be free from injurious defects and shall have a workmanlike finish.

\section{MARKING}

14. Marking.-(a) Sheets shall be properly marked for identification and in accordance with instructions on the purchaser's order.

(b) The name or brand of the manufacturer and the melt number shall be legibly marked on all finished plates.

(c) The melt number shall be legibly stamped on each test specimen that is taken from material that is identified according to melts. 
VII. INSPECTION AND REJECTION

(Inspection and rejection conform to the requirements for galvanized sheets in A. R. A.-M (see 604.32, p. 243) except for the addition in this case of the following requirement:)

All tests (except check analyses) and inspection shall be marle at the place of manufacture, prior to shipment, unless otherwise specified, and shall be so conducted as not to interfere unnecessarily with the operation of the works.

604.29 IRON AND STEEL SHEETS FOR MISCELLANEOUS PURPOSES.

AMERICAN ASSOCIATION OF STATE HIGHWAY OFFICIALS, TENTATIVE STANDARD SPECIFICATION FOR BASE METAL FOR CORRUGATED METAL SHEETS FOR CULVERTS M-36, 1931

METAL PIPE CULVERTS, CORRUGATED

(See 607.5 , p. 488.)

UNITED STATES GOVERNMENT, DEPARTMENT OF COMMERCE, BUREAU OF STANDARDS, IRON AND STEEL ROOFING, SIMPLIFIED PRACTICE RECOMMENDATION R78-28, 1928

IRON AND STEEL ROOFING

(See 604.32, p. 246.)

\subsection{TIN COATED AND ZINC COATED SHEETS.}

604.30 GENERAL ITEMS.

AMERICAN SOCIETY FOR TESTING MATERIAIS, STANDARD METHODS OF DETERMINING WEIGHT OF COATING ON TIN, TERNE, AND LEAD COATED SHEETS, DESIGNATION A91-24, 1924

ANALYSIS OF TIN AND TERNE PLATE

(See 600.3, p. 644.)

604.31 TIN-COATED SHEETS (ROOFING TIN, TERNEPLATE).

UNITED STATES GOVERNMENT, DEPARTMENT OF COMMERCE, BUREAU OF STANDARDS, STANDARD THICKNESSES, WEIGHTS, AND TOLERANCES OF SHEET METAL (CUSTOMARY PRACTICE), CIRCULAR NO. 391, MARCH 24, 1931

\section{TIN-PLATE GAGE}

(See 604.0, p. 224.)

UNITED STATES GOVERNMENT, DEPARTMENT OF COMMERCE, BUREAU OF STANDARDS, ROOFING TERNES, SIMPIIFIED PRACTICE RECOMMENDATION R30-28, 1928

\section{ROOFING TERNES}

In accordance with the unanimous action of a general conference of representative manufacturers, distributors, and users of roofing ternes held on
October 15, 1924, and reviewed by the meeting of the standing committee on October 18, 1927, the industry has adopted, and approved for promulgation by the United States Department of Commerce, a simplified practice recommendation limiting the weights and thicknesses of roofing ternes to the following:

Weights. $-8,15,20,25,30,32$, and 40 pounds.

No roofing ternes to be manufactured lighter than IC thickness for roofing.

UNITED STATES GOVERNMENT, FEDERAL SPECIFICATIONS BOARD, FEDERAL SPECIFICATION FOR TERNEPLATE (ROOFING TIN), QQ-T-201, NOVEMBER 11,1930

\section{TERNEPLATE}

\section{A. APPLICABLE FEDERAL SPECIFICATIONS}

A-1. Federal specification No. 339a (QQ-M-151), general specifications for metals, in effect on date of invitation for bids, shall form a part of this specification, as far as applicable. (See 600.0, p. 3.)

\section{B. TYPE AND GRADE}

B-1. Type.-Terneplate shall be one type, commercially called roofing tin.

B-2. Grade.-Terneplate shall be furnished in prime sheets only.

\section{MATERIAL AND WORKMANSHIP}

C-1. Base metal shall be either Bessemer or open hearth steel of soft grade, or iron, as ordered.

C-2. Black sheets shall be free from blisters, slivers, scale, laminations, open seams, dirt and grease spots, roll and opening marks, ragged edges, holes, pickling patches, stains, burnt edges, bent corners, black or white edges, brittleness, and internal or surface defects impairing their use for the purpose intended.

C-3. Coated sheets shall be free from defects referred to above, and in addition shall be free from bare or imperfectly coated spots, deep scratches or heavy list edges.

\section{GENERAL REQUIREMENTS}

D-1. See section E.

\section{E. DETAIL REQUIREMENTS}

\section{E-1. Base metal.-}

E-1a. Iron.-The carbon, manganese, phosphorus, sulphur, and silicon shall not exceed 0.25 per cent, and if copper is specified the minimum copper content shall be not less than 0.20 per cent.

E-1b. Steel. - The sum of carbon, manganese, phosphorus, sulphur, and silicon not exceeding 0.70 per cent, and copper shall be not less than 0.20 per cent.

E-1c. Sheets shall be cold rolled, double annealed and double pickled. All sheets shall be as nearly uniform in thickness as is practicable and shall be commercially flat.

E-2. Coating shall consist of not less than 25 per cent of tin, and not more than 75 per cent of lead, both by weight. Tin plus lead shall equal not less than 99 per cent of the total coating exclusive of iron. The process of applying coating shall thoroughly amalgamate it with the base sheets, and they shall be as thoroughly and evenly coated on both sides as good mill practice will permit. All sheets shall be clean, and excess oil shall be removed. 
E-3. Resquaring. - All sheets shall be resquared after cold rolling and before coating. They shall have straight edges and shall be rectangular.

E-4. Tolerances.-

E-4a. Tolerances in dimensions.-Sheets shall have no minus tolerance. A plus tolerance of onesixteenth inch in width or length, or out of square will be allowed.

E-4b. Tolerances in net weights of finished product will be allowed as follows:

Unit:

All of one base weight and size in a shipment, or individual package 2.5 per cent plus or minus.

Single sheets in accordance with the minimums and maximums shown in Table 1 .
E-4c. Tolerances in coating. - The average weight of coating shall not fall below that specified. No spot on the sheet shall have a coating less than 75 per cent of the weight specified.

E-5. Bending quality.-Sheets shall be capable of withstanding bending, without fracture on the outside of the bent portion, under the method covered by paragraph $\mathrm{F}-6$.

E-6. Seaming quality. - Sheets shall be capable of withstanding double-seaming, without fracture. (See par. F-6.)

E-7. Weights of sheets shall conform to the limits shown in Table 1.

TABLE 1.-Weights

\begin{tabular}{|c|c|c|c|c|c|c|c|c|c|c|c|c|c|c|}
\hline \multirow{6}{*}{ Trace symbol } & \multirow{5}{*}{\multicolumn{2}{|c|}{$\begin{array}{l}\text { Weight of } \\
\text { black sheets } \\
\text { after } \\
\text { pickling }\end{array}$}} & \multicolumn{12}{|c|}{ Weight of coating, in pounds per box } \\
\hline & & & 8 & & \multicolumn{2}{|c|}{15} & \multicolumn{2}{|c|}{20} & \multicolumn{2}{|c|}{25} & \multicolumn{2}{|c|}{30} & \multicolumn{2}{|c|}{40} \\
\hline & & & \multicolumn{12}{|c|}{ Weight of coating, in ounces per square foot of sheet } \\
\hline & & & \multicolumn{2}{|c|}{0.2939} & \multicolumn{2}{|c|}{0.5510} & \multicolumn{2}{|c|}{0.7347} & \multicolumn{2}{|c|}{0.9184} & \multicolumn{2}{|c|}{1.1020} & \multicolumn{2}{|c|}{1.4694} \\
\hline & & & \multicolumn{12}{|c|}{ Weight of finished sheets } \\
\hline & 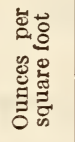 & 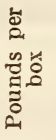 & 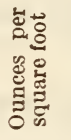 & 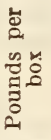 & 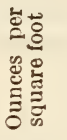 & 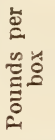 & 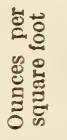 & 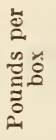 & 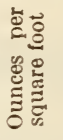 & 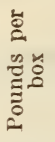 & 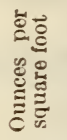 & 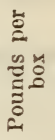 & 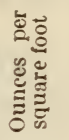 & 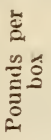 \\
\hline IC ..... $\left\{\begin{array}{l}\text { Minimum.. } \\
\text { Nominal.... } \\
\text { Maximum.. }\end{array}\right.$ & $\begin{array}{l}7.00 \\
8.00 \\
9.00\end{array}$ & $\begin{array}{l}191 \\
218 \\
245\end{array}$ & $\begin{array}{l}\text { 7. } 29 \\
8.29 \\
9.29\end{array}$ & $\begin{array}{l}199 \\
226 \\
253\end{array}$ & $\begin{array}{l}7.55 \\
8.55 \\
9.55\end{array}$ & $\begin{array}{l}206 \\
233 \\
260\end{array}$ & $\begin{array}{l}7.73 \\
8.73 \\
9.73\end{array}$ & $\begin{array}{l}211 \\
238 \\
265\end{array}$ & $\begin{array}{l}7.92 \\
8.92 \\
9.92\end{array}$ & $\begin{array}{l}216 \\
243 \\
270\end{array}$ & $\begin{array}{r}8.10 \\
9.10 \\
10.10\end{array}$ & $\begin{array}{l}220 \\
248 \\
275\end{array}$ & $\begin{array}{r}8.47 \\
9.47 \\
10.47\end{array}$ & $\begin{array}{l}231 \\
253 \\
285\end{array}$ \\
\hline IX..... $\left\{\begin{array}{l}\text { Minimum.. } \\
\text { Nominal.... } \\
\text { Maximum... }\end{array}\right.$ & $\begin{array}{r}9.00 \\
10.00 \\
11.00\end{array}$ & $\begin{array}{l}245 \\
272 \\
299\end{array}$ & $\begin{array}{r}9.29 \\
10.29 \\
11.29\end{array}$ & $\begin{array}{l}253 \\
280 \\
307\end{array}$ & $\begin{array}{r}9.55 \\
10.55 \\
11.55\end{array}$ & $\begin{array}{l}260 \\
287 \\
314\end{array}$ & $\begin{array}{r}9.73 \\
10.73 \\
11.73\end{array}$ & $\begin{array}{l}265 \\
292 \\
319\end{array}$ & $\begin{array}{r}9.92 \\
10.92 \\
11.92\end{array}$ & $\begin{array}{l}270 \\
297 \\
324\end{array}$ & $\begin{array}{l}\text { 10. } 10 \\
11.10 \\
12.10\end{array}$ & $\begin{array}{l}275 \\
302 \\
329\end{array}$ & $\begin{array}{l}10.47 \\
11.47 \\
12.47\end{array}$ & $\begin{array}{l}285 \\
312 \\
339\end{array}$ \\
\hline
\end{tabular}

\section{F. METHODS OF SAMPLING, INSPECTION, AND TESTS}

F-1. Inspection. - The inspector shall take at random one package in each 10 packages or fraction included in the lot and shall weigh and make check measurements on each sheet therein. He shall also inspect each sheet for surface defects and poor coating. If any of these sheets fail to meet the specified requirements, he shall take two additional packages and proceed as before. If any of these sheets fail to meet the specified requirements the entire lot may be rejected.

F-2. Chemical analysis.-When required, chemical analyses shall be made by the manufacturer for each melt or blow, and duplicate copies of analyses shall be given to the Government inspector.

F-3. Chemical analyses may be made by the Government.- The inspector shall obtain drillings from each melt or blow, which may be taken as a composite sample of drillings from three coating test pieces, or pieces adjacent thereto.

F-4a. Test specimens.-Specimens for tests shall be taken by the Government inspector, and, as far as practicable, may be cut at contractor's option from rejected sheets which are imperfect outside of the zones from which the specimens are to be taken, as follows: One sheet shall be taken from each five boxes or fraction thereof, and from each there shall be cut three pieces each 2 inches square, one from the center and the others from diagonally opposite corners and 1 inch from the edges.

F-4b. Retests. - If any test specimen fails to meet the requirements of the specification, a retest may be allowed from the same lot, using specimens from two additional sheets for each original sheet. the test specimens from either of those sheets fail to meet the specified requirements the entire lot may be rejected.

$\mathrm{F}-5$. Weight of coating, determination of iron, determination of tin, determination of lead.-(Methods for determination of weight of coating and of chemical analysis are the same as given in A. S. T. M. standard methods of determining weight of coating on tin, terne, and lead coated sheets, designation A91-24, with corrections in calculation factors due to difference in number of test specimens. See 600.3 , p. 64.)

F-6. Bending and seaming tests.-Sheets shall withstand bending through an angle of $180^{\circ}$, and flattening down with a wooden mallet on a wooden block, without fracture on the outside of the bent portion. Sheets shall also stand double seaming without fracture. Both tests shall be made both with and across the direction of rolling.

\section{G. PACKAGING, PACKING, AND MARKING}

G-1. Packaging.-Commercial custom designates a standard package of roofing ternes as 112 sheets, unless the limiting weight would thereby be exceeded, in which cases fractional units of 56,28 , or 14 sheets are used as standard. Packages containing special sheetages may be ordered if desired. The weight of any single package shall not exceed 302 pounds (nominal net weight), except by specific permission first obtained from the purchaser.

G-2. Packing. - Sheets shall be packed in standard commercial containers so constructed as to insure acceptance by common or other carrier for safe transportation at the lowest rate to point of deliv- 
ery. A sheet of heavy paper shall be placed between outside sheets and the container.

G-3. Marking.-Each sheet shall be stamped with the trade symbol ( $1 \mathrm{C}$ or $1 \mathrm{X}$ ) and the weight of coating, together with identification of manufacturer by name, brand, or trade-mark. Containers shall have the following information clearly stenciled or lettered, making use of a durable marking medium-trade symbol, weight of coating, size of sheets, identification of manufacturer, and requisi. tion, contract, or order numbers as may be directed.

\section{NOTES}

I-1. Box, wherever used in this specification, refers to the double-base box, consisting of 112 sheets 20 by 28 inches, equal to 62,720 square inches or 435.56 square feet of sheet, or equivalent.

I-2. Invitations for bids should specify the base, if desired (open-hearth or Bessemer steel, or iron, and if copper bearing), trade symbol, weight of coating, number of packages, sheets per package, and width and length of sheets, also whether inspection will be made at place of manufacture or after delivery.

I-3. Samples. - It is believed that this specification adequately describes the characteristics necessary to secure the desired material, and that normally no samples will be necessary prior to award to determine compliance with this specification. If, for any particular purpose, samples with bids are necessary, they should be specifically asked for in the invitation for bids, and the particular purpose to be served by the bid sample should be definitely stated, the specification to apply in all other respects.

I-5. This specification governs all United States Government purchases of this commodity.

\subsection{ZIN C - C O A T E D (GALVANIZED) STEEL SHEETS.}

AMERICAN RAILWAY ASSOCIATION, MECHANICAL DIVISION, GALVANIZED SHEETS, RECOMMENDED PRACTICE, 1923

\section{GALVANIZED SHEETS}

1. Scope.-These specifications cover galvanized steel and iron sheets for all purposes.

\section{CHEMICAL PROPERTIES AND TESTS}

2. Chemical composition.- When copper bearing sheets are specified the base metal in the sheet shall conform to the following requirements as to chemical composition:

Copper. Not less than 0.18 per cent.

3. Ladle analysis.-An analysis of each melt or lot of copper-bearing sheets shall be made by the manufacturer to determine the percentage of copper. The chemical composition thus determined shall be reported to the purchaser or his representative, and shall conform to the requirements specified in section 2.

4. Check analysis.-An analysis of copper-bearing sheets may be made by the purchaser from the test specimen specified in section 7 . The copper content thus determined shall conform to the requirements specified in section 2 .

5. Zinc coating.-(a) The weight of the zinc coating shall conform to the requirements specified in Table 1 , the average being taken of the chemical determinations from three specimens $2 \frac{1}{4}$ inches square cut from the ends and middle of the test specimen specified in section 7 ; provided, however, that the coating on any one of the three specimens shall be at least 75 per cent of the minimum weight of coating specified in Table 1 .

(b) The weight of the zinc coating shall be determined by the basic lead acetate, hydrochloric acid-antimony chloride or sulphuric-permanganate method.

(c) The minimum weight of coating specified in Table 1 is the total coating on both sides of a sheet 1 foot square, the numerical value of which in ounces is the same as that determined in grams from a sample $2 \frac{1}{4}$ inches square.

TABLE 1

\begin{tabular}{|c|c|c|c|c|c|}
\hline \multirow{2}{*}{$\begin{array}{l}\text { U. S. } \\
\text { stand. } \\
\text { ard } \\
\text { gage } \\
\text { No.a }\end{array}$} & \multirow{2}{*}{$\begin{array}{c}\text { Nominal } \\
\text { thickness } \\
\text { of base } \\
\text { sheet }\end{array}$} & \multicolumn{3}{|c|}{ Weight of galvanized sheets } & \multirow{2}{*}{$\begin{array}{l}\text { Mini- } \\
\text { mum } \\
\text { weight of } \\
\text { coating c }\end{array}$} \\
\hline & & $\begin{array}{l}\text { Mini- } \\
\text { mum b }\end{array}$ & Nominal & $\begin{array}{l}\text { Maxi- } \\
\text { mum b }\end{array}$ & \\
\hline $\begin{array}{r}8 \\
9 \\
10 \\
11 \\
12\end{array}$ & $\begin{array}{l}\text { Inch } \\
0.172 \\
.156 \\
.141 \\
.125 \\
.109\end{array}$ & $\begin{array}{c}\text { Oz./fft.2 } \\
106.9 \\
97.4 \\
87.9 \\
78.4 \\
68.9\end{array}$ & $\begin{array}{r}\text { Oz./ft. } \\
112.5 \\
102.5 \\
92.5 \\
82.5 \\
72.5\end{array}$ & $\begin{array}{c}\text { Oz./ft. } \\
118.1 \\
107.6 \\
97.1 \\
86.6 \\
76.1\end{array}$ & $\begin{array}{r}\text { Oz./ft. }{ }^{2} \\
1.75 \\
1.75 \\
1.75 \\
1.75 \\
1.75\end{array}$ \\
\hline $\begin{array}{l}13 \\
14 \\
16 \\
18 \\
20\end{array}$ & $\begin{array}{l}.094 \\
.078 \\
.063 \\
.050 \\
.038\end{array}$ & $\begin{array}{l}59.4 \\
49.9 \\
40.4 \\
33.6 \\
25.8\end{array}$ & $\begin{array}{l}62.5 \\
52.5 \\
42.5 \\
34.5 \\
26.5\end{array}$ & $\begin{array}{l}65.6 \\
55.1 \\
44.6 \\
35.4 \\
27.2\end{array}$ & $\begin{array}{l}1.75 \\
1.75 \\
1.75 \\
1.50 \\
1.50\end{array}$ \\
\hline $\begin{array}{l}22 \\
23 \\
24 \\
25\end{array}$ & $\begin{array}{l}.031 \\
.028 \\
.025 \\
.022\end{array}$ & $\begin{array}{l}21.9 \\
20.0 \\
18.0 \\
16.1\end{array}$ & $\begin{array}{l}22.5 \\
20.5 \\
18.5 \\
16.5\end{array}$ & $\begin{array}{l}23.1 \\
21.0 \\
19.0 \\
16.9\end{array}$ & $\begin{array}{l}1.50 \\
1.30 \\
1.30 \\
1.30\end{array}$ \\
\hline $\begin{array}{l}26 \\
27 \\
28 \\
30\end{array}$ & $\begin{array}{l}.019 \\
.017 \\
.016 \\
.013\end{array}$ & $\begin{array}{l}14.1 \\
13.2 \\
12.2 \\
10.2\end{array}$ & $\begin{array}{l}14.5 \\
13.5 \\
12.5 \\
10.5\end{array}$ & $\begin{array}{l}14.9 \\
13.8 \\
12.8 \\
10.8\end{array}$ & $\begin{array}{l}1.10 \\
1.10 \\
1.10 \\
1.10\end{array}$ \\
\hline
\end{tabular}

- See sec. $9(d)$.

bee sec. 10 .

• See sec. 5 .

\section{PHYSICAL PROPERTIES AND TESTS}

6. Bend tests-(a) Base metal tests.-Any portion of the test specimen specified in section 7 , shall bend cold $180^{\circ}$ flat on itself without fracture of the base metal. For steel, the bend may be made in any direction; for iron the line of the bend shall be transverse to the direction of the rolling of the sheet.

(b) Coating test.-Any portion of the test specimen specified in section 7, of sheets lighter than No. 16 gage, shall stand bending in any direction without flaking of the coating on either side when bent flat on itself over four thicknesses of the material, and for sheets ordered to gages 26 to 30 inclusive, when made in the form of a double-lock seam.

(c) A vise with smooth jaws and operated by hand shall be used for making the bend tests. A brake shall be used for making the double-lock seam test.

7. Test specimens.-A rectangular test specimen $2 \frac{1}{2}$ inches wide shall be cut diagonally across the sheet, the ends terminating within 2 inches of the sides and 4 inches of the ends of the sheet.

8. Number of tests.-(a) One each of the tests specified shall be made from one sheet in each lot of 1,000 sheets or fraction thereof of each gage on each order.

(b) If any test specimen fails to meet the requirements of this specification a retest will be allowed from two other sheets in the same lot, both of which shall meet the requirements.

\section{PERMISSIBLE VARIATIONS}

9. Dimensions.-The sheets shall conform to the dimensions specified with following permissible variations.

(a) The width shall be not less than that specified, but may be up to one-fourth inch greater for sheets 
48 inches wide and under, and up to three-eighths inch greater for sheets over 48 inches wide.

(b) The length shall not be less than that specified, but for sheets 96 inches long and under may be up to three-fourths inch greater, to which latter figure add one-fourth inch for each 24 inches or fraction thereof above 96 inches length.

(c) Where sheets are required accurate to size, they shall be specified "to be resquared."

(d) The thickness may vary from the specified gage, the average being controlled by the minimum and maximum weights specified in Table 1 .

10. Weight.-The average weight per square foot of the galvanized sheets as determined by the weighing of lots not exceeding 6,000 pounds, shall conform to the requirements specified in Table 1 which are based on a maximum variation from the nominal weight of 5 per cent for gages 8 to 16 inclusive, and $2 \frac{1}{2}$ per cent for gages lighter than 16 .

\section{FINISH}

11. Finish.-The finished sheets shall be of firstclass commercial quality, free from flaws or mechanical defects such as pin holes, cracks, blisters, and blackened sal-ammoniac or acid spots.

\section{MARKING}

12. Marking. - The top sheet in each bundle, the top sheet in each lot, or a number of sheets when shipped loose shall legibly show the name and brand of manufacturer, point of shipment, amount and size of sheets, lot number, and purchaser's order number.

\section{INSPECTION AND REJECTION}

13. Inspection.-(a) The inspector representing the purchaser shall have free entry, at all times while work on the contract of the purchaser is being performed, to all parts of the manufacturer's works which concern the manufacture of the material ordered. The manufacturer shall afford the in pector, free of charge, all reasonable facilities to satisfy him that the material is being furnished in accordance with these specifications.

(b) The purchaser may make the tests and inspection to govern the acceptance or rejection of the material in his own laboratory or elsewhere. Such tests and inspection shall be made at the expense of the purchaser.

14. Rejection.-(a) Sheets represented by samples which fail to conform to the requirements of these specifications will be rejected.

(b) Sheets which, subsequent to tests and inspection at the mills or elsewhere and their acceptance, show blackened sal-ammoniac or acid spots, improper galvanizing, improper trimming or other defects for which the manufacturer is responsible, will be rejected and shall be replaced by the manufacturer.

15. Rehearing.-Samples tested in accordance with section $8(a)$, which represent rejected material, shall be preserved for two weeks from the date of the test report.

\section{AMERICAN SOCIETY FOR TESTING MATE- RIALS, STANDARD SPECIFICATIONS FOR ZINC-COATED (GALVANIZED) SHEETS, DESIGNATION A93-27, 1927}

[Approved as American Tentative Standard by the American Standards Association, 1931]

\section{ZINC-COATED (GAIVANIZED) SHEETS} SCOPE

1. These specifications cover sheets of Bessemer steel, open-hearth steel, and open-hearth iron, with five classes of zinc coatings, applied by the hot-dip process, as follows:

Class A.-Extra heavily coated sheets that are not intended to be formed other than by corrugating.

Class B.-Heavily coated sheets that are not intended to be formed other than by corrugating and curving to large radii.

Class C.-Moderately heavily coated sheets for moderate bending.

Class D.-Ordinary coated sheets for general utility. These coatings approximate those of class $\mathrm{C}$ except in medium gages in which coatings of class D are appreciably lighter. Class D represents material generally available in warehouse stocks, which is not intended for use where relatively long life, represented by classes $A, B$, and $C$, or severe forming, represented by class $\mathrm{E}$, is required.

Class E.-Sheets having lighter, more tightly adherent coatings to reduce liability of flaking in severe forming. The sheet maker should be made acquainted with the requirements of fabrication.

\section{CHEMICAL PROPERTIES AND TESTS}

2. No detailed requirements for the chemical composition of the base metal are specified, except when base metal containing copper is ordered, in which case it shall conform to the following requirements:

Copper, minimum:
Ladle analysis_- Per cent
Check analysis

3. An analysis of copper-bearing sheets may be made by the purchaser from the test specimens specified in section 6 . The copper content thus determined shall conform to the requirements specified in section 2 .

\section{PHYSICAL PROPERTIES AND TESTS}

4. (a) The weight of the zinc coating shall conform to the requirements specified in Tables 1 and 2. The weight of coating is the total coating, expressed in ounces, on both sides of a sheet 1 foot square.

TABLE 1.-Desired or ordered weight of coatings (in ounces per square foot)

\begin{tabular}{|c|c|c|c|c|c|}
\hline $\begin{array}{c}\text { Gal- } \\
\text { van- } \\
\text { ized } \\
\text { sheet } \\
\text { gage } \\
\text { No. }\end{array}$ & Class A & Class B & Class C & Class D & Class $\mathrm{E}$ \\
\hline $\begin{array}{r}8 \\
9 \\
10 \\
11 \\
12\end{array}$ & $\begin{array}{l}2.75 \\
2.75 \\
2.75 \\
2.75 \\
2.75\end{array}$ & $\begin{array}{l}2.50 \\
2.50 \\
2.50 \\
2.50 \\
2.50\end{array}$ & $\begin{array}{l}2.00 \\
2.00 \\
2.00 \\
2.00 \\
2.00\end{array}$ & \multirow{4}{*}{$\begin{array}{l}\text { No specif } \\
\text { ings. } \\
1 .)\end{array}$} & \\
\hline $\begin{array}{l}13 \\
14 \\
16 \\
18\end{array}$ & $\begin{array}{l}2.75 \\
2.75 \\
2.75 \\
2.75\end{array}$ & $\begin{array}{l}2.50 \\
2.50 \\
2.50 \\
2.50\end{array}$ & $\begin{array}{l}2.00 \\
2.00 \\
2.00 \\
1.75\end{array}$ & & $\begin{array}{l}\text { fied coat- } \\
\text { (See sec. }\end{array}$ \\
\hline $\begin{array}{l}20 \\
22 \\
24 \\
26\end{array}$ & $\begin{array}{r}2.75 \\
2.75 \\
\end{array}$ & $\begin{array}{l}2.50 \\
2.50 \\
2.50 \\
2.25\end{array}$ & $\begin{array}{l}1.75 \\
1.75 \\
1.50 \\
1.25\end{array}$ & & 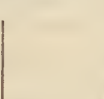 \\
\hline $\begin{array}{l}27 \\
28 \\
29 \\
30\end{array}$ & - & $\begin{array}{l}2.00 \\
1.75 \\
1.50 \\
1.25\end{array}$ & $\begin{array}{l}1.25 \\
1.25 \\
1.25 \\
1.25\end{array}$ & & \\
\hline
\end{tabular}

(b) The weight test is to be preferred and shall consist of a 10-sheet lot of a light gage or a 5-sheet lot of No. 16 gage or heavier. The test lots shall be weighed after pickling, washing, and drying, and again after coating, and the weight of coating calculated from the difference on the basis of the ordered 
size. The scale used shall be sensitive to one-half pound. The average of weight tests shall be determined from not less than 10 successive weight tests that have met the requirements of the specification as provided in section $7(a)$.

(c) The triple spot test shall consist of the average of determinations from the three specimens cut from the test sheet as provided in sections 6 and 7 (b). The average of triple spot tests shall be determined from not less than 10 successive triple spot tests and shall include all such tests made.

The minimum spot test shall be that one of the three specimens of the triple spot test bearing the lightesu coating, or the purchaser may select a single specimen taken from any part of the sheet, at least 2 inches from the side and 4 inches from the end. The average of minimum spot tests shall be determined from not less than 10 successive minimum spot tests and shall include all such tests made.

(d) The weight of the zinc coating by triple and minimum spot tests shall be determined by the hydrochloric acid-antimony chloride, or sulphuricpermanganate method as described in A. S. T. M. A90 (see 600.3, p. 62). The weight of coating, in ounces per square foot, is numerically equal to that determined in grams from a sample $2 \frac{1}{4}$ inches square.

TABLE 2.-Minimum weight-of-coating test limits (ounces per square foot)

\begin{tabular}{|c|c|c|c|c|c|c|}
\hline & \multicolumn{6}{|c|}{ Rejection limits by different tests 1 } \\
$\begin{array}{c}\text { Class ac- } \\
\text { cording } \\
\text { to desired } \\
\text { or } \\
\text { ordered } \\
\text { coating }\end{array}$ & $\begin{array}{c}\text { A verage } \\
\text { of } \\
\text { weight } \\
\text { tests }\end{array}$ & $\begin{array}{c}\text { Any one } \\
\text { weight } \\
\text { test }\end{array}$ & $\begin{array}{c}\text { A verage } \\
\text { of triple } \\
\text { spot } \\
\text { tests }\end{array}$ & $\begin{array}{c}\text { Any one } \\
\text { triple } \\
\text { spot } \\
\text { test }\end{array}$ & $\begin{array}{c}\text { A verage } \\
\text { of mini- } \\
\text { mum } \\
\text { spot } \\
\text { tests }\end{array}$ & $\begin{array}{c}\text { Any one } \\
\text { mini- } \\
\text { mum } \\
\text { spot } \\
\text { test }\end{array}$ \\
\hline & & & & & & \\
\hline 2.75 & 2.60 & 2.45 & 2.40 & 2.30 & 2.20 & 2.00 \\
2.50 & 2.30 & 2.20 & 2.15 & 2.00 & 1.95 & 1.80 \\
2.25 & 2.10 & 2.00 & 1.95 & 1.80 & 1.75 & 1.60 \\
2.00 & 1.90 & 1.80 & 1.75 & 1.60 & 1.55 & 1.40 \\
1.75 & 1.65 & 1.55 & 1.50 & 1.35 & 1.30 & 1.20 \\
1.50 & 1.40 & 1.30 & 1.25 & 1.15 & 1.10 & 1.00 \\
1.25 & 1.15 & 1.10 & 1.05 & 0.95 & 0.90 & 0.85 \\
\hline
\end{tabular}

1 Different methods of sampling and testing give different results as indicated in the above table. The purchaser shall specify which method he will use.

5. (a) Base metal tests.-Any part of the test specimen specified in section 6 shall stand being bent cold through $180^{\circ}$ flat on itself without fracture of the base metal. The bend may be made in any direction.

(b) Coating tests.-Sheets of class A of No. 18 gage and lighter shall stand corrugating without flaking of the coating. Sheets of class B of No. 12 gage and lighter shall stand corrugating and also subsequent longitudinal curving, as given below, without flaking of the coating. The corrugations for these tests shall not be less than $2 \frac{2}{3}$ inches in width and not more than one-half inch in depth.

\begin{tabular}{|c|c|}
\hline $\begin{array}{c}\text { Galvan- } \\
\text { ized sheet } \\
\text { gage No. }\end{array}$ & $\begin{array}{c}\text { Diame- } \\
\text { ter of } \\
\text { circle }\end{array}$ \\
\hline $\begin{array}{l}12 \\
14 \\
16\end{array}$ & $\begin{array}{c}\text { Inches } \\
36 \\
24 \\
18\end{array}$ \\
\hline
\end{tabular}

In the case of sheets of class C, any part of the test specimen specified in section 6 , of sheets lighter than No. 16 gage, shall stand being bent in any direction without flaking of the coating on either side when bent through $180^{\circ}$ over four thicknesses of the material. A vise with smooth jaws and operated by hand shall be used for making the bend tests.

6. For the triple and minimum spot tests, three specimens exactly $2 \frac{1}{4}$ inches square shall be cut from each test sheet, one being cut from the center and the others from diagonally opposite corners, but at least 4 inches from the end and 2 inches from the side. The bend test specimens shall be cut from the test sheet at least 4 inches from the end and 2 inches from the side. These specimens, so far as practicable, shall be cut from sheets that are imperfect outside the zone from which the specimens are to be taken.

7. (a) Weight tests shall be made of not less than 1 per cent of the sheets of each gage and ordered coating, or at approximately hourly intervals. If an inspector representing the purchaser witnesses and accepts weight tests, the triple and minimum spot tests shall not be made. If certificate of weight tests be accepted, the triple and minimum spot tests shall not be made unless doubt arises regarding uniformity of the material.

(b) The bend and spot tests, if required, shall be made from one sheet in each lot of 1,000 sheets or fraction thereof, of each gage and ordered coating.

(c) If any sheet fails to meet the requirements of these specifications, a retest shall be made from two other sheets in the same lot, both of which shall meet the requirements.

\section{PERMISSIBLE VARIATIONS}

8. The sheets shall conform to the dimensions specified with the following permissible variations:

(a) The width shall be not less than that ordered, but may be one-fourth inch over for sheets 48 inches or less in width, and three-eighths inch over for sheets over 48 inches in width.

(b) The length shall be not less than that ordered, but for sheets 96 inches or less in length it may be three-fourths inch greater. For each 24 inches or fraction thereof above 96 inches the permissible variation may be increased one-fourth inch over three-fourths inch.

(c) When sheets are required accurate to size, they shall be ordered "resquared." Resquared sheets shall be not less than the ordered width and length and shall be in excess by not more than onesixteenth inch unless wider than 48 inches or longer than 120 inches, in either of which case the excess tolerance shall be one-eighth inch. Should the sheets be resquared before galvanizing, double the foregoing tolerances are permissible.

9. The gage of galvanized sheets shall be determined by weight only. The weight per square foot of galvanized sheets shall conform to the tolerances given in Table 3 :

TABLE 3

\begin{tabular}{|c|c|c|c|c|c|c|}
\hline \multirow{2}{*}{\multicolumn{2}{|c|}{$\begin{array}{c}\text { Gage range } \\
\text { Weight of sheets } \\
\text { (in ounces per } \\
\text { square foot) }\end{array}$}} & \multicolumn{3}{|c|}{$\begin{array}{l}\text { Permissible tolerances in } \\
\text { weights of sheets, plus } \\
\text { or minus, in percentage } \\
\text { of theoretical weight } 1\end{array}$} & \multirow{2}{*}{\multicolumn{2}{|c|}{$\frac{\text { Gage range }}{\begin{array}{c}\text { Galvanized sheet } \\
\text { gage number }\end{array}}$}} \\
\hline & & \multirow{2}{*}{$\begin{array}{l}\text { All of } \\
1 \text { gage } \\
\text { and size } \\
\text { in ship- } \\
\text { ment } 2\end{array}$} & \multirow{2}{*}{$\begin{array}{l}\text { Single } \\
\text { pack- } \\
\text { ages }\end{array}$} & \multirow{2}{*}{$\begin{array}{l}\text { Single } \\
\text { sheets }\end{array}$} & & \\
\hline Less than & $\begin{array}{c}\text { Not less } \\
\text { than }\end{array}$ & & & & $\begin{array}{c}\text { Lighter } \\
\text { than }\end{array}$ & $\begin{array}{l}\text { Not } \\
\text { lighter } \\
\text { than }\end{array}$ \\
\hline $\begin{array}{l}42.5 \\
22.5\end{array}$ & $\begin{array}{r}42.5 \\
22.5 \\
\end{array}$ & $\begin{array}{l}5.0 \\
3.5 \\
2.5\end{array}$ & $\begin{array}{l}7.0 \\
5.5 \\
4.0\end{array}$ & $\begin{array}{l}10.0 \\
10.0 \\
10.0\end{array}$ & $\begin{array}{l}16 \\
22\end{array}$ & $\begin{array}{l}16 \\
22\end{array}$ \\
\hline
\end{tabular}

See footnotes at end of table. 
TABLE 3-Continued

WEIGHT OF GALVANIZED SHEETS, ALL CLASSES

\begin{tabular}{|c|r|r||c|c|c|}
\hline $\begin{array}{c}\text { Galvan- } \\
\text { ized sheet } \\
\text { gage No. }\end{array}$ & $\begin{array}{c}\text { Ounces } \\
\text { per square } \\
\text { foot }\end{array}$ & $\begin{array}{c}\text { Pounds } \\
\text { per square } \\
\text { foot }\end{array}$ & $\begin{array}{c}\text { Galvan- } \\
\text { ized sheet } \\
\text { gage No. }\end{array}$ & $\begin{array}{c}\text { Ounces } \\
\text { per square } \\
\text { foot }\end{array}$ & $\begin{array}{c}\text { Pounds } \\
\text { per square } \\
\text { foot }\end{array}$ \\
\hline & 112.5 & 7.031 & 20 & 26.5 & 1.656 \\
9 & 102.5 & 6.406 & 22 & 22.5 & 1.406 \\
10 & 92.5 & 5.781 & 24 & 18.5 & 1.156 \\
11 & 82.5 & 5.156 & 26 & 14.5 & .906 \\
12 & 72.5 & 4.531 & 27 & 13.5 & .844 \\
13 & 62.5 & 3.906 & 28 & 12.5 & .781 \\
14 & 52.5 & 3.281 & 29 & 11.5 & .719 \\
16 & 42.5 & 2.656 & 30 & 10.5 & .656 \\
18 & 34.5 & 2.156 & & & \\
\hline
\end{tabular}

1 References are to gross weights of bundled material and to net weights of crated and boxed material. If the minimum or maxiweights of crated and boxed material. If the minimum or maxi-
mum only be ordered double tolerance is to be taken on permissible side.

${ }_{2}^{2}$ All of one gage and size in shipment shall apply to lots of not less than 6,000 pounds.

\section{FINISH}

10. The finished sheets shall be of first-class commercial quality, free from injurious defects, such as blisters, flux, and uncoated spots.

\section{MARKING}

11. The top sheet of each bundle or the top sheet of a number of sheets when shipped loose, shall show legibly the name and brand of the manufacturer, class, gage, and size, unless otherwise ordered.

\section{INSPECTION AND REJECTION}

(Requirements covering inspection, rejection, and rehearing conform to those specified in A. S. T. M. standard specifications for steel. See 605.0, p. 247.)

UNITED STATES GOVERNMENT, DEPARTMENT OF COMMERCE, BUREAU OF STANDARDS, STANDARD THICKNESSES, WEIGHTS, AND TOLERANCES OF SHEET METAL (CUSTOMARY PRACTICE), CIRCULAR NO. 391, MARCH 24, 1931

\section{GALVANIZED SHEET GAGE}

(See 604.0, p. 224.)

UNITED STATES GOVERNMENT, DEPARTMENT OF COMMERCE, BUREAU OF STANDARDS, SIMPLIFIED PRACTICE RECOMMENDATION FOR IRON AND STEEL ROOFING, R78-28, JUNE 1, 1928

\section{IRON AND STEEL ROOFING}

(Simplified practice recommended and accepted by industry establishing a limited list of standard varieties and sizes.)

\section{GALVANIZED OR PAINTED ROOFING}

All styles and forms.-Corrugated, roll, V crimp, and pressed standing steam.

Widths.-Corrugated, 26 and $271 / 2$ inches; roll, $261 / 2$ inches; and all other, 24 inches.

Lengths. $-5,6,7,8,9,10,11$, and 12 feet; roll roofing shall be 50 linear feet.

Gages.-Corrugated, 28, 26, and 24; roll, 29, 28, and 26 ; and all other, 28 and 26 .

Weights.- Standard weight subject to the usual mill tolerances of $2 \frac{1}{2}$ per cent plus or minus.
TABLE 1.-Gages

\begin{tabular}{|c|c|c|c|c|}
\hline \multicolumn{2}{|c|}{$\begin{array}{l}\text { Galvanized sheet } \\
\text { gage }{ }^{1}\end{array}$} & \multicolumn{3}{|c|}{$\begin{array}{l}\text { Painted sheets, United States } \\
\text { standard gage }\end{array}$} \\
\hline $\begin{array}{l}\text { Grge } \\
\text { No. }\end{array}$ & $\begin{array}{l}\text { Weight } \\
\text { per } \\
\text { square } \\
\text { foot }\end{array}$ & $\begin{array}{l}\text { Gage } \\
\text { No. }\end{array}$ & $\begin{array}{l}\text { Thick- } \\
\text { ness }\end{array}$ & $\begin{array}{l}\text { Weight } \\
\text { per } \\
\text { square } \\
\text { foot }\end{array}$ \\
\hline $\begin{array}{l}29 \\
28 \\
26 \\
24\end{array}$ & $\begin{array}{c}\text { Ounces } \\
11.5 \\
12.5 \\
14.5 \\
18.5\end{array}$ & $\begin{array}{l}29 \\
28 \\
26 \\
24\end{array}$ & $\begin{array}{l}\text { Inch } \\
0.014 \\
.016 \\
.019 \\
.025\end{array}$ & $\begin{array}{r}\text { Ounces } \\
9 \\
10 \\
12 \\
16\end{array}$ \\
\hline
\end{tabular}

1 Weights given are identical with requirements of American Society for Testing Materials specification A93-27, zinc-coated (galvanized) sheets. (See above.)

605. STRUCTURAL IRON AND STEEL.

605.0 GENERAL ITEMS.

AMERICAN INSTITUTE OF ARCHITECTS, A FILING SYSTEM FOR ARCHITECTS' OFFICES, 1927

\section{STANDARD CONSTRUCTION CLASSIFICATION FOR FILING}

(A standard classification for the materials, methods, and test data useful in the construction industry by which information reaching the architect may be filed where it is readily available. Advertising matter supplied by manufacturers may then be marked with the proper file number by the manufacturer and be ready for immediate filing on reaching the architect. The classification is principally a use classification with particular reference to use in the construction industry.)

\section{AMERICAN INSTITUTE OF STEEL CON- STRUCTION, STEEL CONSTRUCTION HANDBOOK, APRIL, 1929}

\section{CODE OF STANDARD PRACTICE}

(This code covers standard practice in steel construction which is not included in the A. I. S. C. specification on the design, fabrication, and erection of structural steel) (For the latter specification, see 605.12 , p. 252.) This code includes a classification of all the iron and steel furnished on a building into structural iron and steel, ornamental iron and steel, steel floor joists, and miscellaneous iron and steel. Regulations are given on invoicing of materials by weight and on the type and number of drawings to be furnished by the buyer and seller. Regulations under workmanship include requirements on straightening, punching, reaming, assembling, and riveting. Other regulations cover inspection and delivery, erection, delays in prosecution of work, extra work, standard terms of payment, and standard form of proposal.)

\section{AMERICAN SOCIETY OF MECHANICAI ENGINEERS, BIBIIOGRAPIY ON RIV- ETED JOINTS, MAY, 1924}

\section{BIBIIOGRAPHY ON RIVETED JOINTS}

Riveted joints are used so universally in mechanical and structural engineering work that a study of their strength and other physical properties is of importance to nearly all designers of metal structures. 
The art or practice of riveting has reached such a high state of development that such structures as built-up riveted columns may be greatly deformed before any rivet fails, the tying power of the rivets being sufficient to make the built-up specimen act as if it were one piece.

It is believed that the bibliography contains a list of most of the results of tests on riveted joints that have been published up to the present time. A list of textbooks which contain chapters on design has been included, and there are several articles of a miscellaneous nature that deal either with riveting in general or with some particular phase not important enough to be listed as a subject by itself.

\section{AMERICAN SOCIETY FOR TESTING MA- TERIALS, STANDARD SPECIFICATIONS FOR STEEL.}

Structural steel for bridges, serial designation A7-29, 1929.) See also 605.11, p. 249.)

Structural steel for buildings, serial designation A9-29, 1929. (See also 605.12, p. 252.)

Structural steel for ships, serial designation A12-21, 1921. (See also 605.15, p. 269.)

Rivet steel for ships, serial designation A13-24, 1924. (See also 605.15, p. 269.)

Structural steel for locomotives and cars, serial designation A113-29, 1929. (See also 605.13, p. 266.)

(The following general requirements are in substantial agreement in, and form an integral part of, the specifications listed above, except that in part A of specification A13, paragraphs $3(a)$ and 4 and Figures 1 and 2 do not apply, and in part B of specification A 13, only paragraphs 7 and $8(b)$ apply; also, paragraph $4(a)$ does not apply to specification A113.)

1. Check analyses. - Analyses may be made by the purchaser from finished material representing each melt. The phosphorus and sulphur content thus determined shall not exceed that specified under

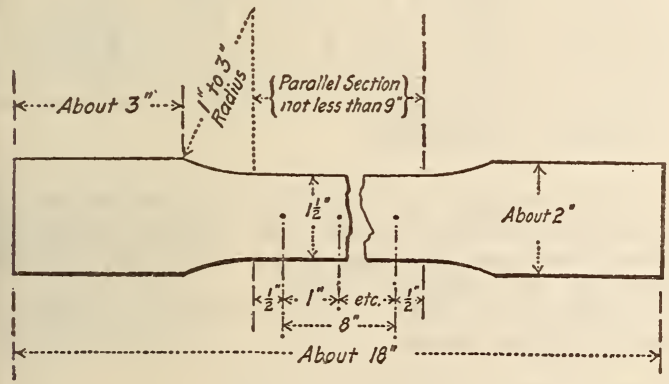

Figure 128.-Test specimen for plates, shapes, and flats

requirements as to chemical composition by more than 25 per cent.

2. Yield point.-The yield point shall be determined by the drop of the beam of the testing machine.

3. Number of tests. - (a) One tension and one bend test shall be made from each melt; except that if material from one melt differs three-eighths inch or more in thickness, one tension and one bend test shall be made from both the thickest and the thinnest material rolled.

(b) If any test specimen shows defective machining or develops flaws, it may be discarded and another specimen substituted. (c) If the percentage of elongation of any tension test specimen is less than that specified in the table of tensile properties and any part of the fracture is more than three-fourths inch from the center of the gage length of a 2-inch specimen or is outside the middle third of the gage length of an 8-inch specimen, as indicated by scribe scratches marked on the specimen before testing, a retest shall be allowed.

4. Permissible variations in weight and thickness of materials.-(a) The cross section or weight of each piece of steel shall not vary more than 2.5 per cent from that specified; except in the case of sheared plates, which shall be covered by the permissible variations specified in paragraphs $(b)$ and $(c)$. (One

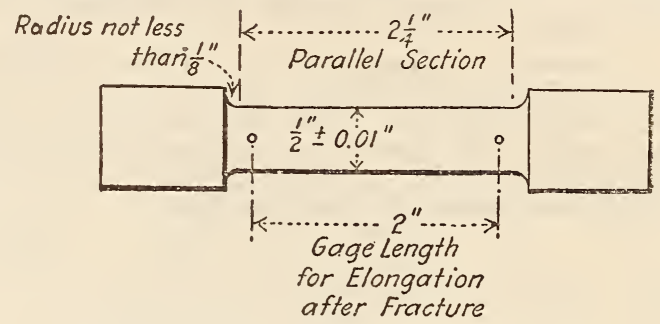

Figure 129.-Tension test specimen

Note.-As shown in the illustration, this tension test specimen has a diameter of onehalf inch, with a tolerance of \pm 0.01 inch. Tension test specimens specified by several other associations show a diameter of 0.505 inch, a difference of 0.005 inch. (See fig. 1 , under $600,0.5$ ) The diameter of 0.505 inch hes been adopted for the purpose of permitting an exact expression for the cross-sectional area of the test specimen.

cubic inch of rolled steel is assumed to weigh 0.2833 pound.)

(b) Sheared plates, when ordered to weight per square foot.-The weight of each lot 54 in each shipment shall not vary from the weight ordered more than the amount given in Table 1 . (See A. A. S. M. below.)

(c) Sheared plates, when ordered to thickness.-The thickness of each plate shall not vary more than 0.01 inch under that ordered.

The overweight of each lot in each shipment shall not exceed the amount given in Table 2. (See A. A. S. M. below.)

5. Finish.-The finished material shall be free from injurious defects and shall have a workmanlike finish.

6. Marking. - The name or brand of the manufacturer and the melt number shall be legibly stamped or rolled on all finished material, except that rivet and lattice bars and other small sections shall, when loaded for shipment, be properly separated and marked for identification. The identification marks shall be legibly stamped on the end of each pin and roller. The melt number shall be legibly marked, by stamping, if practicable, on each test specimen.

7. Inspection.-The inspector representing the purchaser shall have free entry, at all times while work on the contract of the purchaser is being performed, to all parts of the manufacturer's works which concern the manufacture of the material ordered. The manufacturer shall afford the in-

54 The term "lot" as applied to Table 1 means all of the plates of each group width and group weight; as applied to Table 2, it means all of the plates of each group width and group thickness. (For Tables 1 and 2, see A. A. S. M. below.) 
spector, without charge, all reasonable facilities to satisfy him that the material is being furnished in accordance with these specifications. All tests (except check analyses) and inspection shall be made at the place of manufacture prior to shipment, unless otherwise specified, and shall be so conducted as not to interfere unnecessarily with the operation of the works.

8. Rejection.-(a) Unless otherwise specified, any rejection based on tests made in accordance with paragraph 1 shall be reported within five working days from the receipt of samples.

(b) Material which shows injurious defects subsequent to its acceptance at the manufacturer's works will be rejected, and the manufacturer shall be notified.

9. Rehearing.-Samples tested in accordance with paragraph 1, which represent rejected material, shall be preserved for two weeks from the date of the test report. In case of dissatisfaction with the results of the tests, the manufacturer may make claim for a rehearing within that time.
ASSOCIATION OF AMERICAN STEEL MANUFACTURERS, STANDARD SPECIFICA. TIONS FOR STRUCTURAL AND BOILER STEEL, NOVEMBER 24, 1922

\section{PERMISSIBLE VARIATIONS IN WEIGHT AND SIZE OF PLATES AND BARS}

1. When ordered to weight per square foot.-Each lot in each shipment shall not vary from the weight ordered more than the amount given in the following table.

2. This table shall not be used when a minimum edge thickness is required. In such cases the table of permissible variations for plates ordered to thickness shall apply.

3. The weight per square foot of individual plates shall not vary from the ordered weight by more than one and one-third times the amount given in the table.

4. The term "lot" applied to these tables means all of the plates of each group width and each group thickness or weight.

TABLE 1.-Permissible variations of rectangular plates ordered to weight

\begin{tabular}{|c|c|c|c|c|c|c|c|c|c|c|c|c|c|c|c|c|c|c|}
\hline \multirow{3}{*}{$\begin{array}{l}\text { Ordered weight (in pounds } \\
\text { per square foot) }\end{array}$} & \multicolumn{18}{|c|}{$\begin{array}{c}\text { Permissible variations in average weights per square foot of plates for widths given, expressed in percentages } \\
\text { of ordered weights }\end{array}$} \\
\hline & \multicolumn{2}{|c|}{$\begin{array}{l}\text { Under } 48 \\
\text { inches }\end{array}$} & \multicolumn{2}{|c|}{$\begin{array}{l}48 \text { to } 60 \\
\text { inches, } \\
\text { exclusive }\end{array}$} & \multicolumn{2}{|c|}{$\begin{array}{l}60 \text { to } 72 \\
\text { inches, } \\
\text { exclusive }\end{array}$} & \multicolumn{2}{|c|}{$\begin{array}{l}72 \text { to } 84 \\
\text { inches, } \\
\text { exclusive }\end{array}$} & \multicolumn{2}{|c|}{$\begin{array}{l}84 \text { to } 96 \\
\text { inches, } \\
\text { exclusive }\end{array}$} & \multicolumn{2}{|c|}{$\begin{array}{l}96 \text { to } 108 \\
\text { inches, } \\
\text { exclusive }\end{array}$} & \multicolumn{2}{|c|}{$\begin{array}{l}108 \text { to } 120 \\
\text { inches, } \\
\text { exclusive }\end{array}$} & \multicolumn{2}{|c|}{$\begin{array}{l}120 \text { to } 132 \\
\text { inches, } \\
\text { exclusive }\end{array}$} & \multicolumn{2}{|c|}{$\begin{array}{l}132 \text { to } 144 \\
\text { inches } 1\end{array}$} \\
\hline & Over & $\begin{array}{l}\text { Un- } \\
\text { der }\end{array}$ & Over & $\begin{array}{l}\text { Un- } \\
\text { der }\end{array}$ & Over & $\begin{array}{l}\text { Un- } \\
\text { der }\end{array}$ & Over & $\begin{array}{l}\text { Un- } \\
\text { der }\end{array}$ & Over & $\begin{array}{l}\text { Un- } \\
\text { der }\end{array}$ & Over & $\begin{array}{l}\text { Un- } \\
\text { der }\end{array}$ & Over & $\begin{array}{l}\text { Un- } \\
\text { der }\end{array}$ & Over & $\begin{array}{l}\text { Un- } \\
\text { der }\end{array}$ & Over & $\begin{array}{l}\text { Un- } \\
\text { der }\end{array}$ \\
\hline $\begin{array}{l}\text { Under } 5 \\
5 \text { to } 7.5 \text { exclusive } \\
7.5 \text { to } 10 \text { exclusive } \\
10 \text { to } 12.5 \text { exclusive. } \\
12.5 \text { to } 15 \text { exclusive } \\
15 \text { to } 17.5 \text { exclusive. }\end{array}$ & $\begin{array}{l}5 \\
4.5 \\
4 \\
3.5 \\
3 \\
2.5\end{array}$ & $\begin{array}{l}3 \\
3 \\
3 \\
2.5 \\
2.5 \\
2.5\end{array}$ & $\begin{array}{l}5.5 \\
5 \\
4.5 \\
4 \\
3.5 \\
3\end{array}$ & $\begin{array}{l}3 \\
3 \\
3 \\
3 \\
2.5 \\
2.5\end{array}$ & $\begin{array}{l}6 \\
5.5 \\
5 \\
4.5 \\
4 \\
3.5\end{array}$ & $\begin{array}{l}3 \\
3 \\
3 \\
3 \\
3 \\
2.5\end{array}$ & \begin{tabular}{l|}
7 \\
6 \\
5.5 \\
5 \\
4.5 \\
4
\end{tabular} & $\begin{array}{l}3 \\
3 \\
3 \\
3 \\
3 \\
3\end{array}$ & $\begin{array}{l}-\cdots \\
5.5 \\
5 \\
4.5\end{array}$ & $\begin{array}{l}3 \\
3 \\
3 \\
3\end{array}$ & $\begin{array}{l}7 \\
6 \\
5.5 \\
5\end{array}$ & $\begin{array}{l}3 \\
3 \\
3 \\
3\end{array}$ & $\begin{array}{l}8 \\
7 \\
6 \\
5.5\end{array}$ & $\begin{array}{l}3 \\
3 \\
3 \\
3\end{array}$ & $\begin{array}{l}-\overline{8} \\
7 \\
6\end{array}$ & $\begin{array}{l}-3 \\
3 \\
3\end{array}$ & $\begin{array}{l}9 \\
8 \\
7\end{array}$ & $\begin{array}{l}- \\
3 \\
3 \\
3\end{array}$ \\
\hline $\begin{array}{l}17.5 \text { to } 20 \text { exclusive } \\
20 \text { to } 25 \text { exclusive } \\
25 \text { to } 30 \text { exclusive } \\
30 \text { to } 40 \text { exclusive } \\
40 \text { or over. }\end{array}$ & $\begin{array}{l}2.5 \\
2 \\
2 \\
2 \\
2\end{array}$ & $\begin{array}{l}2 \\
2 \\
2 \\
2 \\
2\end{array}$ & $\begin{array}{l}2.5 \\
2.5 \\
2 \\
2 \\
2\end{array}$ & $\begin{array}{l}2.5 \\
2 \\
2 \\
2 \\
2\end{array}$ & $\begin{array}{l}3 \\
2.5 \\
2.5 \\
2 \\
2\end{array}$ & $\begin{array}{l}2.5 \\
2.5 \\
2 \\
2 \\
2\end{array}$ & $\begin{array}{l}3.5 \\
3 \\
2.5 \\
2.5 \\
2.2\end{array}$ & $\begin{array}{l}2.5 \\
2.5 \\
2.5 \\
2 \\
2\end{array}$ & $\begin{array}{l}4 \\
3.5 \\
3 \\
2.5 \\
2.5\end{array}$ & $\begin{array}{l}3 \\
2.5 \\
2.5 \\
2.5 \\
2\end{array}$ & $\begin{array}{l}4.5 \\
4 \\
3.5 \\
3 \\
2.5\end{array}$ & $\begin{array}{l}3 \\
3 \\
3 \\
2.5 \\
2.5\end{array}$ & $\begin{array}{l}5 \\
4.5 \\
4 \\
3.5 \\
3\end{array}$ & $\begin{array}{l}3 \\
3 \\
3 \\
3 \\
2.5\end{array}$ & $\begin{array}{l}5.5 \\
5 \\
4.5 \\
4 \\
3.5\end{array}$ & $\begin{array}{l}3 \\
3 \\
3 \\
3 \\
3\end{array}$ & \begin{tabular}{|l}
6 \\
5.5 \\
5 \\
4.5 \\
4
\end{tabular} & $\begin{array}{l}3 \\
3 \\
3 \\
3 \\
3\end{array}$ \\
\hline
\end{tabular}

1 Replace this column heading by "132 inches or over" for A. S. T. M. and F. S. B. specifications.

8 In F. S. B. specification substitute 2.5 .

5. When ordered to thickness.-Each plate shall not vary more than 0.01 inch under the thickness ordered, and the overweight of each lot in each shipment shall not exceed the amount given in the following table.
6. The weight of individual plates ordered to thickness shall not exceed the nominal weight by more than one and one-third times the amount given in the following table.

7. One cubic inch of rolled steel is assumed to weigh 0.2833 pound.

TABLE 2.-Permissible overweights of rectangular plates ordered to thickness

\begin{tabular}{|c|c|c|c|c|c|c|c|c|c|}
\hline \multirow[b]{2}{*}{ Ordered thickness (in inches) } & \multicolumn{9}{|c|}{$\begin{array}{l}\text { Permissible excess in arerage weights per square foot of plates for widths } \\
\text { given, expressed in percentages of nominal weights }\end{array}$} \\
\hline & $\begin{array}{c}\text { Under } \\
48 \\
\text { inches }\end{array}$ & $\begin{array}{l}48 \text { to } 60 \\
\text { inches, } \\
\text { exclu- } \\
\text { sive }\end{array}$ & $\begin{array}{c}60 \text { to } 72 \\
\text { inches, } \\
\text { exclu- } \\
\text { sive }\end{array}$ & $\begin{array}{l}72 \text { to } 84 \\
\text { inches, } \\
\text { exclu- } \\
\text { sive }\end{array}$ & $\begin{array}{l}84 \text { to } 96 \\
\text { inches. } \\
\text { exclu- } \\
\text { sive }\end{array}$ & $\begin{array}{c}96 \text { to } 108 \\
\text { inches. } \\
\text { exclu- } \\
\text { sive }\end{array}$ & $\begin{array}{l}108 \text { to } \\
120 \\
\text { inches, } \\
\text { exclu- } \\
\text { sive }\end{array}$ & $\begin{array}{l}120 \text { to } \\
132 \\
\text { inches, } \\
\text { exclu- } \\
\text { sive }\end{array}$ & $\begin{array}{c}132 \text { to } \\
144 \\
\text { inches } 1\end{array}$ \\
\hline $\begin{array}{l}\text { Under } 1 / 8 \\
18 \text { to } 3 / 16 \text { exclusive } \\
3 / 16 \text { to } 34 \text { exclusive } \\
14 \text { to } 5 / 16 \text { exclusive } \\
5 / 16 \text { to } 3 / 8 \text { exclusive } \\
3 / 8 \text { to } 7 / 16 \text { exclusive }\end{array}$ & $\begin{array}{l}9 \\
8 \\
7 \\
6 \\
5 \\
4.5\end{array}$ & $\begin{array}{r}10 \\
9 \\
8 \\
7 \\
6 \\
5\end{array}$ & $\begin{array}{r}12 \\
10 \\
9 \\
8 \\
7 \\
6\end{array}$ & $\begin{array}{r}14 \\
12 \\
10 \\
9 \\
8 \\
7\end{array}$ & $\begin{array}{r}12 \\
10 \\
9 \\
8\end{array}$ & $\begin{array}{r}12 \\
10\end{array}$ & $\begin{array}{l}14 \\
12 \\
10\end{array}$ & $\begin{array}{l}16 \\
14 \\
12\end{array}$ & $\begin{array}{l}19 \\
17 \\
15\end{array}$ \\
\hline $\begin{array}{l}7 / 16 \text { to } 1 / 2 \text { exclusive } \\
1 / 2 \text { to } 5 / 8 \text { exclusive } \\
5 / 8 \text { to } 3 / 4 \text { exclusive } \\
3 / 4 \text { to } 1 \text { exclusive } \\
1 \text { or over }\end{array}$ & $\begin{array}{l}4 \\
3.5 \\
3 \\
2.5 \\
2.5\end{array}$ & $\begin{array}{l}4.5 \\
4 \\
3.5 \\
3 \\
2.5\end{array}$ & $\begin{array}{l}5 \\
4.5 \\
4 \\
3.5 \\
3\end{array}$ & $\begin{array}{l}6 \\
5 \\
4.5 \\
4 \\
3.5\end{array}$ & $\begin{array}{l}7 \\
6 \\
5 \\
4.5 \\
4\end{array}$ & $\begin{array}{l}8 \\
7 \\
6 \\
5 \\
4.5\end{array}$ & $\begin{array}{l}9 \\
8 \\
7 \\
6 \\
5\end{array}$ & $\begin{array}{r}10 \\
9 \\
8 \\
7 \\
6\end{array}$ & $\begin{array}{r}13 \\
11 \\
9 \\
8 \\
7\end{array}$ \\
\hline
\end{tabular}

1 Replace this column heading by " 132 inches or over" for A. S. T. M. and F. S. B. specifications. 
8. Allowable variations in the weight of bar sizes of angles, tees, zees, and channels.-For bar sizes of angles, tees, zees, and channels, the following average variations in weight will be permitted for sections of the various dimensions and thicknesses stated, namely:

TABLE 3.-Angles, tees, zees, and channels

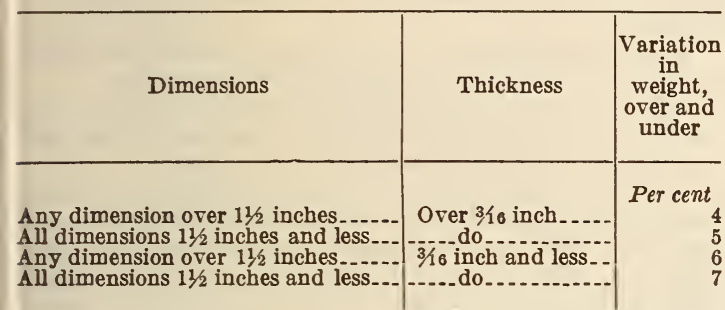

NoTE. - A channel is in "bar" size when its greatest dimension is less than 3 inches. An angle, $T$, or $z$ is in "bar" size when its greatest dimension is less than 3 inches; or when it is 3 inches or more and at the same time the thickness is less than one-fourth inch.

TABLE 4.-Rounds, squares, and hexagons

\begin{tabular}{|c|c|c|}
\hline \multirow{2}{*}{ Size } & \multicolumn{2}{|c|}{$\begin{array}{l}\text { Variation in } \\
\text { size }\end{array}$} \\
\hline & Under & Over \\
\hline 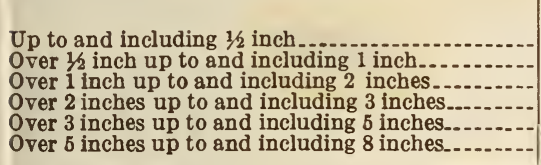 & $\begin{array}{l}\text { Inch } \\
0.007 \\
.010 \\
1 / 64 \\
1 / 32 \\
1 / 32 \\
1 / 10\end{array}$ & $\begin{array}{c}\text { Inch } \\
0.007 \\
.010 \\
1 / 32 \\
3 / 64 \\
3 / 32 \\
18\end{array}$ \\
\hline
\end{tabular}

TABLE 5.-Flats

\begin{tabular}{|c|c|c|c|c|c|c|}
\hline \multirow[b]{3}{*}{ Width of flats } & \multirow{2}{*}{\multicolumn{2}{|c|}{$\begin{array}{l}\text { Variation in } \\
\text { width }\end{array}$}} & \multirow{2}{*}{\multicolumn{4}{|c|}{$\begin{array}{c}\begin{array}{c}\text { Variation in thickness, } \\
\text { under and over }\end{array} \\
\text { Thickness of flats }\end{array}$}} \\
\hline & & & & & & \\
\hline & $\begin{array}{l}\text { Un- } \\
\text { der }\end{array}$ & Over & $\begin{array}{l}\text { 3/16 } \\
\text { inch } \\
\text { and } \\
\text { under }\end{array}$ & $\begin{array}{l}\text { Over } \\
3 / 16 \\
\text { inch } \\
\text { up to } \\
1 / 2 \\
\text { inch }\end{array}$ & $\begin{array}{l}\text { Over } \\
1 / 2 \\
\text { inch } \\
\text { up to } \\
1 \text { inch }\end{array}$ & $\begin{array}{l}\text { Over } \\
1 \text { inch } \\
\text { up to } \\
2 \text { inch- } \\
\text { es }\end{array}$ \\
\hline \multirow{5}{*}{$\begin{array}{l}\text { Up to and including } 1 \\
\text { inch.- } \\
\text { Over } 1 \text { inch up to and } \\
\text { including } 2 \text { inches.-. } \\
\text { Over } 2 \text { inches upto and } \\
\text { including } 4 \text { inches.-. } \\
\text { Over } 4 \text { inches up to and } \\
\text { including } 6 \text { inches..- }\end{array}$} & $\begin{array}{c}\text { Inch } \\
1 / 64\end{array}$ & Inch & $\begin{array}{l}\text { Inch } \\
0.006\end{array}$ & $\begin{array}{l}\text { Inch } \\
0.008\end{array}$ & $\begin{array}{c}\text { Inch } \\
0.010\end{array}$ & Inch \\
\hline & 13. & $3 / 64$ & .008 & 012 & 015 & \\
\hline & & 964 & & & & \\
\hline & $3 / 6$ & $1 / 16$ & .010 & .015 & .020 & $1 / 3$ \\
\hline & $1 / 10$ & $3 / 32$ & .010 & .015 & .020 & $1 / 32$ \\
\hline
\end{tabular}

NATIONAL BOARD OF FIRE UNDERWRITERS, BUILDING CODE, 1931

\section{BUILDING CODE}

(See 605.22, p. 276.)

UNITED STATES GOVERNMENT, DEPARTMENT OF COMMERCE, BUREAU OF STANDARDS, STANDARD THICKNESSES, WEIGHTS, AND TOLERANCES OF SHEET METAL (CUSTOMARY PRACTICE), CIRCULAR NO. 391, MARCH 24, 1931

\section{IRON AND STEEL SHEET AND PLATE}

(Standard gage for sheet and plate steel and iron. See above Bureau of Standards Circular 391 under 604.0, p. 224.)
(Permissible variations in weight and thickness of plates given in this circular are identical with those of the Association of American Steel Manufacturers. See 605.0, p. 248.)

\subsection{STRUCTURAL SHAPES AND PLATES, NOT FABRICATED}

\subsection{STRUCTURAL STEEL FOR BRIDGES.}

\section{AMERICAN RAILWAY ENGINEERING ASSO- CIATION, SPECIFICATIONS FOR STEEL RAILWAY BRIDGES, 1924}

(See also 611.11, p. 636; 611.41, p. 707; and 611.51, p. 753.$)$

\section{STRUCTURAL AND RIVET STEEL}

(This specification is nearly identical with specification A7 of the American Society for Testing Materials (see below) with the exceptions that all reference to copper steel is omitted, there is a slight difference in permissible variation of plate weights, and the following additional material is given:)

1. Speed of testing machine.-The crosshead speed of the testing machine shall be such that the beam of the machine can be kept balanced, but in no case shall the values given in the following table be exceeded:

\begin{tabular}{|c|c|c|}
\hline \multirow{2}{*}{$\begin{array}{c}\text { Gage } \\
\text { length } \\
\text { of } \\
\text { speci- } \\
\text { men }\end{array}$} & \multicolumn{2}{|c|}{$\begin{array}{l}\text { Maximum crosshead } \\
\text { speed in determin- } \\
\text { ing- }\end{array}$} \\
\hline & $\begin{array}{l}\text { Yield } \\
\text { point }\end{array}$ & $\begin{array}{c}\text { Tensile } \\
\text { strength }\end{array}$ \\
\hline $\begin{array}{c}\text { Inches } \\
2 \\
8\end{array}$ & $\begin{array}{c}\text { Inches } \\
\text { per } \\
\text { minute } \\
0.5 \\
2\end{array}$ & $\begin{array}{c}\text { Inches } \\
\text { per } \\
\text { minute } \\
2 \\
6\end{array}$ \\
\hline
\end{tabular}

2. The manufacturer shall furnish, without charge, the test specimens as specified herein, and the labor, testing machines, and tools necessary to make the specimen and full-size tests.

\section{AMERICAN SOCIETY FOR TESTING MA- TERIALS, STANDARD SPECIFICATIONS FOR STRUCTURAL STEEL FOR BRIDGES, SERIAL DESIGNATION A7-29, 1929}

1. Steel castings.-The standard specifications for steel castings, A. S. T. M. A27-24 (see 611.41, p. 707), shall govern the purchase of steel castings for bridges. Unless otherwise specified, class B castings, medium grade, shall be used.

2. Process.-The steel shall be made by the openhearth process.

3. Chemical composition.-The steel shall conform to the following requirements as to chemical composition:

\begin{tabular}{|c|c|c|}
\hline & $\begin{array}{l}\text { Struc- } \\
\text { tural } \\
\text { steel }\end{array}$ & $\begin{array}{l}\text { Rivet } \\
\text { steel }\end{array}$ \\
\hline $\begin{array}{l}\text { Phosphorus: } \\
\text { Acid, not over-- }\end{array}$ & $\begin{array}{c}\text { Per } \\
\text { cent } \\
0.06\end{array}$ & $\begin{array}{c}\text { Per } \\
\text { cent } \\
0.04\end{array}$ \\
\hline $\begin{array}{l}\text { Basic, not over } \\
\text { Sulphur, not over- } \\
\text { Copper, when copper steel is specified, not under- }\end{array}$ & $\begin{array}{l}.04 \\
.05 \\
.20\end{array}$ & $\begin{array}{l}.04 \\
.045\end{array}$ \\
\hline
\end{tabular}


4. Ladle analyses.-An analysis of each melt of steel shall be made by the manufacturer to determine the percentages of carbon, manganese, phosphorus, and sulphur; also copper when copper steel is specified. This analysis shall be made from a test ingot taken during the pouring of the melt. The chemical composition thus determined shall be reported to the purchaser or his representative and the percentages of phosphorus and sulphur, and copper when copper steel is specified, shall conform to the requirements specified under chemical composition.

5. Tension tests.-(a) The material shall conform to the following requirements as to tensile properties:

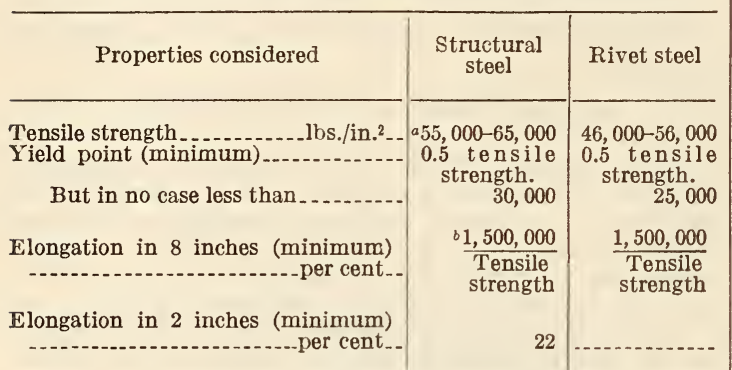

a See par. (b).

${ }^{b}$ See sec. 6 .

(b) In order to meet the required minimum tensile strength of full-size annealed eyebars, the purchaser may determine the tensile strength to be obtained in specimen tests; the range shall not exceed 14,000 lbs./in. ${ }^{2}$, and the maximum shall not exceed 74,000 lbs./in. ${ }^{2}$. The material shall conform to the requirements as to physical properties other than that of tensile strength.

6. Modification in elongation.-(a) For structural steel over three-f ourths inch in thickness or diameter, a deduction from the percentage of elongation in 8 inches specified in table of tensile properties of 0.25 per cent shall be made for each increase of one-thirtysecond inch of the specified thickness or diameter above three-fourths inch, to a minimum of 18 per cent.

(b) For structural steel under five-sixteenths inch in thickness or diameter, a deduction from the percentage of elongation in 8 inches specified in table of tensile properties of 1.25 per cent shall be made for each decrease of one-thirty-second inch in thickness or diameter below five-sixteenths inch.

7. Bend tests. - (a) Bend-test specimens, except as specified below, shall stand being bent cold through $180^{\circ}$ without cracking on the outside of the bent portion, as follows: For material three-fourths inch or under in thickness, flat on itself; for material over three-fourths inch to and including $1 \frac{1}{4}$ inches in thickness, around a pin the diameter of which is equal to the thickness of the specimen; and for material over $1 \frac{1}{4}$ inches in thickness, around a pin the diameter of which is equal to twice the thickness of the specimen.

(b) Bend-test specimens for eyebar flats shall stand being bent cold through $180^{\circ}$ without cracking on the outside of the bent portion as follows: For material three-fourths inch or under in thickness, around a pin the diameter of which is equal to the thickness of the specimen; for material over threefourths inch to and including $11 / 4$ inches in thickness, around a pin, the diameter of which is equal to twice the thickness of the specimen; and for material over $1 \frac{11}{4}$ inches in thickness, around a pin, the diameter of which is equal to three times the thickness of the specimen. (c) Bend-test specimens for rivet steel shall stand being bent cold through $180^{\circ}$ flat on themselves without cracking on the outside of the bent portion.

8. Test specimens.-(a) Test specimens shall be prepared for testing from the material in its rolled or forged condition, except as specified below.

(b) Test specimens for annealed material shall be prepared from the material as annealed for use, or from a short length of a full section similarly treated.

(c) Test specimens for rivet bars which have been cold-drawn shall be normalized before testing.

(d) Test specimens shall be taken longitudinally and, except as specified below, shall be of the full thickness or section of material as rolled.

(e) Test specimens for plates, shapes, and flats may be machined to the form and dimensions shown in Figure 128 standard specifications for metals (see 605.0 , p. 247), or with both edges parallel. Bendtest specimens for eyebar flats may have three rolled sides.

$(f)$ Tension-test specimens for material over $1 \frac{1}{2}$ inches in thickness or diameter, except pins and rollers, may be machined to a thickness or diameter of at least three-fourths inch for a length of at least 9 inches, or they may conform to the dimensions shown in Figure 129 in A. S. T. M. standard specifications for metals (see 605.0, p. 247).

(g) Bend-test specimens for material over $1 \frac{1}{2}$ inches in thickness or diameter, except eyebar flats, pins, and rollers, may be machined to a thickness or diameter of at least three-fourths inch or to 1 by $1 / 2$ inch in section.

(h) Tension-test specimens for pins and rollers shall conform to the dimensions shown in Figure 129 and bend-test specimens shall be 1 by $1 / 2$ inch in section.

(i) Test specimens for pins and rollers shall be taken so that the axis is 1 inch from the surface.

(j) The machined sides of rectangular bend-test specimens may have the corners rounded to a radius not over one-sixteenth inch.

9. (For tables of permissible variations of rectangular plates ordered to weight and permissible overweights of rectangular plates ordered to thickness, see A. A. S. M., Tables 1 and $2(605.0$, p. 248).)

10. (For general requirements relating to check analyses, yield point, number of tests, permissible variations in weight and thickness of plates, finish, marking, inspection, rejection, and rehearing, also test specimen Figures 128 and 129, see A. S. T. M. specifications, 605.0, p. 247.)

\section{ASSOCIATION OF AMERICAN STEEL MAN- UFACTURERS, STANDARD SPECIFICA- TIONS FOR STRUCTURAL AND BOILER STEEL, NOVEMBER 24, 1922}

(See also 604.11, p. 223.)

\section{STRUCTURAL STEEL}

1. Classes.-These specifications cover three classes of structural steel, namely: Class A steel, to be used for railway bridges, ships, cars, and similar structures; class B steel, to be used for buildings, highway bridges, train sheds, and similar structures; and class $\mathrm{C}$ steel, to be used for structural rivets.

2. Process.-(a) Steel for classes A and C shall be made by the open-hearth process. Steel for class B may be made by either or by both the openhearth or Bessemer processes, except as specified in (b).

(b) Steel for class B plates and angles over threefourths inch in thickness which are to be punched, shall be made by the open-hearth process. 
3. Chemical composition.-The steel shall conform to the following requirements as to chemical composition:

\begin{tabular}{|c|c|c|c|}
\hline Elements considered & $\underset{\text { steel }}{\text { Class A }}$ & $\underset{\text { steel }}{\text { Class B }}$ & $\underset{\text { steel }}{\text { Class C }}$ \\
\hline $\begin{array}{l}\text { Phosphorus, maximum: } \\
\text { Basic open-hearth. } \\
\text { Acid open-hearth } \\
\text { Bessemer }\end{array}$ & $\begin{array}{c}\text { Per cent } \\
0.04 \\
.06\end{array}$ & $\begin{array}{c}\text { Per cent } \\
0.06 \\
.08 \\
.11\end{array}$ & $\begin{array}{r}\text { Per sent } \\
0.04 \\
.04 \\
-0.0\end{array}$ \\
\hline Sulphur, maximu & .06 & & \\
\hline
\end{tabular}

4. Ladle analysis.-An analysis of each melt of steel shall be made by the manufacturer to determine the percentages of the elements specified in section 3. This analysis shall be made from a test ingot taken during the pouring of the melt. The chemical composition thus determined shall conform to the requirements specified in section 3 , and shall be reported to the purchaser or his representative, if requested.

5. Tension tests. - The steel shall conform to the following requirements as to tensile properties:

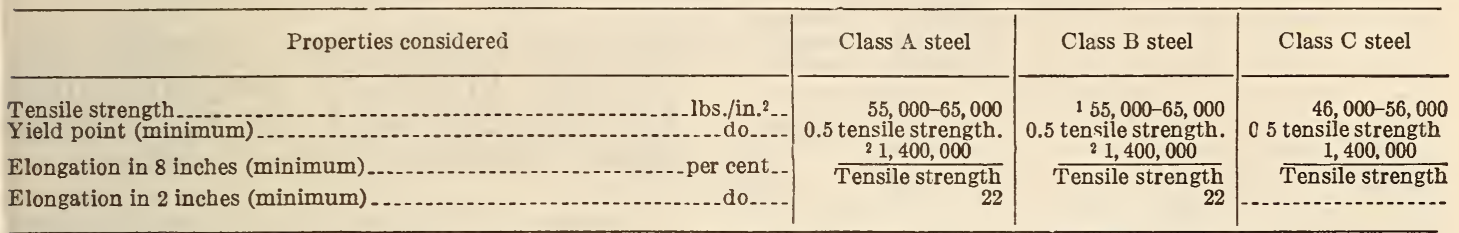

1 See sec. 6.

6. Modification in tensile strength.-Class B steel may have tensile strength up to 70,000 pounds maximum, provided the elongation is not less than the percentage required for 65,000 pounds tensile strength.

7. Bend tests. - The 1 by $1 / 2$ inch test specimen for pins, rollers, and other bars, shall bend cold through $180^{\circ}$ around a pin 1 inch in diameter without cracking on the outside of the bent portion.

8. Test specimens.-(a) Tension-test specimens for plates and eyebar flats over, $1 \frac{1}{2}$ inches in thickness, and bend-test specimens for plates over $1 \frac{1}{2}$ inches in thickness may be machined to a thickness or diameter of at least three-fourths inch for a length of at least 9 inches.

(b) Test specimens for bars over $1 \frac{1}{2}$ inches in thickness may be machined to a thickness or diameter of at least three-fourths inch for a length of at least 9 inches; or tension-test specimens may conform to the dimensions shown in Figure 129. Bend-test specimens may be 1 by $1 / 2$ inch in section.

(c) The tension-test specimen shown in Figure 129 and the 1 by $1 / 2$ inch bend-test specimen for pins and rollers shall be taken so that the axis is 1 inch from the surface; and for other bars over $1 \frac{1}{2}$ inches in thickness, midway between the center and surface.

9. Number of tests.-Plates three-sixteenths inch and under in thickness; shapes less than 1 square inch sectional area; rounds (except rivet rods) under one-half inch in diameter; and squares, hexagons, and flats less than one-half square inch sectional area, shall not be subject to tension tests.

10. Permissible variations. - (a) The sectional area or weight of each structural shape, and of each rolled edge plate up to and including 36 inches in width, shall not vary more than 2.5 per cent from the theoretical or specified amounts.

(b) The thickness or weight of sheared mill plates, and of universal mill plates over 36 inches in width, shall conform to the manufacturers' standard practice governing the permissible variations for sheared mill plates. (See A. A. S. M., 605.0, p. 248, Tables 1 and 2.)

(c) The weights of angles, tees, zees, and channels of bar sizes, and the dimensions of rounds, squares, hexagons, and flats, shall conform to the manufacturers' standard practice governing the allowable variations in size and weight of hot-rolled bars. (See A. A. S. M., 605.0, p. 248, Tables 3, 4, and 5.)

(For requirements relating to check analyses, yield point, modification in tensile strength, modifications in elongation, bend tests, test specimens, number of tests, finish, marking, inspection, rejection, and test specimen figures, see A. S. T. M. A7 (above), paragraphs $5(b), 6,7$, and $8(a),(b),(c)$,
${ }^{2}$ See sections on modifications in elongation.

$(d),(e),(h)$, and $(j)$, and A. S. T. M. specifications (605.0, p. 247,) paragraphs $1,2,3,5,6,7$, and 8 , and Figures 128 and 129.)

\section{UNITED STATES GOVERNMENT, FEDERAL SPECIFICATIONS BOARD, FEDERAL SPECIFICATION FOR STRUCTURAL STEEL FOR BRIDGES, NO. 351a, MARCH 25, 1929. (CURRENT DESIGNATION : QQ-S-711)}

(This specification is, for certain requirements, substantially the same as specification A7 of the American Society for Testing Materials. For requirements relating to process, manufacturers' analyses, tension tests, modifications in elongation, bend tests, test specimens, and copper steel, see A. S. T. M. A7 (above), paragraphs 2, 4, 5, 6, 7, and $8(a),(b),(c),(d),(e),(f),(g),(h)$, and $(j)$, and that part of paragraph 3 which relates to copper steel. For requirements relating to yield point, permissible variations in sheared plates and structural shapes except of bar sizes, finish, and marking, see A. S. T. M. specifications (605.0, p. 247), paragraphs 2, 4, and 5, and the first two sentences in paragraph 6.)

(For tables of permissible variations in plates ordered to weight and thickness, and in angles, tees, zees, channels, rounds, squares, hexagons, and flats, see A. A. S. M. (605.0, p. 248), Tables 1, 2, 3, 4, and 5.)

(The following additional material is given:)

\section{GINERAL SPECIFICATIONS}

1. Federal specification No. 339a (QQ-M-151) for metals, in effect on date of invitation for bids, shall form part of this specification. (See 600.0, p. 3.)

\section{CLASSES}

2. Structural steel for bridges shall be of the following classes: (a) structural steel, noncopper; (b) structural steel, copper; (c) rivet steel, noncopper; and $(d)$ rivet steel, copper.

\section{GENERAL REQUIREMENTS}

3. Struciural shapes of bar sizes.-The weight of each piece shall not vary from the weight specified more than the amount given in Table 3.

4. Rolled edge plates.-Each plate up to and including 36 inches in width, shall not vary more than 2.5 per cent from the cross section (or sectional area) or weight specified; rolled edge plates more than 36 inches in width shall conform to limits specified for sheared plates. 
5. Rounds (including rivet bars), squares, and hexagons.-No piece shall vary from the limits given in Table 4.

6. Flats.-No piece shall vary from the limits given in Table 5 .

\section{DETAIL REQUIREMENTS}

7. Chemical composition.-The amounts of phosphorus and sulphur shall not exceed the following percentages:

\begin{tabular}{l|r|r}
\hline & $\begin{array}{r}\text { Structural } \\
\text { steel, classes } \\
\text { A and B }\end{array}$ & $\begin{array}{r}\text { Rivet steel, } \\
\text { classes C } \\
\text { and D }\end{array}$ \\
\hline $\begin{array}{r}\text { Phosphorus: } \\
\text { Acid }\end{array}$ & $\begin{array}{r}\text { Per cent } \\
0.070\end{array}$ & $\begin{array}{r}\text { Per cent } \\
0.050\end{array}$ \\
Sulphur. & .050 & 050 \\
.075 & .070 \\
\hline
\end{tabular}

\section{METHODS OF TEST}

8. Number of tests. - Two tension and two bend tests shall be made from each melt, unless the finished material from a melt is less than 26 short tons, when one tension and one bend shall be suffcient. If, however, material from one melt differs three-eighths inch or more in thickness, the thickest and thinnest material rolled shall each be represented by one tension and one bend test, regardless of the weight of the melt. Plates and flats threesixteenths inch and less in thickness and shapes less than 1 square inch in section, shall not be subject to tension tests.

9. Test specimens.-For bars over $1 \frac{1}{2}$ inches in thickness or diameter, other than flats, when not tested in the full section as rolled, the tension test specimens shall be taken so that the axis shall be at a point one-half the distance between the center and the surface of the bar.

\section{NOTES}

10. Material covered by this specification is suitable for the following classes of work, among others: Reamed work, oil-tight and water-tight work, railroad and highway bridges, cranes of 40-ton capacity and over, radio towers 300 feet high or more, and any other structures in which there is a considerable and rapid variation of stress intensity.

11. When specifying materials in accordance with this specification, the class or classes (A, B, C, and D) should be stated definitely.

605.12 STRUCTURAL STEEL FOR BUILDINGS.

AMERICAN INSTITUTE OF STEEL CONSTRUCTION, STANDARD SPECIFICA. TION FOR STRUCTURAL STEEL FOR BUILDINGS, 1923

\section{MATERIAL}

(The institute indorses specification A9, of the American Society for Testing Materials, for structural steel for buildings. See below.)

\section{AMERICAN RAILWAY ENGINEERING ASSO- CIATION, SPECIFICATIONS FOR BUILD- INGS FOR RAILWAY PURPOSES, 1929}

\section{STRUCTURAL STEEL}

(The association indorses American Society for Testing Materials specification A9, for structural steel for buildings (below), with the following exceptions:)

1. Structural steel shall be made by the openhearth process.

2. The reference to Bessemer steel in paragraph 2 of A. S. T. M. A9 shall be omitted.

\section{CAST STEEL}

(The association indorses American Society for Testing Materials specification A7, for structural steel for bridges. (See 605.11, p. 249.)

\section{AMERICAN SOCIETY FOR TESTING MA- TERIALS, STANDARD SPECIFICATIONS FOR STRUCTURAL STEEL FOR BUILD- INGS, DESIGNATION A9-29, 1929}

1. Process.-(a) Structural steel, except as noted in paragraph $(b)$, shall be made by either or both the following processes: Bessemer or open hearth.

(b) Rivet steel, and steel for plates or angles over three-fourths inch in thickness which are to be punched, shall be made by the open-hearth process.

2. Chemical composition.-The steel shall conform to the following requirements as to chemical composition:

\begin{tabular}{|c|c|c|}
\hline & \begin{tabular}{|c|} 
Struc- \\
tural \\
steel
\end{tabular} & $\begin{array}{l}\text { Rivet } \\
\text { steel }\end{array}$ \\
\hline $\begin{array}{l}\text { Phosphorus, maximum: } \\
\text { Bessemer }\end{array}$ & \multirow{2}{*}{\begin{tabular}{|c|} 
Per cent \\
0.10 \\
.08
\end{tabular}} & Per cent \\
\hline Open hearth & & 0.08 \\
\hline $\begin{array}{l}\text { Sulphur, maximum } \\
\text { Copper, when copper steel is specified, minimum. }\end{array}$ & & .045 \\
\hline
\end{tabular}

3. Ladle analyses.-(a) A carbon determination, and a copper determination when copper steel is specified, shall be made of each melt of Bessemer steel, and determinations for manganese, phosphorus, and sulphur representing the average of the melts applied for each 12-hour period.

(b) An analysis of each melt of open-hearth steel shall be made to determine carbon, manganese, phosphorus, and sulphur; also copper when copper steel is specified.

(c) These analyses shall be made by the manufacturer from test ingots taken during the pouring of each melt. The chemical composition thus determined shall be reported to the purchaser or his representative and the percentages of phosphorus and sulphur, and also copper when copper steel is specified, shall conform to the requirements specified in section 2 .

4. Tension tests. - The material shall conform to the following requirements as to tensile properties:

\begin{tabular}{|c|c|c|}
\hline Properties considered & $\begin{array}{l}\text { Structural } \\
\text { steel }\end{array}$ & $\begin{array}{l}\text { Rivet } \\
\text { steel }\end{array}$ \\
\hline Tensile strength & $55,000-65,000$ & $46,000-56,000$ \\
\hline $\begin{array}{l}\text { Yield point, minimum } \\
\text { but in no case less than }\end{array}$ & $\begin{array}{l}0.5 \text { tensile } \\
\text { strength. } \\
30,000\end{array}$ & $\begin{array}{l}0.5 \text { tensile } \\
\text { strength. } \\
25,000\end{array}$ \\
\hline Elongation in 8 inches, minimum & 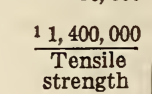 & 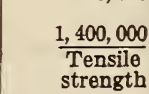 \\
\hline Elongation in 2 inches, minimum & 22 & - n \\
\hline
\end{tabular}

1 See section on modifications in elongation. 
5. (For general requirements relating to check analyses, yield point, number of tests, permissible variations in weight and thickness of plates, finish, marking, inspection, rejection, and rehearing, also test specimen Figures 128 and 129, see A. S. T. M. specifications (605.0, p. 247).)

6. (For requirements relating to modifications in elongation, bend tests, and test specimens. See A. S. T. M. A7 (605.11, p. 249), pars. 6, 7 (a) and (c), and 8.)

7. (For tables of permissible variations of rectangular plates ordered to weight and permissible overweights of rectangular plates ordered to thickness, see A. A. S. M., Tables 1 and 2 (605.0, p. 248).)

ASSOCIATION OF AMERICAN STEEL MANUFACTURERS, STANDARD SPECIFICATIONS FOR STRUCTURAL AND BOILER STEEL, NOVEMBER 24, 1922

\section{STRUCTURAL STEEL}

(See 605.11, p. 250.)
UNITED STATES GOVERNMENT, DEPARTMENT OF COMMERCE, BUREAU OF STANDARDS, SIMPLIFIED PRACTICE RECOMMENDATION, OPEN-WEB STEEL JOISTS, R94-30, 1930

\section{OPEN-WEB STEEL JOISTS}

Prior to 1930 designers found it difficult to specify accurately when using steel joists in construction unless they designated the product of some particular producer. This condition resulted from the then existing variation in capacities of the joists produced by the different manufacturers.

In order to correct this condition and to make the use of steel joists as easy as possible, the manufacturers, through their association, the Steel Joist Institute, drafted a table of sizes and loads. They felt that its use by the architects and contractors throughout the country would do much to eliminate waste and make for more efficient designing of steel-joist construction. (The table thus prepared is given below.)

Open-web steel joists

\begin{tabular}{|c|c|c|c|c|c|c|c|c|c|c|c|c|c|c|}
\hline \multirow{8}{*}{ Span } & \multicolumn{14}{|c|}{ Properties and allowable total loads (in pounds per linear foot); Steel Joist Institute designation } \\
\hline & SJ81 & SJ82 & SJ102 & SJ103 & $\mathrm{S}, \mathrm{I} 104$ & SJ123 & SJ124 & $\mathrm{SJ} 125$ & SJ126 & SJ145 & SJ146 & SJ147 & SJ166 & SJ167 \\
\hline & \multicolumn{14}{|c|}{ Depth (in inches) } \\
\hline & 8 & 8 & 10 & 10 & 10 & 12 & 12 & 12 & 12 & 14 & 14 & 14 & 16 & 16 \\
\hline & \multicolumn{14}{|c|}{ Resisting moment (in inch-pounds) } \\
\hline & 29,500 & 52,500 & 63,000 & 82,000 & 100,000 & 92,000 & 115,000 & 142,000 & 175,000 & 156,000 & 205,000 & 246,000 & 232,000 & 281,000 \\
\hline & \multicolumn{14}{|c|}{ Maximum end reaction (in pounds) } \\
\hline & 1,600 & 1,900 & 1,900 & 1,950 & 2,200 & 2,200 & 2,300 & 2,500 & 2,700 & 2,900 & 3,100 & 3,400 & 3,200 & 3,600 \\
\hline $\begin{array}{c}\text { Feet } \\
4\end{array}$ & 800 & & & & & & & & & & & & & \\
\hline $\begin{array}{l}4 \\
5 \\
6 \\
7 \\
8\end{array}$ & $\begin{array}{l}640 \\
530 \\
402 \\
402\end{array}$ & & & & & & & & & & & & & $\cdots$ \\
\hline $\begin{array}{l}8 \\
9\end{array}$ & 243 & & & & & & & & & & & & & \\
\hline 10 & 197 & 350 & $\cdots$ & & & & & & & 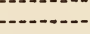 & & & 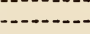 & - \\
\hline $\begin{array}{l}11 \\
12\end{array}$ & $\begin{array}{l}162 \\
137\end{array}$ & $\begin{array}{l}289 \\
243\end{array}$ & 292 & & & & & & & . & & & -- & - \\
\hline 13 & 116 & 207 & 248 & 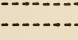 & $\cdots$ & 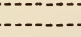 & (t) & - & $\cdots$ & & & & $\cdots$ & \\
\hline 14 & 100 & 178 & 214 & & & & & & & & & & & \\
\hline $\begin{array}{l}15 \\
16\end{array}$ & $\begin{array}{l}87 \\
77\end{array}$ & $\begin{array}{l}155 \\
137\end{array}$ & $\begin{array}{l}187 \\
164\end{array}$ & $\begin{array}{l}243 \\
213\end{array}$ & 260 & $\begin{array}{l}272 \\
240\end{array}$ & & -- & -- & -- & - & & $\cdots$ & 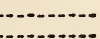 \\
\hline $\begin{array}{l}17 \\
18\end{array}$ & & & 145 & 189 & 230 & 212 & 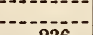 & & 年 & . & & $\ldots$ & 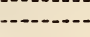 & 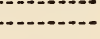 \\
\hline & $-\cdots+\infty$ & -.......... & 130 & 169 & 205 & 189 & 236 & -- & - & 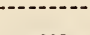 & 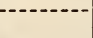 & & $\cdots$ & ..... \\
\hline $\begin{array}{l}19 \\
20\end{array}$ & (n) & -an- & $\begin{array}{l}116 \\
105\end{array}$ & $\begin{array}{l}151 \\
137\end{array}$ & $\begin{array}{l}184 \\
167\end{array}$ & $\begin{array}{l}170 \\
153\end{array}$ & $\begin{array}{l}212 \\
192\end{array}$ & 237 & & $\begin{array}{l}288 \\
260\end{array}$ & & & & \\
\hline 21 & $-\cdots$ & & & & & 139 & 174 & 215 & & 236 & $\begin{array}{c}-2 \\
-2\end{array}$ & [-. & $\cdots$ & 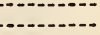 \\
\hline $\begin{array}{l}22 \\
23\end{array}$ & 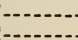 & & & & (n) & $\begin{array}{l}127 \\
116\end{array}$ & $\begin{array}{l}158 \\
145\end{array}$ & $\begin{array}{l}196 \\
179\end{array}$ & $\begin{array}{l}241 \\
221\end{array}$ & $\begin{array}{l}215 \\
197\end{array}$ & 258 & 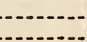 & & $\ldots$ \\
\hline 24 & -....... & |-n-n & 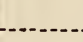 & 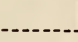 & $\ldots$ & 106 & 133 & 164 & 202 & 180 & 237 & & & \\
\hline $\begin{array}{l}25 \\
26\end{array}$ & 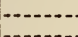 & & & & - & & & - & - & $\begin{array}{l}166 \\
154\end{array}$ & 218 & 262 & 247 & . \\
\hline 27 & (-n) & - & (n) & 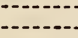 & . & 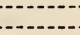 & (n) & (n) & - & 143 & 187 & 225 & 212 & 257 \\
\hline 28 & . & 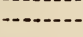 & & & & & & & 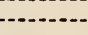 & 133 & 174 & 209 & 197 & 239 \\
\hline $\begin{array}{l}29 \\
30\end{array}$ & & & & & & & & & & & & & 184 & $\begin{array}{l}223 \\
208\end{array}$ \\
\hline $\begin{array}{l}80 \\
32\end{array}$ & & $\cdots$ & 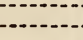 & & & & 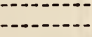 & 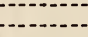 & $\cdots$ & - & $\ldots$ & 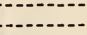 & 161 & 195 \\
\hline 32 & & & & & & & & & & & & & 151 & 183 \\
\hline
\end{tabular}




\section{UNITED STATES GOVERNMENT, FEDERAL SPECIFICATIONS BOARD, FEDERAL SPECIFICATION FOR STRUCTURAL STEEL FOR BUILDINGS, NO. 352a, MARCH 15,1929 . (CURRENT DESIG- NATION : QQ-S-721)}

\section{CHEMICAL COMPOSITION}

1. The amount of phosphorus and sulphur shall not exceed the following percentages:

\begin{tabular}{l|r|r}
\hline & $\begin{array}{c}\text { Struc- } \\
\text { tural } \\
\text { steel } \\
\text { classes } \\
\text { A and B }\end{array}$ & $\begin{array}{c}\text { Rivet } \\
\text { steel } \\
\text { classes } \\
\mathrm{C} \text { and D }\end{array}$ \\
\hline $\begin{array}{c}\text { Phosphorus: } \\
\text { Bessemer }\end{array}$ & $\begin{array}{r}\text { Per cent } \\
0.125 \\
.07\end{array}$ & $\begin{array}{c}\text { Per cent } \\
\text { Sulphur....... hearth.... }\end{array}$ \\
\hline
\end{tabular}

\section{NOTES}

2. Material covered by this specification is suitable for the following classes of work, among others: Building columns and girders, roof trusses, mill buildings, light structures, and all work of a similar character in which the live load is largely static or the construction is light or of a commercial character.

3. (For requirements relating to modification in elongation, bend tests, test specimens, and copper, see A. S. T. M. A7 (605.11, p. 249), paragraphs 6, $7(a)$ and $(c), 8(a),(b),(c),(d),(e),(f),(g),(h)$, and $(j)$, and that part of paragraph 3 dealing with copper.)

4. (For requirements relating to yield point, permissible variations in sheared plates and structural shapes except of bar sizes, finish, and marking, see A. S. T. M. specifications $(605.0$, p. 247$)$, paragraphs $2,4,5$, and 6 .)

5. (For requirements relating to process, manufacturers' analyses, and table of tensile properties, see A. S. T. M. A9 (above), paragraphs 1, 3, and 4.)

6. (For general specifications, classes, permissible variations in structural shapes of bar sizes, rollededge plates, rounds, and flats, number of tests, test specimens, and notes, see F. S. B. 351a (QQ-S-711) (605.11, p. 251), paragraphs 1, 2, 3, 4, 5, 6, 8, 9, and 11. In paragraph 8 , number of tests, change " 26 short tons" to "25 short tons.")

7. (For tables of permissible variations in plates ordered to weight and thickness, and in angles, tees, zees, channels, rounds, squares, hexagons, and flats, see A. A. S. M. (605.0, p. 248), Tables 1, 2, 3, 4, and 5.)

\subsection{STRUCTURAL STEEL FOR CARS AND} IOCOMOTIVES.

AMERICAN RAILWAY ASSOCIATION, MECHANICAL DIVISION, SPECIFICATIONS FOR STRUCTURAL STEEI SHAPES, PLATES AND BARS, 1923

(For general requirements relating to yield point, number of tests, finish, marking, inspection, rejection, and rehearing, also test specimen figures: See A. S. T. M. specifications (605.0, p. 247), paragraphs $2,3(a)$ and $(c), 5,6,7,8(b)$, and 9, and Figures 128 and 129. Omit last sentence in (9). The tension test specimen specified by the A. R. A. carries a diameter of 0.505 inch in order to aid in the computation of the cross-sectional area.)
(For requirements relating to process, ladle analysis, modification in elongation, bend tests for structural steel and plates for cold pressing, and test specimens: See A. S. T. M. A7 (605.11, p. 249), paragraphs $2,4,6,7(a)$ and $(c)$, and $8(d),(e),(f)$, $(g)$, and $(j)$. Add to (4): Drillings for analysis shall be taken not less than one-fourth inch below surface of test ingot.)

(For tables of permissible variations of rectangular plates ordered to weight; permissible overweights of rectangular plates ordered to thickness; permissible variations in weight of bar sizes of angles, tees, zees, and channels; and permissible variations in crosssectional dimensions of rounds, squares, hexagons, and flats, see A. A. S. M., Tables 1, 2, 3, 4, and 5 (605.0, p. 248).)

(The following additional material is given:)

1. Scope.-(a) These specifications apply to all structural and bar size shapes and structural plates and bars for locomotives, freight and passenger equipment cars. Plates will be classed as structural plates when three-sixteenths inch or greater in thickness.

(b) A channel will be considered as in a "bar" size when its greatest dimension is less than 3 inches, and the angle, $T$ or $\mathbf{Z}$ as in a "bar" size when its greatest dimension is less than 3 inches, or when it is 3 inches or more and at the same time the thickness is less than one-fourth inch. Shapes not falling within the above limits will be classed as "structural."

2. Chemical composition.-The steel shall conform to the following requirements as to chemical composition:

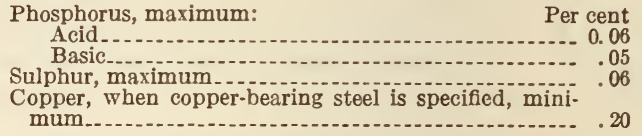

3. Check analysis.-Check analysis may be made by the purchaser from the finished material representing each melt. Such analysis shall meet the requirements specified under chemical composition.

4. Tension tests. - The material shall conform to the following requirements as to tensile properties:

\begin{tabular}{|c|c|c|}
\hline & Structural & $\begin{array}{l}\text { Plates for cold } \\
\text { flanging }\end{array}$ \\
\hline Tensile strength_-lbs./in. ${ }^{2}-$ - & $50,000-65,000$ & $48,000-58,000$ \\
\hline Yield point, minimum & 0.5 tensile strength & 0.5 tensile strength \\
\hline $\begin{array}{l}\text { Elongation in } 8 \text { inches, } \\
\text { minimum }\end{array}$ & $1,500,000$ & $1,500,000$ \\
\hline $\begin{array}{l}\text { Elongation in } 2 \text { inches, } \\
\text { minimum.-.- per cent.- }\end{array}$ & 25 & 25 \\
\hline
\end{tabular}

5. Test specimens.-(a) Tension and bend test specimens shall be taken from the finished rolled product.

(b) The tension test specimen conforms to that shown (fig. 129) in A. S. T. M. standard specifications for steel (see 605.0 p. 247), except as stated above that the diameter is 0.505 inch, and the 1 by $1 / 2$ inch bend test specimen for sections over $11 / 2$ inches in thickness or diameter shall be taken so that the axis is midway between the center and surface of the material as rolled.

6. Number of tests. - Tension tests will not be required for shapes less than 1 square inch in cross section or for sheets three-sixteenths inch or under in thickness.

7. Permissible variations in weight and gage.-(a) The cross-sectional area, or weight, of each structural 
shape and of each rolled-edge plate, up to and including 36 inches in width, shall not vary more than 2.5 per cent from that specified. One cubic inch of steel is assumed to weigh 0.2833 pound.

(b) The variations in weight of bar sizes of angles, tees, zees, and channels shall conform to Table 3 .

(c) When ordered to weight per square foot.-The weight of each lot of universal plates over 36 inches in width, and of each lot of sheared plates, shall not vary from the weight ordered more than the amount shown in Table 1 .

(d) When ordered to thickness. - The thickness of each universal plate over 36 inches in width and of each sheared plate shall not vary more than 0.01 inch under that specified. The overweight of each lot of plates in each shipment shall not exceed the amount given in Table 2 .

(e) Unless covered by special agreement between the purchaser and the manufacturer that the material shall be furnished recut to exact dimensions ordered, the permissible variations under the dimensions ordered shall not be more than one-fourth inch under in length and width, in case of sheared mill plate, and in the case of universal mill plate, the length shall not be more than one-fourth inch under and the width more than one-eighth inch under. The variations over the dimensions ordered shall be the minimum obtainable under the mill conditions and consistent with the thickness and linear dimensions of the material. The same shall apply to structural and bar size shapes.

(f) All bars shall conform to the limits given in Tables 4 and 5 .

8. Inspection. - The purchaser may make the tests and inspection to govern the acceptance or rejection of the material in his own laboratory or elsewhere. Such tests and inspection shall be made at the expense of the purchaser.

AMERICAN RAILWAY ASSOCIATION, MECHANICAL DIVISION, ARCH BARS, COLUMN AND JOURNAL-BOX BOLTS AND COLUMN CASTINGS TO BE USED FOR MAINTENANCE OF EXISTING ARCH-BAR TRUCKS OF 40 AND 50 TON CAPACITY, STANDARD, 1929

\section{ARCH BARS}

1. The dimensions of arch and tie bars to be used for maintenance of existing trucks shall be:

Arch bars.-Either $13 / 4$ by $41 / 2$ inches or $1 \frac{1}{2}$ by 5 inches for 40 -ton trucks; $13 / 4$ by 5 inches or $1 \frac{1}{2}$ by 6 inches for 50-ton trucks.

Tie bars. $-5 / 8$ by $4 \frac{1}{2}$ inches or $5 / 8$ by 5 inches for 40 -ton trucks and $5 / 8$ by 5 inches or $5 / 8$ by 6 inches for 50-ton trucks.

2. When, for any cause, it is necessary to renew an arch bar or a tie bar, and the construction of truck and car body will permit, it shall conform to these requirements, the renewal to be confined to the defective bar or bars.

3. Column castings should be of malleable iron or cast steel and conform to the general design and dimensions as covered by Figure 134 .

4. The shear value of arch-bar connections above the journal box should be increased by one of the optional methods shown in Figures 130, 131, 132, and 133.

5. The shear value of journal-box and tie-bar connections should be increased by turning up the end of the tie bars, as shown on Figures 130, 131, 132 , and 133 .
6. Arch bars and column and journal-box bolts should be manufactured of refined wrought iron or mild steel bars.

7. The use of the oxy-acetylene torch for burning out holes in old or new arch bars is prohibited.

8. Improperly matched bolt holes should be avoided. To assist in proper matching of bolt holes and to obtain the proper fit of arch bars against each other, it is recommended, when only one arch bar is being renewed, that the new bar be fitted to the bar that is retained and the old bar used as a template for drilling holes.

9. No bolts that have been welded should be used in conjunction with arch-bar trucks. Cleancut full-size threads should be insisted upon in order to obtain proper holding power between the holding nut and the bolt and assurance that locking nuts will function properly when used.

\section{AMERICAN RAILWAY ASSOCIATION, ME- CHANICAL DIVISION, TRUCK BOLSTERS, RECOMMENDED PRACTICE, 1929}

\section{TRUCK BOISTERS}

1. Scope.-These specifications cover truck bolsters for freight equipment with either integral or separable center plate.

2. Material.-May be of cast steel, structural steel, or forged iron or steel, in accordance with A. R. A. specifications.

3. Design.-All standard fundamental A. R. A. requirements, not specifically mentioned herein, shall also govern.

The basis for the calculation of maximum combined unit stress shall be the vertical load equal to " $\mathrm{P}$ " as given in Table 1, and the transverse load equal to $0.25 \mathrm{P}$.

TABLE 1

\begin{tabular}{|c|c|r|}
\hline $\begin{array}{c}\text { Size of } \\
\text { journal }\end{array}$ & $\begin{array}{c}\text { Type of } \\
\text { truck }\end{array}$ & $\begin{array}{r}\text { Vertical } \\
\text { load }=P\end{array}$ \\
\cline { 1 - 2 } & Inches & Pounds \\
5 by 9 & $2 \mathrm{C}$ & 62,000 \\
$51 / 2$ by 10 & $2 \mathrm{D}$ & 77,000 \\
6 by 11 & $2 \mathrm{E}$ & 96,000 \\
$61 / 2$ by 12 & $2 \mathrm{~F}$ & 115,000 \\
\hline
\end{tabular}

The vertical load shall be taken as acting on the center line of the bolster anywhere within 8 inches each side of the center of the bolster; also anywhere from the center of spring support to a point 23 inches from the center of the bolster. The section moduli shall decrease uniformly from the section 8 inches from the center to the section 23 inches from the center of the bolster. The transverse load shall be taken as acting on the neutral axis (or, for test, on a line 5 inches below the center plate bearing face), and shall be applied only at the center of the bolster. On this basis of calculation for checking relative designs, the maximum combined unit stress in the design shall not exceed 16,000 pounds per square inch and the maximum unit stress for vertical or transverse loads considered separately shall not exceed 12,500 pounds per square inch for vertical load equal to "P" or horizontal load equal to $0.8 \mathrm{P}$.

4. Test load requirements.- (a) At least two specimens of each new design shall be tested on a suitable static-testing machine. The two specimens selected shall be good average product.

(b) Bolsters shall comply with the requirements in Table 2, the vertical and transverse tests to be made separately. The vertical loads shall be applied separately at center plate, and at one side bearing. 


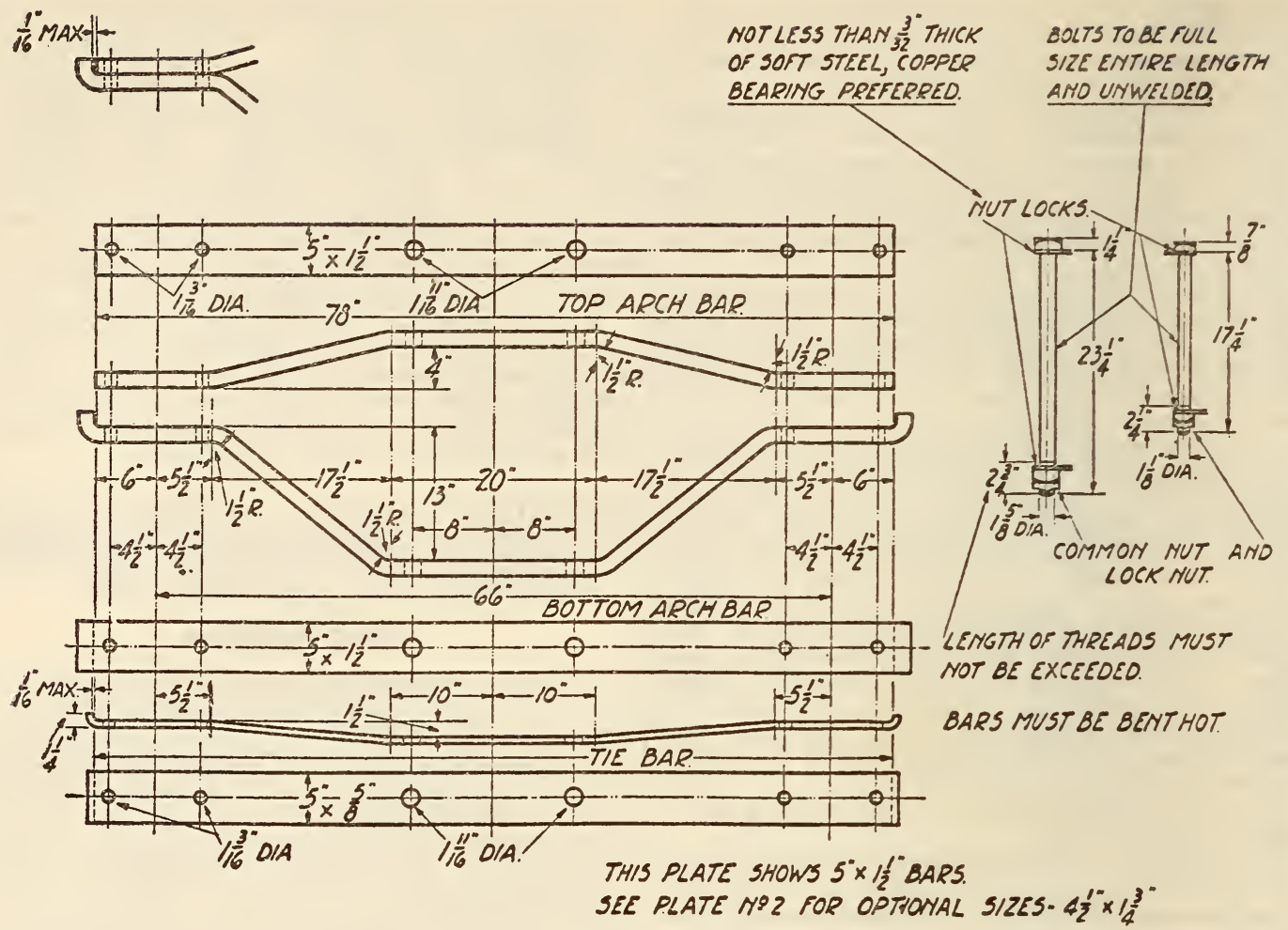

Figdre 130
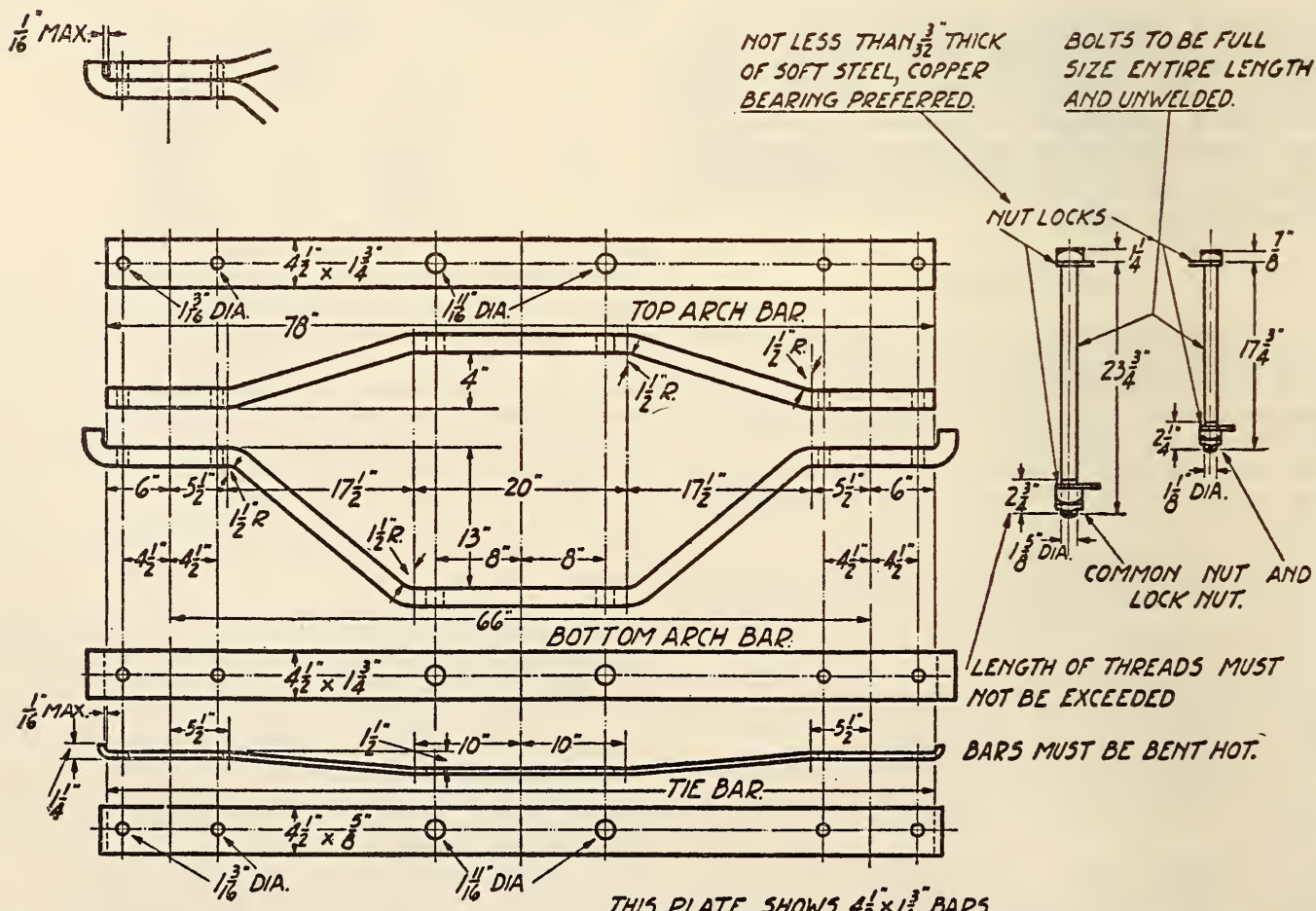

LENGTH OF THREADS MUST NOT BE EXCEEOEO

\section{BARS MUST BE BENT HOT.}




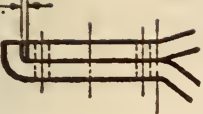

NOT LESS THAN $\frac{3}{32} "$ THICK OF SOFT STEEL, COPPER

BOLTS TO BE FULL BEARING PREFERRED. SIZE EMTIRE LENGTH AMD UNWELDEO

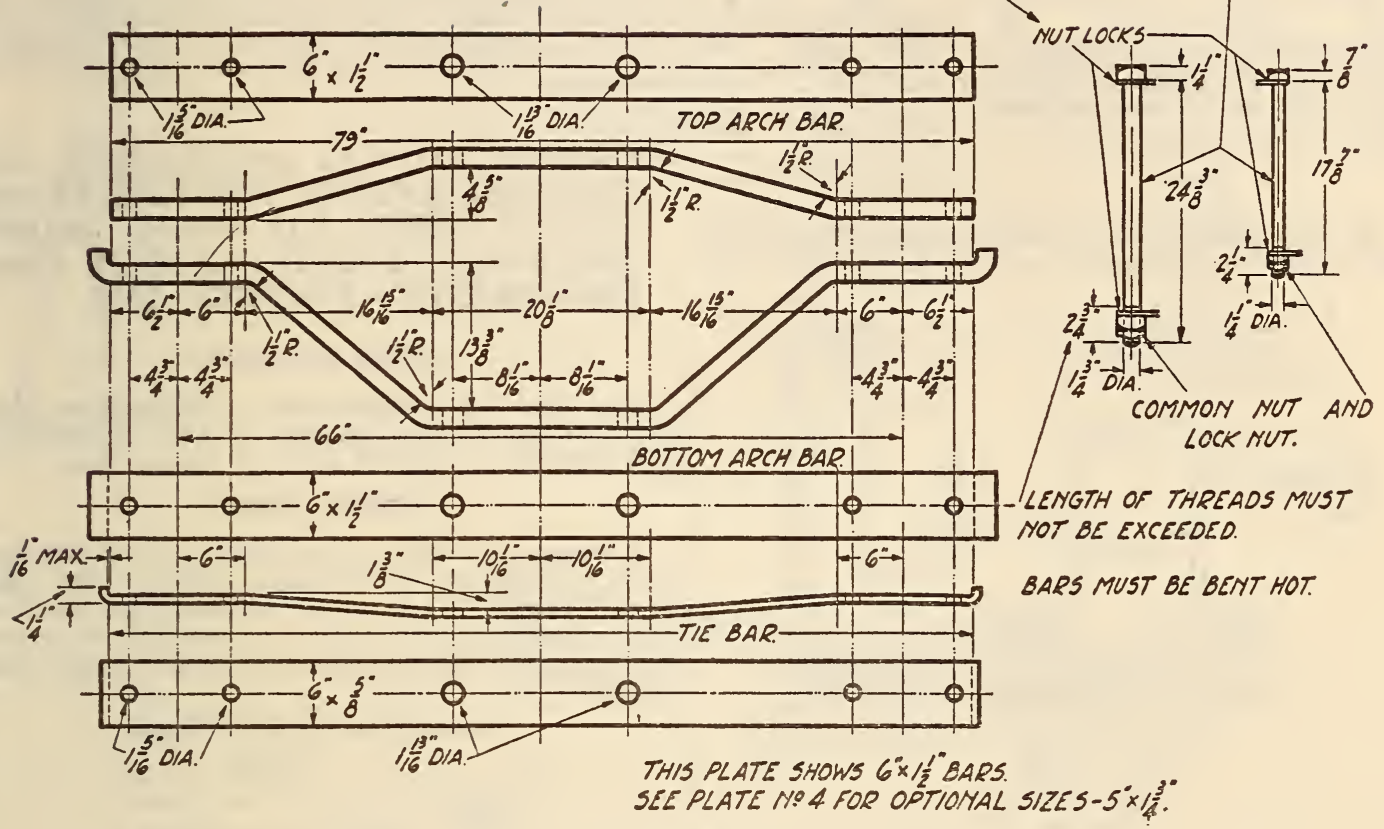

Figure 132

$\frac{1}{16} M A X-1$

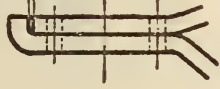

NOTLESS THAN" "THISK OF SOFT STEEL, COPPER BEARIIG PREFERRED.
BOLTS TO BE FULL SIZE EMTIRE LENGTH AND UNWELDEP.
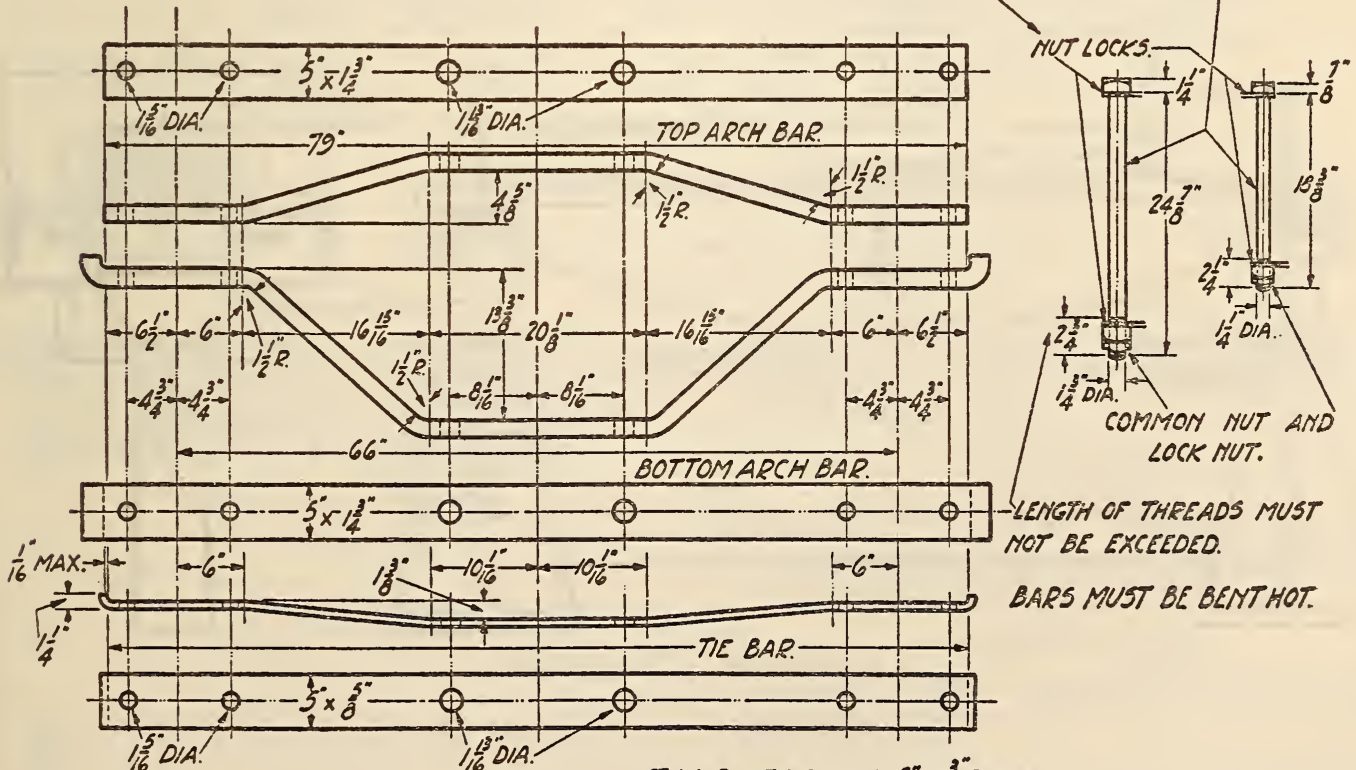

- EENGTH OF THEEADS MUST MOT BE EXCEEDED.

BARS MUST BE BENTHOT.

THIS PLATE SHOWS $5 " \times 13$ "BARS.

SEE PLATE NO 3 FOR OPTIONAL SIZES- $6^{\circ} \times 1 \frac{1}{2}^{\circ}$

Figure 133 
TABLE 2

Load for zero setting of instrument.

Load at 0.055 inch, maximum deflection.

Load at 0.075 inch maximum deflection.

Load at 0.025 inch, maximum permanent

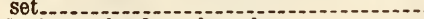

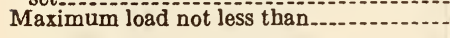

\begin{tabular}{|c|r|r}
\multicolumn{3}{|c|}{ Vertical } \\
\hline $\begin{array}{c}\text { Center } \\
\text { plate }\end{array}$ & $\begin{array}{c}\text { Side } \\
\text { bearing }\end{array}$ & $\begin{array}{c}\text { Trans- } \\
\text { verse }\end{array}$ \\
\hline Pounds & $\begin{array}{r}\text { Pounds } \\
5,000\end{array}$ & $\begin{array}{r}\text { Pounds } \\
5,000 \\
1.5 \mathrm{P}\end{array}$ \\
\hdashline $1.5 \mathrm{P}$ & $\begin{array}{r}5,000 \\
1.0 \mathrm{P}\end{array}$ \\
$3.0 \mathrm{P}$ & $2.5 \mathrm{P}$ & $2.0 \mathrm{P}$ \\
$5.5 \mathrm{P}$ & - & $\ldots . . .-$ \\
\end{tabular}

(c) The deflection measuring instruments shall be located midway between supports of specimen, and

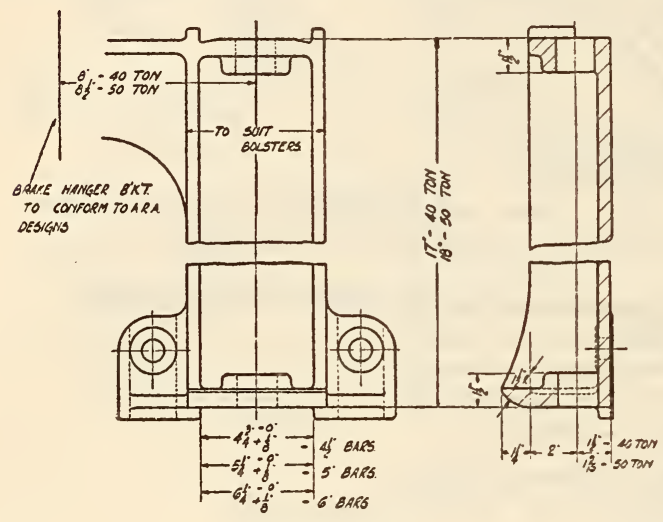

Figure 134

shall be set at zero under an initial load of 5,000 pounds after a load of 20,000 pounds has been applied for five minutes and then released to 5,000 pounds. The load for the deflection measurement shall be 5,000 pounds additional to the load shown in Table 2, but the load for the permanent set and maximum load shall be as given in Table 2.

5. Loading diagrams.-Figure 135 shows the recommended method for vertical test loading and Figure 136 for transverse test loading of truck bolsters.

NoTE.-This specification covers all bolsters, except vertical truss bolster composed of a tension member, a compression member and a counterpost between; for such bolsters the specifications do not apply, until construction of such bolsters can be further investigated. to determine whether or not they should also comply with the same specifications.

AMERICAN RAILWAY ASSOCIATION, MECHANICAL DIVISION, BOLSTERS, TRUCK, PRESSED STEEL, FOR $80,000,100,000$, AND 140,000 POUND CAPACITY CARS, RECOMMENDED PRACTICE, 1915

\section{TRUCK BOISTERS}

The steel for pressed-steel truck bolsters shall conform to the requirements of the specifications and tests for structural steel for freight cars.

\section{LIMITING WEIGHTS}

Bolsters, including center plate and side bearings, shall conform to the weights given in the table shown below. In case the bolsters have met all requirements except that of overweight, they may be accepted at the maximum allowable weight here specified.

\begin{tabular}{|c|c|c|c|}
\hline \multirow{2}{*}{$\begin{array}{l}\text { Car ca- } \\
\text { pacity }\end{array}$} & \multicolumn{3}{|c|}{ Weight } \\
\hline & $\begin{array}{l}\text { Mini- } \\
\text { mum }\end{array}$ & Normal & $\begin{array}{l}\text { Maxi- } \\
\text { mum }\end{array}$ \\
\hline $\begin{array}{r}\text { Pounds } \\
80,000 \\
100,000 \\
140,000\end{array}$ & $\begin{array}{c}\text { Pounds } \\
775 \\
840 \\
1,110\end{array}$ & $\begin{array}{c}\text { Pounds } \\
795 \\
865 \\
1,140\end{array}$ & $\begin{array}{c}\text { Pounds } \\
815 \\
890 \\
1,170\end{array}$ \\
\hline
\end{tabular}

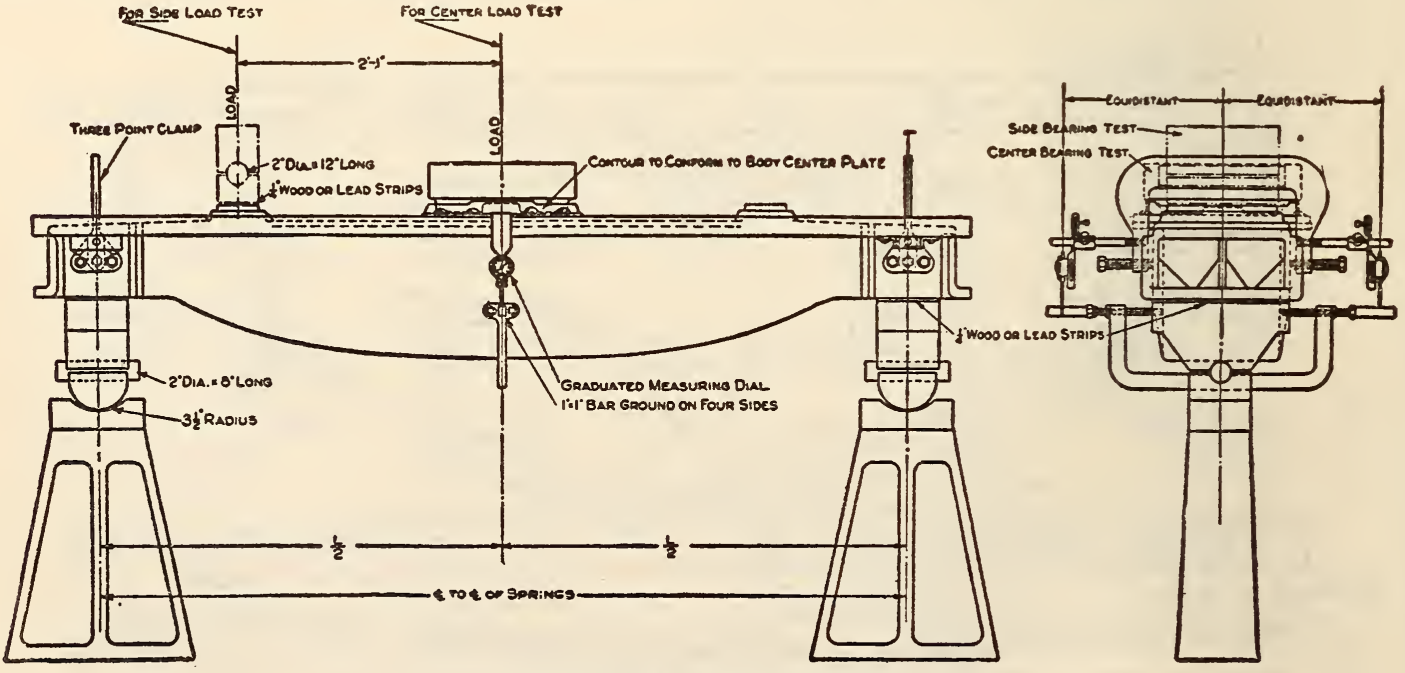

FIGURE 135.-Truck bolsters 

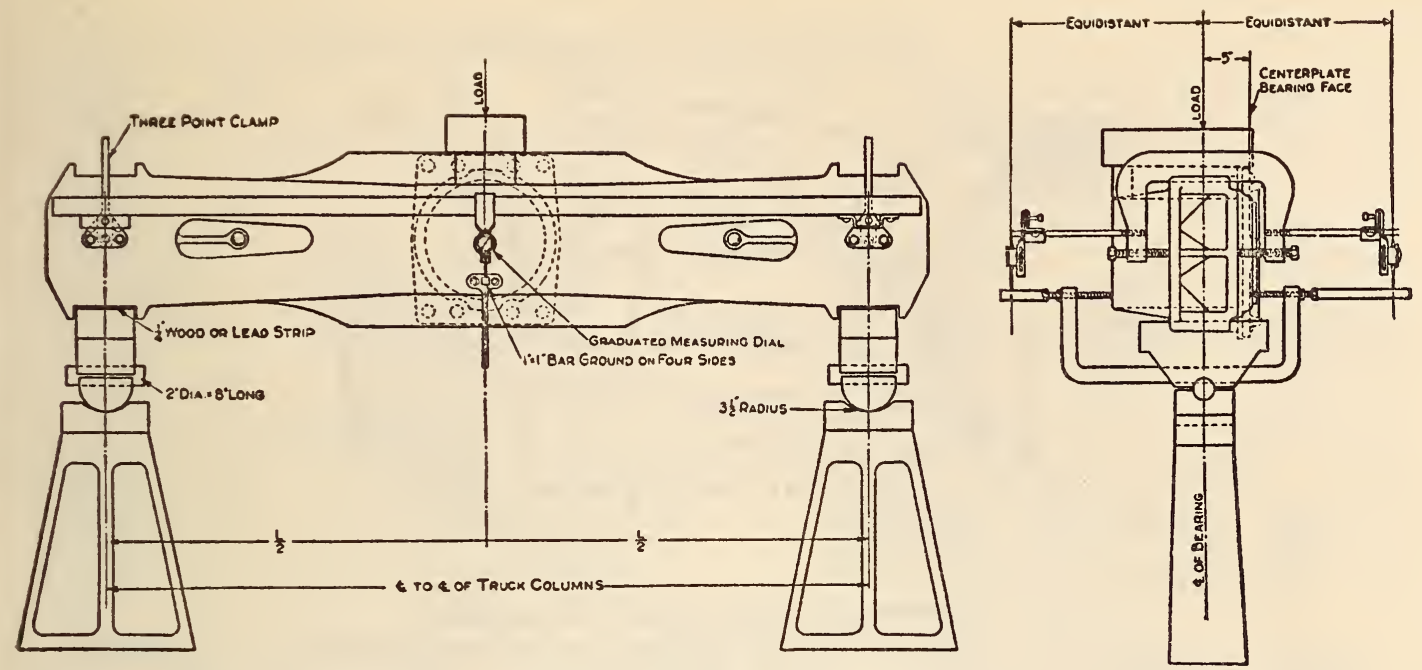

FigURe 136.-Truck bolsters

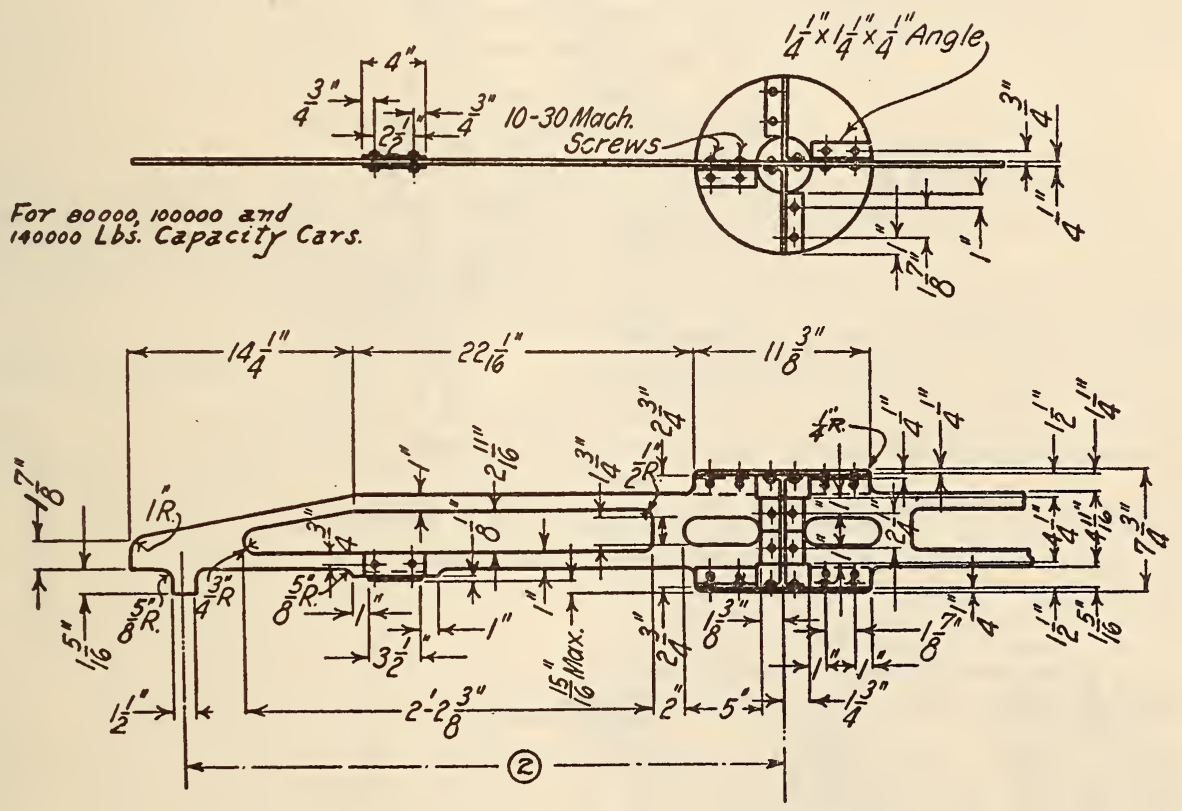

Figdre 137.-Center plate and straight edge gage

Dimensions as in Table 1.

AMERICAN RAILWAY ASSOCIATION, MECHANICAL DIVISION, BOLSTERS, TRUCK, CAST AND PRESSED STEEL, GAGES FOR; RECOMMENDED PRACTICE, 1914

\section{GAGES FOR BOLSTERS}

TABLE 1

\begin{tabular}{|c|c|c|c|c|c|c|c|c|c|}
\hline \multirow{2}{*}{ Bolster } & \multicolumn{9}{|c|}{$\begin{array}{l}\text { Values for dimensions shown by circled numerals are } \\
\text { indicated in Figs. 137, 138, and } 139 .\end{array}$} \\
\hline & 1 & 2 & 3 & 4 & 5 & 6 & 7 & 8 & 9 \\
\hline $\begin{array}{c}\text { Pounds } \\
80,000 \\
100,000 \\
140,000\end{array}$ & \begin{tabular}{cc|}
$F t . i n$. \\
6 & 4 \\
6 & 5 \\
6 & 6
\end{tabular} & $\mid \begin{array}{ll}\text { Ft.in. } \\
3 & 2 \\
3 & 21 / 2 \\
3 & 3\end{array}$ & $\begin{array}{l}\text { In. } \\
51 / 8 \\
518 \\
618\end{array}$ & \begin{tabular}{l|} 
In. \\
$147 / 8$ \\
$147 / 8$ \\
$187 / 8$
\end{tabular} & \begin{tabular}{l|} 
In. \\
$127 / 8$ \\
$127 / 8$ \\
$16^{7} / 8$
\end{tabular} & $\begin{array}{l}I n . \\
1515 \\
1512 \\
1616\end{array}$ & $\begin{array}{l}\text { In. } \\
2516 \\
2516 \\
2618\end{array}$ & $\begin{array}{ll}\text { In. } \\
11278 \\
11278 \\
1167 / 8\end{array}$ & $\begin{array}{rl} & I n . \\
2 & 123 / 4 \\
2 & 1234 \\
2 & 16^{3} / 4\end{array}$ \\
\hline
\end{tabular}

AMERICAN RAILWAY ASSOCIATION, MECHANICAL DIVISION, CENTER PLATES, STANDARD, 1927

\section{CENTER PLATES \\ (See fig. 140, p. 261.)}

AMERICAN RAILWAY ASSOCIATION, MECHANICAL DIVISION, COUPLER YOKES, RECOMMENDED PRACTICE, 1929

\section{COUPLER YOKES}

1. Scope.-These specifications cover coupler yokes of either horizontal or vertical plane types for freight equipment.

2. Material.-May be of cast steel, structural steel, or forged iron or steel, in accordance with A. R. A. specifications. 


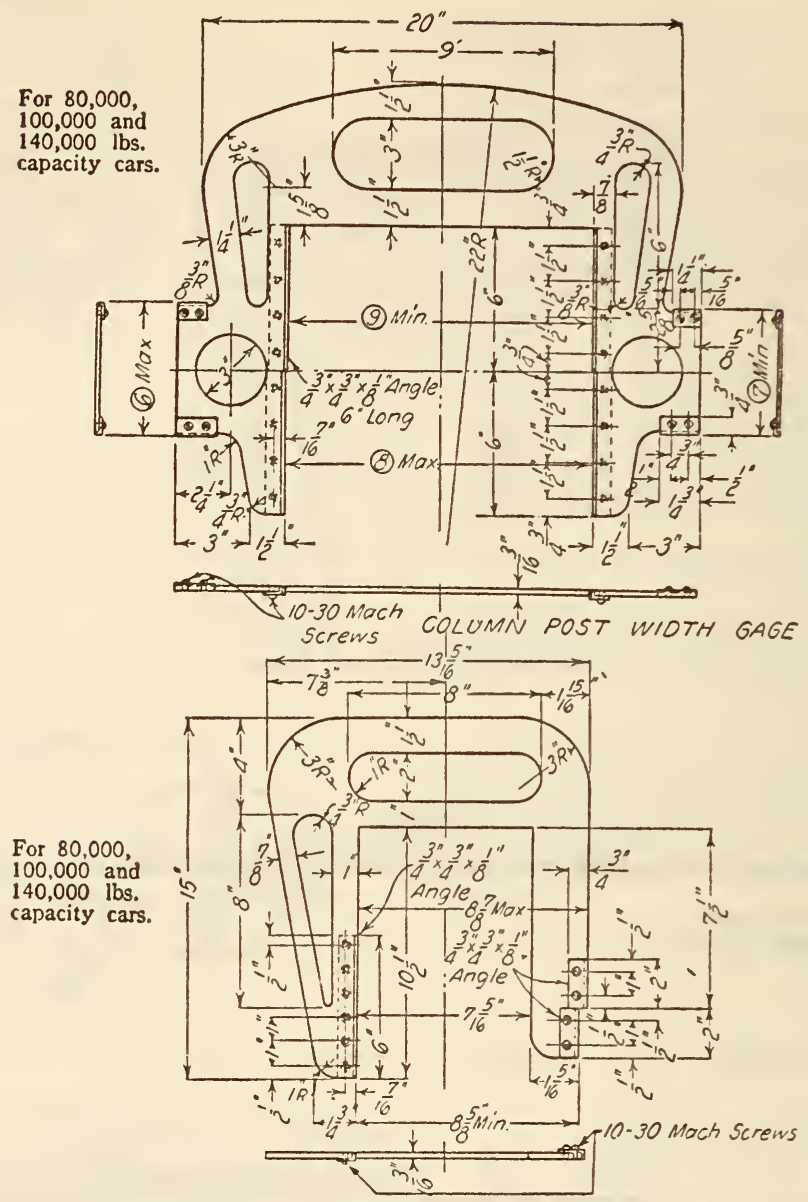

Figure 138.- Spring seat to side bearing gage

Dimensions as in Table 1, p. 259.
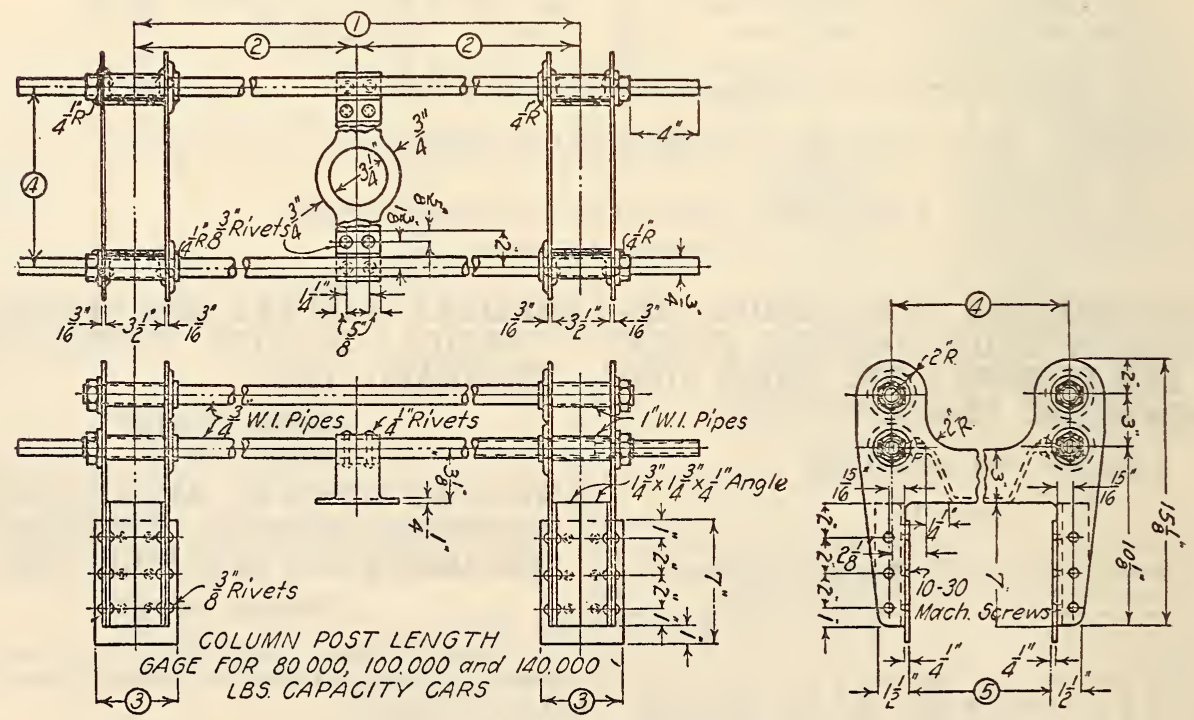

Figdre 139.-Gages for truck bolsters

Dimensions as in Table 1, p. 259. 


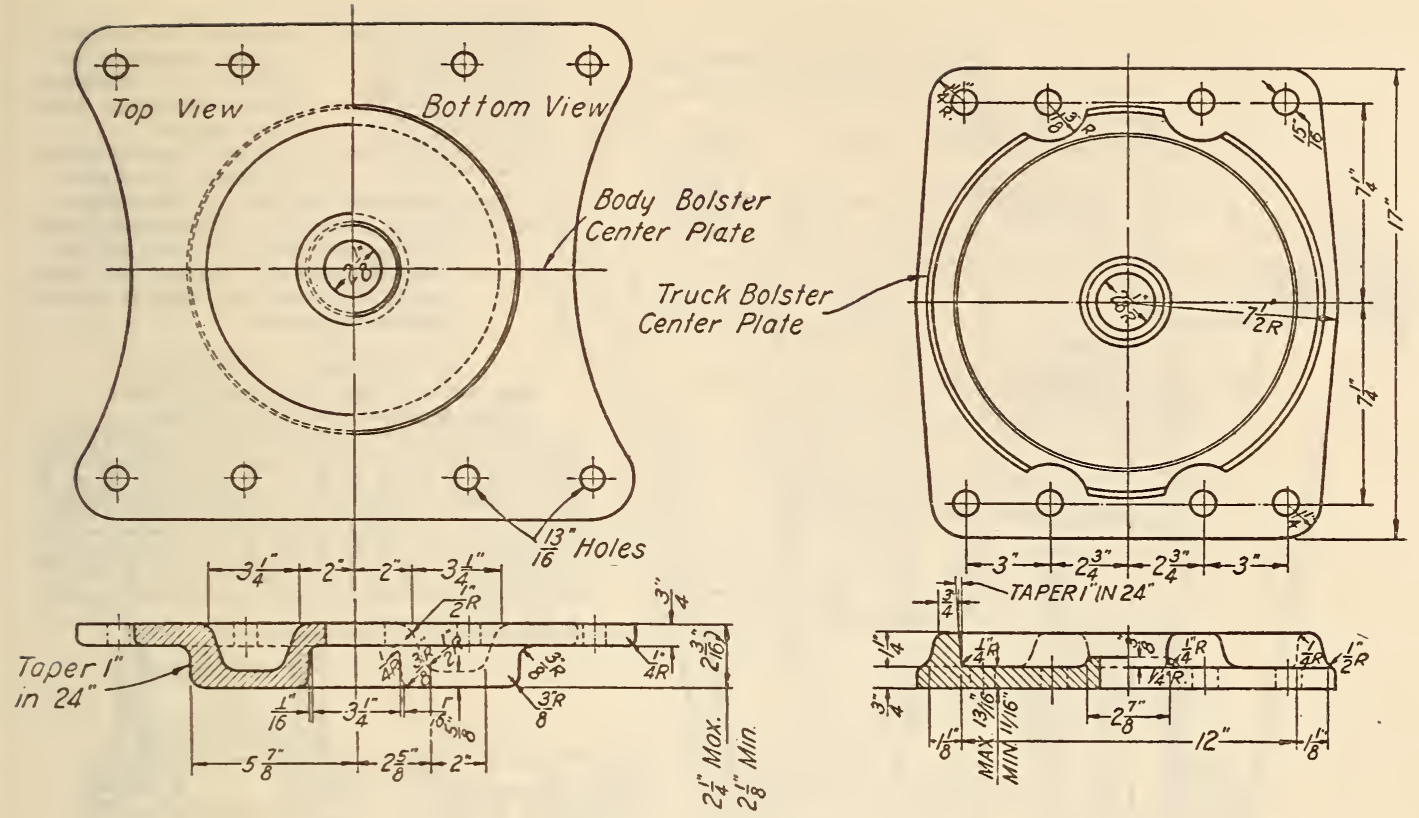

Figdre 140.-Center plates

3. Design.-Each coupler yoke design intended for use with type " $D$ " coupler and 6 by $1 \frac{1}{2}$ inch key, shall meet the following design requirements: If made from grade "A" cast steel, or its equivalent, the tension area shall not be less than 12 square

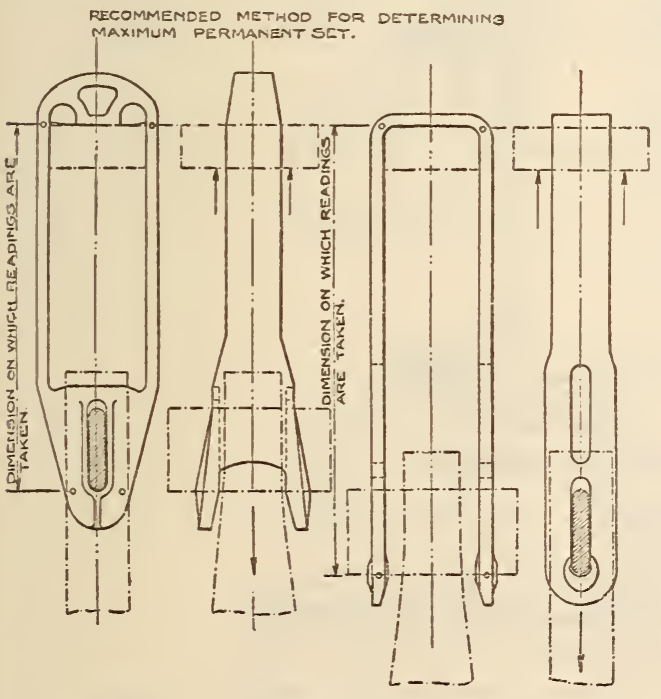

FIGURE 141

inches; and if made of grade "B" steel, or its equivalent, the tension area shall not be less than $10 \frac{1}{2}$ square inches.
4. Test requirements.-(a) At least two specimens of each new design shall be tested on a suitable static testing machine.

(b) The method of support and loading shall be equivalent to service conditions on tangent track. The maximum set shall not exceed 0.031 inch under a load of 325,000 pounds. The breaking load shall not be less than 550,000 pounds. The set shall be taken in the length from the rear follower bearing face on the yoke to the front coupler-key bearing face.

(c) The deflection measuring instruments shall be set at zero under an initial load of 5,000 pounds after a load of 20,000 pounds has been applied for five minutes and then released to 5,000 pounds.

5. Loading diagrams.-Figure 141 indicates the recommended method for testing of yokes and taking the measurement determining the maximum set.

AMERICAN RAILWAY ASSOCIATION, MECHANICAL DIVISION, PEDESTALS FOR PASSENGER-CAR TRUCKS, 1931

PEDESTALS FOR PASSENGER-CAR TRUCKS

(See fig. 142, p. 262)

AMERICAN RAILWAY ASSOCIATION, MECHANICAL DIVISION; SILL, CENTER, STEEL, SECTION FOR; STANDARD, 1922

\section{CENTER SILL SECTION}

(See fig. 143, p. 262) 


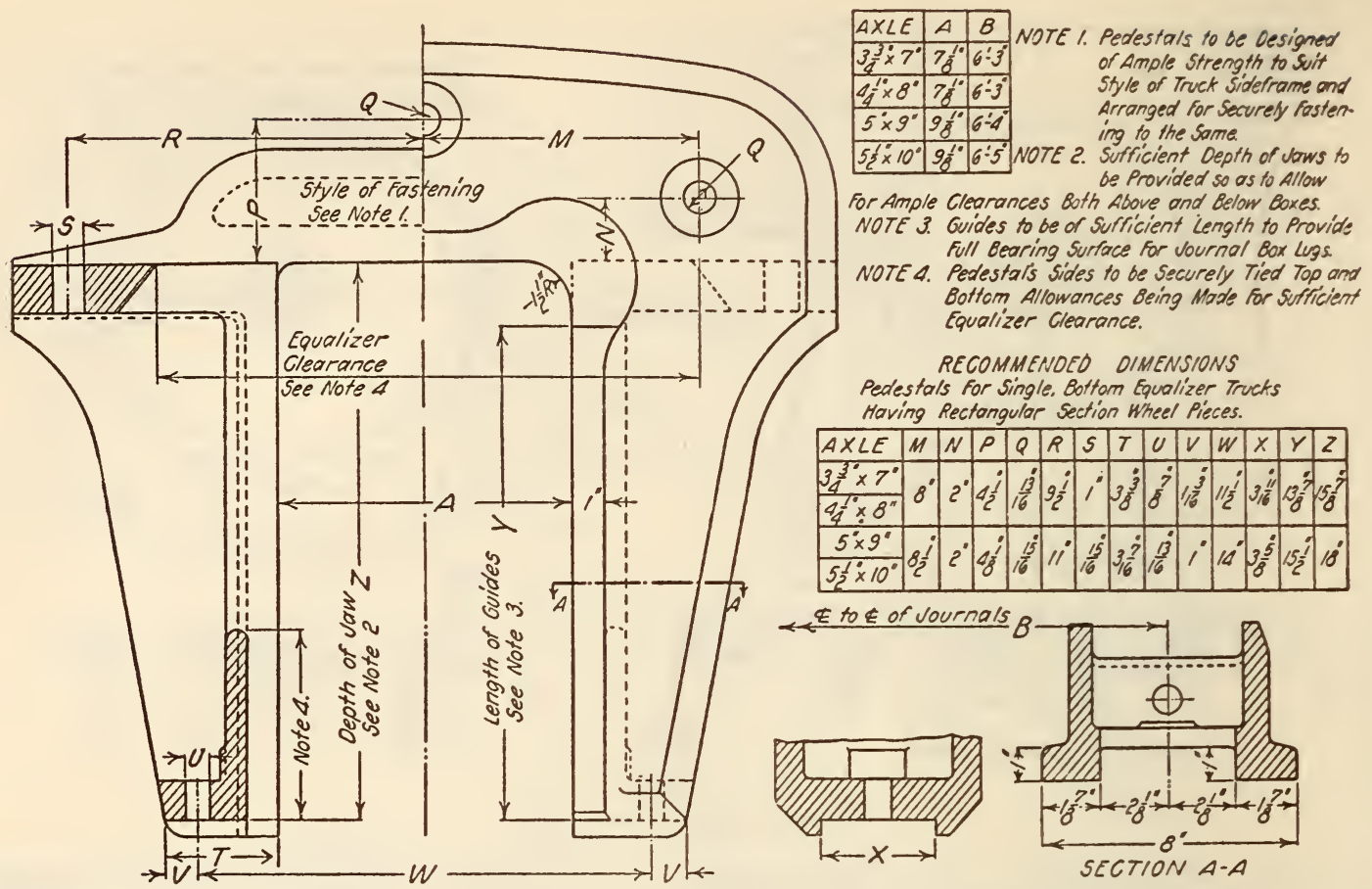

FigdRE 142.-Pedestals for passenger car trucks, 33/4 by 7 inches, 41/4 by 8 inches, 5 by 9 inches, and $51 / 2$ by 10 inches

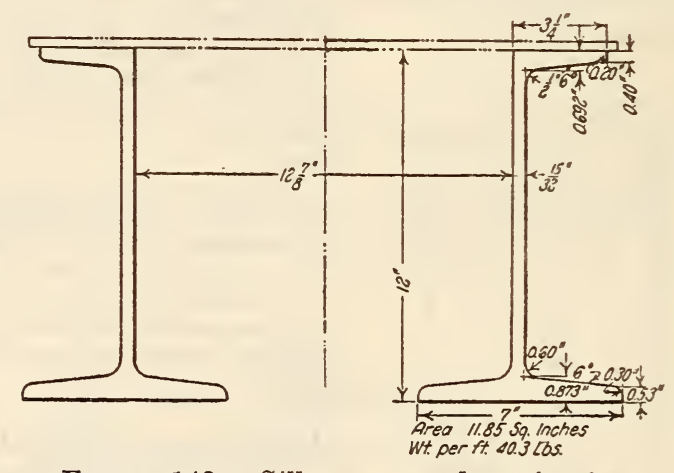

FIGURE 143.-Sill, center, steel, section for

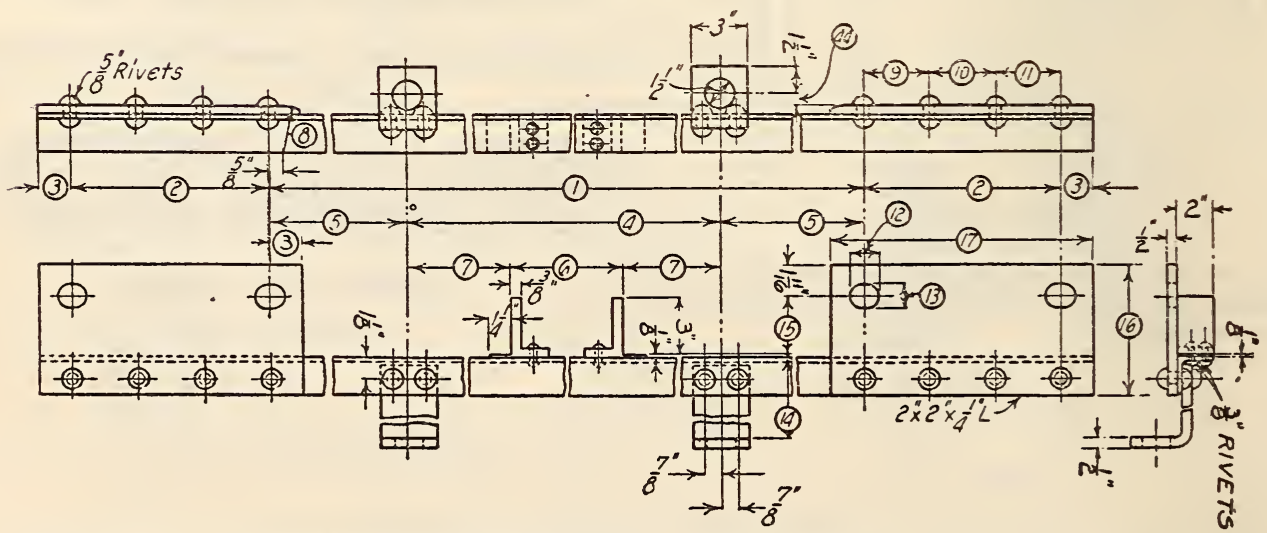

FIGURE 144.-Straightening and journal box bolt spacing gage with brake hanger spacing gage attached (See p. 265.) 


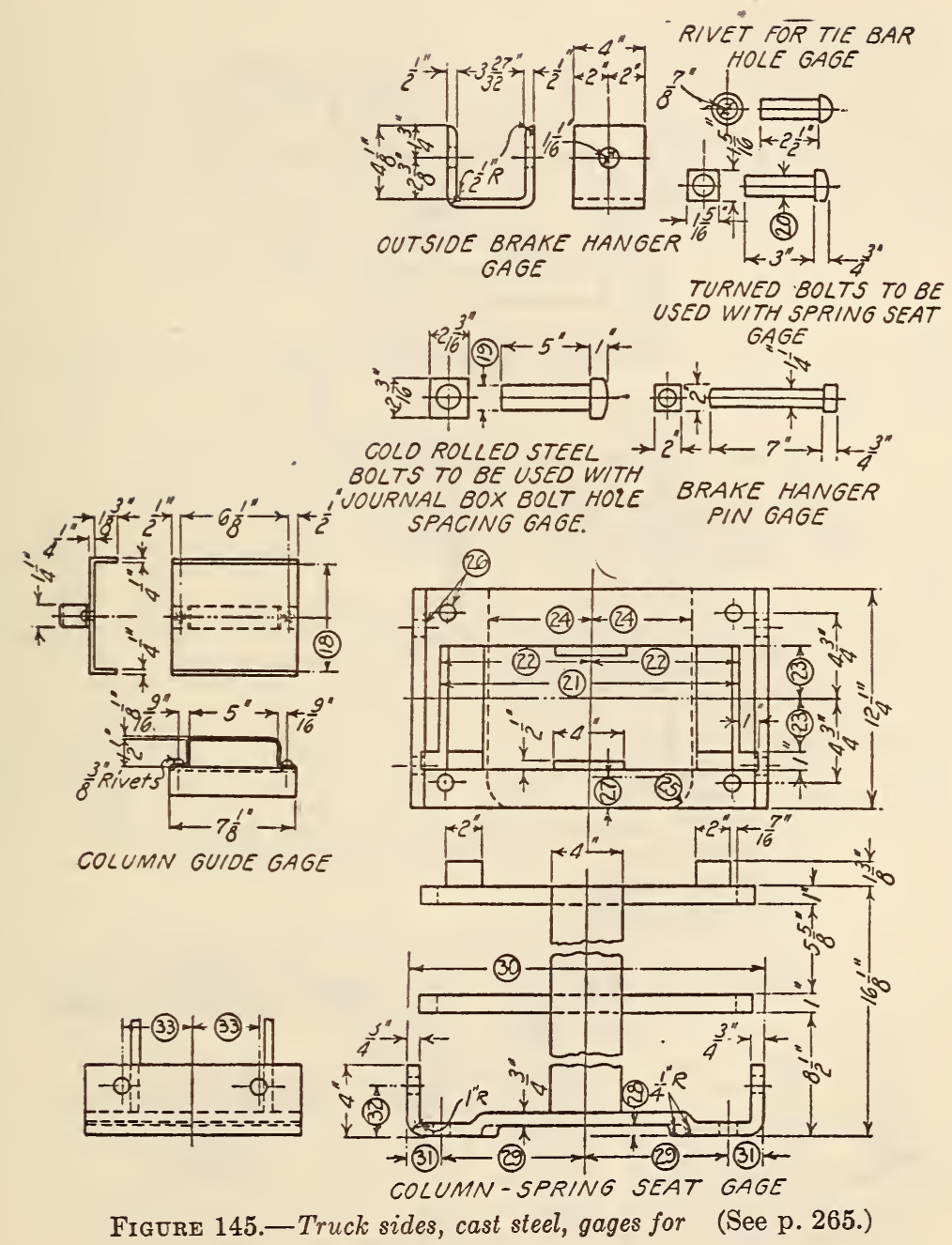



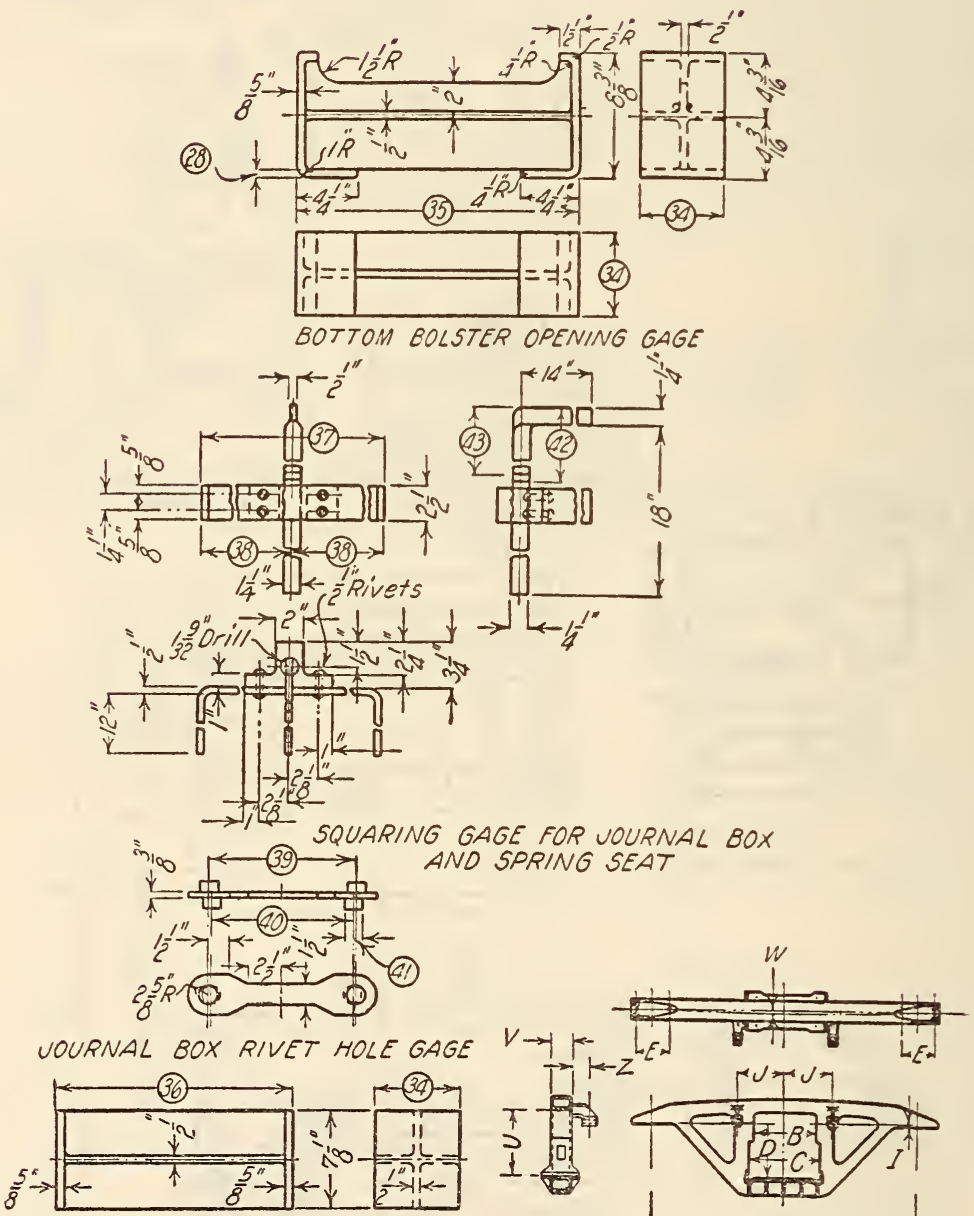

BOLSTER OPENING GAGE
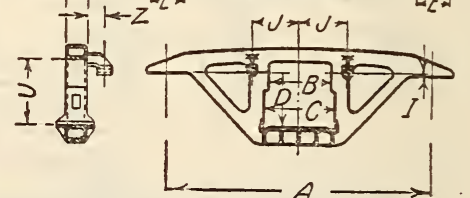

Figdre 146.-Truck sides, cast steel, gages for (See p. 265.) 
AMERICAN RAILWAY ASSOCIATION, MECHANICAL DIVISION; TRUCK SIDES, CAST STEEL, GAGES FOR; RECOMMENDED PRACTICE, 1917

GAGES FOR TRUCK SIDES

\begin{tabular}{|c|c|c|c|c|c|c|c|c|c|c|c|c|c|}
\hline & & & & & & (Dim & ensions in & inch) & & & & & \\
\hline & A & B & C & & $D$ & $\mathrm{E}$ & I & $J$ & $\mathrm{U}$ & $\mathrm{V}$ & $w$ & Z & \\
\hline & $\begin{array}{r}+316 \\
-18\end{array}$ & $\begin{array}{r}+18 \\
-0\end{array}$ & & \begin{tabular}{r|}
-1.4 \\
-0
\end{tabular} & $\begin{array}{r}+3 / 16 \\
-1 / 6\end{array}$ & $\begin{array}{r}136 \\
+132 \\
-1 / 32\end{array}$ & $\begin{array}{l}+18 \\
-18\end{array}$ & $\begin{array}{l}+1 / 4 \\
-1 / 8\end{array}$ & $\begin{array}{r}+3 / 16 \\
-18\end{array}$ & $\begin{array}{l}+1 / 16 \\
-1 / 16\end{array}$ & $\begin{array}{l}+1 \\
-1\end{array}$ & $\begin{array}{l} \pm 1 / 1 \\
-1 / 1\end{array}$ & \\
\hline & & & & & & Table & of toler & ances & & & & & \\
\hline & & & alues & for $c$ & dimens & sions showr & n by circle & d numer & als in Fig & gs. 144,14 & & 146 & \\
\hline Frante & 1 & 2 & & 3 & 4 & 5 & 6 & 7 & 8 & 9 & 10 & 11 & 12 \\
\hline $\begin{array}{c}\text { Tons } \\
40 \\
50 \\
70\end{array}$ & $\begin{array}{rr}\text { Ft. } & \text { i } \\
4 & 9 \\
4 & 8 \\
4 & 11\end{array}$ & \begin{tabular}{r|r}
$n$. & Inch \\
$1 / 2$ & \\
$1 / 2$ & 10 \\
\end{tabular} & $\begin{array}{l}e s \\
1 \frac{1}{2} \\
1 / 2\end{array}$ & $\begin{array}{r}\text { Inches } \\
11 \\
13 \\
13 \\
\end{array}$ & \begin{tabular}{l|l}
$1 / 2$ & Inch \\
132 & \\
$3 / 4$ & \\
\end{tabular} & \begin{tabular}{c|c} 
hes & Inches \\
21 & 18 \\
21 & 173 \\
25 & 171 \\
\end{tabular} & \begin{tabular}{r|r}
8 & Inches \\
& $127 / 8$ \\
$3 / 4$ & $12 \pi / 8$ \\
$1 / 4$ & $167 / 8$
\end{tabular} & $\begin{array}{r}\text { Inches } \\
4916 \\
4916 \\
4916\end{array}$ & \begin{tabular}{|c|} 
Inches \\
$11 / 2$ \\
2 \\
216
\end{tabular} & $\begin{array}{c}\text { Inches } \\
3 \\
35 / 32 \\
31 / 2\end{array}$ & $\begin{array}{c}\text { Inches } \\
3 \\
331 \\
315 \\
\end{array}$ & 6 \begin{tabular}{c|c} 
Inches \\
3 \\
$35 / 32$ \\
$31 / 2$
\end{tabular} & $\begin{array}{l}\text { Inches } \\
1 \\
115 / 32 \\
11 \% / 32\end{array}$ \\
\hline & & & olups & for & d & as showr & n by circle & ed numer & rals in Fig & igs. 144,14 & 45 , and & 146 & \\
\hline 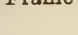 & 13 & 14 & 15 & & 16 & 17 & 18 & 19 & 20 & 21 & 22 & 23 & 24 \\
\hline $\begin{array}{r}\text { Tons } \\
40 \\
50 \\
70\end{array}$ & \begin{tabular}{r|} 
Inches \\
1316 \\
1516 \\
$17 / 16$ \\
\end{tabular} & $\begin{array}{c}\text { Inches } \\
717 / 32 \\
8 \\
8\end{array}$ & $\begin{array}{r}\text { Inch } \\
2 \\
2 \\
3\end{array}$ & $\begin{array}{l}\text { hes } \\
2916 \\
29116 \\
31 / 16\end{array}$ & $\begin{array}{r}\text { Inches } \\
6368 \\
638 \\
678 \\
\end{array}$ & \begin{tabular}{|c|} 
Inches \\
12 \\
$121 / 2$ \\
14
\end{tabular} & $\begin{array}{r}\text { Inches } \\
5116 \\
5116 \\
6116\end{array}$ & $\begin{array}{c}\text { Inches } \\
15 / 32 \\
1932 \\
113 / 32\end{array}$ & \begin{tabular}{r|} 
Inches \\
$3 / 4$ \\
$7 / 8$ \\
$7 / 8$
\end{tabular} & $\begin{array}{r}\text { Inches } \\
127 / 8 \\
12^{7} / 8 \\
16^{7} / 8\end{array}$ & $\begin{array}{c}\text { Inches } \\
67 / 1 \\
671 \\
87 / 1 \\
\end{array}$ & \begin{tabular}{l|r} 
& \multicolumn{1}{|c}{ Inches } \\
6 & $2^{17} 732$ \\
$2^{17} / 32$ \\
6 \\
$617 / 32$ \\
6
\end{tabular} & \begin{tabular}{|r|r} 
& Inches \\
2 & $33 / 4$ \\
2 & 334 \\
2 & $53 / 4$ \\
\end{tabular} \\
\hline & & & alues & f for $d$ & dimens & sions shown & n by circle & d numer & als in Fig & gs. 144,14 & 45 , and & 146 & \\
\hline Frame & 25 & 26 & 27 & & 28 & 29 & 30 & 31 & 32 & 33 & 34 & & 35 \\
\hline $\begin{array}{c}\text { Tons } \\
40 \\
50 \\
70\end{array}$ & \begin{tabular}{r|} 
Inches \\
$33 / 4$ \\
334 \\
134
\end{tabular} & $\begin{array}{r}\text { Inches } \\
13 / 16 \\
13 / 16 \\
13 / 16\end{array}$ & Inch & \begin{tabular}{l|} 
hes \\
23,8 \\
23,8 \\
158
\end{tabular} & $\begin{array}{r}\text { Inches } \\
1 / 2 \\
5.8 \\
5 / 8\end{array}$ & \begin{tabular}{|c|} 
Inches \\
6 \\
6 \\
$81 / 16$
\end{tabular} & $\begin{array}{r}\text { Inches } \\
16 \\
16 \\
20\end{array}$ & $\begin{array}{c}\text { Inches } \\
13 / 4 \\
2 \\
115 / 16\end{array}$ & $\begin{array}{r}\text { Inches } \\
23 / 4 \\
23 / 4 \\
23 / 4\end{array}$ & $\begin{array}{r}\text { Inches } \\
33 / 8 \\
338 \\
37 / 8\end{array}$ & $\begin{array}{r}\text { Inches } \\
5 \\
5 \\
6\end{array}$ & $\begin{array}{l}\text { Inches } \\
1161 / 4 \\
1161 / 4 \\
1201 / 4\end{array}$ & $\mid \begin{array}{c}\text { Inches } \\
216 \\
216 \\
20\end{array}$ \\
\hline & & & ues & $s$ for $d$ & dimens & sions shown & n by circle & numer & als in Fig & igs. 144,1 & 45 , and & 146 & \\
\hline F rame & & 36 & & & 37 & 38 & 39 & 40 & 41 & & 12 & 43 & 44 \\
\hline $\begin{array}{c}\text { T'ons } \\
40 \\
50 \\
70\end{array}$ & $\begin{array}{r}\text { Inches } \\
113 \\
113 \\
117\end{array}$ & \begin{tabular}{l|l} 
& $I n$ \\
$1 / 8$ & \\
$1 / 8$ & \\
$1 / 8$ &
\end{tabular} & $\begin{array}{l}\text { hes } \\
213 \\
213 \\
17 \\
17\end{array}$ & $\begin{array}{r}F t . \\
5 \\
5 \\
5\end{array}$ & $\begin{array}{rr} & \\
5 & \text { in. } \\
5 & 6 \\
5 & 6 \\
5 & 10\end{array}$ & $\begin{array}{rr}\text { Ft. } & \text { in. } \\
2 & 9 \\
2 & 9 \\
2 & 11\end{array}$ & $\begin{array}{c}\text { Inches } \\
191 / 32 \\
1917 / 32 \\
110^{17 / 32}\end{array}$ & $\begin{array}{r}\text { Inches } \\
28^{31 / 3} \\
2915 / 3 \\
1015 / 3\end{array}$ & \begin{tabular}{l|r} 
& Inche \\
32 & 15 \\
32 & 19 \\
32 & 1
\end{tabular} & $\begin{array}{l}e s \\
5 \% 32 \\
9 / 32 \\
13 / 32\end{array}$ & $\begin{array}{l}\text { ches } \\
915 / 16 \\
103 / 16 \\
10^{13} / 16\end{array}$ & $\begin{array}{r}\text { Inches } \\
295 / 8 \\
297 / 8 \\
2101 / 2\end{array}$ & $\begin{array}{r}\text { Inches } \\
23 / 4 \\
21 / 2 \\
17 / 8\end{array}$ \\
\hline
\end{tabular}

1 Maximum.

' Minimum.

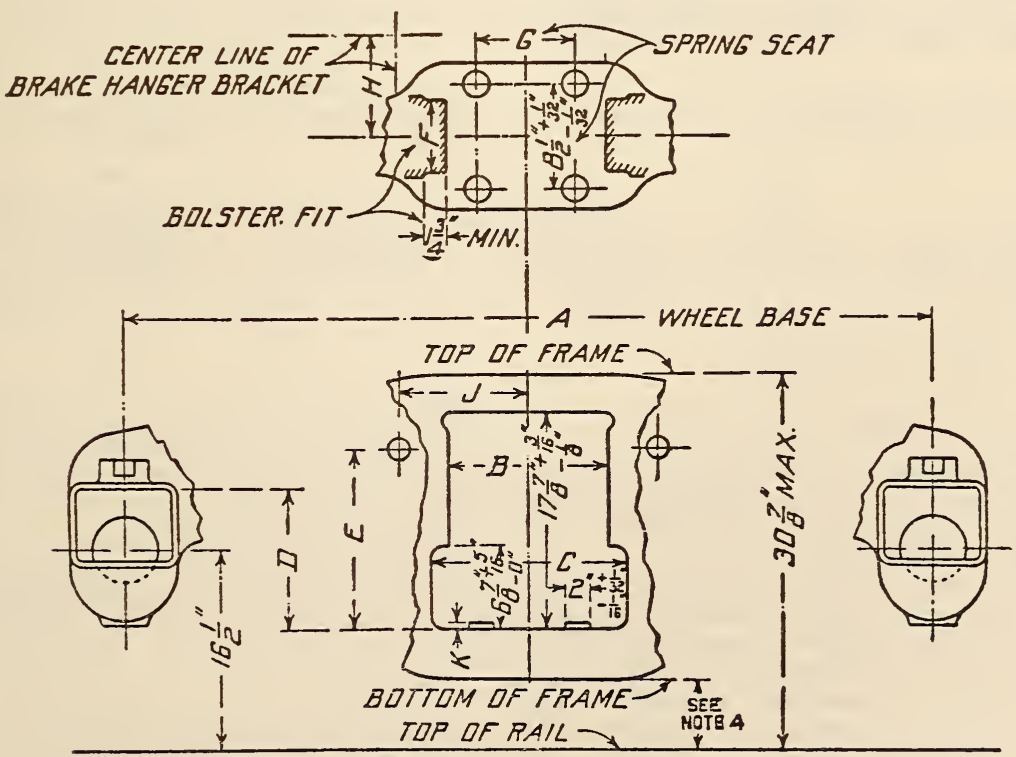

Figure 147.-Truck side frames. (See p. 265.) 


\section{AMERICAN RAILWAY ASSOCIATION, ME- CHANICAL DIVISION, LIMITING DIMEN- SIONS FOR CAST-STEEL TRUCK SIDE FRAMES, STANDARD, 1929}

\section{TRUCK SIDE FRAMES}

(See fig. 247, p. 265)

Table of dimensions and tolerances

\begin{tabular}{|c|c|c|c|c|}
\hline & $\begin{array}{c}2-\mathrm{C} \\
\text { frame } \\
5 \text { by } 9 \\
\text { inch } \\
\text { journal }\end{array}$ & $\begin{array}{c}2-\mathrm{D} \\
\text { frame } \\
532 \text { by } 10 \\
\text { inch } \\
\text { journal }\end{array}$ & $\begin{array}{c}2-\mathrm{E} \\
\text { frame } \\
6 \text { by } 11 \\
\text { inch } \\
\text { journal }\end{array}$ & $\begin{array}{l}\text { Toler- } \\
\text { ances }\end{array}$ \\
\hline A & $\begin{array}{cc}F t . & \text { in. } \\
5 & 6\end{array}$ & $\underset{5}{F t}{ }_{6}^{i n .}$ & $\begin{array}{cc}F t . & \text { in. } \\
5 & 8\end{array}$ & $\begin{array}{l}\text { Inches } \\
+3 / 16\end{array}$ \\
\hline B & 13 & 13 & 17 & +18 \\
\hline C & 16 & 16 & 20 & $+1 / 4$ \\
\hline D & $111 / 8$ & $117 / 16$ & 12 & $+1 / 4$ \\
\hline E & $147 / 8$ & $14^{15} / 16$ & 15 & $\begin{array}{r}+1 / 8 \\
-18\end{array}$ \\
\hline $\mathrm{F}$ & $51 / 2$ & 6 & $61 / 2$ & $+1 / 16$ \\
\hline G & 8 & 8 & 12 & $\begin{array}{r}1132 \\
+13\end{array}$ \\
\hline H & 8 & $81 / 2$ & 9 & +18 \\
\hline $\mathbf{J}$ & $101 / 2$ & $101 / 2$ & $111 / 2$ & $+1 / 4$ \\
\hline K & $5 / 16$ & $3 / 8$ & $7 / 16$ & \\
\hline
\end{tabular}

Note 1.-Inside dimensions of journal box and contained parts must conform to A. R. A. standards.

Note 2.-The side frame must conform to the A. R. A. Specifications for strength.

Note 3.-Alternate designs involving nonstandard bolste-, spring plank, or spring arrangement will be permissible, providing that they conform to A. R. A. specifications for strength, and the truck, as a whole, meets the A. R. A. requirements for interchangeability.

NOTE 4.-The distance from bottom of frame to top of rail should be 5 inches minimum when all parts are new for side frames intended to be used with multiple-wear wheels, and $33 / 4$ inches minimum when all parts are new for side frames intended to be used with 1-wear all parts

\section{AMERICAN SOCIETY FOR TESTING MATE- RIALS, STANDARD SPECIFICATIONS FOR STRUCTURAL STEEL FOR IOCOMO- TIVES AND CARS, SERIAL DESIGNATION A113-29, 1929}

(For general requirements relating to check analyses, yield point, number of tests, variations in weight and thickness of sheared and universal mill plates, finish, marking, inspection, rejection, and rehearing, also test specimen Figures 128 and 129, see A. S. T. M. specifications $(605.0$, p. 247$)$, omitting paragraph $4(a)$.)

(For tables of permissible variations of rectangular plates ordered to weight; permissible overweights of rectangular plates ordered to thickness; permissible variations in weight of bar sizes of angles, tees, zees, and channels; and permissible variations in cross-sectional dimensions of rounds, squares, hexagons, and flats, see A. A. S. M., Tables 1, 2, 3, 4, and 5 (605.0, p. 248).)

(For requirements relating to process, ladle analyses, modifications in elongation, bend tests, and test specimens, see A. S. T. M., A7 (605.11, p. 249), paragraphs $2,4,6,7(a)$ and $(c)$, and $8(a),(c)$, $(d),(e),(f),(g)$, and $(j)$. In paragraph $7(c)$, after "rivet steel," insert: "and plates for cold pressing.")

(The following additional requirements are given:)

1. Scope.-These specifications apply to shapes, plates (except boiler and fire-box plates), rivet steel, and bars.

2. Chemical composition.-Thesteel shall conform to the following requirements as to chemical composition:

\begin{tabular}{|c|c|c|c|c|}
\hline & $\begin{array}{l}\text { Struc- } \\
\text { tural } \\
\text { steel } \\
\text { for } \\
\text { cars }\end{array}$ & \begin{tabular}{|c|} 
Struc- \\
tural \\
steel \\
for \\
locomo- \\
tives
\end{tabular} & $\begin{array}{l}\text { Plates } \\
\text { for } \\
\text { cold } \\
\text { press- } \\
\text { ing }\end{array}$ & $\begin{array}{l}\text { Rivet } \\
\text { steel }\end{array}$ \\
\hline $\begin{array}{l}\text { Phosphorus: } \\
\text { Acid (maximum) } \\
\text { Basic (maximum) } \\
\text { Sulphur (maximum) } \\
\text { Copper, when copper steel is }\end{array}$ & $\begin{array}{c}\text { Per cent } \\
0.06 \\
.04 \\
.06\end{array}$ & $\begin{array}{c}\text { Per cent } \\
0.06 \\
.04 \\
.05\end{array}$ & $\begin{array}{c}\text { Per cent } \\
0.06 \\
.04 \\
.06\end{array}$ & $\begin{array}{c}\text { Per cent } \\
0.04 \\
.04 \\
.045\end{array}$ \\
\hline $\begin{array}{l}\text { Copper, when copper steel is } \\
\text { specified (minimum) }\end{array}$ & & & & .20 \\
\hline
\end{tabular}

3. Tension tests.-(a) The material, except as specified in paragraph (b), shall conform to the following requirements as to tensile properties:

\begin{tabular}{|c|c|c|c|c|}
\hline Properties considered & $\begin{array}{c}\text { Structural steel for } \\
\text { cars }\end{array}$ & $\begin{array}{l}\text { Structural steel for } \\
\text { locomotives }\end{array}$ & $\begin{array}{l}\text { Plates for cold } \\
\text { pressing }\end{array}$ & Rivet steel \\
\hline $\begin{array}{l}\text { Tensile strength } \\
\text { Yield point, minimum } \\
\text { Elongation in } 8 \text {-inch, }{ }^{a} \text { minimum. } \\
\text { Elongation in } 2 \text {-inch, minimum }\end{array}$ & $\begin{array}{r}50,000-65,000 \\
0.5 \text { tensile strength } \\
\frac{1,500,000}{\text { Tensile strength }} \\
22\end{array}$ & $\begin{array}{r}55,000-65,000 \\
0.5 \text { tensile strength } \\
\frac{1,500,000}{\text { Tensile strength }} \\
22\end{array}$ & $\left\{\begin{array}{r}48,000-58,000 \\
0.5 \text { tensile strength } \\
\frac{1,500,000}{\text { Tensile strength }}\end{array}\right.$ & $\begin{array}{r}45,000-60,000 \\
0.5 \text { tensile strength. } \\
\frac{1,500,000}{\text { Tensile strength }}\end{array}$ \\
\hline
\end{tabular}

a See section on modifications in elongation.

(b) Plates three-sixteenths inch and under in thickness, shapes less than 1 square inch in cross section, flats three-sixteenths inch and under in thickness, and other bars, except rivet rods, less than one-half inch in thickness or diameter, need not be subjected to tension tests.

4. Permissible variations.-(a) One cubic inch of rolled steel is assumed to weigh 0.2833 pound. The cross-sectional area or weight of each structuralsize shape shall not vary more than 2.5 per cent from the theoretical or specified amounts. The thickness or weight of rectangular sheared mill plates and of rectangular universal mill plates over 36 inches in width shall conform to the requirements of paragraphs $4(b)$ and (c) of A.S.T. M. (605.0, p. 247): the cross-sectional area or weight of bar size ${ }^{55}$ angles, tees, zees, and channels shall conform to the requirements of paragraph (b); and the cross-sectional dimensions of bars shall conform to the requirements of paragraph $(c)$.

(b) The weight of each lot 56 of bar sizes of angles, tees, zees, and channels in each shipment shall not vary from the weight ordered more than the amounts given in Table 3 under A. A. S. M. 605.0, p. 248.

55 A channel is in "bar" size when its greatest dimension is less than 3 inches. An angle, $T$, or $z$ is in "bar" size when its greatest dimension is less than 3 inches, or when it is 3 inches or more and at the same time the thickness is less than one-fourth inch.

66 The term "lot" here means all of the bars of each group dimen${ }^{56}$ The term "lot" here means all of the bars of each group dimen-
sion and group thickness shown in Table 3. (See A. A. S. M., 605.0, p. 248.) 
(c) The cross-sectional dimension of bars shall not vary from the size ordered more than the amounts given in Tables 4 and 5 under A. A. S. M. 605.0. p. 248 .

5. Except in cases of special agreement between the purchaser and manufacturer that the material shall be furnished recut to exact ordered dimensions, the length of all shapes, plates, and bars shall not vary under the dimensions ordered more than onefourth inch. The width of plates shall not vary under the dimensions ordered more than one-fourth inch. The variation over the dimension ordered shall not be more than is consistent with thickness, length, and good mill practice.

ASSOCIATION OF AMERICAN STEEL MANUFACTURERS, STANDARD SPECIFICATIONS FOR STRUCTURAL AND BOILER STEEL, NOVEMBER 24, 1922

\section{STRUCTURAL STEEL}

(See 605.11, p. 250.)

\section{UNITED STATES GOVERNMENT, FEDERAL SPECIFICATIONS BOARD, FEDERAL SPECIFICATION FOR STRUCTURAL STEEL FOR CARS, NO. 373a, MARCH 25, 1929. (CURRENT DESIGNATION: QQ-S-731)}

(This specification is in some respects substantially the same as F. S. B. 351a (QQ-S-711) (605.11, p. 251). For general specifications, permissible variations in structural shapes of bar sizes, rolled-edge plates, rounds (including rivet bars), squares, hexagons, and flats, and number of tests, see F. S. B. 351a (QQ-S-711), paragraphs 1, 3, 4, 5, 6 , and 8 . Substitute in paragraph 8 , " 25 short tons" for "26 short tons," and add: "Bars, except rivet rods, less than one-half inch in section shall not be subjected to tension tests.")

(For requirements relating to process, manufacturers' analyses, modifications in elongation, bend tests, test specimens, and copper steel, see A. S. T. M., A7 (605.11, p. 249), paragraphs 2, 4, 6, 7 (a) and $(c), 8(a),(b),(c),(d),(e),(f),(g)$, and $(j)$, and that part of paragraph 3 relating to copper steel. In paragraph 7 (c), after "rivet steel," insert: "and plates for cold pressing.")

(For requirements relating to yield point, permissible variations in sheared plates and structural shapes except of bar sizes, finish, and marking, see A. S. T. M. specifications $(605.0$, p. 247$)$, paragraphs 2,4 , and 5 , and the first sentence of paragraph 6.)

(For tables of permissible variations in plates ordered to weight and thickness, and in angles, tees, zees, channels, rounds, squares, hexagons, and flats, see A. A. S. M. (605.0, p. 248), Tables 1, 2, 3, 4, and 5.)

(The following additional material is given:)

1. Classes.- Structural steel for cars shall be of the following classes: A, structural steel, noncopper; B, structural steel, copper; C, steel for cold pressing, noncopper; D, steel for cold pressing, copper; $\mathrm{E}$, rivet steel, noncopper; and $\mathrm{F}$, rivet steel, copper.

2. Chemical composition.-The amounts of phosphorus and sulphur shall not exceed the following percentages:

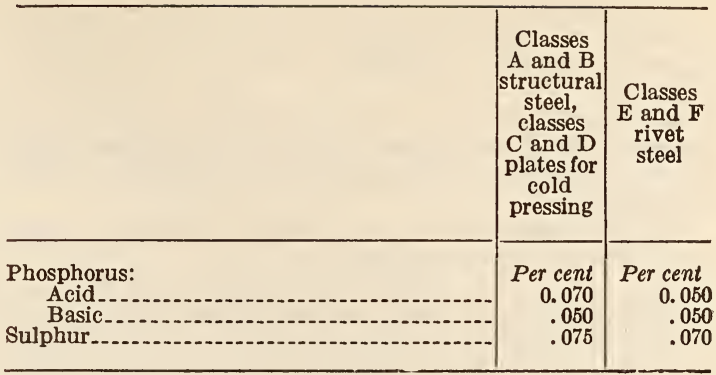

3. Tension tests.-The material shall conform to the following requirements as to tensile properties:

\begin{tabular}{|c|c|c|c|}
\hline Properties considered & $\begin{array}{c}\text { Classes A and } \\
\text { B structural } \\
\text { steel }\end{array}$ & $\begin{array}{l}\text { Classes C and } \\
\mathrm{D} \text { plates for } \\
\text { cold pressing }\end{array}$ & $\begin{array}{l}\text { Classes E } \\
\text { and F } \\
\text { rivet steel }\end{array}$ \\
\hline $\begin{array}{l}\text { Tensile strength } \\
\text { Yield point, mini- } \\
\text { mum.1b./in.2 } \\
\text { Elongation in } 8 \text { inches, } \\
\text { minimum a } \\
\\
\text { Elongation in } 2 \text { inches, } \\
\text { minimum._per cent. }\end{array}$ & $\begin{array}{c}50,000-65,000 \\
0.5 \text { tensile } \\
\text { strength. } \\
\frac{1,500,000}{\text { Tensile }} \\
\text { strength } \\
22\end{array}$ & $\begin{array}{l}\text { 48,000-58,000 } \\
0.5 \text { tensile } \\
\text { strength. } \\
\frac{1,500,000}{\text { Tensile }} \\
\text { strength }\end{array}$ & $\begin{array}{l}45,000-60,000 \\
0.5 \text { tensile } \\
\text { strength. } \\
\frac{1,500,000}{\text { Tensile }} \\
\text { strength }\end{array}$ \\
\hline
\end{tabular}

a See section on modifications in elongation in A.S.T.M. A7., p. 249.

4. Notes.-(a) This specification applies to shapes, plates, and bars over one-eighth inch in thickness or diameter.

(b) When specifying materials in accordance with this specification, the class or classes (A, B, C, D, E, and $F$ ) should be stated definitely.

605.14 STRUCTURAL STEEL FOR MACHINERY.

AMERICAN BUREAU OF SHIPPING, RULES FOR BUILDING AND CLASSING STEEL VESSELS; QUALITY AND TESTING OF MATERIALS, SHIP CONSTRUCTION, 1930

\section{STEEL PLATES, ANGLES, BULB ANGLES,} CHANNELS, ETC.

(See 605.15, p. 268.)

AMERICAN RAILWAY ASSOCIATION, SIGNAL SECTION, SPECIFICATION FOR MACHINERY STEEL, NO. 2111, 1911

\section{MACHINERY STEEL}

\section{MATERIAL}

(a) All sections must be true to size, free from cracks, flaws, and defects of all kinds.

(b) When subjected to a tensile test the steel must show: Tensile strength per square inch 48,000 to 58,000 , pounds. Elongation in 8 inches, not less than 25 per cent.

(c) Phosphorus not to exceed 0.1 per cent, sulphur not to exceed 0.065 per cent. 
(d) When subjected to a bending test, either hot or cold, the steel must bend double over a diameter equal to its own thickness without showing any fracture.

(e) Results of the chemical analysis made at the steel works of each melt used to fill the order must be furnished to the purchaser's inspector when requested.

$(f)$ Mild steel will be inspected and tested at the shops where it is to be used.

(g) If material fails to meet the requirements of this specification, it will be rejected and returned to the manufacturer, who must pay all freight charges.

605.15 STRUCTURAL IRON AND STEEL FOR SHIPS.

AMERICAN BUREAU OF SHIPPING, RULES FOR BUILDING AND CLASSING STEEL VESSELS; QUALITY AND TESTING OF MATERIALS, SHIP CONSTRUCTION, 1930

IRON PLATES AND ANGLES

(See 604.14, p. 234.)

AMERICAN BUREAU OF SHIPPING, RULES FOR BUILDING AND CLASSING STEEL VESSELS; QUALITY AND TESTING OF MATERIALS, SHIP CONSTRUCTION, 1930

\section{STEEL PLATES, ANGLES, BULB ANGLES, CHANNELS, ETC.}

(For general information and requirements see 600.0, p. 2.)

1. The tensile strength and ductility shall be determined from test specimens cut lengthwise or crosswise from the finished material; when material is annealed or otherwise treated before dispatch the test specimens shall be similarly and simultaneously treated before testing. Wherever practicable the rolled surfaces shall be retained on two opposite sides of the test specimens; any straightening of test specimens which may be required shall be done cold. The parallel part of the tension-test piece shall not be less than 9 inches in length; the width shall not exceed $1 \frac{1}{2}$ inches for material more than 0.875 inch in thickness; from 0.875 to 0.375 inch it shall not exceed 2 inches, and under 0.375 inch the width shall not be more than $2 \frac{1}{2}$ inches.

2. Should a tension-test specimen break outside the middle half of its gage length the specimen may, at the maker's option, be discarded and another test made of the same plate or bar. Test specimens for bends shall not be less than $1 \frac{1}{2}$ inches wide and for small bars the whole section may be used. For quench-bend tests the specimens are to be heated to a light cherry red and quenched at once in water the temperature of which is between $80^{\circ}$ and $90^{\circ} \mathrm{F}$. The color shall be judged indoors in the shade. The rough edge caused by the shearing may be removed by filing or grinding from all test specimens for cold bends, and from test specimens for quench bends which are 0.50 inch and above in thickness; specimens 1 inch thick and above may be machined; the test specimens shall receive no other preparation. The specimens are not to be annealed unless the material from which they are cut $i_{i}$ similarly and simultaneously treated.

At least one tension test shall be made from the finished material of each heat; where the heat weight exceeds 56,000 pounds, a second tension test will be required; an additional test shall be made for each variation of 0.15 inch thickness of the material rolled from each heat. A cold or quench bend test is to be made from each plate or bar as rolled; these tests shall be made in about equal numbers from each heat; a cold-bend test shall be made from each plate rolled which is specially marked for cold flanging.

3 . The tensile strength of plates not intended for cold flanging shall be between the limits of 62,000 and 72,000 pounds per square inch of section; sectional material shall be between the limits of 62,000 and 74,000 pounds per square inch; garboard strake plates and other plates intended for cold flanging are to be marked so as to be easily identified by the surveyor at the steel works and in the shipyard, and are to have a tensile strength between the limits of 55,000 and 65,000 pounds per square inch. Material for purposes where tensile strength is not important need not be subjected to tensile tests if specified by the builders and approved by the committee, but will be subjected to bend tests and should be marked so as to be easily identified by the surveyors.

4. (a) The material shall conform to the following requirements:

$$
\begin{aligned}
& \text { Yield point, minimum........ lbs./in. }{ }^{2} \ldots 0.5 \text { tensile strength. } \\
& \text { Elongation in } 8 \text { inches, minimum }
\end{aligned}
$$$$
\text { Tensile strength }
$$

(b) For material over three-fourths inch in thickness, a deduction of 1 from the percentage of elongation specified in paragraph (a) shall be made for each increase of one-eighth inch in thickness above three-fourths inch, to a minimum of 20 per cent.

5. The cold-bend test specimen shall bend cold through $180^{\circ}$ without cracking on the outside of the bent portion, as follows:

For material three-fourths inch or under in thickness, around a pin the diameter of which is equal to twice the thickness of the specimen; for material over three-fourths inch in thickness, around the pin the diameter of which is equal to three times the thickness of the specimen.

6. In the event of any test specimen failing to meet the requirements, unless two further specimens prove satisfactory, the plate or bar represented by such sample specimen shall be rejected. Further tests of specimens taken from the same heat shall then be made and if these fail the whole of the material produced from that heat is to be rejected.

7. All material, which has satisfactorily passed the requirements, must be clearly stamped by the manufacturer in at least two places on each finished plate or bar, thus: $A B$ signifying that the material has satisfactorily complied with the tests above described. Material having tensile strength which is between the limits of 55,000 and 65,000 pounds and complies with the corresponding requirements for elongation, should be marked thus $\frac{A B}{F}$ to signify that it is of "cold flanging" quality; material which has been approved on bend tests only is to be marked thus $\frac{A B}{B}$ so that it may be readily identified. No material bearing these brands is to be forwarded from the steel works until the prescribed tests have been made by the surveyors and the mill sheets signed by them.

8. Before the mill sheets are signed, the manufacturers must furnish the surveyor with a certificate guaranteeing that the material has been made by the open-hearth process and that it has withstood satisfactorily the tests above described. The following form of certificate will be accepted if printed on each mill sheet with the name of the firm and initialed by the manufacturer's chief inspector:

We hereby certify that the material described herein has been made by the open-hearth process and has been tested by us in accordance with the rules of the American Bureau of shipping with satisfactory results.

9. The maker must adopt a system of marking the ingots, billets, slabs, test pieces, and finished 
material which will enable all material to be traced to its tests and original heat. The surveyor must be given every facility for tracing the material to the respective heats from which it is made, and he should be furnished with two copies of advice notes of such material as may have been satisfactorily dealt with, for his signature-one to be forwarded by the manufacturer to the shipbuilder, the other to be retained for the use of the surveyor in attendance at the shipyard.

10. Where steel is not produced in the works at which it is rolled, a certificate shall be supplied to the surveyor, deputed to witness the testing of the material, stating the open-hearth process by which it was manufactured, the name of the steel maker who supplied it, and the number of the heats from which it was made for reference to the books of the steel maker. The number of the heat shall be marked on each plate or bar for the purpose of identification.

11. The foregoing tests shall be made at the place of manufacture prior to the dispatch of the material, but in the event of any of the material proving unsatisfactory in the process of being worked it shall be rejected; such further tests may be made of material from the same heat as the surveyor in attendance may consider desirable and material then found defective shall also be rejected, notwithstanding any previous certificate of satisfactory testing.

\section{AMERICAN SOCIETY FOR TESTING MATE- RIALS, STANDARD SPECIFICATIONS FOR STRUCTURAL STEEL FOR SHIPS, DESIGNATION A12-21, 1921}

(For general requirements relating to $\mathrm{check}$ analyses, yield point, number of tests, permissible variations in weight and thickness of plates, finish, marking, inspection, rejection, and rehearing, also test specimen Figures 128 and 129: See A. S. T. M. specifications $(605.0$, p. 247$)$, omitting, in paragraph 3 (c), reference to 2 -inch specimens, and in paragraph 6 , references to rivet and lattice bars and other small sections, and to pins and rollers.)

(Requirements relating to process, ladle analyses, modifications in elongation, and test specimens are substantially the same as in A. S. T. M. A7 (605.11, p. 249), paragraphs $2,4,6(a)$, and $8(a),(b),(d),(e)$, and $(j)$. In paragraph 4 omit reference to copper steel.)

(For tables of permissible variations of plates ordered to weight, and permissible overweights of plates ordered to thickness, see A. A. S. M., Tables 1 and $2(605.0$, p. 248).)

(The following additional material is given:)

1. Chemical composition.-The steel shall conform to the following requirements as to chemical composition:

$\begin{array}{rr}\text { Phosphorus, maximum: } & \text { Per cent } \\ \text { Acid } & \\ \text { Basic } & \\ \text { Sulphur, maximum } & \end{array}$

2. Tension tests.-The material shall conform to the following requirements as to tensile properties:

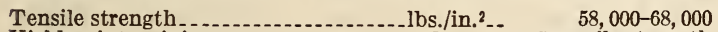
Yield point, minimum .................... do 0.5 tensile strength Elongation in 8 inches, minimum .... per cent. $1,500,000$

(See paragraphs on modifications in elongation.) Tensile strength

3. Modifications in elongation.-For material onefourth inch or under in thickness, the elongation shall be measured on a gage length of twenty-four times the thickness of the specimen.

4. Bend tests. - The test specimen shall bend cold through $180^{\circ}$ without cracking on the outside of the bent portion, as follows: For material three-fourths inch or under in thickness, around a pin, the diam- eter of which is equal to the thickness of the specimen; for material over three-fourths inch to and including $1 \frac{1}{4}$ inches in thickness, around a pin, the diameter of which is equal to one and one-half times the thickness of the specimen; and for material over $1 \frac{1 / 4}{4}$ inches in thickness, around a pin, the diameter of which is equal to twice the thickness of the specimen.

5. Test specimens.-(a) Test specimens for plates over $1 \frac{1}{2}$ inches in thickness may be machined to a thickness or diameter of at least three-fourths inch for a length of at least 9 inches.

(b) Test specimens for bars over $1 \frac{1}{2}$ inches in thickness or diameter may be machined to a thickness or diameter of at least three-fourths inch for a length of at least 9 inches; or tension test specimens may conform to the dimensions shown in Figure 129 (p. 247), in which case the ends shall be of a form to fit the holders of the testing machine in such a way that the load shall be axial. Bend test specimens may be one by one-half inch in section.

(c) The tension test specimen shown in Figure 129 (p. 247), and the 1 by $1 / 2$ inch bend test specimen for bars over $1 \frac{1}{2}$ inches in thickness or diameter shall be taken so that the axis is midway between the center and surface.

\section{AMERICAN SOCIETY FOR TESTING MATE- RIALS, STANDARD SPECIFICATIONS FOR RIVET STEEL FOR SHIPS, DESIGNA- TION A13-24, 1924}

\section{A. REQUIREMENTS FOR ROLLED BARS}

(For general requirements relating to check analyses, yield point, number of tests, finish, marking, inspection, rejection, and rehearing: See A. S. T. M. specifications $(605.0$, p. 247$)$, paragraphs $1,2,3(b)$ and $(c), 5,6,7,8$, and 9 . In paragraph $3(c)$, omit reference to 2 -inch specimen.)

(Requirements relating to process, ladle analyses, and test specimens are substantially the same as in A. S. T. M. A7 (605.11, p. 249), paragraphs 2 , 4, and 8 (c). In paragraph 4 omit reference to copper steel.)

(The following additional material is given:)

1. Chemical composition.-The steel shall conform to the following requirements as to chemical composition:

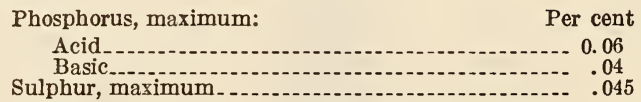

2. Tension tests. - The bars shall conform to the following requirements as to tensile properties:

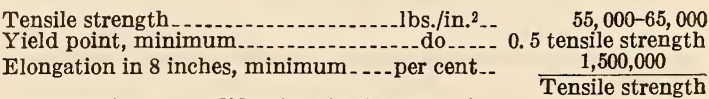

(See section on modifications in elongation.)

3. Modifications in elongation.-For bars over three-fourths inch in diameter, a deduction from the percentage of elongation specified in paragraph 2 of 0.25 per cent shall be made for each increase of one thirty-second inch of the specified diameter above three-fourths inch.

4. Bend tests.-The test specimen shall bend cold through $180^{\circ}$ flat on itself without cracking on the outside of the bent portion.

5. Tesi specimens.-Test specimens shall be of the full diameter of bars as rolled.

6. Number of tests. - Two tension and two bend tests shall be made from each melt, each of which shall conform to the requirements specified; except that if bars from one melt differ three-eighths inch or more in diameter, one tension and one bend test shall be made from both the greatest and the least. diameters rolled. 
7. Permissible variations.-The diameter of bars 1 inch or under in diameter shall not vary more than 0.01 inch from that specified; the diameter of bars over 1 inch to and including 2 inches in diameter shall not vary more than one sixty-fourth inch under nor more than one thirty-second inch over that specified.

\section{B. REQUIREMENTS FOR RIVETS}

(For general requirements relating to inspection and rejection, See A. S. T. M. specifications (605.0, p. 247), paragraphs 7 and $8(b)$.)

(The following additional material is given:)

1. Bend tests. - The rivet shank shall bend cold

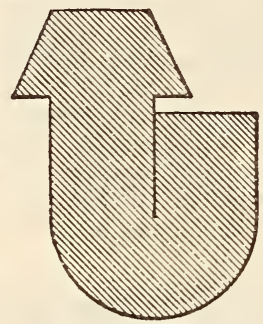

Frgure 148

through $180^{\circ}$ flat on itself, as shown in Figure 148, without cracking on the outside of the bent portion.

2. Flattening tests. - The rivel head shall flatten, while hot, to a diameter two and one-half times the diameter of the shank, as shown in Figure 149, without cracking at the edges.

3. Number of tests. - Three bend and three flattening tests shall be made from each size in each lot of rivets offered for inspection, each of which shall conform to the requirements specified.

4. Workmanship and finish.-The rivets shall be true to form and concentric, shall be made in a work-

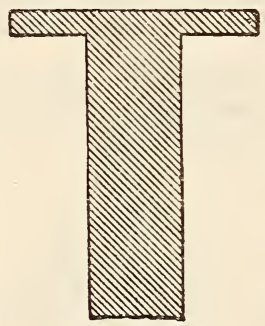

FigURe 149

manlike manner and shall be free from injurious defects.

NOTE.-A tentative revision of A12 and A13, designated A131-31T will, when adopted as standard, rupersede these two specifications

\section{ASSOCIATION OF AMERICAN STEEL MAN- UFACTURERS, STANDARD SPECIFICA- TIONS FOR STRUCTURAL AND BOILER STEEL, NOVEMBER 24, 1922}

\section{STRUCTURAL STEEL}

(See 605.11, p. 250.)
UNITED STATES GOVERNMENT, FEDERAL SPECIFICATIONS BOARD, FEDERAL SPECIFICATION FOR STEEL; STRUCTURAL (INCLUDING STEEL FOR COLD FLANGING), AND STEEL, RIVET, FOR SHIPS OTHER THAN NAVAL VESSELS, QQ-S-751 JUNE 23, 1931.

\section{STRUCTURAL STEEL FOR SHIPS}

\section{A. APPIICABLE FEDERAL SPECIFICATIONS}

A-1. Federal specification No. 339a (current designation $Q Q-M-151$ ), general specifications for metals, in effect on date of invitation for bids, shall form a part of this specification. (See 600.0, p. 3.)

\section{B. GRADE}

B-1. Structural steel, structural steel for cold flanging, and rivet steel for ships other than naval vessels shall be of but one grade each, as defined by paragraphs E-7 and E-8.

\section{MATERIAL AND WORKMANSHIP, ETC.}

C-1. Unless otherwise specified, steel shall be made by the open-hearth process or electric-furnace process.

C-2. The finished material shall be free from welds and injurious defects and shall have a workmanlike finish.

\section{GENERAL REQUIREMENTS}

D-1. See section E.

\section{E. DETAIL REQUIREMENTS}

E-1. Structural shapes other than bar sizes.-The cross section (or sectional area) or weight of each piece shall not vary from that specified more than 2.5 per cent.

E-2. Structural shapes of bar sizes.-The weight of each piece shall not vary from that specified more than the amount given in Table 2.

E-3. Rolled edge plates.-Each plate up to and including 36 inches in width shall not vary more than 2.5 per cent from the cross section (or sectional area) or weight specified; rolled edge plates more than 36 inches in width shall conform to limits specified in paragraph E-4 for sheared plates.

E-4. Sheared plates.-The weight of each lot in each shipment of rectangular plates shall not vary from the limits given in Table 1; the term "lot" meaning all of the plates of each group width and group weight. The weight per square foot of each individual plate shall not vary by more than one and one-third times the percentages allowed by Table 1.

E-5. Rounds (including rivet bars), squares, and hexagons.-No piece shall vary from the limits given in Table 3.

E-6. Flats.-No piece shall vary from the limits given in Table 4.

E-7. Chemical properties.-The amount of phosphorus and sulphur shall not exceed the following percentages: 


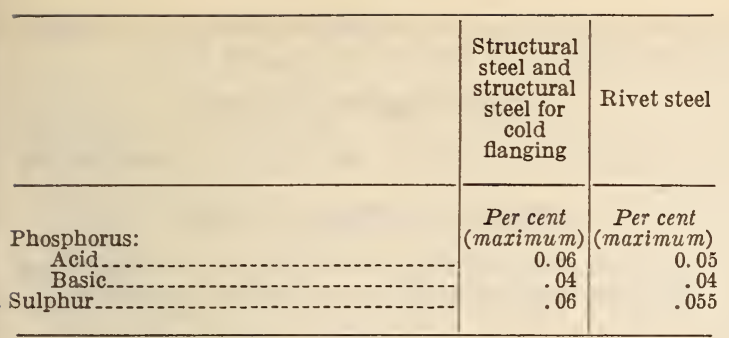

E-8. Physical properties.-The material shall have the following physical properties:

\begin{tabular}{|c|c|c|}
\hline & Structural steel & $\begin{array}{l}\text { Structural steel } \\
\text { for colda flanging, } \\
\text { and rivet steel }\end{array}$ \\
\hline 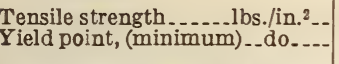 & $\begin{array}{c}60,000-72,000 \\
0.5 \text { tensile }\end{array}$ & $\begin{array}{r}55,000-65,000 \\
0.5 \text { tensile }\end{array}$ \\
\hline $\begin{array}{l}\text { Yield point, in no case less than- } \\
\text { Elongation in } 8 \text { inches (mini- }\end{array}$ & 33,000 & 30,000 \\
\hline mum) & $\begin{array}{l}\frac{1,500,000}{\text { Tensile }} \\
\text { strength }\end{array}$ & $\frac{1,500,000}{\begin{array}{l}\text { Tensile } \\
\text { strength }\end{array}}$ \\
\hline $\begin{array}{l}\text { Elongation in } 2 \text { inches } \\
\text { (minimum) }\end{array}$ & 22 & 25 \\
\hline
\end{tabular}

For material over $3 / 4$ inch in thickness or diameter, a deduction from the percentage of elongation in 8 inches specified above of 0.25 per cent shall be made for each increase of $1 / 32$ inch in the specified thickness or diameter above $3 / 4$ inch, to a mimimum of 16 per cent for structural steel and 18 per cent for structural steel for cold flanging and rivet steel.

For material under $5 / 16$ inch in thickness or diameter, a deduction from the percentage of elongation in 8 inches specified above of 1.25 per cent shall be made for each decrease of $1 / 32$ inch of the specified thickness or diameter below 5/6 inch.

\section{F. METHODS OF SAMPLING, INSPECTION, AND TESTS}

\section{F-1. Number of tests.-}

F-1a. Two tension and two bend tests shall be made from each melt of structural steel and steel for cold flanging unless the finished material from a melt is less than 25 short tons, when one tension and one bend test shall be sufficient. If, however, material from one melt varies 0.15 inch or more in thickness, the thickest and thinnest material rolled shall each be represented by one tension and one bend test, regardless of the weight of the melt. Two tension and two bend tests shall be made from each melt of rivet steel.

F-1b. When structural steel is ordered for cold flanging and also required to conform to ship classification societies' tests and inspection, one bend test specimen shall be taken from each plate as rolled.

F-1c. If any test specimen shows defective machining or develops flaws, it may be discarded and another specimen substituted.

F-2. Test specimens.-

F-2a. Unless specified otherwise, test specimens shall be taken longitudinally as approved by the inspector and, except as specified in paragraphs $F-2 f$ and $F-2 g$, shall be of the full thickness or section of material as rolled.

F-2b. Test specimens shall be prepared for testing from the material in its rolled condition, except as specified in paragraphs $\mathrm{F}-2 \mathrm{c}$ and $\mathrm{F}-2 \mathrm{~d}$.

F-2c. Test specimens for annealed material shall be prepared from the material as annealed for use.

F-2d. Test specimens for rivet bars which have been cold-drawn shall be normalized before testing.

F-2e. Test specimens for plates, shapes, and flats may be machined to conform to type 2, Federal specification QQ-M-151, referred to in paragraph A-1, or with both edges parallel.
F-2f. Tension test specimens for material over $1 \frac{1}{2}$ inches in thickness or diameter, may be machined to a thickness or diameter of at least three-fourths inch for a length of at least 9 inches, or they may conform to type 1, Federal specification QQ-M-151, referred to in paragraph $\mathrm{A}-1$.

F-2g. Bend test specimens for material over $11 / 2$ inches in thickness or diameter may be machined to a thickness or diameter of at least three-fourths inch or to 1 by $1 / 2$ inch in section.

F-2h. For bars over $1 \frac{1}{2}$ inches in thickness or diameter, other than flats, when not tested in the full section as rolled, the tension test specimens shall be taken so that the axis shall be at a point one-half the distance between the center and the surface of the bar.

F-2i. The machined sides of rectangular bend test specimens may have the corners rounded to a radius not over one-sixteenth inch.

$\mathrm{F}-3$. Bend tests.-

F-3a. Bend test specimens for structural steel other than for cold flanging shall bend cold through $180^{\circ}$ without cracking on the outside of the bent portion as follows: For material three-fourths inch or under in thickness, around a pin the diameter of which is equal to the thickness of the specimen; for material over three-fourths inch, to and including $1 \frac{1}{4}$ inches in thickness, around a pin the diameter of which is equal to twice the thickness of the specimen; and for material over $1 \frac{1}{4}$ inches in thickness around the pin, the diameter of which is equal to three times the thickness of the specimen.

F-3b. Bend-test specimens for structural steel for cold flanging and rivet steel shall bend cold through $180^{\circ}$ without cracking on the outside of the bent portion as follows: For material three-fourths inch or under in thickness, flat on itself; for material over three-fourths inch, to and including $11 / 4$ inches in thickness, around a pin the dimaeter of which is equal to the thickness of the specimen; and for material over $11 / 4$ inches in thickness, around a pin the diameter of which is equal to twice the thickness of the specimen.

F-4. The yield point shall be determined by the drop of the beam of the testing machine.

\section{G. PACKAGING, PACKING, AND MARKING}

G-1. The name or brand of the manufacturer and the melt number, if practicable, shall be legibly stamped or rolled on all finished material, except that rivet and lattice bars and other small sections, when loaded for shipment, shall be properly separated and marked for identification. If it is not practicable to stamp or roll the melt number on finished material, other methods of marking may be used so long as the marking is legible.

G-2. Shipments shall be marked with the name of the material, the size, the shape, and the quantity contained therein as defined by the contract or order under which shipment is made, the name of the contractor, and the number of the contract or order.

\section{HOTES}

I-1. The contractor shall furnish for each melt a carbon determination and determinations for manganese, phosphorus, and sulphur representing the average of the melts applied for each 12-hour period.

I-2. When specifying material in accordance with this specification the grade should be definitely siated. When structural steel is for cold flanging, orders should so state.

I-3. It is believed that this specification adequately describes the characteristics necessary to secure the desired material, and that normally no samples will be necessary prior to award to deter- 
mine compliance with this specification. If, for any particular purpose, samples with bids are necessary, they should be specifically asked for in the invitation for bids, and the particular purpose to be served by the bid sample should be definitely stated, the specification to apply in all other respects.

I-4. This specification governs all United States Government purchases of this commodity.

TABLE 1.-Permissible variations in weights per square foot of rectangular sheared plates and universal plates over 36 inches, for widths given, expressed in percentage of ordered weights

(Identical with Table 1 of A. A. S. M. standard specifications for structural and boiler steel. See 605.0, p. 248.)

TABLE 2.-Permissible variations in weights of angles, tees, zees, and channels

(Identical with Table 3 of A. A. S. M. standard specifications for structural and boiler steel. See 605.0, p. 249.)

TABLE 3.-Permissible variations in dimensions of rounds, squares, and hexagons

(Identical with Table 4 of A. A. S. M. standard specifications for structural and boiler steel. See 605.0, p. 249.)

TABLE 4.-Permissible variations in dimensions of flats

(Identical with Table 5 of A. A. S. M. standard specifications for structural and boiler steel. See 605.0, p. 249.)

605.19 MISCELLANEOUS STRUCTURAL STEEL.

AMERICAN ELECTRIC RAILWAY ENGINEERING ASSOCIATION, SPECIFICA. TION FOR MATERIALS FOR USE IN THE MANUFACTURE OF SPECIAL TRACKWORK, W104-28, 1928

[Approved as tentative American Standard by the American Standards Association, January 23, 1929]

SPECIAL TRACKWORK MATERIALS

(See 606.3, p. 342.)

\section{AMERICAN SOCIETY FOR TESTING MATE- RIALS, STANDARD SPECIFICATIONS FOR STEEL PLATES OF STRUCTURAL QUALITY FOR FORGE WELDING, DESIG- NATION A78-30, 1930}

\section{STEEL PLATES FOR FORGE WELDING}

\section{SCOPE}

1. These specifications cover steel plates of structural quality suitable for forge welding without the use of fluxes where the weld is not reinforced.

\section{MAN UFACTURE}

2. The steel shall be made by the open-hearth process.

\section{CHEMICAL PROPERTIES AND TESTS}

(The chemical composition of grade $\mathrm{A}$ is identical with that of flange quality, grade A, including phosphorus (acid) in A. S. T. M. A89-31T. See 604.11, p. 230. Grade B is identical with that of fire-box quality, grade B in A89-31T, except that phosphorus shall not be over 0.06 and sulpnur not over 0.05 per cent. Requirements covering nickel, chro- mium, and silicon, with footnote reference to nickel, are also identical with those in A89-31T.)

\section{LADLE AND CHECK ANALYSES}

(Conform to A. S. T. M. A70-27. See 604.11, p. 227.)

\section{PHYSICAL PROPERTIES AND TESTS}

3. The material shall conform to the following minimum requirements as to tensile properties:

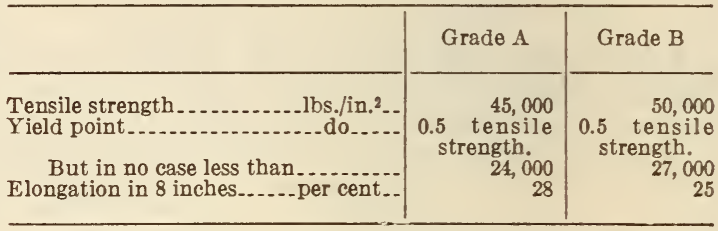

(In respect to determination of yield point and modifications for material over three-fourths inch in thickness, this specification conforms to A. S. T. M. A70-27. In respect to modifications in elongation for material under five-sixteenths inch in thickness, machining of test specimens for plates over $1 \frac{1}{2}$ inches in thickness, rounding corners of rectangular bend test specimens, and retesting defective test specimens, this specification conforms to A. S. T. M. A129-30T. See 604.11, p. 232. Otherwise the test requirements are as follows:)

4. Bend tests. - The test specimen shall stand being bent cold through $180^{\circ}$ flat on itself without cracking on the outside of the bent portion.

5. (a) Test specimens. - Test specimens shall be prepared for testing from the material in its rolled condition.

(b) Test specimens shall be taken longitudinally and, except in the case of test specimens for plates over $1 \frac{1}{2}$ inches in thickness, shall be of the full thickness of the material as rolled. They may be machined to the form and dimensions shown in Figure 128 in A. S. T. M. standard specifications for steel (see 605.0, p. 247), or with both edges parallel.

6. Number of tests.-One tension and one bend test shall be made from each melt; except that if material from one melt differs three-eighths inch or more in thickness, one tension and one bend test shall be made from both the thickest and the thinnest material rolled.

\section{PERMISSIBLE VARIATIONS IN WEIGHT AND THICKNESS}

7. The cross section or weight of plates shall be covered by the following permissible variations. One cubic inch of rolled steel is assumed to weight 0.2833 pound.

(a) When ordered to weight per square foot.-The weight of each lot 57 in each shipment shall not vary from the weight ordered more than the amount given in Table 1 in A. A. S. M. standard specifications for structural and boiler steel. (See 605.0, p. 248.)

(b) When ordered to thickness.-The thickness of each plate shall not vary more than 0.01 inch under that ordered. The overweight of each lot ${ }^{57}$ in each shipment shall not exceed the amount given in Table 2 in A. A. S. M. standard specifications for structural and boiler steel. (See 605.0, p. 248.)

57 The term "lot" as applied to Table 1 in A. A. S. M. standard specifications for structural and boiler steel (see 605.0, p. 248) means all of the plates of each group width and group weight. As applied to Table 2 it means all of the plates of each group width and group thickness. 


\section{MARKING}

8. The name or brand of the manufacturer and the melt number shall be legibly rolled or stamped on all finished material. The melt number shall be legibly marked, by stamping if practicable, on each test specimen.

(In respect to finish, inspection and rejection, and rehearing, this specification conforms to A. S. T. M. standard specifications for steel. (See 605.0, p. 247.)

605.2 STRUCTURAL SHAPES AND PLATES, FABRICATED.

605.20 GENERAL ITEMS.

\section{AMERICAN ELECTRIC RAILWAY ENGI- NEERING ASSOCIATION, RULES FOR INSPECTION AND MAINTENANCE OF BRIDGES, MANUAL SECTION B207-20, 1920}

\section{BRIDGE INSPECTION}

(These recommendations cover the frequency and scope of the inspections of bridges to be made by track force, by the bridge foreman, and by the engineering department in conjunction with the bridge foreman. Suggested forms for reporting inspections and repair work done are included.)

605.21 STEEL BRIDGES AND RAILWAY TURN-
TABLES.

AMERICAN ASSOCIATION OF STATE HIGHWAY OFFICIALS, STANDARD SPECIFICATIONS FOR HIGHWAY BRIDGES AND INCIDENTAL STRUCTURES, 1931

\section{HIGHWAY BRIDGES}

Section 7. STROCTURAL, RIVET, AND EYEBAR STEEL

2. 7. 1. General.-All structural, rivet, and eyebar steel shall conform to the requirements of the standard specifications for structural steel for bridges, designation A7, of the American Society for Testing Materials (see 605.11 , p. 249), supplemented by the following paragraphs:

2. 7. 2. Character of fracture.-Test requirements of structural, rivet, or eyebar steel shall show a fracture having a silky or fine granular structure throughout, with a bluish gray or dove color, and shall be entirely free from granular, black, and brilliant specks.

2. 7. 3. Defects in material.-Finished rolled material shall be free from cracks, flaws, injurious seams, laps, blisters, ragged and imperfect edges, and other defects. It shall have a smooth, uniform finish, and shall be straightened in the mill before shipment.

Material shall be free from loose mill scale, rust pits, or other defects affecting its strength and durability.

2.7.4. Full-size tests.-When full-size tests of built-up structural members and eyebars are required by the contract, the contractor shall supply testing machines of the proper type and capacity and shall provide all facilities and labor incidental to the making of tests. In all tests involving the determination of tensile and compressive strengths, the ultimate strength, deformation, and other pertinent data shall be recorded.

2. 7. 5. Payments for full-size tests.-Any full-size member tested to destruction shall be paid for by the purchaser at the unit contract price, less its scrap value, if the test proves satisfactory. If the test proves the member to be unsatisfactory, the mem- bers represented by it will be rejected. The expense of conducting tests shall be borne by the contractor unless otherwise provided.

\section{Section 8. EYEBARS}

2. 8. 1. Full-size tests.-When tests of full-size bars are required the following conditions and requirements shall supplement the general provisions of section 7 .

2. 8. 2. Number and size of test bars.-The number and size of the bars to be tested shall be designated by the engineer before the mill order is placed. The number shall not exceed 5 per cent of the whole number of bars ordered, with a minimum of two bars on small orders.

$2,8,3$. Selection of test bars. - The test bars shall be of the same section as the bars to be used in the structure and of the same length if within the capacity of the testing machine. They shall be selected by the inspector from the finished bars, preferably after annealing. Test bars representing bars too long for the testing machine shall be selected from the full-length bar material after the heads on one end have been formed and shall have the second head formed upon them after being cut to the greatest length which can be tested.

2. 8. 4. Physical requirements.-Full-size tests of eyebars shall show a yield point of not less than 33,000 pounds per square inch, an ultimate strength of not less than 60,000 pounds per square inch, and an elongation, including fracture, of not less than 12 per cent in a length of 18 feet measured in the body of the bar. The fracture shall show a uniform silky or fine granular structure throughout.

2. 8. 5. Failure to meet requirements.-If a bar fails to fulfill the specified requirements, two additional bars of the same size and from the same melt shall be tested. The bars represented by the test may be reannealed before the additional bars are tested.

If two of the three test bars fail to give satisfactory results, the bars of that size and melt shall be rejected.

2. 8.6. Record of annealing.-A record of the annealing charges shall be furnished the engineer showing the bars included in each charge and the treatment they received.

\section{Section 9. STEEL FORGINGS}

2. 9. 1. General.-Steel forgings from which pins, rollers, trunnions, or other forged parts are to be fabricated shall conform to the requirements of the standard specifications for carbon-steel forgings for locomotives, designation A20, of the American Society for Testing Materials. (See 611.51, p. 757.)

2. 9, 2. Annealing.-All forgings shall be thoroughly annealed prior to being machined to form finished parts.

\section{Section 10. WROUGHT IRON}

2. 10. 1. Wrought iron.-Wrought iron shall conform to the requirements of the standard specifications for refined wrought-iron bars, designation A41, of the American Society for Testing Materials. (See 603.1, p. 139.)

\section{Section 11. STEEL CASTINGS}

2. 11. 1. General.-Steel castings shall conform to the requirements of the standard specifications for steel castings, designation A27, of the American Society for Testing Materials. (See 611.41, p. 707). supplemented by the following: Unless otherwise specified, all castings shall be class B, medium grade. 
2. 11. 2. Annealing.-All steel castings shall be thoroughly annealed unless otherwise provided.

2. 11.3. Structural defects. - Steel castings shall be true to pattern in form and dimensions, free from pouring faults, sponginess, cracks, blowholes and other defects in positions affecting their strength and value for the service intended.

Blowholes appearing upon finished castings shall be so located that a straight line laid in any direction will not cut a total length of cavity greater than 1 inch in any 1 foot, nor shall any single blowhole exceed 1 inch in any dimension or have an area greater than one-half square inch. Blowholes shall not have a depth injuriously affecting the strength of the casting. Minor defects which do not impair the strength may, with the approval of the engineer, be welded by an approved process. The defects shall be removed to solid metal by chipping, drilling, or other satisfactory methods and, after welding, the castings shall be annealed, if required by the engineer. Castings which have been welded without the engineer's permission shall be rejected.

Large castings, if required by the engineer, shall be suspended and hammered all over. No cracks flaws, or other defects shall appear after such treatment.

No sharp unfilleted angles or corners will be allowed.

\section{Section 12. GRAY-IRON CASTINGS}

2. 12. 1. General.-Iron castings shall conform to the requirements of the standard specifications for gray-iron castings, designation A48 of the American Society for Testing Materials. (See 611.11, p. 636.) Castings shall be boldly filleted at angles and the arrises shall be sharp and perfect.

2. 12. 2. Structural defects.-Iron castings shall be true to pattern in form and dimensions, free from pouring faults, sponginess, cracks, blowholes, and other defects in positions affecting their strength and value for the service intended.

\section{Section 13. MALLEABLE CASTINGS}

2. 13. 1. General.-Malleable castings shall conform to the requirements of the standard specifications for malleable castings, designation A47, of the American Society for Testing Materials. (See 611.21, p. 665.) The castings shall be boldly filleted at angles and the arrises shall be sharp and perfect.

The surfaces shall have a workmanlike finish.

2. 13. 2. Structural defects.-(Identical with grayiron castings, given above.)

\section{Section 14 PHOSP}

2. 14. 1. General.-Phosphor-bronze shall conform to the requirements of the standard specifications for bronze bearing metals for turntables and movable railroad bridges, designation $\mathrm{B} 22$, of the American Society for Testing Materials. (See 692.2 , p. 1324.) Grade B metal shall be used.

2. 14. 2. Structural defects.-Bronze castings shall be free from inclusions of foreign material, casting faults, injurious blowholes, or other defects rendering them unsuitable for the service intended.

(Details of structural steel design and erection are included in these specifications.)

\section{AMERICAN RAILWAY ENGINEERING ASSO- CIATION, SPECIIICATIONS FOR MOVA- BLE RAILWAY BRIDGES, 1929}

\section{MOVABLE RAILWAY BRIDGES}

(Materials required for various parts, conditions specified for computation of stresses and machinery power requirements, proportioning of parts and machinery power, friction coefficients, allowable unit stresses, bearing stresses, permissible loads and strength of wire rope, general design requirements for rail end connections, rim girders, counterweights, bearings, shafts, gears, and other bridge parts. Design and type requirements for steam-power equipment, gasoline-engine equipment, electrical power and controllers. Specifications for cast steel, forged steel, tool steel, bronzes, Babbitt metal, workmanship, and erection.)

\section{AMERICAN RAILWAY ENGINEERING ASSO- CIATION, SPECIFICATIONS FOR STEEL RAILWAY BRIDGES, 1929}

STEEL RAILWAY BRIDGES

(See 605.11, p. 249.)

\section{AMERICAN RAILWAY ENGINEERING ASSO- CIATION, STEEL RAILWAY TURN- TABLES, 1924}

\section{RAILWAY TURNTABLES}

(For balanced and 3-point support types; clearances, minimum live loads for design, allowable unit stresses and proportioning of parts, general features of design of center, end trucks, pit, and tracks.)

\section{AMERICAN RAILWAY ENGINEERING ASSO- CIATION, SPECIFICATIONS FOR STEEL HIGHWAY BRIDGES, 1929}

\section{STEEL HIGHWAY BRIDGES}

(Same as American Association of State Highway Officials specification, given above.) In scope these specifications are limited to the field of ordinary highway bridges and do not provide for unusual span lengths and types of construction, for which provision must be made by special supplemental specifications.

\section{AMERICAN SOCIETY OF CIVIL ENGINEERS, SPECIFICATIONS FOR DESIGN AND CON: STRUCTION OF STEEL HIGHWAY BRIDGE SUPERSTRUCTURE, 1924}

\section{HIGHWAY BRIDGES}

(Construction, loading and application of loads, allowable stresses, details of design, tension and bend test requirements for structural steel, permissible variations in weight and dimensions, test requirements for eyebars. Specifications for structural nickel steel to conform to those of the American Society for Testing Materials, A8-29. See 621.33, p. 1126.)

\section{AIERICAN SOCIETY OF CIVIL ENGINEERS, SPECIFICATIONS FOR DESIGN AND CON- STRUCTION OF STEEL RAILWAY BRIDGE SUPERSTRUCTURE, 1923}

\section{RAILWAY BRIDGES}

(Types of loading and other items requiring consideration in the design, allowable stresses in various materials and parts, design requirements, construction, eyebar tests, tension and bend test requirements for the steel, permissible variations in weight and dimensions, data and curves for use in bridge design. The specifications for structural nickel steel to conform to those of the American Society for Testing Materials, A8-29. See 621.33, p. 1126.) 
605.22 STEEL BUILDINGS AND METAL PARTS OF BUILDINGS.

AMERICAN INSTITUTE OF STEEL CONSTRUCTION, STANDARD SPECIFICATION FOR FIREPROOFING STRUCTURAL STEEL BUILDINGS, OCTOBER 8, 1927

\section{FIRE TESTS OF BUILDING CONSTRUCTION AND MATERIALS}

(The test procedure for determining the resistance of buildings and building materials to exposure to fire is the same in this specification as in the A. S. T. M. tentative specification C19-26T for fire tests of building construction and materials. The A. I. S. C. specification provides an alternate method of defining the end point of the tests which is based on the maximum temperatures at which the structural steel is permitted to carry the stresses used in the design. The A. S. T. M. procedure has been approved by the American Standards Association as A2-1926. It prescribes a standard exposing fire of controlled extent and severity. Performance is defined as the period of resistance to standard exposure elapsing before the first critical point in behavior is observed. The alternate method of the A. I. S. C. prescribes a certain placing of thermocouples on the test specimen (column, girder, or beam) and expressing the fire resistance in the number of hours it takes the scandard fire to cause the average readings of these thermocouples to reach $1.000^{\circ} \mathrm{F}$.)

\section{SAFETY FACTOR IN FIREPROOFING BUILDINGS}

Steel buildings whose conditions of exterior exposure and whose contents under fire hazards will not produce a temperature greater than $800^{\circ} \mathrm{F}$. in the steel, shall be considered fire resistive without insulating protection for the steel.

If the steel work has an insulating protection, the safety factor shall be based on the fireproofing material providing protection for a greater period of time than the combustible contents of the building will burn as shown by tests of office occupancy and record storage. The safety factor for all skeleton frame and secondary members shall be one and onehalf. For example: If a building contains 10 pounds of combustible material per square foot of floor, and has a fire hazard of 1-hour duration, the steel work shall be protected against the temperature defined in this specification for one and one-half hours.

\section{ANERICAN INSTITUTE OF STEEL CON- STRUCTION, STANDARD SPECIFICA- TION FOR STRUCTURAL STEEL FOR BUILDINGS, 1923}

\section{STRUCTURAL STEEL FOR BUILDINGS}

(See 605.12.p. 252.)

\section{AMERICAN RAIIWAY ENGINEERING AS- SOCIATION, 1930 SUPPIEMENT TO MANUAL OF 1929, SPECIFICATIONS FOR RAILWAY BUILDINGS, SECTION 12-B}

\section{ORNAMENTAL AND MISCELIANEOUS METAL WORK}

(Among other requirements covering permissible unit stresses in ornamental and miscellaneous metal work, standard A. S. T. IN. specifications for steel, wrought iron, and cast iron, requirements on casiings, construction, and installation of pipe railings, fabric fences, wrought-iron fences, live loads to be used in design of stairs, balconies, ladders, and fire escapes, design and installation requirements for flagpoles, window guards, elevator inclosures, etc., are the following requirements for metal and metalclad doors.)

\section{METAL DOORS AND FIRE DOORS}

Metal doors may be either swinging, rolling, or sliding, and of solid or hollow sections. Doors shall be so counter-weighted and balanced that they may easily be operated by one man. No metal shall be less than No. 22 U. S. standard gage, and if of steel shall be copper bearing. Doors shall be stiff enough to resist bending and warping and when of hollow section shall be absolutely water-tight to prevent internal corrosion. Fire doors shall meet all the requirements specified above for metal doors and shall meet the requirements of the National Board of Fire Underwriters for the particular use intended.

\section{METAI-CLAD DOORS AND FIRE DOORS}

Metal-clad doors shall consist of cores of not less than two ply of sound, kiln dried, nonresinous wood, covered with not less than 20-pound weight prime terneplate. The nonresinous wood may be either cedar, cypress, white pine, northern pine, redwood, eastern spruce, Sitka spruce, yellow poplar, or Douglas fir, but only one kind of wood may be used in any one door. Terneplate shall be I. C. base weight (107 pounds) or heavier, of an alloy of not less than 20 per cent tin and the balance lead, and the 20-pound weight shall be in accordance with the simplified practice recommendation of the U. S. Department of Commerce. R30-28. (See 604.31. p. 241.) All metal shall be made absolutely watertight to protect the wood cores. Each wood core shall be covered separately before doors are assembled. Metal-clad doors shall meet all of the requirements of the National Board of Fire Underwriters.

\section{IIETAL SASH}

Metal sash may be either solid or hollow section as indicated on drawings. No metal shall be less than No. 22 U. S. standard gage, and, if steel, shall be copper bearing.

\section{METAL-COVERED SASH}

(The requirements for metal-covered sash are the same as for metal-clad doors and fire doors given above.)

\section{AMERICAN RAILWAY ENGINEERING AS- SOCIATION, MANUAL, 1929, SPECIFI- CATIONS FOR BUILDINGS FOR RAIL- WAY PURPOSES}

\section{SHEET-METAL WORK}

\section{MATERIALS}

Sheet-metal work shall be copper, galvanized iron, lead, or rolled zinc as shown on the drawings, or as specifically called for in the contract.

Copper.-Copper sheets shall be rolled from copper conforming to the specifications of the A. S. T. M. and marked with the weight and manufacturers' name. Copper flashings, valleys, eave strips, and roof pans shall be 16-ounce soft rolled copper.

Copper rain conductors, eave troughs, molded and hanging gutters, and conductor heads shall be 16-ounce cold-drawn or hard copper. 
Copper cornices shall be 20-ounce cold-rolled or hard copper.

Galvanized iron.-Galvanized iron shall be 24 gage, of one of the following brands: - -

Lead.-Lead used for sheet-metal work shall be 6 -pound rolled sheet lead.

Zinc.-Zinc used for sheet-metal work shall be 11-gage rolled zinc of one of the following brands:

Solder-Solder shall be composed of one-half pig lead and one-half block tin (new metals) and shall conform to the specifications of the A. S. T. M., serial B32.

(The remainder of this specification dealing with sheet-metal work covers general requirements on installation, with limiting dimensions in some cases, for flashings, gutters, eave strips, and gravel stops, cornices, rain conductors, rain-conductor heads, ridge rolls and hip capping, ventilators, skylights.)

\section{AMERICAN RAILWAY ENGINEERING AS- SOCIATION, SPECIFICATIONS FOR BUILDINGS FOR RAILWAY PURPOSES, 1929}

STEEL FOR BUILDINGS

(See 605.12. p. 252.)

\section{ASSOCIATED FACTORY MUTUAL FIRE IN- SURANCE COMPANIES, TIN-CLAD FIRE DOORS AND SHUTTERS, OCTOBER 1, 1925}

\section{TIN-CLAD FIRE DOORS}

Cores must be constructed of white pine, cypress, or other soft wood. Stock must be dry and thoroughly seasoned, sound, and free from sap or loose knots. Boards must be tongued and grooved, dressed both sides to thirteen-sixteenths inch, not beaded, and not over 8 inches wide. The lengths of the boards must be such that not more than two pieces are used in any continuous strip on either exposed face, and at least alternate strips must be full length boards. Shorter lengths may be used in the middle section of 3-ply cores, but in no case shall there be more than three pieces in any of such middle layers.

Three-ply doors must have the outer layers vertical and the inner layer horizontal and 2-ply doors must have one layer vertical and one horizontal. The layers must be thoroughly fastened together by wrought-iron clinch nails not over 8 inches apart, extending clear through the door and clinched on the back side, leaving both surfaces of the door smooth. The edges and corners of the doors must be true and square.

The covering for doors and shutters, whether exposed to dampness or not, should be terneplate. Plates should be of basic open-hearth steel 14 inches by 20 inches and of I. C. thickness, weighing at least 103 pounds net per box of 112 sheets, exclusive of the coating. This should weigh 10 pounds per box additional, and should be an alloy of not less than 25 per cent tin and not more than 75 per cent lead.

The corners of the door should be covered first, using a whole sheet of tin without cutting and with a miter fold nailed under the fold. The edges should next be covered with either regular plates or long sheets. The faces should next be covered beginning at the lower right-hand corner. The top horizontal joint and all vertical ones must be double lock joints of a type illustrated. (Illustrations of the construction of doors and shutters are given in the full text of these specifications.) The remaining joints should be single lock. The finished lap in all cases must be full one-half inch. The folds at the corners and all joints possible must be made to shed water. The plates should be fastened to the core with 2 -inch wire nails barbed full length, five nails being used on the sides and four on the ends of each sheet, and driven close to the center of each joint. Finished seams must be well flattened, no nail heads must be exposed, and no solder must be used. When covering is complete cut a hole 3 inches in diameter through the middle plate on the exposed side of the door but not through the wood core. Secure the tin around this opening with small nails and thoroughly paint the wood thus exposed. (The hole will prevent bulging of the covering and rupture of the joints by permitting the escape of gases generated from the wood core when the door is exposed to fire.)

(The full text of this specification also includes requirements on types, sizes, and installation of hardware; fit and installation of doors; sizes of sills; etc.)

\section{TIN-CLAD FIRE SHUTTERS}

(Construction is the same as for 2-ply core fire doors given above.)

\section{NATIONAL ASSOCIATION SHEET-METAL CONTRACTORS, STANDARD PRACTICE IN SHEET-METAL WORK, 1929}

\section{SHEET-METAL WORK}

(This reference book of standards of practice in fabricating and erecting sheet-metal work for the building industry contains 768 pages, including 494 full-page illustrations. The contents are divided into 12 sections, as follows:

Roofing, gutters, conductors, flashing, and corrugated iron work.

Skylights and ventilators.

Metal cornices.

Metal ceilings.

Warm air furnaces.

Heating and ventilating.

Blowpipe and exhaust systems.

Fire doors, tin clad and kalamein.

Hollow metal doors and trim.

Hollow metal windows.

Restaurant, kitchen, and hotel equipment.

Protective coatings and paints.)

\section{NATIONAL BOARD OF FIRE UNDER- WRITERS, BUILDING CODE, 1931}

\section{BUILDING CODE}

(Covers classification of buildings, tests, and quality of materials, working stresses, cast iron and steel construction, fire doors and windows, fireproof construction, fire tests. See also 611.19, p. 664.)

\section{NATIONAL ELECTRICAL MANUFACTURERS ASSOCIATION, SWITCHGEAR STAND- ARDS, SG8-60. OUTDOOR SUBSTA- TIONS. PUBLICATION NO. 31-10, 1931}

\section{OUTDOOR SUBSTATIONS}

(Structural steel according to specification A9 of the American Society for Testing Materials. (See 605.12 , p. 252.) Requirements on strength of bolt and rivet steel, minimum thickness of metal in structure, minimum sizes of bolts and rivets, zinc 
coating of steel work, allowances for ice and wind loads, permissible stresses, general design of foundations, spacing of rivets and bolts, fabrication, current ratings for bare conductors.)

NATIONAL FIRE PROTECTION ASSOCIATION, SPECIFICATIONS FOR STANDARD INDUSTRIAL BUILDINGS, 1929

\section{INDUSTRIAL BUILDINGS}

Standard industrial buildings shall include all structures more than one story in height used for industrial purposes, such as factories, lofts and manufactories, mills, storage warehouses, workshops, etc.

Buildings of this class, in which the fire hazard is recognized to be from moderate to high, shall conform to the minimum specification requirements for reinforced concrete, slow-burning heavy timber, and protected steel construction.

(The requirements in this specification include permissible height and floor area, number of exits, materials and allowable stresses to be in accordance with the standard specifications quoted, requirements on construction and protection of roof and wall openings and shaft inclosures, on wall thicknesses, on fireproofing of structural members, floors and roofs, on electrical and fire equipment, etc.)

\section{RAILWAY FIRE PROTECTION ASSOCIA-} TION, HANDBOOK OF THE R. F. P. A., 1925

\section{GARAGES AND REPAIR SHOPS FOR RAIL MOTOR CARS}

Garage and repair shop construction should be in conformity with the National Building Code, of fireresistive construction throughout, with no cellar, pit, or depression below the first floor level unless properly ventilated to remove all gas accumulations therefrom. Repair shops should be separated from garage proper by standard fire walls. See report of National Fire Prevention Association committee on bus garages, which regulations should be followed in so far as they apply. Floors shall be of concrete or earth construction. Garage floors of other than earth construction shall drain to an oil separator or trap and if area is extensive a series of drains shall be provided.

(Other recommendations given in the full text of this standard include recommendations on types of lighting and heating equipment, ventilation including recommended capacity of a mechanical ventilafion system, removal of engine exhaust gases from repair shops, operation and maintenance of garages, storage and handling of gasoline and oil, recommended fire-fighting facilities.)

\section{STEEL JOIST INSTITUTE, STEEL JOIST CONSTRUCTION, 1932}

\section{STEEL JOISTS}

(This specification includes construction details for steel joists composed of rolled shapes, riveted or welded bars or rods, or expanded rolled shapes, as used between girders. Steel according to A. S. T. M. specification A9 (see 605.12 , p. 252). Requirements on methods of design and stresses, span, spacing, bridging, etc. Table of open-web steel joists is identical with that in Simplified Practice Recommendation R94-30 of the United States Department of Commerce. See 605.12, p. 253.)
UNDERWRITERS' LABORATORIES, STANDARD FOR TIN-CLAD FIRE DOORS AND SHUTTERS, JUNE, 1930

\section{TIN-CLAD FIRE DOORS AND SHUTTERS}

\section{GENERAT}

These are requirements for tin-clad fire doors and shutters entitled to classification as "standard" for one or more of the situations defined in the Regulations of the National Board of Fire Underwriters for the protection of openings in walls and partitions against fire, as follows:

Class A.-Openings in division walls between separate buildings or sections of buildings (that is, fire walls).

Class B.-Openings in inclosures to vertical communications through buildings (that is, vertical shafts).

Class C.-Openings in corridor and room partitions.

Class D.-Openings in exterior walls subject to severe fire exposure.

Class E.-Openings in exterior walls subject to moderate fire exposure.

Fire doors and shutters conforming to these specifications have been shown by tests at the laboratories, and by records of service, to be capable of furnishing the degree of fire protection entitling them to classification as standard for the following situations when properly mounted with standard hardware:

Three-ply doors for classes A, B, C, D, and E situations.

Two-ply doors for classes B, C, D, and E situations.

Two and three ply shutters for classes D and E situations.

Two and three ply doors and shutters provided with glass panels for classes $\mathrm{C}$ and $\mathrm{E}$ situations.

\section{IUMBER}

The following soft woods listed in Appendix A of the American Lumber Standards given in Simplified Practice Recommendation R16-29 of the United States Department of Commerce may be used, provided only one kind is used in the assembly of a single core: Cedars, all classes; cypress, all classes; Douglas fir; white pine; northern white pine; redwood; eastern spruce; Sitka spruce; and yellow poplar (tupelo).

Lumber used shall be well seasoned so that it has reached its normal moisture content, as specified by the Forest Products Laboratory for its kind. In all cases stocks of lumber shall be stored under cover in the premises of the fire-door manufacturer for at least one month before being used in the manufacture of fire-door cores, and while in storage shall be piled in such manner that the air has free access to all surfaces of each board. Kiln drying will be accepted for the 30-day air-drying period.

The lumber shall be graded according to the American Lumber Standards as No. 1 Common, or better, matched yard lumber, 1-inch standard boards, surfaced on two sides. This specifies boards free from sap, dry-rot, knot or other holes, loose knots, unsound knots, or knots exceeding $2 \frac{1}{2}$ inches in any dimension. Sound boards 10 feet in length shall not exceed in extent or damage three $2 \frac{1}{2} 2$-inch knots.

The matched tongued-and-grooved boards shall be surfaced on two sides as specified for yard lumber, American Lumber Standards, and shall not be beaded or beveled in addition. 
The tolerances in actual thickness for nominal 1-inch yard lumber surfaced on two sides shall be as specified in the American Lumber Standards, which provides a minimum thickness of board of twenty-five thirty-seconds of an inch.

Boards shall be at least 3 inches and not more than 8 inches in width, nominal width, according to American Lumber Standards.

Note.-The nominal width (or stock width) is usually slightly greater than the width over the tongue and groove.

\section{METAL COVERING}

Prime terneplate shall be used. (For the purpose of this specification "terne" shall be understood as indicating an alloy, for the coating, of tin and lead in the proportion of not less than about 20 per cent of tin.)

The plate shall be I. C., base weight 107 pounds or heavier, classified as being of a 20-pound weight as contemplated by Simplified Practice Recommendation No. 30 of the United States Department of Commerce, approved January 1, 1925.

Each "case" package of terneplate of 112 sheets 20 by 28 inches shall have a nominal net weight of not less than 226 pounds with a plus or minus tolerance of approximately 4 per cent.

Each "base box" package of terneplate of 112 sheets 14 by 20 inches shall have a nominal net weight of not less than 113 pounds with a plus or minus tolerance of approximately 4 per cent.

The plates shall have straight edges and square corners and shall be resquared by the plate manufacturer before shipment. (For the purpose of this specification "resquared" shall be understood as meaning that one long side is taken as a base edge without trimming and that the other three sides are trimmed true to it as a base. A deviation of one thirty-second of an inch per foot from square shall be accepted, provided the door manufacturer is able to obtain true, straight joints, and to avoid patching of the rows of sheets in the covering.

Each plate shall be identified as to the actual manufacturer by means of name or registered trademark and the words "Fire Door Standard," "I. C. 20 lbs." In addition, private brand names or marks may be shown if desired.

\section{NAILS}

Core nails shall be cut nails of the clinch type. For 3-ply cores the nails shall be not less than $27 \%$ inches or more than 3 inches long. For 2-ply cores the nails shall be not less than $17 / 8$ inches or more than 2 inches long. Nails for applying the metal covering shall be wire nails with flat heads, either full or quarter barbed. The shank of the nails shall be not less than 0.091 inch nor more than 0.109 inch in diameter. The nails for 3-ply cores shall be 2 inches long and for 2-ply cores shall be not less than $1 \frac{1}{4}$ inches nor more than $1 \frac{1}{2}$ inches long.

\section{CONSTRUCTION}

\section{Assembly of Boards}

Only one stock width of board shall be used in any one core. Boards shall be not less than 1 foot in length with ends cut square. Not more than two pieces shall be used in any continuous strip in any outside layer of a 2-ply or 3-ply core, nor more than three pieces in any middle-layer strip of a 3-ply core. At least alternate strips in outside layers shall be full-length boards.

When glass panels are provided and the panel opening is of such a size that the distance between the opening and the edges of the door is less than 2 feet, all boards bordering the vertical edges of the opening may be laid vertically and all boards border- ing the horizontal edges of the opening may be laid horizontally.

When glass panels are provided, the boards in the normally vertical layers bordering the sides of the panel opening shall be continuous from top to bottom of the door, and boards in the normally horizontal layer bordering the top or bottom of the panel opening shall be continuous from side to side of the door. The distance between the panel opening and the side of the door shall be not less than 7 inches.

Outside layers in a 3-ply core and one layer of a 2-ply core shall be vertical, and the other layer horizontal. The several boards in each layer and the ends of pieces of boards in strips shall make tight joints at edges and ends of boards. The top edge for sliding door or shutter designed to close by gravity shall conform to incline of three-fourths inch per foot. The maximum face width of the top horizontal board of a core having the top edge inclined shall be not less than 3 inches.

\section{Nailing of Cores}

The boards shall be nailed so that the several layers are fastened tightly together with the points of the nails turning back and clinching thoroughly in the face of the core and with no portion of the nails projecting beyond the surfaces of the core.

(The full text of this standard includes requirements on the location of rows of nails in the indivicual boards and the spacing of nails in the nailing of 2-ply and 3-ply cores for various widths of boards used. Illustrations are also given showing nailing details.)

\section{Finished Core}

The finished core shall be not less than $2^{11 / 32}$ inches nor more than $25 / 8$ inches in thickness for a 3 -ply core and not less than $1 \% 16$ inches nor more than $13 / 4$ inches in thickness for a 2-ply core. The cores shall have true corners. All edges shall be finished smooth and square excepting that meeting edges of doors when mounted in pairs may be beveled one quarter inch (not rabbeted).

\section{Size of Plates}

Face plates shall be not larger than the 14 by 20 inch size. Corner plates shall be not over 14 inches wide and of any length that will avoid joints with edge plates coming under miter fold. Edge plates (excepting cap plates) shall be of the same width as corner plates and of any convenient length. Cap plates shall be of any convenient length and equal in width to thickness of core plus $3 \frac{1}{2}$ inches.

\section{Forming of Plates}

Face plates, excepting the face plates used in the row forming the closure, shall have one vertical edge turned five-eighths inch and the other vertical edge doubled under $13 / 16$ inches and the doubled edge then turned up five-eighths inch from cut edge.

Face plates, excepting face plates forming top horizontal seams, shall have both horizontal edges turned five-eighths inch to lock with edge and other face plates.

Face plates forming top horizontal seams, excepting seams formed with a cap, shall have the lower horizontal edge turned five-eighths inch to lock with other face plates, and the other horizontal edge doubled under $13 / 16$ inches and the doubled edge then turned up five-eighths inch from cut edge.

Face plates forming top horizontal seams with a cap shall have both horizontal edges turned up fiveeighths inch to lock with cap and other face plates. Corner plates shall have all edges turned five-eighths inch so as to lock with edge and face plates. Edge plates, excepting cap plates, shall have all edges 
turned five-eighths inch so as to lock with corner, face, and other edge plates. Cap plates shall have edges forming seams with other cap plates turned five-eighths inch. Cap plates shall have edges forming top horizontal seams with face and edge plates doubled under $13 / 16$ inches and the portion next to the cut edge then turned down five-eighths inch so

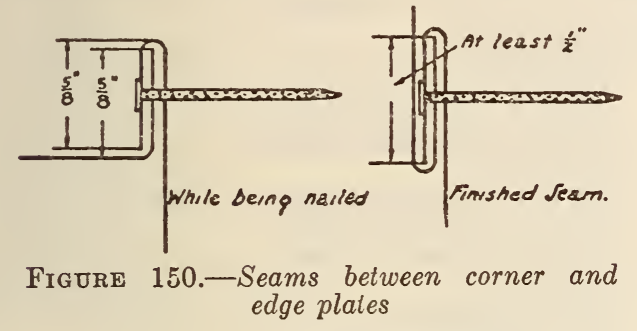

as to lap the edge and face plates as shown in Figure 153.

$$
\text { Application of Plates }
$$

The plates shall fit the core as flatly and tightly as practicable. The plates shall be locked together not less than one-half inch. Both faces of the core shall be covered with plates laid with their longer sides vertical, except that the plates in one vertical row on each face of the core may be laid horizontally.
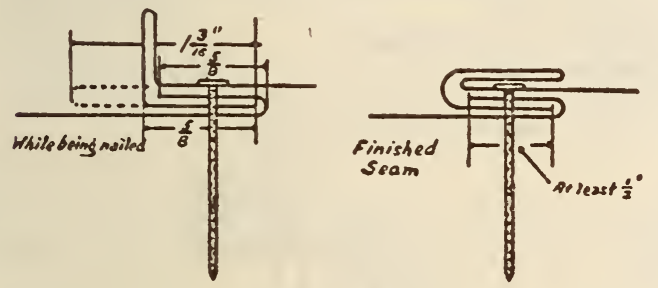

Frgure 151.-Vertical seams of face plates (top view)

Vertical seams formed with face plates to be hook seams with the upper plate having a fold for covering the heads of the nails in the seam as shown in Figure 151. Horizontal seams formed with face plates, excepting top horizontal seams, shall be hook seams as shown in Figure 152. Top horizontal seams, excepting seams formed with a cap, shall have a fold for covering the heads of the nails in the seam as shown in Figure 151. Top horizontal seams formed with a cap shall be lock seams with the locking por-

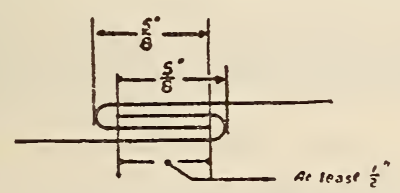

Figure 152. - Horizontal seams of face plates (side view)

tion of the cap having a fold for covering the heads of the nails in the seam as shown in Figure 153 . The upper ends of vertical seams shall be covered by the doubled edges of the top horizontal seams.

Each bottom corner of the core shall be covered with a plate bent over the edges of the core and lapped an equal distance on both faces of the core, making a miter fold (without cutting) on each face, the folds on a door or shutter for use at an opening in an exterior wall being arranged to shed water.
Each upper corner shall be covered the same as the bottom corners if a cap is not used for covering the top edge of the core. The bottom edge and the vertical edges of the core shall be covered with plates bent over the edges of the core and lapped an equal distance on both faces. The plates shall be

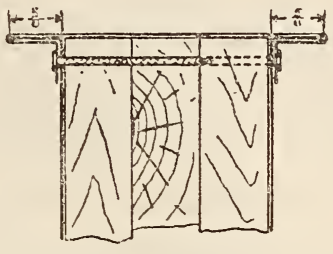

While being nailed

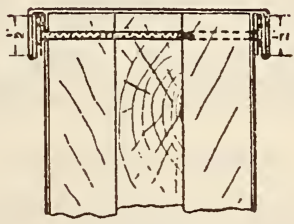

Finished Scam

Figure 153. - Applica-

tion of cap plates at top edge of core

joined to each other and to the corners with hook seams, the seams being made so as to shed water when the door or shutter is for use at an opening in an exterior wall. The top edge of the core shall be covered with a cap when the door or shutter is for use at an exterior wall or when the door or shutter has a segmental head. The cap shall be formed of

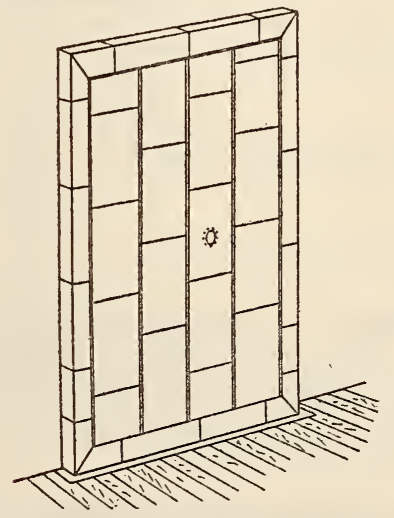

\section{FrgURE 154.-Completed door or shuiter showing vent hole}

plates joined to each other with hook seams as shown in Figure 150.

When glass panels are provided and band or angle iron reinforcement for glass grooves is used, the vertical edges of the panel opening shall be covered with terne plate secured to the face plates by vertical seams. The covering at the horizontal edges of the opening shall be cap seams. 
Nailing of Plates

(This standard includes requirements on the location of nails in the seams, the number of nails on each side of face plate, etc.)

\section{Glass Sizes}

Individual lights in doors bearing "Corridor and room partition" labels shall not exceed 1,296 square inches in exposed area or 54 inches in either dimension. Individual lights in doors bearing "Fire escape" labels shall not exceed 720 square inches in exposed area, 54 inches in height, or 48 inches in width.

Splicing of Doors Made in Sections

(The full text of this standard includes structural requirements on the splicing of doors made in sections, including thickness requirements for the splice sheets and sizes of angles or channels for 3ply and 2-ply doors.)

UNITED STATES GOVERNMENT, DEPARTMENT OF COMMERCE, BUREAU OF STANDARDS, SIMPLIFIED PRACTICE RECOMMENDATION FOR SOLID-SECTION STEEL WINDOWS, R72-32, 1932

\section{STEEL WINDOWS}

(This simplified practice recommendation as accepted by industry establishes a standard simplified nomenclature, standard symbol designations, and a limited list special sizes and types for piroted windows, pro-

\section{Section 1. SIZES OF DOORS}

Designated as jamb-opening sizes. All sizes are measured between rabbets in side jambs and from rabbet in head jamb to finished floor line. (See fig. 155.)

All allowance for clearance and saddles shall be taken off the door.

For single doors:

\section{Semistock}

2 by 7 feet.

2 feet 3 inches by 7 feet.

2 feet 6 inches by 7 feet.

2 feet 9 inches by 7 feet.

3 by 7 feet.

3 feet 3 inches by 7 feet.

3 feet 6 inches by 7 feet.

3 feet 9 inches by 7 feet.

4 by 7 feet.

For resultant pairs:

4 by 7 feet.

4 feet 6 inches by 7 feet.

5 by 7 feet.

5 feet 6 inches by 7 feet.

6 by 7 feet.

6 feet 6 inches by 7 feet.

7 by 7 feet.

7 feet 6 inches by 7 feet.

8 by 7 feet.

\section{Section 2. SEMISTOCX TYPES OF DOORS}

1 panel, solid.

1 panel, open for glass.

2 panels, solid.

2 panels, upper open for 1 light.

2 panels, upper open for 4 lights.
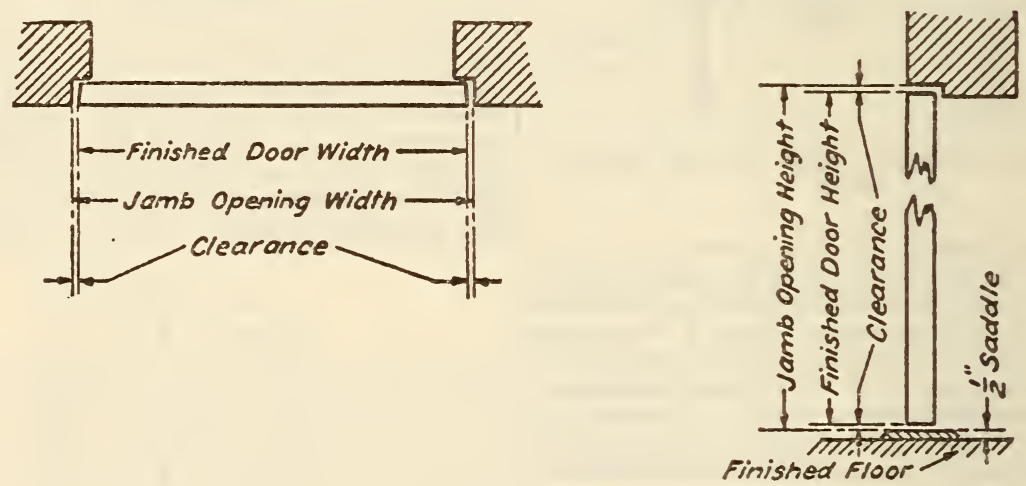

FIGURE 155

jected windows both commercial and architectural, industrial doors, underwriters' labeled windows, continuous windows, and counterbalanced windows. This recommendation as published by the Bureau of Standards includes about 22 plates illustrating these standard sizes and types.)

UNITED STATES GOVERNMENT, DEPARTMENT OF COMMERCE, BUREAU OF STANDARDS, SIMPLIFIED PRACTICE RECOMMENDATION FOR HOLLOW. METAL SINGLE-ACTING SWING DOORS, FRAMES, AND TRIM, R82-28, AUGUST 1, 1928

\section{HOLLOW-METAL DOORS, FRAMES, AND TRIM}

(Simplified practice recommended and accepted by industry establishing a limited list of standard stock sizes and types.)
Semistock sizes of doors, though not carried in stock, are made up from parts which are carried in stock.

\section{Section 3. DOOR CLEARANCES}

$3 / 32$ inch betreen hinge stile and jamb.

$3 / 32$ inch between lock stile and jamb.

$1 / 8$ inch between meeting stiles of doors hung in pairs. $1 / 16$ inch between top rail and head jamb.

$3 / 16$ inch between bottom rail and saddle, assuming use of $1 / 2$-inch saddle.

Note.-All clearances are taken off the door, not from jambopening size. Thus the 3 by 7 foot door actually measures 2 feet $11^{13} / 16$ inches by 6 feet $11 / 4$ inches.

\section{Section 4. DOOR DIMENSIONS}

No. 18 U. S. standard gage ( 0.05 inch) steel for stiles and rails.

No. 20 U. S. standard gage $(0.0375$ inch) steel minimum for panels. 
No. 18 U. S. standard gage $(0.05$ inch) steel minimum for panels over 60 inches in height if over $2 S$ inches wide.

$1{ }^{3}{ }_{4}^{\prime}$-inch finished door thickness.

5 -inch face width of stiles and top rail.

7 -inch face width of lock rail.

$95 / 16$-inch face width of bottom rail.

10 inches height of top edge of bottom rail above finished floor line.

42 inches height of top edge of lock rail, if any, above finished floor line.

3/8-inch bevel in 2 inches on lock stile.

$1 / 8$-inch bevel in 2 inches on hinge stile and top rail, if bevel is used.

$3 / 8$-inch allowance for glass, where used (width of groove).

NoTE.-Width of stiles and rails is exclusire of panel molding and is measured on hinge side of door.

\section{Section 5. BUTT MORTISE DETAILS}

5 inches distance from jamb rabbet to top edge of top butt.

10 inches distance from finished floor line to bottom edge of bottom butt.

$\mathrm{X}$ center butt, if any, to be evenly spaced between other two butts.

$3 \prime 4$ inch distance from face edge of door to edge of butt mortise.

$5 / 16$ inch distance from stop to edge of butt mortise on frame (thus providing $1 / 16$-inch clearance between door and stop).

$\mathrm{X}$ height and depth of individual hinge mortises and the number of mortises shall be governed by the following table:

\section{Template butts}

\begin{tabular}{|c|c|c|c|c|c|}
\hline Width (inches) & Height (inches) & $\begin{array}{l}\text { Num- } \\
\text { ber of } \\
\text { butts }\end{array}$ & Size & $\begin{array}{l}\text { Weight of } \\
\text { butts }\end{array}$ & $\begin{array}{l}\text { Tem- } \\
\text { plate } \\
\text { letter }\end{array}$ \\
\hline To $32 .$. & $\left\{\begin{array}{l}\text { To } 60 \\
\text { Over } 60 \text { to } 90 \\
\text { Over } 90 \text { to } 120 .\end{array}\right.$ & $\begin{array}{l}2 \\
3 \\
4\end{array}$ & $\begin{array}{r}\text { Inches } \\
41 / 2 \\
412 \\
41 / 2\end{array}$ & \begin{tabular}{|l} 
Regular \\
- \\
\end{tabular} & $\begin{array}{l}\mathrm{A} \\
\mathrm{A} \\
\mathrm{A}\end{array}$ \\
\hline Over 32 to $37 \ldots$ & $\left\{\begin{array}{l}\text { To } 60 \\
\text { Over } 60 \text { to } 90 \\
\text { Over } 90 \text { to } 120 .\end{array}\right.$ & $\begin{array}{l}2 \\
3 \\
4 \\
\end{array}$ & $\begin{array}{l}5 \\
5 \\
5\end{array}$ & $\begin{array}{l}\text { [.... do } \\
\\
\end{array}$ & $\begin{array}{l}\mathrm{A} \\
\mathrm{A} \\
\mathrm{A}\end{array}$ \\
\hline Over 37 to 43 & $\left\{\begin{array}{l}\text { To } 60 \\
\text { Over } 60 \text { to } 90\end{array}\right.$ & $\begin{array}{l}2 \\
3\end{array}$ & $\begin{array}{l}5 \\
5\end{array}$ & Extra heavy & $\begin{array}{l}\mathrm{B} \\
\mathrm{B}\end{array}$ \\
\hline $\begin{array}{l}\text { Over } 43 \text { to } 50 \ldots \\
\text { Over } 37 \text { to } 50 \ldots\end{array}$ & Over 90 to $120=$ & $\begin{array}{l}3 \\
4\end{array}$ & $\begin{array}{l}6 \\
6\end{array}$ & -....do... & $\underset{\mathrm{B}}{\mathrm{B}}$ \\
\hline
\end{tabular}

Nots.-The above table is based on underwriters' requirement that 2 butts shall be used for doors measuring 5 feet or less in height and that doors of a greater height shall require 1 butt for each $21 / 2$ feet or fraction thereof of height. Sizes given joint. Template letters refer to templates recommended as standard by the Advisory Committee on Standardization of Hardware for by the Advisory Committee on Stan
hollow-metal doors, December 1, 1923.

(For further details see United States Government, Department of Commerce, Bureau of Standards, Commercial Standard CS9-29. See 617.12, p. 982.)

\section{Section 6. IOCK MORTISE DETAIIS}

No. 16 U. S. standard gage $(-0.0625$ inch $)$ steel, minimum for box placed within lock stile as reinforcement for lock and escutcheons.

$391 / 2$ inches height of center line of lock mortise above finished floor line, or $38^{13} / 16$ inches height of center line of lock mortise above bottom edge of door.

8 inches $(+0.015-0.000)$ height of mortise for lock front.

$11^{\prime}$ inches $(+0.015-0.000)$ width of mortise for lock front.

$7 / 32$ inch depth of mortise for lock front.
$6 y_{4}^{\prime}$ inches height of mortise for lock case (centered on height of mortise for lock front).

$11^{\prime}$ inches width of mortise for lock case (coincident with width of mortise for lock front).

$43^{\prime}$ inches minimum depth of mortise for lock case (clearance for lock inside stile).

$31 / 2$ inches distance from center of beveled door edge to beginning of contact between lock case and spring-leaf reinforcement for rear edge of case.

$43 / 8$ inches distance from center of beveled door edge to termination of contact between lock case and spring-leaf reinforcement for rear edge of case.

$3 / 4$ inch normal distance between contact planes of the two spring-leaf reinforcements for rear edge of lock case.

$1 / 8$ inch maximum distance which each spring reinforcement may be required to be sprung by insertion of lock case.

1 inch approximate width of bearing planes of spring reinforcements.

4 inches height of center line of spring reinforcements above bottom edge of lock front (or coincident with center line of lock.)

$3 / 8$ inch $(+0.005-0.005)$ distance from center of lock front mounting screw holes to top or bottom edge of lock front.

$5 / 8$ inch $(+0.005-0.005)$ distance from center of lock front mounting screw holes to vertical edge of lock front.

No. 12-24 F. H. machine screw for mounting lock front (drill and tap accordingly).

$1{ }^{\prime}$ inch distance from either vertical edge of lock front mortise to face edge of door (thus centering lock front on beveled edge of lock stile).

$23^{\prime}$ inches backset for knobs, cylinders, thumb pieces, etc.

\section{Section 7. STRIKE MORTISE DETAILS}

$391 \frac{1}{2}$ inches height of center line of strike mortise above finished floor line (thus giving strike and lock front a common horizontal center line).

$53^{3 \prime}$ inches $(+0.015-0.000)$ height of mortise for body of strike.

$13^{\prime}$ inches $(+0.015-0.000)$ width of mortise for body of strike.

$3 y_{4}^{\prime}$ inches height of mortise for strike lip (centered on height of mortise for body of strike)

$\mathrm{X}$ mortise for strike lip to provide for carrying lip beyond edge of jamb.

$5 / 32$ inch depth of mortise for flat universal strike.

$3 / 8$ inch $(+0.005-0.005)$ distance from center of strike mounting screw holes to top or bottom edge of strike.

$5 / 8$ inch $(+0.005-0.005)$ distance from center of strike mounting screw holes to vertical edge of body of strike.

No. 12-24 F. H. machine screw for mounting strike (drill and tap accordingly).

${ }^{1} 4$ inch distance from stop to edge of mortise for strike (thus making vertical center line of body of strike coincident with vertical center line of lock front).

NOTE.-Dimensions of strike box to be determined later.

NoTE.-Details for lock mortise and strike mortise will admit NoTE.-Details for lock mortise and strike mortise will admit standard cylinder lock for hollow-metal doors and the universal
strikes approved by the Manufacturers' Advisory Committee on Standardization of Builders' Hardware, CS1007b, April, 1928. (See United States Government, Department of Commerce, Bureau of Standards, Commercial Standard CS9-29, for builders' template hardware. See 617.21 , p. 1020.)

\section{Section 8. TRANSOM DIMENSIONS}

No. 18 U. S. standard gage (0.05 inch) steel, minimum for all stiles and rails.

2 inches face width of all stiles and rails, exclusive of panel molding.

$3 / 32$ inch clearance all around.

$3 / 8$ inch allowance for glass (width of groove). 
2 inches face height of transom bars between rabbets.

$13 \%$ inches thickness of transom.

3 inches from rabbet in side jamb to edge of butt, where butts are used.

NotE.-Transom sizes are measured between rabbets with clearances taken off transom.

Note.-Where butts are used, both leaves are mortised into sash. Size and number of mortises are to be in accordance with the following table.

\begin{tabular}{|c|c|c|c|c|c|}
\hline Width (inches) & Height (inches) & $\mid \begin{array}{l}\text { Num- } \\
\text { ber of } \\
\text { butts }\end{array}$ & Size & $\begin{array}{l}\text { Weight of } \\
\text { butts }\end{array}$ & $\begin{array}{l}\text { Tem } \\
\text { plate } \\
\text { letter }\end{array}$ \\
\hline $\begin{array}{l}\text { To } 48 \\
\text { Over } 48 \text { to } 72 \\
\text { To } 48 \\
\text { Over } 48 \text { to } 72\end{array}$ & $\begin{array}{l}\text { To } 20 \\
\text { Over } 20 \text { to } 36\end{array}$ & $\begin{array}{l}2 \\
3 \\
2 \\
3\end{array}$ & $\begin{array}{c}\text { Inches } \\
3 \\
3 \\
31 / 2 \\
31 / 2\end{array}$ & $\begin{array}{l}\text { Regular... } \\
\\
\end{array}$ & $\begin{array}{l}\text { A } \\
\text { A } \\
\text { A } \\
\text { A }\end{array}$ \\
\hline
\end{tabular}

Section 9. ROUGH BUCK DIMENSIONS

No. 16 U. S. standard gage (0.0625 inch) steel, minimum for rough bucks.

1 inch height of clear opening in excess of jambopening height.

2 inches clear opening width in excess of jamb opening ( 1 inch on a side).

$41 \frac{1}{2}$ inches Standard finished wall thicknesses $7 \frac{1}{2}$ inches $\}$ (bucks cover full thickness of wall).

2 inches width of flanges.

24 inches $\mathrm{O}$. C. approximate spacing for perforated corrugated, or wire anchors.

\section{Section 10. CABINET JAMB DIMENSIONS}

No. 18 U. S. standard gage (0.05 inch) steel, minimum for cabinet jambs.

No. 16 U. S. standard gage $(0.0625 \mathrm{inch})$ steel, minimum for cabinet jambs over $7 \frac{1}{2}$ inches in width.

$1 \frac{15}{16}$ inch door rabbet in jamb.

$5 \%$ inch height of stop.

2 inches width of flanges.

\section{Section 11. CASING DIMENSIONS}

4 inches maximum face width.

$11 / 8$ inches maximum projection.

$3 / 8$ inch setback for trim.

\section{Section 12. COMBINATION FRAME DIMENSIONS}

No. 16 U. S. standard gage (0.0625 inch) steel, minimum for combination frame.

$1 \frac{15 / 16}{1}$ inch door rabbet in jamb.

$5 / 8$ inch height of stop.

24 inches O. C. approximate spacing for perforated, corrugated, or wire anchors.

\section{Section 18. COMBINATION FRAME TYPES}

(a) With 21/2-inch flange finishing flush with plaster and forming flush trim on both sides of wall.

(b) With molded flanges, the molding being formed from the stock of the flange.

(c) With molded flanges planted on, becoming an integral part of the flange.

(d) With standard casings.

\section{UNITED STATES GOVERNMENT, DEPART- MENT OF COMMERCE, BUREAU OF STANDARDS, SIMPLIFIED PRACTICE RECOMMENDATION FOR KALAMEIN SINGLE-ACTING SWING DOORS, FRAMES, AND TRIM, R83-28, APRIL 1, 1928}

\section{KALAMEIN DOORS, FRAMES, AND TRIM}

(Simplified practice recommended and accepted by industry establishing a limited list of standard stock sizes.)
Section 1. SIZES OF DOORS

Designated as jamb-opening sizes.

All sizes as given shall be considered the size between the jambs in the rabbet and from the jamb head in rabbet to the finished floor.

All allowance for clearance and saddles shall be taken off door.

Stock Door Sizes

2 feet 6 inches by 7 feet.

2 feet 9 inches by 7 feet.

3 by 7 feet.

3 feet 6 inches by 7 feet.

\section{Semistock Door Sizes}

For single doors:

2 by 7 feet.

2 feet 3 inches by 7 feet.

3 feet 3 inches by 7 feet.

3 feet 9 inches by 7 feet.

4 by 7 feet.

For resulting pairs:

4 by 7 feet.

4 feet 6 inches by 7 feet.

6 feet 6 inches by 7 feet.

7 feet 6 inches by 7 feet.

8 by 7 feet.

Semistock door sizes, though not carried in stock, are made up from parts which are carried in stock.

Section 2. STOCK TYPES OF DOORS

1 solid panel.

2 solid panels.

2 panels upper open for glass.

1 panel open for glass.

NotE.-Two-panel doors, upper open for four lights of glass, are considered as semistock.

\section{Section 3. DOOR CLEARANCES}

$3 / 32$ inch between hinge stile and jamb.

$3 / 32$ inch between lock stile and jamb.

$1 / 8$ inch between meeting stiles of doors hung in pairs.

$1 / 16$ inch between top rail and head jamb.

$3 / 16$ inch between bottom rail and saddle.

$1 / 2$ inch saddle.

NoтE.-All clearances are taken off the door, not from jamb. opening sizes. Thus the stock 3 foot by 7 foot door actually measures 2 feet $1113 / 10$ inches by 6 feet $111 / 4$ inches.

\section{Section 4. DOOR DIMENSIONS}

$13 \%$-inch finished door thickness.

5 -inch face width of stiles and top rail, exclusive of paneled molding.

7-inch face width of lock rail, exclusive of paneled molding.

$95 / 16$-inch face width of bottom rail, exclusive of paneled molding.

10 -inch height of top edge of bottom rail from finished floor line.

42 -inch height of top edge of lock rail, if any, from finished floor line.

1/8-inch bevel in 2 inches on both hinge and lock stile. NoTE.-Top of top rail and bottom of bottom rail shall be square.

\section{Section 5. STANDARD JAM DETAILS}

Standard door rabbet, $15 / 16$ inches.

Standard height to stop, $5 / 8$ inch for steel bucks.

Standard height of stop, $1 / 2$ inch for metal covered jambs.

Note.-Hinge stile of door when hung will have 1/16-inch clearance between door and jamb stop.

\section{Section 6. GAGE OF METAL}

26 Gage U. S. Standard (0.01875 inch) for commercial doors. 
Section 7. BUTT MORTISE DETAILS

5 inches distance from jamb rabbet to top edge of top butt.

10 inches distance from finished floor line to bottom edge of bottom butt.

$\mathrm{X}$ center butt, if any, to be evenly spaced between other two butts.

For butts 5 inches and larger used with $1^{15} / 16$-inch rabbet and $1{ }^{3}{ }_{4}^{\prime}$-inch doors the distance between stop and rear edge of butt is $5 / 16$-inch on jamb, and from the rear edge of butt to edge of door $1 y_{4}^{\prime}$ inch.

When 4 -inch butts are used with $115 / 16$-inch rabbet, these distances will be $1 / 2$ inch on jamb and $7 / 16$ inch on door.

For spring hinges used with $1^{15} / 16$-inch rabbet these sizes will be:

For 6 -inch single-acting hinge, $9 / 10$ inch on jamb, 162 inch on door.

For 7 -inch single-acting hinge, $7 / 16$ inch on jamb, $3 / 8$ inch on door.

For S-inch single-acting hinge, $5 / 16$ inch on jamb, $1 / 4$ inch on door.

For 10 -inch single-acting hinge, $5 / 16$ inch on jamb, $1^{\prime} y^{\prime}$ inch on door.

\subsection{STEEL TANKS, TOWERS, AND FLUMES.}

AMERICAN PETROLEUM INSTITUTE, SPECIFICATIONS FOR STANDARD RIGS AND DERRICKS, STANDARD NO. 4 (FIFTH EDITION), JULY, 1931

\section{STEEL DERRICKS}

\section{Section 1. MATERIAL COVERED}

1. (a) These specifications cover the major dimensions of steel and wood derricks, including heights, bases, and water-table openings; also of standard rig parts, including Samson posts, walking beams, bull wheels, calf wheels, and band wheels. They cover also the safe working and wind load capacities of derricks and the working capacities of Samson posts and walking beams, together with the methods of computing these capacities.

(b) The standards contained herein are primarily dimensional, but they include also the minimum physical properties of material in so far as the strength computations require the use of unit stress figures, and apply to new material.

\section{Section 2. WORKMANSHIP AND FINISH}

2. (a) Machined surfaces.-All machined surfaces shall be finished in a good and workmanlike manner and shall be free from burrs, rough edges, etc.

(b) Castings. - All castings shall be thoroughly cleaned and all cored holes shall be drifted to insure free passage of proper size bolts.

(c) Protective covering.-All forged or rolled steel parts or sections, and all cast material shall be covered before shipment, with a good grade of commercial paint (or shall be galvanized), except such surfaces as are machined for bearing service, which latter shall be protected with a suitable lubricant or compound.

\section{Section 8. STEEL DERRICK DIMENSIONS}

\section{Definitions of Measurements}

3. (a) Derrick heights.-The height dimension "A" shall be measured along the neutral axis of the derrick leg from the top of the derrick floor joists to the bottom of the water-table beams or bumpers, with a tolerance of plus 6 inches, minus zero.

(b) Derrick bases.-Shall be square, and base square dimensions " $\mathrm{B}$ " shall be the measurement between the neutral axis of adjacent legs measured at the top of the derrick floor joists, with a tolerance of $41 / 2$ inches plus or minus. Base dimension " $\mathrm{B}$ " is a fixed dimension which permits interchangeability of several-height derricks of this group on common-sized bases.

(c) Water-table opening.-Shall be square, and water-table opening dimension " $D$ " shall be the inside or in-the-clear measurement of the opening in the water table, with a tolerance of 2 inches plus or minus.

\section{Steel Derrick Sizes}

(d) Derrick heights, bases, and water-table openings.-The standard sizes of A. P. I. steel derricks shall be as specified in Table 1 . The eight sizes may be referred to by numbers, and each size shall have only the particular combination of height "A," base square "B" and water-table opening "D" as given.

TABLE 1.-A. P. I. steel-derrick standard sizes

\begin{tabular}{|c|c|c|c|c|c|c|}
\hline \multirow{2}{*}{$\begin{array}{l}\text { Size } \\
\text { No. }\end{array}$} & \multicolumn{2}{|c|}{ Heights } & \multicolumn{2}{|c|}{ Base squares } & \multicolumn{2}{|c|}{$\begin{array}{l}\text { Water-table } \\
\text { opening }\end{array}$} \\
\hline & Nominal & $\begin{array}{l}\text { Toler- } \\
\text { ance }\end{array}$ & $\begin{array}{l}\text { Nomi- } \\
\text { nal "B" }\end{array}$ & $\begin{array}{l}\text { Toler- } \\
\text { ance }\end{array}$ & $\begin{array}{l}\text { Nomi-; } \\
\text { nal "D" }\end{array}$ & $\begin{array}{l}\text { Toler- } \\
\text { ance }\end{array}$ \\
\hline $\begin{array}{c}8 \\
9 \\
10 \\
11 \mathrm{~A}\end{array}$ & $\begin{array}{rr}\text { Ft. } & \text { in. } \\
66 & 0 \\
73 & 0 \\
80 & 0 \\
87 & 0\end{array}$ & $\begin{array}{l}\text { Inches } \\
+6-0 \\
+6-0 \\
+6-0 \\
+6-0\end{array}$ & $\begin{array}{cc}\text { Ft. } & \text { in. } \\
20 & 0 \\
20 & 0 \\
20 & 0 \\
20 & 0\end{array}$ & $\begin{array}{c}\text { Inches } \\
\pm 41 / 2 \\
\pm 41 / 2 \\
\pm 41 / 2 \\
\pm 41 / 2\end{array}$ & $\begin{array}{cc}F t . & \text { in. } \\
4 & 4 \\
4 & 4 \\
5 & 6 \\
5 & 6\end{array}$ & $\begin{array}{c}\text { Inches } \\
\quad \pm 2 \\
\pm 2 \\
\pm 2 \\
\pm 2\end{array}$ \\
\hline $\begin{array}{l}11 \\
12 \\
16 \\
18\end{array}$ & $\begin{array}{rr}87 & 0 \\
94 & 0 \\
122 & 0 \\
136 & 0\end{array}$ & $\begin{array}{l}+6-0 \\
+6-0 \\
+6-0 \\
+6-0\end{array}$ & $\begin{array}{ll}24 & 0 \\
24 & 0 \\
24 & 0 \\
26 & 0\end{array}$ & $\begin{array}{l} \pm 41 / 2 \\
\pm 41 / 2 \\
\pm 41 / 2 \\
\pm 41 / 2\end{array}$ & $\begin{array}{ll}5 & 6 \\
5 & 6 \\
5 & 6 \\
5 & 6\end{array}$ & $\begin{array}{l} \pm 2 \\
\pm 2 \\
\pm 2 \\
\pm 2\end{array}$ \\
\hline
\end{tabular}

Steel Derrick Parts Dimensions

(e) Gin pole.-The gin pole shall be constructed so as to extend across the center of the water-table opening. The clearance between the gin pole and the base of the crown block shall be 8 feet 0 inch minimum for derricks under 122 feet in height, and shall be 10 feet 0 inch minimum for derricks 122 feet and over in height.

(f) Window opening of steel derricks for rotary drilling.-On steel derricks for rotary drilling, the minimum window opening height shall be 23 feet 8 inches in the clear, measured parallel to the center line of the derrick side from the top of the derrick floor joists, and a calf wheel girt shall be provided for cable tool work.

(g) Bull wheel girt clearance.-The distance in the clear from the bull wheel girt to the top of the derrick floor joists shall be 9 feet 6 inches $\pm 1 / 8$ inch, measured parallel to the center line of the derrick side.

(h) Calf wheel girt clearance. The distance in the clear from the calf wheel girt to the top of the derrick floor joists shall be 11 feet measured parallel to the center line of the derrick side, with a tolerance of plus or minus one-eighth inch.

(i) Corner foundation bolt settings.-At each A. P. I. steel derrick corner foundation there shall be set two 36 -inch foundation bolts, 1 to $11 / 4$ inches in diameter, with upper ends threaded 6 inches with U. S. standard thread. These bolts shall be set on the sides of a square 12 inches larger than the nominal base square without tolerance, and located 10 inches plus or minus one-eighth inch from the corner of said larger square. Said bolts shall be set to protrude 6 inches above foundations. The foundation bolt holes in the derrick base shall be $1 \frac{3}{8}$ inches in diameter.

(j) Center of drill hole.-The center of the drill hole shall be 6 inches from the center of the derrick base on the center line towards the Samson post, with a tolerance of plus or minus one-half inch. 
Section 4. STEEL DERRICK STRENGTH OR CAPACITY REQUIREMENTS

4. (a, Structural material.-Structural steel used in the construction of A. P. I. steel derricks shall be new and shall conform to the requirements of the American Society for Testing Materials, "Standard Specifications for Structural Steel for Buildings," serial designation A-9-24 (see 605.12, p. 252), or A. S. T. M. "Standard Specifications for Structural Silicon Steel," serial designation "A94-29," as amended to date. (See 621.33, p. 1126.) Tubular steel used in construction of A. P. I. steel derricks shall be new and shall conform to A. P. I. pipe specifications as amended to date. (See A. P. I. Stds. No. 5-A.) (See 607.4, p. 444.) Structural lumber used for reinfolcing legs in A. P. I. steel derricks shall be new and shall conform to the requirements of the American Lumbel Standards for select or dense select grades as given in the revised Simplified $\mathrm{P}_{1}$ actice Recommendation No. 16 of the United States Department of Commerce.

(b) Unreinforced safe-load capacily. - The A. P. I. safe-load capacity of an A. P. I. del rick without leg reinforcing, expressed in pounds, shall be the gross capacity of the derrick, and shall be specified on the name plate. It shall be equal to the sum of the stiengths of the four unreinforced legs at their weakest section.

(c) Reinforced safe-load capacity.-The A. P. I safe-load capacity of an A. P. I. derrick, with leg reinforcing, expressed in pounds, shall be the gross capacity of the del rick when 1 einforced with properly applied reinforcing legs of a particular size. It shall be equal to the sum of the strengths of the four reinforcea legs at their weakest section, the strength of each reinforced leg to be the sum of the stiengths of the two or mole leg elements. Both the sateload capacity when reinforcing legs are used and the size, weight per foot, and type of section of the leg reinforcing shall be specified on the name plate.

Safe-Load Capacity Determinations

(d) (1). The leg members or reinforcing lug members of an A. P. I. derrick shall be classified as "Main compression member," and their $l / r$ ratio shall not exceed 120 . The A. P. I. safe-load capacities of A. P. I. derricks shall be determined by computing the sum of the strengths of the four leg members at their weakest section plus the sum of the strengths of the four reinforcing leg members at their weakest section, if the derrick is reinforced, considering same as columns unsupported between adjacent panel points, and determined in accordance with the column formula of the American Institute of Steel Construction, to wit:

For ratios of $l / r$ up to anc including 60

$$
P / a=15,000
$$

and for ratios of $l / r$ greater than 60 ,

where

$$
P / a=\frac{18,000}{1+\frac{l^{2}}{(18,000)\left(r^{2}\right)}}
$$

$P$ is the total compression load in pounds.

$a$ is the gross cross-sectional area of the column or leg element in square inches.

$l$ is the unsupported length of the column or leg element between adjacent panel points in inches.

$r$ is the corresponding least radius of gyration of the column cross section in inches.

(2) The formula given in paragraph $4-d-1$ shall apply to derricks fabricated of structural steel or steel pipe having a minimum yield point of 30,000 pounds per square inch; structural steel or pipe having a yield point of less than 30,000 pounds per square inch shall not be used in the construction of A. P. I. derricks.
(This specification contains a tentative formula for determining the safe-load capacity of leg members of derricks made of silicon structural steel of A. S. T. M. specification A94-29. Requirements for determining the safe-load capacity of wooden reinforcing legs are also given.)

(e) Wind-load capacity. - The A. P. I. wind-load capacity of an A. P. I. derrick, explessed in miles per hour, shall be the maximum wind velocity that the unboarded derrick can withstand without failure, assuming the four legs to be anchored solidly and the derrick to be unguyed, and shall be specified on the name plate. Every A. P. I. steel derrick shall have a minimum A. P. I. wind-load capacity of 70 miles per hour, as indicated by the United States Weather Bureau's standard Robinson 4-cup anemometer.

(This specification also contains requirements on methods of determining wind-load capacity, requirements on leg joints, determinations of strengths of joints, girts, and braces, design of water-table beams, computation details, standard headboard connections, standard bull wheel and calf wheel post connections, standard dimensions and locations of rig parts such as Samson post, walking beams, bull wheel, bull-wheel shaft, calf wheel, calf-wheel shaft, band wheel, bull-rope grooves, etc., standard rig strength and capacity requirements, marking, and inspection requirements.)

(The inspection requirements include the item given below.)

All tests and inspection shall be made at the place of manufacture prior to shipment, unless otherwise specified, and shall be so conducted as not to interfere unnecessarily with the operation of the works.

\section{AMERICAN PETROLEUM INSTITUTE, SPEC.} IFICATIONS FOR STANDARD TANKS WITH RIVETED SHELIS, STANDARD NO. 12-A WITH SUPPLEMENT NO. 2, JULY, 1931

\section{RIVETED TANKS FOR OIL STORAGE}

(The American Petroleum Institute can supply detail drawings of seven standard tanks from 36 to 144 feet in diameter and also detail drawings for manholes, oil connections, drain connections, roof openings, and stairways. The use of appurtenances shown on these drawings, or designs of equivalent strength and tightness, will be required on tank to winich the A. P. I. monogram is attached.)

The following table shows the range of capacities that can be obtained using 11 different diameters and varying the height:

\begin{tabular}{|c|c|c|c|c|c|c|c|}
\hline \multirow{5}{*}{$\begin{array}{c}\text { Diam- } \\
\text { eter } \\
\text { (feet) }\end{array}$} & \multicolumn{7}{|c|}{ Nominal height (feet) } \\
\hline & 12 & 1734 & $231 / 2$ & $291 / 2$ & 35 & $401 / 2$ & 46 \\
\hline & \multicolumn{7}{|c|}{ Number of courses } \\
\hline & 2 & 3 & 4 & 5 & 6 & 7 & 8 \\
\hline & \multicolumn{7}{|c|}{ Capacity in 42-gallon barrels; approximately level full } \\
\hline $\begin{array}{l}12 \\
18 \\
24 \\
30 \\
36 \\
48\end{array}$ & $\begin{array}{r}240 \\
540 \\
960 \\
1,500 \\
2,160 \\
3,850\end{array}$ & $\begin{array}{r}360 \\
810 \\
1,440 \\
2,440 \\
3,400 \\
5,730\end{array}$ & $\begin{array}{r}480 \\
1,070 \\
1,910 \\
2,980 \\
4,300 \\
7,600\end{array}$ & $\begin{array}{r}590 \\
1,340 \\
2,380 \\
3,710 \\
5,400 \\
9,500\end{array}$ & $\begin{array}{r}720 \\
1,600 \\
2,850 \\
4,450 \\
6,400 \\
11,300\end{array}$ & $\begin{array}{r}7,400 \\
13,200\end{array}$ & $\begin{array}{r}8,400 \\
15,000\end{array}$ \\
\hline $\begin{array}{r}60 \\
78 \\
102 \\
120 \\
144\end{array}$ & 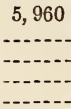 & $\begin{array}{l}8,880 \\
\ldots . . \\
\end{array}$ & $\begin{array}{l}11,800 \\
\end{array}$ & $\begin{array}{l}15,000 \\
25,000 \\
42,500 \\
59,000 \\
\$ 5,000\end{array}$ & $\begin{array}{r}17,500 \\
30,000 \\
51,000 \\
70,000 \\
101,000\end{array}$ & $\begin{array}{r}20,500 \\
35,000 \\
59,000 \\
82,000 \\
118,000\end{array}$ & $\begin{array}{r}23,500 \\
39,500 \\
68,000 \\
93,000 \\
134,000\end{array}$ \\
\hline
\end{tabular}

TABLE 1.-Tank sizes 


\section{PART I. DESIGN SPECIFICATIONS}

Section 1. Material

1. The various materials used shall conform in chemical and physical properties to the following specifications:

2. Plates.-Either class "A" Association of American Steel Manufacturers, current A. S. T. M. A-7 (see 605.11, p. 249) or current A. S. T. M. A-9 (open-hearth steel only) (see 605.12, p. 252).

3. Copper-bearing steel may be used for roof plates if so specified by the purchasers.

4. Plate specification by weight is recommended, and plates purchased on weight specifications shall individually comply with the normal tolerances as they appear in these specifications.

NотE.- Where plates are referred to by thickness in the specification, or on the drawings, the thickness shall be considered as nominal only.

5. Structural steel.-Current A. S. T. M. A-9. (See 605.12, p. 252.)

6. Rivets for hot driving.-Either current A. S. T. M. A-7 (see 605.11 , p. 249) current A. S. T. M. A-31, or A. S. M. E. Boiler Code (see 603.21, p. 144).

7. Rivets for cold driving.-Rivets conforming to either of the above specifications, annealed.

If copper bearing steel is specified for the roof plates, the rivets shall contain the same percentage of copper.

Section 2. Shell Design

Loads and working stresses.-

8. Stresses are to be computed on the assumption that the tank is filled level full of water at $60^{\circ} \mathrm{F}$., and the tension in each ring is to be computed 12 inches above the bottom gage line of the ring in question.

9. In computing net section of plate, rivet boles when punched one-sixteenth inch over nominal diameter for hot driven rivets, are to be assumed oneeighth inch larger than nominal diameter of the rivet; when punched one-thirty-second inch over nominal diameter for cold diven rivets, holes are to be assumed three tbirty-seconds inch larger than nominal diameter of rivet. When drilled, or when subpunched and reamed, holes are to be assumed one-sixteenth inch larger in diameter than nominal diameter of rivet. The net section of rivet to be used for computing shear values should be, in the case of hot driven rivets, nominal diameter plus one-sixteenth inch; in case of cold driven, nominal diameter plus one thirty-second inch.

10. All openings over 4 inches in diameter in the shell of the tank shall be reinforced. This reintorcement may be the flange of the fitting used, or an additional ring of metal, or both flange and ring.

11. The amount of reinforcement shall be determined in accordance with paragraph 1 of Appendix "A," attached to these specifications.

12. The following maximum allowable working stresses shall be used in design:

Maximum tensile stress in net section of plate.21,000 pounds per square inch.

Maximum shear in net secion of rivet. $-16,000$ pounds per square inch.

Maximum bearing stress on plates or rivets. $-35,000$ pounds per square inch when rivets are in double shear; 32,000 pounds per square inch when rivets are in single shear.

Sizes of plate and tank.-

13. Tanks shall be designed so that the diameter is a multiple of 6 feet, using shell plates having a net length (total length minus lap) of $6 \pi$ feet ( 6 times $3.1416)$. The number of plates in each course will thus be equal to the diameter of the tank divided by 6 . Shell plates shall have an over-all width of 72 inches.

14. The minimum thickness of shell plates shall be one-fourth inch in tanks over 48 feet in diameter, and shall be three-sixteenths inch in tanks 48 feet or less in diameter.
(This specification also contains requirements on the arrangement of parts, suggested rivet sizes, requirements on rivet spacing, and spacing of rivetsfrom edge of plate.)

\section{Section 3. Roof Design}

22. Conical steel roof and supporting structure shall be designed to carry a live load of 25 pounds per square foot in addition to the dead load, and the supporting structure shall be designed in accordance with current American Institute of Steel Construction "Standard Specifications for the Design, Fabrication, and Erection of Structural Steel for Buildings," except that the value of $l / r$. shall not exceed 180. Structural shapes shall be used tor roof columns. The slope or the roof shall be one-half inch in 12 inches, except that the slope of the selfsupporting roof of the 12 -foot tank shall be 2 inches in 12 inches.

23. Roof plates shall be three-sixteenths inch thick, 72 inches wide, and shall have the same approximate length as three-sixteenths-inch shell plates. They may be either riveted or welded; if riveted, rivets shall be seven-sixteenths inch at $1 \frac{112-}{2}$ inch pitch.

24. Rafters are to be spaced so that in the outer row there shall be not less than three rafters to each shell plate; spacing on inner rows is not to be greater than $5 \frac{1}{2}$ feet.

\section{Section 4, Bottom Design}

Plate sizes.-

25. All rectangular bottom plates shall be onefourth inch thick, 72 inches wide, and shall have the same approximate lengths as one-fourth-inch shell plates. These may be either riveted or welded.

26. Bottom sketch plates in tanks over 48 feet in diameter shall be five-sixteenths inch thick and 72 inches wide. Sketch plates in tanks 48 feet in diameter and less shall be one-fourth inch thick and 72 inches wide.

Rivets.-

27. For tanks with riveted bottoms the rivets shall be five-eighths inch in diameter.

Welded construction.-

28. Tanks with welded bottoms shall have all plates lapped a minimum of 1 inch and electric welded with a continuous fillet-weld.

29. Not less than 4 inches inside the bottom angle the welded lap joints on the bottom sketch plates shall be changed to welded butt-joints and continue thus underneath the angle to the end of the plate.

30. The bottom angle, for tanks with welded bottoms, shall be placed on the outside of the shell with the horizontal leg turned out. The vertical leg of the angle shall be riveted to the tank shell in accordance with the standard punching in the shell plate. The horizontal leg of the angle shall be welded to bottom plates with continuous filletwelds at toe and heel of angle.

(Regulations concer ning the identification of tanks by means of a special nameplate bearing the A. P. I. monogram are included in this specification.)

(Part II of this specification is made up of fabrication specifications for tanks and Part III covers erection specifications. The required tests for tanks are as given below.)

Testing and lowering of bottom. - The bottom and lower part of the first course of tank, when riveted bottoms are specified, shall be riveted and calked while supported above the grade. Where a welded bottom is specified the bottom shall be welded on the tank grade and one or more shell courses riveted up before the bottom is tested.

Where water is available, the tightness of a riveted bottom shall be tested with 6 inches of water before being lowered to foundation; bottom shall be entirely tight to the satisfaction of the purchaser's inspector before lowering. 
For a tank with welded bottom and where water is not available for a tank with riveted bottom, tightness shall be tested by pumping oil underneath bottom and maintaining a head of 6 inches by holding that depth around the edge inside a temporary dam. The bottom shall be made entirely tight to the satisfaction of the purchaser's inspector. Oil for the above test shall be supplied by the purchaser.

Testing shell and roof.-Upon completion, the entire tank shell shall be tested by filling with oil or water to the level of the top angle. If it is not feasible to fill the tank as indicated, the test may be made by painting all seams on the inside with a highly penetrating oil, such as automobile spring oil, examining the outside of the seams for leakage. All leaks discovered by the above tests shall be stopped.

The roof shall be tested by applying an internal air pressure equivalent to not less than one-half inch of water, and applying strong soap solution to all seams. All leaks discovered shall be stopped.

Before acceptance, entire tank, when filled with oil, must be tight and free from leaks.

(The appendix to this specification contains details of design for small tanks and for large tanks, methods of computing reinforcement for openings in tank shell, method of computing working stresses in riveted butt-strap joints, tables on strength of butt joints and of lap joints.)

AMERICAN PETROLEUM INSTITUTE DIMENSIONAL SPECIFICATIONS FOR STANDARD BOLTED TANKS, STANDARD NO. 12-B, SECOND EDITION, JULY, 1931

\section{BOLTED TANKS}

Section 1. GENERAL DETAILS

\section{Material}

1. (a) Joints are designed on the assumption of 1/16-inch final net thickness of gasket in place. Sup- plier shall furnish gasket material of such texture and thickness that it will provide oil-tight connections in these joints.

(b) Gages of material as shown in Table 1 are minimum thicknesses acceptable under these standards; heavier material may be required at option of purchaser.

\section{Fabrication}

2. (a) Nominal spacing of all bolt holes is 2 inches. In bottoms and decks $3 / 4$-inch minimum edge distance shall be provided for the vertical stave joints. Vertical seams having two or more rows of bolts shall be square pattern, with 2 -inch gage between lines of holes. All bolt holes shall be laid out so that after bending and flanging they conform to the specified dimensions.

(b) Bolt holes shall be one thirty-second inch greater in diameter than the nominal diameter of the bolc.

(c) Bolts in chime of cone roof shall be threeeighths inch for 100 and 200 barrel tanks of which the upper course is 14-gage material, and one-half inch for all other tanks.

(d) Wherever 12-gage or heavier material is used, bolts shall be one-half inch.

\section{Erection}

3. (a) The stave design contemplates that they shall be assembled in a counterclockwise direction.

(b) The dimensions of Table 1 assume that most of the $1 / 32$-inch slack, due to bolt clearances, will be taken up in all stave joints in erecting the tank. Bolts are expected to come nearer bearing in tension on vertical joints, and in compression against the roof and bottom, than being centered in their holes when the tank is fitted together, before filling. At vertical stave joints, some "pinning" will be required to squeeze gasket down to $1 / 16$-inch thickness assumed in design.

Roof Pitch

4. (a) Pitch of cone roof shall be 1 inch in 12 inches.

TABLE 1.-A.P.I. dimensional specifications for bolted tanks

\begin{tabular}{|c|c|c|c|c|c|c|c|c|c|c|c|c|c|c|c|c|}
\hline \multicolumn{2}{|c|}{ Capacity } & \multicolumn{3}{|c|}{ Dimensions } & \multicolumn{5}{|c|}{ Shell } & \multicolumn{2}{|c|}{ Bottom } & \multicolumn{2}{|c|}{$\begin{array}{l}\text { Water-seal } \\
\text { roof }\end{array}$} & \multicolumn{3}{|c|}{ Cone roof } \\
\hline \multirow{2}{*}{$\begin{array}{l}\text { Nominal capacity } \\
\text { (42-gallon barrels) }\end{array}$} & \multirow{2}{*}{$\begin{array}{l}\text { Actual } \\
\text { capacity } \\
\text { level full } \\
\text { (42-gallon } \\
\text { barrels) }\end{array}$} & \multirow{2}{*}{$\begin{array}{l}\text { Inside } \\
\text { diam- } \\
\text { eter } \\
\text { (nom- } \\
\text { inal) } 1\end{array}$} & \multirow{2}{*}{\multicolumn{2}{|c|}{$\begin{array}{l}\text { Roof and } \\
\text { bottom } \\
\text { bolt cir- } \\
\text { cles } \\
\text { (diam- } \\
\text { eter) }\end{array}$}} & \multirow{2}{*}{ Height } & \multirow{2}{*}{$\begin{array}{l}\text { Num- } \\
\text { ber } \\
\text { staves } \\
\text { per } \\
\text { ring }\end{array}$} & \multirow{2}{*}{$\mid \begin{array}{c}\text { U. S. } \\
\text { stand- } \\
\text { ard } \\
\text { gage }\end{array}$} & \multirow{2}{*}{$\begin{array}{l}\text { Num- } \\
\text { ber of } \\
\text { rows } \\
\text { of } \\
\text { bolts }\end{array}$} & \multirow{2}{*}{$\begin{array}{c}\text { Size } \\
\text { of } \\
\text { bolts }{ }^{2}\end{array}$} & \multirow{2}{*}{$\begin{array}{c}\text { U. S. } \\
\text { stand- } \\
\text { ard } \\
\text { gage }\end{array}$} & \multirow{2}{*}{$\begin{array}{c}\text { Size } \\
\text { of } \\
\text { bolts }^{2}\end{array}$} & \multirow{2}{*}{$\begin{array}{l}\text { U. S. } \\
\text { stand- } \\
\text { ard } \\
\text { gage }\end{array}$} & \multirow{2}{*}{$\begin{array}{c}\text { Size } \\
\text { of } \\
\text { bolts }{ }^{2}\end{array}$} & \multirow{2}{*}{$\begin{array}{c}\text { U. S. } \\
\text { stand- } \\
\text { ard } \\
\text { gage }\end{array}$} & \multicolumn{2}{|c|}{ Size of bolts ${ }^{2}$} \\
\hline & & & & & & & & & & & & & & & Chime & Seam \\
\hline $100 \ldots$ & 95.80 & $\begin{array}{cc}F t . & \text { in } \\
9 & 23 / 4\end{array}$ & $\begin{array}{c}F t . \\
9\end{array}$ & in. & Ft. in. & 6 & 14 & 1 & In. & 14 & In & 14 & In. & 14 & In. & $I_{36}$ \\
\hline $200 \ldots \ldots$ & 191.64 & $\begin{array}{ll}9 & 23 / 4\end{array}$ & 9 & $43 / 4$ & 161 & 6 & $\begin{array}{l}14 \\
14\end{array}$ & $\begin{array}{l}1 \\
1\end{array}$ & $\begin{array}{l}3 / 5 \\
3 / 8\end{array}$ & 14 & $3 / 8$ & 14 & 38 & 14 & $3 / 8$ & 36 \\
\hline $250 \ldots$ & 265.28 & $1545 / 8$ & 15 & $65 \%$ & $8 \quad 16$ & 10 & 12 & 1 & 12 & 12 & $1 / 2$ & 12 & 1,2 & 14 & $1 / 2$ & 36 \\
\hline 500 , high...... & 532.56 & $15 \quad 45 / 8$ & 15 & $65 / 8$ & $\begin{array}{ll}16 & 1\end{array}$ & 10 & $\begin{array}{l}12 \\
12\end{array}$ & $\begin{array}{l}1 \\
1\end{array}$ & $\begin{array}{l}1 / 2 \\
1 / 2\end{array}$ & 12 & 112 & 12 & 16 & 14 & 36 & $3 / 6$ \\
\hline 500, low $\ldots . .$. & 522.01 & $21 \quad 61 / 2$ & 21 & $81 / 2$ & $8 \quad 1 / 2$ & 14 & 12 & 1 & $1 / 2$ & 12 & 16 & 12 & $1 / 2$ & 14 & $1 / 2$ & $3 / 8$ \\
\hline 1,000 , high.. & $1,044.02$ & $21 \quad 61 / 2$ & 21 & 81,2 & 161 & 14 & $\begin{array}{l}12 \\
12\end{array}$ & $\begin{array}{l}1 \\
1\end{array}$ & $\begin{array}{l}1 / 2 \\
1 / 2\end{array}$ & 12 & $1 / 2$ & 12 & $1 / 2$ & 14 & $1 / 2$ & 38 \\
\hline 1,000 , low & 993.53 & $2985 / 8$ & 29 & $105 / 8$ & $8 \quad 1 / 2$ & 20 & 12 & 2 & $1 / 2$ & 12 & $1 / 2$ & 12 & $1 / 2$ & 14 & $1 / 2$ & 38 \\
\hline $2,000 \ldots$ & $1,987.06$ & $29 \quad 85 / 8$ & 29 & $105 / 8$ & $16 \quad 1$ & 20 & $\begin{array}{l}12 \\
12 \\
12\end{array}$ & $\begin{array}{l}2 \\
2 \\
2\end{array}$ & $\begin{array}{l}12 \\
1 / 2 \\
1 / 2\end{array}$ & 12 & $1 / 2$ & 12 & $1 / 2$ & 14 & 12 & 38 \\
\hline $5,000 \ldots$ & $5,037.45$ & $\begin{array}{ll}38 & 756\end{array}$ & 38 & $95 / 8$ & $24 \quad 11 / 2$ & 26 & $\begin{array}{l}10 \\
10 \\
10\end{array}$ & 2 & $\begin{array}{l}72 \\
1,2 \\
1,2 \\
16\end{array}$ & 10 & $1 / 2$ & 12 & $1 / 2$ & 14 & $1 / 2$ & 36 \\
\hline $10,000 \ldots$ & $10,218.49$ & $54113 / 4$ & 55 & $13 / 4$ & $24 \quad 2$ & 37 & $\frac{10}{7}$ & $\begin{array}{l}2 \\
2 \\
3\end{array}$ & $\begin{array}{l}1 / 2 \\
1 / 2 \\
1 / 2\end{array}$ & 10 & $1 / 2$ & 12 & $1 / 2$ & 14 & $1 / 2$ & 38 \\
\hline
\end{tabular}

1 Nominal inside diameter is considered as 2 inches less than bolt-circle diameter and actual capacity figured on that basis.

2 Wherever 12-gage or heavier material is used, bolts shall be $1 / 2$ inch. See pars. 2 . (c) and (d). 
(This specification also includes a detail drawing of a standard stave for bolted tanks showing bolt spacings and layouts, not shown herewith. Regulations are also included covering the identification of tanks manufactured in accordance with this specification by the use of the A. P. I. monogram.)

\section{AMERICAN RAILWAY ENGINEERING AS- SOCIATION, STEEL SUBSTRUCTURES FOR WATER TANR, 50,000 AND 100,000 GALLONS CAPACITY, 1921}

\section{STEEL SUBSTRUCTURES}

\section{GENERAI}

1. The structure will consist of a 12-post steel tower, complete in all details for supporting a wooden water tank of the specified size and capacity at the required elevation. The intent of the plans and specifications is to include all material required between the top of foundation and the bottom of tank.

\section{MATERIAL}

2. Except as may be herein noted all metal in the structure will be made in accordance with specifications of the association as given in part second, Iron and Steel Structures, Manual of Recommended Practice.

\section{WOREMANSHIP}

3. Except as may herein be noted, workmanship on the structure will be performed in accordance with the requirements of the association as given in part second, Iron and Steel Structures, Manual of Recommended Practice.

\section{PAINTING}

4. Steel work before leaving the shop shall be thoroughly cleaned and given one good coat of red lead ground in linseed oil or such paint as may be specified by the engineer. Except as herein noted, cleaning and painting shall be done in accorciance with specifications of the association as given in part second, Iron and Steel Structures, Manual of Recommended Practice.

\section{AMERICAN RAILWAY ENGINEERING AS- SOCIATION, SPECIFICATIONS STEEL WATER AND OIL TANKS, 1912}

\section{WATER AND OIL TANKS \\ SCOPE OF SPECIFICATIONS}

1. These specifications are intended for steel tanks requiring plates not more than five-eighths inch thick.

\section{QUALITY OF METAL}

2. The metal in these tanks shall be open-hearth steel. The steel shall conform in physical and chemical properties to the specifications for steel railway bridges. (See 605.11, p. 249.)

\section{IOADING}

3. The weight of water shall be assumed to be 63 pounds, crude oil 56 pounds, and creosote oil 66 pounds per cubic foot. Wind pressure, acting in any direction, shall be assumed to be, in pounds, thirty times the product of the height by two-thirds of the diametel of the tank in feet.

\section{UNIT STRESSES}

4. Unit stresses shall not exceed the following: (a) Tension in plates, 15,000 pounds per square inch on net section; (b) shear plates, 12,000 pounds per square inch on net section; (c) shear on rivets, 12,000 pounds pel square inch on net section; and (a) bearing pressure on field rivets, 20,000 pounds pei square inch.

\section{CYLINDRICAL RINGS}

5. Plates forming the shell of the tank shall be cylindrical and of different diameters, in and out, from course to course.

\section{WOREMANSHIP}

6. Workmanship shall be first class. Plates shall be beveled on all edges for calking after being punched. The punching shall be from the surface to be in contact. The plates shall be formed cold to exact form after punching and beveling. Rivet holes shall be accurately spaced. Drift pins shall be used only for bringing the parts together. They shall not be driven with enough force to deform the metal about the holes. Power riveting and calking should be used. A heavy yoke or pneumatic bucker shall be used for power-driven rivets. Riveting shall draw the joints to full and tight bearing.

\section{CALEING}

7. The tank shall be made water or oil tigbt by calking only. No foreign substance shall be used in the joints. For water tanks, the calking shall preferably be done on the inside of tank and joint only; but for oil tanks the calking should be done on both sides. No form of calking tool or work that injures the abutting plate shall be used.

\section{MINIMUM THICKNESS OF PLATES}

8. The minimum thickness of plates in the cylindrical part of the tank shall be not less than onefourth inch and in flat bottoms not less than fivesixteenths inch. In curved bottoms the thickness of plate shall be not less than that of the lower plate in the cylindrical part.

\section{HORIZONTAL AND RADIAL JOINTS}

9. Lap joints shall generally be used for horizontal seams and splices and for radial seams in curved bottoms.

\section{VERTICAL JOINTS}

10. For vertical seams and splices, lap joints shall be used with plates not more than three-eighths inch thick. With thicker plates, double butt joints with inside and outside straps shall generally be used. The edge of the plate in contact at the intersection of horizontal and vertical lap joints shall be drawn out to a uniform taper and thin edge.

\section{RIVETS, RIVET HOLES, PUNCHING AND PITCH}

11. For plates not more than three-eighths inch thick, $5 / 8$-inch rivets shall be used. For thicker plates, $3 / 4$-inch rivets shall be used. The diameter of rivet holes shall be one-sixteenth inch larger than the diameter of the rivets used. The punching shall conform to the specifications of this association for such work on steel bridges. A close pitch, with due regard for thickness of plate and balanced stress between tension on plates and shear on rivets, is desirable for calking.

\section{TANK SUPPORT}

12. If the tank is supported on a steel substructure, the latter shall conform to the specifications of this association for the manufacture and erection of steel bridges, except that allowance shall be made for wind pressure but not for impact. 


\section{PAINTING}

13. In the shop the metal shall be cleaned of dirt rust, and scale and, except the surfaces to be in contact in the joints of the tank, shall be given a shop coat of paint or metal preservative selected and applied as specified by the railway company.

After being completely erected, calked, and cleaned of dirt, rust, and scale, all exposed metal work shall be painted or treated with such coat or coats of paint or metal preservative as sliall be selected by the railway company.

\section{PLANS AND SPECIFICATIONS}

14. Under these specifications and in conformity thereto the railway company shall cause to be prepared or shall approve detail plans and specifications for tanks, herein specified, as it shall construct. Such plans and specifications shall cover all necessary tank auxiliaries.

\section{AMERICAN SOCIETY OF MECHANICAL EN- GINEERS, RULES FOR CONSTRUCTION OF UNFIRED PRESSURE VESSELS, A. S. II. E. BOILER CONSTRUCTION CODE, 1931}

\section{UNFIRED PRESSURE VESSELS}

[These rules are not intended to apply $(a)$ to pressure vessels which are subject to Federal inspection and control, $(b)$ to locomotives of all types, $(c)$ to vessels for containing only water under pressure for domestic supply, (d) to vessels for flammable liquids and/o gases at temperatures of over $300^{\circ} \mathrm{F}$.]

U-1. The rules in this section apply to unfired pressure vessels over 6 inches in diameter, having a volume of more than 1.5 cubic feet and carrying a pressure of over 30 pounds per square inch, constructed of material herein specified.

\section{SAFETY APPLIANCES}

U-2. All pressure vessels shall be protected by such safety and relief valves and indicating and controlling devices as will insure their safe operation. These devices shall be so constructed, located, and installed that they can not readily be rendered inoperative. The relieving capacity of safety valves shall be such as to prevent a rise of pressure in the vessel of more than 10 per cent above the maximum allowable working pressure, and their discharges shall be carried to a safe place.

\section{SAFETY VALVES FOR OTHER THAN NOXIOUS LIQUIDS OR} NOXIOUS VAPORS

U-3. Safety valves shall be of the direct springloaded type, designed with substantial lifting device so that disk can be lifted from its seat by the spindle not less than one-eighth the diameter of the valve when the pressure on the vessel is 75 per cent of that at which the safety valve is set to blow. If the safety valve is to be used for relief of liquid pressure, no lifting device is required.

U-4. Safety valves having either the seat or disk of cast iron shall not be used.

$\mathrm{U}-5$. If more than one safety valve is used, the discharge capacity shall be taken as the combined capacity of all valves.

U-6. For vessels in which pressure is not generated but is derived from an outside source, each safety valve shall be so connected to the vessel, vessels, or system which it protects, as to prevent a rise in pressure beyond the maximum allowable pressure (see par. U-2) in any vessel protected by the safety valve.

For vessels in which pressure may be generated, the safety valve or valves must be connected directly to the vessel which is to be protected or to a pipe line leading to the vessel; the internal crosssectional area of the pipe line shall be not less than the nominal area of the safety valve or valves used, and without any intervening valve between the vessel and the safety valve or valves protecting it. All vessels, the contents of which are likely to cause interference with the operation of a safety valve if attached directly to the vessel, shall have the safety valve or valves connected in such manner as to avoid such interference. When an escape pipe is used, it shall be full sized and fitted with an open drain, to prevent liquid from lodging in the upper part of the safety valve, and no valve of any description shall be placed on the escape pipe between the safety valve and the atmosphere. When an elbow is placed on an escape pipe, it shall be located close to the safety-valve outlet, or the escape pipe shall be securely anchored and supported. When two or more safety valves are placed on one connection, this connection shall have a cross-sectional area at least equal to the combined area of these safety valves.

U-7. Every safety valve which is exposed to a temperature of $32^{\circ} \mathrm{F}$., or less, shall have a drain at least three-eighths inch in diameter at the lowest point where water can collect.

U-8. Safety-valve springs shall not be adjusted to carry more than 10 per cent greater pressure than that for which the springs were made.

U-9. Each safety valve shall be tested once every day, or oftener, by raising the disk from its seat.

U-10. Safety valves for compressed-air tanks shall not exceed 3 inches in diameter and shall be proportioned for the maximum number of cubic feet of free air that can be supplied per minute as shown in Table U-1.

\section{CORROSIVE SUBSTANCES}

U-11. All pressure vessels which are to contain substances having a corrosive action upon the metal of which the vessel is constructed, shall be designed for the pressure they are to carry, and the thickness of all parts subject to corrosion should be increased by a uniform amount to safeguard against early rejection.

\section{MATERIAL}

U-12. All materials used in the construction of the important parts of unfired pressure vessels, for which specifications are given in Section II of the code, shall conform to such requirements.

U-13. (a) Plates for any part of a riveted vessel required to resist stress produced by internal pressure shall be of flange or fire-box quality steel conforming with specifications $\mathrm{S}-1$ for steel boiler plate and S-2 for steel plates of flange quality for forge welding of Section II of the code, except as provided in (b).

If desired, both flange and fire-box steel of lower tensile strength than specified may be used for an entire boiler or part thereof, the desired tensile limits to be specified with a range of 10,000 pounds per square inch. All such steel shall conform to the specifications for steel boiler plate.

(b) Steel plates for any part of a pressure vessel which is to be constructed with other than riveted joints shall be of the quality specified for the particular kind of joint used.

U-14. In determining the maximum allowable working pressure, the maximum allowable working stress as herein provided shall be used in the computations.

U-15. For resistance to crushing of steel plate, the maximum allowable working stress shall be 19,000 pounds per square inch of cross-sectional area.

U-16. In computing the maximum allowable stress on rivets in shear, the following values in pounds per square inch of the cross-sectional area of the rivet shank shall be used:

Iron rivets in single shear

Iron rivets in double shear 7,600 7,600

Steel rivets in double shear

The cross-sectional area used in the computations shall be that of the rivet shank after driving. 
TABLE U-1.-Maximum free air supplied in cubic feet per minute for different sizes of safety valves at stated pressures

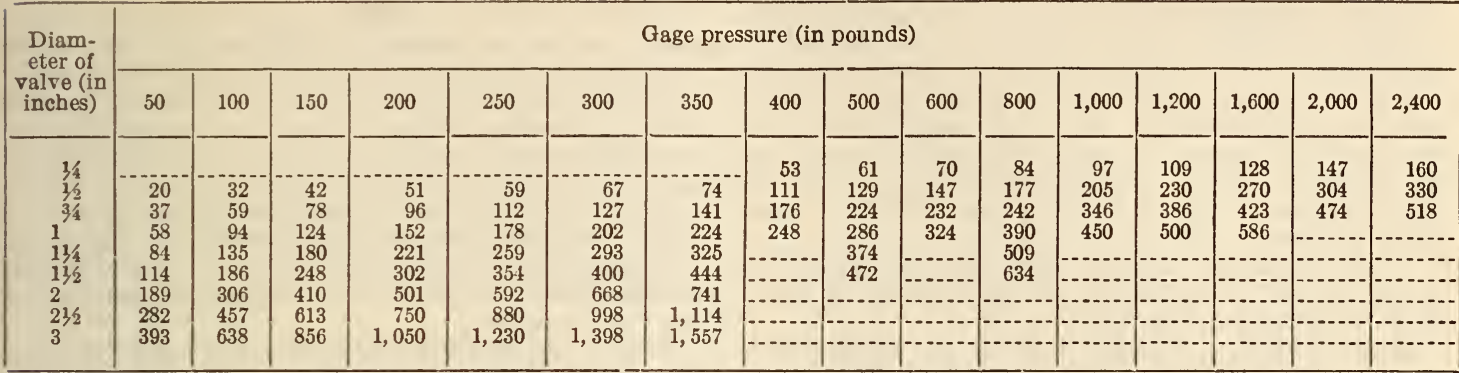

The foregoing table is based on the following formulas:

and

$Q=28 P D l$ for $45^{\circ}$ bevel-seat valves

where

$Q=40 P D l$ for flat-seat valves

$Q=$ discharge in cubic feet of free air per minute.

$P=a b s o l u t e$ pressure at which the safety valve opens (gage pressure plus 14.7 pounds at sea level).

$D=$ diameter, in inches, of the inside edge of the bearing surface between the disk and seat.

$l=$ vertical lift of the safety-valve disk from its seat, in inches, representing the lift for minimum discharge capacity, for satisfactory operation of the valve.

\section{CONSTROCTION AND MAXIMUM ALLOWABLE WORKING}

U-17. For all pressure vessels the minimum thicknesses of shell plates, heads and dome plates after flanging shall be as follows:

When the diameter of shell is-
16 inches diameter and under
Over 16 inches to 24 inches
Over 24 inches to 42 inches
Over 42 inches to 60 inches
Over 60 inches.......

U-18. The minimum thicknesses of butt straps for double-strap riveted joints shall be as given in Table U-2. Intermediate values shall be determined by interpolation. For plate thicknesses exceeding $1 \frac{1}{4}$ inches the thickness of the butt straps shall be not less than two-thirds of the thickness of the plate.

TABLE U-2.-Minimum thicknesses of butt straps

\begin{tabular}{|c|c|c|c|}
\hline $\begin{array}{c}\text { Thickness } \\
\text { of shell } \\
\text { plates }\end{array}$ & $\begin{array}{l}\text { Minimum } \\
\text { thickness } \\
\text { of butt } \\
\text { straps }\end{array}$ & $\begin{array}{c}\text { Thickness } \\
\text { of shell } \\
\text { plates }\end{array}$ & $\begin{array}{l}\text { Minimum } \\
\text { thickness } \\
\text { of butt } \\
\text { straps }\end{array}$ \\
\hline $\begin{array}{c}\text { Inch } \\
3 / 16 \\
14 \\
9 / 32 \\
5 / 16 \\
11 / 32 \\
3,8 \\
13 / 32 \\
7 / 6 \\
15 / 32\end{array}$ & $\begin{array}{l}I n c h \\
3116 \\
14 \\
134 \\
1 / 4 \\
14 \\
5 / 16 \\
5 / 16 \\
38 \\
3 / 8\end{array}$ & $\begin{array}{c}\text { Inches } \\
1 / 2 \\
17 / 32 \\
9 / 16 \\
5 / 8 \\
3 / 4 \\
7 / 8 \\
118 \\
118 \\
11 / 4\end{array}$ & $\begin{array}{c}\text { Inches } \\
7 / 16 \\
7 / 16 \\
7 / 16 \\
1 / 2 \\
1 / 2 \\
5 / 8 \\
1116 \\
34 \\
7 / 8\end{array}$ \\
\hline
\end{tabular}

U-19. The maximum allowable working pressure is the maximum pressure at which a pressure vessel may be operated.

Where the term "maximum allowable working pressure" is used it refers to gage pressure, or the pressure above the atmosphere, in pounds per square inch.

U-20. For internal pressure.-The maximum allowable working pressure on the shell of a pressure vessel shall be determined by the strength of the weakest course computed from the thickness of the plate, the efficiency of the longitudinal joint, the inside diameter of the course, and the maximum allowable unit working stress.

$\frac{S \times t \times E}{R}=$ maximum allowable working pressure, pound per square inch.

${ }^{58}$ For riveted construction the minimum thickness shall be $3 / 16$
nch. where

$S=$ maximum allowable unit working stress in pounds per square inch, taken from Table U-3.

$t=$ minimum thickness of shell plates in weakest course, inches.

$E=$ efficiency of longitudinal joint or of ligaments between openings.

for riveted joints $=$ calculated riveted efficiency.

for fusion-welded joints=efficiency specified under rules for the fusion process of welding.

for seamless shells $=100$ per cent.

for ligaments between openings, the efficiency shall be calculated by the rules given in paragraphs $\mathrm{P}-192$ and $\mathrm{P}-193$ of Section I of the code.

$R=$ inside radius of the weakest course of the shell, inches, provided the thickness of the shell does not exceed 10 per cent of the radius. If the thickness is over 10 per cent of the radius, the outer radius shall be used.

Note. - When the allowable efficiency of the joints is not given, the values for $S \times E$ for the maximum unit joint working stress at right angles to the direction of the joint given in paragraphs U-70, U-82, or U-94 shall be used.

\section{TABLE U-3.-Values for $S$ at various temperatures}

\begin{tabular}{|c|r|r|r|r|r|}
\hline $\begin{array}{c}\text { Maxi- } \\
\text { mum } \\
\text { tem- } \\
\text { pera- } \\
\text { ture } \\
\text { ( }^{\circ} \text { F.) }\end{array}$ & \multicolumn{2}{|c|}{$\begin{array}{c}\text { Minimum of specified range of the tensile strength } \\
\text { of the material (in pounds per square inch) }\end{array}$} \\
\cline { 2 - 6 } & 45,000 & 50,000 & 55,000 & 60,000 & 75,000 \\
\hline 700 & 9,000 & 10,000 & 11,000 & 12,000 & 15,000 \\
750 & 8,220 & 9,110 & 10,000 & 11,200 & 13,000 \\
800 & 6,550 & 7,330 & 8,000 & 9,000 & 10,200 \\
850 & 5,440 & 6,050 & 6,750 & 7,400 & 8,300 \\
900 & 4,330 & 4,830 & 5,500 & 5,600 & 6,000 \\
950 & 3,200 & 3,600 & 4,000 & 4,000 & 4,000 \\
\hline
\end{tabular}

This table applies to all plain carbon steels for which specifications are given in the code. Where the minimum tensile stregnth of the specified range or the temperature differs from those for which values are given in the table, the values for $S$ for such materials may be determined by interpolation.

\section{JOINTS}

U-21. The joints of pressure vessels, if of riveted construction, shall conform to the requirements of paragraphs U-26 to U-35. 
U-22. Pressure vessels may be fabricated by means of fusion or forge welding, or brazing, provided the rules governing the method adopted and as given in paragraphs U-67 to U-96 are followed.

U-23. Pressure vessels may be fabricated by means of fusion welding under the rules given for the fused process of welding, provided the construction is in accordance with the requirements for material and design as required by this code and the fusion welding process used conforms to the specifications for the welding indicated for each class of vessel.

U-24. Pressure vessels may be fabricated by means of forge welding when the rules given under forge welding are followed.

U-25. Pressure vessels for use at any temperature not exceeding $406^{\circ} \mathrm{F}$., may be fabricated by means of the brazing process when the rules given under brazing are followed.

\section{RIVETED JOINTS}

U-26. The efficiency of a joint is the ratio which the strength of the joint bears to the strength of a solid plate. In the case of a riveted joint this is determined by calculating the breaking strength of a unit section of the joint, considering each possible mode of failure separately, and dividing the lowest result by the breaking strength of a length of a solid plate equal to that of the section considered.

U-27. The distance between the center lines of any two adjacent rows of rivets, or the "back pitch" measured at right angles to the direction of the joint, shall have the following minimum values:

(a) If $\frac{P}{d}$ is 4 or less, the minimum value shall be $13 / 4 d$

(b) If $\frac{P}{d}$ is over 4 , the minimum value shall be:

$$
13 / 4 d+0.1(P-4 d)
$$

where $P=$ pitch of rivets in outer row when a rivet in the inner row comes midway between two rivets in the outer row, inches.

$=$ pitch of rivets in the outer row less pitch of rivets in the inner row when two rivets in the inner row come between two rivets in the outer row, inches.

(It is here assumed that the joints are of the usual construction when the rivets are symmetrically spaced.)

\section{$d=$ diameter of the rivet holes, inches.}

The back pitch of rivets in circumferential joints may be less than that called for by the above formulas provided the ligaments between rivets in a circumferential direction, as well as those in a diagonal direction as determined by the rules in paragraph P-193 of Section I of the code, are sufficient to withstand the stress due to pressure, together with any stress due to weight components in support of the vessel structure, with a factor of safety of five.

The back pitch of rivets shall be measured either on the flat plate before rolling, or on the median line after rolling, and the back pitch as there measured shall govern the locations of rivet holes in the butt straps.

U-28. On longitudinal joints, the distance from the centers of rivet holes to the edges of the plates, except rivet holes in the ends of butt straps, shall be not less than one and one-half and not more than one and three-fourths times the diameter of the rivet holes; this distance to be measured from the center of the rivet holes to the calking edge of the plate before calking. The corresponding distance for circumferential seams shall be not less than one and one-fourth times the diameter of the rivet holes.

U-29. (a) The strength of circumferential joints of pressure vessels, the heads of which are not stayed by tubes or through braces, shall be at least 50 per cent of that required for the longitudinal joints of the same structure.

(b) When 50 per cent or more of the load which would act on an unstayed solid head of the same diameter as the shell, is, in consequence of the holding power of the tubes and stays, relieved by the effect of through tubes or stays the strength of the circumferential joints in the shell shall be at least 35 per cent of that required for the longitudinal joints.

U-30. The riveted longitudinal joints of a shell which exceeds one-half inch in thickness, shall be of butt-and-double-strap construction. This rule does not apply to the portion of a shell which is stay bolted to an inner sheet.

U-31. The longitudinal joints of a shell not more than one-half inch in thickness, with the exception given below, may be of lap-riveted construction; but the maximum allowable working pressure of such construction shall not exceed 200 pounds per square inch for vessels less than 24 inches in diameter nor 150 pounds per square inch for vessels 24 inches in diameter or over.

When a vessel is used for a purpose that makes it necessary to provide in its construction for extraordinary wear, corrosion, or other deterioration in service and plates of greater thickness are used than would otherwise be required, the longitudinal joints of shells exceeding 48 inches in diameter may be lapriveted if the following conditions are met:

The operating pressure shall not exceed 50 pounds per square inch.

The plate thickness shall be at least 1.8 times the required plate thickness.

Telltale holes one-eighth inch to one-fourth inch in diameter shall be drilled to a depth of at least 60 per cent of the required plate thickness in those surfaces opposite the surfaces subjected to wear or other deterioration, with the spacing of the telltale holes not over 2 feet apart.

The spherical portion of vessels of any diameter which are wholly spherical or partly hemispherical, may be constructed with lap joints, provided that if the plate thickness exceeds three-eighths inch, the several spherical sections of plate shall be hot pressed to the proper radius of curvature. When the vessel can not be completed in the shop, the whole structure shall be carefully and completely fitted up readv for riveting before shipment.

U-32. Butt straps and the ends of shell plates forming the longitudinal joints shall be rolled or formed to the proper curvature by pressure and not by blows.

U-33. The longitudinal joint of a dome 24 inches or over in inside diameter shall be of butt-anddouble-strap construction or made without a seam of one piece of steel pressed into shape, and its flange shall be double-riveted to the shell. In the case of a dome less than 24 inches in diameter, for which the product of the inside diameter and the maximum allowable working pressure does not exceed 4,000 inch-pounds, its flange may be single-riveted to the shell and the longitudinal joint may be of the lap type, provided it is computed with a factor of safety not less than 8.

When shells are cut to apply domes or manholes the net area of metal, after rivet holes are deducted, in flange and liner, if used, must not be less than the area required by these rules for a length of shell equal to the length removed. A height of vertical flange equal to three times the thickness of the flange may be included in the area of the flange.

U-34. Rivet and stay bolt holes.-All holes for rivets or stay bolts in plates, butt straps, heads, braces, and lugs shall be drilled; or they may be punched at least one-eighth inch less than full diameter for material not over five-sixteenths 
inch in thickness and at least one-fourth inch less than full diameter for material over five-sixteenths inch.

Such holes shall not be punched in material more than five-eighths inch in thickness.

For final drilling or reaming the holes to full diameter, the parts shall be firmly bolted in position by tack bolts.

The finished holes must be true, clean, and concentric.

U-35. Rivets shall be of sufficient length to completely fill the rivet holes and form heads at least equal in strength to the bodies of the rivets. Forms of finished rivet heads that will be acceptable are shown in Figure 156.

(Other structural requirements include a formula for computing the thickness of blank unstayed dished heads, their dimensions, working pressures, manhole requirements; a formula for computing the thickness of unstayed flat heads; formula for determining the maximum allowable working pressure for various thicknesses of braced and stayed flat plates, design of stay bolts, structural reinforcements, maximum allowable pitch, in inches, of screwed stay bolts, ends riveted over, and maximum allowable stresses for stay bolts and stays or braces. The following requirements for calking form part of the rules:)

U-52. Calking.-(a) The calking edges of plates, butt straps, and heads shall be beveled to an angle not sharper than $70^{\circ}$ to the plane of the plate, and as near thereto as practicable. Every portion of the unfinished surfaces of the calking edges of plates, butt straps, and heads shall be planed, milled, or chipped to a depth of not less than oneeighth inch. Calking shall be done with a tool of such form that there is no danger of scoring or damaging the plate underneath the calking edge, or splitting the calked sheet.

(b) Fusion welding may be used to seal the calking edges of riveted joints and rivet heads of unfired pressure vessels, provided the plates do not exceed 0.30 per cent carbon and that the thickness of the plate or head affected by the welding is at least one-eighth inch more than that required for a seamless shall of the same diameter, the same working pressure and the same grade of material. Such seal welding shall not be applied until after the vessel is made tight as evidenced by the regular hydrostatic pressure test prescribed. Seal welding may be used on nozzles and their reinforcing plates under the the same conditions. On unstayed dished heads, seal welding shall not be applied closer than one-half inch to the point of tangency of the knuckle of the flange. Seal welding may be applied only when the weld metal is deposited in a single layer having a throat thickness of not less than three-sixteenths inch nor more than fivesixteenths inch. The heat from welding shall not distort the plate or loosen the rivets in such a manner as to break the initial bond effected in the riveted joint. After seal welding, the vessel shall be resubjected to the prescribed hydrostatic test.

\section{MANHOLES AND HANDHOLES}

U-53. All vessels for use with compressed air, or subject to internal corrosion, 18 inches in diameter or over, and not exceeding 36 inches in diameter, shall have a handhole in the shell or head, or a manhole or at least two plugged threaded openings of not less than 2 inches pipe size. When two threaded openings are used, they should be located in the heads or in the shell near the heads.

All such vessels less than 18 inches in diameter shall be provided with at lest two handholes, or two inspection holes, properly located for inspection, the inspection holes to be not less than $1 \frac{1}{2}$ inches

pipe size, unless the vessels have removable heads or cuver plates.

In special cases where vessels 16 inches or less in diameter are located so that inspections can not be made without dismantling or removing same, special openings need not be provided if the tapping

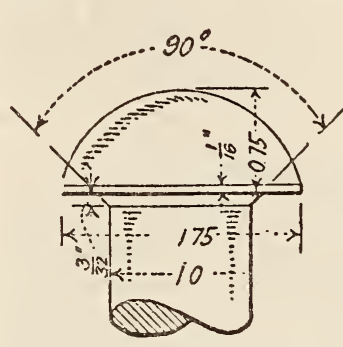

Straight Base Button Head

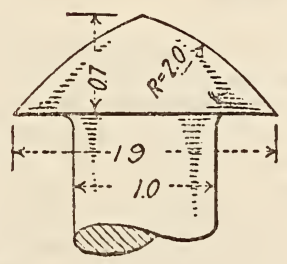

Double Radius Button Head Steeple Head

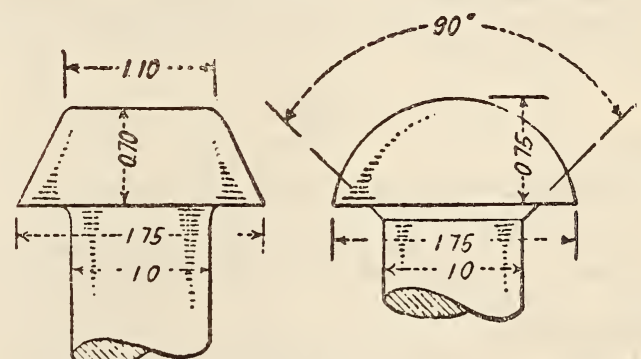

Pan Head

Button Head

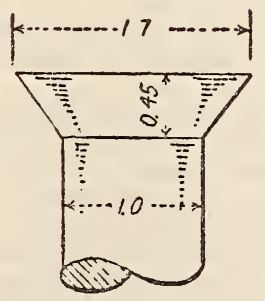

Countersunk Head

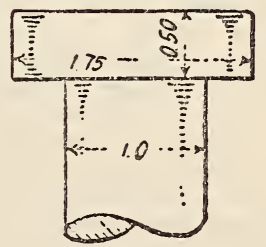

Fiat Head

Figure 156.-Acceptable forms of finished rivet heads

for pipe connections properly located for inspection purposes is not less than $1 \frac{1}{2}$-inch pipe size.

U-54. Vessels for use with compressed air, or subject to internal corrosion, over 36 inches in diameter, shall have a manhole, excepting those whose shape or use make it impracticable, in which case they shall have a 4 by 6 -inch handhole or its equivalent in area, located as near each head as 
practicable. An elliptical manhole opening shall be not less than 11 by 15 inches or 10 by 16 inches in size. A circular manhole opening shall be not less than 15 inches in diameter.

U-55. A manhole reinforcing ring, when used, shall be of rolled, forged, or cast steel, shall be at least as thick as the shell plate, and shall have a net cross-sectional area, on a line parallel to the axis of the shell, not less than the cross-sectional area of shell plate removed on the same line.

(Additional requirements cover strength of rivets in shear and in tension, spacing of rivets attaching nozzles, material of manhole plates, pipe threads for nozzle openings, formulas for determining maximum diameter of unreinforced circular openings, types of nozzle fittings, supporting vessels so as properly to distribute the stresses due to weight of vessel and contents, hydrostatic tests, and inspection. Rules for the fusion process of welding, for forge welding, and for brazing are contained in three separate chapters. The following provisions are made for enameled vessels:)

\section{ENAMELED VESSELS}

U-97. Material.-All pressure vessels intended to be coated with glass or other enamel shall be made of steel or iron not under three-sixteenth inch nor more than five-eighth inch in thickness and may be welded by the oxyacetylene, electric, or forgewelding processes. If forge welding is used the requirements of paragraphs U-80 to U-90 shall be followed. All plates shall conform to the Specifications for Steel Plates of Flange Quality for Forge Welding, Specifications S-2, and any plate not conforming thereto may be rejected.

U-98. The maximum allowable working pressure for single-shell vessels shall be determined by the following formula:

where

$$
P=\frac{5000 t}{R}
$$

$t=$ thickness of plate, inches, and $R=$ inside radius, inches

U-99. The formula given in paragraph U-36 shall be used in calculating the minimum thickness of heads necessary for the various pressures and diameters.

$\mathrm{U}-100$. The ratio of diameter of the vessel to thickness of plate shall in no case exceed 320 .

U-101. Any brand of welding wire which has been found by practice to give good results may be used, and mav be bare, coated or covered.

U-102. Vessels of this class will be considered under two types, viz, single-shell vessels and jacketed vessels.

U-103. Longitudinal seams of single-shell vessels welded by either the oxyacetylene or electric processes or both shall be double $\mathbf{V}$ welded.

The weld on all surfaces to be enameled may be ground flush with the surface of the plate.

U-104. Jacketed or double-shell vessels may be of two types, one in which one of the heads of the inner vessel forms the sealer apron for the jacket, and one in which the sealer apron is joined to the shell of the inner vessel at some point between the heads, forming a partially jacketed vessel.

U-105. The inner shell of a jacketed vessel, when not stayed to the outer shell, should be of such construction as to safely withstand both the pressure in the jacket which tends to collapse it, and the pressure on the inside which tends to burst it, when either of these pressures acts singly.

U-106. The longitudinal seam of the jacket shall be double $V$ welded. The circumferential seam in the jacket joining the head to the shell may be single $V$ welded.

U-107. In jacketed vessels where the sealer apron is welded to the shell of the inner vessel, and also where the sealer apron is welded to the shell of the jacket, the weld may be made from outzide only, provided the thickness of the metal deposited is equal to or greater than the thickness of the apron or plate.

U-108. In jacketed vessels where the top head of the inner shell forms the sealer apron, the head may be welded to the shell of the inner vessel from the inside only, provided the thickness of the weld after grinding is equal to or greater than the thickness of the shell. ing.

U-109. All cylinders shall be rerolled after weld-

ASSOCIATED FACTORY MUTUAL FIRE INSURANCE COMPANIES, GRAVITY WATER TANKS AND STEEL TOWERS, STRUCTURAL DETAILS, 1931

\section{WATER TANKS AND STEEL TOWERS}

(For steel tanks for fire protection, standard sizes; plates, shapes, and rivets according to A. S. T. M. specifications; thickness of plates and design details. Also covers steel towers for supporting water tanks, minimum number of columns; materials according to A. S. T. M. specifications; computation of dead, live, and wind load. Under piping, fittings, and heating systems this specification contains requirements for discharge pipe and fittings, expansion joint, overflow, clean out and drain, mill-use connections, telltale, valve pit or valve house, and frost-proof casings for pipes. General requirements for steam heaters and for coal-burning heaters.)

\section{ASSOCIATED FACTORY MUTUAL FIRE IN- SURANCE COMPANIES, STORAGE AND HANDLING OF FLAMMABLE LIQUIDS, 1930}

\section{STORAGE TANKS}

Horizontal, cylindrical steel tanks are most commonly and economically used for underground storage. They may be obtained readily in sizes up to 25,000 gallons capacity, this size usually being about 12 feet in diameter by 42 feet long.

(In respect to materials this specification is identical with that of the National Board of Fire Underwriters specification for containers for storing and handling flammable liquids, given below.)

Note.-For tanks of 1,100 gallons and more a variation of 10 per cent in capacity may be allowed from the tabulated sizes. (And, as stated in the N. B. F. U. specification referred to in the preceding paragraph, if adequate internal bracing is provided, tanks from 12,000 to 30,000 gallons capacity may be built of $1 / 4$-inch plate.)

\section{NATIONAL BOARD OF FIRE UNDERWRIT- ERS, OIL-BURNING EQUIPMENTS, 1928}

\section{OIL-BURNING EQUIPMENTS}

(Requirements covering material, construction, and tests are identical with those for containers for storing and handling flammable liquids, given below. except that in this specification no provision is made for portable tanks.) 
NATIONAL BOARD OF FIRE UNDERWRITERS, CONTAINERS FOR STORING AND HANDLING FLAMMABLE LIQUIDS, 1927

\section{UNDERGROUND TANKS}

Tanks shall be constructed of galvanized steel, open-hearth steel, or wrought iron of a minimum gage (U. S. standard), depending upon the capacity as given in Table 1 . For liquids heavier than $35^{\circ}$ Baumé, tanks may be of reinforced concrete.

TABLE 1

\begin{tabular}{|c|c|c|}
\hline Capacity (in gallons) & Gage & $\begin{array}{l}\text { Minimum } \\
\text { weight of } \\
\text { material }\end{array}$ \\
\hline $\begin{array}{l}1 \text { to } 285 \\
286 \text { to } 560 \\
561 \text { to } 1,100 \\
1,101 \text { to } 4,000 \\
4,001 \text { to } 12,000 \\
12,001 \text { to } 20,000 \\
20,001 \text { to } 30,000\end{array}$ & 14 inch..... & $\begin{array}{c}\text { Lbs./in. } 2 \\
2.50 \\
3.125 \\
4.375 \\
7.50 \\
10.00 \\
12.50 \\
15.00\end{array}$ \\
\hline
\end{tabular}

Tanks of open-hearth steel or wrought iron thinner than No. 7 gage shall be galvanized.

For class 3 liquids, if adequate internal bracing is provided, tanks from 12,001 to 30,000 gallons capacity may be built of $1 / 4$-inch plate.

All joints of tanks shall be riveted and calked, brazed, welded, or made tight by some equally satisfactory process. Tanks shall be tight and sufficiently strong to bear without injury the most severe strains to which they may be subjected in practice. Shells of tanks shall be properly reinforced where connections are made, and all connections made through the top of tank above the liquid level.

Note.-Tanks for systems under pressure shall be designed for four times the working pressure and tested to twice the maximum working pressure.

(Provision is also made for venting of tanks, setting, location of filling pipe, manholes, gaging devices, withdrawal of liquids, care, and attendance.)

\section{PORTABLE TANKS}

Capacity of tank shall not exceed 65 gallons. Tank shall be made of iron or steel plate not less than three-sixteenths inch, with joints eicher riveted and calked, welded, brazed, or made tight by some equivalent method. Where necessary, tanks shall be substantially braced.

Tanks shall be made with as few openings as practicable and suitably reinforced where necessary. Openings for fittings shall be made in top above liquid level.

(Provision is also made for tank supports, wi ihdrawal of liquid, stability, vents, wheels, fittings, and care and attendance.)

\section{STATIONARY TANKS IN BUILDINGS}

Rectangular tanks shall be made of sheet iron or steel not less than No. 14 U. S. standard gage in thickness. Cylindrical tanks not exceeding 120 gallons capacity shall be made of sheet iron or steel not less than No. 20 U. S. standard gage. Material lighter than No. 16 U. S. gage shall be galvanized. All joints of tanks shall be riveted, brazed, welded, or made tight by some equally satisfactory process. Joints shall be tight and sufficiently strong to bear without injury the most severe strains to which they are liable to be subjected in practice. The shells of tanks shall be properly reinforced where connections are made, and all connections made through upper side of tanks above the liquid level.

Tanks shall be thoroughly braced where necessary, and designed to withstand extreme service conditions.

All attachments shall be made at top of tank by means of suitable flanges.

(Fittings, care and attendance, and withdrawal of liquid are also provided for.)

\section{ABOVEGROUND TANKS}

Tanks, including top, shall be constructed of open-inearth steel or wrought iron of a tilickness depending upon the capacity, and to be figured in accordance with the following table or formula. For liquids of $35^{\circ}$ Baumé or heavier, tanks may be constructed of concrete in accordance with the regulations for the design and construction of concrete fueloil storage tanks.

1. Horizontal or vertical tanks not over 1,100 gallons capacity.-

\begin{tabular}{|c|c|}
\hline Capacity (in gallons) & $\begin{array}{c}\text { Minimum } \\
\text { thickness } \\
\text { of material }\end{array}$ \\
\hline & Gage \\
1 to 60 & 18 \\
61 to 350 & 16 \\
351 to 560 & 14 \\
561 to 1,100 & 12 \\
\hline
\end{tabular}

2. Horizontal tanks over 1,100 gallons capacity.Tanks having a diameter of not over 6 feet shall be made of at least three-sixteenth inch steel.

Tanks having a diameter of over 6 feet and less than $11 \frac{1}{2}$ feet shall be of at least $1 / 4$-inch steel.

3. Vertical tanks over 1,100 gallons capacity.Tanks of this class shall be of such material and so constructed as to give a factor of safety of at least 2.5.

The minimum thickness of shell or bottom shall be three-sixteenth inch.

The minimum thickness of roof shall be one-eighth inch.

The thickness of plates shall be figured in accordance with the following formula:

Where

$$
t=\frac{2.604 \times H \times D \times F \times S}{T \times E}
$$

$t=$ thickness of plate in inches;

$H=$ height of tank in feet above the bottom of the ring under consideration;

$D=$ diameter of the tank in feet;

$F=$ factor of safety (taken as 2.5 );

$S=$ specific gravity of liquid, taking water equal to 1 ;

$T=$ tensile strength of plate in pounds per square inch;

$E=$ efficiency of vertical joint in ring under consideration.

The tensile strength of the steel shall be taken as 55,000 pounds per square inch, while the shearing strength of rivets shall be taken as 40,000 pounds per square inch.

Tanks shall be riveted, welded, or brazed, and shall be calked or otherwise made tight in a mechanical and workmanlike manner. The top of tank shall be securely fastened to top ring with joints of practically equal tightness to those between the rings.

(Provision is also made for rusting, vents, setting of tanks, piping, embankments and dykes, withdrawal of liquid, care and attendance.) 
NATIONAL BOARD OF FIRE UNDERWRITERS; TANKS, GRAVITY AND PRESSURE, TOWERS, ETC., 1926

\section{STEEL GRAVITY WATER TANKS}

\author{
Section 1. GENERAI
}

101. These specifications define the recommended practice for the design, fabrication, and erection of steel gravity water tanks, elevated or on concrete foundations.

102. Capacity.-The capacity of the tank is the number of U. S. gallons available above the outlet opening.

103. Standard sizes.-The standard sizes of steel tanks are: 5,000, 10,000, 15,000, 20,000, 25,000, $30,000,40,000,50,000,60,000,75,000,100,000$, $150,000,200,000,300,000$, and 500,000 U. S. gallons net capacity. Tanks of other sizes are built occasionally.

104. Form.-The form of a steel tank shall be cylindrical with vertical axis. If supported by a steel tower without beams, a hemispherical or other acceptable form of suspended bottom shall be provided. The tank may have a flat bottom when placed on steel or concrete beams, or on a slab. Tanks of special shapes are sometimes permitted.

105. Strength. - The material as specified shall be without defects affecting its strength or service. The workmanship shall be good, so that defects or injuries are not produced during manufacture or erection. Unit stresses as specified shall not be exceeded. The structure and its details shall possess the requisite strength and rigidity.

\section{Section 2. MATERIAL}

201. Plates and shapes.-The steel for structural shapes and plates except in suspended bottoms, shall conform to current specification A-7 structural steel of the American Society for Testing Materials. (See 605.11 , p. 249.) For suspended bottoms, steel not milder than $\mathrm{A}-7$ rivet steel may be used provided that unit tension specified in article 402 is reduced in proportion to the reduction in the ultimate strength.

202. Rivets. - The steel for rivets shall conform to current specification A-7 rivet steel of the American Society for Testing Materials.

203. Anchor bolts. - Anchor bolts shall be of iron or steel conforming to current specification A-84, for engine bolt iron (see 603.1, p. 139), or current specification A-7 of the A. S. T. M. Welds are not permitted.

\section{Section 3. IOADS}

301. Dead load.-The dead load shall be the estimated weight of all permanent construction and fittings. The unit weight of steel shall be considered 490 pounds, and of concrete 144 pounds, per cubic foot.

302. Live load.-The live load shall be the weight of all the liquid when overflowing the top of the tank. The unit weight of water shall be considered as 62.5 pounds per cubic foot. Proper provision must be made for temporary stresses during erection. When roofs have a slope of less than 30 degrees, they shall be designed to support a uniform weight of snow of 25 pounds per square foot on the horizontal projection.

303. Wind load.-The wind pressure shall be assumed to be 30 pounds per square foot on a vertical plane surface. In calculating the wind load on a cylindrical surface, six-tenths of the above pressure shall be applied to the total area of the vertical projection, and the point of application of the load shall be at the center of gravity of the projected area.

\section{Section 4. UNIT STRESSES}

401. General.-The maximum stresses in pounds per square inch produced by the above loads shall not exceed the following:

402. Tension on net section, rolled steel, 15,000; anchor bolts, 18,000.

403. Shearing: Rivets, 11,250.

404. Bearing: Rivets, 22,500.

405. Bending: Extreme fibers of rolled shapes and built-up sections, net sections, 16,000 .

When the laterally unsupported length $l$ exceeds ten times $b$, the width of the compression flange, the stress in the latter shall not exceed

$$
16,000-150 \frac{l}{b}
$$

The laterally unsupported length of beams and girders shall not exceed forty times $b$, the width of the compression flange.

406. Wind allowance.-When the wind load is considered in calculating stresses the permissible working unit stresses may be increased 25 per cent provided that the resulting section is not less than that required for dead and live loads alone.

\section{Section 5. DETAILS OF DESIGN}

501. Minimum thickness.-The minimum thickness of metal shall be one-quarter inch except in roofs, railings, and webs of channels and I beams.

502. Thickness for corrosion.-Interior bracing, if unavoidable, shall always have one-sixteenth inch additional thickness added to the calculated sections. The sections shall be open to facilitate cleaning and painting. The plates of tanks to contain salt or alkaline water shall be one-sixteenth inch thicker than calculated.

503. Thickness of bottom cylindrical courses.-The thickness of plates in the lowest cylindrical courses of tanks with suspended bottoms shall be not less than five-sixteenths inch for 100,000 and 150,000 gallons, three-eighths inch for 200,000-gallon tanks, and for larger tanks shall be at least one-sixteenth inch greater than that calculated.

504. Thickness of flat bottoms. - The thicknesses of plates in flat bottoms shall be not less than those given in Table 1.

TABLE 1.-Thickness, in inches, of bottom plates for flat-bottom tanks

\begin{tabular}{|c|c|c|c|c|c|c|c|c|c|c|c|c|c|}
\hline \multirow{2}{*}{\multicolumn{2}{|c|}{ Type of support }} & \multicolumn{12}{|c|}{ Depth of water (in feet) } \\
\hline & & 10 & 12 & 14 & 16 & 18 & 20 & 22 & 24 & 26 & 28 & 30 & 40 \\
\hline $\begin{array}{l}\text { Distance in clear between steel } \\
\text { or concrete beams (in inches) } \\
\text { Concrete slab }\end{array}$ & $\left\{\begin{array}{l}12 \\
14 \\
16 \\
18 \\
20 \\
24\end{array}\right.$ & $\begin{array}{l}5 / 16 \\
5 / 16 \\
5 / 16 \\
5 / 16 \\
5 / 16 \\
3 / 8 \\
1 / 4\end{array}$ & $\begin{array}{l}5 / 16 \\
5 / 16 \\
5 / 16 \\
5 / 16 \\
5 / 16 \\
3 / 8 \\
114\end{array}$ & $\begin{array}{l}5 / 16 \\
5 / 16 \\
5 / 16 \\
5 / 16 \\
3 / 8 \\
7 / 16 \\
1 / 4\end{array}$ & $\begin{array}{l}5 / 16 \\
5 / 16 \\
5 / 16 \\
5 / 16 \\
3 / 8 \\
7 / 16 \\
1 / 4\end{array}$ & $\begin{array}{l}5 / 16 \\
5 / 16 \\
5 / 16 \\
3 / 8 \\
3 / 8 \\
7 / 16 \\
1 / 4\end{array}$ & $\begin{array}{l}5 / 16 \\
5 / 16 \\
5 / 16 \\
3 / 8 \\
7 / 16 \\
1 / 2 \\
1 / 4\end{array}$ & $\begin{array}{l}5 / 16 \\
5 / 16 \\
3 / 8 \\
3 / 8 \\
7 / 16 \\
1 / 2 \\
1 / 4\end{array}$ & $\begin{array}{l}5 / 16 \\
5 / 16 \\
3 / 8 \\
3 / 8 \\
7 / 16 \\
1 / 2 \\
1 / 4\end{array}$ & $\begin{array}{l}5 / 16 \\
5 / 16 \\
3 / 8 \\
7 / 16 \\
7 / 16 \\
9 / 16 \\
1 / 4\end{array}$ & $\begin{array}{l}5 / 16 \\
3 / 8 \\
3 / 8 \\
7 / 16 \\
1 / 2 \\
9 / 16 \\
1 / 4\end{array}$ & $\begin{array}{l}5 / 16 \\
3 / 8 \\
3 / 8 \\
7 / 16 \\
1 / 2 \\
9 / 16 \\
1 / 4\end{array}$ & $\begin{array}{l}3 / 8 \\
7 / 16 \\
7 / 16 \\
1 / 2 \\
9 / 16 \\
11 / 16 \\
1 / 1\end{array}$ \\
\hline
\end{tabular}


505. Accessibility of bottoms.-Grillages shall be designed so that the tank bottom and beams will be accessible for inspection and painting.

506. Net sections. - Net sections shall be used in calculating the tensile stress in plates and members. In deducting the rivet holes their diameter shall be taken one-eighth inch larger than the undriven rivets for punched holes and one-sixteenth inch larger for reamed or drilled holes.

507. Rivet size considered.-Nominal diameters shall be used in proportioning rivets in punched work. Diameters of the rivet holes may be used in drilled or reamed work.

508. Edge distance.-The minimum distance from the center of any rivet hole to a sheared edge shall be at least one and one-half times the nominal rivet diameter. This distance is to be measured from the center of the bevel of bevel-sheared plates greater than three-eighths inch in thickness.

509. Spacing between rows. - When the rivets of adjacent parallel rows are staggered, the distance between center lines of adjacent rows shall be at least twice the diameter of the rivets, and the section of the plate between two rivets in adjacent rows shall be at least six-tenths of the section between two rivets in the line of stress.

510. Pitch of rivets.-The minimum distance between center of rivet holes shall be at least two and one-half diameters of the rivet, but should preferably be not less than $17 / 8$ inches for $5 / 8$-inch rivets, $21 / 4$ inches for $3 / 4$-inch rivets, and $25 / 8$ inches fur $7 / 8$-inch rivets.

511. Rivet pitch for calking.-The maximum distance between centers of rivet holes along calking edges of plates, except at the column connections, shall not exceed ten times the thickness of the thinnest plate for single-riveted joints or twelve times the thickness of the thinnest plate in joints having more than one row of rivets.

512. Load location.-Vertical joints shall be designed with the load applied at the center of plates for plates not exceeding 7 feet in width, except as specified in article 503.

513. Opening reinforcement.-Manholes, pipe openings, and connections for attachments shall be reinforced by additional steel plates of sufficient thickness and properly riveted to carry the total load that would be carried by the cut-out portion of the plate, without overstressing, when the average tensile stress in the net section of the cut plate exceeds 13,000 pounds per square inch

514. Curved-bottom plate location.-Suspended bottom plates shall lap inside the plates of the lowest cylindrical course.

515. Stiffening angle.-Stiffening angles shall be provided around the tops of tanks without roofs.

516. Bottom angle. - The sides and bottoms of flat-bottom tanks over 20 feet in diameter shall be connected by spliced angles calked along both legs.

\section{Section 6. WORKMANSHIP}

601. Shearing.-All shearing shall be done neatly, and material exceeding three-fourths inch in thickness shall have one-eighth inch planed from the sheared edges. All calking edges shall be bevel sheared or planed to an angle of not less than $60^{\circ}$ with the plane of the plate.

602. Punching. - Rivet holes in plates one-fourth inch up to five-eighths inch in thickness may be punched. The diameter of the punch shall be not more than one-sixteenth inch larger than that of the rivet, and the diameter of the die not more than three-thirty-seconds inch larger than that of the punch.

603. Subpunching.-Rivet holes in plates thicker than five-eighths inch but not exceeding seveneighths inch shall be drilled or subpunched and reamed. The diameter of the punch shall be at least three-sixteenths inch less than that of the undriven rivets. The diameter of the finished holes shall not be more than one-sixteenth inch greater than that of the rivets.

604. Drilling. - Rivet holes in plates over seveneighths inch thick shall be drilled to a diameter not more than one-sixteenth inch greater than that of the rivets.

605. Removing burrs.-After drilling or reaming holes, the burrs shall be completely removed.

606. Shaping.-All plates shall be shaped to the proper curvature by cold working without reducing the elastic limit of the metal.

607. Use of rivets. - All joints between tank plates shall be riveted.

608. Heads of rivets.-Driven rivets over one-half inch in size shall have button, cone, or steeple heads central on the shank. The heads shall grip the assembled pieces firmly and be neat and of equal size. Heads shall be formed on the side of the plate which is calked.

609. Assembling.-Plates shall be bolted firmly together before riveting. Drift pins should preferably not be used for bringing the parts together and shall never be used to enlarge unfair holes.

610. Driving of rivets. - Rivets of greater diameter than one-half inch shall be uniformly heated to a light cherry red heat. Shop rivets shall be driven by pressure. Rivets in butt joints shall be driven with pneumatic hammers. Recupping and calking is not allowed. Loose, burned, or otherwise defective rivets shall be cut or drilled out and replaced without injuring the adjacent metal.

611. Calking. - The tank shall be made watertight by calking the edges of the plates with a round-nosed tool before painting. Foreign materials such as lead, copper filings, cement, etc., shall not be used in the joints.

612. Fitting roofs. - The roof shall fit tightly to the top of the tank to prevent circulation of air over the surface of the water. To assist in obtaining a tight fit, a grid or spider consisting of steel rods spaced preferably not over 42 inches apart should be placed around the top of the tank running radially inward to a ring. The spider shall not obstruct the flow of water into the overflow inlet.

613. Preventing ice damage.-The contractor shall keep the tank, structure, and building roofs free of ice caused by leakage during the construction and until the tank equipment is made water-tight.

\section{Section 7. ACCESSORIES}

701. Connections.-Connections shall be provided on the tank for the necessary pipes, braces, frostcasing, and walkway supports.

702. Roof (general). - A conical steel roof shall be provided when the tank is out of doors. The roof plates shall be at least one-eighth inch thick, joined together with rivets spaced not more than 6 inches apart. Conical roofs with a minimum slope of $30^{\circ}$ are self supporting when roof plates are one-eighth inch thick on tanks not over 30 feet in diameter and when plates are three sixteenths inch thick on tanks not over 40 feet in diameter. Flatter or larger roofs shall be supported by steel rafters. A cover may also be required when the tank is inside of a building.

703. Roof anchorage.-Each roof plate shall be securely fastened to the top of the tank by bolts or rivets at least one-half inch in diameter. At least two bolts or rivets shall be placed in each roof plate.

704. Roof hatch.-An easily accessible hatch not less than 20 by 22 inches shall be provided in the roof. The hatch cover shall be built of steel plate at least one-eighth inch thick and shall open to the 
right on heavy hinges with noncorrodible pins. A substantial catch shall be provided to keep the cover closed. Other forms of hatch covers may be accepted but approval of the design shall first be obtained from the inspection department having jurisdiction.

705. Ladders (general).- -Inside and outside steel ladders arranged for convenient passage from one to the other and through the roof hatch shall be provided. Ladders shall not interfere with opening the hatch cover and shall not incline outward from the vertical at any point. Ladders riveted to the tank side are preferred.

706. Outside fixed ladder.-The outside tank ladder should preferably be fixed at least 6 inches from the tank side and rigidly bolted to brackets riveted to the tank plates not over 12 feet apart. The top bracket shall not be more than 1 foot below the top of the shell and the bottom bracket not more than 6 feet above the base of the tank cylinder. The fixed ladder shall be in line with the roof hatch and there shall be at least 1 foot clearance at the sides and front of the ladder at the balcony.

707. Outside revolving ladder.-Outside revolving ladders, if provided, in lieu of outside fixed ladders, shall not exceed 30 feet in length and shall extend from tower ladder to painter's trolley. The ladder shall be rigidly bolted, at the top and bottom, in its normal position in line with the tower ladder and just to the left of the roof hatch.

708. Inside ladder. - The inside ladder shall be riveted to the cylindrical shell by brackets not over 12 feet apart, the upper bracket being located at the top of the tank. The portion of the ladder in the suspended bottom shall extend to the lowest point, and be provided with brackets not over 12 feet apart, which rest upon the tank without scraping off the paint. The brackets shall not be connected to the suspended bottom plates.

709. Ludder bars and rungs.-Ladder side bars shall be not less than 2 inches by one-fourth inch flat steel for fixed ladders and $21 / 2$ inches by three-eighths inch for movable ladders. Side bars shall be spaced 14 inches apart. Rungs shall be at least fiveeighths inch round or square steel spaced 12 inches on centers. The rungs shall be firmly riveted to the side bars. Ladders and connections shall be designed to support a concentrated load of 350 pounds.

710. Painter's trolley. - Some form of trolley or other acceptable device shall be provided to facilitate repainting the outside of the tank. The connection of trolley at the top of the tank shall be substantial.

711. Painting inaccessible parts.-Parts inacessible after fabrication shall be protected by paint before assembling.

712. Shop painting. - All steel, except where encased in concrete, shall be thoroughly cleaned and given a shop coat of red-lead paint, mixed with 100 pounds of red-lead paste $\left(94\right.$ per cent $\mathrm{Pb}_{3} \mathrm{O}_{4}$ ) to $21 / 2$ gallons of linseed oil (if raw oil is used add one-half pint of dryer to every gallon of paint); or an approved ready-mixed red-lead paint.

713. Field painting. - After erection, a patch coat of the same kind of paint as the shop coat shall be applied to all surfaces from which the shop coat has become removed. An over-all field coat of a good grade of metallic paint of color specified by the purchaser shall be applied after the tank has been made water-tight.

714. Painting conditions.-All steel shall be thoroughly cleaned of mill scale, rust, and grease before painting by scraping, wire brushing and washing with carbon tetrachloride or other nonhazardous cleaning agent except soda compounds. Painting shall not be done out of doors during wet or freezing weather. The coats of paint shall completely cover the steelwork.
PRESSURE TANKS

Section 36. CONSTRUCTION AND GENERAL REQUIREMENTS

3601. Service. - Pressure tanks shall be used only as a supply to automatic sprinkler systems, unless otherwise approved by the inspection department having jurisdiction.

3602. Capacity. - The capacity shall be approved by the inspection department having jurisdiction. The capacity is the total contents, both air and water, in the cylinder, not including dished ends, and should generally be not less than 4,500 gallons nor more than 9,000 gallons in one tank.

3606. Construction-(a) General.-Except as herein specified to the contrary, pressure tanks shall be constructed in accordance with the current rules for the construction of unfired pressure vessels, Section VIII of the American Society of Mechanical Engineers, Boiler Construction Code. (See above.)

(b) Allowance for corrosion. - The computed or minimum required thickness of tank plates shall be increased one-sixteenth inch to allow for corrosion.

(c) Rivet diameter. - The rivet diameter used in computations shall be not more than one-sixteenth inch greater than the nominal diameter.

(d) Tests.-Each pressure tank shall be given the tests specified by the A. S. M. E. rules before painting, except that the hydrostatic test pressure shall in no case be less than 150 pounds per square inch.

In addition to the A. S. M. E. tests, each pressure tank shall be filled two-thirds full and tested at the normal working pressure with all valves closed, and shall not lose more than one-half pound pressure in 24 hours.

A certificate signed by the manufacturer shall be filed with the inspection department having jurisdiction, certifying that the foregoing tests have been made.

A repetition of the above tests may be required after the tank has been set in place and connected. In all cases where conditions do not permit shipping the tank assembled, the above tests shall be conducted after erection in the presence of a representative of the inspection department having jurisdiction.

(e) Supports. - The supports shall be steel or reinforced concrete located so as to prevent sagging or vibration, and properly to distribute the loads due to the weight of the vessel completely full of water. Stresses in steel supports shall not exceed those permitted by section 11 for steel towers. The stresses in rivets fastening lugs or brackets, if used, to the vessel shall not exceed 3,800 pounds per square inch for single shear, or, 7,600 pounds per square inch for double shear or bearing. For horizontal tanks there shall be at least one support for each course of the tank and the ends shall not overhang more than 4 feet. Lugs, brackets, or cradles shall be properly fitted and cradles or chucks braced to prevent movement. Tne supports shall be arranged to permit convenient inspection and maintenance of all parts of the equipment and shall not interfere with the piping or tank seams.

(Other requirements in this specification cover painting, care and maintenance, marking, name plate, water-level indicator, housing, and details connected with pipe connections and fittings.)

\section{STEEL TOWERS}

Section 8. GENERAI

801. These specifications define the recommended practice for the design, fabrication and erection of steel towers to support water tanks.

802. Height. - The height of tower is the vertical distance from the top of the foundations to the bottom capacity line of the tank. 
S03. Number of columns.-The minimum permissible number of columns for tanks with suspended bottoms is 4 for tanks of 50,000 to 200,000 gallons capacity, inclusive, 6 for tanks of over 200,000 gallons and including 300,000 gallons, and 8 for tanks of over 300,000 gallons capacity.

804. Strength. - The material as specified shall be without defects affecting the strength or service of the structure. The workmanship shall be good so that defects or injuries are not produced in the manufacture or erection. Unit stresses as specified shall not be exceeded. The structure and its details shall possess the requisite strength and rigidity.

\section{Section 9. MATERIAI}

901. Shapes and plates.-Structural steel for towers shall conform in its chemical and physical properties to current specification $A-7$, structural steel of the American Society for Testing Materials. (See 605.11, p. 249.)

902. Rivets. - Steel for rivets shall conform in its chemical and physical properties to current specification A-7, rivet steel of the American Society for Testing Materials.

903. Rods and bolts.-Adjustable tension members and anchor bolts shall be of wrought iron or steel. Wrought iron shall conform in its chemical and physical properties to current specification A-84, engine-bolt iron of the American Society for Testing Materials. (See 603.1, p. 139.) Steel shall conform in its chemical and physical properties to current specification A-7 of the American Society for Testing Materials. Welding by oxyacetylene or electric-arc processes is not permitted in members carrying calculated stress.

\section{Section 10. LOADS}

(Requirements covering dead load, live load, and wind load conform to those specified for steel gravity water tanks, given above. In the case of wind load, the load on the tower shall be assumed to be concentrated at the panel points. No provision is, of course, made for roof loads. Otherwise this section provides for the following:)

1003. Live load (large risers).-The water contained in a cylinder extending from the water surface to the base of the riser need not be included in the load supported by the tower. The diameter of the cylinder shall be taken as that at the contact between the riser and the foundation.

1005. Walkway and balcony loads. - The walkway under the tank and the balcony around the tank shall be designed to carry a 500-pound and a 1,000 -pound concentrated load respectively, at any point without overstressing.

\section{Section 11. UNIT STRESSES}

(This section covers the maximum stresses in pounds per square inch produced by the above loads. The stresses include axial tension, axial compression, slenderness ratio of both main and secondary members, bending, shearing, bearing, combined stresses, wind allowance, and initial tension.)

\section{Section 12. DETAILS OF DESIGN}

1201. Sections.-Sections shall preferably be symmetrical. The metal shall be concentrated as much as possible in webs and flanges and the members shall be built of standard or Bethlehem structural shapes. Members shall be designed with open sections to permit painting all surfaces exposed to air or moisture and subject to corrosion.

1202. Minimum thickness (general).-The minimum thickness of metal shall be one-fourth inch except in the webs of channels and I-beams and in minor parts which carry no load. The minimum size of sway rods shall be three-fourths inch.

1203. Thickness of cover plates.-The thickness of cover plates shall be at least one-fourth inch and not less than one-sixtieth of the distance between rivet lines. A cover plate may be used on but one side of a column.

1204. Net sections.-To calculate net sections of tension members the diameters of the rivet holes shall be taken one-eighth inch larger than the nominal diameters of the rivets. For compression members all bolt holes and openings other than rivet holes shall be deducted from the cross-sectional area. For a distance of twice the maximum width of the member from the end, the allowable unit compression on the net section may be taken as the unit compression on short lengths. Article 1103.

1205. Rivet sizes.-Nominal diameters shall be used in proportioning rivets. The diameters of rivets in the flanges of channels except in minor and unimportant parts shall be one-half inch or fiveeighths inch for 6-inch and 5-inch channels, fiveeighths inch for 7-inch and 8-inch channels, threefourths inch for 9,10 , and 12 inch channels, and seven-eighths inch or three-fourths inch for larger channels. The diameters of rivets in the flanges of I-beams shall not be greater than five-eighths inch for flanges less than 4 inches in width, threefourths inch from 4 to 6 inches, inclusive, and seveneighths inch for widths greater than 6 inches.

1206. Rivet edge distance. - The distance from the center of any rivet hole to an edge, except rolled edges of structural shapes, shall be at least one and one-half times the nominal diameter of the rivet.

1207. Minimum rivet pitch.-The distance between centers of rivet holes shall be at least three diameters of the rivet.

1208. Maximum rivet pitch.-The distance between centers of rivet holes in the line of stress for members composed of plates and shapes shall not exceed sixteen times the thickness of the thinnest plate but shall be not more than 12 inches. When two rows of rivets are used and the rivets are staggered, the maximum distance between rivet holes in each line shall not exceed twice the above.

1209. Riveting at ends of cover plates.-The pitch of rivets at the ends of cover plates in built-up compression members shall not exceed four diameters of the rivet for a length equal to one and one-half times the maximum width of the member.

1210. Lattice.-The open sides of compression members shall be provided with lattice. The thickness of lattice bars shall be at least one-fortieth of their length between the centers of end rivets for single and one-sixtieth for double latticing. The minimum width of lattice bars shall be as follows:

For 15 -inch channels or built sections with $3 \frac{1}{2-}$ inch or 4 -inch angles-21/4 inches ( $3 / 4$-inch rivets) or $21 / 2$ inches ( $7 / 8$-inch rivets).

For 12-inch, 10-inch, and 9-inch channels or built sections with 3 -inch angles $-2 \frac{1}{4}$ inches $(3 / 4$-inch rivets).

For 8 -inch and 7 -inch channels or built sections with $21 / 2$-inch angles -2 inches ( $5 / 8$-inch rivets).

For 6-inch and 5-inch channels or built sections with 2 -inch angles- $1 \frac{1}{2}$ inches (1/2-inch rivets) or $13 / 4$ inches ( $5 / 8$-inch rivets).

The inclination of lattice bars with the axis of the member shall not be less than $45^{\circ}$. Lattice bars shall be so spaced that the ratio $l / r$ of one flange of the member included between two adjacent connections of lattice bars is not over three-fourths of that of the unsupported length of the member as a whole.

(Other details of design concern themselves with the dimensions and locations of batten plates, combined shapes, starred angles, pins, pin plate, clevises, 
tank connections, tie plates, tension members, column bases and caps, circular girders, interior bracing, top struts, grillage, base braces, and web stiffeners.)

\section{Section 13. WORKMANSHIP}

(Covers requirements for shearing, punching, subpunching and drilling, straightening and fitting, use of rivets and bolts, heads of rivets, driving of rivets, drifting, lattice bars, bearing surfaces, and threads and nuts.)

\section{Section 14. ACCESSORIES}

(Covers requirements for connections, ladders, walkways, painting inaccessible parts, shop painting, field painting, and painting conditions. All painting requirements are the same as for steel gravity water tanks, given above.)

\section{NATIONAI FIRE PROTECTION ASSOCIA- TION, SUGGESTED ORDINANCE REGU- LATING THE USE, HANDLING, STORAGE, AND SALE OF FLAMMABLE IIQUIDS, 1926}

\section{STORAGE TANKS}

(Contains specifications for storage tanks for flammable liquids, both vertical and horizontal types, material (galvanized steel, open-hearth steel, and wrought iron); capacity and location; thickness and weights of metal; tensile strength; piping; and valve requirements.)

\section{UNDER WRITERS' IABORATORIES, STAND- ARD REQUIREMENTS FOR CONSTRUC- TION OF INSIDE TANK EQUIPMENT FOR OIL BURNERS, 1927}

\section{TANKS FOR OIL BURNERS}

\section{SCOPE}

1. These requirements apply to steel tanks of capacities of 275 U. S. gallons and less, used in basements of buildings for the storage and supply of oil fuels for oil burners.

2. Group A.- Individual storage tanks and gravity tanks up to $275 \mathrm{U}$. S. gallons capacity.

3. Group B.-Combination storage and gravity tank systems with pump for transferring oil from storage tank to gravity tank. The aggregate capacity of these systems shall not be over 275 U. S. gallons.

\section{Group A. INDIVIDUAL TANKS}

4. Size.-Tanks of this class shall not have capacities exceeding 275 U. S. gallons.

5. Form.-These tanks may be cylindrical or of special forms. Special designs will usually require special tests for strength and tightness to determine acceptability.

6. Material.-Open-hearth steel or wrought iron shall be used as material for these tanks.

7. Thickness. - The shell and heads of tanks up to and including $60 \mathrm{U}$. S. gallons shall be not less than No. 14 U. S. standard gage (0.078 inch) in thickness. Tanks of larger than $60 \mathrm{U}$. S. gallons capacity shall be made of metal not less than No. 12 U. S. standard gage (0.109 inch).

8. Joints. - The joints of these tanks shall be welded, brazed, or riveted and welded. (Acceptable designs are shown in sketches accompanying this specification.)

9. Pipe connections.-Pipe connections shall be made by welding or riveting standard taper threaded pipe couplings, flanges, or spuds to the tanks. Riveted flanges shall be secured by not less than four rivets on a pitch not to exceed 2 inches. A pipe connection may be provided in or near the bottom of the tank for supply to burner, and at least two should be provided in or near the top for fill and vent pipes. The vent pipe should be of ample size to prevent abnormal pressure in case of fire or when filling, and in no case to be less than 1-inch pipe size. Means shall be provided for removing water and sediment from both gravity and storage tanks. The threaded portions of pipe connections shall not be less than shown in the following table:

\begin{tabular}{|c|c|}
\hline $\begin{array}{c}\text { Trade } \\
\text { size of } \\
\text { pipe }\end{array}$ & $\begin{array}{c}\text { Minimum } \\
\text { length of } \\
\text { threaded } \\
\text { part }\end{array}$ \\
\hline Inches & Inch \\
$1 / 2$ & 0.348 \\
$3 / 4$ & .348 \\
1 & .348 \\
$11 / 4$ & .348 \\
$11 / 2$ & .435 \\
2 & .435 \\
$21 / 2$ & .875 \\
\hline
\end{tabular}

10. Gages.-Each tank shall be provided with a suitable listed liquid level gage or equivalent.

11. Coating.-Each tank shall be galvanized or coated outside with a suitable rust-resisting paint.

12. Test.-Each tank, after assembly and before being painted, shall be tested and proven tight against leakage under a test pressure of not less than 5 nor more than 10 pounds per square inch.

13. Strength.-These tanks shall be so designed and constructed that a strength test of 25 pounds per square inch will not rupture them, nor cause leakage.

14. Marking.-The inspection label shall be attached to one side of the tank near the top. In addition to the inspection label, each tank shall bear the name of the manufacturer.

15. Shipping.-Each tank, before shipping, shall have all pipe connection openings closed with suitable wood or metal plugs or caps to protect them and keep out foreign matter during shipping.

\section{Group B. COMBINATION GRAVITY AND STORAGE TANES
WITH POMP}

(In respect to size, form, material, thickness, joints, pipe connections, gages, coating, test, strength, marking, and shipping, this part of the specification conforms to the requirements for group A tanks, given above. Other provisions are:)

16. Two-compartment tanks.- Single combination tanks having gravity and storage sections separated by a compartment head shall have the overflow opening in the head located as near the top as possible, and provided with a threaded fitting which can be tightly closed for testing.

17. Attachment.-The gravity tank shall be securely attached to the storage tank by substantial metal supports, special attention being given to the load required to be sustained and to the manner in which it is supported.

18. Pump. - A suitable listed hand-operated oil pump or its equivalent shall be provided for transferring oil from storage to gravity tank.

19. Piping. - An overflow pipe shall be provided connecting the gravity tank with the top of the storage tank for returning excess oil pumped. This pipe shall be one pipe-size larger than discharge pipe from hand pump. Standard wrought-iron pipe shall be used. 
UNDERWRITERS' LABORATORIES, STANDARD FOR DESIGN AND CONSTRUCTION OF UNDERGROUND STORAGE TANKS, 1929

\section{UNDERGROUND STORAGE TANKS}

\section{GENERAI}

This standard covers the design and construction of underground storage tanks to be employed for the storage of flammable liquids, such as gasoline, kerosene, fuel oil, naphtha, etc., not under pressure, in accordance with the regulations of the National Board of Fire Underwriters for the installation of containers for storing and handling flammable liquids.

\section{CAPACITY AND SIZE-HORIZONTAL TANKS}

1. Horizontal tanks shall not exceed the maximum capacities, diameters, or lengths for the corresponding gages of metal outlined in the following table, except as noted below.

TABLE 1

\begin{tabular}{|c|c|c|c|c|c|}
\hline $\begin{array}{l}\text { U. S. standard } \\
\text { metal gage }\end{array}$ & $\begin{array}{l}\text { Approxi- } \\
\text { mate } \\
\text { thickness }\end{array}$ & $\begin{array}{l}\text { Maxi- } \\
\text { mum } \\
\text { capacity }\end{array}$ & $\begin{array}{l}\text { Equiva- } \\
\text { lent } \\
\text { capacity }\end{array}$ & $\begin{array}{c}\text { Maxi- } \\
\text { mum } \\
\text { diameter }\end{array}$ & $\begin{array}{l}\text { Maxi- } \\
\text { mum } \\
\text { length of } \\
\text { shell }\end{array}$ \\
\hline 000 & $\begin{aligned} & \text { Inch } \\
& 1 / 16 \\
& 5,64 \\
& 7 / 64 \\
& 3 / 16 \\
& 14 \\
& 5 / 16 \\
& 3 / 8\end{aligned}$ & $\begin{array}{r}\text { U.S. } \\
\text { gallons } \\
285 \\
560 \\
1,100 \\
4,000 \\
12,000 \\
20,000 \\
30,000\end{array}$ & $\begin{array}{r}\text { Imperial } \\
\text { gallons } \\
238 \\
467 \\
917 \\
3,333 \\
10,000 \\
16,667 \\
25,000\end{array}$ & $\begin{array}{r}\text { Inches } \\
38 \\
46 \\
56 \\
84 \\
126 \\
132 \\
132\end{array}$ & $\begin{array}{rr}\text { Feet } \\
8 \\
11 \\
14 \\
22 \\
32 \\
42 \\
50\end{array}$ \\
\hline
\end{tabular}

NotE.-To take care of miscalculations and mistakes in fabrication for tanks made of No. 7 or heavier gage metal, a tolerance of 5 per cent in either the diameter or the length will be permitted.

(As stated above in N. B. F. U. specification for containers for storing and handling flammable liquids, if adequate internal bracing is provided, tanks up to 30,000 gallons capacity may be made of $1 / 4$-inch material.)

\section{CAPACITY AND SIZE, VERTICAL CONE-BOTTOM TANKS}

2. Vertical cone-bottom tanks shall not exceed the maximum capacities, diameters, or lengths for the corresponding gages of metal outlined in the following table except as indicated in the note under Table 1 .

(Table 2 is identical with Table 1 in respect to gage, thickness, and capacity in both U. S. and Imperial gallons. It differs from Table 1 in the following respects:)

TABLE 2

\begin{tabular}{|c|r|r|r|}
\hline $\begin{array}{c}\text { U. S. standard } \\
\text { metal gage }\end{array}$ & $\begin{array}{c}\text { Maximum } \\
\text { diameter }\end{array}$ & $\begin{array}{c}\text { Maximum } \\
\text { length of } \\
\text { cylinder }\end{array}$ & $\begin{array}{c}\text { Maximum } \\
\text { diameter } \\
\text { unbraced } \\
\text { flat tops } \\
\text { (no toler- } \\
\text { ance) }\end{array}$ \\
\hline & Inches & Feet & Inches \\
16 & 43 & 10 & 36 \\
14 & 68 & 12 & 43 \\
7 & 105 & 18 & 72 \\
3 & 132 & 24 & 96 \\
000 & 132 & 35 & 120 \\
& 132 & & 132 \\
\hline
\end{tabular}

3. Flat tops, larger in diameter than shown by the last column of the above table shall be suitably braced. Bracing details are included in the Appendix.

\section{STANDARD SIZES}

4. The following are suggested as standard sizes, and wherever possible the manufacturer should make his tanks conform to these capacity denominations:

\begin{tabular}{|c|c|c|c|c|c|}
\hline Size & $\begin{array}{l}\text { Capacity } \\
\text { in U. S. } \\
\text { gallons }\end{array}$ & $\begin{array}{l}\text { Equiva- } \\
\text { lent } \\
\text { capacity } \\
\text { in } \\
\text { imperial } \\
\text { gallons }\end{array}$ & Size & $\begin{array}{l}\text { Capacity } \\
\text { in U. S. } \\
\text { gallons }\end{array}$ & $\begin{array}{l}\text { Equiva- } \\
\text { lent } \\
\text { capacity } \\
\text { in } \\
\text { imperial } \\
\text { gallons }\end{array}$ \\
\hline $\begin{array}{l}\mathrm{A} \\
\mathrm{B} \\
\mathrm{C} \\
\mathrm{D} \\
\mathrm{D}\end{array}$ & $\begin{array}{r}65 \\
120 \\
280 \\
550 \\
800 \\
1,000 \\
1,500 \\
2,000\end{array}$ & $\begin{array}{r}54 \\
100 \\
233 \\
450 \\
666 \\
833 \\
1,250 \\
1,667\end{array}$ & $\begin{array}{l}\mathrm{I} \\
\mathrm{J}_{-} \\
\mathrm{K} \\
\mathrm{L}_{-\ldots} \\
\mathrm{M} \\
\mathrm{N} \\
\mathrm{O}_{\ldots} \\
\mathrm{P}_{\ldots}\end{array}$ & $\begin{array}{r}3,000 \\
4,000 \\
5,000 \\
10,000 \\
12,000 \\
15,000 \\
20,000 \\
30,000\end{array}$ & $\begin{array}{r}2,500 \\
3,333 \\
4,166 \\
8,333 \\
10,000 \\
12,500 \\
16,667 \\
25,000\end{array}$ \\
\hline
\end{tabular}

MATERIALS

5. Tanks shall be constructed of open-hearth steel or wrought iron. No seconds shall be used. All material lighter than No. 7 U. S. standard gage shall be galvanized.

(Construction details covering shell joints, heads and head joints, dished heads for horizontal tanks, cone heads for horizontal tanks and bottoms for cone-bottom tanks; flat, dished or cone tops of vertical cone-bottom tanks, joints of cone heads, tops or bottoms, pipe connections, and manholesall are illustrated in sketches accompanying this specification.)

(An appendix contains tabular matter showing the number of both U. S. and imperial gallons in tanks 1 foot long with diameters ranging from 24 to 132 inches. Other tables show the maximum and minimum thickness limits for uncoated sheet and plate steel, also weights in pounds per square foot, for material ranging in size from $6 / 0$ to $40 \mathrm{U}$. S. standard gage; and maximum and minimum thickness limits for galvanized sheet steel, with weights in pounds per square foot, for material ranging in size from 8 to 34 U. S. standard gage.)

\section{UNDERWRITERS' LABORATORIES, STAND- ARD SPECIFICATIONS FOR HORIZONTAL AND VERTICAL ABOVEGROUND STOR- AGE TANKS FOR HAZARDOUS IIQUIDS, 1922}

\section{ABOVEGROUND STORAGE TANKS}

\section{GENERAI}

These specifications cover only cylindrical tanks of a size that can be completely assembled and tested at the factory before shipment. These tanks are intended for the storage of hazardous liquids not normally under pressure and are not to be used in connection with pressure systems.

\section{HORIZONTAL TANKS}

CAPACITY

Capacity to be not less than 2,500 gallons nor greater than 35,000 gallons.

\section{DIMENSIONS}

These tanks may be of any diameter from 4 feet up to 11 feet inclusive, and any length that can be 
shipped on a single railroad car, provided these dimensions give capacities within the limits specified above. In no case must the diameter be greater than the length, or the length be more than six times the diameter.

\section{MATERIAL}

Standard open-hearth steel tank plate is to be used in the construction of these tanks. The minimum thickness of metal required for shell and heads of tanks from 48 to 72 inches diameter is threesixteenths inch, and from 73 to 132 inches diameter, one-fourth inch.

\section{PIPE CONNECTIONS}

Standard wrought-iron pipe couplings may be welded to the tank. Standard boiler flanges may be welded or riveted to the tanks.

(Various methods of attaching pipe connections are shown in blue prints accompanying this specification.)

All pipe connection openings in the tank must be protected with wooden plugs, metal covers, or their equivalent before the tank is shipped.

Each tank must have provision for the attachment of not less than a 2 -inch vent. In case the largest inlet or outlet pipe has a cross-sectional area greater than four times that of a 2 -inch vent, the area of the vent must be increased to at least onefourth this area.

\section{HEADS AND HEAD JOINTS}

Heads may be in one or two pieces. If made of two pieces, the seam joining the two pieces together must be made in the same manner as the longitudinal shell seams are made. (Various types of heads and joints are shown in blue prints accompanying this specification.)

\section{MANBOLES}

All tanks over 5,000 gallons capacity must be provided with manholes. Standard malleable iron screw-cover manholes may be used. Face of collar and cover must be finished to a close fit. Gasket not required.

Bolted cover manholes may be used. Sheet packing gaskets not less than one-eighth inch thick must be used. These gaskets must be of a material listed by Underwriters' Laboratories for this purpose, or of some material authorized especially for this particular use.

Manholes located below the liquid level must be of the bolted-cover type. (Various types of manholes are shown in blue prints accompanying this specification.)

\section{TESTING}

Each tank must be tested and proven tight under a pressure of approximately one and one-half times the pressure exerted on the bottom when the tank is filled with water.

\section{MARKING}

Each tank must be plainly and durably marked with the name of the manufacturer in letters not less than $1 \frac{1}{2}$ inches high.

\section{VERTICAL TANKS}

CAPACITY

These tanks shall have a capacity of more than 2,500 gallons and less than 25,000 gallons.

\section{DIMENSIONS}

These tanks are cylindrical in shape, the height never being more than four times the diameter. A maximum diameter of 11 feet and a maximum height of 35 feet are permissible.

\section{MATERIAL}

Standard sheets of open-hearth steel tank plate must be used in the construction of these tanks.

\section{BоTTOM}

The bottoms of these tanks shall be in one or two pieces and not less than three-sixteenths inch thick. They may be riveted or welded to the shell. (Various types of rivetings and weldings are shown in blue prints accompanying this specification.)

\section{SHELL}

The shell must be not less than three-sixteenths inch thick for tanks up to 25 feet in height. For tanks from 25 to 35 feet high the first ring must be not less than 1.4 inches thick and must be not less than 5 feet wide. The rings above the first must not be less than three-sixteenths inch thick. Tanks between 30 and 35 feet high must have first two rings not less than one-fourth inch thick. Each of these $3 / 4$-inch rings must be not less than 5 feet wide; the remaining rings must be not less than threesixteenths inch thick. The seams of the shell may be riveted or welded. (Various types of rivetings and weldings are shown in blue prints accompanying this specification.)

\section{TOP}

The tops of these tanks must either be dished or cone shaped, and of No. 10 U. S. gage steel. (Various methods of fastening tops to shells are shown in blue prints accompanying this specification.)

\section{PIPE CONNECTIONS}

Pipe connections may be either standard steel flanges or standard wrought-iron pipe couplings. (Methods for securing couplings to shell are shown in blue prints accompanying this specification. In other respects this part of this specification conforms to the pipe connections for horizontal tanks, given above.)

(Manhole, test, and marking requirements are in conformity with those for horizontal tanks, given above.)

\section{UNITED STATES GOVERNMENT, DEPART- MENT OF COMMERCE, STEAMBOAT IN- SPECTION SERVICE, GENERAL RULES AND REGULATIONS, MAY 4, 1929}

\section{STEEL TANKS FOR STORAGE}

(Requirements covering steel tanks for storage of fuel oil on passenger vessels are contained in the manuals for ocean, bay, lake, and river vessels published under the supervision of the Board of Supervising Inspectors. The requirements include riveting of joints, minimum thickness of plates, hydrostatic test, size of vent pipe, attachment of fittings, and installation of heaters.)

605.24. METAL LATH.

\section{ASSOCIATED METAL IATH MANUFAC- TURERS, METAL LATH CONSTRUCTION, 1929}

\section{WOOD STUD AND JOIST CONSTRJCTION}
(a) NAILED TO WOOD STUD PARTITIONS

The minimum weight of expanded metal lath for studs with a maximum spacing of 16 inches on centers shall be 2.5 pounds per square yard. 
Flat rib lath for studs with a maximum spacing of 16 and 19 inches on centers shall weight not less than 2.75 and 3 pounds per square yard, respectively.

Three-eighths inch rib lath for studs with a maximum spacing of 19,24 , and $31 \frac{1}{2}$ inches on centers shall weigh not less than $2.5,2.75$, and 3.4 pounds per square yard, respectively.

Sheet lath for studs with a maximum spacing of 24 inches on centers shall weigh not less than 4.5 pounds per square yard.

\section{(b) FOR CEIUINGS NAILED TO TNDERSIDE OF WOOD JOISTS}

Expanded metal lath for joists with a maximum spacing of $13 \frac{1}{2}$ and 16 inches on centers shall weigh not less than 3 and 3.4 pounds per square yard, respectively.

Flat rib lath for joists with a maximum spacing of 16 and 19 inches on centers shall weigh not less than 2.75 and 3 pounds per square yard, respectively.

Sheet lath for joists with a maximum spacing of 24 inches on centers shall weigh not less than 4.5 pounds per square yard.

(Requirements covering nails, staples, and tie wires are also covered in this specification.)

\section{SOIID METAL LATH AND PLASTER PARTITIONS}

(a) Flat expanded metal lath shall weigh not less than 2.2 pounds per square yard for channels 12 inches on centers, 3 pounds per square yard for spacing of $13 \frac{1}{2}$ inches on centers, or 3.4 pounds for 16-inch centers.

(Requirements covering flat rib lath and threeeighths inch and sheet lath are the same as for wood stud and joist construction, given above. Provision is also made for size and spacing of channel studs, and for tie wire and spacing of ties.)

\section{HOLLOW METAL LATH AND PLASTER PARTITIONS}

(a) Flat expanded metal lath shall weigh not less than 2.5 pounds per square yard for channels 12 inches on centers, or 3 pounds per square yard for spacing not exceeding $131 / 2$ inches on centers.

(Requirements covering flat rib lath and threeeighths inch and sheet lath are the same as for wood stud and joist construction, given above. Provision is also made for size, spacing, and bracing of channel studs, and for tie wire and spacing of ties.)

\section{SOUND-PROOF DOUBLE-METAL LATH AND PLASTER PARTITIONS}

(Same as for solid metal lath and plaster partitions, given above.)

\section{CONTRACTING PLASTERERS' INTERNA- TIONAL ASSOCIATION, OUTLINE SPEC- IFICATIONS FOR LATHING AND PLAS- TERING, 1930}

\section{WIRE LATH AND METAL LATH}

\section{WIRE OR METAL LATH}

All wire lath to be No. 18 gage, $(0.0475)$ 3/8-inch mesh painted wire lath, weighing 3.3 pounds per square yard; or No. 24 gage metal lath painted, weighing no less than 3.4 pounds per square yard.

(Provision is also made in this specification for nails and staples, band iron furring, standard steel shapes for suspended ceilings, galvanized hangers and steel channels for furred or attached ceilings to concrete, metal corner beads, and metal lathing for exterior stucco work on frame construction.)
UNITED STATES GOVERNMENT, DEPARTMENT OF COMMERCE, BUREAU OF STANDARDS, SIMPLIFIED PRACTICE RECOMMENDATION FOR METAL LATH, R3-28, JANUARY 1, 1928

\section{METAL IATH}

(Simplified practice recommended and accepted by industry establishing the following limited list of standard stock sizes and varieties.)

TABLE 1.-Flat expanded metal lath

\begin{tabular}{|c|c|c|c|c|}
\hline \multicolumn{3}{|c|}{$\begin{array}{l}\text { Weight in pounds per square } \\
\text { yard of - }\end{array}$} & \multicolumn{2}{|c|}{$\begin{array}{l}\text { Gages of sheets } \\
\text { (U. S. standard) }\end{array}$} \\
\hline $\begin{array}{l}\text { Painted } \\
\text { steel }\end{array}$ & $\begin{array}{l}\text { Special } \\
\text { metal }\end{array}$ & $\begin{array}{l}\text { Srieets } \\
\text { galvan- } \\
\text { ized be- } \\
\text { fore } \\
\text { fabri- } \\
\text { cation }\end{array}$ & $\begin{array}{l}\text { Mini- } \\
\text { mum }\end{array}$ & $\begin{array}{l}\text { Maxi- } \\
\text { mum }\end{array}$ \\
\hline $\begin{array}{r}2.2 \\
2.5 \\
23.0 \\
3.4\end{array}$ & $\begin{array}{r}22.2 \\
2.5 \\
23.0 \\
3.4\end{array}$ & $\frac{2.5}{3.4}$ & $\begin{array}{l}28 \\
28 \\
28 \\
26\end{array}$ & $\begin{array}{l}26 \\
26 \\
24 \\
24\end{array}$ \\
\hline
\end{tabular}

1 United States Standard Gage for Sheet and Plate Iron and Steel. 2 Included in the present schedule with the recommendation by the standing committee, that the industry work toward the eventual elimination of this weight.

TABLE 2.-3/8-inch rib expanded metal lath

\begin{tabular}{|c|c|c|c|c|}
\hline \multicolumn{3}{|c|}{$\begin{array}{l}\text { Weight in pounds per square } \\
\text { yard of - }\end{array}$} & \multicolumn{2}{|c|}{$\begin{array}{l}\text { Gages of sheets } \\
\text { (U. S. standard) }\end{array}$} \\
\hline $\begin{array}{l}\text { Painted } \\
\text { steel }\end{array}$ & $\begin{array}{l}\text { Special } \\
\text { metal }\end{array}$ & $\begin{array}{l}\text { Sheets } \\
\text { galvan- } \\
\text { ized be- } \\
\text { fore } \\
\text { fabri- } \\
\text { cation }\end{array}$ & $\begin{array}{l}\text { Mini- } \\
\text { mum }\end{array}$ & $\begin{array}{l}\text { Maxi- } \\
\text { mum }\end{array}$ \\
\hline $\begin{array}{ll}2 & 2.5 \\
2 & 2.75 \\
3.0 \\
2 \\
2.4 \\
4.0\end{array}$ & $\begin{array}{ll}2 & 2.5 \\
2 & 2.75 \\
3.0 \\
2 \\
2.4 \\
4.0\end{array}$ & 23.6 & $\begin{array}{l}28 \\
28 \\
28 \\
28 \\
26\end{array}$ & $\begin{array}{l}26 \\
26 \\
26 \\
24 \\
24\end{array}$ \\
\hline
\end{tabular}

1 United States Standard Gage for Sheet and Plate Iron and Steel. 2 Included in the present schedule with the recommendation by the standing committee, that the industry work toward the eventual elimination of this weight.

\section{TABLE 3.-Flat rib expanded metal lath}

\begin{tabular}{|c|c|c|c|c|}
\hline \multicolumn{3}{|c|}{$\begin{array}{l}\text { Weight in pounds per square } \\
\text { yard of- }\end{array}$} & \multicolumn{2}{|c|}{$\begin{array}{l}\text { Gages of sheets } \\
\text { (U. S. Standard)1 }\end{array}$} \\
\hline $\begin{array}{l}\text { Painted } \\
\text { steel }\end{array}$ & $\begin{array}{l}\text { Special } \\
\text { metal }\end{array}$ & $\begin{array}{l}\text { Sheets } \\
\text { galvan- } \\
\text { ized be- } \\
\text { fore } \\
\text { fabri- } \\
\text { cation }\end{array}$ & $\begin{array}{l}\text { Mini- } \\
\text { mum }\end{array}$ & $\begin{array}{l}\text { Maxi- } \\
\text { mum }\end{array}$ \\
\hline $\begin{array}{c}2.75 \\
3.0 \\
3.4 \\
24.0\end{array}$ & $\begin{array}{l}2.75 \\
3.0 \\
3.4\end{array}$ & 23.6 & $\begin{array}{l}30 \\
30 \\
30 \\
28\end{array}$ & $\begin{array}{l}28 \\
28 \\
24 \\
24\end{array}$ \\
\hline
\end{tabular}

1 United States Standard Gage for Sheet and Plate Iron and Steel. 2 Included in the present schedule with the recommendation by the standing committee, that the industry work toward the eventual elimination of this weight. 
All types of lath are to be specified and sold by weight per square yard. Manufacturers accepting this recommendation, and who so desire, may stamp or tag their product "Made in accordance with United States Department of Commerce Simplified Practice Recommendation R3."

All copper bearing, special analysis, and pure iron lath to be designated by an undetachable metallic seal, piercing all sheets of lath in each bundle. The above lath to be painted with the same material as open-hearth sheets.

Sheet lath.-Painted steel sheet lath is to weigh not less than 4.5 pounds per square yard, with corresponding minimum weights in this type of lath made from special metals or from sheets galvanized before fabrication.

Expanded and sheets.-Metal lath in all types and weights galvanized after fabrication is to be eliminated.

\section{UNITED STATES GOVERNMENT, FEDERAL SPECIFICATIONS BOARD, FEDERAL SPECIFICATION FOR BASES, METAL; FOR PLASTER AND STUCCO CONSTRUC- TION, QQ-B-101, AUGUST 19, 1930}

\section{METAL BASES}

\section{A. Applicable Federal Specifications}

A-1. There are no other Federal specifications applicable to this specification.

\section{B. Types}

B-1. Metal bases sold under this specification may be of any one or more of the 14 following types, as stated in the invitation for bids.

B-2. Type E-Expanded metal base.-Expanded metal is formed by slitting and expanding sheets of metal.

B-2a. Class EF-Flat expanded metal lath.-Flat expanded metal lath has no further forming than that indicated in the preceding paragraph.

B-2b. Class EFR-Flat rib lath.-Flat rib lath is a combination of metal lath and ribs in which the rib has a total depth of less than three-sixteenths of an inch over all.

B-2c. Class ESF-Self-furring lath.-Self-furring lath is so formed that portions of it expand from the face of the lath to separate the face at least onefourth inch from the background to which the lath is attached.

B-2d. Class E3/8R-Three-eighths inch rib lath.Three-eighths inch rib lath is a combination of metal lath and ribs in which the rib has a total depth of approximately three-eighths of an inch over all. The rib is an unexpanded portion of the metal lath which leaves the plane of the lath at a certain angle and returns at the same angle.

B-2e. Class E3/4R-Three-fourths inch rib lath.Three-fourths inch rib lath is a combination of metal lath and ribs, in which the rib has a total depth of approximately three-fourths of an inch over all. The rib is an unexpanded portion of the metal lath which leaves the plane of the lath at a certain angle and returns at the same angle.

B-2f. Class EMR-Expanded metal reinforcement.- Expanded metal reinforcement is formed by slitting and expanding sheets of metal, differing from expanded metal lath in that the opening is many times larger.

B-3. Type S-Sheet-metal base.-Metal sheet lath is formed by perforating sheets of metal.

B-3a. Class SMF-Flat sheet-metal lath.-Flat sheet-metal lath without ribs.
B-3b. Class SMR-Ribbed sheet-metal lath.-Flat sheet-metal lath further formed to provide ribs as described in $\mathrm{B}-2 \mathrm{~d}$.

B-4. Type W-Wire base.-Wire base is formed by weaving or welding wires.

B-4a. Class WOL-Woven-wirelath.-Woven-wire lath is woven from wires of light gage with square or triangular openings, usually less than 1 inch in greatest dimension. The lath has no ribs.

B-4b. Class WOLR-Ribbed woven-wire lath.Woven-wire lath with small solid round rods acting as ribs.

B-4c. Class WOLV-Ribbed woven-wire lath.Woven-wire lath with $V$-shaped metal strips acting as ribs.

B-4d. Class WOF-Woven-wire fabric.-Wovenwire fabric is usually made with longitudinal wires spaced by means of diagonal cross wires so arranged as to form a series of triangles between the longitudinal wires. The longitudinal wires are usually heavier in gage and spaced at greater distances than the cross wires.

B-4e. Class WEF-Welded-wire fabric.-Weldedwire fabric is formed by spot welding wires of the same or different gages at right angles to each other forming rectangular openings. Often one set of wires are crimped to provide furring and the fabric may be either without backing or have an integral paper or screen wire backing.

B-4f. Class WFG.-Galvanized hexagonal mesh wire fabric.

\section{Material and Workmanship}

C-1. The manufacturer is given a wide range in the selection of materials and processes of manufacture in order that metal bases of the prescribed quality may be produced.

\section{General Requirements}

D-1. All metal bases shall be specified by thickness of sheet or diameter of wire and weight per square yard and shall be furnished in the particular grade or grades listed below finished with the specified coating as ordered by the purchasing agent.

D-2. Sizes. - The thickness of sheet metal specified herein shall be United States standard gage for sheet and plate iron and steel (Bureau of Standards Circular No. 391, see 604.0, p. 224), and all diameters of wire shall be steel wire gages (Stl. W. G.) (Bureau of Standards Circular No.67, see 603.40, p. 153).

Gage number, weight, and thickness of sheet metal

\begin{tabular}{|c|c|c|}
\hline $\begin{array}{c}\text { Gage } \\
\text { No. }\end{array}$ & $\begin{array}{c}\text { Minimum } \\
\text { weight } \\
\text { (pounds } \\
\text { per square } \\
\text { foot) }\end{array}$ & $\begin{array}{c}\text { Approxi- } \\
\text { mate } \\
\text { thickness } \\
\text { (inches) }\end{array}$ \\
\hline & 1.00 & 0.025 \\
24 & .87 & .022 \\
25 & .75 & .019 \\
26 & .68 & .017 \\
27 & .62 & .016 \\
28 & .56 & .014 \\
29 & .50 & .012 \\
30 & .50 \\
\hline
\end{tabular}

D-3. Wires for lath or fabric.-All wires used in the manufacture of woven-wire lath and fabric shall be cold-drawn mild steel having a minimum ultimate tensile strength of 60,000 pounds per square inch.

D-4. Attached ribs. - All attached V ribs for metal or wire lath shall be made of metal at least 0.025 inch in thickness and all round ribs shall be of mild steel. Metal ribs for all lath shall be spaced not more than 8 inches on centers. They shall be formed in the same sheet or be securely attached to the 
lath in such a manner as will prevent their loss or displacement before the lath is in place.

D-5. Paper.- The paper used for backing any type of lath or fabric shall be of good quality, free from volatile oil and have a strength of not less than 80 pounds as determined by the bursting strength test. Where the fabric is to be used for stucco or other exterior work the paper shall be waterproof unless otherwise specified. Backing paper for any type of base shall be securely attached to the base in such a manner as to prevent tearing during handling and erection.

D-6. Galvanized wire mesh shall be galvanized after weaving.

D-7. Lath.-All metal lath shall be cut from galvanized sheets or shall be heavily coated after expansion by the use of a suitable paint.

D-8. Bases made of copper-bearing metais are within the scope of this specification.

\section{E. Detail Requiremerts}

E-1. Type E.-(Expanded metal base.)

E-1a. Class EF (flat expanded metal lath) and class ESF (self-furring lath). -

\begin{tabular}{l|r|r}
\hline Subclass & $\begin{array}{c}\text { Minimum } \\
\text { weight } \\
\text { pounds } \\
\text { per square } \\
\text { yard) }\end{array}$ & $\begin{array}{c}\text { Minimum } \\
\text { thickness } \\
\text { (inches) }\end{array}$ \\
\hline & 2.20 & 0.016 \\
& 2.50 & .016 \\
& 3.00 & .016 \\
.019 \\
\hline
\end{tabular}

E-1b. Class E3/8R (3/8-inch rib).-
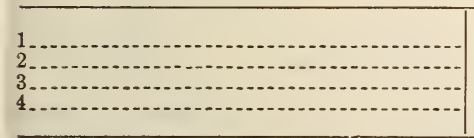

\begin{tabular}{r|r}
2.75 & 0.016 \\
3.00 & .016 \\
3.40 & .016 \\
4.00 & .019
\end{tabular}

E-1c.-Class E3/4R (3/4-inch rib).-

\begin{tabular}{r|r|r}
\hline 1 & 4.10 & 0.016 \\
3 & 5.00 & .019 \\
\hline
\end{tabular}

E-1d. Class EFR (flat ribbed).-

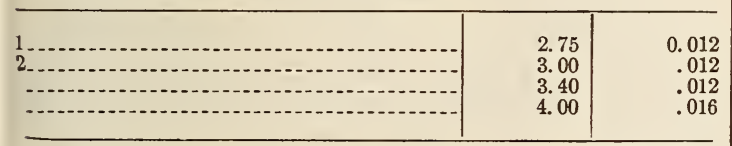

E-1e. Class EMR (expanded metal reinforcement).-

1

\begin{tabular}{|c|c|c|}
\hline 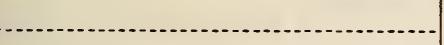 & 1.80 & 0.025 \\
\hline
\end{tabular}

E-2. Type S (sheet-metal lath).

E-2a. Class SMR (ribbed).-
E-3. Type W (wire base).

E-3a. Class WOL (woven-wire lath, flat).-

\begin{tabular}{r|r|r|r|r}
\hline \multirow{2}{*}{ Subclass } & $\begin{array}{c}\text { Meshes per } \\
\text { inch }\end{array}$ & \multicolumn{2}{|c|}{$\begin{array}{l}\text { Minimum weight } \\
\text { (pounds per square } \\
\text { yard) }\end{array}$} & $\begin{array}{r}\text { Minimum } \\
\text { diameter } \\
\text { of wire } \\
\text { (inches) }\end{array}$ \\
\cline { 3 - 5 } & & Galvanized & Painted & \\
\hline & $21 / 2$ & 2.48 & 1.80 & 0.035 \\
2 & 2.15 & .041 \\
3 & $21 / 2$ & 4.05 & 3.23 & .047 \\
\hline
\end{tabular}

E-3b. Class WOLR (woven-wire lath, three-sixteenths round ribbed).-

\begin{tabular}{l|l|r|r|r}
\hline $1.21 / 2$ & 3.60 & 2.93 & .035 \\
\hline
\end{tabular}

E-3c. Class WOLV (woven-wire lath, threeeighths inch $\mathrm{V}$ ribbed).-

\begin{tabular}{l|r|r|r|r}
\hline & $21 / 2$ & 3.30 & 2.35 & 0.032 \\
\hline 3 & $21 / 2$ & 3.75 & 2.60 & .035 \\
4 & $21 / 2$ & 4.35 & 3.30 & .041 \\
& $21 / 2$ & 5.25 & 4.00 & .047 \\
\hline
\end{tabular}

E-3d. Class WOF (woven-wire fabric).-

\begin{tabular}{|c|c|c|c|c|}
\hline \multirow{2}{*}{ Subclass } & \multirow{2}{*}{$\begin{array}{l}\text { Mesh (triangu- } \\
\text { lar) (inches) }\end{array}$} & \multirow{2}{*}{$\begin{array}{l}\text { Minimum } \\
\text { weight } \\
\text { (pounds } \\
\text { per square } \\
\text { yard) }\end{array}$} & \multicolumn{2}{|c|}{$\begin{array}{l}\text { Minimum diameter of } \\
\text { wire (inches) }\end{array}$} \\
\hline & & & Length & Transverse \\
\hline & $\begin{array}{l}4 \text { by } 4 \\
2 \text { by } 4 \ldots \\
2 \text { by } 4\end{array}$ & $\begin{array}{l}1.94 \\
2.10 \\
2.84\end{array}$ & $\begin{array}{r}0.105 \\
.080 \\
.105\end{array}$ & $\begin{array}{r}0.080 \\
.080 \\
.080\end{array}$ \\
\hline
\end{tabular}

E-3e. Class WEF (welded-wire fabric. Flat and self-furring).-

\begin{tabular}{r|r|r|r|r}
\hline $1 \ldots \ldots \ldots$ & 2 by $4 \ldots \ldots \ldots$ & 1.50 & 0.080 & 0.080 \\
2 by $2 \ldots \ldots$ & .080 & .080 \\
2 by $4 \ldots \ldots$ & 2.00 & .105 & .105 \\
\hline
\end{tabular}

E-3f. Class WFG (galvanized hexagonal mesh wire fabric).-

\begin{tabular}{r|r|r|r} 
Subclass & $\begin{array}{c}\text { Mesh (hexagonal) } \\
\text { (inches) }\end{array}$ & $\begin{array}{c}\text { Minimum } \\
\text { weight } \\
\text { (pounds } \\
\text { per square } \\
\text { yard) }\end{array}$ & $\begin{array}{c}\text { Minimum } \\
\text { diameter } \\
\text { of wire } \\
\text { (inches) }\end{array}$ \\
\hline & $1 . \ldots 047$ \\
\hline
\end{tabular}

E-4. Nоте.-Backing paper will add from onehalf to three-quarters of a pound per square yard to the weights given above. Screen wire will add approximately 1 pound to the above weights.

\section{F. Method of Inspection and Test}

F-1. Inspection.-One sheet shall be taken from every 10 bundles or every 10 rolls for inspection and test. The number of sheets taken shall not exceed five from every carload shipment.

F-2. Rejection.-Materials shall be rejected when 10 per cent or more of that examined fails to meet one or more of the requirements of the specification. 


\section{G. Packaging, Packing, and Marking}

G-2. Packaging.-Unless otherwise specified commerical packages are acceptable under this specification.

G-2a. Expanded and sheet-metal lath.-All bundles of expanded and sheet-metal lath on any contract shall contain an equal number of sheets of definite area which may be crated or securely wired together.

G-2b. Woven-wire lath and fabric.-Woven-wire lath and fabric shall be furnished in rolls of definite area or in bundles of equal number of sheets of definite area, which may be crated or securely wired together.

G-3. Packing.-There are no special requirements.

G-4. Marking.-All bundles and rolls of material shall be marked with the name of the material, the size, type, class, and the quantity contained therein, as defined by the contract or order, the name of the contractor, and the number of the contract or order.

\section{Notes}

I-1. It is believed that this specification adequately describes the characteristis necessary to secure the desired material, and that normally no samples will be necessary prior to award to determine compliance with this specification. If, for any particular purpose, samples with bids are necessary, they should be specifically asked for in the invitation for bids, and the particular purpose to be served by the bid sample should be definitely stated, the specification to apply in all other respects.

I-2. This specification governs all United States Government purchases of this commodity.

605.25 STEEL FOR CONCRETE REINFORCEMENT.

$\begin{array}{lll}\text { AMERICAN } & \text { ASSOCIATION } & \text { OF STATE } \\ \text { HIGHWAY } & \text { OFFICIALS, } & \text { TENTATIVE } \\ \text { STANDARD } & \text { SPECIFICATION FOR BIL- } \\ \text { LET-STEEL } & \text { CONCRETE } & \text { REINFORCE- } \\ \text { MENT BARS, M-31, 1924 } & \end{array}$

\section{BILLET-STEEL CONCRETE REINFORCEMENT BARS}

\section{MATERIAL COVERED}

1. (a) These specifications cover two classes of billet-steel concrete reinforcement bars, namely, plain and deformed.

(b) Plain and deformed bars are of two grades, namely, structural steel and intermediate. Structural grade shall be used unless otherwise specified.

2 . The structural steel grade made by the openhearth process shall be used unless otherwise specified.

\section{MANUFACTURE}

3. (a) These specifications include steel made by either the Bessemer or open-hearth process, but open-hearth steel shall be used unless otherwise specified.

(b) The bars shall be rolled from new billets. No high-carbon or rerolled material will be accepted.

(In all other respects this specification conforms to that of the Association of American Steel Manufacturers, given below, except that here the tensile strength in pounds per square inch for both plain and deformed bars of the intermediate grade ranges from 70,000 to 85,000 instead of from 70,000 to 90,000 ; that cold-twisted bars are omitted, and that test specimens shall not be less than 24 inches long.)

(Requirements covering finish, inspection, rejection, and rehearing conform to those specified in A. S. T. M. standard specifications for steel. See 605.0 , p. 247. )

\section{AMERICAN ASSOCIATION OF STATE HIGHWAY OFFICIALS, TENTATIVE STANDARD SPECIFICATIONS FOR COLD. DRAWN STEEL WIRE FABRIC FOR CON- CRETE FOR REINFORCEMENT, M-32, 1924}

\section{WIRE FABRIC FOR CONCRETE REINFORCEMENT}

\section{MATERIAL COVERED}

1. These specifications cover cold-drawn steel wire to be used as such, or in fabricated form, for the reinforcement of concrete, in gages not less than 0.080 inch nor greater than 0.500 inch.

\section{GENERAL REQUIREMENTS}

2. When wire is ordered by gage number the following relation between number and diameter, in inches, shall apply unless otherwise specified.

\begin{tabular}{|r|r||r|c|}
\hline Gage No. & $\begin{array}{c}\text { Equivalent } \\
\text { diameter }\end{array}$ & Gage No. & $\begin{array}{c}\text { Equivalent } \\
\text { diameter }\end{array}$ \\
\cline { 1 - 2 } & Inch & & Inch \\
0000000 & 0.4900 & 5 & 0.2070 \\
000000 & .4615 & 6 & .1920 \\
00000 & .4305 & 7 & .1770 \\
0000 & .3938 & 8 & .1620 \\
000 & .3625 & 9 & .1483 \\
00 & .3310 & 10 & .1350 \\
0 & .3065 & 11 & .1205 \\
1 & .2830 & 12 & .1055 \\
2 & .2625 & 13 & .0915 \\
3 & .2437 & 14 & .0800 \\
4 & .2253 & & \\
\hline
\end{tabular}

\section{MANUFACTURE}

3. (a) The steel shall be made by either or both the following processes: Bessemer or open-hearth.

(b) The wire shall be cold drawn from rods hot rolled from billets.

\section{PHYSICAL PROPERTIES AND TESTS}

4. (a) The wire, except as specified in paragraphs (b) and (c), shall conform to the following minimum requirements as to tensile properties:

Tensile strength Ibs./in. ${ }^{2}--80,000$

Reduction of area

(b) For wire to be used in the fabrication of mesh or fabric a minimum tensile strength of 70,000 pounds per square inch after fabrication shall be permitted.

(c) For wire with a tensile strength over 100,000 pounds per square inch the reduction of area shall be not less than 25 per cent.

5 . The test specimen shall withstand being bent cold through $180^{\circ}$ around a pin, the diameter of which is equal to the diameter of the specimen, without cracking on the outside of the bent portion.

6 . Tension and bend test specimens shall be of the full size section of the wire, cut from fabricated material, with a minimum length of 24 inches.

7. (a) One tension and one bend test shall be made for each 10 tons or less of each size of wire.

(b) If any test specimen shows defects or develops flaws, it may be discarded and another specimen substituted.

8. If the fabricated material is specified to be of galvanized material, each wire shall be galvanized by the hot-dip method and shall have a continuous coating of zinc of uniform thickness. 
PERMISSIBLE VARIATIONS IN GAGE

9. The diameter of the wire shall not vary more than 0.003 inch from the size ordered.

(Requirements covering workmanship and finish, inspection, rejection, and rehearing conform to those specified in A. S. T. M. standard specifications for steel. See 605.0, p. 247.)

\section{SAMPIING AND TESTING}

10. Sampling and testing of cold-drawn steel wire fabric for concrete reinforcement shall be done in accordance with the tentative standard methods for sampling and testing of the American Association of State Highway Officials.

\section{AMERICAN RAILWAY ENGINEERING ASSO- CIATION, SPECIFICATIONS FOR BILLET- STEEL CONCRETE REINFORCEMENT BARS, 1920}

\section{BILLET-STEEL CONCRETE REINFORCEMENT BARS}

\section{MATERIAL COVERED}

1. (a) These specifications cover two classes of billet-steel concrete reinforcement bars, namely, plain and deformed.

(b) Plain and deformed bars are of three grades, namely, structural-steel, intermediate, and hard.

(c) Twisted bars will not be accepted under these specifications.

\section{BASIS OF PURCHASE}

2. The structural-steel grade shall be used unless otherwise specified.

Process-

\section{MANUFACTURE}

3. (a) The steel shall be made by the openhearth process.

(b) The bars shall be rolled from new billets. No rerolled material will be accepted.

\section{IADLE AND CHECX ANALYSES}

(Conform to Association of American Steel Manufacturers, below.)

\section{TENSILE PROPERTIES}

(Conform to Association of American Steel Manufacturers, below, except that here the tensile strength in pounds per square inch for both plain and deformed bars of the intermediate grade ranges from 70,000 to 85,000 instead of from 70,000 to 90,000 .)

\section{MODIFICATIONS IN ELONGATION}

(Conform to Association of American Steel Manufacturers, below.)

\section{BEND TESTS AND BEND-TEST RIGUIREMENTS}

(Conform to Association of American Steel Manufacturers, below, except that cold-twisted bars are omitted from the A. P. E. A. specification.)

\section{TEST SPECIMENS}

(Conform to Association of American Steel Manufacturers, below, except for the omission of machining in the A. R. E. A. specification.)

\section{NUMBER OF TESTS}

(Conform to Association of American Steel Manufacturers, below, except for the omission of substitution of another test specimen in the A. R. E. A. specification.)
PERMISSIBLE VARIATIONS IN WEIGHT

(Conform to Association of American Steel Manufacturers, below.)

(Requirements covering finish, inspection, rejection, and rehearing conform to those specified in A. S. T. M. standard specifications for steel. See 605.0, p. 247. )

AIIERICAN SOCIETY FOR TESTING MATERIALS, STANDARD SPECIFICATIONS FOR BIIIET-STEEL CONCRETE REINFORCEMENT BARS, DESIGNATION A15-30, 1930

\section{BILLET-STEEL REINFORCEMENT BARS}

(Same as Association of American Steel Manufacturers' specification, given below.)

ANIERICAN SOCIETY FOR TESTING MATERIAIS, STANDARD SPECIFICATIONS FOR RAIL-STEEL CONCRETE REINFORCEMENT BARS, DESIGNATION A16-14, 1914

\section{RAIL-STEEL REINFOPCEMENT BARS}

(Same as Association of American Steel Manufacturers' specification, given below.)

AMERICAN SOCIETY FOR TESTING MATERIALS, STANDARD SPECIFICATIONS FOR COID-DRAWN STEEL WIRE FOR CONCRETE REINFORCEMENT, DESIGNATION A82-27, 1927

\section{STEEL WIRE FOR CONCRETE REINFORCEMENT} SCOPE

1. These specifications cover cold-drawn steel wire to be used as such, or in fabricated form, for the reinforcement of concrete, in gages not less than 0.080 inch nor greater than 0.625 inch.

\section{BASIS OF PURCHASE}

2. When wire is ordered by gage number the following relation between number and diameter, in inches, shall apply unless otherwise specified:

\begin{tabular}{|c|c|c|c|}
\hline Gage No. & $\begin{array}{l}\text { Equiva- } \\
\text { lent } \\
\text { diameter }\end{array}$ & Gage No. & $\begin{array}{l}\text { Equiva- } \\
\text { lent } \\
\text { diameter }\end{array}$ \\
\hline $\begin{array}{r}0000000 \\
000000 \\
00000 \\
0000 \\
000 \\
00 \\
0 \\
1 \\
2 \\
3 \\
4\end{array}$ & $\begin{array}{l}\text { Inch } \\
0.4900 \\
.4615 \\
.4305 \\
.3938 \\
.3625 \\
.3310 \\
.3065 \\
.2830 \\
.2625 \\
.2437 \\
.2253\end{array}$ & $\begin{array}{r}5 \\
6 \\
7 \\
8 \\
9 \\
10 \\
11 \\
12 \\
13 \\
14\end{array}$ & $\begin{array}{l}\text { Inch } \\
0.2070 \\
.1920 \\
.1770 \\
.1620 \\
.1483 \\
.1350 \\
.1205 \\
.1055 \\
.0915 \\
.0800\end{array}$ \\
\hline
\end{tabular}

\section{MANUFACTORE}

3. (a) The steel shall be made by either or both the following processes: Bessemer or open-hearth.

(b) The wire shall be cold-drawn from rods hotrolled from billets.

\section{PHYSICAL PROPERTIES AND TESTS}

4. (a) The wire, except as specified in paragraphs (b) and (c), shall conform to the following minimum requirements as to tensile properties:

Tensile strength. lbs./in. ${ }^{2}-80,000$ Reduction of area. 30 
(b) For wire to be used in the fabrication of mesh a minimum tensile strength of 70,000 pounds per square inch shall be permitted.

(c) For wire testing over 100,000 pounds per square inch tensile strength, the reduction of area shall be not less than 25 per cent.

5. The test specimen shall stand being bent cold through $180^{\circ}$ without cracking on the outside of the bent portion, as follows:

For wire 0.3 inch in diameter or under, around a pin the diameter of which is equal to the diameter of the specimen.

For wire over 0.3 inch in diameter, around a pin the diameter

of which is equal to twice the diameter of the specimen.

6. Tension and bend test specimens shall be of the full-size section of the wire as drawn.

7. (a) One tension and one bend test shall be made for each 10 tons or less of each size of wire.

(b) If any test specimen shows defects or develops flaws, it may be discarded and another specimen substituted.

\section{PERMISSIBLE VARIATIONS IN GAGE}

8. The diameter of the wire shall not vary more than 0.003 inch from the size ordered.

\section{FINISE}

9. The finished wire shall be free from injurious defects and shall have a workmanlike finish with smooth surface.

\section{INSPECTION AND REJECTION}

10. The manufacturer shall afford the inspector, without charge, all reasonable facilities to satisfy him that the wire is being furnished in accordance with these specifications. All tests and inspection shall be made at the place of manufacture prior to shipment, unless otherwise specified, and shall be so conducted as not to interfere unnecessarily with the operation of the works.

11. Wire which shows injurious defects subsequent to its acceptance at the manufacturer's works will be rejected, and the manufacturer shall be notified.

\section{ASSOCIATION OF AMERICAN STEEL MAN- UFACTURERS, STANDARD SPECIFICA- TIONS FOR CONCRETE REINFORCEMENT BARS ROLIED FROM BILIETS, 1928}

\section{BILLET-STEEL REINFORCEMENT BARS}

1. Scope.-(a) These specifications cover three classes of billet-steel concrete reinforcement bars: Plain, deformed, and cold-twisted.

(b) Plain and deformed bars are of three grades: Structural-steel, intermediate, and hard.

2. Purchase conditions.-(a) Orders should specify the class and grade desired.

(b) Open-hearth and Bessemer steel may be furnished.

(c) If desired, the acceptance of cold-twisted bars may be based on testing the bars before twisting. in which case the requirements for plain bars of structural-steel grade shall govern and orders shall so specify.

3. Manufacture.-(a) The steel shall be made by the open-hearth or the Bessemer process.

(b) The bars shall be rolled from billets.

(c) Cold-twisted bars shall be twisted cold with one complete twist in a length not over twelve times the thickness of the bar.

4. Chemical composition. - The steel shall conform to the following requirements as to chemical composition:

Phosphorus:

Bessemer, not over

Per cent

Open-hearth basic

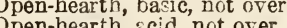

5. Ladle analysis.-(a) For Bessemer steel, a carbon determination shall be made of each melt; and determinations for manganese, phosphorus and sulphur, representing the average of the melts made in each 12-hour period.

(b) For open-hearth steel an analysis of each melt shall be made for carbon, manganese, phosphorus, and sulphur.

(c) These analyses shall be made by the manufacturer from test ingots taken during the pouring of each melt. The chemical composition thus determined shall be reported to the purchaser or his representative. The phosphorus content shall conform to the requirements of section 4 .

6. Check analyses.-Analyses may be made by the purchaser from finished bars representing each melt of open-hearth steel, and each melt, or lot of 10 tons, of Bessemer steel. The phosphorus content thus determined shall not exceed that specified in section 4 by more than 25 per cent.

7. Tension tests.-(a) The bars shall conform to the following requirements as to tensile properties:

\section{Tensile properties}

\begin{tabular}{|c|c|c|c|}
\hline Classes and grades & $\begin{array}{l}\text { Yield } \\
\text { point }\end{array}$ & $\begin{array}{l}\text { Tensile } \\
\text { strength }\end{array}$ & $\begin{array}{l}\text { Elongation in } \\
8 \text { inches }^{a}\end{array}$ \\
\hline \multirow{2}{*}{$\begin{array}{l}\text { Plain bars: } \\
\quad \text { Structural-steel .... }\end{array}$} & Lbs./in. ${ }^{2}$ & Lbs./in. ${ }^{2}$ & Per cent \\
\hline & 33,000 & \multirow[t]{2}{*}{$55,000-70,000$} & $1,400,000$ \\
\hline \multirow{2}{*}{ Intermediate } & \multirow{2}{*}{40,000} & & $\begin{array}{c}\text { Tensile strength } \\
1,300,000\end{array}$ \\
\hline & & $70,000-90,000$ & $\begin{array}{l}\text { Tensile strength } \\
\text { minimum } \\
16 \text { per cent. } \\
1,200,000 \\
\end{array}$ \\
\hline \multirow{2}{*}{$\begin{array}{l}\text { Hard } \\
\text { Deformed bars: } \\
\text { Structural-steel .... }\end{array}$} & 50,000 & (Min.) 80,000 & $\begin{array}{c}\text { Tensile strength } \\
1,250,000\end{array}$ \\
\hline & 33,000 & $55,000-70,000$ & Tensile strength \\
\hline \multirow[t]{2}{*}{ Intermediate........ } & \multirow[t]{2}{*}{40,000} & \multirow[t]{2}{*}{$70,000-90,000$} & $1,125,000$ \\
\hline & & & $\begin{array}{l}\text { Tensile strength } \\
\text { minimum } \\
14 \text { per cent. } \\
1,000,000\end{array}$ \\
\hline Hard................ & 50,000 & (Min.) 80,000 & Tensile strength \\
\hline Cold-twisted bars.. & 55,000 & (b) & 5 \\
\hline
\end{tabular}

a See sec. 8.

b Recorded only.

(b) The yield point shall be determined by the drop of the beam of the testing machine.

8. Modifications in elongation.-(a) For plain and deformed bars over three-fourths inch in thickness or diameter, a deduction from the percentages of elongation specified in section $7(a)$ of 0.25 per cent shall be made for each increase of one-thirty-second inch of the specified thickness or diameter above three-fourths inch.

(b) For plain and deformed bars under sevensixteenths inch in thickness or diameter, a deduction from the percentages of elongation specified in section $7(a)$ of 0.5 per cent shall be made for each decrease of one-thirty-second inch of the specified thickness or diameter below seven-sixteenths inch.

9. Bend tests. - The test speciment shall bend cold around a pin, without cracking on the outside of the bent portion, as follows:

\section{Bend test requirements}

\begin{tabular}{|c|c|c|c|c|c|c|c|}
\hline \multirow[b]{2}{*}{$\begin{array}{c}\text { Thickness or } \\
\text { diameter of } \\
\text { bar }\end{array}$} & \multicolumn{3}{|c|}{ Plain bars } & \multicolumn{3}{|c|}{ Deformed bars } & \multirow[b]{2}{*}{$\begin{array}{l}\text { Cold- } \\
\text { twist- } \\
\text { ed } \\
\text { bars }\end{array}$} \\
\hline & $\begin{array}{l}\text { Struc- } \\
\text { tural- } \\
\text { steel } \\
\text { grade }\end{array}$ & $\begin{array}{l}\text { Inter- } \\
\text { medi- } \\
\text { ate } \\
\text { grade }\end{array}$ & $\begin{array}{l}\text { Hard } \\
\text { grade }\end{array}$ & $\begin{array}{l}\text { Struc- } \\
\text { tural- } \\
\text { steel } \\
\text { grade }\end{array}$ & $\begin{array}{l}\text { Inter- } \\
\text { medi- } \\
\text { ate } \\
\text { grade }\end{array}$ & $\begin{array}{l}\text { Hard } \\
\text { grade }\end{array}$ & \\
\hline $\begin{array}{l}\text { Uncer } 3 / 4 \text { inch.- } \\
3 / 4 \text { inch or over. }\end{array}$ & $\begin{array}{l}180^{\circ} \\
d=t \\
180^{\circ} \\
d=t\end{array}$ & $\begin{array}{r}180^{\circ} \\
d=2 t \\
90^{\circ} \\
d=2 t\end{array}$ & $\begin{array}{r}180^{\circ} \\
d=3 t \\
90^{\circ} \\
d=3 t\end{array}$ & $\begin{array}{c}180^{\circ} \\
d=t \\
180^{\circ} \\
d=2 t\end{array}$ & $\begin{array}{r}180^{\circ} \\
d=3 t \\
90^{\circ} \\
d=3 t\end{array}$ & $\begin{array}{r}180^{\circ} \\
d=4 t \\
90^{\circ} \\
d=4 t\end{array}$ & $\begin{array}{r}180^{\circ} \\
d=2 t \\
180^{\circ} \\
d=3 t\end{array}$ \\
\hline
\end{tabular}

$d=$ the diameter of pin about which the specimen is bent. $t=$ the thickness or diameter of the specimen. 
10. Test specimens.-(a) Tension and bend test specimens for plain and deformed bars shall be taken from the finished bars, and shall be of the full thickness or diameter of bars as rolled; except that the specimens for deformed bars may be machined for a length of at least 9 inches, if deemed necessary by the manufacturer to obtain uniform cross section.

(b) Tension and bend test specimens for coldtwisted bars shall be taken from the finished bars, without further treatment; except as specified in section $2(c)$.

11. Number of tests.-(a) One tension and one bend test shall be made from each melt of openhearth steel, and from each melt, or lot of 10 tons of Bessemer steel; except that if material from any such test lots differs three-eighths inch or more in thickness or diameter, one tension and one bend test shall be made from both the thickest and the thinnest material rolled.

(b) If any test specimen shows defective machining or develops flaws, it may be discarded and another specimen substituted.

(c) If the percentage of elongation of any tension test specimen is less than that specified in section 7 (a) and any part of the fracture is outside the middle third of the gage length, as indicated by scribe scratches marked on the specimen before testing, a retest shall be allowed.

12. Permissible variations. - The weight of any lot of bars shall not vary more than 5 per cent from the theoretical weight of that lot.

Note.-The term "lot" used in this paragraph means all the bars of the same nominal weight per linear foot in a carload.

(Requirements covering finish, inspection, rejection, and rehearing conform with those specified in A. S. T. M. standard specifications for steel. See 605.0, p. 247.)

ASSOCIATION OF AMERICAN STEEL MANUFACTURERS, STANDARD SPECIFICATIONS FOR RAIL-STEEL CONCRETE REINFORCEMENT BARS, 1914

\section{RAIL-STEEL REINFORCEMENT BARS}

MANUFACTURE

1. All steel shall be rolled from standard section $T$ rails.

\section{PHYSICAL PROPERTIES}

2. The physical properties shall conform to the following limits:

\begin{tabular}{|c|c|c|}
\hline \multirow{2}{*}{ Properties considered } & \multicolumn{2}{|c|}{ Rail steel, grade } \\
\hline & Plain bars & $\begin{array}{l}\text { Deformed and } \\
\text { hot-twisted bars }\end{array}$ \\
\hline 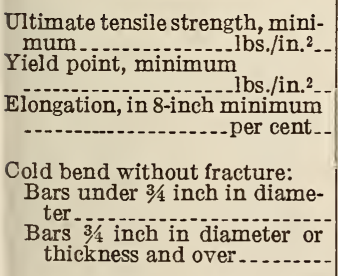 & $\begin{array}{r}80,000 \\
50,000 \\
1,200,000 \\
\text { Tensile strength }\end{array}$ & $\begin{array}{r}80,000 \\
50,000 \\
1,000,000 \\
\text { Tensile strength }\end{array}$ \\
\hline
\end{tabular}

YIELD POINT

3. For the purposes of these specifications, the yield point shall be determined by careful observation of the drop of the beam of the testing machine or by other equally accurate method.

\section{FORM OF SPECIMENS}

4. (a) Tensile and bending test specimens may be cut from the bars as rolled, but tensile and bending test specimens of deformed bars may be planed or turned for a length of at least 9 inches if deemed necessary by the manufacturer in order to obtain uniform cross section.

(b) Tensile and bending test specimens of hottwisted bars shall be cut from the bars after twisting and shall be tested in full size without further treatment, unless otherwise specified.

\section{NUMBER OF TESTS}

5. (a) One tensile and one bending test shall be made from each lot of 10 tons or less of each size of bar rolled from rails varying not more than 10 pounds per yard in nominal weight. Should a test specimen develop flaws, or should the tensile-test specimen break outside of the middle third of its gaged length, it may be discarded and another test specimen substituted therefor. In case a tensile specimen does not meet the specifications, an additional test may be made.

(b) The bending test may be made by pressure or by light blows.

\section{MODIFICATIONS IN EIONGATION FOR THIN AND THICK}

6. For bars less than seven-sixteenths inch and more than three-fourths inch nominal diameter or thickness, the following modifications shall be made in the requirements for elongation:

(a) For each increase of one-eighth inch in diameter or thickness above three-fourths inch, a deduction of 1 shall be made from the specified percentage of elongation.

(b) For each decrease of one-sixteenth inch in diameter or thickness below seven-sixteenths inch, a deduction of 1 shall be made from the specified percentage of elongation.

\section{NOMPER OF TWISTS}

7. Hot-twisted bars of rail carbon steel shall be twisted with one complete twist in a length equal to not more than twelve times the thickness of the bar.

\section{FINISH}

8. Material must be free from injurious seams, flaws, or cracks, and have a workmanlike finish.

\section{VARIATION IN WEIGHT}

9. Bars for reinforcement are subject to rejection if the actual weight of any lot varies more than 5 per cent over or under the theoretical weight of that lot.

\section{CONCRETE REINFORCING STEEL INSTI- TUTE, STANDARDS FOR CONCRETE-RE- INFORCING BARS; TABLE OF STAND- ARD SIZES, AREAS, AND WEIGHTS, 1930}

\section{CONCRETE-REINFORCING BARS}

(Conforms to Simplified Practice Recommendation No. R26-30 of the United States Department of Commerce in respect to area and size, see below. The weights for squares and rounds are as follows:)

\begin{tabular}{|c|c|c|c|}
\hline Size (in inches) & Weight & Size (in inches) & Weight \\
\hline 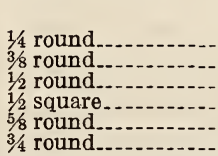 & $\begin{array}{r}\text { Lbs./ft. } \\
0.17 \\
.38 \\
.68 \\
.86 \\
1.06 \\
1.52\end{array}$ & $\begin{array}{l}\text { 7/8 round } \\
1 \text { round..... } \\
1 \text { square } \\
1118 \text { square.... } \\
11 / 4 \text { square }\end{array}$ & $\begin{array}{r}\text { Lbs.lft. } \\
2.07 \\
2.70 \\
3.44 \\
4.35 \\
5.37\end{array}$ \\
\hline
\end{tabular}


UNITED STATES GOVERNMENT, DEPARTMENT OF COMMERCE, BUREAU OF STANDARDS, SIMPLIFIED PRACTICE RECOMMENDATION R26-30, APRIL 1, 1930

\section{STEEL REINFORCING BARS}

The industry has accepted the following simplified list of cross-sectional areas:

Cross-sectional areas

[In square inches]

\begin{tabular}{|l|l|l|}
\hline 0.05 & 0.31 & 1.00 \\
.11 & .44 & 1.27 \\
.20 & .60 & 1.56 \\
.25 & .79 & - \\
\hline
\end{tabular}

Table of typical equivalents

(The following table is for informational purposes and is not a part of the simplified practice recommendation:)

\begin{tabular}{|c|c|}
\hline $\begin{array}{c}\text { Area (in } \\
\text { square } \\
\text { inches) }\end{array}$ & $\begin{array}{c}\text { Sizes of square } \\
\text { and round bars } \\
\text { (in inches) }\end{array}$ \\
\hline 0.05 & $1 / 4$ round. \\
.11 & $3 / 8$ round. \\
.20 & $1 / 2$ round. \\
.25 & $1 / 2$ square. \\
.31 & $5 / 8$ round. \\
.44 & $3 / 4$ round. \\
.60 & $7 / 8$ round. \\
.79 & 1 round. \\
1.00 & 1 square. \\
1.27 & $11 / 8$ square. \\
1.56 & $11 / 4$ square. \\
\hline
\end{tabular}

This recommendation, subject to annual revision by the standing committee of the industry, is effective from September 2, 1930.

UNITED STATES GOVERNMENT, DEPARTMENT OF COMMERCE, BUREAU OF STANDARDS, SIMPLIFIED PRACTICE RECOMMENDATION FOR STEEL SPIRAI RODS FOR CONCRETE REINFORCEMENT, R53-31, SEPTEMBER 30, 1931

\section{SPIRAL REINFORCEMENT RODS}

(Simplified practice recommended and accepted by industry establishing a limited list of standard sizes of round bars for reinforcing spirals.)

\section{Diameters and areas of spiral rods (plain rounds)}

\begin{tabular}{|c|c|}
\hline $\begin{array}{c}\text { Diameters } \\
\text { of round } \\
\text { bars }\end{array}$ & Areas \\
\hline Inch & Square inch \\
$1 / 4$ & 0.05 \\
$3 / 8$ & .11 \\
$1 / 2$ & .20 \\
$5 / 8$ & .31 \\
\hline
\end{tabular}

Note.-Seefollowing table of rod spacings which is for informational purposes and corers the normal conditions for use of spiral rods.

Pitch of spirals for various percentages of four standard rod sizes

[The following table shows the application of the sizes of simplified reinforcing spirals to all normal column core diameters with $1 / 4$-inch intervals of vertical spacing between successive turns]

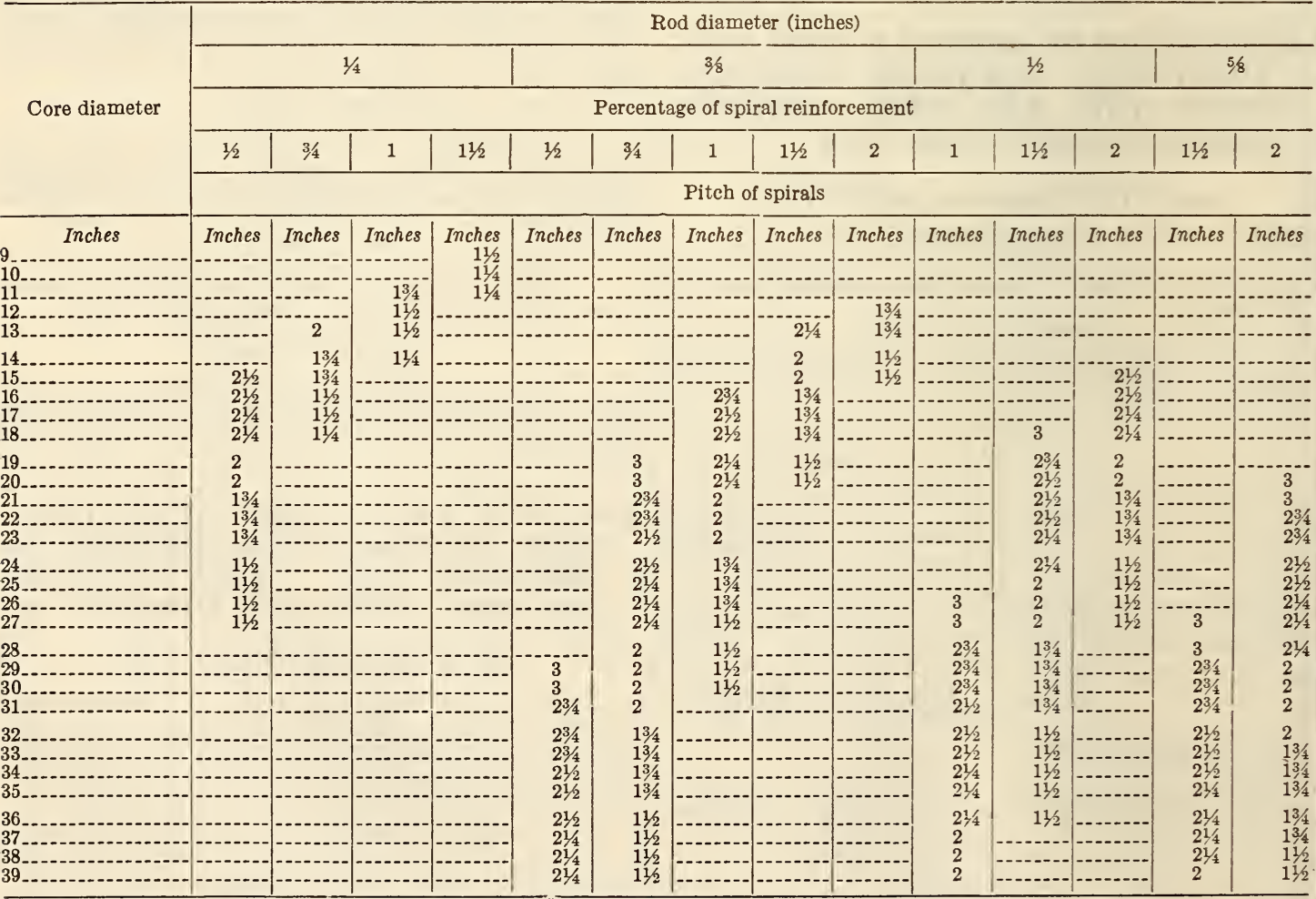

NOTES-Pitches not greater than one-sixth of core diameter nor greater than 3 inches. (See joint building code of American Concrete Institute and Concrete Reinforcing Steel Institute)

Pitches not shown for smaller core diameters must be checked by above rule to determine minimum spacing.

Pitches less than 113 inches not recommended except for $1 / 1$-inch rods.

Pitch of $1 \frac{1}{4}$ inches may be used for $1 / 4$-inch rods where absolutely necessary but no smaller spacing recommended.

Pitch variation by $1 / 4$-inch intervals recommended. 
UNITED STATES GOVERNMENT, FEDERAL SPECIFICATIONS BOARD, FEDERAL SPECIFICATION FOR BARS, REINFORCEMENT, CONCRETE, NO. 350a, 1929. (CURRENT DESIGNATION, QQ-B-71)

\section{REINFORCEMENT BARS}

\section{GENERAI SPECIFICATIONS}

United States Government general specification for metals, Federal Specifications Board specification No. 339a (QQ-M-151), in effect on date of invitation for bids, shall form part of this specification. (See 600.0, p. 3.)

\section{TYPES, GRADES, AND CLASSES}

1. Concrete reinforcement bars shall be of four types: A, plain; B, deformed; C, hot twisted; and $D$, cold twisted.

2. Concrete reinforcement bars shall be of four grades: (1) Structural billet steel, (2) intermediate billet steel, (3) hard billet steel, and (4) hard rail steel.

3. Concrete reinforcement bars shall be of three classes: (a) Open-hearth steel, basic; (b) open-hearth steel, acid; and (c) Bessemer steel.

\section{MATERIAL AND WORKMANSHIP}

The finished material shall be free from injurious defects and shall have a workmanlike finish.

\section{GENERAI REQUIREMENTS}

1. Bars of grades 1, 2, and 3 shall be rolled only from billets. Bars of grade 4 shall be rolled only from standard section $T$ rails.

2. Cold-twisted bars shall be twisted cold and hot-twisted bars shall be twisted hot. Each shall have one complete twist in a length of not over twelve times the thickness of the bar.

3. Permissible variations. - The weight of any lot of bars shall not vary more than 5 per cent from the theoretical weight of that lot. No bar shall have a minimum cross section over 6 per cent less than the theoretical cross section. If 5 per cent or more of the bars in any lot fail to meet this requirement, the lot shall be rejected. The term "lot" in this section shall be taken to include all bars of the same nominal weight per linear foot contained in a carload and represented by one invoice.

\section{DETAIL REQUIREMENTS}

1. Chemical composition.-The phosphorus content of grades 1, 2, and 3 steel shall not exceed $0.070,0.100$, and 0.125 per cent for classes $(a),(b)$, and (c), respectively. Grade 4 steel shall not be subjected to any chemical tests.

2. Manufacturers' analyses.-For grades 1, 2, and 3 , the manufacturer shall furnish the analyses given in Section V, $2(a)$ and (b). The phosphorus content shall not exceed that specified in Section V, 1.

(a) Open-hearth steel.-An analysis of each melt of steel showing the percentages of carbon, manganese, phosphorus, and sulphur.

(b) Bessemer steel. - An analysis of each melt, or lot of 10 tons, showing the percentage of carbon, and also an analysis for each 12 -hour period showing the percentages of manganese, phosphorus, and sulphur. This shall be considered as representing all melts poured during this period.

3. Tension and bend tests. - (a) The bars shall conform to the requirements given in Tables 1 and 2 as to tension properties and bend test requirements, respectively.

(b) The yield point shall be determined by the drop of the beam of the testing machine.

\section{METHODS OF INSPECTION AND TESTS}

1. Number of tests.-(a) For billet steel, two tension and two bend tests shall be made from each melt, unless the finished material from a melt is less than 25 short tons, when one tension and one bend test shall be sufficient. For Bessemer billet steel, one tension and one bend test shall be made from each lot of 10 short tons or less when the melts can not be identified. If, however, material from one melt or test lot differs three-eighths inch or more in thickness, the thickest and thinnest material rolled shall each be represented by one tension and one bend test, regardless of the weight of the melt or test lot.

(b) For rail steel, one tension and one bend test shall be made from each lot of 10 short tons or less of each size of bar rolled from rails varying not more than 10 pounds per yard in nominal weight.

2. Test specimens.- (a) Tension and bend test specimens for plain and deformed bars shall be taken from the finished bars, and shall be of the full thickness or diameter of bars as rolled; except that the specimens for deformed bars may be machined for a length of at least 9 inches, if deemed necessary to obtain uniform cross section.

(b) Tension and bend test specimens for coldtwisted bars shall be taken from the finished bars without further treatment, except as specified in Section VIII, 3. Tension and bend test specimens for hot-twisted bars shall be taken from the finished bars without further treatment.

3. Bend tests.-The test specimen shall bend cold around a pin without cracking on the outside of the bent portion, as specified in Table 2 .

\section{TABLE 1.-Tensile properties}

(This table is the same as the two tables given in the two specifications of the Association of American Steel Manufacturers for billet steel and for rail steel reinforcement bars, tensile properties. See 605.25, above.)

\section{TABLE 2.-Bend-test requirements}

(This table is the same as the two tables given for bend-test requirements in the two specifications of the A. A. S. M. for billet steel and for rail steel reinforcement bars. See 605.25, above.)

\section{PACKING AND MARKING OF SHIPMENTS}

Bars shall be made up in bundles, securely wired, as specified in the contract or purchase order. A metal tag shall be affixed to each bundle upon which shall be stamped data, as directed.

\section{NOTES}

1. When specifying material in accordance with this specification, the type (A, B, C, or D), the grade $(1,2,3$, or 4$)$, and the class $(a, b$, or $c)$, should be definitely stated.

2. Type A, plain bars and type B, deformed bars, may be obtained in any of the four grades specified in Section II, 2.

3. Type D, cold-twisted bars, in addition to being obtainable in accordance with the properties after twisting as specified herein, may be purchased on the basis of tests of the hot-rolled bars before twisting, in which case these latter tests shall govern and shall conform to the requirements specified for type A, plain bars of grade 1, structural steel.

4. While type $\mathbf{C}$, hot-twisted bars, are obtainable in any of the four grades, 1, 2, 3, and 4, they are usually purchased only in grades 3 and 4 .

5 . The areas (with their equivalent sizes of square and round bars) given in Simplified Practice Recommendation No. R26-30 of the United States Department of Commerce are given above. 


\section{RAILWAY TRACK MATERIAL}

\subsection{GENERAL ITEMS.}

\section{AMERICAN ELECTRIC RAILWAY ENGI- NEERING ASSOCIATION, STANDARDS, RECOMMENDATIONS, AND MISCELLA- NEOUS METHODS AND PRACTICES DEALING WITH THE GENERAL SUBJECT OF WAY MATTERS}

\section{TRACK CONSTRUCTION}

(These specifications do not concern themselves with the manufacture of materials, but with the utilization of manufactured materials in track-work construction.)

\begin{tabular}{|c|c|c|}
\hline Serial No. & Date & Subject matter \\
\hline W2-15. & 1915 & Definitions of bond and third-rail gage. \\
\hline W3 & 1915 & $\begin{array}{l}\text { Location of and clearances for third-rail working } \\
\text { conductors, structures, and rolling equipment. }\end{array}$ \\
\hline W10-2 & 1921 & Tongue switch construction. \\
\hline W12-23. & 1923 & Method of designating compromise joints. \\
\hline W 13-28. & 1928 & Special track-work layouts. \\
\hline W14-22. & 1922 & Location of tongue switches. \\
\hline W35-28 & 1928 & Uniform track center distances. \\
\hline W4 & 1928 & $\begin{array}{l}\text { Determination of flange ways and gages on } \\
\text { curves. }\end{array}$ \\
\hline W101-15_- & 1915 & Third-rail guards. \\
\hline W113-28 & 1928 & Street railway track construction. \\
\hline
\end{tabular}

AMERICAN MINING CONGRESS, MINE TRACKS, SIGNALS, AND SWITCHES, M7A-1927

[Approved as American Tentative Standard by the American Standards Association, May 13, 1927]

\section{MINE TRACKS, SIGNALS, AND SWITCHES}

\section{GAGE}

1. The standard track gage shall be 42 inches.

2. A 42-inch gage forms the best radii of curves with useful frog angles to suit the majority of coalmine work.

3. Under certain conditions, such as demands the use of cars to meet low-roof mines, etc., a gage of 42 inches may be prohibitive, and if necessary that it be other than standard the alternatives shall be in steps of 6 inches above or below, such as 30,36 , or 48 inches.

4. In addition to the above, an extra alternate of 4 feet $81 / 2$ inch gage is allowed, as some mines find it an advantage to have equipment that will roll on standard railroad tracks. Regardless of the alternates, the standard gage of 42 inches shall be used whenever possible.

RAIL

5. The rails covered by these standards shall be from 16 to 70 pounds per yard, inclusive, with room tracks limited to 45 pounds and below.

\section{DRILLING}

6. The drilling of rails or spacing and size of holes for splice bars shall be as follows:

\begin{tabular}{|c|c|c|}
\hline Rails (in pounds) & Spacing & $\begin{array}{l}\text { Diam- } \\
\text { eter of } \\
\text { hole }\end{array}$ \\
\hline $\begin{array}{l}16 \text { to } 25 \\
30 \text { and } 35 \\
40 \text { and } 45 \\
50 \text { and } 70\end{array}$ & $\begin{array}{ll}\text { Inches } \\
2 & 4 \\
2 & 4 \\
21 / 2 & 5 \\
21 / 2 & 5\end{array}$ & $\begin{array}{r}\text { Inch } \\
5 / 8 \\
3 / 4 \\
7 / 8 \\
1\end{array}$ \\
\hline
\end{tabular}

7. In the spacing given, the first dimension is the distance from end of rail to center of first hole, and the second dimension is the distance from center of first to center of second hole.

\section{FROG NOMBERS (ANGLES)}

8. Standard frog numbers and angles shall be as follows:

\begin{tabular}{|c|c|c|c|}
\hline No. & Degrees & Minutes & Seconds \\
2 & 28 & 04 & 21 \\
3 & 18 & 55 & 29 \\
4 & 14 & 15 & 00 \\
5 & 11 & 25 & 16 \\
6 & 9 & 31 & 38 \\
\hline
\end{tabular}

No. 2 frog is for room turnouts. No. 3 frog is for turnouts between room entries and face or panel entries. No. 4 frog is for turnouts between face and panel entries and in some cases for main haulage. Nos. 5 and 6 frogs are for turnouts for main-line haulage only, and especially where traffic is fast.

\section{SWITCH LENGTHS}

9. Lengths of switches and the number of frog with which they are to be used shall be as follows:

3 foot 6 inch switch for use with No. 2 frog.

5 -foot switch for use with Nos. 3 and 4 frogs.

7 foot 6 inch switch for use with Nos, 5 and 6 frogs.

\section{SWITCH HEEL SPREADS}

10. Heel spreads of switches shall be such as to present the sharpest angle and still allow room for spike holes in heel plates. The spreads are governed by size of rail and not by lengths of switches, and shall be as listed below:

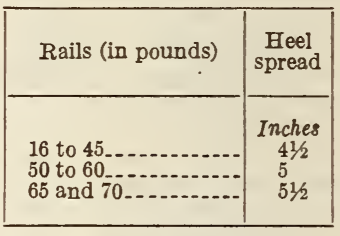

\section{SWITCH TYPES}

11. Switches shall be of two types-light and heavy duty. The light-duty switch shall be only for rails from 16 to 35 pounds and lengths of 3 feet 6 inches and 5 feet.

AMERICAN RAILWAY ASSOCIATION, MECHANICAL DIVISION, WHEELS AND TRACK, TERMS AND GAGING POINTS FOR, 1894

\section{WHEELS AND TRACK GAGE}

1. Track rails are the two main rails forming the track.

2. Gage of track is the shortest distance between the heads of track rails.

3. Base line, for wheel gages, is a line parallel to the axis of the wheels drawn through the point of intersection of tread with a line perpendicular to the axis, and passing through the center of the throat curve.

4. Inside gage of flanges is the distance between backs of flanges of a pair of mounted wheels measured on the base line.

5. Gage of wheels is the distance between the outside face of flanges of a pair of mounted wheels 
measured on a line parallel to the base line, but five-eighths inch farther from the axis of the wheels.

6. Thickness of flange is the distance measured parallel to the base line between two lines perpendicular thereto, one drawn through the point of measurement of "inside gage of flanges," and the other drawn through the point of measurement of "gage of wheels."

\begin{tabular}{|c|c|c|}
\hline & Feet & Inches \\
\hline $\begin{array}{l}\text { Inside gage of flanges } \\
\text { Gage of wheels } \\
\text { Thickness of flange. } \\
\text { Width of tread } \\
\text { Check gage distance. } \\
\text { Over-all gage. }\end{array}$ & \begin{tabular}{r}
4 \\
4 \\
\hdashline-1 \\
-4 \\
-4
\end{tabular} & $\begin{array}{r}57 / 32 \\
711 / 16 \\
115 \% 64 \\
411 / 32 \\
62964 \\
41 / 243 \%\end{array}$ \\
\hline
\end{tabular}

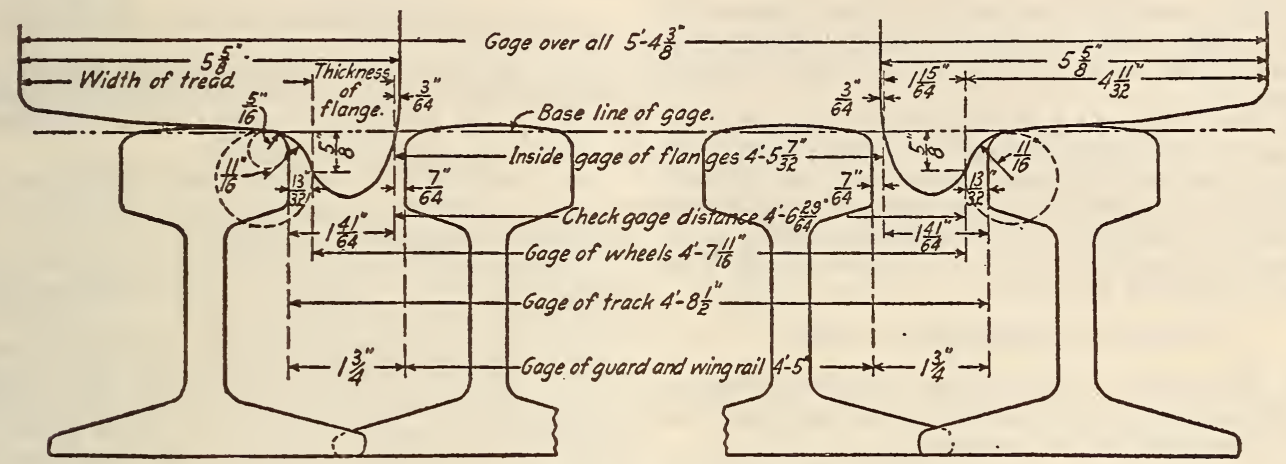

Terms and Gaging Points For Wheels and Track.

FIGURE 157. -Wheel tread worn hollow gage

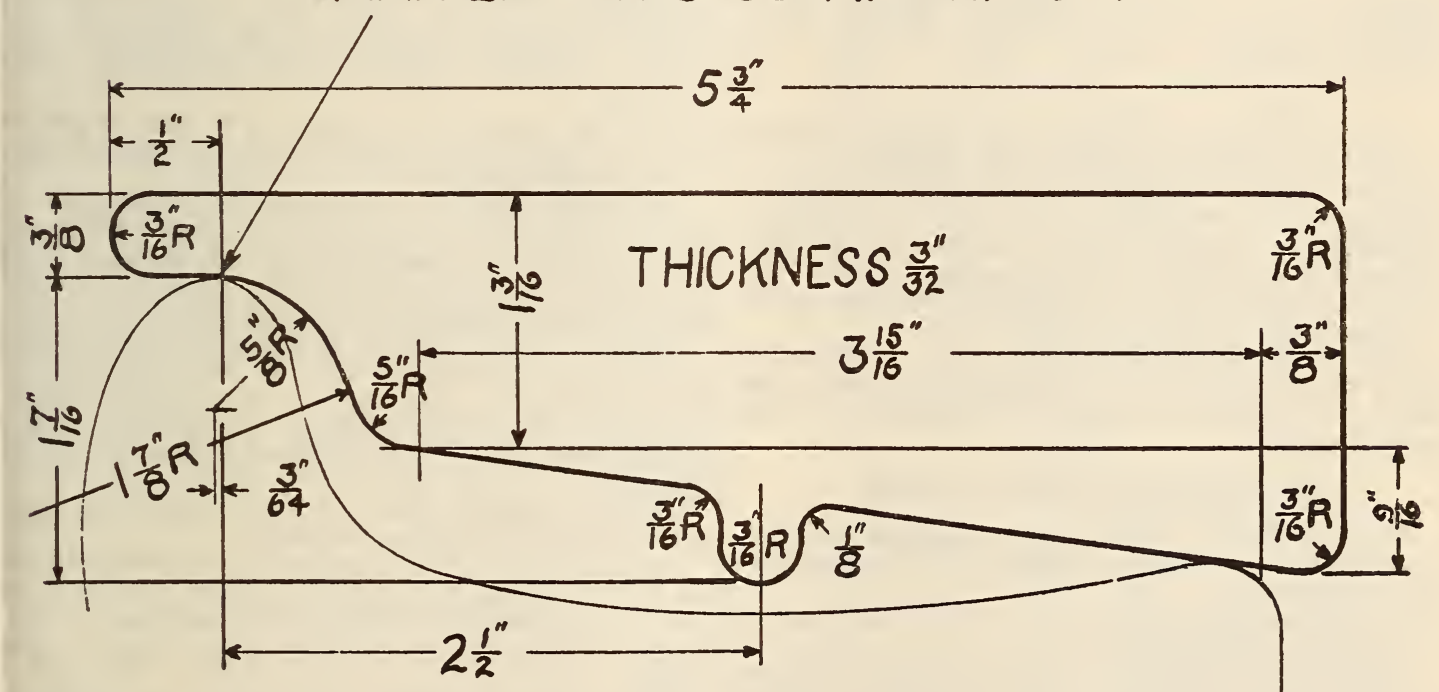

FIGURE 158.-Wheel tread worn hollow gage

7. Width of tread is the distance measured parallel to the base line from a line perpendicular thereto, drawn through the point of measurement of "gage of wheels" to the outer edge of tread.

8. Check gage distance is the distance measured parallel to the base line between two lines perpendicular thereto, one drawn through the point of measurement of "inside gage of flanges" on either wheel, and the other drawn through point of measurement of "gage of wheels" on mate wheel.

9. Over-all gage is the distance parallel to base line from outer edge of one wheel to the outer edge of mate wheel.

The above-mentioned wheel gage distances are either directly or by inference as follows, having been modified in 1909 :
606.1 STEEL RAILS, TEE, AND GIRDER

AMERICAN ELECTRIC RAILWAY ENGINEERING ASSOCIATION, STANDARD SPECIFICATION FOR THE MANUFACTURE OF OPEN-HEARTH STEEL GIRDER RAILS OF PLAIN, GROOVED, AND GUARD TYPES, W1-31, 1931

\section{STEEL GIRDER RAILS}

These specifications are intended to cover the manufacture of open-hearth steel girder rails of plain, grooved, and guard types of the classes specified. 
Girder-guard rails shall be class A.

Plain and grooved girder rails under 135 pounds in weight per yard shall be specified as either class A or class B.

Plain and grooved girder rails of 135 pounds in weight per yard and heavier shall be class $C$, unless otherwise specified.

\section{MANUFACTURE}

101. The steel shall be made by the open-hearth process. The entire process of manufacture and testing shall be in accordance with the best current practice.

102. Bled ingots and ingots or blooms which show the effects of injurious treatment shall not be used.

103. A sufficient discard from the top of each ingot shall be made at any stage of the manufacture to obtain sound rails. When finished rails show piping, they may be cut to shorter lengths until all evidence of this is removed.

\section{CHENICAL PROPERTIES AND TESTS}

201. The steel shall conform to the following requirements as to chemical compositions, according to the class specified in the order:

\begin{tabular}{|c|c|c|c|}
\hline & Class A & Class B & Class C \\
\hline $\begin{array}{l}\text { Carbon } \\
\text { Manganese } \\
\text { Silicon } \\
\text { Phosphorus, not over........ }\end{array}$ & $\begin{array}{r}\text { Per cent } \\
0.60-0.75 \\
.60-.90 \\
.15-.40 \\
.04\end{array}$ & $\begin{array}{r}\text { Per cent } \\
0.70-0.85 \\
.60-.90 \\
.15-.40 \\
.04\end{array}$ & $\begin{array}{r}\text { Per cent } \\
0.75-0.90 \\
.60-.90 \\
.15-.40 \\
.04\end{array}$ \\
\hline
\end{tabular}

202. To determine whether the material conforms to the requirements specified in section 201, an analysis shall be made by the manufacturer from a test ingot taken during the pouring of each melt. Drillings for analysis shall be taken not less than one-eighth inch beneath the surface of the test ingot. A copy of this analysis shall be given to the purchaser or his representative.

203. A check analysis may be made as information from time to time by the purchaser from a test ingot or drillings therefrom furnished by the manufacturer.

\section{PHYSICAL PROPERTIES AND TESTS}

301. (a) Four representative sections of rail from each melt shall be selected by the inspector as test specimens.

(b) Excess scale on the head or web of the sections shall be carefully removed.

302. (a) The head of each specimen shall be subjected to a pressure of 100,000 pounds $(45,359$ $\mathrm{kg}$ ) for a period of 15 seconds, applied through a hardened steel ball 0.75 inch $(19.05 \mathrm{~mm})$ in diameter.

(b) The steel test ball shall have a minimum Brinell hardness of 600 and it shall not be possible to attack the surface of the ball with an American Swiss pillar file No. 2.

(c) When fractured, the ball shall show a fine uniform grain, and the fracture shall resist file attack for at least one-third of its depth from the surface of the ball. The permissible variation in the diameter of the ball shall not be greater than 0.002 inch over or under the standard size, and the permanent deformation under the required loading shall not be greater than 0.003 inch.

(d) The average depth of impression obtained on the four specimens shall not be more than 0.1496 inch (3.8 mm) for class A rails, 0.1391 inch (3.5 $\mathrm{mm}$ ) for class $B$ rails, and 0.1338 inch $(3.4 \mathrm{~mm})$ for class $\mathbf{C}$ rails.
303. If the average of the impression tests on the head of the section from any melt fails to conform to the requirements specified in section $302(d)$ the manufacturer may at his option test each rail from such melt by making an impression test on the web, as described in section $302(a)$. Rails so retested which conform to the requirements as to depths of impression specified in section $302(d)$ shall be accepted.

\section{STANDARD SECTIONS, LENGTHS, AND WEIGETS}

401. (a) The cold template of the manufacturer shall conform to the specified section as snown in detail on the drawing of the purchaser and shall at all times be maintained perfect.

(b) The section of the rail shall conform as accurately as possible to the template, and witnin the following permissible variations:

(1) The neight shall not vary more than onesixty-fourth inch under nor more than one-thirtysecond inch over that specified.

(2) The over-all width of head and tram shall not vary more than one-eighth inch from that specified. Any variation which would affect the gage line more than one-thirty-second inch will not be allowed.

(3) The over-all width of base shall not vary more than one-eighth inch under that specified for widths less than $6 \frac{1}{2}$ inches; three-sixteenths inch under for a width of $6 \frac{1}{2}$ inches, and one-fourth inch under for a width of 7 inches.

(4) No changes will be allowed in dimensions affecting the fit of splice bars except that the fishing template approved by the purchaser may stand out not to exceed three-thirty-seconds inch laterally.

(5) The base of the rail shall be at right angles to the web; and the convexity shall not exceed onethirty-second inch.

(c) When necessary on account of the type of track construction, and notice to that effect has been given to the manufacturer, special care shall be taken to maintain the proper position of the gage line with respect to the outer edge of the base.

402. (a) Unless otherwise specified, the lengths of rails at a temperature of $60^{\circ} \mathrm{F}$. (15.5 $5^{\circ} \mathrm{C}$.) shall be 60 and 62 feet for those sections in which the weight per yard will permit; except girder-guard rails, which shall be 30 and 32 feet, unless otherwise specified.

(b) The lengths shall not vary more than onefourth inch from those specified.

(c) Shorter lengths, varying by 1 foot down to 40 feet for plain and grooved girder rails and 24 feet for girder-guard rails, will be accepted to the extent of 10 per cent by weight of each class on the order.

403. (a) The weight of the rails per yard as calculated shall be maintained as nearly as possible after conforming to the requirements specified in section 401 .

(b) A variation of 0.5 per cent from the calculated weight of section as applied to the entire order will be allowed.

(c) Rails accepted will be paid for according to actual weight.

\section{WORKMANSHIP AND FINISH}

501. (a) Rails on the hot beds shall be protected from water or snow and shall be carefully handled to minimize cold straightening.

(b) The distance between the rail supports in the cold-straightening presses shall not be less than 42 inches, except as may be necessary near the ends of the rails. The gag shall have rounded corners to avoid injury to the rails. 
(c) Rails heard to snap or check while being straightened shall be at once rejected.

502. (a) Rails shall be smooth on the head, straight in line and surface without any twists, waves, or kinks, particular attention being given to having the ends without kinks or droop.

(b) All burrs or flow caused by drilling or sawing shall be carefully removed.

(c) Rails shall be free from gag marks and other injurious defects of cold straightening.

(d) Any rails to be cold straightened showing sharp kinks or greater camber than that indicated by the middle ordinate of 18 inches in 60 feet shall be classed as No. 2 rails.

\section{DRILLING, MILIING, AND PUNCHING}

601. (a) Circular holes for splice bar bolts, bonds, and tie rods shall be drilled to conform to the drawings and dimensions furnished by the purchaser and within the following permissible variations:

(b) The diameter of bolt holes shall not vary more than one-thirty-second inch over or under that specified. The diameter of bond holes shall not be over the size specified, but may be one-thirtysecond inch under. The diameter of the tie-rod holes shall not be less than that specified but may be one-sixteenth inch over.

(c) The location of bolt and bond holes shall not vary more than one-sixteenth inch either longitudinally or vertically from that specified. The location of the tie-rod holes shall not vary more than one-fourth inch vertically and not more than onehalf inch longitudinally from that specified.

(d) Bond holes shall be truly cylindrical and not conical.

602. The ends of the rail shall be milled. The plane of the finished end surface shall, in the direction of the width of the rail, be at right angles to the gage with a permissible variation of one-thirtysecond inch in 6 inches, and in the direction of the height of the rail, be inclined to the plane of the base so that the top edge of the head will project beyond the bottom edge of the base not less than one-thirty-second inch nor more than three-thirtyseconds inch.

603. Unless otherwise specified by the purchaser, the tie-rod holes in class A rails may be punched, and, when so made, they shall be free from burrs, fins, etc. Punched tie-rod holes shall not be less in diameter than specified but may be not over oneeighth inch oversize.

\section{CLASSIFICATION OF RAILS}

701. Rails which are free from injurious defects and flaws of all kinds shall be classed as No. 1 rails. 702. (a) Rails which are rough on the head or which by reason of surface or other imperfections are not classed as No. 1 rails shall be classed as No. 2 rails; provided they do not, in the judgment of the inspector, contain imperfections in such number and of such character as to render them unfit for No. 2 rail uses, and provided they conform to the requirements specified in section 401 .

(b) No. 2 rails will be accepted to the extent of 10 per cent by weight of the entire order.

\section{MARKING AND LOADING}

801. (a) The name or brand of the manufacturer, the year and month of manufacture, the letters "O. H.," the weight of the rail, and the section number shall be legibly rolled in raised letters and figures on the web. The melt number shall be legibly stamped on each rail where it will not be covered subsequently by the splice bars.

(b) Both ends of all short-length No. 1 rails shall be painted green. Both ends of all No. 2 rails shall be painted white and shall have two heavy centerpunch marks on the web at each end at such a distance from the end that they will not be covered subsequently by the splice bars.

802. (a) Rails shall be loaded in the presence of the inspector, and shall be handled in such a manner as not to bruise the flanges or cause other injuries.

(b) Rails of each class shall be placed together in loadings.

(c) Rails shall be paired as to length before shipment.

IX. INSPECTION

(Conforms with paragraph 7 in A. S. T. M. standard specifications for steel. See 605.0, p. 247.)

\section{AMERICAN ELECTRIC RAILWAY ENGI- NEERING ASSOCIATION, STANDARD DESIGN FOR \%-INCH GIRDER-GROOVED RAIL, W6-31, 1931}

\section{7-INCH GIRDER-GROOVED RAIL}

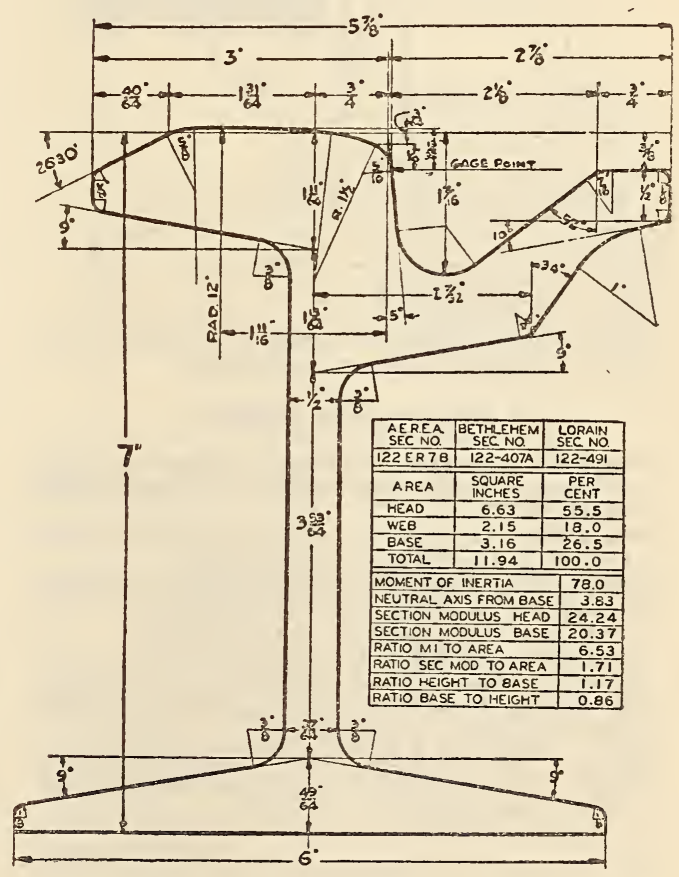

Figure 159.-Siandard design for 7 -inch 122pound girder grooved rail 


\section{AMERICAN ELECTRIC RAILWAY ENGI- NEERING ASSOCIATION, STANDARD DESIGN FOR 9-INCH GIRDER-GROOVED RAIL, W7-31，1931}

\section{9-INCH GIRDER-GROOVED RAIL}

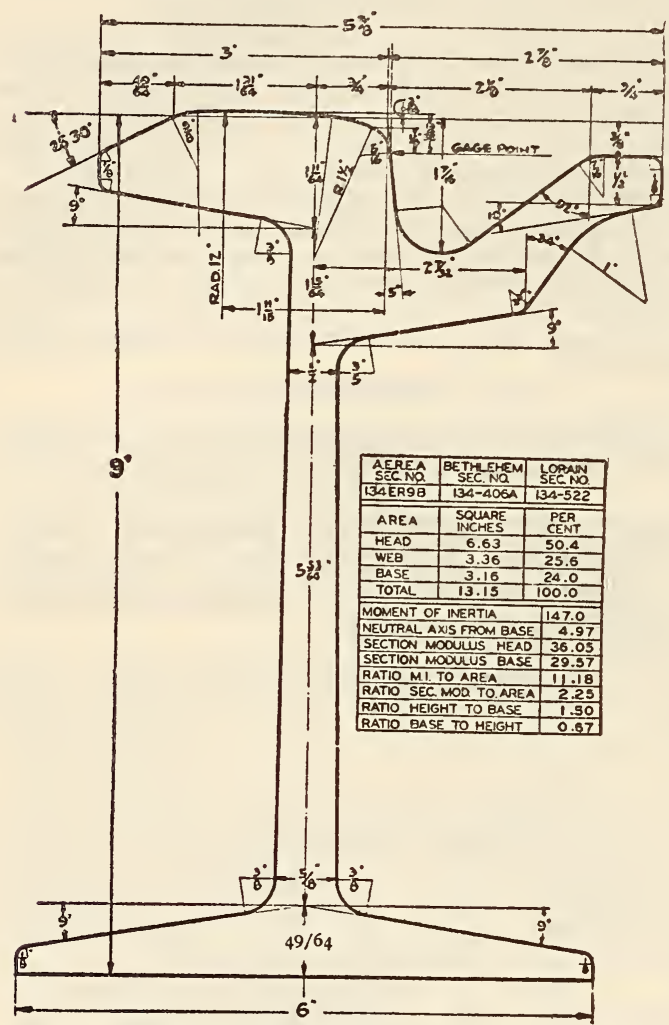

FIGURE 160.-Standard design for 9-inch 134pound girder grooved rail

Section Number 134 ER 9B

Note 1.-If necessary to refer to the preceding design covered by specification Wr $3 \mathrm{~b}$ its section number would be 134 ER 9 A.

Note 2.-The ER in above section numbers indicates the A. E. R. E. A. as the sponsor organization.

NOTE 3.-This rail fishes with section $152 \mathrm{ER} 9 \mathrm{~A}$ and Bethle. hem 125-273; $134-406$ and Lorain $125-427 ; 134-521$. It is the same as Bethelhem 134-406A and Lorain 134-522.

Note 4.-The position of the gaging point is somewhat difficult to locate on curved head rails, and special gages should be used which will insure exact track gage at the point indicated in the drawing.

Note 5.-These rails should be laid on straight track to a distance between gage points of rail, one-eighth inch less than the nominal gage.
AMERICAN ELECTRIC RAILWAY ENGINEERING ASSOCIATION, STANDARD DESIGN FOR \%-INCH GIRDER GUARD RAIL, W8-31, 1931

\section{7-INCH GIRDER GUARD RAIL}

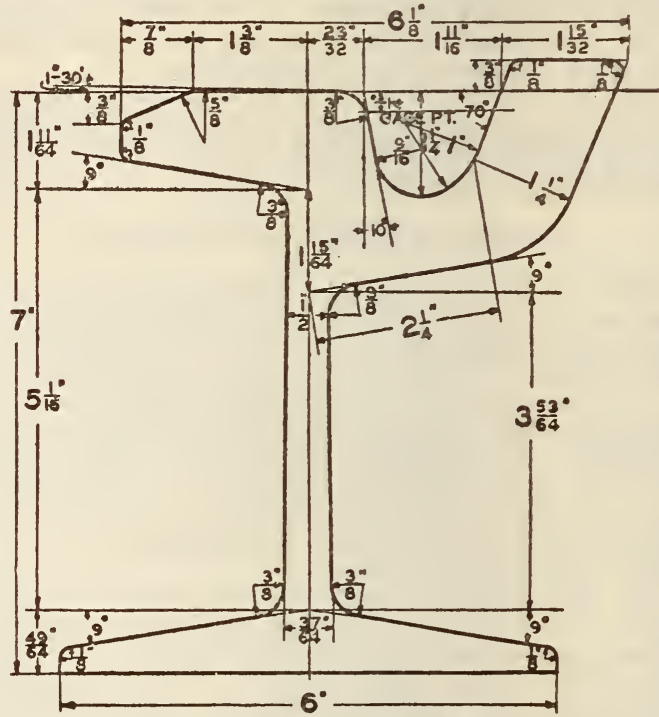

Figure 161. - Standard design for 7-inch 140. pound girder guard rail

Section Number 140 ER 7B

Note 1.-The $\mathrm{ER}$ in above section numbers indicates the A. E. R E. A. as the sponsor organization.

NOTE 2-This rail fishes with section $122 \mathrm{ER} 7 \mathrm{~B}$ or $122 \mathrm{ER}$ $7 \mathrm{~A}$ and is the same as Bethlehem 140-411 and Lorain $140-468$.

NOTE 3.-These rails should be laid on straight track to a distance between gage points equal to the nominal gage. From the junction of this rail with curved head rail the gage should be gradually reduced to one-eighth inch less than the nominal gage.

\begin{tabular}{|c|c|c|}
\hline A. E. R. E. A. Sec. No. & \begin{tabular}{|c|} 
Bethle- \\
hem Sec. \\
No.
\end{tabular} & $\begin{array}{l}\text { Lorain } \\
\text { Sec. No. }\end{array}$ \\
\hline $140 \mathrm{ER} 7 \mathrm{~B}$ & $140-411$ & $140-546$ \\
\hline $\begin{array}{l}\text { Area: } \\
\text { Head } \\
\text { Web } \\
\text { Base.............. }\end{array}$ & $\begin{array}{l}\text { Square } \\
\text { inches } \\
8.41 \\
2.15 \\
3.16\end{array}$ & $\begin{array}{c}\text { Per cent } \\
61.3 \\
15.7 \\
23.0\end{array}$ \\
\hline Total.. & 13. 72 & 100.0 \\
\hline $\begin{array}{l}\text { Moment of inertia } \\
\text { Neutral axis from base } \\
\text { Section modulus head...... } \\
\text { Section modulus base..... } \\
\text { Ratio, moment of inertia to } \\
\text { Ratio, section modulus to ar } \\
\text { Ratio, height to base......... } \\
\text { Ratio, base to height......... }\end{array}$ & ea & $\begin{array}{r}89.2 \\
4.18 \\
27.92 \\
21.34 \\
6.50 \\
1.56 \\
1.17 \\
0.86\end{array}$ \\
\hline
\end{tabular}


AMERICAN ELECTRIC RAILWAY ENGINEERING ASSOCIATION, STANDARD DESIGN FOR 9-INCH GIRDER GUARD RAIL, W9-31, 1931

\section{9-INCH GIRDER GUARD RAIL}

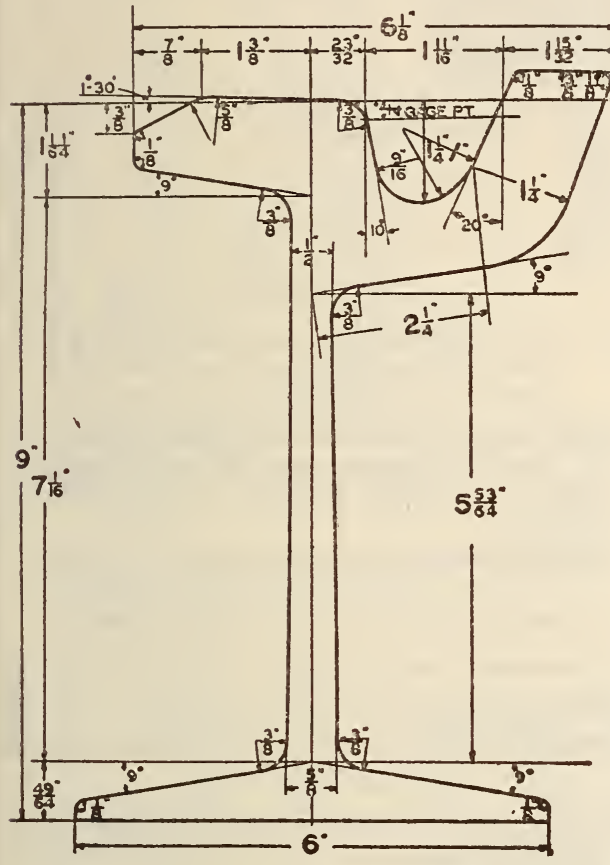

FIGURE 162.-Standard design for 9-inch 152pound girder guard rail

Section Number 152 ER 9B

Note 1.-The $\mathrm{ER}$ in above section numbers indicates the A. E. R. E. A. as the sponsor organization.

NOTE 2.-This rail fishes with sections $134 \mathrm{ER} 7 \mathrm{~B}$ or $134 \mathrm{ER}$ $7 \mathrm{~A}$ and is the same as Bethlehem 152-413 and Lorain 152520

NOTE 3.-These rails should be laid on straight track to a distance between gage points equal to the nominal gage. From the junction of this rail with curved head rail the gage should be gradually reduced to one-eighth inch less than the nominal gage.

\begin{tabular}{|c|c|c|}
\hline A. E. R. E. A. Sec. No. & $\begin{array}{l}\text { Bethle- } \\
\text { hem Sec. } \\
\text { No. }\end{array}$ & $\begin{array}{c}\text { Lorain } \\
\text { Sec. No. }\end{array}$ \\
\hline 152E R9B & $152-413$ & $152-547$ \\
\hline $\begin{array}{l}\text { Area: } \\
\text { Head } \\
\text { Web } \\
\text { Base }\end{array}$ & $\begin{array}{c}\text { Square } \\
\text { inches } \\
8.41 \\
3.36 \\
3.16\end{array}$ & $\begin{array}{c}\text { Per cent } \\
56.3 \\
22.5 \\
21.2 \\
\end{array}$ \\
\hline Total.... & 14.93 & 100.0 \\
\hline $\begin{array}{l}\text { Moment of inertia } \\
\text { Neutral axis from base } \\
\text { Section modulus head. } \\
\text { Section modulus base } \\
\text { Ratio, moment of inertia to } \\
\text { Ratio, section modulus to a } \\
\text { Ratio, height to base } \\
\text { Ratio, base to height }\end{array}$ & area & $\begin{array}{r}167.4 \\
5.38 \\
41.90 \\
31.12 \\
11.21 \\
2.08 \\
1.50 \\
0.67\end{array}$ \\
\hline
\end{tabular}

AMERICAN ELECTRIC RAILWAY ENGINEERING ASSOCIATION, STANDARD SPECIFICATION FOR CARBON-STEEL RAILS OF STANDARD SECTION, W16-29, 1929

\section{CARBON-STEEL RAILS}

(Same as A. S. T. M. A1-30, given below, except for additions and modifications of paragraphs to cover 62-foot rails and bond and tie rod holes and orders for small lots of rails.)

\section{AMERICAN ELECTRIC RAILWAY ENGI- NEERING ASSOCIATION, STANDARD DESIGN FOR IIGHT \%-INCH GIRDER- GROOVED RAIL SECTION, W28-31, 1931}

\section{LIGHT \%-INCH GIRDER-GROOVED RAIL SECTION}

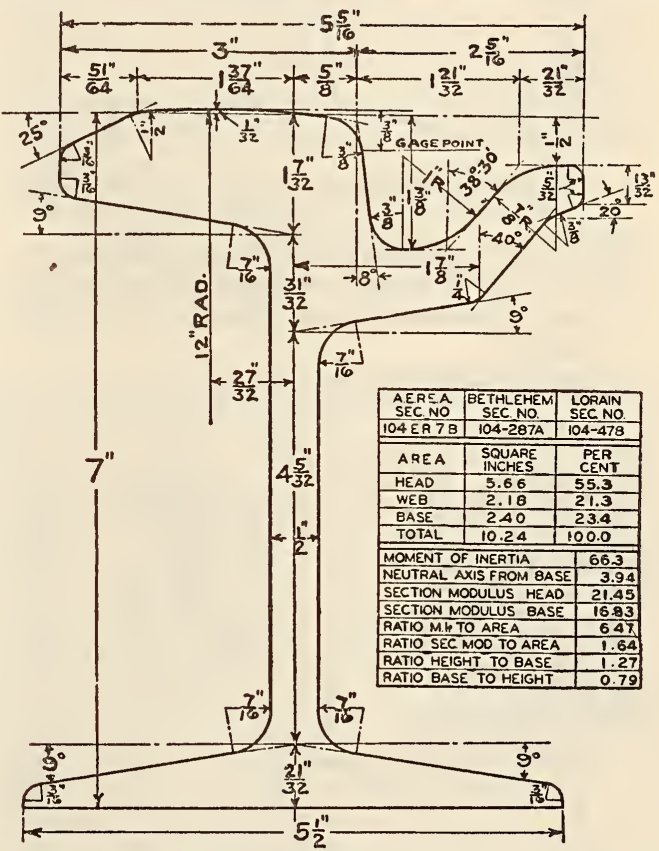

FIGURE 163.-Standard design for 7-inch 104pound girder-grooved rail

Section Number 104 ER 7B

Note 1. -If necessary to refer to the preceding design with plane head as covered by Bethlehem 103-287 and Lorain 103-426, its section number would be 103 ER 7A.

Note 2.-The ER in above section numbers indicates the A. E. R. E. A. as the sponsor organization.

Note 3.-This rail is the same as Bethlehem 103-287A and Lorain 103-478.

NoTE 4.-The position of the gaging point is somewhat difficult to locate on curved head rails, and special gages should be used which will insure exact track gage at the point ind cated on the drawing. 


\section{AMERICAN ELECTRIC RAILWAY ENGI- NEERING ASSOCIATION, DESIGN OF PLAIN GIRDER RAILS AND SPLICE BARS, W31-31, 1931}

\section{PLAIN GIRDER RAILS AND SPLICE BARS}

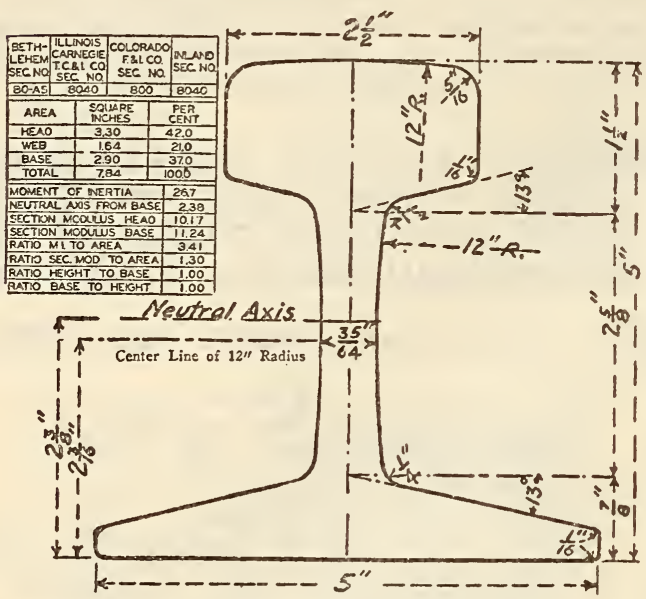

FIGURe 164.-80-pound standard section ( $A$. S. C. E. 80-pound) rail for use in private right of way and in macadam or other shallow paving

Calculated weight, 80 pounỏs per yard.

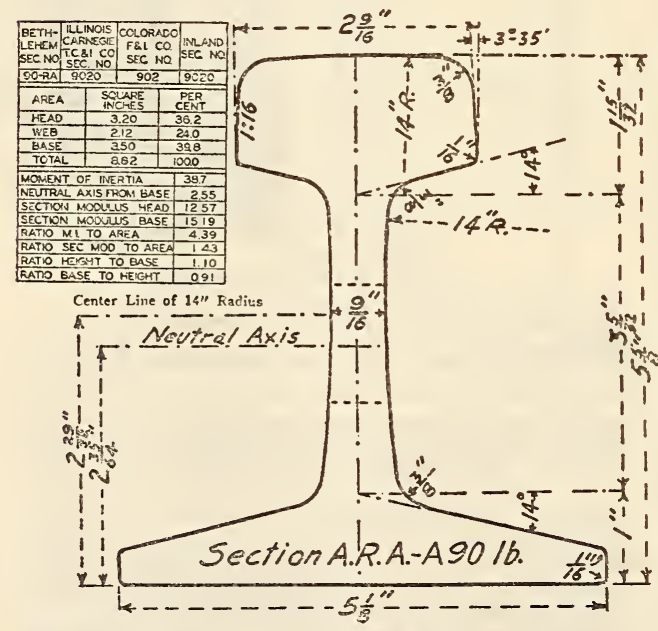

FigURE 165.-90-pound standard section ( $A$. R. A.-A 90-pound) rail for use in private right of way and in macadam or other shallow paving

Calculated weight, 90 pounds per yard.

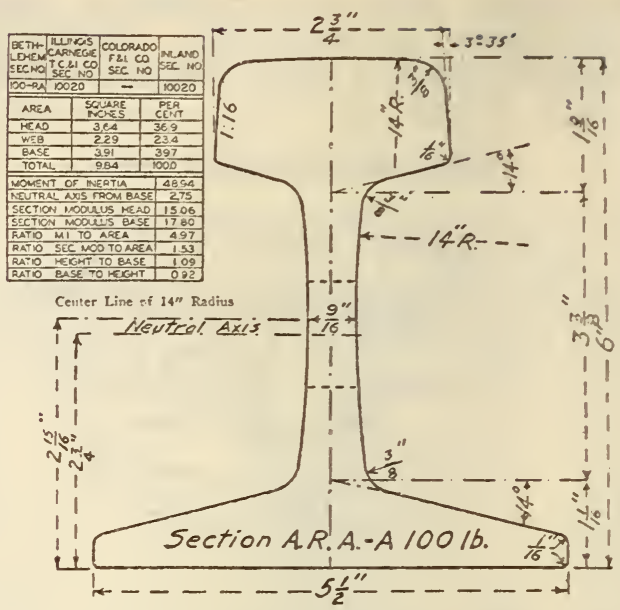

FigURE 166.-100-pound standard section ( $A$. R. A.-A 100-pound) rail for use in private right of way and in macadam or other shallow paving

Calculated weight, 100.4 pounds per yard.

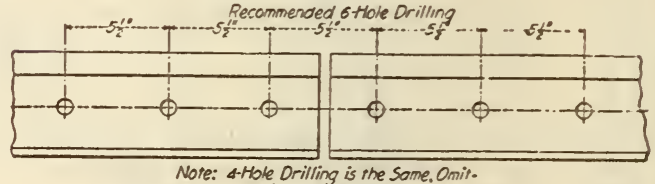
ting 2 Outer Holes.

FIGURE 167.-Recommended 6-hole drilling for 80-pound, 90-pound, and 100-pound standard rail sections

NorE. - This drilling is identical with that adopted as standard by the American Railway Engineering Associa. tion.

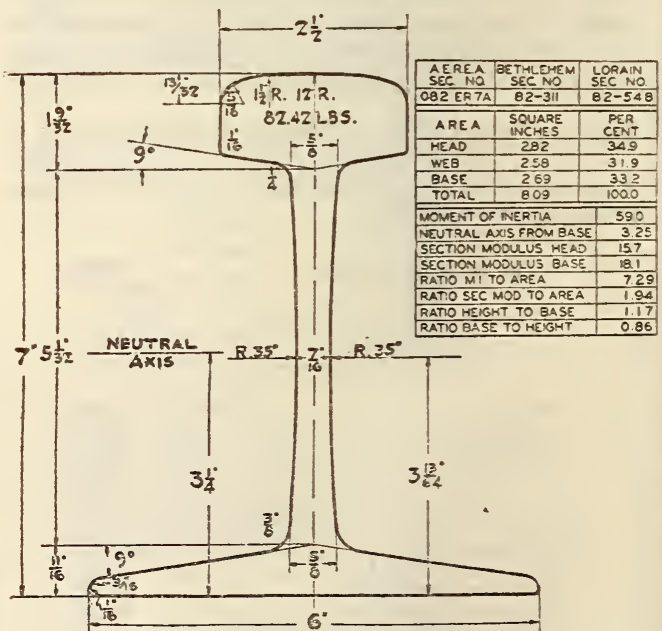

FIGURE 168.-7-inch, 82-pound plain girder rail for use in paved streets 


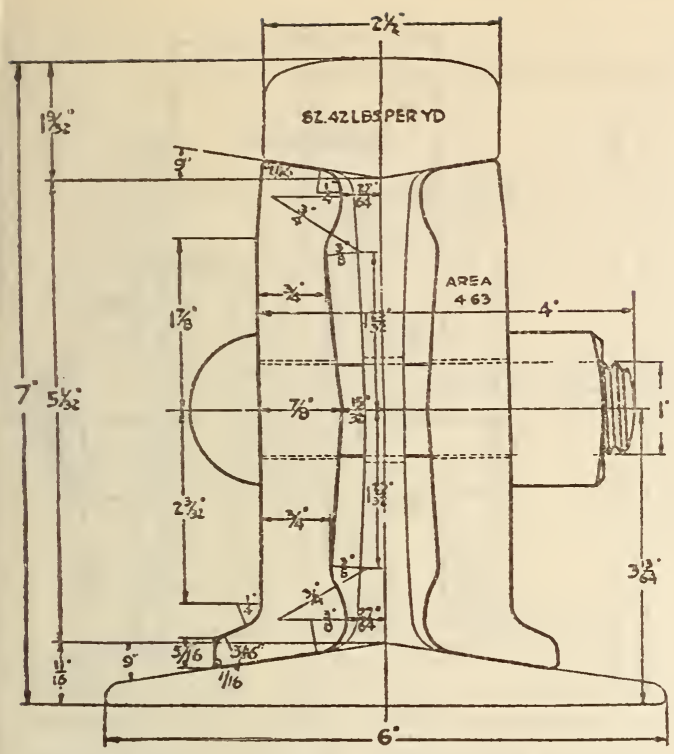

FIgURE 169.-Splice bar for use with design of 7 -inch, 82-pound plain girder rail shown in Figure 168

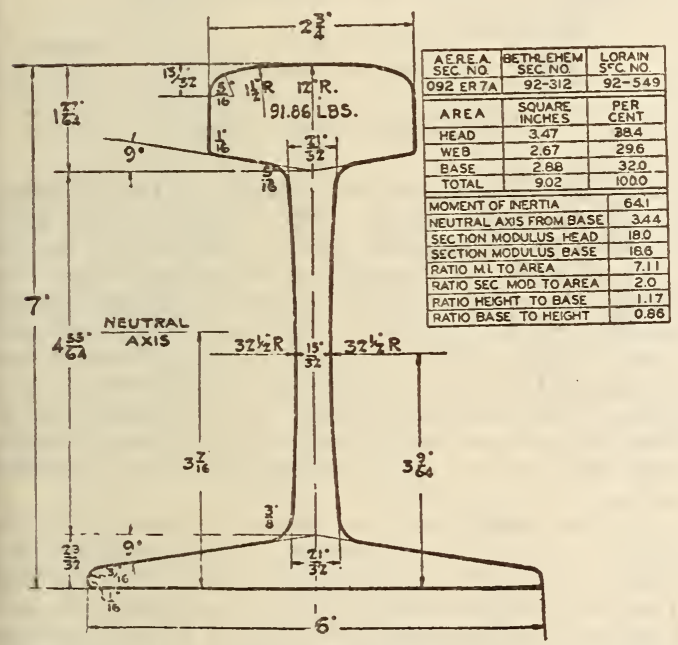

FIGURE 170.-7-inch, 92-pound plain girder rail for use in paved streets

Nore.- These rails should be laid on straight track to a distance between gage points of rail, one-eighth inch less than the nominal gage.

\section{AMERICAN RAILWAY ENGINEERING AS- SOCIATION, SPECIFICATIONS DROP-TEST MACHINE, 1910}

\section{DROP-TEST MACHINE}

A drop-test machine conforming essentially to the manufacturers' plans and specifications and in general accord with the following requirements will give satisfactory results:

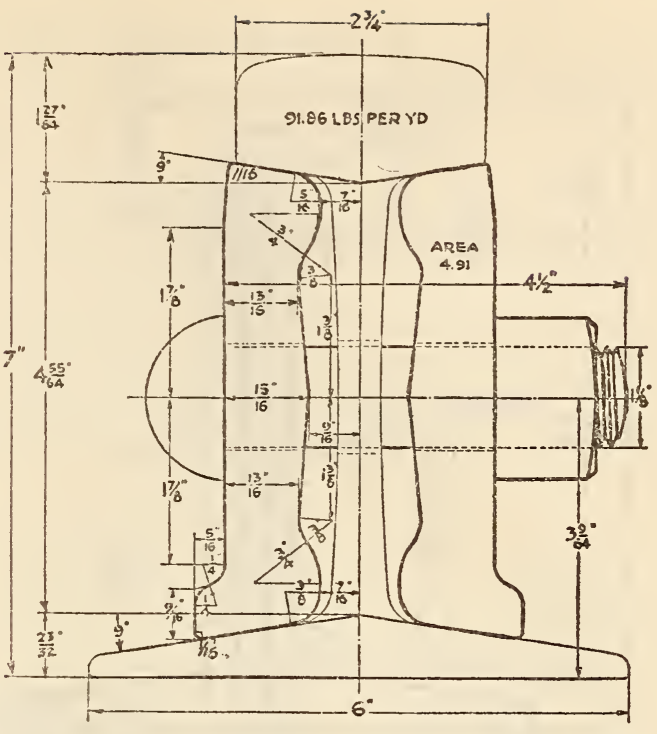

Figure 171.-Splice bar for use with design of 7-inch, 92-pound plain girder rail shown in Figure 170

Standard oval or elliptical bolt-holes in both bars.

Number of bolts, 6 .

Size of bolts, 1 by 4 inches.

Weight, 2 bars, each 26 inches long Pounds Weight of bolts 65.5

Total weight, 2 bars and 6 bolts..........

1. The machine shall be arranged to allow a 2,000 -pound tup to fall freely at least 25 feet on the center of a rail resting on supports that can be adjusted to spans varying from 3 feet to 4 feet 6 inches.

2. The anvil shall be a solid casting, weighing, with the attachments that move with it, 20,000 pounds. It shall be free to move vertically independent of the lead columns. It shall be supported on 20 springs known as the standard " $\mathrm{C}$ " spring, without center coil, as employed by the A. R. A. mechanical division. (See fig. 175.) This spring has a free length of $81 / 4$ inches, an outside diameter of $5 \% 16$ inches, and is made from a bar having a diameter of $13 / 16$ inches. These springs shall be arranged in groups of five at each corner of the anvil. They shall be held in place by hubs raised on the top of the base plate, and by circular pockets on the underside of the anvil. The anvil shall be guided in its vertical movement by removable finished wearing strips. These wearing strips shall be suitably attached to the finished edges of the column base.

3 . The base plate shall be of cast iron or cast steel 8 inches thick in the area covered by the anvil. It shall be firmly secured to the substructure by four bolts 2 inches in diameter.

4. The substructure shall consist of a timber grillage resting on a masonry foundation. The grillage shall project 9 inches beyond the ends of the base plate, and clear the columns at the side. It shall consist of one course of 12 by 12 inch sound oak or southern yellow pine, preferably creosoted, laid close and well bolted together. The masonry, preferably concrete, shall be not less than 5 feet deep below the grillage, suitably supported on the subsoil. 


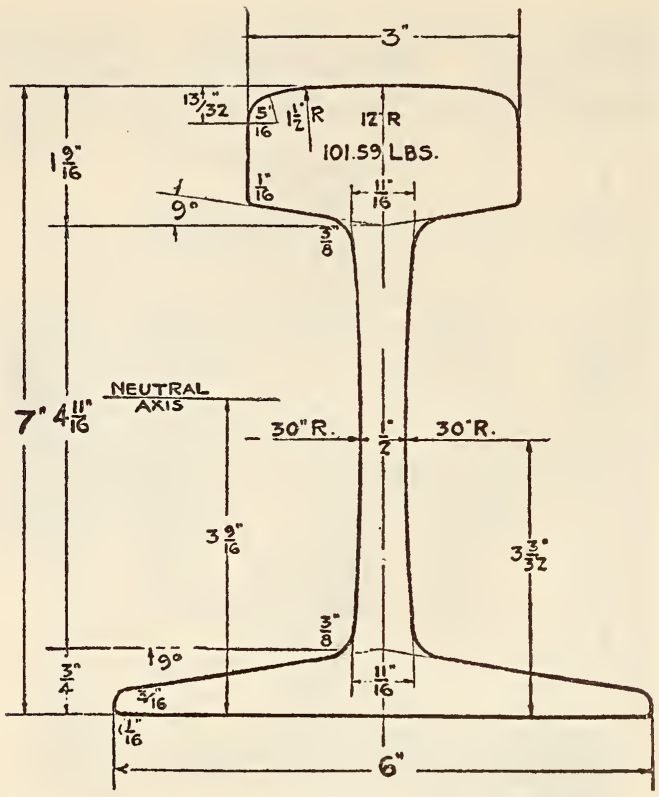

FIGURE 172.-7-inch, 102-pound plain girder rail for use in paved streets

\begin{tabular}{|c|c|c|}
\hline A. E. R. E. A. Sec. No. & $\begin{array}{c}\text { Bethle- } \\
\text { hem Sec. } \\
\text { No. }\end{array}$ & $\begin{array}{l}\text { Lorain } \\
\text { Sec. No. }\end{array}$ \\
\hline 102ER7A & $102-313$ & $102-550$ \\
\hline 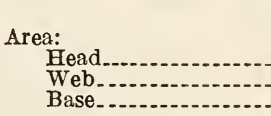 & $\begin{array}{r}\text { Square } \\
\text { inches } \\
4.18 \\
2.73 \\
3.06\end{array}$ & $\begin{array}{c}\text { Per cent } \\
41.9 \\
27.4 \\
30.7\end{array}$ \\
\hline Total_. & 9.97 & 100.0 \\
\hline \multicolumn{2}{|c|}{$\begin{array}{l}\text { Moment of inertia } \\
\text { Neutral axis from base } \\
\text { Section modulus head } \\
\text { Section modulus base } \\
\text { Ratio M. I. to area } \\
\text { Ratio section modulus to area } \\
\text { Ratio height to base. } \\
\text { Ratio base to height. }\end{array}$} & $\begin{array}{l}70.6 \\
3.56 \\
20.5 \\
19.9 \\
7.08 \\
1.99 \\
1.17 \\
0.86\end{array}$ \\
\hline
\end{tabular}

Note.-These rails should be laid on straight track to a distance between gage points of rail, oneeighth inch less than the nominal gage.

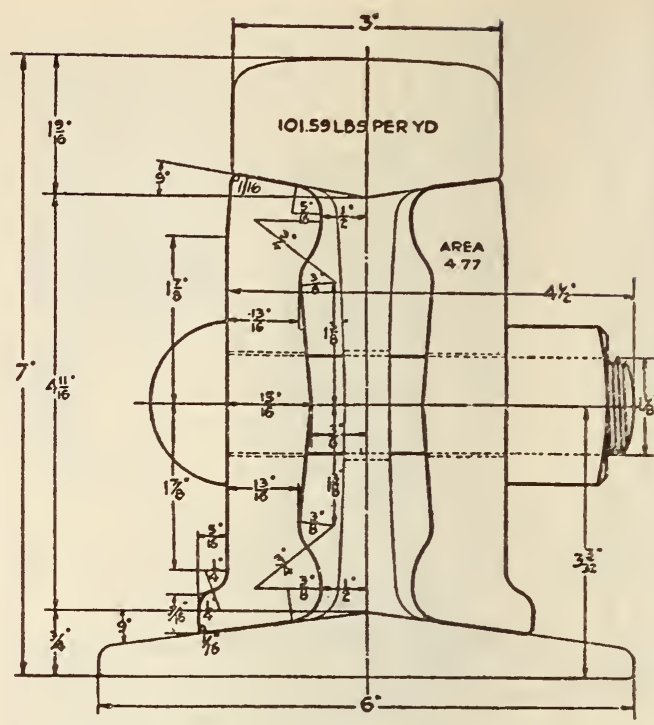

FigURe 173.- Splice bar for use with design of 7-inch, 102-pound plain girder rail shown in Figure 172

Standard oval or elliptical bolt-holes in both bars. No. of bolts, 6 .

Size of bolts, 1 by 4 inches.

Weight, 2 bars, each 26 inches long............ Pounds 65.5 Weight of bolts..................................... 11.5

Total weight, 2 bars and 6 bolts.........- 77.0

5. The pedestals for supporting the test rail shall be substantial castings. The surface of the anvil between these pedestals shall be formed to receive a wooden block to absorb shock under broken test pieces, the rail having an upper cylindrical bearing surface, with a radius of 5 inches. The pedestals shall be adjustable to spans varying from 3 feet minimum to 4 feet 6 inches maximum between centers. They shall be securely held together, and so fixed to the anvil as to insure that the center of the span shall always coincide with the center between leads.

6. The leads shall be firmly connected to the column base and well braced. They shall be long enough to provide the prescribed free fall of the tup. They shall be provided with a convenient ladder and a plainly marked gage, divided into 1 -foot intervals. The zero of this gage shall be $51 / 4$ inches above the top of the rail support. The specified height of drop shall

\section{LENGTH OF SPLICE BARS AND SPACING OF HOLES}
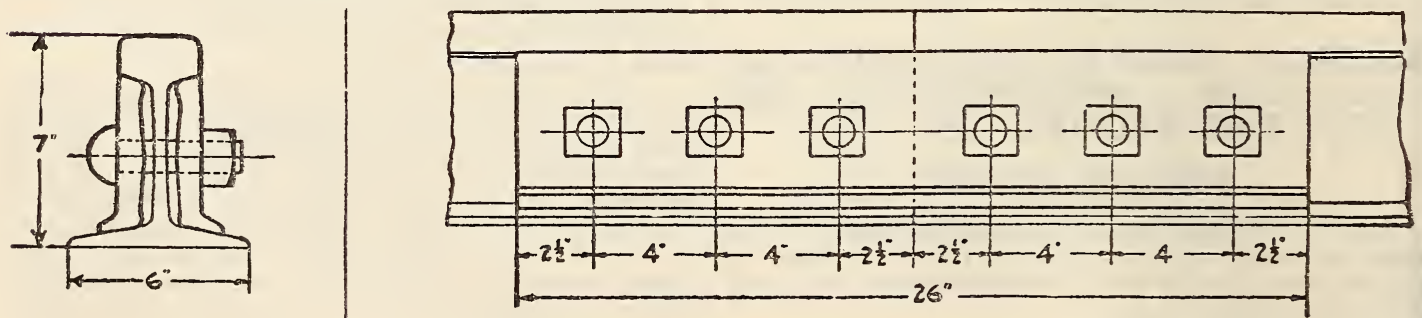

FIGURE 174.-26-inch splice bar for 7-inch plain girder rails (figures 168, 170 and 172) showing hole spacing and size of bolts 


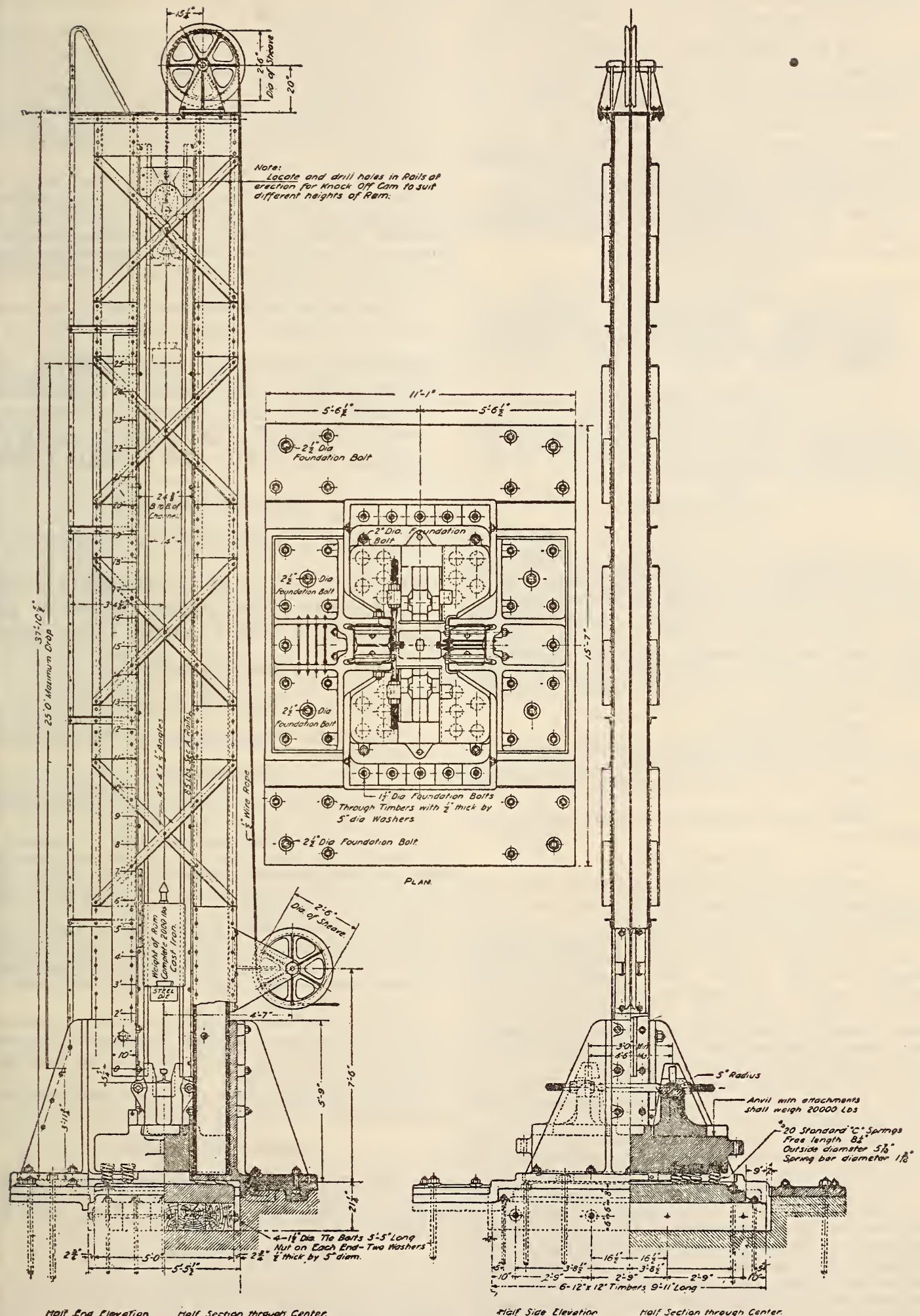

FIGURE 175.-Standard drop test machine 
be measured from this zero, irrespective of the height of the rail being tested. One of the guides shall have a movable section 6 feet long at the bottom, so that the tup or tripping block may be readily removed.

7. The tup shall weigh, with the accessories that drop with it, 2,000 pounds. The striking die shall be of steel, having a cylindrical striking face, with a radius of 5 inches and a length of 12 inches. The guide grooves shall have finished surfaces. The tripping head shall allow a grip of the tongs that will release at the exact height for which the tripping device is set, and that will be safe from accidental release while the test piece is being shifted.

8. The tongs and tripping device shall be arranged to release the tup automatically only. No manual releasing will be allowed. The tripping device shall be easily adjusted at 1 -foot intervals.

\section{AHERICAN RAILWAY ENGINEERING AS- SOCIATION, STANDARD SPECIFICA- TIONS FOR OPEN-HEARTH CARBON- STEEI RAIIS, 1925}

\section{CARBON-STEEL RAILS}

(Identical in substance with A. S. T. M. A1-30, given below, with the addition of the following requirements covering inspection:)

\section{RAIL INSPECTION}

\section{Inspection Force}

Rail inspectors shall be selected with care and men not familiar with rolling-mill practice shall not be sent to inspect rails except under the supervision of an experienced rail inspector. As far as possible the inspections shall be made by the same men, as they then become familiar with the methods of manufacture peculiar to each mill, for no two mills follow the same procedure even though owned by the same company.

1. Chief rail inspector.

2. Three or more assistant inspectors as size of mill and rate of rolling may require.

3. One or more checkers, depending upon rapidity of loading.

4. One chemist if check analyses are made in mill laboratory.

\section{Duties of Inspectors}

1. The chief rail inspector shall supervise the inspection force, mill practice, drop tests and make records.

2. One assistant inspector shall follow the mill practice closely, i. e., time of charging, time of tapping, cutting of tests, etc.; record any irregularities, such as too rapid pouring, charging cold ingots, rolling cold bloom, low finishing temperature, behavior of rails under straightening presses or any other departures from good mill practice which may affect the service of the rails.

3. One assistant inspector for night duty shall make drop tests if necessary, and follow the mill practice.

4. One or more assistant inspectors shall inspect the rails on the loading beds for surface defects, straightness, etc.

5 . One or more checkers shall record the number of rails of each heat accepted and loaded in each car. A record of the car number and the number of rails of each heat in the car shall be sent to the division engineer, the supervisor, the road master or other officer to whom the rails are consigned. This prevents the loading of rails rolled from odd ingots, or the loading of more rails than were originally rolled in a heat, or the loading of rails from rejected heats. It also furnishes a check record of the location of the rails if at any time in the future it is desired to remove the rails of a particular heat for any cause whatever.

6. A chemist shall make check analysis of drillings taken from corner of head of the rail, no rails being loaded until the check analysis is finished.

7. The office to which the rail inspection force reports shali keep a record of the history of each heat in a convenient form, so the results obtained from the service of the rails may be traced to the manufacture of the steel or rails.

8. Acceptance of heats meeting the technical requirements of specifications, but in the judgment of the inspector of inferior quality, shall be deferred pending decision of engineer of tests after full report and review of conditions; conversely, rejections of heats whose deficiencies may be technical only shall be similarly governed.

9. The inspectors can not be too careful and must exercise good judgment and all possible tact.

AMERICAN RAILWAY ENGINEERING ASSOCIATION, STANDARD SPECIFICATIONS FOR THE MANUFACTURE OF OPEN-HEARTH STEEL GIRDER RAILS OF PLAIN, GROOVED, AND GUARD TYPES, 1929

\section{STEEL GIRDER RAILS}

(Same as A. E. R. E. A., W1-31, given above.)

AMERICAN RAILWAY ENGINEERING ASSOCIATION, SPECIFICATIONS FOR REIAYER RAIL FOR VARIOUS USES, 1920

\section{RELAYER RAIL}

\section{DEFINITION}

1. Worn rails suitable for use in track are known as relayer rails.

\section{CLASSIFICATION}

2. Relayer rails shall be classified according to the wear on the side and top of the head:

(a) Side wear shall be represented by figures: 0 representing no side wear; 1,2 , 3, etc., representing the number of sixteenths of an inch wear on the side of the head at the gage five-eighths inch below the original top of the rail.

(b) Top wear shall be represented by capital letters: O representing no top wear; A representing one-thirty-second inch; B, two-thirty-second inch; $\mathrm{C}$, three-thirty-second inch, etc., wear, measured at the center of the rail.

(c) If the rail head is worn on both sides, the first figure shall represent the side showing the greater wear, the letter the top wear, and the figure following the lesser side wear.

(d) The maximum wear allowed in each case shall be specified by the user or the purchaser.

\section{PROCESS AND SECTION}

3. The process, as Bessemer, open-hearth, special alloy, or process steel, the section, the original weight per yard, and the splice drilling shal! be specified.

\section{MAIN-TRACK RELAYER RAILS}

4. Grade 1.-Used rails suitable for main track on main or branch lines. They must be free from all physical defects and shall pass the A. R. E. A. specifications for carbon-sieel rail (see above) in all re- 
spects except wear. The surface of the rails of this grade must be fairly smooth and shall not have flat spots or wheel burns. They shall be sawed at the ends, not cut with a chisel. Drillings shall be uniform. Chemical analysis, or the specifications under which the rails were originally purchased, shall be furnished when requested by the purchaser. There shall be full-length pieces, 30,33 , or 39 feet long, and not over 10 per cent of shorts varying in length by one foot, with a minimum length of 22 feet.

\section{RESA WING RAILS}

5. Grade 2.-All rails with battered or otherwise defecive ends, which if resawed would meet the grade 1 requirements, shall be graded as "resawed main-track relayer rails." In this case 90 per cent of the rails shall be of uniform length, 27 feet or over.

\section{SIDE-TRACK RELAYER RAILS}

6. Grade 3.-All used rails suitable for side tracks shall be included in this grade. They shall be not less than 15 feet long. Rails too badly out of line or surface to be included in grade 1, but straight enough to be easily spiked to line and gage shall be accepted. Drilling shall be uniform. Base shall be full or uniform width.

Any physical defects, such as broken lower flange, corroded, curved ends, burnt, ends battered, ends down, flat spots, head flow, line bent, piped, pitted, short, split end, split head, surface bent, twisted, worn under head, shall be specified where they occur and the extent of the defect shall be represented by the use of the term "slight," "moderate," or "bad."

\section{SCRAP RAILS (ROLLING-MILL RAILS)}

7. Grade 4--Rails of standard section not suitable for use as relayer rails. They shall be not less than 6 feet long and shall be free from pipes, split heads and similar defects. Badly twisted rails or bent rails, frogs, switches, and guard rails will not be accepted.

\section{SCRAP RAILS}

8. Grade 5.-All used rails of any length or condition not meeting the above specifications shall be designated as grade 5 .

\section{MARKING AND SHIPPING}

9. Rails when classified shall be marked with white paint on the web about 3 feet from the end. The figure and letter representing the side and top wear shall be painted first, followed by a dash (-) and the grade number, except scrap rails shall be marked by the grade number only. No two grades of rails shall be loaded in the same car.

\section{EXAMPLES}

10. (a) Main-track relayer rails having $3 / 16$-inch side wear and $3 / 32$-inch top wear would be classified and marked " $3 \mathrm{C}-1$."

(3) Resawed main-track relayer rails having 1/4inch side wear and $3 / 32$-inch top wear would be classified and marked " $4 \mathrm{C}-2$."

(c) Side-track relayer rails having $1 / 2$-inch side wear and $1 / 8$-inch top wear would be classified and marked "8 D-." If there are any physical defects, as curved ends, line bent, pitted, etc., they should be so classified and the extent of the defects specified as "slight," "moderate," or "bad."

(d) Side-track relayer rails having $1 / 2$-inch side wear on one side and 3/8-inch side wear on the other side, $1 / 8$-inch top wear, would be classified and marked "8 D 6-3." If there are any physical defects, as curved ends, line bent, pitted, etc., they should be so classified and the extent of the defect specified as "slight," "moderate," or "bad."
AMERICAN RAILWAY ENGINEERING ASSOCIATION, STANDARD RAIL SECTIONS, 1924

\section{STANDARD RAIL SECTIONS}

(Rail section A.-R. A. A-90 pounds is identical with that of A. E. R. E. A. shown in Figure 165.)

(See figs. 176 to 181 , inclusive.)

\section{AMERICAN SOCIETY FOR TESTING MATE- RIAIS, STANDARD SPECIFICATIONS FOR OPEN-HEARTH CARBON-STEEL RAILS, DESIGNATION A1-30, 1930}

\section{CARBON-STEEL RAILS}

\section{MANUFACTORE}

1. (a) The entire process of manufacture shall be in accordance with the best current state of the art. It is expected that thoroughly deoxidized dead setting steel will be furnished and that in every stage of manufacture strict adherence to the standards of best practice of the individual mill will be observed.

(b) Should anything occur in the process of manufacture, which, in the judgment of the inspector, varies detrimentally from regular care and practice, the inspector will immediately notify the chief inspector and the general superintendent of the mill, confirming such report in writing. If, after full investigation, the inspector is not satisfied as to the good quality of the rails in question, and the rail, complying in other respects to these specifications, be loaded for shipment, the inspector will immediately forward a copy of this report to the purchaser.

2. Sufficient discard shall be taken from the ingot to insure freedom from injurious segregation and pipe.

\section{CHEMTCAL PROPERTIES AND TESTS}

3. The steel shall conform to the following requirements as to chemical composition:

\begin{tabular}{|c|c|c|c|c|c|}
\hline \multirow{2}{*}{$\begin{array}{l}\text { Elements con- } \\
\text { sidered }\end{array}$} & \multicolumn{5}{|c|}{ Weight (pounds per yard) } \\
\hline & $50-69$ & $70-84$ & $85-100$ & $101-120$ & $121-140$ \\
\hline $\begin{array}{l}\text { Carbon } \\
\text { Manganese } \\
\text { Phosphorus, maxi- } \\
\text { mum, } \\
\text { Silicon, minimum. }\end{array}$ & $\begin{array}{r}\text { Per cent } \\
0.50-0.63 \\
.60-.90 \\
.04 \\
.15\end{array}$ & $\left\{\begin{array}{r}\text { Per cent } \\
0.53-0.70 \\
.60-.90 \\
.04 \\
.15\end{array}\right.$ & $\begin{array}{r}\text { Per cent } \\
0.62-0.77 \\
.60-.90 \\
.04 \\
.15\end{array}$ & $\begin{array}{r}\text { Per cent } \\
0.67-0.83 \\
.50-.90 \\
.04 \\
.15\end{array}$ & $\begin{array}{r}\text { Per cent } \\
0.72-0.89 \\
.50-.90 \\
.04 \\
.15\end{array}$ \\
\hline
\end{tabular}

4. (a) In any rolling, it is desired that the number of heats above the mean carbon percentage of the specific range shall be at least equal to the number of heats below the mean, and that the average carbon shall be as high as the mean.

(b) For information only, the manufacturer shall furnish the carbon and manganese analysis on drillings taken from both the "O" and "M" positions of the head at the top end of the "A" rail of the last full ingot rolled on each tenth heat.

5. Separate analyses shall be made from drillings taken from test ingots representing the second and one of the last full ingots of the heat to determine the percentages of carbon and manganese. The percentages of phosphorus, sulphur, and silicon shall be determined on equally mixed drillings from the test ingots. The average analysis of the ladle test ingots shall conform to the chemical requirements. A portion of the drillings shall be furnished to the inspector upon request for check analysis. 


\section{PHYSICAL PROPERTIES AND TESTS}

6. (a) Ductility and resistance to impact will be determined by the standard A. R. E. A. drop-testing machine, with test specimens from 4 feet to 6 feet long cut from the top of the "A" rails from the second, middle, and last full ingots of each heat. These specimens shall be marked on the base with gage marks 1 inch apart for 3 inches each side of the center of the test specimens for measuring the ductility of the metal. The distance between supports shall be 3 feet for sections under 106 pounds. For sections 106 pounds and over it shall be 4 feet.
8. (a) If all three of the specimens endure the above tests without fracture, all the rails of the melt will be accepted subject to final inspection for surface, section, and finish.

(b) If one of the three specimens fails, all the " $A$ " rails of the heat will be rejected. Specimens shall then be cut from the bottom end of the same "A" rails or the top end of the "B" rails and tested.

(c) If any of these test specimens fail, the "B" rails of the heat will be rejected. Three additional specimens shall then be taken from the bottom end of the " $B$ " rails or the top end of the " $C$ " rails. If

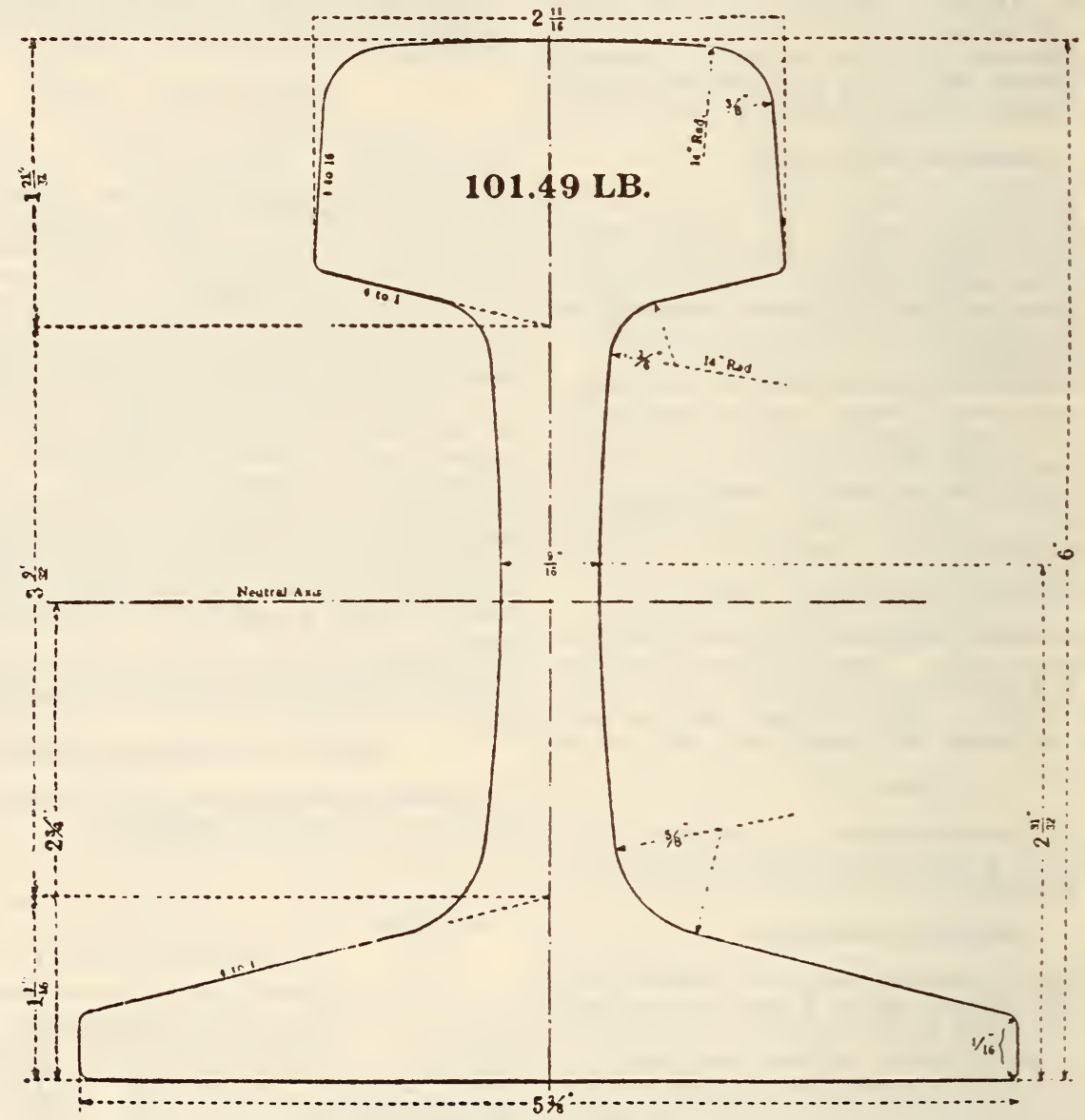

FIGURE 176.-Rail section, R. E., 100 pounds

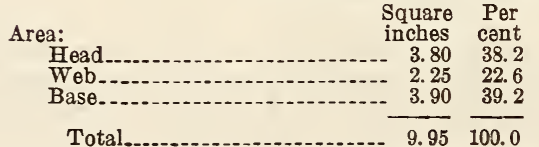

(b) The temperature of the test specimens shall not exceed $38^{\circ}$ C. (100 ${ }^{\circ}$ F.)

7. The test specimens shall be placed head upwards on the supports and subjected to one blow from the tup falling free from the following heights:

\begin{tabular}{|c|c|}
\hline $\begin{array}{l}\text { Weight of rail (in pounds per } \\
\text { yard) }\end{array}$ & $\begin{array}{l}\text { Height } \\
\text { of drop }\end{array}$ \\
\hline $\begin{array}{l}50 \text { to } 60 \text {, inclusive..... } \\
61 \text { to } 80 \text {, inclusive...... } \\
81 \text { to } 90 \text {, inclusive..... } \\
91 \text { to } 100 \text {, inclusive..... } \\
101 \text { to } 120 \text {, inclusive.... } \\
121 \text { to } 140 \text {, inclusive... }\end{array}$ & $\begin{array}{r}\text { Feet } \\
16 \\
17 \\
18 \\
19 \\
20\end{array}$ \\
\hline
\end{tabular}

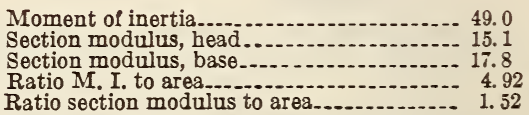

Ratio section modulus to area

all these test specimens endure the test, the balance of the heat will be accepted. If any of these tests specimens fail, the entire heat shall be rejected.

9. (a) One of the three test specimens shall be given a sufficient number of blows to determine, for information only, the exhausted ductility, reported inch by inch over the entire 6 inches gaged. No ductility readings will be taken between blows. The other two specimens shall be nicked and broken. The fracture of each specimen shall be examined to determine if the fracture conforms to the requirements of section 10 .

(b) For information only, the permanent set measured by middle ordinate in inches in length of 3 feet shall be recorded after the first blow on all test specimens. 
(c) For information only, one of the three test specimens shall be tested by Brinell indentation upon the head of the rail, and the diameter of the indentation shall be entered on the test record. The ball shall be $19 \mathrm{~mm}$ in diameter and the pressure 100,000 pounds.

10. If the fracture on any test specimen exhibits seams, laminations, cavities, interposed foreign matter, or a distinctly bright or fine-grained struc- that on 15 per cent of the order a variation of seven-sixteenths inch will be allowed.

When the specified length is 33 feet, 10 per cent of the entire order will be accepted in shorter lengths varying by 1 foot from 32 feet to 25 feet, and a variation of one-fourth inch from the specified length will be allowed, except that on 15 per cent of the order a variation of three-eighths inch will be allowed.

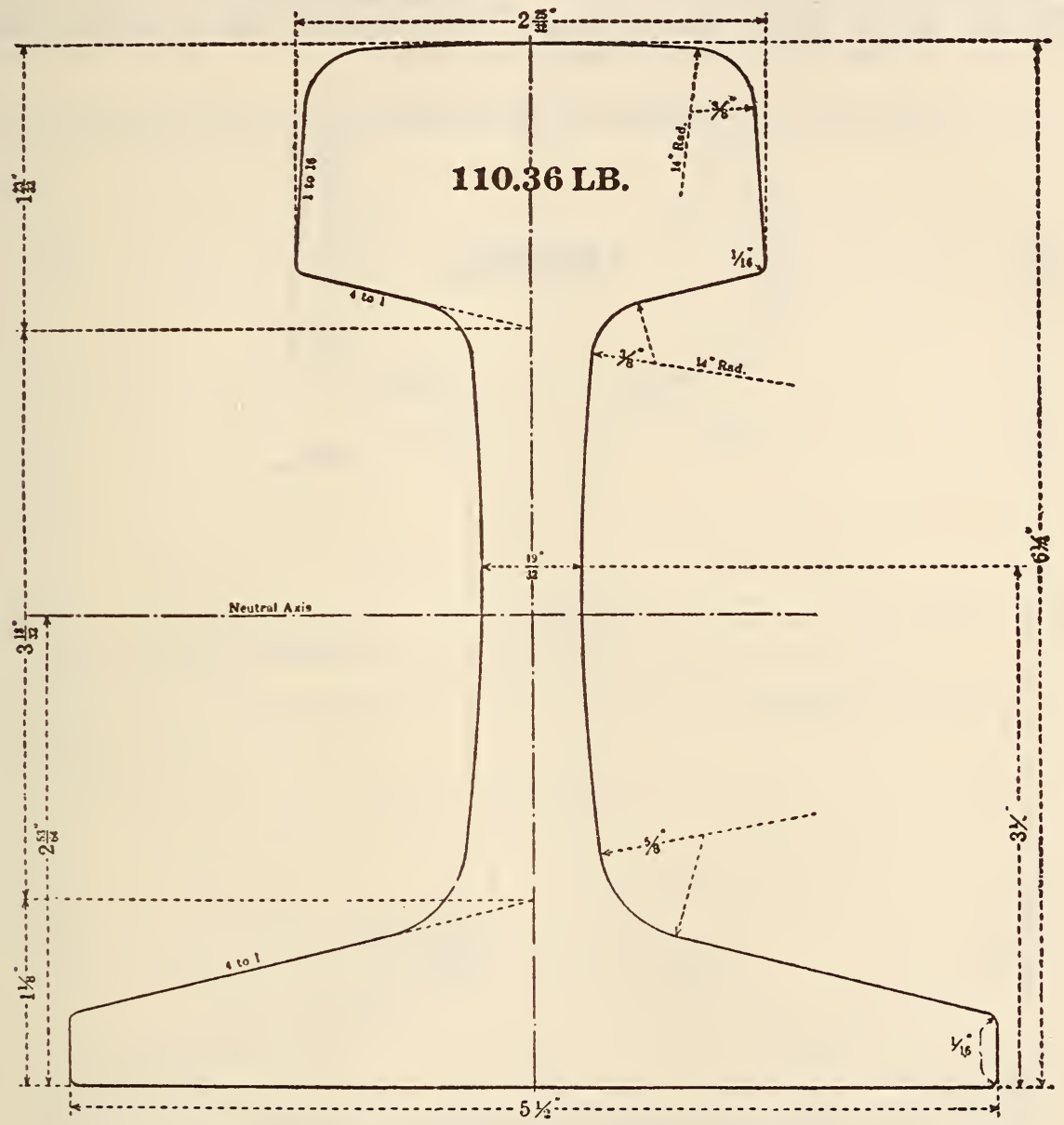

FIgURE 177.-Rail section, R. E., 110 pounds

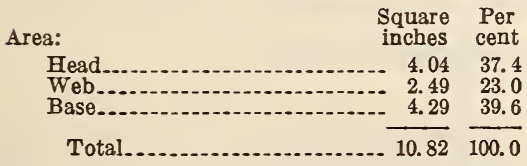

ture, "X-rayls."

\section{STANDARD SECTION, IENGTH AND WEIGHT}

11. The standard length of rails shall be 39 feet or $333_{\mathrm{f}}^{7}$ feet, as ordered, when measured at a temperature $15.5^{\circ} \mathrm{C}$. $\left(60^{\circ} \mathrm{F}\right.$.). When the length specified is 39 sfeet, 11 per cent of the entire order will be accepted in shorter lengths varying by 1 foot from 38 feet to 25 feet, and a variation of three-eighths inch from the specified length will be allowed, except

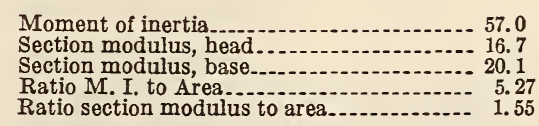

12. The section of the rails shall conform as accurately as possible to the templets furnished by the purchaser. A variation of one sixty-fourth inch less or one thirty-second inch greater than the specified height will be permitted. A variation of one-sixteenth inch in the length of either flange will be permitted, but the variation in total width of base must not exceed one sixteenth inch. No variation will be allowed in dimensions affecting the fit of the joint bars, except that the fishing template approved by the purchaser may stand out not to exceed onesixteenth inch laterally. 
13. A variation of 0.5 per cent from the calculated weight of section as applied to the entire order will be allowed.

\section{WORKNIANSHIP AND FINISE}

14. Circular holes for joint bolts shall be drilled to conform to the drawings and dimensions furnished by the purchaser. A variation of one thirty-second inch in the size and location of bolt holes will be allowed.

15. (a) All rails shall be smooth on the heads, straight in line, and without twists, waves, or kinks. be reconditioned by the mill, provided they can be made fully to meet the requirements.

(c) When any finished rail shows conditions as described in section 10 at either end or at any drilled hole, it shall be cut back to sound metal, and accepted as an "X-rayl."

\section{CLASSIFICATION OF RAILS}

16. No. 1 rails shall be free from injurious defects and flaws of all kinds.

17. X-rayls are rails as described in sections 10 and $15(\mathrm{c})$.

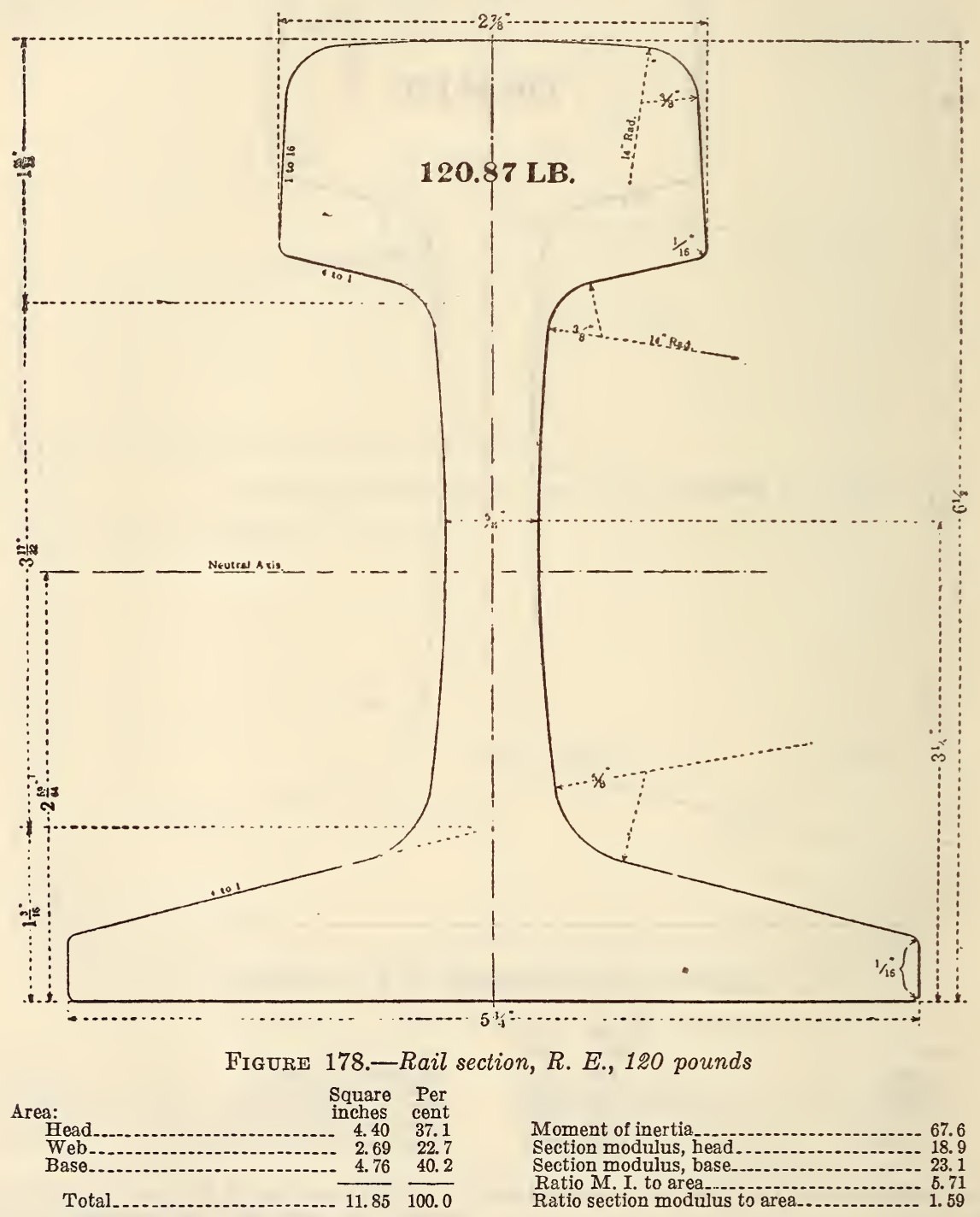

The supports for rails in the straightening presses shall have flat surfaces and be free from hollow places, bends, or crooks, and shall be spaced not less than 60 inches. When placed head up on a horizontal surface, rails that are slightly higher at the ends than the middle will be accepted, provided they contain a uniform sweep, the middle ordinate of which does not exceed one and one-fourth inches in 39 feet. They shall be sawed square at the ends, a variation of not more than one thirty-second inch being allowed, and burrs shall be entirely removed.

(b) Rails presented for inspection which do not conform to the requirements of paragraph (a) may
18. Rails which conform to the following requirements will be accepted as No. 2 rails:

(a) Rails which do not contain surface imperfections in such number or of such character as will in the judgment of the inspector render them unfit for recognized uses.

(b) Rails of A. R. A. type of sections arriving at the straightening presses with sharp kinks or greater camber than that indicated by a middle ordinate of 6 inches in 39 feet or 4 inches in 33 feet. Rails of A. S. C. E. type of sections arriving at the straightening presses with sharp kinks or greater camber than 
that indicated by a middle ordinate of 7 inches in 39 feet or 5 inches in 33 feet.

\section{MARIING}

19. Brands made so plain and sharp that they may be read as long as the rails are in service shall be rolled on or hot stamped into the side of the web of each rail in accordance with the following requirements, and to indicate:

(a) Name of the manufacturer, the month and year of manufacture, and the weight and type of section of rail as rolled. (d) All rails shall be branded "O. H." in addition to other marks.

20. (a) Rails accepted as No. 2 rails shall have the ends painted white and shall be stamped with the figure 2 on both end faces.

(b) Rails accepted as "X-rayls" shall have the ends painted brown and shall be stamped with the letter " $X$ " on both end faces.

(c) "A" rails shall have both ends painted yellow.

(d) No. 1 rails less than 39 feet long shall have both ends painted green.

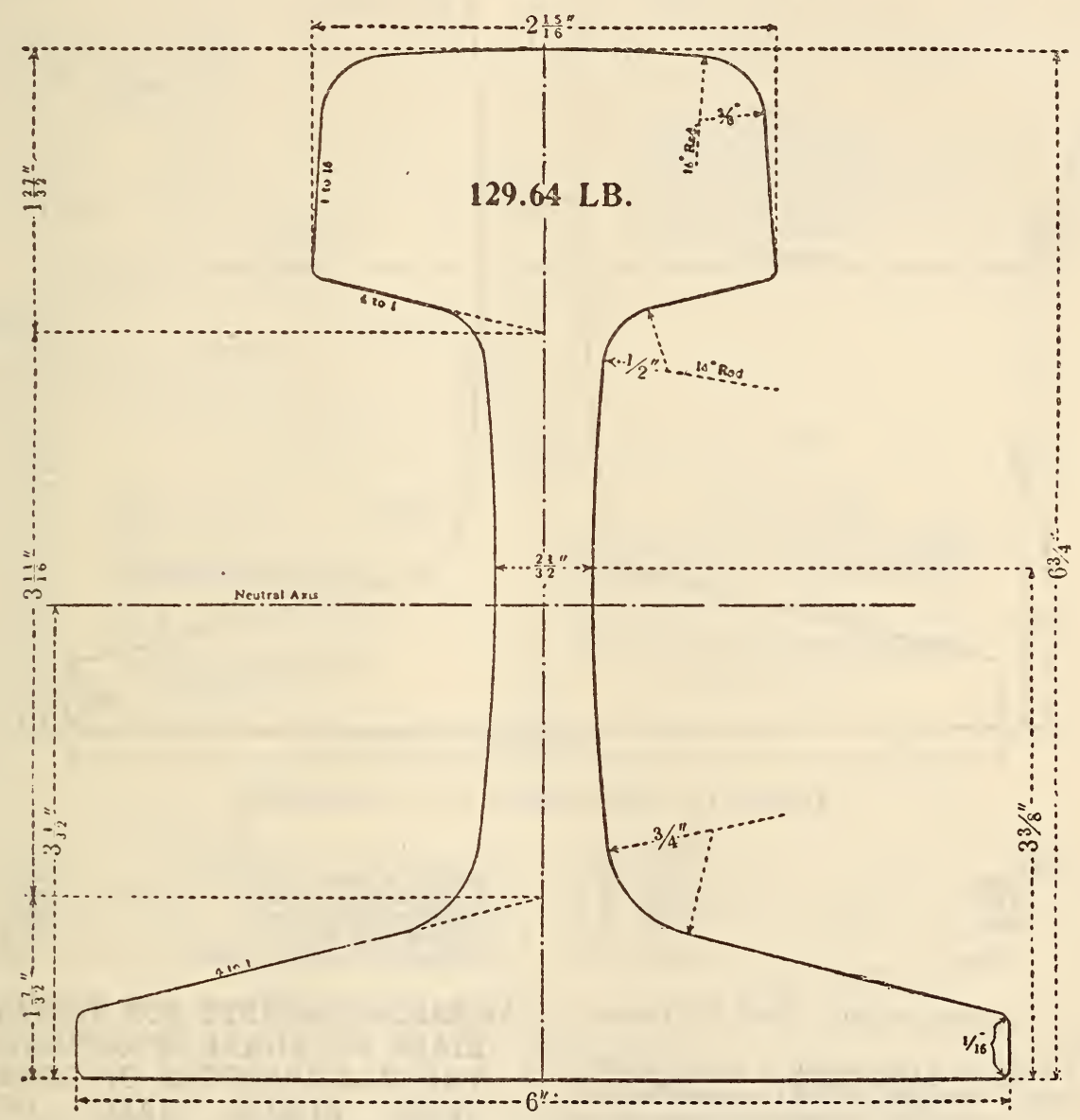

FigURe 179.-Rail section, R. E., 130 pounds

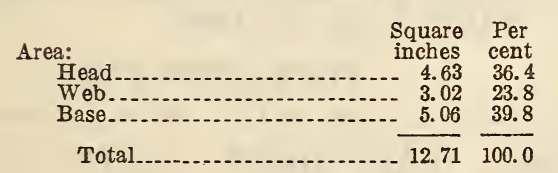

(b) The heat number and the ingot number as rolled shall be stamped in the web of each rail where it will not be covered by the joint bars.

(c) The top rails shall normally be lettered " $A$ " and the succeeding ones "B," "C," "D," "E," etc., consecutively, but in case the top discard is greater than normal, the rail lettering shall conform to the amount of discard, the top rail becoming "B" or other succeeding letter to suit the condition.

Only the "A," "B," and "C" rails are required to be lettered when the normal weight per yard is less than 70 pounds.

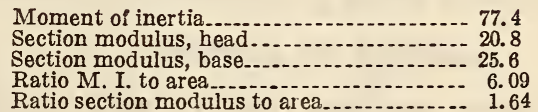

(e) All rails of heats the carbon content of which exceeds the mean carbon percentage of the specific range shall have both ends painted blue.

Individual rails shall be painted only one color, according to the order of precedence listed above.

\section{LOADING}

21. Rails shall be carefully handled in such manner as to avoid injury and shall be loaded as follows:

(a) No. 1 low-carbon rails shall be loaded in separate cars. 


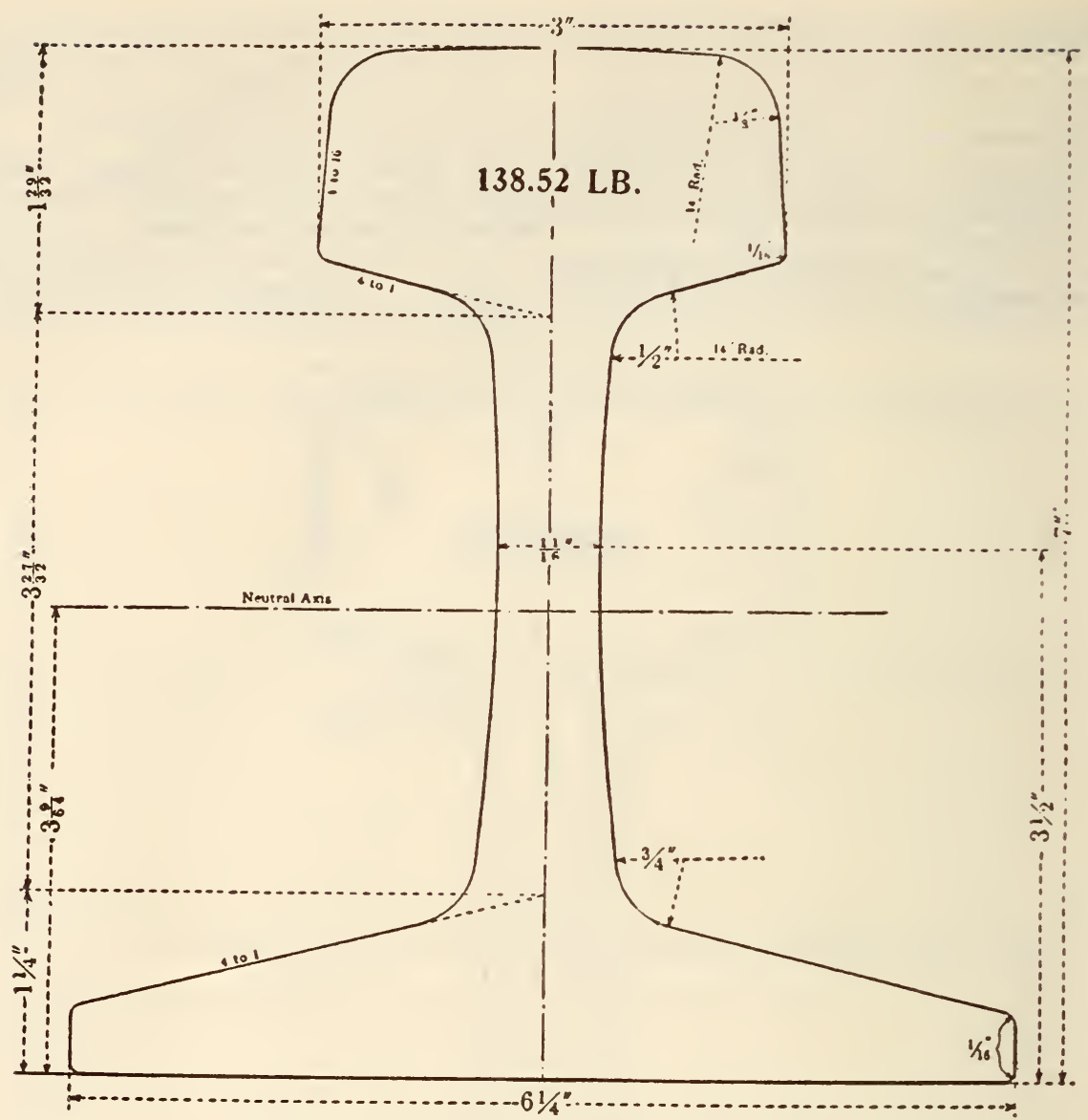

FigURe 180.-Rail section, R. E., 140 pounds

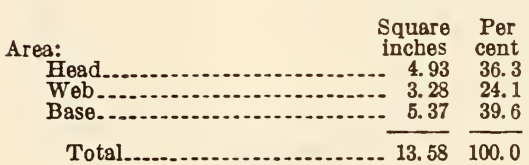

(b) No. 1 high-carbon rails shall be loaded in separate cars.

(c) No. 2 rails shall be loaded in separate cars.

(d) "X-rayls" shall be loaded in separate cars.

(e) No. 1 "A" rails shall be loaded in separate cars.

$(f)$ No. 1 short rails shall be loaded in separate cars. No subdivision by classification markings other than listed above is necessary for separate loading.

\section{INSPECTION AND REJECTION}

22. Inspection and tests shall be made at the works of the manufacturer before shipment, and the works management shall afford all reasonable facilities for determining the satisfactory quality of the rails accepted.

23. (a) In order to be accepted, the rails offered shall fulfill all the requirements of these specifications.

(b) No. 2 rails will be accepted to the extent of 8 per cent of the whole order of 39-foot standard length rails and 5 per cent of the whole order of 33-foot standard length rails.

(c) Rails accepted will be paid for according to actual weights.

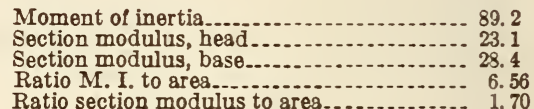

AMERICAN SOCIETY FOR TESTING MATE. RIALS, STANDARD SPECIFICATIONS FOR THE MANUFACTURE OF OPEN-HEARTH STEEL GIRDER RAILS OF PLAIN GROOVED, AND GUARD TYPES, DESIG. NATION A2-27, 1927

\section{STEEL GIRDER RAILS}

(Same as A. E. R. E. A. W1-31, given above.)

606.2 RAIL JOINT PLATES AND TIE PLATES.

\section{AMERICAN ELECTRIC RAILWAY ENGI- NEERING ASSOCIATION, STANDARD DESIGN FOR JOINT PLATES FOR 7- INCH GIRDER GROOVED AND GUARD RAILS, SERIAL NO. W4-22, 1922}

[Approved as American standard by the American Standards Association, February 5, 1923]

(See fig. 182, p. 32.) 


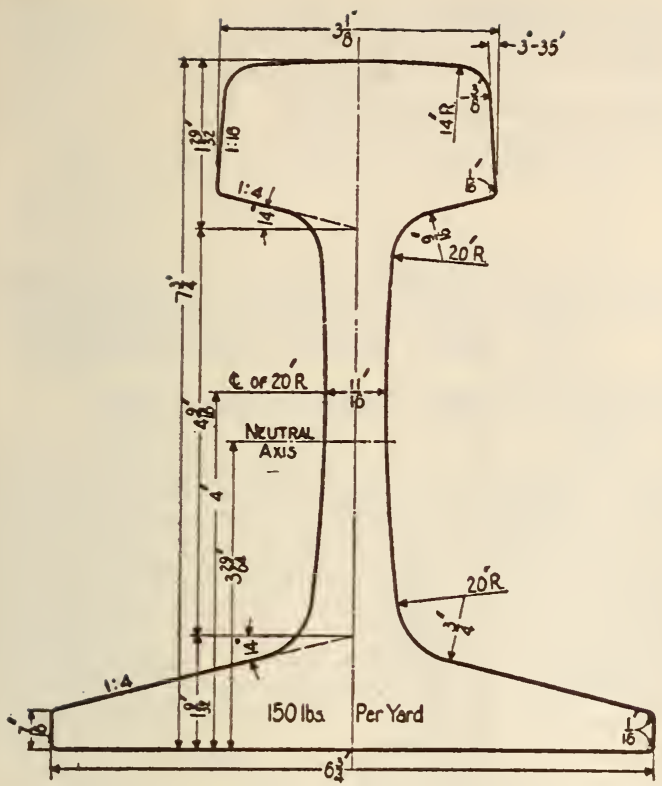

\begin{tabular}{|c|c|c|}
\hline Area & $\begin{array}{l}\text { Square } \\
\text { inches }\end{array}$ & Per cent \\
\hline 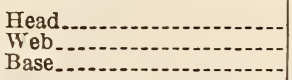 & $\begin{array}{l}5.12 \\
3.85 \\
5.78\end{array}$ & $\begin{array}{l}34.7 \\
26.1 \\
39.2\end{array}$ \\
\hline Total. & 14.75 & 100.0 \\
\hline $\begin{array}{l}\text { Moment of inertia } \\
\text { Section modulus, head. } \\
\text { Section modulus, base. } \\
\text { Ratio M. I. to area } \\
\text { Ratio section modulus to ar } \\
\text { Ratio height to base. } \\
\text { Ratio base to height...... }\end{array}$ & - & $\begin{array}{r}121.1 \\
28.2 \\
35.1 \\
8.21 \\
1.91 \\
1.15 \\
0.87\end{array}$ \\
\hline
\end{tabular}

Figure 181.-Rail section, R. E., 150 pounds

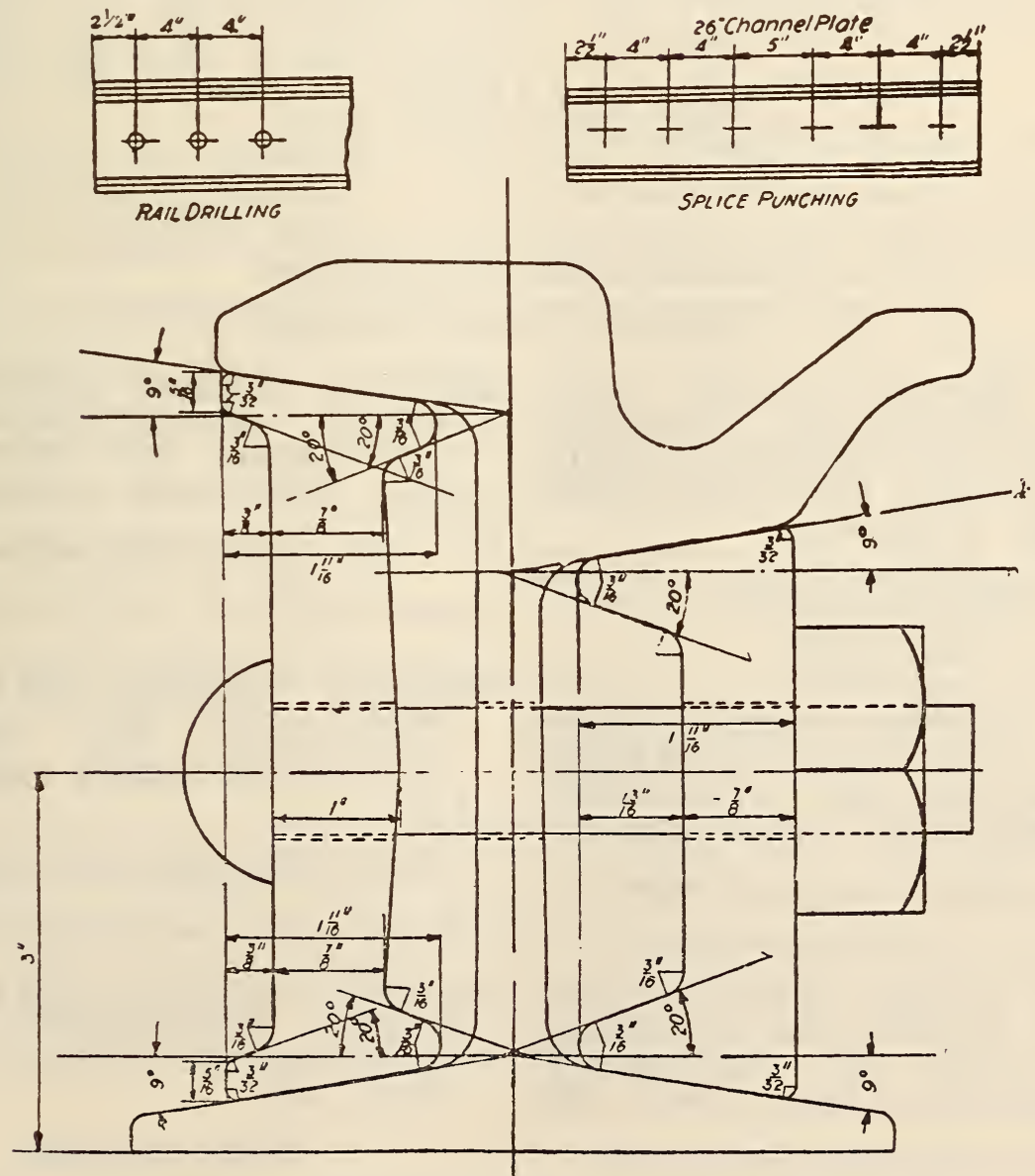

FIGURE 182.-Standard joint-plates for use with the standard 7-inch girder-grooved and guard rails

NoTE. - The size and shape of bolts and bolt-holes shown in this figure are not covered or included in the committee recommendation 


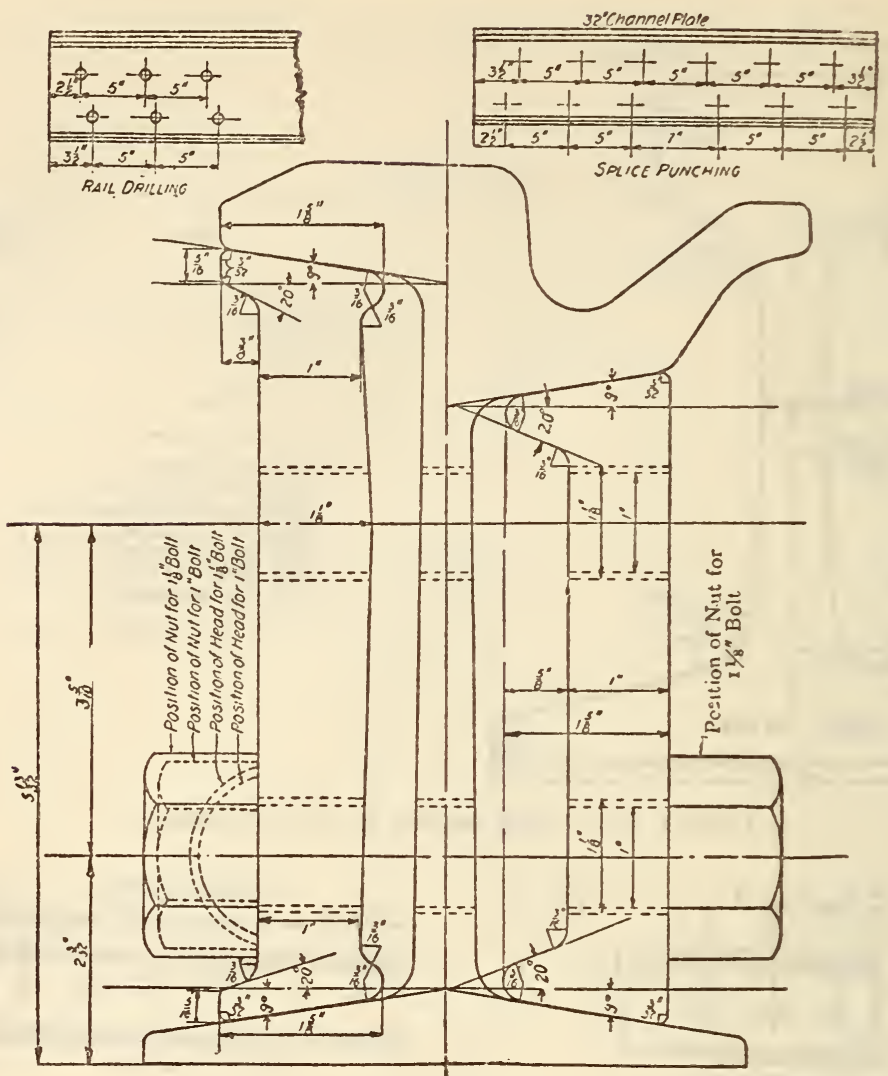

FIGURE 183.- Standard joint-plates for use with the standard 9-inch girder-grooved and girder-guard rails

Nore.-The size and shape of bolts and bolt-holes shown in this figure are not covered or included in the committee's recommendation

AMERICAN ELECTRIC RAILWAY ENGINEERING ASSOCIATION, STANDARD DESIGN FOR JOINT PLATES FOR 9-INCH GIRDER GROOVED AND GUARD RAILS, SERIAI NO. W5-22, 1922

[Approved as American standard by the American Standards Association, February 5, 1923]

\section{JOINT PLATES}

(See fig. 183 above)

AMERICAN ELECTRIC RAILWAY ENGINEERING ASSOCIATION, STANDARD SPECIFICATION FOR LOW-CARBONSTEEL SPLICE BARS, W17-22, 1922

\section{LOW-CARBON-STEEL SPLICE BARS}

(Same as A. S. T. M., A3-24, given below.)

AMERICAN ELECTRIC RAILWAY ENGINEERING ASSOCIATION, STANDARD SPECIFICATION FOR MEDIUM-CAR BON-STEEL SPLICE BARS, W18-22, 1922

MEDIUM-CARBON-STEEL SPLICE BARS

(Same as A. S. T. M., A4-14, given below.)

\section{AMERICAN ELECTRIC RAILWAY ENGI- NEERING ASSOCIATION, STANDARD SPECIFICATION FOR HIGH-CARBON- STEEL SPLICE BARS, W19-22, 1922}

\section{HIGH-CARBON-STEEL SPLICE BARS}

(Same as A. S. T. M., A5-14, given below.)

AMERICAN ELECTRIC RAILWAY ENGINEERING ASSOCIATION, STANDARD SPECIFICATION FOR EXTRA-HIGHCARBON-STEEL SPLICE BARS, W20-22, 1922

\section{EXTRA-HIGH-CARBON-STEEL SPIICE BARS}

(Same as A. S. T. M., A6-14, given below.)

AMERICAN ELECTRIC RAILWAY ENGINEERING ASSOCIATION, STANDARD SPECIFICATION FOR STEEL TIE PLATES, W23-23, 1923

\section{STEEL TIE PLATES}

(Same as A. S. T. M., A67-30, given below.) 
AMERICAN ELECTRIC RAILWAY ENGINEERING ASSOCIATION, DESIGN OF PLAIN GIRDER RAILS AND SPIICE BARS, W31-31, 1931

\section{PLAIN GIRDER RAILS AND SPIICE BARS}

(See 606.1, p. 316.)

\section{AMERICAN RAILWAY ENGINEERING AS- SOCIATION, SPECIFICATIOISS FOR HIGH- CARBON-STEEL JOINT BARS, 1915}

\section{HIGH-CARBON-STEEL JOINT BARS}

(In respect to material, chemical properties, physical properties and tests, and inspection, this specification conforms to A. S. T. M., A5, given below, except that under chemical composition no reference is made to percentage of carbon content, and under physical properties the minimum elongation in 2 inches is 16 per cent instead of 14 per cent as in A5. It also carries the same reference to ladle and check analysis as A5. Otherwise it provides as follows:)

\section{GENERAL REQOIRIMENTS}

8. The different sections of joint bars shall be rolled to dimensions specified in drawing furnished by the purchaser. No variation will be allowed in the dimensions affecting the fit and the fishing spaces of the rail. The maximum camber on either plane shall not exceed one-thirty-second inch in 24 inches.

9. The joint bars shall be sheared to the length prescribed by the purchaser and shall not vary therefrom by more than one-eighth inch.

10. (a) All joint bars shall be punched, slotted, and shaped at a temperature of not less than $1,470^{\circ}$ F. $\left(800^{\circ}\right.$ C. $)$.

(b) All bolt holes shall be punched in one operation, without bulging or distorting the section, and the bars shall be slotted for spikes when required, in accordance with the drawings, the slotting being done in one operation; a variation of one thirtysecond inch in the size and location of the holes will be allowed.

11. All joint bars must be finished smooth and true, without swelling over or under the bolt holes, and be free from flaws, seams, checks, or fins, and the fishing angles must be fully maintained.

12. The manufacturer's identification symbol, kind of material, month and year rolled, and number of design shall be rolled in raised letters and figures on each bar. The number of the melt shall be plainly stenciled on each lot of joint bars.

\section{INSPECTION}

13. The joint bars from each melt shall be piled separately until tested and inspected by the purchaser's inspector. One joint bar for tension test shall be selected by the inspector for each melt represented in finished bars, or by agreement specimens for tension test may be cut from the bar as rolled. One joint bar for bend test shall be selected by the inspector for each lot of 1,000 bars or less presented.
AMERICAN RAILWAY ENGINEERING ASSOCIATION, SPECIFICATIONS FOR MALLEABIE-IRON TIE PLATES, 1920

\section{MALLEABIE-IRON TIE PLATES}

\section{MATERIAI}

1. Plates shall be made from furnace malleable iron.

\section{FHYSICAI REQUTREMENTS}

2. Tension tests.-The tension-test specimens specified in section 4 shall conform to the following minimum requirements as to tensile properties:

Tensile strength lbs./in. ${ }^{2}--45,000$

Elongation in 2 inches._._.

3. Special tests.-(a) All tie plates shall have cast thereon test lugs of a size proportional to the thickness of the tie plate, but not exceeding $5 / 8$ by $3 / 4$ inch in cross section. These lugs shall be attached to the tie plate at such a point that they will not interfere with the assembling of the tie plates, and may be broken off by the inspector.

(b) If the purchaser or his representative so desires, a tie plate may be tested to destruction.

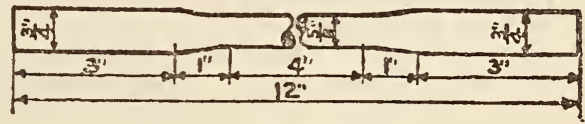

Figure 184

Such a tie plate shall show good, tough malleable iron.

4. Tension-test specimens.-(a) Tension-test specimens shall be of the form and dimensions shown in figure 184. Specimens whose mean diameter at the smallest section is less than nineteen thirty-seconds inch will not be accepted for test.

(b) A set of three tension-test specimens shall be cast from each melt, without chills, using risers of sufficient height to secure sound bars.

The specimens shall be suitably marked for identification with the melt. Each set of specimens so cast shall be placed in some one oven containing tie plates to be annealed.

5. Number of tests.-(a) After annealing, three tension-test specimens shall be selected by the inspector as representing the tie plates in the oven from which these specimens are taken.

(b) If the first specimen conforms to the specified requirements, or if, in the event of failure of the first specimen, the second and third specimens conform to the requirements, the tie plates in that oven shall be accepted, except that any tie plate may be rejected if its test lug shows that it has not been properly annealed. If either the second or third specimen fails to conform to the requirements, the entire contents of that oven shall be rejected.

6. Reannealing.-Any tie plates rejected for insufficient annealing may be reannealed once. The reannealed tie plates shall be inspected and if the remaining test lugs or tie plates broken as specimens show the tie plates to be thoroughly annealed, they shall be accepted; if not, they shall be finally rejected. 


\section{DESIGN}

7. Plan.-Tie plates shall conform to the drawing submitted to the manufacturer, with the following permissible variations:

Variation.-(a) The length and width shall not vary more than one-eighth inch from the dimensions shown.

(b) The thickness shall not vary more than one thirty-second inch from the dimensions shown.

\section{MANUFACTURE}

8. Workmanship.-The plates shall be straight and out of wind on the surface which forms the bearing for the rail, and shall be made in a workmanlike manner.

9. Finish.-The finished tie-plate shall be well cleaned and free from warping, shrinkage, cracks, blowholes, fins, and other imperfections.

10. Marking. - The manufacturer's identification mark and the pattern numbers assigned by the purchaser shall be cast on all tie-plates in such positions that they will not interfere with the service of the tie plate.

\section{INSPECTION}

11. Inspection.-(a) (Same as paragraph 7 of A. S. T. M. standard specifications for steel. See 605.0 , p. 247.$)$

(b) The manufacturer shall be required to keep a record of each melt, from which tie-plates are produced, showing tensile strength and elongation of test specimens cast from such melts. These records shall be available and shown to the inspector whenever required.

12. Rejection.-Tie-plates which show injurious defects subsequent to their acceptance at the manufacturer's works may be rejected, and, if rejected, shall be replaced by the manufacturer free of cost to the purchaser.

\section{SHIPMENT}

Packing.-(Same as for wrought-iron tie-plates, given below.)

Table of dimensions for tie-plates

\begin{tabular}{|c|c|c|c|c|c|c|c|c|c|}
\hline \multirow{2}{*}{$\begin{array}{l}\text { Length } \\
\text { of } \\
\text { plates }\end{array}$} & \multirow{2}{*}{$\begin{array}{c}\text { Range of } \\
\text { rail } \\
\text { bases }\end{array}$} & \multicolumn{3}{|c|}{$\begin{array}{l}\text { Same for flat } \\
\text { and canted } \\
\text { plates }\end{array}$} & \multirow{2}{*}{$\frac{\begin{array}{c}\text { Flat } \\
\text { plates }\end{array}}{\mathrm{D}_{\mathrm{E}} \text { and }}$} & \multicolumn{2}{|c|}{$\underset{40}{\text { Canted } 1 \text { in }}$} & \multicolumn{2}{|c|}{$\underset{20}{\text { Canted }} 1$ in } \\
\hline & & A & B & $\mathrm{C}$ & & $\mathrm{D}$ & $\mathbf{E}$ & $\mathrm{D}$ & $\mathrm{E}$ \\
\hline $\begin{array}{c}\text { inches } \\
9 \\
91 / 2 \\
10 \\
101 / 2 \\
11 \\
111 / 2 \\
12\end{array}$ & $\begin{array}{l}\text { inches } \\
43 / 4-5 \\
43 / 4-51 / 8 \\
47 / 8-51 / 2 \\
51 / 8-53 / 4 \\
53 / 8-6 \\
53 / 4-61 / 4 \\
6 \quad-61 / 2\end{array}$ & $\begin{array}{l}\text { ins. } \\
23 / 8 \\
25 / 8 \\
27 / 8 \\
3 \\
31 / 4 \\
31 / 2 \\
35 \% 8\end{array}$ & $\begin{array}{c}\text { ins. } \\
53 / 8 \\
59 / 16 \\
53 / 4 \\
5^{3} 5 / 16 \\
618 \\
65 / 16 \\
61 / 2\end{array}$ & $\begin{array}{l}\text { in. } \\
5 / 16 \\
5 / 16 \\
5 / 16 \\
3 / 8 \\
3 / 8 \\
3 / 8 \\
3 / 8\end{array}$ & $\begin{array}{r}\text { inch } \\
9 / 16 \\
5 / 8 \\
5 / 8 \\
11 / 16 \\
1116 \\
3 / 4 \\
3 / 4\end{array}$ & $\begin{array}{r}\text { inch } \\
9 / 16 \\
5 / 8 \\
5 / 8 \\
11 / 16 \\
11 / 16 \\
3 / 4 \\
3 / 4\end{array}$ & $\begin{array}{r}\text { inch } \\
7 / 16 \\
1 / 2 \\
15 / 32 \\
17 / 32 \\
17 / 32 \\
19 / 32 \\
19 / 32\end{array}$ & $\begin{array}{r}\text { inch } \\
3 / 4 \\
3 / 4 \\
3 / 4 \\
13 / 16 \\
13 / 16 \\
13 / 16 \\
13 / 16\end{array}$ & $\begin{array}{l}\text { inch } \\
15 / 32 \\
15 / 32 \\
15 / 32 \\
17 / 32 \\
1 / 2 \\
1 / 2 \\
1 / 2\end{array}$ \\
\hline
\end{tabular}

\section{AMERICAN RAILWAY ENGINEERING AS- SOCIATION, SPECIFICATIONS FOR QUENCHED CARBON-STEEL JOINT BARS, 1924}

\section{QUENCHED CARBON-STEEL JOINT BARS}

(This specification conforms to the A. S. T. M. specifications given below and to B11 in 641.21 , p. 1176 , in respect to the following items in each specification:)

A3. . . . . . Ladle and check analyses; marking; finish.

A4........ Test specimens; number of tests.

A5...... Bend tests; optional bend tests.

A49....... Material; chemical requirements; retests.

B11........... Inspection and rejection.
(The text of specifications A3, A4, A5, and A49 is given below. Otherwise this specification provides as follows:)

\section{PHYSICAL REQUIREMEITS}

5. (a) The joint bars shall conform to the following minimum requirements as to tensile properties:

\begin{tabular}{|c|c|}
\hline $\begin{array}{l}\text { Tensile strength } \\
\text { Yield point. }\end{array}$ & $\begin{array}{r}100,000 \\
70,000\end{array}$ \\
\hline Elongation in 2 inches, ... & \\
\hline But in no case under 12 per cent. & \\
\hline Reduction of area... & $\frac{3,500,000}{\text { Tensile }}$ \\
\hline
\end{tabular}

(b) The yield point shall be determined by the drop of the beam of the testing machine.

\section{DESIGN AND TOLERANCE}

11. The splice bars shall be smoothly rolled, true to templet, and shall accurately fit the rails for which they are intended. The bars shall be sheared to length, and the punching and notching shall conform to the dimensions specified by the purchaser. A variation of one thirty-second inch from the specified size of holes, of one-sixteenth inch from the specified location of holes, and of oneeighth inch from the specified length of splice bar, will be permitted. Bars shall be straight without camber in either plane and with outside surface of web parallel to the axis of rail.

\section{MANUFACTURE}

13. Joint bars shall be punched, slotted, and shaped at a temperature of not less than $1,470^{\circ} \mathrm{F}$. $\left(800^{\circ}\right.$ C. $)$ and subsequently quenched from a temperature of $810^{\circ} \mathrm{C}$. $\left(1,490^{\circ} \mathrm{F}\right.$. $)$.

AMERICAN RAILWAY ENGINEERING ASSOCIATION, SPECIFICATIONS FOR STEEL TIE PLATES, 1926

\section{STEEL TIE PLATES}

(Identical with A. S. T. M., A67-30, given below.)

\section{AMERICAN RAILWAY ENGINEERING AS- SOCIATION, SPECIFICATIONS FOR WROUGHT-IRON TIE PLATES, 1920}

\section{WROUGHT-IRON TIE PLATES}

\section{MATERIAL}

1. Plates shall be made of all-pig puddled iron.

\section{PHYSICAL REQUIREMENTS}

2. The material shall conform to the following minimum requirements as to tensile properties:

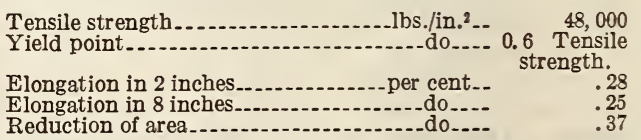

3. Bend tests. - The bend-test specimen shall bend cold through $180^{\circ}$ without fracture around a pin, the diameter of which is equal to the thickness of the specimen.

4. Test specimens.-(a) The tension-test specimen shall be taken from the finished tie plates, or from the rolled bar. They shall be cut so that the sides of the specimens are parallel to the direction in which the tie plates have been rolled. 
(b) Tension-test specimens may conform to the essential dimensions shown in Figures 128 and 129 in A. S. T. M. standard specifications for steel (see 605.0 , p. 247). The 2-inch specimen shall have filleted shoulders, or threaded ends, to fit into the holders on the testing machine in such a way that the line of action of the force exerted by the testing machine shall coincide with the axis of the specimen.

5. Number of tests.- (a) One tension test and one bend test shall be made from each lot of 1,000 tie plates.

(b) If any test specimen from either of the bars originally selected to represent a lot of material contains surface defects not visible before testing, but visible after testing, or if a tension-test specimen breaks outside the middle third of the gage length, the individual bar shall be rejected and one retest from a different bar will be allowed.

\section{DESIGN}

6. Plan.-The tie plates shall conform to the drawings submitted to the manufacturer, with the following permissible variations:

Variations.- (a) For plates with shoulders parallel to the direction of rolling, a variation of one-thirtysecond inch in thickness, one-eighth inch in rolled width, and three-sixteenths inch in sheared length will be permitted.

(b) For plates with shoulders perpendicular to the direction of rolling, a variation of one thirty-second inch in thickness, one-eighth inch in rolled width and one-fourth inch in sheared length will be permitted. The distance from the face of the shoulder to the outside end of plate shall not vary more than one-fourth inch, and from the face of the shoulder to the inside end not more than one-half inch.

\section{MANUFACTURE}

7. Workmanship. - The tie plate shall be smoothly rolled, true to templet, and shall be straight and out of wind on the surface which will form the bearings for the rail, and have a workmanlike finish.

8. Finish.-The finished tie plates shall be free from burs and other surface deformations caused by the shearing and punching; they shall also be free from slivers, depressions, seams, crop ends, and evidences of being burnt.

Marking.-(Same as A. S. T. M., A3, given below.)

Inspection.- (Same as paragraph 7 of A. S. T. M. standard specifications for steel. See 605.0, p. 247.)

11. Rejection. - If either of the test bars selected to represent a lot does not conform to the requirements specified in sections $2,3,4$, and 5 , the lot will be rejected.

12. Packing.- Tie plates shall be wired together in bundles of uniform number, weighing not to exceed 100 pounds for shipment, unless otherwise specified.

AMERICAN SOCIETY FOR TESTING MATERIALS, STANDARD SPECIFICATIONS FOR IOW - CARBON-STEEL SPLICE BARS, DESIGNATION A3-24, 1924

\section{LOW-CARBON-STEEL SPLICE BARS}

\section{MANUFACTURE}

1. The steel shall be made by either or both the following processes: Bessemer or open hearth.

\section{CHEMICAL PROPERTIES AND TESTS}

2. The steel shall conform to the following requirements as to chemical composition:

\section{Phosphorus:}

Bessemer, not over

Per cent

Open hearth, not ore
3. (a) A carbon determination shall be made of each melt of Bessemer steel, and two analyses every 24 hours representing the average of the elements carbon, manganese, phosphorus, and sulphur contained in the steel, one for each 12-hour period, respectively. These analyses shall be made from drillings taken at least one-eighth inch beneath the surface of a test ingot obtained during the pouring of the melts. The chemical composition thus determined shall be reported to the purchaser or his representative and shall conform to the requirements specified in section 2 .

(b) An analysis of each melt of open-hearth steel shall be made by the manufacturer to determine the percentages of carbon, manganese, phosphorus, and sulphur. This analysis shall be made from drillings taken at least one-eighth inch beneath the surface of a test ingot obtained during the pouring of the melt. The chemical composition thus determined shall be reported to the purchaser or his representative and shall conform to the requirements specified in section 2

4. An analysis may be made by the purchaser from a finished splice bar representing each melt. The phosphorus content thus determined shall not exceed that specified in section 2 by more than 25 per cent.

\section{PHYSICAL PROPERTIES AND TESTS}

5. The splice bars shall conform to the following requirements as to tensile properties:

Tensile strength lbs./in. ${ }^{2}-55,000-65,000$ Elongation in 8-inch, minimum .....per cent.. 25

6 . The test specimen shall stand being bent cold through $180^{\circ}$ flat on itself without cracking on the outside of the bent portion.

7. Tension and bend test specimens shall be taken from the finished rolled bars. Tension-test specimens shall be of eight-inch gage length. Bend tests may be made of an unpunched splice bar, flattened if necessary.

8. (a) One tension and one bend test shall be made from each melt.

(b) If any test specimen shows defective machining or develops flaws, it may be discarded and another specimen substituted.

(c) If the percentage of elongation of any tensiontest specimen is less than that specified in section 5 and any part of the fracture is outside the middle third of the gage length, as indicated by scribe scratches marked on the specimen before testing, a retest shall be allowed.

\section{WORKMANSHIP AND FINISH}

9. (a) The splice bars shall be smoothly rolled, true to template, and shall accurately fit the ralls for which they are intended. The bars shall be sheared to length, and the punching and notching shall conform to the dimensions specified by the purchaser. A variation of one thirty-second inch from the specified size and location of holes and of one-eighth inch from the specified length of splice bar will be permitted. Any variation from a straight line in a vertical plane shall be such as will make the bars high in the center. The maximum camber in either plane shall not exceed onesixteenth inch in 24 inches, except as specified in paragraph $(b)$.

(b) For splice bars for girder and high $T$ rails, any variation from a straight line in a vertical plane shall be such as will make the bars high in the center, and the maximum camber in this plane shall not exceed three sixty-fourths inch in 24 inches. Any variation from a straight line in a horizontal plane shall be such as will make the bars convex toward the web of the rail, and the maximum camber in this plane shall not exceed one-sixteenth inch in 24 inches. 
10. The finished splice bars shall be free from injurious defects and shall have a workmanlike finish.

\section{MARIING}

11. The name or brand of the manufacturer and the year of manufacture shall be rolled in raised letters and figures on the side of the rolled bars, and a portion of this marking shall appear on each finished splice bar.

\section{INSPECTION AND REJECTION}

(Same as A. S. T. M., B11-18. See 641.21, p. 1176.)

\section{AMERICAN SOCIETY FOR TESTING MATE- RIAIS, STANDARD SPECIFICATIONS FOR MEDIUM-CARBON-STEEL SPLICE BARS, DESIGNATION A4-14, 1914}

\section{MEDIUM-CARBON-STEEL SPLICE BARS}

\section{MANUFACTORE}

1. The steel shall be made by the open-hearth process.

2. (a) The splice bars may be punched, slotted, and, in the case of special designs, shaped either hot or cold.

(b) Bars that are punched, slotted, or shaped cold shall be subsequently annealed.

\section{CHEMICAL PROPERTIES AND TESTS}

3. The steel shall conform to the following requirements as to chemical composition:

$\begin{array}{lr}\text { Carbon, not under } & \text { Per cent } \\ \text { Phosphorus, not over. } & 0.30\end{array}$

\section{IADLE AND CHECK ANALYSES}

(Same as A. S. T. M., A3, given above.)

\section{PHYSICAL PROPERTIES AND TESTS}

6. The splice bars shall conform to the following minimum requirements as to tensile properties:

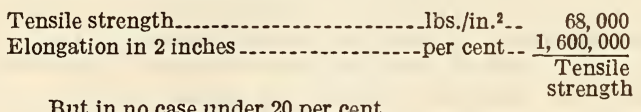

7. The bend-test specimen specified in section 8 shall bend cold through $180^{\circ}$ around a pin, the diameter of which is equal to twice the thickness of the specimen, without cracking on the outside of the bent portion.

8. Tension and bend-test specimens shall be taken from the finished bars. Tension-test specimens shall conform to the dimensions shown in Figure 129 in A. S. T. M. standard specifications for steel. (See 605.0, p. 247.) The ends shall be of a form to fit the holders of the testing machine in such a way that the load shall be axial. Bend-test specimens may be one-half inch square in section or rectangular in section with two parallel faces as rolled.

9. If preferred by the manufacturer and approved by the purchaser, the following bend test may be substituted for that described in section 7: $\mathrm{A}$ piece of the finished bar shall bend cold through $90^{\circ}$ around a pin the diameter of which is equal to twice the greatest thickness of the section, without cracking on the outside of the bent portion.

10. (a) One tension and one bend test shall be made from each melt. (b) If any test specimen shows defective machining or develops flaws, it may be discarded and another specimen substituted.

(c) If the percentage of elongation of any tensiontest specimen is less than that specified in section 6 and any part of the fracture is more than threefourth inch from the center of the gage length, as indicated by scribe scratches marked on the specimen before testing, a retest shall be allowed.

WORKMANSHIP AND FINISH, AND MAREITG

(Same as A. S. T. M., A3, given above.) INSPECTION AND REJECTION

(Same as A. S. T. M,. B11-18. See 641.21, p. 1176.)

AMERICAN SOCIETY FOR TESTING MATERIALS, STANDARD SPECIFICATIONS FOR HIGH-CARBON-STEEL SPLICE BARS, DESIGNATION A5-14, 1914

\section{HIGH-CARBON-STEEL SPLICE BARS}

1. The steel shall be made by the open-hearth process.

2. The splice bars shall be punched, slotted, and in the case of special designs, shaped at a temperature not less than $750^{\circ} \mathrm{C}$.

\section{CHEMICAL PROPERTIES AND TESTS}

3 . The steel shall conform to the following requirements as to chemical composition:

Carbon, not under Per cent

Phosporus, not over

\section{IADLE AND CHECK ANALYSES}

(Same as A. S. T. M. A3, given above.)

\section{PHYSICAL PROPERTIES AND TESTS}

6. The splice bars shall conform to the following minimum requirements as to tensile properties:

Tensile strength. lbs./in. ${ }^{2}--85,000$

Elongation in 2 inches......... 14

7. The bend-test specimen specified in section 8 shall stand being bent cold through $90^{\circ}$ around a pin the diameter of which is equal to three times the thickness of the specimen, without cracking on the outside of the bent portion.

(Paragraph 8 is identical with paragraph 8 in A4, given above.)

9. If preferred by the manufacturer and approved by the purchaser, the following bend test may be substituted for that described in section 7: A piece of the finished bar shall stand being bent cold through $45^{\circ}$ around a pin the diameter of which is equal to three times the greatest thickness of the section, without cracking on the outside of the bent portion.

NUMBER OF TESTS

(Identical with paragraphs $10(a),(b)$, and (c) in A4, given above.)

$$
\text { WORZMANSLIP AND FINISE, AND MARKING }
$$

(Same as A. S. T. M. A3, given above.) INSPECTION AND REJECTION

(Same as A. S. T. M. B11-18. See 641.21, p. 1176.) 
AMERICAN SOCIETY FOR TESTING MATERIALS, STANDARD SPECIFICATIONS FOR Q U E N C H E D HIGH - CARBON - STEEL SPLICE BARS, DESIGNATION A49-21, 1921

\section{QUENCHED HIGH-CARBON-STEEL SPLICE BARS}

1. The steel shall be made by the open-hearth process.

2. The splice bars shall be punched, slotted, and, in the case of special designs, shaped at a temperature not less than $750^{\circ} \mathrm{C}$., and subsequently quenched.

\section{CHEMICAL PROPERTIES AND TESTS}

3. The steel shall conform to the following requirements as to chemical composition:

Carbon, not over

Manganese, not over

Phosphorus, not over .04

LADLE AND CHECK ANALYSES

(Same as A. S. T. M. A3, given above.)

\section{PHYSICAL PROPERTIES AND TESTS}

6. (a) The splice bars shall conform to the following minimum requirements as to tensile properties:

Tensile strength. Ibs./in. ${ }^{2}-100,000$

Yield point lbs./in. $2-100,000$

Elongation in 2 inches,

(b) The yield point shall be determined by the drop of the beam of the testing machine.

(Paragraph 7 is identical with paragraph 7 in A5, given above.)

(Paragraph 8 is identical with paragraph 8 in A4, given above, except that bend test specimens have the corners rounded to a radius not over $1 / 16$ inch.)

(Paragraph 9 is identical with paragraph 9 in A5, given above.)

\section{NUMBER OF TESTS}

(Identical with paragraphs $10(a),(b)$, and $(c)$ in A4, given above.)

\section{RETESTS}

11. If the results of the physical tests of any test lot do not conform to the requirements specified, the manufacturer may re-treat such lot one or more times, in which case two additional tension and two additional bend tests shall be made from such lot, all of which shall conform to the requirements specified.

\section{WORKMANSHIP AND FINISH, AND MARKING}

(Same as A. S. T. M. A3, given above, except that no reference is made to splice bars for girder and high $T$ rails.)

\section{INSPECTION}

(Same as A. S. T. M. B11-18. See 641.21, p. 1176.)

AMERICAN SOCIETY FOR TESTING MATERIALS, STANDARD SPECIFICATIONS FOR STEEL TIE PLATES, DESIGNATION A6730,1930

\section{STEEL TIE PLATES}

1. These specifications cover two grades of steel tie plates, namely, soft and medium. The soft grade will be used unless otherwise specified.

\section{MAN UFACTURE}

2. The steel shall be made by one or more of the following processes: Open-hearth, electric furnace, or acid Bessemer.

\section{CHEMICAL PROPERTIES AND TESTS}

3. (a) The steel shall conform to the following requirement as to limitation of phosphorus content:

\begin{tabular}{l|c|c}
\hline & $\begin{array}{c}\text { Besse- } \\
\text { mer }\end{array}$ & $\begin{array}{c}\text { Open- } \\
\text { hearth }\end{array}$ \\
\hline $\begin{array}{c}\text { Per } \\
\text { cent } \\
0.10\end{array}$ & $\begin{array}{c}P_{e r} \\
\text { cent } \\
0.05\end{array}$ \\
$\begin{array}{l}\text { Phosphorus, not over- } \\
\text { Copper, when copper-steel is specified, per cent, } \\
\text { not under 0.20. }\end{array}$ & & \\
\hline
\end{tabular}

(b) Unless otherwise specified, the steel shall be furnished according to chemical composition only, in which case the minimum carbon content shall be as follows:

\begin{tabular}{|c|c|c|}
\hline & $\begin{array}{c}\text { Besse- } \\
\text { mer }\end{array}$ & $\begin{array}{l}\text { Open- } \\
\text { hearth }\end{array}$ \\
\hline 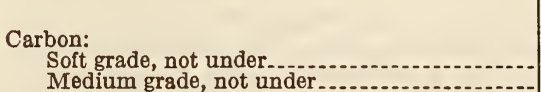 & $\begin{array}{l}\text { Per } \\
\text { cent } \\
0.08 \\
.12\end{array}$ & $\begin{array}{l}P e r \\
c \in n t \\
0.15\end{array}$ \\
\hline
\end{tabular}

4. (a) A carbon determination shall be made of each melt of Bessemer steel, and three analyses every 24 hours representing the average of the elements carbon and phosphorus, and also copper, where copper-steel is specified, contained in the steel, one for each 8-hour turn respectively. These analyses shall be made from drillings taken at least one-eighth inch beneath the surface of a test ingot obtained during the pouring of the melts. The chemical composition thus determined shall be reported to the purchaser or his representative, and shall conform to the requirements specified in section 3 .

(b) An analysis of each melt of open-hearth steel shall be made by the manufacturer to determine the percentages of carbon and phosphorus; also copper when copper-steel is specified. This analysis shall be made from drillings taken at least one-eighth inch beneath the surface of a test ingot obtained during the pouring of the melt. The chemical composition thus determined shall be reported to the purchaser or his representative, and shall conform to the requirements specified in section 3 .

5. An analysis may be made by the purchaser from a finished tie plate representing each melt of open-hearth steel, and each melt or lot of 10 tons of Bessemer steel. The carbon content thus determined shall not be less than that specified in section 3 , and the phosphorus content shall not exceed that specified in section 3 by more than 25 per cent.

\section{PHYSICAL PROPERTIES AND TESTS}

6. The bend-test specimens specified in section 7 shall stand being bent cold through $180^{\circ}$ around a pin the diameter of which is equal to the thickness of the specimen for the soft grade, and to twice the thickness of the specimen for the medium grade, without cracking on the outside of the bent portion.

7. Bend-test specimens shall be taken from the finished tie plates, or from the rolled bars, and longitudinally with the rolling. They shall be rectangular in section, not less than one-half inch in width between the planed sides, and shall have two faces as rolled. They shall be free from ribs or projections. Where the design of the tie plates 
is such that the specimen can not be taken between the ribs or projections, these ribs or projections shall, in preparing the specimen, be planed off even with the main surface of the tie plate.

8. If preferred by the manufacturer and approved by the purchaser, the following bend test may be substituted for that described in section 6 :

A piece of the rolled bar shall stand being bent cold through $90^{\circ}$ around a pin the diameter of which is equal to the thickness of the section where bent for the soft grade, and to twice the thickness of the section where bent for the medium grade, without cracking on the outside of the bent portion.

9. (a) One bend test shall be made from each melt of open-hearth steel, or from each melt or lot of 10 tons of Bessemer steel.

(b) If any test specimen shows defective machining or develops flaws, it may be discarded and another specimen substituted.

\section{WORKMANSHIP AND FINISH}

10. The tie-plates shall conform to the dimensions specified by the purchaser, with the following permissible variations:

(a) For plates with shoulders parallel to the direction of rolling, a variation of one thirty-second inch in thickness, one-eighth inch in rolled width, and threesixteenths inch in sheared length will be permitted.

(b) For plates with shoulders perpendicular to the direction of rolling, a variation of one twenty-second inch in thickness, three-sixteenths inch in rolled width, and one-fourth inch in sheared length will be permitted. The distance from the face of shoulder to the outside end of the plate shall not vary more than one-eighth inch, and from the face of shoulder to the inside end not more than one-fourth inch.

(c) A variation of not more than one thirtysecond inch in the location of spike holes and in the length of their sides will be permitted.

11. The tie-plate shall be smoothly rolled, true to template, and shall be straight and out of wind on the surface which will form the bearings for the rail.

12. The finished tie-plates shall be free from burrs and other surface deformities caused by the shearing and punching; they shall also be free from other injurious defects and shall have a workmanlike finish.

\section{MARKING}

13. The name or brand of the manufacturer, the section of the tie-plate and the year of manufacture shall be rolled in raised letters and figures on the outside of the shoulder of the plates, and a portion of this marking shall appear on each finished tie-plate.

(Requirements covering inspection, rejection, and rehearing conform with those specified in A. S. T. M. standard specifications for steel. (See 605.0, p. 247.)

\section{ASSOCIATION OF AMERICAN STEEL MAN- UFACTURERS, STANDARD SPECIFICA- TIONS FOR STEEL TIE-PLATES, 1923}

\section{STEEL TIE-PLATES}

1. Material covered.-These specifications cover three grades of steel tie-plates, namely, soft, medium, and hard. The purchaser shall specify on the order which grade is desired.

2. Process.- Steel for the soft and medium grades may be made by either or by both the open hearth or Bessemer processes. Steel for the hard grade shall be made by the open-hearth process.

3. Finishing and heat treatment.-Tie-plates of the soft and medium grades may be sheared, slotted, and punched cold, but tie-plates of the hard grade shall be sheared, slotted, and punched hot, at a temperature that will prevent injury to the steel and shall immediately thereafter be put into a closed chamber or box which will prevent rapid cooling.

4. Chemical composition.-The steel shall conform to the following requirements as to chemical composition, based on ladle analysis:

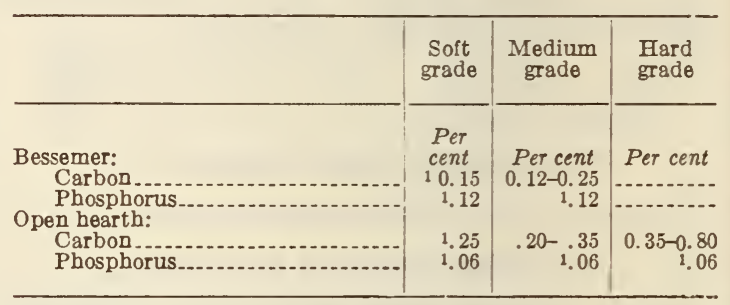

1 Maximum.

(Workmanship and finish conform with A. S. T. M. A67-30, given below, except that for tie-plates with shoulders parallel to the direction of rolling the variation in width permitted is three-sixteenths instead of one-eighth inch; and for tie-plates with shoulders perpendicular to the direction of rolling the distance from the face of the shoulder to the outside end of the plates shall not vary more than one-fourth inch, and from the face of the shoulder to the inside end not more than one-half inch.)

(Marking conforms with A. S. T. M. A67-30, except for the addition of the following:) Each individual tie-plate made from the hard grade of steel, hot worked, shall be stamped or branded with the letters $\mathrm{H}$. W.

(Requirements covering inspection and rejection conform with those specified in A. S. T. M. standard specifications for steel. See 605.0, p. 247.)

\subsection{SWITCHES AND SPECIAL TRACK WORK.}

\section{AMERICAN ELECTRIC RAILWAY ENGI- NEERING ASSOCIATION, STANDARD DIMENSIONS OF SWITCHES AND MATES, W11-30, 1930}

\section{STANDARD DIMENSIONS OF SWITCHES AND MATES}

(Identical with paragraph 406 in A. E. R. E. A. W33, given below.) 
AMERICAN ELECTRIC RAILWAY ENGINEERING ASSOCIATION, STANDARD DIMENSIONS OF FROGS FOR CROSSOVERS AND TURNOUTS, W15-22, 1922

\section{FROGS FOR CROSSOVERS AND TURNOUTS}

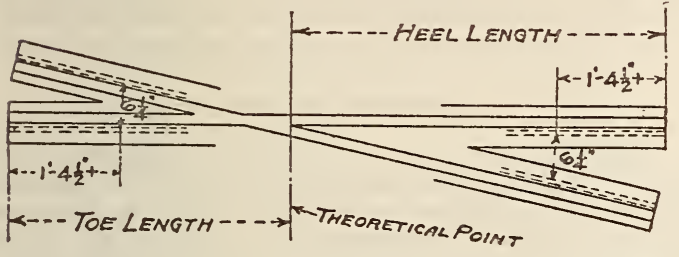

Figure 185.-Dimensions of turnout and crossover frogs for girder-grooved rails and girder guard rails

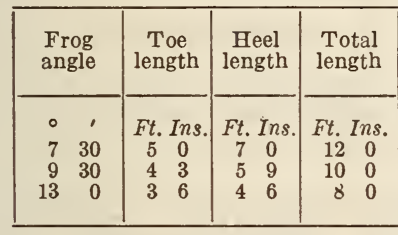

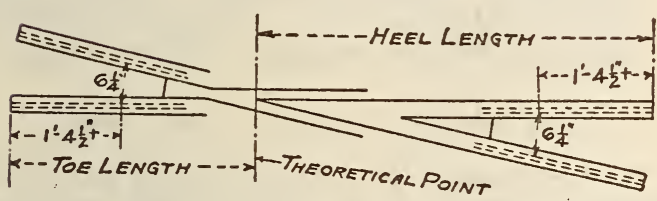

FIGURE 186.-Dimensions of turnout and crossover frogs for plain girder rails and standard section rails (flange bearing frogs)

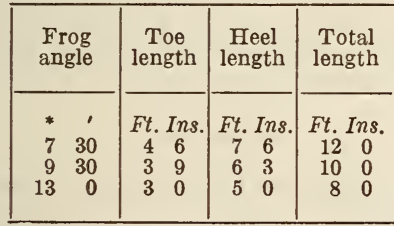

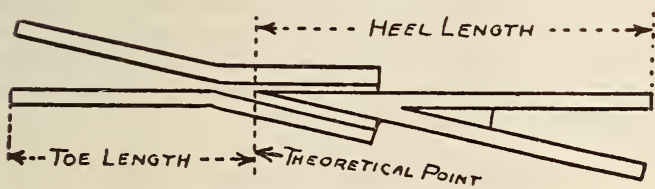

FIGURE 187.-Dimensions of frogs for standard section rails and for $M$. C. B. wheels (corresponding with $A$. R. E. A. standard dimensions).

\begin{tabular}{|c|c|c|c|c|}
\hline $\begin{array}{l}\text { Frog } \\
\text { No. }\end{array}$ & Frog angle & $\begin{array}{c}\text { Toe } \\
\text { length }\end{array}$ & $\underset{\text { length }}{\text { Heel }}$ & $\begin{array}{c}\text { Total } \\
\text { length }\end{array}$ \\
\hline $\begin{array}{r}4 \\
5 \\
6 \\
7 \\
8 \\
10\end{array}$ & \begin{tabular}{rrl}
\multicolumn{1}{c}{$\circ$} & \multicolumn{1}{c}{$\prime$} \\
14 & 15 & 00 \\
11 & 25 & 16 \\
9 & 31 & 38 \\
8 & 10 & 16 \\
7 & 09 & 10 \\
5 & 43 & 29
\end{tabular} & $\begin{array}{cc}\text { Ft. } & \text { Ins. } \\
3 & 2 \\
3 & 4 \\
3 & 6 \\
4 & 5 \\
4 & 9 \\
6 & 0\end{array}$ & $\begin{array}{rc}\text { Ft. } & \text { Ins. } \\
4 & 10 \\
5 & 8 \\
6 & 6 \\
7 & 7 \\
8 & 3 \\
10 & 6\end{array}$ & $\begin{array}{rc}\text { Fl. } & \text { Ins. } \\
8 & 0 \\
9 & 0 \\
10 & 0 \\
12 & 0 \\
13 & 0 \\
16 & 6\end{array}$ \\
\hline
\end{tabular}

\section{AMERICAN ELECTRIC RAILWAY ENGI- NEERING ASSOCIATION, STANDARD SPECIFICATION FOR SOLID MANGA- NESE-STEEL SPECIAL TRACK WORK FOR GIRDER RAIL, W32-30, 1930}

\section{MANGANESE-STEEL TRACK WORK}

(In respect to materials (except for the use of manganese steel), chemical properties and tests, physical properties and tests, design (including standard dimensions of switches and mates), workmanship, assembly for inspection, marking, and shipping, and inspection, this specification conforms to A. E. R. E. A. W33, given below, except for the following provisions:)

These specifications are intended to cover the manufacture of special track work wherein the tongue switches, mates, frogs, and crossings are made of solid manganese steel. The term "solid manganese" shall be considered as distinguishing such special track work from other types wherein manganese steel is used only for inserts at points receiving the principal wear.

\section{MANGANESE-STEEL CASTINGS}

407. Tongue switches, mates, frogs, and crossings (not steam-railroad crossings) shall be made of manganese-steel castings of uniform design, with extremities to conform to the connecting rail. The castings shall be uniform in depth of 6,7 , or 9 inches and of not less depth than the highest of the connecting rails and of a cross section in which the thickness of metal will not be under the following dimensions:

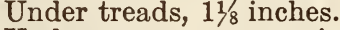

Under grooves, seven-eighths inch.

Under grooves at flange bearing, 11/8 inches.

Vertical walls or webs, five-eighths inch in double webs.

Vertical walls or webs, three-quarters inch in single web.

Base flange, one-half inch at outer edge; threequarters inch at web.

Base flanges shall be not less than 3 inches wide on each side of double-web sections and 5 inches wide on single-web sections. Angles in castings are not permissible, all must have fillets. All holes shall be cored, and smooth and accurately located. Where drilled holes are required, soft iron or steel plugs may be inserted in the casting.

\section{HEEL OPENINGS}

408. Tongue switch and mate castings shall be open on top at the heel ends for convenience of setting joint bars.

\section{TONGOE SWITCHES}

409. The tongues in tongue switches shall be provided with lateral flanges to promote lateral stiffness, with a construction at the heel that will furnish adequate bearing and give a secure and adjustable fastening. There shall be provision for attaching throwing or controlling mechanism and the box containing same to either side of the switch. The tongue shall be arranged for such attachment when specified. Provision shall also be made for attaching a cleaning box to the switch casting opposite the throwing device and openings shall be provided in the tongue bed for proper drainage. All bearing parts of the tongue and switch bed shall be properly 
finished to a good surface. The tread of the tongue shall be at least one-quarter inch above the curved tread of the switch, from 1 foot from tongue point to give an effective guard where required. Switch tongues shall be finished so that practical interchangeability is assured.

FROGS AND CROSSINGS; IENGTH OF ARNS AND CASTINGS

410. (a) The length of frog and crossing arms shall be sufficient to give at least $6 \frac{1}{4}$ inches clearance between vertical webs at end of joint plates with a minimum length of 3 feet unless adjoining piece will not permit. The length and angles of frogs for turnouts and crossovers, shall be as provided in specification W15-22. (Dimensions of frogs for crossovers and turnouts given above.)

(b) Single crossings shall be cast in two pieces in angles from $90^{\circ}$ to $60^{\circ}$ and in four pieces for angles under $60^{\circ}$. In 2-piece crossings or compound pieces the length of arms shall be kept under 4 feet 6 inches. Castings shall not exceed a maximum of 16 feet in length.

\section{JOINTS}

411. Where castings connect directly with rails they shall be finished for a tight fit of standard splice bars throughout the length of such bars, and projecting portions of castings taking the place of joint plates shall be finished to a splice-bar fit. When joints are bolted up tight, the rail shall align correctly with castings with no greater variation than one thirty-second inch in either line or level. For joints between castings, special splice bars may be used only when it is impossible to apply standard bars and the finish shall then be maintained equally good. Joints shall be tight. Occasional open joints shall not exceed one-eighth inch. Manufacturers shall make note of such open joints on drawings.

\section{COMPROMISE JOINTS}

412. (a) Compromise joints shall be avoided wherever possible by shaping the ends of special pieces to match the adjoining rail, or compromise rails shall be used consisting either of steel castings or two pieces of rails connected by cast welding or other welding method, and shaped on the two ends to suit the respective sections to be joined. Such compromise rails shall be not less than 5 feet long over all.

(b) Where the foregoing practice is not specified or practicable, compromise joints shall be of forged or cast steel at least equivalent in strength to the joint for the lower rail and must be finished to a good fit so that good line and surface are obtained.

(Paragraphs $(c)$ and $(d)$, covering shape of guard rail and designation of compromise joints, conform to paragraphs $(b)$ and $(c)$, section 415 , in A. E. R. E. A. W33, given below.)

\section{PAVING FACE}

413. In work specified for paved streets the angles in frogs and crossings shall be filled to provide paving faces to a width of not less than 4 inches and in switches and mates to not less than 3 inches by carrying out the castings forming the body of the piece where length of arms and points will permit. Where this is not possible, paving chocks shall be used consisting of separate iron castings which must have ample bearing on the bottom flanges of the special pieces and rails to prevent their being depressed under vehicular traffic and be so constructed as to permit their being placed and solidly grouted after the joint plates are applied.

(In respect to finish of castings, this specification conforms to A. R. E. A. track-work plans, given below, except for the following provisions:)
502. (b) Running surfaces of castings shall be finished with as good surfaces as those of rolled rail.

(c) That portion of castings which rests on ties shall be straight and out of wind and shall be free from lumps or such imperfections as would prevent a good bearing. The wind or warp in bottom surfaces of castings shall not exceed one-eighth inch in 5 feet and when work is specified for use with steeltie structures exceptional care shall be exercised to secure practically true bearing surfaces.

\section{FINISHING OF ENDS}

503. All ends of frogs, switches, mates, crossings, or rail lengths shall be finished square to the axis of the rail unless otherwise specified. A variation of more than one thirty-second inch in the width of the head out of square will not be accepted. There shall be no variation of more than one-fourth inch from theoretical lengths, and such variations shall be noted on plans.

\section{CURVING OF RAILS}

504. All curved rails shall be so bent true to templet throughout their entire length and depth that they may rest in a horizontal plane.

\section{AMERICAN ELECTRIC RAILWAY ENGI- NEERING ASSOCIATION, STANDARD SPECIFICATION FOR PLAIN BOLTED SPECIAL TRACK WORK, W33-30, 1930}

\section{PLAIN BOLTED SPECIAL TRACK WORK}

This specification is intended to cover the manufacture of special track work wherein switches, mates, frogs, crossings, curves, guard rails and connecting rails are made of rolled steel rails properly shaped by machining or grinding and secured together by means of cast iron, cast, forged or rolled steel fillers, bolts, rivets, and plates. The term "plain bolted" shall be considered as being synonymous with the term "built up."

\section{MATERIALS}

101. Conformity with association specifications.All work furnished under these specifications shall be manufactured of materials which are in accordance with the recommended specifications of the American Electric Railway Engineering Association for materials for use in the manufacture of special track work, W104, given below.

\section{CHEMICAL PROPERTIES AND TESTS}

201. Conformity with association specifications.The chemical composition and tests covering each of the several materials in the special track work shall conform to the requirements of the recommended specifications of the American Electric Railway Engineering Association for materials for use in the manufacture of special track work, W104, given below.

\section{PHYSICAL PROPERTIES AND TESTS}

301. Conformity with association specifications.The physical properties and tests covering each of the several materials in the special track work shall conform to the requirements of the recommended specifications of the American Electric Railway Engineering Association for materials for use in the manufacture of special track work, W104, given below. 


\section{DESIGN}

(A) General Specifications

401. Engineering data and drawings.-(a) The manufacturer shall obtain all necessary engineering data either in the field or from such plans and data as may be furnished by the purchaser. The manufacturer shall be held responsible only for the accuracy of such data as he may obtain which is not furnished by the purchaser.

(b) The manufacturer shall submit working drawings, showing engineering data, piece numbers, and lengths, when so specified.

(c) The drawings of the purchaser and the manufacturer's drawings approved by the purchaser shall be a part of the specifications. Anything not shown on the drawings but which is mentioned in the specifications, or vice versa, and anything not specifically set forth in either but which is reasonably implied shall be furnished the same as if specifically shown and mentioned in both. Any omissions from drawings or specifications necessary for a clear understanding of the work shall be called to the purchaser's attention promptly by the manufacturer.

402. Grooves, flangeways, and gage.-(a) Grooves, flangeways, and gage shall conform to the recommended practice of the American Electric Railway Engineering Association.

(b) Flangeways shall not vary from the widths and depths specified by greater amounts than allowed by the following permissible variations:

\begin{tabular}{|c|c|c|}
\hline & Under & Over \\
\cline { 1 - 2 } & Inch & Inch \\
Width of flangeway.......- & None. & $3 / 32$ \\
Depth of flangeway: & $1 / 10$ & $1 / 32$ \\
Flange bearing ....... & $1 / 16$ & $3 / 16$ \\
\hline Nonflange bearing..... & $1 / 6$ & \\
\hline
\end{tabular}

(c) When M. C. B. flangeways are specified, the width and depth shall not be less than $13 \%$ inches.

403. Flange bearing. - When flange bearing is required the depth of groove at intersections and on curved side of mate points shall be one-eighth inch less than the depth of the wheel flange except that the minimum depth of flangeway shall be nine-sixteenths inch. Wherever possible the flange bearing shall be carried to a point where the wheel will have not less than 2 inches tread bearing. The effective part of risers approaching the flange bearing shall not be steeper than one-sixteenth inch in 1 inch of length and shall be made a maximum of one thirtysecond inch in 1 inch wherever possible. When so specified, continuous flange bearing shall be provided through crossings and involved special track work, except on the inner rails of curves.

404. Wheel clearance.-Tread clearance shall be 4 inches in width for A. E. R. E. A. wheels and $4 \frac{1}{2}$ inches wide for M. C. B. wheels, unless otherwise specified.

405. Line and surface.-The line and surface of all work shall be practically true and uniform throughout, with no greater variation than onesixteenth inch in 5 feet of length.

406. Lengths of tongue switches and mates.-The lengths of tongue switches and mates shall be in accordance with the following table of standard dimensions of switches and mates:

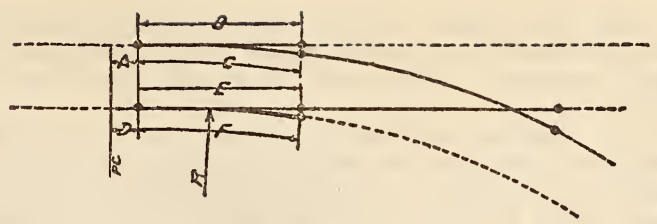

Figure 188

Standard dimensions of switches and mates

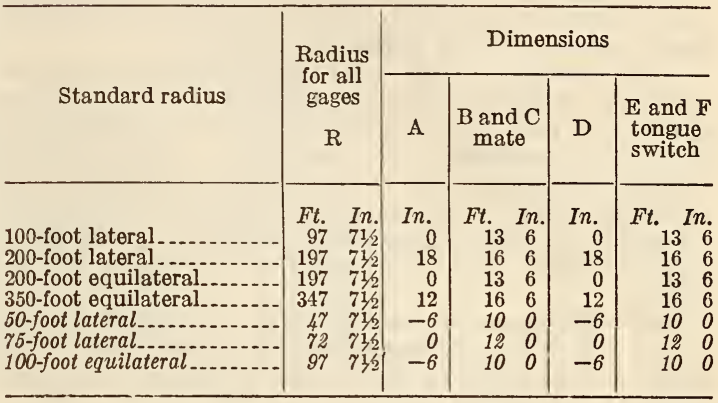

Note.-Radii and dimensions shown in italic type are not standard and should be used only where special conditions will not permit the use of radii and dimensions shown in roman type. As it will rarely be found necessary to use other than a standard, it is recommended that the 100 and 200 foot radius lateral and 200 -foot radius equilateral be used wherever practicable.

407. Guards-For frogs and curves.-(a) Frog guards shall be furnished when specified. They shall be similar in design to regular rolled rail guards in all respects. They shall be suitably flared at ends and provided with spacing blocks and braces as called for. They may be made of second-quality rails.

(b) Frog guards shall not be less than 8 feet 3 inches nor more than 16 feet 6 inches long over all.

(c) Guards for standard section rails on curves shall be of standard section rails unless rolled-shaped guards or flat-bar guards are definitely specified. If of standard section rail on sharp curves of 100foot radius or less for wheels with flanges shallower than M. C. B. flanges, unless otherwise specified the top of the guard rail shall be raised above the level of the running rail at least five-sixteenths inch by the use of step tie-plates if guard section is same as running rail or by the use of suitable higher rail with bases set level. For M. C. B. flanges the guards shall be level with the running rail. The guard rails shall be fastened to the running rails by through bolts as specified in section 413 (a) and cast-iron or steel spacing blocks not less than 3 inches long and spaced not more than 30 inches on centers.

(d) Guard-rail joints shall have an overlapping filler with two bolts with short splice bars on the outside or joints may be the same as those used on the running rail.

408. Rail braces.-Rail braces shall be of pressed steel or malleable iron not less than three-eighths inch in thickness unless cast iron is specified. They shall be of approved design and have a good fit under the head and against the web of rail.

409. Foot guards.-Foot guards shall be furnished only when specified and shall be of approved design. 
410. Cotter pins.-Cotter pins shall be used in all vertical bolts, pins, and through body bolts in frogs and crossings. They shall be placed close up to the nut.

411. Washers, head locks, and nut locks.-(a) Flat washers under the nut shall be not less than threeeighths inch thick. Angle or beveled washers and head locks shall be not less than one-eighth inch thick at the thin end. Washers other than springtype lock washers shall be not less than $2 \frac{1}{2}$ inches long and of ample width to provide bearing against the head or base of rail.

(b) All main or body bolts and through bolts shall be provided with self-locking heads or with separate head locks and with approved nut locks.

412. Sawing of rails-All rails whether furnished in frogs, switches, mates, crossings, or rail lengths shall be sawed square to the axis of the rail unless otherwise specified. A variation of more than one thirty-second inch in the width of the head out of square will not be accepted. There shall be no variation of more than one-fourth inch from theoretical lengths, and such variation shall be noted on plans.

413. Minimum diameters-bolts, rivets, and rivet holes-(a) The minimum diameters of through bolts shall be controlled by the depth of the fishing space measured along the vertical center line of the rail as follows:

\begin{tabular}{|c|c|c|}
\hline \multicolumn{2}{|c|}{$\begin{array}{c}\text { Depth of fishing } \\
\text { space }\end{array}$} & \multirow{2}{*}{$\begin{array}{l}\text { Mini- } \\
\text { mum } \\
\text { diame } \\
\text { ters of } \\
\text { bolts }\end{array}$} \\
\hline From- & To- & \\
\hline Inches & \multirow{2}{*}{$\begin{array}{c}\text { Inches } \\
218 \\
25 \% \\
3\end{array}$} & \multirow{2}{*}{$\begin{array}{c}\text { Inches } \\
7 / 8 \\
1 \\
118 \\
11 / 4\end{array}$} \\
\hline $\begin{array}{l}25 / 64 \\
241 / 64 \\
31 / 64\end{array}$ & & \\
\hline
\end{tabular}

(b) Joint bolts shall be of mill standard for section of rail used unless otherwise specified.

(c) The minimum diameter of rivets and bolts used for any purpose shall be five-eighths inch. The diameters of rivet holes shall not be more than one-sixteenth inch greater than the diameters of corresponding rivets.

414. Joints-Joints for bolted work in streets shall be tight. Occasional open joints shall not exceed one-eighth inch. Joints for work for open tracks need not be tight, but no joint shall be more than one-eighth inch open, excepting joints designated as insulated joints. When joints are bolted tightly, the rails shall align correctly with each other with no greater variation than one thirty-second inch in either line or level. Occasional open joints in guard rails shall not exceed one-fourth inch. The manufacturer shall make note of such open joints on plans.

415. Compromise joints-(a) Compromise rails shall be used instead of compromise joints wherever possible. They shall be made of steel castings, forged taper rails, or two pieces of rail connected by cast welding or other welding methods. Where this practice is not specified or practicable, compromise joints shall be of forged or cast steel at least equivalent in strength to the joint for the lower rail and must be finished to a good fit so that good line and surface are obtained.

(b) Where guard rails connect with other sections the shape of the guard shall be modified in a distance of 6 to 9 inches to conform gradually and as nearly as practicable to the contour of the connecting section. (c) Compromise joints shall be designated in accordance with the standard method of the American Electric Railway Engineering Association W12. (See 606.0, p. 310.)

416. Paving face-In work specified for paved streets the angles in switches, mates, and frogs shall be filled to provide paving faces to a width of not less than 4 inches, by means of paving chocks consisting of iron castings which must have ample bearing on the bottom flanges of the special pieces and rails to prevent their being depressed under vehicular traffic. They shall be so constructed as to permit their being placed and solidly grouted after the joint plates are applied.

417. Split switch design-(a) The design shall be in accordance with Figure 189.

(b) The fixtures shall conform to the A. R. E. A. Details as per A. R. E. A. plans 201 and 202. (See A. R. E. A. track work plans given below.)

The number and kind of fixtures required shall be specified by a selection from the bill of materials applicable to the length of switch desired, given on A. R. E. A. plans Nos. 210 and 211. (See A. R. E. A. track work plans, given below.)

\section{(B) Frogs and Accessories}

418. Lengths of frogs.- (a) The lengths and angles of frogs for crossings and turnouts shall be in accordance with those provided in W15, given above.

(b) The lengths of spring rail frogs shall be in accordance with the standard lengths of spring rail frogs of the American Railway Engineering Association.

419. Frog and crossing arms.-Frog and crossing arms, except as provided in section 418 (a) and (b), shall in all cases be long enough to give $61 / 4$ inches clearance between webs at end of splice bars. When adjoining pieces or combination pieces prevent a length of arm for proper joining, special pieces made of cast steel may be substituted. Minimum arm length for frogs shall be 3 feet and for crossing arms 4 feet unless adjoining pieces or other conditions will not permit.

420. Frog points, body fillers, and wing rails.(a) Frogs shall be made with points formed of rails suitably planed and fitted together and securely held in correct position by main body fillers of rolled steel or cast iron of sufficient length to provide for not less than two through body bolts passing through both point rails and wing rails. Through bolts shall not be spaced more than 10 inches center to center. Wing rails shall lap the point not less than 20 inches and for frogs having an angle of $10^{\circ}$ or less, the lap of the wing rail shall be the same as that provided in the American Railway Engineering Association standards.

(b) The body fillers shall be of sufficient length to provide proper flange bearings where required and the surfaces for such flange bearings shall be steel, properly treated, or cast iron with chilled surface to provide hard wearing surface.

421. Spring rail frogs.-The design shall be in accordance with Figure 190.

422. Girder rail frogs.-Frogs made of grooved girder and girder guard rails shall be made with points formed by rails properly planed, shaped, and machined, securely held together by means of body fillers and through bolts. Further strength shall be provided by means of rolled steel plates not less than three-fourths inch thick, planed to fit the fishing section on the outside of the rails and throughbolted. Flange bearing when required may be provided by suitably shaping the rails. 



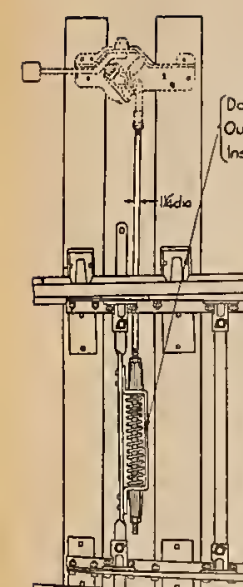

(Double Coll Soring

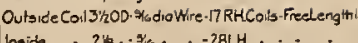

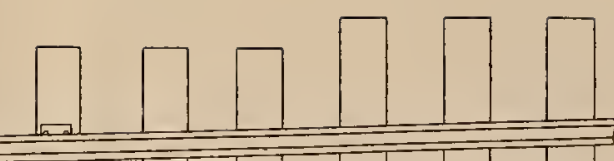
$\{$ की

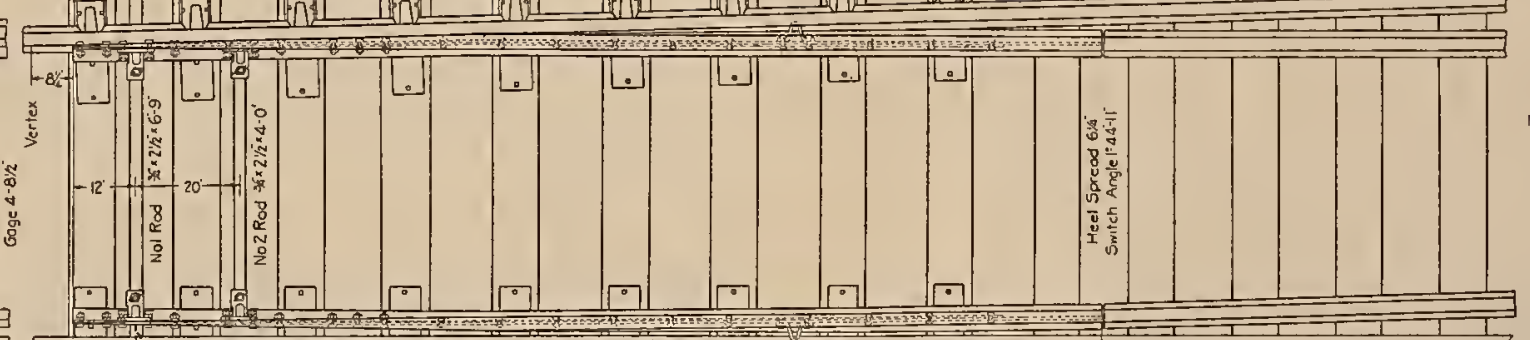

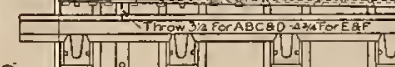

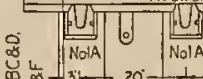

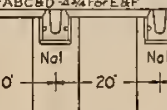

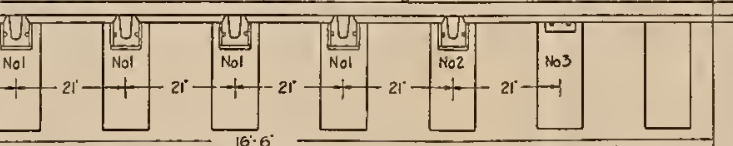

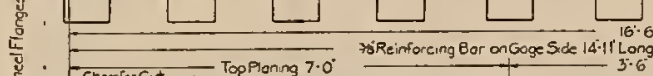

\section{6}
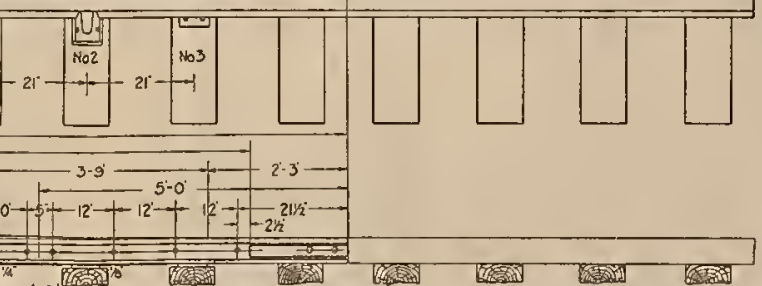

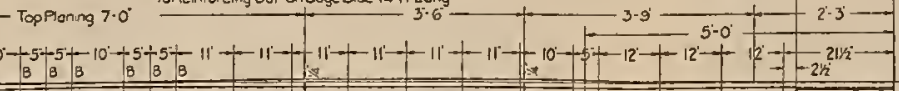

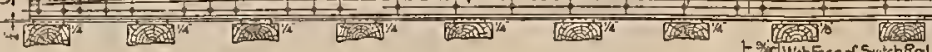

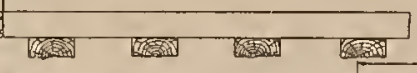

Nete-Shope of Dotled Broce Optiond

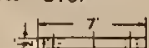
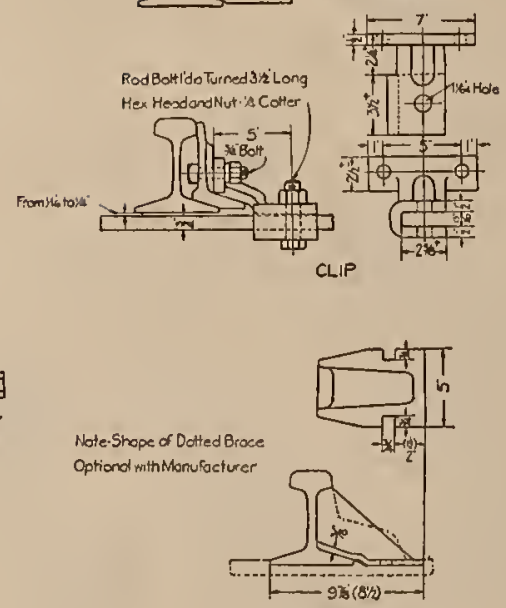

BRACE

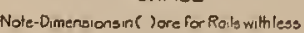
than 5 width of Bose.

ChamferCut-Moke Swîkeh Pont's Thick rivinOututis From End of tont andRound Paint to Shorp Edge

Note-\% Reenforcing Secured by 3 Rivets in Center Line of Web, Except Where Batty ore tobe Usedos Indicated byletter $B$ Alternate-Rivets maybe Usedo on on Points Morked when 305 pecilied

I'if For 7 i 8 ofts For First Elesten Hodes 1
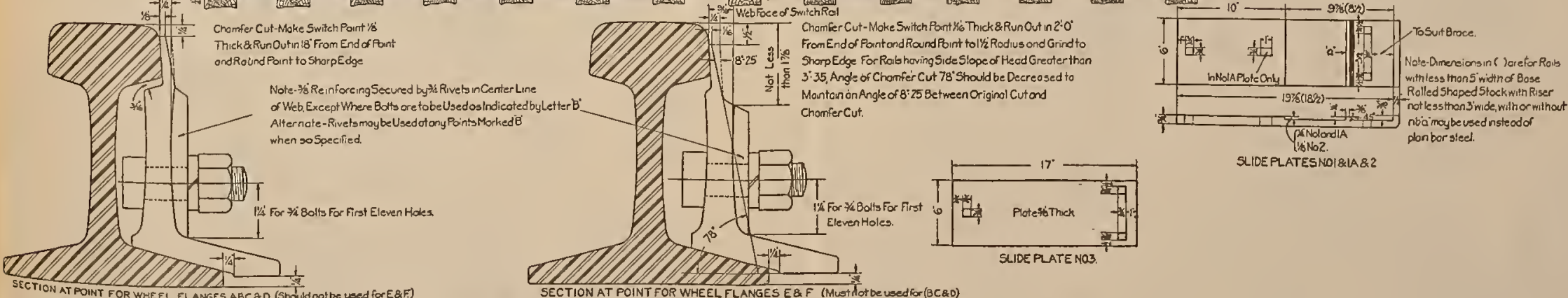

(C) Tongue Switches, Mates, and Crossings

423. Tongue switches. - Tongue switches shall have a cast iron, cast steel, or rolled steel bed underneath the tongue. The tongue shall be of hard steel not less than 2 inches thick and have a substantial pivot pin. The pivot pin shall be of sufficient size to provide ample strength and not less than $27 / 16$ inches in diameter and fitted accurately in a bored hole in the bed. The base of the tongue and the surface of the bearing bed shall be finished true and smooth so that the tongue will maintain a true bearing in any position. The tread of the tongue shall be at least onefourth inch above the curved tread of the switch from a point 1 foot back from the point of the tongue toward the heel to provide an efficient guard where required. Tongues of switches of 200foot radius or over may have their tops level with the curved tread surface. Provision for attachment bolts, rivets, and bearing plates or corner plates as may be required. One line of rails shall be carried through the crossing with the heads of the through rails notched for the flangeways of the intersecting rails. The through rails may be cut through vertically at the middle of the notch. Flange bearing, when required, shall be provided by hard-steel fillers, by cast-iron fillers having chilled top surfaces, or by suitably planing the guard rails.

(b) Single crossings may be made in one or two pieces at the manufacturer's option unless otherwise specified.

(c) Crossings having an angle of $35^{\circ}$ or less shall be made with frog-point construction as provided in section 420 .

(d) Easer rails in steam tracks shall be of the same section as the main running rails and shall be full headed throughout except that the ends of easers
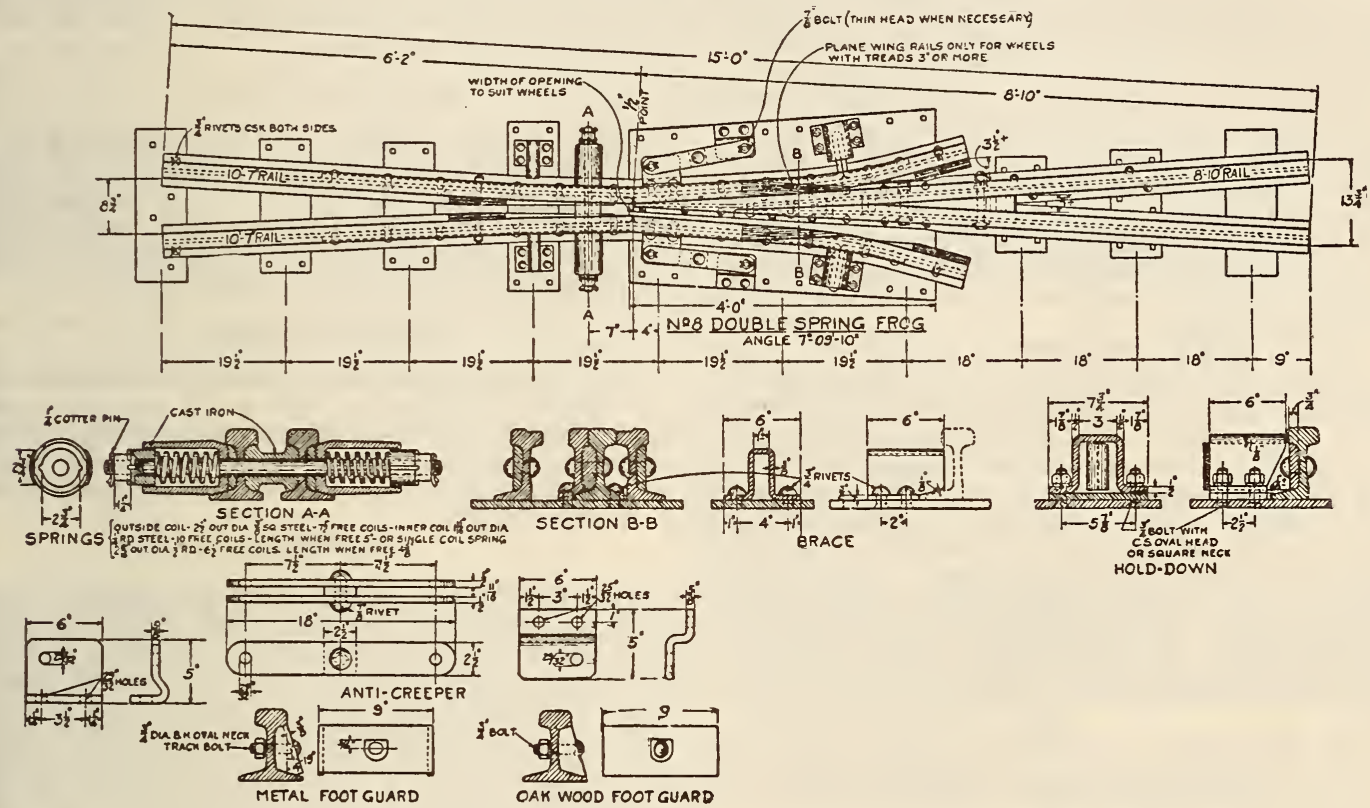

FIGURE 190.-No. 8 double spring frog. (But only partially spring acting)

of switch throwing or locking devices shall only be made when specified.

424. Mates.-(a) Mates shall be made with guards of sufficient length to extend beyond the point toward the heel not less than 20 inches and sufficiently toward the P. C. to guard the point of the tongue properly. Where flange bearing is required the surfaces for such flange bearing shall be steel properly treated or cast iron with chilled surface to provide a hard-wearing surface.

(b) Body fillers shall be of sufficient length properly to protect the mate point and hold it in position, with not less than four through body bolts passing through the rail forming the point.

(c) Mates made of girder grooved and girder guard rails shall be made with points formed of rails properly planed, shaped, and machined, securely held together by body fillers and through bolts. Flange bearing may be provided by suitably shaping the rails.

425. Crossings.-(a) Crossings of angles from $90^{\circ}$ down to $35^{\circ}$ shall be made of double or triple rails as specified. They shall be made of rails suitably shaped, planed, and fitted together and securely held by such main body fillers, heavy forged-steel reinforced knees, rolled-steel angle knees, through shall be planed down one-half inch in 12 inches. They shall extend not less than 21 inches from the gage-line intersection.

\section{WORKMANSHIP, ASSEMBLY, AND FINISH}

501. Workmanship. - Workmanship shall be first class in accordance with best current practice and the assembly of the several parts shall be such that uniformity of detail and finish will result.

502. Bolt holes, method of drilling.-Main or body bolts shall have a driving fit in straight true holes and surrounding metal shall be free from burrs or cracks. Holes in carbon-steel rail shall be drilled and shall be cylindrical and not conical. Drilling shall be accurately done on bevel where necessary and no punching shall be permitted except in bearing plates, tie plates, and washers. Holes for main bolts shall be one-sixteenth inch less in diameter than bolts to be used. The parts shall then be assembled and holes reamed straight and true with no offsets between adjacent parts and of such size as will give the driving fit. In lieu of this method the parts may be assembled and accurately fitted before any drilling is done; after the parts have been securely clamped in correct position the holes may 
be drilled of such size as will give driving fit for their entire length.

All bolt holes through rails and fillers shall be drilled with a minimum clearance for rough bolts and they shall conform accurately to the required dimensions.

503. Rivets and riveting.-Rivets shall be of full diameter called for on plans, and rivet holes shall not be more than one-sixteenth inch greater in diameter. When not otherwise called for by plans or specifications, rivets shall have standard button or cone heads of uniform size for the same size of rivet. The heads shall be concentric with the holes. Countersunk rivets shall be flush with the surface and fill the countersink except that rivets through base and tie plates may have a crown of not more than one-eighth inch below the lower surface of the plate.

504. Curving of rails.-All curved rails shall be so bent true to templet throughout their entire length and depth that when ready for installation all parts of the undersurface of the base will rest in a plane.

505. Set-in guards. - Set-in guards shall be carefully fitted to the back of switch points to insure perfect line, surface, and flangeway when point is open, and accurate fit against the abutting rail when the point is closed.

506. Bolt and rivet clearance in split switches.-Care must be taken to provide at least one-eighth inch clearance between heads on all rivets and bolts and the adjacent rails.

507. Fit of fillers, braces, and spacers.-All abutting parts and pieces shall have accurate true fit. All filler and spacer blocks shall fit in the bases, heads, and webs of rails for a distance of one-half inch above the base and below the head and maintain required flangeway. Throat filler blocks shall fit sufficiently well to maintain required spacing. When the raised brand of rail interferes with fit of fillers the brand shall be removed. Fillers shall be grooved or cut to fit over rivet heads and bolt heads.

508. Heating of rails.-Open-hearth steel rails shall not be heated to more than a dull red color for the purpose of kinking, bending, drilling, or punching.

509. Assembly for inspection.-Before any of the work is shipped, the several parts shall be assembled and inspected and checked to insure proper fit and conformity with plans.

\section{MARKING AND SHIPPING}

601. All material shall be given suitable shop marks by painting and die stamping for ready identification, corresponding to marks given on plans. Die-stamping figures shall not be less than fiveeighths inch high. This marking shall be carefully arranged to insure proper and easy assembly of material in the field. No paint, tar, or other covering shall be used before inspection and not at any time unless definitely required by the specifications.

\section{INSPECTION}

701. The inspector representing the purchaser shall have all reasonable facilities afforded him by the manufacturer to satisfy himself that the finished material is furnished in accordance with these specifications. All inspections shall be made at place of manufacture, unless otherwise arranged.

\section{AMERICAN ELECTRIC RAILWAY ENGI- NEERING ASSOCIATION, STANDARD SPECIFICATION FOR RAIL-BOUND INSERT TYPE SPECIAL TRACK WORK, W34-30, 1930}

\section{RAIL-BOUND SPECIAL TRACK WORK}

(In respect to materials, chemical properties and tests, physical properties and tests, design (including standard dimensions of switches and mates), workmanship, heating of rails and inserts, assembly for inspection, marking and shipping, and inspection, this specification conforms to A. E. R. E. A., W33, given above, except for the following provisions:)

This specification is intended to cover the manufacture of special track work wherein the switches, mates, frogs, and crossings are made of cast manganese steel inserts, at the points of greatest wear, secured in place by rolled-steel rails properly machined and bolted thereto.

\section{FLANGE-WAY FILIERS}

406. Flange-way fillers when required shall be of rolled or forged steel, cast steel, or wrought iron, or of good quality gray cast iron, as may be specified. They shall fit in the fishing space of the rail and into the fillets of the web for not less than one-half inch below the head and above the base flange.

\section{MANGANESE INSERTS}

408. The cast manganese steel inserts shall be the full height of the rail, or have a full support on the base of the rail. The sectional area shall be not less than the minimum recommended by the Manganese Track Society and the Manganese Steel Founders Society.

\section{BASE PLATES}

410. Base plates under frogs or crossing intersections shall only be furnished when specified. They shall lap the joint between the rolled rail and the manganese-steel insert, and shall not be shorter than the manganese-steel casting. They may be fastened to the structure by rivets or clip bolts. The thickness of the plate shall be not less than five-eighths inch for rails weighing less than 100 pounds per yard and three-fourths inch for rails weighing 100 pounds per yard and over.

\section{TONGUE SWITCHES}

417. (a) Tongue switches shall be made of standard rails suitably bent and fitted together to form a running surface for the straight and curved tracks, with a guard of same section bolted thereto forming a pocket for the tongue. The tongue shall be made of manganese steel or other hard steel as may be specified, not less than 2 inches thick and have a substantial pivot pin fitted accurately in a bored hole, or other approved fastening. The bed shall be of cast iron, cast steel, or rolled steel underneath the tongue. The base of the tongue and the surface of the bearing bed shall be finished true and smooth so that the tongue will maintain a true bearing in any position. The tread of the tongue shall be at least one-fourth inch above the curved tread of the switch from a point 1 foot back from the point of the tongue toward the heel to provide an efficient guard 
where required. Tongues of switches of 200 feet radius or over may have their tops level with the curved tread surface. Provision for attachment of switch throwing or locking devices shall only be made when specified.

(b) Manganese-steel inserts shall be provided at either the point or the heel of the tongue, or both, as may be specified. The insert at the point shall form a pocket for the tongue and extend in front of the point not more than 6 inches, and toward the heel about one-fourth the length of the tongue and be securely bolted to main and guard rails through the webs by not less than four bolts.

The insert at the heel of the tongue shall continue the running surface of the tongue and form the junction of the running rails and guard.

\section{MATES}

418. Mates shall be made of main and guard rails, enveloping a cast manganese steel insert, and of such length as to insure wheel flange bearing in the grooves, and tread bearing on the head. The insert shall extend back of the head a sufficient distance properly to protect the mate point and hold it firmly in position by not less than four through bolts or it shall be made with a cast manganesesteel insert with guard forming integral part thereof extending in front of the point to insure full wheel tread bearing, and back of the point to the full width of the rail head and be securely bolted to the rail.

The straight rail of the mate shall be recessed in the head by planing or bending to provide a bearing on the manganese-steel insert opposite the point, for the outside edge of the wheel tread on the curve run.

(Paragraph 419, lengths of tongue switches and mates, is identical with paragraph 406 (including accompanying note) in W33, given above.)

\section{FROGS}

420. Frogs shall be made with a manganesesteel insert set between the wing rails, and securely fastened together with through bolts spaced not more than 10 inches center to center. The manganese-steel insert shall have a width across the head at the heel end of not less than 4 inches, shall extend between rails at the heel not less than 8 inches, and shall be secured to the heel rails with not less than two bolts.

The wing rails shall be recessed in the head to receive the insert and to provide a bearing on the manganese steel for the outside edge of the wheel tread opposite the point on both runs, and shall extend in front of the throat to a spread between gage lines one and one-half times the width of the groove. Where it is necessary to form a guard point on the insert, the latter shall be suitably extended.

At the heel end the wing rails shall be flared to an opening twice the width of the throat, and rolled steel or cast iron fillers shall be extended to the end of the wing rails and securely bolted thereto.

\section{CROSSINGS}

421. (a) Crossings of angles of $90^{\circ}$ down to but not including $35^{\circ}$ shall be made of double or triple rails as specified on either or both runs. The portion at intersections shall consist of manganese-steel inserts surrounded by the rolled rails and forged or cast steel knees, or fastened to base plates. The insert shall extend on the running surfaces to the spreads between gage lines of not less than 4 inches on electric railway track and $43 / 4$ inches on steam railway track, and form the guard points where necessary. The rolled rails and knees shall be suitably shaped and fitted to the manganese-steel inserts and secured together by through bolts and by clip bolts to the base plate when furnished.
Single crossings may be made in one or two pieces at the option of the manufacturer, unless otherwise specified.

(b) Crossings having an angle of $35^{\circ}$ or less shall be made of frog construction, as provided in section 420 . On steam railway lines the manganese-steel insert shall extend into the wings opposite the point not less than 4 inches measured from gage lines.

\section{FROG AND CROSSING ARMS}

422. Frog and crossing arms shall in all cases be long enough to give $61 / 4$-inch clearance between webs at end of splice bars. When adjoining pieces or combination pieces prevent a length of arm for proper joining, special pieces made of cast steel may be substituted. Minimum arm length for frogs shall be 3 feet and for crossing arms 4 feet, unless adjoining pieces or other conditions will not permit. The lengths and angles of frogs for crossovers and turnouts shall be as provided in Engineering Manual Specification W15, Dimensions of Frogs for Crossovers and Turnouts. (See figs. 185 to 187.)

(In respect to finish of castings, this specification conforms to A. R. E. A. track-work plans, given below, except for the following provisions:)

502. (b) The running surface of manganesesteel inserts shall be finished with as good surfaces as those of the rolled rail, and portions fitting into rails or other parts shall be ground to a good fit.

(Paragraphs 503 and 504 are identical with paragraphs 503 and 504 in A. E. R. E. A., W32, given above.)

\section{HEATING OF RAILS AND INSERTS}

(Paragraph $505(a)$ is identical with paragraph 508 in A. E. R. E. A. W33, given above. Paragraph $505(b)$ is as follows:)

(b) Manganese steel inserts shall under no circumstances be heated to more than a light blue temper color.

\section{AMERICAN ELECTRIC RAILWAY ENGI- NEERING ASSOCIATION, STANDARD DESIGN OF BOLTED RAIL CROSSINGS; STEAM RAILROAD OVER ELECTRIC RAIL- WAY, W39-28, 1928}

\section{BOLTED RAIL CROSSINGS}

\section{BOLTS}

Through bolts shall be high tensile steel- $13 / 8$ inch diameter bolts for 120-pound rail and heavier; $1 \frac{1}{4}$-inch diameter bolts for rails less than 120 pounds and down to rails having 3 -inch fishing height; 11/8-inch diameter bolts for rails having less than 3 -inch fishing height, down to and including 80 -pound rail. Fishing height shall be measured on vertical center line of rail.

All through bolts to have tight fit. Through bolts to have square heads and nuts, with U. S. standard thread, fitted with head locks, nut locks, and bevel washers where necessary to afford square bearing.

Suitable washers in any case shall be placed under nuts to bring same out from under head of rail, so that they may be tightened with a standard wrench.

\section{FIILERS}

Flangeway fillers shall be of rolled or forged steel on steam railroad, and on electric railway may be of rolled steel, forged steel, or cast iron, the latter to be used only when flange bearing is omitted. They shall fit closely into the fishing space of the rail, and tightly into the fillets of the web for not less than one-half inch below the head and above the base.

Third rail through fillers shall be of rolled or forged steel. Other fillers and spacer blocks may be of cast iron. 


\section{PLATES}

Crossings shall be provided with base plates unless otherwise specified. Plates shall be threefourths inch thick, of length to suit angle of crossing, and of sufficient width for attaching to crossing by clips with three-fourths-inch diameter countersunk-head bolts.

\section{CORNER BRACES}

Forged steel or iron corner braces with heavy reinforced corners shall be provided, and shall be planed to fit closely into the fishing space of the rail, and into the fillets of the web for not less than one-half inch below the head and above the base flange.

Spaces marked " $\mathrm{X}$ " in flangeway between rolled steel fillers on steam railroad run may be filled with either rolled-steel or cast-iron fillers, if so specified.

Third-rail fillers at " $\mathrm{Y}$ " may be continuous if so specified. Flange bearing on electric railway run may be omitted when so specified.

(For illustrative details of bolted rail crossings, steam railroad over electric railway, see A. R. E. A. track-work plans, plan No. 716.)

\section{AMERICAN ELECTRIC RAILWAY ENGI- NEERING ASSOCIATION, STANDARD DESIGN OF BOLTED RAIL CROSSINGS ; STEAM RAILROAD OVER ELECTRIC RAIL- WAY, FOR ANGLES BELOW $50^{\circ}$ TO $30^{\circ}$, INCLUSIVE, W43-28, 1928}

\section{BOLTED RAIL CROSSINGS}

(Identical with A. R. E. A. track-work plans, plan No. 717.)

NotE.-Specifications for the general character and quality of the materials and workmanship entering into bolted rail crossings are contained in specification W33-29, given above.

\section{AMERICAN ELECTRIC RAILWAY ENGI- NEERING ASSOCIATION, STANDARD DESIGN OF BOLTED MANGANESE-STEEL CROSSINGS; STEAM RAILROAD OVER ELECTRIC RAILWAY, FOR ANGLES BE- LOW $45^{\circ}$ TO $30^{\circ}$, INCLUSIVE, W44-28, 1928}

\section{BOLTED MANGANESE-STEEL CROSSINGS}

(Identical with A. R. E. A. track-work plans, plan No. 778.)

Note - Specifications for the general character and quality of the materials and workmanship entering into these crossings are contained in specification W34-29, given above.

\section{AMERICAN ELECTRIC RAILWAY ENGI- NEERING ASSOCIATION, SPECIFICATION FOR MATERIALS FOR USE IN THE MANUFACTURE OF SPECIAL TRACK WORK, W104-28, 1928}

[Approved as American Tentative Standard by the American Standards Association, January 23, 1929]

\section{SPECIAL TRACK WORK MATERIALS}

\section{Part I.-INTRODUCTORY}

1. Definitions-Scope-Inspection and Tests

101. Definition of special track-work.-All rails, track structures and fittings, other than plain unguarded track neither curved nor fabricated before laying.
102. Scope of specifications.-These specifications are intended to cover the manufacture, chemical and physical properties, and tests of materials entering into the manufacture and assembly of special track work for steam, electric and industrial tracks of all gauges and types, for heavy rail of 50 pounds per yard and over, under any motive power, and in either open or paved track.

103. Principal structural items in special track work.-Switches; mates; frogs; crossings; guarded, curved, and fabricated rails; appurtenances and fittings for the preceding items, such as plates, rods, fillers, braces, knees, guards, risers, stops, springs, housings, clips, bolts, nuts, washers, rivets, and splice bars.

104. Principal materials used for structural items.-

(a) Rolled rails:

Carbon-steel rails-

Standard T-sections.

Plain girder sections.

Girder grooved sections.

Girder guard sections.

Manganese steel rails.

(b) Castings:

Manganese-steel castings.

Carbon-steel castings-general.

Carbon-steel castings-hard.

Gray-iron castings-general.

Gray-iron castings-high test.

Malleable castings.

(c) Rolled or forged material:

Steel forgings-medium.

Steel forgings-hard.

Mild-steel bars and shapes.

Rolled-steel frog fillers.

Wrought iron-common.

(d) Springs:

Helical springs.

(e) Fastenings:

Splice bars.

Bolts and nuts-general.

Bolts and nuts - high tensile.

Washers.

Nut locks.

Rivets.

105. Selection of material to be used.-The kind of material to be used for the various parts of special track work shall be as specified by the purchaser. If not so specified, and two or more kinds are applicable to a particular part, the selection shall be optional with the manufacturer.

106. Application of specifications.-Any material entering into the manufacture of special track work or parts thereof and which is covered by specifications contained (or referred to) herein, shall conform to the requirements of such specifications, unless otherwise specified by purchaser.

107. Reference to established specifications.-The specifications contained (or referred to) herein are based, as far as possible, on established specifications of various national associations with such additional or modified requirements, necessary to adapt the material to the manufacturing of special track work, as are particularly specified herein.

108. Inspection and tests.-(a) The records of any tests made by the manufacturer as his regular routine in the manufacture of materials entering into the work of the purchaser shall be open, free of charge, for the examination of the inspector representing the purchaser.

(b) The inspector representing the purchaser shall have free entry, at all times while work on the contract of the purchaser is being performed, to all parts of the manufacturer's works, which concern the manufacture of the materials ordered. The manufacturer shall afford the inspector, without charge, all reasonable facilities to examine the work during 
its progress as well as the finished product to satisfy him that the materials comply with these specifications.

(c) Other chemical and physical tests covered by the specifications shall be made when required by the purchaser. For materials originally manufactured by the special track-work manufacturer for a specific order such tests shall be made at the expense of the manufacturer when the amount of the particular kind of material is 50 tons or more; if less than 50 tons the purchaser shall pay for the expense of such tests, unless the material fails to meet the specifications, in which case the manufacturer shall bear the expense.

(d) For materials or parts of the work taken from stock or purchased from other manufacturers the special track-work manufacturer, when required, shall certify that the material conforms with these specifications. If the purchaser desires any chemical or physical tests of such materials, he shall pay the expense of such tests, unless the materials fail to meet the specifications, in which case the special track-work manufacturer shall pay the expense. For the rails used in the work the manufacturer, when requested, shall supply the purchaser with a certificate of inspection from the rail manufacturer.

(e) All inspection and tests shall be made at the place of manufacture prior to shipment and shall be so conducted as not to interfere unnecessarily with the operation of the works or the progress of the work on the purchaser's order. Chemical and physical tests may be made at a testing laboratory outside of the manufacturer's works to be agreed upon between the manufacturer and the purchaser.

(f) The acceptance of any material by an inspector shall not prevent subsequent rejection if found defective after delivery.

(g) No guarantee of specific length of service will be required. A claim for defective material and/or workmanship shall cover only such defects as will impair the life of the work.

(h) No claim for free replacement of defective material and/or workmanship shall be recognized unless made promptly and based on defects appearing within three months from date of installation and in any event within two years from date of shipment.

(i) The foregoing paragraphs cover the inspection and tests of the materials when used for special track work and take precedence over the inspection clauses contained in the various established specifications for similar materials referred to in section 107.

\section{Part II. SPECIFICATIONS}

\section{RAILS}

\section{Carbon Steel Rails of Standard T-Rail Section}

101. Material covered.-Rails of standard T-rail section weighing 50 pounds per yard or over, and of open-hearth steel (Bessemer-steel rails are not desired for special track work).

102. Manufacture.-Unless otherwise specified by the purchaser the rails shall be manufactured, at the option of the special track-work manufacturer, according to any one of the specifications of the American Society for Testing Materials, A1-30, the American Railway Engineering Association, 1925, or the American Electric Railway Engineering Association, W16-29 (see 606.1, p. 315), for openhearth steel rails of the weight and section specified and conform to the following requirements:

103. Quality.-First quality No. 1 rails only shall be used in special track work, except that No. 2 rails may be used for guard rails and for parts of track structures where the necessary machining of the rail entirely removes any flaws or imperfections.
104. Length. - The standard length of full-length rails in special track work shall, at $60^{\circ} \mathrm{F}$., be 30 feet, 33 feet, and 39 feet, as may be specified, and within the variations permitted by the specifications under which the rails were rolled.

105. Drilling.-(a) When circular holes for joint bolts, electric bonds, or tie-rods are required they shall be drilled to conform to the specifications of the purchaser and be within the following permissible variations:

(b) The diameter of the bolt holes shall not vary more than one thirty-second inch over or under that specified. The diameter of the bond holes shall not vary more than one thirty-second inch under that specified. The diameter of the tie-rod holes shall not vary more than one-sixteenth inch over or under that specified.

(c) The location of the bolt and bond holes shall not vary more than one-sixteenth inch either longitudinally or vertically from that specified. The location of the tie-rod holes shall not vary more than one-eighth inch vertically and not more than onehalf inch longitudinally from that specified.

(d) The bond holes shall be truly cylindrical and not conical.

106. Punching.-Unless otherwise specified by the purchaser, the tie-rod holes may be punched, and when so made they shall be free from burrs, fins, etc. The permissible variation in the diameter of punched tie-rod holes shall be not more than oneeighth inch over or under that specified.

II. Open-Hearth Steel Girder Rails of the Plain, Grooved, and Guard Types

201. Material covered.-Girder rails of the different types for curves, turnouts, and other parts of special track work for use in paved streets.

202. Manufacture.-Unless otherwise specified by the purchaser, the rails shall be manufactured according to the standard specifications for openhearth girder rails of the American Electric Railway Engineering Association W1-31, the American Society for Testing Materials A2-27, or the American Railway Engineering Association, 1926. (See 606.1, p. 311.) Where the class of the rail is optional, the special track-work manufacturer may use either class.

203. Quality.-First quality No. 1 rails only shall be used in special track work, except that No. 2 rails may be used for parts of track structures where the necessary machining of the rail entirely removes any flaws or imperfections.

204. Length. - The standard length of full-length rails in special track work shall be 30 feet and 32 feet at $60^{\circ} \mathrm{F}$. and within the variations permitted by the specifications under which the rails are rolled.

205. Drilling and punching.-The drilling of holes for joint bolts and electric bonds and the drilling or punching of tie-rod holes shall conform to the specifications of the purchaser and be within the limits of variations permitted by the standard specifications referred to in section 202.

206. Sawing. - The ends of the rails shall be sawed at right angles to the gage with a permissible variation of one-sixteenth inch in 6 inches and on an incline from the vertical so that the top edge of the head will project beyond the bottom edges of the base not less than one thirty-second inch, nor more than three-sixteenths inch. The ends of the rails in special track work need not be milled unless specially called for.

\section{Rolled Manganese-Steel Rails}

301. Material covered.-Rails rolled of manganese steel for sections corresponding to sections of openhearth rails. 
302. Manufacture.-The process of manufacture shall be in accordance with the best current state of the art and the rail manufacturer's approved specifications No. I-B. The rails shall comply with the following requirements.

303. Chemical compositions.-The steel shall conform to the following requirements as to chemical composition:

Per cent

Manganese 10. $50-15.00$

Carbon. $.95-1.35$

Phosphorus, not over

304. Physical properties and tests.-A test of the proper toughness of the rail, when required by the purchaser may consist of taking a 2-inch-long section of the rail and bending the same cold under a hammer through the flat part of the web until the head stands at an angle of $90^{\circ}$ to its original normal position without fracture at the bend.

305. Workmanship and finish.-The general workmanship, finish, and classification of the rails shall be the same as required for carbon-steel rails in the recommended specifications for open-hearth steel rails of the American Society for Testing Materials, A1-30, or of the American Electric Railway Engineering Association, W16-29. (See 606.1, p. 315.)

306. Quality.-First class No. 1 rails only shall be used in special track work, except that No. 2 rails may be used for guard rails and for parts of track-work structures where the necessary machining entirely removes any flaws or imperfections.

307. Punching.-(a) Holes for joint bolts or tierods may be punched or drilled in location and of size specified by the purchaser.

(b) The diameter of the bolt holes shall not vary more than one thirty-second inch over or under that specified. The diameter of the bond holes shall not vary more than one thirty-second inch under that specified. The diameter of the tie-rod holes shall not vary more than one-sixteenth inch over or under that specified.

(c) The location of the bolt and bond holes shall not vary more than one-sixteenth inch either longitudinally or vertically from that specified. The location of the tie-rod holes shall not vary more than one-eighth inch vertically and not more than one-half inch longitudinally from that specified.

\section{CASTINGS}

\section{Manganese-Steel Track Castings}

401. Material covered.-Manganese-steel castings for solid manganese-steel track structures or wearing parts of track structures.

402. Manufacture.-The entire process of manufacture and heat treatment shall be in accordance with the best current practice.

403. Chemical requirements.-The chemical composition of cast manganese steel shall be within the following limits:

\begin{tabular}{|c|c|c|}
\hline Elements & Minimum & Maximum \\
\hline & Per cent & Per cent \\
& 10.00 & 14.00 \\
Manganese_...... & 1.40 \\
Carbon_... 00 & .14 \\
\hline Phosphorus........ & & \\
\hline
\end{tabular}

404. Check analysis.-Drillings from the finished articles shall be furnished for check analysis if so specified by the purchaser.

405. Physical requirements and tests.-When specified, a test coupon shall be cast from the same heat, separate from the casting, key-marked by the inspector and subject to equivalent heat treatment as the special track-work casting. The coupon shall be 6 to 12 inches long by one-half by three-quarters inch; shall bend cold on the flat side around a diameter of 1 inch to an angle of $160^{\circ}$ without fracture. The bending may be accomplished by pressure or by blows.

406. Bend test.-Bending test demonstration from the test coupon, if specified by the purchaser, shall be made in the presence of his representative at the foundry where the castings are made.

407. General conditions. - (a) Castings shall be reasonably smooth and true to patterns in accordance with good foundry practice. Large lumps, sharp fins, sand, and chills on the outside of the casting shall be removed. The castings shall be free from such blowholes, sand holes, cracks, cold shuts, and other defects which would impair their serviceability and as further specified below. Castings must be out of twist and reasonably true, both as to general surface and alignment, and must not show any signs of straining or undue denting produced in the straightening process.

(b) The bottom part of castings which rest on ties shall be reasonably straight and out of twist, and shall be free from lumps or such imperfections as would prevent a good bearing.

408. Imperfections.-Tread surface within $21 / 2$ inches of gage line and side of groove 1 inch down from thread shall be free from physical defects, such as shrinkage cracks, sand holes, blowholes, cold shuts or segregation of metal, unless such defects are so small that they have been practically removed by the finish grinding, and there must be no indication of unsoundness of the metal. Shrinkage cracks, cold shuts or segregations of metal will not be allowed in any part of the tread surfaces. Sand holes, blow holes, and cold shuts in portions of the casting where they will not appreciably weaken the casting, or impair its wearing qualities, will be permitted. Castings must be free from shrinkage cracks running vertically in web members of solid work or horizontally at or near the same or in corners of junction of projecting members or longitudinally in grooves. Other small shrinkage cracks which do not materially weaken the castings will be acceptable.

\section{v. Carbon-Steel Castings}

501. Material covered.-(a) Steel castings for general service in track work for which no physical requirements are specified, appurtenances and fittings, braces, washers, switch heel blocks, spring housings, and other parts not exposed to wheel wear, designated as "class A" castings.

(b) Carbon-steel castings for solid cast track structures or parts of track structures exposed to wheel wear, or special splice bars designated as "class B-hard" castings.

502. Manufacture.-(a) The steel shall be made by one or more of the following processes: Openhearth, electric furnace, converter, or crucible.

(b) The castings shall conform in general character to the standard specifications of the American Society for Testing Materials A27-24 (see 611.41, p. 707) for steel castings of "class A" or "class Bhard" as designated and comply with the following requirements:

503. Chemical composition.-The chemical composition shall be as follows:

(a) For class A castings:

Per cent

Carbon, not over. 0.45 Phosphorus:

Acid process, not over

Basic process, not over .07 
(b) For class B-hard castings:

\begin{tabular}{|c|c|c|}
\hline & $\begin{array}{l}\text { Not less } \\
\text { than- }\end{array}$ & $\begin{array}{c}\text { Not more } \\
\text { than- }\end{array}$ \\
\hline $\begin{array}{l}\text { Carbon.... } \\
\text { Silicon } \\
\text { Manganese.... } \\
\text { Sulphur.... } \\
\text { Phosphorus.... }\end{array}$ & $\begin{array}{c}\text { Per cent } \\
0.45 \\
.30 \\
.60 \\
-60\end{array}$ & $\begin{array}{c}\text { Per cent } \\
0.55 \\
.45 \\
.85 \\
.06 \\
.05\end{array}$ \\
\hline
\end{tabular}

504. Check analysis.-Check analysis may be made by the purchaser. The phosphorus and sulphur contents thus determined shall not exceed that specified by more than 20 per cent. Drillings for the analysis shall be taken not less than one-fourth inch beneath the surface and in such a way as not to injure the strength or usefulness of the castings.

505. Physical requirements.-(a) For class A castings, no test for physical properties shall be required unless otherwise specified.

(b) Class B-hard castings shall conform to the following minumim requirements as to tensile properties:

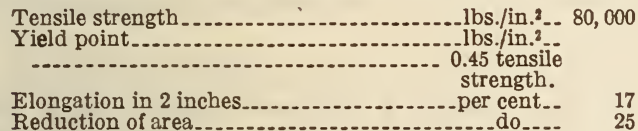

Note. No maximum limit shall be placed on the tensile strength.

506. Bend test.-Bend test shall not be required in either class of castings.

507. Tension test and alternative test. - Test on class $B$ castings, when required, to determine the tensile properties of the castings or alternative test therefor shall be made in accordance with requirements and method of procedure as per A. S. T. M. specifications referred to in section 502 (b).

508. Annealing and heat treatment.-(a) Class A castings coming within the desired chemcial composition, need not be annealed nor heat treated unless so specified. Castings with carbon content above 0.30 , but not over 0.45 may be accepted, when properly annealed.

(b) Class B-hard castings shall receive proper heat treatment in accordance with the specifications of the American Society for Testing Materials referred to in section $502(b)$; the treatment depending upon the design of the casting.

509. Workmanship. - The castings shall conform substantially to the shapes and sizes indicated by the patterns and drawings.

510. Finish.-The castings shall be free from injurious defects.

511. Welding.-Defects which do not impair the strength or wearing qualities of the castings, except those located on the tread surface within $1 \frac{1}{2}$ inches of the gage line, may be welded by an approved process. The defects shall be cleaned out to the solid metal, before welding, and when so required by the inspector, shall be submitted to him in this condition for his approval. When required by the inspector, important castings shall be heat treated after welding.

\section{Gray Iron Castings}

601. Material covered.-(a) "General" gray iron castings for switches, fillers, braces, and other general appurtenances for track work not requiring high strength.

(b) "High test" gray iron castings for parts of track work requiring high strength to sustain weight and shock of car-wheel traffic and not requiring to be readily machinable including those generally known as "semisteel" castings.

602. Manufacture. - The castings may be made by the cupola or any other approved process.

603. Chemical composition.-(a) For "general" gray-iron castings the sulphur content of the metal shall not be over 0.10 per cent.

(b) Sulphur determination when required shall be made in accordance with the standard method of sampling and chemical analysis of pig and cast iron of the American Society for Testing Materials, A64-27. (See 601.20, p. 119.)

(c) For "high test" gray-iron casting no specific chemical composition shall be required.

604. Physical properties and tests.-(a) For "general" gray-iron castings no test for physical properties shall be required.

(b) "High test" gray-iron castings shall be subject to the following test when required:

(c) Transverse test specimens poured from the same heat as the castings corresponding to the standard arbitration tests bars of the American Society for Testing Materials when placed horizontally upon supports 12 inches apart and tested under a centrally applied load shall withstand a minimum load of 3,800 pounds and deflect under this load at least 0.12 inches at the center.

(d) The rate of the application of the load shall be such that the central deflection of 0.10 inch is produced in from 20 to 40 seconds.

(e) One test bar of each set of castings shall conform to the above requirements, otherwise the castings represented by such bars may be rejected.

605. Workmanship and finish.-The castings shall be true to pattern and free from gas holes, cracks, flaws, and excessive shrinkage and in all other respects they shall conform to whatever points may be specially agreed upon between the manufacturer and the purchaser.

\section{Malleable-Iron Castings}

701. Material covered.-Malleable-iron castings for fittings and appurtenances for track work, braces, washers, switch clips, spring housings, and foot guards.

702. Manufacture.-(a) The castings shall be produced by either air furnace, open hearth, or electric furnace process.

(b) The castings shall be thoroughly annealed. Castings rejected for insufficient annealing may be reannealed once.

(c) When so specified by the purchaser the castings shall be manufactured in accordance with the standards specifications of the A. S. T. M. for malleable castings, A47-30. (See 611.21, p. 665.)

703. Physical properties and tests.-(a) If the purchaser or his representative so desires, a casting may be tested to destruction. Such casting shall show good, tough malleable iron. No other test shall be required unless otherwise specified.

(b) When so specified by purchaser the castings shall be subject to the test prescribed by the specification of the A. S. T. M. referred to in section 702 (c) and shall conform to the following minimum requiremen is as to the tensile properties:

(c) Tensile strength -1bs./in. ${ }^{2}$ - 50,000 Elongation in 2 inches......... 10.0 Yield point.

704. Workmanship and finish.-(a) The castings shall conform substantially to the patterns or drawings.

(b) The castings shall be made in a workmanlike manner and shall be free from injurious defects. 


\section{ROLLED OR FORGED MATERIALS}

\section{Steel Forgings}

801. Material "covered.-(a) Forgings of mildcarbon steel for knee braces, fillers and other heavy parts of trackwork not exposed to wheel wear.

(b) Forgings of hard steel for riser blocks, switch tongues, and other parts of track work exposed to wheel wear.

802. Manufacture.-(a) The forgings shall be made from billets or slabs manufactured in accordance with the standard specification of the American Society for Testing Materials A17-29. (A. S. A. tentative American Standards G9-1924), for carbon-steel blooms, billets, and slabs for forgings. (See 602.2, p. 131.)

(b) Hard-steel forgings if desired may be made from rail steel of the respective qualities specified for carbon steel $T$ rails of standard sections.

803. Chemical composition.-The steel shall conform to the following requirements as to chemical composition:

\begin{tabular}{|c|r|r|}
\hline & Mild steel & Hard steel \\
\cline { 2 - 3 } & Per cent & Per cent \\
Carbon._...... & $0.15-0.30$ & 0.45 \\
Manganese...... & $.40-.80$ & $0.60-0.90$ \\
Phosphorus...... & 2.05 & 2.05 \\
Sulphur....... & 2.05 & 2.05 \\
\hline 1 Minimum. & \multicolumn{2}{|c|}{ 2 Maximum. } \\
\hline
\end{tabular}

804. Heat treatment.-(a) For mild-steel forgings heat treatment shall not be required unless otherwise specified

(b) For hard-steel forgings heat treatment, when required, shall consist of annealing or quenching and tempering as specified.

805. Physical properties.-Forgings except when made from rail steel shall conform after final treatment to the following requirements as to tensile properties:

\begin{tabular}{|c|c|c|}
\hline & $\begin{array}{c}\text { Mild steel } \\
\text { (minimum) }\end{array}$ & $\begin{array}{l}\text { Hard steel } \\
\text { (minimum) }\end{array}$ \\
\hline Tensile strength...........lbs./in. ${ }^{2}$ & 60,000 & 80,000 \\
\hline Yield point & 30,000 & 40,000 \\
\hline 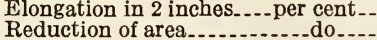 & & $\begin{array}{l}15 \\
22\end{array}$ \\
\hline
\end{tabular}

806. Tests.-(a) Tension test may be made when so specified.

(b) Test specimens shall be taken from a full-size prolongation of any forging and shall conform in dimensions to A. S. T. M. standard 2-inch test abr.

807. Hardness.-(a) Forgings exposed to wheel wear in track work shall be equal in hardness to rai steel of the weight and type of rail with which they are used.

(b) A hardness test may be made when so specified. The details of such test shall be agreed upon between the manufacturer and the purchaser.

808. Workmanship and finish.-The forgings shall be free from injurious defects and shall have a workmanlike finish.

\section{Mild Steel}

901. Material covered.-Rolled-steel bars and shapes for parts of track work, hammered, pressed, or machined from such semifinished materials for fittings and appurtenances, reinforcements, tie-rods, tie-plates, base-plates, etc.

902. Manufacture.-(a) When made from bar steel, the same shall be of open-hearth steel conforming, in general, with standard specifications of the American Society for Testing Materials for com- mercial quality hot-rolled bar steel A107-30 (see 603.23 , p. 147), and/or the specifications of the American Railway Association, Mechanical Division, for structural steel shapes, plates, and bars, 1923. (See 605.13 , p. 254.)

(b) If made from structural steel, rolled shapes, or plates, they shall be of Bessemer or open-hearth steel conforming, in general, with the standard specifications of the American Society for Testing Materials for structural steel for buildings A9-29 (see 605.12 , p. 252), and complying with the following requirements:

903. Chemical requirements.-(a) For bar steel the chemical requirements shall be as follows:

\section{Per cent}

Carbon $0.15-0.30$

Phosphorus, not over... 045

Sulphur, not over.

(b) For structural shapes and plates the chemical requirements shall be as follows:

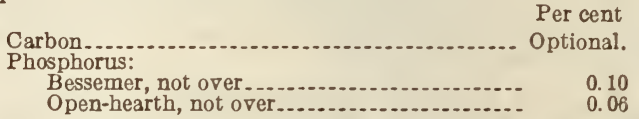

904. Physical requirements.-(a) The material shall conform to the following requirements as to tensile properties:

Tensile strength...................... bs./in. ${ }^{2}$ 50, 000-65, 000

(b) Test specimens shall bend cold through $180^{\circ}$ without cracking on the outside of the bent portion for material up to and including $1 \frac{1}{4}$ inches in thickness around a pin, the diameter of which is equal to the thickness of the specimen; and for material over $11 / 4$ inches in thickness around a pin of a diameter equal to twice the thickness of the specimen.

905. Tests. - All tests, when required, shall be made in accordance with the respective specifications referred to in sections $902 \mathrm{a}$ and $902 \mathrm{~b}$.

906. Workmanship and finish.- (a). The permissible variations in the size and dimensions of the rolled bars and shapes shall be within the limits of the specifications referred to in sections $2 \mathrm{a}$ and $2 \mathrm{~b}$.

(b) The finished articles shall be free from injurious defects and accurately machined and otherwise finished for the purposes intended.

\section{Rolled Steal Frog Fillers}

1001. Material covered.-Rolled-steel fillers of various sections to fit $T$ rails of standard sections.

1002. Manufacture.-(a) The steel shall be made by either one or both of the following processes: Bessemer or open-hearth.

(b) Bars shall be hot rolled.

1003. Chemical composition.-The steel shall conform to A. S. T. M. specification A107-30 (See 603.23 , p. 147) for commercial steel bars of classes applicable hereto in the following grades:

\begin{tabular}{|c|c|c|}
\hline & $\begin{array}{c}\text { Open- } \\
\text { hearth }\end{array}$ & Bessemer \\
\hline Carbon_.......... & $\begin{array}{c}\text { Per cent } \\
0.15-0.30\end{array}$ & $\begin{array}{l}\text { Per cent } \\
0.12-0.25\end{array}$ \\
\hline
\end{tabular}

1004. Workmanship and finish--(a) The bars shall be smooth and straight and within the following permissible variations and dimensions:

The finishing heights of the fillers shall not exceed that called for by the rail section and shall not underrun by more than three sixty-fourths inch.

Width of fillers between rail webs shall not be under, nor more than one-sixteenth inch over the dimensions called for.

Depth of the flangeway groove may be one-sixteenth inch under or one-eighth inch over dimensions called for. 
XI. Wrought Iron

1101. Material covered.-These specifications cover wrought iron common or refined, as may be specified, for parts of track work such as rods, reinforcing, bars, fillers, and small forgings hammered or pressed.

1102. Manufacture.-(a) Common wrought-iron bars shall be manufactured in accordance with the standard specifications of the American Society for Testing Materials for common iron bars, A85-27. (See 603.1, p. 140.)

(b) Refined wrought iron bars shall be manufactured in accordance with the standard specifications of the American Society for Testing Materials A41-18 and/or the American Railway Association, Mechanical Division, for refined wrought iron bars. (See 603.1, p. 139).

1103. Physical requirements.-The bars shall have a minimum tensile strength of 45,000 pounds per square inch.

1104. Tests.-(a) Tests for tensile strength and elongation may be made in accordance with the specifications referred to in section $1102 \mathrm{~b}$.

(b) No other test shall be required.

1105. Workmanship and finish.-(a) The permissible variations in size of the rolled bars shall be within the limits of the specifications referred to in section 1102.

(b) The rolled bars and the finished articles shall be smooth and free from slivers, depressions, seams, crop ends, evidence of being burned and other injurious defects.

\section{SPRINGS}

\section{Helical Springs}

1201. Material covered.-These specifications cover helical springs for spring rail frogs and for actuating devices for switches.

1202 Manufacture.-The springs shall be manufactured in accordance with the specifications for helical springs for railways of the American Society for Testing Materials A61-16. (See 611.54, p. 801.)

1203. Chemical requirements. - The steel for the bars shall conform to the following chemical requirements:

Carbon.

Per cent $0.90-1.10$

Manganese, maximum .50

Phosphorus, maximum 05

1204. Workmanship and finish.-(a) The springs shall be of uniform pitch with ends tapered to give a reasonably firm square bearing. The point of the bars shall not protrude beyond the outside diameter of the springs.

(b) The free heights of the springs shall not vary more than one-eighth inch from that specified.

(c) The outside diameters of the springs excepting the heights shall not vary more than one-sixteenth inch from those specified.

\section{FASTENINGS}

\section{Splice Bars}

1301. Material covered.-(a) Rolled steel splice bars of standard design for joints in rails of standard section or girder rail sections and of the following grades:

Low-carbon-steel splice bars.

Medium-carbon-steel splice bars.

High-carbon-steel splice bars.

Extra-high-carbon-steel splice bars.

(b) Special splice bars, when standard rolled sections of splice bars are not applicable in special track work including compromise splice bars or joint fixtures.
1302. Manufacture.-(a) Splice bars of standard section may be manufactured in accordance with any of the standard specifications for the different grades of the A. S. T. M. A3-24, A4-14, A5-14, A6-14; A. E. R. E. A. W17-22, W18-22, W19-22, W20-22, or the A. R. E. A. (see 606.2 , p. 331), as may be specified by the purchaser. When not so specified it shall be optional with the special trackwork manufacturer to use bars made to either of these specifications as may be available to fit the rail sections called for at the time when the special track work is being manufactured.

(b) Special splice bars used in special track work may be machined from hard steel or may be of cast steel according to design required.

1303. Chemical and physical properties.-The special splice bars shall conform to the specifications herein contained or referred to, for hard steel or for steel castings-class $\mathrm{B}$, according to the material that is used.

1304. Tests.-(a) Tests, when required, shall be made in accordance with the specifications under which the splice bars are manufactured or in case of the special splice bars according to the test required for the material from which they are made.

(b) No test shall be required for low-carbon-steel splice bars or when no higher grade is specified.

\section{Bolts and Nuts}

1401. Material covered.-(a) Low-carbon-steel bolts and nuts for general use in special track work including track bolts for joints as well as bolts with square or hexagon heads or other shapes of head as may be required for the various purposes.

(b) High-tensile-strength bolts and nuts of carbon steel or alloy steel whether track bolts for joints or bolts with square, hexagon, or other shaped head as may be required for the various purposes, when so specified.

(c) Low-carbon-steel bolts shall be furnished unless high-tensile-strength bolts are called for.

1402. Manufacture.-The manufacture of the bolts shall conform in general and as far as applicable to A. S. T. M. specification for low-carbon-steel track bolts (A76-27); high-tensile-strength bolts, either quenched-carbon-steel bolts (A50-24), or alloy-steel track bolts (A51-21); or to similar specifications of A. E. R. E. A. or A. R. E. A. (see 606.4, p. 352), at the option of the special track-work manufacturer.

XV. Nut Locks

1501. Material covered.-Helical spring washers. Unless otherwise specified helical spring washers shall be furnished where nut locks are called for in special track work. They shall be of two classes: High spring pressure and low spring pressure.

1502. Manufacture.-(a) The spring washers shall be of carbon steel or an acceptable alloy steel and shall be from lots that have passed satisfactory tests at the place of manufacture in accordance with the specifications for spring washers of the American Railway Engineering Association, adopted 1926.

(b) High-spring-pressure washers shall be used in connection with high-tensile bolts, low-spring-pressure washers with all other bolts unless otherwise specified.

XVI. Rivets

1601. Material covered.-These specifications cover steel rivets for general use in special track work.

1602. Manufacture.-The rivets shall conform in general requirements to the specification of the American Society for Testing Materials for rivets in structural steel for buildings A9-29. (See 605.12, p. 252.) 


\section{AMERICAN ELECTRIC RAILWAY ENGI- NEERING ASSOCIATION, RECOMMENDED SPECIFICATION FOR CAST-STEEL CON- STRUCTION HARD CENTER SPECIAL TRACK WORK, W106-27, 1927}

\section{CAST-STEEL SPECIAL TRACK WORK}

(In respect to materials, chemical properties and tests, physical properties and tests, design (including standard dimensions of switches and mates), workmanship, assembly for inspection, marking and shipping, and inspection, this specification conforms to A. E. R. E. A., W33, given above, except for the following provisions:)

These specifications are intended to cover the manufacture of special track work wherein the tongue switches, mates, frogs, and crossings are made of steel castings with hard centers of special steel inserted at points receiving the principal wear.

\section{FLANGE BEARING}

403. The depth of grooves at intersections and on curved side of mate points shall be one-eighth inch less than the depth of the wheel flange except that the minimum depth of flangeway shall be nine-sixteenths inch. Flange bearings and risers shall conform to the requirements of Figure 191 of A. E. R. E. A., W122, minimum dimensions for center plates for single and middle frogs, given below.

\section{CAST-STEEL BODY DIMENSIONS}

407. (a) Tongue switches, mates, frogs, and crossings (not steam railroad crossings) shall consist of cast-steel bodies, the extremities conforming to the adjoining rail and have hard steel centers secured to the bodies. The bodies shall be of uniform depth of 6,7 , or 9 inches, and be provided with a base flange not less than 3 inches wide on each side of double web sections and 5 inches wide on single web sections.

(b) The cross sections shall provide minimum thickness of metal as follows:

Thickness under groove, three-fourths inch.

Thickness under center, three-fourths inch.

Thickness of vertical walls or webs, five-eighths inch for double webs.

Thickness of vertical walls or webs, three-fourths inch single webs.

Thickness of base, one-half inch at outer edge, three-fourths inch at web.

\section{HARD CENTERS}

408. (a) Hard-center inserts shall be made of manganese steel or other hard steel as approved by the purchaser.

(b) For centers fastened along the center line of the plate, the depth shall be not less than 2 inches, and for centers designed to have fastenings on the edges of the plates the centers shall be not less than $2 \frac{1}{2}$ inches thick. In all cases the minimum thickness underneath the grooves shall be three-fourths inch. Minimum dimensions of all center plates shall conform to the requirements of Figure 191, W122, minimum dimensions for center plates for single and middle frogs, given below. The centers shall be fastened to the bodies by an efficient method, and in frogs and crossings shall be renewable. Hardcenter inserts shall have either a partial or full machine bearing when specified. The running surface of hard centers must be finished to a true level with the adjoining body castings.

\section{TONGUE SWITCHES}

409. The switches shall be provided with a hard steel center at the point of, and a hard steel bed under the entire length of, the tongue.

(Otherwise tongue switches are identical with paragraph 409 in A. E. R. E. A., W32, given above, except that tongue is of hard steel instead of manganese steel.)

\section{MATES}

410. Mates shall have a hard steel wearing plate which shall include the guard as well as the running surfaces and be of sufficient length to provide at least a 2-inch tread at both ends. The hard steel wearing plate in mates need not be renewable.

(In respect to frogs and crossings, length of arms and castings, joints, compromise joints (including reference to paragraphs $(b)$ and $(c)$, section 415 , in A. E. R. E. A., W33), paving face, finish of castings (including reference to A. R. E. A. track-work plans), finishing of ends (except that in W106 an allowable variation of three-fourths inch from theoretical lengths is provided for), and curving of rails, this specification conforms to A. E. R. E. A., W32, given above.)

\section{AMERICAN ELECTRIC RAILWAY ENGI- NEERING ASSOCIATION, RECOMMENDED SPECIFICATION FOR IRON - BOUND HARD-CENTER SPECIAL TRACK WORK, W107-29, 1929}

\section{IRON-BOUND HARD-CENTER SPECIAL TRACK WORK}

(In respect to materials, chemical properties and tests, physical properties and tests, design (including standard dimensions of switches and mates), workmanship, assembly for inspection, marking and shipping, and inspection, this specification conforms to A. E. R. E. A., W33, given above, except for the following provisions):

These specifications are intended to cover the manufacture of special track work wherein the tongue switches, mates, frogs, and crossings are made principally of rolled rail arms bound together by cast-iron bodies having recesses to receive the hard steel centers which form the running portions between the rolled rail arms, and at points receiving the principal wear.

\section{CONSTRUCTION OF BODY CASTINGS; MINIMUM}

407. (a) Tongue switches, mates, frogs, and crossings shall consist of rolled rail arms bound together by a cast body with a recess to receive a hard steel center which shall form the running portions of the structure between the rolled rail arms. The body portion shall be of a cross section giving not less than $1 \frac{1}{4}$ inches thickness of metal underneath the hard steel center, not less than $13 / 8$ inches thickness of walls, and not less than 6 inches total width of bearing surface on the ties. It shall be provided with a spiking ledge on the outside. The minimum depth of the body portion shall be 7 inches.

(b) The rail arms must be secured in the body casting by proper means and an adequate amount of the cast metal along the webs of the rail to assure their being held absolutely tight and to give proper strength to both the rail arms and the body casting. 
FROGS AND CROSSINGS; LENGTH OF ARMS

411. (a) Frog and crossing arms shall be long enough to give $61 / 4$ inches clearance between webs at end of joint plates. When adjoining pieces, or a combination of pieces, prevent a length of arm for proper joining, pieces made of cast steel with hard steel center, or solid manganese steel, may be substituted at the manufacturer's option. The length and angles of frogs for crossovers and turnouts shall be as provided in W15-22, dimensions of frogs for crossovers and turnouts, given above.

(b) Crossings shall be in 1 and 2 pieces, or, in small angles, in 4 pieces, at manufacturer's option. Outside crossing arms shall be not less than 3 feet in length unless joined to other pieces necessitating shorter lengths.

(c) The hard steel center plate shall be of sufficient length in all cases to provide a full tread at the junction with the rolled rails, and no center shall be less than 12 inches long, measured on the gage line.

(In respect to flange bearing, tongue switches (including reference to A. E. R. E. A., W32), hard centers, and mates, this specification conforms to A. E. R. E. A., W106, given above.)

(In respect to joints, compromise joints (including reference to paragraphs $(b)$ and $(c)$, section 415 , in A. E. R. E. A., W32), paving face, finish of castings (including reference to A. R. E. A. track-work plans), sawing of rails, and curving of rails, this specification conforms to A. E. R. E. A., W32, given above.)

\section{AMERICAN RAILWAY ENGINEERING AS- SOCIATION, SPECIFICATIONS FOR FROG FILLER SECTIONS, 1926}

\section{FROG FILLER SECTIONS}

\section{MANUFACTURE}

\section{Process}

1. The steel shall be made by either or both the following processes: Bessemer or open-hearth.

2. Bars shall be hot rolled.

\section{CHEMICAL PROPERTIES}

3. Steel shall conform to A. S. T. M. specifications for commercial bar steel, in so far as they apply to this product, A. S. T. M. A107 and A108 (see 603.23, p. 147) of the following grades:

\begin{tabular}{|c|c|c|}
\hline & Open-hearth & Bessemer \\
\hline Carbon ................ & $\begin{array}{c}\text { Per cent } \\
0.15-0.30\end{array}$ & $\begin{array}{c}\text { Per cent } \\
0.12-0.25\end{array}$ \\
\hline
\end{tabular}

III. PERMISSIBLE VARIATIONS IN DIMENSIONS

4. The fishing height of filler shall not exceed that shown in drawings by more than one sixty-fourth inch, and shall not underrun by more than one thirty-second inch.

at Width of filler between rail webs shall be nothing under to one-sixteenth inch over dimensions shown.

Depth of flange-way groove may be one-sixteenth inch under or one-eighth over dimensions given on drawing.

\section{GROOPIYG}

In order to reduce the large number of sections now rolled, fillers recommended as standard have been grouped as shown on plan 325 in A. R. E. A. track-work plans.
AMERICAN ELECTRIC RAILWAY ENGINEERING ASSOCIATION, RECOMMENDED DESIGN, MINIMUM DIMENSIONS FOR CENTER PLATES FOR SINGLE AND MIDDLE FROGS, W122-27, 1927

\section{CENTER PLATES FOR SINGLE AND MIDDLE FROGS}
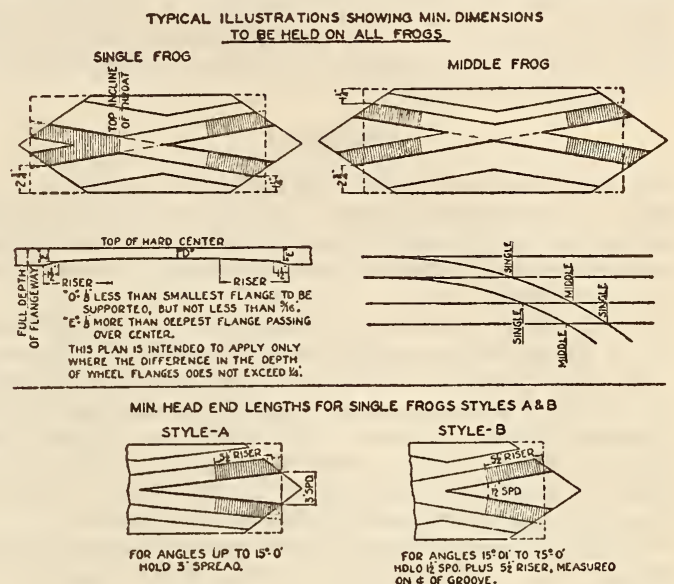

MUN GUARD END LENGTHS FOR SINGLE FROGS

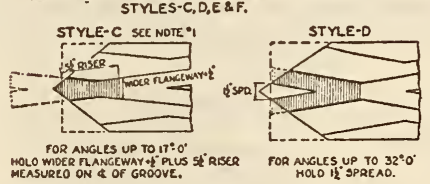

MIN. LENGTHS FOR MIDOLE FROGS STYLE-H

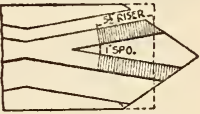

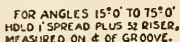

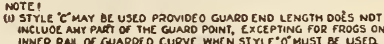
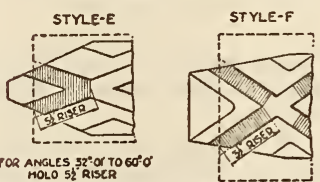

FOR ANGLES GOOCITO $75^{\circ} 0^{\circ}$

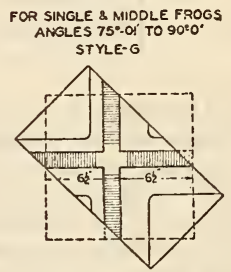

HOLO MINIMUM LENGTH OF $65^{\circ}$ CENTEQ

FIgURe 191.-Minimum dimensions for center plates for single and middle frogs

\section{AMERICAN RAILWAY ENGINEERING ASSOCIATION, SPECIFICATIONS FOR SWITCHES, FROGS, CROSSINGS, AND GUARD RAILS, A. R. E. A. TRACK-WORK PLANS, 1929}

\section{TRACK WORK}

\section{GENERAL INSTROCTION}

1. The purchaser will furnish the manufacturer specifications and drawings, giving rail sections, splice drilling, angles, alinement, and general dimensions, and such special details as may be required.

2. Unless otherwise specified the construction, design, and details shall conform to the plans adopted by the American Railway Engineering Association as recommended practice. For track structures for which no such plans have been adopted, the manufacturer shall, when requested, submit for approval detail drawings. 
3. The drawings shall be part of the specifications. Anything that is not shown on the drawings, but which is mentioned in the specifications, or vice versa, or anything not expressly set forth in either, but which is reasonably implied, shall be furnished the same as if specifically shown and mentioned in both. Should anything be omitted from the drawings or specifications that is necessary for a clear understanding of the work, or should any error appear in either the drawings or specifications affecting the work, the manufacturer shall notify the purchaser and shall not proceed with the work until instructed to do so.

\section{MATERIEI}

4. Rail.-The rail used shall be first quality openhearth steel of the section called for, manufactured according to A. R. E. A. specifications or to rail manufacturer's standard specifications, unless otherwise specified.

5. Gray iron castings.-Gray iron castings shall be of a good commercial grade of medium gray iron.

6. Steel castings.-Steel castings shall be of good commercial grade manufactured in accordance with standard specifications of the American Society for Testing Materials for steel castings, class B, except that steel castings exposed to wheel wear shall have a hardness approximately that of rail steel.

7. Manganese steel track castings. - The cast manganese steel shall conform to the following specifications.

(a) Chemical composition.-The chemical composition shall be within the following limits:

\begin{tabular}{|c|c|c|}
\hline \multirow[b]{2}{*}{ Elements } & \multicolumn{2}{|c|}{ Percentages } \\
\hline & $\begin{array}{l}\text { Not less } \\
\text { than- }\end{array}$ & Not more \\
\hline $\begin{array}{l}\text { Manganese } \\
\text { Carbon } \\
\text { Phosphorus... }\end{array}$ & $\begin{array}{c}10.00 \\
1.00 \\
-0\end{array}$ & $\begin{array}{r}14.00 \\
1.40 \\
.10\end{array}$ \\
\hline
\end{tabular}

Drillings from the finished articles shall be furnished to the inspector from time to time on request for check analysis.

(b) Physical qualities. - A rough cast specimen $3 / 4$ by $1 / 2$ inch shall bend cold on the flat side around a diameter of 1 inch to an angle of $180^{\circ}$ without breaking. The bending may be accomplished by pressure or by blows.

Bending test demonstrations from test bars poured from the same heat as the castings, when required, shall be made in the presence of the inspector at the foundry where the castings are made.

(c) General conditions. - Castings shall be reasonably smooth and true to pattern in accordance with good foundry practice. Large lumps, sharp fins, sand, and chills on the outside of castings shall be removed. The castings shall be free from such blowholes, sand holes, cracks, cold shuts, and other defects which would impair their serviceability and as further specified below. Castings must be out of twist and reasonably true, both as to general service and alignment, and must not show any signs of straining or undue denting produced in the straightening process.

The bottom part of castings which rest on ties shall be reasonably straight and out of twist, and shall be free from lumps or such imperfections as would prevent a good bearing.

(d) Imperfections.-Tread surfaces within $2 \frac{1}{2}$ inches of gage line and side of groove 1 inch down from tread shall be free from physical defects such as shrinkage cracks, sand holes, blowholes, cold shuts, or segregation of metal, unless such defects are so small that they have been practically removed by the finish grinding, and there must be no indication of unsoundness of the metal. Shrinkage cracks, cold shuts, or segregation of metal will not be allowed in any part of the tread surfaces. Sand holes, blowholes, and cold shuts in portions of the casting where they will not appreciably weaken the casting, or impair its wearing qualities, will be permitted. Castings must be free from shrinkage cracks running vertically in web members of solid work, or horizontally at or near the ends, or in corners of junction of projecting members, or longitudinally in grooves. Other small shrinkage cracks which do not materially weaken the casting will be acceptable.

8. Malleable iron castings.-Malleable iron castings shall be of a good commercial grade, properly annealed.

9. Rolled or forged steel.-Rolled or forged steel parts shall be of a medium grade of commerical mild steel. Parts exposed to wheel wear shall be equal in hardness to rail steel.

10. Fillers.-Fillers shall be of rolled or forged steel, wrought iron or of good quality gray cast iron as called for on plans and as specified.

11. Heel risers.-Heel risers shall be as called for on plans and provide wearing surface equal in hardness to rail steel.

12. Foot guards.-Metal foot guards as shown on plans shall be of rolled steel or malleable iron. Wooden foot guards shall be good quality hard wood. Filler blocks when acting as foot guards may be of gray iron.

13. Bolts.-Bolts, other than where heat-treated bolts are called for on plans or specified, shall be of mild carbon steel and shall have a tensile strength of not less then 50,000 pounds per square inch and an elongation of not less than 15 per cent in 8 incbes.

Heat treated or high tensile bolts shall be of carbon or alloy steel and conform to the following minimum requirements.

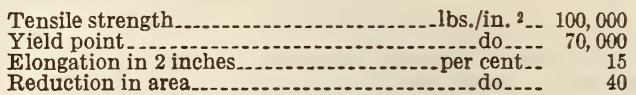

Full-size bolts shall bend cold without cracking through $180^{\circ}$ around a pin of the same diameter as the bolt. The yield point, elongation, and reduction of area may be determined from a finished bolt or from a test piece $1 / 2$ by 2 inches turned from a finished bolt. Nuts may be Bessemer or open-hearth carbon steel not heat treated and shall be of sufficient thickness to develop the full strength of the bolt. Bolts with countersunk heads, button neads, or coneheads shall be provided with locking necks or other effective locking means to keep bolts from turning.

14. Rivets.-Rivets shall be made of steel manufactured in accordance with the standard specifications of the American Society for Testing Materials for rivet steel for ship or structural work.

15. Reinforcing bars.-Reinforcing bars shall be of wrought iron or mild open-hearth steel.

16. Plates. - Switch plates, special frog tie-plates, and bearing plates shall be of mild open-hearth steel.

17. Switch clips. - Switch clips shall be of mild open-hearth steel, except special designs which may be of cast steel or malleable iron.

18. Switch rods. - Switch rods shall be of mild rolled steel or wrought iron.

19. Stops and hold-downs. - Stops and hold-downs shall be of mild rolled steel or wrought iron.

20. Anticreeping device.-Anticreeping devices shall be of mild rolled steel or wrought iron.

21. Braces.-Braces shall be of mild rolled steel, malleable iron, or cast steel. 
22. Washers.-Washers shall be of mild rolled steel, malleable iron, or cast steel.

23. Spring washers.-For heat-treated or hightensile bolts, spring washers shown on track-work plans shall conform to specifications of the American Railway Engineering Association for high spring pressure, and where nut locks (N. L.) are specified on present track-work plans the term "spring washers" shall be understood.

24. Switch heel blocks.- Switch heel blocks shall be of gray iron, cast or forged steel, as specified.

25. Springs. - The steel in springs shall conform to the standard specifications of the American Society for Testing Materials for carbon steel bars for railway springs. Springs when forced down solid and held in the compressed position for 30 seconds, upon release, must not vary from their original free length.

26. Spring housings.--Spring housings shall be of gray cast iron, malleable iron, or cast steel.

27. Forged crossing knees.-Forged crossing knees shall be of mild rolled open-hearth steel or wrought iron.

28. Special splice bars.-Special splice bars shall be made of mild rolled steel or cast steel.

\section{WORXMANSHIP}

29. Workmanship.-Workmanship shall be first class and in accordance with best current practice. The assembly of the several parts shall be such that uniformity of detail and finish will result.

30. Alignment and surface.-The alignment and surface of all finished work shall be even and true and conform to the angles specified.

31. Length. - Length of frogs and crossing arms shall not vary more than one-fourth inch from lengths specified. Switch-point rails and guard rails shall not vary more than one-half inch from length specified. Rail ends shall be cut square to the axis of the rail unless otherwise required.

32. Gages and flange ways. - Track gage may be one-eighth inch under or one-sixteenth inch over that specified for manganese construction, and onesixteenth inch under or over for bolted construction.

Guard gage (distance between guards) shall not exceed that specified, but may be one-eighth inch less. The width of flange ways may not be less than specified nor more than one-sixteenth inch wider than specified. All the above measured on the level of gage line five-eighth inch below tread surface.

Flange ways shall be not less than $17 / 8$ inches deep measured from top of thread surfaces, unless otherwise specified.

33. Bending. - Bends shall be made accurately in ares of circles and without injury to the material. It is desired that rails be bent cold. If heating of the rails is resorted to it must be done in a manner so as not to injure the metal.

34. Planing.-All planing must be true and all abutting surfaces must fit accurately.

35. Grinding.-Running surfaces of the manganese steel parts shall be ground to practically as good a surface as that of the rolled rail. Manganese steel portions fitting into rails or other parts shall be ground to a good fit.

36. Drilling and punching.-All holes in carbon steel rails must be drilled. In other parts all holes for turned pins or bolts and for tight fit of rough bolts must be drilled. Drilling to be done accurately, on bevel where necessary. Punching will be permitted only in wrought iron or mild steel parts for rivets, loose rough bolts, and spikes, except when such holes come so close together or close to the edge of the piece that the metal between holes or between hole and edge is less in width than the thickness of the material, in which case holes must be drilled.
37. Fit of bolts.-Main bolts (also referred to on plans as "through" or "body" bolts) in bolted rigid frogs and bolted rail crossings shall have a tight fit in straight, true holes. Heads and nuts shall have a square bearing. Other bolts not requiring a tight fit, unless otherwise specified, shall have a clearance of not more than one-sixteenth inch in drilled or punched holes and not more than one-eighth inch in cored holes. Holes in solid manganese steel frogs and crossings to be one-fourth inch larger than bolt diameter as specified on plans. Threads must be United States standard, accurately cut within tolerance of best practice for cut threads. Nuts must have a tight fit.

38. Rivets.-Rivets shall be of full diameter called for on plans, and rivet holes shall not be more than one-sixteenth inch greater in diameter. When not otherwise called for by plans or specifications, rivets shall have standard button or cone heads of uniform size for the same size rivet. The heads shall be concentric with the holes. Countersunk rivets shall be flush with the surface and fill the countersink, except that rivets through base plates and tie-plates may have a crown of not more than one-eighth inch below the lower surface of the plate.

39. Fit of fillers, braces, and reinforcing bars.Fillers, except as otherwise called for or permitted by plans and specifications, shall fit closely into the fishing space of the rail and into the fillets of the web for not less than one-half inch below the head and above the base flange. When the raised brand of the rail interferes with fit of filler the brand shall be removed. Fillers shall be grooved or cut to clear rivet heads and boltheads.

Switch braces shall fit the fishing space of the rail when the brace is tight against the shoulder of the switch plate.

Reinforcing bars shall fill the height of the fishing space of the rail.

40. Plates and bars.-All plates must be flat and true to surface. Bars must be straight and of the full size called for.

41. Painting.-No paint, tar, or other covering shall be used unless specified, and, in any case, shall not be applied before final inspection.

42. Welding.-No welding shall be permitted on rails or on surfaces of other parts exposed to wheel wear. Welding in other portions may be permitted if in the judgment of the inspector the strength of the piece is not impaired.

43. Marking. - The finished articles shall be plainly stamped with 3/4-inch figures and letters for identification. The manufacturer's name or initials, section and weight of rail, and month and year of manufacture must be stamped on a rail portion of the structure not exposed to wheel wear and where marking can be plainly seen, or may be stamped on a separate rust-proof plate, riveted to the web of the rail, in which case smaller letters may be used. Frogs must be marked with the frog number. All loose parts or fixtures shall be similarly stamped with suitable size letters and figures, the stamping also to show the numbers of the parts appearing on the plan and detail number, where established. On cast parts all or part of the lettering may be cast on the piece. All heat-treated bolts shall be marked on the head with letter or symbol indicating the manufacturer.

\section{INSPECTION}

44. Material and workmanship shall be at all times subject to inspection by a duly authorized representative of the purchaser. The inspector shall have all reasonable facilities afforded to him by the manufacturer to examine the work during its progress, as well as the finished product, to satisfy himself that the work is manufactured and finished in accordance with these specifications. 
45. All inspection shall be made at the place of manufacture. Tests of material may be made at the expense of the manufacturer if the amount of any particular kind of material is 50 tons or more. If less than 50 tons, the manufacturer shall certify that it is furnished in accordance with this specification; however, if the purchaser desires that a test be made he shall bear the expense of same, unless the material fails to meet the specifications, in which case the manufacturer shall stand the expense of such test. For the rails used in the work the manufacturer, when requested, shall supply the purchaser with a certificate of inspection from the rail manufacturer.

46. The acceptance of any material by an inspector shall not prevent subsequent rejection if found defective after delivery. No guarantee of specific length of service will be required. A claim for defective material and/or workmanship shall cover only such defects as will impair the life of the work.

No claim for free replacement of defective material and/or workmanship shall be recognized unless made promptly and based on defects appearing within three months from date of installation and in any event within two years from date of shipment.

(As provided for in paragraph 3 above, these specifications are accompanied by drawings which form part of the specifications. There are 190 such drawings, covering switches, rail braces, switch stands, frogs, guard rails, crossings, gages and flange ways, double-slip switches, turnouts and crossovers, movable point crossings, and track construction details. Characteristic of these drawings is drawing No. 279, showing A. R. E. A. No. 12 frogs for heavy rails, angle $4^{\circ} 46^{\prime} 19^{\prime \prime}$, rail-bound manganese steel, bolted rigid, spring rail, and solid manganese steel. Part of this drawing is appended as Figure 192.

\section{NOTES TO DRAWING NO. 279}

Frogs on this plan designed for heavy rails (combined width of head and base more than $85 \% 8$ inches but not exceeding $9^{13 / 16}$ inches); also recommended for other heavy rails $61 / 2$ inches high and over.

The uniform tie layout shown is suitable for any type of guard rail, including 6-tie 1-piece designs with spacing 19 to 20 inches, to accommodate which the distances underlined thus (191/2 inches) must be adhered to.

The use of plates on all ties is recommended. When specified the tie-plates shown in dotted lines shall be furnished with frog, in which event railway company shall supply details of splice bars showing spike slotting, if any. Distance $X$ to suit spike slotting.

MANGANESE TRACK SOCIETY, SPECIFICATIONS FOR SWITCHES, FROGS, CROSSINGS, AND GUARD RAILS, TRACK-WORK PLANS, 1929

\section{TRACK WORK}

(See A. R. E. A., given above.)

606.4 TRACK BOLTS, NUTS, AND WASHERS. AMERICAN ELECTRIC RAILWAY ENGINEERING ASSOCIATION, STANDARD SPECIFICATION FOR QUENCHED CARBON-STEEL TRACK BOLTS, SERIAL NO. W21-23, 1923

\section{TRACK BOLTS}

(Identical in substance with A. S. T. M. A50, given below, including the references in A50 to A. S. T. M. A3, B11, and to standard test specimen in standard specifications for steel under 605.0.)
AMERICAN ELECTRIC RAILWAY ENGINEERING ASSOCIATION, STANDARD SPECIFICATION FOR QUENCHED, ALLOYSTEEL TRACK BOLTS, W22-23, 1923

\section{ALLOY-STEEL TRACK BOLTS}

(Identical in substance with A. S. T. M. A51, given below, including the references in A51 to A. S. T. M. A3, A50, B11, and to standard test specimen in standard specifications for steel under 605.0.)

\section{AMERICAN ELECTRIC RAILWAY ENGI- NEERING ASSOCIATION, STANDARD SPECIFICATION FOR LOW CARBON- STEEL TRACK BOLTS, W36-28, 1928}

\section{TRACK BOLTS}

(Identical in substance with A. S. T. M. A76, given below, including the references in A76 to A. S. T. M. A3, A50, and to standard test specimen, and inspection and rejection, in standard specifications for steel under 605.0.)

\section{AMERICAN RAILWAY ENGINEERING ASSOCIATION, SPECIFICATIONS FOR QUENCHED CARBON-STEEL AND ALLOY- STEEL TRACK BOLTS, 1924}

\section{TRACK BOLTS}

(This specification is substantially the same as A. S. T. M. A50, given below, including the references in A50 to paragraphs 3 (b) and 4 in A. S. T. M. $\mathrm{A} 3$, and inspection and rejection in A. S. T. M. B11. It differs from A50 in the following additional requirements:)

\section{PHYSICAL REQUIREMENTS}

1. (a) The bolts shall conform to the following minimum requirements as to tensile properties:

\begin{tabular}{|c|c|c|}
\hline & $\begin{array}{c}\text { Carbon } \\
\text { steel }\end{array}$ & Alloy steel \\
\hline $\begin{array}{l}\text { Ultimate tensile strength........lbs./in. } \\
\text { Yield point } \\
\text { Elongation in } 2 \text { inches.............. }\end{array}$ & $\begin{array}{r}100,000 \\
75,000 \\
10\end{array}$ & $\begin{array}{r}110,000 \\
85,000 \\
12\end{array}$ \\
\hline
\end{tabular}

(b) The yield point shall be determined by the drop of the beam or by the dividers, the method being optional with the inspector and at a crosshead speed not to exceed one-eighth inch per minute. The tensile strength shall be determined at a speed not to exceed $1 \frac{1}{2}$ inches per minute.

(c) The capacity of the nut in developing the full strength of the bolt shall be determined by a strip test. The threads must not strip when the bolt, with nut fully mounted, is tested in tension to its yield point, the load being applied to the head and the outside of the nut.

\section{DESIGN AND TOLERANCE}

2. (a) The nominal size of rolled thread bolts shall be the over-all diameter of the rolled threads.

\section{MARIING}

3. (a) A letter or brand indicating the manufacturer and the initials " $\mathrm{H} T$ " shall be pressed on the head of the bolt when it is formed.

(b) All containers shall be marked by the manufacturer as follows: (1) Name of the manufacturer, (2) material (carbon or alloy steel), (3) size of bolts (diameter and length), and (4) weight. 


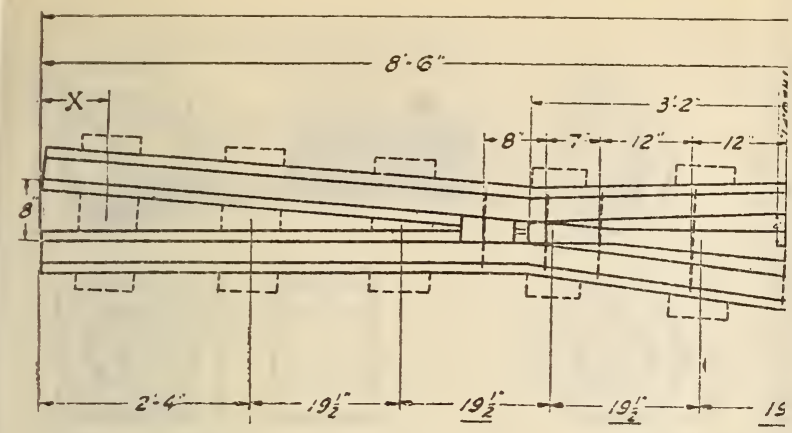

NO. 12 RAILE

FOR RULES FOR

AND ALTERNATE
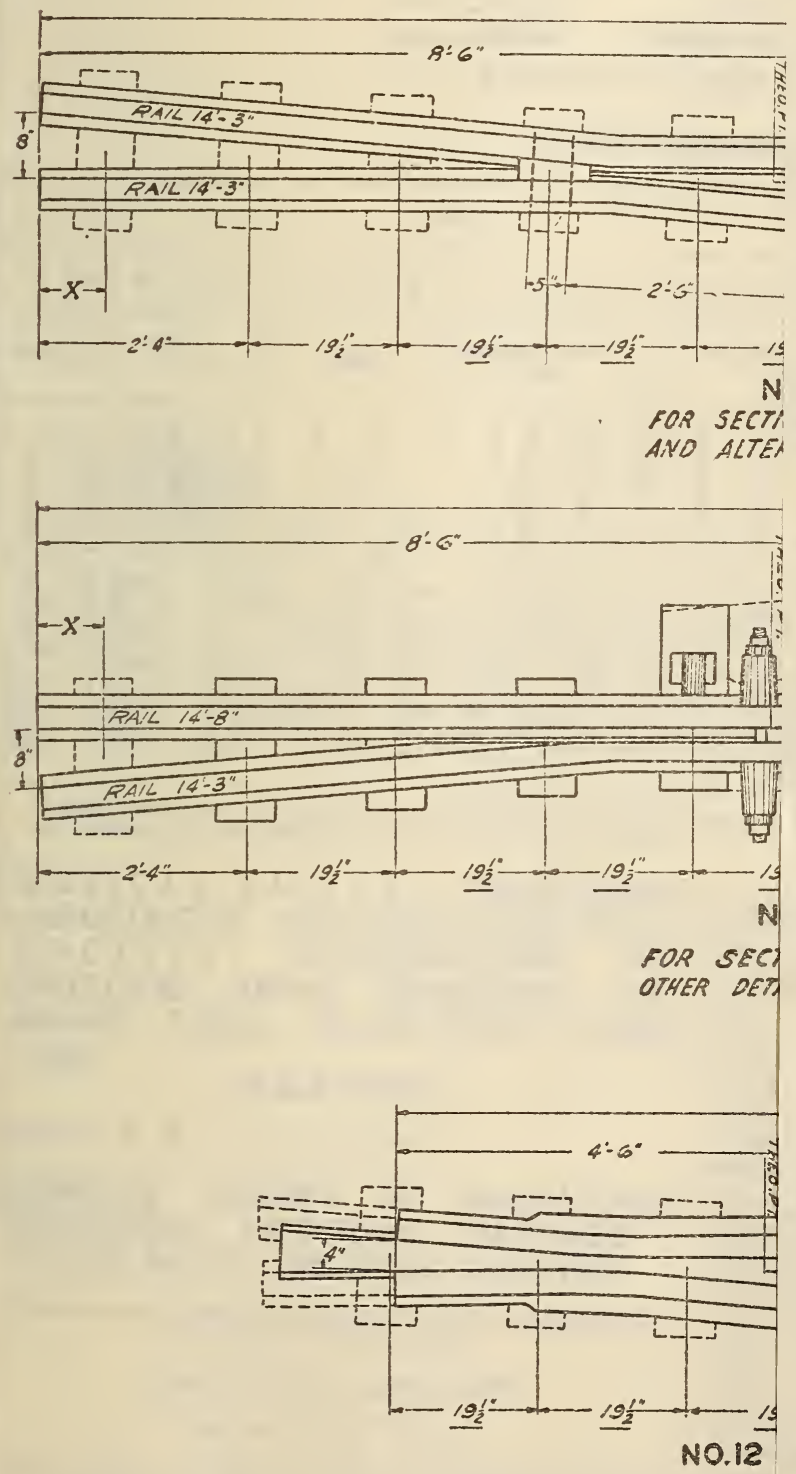

FOR SECTIONS

OTHEA DETA

Figdre 192.-A. R. E. A. No: 12 frogs for heavy rails 
352 

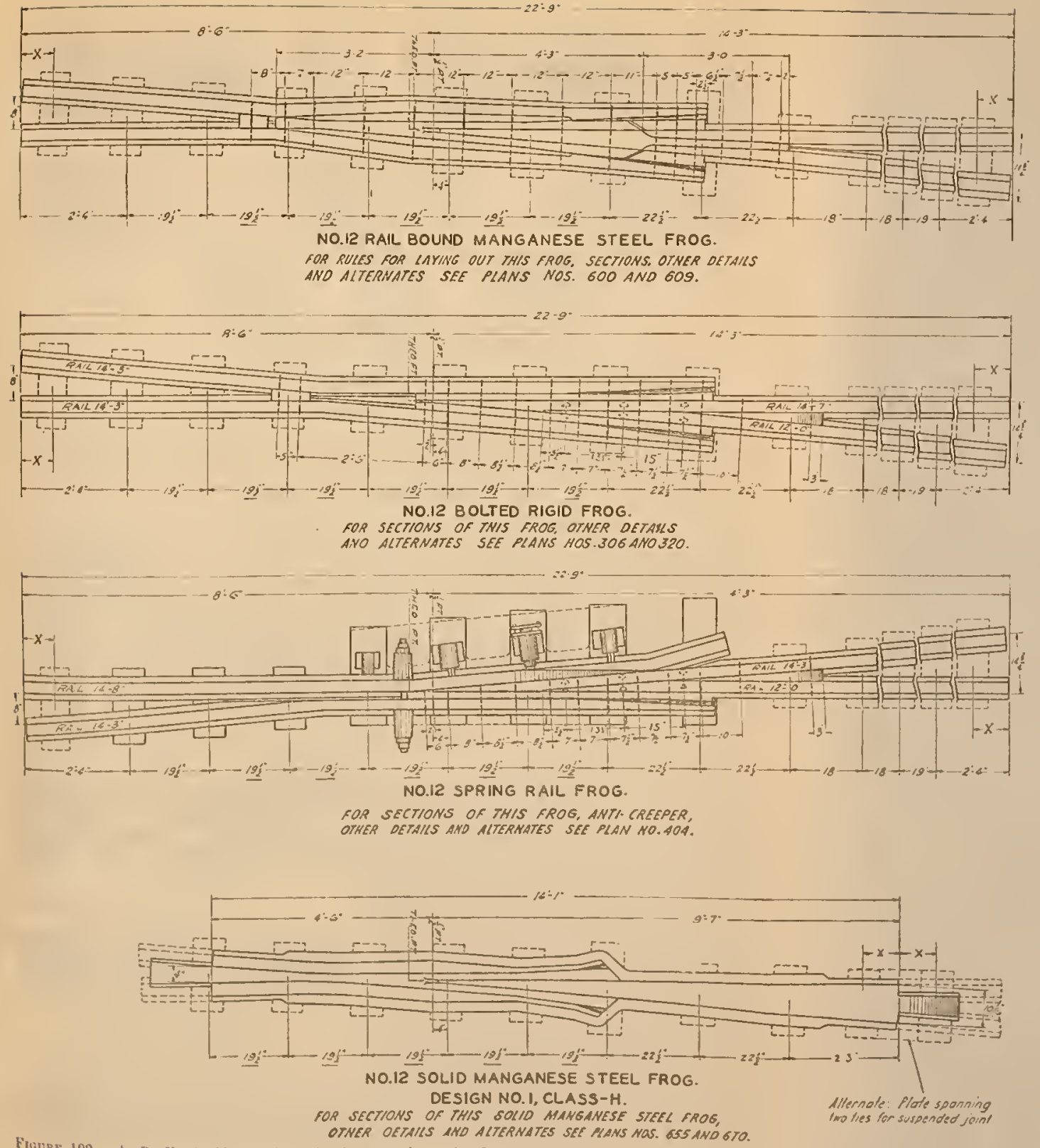

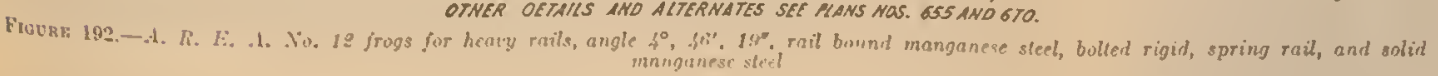




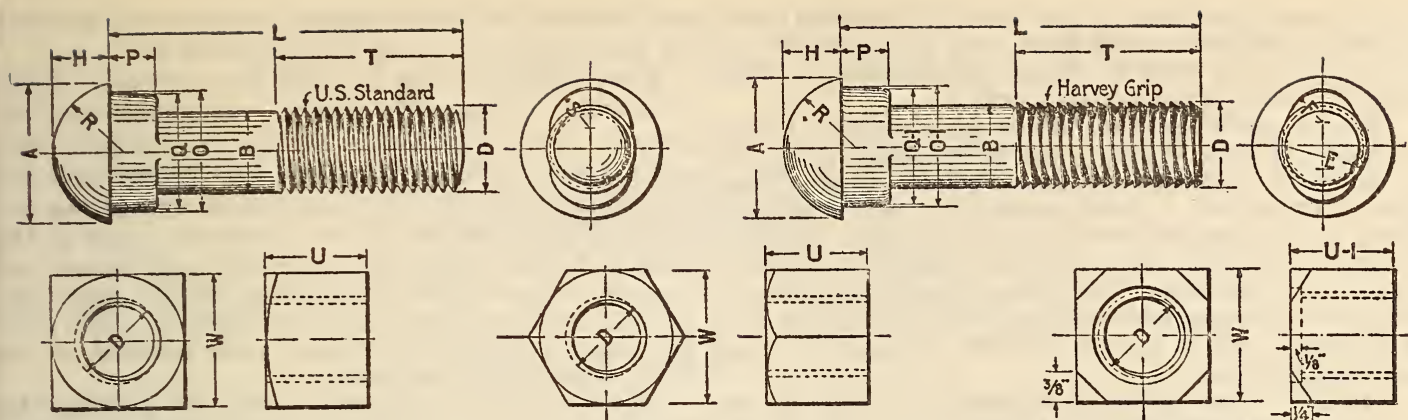

SQUARE

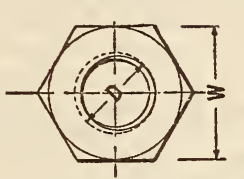

HEXAGON

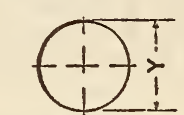

ROUND PUNCHING
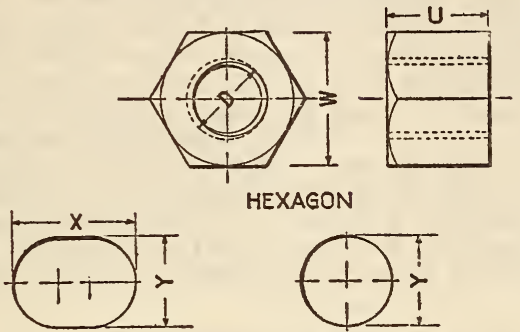

OVAL PUNCHING

FrguRe 193.-Design for track bolts

Table of dimensions (inches)

\begin{tabular}{|c|c|c|c|c|c|c|c|c|c|c|c|c|c|c|c|c|c|c|c|c|c|c|c|}
\hline \multicolumn{16}{|c|}{ Bolt } & \multicolumn{3}{|c|}{ Nut 1} & \multicolumn{5}{|c|}{ Bar punching } \\
\hline D & $\mathrm{N}$ & B & $\mathbf{L}$ & $\mathrm{T}$ & A & H & $\mathbf{R}$ & $\mathrm{s}$ & 0 & $\mathbf{Q}$ & $\mathbf{P}$ & $0-1$ & Q-1 & $\mathbf{E}$ & $\mathbf{F}$ & U & U-1 & w & $\mathrm{X}$ & $\mathbf{Y}$ & $\mathrm{X}-1$ & $\mathrm{E}-1$ & F-1 \\
\hline \multicolumn{2}{|c|}{ Threars } & \multicolumn{3}{|c|}{ Body } & \multicolumn{3}{|c|}{ Head } & \multicolumn{3}{|c|}{$\underset{\text { type }}{\text { Shoulder-oval }}$} & & \multicolumn{4}{|c|}{$\begin{array}{c}\text { Shoulder-elliptic } \\
\text { type }\end{array}$} & \multicolumn{2}{|c|}{ Thickness } & \multirow{2}{*}{ 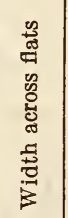 } & \multirow[b]{2}{*}{ 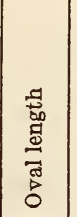 } & \multirow{2}{*}{ 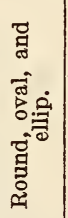 } & \multicolumn{3}{|c|}{ Elliptical } \\
\hline 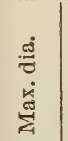 & 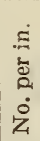 & 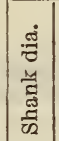 & 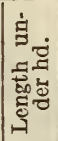 & 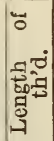 & $\stackrel{\oplus}{\vec{A}}$ & 泾 & تृّ & 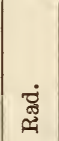 & 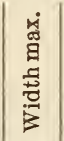 & 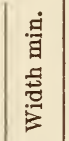 & 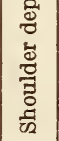 & 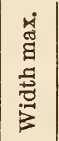 & 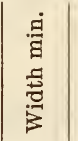 & 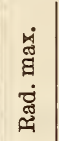 & 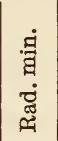 & 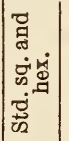 & 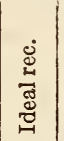 & & & & 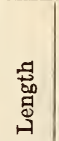 & 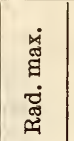 & 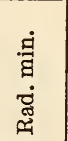 \\
\hline $\begin{array}{l}3 / 4 \\
13 / 16 \\
7 / 8 \\
15 / 16 \\
1 \\
11 / 16 \\
11 / 8 \\
13 / 16 \\
11 / 4\end{array}$ & $\begin{array}{r}10 \\
10 \\
9 \\
9 \\
8 \\
8 \\
7 \\
7 \\
7\end{array}$ & $\begin{array}{l}11 / 16 \\
3 / 4 \\
13 / 16 \\
7 / 8 \\
15 / 16 \\
1 \\
11116 \\
118 \\
13 / 16\end{array}$ & (2) & $\begin{array}{l}13 / 4 \\
13 / 4 \\
2 \\
2 \\
21 / 4 \\
21 / 4 \\
21 / 2 \\
21 / 2 \\
21 / 2\end{array}$ & $\begin{array}{l}11 / 4 \\
111 / 32 \\
17 / 16 \\
117 / 32 \\
15 \% \\
123 / 32 \\
113 / 16 \\
129 / 32 \\
2\end{array}$ & $\begin{array}{l}15 / 32 \\
3364 \\
9 / 16 \\
3964 \\
2132 \\
4564 \\
3 / 4 \\
5164 \\
27 / 32 \\
\end{array}$ & $\begin{array}{c}21 / 32 \\
11 / 16 \\
4764 \\
25 / 32 \\
53 / 64 \\
7 / 8 \\
59 / 64 \\
31 / 32 \\
11 / 64\end{array}$ & $\begin{array}{l}11 / 32 \\
3 / 8 \\
13 / 32 \\
7 / 16 \\
15 / 32 \\
1 / 2 \\
17 / 32 \\
9 / 16 \\
19 / 32\end{array}$ & $\begin{array}{l}11 / 16 \\
1964 \\
17 / 32 \\
11964 \\
13 / 8 \\
12964 \\
11732 \\
13964 \\
11116\end{array}$ & $\begin{array}{l}11 / 32 \\
1764 \\
1316 \\
11 / 64 \\
11 / 32 \\
12764 \\
11 / 2 \\
13764 \\
121 / 32\end{array}$ & \begin{tabular}{|c}
$7 / 16$ \\
$15 / 32$ \\
112 \\
$17 / 32$ \\
$9 / 16$ \\
$19 / 32$ \\
$5 / 8$ \\
$21 / 32$ \\
$11 / 16$
\end{tabular} & \begin{tabular}{|l|}
$11 / 16$ \\
118 \\
$13 / 16$ \\
$11 / 4$ \\
138 \\
$17 / 16$ \\
$11 / 2$ \\
$19 / 16$ \\
$15 / 8$
\end{tabular} & $\begin{array}{l}11 / 32 \\
13 / 32 \\
15 / 32 \\
17 / 32 \\
111 / 32 \\
113 / 32 \\
115 / 32 \\
117 / 32 \\
119 / 32\end{array}$ & $\begin{array}{l}21 / 32 \\
11 / 16 \\
23 / 32 \\
3 / 4 \\
15 / 16 \\
31 / 32 \\
1 \\
11 / 32 \\
11 / 16\end{array}$ & $\begin{array}{l}7 / 32 \\
1 / 4 \\
9 / 32 \\
5 / 16 \\
3 / 8 \\
13 / 32 \\
7 / 16 \\
15 / 32 \\
1 / 2\end{array}$ & \begin{tabular}{|l|}
1 \\
1 \\
$11 / 8$ \\
118 \\
$11 / 4$ \\
114 \\
138 \\
138 \\
$11 / 2$
\end{tabular} & $\begin{array}{l}116 \\
118 \\
1114 \\
113 \\
138 \\
138 \\
113 \\
112 \\
158\end{array}$ & $\begin{array}{l}11 / 4 \\
11 / 4 \\
17 / 10 \\
1716 \\
15 / 8 \\
15 / 8 \\
113 / 16 \\
113 / 16 \\
2\end{array}$ & $\begin{array}{l}13 / 32 \\
11164 \\
11 / 4 \\
1^{21} 164 \\
13 / 32 \\
131 / 64 \\
196 \\
111 / 64 \\
123 / 32\end{array}$ & $\begin{array}{l}13 / 16 \\
7 / 8 \\
15 / 16 \\
1 \\
11 / 16 \\
118 \\
13 / 16 \\
11 / 4 \\
15 / 16\end{array}$ & $\begin{array}{l}11 / 16 \\
118 \\
13 / 16 \\
114 \\
13 / 8 \\
17 / 16 \\
112 \\
1916 \\
15 \% 8\end{array}$ & $\begin{array}{l}5 / 8 \\
21 / 32 \\
11 / 16 \\
23 / 32 \\
7 / 8 \\
29 / 32 \\
15 / 16 \\
31 / 32 \\
1\end{array}$ & $\begin{array}{l}5 / 16 \\
11 / 32 \\
3 / 8 \\
13 / 32 \\
15 / 32 \\
1 / 2 \\
17 / 32 \\
9 / 16 \\
19 / 32\end{array}$ \\
\hline
\end{tabular}

1 When other than heat treated bolts are used nut to be $1 / 4$ inch less in thickness.

2 As specified oil quenched to be $1 / 4$ inch longer than common.

A MI ERICA N RAIL W A Y ENGINEERING ASSOCIATION AND MANGANESE TRACK SOCIETY, SPECIFICATIONS FOR SWITCHES, FROGS, CROSSINGS, AND GUARD RAILS, TRACK-WORK PLANS, 1929

\section{TRACK WORK}

(See A. R. E. A., 606.3, p. 349.)

\section{AMERICAN SOCIETY OF MECHANICAL ENGINEERS, AMERICAN STANDARD TRACK BOLTS AND NUTS, B18d-1930}

[Approved as American standard by the American Standards Association, November, 1930]

\section{TRACK BOLTS AND NUTS}

\section{INTRODJCTORY NOTES}

Sizes.-The designation for standard track bolts is considered as the nominal diameter over the thread. The sizes vary by $1 / 8$-inch steps, but as large quantities of certain $1 / 16$-inch sizes are in general use, dimensions are included for these $1 / 16$-inch sizes. This last-named group is designated as "special sizes for use during transition period," and are for replacements in present splice bars. On new construction the $1 / 8$-inch sizes should be used so that the standard sizes may come into general use as quickly as possible.

Heads.-Users of track bolts expressed a preference for keeping the size of the head as small as possible, and still they wish to insure that the full strength of the thread could be developed. A type of head commonly referred to as of round or button type, in which a section has the top formed to a single radius, was considered first. Tests showed that a stronger head of the same diameter would result by increasing the thickness of metal at the edges. This has been done in the present standards by use of what has been called a mushroom-type head, in which a section has the top formed by three radii. Tests with this type of head showed that the full strength of the thread could be developed without shearing off the edges of the head.

Necks.-The types of track bolts are designated as "oval neck" and "elliptic neck." The oval-neck type is in most general use, but some large systems 
have found advantages in the use of the elliptic neck for their particular conditions, and this type is included to take care of their requirements. The heads for both types are the same.

Body diameters. - The tables of standard track bolts do not have the body diameter designated due to different methods of manufacture used. The type of track bolt in most general use has a thread rolled to a larger diameter than the body. Bodies of these bolts are approximately one-sixteenth inch smaller in diameter than the threaded portion. When cut threads are used the body diameter is approximately equal to the outside diameter of the threaded portion.

Threads.-Two types of threads are referred to, viz, the American National Standard with fit as specified by the customer and the Harvey grip thread. The latter has an included angle of $55^{\circ}$, but the angles wnich the two faces of the thread make with a line perpendicular to the axis of the bolt are not equal, one being $10^{\circ}$ the other $45^{\circ}$.

Nuts.-Three types of nuts are included in the standards. These are square, hexagonal, and recessed. Two thicknesses are given, common and extra thick. The width across flats agrees with the American standard for rough square and hexagonal nuts. The thickness of the "common" nut is the same as for the American standard rough square and hexagonal nuts, and the "extra thick" and "recessed" nuts are one-fourth inch thicker. The present general practice is to have nuts screwed on the track bolts before shipping.

Material.-The American Society for Testing Materials has three specifications that appear satisfactory. These cover low-carbon steel track bolts, quenched carbon-steel track bolts, and quenched alloy-steel track bolts-all of which are given below.

TABLE 1.-Oval neck track bolts

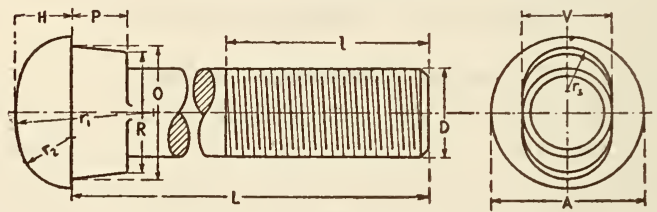

Figure 194.-Oval neck track bolt

[All dimensions are given in inches unless otherwise noted]

\begin{tabular}{|c|c|c|c|c|c|c|c|c|c|c|c|c|c|c|}
\hline$D$ & $\boldsymbol{A}$ & $H$ & $r_{1}$ & $r_{2}$ & $O$ & $R$ & $P$ & $r_{8}$ & $V$ & $E$ & $K$ & $L$ & $\iota$ & \\
\hline $\begin{array}{l}\text { Nomi- } \\
\text { nal di- } \\
\text { ameter } \\
\text { over } \\
\text { thread }\end{array}$ & \multicolumn{4}{|c|}{ Head } & \multicolumn{5}{|c|}{ Neck } & $\begin{array}{l}\text { Bear- } \\
\text { ing } \\
\text { area } 1\end{array}$ & $\begin{array}{c}\text { Shear- } \\
\text { ing } \\
\text { area }^{2}\end{array}$ & $\begin{array}{l}\text { Length } \\
\text { under } \\
\text { head }\end{array}$ & $\begin{array}{l}\text { Mini- } \\
\text { mum } \\
\text { thread } \\
\text { length }\end{array}$ & $\begin{array}{l}\text { Threads } \\
\text { per inch }\end{array}$ \\
\hline \multicolumn{15}{|c|}{ SPECIAL SIZES, FOR USE DURING TRANSITION PERIOD } \\
\hline $\begin{array}{c}7 / 16 \\
9 / 16 \\
11 / 16 \\
13 / 16 \\
15 / 16 \\
11 / 16\end{array}$ & $\begin{array}{c}25 / 32 \\
63 / 64 \\
13 / 16 \\
125 / 64 \\
119 / 32 \\
151 / 64\end{array}$ & $\begin{array}{l}9 / 32 \\
23 / 64 \\
7 / 16 \\
33 / 64 \\
19 / 32 \\
43 / 64\end{array}$ & $\begin{array}{l}37 / 64 \\
13 / 16 \\
13 / 64 \\
19 / 32 \\
133 / 64 \\
13 / 4\end{array}$ & $\begin{array}{l}1 / 4 \\
21 / 64 \\
13 / 32 \\
31 / 64 \\
9 / 16 \\
4164\end{array}$ & $\begin{array}{c}35 / 64 \\
45 / 64 \\
63 / 64 \\
19 \% 64 \\
11964 \\
129464\end{array}$ & $\begin{array}{c}33 / 64 \\
43 / 64 \\
61 / 64 \\
17 / 64 \\
11764 \\
127,64\end{array}$ & $\begin{array}{l}9 / 32 \\
11 / 32 \\
13 / 32 \\
1532 \\
17 / 32 \\
19 / 32\end{array}$ & $\begin{array}{l}\text { One-half } \\
\text { body di- } \\
\text { ameter } \\
\text { of bolt. }\end{array}$ & $\begin{array}{c}\text { Same } \\
\text { as } \\
\text { body } \\
\text { diame- } \\
\text { ter of } \\
\text { bolt. }\end{array}$ & $\begin{array}{r}0.227 \\
.364 \\
.532 \\
.734 \\
.968 \\
1.191\end{array}$ & $\begin{array}{r}0.35 \\
.58 \\
.88 \\
1.23 \\
1.63 \\
2.12\end{array}$ & $\begin{array}{l}\text { Length } \\
\text { as speci- } \\
\text { fied. }\end{array}$ & $\begin{array}{l}11 / 16 \\
13 / 16 \\
15 / 8 \\
17 / 8 \\
21 / 8 \\
23 / 8\end{array}$ & $\begin{array}{r}14 \\
12 \\
11 \\
10 \\
9 \\
8\end{array}$ \\
\hline
\end{tabular}

1 Bearing area is equal to the area of bolthead minus the area of the oval hole in the splice bar.

2 Shear area is equal to the periphery of the oval hole in the splice bar times the mean height of the head above the bearing surface.

Screw threads. - The screw threads on track bolts shall conform to the American national standard form, with fit as specified, or have the Harvey grip thread. They may be formed by cutting, drawing, or rolling.

Tolerances-Length $(L)$ plus or minus 1/8 inch. Neck $(V, O$, and $R$ ) plus or minus $1 / 32$ inch. Head $(A$ and $H$ ) plus or minus $1 / 16$ inch.
$A=15 / 8 D+1 / 16$.

FORMULAS

$H=58 D$.

$r_{1}=17 / 8 \dot{D}-1 / 4$

$0=\{11 / 4 D$ (for $7 / 16$ to $5 / 8$ inch inclusive)

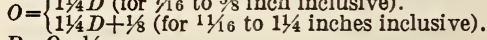
$R=O-1 / 32$
$P=1 / 2 D+1 / 1$ 
TABLE 2.-Elliptic neck track bolts

SPECIAL TYPE AND SIZES, FOR USE DURING TRANSITION PERIOD
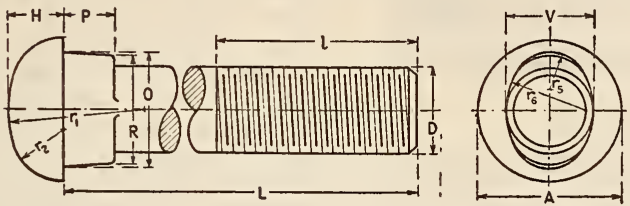

FIGURE 195.-Elliptic neck track bolt

[All dimensions are given in inches]

\begin{tabular}{|c|c|c|c|c|c|c|c|c|c|c|c|c|c|}
\hline$D$ & $A$ & $H$ & $r_{1}$ & $r_{2}$ & 0 & $R$ & $P$ & $r_{5}$ & $r_{0}$ & $V$ & $L$ & $l$ & \\
\hline $\begin{array}{l}\text { Nomi- } \\
\text { nal di- } \\
\text { ameter } \\
\text { over } \\
\text { thread }\end{array}$ & \multicolumn{4}{|c|}{ Head } & \multicolumn{6}{|c|}{ Neck } & $\begin{array}{l}\text { Length under } \\
\text { head }\end{array}$ & $\begin{array}{l}\text { Mini- } \\
\text { mum } \\
\text { thread } \\
\text { length }\end{array}$ & $\begin{array}{l}\text { Threads } \\
\text { per inch }\end{array}$ \\
\hline $\begin{array}{l}3 / 4 \\
13 / 16 \\
7 / 8 \\
15 / 16 \\
1118 \\
114\end{array}$ & $\begin{array}{l}19 / 32 \\
125,64 \\
13164 \\
119 / 32 \\
111 / 16 \\
153 / 64 \\
23 / 32\end{array}$ & $\begin{array}{l}15 / 32 \\
33 / 34 \\
35 \% 4 \\
19 / 32 \\
5 / 3 \\
45 / 64 \\
25 / 32\end{array}$ & $\begin{array}{l}15 / 32 \\
1 \% 32 \\
12525 \\
13364 \\
158 \\
15564 \\
23 / 32\end{array}$ & $\begin{array}{l}7 / 16 \\
3164 \\
3364 \\
916 \\
19 / 32 \\
4364 \\
3 / 4\end{array}$ & $\begin{array}{l}63 / 64 \\
11 / 8 \\
13 / 6 \\
17 / 32 \\
13 / 8 \\
11 / 2 \\
15 \%\end{array}$ & $\begin{array}{l}61 / 64 \\
13 / 32 \\
15 / 32 \\
13 / 16 \\
111 / 32 \\
115 / 32 \\
11 \% / 32\end{array}$ & $\begin{array}{l}7 / 16 \\
15,32 \\
132 \\
17 / 32 \\
9916 \\
5,8 \\
11 / 16\end{array}$ & $\begin{array}{l}9 / 32 \\
1964 \\
5 / 16 \\
2364 \\
3,8 \\
7 / 16 \\
1 / 2\end{array}$ & $\begin{array}{l}4164 \\
2532 \\
5162 \\
27 / 32 \\
15 / 16 \\
11 / 16 \\
11 / 16\end{array}$ & $\begin{array}{l}\text { Same as } \\
\text { body di- } \\
\text { ameter of } \\
\text { bolt. }\end{array}$ & $\begin{array}{l}\text { Length } 3 \text { inches } \\
\text { to } 6 \text { inches by } \\
\text { steps of } 14 \text { inch } \\
\text { and } 6 \text { inches to } \\
10 \text { inches by } \\
\text { steps of } 1 / 2 \text { inch. }\end{array}$ & $\begin{array}{l}13 / 4 \\
13 / 4 \\
2 \\
2 \\
21 / 4 \\
21 / 2 \\
21 / 2\end{array}$ & $\begin{array}{r}10 \\
10 \\
9 \\
9 \\
8 \\
7 \\
7\end{array}$ \\
\hline
\end{tabular}

Tolerances-Length $(L)$ plus or minus $1 / 8$ inch.

Neck $(V, O$, and $R$ ) plus or minus $1 / 32$ inch.

Screw threads. - The screw threads on track bolts shall have either the American national standard form with fit as specified or have the Harvey grip thread. They may be formed by cutting, drawing, or rolling.

TABLE 3.-Square and hexagonal nuts for track bolts

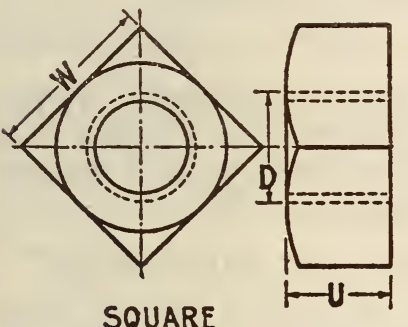

SQUARE

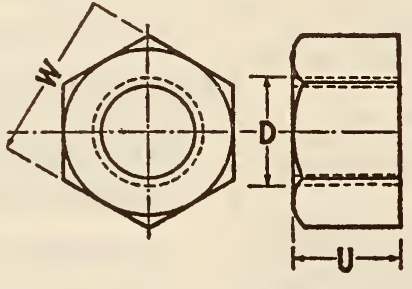

HEXAGONAL

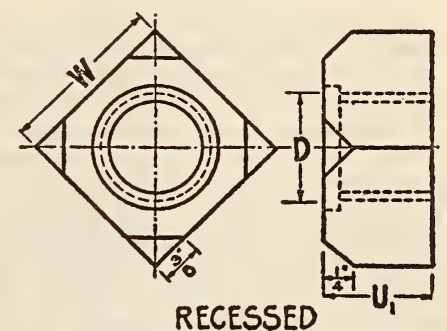

RECESSED

FiguRE 196.-Square and hexagonal nuts for track bolts

[All dimension are given in inches]

\begin{tabular}{|c|c|c|c|c|c|c|c|c|c|c|c|c|c|}
\hline \multirow{3}{*}{\multicolumn{2}{|c|}{$\begin{array}{c}\text { Diameter of } \\
\text { bolt } \\
\text { D }\end{array}$}} & \multicolumn{3}{|c|}{$\begin{array}{l}\text { Width across flats } \\
\text { W }\end{array}$} & \multirow{2}{*}{\multicolumn{3}{|c|}{$\begin{array}{c}\begin{array}{c}\text { Thickness } \\
\text { U }\end{array} \\
\text { Common }^{8}\end{array}$}} & \multicolumn{6}{|c|}{$\begin{array}{c}\text { Thickness }^{2} \\
U_{1}\end{array}$} \\
\hline & & \multirow{2}{*}{\multicolumn{2}{|c|}{ Maximum }} & \multirow{2}{*}{$\begin{array}{l}\text { Mini- } \\
\text { mum }\end{array}$} & & & & \multicolumn{3}{|c|}{ Extra thick } & \multicolumn{3}{|c|}{ Recessed 4} \\
\hline & & & & & $\begin{array}{l}\text { Nomi- } \\
\text { nal }\end{array}$ & $\begin{array}{l}\text { Maxi- } \\
\text { mum }\end{array}$ & $\begin{array}{l}\text { Mini- } \\
\text { mum }\end{array}$ & $\begin{array}{c}\text { Nomi. } \\
\text { nal }\end{array}$ & $\begin{array}{l}\text { Maxi- } \\
\text { mum }\end{array}$ & $\begin{array}{l}\text { Mini- } \\
\text { mum }\end{array}$ & $\begin{array}{l}\text { Nomi- } \\
\text { nal }\end{array}$ & $\begin{array}{l}\text { Maxi- } \\
\text { mum }\end{array}$ & $\begin{array}{l}\text { Mini- } \\
\text { mum }\end{array}$ \\
\hline $\begin{array}{l}1 / 2 \\
5 / 8 \\
3 / 4 \\
7 / 8 \\
1 \\
11 / 8 \\
114\end{array}$ & $\begin{array}{r}0.5000 \\
.6250 \\
.7500 \\
.8750 \\
1.0000 \\
1.1250 \\
1.2500\end{array}$ & $\begin{array}{l}13 / 16 \\
1 \\
11 / 8 \\
15 / 16 \\
11 / 2 \\
111 / 16 \\
17 / 8\end{array}$ & $\begin{array}{l}0.8125 \\
1.0000 \\
1.1250 \\
1.3125 \\
1.5000 \\
1.6875 \\
1.8750\end{array}$ & $\begin{array}{r}0.788 \\
.969 \\
1.088 \\
1.269 \\
1.450 \\
1.631 \\
1.813\end{array}$ & $\begin{array}{l}7 / 16 \\
35 / 64 \\
21 / 32 \\
49 / 64 \\
7 / 8 \\
1 \\
13 / 32\end{array}$ & $\begin{array}{r}0.458 \\
.569 \\
.680 \\
.792 \\
.903 \\
1.030 \\
1.126\end{array}$ & $\begin{array}{r}0.418 \\
.525 \\
.632 \\
.740 \\
.847 \\
.970 \\
1.062\end{array}$ & $\begin{array}{l}11 / 16 \\
5,1 / 64 \\
29 / 32 \\
11 / 64 \\
11 / 8 \\
11 / 4 \\
111 / 32\end{array}$ & $\begin{array}{r}0.708 \\
.819 \\
.930 \\
1.042 \\
1.153 \\
1.280 \\
1.376\end{array}$ & $\begin{array}{r}0.668 \\
.775 \\
.882 \\
.990 \\
1.097 \\
1.220 \\
1.312\end{array}$ & $\begin{array}{l}11 / 16 \\
51 / 64 \\
29 / 32 \\
11 / 64 \\
11 / 8 \\
11 / 4 \\
111 / 32\end{array}$ & $\begin{array}{r}0.708 \\
.819 \\
.930 \\
1.042 \\
1.153 \\
1.280 \\
1.376\end{array}$ & $\begin{array}{r}0.668 \\
.775 \\
.882 \\
.990 \\
1.097 \\
1.220 \\
1.312\end{array}$ \\
\hline \multicolumn{14}{|c|}{ SPECIAL SIZES, FOR USE DURING TRANSITION PERIOD } \\
\hline $\begin{array}{l}7 / 16 \\
9 / 16 \\
11 / 16 \\
13 / 16 \\
15 / 16 \\
11 / 16\end{array}$ & $\begin{array}{r}0.4375 \\
.5625 \\
.6875 \\
.8125 \\
.9375 \\
1.0625\end{array}$ & $\begin{array}{l}3 / 4 \\
7 / 8 \\
11 / 8 \\
15 / 16 \\
11 / 2 \\
111 / 16\end{array}$ & $\begin{array}{l}0.7500 \\
.8750 \\
1.1250 \\
1.3125 \\
1.5000 \\
1.6875\end{array}$ & $\begin{array}{r}0.728 \\
.847 \\
1.088 \\
1.269 \\
1.450 \\
1.631\end{array}$ & $\begin{array}{l}3 / 8 \\
1 / 2 \\
21 / 32 \\
496 \\
7 / 8 \\
1\end{array}$ & $\begin{array}{r}0.394 \\
.521 \\
.680 \\
.792 \\
.903 \\
1.030\end{array}$ & $\begin{array}{l}0.356 \\
.479 \\
.632 \\
.740 \\
.847 \\
.970\end{array}$ & $\begin{array}{l}5 / 8 \\
3 / 4 \\
29 / 32 \\
11 / 64 \\
11 / 8 \\
11 / 4\end{array}$ & $\begin{array}{r}0.644 \\
.771 \\
.930 \\
1.042 \\
1.153 \\
1.280\end{array}$ & $\begin{array}{r}0.606 \\
.729 \\
.882 \\
.990 \\
1.097 \\
1.220\end{array}$ & $\begin{array}{l}5 / 8 \\
3 / 4 \\
29 / 32 \\
11 / 64 \\
11 / 8 \\
11 / 4\end{array}$ & $\begin{array}{r}0.644 \\
.771 \\
.930 \\
1.042 \\
1.153 \\
1.280\end{array}$ & $\begin{array}{r}0.606 \\
.729 \\
.882 \\
.990 \\
1.097 \\
1.220\end{array}$ \\
\hline
\end{tabular}

1 The tolerance for the width across flats (W) shall be minus $0.050 \mathrm{D}$ inches.

The tolerance for the thickness of nuts ( $U$ and $U_{1}$ ) shall be plus or minus $(0.016 D+0.012)$ inches.

American standard rough square and hexagonal nuts.

4 The recessed type of nut shall have a recess of $1 / 8$ inch minimum and $3 / 16$ inch maximum.

Note.-Formulas for the width across flats are the same as given in the American standard wrench head bolts and nuts. For special sizes, the width across fiats is the same as for the standard of $1 / 16$ inch greater bolt diameter. 
AMERICAN SOCIETY FOR TESTING MATERIALS, STANDARD SPECIFICATIONS FOR QUENCHED, CARBON-STEEL TRACK BOLTS, DESIGNATION A50-24, 1924

\section{CARBON-STEEL TRACK BOLTS}

\section{MANUFACTURE}

1. (a) The steel for the bolts shall be made by the open-hearth process.

(b) The steel for the nuts shall be made by either or both the following processes: Bessemer or openhearth.

2. The bolts shall enter the quenching medium at a temperature not less than $790^{\circ} \mathrm{C}$. The threads may be rolled either hot or cold.

\section{CHEMICAL PROPERTIES AND TESTS}

3. The steel for the bolts shall conform to the following requirements as to chemical composition:

Carbon, not under Per cent

Phosphorus, not over 0.30

(Ladle and check analyses are identical with paragraphs $3(a), 3(b)$, and 4 in A. S. T. M. A3. See 606.2 , p. 331.)

\section{PEYSICAL PROPERTIES AND TESTS}

6. (a) The bolts shall conform to the following minimum requirements as to tensile properties:

Tensile strength

Yield point lbs./in. ${ }^{2}--100,000$

70,000

Elongation in 2 inches.................... per cent.- 12

(b) Nuts shall be capable of developing the strength of the finished bolt up to its yield point.

(c) The yield point shall be determined by the drop of the beam of the testing machine.

7. Full-size bolts shall stand being bent cold through $45^{\circ}$ around a pin the diameter of which is equal to the diameter of the bolt, without cracking on the outside of the bent portion.

8. Tension-test specimens shall be taken from the finished bolts and shall conform to the dimensions shown in Figure 129 in A. S. T. M. standard specifications for metals. (See 605.0, p. 247.) The ends shall be of a form to fit the holders of the testing machine in such a way that the load shall be axial.

9. (a) One tension and one bend test shall be made from each lot of 50 kegs or fraction thereof.

(b) If any test specimen shows defective machining or develops flaws, it may be discarded and another specimen substituted.

(c) If the percentage of elongation of any tensiontest specimen is less than that specified in section $6(a)$ and any part of the fracture is more than three-fourths inch from the center of the gage length, as indicated by scribe scratches marked on the specimen before testing, or if the bend-test specimen breaks in the threaded portion, a retest shall be allowed.

10. If the results of the physical tests of any test lot do not conform to the requirements specified, the manufacturer may re-treat such lot one or more times in which case two additional tension and two additional bend tests shall be made from such lot, all of which shall conform to the requirements specified.

\section{PERMISSIBLE VARIATIONS}

11. The bolts and nuts shall conform to the dimensions specified by the purchaser subject to the following variations: A variation of one thirtysecond inch under and one sixty-fourth inch over the specified diameter of the shank of the bolt will be permitted. The diameter of rolled thread shall not exceed the diameter of the shank more than one-sixteenth inch for bolts seven-eighths inch in diameter and under, nor more than three thirtysecond inch for bolts 1 inch in diameter and over. The outside diameter of the finished rolled threads shall not exceed the diameter of the shank of a cutthread bolt of corresponding size. The length of the bolt under the head shall not vary more than one-eighth inch from that specified. A variation in the dimensions of the elliptical shoulders under the head of one thirty-second inch and a taper of the shoulder of one thirty-second inch will be permitted.

\section{WORKIIANSHIP AND FINISH}

12. The bolts and nuts shall be neatly formed and free from fins or nicking. The head of the bolt shall be concentric with and firmly joined to the shank, with the underside at right angles to the axis of the bolt. The threads shall be sharp and true to gage and of the design specified by the purchaser. The nuts shall have a hand free fit on the bult from two to six turns, and wrench tight the balance of the screw length without distorting the threads or twisting the shank. They shall be screwed on the bolts before packing, a sufficient number of turns to hold them in place until used.

13. The finished bolts and nuts shall be free from injurious defects and shall have a workmanlike finish.

\section{MARKING}

14. A latter or brand indicating the manufacturer shall be pressed on the head of the bolt while it is being formed.

\section{INSPECTION AND REJECTION}

(Conform to A. S. T. M. B11. See 641.21, p. 1176.)

\section{AMERICAN SOCIETY FOR TESTING MATE- RIALS, STANDARD SPECIFICATIONS FOR QUENCHED ALIOY-STEEL TRACK BOLTS, DESIGNATION A51-21, 1921}

\section{QUENCHED ALLOY-STEEL TRACK BOLTS}

(This specification is identical in form with A. S. T. M. A50, given above, including references in A50 to A. S. T. M. A3, paragraphs $3(b)$ and 4; standard test specimen in A. S. T. M. standard specifications for steel under 605.0; and inspection and rejection in B11. It differs from $\mathrm{A} 50$ in the following particulars:)

\section{MANUFACTURE}

1. (a) The steel for the bolts shall be made by either or both the following processes: Open hearth or electric furnace.

\section{CHENICAI PROFERTIES}

2. The steel for the bolts shall conform to the following requirements as to chemical compositions:

Phosphorus, not over-10r 0.035

\section{PEYSICAL PROPERTIES AND TESTS}

3. (a) The bolts shall conform to the following minimum requirements as to tensile properties:

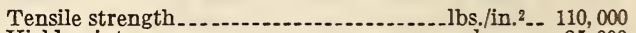
Yield point Elongation in 2 inches..- 12

4. Full-size bolts shall stand being bent cold through $90^{\circ}$ around a pin the diameter of which is equal to the diameter of the bolt, without cracking on the outside of the bent portion. 
AMERICAN SOCIETY FOR TESTING MATERIALS, STANDARD SPECIFICATIONS FOR LOW-CARBON-STEEL TRACK BOLTS, DESIGNATION A76-27, 1927

\section{LOW-CARBON-STEEL TRACK BOLTS}

\section{MAN OFACTURE}

1. (a) The steel shall be made by either or both the following processes: Bessemer or open hearth.

(b) The threads may be cut or rolled.

\section{CHEMICAL PROPERTIES AND TESTS}

2. (a) The steel for the bolts shall conform to the following requirements as to chemical composition:

Phosphorus: Per cent

Bessemer, not over 0.10

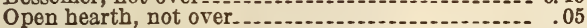

(b) The steel for the nuts shall be soft steel.

(Ladle and check analyses are identical with paragraphs $3(a), 3(b)$, and 4 in A. S. T. M., A3. See 606.2, p. 331.)

\section{PHYSICAL PROPERTIES AND TESTS}

5. The stock from which the bolts are made shall conform to the following requirements as to tensile properties:

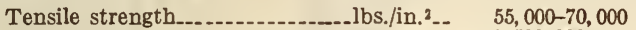

Elongation in 8 inches, minimum. per cent.- $1,500,000$

Elongation in 2 inches, minimum...do.... Tensile strength

6. The test specimen shall stand being bent cold through $180^{\circ}$ flat on itself without cracking on the outside of the bent portion.

7. Tension and bend test specimens shall be taken from a finished bolt or from the full-size rolled bars. Tension-test specimens shall be of 8-inch gage lengtn when taken from the rolled bars. When taken from a finished bolt the tension-test specimen shall conform to the dimensions shown in Figure 129 in A. S. T. M., standard specifications for metals. (See 605.0, p. 247.)

8. (a) One tension and one bend test shall be made from each melt of steel. When the melts can not be identified, one tension and one bend test shall be made from each lot of 10 tons or fraction thereof.

(b) If any test specimen develops flaws, it may be discarded and another specimen substituted.

(c) If the percentage of elongation of any tensiontest specimen is less than that specified in section 5 and any part of the fracture is outside the middle third of the gage length, as indicated by scribe scratches marked on the specimen before testing, a retest shall be allowed.

(Retests, permissible variations, workmanship and finish, and marking are the same as in A. S. T. M., A50, given above, with the addition of the following requirement as to marking:)

Bolts shall be packed in serviceable packages. All packages shall be plainly marked as to material, size of bolts, and name of manufacturer.

\section{INSPECTION AND REJECTION}

(Conform to A. S. T. M. standard specifications for steel. See 605.0 p. 247.)
606.5 TRACK SPIKES.

AMERICAN ELECTRIC RAILWAY ENGINEERING ASSOCIATION, STANDARD SPECIFICATION FOR STEEL SCREW SPIKES, W24-23, 1923

\section{STEEL SCREW SPIKES}

(Identical in substance with A. S. T. M., A6621, given below, including references in A66 to A. S. T. M. A65 and B11.)

\section{ANERICAN ELECTRIC RAILWAY ENGI- NEERING ASSOCIATION, STANDARD SPECIFICATION FOR STEEL TRACK SPIKES, W25-30, 1930}

\section{STEEL TRACK SPIKES}

(Identical in substance with A. S. T. M., A65$26 \mathrm{~T}$, given below, including reference in $\mathrm{A} 65$ to A. S. T. M., B11.)

\section{AMERICAN ELECTRIC RAILWAY ENGI- NEERING ASSOCIATION, STANDARD DESIGN FOR 51/2 BY 9116 INCH TRACK SPIKE, W41-30, 1930}

\section{STANDARD TRACK SPIKE}

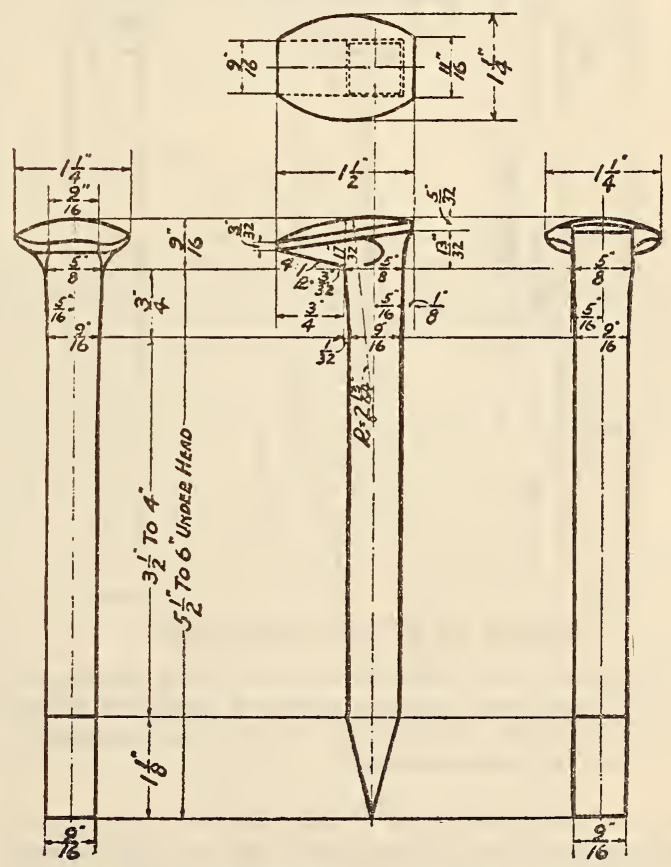

FIGURE 197.-Recommended design for $5 \frac{1}{2}$ by \%/16 inch track spike 
AMERICAN RAILWAY ENGINEERING ASSOCIATION SPECIFICATIONS FOR SOFT-STEEL CUT TRACK SPIKES, 1926

\section{SOFT-STEEL CUT TRACK SPIKES}

\section{MANJFACTURE}

1. The steel may be made by the Bessemer or open-hearth process, or both.

\section{CHEMICAL PROPERTIES AND TESTS}

2. (a) The steel shall conform to the following requirements as to chemical composition:

\begin{tabular}{|c|c|c|}
\hline & Bessemer & $\begin{array}{c}\text { Open- } \\
\text { hearth }\end{array}$ \\
\hline Carbon, not under_...- & $\begin{array}{c}\text { Per cent } \\
0.06\end{array}$ & $\begin{array}{c}\text { Per cent } \\
0.12\end{array}$ \\
\hline
\end{tabular}

(b) A carbon determination shall be made of each melt of Bessemer steel and three determinations

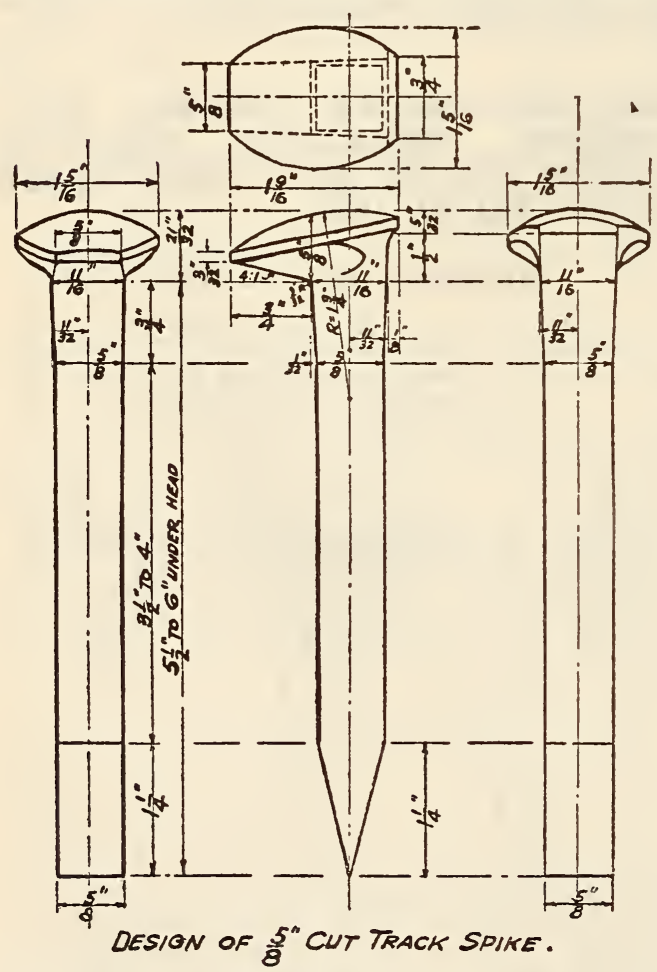

Figure 198.-(The dimensions of the A.R.E. A. $9 / 16$ inch cut track spike are identical with those of the A.E. R. E. A. $9 / 16$ inch standard track spike shown above)

shall be made every 24 hours, one for each 8-hour turn, respectively, representing the average carbon content in the steel. These analyses shall be made from drillings taken at least one-eighth inch beneath the surface of a test ingot obtained during the pouring of the melts. The chemical composition thus determined shall be reported to the purchaser or his representative, and shall conform to the requirements specified in section 2 .

(c) An analysis of each melt of open-hearth steel shall be made by the manufacturer to determine the percentage of carbon. This analysis shall be made from drillings taken at least one-eighth inch beneath the surface of a test ingot obtained during the pouring of the melt. The chemical composition thus determined shall be reported to the purchaser or his representative, and shall conform to the requirements in section 2 .

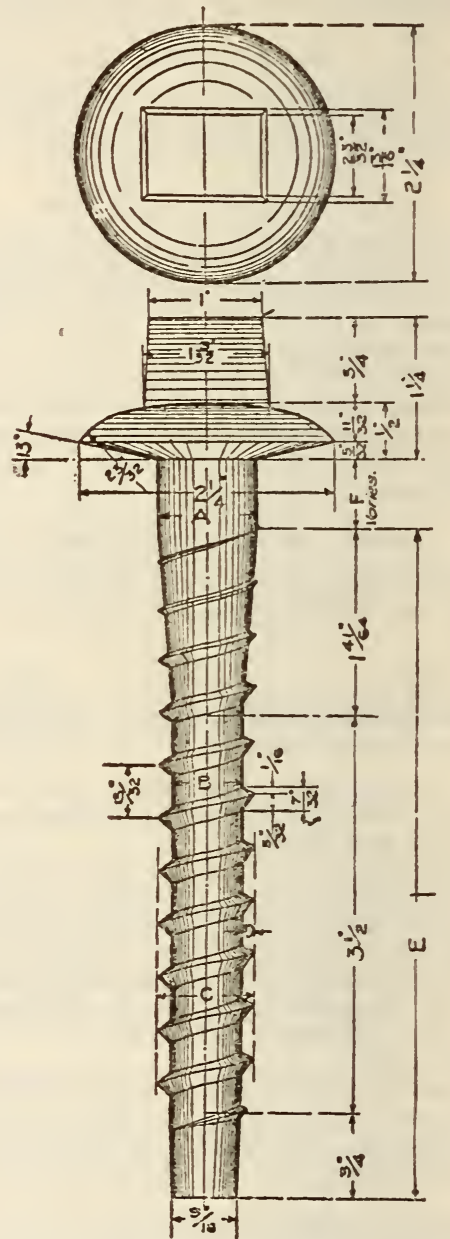

Frgure 199.-Design of screw spike

\begin{tabular}{|c|c|c|c|c|}
\hline & $\begin{array}{l}\text { Let- } \\
\text { ter }\end{array}$ & $\begin{array}{l}\text { Maxi- } \\
\text { mum }\end{array}$ & $\begin{array}{l}\text { Stand- } \\
\text { ard }\end{array}$ & $\begin{array}{l}\text { Mini- } \\
\text { mum }\end{array}$ \\
\hline $\begin{array}{l}\text { Under head. } \\
\text { Root } \\
\text { Over thread } \\
\text { Thread. } \\
\text { Length } \\
\text { Length under head... }\end{array}$ & $\begin{array}{l}\mathrm{A} \\
\mathrm{B} \\
\mathrm{C} \\
\mathrm{D} \\
\mathrm{E} \\
\mathrm{F}\end{array}$ & $\begin{array}{c}29 / 32 \\
2132 \\
2932 \\
964 \\
6164 \\
\text { Varies } \\
\text { thick } \\
\text { base }\end{array}$ & $\begin{array}{r}7 / 8 \\
5 / 8 \\
7 / 8 \\
1 / 8 \\
557 / 64 \\
\text { accord } \\
\text { kness } 0 \\
\text { and tie }\end{array}$ & $\begin{array}{r}7 / 8 \\
5 / 8 \\
7 / 8 \\
1 / 8 \\
54964 \\
\text { ing to } \\
\text { f rail } \\
\text { plate. }\end{array}$ \\
\hline
\end{tabular}

\section{PHYSICAL PROPERTIES AND TESTS}

(Same as A. S. T. M., A65, given below, in respect to bend tests. No tension tests are specified, but one bend test of each kind shall be made from each lot of 5 tons or fraction thereof.)

\section{WORKMANSHIP AND FINISH}

5. (a) The spikes shall conform to the dimensions specified by the purchaser, with the following permissible variations: 
(b) A variation of one thirty-second inch over and one sixty-fourth inch under the specified crosssection dimensions of the body of the spike will be permitted.

(c) A variation of three thirty-seconds inch over and one thirty-second inch under the specified dimensions of the head of the spike will be permitted.

(d) A variation of one-eighth inch from the specified length of the spike measured from the underside of the head to the point will be permitted.

(e) A variation of $1^{\circ}$ in the specified angle of the underside of the head of the spike will be permitted.

6. The finished spikes shall be free from injurious defects and shall have a workmanlike finish.

\section{IISSPECTION}

(Same in substance as A. S. T. M., B11, paragraphs 12 and 13 (b). See 641.21, p. 1176.)

\section{AMERICAN RAILWAY ENGINEERING ASSOCIATION, SPECIFICATIONS FOR STEEL SCREW TRACK SPIKES, 1920}

\section{STEEL SCREW TRACK SPIKES \\ (See fig. 199, p. 358)}

(Identical in substance with A. S. T. M., A66, given below, including the references in A66 to A. S. T. M., A65 and B11, except for the following additions:)

\section{SHIPMENT}

When spikes are shipped they shall be properly oiled to prevent rusting and shall be packed in good, serviceable packages. All packages shall be plainly marked as to material, size of spike, and name of manufacturer unless otherwise specified.

\section{AMERICAN SOCIETY FOR TESTING MATE- RIALS, STANDARD SPECIFICATIONS FOR STEEL TRACK SPIKES, DESIGNATION A65-24, 1924}

\section{STEEL TRACK SPIKES}

\section{MANUFACTURE}

1. The steel shall be made by either or both the following processes: Bessemer or open hearth.

\section{PHYSICAL PROPERTIES AND TESTS}

2. The full-size finished spikes, or the full-size bars from which the spikes are made, shall conform to the following minimum requirements as to tensile properties:

Tensile strength

55,000

Yield point 0.5 ten

3. (a) The body of the full-size finished spike shall stand being bent through $180^{\circ}$ flat on itself without cracking on the outside of the bent portion.

(b) The head of the full-size finished spike shall stand being bent backward to the line of the face of the spike without cracking on the outside of the bent portion.

4. (a) One tension and one bend test of each kind shall be made from each lot of 10 tons or fraction thereof.

(b) If any test specimen develops flaws it may be discarded and another specimen substituted.

5. If any tension test specimen breaks more than three-fourths inch from the center of the gage length a retest shall be allowed.
WORKMANSHIP AND FINISH

6. The spikes shall conform to the dimensions specified by the purchaser except that a variation of one thirty-second inch over and one sixty-fourth inch under the specified dimension of the body of the spike, and a variation of one-eighth inch over and one-sixteenth inch under the specified dimension of the head of the spike will be permitted. A variation of one-fourth inch over and nothing under the specified length of the spike, measured from the underside of the head to the point, will be permitted. A variation of $1^{\circ}$ in the specified angle of the underside of the head of the spike will be permitted.

7. The finished spikes shall be free from injurious defects and shall have a workmanlike finish.

\section{INSPECTION AND REJECTION}

(Conform to A. S. T. M., B11, paragraphs 12 and $13(\mathrm{~b})$. See 641.21, p. 1176.)

\section{AMERICAN SOCIETY FOR TESTING MATE- RIALS, TENTATIVE SPECIFICATIONS FOR SOFT-STEEL TRACK SPIKES, DES- IGNATION A65-26T, 1926}

\section{SOFT-STEEL TRACK SPIKES}

\section{MANUFACTORE}

1. The steel shall be made by either or both of the following processes: Bessemer or open hearth.

\section{CHEMICAL PROPERTIES AND TESTS}

2. (a) The steel shall conform to the following requirements as to chemical composition:

\begin{tabular}{|l|r|r|}
\hline & Bessemer & Open hearth \\
\hline Carbon, minimum...................... & $\begin{array}{r}\text { Per cent } \\
0.06\end{array}$ & $\begin{array}{r}\text { Per cent } \\
0.12\end{array}$ \\
\hline
\end{tabular}

3. (a) A carbon determination shall be made of each melt of Bessemer steel and three determinations shall be made every 24 hours, one for each 8-hour turn, respectively, representing the average carbon content in the steel. These analyses shall be made from drillings taken at least one-eighth inch beneath the surface of a test ingot obtained during the pouring of the melts. The chemical composition thus determined shall be reported to the purchaser or his representative, and shall conform to the requirements specified in section 2 .

(b) An analysis of each melt of open-hearth steel shall be made by the manufacturer to determine the percentage of carbon. This analysis shall be made from drillings taken at least one-eighth inch beneath the surface of a test ingot obtained during the pouring of the melt. The chemical composition thus determined shall be reported to the purchaser or his representative, and shall conform to the requirements specified in section 2 .

\section{PHYSICAL PROPERTIES AND TESTS}

4. (a) The body of the full-size finished spike shall stand being bent cold through $180^{\circ}$ flat on itself, without cracking on the outside of the bent portion.

(b) The head of the full-size finished spike shall stand being bent backward to the line of the face of the spike, without cracking on the outside of the bent portion.

5. One bend test of each kind shall be made from each lot of 5 tons or fraction thereof. 
IV. WORKMANSEIP AND FINISH

6. The spikes shall conform to the dimensions specified by the purchaser with the following permissible variations:

(a) A variation of one thirty-second inch over and one sixty-fourth inch under the specified crosssection dimensions of the body of the spike will be permitted.

(b) A variation of three thirty-seconds inch over and one thirty-second inch under the specified dimensions of the head of the spike will be permitted.

(c) A variation of one-eighth inch from the specified length of the spike measured from the underside of the head to the point will be permitted.

(d) A variation of 1 degree in the specified angle of the underside of the head of the spike will be permitted.

7. The finished spikes shall be free from injurious defects and shall have a workmanlike finish.

(Requirements covering inspection and rejection conform to paragraphs 7 and 8 of A. S. T. M. standard specifications for steel. See 605.0, p. 247.)

\section{AMERICAN SOCIETY FOR TESTING MATE- RIALS, SPECIFICATION FOR STEEL SCREW SPIKES, DESIGNATION A66-21, 1921}

\section{STEEL SCREW SPIKES}

(In respect to process, number of tests, retests, and finish, this specification is the same as A65-24 given above, except that under retests if the percentage of elongation of any tension test spike is less than that specified in section 3 , given below, a retest shall be allowed. As in A65, inspection and rejection conform to A. S. T. M., B11, paragraphs 12 and 13 (b). Otherwise this specification provides that-

\section{MANUFACTURE}

2. The heads of the spikes shall be formed and the threads rolled at a temperature not less than $750^{\circ} \mathrm{C}$.

\section{PHYSICAL PROPERTIES AND TESTS}

3. The full-size finished spikes shall conform to the following minimum requirements as to tensile properties:

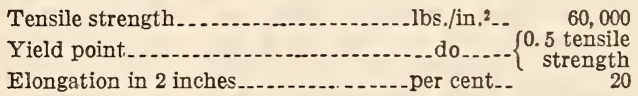

4. The full-size finished spikes shall bend cold through $90^{\circ}$ around a pin the diameter of which is equal to three times the diameter of the spike, without cracking on the outside of the bent portion. One tension and one bend test shall be made from each lot of $100 \mathrm{kegs}$ or fraction thereof.

\section{WORKMANSHIP AND FINISE}

7. The spikes shall conform to the dimensions specified by the purchaser. The head shall be concentric with, and firmly joined to, the body of the spike. The threads snall be sharp and true to gage and of the design specified by the purchaser. A variation of one thirty-second inch over the specified diameter of the unthreaded portion of the body of the spike will be permitted. A variation of one thirty-second inch over the specified diameter of the threaded portion of the spike will be permitted. A variation of one-sixteenth inch under and one-eighth inch over in the reach of the head of the spike will be permitted. A variation of one-eighth inch from the specified length of the spike will be permitted.

8. The finished spikes shall be free from injurious defects and shall have a workmanlike finish.
MARIING

9. A letter or brand indicating the manufacturer shall be pressed on the head of the spike while it is being formed.

UNITED STATES GOVERNMENT, FEDERAI SPECIFICATIONS BOARD, FEDERAL SPECIFICATION FOR NAILS, SPIKES, TACKS, AND STAPIES, FF-N-101, IMAY 3, 1932

\author{
TRACK SPIKES
}

(See 608.11, p. 560.)

\subsection{TRACK TIE RODS.}

\section{AMERICAN ELECTRIC RAILWAY ENGI- NEERING ASSOCIATION, STANDARD SPECIFICATION FOR DESIGN AND MANUFACTURE OF TIE-RODS, W42-31, 1931 \\ TIE-RODS}

This specification is intended to cover the design and manufacture of low-carbon-steel tie-rods of the round or flat types, for use in street-railway track.

\section{MANUFACTURE}

101. Process-The steel shall be made by either or both the Bessemer or open hearth process.

\section{CHEMICAL PROPERTIES}

201. Tie-rods shall be manufactured from commercial-quality hot-rolled bar steels conforming to A. S. T. M. specification A107-30, screw steel grades, for both open hearth and Bessemer steels, the chemical properties requirements of which are as follows:

\begin{tabular}{|c|c|c|c|c|}
\hline 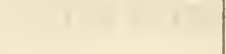 & Carbon & $\begin{array}{l}\text { Manga- } \\
\text { nese }\end{array}$ & Phosphorus & Sulphur \\
\hline $\begin{array}{l}\text { Bessemer-screw } \\
\text { Open-hearth screw.- }\end{array}$ & $\begin{array}{l}\text { Per cent } \\
0.08-0.16 \\
.15-.25\end{array}$ & $\begin{array}{l}\text { Per cent } \\
0.60-0.90 \\
.60-.90\end{array}$ & $\begin{array}{c}\text { Per cent } \\
0.09-0.13 \\
\text { (Max.).06 }\end{array}$ & $\begin{array}{c}\text { Per cent } \\
0.10-0.15 \\
.075-.150\end{array}$ \\
\hline
\end{tabular}

\section{WORKMANSHIP AND FINISH}

401. Workmanship.-(a) Flat-type tie rods, if manufactured from flat bars, shall be three-eighths inch by 2 inches, with the permissible variations allowed in A. S. T. M., A107 (these permissible variations will be found under Association of American Steel Manufacturers, hot-rolled bars, in $600.7, \mathrm{p} .116)$. If rolled from round stock, the thickness shall be not less than that permitted by the above specification.

(b) Tie rods of the round type and the round portion of the rods of flat type shall have an area in the unthreaded portion of not less than the area at the root of the threads.

(c) The terminals of the rods shall be fully threaded with a seven-eighths U. S. standard thread, 3 inches on one end and 6 inches on the other. The threads on the terminals shall be U. S. standard cut threads, so threaded that the inner nuts may be turned down the full length of threading specified. The full section threading of the terminal shall extend to within one-eighth inch from the end of the rod.

(d) Unless otherwise specified, each terminal shall be fitted with two hot-pressed U. S. standard square nuts, four nuts per tie rod. Nuts shall be free from burrs and fins. Inner nuts shall turn by hand; 
outer nuts shall have wrench-tight fit. The steel for the nuts shall be soft steel.

402. Lengths. - The dimensions shall be as follows, and in accordance with Figure 200.

(a) Tie rods for use with girder rails for 4 feet 8/2-inch gage shall be 61 inches long over all.

(b) Tie rods for use with plain girder and standard section rails for 4 feet $8 \frac{1}{2}$-inch gage shall be 63 inches long over all.

(c) Tie rods for all gages other than 4 feet $8 \frac{1}{2}$ inches shall have increased or decreased lengths to correspond with the increase or decrease in the track gage as specified by the purchaser.

403. Permissible variations. - (a) The length of the tie rod shall be not more than one-eighth inch
607.0 GENERAI ITEMS.

AMERICAN GAS ASSOCIATION, AMIERICAN STANDARD GAS SAFETY CODE FOR INSTALLATIONS AND WORK IN BUILD. INGS, K2-1927

[Approved as American Standard by the American Standard Association, 1927]

\section{GAS SAFETY CODE}

(Joint sponsor with the Bureau of Standards for safeguarding the lives, health, and property of the users of manufactured gas. This code contains

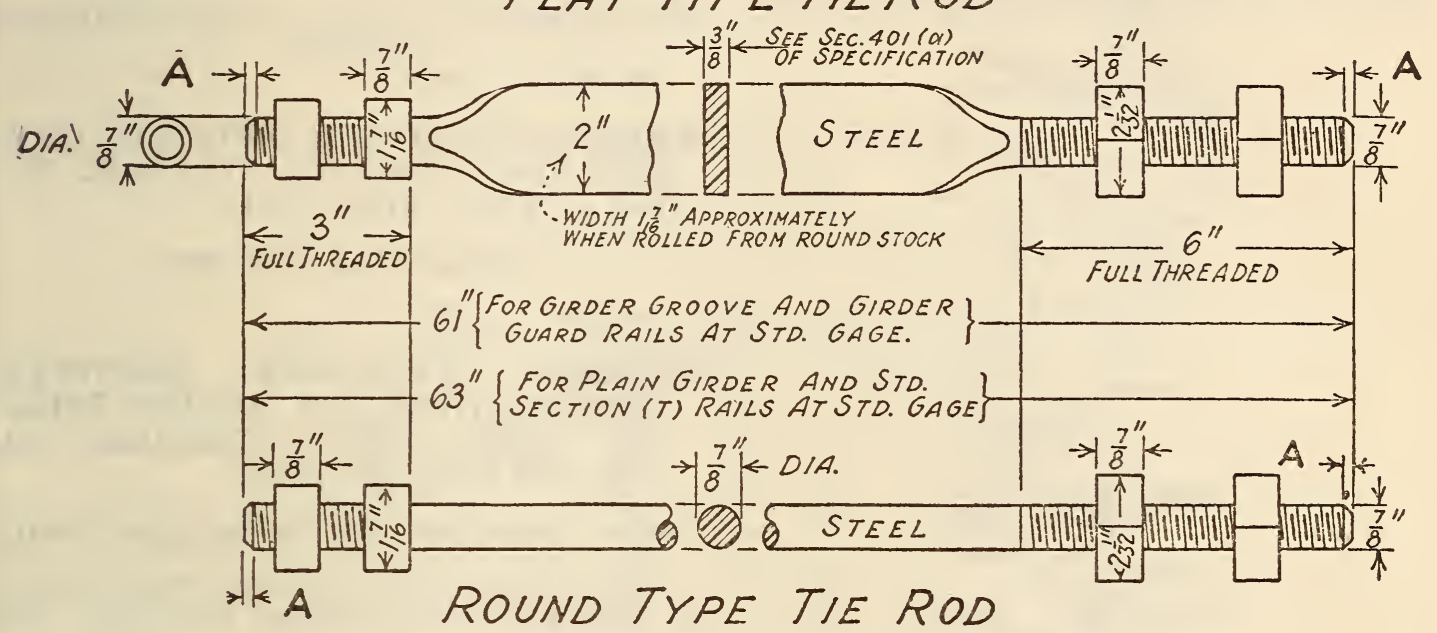

NOTES: DIMENSION A NOT MORE THAN IIN.

U.S.STD. SO. NUTS REOUIRED UNLESS OTHERWISE SPECIFIED.

U.S.STD. TIN. CUT THREADS REQUIRED.

FIGURE 200.-Dimensions of tie rods

shorter nor more than one-fourth inch longer than that shown on the drawing.

(b) The diameter of the threaded portion or terminal shall not vary more than one sixty-fourth inch from the diameter shown on the drawing.

(c) The lengths of the threaded portions or terminal of the rod shall not vary more than one-eighth inch from those shown on the drawing.

(d) The outside dimensions of the nuts shall not vary more than one thirty-second inch from those shown on the drawing.

\section{MARIING AND SHIPPING}

501. Marking and shipping.-When the rods are shipped, they shall have the nuts applied for at least the length of the nut, and shall be properly oiled to prevent rusting. They shall be securely wired together in bundles of five rods each, and eacb bundle shall be plainly marked as to material, name of manufacturer, length of rods, and name and shipping address of consignee.

\section{INSPECTION}

(Conforms to A. S. T. M., B11, paragraph 12. See 641.21, p. 1176.)

607. TUBULAR PRODUCTS AND FITTINGS, COCKS, AND VALVES mandatory rules which must be followed to attain the greatest measure of safety, supplemented by a series of notes on these rules citing the dangers to be guarded against.)

AMERICAN GAS ASSOCIATION, REQUIREMENTS FOR HOUSE PIPING AND AP. PLIANCE INSTALLATION, 1928

\section{GAS PIPING}

(Basic requirements for house piping and appliance installation to insure safe and satisfactory service. Includes material and size of pipe, maximum gas demand required for household gas-consuming appliances, location of such appliances, recommended pracíce for flue connections, and diagrams picturing relative positions of gas-operated and auxiliary appliances.)

\section{AMERICAN MARINE STANDARDS COM- MITTEE, DISTINCTIVE MARKINGS FOR PIPING, 0 NO. 10-1926}

\section{PIPE MARKINGS}

(Scheme for indicating the purpose of conveyor pipes aboard ship by suitable markings. These markings occur at intervals throughout the ship, 
and involve not only the pipe itself but also the edges of flanges adjacent to valves and on such other flanges as will identify the function of the pipe. These markings are based on and coincide with the standard marking system of the U. S. Navy.)

\section{AMERICAN PETROLEUM INSTITUTE, DIMENSIONAL STANDARDS FOR CABLE- DRILLING TOOLS, A. P. I. STANDARDS NO. 3, 1930}

\section{BASIC THREAD FORM}

Profile.-The angle between the sides of the thread is $60^{\circ}$ measured in the axial plane, and the thread is perpendicular to the axis of the joint.

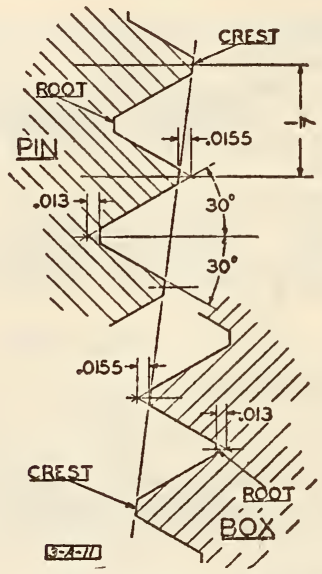

Figure 201.-Basic form, 7 -thread

The crest on seven threads per inch is truncated parallel to the taper an amount equal to 0.0155 inch, while the root is truncated an amount equal to 0.013 inch parallel to the axis. (See fig. 201.)

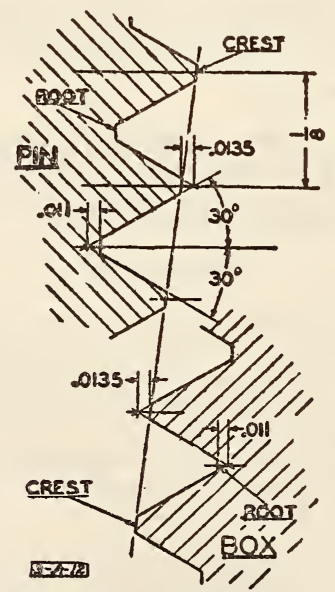

Figure 202 . Basic form, 8-thread

The crest on eight threads per inch is truncated parallel to the taper an amount equal to 0.0135 inch, while the root is truncated an amount equal to 0.011 inch, parallel to the axis. (See fig. 202.)

Pitch.-The number of threads per inch is seven on joints $15 / 8$ by $25 /$ inches and larger and the number of threads per inch is eight on joints $1 \frac{1 / 2}{2}$ by $2 \frac{1}{4}$ inches and smaller, both conforming to United States standard, measured in a direction parallel to the axis of the thread.

Taper of joint.- The taper of the joint is 3 inches per foot, or 1 in 4 measured on the diameter.

(Recommended practice for turning threads is included in this specification. While not forming part of the specification, these recommendations are generally accepted as productive of satisfactory results. They include setting the taper of the lathe and also setting the threading tool.)

AMERICAN PETROLEUM INSTITUTE, PIPE SPECIFICATIONS, STANDARD NO. 5-A (7TH ED.), JULY, 1931

PIPE THREADS FOR OIL COUNTRY TUBULAR (See 607.4, p. 444.)

AMERICAN PETROLEUM INSTITUTE, LINEPIPE SPECIFICATIONS, STANDARD NO. 5-L (4TH ED.), JULY, 1931

\section{THREADS FOR LINE PIPE}

(See 607.4, p. 457.)

\section{AMERICAN PETROLEUM INSTITUTE, SPECIFICATIONS FOR ROTARY DRILL- ING TAPER JOINTS, STANDARD NO. 7-B (4TH ED.), JULY, 1931}

\section{TAPER JOINTS FOR ROTARY DRILLING TOOLS}

(This specification includes dimensional standards for taper joints, tool joints and fishtail bits, drill

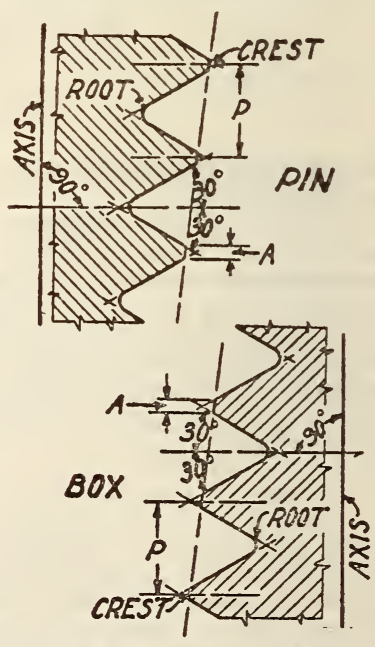

Figure 203.-Basic thread form

$A=0.040$ inch on 5 -thread $A=0.050$ inch on 4 -thread

Truncation is parallel to taper, measured parallel to.axis

collars, grief stems and couplings, and swivel gooseneck connections. Below are given the basic thread form and the dimensional standards of the standard rotary taper joint.)

\section{TAPER JOINTS}

9. (a) The sizes of taper joints shown in Table 1 and Figures 203 and 204 are standard. 
(b) Basic thread form, profile.-The angle between the sides of the thread is $60^{\circ}$, measured in the axial plane, and the line bisecting this angle is perpendicular to the axis of the joint. The crest is truncated parallel to the taper to produce a flat of 0.050 inch (see "A," fig. 203) on 4-thread and similarly truncated to produce a flat of 0.040 inch on 5 -thread (see "A," fig. 203), while the root is rounded a sufficient amount to clear the mating threads on both four and five threads. Both flats are measured parallel to the axis.

(c) Pitch.-The number of threads per inch on all sizes up to and including the $3 \% 16$ by $45 \% 8$ inches is five, and on all sizes over $3 \% 16$ by $4 \% / 8$ inches is four, as specified, measured in a direction parallel to the axis of the thread.

(d) Taper of joints.-The taper of all joints is 3 inches per foot, or 1 in 4 , measured on the diameter except the 5 by $6-4$, which is 2 inches per foot, or 1 in 6 , measured on the diameter.

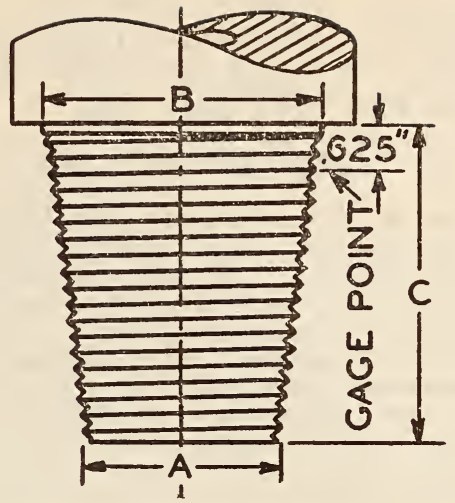

FIGURE 204

TABle 1.-A.P. I. standard rotary taper joints

[See figs. 203 and 204]

[Taper joints shall be identified by the drill-pipe size. All dimensions in inches at $68^{\circ} \mathrm{F}$.]

\begin{tabular}{|c|c|c|c|c|c|c|c|}
\hline $\begin{array}{l}\text { Size } \\
\text { of drill } \\
\text { pipe }\end{array}$ & $\begin{array}{c}\text { Nominal size of } \\
\text { taper joint }\end{array}$ & $\begin{array}{l}\text { Small di- } \\
\text { ameter of } \\
\text { pin "A" }\end{array} \mid$ & $\begin{array}{c}\text { Large } \\
\text { diame- } \\
\text { ter of } \\
\text { pin } \\
\text { "B" }\end{array}$ & $\begin{array}{l}\text { Length } \\
\text { of pin } \\
\text { " } \mathrm{C} "\end{array}$ & $\begin{array}{c}\text { Num- } \\
\text { ber of } \\
\text { threads } \\
\text { per inch }\end{array}$ & $\begin{array}{c}\text { Taper } \\
\text { of joint } \\
\text { inches } \\
\text { per } \\
\text { foot }\end{array}$ & $\begin{array}{l}\text { Form of } \\
\text { thread }\end{array}$ \\
\hline $\begin{array}{l}23 / 8 \\
27 / 8 \\
31 / 2 \\
41 / 2\end{array}$ & $\begin{array}{l}17 / 8 \text { by } 25 / 8-5 \\
21 / 8 \text { by } 3-5 \\
29,16 \text { by } 31 / 2-5 \\
396 \text { by } 45 / 8-5\end{array}$ & $\begin{array}{l}1.875 \\
2.125 \\
2.562 \\
3.562\end{array}$ & $\begin{array}{l}25 / 8 \\
3 \\
31 / 2 \\
45 / 8\end{array}$ & $\begin{array}{l}3 \\
31 / 2 \\
33 / 4 \\
41 / 4\end{array}$ & $\begin{array}{l}5 \\
5 \\
5 \\
5\end{array}$ & $\begin{array}{l}3 \\
3 \\
3 \\
3\end{array}$ & $\begin{array}{l}\text { V-.040 flat. } \\
\text { V-.040 flat. } \\
\text { V-.040 flat. } \\
\text { V-.040 flat. }\end{array}$ \\
\hline $\begin{array}{l}59 / 16 \\
65 \% 8 \\
75 \% 8 \\
85 \% 8\end{array}$ & $\begin{array}{l}41 / 2 \text { by } 51 / 2-4 \\
5 \text { by } 6-4 \\
6 \text { by } 7-4 \\
7 \text { by } 8-4\end{array}$ & $\begin{array}{l}4.333 \\
5.159 \\
5.6875 \\
6.608\end{array}$ & $\begin{array}{l}51 / 2 \\
6 \\
7 \\
8\end{array}$ & $\begin{array}{l}43 / 4 \\
5 \\
51 / 4 \\
53 / 8\end{array}$ & $\begin{array}{l}4 \\
4 \\
4 \\
4\end{array}$ & $\begin{array}{l}3 \\
2 \\
3 \\
3\end{array}$ & $\begin{array}{l}\text { V-.050 flat. } \\
V-.050 \text { flat. } \\
\text { V-.050 flat. } \\
\text { V-.050 flat. }\end{array}$ \\
\hline
\end{tabular}

NotE,-Dimensions " $A$ " and " $C$ " for all sizes except the $5 \% 16,65 \%$, and $85 \%$ inches are absolute dimensions from which gage values are computed. Dimensions " $\mathrm{A}$ " and " $\mathrm{C}$ " for the $5 \% 16,65 \%$, and $85 \% 8$ sizes are approximately accurate.

\section{AMERICAN SOCIETY OF MECHANICAL ENGINEERS, SCHEME FOR THE IDENTI- FICATION OF PIPING SYSTEMS, A13- 1928}

[Approved as American recommended practice by the American Standards Association, 1928]

\section{IDENTIFYING PIPING SYSTEMS}

(Joint sponsor with the National Safety Council for a system to indicate the purpose of conveyor pipes by suitable markings. The scheme is limited to piping systems in industrial and power plants, exclusive of electrical conduits and pipes buried in the ground. For identification purposes the piping systems are divided into five classes, viz, fire protection, dangerous materials, safe materials, protective materials, and extra valuable materials. The colors attaching to each of these classes are, respectively, red, yellow or orange, green (or the achromatic colors white, black, gray, or aluminum), bright blue, or deep purple. An appendix lists the identifying trade names of the specific five basic colors which are least affected chemically and physically by abnormal exposure conditions.)

\section{AMERICAN SOCIETY OF MECHANICAL ENGINEERS, PIPE THREAD, NO. 3, 1919}

[Approved as American standard (B2-1919) by the American Standards Association]

\section{PIPE THREADS}

(Joint sponsor with the American Gas Association under the asupices of the American Standards Association. Substantially the same as the National Screw Thread Commission report, given below.)

\section{ASSOCIATED FACTORY MUTUAL FIRE INSURANCE COMPANIES, SPECIFICA- TIONS FOR FIRE HOSE, COUPIINGS, AND PLAY PIPES, 1930}

(See also 645.4 , p. 1232.)

\section{THREADS FOR HOSE COUPLINGS}

Threads of $2 \frac{1}{2} 2^{-i n c h}$ hose couplings should conform to the national (American) standard thread. (See National Screw Thread Commission, given below.) 


\section{NATIONAL BOARD OF FIRE UNDER- WRITERS, INSTALLATION, MAINTE- NANCE, AND USE OF PIPING AND FIT- TINGS FOR CITY GAS, 1920}

\section{PIPING FOR CITY GAS}

All pipe shall be cut square with its length, and the exact dimensions as given on the piping plans shall be followed. Pipe shall be threaded with clean-cut threads, and all burrs or other obstructions shall be removed from the pipe.

Nominal ordinary pipe sizes and Briggs' standard are understood in these regulations for all pipes and threads where not otherwise specified.

The following table specifies the number of threads to be cut and the length of section to be threaded for each size of pipe, based on Briggs' standard:

\begin{tabular}{|c|c|c|}
\hline $\begin{array}{c}\text { Size of } \\
\text { pipe }\end{array}$ & $\begin{array}{c}\text { Approxi- } \\
\text { mate } \\
\text { length of } \\
\text { threaded } \\
\text { portion }\end{array}$ & $\begin{array}{c}\text { Approxi- } \\
\text { mate } \\
\text { number } \\
\text { of threads } \\
\text { to be cut }\end{array}$ \\
\hline Inches & $\begin{array}{c}\text { Inches } \\
3 / 8\end{array}$ & \\
$11 / 2$ & $9 / 10$ & 10 \\
$3 / 4$ & $3 / 4$ & 10 \\
11 & 718 & 10 \\
$11 / 4$ & 1 & 11 \\
$11 / 2$ & 1 & 11 \\
2 & 1 & 11 \\
2112 & $11 / 2$ & 12 \\
3 & $11 / 2$ & 12 \\
4 & 158 & 13 \\
\hline
\end{tabular}

Pipe with threads stripped, chipped, or damaged, or which has crooked threads, shall not be used. If the weld opens during the operation of cutting or threading, that portion of the pipe shall not be used.

When an approved jointing compound is used, it should always be applied sparingly and only to the male thread of the joint. Sealing wax or any material or compound known as "gas-fitter's cement" shall not be used in making up joints in piping systems. If material containing lead is used for a jointing material, it shall not be applied with the hand because of its poisonous nature.

\section{POWER PIPING SOCIETY, STANDARD SPECIFICATIONS FOR POWER PIPING, 1928}

\section{POWER PIPING}

(This specification contains requirements for a safe, economical, commercial installation. It has been compiled as a guide for framing actual specifications. The materials and workmanship are of higher grade than safety alone or service under the most favorable conditions might dictate, but are recommended by the Power Piping Society as the minimum permitted by good practice. The text consists of excerpts from standard specifications, each paragraph carrying an identifying letter and number. This is supplemented by a materialservice index which indicates at a glance the powerpiping requirements for different kinds of service. For example, superheated steam at pressures of 100 to 600 pounds per square inch, or saturated steam at a pressure of 100 pounds per square inch, requires for safe and economical service certain standards in regard to materials, dimensions, finish and tolerances, hydrostatic tests, gate valves, globe and angle valves, nonreturn valves, blow-off valves, fittings, loose flanges, screwed unions, wrought pipe, bolting, gaskets, welding, bends, expansion, and supports. The standard requirements in each case are indicated by paragraph number and letter. By paragraph number and letter are also indicated the powerpiping requirements as detailed above for superheated steam at other pressures, saturated steam at other pressures, boiler-feed water at various pressures, exhaust, service water, suction and lowpressure water, live-steam drains, low-pressure drains, boiler blow-off, fuel-oil discharge, transformer circulating oil, and fuel-oil suction. Under the head of gate valves it is provided that):

The bodies of steel gate valves of the sizes listed below shall have three bosses on each connecting end, one such boss on each side and one on the bottoin. Each boss shall be of a diameter and height to permit tapping of American standard pipe threads of the following sizes. (Standard practice of the Manufacturers' Standardization Society of the valve and fitting industry.)

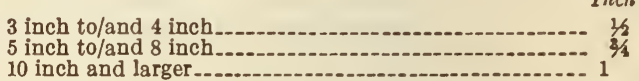

\section{SOCIETY OF AUTOMOTIVE ENGINEERS, SCREW THREADS, S. A. E. (AMERICAN) STANDARD, FEBRUARY 1924}

\section{[Approved as American Standard (B1a-1924) by the American Standards Association]}

(Joint sponsor with the American Society of Mechanical Engineers under the auspices of the American Standards Association. Substantially the same as corresponding tables in United States Government, National Screw Thread Commission, Bureau of Standards Miscellaneous Publication No. 89 (see 608.0, p. 529), except for data on extra-fine-thread series, and fine-thread series over $1 \frac{1}{2}$ inches in diameter, for which see S. A. E. (608.0, p. 517).)

\section{SOCIETY OF AUTOMOTIVE ENGINEERS, SCREW THREADS; SCREW-THREAD-FIT APPLICATIONS, JULY, 1926}

\section{SCREW-THREAD-FIT APPLICATIONS}

(A system of symbols for readily identifying the threads per inch, the series, and the fit of screw threads to be used in the different S. A. E. standards and recommended practices which do not specify screw-thread fits. These standards and recommended practices include carburetor flanges, oil and grease-cup threads, tachometer drive, and fruntaxle hubs.)

\section{SOCIETY OF AUTOMOTIVE ENGINEERS, SCREW THREADS ; TAPER PIPE THREADS, S. A.
MARCH,
1921 (AMERICAN) STANDARD,}

\section{TAPER PIPE THREADS}

(Same as Table 44 of the National Screw Thread Commission, given below, from which it is ab stracted, in pipe sizes ranging from one-eighth to 6 inches.) 
UNITED STATES GOVERNMENT, DEPARTMENT OF COMMERCE, BUREAU OF STANDARDS, WROUGHT-IRON AND WROUGHT-STEEL PIPE, VALVES, AND FITTINGS, SIMPLIFIED PRACTICE RECOMMENDATION NO. 57, 1926

\section{WROUGHT-IRON AND WROUGHT-STEEL PIPE, VALVES, AND FITTINGS}

The United States Department of Commerce, through the Bureau of Standards, recommends that the sizes and dimensions of wrought-iron and wrought-steel pipe and the sizes of valves and fittings conform to the following simplified lists:

TABLE 1. - "Standard weight" pipe

\begin{tabular}{|c|c|c||c|c|c}
\hline $\begin{array}{c}\text { Nominal } \\
\text { inside } \\
\text { diameter }\end{array}$ & $\begin{array}{c}\text { Outside } \\
\text { diameter }\end{array}$ & $\begin{array}{c}\text { Approxi- } \\
\text { mate } \\
\text { thickness }\end{array}$ & $\begin{array}{c}\text { Nominal } \\
\text { inside } \\
\text { diameter }\end{array}$ & $\begin{array}{c}\text { Outside } \\
\text { diameter }\end{array}$ & $\begin{array}{c}\text { Approxi- } \\
\text { mate } \\
\text { thickness }\end{array}$ \\
\cline { 1 - 2 } Inches & Inches & Inch & Inches & Inches & Inch \\
$1 / 8$ & 0.405 & 0.068 & $21 / 2$ & 2.875 & 0.203 \\
114 & .540 & .088 & 3 & 3.500 & .216 \\
38 & .675 & .091 & $31 / 2$ & 4.000 & .226 \\
$1 / 2$ & .840 & .109 & 4 & 4.500 & .237 \\
$3 / 4$ & 1.050 & .113 & 5 & 5.563 & .258 \\
1 & 1.315 & .133 & 6 & 6.625 & .280 \\
114 & 1.660 & .140 & 8 & 8.625 & .322 \\
$11 / 2$ & 1.900 & .145 & 10 & 10.750 & .365 \\
2 & 2.375 & .154 & 12 & 12.750 & .375 \\
\hline
\end{tabular}

TABLE 2. - "Extra strong" pipe

\begin{tabular}{|c|c|c|c|c|c|}
\hline $\begin{array}{l}\text { Nominal } \\
\text { inside } \\
\text { diameter }\end{array}$ & $\begin{array}{l}\text { Outside } \\
\text { diameter }\end{array}$ & $\begin{array}{l}\text { Approxi- } \\
\text { mate } \\
\text { thickness }\end{array}$ & $\begin{array}{l}\text { Nominal } \\
\text { inside } \\
\text { diameter }\end{array}$ & $\begin{array}{l}\text { Outside } \\
\text { diameter }\end{array}$ & $\begin{array}{l}\text { Approxi- } \\
\text { mate } \\
\text { thickness }\end{array}$ \\
\hline $\begin{array}{r}\text { Inches } \\
1 / 8 \\
1 / 4 \\
3 / 8 \\
1 / 2 \\
3 / 4 \\
1 \\
114 \\
11 / 2 \\
2\end{array}$ & $\begin{array}{r}\text { Inches } \\
0.405 \\
.540 \\
.675 \\
.840 \\
1.050 \\
1.315 \\
1.660 \\
1.900 \\
2.375\end{array}$ & $\begin{array}{l}\text { Inch } \\
0.095 \\
.119 \\
.126 \\
.147 \\
.154 \\
.179 \\
.191 \\
.200 \\
.218\end{array}$ & $\begin{array}{c}\text { Inches } \\
21 / 2 \\
3 \\
31 / 2 \\
4 \\
5 \\
6 \\
8 \\
10 \\
12\end{array}$ & $\begin{array}{r}\text { Inches } \\
2.875 \\
3.500 \\
4.000 \\
4.500 \\
5.563 \\
6.625 \\
8.625 \\
10.750 \\
12.750\end{array}$ & $\begin{array}{l}\text { Inch } \\
0.276 \\
.300 \\
.318 \\
.337 \\
.375 \\
.432 \\
.500 \\
.500 \\
.500\end{array}$ \\
\hline
\end{tabular}

TABLE 3. - "Double extra strong" pipe

\begin{tabular}{|r|c|c||c|c|c|}
\hline $\begin{array}{c}\text { Nominal } \\
\text { inside } \\
\text { diameter }\end{array}$ & $\begin{array}{c}\text { Outside } \\
\text { diameter }\end{array}$ & $\begin{array}{c}\text { Approxi- } \\
\text { mate } \\
\text { thickness }\end{array}$ & $\begin{array}{c}\text { Nominal } \\
\text { inside } \\
\text { diameter }\end{array}$ & $\begin{array}{c}\text { Outside } \\
\text { diameter }\end{array}$ & $\begin{array}{c}\text { Approxi- } \\
\text { mate } \\
\text { thickness }\end{array}$ \\
\hline Inches & Inches & Inch & Inches & Inches & Inch \\
$1 / 2$ & 0.840 & 0.294 & 3 & 3.500 & 0.600 \\
$3 / 4$ & 1.050 & .308 & $31 / 2$ & 4.000 & .636 \\
$111 / 4$ & 1.315 & .358 & 4 & 4.500 & .674 \\
11.660 & .382 & 5 & 5.563 & .750 \\
$11 / 2$ & 1.900 & .400 & 6 & 6.625 & .864 \\
2112 & 2.375 & .436 & 8 & 8.625 & .875 \\
2.875 & .552 & & & \\
\hline
\end{tabular}

Nоте.-Random lengths according to present pipe-mill practice.

UNITED STATES GOVERNMENT, FEDERAI SPECIFICATIONS BOARD, FEDERAI SPECIFICATIONS FOR PIPE THREADS, NO. 238, NOVEMBER 5, 1924. (CURRENT DESIGNATION GGG-P-351)

\section{PIPE THREADS}

TYPES

I. This specification covers taper and straight pipe threads.

MATERIAL AND WORKMANSHIP

II. 1. Threads shall be full and clean cut in conformity with best commercial practice.
GENERAL REQUIREMENTS

III. No details.

\section{DETAIL REQUIREMENTS}

IV. 1. Unless otherwise specified taper pipe threads shall apply to all pipe and pipe fittings.

2. Taper pipe threads.

(The illustrative sketch and table of taper pipe thread dimensions given in this specification are identical with the sketch and table of taper pipe thread dimensions given in the standards of the National Screw Thread Commission below, for pipe sizes up to and including 12 inches.)

3. Where straight pipe threads are specified, the following shall apply.

(a) The sizes and basic dimensions of straight pipe threads shall be as given in columns $1,2,3$, and 11 of the N. S. T. C. table of taper-pipe-thread dimensions.

(b) Diameters of straight threads. (Determined by same formula as given in standards of N. S. T. C., see below.)

\section{NETHOD OF INSPECTION}

V. 1. Gages within the tolerances specified shall be used for inspecting pipe threads. Gages approved by the purchaser shall be supplied by the vendor.

2. Gages used for inspection shall not vary from the basic dimensions more than one-half turn either way. Proper allowance shall be made for any such variation in using the gage.

3 . The thread is within the extreme tolerance in the case of internal threads when the plug gage notch is within $1 \frac{11}{2}$ turns of the coupling or fitting face, or if chamfered at the bottom of chamfer when screwed in tightly by hand. In the case of external threads the small end of the ring gage shall be within $1 \frac{1}{2}$ turns of the end of the pipe when screwed on tightly by hand.

\section{PACKING AND MARKING}

VI. 1. Details for packing and marking are given in the specifications for the pipe.

\section{ADDITIONAL INFORMATION}

VII. 1. For new inspection gages, a tolerance of plus or minus 0.0005 inch is allowed on the distance between the gaging notch and the small end of the inspection plug gage, or on the thickness of the inspection ring gage.

2. (For table of tolerances for use in purchasing new inspection gages, see National Screw Commission, 615.82 , p. 921.)

UNITED STATES GOVERNMENT, FEDERAL SPECIFICATIONS BOARD, SPECIFICATION FOR PLUMBING FIXTURES (FOR LAND USE), NO. 448, NOVEMBER 22, 1926. (CURRENT DESIGNATION : FF-P451)

(See 617.73, p. 1062 and 600.3, p. 70.)

$$
\text { PLUMBING FIXTURES (FOR LAND USE) }
$$

UNITED STATES GOVERNMENT, NATIONAL SCREW THREAD COMMISSION, AMERICAN NATIONAL PIPE THREADS, BUREAU OF STANDARDS MISCELIANEOUS PUBLICATION NO. 89, JUNE 22, 1928

\section{PIPE THREADS}

The American national pipe-thread standard for taper-threaded pipe joints was formulated prior 
to the year 1882 by Robert Briggs, of Philadelphia, Pa. This standard, with certain modifications and additions, is now in general use throughout the United States and Canada.

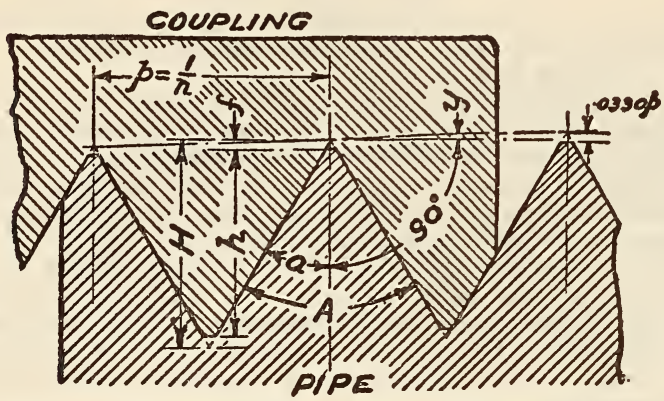

FIgURE 205.-American national taper pipe thread form and notation

$A=60^{\circ}$ angle of thread.

$a=30^{\circ}$ one-half angle of thread.

$y=1^{\circ} 47^{\prime}$ approximate taper angle $=$ one-sixteenth inch per inch on diameter.

$H=0.866025 p$ depth of $60^{\circ}$ sharp $\vee$ thread.

$h\{=0.800000 p\}$ depth of thread on work

$=0.923761 H$

$f\left\{\begin{array}{l}=0.033012 \\ =0.038120 \\ H\end{array}\right\}$ depth of truncation.

$=0.041266 \mathrm{~h}$

$p=1 / n$ pitch

$n=$ number of threads per inch.

For a symmetrical straight screw thread, $H=p$ cot $a$. For a symmetrical taper screw thread $H=\frac{p}{2}\left(\cot ^{2} a-\tan ^{2} y \tan a\right)$, so that the exact value for an American National taper pipe thread is $H=0.865743 p$ as against $H=0.866025 p$, the value given above. For an 8-pitch thread, which is the coarsest standard taper pipe thread pitch, the corresponding values of $H$ are 0.108218 inch and 0.108253 inch, respectively, the difference being 0.000035 inch. This difference being too small to be significant, the value of $H=0.866025 p$ continues in use for threads of three-fourths inch, or less, taper per foot.

\section{FORM OF THREAD}

(a) Specifications

1. Angle of thread.-The angle between the sides of the thread is $60^{\circ}$ when measured in an axial plane.

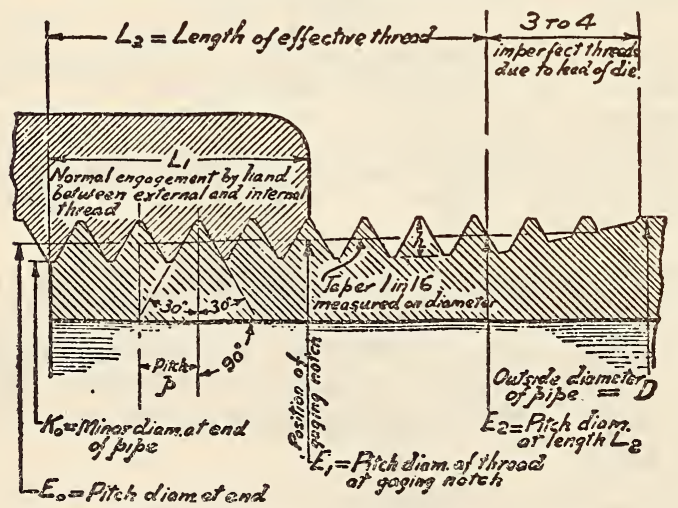

Figure 206.-American national taper pipe thread notation

NOTATION

$E_{0}=D-(0.05 D+1.1)$

$E_{1}=E_{0}+0.0625 L_{1}$.

$L_{2}=p(0.8 \mathrm{D}+6.8)$.

$\begin{aligned} L & =p \\ h & =0.8 \mathrm{p} \text {. }\end{aligned}$

and the line bisecting this angle is perpendicular to the axis of the pipe for either taper or straight threads.
2. Depth of thread. ${ }^{68}$ - The crest and root of the thread form are truncated an amount equal to $0.0330 p$; the depth of thread is, therefore, equal to $0.8 p$.

3. Taper of thread.-The taper of the thread is 1 in 16, or three-fourths inch per foot, measured on the diameter.

\section{(b) Illustration}

There are shown in Figure 205 the relations as specified herein for form of thread and general notation. Special notation is given in Figures 206, 207, and 209

\section{SYMBOLS}

(The significance of symbols used in the several formulas given below are as shown in the following tabulation:)

Pitch diameter of thread at end of pipe

Pitch diameter of thread at gaging notch

Pitch diameter of thread at $L_{2}$ from end of pipe........... $E_{2}$

Maximum pitch diameter, external locknut thread........E.

Minimum pitch diameter, internal locknut thread ....

Distance from gaging notch to end of pipe=normal engage-

ment by hand

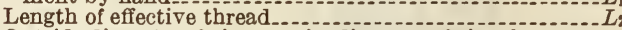

Outside diameter of pipe=major diameter of pipe thread at $L_{2}$ from end of pipe.

Internal diameter of pipe...

\section{THREAD SERIES}

\section{(a) American National Taper-Pipe Threads}

Taper external and internal pipe threads are recommended for threaded pipe joints and pipe fittings for any service. The sizes and basic dimensions of the "American national taper pipe threads" are specified in Table 44.

1. Outside diameter of pipe.-The outside diameters of pipe are given in column 5 of Table 44 .

2. Diameters of taper threads. - The pitch diameters of the taper threads are determined by formulas based on the outside diameter of pipe and the pitch of thread. These are as follows ${ }^{50}$ (see Symbols above):

$$
\begin{aligned}
& E_{0}=D-(0.05 D+1.1) p \\
& E_{1}=E_{0}+0.0625 L_{1}
\end{aligned}
$$

3. Length of thread.-The length of the taper external thread is determined by a formula based on the outside diameter of pipe and the pitch of the thread. This is as follows ${ }^{59}$ (see Symbols above):

$$
L_{2}=(0.8 D+6.8) p
$$

4. Length of engagement.-The normal length of engagement between taper external and internal threads, when screwed together by hand, is shown in column 6 of Table 44 . This length is controlled by means of gages.

5. Tolerances.-The tolerance on diameter is the equivalent of the variation in diameter due to taper over one and one-half turns either way from the basic dimensions. 60

58 While $\mathrm{Mr}$. Briggs originally advocated a slightly rounded crest and root, cutting tools are actually slightly flattened at the crest and root.

${ }_{59}$ These formulas are not expressed in the same terms as the formulas originally established by Mr. Briggs, because they are used to las originally established thread, which includes two threads slightly imperfect at the crest; thread, which includes two threads slightly imperfect at the crest; whereas the Briggs formulas determined the major diameter and the being included in the formula. However, both forms give identical results.

$60 \mathrm{On}$ account of the gage tolerance of one-half turn on working gages, this is equivalent to one turn plus or one turn minus from the gaging notch when using working gages. 
TABLE 44.-Dimensions of American national taper-pipe threads

[For notation, see fig. 206]

\begin{tabular}{|c|c|c|c|c|c|c|c|c|c|c|c|}
\hline \multirow{3}{*}{$\begin{array}{l}\text { Nominal size } \\
\text { of pipe in } \\
\text { inches }\end{array}$} & \multirow{3}{*}{$\begin{array}{c}\text { Number } \\
\text { of } \\
\text { threads } \\
\text { per inch, } \\
n\end{array}$} & \multirow{3}{*}{$\begin{array}{l}\text { Pitch, } \\
\quad p\end{array}$} & \multirow{3}{*}{$\begin{array}{c}\text { Depth of } \\
\text { thread, } \\
\quad h\end{array}$} & \multirow{3}{*}{$\begin{array}{c}\text { Outside } \\
\text { diameter } \\
\text { of pipe, } \\
D\end{array}$} & \multirow{3}{*}{$\begin{array}{l}\text { Length } \\
\text { of normal } \\
\text { engage- } \\
\text { ment by } \\
\text { hand } \\
L_{1}\end{array}$} & \multirow{3}{*}{$\begin{array}{c}\text { Length } \\
\text { of effec- } \\
\text { tive } \\
\text { thread, } \\
L_{2}\end{array}$} & \multirow{3}{*}{$\begin{array}{c}\begin{array}{c}\text { Increase } \\
\text { in } \\
\text { diameter } \\
\text { per } \\
\text { thread, }\end{array} \\
\frac{0.0625}{n}\end{array}$} & \multicolumn{4}{|c|}{ Pitch diameters } \\
\hline & & & & & & & & $\begin{array}{c}\text { At end of pipe, } \\
\text { or at length } L_{1} \\
\text { from end of } \\
\text { coupling, } \\
E_{0}=D-0.05 D+1.1\end{array}$ & $\begin{array}{r}\text { At lengt } \\
\text { en }\end{array}$ & $\begin{array}{l}L_{1} \text { on } \mathrm{pi} \\
\text { of coupl } \\
=E_{0}+\frac{L_{1}}{16}\end{array}$ & e, or at \\
\hline & & & & & & & & Basic & $\begin{array}{c}\text { Maxi- } \\
\text { mum }\end{array}$ & Basic & $\underset{\text { mumi- }}{\text { Mini- }}$ \\
\hline 1 & 2 & 3 & 4 & 5 & 6 & 7 & 8 & 9 & 10 & 11 & 12 \\
\hline $\begin{array}{l}1 / 8- \\
145- \\
38- \\
1 / 2- \\
3 / 2-\end{array}$ & $\begin{array}{l}27 \\
18 \\
18 \\
14 \\
14\end{array}$ & $\begin{array}{l}\text { Inch } \\
0.03704 \\
.05556 \\
.05556 \\
.07143 \\
.07143\end{array}$ & $\begin{array}{l}\text { Inch } \\
0.02963 \\
.04444 \\
.04444 \\
.05714 \\
.05714\end{array}$ & $\begin{array}{r}\text { Inches } \\
0.405 \\
.540 \\
.675 \\
.840 \\
1.050\end{array}$ & $\begin{array}{c}\text { Inches } \\
0.180 \\
.200 \\
.240 \\
.320 \\
.339\end{array}$ & $\begin{array}{l}\text { Inches } \\
0.26385 \\
.40178 \\
.40778 \\
.53371 \\
.54571\end{array}$ & $\begin{array}{l}\text { Inch } \\
0.00231 \\
.00347 \\
.00347 \\
.00446 \\
.00446\end{array}$ & $\begin{array}{l}\text { Inches } \\
0.36351 \\
.47739 \\
.61201 \\
.75843 \\
.96768\end{array}$ & $\begin{array}{l}\text { Inches } \\
0.37823 \\
.49510 \\
.63222 \\
.78513 \\
.99557\end{array}$ & $\begin{array}{l}\text { Inches } \\
0.37476 \\
.48989 \\
.62701 \\
.77843 \\
.98887\end{array}$ & $\begin{array}{l}\text { Inches } \\
0.37129 \\
.48468 \\
.62181 \\
.77173 \\
.98217\end{array}$ \\
\hline $\begin{array}{l}19 \\
1134 \\
1132 \\
2132 \\
2132\end{array}$ & $\begin{array}{c}111 / 2 \\
111 / 2 \\
111 / 2 \\
111 / 2 \\
8\end{array}$ & $\begin{array}{l}.08696 \\
.08696 \\
.08696 \\
.08696 \\
.12500\end{array}$ & $\begin{array}{l}.06957 \\
.06957 \\
.06957 \\
.06957 \\
.10000\end{array}$ & $\begin{array}{l}1.315 \\
1.660 \\
1.900 \\
2.375 \\
2.875\end{array}$ & $\begin{array}{l}.400 \\
.420 \\
.420 \\
.436 \\
.682\end{array}$ & $\begin{array}{r}.68278 \\
.70678 \\
.72348 \\
.75652 \\
1.13750\end{array}$ & $\begin{array}{l}.00543 \\
.00543 \\
.00543 \\
.00543 \\
.00781\end{array}$ & $\begin{array}{l}1.21363 \\
1.55713 \\
1.79609 \\
2.26902 \\
2.71953\end{array}$ & $\begin{array}{l}1.24678 \\
1.59153 \\
1.83049 \\
2.30442 \\
2.77388\end{array}$ & $\begin{array}{l}1.23863 \\
1.58338 \\
1.82234 \\
2.29627 \\
2.76216\end{array}$ & $\begin{array}{l}1.23048 \\
1.57523 \\
1.81418 \\
2.28812 \\
2.75044\end{array}$ \\
\hline $\begin{array}{l}3 \ldots \ldots \\
313 \ldots \\
413 \ldots \\
412 \ldots \\
5\end{array}$ & $\begin{array}{l}8 \\
8 \\
8 \\
8 \\
8\end{array}$ & $\begin{array}{l}.12500 \\
.12500 \\
.12500 \\
.12500 \\
.12500\end{array}$ & $\begin{array}{l}.10000 \\
.10000 \\
.10000 \\
.10000 \\
.10000\end{array}$ & $\begin{array}{l}3.500 \\
4.000 \\
4.500 \\
5.000 \\
5.563\end{array}$ & $\begin{array}{l}.766 \\
.821 \\
.844 \\
.875 \\
.937\end{array}$ & $\begin{array}{l}1.20000 \\
1.25000 \\
1.30000 \\
1.35000 \\
1.40630\end{array}$ & $\begin{array}{l}.00781 \\
.00781 \\
.00781 \\
.00781 \\
.00781\end{array}$ & $\begin{array}{l}3.34062 \\
3.83750 \\
4.33438 \\
4.83125 \\
5.39073\end{array}$ & $\begin{array}{l}\text { 3. } 40022 \\
\text { 3. } 90053 \\
4.39884 \\
4.89766 \\
5.46101\end{array}$ & $\begin{array}{l}\text { 3. } 38850 \\
\text { 3. } 88881 \\
4.38712 \\
4.88594 \\
5.44929\end{array}$ & $\begin{array}{l}\text { 3. } 37678 \\
3.87709 \\
4.37541 \\
4.87422 \\
5.43757\end{array}$ \\
\hline $\begin{array}{l}6 \\
7 \\
8 \\
8 \\
9 \\
10 \\
9\end{array}$ & $\begin{array}{l}8 \\
8 \\
8 \\
8 \\
8\end{array}$ & $\begin{array}{l}.12500 \\
.12500 \\
.12500 \\
.12500 \\
.12500\end{array}$ & $\begin{array}{l}.10000 \\
.10000 \\
.10000 \\
.10000 \\
.10000\end{array}$ & $\begin{array}{r}6.625 \\
7.625 \\
8.625 \\
9.625 \\
10.750\end{array}$ & $\begin{array}{l}.958 \\
1.000 \\
1.063 \\
1.130 \\
1.210\end{array}$ & $\begin{array}{l}1.51250 \\
1.61250 \\
1.71250 \\
1.81250 \\
1.92500\end{array}$ & $\begin{array}{l}.00781 \\
.00781 \\
.00781 \\
.00781 \\
.00781\end{array}$ & $\begin{array}{r}6.44609 \\
7.43984 \\
8.43359 \\
9.42734 \\
10.54531\end{array}$ & $\begin{array}{r}6.51769 \\
7.51406 \\
8.51175 \\
9.50969 \\
10.63266\end{array}$ & $\begin{array}{r}6.50597 \\
7.50234 \\
8.50003 \\
9.49797 \\
10.62094\end{array}$ & $\begin{array}{r}6.49425 \\
7.49062 \\
8.48831 \\
9.48625 \\
10.60922\end{array}$ \\
\hline 150.0 & $\begin{array}{l}8 \\
8 \\
8 \\
8 \\
8\end{array}$ & $\begin{array}{l}.12500 \\
.12500 \\
.12500 \\
.12500 \\
.12500\end{array}$ & $\begin{array}{l}.10000 \\
.10000 \\
.10000 \\
.10000 \\
.10000\end{array}$ & $\begin{array}{l}11.750 \\
12.750 \\
14.000 \\
15.000 \\
16.000\end{array}$ & $\begin{array}{l}1.285 \\
1.360 \\
1.562 \\
1.687 \\
1.812\end{array}$ & $\begin{array}{l}2.02500 \\
2.12500 \\
2.25000 \\
2.35000 \\
2.45000\end{array}$ & $\begin{array}{l}.00781 \\
.00781 \\
.00781 \\
.00781 \\
.00781\end{array}$ & $\begin{array}{l}11.53906 \\
12.53281 \\
13.77500 \\
14.76875 \\
15.76250\end{array}$ & $\begin{array}{l}11.63109 \\
12.62953 \\
13.88434 \\
14.88591 \\
15.88747\end{array}$ & $\begin{array}{l}11.61938 \\
12.61781 \\
13.87262 \\
14.87419 \\
15.87575\end{array}$ & $\begin{array}{l}11.60766 \\
12.60609 \\
13.86091 \\
14.86247 \\
15.86403\end{array}$ \\
\hline $\begin{array}{lll}17 & \mathrm{O} . & \mathrm{D} \\
18 & \mathrm{O} & \mathrm{D}- \\
20 & \mathrm{O} & \mathrm{D}- \\
22 & \mathrm{O} . & \mathrm{D}- \\
24 & \mathrm{O} . \mathrm{D}\end{array}$ & $\begin{array}{l}8 \\
8 \\
8 \\
8 \\
8\end{array}$ & $\begin{array}{l}.12500 \\
.12500 \\
.12500 \\
.12500 \\
.12500\end{array}$ & $\begin{array}{r}.10000 \\
10000 \\
.10000 \\
.10000 \\
.10000\end{array}$ & $\begin{array}{l}17.000 \\
18.000 \\
20.000 \\
22.000 \\
24.000\end{array}$ & $\begin{array}{l}1.900 \\
2.000 \\
2.125 \\
2.250 \\
2.375\end{array}$ & $\begin{array}{l}2.55000 \\
2.65000 \\
2.85000 \\
3.05000 \\
3.25000\end{array}$ & $\begin{array}{l}.00781 \\
.00781 \\
.00781 \\
.00781 \\
.00781\end{array}$ & $\begin{array}{l}16.75625 \\
17.75000 \\
19.73750 \\
21.72500 \\
23.71250\end{array}$ & $\begin{array}{l}16.88672 \\
17.88672 \\
19.88203 \\
21.87734 \\
23.87266\end{array}$ & $\begin{array}{l}16.87500 \\
17.87500 \\
19.87031 \\
21.86562 \\
23.86094\end{array}$ & $\begin{array}{l}16.86328 \\
17.86328 \\
19.85859 \\
21.85391 \\
23.84922\end{array}$ \\
\hline $\begin{array}{lll}26 & 0 . & \mathrm{D}-- \\
28 & \mathrm{O} & \mathrm{D} \\
30 & \mathrm{O} & \mathrm{D} \\
\mathrm{D} & --\end{array}$ & $\begin{array}{l}8 \\
8 \\
8\end{array}$ & $\begin{array}{l}.12500 \\
.12500 \\
.12500\end{array}$ & $\begin{array}{l}.10000 \\
.10000 \\
.10000\end{array}$ & $\begin{array}{l}26.000 \\
28.000 \\
30.000\end{array}$ & $\begin{array}{l}2.500 \\
2.625 \\
2.750\end{array}$ & $\begin{array}{l}3.45000 \\
3.65000 \\
3.85000\end{array}$ & $\begin{array}{l}.00781 \\
.00781 \\
.00781\end{array}$ & $\begin{array}{l}25.70000 \\
27.68750 \\
29.67500\end{array}$ & $\begin{array}{l}25.86797 \\
27.86328 \\
29.85859\end{array}$ & $\begin{array}{l}25.85625 \\
27.85156 \\
29.84688\end{array}$ & $\begin{array}{l}25.84453 \\
27.83984 \\
29.83516\end{array}$ \\
\hline
\end{tabular}

(b) American National Straight-Pipe Threads.

The specified sizes and basic dimensions on the "American national straight-pipe threads" are given in Table 45.

1. Diameters of straight threads.-The basic pitch diameter of the straight thread is equal to the diameter at the gaging notch of American national taper-pipe thread, and is determined by the following formula based on the outside diameter of pipe and the pitch of thread (see Symbols above):

$$
E_{1}=D-(0.05 D+1.1) p+0.0625 L_{1}
$$

2. Tolerances.-The tolerance on pitch diameter of a straight-pipe thread is the equivalent of the variation in diameter over one and one-half turns either way from the gaging notch of the American national taper-pipe thread.61 (See columns 4 and 6 of Table 45.)

3. Application to internal threads.-Straightthreaded internal wrought-iron or wrought-steel couplings of the weight known as standard may be

${ }^{61}$ The coupling thread may be gaged with a taper-threaded plug gage. On account of the gage tolerance of one-half turn on working taper-pipe-thread gages, the working tolerance is equivalent to one turn either way from the gaging notch. In gaging, care must be taken to gage at the first thread and not at the end of the coupling when the thread is chamfered.

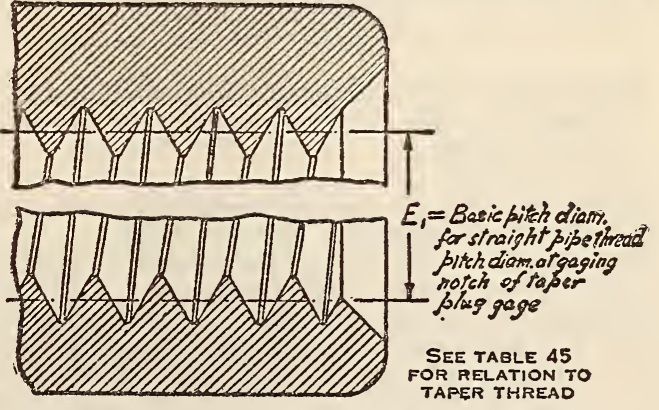

Figure 207.-American national straight pipe thread notation (internal)

Note.-This thread is gaged with the taper threaded plug gage and should gage flush at the face with the gaging notch, allowing a maximum variation of one turn plus or minus from the notch.

used with taper-threaded pipe for ordinary pressures, as they are sufficiently ductile to adjust themselves to the taper external thread when properly screwed together.

For high pressures, only taper external and internal threads should be used. 
4. Application to external threads.-Straight external threads are recognized only for special applications, such as long screws and tank nipples.

TABLE 45.-Dimensions of American national straight pipe threads (for couplings)

[For notation see fig. 207]

\begin{tabular}{|c|c|c|c|c|c|c|}
\hline \multirow{2}{*}{$\begin{array}{l}\text { Nom- } \\
\text { inal } \\
\text { size }\end{array}$} & \multirow{2}{*}{$\begin{array}{l}\text { Threads } \\
\text { per inch }\end{array}$} & \multirow{2}{*}{$\begin{array}{l}\text { Major } \\
\text { diam- } \\
\text { eter, } 1 \\
\text { basic }\end{array}$} & \multicolumn{3}{|c|}{ Pitch diameter } & \multirow{2}{*}{$\frac{\begin{array}{c}\text { Minor } \\
\text { diameter }\end{array}}{\text { Basic }}$} \\
\hline & & & $\begin{array}{l}\text { Maxi- } \\
\text { mum }\end{array}$ & Basic & $\begin{array}{l}\text { Mini- } \\
\text { mum }\end{array}$ & \\
\hline 1 & 2 & 3 & 4 & 5 & 6 & 7 \\
\hline $\begin{array}{c}\text { Inches } \\
1 / 8 \\
1 / 4 \\
3 / 8 \\
1 / 2 \\
3 / 4\end{array}$ & $\begin{array}{l}27 \\
18 \\
18 \\
14 \\
14\end{array}$ & $\begin{array}{r}\text { Inches } \\
0.40439 \\
.53433 \\
.67145 \\
.83557 \\
1.04600\end{array}$ & $\begin{array}{r}\text { Inches } \\
0.37823 \\
.49510 \\
.63222 \\
.78513 \\
.99557\end{array}$ & $\begin{array}{c}\text { Inches } \\
0.37476 \\
.48989 \\
.62701 \\
.77843 \\
.98887\end{array}$ & $\begin{array}{l}\text { Inches } \\
0.37129 \\
.48468 \\
.62181 \\
.77173 \\
.98217\end{array}$ & $\begin{array}{r}\text { Inches } \\
0.34513 \\
.44544 \\
.58257 \\
.72129 \\
.93172\end{array}$ \\
\hline $\begin{array}{l}1 \\
11 / 4 \\
11 / 2 \\
2 \\
21 / 2\end{array}$ & $\begin{array}{l}111 / 2 \\
111 / 2 \\
111 / 2 \\
111 / 2 \\
8\end{array}$ & $\begin{array}{l}1.30819 \\
1.65294 \\
1.89190 \\
2.36583 \\
2.86216\end{array}$ & $\begin{array}{l}1.24678 \\
1.59153 \\
1.83049 \\
\text { 2. } 30442 \\
2.77388\end{array}$ & $\begin{array}{l}\text { 1. } 23863 \\
\text { 1. } 58338 \\
\text { 1. } 82234 \\
\text { 2. } 29627 \\
\text { 2. } 76216\end{array}$ & $\begin{array}{l}1.23048 \\
1.57523 \\
1.81418 \\
2.28812 \\
\text { 2. } 75044\end{array}$ & $\begin{array}{l}\text { 1. } 16907 \\
\text { 1. } 51382 \\
\text { 1. } 75277 \\
\text { 2. } 22671 \\
\text { 2. } 66216\end{array}$ \\
\hline $\begin{array}{l}3 \\
31 / 2 \\
4 \\
41 / 2 \\
5\end{array}$ & $\begin{array}{l}8 \\
8 \\
8 \\
8 \\
8\end{array}$ & $\begin{array}{l}\text { 3. } 48850 \\
\text { 3. } 98881 \\
\text { 4. } 48713 \\
\text { 4. } 98594 \\
\text { 5. } 54929\end{array}$ & $\begin{array}{l}\text { 3. } 40022 \\
\text { 3. } 90053 \\
\text { 4. } 39884 \\
\text { 4. } 89766 \\
\text { 5. } 46101\end{array}$ & $\begin{array}{l}\text { 3. } 38850 \\
3.88881 \\
\text { 4. } 38712 \\
\text { 4. } 88594 \\
\text { 5. } 44929\end{array}$ & $\begin{array}{l}3.37678 \\
\text { 3. } 87709 \\
4.37541 \\
4.87422 \\
5.43757\end{array}$ & $\begin{array}{l}\text { 3. } 28850 \\
\text { 3. } 78881 \\
\text { 4. } 28713 \\
\text { 4. } 78594 \\
\text { 5. } 34929\end{array}$ \\
\hline 6 & 8 & 6. 60597 & 6.51769 & 6.50597 & 6.49425 & 6.40597 \\
\hline
\end{tabular}

1 The American national pipe thread form is maintained; therefore, the major and minor diameters vary with the pitch diameter and are determined by the threading tools.

5. Application to long screw joints.-Long screw joints are used to a limited extent. This joint is not considered satisfactory when subjected to high temperature or pressure. In this application the coupling has a straight thread and must make a joint with an American national taper pipe thread. (See fig. 207.) It is necessary that the coupling be screwed on the straight external thread for the full length of the coupling and then back until it engages the taper external thread. The straight thread on the pipe enters the coupling freely by hand, the

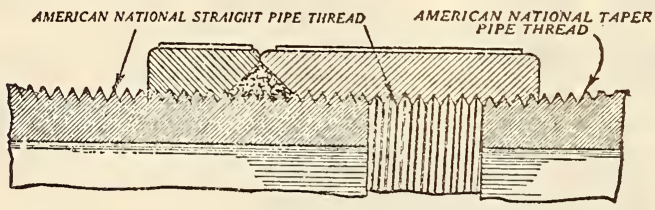

Figure 208.-Illustration of "long screw" joint between straight threaded coupling and taper threaded pipe

joint being made by a packing material between the locknut and the coupling. (See fig. 208.)

On account of the long engagement of thread, imperfections in pitch affect the fit when the coupling is screwed on the pipe its full length. Refinements of manufacture and gaging to insure a properly interchangeable product are more costly than the commercial use warrants; therefore, the use of this type of joint is not recommended. For this reason, specifications for tolerances and gaging are not included herein.

\section{(c) American National Locknut Threads}

Occasional requirements make it advisable to have a straight thread of the largest diameter it is possible to cut on a pipe. This practice has been standardized and is known as "maximum external and minimum internal locknut threads." For dimensions, see Table 46. The "tank nipple" shown in
Figure 210 is an example of this thread. In this application an American national standard-taper

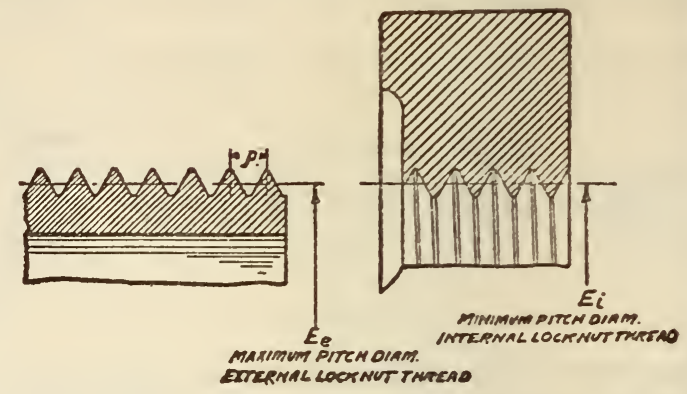

Figdre 209.-American national locknut thread notation

\section{NOTATION}

$E_{1}=$ pitch diameter at gaging notch of American national taper plug gage.

$E_{\mathrm{e}}=E_{1}+(4 \mathrm{p} \times 0.0625)$

$E_{1}=E_{1}+(5 \mathrm{p} \times 0.0625)$

NOTE.-See Table 46 for relation to taper pipe thread.

pipe thread is cut on the end of the pipe after having first cut the "external locknut thread."

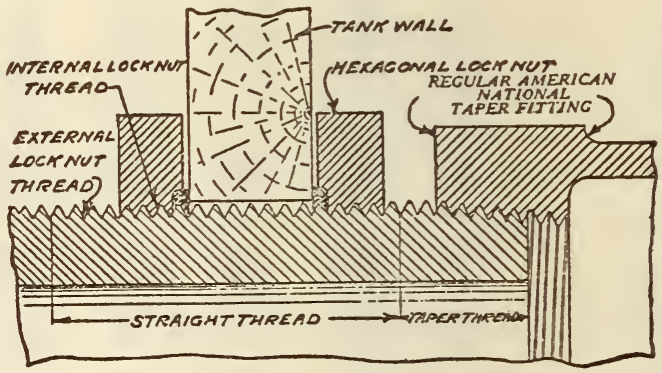

FIgURE 210.-Illustration of "tank nipple" thread TABLE 46.-Dimensions of American national
locknut threads

[For notation, see fig. 209]

\begin{tabular}{|c|c|c|c|c|}
\hline $\begin{array}{l}\text { Nom- } \\
\text { inal } \\
\text { size }\end{array}$ & $\begin{array}{l}\text { Threads } \\
\text { per inch }\end{array}$ & $\begin{array}{c}E_{\mathrm{e}} \text { (Maxi- } \\
\text { mum) } 1\end{array}$ & $\begin{array}{l}E_{\mathrm{i}} \text { (Mini- } \\
\text { mum) }\end{array}$ & $\begin{array}{l}\text { Depth of } \\
\text { thread }\end{array}$ \\
\hline 1 & 2 & 3 & 4 & 5 \\
\hline $\begin{array}{c}\text { Inches } \\
16 \\
1 / 4 \\
36 \\
1 / 2 \\
3 / 4\end{array}$ & $\begin{array}{l}27 \\
18 \\
18 \\
14 \\
14\end{array}$ & $\begin{array}{l}\text { Inches } \\
0.38402 \\
.50378 \\
.64090 \\
.79629 \\
1.00672\end{array}$ & $\begin{array}{l}\text { Inches } \\
0.38633 \\
.50725 \\
.64437 \\
.80075 \\
1.01119\end{array}$ & $\begin{array}{l}\text { Inch } \\
0.02963 \\
.04444 \\
.04444 \\
.05714 \\
.05714\end{array}$ \\
\hline $\begin{array}{l}1 \\
11 / 4 \\
11 / 2 \\
2 \\
21 / 2\end{array}$ & $\begin{array}{l}111 / 2 \\
111 / 2 \\
111 / 2 \\
111 / 2 \\
8\end{array}$ & $\begin{array}{l}\text { 1. } 26037 \\
\text { 1. } 60512 \\
\text { 1. } 84408 \\
\text { 2. } 31801 \\
\text { 2. } 79341\end{array}$ & $\begin{array}{l}1.26580 \\
1.61055 \\
1.84951 \\
\text { 2. } 32345 \\
\text { 2. } 80122\end{array}$ & $\begin{array}{l}.06957 \\
.06957 \\
.06957 \\
.06957 \\
.10000\end{array}$ \\
\hline $\begin{array}{l}3 \\
31 / 2 \\
4 \\
41 / 2\end{array}$ & $\begin{array}{l}8 \\
8 \\
8 \\
8\end{array}$ & $\begin{array}{l}\text { 3. } 41975 \\
\text { 3. } 92006 \\
4.41838 \\
4.91719\end{array}$ & $\begin{array}{l}3.42756 \\
3.92787 \\
4.42619 \\
4.92500\end{array}$ & $\begin{array}{l}.10000 \\
.10000 \\
.10000 \\
.10000\end{array}$ \\
\hline $\begin{array}{l}5 \\
6 \\
7 \\
8\end{array}$ & $\begin{array}{l}8 \\
8 \\
8 \\
8\end{array}$ & $\begin{array}{l}5.48054 \\
6.53722 \\
7.53359 \\
8.53128\end{array}$ & $\begin{array}{l}5,48836 \\
6.54503 \\
7.54141 \\
8.53909\end{array}$ & $\begin{array}{l}.10000 \\
.10000 \\
.10000 \\
.10000\end{array}$ \\
\hline $\begin{array}{r}9 \\
10 \\
11 \\
12\end{array}$ & $\begin{array}{l}8 \\
8 \\
8 \\
8\end{array}$ & $\begin{array}{r}9.52922 \\
10.65219 \\
11.65063 \\
12.64906\end{array}$ & $\begin{array}{r}9.53703 \\
10.66600 \\
11.65844 \\
12.65688\end{array}$ & $\begin{array}{l}.10000 \\
.10000 \\
.10000 \\
.10000\end{array}$ \\
\hline
\end{tabular}

1 A tolerance equivalent to $11 / 2$ turns of the American national taper pipe thread is recommended, the tolerance being minus on $E_{\bullet}$ and plus on $E_{\mathrm{i}}$. 
607.1 CAST-IRON PIPE, FITTINGS, AND CONNECTIONS.

607.10 GENERAL ITEMS.

ASSOCIATED FACTORY MUTUAL FIRE INSURANCE COMPANIES, RULES FOR LAYING CAST-IRON WATER PIPES IN FACTORY YARDS, 1931

\section{WATER PIPES FOR FIRE PROTECTION}

(These rules specify that underground pipe for fire protection should be made in accordance with the standards of the New England Water Works Association or of the American Water Works Association, class $\mathrm{C}$, or the equivalent. The rules cover the depth of laying of pipe, protection against frost, care in laying, amount of jute yarn and lead required for making joints, installation of hydrants and gate valves, connections from water supplies, and testing of piping and hydrants.)

607.11 CAST-IRON PIPE CULVERTS, DRAINS, AND SEWERS.

AMERICAN ASSOCIATION OF STATE HIGHWAY OFFICIALS, METHODS OF TESTING CULVERT PIPE, TENTATIVE METHOD T-33, 1931

\section{TESTING CAST-IRON CULVERT PIPE PHYSICAL TESTS}

Strength tests. - These methods of making strength tests are intended to apply to cast iron, vitrified clay, and concrete culvert pipe.

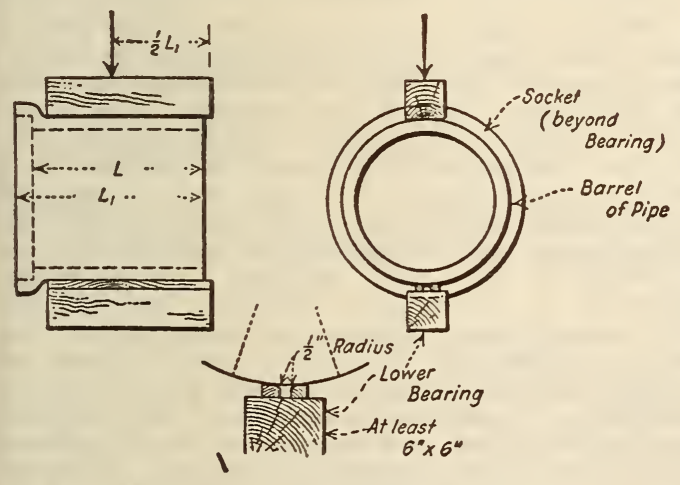

FIGURE 211.-Three-edge bearings

Pipe may be tested for strength by either the 3edge bearing or sand-bearing method.

\section{THREE-EDGE BEARING METHOD}

When the 3-edge bearing method is used, the lower bearing for the pipe shall consist of two wooden strips with vertical sides having their interior top corners rounded to a radius of approximately onehalf inch. The strips shall be straight and shall be securely fastened to a rigid block with the interior vertical sides spaced a distance apart not less than one-half inch or more than one inch for each foot of pipe diameter. The upper bearing shall be a rigid wooden block, straight and true from end to end. The upper and lower bearings shall extend the full length of pipe exclusive of bell. The pipe shall be placed symmetrically between the two bearings as illustrated in Figures 211 and 212. In testing pipe which is "out of line" the lines of the bearings chosen shall be from those which appear to give the most favorable conditions for fair test.
When the sand-bearing method is used (see figs. 213 and 214), the ends of each specimen of pipe shall be accurately marked prior to the test in quarters of the circumference. Specimens shall be carefully bedded, above and below, in sand, for

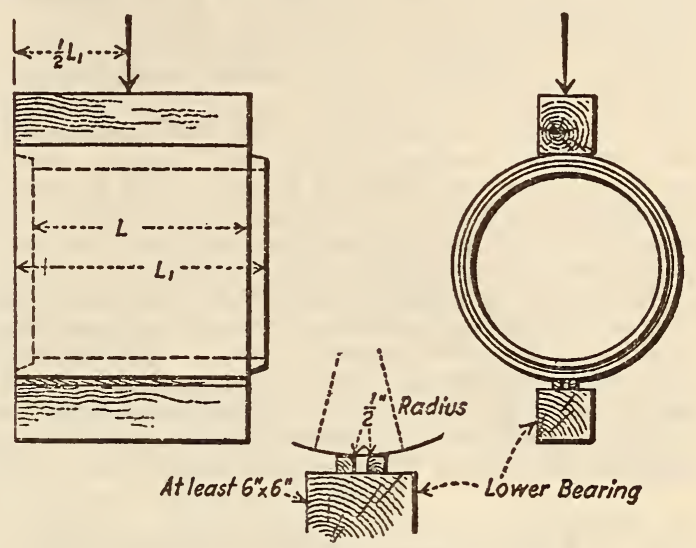

FIguRE 212.-Three-edge bearings

one-fourth the circumference of the pipe measured on the middle line of the barrel. The depth of bedding above and below the pipe at the thinnest points shall be one-half the radius of the middle line of the barrel.

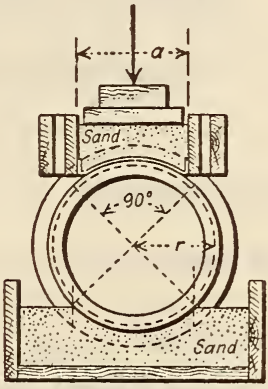

Bedding of Barrel.

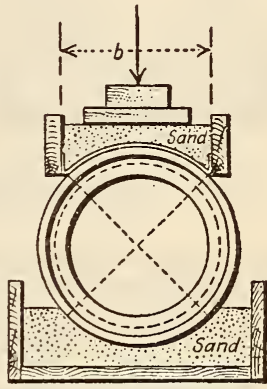

Bedding of Socket.

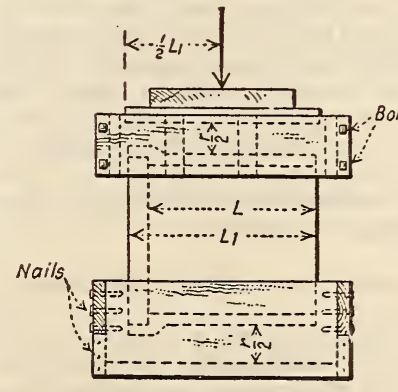

Side View.

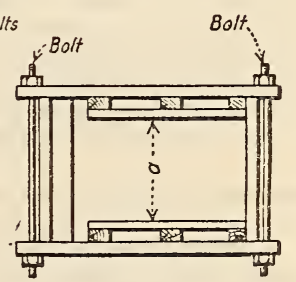

Upper

Bearing Frame.
FIGURE 213.- Sand bearings

The sand used shall be clean and moist, and shall be such as will pass a 4,760 micron (No. 4) sieve. The sand in the lower bearing shall be loose when the pipe is placed.

The top bearing frame shall not be allowed to come into contact with the pipe nor with the top bearing plate. The upper surface of the sand in the top bearing shall be struck off level with a straight- 
edge, and shall be covered with a rigid top bearing plate, with lower surface a true plane, made of heavy timbers or other rigid material, capable of distributing the test load uniformly without appreciable bending. The test load shall be applied at the exact center of this top bearing plate, or in such manner as to produce uniform deflection throughout the full length of the pipe. For this purpose a spherical bearing is preferred, but two rollers at right angles may be used. The test may be made without the use of a testing machine, by piling weights directly on a platform resting on the top bearing plate; provided, however, that the weights shall be piled symmetrically about a vertical line through the center of the pipe, and that the platform shall not be allowed to touch the top bearing frame.

The frames of the top and bottom bearings shall be made of timbers so heavy as to avoid appreciable bending by the side pressure of sand. The interior surfaces of the frames shall be dressed. No frame shall come in contact with the pipe during the test. A strip of cloth may, if desired, be attached to the inside of the upper frame on each side, along the

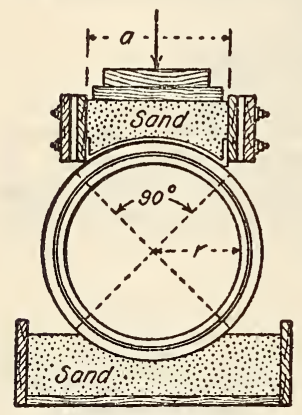

Bedding of Borrel

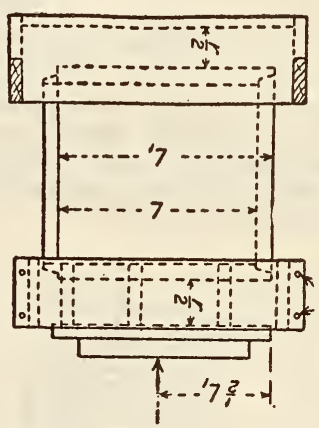

FIgURE 214.-Sand bearings

lower edge, to prevent the escape of sand between the frame and the pipe.

\section{TESTING APPARATUS}

It is desirable that the testing machine used for the strength tests shall give a uniform deflection throughout the full length of the pipe. Any mechanical or hand-power device may be used in which the head that applies the load moves at a speed of not more than 0.05 inch per minute while making the test. The testing machine shall be substantial and rigid throughout, so that the distribution of the load will not be affected appreciably by the deformation or yielding of any part. The load shall be applied continuously until the ultimate strength of the pipe is reached.

\section{PRELIMINARY TESTS AND TESTS FOR EXTENDED DELIVERIES}

Preliminary to placing an order, a purchaser of pipe whose needs require shipments at intervals over extended periods of time shall be entitled to test not more than 10 pieces of pipe covering the size in which he is interested. The test specimens shall be selected in approximately equal numbers from the larger and smaller sizes of pipe. The acceptability of the larger sizes of pipe shall not be based on the results of tests in smaller sizes. After these preliminary tests, a purchaser shall be entitled to additional tests in such numbers and at such times as he may deem necessary, provided that the total number of pipe tested shall not exceed 2 per cent of the total deliveries.

\section{TESTS FOR OCCASIONAL ORDERS}

A purchaser who places occasional orders shall be entitled to test a number of pipe equal to 2 per cent of an order but not to exceed five pieces of any one size; otherwise the number of pipe desired for testing shall be included in the order.

\section{SELECTING TEST SPECIMENS}

All pipe for testing purposes shall be selected at random by the purchaser from the stock of the manufacturer and shall be pipe which would not otherwise be rejected under these specifications. The pipe shall be free from visible moisture when tested and they shall not have been exposed to a temperature below $40^{\circ} \mathrm{F}$. for the 24 hours immediately preceding the test.

Pipe shall be acceptable under the strength tests when all test specimens conform to the test requirements. Should less than 3 of the 10 preliminary test specimens, or any one of the additional test specimens provided for above in the preliminary tests, or any one specimen provided for above in tests for occasional orders, fail to meet test requirements, then the manufacturer will be allowed a retest on two like specimens for each specimen that failed, and the pipe shall be acceptable only when all of these retest specimens meet the test requirements. No further retests shall be permitted.

\section{AMERICAN RAILWAY ENGINEERING ASSO- CIATION, SPECIFICATIONS FOR CAST- IRON CULVERT PIPE, 1930}

\section{CAST-IRON CULVERT PIPE}

\section{MANOFACTURE}

The pipe shall be manufactured of cast iron of good quality and of such character as to be strong, tough, and of even grain, but soft enough to admit of satisfactory drilling and cutting. The metal shall be made without any admixture of cinder iron or other inferior metal and shall be remelted in a cupola or air furnace. The pipe shall be hub and spigot style of cast-iron water pipe or cast-iron culvert pipe, or spiral corrugated cast-iron pipe, or a ribbed or corrugated cast-iron pipe of design approved by the railroad company, smooth, free from scales, lumps, blisters, and other defects impairing its strength or durability, and be round, of uniform physical character, of close grain, and cast vertically. It shall be manufactured in lengths of not less than 3 feet, and the inner and outer surfaces shall be true, whole, concentric cylinders. This pipe shall be heated to $300^{\circ} \mathrm{F}$. and immersed in coal tar varnish so that the result will be a smooth coating on both the inside and outside of the pipe. This coating must be of such a nature that it will be tough, tenacious-not brittle-or have any tendency to scale when cold, and such results shall be consistent.

\section{PHYSICAL PROPERTIES AND TESTS}

Cast-iron culvert pipe shall have minimum thickness of pipe as follows:

\begin{tabular}{|c|c||c|c|}
\hline $\begin{array}{c}\text { Inside } \\
\text { diam- } \\
\text { eter }\end{array}$ & Thickness & $\begin{array}{c}\text { Inside } \\
\text { diam- } \\
\text { eter }\end{array}$ & Thickness \\
\hline Inches & Inch & Inches & Inch \\
12 & $7 / 16$ & 24 & 9116 \\
14 & $7 / 16$ & 30 & $11 / 16$ \\
16 & $1 / 2$ & 36 & $13 / 16$ \\
18 & 112 & 42 & $15 / 16$ \\
20 & $1 / 2$ & 48 & $11 / 4$ \\
\hline
\end{tabular}


No pipe shall be accepted the thickness of which is one-sixteenth inch less than the thickness herein specified.

The transverse test shall be used to determine the physical properties. The test bar shall be the 1 by 2 by 28 inch American Foundrymen's Association standard rectangular bar. This bar shall be cast vertically in a dry sand mold, coated with a bituminous facing and not cast until the mold is cold. The test bar shall not be tumbled or otherwise treated, but simply brushed off before testing.

The test shall be transverse with the bar placed horizontally upon supports 24 inches apart and a concentrated load applied midway between the supports.

The test shall conform to the following requirements as a minimum:

Average load at the center

Average deflection at center.

pounds_ 2,200

From each melt, test bars shall be poured, the first one five minutes after the first tap and then one bar from each 2-hour interval or fraction thereof, throughout the heat.

The pipe shall have sufficient strength so that when tested by the 3-edge bearing method as specified in the standard specifications for drain tile C14-24, of the American Society for Testing Materials, will show a load-supporting capacity in pounds per linear foot equal to or greater than that obtained by the use of the formula, $W$ equals $1,500 D$, in which $W$ equals pounds per foot and $D$ equals the inside diameter of pipe in feet. All pipe shall be carefully examined for defects and sounded with a hammer before shipment. No fillings with metal, cement, or other materials, or so-called burning on of iron, will be permitted. The castings shall be sound and free from cracks, sand holes, blowholes, and cold shuts.

\section{INSPECTION AND REJECTION}

(Conform with A. S. T. M. standard specifications for steel, paragraph 7 . See 605.0, p. 247.)

\section{AMERICAN RAILWAY ENGINEERING AS- SOCIATION, PLUMBING FOR RAILWAY BUILDINGS, 1929}

\section{CAST-IRON PIPE AND FITTINGS}

Concealed soil, waste, drain, sewer, and vent pipes in buildings shall be extra heavy cast-iron soil pipe. Pipe shall have the maker's name cast thereon and shall be in lengths not less than 5 feet. It shall be sound and free from defects and with the inner and outer surfaces concentric and smooth and of the following weights per linear foot:

\begin{tabular}{|r|c||r|l|}
\hline Inches & Pounds & Inches & Pounds \\
\cline { 1 - 3 } 2 & $51 / 2$ & 6 & 20 \\
3 & $91 / 2$ & 7 & 27 \\
4 & 13 & 8 & $331 / 2$ \\
5 & 17 & 10 & 45 \\
\hline
\end{tabular}

Fittings for cast-iron pipe shall be extra heavy cast iron of the same make as the soil pipe, and shall be of the same inside diameter as the pipe with which they are connected. Unless otherwise specified, cast-iron pipe and fittings must be coated with hot asphaltum both inside and outside by dipping. Fittings for the junction of cast and wrought iron pipe shall be cut at one end with full threads; and fittings supporting risers shall have proper shoes cast on them.

Water supply pipes larger than $21 / 2$ inches diameter located below ground shall be class $B$ cast-iron pipe, in accordance with the specifications of the American Water Works Association (see 607.12, p. 379), unless local conditions require the use of heavier pipe.

\section{AMERICAN SOCIETY FOR TESTING MATE- RIALS, STANDARD SPECIFICATIONS FOR CAST-IRON SOIL PIPE AND FITTINGS, DESIGNATION A74-29, 1929}

\section{CAST-IRON SOIL PIPE AND FITTINGS}

\section{MANUFACTURE}

1. The cast iron from which the pipe and fittings are made shall be of such composition, and the conditions of manufacture so maintained, that the castings will be of uniform physical character, close grain, and not hard, brittle, nor difficult to cut with file or chisel.

2. (a) When pipe or fittings are to be coated, coaltar pitch shall be used, which shall contain sufficient oil to make a smooth coating. The pitch shall be tough and tenacious when cold, and not brittle nor having any tendency to scale.

(b) The varnish shall be heated to about $300^{\circ} \mathrm{F}$. and shall remain at this temperature during the time the casting is immersed.

(c) Each casting shall be heated to a uniform temperature of about $300^{\circ} \mathrm{F}$. immediately before it is dipped, and shall possess this temperature at the time it is put in the bath.

(d) Each casting shall remain in the bath at least two minutes.

(e) Fresh pitch and oil shall be added when necessary to keep the mixture of the proper consistency, and the vat shall be emptied of its contents and refilled with fresh pitch whenever the accumulation of sand or carbonaceous matter renders this desirable, as can be seen by the solids adhering to the under side or lower ends of the castings.

(f) After being coated, the pipe and fittings shall be carefully drained of the surplus varnish.

\section{CHEMICAL PROPERTIES AND TESTS}

3. Drillings taken from the fractured end of the arbitration test bar shall not contain over 0.10 per cent of sulphur.

\section{PHYSICAL PROPERTIES AND TESTS}

4. The transverse test speciment (arbitration test bars) specified in section 6 , when placed horizontally upon supports 18 inches apart and tested under a centrally applied load, shall conform to the following minimum requirements:

$$
\text { Average load at center................. pound .. 1, } 500
$$

Average deflection at center............ inch.. 0.20

5. All pipe shall be tested to a hydrostatic pressure of not less than 50 pounds per square inch

TABLE 1.-Weights of soil pipe

\begin{tabular}{|c|c|c|c|}
\hline & \multicolumn{2}{|c|}{ Single hub } & $\begin{array}{c}\text { Double } \\
\text { hub }\end{array}$ \\
\cline { 2 - 4 } Size & $\begin{array}{c}\text { Per 5-foot } \\
\text { length }\end{array}$ & $\begin{array}{c}\text { Per foot, } \\
\text { including } \\
\text { hub }\end{array}$ & $\begin{array}{c}\text { Per 5-foot } \\
\text { length }\end{array}$ \\
\cline { 2 - 4 } Inches & Pounds & Pounds & Pounds \\
2 & $271 / 2$ & $51 / 2$ & $271 / 2$ \\
3 & $471 / 2$ & $91 / 2$ & $471 / 2$ \\
4 & 65 & 13 & 65 \\
5 & 85 & 17 & 85 \\
6 & 100 & 20 & 100 \\
\hline
\end{tabular}

before coating. Any casting showing defects under this hydrostatic test shall be promptly broken and returned to the cupola. 
6. The arbitration test bar shall be in accordance with the standard specifications for the arbitration test bar and tension test specimen for cast iron in A. S. T. M. A124 (see. 600.1, p. 18).

7. From each melt of metal not less than three test specimens (arbitration test bars) shall be poured, the first of which shall be poured within five minutes after the first ladle is tapped and the remainder at intervals not exceeding one hour throughout the melt.
STANDARD SIZES AND WZIGHTS

8. (a) The inside diameter of the barrel of any pipe or fittings or branch thereof shall not vary more than one-eighth inch under the nominal size of pipe.

(b) The outside diameter of the barrel of pipe and fittings shall be one-half inch greater that its nominal inside diameter. A variation in the outside diameter of one-eighth inch over or under these figures will be permitted.

\section{TABLE 2.-Weights of soil pipe fittings}

[Only the staple fittings are shown. From the data herewith the weights of other fittings may be calculated]

[All values are in pounds]

\begin{tabular}{|c|c|c|c|c|c|c|c|c|c|c|c|c|c|c|c|}
\hline \multirow[b]{2}{*}{ Fittings } & \multicolumn{15}{|c|}{ Size of fittings (in inches) } \\
\hline & 2 & 3 & 4 & 5 & 6 & $\begin{array}{c}3 \\
\text { by } \\
2\end{array}$ & $\begin{array}{c}4 \\
\text { by } \\
2\end{array}$ & $\begin{array}{c}4 \\
\text { by } \\
3\end{array}$ & $\begin{array}{c}5 \\
\text { by } \\
2\end{array}$ & $\begin{array}{c}5 \\
\text { by } \\
3\end{array}$ & $\begin{array}{c}5 \\
\mathrm{by} \\
4\end{array}$ & $\begin{array}{c}6 \\
\text { by } \\
2\end{array}$ & $\begin{array}{c}6 \\
\text { by } \\
3\end{array}$ & $\begin{array}{c}6 \\
\text { by } \\
4\end{array}$ & $\begin{array}{c}6 \\
\text { by } \\
5\end{array}$ \\
\hline $\begin{array}{l}1 / 4 \text { bends, regular } \\
1 / 4 \text { bends, short sweep } \\
1 / 4 \text { bends, long sweep } \\
1 / 5 \text { bends. } \\
1 / 6 \text { bends. }\end{array}$ & $\begin{array}{r}63 / 4 \\
81 / 4 \\
101 / 4 \\
61 / 4 \\
6\end{array}$ & $\begin{array}{r}101 / 4 \\
121 / 2 \\
153 / 4 \\
93 / 4 \\
91 / 4\end{array}$ & $\begin{array}{l}15 \\
173 / 4 \\
22 \\
133 / 4 \\
13\end{array}$ & $\begin{array}{l}19 \\
221 / 2 \\
271 / 2 \\
171 / 2 \\
161 / 2\end{array}$ & $\begin{array}{l}231 / 2 \\
271 / 2 \\
331 / 2 \\
211 / 2 \\
20\end{array}$ & & & & & & & & & & 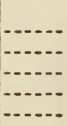 \\
\hline $\begin{array}{l}1 / 8 \text { bends } \\
1 / 16 \text { bends. } \\
\text { Return bends. }\end{array}$ & $\begin{array}{l}51 / 2 \\
5 \\
83 / 4\end{array}$ & $\begin{array}{c}81 / 2 \\
73 / 4 \\
14\end{array}$ & $\begin{array}{l}121 / 4 \\
103 / 4 \\
201 / 4\end{array}$ & $\begin{array}{l}151 / 4 \\
131 / 4 \\
261 / 2\end{array}$ & $\begin{array}{l}181 / 4 \\
153 / 4 \\
331 / 2\end{array}$ & & & & & & & & & & \\
\hline Tapped tees (tapped up to 2 inches) & $\begin{array}{r}101 / 4 \\
83 / 4\end{array}$ & $151 / 4$ & 21 & $261 / 2$ & $32 \frac{1}{2}$ & $\begin{array}{l}131 / 4 \\
113 / 4\end{array}$ & $\begin{array}{l}16 \overline{1 / 2} \\
15\end{array}$ & $183 / 4$ & $19 \overline{1} / 4$ & 22 & $24 \frac{11 / 2}{2}$ & $\begin{array}{l}221 / 2 \\
2016\end{array}$ & 25 & $27 \overline{15}$ & 30 \\
\hline $\begin{array}{l}\text { Sanitary tees } \\
\text { inches) }\end{array}$ & \begin{tabular}{l|}
11 \\
9
\end{tabular} & $161 / 4$ & $221 / 2$ & 28 & $341 / 2$ & $\begin{array}{l}14 \\
12\end{array}$ & $\begin{array}{l}171 / 4 \\
151 / 4\end{array}$ & $193 / 4$ & $\begin{array}{l}20 \\
18\end{array}$ & 23 & $251 / 2$ & $\begin{array}{l}23 \\
211 / 4\end{array}$ & 26 & 29 & $311 / 2$ \\
\hline Y/2 Y Y branch & $\begin{array}{l}11 \\
101 / 4\end{array}$ & 17 & $211 / 2$ & $\begin{array}{l}311 / 2 \\
271 / 2\end{array}$ & $\begin{array}{l}391 / 2 \\
34\end{array}$ & $\begin{array}{l}14 \\
131 / 2\end{array}$ & $\begin{array}{l}171 / 4 \\
161 / 2\end{array}$ & $201 / 2$ & $\begin{array}{l}20 \\
191 / 2\end{array}$ & 2316 & $27 \frac{16}{25}$ & $\begin{array}{l}23 \\
221 / 2\end{array}$ & 25162 & $281 / 2$ & 31 \\
\hline Tapped inverted $Y$ branch (tapped up to & $101 / 4$ & $133 / 4$ & $171 / 2$ & 21 & 24 & & & & & & & & & & \\
\hline Inverted $Y$ branch & 1116 & 18 & $251 / 2$ & 33 & $411 / 2$ & 15 & $183 / 4$ & 22 & 22 & $251 / 2$ & $291 / 2$ & $251 / 2$ & $291 / 2$ & 33 & 37 \\
\hline $\begin{array}{l}\text { Combination } Y \text { and } 18 \text { bend } \\
\text { Upright } Y \text { branch }\end{array}$ & $\begin{array}{l}12 \\
121 / 2\end{array}$ & $\begin{array}{l}183 / 4 \\
191 / 2\end{array}$ & $\begin{array}{l}27 \\
28\end{array}$ & $\begin{array}{l}35 \\
361 / 2\end{array}$ & $\begin{array}{l}4416 \\
46\end{array}$ & 15 & 1814 & $221 / 2$ & 21 & 2516 & 3012 & 24 & 29 & 34 & $\begin{array}{l}39 \\
40\end{array}$ \\
\hline & & & & & & $15 \%$ & $18 \%$ & 23 & 2142 & 2612 & 3112 & $241 / 2$ & 2912 & 35 & 40 \\
\hline
\end{tabular}

(c) All pipe and fittings shall be of uniform thickness of wall and present true circles at the hub and spigot ends. A variation of one-sixteenth inch under the following dimensions will be permitted, but only when the actual weight is not less than the variation of the marked or estimated weight as given in Tables 1 and 2:

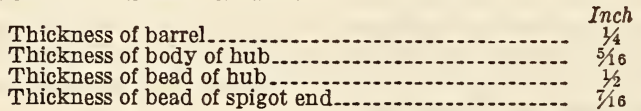

9. (a) Weights and measurements of pipe and fittings shall be taken as those of plain uncoated pipe. All weights shall be given in pounds.

(b) Individual lengths of pipe and fittings may weigh 5 per cent less than designated in Tables 1 and 2, but only when the average weight of a given size and weight of pipe and fittings selected at random, is not less than that shown in Tables 1 and 2.

(c) The regular length of pipe shall be such as to lay 5 feet, including hub.

(d) The average weights of soil pipe and fittings shall not be less than those given in Tables 1 and 2 .

\section{WORKMANSHIP AND FINISH}

10. (a) All pipe and fittings shall be practically straight and cylindrical and fittings true to pattern. The specified sizes shall be for the inside diameter and shall conform, within the allowable variation, to the dimensions given in the tables.

(b) All pipe and fittings shall be carefully examined for defects and sounded with a hammer before shipment. No fillings with metal, cement, or other material, or so-called burning on of iron will be permitted. The castings shall be sound and free from cracks, sand holes, blowholes, and cold shots.

\section{MARKING}

11. All pipe and fittings shall be marked with the name of the manufacturer, or appropriate initial. Each casting shall have cast upon it the minimum or estimated weight of same as shown in Tables 1 and 2 .

\section{INSPECTION AND REJECTION}

(Inspection and rejection conform to the requirements specified in A. S. T. M. standard specifications for metals. See 605.0 , p. 247.)

\section{UNITED STATES GOVERNMENT, FEDERAI SPECIFICATIONS BOARD, FEDERAL SPECIFICATION FOR PIPE AND PIPE FITTINGS, SOIL, CAST IRON, COATED AND UNCOATED, NO. 343a, AUGUST 22, 1928. (CURRENT DESIGNATION: WW- $\mathrm{P}-401)$}

\section{CAST-IRON SOIL PIPE AND FITTINGS}

(This specification conforms to A. S. T. M. A74, given above, except for the additional weights of fittings, the laying lengths of fittings, and the dimensions of hubs and spigots shown in the annexed tables. F. S. B. No. 343a $(\mathrm{WW}-\mathrm{P}-401)$ also contains the following provisions:) 


\section{GENERAL REQUIREMENTS}

Unless otherwise specified, the pipe shall be provided with a lead groove inside the hub opposite the hub band. A variation in straightness not

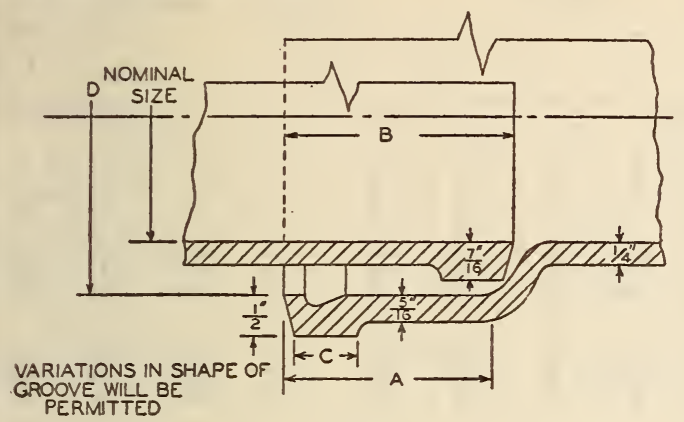

Figure 215

exceeding one-fourth inch per 5 feet length of pipe will be permitted,

\section{DETAIL REQUIREMENTS}

The depth of hub (see dimension A, fig. 215) shall be the distance, measured parallel to the axis of the opening, from the end of the hub to the beginning
1 of any offset or change of direction of the inside wall of same.

The lettered dimensions indicated in Figure 215 shall conform to the requirements as listed in Table 1.

TABLE 1

\begin{tabular}{|c|c|c|c|c|c|c|}
\hline \multirow{2}{*}{ Dimension } & \multicolumn{6}{|c|}{ Nominal size (inches) } \\
\hline & 2 & 3 & 4 & 5 & 6 & 8 \\
\hline $\begin{array}{l}\text { Depth of hub } A \text {, shall be } \\
\text { not less than }\end{array}$ & \multirow{4}{*}{$\begin{array}{r}\text { Ins. } \\
21 / 4 \\
212 \\
5, \\
5,8 \\
3316 \\
\pm 1 / 16\end{array}$} & \multirow{4}{*}{$\begin{array}{r}\text { Ins. } \\
21 / 2 \\
23 / 4 \\
11 / 16 \\
4316 \\
\pm 1 / 16\end{array}$} & \multirow{4}{*}{$\begin{array}{c}\text { Ins. } \\
23 / 4 \\
3 \\
3 / 4 \\
53 / 16 \\
\pm 1 / 16\end{array}$} & \multirow{4}{*}{$\begin{array}{c}\text { Ins. } \\
23 / 4 \\
3 \\
3 / 4 \\
63 / 16 \\
\pm 1 / 16\end{array}$} & \multirow{4}{*}{$\begin{array}{c}\text { Ins. } \\
23 / 4 \\
3 \\
3 / 4 \\
7316 \\
\pm 116\end{array}$} & \multirow{4}{*}{$\begin{array}{c}\text { Ins. } \\
3 \\
31 \\
1 \\
1 \\
19.62 \\
39.37\end{array}$} \\
\hline $\begin{array}{l}\text { Spigot end shall telescope } \\
\mathrm{B} \text { not less than }\end{array}$ & & & & & & \\
\hline $\begin{array}{l}\text { Width of hub bead C, shall } \\
\text { be not less than }\end{array}$ & & & & & & \\
\hline $\begin{array}{l}\text { Inside diameter of hub } \mathrm{D} \\
\text { shall be as follows.--- }\end{array}$ & & & & & & \\
\hline
\end{tabular}

The laving length of fittings shall be in accordance with dimensions given in Table 3 , with a tolerance of $\pm 1 / 4$ inch.

Calking room.- The spigot end, including bead, of every fitting must be straight without offset or change in direction for at least 4 inches, except that on 3 -inch bends this may be $3 \frac{1}{2}$ inches, and on 2 -inch bends, 3 inches. On all traps this must be at least 5 inches.

TABLE 2.-Weight of soil pipe fittings

[Only the single hub staple fittings are listed. From the data herewith the weights of other fittings may be calculated. All weights are in pounds. Sizes are nominal inside diameter]

\begin{tabular}{|c|c|c|c|c|c|c|c|c|c|c|c|c|}
\hline \multirow{2}{*}{ Fittings } & \multicolumn{12}{|c|}{ Size of fittings (inches) } \\
\hline & 2 & 3 & 4 & 5 & 6 & 8 & 8 by 2 & 8 by 3 & 8 by 4 & 8 by 5 & 8 by 6 & 8 by 8 \\
\hline 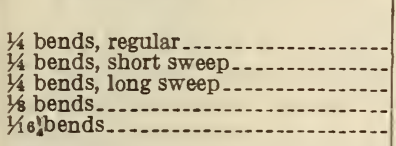 & Pounds & Pounds & Pounds & Pounds & Pounds & \begin{tabular}{|r|} 
Pounds \\
45 \\
58 \\
78 \\
35 \\
28
\end{tabular} & \begin{tabular}{|c|} 
Pounds \\
\\
\\
\end{tabular} & \begin{tabular}{|l} 
Pounds \\
\\
\\
\end{tabular} & 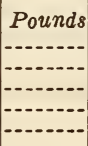 & 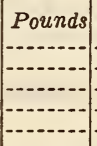 & $\begin{array}{c}\text { Pounds } \\
\\
\end{array}$ & $\begin{array}{c}\text { Pounds } \\
\\
\\
\\
\end{array}$ \\
\hline Tees. & & & & & & & $\begin{array}{r}35 \\
30\end{array}$ & 43 & 46 & 50 & 54 & 62 \\
\hline $\begin{array}{l}\text { Sanitary tees } \\
\text { inches) }\end{array}$ & A. S. & $\begin{array}{l}\text { Tons lde } \\
\text { T. M. A }\end{array}$ & 74 , given & $\begin{array}{l}\text { With tnc } \\
\text { above. }\end{array}$ & ose in & & $\begin{array}{l}37 \\
29\end{array}$ & 40 & 48 & 51 & 61 & 81 \\
\hline $\begin{array}{l}Y \text { branch } \\
1 / 2 \mathrm{Y} \text { branch } \\
\text { Tapped inverted } \mathrm{Y} \text { branch (tapped } \\
\text { to } 2 \text { inches) }\end{array}$ & & & & & & & $\begin{array}{l}41 \\
39 \\
25\end{array}$ & $\begin{array}{l}44 \\
47\end{array}$ & $\begin{array}{l}53 \\
50\end{array}$ & $\begin{array}{l}58 \\
56\end{array}$ & $\begin{array}{l}67 \\
58\end{array}$ & $\begin{array}{l}80 \\
65\end{array}$ \\
\hline Inverted $Y$ branch & & & & & & & 44 & 48 & 57 & 60 & 69 & 82 \\
\hline $\begin{array}{l}\text { Combination } Y \text { and one-eighth bend. } \\
\text { Running trap with single hub vent-- } \\
\text { Running trap with double hub vent- } \\
\text { Ruaning trap......................... }\end{array}$ & $\begin{array}{l}14.5 \\
17.5\end{array}$ & 27.5 & $\begin{array}{l}33 \\
38.5 \\
27.5\end{array}$ & $\begin{array}{l}43.5 \\
50 \\
37\end{array}$ & $\begin{array}{l}55.5 \\
63 \\
48\end{array}$ & 141 & 53 & 56 & $\begin{array}{r}64 \\
112 \\
125\end{array}$ & 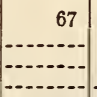 & $\begin{array}{r}77 \\
117 \\
145\end{array}$ & 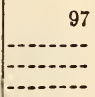 \\
\hline
\end{tabular}


TABLE 3.-Laying lengths of fittings

[The laying length is over-all length less telescoping. All laying lengths and sizes are in inches]

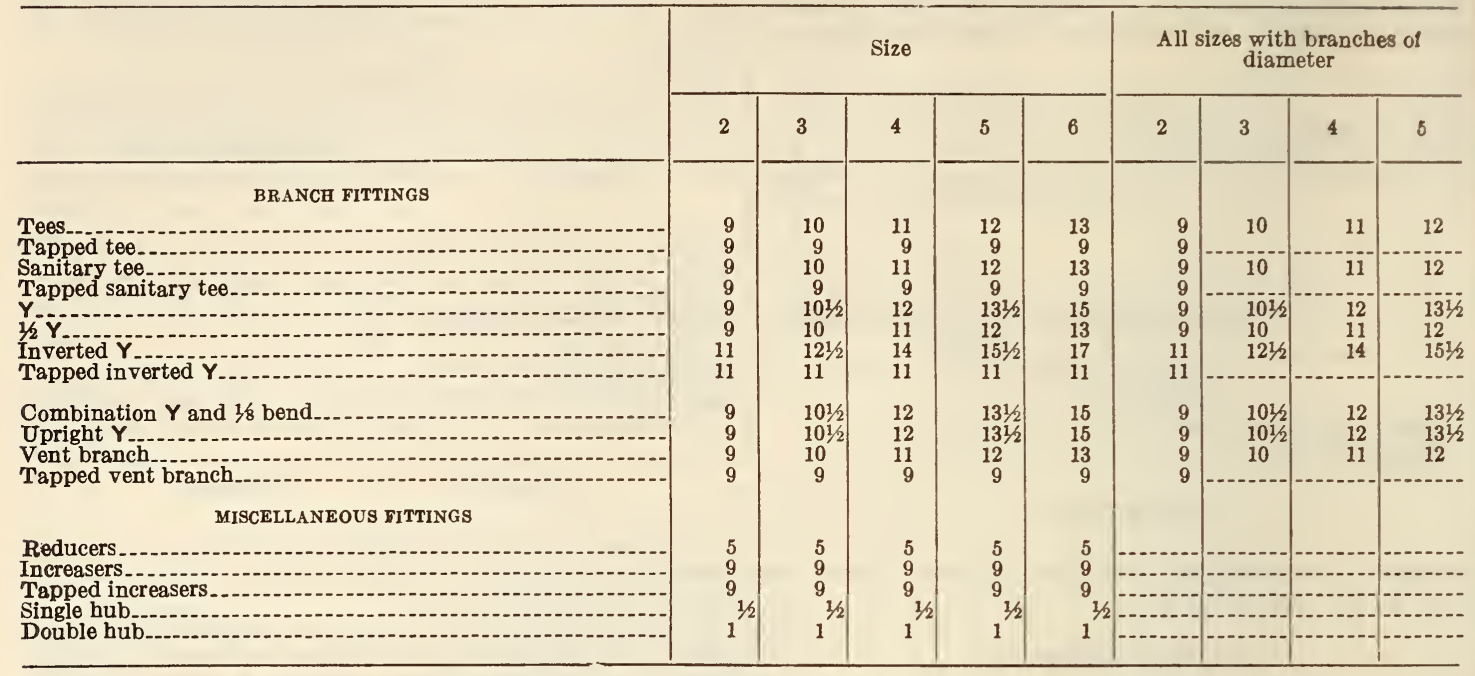

\section{METHOD OF INSPECTION AND TESTS}

All pipe before coating shall be tested to a hydrostatic pressure of not less than 50 pounds per square inch. Pipe while under pressure shall be struck in three places-body, spigot, and hubwith a 1-pound hammer, using a full elbow swing. The strenth of the blow shall be such as to disclose brittleness, and not break good pipe. Any casting showing defects under this test shall be rejected.

All pipe shall be surface inspected for defects before coating by rolling on suitable supports, so that the entire surface of each pipe may be properly inspected.

All fittings shall be subjected to the hammer test, striking each piece in body, spigots, and hubs

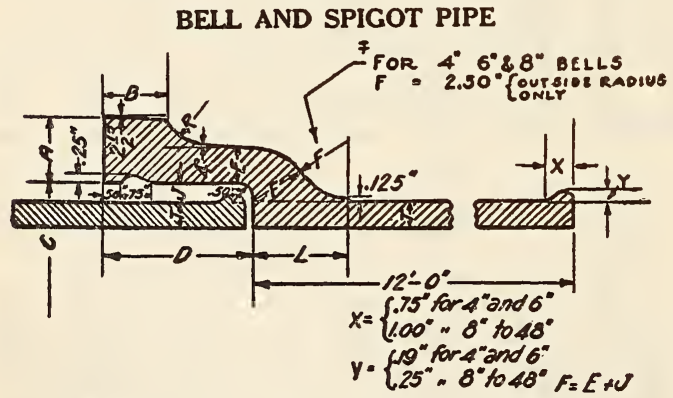

Figure 216.-Dimensions of bell No. 1

with a 1-pound hammer. Any casting showing defects under this test shall be rejected.

NOTES

In the event that sizes larger than those covered by this specification are required, the department or bureau concerned should include such requirement in its purchase specification. Cast-iron soil pipe and fittings should be purchased by the piece and not by weight.

\subsection{CAST-IRON GAS AND WATER PIPE.}

\section{AMERICAN GAS ASSOCIATION STAND- ARD SPECIFICATIONS FOR CAST-IRON PIPE AND SPECIAL CASTINGS, 1925}

\section{CAST-IRON PIPE AND SPECIAL CASTINGS}

(Text of specifications conforms with A. S. T. M. A44-04, given below. Weights and dimensions of bell and spigot pipe are as shown in accompanying tables. Additional tables in this specification give the weights and dimensions of the following special castings:)

Branches, $Y$,

Bushings.

Caps.

Concentric reducers.

Crosses and tees.

Eccentric reducers.

Eighth bends, large.

Eighth bends, long radius.

Eighth curves, long radius.

Flanged pipe.

Hat flanges.

Line drips.

Offsets.

Plugs.

Quarter bends.

Quarter bends, long radius.

Side pots.

Sixteenth bends, large.

Sixteenth bends, long radius.

Sixteenth curves, long radius.

Sleeves, hub.

Sleeves, service.

Sleeves, solid.

Sleeves, split. 
Bell No. 1.-Standard bell

\begin{tabular}{|c|c|c|c|c|c|c|c|c|c|c|c|c|c|c|c|}
\hline \multirow{2}{*}{$\begin{array}{c}\text { Nomi- } \\
\text { nal } \\
\text { diam- } \\
\text { eter }\end{array}$} & \multirow{2}{*}{$\begin{array}{l}\text { Actual } \\
\text { outside } \\
\text { diam- } \\
\text { eter }\end{array}$} & \multirow{2}{*}{$\mathbf{T}$} & \multirow{2}{*}{$\begin{array}{c}\text { Actual } \\
\text { inside } \\
\text { diam- } \\
\text { eter }\end{array}$} & \multicolumn{9}{|c|}{ Dimensions } & \multicolumn{3}{|c|}{ Approximate weight } \\
\hline & & & & A & B & C & D & $\mathbf{E}$ & $\mathbf{F}$ & $\mathbf{J}$ & $\mathbf{L}$ & $\mathbf{R}$ & Bell 1 & $\begin{array}{l}\text { Per } \\
\text { foot } 2\end{array}$ & $\begin{array}{l}\text { 12-foot } \\
\text { length }\end{array}$ \\
\hline $\begin{array}{r}\text { Inches } \\
4 \\
6 \\
8 \\
10\end{array}$ & $\begin{array}{r}\text { Inches } \\
4.80 \\
6.90 \\
9.05 \\
11.10\end{array}$ & $\begin{array}{r}\text { Inches } \\
0.40 \\
.43 \\
.45 \\
.49\end{array}$ & $\begin{array}{r}\text { Inches } \\
4.00 \\
6.04 \\
8.15 \\
10.12\end{array}$ & $\begin{array}{r}\text { Inches } \\
1.30 \\
1.40 \\
1.50 \\
1.50\end{array}$ & $\begin{array}{r}\text { Inches } \\
1.50 \\
1.50 \\
1.50 \\
1.50\end{array}$ & $\begin{array}{r}\text { Inches } \\
5.80 \\
7.90 \\
10.05 \\
12.10\end{array}$ & $\begin{array}{r}\text { Inches } \\
4.00 \\
4.00 \\
4.00 \\
4.00\end{array}$ & $\begin{array}{r}\text { Inches } \\
0.59 \\
.62 \\
.69 \\
.69\end{array}$ & $\begin{array}{r}\text { Inches } \\
1.09 \\
1.12 \\
1.19 \\
1.19\end{array}$ & $\begin{array}{r}\text { Inch } \\
0.50 \\
.50 \\
.50 \\
.50\end{array}$ & $\begin{array}{r}\text { Inches } \\
3.25 \\
3.25 \\
3.25 \\
2.10\end{array}$ & $\begin{array}{r}\text { Inches } \\
0.75 \\
.80 \\
.80 \\
.90\end{array}$ & $\begin{array}{c}\text { Pounds } \\
27.0 \\
39.5 \\
52.8 \\
57.93\end{array}$ & $\begin{array}{r}\text { Pounds } \\
19.33 \\
30.25 \\
42.08 \\
55.91\end{array}$ & $\begin{array}{c}\text { Pounds } \\
234 \\
367 \\
509 \\
671\end{array}$ \\
\hline $\begin{array}{l}12 \\
16 \\
20 \\
24\end{array}$ & $\begin{array}{l}13.20 \\
17.40 \\
21.60 \\
25.80\end{array}$ & $\begin{array}{l}.54 \\
.62 \\
.68 \\
.76\end{array}$ & $\begin{array}{l}12.12 \\
16.16 \\
20.24 \\
24.28\end{array}$ & $\begin{array}{l}1.60 \\
1.80 \\
2.00 \\
2.10\end{array}$ & $\begin{array}{l}1.50 \\
1.75 \\
1.75 \\
2.00\end{array}$ & $\begin{array}{l}14.20 \\
18.40 \\
22.85 \\
27.05\end{array}$ & $\begin{array}{l}4.50 \\
4.50 \\
\text { 4. } 50 \\
\text { 5. } 00\end{array}$ & $\begin{array}{r}.75 \\
.90 \\
.97 \\
1.05\end{array}$ & $\begin{array}{l}1.25 \\
1.40 \\
1.60 \\
1.68\end{array}$ & $\begin{array}{l}.50 \\
.50 \\
.63 \\
.63\end{array}$ & $\begin{array}{l}2.20 \\
2.50 \\
2.80 \\
2.80\end{array}$ & $\begin{array}{l}1.00 \\
1.10 \\
1.15 \\
1.25\end{array}$ & $\begin{array}{r}79.47 \\
125.18 \\
169.10 \\
235.10\end{array}$ & $\begin{array}{r}73.83 \\
112.58 \\
153.83 \\
206.41\end{array}$ & $\begin{array}{r}886 \\
1,351 \\
1,846 \\
2,477\end{array}$ \\
\hline $\begin{array}{l}30 \\
36 \\
42 \\
48\end{array}$ & $\begin{array}{l}31.74 \\
37.96 \\
44.20 \\
50.50\end{array}$ & $\begin{array}{r}.85 \\
.95 \\
1.07 \\
1.26\end{array}$ & $\begin{array}{l}30.04 \\
36.06 \\
42.06 \\
47.98\end{array}$ & $\begin{array}{l}2.30 \\
2.50 \\
2.80 \\
3.00\end{array}$ & $\begin{array}{l}2.00 \\
2.00 \\
2.00 \\
2.00\end{array}$ & $\begin{array}{l}32.99 \\
39.21 \\
45.45 \\
51.75\end{array}$ & $\begin{array}{l}5.00 \\
5.00 \\
5.00 \\
5.00\end{array}$ & $\begin{array}{l}1.15 \\
1.25 \\
1.40 \\
1.50\end{array}$ & $\begin{array}{l}1.78 \\
1.88 \\
2.03 \\
2.13\end{array}$ & $\begin{array}{l}.63 \\
.63 \\
.63 \\
.63\end{array}$ & $\begin{array}{l}3.00 \\
3.20 \\
3.40 \\
3.60\end{array}$ & $\begin{array}{l}1.30 \\
1.40 \\
1.45 \\
1.60\end{array}$ & $\begin{array}{l}315.2 \\
410.2 \\
537.5 \\
657.0\end{array}$ & $\begin{array}{l}284.0 \\
379.25 \\
497.66 \\
663.5\end{array}$ & $\begin{array}{l}3,408 \\
4,551 \\
5,972 \\
7,962\end{array}$ \\
\hline
\end{tabular}

1 Weight of bell includes only metal beyond outside diameter of pipe.

Weight per foot includes bell and bead.

NотE.-Pipe heavier than these standards may be made by reducing the cores, or internal diameters $\mathrm{C}$ and $\mathrm{D}$; same for specials.

Dimension $\mathrm{L}$ for sizes 4,6 , and 8 inches, and dimension $\mathrm{F}$, shown in sketch as 2.50 (outside radius only), apply to bells of straight pipe only. For bells of special castings the correct dimensions for $\mathrm{F}$ and $\mathrm{L}$ are:

\begin{tabular}{|r|c|c|}
\hline Size & F & L \\
\cline { 1 - 2 } 4 & 1.09 & 1.90 \\
6 & 1.12 & 2.00 \\
8 & 1.19 & 2.10 \\
\hline
\end{tabular}

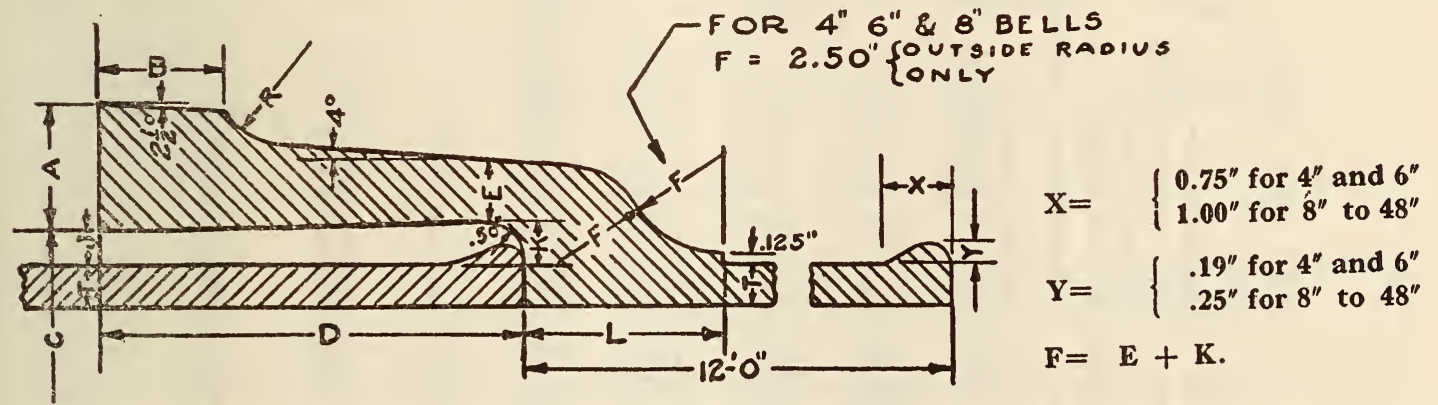

Figure 217.-Dimensions of bell No. 2

Bell No. 2.-Alternate bell for cement and combination joints

\begin{tabular}{|c|c|c|c|c|c|c|c|c|c|c|c|c|c|c|c|c|c|c|}
\hline \multirow{2}{*}{$\begin{array}{c}\text { Nomi- } \\
\text { nal } \\
\text { diam- } \\
\text { eter }\end{array}$} & \multirow{2}{*}{$\begin{array}{c}\text { Actual } \\
\text { outside } \\
\text { diam- } \\
\text { eter }\end{array}$} & \multirow{2}{*}{$\mathrm{T}$} & \multirow{2}{*}{$\begin{array}{l}\text { Actual } \\
\text { inside } \\
\text { diam- } \\
\text { eter }\end{array}$} & \multicolumn{10}{|c|}{ Dimensions } & \multicolumn{3}{|c|}{ Approximate weight } & \multirow{2}{*}{$\begin{array}{c}\text { Ultimate } \\
\text { tensile } \\
\text { strength } \\
\text { of pipe } \\
M \\
\text { pounds }\end{array}$} & \multirow{2}{*}{$\begin{array}{l}\text { Ultimate } \\
\text { strength } \\
\text { all ce- } \\
\text { ment } \\
\text { joint M } \\
\text { pounds }\end{array}$} \\
\hline & & & & A & B & C & D & $\mathbf{E}$ & $\mathbf{F}$ & $\mathbf{J}$ & K & L & $\mathbf{R}$ & Bell & $\begin{array}{l}\text { Per } \\
\text { foot }\end{array}$ & $\begin{array}{l}\text { 12-foot } \\
\text { length }\end{array}$ & & \\
\hline $\begin{array}{r}\text { Inches } \\
4 \\
6 \\
8 \\
10 \\
12 \\
16 \\
20 \\
24 \\
30 \\
36 \\
42 \\
48\end{array}$ & $\begin{array}{r}\text { Inches } \\
4.80 \\
6.90 \\
9.05 \\
11.10 \\
13.20 \\
17.40 \\
21.60 \\
25.80 \\
31.74 \\
37.96 \\
44.20 \\
50.50\end{array}$ & $\begin{array}{c}\text { Inches } \\
0.40 \\
.43 \\
.45 \\
.49 \\
.54 \\
.62 \\
.68 \\
.76 \\
.85 \\
.95 \\
1.07 \\
1.26\end{array}$ & $\begin{array}{r}\text { Inches } \\
4.00 \\
6.04 \\
8.15 \\
10.12 \\
12.12 \\
16.16 \\
20.24 \\
24.28 \\
30.04 \\
36.06 \\
42.06 \\
47.98\end{array}$ & \begin{tabular}{|c|} 
Inches \\
1.30 \\
1.40 \\
1.50 \\
1.50 \\
1.60 \\
1.80 \\
2.00 \\
2.10 \\
2.30 \\
2.50 \\
2.80 \\
3.00
\end{tabular} & \begin{tabular}{|c|} 
Inches \\
1.50 \\
1.50 \\
1.50 \\
1.50 \\
1.50 \\
1.75 \\
1.75 \\
2.00 \\
2.00 \\
2.00 \\
2.00 \\
2.00
\end{tabular} & $\begin{array}{c}\text { Inches } \\
5.80 \\
7.90 \\
10.05 \\
12.10 \\
14.20 \\
18.40 \\
22.85 \\
27.05 \\
32.99 \\
39.21 \\
45.45 \\
51.75\end{array}$ & $\begin{array}{l}\text { Inches } \\
4.00 \\
4.00 \\
4.50 \\
5.00 \\
5.00 \\
6.00 \\
6.00 \\
6.00 \\
6.50 \\
6.50 \\
6.75 \\
7.00\end{array}$ & $\begin{array}{c}\text { Inches } \\
0.59 \\
.62 \\
.69 \\
.69 \\
.75 \\
.90 \\
.97 \\
1.05 \\
1.15 \\
1.25 \\
1.40 \\
1.50\end{array}$ & \begin{tabular}{|c|} 
Inches \\
1.22 \\
1.25 \\
1.32 \\
1.32 \\
1.38 \\
1.53 \\
1.72 \\
1.80 \\
1.90 \\
2.00 \\
2.15 \\
2.25
\end{tabular} & $\begin{array}{l}\text { Inch } \\
0.50 \\
.50 \\
.50 \\
.50 \\
.50 \\
.50 \\
.63 \\
.63 \\
.63 \\
.63 \\
.63 \\
.63\end{array}$ & $\begin{array}{l}\text { Inch } \\
0.63 \\
.63 \\
.63 \\
.63 \\
.63 \\
.63 \\
.75 \\
.75 \\
.75 \\
.75 \\
.75 \\
.75\end{array}$ & \begin{tabular}{|c|} 
Inches \\
3.25 \\
3.25 \\
3.25 \\
2.10 \\
2.20 \\
2.50 \\
2.80 \\
2.80 \\
3.00 \\
3.20 \\
3.40 \\
3.60
\end{tabular} & \begin{tabular}{|c|} 
Inches \\
0.75 \\
.80 \\
.80 \\
.90 \\
1.00 \\
1.10 \\
1.15 \\
1.25 \\
1.30 \\
1.40 \\
1.45 \\
1.60
\end{tabular} & \begin{tabular}{|c|}
\multicolumn{1}{c}{$L b s}$. \\
27 \\
39.5 \\
56.0 \\
64.7 \\
84.2 \\
146.8 \\
198.3 \\
259.5 \\
363.7 \\
472.7 \\
631.8 \\
788.3
\end{tabular} & $\begin{array}{l}\text { Lbs. } \\
19.3 \\
30.3 \\
42.3 \\
56.5 \\
74.3 \\
115.3 \\
156.3 \\
208.4 \\
286.3 \\
384.4 \\
505.5 \\
674.6\end{array}$ & $\begin{aligned} \text { Lbs. } \\
234 \\
367 \\
512 \\
678 \\
891 \\
1,373 \\
1,875 \\
2,501 \\
3,456 \\
4,613 \\
6,066 \\
8,093\end{aligned}$ & \begin{tabular}{|c|} 
Pounds \\
66.4 \\
104.8 \\
146.0 \\
196.5 \\
257.3 \\
393.7 \\
533 \\
721 \\
995 \\
1,338 \\
1,716 \\
2,351
\end{tabular} & $\begin{array}{c}\text { Pounds } \\
135.8 \\
195.2 \\
298.6 \\
418.8 \\
498 \\
820 \\
1,018 \\
1,216 \\
1,646 \\
1,968 \\
2,397 \\
2,857\end{array}$ \\
\hline
\end{tabular}


AMERICAN RAILWAY ENGINEERING ASSOCIATION, STANDARD SPECIFICATIONS FOR CAST-IRON PIPE AND SPECIAI CASTINGS, MANUAL, 1922

\section{CAST-IRON PIPE AND SPECIAL CASTINGS}

(Identical with A. S. T. M., A44-04, given below.)

\section{AMERICAN SOCIETY FOR TESTING MATE- RIALS, STANDARD SPECIFICATIONS FOR CAST-IRON PIPE AND SPECIAL CAST- INGS, DESIGNATION A44-04, 1904}

\section{CAST-IRON PIPE AND SPECIAL CASTINGS}

\section{DESCRIPTION OF PIPES}

Section 1.-The pipes shall be made with hub and spigot joints, and shall accurately conform to the dimensions given in Tables 1 and 2. They shall be straight and shall be true circles in section, with their inner and outer surfaces concentric, and shall be of the specified dimensions in outside diameter. They shall be at least 12 feet in length, exclusive of socket. For pipes of each size from 4 to 24 inches, inclusive, there shall be two standards of outside diameter, and for pipes from 30 to 60 inches, inclusive, there shall be four standards of outside diameter, as shown by Table 1.

All pipes having the same outside diameter shall have the same inside diameter at both ends. The inside diameter of the lighter pipes of each standard outside diameter shall be gradually increased for a distance of about 6 inches from each end of the pipe

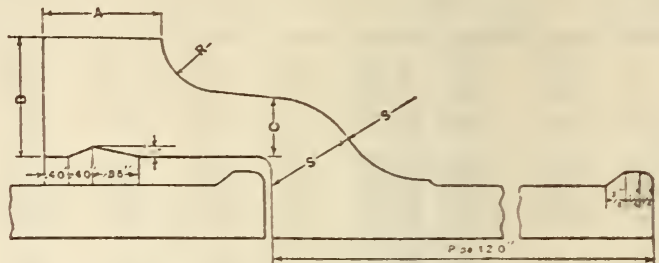

Figure 218.-General dimensions of pipes

so as to obtain the required standard thickness and weight for each size and class of pipe.

Pipes whose standard thickness and weight are intermediate between the classes in Table 2 shall be made of the same outside diameter as the next heavier class. Pipes whose standard thickness and weight are less than shown by Table 2 shall be made of the same outside diameter as the class A pipes, and pipes whose thickness and weight are more than shown by Table 2 shall be made of the same outside diameter as the class $\mathrm{D}$ pipes.

TABLE 1.-General dimensions of pipes

\begin{tabular}{|c|c|c|c|c|c|c|c|c|c|}
\hline \multirow{2}{*}{$\begin{array}{l}\text { Nomi- } \\
\text { nal } \\
\text { diam- } \\
\text { eter }\end{array}$} & \multirow{2}{*}{ Classes } & \multirow{2}{*}{$\begin{array}{c}\text { Actual } \\
\text { outside } \\
\text { diameter }\end{array}$} & \multicolumn{2}{|c|}{ Diameter of sockets } & \multicolumn{2}{|c|}{ Depth of sockets } & \multirow{2}{*}{ A } & \multirow{2}{*}{ B } & \multirow{2}{*}{ C } \\
\hline & & & Pipe & $\begin{array}{l}\text { Special } \\
\text { castings }\end{array}$ & Pipe & $\begin{array}{l}\text { Special } \\
\text { castings }\end{array}$ & & & \\
\hline $\begin{array}{c}\text { Inches } \\
4 \\
4 \\
6 \\
6 \\
8\end{array}$ & $\begin{array}{l}A-B \\
C-D \\
A-B \\
C-D \\
A-B\end{array}$ & $\begin{array}{r}\text { Inches } \\
4.80 \\
5.00 \\
6.90 \\
7.10 \\
9.05\end{array}$ & $\begin{array}{r}\text { Inches } \\
5.60 \\
5.80 \\
7.70 \\
7.90 \\
9.85\end{array}$ & $\begin{array}{r}\text { Inches } \\
5.70 \\
5.70 \\
7.80 \\
7.80 \\
10.00\end{array}$ & $\begin{array}{r}\text { Inches } \\
3.50 \\
3.50 \\
3.50 \\
3.50 \\
4.00\end{array}$ & $\begin{array}{r}\text { Inches } \\
4.00 \\
4.00 \\
4.00 \\
4.00 \\
4.00\end{array}$ & $\begin{array}{l}1.50 \\
1.50 \\
1.50 \\
1.50 \\
1.50\end{array}$ & $\begin{array}{l}1.30 \\
1.30 \\
1.40 \\
1.40 \\
1.50\end{array}$ & $\begin{array}{r}0.65 \\
.65 \\
.70 \\
.70 \\
.75\end{array}$ \\
\hline $\begin{array}{r}8 \\
10 \\
10 \\
12 \\
12\end{array}$ & $\begin{array}{l}C-D \\
A-B \\
C-D \\
A-B \\
C-D\end{array}$ & $\begin{array}{r}9.30 \\
11.10 \\
11.40 \\
13.20 \\
13.50\end{array}$ & $\begin{array}{l}10.10 \\
11.90 \\
12.20 \\
14.00 \\
14.30\end{array}$ & $\begin{array}{l}10.00 \\
12.10 \\
12.10 \\
14.20 \\
14.20\end{array}$ & $\begin{array}{l}4.00 \\
4.00 \\
4.00 \\
4.00 \\
4.00\end{array}$ & $\begin{array}{l}4.00 \\
4.00 \\
4.00 \\
4.00 \\
4.00\end{array}$ & $\begin{array}{l}1.50 \\
1.50 \\
1.50 \\
1.50 \\
1.50\end{array}$ & $\begin{array}{l}1.50 \\
1.50 \\
1.60 \\
1.60 \\
1.70\end{array}$ & $\begin{array}{l}.75 \\
.75 \\
.80 \\
.80 \\
.85\end{array}$ \\
\hline $\begin{array}{l}14 \\
14 \\
16 \\
16 \\
18\end{array}$ & $\begin{array}{l}A-B \\
C-D \\
A-B \\
C-D \\
A-B\end{array}$ & $\begin{array}{l}15.30 \\
15.65 \\
17.40 \\
17.80 \\
19.50\end{array}$ & $\begin{array}{l}16.10 \\
16.45 \\
18.40 \\
18.80 \\
20.50\end{array}$ & $\begin{array}{l}16.10 \\
16.45 \\
18.40 \\
18.80 \\
20.50\end{array}$ & $\begin{array}{l}4.00 \\
4.00 \\
4.00 \\
4.00 \\
4.00\end{array}$ & $\begin{array}{l}4.00 \\
4.00 \\
4.00 \\
4.00 \\
4.00\end{array}$ & $\begin{array}{l}1.50 \\
1.50 \\
1.75 \\
1.75 \\
1.75\end{array}$ & $\begin{array}{l}1.70 \\
1.80 \\
1.80 \\
1.90 \\
1.90\end{array}$ & $\begin{array}{r}.85 \\
.90 \\
.90 \\
1.00 \\
.95\end{array}$ \\
\hline $\begin{array}{l}18 \\
20 \\
20 \\
24 \\
24\end{array}$ & $\begin{array}{l}C-D \\
A-B \\
C-D \\
A-B \\
C-D\end{array}$ & $\begin{array}{l}19.92 \\
21.60 \\
22.06 \\
25.80 \\
26.32\end{array}$ & $\begin{array}{l}20.92 \\
22.60 \\
23.06 \\
26.80 \\
27.32\end{array}$ & $\begin{array}{l}20.92 \\
22.60 \\
23.06 \\
26.80 \\
27.32\end{array}$ & $\begin{array}{l}4.00 \\
4.00 \\
4.00 \\
4.00 \\
4.00\end{array}$ & $\begin{array}{l}4.00 \\
4.00 \\
4.00 \\
4.00 \\
4.00\end{array}$ & $\begin{array}{l}1.75 \\
1.75 \\
1.75 \\
2.00 \\
2.00\end{array}$ & $\begin{array}{l}2.10 \\
2.00 \\
2.30 \\
2.10 \\
2.50\end{array}$ & $\begin{array}{l}1.05 \\
1.00 \\
1.15 \\
1.05 \\
1.25\end{array}$ \\
\hline $\begin{array}{l}30 \\
30 \\
30 \\
30 \\
36\end{array}$ & $\begin{array}{l}\mathrm{A} \\
\mathrm{B} \\
\mathrm{C} \\
\mathrm{D} \\
\mathrm{A}\end{array}$ & $\begin{array}{l}31.74 \\
32.00 \\
32.40 \\
32.74 \\
37.96\end{array}$ & $\begin{array}{l}32.74 \\
33.00 \\
33.40 \\
33.74 \\
38.96\end{array}$ & $\begin{array}{l}32.74 \\
33.00 \\
33.40 \\
33.74 \\
38.96\end{array}$ & $\begin{array}{l}4.50 \\
4.50 \\
4.50 \\
4.50 \\
4.50\end{array}$ & $\begin{array}{l}4.50 \\
4.50 \\
4.50 \\
\text { 4. } 50 \\
4.50\end{array}$ & $\begin{array}{l}2.00 \\
2.00 \\
2.00 \\
2.00 \\
2.00\end{array}$ & $\begin{array}{l}2.50 \\
2.30 \\
2.60 \\
3.00 \\
2.50\end{array}$ & $\begin{array}{l}1.15 \\
1.15 \\
1.32 \\
1.50 \\
1.25\end{array}$ \\
\hline $\begin{array}{l}36 \\
36 \\
36 \\
42 \\
42\end{array}$ & $\begin{array}{l}\mathrm{B} \\
\mathrm{C} \\
\mathrm{D} \\
\mathrm{A} \\
\mathrm{B}\end{array}$ & $\begin{array}{l}38.30 \\
38.70 \\
39.16 \\
44.20 \\
44.55\end{array}$ & $\begin{array}{l}39.30 \\
39.70 \\
40.16 \\
45.20 \\
45.50\end{array}$ & $\begin{array}{l}39.30 \\
39.70 \\
40.16 \\
45.20 \\
45.50\end{array}$ & $\begin{array}{l}4.50 \\
4.50 \\
4.50 \\
5.00 \\
5.00\end{array}$ & $\begin{array}{l}4.50 \\
4.50 \\
4.50 \\
5.00 \\
5.00\end{array}$ & $\begin{array}{l}2.00 \\
2.00 \\
2.00 \\
2.00 \\
2.00\end{array}$ & $\begin{array}{l}2.80 \\
3.10 \\
3.40 \\
2.80 \\
3.00\end{array}$ & $\begin{array}{l}1.40 \\
1.60 \\
1.80 \\
1.40 \\
1.50\end{array}$ \\
\hline $\begin{array}{l}42 \\
42 \\
48 \\
48 \\
48\end{array}$ & $\begin{array}{l}\mathrm{C} \\
\mathrm{D} \\
\mathrm{A} \\
\mathrm{B} \\
\mathrm{C}\end{array}$ & $\begin{array}{l}45.10 \\
45.58 \\
50.50 \\
50.80 \\
51.40\end{array}$ & $\begin{array}{l}46.10 \\
46.58 \\
51.50 \\
51.80 \\
52.40\end{array}$ & $\begin{array}{l}46.10 \\
46.58 \\
51.50 \\
51.80 \\
52.40\end{array}$ & $\begin{array}{l}5.00 \\
5.00 \\
5.00 \\
5.00 \\
5.00\end{array}$ & $\begin{array}{l}5.00 \\
5.00 \\
5.00 \\
5.00 \\
5.00\end{array}$ & $\begin{array}{l}2.00 \\
2.00 \\
2.00 \\
2.00 \\
2.00\end{array}$ & $\begin{array}{l}3.40 \\
3.80 \\
3.00 \\
3.30 \\
3.80\end{array}$ & $\begin{array}{l}1.75 \\
1.95 \\
1.50 \\
1.65 \\
1.95\end{array}$ \\
\hline $\begin{array}{l}48 \\
54 \\
54 \\
54 \\
54\end{array}$ & $\begin{array}{l}\mathrm{D} \\
\mathrm{A} \\
\mathrm{B} \\
\mathrm{C} \\
\mathrm{D}\end{array}$ & $\begin{array}{l}51.98 \\
56.66 \\
57.10 \\
57.80 \\
58.40\end{array}$ & $\begin{array}{l}\mathbf{5 2 .} 98 \\
\mathbf{5 7 .} 66 \\
58.10 \\
58.80 \\
59.40\end{array}$ & $\begin{array}{l}52.98 \\
57.66 \\
58.10 \\
58.80 \\
59.40\end{array}$ & $\begin{array}{l}5.00 \\
5.50 \\
5.50 \\
5.50 \\
5.50\end{array}$ & $\begin{array}{l}5.00 \\
5.50 \\
5.50 \\
5.50 \\
5.50\end{array}$ & $\begin{array}{l}2.00 \\
2.25 \\
2.25 \\
2.25 \\
2.25\end{array}$ & $\begin{array}{l}4.20 \\
3.20 \\
3.60 \\
4.00 \\
4.40\end{array}$ & $\begin{array}{l}2.20 \\
1.60 \\
1.80 \\
2.15 \\
2.45\end{array}$ \\
\hline $\begin{array}{l}60 \\
60\end{array}$ & $\begin{array}{l}\mathrm{A} \\
\mathrm{B} \\
\mathrm{C} \\
\mathrm{D}\end{array}$ & $\begin{array}{l}62.80 \\
63.40 \\
64.20 \\
64.82\end{array}$ & $\begin{array}{l}63.80 \\
64.40 \\
65.20 \\
65.82\end{array}$ & $\begin{array}{l}63.80 \\
64.40 \\
65.20 \\
65.82\end{array}$ & $\begin{array}{l}5.50 \\
5.50 \\
5.50 \\
5.50\end{array}$ & $\begin{array}{l}5.50 \\
5.50 \\
5.50 \\
5.50\end{array}$ & $\begin{array}{l}2.25 \\
2.25 \\
2.25 \\
2.25\end{array}$ & $\begin{array}{l}\text { 3. } 40 \\
3.70 \\
\text { 4. } 20 \\
\text { 4. } 70\end{array}$ & $\begin{array}{l}1.70 \\
1.90 \\
2.25 \\
2.60\end{array}$ \\
\hline
\end{tabular}


For pipes 4 to 12 inches, inclusive, one class of special castings shall be furnished, made from class D pattern. Those having spigot ends shall have outside diameters of spigot ends midway between the two standards of outside diameter as shown by Table 1, and shall be tapered back for a distance of 6 inches. For pipes from 14 to 24 inches, inclusive, two classes of special castings shall be furnished, class $B$ special castings with classes $A$ and $B$ pipes, and class $\mathrm{D}$ special castings with classes $\mathrm{C}$ and $\mathrm{D}$ pipes, the former to be stamped "AB" and the latter to be stamped "CD." For pipes 30 to 60 inches, inclusive, four classes of special castings shall be furnished, one for each class of pipe, and shall be stamped with the letter of the class to which they belong.

\section{ALLOWABLE VARIATION IN DIAMETER OF PIPES AND}

Section 2.-Special care shall be taken to have the sockets of the required size. The sockets and spigots will be tested by circular gages, and no pipe will be received which is defective in joint room from any cause. The diameters of the sockets and the outside diameters of the bead ends of the pipe shall not vary from the standard dimensions by more than 0.06 inch for pipes 16 inches or less in diameter; 0.08 inch for 18-inch, 20-inch, and 24-inch pipes; 0.10 inch for 30-inch, 36-inch, and 42-inch pipes; 0.12 inch for 48 -inch pipes; and 0.15 inch for $54-$ inch and 60-inch pipes.

\section{ALLOWABLE VARIATION IN THICKNESS}

Section 3.-For pipes whose standard thickness is less than 1 inch, the thickness of metal in the body of the pipe shall not be more than 0.08 inch less than the standard thickness; and for pipes whose standard thickness is 1 inch or more, the variation shall not exceed 0.10 inch, except that for spaces not exceeding 8 inches in length in any direction, variations from the standard thickness of 0.02 inch in excess of the allowance above given shall be permitted.

TABLE 2.-Standard thicknesses and weights of cast-iron pipe

\begin{tabular}{|c|c|c|c|c|c|c|c|c|c|c|c|c|c|}
\hline \multirow{3}{*}{$\begin{array}{l}\text { Nom- } \\
\text { inal } \\
\text { inside } \\
\text { diam- } \\
\text { eter }\end{array}$} & \multicolumn{3}{|c|}{$\begin{array}{c}\text { Class } A-100 \text {-foot head, } 43 \\
\text { pounds pressure }\end{array}$} & \multicolumn{3}{|c|}{$\begin{array}{c}\text { Class B-200-foot head, } 86 \\
\text { pounds pressure }\end{array}$} & \multicolumn{3}{|c|}{$\begin{array}{l}\text { Class } \mathrm{C}-300 \text {-foot head, } 130 \\
\text { pounds pressure }\end{array}$} & \multicolumn{3}{|c|}{$\begin{array}{c}\text { Class } \mathrm{D}-400 \text {-foot head, } 173 \\
\text { pounds pressure }\end{array}$} & \multirow{3}{*}{$\begin{array}{l}\text { Nomi- } \\
\text { nal in- } \\
\text { side } \\
\text { diam- } \\
\text { eter }\end{array}$} \\
\hline & \multirow{2}{*}{$\begin{array}{l}\text { Thick- } \\
\text { ness }\end{array}$} & \multicolumn{2}{|c|}{ Weight per- } & \multirow{2}{*}{$\begin{array}{l}\text { Thick- } \\
\text { ness }\end{array}$} & \multicolumn{2}{|c|}{ Weight per- } & \multirow{2}{*}{$\begin{array}{c}\text { Thick- } \\
\text { ness }\end{array}$} & \multicolumn{2}{|c|}{ Weight per- } & \multirow{2}{*}{$\begin{array}{l}\text { Thick- } \\
\text { ness }\end{array}$} & \multicolumn{2}{|c|}{ Weight per- } & \\
\hline & & Foot & Length & & Foot & Length & & Foot & Length & & Foot & Length & \\
\hline $\begin{array}{c}\text { Inches } \\
4 \\
6 \\
8 \\
10\end{array}$ & $\begin{array}{c}\text { Inches } \\
0.42 \\
.44 \\
.46 \\
.50\end{array}$ & $\begin{array}{c}\text { Pounds } \\
20.0 \\
30.8 \\
42.9 \\
57.1\end{array}$ & $\begin{array}{c}\text { Pounds } \\
240 \\
370 \\
515 \\
685\end{array}$ & $\begin{array}{c}\text { Inches } \\
0.45 \\
.48 \\
.51 \\
.57\end{array}$ & $\begin{array}{c}\text { Pounds } \\
21.7 \\
33.3 \\
47.5 \\
63.8\end{array}$ & $\begin{array}{c}\text { Pounds } \\
260 \\
400 \\
570 \\
765\end{array}$ & $\begin{array}{c}\text { Inches } \\
0.48 \\
.51 \\
.56 \\
.62\end{array}$ & $\begin{array}{r}\text { Pounds } \\
23.3 \\
35.8 \\
52.1 \\
70.8\end{array}$ & $\begin{array}{c}\text { Pounds } \\
280 \\
430 \\
625 \\
850\end{array}$ & $\begin{array}{c}\text { Inches } \\
0.52 \\
.55 \\
.60 \\
.68\end{array}$ & $\begin{array}{r}\text { Pounds } \\
25.0 \\
38.3 \\
55.8 \\
76.7\end{array}$ & $\begin{array}{c}\text { Pounds } \\
300 \\
460 \\
670 \\
920\end{array}$ & $\begin{array}{c}\text { Inches } \\
4 \\
6 \\
8 \\
10\end{array}$ \\
\hline $\begin{array}{l}12 \\
14 \\
16 \\
18\end{array}$ & $\begin{array}{l}.54 \\
.57 \\
.60 \\
.64\end{array}$ & $\begin{array}{r}72.5 \\
89.6 \\
108.3 \\
129.2\end{array}$ & & $\begin{array}{l}.62 \\
.66 \\
.70 \\
.75\end{array}$ & & & $\begin{array}{l}.68 \\
.74 \\
.80 \\
.87\end{array}$ & & & $\begin{array}{l}.75 \\
.82 \\
.89 \\
.96\end{array}$ & & & $\begin{array}{l}12 \\
14 \\
16 \\
18\end{array}$ \\
\hline $\begin{array}{l}20 \\
24 \\
30 \\
36\end{array}$ & $\begin{array}{l}.67 \\
.76 \\
.88 \\
.99\end{array}$ & & & $\begin{array}{r}.80 \\
.89 \\
1.03 \\
1.15\end{array}$ & & & & & & & & & $\begin{array}{l}20 \\
24 \\
30 \\
36\end{array}$ \\
\hline $\begin{array}{l}42 \\
48 \\
54 \\
60\end{array}$ & $\begin{array}{l}1.10 \\
1.26 \\
1.35 \\
1.39\end{array}$ & $\begin{array}{l}512.5 \\
666.7 \\
800.0 \\
916.7\end{array}$ & $\begin{array}{r}6,150 \\
8,000 \\
9,600 \\
11,000\end{array}$ & $\begin{array}{l}1.28 \\
1.42 \\
1.55 \\
1.67\end{array}$ & $\begin{array}{r}591.7 \\
750.0 \\
933.3 \\
1,104.2\end{array}$ & $\begin{array}{r}7,100 \\
9,000 \\
11,200 \\
13,250\end{array}$ & $\begin{array}{l}1.54 \\
1.71 \\
1.90 \\
2.00\end{array}$ & $\begin{array}{r}716.7 \\
908.3 \\
1,141.7 \\
1,341.7\end{array}$ & & $\begin{array}{l}1.78 \\
1.96 \\
2.23 \\
2.38\end{array}$ & $\begin{array}{r}825.0 \\
1,050.0 \\
1,341.7 \\
1,583.3\end{array}$ & & $\begin{array}{l}42 \\
48 \\
54 \\
60\end{array}$ \\
\hline
\end{tabular}

Nоте-The above weights are for 12-foot laying lengths and standard sockets; proportionate allowance to be made for any variation therefrom.

For special castings of standard patterns a variation of 50 per cent greater than allowed for straight pipe shall be permitted.

\section{DEFECTIVE SPIGOTS MAY BE CUT}

Section 4.-Defective spigot ends on pipes 12 inches or more in diameter may be cut off in a lathe, and a half-round wrought-iron band shrunk into a groove cut in the end of the pipe. Not more than 12 per cent of the total number of accepted pipes of each size shall be cut and banded, and no pipe shall be banded which is less than 11 feet in length, exclusive of the socket.

In case the length of a pipe differs from 12 feet, the standard weight of the pipe given in Table 2 shall be modified in accordance therewith.

\section{SPECIAL CASTINGS}

Section 5.-All special castings shall be made in accordance with the cuts and the dimensions given in the table forming a part of these specifications.

The diameters of the sockets and the external diameters of the bead ends of the special castings shall not vary from the standard dimensions by more than 0.12 inch for castings 16 inches or less in diameter; 0.15 inch for 18-inch, 20 -inch, and 24inch pipes; 0.20 inch for 30 -inch, 36 -inch, and 42 inch pipes; and 0.24 inch for 48-inch, 54 -inch, and 60 -inch pipes. These variations apply only to special castings made from standard patterns.

The flanges on all manhole castings and manhole covers shall be faced true and smooth, and drilled to receive bolts of the sizes given in the tables. The manufacturer shall furnish and deliver all bolts for bolting on the manhole covers, the bolts to be of the sizes shown on plans and made of the best quality of mild steel, with hexagonal heads and nuts and sound well-fitting threads.

\section{MARKING}

Section 6.-Every pipe and special casting shall have distinctly cast upon it the initials of the maker's name. When cast especially to order, each pipe and special casting larger than 4 inches may also have cast upon it figures showing the year in which it was cast and a number signifying the order in 
point of time in which it was cast, the figures denoting the year being above and the number below, thus:

\begin{tabular}{|c|c|c|}
\hline 1901 & 1901 & 1901 \\
\hline 1 & 2 & 3 \\
\hline
\end{tabular}

etc., also any initials, not exceeding four, which may be required by the purchaser. The letters and figures shall be cast on the outside and shall be not less than 2 inches in length and one-eighth inch in relief for pipes 8 inches in diameter and larger. For smaller sizes of pipes the letters may be 1 inch in length. The weight and the class letter shall be conspicuously painted in white on the inside of each pipe and special casting after the coating has become hard.

\section{ALLOWABLE PERCENTAGE OF VARIATION IN WEIGHT}

Section 7.-No pipe shall be accepted the weight of which shall be less than the standard weight by more than 5 per cent for pipes 16 inches or less in diameter, and 4 per cent for pipes more than 16 inches in diameter; and no excess above the standard weight of more than the given percentages for the several sizes shall be paid for. The total weight to be paid for shall not exceed for each size and class of pipe received the sum of the standard weights of the same number of pieces of the given size and class by more than 2 per cent.

No special casting shall be accepted the weight of which shall be less than the standard weight by more than 10 per cent for pipes 12 inches or less in diameter, and 8 per cent for larger sizes, except that curves, $Y$ pieces, and breeches pipe may be 12 per cent below the standard weight, and no excess above the standard weight of more than the above percentages for the several sizes will be paid for. These variations apply only to castings made from the standard patterns.

\section{QUALITY OF IRON}

Section 8.-All pipes and special castings shall be made of cast iron of good quality, and of such character as shall make the metal of the castings strong, tough, and of even grain, and soft enough satisfactorily to admit of drilling and cutting. The metal shall be made without any admixture of cinder iron or other inferior metal, and shall be remelted in a cupola or air furnace.

\section{TESTS OF MATERIALS}

Section 9.-Specimen bars of the metal used, each being 26 inches long by 2 inches wide and 1 inch thick, shall be made without charge as often as the engineer may direct, and, in default of definite instructions, the contractor shall make and test at least one bar from each heat or run of metal. The bars, when placed flatwise upon supports 24 inches apart and loaded in the center, shall, for pipes 12 inches or less in diameter, support a load of 1,900 pounds, and show a deflection of not less than 0.30 inch before breaking; and, for pipes of sizes larger than 12 inches, they shall support a load of 2,000 pounds and show a deflection of not less than 0.32 inch. The contractor shall have the right to make and break three bars from each heat or run of metal, and the test shall be based upon the average results of the three bars. Should the dimensions of the bars differ from those above given, a proper allowance therefor shall be made in the results of the tests.

\section{CASTING OF PIPES}

Section 10.-The straight pipes shall be cast in dry sand molds in a vertical position. Pipes 16 inches or less in diameter shall be cast with the hub end up or down as specified in the proposal. Pipes 18 inches or more in diameter shall be cast with the hub end down.

The pipes shall not be stripped or taken from the pit while showing color of heat, but shall be left in the flasks for a sufficient length of time to prevent unequal contraction by subsequent exposure.

\section{QUALITY OF CASTINGS}

Section 11.-The pipes and special castings shall be smooth, free from scales, lumps, blisters, sand holes, and defects of every nature which unfit them for the use for which they are intended. No plugging or filling will be allowed.

\section{CLEANING AND INSPECTION}

Section 12.-All pipes and special castings shall be thoroughly cleaned and subjected to a careful hammer inspection. No casting shall be coated unless entirely clean and free from rust, and approved in these respects by the engineer immediately before being dipped.

\section{COATING}

Section 13.-Every pipe and special casting shall be coated inside and out with coal-tar-pitch varnish. The varnish shall be made from coal tar. To this material sufficient oil shall be added to make a smooth coating, tough and tenacious when cold, and not brittle nor with any tendency to scale off.

Each casting shall be heated to a temperature of $300^{\circ} \mathrm{F}$. immediately before it is dipped, and shall possess not less than this temperature at the time it is put in the vat. The ovens in which the pipes are heated shall be so arranged that all portions of the pipe shall be heated to an even temperature. Each casting shall remain in the bath at least five minutes.

The varnish shall be heated to a temperature of $300^{\circ} \mathrm{F}$. (or less if the engineer shall so order), and shall be maintained at this temperature during the time the casting is immersed.

Fresh pitch and oil shall be added when necessary to keep the mixture at the proper consistency, and the vat shall be emptied of its contents and refilled with fresh pitch when deemed necessary by the engineer. After being coated, the pipes shall be carefully drained of the surplus varnish. Any pipe or special casting that is to be recoated shall first be thoroughly scraped and cleaned.

\section{HYDROSTATIC TEST}

Section 14.- When the coating has become hard, the straight pipes shall be subjected to a proof by hydrostatic pressure, and, if required by the engineer, they shall also be subjected to a hammer test under this pressure.

The pressure to which the different sizes and classes of pipes shall be subjected are as follows:

\begin{tabular}{|c|c|c|}
\hline & $\begin{array}{l}20 \text { inches } \\
\text { diameter } \\
\text { and larger }\end{array}$ & $\begin{array}{l}\text { Less than } \\
20 \text { inches } \\
\text { diameter }\end{array}$ \\
\hline $\begin{array}{l}\text { Class A pipe } \\
\text { Class B pipe } \\
\text { Class C pipe } \\
\text { Class D pipe }\end{array}$ & $\begin{array}{r}\text { Lbs./in. } \\
150 \\
200 \\
250 \\
300\end{array}$ & $\begin{array}{r}\text { Lbs./in. }{ }^{2} \\
300 \\
300 \\
300 \\
300\end{array}$ \\
\hline
\end{tabular}


WEIGHING

Section 15.-The pipes and special castings shall be weighed for payment under the supervision of the engineer after the application of the coal-tar-pitch varnish. If desired by the engineer the pipes and special castings shall be weighed after their delivery, and the weights so ascertained shall be used in the final settlement, provided such weighing is done by a legalized weighmaster. Bids shall be submitted and a final settlement made on the basis of a ton of 2,000 pounds.

\section{CONTRACTOR TO FURNISH MEN AND MATERIALS}

Section 16. -The contractor shall provide all tools, testing machines, materials, and men necessary for the required testing, inspection, and weighing at the foundry of the pipes and special castings; and should the purchaser have no inspector at the works, the contractor shall, if required by the engineer, furnish a sworn statement that all of the tests have been made as specified, this statement to contain the results of the tests upon the test bars.

\section{POWER OF ENGINEER TO INSPECT}

Section 17.-The engineer shall be at liberty at all times to inspect the material at the foundry, and the molding, casting, and coating of the pipes and special castings. The forms, sizes, uniformity, and conditions of all pipes and other castings herein referred to shall be subject to his inspection and approval, and he may reject, without proving, any pipes or other castings which are not in conformity with the specifications or drawings.

\section{INSPECTOR TO REPORT}

Section 18.-The inspector at the foundry shall report daily to the foundry office all pipes and special castings rejected, with the causes for rejection.

\section{CASTINGS TO BE DELIVERED SOUND AND PERFECT}

Section 19. - All the pipes and other castings must be delivered in all respects sound and conformable to these specifications. The inspection shall not relieve the contractor of any of his obligations in this respect, and any defective pipe or other castings which may have passed the engineer at the works or elsewhere shall be at all times liable to rejection when discovered until the final completion and adjustment of the contract, provided, however, that the contractor shall not be held liable for pipes or special castings found to be cracked after they have been accepted at the agreed point of delivery. Care shall be taken in handling the pipes not to injure the coating, and no pipes or other material of any kind shall be placed in the pipes during transportation or at any time after they receive the coating.
DEFINITION OF THE WORD " ENGINEER"

Section 20.-Wherever the word "engineer" is used herein, it shall be understood to refer to the engineer or inspector acting for the purchaser and to his properly authorized agents, limited by the particular duties intrusted to them.

\section{AMERICAN WATER WORKS ASSOCIATION, STANDARD SPECIFICATIONS FOR CAST- IRON WATER PIPE AND SPECIAL CAST. INGS, 1908}

\section{CAST-IRON WATER PIPE AND SPECIAL CASTINGS}

(Text of specifications identical with A. S. T. M., A44-04, given above, with the addition, in tabular form, of weights and dimensions of 72-inch and 84inch pipe, weights and dimensions of pipe for highpressure service, as well as weights and dimensions of the following special castings:)

Branches, blow-off.

Branches, blow-off with manhole.

Branches, standard.

Branches, $Y$.

Caps.

Curves, bell and spigot.

Curves, bell and spigot, offsets.

Lugs.

Manhole pipe.

Plugs.

Reducers and increasers.

Sleeves.

STANDARD DIMENSIONS OF PIPE

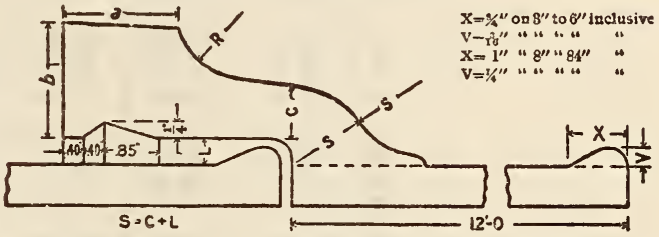

FIgURE 219.-Standard dimensions of pipes

Table 1.-Classes $A, B, C$,

\begin{tabular}{|c|c|c|c|c|c|c|c|c|c|}
\hline \multirow{2}{*}{$\begin{array}{c}\text { Nom- } \\
\text { inal } \\
\text { diam- } \\
\text { eter }\end{array}$} & \multirow[b]{2}{*}{ Classes } & \multirow{2}{*}{$\begin{array}{l}\text { Act- } \\
\text { ual } \\
\text { out- } \\
\text { side } \\
\text { diam- } \\
\text { eter }\end{array}$} & \multicolumn{2}{|c|}{$\begin{array}{l}\text { Diameter of } \\
\text { sockets }\end{array}$} & \multicolumn{2}{|c|}{$\begin{array}{l}\text { Depth of } \\
\text { sockets }\end{array}$} & \multirow[b]{2}{*}{ a } & \multirow[b]{2}{*}{ b } & \\
\hline & & & Pipe & $\begin{array}{l}\text { Spe- } \\
\text { cial } \\
\text { cast- } \\
\text { ings }\end{array}$ & Pipe & $\begin{array}{l}\text { Spe- } \\
\text { cial } \\
\text { cast- } \\
\text { ings }\end{array}$ & & & \\
\hline $\begin{array}{r}\text { In. } \\
72 \\
72 \\
72\end{array}$ & $\begin{array}{l}\mathrm{A} \\
\mathrm{B} \\
\mathrm{C}\end{array}$ & $\begin{array}{l}\text { In. } \\
75.34 \\
76.00 \\
76.88\end{array}$ & $\begin{array}{l}\text { In. } \\
76.34 \\
77.00 \\
77.88\end{array}$ & $\begin{array}{l}\text { In. } \\
76.34 \\
77.00 \\
77.88\end{array}$ & \begin{tabular}{|c|} 
In. \\
5.50 \\
5.50 \\
5.50
\end{tabular} & $\begin{array}{l}\text { In. } \\
5.50 \\
5.50 \\
5.50\end{array}$ & $\begin{array}{l}\text { In. } \\
2.25 \\
2.25 \\
2.25\end{array}$ & $\begin{array}{l}\text { In. } \\
3.80 \\
4.20 \\
4.60\end{array}$ & $\begin{array}{l}\text { In. } \\
1.87 \\
2.20 \\
2.64\end{array}$ \\
\hline $\begin{array}{l}84 \\
84\end{array}$ & $\stackrel{A}{B}$ & $\begin{array}{l}87.54 \\
88.54\end{array}$ & $\begin{array}{l}88.54 \\
89.54\end{array}$ & $\begin{array}{l}88.54 \\
89.54\end{array}$ & $\begin{array}{l}5.50 \\
5.50\end{array}$ & $\begin{array}{l}5.50 \\
5.50\end{array}$ & $\begin{array}{l}2.50 \\
2.50\end{array}$ & $\begin{array}{l}4.10 \\
4.50\end{array}$ & $\begin{array}{l}2.10 \\
2.60\end{array}$ \\
\hline
\end{tabular}

STANDARD THICKNESSES AND WEIGHTS OF CAST-IRON PIPE

Table 2.-Classes $A, B, C, D$

\begin{tabular}{|c|c|c|c|c|c|c|c|c|c|c|c|c|c|}
\hline \multirow{3}{*}{$\begin{array}{l}\text { Nomi- } \\
\text { nal in- } \\
\text { side } \\
\text { diam- } \\
\text { eter }\end{array}$} & \multicolumn{3}{|c|}{$\begin{array}{l}\text { Class } A-100 \text {-foot head, } \\
43 \text { pounds pressure }\end{array}$} & \multicolumn{3}{|c|}{$\begin{array}{l}\text { Class B-200-foot head, } \\
86 \text { pounds pressure }\end{array}$} & \multicolumn{3}{|c|}{$\begin{array}{l}\text { Class } C-300 \text {-foot head, } \\
130 \text { pounds pressure }\end{array}$} & \multicolumn{3}{|c|}{$\begin{array}{l}\text { Class } D-400 \text {-foot head, } \\
173 \text { pounds pressure }\end{array}$} & \multirow{3}{*}{$\begin{array}{l}\text { Nomi- } \\
\text { nal in } \\
\text { side } \\
\text { diam- } \\
\text { eter }\end{array}$} \\
\hline & \multirow{2}{*}{$\begin{array}{l}\text { Thick- } \\
\text { ness }\end{array}$} & \multicolumn{2}{|c|}{ Weight per- } & \multirow{2}{*}{$\begin{array}{l}\text { Thick- } \\
\text { ness }\end{array}$} & \multicolumn{2}{|c|}{ Weight per- } & \multirow{2}{*}{$\begin{array}{l}\text { Thick- } \\
\text { ness }\end{array}$} & \multicolumn{2}{|c|}{ Weight per- } & \multirow{2}{*}{$\begin{array}{c}\text { Thick- } \\
\text { ness }\end{array}$} & \multicolumn{2}{|c|}{ Weight per - } & \\
\hline & & Foot & Length & & Foot & Length & & Foot & Length & & Foot & Length & \\
\hline $\begin{array}{r}\text { Inches } \\
72 \\
84\end{array}$ & $\begin{array}{r}\text { Inches } \\
1.62 \\
1.72\end{array}$ & $\begin{array}{r}\text { Pounds } \\
1,283.4 \\
1,633.1\end{array}$ & $\begin{array}{r}\text { Pounds } \\
15,400 \\
19,600\end{array}$ & $\begin{array}{r}\text { Inches } \\
1.95 \\
2.22\end{array}$ & $\begin{array}{r}\text { Pounds } \\
1,545.8 \\
2,104.2\end{array}$ & $\begin{array}{r}\text { Pounds } \\
18,550 \\
25,250\end{array}$ & $\begin{array}{r}\text { Inches } \\
2.39\end{array}$ & $\begin{array}{c}\text { Pounds } \\
1,904.2\end{array}$ & $\begin{array}{r}\text { Pounds } \\
22,850\end{array}$ & Inches & Pounds & Pounds & $\begin{array}{c}\text { Inches } \\
72 \\
84\end{array}$ \\
\hline
\end{tabular}
tion.

Nork. - The above weights are per length to lay 12 feet, including standard sockets; proportionate allowance to be made for any varia- 
STANDARD DIMENSIONS OF PIPE

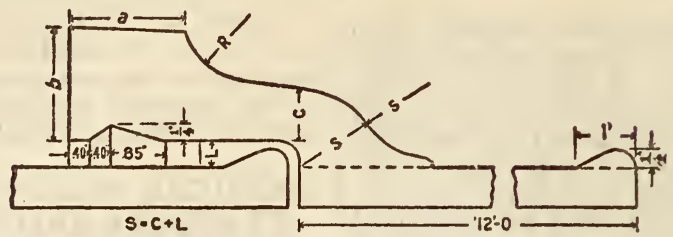

Figure 220.-Standard dimensions of pipe, highpressure service

High-Pressure Service

Table 3.-Classes $E, F, G, H$

\begin{tabular}{|c|c|c|c|c|c|c|c|c|c|}
\hline \multirow{2}{*}{$\begin{array}{l}\text { Nomi- } \\
\text { nal } \\
\text { diam- } \\
\text { eter }\end{array}$} & \multirow[b]{2}{*}{ Classes } & \multirow{2}{*}{$\begin{array}{c}\text { Actual } \\
\text { outside } \\
\text { diameter }\end{array}$} & \multicolumn{2}{|c|}{ Pipe and specials } & \multirow[b]{2}{*}{$\mathbf{A}$} & \multirow[b]{2}{*}{ B } & \multirow[b]{2}{*}{ C } & \multirow[b]{2}{*}{$\mathbf{R}$} & \multirow{2}{*}{$\begin{array}{c}\text { Nomi- } \\
\text { nal } \\
\text { diam. } \\
\text { eter }\end{array}$} \\
\hline & & & $\begin{array}{l}\text { Diameter } \\
\text { of sockets }\end{array}$ & $\begin{array}{c}\text { Depth } \\
\text { of } \\
\text { sockets }\end{array}$ & & & & & \\
\hline $\begin{array}{c}\text { Inches } \\
6 \\
6 \\
8 \\
8 \\
10\end{array}$ & $\begin{array}{l}E-F \\
G-H \\
E-F \\
G-H \\
E-F\end{array}$ & $\begin{array}{r}\text { Inches } \\
7.22 \\
7.38 \\
9.42 \\
9.60 \\
11.60\end{array}$ & $\begin{array}{r}\text { Inches } \\
8.02 \\
8.18 \\
10.22 \\
10.40 \\
12.40\end{array}$ & $\begin{array}{c}\text { Inches } \\
4.00 \\
4.00 \\
4.00 \\
4.00 \\
4.50\end{array}$ & $\begin{array}{c}\text { Inches } \\
1.50 \\
1.50 \\
1.50 \\
1.50 \\
1.75\end{array}$ & $\begin{array}{c}\text { Inches } \\
1.75 \\
1.85 \\
1.85 \\
1.95 \\
1.95\end{array}$ & $\begin{array}{c}\text { Inches } \\
0.75 \\
.85 \\
.85 \\
.95 \\
.95\end{array}$ & $\begin{array}{c}\text { Inches } \\
1.10 \\
1.10 \\
1.10 \\
1.10 \\
1.10\end{array}$ & $\begin{array}{c}\text { Inches } \\
6 \\
6 \\
8 \\
8 \\
10\end{array}$ \\
\hline $\begin{array}{l}10 \\
12 \\
12 \\
14\end{array}$ & $\begin{array}{l}\mathrm{G}-\mathrm{H} \\
\mathrm{E}-\mathrm{F} \\
\mathrm{G}-\mathrm{H} \\
\mathrm{E}-\mathrm{F}\end{array}$ & $\begin{array}{l}11.84 \\
13.78 \\
14.08 \\
15.98\end{array}$ & $\begin{array}{l}12.64 \\
14.58 \\
14.88 \\
16.78\end{array}$ & $\begin{array}{l}4.50 \\
4.50 \\
4.50 \\
4.50\end{array}$ & $\begin{array}{l}1.75 \\
1.75 \\
1.75 \\
2.00\end{array}$ & $\begin{array}{l}2.05 \\
2.05 \\
2.20 \\
2.15\end{array}$ & $\begin{array}{l}1.05 \\
1.05 \\
1.20 \\
1.15\end{array}$ & $\begin{array}{l}1.10 \\
1.10 \\
1.10 \\
1.10\end{array}$ & $\begin{array}{l}10 \\
12 \\
12 \\
14\end{array}$ \\
\hline $\begin{array}{l}14 \\
16 \\
16 \\
18\end{array}$ & $\begin{array}{l}\mathrm{G}-\mathrm{H} \\
\mathrm{E}-\mathrm{F} \\
\mathrm{G}-\mathrm{H} \\
\mathrm{E}-\mathrm{F}\end{array}$ & $\begin{array}{l}16.32 \\
18.16 \\
18.54 \\
20.34\end{array}$ & $\begin{array}{l}17.12 \\
18.96 \\
19.34 \\
21.14\end{array}$ & $\begin{array}{l}4.50 \\
4.50 \\
4.50 \\
4.50\end{array}$ & $\begin{array}{l}2.00 \\
2.00 \\
2.00 \\
2.25\end{array}$ & $\begin{array}{l}2.35 \\
2.30 \\
2.55 \\
2.45\end{array}$ & $\begin{array}{l}1.35 \\
1.25 \\
1.45 \\
1.40\end{array}$ & $\begin{array}{l}1.10 \\
1.15 \\
1.15 \\
1.15\end{array}$ & $\begin{array}{l}14 \\
16 \\
16 \\
18\end{array}$ \\
\hline $\begin{array}{l}18 \\
20 \\
20 \\
24\end{array}$ & $\begin{array}{l}\mathrm{G}-\mathrm{H} \\
\mathrm{E}-\mathrm{F} \\
\mathrm{G}-\mathrm{H} \\
\mathrm{E}-\mathrm{F}\end{array}$ & $\begin{array}{l}20.78 \\
22.54 \\
23.02 \\
26.90\end{array}$ & $\begin{array}{l}21.58 \\
23.34 \\
23.82 \\
27.90\end{array}$ & $\begin{array}{l}4.50 \\
4.50 \\
4.50 \\
5.00\end{array}$ & $\begin{array}{l}2.25 \\
2.25 \\
2.25 \\
2.25\end{array}$ & $\begin{array}{l}2.75 \\
2.55 \\
2.85 \\
2.85\end{array}$ & $\begin{array}{l}1.65 \\
1.50 \\
1.75 \\
1.70\end{array}$ & $\begin{array}{l}1.15 \\
1.15 \\
1.20 \\
1.20\end{array}$ & $\begin{array}{l}18 \\
20 \\
20 \\
24\end{array}$ \\
\hline $\begin{array}{l}30 \\
30 \\
36 \\
36\end{array}$ & $\begin{array}{l}\mathrm{E} \\
\mathrm{F} \\
\mathrm{E} \\
\mathrm{F}\end{array}$ & $\begin{array}{l}33.10 \\
33.46 \\
39.60 \\
40.04\end{array}$ & $\begin{array}{l}34.10 \\
34.36 \\
40.60 \\
41.04\end{array}$ & $\begin{array}{l}5.00 \\
5.00 \\
5.00 \\
5.00\end{array}$ & $\begin{array}{l}2.25 \\
2.25 \\
2.25 \\
2.25\end{array}$ & $\begin{array}{l}3.25 \\
3.50 \\
3.70 \\
4.00\end{array}$ & $\begin{array}{l}1.80 \\
2.00 \\
2.05 \\
2.30\end{array}$ & $\begin{array}{l}1.50 \\
1.55 \\
1.70 \\
1.80\end{array}$ & $\begin{array}{l}30 \\
30 \\
36 \\
36\end{array}$ \\
\hline
\end{tabular}

STANDARD THICKNESSES AND WEIGHTS OF CAST-IRON PIPE

For Fire Lines and Other High-Pressure Service

Table 4.-Classes $E, F, G, H$

\begin{tabular}{|c|c|c|c|c|c|c|c|c|c|c|c|c|c|}
\hline \multirow{3}{*}{$\begin{array}{l}\text { Nomi- } \\
\text { nal in- } \\
\text { side } \\
\text { diam- } \\
\text { eter }\end{array}$} & \multicolumn{3}{|c|}{$\begin{array}{l}\text { Class } \mathrm{E}-500 \text {-foot head, } \\
217 \text { pounds pressure }\end{array}$} & \multicolumn{3}{|c|}{$\begin{array}{l}\text { Class } F-600 \text {-foot head, } \\
260 \text { pounds pressure }\end{array}$} & \multicolumn{3}{|c|}{$\begin{array}{l}\text { Class G-700-foot head, } \\
304 \text { pounds pressure }\end{array}$} & \multicolumn{3}{|c|}{$\begin{array}{l}\text { Class H-800-foot head, } \\
347 \text { pounds pressure }\end{array}$} & \multirow{3}{*}{$\begin{array}{l}\text { Nomi- } \\
\text { nal in- } \\
\text { side } \\
\text { diam. } \\
\text { eter }\end{array}$} \\
\hline & \multirow{2}{*}{$\begin{array}{l}\text { Thick- } \\
\text { ness }\end{array}$} & \multicolumn{2}{|c|}{ Weight per- } & \multirow{2}{*}{$\begin{array}{c}\text { Thick- } \\
\text { ness }\end{array}$} & \multicolumn{2}{|c|}{ Weight per- } & \multirow{2}{*}{$\begin{array}{l}\text { Thick- } \\
\text { ness }\end{array}$} & \multicolumn{2}{|c|}{ Weight per- } & \multirow{2}{*}{$\begin{array}{l}\text { Thick- } \\
\text { ness }\end{array}$} & \multicolumn{2}{|c|}{ Weight per- } & \\
\hline & & Foot & Length & & Foot & Length & & Foot & Length & & Foot & Length & \\
\hline $\begin{array}{c}\text { Inches } \\
6 \\
8 \\
10 \\
12\end{array}$ & $\begin{array}{c}\text { Inches } \\
0.58 \\
.66 \\
.74 \\
.82\end{array}$ & $\begin{array}{c}\text { Pounds } \\
41.7 \\
61.7 \\
86.3 \\
113.8\end{array}$ & $\begin{array}{c}\text { Pounds } \\
500 \\
740 \\
1,035 \\
1,365\end{array}$ & $\begin{array}{c}\text { Inches } \\
0.61 \\
.71 \\
.80 \\
.89\end{array}$ & $\begin{array}{c}\text { Pounds } \\
43.3 \\
65.7 \\
92.1 \\
122.1\end{array}$ & $\begin{array}{c}\text { Pounds } \\
520 \\
790 \\
1,105 \\
1,465\end{array}$ & $\begin{array}{c}\text { Inches } \\
0.65 \\
.75 \\
.86 \\
.97\end{array}$ & $\begin{array}{c}\text { Pounds } \\
47.1 \\
70.8 \\
100.9 \\
135.4\end{array}$ & $\begin{array}{r}\text { Pounds } \\
565 \\
850 \\
1,210 \\
1,625\end{array}$ & $\begin{array}{c}\text { Inches } \\
0.69 \\
.80 \\
.92 \\
1.04\end{array}$ & $\begin{array}{c}\text { Pounds } \\
49.6 \\
75.0 \\
106.7 \\
143.8\end{array}$ & $\begin{array}{c}\text { Pounds } \\
595 \\
900 \\
1,280 \\
1,725\end{array}$ & $\begin{array}{c}\text { Inches } \\
6 \\
8 \\
10 \\
12\end{array}$ \\
\hline $\begin{array}{l}14 \\
16 \\
18 \\
20\end{array}$ & $\begin{array}{r}.90 \\
.98 \\
1.07 \\
1.15\end{array}$ & & $\begin{array}{l}1,7 \\
2,1 \\
2,6 \\
3,1\end{array}$ & $\begin{array}{r}.99 \\
1.08 \\
1.17 \\
1.27\end{array}$ & & & $\begin{array}{l}1.07 \\
1.18 \\
1.28 \\
1.39\end{array}$ & & & & & & $\begin{array}{l}14 \\
16 \\
18 \\
20\end{array}$ \\
\hline $\begin{array}{l}24 \\
30 \\
36\end{array}$ & $\begin{array}{l}1.31 \\
1.55 \\
1.80\end{array}$ & $\begin{array}{l}359.6 \\
521.7 \\
725.0\end{array}$ & $\begin{array}{l}4,315 \\
6,260 \\
8,700\end{array}$ & $\begin{array}{l}1.45 \\
1.73 \\
2.02\end{array}$ & $\begin{array}{l}392.9 \\
585.4 \\
820.0\end{array}$ & & & & & & & & $\begin{array}{l}24 \\
30 \\
36\end{array}$ \\
\hline
\end{tabular}

NorE.-The above weights are per length to lay 12 feet, including standard sockets; proportionate allowance to be made for any variation. For High-pressure pipe from 6 to 24 inches inclusive, one class of special castings shall be furnished for classes $\mathrm{E}$ and $\mathrm{F}$ pipe, and one class of special castings for classes $\mathrm{G}$ and $\mathrm{H}$ pipe. For 30-inch and 36 -inch pipe, one class of special castings shall be furnished for each class of pipe. 
UNITED STATES GOVERNMENT, FEDERAL SPECIFICATIONS BOARD, FEDERAL SPECIFICATION FOR PIPE; WATER, CAST-IRON (BELL AND SPIGOT), WW-P-421, JULY 21, 1931

\section{CAST-IRON WATER PIPE}

\section{A. APPLICABLE FEDERAL SPECIFICATIONS}

A-1. The following Federal specifications, or parts thereof that are indicated below, of the issue in effect on date of invitation for bids, shall form a part of this specification:

F. S. SS-C-191, Portland cement.

F. S. QQ-M-151 (F. S. B. No. 339), general specifications for metals. (See 600.0, p. 3.)

\section{B. TYPES AND CLASSES}

B-1. This specification covers bell and spigot castiron water pipe in the following types:

Type I.-Centrifugally cast in metal-contact molds in 12-foot and 18-foot lengths.

Type II. - Centrifugally cast in sand-lined molds in 16-foot, $16 \frac{1}{2}$-foot and 20-foot lengths.

Type III. - Horizontally cast in green-sand molds in 12-foot and 16-foot lengths.

B-2. This specification covers the following classes:

Class 150 for 150 pounds maximum water working pressure. (See par. I-6.)

Class 250 for 250 pounds maximum water working pressure. (See par. I-6.)

B-3. This specification covers sizes from 4 to 24 inches, inclusive, for Types I and II, and from 4 to 12 inches, inclusive, for Type III.

B-4. Pipe shall be furnished coated, unless cement-lined or uncoated pipe is required in the invitation for bids.

\section{MATERIAL AND WORKMANSHIP}

C-1. All pipe shall be made of cast iron of good quality and of such character as shall make the pipe strong, tough, and of even grain, and soft enough to admit of satisfactory drilling and cutting. Each pipe shall be smooth, free from cold shuts, scale, lumps, blisters, and sand holes, and defects of every nature which unfit it for the use intended. It shall be straight and shall be true circles in section with its inner and outer surfaces concentric. No plugging, filling, "burning in", or welding will be allowed.

\section{GENERAL REQUIREMENTS}

D-1. Lengths.-Pipe shall have nominal laying lengths of $12,16,161 / 2,18$, or 20 feet. Not more than 10 per cent of cut pipe shall be accepted. Such cut pipe shall be not more than 1 foot under nominal laying length.

D-2. Cleaning. - All pipe shall be thoroughly cleaned, and any rough spots in bells or on spigot ends shall be removed before coating or lining.

D-3. Coating.-Unless otherwise specified all pipe shall be completely coated inside and out with coaltar-pitch varnish, to which sufficient oil shall be added to make a smooth coating, tough and tenacious when cold, not "tacky" and not brittle.

D-4. Cement mortar lining. - When required in the contract or purchase order, pipe shall be lined with cement mortar. No coating shall be applied to the outside of pipe unless specifically provided for in the contract or order.

D-4a. Preparation of pipe for lining.-Pipe to be lined with cement mortar shall not be coated inside with tar or other asphaltum products. Its interior surface shall be thoroughly cleaned of all core sand, mud, grease, foreign materials, or any sharp projections of iron which might project through the lining. Pipe shall be tested hydrostatically before being lined.

D-4b. Cement. - The cement used for making cement mortar shall be standard Portland cement, complying in all respects with the requirements of Federal specification SS-C-191 for Portland cement, referred to in paragraph $\mathrm{A}-1$.

D-4c. Sand.-The sand for mortar shall be clean, sharp, hard, and silicious sand, free from loam, clay, organic matter, or other foreign substance considered as deleterious for good mortar. The sand shall be well graded and when tested by laboratory sieves shall meet the following specifications:

Total passing 12-mesh sieve, 100 per cent.

Total passing $100-$ mesh sieve, not over 5 per cent.

D-4d. Water. - The water for tempering the cement mortar shall be free from harmful amounts of oil, acid, alkali, organic, or vegetable matter.

D-4e. Mortar. - The cement mortar used for lining pipe shall be a mixture of the above-specified sand and cement in such proportions as to obtain a good, hard, dense lining, reasonably well bonded to the pipe, and with a smooth interior surface. (A mixture which has been found to give very satisfactory results consist of three parts cement to one part sand, by volume.) The cement mortar shall be thoroughly mixed, only sufficient water being added to form a workable mixture for placing in the pipe. Only sufficient cement mortar shall be mixed for the immediate requirements of lining.

D-4f. Application of lining.-Sufficient cement mortar shall be introduced to produce the required thickness of lining and shall be spread evenly over the interior surface of the pipe by any suitable means. A careful examination shall be made after this operation is completed to see that the inner surface of the pipe is completely covered with cement mortar. The shoulder of the bell and the end of the spigot may be covered with cement mortar by applying with a brush. Surplus cement mortar shall be removed from the interior of the bell so as not to interfere with proper keying of the joint. The work of lining the pipe shall be done in a building where the product shall be protected from the direct rays of the sun, and from extreme weather conditions, such as rain, frost, etc. The product shall not be put on the yard until the cement has set sufficiently to avoid injury or damage thereto. Patching of improperly lined pipe will not be permitted.

D-4g. Smoothness of lining.-The lining of pipe shall be smooth and substantially free from noticeable ridges, corrugations, projections, or depressions.

D-4h. Thickness of lining. - The thickness of lining shall be $1 / 8$ inch $+1 / 8,-0$ inch for sizes 12 inches and under, and $3 / 16$ inch $+1 / 8,-0$ inch for sizes over 12 inches. At the end of the pipe where the lining naturally tends to taper off to a thin edge, the full thickness of lining shall extend to within 1 inch of end of pipe. Linings of greater thickness shall be furnished when specified.

D-4i. Curing of lining.- Immediately after pipe is lined with cement mortar, it shall be protected in a suitable manner to prevent the too rapid withdrawal of moisture from the cement mortar and, if necessary, suitable means shall be provided to keep lining damp for a period of at least 24 hours after lining.

D-4j. No pipe shall be shipped until the lining is thoroughly set.

D-5. Weighing.-Each length of pipe shall be weighed and the weight plainly and indelibly marked on the pipe. Coated pipe shall be weighed after coating. Cement-lined pipe shall be weighed before 
lining and the mark shall be placed on the outside surface.

D-6. Hardness.-The average Rockwell number shall not exceed 95 when tested in accordance with the method described in paragraph $\mathrm{F}-6$.

D-7. Handling. - Care shall be taken in handling the pipes not to injure the coating, and no pipes or other material shall be placed in the pipes during transportation, or at any time after they have been coated.

D-8. Marking. - In addition to the weight being marked on the pipe as previously specified, each pipe shall have distinctly cast or stamped into the metal on the face or outside surface of the bell or on body near the bell the manufacturer's mark, and the year in which the pipe was cast. When so specified in the

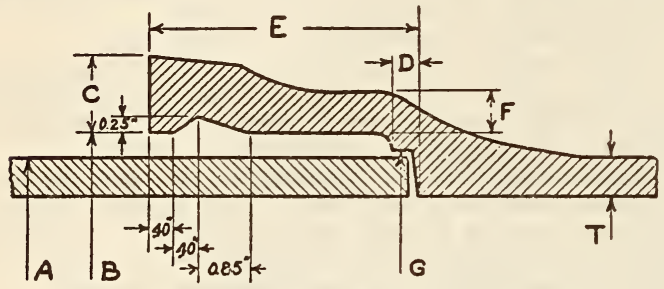

Figure 221.-Type I pipe, end details

invitation for bids each pipe shall also have cast upon it, or stamped into the metal, a designated symbol.

\section{E. DETAIL REQUIREMENTS}

E-1. Tolerances in thickness.-

E-1a. The tolerances in thickness of pipe, plus or minus, shall not exceed those listed below.

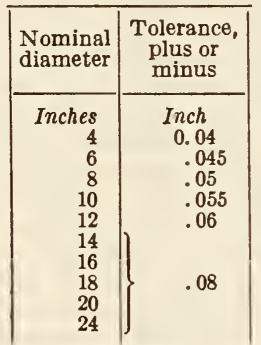

E-1b. For all sizes of pipe, tolerances not exceeding 0.02 inch additional to above will be allowed for spaces not exceeding 8 inches in length in any direction.

$\mathrm{E}-2$. Tolerance in weight. - The weight of no single pipe shall be less than the nominal tabulated weight by more than 5 per cent. The total weight of any order shall be not more than 2 per cent under nominal weight.

E-3. Type I.-Centrifugally cast in metal-contact molds in 12 and 18 foot lengths.-

E-3a. Type I pipe shall be of the bell and spigot type, centrifugally cast in metal-contact molds with plain spigot end, and with lead groove and selfcentering shoulder in the bell.

E-3b. Annealing.-Type I pipe, after withdrawing from machines, shall be annealed to meet the hardness limits specified in paragraph $\mathrm{F}-6$.

E-3c. Physical requirements.-Type I pipe shall have a secant modulus of elasticity (see par. F-4b) not to exceed $15,000,000$ pounds per square inch, with a corresponding modulus of rupture not less than 45,000 pounds per square inch for sizes 12 inches and under, and 40,000 pounds per square inch for sizes over 12 inches.

E-3d. Tolerances in diameter. - The inside diameters of the bells and the outside diameters of the spigot ends of Type I pipe shall not vary plus or minus from the tabulated dimensions by more than
0.06 inch for pipe 12 inches and less in nominal diameter and 0.08 inch for pipe 14 inches and larger.

E-3e. Dimensions and weights. - Type I pipe shall conform to the dimensions and weights given in Figure 221 and Tables 1 and 2 for the respective classes and subject to the tolerances given in paragraphs E-1a, E-1b, E-2, and E-3d.

\section{TABLE 1.-Dimensions of Type I pipe}

\begin{tabular}{|c|c|c|c|c|c|c|c|c|c|c|c|}
\hline \multirow{4}{*}{ 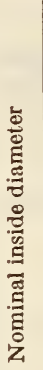 } & \multirow{3}{*}{ 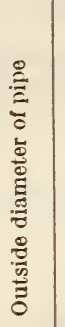 } & \multirow{3}{*}{ 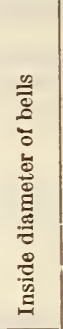 } & \multirow{3}{*}{ 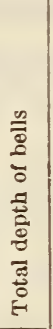 } & \multirow{3}{*}{ 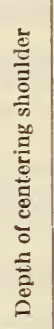 } & \multirow{3}{*}{ 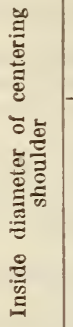 } & \multicolumn{3}{|c|}{$\begin{array}{l}\text { Class } 150 \text {-150 } \\
\text { pounds work- } \\
\text { ing pressure, } \\
346 \text {-foot head }\end{array}$} & \multicolumn{3}{|c|}{$\begin{array}{l}\text { Class } 250-250 \\
\text { pounds work- } \\
\text { ing pressure, } \\
576 \text {-foot head }\end{array}$} \\
\hline & & & & & & \multirow{2}{*}{ 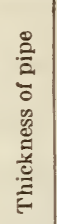 } & \multicolumn{2}{|c|}{$\begin{array}{c}\text { Thickness } \\
\text { of bells }\end{array}$} & \multirow{2}{*}{ 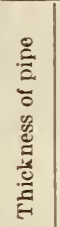 } & \multicolumn{2}{|c|}{$\begin{array}{l}\text { Thickness } \\
\text { of bells }\end{array}$} \\
\hline & & & & & & & 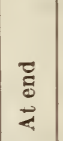 & $\begin{array}{l}\frac{b}{0} \\
\frac{0}{3} \\
\stackrel{0}{0} \\
\stackrel{\leftrightarrow}{4}\end{array}$ & & 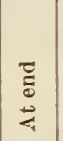 & 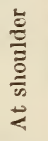 \\
\hline & A & B & $\mathrm{E}$ & D & $\mathrm{G}$ & $\mathrm{T}$ & C & $\mathrm{F}$ & $\mathrm{T}$ & C & $\mathrm{F}$ \\
\hline $\begin{array}{r}\text { In. } \\
4 \\
6 \\
8 \\
10 \\
12\end{array}$ & $\begin{array}{r}\text { In. } \\
4.80 \\
6.90 \\
9.05 \\
11.10 \\
13.20\end{array}$ & $\begin{array}{r}\text { In. } \\
5.60 \\
7.70 \\
9.85 \\
11.90 \\
14.00\end{array}$ & $\begin{array}{l}\text { In. } \\
\text { 3. } 30 \\
3.88 \\
\text { 4. } 38 \\
\text { 4. } 38 \\
4.38\end{array}$ & $\begin{array}{l}\text { In. } \\
0.30 \\
.38 \\
.38 \\
.38 \\
.38\end{array}$ & \begin{tabular}{r}
\multicolumn{1}{c}{ In. } \\
4.93 \\
7.18 \\
9.31 \\
11.43 \\
13.43
\end{tabular} & $\begin{array}{l}\text { In. } \\
0.34 \\
.37 \\
.42 \\
.47 \\
.50\end{array}$ & $\begin{array}{l}\text { In. } \\
1.06 \\
1.13 \\
1.18 \\
1.23 \\
1.28\end{array}$ & $\begin{array}{l}\text { In. } \\
0.48 \\
.52 \\
.57 \\
.63 \\
.69\end{array}$ & $\begin{array}{l}\text { In. } \\
0.38 \\
.43 \\
.50 \\
.57 \\
.62\end{array}$ & $\begin{array}{l}\text { In. } \\
1.06 \\
1.13 \\
1.18 \\
1.23 \\
1.28\end{array}$ & $\begin{array}{l}\text { In. } \\
0.48 \\
.52 \\
.57 \\
.63 \\
.69\end{array}$ \\
\hline $\begin{array}{l}14 \\
16 \\
18 \\
20 \\
24\end{array}$ & $\begin{array}{l}15.65 \\
17.80 \\
19.92 \\
22.06 \\
26.32\end{array}$ & $\begin{array}{l}16.45 \\
18.80 \\
20.92 \\
23.06 \\
27.32\end{array}$ & $\begin{array}{l}\text { 4. } 50 \\
\text { 4. } 50 \\
\text { 4. } 50 \\
\text { 4. } 50 \\
\text { 4. } 50\end{array}$ & $\begin{array}{l}.50 \\
.50 \\
.50 \\
.50 \\
.50\end{array}$ & $\begin{array}{l}15.85 \\
18.00 \\
20.12 \\
22.26 \\
26.53\end{array}$ & $\begin{array}{l}.55 \\
.60 \\
.65 \\
.68 \\
.76\end{array}$ & $\begin{array}{l}1.35 \\
1.43 \\
1.53 \\
1.63 \\
1.83\end{array}$ & $\begin{array}{r}.73 \\
.77 \\
.82 \\
.87 \\
1.03\end{array}$ & $\begin{array}{r}.69 \\
.75 \\
.83 \\
.88 \\
1.00\end{array}$ & $\begin{array}{l}1.56 \\
1.63 \\
1.68 \\
1.75 \\
2.00\end{array}$ & $\begin{array}{r}.93 \\
1.00 \\
1.09 \\
1.18 \\
1.38\end{array}$ \\
\hline
\end{tabular}

TABLE 2.-Nominal weights of Type I pipe

\begin{tabular}{|c|c|c|c|c|c|c|c|c|}
\hline \multirow{3}{*}{ 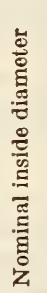 } & \multicolumn{4}{|c|}{$\begin{array}{l}\text { Class } 150-150 \text { pounds work- } \\
\text { ing pressure, } 346-\text { foot head }\end{array}$} & \multicolumn{4}{|c|}{$\begin{array}{l}\text { Class } 250-250 \text { pounds work- } \\
\text { ing pressure, } 576 \text {-foot head }\end{array}$} \\
\hline & \multicolumn{2}{|c|}{$\begin{array}{l}\text { 12-foot nomi- } \\
\text { nal laying } \\
\text { length }\end{array}$} & \multicolumn{2}{|c|}{$\begin{array}{l}\text { 18-foot nomi- } \\
\text { nal laying } \\
\text { length }\end{array}$} & \multicolumn{2}{|c|}{$\begin{array}{l}\text { 12-foot nomi- } \\
\text { nal laying } \\
\text { length }\end{array}$} & \multicolumn{2}{|c|}{$\begin{array}{l}\text { 18-foot nomins] } \\
\text { laying length }\end{array}$} \\
\hline & 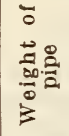 & 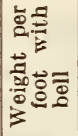 & $\begin{array}{l}\overrightarrow{0} \\
\vec{a} 0 \\
\overrightarrow{0} 0 \\
\overrightarrow{0}\end{array}$ & 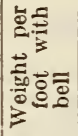 & 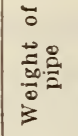 & 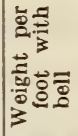 & $\begin{array}{l}\overline{0} \\
\overrightarrow{0} 0 \\
\frac{0}{0} \\
\overrightarrow{0}\end{array}$ & 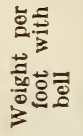 \\
\hline $\begin{array}{r}\text { In. } \\
4 \\
6 \\
8 \\
10 \\
12\end{array}$ & $\begin{array}{r}\text { Lbs. } \\
195 \\
315 \\
475 \\
640 \\
810\end{array}$ & $\begin{array}{l}\text { Lbs. } \\
16.4 \\
26.3 \\
39.4 \\
53.3 \\
67.4\end{array}$ & $\begin{array}{r}\text { Lbs. } \\
285 \\
460 \\
690 \\
935 \\
1,180\end{array}$ & $\begin{array}{l}\text { Lbs. } \\
15.9 \\
25.5 \\
38.3 \\
51.8 \\
65.6\end{array}$ & $\begin{array}{r}\text { Lbs. } \\
220 \\
350 \\
545 \\
760 \\
990\end{array}$ & $\begin{array}{l}\text { Lbs. } \\
18.4 \\
29.3 \\
45.5 \\
63.3 \\
82.5\end{array}$ & $\begin{array}{r}\text { Lbs. } \\
325 \\
515 \\
800 \\
1,115 \\
1,450\end{array}$ & $\begin{array}{l}\text { Lbs. } \\
17.9 \\
28.5 \\
44.3 \\
61.9 \\
80.7\end{array}$ \\
\hline $\begin{array}{l}14 \\
16 \\
18 \\
20 \\
24\end{array}$ & $\begin{array}{l}1,060 \\
1,320 \\
1,595 \\
1,860 \\
2,480\end{array}$ & $\begin{array}{r}88.5 \\
110.2 \\
132.8 \\
155.0 \\
206.8\end{array}$ & $\begin{array}{l}1,555 \\
1,935 \\
2,330 \\
2,720 \\
3,630\end{array}$ & $\begin{array}{r}86.3 \\
107.4 \\
129.6 \\
151.0 \\
201.6\end{array}$ & $\begin{array}{l}1,320 \\
1,635 \\
2,015 \\
2,365 \\
3,200\end{array}$ & $\begin{array}{l}110.0 \\
136.1 \\
168.0 \\
197.1 \\
266.8\end{array}$ & & $\begin{array}{l}107.3 \\
132.7 \\
163.9 \\
192.4 \\
260.6\end{array}$ \\
\hline
\end{tabular}

Note.-Calculated weight of pipe has been rounded off to nearest 5 pounds. Weight per foot is based on weight of pipe computed to nearest pound.

E-4. Type II.-Centrifugally cast in sand-lined molds in 16, 1612, and 20-foot lengths.-

E-4a. Type II pipe shall be of the bell and spigot type, centrifugally cast in sand-lined molds, with bead cast on spigot end, and with lead groove in the bell.

E-4b. Physical requirements.-Type II pipe shall have a secant modulus of elasticity (see par. F-4b) not to exceed $10,000,000$ pounds per square inch, with a corresponding modulus of rupture not less than 40,000 pounds per square inch.

E-4c. Tolerances in diameter. - The inside diameters of the bells and the outside diameters of the spigot ends (exclusive of bead) of Type II pipe shall not vary plus or minus from the tabulated dimensions by more than 0.06 inch for pipe 12 inches and less in nominal diameter, and 0.08 inch for pipe 14 inches and larger. 
E-4d. Dimensions and weights.-Type II pipe shall conform to the dimensions and weights given in Figure 222 and Tables 3 and 4 for the respective classes and subject to the tolerances given in paragraphs E-1a, E-1b, E-2, and E-4c.

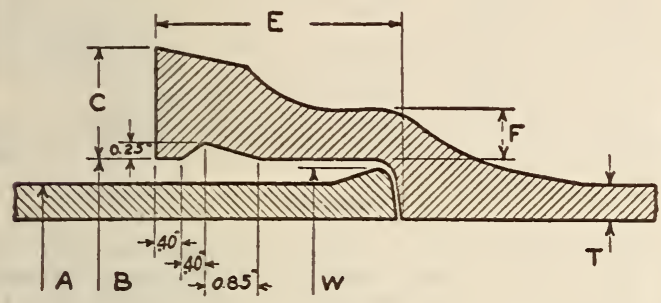

FIgURe 222.-Type II and III pipe, end details

TABLE 3.-Dimensions of Type II pipe

[All dimensions given in inches]

\begin{tabular}{|c|c|c|c|c|c|c|c|c|c|c|}
\hline \multirow{4}{*}{ 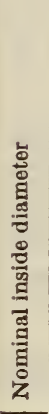 } & \multirow{3}{*}{ 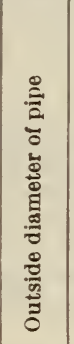 } & \multirow{3}{*}{ 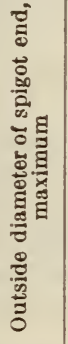 } & \multirow{3}{*}{ 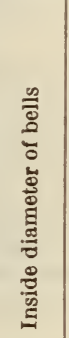 } & \multirow[b]{3}{*}{ 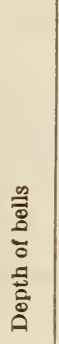 } & \multicolumn{3}{|c|}{$\begin{array}{c}\text { Class } 150-150 \\
\text { pounds working } \\
\text { pressure, } 346-\text { foot } \\
\text { head }\end{array}$} & \multicolumn{3}{|c|}{$\begin{array}{c}\text { Class 250-250 } \\
\text { pounds working } \\
\text { pressure, 576-foot } \\
\text { head }\end{array}$} \\
\hline & & & & & \multirow{2}{*}{ 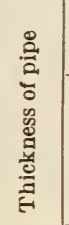 } & \multicolumn{2}{|c|}{$\begin{array}{l}\text { Thickness } \\
\text { of bells }\end{array}$} & \multirow{2}{*}{ 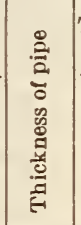 } & \multicolumn{2}{|c|}{$\begin{array}{l}\text { Thickness } \\
\text { of bells }\end{array}$} \\
\hline & & & & & & 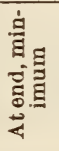 & 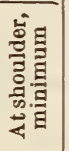 & & 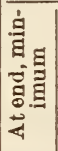 & 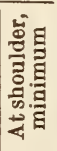 \\
\hline & $\mathbf{A}$ & W & B & $\mathbf{E}$ & $\mathbf{T}$ & C & F & $T$ & C & F \\
\hline & & & & & $\begin{array}{l}0.34 \\
37\end{array}$ & & 44 & $\begin{array}{l}0.38 \\
43\end{array}$ & 1.2 & 0.44 \\
\hline & & & & & & & • & 50 & & \\
\hline 10 & 11. & 11. & & & & & 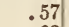 & .57 & & . \\
\hline 12 & 13. & 13. & 14.0 & & .5 & & 4 & .62 & 1. & \\
\hline 14 & 15 & 16 & 1 & 4. & .55 & & 6 & 69 & & .77 \\
\hline 16 & & & & & 6 & & 7 & .75 & & .8 \\
\hline 1 & & & & & & & • & .83 & & $\because 8$ \\
\hline $2 x$ & & & 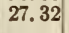 & 4. & 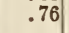 & & .8 & 1.00 & 2. & .9 \\
\hline
\end{tabular}

TABLE 4.-Nominal weights of Type II pipe

\begin{tabular}{|c|c|c|c|c|c|c|c|c|c|c|c|c|}
\hline & \multicolumn{5}{|c|}{$\begin{array}{l}\text { Class } 150-150 \text { pounds work- } \\
\text { ing pressure, } 346 \text {-foot head }\end{array}$} & \multicolumn{6}{|c|}{$\begin{array}{l}\text { Class } 250-250 \text { pounds work- } \\
\text { ing pressure, } 576 \text {-foot head }\end{array}$} \\
\hline & \multicolumn{2}{|c|}{$\begin{array}{l}\text { 16-foot } \\
\text { nominal } \\
\text { laying } \\
\text { length }\end{array}$} & \multicolumn{2}{|c|}{$\begin{array}{c}161 / 2-\text {-foot } \\
\text { nominal } \\
\text { laying } \\
\text { length }\end{array}$} & \multicolumn{2}{|c|}{$\begin{array}{c}20 \text {-foot } \\
\text { nominal } \\
\text { laying } \\
\text { length }\end{array}$} & \multicolumn{2}{|c|}{$\begin{array}{c}\text { 16-foot } \\
\text { nominal } \\
\text { laying } \\
\text { length }\end{array}$} & \multicolumn{2}{|c|}{$\begin{array}{c}161 / 2 \text {-foot } \\
\text { nominal } \\
\text { laying } \\
\text { length }\end{array}$} & \multicolumn{2}{|c|}{$\begin{array}{l}20 \text {-foot } \\
\text { nominal } \\
\text { laying } \\
\text { length }\end{array}$} \\
\hline 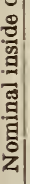 & 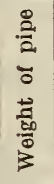 & 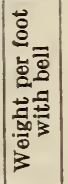 & 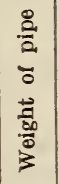 & 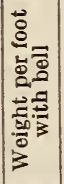 & 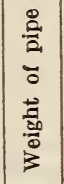 & 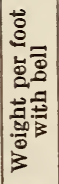 & 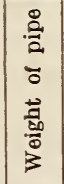 & 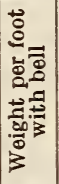 & 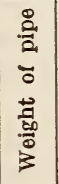 & 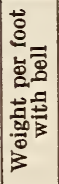 & 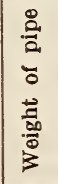 & 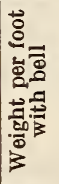 \\
\hline & $\begin{array}{r}\text { Lbs. } \\
255 \\
410 \\
615 \\
835 \\
1,055\end{array}$ & $\begin{array}{l}L b s . \\
16.1 \\
25.7 \\
38.6 \\
52.2 \\
66.1\end{array}$ & $\begin{array}{r}L b s . \\
265 \\
425 \\
635 \\
860 \\
1,090\end{array}$ & $\begin{array}{l}\text { Lbs. } \\
16.0 \\
25.6 \\
38.5 \\
52.1 \\
65.9\end{array}$ & \begin{tabular}{c}
$L b 8$. \\
\hdashline
\end{tabular} & Lbs. & $\begin{array}{r}L b s . \\
290 \\
460 \\
715 \\
995 \\
1,300\end{array}$ & $\begin{array}{l}L b s . \\
18.1 \\
28.7 \\
44.6 \\
62.3 \\
81.1\end{array}$ & $\begin{array}{r}L b s . \\
300 \\
475 \\
735 \\
1,025 \\
1,335\end{array}$ & $\begin{array}{l}\text { Lbs. } \\
18.0 \\
28.6 \\
44.5 \\
62.2 \\
81.0\end{array}$ & Lbs. & 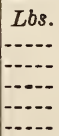 \\
\hline & $\begin{array}{l}1,390 \\
1,730 \\
2,085 \\
2,430 \\
3,245\end{array}$ & $\begin{array}{r}86.9 \\
108.1 \\
130.4 \\
152.0\end{array}$ & $\begin{array}{l}1,430 \\
1,780 \\
2,150 \\
2,505 \\
3,340\end{array}$ & $\begin{array}{r}86.7 \\
107.9 \\
130.2 \\
151.7 \\
202.5\end{array}$ & $\left\{\begin{array}{l}2,14 \\
2,58 \\
3,00 \\
4,01\end{array}\right.$ & 12 & $\begin{array}{l}1,730 \\
2,135 \\
2,640 \\
3,095 \\
4,195\end{array}$ & $\begin{array}{l}108.0 \\
133.6 \\
164.9 \\
193.6 \\
262.1\end{array}$ & $\begin{array}{l}1,780 \\
2,200 \\
2,715 \\
3,190 \\
4,320\end{array}$ & & $\begin{array}{l}2,64 \\
3,26 \\
3,83 \\
5,18\end{array}$ & $\begin{array}{l}132.1 \\
163.2 \\
191.5 \\
259.3\end{array}$ \\
\hline
\end{tabular}

E-5. Type III.-Horizontally cast in green-sand molds in 12 and 16 foot lengths.-

E-5a. Type III pipe shall be of che bell and spigot type, horizontally cast in green-sand molds with green-sand cores and multiple gates, and with spigot end bead and lead groove in the bell.

E-5b. Physical requirements.-Type III pipe shall have a secant modulus of elasticity (see par. F-4b) not to exceed $12,500,000$ pounds per square inch, with a corresponding modulus of rupture not less than 42,500 pounds per square inch.

$\mathrm{E}-5 \mathrm{c}$. Tolerances in diameter.-The inside diameters of bells and the outside diameters of the spigot ends (exclusive of bead) of Type III pipe shall not vary plus or minus from the tabulated dimensions by more than 0.06 inch for pipe 12 inches and less in nominal diameter.

E-5d. Dimensions and weights.-Type III pipe shall conform to the dimensions and weights given in Figure 222 and Tables 5 and 6 for the respective classes and subject to the tolerances given in paragraphs $\mathrm{E}-1 \mathrm{a}, \mathrm{E}-1 \mathrm{~b}, \mathrm{E}-2$, and $\mathrm{E}-5 \mathrm{c}$.

TABLE 5.-Dimensions of Type III pipe

\begin{tabular}{|c|c|c|c|c|c|c|c|c|}
\hline \multirow{3}{*}{$\begin{array}{l}\text { Nomi- } \\
\text { nal } \\
\text { inside } \\
\text { diam- } \\
\text { eter }\end{array}$} & \multirow{2}{*}{$\begin{array}{l}\text { Out- } \\
\text { side } \\
\text { diam- } \\
\text { eter of } \\
\text { pipe }\end{array}$} & \multirow{2}{*}{$\begin{array}{l}\text { Out- } \\
\text { side } \\
\text { diam- } \\
\text { eter of } \\
\text { spigot } \\
\text { end, } \\
\text { maxi- } \\
\text { mum }\end{array}$} & \multirow{2}{*}{$\begin{array}{l}\text { Inside } \\
\text { diam- } \\
\text { eter of } \\
\text { bell }\end{array}$} & \multicolumn{2}{|c|}{$\begin{array}{l}\text { Thickness } \\
\text { of pipe }\end{array}$} & \multirow{2}{*}{$\begin{array}{l}\text { Depth } \\
\text { of bell }\end{array}$} & \multirow{2}{*}{$\begin{array}{l}\text { Thick- } \\
\text { ness of } \\
\text { bell at } \\
\text { end }\end{array}$} & \multirow{2}{*}{$\begin{array}{l}\text { Thick- } \\
\text { ness of } \\
\text { bell at } \\
\text { shoul- } \\
\text { der }\end{array}$} \\
\hline & & & & $\underset{150}{\text { Class }}$ & $\begin{array}{l}\text { Class } \\
250\end{array}$ & & & \\
\hline & A & $W$ & B & $\mathrm{T}$ & $\mathrm{T}$ & $\mathrm{E}$ & C & F \\
\hline $\begin{array}{c}\text { Inches } \\
4 \\
6 \\
8 \\
10 \\
12\end{array}$ & $\begin{array}{c}\text { Inches } \\
4.80 \\
6.90 \\
9.05 \\
11.10 \\
13.20\end{array}$ & $\begin{array}{c}\text { Inches } \\
5.24 \\
7.34 \\
9.61 \\
11.66 \\
13.76\end{array}$ & $\begin{array}{r}\text { Inches } \\
5.60 \\
7.70 \\
9.85 \\
11.90 \\
14.00\end{array}$ & $\begin{array}{r}\text { Inch } \\
0.34 \\
.37 \\
.42 \\
.47 \\
.50\end{array}$ & $\begin{array}{r}\text { Inch } \\
0.38 \\
.43 \\
.50 \\
.57 \\
.62\end{array}$ & $\begin{array}{c}\text { Inches } \\
3.50 \\
3.50 \\
4.00 \\
4.00 \\
4.00\end{array}$ & $\begin{array}{c}\text { Inches } \\
0.88 \\
1.00 \\
1.13 \\
1.25 \\
1.38\end{array}$ & $\begin{array}{l}\text { Inch } \\
0.55 \\
.60 \\
.65 \\
.70 \\
.75\end{array}$ \\
\hline
\end{tabular}

TABLE 6.-Nominal weights of Type III pipe

\begin{tabular}{|c|c|c|c|c|c|c|c|c|}
\hline \multirow{3}{*}{ 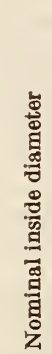 } & \multicolumn{4}{|c|}{$\begin{array}{l}\text { Class } 150-150 \text { pounds work- } \\
\text { ing } \\
\text { head }\end{array}$} & \multicolumn{4}{|c|}{$\begin{array}{l}\text { Class } 250-250 \text { pounds work- } \\
\text { ing pressure, } 576 \text {-foot head }\end{array}$} \\
\hline & \multicolumn{2}{|c|}{$\begin{array}{l}\text { 12-foot nomi- } \\
\text { nal laying } \\
\text { length }\end{array}$} & \multicolumn{2}{|c|}{$\begin{array}{l}\text { 16-foot nomi- } \\
\text { nal laying } \\
\text { length }\end{array}$} & \multicolumn{2}{|c|}{$\begin{array}{c}\text { 12-foot nomi- } \\
\text { nal laying } \\
\text { length }\end{array}$} & \multicolumn{2}{|c|}{$\begin{array}{l}\text { 16-foot nomi- } \\
\text { nal laying } \\
\text { length }\end{array}$} \\
\hline & 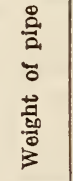 & 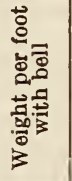 & 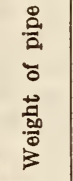 & 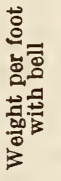 & 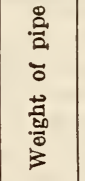 & 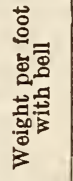 & 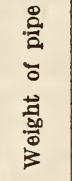 & 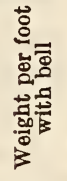 \\
\hline $\begin{array}{r}8 \\
10 \\
12\end{array}$ & $\begin{array}{r}\text { Lbs. } \\
195 \\
315 \\
475\end{array}$ & $\begin{array}{c}\text { Lbs. } \\
16.4 \\
26.3 \\
39.4\end{array}$ & $\begin{array}{r}L b s . \\
255 \\
410 \\
615 \\
835 \\
1,055\end{array}$ & $\begin{array}{l}L b s . \\
16.1 \\
25.7 \\
38.6 \\
52.2 \\
66.1\end{array}$ & $\begin{array}{r}\text { Lbs. } \\
220 \\
350 \\
545\end{array}$ & $\begin{array}{l}L b s . \\
18.4 \\
29.3 \\
45.5\end{array}$ & $\begin{array}{r}\text { Lbs. } \\
290 \\
460 \\
715 \\
995 \\
1,300\end{array}$ & $\begin{array}{c}\text { Lbs. } \\
18.1 \\
28.7 \\
44.6 \\
62.3 \\
81.1\end{array}$ \\
\hline
\end{tabular}

NoTE.-Calculated weight of pipe has been rounded off to nearest 5 pounds. Weight per foot is based on weight of pipe computed to nearest pound.

\section{F. METHOD OF SAMPLING, INSPECTION, AND TESTS}

F-2. Casting record.-A record of the melting and pouring temperatures of the iron shall be furnished when requested.

F-3. Chemical analysis shall be made by the manufacturer from each heat to determine graphitic and combined carbon or total carbon if the separate determinations are not available, manganese, phosphorus, sulphur, and silicon, and duplicate copies of test reports shall be furnished when requested. Sulphur shall not exceed 0.10 per cent and phosphorus 0.90 per cent in either ladle or inspection analysis. 


\section{F-4. Tests of material.-}

F-4a. From each 300 lengths of pipe, or fraction thereof, of each size in the contract or order, one length of pipe shall be selected by the inspector before coating. From each sample pipe there shall be cut and machined one test strip 12 inches long, 0.50 inch deep, and the full thickness of the shell in width. This shall be tested as a beam (with machined surfaces top and bottom) on supports 10 inches apart with load applied at two points $3 \frac{1}{3}$ inches from the supports. The strip shall be accurately calipered at point of rupture and stress calculated by the formula

$$
S=\frac{P L c}{6 I} \text {, or for the above specimen } S=\frac{40 P}{b}
$$

F-4b. The secant modulus of elasticity at the breaking load shall be calculated by the formula

$$
E=\frac{23 P L^{3}}{1,296 I y}, \text { or for the above specimen } E=S \frac{42.6}{y}
$$

In these formulas -

$S=$ modulus of rupture.

$E=$ modulus of elasticity.

$P=$ total load.

$L=$ length of span.

$c=$ distance to extreme fiber.

$I=$ moment of inertia.

$b=$ width of specimen (thickness of pipe).

$d=$ depth of specimen.

$y=$ center deflection at load $P$.

F-5. Hydrostatic tests.-Each length of pipe shall be subjected to a hydrostatic pressure of 500 pounds per square inch. The pipe shall be under this pressure at least one-half minute, and while under pressure shall be subjected to a hammer test. Any pipes showing defects by leaking, sweating, or otherwise shall be rejected.

F-6. Rockwell tests.-Upon the machined edges of each test strip there shall be made at well-distributed points not less than three determinations, using a 1.59-millimeter (one-sixteenth inch) ball and 100 kilogram load. The average Rockwell number shall not exceed 95. An additional determination $\mathrm{s}^{\prime}$ all also be made for pipe cast in metal contact molds, upon the outside of each pipe, using a portable type machine. The equivalent Rockwell number for the exterior shall not exceed $\mathbf{9 5}$.

F-7. Cement lining. - Not less than 1 per cent of pipe shall be tested, and in the event defects are discovered in the lining, testing shall proceed until the lining complies with the specification.

F-7a. The thickness of lining may be determined by means of spear measurements, using a hardened steel point not greater than one-sixteenth inch in diameter. The inspector shall pierce the lining immediately after it is placed in the pipe, and before cement has set, at four diametrically opposite points of the pipe at bell and spigot ends, making two sets of measurements at each end. The first set shall be not greater than 4 inches from the respective ends of the pipe and the second set shall be made as far into the interior of the pipe as can readily be obtained by reaching into the pipe without injuring the lining. All measurements shall be within the limits specified.

F-7b. For lining of the above-specified thickness or of greater thickness, failure of the lining to adhere completely to the wall of the pipe shall not be cause for rejection, if the lining conforms to these specifications in all other respects.

F-8. If any test specimen shows defective machining or obvious lack of continuity of metal, it may be discarded and replaced by another specimen selected by the inspector. If any of the test specimens selected fail to meet the requirements specified and the purchaser permits a retest, at least twice the number of specimens that failed shall be selected by the inspector for replacement, all of which shall meet the requirements.

\section{G. PACKING AND MARKING OF SHIPMENTS}

G-2. Packing.-Unless otherwise specified, the subject commodity shall be prepared for standard commercial shipment so as to insure acceptance by common or other carriers, for safe transportation, at the lowest rate, to the point of delivery.

G-3. Marking.-Unless otherwise specified, shipments shall be marked with the number of the contract or order.

\section{NOTES}

I-1. Invitations for bids should state the number of linear feet of pipe desired and payment should be made on a per foot basis. No bids should be accepted on a tonnage basis.

I-2. Award of contract ordinarily will be made on the basis of evaluated cost per foot, in order to take into account the cost of jointing materials required.

I-2a. The cost per foot may be evaluated from the price bid per foot by the following formula:

in which

$$
E=A+K P
$$

$E=$ evaluated cost per foot in dollars.

$A=$ bid price per foot in dollars.

$K=$ constant found in Table 7 opposite diameter of pipe and under length of pipe bid upon.

$P=$ The price of lead ${ }^{62}$ in cents per pound current upon date of invitation for bids. The current price of lead may be obtained from the publication "Iron Age." Only New York prices shall be considered.

TABLE 7.-Values of constant " $K$ " for evaluating prices bid per foot of cast-iron water pipe

[Based on total weight of lead and jute at 1 cent per pound required for one joint]

\begin{tabular}{|c|c|c|c|c|c|}
\hline $\begin{array}{c}\text { Nomi- } \\
\text { nal } \\
\text { pipe } \\
\text { diam- } \\
\text { eter }\end{array}$ & \multicolumn{5}{|c|}{ Length of pipe (in feet) } \\
\cline { 2 - 6 } & 12 & 16 & $161 / 2$ & 18 & 20 \\
\hline $\begin{array}{c}\text { Inches } \\
4\end{array}$ & 0.0064 & 0.0048 & 0.0047 & 0.0043 & 0.0038 \\
6 & .0088 & .0066 & 0064 & .0059 & .0053 \\
8 & .0114 & .0086 & .0083 & .0076 & .0068 \\
10 & .0138 & .0103 & .0101 & .0092 & .0083 \\
12 & .0163 & .0123 & .0120 & .0109 & .0098 \\
14 & .0190 & .0143 & .0139 & .0127 & .0114 \\
16 & .0258 & .0193 & .0189 & .0172 & .0155 \\
18 & .0290 & .0218 & .0212 & .0193 & .0174 \\
20 & .0319 & .0239 & .0234 & .0212 & .0191 \\
24 & .0379 & .0284 & .0278 & .0252 & .0227 \\
\hline
\end{tabular}

Example.-If the bid price on 12-inch diameter pipe in 18-foot lengths is $\$ 1.25$ per foot, and the current price of lead is $61 / 2$ cents per pound, the evaluated price per foot of pipe.

$$
E=\$ 1.25+(\$ .0109 \times 61 / 2)=\$ 1.25+0.0708=\$ 1.3208
$$

I-3. If special symbol is desired (see par. D-8), it should be so stated or indicated in the invitation for bids. If not so stated, it will be assumed that none is desired.

I-4. For lines for water known to cause serious tuberculation, it is recommended that pipe be not coated, and that it be lined with cement mortar.

I-4a. When linings other than those specified in section $\mathrm{D}-4$ are required, it should be so stated in the invitation for bids.

I-5. When American Water Works Association pipe is specifically required by the requisition or

62 The price of jute need not be considered. 
order, such pipe shall conform to the requirements and tests of the A. W. W. A. specification. In such cases the desired A. W. W. A. class should be stated.

I-6. This specification is designed for average waterworks construction conditions. Where construction conditions involving excessive vibration, shock, bad trench conditions, corrosion, etc., are contemplated, due consideration should be given to the use of pipe of heavier weight.

I-6a. Cast-iron pipe of lighter or heavier weight than specified herein or with other than bell and spigot joint should be purchased under a separate specification.

I-7. As far as known at the time of promulgation of this specification type 3 pipe was produced regularly by but one company.

I-8. Bids shall clearly indicate the type and length of pipe which bidders will furnish.

I-9. It is believed that this specification adequately describes the characteristics necessary to secure the desired material, and that normally no samples will be necessary prior to award to determine compliance with this specification. If for any particular purpose samples with bids are necessary, they should be specifically asked for in the invitation for bids and the particular purpose to be served by the bid sample should be definitely stated, the specification to apply in all other respects.

I-10. This specification governs all United States Government purchases of this commodity.

\subsection{CAST-IRON PIPE FOR RAILINGS AND POLES.}

(No nationally recognized specifications available.) 607.14 CAST-IRON PIPE FITTINGS AND CONNEC-
TIONS.

\section{AMERICAN BUREAU OF SHIPPING, RULES FOR BUILDING AND CLASSING STEEL VESSELS ; PUMPS AND PIPING SYSTEMS, 1930}

\section{PIPE FITTINGS}

(For general requirements see 607.4, p. 435.)

1. Cast iron and semisteel of the physical characteristics specified under iron castings (see 611.11, p. 635) may be used in the construction of valves and fittings for pressures not exceeding 300 pounds per square inch, and for temperatures up to $450^{\circ} \mathrm{F}$. The minimum thickness in inches shall be determined by the formula:

$$
t=\frac{\text { Working pressure } \times \text { internal diameter }}{3,000}+0.25
$$

AMERICAN SOCIETY OF MECHANICAL ENGINEERS, ANERICAN STANDARD CASTIRON PIPE FLANGES AND FLANGED FITTINGS FOR MAXIMUM WORKING SATU. RATED STEAM PRESSURE OF 125 POUNDS PER SQUARE INCH (GAGE), B16a-1928 Approved as American Standard by the American Standards Asso-
ciation, ${ }^{63}$ February, 1928]

CAST-IRON PIPE FLANGES AND FLANGED FITTINGS 125 POUNDS PER SQUARE INCH

\section{INTRODUCTORY NOTES}

1. Sizes.-The sizes of the fittings in the following tables will be identified by the corresponding nominal pipe size.

63 Sponsor organizations: American Society of Mechanical Engineers, Manufacturers' Standardization Society of Valve and Fitting Industry, and Heating and Piping Contractors National Association.
2. Pressure rating.-It is recommended that in addition to the maximum working steam pressure for which these fittings are intended, the sizes 12 inches and smaller may also be used for maximum nonshock working hydraulic pressure of 175 pounds per square inch (gage), at or near the ordinary range of air temperatures.

3. Markings. - All fittings must have marks cast on them indicating the manufacturer and the figures 125 indicating the maximum working steam pressure for which the fittings are intended.

4. Material.-The dimensional standards for cast-iron fittings covered herein are based on a highgrade product. The physical and chemical requirements of these flanges and fittings shall be in accordance with Table 1 . These requirements are recommended, in the absence of similar data in the specifications of the A. S. T. M. applicable to cast iron intended for the manufacture of pipe flanges and fittings, pending the preparation of an acceptable specification.

TABLE 1.-Physical and chemical (cast test bar) requirements for cast iron for flanges and flanged fittings

\begin{tabular}{|c|c|c|}
\hline Casting & $\begin{array}{c}\text { Tensile } \\
\text { strength, } \\
\text { minimum }\end{array}$ & $\begin{array}{l}\text { Sulphur, } \\
\text { maxi- } \\
\text { mum }\end{array}$ \\
\hline $\begin{array}{l}\text { Light..... } \\
\text { Medium } \\
\text { Heavy }\end{array}$ & $\begin{array}{l}\text { Lbs./in. }{ }^{2} \\
20,000 \\
21,000 \\
24,000\end{array}$ & $\begin{array}{c}\text { Per cent } \\
0.12 \\
.12 \\
.12\end{array}$ \\
\hline
\end{tabular}

Light castings are those having any section less than 1/2-inch thickness.

Heavy castings are those in which no section is less than 2 inches in thickness.

Medium castings are those not included in either of the above classes.

5. Facing.-All 125-pound cast-iron flanges and flanged fittings shall be plain faced; that is, without projection or raised face.

An inspection limit of plus or minus one thirtysecond inch shall be allowed on all center to contact surface dimensions for sizes up to and including 10 inches and plus or minus one-sixteenth inch on sizes larger than 10 inches. An inspection limit of plus or minus one-sixteenth inch shall be allowed on all contact surface to contact surface dimensions for sizes up to and including 10 inches and plus or minus one-eighth inch on sizes larger than 10 inches.

6. Bolting.-Drilling templates are in multiples of four, so that fittings may be made to face in any quarter.

Bolt holes shall straddle the center line. For bolts smaller than $1 \frac{3}{4}$ inches the bolt holes shall be drilled one-eighth inch larger in diameter than the nominal size of the bolt. Holes for bolts $13 / 4$ inches and larger shall be drilled one-fourth inch larger than nominal diameter of bolts.

Bolts shall be of steel with standard rough square heads and the nuts shall be of steel with standard rough hexagonal dimensions; all as given in the Tentative American Standard on wrench head bolts and nuts and wrench openings. For bolts, $13 / 4$ inches in diameter and larger, bolt studs with $a$ nut on each end are recommended.

Hexagonal nuts for pipe sizes 1 to 48 inches can be conveniently pulled up with open wrenches of minimum design of heads. Hexagonal nuts for pipe sizes 48 to 96 inches can be conveniently pulled up with box wrenches.

7. Spot facing. - The bolt holes of 125-pound castiron flanges and flanged fittings shall not be spot faced for ordinary service. When required the flanges and fittings in sizes 36 inches and larger may be spot faced or back faced to the minimum thickness of flange with a plus tolerance of one-eighth inch. 
8. Reducing fittings.-Reducing elbows and side outlet elbows carry same dimensions center to face as straight size elbows corresponding to the size of the larger opening.

Tees, side outlet tees, crosses, and laterals, sizes 16 inches and smaller, reducing on the outlet or branch, have the same dimension, center to face and face to face as straight size fittings corresponding to the size of the larger opening. Sizes 18 inches and larger, reducing on the outlet or branch, are made in two lengths depending on the size of the outlet as given in the tables of dimensions.

Tees, crosses, and laterals, reducing on the run only, have the same dimensions center to face and face to face as straight size fittings corresponding to the size of the larger opening.

Reducers and eccentric reducers for all reductions have the same face-to-face dimensions for the larger opening as given in Table 7 .

Reducing fittings listed in this standard shall be ordered by the designation of the outlets in their proper sequence as indicated in the sketches for steel flanged fittings. (See 607.4, p. 467.)

Special double branch elbows, whether straight or reducing, have the same dimension center to face as straight size elbows corresponding to the size of the larger opening.

Side outlet elbow's and side outlet tees shall have all openings on intersecting center lines.

9. Elbows.- Special degree elbows ranging from 1 to $45^{\circ}$ inclusive have the same center to face dimension given for $45^{\circ}$ elbows and those over $45^{\circ}$ and up to $90^{\circ}$, inclusive, shall have the same center to face dimensions given for $90^{\circ}$ elbows. The angle designation of an elbow is its deflection from straight line flow and is the angle between the flange faces.

10. True $Y^{\prime} s-$ The dimensions of true Y's straignt sizes, are given in Table 6 . Other forms are considered special and should be made to suit conditions:

11. Laterals. - Laterals ( $Y$ branches), botn straight and reducing, sizes 8 inches and larger, shall be reinforced to compensate for the inherent weakness in the casting design.

(Illustrations showing method of designating outlets of reducing fittings when ordering are identical with those for steel flanged fittings 250, 400, 600,900 , and 1,350 pounds, except that in this case there is no provision for the raised face required for higher steam pressure steel flanged fittings. See 607.4, p. 467.)

\section{TABLE 2.-Templates for drilling}

[All dimensions given in inches]

\begin{tabular}{|c|c|c|c|c|c|c|c|c|c|c|c|}
\hline $\begin{array}{l}\text { Nomi- } \\
\text { nal } \\
\text { pipe } \\
\text { size }\end{array}$ & $\begin{array}{l}\text { Diameter } \\
\text { of flange }\end{array}$ & $\begin{array}{l}\text { Thick- } \\
\text { ness of } \\
\text { flange }{ }^{1} \\
\text { (mini- } \\
\text { mum) }\end{array}$ & $\begin{array}{c}\text { Diameter } \\
\text { of bolt } \\
\text { circle }\end{array}$ & $\begin{array}{l}\text { Number } \\
\text { of bolts ? }\end{array}$ & $\begin{array}{l}\text { Diameter } \\
\text { of bolts }\end{array}$ & $\begin{array}{c}\text { Diameter } \\
\text { of drilled } \\
\text { bolt } \\
\text { holes }\end{array}$ & $\begin{array}{l}\text { Length } \\
\text { of bolts }\end{array}$ & $\begin{array}{c}\text { Length } \\
\text { of bolt } \\
\text { stud with } \\
2 \text { nuts }\end{array}$ & $\begin{array}{l}\text { Total } \\
\text { effective } \\
\text { area bolt } \\
\text { metal }\end{array}$ & $\begin{array}{c}\text { Stress } \\
\text { pounds } \\
\text { per } \\
\text { square } \\
\text { inch bolt } \\
\text { metal }\end{array}$ & $\begin{array}{l}\text { Size of ring } \\
\text { gasket }\end{array}$ \\
\hline $\begin{array}{l}1 \\
11 / 4 \\
11 / 2 \\
2 \\
21 / 2\end{array}$ & $\begin{array}{l}41 / 4 \\
45 / 8 \\
5 \\
6 \\
7\end{array}$ & $\begin{array}{l}7 / 16 \\
1 / 2 \\
9 / 16 \\
5 / 8 \\
11 / 16\end{array}$ & $\begin{array}{l}31 / 8 \\
31 / 2 \\
37 / 8 \\
43 / 4 \\
51 / 2\end{array}$ & $\begin{array}{l}4 \\
4 \\
4 \\
4 \\
4\end{array}$ & $\begin{array}{l}1 / 2 \\
1 / 2 \\
1 / 2 \\
5 / 8 \\
5,8\end{array}$ & $\begin{array}{l}5 / 8 \\
5 / 8 \\
5 / 8 \\
3 / 4 \\
3 / 4\end{array}$ & $\begin{array}{l}11 / 2 \\
11 / 2 \\
13 / 4 \\
2 \\
21 / 4\end{array}$ & & $\begin{array}{l}0.504 \\
.504 \\
.504 \\
.808 \\
.808\end{array}$ & $\begin{array}{l}1,340 \\
1,755 \\
2,215 \\
2,065 \\
2,885\end{array}$ & $\begin{array}{ll}1 & \text { by } 25 / 8 \\
11 / 4 & \text { by } 3 \\
11 / 2 & \text { by } 33 / 8 \\
2 & \text { by } 418 \\
21 / 2 & \text { by } 47 / 8\end{array}$ \\
\hline $\begin{array}{l}3 \\
31 / 2 \\
4 \\
5 \\
6\end{array}$ & $\begin{array}{l}71 / 2 \\
81 / 2 \\
9 \\
10 \\
11\end{array}$ & $\begin{array}{l}3 / 4 \\
13 / 16 \\
15 / 16 \\
15 / 16 \\
1\end{array}$ & $\begin{array}{l}6 \\
7 \\
71 / 2 \\
81 / 2 \\
91 / 2\end{array}$ & $\begin{array}{l}4 \\
8 \\
8 \\
8 \\
8\end{array}$ & $\begin{array}{l}5 / 8 \\
5 / 8 \\
5 / 8 \\
3 / 4 \\
3 / 4\end{array}$ & $\begin{array}{l}3 / 4 \\
3 / 4 \\
3 / 4 \\
7 / 8 \\
7 / 8\end{array}$ & $\begin{array}{l}21 / 4 \\
21 / 2 \\
23 / 4 \\
23 / 4 \\
3\end{array}$ & 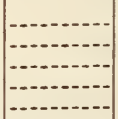 & $\begin{array}{l}.808 \\
1.616 \\
1.616 \\
2.416 \\
2.416\end{array}$ & $\begin{array}{l}3,510 \\
2,410 \\
2,870 \\
2,440 \\
3,110\end{array}$ & $\begin{array}{ll}3 & \text { by } 536 \\
31 / 2 & \text { by } 636 \\
4 & \text { by } 67 / 8 \\
5 & \text { by } 73 / 4 \\
6 & \text { by } 83 / 4\end{array}$ \\
\hline $\begin{array}{r}8 \\
10 \\
12 \\
\times 14 \\
016\end{array}$ & $\begin{array}{l}131 / 2 \\
16 \\
19 \\
21 \\
231 / 2\end{array}$ & $\begin{array}{l}11 / 8 \\
13 / 16 \\
11 / 4 \\
13 / 8 \\
17 / 16\end{array}$ & $\begin{array}{l}113 / 4 \\
141 / 4 \\
17 \\
183 / 4 \\
211 / 4\end{array}$ & $\begin{array}{r}8 \\
12 \\
12 \\
12 \\
16\end{array}$ & $\begin{array}{l}3 / 4 \\
7 / 8 \\
7 / 6 \\
1 \\
1\end{array}$ & $\begin{array}{l}7 / 8 \\
1 \\
1 \\
118 \\
118\end{array}$ & $\begin{array}{l}31 / 4 \\
31 / 2 \\
31 / 2 \\
4 \\
41 / 4\end{array}$ & - & $\begin{array}{l}2.416 \\
5.04 \\
5.04 \\
6.60 \\
8.80\end{array}$ & $\begin{array}{l}4,915 \\
3,485 \\
5,065 \\
4,685 \\
4,575\end{array}$ & $\begin{array}{l}8 \text { by } 11 \\
10 \text { by } 133 / 8 \\
12 \text { by } 1618 \\
14 \text { by } 173 / 4 \\
16 \text { by } 201 / 4\end{array}$ \\
\hline $\begin{array}{ll}0 & 18 \\
0 & 20 \\
0 & 24 \\
6 & 30\end{array}$ & $\begin{array}{l}25 \\
271 / 2 \\
32 \\
383 / 4\end{array}$ & $\begin{array}{l}19 / 16 \\
11 / 16 \\
17 / 8 \\
21 / 8\end{array}$ & $\begin{array}{l}223 / 4 \\
25 \\
291 / 2 \\
36\end{array}$ & $\begin{array}{l}16 \\
20 \\
20 \\
28\end{array}$ & $\begin{array}{l}11 / 8 \\
11 / 8 \\
11 / 4 \\
11 / 4\end{array}$ & $\begin{array}{l}11 / 4 \\
11 / 4 \\
13 / 8 \\
13 / 8\end{array}$ & $\begin{array}{l}41 / 2 \\
43 / 4 \\
51 / 4 \\
53 / 4\end{array}$ & 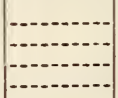 & $\begin{array}{l}11.10 \\
13.88 \\
17.86 \\
25.00\end{array}$ & $\begin{array}{l}4,135 \\
4,030 \\
4,385 \\
4,700\end{array}$ & $\begin{array}{l}18 \text { by } 215 / 8 \\
20 \text { by } 237 / 8 \\
24 \text { by } 281 / 4 \\
30 \text { by } 315 / 8\end{array}$ \\
\hline $\begin{array}{l}036 \\
042 \\
648 \\
054\end{array}$ & $\begin{array}{l}46 \\
53 \\
591 / 2 \\
661 / 4\end{array}$ & $\begin{array}{l}23 / 8 \\
25 / 8 \\
23 / 4 \\
3\end{array}$ & $\begin{array}{l}423 / 4 \\
491 / 2 \\
56 \\
623 / 4\end{array}$ & $\begin{array}{l}32 \\
36 \\
44 \\
44\end{array}$ & $\begin{array}{l}11 / 2 \\
11 / 2 \\
11 / 2 \\
13 / 4\end{array}$ & $\begin{array}{l}15 / 8 \\
15 \% \\
15 / 8 \\
2\end{array}$ & $\begin{array}{l}61 / 2 \\
71 / 4 \\
71 / 2 \\
81 / 4\end{array}$ & $\begin{array}{r}91 / 2 \\
91 / 2 \\
101 / 2\end{array}$ & $\begin{array}{l}41.41 \\
46.57 \\
56.93 \\
76.82\end{array}$ & $\begin{array}{l}4,035 \\
4,810 \\
5,200 \\
4,755\end{array}$ & $\begin{array}{l}36 \text { by } 411 / 4 \\
42 \text { by } 477 / 8 \\
48 \text { by } 543 / 8 \\
54 \text { by } 61\end{array}$ \\
\hline $\begin{array}{l}660 \\
672 \\
6784 \\
689 \\
696\end{array}$ & $\begin{array}{c}73 \\
861 / 2 \\
993 / 4 \\
1131 / 4\end{array}$ & $\begin{array}{l}31 / 8 \\
31 / 2 \\
37 / 8 \\
41 / 4\end{array}$ & $\begin{array}{r}691 / 4 \\
821 / 2 \\
951 / 2 \\
1081 / 2\end{array}$ & $\begin{array}{l}52 \\
60 \\
64 \\
68\end{array}$ & $\begin{array}{l}13 / 4 \\
13 / 4 \\
2 \\
21 / 4\end{array}$ & $\begin{array}{l}2 \\
2 \\
21 / 4 \\
21 / 2\end{array}$ & $\begin{array}{r}81 / 2 \\
91 / 2 \\
101 / 2 \\
111 / 2\end{array}$ & $\begin{array}{l}11 \\
12 \\
13 \\
141 / 2\end{array}$ & $\begin{array}{r}90.79 \\
104.70 \\
147.33 \\
205.56\end{array}$ & $\begin{array}{l}4,925 \\
6,150 \\
5,825 \\
5,415\end{array}$ & $\begin{array}{r}60 \text { by } 671 / 2 \\
72 \text { by } 805 / 8 \\
84 \text { by } 931 / 2 \\
96 \text { by } 1061 / 4\end{array}$ \\
\hline
\end{tabular}

1 All 125-pound cast-iron standard flanges have a plain face.

2 Drilling templates are in multiples of 4 , so that fittings may be made to face in any quarter, and bolt holes straddle the center line. For bolts smaller than $13 / 4$ inches the bolt holes shall be drilled $1 / 8$ inch larger in diameter than the nominal diameter of the bolt. Holes for bolts $13 / 4$ inches and larger shall be drilled $1 / 4$ inch larger than nominal diameter of bolts.

3 The bolt holes on cast-iron flanged fittings are not spot faced for ordinary service. When required, the fittings and flanges in sizes 36 inches and larger can be spot faced or back faced, so that standard length bolts can be used.

Bolts shall be of steel with standard rough square heads, and the nuts shall be of steel with standard rough hexagonal dimensions: all as given in the American Tentative Standard on wrench head bolts and nuts, and wrench openings. For bolts $13 / 4$ inches diameter and larger, bolt studs with a nut on each end are recommended. Hexagonal nuts for pipe sizes 1 inch to 48 inches can be conveniently pulled up with open wrenches of minimum design of heads. Hexagonal nuts for pipe sizes 48 inches to 96 inches can be conveniently pulled up with box wrenches.

5 The stress shown is that of internal pressure only, assumed to act on a circular area equal in diameter to the outside diameter of a ring gasket covering the flange to the inside of bolts.

Outside diameter. 
TABLE 3.-Dimensions and theoretical weights (pounds) of screwed companion and blind flanges 1
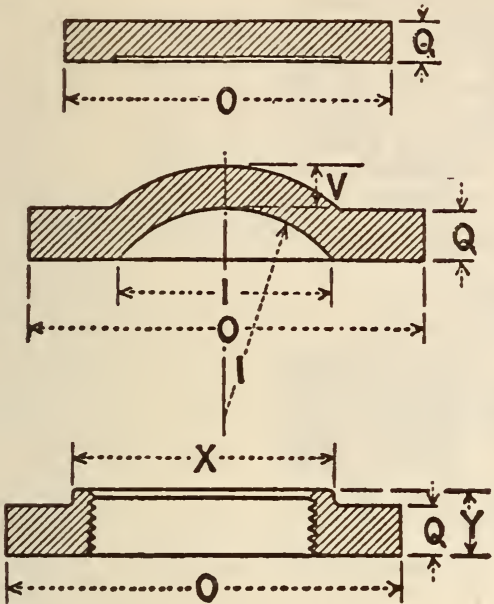

FIgURE 223.-Screwed companion and blind flanges

[All dimensions given in inches]

\begin{tabular}{|c|c|c|c|c|c|c|c|}
\hline \multirow{2}{*}{$\begin{array}{l}\text { Nomi } \\
\text { nal } \\
\text { pipe } \\
\text { size:3 } \\
\text { I }\end{array}$} & \multirow{2}{*}{$\begin{array}{l}\text { Diam- } \\
\text { eter of } \\
\text { flange } \\
0\end{array}$} & \multirow{2}{*}{$\begin{array}{l}\text { Thick- } \\
\text { ness of } \\
\text { flange } \\
\text { (mini- } \\
\text { mum) } \\
\text { Q }\end{array}$} & \multirow{2}{*}{$\begin{array}{l}\text { Metal } \\
\text { thick- } \\
\text { ness }{ }^{3} \\
\text { (mini- } \\
\text { mum) } \\
\text { V }\end{array}$} & \multirow{2}{*}{$\begin{array}{c}\text { Diame- } \\
\text { ter hub } \\
\text { (mini- } \\
\text { mum) } \\
\text { X }\end{array}$} & \multirow{2}{*}{$\mid \begin{array}{c}\text { Length } \\
\text { of hub } \\
\text { and } \\
\text { threads } \\
\text { (mini- } \\
\text { mum) } \\
\text { Y }\end{array}$} & \multicolumn{2}{|c|}{$\begin{array}{c}\text { Theoretical } \\
\text { weights }\end{array}$} \\
\hline & & & & & & $\begin{array}{l}\text { Com- } \\
\text { panion } \\
\text { flanges }\end{array}$ & $\begin{array}{l}\text { Blind } \\
\text { flanges }\end{array}$ \\
\hline $\begin{array}{l}1 \\
11 / 4 \\
11 / 2 \\
2 \\
21 / 2\end{array}$ & $\begin{array}{l}41 / 4 \\
458 \\
5 \\
6 \\
7\end{array}$ & $\begin{array}{l}7 / 16 \\
112 \\
9 / 16 \\
568 \\
11 / 16\end{array}$ & & $\begin{array}{l}115 / 10 \\
25 / 10 \\
29160 \\
31 / 10 \\
3 \% 10\end{array}$ & $\begin{array}{r}0.68 \\
.76 \\
.87 \\
1.00 \\
1.14\end{array}$ & $\begin{array}{l}2 \\
2 \\
3 \\
5 \\
7\end{array}$ & $\begin{array}{l}2 \\
3 \\
3 \\
5 \\
7\end{array}$ \\
\hline $\begin{array}{l}3 \\
31 / 2 \\
4 \\
5 \\
6\end{array}$ & $\begin{array}{l}71 / 2 \\
81 / 2 \\
9 \\
10 \\
11\end{array}$ & $\begin{array}{l}3 / 4 \\
13 / 10 \\
15 / 10 \\
15 / 10 \\
1^{150}\end{array}$ & & $\begin{array}{l}41 / 4 \\
413 / 16 \\
55 / 10 \\
67 / 16 \\
7 \% 10\end{array}$ & $\begin{array}{l}1.20 \\
1.25 \\
1.30 \\
1.41 \\
1.51\end{array}$ & $\begin{array}{r}8 \\
11 \\
14 \\
17 \\
22\end{array}$ & $\begin{array}{r}9 \\
12 \\
16 \\
20 \\
25\end{array}$ \\
\hline $\begin{array}{r}8 \\
10 \\
12 \\
614\end{array}$ & $\begin{array}{l}131 / 2 \\
16 \\
19 \\
21\end{array}$ & $\begin{array}{l}11 / 8 \\
13 / 160 \\
114 \\
13 \%\end{array}$ & $\begin{array}{l}13 / 16 \\
7 / 8\end{array}$ & $\begin{array}{l}911 / 16 \\
11^{15 / 16} \\
141 / 10 \\
153 \%\end{array}$ & 1. 71 & $\begin{array}{l}31 \\
45 \\
63 \\
82\end{array}$ & $\begin{array}{r}42 \\
63 \\
88 \\
115\end{array}$ \\
\hline $\begin{array}{l}816 \\
818 \\
820 \\
0 \\
0\end{array}$ & $\begin{array}{l}231 / 2 \\
25 \\
271 / 2 \\
32\end{array}$ & $\begin{array}{l}17 / 16 \\
1910 \\
111 / 16 \\
17 / 8\end{array}$ & $\begin{array}{l}1 \\
11110 \\
118 \\
114\end{array}$ & $\begin{array}{l}171 / 2 \\
1958 \\
213 / 4 \\
26\end{array}$ & 3. 25 & $\begin{array}{l}105 \\
120 \\
150 \\
220\end{array}$ & $\begin{array}{l}160 \\
190 \\
250 \\
370\end{array}$ \\
\hline $\begin{array}{l}830 \\
836 \\
842 \\
048\end{array}$ & $\begin{array}{l}383 / 4 \\
46 \\
53 \\
591 / 2\end{array}$ & $21 / 8$ & $\begin{array}{l}17 / 6 \\
15 / 8 \\
1^{13 / 16}\end{array}$ & & & & $\begin{array}{r}620 \\
990 \\
1,470 \\
2,000\end{array}$ \\
\hline
\end{tabular}

1 All 125-pound standard flanges have a plain face.

1 Sizes 14 inches and larger are to be used with outside diameter pipe of the same sizes.

${ }^{3}$ All blind flanges for sizes 12 inches (19 inches outside diameter) and larger must be dished, with inside radius equal to the port diameter.

This column is the same as column for effective thread (E) in Table 1 of the American Pipe Thread Standard, published by the American Standards Association except in sizes 11/4, 11/2, and 2 inches.

3 All weights listed are for flanges faced only, based upon minimum thicknesses given in the table above without allowances for variation. Cast iron is considered to weigh 0.26 pound per cubic inch. $\checkmark$ Outside diameter.
TABLE 4.-Dimensions of elbows
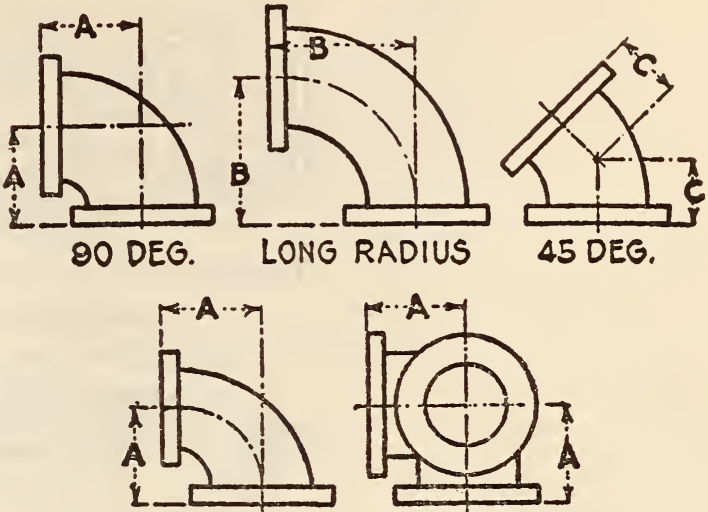

REDUCING

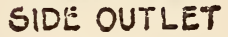

Figure 224.-Dimensions of elbows

[All dimensions given in inches]

\begin{tabular}{|c|c|c|c|c|c|c|}
\hline $\begin{array}{l}\text { Nominal } \\
\text { pipe } \\
\text { size }^{1}\end{array}$ & $\begin{array}{c}\text { Center } \\
\text { to face } \\
\text { elbow'31 } \\
\text { A }\end{array}$ & $\begin{array}{c}\text { Center } \\
\text { to face } \\
\text { long } \\
\text { radius } \\
\text { elbow }{ }^{234} \\
\text { B }\end{array}$ & $\begin{array}{c}\text { Center } \\
\text { to face } \\
45^{\circ} \\
\text { elbow }{ }^{3} \\
\\
\text { C }\end{array}$ & $\begin{array}{l}\text { Diam- } \\
\text { eter of } \\
\text { flange }\end{array}$ & $\begin{array}{l}\text { Thick: } \\
\text { ness of } \\
\text { flange } \\
\text { (mini- } \\
\text { mum) }\end{array}$ & $\begin{array}{l}\text { Metal } \\
\text { thickness } \\
\text { of body } \\
\text { (mini- } \\
\text { mum) }\end{array}$ \\
\hline $\begin{array}{l}1 \\
11 / 4 \\
11 / 2 \\
21 / 2 \\
21 / 2\end{array}$ & $\begin{array}{l}31 / 2 \\
33 / 4 \\
4 \\
41 / 2 \\
5\end{array}$ & $\begin{array}{l}5 \\
51 / 2 \\
6 \\
61 / 2 \\
7\end{array}$ & $\begin{array}{l}13 / 4 \\
2 \\
21 / 4 \\
21 / 2 \\
3\end{array}$ & $\begin{array}{l}41 / 4 \\
45 / 8 \\
5 \\
6 \\
7\end{array}$ & $\begin{array}{l}7 / 10 \\
1 / 2 \\
9 / 16 \\
5 / 8 \\
11 / 16 .\end{array}$ & $\begin{array}{l}7 / 10 \\
7 / 10 \\
7 / 10 \\
7 / 10 \\
7 / 10\end{array}$ \\
\hline $\begin{array}{l}3 \\
31 / 2 \\
4 \\
5 \\
6\end{array}$ & $\begin{array}{l}51 / 2 \\
6 \\
61 / 2 \\
71 / 2 \\
8\end{array}$ & $\begin{array}{c}73 / 4 \\
81 / 2 \\
9 \\
101 / 4 \\
111 / 2\end{array}$ & $\begin{array}{l}3 \\
31 / 2 \\
41 / 2 \\
41 / 2 \\
5\end{array}$ & $\begin{array}{l}71 / 2 \\
81 / 2 \\
9 \\
10 \\
11\end{array}$ & $\begin{array}{l}3 / 4 \\
13 / 16 \\
15 / 10 \\
15 / 16 \\
1\end{array}$ & $\begin{array}{l}7 / 10 \\
7 / 10 \\
1 / 2 \\
1 / 2 \\
9 / 10\end{array}$ \\
\hline $\begin{array}{r}8 \\
10 \\
12 \\
814\end{array}$ & $\begin{array}{r}9 \\
11 \\
12 \\
14\end{array}$ & $\begin{array}{l}14 \\
161 / 2 \\
19 \\
211 / 2\end{array}$ & $\begin{array}{l}51 / 2 \\
61 / 2 \\
71 / 2 \\
71 / 2\end{array}$ & $\begin{array}{l}131 / 2 \\
16 \\
19 \\
21\end{array}$ & $\begin{array}{l}11 / 8 \\
13 / 16 \\
11 / 4 \\
13 / 8\end{array}$ & $\begin{array}{l}5 / 8 \\
3 / 4 \\
13 / 16 \\
7 / 8\end{array}$ \\
\hline 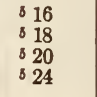 & $\begin{array}{l}15 \\
161 / 2 \\
18 \\
22\end{array}$ & $\begin{array}{l}24 \\
261 / 2 \\
29 \\
34\end{array}$ & $\begin{array}{l}8 \\
81 / 2 \\
91 / 2 \\
11\end{array}$ & $\begin{array}{l}231 / 2 \\
25 \\
271 / 2 \\
32\end{array}$ & $\begin{array}{l}17 / 16 \\
1916 \\
11 / 16 \\
17 \% 8\end{array}$ & $\begin{array}{l}1 \\
11 / 10 \\
11 / 8 \\
11 / 4\end{array}$ \\
\hline 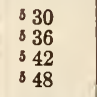 & $\begin{array}{l}25 \\
28 \\
31 \\
34\end{array}$ & $\begin{array}{l}411 / 2 \\
49 \\
561 / 2 \\
64\end{array}$ & $\begin{array}{l}15 \\
18 \\
21 \\
24\end{array}$ & $\begin{array}{l}383 / 4 \\
46 \\
53 \\
591 / 2\end{array}$ & $\begin{array}{l}21 / 8 \\
23 / 8 \\
25 / 8 \\
23 / 4\end{array}$ & $\begin{array}{l}17 / 16 \\
1^{15 / 8} \\
2^{13 / 10}\end{array}$ \\
\hline
\end{tabular}

1 Size of all fittings listed indicates nominal inside diameter of port.

2 Reducing elbows and side 'outlet elbows carry same dimensions center to face as straight size elbows, corresponding to the size of the larger opening.

${ }_{3}$ Special degree elbows, ranging from $1^{\circ}$ to $45^{\circ}$ inclusive, have the same center to face dimensions given in $45^{\circ}$ elbows and those over $45^{\circ}$ and up to $90^{\circ}$ inclusive shall have the same center to face dimen. sions given for $90^{\circ}$ elbows. The angle designation of an elbow is its deflection from straight line flow and is the angle between the flange faces.

1 Side outlet elbows shall have all openings on intersecting center lines.

Outside diameter. 
TABLE 5.-Dimensions of tees and crosses (straight sizes)

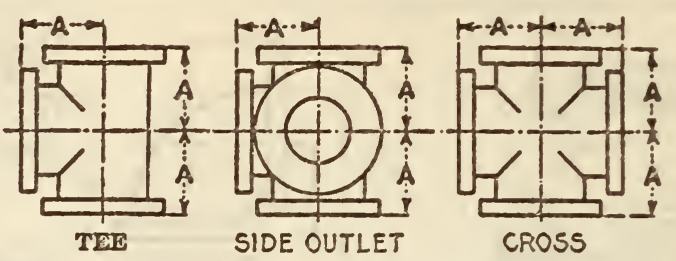

FIGURE 225.-Tees and crosses (straight sizes)

[All dimensions given in inches]

\begin{tabular}{|c|c|c|c|c|c|}
\hline $\begin{array}{l}\text { Nominal } \\
\text { pipe size }{ }^{12}\end{array}$ & $\begin{array}{l}\text { Center to } \\
\text { face tees and } \\
\text { crosses } 23 \\
\text { A }\end{array}$ & $\begin{array}{c}\text { Face to } \\
\text { face tees } \\
\text { and } \\
\text { crosses } 23 \\
\text { A A }\end{array}$ & $\begin{array}{l}\text { Diameter } \\
\text { of flange }\end{array}$ & $\begin{array}{l}\text { Thick- } \\
\text { ness of } \\
\text { flange } \\
\text { (mini- } \\
\text { mum) }\end{array}$ & $\begin{array}{l}\text { Metal } \\
\text { thick- } \\
\text { ness of } \\
\text { of body } \\
\text { (mini- } \\
\text { mum) }\end{array}$ \\
\hline $\begin{array}{l}\text { Same as in } \\
\text { Table } 4 .\end{array}$ & $\left\{\begin{array}{c}\text { Same as cen- } \\
\text { ter to face } \\
\text { elbow (A) } \\
\text { in Table 4. }\end{array}\right.$ & $\begin{array}{l}7 \\
71 / 2 \\
8 \\
9 \\
10 \\
11 \\
12 \\
13 \\
15 \\
16 \\
18 \\
22 \\
24 \\
28 \\
30 \\
33 \\
36 \\
44 \\
50 \\
56 \\
62 \\
68\end{array}$ & Same & as in Tat & le 4 . \\
\hline
\end{tabular}

1 Size of all fittings listed indicates nominal inside diameter of port. 2 Tees, side outlet tees, and crosses, 16 inches and smaller, reducing on the outlet, have the same dimensions center to face and face to face as straight size fittings, corresponding to the size of the larger opening. Sizes 18 inches and larger, reducing on the outlet, are made in 2 lengths, depending on the size of the outlet as given in Table 9.

3 Tees and crosses, reducing on run only, carry same dimensions center to face and face to face as a straight size fitting of the larger opening. 
TABLE 6.-Dimensions of reducing tees and reducing crosses ${ }^{156}$ (short body patterns)
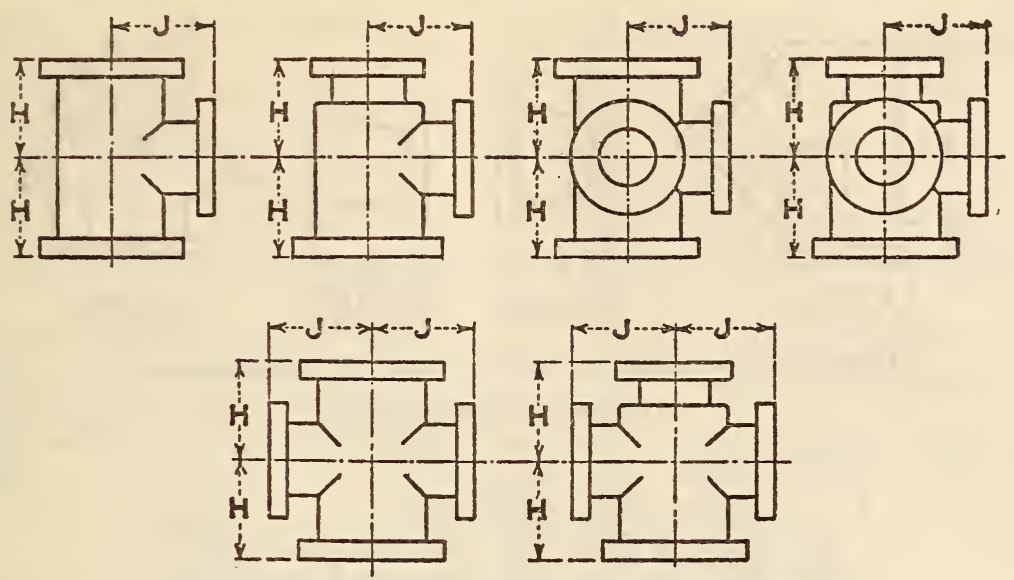

FIGURE 226.-Dimensions of reducing tees and reducing crosses (short
body patterns)

[All dimensions given in inches]

\begin{tabular}{|c|c|c|c|c|}
\hline $\begin{array}{l}\text { Nominal } \\
\text { pipe size }\end{array}$ & $\begin{array}{l}\text { Size of } \\
\text { outlet } \\
\text { and } \\
\text { smaller }\end{array}$ & $\begin{array}{c}\text { Center } \\
\text { to face } \\
\text { run } \\
\text { H }\end{array}$ & $\begin{array}{c}\text { Face to } \\
\text { face run } \\
\text { HH }\end{array}$ & $\begin{array}{c}\text { Center } \\
\text { to face } \\
\text { outlet } \\
\text { J }\end{array}$ \\
\hline $\begin{array}{l}1 \\
11 / 4 \\
11 / 2 \\
2\end{array}$ & $\begin{array}{l}1 \\
11 / 4 \\
11 / 2 \\
2\end{array}$ & $\begin{array}{l}31 / 2 \\
33 / 4 \\
4 \\
41 / 2\end{array}$ & $\begin{array}{l}7 \\
71 / 2 \\
8 \\
9\end{array}$ & $\begin{array}{l}31 / 2 \\
33 / 4 \\
4 \\
41 / 2\end{array}$ \\
\hline $\begin{array}{l}21 / 2 \\
3 \\
31 / 2 \\
4\end{array}$ & $\begin{array}{l}21 / 2 \\
3 \\
31 / 2 \\
4\end{array}$ & $\begin{array}{l}5 \\
51 / 2 \\
6 \\
61 / 2\end{array}$ & $\begin{array}{l}10 \\
11 \\
12 \\
13\end{array}$ & $\begin{array}{l}5 \\
51 / 2 \\
6 \\
61 / 2\end{array}$ \\
\hline $\begin{array}{r}5 \\
6 \\
8 \\
10\end{array}$ & $\begin{array}{r}5 \\
6 \\
8 \\
10\end{array}$ & $\begin{array}{l}71 / 2 \\
8 \\
9 \\
11\end{array}$ & $\begin{array}{l}15 \\
16 \\
18 \\
22\end{array}$ & $\begin{array}{l}71 / 2 \\
8 \\
9 \\
11\end{array}$ \\
\hline $\begin{array}{r}12 \\
414 \\
116 \\
18\end{array}$ & $\begin{array}{l}12 \\
14 \\
16 \\
12\end{array}$ & $\begin{array}{l}12 \\
14 \\
15 \\
13\end{array}$ & $\begin{array}{l}24 \\
28 \\
30 \\
26\end{array}$ & $\begin{array}{l}12 \\
14 \\
15 \\
151 / 2\end{array}$ \\
\hline $\begin{array}{l}+20 \\
+24 \\
430 \\
436\end{array}$ & $\begin{array}{l}14 \\
16 \\
20 \\
24\end{array}$ & $\begin{array}{l}14 \\
15 \\
18 \\
20\end{array}$ & $\begin{array}{l}28 \\
30 \\
36 \\
40\end{array}$ & $\begin{array}{l}17 \\
19 \\
23 \\
26\end{array}$ \\
\hline
\end{tabular}

1 Short body patterns are used for sizes 18 inches and larger.

2 Long body patterns are used when outlets are larger than given in the above table, and, therefore, have the same dimensions as straight size fittings.

${ }^{3}$ In a side outlet tee the larger of the 2 side outlets governs the center to face dimension " $\mathrm{J}$."

4 Outside diameter.

$\checkmark$ Fittings reducing on the run only carry same dimensions center to face and face to face as straight size fittings corresponding to size of the larger opening. Tees increasing on outlet, known as bull head tees, will have same center to face and face to face dimensions as a straight fitting of the size of the outlet. For example, a 12 by 12 by 18 inch tee will be governed by the dimensions of the 18-inch long body tee given in Table 5 , namely, $161 / 2$ inches center to face of all openings and 33 inches face to face.

6 Side outlet tees, with outlet at $90^{\circ}$ or any other angle, straight or reducing, carry same dimensions center to face and face to face as regular tees having same reductions. 
TABLE 7.-Dimensions of laterals, reducers, true Y's (straight sizes)

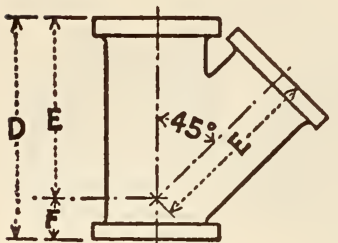

LATERAL

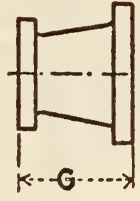

REDUCER
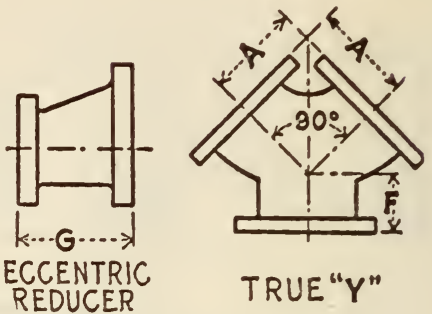

TRUE " $Y$ "

Figure 227.-Laterals, reducers, true Y's (straight sizes)

[All dimensions given in inches]

\begin{tabular}{|c|c|c|c|c|c|c|c|c|}
\hline $\begin{array}{l}\text { Nom- } \\
\text { inal } \\
\text { pipe } \\
\operatorname{size}^{1} 23\end{array}$ & $\begin{array}{c}\text { Cen- } \\
\text { ter to } \\
\text { face } \\
\text { true } \\
Y \\
\\
\\
\text { A }\end{array}$ & $\begin{array}{c}\text { Face } \\
\text { to } \\
\text { face } \\
\text { later- } \\
\text { al } 84 \\
\\
D\end{array}$ & $\begin{array}{l}\text { Cen- } \\
\text { ter to } \\
\text { face } \\
\text { later- } \\
\text { al } 34 \\
\\
\text { E }\end{array}$ & $\begin{array}{c}\text { Cen- } \\
\text { ter to } \\
\text { face } \\
\text { true } \\
Y \\
\text { and } \\
\text { later- } \\
\text { al }^{34} \\
\text { F }\end{array}$ & $\begin{array}{c}\text { Face } \\
\text { to } \\
\text { face } \\
\text { re- } \\
\text { ducer }{ }^{3} \\
\\
\text { G }\end{array}$ & $\begin{array}{l}\text { Diam } \\
\text { eter of } \\
\text { flange }\end{array}$ & $\begin{array}{l}\text { Thick- } \\
\text { ness of } \\
\text { flange } \\
\text { (mini- } \\
\text { mum) }\end{array}$ & $\begin{array}{l}\text { Metal } \\
\text { thick- } \\
\text { ness of } \\
\text { body } \\
\text { (mini- } \\
\text { mum) }\end{array}$ \\
\hline $\begin{array}{l}1 \\
11 / 4 \\
11 / 2 \\
2 \\
21 / 2\end{array}$ & $\begin{array}{l}31 / 2 \\
33 / 4 \\
4 \\
41 / 2 \\
5\end{array}$ & $\begin{array}{l}71 / 2 \\
8 \\
9 \\
101 / 2 \\
12\end{array}$ & $\begin{array}{l}53 / 4 \\
61 / 4 \\
7 \\
8 \\
91 / 2\end{array}$ & $\begin{array}{l}13 / 4 \\
13 / 4 \\
2 \\
21 / 2 \\
21 / 2\end{array}$ & $\begin{array}{l}5 \\
51 / 2\end{array}$ & $\begin{array}{l}41 / 4 \\
4558 \\
5 \\
6 \\
7\end{array}$ & $\begin{array}{l}7 / 16 \\
1 / 2 \\
9 / 16 \\
5 / 8 \\
1116\end{array}$ & $\begin{array}{l}7 / 16 \\
7 / 16 \\
7 / 16 \\
7 / 16 \\
7 / 16\end{array}$ \\
\hline $\begin{array}{l}3 \\
31 / 2 \\
4 \\
5 \\
6\end{array}$ & $\begin{array}{l}51 / 2 \\
6 \\
61 / 2 \\
71 / 2 \\
8\end{array}$ & $\begin{array}{l}13 \\
141 / 2 \\
15 \\
17 \\
18\end{array}$ & $\begin{array}{l}10 \\
111 / 2 \\
12 \\
131 / 2 \\
141 / 2\end{array}$ & $\begin{array}{l}3 \\
3 \\
3 \\
31 / 2 \\
31 / 2\end{array}$ & $\begin{array}{l}6 \\
61 / 2 \\
7 \\
8 \\
9\end{array}$ & $\begin{array}{l}71 / 2 \\
81 / 2 \\
9 \\
10 \\
11\end{array}$ & $\begin{array}{l}3 / 4 \\
13 / 16 \\
15 / 16 \\
15 / 16 \\
1\end{array}$ & $\begin{array}{l}7 / 16 \\
7 / 16 \\
1 / 2 \\
1 / 2 \\
9 / 16\end{array}$ \\
\hline $\begin{array}{r}8 \\
10 \\
12 \\
614 \\
616\end{array}$ & $\begin{array}{r}9 \\
11 \\
12 \\
14 \\
15\end{array}$ & $\begin{array}{l}22 \\
251 / 2 \\
30 \\
33 \\
361 / 2\end{array}$ & $\begin{array}{l}171 / 2 \\
201 / 2 \\
241 / 2 \\
27 \\
30\end{array}$ & $\begin{array}{l}41 / 2 \\
5 \\
51 / 2 \\
6 \\
61 / 2\end{array}$ & $\begin{array}{l}11 \\
12 \\
14 \\
16 \\
18\end{array}$ & $\begin{array}{l}131 / 2 \\
16 \\
19 \\
21 \\
231 / 2\end{array}$ & $\begin{array}{l}11 / 8 \\
13 / 16 \\
11 / 4 \\
1368 \\
17 / 16\end{array}$ & $\begin{array}{l}5 / 8 \\
3 / 4 \\
13 / 16 \\
7 / 8 \\
1^{7 / 8}\end{array}$ \\
\hline $\begin{array}{ll}6 & 18 \\
6 & 20 \\
6 & 24 \\
6 & 30 \\
6 \\
6 & 36\end{array}$ & $\begin{array}{l}161 / 2 \\
18 \\
22 \\
25\end{array}$ & $\begin{array}{l}39 \\
43 \\
491 / 2 \\
59\end{array}$ & $\begin{array}{l}32 \\
35 \\
401 / 2 \\
49\end{array}$ & $\begin{array}{r}7 \\
8 \\
9 \\
10\end{array}$ & $\begin{array}{l}19 \\
20 \\
24 \\
30 \\
36\end{array}$ & $\begin{array}{l}25 \\
271 / 2 \\
32 \\
3834 \\
46\end{array}$ & $\begin{array}{l}19 / 16 \\
111 / 16 \\
17 / 8 \\
218 \\
238\end{array}$ & $\begin{array}{l}11 / 16 \\
118 \\
11 / 4 \\
17 / 16 \\
156\end{array}$ \\
\hline $\begin{array}{l}642 \\
648\end{array}$ & & & & & $\begin{array}{l}42 \\
48\end{array}$ & $\begin{array}{l}53 \\
591 / 2\end{array}$ & $\begin{array}{l}25 / 8 \\
23 / 4\end{array}$ & $2^{1316}$ \\
\hline
\end{tabular}

1 Size of all fittings listed indicates nominal inside diameter of port. 2 Laterals both straight and reducing sizes 8 inches and larger, should be reinforced to compensate for the inherent weakness in the casting design.

3 Laterals, 16 inches and smaller, reducing on the outlet or branch, use the same dimensions center to face and face to face as straight size fittings corresponding to size of the larger opening. Sizes 18 inches and larger, reducing on the outlet or branch, are made in 2 lengths depending on the size of the outlet as given in Table 8.

1 Laterals, reducing on the run only, carry same dimensions center to face and face to face as straight size fittings corresponding to size of the larger opening.

s Reducers, and eccentric reducers, for all reductions, use the same face to face dimensions given in the above table of dimensions for the larger opening.

6 Outside diameter. 
TABLE 8.-Reducing laterals (short body pattern) ${ }^{1}$
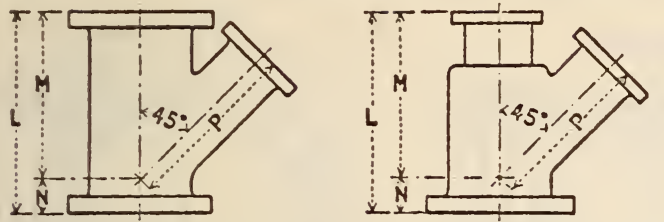

FIGURE 228. - Reducing laterals (short body pattern)

[All dimensions given in inches]

\begin{tabular}{|c|c|c|c|c|c|}
\hline $\begin{array}{c}\text { Nominal } \\
\text { pipe } \\
\text { size } 234\end{array}$ & $\begin{array}{c}\text { Size of } \\
\text { branch } \\
\text { and } \\
\text { smaller \& }\end{array}$ & $\begin{array}{c}\text { Face to } \\
\text { face run } \\
\text { L }\end{array}$ & $\begin{array}{c}\text { Center } \\
\text { to face } \\
\text { run } \\
\text { M }\end{array}$ & $\begin{array}{c}\text { Center } \\
\text { to face } \\
\text { run } \\
\mathrm{N}\end{array}$ & $\begin{array}{c}\text { Center } \\
\text { to face } \\
\text { branch } \\
\text { P }\end{array}$ \\
\hline $\begin{array}{l}1 \\
11 / 4 \\
11 / 2 \\
2 \\
21 / 2\end{array}$ & $\begin{array}{l}1 \\
11 / 4 \\
11 / 2 \\
2 \\
21 / 2\end{array}$ & $\begin{array}{l}71 / 2 \\
8 \\
9 \\
101 / 2 \\
12\end{array}$ & $\begin{array}{l}53 / 4 \\
61 / 1 \\
7 \\
8 \\
91 / 2\end{array}$ & $\begin{array}{l}13 / 4 \\
13 / 4 \\
2 \\
2312 \\
21 / 2\end{array}$ & $\begin{array}{l}53 / 4 \\
61 / 4 \\
7 \\
8 \\
91 / 2\end{array}$ \\
\hline $\begin{array}{l}3 \\
31 / 2 \\
4 \\
5 \\
6\end{array}$ & $\begin{array}{l}3 \\
31 / 2 \\
4 \\
5 \\
6\end{array}$ & $\begin{array}{l}13 \\
141 / 2 \\
15 \\
17 \\
18\end{array}$ & $\begin{array}{l}10 \\
111 / 2 \\
12 \\
131 / 2 \\
141 / 2\end{array}$ & $\begin{array}{l}3 \\
3 \\
3 \\
31 / 2 \\
31 / 2\end{array}$ & $\begin{array}{l}10 \\
111 / 2 \\
12 \\
131 / 2 \\
141 / 2\end{array}$ \\
\hline $\begin{array}{r}8 \\
10 \\
12 \\
014 \\
616\end{array}$ & $\begin{array}{r}8 \\
10 \\
12 \\
14 \\
16\end{array}$ & $\begin{array}{l}22 \\
251 / 2 \\
30 \\
33 \\
361 / 2\end{array}$ & $\begin{array}{l}171 / 2 \\
201 / 2 \\
241 / 2 \\
27 \\
30\end{array}$ & $\begin{array}{l}41 / 2 \\
5 \\
51 / 2 \\
6 \\
61 / 2\end{array}$ & $\begin{array}{l}171 / 2 \\
201 / 2 \\
241 / 2 \\
27 \\
30\end{array}$ \\
\hline 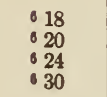 & $\begin{array}{r}8 \\
10 \\
12 \\
14\end{array}$ & $\begin{array}{l}26 \\
28 \\
32 \\
39\end{array}$ & $\begin{array}{l}25 \\
27 \\
311 / 2 \\
39\end{array}$ & $\begin{array}{l}1 \\
1_{1 / 2}\end{array}$ & $\begin{array}{l}271 / 2 \\
291 / 2 \\
341 / 2 \\
42\end{array}$ \\
\hline
\end{tabular}

1 Long body patterns shall be used for fittings which are reducing on the run only.

on the run only. to face dimensions as straight size fittings.

3 Laterals both straight and reducing sizes, 8 inches and larger, shall be reinforced to compensate for the inherent weakness in the casting design.

- Size of all fittings listed indicates nominal inside diameter of port.

- Long body patterns are used when branches are larger than given in the above table, therefore have same dimensions as straight size fittings.

Outside diameter.
TABLE 9.-Dimensions of base elbows and base T's 123

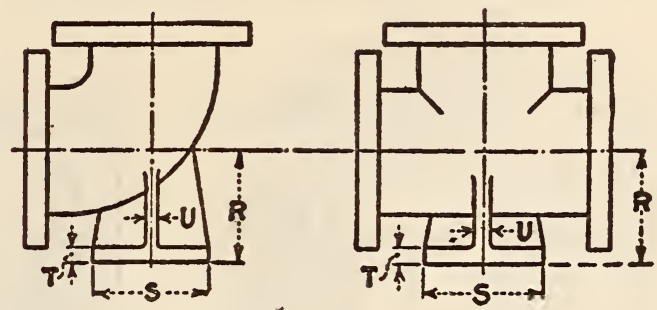

FiguRe 229.-Base elbows and base T's

[All dimensions given in inches]

\begin{tabular}{|c|c|c|c|c|c|}
\hline $\begin{array}{l}\text { Nominal } \\
\text { pipe } \\
\text { size }\end{array}$ & $\begin{array}{c}\text { Center } \\
\text { to base } \\
\mathrm{R}\end{array}$ & $\begin{array}{c}\text { Diameter } \\
\text { of round } \\
\text { base }{ }^{16} 6 \\
\mathrm{~S}\end{array}$ & $\begin{array}{c}\text { Thick- } \\
\text { ness of } \\
\text { base } \\
\text { T }\end{array}$ & $\begin{array}{c}\text { Thick- } \\
\text { ness of } \\
\text { ribs } \\
\text { U }\end{array}$ & $\begin{array}{l}\text { Size of } \\
\text { support- } \\
\text { ing pipe } \\
\text { for base }\end{array}$ \\
\hline $\begin{array}{l}1 \\
11 / 4 \\
11 / 2 \\
2\end{array}$ & $\begin{array}{l}31 / 2 \\
358 \\
33 / 4 \\
418\end{array}$ & $\begin{array}{l}31 / 2 \\
31 / 2 \\
41 / 4 \\
45 / 8\end{array}$ & $\begin{array}{l}7 / 16 \\
7 / 16 \\
7 / 16 \\
1 / 2\end{array}$ & $\begin{array}{l}3 / 8 \\
3 / 8 \\
1 / 2 \\
1 / 2\end{array}$ & $\begin{array}{l}3 / 4 \\
3 / 4 \\
11 / 4 \\
11 / 4\end{array}$ \\
\hline $\begin{array}{l}21 / 2 \\
3 \\
31 / 2 \\
4 \\
5\end{array}$ & $\begin{array}{l}41 / 2 \\
478 \\
51 / 4 \\
51 / 2 \\
614\end{array}$ & $\begin{array}{l}45 / 8 \\
5 \\
5 \\
6 \\
7\end{array}$ & $\begin{array}{l}1 / 2 \\
9 / 18 \\
9 / 16 \\
58 \\
11 / 16\end{array}$ & $\begin{array}{l}1 / 2 \\
1 / 2 \\
1 / 2 \\
1 / 2 \\
5 / 8\end{array}$ & $\begin{array}{l}11 / 4 \\
11 / 2 \\
11 / 2 \\
2 \\
21 / 2\end{array}$ \\
\hline $\begin{array}{r}6 \\
8 \\
10 \\
12 \\
714\end{array}$ & $\begin{array}{c}7 \\
83 / 8 \\
93 / 4 \\
111 / 4 \\
121 / 2\end{array}$ & $\begin{array}{r}7 \\
9 \\
9 \\
11 \\
11\end{array}$ & $\begin{array}{l}11 / 16 \\
15 / 16 \\
15 / 16 \\
1^{15}\end{array}$ & $\begin{array}{l}5 / 8 \\
7 / 8 \\
1^{7 / 8} \\
1\end{array}$ & $\begin{array}{l}21 / 2 \\
4 \\
4 \\
6 \\
6\end{array}$ \\
\hline $\begin{array}{l}716 \\
7118 \\
7 \\
7 \\
7 \\
7\end{array} 24$ & $\begin{array}{l}133 / 4 \\
15 \\
16 \\
181 / 2\end{array}$ & $\begin{array}{l}11 \\
131 / 2 \\
1312 \\
131 / 2\end{array}$ & $\begin{array}{l}1 \\
118 \\
118 \\
118\end{array}$ & $\begin{array}{l}1 \\
118 \\
118 \\
118\end{array}$ & $\begin{array}{l}6 \\
8 \\
8 \\
8\end{array}$ \\
\hline
\end{tabular}

1 Bases, when drilled, shoul 1 be drilled to the template of the flange of the supporting pipe size.

${ }^{2}$ Dimensions for base fittings apply to straight and reducing sizes, and long and short body patterns.

$\checkmark$ Bases not finished unless so ordered.

- For sizes larger than 24 inches, anchorage fittings are recommended.

3 Dimensions center to base is the same as the center to base for anchorage fittings for the same size fittings.

6 Size of base determined by size of largest opening of fitting. $?$ Outside diameter. 
TABLE 10.-Dimensions of anchorage bases for tees (straight sizes) 12

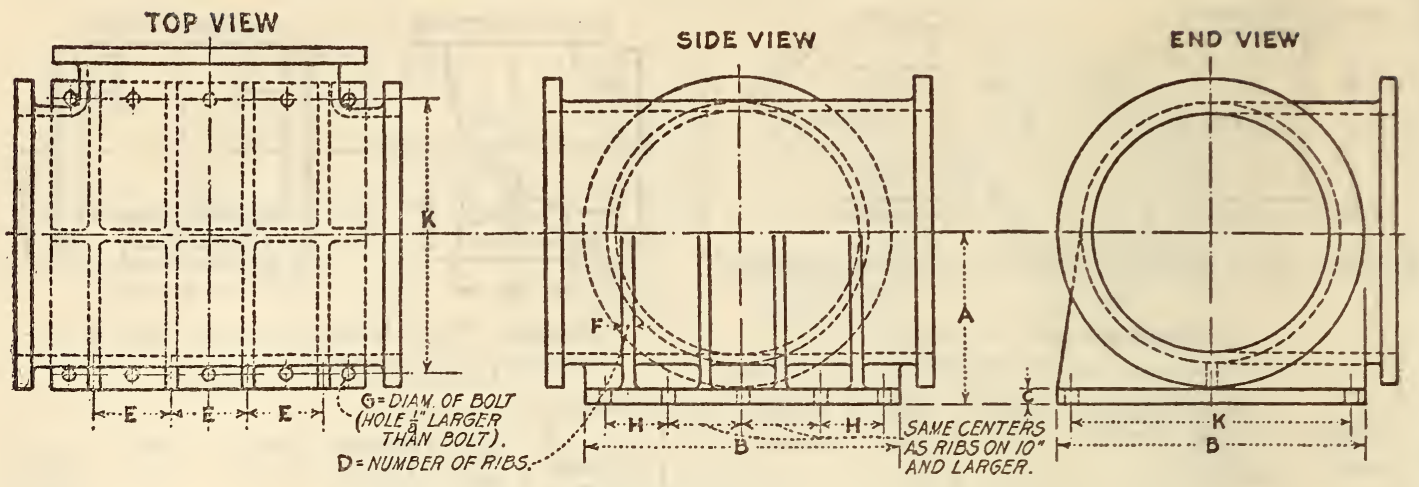

Figure 230.-Anchorage bases for tees (straight sizes)

[All dimensions given in inches]

\begin{tabular}{|c|c|c|c|c|c|c|c|c|c|}
\hline $\begin{array}{l}\text { Nominal } \\
\text { pipe } \\
\text { size } 1\end{array}$ & $\begin{array}{c}\text { Center } \\
\text { to base } \\
\text { A }\end{array}$ & $\begin{array}{c}\text { Length } \\
\text { of square } \\
\text { base }^{3} \\
\text { B }\end{array}$ & $\begin{array}{l}\text { Thick- } \\
\text { ness of } \\
\text { base } \\
\text { C }\end{array}$ & $\begin{array}{c}\text { Number } \\
\text { of ribs } \\
\text { D }\end{array}$ & $\begin{array}{c}\text { Centers } \\
\text { of ribs } \\
\text { and } \\
\text { inside } \\
\text { bolts } \\
\text { E }\end{array}$ & $\begin{array}{c}\text { Thick- } \\
\text { ness of } \\
\text { ribs } \\
\text { F }\end{array}$ & $\begin{array}{c}\begin{array}{c}\text { Diameter } \\
\text { of bolts }\end{array} \\
G\end{array}$ & $\begin{array}{c}\text { Longitu- } \\
\text { dinal } \\
\text { centers } \\
\text { from end } \\
\text { to second } \\
\text { bolt } \\
\text { H }\end{array}$ & $\begin{array}{c}\text { Trans- } \\
\text { verse } \\
\text { bolt } \\
\text { centers } \\
\\
\mathbf{K}\end{array}$ \\
\hline $\begin{array}{l}21 / 2 \\
3 \\
31 / 2 \\
4 \\
5\end{array}$ & $\begin{array}{l}41 / 2 \\
47 / 8 \\
51 / 4 \\
51 / 2 \\
61 / 4\end{array}$ & $\begin{array}{l}7 \\
71 / 2 \\
81 / 2 \\
9 \\
10\end{array}$ & $\begin{array}{l}11 / 16 \\
3 / 4 \\
13 / 16 \\
15 / 16 \\
15 / 16\end{array}$ & $\begin{array}{l}1 \\
1 \\
1 \\
2 \\
2\end{array}$ & 5 & $\begin{array}{l}7 / 16 \\
7 / 16 \\
7 / 16 \\
1 / 2 \\
1 / 2\end{array}$ & $\begin{array}{l}5 / 8 \\
5 / 8 \\
5 / 8 \\
5 / 8 \\
7 / 8\end{array}$ & $\begin{array}{l}41 / 2 \\
5 \\
6 \\
31 / 4 \\
33 / 4\end{array}$ & $\begin{array}{l}41 / 2 \\
5 \\
6 \\
61 / 2 \\
71 / 2\end{array}$ \\
\hline $\begin{array}{r}6 \\
8 \\
10 \\
12 \\
414\end{array}$ & $\begin{array}{l}7 \\
83 / 8 \\
93 / 4 \\
111 / 4 \\
121 / 2\end{array}$ & $\begin{array}{l}11 \\
131 / 2 \\
16 \\
19 \\
21\end{array}$ & $\begin{array}{l}1 \\
118 \\
13 / 16 \\
11 / 4 \\
138\end{array}$ & $\begin{array}{l}2 \\
2 \\
3 \\
3 \\
3\end{array}$ & $\begin{array}{l}6 \\
8 \\
47 / 8 \\
53 / 4 \\
63 / 4\end{array}$ & $\begin{array}{c}9 / 16 \\
5 / 8 \\
3 / 4 \\
13 / 16 \\
7 / 8\end{array}$ & $\begin{array}{l}7 / 8 \\
1 \\
11 / 8 \\
11 / 4 \\
11 / 4\end{array}$ & $\begin{array}{l}43 / 8 \\
51 / 2 \\
41 / 4 \\
47 / 8 \\
51 / 2\end{array}$ & $\begin{array}{l}83 / 4 \\
11 \\
133 / 8 \\
151 / 2 \\
173 / 4\end{array}$ \\
\hline $\begin{array}{l}416 \\
418 \\
420 \\
424\end{array}$ & $\begin{array}{l}13^{3 / 4} \\
15 \\
16 \\
181 / 2\end{array}$ & $\begin{array}{l}231 / 2 \\
25 \\
271 / 2 \\
32\end{array}$ & $\begin{array}{l}17 / 16 \\
19116 \\
11116 \\
178\end{array}$ & $\begin{array}{l}3 \\
3 \\
3 \\
3\end{array}$ & $\begin{array}{r}73 / 4 \\
81 / 2 \\
91 / 2 \\
113 / 8\end{array}$ & $\begin{array}{l}1 \\
11 / 16 \\
118 \\
134\end{array}$ & $\begin{array}{l}13 / 8 \\
13 / 8 \\
11 / 2 \\
158\end{array}$ & $\begin{array}{l}6 \\
65 / 8 \\
71 / 4 \\
81 / 2\end{array}$ & $\begin{array}{l}193 / 4 \\
213 / 4 \\
24 \\
283 / 8\end{array}$ \\
\hline $\begin{array}{l}130 \\
436 \\
442 \\
448\end{array}$ & $\begin{array}{l}22 \\
251 / 2 \\
291 / 4 \\
323 / 4\end{array}$ & $\begin{array}{l}383 / 4 \\
46 \\
53 \\
591 / 2\end{array}$ & $\begin{array}{l}21 / 8 \\
23 / 8 \\
258 \\
23 / 4\end{array}$ & $\begin{array}{l}4 \\
4 \\
4 \\
4\end{array}$ & $\begin{array}{l}93 / 8 \\
111 / 4 \\
13 \\
147 / 8\end{array}$ & $\begin{array}{l}17 / 16 \\
15 / 8 \\
1^{13 / 16}\end{array}$ & $\begin{array}{l}13 / 4 \\
17 / 8 \\
2 \\
21 / 4\end{array}$ & $\begin{array}{r}77 / 8 \\
91 / 8 \\
103 / 8 \\
113 / 4\end{array}$ & $\begin{array}{l}341 / 2 \\
403 / 4 \\
463 / 4 \\
531 / 4\end{array}$ \\
\hline
\end{tabular}

1 All reducing fittings, 16 inches and smaller, with all reductions, have same base as straight sizes.

2 Bases not finished unless so ordered.

3 Size of base determined by size of largest opening fittings.

1 Outside diameter. 
TABLE 11.-Dimensions of anchorage bases for reducing tees ${ }^{12}$

TOP VIEW
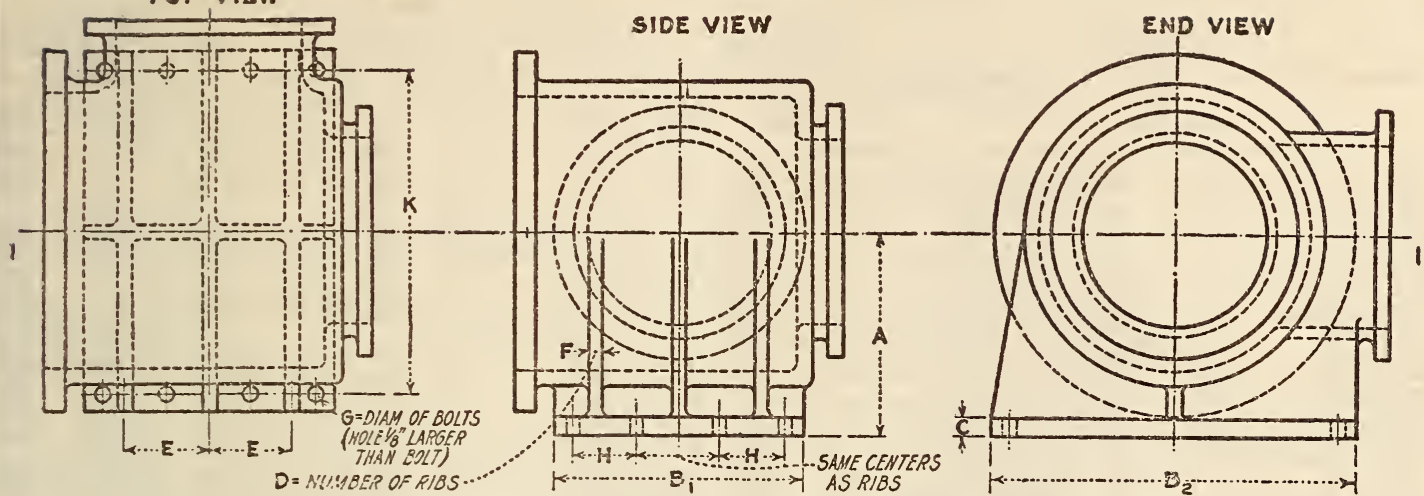

FigURE 231.-Anchorage bases for reducing tees

[All dimensions given in inches]

\begin{tabular}{|c|c|c|c|c|c|c|c|c|c|c|c|}
\hline $\begin{array}{l}\text { Nomi- } \\
\text { nal } \\
\text { pipe } \\
\text { size }\end{array}$ & $\begin{array}{l}\text { Outlet } \\
\text { sizes } \\
\text { and } \\
\text { smaller }\end{array}$ & $\begin{array}{c}\text { Center } \\
\text { to base } \\
\text { A }\end{array}$ & $\begin{array}{c}\text { Length } \\
\text { of } \\
\text { square } \\
\text { base } \\
\\
B_{1}\end{array}$ & $\begin{array}{c}\text { Width } \\
\text { of base } \\
\mathrm{B}_{3}\end{array}$ & $\begin{array}{c}\text { Thick- } \\
\text { ness } \\
\text { of base } \\
\text { C }\end{array}$ & $\begin{array}{l}\text { Num- } \\
\text { ber of } \\
\text { ribs }\end{array}$ & $\begin{array}{l}\text { Centers } \\
\text { of ribs } \\
\text { and } \\
\text { inside } \\
\text { bolts } \\
\text { E }\end{array}$ & $\begin{array}{l}\text { Thick- } \\
\text { ness of } \\
\text { ribs } \\
\text { F }\end{array}$ & $\begin{array}{c}\begin{array}{c}\text { Dia- } \\
\text { meter } \\
\text { of bolts }\end{array} \\
\quad \text { G }\end{array}$ & $\begin{array}{c}\text { Longi- } \\
\text { tudinal } \\
\text { center } \\
\text { from end } \\
\text { to } \\
\text { second } \\
\text { bolt } \\
\text { H }\end{array}$ & $\begin{array}{c}\text { Trans- } \\
\text { verse } \\
\text { bolt } \\
\text { centers } \\
\\
\mathrm{K}\end{array}$ \\
\hline $\begin{array}{l}18 \\
? 20 \\
+24\end{array}$ & $\begin{array}{l}12 \\
14 \\
16\end{array}$ & $\begin{array}{l}151 / 2 \\
16 \\
181 / 2\end{array}$ & $\begin{array}{l}19 \\
21 \\
231 / 2\end{array}$ & $\begin{array}{l}25 \\
271 / 2 \\
32\end{array}$ & $\begin{array}{l}19 / 16 \\
111 / 16 \\
17 / 8\end{array}$ & $\begin{array}{l}3 \\
3 \\
3\end{array}$ & $\begin{array}{l}51 / 4 \\
6 \\
7\end{array}$ & $\begin{array}{l}1116 \\
11 / 8 \\
114\end{array}$ & $\begin{array}{l}11 / 4 \\
11 / 4 \\
13 / 8\end{array}$ & $\begin{array}{l}51 / 8 \\
53 / 8 \\
61 / 4\end{array}$ & $\begin{array}{l}211 / 2 \\
231 / 4 \\
28\end{array}$ \\
\hline $\begin{array}{l}+30 \\
436 \\
442 \\
+48\end{array}$ & $\begin{array}{l}20 \\
24 \\
24 \\
30\end{array}$ & $\begin{array}{l}22 \\
251 / 2 \\
291 / 4 \\
323 / 4\end{array}$ & $\begin{array}{l}271 / 2 \\
32 \\
361 / 2 \\
413 / 4\end{array}$ & $\begin{array}{l}383 / 4 \\
46 \\
53 \\
591 / 2\end{array}$ & $\begin{array}{l}21 / 8 \\
238 \\
25 / 8 \\
23 / 4\end{array}$ & $\begin{array}{l}3 \\
3 \\
4 \\
4\end{array}$ & $\begin{array}{c}9 \\
103 / 4 \\
81 / 2 \\
93 / 4\end{array}$ & $\begin{array}{l}17 / 16 \\
1558 \\
1_{2}^{13 / 16}\end{array}$ & $\begin{array}{l}11 / 2 \\
11 / 2 \\
158 \\
15 / 8\end{array}$ & $\begin{array}{l}71 / 4 \\
833 \\
71 / 2 \\
81 / 4\end{array}$ & $\begin{array}{l}343 / 4 \\
41 / 2 \\
481 / 2 \\
53^{3} / 4\end{array}$ \\
\hline
\end{tabular}

1 All reducing fittings, 16 inches and smaller, with all reductions, have same base as straight sizes. (See Table 10.) 2 Bases not finished unless so ordered.

3 Size of base determined by size of largest opening of fitting.

- Outside diameter.

TABLE 12.-Theoretical weights in pounds of elbows, crosses, tees, side outlet tees, and laterals ${ }^{1}$

\begin{tabular}{|c|c|c|c|c|c|c|c|}
\hline $\begin{array}{l}\text { Nomi- } \\
\text { nal pipe } \\
\text { size }\end{array}$ & $\begin{array}{l}90^{\circ} \\
\text { elbow }\end{array}$ & $\begin{array}{l}45^{\circ} \\
\text { elbow }\end{array}$ & $\begin{array}{c}90^{\circ} \text { long } \\
\text { radius } \\
\text { elbow }\end{array}$ & $\begin{array}{l}\text { Side } \\
\text { outlet } \\
\text { elbow }\end{array}$ & Tees & \begin{tabular}{|c|} 
Cross \\
and side \\
outlet \\
tees
\end{tabular} & $\begin{array}{c}\text { Lat- } \\
\text { erals }{ }^{2} \\
\text { (not } \\
\text { ribbed) }\end{array}$ \\
\hline $\begin{array}{l}1 \\
11 / 1 / 2 \\
11 / 2 \\
2 \\
21 / 2\end{array}$ & $\begin{array}{r}5 \\
7 \\
9 \\
14 \\
19\end{array}$ & $\begin{array}{l}4 \\
6 \\
8 \\
12 \\
17\end{array}$ & $\begin{array}{r}7 \\
9 \\
11 \\
16 \\
23\end{array}$ & $\begin{array}{r}8 \\
10 \\
13 \\
20 \\
28\end{array}$ & $\begin{array}{r}9 \\
11 \\
15 \\
21 \\
30\end{array}$ & $\begin{array}{l}11 \\
15 \\
19 \\
28 \\
39\end{array}$ & $\begin{array}{l}10 \\
13 \\
17 \\
25 \\
36\end{array}$ \\
\hline $\begin{array}{l}3 \\
31 / 2 \\
4 \\
5 \\
6\end{array}$ & $\begin{array}{l}24 \\
31 \\
41 \\
52 \\
68\end{array}$ & $\begin{array}{l}20 \\
27 \\
36 \\
45 \\
60\end{array}$ & $\begin{array}{l}28 \\
37 \\
48 \\
62 \\
85\end{array}$ & $\begin{array}{l}34 \\
46 \\
59 \\
74 \\
96\end{array}$ & $\begin{array}{r}37 \\
49 \\
64 \\
81 \\
105\end{array}$ & $\begin{array}{r}48 \\
63 \\
82 \\
105 \\
135\end{array}$ & $\begin{array}{r}44 \\
59 \\
75 \\
96 \\
125\end{array}$ \\
\hline $\begin{array}{r}8 \\
10 \\
12 \\
314\end{array}$ & $\begin{array}{l}110 \\
175 \\
250 \\
350\end{array}$ & $\begin{array}{r}94 \\
145 \\
220 \\
270\end{array}$ & $\begin{array}{l}145 \\
230 \\
350 \\
470\end{array}$ & $\begin{array}{l}150 \\
240 \\
340 \\
470\end{array}$ & $\begin{array}{l}165 \\
270 \\
380 \\
530\end{array}$ & $\begin{array}{l}210 \\
330 \\
470 \\
650\end{array}$ & $\begin{array}{l}210 \\
340 \\
520 \\
680\end{array}$ \\
\hline 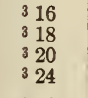 & $\begin{array}{r}470 \\
580 \\
740 \\
1,160\end{array}$ & $\begin{array}{l}360 \\
420 \\
540 \\
800\end{array}$ & $\begin{array}{r}670 \\
840 \\
1,080 \\
1,640\end{array}$ & $\begin{array}{r}620 \\
760 \\
970 \\
1,510\end{array}$ & $\begin{array}{r}700 \\
860 \\
1,100 \\
1,730\end{array}$ & $\begin{array}{r}850 \\
1,040 \\
1,330 \\
2,080\end{array}$ & $\begin{array}{r}950 \\
1,150 \\
1,480 \\
2,080\end{array}$ \\
\hline 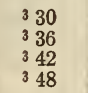 & $\begin{array}{l}1,850 \\
2,800 \\
4,010 \\
5,400\end{array}$ & $\begin{array}{l}1,430 \\
2,280 \\
3,380 \\
4,680\end{array}$ & $\begin{array}{l}2,800 \\
4,450 \\
6,610 \\
9,250\end{array}$ & $\begin{array}{l}2,350 \\
3,500 \\
4,930 \\
6,520\end{array}$ & $\begin{array}{l}2,710 \\
4,050 \\
5,790 \\
7,620\end{array}$ & $\begin{array}{l}3,210 \\
4,750 \\
6,710 \\
8,740\end{array}$ & 3,680 \\
\hline
\end{tabular}

1 All weights listed are for fittings faced and drilled, based on minimum thicknesses and dimensions given in preceding tables, without allowances for variation. Cast iron is assumed to weigh 0.26 pound per cubic inch.

2 Weights of laterals do not include reinforcing ribs.

3 Outside diameter.
TABLE 13.-Theoretical weights in pounds of reducing elbows, reducers, and eccentric reducers 1

\begin{tabular}{|c|c|c|c|c|c|}
\hline$\underset{\text { size }}{\text { Nominal pipe }}$ & $\begin{array}{l}\text { Reduc- } \\
\text { ing el- } \\
\text { bows }\end{array}$ & $\begin{array}{l}\text { Re- } \\
\text { ducers } \\
\text { and } \\
\text { eccen- } \\
\text { tric re- } \\
\text { ducers }\end{array}$ & $\underset{\text { size }}{\text { Nominal pipe }}$ & $\begin{array}{l}\text { Reduc- } \\
\text { ing el- } \\
\text { bows }\end{array}$ & $\begin{array}{l}\text { Re- } \\
\text { ducers } \\
\text { and } \\
\text { eccen- } \\
\text { tric re- } \\
\text { ducers }\end{array}$ \\
\hline 3 by & 22 & 19 & 12 by 10 & 220 & \\
\hline $\begin{array}{l}3 \\
3\end{array}$ & 19 & 16 & 1 & 190 & $\begin{array}{l}155 \\
140\end{array}$ \\
\hline $3^{1 / 2}$ & 28 & 24 & 14 by 12 & $\begin{array}{l}165 \\
320\end{array}$ & 140 \\
\hline & 24 & 20 & $14 \mathrm{~b}$ & 280 & 2 \\
\hline & 37 & 31 & & 240 & \\
\hline & 38 & 2 & & 42 & 3 \\
\hline $4 \mathrm{~b}$ & 31 & 26 & 16 by 12 & $3 \varepsilon$ & 3 \\
\hline $4 \mathrm{~b}$ & 29 & 24 & 16 & 340 & \\
\hline & 48 & 39 & & 300 & \\
\hline & 40 & 32 & 18 & 540 & \\
\hline & 37 & 31 & 18 & 480 & \\
\hline & & 5 & 18 & & \\
\hline & 56 & 47 & 18 & & 32 \\
\hline 63 & 51 & 43 & 2 & 6 & 520 \\
\hline 6 ? & 47 & 39 & 2 & 6 & 490 \\
\hline & 90 & 77 & 2 & 5 & 450 \\
\hline & 82 & 71 & 201 & 520 & 410 \\
\hline & 77 & 66 & $24 \mathrm{~b}$ & 1,010 & 760 \\
\hline & 10 & 120 & 24 by 18 & 930 & 700 \\
\hline & & 100 & & $8 \varepsilon$ & 670 \\
\hline & 115 & 95 & 24 by 12 & 740 & \\
\hline
\end{tabular}

1 All weights listed are for fittings faced and drilled, based on minimum thicknesses and dimensions given in preceding tables, without mum thicknesses and dimensions given in preceding tables, without
allowances for variation. Cast iron is assumed to weigh 0.26 pound per cubic inch. 
TABLE 14.-Theoretical weights in pounds of reducing laterals 1

\begin{tabular}{|c|c|c|c|}
\hline Nominal pipe size & $\begin{array}{l}\text { Lateral } \\
\text { reducing } \\
\text { outlet }{ }^{2} \\
\text { (not } \\
\text { ribbed) }\end{array}$ & Nominal pipe size & $\begin{array}{l}\text { Lateral } \\
\text { reducing } \\
\text { outlet }{ }^{2} \\
\text { (not } \\
\text { ribbed) }\end{array}$ \\
\hline $\begin{array}{l}3 \text { by } 3 \text { by } 21 / 2 \\
3 \text { by } 3 \text { by } 2 \\
3 \text { by } 3 \text { by } 11 / 2 \\
31 / 2 \text { by } 31 / 2 \text { by } 3 \\
31 / 2 \text { by } 31 / 2 \text { by } 21 / 2 \\
31 / 2 \text { by } 31 / 2 \text { by } 2 \\
4 \text { by } 4 \text { by } 31 / 2 \\
4 \text { by } 4 \text { by } 3 \text { by } 4 \text { by } 21 / 2 \\
4 \text { by } 4 \text { by } 2 \\
5 \text { by } 5 \text { by } 4 \\
5 \text { by } 5 \text { by } 31 / 2 \\
5 \text { by } 5 \text { by } 3 \\
5 \text { by } 5 \text { by } 21 / 2 \\
6 \text { by } 6 \text { by } 5 \\
6 \text { by } 6 \text { by } 4 \\
6 \text { by } 6 \text { by } 31 / 2 \\
6 \text { by } 6 \text { by } 3 \\
8 \text { by } 8 \text { by } 6 \\
8 \text { by } 8 \text { by } 5 \\
8 \text { by } 8 \text { by } 4 \\
10 \text { by } 10 \text { by } 8 \\
10 \text { by } 10 \text { by } 6 \\
10 \text { by } 10 \text { by } 5\end{array}$ & $\begin{array}{r}42 \\
39 \\
36 \\
55 \\
52 \\
49 \\
70 \\
66 \\
63 \\
60 \\
93 \\
86 \\
82 \\
79 \\
120 \\
115 \\
105 \\
105 \\
195 \\
180 \\
175 \\
310 \\
280 \\
270\end{array}$ & $\begin{array}{l}12 \text { by } 12 \text { by } 10 \\
12 \text { by } 12 \text { by } 8 \\
12 \text { by } 12 \text { by } 6 \\
14 \text { by } 14 \text { by } 12 \\
14 \text { by } 14 \text { by } 10 \\
14 \text { by } 14 \text { by } 8 \\
16 \text { by } 16 \text { by } 14 \\
16 \text { by } 16 \text { by } 12 \\
16 \text { by } 16 \text { by } 10 \\
16 \text { by } 16 \text { by } 8 \\
18 \text { by } 18 \text { by } 16 \\
18 \text { by } 18 \text { by } 14 \\
18 \text { by } 18 \text { by } 12 \\
18 \text { by } 18 \text { by } 10 \\
20 \text { by } 20 \text { by } 18 \\
20 \text { by } 20 \text { by } 16 \\
20 \text { by } 20 \text { by } 14 \\
20 \text { by } 20 \text { by } 12 \\
20 \text { by } 20 \text { by } 10 \\
24 \text { by } 24 \text { by } 20 \\
24 \text { by } 24 \text { by } 18 \\
24 \text { by } 24 \text { by } 16 \\
24 \text { by } 24 \text { by } 14 \\
24 \text { by } 24 \text { by } 12\end{array}$ & $\begin{array}{r}470 \\
430 \\
400 \\
640 \\
590 \\
550 \\
880 \\
830 \\
790 \\
740 \\
1,100 \\
1,030 \\
980 \\
930 \\
1,400 \\
1,350 \\
1,270 \\
1,220 \\
3840 \\
2,040 \\
1,950 \\
1,890 \\
1,810 \\
1,250\end{array}$ \\
\hline
\end{tabular}

1 All weights listed are for fittings faced and drilled, based on minimum thicknesses and dimensions given in preceding tables, without allowances for variation. Cast iron is assumed to weigh 0.26 pound per cubic inch.

2 Weights of laterals do not include reinforcing ribs.

3 These sizes made in the short body pattern only.

TABLE 15.-Theoretical weights in pounds of reducing tees $^{1}$

\begin{tabular}{|c|c|c|c|}
\hline Size & Weight & Size & Weight \\
\hline $\begin{array}{l}3 \text { by } 3 \text { by } 21 / 2 \ldots \\
3 \text { by } 3 \text { by } 2 \\
3 \text { by } 3 \text { by } 11 / 2 \ldots \\
3 \text { by } 21 / 2 \text { by } 3 \\
3 \text { by } 21 / 2 \text { by } 21 / 2 \ldots\end{array}$ & $\begin{array}{l}36 \\
33 \\
31 \\
35 \\
34\end{array}$ & $\begin{array}{l}5 \text { by } 4 \text { by } 3 \\
5 \text { by } 4 \text { by } 21 / 2 \ldots \\
5 \text { by } 3 \text { by } 5 \text { by } 3 \text { by } 4 \\
5 \text { by } 3 \text { by } 3 \ldots\end{array}$ & $\begin{array}{l}68 \\
66 \\
72 \\
68 \\
61\end{array}$ \\
\hline $\begin{array}{l}3 \text { by } 21 / 2 \text { by } 2 \\
3 \text { by } 21 / 2 \text { by } 11 / 2 \ldots \\
3 \text { by } 2 \text { by } 3 \\
3 \text { by } 2 \text { by } 21 / 2 \ldots \\
3 \text { by } 2 \text { by } 2\end{array}$ & $\begin{array}{l}31 \\
29 \\
33 \\
32 \\
29\end{array}$ & $\begin{array}{l}5 \text { by } 3 \text { by } 21 / 2 \\
5 \text { by } 21 / 2 \text { by } 5 \\
5 \text { by } 21 / 2 \text { by } 4 \\
5 \text { by } 21 / 2 \text { by } 3 \\
5 \text { by } 21 / 2 \text { by } 21 / 2\end{array}$ & $\begin{array}{l}59 \\
70 \\
67 \\
60 \\
58\end{array}$ \\
\hline $\begin{array}{l}3 \text { by } 2 \text { by } 11 / 2 \ldots \ldots \\
3 \text { by } 11 / 2 \text { by } 3 \text { by } 11 / 2 \text { by } 21 / 2 \ldots \\
3 \text { by } 11 / 2 \text { by } 2 \\
3 \text { by } 11 / 2 \text { by } 11 / 2 \ldots\end{array}$ & $\begin{array}{l}27 \\
32 \\
30 \\
27 \\
25\end{array}$ & $\begin{array}{l}6 \text { by } 6 \text { by } 5 \\
6 \text { by } 6 \text { by } 4 \\
6 \text { by } 6 \text { by } 31 / 2 \\
6 \text { by } 6 \text { by } 3 \\
6 \text { by } 5 \text { by } 6 .\end{array}$ & $\begin{array}{r}99 \\
96 \\
92 \\
89 \\
100\end{array}$ \\
\hline $\begin{array}{l}31 / 2 \text { by } 31 / 2 \text { by } \\
31 / 2 \text { by } 31 / 2 \text { by } \\
31 / 2 \text { by } 31 / 2 \text { by } \\
4 \text { by } 4 \text { by } 31 / 2 \\
4 \text { by } 4 \text { by } 3 . .\end{array}$ & $\begin{array}{l}46 \\
44 \\
42 \\
60 \\
57\end{array}$ & $\begin{array}{l}6 \text { by } 5 \text { by } 5 \\
6 \text { by } 5 \text { by } 4 \\
6 \text { by } 5 \text { by } 3 \\
6 \text { by } 4 \text { by } 6 \text { by } 4 \text { by } 5 \\
6 \text { by }\end{array}$ & $\begin{array}{l}95 \\
92 \\
84 \\
98 \\
93\end{array}$ \\
\hline $\begin{array}{l}4 \text { by } 4 \text { by } 21 \\
4 \text { by } 4 \text { by } 2- \\
4 \text { by } 3 \text { by } 4- \\
4 \text { by } 3 \text { by } 3 \\
4 \text { by } 3 \text { by } 21\end{array}$ & $\begin{array}{l}55 \\
53 \\
57 \\
50 \\
49\end{array}$ & $\begin{array}{l}6 \text { by } 4 \text { by } 4 \\
6 \text { by } 4 \text { by } 3 \text { by } 3 \text { by } 6 \\
6 \text { by } 3 \text { by } 5 \\
6 \text { by } 3 \text { by } 4\end{array}$ & $\begin{array}{l}89 \\
82 \\
92 \\
86 \\
83\end{array}$ \\
\hline $\begin{array}{l}4 \text { by } 3 \text { by } 2 \\
4 \text { by } 21 / 2 \text { by } 4 \\
4 \text { by } 21 / 2 \text { by } 3 \\
4 \text { by } 21 / 2 \text { by } 21 / 2 \\
4 \text { by } 21 / 2 \text { by } 2\end{array}$ & $\begin{array}{l}46 \\
56 \\
49 \\
47 \\
45\end{array}$ & $\begin{array}{l}6 \text { by } 3 \text { by } 3 \\
8 \text { by } 8 \text { by } 6 \text { by } 8 \text { by } 5 \\
8 \text { by } 8 \text { by } 4 \\
8 \text { by } 6 \text { by } 8\end{array}$ & $\begin{array}{r}76 \\
150 \\
145 \\
145 \\
155\end{array}$ \\
\hline $\begin{array}{l}4 \text { by } 2 \text { by } 4- \\
4 \text { by } 2 \text { by } 3- \\
4 \text { by } 2 \text { by } 21 \\
4 \text { by } 2 \text { by } 2- \\
5 \text { by } 5 \text { by } 4 .\end{array}$ & $\begin{array}{l}54 \\
47 \\
45 \\
43 \\
78\end{array}$ & $\begin{array}{l}8 \text { by } 6 \text { by } 6 \\
8 \text { by } 6 \text { by } 5 \text { by } 6 \text { by } 4 \\
8 \text { by } 5 \text { by } 8 \\
8 \text { by } 5 \text { by } 6 \ldots\end{array}$ & $\begin{array}{l}140 \\
135 \\
130 \\
150 \\
135\end{array}$ \\
\hline $\begin{array}{l}5 \text { by } 5 \text { by } 31 / 2 \\
5 \text { by } 5 \text { by } 3 \\
5 \text { by } 5 \text { by } 21 / 2 \\
5 \text { by } 4 \text { by } 5 \\
5 \text { by } 4 \text { by } 4 .\end{array}$ & $\begin{array}{l}74 \\
70 \\
68 \\
78 \\
75\end{array}$ & $\begin{array}{l}8 \text { by } 5 \text { by } 5 \\
8 \text { by } 5 \text { by } 4 \text { by } 4 \text { by } 8 \\
8 \text { by } 4 \text { by } 6 \\
8 \text { by } 4 \text { by } 5\end{array}$ & $\begin{array}{l}130 \\
125 \\
150 \\
135 \\
130\end{array}$ \\
\hline
\end{tabular}

1 All weights listed are for fittings faced and drilled, based on minimum thicknesses and dimensions given in preceding tables, without allowances for variation. Cast iron is assumed to weigh 0.26 pound per cubic inch.
TABLE 15.-Theoretical weights in pounds of reducing tees-Continued

\begin{tabular}{|c|c|c|c|}
\hline Size & Weight & Size & Weight \\
\hline $\begin{array}{l}8 \text { by } 4 \text { by } 4 \\
10 \text { by } 10 \text { by } 8 \\
10 \text { by } 10 \text { by } 6 \\
10 \text { by } 10 \text { by } 5 \\
10 \text { by } 8 \text { by } 10\end{array}$ & $\begin{array}{l}125 \\
250 \\
240 \\
230 \\
260\end{array}$ & $\begin{array}{l}16 \text { by } 16 \text { by } 14 \\
16 \text { by } 16 \text { by } 12 \\
16 \text { by } 16 \text { by } 10 \ldots \\
16 \text { by } 16 \text { by } 8 \\
16 \text { by } 14 \text { by } 16 . \ldots\end{array}$ & $\begin{array}{l}670 \\
650 \\
620 \\
610 \\
650\end{array}$ \\
\hline $\begin{array}{l}10 \text { by } 8 \text { by } 8 \\
10 \text { by } 8 \text { by } 6 \\
10 \text { by } 6 \text { by } 10 \\
10 \text { by } 6 \text { by } 8 \\
10 \text { by } 6 \text { by } 6\end{array}$ & $\begin{array}{l}240 \\
220 \\
250 \\
230 \\
210\end{array}$ & $\begin{array}{l}16 \text { by } 14 \text { by } 14 \ldots \\
16 \text { by } 14 \text { by } 12 \ldots \\
16 \text { by } 14 \text { by } 10 \ldots \ldots \\
16 \text { by } 14 \text { by } 8 . \ldots \ldots \\
16 \text { by } 12 \text { by } 16 \ldots \ldots\end{array}$ & $\begin{array}{l}650 \\
630 \\
600 \\
580 \\
670\end{array}$ \\
\hline $\begin{array}{l}12 \text { by } 12 \text { by } 10 \\
12 \text { by } 12 \text { by } 8 \\
12 \text { by } 12 \text { by } 6 \\
12 \text { by } 10 \text { by } 12 \\
12 \text { by } 10 \text { by } 10\end{array}$ & $\begin{array}{l}360 \\
340 \\
320 \\
370 \\
340\end{array}$ & $\begin{array}{l}16 \text { by } 12 \text { by } 14 \ldots \\
16 \text { by } 12 \text { by } 12 \ldots \ldots \\
16 \text { by } 12 \text { by } 10 \ldots \ldots \\
16 \text { by } 12 \text { by } 8 \ldots \ldots \\
16 \text { by } 10 \text { by } 16 \ldots \ldots \ldots\end{array}$ & $\begin{array}{l}640 \\
620 \\
590 \\
570 \\
650\end{array}$ \\
\hline $\begin{array}{l}12 \text { by } 10 \text { by } 8 \\
12 \text { by } 10 \text { by } 6 \\
12 \text { by } 8 \text { by } 12 \\
12 \text { by } 8 \text { by } 10 \\
12 \text { by } 8 \text { by } 8\end{array}$ & $\begin{array}{l}320 \\
310 \\
350 \\
330 \\
310\end{array}$ & $\begin{array}{l}16 \text { by } 10 \text { by } 14 \\
16 \text { by } 10 \text { by } 12 \\
16 \text { by } 10 \text { by } 10 \ldots \\
16 \text { by } 10 \text { by } 8 \\
16 \text { by } 8 \text { by } 16 \ldots\end{array}$ & $\begin{array}{l}620 \\
600 \\
570 \\
550 \\
640\end{array}$ \\
\hline $\begin{array}{l}12 \text { by } 8 \text { by } 6 \\
12 \text { by } 6 \text { by } 12 \\
12 \text { by } 6 \text { by } 10 \\
12 \text { by } 6 \text { by } 8 \\
12 \text { by } 6 \text { by } 6 \ldots \ldots\end{array}$ & $\begin{array}{l}300 \\
340 \\
320 \\
300 \\
280\end{array}$ & $\begin{array}{l}36 \text { by } 8 \text { by } 14 \\
16 \text { by } 8 \text { by } 12 \ldots \ldots \\
16 \text { by } 8 \text { by } 10 \ldots \ldots \\
16 \text { by } 8 \text { by } 8 \\
18 \text { by } 18 \text { by } 16 \ldots \ldots \ldots\end{array}$ & $\begin{array}{l}610 \\
580 \\
560 \\
540 \\
860\end{array}$ \\
\hline $\begin{array}{l}14 \text { by } 14 \text { by } 12 \\
14 \text { by } 14 \text { by } 10 \\
14 \text { by } 14 \text { by } 8 \\
14 \text { by } 12 \text { by } 14 \\
14 \text { by } 12 \text { by } 12 \ldots\end{array}$ & $\begin{array}{l}500 \\
480 \\
460 \\
510 \\
490\end{array}$ & $\begin{array}{l}18 \text { by } 18 \text { by } 14 \\
18 \text { by } 18 \text { by } 12 \\
18 \text { by } 18 \text { by } 10 \ldots \\
20 \text { by } 20 \text { by } 18 \ldots \\
20 \text { by } 20 \text { by } 16 . \ldots\end{array}$ & $\begin{array}{l}820 \\
2660 \\
2640 \\
1,060 \\
1,040\end{array}$ \\
\hline $\begin{array}{l}14 \text { by } 12 \text { by } 10 \\
14 \text { by } 12 \text { by } 8 \\
14 \text { by } 10 \text { by } 14 \\
14 \text { by } 10 \text { by } 12 \\
14 \text { by } 10 \text { by } 10 . \ldots\end{array}$ & $\begin{array}{l}460 \\
440 \\
490 \\
470 \\
450\end{array}$ & $\begin{array}{l}20 \text { by } 20 \text { by } 14 \\
20 \text { by } 20 \text { by } 12 \ldots \\
20 \text { by } 20 \text { by } 10 \ldots \\
24 \text { by } 24 \text { by } 20 \\
24 \text { by } 24 \text { by } 18 \ldots\end{array}$ & $\begin{array}{l}2840 \\
2820 \\
1790 \\
1,640 \\
1,600\end{array}$ \\
\hline $\begin{array}{l}14 \text { by } 10 \text { by } 8 \\
14 \text { by } 8 \text { by } 14 \\
14 \text { by } 8 \text { by } 12 \ldots \\
14 \text { by } 8 \text { by } 10 \ldots \\
14 \text { by } 8 \text { by } 8\end{array}$ & $\begin{array}{l}420 \\
480 \\
460 \\
430 \\
410\end{array}$ & $\begin{array}{l}24 \text { by } 24 \text { by } 16 \\
24 \text { by } 24 \text { by } 14 \ldots \\
24 \text { by } 24 \text { by } 12\end{array}$ & $\begin{array}{r}21,170 \\
21,140 \\
21,110 \\
\hdashline \ldots\end{array}$ \\
\hline
\end{tabular}

2 These sizes made in short body pattern only.

TABLE 16.-Theoretical weights in pounds of reducing crosses 1

\begin{tabular}{|c|c|c|c|}
\hline Size & Weight & Size & Weight \\
\hline $\begin{array}{l}3 \text { by } 3 \text { by } 21 / 2 \text { by } 21 / 2 \\
3 \text { by } 3 \text { by } 2 \text { by } 2 \\
3 \text { by } 3 \text { by } 11 / 2 \text { by } 11 / 2 \\
31 / 2 \text { by } 31 / 2 \text { by } 3 \text { by } 3 \\
31 / 2 \text { by } 31 / 2 \text { by } 21 / 2 \text { by } 21 / 2 \\
31 / 2 \text { by } 31 / 2 \text { by } 2 \text { by } 2 \\
4 \text { by } 4 \text { by } 31 / 2 \text { by } 31 / 2 \\
4 \text { by } 4 \text { by } 3 \text { by } 3 \\
4 \text { by } 4 \text { by } 21 / 2 \text { by } 21 / 2 \\
4 \text { by } 4 \text { by } 2 \text { by } 2 \\
5 \text { by } 5 \text { by } 4 \text { by } 4 \\
5 \text { by } 5 \text { by } 31 / 2 \text { by } 31 / 2 \\
5 \text { by } 5 \text { by } 3 \text { by } 3 \\
5 \text { by } 5 \text { by } 21 / 2 \text { by } 21 / 2 \\
6 \text { by } 6 \text { by } 5 \text { by } 5 \\
6 \text { by } 6 \text { by } 4 \text { by } 4 \\
6 \text { by } 6 \text { by } 31 / 2 \text { by } 31 / 2 \\
6 \text { by } 6 \text { by } 3 \text { by } 3 \\
8 \text { by } 8 \text { by } 6 \text { by } 6 \\
8 \text { by } 8 \text { by } 5 \text { by } 5 \\
8 \text { by } 8 \text { by } 4 \text { by } 4 \\
10 \text { by } 10 \text { by } 8 \text { by } 8 \\
10 \text { by } 10 \text { by } 6 \text { by } 6 \\
10 \text { by } 10 \text { by } 5 \text { by } 5 \\
12 \text { by } 12 \text { by } 10 \text { by } 10\end{array}$ & $\begin{array}{r}44 \\
40 \\
36 \\
57 \\
53 \\
47 \\
74 \\
68 \\
64 \\
59 \\
96 \\
89 \\
82 \\
78 \\
120 \\
115 \\
105 \\
100 \\
190 \\
175 \\
165 \\
300 \\
270 \\
250 \\
420\end{array}$ & 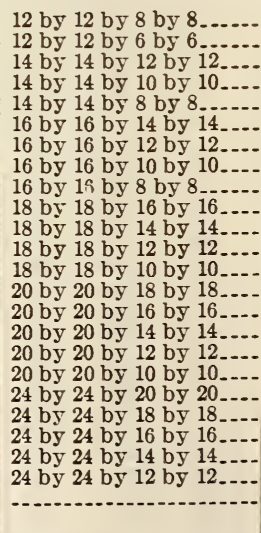 & $\begin{array}{r}380 \\
350 \\
600 \\
550 \\
500 \\
790 \\
740 \\
690 \\
650 \\
1,000 \\
930 \\
2750 \\
2700 \\
1,250 \\
1,200 \\
2960 \\
2910 \\
2860 \\
1,900 \\
1,810 \\
21,310 \\
21,250 \\
21,210 \\
-2 \ldots\end{array}$ \\
\hline
\end{tabular}

${ }_{1}$ All weights listed are for fittings faced and drilled, based on minimum thicknesses and dimensions given in preceding tables, without allowances for variation. Cast iron is assumed to weigh 0.26 pound per cubic inch.

2 These sizes made in the short body pattern only. 
AMERICAN SOCIETY OF MECHANICAL ENGINEERS, AMERICAN STANDARD CASTIRON PIPE FLANGES AND FLANGED FITTINGS FOR MAXIMUM WORKING SATURATED STEAM PRESSURE OF 250 POUNDS PER SQUARE INCH (GAGE), B16b-1928

[A pproved as American standard by the American Standards Association, ${ }^{63}$ February, 1925]

\section{CAST-IRON PIPE FLANGES AND FLANGED FIT- TINGS, 250 POUNDS PER SQUARE INCH}

(This specification is identical with A. S. M. E., B16a, given above, except in the following particulars:)

\section{INTRODUCTORY NOTES}

1. Sizes.-The sizes of the fittings in the following tables will be identified by the corresponding nominal pipe size. This nominal pipe size is the same as the port diameter of fittings for pipe having inside diameters of 12 inches and smaller. For pipe 14 inches and larger the corresponding outside diameeter of the pipe is given, and consequently the fittings will have a smaller port diameter.

2. Pressure rating.-It is recommended that in addition to the maximum working steam pressure

63 Sponsor organizations: American Society of Mechanical Engineers, Manufacturers' Standardization Soc ety of Valve and Fittings Industry, and Heating and Piping Contractors' National Assoziation. for which these fittings are intended, the sizes 10 inches and smaller may also be used for maximum nonshock working hydraulic pressure of 325 pounds per square inch at a temperature of $250^{\circ} \mathrm{F}$., and for maximum nonshock working hydraulic pressures of 400 pounds per square inch at or near the ordinary range of air temperatures.

3. Marking.-All fittings must have marks cast on them indicating the manufacturer and the figures 250 indicating the maximum working steam pressure for which the fittings are intended.

5. Facing.-All 250-pound cast-iron flanges and flanged fittings shall have a raised face one-sixteenth inch high of the diameters given in Table 2. The raised face is included in the minimum flange thickness and center to face dimensions.

6. Bolting.-Hexagonal nuts for pipe sizes 1 to 16 inches can be conveniently pulled up with open wrenches of minimum design of heads. Hexagonal nuts for pipe sizes 18 to 48 inches can be conveniently pulled up with box wrenches.

7. Spot facing. - The bolt holes of 250-pound cast-iron flanged fittings and flanges shall not be spot faced for ordinary service. When required, the fittings and flanges in sizes 36 inches and larger may be spot faced or back faced, to the minimum thickness of flanges with a plus tolerance of one-eighth inch.

(Illustrations showing method of designating outlets of reducing fittings when ordering are identical with those for steel-flanged fittings 250, 400, 600, 900 , and 1,350 pounds. (See 607.4, p. 467. )

\section{TABLE 2.-Templates for drilling}

[All dimensions given in inches]

\begin{tabular}{|c|c|c|c|c|c|c|c|c|c|c|c|c|}
\hline $\begin{array}{l}\text { Nominal } \\
\text { pipe size }\end{array}$ & $\begin{array}{l}\text { Diam- } \\
\text { eter of } \\
\text { flange }\end{array}$ & \begin{tabular}{|} 
Thick- \\
ness of \\
flange \\
(mini- \\
mum $)^{12}$
\end{tabular} & $\begin{array}{l}\text { Diame- } \\
\text { ter of } \\
\text { raised } \\
\text { face }\end{array}$ & $\begin{array}{l}\text { Diam- } \\
\text { eter of } \\
\text { bolt } \\
\text { circle }\end{array}$ & $\begin{array}{l}\text { Num- } \\
\text { ber of } \\
\text { bolts }\end{array}$ & $\begin{array}{l}\text { Diam- } \\
\text { eter of } \\
\text { bolts }\end{array}$ & $\begin{array}{l}\text { Diam- } \\
\text { eter of } \\
\text { drilled } \\
\text { bolt } \\
\text { holes }\end{array}$ & $\begin{array}{l}\text { Length } \\
\text { of } \\
\text { bolts } 4 \mathrm{~s}\end{array}$ & $\begin{array}{l}\text { Length } \\
\text { of bolt } \\
\text { studs } \\
\text { with } \\
2 \text { nuts } 5\end{array}$ & $\begin{array}{l}\text { Total } \\
\text { effective } \\
\text { area bolt } \\
\text { metal }\end{array}$ & $\begin{array}{l}\text { Stress } \\
\text { pounds } \\
\text { per } \\
\text { square } \\
\text { inch } \\
\text { bolt } \\
\text { metal } 6\end{array}$ & $\begin{array}{l}\text { Size of ring } \\
\text { gasket }\end{array}$ \\
\hline $\begin{array}{l}1 \\
11 / 4 \\
11 / 2 \\
2 \\
21 / 2\end{array}$ & $\begin{array}{l}47 / 8 \\
51 / 4 \\
618 \\
61 / 2 \\
71 / 2\end{array}$ & $\begin{array}{r}13 / 16 \\
3 / 4 \\
13 / 16 \\
7 / 8 \\
1 \quad\end{array}$ & $\begin{array}{l}211 / 16 \\
31 / 16 \\
39 / 16 \\
43 / 16 \\
415 / 16\end{array}$ & $\begin{array}{l}31 / 2 \\
37 / 8 \\
41 / 2 \\
5 \\
57 / 8\end{array}$ & $\begin{array}{l}4 \\
4 \\
4 \\
8 \\
8\end{array}$ & $\begin{array}{l}5 / 8 \\
5 / 8 \\
3 / 4 \\
5 / 8 \\
3 / 4\end{array}$ & $\begin{array}{l}3 / 4 \\
3 / 4 \\
7 / 8 \\
3 / 4 \\
7 / 8\end{array}$ & $\begin{array}{l}21 / 4 \\
21 / 2 \\
21 / 2 \\
21 / 2 \\
3\end{array}$ & $\ldots$ & $\begin{array}{r}0.808 \\
.808 \\
1.208 \\
1.616 \\
2.416\end{array}$ & $\begin{array}{r}970 \\
1,520 \\
1,345 \\
1,595 \\
2,090\end{array}$ & $\begin{array}{lll}1 & \text { by } & 27 / 8 \\
11 / 4 & \text { by } & 31 / 4 \\
11 / 2 & \text { by } & 33 / 4 \\
2 & \text { by } & 43 / 8 \\
21 / 2 & \text { by } & 51 / 8\end{array}$ \\
\hline $\begin{array}{l}3 \\
31 / 2 \\
4 \\
5 \\
6\end{array}$ & $\begin{array}{l}81 / 4 \\
9 \\
10 \\
11 \\
121 / 2\end{array}$ & $\begin{array}{l}11 / 8 \\
13 / 16 \\
11 / 4 \\
13 / 8 \\
17 / 16\end{array}$ & $\begin{array}{l}5^{11 / 16} \\
6^{5} / 16 \\
6^{15} / 16 \\
85 / 16 \\
9^{11} 1 / 16\end{array}$ & $\begin{array}{r}65 / 8 \\
71 / 4 \\
77 / 8 \\
91 / 4 \\
105 / 8\end{array}$ & $\begin{array}{r}8 \\
8 \\
8 \\
8 \\
12\end{array}$ & $\begin{array}{l}3 / 4 \\
3 / 4 \\
3 / 4 \\
3 / 4 \\
3 / 4\end{array}$ & $\begin{array}{l}7 / 8 \\
7 / 8 \\
7 / 8 \\
7 / 8 \\
7 / 8\end{array}$ & $\begin{array}{l}31 / 4 \\
31 / 4 \\
31 / 2 \\
33 / 4 \\
334\end{array}$ & 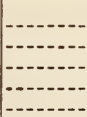 & $\begin{array}{l}2.416 \\
2.416 \\
2.416 \\
2.416 \\
3.624\end{array}$ & $\begin{array}{l}2,030 \\
2,460 \\
3,120 \\
4,385 \\
3,915\end{array}$ & $\begin{array}{lll}3 & \text { by } & 57 / 8 \\
31 / 2 & \text { by } & 61 / 2 \\
4 & \text { by } & 71 / 8 \\
5 & \text { by } & 81 / 2 \\
6 & \text { by } & 97 / 8\end{array}$ \\
\hline $\begin{array}{r}8 \\
10 \\
12 \\
714\end{array}$ & $\begin{array}{l}15 \\
171 / 2 \\
201 / 2 \\
23\end{array}$ & $\begin{array}{l}15 / 8 \\
17 / 8 \\
2 \\
216\end{array}$ & $\begin{array}{l}1115 / 16 \\
141 / 16 \\
167 / 16 \\
18^{15 / 16}\end{array}$ & $\begin{array}{l}13 \\
151 / 4 \\
173 / 4 \\
201 / 4\end{array}$ & $\begin{array}{l}12 \\
16 \\
16 \\
20\end{array}$ & $\begin{array}{l}1^{7 / 8} \\
11 / 8 \\
11 / 8\end{array}$ & $\begin{array}{l}1 \\
118 \\
11 / 4 \\
11 / 4\end{array}$ & $\begin{array}{l}41 / 4 \\
5 \\
51 / 2 \\
53 / 4\end{array}$ & & $\begin{array}{r}5.04 \\
8.80 \\
11.10 \\
13.88\end{array}$ & $\begin{array}{l}4,400 \\
3,625 \\
3,975 \\
3,735\end{array}$ & $\begin{array}{cc}8 & \text { by } 121 / 8 \\
10 & \text { by } 141 / 4 \\
12 & \text { by } 165 / 8 \\
131 / 4 & \text { by } 191 / 8\end{array}$ \\
\hline $\begin{array}{l}716 \\
718 \\
720 \\
724\end{array}$ & $\begin{array}{l}251 / 2 \\
28 \\
301 / 2 \\
36\end{array}$ & $\begin{array}{l}21 / 4 \\
23 / 8 \\
21 / 2 \\
23 / 4\end{array}$ & $\begin{array}{l}211 / 16 \\
235 / 16 \\
25 \% 16 \\
305 / 16\end{array}$ & $\begin{array}{l}221 / 2 \\
243 / 4 \\
27 \\
32\end{array}$ & $\begin{array}{l}20 \\
24 \\
24 \\
24\end{array}$ & $\begin{array}{l}11 / 4 \\
11 / 4 \\
11 / 4 \\
11 / 2\end{array}$ & $\begin{array}{l}13 / 8 \\
138 \\
138 \\
15 / 8\end{array}$ & $\begin{array}{l}6 \\
61 / 4 \\
61 / 2 \\
71 / 2\end{array}$ & - & $\begin{array}{l}17.86 \\
21.43 \\
21.43 \\
31.06\end{array}$ & $\begin{array}{l}2,255 \\
4,505 \\
4,845 \\
4,500\end{array}$ & $\begin{array}{l}151 / 4 \text { by } 211 / 4 \\
17 \text { by } 231 / 2 \\
19 \text { by } 253 / 4 \\
23 \text { by } 301 / 2\end{array}$ \\
\hline $\begin{array}{l}730 \\
736 \\
742 \\
748\end{array}$ & $\begin{array}{l}43 \\
50 \\
57 \\
65\end{array}$ & $\begin{array}{l}3 \\
3^{3 / 8} \\
3^{111 / 16} \\
4\end{array}$ & $\begin{array}{l}373 / 16 \\
43^{11} 1 / 16 \\
507 / 16 \\
587 / 16\end{array}$ & $\begin{array}{l}391 / 4 \\
46 \\
523 / 4 \\
60^{3} / 4\end{array}$ & $\begin{array}{l}28 \\
32 \\
36 \\
40\end{array}$ & $\begin{array}{l}13 / 4 \\
2 \\
2 \\
2\end{array}$ & $\begin{array}{l}2 \\
21 / 4 \\
21 / 4 \\
21 / 4\end{array}$ & $\begin{array}{r}81 / 4 \\
91 / 4 \\
93 / 4 \\
101 / 2\end{array}$ & $\begin{array}{l}101 / 2 \\
111 / 2 \\
12 \\
13\end{array}$ & $\begin{array}{l}48.89 \\
73.70 \\
82.90 \\
92.08\end{array}$ & $\begin{array}{l}5,590 \\
5,355 \\
5,945 \\
7,315\end{array}$ & $\begin{array}{l}29 \text { by } 371 / 2 \\
341 / 2 \text { by } 44 \\
401 / 4 \text { by } 503 / 4 \\
46 \text { by } 583 / 4\end{array}$ \\
\hline
\end{tabular}

1 All 250-pound cast-iron standard flanges have a $1 / 16$-inch raised face. This raised face is included in the face-to-face, center-to-face, and the minimum thickness of flange dimensions.

${ }^{2}$ For tongue-groove and male-female facings it is recommended that the dimensions given in Table 5 of the American tentative standards for stoel pipe flanges and flanged fittings be used.

Drilling templates are in multiples of 4, so that fittings may be made to face in any quarter, and bolt holes straddle the center line. For bolts smaller than 13 inch the bolt holes shall be drilled 18 inch larger in diameter than the nominal size of the bolt. Holes for bolts 134 inches and larger shall be drilled $1 / 4$ inch larger than nominal diameter of bolts.

4 The bolt holes on cast-iron fanged fittings are not spot faced for ordinary service. When required, the fittings and fianges in sizes 36 inches and larger can be spot faced or back faced, so that standard length bolts can be used.

5 Bolts shall be of steel with standard rough square heads and the nuts shall be of steel with standard rough hexagonal dimensions; all as given in the American tentative standard on wrench head bolts and nuts, and wrench openings. For bolts 13/4 inches diameter and larger, bolt-studs with a nut on each end are recommended. Hexagonal nuts for pipe sizes 1 to 16 inches can be conveniently pulled up with open wrenches of minimum design of heads. Hexagonal nuts for pipe sizes 18 to 48 inches can be conveniently pulled up with box wrenches.

- The stress shown is that of internal pressure only assumed to act on a circular area equal in diameter to the outside diameter of the raised face.

7 Outside diameter.

(Illustrations showing dimensions of screwed companion and blind flanges are identical with those for 250-pound steel flanges. See 607.4, p. 465.) 
TABLE 3.-Dimensions and theoretical weights (pounds) of screwed companion and blind flanges ${ }^{1}$ [All dimensions given in inches]

\begin{tabular}{|c|c|c|c|c|c|c|c|c|c|c|}
\hline \multirow{2}{*}{$\begin{array}{l}\text { Nominal } \\
\text { pipe size }\end{array}$} & \multirow{2}{*}{$\begin{array}{c}\text { Diam- } \\
\text { eter of } \\
\text { port } 23\end{array}$} & \multirow{2}{*}{$\begin{array}{c}\text { Diam. } \\
\text { eter of } \\
\text { flange } \\
\text { O }\end{array}$} & \multirow{2}{*}{$\begin{array}{c}\text { Thick- } \\
\text { ness of } \\
\text { flange } \\
\text { (mini- } \\
\text { mum) } 1 \\
\text { Q }\end{array}$} & \multirow{2}{*}{$\begin{array}{c}\text { Metal } \\
\text { thick- } \\
\text { ness } \\
\text { (mini- } \\
\text { mum) } \\
\text { V }\end{array}$} & \multirow{2}{*}{$\begin{array}{l}\text { Diam- } \\
\text { eter hub } \\
\text { (mini- } \\
\text { mum) } \\
\text { X }\end{array}$} & \multirow{2}{*}{$\begin{array}{c}\text { Length } \\
\text { through } \\
\text { hub } \\
\text { (mini- } \\
\text { mum) } \\
\text { Y }\end{array}$} & \multirow{2}{*}{$\begin{array}{l}\text { Length } \\
\text { of } \\
\text { threads } \\
\text { (mini- } \\
\text { mum) } \\
\text { Z }\end{array}$} & \multirow{2}{*}{$\begin{array}{l}\text { Diam- } \\
\text { eter of } \\
\text { raised } \\
\text { face } 1\end{array}$} & \multicolumn{2}{|c|}{$\begin{array}{l}\text { Theoretical } \\
\text { weights } 5\end{array}$} \\
\hline & & & & & & & & & $\begin{array}{l}\text { Com- } \\
\text { panion } \\
\text { flanges }\end{array}$ & $\begin{array}{l}\text { Blind } \\
\text { flanges }\end{array}$ \\
\hline $\begin{array}{l}1 \\
11 / 4 \\
11 / 2 \\
2 \\
21 / 2\end{array}$ & $\begin{array}{l}1 \\
11 / 4 \\
11 / 2 \\
2 \\
21 / 2\end{array}$ & $\begin{array}{l}47 / 8 \\
51 / 4 \\
61 / 8 \\
61 / 2 \\
71 / 2\end{array}$ & $\begin{array}{l}11 / 16 \\
3 / 4 \\
13 / 16 \\
7 / 8 \\
1^{7 / 8}\end{array}$ & - & $\begin{array}{l}21 / 16 \\
21 / 2 \\
23 / 4 \\
35 / 16 \\
315 / 16\end{array}$ & $\begin{array}{l}7 / 8 \\
1 \\
11 / 8 \\
11 / 4 \\
17 / 16\end{array}$ & $\begin{array}{r}0.68 \\
.76 \\
.87 \\
1.00 \\
1.14\end{array}$ & $\begin{array}{l}2^{11} 116 \\
31 / 16 \\
39 / 16 \\
43 / 16 \\
4^{15} / 16\end{array}$ & $\begin{array}{r}3 \\
4 \\
6 \\
7 \\
11\end{array}$ & $\begin{array}{r}3 \\
4 \\
6 \\
8 \\
12\end{array}$ \\
\hline $\begin{array}{l}3 \\
31 / 2 \\
4 \\
5 \\
6\end{array}$ & $\begin{array}{l}3 \\
31 / 2 \\
4 \\
5 \\
6\end{array}$ & $\begin{array}{l}81 / 4 \\
9 \\
10 \\
11 \\
121 / 2\end{array}$ & $\begin{array}{l}11 / 8 \\
13 / 16 \\
11 / 4 \\
13 / 8 \\
17 / 16\end{array}$ & 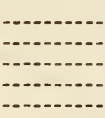 & $\begin{array}{l}45 / 8 \\
51 / 4 \\
53 / 4 \\
7 \\
81 / 8\end{array}$ & $\begin{array}{l}19 / 16 \\
15 / 8 \\
13 / 4 \\
17 / 8 \\
115 / 16\end{array}$ & $\begin{array}{l}1.20 \\
1.25 \\
1.30 \\
1.41 \\
1.51\end{array}$ & $\begin{array}{l}511 / 16 \\
65116 \\
615 / 16 \\
85 / 16 \\
9^{11} 1 / 16\end{array}$ & $\begin{array}{l}14 \\
18 \\
23 \\
29 \\
37\end{array}$ & $\begin{array}{l}16 \\
20 \\
26 \\
34 \\
46\end{array}$ \\
\hline $\begin{array}{r}8 \\
10 \\
12 \\
614 \\
616\end{array}$ & $\begin{array}{l}8 \\
10 \\
12 \\
131 / 4 \\
151 / 4\end{array}$ & $\begin{array}{l}15 \\
171 / 2 \\
201 / 2 \\
23 \\
251 / 2\end{array}$ & $\begin{array}{l}15 / 8 \\
17 / 8 \\
2 \\
21 / 8 \\
21 / 4\end{array}$ & $\begin{array}{l}15 / 16 \\
11 / 8 \\
11 / 4\end{array}$ & $\begin{array}{l}101 / 4 \\
125 / 8 \\
143 / 4 \\
161 / 4 \\
183 / 8\end{array}$ & $\begin{array}{l}23 / 16 \\
23 / 8 \\
29 / 16 \\
2^{1} 1 / 16 \\
2^{7} / 8\end{array}$ & $\begin{array}{l}1.71 \\
1.92 \\
2.12 \\
2.25 \\
2.45\end{array}$ & $\begin{array}{l}1115 / 16 \\
141 / 16 \\
167 / 16 \\
1815 / 16 \\
211 / 16\end{array}$ & $\begin{array}{r}56 \\
81 \\
115 \\
155 \\
195\end{array}$ & $\begin{array}{r}75 \\
120 \\
155 \\
210 \\
270\end{array}$ \\
\hline $\begin{array}{ll}\begin{array}{l}6 \\
6\end{array} & 18 \\
6 & 20 \\
6 & 24 \\
6 & 30\end{array}$ & $\begin{array}{l}17 \\
19 \\
23 \\
29\end{array}$ & $\begin{array}{l}28 \\
301 / 2 \\
36 \\
43\end{array}$ & $\begin{array}{l}23 / 8 \\
21 / 2 \\
23 / 4 \\
3\end{array}$ & $\begin{array}{l}13 / 8 \\
11 / 2 \\
15 \% 8 \\
2\end{array}$ & $\begin{array}{l}205 / 8 \\
223 / 4 \\
273 / 8\end{array}$ & $\begin{array}{l}31 / 8 \\
35 / 16 \\
3^{1} 1 / 16\end{array}$ & $\begin{array}{l}2.65 \\
2.85 \\
3.25 \\
\end{array}$ & $\begin{array}{l}235 / 16 \\
25 \% / 16 \\
305 / 16 \\
373 / 16\end{array}$ & $\begin{array}{r}240 \\
300 \\
450 \\
\end{array}$ & $\begin{array}{r}350 \\
440 \\
670 \\
1,070\end{array}$ \\
\hline
\end{tabular}

${ }_{1}^{1}$ All 250-pound cast-iron standard flanges have a $1 / 16$-inch raised face. This raised face is included in the minimum thickness of flange dimensions.

'Sizes 14 inches and larger are to be used with outside diameter pipe of the same sizes.

3 All blind flanges for sizes 10 inches (171/2 inches outside diameter) and larger must be dished, with inside radius equal to the port diameter.

4 This column is the same as column for effective thread (E) in Table 1 of the American Pipe Thread Standard, published by the American ${ }^{2}$ Standards Association except in sizes 11/4, 132, and 2 inches.

$s$ All weights listed are for flanges faced only, based upon minimum thicknesses given in the table above without allowances for variation. Cast iron is considered to weigh 0.26 pound per cubic inch.

6 Outside diameter.

\section{TABLE 4.-Dimensions of elbows}
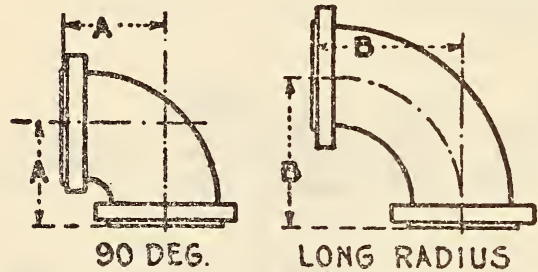

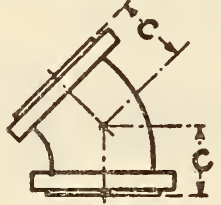

45 DEG.

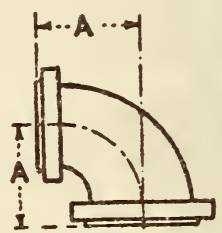

REDUCING

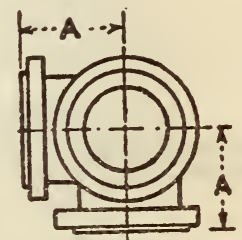

SIDE OUTLET

Figdre 232

[All dimensions given in inches]

\begin{tabular}{|c|c|c|c|c|c|c|c|c|}
\hline $\begin{array}{l}\text { Nominal } \\
\text { pipe } \\
\text { size }\end{array}$ & $\begin{array}{c}\text { Mini- } \\
\text { mum } \\
\text { inside } \\
\text { diameter } \\
\text { of fitting }\end{array}$ & $\begin{array}{c}\text { Center to } \\
\text { face el- } \\
\text { bow } 1234 \\
\text { A }\end{array}$ & $\begin{array}{l}\text { Center } \\
\text { to face } \\
\text { long- } \\
\text { radius } \\
\text { elbow }{ }^{1} \\
\text { B }\end{array}$ & $\begin{array}{l}\text { Center } \\
\text { to face } \\
45^{\circ} \text { el- } \\
\text { bow } 13 \\
\text { C }\end{array}$ & $\begin{array}{l}\text { Diam- } \\
\text { eter of } \\
\text { flange } \\
\text { D }\end{array}$ & $\begin{array}{l}\text { Thick- } \\
\text { ness of } \\
\text { flange } \\
\text { (mini- } \\
\text { mum) } 1\end{array}$ & $\begin{array}{l}\text { Diam- } \\
\text { eter of } \\
\text { raised } \\
\text { face } 1\end{array}$ & $\begin{array}{l}\text { Metal } \\
\text { thick- } \\
\text { ness of } \\
\text { body } \\
\text { (mini- } \\
\text { mum) }\end{array}$ \\
\hline $\begin{array}{l}1 \\
11 / 4 \\
11 / 2 \\
2 \\
21 / 2\end{array}$ & $\begin{array}{l}1 \\
11 / 4 \\
11 / 2 \\
2 \\
21 / 2\end{array}$ & $\begin{array}{l}4 \\
41 / 4 \\
41 / 2 \\
5 \\
51 / 2\end{array}$ & $\begin{array}{l}5 \\
51 / 2 \\
6 \\
61 / 2 \\
7\end{array}$ & $\begin{array}{l}2 \\
21 / 2 \\
23 / 4 \\
3 \\
31 / 2\end{array}$ & $\begin{array}{l}47 / 8 \\
51 / 4 \\
61 / 8 \\
61 / 2 \\
71 / 2\end{array}$ & $\begin{array}{l}11 / 16 \\
3 / 4 \\
13 / 16 \\
7 / 8 \\
1^{7 / 8}\end{array}$ & $\begin{array}{l}2^{11} 1 / 16 \\
31 / 16 \\
39 / 16 \\
43 / 16 \\
415 / 16\end{array}$ & $\begin{array}{l}1 / 2 \\
1 / 2 \\
1 / 2 \\
1 / 2 \\
9 / 16\end{array}$ \\
\hline $\begin{array}{l}3 \\
31 / 2 \\
4 \\
5 \\
6\end{array}$ & $\begin{array}{l}3 \\
31 / 2 \\
4 \\
5 \\
6\end{array}$ & $\begin{array}{l}6 \\
61 / 2 \\
7 \\
8 \\
81 / 2\end{array}$ & $\begin{array}{l}73 / 4 \\
81 / 2 \\
9 \\
101 / 4 \\
111 / 2\end{array}$ & $\begin{array}{l}31 / 2 \\
4 \\
41 / 2 \\
5 \\
51 / 2\end{array}$ & $\begin{array}{l}81 / 4 \\
9 \\
10 \\
11 \\
121 / 2\end{array}$ & $\begin{array}{l}11 / 8 \\
13 / 16 \\
11 / 4 \\
13 / 8 \\
17 / 16\end{array}$ & $\begin{array}{l}5^{11} / 16 \\
65 / 16 \\
6^{15} / 16 \\
85 / 16 \\
9^{11} 1 / 16\end{array}$ & $\begin{array}{l}9 / 16 \\
9 / 16 \\
5 / 8 \\
11 / 16 \\
3 / 4\end{array}$ \\
\hline $\begin{array}{r}8 \\
10 \\
12 \\
514 \\
516\end{array}$ & $\begin{array}{l}8 \\
10 \\
12 \\
131 / 4 \\
151 / 4\end{array}$ & $\begin{array}{l}10 \\
111 / 2 \\
13 \\
15 \\
161 / 2\end{array}$ & $\begin{array}{l}14 \\
161 / 2 \\
19 \\
211 / 2 \\
24\end{array}$ & $\begin{array}{l}6 \\
7 \\
8 \\
81 / 2 \\
91 / 2\end{array}$ & $\begin{array}{l}15 \\
171 / 2 \\
201 / 2 \\
23 \\
251 / 2\end{array}$ & $\begin{array}{l}15 / 8 \\
17 / 8 \\
2 \\
21 / 8 \\
21 / 4\end{array}$ & $\begin{array}{l}1115 / 16 \\
141 / 16 \\
167 / 16 \\
1815 / 16 \\
211 / 16\end{array}$ & $\begin{array}{l}13 / 16 \\
15 / 16 \\
1 \\
138 \\
134\end{array}$ \\
\hline $\begin{array}{l}518 \\
520 \\
524 \\
530\end{array}$ & $\begin{array}{l}17 \\
19 \\
23 \\
29\end{array}$ & $\begin{array}{l}18 \\
191 / 2 \\
221 / 2 \\
271 / 2\end{array}$ & $\begin{array}{l}261 / 2 \\
29 \\
34 \\
411 / 2\end{array}$ & $\begin{array}{l}10 \\
101 / 2 \\
12 \\
15\end{array}$ & $\begin{array}{l}28 \\
301 / 2 \\
36 \\
43\end{array}$ & $\begin{array}{l}23 / 8 \\
21 / 2 \\
23 / 4 \\
3\end{array}$ & $\begin{array}{l}235 / 16 \\
259 / 16 \\
305 / 16 \\
373 / 16\end{array}$ & $\begin{array}{l}13 / 8 \\
11 / 2 \\
15 / 8 \\
2\end{array}$ \\
\hline
\end{tabular}

1 All 250-pound cast-iron standard flanges have a 1/16-inch raised face. This raised face is included in the face-to-face, center-to-face, and the minimum thickness of flange dimensions.

Reducing elbows and side-outlet elbows carry the same dimensions center to face as regular straight size-elbows corresponding to the

size of the larger opening. and up to $90^{\circ}$ inclusive shall have the same center-to-face dimensions given for $90^{\circ}$ elbows. The angle designation of an elbow is its deflection from straight-line flow and is the angle between the flange faces.

1 Side-outlet elbows shall have all openings on intersecting center lines.

outside diameter. 
(Illustrations showing dimensions of tees and crosses (straight sizes) are identical with those for 125-pound cast-iron fittings (see fig. 227) except for the addition, in the case of 250-pound fittings, of lines showing raised faces, similar to the raised faces shown in the illustrations accompanying Table 4 in this specification.)

TABLE 5.-Dimensions of tees and crosses (straight

[All dimensions given in inches]

\begin{tabular}{|c|c|c|c|c|c|c|c|}
\hline $\begin{array}{l}\text { Nom- } \\
\text { inal } \\
\text { pipe } \\
\text { size 1 }\end{array}$ & $\begin{array}{l}\text { Mini- } \\
\text { mum } \\
\text { inside } \\
\text { diam- } \\
\text { eter of } \\
\text { fitting }\end{array}$ & $\begin{array}{l}\text { Center-to- } \\
\text { face tees } \\
\text { and } \\
\text { crosses } 198 \\
\text { A }\end{array}$ & $\begin{array}{c}\text { Face-to- } \\
\text { face tees } \\
\text { and } \\
\text { crosses } 198 \\
\text { AA }\end{array}$ & $\begin{array}{l}\text { Diam- } \\
\text { eter of } \\
\text { flange }\end{array}$ & $\begin{array}{l}\text { Thick- } \\
\text { ness } \\
\text { of } \\
\text { flange } \\
\text { (mini- } \\
\text { mum) }\end{array}$ & $\begin{array}{c}\text { Diam- } \\
\text { eter } \\
\text { of } \\
\text { raised } \\
\text { face }^{2}\end{array}$ & $\begin{array}{l}\text { Metal } \\
\text { thick- } \\
\text { ness } \\
\text { of } \\
\text { body } \\
\text { (mini- } \\
\text { mum) }\end{array}$ \\
\hline \multicolumn{2}{|c|}{$\begin{array}{c}\text { Same as in } \\
\text { Table } 4 .\end{array}$} & $\begin{array}{l}\text { Same as } \\
\text { center to } \\
\text { face el- } \\
\text { bow (A) } \\
\text { in Table } \\
4 .\end{array}$ & $\begin{array}{l}8 \\
8132 \\
9 \\
10 \\
11 \\
12 \\
13 \\
14 \\
16 \\
17 \\
20 \\
23 \\
26 \\
30 \\
33 \\
36 \\
39 \\
45 \\
55\end{array}$ & \multicolumn{4}{|c|}{ Same as in Table 4.} \\
\hline
\end{tabular}

1 Tees, side-outlet tees, and crosses, 16 inches and smaller, reducing on the outlet, have the same dimensions center to face, and face to face as straight-size fittings, corresponding to the size of the larger opening. Sizes 18 inches and larger, reducing on the outlet, are made in two lengths, depending on the size of the outlet as given in Table 6.

3 All 250 -pound cast-iron standard flanges have a $1 / 16$-inch raised face. This raised face is included in the face-to-face, center-to-face, and the minimum thickness of flange dimensions.

$\checkmark$ Tees and crosses, reducing on run only, carry same dimensions center to face and face to face as straight-size fittings corresponding to size of the larger opening.

(Illustrations showing dimensions of reducing tees and reducing crosses (short-body patterns) are identical with those for 125-pound cast-iron fittings (see fig. 226) except for the addition, in the case of 250-pound fittings, of lines showing raised faces, similar to the raised faces shown in the illustrations accompanying Table 4 in this specification.)

\section{TABLE 6.-Dimensions of reducing tees and reducing crosses (short-body patterns) 123}

[All dimensions given in inches]

\begin{tabular}{|c|c|c|c|c|}
\hline $\begin{array}{l}\text { Nominal } \\
\text { pipe size } e^{3}\end{array}$ & $\begin{array}{l}\text { Size of } \\
\text { outlet } \\
\text { and } \\
\text { smaller } 4\end{array}$ & $\begin{array}{c}\text { Center- } \\
\text { to-face } \\
\text { run }{ }^{5} \\
\text { H }\end{array}$ & $\begin{array}{l}\text { Face-to- } \\
\text { face run } \\
\text { НH } \\
\text { НH }\end{array}$ & $\begin{array}{c}\text { Center- } \\
\text { to-face } \\
\text { outlet }{ }^{5} \text { B }\end{array}$ \\
\hline $\begin{array}{l}1 \\
11 / 4 \\
11 / 2 \\
2 \\
21 / 2\end{array}$ & $\begin{array}{l}1 \\
11 / 4 \\
11 / 2 \\
21 / 2 \\
21 / 2\end{array}$ & $\begin{array}{l}4 \\
41 / 4 \\
41 / 2 \\
5 \\
51 / 2\end{array}$ & $\begin{array}{l}8 \\
81 / 2 \\
9 \\
10 \\
11\end{array}$ & $\begin{array}{l}4 \\
41 / 4 \\
41 / 2 \\
5 \\
51 / 2\end{array}$ \\
\hline $\begin{array}{l}3 \\
31 / 2 \\
4 \\
5 \\
6\end{array}$ & $\begin{array}{l}3 \\
31 / 2 \\
4 \\
5 \\
6\end{array}$ & $\begin{array}{l}6 \\
61 / 2 \\
7 \\
8 \\
81 / 2\end{array}$ & $\begin{array}{l}12 \\
13 \\
14 \\
16 \\
17\end{array}$ & $\begin{array}{l}6 \\
61 / 2 \\
7 \\
8 \\
81 / 2\end{array}$ \\
\hline $\begin{array}{r}8 \\
10 \\
12 \\
714 \\
716\end{array}$ & $\begin{array}{r}8 \\
10 \\
12 \\
14 \\
16\end{array}$ & $\begin{array}{l}10 \\
111 / 2 \\
13 \\
15 \\
161 / 2\end{array}$ & $\begin{array}{l}20 \\
23 \\
26 \\
30 \\
33\end{array}$ & $\begin{array}{l}10 \\
111 / 2 \\
13 \\
15 \\
161 / 2\end{array}$ \\
\hline $\begin{array}{l}? 18 \\
? 20 \\
? 24 \\
730\end{array}$ & $\begin{array}{l}12 \\
14 \\
16 \\
20\end{array}$ & $\begin{array}{l}14 \\
151 / 2 \\
17 \\
201 / 2\end{array}$ & $\begin{array}{l}28 \\
31 \\
34 \\
41\end{array}$ & $\begin{array}{l}17 \\
181 / 2 \\
211 / 2 \\
251 / 2\end{array}$ \\
\hline
\end{tabular}

All above reducing fittings, 1 to 16 inches inclusive, have the same center-to-face dimensions as straight fittings of the larger size.

3 Fittings reducing on the run only carry same dimensions center to face and face to face as straight fittings corresponding to size of the larger opening. Tees increasing on outlet, known as bull-head tees, will have same center-to-face and face-to-face dimensions as a straight fitting of the size of the outlet. For example: A 12 by 12 by 18 inch tee will be governed by the dimensions of the 18-inch long-body tee, given in Table 5; namely 18 inches center to face of all openings and 36 inches face to face.

3 Short-body patterns are used for sizes 18 inches and larger.

1 Long-body patterns are used when outlets are larger than given in the above table, and, therefore have the same dimensions as straight-size fittings.

5 All 250 -pound cast-iron standard flanges have a $1 / 16$-inch raised face. This raised face is included in the face-to-face, center-to-face, and the minimum thickness of flange dimensions.

6 In a side-outlet tee the larger of the two side outlets governs the center to face dimension $J$.

Outside diameter.

(Illustrations showing dimensions of laterals, reducers, and true Y's (straight sizes) are identical with those for 125-pound cast-iron fittings (see fig. 227) except for the addition, in the case of 250pound fittings, of lines showing raised faces, similar to the raised faces shown in illustrations accompanying Table 4 in this specification.) 
TABLE 7.-Dimensions of laterals, reducers, and true $\mathrm{Y}^{\prime}$ 's (straight sizes)

[All dimensions given in inches]

\begin{tabular}{|c|c|c|c|c|c|c|c|c|c|c|}
\hline $\begin{array}{l}\text { Nominal } \\
\text { pipe size }\end{array}$ & $\begin{array}{l}\text { Mini- } \\
\text { mum } \\
\text { inside } \\
\text { diameter } \\
\text { of fitting }\end{array}$ & $\begin{array}{c}\text { Center- } \\
\text { to-face } \\
\text { true Y } 1 \\
\\
\text { A }\end{array}$ & $\begin{array}{c}\text { Face-to- } \\
\text { face lat- } \\
\text { eral } 12 \\
\text { D }\end{array}$ & $\begin{array}{c}\text { Center- } \\
\text { to-face } \\
\text { lateral } \\
1234 \\
\\
\text { E }\end{array}$ & $\begin{array}{c}\text { Center- } \\
\text { to-face } \\
\text { lateral } \\
\text { true } Y \\
134 \\
F\end{array}$ & $\begin{array}{l}\text { Face-to- } \\
\text { face re- } \\
\text { ducer is } \\
\text { G }\end{array}$ & $\begin{array}{l}\text { Diameter } \\
\text { of flange }\end{array}$ & $\begin{array}{l}\text { Thick- } \\
\text { ness of } \\
\text { flange }{ }^{2} \\
\text { (mini- } \\
\text { mum) }\end{array}$ & $\begin{array}{c}\text { Diameter } \\
\text { of raised } \\
\text { face } 1\end{array}$ & $\begin{array}{c}\text { Metal } \\
\text { thickness } \\
\text { of body } \\
\text { (mini- } \\
\text { mum) }\end{array}$ \\
\hline $\begin{array}{l}1 \\
11 / 4 \\
11 / 2 \\
2 \\
21 / 2\end{array}$ & $\begin{array}{l}1 \\
11 / 4 \\
11 / 2 \\
2 \\
21 / 2\end{array}$ & $\begin{array}{l}4 \\
41 / 4 \\
41 / 2 \\
5 \\
51 / 2\end{array}$ & $\begin{array}{l}81 / 2 \\
91 / 2 \\
11^{11 / 2} \\
11^{1 / 2} \\
13\end{array}$ & $\begin{array}{c}61 / 2 \\
71 / 4 \\
81 / 2 \\
9 \\
101 / 2\end{array}$ & $\begin{array}{l}2 \\
21 / 4 \\
21 / 2 \\
21 / 2 \\
21 / 2\end{array}$ & $\begin{array}{l}5 \\
51 / 2\end{array}$ & $\begin{array}{l}47 / 8 \\
51 / 4 \\
61 / 8 \\
61 / 2 \\
71 / 2\end{array}$ & $\begin{array}{r}11 / 16 \\
3 / 4 \\
13 / 16 \\
7 / 8 \\
1\end{array}$ & $\begin{array}{l}211 / 16 \\
31 / 16 \\
3916 \\
43116 \\
415 / 16\end{array}$ & $\begin{array}{l}1 / 2 \\
1 / 2 \\
1 / 2 \\
1 / 2 \\
\% 16\end{array}$ \\
\hline $\begin{array}{l}3 \\
31 / 2 \\
4 \\
5 \\
6\end{array}$ & $\begin{array}{l}3 \\
31 / 2 \\
4 \\
5 \\
6\end{array}$ & $\begin{array}{l}6 \\
61 / 2 \\
7 \\
8 \\
81 / 2\end{array}$ & $\begin{array}{l}14 \\
151 / 2 \\
161 / 2 \\
181 / 2 \\
211 / 2\end{array}$ & $\begin{array}{l}11 \\
121 / 2 \\
131 / 2 \\
15 \\
171 / 2\end{array}$ & $\begin{array}{l}3 \\
3 \\
3 \\
31 / 2 \\
4\end{array}$ & $\begin{array}{l}6 \\
61 / 2 \\
7 \\
8 \\
9\end{array}$ & $\begin{array}{l}81 / 4 \\
9 \\
10 \\
11 \\
121 / 2\end{array}$ & $\begin{array}{l}11 / 8 \\
13 / 10 \\
11 / 4 \\
138 \\
17 / 16\end{array}$ & $\begin{array}{l}511 / 16 \\
65 / 16 \\
61516 \\
8516 \\
911 / 16\end{array}$ & $\begin{array}{l}9 / 16 \\
9 / 16 \\
58 \\
11 / 16 \\
3 / 4\end{array}$ \\
\hline $\begin{array}{rr} & 8 \\
& 10 \\
& 12 \\
614 \\
616\end{array}$ & $\begin{array}{l}8 \\
10 \\
12 \\
131 / 4 \\
151 / 4\end{array}$ & $\begin{array}{l}10 \\
111 / 2 \\
13 \\
15 \\
161 / 2\end{array}$ & $\begin{array}{l}251 / 2 \\
291 / 2 \\
331 / 2 \\
371 / 2 \\
42\end{array}$ & $\begin{array}{l}201 / 2 \\
24 \\
271 / 2 \\
31 \\
341 / 2\end{array}$ & $\begin{array}{l}5 \\
51 / 2 \\
6 \\
61 / 2 \\
71 / 2\end{array}$ & $\begin{array}{l}11 \\
12 \\
14 \\
16 \\
18\end{array}$ & $\begin{array}{l}15 \\
171 / 2 \\
201 / 2 \\
23 \\
251 / 2\end{array}$ & $\begin{array}{l}15 / 8 \\
17 / 8 \\
2 \\
21 / 8 \\
21 / 4\end{array}$ & $\begin{array}{l}11^{15} / 16 \\
141 / 16 \\
167 / 16 \\
1815 / 16 \\
211 / 16\end{array}$ & $\begin{array}{l}13 / 10 \\
15 / 10 \\
1 \\
11 / 8 \\
11 / 4\end{array}$ \\
\hline 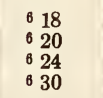 & $\begin{array}{l}17 \\
19 \\
23 \\
29\end{array}$ & $\begin{array}{l}18 \\
191 / 2 \\
221 / 2\end{array}$ & $\begin{array}{l}451 / 2 \\
49 \\
571 / 2\end{array}$ & $\begin{array}{l}371 / 2 \\
401 / 2 \\
471 / 2\end{array}$ & $\begin{array}{l}8 \\
81 / 2 \\
10\end{array}$ & $\begin{array}{l}19 \\
20 \\
24 \\
30\end{array}$ & $\begin{array}{l}28 \\
301 / 2 \\
36 \\
43\end{array}$ & $\begin{array}{l}23 / 8 \\
21 / 2 \\
23 / 4 \\
3\end{array}$ & $\begin{array}{l}235 / 16 \\
25 \% / 16 \\
305 / 16 \\
373 / 16\end{array}$ & $\begin{array}{l}13 / 8 \\
11 / 2 \\
15 / 8 \\
2\end{array}$ \\
\hline
\end{tabular}

1 All 250-pound cast-iron standard flanges have a $1 / 16$-inch raised face. This raised face is included in the face-to-face, center-to-face, and the minimum thickness of flang

${ }^{2}$ Laterals, both straight and reducing, sizes 8 inches and larger, shall be reinforced to compensate for the inherent weakness in the casting design.

${ }_{3}$ Laterals, 16 inches and smaller, reducing on the outlet or branch, use the same dimensions center-to-face and face-to-face as straight-size fittings corresponding to size of the larger opening. Sizes 18 inches and larger reducing on the outlet or branch, are made in two lengths depending on the size of the outlet as given in Table 8.

- Laterals, reducing on the run only, carry same dimensions center-to-face and face-to-face as straight-size fittings corresponding to size of the larger opening.

8 R'educers and eccentric reducers, for all reductions, use the same face-to-face dimensions given in the above table of dimensions for the larger opening.

outside diameter.

(Illustrations showing dimensions of reducing laterals (short-body pattern) are identical with those for 125-pound cast-iron fittings (see fig. 228) except for the addition, in the case of 250-pound fittings, of lines showing raised faces, similar to the raised faces shown in illustrations accompanying Table 4 in this specification.)

TABLE 8.-Dimensions of reducing laterals (shortbody pattern)

[All dimensions given in inches]

\begin{tabular}{|c|c|c|c|c|c|}
\hline $\begin{array}{l}\text { Nominal } \\
\text { pipe size }\end{array}$ & $\begin{array}{l}\text { Size of } \\
\text { branch } \\
\text { and } \\
\text { smaller }\end{array}$ & $\begin{array}{c}\text { Face-to- } \\
\text { face run } \\
\text { L }\end{array}$ & $\begin{array}{c}\text { Center- } \\
\text { to-face } \\
\text { run } \\
\text { M }\end{array}$ & $\begin{array}{c}\text { Center- } \\
\text { to-face } \\
\text { run } \\
\mathrm{N}\end{array}$ & $\begin{array}{c}\text { Center- } \\
\text { to-face } \\
\text { branch } \\
\text { P }\end{array}$ \\
\hline $\begin{array}{l}1 \\
11 / 4 \\
11 / 2 \\
2 \\
21 / 2\end{array}$ & $\begin{array}{l}1 \\
11 / 4 \\
11 / 2 \\
2 \\
21 / 2\end{array}$ & $\begin{array}{l}81 / 2 \\
91 / 2 \\
11 \\
111 / 2 \\
13\end{array}$ & $\begin{array}{c}61 / 2 \\
71 / 4 \\
81 / 2 \\
9 \\
101 / 2\end{array}$ & $\begin{array}{l}2 \\
21 / 4 \\
21 / 2 \\
21 / 2 \\
21 / 2\end{array}$ & $\begin{array}{c}61 / 2 \\
71 / 4 \\
81 / 2 \\
9 \\
101 / 2\end{array}$ \\
\hline $\begin{array}{l}3 \\
31 / 2 \\
4 \\
5 \\
6\end{array}$ & $\begin{array}{l}3 \\
31 / 2 \\
4 \\
5 \\
6\end{array}$ & $\begin{array}{l}14 \\
151 / 2 \\
161 / 2 \\
181 / 2 \\
211 / 2\end{array}$ & $\begin{array}{l}11 \\
121 / 2 \\
131 / 2 \\
15 \\
171 / 2\end{array}$ & $\begin{array}{l}3 \\
3 \\
3 \\
31 / 2 \\
4\end{array}$ & $\begin{array}{l}11 \\
121 / 2 \\
131 / 2 \\
15 \\
171 / 2\end{array}$ \\
\hline $\begin{array}{r}8 \\
10 \\
12 \\
114\end{array}$ & $\begin{array}{r}8 \\
10 \\
12 \\
14\end{array}$ & $\begin{array}{l}251 / 2 \\
291 / 2 \\
331 / 2 \\
371 / 2\end{array}$ & $\begin{array}{l}201 / 2 \\
24 \\
271 / 2 \\
31\end{array}$ & $\begin{array}{l}5 \\
51 / 2 \\
6 \\
61 / 2\end{array}$ & $\begin{array}{l}201 / 2 \\
24 \\
271 / 2 \\
31\end{array}$ \\
\hline $\begin{array}{l}116 \\
1118 \\
3120 \\
124\end{array}$ & $\begin{array}{r}16 \\
8 \\
10 \\
12\end{array}$ & $\begin{array}{l}42 \\
34 \\
37 \\
44\end{array}$ & $\begin{array}{l}341 / 2 \\
31 \\
34 \\
41\end{array}$ & $\begin{array}{l}71 / 2 \\
3 \\
3 \\
3\end{array}$ & $\begin{array}{l}341 / 2 \\
321 / 2 \\
36 \\
43\end{array}$ \\
\hline
\end{tabular}

1 Outside diameter.

(Notes are identical with those for 125-pound fittings, with the following addition:) All 250-pound cast-iron standard flanges have a 1/16-inch raised face. This raised face is included in the face-to-face, center-to-face, and the minimum thickness of flange dimensions.

(Illustrations showing dimensions of base elbows and base tees are identical with those for 125-pound cast-iron fittings (see fig. 229) except for the addition, in the case of 250 -pound fittings, of lines showing raised faces, similar to the raised faces shown in illustrations accompanying Table 4 in this specification.)

\section{TABLE 9.-Dimensions of base elbows and base tees} [All dimensions given in inches]

\begin{tabular}{|c|c|c|c|c|c|}
\hline $\begin{array}{l}\text { Nominal } \\
\text { pipe size }\end{array}$ & $\begin{array}{c}\text { Center } \\
\text { to base } \\
\text { R }\end{array}$ & $\begin{array}{c}\text { Diame- } \\
\text { ter of } \\
\text { round } \\
\text { base } \\
\mathrm{S}\end{array}$ & $\begin{array}{c}\text { Thick- } \\
\text { ness of } \\
\text { base } \\
\text { T }\end{array}$ & $\begin{array}{l}\text { Thick- } \\
\text { ness of } \\
\text { ribs } \\
\text { U }\end{array}$ & $\begin{array}{l}\text { Size of } \\
\text { support- } \\
\text { ing pipe } \\
\text { for base }\end{array}$ \\
\hline $\begin{array}{l}1 \\
11 / 4 \\
11 / 2 \\
2 \\
21 / 2\end{array}$ & $\begin{array}{l}33 / 4 \\
4 \\
41 / 8 \\
41 / 2 \\
43 / 4\end{array}$ & $\begin{array}{l}4 \\
4 \\
47 / 8 \\
51 / 4 \\
51 / 4\end{array}$ & $\begin{array}{r}5 / 8 \\
5 / 8 \\
11 / 16 \\
3 / 4 \\
3 / 4\end{array}$ & $\begin{array}{l}1 / 2 \\
1 / 2 \\
1 / 2 \\
1 / 2 \\
1 / 2\end{array}$ & $\begin{array}{l}3 / 4 \\
3 / 4 \\
1^{31 / 4} \\
11 / 4\end{array}$ \\
\hline $\begin{array}{l}3 \\
31 / 2 \\
4 \\
5 \\
6\end{array}$ & $\begin{array}{l}51 / 4 \\
55 / 8 \\
6 \\
63 / 4 \\
71 / 2\end{array}$ & $\begin{array}{l}61 / 8 \\
618 \\
61 / 2 \\
71 / 2 \\
71 / 2\end{array}$ & $\begin{array}{r}13 / 16 \\
13 / 16 \\
7 / 8 \\
1 \\
1\end{array}$ & $\begin{array}{l}5 / 8 \\
5 / 8 \\
5 / 8 \\
3 / 4 \\
3 / 4\end{array}$ & $\begin{array}{l}11 / 2 \\
11 / 2 \\
2 \\
21 / 2 \\
21 / 2\end{array}$ \\
\hline $\begin{array}{r}8 \\
10 \\
12 \\
114\end{array}$ & $\begin{array}{l}9 \\
101 / 2 \\
12 \\
131 / 2\end{array}$ & $\begin{array}{l}10 \\
10 \\
121 / 2 \\
121 / 2\end{array}$ & $\begin{array}{l}11 / 4 \\
11 / 4 \\
17 / 16 \\
17 / 16\end{array}$ & $1^{7 / 8}$ & $\begin{array}{l}4 \\
4 \\
6 \\
6\end{array}$ \\
\hline $\begin{array}{ll}1 & 16 \\
1 & 18 \\
1 & 20 \\
1 & 24\end{array}$ & $\begin{array}{l}143 / 4 \\
161 / 4 \\
177 / 8 \\
203 / 4\end{array}$ & $\begin{array}{l}121 / 2 \\
15 \\
15 \\
171 / 2\end{array}$ & $\begin{array}{l}17 / 16 \\
15 / 8 \\
15 / 8 \\
17 / 8\end{array}$ & $\begin{array}{l}11 / 8 \\
11 / 8 \\
114 \\
11 / 4\end{array}$ & $\begin{array}{r}6 \\
8 \\
8 \\
10\end{array}$ \\
\hline
\end{tabular}

1 Outside diameter.

(Notes are identical with those for 125-pound fittings.

(Illustrations showing dimensions of anchorage bases for tees (straight sizes) are identical with those for 125-pound cast-iron fittings (see fig. 230) except for the addition, in the case of 250-pound fittings, of lines showing raised faces, similar to the raised faces shown in illustrations accompanying Table 4 in this specification.) 
TABLE 10.-Dimensions of anchorage bases for tees (straight sizes)

[All dimensions given in inches]

\begin{tabular}{|c|c|c|c|c|c|c|c|c|c|}
\hline $\begin{array}{l}\text { Size of } \\
\text { run }\end{array}$ & $\begin{array}{c}\text { Center } \\
\text { to base } \\
\text { A }\end{array}$ & $\begin{array}{c}\text { Length } \\
\text { of square } \\
\text { base }\end{array}$ & $\begin{array}{l}\text { Thick- } \\
\text { ness of } \\
\text { of base } \\
\text { C }\end{array}$ & $\begin{array}{c}\text { Number } \\
\text { of ribs } \\
\text { D }\end{array}$ & $\begin{array}{l}\text { Centers } \\
\text { of ribs } \\
\text { and } \\
\text { inside } \\
\text { bolts } \\
\text { E }\end{array}$ & $\begin{array}{l}\text { Thick- } \\
\text { ness of } \\
\text { ribs } \\
\text { F }\end{array}$ & $\begin{array}{c}\text { Diameter } \\
\text { of bolts } \\
\text { G }\end{array}$ & $\begin{array}{c}\text { Longitu- } \\
\text { dinal } \\
\text { centers } \\
\text { from end } \\
\text { to second } \\
\text { bolt } \\
\text { H }\end{array}$ & $\begin{array}{c}\text { Trans- } \\
\text { verse } \\
\text { bolt } \\
\text { centers } \\
\text { K }\end{array}$ \\
\hline $\begin{array}{l}21 / 2 \\
3 \\
31 / 2 \\
4 \\
5\end{array}$ & $\begin{array}{l}43 / 4 \\
51 / 4 \\
55 / 8 \\
6 \\
63 / 4\end{array}$ & $\begin{array}{l}71 / 2 \\
81 / 4 \\
9 \\
10 \\
11\end{array}$ & $\begin{array}{r}3 / 4 \\
13 / 10 \\
15 / 16 \\
1516 \\
1^{1516}\end{array}$ & $\begin{array}{l}1 \\
1 \\
1 \\
2 \\
2\end{array}$ & $\begin{array}{r}4 \\
5\end{array}$ & $\begin{array}{r}9 / 10 \\
9 / 16 \\
916 \\
5 / 8 \\
11 / 10\end{array}$ & $\begin{array}{l}58 \\
58 \\
58 \\
58 \\
78\end{array}$ & $\begin{array}{l}3 / 4 \\
61 / 2 \\
71 / 4 \\
37 / 8 \\
43 / 8\end{array}$ & $\begin{array}{l}53 / 4 \\
61 / 2 \\
71 / 4 \\
73 / 4 \\
83 / 4\end{array}$ \\
\hline $\begin{array}{r}6 \\
8 \\
10 \\
12 \\
114\end{array}$ & $\begin{array}{c}71 / 2 \\
9 \\
101 / 2 \\
12 \\
131 / 2\end{array}$ & $\begin{array}{l}121 / 2 \\
15 \\
171 / 2 \\
201 / 2 \\
23\end{array}$ & $\begin{array}{l}11110 \\
118 \\
11 / 4 \\
11 / 4 \\
13 / 8\end{array}$ & $\begin{array}{l}2 \\
2 \\
3 \\
3 \\
3\end{array}$ & $\begin{array}{l}6 \\
8 \\
5 \\
6 \\
7\end{array}$ & $\begin{array}{r}3 / 4 \\
13 \frac{16}{15} \\
1516 \\
1138\end{array}$ & $\begin{array}{l}1^{7 / 8} \\
118 \\
11 / 4 \\
11 / 4\end{array}$ & $\begin{array}{l}51 / 8 \\
61 / 4 \\
47 / 8 \\
53 / 4 \\
61 / 2\end{array}$ & $\begin{array}{l}101 / 4 \\
121 / 2 \\
143 / 4 \\
171 / 2 \\
20\end{array}$ \\
\hline $\begin{array}{ll}1 & 16 \\
1 & 18 \\
1 & 20 \\
1 & 24 \\
1 & 30\end{array}$ & $\begin{array}{l}143 / 4 \\
161 / 4 \\
177 / 8 \\
203 / 4 \\
243 / 4\end{array}$ & $\begin{array}{l}251 / 2 \\
28 \\
301 / 2 \\
36 \\
43\end{array}$ & $\begin{array}{l}19 / 16 \\
111 / 16 \\
17 / 8 \\
21 / 16 \\
25 / 16\end{array}$ & $\begin{array}{l}3 \\
3 \\
3 \\
3 \\
4\end{array}$ & $\begin{array}{r}8 \\
9 \\
10 \\
12 \\
10\end{array}$ & $\begin{array}{l}11 / 4 \\
13 / 8 \\
11 / 2 \\
15 / 8 \\
2\end{array}$ & $\begin{array}{l}13 / 8 \\
13 / 8 \\
11 / 2 \\
158 \\
13 / 4\end{array}$ & $\begin{array}{l}7 \\
75 / 8 \\
83 / 8 \\
97 / 8 \\
9\end{array}$ & $\begin{array}{l}22 \\
241 / 4 \\
263 / 4 \\
313 / 4 \\
38\end{array}$ \\
\hline
\end{tabular}

1 Outside diameter.

(Notes are identical with those for 125-pound fittings.)

(Illustrations showing dimensions of anchorage/lines showing raised faces, similar to the raised faces bases for reducing tees are identical with those for shown in illustrations accompanying Table 4 in 125-pound cast-iron fittings (see fig. 231) except this specification.)

for the addition, in the case of 250-pound fittings, of

TABLE 11.-Dimensions of anchorage bases for reducing tees

[All dimensions given in inches]

\begin{tabular}{|c|c|c|c|c|c|c|c|c|c|c|c|}
\hline $\begin{array}{l}\text { Size of } \\
\text { run }\end{array}$ & $\begin{array}{l}\text { Outlet } \\
\text { sizes and } \\
\text { smaller }\end{array}$ & $\begin{array}{c}\text { Center } \\
\text { to base } \\
\text { A }\end{array}$ & $\begin{array}{c}\begin{array}{c}\text { Length } \\
\text { of square } \\
\text { base }\end{array} \\
\text { B } 1_{1}\end{array}$ & $\begin{array}{c}\text { Width } \\
\text { of base } \\
\mathrm{B}_{2}\end{array}$ & $\begin{array}{c}\text { Thick- } \\
\text { ness of } \\
\text { base } \\
\text { C }\end{array}$ & $\begin{array}{c}\begin{array}{c}\text { Number } \\
\text { of ribs }\end{array} \\
\text { D }\end{array}$ & $\begin{array}{c}\begin{array}{c}\text { Centers } \\
\text { of ribs } \\
\text { and inside } \\
\text { bolts }\end{array} \\
\text { E }\end{array}$ & $\begin{array}{c}\text { Thick- } \\
\text { ness of } \\
\text { ribs } \\
\text { F }\end{array}$ & $\begin{array}{c}\begin{array}{c}\text { Diameter } \\
\text { of bolts }\end{array} \\
\text { G }\end{array}$ & $\begin{array}{l}\text { Longitu- } \\
\text { dinal cen- } \\
\text { ters from } \\
\text { end to } \\
\text { second } \\
\text { bolt } \\
\text { H }\end{array}$ & $\begin{array}{c}\text { Trans- } \\
\text { verse bolt } \\
\text { centers } \\
\text { K }\end{array}$ \\
\hline $\begin{array}{ll}1 & 18 \\
1 & 20 \\
1 & 24 \\
1 & 30\end{array}$ & $\begin{array}{l}12 \\
14 \\
16 \\
20\end{array}$ & $\begin{array}{l}161 / 4 \\
1778 \\
203 / 4 \\
243 / 4\end{array}$ & $\begin{array}{l}201 / 2 \\
23 \\
251 / 2 \\
301 / 2\end{array}$ & $\begin{array}{l}28 \\
301 / 2 \\
36 \\
43\end{array}$ & $\begin{array}{l}111 / 16 \\
17 / 8 \\
21 / 16 \\
25 / 16\end{array}$ & $\begin{array}{l}3 \\
3 \\
3 \\
4\end{array}$ & $\begin{array}{l}61 / 2 \\
7 \\
81 / 8 \\
65 / 8\end{array}$ & $\begin{array}{l}13 / 8 \\
11 / 2 \\
15 / 8 \\
2\end{array}$ & $\begin{array}{l}13 / 8 \\
112 \\
158 \\
134\end{array}$ & $\begin{array}{l}51 / 4 \\
6 \\
6^{11 / 16} \\
61 / 8\end{array}$ & $\begin{array}{l}241 / 2 \\
261 / 2 \\
32 \\
38\end{array}$ \\
\hline
\end{tabular}

1 Outside diameter.

(Notes are identical with those for 125-pound fittings.)

TABLE 12.-Theoretical weights, in pounds, of elbows, crosses, tees, side-outlet tees, and laterals

\begin{tabular}{|c|c|c|c|c|c|c|c|}
\hline $\begin{array}{l}\text { Nom- } \\
\text { inal } \\
\text { pipe } \\
\text { size }\end{array}$ & $\begin{array}{l}90^{\circ} \\
\text { elbow }\end{array}$ & $\begin{array}{l}45^{\circ} \\
\text { elbow }\end{array}$ & $\begin{array}{l}90^{\circ} \\
\text { long- } \\
\text { radius } \\
\text { elbow }\end{array}$ & $\begin{array}{l}\text { Side- } \\
\text { outlet } \\
\text { elbow }\end{array}$ & $\begin{array}{l}\text { Regu- } \\
\text { lar and } \\
\text { single } \\
\text { and } \\
\text { double } \\
\text { sweep } \\
\text { tees }\end{array}$ & $\begin{array}{l}\text { Cross } \\
\text { and } \\
\text { side } \\
\text { outlet } \\
\text { tees }\end{array}$ & $\begin{array}{l}\text { Later- } \\
\text { als (not } \\
\text { ribbed) }\end{array}$ \\
\hline $\begin{array}{l}1 \\
11 / 4 \\
11 / 2 \\
2 \\
21 / 2\end{array}$ & $\begin{array}{r}9 \\
11 \\
16 \\
20 \\
30\end{array}$ & $\begin{array}{r}7 \\
10 \\
15 \\
18 \\
28\end{array}$ & $\begin{array}{l}10 \\
13 \\
18 \\
23 \\
34\end{array}$ & $\begin{array}{l}13 \\
17 \\
24 \\
30 \\
43\end{array}$ & $\begin{array}{l}14 \\
18 \\
25 \\
32 \\
46\end{array}$ & $\begin{array}{l}18 \\
23 \\
32 \\
41 \\
58\end{array}$ & $\begin{array}{l}15 \\
20 \\
30 \\
37 \\
57\end{array}$ \\
\hline $\begin{array}{l}3 \\
31 / 2 \\
4 \\
5 \\
6\end{array}$ & $\begin{array}{r}40 \\
49 \\
65 \\
87 \\
115\end{array}$ & $\begin{array}{r}35 \\
44 \\
58 \\
76 \\
105\end{array}$ & $\begin{array}{r}44 \\
55 \\
72 \\
98 \\
135\end{array}$ & $\begin{array}{r}55 \\
71 \\
94 \\
125 \\
170\end{array}$ & $\begin{array}{r}58 \\
76 \\
99 \\
135 \\
180\end{array}$ & $\begin{array}{r}74 \\
94 \\
130 \\
170 \\
230\end{array}$ & $\begin{array}{r}73 \\
91 \\
120 \\
165 \\
230\end{array}$ \\
\hline $\begin{array}{r}8 \\
10 \\
12 \\
114 \\
116\end{array}$ & $\begin{array}{l}185 \\
290 \\
410 \\
560 \\
750\end{array}$ & $\begin{array}{l}155 \\
240 \\
340 \\
440 \\
620\end{array}$ & $\begin{array}{l}220 \\
350 \\
510 \\
710 \\
960\end{array}$ & $\begin{array}{r}260 \\
400 \\
560 \\
790 \\
1,040\end{array}$ & $\begin{array}{r}280 \\
430 \\
620 \\
870 \\
1,150\end{array}$ & $\begin{array}{r}350 \\
540 \\
770 \\
1,090 \\
1,430\end{array}$ & $\begin{array}{r}360 \\
570 \\
820 \\
1,180 \\
1,610\end{array}$ \\
\hline $\begin{array}{l}118 \\
120 \\
124 \\
130\end{array}$ & $\begin{array}{r}970 \\
1,220 \\
1,840 \\
3,120\end{array}$ & $\begin{array}{r}780 \\
960 \\
1,430 \\
2,230\end{array}$ & $\begin{array}{l}1,260 \\
1,630 \\
2,470 \\
4,290\end{array}$ & $\begin{array}{l}1,330 \\
1,670 \\
2,490 \\
4,150\end{array}$ & $\begin{array}{l}1,490 \\
1,880 \\
2,800 \\
4,740\end{array}$ & $\begin{array}{l}1,840 \\
2,320 \\
3,450 \\
5,760\end{array}$ & $\begin{array}{l}2,100 \\
2,670 \\
4,020\end{array}$ \\
\hline
\end{tabular}

1 Outside diameter.

(Notes are identical with those for 125-pound fittings.)
TABLE 13.-Theoretical weights, in pounds, of reducing elbows, reducers, and eccentric reducers

\begin{tabular}{|c|c|c|c|c|c|}
\hline$\underset{\text { size }}{\text { Nominal pipe }}$ & $\begin{array}{l}\text { Reduc- } \\
\text { ing el- } \\
\text { bow }\end{array}$ & $\begin{array}{l}\text { Reduc- } \\
\text { ers and } \\
\text { eccen- } \\
\text { tric re- } \\
\text { ducers }\end{array}$ & $\underset{\text { size }}{\text { Nominal pipe }}$ & $\begin{array}{l}\text { Reduc- } \\
\text { ing el- } \\
\text { bow }\end{array}$ & $\begin{array}{l}\text { Reduc- } \\
\text { ers and } \\
\text { eccen- } \\
\text { tric re- } \\
\text { ducers }\end{array}$ \\
\hline $\begin{array}{l}3 \text { by } 21 / 2 \\
3 \text { by } 2 \\
3 \text { by } 11 / 2 \\
31 / 2 \text { by } 3 \\
31 / 2 \text { by } 2 \\
4 \text { by } 31 / 2 \\
4 \text { by } 3 \\
4 \text { by } 21 / 2 \\
4 \text { by } 2 \\
5 \text { by } 4 \\
5 \text { by } 3 \\
5 \text { by } 21 / 2 \\
6 \text { by } 5 \\
6 \text { by } 4 \\
6 \text { by } 31 / 2 \\
6 \text { by } 3 \\
8 \text { by } 6 \\
8 \text { by } 5 \\
8 \text { by } 4 \\
10 \text { by } 8 \\
10 \text { by } 6 \\
10 \text { by } 5\end{array}$ & $\begin{array}{r}35 \\
30 \\
28 \\
44 \\
35 \\
56 \\
52 \\
48 \\
43 \\
78 \\
65 \\
60 \\
100 \\
93 \\
89 \\
82 \\
155 \\
140 \\
130 \\
240 \\
210 \\
190\end{array}$ & $\begin{array}{r}29 \\
25 \\
24 \\
36 \\
29 \\
48 \\
44 \\
40 \\
36 \\
63 \\
54 \\
50 \\
85 \\
77 \\
71 \\
67 \\
130 \\
115 \\
105 \\
190 \\
170 \\
155\end{array}$ & $\begin{array}{l}12 \text { by } 10 \\
12 \text { by } 8 \\
12 \text { by } 6 \\
14 \text { by } 12 \\
14 \text { by } 10 \\
14 \text { by } 8 \\
16 \text { by } 14 \\
16 \text { by } 12 \\
16 \text { by } 10 \\
16 \text { by } 8 \\
18 \text { by } 16 \\
18 \text { by } 14 \\
18 \text { by } 12 \\
18 \text { by } 10 \\
20 \text { by } 18 \\
20 \text { by } 16 \\
20 \text { by } 14 \\
20 \text { by } 12 \\
24 \text { by } 20 \\
24 \text { by } 18 \\
24 \text { by } 16 \\
24 \text { by } 12\end{array}$ & $\begin{array}{r}360 \\
300 \\
280 \\
490 \\
440 \\
390 \\
670 \\
600 \\
530 \\
470 \\
870 \\
790 \\
710 \\
650 \\
1,120 \\
1,020 \\
930 \\
840 \\
1,590 \\
1,460 \\
1,350 \\
1,150\end{array}$ & $\begin{array}{r}290 \\
250 \\
220 \\
410 \\
360 \\
320 \\
550 \\
490 \\
440 \\
390 \\
670 \\
640 \\
580 \\
520 \\
880 \\
800 \\
730 \\
660 \\
1,260 \\
1,170 \\
1,070 \\
920\end{array}$ \\
\hline
\end{tabular}

(Note is identical with that for 125-pound fittings.) 
TABLE 14.-Theoretical weights in pounds, of reducing lateral

\begin{tabular}{|c|c|c|c|}
\hline Nominal pipe size & $\begin{array}{l}\text { Lateral } \\
\text { reducing } \\
\text { outlet } \\
\text { (not } \\
\text { ribbed) }\end{array}$ & Nominal pipe size & $\begin{array}{l}\text { Lateral } \\
\text { reducing } \\
\text { outlet } \\
\text { (not } \\
\text { ribbed) }\end{array}$ \\
\hline $\begin{array}{l}3 \text { by } 3 \text { by } 21 / 2 \\
3 \text { by } 3 \text { by } 21 \% \\
3 \text { by } 3 \text { by } 11 / 2 \\
31 / 2 \text { by } 31 / 2 \text { by } 3 \\
31 / 2 \text { by } 31 / 2 \text { by } 21 / 2 . \\
31 / 2 \text { by } 31 / 2 \text { by } 2 \\
4 \text { by } 4 \text { by } 31 / 2 \\
4 \text { by } 4 \text { by } 3 \\
4 \text { by } 4 \text { by } 21 / 2 \\
4 \text { by } 4 \text { by } 2 \\
5 \text { by } 5 \text { by } 4 \\
5 \text { by } 5 \text { by } 31 / 2 \\
5 \text { by } 5 \text { by } 3 \\
5 \text { by } 5 \text { by } 21 / 2 \\
6 \text { by } 6 \text { by } 5 \\
6 \text { by } 6 \text { by } 4 \\
6 \text { by } 6 \text { by } 31 / 2 \\
6 \text { by } 6 \text { by } 3 \\
8 \text { by } 8 \text { by } 6 \\
8 \text { by } 8 \text { by } 5 \\
8 \text { by } 8 \text { by } 4 \\
10 \text { by } 10 \text { by } 8 \\
10 \text { by } 10 \text { by } 6 \ldots \\
10 \text { by } 10 \text { by } 5\end{array}$ & $\begin{array}{r}68 \\
60 \\
58 \\
85 \\
80 \\
73 \\
110 \\
105 \\
98 \\
92 \\
145 \\
140 \\
135 \\
130 \\
210 \\
195 \\
185 \\
180 \\
320 \\
300 \\
290 \\
510 \\
470 \\
450\end{array}$ & $\begin{array}{l}12 \text { by } 12 \text { by } 10 \\
12 \text { by } 12 \text { by } 8 \\
12 \text { by } 12 \text { by } 6 \\
14 \text { by } 14 \text { by } 12 \\
14 \text { by } 14 \text { by } 10 \\
14 \text { by } 14 \text { by } 8 \\
16 \text { by } 16 \text { by } 14 \\
16 \text { by } 16 \text { by } 12 \\
16 \text { by } 16 \text { by } 10 \\
16 \text { by } 16 \text { by } 8 \\
18 \text { by } 18 \text { by } 16 \\
18 \text { by } 18 \text { by } 14 \\
18 \text { by } 18 \text { by } 12 \\
18 \text { by } 18 \text { by } 10 \\
20 \text { by } 20 \text { by } 18 \\
20 \text { by } 20 \text { by } 16 \\
20 \text { by } 20 \text { by } 14 \\
20 \text { by } 20 \text { by } 12 \\
20 \text { by } 20 \text { by } 10 \\
24 \text { by } 24 \text { by } 20 \\
24 \text { by } 24 \text { by } 18 \\
24 \text { by } 24 \text { by } 16 \\
24 \text { by } 24 \text { by } 14 \\
24 \text { by } 24 \text { by } 12 \ldots\end{array}$ & $\begin{array}{r}750 \\
690 \\
640 \\
1,050 \\
980 \\
910 \\
1,440 \\
1,350 \\
1,270 \\
1,190 \\
1,870 \\
1,760 \\
1,660 \\
1,580 \\
2,390 \\
2,260 \\
2,140 \\
2,040 \\
11,620 \\
3,520 \\
3,350 \\
3,200 \\
3,100 \\
12,470\end{array}$ \\
\hline
\end{tabular}

1 These sizes made in the short-body pattern only.

(Notes are identical with those for 125-pound fittings.)

TABLE 15.-Theoretical weights, in pounds, of reducing tees

\begin{tabular}{|c|c|c|c|}
\hline Nominal pipe size & Weight & Nominal pipe size & Weight \\
\hline $\begin{array}{l}3 \text { by } 3 \text { by } 21 / 2- \\
3 \text { by } 3 \text { by } 2 \\
3 \text { by } 3 \text { by } 11 / 2-- \\
3 \text { by } 21 / 2 \text { by } 3- \\
3 \text { by } 21 / 2 \text { by } 21 / 2\end{array}$ & $\begin{array}{l}58 \\
53 \\
51 \\
58 \\
54\end{array}$ & 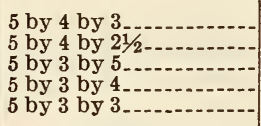 & $\begin{array}{l}110 \\
105 \\
120 \\
110 \\
100\end{array}$ \\
\hline $\begin{array}{l}3 \text { by } 21 / 2 \text { by } 2 \\
3 \text { by } 21 / 2 \text { by } 11 \\
3 \text { by } 2 \text { by } 3 \\
3 \text { by } 2 \text { by } 21 / 2- \\
3 \text { by } 2 \text { by } 2 \ldots\end{array}$ & $\begin{array}{l}50 \\
48 \\
54 \\
50 \\
46\end{array}$ & $\begin{array}{l}5 \text { by } 3 \text { by } 21 / 2 \\
5 \text { by } 21 / 2 \text { by } 5 \\
5 \text { by } 21 / 2 \text { by } 4 \\
5 \text { by } 21 / 2 \text { by } 3 \\
5 \text { by } 21 / 2 \text { by } 21 / 2 \ldots\end{array}$ & $\begin{array}{r}95 \\
115 \\
105 \\
96 \\
92\end{array}$ \\
\hline $\begin{array}{l}3 \text { by } 2 \text { by } 11 / 2 \\
3 \text { by } 11 / 2 \text { by } \\
3 \text { by } 11 / 2 \text { by } 2 \\
3 \text { by } 11 / 2 \text { by } 2 \\
3 \text { by } 11 / 2 \text { by } 1\end{array}$ & $\begin{array}{l}44 \\
52 \\
48 \\
44 \\
42\end{array}$ & $\begin{array}{l}6 \text { by } 6 \text { by } 5 \\
6 \text { by } 6 \text { by } 4 \\
6 \text { by } 6 \text { by } 31 / 2 \\
6 \text { by } 6 \text { by } 3 \text { by } 5 \text { by } 6 \ldots\end{array}$ & $\begin{array}{l}170 \\
160 \\
150 \\
150 \\
175\end{array}$ \\
\hline $\begin{array}{l}31 / 2 \text { by } 31 / 2 \text { by } \\
31 / 2 \text { by } 31 / 2 \text { by } \\
31 / 2 \text { by } 31 / 2 \text { by } \\
4 \text { by } 4 \text { by } 31 / 2 \\
4 \text { by } 4 \text { by } 3 .\end{array}$ & $\begin{array}{l}72 \\
68 \\
63 \\
93 \\
89\end{array}$ & $\begin{array}{l}6 \text { by } 5 \text { by } 5 \\
6 \text { by } 5 \text { by } 4 \\
6 \text { by } 5 \text { by } 3 \\
6 \text { by } 4 \text { by } 6 \ldots \\
6 \text { by } 4 \text { by } 5\end{array}$ & $\begin{array}{l}160 \\
155 \\
145 \\
165 \\
155\end{array}$ \\
\hline $\begin{array}{l}4 \text { by } 4 \text { by } 21 / 2 \\
4 \text { by } 4 \text { by } 2-- \\
4 \text { by } 3 \text { by } 4-- \\
4 \text { by } 3 \text { by } 3- \\
4 \text { by } 3 \text { by } 21 / 2\end{array}$ & $\begin{array}{l}85 \\
80 \\
90 \\
80 \\
76\end{array}$ & 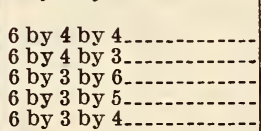 & $\begin{array}{l}145 \\
135 \\
155 \\
145 \\
140\end{array}$ \\
\hline $\begin{array}{l}4 \text { by } 3 \text { by } 2 \\
4 \text { by } 21 / 2 \text { by } 4 \\
4 \text { by } 21 / 2 \text { by } 3 \\
4 \text { by } 21 / 2 \text { by } 2 \\
4 \text { by } 21 / 2 \text { by } 2\end{array}$ & $\begin{array}{l}71 \\
86 \\
76 \\
72 \\
68\end{array}$ & $\begin{array}{l}6 \text { by } 3 \text { by } 3 \\
8 \text { by } 8 \text { by } 6 \\
8 \text { by } 8 \text { by } 5 \\
8 \text { by } 8 \text { by } 4 \text { by } 6 \text { by } 8\end{array}$ & $\begin{array}{l}125 \\
260 \\
240 \\
240 \\
260\end{array}$ \\
\hline $\begin{array}{l}4 \text { by } 2 \text { by } 4 \\
4 \text { by } 2 \text { by } 3- \\
4 \text { by } 2 \text { by } 21 / 2 \\
4 \text { by } 2 \text { by } 2- \\
5 \text { by } 5 \text { by } 4 .\end{array}$ & $\begin{array}{r}82 \\
72 \\
68 \\
64 \\
125\end{array}$ & 8 by 6 by 6 & $\begin{array}{l}240 \\
230 \\
220 \\
250\end{array}$ \\
\hline $\begin{array}{l}5 \text { by } 5 \text { by } 31 \\
5 \text { by } 5 \text { by } 3 \\
5 \text { by } 5 \text { by } 21 \\
5 \text { by } 4 \text { by } 5- \\
5 \text { by } 4 \text { by } 4 .\end{array}$ & $\begin{array}{l}120 \\
115 \\
110 \\
125 \\
120\end{array}$ & $\begin{array}{l}8 \text { by } 5 \text { by } 6 \ldots \\
8 \text { by } 5 \text { by } 5 \ldots \\
8 \text { by } 5 \text { by } 4 \ldots \\
8 \text { by } 4 \text { by } 8 \ldots \\
8 \text { by } 4 \text { by } 6 \ldots\end{array}$ & $\begin{array}{l}230 \\
215 \\
210 \\
240 \\
220\end{array}$ \\
\hline
\end{tabular}

TABLE 15.-Theoretical weights, in pounds, of reducing tees-Continued

\begin{tabular}{|c|c|c|c|}
\hline Nominal pipe size & Weight & Nominal pipe size & Weight \\
\hline $\begin{array}{l}8 \text { by } 4 \text { by } 5 \\
8 \text { by } 4 \text { by } 4 \\
10 \text { by } 10 \text { by } 8 \\
10 \text { by } 10 \text { by } 6 \\
10 \text { by } 10 \text { by } 5\end{array}$ & $\begin{array}{l}210 \\
200 \\
400 \\
370 \\
360\end{array}$ & $\begin{array}{l}14 \text { by } 8 \text { by } 8 \\
16 \text { by } 16 \text { by } 14 \\
16 \text { by } 16 \text { by } 12 \\
16 \text { by } 16 \text { by } 10 \\
16 \text { by } 16 \text { by } 8\end{array}$ & $\begin{array}{r}630 \\
1,090 \\
1,040 \\
990 \\
950\end{array}$ \\
\hline $\begin{array}{l}10 \text { by } 8 \text { by } 10 \\
10 \text { by } 8 \text { by } 8 \\
10 \text { by } 8 \text { by } 6 \\
10 \text { by } 6 \text { by } 10 \\
10 \text { by } 6 \text { by } 8\end{array}$ & $\begin{array}{l}400 \\
360 \\
340 \\
380 \\
350\end{array}$ & $\begin{array}{l}16 \text { by } 14 \text { by } 16 . \\
16 \text { by } 14 \text { by } 14 .- \\
16 \text { by } 14 \text { by } 12 . . \\
16 \text { by } 14 \text { by } 10 \ldots \\
16 \text { by } 14 \text { by } 8 . .\end{array}$ & $\begin{array}{r}1,100 \\
1,040 \\
990 \\
950 \\
910\end{array}$ \\
\hline $\begin{array}{l}10 \text { by } 6 \text { by } 6 \\
12 \text { by } 12 \text { by } 10 . \\
12 \text { by } 12 \text { by } 8 \\
12 \text { by } 12 \text { by } 6 \\
12 \text { by } 10 \text { by } 12 . .\end{array}$ & $\begin{array}{l}320 \\
570 \\
540 \\
510 \\
570\end{array}$ & $\begin{array}{l}16 \text { by } 12 \text { by } 16 \\
16 \text { by } 12 \text { by } 14 . \\
16 \text { by } 12 \text { by } 12 . \\
16 \text { by } 12 \text { by } 10 . \\
16 \text { by } 12 \text { by } 8 .\end{array}$ & $\begin{array}{r}1,050 \\
1,000 \\
950 \\
900 \\
860\end{array}$ \\
\hline $\begin{array}{l}12 \text { by } 10 \text { by } 10 \ldots \\
12 \text { by } 10 \text { by } 8 .- \\
12 \text { by } 10 \text { by } 6-. \\
12 \text { by } 8 \text { by } 12 \\
12 \text { by } 8 \text { by } 10 .\end{array}$ & $\begin{array}{l}530 \\
500 \\
470 \\
560 \\
510\end{array}$ & $\begin{array}{l}16 \text { by } 10 \text { by } 16 .- \\
16 \text { by } 10 \text { by } 14 .- \\
16 \text { by } 10 \text { by } 12- \\
16 \text { by } 10 \text { by } 10 .- \\
16 \text { by } 10 \text { by } 8 . .\end{array}$ & $\begin{array}{r}1,030 \\
970 \\
900 \\
870 \\
830\end{array}$ \\
\hline $\begin{array}{l}12 \text { by } 8 \text { by } 8 \\
12 \text { by } 8 \text { by } 6 \\
12 \text { by } 6 \text { by } 12 \\
12 \text { by } 6 \text { by } 10 \\
12 \text { by } 6 \text { by } 8\end{array}$ & $\begin{array}{l}480 \\
450 \\
540 \\
500 \\
460\end{array}$ & $\begin{array}{l}16 \text { by } 8 \text { by } 16 \\
16 \text { by } 8 \text { by } 14 \ldots \\
16 \text { by } 8 \text { by } 12 \ldots \ldots \\
16 \text { by } 8 \text { by } 10 \ldots \ldots \\
16 \text { by } 8 \text { by } 8 \ldots \ldots\end{array}$ & $\begin{array}{r}1,000 \\
940 \\
890 \\
850 \\
800\end{array}$ \\
\hline $\begin{array}{l}12 \text { by } 6 \text { by } 6 \\
14 \text { by } 14 \text { by } 12 \\
14 \text { by } 14 \text { by } 10 \\
14 \text { by } 14 \text { by } 8 \\
14 \text { by } 12 \text { by } 14\end{array}$ & $\begin{array}{l}440 \\
820 \\
770 \\
730 \\
820\end{array}$ & $\begin{array}{l}18 \text { by } 18 \text { by } 16 \\
18 \text { by } 18 \text { by } 14-.- \\
18 \text { by } 18 \text { by } 12-- \\
18 \text { by } 18 \text { by } 10 . \\
20 \text { by } 20 \text { by } 18 . .\end{array}$ & $\begin{array}{r}1,420 \\
1,360 \\
11,130 \\
11,080 \\
1,800\end{array}$ \\
\hline $\begin{array}{l}14 \text { by } 12 \text { by } 12 \\
14 \text { by } 12 \text { by } 10 \\
14 \text { by } 12 \text { by } 8 \\
14 \text { by } 10 \text { by } 14 \\
14 \text { by } 10 \text { by } 12\end{array}$ & $\begin{array}{l}770 \\
730 \\
690 \\
790 \\
730\end{array}$ & $\begin{array}{l}20 \text { by } 20 \text { by } 16 \\
20 \text { by } 20 \text { by } 14-\ldots . \\
20 \text { by } 20 \text { by } 12 . \\
20 \text { by } 20 \text { by } 10 \\
24 \text { by } 24 \text { by } 20\end{array}$ & $\begin{array}{l}1,730 \\
11,460 \\
11,410 \\
11,360\end{array}$ \\
\hline $\begin{array}{l}14 \text { by } 10 \text { by } 10 \\
14 \text { by } 10 \text { by } 8 \\
14 \text { by } 8 \text { by } 14 \\
14 \text { by } 8 \text { by } 12 \\
14 \text { by } 8 \text { by } 10\end{array}$ & $\begin{array}{l}690 \\
650 \\
760 \\
710 \\
660\end{array}$ & $\begin{array}{l}24 \text { by } 24 \text { by } 18 \\
24 \text { by } 24 \text { by } 16 \ldots \\
24 \text { by } 24 \text { by } 14 \\
24 \text { by } 24 \text { by } 12 \ldots . . . \\
\end{array}$ & $\begin{array}{r}2,020 \\
2,540 \\
12,090 \\
12,030 \\
11,970\end{array}$ \\
\hline
\end{tabular}

1 These sizes made in the short-body pattern only.

(Notes are identical with those for 125-pound fittings.)

TABLE 16.-Theoretical weights in pounds of reducing crosses

\begin{tabular}{|c|c|c|c|}
\hline Nominal pipe size & Weight & Nominal pipe size & Weight \\
\hline & & 12 by 12 by 10 by 10 & \\
\hline & & & 620 \\
\hline 67 & $\begin{array}{l}59 \\
89\end{array}$ & 12 by 12 & $\begin{array}{l}570 \\
990\end{array}$ \\
\hline $\begin{array}{l}20 \\
2 \mathrm{~b}\end{array}$ & 82 & $14 \mathrm{~b}$ & 900 \\
\hline 2 by & 74 & 14 by 14 by 8 by 8 & 820 \\
\hline$y$ & 115 & 16 by 16 & 1,320 \\
\hline & 110 & 16 & 1,210 \\
\hline & 100 & 16 & 1,12 \\
\hline by & 91 & 16 & 1,040 \\
\hline by & 155 & 18 & 1,710 \\
\hline $\begin{array}{l}5 \text { by } 5 \\
5 \text { by } 5\end{array}$ & $\begin{array}{l}145 \\
135\end{array}$ & $\begin{array}{l}18 \\
18\end{array}$ & $\begin{array}{r}1,590 \\
11,320\end{array}$ \\
\hline by & 125 & $18 \mathrm{~b}$ & 11,210 \\
\hline & 20 & $20 \mathrm{~b}$ & 2,170 \\
\hline by & 190 & 20 & 2,030 \\
\hline & 175 & 20 & 11,680 \\
\hline & 170 & 20 & 11,580 \\
\hline & 31 & 20 by 20 by 10 & 11,490 \\
\hline & 28 & 24 by 24 by 20 b & 3,080 \\
\hline & 270 & & 2,920 \\
\hline 10 by & 470 & 24 by 24 by 16 by 16 & 12,380 \\
\hline & 42 & 24 by 24 by 14 & 12,270 \\
\hline & & & 12,150 \\
\hline
\end{tabular}

1 These sizes made in the short-body pattern only.

(Notes are identical with those for 125-pound fittings.) 
AMERICAN SOCIETY OF MECHANICAL ENGINEERS, CAST-IRON PIPE FLANGES AND FLANGED FITTINGS FOR MAXIMUM NON-SHOCK WORKING HYDRAULIC PRESSURE OF 800 POUNDS PER SQUARE INCH (GAGE), AT ORDINARY AIR TEMPERATURES, B16b1-1931

[Approved by American Standards Association 64 as American Standard B16b1-1931]

\section{CAST-IRON PIPE FLANGED FITTINGS, 800-POUND}

\section{INTRODUCTORY NOTES}

1. Sizes.-The sizes of the fittings in the following tables will be identified by the corresponding "nominal pipe size."

2. Pressure rating.-This standard shall be known as the "American 800-pound hydraulic cast-iron flange standard," said pressure designation being the recommended rating at ordinary air temperatures.

3. Marking. - All fittings shall have marks cast, engraved, or otherwise permanently attached identifying the manufacturer and including the figures "800," together with a suitable mark indicating that this is not a steam rating.

(This section will be revised later to harmonize with the system which is being developed by subcommittee No. 8 on marking of the sectional committee on pipe flanges and fittings.)

4. Materials. - The dimensions prescribed in this standard are based on gray iron castings of high quality produced under regular control of chemical and physical properties by a recognized process. The manufacturer shall be prepared to certify that his product has been so produced and that the

" Sponsor organizations; American Society of Mechanical Engineers, Heating and Piping Contractors National Association, and Manufacturers' Standardization Society of the Valve and Fittings Industry. chemical and physical properties thereof, as proved by test specimens, are equal at least to the minimum requirements specified in A. S. T. M. Specifications A-126-30 for class B, higher strength gray iron, viz:

Sulphur, maximum _.._. 0.12

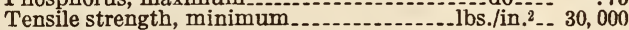

5. Facing.-All 800-pound cast-iron flanges and flanged fittings shall have a $1 / 4$-inch raised face not included in the flange thickness and of a diameter shown in Table 1. The illustrations above Table 1 show the application of raised face and male-female facings.

6. Bolting.-Drilling templates are in multiples of four, so that fittings may be made to face in any quarter.

Bolt holes shall straddle the center line. All bolt holes shall be drilled one-eighth inch larger in diamter than the nominal size of the bolt.

Bolts may be of steel or wrought iron with "rough square heads," and hexagon nuts.

7. Spot facing. - The bolt holes of 800-pound cast-iron flanges and flanged fittings need not be spot faced, unless so ordered.

8. Reducing fittings. - All reducing fittings, whether ells, tees, crosses, or reducers, and all double branch and side outlet fittings, shall have the same center to face and face to face dimensions throughout as for the largest opening.

9. Elbows.- Special degree elbows ranging from $1^{\circ}$ to $45^{\circ}$, inclusive, shall have the same center to face dimension given for $45^{\circ}$ elbows, and those over $45^{\circ}$ and up to $90^{\circ}$, inclusive, shall have the same center to face dimensions given for $90^{\circ}$ elbows. The angle designation of an elbow is its deflection from straight line flow and is the angle between the flange faces.

10. Laterals. - The use of this type of fitting when made of cast iron is discouraged. Where necessary use should be made of 600-pound steel-flanged laterals (B16e-1928) of which the flange dimensions and bolting are interchangeable with this standard

TABLE 1.-Facing dimensions for the American 800-pound hydraulic cast-iron flanges
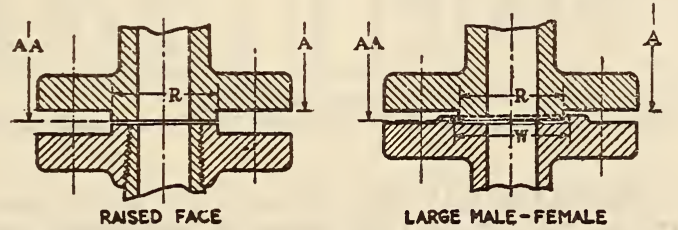

FIGURE 233.-800 pound hydraulic cast iron

flanged fittings

[All dimensions given in inches]

\begin{tabular}{|c|c|c|c|c|c|c|c|c|c|c|}
\hline $\begin{array}{l}\text { Nominal } \\
\text { pipe size }\end{array}$ & $\begin{array}{l}\text { Inside } \\
\text { diameter } \\
\text { of fitting }\end{array}$ & $\begin{array}{c}\text { Outside } \\
\text { diameter } \\
\text { of male } 1 \\
\text { R }\end{array}$ & $\begin{array}{c}\text { Outside } \\
\text { diameter } \\
\text { of large } \\
\text { female } \\
\text { W }\end{array}$ & $\begin{array}{l}\text { Metal } \\
\text { thickness } \\
\text { of fitting } \\
\text { (mini- } \\
\text { mum) }\end{array}$ & $\begin{array}{l}\text { Outside } \\
\text { diameter } \\
\text { of flange }\end{array}$ & $\begin{array}{l}\text { Thick- } \\
\text { ness of } \\
\text { flange } \\
\text { (mini- } \\
\text { mum) } 12\end{array}$ & $\begin{array}{l}\text { Diameter } \\
\text { of bolt } \\
\text { circle }\end{array}$ & $\begin{array}{l}\text { Number } \\
\text { of bolts }\end{array}$ & $\begin{array}{l}\text { Size of } \\
\text { bolts }\end{array}$ & $\begin{array}{c}\text { Diameter } \\
\text { of bolt } \\
\text { holes }\end{array}$ \\
\hline $\begin{array}{l}2 \\
21 / 2 \\
3 \\
31 / 2 \\
4\end{array}$ & $\begin{array}{l}2 \\
21 / 2 \\
3 \\
31 / 2 \\
4\end{array}$ & $\begin{array}{l}35 / 8 \\
41 / 8 \\
5 \\
51 / 2 \\
63 / 16\end{array}$ & $\begin{array}{l}311 / 16 \\
43 / 16 \\
5116 \\
5916 \\
634\end{array}$ & $\begin{array}{l}9 / 16 \\
5 / 8 \\
5 / 8 \\
3 / 4 \\
7 / 8\end{array}$ & $\begin{array}{c}61 / 2 \\
71 / 2 \\
81 / 4 \\
9 \\
103 / 4\end{array}$ & $\begin{array}{l}11 / 4 \\
13 / 8 \\
11 / 2 \\
15 / 8 \\
17 / 8\end{array}$ & $\begin{array}{l}5 \\
57 / 8 \\
658 \\
71 / 4 \\
81 / 2\end{array}$ & $\begin{array}{l}8 \\
8 \\
8 \\
8 \\
8\end{array}$ & $\begin{array}{l}5 / 8 \\
3 / 4 \\
3 / 4 \\
7 / 8 \\
7 / 8\end{array}$ & $\begin{array}{l}3 / 4 \\
7 / 8 \\
7 / 8 \\
1 \\
1\end{array}$ \\
\hline $\begin{array}{r}5 \\
6 \\
8 \\
10 \\
12\end{array}$ & $\begin{array}{l}5 \\
6 \\
77 / 8 \\
93 / 4 \\
113 / 4\end{array}$ & $\begin{array}{l}75 / 16 \\
81 / 2 \\
105 / 8 \\
123 / 4 \\
15\end{array}$ & $\begin{array}{c}73 / 8 \\
89 / 16 \\
1011 / 16 \\
121316 \\
151 / 16\end{array}$ & $\begin{array}{l}11 / 8 \\
118 \\
13 / 8 \\
158 \\
13 / 4\end{array}$ & $\begin{array}{l}13 \\
14 \\
161 / 2 \\
20 \\
22\end{array}$ & $\begin{array}{l}21 / 8 \\
21 / 4 \\
21 / 2 \\
27 / 8 \\
3\end{array}$ & $\begin{array}{l}101 / 2 \\
111 / 2 \\
133 / 4 \\
17 \\
191 / 4\end{array}$ & $\begin{array}{r}8 \\
12 \\
12 \\
16 \\
20\end{array}$ & $\begin{array}{l}1 \\
1 \\
11 / 8 \\
11 / 4 \\
11 / 4\end{array}$ & $\begin{array}{l}11 / 8 \\
11 / 8 \\
11 / 4 \\
13 / 8 \\
13 / 8\end{array}$ \\
\hline
\end{tabular}

1 A tolerance of plus or minus 0.016 inch $(1 / 64 \mathrm{inch}$ ) is allowed on the inside and outside diameters of all facings.

The raised face of $1 / 4$ inch is not included in the "Thickness of flange (minimum)." The depth of the female facing is to be $3 / 16$ inch. 
TABLE 2.-Center to contact surface dimensions for maximum nonshock working hydraulic pres8ure of 800 pounds per square inch at or near the ordinary range of air temperatures

[All dimensions given in inches]

\begin{tabular}{|c|c|c|c|c|c|c|c|c|c|c|c|c|}
\hline & & & & & & & & $\mathbf{A A}$ & $\mathrm{CC}$ & EE & FF & GG \\
\hline $\begin{array}{c}\text { Nom- } \\
\text { inal } \\
\text { pipe } \\
\text { size }\end{array}$ & $\begin{array}{l}\text { Inside } \\
\text { diam- } \\
\text { eter of } \\
\text { fitting }\end{array}$ & $\begin{array}{l}\text { Metal } \\
\text { thick- } \\
\text { ness of } \\
\text { fitting } \\
\text { (mini- } \\
\text { mum) }\end{array}$ & $\begin{array}{l}\text { Out- } \\
\text { side } \\
\text { diam- } \\
\text { eter of } \\
\text { flange }\end{array}$ & $\begin{array}{l}\text { Thick- } \\
\text { ness of } \\
\text { flange } \\
\text { (mini- } \\
\text { mum) }\end{array}$ & $\begin{array}{l}\text { Diam- } \\
\text { eter of } \\
\text { bolt } \\
\text { circle }\end{array}$ & $\begin{array}{l}\text { Num- } \\
\text { ber of } \\
\text { bolts }\end{array}$ & $\begin{array}{c}\text { Size of } \\
\text { bolts }\end{array}$ & $\begin{array}{l}\text { Center } \\
\text { to con- } \\
\text { tact sur } \\
\text { face of } \\
\text { raised } \\
\text { face, } \\
\text { elbow, } \\
\text { tee, } \\
\text { cross } 12\end{array}$ & $\begin{array}{l}\text { Center } \\
\text { to con- } \\
\text { tact sur- } \\
\text { face of } \\
\text { raised } \\
\text { face, } \\
45^{\circ} \text { ell } 1\end{array}$ & $\begin{array}{l}\text { Long } \\
\text { center } \\
\text { to con- } \\
\text { tact sur- } \\
\text { face of } \\
\text { raised } \\
\text { face, } \\
\text { lateral } 1\end{array}$ & $\begin{array}{c}\text { Short } \\
\text { center } \\
\text { to con- } \\
\text { tact sur- } \\
\text { face of } \\
\text { raised } \\
\text { face, } \\
\text { lateral }\end{array}$ & $\begin{array}{l}\text { Con- } \\
\text { tact sur- } \\
\text { face to } \\
\text { con- } \\
\text { tact sur- } \\
\text { face re- } \\
\text { ducer } 1\end{array}$ \\
\hline $\begin{array}{l}2 \\
21 / 2 \\
3 \\
31 / 2 \\
4\end{array}$ & $\begin{array}{l}2 \\
21 / 2 \\
3 \\
31 / 2 \\
4\end{array}$ & $\begin{array}{l}9 / 16 \\
5 / 8 \\
5 / 8 \\
3 / 4 \\
7 / 8\end{array}$ & $\begin{array}{c}61 / 2 \\
71 / 2 \\
81 / 4 \\
9 \\
103 / 4\end{array}$ & $\begin{array}{l}11 / 4 \\
13 / 8 \\
11 / 2 \\
158 \\
17 / 8\end{array}$ & $\begin{array}{l}5 \\
57 / 8 \\
65 / 8 \\
71 / 4 \\
81 / 2\end{array}$ & $\begin{array}{l}8 \\
8 \\
8 \\
8 \\
8\end{array}$ & $\begin{array}{l}5 / 8 \\
3 / 4 \\
3 / 4 \\
7 / 8 \\
7 / 8\end{array}$ & $\begin{array}{l}53 / 4 \\
61 / 2 \\
7 \\
71 / 2 \\
81 / 2\end{array}$ & $\begin{array}{l}41 / 4 \\
41 / 2 \\
5 \\
51 / 2 \\
6\end{array}$ & $\begin{array}{l}101 / 4 \\
111 / 2 \\
123 / 4 \\
14 \\
161 / 2\end{array}$ & $\begin{array}{l}31 / 2 \\
31 / 2 \\
4 \\
41 / 2 \\
41 / 2\end{array}$ & $\begin{array}{l}6 \\
63 / 4 \\
71 / 4 \\
73 / 4 \\
83 / 4\end{array}$ \\
\hline $\begin{array}{r}5 \\
6 \\
8 \\
10 \\
12\end{array}$ & $\begin{array}{l}5 \\
6 \\
77 / 8 \\
93 / 4 \\
113 / 4\end{array}$ & $\begin{array}{l}11 / 8 \\
11 / 8 \\
13 / 8 \\
158 \\
13 / 4\end{array}$ & $\begin{array}{l}13 \\
14 \\
161 / 2 \\
20 \\
22\end{array}$ & $\begin{array}{l}21 / 8 \\
21 / 4 \\
21 / 2 \\
27 / 8 \\
3\end{array}$ & $\begin{array}{l}101 / 2 \\
111 / 2 \\
133 / 4 \\
17 \\
191 / 4\end{array}$ & $\begin{array}{r}8 \\
12 \\
12 \\
16 \\
20\end{array}$ & $\begin{array}{l}1 \\
1 \\
11 / 8 \\
11 / 4 \\
11 / 4\end{array}$ & $\begin{array}{l}10 \\
11 \\
13 \\
151 / 2 \\
161 / 2\end{array}$ & $\begin{array}{l}7 \\
71 / 2 \\
81 / 2 \\
91 / 2 \\
10\end{array}$ & $\begin{array}{l}191 / 2 \\
21 \\
241 / 2 \\
291 / 2 \\
311 / 2\end{array}$ & $\begin{array}{l}6 \\
61 / 2 \\
7 \\
8 \\
81 / 2\end{array}$ & $\begin{array}{l}101 / 4 \\
111 / 4 \\
131 / 4 \\
153 / 4 \\
16^{3} / 4\end{array}$ \\
\hline
\end{tabular}

1 The raised face of $1 / 4$ inch is not included in "Thickness of flange (minimum)" but is included in "Center to contact surface of raised face" dimensions and "Contact surface to contact surface" dimensions.

"Face to face" dimensions on tees and crosses are double the "Center to face" dimensions given in the above table.

TABLE 3.-Center to flange edge dimensions for maximum nonshock working hydraulic pressure of 800 pounds per square inch at or near the ordinary range of air temperatures

[All dimensions given in inches]

\begin{tabular}{|c|c|c|c|c|c|c|c|c|c|c|c|c|}
\hline \multirow[b]{2}{*}{$\begin{array}{l}\text { Nom- } \\
\text { inal } \\
\text { pipe } \\
\text { size }\end{array}$} & \multirow[b]{2}{*}{$\begin{array}{l}\text { Inside } \\
\text { diam- } \\
\text { eter of } \\
\text { fitting }\end{array}$} & \multirow[b]{2}{*}{$\begin{array}{l}\text { Metal } \\
\text { thick- } \\
\text { ness of } \\
\text { fitting } \\
\text { (mini- } \\
\text { mum) }\end{array}$} & \multirow[b]{2}{*}{$\begin{array}{l}\text { Out- } \\
\text { side } \\
\text { diam- } \\
\text { eter of } \\
\text { flange }\end{array}$} & \multirow[b]{2}{*}{$\begin{array}{l}\text { Thick- } \\
\text { ness of } \\
\text { flange } \\
\text { (mini- } \\
\text { imum } 1\end{array}$} & \multirow[b]{2}{*}{$\begin{array}{l}\text { Diam- } \\
\text { eter of } \\
\text { bolt } \\
\text { circle }\end{array}$} & \multirow[b]{2}{*}{$\begin{array}{l}\text { Num- } \\
\text { ber of } \\
\text { bolts }\end{array}$} & \multirow[b]{2}{*}{$\begin{array}{c}\text { Size of } \\
\text { bolts }\end{array}$} & A & C & E & F & G \\
\hline & & & & & & & & $\begin{array}{l}\text { Center } \\
\text { to } \\
\text { flange } \\
\text { edge } \\
\text { elbow, } \\
\text { tee, and } \\
\text { cross? }\end{array}$ & $\begin{array}{c}\text { Center } \\
\text { to } \\
\text { flange } \\
\text { edge, } \\
45^{\circ} \text { ell }\end{array}$ & $\begin{array}{c}\text { Long } \\
\text { center } \\
\text { to } \\
\text { flange } \\
\text { edge } \\
\text { lateral }\end{array}$ & $\begin{array}{c}\text { Short } \\
\text { center } \\
\text { to } \\
\text { flange } \\
\text { edge } \\
\text { lateral }\end{array}$ & $\begin{array}{c}\text { Flange } \\
\text { edge to } \\
\text { flange } \\
\text { edge re- } \\
\text { ducer }\end{array}$ \\
\hline $\begin{array}{l}2 \\
21 / 2 \\
3 \\
31 / 2 \\
4\end{array}$ & $\begin{array}{l}2 \\
21 / 2 \\
3 \\
31 / 2 \\
4\end{array}$ & $\begin{array}{l}9 / 16 \\
5 / 8 \\
5 / 8 \\
3 / 4 \\
7 / 8\end{array}$ & $\begin{array}{c}61 / 2 \\
71 / 2 \\
81 / 4 \\
9 \\
103 / 4\end{array}$ & $\begin{array}{l}11 / 4 \\
13 / 8 \\
11 / 2 \\
15 / 8 \\
17 / 8\end{array}$ & $\begin{array}{l}5 \\
57 / 8 \\
658 \\
71 / 4 \\
81 / 2\end{array}$ & $\begin{array}{l}8 \\
8 \\
8 \\
8 \\
8\end{array}$ & $\begin{array}{l}5 / 8 \\
3 / 4 \\
3 / 4 \\
778 \\
7 / 8\end{array}$ & $\begin{array}{l}51 / 2 \\
61 / 4 \\
63 / 4 \\
71 / 4 \\
81 / 4\end{array}$ & $\begin{array}{l}4 \\
41 / 4 \\
43 / 4 \\
51 / 4 \\
53 / 4\end{array}$ & $\begin{array}{l}10 \\
111 / 4 \\
121 / 2 \\
133 / 4 \\
161 / 4\end{array}$ & $\begin{array}{l}31 / 4 \\
31 / 4 \\
33 / 4 \\
41 / 4 \\
41 / 4\end{array}$ & $\begin{array}{l}51 / 2 \\
61 / 4 \\
63 / 4 \\
71 / 4 \\
81 / 4\end{array}$ \\
\hline $\begin{array}{r}5 \\
6 \\
8 \\
10 \\
12\end{array}$ & $\begin{array}{l}5 \\
6 \\
77 / 8 \\
93 / 4 \\
113 / 4\end{array}$ & $\begin{array}{l}11 / 8 \\
11 / 8 \\
13 / 8 \\
15 / 8 \\
13 / 4\end{array}$ & $\begin{array}{l}13 \\
14 \\
161 / 2 \\
20 \\
22\end{array}$ & $\begin{array}{l}21 / 8 \\
21 / 4 \\
21 / 2 \\
27 / 8 \\
3\end{array}$ & $\begin{array}{l}101 / 2 \\
111 / 2 \\
133 / 4 \\
17 \\
191 / 4\end{array}$ & $\begin{array}{r}8 \\
12 \\
12 \\
16 \\
20\end{array}$ & $\begin{array}{l}1 \\
1 \\
11 / 8 \\
11 / 4 \\
11 / 4\end{array}$ & $\begin{array}{r}93 / 4 \\
103 / 4 \\
123 / 4 \\
151 / 4 \\
161 / 4\end{array}$ & $\begin{array}{l}63 / 4 \\
71 / 4 \\
81 / 4 \\
91 / 4 \\
93 / 4\end{array}$ & $\begin{array}{l}191 / 4 \\
203 / 4 \\
241 / 4 \\
291 / 4 \\
311 / 4\end{array}$ & $\begin{array}{l}53 / 4 \\
61 / 4 \\
63 / 4 \\
73 / 4 \\
81 / 4\end{array}$ & $\begin{array}{r}93 / 4 \\
103 / 4 \\
123 / 4 \\
151 / 4 \\
161 / 4\end{array}$ \\
\hline
\end{tabular}

1 The raised face of 14 inch is not included in "Thickness of lange (minimum)."
2 "Flange edge to flange edge" dimensions on tees and crosses are double the "Center to flange edge" dimensions given in the above table.

AMERICAN SOCIETY OF MECHANICAI ENGINEERS, AMERICAN TENTATIVE STANDARD CAST-IRON PIPE FLANGES AND FLANGED FITTINGS FOR MAXIMUM WORKING SATURATED STEAM PRESSURE OF 25 POUNDS PER SQUARE INCH (GAGE), B16b2-1931

[Approved as American Tentative Standard by the American Standards Association, 65 April, 193i]

\section{CAST-IRON PIPE FLANGES AND FLANGED FIT- TINGS 25 POUNDS PER SQUARE INCH}

\section{INTRODUCTORY NOTES}

1. Size.-The term size is used in all the following tables to indicate the nominal inside diameter of port.

2. Pressure rating.-It is recommended that in addition to the maximum working saturated steam

es Sponsor organizations: American Society of Mechanical Engineers, Manufacturers' Standardization Society of Valve and Fittings Industry, and Heating and Piping Contractors' National Association. pressure for which these fittings are intended, the sizes 36 inches and smaller may also be used for maximum nonshock working hydraulic pressure of 43 pounds per square inch (gauge), or a maximum gas pressure of 25 pounds per square inch (gauge), at or near the ordinary range of air temperatures.

3. Marking.-All fittings must have marks cast on them indicating the manufacturer and the figures 25 indicating the maximum working steam pressure for which the fittings are intended.

4. Material.-The dimensions prescribed in this standard are based upon gray iron castings of high quality produced under regular control of chemical and physical properties by a recognized process. The manufacturer shall be prepared to certify that his product has been so produced and that the chemical and physical properties thereof, as proved by test specimens, are at least equal to the minimum requirements specified in A. S. T. M. specifications A-126 for class A, regular gray iron (see 611.11, p. 637), viz:

Sulphur, maximum Phosphorus, maximum Tensile strength, minimum 
5. Dimensions.-The flange diameters, bolt circles, and number of bolts are the same as for the 125pound American standard A. S. A. B16a-1928), given above, with a reduction in the thickness of flanges and bolt diameters as shown in Table 3 , thereby maintaining interchangeability between the two standards.

The center to face and face to face dimensions of fittings are the same as for the 125-pound American standard cast-iron flanged fittings.

6. Facing.-All 25-pound cast-iron flanges and flanged fittings shall be plain faced; that is, without projection or raised face.

An inspection limit of plus or minus one-sixteenth inch shall be allowed on all center to face dimensions, and plus or minus one-eighth inch on all face to face dimensions.

7. Bolting.-Drilling templates are in multiples of four, so that fittings may be made to face in any quarter.

Bolt holes shall straddle the center line, and are drilled one-eighth inch larger in diameter than the nominal size of the bolt.

Bolts shall be of steel with standard rough square heads and the nuts shall be of steel with standard rough hexagonal dimensions.

8. Spot facing. - The bolt holes of 25-pound castiron flanges and flanged fittings shall not be spot faced for ordinary service. When required the flanges and fittings in sizes 36 inches and larger may be spot faced or back faced to the minimum thickness of flange with a plus tolerance of one-eighth inch.

9. Reducing fittings.-(Same as for steam pressure of 125 pounds per square inch in B16a-1928, given above, except that in $\mathrm{B} 16 \mathrm{~b} 2-1931$ no reference is made to laterals, reducers, or eccentric reducers.)

10. Elbows.-(Same as for steam pressure of 125 pounds per square inch in $\mathrm{B} 16 \mathrm{a}-1928$, given above, with the following additional requirement:)

Where long radius fittings are specified it has reference only to elbows, which are made in two center to face dimensions and known as elbows and long radius elbows, the latter being used only when so specified.

11. Screwed companion flanges.-Screwed companion flanges shall not be thinner than the 125pound American standard thickness on sizes 24 inches and smaller. Other types of flanges may have thicknesses as given in the table.

(Illustrations showing method of designating outlets of reducing fittings when ordering are identical with those for steel flanged fittings $250,400,600$, 900 , and 1,350 pounds, except that in this case reducing laterals are omitted and there is no provision for the raised face required for higher steam pressure steel flanged fittings. See 607.4, p. 467.)

\section{TABLE 1.-Templates for drilling}

[All dimensions given in inches]

\begin{tabular}{|c|c|c|c|c|c|c|c|c|c|c|}
\hline $\begin{array}{l}\text { Nomi- } \\
\text { nal } \\
\text { pipe } \\
\text { size }\end{array}$ & Diameter of flange & $\begin{array}{c}\text { Mini- } \\
\text { mum } \\
\text { thickness } \\
\text { of } \\
\text { flange 12 }\end{array}$ & $\begin{array}{c}\text { Diameter } \\
\text { of bolt } \\
\text { circle }\end{array}$ & $\begin{array}{l}\text { Number } \\
\text { of bolts }{ }^{3}\end{array}$ & $\begin{array}{l}\text { Diam- } \\
\text { eter of } \\
\text { bolts }\end{array}$ & $\begin{array}{l}\text { Diam- } \\
\text { eter of } \\
\text { bolts } \\
\text { holes }\end{array}$ & $\begin{array}{c}\text { Length } \\
\text { of } \\
\text { bolts } 45\end{array}$ & $\begin{array}{l}\text { Total ef- } \\
\text { fective } \\
\text { area bolt } \\
\text { metal }\end{array}$ & $\begin{array}{c}\text { Stress } \\
\text { pound } \\
\text { per } \\
\text { square } \\
\text { inch bolt } \\
\text { metal so }\end{array}$ & $\begin{array}{c}\text { Size of ring } \\
\text { gasket }\end{array}$ \\
\hline $\begin{array}{r}4 \\
5 \\
6 \\
8 \\
10\end{array}$ & \multirow{4}{*}{$\begin{array}{l}\text { Same as for 125- } \\
\text { pound fittings. }\end{array}$} & $\begin{array}{l}3 / 4 \\
3 / 4 \\
3 / 4 \\
3 / 4 \\
7 / 8\end{array}$ & \multirow{4}{*}{\multicolumn{2}{|c|}{$\begin{array}{l}\text { Same as for 125- } \\
\text { pound fittings. }\end{array}$}} & $\begin{array}{l}5 / 8 \\
58 \\
5 / 8 \\
5 / 8 \\
5 / 8\end{array}$ & $\begin{array}{l}3 / 4 \\
3 / 4 \\
3 / 4 \\
3 / 4 \\
3 / 4\end{array}$ & $\begin{array}{l}21 / 4 \\
21 / 4 \\
21 / 4 \\
21 / 4 \\
21 / 2\end{array}$ & $\begin{array}{l}1.616 \\
1.616 \\
1.616 \\
1.616 \\
2.424\end{array}$ & $\begin{array}{r}570 \\
750 \\
930 \\
1,470 \\
1,440\end{array}$ & $\begin{array}{rrr}4 \text { by } & 67 / 8 \\
5 \text { by } & 77 / 8 \\
6 \text { by } & 83 / 4 \\
8 \text { by } & 11 \\
10 \text { by } & 133 / 8\end{array}$ \\
\hline $\begin{array}{l}12 \\
14 \\
16 \\
18 \\
20\end{array}$ & & $\begin{array}{l}1 \\
11 / 8 \\
11 / 8 \\
11 / 4 \\
11 / 4\end{array}$ & & & $\begin{array}{l}5 / 8 \\
3 / 4 \\
3 / 4 \\
3 / 4 \\
3 / 4\end{array}$ & $\begin{array}{l}3 / 4 \\
7 / 8 \\
7 / 8 \\
78 \\
7 / 8\end{array}$ & $\begin{array}{l}23 / 4 \\
31 / 4 \\
31 / 4 \\
31 / 2 \\
31 / 2\end{array}$ & $\begin{array}{l}2.424 \\
3.62 \\
4.83 \\
4.83 \\
6.04\end{array}$ & $\begin{array}{l}2,195 \\
1,750 \\
1,710 \\
1,965 \\
1,920\end{array}$ & $\begin{array}{ll}12 \text { by } & 161 / 8 \\
14 \text { by } & 18 \\
16 \text { by } & 201 / 2 \\
18 \text { by } & 22 \\
20 \text { by } & 241 / 4\end{array}$ \\
\hline $\begin{array}{l}24 \\
30 \\
36 \\
42 \\
48\end{array}$ & & $\begin{array}{l}13 / 8 \\
11 / 2 \\
15 / 8 \\
13 / 4 \\
2\end{array}$ & & & $\begin{array}{l}3 / 4 \\
7 / 8 \\
7 / 8 \\
1^{7}\end{array}$ & $\begin{array}{l}7 / 8 \\
1 \\
1 \\
11 / 8 \\
11 / 8\end{array}$ & $\begin{array}{l}33 / 4 \\
41 / 4 \\
5 \\
51 / 4 \\
51 / 2\end{array}$ & $\begin{array}{r}6.04 \\
11.76 \\
13.44 \\
19.80 \\
24.20\end{array}$ & $\begin{array}{l}2,690 \\
2,030 \\
2,610 \\
2,315 \\
2,475\end{array}$ & 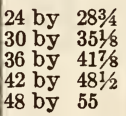 \\
\hline $\begin{array}{l}54 \\
60 \\
72 \\
84 \\
96\end{array}$ & & $\begin{array}{l}21 / 4 \\
21 / 4 \\
21 / 2 \\
23 / 4 \\
3\end{array}$ & & & $\begin{array}{l}1 \\
11 / 8 \\
11 / 8 \\
11 / 4 \\
11 / 4\end{array}$ & $\begin{array}{l}11 / 8 \\
11 / 4 \\
11 / 4 \\
138 \\
13 / 8\end{array}$ & $\begin{array}{l}53 / 4 \\
6 \\
61 / 4 \\
71 / 4 \\
73 / 4\end{array}$ & $\begin{array}{l}24.20 \\
36.02 \\
41.57 \\
57.14 \\
60.57\end{array}$ & $\begin{array}{l}3,195 \\
2,515 \\
3,120 \\
3,005 \\
3,705\end{array}$ & $\begin{array}{l}54 \text { by } 613 / 4 \\
60 \text { by } 6818 \\
72 \text { by } 813 / 8 \\
84 \text { by } 941 / 4 \\
96 \text { by } 1071 / 4\end{array}$ \\
\hline
\end{tabular}

1 All 25-pound cast-iron standard flanges have plain faces.

${ }^{2}$ Screwed companion flanges "should not be thinner than the '125-pound American standard' thickness on sizes 24 inches and smaller. Other types of flanges may have thicknesses as given in the table above."

${ }^{3}$ Drilling templates are in multiples of 4 , so that fit tings may be made to face in any quarter, and bolt holes straddle the center line. Bolt holes are drilled 1/8 inch larger in diameter than the nominal diameter of the bolt.

- The bolt holes on cast-iron flanged fittings are not spot faced for ordinary service. When required, the fittings and flanges in sizes 36 inches and larger may be spot faced or back faced to minimum thickness of flange with a plus tolerance of $1 / 8$ inch, so that standard length bolts can be used.

8 Bolts shall be of steel with standard rough square heads and the hexagon nuts shall be of steel with United States standard dimensions.

o The stress shown is that of internal pressure only, assumed to act on a circular area equal in diameter to the outside diameter of a ring gasket covering the flange to the inside of bolts.

(Illustrations showing dimensions of elbows are fittings, in B16a, fig. 224, except that the side outlet is identical with ihose for 125 -pound cast-iron flanged 1 omitted in this specification.) 
TABLE 2.-Dimensions of elbows

[All dimensions given in inches]

\begin{tabular}{|c|c|c|c|c|c|c|}
\hline $\begin{array}{l}\text { Nomi- } \\
\text { nal } \\
\text { pipe } \\
\text { size } 12\end{array}$ & $\begin{array}{c}\text { Center } \\
\text { to face } \\
\text { elbow } \\
345 \\
\text { A }\end{array}$ & $\begin{array}{c}\text { Center } \\
\text { to face } \\
\text { long } \\
\text { radius } \\
\text { elbow } \\
\text { B }\end{array}$ & $\begin{array}{c}\text { Center } \\
\text { to face } \\
45^{\circ} \\
\text { elbow }{ }^{3} \\
\text { C }\end{array}$ & $\begin{array}{l}\text { Diam- } \\
\text { eter of } \\
\text { flange }\end{array}$ & $\begin{array}{l}\text { Thick- } \\
\text { ness of } \\
\text { flange } \\
\text { (mini- } \\
\text { mum) }\end{array}$ & $\begin{array}{l}\text { Metal } \\
\text { thick- } \\
\text { ness of } \\
\text { body } \\
\text { (mini- } \\
\text { mum) }\end{array}$ \\
\hline $\begin{array}{r}4 \\
5 \\
6 \\
8 \\
10\end{array}$ & \multirow{3}{*}{\multicolumn{4}{|c|}{$\begin{array}{l}\text { Dimensions identical with those } \\
\text { for } 125 \text {-pound cast-iron flanged } \\
\text { fittings. }\end{array}$}} & $\begin{array}{l}3 / 4 \\
3 / 4 \\
3 / 4 \\
3 / 4 \\
7 / 8\end{array}$ & $\begin{array}{r}0.42 \\
.44 \\
.44 \\
.46 \\
.50\end{array}$ \\
\hline $\begin{array}{l}12 \\
14 \\
16 \\
18 \\
20\end{array}$ & & & & & $\begin{array}{l}1 \\
11 / 8 \\
11 / 8 \\
11 / 4 \\
11 / 4\end{array}$ & $\begin{array}{l}.54 \\
.57 \\
.60 \\
.64 \\
.67\end{array}$ \\
\hline $\begin{array}{l}24 \\
30 \\
36 \\
42\end{array}$ & & & & & $\begin{array}{l}13 / 8 \\
11 / 2 \\
15 / 8 \\
13 / 4\end{array}$ & $\begin{array}{r}.76 \\
.88 \\
.99 \\
1.10\end{array}$ \\
\hline $\begin{array}{l}48 \\
54 \\
60 \\
72\end{array}$ & $\begin{array}{l}39 \\
44 \\
53\end{array}$ & $\begin{array}{l}711 / 2 \\
79 \\
94\end{array}$ & $\begin{array}{l}27 \\
30 \\
36\end{array}$ & $\begin{array}{l}661 / 4 \\
73 \\
861 / 2\end{array}$ & $\begin{array}{l}2 \\
21 / 4 \\
21 / 4 \\
21 / 2\end{array}$ & $\begin{array}{l}\text { 1. } 26 \\
1.35 \\
1.39 \\
1.62\end{array}$ \\
\hline
\end{tabular}

1 Size of all fittings listed indicates nominal inside diameter of port. 1 The flange diameters, bolt circles, and number of bolts are the same as the 125-pound American standard (A. S. A. B16a-1928) with a reduction in the thickness of flanges and the bolt diameters as shown in Table 1, thereby maintaining interchangeability with the 125-pound American standard flanges.

3 The center to face dimensions of fittings are the same as the 125 pound American standard cast-iron flanged fittings.

4 Special degree elbows ranging from $1^{\circ}$ to $45^{\circ}$ inclusive shall have the same center to face dimensions given in $45^{\circ}$ elbows, and those over $45^{\circ}$ and up to $90^{\circ}$ inclusive shall have the same center to face dimensions as given for $90^{\circ}$ elbows. The angle designation of an elbow is its deflection from straight line flow and is the angle between the ils deflection

flange faces. intersecting center lines.

6 Screwed companion flanges should not be thinner than the 125pound American standard thickness on sizes 24 inches and smaller. Other types of flanges may have thicknesses as given in the table above.

(Illustrations showing dimensions of tees and crosses (straight sizes) are identical with those for 125-pound cast-iron flanged fittings in B16a, fig. 225.)

TABLE 3.-Dimensions of tees and crosses (straight sizes) [All dimentions given in inches]

\begin{tabular}{|c|c|c|c|c|c|}
\hline $\begin{array}{l}\text { Nomi- } \\
\text { nal } \\
\text { pipe } \\
\text { size }\end{array}$ & $\begin{array}{c}\text { Center } \\
\text { to face } \\
\text { tee and } \\
\text { cross } 12 \\
\text { A }\end{array}$ & $\begin{array}{c}\text { Face to } \\
\text { face tees } \\
\text { and } \\
\text { crosses } 12 \\
\text { AA }\end{array}$ & $\begin{array}{l}\text { Diam- } \\
\text { eter of } \\
\text { flange }\end{array}$ & $\begin{array}{l}\text { Thick- } \\
\text { ness of } \\
\text { flange, } \\
\text { (mini- } \\
\text { mum) }\end{array}$ & $\begin{array}{l}\text { Metal } \\
\text { thick- } \\
\text { ness of } \\
\text { body } \\
\text { (mini- } \\
\text { mum) }\end{array}$ \\
\hline $\begin{array}{r}4 \\
5 \\
6 \\
8 \\
10\end{array}$ & $\begin{array}{l}61 / 2 \\
71 / 2 \\
8 \\
9 \\
11\end{array}$ & $\begin{array}{l}13 \\
15 \\
16 \\
18 \\
22\end{array}$ & $\begin{array}{l}9 \\
10 \\
11 \\
131 / 2 \\
16\end{array}$ & \multirow{4}{*}{\multicolumn{2}{|c|}{$\begin{array}{l}\text { Dimensions same } \\
\text { as for elbows in } \\
\text { Table } 2 \text {. }\end{array}$}} \\
\hline $\begin{array}{l}12 \\
14 \\
16 \\
18 \\
20\end{array}$ & $\begin{array}{l}12 \\
14 \\
15 \\
161 / 2 \\
18\end{array}$ & $\begin{array}{l}24 \\
28 \\
30 \\
33 \\
36\end{array}$ & $\begin{array}{l}19 \\
21 \\
231 / 2 \\
25 \\
271 / 2\end{array}$ & & \\
\hline $\begin{array}{l}24 \\
30 \\
36 \\
42\end{array}$ & $\begin{array}{l}22 \\
25 \\
28 \\
31\end{array}$ & $\begin{array}{l}44 \\
50 \\
56 \\
62\end{array}$ & $\begin{array}{l}32 \\
383 / 4 \\
46 \\
53\end{array}$ & & \\
\hline $\begin{array}{l}48 \\
54 \\
60 \\
72 \\
\end{array}$ & $\begin{array}{l}34 \\
39 \\
44 \\
53 \\
\end{array}$ & $\begin{array}{r}68 \\
78 \\
88 \\
106 \\
\end{array}$ & $\begin{array}{l}591 / 2 \\
661 / 4 \\
73 \\
861 / 2 \\
\end{array}$ & & \\
\hline
\end{tabular}

(Notes same as for elbows in Table 2, except that Note 3 includes face-to-face dimensions, and for notes 4 and 5 in Table 2, substitute the following:)

1 Tees, side outlet tees, and crosses, 16 inches and smaller, reducing on the outlet, have the same dimensions center to face and face to face as straight size fittings, corresponding to the size of the larger opening. Sizes 18 inches and larger, reducing on the outlet, are made in two lengths, depending on the size of the outlet as given in Table 4.

8 Tees and crosses, reducing on run only, carry same dimensions center to face and face to face as a straight size fitting of the larger opening.
(Illustrations showing dimensions of reducing tees and reducing crosses (short body patterns) are identical with those for 125-pound cast-iron flanged fittings in B16a, fig. 226, except that the side outlet type is omitted in this specification.)

TABLE 4.-Dimensions of reducing tees and reducing crosses (short body patterns)

[All dimensions given in inches]

\begin{tabular}{|c|c|c|c|c|}
\hline $\begin{array}{l}\text { Nom- } \\
\text { inal } \\
\text { pipe } \\
\text { size }\end{array}$ & $\begin{array}{l}\text { Size of } \\
\text { outlet } \\
\text { and } \\
\text { smaller }\end{array}$ & $\begin{array}{c}\text { Center } \\
\text { to face } \\
\text { run } \\
\text { H }\end{array}$ & $\begin{array}{c}\text { Face to } \\
\text { face } \\
\text { run } \\
\text { HH }\end{array}$ & $\begin{array}{c}\text { Center } \\
\text { to face } \\
\text { outlet } \\
\text { J }\end{array}$ \\
\hline & \multicolumn{4}{|c|}{$\begin{array}{l}\text { All reducing fittings sizes } 16 \\
\text { inches and smaller have same } \\
\text { center to face dimensions as } \\
\text { straight size fittings }\end{array}$} \\
\hline $\begin{array}{l}18 \\
20 \\
24 \\
30 \\
36\end{array}$ & \multicolumn{4}{|c|}{$\begin{array}{l}\text { Dimensions identical with those } \\
\text { for } 125 \text {-pound cast-iron flanged } \\
\text { fittings. }\end{array}$} \\
\hline $\begin{array}{l}42 \\
48 \\
51 \\
60 \\
72\end{array}$ & $\begin{array}{l}24 \\
30 \\
36 \\
40 \\
48\end{array}$ & $\begin{array}{l}23 \\
26 \\
29 \\
33 \\
40\end{array}$ & $\begin{array}{l}46 \\
52 \\
58 \\
66 \\
80\end{array}$ & $\begin{array}{l}30 \\
34 \\
37 \\
41 \\
48\end{array}$ \\
\hline
\end{tabular}

(Notes identical with those for 125-pound cast-iron flanged fittings given above.)

TABLE 5.-Theoretical weights in pounds of elbows, tees, and crosses ${ }^{1}$

\begin{tabular}{|c|r|r|r|r|r|}
\hline $\begin{array}{c}\text { Nomj- } \\
\text { nal } \\
\text { pipe } \\
\text { size }\end{array}$ & $\begin{array}{c}90^{\circ} \\
\text { elbow }\end{array}$ & $\begin{array}{c}45^{\circ} \\
\text { elbow }\end{array}$ & $\begin{array}{c}90^{\circ} \text { long } \\
\text { radius } \\
\text { elbcw }\end{array}$ & Tees & Crosses \\
\hline 4 & 35 & 30 & 45 & 50 & 65 \\
\hline 5 & -55 & 50 & 65 & 85 & 100 \\
6 & 80 & 65 & 105 & 120 & 150 \\
8 & 135 & 100 & 160 & 185 & 225 \\
10 & 185 & 160 & 245 & 270 & 325 \\
12 & 250 & 195 & 330 & 370 & 450 \\
14 & 340 & 240 & 425 & 450 & 550 \\
16 & 355 & 255 & 530 & 550 & 670 \\
18 & 465 & 350 & 665 & 660 & 780 \\
20 & 695 & 490 & 950 & 960 & 1,130 \\
24 & 1,050 & 840 & 1,550 & 1,500 & 1,750 \\
30 & 1,620 & 1,350 & 2,450 & 2,275 & 2,600 \\
36 & 2,325 & 2,000 & 3,620 & 3,200 & 3,675 \\
42 & 2,050 & & \\
48 & 3,205 & 2,850 & 5,300 & 4,300 & 4,880 \\
54 & 4,565 & 3,970 & 7,500 & 6,250 & 6,880 \\
60 & 6,000 & 5,140 & 9,675 & 8,000 & 10,250 \\
72 & 9,320 & 7,525 & 14,175 & 12,150 & 13,450 \\
\hline
\end{tabular}

1 All weights listed are for fittings faced and drilled, based upon minimum thicknesses and dimensions given in preceding table without allowances for variation. Cast iron is considered to weigh 0.26 pound per cubic inch.

\section{AMERICAN SOCIETY OF MECHANICAL EN- GINEERS, AMERICAN TENTATIVE STANDARD CAST-IRON SCREWED FIT- TINGS FOR MAXIMUM WORKING SATU. RATED STEAM PRESSURES OF 125 AND 250 POUNDS PER SQUARE INCH (GAGE), B16d-1927}

[Approved as American Tentative Standard by the American Standards Association, 60 December, 1927]

\section{CAST-IRON SCREWED FITTINGS \\ INTRODUCTORY NOTES}

1. Pressure ratings.-These standards shall be known as the American 125 and 250 pound cast-

66 Sponsor organizations: American Society of Mechanical Engineers, Manufacturers' Standardization Society of Valve and Fittings Industry, and Heating and Piping Contractors, National Association. 
iron screwed fittings standards, said pressure designation being the recommended maximum working saturated steam rating.

2. Size.-The size of the fittings scheduled in the following tables is identified by the corresponding nominal pipe size. For pipe 14 inches and larger, the corresponding outside diameter of the pipe is given.

In the case of reducing tees and crosses, the size of the largest run opening shall be given first, followed by the size of the opening at the opposite end of the run. Where the fitting is a tee, the size of the outlet is given next. Where the fitting is a cross, the largest opening of outlet is the tnird dimension given followed by the opening opposite. The straight line sketches (fig. 257) illustrate how the reducing fittings are read.
3. Marking.-Each fitting of the American 125pound cast-iron screwed fitting standard shall be marked for identification with the make's name or symbol.

Each fitting of the American 250-pound castiron screwed fitting standard shall be marked for identification by means of (a) maker's name or symbol, and (b) the numerals 250 .

(In respect to metal thickness, threading, ribs, and fittings dimensions, this specification conforms to the A. S. M. E. specification for malleable iron screwed fittings, B16c (see 607.2, p. 422), except that in B16d laterals are omitted, there is no reference to the chamfering of male threads, and the inspection limits for center to end dimensions are as follows:)

\section{Inspection limits for center to end dimensions}

[All dimensions given in inches]

\begin{tabular}{|c|c|c|c|c|c|c|c|c|c|c|}
\hline Nominal pipe size. & 34 & $3 / 8$ & 1,2 & $3 / 4$ & 1 & 114 & $11 / 2$ & 2 & $21 / 2$ & 3 \\
\hline Center to end \pm .. & 0.04 & 0.05 & 0.06 & 0.08 & 0.07 & 0.07 & 0.08 & 0.08 & 0.10 & 0.10 \\
\hline Nominal pipe size. & & $31 / 2$ & 4 & 5 & 6 & 8 & 10 & 12 & 114 & 116 \\
\hline Center to end \pm . & & 0.10 & 0.12 & 0.12 & 0.14 & 0.16 & 0.19 & 0.21 & 0.23 & 0.25 \\
\hline
\end{tabular}

1 Outside diameter.

TABLE 1.-Dimensions of elbows, $45^{\circ}$ elbows, tees, and crosses (straight sizes)

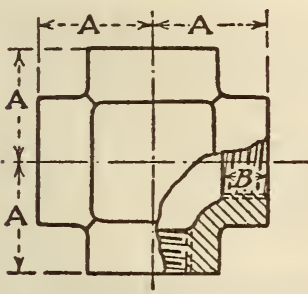

CROSS

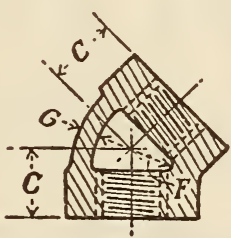

$45^{\circ}$ ELBOW

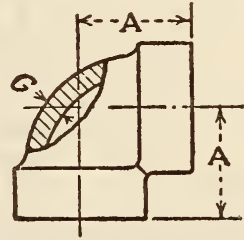

ELBOW

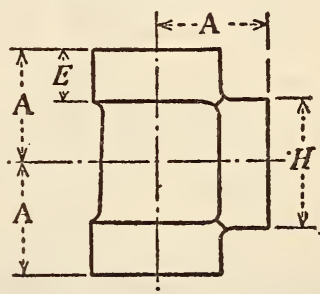

TEE

Figdre 234.-Elbows, $45^{\circ}$ elbows, tees, and crosses (straight sizes)

[All dimensions given in inches]

\begin{tabular}{|c|c|c|c|c|c|c|c|c|}
\hline \multirow{3}{*}{$\begin{array}{l}\text { Nominal } \\
\text { pipe size }\end{array}$} & A & $\mathrm{C}$ & B & $\mathrm{E}$ & \multicolumn{2}{|c|}{$\mathbf{F}$} & $\mathrm{G}$ & $\mathrm{H}$ \\
\hline & \multirow{2}{*}{$\begin{array}{l}\text { Center } \\
\text { to end, } \\
\text { elbows, } \\
\text { tees, and } \\
\text { crosses }\end{array}$} & \multirow{2}{*}{$\begin{array}{l}\text { Center } \\
\text { to end, } \\
45^{\circ} \\
\text { elbows }\end{array}$} & \multirow{2}{*}{$\begin{array}{l}\text { Length } \\
\text { of } \\
\text { thread, } \\
\text { mini- } \\
\text { mum }\end{array}$} & \multirow{2}{*}{$\begin{array}{l}\text { Width } \\
\text { of band, } \\
\text { mini- } \\
\text { mum }\end{array}$} & \multicolumn{2}{|c|}{$\begin{array}{l}\text { Inside diameter } \\
\text { of fitting }\end{array}$} & \multirow{2}{*}{$\begin{array}{l}\text { Metal } \\
\text { thick- } \\
\text { ness, } \\
\text { maxi- } \\
\text { mum }\end{array}$} & \multirow{2}{*}{$\begin{array}{l}\text { Outside } \\
\text { diameter } \\
\text { of band, } \\
\text { mini- } \\
\text { mum }\end{array}$} \\
\hline & & & & & $\begin{array}{l}\text { Mini- } \\
\text { mum }\end{array}$ & $\begin{array}{l}\text { Maxi- } \\
\text { mum }\end{array}$ & & \\
\hline $\begin{array}{l}1 / 4 \\
38 \\
1 / 2 \\
3 / 4 \\
1^{3 / 4}\end{array}$ & \multicolumn{2}{|c|}{$\begin{array}{l}\text { Dimensions same as } \\
\text { able-iron screwed } \\
\text { (See } 607.2, \text { p. } 422 .)\end{array}$} & \multirow{3}{*}{$\begin{array}{c}\text { for malle- } \\
\text { fittings. }\end{array}$} & $\begin{array}{r}0.38 \\
.44 \\
.50 \\
.56 \\
.62\end{array}$ & \multicolumn{2}{|c|}{$\begin{array}{l}\text { Same as for mal- } \\
\text { l e a b l e-i r on } \\
\text { screwed fittings. }\end{array}$} & $\begin{array}{r}0.110 \\
.120 \\
.130 \\
.155 \\
.170\end{array}$ & $\begin{array}{l}0.93 \\
1.12 \\
1.34 \\
1.63 \\
1.95\end{array}$ \\
\hline $\begin{array}{l}11 / 4 \\
11 / 2 \\
2 \\
21 / 2 \\
3\end{array}$ & & & & $\begin{array}{r}.69 \\
.75 \\
.84 \\
.94 \\
.100\end{array}$ & & & $\begin{array}{l}.185 \\
.200 \\
.220 \\
.240 \\
.260\end{array}$ & $\begin{array}{l}2.39 \\
2.68 \\
3.28 \\
3.86 \\
4.62\end{array}$ \\
\hline $\begin{array}{l}31 / 2 \\
4 \\
5 \\
6 \\
8\end{array}$ & & & & $\begin{array}{l}1.06 \\
1.12 \\
1.18 \\
1.28 \\
1.47\end{array}$ & & & $\begin{array}{l}.280 \\
.310 \\
.380 \\
.430 \\
.550\end{array}$ & $\begin{array}{r}5.20 \\
5.79 \\
7.05 \\
8.28 \\
10.63\end{array}$ \\
\hline $\begin{array}{r}10 \\
12 \\
114 \\
116\end{array}$ & $\begin{array}{r}8.08 \\
9.50 \\
10.40 \\
11.82\end{array}$ & $\begin{array}{r}5.16 \\
5.97 \\
\\
\end{array}$ & $\begin{array}{l}1.68 \\
1.88 \\
2.00 \\
2.20\end{array}$ & $\begin{array}{l}1.68 \\
1.88 \\
2.00 \\
2.20\end{array}$ & $\begin{array}{l}10.750 \\
12.750 \\
14.000 \\
16.000\end{array}$ & $\begin{array}{l}10.850 \\
12.850 \\
14.100 \\
16.100\end{array}$ & $\begin{array}{r}.690 \\
.800 \\
.880 \\
1.000\end{array}$ & $\begin{array}{l}13.12 \\
15.47 \\
16.94 \\
19.30\end{array}$ \\
\hline
\end{tabular}

1 Outside diameter.

Dimensions for reducing elbows and reducing tees are given in Tables 2 and 3, respectively. 
TABLE 2.-Dimensions of elbows (reducing sizes)

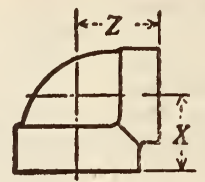

\section{Frgure 235.- Dimensions of elbows (reducing sizes)}

[All dimensions given in inches]

\begin{tabular}{|c|c|c|c|c|c|}
\hline \multirow{2}{*}{$\begin{array}{l}\text { Nominal pipe } \\
\text { sizes }\end{array}$} & \multicolumn{2}{|c|}{ Center to end } & \multirow{2}{*}{$\begin{array}{l}\text { Nominal nipe } \\
\text { sizes }\end{array}$} & \multicolumn{2}{|c|}{ Center to end } \\
\hline & $x$ & $Z$ & & $\mathrm{X}$ & $Z$ \\
\hline $\begin{array}{l}1 / 2 \text { by } 3 / 8 \\
3 / 4 \text { by } 1 / 2 \\
1 \text { by } 3 / 4 \\
1 \text { by } 1 / 2 \\
11 / 4 \text { by } 1 \\
11 / 4 \text { by } 3 / 4 \\
11 / 2 \text { by } 11 / 4 \\
11 / 2 \text { by } 1 \\
2 \text { by } 11 / 2 \\
2 \text { to } 11 / 4 \\
2 \text { by } 1\end{array}$ & $\begin{array}{l}1.04 \\
1.20 \\
1.37 \\
1.26 \\
1.58 \\
1.45 \\
1.82 \\
1.65 \\
2.02 \\
1.90 \\
1.73\end{array}$ & $\begin{array}{l}1.03 \\
1.22 \\
1.45 \\
1.36 \\
1.67 \\
1.62 \\
1.88 \\
1.80 \\
2.16 \\
2.10 \\
2.02\end{array}$ & $\begin{array}{l}21 / 2 \text { by } 2 \\
21 / 2 \text { by } 11 / 2 \\
3 \text { by } 21 / 2 \\
3 \text { by } 2 \\
4 \text { by } 3 \\
4 \text { by } 2 \\
5 \text { by } 4 \\
6 \text { by } 5 \\
6 \text { by } 4 \\
8 \text { by } 6 \\
10 \text { by } 8\end{array}$ & $\begin{array}{l}2.39 \\
2.16 \\
2.83 \\
2.52 \\
3.30 \\
2.74 \\
4.00 \\
4.63 \\
4.13 \\
5.56 \\
7.02\end{array}$ & $\begin{array}{l}2.60 \\
2.51 \\
2.99 \\
2.89 \\
3.60 \\
3.41 \\
4.41 \\
5.03 \\
4.94 \\
6.37 \\
7.87\end{array}$ \\
\hline
\end{tabular}

TABLE 3.-Dimensions of tees (reducing sizes)

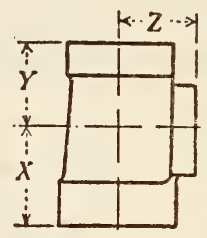

\section{FIGURE 236.- Tees (reduc- ing sizes)}

(Dimensions of cast-iron tees (reducing sizes) are identical with those for malleable iron tees (reducing sizes) in Table 3B, A. S. M. E. B16c. (See 607.2, p. 422.) Additional cast-iron tee dimensions are given in the following table:)

\begin{tabular}{|c|c|c|c|c|c|c|c|c|c|c|c|}
\hline \multirow{2}{*}{ Jominal pipe sizes } & \multicolumn{3}{|c|}{ Center to end } & \multirow{2}{*}{ Nominal pipe sizes } & \multicolumn{3}{|c|}{ Center to end } & \multirow{2}{*}{ Nominal pipe sizes } & \multicolumn{3}{|c|}{ Center to end } \\
\hline & $\mathrm{X}$ & $Y$ & $Z$ & & $\mathrm{X}$ & $\mathrm{Y}$ & $\mathrm{Z}$ & & $\mathrm{X}$ & $Y$ & $\mathrm{Z}$ \\
\hline $\begin{array}{l}1 / 4 \text { by } 11 / 4 \text { by } \\
1 / 4 \text { by } 1 \text { by } 11 / 2 \\
1 / 4 \text { by } 1 \text { by } 1 / 2 \\
1 / 4 \text { by } 1 / 2 \text { by } 11\end{array}$ & $\begin{array}{l}1.50 \\
2.10 \\
1.88 \\
1.34 \\
1.75\end{array}$ & $\begin{array}{l}1.27 \\
2.10 \\
1.80 \\
1.26 \\
1.53\end{array}$ & $\begin{array}{l}1.50 \\
1.90 \\
1.82 \\
1.53 \\
1.75\end{array}$ & $\begin{array}{l}\text { by } 2 \text { by } 11 / 2 .- \\
\text { by } 11 / 2 \text { by } 3 . \\
\text { by } 1 \text { by } 3 \ldots\end{array}$ & $\begin{array}{l}2.00 \\
2.83 \\
2.29 \\
3.08 \\
3.08\end{array}$ & $\begin{array}{l}1.87 \\
2.60 \\
2.02 \\
2.80 \\
2.66\end{array}$ & $\begin{array}{l}2.66 \\
2.99 \\
2.80 \\
3.08 \\
3.08\end{array}$ & $\begin{array}{l}\text { by } 2.2 \\
\text { by } 11 / 2 \\
\text { by } 5 .-\end{array}$ & $\begin{array}{l}3.75 \\
3.26 \\
2.95 \\
2.72 \\
4.50\end{array}$ & $\begin{array}{l}3.75 \\
3.26 \\
2.95 \\
2.72 \\
4.41\end{array}$ & $\begin{array}{l}\text { 4. } 31 \\
\text { 4. } 13 \\
\text { 4. } 03 \\
\text { 3. } 94 \\
\text { 4. } 50\end{array}$ \\
\hline $\begin{array}{l}1 / 2 \text { by } 11 / 4 \text { by } \\
1 / 2 \text { by } 11 / 4 \text { by } \\
1 / 2 \text { by } 1 \text { by } 2 \\
1 / 2 \text { by } 1 \text { by } 11 / 4 \\
1 / 2 \text { by } 3 / 4 \text { by } 1\end{array}$ & $\begin{array}{l}1.52 \\
1.41 \\
2.16 \\
1.82 \\
1.82\end{array}$ & $\begin{array}{l}1.45 \\
1.34 \\
2.02 \\
1.67 \\
1.62\end{array}$ & $\begin{array}{l}1.75 \\
1.66 \\
2.02 \\
1.89 \\
1.88\end{array}$ & $/ 2 \mathrm{by} 31 / 2 \mathrm{by}$ & $\begin{array}{l}3.18 \\
2.62 \\
2.39 \\
2.27 \\
3.18\end{array}$ & $\begin{array}{l}3.18 \\
2.62 \\
2.39 \\
2.27 \\
3.08\end{array}$ & $\begin{array}{l}3.33 \\
3.14 \\
3.05 \\
2.99 \\
3.33\end{array}$ & $\begin{array}{l}\text { by } 4 \\
\text { by } 3 \\
\text { by } 2 \\
\text { by } 5 \\
\text { by } 4\end{array}$ & $\begin{array}{l}4.00 \\
3.51 \\
2.95 \\
4.50 \\
4.00\end{array}$ & $\begin{array}{l}79 \\
30 \\
74 \\
22 \\
60\end{array}$ & $\begin{array}{l}4.41 \\
4.22 \\
4.03 \\
4.50 \\
4.41\end{array}$ \\
\hline $\begin{array}{l}y \quad 21 / 2 \ldots \\
y \quad 1 \\
y \quad 3 / 4\end{array}$ & $\begin{array}{l}1.94 \\
2.89 \\
2.60 \\
1.73 \\
1.60\end{array}$ & $\begin{array}{l}1.66 \\
2.89 \\
2.51 \\
1.65 \\
1.52\end{array}$ & $\begin{array}{l}1.94 \\
2.52 \\
2.39 \\
2.02 \\
1.97\end{array}$ & (n) & $\begin{array}{l}2.93 \\
2.62 \\
2.39 \\
3.42 \\
3.42\end{array}$ & $\begin{array}{l}2.83 \\
2.52 \\
2.29 \\
3.14 \\
3.05\end{array}$ & $\begin{array}{l}3.24 \\
3.14 \\
3.05 \\
3.42 \\
3.42\end{array}$ & $\begin{array}{lll}4 & 8 \\
4 & 5\end{array}$ & $\begin{array}{l}3.51 \\
4.50 \\
6.37 \\
4.63 \\
4.63\end{array}$ & $\begin{array}{l}3.08 \\
4.03 \\
6.37 \\
4.63 \\
4.50\end{array}$ & $\begin{array}{l}4 . \\
4 . \\
5 . \\
5 . \\
5 .\end{array}$ \\
\hline $\begin{array}{l}2 \text { by } 11 / 2 \text { by } 1 / 2 \\
2 \text { by } 11 / 4 \text { by } 1 / 2 \\
2 \text { by } 11 / 4 \text { by } 1 \\
21 / 2 \text { by } 21 / 2 \text { by } \\
21 / 2 \text { by } 2 \text { by } 11\end{array}$ & $\begin{array}{l}1.49 \\
1.90 \\
1.73 \\
3.51 \\
2.16\end{array}$ & $\begin{array}{l}1.41 \\
1.75 \\
1.58 \\
3.51 \\
2.02\end{array}$ & $\begin{array}{l}1.88 \\
2.10 \\
2.02 \\
3.05 \\
2.51\end{array}$ & $\begin{array}{l}6 \\
6 \\
31 / 2- \\
11 / 2- \\
11 / 4-\end{array}$ & $\begin{array}{l}4.94 \\
4.41 \\
3.54 \\
2.51 \\
2.39\end{array}$ & $\begin{array}{l}4.94 \\
4.41 \\
3.54 \\
2.51 \\
2.39\end{array}$ & $\begin{array}{l}4.13 \\
4.00 \\
3.69 \\
3.32 \\
3.26\end{array}$ & -........ & $\begin{array}{l}4.13 \\
5.13 \\
4.13 \\
5.13 \\
5.13\end{array}$ & $\begin{array}{l}4.00 \\
4.94 \\
\text { 3. } 79 \\
\text { 4. } 75 \\
4.56\end{array}$ & $\begin{array}{l}4.94 \\
5.13 \\
4.94 \\
5.13 \\
5.13\end{array}$ \\
\hline $\begin{array}{l}2 \text { by } 11 / 4 \\
2 \text { by } 1 \\
2 \text { by } 3 / 4 \\
2 \text { by } 1 / 2 \\
11 / 2 \text { by } 2\end{array}$ & $\begin{array}{l}2.04 \\
1.87 \\
1.74 \\
1.63 \\
2.39\end{array}$ & $\begin{array}{l}1.90 \\
1.73 \\
1.60 \\
1.49 \\
2.16\end{array}$ & $\begin{array}{l}2.45 \\
2.37 \\
2.32 \\
2.23 \\
2.60\end{array}$ & $\begin{array}{l}1 \\
\text { y } 3 \\
\text { y } 21 \\
4 \\
4\end{array}$ & $\begin{array}{l}2.22 \\
3.30 \\
3.05 \\
2.74 \\
3.79\end{array}$ & $\begin{array}{l}2.22 \\
3.18 \\
2.93 \\
2.62 \\
3.60\end{array}$ & $\begin{array}{l}\text { 3. } 18 \\
3.60 \\
3.51 \\
3.41 \\
3.79\end{array}$ & $\begin{array}{l}4 \\
3 \\
21 / 2\end{array}$ & $\begin{array}{l}\text { 5. } 56 \\
5.03 \\
4.50 \\
4.00 \\
3.69\end{array}$ & $\begin{array}{l}\text { 5. } 56 \\
5.03 \\
4.50 \\
4.00 \\
3.69\end{array}$ & $\begin{array}{l}6.37 \\
6.27 \\
6.17 \\
6.07 \\
6.01\end{array}$ \\
\hline $\begin{array}{l}11 / 2 \text { by } 11 / 2 \\
1 \text { by } 21 / 2 \\
\text { by } 4 \\
\text { by } 31 / 2 \\
\text { by } 3 / 4\end{array}$ & $\begin{array}{l}2.16 \\
2.70 \\
3.60 \\
3.33 \\
1.87\end{array}$ & $\begin{array}{l}1.94 \\
2.37 \\
3.60 \\
3.33 \\
1.87\end{array}$ & $\begin{array}{l}2.51 \\
2.70 \\
3.30 \\
3.18 \\
2.61\end{array}$ & y 4 1 4 (1) & $\begin{array}{l}\text { 3. } 30 \\
2.74 \\
3.79 \\
3.05 \\
3.79\end{array}$ & $\begin{array}{l}2.52 \\
3.51 \\
2.70 \\
3.41\end{array}$ & $\begin{array}{l}\text { 3. } 60 \\
3.41 \\
3.79 \\
3.51 \\
3.79\end{array}$ & $2 \ldots$ & $\begin{array}{l}3.44 \\
6.56 \\
5.56 \\
7.02 \\
6.02\end{array}$ & $\begin{array}{l}3.44 \\
6.37 \\
5.13 \\
7.02 \\
6.02\end{array}$ & $\begin{array}{l}5.84 \\
6.56 \\
6.37 \\
7.87 \\
7.68\end{array}$ \\
\hline $\begin{array}{l}3 \text { by } 21 / 2 \text { by } 21 / 2 \\
3 \text { by } 21 / 2 \text { by } 2 \\
3 \text { by } 21 / 2 \text { by } 11 / 2- \\
3 \text { by } 21 / 2 \text { by } 11 / 4\end{array}$ & $\begin{array}{l}2.83 \\
2.52 \\
2.29 \\
2.17\end{array}$ & $\begin{array}{l}2.70 \\
2.39 \\
2.16 \\
2.04\end{array}$ & $\begin{array}{l}2.99 \\
2.89 \\
2.80 \\
2.74\end{array}$ & $\begin{array}{l}4 \text { by } 11 / 2 \text { by } 4 \\
4 \text { by } 11 / 4 \text { by } 4 \\
5 \text { by } 5 \text { by } 6 \\
5 \text { by } 5 \text { by } 4\end{array}$ & $\begin{array}{l}\text { 3. } 79 \\
3.79 \\
5.03 \\
4.00\end{array}$ & $\begin{array}{l}\text { 3. } 32 \\
\text { 3. } 26 \\
\text { 5. } 03 \\
\text { 4. } 00\end{array}$ & $\begin{array}{l}\text { 3. } 79 \\
\text { 3. } 79 \\
\text { 4. } 63 \\
\text { 4. } 41\end{array}$ & $\begin{array}{l}10 \text { by } 10 \text { by } 4 \\
12 \text { by } 12 \text { by } 8 \\
12 \text { by } 12 \text { by } 6\end{array}$ & $\begin{array}{l}4.96 \\
7.44 \\
6.44\end{array}$ & $\begin{array}{l}4.96 \\
7.44 \\
6.44\end{array}$ & $\begin{array}{l}7.48 \\
9.09 \\
8.90\end{array}$ \\
\hline
\end{tabular}


TABLE 4.-Dimensions of elbows, $45^{\circ}$ elbows, tees, be applied to representative fittings selected at and crosses (straight sizes)
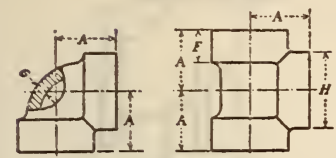

TEE

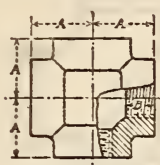

CROSS

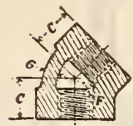

$45^{\circ}$ ELBOW

Figure 237.-Elbows, $45^{\circ}$ elbows, tees, and crosses (straight sizes)

[All dimensions given in inches]

\begin{tabular}{|c|c|c|c|c|c|c|c|c|}
\hline \multirow{3}{*}{ 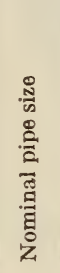 } & \multirow{3}{*}{ 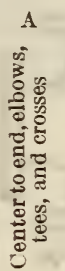 } & \multirow{3}{*}{ 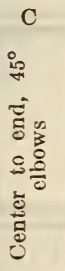 } & \multirow{3}{*}{ 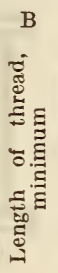 } & \multirow{3}{*}{ 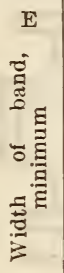 } & & \multirow{3}{*}{ 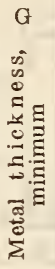 } & \multirow{3}{*}{ 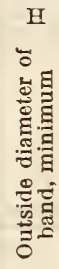 } \\
\hline & & & & & \multicolumn{2}{|c|}{$\begin{array}{l}\text { Inside diameter } \\
\text { of fitting }\end{array}$} & & \\
\hline & & & & & $\stackrel{\Xi}{\Xi}$ & 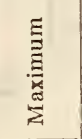 & & \\
\hline $\begin{array}{l}1 / 4 \\
3 / 8 \\
1 / 2 \\
3 / 4 \\
1^{3 / 4}\end{array}$ & $\begin{array}{l}0 . \\
1 . \\
1 . \\
1 . \\
1 .\end{array}$ & $\begin{array}{r}0.81 \\
.88 \\
1.00 \\
1.13 \\
1.31\end{array}$ & $\begin{array}{r}0.43 \\
.47 \\
.57 \\
.64 \\
.75\end{array}$ & $\begin{array}{r}0.49 \\
.55 \\
.60 \\
68 \\
.76\end{array}$ & $\begin{array}{r}0.540 \\
.675 \\
.840 \\
1.050 \\
1.315\end{array}$ & & .2 & $\begin{array}{l}1.17 \\
1.36 \\
1.59 \\
1.88 \\
2.24\end{array}$ \\
\hline $\begin{array}{l}134 \\
11 / 2 \\
2 \\
21 / 2 \\
3\end{array}$ & & & $\begin{array}{r}.84 \\
.87 \\
1.00 \\
1.17 \\
1.23\end{array}$ & & & & $\begin{array}{l}.43 \\
.48\end{array}$ & $\begin{array}{l}2.73 \\
3.07 \\
3.74 \\
4.60 \\
5.36\end{array}$ \\
\hline $\begin{array}{l}31 / 2 \\
4 \\
5 \\
6 \\
8\end{array}$ & $\begin{array}{l}4 . \\
4 . \\
5 . \\
7 .\end{array}$ & $\begin{array}{l}2 . \\
2 . \\
3 . \\
3 . \\
4 .\end{array}$ & $\begin{array}{l}1.28 \\
1.33 \\
1.43 \\
1.53 \\
1.72\end{array}$ & $\begin{array}{l}1.49 \\
1.57 \\
1.74 \\
1.91 \\
2.24\end{array}$ & & $\begin{array}{l}5.663 \\
6.725 \\
8.725\end{array}$ & $\begin{array}{l}.00 \\
.74 \\
.90\end{array}$ & $\begin{array}{r}5.98 \\
6.61 \\
7.92 \\
9.24 \\
11.73\end{array}$ \\
\hline $\begin{array}{r}10 \\
12 \\
114 \\
116\end{array}$ & $\begin{array}{r}8.63 \\
10.00 \\
11.00 \\
12.50\end{array}$ & $\begin{array}{l}\text { 5. } 19 \\
6.00\end{array}$ & $\begin{array}{l}\text { 1. } 2.13 \\
2.25 \\
2.45\end{array}$ & $\begin{array}{l}2.58 \\
2.91 \\
3.10 \\
3.45\end{array}$ & $\begin{array}{l}10.750 \\
12.750 \\
14.000 \\
16.000\end{array}$ & $\begin{array}{l}14.100 \\
16.100\end{array}$ & $\begin{array}{l}1.08 \\
1.24 \\
1.33 \\
1.50\end{array}$ & $\begin{array}{l}14.37 \\
16.84 \\
18.40 \\
20.88\end{array}$ \\
\hline
\end{tabular}

1 Outside diameter.

The 250-pound standard for screwed fittings covers only the straight sizes of $90^{\circ}$ and $45^{\circ}$ elbows, tees, and crosses.

\section{AMERICAN SOCIETY OF MECHANICAL EN- GINEERS, AMERICAN STANDARD CAST- IRON LONG-TURN SPRINKLER FITTINGS (SCREWED AND FLANGED) FOR MAXI- MUM HYDRAULIC WORKING PRESSURES OF 150 AND 250 POUNDS PER SQUARE INCH (GAGE), B16g-1929}

[Approved as American Standard by the American Standards Association, ${ }^{67}$ A pril, 1929]

\section{CAST-IRON IONG-TURN SPRINKLER FITTINGS}

\section{INTRODUCTORY NOTES}

1. Pressure ratings.-These standards shall be known as the American 150 and 250 pound castiron long-turn sprinkler fittings, said pressure designations being the maximum recommended ratings.

The following momentary test pressures shall be applied by insurance companies, underwriters, etc., only when passing upon the acceptability of this material for sprinkler service. This test shall

6i Sponsor organizations: American Society of Mechanical Engineers, Manufacturers' Standardization Society of Valve and Fittings Industry, and Heating and Piping Contractors National Association.

random, the fittings being tested to destruction.

The 150-pound fittings, sizes $2 \frac{1}{2}$ to 6 inches, inclusive, shall be able to resist a momentary pressure of 750 pounds per square inch and the 8-inch size shall be able to resist a momentary pressure of 600 pounds per square inch.

The 250-pound fittings, sizes $2 \frac{1}{2}$ to 6 inches, inclusive, shall be able to resist a momentary pressure of 1,250 pounds per square inch and the 8 -inch size shall be able to resist a momentary pressure of 1,000 pounds per square inch.

2. Size.-The term size shall be used in all the following tables to indicate the nominal inside diameter of port. These specifications are for long-turn cast-iron fittings for sizes $2 \frac{1}{2}$ to 8 inches, inclusive, for use in sprinkler work inside of buildings.

The sizes smaller than $2 \frac{1}{2}$ inches are the American 125 and 250 pound cast-iron screwed fittings standards, while the 8-inch sizes are the American 125 and 250 pound cast-iron flanged fittings standards.

3. Markings.-Each fitting produced with the intent of meeting the specifications of these American cast-iron long-turn sprinkler fittings, shall have marks cast on them indicating legibly:

(1) Maker's name or trade-mark.

(2) Identification symbol by means of which this line of fittings can be distinguished.

(3) Working pressure.

4. Types.-The various styles of elbows, tees, and crosses included in this standard are shown in Figure 238.

When one end of the fitting is flanged, it shall be the largest opening unless otherwise specified.

5. Material.-(Identical with that for cast-iron pipe flanges and flanged fittings for 125 pounds pressure in $\mathrm{B} 16 \mathrm{a}$, given above.)

6. Facings.-All the flanges for the 150-pound standard shall be plain faced. All the flanges for the 250-pound standard shall have a raised face one-sixteenth inch high of the diameters given in Table 5 . The raised face is included in the flange thickness and center to face dimensions.

7. Bolting.-Drilling templates shall be in multiples of four, so that fittings may be made to face in any quarter. Bolt holes shall straddle the center line and shall be drilled one-eighth inch larger than the nominal diameter of bolts. Drilling templates are those of the American 125-pound and 250-pound cast-iron flanged fittings standards.

Bolts shall be of steel with standard rough square heads and the nuts shall be of steel with standard rough hexagonal dimensions; all as given in the tentative American standard on wrenchhead bolts and nuts and wrench openings.

8. Metal thickness.-It is recognized that some variations are unavoidable in the making of patterns and castings. In order to define what may be considered a reasonable degree of accuracy in body thickness measurements of these fittings, the following inspection limits shall be allowed:

Body thickness measurements taken on any fitting at points diametrically opposite shall, when added together and divided by two, equal or exceed the minimum metal thicknesses given in these standards. Body thicknesses at no point shall be more than 10 per cent below the minimum metal thicknesses given in these standards.

The minimum wall thickness of each outlet of reducing fittings shall be that given in Tables 2 and 5. Any changes of thickness necessary shall be made gradually.

9. Reducing fittings.-Fittings may be reduced by bushing in the sand any of the outlets one size smaller, but in reducing fittings, the total reduction of any outlet shall not be greater than 50 per cent 


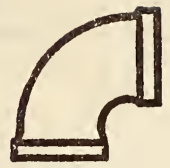

STYLE-A

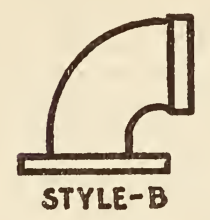

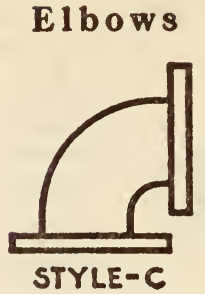
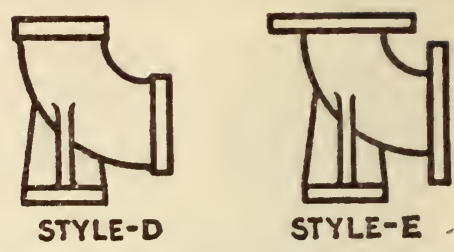

\section{Tees}

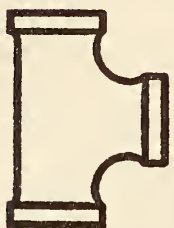

STYLE-G
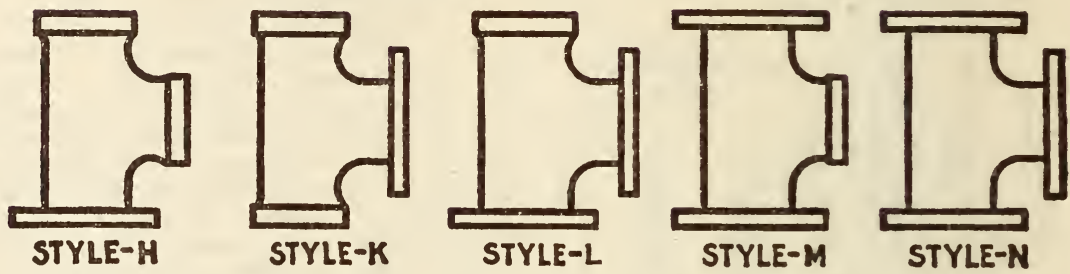

Crosses
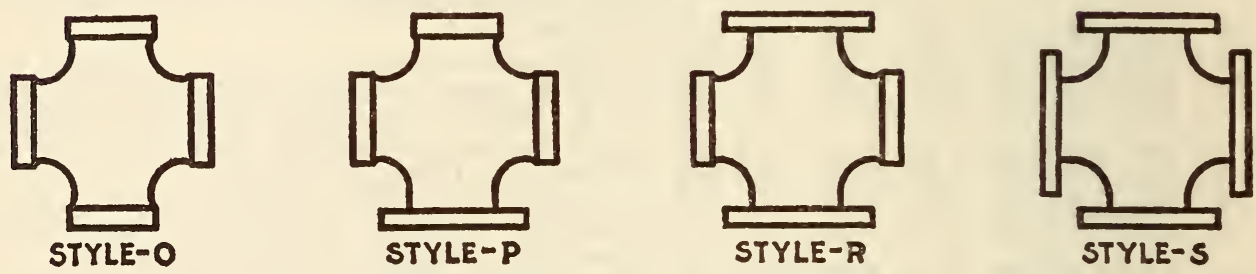

FigURE 238.-Elbows, tees, and crosses

of the nominal diameter of the largest opening.

Reducing tees and crosses for 150 pounds pressure on sizes from $2 \frac{1}{2}$ to 6 inches, inclusive, shall have center-to-end dimensions as shown in Tables 3 and 4. Reducing sizes for 8-inch fittings have the same center-to-end dimensions as the straight sizes.

10. Bosses, ribs, and lugs.-Tapping through the bodies shall be permitted only for drain or test

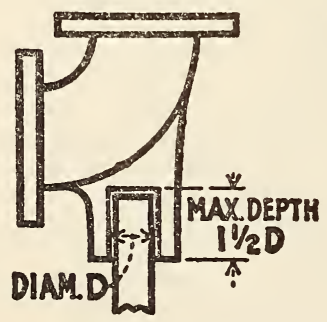

FIgURE 239.-Slip boss with blind hole

purposes, in which case fittings must be provided with bosses for tapping when required and when the location and size of tapping are specified. Slip bosses having blind holes shall be provided when required to act as sockets for support. (See fig. 239)

Fittings for sizes from $2 \frac{1}{2}$ to 6 inches, inclusive, may be tapped one-half inch on the side without a boss, and fittings larger than 6 inches may be tapped three-quarters inch without a boss.
The addition of ribs or lugs is not probihited on any screwed fitting.

11. Threading.- Screwed fittings shall be threaded according to the American standard for pipe thread. (See A. S. A., B2-1919, and the 1924 Report of the National Screw Thread Commission.)

It is recommended that all internal threads be countersunk a distance equal to one-half the pitch of the thread and at an angle of $45^{\circ}$ with the axis of the thread.

The lenght of the threads specified in Tables 2 and 5 shall be measured to include the countersink.

12. Fitting dimensions.-Screwed, flanged, and combination screwed and flanged fittings for 150 and 250 pound pressures shall have dimensions as shown in Tables 2 and 5, respectively. The 8-inch fitting s are the regular American standard flanged fittings.

In describing tees and crosses, the run is first named and then the outlet or outlets.

Due to the unavoidable variations in the making of patterns and castings and in order to establish a reasonable degree of accuracy in the center-to-end dimensions of all screwed fittings, the following inspection limits shall be allowed.

[All dimensions given in inches]

\begin{tabular}{|c|c|c|c|c|c|c|c|c|}
\hline Nominal pipe size.... & 2 & $21 / 2$ & 3 & $31 / 2$ & 4 & 5 & 6 & 81 \\
\hline Center & 0.08 & 0.10 & 0.10 & 0.10 & 0.12 & 0.12 & 0.14 & \\
\hline
\end{tabular}

1 See introductory notes, par. 2. 
TABLE 2.-General dimensions for cast-iron long-turn fittings for maximum hydraulic working pressure of 150 pounds per square inch
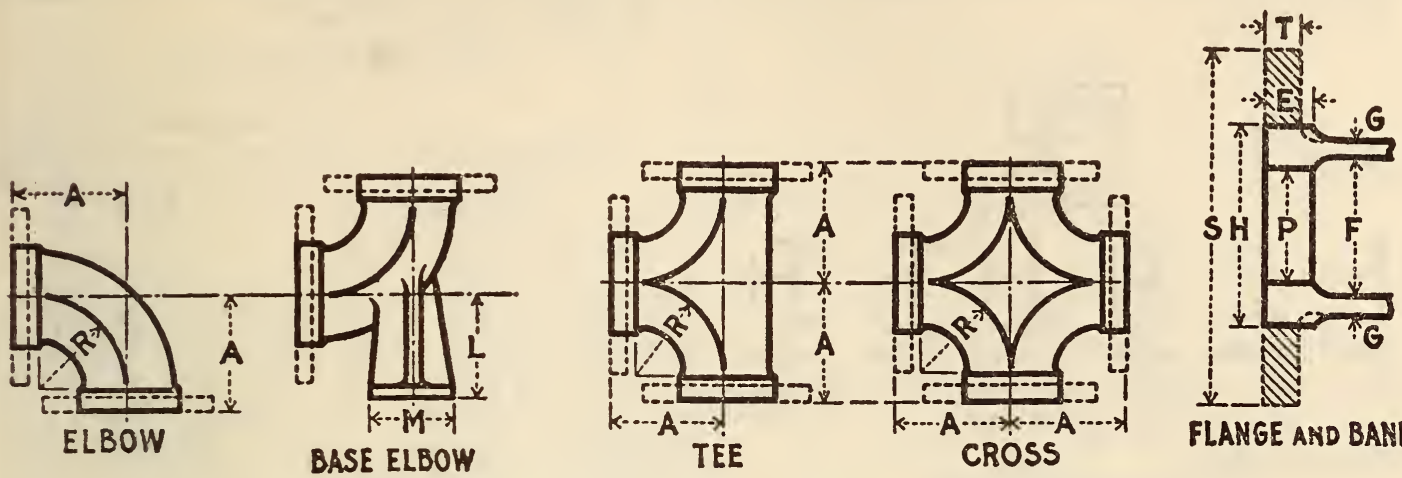

Figure 240.-Cast-iron long-turn fittings 150 pounds per square inch

FLANGE AND BAND

[All dimensions given in inches]

\begin{tabular}{|c|c|c|c|c|c|c|c|c|c|}
\hline & & \multicolumn{8}{|c|}{ Sizes } \\
\hline & & 2 & 21,2 & 3 & $31 / 2$ & 4 & 5 & 6 & 81 \\
\hline $\begin{array}{l}\mathbf{A} \\
\mathrm{L}\end{array}$ & $\begin{array}{l}\text { Center to end or face...... } \\
\text { Center to base }\end{array}$ & \multirow{2}{*}{$\begin{array}{c}\text { Outlets } \\
\text { for } \\
\text { reducing } \\
\text { sizes } \\
6 \\
5 \%\end{array}$} & \multirow{2}{*}{$\begin{array}{l}41 / 2 \\
41 / 2 \\
7 \\
11 / 16 \\
41 / 2\end{array}$} & $\begin{array}{l}5 \\
478\end{array}$ & $\begin{array}{l}53 / 4 \\
51 / 4\end{array}$ & $\begin{array}{l}61 / 2 \\
51 / 2\end{array}$ & $\begin{array}{l}71 / 2 \\
61 / 4\end{array}$ & $\begin{array}{l}9 \\
7\end{array}$ & $\begin{array}{l}9 \\
83 / 8\end{array}$ \\
\hline $\begin{array}{l}\mathrm{S} \\
\mathrm{T} \\
\mathrm{M}\end{array}$ & $\begin{array}{l}\text { Diameter of flange } \\
\text { Thickness of flange } \\
\text { Diameter of base flange. }\end{array}$ & & & $\begin{array}{l}71 / 2 \\
3 / 4 \\
5\end{array}$ & $\begin{array}{l}81 / 2 \\
1316\end{array}$ & ${ }^{9} 15 / 16$ & $7^{15 / 16}$ & $\begin{array}{r}11 \\
1 \\
7\end{array}$ & $\begin{array}{l}131 / 2 \\
11 / 8 \\
9\end{array}$ \\
\hline F & $\begin{array}{l}\text { Inside diameter of }\left\{\begin{array}{l}\text { maximum } \\
\text { body }\end{array}\right.\end{array}$ & $\begin{array}{l}2.445 \\
2.375\end{array}$ & $\begin{array}{l}2.975 \\
2.875\end{array}$ & $\begin{array}{l}\text { 3. } 600 \\
\text { 3. } 500\end{array}$ & $\begin{array}{l}\text { 4. } 100 \\
4.000\end{array}$ & $\begin{array}{l}4.600 \\
4.500\end{array}$ & $\begin{array}{l}5.663 \\
5.563\end{array}$ & $\begin{array}{l}6.725 \\
6.625\end{array}$ & \}Nominal. \\
\hline $\mathbf{P}$ & Diameter of port for $\left\{\begin{array}{l}\text { screwed. } \\
\text { flanged.- }\end{array}\right.$ & \multicolumn{8}{|c|}{ Rough tapping diameter } \\
\hline \multirow[t]{4}{*}{$\begin{array}{l}\mathrm{H} \\
\mathbf{E} \\
\mathrm{G} \\
\mathrm{R}\end{array}$} & $\begin{array}{l}\text { Band diameter, }{ }^{2} \text { minimum } \\
\text { Band width, }{ }^{2} \text { minimum } \\
\text { Metal thickness, minimumm } \\
\text { Radius of sweep, minimum }\end{array}$ & $\begin{array}{r}3.28 \\
.84 \\
.22\end{array}$ & $\begin{array}{r}3.86 \\
.94 \\
.30 \\
3.50\end{array}$ & $\begin{array}{l}4.62 \\
1.00 \\
.32 \\
3.87\end{array}$ & $\begin{array}{r}5.20 \\
1.06 \\
.34 \\
4.53\end{array}$ & $\begin{array}{r}5.79 \\
1.12 \\
.36 \\
5.19\end{array}$ & $\begin{array}{l}7.05 \\
1.18 \\
.41 \\
6.06\end{array}$ & $\begin{array}{l}8.28 \\
1.28 \\
.46 \\
7.44\end{array}$ & 62 \\
\hline & $\begin{array}{l}\text { Length of thread, }{ }^{3} \text { minimum } \\
\text { Diameter of bolt circle... } \\
\text { Number of bolts. } \\
\text { Diameter of bolts.. }\end{array}$ & $\begin{array}{l}.75 \\
43 / 4 \\
4 \\
5 / 8\end{array}$ & $\begin{array}{l}592 \\
4 \frac{152}{5 / 8}\end{array}$ & $\begin{array}{l}6^{.98} \\
4 \\
5 / 8\end{array}$ & $\begin{array}{l}1.03 \\
7 \\
8 / 8\end{array}$ & $\begin{array}{l}1.08 \\
71 / 2 \\
8 \\
5 / 8\end{array}$ & $\begin{array}{l}1.18 \\
81 / 2 \\
8 \\
3 / 4\end{array}$ & $\begin{array}{l}1.28 \\
91 / 2 \\
8 \\
3 / 4\end{array}$ & $\begin{array}{r}113 / 4 \\
8 / 4\end{array}$ \\
\hline & $\begin{array}{l}\text { Diameter of drilled bolt holes } \\
\text { Style elbows recommended for } \\
\text { stock }\end{array}$ & $3 / 4$ & A, B, C & $\mathrm{A}, \mathrm{B}, \mathrm{C}^{3 / 4}$ & $\mathrm{~A}, \mathrm{~B}, \mathrm{C}^{3 / 4}$ & $\mathrm{~A}, \mathrm{~B}, \mathrm{C}^{3 / 4}$ & $\mathrm{~A}, \mathrm{~B}, \mathrm{C}^{7 / 8}$ & $\mathrm{~A}, \mathrm{~B}, \mathrm{C}^{7 / 8}$ & $7 / 8$ \\
\hline & $\begin{array}{l}\text { Style tees recommended for stock } \\
\text { Style crosses recommended for } \\
\text { stock... }\end{array}$ & & $\mathrm{G}, \mathrm{H}, \mathrm{L}$ & $\mathrm{G}, \mathrm{H}, \mathrm{L}, \mathrm{N}$ & $\begin{array}{l}\text { G, H, L, N } \\
P\end{array}$ & $\begin{array}{l}\text { G, H, L, N } \\
P\end{array}$ & $\begin{array}{l}\text { G, H, L, N } \\
P\end{array}$ & $\begin{array}{l}\mathrm{G}, \mathrm{H}, \mathrm{L}, \mathrm{N} \\
\mathrm{P}\end{array}$ & \\
\hline
\end{tabular}

1 The 8-inch size is an American standard 125-pound cast-iron flanged fitting.

2 Fittings may be banded or have equivalent bead and band.

8 The minimum length of thread shall be measured to include countersink.

- Drilling templates are in multiples of 4 , so that fittings may be made to face in any quarter and bolt holes straddle the center line. 
TABLE 3.-Dimensions of cast-iron reducing tees for a maximum hydraulic working pressure of 150 pounds per square inch
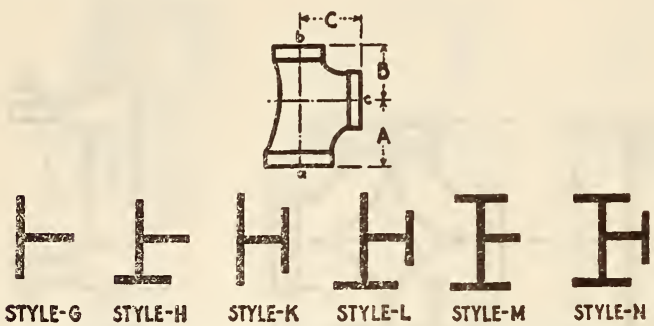

Figure 241.-Cast-iron reducing tees 150 pounds per square inch

[All dimensions given in inches]

\begin{tabular}{l} 
Nominal pipe sizes \\
\hline
\end{tabular}

\begin{tabular}{|c|c|c|c|}
\hline \multicolumn{3}{|c|}{ Center to end } & \multirow{2}{*}{$\begin{array}{l}\text { Styles } \\
\text { recom- } \\
\text { mended } \\
\text { for stock }\end{array}$} \\
\hline A & B & $\mathrm{C}$ & \\
\hline $\begin{array}{l}51 / 8 \\
413 / 16 \\
413 / 16 \\
41 / 2 \\
51 / 2\end{array}$ & $\begin{array}{l}51 / 8 \\
413 / 16 \\
41 / 2 \\
4316 \\
51 / 2\end{array}$ & $\begin{array}{l}411 / 16 \\
49 / 10 \\
49116 \\
413 \\
53 / 16\end{array}$ & $\begin{array}{l}G \\
G, K \\
G \\
G, H \\
G\end{array}$ \\
\hline $\begin{array}{l}51 / 4 \\
4916 \\
51 / 4 \\
5 \\
4916\end{array}$ & $\begin{array}{l}51 / 4 \\
4916 \\
518 \\
413 / 16 \\
41 / 2\end{array}$ & $\begin{array}{l}5 \\
4^{13} / 16 \\
5 \\
5 \\
4^{13} / 16\end{array}$ & $\begin{array}{l}\text { G, K } \\
\text { G, H } \\
\text { G } \\
\text { G, H } \\
\text { G, H }\end{array}$ \\
\hline $\begin{array}{l}51 / 4 \\
5 \\
69 / 16 \\
6 \\
5\end{array}$ & $\begin{array}{l}413 / 16 \\
41 / 2 \\
69 / 16 \\
6 \\
5\end{array}$ & $\begin{array}{l}5 \\
5 \\
515 / 16 \\
5^{13} / 16 \\
51 / 4\end{array}$ & $\begin{array}{l}G \\
G \\
G \\
G, K \\
G, H\end{array}$ \\
\hline $\begin{array}{l}4^{11 / 16} \\
6 \\
5^{334} \\
5 \\
4^{11 / 16}\end{array}$ & $\begin{array}{l}411 / 16 \\
51 / 2 \\
51 / 4 \\
5 \\
4916\end{array}$ & $\begin{array}{l}51 / 8 \\
513,16 \\
53 / 4 \\
51 / 4 \\
51 / 8\end{array}$ & $\begin{array}{l}\text { G, H } \\
\text { G } \\
\text { G, H } \\
\text { G, H } \\
\text { G }\end{array}$ \\
\hline $\begin{array}{l}6 \\
5^{3 / 4} \\
5 \\
5 \\
5^{3 / 4}\end{array}$ & $\begin{array}{l}53 / 8 \\
518 \\
41316 \\
413 / 16 \\
4^{13} / 16\end{array}$ & $\begin{array}{l}513 / 16 \\
53 / 4 \\
51 / 4 \\
514 \\
53 / 4\end{array}$ & $\begin{array}{l}G \\
G \\
G \\
G \\
G \\
G\end{array}$ \\
\hline $\begin{array}{l}5^{3} / 4 \\
71 / 8 \\
51316 \\
53 / 16 \\
61 / 2\end{array}$ & $\begin{array}{l}413 / 16 \\
71 / 8 \\
513 / 16 \\
53 / 16 \\
6\end{array}$ & $\begin{array}{l}53 / 4 \\
65 / 8 \\
6 \\
51 / 2 \\
61 / 2\end{array}$ & $\begin{array}{l}\text { G } \\
\text { G } \\
\text { G, H, L } \\
\text { H } \\
\text { G, H, L }\end{array}$ \\
\hline $\begin{array}{l}513 / 16 \\
61 / 2 \\
513 / 16 \\
53 / 16 \\
61 / 2\end{array}$ & $\begin{array}{l}53 / 4 \\
51 / 2 \\
51 / 4 \\
5 \\
53 / 8\end{array}$ & $\begin{array}{l}6 \\
61 / 2 \\
6 \\
51 / 2 \\
61 / 2\end{array}$ & $\begin{array}{l}G, H \\
G \\
G \\
G \\
G \\
G\end{array}$ \\
\hline $\begin{array}{l}61 / 2 \\
81 / 16 \\
658 \\
71 \frac{1}{2} \\
71 / 2\end{array}$ & $\begin{array}{l}53 / 8 \\
8116 \\
658 \\
718 \\
6916\end{array}$ & $\begin{array}{l}61 / 2 \\
79116 \\
71 / 8 \\
71 / 2 \\
71 / 2\end{array}$ & $\begin{array}{l}\mathrm{G} \\
\mathrm{G} \\
\mathrm{H}, \mathrm{L} \\
\mathrm{K} \\
\mathrm{G}\end{array}$ \\
\hline
\end{tabular}

1 These fittings may be made by bushing in the sand the sizes listed immediately above them.
TABLE 3.-Dimensions of cast-iron reducing tees for a maximum hydraulic working pressure of 150 pounds per square inch-Continued

[All dimensions giren in inches]

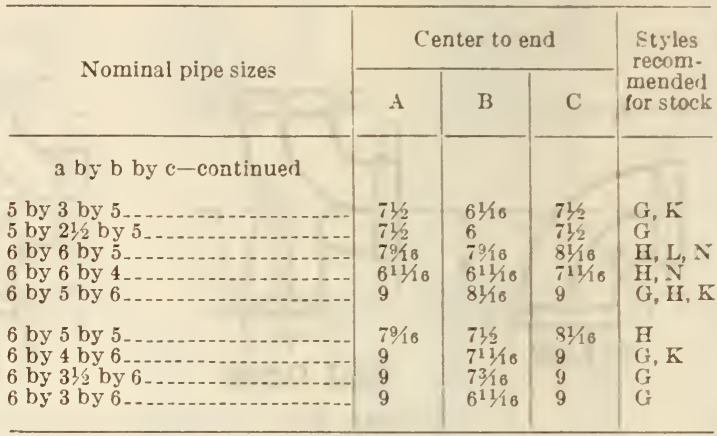

TABLE 4.-Dimensions of cast-iron reducing crosses for a maximum hydraulic working pressure of 150 pounds per square inch

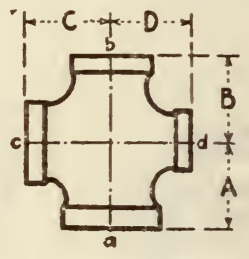

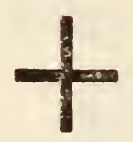

STYLE-O

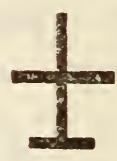

STYLE-P

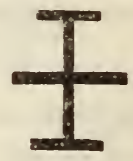

STYLE-R

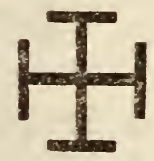

STYLE-S
Figure 242.-Cast-iron reducing crosses 150 pounds per square inch

[All dimensions given in inches]

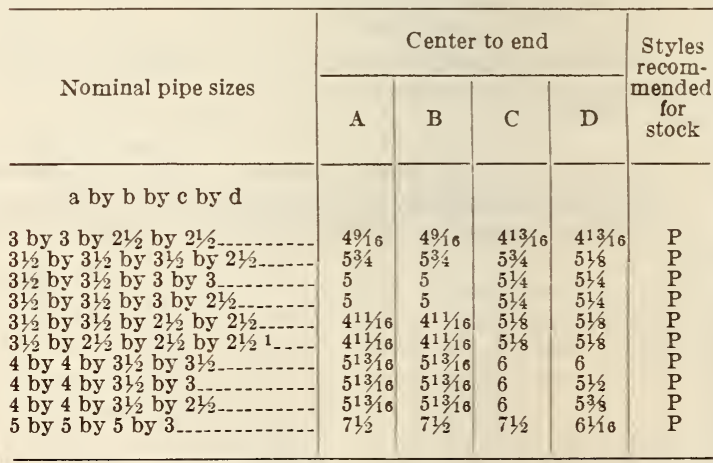

1 This fitting may be made by bushing in the sand the size listed immediateiy above. 
TABLE 5.-General dimensions for cast iron long turn fittings for maximum hydraulic working pressure of 250 pounds per square inch
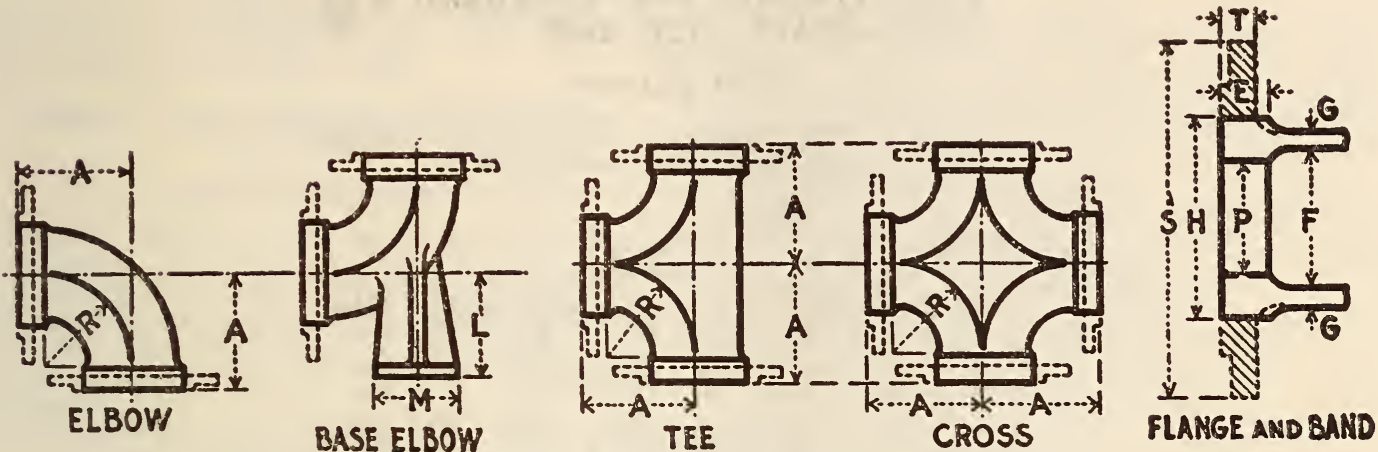

FIGURE 243.-Cast-iron long-turn fittings 250 pounds per square inch

[All dimensions given in inches]

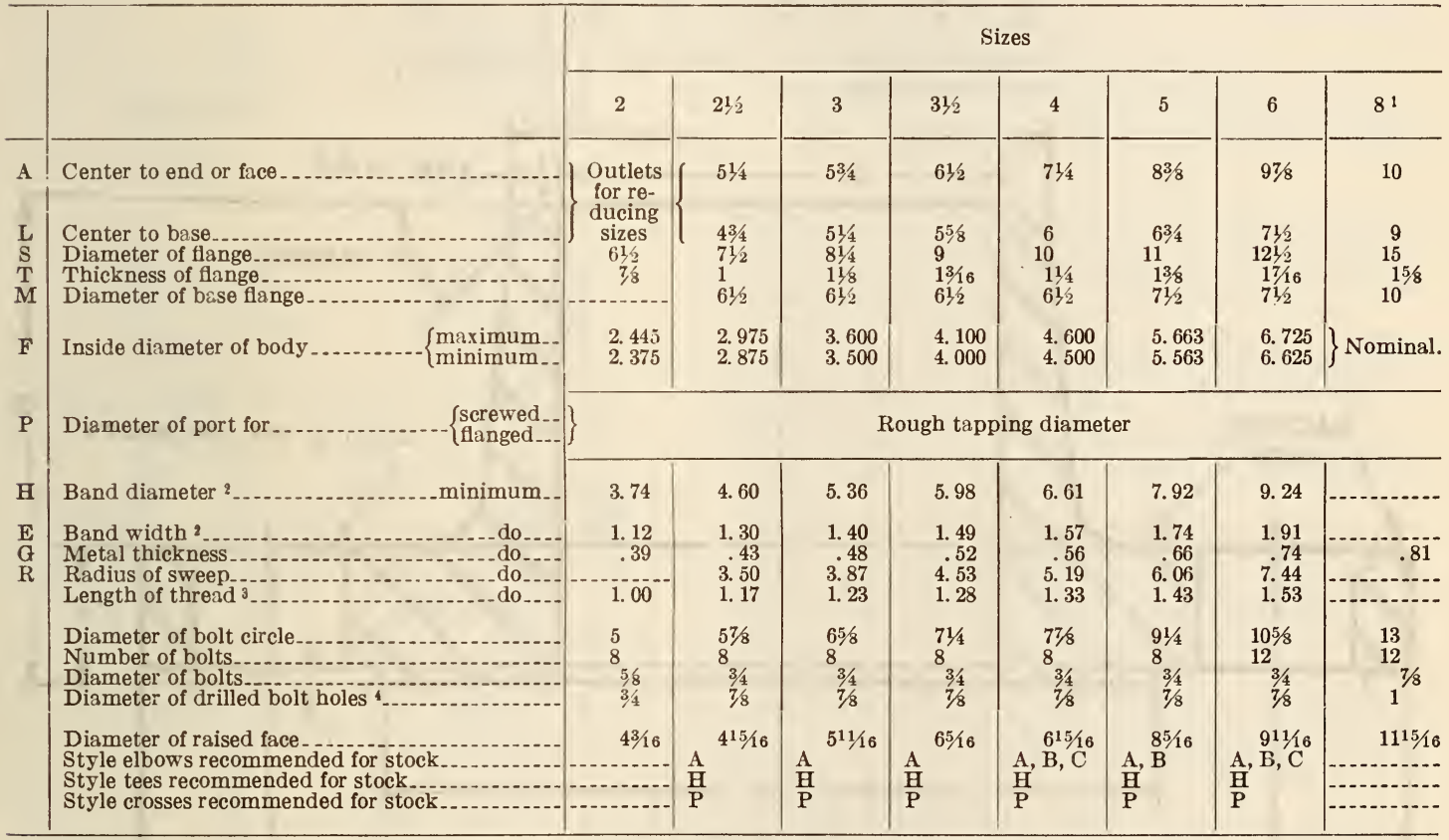

1 The 8-inch size is an American Standard 250 pound cast-iron flanged fitting.

${ }^{2}$ Fittings may be banded or have equivalent bead and band.

3 The minimum length of thread shall be measured to include countersink.

- Drilling templates are in multiples of 4 , so that fittings may be made to face any quarter, and bolt holes straddle the center line.

\section{AMERICAN SOCIETY FOR TESTING MATE- RIALS, STANDARD SPECIFICATIONS FOR CAST-IRON SOIL PIPE AND FITTINGS, DESIGNATION A74-29, 1929}

\section{CAST-IRON SOIL PIPE AND FITTINGS}

(See 607.11, p. 371.)
AMERICAN SOCIETY FOR TESTING MATERIALS, STANDARD SPECIFICATIONS FOR GRAY-IRON CASTINGS FOR VALVES, FLANGES, AND PIPE FITTINGS, DESIGNATION A126-30, 1930

\section{GRAY-IRON CASTINGS FOR VALVES}

(See 611.11, p. 637.) 
HEATING AND PIPING CONTRACTORS NATIONAL ASSOCIATION, WELDING NECK FLANGES FOR STANDARD AND EXTRA-HEAVY PIPE, 1929

\section{PIPE FLANGES}

TABLE 1.-Dimensions of standard-weight welding neck flanges for standard pipe

\begin{tabular}{|c|c|c|c|c|c|c|c|}
\hline \multirow{2}{*}{ Size } & \multirow{2}{*}{ A } & \multicolumn{2}{|c|}{ Drilling } & \multirow{2}{*}{$\begin{array}{c}\text { E 1 } \\
\text { Stand- } \\
\text { ard pipe }\end{array}$} & \multirow{2}{*}{ F } & \multirow{2}{*}{ G } & \multirow{2}{*}{ H } \\
\hline & & B & $\mathrm{C}$ & & & & \\
\hline $\begin{array}{c}\text { Inches } \\
2 \\
21 / 2 \\
3 \\
4\end{array}$ & $\begin{array}{c}\text { Inches } \\
6 \\
7 \\
71 / 2 \\
9\end{array}$ & $\begin{array}{c}\text { Inches } \\
43 / 4 \\
51 / 2 \\
6 \\
71 / 2\end{array}$ & $\begin{array}{r}\text { Inches } \\
4-5 / 8 \\
4-5 / 8 \\
4-5 / 8 \\
8-5 / 8\end{array}$ & $\begin{array}{l}\text { Inches } \\
21 / 16 \\
215 / 32 \\
31 / 16 \\
41 / 32\end{array}$ & $\begin{array}{c}\text { Inches } \\
5 / 8 \\
11 / 16 \\
3 / 4 \\
15 / 10\end{array}$ & $\begin{array}{r}\text { Inches } \\
23 / 2 \\
23 / 4 \\
23 / 4 \\
3\end{array}$ & $\begin{array}{l}\text { Inches } \\
21 / 2 \\
3 \\
35 / 8 \\
45 / 8\end{array}$ \\
\hline $\begin{array}{r}5 \\
6 \\
8 \\
10\end{array}$ & $\begin{array}{l}10 \\
11 \\
131 / 2 \\
16\end{array}$ & $\begin{array}{r}81 / 2 \\
91 / 2 \\
113 / 4 \\
141 / 4\end{array}$ & $\begin{array}{r}8-3 / 4 \\
8-3 / 4 \\
8-3 / 4 \\
12-7 / 8\end{array}$ & $\begin{array}{l}51 / 10 \\
61 / 10 \\
8 \\
10\end{array}$ & $\begin{array}{l}15 / 10 \\
116 \\
13 / 16\end{array}$ & $\begin{array}{l}31 / 2 \\
31 / 2 \\
4 \\
4\end{array}$ & $\begin{array}{l}511 / 10 \\
63 / 4 \\
83 / 4 \\
107 / 8\end{array}$ \\
\hline $\begin{array}{l}12 \\
14 \\
16 \\
16\end{array}$ & $\begin{array}{l}19 \\
21 \\
231 / 2 \\
25\end{array}$ & $\begin{array}{l}17 \\
183 / 4 \\
211 / 4 \\
223 / 4\end{array}$ & $\begin{array}{l}12-7 / 8 \\
12-1 \\
16-1 \\
16-11 / 8\end{array}$ & $\begin{array}{l}12 \\
\text { (1) } \\
(1) \\
(1)\end{array}$ & $\begin{array}{l}11 / 4 \\
13 / 8 \\
17 / 16 \\
1 \% 16\end{array}$ & $\begin{array}{l}41 / 2 \\
5 \\
5 \\
51 / 2\end{array}$ & $\begin{array}{l}127 / 8 \\
143 / 16 \\
161 / 4 \\
181 / 4\end{array}$ \\
\hline
\end{tabular}

1 Orders or inquiries should specify diameter of bore E required.

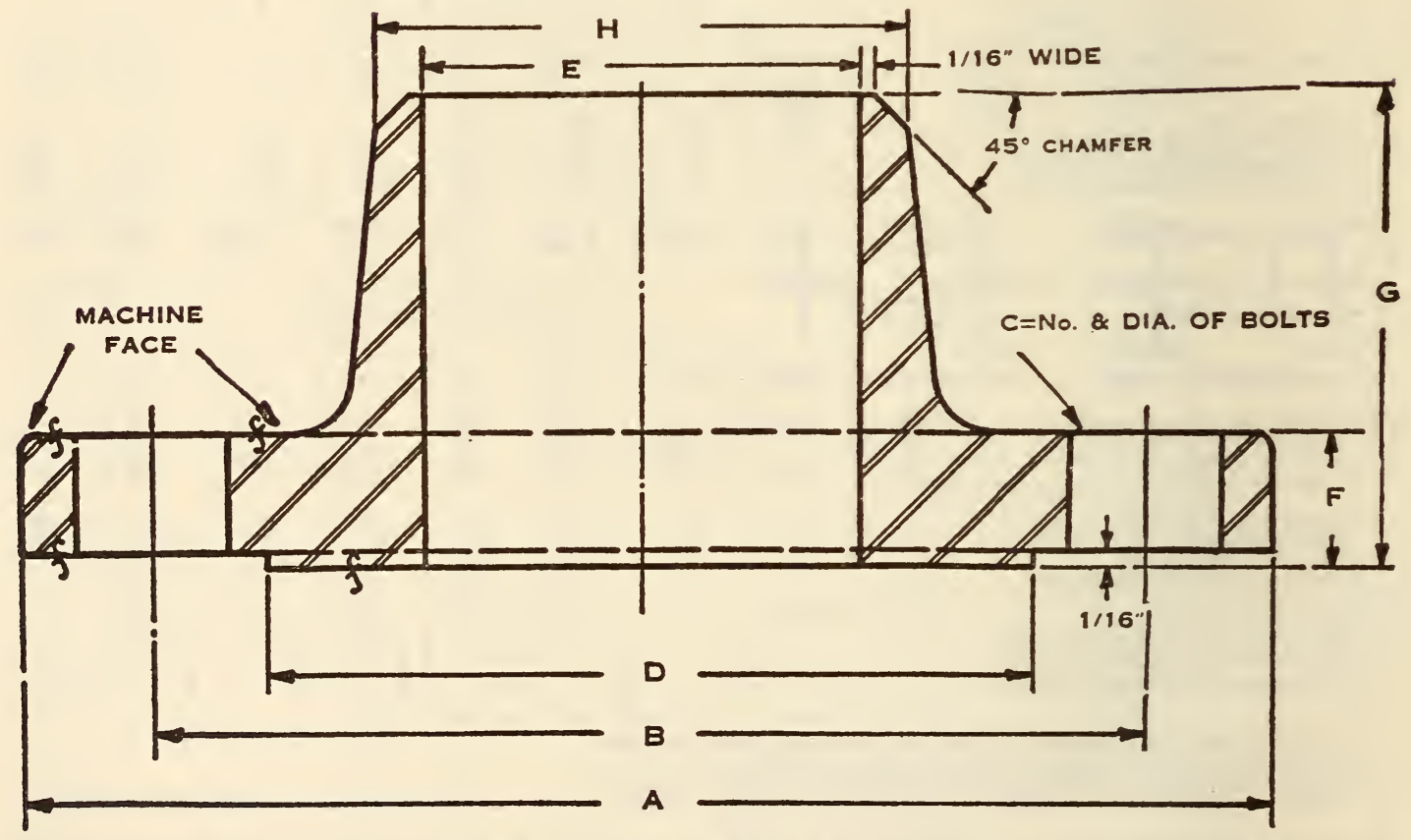

FIGURE 244

TABLE 2. Dimensions of extra-heavy welding neck flanges for standard and extra-heavy pipe

\begin{tabular}{|c|c|c|c|c|c|c|c|c|c|}
\hline \multirow[b]{2}{*}{ Size } & \multirow[b]{2}{*}{ A } & \multicolumn{2}{|c|}{ Drilling } & \multirow[b]{2}{*}{$\mathrm{D}$} & $\mathrm{E}^{1}$ & $\mathrm{E}^{1}$ & \multirow[b]{2}{*}{$F$} & \multirow{2}{*}{ G } & \multirow[b]{2}{*}{ H } \\
\hline & & B & C & & $\begin{array}{l}\text { Standard } \\
\text { pipe }\end{array}$ & $\begin{array}{c}\text { Extra } \\
\text { heavy } \\
\text { pipe }\end{array}$ & & & \\
\hline $\begin{array}{c}\text { Inches } \\
2 \\
21 / 2 \\
3 \\
4\end{array}$ & $\begin{array}{c}\text { Inches } \\
61 / 2 \\
71 / 2 \\
81 / 4 \\
10\end{array}$ & $\begin{array}{c}\text { Inches } \\
5 \\
57 / 8 \\
65 / 8 \\
77 / 8\end{array}$ & $\begin{array}{r}\text { Inches } \\
8-5 / 8 \\
8-3 / 4 \\
8-3 / 4 \\
8-3 / 4\end{array}$ & $\begin{array}{c}\text { Inches } \\
35 / 8 \\
41 / 8 \\
5 \\
63 / 16\end{array}$ & $\begin{array}{c}\text { Inches } \\
21 / 16 \\
215 / 32 \\
31 / 16 \\
41 / 32\end{array}$ & $\begin{array}{c}\text { Inches } \\
1^{15 / 16} \\
25 / 16 \\
27 / 8 \\
3^{13 / 16}\end{array}$ & $\begin{array}{c}\text { Inches } \\
7 / 8 \\
1 \\
11 / 8 \\
11 / 4\end{array}$ & $\begin{array}{r}\text { Inches } \\
23 / 4 \\
318 \\
318 \\
338\end{array}$ & $\begin{array}{c}\text { Inches } \\
21 / 2 \\
3 \\
35 / 8 \\
45 / 8\end{array}$ \\
\hline $\begin{array}{r}5 \\
6 \\
8 \\
10\end{array}$ & $\begin{array}{l}11 \\
121 / 2 \\
15 \\
171 / 2\end{array}$ & $\begin{array}{c}91 / 4 \\
105 / 8 \\
13 \\
151 / 4\end{array}$ & $\begin{array}{l}8-3 / 4 \\
12-3 / 4 \\
12-7 / 8 \\
16-1\end{array}$ & $\begin{array}{c}75 / 16 \\
812 \\
1058 \\
123 / 4\end{array}$ & $\begin{array}{c}51 / 16 \\
6116 \\
8 \\
10\end{array}$ & $\begin{array}{l}413 / 16 \\
53 / 4 \\
75 / 8 \\
93 / 4\end{array}$ & $\begin{array}{l}13 / 8 \\
17 / 6 \\
158 \\
17 / 8\end{array}$ & $\begin{array}{l}37 / 8 \\
37 / 8 \\
458 \\
45 / 8\end{array}$ & $\begin{array}{c}511 / 16 \\
63 / 4 \\
83 / 4 \\
107 / 8\end{array}$ \\
\hline $\begin{array}{ll} & 12 \\
2 & 14 \\
2 & 16 \\
2 & 18\end{array}$ & $\begin{array}{l}203 / 2 \\
23 \\
251 / 2 \\
28\end{array}$ & $\begin{array}{l}173 / 4 \\
201 / 4 \\
221 / 2 \\
243 / 4\end{array}$ & $\begin{array}{l}16-11 / 8 \\
20-118 \\
20-11 / 4 \\
24-11 / 4\end{array}$ & $\begin{array}{l}15 \\
161 / 4 \\
181 / 2 \\
21\end{array}$ & $\begin{array}{r}12 \\
(1) \\
(1) \\
(1)\end{array}$ & $\begin{array}{r}113 / 4 \\
(1) \\
(1) \\
(1)\end{array}$ & $\begin{array}{l}2 \\
21 / 8 \\
21 / 4 \\
23 / 8\end{array}$ & $\begin{array}{l}51 / 8 \\
53 / 4 \\
53 / 4 \\
61 / 4\end{array}$ & $\begin{array}{l}127 / 8 \\
143 / 16 \\
161 / 4 \\
181 / 4\end{array}$ \\
\hline
\end{tabular}

1 Orders or inquiries should specify diameter of bore $\mathrm{E}$ required. 
REFRIGERATING MACHINERY ASSOCIATION, AMMONIA PIPE FLANGES AND FLANGED FITTINGS FOR MAXIMUM WORKING PRESSURES OF 300 POUNDS PER SQUARE INCH, 1931

\section{AMMONIA PIPE FLANGES AND FLANGED FITTINGS}

\section{INTRODUCTORY NOTES}

1. Rating.-These standards shall be known as the American ammonia pipe flanges and flanged fittings standards. The maximum working pressure shall not exceed 300 pounds per square inch.

2. Size.-The sizes of fittings in the following tables are identified by the corresponding nominal pipe size.

3. Materials.-The dimensional standards covered by the following tables are based on a high-grade metal equal to that given in A. S. T. M., A-126, specification for gray-iron castings for valves,
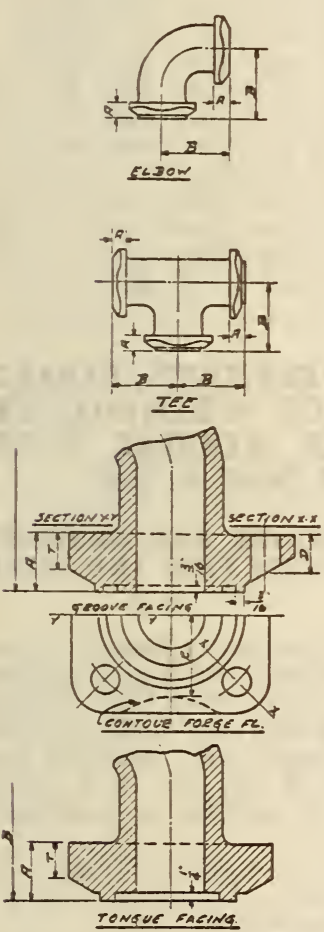

FIGURE 245
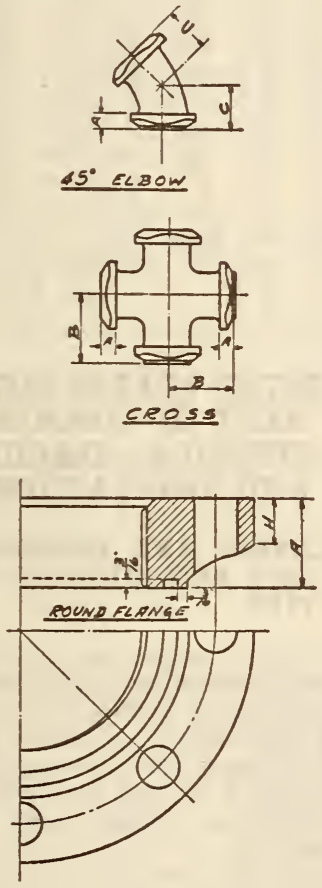

flanges,

(a) Chemical composition.-

Sulphur, not over.

Phosphorus, not over

(b) Physical properties.-A tension-test specimen shall not bave less tensile strength than 30,000 pounds per square inch. However, in designing, the tensile strength calculated shall not exceed:

25,000 pounds per square inch for light castings of less than $1 / 2$ inch thickness.

27,000 pounds per square inch for medium castings between $1 / 2$ and 2 inch section.

30,000 pounds per square inch for heavy castings in which no section is less than 2 inches in thickness. (c) Drop-forged companion flanges are permissible as long as no change in the principal dimensions is made.

4. Bolting material.-Standard commercial steel bolts with rough square heads and rough or coldpunched hexagonal nuts, tensile strength 50,000 pounds per square inch, safety factor 10 . Bolt holes in castings to be three-sixteenths inch larger than the bolt diameter and one-eighth inch larger for drop forgings and drilled holes.

5. Metal thickness.-Minimum metal thickness to be not less than one-fourth inch, all other sizes based on the modified Barlow formula of the A. S. M. E. boiler construction code:

For pipe size one-fourth inch to 5 inches

$$
P=\frac{2 S}{D} \times(t-0.065)-125
$$

For diameters over 5 inches

Where

$$
P=\frac{2 S}{D} \times(t-0.1)
$$

$P=$ working pressure, pounds per square inch, 300 pounds.

$t=$ thickness of fitting wall, inches.

$D=$ actual inside diameter of fitting, inches.

$S=2,500,2,700$, or 3,000 pounds per square inch depending on minimum dimensions of walls.

Where cylindrical parts meet in fittings like tees and crosses, the wall thickness is to be increased 50 per cent and be permitted gradually to run out into standard wall thickness.

6. Fitting dimensions. - In the design of fittings a fixed position of the flange must be maintained with reference to the body of the fitting as indicated in sketches and table.

(a) Note that the flange edge in one case is the face of the tongue in the male fitting as indicated on sketches.

(b) Elbows and angle valves to be provided with tongue and groove, ikewise all straight runs of tees, crosses, and tee and globe valves. The lateral flange of tees will be provided with a tongue and the flange next to the underside of the valve disk will have a groove.

(c) An inspection limit of plus or minus onethirty-second inch shall be allowed on all center to contact surface dimensions for sizes up to and including 10-inch fittings; and one-sixteenths inch for 12 -inch fittings.

7. Valve dimensions.-The center to contact surface and contact surface to contact surface dimensions of globe, angle, tee, and check valves shall be the same as those prescribed for tees and elbows.

8. Reducing fittings.--Reducing fittings shall have same center to flange edge dimensions as those of straight-size fittings of the largest opening and tongues and grooves as under section 6 .

9. Side-outlet fittings. - All side-outlet fittings shall have all openings on the intersection of center lines.

10. Method of designating outlets of reducing fittings.- It is recommended for manufacturers of such fittings to follow A. S. A. recommendation for standards B16d-1927, above. To maintain a minimum fitting stock, the use of reducing flanges is recommended.

11. Marking.-For marking of fittings, recommend following decisions of A. S. M. E. subcommittee No. 8 on marking of pipe fittings.

12. Special flanges.-To save space, special dropforged flanges may be made for making up cooling coils as incorporated in tables and sketches. 
[All dimensions given in inches]

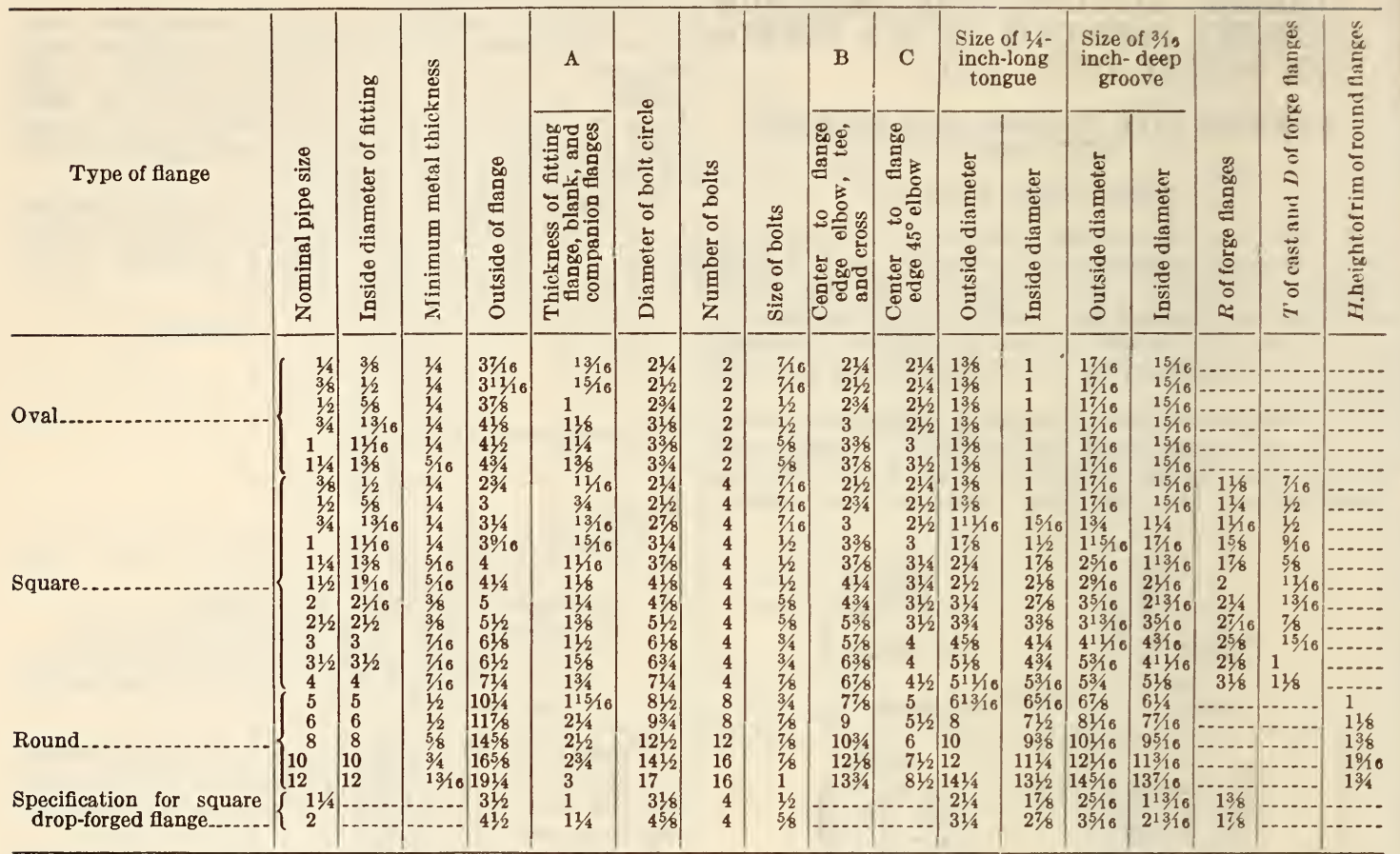

SOCIETY OF AUTOMOTIVE ENGINEERS, POWER PLANT; WATER-PIPE FLANGES, S. A. E. RECOMMENDED PRACTICE, JULY, 1926

WATER-PIPE FLANGES

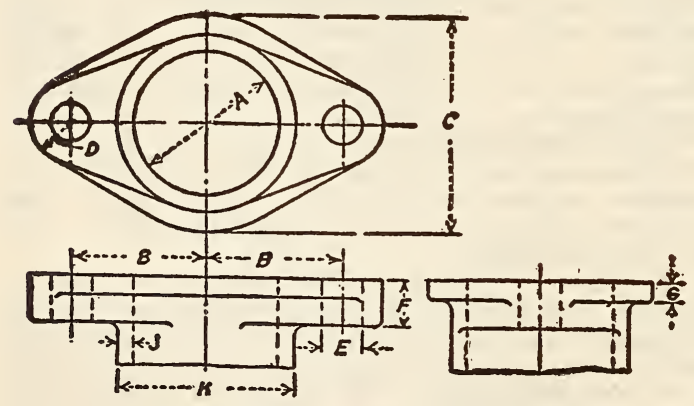

Figure 246.-Water pipe flanges

[All dimensions given in inches]

\begin{tabular}{|c|c|c|c|c|c|}
\hline \multirow{2}{*}{ Nominal flange } & \multicolumn{5}{|c|}{ Size } \\
\hline & $13 / 16$ & $17 / 16$ & $111 / 16$ & $115 / 16$ & $23 / 16$ \\
\hline $\begin{array}{l}\text { Outside diameter } \\
(K) \\
\text { Inside diameter }(A) \\
B \\
C \\
D \\
D E \\
F \\
G \\
G \text { Length, } 1 \text { minimum. } \\
J \\
\text { Cap-screws, NC-2 }\end{array}$ & \begin{tabular}{|l|l}
$11 / 2$ \\
$13 / 16$ \\
$13 / 16$ \\
$17 / 8$ \\
$11 / 32$ \\
$11 / 32$ \\
$13 / 32$ \\
$7 / 32$ \\
$11 / 4$ \\
$5 / 32$ \\
$5 / 16-18$
\end{tabular} & \begin{tabular}{|l|l}
$13 / 4$ \\
$17 / 16$ \\
$111 / 32$ \\
$23 / 16$ \\
$13 / 32$ \\
$13 / 32$ \\
$15 / 32$ \\
$1 / 4$ \\
$11 / 4$ \\
$5 / 32$ \\
$3 / 8-16$
\end{tabular} & \begin{tabular}{|l|}
2 \\
$111 / 16$ \\
$115 / 32$ \\
$21 / 2$ \\
$13 / 32$ \\
$13 / 32$ \\
$15 / 32$ \\
$1 / 4$ \\
$11 / 4$ \\
$5 / 32$ \\
$3 / 8-16$
\end{tabular} & \begin{tabular}{|l|}
$21 / 4$ \\
$115 / 16$ \\
$121 / 32$ \\
$213 / 16$ \\
$15 / 32$ \\
$15 / 32$ \\
$9 / 16$ \\
932 \\
$11 / 4$ \\
$5 / 32$ \\
$7 / 16-14$
\end{tabular} & $\begin{array}{l}21 / 2 \\
23 / 16 \\
125 / 32 \\
31 / 8 \\
15 / 32 \\
15 / 32 \\
9 / 16 \\
9 / 32 \\
11 / 2 \\
5 / 32 \\
7 / 16-14\end{array}$ \\
\hline
\end{tabular}

1 Length of fitting for lap of hose.
UNITED STATES GOVERNMENT, DEPARTMENT OF COMMERCE, STEAMBOAT INSPECTION SERVICE, GENERAL RULES AND REGULATIONS, MAY 4, 1929

\section{FLANGES AND CONNECTIONS FOR WROUGHT- IRON HOMOGENEOUS-STEEL FEED AND STEAM PIPE}

The terminal and intermediate flanges of all wrought-iron and homogeneous-steel feed and steam pipe over 2 inches in diameter, other than on pipe or coil boilers or steam generators, shall be made of wrought iron, homogeneous steel, malleable iron having a tensile strength of not less than 30,000 pounds per square inch of section, or equivalent material.

All such flanges shall have a depth through the bore of not less than one-half of the diameter of the pipe to which the flange is attached.

The bore shall increase slightly toward the face of the flange, and the end of the pipe shall be enlarged to fit the bore of the flange and shall be substantially beaded over or outward into a recess in the face of the flange.

Flanges on extra-heavy lapwelded steam pipe up to and including 5 inches diameter may be attached with screw threads and the joints in bends may be made with extra-heavy malleable-iron elbows or equivalent material.

Feed and steam pipe up to and including 3 inches diameter may be connected at intermediate joints by being screwed into flanges or extra-heavy fittings.

Flanges of cast iron, semisteel, or ferrosteel, having a tensile strength of not less than 20,000 pounds per square inch of section, may be employed in connecting extra-heavy lapwelded pipe by screw threads. Such flanges shall be extra heavy and have a depth through the bore of not less than the U. S. standard length of thread for the pipe to which the flange is attached plus depth of counter- 
bore. The thickness of the flange, excluding hub, shall be not less than three-tenths of the diameter of the pipe plus 0.25 of an inch. The pipe shall fit snugly in the counterbore, which shall be not less than one-fourth the diameter of the pipe in depth.

Flanges of wrought iron or steel grooved in the bore to a depth equal to the thickness of the material in the pipe shall be allowed for use on all steam and feed pipe when the end of the pipe has been thoroughly annealed and expanded into the flange by approved machinery.

Flanges with hubs projecting not less than $13 / 4$ inches from the back of the flange may be shrunk on lapwelded or riveted iron or steel, or seamlessdrawn steel, steam pipes over $5 \frac{1}{2}$ inches diameter where the pipe is brought to a true and parallel circle at the end and beaded over into a recess at the face of the flange, or flared to an angle of approximately $20^{\circ}$, or the bore of the fiange may be tapered and the pipe expanded into the bore.

Flanges of cast steel, wrought iron, or homogeneous steel, equal in strength to the pipe to which it is attached, may be riveted to the pipe.

Flanges may be secured to pipe by expanding the pipe by proper and approved machinery and fiaring the end of the pipe to an angle not exceeding $20^{\circ}$, taken in the direction of the length of the pipe, and having a depth of flare equal to at least one and onehalf times the thickness of the pipe.

Flanges of cast steel, forged steel, or wrought iron may be used in any process of expanding the pipe into grooves in the bore of the flange and flaring the end of the pipe to an angle of $20^{\circ}$.

Flanges welded to lapwelded steam pipes of iron or steel may be used when the manufacturer furnishes an affidavit that the pipe has been properly annealed after all work requiring fire has been finished.

All flange connections made by the process of expanding, welding, or shrinking shall be subjected to a hydrostatic test of twice the working pressure without showing signs of weakness.

\section{FLANGES ON COPPER PIPE}

The flanges of all copper steam and feed pipe over 3 inches diameter shall be made of brass or bronze composition, forged iron or steel, or open-hearth steel castings. The end of the pipe shall be flanged over or outward to a width of not less than twice the thickness of the pipe and to a radius of not more than the thickness of the pipe, and the flange securely brazed or riveted to the pipe. If the pipe is properly formed with a fully reinforced taper through the flange the riveting or brazing may be dispensed with. The thickness of flanges shall be not less than four times the required thickness of the pipe plus 0.25 of an inch.

Any form of expanded, grooved, and flared joint will be allowed for copper pipe under the same regulations, restrictions, and tests applying to iron or steel pipe.

All flange joints shall be fitted with such number of bolts or rivets as will make the joint equal in strength to all other parts of the pipe.

Any form of joint which, in the judgment of local inspectors, is equal in strength and efficiency to the flange connections required by this rule shall be allowed on any and all classes of pipe.
UNITED STATES GOVERNMENT, FEDERAI SPECIFICATIONS BOARD, FEDERAL SPECIFICATION FOR PIPE AND PIPE FITTINGS, SOIL, CAST IRON (COATED AND UNCOATED), NO. 343a, AUGUST 22, 1928. (CURRENT DESIGNATION: WWP-401)

\section{CAST-IRON SOIL PIPE AND FITTINGS}

(See 607.11, p. 372.)

\section{UNITED STATES GOVERNMENT, FEDERAL SPECIFICATIONS BOARD, FEDERAI SPECIFICATION FOR PIPE FITTINGS, CAST IRON (THREADED), NO. 489, $192 \%$. (CURRENT DESIGNATION : WW-P-451)}

\section{THREADED CAST-IRON PIPE FITTINGS}

(This specification conforms to A. S. M. E., B16d, given above, except for the addition of dimensions of end-to-end straight fittings of both the 125 and 250 pound classes, as shown below, and also the following:)

\begin{tabular}{|c|c|c|c|c|}
\hline \multicolumn{5}{|c|}{ Dimensions for straight fittings } \\
\hline \multicolumn{3}{|c|}{ Type A-125 pounds } & \multicolumn{2}{|c|}{$\underset{\text { pounds }}{\text { Type } B-250}$} \\
\hline \multirow{2}{*}{$\begin{array}{l}\text { Nominal } \\
\text { pipe size }\end{array}$} & \multicolumn{2}{|c|}{ End to end } & \multirow{2}{*}{$\begin{array}{l}\text { Nominal } \\
\text { pipe size }\end{array}$} & $\begin{array}{c}\text { End to } \\
\text { end }\end{array}$ \\
\hline & $45^{\circ} \mathrm{Y}$ & Coupling & & $45^{\circ} \mathrm{Y}$ \\
\hline $\begin{array}{r}\text { Inches } \\
1 / 4 \\
3 / 8\end{array}$ & $\begin{array}{r}\text { Inches } \\
2.125 \\
2.250\end{array}$ & $\begin{array}{c}\text { Inches } \\
2.250\end{array}$ & \multirow{2}{*}{$\begin{array}{r}\text { Inches } \\
1 / 4 \\
3 / 8 \\
1 / 2 \\
3 / 4\end{array}$} & Inches \\
\hline $1^{3 / 4}$ & $\begin{array}{l}3.000 \\
3.500 \\
4.000\end{array}$ & $\begin{array}{l}2.250 \\
2.500 \\
2.750\end{array}$ & & \\
\hline $\begin{array}{l}11 / 2 \\
2 \\
21 / 2 \\
3 \\
31 / 2 \\
4\end{array}$ & $\begin{array}{l}5.000 \\
5.750 \\
6.500 \\
7.750 \\
8.875 \\
9.750\end{array}$ & $\begin{array}{l}3.000 \\
3.500 \\
3.500 \\
3.500 \\
4.000 \\
4.500\end{array}$ & $\begin{array}{l}11 / 4 \\
11 / 2 \\
2 \\
21 / 2 \\
3 \\
31 / 2 \\
4 \\
5 \\
6\end{array}$ & $\begin{array}{r}43 / 8 \\
53 / 4 \\
63 / 4 \\
77 / 8 \\
87 / 8 \\
93 / 4 \\
105 / 8 \\
151 / 4\end{array}$ \\
\hline
\end{tabular}

\section{METHOD OF INSPECTION AND TESTS}

One fitting shall be selected by the inspector from each 20 of the same size and kind and subjected to an air pressure of 20 pounds per square inch under water. When impractical to make this test, fittings selected as above shall be subjected to the hydrostatic test stated below for one minute. Unless otherwise specified the following pressures shall be used.

For type A, 125-pound fittings............Lbs./in.2.- $\quad 250$

For type B, 250-pound fittings....................... 500

PACKING AND MARKING OF SHIPMENTS

All shipments shall be marked with the name of the material, the name of the vendor, and the number of the particular contract or order. 
Unless otherwise specified, 10 fittings of the same kind and size up to and including 1 inch shall be securely tied together, or a like number shall be packed in a suitable container.

Unless otherwise specified, five fittings of the same kind and size above 1 inch shall be securely tied together, or a like number shall be packed in a suitable container.

\section{NOTES}

Type A, 125-pound (standard) fittings are for use on steam and other systems where the working pressure does not exceed 125 pounds, or the total temperature does not exceed $450^{\circ} \mathrm{F}$

Type B, 250-pound (extra-heavy) fittings are for use on steam or other systems where the working pressure does not exceed 250 pounds, or the total temperature does not exceed $450^{\circ} \mathrm{F}$.

Fittings commonly known as standard weight should be ordered as tipe A, 125-pound. Fittings commonly known as extra heavy should be ordered as type B, 250-pound.

60\%.2 MALIEABLE IRON PIPE, FITTINGS, AND CONNECTIONS

\section{AMERICAN RAILWAY ASSOCIATION, ME- CHANICAL DIVISION, PIPE UNIONS, STANDARD, 1916}

\section{PIPE UNIONS}

Dimensions for standard pipe unions

(See fig. 247.)

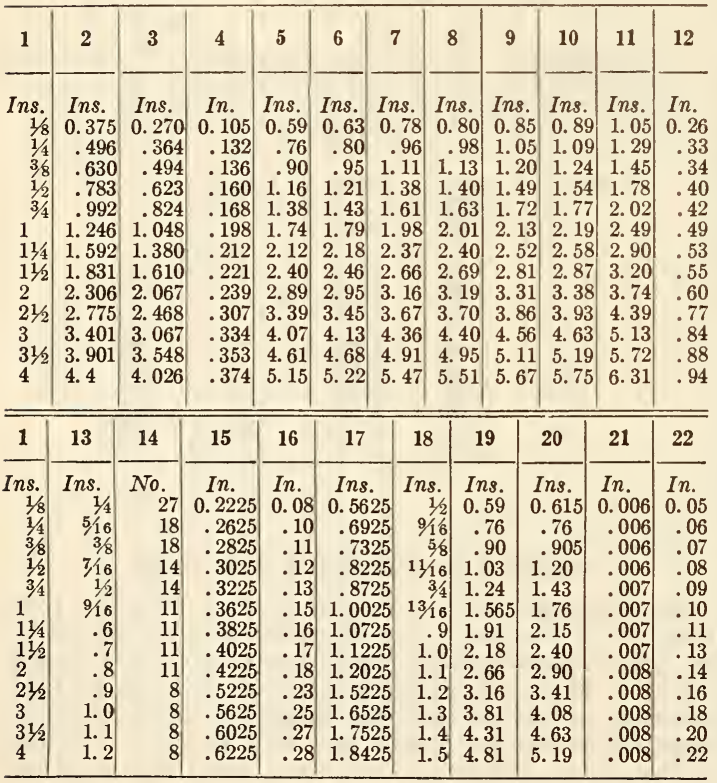

AMERICAN RAILWAY ASSOCIATION, MECHANICAI DIVISION, UNIONS AND COMBINATION UNION FITTINGS, BLACK AND GALVANIZED, FOR 300 POUNDS PRESSURE, RECOMMENDED PRACTICE, 1930

\section{UNIONS AND COMBINATION UNION FITTINGS}

1. Scope.-This specification covers malleableiron and steel pipe unions and combination union fittings for use on locomotives under working pressures up to 300 pounds per square inch.

\section{MANUFACTURE}

2. Process. - The malleable iron shall be made in accordance with A. R. A. specifications for malleable iron, best commercial practice with respect to composition, melting, and annealing. Steel shall be made by the open-hearth or electric process. All castings shall be thoroughly annealed. Rolled steel to be used in forgings or manufacture by machining shall be solid and free from segregation. Nonferrous inserts shall be made of sound, tough brass or bronze. All inserts shall be securely attached to their supporting members. Galvanized unions shall be thoroughly and smuothly coated with zinc by the hot-dipping process.

3. Proof test.-Each individual union shall be tested by the manufacturer under water or oil with air at 150 pounds pressure. No copper or rusting solution, cement, or welding will be permitted.

\section{PROPERTIES AND TESTS}

4. Finished unions. - The weights and strengths of unions shall not be less than those shown in Table 1.

\section{TABLE 1}

\begin{tabular}{|c|c||c|c|}
\hline $\begin{array}{c}\text { Size of } \\
\text { pipe }\end{array}$ & $\begin{array}{c}\text { Breaking } \\
\text { load }\end{array}$ & $\begin{array}{c}\text { Size of } \\
\text { pipe }\end{array}$ & $\begin{array}{c}\text { Breaking } \\
\text { load }\end{array}$ \\
\hline Inches & Pounds & Inches & Pounds \\
$1 / 8$ & 5,000 & $11 / 4$ & 23,000 \\
$1 / 4$ & 7,000 & $11 / 2$ & 28,000 \\
$3 / 8$ & 9,000 & 2 & 35,000 \\
$1 / 2$ & 11,000 & $21 / 2$ & 45,000 \\
$3 / 4$ & 14,000 & 3 & 60,000 \\
1 & 18,000 & & \\
\hline
\end{tabular}

5. Method of test.-Solid bars shall be screwed into both ends of union for gripping in tensile machine. The union nut shall be tightened solidly without undue strain. The assembly shall then be pulled at a crosshead speed of not more than oneeighth inch per minute. The load at failure shall be not less than that shown in Table 1.

6. Number of tests.-Three unions or union fittings of each size will be tested for the first 1,000 , or less, of each size in each shipment, and one additional for each additional 1,000. If any fail to meet the required minimum load, two more will be tested for each one which fails. Both of these shall meet the requirements.

\section{WORZMANSHIP AND FINISH}

7. Dimensions.-Unions shall conform to the limiting dimensions shown on drawings forming a part of this specification.

8. Finish. - Surfaces which are to remain in the cast condition shall be reasonably smooth and free from scale, fins, lumps, cracks, cavities and other injurious defects. Machined surfaces shall be smooth and fit properly against the adjoining parts. All parts shall be finished and assembled in a workmanlike manner. All unions shall be well oiled, to prevent rust in transit or storage.

\section{IMARKING}

9. Marking.-Unions shall bear maker's name or symbol, the size, the designation 300-pounds and A. R. A. clearly cast or stamped into the metal.

\section{INSPECTION AND REJECTION}

(Inspection and rejection conform to the requirements specified in A. R. A., specifications for galvanized sheets. See 604.32 , p. 243.) 

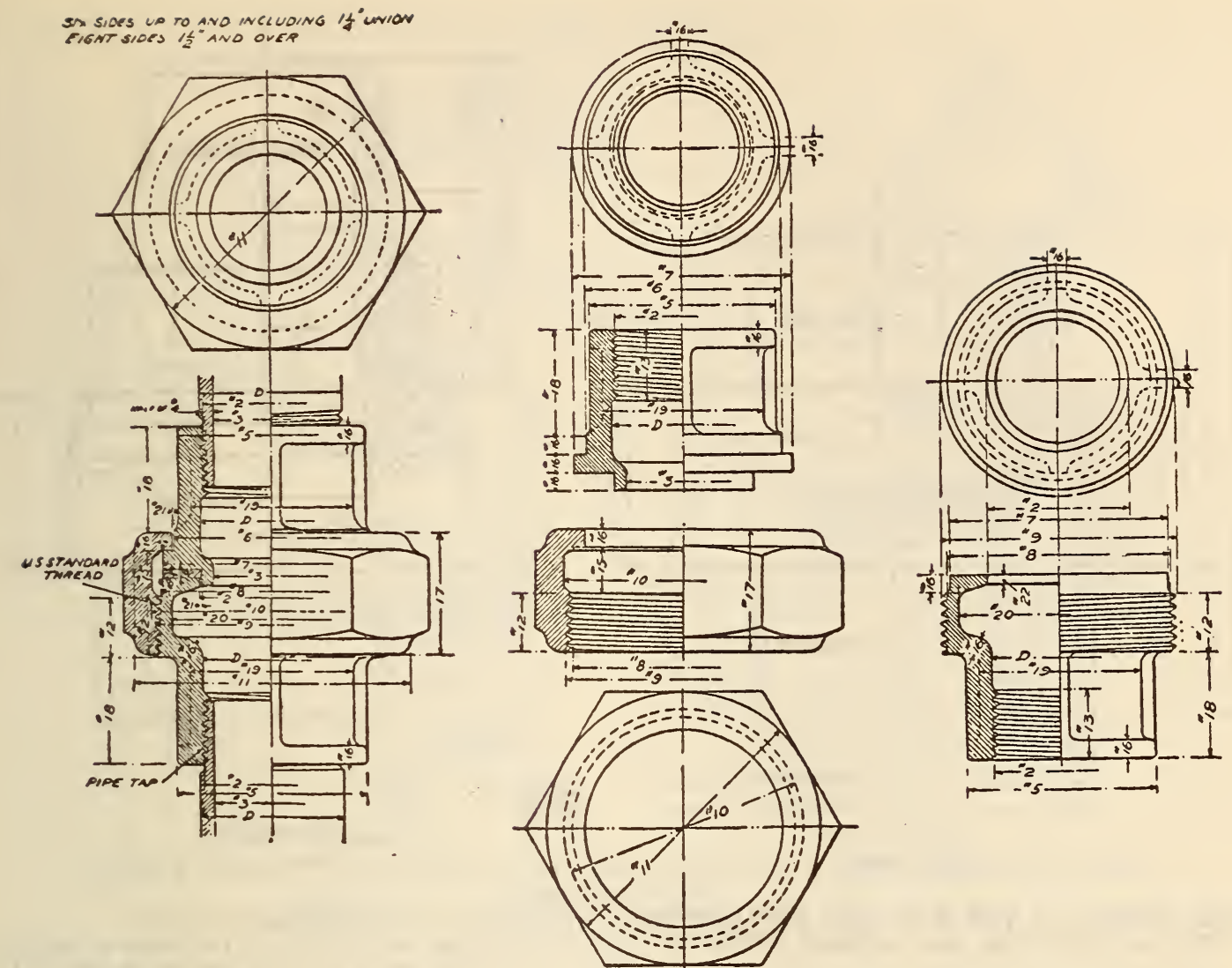

FIGURE 247.-Pipe unions

\section{DESCRIPTION ACCOMPANYING TABLE OF MALLEABLE PIPE UNIONS}

(For table see p. 416.)

Numbers at the Head of the Columns Above are Those Given in the Dimension Lines

Column No. 1 in table represents the nominal diameter of pipe.

Column No. 2 represents diameter of pipe at one-half the height of full thread nearest solid section of pipe.

Column No. 3 represents the internal diameter of the pipe.

Column No. 4 represents the difference between columns Nos. 2 and 3 , and is equal to twice the thickness of metal in pipe measured from inside line to one-half the height of thread as specified before. Column No. 5 represents the outside diameter of end of pipe union and is taken as No. 2, plus twice No. 4, plus an arbitrary increment. Column No. 6 is equal to No. 5 plus an increment varying from 0.04 to 0.07 of an inch. This increment was allowed for the purpose of being able to slip the nut over upper swivel end of union.

Column No. 7 is No. 6 plus an amount varying between 0.15 and 0.25 . This lip created is considerably in excess of what exists on present pipe unions for the reason that we find the surface between the lip and the corresponding part of nut is often damaged, and the bearing surface, when the full strength of the man is used on the wrench, is sufficient. We assume that a man would pull about 30 pounds on a wrench, with a possibility of using less force on pipes of small diameters. For that reason we made a variation in the width of lip, which lip, theoretically, would be uniform for all sizes of pipe. The nut itself has been strengthened to prevent the lip from deflecting upward.
Column No. 8 is No. 7 plus an increment varying from 0.02 to 0.04 of an inch.

Column No. 9 is No. 8 , plus twice the height of the thread.

Column No. 10 is No. 9, plus an increment varying between 0.04 and 0.08 of an inch.

Column No. 11 is No. 10, plus one and one-half times No. 4. Column No. 12 is two and one-half times No. 4, and was figured especially for bearing surface, so that the thread would not wear espay too rapidly when the nut is occasionally removed.

awa too rapidly when the nut is occasionally removed. greater than the length of full thread on standard pipe.

Column No. 14 represents the number of threads per inch in length of nut. This thread, we believe, should be U. S. standard form and not sharp thread.

Column No. 15 is taken arbitrarily, but is based on the probable requirements of manufactures for tapping out the nut.

Column No. 16 is three-fourths of No. 4

Column No. 17 represents the full height of nut, and is equal to No. 12 , plus No. 15 , plus No. 16.

Column No. 18 is the amount of projection outside of nut.

Column No. 19 is No. 2 , plus No. 4, plus an arbitrary increment. Column No. 20 is No. 7, less No. 16, with slight modifications.

Column No. 21 represents the clearance at several points, as indicated on print

Column No. 22 is assumed arbitrarily.

NOTE.-Pipe unions should be branded A. R. A.

All wrought-iron pipe for car work chall be threaded with a standard total taper of three-fourth inch in 1 foot, and all pipe fittings shall be tapped to suit the standard pipe thread with a total taper of three-fourths inch in 1 foot, so that the thread on pipe and fittings will be uniform and taper tight. 

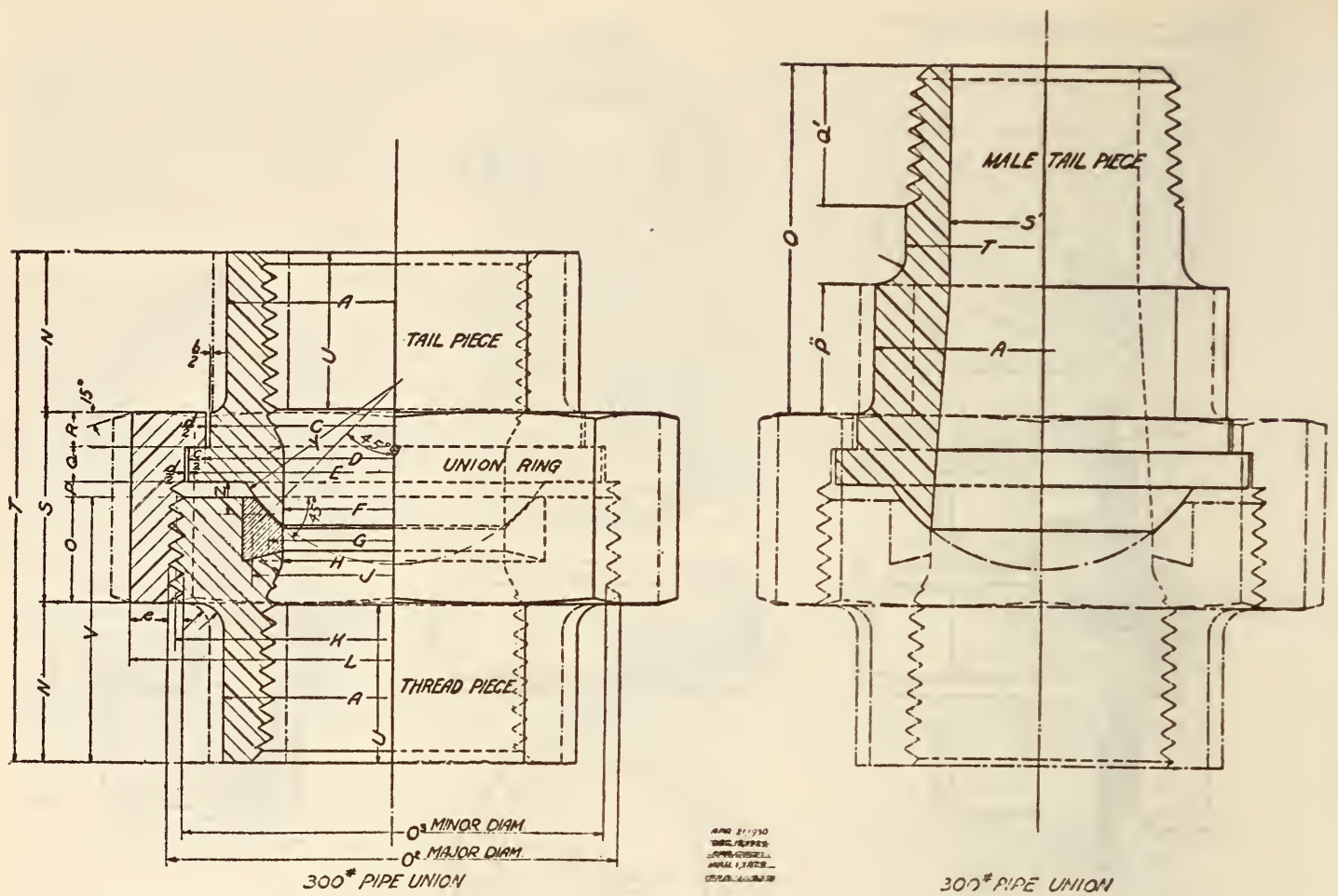

Figure 248.-Pipe unions of the nut and nipple type for locomotives, 300-pounds pressure

\section{PIPE UNIONS OF THE NUT AND NIPPLE TYPE FOR LOCOMOTIVES, 300 POUNDS PRESSURE}

The following formula is used to obtain the dimensions for the design of union fittings for maximum working pressure of 300 pounds steam:

$A=$ center to end, elbows and tees.

$B=$ length of thread.

$C=$ center to end, $45^{\circ}$ elbows.

$F=$ inside diameter of fitting.

Same as 250-pound cast-iron American standard screwed fittings, the idea being that manufacturers can use the same core-box equipment both for cast iron as well as for this proposed 300-pound malleable-iron fitting. Note that this is now done on the 125-pound cast-iron and the 150-pound malleable-iron American standard screwed fittings. This applies to the screwed fittings.

$E=$ width of band.

$H=$ outside diameter of band.

Same as the 125-pound cast-iron American standard screwed fittings.

$G=$ thickness of metal.

The 250-pound cast-iron American standard screwed fitting metal thickness is approximately 150 per cent heavier than the 125-pound cast-iron American standard screwed fitting. The rating is double. The proposed 300-pound malleable-iron fitting is $\mathbf{1 5 0}$ per cent heavier than the 150-pound malleable-iron standard screwed fitting and this rating is also doubled.

$M=$ center to end of union elbow and tee.

$1 / 2$ diameter " $H$ " + " $O$ " + distance " $O$ " for die clearance when threading union end.
$\Lambda=$ center to end of union $45^{\circ}$ elbow.

These are layouts on the bases of the $90^{\circ}$ ells so as to have proper clearance for die to cut the thread.

$J=$ diameter of $45^{\circ}$ seat bevel

$J^{1}=$ inside diameter of union end.

$K=$ pitch diameter.

$O=$ length of thread.

$O^{1}=$ threads per inch.

$\mathrm{O}^{2}=$ major diameter.

Notes.-The inspection limits for the center-to-end dimensions are those adopted by the American standard for all screwed fittings regardless of the working pressure.

All female threads shall be countersunk as provided in the American standard for all screwed fittings.

The American standards for screwed fittings referred to, are publications approved by the American Standards Association and are as follows:

150-pound malleable-iron screwed fittings ....... B-16c-1927 125-pound and 250-pound cast-iron screwed fit-

tings

The following formula is used to obtain the dimen. sions for the design of the pipe unions for maximum working pressure of 300 pounds steam:

$A=$ across flats of octagon $=$ the minimum possible to provide strength for the union ring and tailpiece.

$C=$ diameter of small shoulder.

$D=$ diameter of bore.

$E=$ diameter of shoulder.

$F=$ inside diameter

$G=$ diameter of seat $\operatorname{contact}=$ midway between $H$ and $J$.

$H=$ inside diameter.

$J=$ outside diameter of $45^{\circ}$ seat.

$K=$ pitch diameter.

$L=$ across flats of octagon. 

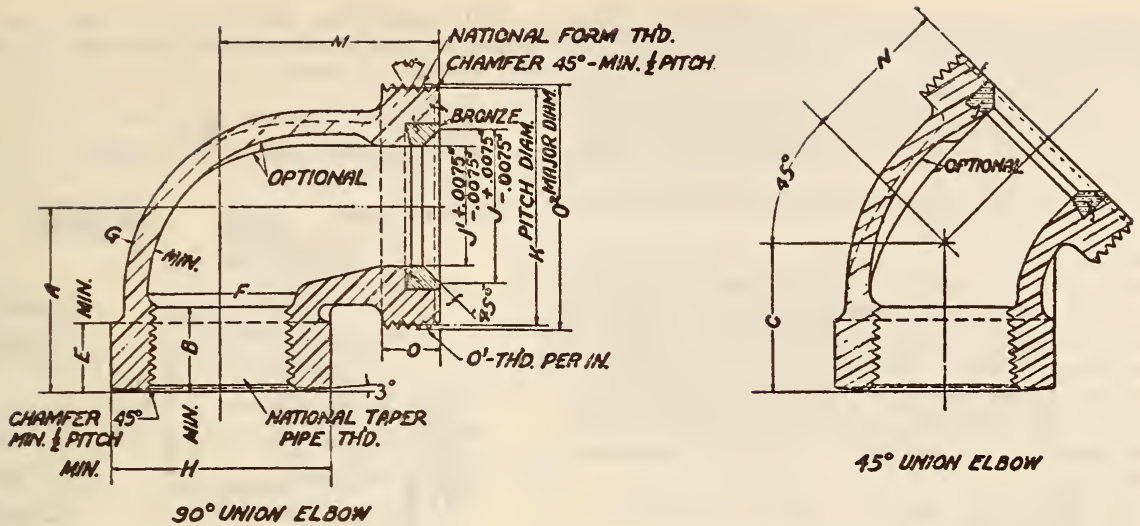

$45^{\circ}$ unON FLOOW
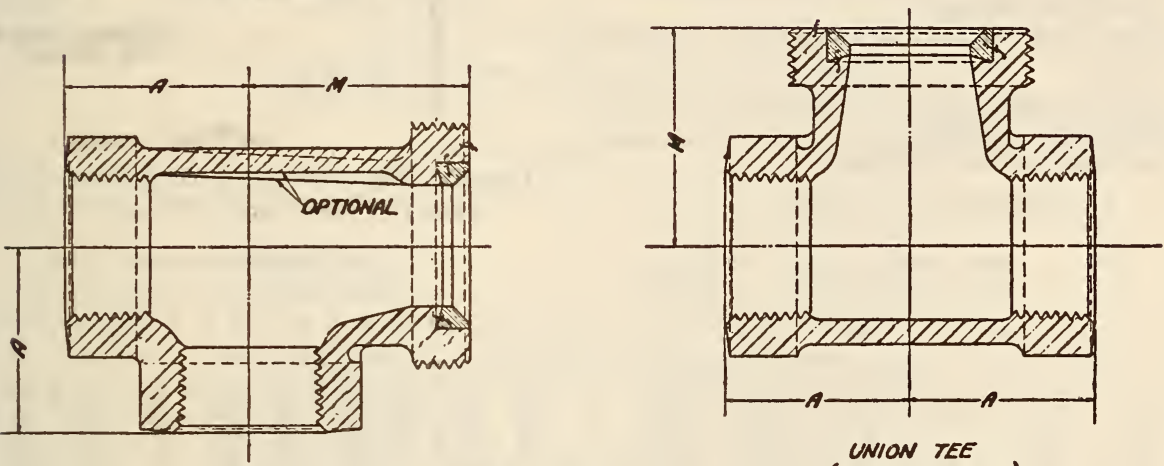

UNION TEE

MREING AS FOLLOWS;

(UNION ON RUN)

\section{a- MAKER'S MAME aL SYMB CL \\ b- THE NUMERALS "300"AND "WRA" C-SRE OF FITTING.}

FigURE 249.-Pipe unions of the nut and nipple type for locomotives, 300-pound pressure

\section{UNION FITTINGS FOR MAXIMUM WORKING PRESSURE OF 300 POUNDS STEAM}

[All dimensions given in inches]

\begin{tabular}{|c|c|c|c|c|c|c|c|c|c|}
\hline \multirow{2}{*}{ Size } & \multirow[b]{2}{*}{$A$} & \multirow[b]{2}{*}{$B$} & \multirow[b]{2}{*}{$C$} & \multirow[b]{2}{*}{$E$} & \multicolumn{2}{|c|}{$F$} & \multirow[b]{2}{*}{$G$} & \multirow[b]{2}{*}{$H$} & \multirow[b]{2}{*}{$J$} \\
\hline & & & & & $\begin{array}{l}\text { Maxi- } \\
\text { mum }\end{array}$ & $\begin{array}{l}\text { Mini- } \\
\text { mum }\end{array}$ & & & \\
\hline $\begin{array}{l}1 / 4 \\
3 / 8 \\
11 / 2 \\
3 / 4 \\
1 \\
11 / 4 \\
11 / 2 \\
2 \\
21 / 2 \\
3\end{array}$ & \multicolumn{8}{|c|}{$\begin{array}{l}\text { These dimensions are identical with those for } \\
300 \text {-pound screwed fittings, elbows, } 45^{\circ} \text { el- } \\
\text { bows, tees, and crosses, given below. }\end{array}$} & $\begin{array}{r}0.58 \\
.75 \\
.93 \\
.07 \\
1.36 \\
1.64 \\
1.92 \\
2.36 \\
2.89 \\
3.51\end{array}$ \\
\hline
\end{tabular}

$N=$ length of octagon $=$ same length of band of the 250-pound cast-iron American standard screwed fitting.

$O=$ length of thread $=$ is based on $5 \frac{1 / 2}{2}$ threads for $1 / 4$-inch to 2 -inch sizes ( 5 threads will be total allowing one-half thread for chamfer), 6 threads for $2 \frac{1}{2}$-inch and 3 -inch sizes because there are 8 threads per inch in the last two sizes.

$\mathrm{O}^{1}=$ threads per inch. (National form thread.)

$\mathrm{O}^{2}=$ major diameter. (National form thread.)

$\mathrm{O}^{3}=$ minor diameter. (National form thread.)

$P=$ clearance $=$ is reasonably provided to allow proper seating on ball joint.

$Q=$ thickness of shoulder. (For tolerance see detail.)
[All dimensions given in inches]

\begin{tabular}{|c|c|c|c|c|c|c|c|c|c|c|}
\hline \multirow[b]{2}{*}{ Size } & \multirow[b]{2}{*}{$J^{1}$} & \multicolumn{2}{|c|}{$K$} & \multirow[b]{2}{*}{$M$} & \multirow[b]{2}{*}{$N$} & \multirow[b]{2}{*}{$O$} & \multirow[b]{2}{*}{$0^{1}$} & \multicolumn{2}{|c|}{$O^{2}$} & \multirow{2}{*}{$\begin{array}{c}\text { Tol- } \\
\text { er- } \\
\text { ance, } \\
\text { center } \\
\text { to } \\
\text { end, } \\
\pm\end{array}$} \\
\hline & & $\begin{array}{l}\text { Maxi- } \\
\text { mum }\end{array}$ & $\begin{array}{l}\text { Mini- } \\
\text { mumn }\end{array}$ & & & & & $\begin{array}{l}\text { Maxi- } \\
\text { mum }\end{array}$ & $\begin{array}{l}\text { Mini- } \\
\text { mum }\end{array}$ & \\
\hline $\begin{array}{l}1 / 4 \\
3 / 8 \\
1 / 2 \\
3 / 4 \\
1 \\
11 / 4 \\
11 / 2 \\
2 \\
21 / 2 \\
3\end{array}$ & $\begin{array}{r}0.38 \\
.54 \\
.68 \\
.85 \\
1.10 \\
1.40 \\
1.63 \\
2.06 \\
2.54 \\
3.05\end{array}$ & $\begin{array}{l}1.050 \\
1.230 \\
1.440 \\
1.770 \\
2.100 \\
2.530 \\
2.850 \\
3.450 \\
4.140 \\
4.920\end{array}$ & $\begin{array}{l}1.047 \\
1.227 \\
1.437 \\
1.766 \\
2.096 \\
2.526 \\
2.846 \\
3.446 \\
4.135 \\
4.915\end{array}$ & $\begin{array}{l}1.16 \\
1.34 \\
1.46 \\
1.81 \\
1.97 \\
2.29 \\
2.44 \\
2.74 \\
3.43 \\
3.81\end{array}$ & $\begin{array}{r}0.78 \\
.86 \\
.99 \\
1.12 \\
1.30 \\
1.49 \\
1.66 \\
1.93 \\
2.19 \\
2.62\end{array}$ & $\begin{array}{r}0.35 \\
.39 \\
.39 \\
.50 \\
.50 \\
.55 \\
.55 \\
.55 \\
.75 \\
.75\end{array}$ & $\begin{array}{r}16-\mathrm{N} \\
14-\mathrm{N} \\
14-\mathrm{N} \\
11-\mathrm{N} \\
11-\mathrm{N} \\
10-\mathrm{N} \\
10-\mathrm{N} \\
10-\mathrm{N} \\
8-\mathrm{N} \\
8-\mathrm{N}\end{array}$ & $\begin{array}{l}1.090 \\
1.276 \\
1.486 \\
1.829 \\
2.159 \\
2.595 \\
2.915 \\
3.515 \\
4.221 \\
5.001\end{array}$ & $\begin{array}{l}1.081 \\
1.266 \\
1.476 \\
1.817 \\
2.147 \\
2.582 \\
2.902 \\
3.502 \\
4.206 \\
4.986\end{array}$ & $\begin{array}{l}0.04 \\
.05 \\
.06 \\
.06 \\
.07 \\
.07 \\
.08 \\
.08 \\
.10 \\
.10\end{array}$ \\
\hline
\end{tabular}

$R=$ thickness of shoulder. (For tolerance see detail.)

$S=$ height of union ring.

$T=$ height of pipe union.

$U=$ depth of pipe tap=same length as 250-pound cast-iron American standard screwed fitting. This depth provides sufficient amount for a screwed-tight joint connection.

$V=$ height of thread piece.

$Y=$ radius of ball seat.

$Z=$ height of contact for seat.

$b=$ nominal clearance.

$c=$ total nominal width seat bearing.

$d=$ nominal clearance over collar. 
Notes.-The ball seat will make a better and tighter joint because there is a narrow seating contact between the ball seat and the $45^{\circ}$ seat. When there is a broad contact between the seating surfaces, it is more difficult to obtain a tight joint.

Bronze seat ring thickness and depth were not dimensioned so that manufacturers can use their own bronze seat rings.

Inspection limits on all parts for over-all lengths are those adopted by the American standard for all screwed fittings regardless of the working pressure.

All female threads shall be countersunk as provided in the American standard for all screwed fittings.

Male tailpiece.

$O=$ length of male tailpiece from small shoulder.

$P=$ length of octagon=same as width of band on the 125-pound cast-iron American standard screwed fitting. Also used on union fittings.

$Q^{1}=$ length of thread= same as length of thread on street ells and tees of the 150-pound malleable-iron American standard screwed fittings.

$S^{1}=$ inside diameter $=$ the maximum possible to provide for strength.

$T=$ outside diameter $=$ same as outside diameter of pipe.

Notes.-The derivations of dimensions not shown on the male tailpiece. See dimensions on tailpiece.

All male threads shall be chamfered at an angle of $45^{\circ}$ as provided in the American standard for screwed fittings.

[All dimensions given in inches]

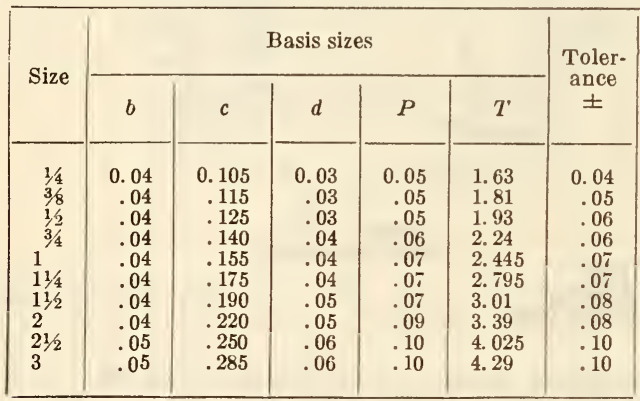

The American standards for screwed fittings referred to, are publications approved by the American Standards Association and are as follows:

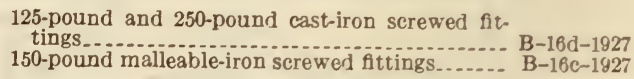

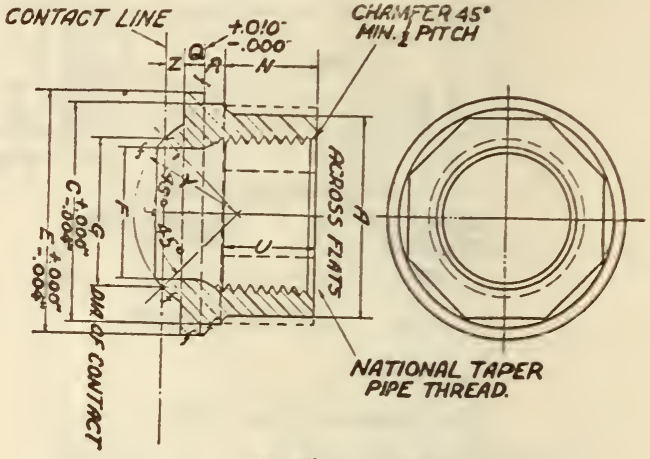

300* TAIL PIECE

Figure 250.-Pipe unions of the nut and nipple type for locomotives, 300-pound pressure

[All dimensions given in inches]

\begin{tabular}{r|c|c|c|c|c|c|c|c|c|c|c}
\hline Size & $A$ & $C$ & $E$ & $F$ & $G$ & $Q$ & $R$ & $N$ & $Z$ & $Y$ & $U$ \\
\hline $1 / 4$ & 0.75 & 0.83 & 0.965 & 0.38 & 0.48 & 0.125 & 0.125 & 0.49 & 0.11 & 0.34 & 0.43 \\
$3 / 8$ & .90 & .99 & 1.135 & .54 & .645 & .135 & .135 & .55 & .11 & .45 & .47 \\
112 & 1.09 & 1.19 & 1.345 & .68 & .805 & .145 & .145 & .60 & .11 & .56 & .57 \\
$3 / 4$ & 1.35 & 1.47 & 1.65 & .85 & .96 & .16 & .16 & .68 & .12 & .68 & .64 \\
1 & 1.63 & 1.78 & 1.975 & 1.10 & 1.23 & .18 & .175 & .76 & .13 & .87 & .75 \\
1314 & 2.00 & 2.18 & 2.395 & 1.40 & 1.52 & .21 & .205 & .88 & .13 & 1.07 & .84 \\
1132 & 2.27 & 2.47 & 2.71 & 1.63 & 1.775 & .23 & .22 & .97 & .14 & 1.25 & .87 \\
2 & 2.80 & 3.04 & 3.31 & 2.06 & 2.21 & .26 & .25 & 1.12 & .17 & 1.56 & 1.00 \\
$21 / 2$ & 3.37 & 3.66 & 3.97 & 2.54 & 2.715 & .295 & .28 & 1.30 & .19 & 1.92 & 1.17 \\
3 & 4.05 & 4.40 & 4.745 & 3.05 & 3.28 & .325 & .315 & 1.40 & .21 & 2.31 & 1.23 \\
& & & & & & & & & & & \\
\hline
\end{tabular}

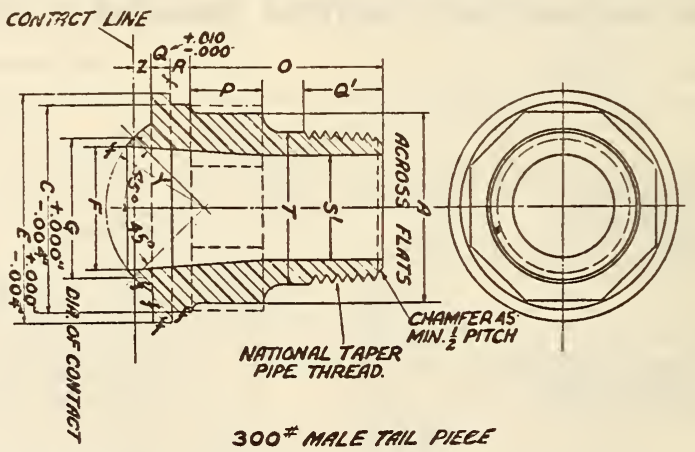

Figure 251.-Pipe unions of the nut and nipple type for locomotives, 300-pound pressure

[All dimensions given in inches]

\begin{tabular}{|c|c|c|c|c|c|c|c|c|c|c|c|c|c|c|}
\hline Size & $A$ & $C$ & $E$ & $F$ & $G$ & $Q$ & $R$ & 0 & $Z$ & $Y$ & $Q^{1}$ & $P$ & $S^{1}$ & $T$ \\
\hline $\begin{array}{l}1 / 4 \\
3 / 8 \\
1 / 2 \\
3 / 4 \\
1 \\
11 / 4 \\
11 / 2 \\
2 \\
21 / 2 \\
3\end{array}$ & $\begin{array}{l}0.75 \\
.90 \\
1.09 \\
1.35 \\
1.63 \\
2.00 \\
2.27 \\
2.80 \\
3.37 \\
4.05\end{array}$ & $\begin{array}{r}0.83 \\
.99 \\
1.19 \\
1.47 \\
1.78 \\
2.18 \\
2.47 \\
3.04 \\
3.66 \\
4.40\end{array}$ & $\begin{array}{l}0.965 \\
1.135 \\
1.345 \\
1.65 \\
1.975 \\
2.395 \\
2.71 \\
\text { 3. } 31 \\
\text { 3. } 97 \\
4.745\end{array}$ & $\begin{array}{r}0.38 \\
.54 \\
.68 \\
.85 \\
1.10 \\
1.40 \\
1.63 \\
2.06 \\
2.54 \\
3.05\end{array}$ & $\begin{array}{l}0.48 \\
.645 \\
.805 \\
.96 \\
1.23 \\
1.52 \\
1.775 \\
2.21 \\
2.715 \\
3.28\end{array}$ & $\begin{array}{l}0.125 \\
.135 \\
.145 \\
.16 \\
.18 \\
.21 \\
.23 \\
.26 \\
.295 \\
.325\end{array}$ & $\begin{array}{l}0.125 \\
.135 \\
.145 \\
.16 \\
.175 \\
.205 \\
.22 \\
.25 \\
.28 \\
.315\end{array}$ & $\begin{array}{l}1.11 \\
1.22 \\
1.35 \\
1.53 \\
1.67 \\
1.83 \\
1.91 \\
2.04 \\
2.30 \\
2.63\end{array}$ & $\begin{array}{l}0.11 \\
.11 \\
.11 \\
.12 \\
.13 \\
.13 \\
.14 \\
.17 \\
.19 \\
.21\end{array}$ & $\begin{array}{r}0.34 \\
.45 \\
.56 \\
.68 \\
.87 \\
1.07 \\
1.25 \\
1.56 \\
1.92 \\
2.31\end{array}$ & $\begin{array}{r}0.401 \\
.407 \\
.533 \\
.545 \\
.682 \\
.706 \\
.723 \\
.756 \\
1.137 \\
1.200\end{array}$ & $\begin{array}{r}0.38 \\
.44 \\
.50 \\
.56 \\
.62 \\
.69 \\
.75 \\
.84 \\
.94 \\
1.00\end{array}$ & $\begin{array}{r}0.26 \\
.36 \\
.49 \\
.67 \\
.88 \\
1.16 \\
1.35 \\
1.75 \\
2.16 \\
2.67\end{array}$ & $\begin{array}{l}0.54 \\
.675 \\
.84 \\
1.05 \\
1.315 \\
1.66 \\
1.90 \\
2.375 \\
2.875 \\
3.50\end{array}$ \\
\hline
\end{tabular}




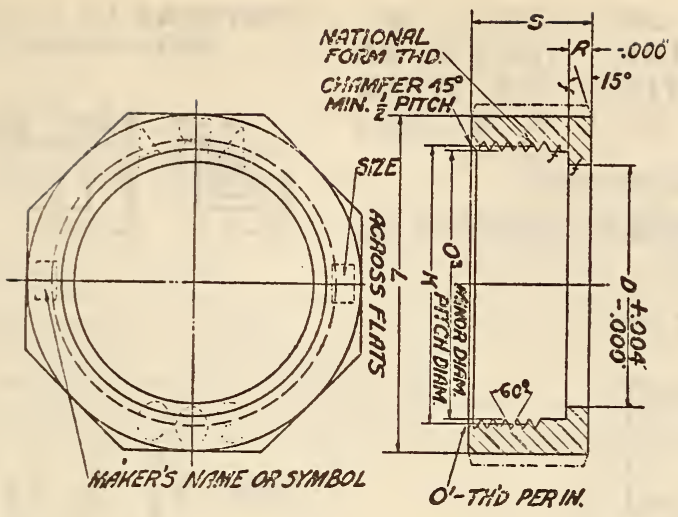

300 " UNION RING

Frgure 252.-Pipe unions of the nut and nipple type for locomotives, 300-pound pressure

[All dimensions given in inches]

\begin{tabular}{|c|c|c|c|c|c|c|c|c|c|}
\hline \multirow{2}{*}{ Size } & \multicolumn{2}{|c|}{$K$} & \multirow{2}{*}{$L$} & \multirow{2}{*}{$R$} & \multirow{2}{*}{$S$} & \multirow{2}{*}{$D$} & \multirow{2}{*}{$O^{1}$} & \multicolumn{2}{|c|}{$O^{3}$} \\
\hline & $\begin{array}{l}\text { Maxi- } \\
\text { mum }\end{array}$ & $\begin{array}{l}\text { Mini- } \\
\text { mum }\end{array}$ & & & & & & $\begin{array}{l}\text { Maxi- } \\
\text { mum }\end{array}$ & $\begin{array}{l}\text { Mini- } \\
\text { mum }\end{array}$ \\
\hline $\begin{array}{l}1 / 4 \\
318 \\
132 \\
3 / 4 \\
1 \\
11 / 4 \\
11 / 2 \\
2 \\
214 \\
3\end{array}$ & $\begin{array}{l}1.055 \\
1.235 \\
1.445 \\
1.776 \\
2.106 \\
2.536 \\
2.856 \\
3.456 \\
4.147 \\
4.927\end{array}$ & $\begin{array}{l}1.052 \\
1.232 \\
1.442 \\
1.772 \\
2.102 \\
2.532 \\
2.852 \\
3.452 \\
4.142 \\
4.922\end{array}$ & $\begin{array}{l}1.35 \\
1.55 \\
1.78 \\
2.18 \\
2.55 \\
3.04 \\
3.39 \\
4.05 \\
4.83 \\
5.69\end{array}$ & $\begin{array}{l}0.125 \\
.135 \\
.145 \\
.16 \\
.175 \\
.205 \\
.22 \\
.25 \\
.28 \\
.315\end{array}$ & $\begin{array}{l}0.65 \\
.71 \\
.73 \\
.88 \\
.925 \\
1.035 \\
1.07 \\
1.15 \\
1.425 \\
1.49\end{array}$ & $\begin{array}{l}0.86 \\
1.02 \\
1.22 \\
1.51 \\
1.82 \\
2.22 \\
2.52 \\
3.09 \\
3.72 \\
4.46\end{array}$ & $\begin{array}{l}16-\mathrm{N} \\
14-\mathrm{N} \\
14-\mathrm{N} \\
11-\mathrm{N} \\
11-\mathrm{N} \\
10-\mathrm{N} \\
10-\mathrm{N} \\
10-\mathrm{N} \\
8-\mathrm{N} \\
8-\mathrm{N}\end{array}$ & $\begin{array}{l}1.030 \\
1.207 \\
1.417 \\
1.740 \\
2.070 \\
2.497 \\
2.817 \\
3.417 \\
4.099 \\
4.879\end{array}$ & $\begin{array}{l}1.023 \\
1.199 \\
1.409 \\
1.730 \\
2.060 \\
2.486 \\
2.806 \\
3.406 \\
4.086 \\
4.866\end{array}$ \\
\hline
\end{tabular}

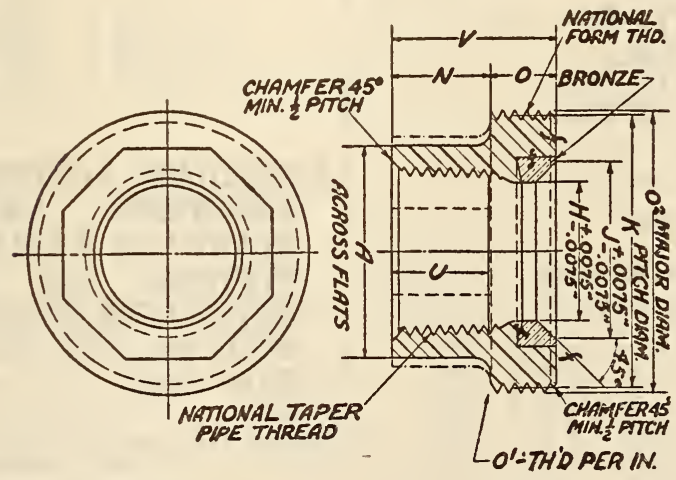

300 "THREAD PIECE

FigURE 253.-Pipe unions of the nut and nipple type for locomotives, 300-pound pressure

[All dimensions given in inches]

\begin{tabular}{|c|c|c|c|c|c|c|c|c|c|c|c|c|}
\hline \multirow[b]{2}{*}{ Size } & \multirow[b]{2}{*}{$A$} & \multirow[b]{2}{*}{$H$} & \multirow[b]{2}{*}{$J$} & \multicolumn{2}{|c|}{$K$} & \multirow{2}{*}{$N$} & \multirow[b]{2}{*}{0} & \multirow[b]{2}{*}{$V$} & \multirow[b]{2}{*}{$U$} & \multirow[b]{2}{*}{$O^{1}$} & \multicolumn{2}{|c|}{$O^{2}$} \\
\hline & & & & $\begin{array}{l}\text { Maxi- } \\
\text { mum }\end{array}$ & $\begin{array}{l}\text { Mini- } \\
\text { mum }\end{array}$ & & & & & & $\begin{array}{l}\text { Maxi- } \\
\text { mum }\end{array}$ & $\begin{array}{l}\text { Mini- } \\
\text { mum }\end{array}$ \\
\hline $\begin{array}{l}1 / 4 \\
3 / 8 \\
112 \\
3 / 4 \\
1^{1} \\
11 / 4 \\
11 / 2 \\
2 \\
21 / 2 \\
3\end{array}$ & $\begin{array}{l}0.75 \\
.90 \\
1.09 \\
1.35 \\
1.63 \\
2.00 \\
2.27 \\
2.80 \\
3.37 \\
4.05\end{array}$ & $\begin{array}{r}0.38 \\
.54 \\
.68 \\
.85 \\
1.10 \\
1.40 \\
1.63 \\
2.06 \\
2.54 \\
3.05\end{array}$ & $\begin{array}{r}0.58 \\
.75 \\
.93 \\
1.07 \\
1.36 \\
1.64 \\
1.92 \\
2.36 \\
2.89 \\
3.51\end{array}$ & $\begin{array}{l}1.050 \\
1.230 \\
1.440 \\
1.770 \\
2.100 \\
2.530 \\
2.850 \\
3.450 \\
4.140 \\
4.920\end{array}$ & $\begin{array}{l}1.047 \\
1.227 \\
1.437 \\
1.766 \\
2.096 \\
2.526 \\
2.846 \\
3.446 \\
4.135 \\
4.915\end{array}$ & $\begin{array}{r}0.49 \\
.55 \\
.60 \\
.68 \\
.76 \\
.88 \\
.97 \\
1.12 \\
1.30 \\
1.40\end{array}$ & $\begin{array}{l}0.35 \\
.39 \\
.39 \\
.50 \\
.50 \\
.55 \\
.55 \\
.55 \\
.75 \\
.75\end{array}$ & $\begin{array}{r}0.84 \\
.94 \\
.99 \\
1.18 \\
1.26 \\
1.43 \\
1.52 \\
1.67 \\
2.05 \\
2.15\end{array}$ & $\begin{array}{r}0.43 \\
.47 \\
.57 \\
.64 \\
.75 \\
.84 \\
.87 \\
1.00 \\
1.17 \\
1.23\end{array}$ & $\begin{array}{l}16-\mathrm{N} \\
14-\mathrm{N} \\
14-\mathrm{N} \\
11-\mathrm{N} \\
11-\mathrm{N} \\
10-\mathrm{N} \\
10-\mathbf{N} \\
10-\mathrm{N} \\
8-\mathrm{N} \\
8-\mathrm{N}\end{array}$ & $\begin{array}{l}1.090 \\
1.276 \\
1.486 \\
1.829 \\
2.159 \\
2.595 \\
2.915 \\
3.515 \\
4.221 \\
5.001\end{array}$ & $\begin{array}{l}1.081 \\
1.266 \\
1.476 \\
1.817 \\
2.147 \\
2.582 \\
2.902 \\
3.502 \\
4.206 \\
4.986\end{array}$ \\
\hline
\end{tabular}


AMERICAN RAILWAY ASSOCIATION, MECHANICAL DIVISION, 300-POUND SCREWED PIPE FITTINGS FOR STEAM IOCOMOTIVES, CIR. NO. D. V.-741, 1931

\section{SCREWED PIPE FITTINGS}

300-POUND SCREWED FITTINGS, REDUCING ELBOWS

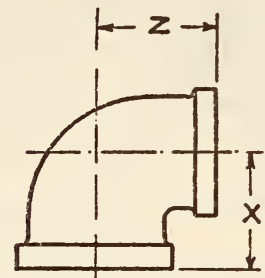

FIgURE 254

[All dimensions given in inches]

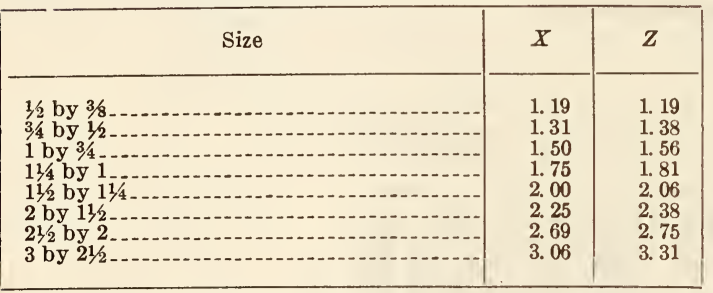

300-POUND SCREWED FITTINGS, REDUCING TEES

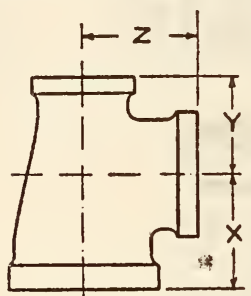

Figure 255

[All dimensions given in inches]

\begin{tabular}{|c|c|c|c|}
\hline Size & $X$ & $Y$ & $Z$ \\
\hline $\begin{array}{l}1 / 2 \text { by } 1 / 2 \text { by } 3 / 8 . \\
1 / 2 \text { by } 3 / 8 \text { by } 1 / 2 . \\
3 / 4 \text { by } 3 / 4 \text { by } 1 / 2- \\
3 / 4 \text { by } 1 / 2 \text { by } 3 / 4 \ldots \\
1 \text { by } 1 \text { by } 3 / 4 \ldots\end{array}$ & $\begin{array}{l}1.19 \\
1.25 \\
1.31 \\
1.44 \\
1.50\end{array}$ & $\begin{array}{l}1.19 \\
1.19 \\
1.31 \\
1.38 \\
1.50\end{array}$ & $\begin{array}{l}1.19 \\
1.25 \\
1.38 \\
1.44 \\
1.56\end{array}$ \\
\hline $\begin{array}{l}1 \text { by } 1 \text { by } 1 / 2 \\
1 \text { by } 1 \text { by } 3 / 8 \\
1 \text { by } 3 / 4 \text { by } 1 \\
11 / 4 \text { by } 11 / 4 \text { by } 1 \\
11 / 4 \text { by } 11 / 4 \text { by } 3 / 4\end{array}$ & $\begin{array}{l}1.44 \\
1.31 \\
1.63 \\
1.75 \\
1.63\end{array}$ & $\begin{array}{l}1.44 \\
1.31 \\
1.56 \\
1.75 \\
1.63\end{array}$ & $\begin{array}{l}\text { 1. } 50 \\
\text { 1. } 44 \\
\text { 1. } 63 \\
\text { 1. } 61 \\
\text { 1. } 75\end{array}$ \\
\hline $\begin{array}{l}11 / 4 \text { by } 11 / 4 \text { by } 1 / 2 \\
11 / 4 \text { by } 1 \text { by } 11 / 4 \\
11 / 2 \text { by } 11 / 2 \text { by } 11 \\
11 / 2 \text { by } 11 / 2 \text { by } 1 \\
11 / 2 \text { by } 11 / 2 \text { by } 3 / 4\end{array}$ & $\begin{array}{l}1.50 \\
1.94 \\
2.00 \\
1.81 \\
1.69\end{array}$ & $\begin{array}{l}1.50 \\
1.81 \\
2.00 \\
1.81 \\
1.69\end{array}$ & $\begin{array}{l}1.69 \\
1.94 \\
2.06 \\
2.00 \\
1.88\end{array}$ \\
\hline $\begin{array}{l}11 / 2 \text { by } 11 / 2 \text { by } 1 / 2 \\
11 / 2 \text { by } 11 / 4 \text { by } 11 \\
2 \text { by } 2 \text { by } 11 / 2 \\
2 \text { by } 2 \text { by } 11 / 4 \ldots \\
2 \text { by } 2 \text { by } 1 .\end{array}$ & $\begin{array}{l}1.63 \\
2.13 \\
2.25 \\
2.13 \\
2.00\end{array}$ & $\begin{array}{l}1.63 \\
2.06 \\
2.25 \\
2.13 \\
2.00\end{array}$ & $\begin{array}{l}1.81 \\
2.13 \\
2.38 \\
2.31 \\
2.25\end{array}$ \\
\hline $\begin{array}{l}2 \text { by } 2 \text { by } 3 / 4 \\
2 \text { by } 2 \text { by } 1 / 2 \\
2 \text { by } 11 / 2 \text { by } 2 \\
21 / 2 \text { by } 21 / 2 \text { by } 2 \\
21 / 2 \text { by } 21 / 2 \text { by } 1\end{array}$ & $\begin{array}{l}1.81 \\
1.75 \\
2.50 \\
2.69 \\
2.44\end{array}$ & $\begin{array}{l}1.81 \\
1.75 \\
2.38 \\
2.69 \\
2.44\end{array}$ & $\begin{array}{l}2.13 \\
2.06 \\
2.50 \\
2.75 \\
2.63\end{array}$ \\
\hline $\begin{array}{l}21 / 2 \text { by } 2 \text { by } 21 / 2- \\
3 \text { by } 3 \text { by } 21 / 2 \\
3 \text { by } 3 \text { by } 2 \\
3 \text { by } 21 / 2 \text { by } 3\end{array}$ & $\begin{array}{l}2.94 \\
3.06 \\
2.81 \\
3.38\end{array}$ & $\begin{array}{l}2.75 \\
3.06 \\
2.81 \\
3.31\end{array}$ & $\begin{array}{l}2.94 \\
3.31 \\
3.13 \\
\text { 3. } 38\end{array}$ \\
\hline
\end{tabular}

DIMENSIONS OF ELBOWS, $45^{\circ}$ ELBOWS, TEES, AND CROSSES (STRAIGHT SIZES)
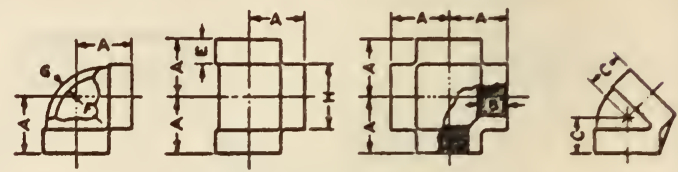

FIGURE 256

[All dimensions given in inches]

\begin{tabular}{|c|c|c|c|c|c|c|c|c|}
\hline \multirow{3}{*}{$\begin{array}{l}\text { Nominal } \\
\text { pipe size }\end{array}$} & $A$ & $C$ & $B$ & $E$ & & & $G$ & $H$ \\
\hline & \multirow{2}{*}{ 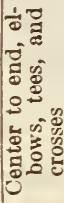 } & \multirow{2}{*}{ 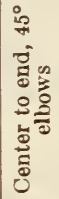 } & \multirow{2}{*}{ 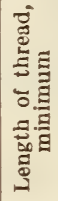 } & \multirow{2}{*}{ 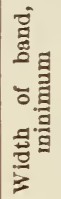 } & \multicolumn{2}{|c|}{$\begin{array}{l}\text { Inside di- } \\
\text { ameter of fit- } \\
\text { ting }\end{array}$} & \multirow{2}{*}{ 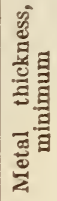 } & \multirow{2}{*}{ 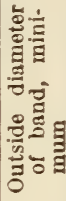 } \\
\hline & & & & & 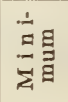 & 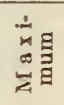 & & \\
\hline & $\begin{array}{l}0.94 \\
1.06 \\
1.25 \\
1.44 \\
1.63\end{array}$ & $\begin{array}{l}0.81 \\
.88 \\
1.00 \\
1.13 \\
1.31\end{array}$ & $\begin{array}{r}0.43 \\
.47 \\
.57 \\
.64 \\
.75\end{array}$ & $\begin{array}{r}0.38 \\
.44 \\
.50 \\
.56 \\
.62\end{array}$ & $\begin{array}{r}0.540 \\
.675 \\
.840 \\
1.050 \\
1.315\end{array}$ & $\begin{array}{r}0.584 \\
.719 \\
.897 \\
1.107 \\
1.385\end{array}$ & $\begin{array}{r}0.14 \\
.15 \\
.16 \\
.18 \\
.20\end{array}$ & $\begin{array}{l}0.93 \\
1.12 \\
1.34 \\
1.63 \\
1.95\end{array}$ \\
\hline 23 & $\begin{array}{l}1.94 \\
2.13 \\
2.50 \\
2.94 \\
3.38\end{array}$ & $\begin{array}{l}1.50 \\
1.69 \\
2.00 \\
2.25 \\
2.50\end{array}$ & $\begin{array}{r}.84 \\
.87 \\
1.00 \\
1.17 \\
1.23\end{array}$ & $\begin{array}{r}.69 \\
.75 \\
.84 \\
.94 \\
1.00\end{array}$ & $\begin{array}{l}\text { 1. } 660 \\
\text { 1. } 900 \\
\text { 2. } 375 \\
\text { 2. } 875 \\
\text { 3. } 500\end{array}$ & $\begin{array}{l}1.730 \\
\text { 1. } 970 \\
\text { 2. } 445 \\
\text { 2. } 975 \\
\text { 3. } 600\end{array}$ & $\begin{array}{r}.22 \\
.24 \\
.26 \\
.31 \\
.35\end{array}$ & $\begin{array}{l}\text { 2. } 39 \\
\text { 2. } 68 \\
\text { 3. } 28 \\
\text { 3. } 86 \\
\text { 4. } 62\end{array}$ \\
\hline
\end{tabular}

Inspection limits for center-to-end dimensions

[All dimensions given in inches]

\begin{tabular}{|r|r||r|r|}
\hline $\begin{array}{r}\text { Nominal } \\
\text { pipe size }\end{array}$ & $\begin{array}{c}\text { Center to } \\
\text { end }\end{array}$ & $\begin{array}{r}\text { Nominal } \\
\text { pipe size }\end{array}$ & $\begin{array}{r}\text { Center to } \\
\text { end }\end{array}$ \\
\cline { 1 - 2 } 13 & 0.04 & $11 / 4$ & 0.07 \\
$3 / 8$ & .05 & $11 / 2$ & .08 \\
$1 / 2$ & .06 & 2 & .08 \\
$3 / 4$ & .06 & $21 / 2$ & .10 \\
1 & .07 & 3 & .10 \\
\hline
\end{tabular}

AMERICAN SOCIETY OF MECHANICAL ENGINEERS, AMERICAN TENTATIVE STANDARD MALLEABLE-IRON SCREWED FITTINGS FOR MAXIMUM WORKING SATURATED STEAM PRESSURES OF 150 POUNDS PER SQUARE INCH (GAGE), B16c-1927

[Approved as American Tentative Standard by the American Standards Association, ${ }^{68}$ December, 1927]

\section{MALLEABLE-IRON SCREWED FITTINGS}

\section{POUNDS PER SQUARE INCH}

\section{INTRODUCTORY NOTES}

1. Pressure ratings.-This standard shall be known as the American 150-pound malleable-iron screwed fittings standard, said pressure designation being the recommended maximum working saturated steam rating.

2. Size.-The size of the fittings scheduled in the following tables is identified by the corresponding nominal pipe size.

In the case of reducing tees, crosses, and $Y$ branches (laterals), the size of the largest run open-

${ }_{68}$ Sponsor organizations American Society of Mechanical En. gineers, Manufacturers' Standardization Society of Valve and Fittings Industry, and Heating and Piping Contractors National Association. 
ing shall be given first, followed by the size of the opening at the opposite end of the run. Where the fitting is a $T$ or $Y$ branch (lateral), the size of the outlet is given next. Where the fitting is a cross, the largest opening of outlet is the third dimension given followed by the opening opposite. The straight-line sketches, Figure 257, illustrate how the reducing fittings are read.

Where an external thread is wanted, the word "male" shall follow the size of that opening.

3. Marking.-Each fitting shall be marked for identification with the maker's name or symbol.

4. Metal thickness.-It is recognized that some variations are absolutely unavoidable in the making of patterns and castings. In order to define what may be considered a reasonable degree of accuracy
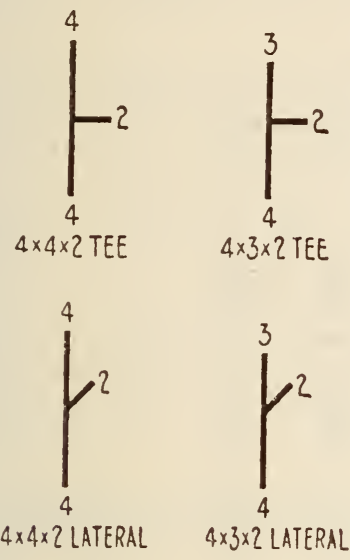

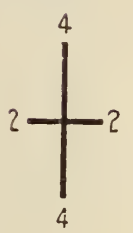

$4 \times 4 \times 2 \times 2$

CROSS

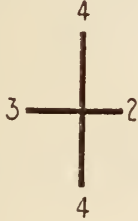

$4 \times 4 \times 3 \times 2$

CROSS

FIGURE 257 in body-thickness measurements of these fittings, the following inspection limits shall be allowed:

Body-thickness measurements taken on any fittings at points diametrically opposite shall, when added together and divided by two, equal or exceed the minimum metal thicknesses set forth in this standard. Body thickness at no point shall be more than 10 per cent below the minimum metal thicknesses set forth in this standard.

5. Threading.- American standard pipe threads shall be used in threading fittings and variations in tapping and dieing shall be limited to one turn either way from the standard size as determined by the working gages prescribed by the American standard pipe thread (No. 3-1919) or its latest revision.

All female threads shall be countersunk a distance not less than one-half the pitch of the thread and at an angle of $45^{\circ}$ with the axis of the thread, and all male threads shall be chamfered at an angle of $45^{\circ}$ both for the purpose of easier entrance in making a joint and for protection of the thread.

The length of threads specified in all tables shall be measured to include the countersink or chamfer.
6. Ribs.-The addition of ribs or lugs shall not be prohibited on any screwed fittings.

7. Fitting dimensions.-Tables of center-to-end dimensions are given for both straight and reducing fittings.

The dimensions in the tables of reducing fittings are for use only when making patterns for the specific reducing fitting in question and do not apply when a larger size pattern is bushed to make the reducing fitting wanted.

It is recognized that some variations are absolutely unavoidable in the making of patterns and castings. In order to define what may be considered a reasonable degree of accuracy in the center-to-end measurements of these fittings, the following inspection limits shall be allowed:

Inspection limits for center-to-end dimensions

[All dimensions given in inches]

\begin{tabular}{l|c|c|c|c|c|c|c|c}
\hline Nominal pipe size ..... & $1 / 8$ & $1 / 4$ & $3 / 8$ & $1 / 2$ & $3 / 4$ & 1 & $11 / 4$ & $11 / 2$ \\
\hline Center to end $\pm \ldots \ldots . .$. & 0.03 & 0.04 & 0.05 & 0.06 & 0.06 & 0.07 & 0.07 & 0.08 \\
\hline \hline Nominal pipe size ..... & 2 & $21 / 2$ & 3 & $31 / 2$ & 4 & 5 & 6 & 8 \\
\hline Center to end $\pm \ldots \ldots . .$. & 0.08 & 0.10 & 0.10 & 0.10 & 0.12 & 0.12 & 0.14 & 0.16
\end{tabular}

TABLE 1.-Dimensions of elbows, tees, crosses, and $45^{\circ}$ elbows (straight sizes)

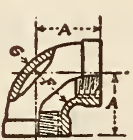

ELBOW

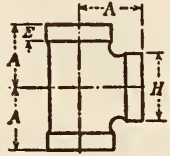

TEE

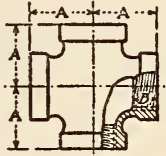

CROSS

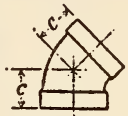

$45^{\circ}$ ELBOW
Figure 258.-Elbows, tees, crosses, and $45^{\circ}$ elbows (straight sizes)

[All dimensions given in inches]

\begin{tabular}{|c|c|c|c|c|c|c|c|c|}
\hline \multirow{3}{*}{$\begin{array}{l}\text { Nom- } \\
\text { inal- } \\
\text { pipe } \\
\text { size }\end{array}$} & \multirow{3}{*}{$\begin{array}{c}\text { A } \\
\text { Center } \\
\text { to } \\
\text { end, } \\
\text { el- } \\
\text { bows, } \\
\text { tees, } \\
\text { and } \\
\text { cros- } \\
\text { ses }\end{array}$} & \multirow{3}{*}{$\begin{array}{c}\text { C } \\
\\
\text { Center } \\
\text { to } \\
\text { end, } \\
45^{\circ} \\
\text { el- } \\
\text { bows }\end{array}$} & \multirow{3}{*}{$\begin{array}{c}B \\
\\
\text { Length } \\
\text { of } \\
\text { thread, } \\
\text { mini- } \\
\text { mum }\end{array}$} & \multirow{3}{*}{$\begin{array}{c}\mathrm{E} \\
\\
\text { Width } \\
\text { of } \\
\text { band, } \\
\text { mini- } \\
\text { mum }\end{array}$} & \multicolumn{2}{|c|}{$\mathbf{F}$} & \multirow{3}{*}{$\begin{array}{c}\text { G } \\
\\
\text { Metal } \\
\text { thick- } \\
\text { ness, } \\
\text { mini- } \\
\text { mum }\end{array}$} & \multirow{3}{*}{$\begin{array}{c}\text { H } \\
\\
\text { Outside } \\
\text { diam- } \\
\text { eter } \\
\text { of } \\
\text { band, } \\
\text { mini- } \\
\text { mum }\end{array}$} \\
\hline & & & & & \multicolumn{2}{|c|}{$\begin{array}{l}\text { Inside diame- } \\
\text { ter of fitting }\end{array}$} & & \\
\hline & & & & & $\begin{array}{l}\text { Mini- } \\
\text { mum }\end{array}$ & $\begin{array}{l}\text { Maxi- } \\
\text { mum }\end{array}$ & & \\
\hline $\begin{array}{l}1 / 8 \\
1 / 4 \\
3 / 8 \\
1 / 2\end{array}$ & $\begin{array}{r}0.69 \\
.81 \\
.95 \\
1.12\end{array}$ & $\begin{array}{r}0.68 \\
.73 \\
.80 \\
.88\end{array}$ & $\begin{array}{r}0.25 \\
.32 \\
.36 \\
.43\end{array}$ & $\begin{array}{r}0.200 \\
.215 \\
.230 \\
.249\end{array}$ & $\begin{array}{r}0.405 \\
.540 \\
.675 \\
.840\end{array}$ & $\begin{array}{r}0.435 \\
.584 \\
.719 \\
.897\end{array}$ & $\begin{array}{r}0.090 \\
.095 \\
.100 \\
.105\end{array}$ & $\begin{array}{r}0.693 \\
.844 \\
1.015 \\
1.197\end{array}$ \\
\hline $\begin{array}{l}3 / 4 \\
11 / 4 \\
11 / 2\end{array}$ & $\begin{array}{l}1.31 \\
1.50 \\
1.75 \\
1.94\end{array}$ & $\begin{array}{r}.98 \\
1.12 \\
1.29 \\
1.43\end{array}$ & $\begin{array}{l}.50 \\
.58 \\
.67 \\
.70\end{array}$ & $\begin{array}{l}.273 \\
.302 \\
.341 \\
.368\end{array}$ & $\begin{array}{l}1.050 \\
1.315 \\
1.660 \\
1.900\end{array}$ & $\begin{array}{l}\text { 1. } 107 \\
\text { 1. } 385 \\
\text { 1. } 730 \\
1.970\end{array}$ & $\begin{array}{l}.120 \\
.134 \\
.145 \\
.155\end{array}$ & $\begin{array}{l}1.458 \\
1.771 \\
2.153 \\
2.427\end{array}$ \\
\hline $\begin{array}{l}2 \\
21 / 2 \\
3 \\
31 / 2\end{array}$ & $\begin{array}{l}2.25 \\
2.70 \\
3.08 \\
3.42\end{array}$ & $\begin{array}{l}1.68 \\
1.95 \\
2.17 \\
2.39\end{array}$ & $\begin{array}{r}.75 \\
.92 \\
.98 \\
1.03\end{array}$ & $\begin{array}{r}.422 \\
.478 \\
.548 \\
.604\end{array}$ & $\begin{array}{l}2.375 \\
2.875 \\
3.500 \\
4.000\end{array}$ & $\begin{array}{l}2.445 \\
2.975 \\
3.600 \\
4.100\end{array}$ & $\begin{array}{r}.173 \\
.210 \\
.231 \\
.248\end{array}$ & $\begin{array}{l}\text { 2. } 963 \\
\text { 3. } 589 \\
\text { 4. } 285 \\
4.843\end{array}$ \\
\hline $\begin{array}{l}4 \\
5 \\
6 \\
8\end{array}$ & $\begin{array}{l}3.79 \\
4.50 \\
5.13\end{array}$ & $\begin{array}{l}2.61 \\
3.05 \\
3.46 \\
\end{array}$ & $\begin{array}{l}1.08 \\
1.18 \\
1.28 \\
1.47\end{array}$ & $\begin{array}{r}.661 \\
.780 \\
.900 \\
1.125\end{array}$ & $\begin{array}{l}4.500 \\
5.563 \\
6.625 \\
8.625\end{array}$ & $\begin{array}{l}4.600 \\
5.663 \\
6.725 \\
8.725\end{array}$ & $\begin{array}{l}.265 \\
.300 \\
.336 \\
.403\end{array}$ & $\begin{array}{l}5.401 \\
6.583 \\
7.767 \\
9.995\end{array}$ \\
\hline
\end{tabular}

Dimensions for reducing elbows and reducing crosses are given in Table 2, and dimensions for reducing tees in Tables $3 \mathrm{~A}$ and $3 \mathrm{~B}$. 
TABLE 2.-Dimensions of elbows and crosses (reducing sizes)

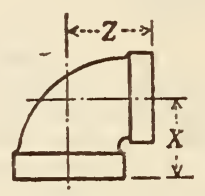

ELBOW

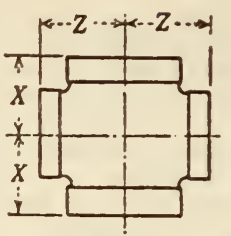

CROSS

Figure 259.-Elbows and crosses (reducing sizes)

[All dimensions giren in inches]

\begin{tabular}{|c|c|c|c|c|c|}
\hline \multicolumn{3}{|c|}{ Elbows } & \multicolumn{3}{|c|}{ Crosses } \\
\hline \multirow{2}{*}{$\begin{array}{l}\text { Nominal pipe } \\
\text { sizes }\end{array}$} & \multicolumn{2}{|c|}{ Center to end } & \multirow{2}{*}{$\begin{array}{l}\text { Nominal pipe } \\
\text { sizes }\end{array}$} & \multicolumn{2}{|c|}{ Center to end } \\
\hline & $\mathrm{X}$ & $\mathbf{Z}$ & & $\mathrm{x}$ & $\mathbf{Z}$ \\
\hline $\begin{array}{l}3 / 8 \text { by } 1 / 4 \ldots \\
1 / 2 \text { by } 3 / 8 \ldots \\
34 \text { by } 16 \ldots \\
3 / 4 \text { by } 3 / 8 \ldots \ldots \\
1 \text { by } 3 / 4 \ldots\end{array}$ & $\begin{array}{l}0.88 \\
1.04 \\
1.20 \\
1.12 \\
1.37\end{array}$ & $\begin{array}{l}0.90 \\
1.03 \\
1.22 \\
1.13 \\
1.45\end{array}$ & $\begin{array}{l}3 / 4 \text { by } 1 / 2 \ldots \ldots \\
1 \text { by } 3 / 4 \ldots \\
1 \text { by } 16 \\
11 / 4 \text { by } 1 \ldots \\
11 / 4 \text { by } 3 / 4 \ldots\end{array}$ & $\begin{array}{l}\text { 1. } 20 \\
\text { 1. } 37 \\
\text { 1. } 26 \\
\text { 1. } 58 \\
\text { 1. } 45\end{array}$ & $\begin{array}{l}\text { 1. } 22 \\
\text { 1. } 45 \\
\text { 1. } 36 \\
1.67 \\
1.62\end{array}$ \\
\hline $\begin{array}{l}1 \text { by } 1 / 2 \ldots \\
1144 \text { by } 1 \ldots \\
11 / 4 \text { by } 3 / 4 \ldots \\
116 \text { by } 114 . \ldots \\
116 \text { by } 1 \ldots \ldots\end{array}$ & $\begin{array}{l}\text { 1. } 26 \\
\text { 1. } 58 \\
\text { 1. } 45 \\
1.82 \\
\text { 1. } 65\end{array}$ & $\begin{array}{l}1.36 \\
1.67 \\
1.62 \\
1.88 \\
1.80\end{array}$ & $\begin{array}{l}11 / 2 \text { by } 1114 \ldots \\
11 / 2 \text { by } 1 \\
11 / 2 \text { by } 3 / 4 \ldots \ldots \\
2 \text { by } 114 \ldots \ldots \\
2 \text { by } 11 / 4 \ldots \ldots\end{array}$ & $\begin{array}{l}1.82 \\
1.65 \\
1.52 \\
2.02 \\
1.90\end{array}$ & $\begin{array}{l}\text { 1. } 88 \\
\text { 1. } 80 \\
\text { 1. } 75 \\
\text { 2. } 16 \\
\text { 2. } 10\end{array}$ \\
\hline $\begin{array}{l}132 \text { by } 3 / 4 \ldots \\
2 \text { by } 1132 \ldots \\
2 \text { by } 11 / 4 \ldots \\
2 \text { by } 1\end{array}$ & $\begin{array}{l}1.52 \\
2.02 \\
1.90 \\
1.73\end{array}$ & $\begin{array}{l}\text { 1. } 75 \\
\text { 2. } 16 \\
\text { 2. } 10 \\
\text { 2. } 02\end{array}$ & $\begin{array}{l}2 \text { by } 1 \\
2 \text { by } 3 / 4 \ldots \\
21 / 2 \text { by } 2 \ldots \ldots \\
3 \text { by } 2 \ldots \ldots\end{array}$ & $\begin{array}{l}\text { 1. } 73 \\
\text { 1. } 60 \\
\text { 2. } 39 \\
\text { 2. } 52\end{array}$ & $\begin{array}{l}\text { 2. } 02 \\
1.97 \\
\text { 2. } 60 \\
2.89\end{array}$ \\
\hline $\begin{array}{l}21,2 \text { by } 2 \ldots \\
3 \text { by } 21, \ldots \ldots \\
3 \text { by } 2 \ldots \ldots \\
4 \text { by } 3 \ldots \ldots\end{array}$ & $\begin{array}{l}\text { 2. } 39 \\
\text { 2. } 83 \\
\text { 2. } 52 \\
\text { 3. } 30\end{array}$ & $\begin{array}{l}\text { 2. } 60 \\
\text { 2. } 99 \\
\text { 2. } 89 \\
\text { 3. } 60\end{array}$ & & & \\
\hline
\end{tabular}

TABLE 3A.-Dimensions of tees (reducing sizes)

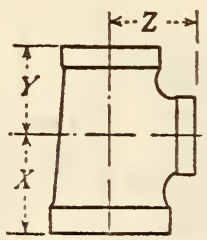

$$
\begin{aligned}
& \text { Figure 260.- } \\
& \text { Tee (reducing } \\
& \text { size) }
\end{aligned}
$$

\begin{tabular}{|c|c|c|c|c|c|c|c|c|c|c|c|}
\hline \multirow{2}{*}{ Nominal pipe sizes } & \multicolumn{3}{|c|}{ Center to end } & \multirow{2}{*}{ Nominal pipe sizes } & \multicolumn{3}{|c|}{ Center to end } & \multirow{2}{*}{ Nominal pipe sizes } & \multicolumn{3}{|c|}{ Center to end } \\
\hline & $\mathrm{x}$ & $\mathrm{Y}$ & $\mathrm{Z}$ & & $\mathrm{x}$ & $\mathrm{Y}$ & $\mathrm{Z}$ & & $\mathrm{x}$ & $\mathrm{Y}$ & Z \\
\hline $\begin{array}{l}1 / 4 \text { by } 1 / 4 \text { by } 3 / 8 \\
3 / 8 \text { by } 3 / 8 \text { by } 112 \\
8 / 8 \text { by } 3 / 8 \text { by } 1 / 4 \\
3 / 8 \text { by } 3 / 8 \text { by } 118 \\
3 / 8 \text { by } 1 / 4 \text { by } 3 / 8\end{array}$ & $\begin{array}{r}0.90 \\
1.03 \\
.88 \\
.81 \\
.95\end{array}$ & $\begin{array}{l}0.90 \\
1.03 \\
.88 \\
.81 \\
.90\end{array}$ & $\begin{array}{l}0.88 \\
1.04 \\
.90 \\
.85 \\
.95\end{array}$ & $\begin{array}{l}3 / 8 \text { by } 1 / 4 \text { by } 1 / 4-\cdots \\
1 / 2 \text { by } 1 / 2 \text { by } 1 / 4- \\
1 / 2 \text { by } 3 / 8 \text { by } 1 / 2 \ldots \\
1 / 2 \text { by } 3 / 8 \text { by } 3 / 8 . \\
3 / 4 \text { by } 3 / 4 \text { by } 1 / 4 \ldots\end{array}$ & $\begin{array}{l}0.88 \\
.97 \\
1.12 \\
1.04 \\
1.05\end{array}$ & $\begin{array}{l}0.81 \\
.97 \\
1.03 \\
.95 \\
1.05\end{array}$ & $\begin{array}{l}0.90 \\
.98 \\
1.12 \\
1.03 \\
1.08\end{array}$ & $\begin{array}{l}3 / 4 \text { by } 1 / 2 \text { by } 3 / 8 \ldots . \\
1 \text { by } 1 / 2 \text { by } 1 / 2-\ldots \\
11 / 4 \text { by } 11 / 4 \text { by } 3 / 8 . \\
11 / 4 \text { by } 3 / 4 \text { by } 3 / 4 .\end{array}$ & $\begin{array}{l}1.12 \\
1.26 \\
1.26 \\
1.45\end{array}$ & $\begin{array}{l}1.04 \\
1.12 \\
1.26 \\
1.31\end{array}$ & $\begin{array}{l}1.13 \\
1.36 \\
1.44 \\
1.62\end{array}$ \\
\hline
\end{tabular}

[All dimensions given in inches] 
TABLE 3B.-Dimensions of tees (reducing sizes)

[All dimensions given in inches]

\begin{tabular}{|c|c|c|c|c|c|c|c|c|c|c|c|}
\hline \multirow{2}{*}{ Nominal pipe sizes } & \multicolumn{3}{|c|}{ Center to end } & \multirow{2}{*}{ Nominal pipe sizes } & \multicolumn{3}{|c|}{ Center to end } & \multirow{2}{*}{ Nominal pipe sizes } & \multicolumn{3}{|c|}{ Center to end } \\
\hline & $\boldsymbol{X}$ & $Y$ & $\mathrm{Z}$ & & $\mathrm{x}$ & $\mathrm{Y}$ & $Z$ & & $\mathrm{x}$ & $\mathrm{Y}$ & $\mathrm{Z}$ \\
\hline $\begin{array}{l}1 / 2 \text { by } 1 / 2 \text { by } 3 / 4 \\
1 / 2 \text { by } 1 / 2 \text { by } 3 / 8 \\
3 / 4 \text { by } 3 / 4 \text { by } 13 \\
3 / 4 \text { by } 3 / 4 \text { by } 1 / 2 \ldots \\
3 / 4 \text { by } 3 / 4 \text { by } 3 / 8\end{array}$ & $\begin{array}{l}1.22 \\
1.04 \\
1.45 \\
1.20 \\
1.12\end{array}$ & \begin{tabular}{l|}
1.22 \\
1.04 \\
1.45 \\
1.20 \\
1.12
\end{tabular} & $\begin{array}{l}1.20 \\
1.03 \\
1.37 \\
1.22 \\
1.13\end{array}$ & $\begin{array}{l}11 / 2 \text { by } 11 / 2 \text { by } 2 \\
11 / 2 \text { by } 11 / 2 \text { by } 11 / 4- \\
11 / 2 \text { by } 11 / 2 \text { by } 1- \\
11 / 2 \text { by } 11 / 2 \text { by } 3 / 4- \\
11 / 2 \text { by } 11 / 2 \text { by } 1 / 2-\end{array}$ & $\begin{array}{l}2.16 \\
1.82 \\
1.65 \\
1.52 \\
1.41\end{array}$ & $\begin{array}{l}2.16 \\
1.82 \\
1.65 \\
1.52 \\
1.41\end{array}$ & $\begin{array}{l}2.02 \\
1.88 \\
1.80 \\
1.75 \\
1.66\end{array}$ & $\begin{array}{l}21 / 2 \text { by } 21 / 2 \text { by } \\
21 / 2 \text { by } 21 / 2 \text { by } \\
21 / 2 \text { by } 21 / 2 \text { by } \\
21 / 2 \text { by } 21 / 2 \text { by } \\
21 / 2 \text { by } 21 / 2 \text { by }\end{array}$ & $\begin{array}{l}2.39 \\
2.16 \\
2.04 \\
1.87 \\
1.74\end{array}$ & $\begin{array}{l}2.39 \\
2.16 \\
2.04 \\
1.87 \\
1.74\end{array}$ & $\begin{array}{l}2.60 \\
2.51 \\
2.45 \\
2.37 \\
2.32\end{array}$ \\
\hline $\begin{array}{l}3 / 4 \text { by } 1 / 2 \text { by } 3 / 4 \\
3 / 4 \text { by } 1 / 2 \text { by } 1 / 2 \ldots \\
1 \text { by } 1 \text { by } 11 / 2 \\
1 \text { by } 1 \text { by } 11 / 4 \ldots \\
1 \text { by } 1 \text { by } 3 / 4\end{array}$ & $\begin{array}{l}1.31 \\
1.20 \\
1.80 \\
1.67 \\
1.37\end{array}$ & $\begin{array}{l}1.22 \\
1.12 \\
1.80 \\
1.67 \\
1.37\end{array}$ & $\begin{array}{l}1.31 \\
1.22 \\
1.65 \\
1.58 \\
1.45\end{array}$ & $\begin{array}{l}11 / 2 \text { by } 11 / 4 \text { by } 11 \\
11 / 2 \text { by } 11 / 4 \text { by } 11 / \\
11 / 2 \text { by } 11 / 4 \text { by } 1 \\
11 / 2 \text { by } 1 \text { by } 11 / 2 \\
11 / 2 \text { by } 1 \text { by } 1 . .\end{array}$ & $\begin{array}{l}1.94 \\
1.82 \\
1.65 \\
1.94 \\
1.65\end{array}$ & $\begin{array}{l}1.88 \\
1.75 \\
1.58 \\
1.80 \\
1.50\end{array}$ & $\begin{array}{l}1.94 \\
1.88 \\
1.80 \\
1.94 \\
1.80\end{array}$ & $\begin{array}{l}21 / 2 \text { by } 2 \text { by } 21 \\
21 / 2 \text { by } 2 \text { by } 2 \\
21 / 2 \text { by } 11 / 2 \text { by } \\
3 \text { by } 3 \text { by } 21 / 2- \\
3 \text { by } 3 \text { by } 2\end{array}$ & $\begin{array}{l}2.70 \\
2.39 \\
2.70 \\
2.83 \\
2.52\end{array}$ & $\begin{array}{l}2.60 \\
2.25 \\
2.51 \\
2.83 \\
2.52\end{array}$ & $\begin{array}{l}2.70 \\
2.60 \\
2.70 \\
2.99 \\
2.89\end{array}$ \\
\hline 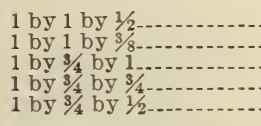 & $\begin{array}{l}1.26 \\
1.18 \\
1.50 \\
1.37 \\
1.26\end{array}$ & $\begin{array}{l}1.26 \\
1.18 \\
1.45 \\
1.31 \\
1.20\end{array}$ & $\begin{array}{l}1.36 \\
1.27 \\
1.50 \\
1.45 \\
1.36\end{array}$ & $\begin{array}{l}11 / 2 \text { by } 8 / 4 \text { by } 11 / 2 \\
2 \text { by } 2 \text { by } 21 / 2 \ldots \\
2 \text { by } 2 \text { by } 11 / 2 \ldots \\
2 \text { by } 2 \text { by } 11 / 4 \ldots \\
2 \text { by } 2 \text { by } 1 . .\end{array}$ & $\begin{array}{l}\text { 1. } 94 \\
2.60 \\
2.02 \\
1.90 \\
1.73\end{array}$ & $\begin{array}{l}1.75 \\
2.60 \\
2.02 \\
1.90 \\
1.73\end{array}$ & $\begin{array}{l}1.94 \\
2.39 \\
2.16 \\
2.10 \\
2.02\end{array}$ & $\begin{array}{l}3 \text { by } 3 \text { by } 11 / 2 \\
3 \text { by } 3 \text { by } 11 / 4- \\
3 \text { by } 3 \text { by } 1 \\
3 \text { by } 21 / 2 \text { by } 3 \\
3 \text { by } 2 \text { by } 3 \\
\end{array}$ & $\begin{array}{l}\text { 2. } 29 \\
2.17 \\
\text { 2. } 00 \\
\text { 3. } 08 \\
\text { 3. } 08\end{array}$ & $\begin{array}{l}2.29 \\
2.17 \\
2.00 \\
2.99 \\
2.89\end{array}$ & $\begin{array}{l}2.80 \\
2.74 \\
2.66 \\
3.08 \\
3.08\end{array}$ \\
\hline $\begin{array}{l}1 \text { by } 1 / 2 \text { by } 1 \\
1 \text { by } 1 / 2 \text { by } 3 / 4- \\
11 / 4 \text { by } 11 / 4 \text { by } 11 / 2 \\
11 / 4 \text { by } 11 / 4 \text { by } 1 \\
11 / 4 \text { by } 11 / 4 \text { by } 3 / 4-\end{array}$ & $\begin{array}{l}1.50 \\
1.37 \\
1.88 \\
1.58 \\
1.45\end{array}$ & $\begin{array}{l}1.36 \\
1.22 \\
1.88 \\
1.58 \\
1.45\end{array}$ & $\begin{array}{l}1.50 \\
1.45 \\
1.82 \\
1.67 \\
1.62\end{array}$ & $\begin{array}{l}2 \text { by } 2 \text { by } 3 / 4 \\
2 \text { by } 2 \text { by } 1 / 2 \ldots \\
2 \text { by } 11 / 2 \text { by } 2- \\
2 \text { by } 11 / 2 \text { by } 11 / 2 \\
2 \text { by } 11 / 2 \text { by } 11 / 4-\end{array}$ & $\begin{array}{l}1.60 \\
1.49 \\
2.25 \\
2.02 \\
1.90\end{array}$ & $\begin{array}{l}1.60 \\
1.49 \\
2.16 \\
1.94 \\
1.82\end{array}$ & $\begin{array}{l}1.97 \\
1.88 \\
2.25 \\
2.16 \\
2.10\end{array}$ & $\begin{array}{l}3 \text { by } 2 \text { by } 2 \\
31 / 2 \text { by } 31 / 2 \text { by } 21 / \\
4 \text { by } 4 \text { by } 3 \\
4 \text { by } 4 \text { by } 21 / 2 \\
4 \text { by } 4 \text { by } 2\end{array}$ & $\begin{array}{l}2.52 \\
2.93 \\
3.30 \\
3.05 \\
2.74\end{array}$ & $\begin{array}{l}2.25 \\
2.93 \\
3.30 \\
3.05 \\
2.74\end{array}$ & $\begin{array}{l}2.89 \\
3.24 \\
3.60 \\
3.51 \\
3.41\end{array}$ \\
\hline $\begin{array}{l}11 / 4 \text { by } 11 / 4 \text { by } 1 / 2- \\
11 / 4 \text { by } 1 \text { by } 11 / 4- \\
11 / 4 \text { by } 1 \text { by } 1 \\
11 / 4 \text { by } 1 \text { by } 3 / 4 \\
11 / 4 \text { by } 3 / 4 \text { by } 11 / 4 \\
11 / 4 \text { by } 3 / 4 \text { by } 1 \ldots\end{array}$ & $\begin{array}{l}1.34 \\
1.75 \\
1.58 \\
1.45 \\
1.75 \\
1.58\end{array}$ & $\begin{array}{l}1.34 \\
1.67 \\
1.50 \\
1.37 \\
1.62 \\
1.45\end{array}$ & $\begin{array}{l}1.53 \\
1.75 \\
1.67 \\
1.62 \\
1.75 \\
1.67\end{array}$ & $\begin{array}{l}2 \text { by } 11 / 4 \text { by } 2 \\
2 \text { by } 11 / 4 \text { by } 11 / 2 \\
2 \text { by } 1 \text { by } 2 \\
2 \text { by } 1 \text { by } 11 / 2 \\
2 \text { by } 3 / 4 \text { by } 2 \\
21 / 2 \text { by } 21 / 2 \text { by } 3\end{array}$ & $\begin{array}{l}2.25 \\
\text { 2. } 02 \\
2.25 \\
2.02 \\
2.25 \\
2.99\end{array}$ & $\begin{array}{l}2.10 \\
1.88 \\
2.02 \\
1.80 \\
1.97 \\
2.99\end{array}$ & \begin{tabular}{l|}
2.25 \\
2.16 \\
2.25 \\
2.16 \\
2.25 \\
2.83
\end{tabular} & $\begin{array}{l}5 \text { by } 5 \text { by } 3 .- \\
6 \text { by } 6 \text { by } 4 .- \\
6 \text { by } 6 \text { by } 3- \\
6 \text { by } 6 \text { by } 21 / 2- \\
6 \text { by } 6 \text { by } 2\end{array}$ & $\begin{array}{l}\text { 3. } 51 \\
\text { 4. } 13 \\
\text { 3. } 64 \\
3.39 \\
3.08\end{array}$ & $\begin{array}{l}3.51 \\
4.13 \\
3.64 \\
3.39 \\
\\
3.08\end{array}$ & $\begin{array}{l}4.22 \\
4.94 \\
4.75 \\
4.66 \\
4.56\end{array}$ \\
\hline
\end{tabular}

(Table 3A contains sizes of reducing tees that are tees that are standard for both malleable-iron standard for malleable-iron fittings, but not for cast- fittings and cast-iron fittings.)

iron fittings. Table $3 \mathrm{~B}$ contains sizes of reducing

TABLE 4.-Dimensions of $45^{\circ} \mathrm{Y}$ branches (straight sizes)

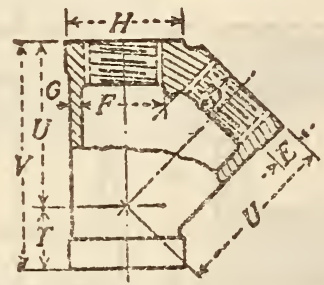

\section{FI G URE $261 .-45^{\circ} \mathrm{Y}$ \\ branch (straight sizes)}

[All dimensions given in inches]

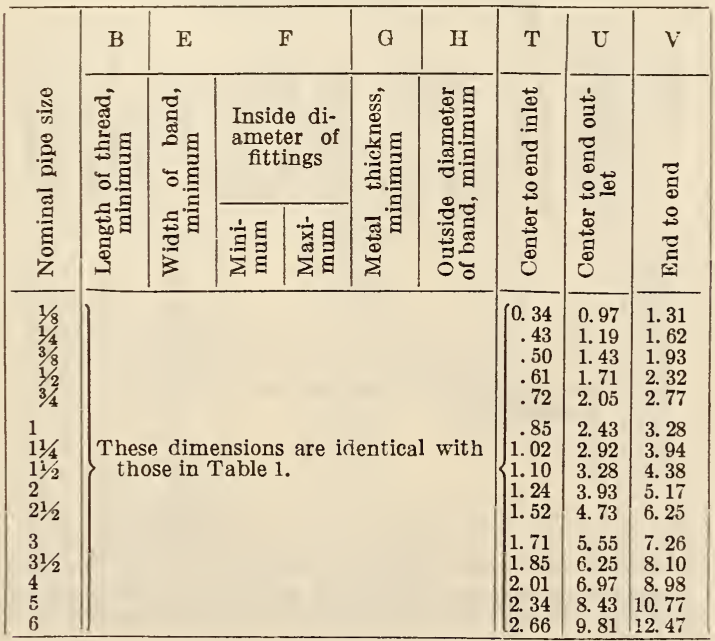


TABLE 5.-Dimensions of service or street tees and $90^{\circ}$ and $45^{\circ}$ elbows (straight sizes)

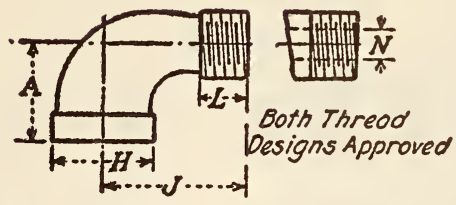

STREET ELBOW

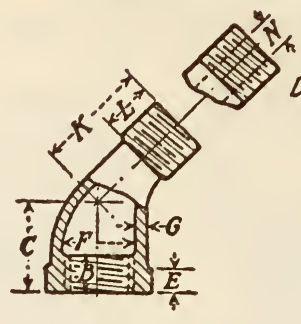

$45^{\circ}$ STREET ELBOW
Both Thread

Designs Approved

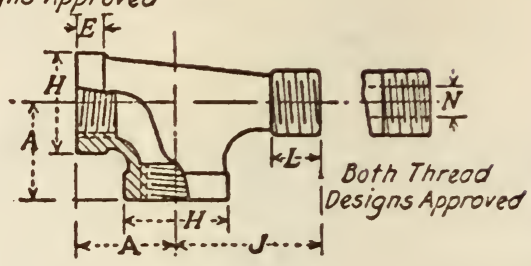

STREET TEE

Figure 262.-Service or street tees and $90^{\circ}$ and $45^{\circ}$ elbows (straight sizes)

[All dimensions given in inches]

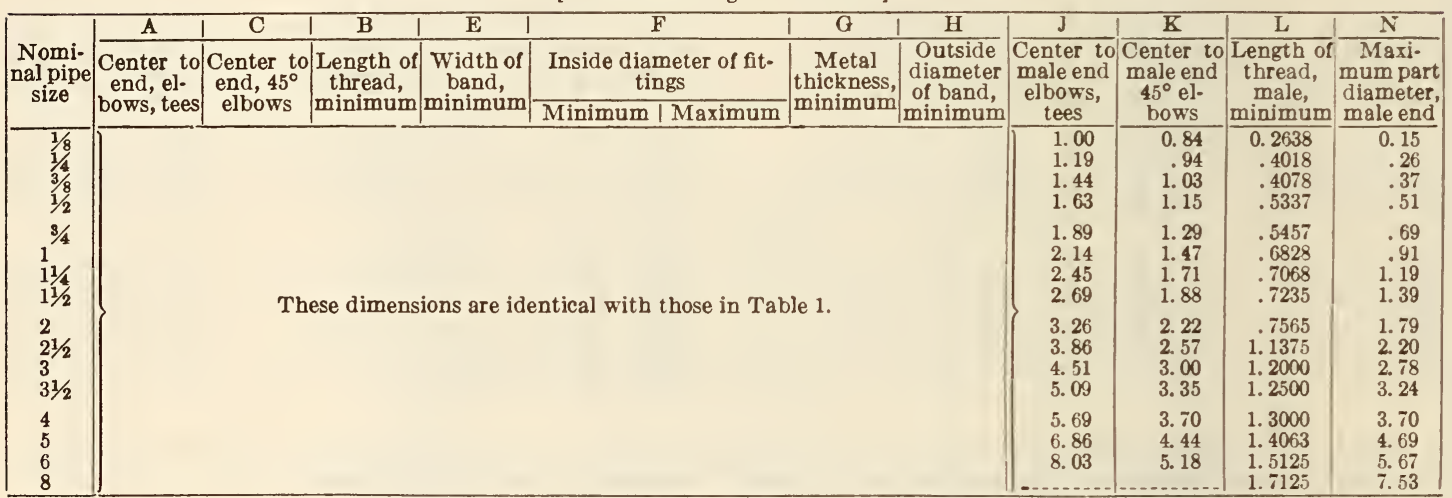

TABLE 6.-Dimensions of couplings (straight and reducing sizes)
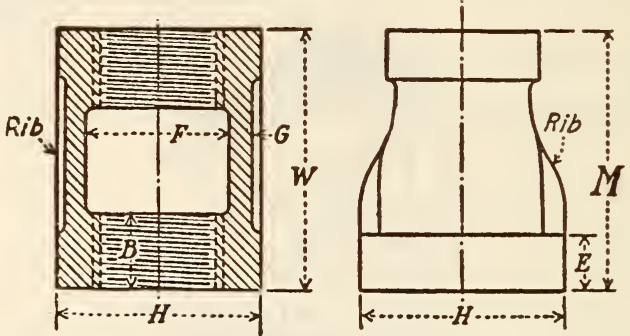

Figure 263.-Couplings (straight and reducing sizes)

[All dimensions given in inches]

\begin{tabular}{|c|c|c|c|c|c|c|c|c|c|}
\hline \multirow{3}{*}{ 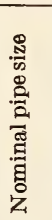 } & B & $\mathbf{E}$ & \multicolumn{2}{|c|}{$\bar{F}$} & $\mathrm{G}$ & $\mathrm{H}$ & \multirow{3}{*}{ 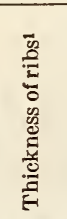 } & \multirow{3}{*}{ 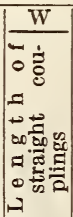 } & \multirow{3}{*}{ 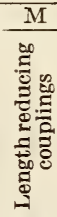 } \\
\hline & \multirow{2}{*}{ 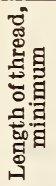 } & \multirow{2}{*}{ 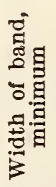 } & \multicolumn{2}{|c|}{$\begin{array}{l}\text { Inside di- } \\
\text { ameter of } \\
\text { fitings }\end{array}$} & \multirow{2}{*}{ 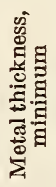 } & \multirow{2}{*}{ 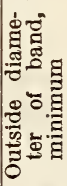 } & & & \\
\hline & & & 宾 & 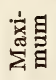 & & & & & \\
\hline $\begin{array}{c}1 / 8 \\
1 / 4 \\
3 / 8 \\
1 / 2 \\
3 / 4 \\
1 \\
11 / 4 \\
11 / 2 \\
2 \\
21 / 2 \\
3 \\
31 / 2 \\
4 \\
5 \\
6 \\
8\end{array}$ & The & dim & $\begin{array}{l}\text { asion } \\
\text { able }\end{array}$ & are id & ntical & with & $\begin{array}{l}0.090 \\
.095 \\
.100 \\
.105 \\
.120 \\
.134 \\
.145 \\
.155 \\
.173 \\
.210 \\
.231 \\
.248 \\
.265 \\
.300 \\
.336 \\
.403\end{array}$ & $\begin{array}{l}0.96 \\
1.06 \\
1.16 \\
1.34 \\
1.52 \\
1.67 \\
1.93 \\
2.15 \\
2.53 \\
2.88 \\
3.18 \\
3.43 \\
3.69 \\
4.22 \\
4.75 \\
5.75\end{array}$ & $\begin{array}{l}1.00 \\
1.13 \\
1.25 \\
1.44 \\
1.69 \\
2.06 \\
2.31 \\
2.81 \\
\text { 3. } 25 \\
3.69 \\
4.00 \\
4.38 \\
5.12 \\
5.86 \\
7.25\end{array}$ \\
\hline
\end{tabular}

1 Right-hand couplings have 2 ribs and right and left hand couplings have 4 ribs on sizes smaller than 1 inch and 6 ribs on sizes 1 inch and larger. 
TABLE 7.-Dimensions of caps

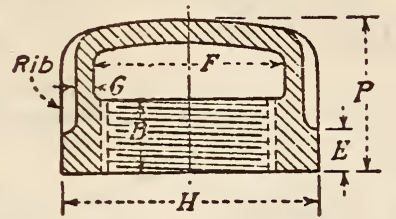

Figdre 264.-Cap

[All dimensions given in inches]

\begin{tabular}{|c|c|c|c|c|c|c|c|c|}
\hline \multirow{3}{*}{ 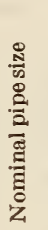 } & \multirow{3}{*}{$\frac{B}{\text { D. }}$} & \multirow{3}{*}{ 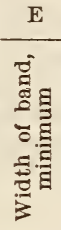 } & \multicolumn{2}{|c|}{ F } & \multirow{3}{*}{ 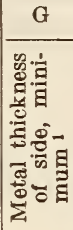 } & \multirow{3}{*}{ 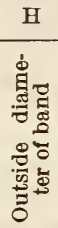 } & $\mathbf{P}$ & \multirow{3}{*}{ 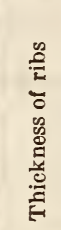 } \\
\hline & & & \multicolumn{2}{|c|}{$\begin{array}{l}\text { Inside } \\
\text { diameter } \\
\text { of fittings }\end{array}$} & & & \multirow[b]{2}{*}{ 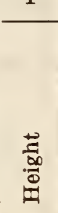 } & \\
\hline & & & 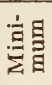 & 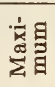 & & & & \\
\hline $\begin{array}{l}1 \\
11 / 1 \\
11 / 2 \\
2 \\
21 / 2 \\
3 \\
31 / 2 \\
4 \\
5 \\
6\end{array}$ & $\left\{\begin{array}{l}\text { Thes } \\
\text { in }\end{array}\right.$ & $\begin{array}{l}\text { dime } \\
\text { able } 1\end{array}$ & ons a & ident & cal witl & those & $\left\{\begin{array}{l}1.16 \\
1.28 \\
1.33 \\
1.45 \\
1.70 \\
1.80 \\
1.90 \\
2.08 \\
2.32 \\
2.55\end{array}\right.$ & $\begin{array}{r}0.134 \\
.145 \\
.155 \\
.173 \\
.210 \\
.231 \\
.248 \\
.265 \\
.300 \\
.336\end{array}$ \\
\hline
\end{tabular}

1 The outside radius of top is equal to $3 \times \mathrm{F}$.

TABLE 8.-Dimensions of close, medium, and open patterns return bends ${ }^{1}$

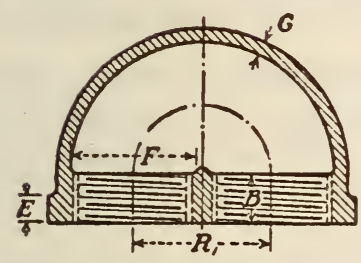

CLOSE

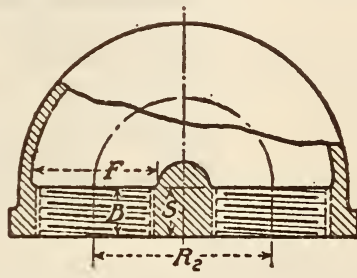

MEDIUM

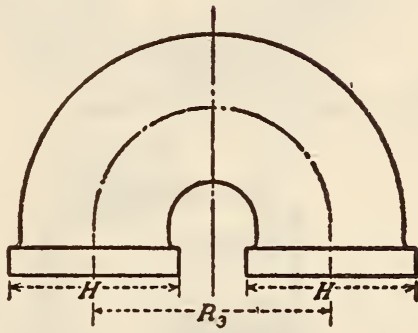

OPEN

FIGURE 265.-Close, medium, and open pattern return bends

[All dimensions in inches]

\begin{tabular}{|c|c|c|c|c|c|c|c|c|c|}
\hline \multirow{3}{*}{ 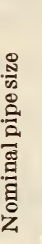 } & $\mathrm{B}^{2}$ & $\mathrm{E}$ & & $F$ & G & H & $\mathrm{R}_{1}$ & $\mathrm{R}_{2}$ & $\mathrm{R}_{3}$ \\
\hline & \multirow{2}{*}{ 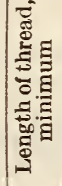 } & \multirow{2}{*}{ 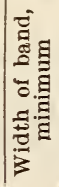 } & \multicolumn{2}{|c|}{$\begin{array}{c}\text { Inside } \\
\text { diameter } \\
\text { of fittings }\end{array}$} & \multirow{2}{*}{ 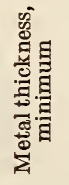 } & \multirow{2}{*}{ 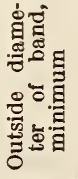 } & \multirow{2}{*}{ 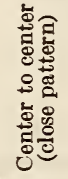 } & \multirow{2}{*}{ 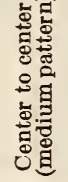 } & \multirow{2}{*}{ 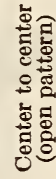 } \\
\hline & & & 要 & 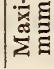 & & & & & \\
\hline $\begin{array}{l}1 / 2 \\
3 / 4 \\
1^{31} \\
11 / 4 \\
11 / 2 \\
2 \\
21 / 2 \\
3 \\
31 / 2 \\
4 \\
5 \\
6\end{array}$ & $\begin{array}{l}\text { The } \\
\text { ar } \\
\text { th }\end{array}$ & $\begin{array}{l}\text { ese d } \\
\text { re ider } \\
\text { aose ir }\end{array}$ & $\begin{array}{l}\text { limen } \\
\text { atical } \\
\text { a Tab }\end{array}$ & $\begin{array}{l}\text { sions } \\
\text { with } \\
\text { le } 1 .\end{array}$ & $\left\{\begin{array}{c}0.116 \\
.133 \\
.150 \\
.165 \\
.178 \\
.201 \\
.244 \\
.272 \\
.295 \\
.318 \\
.360 \\
.403\end{array}\right.$ & $\left\{\begin{array}{l}\text { Same } \\
\text { as in } \\
\text { Table } \\
1 .\end{array}\right.$ & $\left\{\begin{array}{l}1.000 \\
1.250 \\
1.500 \\
1.750 \\
2.188 \\
2.625 \\
3.250 \\
4.000 \\
4.500 \\
5.000\end{array}\right.$ & $\begin{array}{l}1.25 \\
1.50 \\
1.875 \\
2.25 \\
2.50 \\
3.000 \\
3.75 \\
4.50 \\
5.00 \\
5.50 \\
6.50 \\
7.50\end{array}$ & $\begin{array}{l}1.50 \\
2.00 \\
2.50 \\
3.00 \\
3.50 \\
4.00 \\
4.50 \\
5.00 \\
5.50 \\
6.00 \\
7.50 \\
8.50\end{array}$ \\
\hline
\end{tabular}

1 It is regular practice to furnish close pattern return bends not banded. Close pattern return bends will not make up parallel coils, as the distance center to center of 2 adjacent bends is greater than the center to center of openings of a single bend.

2 Dimensions "S" in cut is "face to point of radius" dimension and is same as dimension " $B$ " in table. 
HEATING AND PIPING CONTRACTORS NATIONAL ASSOCIATION, WELDING NECK FLANGES FOR STANDARD AND EXTRA HEAVY PIPE, 1929

\section{PIPE FLANGES}

(See 607.14, p. 412.)

\section{UNITED STATES GOVERNMENT, DEPART- MENT OF COMMERCE, STANDARD WEIGHT MALLEABLE IRON OR STEEL SCREWED UNIONS, COMMERCIAL STANDARD CS7-29, NOVEMBER 1, 1929}

\section{IRON OR STEEL SCREWED UNIONS}

$$
\text { I. TYPES }
$$

Malleable iron or steel unions for use with standard-weight pipe are furnished in the following types: Black, brass seated; galvanized, brass seated.

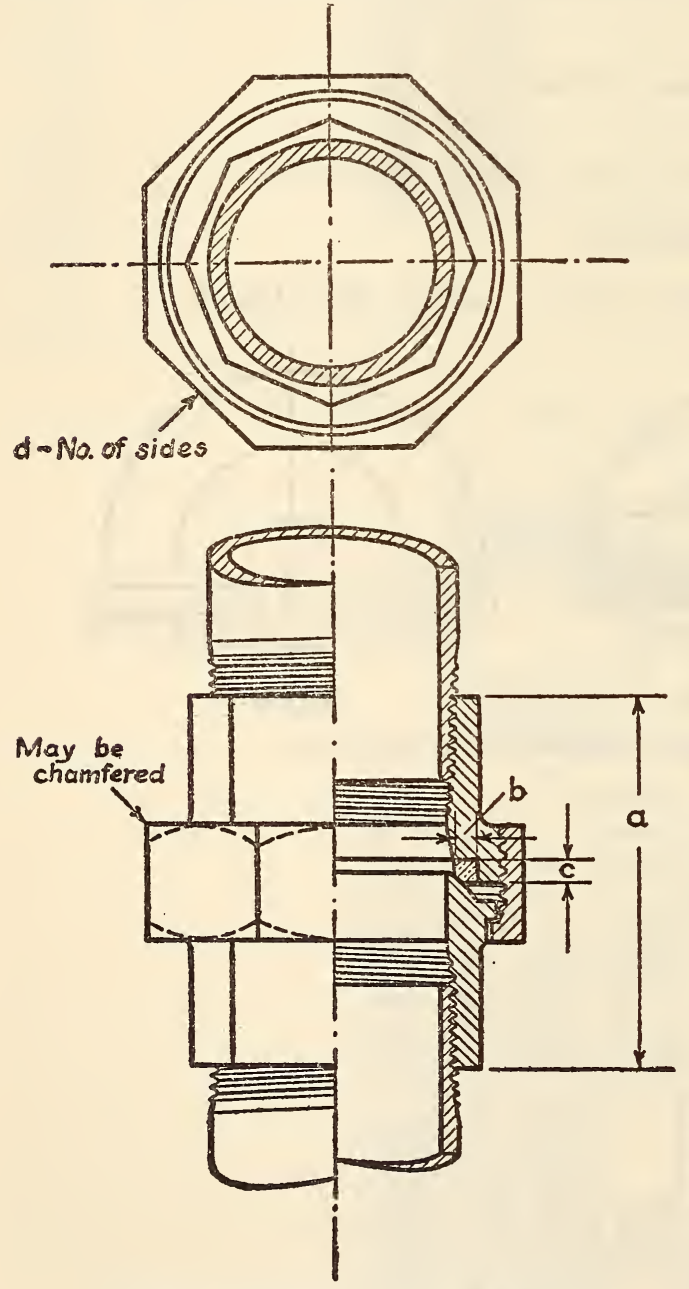

FiguRe 266.-Standard weight union

\section{MATERIAL AND WORKMANSHIP}

With the exception of the brass seats, all parts shall be made of a high-grade malleable iron thoroughly annealed, or of mild steel. No copper or rusting solution shall be used. Workmanship shall be first class in every particular.

\section{GENERAL REQUIREMENTS}

All parts shall be sound, true to form, and free from defects that may affect their serviceability. They shall be thoroughly cleaned from sand and scale.

Unions shall be tapped with taper threads in accordance with American Standard B2-1919 for pipe threads.

Seat and shoulder contact surfaces shall be finished smooth and true.

Union pipe ends may be polygonal, round, or round with lugs.

Representative unions from each lot shall be subjected to not less than 20 pounds air pressure under oil or water during production, and unions that show any leakage shall be rejected. Additional pressure tests shall be at the expense of the purchaser.

\section{DETAIL REQUIREMENTS}

1. Dimensions shall conform to Table 1 and Figure 266, which form part of this specification.

2 . Unions shall be of either malleable iron or mild steel. A brass ring shall be secured in the female portion of the union so as to become an integral part of same and to form a bearing for the male portion, or the female portion may be manufactured of brass or bronze. The seat of the male portion may be either brass, malleable iron, or mild steel. Unions shall be furnished with either ball and socket, ball to cone, or cone to cone joint.

3. Galvanized unions shall be thoroughly zinc coated by the hot-dipped process and so treated as to assure a smooth surface. They shall be galvanized inside and outside before machining and thread cutting.

\section{TABLE 1}

\begin{tabular}{|c|c|c|c|c|c|c|c|c|c|}
\hline \multirow{2}{*}{$\begin{array}{l}\text { Union dimen- } \\
\text { sions (see illus- } \\
\text { tration) }\end{array}$} & \multicolumn{9}{|c|}{ Size of pipe (in inches) } \\
\hline & $1 / 8$ & $1 / 4$ & $3 / 8$ & $1 / 2$ & $3 / 4$ & 1 & $11 / 4$ & $11 / 2$ & 2 \\
\hline $\begin{array}{l}a, \pm 0.125 \text { inch } \\
b \text {, Minimum } \\
c \text {, Minimum } \\
d \text {, Number of } \\
\text { sides, maxi- } \\
\text { mum }\end{array}$ & $\begin{array}{r}1.484 \\
.085 \\
.105\end{array}$ & $\begin{array}{r}1.641 \\
.090 \\
.105\end{array}$ & $\begin{array}{r}1.766 \\
.095 \\
.109\end{array}$ & $\begin{array}{r}2.000 \\
.105 \\
.114\end{array}$ & $\begin{array}{r}2.141 \\
.110 \\
.119\end{array}$ & $\begin{array}{r}2.500 \\
.120 \\
.127\end{array}$ & $\begin{array}{r}2.703 \\
.130 \\
.133\end{array}$ & $\begin{array}{r}2.875 \\
.140 \\
.143\end{array}$ & $\begin{array}{r}3.234 \\
.165 \\
.171\end{array}$ \\
\hline
\end{tabular}

Note.-While dimensions for $21 / 2$ and 3 inch sizes are not given above, provision for these sizes is made in the tensile test requirements of Sec. V.

\section{TENSILE TEST}

Unions shall conform to the minimum tensile test requirements given in Table 2 . The tensile test shall be conducted by inserting solid bars corresponding in outside diameter to the pipe, threaded and screwed into each end of the union.

TABLE 2

\begin{tabular}{|c|c||c|c|}
\hline Size & $\begin{array}{c}\text { Ultimate } \\
\text { breaking } \\
\text { load }\end{array}$ & Size & $\begin{array}{c}\text { Ultimate } \\
\text { breaking } \\
\text { load }\end{array}$ \\
\hline Inch & Pounds & Inches & Pounds \\
$1 / 4$ & 3,800 & $11 / 4$ & 21,300 \\
$3 / 8$ & 5,300 & $11 / 2$ & 25,800 \\
$1 / 2$ & 7,700 & 2 & 30,000 \\
$3 / 4$ & 10,600 & $21 / 2$ & 35,000 \\
1 & 15,500 & 3 & 40,000 \\
\hline
\end{tabular}


Purchaser, at his own expense, may require the testing, in accordance with above paragraph, of representative samples from any lot purchased. For each sample rejected by such test, two additional samples shall be tested, failure of either of which shall cause rejection of the entire lot represented by the samples.

\section{PACKING AND MARKING}

The manufacturer's name, brand name, or trademark shall be permanently marked on each union

\section{NOTES}

American Petroleum Institute line pipe threads are longer than commercial standard threads although of the same pitch diameter. (Reference A. P. I. Standards 5-L. Procurable from A. P. I. division of standardization, 1508 Kirby Building, Dallas, Tex.) Care should be exercised in furnishing fittings to the oil industry, that no interference exists in the threaded portion of the fitting that will prevent the projection of the longer A. P. I. line pipe thread beyond the end of the commercial standard thread in the fitting.

\section{UNITED STATES GOVERNMENT, FEDERAL SPECIFICATIONS BOARD, FEDERAL SPECIFICATION FOR UNIONS, MALLE- ABLE IRON OR STEEL, NO. 393, 1926. (CURRENT DESIGNATION : WW-U-531)}

\section{MALLEABLE IRON OR STEEL UNIONS}

(Conforms with Commercial Standard CS7-29, given above, with the addition of tabulated dimensions on $2 \frac{1}{2}$ and 3 inch sizes.)

\section{UNITED STATES GOVERNMENT, FEDERAL SPECIFICATIONS BOARD, FEDERAL SPECIFICATION FOR UNIONS, 300 POUNDS, MALLEABLE IRON OR STEEL, WW-U-536, MARCH, 1, 1932}

\section{MALLEABLE IRON OR STEEL UNIONS}

\section{A. APPLICABLE FEDERAL SPECIFICATIONS}

A-1. The following Federal specifications, of the issue in effect on date of invitation for bids, shall form a part of this specification (including such applicable cross references to other specifications as may appear in these Federal specifications):

\begin{tabular}{c|r|c|c|c}
\hline $\begin{array}{c}\text { Federal } \\
\text { specification }\end{array}$ & $\begin{array}{c}\text { Former } \\
\text { No. }\end{array}$ & \multicolumn{2}{|c|}{ Title } & See- \\
\cline { 1 - 3 } & 238 & $\begin{array}{l}\text { Standard Pipe Threads for } \\
\text { Pipe and Pipe Fittings. } \\
\text { General Specifications for } \\
\text { Metals. }\end{array}$ & $\begin{array}{c}607.0, \text { p.365. } \\
\text { QQ-M-151 }\end{array}$ & 339.0, p. 3. \\
\hline
\end{tabular}

\section{B. TYPES}

B-1. Three hundred-pound malleable iron or steel unions shall be furnished in the following types as required:

Type XA, black, brass seated.

Type XB, galvanized, brass seated.

Type XC, black, all iron or steel.

Type XD, galvanized, all iron or steel.

\section{MATERIAL AND WORKMANSHIP}

C-1. With the exception of the brass seats, all parts shall be made of high-grade malleable iron or of steel. Workmanship shall be first class in every particular.

C-2. All parts shall be sound, true to form, and free from injurious blowholes, porosity, embrittlement, seams, or other defects that may affect their serviceability. They shall be thoroughly cleaned of sand, scale, and all irregularities, and shall present a surface as smooth as that produced by thorough tumbling.

\section{GENERAL REQUIREMENTS}

D-1. Unions shall be tapped with threads in accordance with Federal specification for standard pipe threads for pipe and pipe fittings, referred to in paragraph $\mathrm{A}-1$.

D-2. When union is closed, not less than four full threads shall be engaged by the nut.

D-3. All surfaces subjected to pressure in tightening the unions shall be finished smooth and true. Each union shall be ground or machined to insure proper seating.

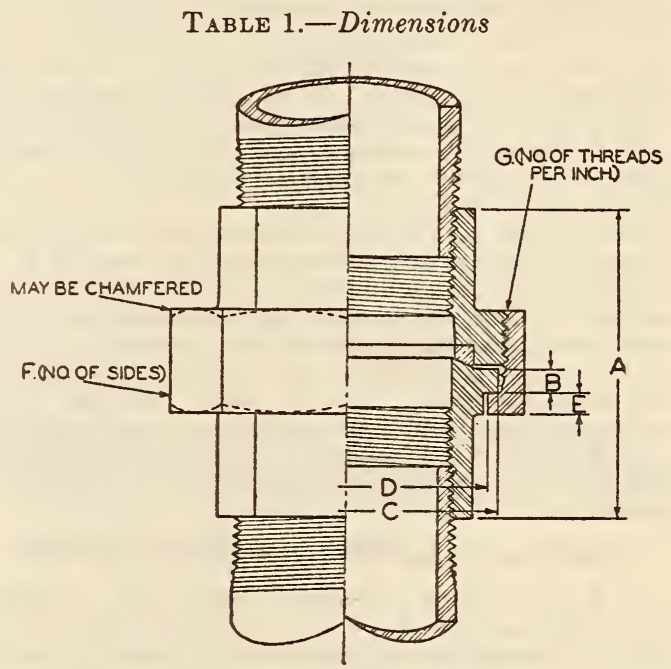

FIGURE 267.-Unions, $300 \mathrm{lb}$., malleable iron or steel

\begin{tabular}{|c|c|c|c|c|c|c|}
\hline \multirow{2}{*}{$\begin{array}{l}\text { Nomi- } \\
\text { nal pipe } \\
\text { size }\end{array}$} & \multicolumn{6}{|c|}{ Union dimensions (see fig. 267) } \\
\hline & $\underset{1 / 8 \text { inch) }}{A( \pm}$ & $\begin{array}{c}\mathrm{B} \\
\text { (Mini- } \\
\text { mum) }\end{array}$ & $\begin{array}{l}\text { C minus } \\
\mathrm{D} \text { (min- } \\
\text { imum) }\end{array}$ & $\underset{\text { (mini- }}{\mathrm{F}}$ & $\begin{array}{c}\mathrm{F} \\
\text { (mini- } \\
\text { mum) }\end{array}$ & $\begin{array}{c}\mathrm{G} \\
\text { (maxi- } \\
\text { mum) }\end{array}$ \\
\hline $\begin{array}{c}\text { Inches } \\
1 / 4 \\
3 / 8 \\
1 / 2 \\
1^{3 / 4}\end{array}$ & $\begin{array}{l}\text { Inches } \\
1.63 \\
1.81 \\
1.93 \\
2.24 \\
2.445\end{array}$ & $\begin{array}{l}\text { Inch } \\
0.124 \\
.128 \\
.138 \\
.151 \\
.169\end{array}$ & $\begin{array}{c}\text { Inch } \\
0.086 \\
.100 \\
.115 \\
.133 \\
.157\end{array}$ & $\begin{array}{c}\text { Inch } \\
0.124 \\
.128 \\
.138 \\
.151 \\
.169\end{array}$ & $\begin{array}{c}\text { Inches } \\
6 \\
6 \\
6 \\
6 \\
6\end{array}$ & $\begin{array}{c}\text { Inches } \\
16 \\
16 \\
14 \\
12 \\
11\end{array}$ \\
\hline $\begin{array}{l}11 / 4 \\
11 / 2 \\
2 \\
21 / 2 \\
3\end{array}$ & $\begin{array}{l}2.795 \\
\text { 3. } 01 \\
\text { 3. } 39 \\
4.025 \\
4.29\end{array}$ & $\begin{array}{l}.197 \\
.216 \\
.250 \\
.280 \\
.315\end{array}$ & $\begin{array}{l}.184 \\
.203 \\
.240 \\
.284 \\
.332\end{array}$ & $\begin{array}{l}.197 \\
.216 \\
.250 \\
.280 \\
.315\end{array}$ & $\begin{array}{l}6 \\
6 \\
6 \\
8 \\
8\end{array}$ & $\begin{array}{r}10 \\
10 \\
8 \\
8 \\
8\end{array}$ \\
\hline
\end{tabular}

$\mathrm{D}-4$. The minimum tensile strength of the unions when tested in accordance with section F-4 shall be as follows:

\begin{tabular}{|c|c||c|c|}
\hline $\begin{array}{c}\text { Iron } \\
\text { pipe } \\
\text { size }\end{array}$ & $\begin{array}{c}\text { Ultimate } \\
\text { load }\end{array}$ & $\begin{array}{c}\text { Iron } \\
\text { pipe } \\
\text { size }\end{array}$ & $\begin{array}{c}\text { Ultimate } \\
\text { load }\end{array}$ \\
\cline { 1 - 2 } Inches & Pounds & Inches & Pounds \\
$1 / 4$ & 5,300 & $11 / 4$ & 27,000 \\
$3 / 8$ & 7,800 & $11 / 2$ & 35,000 \\
$1 / 2$ & 10,800 & 2 & 50,000 \\
$3 / 4$ & 15,400 & $21 / 2$ & 60,000 \\
1 & 22,000 & 3 & 75,000 \\
\hline
\end{tabular}


D-5. All unions shall pass the tests required in section F-2 without showing any leakage of air.

D-6. Union pipe ends may be polygonal, round, or round with lugs.

\section{E. DETAIL REQUIREMENTS}

E-1. Dimensions shall conform to Table 1 and Figure 267, which form part of this specification.

E-2. Type XA unions shall be of either malleable iron or steel. A brass ring shall be secured to the female portion of the union so as to become an integral part of same and to form a bearing for the male portion, or the female portion may be manufactured of brass or bronze. The seat of the male portion may be of either brass, malleable iron, or steel. Figure 267 indicates location of seat but not method of securing. Type XA unions shall be furnished with either ball or ball to cone joint.

E-3. Type XB unions shall be zinc coated (galvanized) by the hot process and so treated as to be thoroughly coated with a smooth, bright, solid surface of zinc, free from blisters, dross, dirt, bare spots, rust, and other defects in the coating. They shall be zinc coated inside and outside before machining and threading. In all other respects these unions shall conform to the requirements of type XA.

$\mathrm{E}-4$. Type XC unions shall be of either malleable iron or steel with either ball or ball to cone joint.

E-5. Type XD unions shall be zinc coated (galvanized) by the hot process and so treated as to be thoroughly coated with a smooth, bright, solid surface of zinc, free from blisters, dross, dirt, bare spots, rust, and other defects in the coating. They shall be zinc coated inside and outside before machining and threading. In all other respects these unions shall conform to the requirements of type XC.

E-6. The manufacturer's name or trade-mark and the figure 300 shall be permanently marked on each union.

\section{F. METHODS OF SAMPLING, INSPECTION, AND TESTS}

F-2. All unions furnished under this specification shall be tested by the manufacturer with at least 60 pounds per square inch air pressure under water or light oil.

F-3. Representative unions shall be selected by the inspector and the dimensions checked with Table 1 .

F-4. Two unions shall be selected by the inspector from each lot of 500 unions or less of the same size and subjected to the tension-test requirements of section D4. This test shall be conducted by inserting threaded bars, corresponding in outside diameter to the pipe.

F-5. For each sample rejected under F-4 above, two additional samples may be tested at the option of the inspector. Failure of either shall cause rejection of the entire lot represented by the samples.

F-6. Representative samples of malleable iron unions shall be selected by the inspector and tested by hammering or crushing to determine that the material has been properly heat treated and is free from embrittlement due to galvanizing.

\section{G. PACKAGING, PACKING, AND MARKING OF SHIPMENTS}

G-2. Unless otherwise specified 10 unions of the same size up to and including 1 inch shall be securely tied together or a like number shall be packed in a suitable container.

$\mathrm{G}-3$. Unless otherwise specified five unions of the same size above 1 inch shall be securely tied together or a like number shall be packed in a suitable container.

\section{NOTES}

I-1. Purchasers should exercise all options offered herein and should specify type required.

I-2. The term 300 pounds as applied to unions herein is a commercial designation only and is not a limitation of working pressure. This specification covers all requirements for extra heavy unions.

I-3. Unions specified herein are intended for use up to pressures for which extra heavy pipe is applicable under conditions requiring frequent opening of the lines or when subject to vibration. Thev are intended for use with extra heavy pipe on oil filling, oil fuel, gas, air, fresh water, and steam lines.

I-4. Type XA and XB unions are not intended for use where the temperature exceeds $425^{\circ} \mathrm{F}$.

I-5. Unions in accordance with this specification should be purchased by the piece.

\section{UNITED STATES GOVERNMENT, FEDERAL} SPECIFICATIONS BOARD, FEDERAL SPECIFICATION FOR PIPE FITTINGS, MALLEABLE IRON (THREADED), 150 POUND, NO. 535, NOVEMBER, 1927. (CURRENT DESIGNATION : WW-P-471)

\section{MALLEABLE IRON PIPE FITTINGS, THREADED}

(This specification conforms to A. S. M. E. B16c, given above, with the following additional requirements in respect to method of inspection and test:)

One fitting shall be selected by the inspector from each lot of 20 of the same size and kind and subjected to an air pressure of 20 pounds per square inch under water. When impracticable to make this test, fittings selected as above shall be subjected to a hydrostatic test of 300 pounds per square inch. Under this test the fittings shall show no weakness or defect. In the event of failure of any fitting to pass either test, the lot represented shall be rejected. When 500 or more fittings of the same size and kind are submitted for inspection, 1 per cent shall be selected for test in lieu of the number required above.

Representative samples from each malleableizing heat where practicable, shall be taken by the inspector and tested by hammering or crushing to determine that the material has been properly heat treated and is free from embrittlement due to the zinc coating process.

\subsection{WROUGHT-IRON PIPE, FITTINGS,} AND CONNECTIONS.

AMERICAN PETROLEUM INSTITUTE, PIPE SPECIFICATIONS, STANDARD NO. 5-A (7TH ED.), JULY, 1931

\section{STEEL AND IRON PIPE FOR OIL COUNTRY TUBULAR GOODS}

(See 607.4, p. 444.)

AMERICAN PETROLEUM INSTITUTE, LINE PIPE SPECIFICATIONS, STANDARD NO. 5-L (4TH ED.), JULY, 1931

\section{LINE PIPE}

(Line pipe for the transportation of oil or gas, including iron line pipe, will be found under A. P. I., under classification number 607.4, p. 457.) 
AMERICAN RAILWAY ASSOCIATION, MECHANICAL DIVISION, SPECIFICATIONS FOR WELDED WROUGHT-IRON PIPE, 1925

\section{WELDED WROUGHT-IRON PIPE}

\section{SCOPE}

1. These specifications cover standard weight, extra strong, and double extra strong black and galvanized welded wrought-iron pipe. All pipes to be used on locomotives and cars shall be of coiling or bending quality.

\section{MANUFACTURE}

2. (a) The iron shall be made from muck bars, made from all pig puddled iron, free from any admixture of iron scrap or steel.

(b) Pipe up to and including 1 inch may be butt welded. Pipe $1 \frac{1}{4}$ and $1 \frac{1}{2}$ inches shall be furnished lap-welded, unless butt-welded is specified. Pipe 2 inches and over in diameter shall be lap-welded.

\section{ETCH TEST}

3. A cross section of the test specimen shall be polished and etched for a sufficient period to develop the structure. This test shall show the material to be free from steel. If there is any doubt as to the structure, microscopic examination may be made.

\section{TEST SPECIMENS}

4. (a) Test specimens shall consist of sections cut from a pipe. They shall be smooth on the ends and free from burrs.

(b) Tension-test specimens shall be longitudinal and shall consist of the whole pipe when it is within the capacity of the available testing machine. For larger sizes the test will be made on strips not less than three-fourths inch wide, machined from the pipe.

(c) All specimens shall be tested cold

(In respect to definition of scrap, tensile properties, determination of yield point, hydrostatic tests, fracture tests, and bend tests, this specification conforms with A. S. T. M. A72-30, given below. It conforms with A. S. T. M. A53-30 (see 607.4, p. 476) in respect to number of tests, retests, standard weights, permissible variations, lengths, workmanship, ends, threads, couplings, finish, inspection, rejection, and rehearing, with the addition of weights, dimensions, and hydrostatic pressures on 7, 9, and 11 inch pipes.)

\section{AMERICAN RAILWAY ASSCCIATION, SIG- NAL SECTION, 1-INCH WROUGHT-IRON SIGNAL PIPE, SPECIFICATION 12324, JULY, 1924}

\section{WROUGHT-IRON SIGNAL PIPE}

(In respect to manufacture, tensile properties, and bend test, this specification conforms with A. S. T.M. A72-30, given below; in other respects it provides as follows:

The tensile strength, limit of elasticity and ductility, and weld, shall be determined from a test piece cut from finished pipe. When required by the purchaser, the following tests shall be made: From each 1,000 feet of finished pipe, a piece 6 feet long shall be cut from a length selected at random, and subjected to the following tests:

(a) One specimen cut by hand, with standard stock and die, $1 \frac{1}{2}$ inches of thread. (b) Punch two 1/4-inch holes on welded seam, 2 inches and 4 inches from threaded end, without use of plug or filler piece.

(c) Twist piece through angle of $180^{\circ}$ by clamping one end and turning the other end. After tests, if 20 per cent of the pieces show split or fracture at weld, the lot will be rejected.

The weight of 1 foot of 1 -inch pipe before galvanizing should be 1.71 pounds, and in no case will pipe be accepted weighing less than 1.63 pounds per foot, weight of plug and coupling not included.

The outside diameter of pipe must conform to Briggs' standard. Any pipe less than 1.31 inches in diameter to result in flat thread will be rejected.

The manufacturer shall furnish all necessary facilities for making tests, and the tests shall be made at the mill.

Inside diameter of all pipe must be large enough to receive a hardened steel plug of sixty-three-sixtyfourths-inch diameter for a length of 6 inches. Not more than 1 per cent of pipe less than 15 feet long will be accepted; lengths of 17 feet and over preferred.

\section{COUPLINGS}

Pipe couplings must be galvanized, must be $2 \frac{1}{4}$ inches long and $13 / 4$ inches outside diameter, of wrought iron, free from defects, faced at ends, tapped straight through, pitch diameter of thread to be such as to fit pipe as specified under threads in A. R. A.-S, specification 12227 (see 607.4, p. 463), 1.26 inches, varying not more than 0.003 inch.

(In respect to ends, threads, plugs, rivets, and drawings this specification conforms with A. R. A.S, specification 12227 , 1-inch welded steel pipe. See 607.4 , p. 463.)

\section{AMERICAN SOCIETY FOR TESTING MATE- RIALS, STANDARD SPECIFICATIONS FOR WELDED WROUGHT-IRON PIPE, DESIG- NATION A72-31, 1931}

\section{WELDED WROUGHT-IRON PIPE}

(This specification conforms with A. S. T. M. A53-30 (see 607.4, p. 476) except in the following respects:)

\section{SCOPE}

These specifications cover standard weight, extra strong, and double extra strong welded wroughtiron pipe. Pipe ordered under these specifications is intended for coiling, bending, flanging, and other special purposes. Butt-welded pipe is not intended for flanging and is not recommended for bending or coiling in sizes $1 \frac{1}{4}$ inches or over.

\section{MANUFACTURE}

The pipe shall be made from wrought iron ${ }^{69}$ and shall be free from any admixture of iron scrap or steel.

Iron scrap.-This term applies only to foreign or bought scrap and does not include local mill products free from foreign or bought scrap.

\section{PHYSICAL PROPERTIES AND TESTS}

The material shall conform to the following minimum requirements as to tensile properties:

Tensile strength $\ldots$.
Yield point

Elongation in 8 inches......... 12

The yield point shall be determined by the drop of the beam of the testing machine. The speed of

69 Wrought iron.-A ferrous material aggregated from a solidifying mass of pasty particles of highly refined metallic iron with which without subsequent fusion, is incorporated a minutely and uniformly distributed quantity of slag. 
the crosshead of the machine shall not exceed threefourths inch per minute.

A section of pipe 6 inches in length shall be flattened until broken by repeated light blows of a hammer or by pressure. The fracture developed shall have a fibrous appearance.

For pipe 2 inches or under in diameter, a sufficient length of coiling or bending pipe shall stand being bent cold through $90^{\circ}$ around a cylindrical mandrel the diameter of which is 15 times the nominal diameter of the pipe, without developing cracks at any portion and without opening the weld. Double extra strong pipe over $1 \frac{1}{4}$ inches in nominal size need not be subjected to the bend test.

In case of doubt as to the presence of steel, the purchaser may, at his own expense, make micrographic examination and chemical analysis to assist in determining whether the material meets the requirements.

For pipe up to and including 2 inches in nominal size, tension-test specimens shall be of the full section of the pipe. For pipe larger than 2 inches in nominal size, tension-test specimens may be strips not less than three-fourths inch in width, cut longitudinally from the pipe. The sides of such specimens shall be parallel and not flattened between gage marks.

All specimens shall be tested at room temperature. (This specification carries no reference to permissible variation of thickness of wall. The thickness, in inches, of the various kinds of pipe is as follows:)

\begin{tabular}{|c|c|c|c|c|c|c|c|}
\hline $\begin{array}{c}\text { Size } \\
\text { (nomi- } \\
\text { nal } \\
\text { inside } \\
\text { diam- } \\
\text { eter) }\end{array}$ & $\begin{array}{c}\text { "Stand- } \\
\text { ard } \\
\text { weight" } \\
\text { pipe } \\
\text { (thick- } \\
\text { ness) }\end{array}$ & $\begin{array}{c}\text { "Extra } \\
\text { strong" } \\
\text { pipe } \\
\text { (thick- } \\
\text { ness) }\end{array}$ & $\begin{array}{l}\text { "Dou- } \\
\text { ble } \\
\text { extra } \\
\text { strong" } \\
\text { pipe } \\
\text { (thick- } \\
\text { ness) }\end{array}$ & $\begin{array}{c}\text { Size } \\
\text { (nomi- } \\
\text { nal } \\
\text { inside } \\
\text { diam- } \\
\text { eter) }\end{array}$ & $\begin{array}{l}\text { "Stand- } \\
\text { ard } \\
\text { weight" } \\
\text { pipe } \\
\text { (thick- } \\
\text { ness) }\end{array}$ & $\mid \begin{array}{c}\text { "Extra } \\
\text { strong" } \\
\text { pipe } \\
\text { (thick- } \\
\text { ness) }\end{array}$ & $\begin{array}{l}\text { "Dou- } \\
\text { ble } \\
\text { extra } \\
\text { strong" } \\
\text { pipe } \\
\text { (thick- } \\
\text { ness) }\end{array}$ \\
\hline $\begin{array}{c}\text { Inches } \\
1 / 8 \\
1 / 4 \\
3 / 3 \\
1 / 2 \\
3 / 4 \\
1 \\
11 / 4 \\
11 / 2 \\
2 \\
21 / 2 \\
3\end{array}$ & $\begin{array}{l}\text { Inch } \\
0.070 \\
.090 \\
.093 \\
.111 \\
.115 \\
.136 \\
.143 \\
.148 \\
.158 \\
.208 \\
.221\end{array}$ & $\begin{array}{r}\text { Inch } \\
0.098 \\
.122 \\
.129 \\
.151 \\
.157 \\
.183 \\
.195 \\
.204 \\
.223 \\
.282 \\
.306\end{array}$ & \begin{tabular}{|c} 
Inch \\
0.307 \\
.318 \\
.369 \\
.393 \\
.411 \\
.447 \\
.567 \\
.615
\end{tabular} & $\begin{array}{c}\text { Inches } \\
31 / 2 \\
4 \\
5 \\
6 \\
8 \\
8 \\
10 \\
10 \\
10 \\
12 \\
12\end{array}$ & $\begin{array}{r}\text { Inch } \\
0.231 \\
.242 \\
.263 \\
.286 \\
.283 \\
.329 \\
.284 \\
.313 \\
.372 \\
.336 \\
.382\end{array}$ & $\begin{array}{c}\text { Inch } \\
0.325 \\
.344 \\
.383 \\
.441 \\
.510 \\
.510 \\
.510\end{array}$ & $\begin{array}{r}\text { Inch } \\
0.651 \\
.690 \\
.768 \\
.884 \\
.895 \\
\end{array}$ \\
\hline
\end{tabular}

One cubic inch of wrought iron weighs 0.2776 pound.

HEATING AND PIPING CONTRACTORS NATIONAI ASSOCIATION, EXTRA-HEAVY WELDING NECK FLANGES FOR STAND. ARD AND EXTRA-HEAVY PIPE, 1929

PIPE FIANGES

(See 607.14, p. 412.)

\section{UNITED STATES GOVERNMENT, DEPART- MENT OF COMMERCE, BUREAU OF STANDARDS, COMMERCIAL STANDARD FOR WROUGHT-IRON PIPE NIPPLES, CS6-31, MAY 1, 1931}

\section{WROUGHT-IRON PIPE NIPPLES}

(A commercial standard that has been selected and accepted by industry.)

\section{GENERAL REQUIREMENTS}

Pipe nipples shall be made only from tested new black or zinc coated (galvanized) wrought-iron pipe, of full weight, in full or short lengths that are free from buckles, bends, kinks, cracks, laminations, imperfect welds, rust, injurious sand marks, or any other defects which might affect its strength or serviceability.

The pipe shall be made from wrought iron and shall be free from any admixture of iron scrap or steel. Pipe shall also conform in all respects to American Society for Testing Materials Specification for Welded Wrought-Iron Pipe, A72-31. (See above.)

Note.-Wrought iron is a ferrous material, aggregated from a solidifying mass of pasty particles of highly refined metallic iron with which, without subsequent fusion, is incorporated a minutely and uniformly distributed quantity of slag.

Nipples shall be reamed and chamfered at an angle of $25^{\circ}$ to $45^{\circ}$ to the central axis.

Nipples shall not exceed one-sixteenth of an inch under or over the specified length.

(Permissible diameter variations and permissible weight variations of the pipe from which wroughtiron pipe nipples are made are as given in Federal specification WW-P-441 for wrought-iron pipe. See United States Government Federal Specifications Board, WW-P-441, below.)

The wall thickness shall be not more than $12 \frac{1}{2}$ per cent under the nominal wall thickness at any point.

(Dimensions of standard weight, extra strong, and double extra strong pipe from which wrought-iron pipe nipples are made are the same as given in Federal specification WW-P-441 below.)

(Threading for both ends of pipe nipples is required to be in conformity with the taper pipe thread dimensions given in the standards of the National Screw Thread Commission. See 607.0, p. 365.)

(Stock sizes and lengths of wrought-iron pipe nipples are the same as given in Federal Specification WW-N-351 for wrought-iron pipe nipples. See United States Government, Federal Specifications Board, WW-N-351, under 607.4, p. 485.)

(In addition, this standard includes the following requirements on packing.)

TABLE 8.-Packing of standard-weight black or galvanized nipples

[Number contained in carton]

\begin{tabular}{|c|c|c|c|c|c|c|c|c|c|c|c|c|}
\hline \multirow{3}{*}{$\begin{array}{l}\text { Diam- } \\
\text { eter }\end{array}$} & \multirow{3}{*}{ Close } & \multicolumn{10}{|c|}{ Sizes in inches } & \multirow{3}{*}{ Total } \\
\hline & & \multirow{2}{*}{$\frac{\text { Short }}{11 / 2}$} & \multicolumn{4}{|c|}{ Long } & \multicolumn{5}{|c|}{ Extra long } & \\
\hline & & & 2 & $21 / 2$ & 3 & $31 / 2$ & 4 & $41 / 2$ & 5 & $51 / 2$ & 6 & \\
\hline Inch & & & & & & & & & & & & \\
\hline $3 / 8$ & 20 & 10 & 15 & 10 & 10 & $\begin{array}{r}5 \\
5\end{array}$ & 10 & 5 & 5 & 5 & 5 & 100 \\
\hline 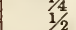 & 20 & $\begin{array}{l}10 \\
10\end{array}$ & 15 & $\begin{array}{l}10 \\
10\end{array}$ & 10 & $\begin{array}{l}5 \\
5\end{array}$ & 10 & $\begin{array}{l}5 \\
5\end{array}$ & 5 & $\begin{array}{l}0 \\
5\end{array}$ & $\begin{array}{l}5 \\
5\end{array}$ & 100 \\
\hline $3 / 4$ & 20 & 10 & 15 & 10 & 10 & 5 & 10 & 5 & 5 & 5 & 5 & 100 \\
\hline 1 & 15 & & 12 & 5 & 12 & 3 & 12 & 3 & 5 & 3 & 5 & 75 \\
\hline
\end{tabular}

UNITED STATES GOVERNMENT, DEPARTMENT OF COMMERCE, BUREAU OF STANDARDS, WROUGHT-IRON AND WROUGHT-STEEL PIPE, VALVES, AND FITTINGS, SIMPLIFIED PRACTICE RECOMMENDATION NO. 57, 1926

WROUGHT-IRON AND WROUGHT-STEEL PIPE, VALVES, AND FITTINGS

(See 607.0, p. 365.) 
UNITED STATES GOVERNMENT, DEPARTMENT OF COMMERCE, STEAMBOAT INSPECTION SERVICE, GENERAL RULES AND REGULATIONS PRESCRIBED BY THE BOARD OF SUPERVISING INSPECTORS, MAY 4, 1929

\section{WELDED AND SEAMLESS STEAM AND WATER}

(See 607.4, p. 483.)

UNITED STATES GOVERNMENT, FEDERAI SPECIFICATIONS BOARD, FEDERAI SPECIFICATION FOR PIPE, WROUGHTIRON, WELDED, BLACK AND GALVANIZED, WW-P-441, AUGUST 18, 1931

\section{WROUGHT-IRON PIPE}

\section{A. APPLICABLE FEDERAL SPECIFICATIONS}

A-1. The following Federal specifications, or parts thereof that are below indicated, of the issue in effect on date of invitation for bids, shall form a part of this specification:

\begin{tabular}{|c|c|c|c|}
\hline $\begin{array}{c}\text { Federal } \\
\text { specification }\end{array}$ & $\begin{array}{c}\text { Former } \\
\text { number }\end{array} \mid$ & Title & $\mathrm{See}-$ \\
\hline $\begin{array}{r}\text { GGG-P-351 } \\
\text { QQ-M-151 }\end{array}$ & $\begin{array}{l}238 \\
339\end{array}$ & $\begin{array}{l}\text { Pipe threads. } \\
\text { General specifications for } \\
\text { metals. }\end{array}$ & $\begin{array}{r}607.0, \text { p. } 365 . \\
600.0, \text { p. } 3\end{array}$ \\
\hline
\end{tabular}

\section{B. CLASSES}

B-1. Wrought-iron pipe shall be furnished in the following classes, as required: Class $A$, standard weight; class B, extra strong; class C, double extra strong.

B-2. Wrought-iron pipe shall be furnished black or zinc coated (galvanized), as required.

\section{MATERIAL AND WORKMANSHIP}

C-1. The pipe shall be made from wrought iron and shall be free from any admixture of iron scrap or steel, except that the manufacturer's own iron skelp crop ends and finished iron pipe ends may be used.

C-2. Wrought iron is a ferrous material, aggregated from a solidifying mass of pasty particles of highly refined metallic iron with which, without subsequent fusion, is incorporated a minutely and uniformly distributed quantity of slag.

C-3. Pipe shall be straight and free from buckles, kinks, bends, cracks, blisters, laminations, imperfect welds, rust, injurious sand marks, or any other defects which might affect its strength or serviceability. All burrs at the ends of the pipe shall be removed. (Ordinary liquor marks incident to manufacture of welded material will not be considered cause for rejection.)

C-4. All pipe 1-inch iron pipe size and smaller shall be butt-welded; all $2 \frac{1}{2}$ inches and larger shall be lap-welded; $11 / 4,11 / 2$, and 2 inch pipe may be either butt-welded or lap-welded unless pipe made by one of these processes is specifically ordered.

\section{GENERAL REQUIREMENTS}

D-2. The material shall conform to the following minimum requirements with respect to tensile properties:

Tensile strength
Yield point

Yield point
(Tensile properties are the same as required in A. S. T. M. specification A72-31, above.)

$\mathrm{D}-3$. When required by the contract or order, the material shall conform to the following chemical requirements:

Carbon not over 0.08 per cent.

Manganese not over 0.08 per cent.

D-4. For pipe $1 \frac{1}{2}$ inches iron pipe size or under the outside diameter at any point shall vary not more than one-sixtyfourth inch over nor more than one-thirtysecond inch under the outside diameter as specified in Tables 1, 2, and 3. For pipe 2 inches iron pipe size or over, the outside diameter shall vary not more than 1 per cent over or under the outside diameter as specified in Tables 1, 2, and 3 .

D-5. The weight of black pipe shall vary not more than 5 per cent over or under that specified in Tables 1 (class A, standard weight) and 2 (class $\mathbf{B}$, extra strong), nor more than 10 per cent over or under that specified in Table 3 (class $\mathrm{C}$, double extra strong).

D-6. The weight of zinc-coated pipe shall vary not more than 3 per cent under that given in Table 1 (class A, standard weight) and Table 2 (class B, extra strong), nor more than 8 per cent under that specified in Table 3 (class C, double extra strong).

D-7. Zinc-coated pipe shall be thoroughly and evenly coated with zinc by the hot process, both inside and outside, and shall be free from blisters and other defects. The weight of zinc coating shall be not less than 2 ounces per square foot of surface covered. The zinc bath shall contain not more than 2 per cent of elements other than zinc as determined from samples taken at a point half the depth of the bath. Unless zinc coating is specified in the invitation for bids black pipe shall be furnished.

D-8. All threads shall be in accordance with the standard threads for pipe as given in the Federal specification for pipe threads, GGG-P-351 referred to in paragraph $\mathrm{A}-1$.

D-9. Before shipment all threads shall be adequately protected. Tapped rings or half-length couplings shall be provided on the end not protected by the coupling on all sizes of threaded pipe 2 inches iron pipe size or larger. Threads on smaller sizes shall be protected by having ends of bundles securely wrapped with burlap unless otherwise specified.

D-10. Unless exact lengths are specified, pipe shall be furnished in lengths of from 16 to 22 feet for standard weight pipe and 12 to 22 feet for extra strong and double extra strong pipe. When ordered in carload lots a maximum length of 24 feet will be accepted. Unless otherwise specified, not over 5 per cent of jointers, which are two pieces coupled together, will be accepted in standard weight pipe. When exact lengths are specified, no jointers will be permitted. Five per cent of extra strong and double extra strong pipe may be in lengths of 6 to 12 feet.

\section{E. DETAII REQUIREMENTS}

E-1. Standard weight pipe shall, unless otherwise specified, be threaded at both ends and have a standard weight wrought-iron coupling tightly screwed on one end. Extra strong and double extra strong pipe shall, unless otherwise specified, be furnished with plain ends and without couplings. Zinc-coated (galvanized) couplings shall be furnished with zinc-coated pipe.

E-2. Each length of pipe over one-eighth inch I. P. S. shall have the name of the manufacturer rolled onto the pipe.

E-3. The dimensions, weights, and test pressures of standard weight, extra strong, and double extra strong pipe shall be as shown in Tables 1,2 , and 3 . 
TABLE 1.-Requirements for class $A$ standard weight pipe

TABLE 2.-Requirements for class $B$ extra strong pipe

TABLE 3.-Requirements for class $C$ double extra strong pipe

(Sizes, outside diameters, and weights are the same as given in A. S. T. M. specification A53-30, Table 2. Test pressures are the same as specified in Table 1 of A53-30. See 607.4, p. 476. Wall thicknesses are as given in A. S. T. M. specification A72-31. See 607.3 , p. 431 .)

(Table 1 also includes weights of standard weight pipe with plain ends which does not appear in above references. See following.)

Weight requirements for class A standard weight pipe with plain ends:

\begin{tabular}{|c|c|c|c|}
\hline $\begin{array}{l}\text { Iron } \\
\text { pipe } \\
\text { size }\end{array}$ & $\begin{array}{l}\text { Weight } \\
\text { per foot }\end{array}$ & $\begin{array}{l}\text { Iron } \\
\text { pipe } \\
\text { size }\end{array}$ & $\begin{array}{l}\text { Weight } \\
\text { per foot }\end{array}$ \\
\hline \begin{tabular}{r|} 
Inches \\
$1 / 8$ \\
$1 / 4$ \\
$3 / 8$ \\
$1 / 2$ \\
$3 / 4$
\end{tabular} & $\begin{array}{c}\text { Pounds } \\
0.244 \\
.424 \\
.567 \\
.850 \\
1.130\end{array}$ & $\begin{array}{c}\text { Inches } \\
3 \\
31 / 2 \\
4 \\
5 \\
6\end{array}$ & $\begin{array}{r}\text { Pounds } \\
7.575 \\
9.109 \\
10.790 \\
14.617 \\
18.974\end{array}$ \\
\hline $\begin{array}{l}1 \\
11 / 4 \\
11 / 2 \\
2 \\
21 / 2\end{array}$ & $\begin{array}{l}1.678 \\
2.272 \\
2.717 \\
3.652 \\
5.793\end{array}$ & $\begin{array}{r}8 \\
10 \\
12 \\
12\end{array}$ & $\begin{array}{l}28.554 \\
40.483 \\
49.562 \\
43.773\end{array}$ \\
\hline
\end{tabular}

\section{F. METHOD OF INSPECTION AND TESTS}

F-1. Hydrostatic test.-Each pipe shall be tested at the mill to an internal hydrostatic pressure not less than that indicated in Tables 1, 2, and 3, respectively, for the classes specified. Any pipe which develops any defects shall be rejected. Pipe, while under pressure, shall be jarred with a hammer not less than 1 pound in weight.

F-1a. Hydrostatic tests shall be made in the presence of the inspector unless inspection is specifically waived, in which case the manufacturer shall furnish a certificate to the effect that the pipe has withstood satisfactorily the hydrostatic test required.

F-2. Surface inspection.-All pipe shall be surface inspected for defects by "rolling" on suitable supports, so that entire surface of each pipe may be properly inspected.

F-3. Number of tests (regular).-Unless otherwise specified one each of the tests specified in paragraphs $\mathrm{F}-4, \mathrm{~F}-5, \mathrm{~F}-6$, and $\mathrm{F}-7$ shall be made on a length of pipe selected by the inspector from each lot or fraction thereof as defined in Table 4 to insure that the pipe is of the material and quality required.

TABLE 4.- Lots for regular number of tests

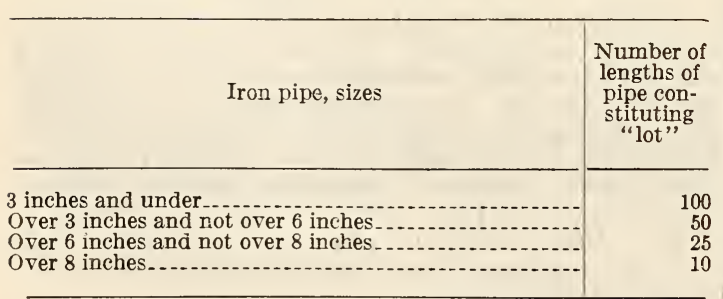

F-3a. Number of tests (alternate).-When required in the order or invitation for bids, one each of the tests specified in paragraphs $\mathrm{F}-4, \mathrm{~F}-5$, and F-6 shall be made on a length of pipe selected by the inspector from each lot of 500 lengths or fraction thereof of each size.

F-4. Flattening test.-A section of pipe 6 inches in length shall be flattened until broken by repeated light blows of a hammer or by pressure, having the weld at $90^{\circ}$ from the point of application of load. The test piece shall show a sound weld and the fracture shall have a fibrous appearance. If any section fails to meet these requirements, two additional lengths may be selected by the inspector and samples taken. Should either of these fail, the lot of pipe shall be rejected.

F-5. Tension test.-For pipe up to and including 2 -inch iron pipe size, tension-test specimens inay be of the full section of the pipe. For pipe larger than 2 inches I. P. S., tension-test specimens may be strips not less than three-fourths inch in width, cut longitudinally from the pipe. The sides of such specimens shall be parallel, and specimens shall not be flattened between gage marks.

F-6. Bend test.-Pipe 2 inches I. P. S. or under shall withstand being bent cold under slowly applied pressure through $90^{\circ}$ around a cylindrical mandrel the diameter of which is fifteen times the nominal diameter (I. P. S.) of the pipe, without developing cracks at any portion and without opening the weld. The bend test will not be required on double extra strong pipe over $1 \frac{1}{4}$ inches I. P. S.

F-7. Macroscopic examination (etch test).-After removing all marks of the cutting-off tool and making sure that the end of the pipe is smooth, the end of the sample shall be suspended in a solution of 10 per cent hydrochloric acid (specific gravity 1.19), 30 per cent sulphuric acid (specific gravity 1.81 ), and 60 per cent water; or 25 per cent nitric acid (specific gravity 1.42), and 75 per cent water. After the end of the pipe has been immersed about an hour, the piece shall be removed, washed off and dried quickly with a soft rag. The end shall show ridges or rings indicating the silicious slag content of wrought iron.

F-8. Microscopic examination and chemical analysis.-Microscopic examination and chemical analysis may be made when required by the contract to assist in determining whether the material meets the requirements. These additional tests shall be at the expense of the Government. If upon microscopic examination of a test specimen, distinct pearlitic areas or improper distribution of slag is revealed, the lot represented shall be rejected.

F-8a. Drillings, millings, or clippings for chemical analysis shall be taken from several points around each piece selected for physical tests, and the chips from each lot combined and thoroughly mixed. The resultant sample shall be free from dirt, grit, and other foreign matter and shall consist of at least 2 ounces.

F-9. Test for coating.-If impracticable to obtain samples from the bath or to weigh pipe immediately before coating, the quality of the zinc coating and the weight per square foot may be determined from the average of samples 6 inches long taken from both ends of finished pipe. When quality of zinc is determined by the stripping test, iron shall not be counted as an impurity.

\section{G. PACKING AND MARKING OF SHIPMENTS}

G-1. Packing.-Unless otherwise specified, the subject commodity shall be delivered in such manner as to insure acceptance by common or other carriers for safe transportation, at the lowest rate, to the point of delivery.

G-2. Marking.-Unless otherwise specified, shipments shall be marked with the name of the material, the size, the class, and the quantity contained therein, as defined by the contract or order under 
which the shipment is made, the name of the contractor, and the number of the contract or order.

\section{NOTES}

I-1. Pipe is regularly designated by iron-pipe size class (standard weight, extra strong, or double extra strong), black or zinc coated (galvanized).

I-2. Pipe under this specification should be ordered by number of feet required. In the case of pipe furnished with couplings the length includes the coupling when tightly screwed on pipe.

I-3. The pipe described in this specification is suitable in general for conveying steam, water, air, gas, and oil. The specification is not intended to cover pipe for oil country tubular goods.

I-4. Pipe 11/4 inches to 2 inches I. P. S., inclusive, as made by both the lap-welding and butt-welding processes, is covered by this specification. If either is specifically desired, the invitation for bids should so state, otherwise either lap-welded or butt-welded pipe of these sizes may be furnished and will be acceptable.

I-5. Standard weight couplings normally furnished with standard weight pipe have straight threads up to and including the $1 \frac{1}{2}$-inch size. Federal Specifications Nos. WW-P-501 and WW-P-521 cover cast-iron and malleable-iron couplings with tapered threads which should be used when such threads are required by practice or regulation.

I-6. It is believed that this specification adequately describes the characteristics necessary to secure the desired material, and that normally no samples will be necessary prior to award to determine compliance with this specification. If, for any particular purpose, samples with bids are necessary, they should be specifically asked for in the invitation for bids and the particular purpose to be served by the bid sample should be definitely stated, the specification to apply in all other respects.

\section{UNITED STATES GOVERNMENT, FEDERAI} SPECIFICATIONS BOARD, FEDERAI SPECIFICATION FOR NIPPLES, PIPE, BRASS, STEEL, AND WROUGHT IRON, WW-N-351, JUNE 24, 1930

\section{WROUGHT-IRON NIPPLES}

(See 607.4, p. 485.)

607.4 STEEL PIPE, FITTINGS, AND CONNECTIONS.

\section{AMERICAN BUREAU OF SHIPPING, RULES FOR BUILDING AND CLASSING STEEL VESSELS; PUMPS AND PIPING SYSTEMS, 1930}

(This organization has prepared specifications relating to steel ship construction, including ship parts from keel to masts, boilers, propelling and other machinery, and electrical equipment. Specifications contained in the publication relating to ferrous and nonferrous metals have been placed in this compilation under their proper commodity headings, as follows:)

\begin{tabular}{|c|c|c|}
\hline $\begin{array}{l}\text { Com- } \\
\text { modity } \\
\text { desig- } \\
\text { nation }\end{array}$ & Material & Page \\
\hline $\begin{array}{l}607.14 \\
607.4 \\
607.6 \\
642.23 \\
646.41\end{array}$ & $\begin{array}{l}\text { Pipe fittings } \\
\text { General } \\
\text { Valves } \\
\text { Copper and brass pipes. } \\
\text { Bronze castings. }\end{array}$ & $\begin{array}{r}385 \\
435 \\
492 \\
1182 \\
1250\end{array}$ \\
\hline
\end{tabular}

AMERICAN BUREAU OF SHIPPING, RULES FOR BUILDING AND CLASSING STEEL VESSELS; PUMPS AND PIPING SYSTEMS, 1930

\section{PIPE FITTINGS}

1. All pipe fittings over 2 inches in diameter for working pressures over 150 pounds per square inch, and fittings over 3 inches in diameter for pressures over 100 pounds should be cast with flanged ends. For boiler mountings this requirement is to apply to fittings $1 \frac{1}{2}$ inches in diameter and over.

2. Fittings of 3 inches diameter and over for working pressures over 100 pounds, and fittings of all sizes for pressures in excess of 300 pounds, are to be subjected by the manufacturer to a hydrostatic test of not less than three times the working pressure. They should also be stamped with the trade-mark of the manufacturer and the maximum allowable working pressure.

3. Flanges.-Pipes $2 \frac{1}{2}$ inches diameter and over for working pressures over 150 pounds per square inch should be expanded into steel flanges, or, in the case of copper pipes, brazed to composition or steel flanges. On other pipes screwed flanges should be fitted in preference to screw unions. The screwed pipes of high pressure oil and steam lines should be expanded into the flanges in order to secure uniformly tight threads.

4. Material of valves and fittings.-Cast steel may be used in the construction of valves and fittings of any system and for all pressures and temperatures. The physical characteristics should be in accordance with those specified for steel castings (see 611.41, p. 706, par. 1). Manufacturers should make physical tests of each heat, and when required submit the results of such tests for the bureau's information. All steel castings must be thoroughly annealed. The minimum thickness in inches shall be determined by the formula:

$$
t=\frac{\text { working pressureXinternal diameter }}{7,000}+0.188
$$

5. Cast iron and semisteel of the physical characteristics specified in the A. B. S. specification for iron castings (see 611.11 , p. 635), may be used in the construction of valves and fittings for pressures not exceeding 300 pounds per square inch and for temperatures up to $450^{\circ} \mathrm{F}$. The minimum thickness in inches shall be determined by the formula:

$$
t=\frac{\text { working pressureXinternal diameter }}{3,000}+0.25
$$

6. Composition having the physical characteristics for grade 1 material specified in the A. B. S. specification for brass and bronze castings (see 646.41, p. 1250), may be used in the construction of valves and fittings for pressures not exceeding 300 pounds per square inch and temperatures up to $470^{\circ} \mathrm{F}$. The minimum thickness in inches shall be determined by the formula:

$$
t=\frac{\text { working pressure Xinternal diameter }}{5,000}+0.125
$$

7. Bronze having the physical characteristics for grade 2 material specified for brass and bronze castings (p. 1250), or other approved material may be used in the construction of valves and fittings for pressures over 300 pounds per square inch and temperatures up to $550^{\circ} \mathrm{F}$.

8. Pipes for working pressures over 150 pounds per square inch are to be tested and inspected by the bureau's surveyors in accordance with the requirements for steel pipes given above in materials for machinery, boilers, and piping.

9. Steel pipes for pressures up to 350 pounds per square inch may be lap-welded; for higher pressures they must be seamless drawn. 
10. Butt-welded steel pipes over 2 inches in diameter are not to be used for pressures over 100 pounds per square inch.

11. The minimum thicknesses of pipes shall be determined by the following formulas:

Lap-welded iron and steel pipe,

$$
t=\frac{\mathrm{DW}}{10,000}+C
$$

Seamless steel pipe, grade 1 ,

$$
t=\frac{\mathrm{DW}}{12,000}+C
$$

Seamless steel pipe, grade 2,

$$
t=\frac{\mathrm{DW}}{14,000}+C
$$

where $t=$ thickness in inches.

$D=$ internal diameter in inches.

$W=$ working pressure in pounds per square inch. For feed pipes this should be 1.2 times the boiler pressure.

$C=0.12$ inch for steam and water; 0.06 inch for air and oil.

12. The reduction in thickness at the outer radius of bent pipes is to be considered in determining the required thickness.

\section{AMERICAN BUREAU OF SHIPPING, RULES FOR BUILDING AND CLASSING STEEL VESSELS; MATERIALS FOR MACHINERY, BOILERS, AND PIPING, 1930}

\section{STEEL PIPES}

(For general information and requirements see 600.0 , p.1. For test requirements see 600.1, p. 7.)

1. Lapwelded steel pipes over 2 inches inside diameter, for a working pressure over 150 pounds per square inch are required to be tested and inspected by the surveyors in accordance with the following requirements:

2. The material shall be of a soft weldable quality. Pipes must be free from seams, cracks, or other defects. Test specimens are to be cut from pipes selected by the surveyor from each lot. At least one set of the following tests is to be made for each lot of 2,000 pounds or less.

3. Tension test. - A test piece 8 inches long, 11/2 inches wide, shall show a minimum tensile strength of 45,000 pounds per square inch, a yield point of 55 per cent of the tensile strength, and a minimum elongation of 20 per cent.

4. Flattening test.-A section of pipe not less than 4 inches long shall stand flattening until the outside distance between the flat sides is not more than onethird the outside diameter of the pipe without showing cracks or flaws. The weld must be located at right angles to the flat sides.

5. Hydrostatic test.-All pipes shall be tested to a hydrostatic pressure of not less than three times the working pressure.

6. Gaging and marking.-The minimum thickness shall not be less than specified by the purchaser and required by the rules.

7. Upon satisfactory compliance with the requirements all pipes are to be legibly stenciled with the identification mark of the manufacturer, the test pressure, and A. B., showing that they have been satisfactorily tested and inspected by a surveyor to the bureau. Pipes showing defects previous to or during their installation shall be rejected notwithstanding any previous acceptance.

\section{SEAMLESS STEEL PIPES}

8. Seamless steel pipes for a working pressure of over 150 pounds are to be tested and inspected by the surveyors in accordance with the following requirements: Pipes are to be uniform in thickness and of good ductile material. They are to be annealed as a final process. Test tubes are to be selected by the surveyor, and at least one set of tests is to be made for each lot of 2,000 pounds or less.

9. Physical test.-A standard test piece must

\begin{tabular}{|c|c|c|c|}
\hline Grade & $\begin{array}{l}\text { Tensile } \\
\text { strength }\end{array}$ & Yield point & $\begin{array}{l}\text { Mini- } \\
\text { mum } \\
\text { elonga- } \\
\text { tion }\end{array}$ \\
\hline 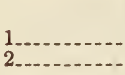 & $\begin{array}{c}\text { Lbs./in. }{ }^{2} \\
48,000-60,000 \\
60,000-70,000\end{array}$ & $\begin{array}{l}55 \text { per cent of tensile strength.- } \\
55 \text { per cent of tensile strength.. }\end{array}$ & $\begin{array}{r}\text { Per cent } \\
20 \\
18\end{array}$ \\
\hline
\end{tabular}
show the material to be of either of the following characteristics:

(Flattening test, hydrostatic test, and gaging and marking are the same as those specified above for lapwelded steel pipes.)

\section{AMERICAN ELECTRIC RAILWAY ENGI- NEERING ASSOCIATION, SPECIFICA- TION FOR TUBULAR STEEL POLES, D11-26, 1926}

[A pproved as American Tentative Standard C13-1926, by the American Standards Association]

\section{TUBULAR STEEL POLES}

\section{SCOPE}

1. These specifications ${ }^{70}$ cover material for, and the manufacture of, tubular steel poles for electric line construction.

\section{CONSTROCTION OF POLE}

2. Selection of material.-The material used in pole construction shall consist of lapwelded or seamless pipe conforming to the current standard specification of the American Society for Testing Materials for standard and extra strong pipe. (See A53-30, below.)

3. Design.-Each pole shall be built up of three pipe sections, butt, middle, and top, arranged as per Figure 268, with overlapping joints 18 inches long. The lapweld seams in the sections shall be set at an angle of $120^{\circ}$ to each other. Each section shall be composed of steel pipe of the respective sizes and lengths shown in Table 1 for the pole selected. ${ }^{71}$

When specified, a ground sleeve shall be attached to the butt section of each pole consisting of a section of steel pipe 2 feet long and of one-half inch nominal thickness after attachment to the pole, with its bottom 5 feet 4 inches from the base of the pole, unless otherwise specified by the purchaser. Its top shall be beveled sufficiently to shed water and the joint shall be water-tight.

The use of a pole top is recommended to protect the inside of the pole from exposure to the weather.

4. Method of fabrication.-Overlapping joints may be made either by swaging or by drawing through a die. If the swaging method is used, the larger section shall be heated and the smaller one while cold inserted therein, che larger section while hot being then swaged tightly on the smaller one. If the die method is used, the larger section shall be drawn over the smaller one while they are either hot or

70 The specifications include also a carefully selected list of pole sizes which the committee recommends for general use.

71 If any other design of pole is desired, its dimensions and test requirements shall be specified in the order. 
cold, or the larger one may be drawn down to suitable size while hot and the smaller one forced into it while cold. There shall be no irregularities in the shape of the joint after swaging or drawing that are indicative of lack of contact between the larger and smaller sections, and the thickness of the larger section shall not be reduced by these operations. No mechanical device shall be used that would tend to waaken the joint in any way. The top of the outer pipe shall be beveled to shed water and the completed joint shall be water-tight.

If ground sleeves are specified they may be attached by any of the above methods.

\section{TESTS}

5. Drop test.-Each test pole shall be dropped butt downwards three times in succession from a height of 6 feet upon a solid wooden block 6 inches thick supported upon a rigid base at least five times the weight of the pole, without showing signs of telescoping or loosening of the joints.

6. Deflection test.-Each test pole shall be rigidly supported for 6 feet from its base, and subjected as a cantilever to the respective loads given in Table 1, these loads being applied 18 inches from top of pole and at right angles to its longitudinal axis.

Under the deflection load, $L$, the pole at point of loading shall not show a deflection exceeding the limit, $D$, given in Table 1 .

Under the set load, $P$, the temporary deflection shall be measured and after the removal of the set load the pole at point of loading shall not show a permanent set exceeding 10 per cent of its temporary deflection under this load, but the permanent set shall never be required to be less than one-half inch.

7. Number of tests. - If required by the purchaser or his representarive the tests specified in sections 5 and 6 must be made on 5 per cent of the poles in any lot. By lot is meant the number of poles of any one design on each order.

8. Retests. - If the results of the physical tests of any lot do not conform to the requirements specified in section 5 or 6 , an additional 5 per cent of the poles from the same lot shall be subjected to the test or tests not previously met. In case any one of these fail to meet the requirements specified, the entire lot shall be subject to rejection, except that the manufacturer may at his option subject each pole in the lot in question to the tests not previously met, and those which fullfill the requirements shall be accepted.

\section{PERMISSIBLE VARIATIONS IN WEIGHT AND DIMENSIONS}

9. The weight of the pole after fabrication shall not exceed 5 per cent over or under the weight specified in Table 1. The various pipe sections used shall be within the permissible variations in weight and dimensions required in the standard pipe specification referred to in section 2 .

\section{WORKMANSHIP AND FINISH}

10. Defects.-The finished pole shall be reasonably straight and free from injurious defects. The ends shall be free from burrs.

11. Painting.-Unless otherwise specified, the outside of all poles shall receive one coat of good red lead and linseed oil paint and allowed to dry before

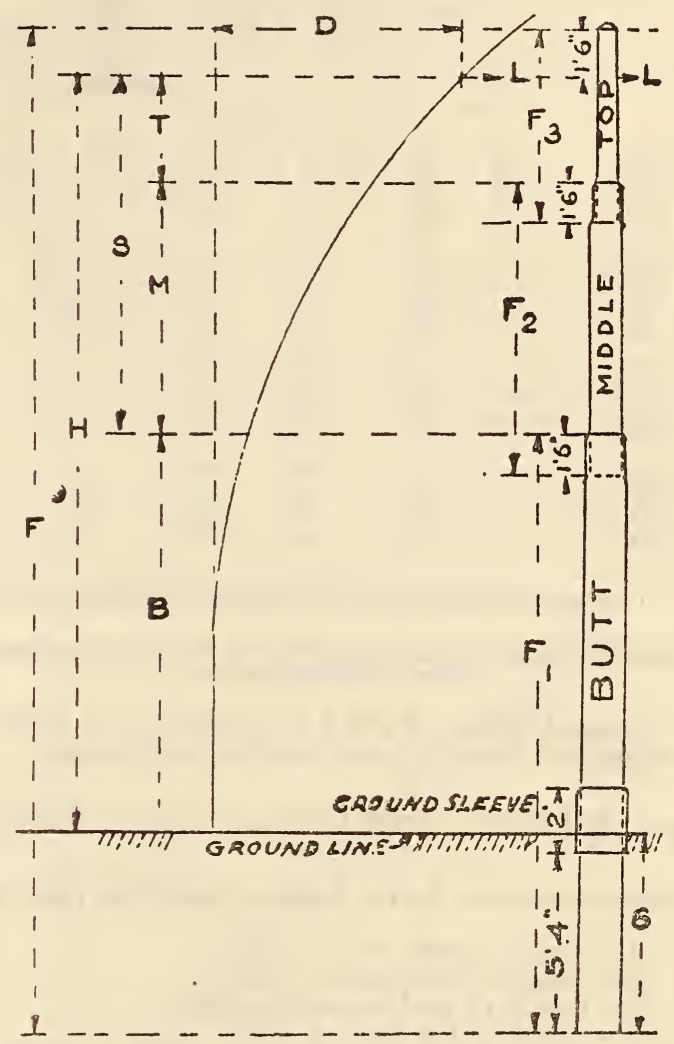

FIGURE 268.-Pole assembly and deflection diagram

loading on cars for shipment. The paint shall be well mixed and consist of 12 pounds of red lead to 1 gallon of boiled linseed oil. Poles shall be dry and cleaned free from dirt, oil, or loose scale before painting.

\section{INSPECTION AND REJECTION}

(Requirements covering inspection and rejection conform with those specified in A. S. T. M. standard specifications for steel. (See 605.0, p. 247.) 
TABLE 1.-Three-section tubular steel poles (deflections and loads)

\begin{tabular}{|c|c|c|c|c|c|c|c|c|c|c|c|c|}
\hline \multirow{3}{*}{ Pole No. } & \multirow{3}{*}{$\begin{array}{l}\text { Weight } \\
\text { of pole } \\
\text { without } \\
\text { ground } \\
\text { sleeve }\end{array}$} & \multicolumn{8}{|c|}{ Description of poles } & \multicolumn{3}{|c|}{ Test requirements ? } \\
\hline & & \multirow{2}{*}{$\begin{array}{c}\text { Over-all } \\
\text { length } \\
\text { in feet } \\
(F)\end{array}$} & \multirow{2}{*}{$\begin{array}{l}\text { Approxi- } \\
\text { mate } \\
\text { weight of } \\
\text { ground } \\
\text { sleeves, }\end{array}$} & \multicolumn{3}{|c|}{$\begin{array}{l}\text { Nominal inside diameter } \\
\text { pipe }\end{array}$} & \multicolumn{3}{|c|}{ Section lengths } & \multirow{2}{*}{$\begin{array}{c}\text { Load for } \\
\text { perms- } \\
\text { nent set } \\
\text { (pounds) } \\
P\end{array}$} & \multirow{2}{*}{$\begin{array}{c}\text { Load for } \\
\text { deflection } \\
D \\
\text { (pounds) } \\
L\end{array}$} & \multirow{2}{*}{$\begin{array}{l}\text { Deflec- } \\
\text { tion for } \\
\text { Load L } \\
\text { (inches) } \\
D\end{array}$} \\
\hline & & & & $\begin{array}{c}\text { Butt } \\
\text { standard } \\
\text { and } \\
\text { extra } \\
\text { strong }\end{array}$ & $\begin{array}{l}\text { Middle } \\
\text { standard }\end{array}$ & $\begin{array}{l}\text { Top } \\
\text { stand- } \\
\text { ard }\end{array}$ & Butt & Middle & Top & & & \\
\hline $\begin{array}{l}286 \\
286 \mathrm{X} \\
287 \\
287 \mathrm{X}\end{array}$ & $\begin{array}{l}509 \\
681 \\
644 \\
905\end{array}$ & $\begin{array}{l}28 \\
28 \\
28 \\
28\end{array}$ & $\begin{array}{c}\text { Pounds } \\
76 \\
76 \\
87 \\
87\end{array}$ & $\begin{array}{r}\text { Inches } \\
6 \\
6 \\
7 \\
7\end{array}$ & $\begin{array}{r}\text { Inches } \\
5 \\
5 \\
6 \\
6\end{array}$ & $\begin{array}{c}\text { Inches } \\
4 \\
4 \\
5 \\
5\end{array}$ & $\begin{array}{r}\text { Feet } \\
18 \\
18 \\
18 \\
18\end{array}$ & $\begin{array}{r}\text { Feet } \\
7 \\
7 \\
7 \\
7\end{array}$ & $\begin{array}{r}\text { Feet } \\
6 \\
6 \\
6 \\
6\end{array}$ & $\begin{array}{r}930 \\
1,340 \\
1,340 \\
2,050\end{array}$ & $\begin{array}{r}620 \\
890 \\
890 \\
1,370\end{array}$ & $\begin{array}{l}4.68 \\
4.94 \\
4.00 \\
4.25\end{array}$ \\
\hline $\begin{array}{l}307 \\
307 \bar{X}-. \\
308 \\
308 \mathrm{X} \\
\end{array}$ & $\begin{array}{r}682 \\
958 \\
840 \\
1,122\end{array}$ & $\begin{array}{l}30 \\
30 \\
30 \\
30\end{array}$ & $\begin{array}{l}87 \\
87 \\
97 \\
97\end{array}$ & $\begin{array}{l}7 \\
7 \\
8 \\
8\end{array}$ & $\begin{array}{l}6 \\
6 \\
7 \\
7\end{array}$ & $\begin{array}{l}5 \\
5 \\
6 \\
6\end{array}$ & $\begin{array}{l}19 \\
19 \\
19 \\
19\end{array}$ & $\begin{array}{l}7 \\
7 \\
7 \\
7\end{array}$ & $\begin{array}{l}7 \\
7 \\
7 \\
7\end{array}$ & $\begin{array}{l}1,220 \\
1,870 \\
1,680 \\
2,450\end{array}$ & $\begin{array}{r}810 \\
1,250 \\
1,120 \\
1,630\end{array}$ & $\begin{array}{l}4.85 \\
5.18 \\
4.25 \\
4.48\end{array}$ \\
\hline $\begin{array}{l}327 \\
327 \overline{\mathrm{X}}-. . \\
328 \\
328 \overline{\mathrm{X}} \\
\end{array}$ & $\begin{array}{r}720 \\
996 \\
887 \\
1,169\end{array}$ & $\begin{array}{l}32 \\
32 \\
32 \\
32\end{array}$ & $\begin{array}{l}87 \\
87 \\
97 \\
97\end{array}$ & $\begin{array}{l}7 \\
7 \\
8 \\
8\end{array}$ & $\begin{array}{l}6 \\
6 \\
7 \\
7\end{array}$ & $\begin{array}{l}5 \\
5 \\
6 \\
6\end{array}$ & $\begin{array}{l}19 \\
19 \\
19 \\
19\end{array}$ & $\begin{array}{l}9 \\
9 \\
9 \\
9\end{array}$ & $\begin{array}{l}7 \\
7 \\
7 \\
7\end{array}$ & $\begin{array}{l}1,120 \\
1,660 \\
1,540 \\
2,250\end{array}$ & $\begin{array}{r}750 \\
1,110 \\
1,030 \\
1,500\end{array}$ & $\begin{array}{l}5.84 \\
6.16 \\
5.11 \\
5.48\end{array}$ \\
\hline $\begin{array}{l}358 \\
358 \bar{X} \\
359 \\
359 \times \bar{X}\end{array}$ & $\begin{array}{r}968 \\
1,279 \\
1,162 \\
1,474\end{array}$ & $\begin{array}{l}35 \\
35 \\
35 \\
35\end{array}$ & $\begin{array}{r}97 \\
97 \\
108 \\
108\end{array}$ & $\begin{array}{l}8 \\
8 \\
9 \\
9\end{array}$ & $\begin{array}{l}7 \\
7 \\
8 \\
8\end{array}$ & $\begin{array}{l}6 \\
6 \\
7 \\
7\end{array}$ & $\begin{array}{l}21 \\
21 \\
21 \\
21\end{array}$ & $\begin{array}{l}10 \\
10 \\
10 \\
10\end{array}$ & $\begin{array}{l}7 \\
7 \\
7 \\
7\end{array}$ & $\begin{array}{l}1,370 \\
2,000 \\
1,830 \\
2,540\end{array}$ & $\begin{array}{r}920 \\
1,340 \\
1,220 \\
1,700\end{array}$ & $\begin{array}{l}6.40 \\
6.81 \\
5.69 \\
5.99\end{array}$ \\
\hline
\end{tabular}

1 See notes under heading General information supplementing Table I, Figure 268, and text. General Information Supplementing Table 1, Figure 268, and Text-
General Deflection Formula

Computations in Table 1 were made by the following general formula for a 3 -section tubular pole:

$D=\frac{11.52 L}{10_{0}}\left[\frac{B^{2}(2 H+S)+3 B S(H+S)}{I_{2}}+\frac{3 M\left(S^{2}+T^{2}\right)-M^{3}}{I_{2}}+\frac{2 T^{3}}{I_{8}}\right]$

Definition of Symbols Used in Formula, Table 1, and Figure 268

$F=$ length of pole, feet.

$F_{1}=$ length of butt section, feet.

$F_{2}=$ length of middle section, feet.

$F_{3}=$ length of top section, feet.

$H=$ height from ground line to point of application of load, feet.

$B=$ length of butt section above ground, feet.

$M=$ length of middle section above butt, feet.

$T=$ length of top section above middle to point of application of load, feet.

$S=$ length of pole above butt section to point of application of load, feet, $S=M+T$.

$I_{1}=$ moment of inertia of butt section, a constant for any pole.

$I_{2}=$ moment of inertia of middle section, a constant for any pole.

$I_{3}=$ moment of inertia of top section, a constant for any pole.

$L=$ load applied 18 inches from top of pole, pounds.

$D=$ Deflection of pole at point of application of load, inches.

$E=$ modulus of elasticity, 29,000,000.

$P=$ load for permanent set, pounds.

\section{NOTES}

The above general deflection formula for a 3section tubular pole was derived from the basic formula $D=\frac{L H^{3}}{3 E I}$ for a single-section pole, in which the symbols have the same meaning as above except that $H$ is in inches and $I$ is the moment of inertia of the single section.

In deriving the general formula, a factor of variation has been introduced to allow for variations in material, dimensions, and quality. This allowance is equivalent to the use of a modulus of elasticity of $25,000,000$.

The load for permanent set, $P$, has been computed by using 27,000 pounds per square inch fiber stress and the normal section; that is, a pipe that is absolutely true to weight and shape, and free from all variations.

The deflection load, $L$, is approximately twothirds of the set load.

\section{AMERICAN MARINE STANDARDS COM- MITTEE, TUBULAR STEEL CARGO BOOMS FOR SHIPS, H NO. 21-1927 TO H NO. 25-1927}

\section{TUBULAR STEEL CARGO BOOMS FOR SHIPS}

\section{(Capacities 5, 10, 15, 20, and 30 tons)}

\section{GENERAL NOTES}

1. These standards are believed to cover the requirements for ordinary installations, but, where extraordinary conditions exist, due allowance should be made therefor in selecting or determining the boom to be provided. Eccentric loading and liability to bending and shock under live loads would be conditions requiring special consideration. Therefore the standards should be used only with due consideration to the actual conditions prevailing in each individual case.

2 . In the calculations upon which the design of these standard booms is based, the following assumptions, formulas, and methods were used:

(a) Strength of booms.-

The booms were consiaered as columns with ends free or round. 
The Rankine-Gordon formula was used, viz.:

$P=$ ultimate strength of column in pounds per square inch.

$f=$ crushing strength of material in pounds per square inch.

$L=$ length of column in inches.

$r=$ least radius of gyration of column in inches.

$a=$ constants; that is, $\frac{1}{9000}$ for mild steel, with ends free or round.

$P=\frac{f}{1 \text { plus } a\left(\frac{L}{r}\right)^{2}}$ and assuming $f$ as 50,000

pounds per square incn for lapwelded pipe,

$$
P=\frac{50,000}{1 \text { plus } \frac{1}{9000}\left(\frac{L}{r}\right)^{2}} .
$$

A curve was made up for $P$ for various values of $\frac{L}{r}$ from 0 to 200 , from which the ultimate strength

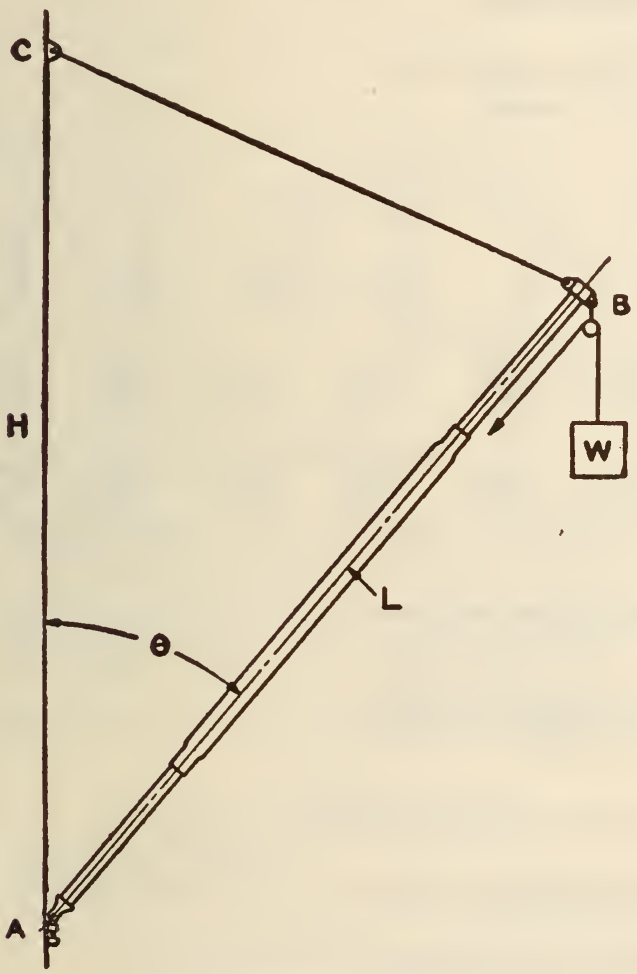

FIGURE 269

could be read off for any value of $\frac{L}{r}$. In figuring the strength of the booms a factor of safety of 5 was primarily used to determine the allowable working stress, but the pipes or tubes adopted for the various parts being the nearest commercial sizes over the calculated requirements the resultant factor of safety actually exceeds 5 . This has been augmented in some cases by minimizing the variety of parts for the construction of the 26 different booms comprised in the standards by using identical sizes so far as deemed practicable and economical. Half the estimated weight of the boom was included in the load factor. (b) Thrust in booms.-The thrust was calculated on the following basis. (Fig. 269.) This formula gives the thrust due to the dead load and the additional thrust due to the pull of the cargo whip:

$W=$ load on boom.

$P=$ thrust in boom

$L=$ length of boom $(A B)$.

$H=$ height of topping lift above boom support $(A C)$.

$n=$ number of parts in cargo whip.

(Nore.-If height $A C$ is kept constant, the thrust in the boom is the same at all angles of $\theta$.)

$$
P=W\left(\frac{L}{H} \text { plus } \frac{1}{n}\right)
$$

For these standard booms the value of $n$ was assumed as follows:

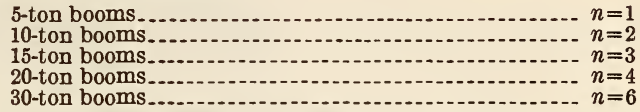

The allowable thrust in boom is the permissible working stress multiplied by the sectional area. It was calculated for three thicknesses of various sizes of pipe for three lengths with ratios of $\frac{L}{r}$ ranging from 100 to about 180 . Curves were plotted for each size and thickness of pipe, giving allowable

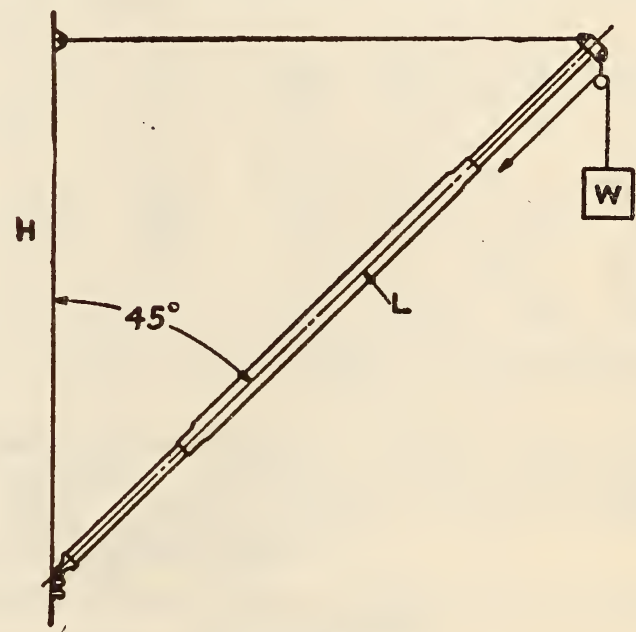

FIGURE 270

thrust for various lengths. These curves were used to determine the diameter and thickness at the middle of the boom. Curves were also plotted for various values of $\frac{L}{r}$ for allowable stress at different points in the length of the boom, from which to determine the permissible reductions of section at points away from the middle.

In standardizing booms for various loads and lengths some ratio of $\frac{L}{H}$ must be assumed, and as a basis for these standards the position of the boom was taken at $45^{\circ}$ with the topping lift horizontal, as per Figure 270 , viz:

$$
\frac{L}{H}=\frac{1}{\cos . \theta}=\operatorname{secant} \text { of } 45^{\circ}=1.41421
$$

When $\frac{L}{H}$ is greater than 1.414 , the strength of the boom must be increased above standard. 
When $\frac{L}{H}$ is less than 1.414, the strength of the boom may be decreased below standard.

3. Joints of pipe sections.- The sections are joined by swaging and shrinking. The joints are made by inserting the end of the smaller pipe cold into the end of the larger pipe heated to a full red heat, and while in this position swaging the larger pipe into close contact with the inserted cold pipe. The joint so swaged is then allowed to cool and shrink.
4. Materials and workmanship.-The pipe or tubing shall be open-hearth steel having a tensile strength of not less than 50,000 pounds per square inch and an elongation in 8 inches not under 20 per cent. The dimensions of the pipe or tubing as given are commercial standards which are subject to the manufacturing tolerances allowed by the shipclassification authorities. The finished booms must meet the requirements of the ship-classification authorities.

\section{TUBULAR STEEL CARGO BOOMS, 5 TONS CAPACITY}

$W=$ commercial designation.

$X=$ outside diameter.

$Y=$ inside diameter. $\}$ Commercial-standard dimensions, subject to manufacturing tolerances.

$T=$ thickness.

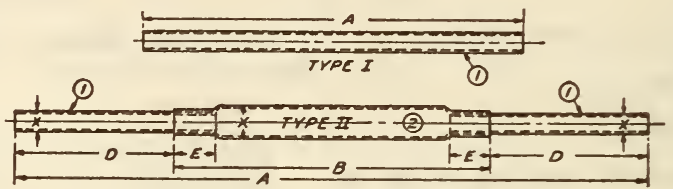

FiguRe 271.- Tubular steel cargo booms, 5 tons capacity

Particulars of pipe and tubing

[See general notes for grade of material]

\begin{tabular}{|c|c|c|c|c|c|c|c|c|}
\hline & \multicolumn{2}{|c|}{ Type I } & \multicolumn{5}{|c|}{ Type II } & \\
\hline $\begin{array}{l}\text { A } \\
\text { B } \\
\text { D } \\
\text { E } \\
\text { Part 1: }\end{array}$ & 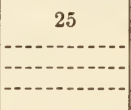 & 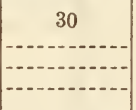 & $\begin{array}{l}35 \\
11 \\
12 \\
11 / 2\end{array}$ & $\begin{array}{l}40 \\
16 \\
12 \\
11 / 2\end{array}$ & $\begin{array}{l}45 \\
20 \\
121 / 2 \\
11 / 2\end{array}$ & $\begin{array}{l}50 \\
25 \\
121 / 2 \\
11 / 2\end{array}$ & $\begin{array}{l}55 \\
29 \\
131 / 2 \\
11 / 2\end{array}$ & $\begin{array}{l}60 \\
32 \\
14 \\
11 / 2\end{array}$ \\
\hline Parl ${ }_{\mathrm{X}}^{\mathrm{W}}$ & $\begin{array}{r}8.625 \\
8.071 \\
.277\end{array}$ & $\begin{array}{r}8.625 \\
8.071 \\
.277\end{array}$ & $\begin{array}{r}8.625 \\
8.071 \\
.277\end{array}$ & $\begin{array}{r}\text { Standard-w } \\
8.625 \\
8.071 \\
.277\end{array}$ & $\begin{array}{l}\text { eight pipe } \\
8.625 \\
8.071 \\
.277\end{array}$ & $\begin{array}{r}8.625 \\
8.071 \\
.277\end{array}$ & $\begin{array}{r}8.625 \\
8.071 \\
.277\end{array}$ & $\begin{array}{r}8.625 \\
8.071 \\
.277\end{array}$ \\
\hline 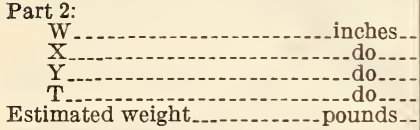 & - 612 & $\mid$ & $\begin{array}{r}10.750 \\
10.020 \\
1,112^{.365}\end{array}$ & $\begin{array}{r}10.750 \\
10.020 \\
1,315^{.365}\end{array}$ & $\begin{array}{l}\text { Standard-w } \\
10.750 \\
10.020 \\
1,501\end{array}$ & $\begin{array}{l}\text { ight pipe } \\
10.750 \\
10.020 \\
1,704\end{array}$ & $\begin{array}{r}12.750 \\
12.090 \\
1,967^{.330}\end{array}$ & $\begin{array}{r}12.750 \\
12.090 \\
2,166^{.330}\end{array}$ \\
\hline
\end{tabular}

TOBULAR STEEL CARGO BOOMS, 10 TONS CAPACITY

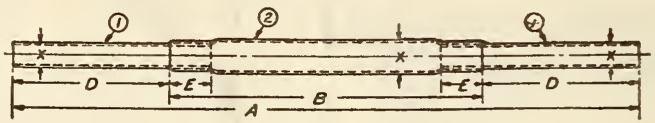

Figdre 272.-Tubular steel cargo booms, 10 tons capacity

$W=$ commercial designation

Particulars of pipe and tubing

$X=$ outside diameter

$\left.\begin{array}{l}X=\text { inside diameter. } \\ T=\text { thickness. }\end{array}\right\}$ Commercial-standard dimensions, subject to manufacturing tolerances.

[See general notes for grade of material]

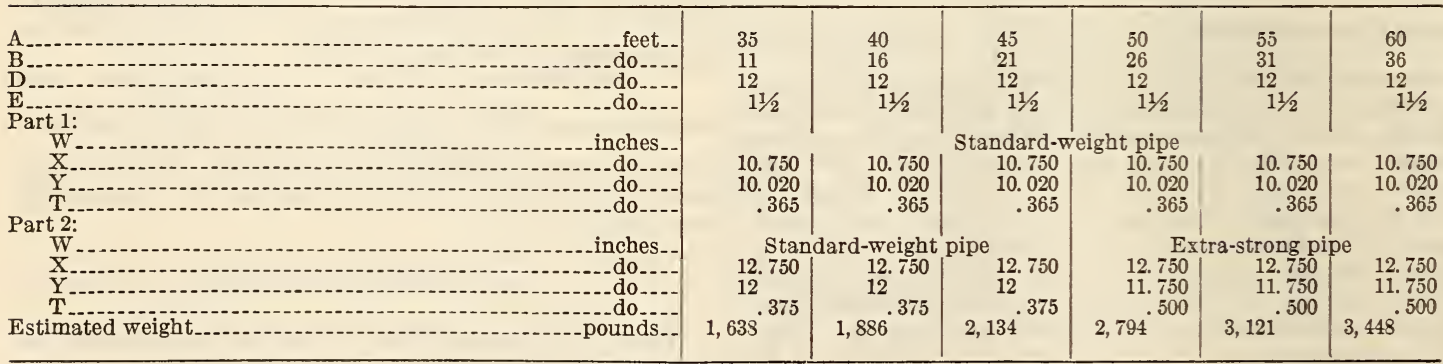


TUBOLAR STEEL CARGO BOOMS, 15 TONS CAPACITY

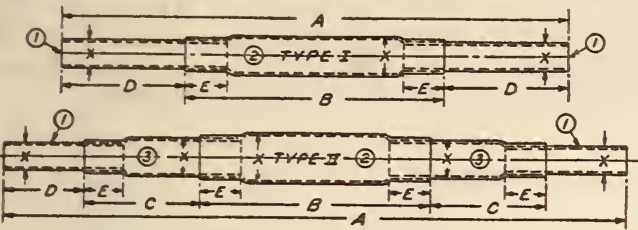

FIGURE 273.-Tubular steel cargo booms, 15 tons capacity

Particulars of pipe and tubing

$W=$ commercial designation.

$X=$ outside diameter. Commercial-standard dimensions, sub $Y=$ inside diameter.
$T=$ thickness. ject to manufacturing tolerances.

[See general notes for grade of material]

\begin{tabular}{|c|c|c|c|c|}
\hline \multirow{3}{*}{ B } & \multicolumn{2}{|c|}{ Type I } & \multicolumn{2}{|c|}{ Type II } \\
\hline & $\begin{array}{l}45 \\
23\end{array}$ & $\begin{array}{l}50 \\
28\end{array}$ & $\begin{array}{l}55 \\
16\end{array}$ & \\
\hline & 11 & & $81 / 2$ & $\begin{array}{l}10 \\
11\end{array}$ \\
\hline do & $11 / 2$ & $11 / 2$ & $11 / 2$ & $11 / 2$ \\
\hline $\begin{array}{l}\text { Part 1: } \\
\text { w. }\end{array}$ & & Standard. & veight pipe & \\
\hline Y & $\begin{array}{r}10.750 \\
10.020 \\
.365\end{array}$ & $\begin{array}{r}10.750 \\
10.020 \\
.365\end{array}$ & $\begin{array}{r}10.750 \\
10.020 \\
.365\end{array}$ & $\begin{array}{r}10.750 \\
10.020 \\
.365\end{array}$ \\
\hline $\begin{array}{l}\text { Part 2: } \\
W\end{array}$ & Extra-st & rong pipe & Tul & .000 \\
\hline $\begin{array}{l}\mathrm{X} \\
\mathrm{Y} \\
\mathrm{T}\end{array}$ & $\begin{array}{r}12.750 \\
11.750 \\
.500\end{array}$ & $\begin{array}{r}12.750 \\
11.750 \\
.500\end{array}$ & $\begin{array}{l}14.125 \\
13.125 \\
.4375\end{array}$ & ${ }^{14}{ }_{.500}$ \\
\hline $\begin{array}{l}\text { Part } 3: \\
\text { W }\end{array}$ & & & $\begin{array}{r}.4315 \\
\text { Extra-str }\end{array}$ & ong pipe \\
\hline inches. & & & $\begin{array}{l}12.750 \\
11.750\end{array}$ & $\begin{array}{l}12.750 \\
11.750\end{array}$ \\
\hline Estimated weight & 2,517 & 2,844 & 3,334 & 3,814 \\
\hline
\end{tabular}

TOBOLAR STEEL CARGO BOOMS, 20 TONS CAPACITY

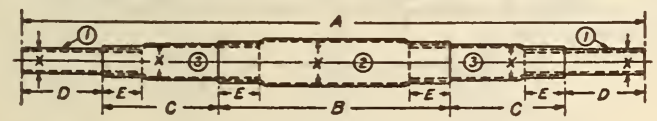

FIGURE 274.-Tubular steel cargo booms, 20 tons capacity

\section{Particulars of pipe and tubing}

$W=$ commercial designation.

$X=$ outside diameter.
$Y=$ inside diameter. Commercial-standard dimensions, sub $T=$ thickness.

[See general notes for grade of material],

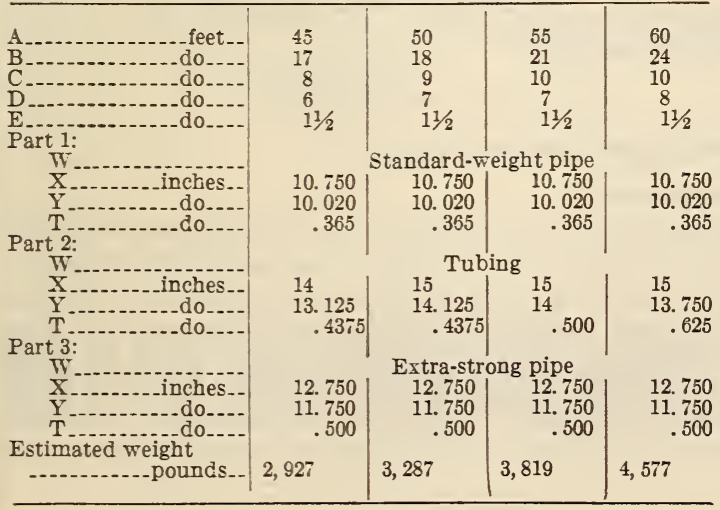

TUBULAR STEEL CARGO BOOMS, SO TONS CAPACITY

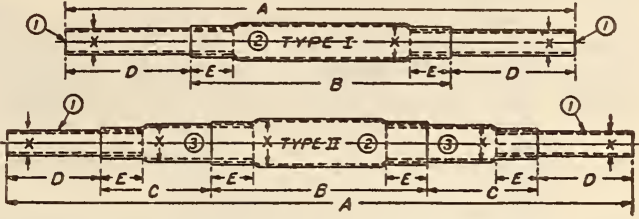

FIGURE 275.-Tubular steel cargo booms, 30 tons capacity

\section{Particulars of pipe and tubing}

$W=$ commercial designation.

$Y=$ outside diameter. Commercial-standard dimensions, sub$\left.\begin{array}{l}Y=\text { inside diameter. } \\ T=\text { thickness. }\end{array}\right\} \begin{aligned} & \text { ject to manufacturing tolerances. } \\ & \text { jering }\end{aligned}$

[See general notes for grade of material]

\begin{tabular}{|c|c|c|c|c|}
\hline & Type I & \multicolumn{3}{|c|}{ Type II } \\
\hline -...feet. & 45 & \multirow{4}{*}{$\begin{array}{l}50 \\
18 \\
9 \\
7 \\
11 / 2\end{array}$} & \multirow{4}{*}{$\begin{array}{l}55 \\
21 \\
10 \\
7 \\
11 / 2\end{array}$} & \multirow{4}{*}{$\begin{array}{l}60 \\
23 \\
101 / 2 \\
8 \\
11 / 2\end{array}$} \\
\hline -.-do-.. & 24 & & & \\
\hline do... & $101 \%$ & & & \\
\hline $\begin{array}{l}\text { E.........- do } \\
\text { Part 1: }\end{array}$ & $11 / 2$ & & & \\
\hline$\vec{W}$ & \multicolumn{4}{|c|}{ Extra-strong pipe } \\
\hline -. inches. & 12. 750 & 12.750 & 12.750 & 12.750 \\
\hline 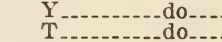 & 11. 750 & 11.750 & 11.750 & 11. 750 \\
\hline $\begin{array}{l}\operatorname{art} 2: \\
\mathrm{W}\end{array}$ & \multirow{2}{*}{\multicolumn{4}{|c|}{ Tubing }} \\
\hline .....-inches & & & & \\
\hline Y & 13. 750 & 14. 875 & 14. 750 & 17 \\
\hline Part 3: & & .5625 & .025 & .50 \\
\hline $\mathrm{W}$ & -- & & $\underset{14}{\text { Tubing }}$ & \\
\hline .... do & & 12. 875 & 12.875 & 13. 875 \\
\hline & & & & .5625 \\
\hline 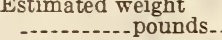 & 3,873 & 4,477 & 5,124 & 5,474 \\
\hline
\end{tabular}

\section{AMERICAN MARINE STANDARDS COM- MITTEE, FITTINGS FOR TUBULAR STEEL CARGO BOOMS, H NO. 30-1928, AND H NO. 31-1929}

\section{HEEL FITTINGS AND CAPS FOR 5, 10, 15, 20, AND 30 TON BOOMS}

\section{GENERAL NOTES}

These standards are believed to provide safely for ordinary installations and the loads specified for the tubular steel booms to which they apply. Where extraordinary conditions exist, due allowance should be made therefor by installing larger booms and fittings. Eccentric loading and liability to extraordinary shock under live loads would be conditions requiring special consideration. Therefore, the standards should be used with due regard to the prevailing conditions in each individual case.

The quality of the materials and workmanship shall equal or exceed the requirements of the American Bureau of Shipping. The materials and the finished booms shall be subject to inspection and acceptance by that bureau or by other ship classification or inspection authorities as may be required by rules and regulations or by the contract or purchase order.

The method indicated for lubrication of the pin of the heel fitting should be regarded as typical. 
Other means of lubrication having equal or superior $\mid$ and approved by the American Standards Associamerit may be substituted.

The American (National) standard of screw threads noted on Standard H No. 30-1928 is that sponsored by the Society of Automotive Engineers and the American Society of Mechanical Engineers,

tion in May, 1924, as standard B1a-1924. It is in complete accordance with the report of the $\mathrm{Na}$ tional Screw Thread Commission (Miscellaneous Publication No. 89, Bureau of Standards).

CAPS FOR 5, 10, 15, 20, AND 30 TON BOOMS

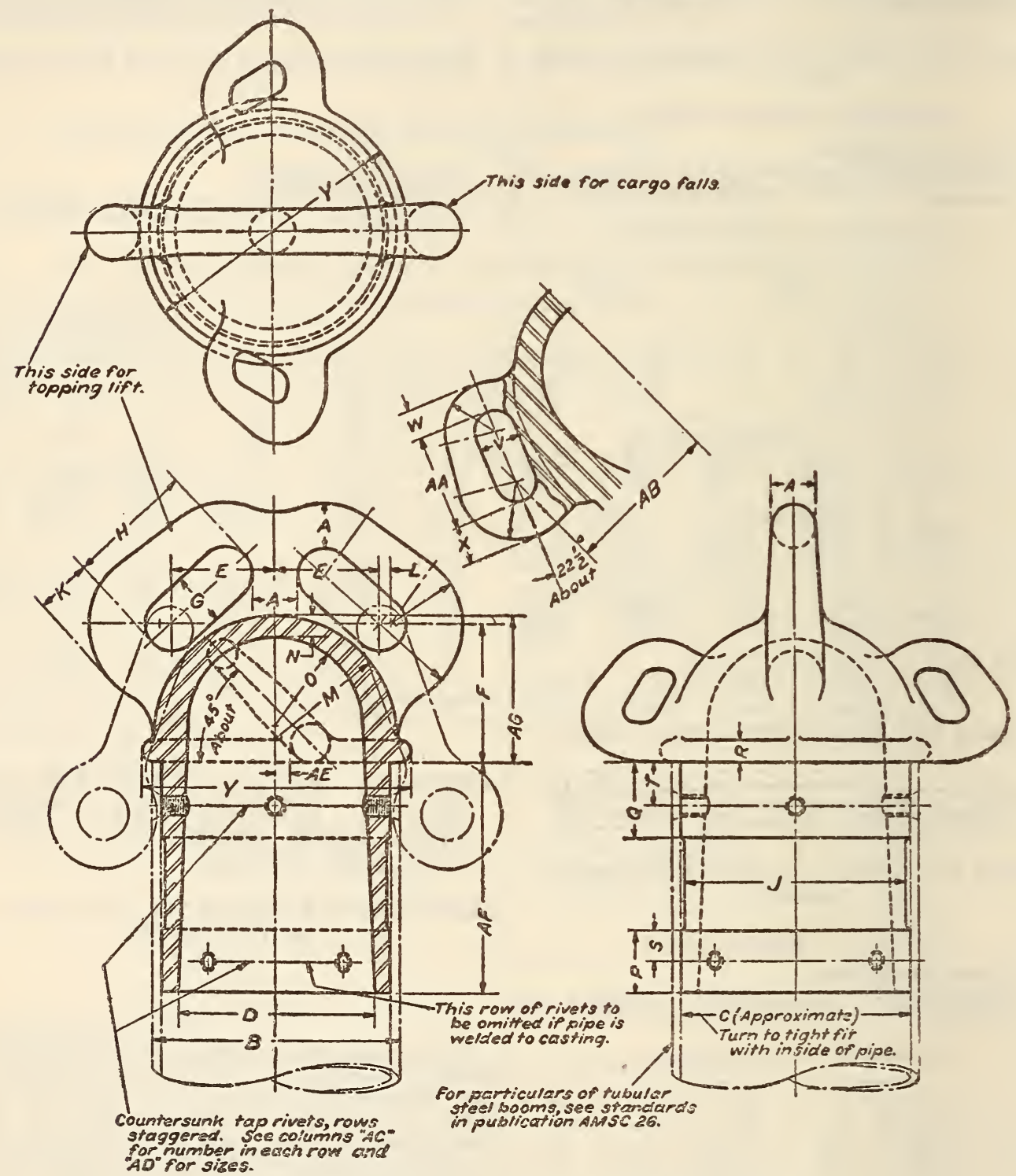

Figure 276.-Caps for 5, 10,15, 20, and 30 ton booms

[All dimensions in inches]

\begin{tabular}{|c|c|c|c|c|c|c|c|c|c|c|c|c|c|c|c|c|c|c|c|c|c|c|c|c|c|c|c|c|c|c|}
\hline B & A & B & C & D & $\mathrm{E}$ & $\mathrm{F}$ & G & $\mathrm{H}$ & $\mathrm{J}$ & K & $\mathbf{L}$ & $\mathrm{M}$ & $\mathrm{N}$ & 0 & $\mathrm{P}$ & Q & $\mathrm{R}$ & $\mathrm{S}$ & $\mathrm{T}$ & $\mathrm{V}$ & $\mathrm{W}$ & $\mathrm{x}$ & $\mathrm{Y}$ & $\mathrm{AA}$ & $\mathrm{AB}$ & $\mathrm{AC}$ & $\mathrm{AD}$ & $\mathrm{AE}$ & $\mathrm{AF}$ & $A G$ \\
\hline & $\begin{array}{l}11 / 2 \\
13 / 4 \\
2 \\
21 / 4 \\
23 / 4\end{array}$ & $\begin{array}{r}85 / 8 \\
103 / 4 \\
103 / 4 \\
103 / 4 \\
123 / 4\end{array}$ & $\begin{array}{l}81 / 8 \\
101 / 16 \\
101 / 16 \\
101 / 16 \\
113 / 4\end{array}$ & \begin{tabular}{|c|c}
$63 / 4$ \\
8112 \\
$81 / 2$ \\
$81 / 2$ \\
10
\end{tabular} & $\begin{array}{l}31 / 2 \\
41 / 4 \\
41 / 2 \\
43 / 4 \\
6\end{array}$ & $\begin{array}{l}5 \\
6 \\
6 \\
6\end{array}$ & $\begin{array}{l}13 / 4 \\
2 \\
21 / 4 \\
21 / 2 \\
3\end{array}$ & \begin{tabular}{|l|}
$41 / 4$ \\
$43 / 4$ \\
$53 / 8$ \\
6 \\
$71 / 4$ \\
\end{tabular} & $\begin{array}{r}73 / 4 \\
93 / 4 \\
93 / 4 \\
93 / 4 \\
1132 \\
\end{array}$ & \begin{tabular}{|l|}
2 \\
$21 / 4$ \\
$21 / 2$ \\
$23 / 4$ \\
314 \\
\end{tabular} & $\begin{array}{l}52 \\
5 / 8 \\
7 / 8\end{array}$ & $\begin{array}{l}45 / 16 \\
53 / 8 \\
53 / 8 \\
53 / 8 \\
63 / 8\end{array}$ & $\begin{array}{l}1^{7 / 8} \\
1 \\
1 \\
11 / 4\end{array}$ & $\begin{array}{l}3 \\
33 / 4 \\
33 / 4 \\
33 / 4 \\
438\end{array}$ & $\mid \begin{array}{l}21 / 2 \\
23 / 4 \\
23 / 4 \\
23 / 4 \\
3\end{array}$ & \begin{tabular}{|l}
3 \\
$31 / 4$ \\
314 \\
314 \\
$31 / 2$ \\
31
\end{tabular} & $\mid \begin{array}{l}3 / 4 \\
1 \\
1 \\
1 \\
1\end{array}$ & \begin{tabular}{l|l}
113 & 1 \\
138 & 1 \\
138 & 1 \\
133 & 1 \\
$11 / 2$ & 2
\end{tabular} & \begin{tabular}{|l|l}
$13 / 4$ \\
178 \\
178 \\
178 \\
2
\end{tabular} & $13 / 4$ & & \begin{tabular}{|l|}
$11 / 2$ \\
$15 / 8$ \\
$13 / 4$ \\
178 \\
$21 / 8$
\end{tabular} & & $\begin{array}{l}31 / 4 \\
315 \\
4 \\
41 / 4 \\
43 / 4\end{array}$ & $\begin{array}{l}53 / 8 \\
65 / 8 \\
634 \\
67 / 8 \\
8\end{array}$ & $\begin{array}{l}3 \\
3 \\
4 \\
4 \\
4\end{array}$ & $\begin{array}{c}3 / 4 \\
7 / 8 \\
7 / 8 \\
7 / 8 \\
1\end{array}$ & $\begin{array}{l}13 \\
132 \\
5 / 8 \\
58 \\
3 / 4 \\
\end{array}$ & $\begin{array}{r}8 \\
10 \\
10 \\
10 \\
12\end{array}$ & $\begin{array}{l}51 / 8 \\
63 \% \\
63 \% \\
63 \% \\
75 \%\end{array}$ \\
\hline
\end{tabular}


IRON AND STEEL

HEEL FITTINGS FOR 5, 10, 15, 20, AND 30 TON BOOMS
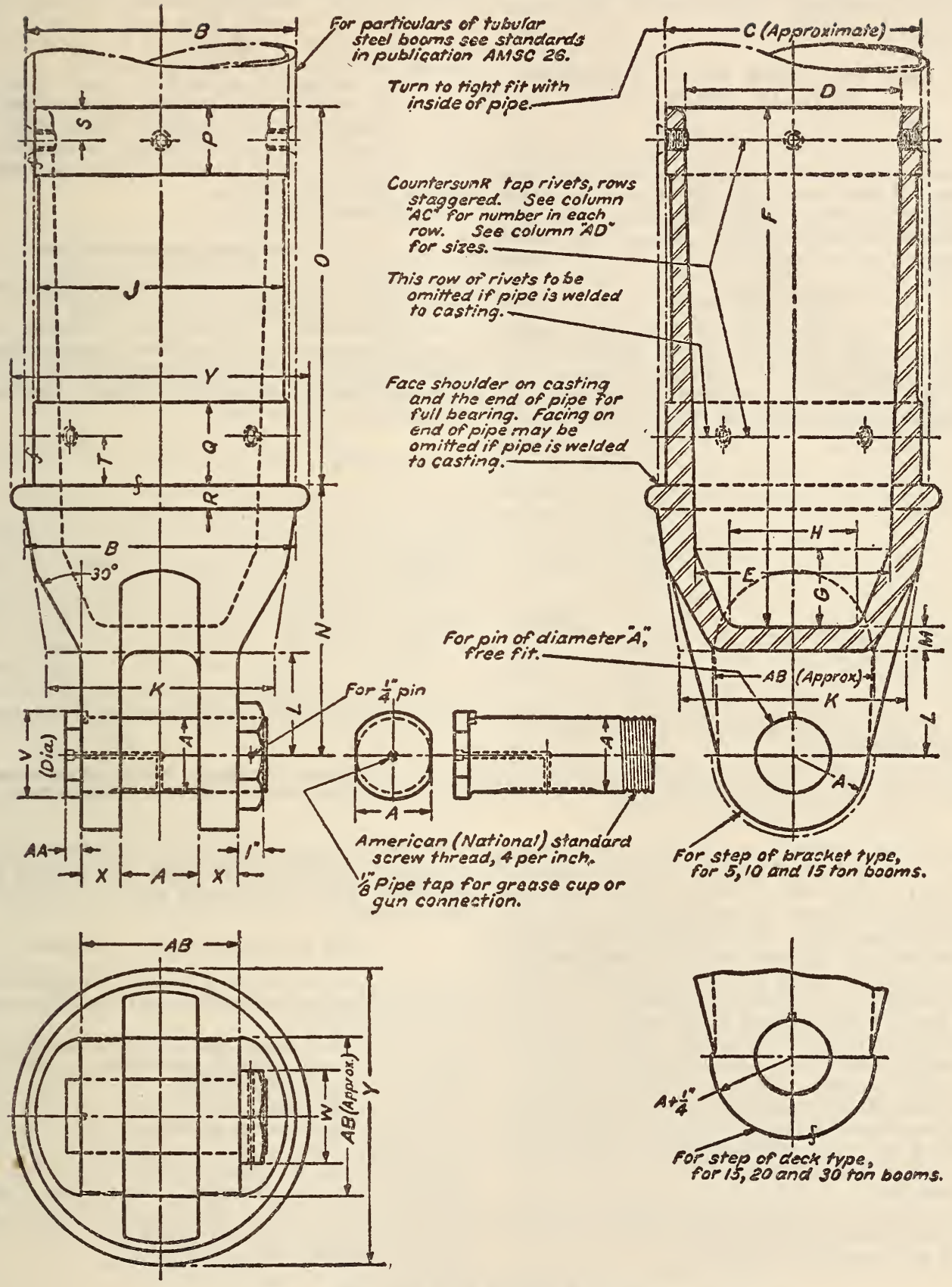

Figure 277.-Heel fittings for 5, 10, 15, 20, and 30 ton booms

[All dimensions in inches]

\begin{tabular}{|c|c|c|c|c|c|c|c|c|c|c|c|c|c|c|c|c|c|c|c|c|c|c|c|c|c|c|c|}
\hline & A & B & C & D & $\mathrm{E}$ & $F$ & G & H & $\mathrm{J}$ & K & $\mathrm{L}$ & $\mathrm{M}$ & $\mathrm{N}$ & 0 & $\mathrm{P}$ & Q & $\mathrm{R}$ & $\mathrm{S}$ & $\mathrm{T}$ & $\mathrm{V}$ & W & $x$ & $\mathrm{Y}$ & $\mathrm{AA}$ & $\mathrm{AB}$ & $\mathrm{AC}$ & AI \\
\hline & \begin{tabular}{|l|}
$21 / 2$ \\
$23 / 4$ \\
3 \\
$31 / 4$ \\
$33 / 4$
\end{tabular} & $\begin{array}{r}85 / 8 \\
10^{3} / 4 \\
10^{3} / 4 \\
10^{3} / 4 \\
12^{3} / 4\end{array}$ & $\begin{array}{l}81 / 8 \\
101 / 16 \\
101 / 16 \\
101 / 16 \\
113 / 4\end{array}$ & $\begin{array}{l}63 / 4 \\
81 / 2 \\
81 / 2 \\
81 / 2 \\
10\end{array}$ & \begin{tabular}{|l|}
6 \\
$75 / 8$ \\
$75 / 8$ \\
$75 / 8$ \\
9
\end{tabular} & $\begin{array}{l}161 / 8 \\
203 / 4 \\
20^{3} / 4 \\
20^{3} / 4 \\
24^{3} / 4\end{array}$ & & $\begin{array}{l}41 / 4 \\
45 / 8 \\
5 \\
53 / 8 \\
61 / 4 \\
\end{array}$ & \begin{tabular}{|r|}
$73 / 4$ \\
$93 / 4$ \\
$93 / 4$ \\
$93 / 4$ \\
$111 / 2$ \\
1
\end{tabular} & \begin{tabular}{|c|}
$71 / 2$ \\
$81 / 4$ \\
9 \\
$93 / 4$ \\
$111 / 4$
\end{tabular} & $\begin{array}{l}31 / 2 \\
33 / 4 \\
4114 \\
434 \\
4^{3} / 4\end{array}$ & $\begin{array}{lll}1^{7 / 8} & 1 \\
1 & 1 \\
1 & 1 & 1 \\
11 / 4 & 1\end{array}$ & \begin{tabular}{|c|}
$81 / 2$ \\
$101 / 2$ \\
$103 / 4$ \\
11 \\
$123 / 4$
\end{tabular} & \begin{tabular}{l|}
12 \\
15 \\
15 \\
15 \\
18
\end{tabular} & $\begin{array}{l}21 / 2 \\
23 / 4 \\
23 / 4 \\
2^{33} / 4 \\
3\end{array}$ & \begin{tabular}{l|}
3 \\
$31 / 4$ \\
$31 / 4$ \\
$31 / 4$ \\
$31 / 2$
\end{tabular} & $\begin{array}{l}1^{3 / 4} \\
1 \\
1 \\
11 / 4\end{array}$ & $\begin{array}{l}11 / 4 \\
13 / 8 \\
13 / 8 \\
13 / 8 \\
11 / 2\end{array}$ & $\begin{array}{l}13 / 4 \\
17 / 8 \\
17 / 8 \\
17 / 8 \\
2\end{array}$ & \begin{tabular}{|l|}
3 \\
$31 / 4$ \\
$31 / 2$ \\
$33 / 4$ \\
$41 / 4$ \\
\end{tabular} & \begin{tabular}{|}
$33 / 8$ \\
$33 / 4$ \\
$33 / 4$ \\
$41 / 8$ \\
$41 / 2$
\end{tabular} & \begin{tabular}{|l|}
$13 / 8$ \\
$11 / 2$ \\
$15 / 8$ \\
$13 / 4$ \\
2
\end{tabular} & & $\begin{array}{l}1 / 2 \\
1 / 2 \\
5 / 8 \\
5 / 8 \\
3 / 4\end{array}$ & \begin{tabular}{|}
$51 / 4$ \\
$5^{3} / 4$ \\
$61 / 4$ \\
633 \\
$7^{3} / 4$ \\
\end{tabular} & \begin{tabular}{l|l}
3 \\
3 \\
4 \\
4 \\
4
\end{tabular} & 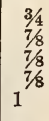 \\
\hline
\end{tabular}


AMERICAN PETROLEUM INSTITUTE, PIPE SPECIFICATIONS, STANDARD NO. 5-A (7TH ED.), JULY, 1931

\section{STEEL AND IRON PIPE FOR OIL COUNTRY TUBULAR GOODS}

\section{MATERIAL COVERED}

1. These specifications apply to lapwelded and seamless steel and iron tubular goods for oil country purposes; covering drill pipe, casing, and tubing. Where the term "pipe" is used in these specifications it is intended to cover all classes referred to.

Section I.-Manufacture

Process.-2. (a) The steel for casing and tubing shall be of good welding quality made by the Bessemer, open-hearth or electric process. The steel for lapwelded drill pipe shall be made by the open-hearth process, and the steel for all seamless drill pipe shall be made by the electric or openhearth process. Seamless drill pipe and its couplings shall be made of grade B or grade C steel. Upset end drill pipe to have upset of the diameters and lengths as indicated in tables appended.

(b) The wrought iron shall be made from muck bars made entirely from puddled pig iron, free from any admixture of iron scrap or steel. The term iron scrap applies only to foreign or bought scrap and does not include local mill products free from foreign or bought scrap.

(c) Open-hearth iron.

\section{Section II.-Chemical Properties and Tests}

3. (a) The steel shall conform to requirements as to chemical composition given in Table 1 .

(b) Open-hearth iron shall not contain a total impurity content of more than sixteen one-hundredths of 1 per cent considering the elements carbon, sulphur, phosphorus, silicon, copper, and manganese as impurities.

TABLE 1.-Chemical properties

\begin{tabular}{|c|c|c|c|c|c|c|c|}
\hline & \multirow[b]{2}{*}{$\begin{array}{l}\text { Bessemer } \\
\text { welded }\end{array}$} & \multicolumn{2}{|c|}{ Open-hearth welded } & \multicolumn{4}{|c|}{ Seamless } \\
\hline & & Class I & $\begin{array}{c}\text { Class II } \\
\begin{array}{c}\text { (rephosphor- } \\
\text { ized) } 1\end{array}\end{array}$ & Grade A & Grade B & Grade C & Grade D \\
\hline $\begin{array}{l}\text { Manganese } \\
\text { Phosphorus, not over. } \\
\text { Not less than }\end{array}$ & $\begin{array}{r}\text { Per cent } \\
0.30-0.60 \\
.11\end{array}$ & $\begin{array}{r}\text { Per cent } \\
0.30-0.60 \\
.045\end{array}$ & $\begin{array}{r}\text { Per cent } \\
0.30-0.60 \\
.08 \\
.045\end{array}$ & $\begin{array}{r}\text { Per cent } \\
0.30-0.60 \\
.04\end{array}$ & $\begin{array}{r}\text { Per cent } \\
0.35-1.50 \\
.04\end{array}$ & $\begin{array}{r}\text { Per cent } \\
0.35-1.50 \\
.04\end{array}$ & $\begin{array}{l}\text { Per cent } \\
\text { (2) } \\
0.04\end{array}$ \\
\hline Sulphur, not over & .065 & .06 & .06 & .06 & .06 & .06 & $.06^{-1}$ \\
\hline
\end{tabular}

1 Rephosphorized open-hearth pipe shall be marked by the letter " $\mathrm{R}$ " stamped with a steel die directly alongside the A. P. I. monogram, the size of the letter to be the same as the A. P. I. monogram.

2 Manufacturer's option.

It will be noted in the table under "Physical properties and tests" that rephosphorized open-hearth welded pipe has higher tensile strength and higher yield point than Class I open-hearth welded pipe, and as a consequence is probably somewhat harder to bend. It probably has better threading and lap-welding properties.

4. (a) Check analysis.-Analyses of two lengths of pipe in each lot of 400 or less of each size under 6 inches, and in each lot of 200 or less of each size 6 inches or over, may be made by the purchaser from the finished pipe. By agreement between the manufacturer and the purchaser, the analyses may be taken from the skelp in cases of lapwelded pipe. When taken from the skelp, the number of analyses per lot shall be determined in the same manner as when taken from the finished pipe, except in no case shall there be less than one analysis for each heat.

(b) If the analysis of only one length of pipe or piece of skelp does not conform to the requirements specified, analyses of two additional lengths of pipe or pieces of skelp from the same lot or heat shall be made, each of which shall conform to the requirements specified.

(c) Drillings for analyses shall be taken from several points around each length of pipe or across the pieces of skelp selected for test. (d) Ladle analyses on each heat of steel shall be furnished by the manufacturer if requested by the purchaser, and shall comply with the requirements of paragraph $3(a)$.

\section{Section III.-Physical Properties and Tests}

5. (a) Tension tests.- The material shall conform to the minimum requirements as to tensile properties given in Table 2.

(b) The yield point shall be determined by the drop of the beam of testing machine, by dividers, or any other approved methods.

5. (a) Hydrostatic tests.-Each length of oil country tubular goods, whether butt or lap welded, or seamless, shall be tested at the mill to the hydrostatic pressure specified in tables appended, which pressure shall be maintained for not less than five seconds. Butt or lap welded pipe shall be struck near both ends, while under pressure, a blow with a 2 -pound hammer or its equivalent.

TABLE 2.-Physical properties

\begin{tabular}{|c|c|c|c|c|c|c|c|c|c|}
\hline & \multicolumn{7}{|c|}{ Steel } & \multirow{3}{*}{$\begin{array}{l}\text { Wrought } \\
\text { iron }\end{array}$} & \multirow{3}{*}{$\begin{array}{l}\text { Open- } \\
\text { hearth } \\
\text { iron }\end{array}$} \\
\hline & \multirow[b]{2}{*}{$\begin{array}{l}\text { Bessemer } \\
\text { welded }\end{array}$} & \multicolumn{2}{|c|}{ Open-hearth welded } & \multicolumn{4}{|c|}{ Seamless } & & \\
\hline & & Class I & $\left|\begin{array}{c}\text { Class II } \\
\text { (rephos- } \\
\text { phorized) }\end{array}\right|$ & Grade A & Grade B & Grade C & Grade D & & \\
\hline 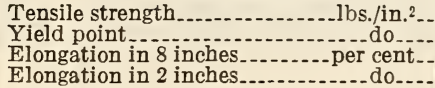 & $\begin{array}{r}50,000 \\
30,000 \\
20\end{array}$ & $\begin{array}{r}45,000 \\
25,000 \\
22\end{array}$ & $\begin{array}{r}48,000 \\
28,000 \\
20\end{array}$ & $\begin{array}{r}48,000 \\
30,000 \\
-40\end{array}$ & $\begin{array}{l}70,000 \\
40,000 \\
-25\end{array}$ & $\begin{array}{r}75,000 \\
45,000 \\
-20\end{array}$ & $\begin{array}{r}95,000 \\
55,000 \\
18\end{array}$ & $\begin{array}{r}42,000 \\
24,000 \\
12\end{array}$ & $\begin{array}{r}42,000 \\
24,000 \\
20\end{array}$ \\
\hline
\end{tabular}

Due to the necessity for placing carbon limits on line pipe for welding, it should be noted that the physical properties of grade B line pipe are slightly lower than for grade B material in A. P. I. standards No. 5-A. 
(b) The hydrostatic test pressure on sizes or weights not listed should not exceed those required by the formula:

$$
P=\frac{2 S t}{D}
$$

in which

$P=$ the pressure in pounds per square inch.

$S=$ allowable fiber stress, as follows:

\begin{tabular}{|c|c|}
\hline Grade & $\begin{array}{l}\text { Pounds per } \\
\text { square inch }\end{array}$ \\
\hline 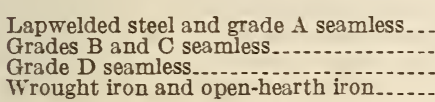 & $\begin{array}{l}14,000-16,000 \\
18,000-20,000 \\
24,000-26,000 \\
12,500-14,000\end{array}$ \\
\hline
\end{tabular}

For grade D seamless pipe in sizes up to and including 958 inches outside diameter, no test pressure need exceed 2,800 pounds per square inch, and for all other sizes and grades no test pressure need exceed 2,500 pounds per square inch.

$t=$ thickness of the wall in inches.

$D=$ outside diameter in inches.

7. (a) Flattening tests.-The crop end cut from each end of each length of steel or open-hearth iron lapwelded pipe shall be flattened between parallel plates, with the lap weld at the point of maximum bending, until opposite walls of the pipe meet. No opening in the weld shall take place until the distance between the plates is less than two-thirds of the original outside diameter of the pipe. No cracks or breaks in the metal elsewhere than in the lap weld shall occur until the distance between the plates is less than one-third of the original outside diameter of the pipe. Evidence of laminations or burnt material shall not develop during the entire flattening process. If any section fails to meet these requirements, another piece may be cut. If the second test fails, additional tests may be made until the requirements are met or the length rejected, except that the finished pipe shall not be shorter than 80 per cent of its length after the first crop was cut. Precautions shall be taken so that crop ends can be identified with respect to the length from which they are cut.

(b) The crop ends cut from each length of lapwelded wrought-iron pipe shall be flattened until broken. The test shall be made with the weld approximately $45^{\circ}$ from the point of maximum bend. The test piece must show a good lap weld and the fracture shall have a fibrous appearance. If any section fails to meet these requirements, another piece may be cut. If the second test should fail, additional tests may be made until the test meets the requirements, provided that the finished pipe shall not be shorter than 80 per cent of its length after the first crop has been made. Precautions shall be taken so that crop ends can be identified with respect to the length from which they are cut.

8. (a) Drift tests.-Each length of casing shall be drifted with cylindrical mandrels of the following dimensions:

\begin{tabular}{|c|c|c|}
\hline & Diameter & Length \\
\hline $\begin{array}{l}\text { For sizes up to } 85 / 8 \text { inches } \\
\text { outside diameter, inclu- } \\
\text { sive. }\end{array}$ & $\begin{array}{l}1 / 8 \text { inch smaller than nomi- } \\
\text { nal inside diameter of pipe. }\end{array}$ & $\begin{array}{l}\text { Inches } \\
6\end{array}$ \\
\hline $\begin{array}{l}\text { For sizes over } 85 / 8 \text { inches out- } \\
\text { side diameter to } 14 \text { inches } \\
\text { outside diameter, inclu- } \\
\text { sive. }\end{array}$ & $\begin{array}{l}5 / 32 \text { inch smaller than nomi- } \\
\text { nal inside diameter of pipe. }\end{array}$ & 12 \\
\hline $\begin{array}{l}\text { For sizes over } 14 \text { inches out- } \\
\text { side diameter. }\end{array}$ & $\begin{array}{l}3 / 16 \text { inch smaller than nomi- } \\
\text { nal inside diameter of pipe. }\end{array}$ & 12 \\
\hline
\end{tabular}

(b) All tubing up to and including $2 \frac{1}{2}$ inches shall be drifted to insure free passage of a plunger 42 inches long and three thirty-seconds of an inch smaller than tabulated inside diameter of tubing. On sizes larger than $2 \frac{1}{2}$ inches the plunger shall be one-eighth inch smaller than tabulated inside diameter of tubing.

9. (a) Test specimens.-Tension-test specimens shall consist of sections cut longitudinally from either end of the pipe (when not pulled in full sections, as would probably be the case in the smaller sizes of pipe). They shall not be flattened before testing. The specimens from welded pipe shall be taken from a point approximately $90^{\circ}$ from the weld, since this is to be a test of the material and not the weld. By agreement between the manufacturer and purchaser, the test specimens for welded pipe may be taken from the skelp.

(b) All testing shall be done cold.

10. Number of tests.- One of each of the tests specified in paragraph 5 (a) may be made on a length in each lot of 400 or less, of each size under 6 inches, and in each lot of 200 or less, of each size 6 inches or over. When taken from the skelp the number of tests shall be determined in the same manner as when taken from the finished pipe.

11 (a) Retests. - If the result of the physical tests of any lot or heat does not conform to the requirements specified in paragraph $5(a)$, retests of two additional lengths or pieces of skelp shall be made, each of which shall conform to the requirements specified.

(b) If any specimen shows defective machining and develops flaws, it may be discarded and another specimen substituted. If the elongation of any tension-test specimen is less than that specified and any part of the fracture is outside of the middle third of the gage length as indicated by scribe scratches marked on the specimen before testing, a retest shall be allowed.

\section{Section IV.-Standard Weights and Lengths}

12. (a) The standard weights for oil country tubular material of A. P. I. sizes are given in tables appended.

(b) Permissible variations.-The weight of any length of pipe shall not vary more than $31 / 2$ per cent under or $6 \frac{1}{2}$ per cent over that specified; but the carload weight shall not be more than $13 / 4$ per cent 72 under the nominal weight. On upset pipe the nominal weight is based on 20-foot length and due allowance must be made for longer lengths when same are furnished. (The mills will prepare tables for the guidance of inspectors.) Each joint shall be weighed separately with couplings screwed on, but with thread protector removed. In arriving at carload weight proper allowances are to be made for thread protectors.

13. (a) Lengths.-Unless otherwise specified pipe shall be furnished as to minimum lengths in accordance with the following:

(b) Tubing and casing shall have an average length of not less than 20 feet for every carload shipped.

(c) On tubing, not over 5 per cent of any carload shall be under 20 feet in length, with a minimum of 18 feet for the length of any joint.

(d) On casing, not over 5 per cent of any carload shall be under 18 feet in length, with a minimum of 16 feet for the length of any joint. (See footnote.)

72 The weight of 1 cubic inch of steel is 0.2833 pound and that of wrought iron is 0.2778 pound, therefore a length of wrought-iron pipe made to full dimensions for thickness and diameter will weight approximately 2 per cent less than a similar length of pipe made from steel, and due allowance should be made in the "weights per from steel, and due allowance should be made in the "weights per for wrought-iron pipe shall not be more than 312 per cent under the nominal weight. 
(e) Drill pipe shall be manufactured in two average lengths, of 20 to 22 feet or of 27 to 30 feet. On the 20 to 22 foot average length, not more than 5 per cent of any carload shall be less than 20 feet in length with a minimum of 18 feet and no single joint shall exceed 22 feet. On the 27 to 30 foot average length, not more than 5 per cent of any carload shall be under 27 feet with a minimum of 26 feet, and no single joint shall exceed 30 feet.

$(f)$ Jointers (two short lengths: connected by couplings), the minimum length of the shorter piece to be 5 feet, may be shipped to a maximum of 5 per cent of the order for casing. No jointers shall be shipped on tubing or rotary drill pipe orders.

Note.-It is the sense of this committee that within a reasonable time the specifications as to the lengths of joints will be changed to give longer average lengths on all joints, and a longer minimum length of any joint of tubular goods, included in this specification; that great advantages could be effected for the consumer as well as the manufacturer if the lengths of tubular goods used in the oil business averaged 30 feet; and that due consideration should be given this recommendation by the pipe mills, as they have opportunities to make changes in their furnaces.

\section{SECTION V. WORKMANSHIP AND FINISH}

14. (a) All skelp entering into welded oil field tubular goods, whether casing, tubing, or drill pipe, must be scarfed.

(b) For pipe $1 \frac{1}{2}$ inches or under in nominal inside diameter, the outside diameter at any point shall not vary more than one sixty-fourth inch over nor more than one thirty-second inch under the standard size. For pipe 2 inches or over in nominal inside diameter, the outside diameter shall not vary more than 1 per cent over or under the standard size. The wall thickness of welded and seamless pipe shall not be more than $12 \frac{1}{2}$ per cent under the nominal wall thickness at any point. The thickness shall be determined from measurements on the body of the pipe, and the outside diameter of that portion of the pipe used for threading must be governed by the closer tolerances called for by the threading specifications.

(c) The finished pipe shall be reasonably straight and free from injurious defects, such as burnt material, bad welds, sand pits, ball cuts, pits, cinder spots, liquor marks, blisters, slivers, and laminations. Seamless pipe shall also be free from injurious seams. When the depth of sand pits, liquor marks, and cinder spots is in excess of $12 \frac{1}{2}$ per cent of the nominal wall thickness they shall be considered injurious defects; welding or patching of such defects will not be permitted. The threading on the pipe must be free from tears, shoulders, cuts, or any defects which may break the continuity of the thread.

(d) The inside diameter of the upset ends on drill pipe shall not vary more than $\pm 1 / 16$ inch from the dimensions specified in Table 9 .

15. (a) Ends.-Unless otherwise specified, pipe shall conform to the following regular practice:

(b) Each end of pipe shall be threaded and reamed to remove all burrs.

(c) The inside ends of casing and tubing, and the outside and inside ends of couplings therefor, shall be chamfered sufficiently to produce a round corner to prevent pipe or tools from hanging up in the hole. (See figs. 280, 283, and 284.) This chamfer shall not materially reduce dimension " $\mathrm{R}$," width of bearing face on couplings.

16. Threads and gages. - The threads shall be in accordance with the American standard (as illustrated in thread and gage tables appended), and cut so as to make a tight joint when the pipe is tested at the mill to the specified internal hydrostatic pressure. The variation from the standard, when tested with the standard working gage, shall not exceed a maximum of one turn either way on 8 thread, $3 / 4$-inch taper, and one and one-half turns either way on 10 thread and $11 \frac{1}{2}$ thread pipe. For thread diameters of A. P. I. tubular material, see thread tables appended.

$(a)$. The crests and roots of all drill pipe threads shall be rounded, the radius at the crests being slightly greater than that at the root, to insure full flank contact, as illustrated and specified in Figure 282 .

17. Threads in couplings and on casing and pipe shall be so nearly alike in form and size, and so well finished, that joints can be screwed together with suitable lubricant, far enough to make a tight joint, and unscrewed four times without injury to the threads. At the discretion of inspector, where the purchaser is represented by an inspector, at least one joint may be so tested out of a lot of 100 or more after joints have been made up at coupling screwing machine.

18. Pipe shall not be rounded out by hammering in order to get a full thread. There shall not be more than two black threads for $3 / 4$-inch taper nor more than five black threads for $3 / 8$-inch taper among the perfect threads. (See dimension "E" in various A. P. I. thread tables.) Black threads should not be confused with imperfect threads such as torn, shaven, or broken threads.

19. Each manufacturer of A. P. I. tubular threaded material shall provide himself with certified plug and ring reference gages which have been manufactured in accordance with A. P. I. gage stipulations. (See 615.82, p. 894.)

20. Gaging shall be done to conform with A. P. I. gaging practice.

21. (a) Taper tests. - The taper of threads on both pipe and couplings shall be tested by approved methods at intervals sufficient to insure that taps and dies are in proper adjustment.

(b) The variations in taper shall not exceed the following limits:

\begin{tabular}{|c|c|c|c|}
\hline & \multicolumn{3}{|c|}{ When taper ${ }^{1}$ per foot is specified as- } \\
\hline & $3 / 16$ inch & $38 \mathrm{inch}$ & 3/4 inch \\
\hline $\begin{array}{l}\text { Maximum taper } \\
\text { Minimum taper_.......... }\end{array}$ & 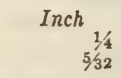 & $\begin{array}{l}\text { Inch } \\
7 / 16 \\
11 / 32\end{array}$ & $\begin{array}{l}\text { Inch }_{13 / 10} \\
23 / 32\end{array}$ \\
\hline
\end{tabular}

1"Taper" as used herein means "total," or measured on the diameter.

22. (a) Thread protectors.-Solid tapped rings or the equivalent, designed to cover the full thread length of all casing, drill pipe, and tubing 2 inches and larger shall be provided. They must prevent water and dirt from accumulating around the thread and must protect the thread and end of pipe from damage during handling, transportation, and storage. On sizes under 2 inches for export shipment the ends shall be protected by suitable fabric wrappings.

(b) Threaded nipples or the equivalent to cover the total pipe thread length of all couplings for casing, drill pipe, and tubing shall be provided. They must prevent water and dirt from accumulating around the threads and must protect the threads from damage during handling, transportation, and storage.

23. Couplings.-Each length of threaded pipe shall be provided with one coupling having clean-cut threads of such a pitch diameter as to make a tight joint. Couplings may be made of wrought iron or steel, seamless or welded, and shall be free from blisters, pits, cinder marks, and any other injurious defects that would impair the efficiency of the coupling and break the continuity of the thread. When couplings are made of steel, they shall be 
electroplated on the threads, heat treated or treated by some other method of manufacture which will minimize the chance of galling.

(a) The ends of couplings to be faced true at right angles to the axis and outside beveled so as to prevent catching on the ends of the joints of pipe through which they pass, but enough thickness should be left at the end of coupling to safely support the weight of the pipe on the elevator.

24. No tapers of couplings to be secured by expanding.

25. When couplings are screwed on at the mill, a high-grade lubricant shall be applied to coupling and pipe threads before making up the joint; and to prevent rust all exposed threads shall be thoroughly greased.

26. Alignment test.-The couplings shall be tapped concentric so that the axes of the threads of the two ends are closely in line. Couplings shall be tested for alignment by screwing onto a threaded piece carefully centered in a lathe; in the outer end of coupling is screwed a lathe-turned piece which will give a measured length of about 1 foot on which the alignment of the coupling threads may be determined by an alignment indicator when rotated in the lathe. Other approved means of measuring alignment may be used. Couplings shall be in line within three-fourths inch in a length of 20 feet on all tubular goods except drill pipe. Couplings on drill pipe shall be in line within one-half inch in a length of 20 feet. At least one coupling may be

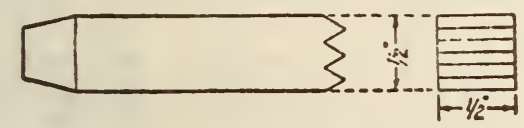

FIgURe 278.-Canceling die

taken from each lot of 100 or less, but this shall be left to the discretion of the inspector, where the purchaser is represented by an inspector. In case this coupling fails, two more couplings may be taken from the same lot, each of which must be within the alignment specified, otherwise the lot shall be rejected. In any case the manufacturer may test rejected couplings individually if desired.

27. (a) Marking.-The length, the test pressure in pounds, and class of material shall be stenciled on each length of pipe; and on sizes 6 inches and larger, except dipped or coated pipe, the weight of each length shall be stenciled. Approved abbreviations may be used. Weight per foot and A. P. I. monogram to be stamped with steel dies on each joint 1 foot from coupling on the shop end. The A. P. I. monogram shall also be stamped with a steel die on all couplings, the size of the monogram to be one-fourth inch high on all sizes of pipe, threeeighths inch high on couplings for pipe sizes from 2 to $6 \frac{5}{8}$ inches, and one-half inch high on couplings for pipe sizes over $65 / 8$ inches. (The upper and lower crossbar on the letter "I" in the A. P. I. monogram may be omitted when applied to pipe. See Appendix "E".) In addition to the above markings and in letters of similar size, the grades A, B, C, and D of seamless pipe shall be stamped with a steel die.

(b) All pipe manufactured in accordance with these specifications shall be marked, by stamping or rolling, with the manufacturer's private identifying mark. The manufacturer's mark or monogram should be stamped within 3 inches of the thread on the coupling end; the size of the mark or monogram to be in conformity with the A. P. I. specifications for marking.

(c) Any manufacturer authorized to use the A. P. I. monogram on his product shall forfeit this right if the monogram is not used on material manufactured or sold in accordance with these specifications within a period of 12 months after such authority has been granted. It will be necessary for the manufacturer to make another application in order to be reinstated.

28. An item of equipment (such as drilling valves, casing heads, etc.) for which no specifications are given herein, but which carries a standard A. P. I. thread, may be marked with the letters "A. P. I.", the size, the manufacturer's name or mark, and the word "threads." For example, an item with a 53/4inch casing ${ }^{73}$ thread could be marked as follows:

$$
\text { (or mark) } \quad 5 \frac{3}{4} \text { A. P. I. csg. thds. }
$$

When so marked, the threads on such equipment must be manufactured and gaged in accordance with the A. P. I. thread specifications thereon and the manufacturer must have in his possession duly certified gages. ${ }^{74}$

The use of the A. P. I. monogram on such articles is expressly prohibited.

\section{Section VI-Inspection and Rejection}

29. (a) Inspection.-The inspector representing the purchaser shall have free entry at all times while work on the contract of the purchaser is being performed, to all parts of the manufacturer's works which will concern the manufacture of the pipe ordered. The manufacturer shall afford the inspector, free of charge, all reasonable facilities to satisfy him that the pipe is being furnished in accordance with these specifications. All tests and inspection shall be made at the place of manufacture prior to shipment, unless otherwise specified, and shall be so conducted as not to interfere unnecessarily with the operation of the works.

(b) Where inspector representing purchaser desires to witness these tests, reasonable notice shall be given of the time at which the run is to be made.

30. Rejection.-Material which shows injurious defects subsequent to acceptance at manufacturer's works, or which proves defective when properly applied in service, may be rejected and the manufacturer shall be notified. No rejections, under these or any other specifications, are to be stamped with the A. P. I. monogram or sold as A. P. I. pipe, except where said pipe fails to comply with the weight specification alone, in which case it may be sold under a weight specification with which it does comply. When pipe stamped with the A. P. I. monogram is rejected, a canceling die, one-half inch square, per the following figure, shall be applied to such monogram in the following manner: The canceling die shall be applied twice, first with the lines of the die parallel to the axis of the pipe, the die then turned $90^{\circ}$, and applied the second time. 73 When threaded for upset casing, mark "Up. Csg." When
threaded for drill pipe, mark "D. P." When threaded for plain tubing, mark "Tbg." When threaded for upset tubing, mark "Up. Tbg."

${ }_{74}$ It is not necessary that authority to use the A. P. I. monogram be procured to purchase such gages, but the purchaser of such gages be procured to purchase such gages, but the purchaser of such gages
is subject to all the stipulations on certification and retest as given is subject to all the stipulations
in the governing specifications. 
TABLE 3.-A. P. I. standard casing

["Size" of A. P. I. casing indicates outside diameter. All dimensions in inches at $68^{\circ}$ F.]

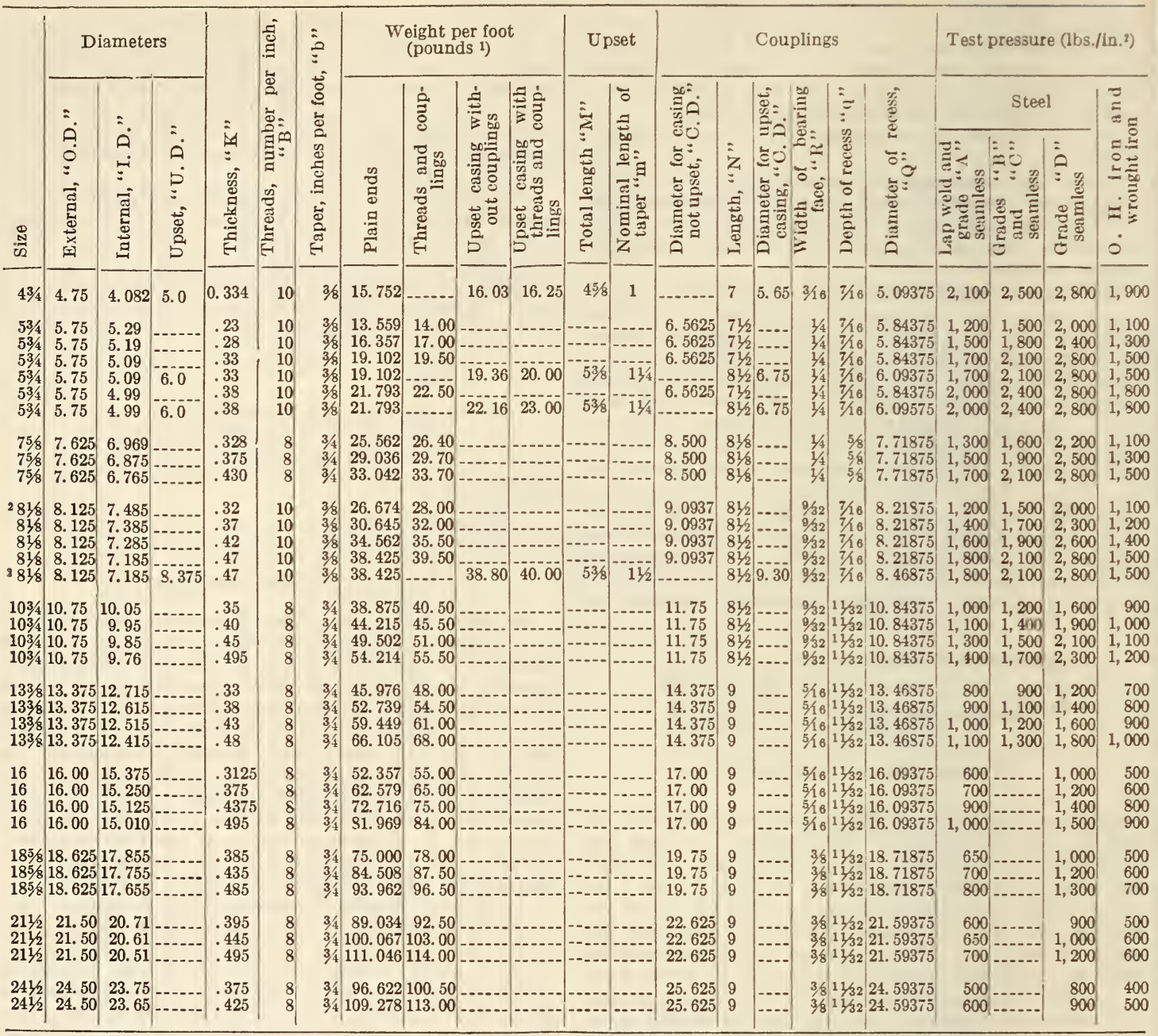

1 See par. 12. (b).

8 This size inactive; arailable on special order only.

See notes to Table 3, following on p. 449. 
TABLE 4.-A. P. I. supplementary standard casing

[Special sizes selected from those now in use and added on request of certain districts]

["Size" of A. P. I. casing indicates outside diameter. All dimensions in inches at $68^{\circ} \mathrm{F}$.]

\begin{tabular}{|c|c|c|c|c|c|c|c|c|c|c|c|c|c|c|c|c|c|c|c|c|}
\hline \multirow[b]{3}{*}{$\frac{\pi}{\pi}$} & \multicolumn{3}{|c|}{ Diameters } & \multirow[b]{3}{*}{ 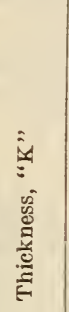 } & \multirow{3}{*}{ 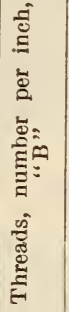 } & \multirow{3}{*}{ 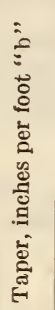 } & \multicolumn{4}{|c|}{$\begin{array}{l}\text { Weight per foot } \\
\text { (pounds 1) }\end{array}$} & \multicolumn{6}{|c|}{ Couplings } & \multicolumn{4}{|c|}{ Test pressure (lbs./in. $\left.{ }^{2}\right)$} \\
\hline & \multirow[b]{2}{*}{ 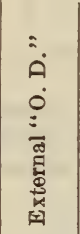 } & \multirow[b]{2}{*}{ 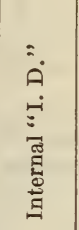 } & \multirow[b]{2}{*}{$\begin{array}{l}a \\
\dot{\theta} \\
\dot{0} \\
\vdots \\
\dot{0} \\
0 \\
0 \\
0\end{array}$} & & & & & $\stackrel{2}{3}$ & 空 & 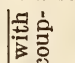 & 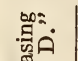 & & 诘: & $\stackrel{\infty}{E}$ & $\begin{array}{l}\approx \\
=\end{array}$ & ఫ్రి & & Steel & & \\
\hline & & & & & & & 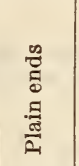 & 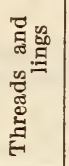 & 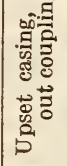 & 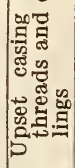 & 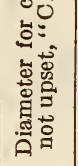 & 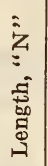 & 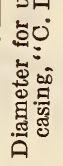 & 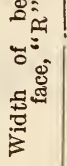 & 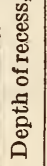 & 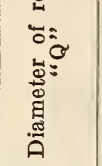 & 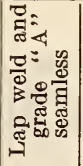 & 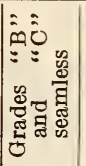 & 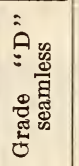 & 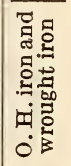 \\
\hline $43 / 4$ & 4. 75 & 4.082 & & 0.334 & 10 & $3 / 8$ & 15.752 & 16.00 & & & 5.364 & $65 \%$ & & $3 / 16$ & $5 / 8$ & 4. 84375 & 2,100 & 2,500 & 2,800 & 1,900 \\
\hline $65 \%$ & 6. 625 & 6.049 & & .288 & 10 & $3 / 8$ & 19.491 & 20.00 & & & 7. 390 & $75 / 8$ & & $1 / 4$ & $5 / 8$ & 6. 71875 & 1,500 & 1,600 & 2,200 & 1,200 \\
\hline 658 & 6.625 & 5. 921 & & .352 & 10 & $3 / 8$ & 23. 582 & 24.00 & & & 7. 390 & $75 \%$ & & $1 / 4$ & $5 / 8$ & 6. 71875 & 1,600 & 2,000 & 2,700 & 1,400 \\
\hline 658 & 6. 625 & 5.855 & & .385 & 10 & $3 / 8$ & 25.658 & 26.00 & & & 7. 390 & $75 \%$ & & $1 / 4$ & 5,8 & 6. 71875 & 1,700 & 2,100 & 2,800 & 1,500 \\
\hline $65 \%$ & 6.625 & 5. 791 & & .417 & 10 & $3 / 8$ & 27.648 & 28.00 & & & 7.390 & $75 / 8$ & & $1 / 4$ & $5 / 8$ & 6.71875 & 1,900 & 2,300 & 2,800 & 1,700 \\
\hline 7 & 7.000 & 6. 456 & & .272 & 10 & $3 / 8$ & 19.544 & 20.00 & & & 7. 656 & 758 & & $3 / 16$ & $5 / 8$ & 7.09 & 1,200 & 1,400 & 1,900 & 1,000 \\
\hline 7 & 7.000 & 6. 398 & & .301 & 10 & $3 / 8$ & 21. 535 & 22.00 & & & 7.656 & $75 \%$ & & $3 / 16$ & $5 / 8$ & 7. 09375 & 1,300 & 1,600 & 2,200 & 1,100 \\
\hline 7 & 7.000 & 6. 336 & & .332 & 10 & 38 & 23.643 & 24.00 & & & 7.656 & $75 / 8$ & & $3 / 16$ & $5 / 8$ & 7.09375 & 1,400 & 1,800 & 2,400 & 1,300 \\
\hline 7 & 7.000 & 6.276 & & .362 & 10 & $3 / 8$ & 25.663 & 26.00 & & & 7.656 & $75 \%$ & & $3 / 16$ & $5 / 8$ & 7. 09375 & 1,600 & 1,900 & 2,600 & 1,400 \\
\hline 7 & 7.000 & 6.214 & & .393 & 10 & 3,8 & 27. 731 & 28.00 & & & 7. 656 & $75 \%$ & & $3 / 16$ & $5 / 8$ & 7.09375 & 1,700 & 2,100 & 2,800 & 1,50 \\
\hline 7 & 7.000 & 6.154 & & .423 & 10 & $3 / 8$ & 29.712 & 30.00 & & & 7.656 & $75 \%$ & & 36 & 56 & 7.09375 & 1,800 & 2,200 & 2,800 & 1,600 \\
\hline $85 \%$ & 8.625 & 8.017 & & .304 & 8 & $3 / 4$ & 27.016 & 28.00 & & & 9.593 & $81 / 8$ & & $9 / 32$ & $5 / 8$ & 8. 71 & 1,100 & 1,300 & 1,800 & 1,000 \\
\hline 858 & 8.625 & 7.921 & & .352 & 8 & $3 / 4$ & 31. 101 & 32.00 & & & 9.593 & $81 / 8$ & & $9 / 32$ & $5 / 8$ & 8. 71875 & 1,300 & 1,500 & 2,000 & 1,100 \\
\hline $85 \%$ & 8.625 & 7.825 & & .400 & 8 & $3 / 4$ & 35.137 & 36.00 & & & 9.593 & 818 & & $9 / 32$ & $5 / 8$ & 8.71875 & 1,400 & 1,700 & 2,300 & 1,200 \\
\hline $85 \%$ & 8. 625 & 7.775 & & .425 & 8 & $3 / 4$ & 37.220 & 38.00 & & & 9.593 & 818 & & $9 / 32$ & $5 / 8$ & 75 & 1,500 & 1,800 & 2,500 & 1,300 \\
\hline $85 \%$ & 8.625 & 7. 651 & & .487 & 8 & $3 / 4$ & 42.327 & 43.00 & & & 9.593 & 816 & & $9 / 3$ & & 1875 & 1,700 & 2,100 & 2,800 & 1,500 \\
\hline 9 & 9.000 & 8.290 & & .355 & 8 & $3 / 4$ & 32.777 & 34.00 & & & 10.010 & $81 / 8$ & & $9 / 32$ & $5 / 8$ & 9.09375 & 1,200 & 1,500 & 2,000 & 1,100 \\
\hline 9 & 9.000 & 8. 196 & & .402 & 8 & $3 / 4$ & 36.914 & 38.00 & & & 10.010 & $81 / 8$ & & $9 / 32$ & 5,8 & 9.09375 & 1,300 & 1,700 & 2,200 & 1,200 \\
\hline 9 & 9.000 & 8.150 & & .425 & 8 & 34 & 38.922 & 40.00 & & & 10.010 & 818 & & $9 / 32$ & 5,8 & 9.09375 & 1,400 & 1,700 & 2,400 & 1,200 \\
\hline 9 & 9.000 & 8.032 & & .484 & 8 & 31 & 44.020 & 45.00 & & & 10.010 & 818 & & $9 / 32$ & 56 & 9.09375 & 1,600 & 2,000 & 2,700 & 1,400 \\
\hline 958 & 9.625 & 8.921 & & .352 & 8 & $3 / 4$ & 34.861 & 36.00 & & & 10.625 & $81 / 8$ & & $9 / 32$ & $5 / 8$ & 9.71875 & 1,100 & 1,400 & 1,800 & 1,000 \\
\hline 958 & 9.625 & 8.835 & & .395 & 8 & 34 & 38.938 & 40.00 & & & 10.625 & 818 & & $9 / 32$ & $5 / 8$ & 9.71875 & 1,200 & 1,600 & 2,100 & 1,100 \\
\hline 958 & 9.625 & 8.755 & & .435 & 8 & $3 / 4$ & 42.695 & 43.50 & & & 10.625 & $81 / 8$ & & 9,32 & 56 & 9.71875 & 1,400 & 1,700 & 2,300 & 1,200 \\
\hline $113 / 4$ & 11. 75 & 11.000 & & .375 & 8 & $3 / 4$ & 45. 557 & 47.00 & & & 12.866 & $81 / 8$ & & $9 / 32$ & $5 / 8$ & 11.84375 & 1,000 & 1,200 & 1,600 & 800 \\
\hline & 11. 75 & 10.880 & & . 435 & 8 & $3 / 4$ & 52.568 & 54.00 & & & 12.866 & 818 & & $9 / 32$ & $9 / 8$ & 11.84375 & 1,100 & & & 1,000 \\
\hline & 11.75 & 10.772 & & .489 & 8 & $3 / 4$ & 58.811 & 60.00 & & & 12.866 & 81,8 & & $9 / 32$ & $5 / 8$ & 11.84375 & 1,200 & 1,500 & 2,100 & 1,100 \\
\hline
\end{tabular}

1 See par. 12. (b).

See additional notes to Table 4 below.

NOTE.-The sizes given in Table 4 have been added from time to time on requests from various distiicts and were selected from sizes now in use and size changed to outside diameter. No attempt was made to change the thr eads or couplings (except as to thread classification) and the joints and threads are identical with diamond BX casing for (10 thread) and diamond B8 (for 8 thread). Complete information on threads, gages, etc., is contained herein.

\section{A. P. I. Standard Casing}

NOTES TO TABLES 3 AND 4

The column headed "Depth in feet for compression factor of safety of 2 for lap-weld steel" in the fourth edition has been eliminated and will be incorporated in greater detail in the A. P. I. Code on the Proper Care and Use of Oil Country Tubular Goods.

When joints are screwed tight the ends of casing shall be fiveeighths inch from center of coupling and, when in this position, the outside diameter of thread in end of coupling to be that of the nominal outside diameter of casing. Beyond the threads at the end of coupling there shall be 2 threads perfect at the bottom on 8 -thread and 3 threads on 10-thread casing. A slight variation from this provision is permitted in the supplementary sizes given in Table 4, as follows:

\begin{tabular}{|c|c|c|c|}
\hline Sizes- & $\begin{array}{l}\text { Number } \\
\text { of threads } \\
\text { per inch }\end{array}$ & $\begin{array}{c}\text { Distance } \\
\text { end of } \\
\text { pipe from } \\
\text { center of } \\
\text { coupling }\end{array}$ & $\begin{array}{l}\text { Number } \\
\text { of threads } \\
\text { perfect at } \\
\text { bottom } \\
\text { exposed } \\
\text { beyond } \\
\text { the } \\
\text { threads } \\
\text { in the } \\
\text { coupling }\end{array}$ \\
\hline 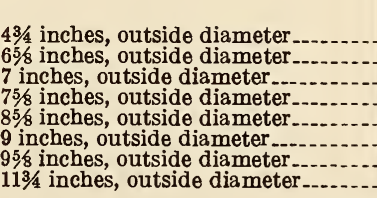 & $\begin{array}{r}10 \\
10 \\
10 \\
8 \\
8 \\
8 \\
8 \\
8\end{array}$ & $\begin{array}{l}\text { Inch } \\
0.5625 \\
.6125 \\
.6125 \\
.6875 \\
.6875 \\
.6875 \\
.6875 \\
.6875\end{array}$ & $\begin{array}{l}2 \\
2 \\
2 \\
3 \\
3 \\
3 \\
3 \\
3\end{array}$ \\
\hline
\end{tabular}

Bevel on coupling recess to be $35^{\circ}$ to line parallel to axis of casing. 


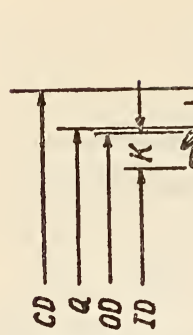

3 Yanishing Threads 2 Threads Impersect as Top, perfect at bolfom.

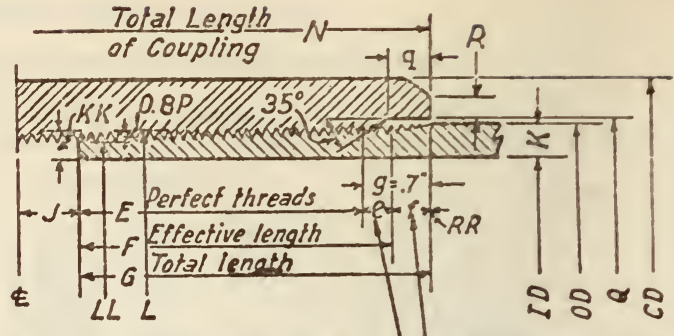

10-Threads per Inch. Taper $1 / 8^{\prime \prime}$ pes foot.

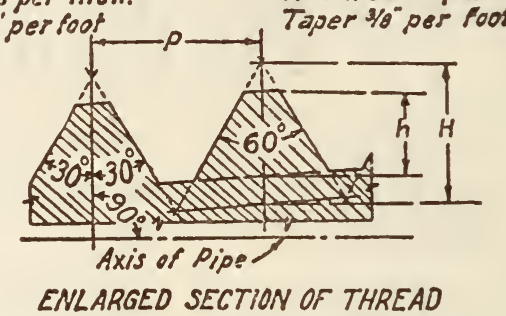

8-Threads per inch.

Taper $3 / 4$ " perfoot

Figure 279.-A. P. I. standard casing

See Figure 280 for chamfer on ends of coupling and pipe.

TABLE 5.-A. P. I. standard casing threads (See fig. 279)

[All dimensions in inches at $68^{\circ} \mathrm{F}$. All weights in pounds]

\begin{tabular}{|c|c|c|c|c|c|c|c|c|c|c|c|c|c|c|}
\hline \multicolumn{3}{|c|}{ Casing } & \multicolumn{7}{|c|}{ Threads } & \multirow{2}{*}{$\begin{array}{l}\text { Diam- } \\
\text { eter of } \\
\text { casing at } \\
\text { root of } \\
\text { threads, } \\
\text { "LL" }\end{array}$} & \multirow[b]{2}{*}{$\begin{array}{l}\text { Diam- } \\
\text { eter of } \\
\text { casing } \\
\text { at top of } \\
\text { threads, } \\
\text { "L" }\end{array}$} & \multirow[b]{2}{*}{$\begin{array}{l}\text { End of } \\
\text { pipe to } \\
\text { center of } \\
\text { coupling, } \\
\text { made up, } \\
\text { "J" " }\end{array}$} & \multirow{2}{*}{$\begin{array}{c}\text { Distance } \\
\text { end of } \\
\text { coupling } \\
\text { beyond } \\
\text { last } \\
\text { scratch, } \\
\text { made up, } \\
\text { "R. R." }\end{array}$} & \multirow[b]{2}{*}{$\begin{array}{l}\text { Stand- } \\
\text { off of } \\
\text { coupling } \\
\text { at hand- } \\
\text { tight fit, } \\
\text { "A"s" }\end{array}$} \\
\hline Size & $\begin{array}{c}\text { External } \\
\text { diam- } \\
\text { eter, } \\
\text { "O. D." }\end{array}$ & $\begin{array}{l}\text { Weight } \\
\text { per foot, } \\
\text { com- } \\
\text { plete }\end{array}$ & $\begin{array}{l}\text { Num- } \\
\text { ber per } \\
\text { inch, } \\
\text { "B" }\end{array}$ & $\begin{array}{c}\text { In- } \\
\text { cluded } \\
\text { taper, } \\
\text { inches } \\
\text { per foot, } \\
\text { "b" }\end{array}$ & $\begin{array}{l}\text { Length } \\
\text { of } \\
\text { perfect, } \\
\text { "E" }\end{array}$ & $\begin{array}{l}\text { Num- } \\
\text { ber of } \\
\text { perfect }\end{array}$ & $\begin{array}{c}\text { Effec- } \\
\text { tive } \\
\text { length, } \\
\text { "F" }\end{array}$ & $\begin{array}{l}\text { Total } \\
\text { length, } \\
\text { "G" }\end{array}$ & $\begin{array}{l}\text { Height } \\
\text { of } \\
\text { thread } \\
\text { trun- } \\
\text { cated, } \\
\text { "h" }\end{array}$ & & & & & \\
\hline $\begin{array}{l}53 / 4 \\
75 / 8 \\
881 / 8 \\
103 / 4 \\
133 / 8 \\
16 \\
185 / 8 \\
211 / 2 \\
241 / 2\end{array}$ & $\begin{array}{r}5.750 \\
7.625 \\
8.125 \\
10.750 \\
13.375 \\
16.000 \\
18.625 \\
21.500 \\
24.500\end{array}$ & $\begin{array}{l}\text { All. } \\
\text { All. } \\
\text { All. } \\
\text { All. } \\
\text { All. } \\
\text { All. } \\
\text { All. } \\
\text { All. } \\
\text { All. }\end{array}$ & $\begin{array}{r}10 \\
8 \\
10 \\
8 \\
8 \\
8 \\
8 \\
8 \\
8\end{array}$ & $\begin{array}{l}3 / 8 \\
3 / 4 \\
3 / 8 \\
3 / 4 \\
3 / 4 \\
3 / 4 \\
3 / 4 \\
3 / 4 \\
3 / 4\end{array}$ & $\begin{array}{l}2.4250 \\
2.6250 \\
2.9250 \\
3.0000 \\
\text { 3. } 2500 \\
\text { 3. } 2500 \\
\text { 3. } 2500 \\
3.2500 \\
\text { 3. } 2500\end{array}$ & $\begin{array}{l}24.25 \\
21.00 \\
29.25 \\
24.00 \\
26.00 \\
26.00 \\
26.00 \\
26.00 \\
26.00\end{array}$ & $\begin{array}{l}2.725 \\
2.875 \\
3.225 \\
3.250 \\
3.500 \\
3.500 \\
3.500 \\
3.500 \\
3.500\end{array}$ & $\begin{array}{l}3.125 \\
3.250 \\
3.625 \\
3.625 \\
3.875 \\
3.875 \\
3.875 \\
3.875 \\
3.875\end{array}$ & $\begin{array}{r}0.0800 \\
.1000 \\
.0800 \\
.1000 \\
.1000 \\
.1000 \\
.1000 \\
.1000 \\
.1000\end{array}$ & $\begin{array}{r}5.514 \\
7.261 \\
7.874 \\
10.363 \\
12.972 \\
15.597 \\
18.222 \\
21.097 \\
24.097\end{array}$ & $\begin{array}{r}5.674 \\
7.461 \\
8.034 \\
10.563 \\
13.172 \\
15.797 \\
18.422 \\
21.297 \\
24.297\end{array}$ & $\begin{array}{l}0.625 \\
.6875 \\
.625 \\
.625 \\
.625 \\
.625 \\
.625 \\
.625 \\
.625\end{array}$ & $\begin{array}{l}0.000 \\
.125 \\
.000 \\
.000 \\
.000 \\
.000 \\
.000 \\
.000 \\
.000\end{array}$ & 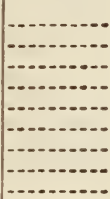 \\
\hline
\end{tabular}

1 Column " $A$ " to be filled in at some future date, to be based on actual experience. 2 This size inactive; available on special order only. 


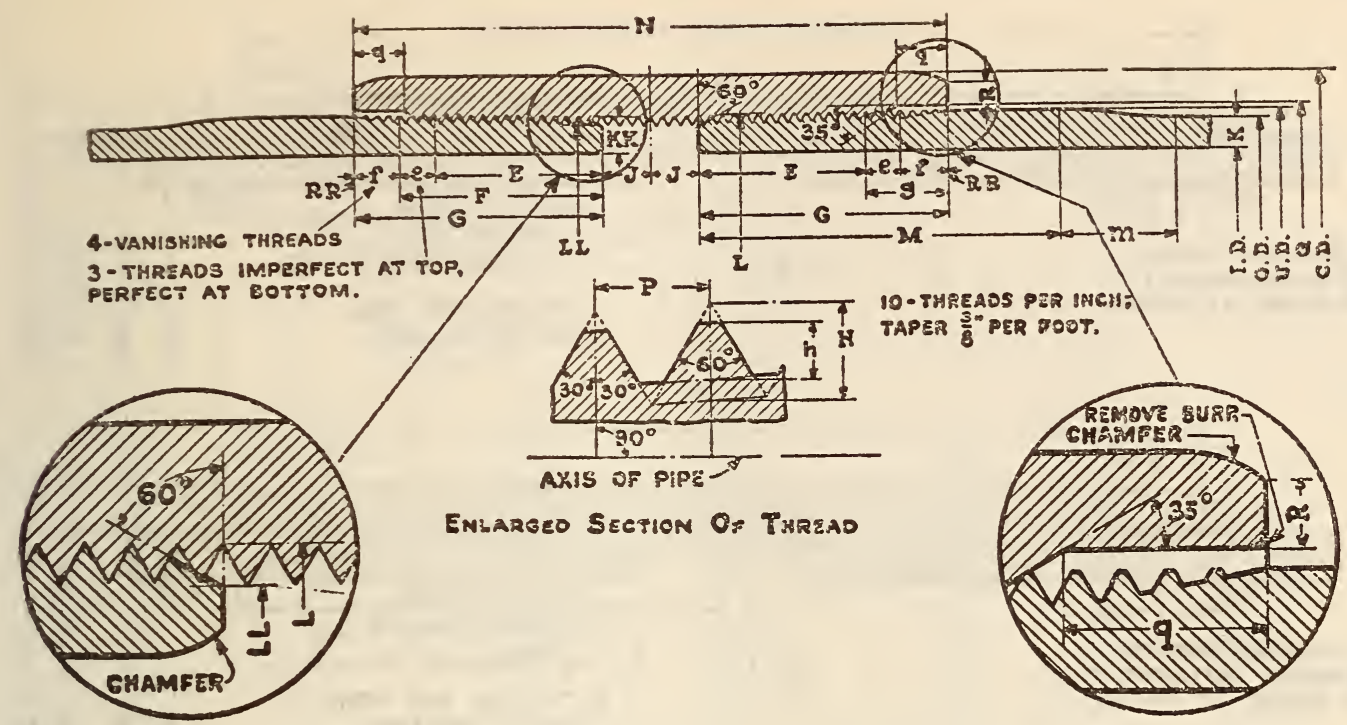

FIGURE 280.-A. P. I. standard casings, external upset

TABLE 6.-A. P. I. standard external upset casing thread

(See fig. 280)

[All dimensions in inches at $68^{\circ} \mathrm{F}$. All weights in pounds]

\begin{tabular}{|c|c|c|c|c|c|c|c|c|c|c|c|c|c|c|c|}
\hline \multicolumn{4}{|c|}{ Casing } & \multicolumn{7}{|c|}{ Threads } & \multirow{2}{*}{$\begin{array}{l}\text { Diam- } \\
\text { eter of } \\
\text { casing } \\
\text { at root } \\
\text { of } \\
\text { threads, } \\
\text { "LL" }\end{array}$} & \multirow{2}{*}{$\begin{array}{l}\text { Diam- } \\
\text { eter of } \\
\text { casing } \\
\text { at top } \\
\text { of } \\
\text { threads, } \\
\text { "L" }\end{array}$} & \multirow{2}{*}{$\begin{array}{c}\text { End of } \\
\text { pipe to } \\
\text { center } \\
\text { of cou- } \\
\text { pling, } \\
\text { made up, }\end{array}$} & \multirow{2}{*}{$\begin{array}{c}\text { Distance } \\
\text { end of } \\
\text { coupling } \\
\text { beyond } \\
\text { last } \\
\text { scratch, } \\
\text { made } \\
\text { up, } \\
\text { "R. R." }\end{array}$} & \multirow{2}{*}{$\begin{array}{l}\text { Stand. } \\
\text { off of } \\
\text { coupling } \\
\text { at hand } \\
\text { tight } \\
\text { fit, } \\
\text { "A" t }\end{array}$} \\
\hline Size & $\begin{array}{c}\text { Ex- } \\
\text { ternal } \\
\text { diam- } \\
\text { eter, } \\
\text { "O.D." }\end{array}$ & $\begin{array}{c}\text { Ex- } \\
\text { ternal } \\
\text { diam- } \\
\text { eter of } \\
\text { upset, } \\
\text { "U.D." }\end{array}$ & $\begin{array}{l}\text { Weight } \\
\text { perfoot, } \\
\text { com- } \\
\text { plete }\end{array}$ & $\begin{array}{c}\text { Num- } \\
\text { ber per } \\
\text { inch, } \\
\text { "B" }\end{array}$ & $\begin{array}{c}\text { In- } \\
\text { cluded } \\
\text { taper, } \\
\text { inches } \\
\text { per foot, } \\
\text { "b" }\end{array}$ & $\begin{array}{l}\text { Length } \\
\text { of } \\
\text { perfect, } \\
\text { "E" }\end{array}$ & $\begin{array}{l}\text { Num- } \\
\text { ber of } \\
\text { perfect }\end{array}$ & $\begin{array}{l}\text { Effec- } \\
\text { tive } \\
\text { length, } \\
\text { "F"" }\end{array}$ & $\begin{array}{l}\text { Total } \\
\text { length, } \\
\text { "G" }\end{array}$ & $\begin{array}{l}\text { Height } \\
\text { of } \\
\text { thread } \\
\text { trun- } \\
\text { cated, } \\
\text { "h" }\end{array}$ & & & & & \\
\hline $\begin{array}{r}43 / 4 \\
53 / 4 \\
2816\end{array}$ & $\begin{array}{l}4.750 \\
5.750 \\
8.125\end{array}$ & $\begin{array}{l}5.000 \\
6.000 \\
8.375\end{array}$ & $\begin{array}{r}16.250 \\
\text { All. } \\
40.000\end{array}$ & $\begin{array}{l}10 \\
10 \\
10\end{array}$ & $\begin{array}{l}36 \\
36 \\
3,8\end{array}$ & $\begin{array}{l}2.1750 \\
2.9250 \\
2.9250\end{array}$ & $\begin{array}{l}21.75 \\
29.25 \\
29.25\end{array}$ & $\begin{array}{l}2.475 \\
3.225 \\
3.225\end{array}$ & $\begin{array}{l}2.875 \\
3.625 \\
3.625\end{array}$ & $\begin{array}{r}0.0800 \\
.0800 \\
.0800\end{array}$ & $\begin{array}{l}4.772 \\
5.749 \\
8.124\end{array}$ & $\begin{array}{l}4.932 \\
5.909 \\
8.284\end{array}$ & $\begin{array}{r}0.625 \\
.625 \\
.625\end{array}$ & $\begin{array}{r}0.000 \\
.000 \\
.000\end{array}$ & \\
\hline
\end{tabular}

1 Column " $A$ " to be filled in at some future date, to be based on actual experience.

This size inactive; available on special order only. 


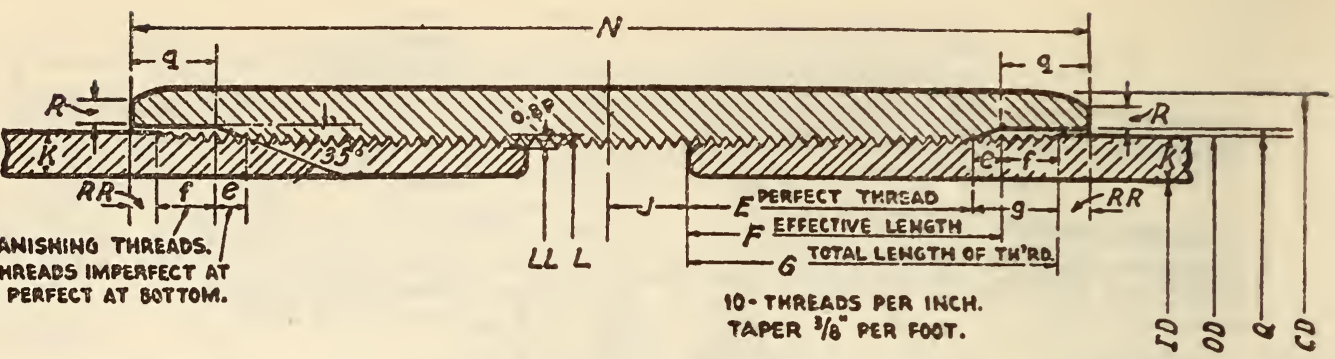

\section{TOP, PERFECT AT BOTTOM. \\ TAPER $3 / 8^{\circ}$ PER FOOT.}

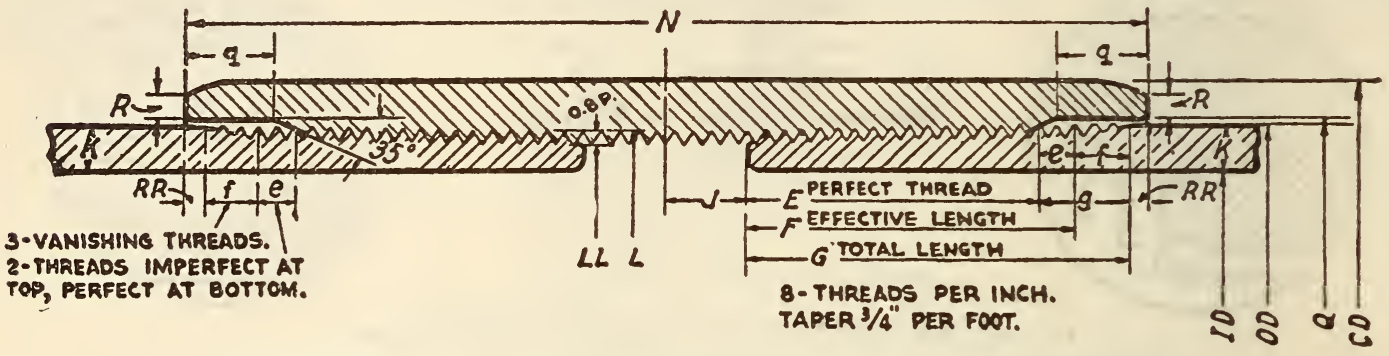

FigURE 281.-A. P. I. supplement casing threads, DBX and DBS sizes

See Figure 280 for chamfer on ends of coupling and pipe

TABLE 7.-Threads, A. P. I. supplementary standard casing

[See fig. 281]

[All dimensions in inches at $68^{\circ} \mathrm{F}$. All weights in pounds]

JThe values given in the following table and in fig. 281 apply to the $43 / 4$-inch, $65 / 8$-inch, and 7-inch, 10-thread, and to the 85/8-inch, 9-inch, 95/8-inch, and 113/4-inch, 8-thread, sizes only. These sizes are standard at present and are copied from lists now in use]

\begin{tabular}{|c|c|c|c|c|c|c|c|c|c|c|c|c|c|c|c|}
\hline \multicolumn{4}{|c|}{ Casing } & \multicolumn{7}{|c|}{ Threads } & \multirow[b]{2}{*}{$\begin{array}{l}\text { Diam- } \\
\text { eter of } \\
\text { casing } \\
\text { at root } \\
\text { of } \\
\text { threads, } \\
\text { "L. L." }\end{array}$} & \multirow[b]{2}{*}{$\begin{array}{l}\text { Diam- } \\
\text { eter of } \\
\text { casing at } \\
\text { top of } \\
\text { threads, } \\
\text { "L"" }\end{array}$} & \multirow[b]{2}{*}{$\begin{array}{l}\text { End of } \\
\text { pipe to } \\
\text { center } \\
\text { coupling, } \\
\text { made } \\
\text { up, "J " }\end{array}$} & \multirow[b]{2}{*}{$\begin{array}{l}\text { Distance } \\
\text { end of } \\
\text { coupling } \\
\text { beyond } \\
\text { last } \\
\text { scratch, } \\
\text { made } \\
\text { up, ", } \\
\text { "R.R." }\end{array}$} & \multirow[b]{2}{*}{$\begin{array}{l}\text { Stand- } \\
\text { off of } \\
\text { coupling } \\
\text { at hand- } \\
\text { tight } \\
\text { fit, "A."1 } \\
\text {. }\end{array}$} \\
\hline Size & $\begin{array}{l}\text { Exter- } \\
\text { nal di- } \\
\text { ameter, } \\
\text { "O.D.' }\end{array}$ & $\begin{array}{c}\text { Exter- } \\
\text { nal di- } \\
\text { ameter, } \\
\text { of up- } \\
\text { set, } \\
\text { "U. D." }\end{array}$ & $\begin{array}{l}\text { Weight } \\
\text { per } \\
\text { foot, } \\
\text { com- } \\
\text { plete }\end{array}$ & $\begin{array}{l}\text { Num- } \\
\text { ber per } \\
\text { inch, } \\
\text { "B" }\end{array}$ & $\begin{array}{c}\text { In- } \\
\text { cluded } \\
\text { taper } \\
\text { inches } \\
\text { per } \\
\text { foot, } \\
\text { "b" }\end{array}$ & $\begin{array}{l}\text { Length } \\
\text { of per- } \\
\text { fect, } \\
\text { "E" }\end{array}$ & $\begin{array}{l}\text { Num- } \\
\text { ber of } \\
\text { perfect }\end{array}$ & $\begin{array}{c}\text { Effec- } \\
\text { tive } \\
\text { length, } \\
\text { "F"" }\end{array}$ & $\begin{array}{c}\text { Total } \\
\text { length, } \\
\text { "G", }\end{array}$ & $\begin{array}{l}\text { Height } \\
\text { of } \\
\text { thread } \\
\text { trun- } \\
\text { cated, } \\
\text { "h" }\end{array}$ & & & & & \\
\hline $\begin{array}{l}243 / 4 \\
265 / 8 \\
27 \\
385 / 8 \\
9 \\
395 / 8 \\
113 / 4\end{array}$ & $\begin{array}{r}4.750 \\
6.625 \\
7.000 \\
8.625 \\
9.000 \\
9.625 \\
11.750\end{array}$ & \begin{tabular}{l}
$-\cdots$ \\
.-- \\
.-- \\
\hdashline.- \\
\hdashline.- \\
-
\end{tabular} & $\begin{array}{r}\text { 16. } 000 \\
\text { All. } \\
\text { All. } \\
\text { All. } \\
\text { All. } \\
\text { All. } \\
\text { All. }\end{array}$ & $\begin{array}{r}10 \\
10 \\
10 \\
8 \\
8 \\
8 \\
8\end{array}$ & $\begin{array}{l}3 / 8 \\
3 / 8 \\
3 / 8 \\
3 / 4 \\
3 / 4 \\
3 / 4 \\
3 / 4\end{array}$ & $\begin{array}{l}1.9500 \\
2.4000 \\
2.4000 \\
2.6250 \\
2.6250 \\
2.6250 \\
2.6250\end{array}$ & $\begin{array}{l}19.50 \\
24.00 \\
24.00 \\
21.00 \\
21.00 \\
21.00 \\
21.00\end{array}$ & $\begin{array}{l}2.150 \\
2.600 \\
2.600 \\
2.875 \\
2.875 \\
2.875 \\
2.875\end{array}$ & $\begin{array}{l}\text { 2. } 550 \\
\text { 3. } 000 \\
3.000 \\
3.250 \\
3.250 \\
\text { 3. } 250 \\
\text { 3. } 250\end{array}$ & $\begin{array}{r}0.0800 \\
.0800 \\
.0800 \\
.1000 \\
.1000 \\
.1000 \\
.1000\end{array}$ & $\begin{array}{r}4.529 \\
6.390 \\
6.765 \\
8.261 \\
8.636 \\
9.261 \\
11.386\end{array}$ & $\begin{array}{r}4.689 \\
6.550 \\
6.925 \\
8.461 \\
8.836 \\
9.461 \\
11.586\end{array}$ & $\begin{array}{r}0.5625 \\
.6125 \\
.6125 \\
.6875 \\
.6875 \\
.6875 \\
.6875\end{array}$ & $\begin{array}{r}0.200 \\
.200 \\
.200 \\
.125 \\
.125 \\
.125 \\
.125\end{array}$ & $\begin{array}{l}-- \\
-- \\
--. \\
--. \\
-\cdots \\
--.\end{array}$ \\
\hline
\end{tabular}

1 Column "A" to be filled in at some future date; to be based on actual experience.

2 Same joint and threading as diamond BX casing.

Same joint and threading as diamond $\mathrm{B} 8$ casing. 
TABLE 9.-A. P. I. drill pipe

[See fig. 282]

[Size of A. P. I. drill pipe indicates outside diameter. All dimensions in inches at $68^{\circ} \mathrm{F}$. All weights in pounds]

\begin{tabular}{|c|c|c|c|c|c|c|c|c|c|c|c|c|c|c|c|c|c|}
\hline \multirow[b]{2}{*}{$\begin{array}{l}2 \\
\dot{A} \\
\dot{0} \\
\dot{0} \\
\dot{N}\end{array}$} & \multirow{2}{*}{ 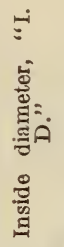 } & \multirow{2}{*}{ 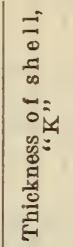 } & \multicolumn{2}{|c|}{ Weight per foot 1} & \multicolumn{2}{|c|}{ Threads } & \multirow{2}{*}{ 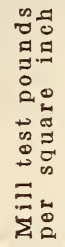 } & \multicolumn{4}{|c|}{ Upset 2} & \multicolumn{6}{|c|}{ Couplings } \\
\hline & & & 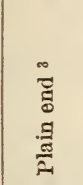 & 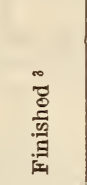 & 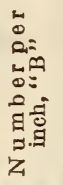 & 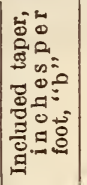 & & 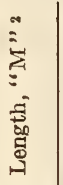 & 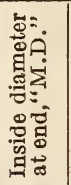 & 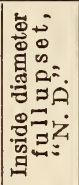 & 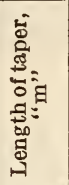 & 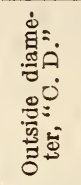 & 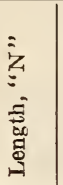 & 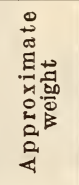 & 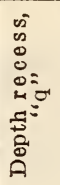 & 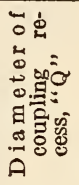 & 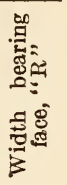 \\
\hline 4236 & 2.000 & 1875 & 4.380 & 4.800 & 10 & 38 & 2,500 & $31 / 4$ & $17 / 1$ & $17 /$ & $11 / 2$ & 3.125 & 516 & 5.57 & & 2.500 & \\
\hline $23 \%$ & 1.815 & 2800 & 6. 264 & 6.650 & 10 & $3 / 4$ & 2,500 & & 13 & 11 & 1 & 3.1 & 5 & 5. & & 2.500 & \\
\hline $2^{7 / 8}$ & 2. 469 & 2030 & 5. 793 & 6.450 & 8 & $3 / 4$ & 2,500 & 3 & 17 & 17 & & 3.7 & $61 / 2$ & 9.2 & & 3. 000 & \\
\hline $27 / 8$ & 2. 323 & .2760 & 7.661 & 8. 350 & 8 & $3 / 4$ & 2,500 & $31 / 2$ & $17 / 8$ & $15 \%$ & 1 & 3.7 & $61 / 2$ & 9. & & 00 & \\
\hline 278 & 2. 151 & .3620 & 9.715 & 10.400 & 8 & $3 / 4$ & 2,500 & 31 & $1 \%$ & 13 & & 3. & 6 & 9. & & 3. & \\
\hline $31 / 2$ & 3.063 & 2187 & 7.665 & 8.500 & 8 & $3 / 4$ & 2,250 & 3 & 27 & 27 & 1 & 4.2 & 61 & 9. & & 3. & \\
\hline 316 & 2. 900 & .3000 & 10. 252 & 11.200 & 8 & $3 / 4$ & 2,500 & 31 & 23 & 21 & 13 & 4. & $61 / 2$ & 9.7 & & 3.625 & \\
\hline 316 & 2. 764 & .3680 & 12 & 13 & 8 & $3 / 4$ & 2,50 & 31 & & 1 & 1 & & 61 & 9. & & 3. & \\
\hline $1 / 2$ & 4.0 & .2500 & 1. & & 8 & 3 & 2,0 & & 3 & & & & 8 & 19. & & 4. & \\
\hline & & .2710 & 240 & 50 & 8 & $3 / 4$ & 2,200 & 5 & $3 \%$ & 35 & 2 & & 8 & 19. & & 4. & \\
\hline 16 & 3.8 & .3370 & & & 8 & $3 / 4$ & 2,50 & 5 & 31 & $2^{13}$ & $?$ & & 8 & 19. & & & \\
\hline $5 \%$ & 4. & .2940 & 1 & 1 & 8 & $3 / 4$ & 1,90 & 5 & 49 & 41 & $\overline{2}$ & & $81 / 2$ & 30.7 & & 875 & \\
\hline $5 \%$ & & .3520 & 590 & 00 & 8 & $3 / 4$ & 2,250 & 5 & 45 & $3^{13 / 16}$ & 2 & & $81 / 2$ & 30.772 & & 5. 6875 & \\
\hline 16 & 4. & .4150 & 22 & & 0 & $3 / 4$ & 2,500 & & & 316 & 2 & & $81 / 2$ & 30.772 & & & \\
\hline 56 & 6. & .280 & 18 & 22 & 8 & 3 & 1,6 & & 5 & 53 & 2 & & & 36. & & & \\
\hline 58 & & .3300 & 22. & 25 & 8 & $3 / 4$ & 1,800 & 5 & $5^{11} / 3$ & & 2 & & 9 & 36. & 38 & 6.750 & \\
\hline $65 \%$ & & .4320 & 28 & 31. & 8 & $3 / 4$ & 2,400 & 5 & $51 / 1$ & 45 & 2 & & ( & & & & \\
\hline & & 32 & & & 8 & $3 / 4$ & 1,6 & 51 & & & 2 & & $93 / 4$ & 49. & & & \\
\hline & & .4000 & 35.137 & 40.000 & 8 & $3 / 4$ & 1,800 & 01 & & $65 / 8$ & 2 & 10.000 & $101 / 2$ & 70.800 & 38 & 8.750 & \\
\hline
\end{tabular}

1 See par. $12(b)$.

2 Lengths of upset are minimum, measured from end of pipe to commencement of radius.

"Weight per foot, finished" includes threads and couplings. "Weight per foot, plain end" is without threads, couplings, and upsets. - On special order only.

- Outside diameter of $5 \%$ size is 5.563 inches.

6 Tentative; special order only; see note 2 under Table 10 . Bevel on coupling recess to be $15^{\circ}$ to line parallel to axis of pipe. Bevel on and of drill pipe to be $65^{\circ}$ to line perpendicular to axis of pipe. 

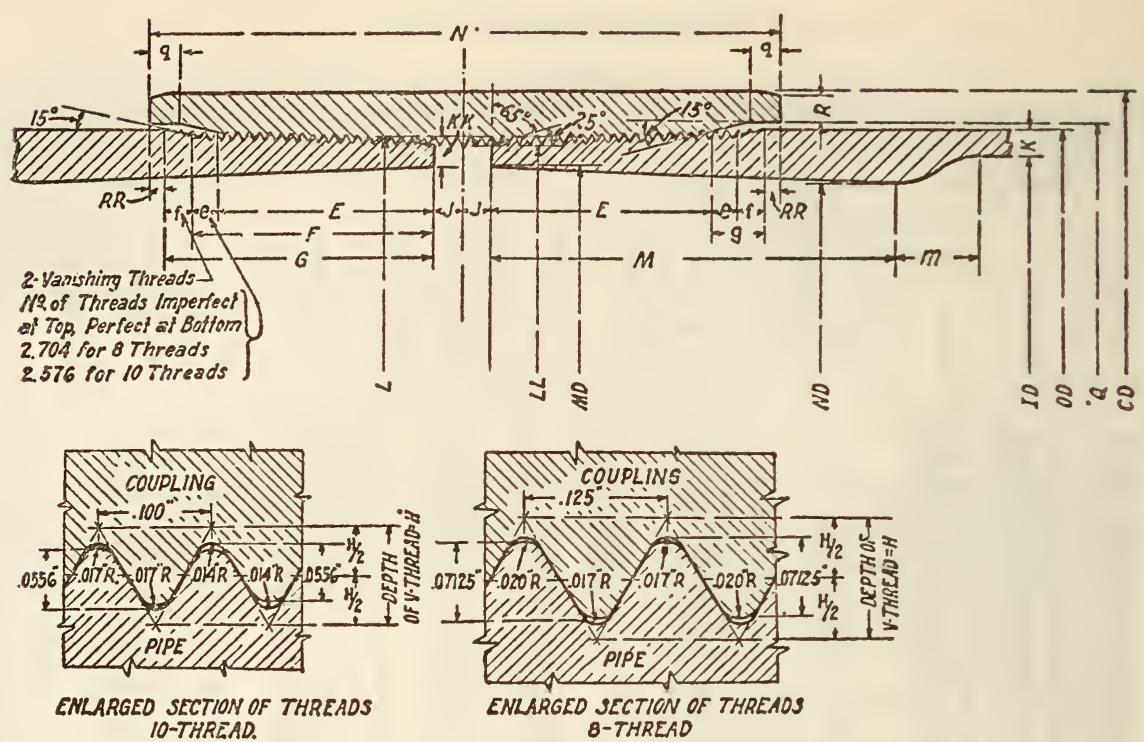

FIGURE 282.-Drill pipe thread

TABLE 10.-A. P. I. standard drill pipe threads

[See fig. 282]

["Size" of drill pipe indicates outside diameter. All dimensions in inches at $68^{\circ} \mathrm{F}$.]

\begin{tabular}{|c|c|c|c|c|c|c|c|c|c|c|c|c|c|c|}
\hline Size & $\begin{array}{l}\text { Outside } \\
\text { diam- } \\
\text { eter, } \\
\text { "O.D." }\end{array}$ & $\begin{array}{c}\text { Weight, } \\
\text { pounds } \\
\text { per } \\
\text { foot, } \\
\text { finished }\end{array}$ & $\begin{array}{l}\text { Num- } \\
\text { ber of } \\
\text { threads } \\
\text { per } \\
\text { inch- } \\
\text { "B" }\end{array}$ & $\begin{array}{l}\text { In- } \\
\text { cluded } \\
\text { taper, } \\
\text { inches } \\
\text { per } \\
\text { foot, } \\
\text { "b") }\end{array}$ & $\begin{array}{l}\text { Total } \\
\text { length } \\
\text { of } \\
\text { threads, } \\
\text { "G" }\end{array}$ & $\begin{array}{c}\text { Length of } \\
\text { threads } \\
\text { to gage } \\
\text { point, } \\
\text { "length } \\
\text { of perfect } \\
\text { threads," } \\
\text { "E" }\end{array}$ & $\begin{array}{l}\text { Length } \\
\text { of van- } \\
\text { ishing } \\
\text { plus im- } \\
\text { perfect } \\
\text { threads, } \\
\text { "g" }\end{array}$ & $\begin{array}{l}\text { Height } \\
\text { of } \\
\text { threads } \\
\text { (trun- } \\
\text { cated), } \\
\text { "h" }\end{array}$ & $\begin{array}{l}\text { Diameter } \\
\text { at end of } \\
\text { pipe to } \\
\text { root of } \\
\text { threads, } \\
\text { "L.L." }\end{array}$ & $\begin{array}{l}\text { Diameter } \\
\text { at end of } \\
\text { pipe to } \\
\text { crest of } \\
\text { threads, } \\
\text { "L" }\end{array}$ & $\begin{array}{c}\text { Distance } \\
\text { end of } \\
\text { coupling } \\
\text { beyond } \\
\text { last } \\
\text { scratch, } \\
\text { made up } \\
\text { by power, } \\
\text { "R.R." }\end{array}$ & $\begin{array}{l}\text { Threads } \\
\text { allowed } \\
\text { for } \\
\text { make- } \\
\text { up and } \\
\text { creep }\end{array}$ & $\begin{array}{l}\text { Dis- } \\
\text { tance } \\
\text { end of } \\
\text { pipe to } \\
\text { center } \\
\text { of cou- } \\
\text { pling, } \\
\text { made } \\
\text { up, "J" }\end{array}$ & $\begin{array}{l}\text { Stand-off of } \\
\text { coupling at } \\
\text { hand-tight } \\
\text { fit, "A" }\end{array}$ \\
\hline $\begin{array}{r}1236 \\
236 \\
27 / 8 \\
31 / 2 \\
41 / 2 \\
25916 \\
65 \% 8 \\
375 \% 8 \\
3858\end{array}$ & $\begin{array}{l}2.375 \\
2.375 \\
2.875 \\
3.500 \\
4.500 \\
5.563 \\
6.625 \\
7.625 \\
8.625\end{array}$ & $\begin{array}{l}4.800 \\
6.650 \\
\text { All. } \\
\text { All. } \\
\text { All. } \\
\text { All. } \\
\text { All. } \\
\text { All. } \\
\text { All. }\end{array}$ & $\begin{array}{r}10 \\
8 \\
8 \\
8 \\
8 \\
8 \\
8 \\
8 \\
8\end{array}$ & $\begin{array}{l}3 / 8 \\
3 / 4 \\
3 / 4 \\
3 / 4 \\
3 / 4 \\
3 / 4 \\
3 / 4 \\
3 / 4 \\
3\end{array}$ & $\begin{array}{l}2.125 \\
2.125 \\
2.625 \\
2.625 \\
3.375 \\
3.625 \\
3.875 \\
4.250 \\
4.625\end{array}$ & $\begin{array}{l}1.667 \\
1.537 \\
2.037 \\
2.037 \\
2.787 \\
3.037 \\
3.287 \\
3.662 \\
4.037\end{array}$ & $\begin{array}{l}0.458 \\
.588 \\
.588 \\
.588 \\
.588 \\
.588 \\
.588 \\
.588 \\
.588\end{array}$ & $\begin{array}{r}0.05560 \\
.07125 \\
.07125 \\
.07125 \\
.07125 \\
.07125 \\
.07125 \\
.07125 \\
.07125\end{array}$ & $\begin{array}{l}2.206 \\
2.130 \\
2.599 \\
3.224 \\
4.177 \\
5.225 \\
6.271 \\
7.248 \\
8.224\end{array}$ & $\begin{array}{l}2.317 \\
2.273 \\
2.742 \\
3.367 \\
4.320 \\
5.367 \\
6.414 \\
7.390 \\
8.367\end{array}$ & $\begin{array}{l}1 / 16 \\
1 / 16 \\
1 / 16 \\
1 / 16 \\
1 / 16 \\
1 / 16 \\
116 \\
1 / 16 \\
1 / 16\end{array}$ & $\begin{array}{l}7 \\
7 \\
7 \\
7 \\
7 \\
7 \\
7 \\
7 \\
7\end{array}$ & $\begin{array}{l}9 / 16 \\
\% 16 \\
916 \\
9 / 16 \\
916 \\
916 \\
916 \\
916 \\
916\end{array}$ & $\begin{array}{l}41 / 2 \text { threads. } \\
\text { Do. } \\
\text { Do. } \\
\text { Do. } \\
\text { Do. } \\
\text { Do. } \\
\text { Do. } \\
\text { Do. } \\
\text { Do. }\end{array}$ \\
\hline
\end{tabular}

1 On special order only. $\quad 2$ Outside diameter of $5 \%$-inch is 5.563 inches. $\quad{ }^{3}$ Tentative; special order only. (See special note below.)

SPECIAL NOTE

Dimension "A."-The stand-off "A" specified does not apply to materials used with drill pipe, such as tool joints, drill collars, etc., except where such materials have the same recess dimensions as the drill pipe couplings.

SPECIAL NOTE ON 75/8-INCH AND 85/8-INCH DRILL PIPE. SEE TABLES 9 AND 10

Data on the 75/8-inch and $85 \%$-inch size published for general information only. Due to the very limited demand for these sizes manu. facturers are not required to supply themselves with certified master gages unless they desire to do so. Manufacturers will furnish these sizes with threads made as closely as possible to dimensions given, without certifled gages. The A. P. I. monogram can not be used on these sizes unless the manufacturer has the necessary master gages. One weight only listed; consult manufacturers if other weights are desired. 


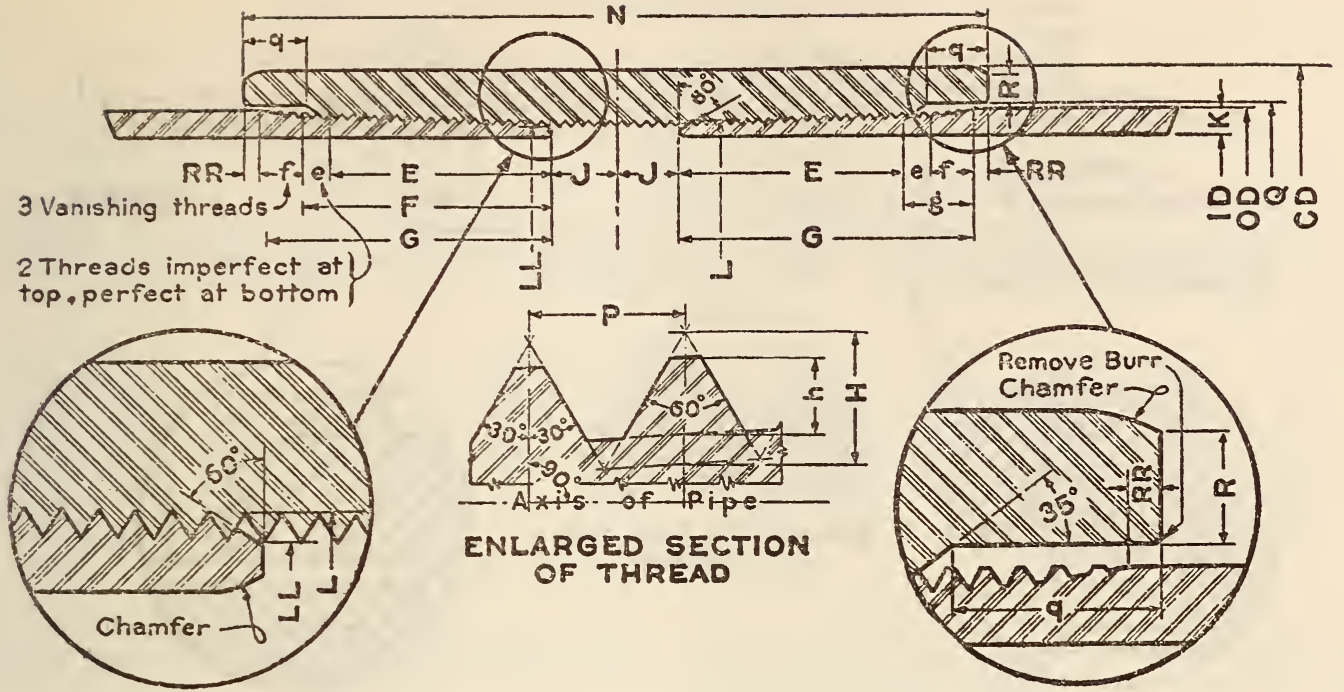

Figure 283.-A. P. I. standard tubing-plain

TABLE 12.-A. P. I. standard tubing-plain

[See fig. 283]

["Size" of A. P. I. tubing indicates nominal diameter. All dimensions in inches at $68^{\circ} \mathrm{F}$.]

\begin{tabular}{|c|c|c|c|c|c|c|c|c|c|c|c|c|c|c|c|}
\hline \multirow[b]{2}{*}{ Size } & \multicolumn{2}{|c|}{ Diameter } & \multirow[b]{2}{*}{$\begin{array}{l}\text { Thick- } \\
\text { ness of } \\
\text { shell, } \\
\text { " } \mathrm{K} \text { " }\end{array}$} & \multicolumn{2}{|c|}{$\begin{array}{l}\text { Weight, }{ }^{1} \text { pounds } \\
\text { per foot }\end{array}$} & \multicolumn{2}{|c|}{ Threads } & \multicolumn{2}{|c|}{$\begin{array}{l}\text { Mill test, } \\
\text { pounds per } \\
\text { square inch }\end{array}$} & \multicolumn{6}{|c|}{ Couplings } \\
\hline & $\begin{array}{l}\text { Out- } \\
\text { side, } \\
\text { "O.D." }\end{array}$ & "Inside, & & $\begin{array}{l}\text { Plain } \\
\text { end } 2\end{array}$ & Finished ${ }^{3}$ & $\begin{array}{c}\text { Num- } \\
\text { ber } \\
\text { per } \\
\text { inch, } \\
\text { "B" }\end{array}$ & $\begin{array}{c}\text { In- } \\
\text { cluded } \\
\text { taper, } \\
\text { inches } \\
\text { per } \\
\text { foot, } \\
\text { "b" }\end{array}$ & $\begin{array}{l}\text { Lap } \\
\text { weld }\end{array}$ & $\begin{array}{c}\text { Seam- } \\
\text { less }\end{array}$ & $\begin{array}{l}\text { Outside } \\
\text { diam- } \\
\text { eter, } \\
\text { "C.D." }\end{array}$ & Length, & Weight & $\begin{array}{l}\text { Depth } \\
\text { of } \\
\text { recess, } \\
\text { "q" }\end{array}$ & $\begin{array}{l}\text { Diam- } \\
\text { eter of } \\
\text { recess, } \\
\text { "Q" }\end{array}$ & $\begin{array}{l}\text { Width } \\
\text { of } \\
\text { bearing } \\
\text { face, } \\
\text { " } \mathrm{R} \text { " }\end{array}$ \\
\hline $\begin{array}{l}11 / 2 \\
2 \\
21 / 2 \\
3 \\
3 \\
31 / 2 \\
4\end{array}$ & $\begin{array}{l}1.900 \\
2.375 \\
2.875 \\
3.500 \\
3.500 \\
4.000 \\
4.500\end{array}$ & $\begin{array}{l}1.610 \\
1.995 \\
2.441 \\
2.992 \\
2.922 \\
3.548 \\
3.958\end{array}$ & $\begin{array}{r}0.145 \\
.190 \\
.217 \\
.254 \\
.289 \\
.226 \\
.271\end{array}$ & $\begin{array}{r}2.717 \\
4.433 \\
6.160 \\
8.805 \\
9.910 \\
9.109 \\
12.240\end{array}$ & $\begin{array}{r}2.750 \\
4.600 \\
6.400 \\
9.200 \\
10.200 \\
9.500 \\
12.600\end{array}$ & $\begin{array}{l}113 / 2 \\
1112 \\
111 / 2 \\
111 / 2 \\
111 / 2 \\
10 \\
10\end{array}$ & $\begin{array}{l}3 / 4 \\
3 / 4 \\
3 / 4 \\
3 / 4 \\
3 / 4 \\
3 / 4 \\
3 / 4\end{array}$ & $\begin{array}{l}2,300 \\
2,500 \\
2,300 \\
2,200 \\
2,500 \\
1,700 \\
1,800\end{array}$ & $\begin{array}{l}2,500 \\
2,500 \\
2,500 \\
2,500 \\
2,500 \\
2,100 \\
2,200\end{array}$ & $\begin{array}{l}2.169 \\
2.870 \\
3.517 \\
4.200 \\
4.200 \\
4.750 \\
5.216\end{array}$ & $\begin{array}{l}3.375 \\
4.250 \\
5.250 \\
5.875 \\
5.875 \\
6.000 \\
6.375\end{array}$ & $\begin{array}{r}1.100 \\
2.769 \\
5.352 \\
7.939 \\
7.939 \\
9.956 \\
11.324\end{array}$ & $\begin{array}{l}0.250 \\
.500 \\
.500 \\
.500 \\
.500 \\
.500 \\
.500\end{array}$ & $\begin{array}{l}1.9625 \\
2.4375 \\
2.9375 \\
3.5625 \\
3.5625 \\
4.0625 \\
4.5625\end{array}$ & $\begin{array}{r}0.0625 \\
.1875 \\
.2500 \\
.2500 \\
.2500 \\
.2500 \\
.2500\end{array}$ \\
\hline
\end{tabular}

1 See par. $12(b)$.

"Weights per foot, plain end," is without threads or couplings. Weights are estimated only; wall thickness is specified exactly. 8 "Weights per foot, finished," includes threads and couplings.

- On special order only.

NoTE.-Bevel on coupling recess to be $35^{\circ}$ to line parallel to axis of tubing. Bevel on end of tubing to be $60^{\circ}$ to line perpendicular to axis of tubing. 


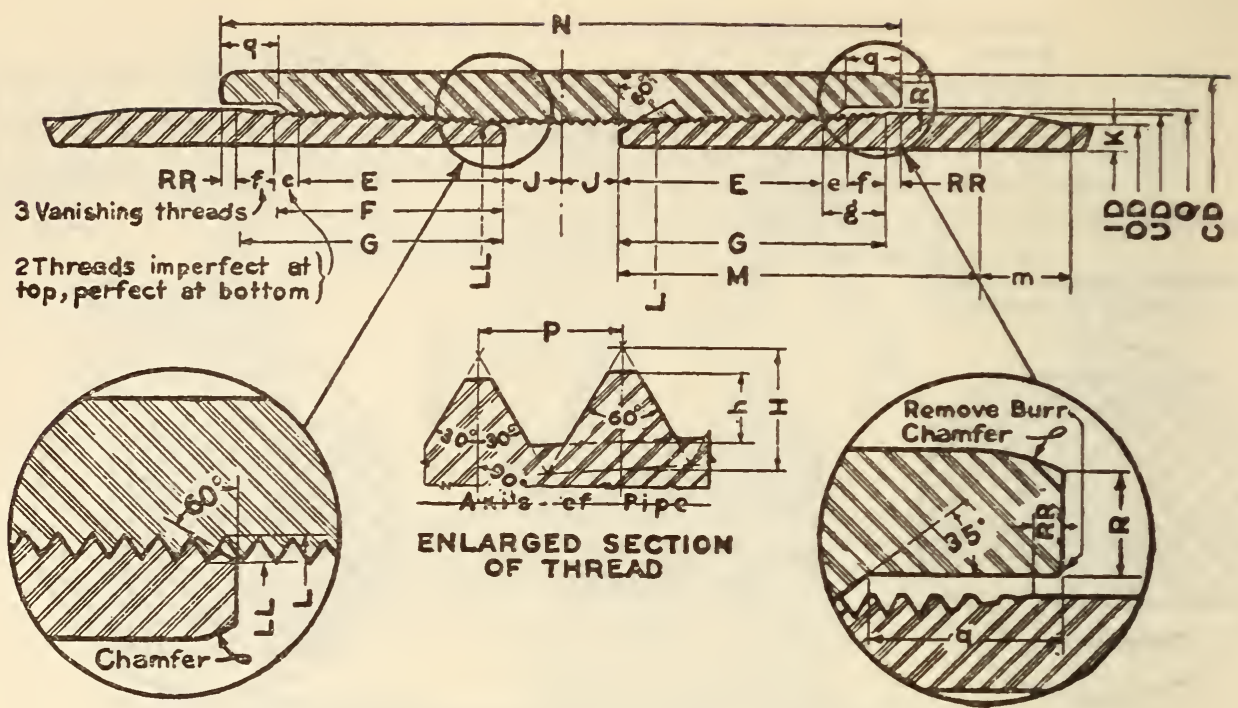

FIgURE 284.-A. P. I. standard tubing-external upset

TABLE 13.-A. P. I. standard tubing-external upset

[See fig. 284]

["Size of A. P. I. tubing indicates nominal diameter. All dimensions in inches at $68^{\circ} \mathrm{F}$.]

\begin{tabular}{|c|c|c|c|c|c|c|c|c|c|c|c|c|c|c|c|c|c|c|}
\hline & Dian & eters & $\stackrel{\bar{z}}{=}$ & $\begin{array}{l}\text { Wei } \\
\text { poun }\end{array}$ & $\begin{array}{l}t, 1 \\
\text { per }\end{array}$ & & Upset & & Thr & reads & $\begin{array}{l}\text { Mill } \\
\text { poun } \\
\text { squar }\end{array}$ & $\begin{array}{l}\text { st, } \\
\text { per } \\
\text { ach }\end{array}$ & & & Coup & ings & & \\
\hline$\stackrel{8}{8}$ & 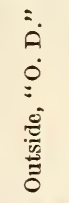 & 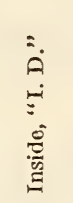 & 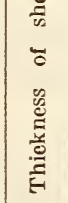 & 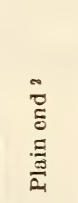 & 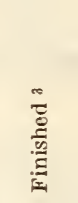 & 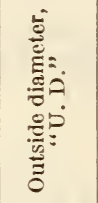 & 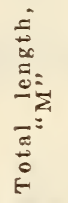 & 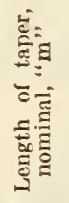 & 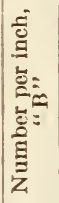 & 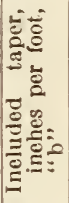 & 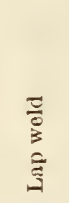 & 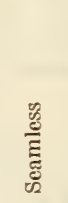 & 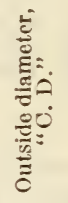 & 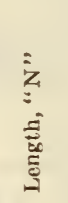 & $\overrightarrow{\vec{E}}$ & 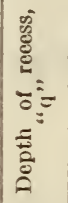 & 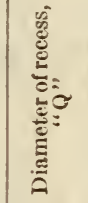 & 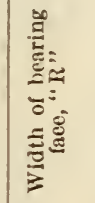 \\
\hline $\begin{array}{l}11 / 4 \\
11 / 2 \\
2 \\
21 / 2 \\
3 \\
31 / 2\end{array}$ & $\begin{array}{l}1.660 \\
1.900 \\
2.375 \\
2.875 \\
3.500 \\
4.000 \\
4.500\end{array}$ & $\begin{array}{l}1.380 \\
1.610 \\
1.995 \\
2.441 \\
2.992 \\
3.476 \\
3.958\end{array}$ & $\begin{array}{l}0.140 \\
.145 \\
.190 \\
.217 \\
.254 \\
.262 \\
.271\end{array}$ & $\begin{array}{r}2.272 \\
2.717 \\
4.433 \\
6.160 \\
8.805 \\
10.459 \\
12.240\end{array}$ & $\begin{array}{r}2.400 \\
2.900 \\
4.700 \\
6.500 \\
9.300 \\
11.000 \\
12.750\end{array}$ & $\begin{array}{l}1.81250 \\
2.09375 \\
2.59375 \\
3.09375 \\
3.75000 \\
4.25000 \\
4.75000\end{array}$ & $\begin{array}{l}2.000 \\
2.250 \\
3.500 \\
3.750 \\
4.000 \\
4.000 \\
4.250\end{array}$ & $\begin{array}{l}1.00 \\
1.00 \\
1.00 \\
1.00 \\
1.00 \\
1.000 \\
1.000\end{array}$ & $\begin{array}{l}111 / 2 \\
1112 \\
10 \\
10 \\
10 \\
10 \\
10\end{array}$ & $\begin{array}{l}3 / 4 \\
3 / 4 \\
3 / 4 \\
3 / 4 \\
3 / 4 \\
3 / 4 \\
3 / 4\end{array}$ & $\begin{array}{l}2,500 \\
2,300 \\
2,500 \\
2,300 \\
2,200 \\
2,000 \\
1,800\end{array}$ & $\begin{array}{l}2,500 \\
2,500 \\
2,500 \\
2,500 \\
2,500 \\
2,400 \\
2,200\end{array}$ & $\begin{array}{l}\text { 2. } 200 \\
\text { 2. } 500 \\
\text { 3. } 060 \\
3.668 \\
4.504 \\
5.000 \\
\text { 5. } 563\end{array}$ & $\begin{array}{l}\text { 3. } 500 \\
\text { 3. } 750 \\
4.875 \\
5.375 \\
6.000 \\
6.250 \\
6.500\end{array}$ & $\begin{array}{r}1.421 \\
1.824 \\
3.263 \\
5.379 \\
9.351 \\
11.015 \\
13.787\end{array}$ & $\begin{array}{l}0.250 \\
.250 \\
.500 \\
.500 \\
.500 \\
.500 \\
.500\end{array}$ & $\begin{array}{l}1.87500 \\
2.15625 \\
2.65625 \\
3.15625 \\
3.81250 \\
4.3125 \\
4.8125\end{array}$ & $\begin{array}{r}0.12500 \\
.12500 \\
.15625 \\
.21875 \\
.25000 \\
.25000 \\
.25000\end{array}$ \\
\hline
\end{tabular}

1 See par. $12(b)$.

2 "Weight per foot, plain end," without threads, couplings, and upset. Weights are estimated only; wall thickness is specified exactly.

3 "Weight per foot, finished," includes threads and couplings.

Note.- Bevel on coupling recess to be $35^{\circ}$ to line parallel to axis of tubing. Bevel on end of tubing to be $60^{\circ}$ to line perpendicular to axis of tubing.

TABLE 14.-A. P. I. standard tubing threads-plain

[See fig. 283]

[All dimensions in inches at $68^{\circ} \mathrm{F}$. "Size" of A. P. I. tubing indicates nominal diameter]

\begin{tabular}{|c|c|c|c|c|c|c|c|c|c|c|c|c|c|}
\hline $\mathrm{Siz}$ & $\begin{array}{l}\text { Outside } \\
\text { diameter, } \\
\text { "O. D." }\end{array}$ & $\begin{array}{l}\text { Weight, } \\
\text { pounds } \\
\text { per foot, } \\
\text { finished }\end{array}$ & $\begin{array}{c}\text { Number } \\
\text { of } \\
\text { threads } \\
\text { per inch, } \\
\text { "B" }\end{array}$ & $\begin{array}{l}\text { Included } \\
\text { taper, } \\
\text { inches } \\
\text { per foot, } \\
\text { "b" }\end{array}$ & $\begin{array}{l}\text { Total } \\
\text { length of } \\
\text { threads, } \\
\text { "G" }\end{array}$ & $\begin{array}{l}\text { Length of } \\
\text { thread to } \\
\text { gage } \\
\text { point, } \\
\text { "length } \\
\text { of perfect } \\
\text { thread," } \\
\text { "E" }\end{array}$ & $\begin{array}{l}\text { Length of } \\
\text { imperfect } \\
\text { plus } \\
\text { vanishing } \\
\text { threads, } \\
\text { "g" }\end{array}$ & $\begin{array}{l}\text { Height of } \\
\text { threads } \\
\text { (trun- } \\
\text { cated), } \\
\text { " } \mathrm{h} \text { " }\end{array}$ & $\begin{array}{l}\text { Diameter } \\
\text { at end of } \\
\text { tubing } \\
\text { to root of } \\
\text { threads, } \\
\text { "L.L." }\end{array}$ & $\begin{array}{l}\text { Diameter } \\
\text { at end of } \\
\text { tubing } \\
\text { to crest } \\
\text { of } \\
\text { threads, } \\
\text { "L" }\end{array}$ & $\begin{array}{l}\text { End of } \\
\text { tubing } \\
\text { from } \\
\text { center of } \\
\text { eoupling, } \\
\text { made up, } \\
\text { "J" }\end{array}$ & $\begin{array}{l}\text { Distance } \\
\text { end of } \\
\text { coupling } \\
\text { beyond } \\
\text { last } \\
\text { scratch, } \\
\text { made up } \\
\text { "R. R." }\end{array}$ & $\begin{array}{l}\text { Stand-off } \\
\text { of cou- } \\
\text { pling at } \\
\text { hand- } \\
\text { tight fit, } \\
\text { "A"1. }\end{array}$ \\
\hline $\begin{array}{l}11 / 2 \\
2 \\
21 / 2 \\
3 \\
31 / 2 \\
4\end{array}$ & $\begin{array}{l}1.900 \\
2.375 \\
2.875 \\
3.500 \\
4.000 \\
4.500\end{array}$ & $\begin{array}{l}\text { All. } \\
\text { All. } \\
\text { All. } \\
\text { All. } \\
\text { All. } \\
\text { All. }\end{array}$ & $\begin{array}{l}111 / 1 \\
1112 \\
111 / 2 \\
111 / 2 \\
10 \\
10\end{array}$ & $\begin{array}{l}3 / 4 \\
3 / 4 \\
3 / 4 \\
3 / 4 \\
3 / 4 \\
3 / 4\end{array}$ & $\begin{array}{l}1.3748 \\
1.6250 \\
2.0625 \\
2.3125 \\
2.3750 \\
2.5625\end{array}$ & $\begin{array}{l}0.940 \\
1.1902 \\
1.6277 \\
1.8777 \\
1.8750 \\
2.0625\end{array}$ & $\begin{array}{l}0.4348 \\
.4348 \\
.4348 \\
.4348 \\
.5000 \\
.5000\end{array}$ & $\begin{array}{r}0.06956 \\
.06956 \\
.06956 \\
.06956 \\
.08000 \\
.08000\end{array}$ & $\begin{array}{l}\text { 1. } 7021 \\
\text { 2. } 1615 \\
\text { 2. } 6341 \\
\text { 3. } 2435 \\
\text { 3. } 7228 \\
4.2111\end{array}$ & \begin{tabular}{l|}
1.8412 \\
2. 3006 \\
2.7733. \\
3.3826 \\
3. 8828 \\
4.3711
\end{tabular} & $\begin{array}{l}5 / 16 \\
3 / 8 \\
7 / 16 \\
1 / 2 \\
1 / 2 \\
1 / 2\end{array}$ & $\begin{array}{l}0 \\
18 \\
18 \\
1 / 8 \\
18 \\
18\end{array}$ & 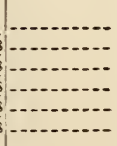 \\
\hline
\end{tabular}

1 Column " $A$ " to be filled in at some future date; to be based on actual experience. 
[See fig. 284]

["Size" of A. P. I. tubing indicates nominal diameter. All dimensions in inches at $68^{\circ} \mathrm{F}$.]

\begin{tabular}{|c|c|c|c|c|c|c|c|c|c|c|c|c|c|c|}
\hline Size & $\begin{array}{l}\text { External } \\
\text { diameter } \\
\text { of tube, } \\
\text { "O.D." }\end{array}$ & $\begin{array}{c}\text { Outside } \\
\text { diameter } \\
\text { of } \\
\text { external } \\
\text { upset, } \\
\text { "U.D." }\end{array}$ & $\begin{array}{l}\text { Weight, } \\
\text { pounds } \\
\text { per foot, } \\
\text { finished }\end{array}$ & $\begin{array}{l}\text { Number } \\
\text { of } \\
\text { threads } \\
\text { per } \\
\text { inch, } \\
\text { "B" }\end{array}$ & $\begin{array}{l}\text { Includ- } \\
\text { ed taper, } \\
\text { inches } \\
\text { per foot, } \\
\text { "b" }\end{array}$ & $\begin{array}{c}\text { Total } \\
\text { length of } \\
\text { threads, } \\
\text { "G" }\end{array}$ & $\begin{array}{l}\text { Length of } \\
\text { thread to } \\
\text { gage } \\
\text { point, } \\
\text { "length } \\
\text { of perfect } \\
\text { thread," } \\
\text { "E" }\end{array}$ & $\begin{array}{l}\text { Length of } \\
\text { imperfect } \\
\text { plus } \\
\text { vanishing } \\
\text { threads, } \\
\text { "g"g" }\end{array}$ & $\begin{array}{l}\text { Height of } \\
\text { threads } \\
\text { (trun- } \\
\text { cated), } \\
\text { "h"' }\end{array}$ & $\begin{array}{l}\text { Diameter } \\
\text { at end of } \\
\text { tubing } \\
\text { to root of } \\
\text { threads, } \\
\text { "L.L." }\end{array}$ & $\begin{array}{l}\text { Diameter } \\
\text { at end of } \\
\text { tubing } \\
\text { to crest } \\
\text { of } \\
\text { threads, } \\
\text { "L"" }\end{array}$ & $\begin{array}{c}\text { End of } \\
\text { tubing } \\
\text { from } \\
\text { center of } \\
\text { coupling, } \\
\text { made up, } \\
\text { "JJ" }\end{array}$ & $\begin{array}{c}\text { Distance } \\
\text { end of } \\
\text { coupling } \\
\text { beyond } \\
\text { last } \\
\text { scratch, } \\
\text { "R.R." }\end{array}$ & $\begin{array}{l}\text { Stand- } \\
\text { off of } \\
\text { cou- } \\
\text { pling at } \\
\text { hand- } \\
\text { tight fit } \\
\text { "A" 1 }\end{array}$ \\
\hline $\begin{array}{l}11 / 4 \\
11 / 2 \\
2 \\
21 / 2 \\
3 \\
31 / 2 \\
4\end{array}$ & $\begin{array}{l}1.660 \\
1.900 \\
2.375 \\
2.875 \\
3.500 \\
4.000 \\
4.500\end{array}$ & $\begin{array}{l}1.81250 \\
2.09375 \\
\text { 2. } 59375 \\
\text { 3. } 09375 \\
\text { 3. } 75000 \\
\text { 4. } 25000 \\
\text { 4. } 75000\end{array}$ & $\begin{array}{l}\text { All. } \\
\text { All. } \\
\text { All. } \\
\text { All. } \\
\text { All. } \\
\text { All. } \\
\text { All. }\end{array}$ & $\begin{array}{l}111 / 2 \\
111 / 2 \\
10 \\
10 \\
10 \\
10 \\
10\end{array}$ & $\begin{array}{l}3 / 4 \\
3 / 4 \\
3 / 4 \\
3 / 4 \\
3 / 4 \\
3 / 4 \\
3 / 4 \\
3 / 4\end{array}$ & $\begin{array}{l}1.3750 \\
1.4375 \\
1.9375 \\
2.1250 \\
2.3750 \\
2.5000 \\
2.6250\end{array}$ & $\begin{array}{l}0.9402 \\
1.0027 \\
1.4375 \\
1.6250 \\
1.8750 \\
2.0000 \\
\text { 2. } 1250\end{array}$ & $\begin{array}{r}0.4348 \\
.4348 \\
.5000 \\
.5000 \\
.5000 \\
.5000 \\
.5000\end{array}$ & $\begin{array}{r}0.06956 \\
.06956 \\
.08000 \\
.08000 \\
.08000 \\
.08000 \\
.08000\end{array}$ & $\begin{array}{l}\text { 1. } 6146 \\
1.8919 \\
2.3439 \\
2.8322 \\
3.4728 \\
3.9650 \\
4.4572\end{array}$ & $\begin{array}{l}\text { 1. } 7537 \\
2.0311 \\
\text { 2. } 5039 \\
\text { 2. } 9922 \\
\text { 3. } 6328 \\
\text { 4. } 1250 \\
\text { 4. } 6172\end{array}$ & $\begin{array}{l}5 / 16 \\
5 / 16 \\
3 / 8 \\
7 / 16 \\
1 / 2 \\
12 \\
1 / 2\end{array}$ & $\begin{array}{l}1 / 16 \\
1 / 8 \\
1 / 8 \\
1 / 8 \\
1 / 8 \\
1 / 8 \\
1 / 8\end{array}$ & |-n \\
\hline
\end{tabular}

${ }^{1}$ Column " $A$ " to be filled in at some future date; to be based on actual experience.

AMERICAN PETROLEUM INSTITUTE, LINEPIPE SPECIFICATIONS, STANDARD NO. 5-L (4TH ED.), JULY, 1931

\section{STEEL AND IRON PIPE FOR LINE-PIPE PURPOSES}

\section{MATERIAL COVERED}

1. These specifications apply to welded and seamless steel, and welded iron tubular goods for linepipe purposes, commonly used to convey gas, water, or oil, and include light-weight, standard, and extra-strong classes.

\section{Section I. MANOFACTORE}

2. (a) Process.-The steel for welded pipe shall be of good welding quality made by the Bessemer, open-hearth, or electric process. The steel for all seamless pipe shall be made by the electric or openhearth process.

2. (b) The wrought iron shall be made from muck bars made entirely from puddled pig iron, free from any admixture of iron scrap or steel. The term iron scrap applies only to foreign or bought scrap and does not include local mill products free from foreign or bought scrap.

(c) Welded pipe, 3 inches or under in nominal diameter, may be butt-welded unless otherwise specified. Welded pipe over 3 inches in nominal diameter shall be lap-welded.

(d) Open-hearth iron.

\section{Section II. CHEMICAL PROPERTIES AND TESTS}

(Chemical properties and tests are the same as specified for pipe given in A. P. I. standard No. 5-A above, with the following exceptions.)

(Table 1 of chemical properties given in A. P. I. standard No. 5-A above includes a grade D for seamless pipe which is not included as a grade in this specification for line pipe. Also a carbon content of 0.30 per cent is permitted on grade B line pipe because of field welding. In case grade $\mathrm{C}$ is to be joined by welding, the purchaser may wish to stipulate the carbon content by special agreement. It is contemplated that a limit will be included in this specification for this grade when engineering experience has established the highest satisfactory maximum.)

\section{Section III. PHYSICAL PROPERTIES AND TESTS}

(Tension-test requirements for line pipe are the same as given in A. P. I. standard 5-A, above, with the exception of the following differences in Table 2. Grade B of seamless steel line pipe shall have a minimum tensile strength of 65,000 pounds per square inch and a yield point of 38,000 pounds per square inch. The elongation for Bessemer steel line pipe, welded, shall be as follows: For large sizes, 18 per cent in 8 inches; for $3 / 4$-inch and $1 / 2$-inch butt-welded sizes, 18 per cent in 6 inches; for $3 / 8$-inch and $1 / 4$-inch butt-welded sizes, 18 per cent in 4 inches; and for 1/8-inch butt-welded size, 18 per cent in 2 inches.)

(Hydrostatic tests and flattening tests for line pipe are the same as specified in A. P. I. standard 5-A, above, paragraphs $6(a),(b), 7(a)$, and $(b)$. No drift tests are required for line pipe. Additional tests required of line pipe are as given below.)

7. (c) For butt-welded steel or open-hearth iron pipe over 2 inches in diameter, a section of pipe 6 inches long shall be flattened between parallel plates until the distance between the plates is 60 per cent of the outside diameter of the pipe with the weld located $45^{\circ}$ from the point of maximum bend, without developing cracks. In the case of buttwelded wrought-iron pipe over 2 inches in diameter, a section of pipe 6 inches long shall be flattened until broken. The test piece must show a good weld and the fracture shall have a fibrous appearance.

8. Bend tests.-For butt-welded steel pipe 2 inches or under in diameter, a sufficient length of pipe shall stand being bent cold through $90^{\circ}$ around a cylindrical mandrel, the diameter of which is twelve times the nominal diameter of the pipe, without developing cracks at any portion and without opening the weld. In the case of butt-welded iron pipe, the cylindrical mandrel for this test may be fifteen times the nominal diameter of the pipe. The weld shall be placed approximately $45^{\circ}$ from the inside radius of the axis of bending.

(Selection of test specimens, number of tests, and regulations for retests are the same for line pipe as given in A. P. I. standard 5-A, above, paragraphs $9(a),(b), 10,11(a)$, and $(b)$, with the following additional requirement with respect to the number of tests.)

One each of the tests specified in paragraphs 7 (c) and 8 may be made on a length in each lot of 400 or less, of each size.

\section{Section IV. STANDARD WEIGHTS AND LENGTHS}

12. (a) The standard sizes and weights for pipe are given in tables appended. (See Tables 3, 6, 7, and 8.)

(b) Permissible variations.-The weight of any length of pipe listed in Tables 3,6 , and 8 shall not vary more than $3 \frac{1}{2}$ per cent under or 10 per cent over that specified, but the carload weight shall not 
be more than $1 \frac{3}{4}$ per cent 75 under the nominal weight. The weight of any length of pipe listed in Table 7 shall not vary more than 5 per cent under or 10 per cent over that specified. Unless otherwise agreed upon, pipe sizes 4 inches and larger shall be weighed separately. Sizes smaller than 4 inches may be weighed in convenient lots. In the case of threaded line pipe, the pipe will be weighed with coupling screwed on but with thread protectors removed.

13. (a) Lengths.-Unless otherwise specified, threaded line pipe in standard weights and thicknesses, as listed in Table 3 , shall be furnished with not over 5 per cent under 18 feet in length, with a minimum of 16 feet for the length of any joint. On this class of goods, jointers (two short lengths connected by a coupling), the minimum length of the shorter piece to be 5 feet, may be shipped to a maximum of 5 per cent of the order.

(b) Unless otherwise specified, plain-end line pipe as listed in Table 6 shall have an average length of not less than 17 feet 6 inches and no length shorter than 9 feet may be shipped. Plain-end pipe as listed in Table 7 in sizes up to 20 inches outside diameter shall have an average length of not less than 17 feet and no length shorter than 9 feet may be shipped. On sizes larger than 20 inches outside diameter the minimum and average lengths shall be furnished as agreed upon between purchaser and manufacturer at time of purchase.

(c) When the average length agreed upon by the purchaser and manufacturer for either welded or seamless line pipe is in excess of 20 feet, not over 10 per cent of the number of pieces furnished on the order shall be less than 75 per cent of the average length agreed upon, and no pipe will be accepted which is less than 40 per cent of the average length. The average length, however, shall be maintained. When specified by the purchaser the foregoing stipulation shall apply to each carload.

\section{SECTION V. WORKMANSHIP AND FINISH}

14. (a) All skelp entering into lapwelded pipe must be scarfed.

(b) For pipe $1 \frac{1}{2}$ inches or under in nominal inside diameter, the outside diameter at any point shall not vary more than $1 / 64$ inch over nor more than $1 / 32$ inch under the standard size. For pipe 2 inches or over in nominal inside diameter, the outside diameter shall not vary more than 1 per cent over or under the standard size. The wall thickness of welded and seamless pipe shall not be more than $12 \frac{1}{2}$ per cent under the nominal wall thickness at any point. The thickness shall be determined from measurements on the body of the pipe and the outside diameter of that portion of the pipe used for threading must be governed by the closer tolerances called for by the threading specifications.

(c) The finished welded pipe shall be reasonably straight and free from injurious defects, such as bad welds, sand pits, ball cuts, pits, cinder spots, liquor marks, blisters, slivers, and laminations. Seamless pipe shall be reasonably straight and free from injurious seams and defects. When the depth of defect of either seamless or welded pipe is in excess of $12 \frac{1}{2}$ per cent of the nominal wall thickness, such defects shall be considered injurious. The welding of injurious defects shall be permitted at the discretion of the manufacturer, provided the depth does not exceed $33 \frac{1}{3}$ per cent of the nominal wall thick-

75 The weight of 1 cubic inch of steel is 0.2833 pound, and that of wrought iron is 0.2788 pound, therefore a length of wrought-iron pipe made to full dimensions for thickness and diameter will weigh approximately 2 per cent less than a similar length of pipe made from steel, and due allowance should be made in the "weights per foot" given in tables. In view of this difference the carload weight for wrought-iron pipe should not be more than $3 \frac{1}{2}$ per cent under the nominal weight. ness, and provided the length of the repair in seamless pipe does not exceed 25 per cent of the nominal diameter of the pipe. On welded pipe repairing shall not be done closer than 1 inch to the weld. Defects must be thoroughly chipped out before welding. It should be understood that hydrostatic tests made on pipe repaired in this manner shall be made on the finished pipe. Welding of any defects in welded or seamless material is subject to the approval of the inspector, when the purchaser is represented by an inspector. The threading on the pipe must be free from tears, shoulders, cuts, or any defects that may break the continuity of the thread.

15. (a) Ends.-Unless otherwise specified, pipe shall conform to the following regular practice:

(b) Each end of threaded pipe shall be reamed to remove all burrs. Plain-end pipe for use with the Dresser or Dayton type coupling shall be reamed both inside and outside just sufficiently to remove any burrs or shall be beveled on the outside to an angle of $45^{\circ}$ and with an average width of flat at the end of pipe of $1 / 16$ inch $\pm 1 / 32$ inch. All such pipe shall be sufficiently free from indentations, projections, or roll marks for a distance of 8 inches from the end of the pipe to make a tight joint with the rubber-gasket type of coupling. All plain-end line pipe intended for Dresser or Dayton type joints, or for welding sizes $103 / 4$ inches outside diameter and smaller, shall not be more than one sixty-fourth inch smaller than the nominal outside diameter for a distance of 8 inches from the end of the pipe and shall permit the passing for a distance of 8 inches of a ring gage which has a bore one-sixteenth inch larger than the nominal outside diameter of the pipe. Sizes larger than $103 / 4$ inches outside diameter shall not be more than one thirty-second inch smaller than the nominal outside diameter for a distance of 8 inches from the end of the pipe and shall permit the passing for a distance of 8 inches of a ring gage which has bore three thirty-seconds inch larger than the nominal outside diameter of the pipe.

(c) Plain-end pipe for welding, unless otherwise specified, shall be beveled to an angle of $45^{\circ}$ and with a width of flat at the end of the pipe $1 / 16$ inch \pm $1 / 32$ inch. This class of material shall be reamed inside at the ends sufficiently to remove all burrs. Where material is ordered beveled to any other than a $45^{\circ}$ angle, it shall be understood that the angle is to be measured from a line drawn perpendicular to the axis of the pipe. This means that a greater amount of material is removed with a $60^{\circ}$ angle than with a $45^{\circ}$ angle.

(d) Extra-strong pipe shall be furnished with plain ends, unless otherwise specified.

16 (a) Threads and gages. - When threaded line pipe is ordered, as specified in Table 3 , the threads shall be in accordance with Table 4 and cut so as to make a tight joint when the pipe is tested at the mill to the specified internal hydrostatic pressure. The variation from the standard, when tested with the standard working gage, shall not exceed a maximum of one and one-half turns either way.

(b) When extra-strong pipe is ordered threaded, the threads shall be in accordance with Table 4 and shall be subject to the same requirements and tests as threads on line pipe.

(Other requirements for threads and gages are the same as given in paragraphs 17 to 20 , inclusive, of A. P. I. standard No. 5-A, above, except that no requirements are included in this specification for $3 / 8$-inch taper because line pipe is all $3 / 4$-inch taper. Requirements on taper tests, thread protectors, and couplings are the same as given in A. P. I. standard No. 5-A, above, paragraphs 22 (a), 23, 24, and 25, with the following additional requirement for couplings: When couplings are furnished with extrastrong pipe, they shall be of the same weight and dimensions as the couplings specified for line pipe. 
The alignment test is the same as specified in inclusive, and one-half inch high on couplings for paragraph 26 of A. P. I. standard No. 5-A, above.) 27. (a) Marking.- The length, test pressure in pounds, and class of material shall be stenciled on each length of pipe, sizes 2 inches and larger, and on sizes 4 inches and larger; except dipped or coated pipe, the weight of each length shall be stenciled. Approved abbreviations may be used. The A.P.I. monogram shall be stamped with steel die on each joint, sizes 2 inches and larger, 1 foot from couplings on the shop end of threaded line pipe and about 1 foot from either end on plain-end line pipe. The A. P. I. monogram shall also be stamped with a steel die on all couplings for pipe sizes 2 inches and larger. The size of monogram to be one-fourth inch high on all sizes of pipe; three-eighths inch high on couplings for pipe sizes from 2 inches to 6 inches, pipe sizes over 6 inches. (The upper and lower crossbar on the letter "I" in the A.P. I. monogram may be omitted. On pipe sizes smaller than 2 inches, which are regularly bundled, the A. P. I. monogram will not be stamped on either the pipe or the couplings but will be stamped on the metal measurement tag which is attached to each bundle. In addition to the above markings and in letters of similar size, the grades A, B, and C of seamless pipe shall be stamped with a steel die.

(Other requirements under "marking" are the same as given in A. P. I. standard No. 5-A, above, paragraphs $27(b),(c)$, and 28.)

(Requirements on inspection and rejection are the same as given in A. P. I. standard No. 5-A, above, paragraphs $29(a),(b)$, and 30 .)

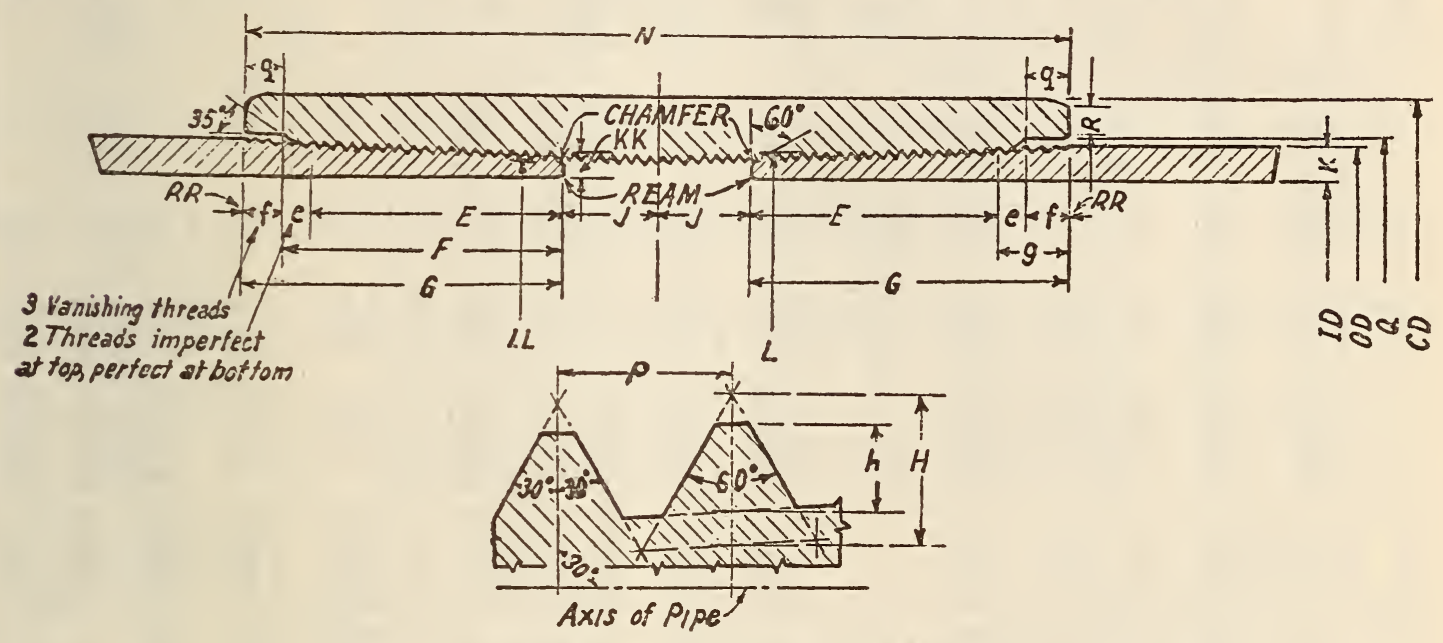

ENLARGEO SECTION OF THREAD

FIGURE 285.-A. P. I. line pipe 
TABle 3.-A.P.I. line pipe (threaded)

[See Fig. 285]

[All dimensions in inches at $68^{\circ} \mathrm{F}$.]

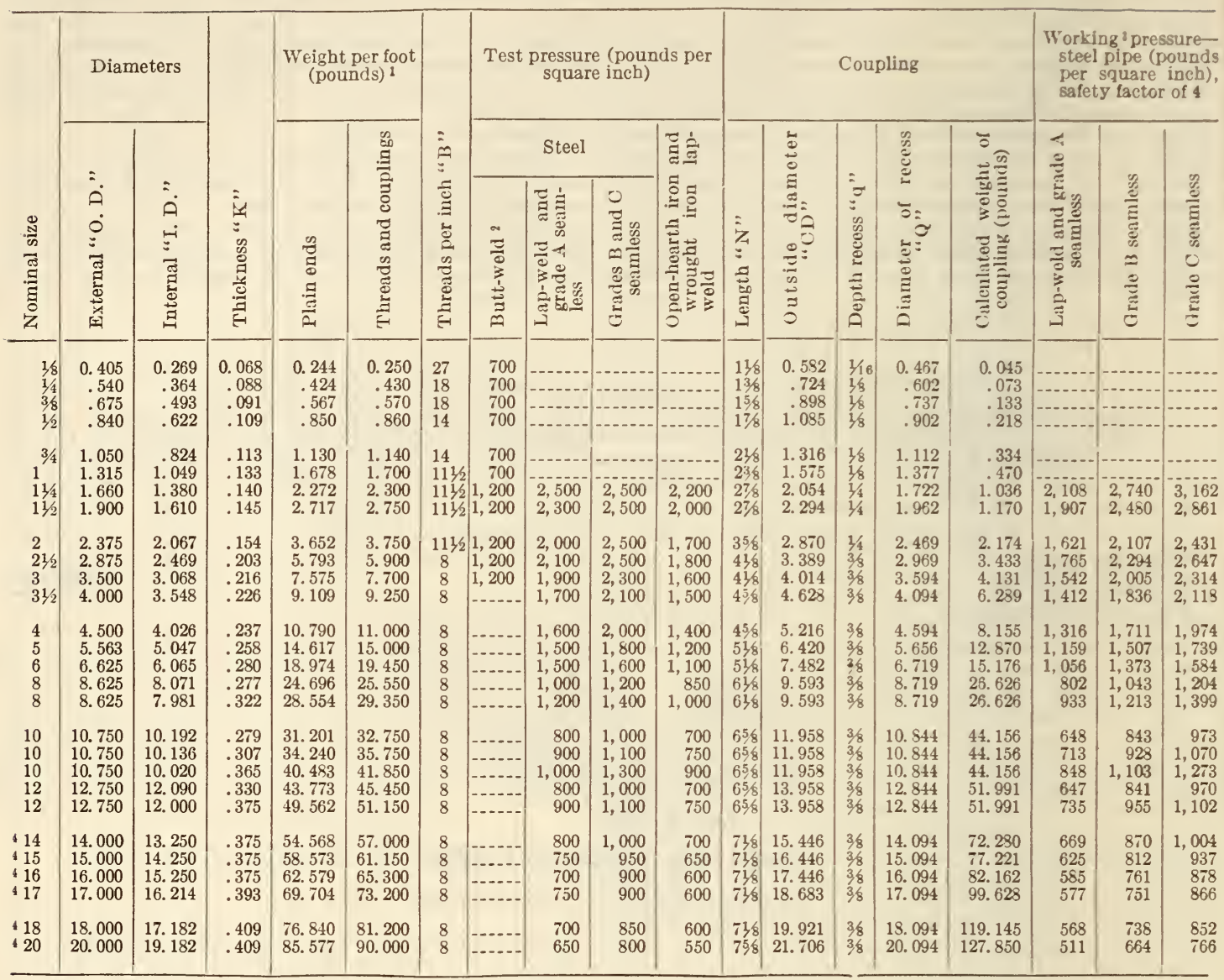

1 See par. $12(b)$.

2 Standard test pressures for butt-weld pipe are not figured by formula.

3 The indicated working pressures are given for reference purposes only. They apply for a safety factor of 4 throughout, and do not take into account differences in pipe fabrication or in service requirements. To obtain the working pressures for other assumed factors of safety, multiply these pressures by 4 and divide by the safety factor desired.

4 Outside diameter.

Note.-(Values in the first four and the seventh columns of Table 3 are the same as given in A. S. T. M. specification A53-30 for welded and seamless steel pipe, standard weight. See A. S. T. M. below. Column 5 of Table 3 agrees with Federal specification W W-P-431 given below under United States Government, Federal Specifications Board.) 
TABLE 4.-Threads, American Petroleum Institute line pipe

[See Fig. 285]

[All dimensions in inches at $68^{\circ}$ ]

\begin{tabular}{|c|c|c|c|c|c|c|c|c|c|c|c|c|c|c|}
\hline \multicolumn{3}{|c|}{ Pipe } & \multicolumn{7}{|c|}{ Threads } & \multirow{2}{*}{$\begin{array}{l}\text { Diam- } \\
\text { eter of } \\
\text { pipe at } \\
\text { root of } \\
\text { threads, } \\
\text { "L. L." }\end{array}$} & \multirow{2}{*}{$\begin{array}{l}\text { Diam- } \\
\text { eter of } \\
\text { pipe at } \\
\text { top of } \\
\text { threads } \\
\text { "L" }\end{array}$} & \multirow{2}{*}{$\begin{array}{l}\text { End of } \\
\text { pipe to } \\
\text { center } \\
\text { of cou- } \\
\text { pling, } \\
\text { made } \\
\text { up, } \\
\text { "J", }\end{array}$} & \multirow{2}{*}{$\begin{array}{c}\text { Distance } \\
\text { end of } \\
\text { coupling } \\
\text { beyond } \\
\text { last } \\
\text { scratch } \\
\text { made up, } \\
\text { "R. R." }\end{array}$} & \multirow{2}{*}{$\begin{array}{l}\text { Stand- } \\
\text { off of } \\
\text { cou- } \\
\text { pling at } \\
\text { hand- } \\
\text { tight } \\
\text { fit, } \\
\text { "A" }\end{array}$} \\
\hline Size & $\begin{array}{c}\text { Exter- } \\
\text { nal } \\
\text { dism- } \\
\text { eter, } \\
\text { "O.D." }\end{array}$ & $\begin{array}{l}\text { Weight } \\
\text { per } \\
\text { foot } \\
\text { com- } \\
\text { plete }\end{array}$ & $\begin{array}{l}\text { Num. } \\
\text { ber } \\
\text { per } \\
\text { inch, } \\
\text { "B" }\end{array}$ & $\begin{array}{l}\text { Taper, } \\
\text { inches } \\
\text { per } \\
\text { foot, } \\
\text { "b" }\end{array}$ & $\begin{array}{l}\text { Length } \\
\text { of } \\
\text { perfect, } \\
\text { "E" }\end{array}$ & $\begin{array}{c}\text { Number } \\
\text { of } \\
\text { perfect }\end{array}$ & $\begin{array}{l}\text { Effec- } \\
\text { tive } \\
\text { length, } \\
\text { "F" }\end{array}$ & $\begin{array}{l}\text { Total } \\
\text { lengtb } \\
\text { "G" }\end{array}$ & $\begin{array}{l}\text { Height } \\
\text { of } \\
\text { thread } \\
\text { trun- } \\
\text { cated, } \\
\text { "h" }\end{array}$ & & & & & \\
\hline $\begin{array}{l}1 / 8 \\
1 / 4 \\
3 / 8\end{array}$ & $\begin{array}{l}0.405 \\
.540 \\
.675 \\
.840\end{array}$ & & $\begin{array}{l}27 \\
18 \\
18 \\
14\end{array}$ & \begin{tabular}{c|c}
$3 / 4$ \\
$3 / 4$ \\
$3 / 4$ \\
$3 / 4$
\end{tabular} & $\begin{array}{r}0.1 \\
.2 \\
.2 \\
.3\end{array}$ & & $\begin{array}{l}0.2 \\
.4 \\
.4 \\
.5\end{array}$ & & & & & $\begin{array}{r}0.187 \\
.119 \\
.238 \\
.189\end{array}$ & $\begin{array}{r}0.000 \\
.000 \\
.000 \\
.000\end{array}$ & \\
\hline $\begin{array}{l}8 / 4 \\
11 / 4 \\
11 / 2\end{array}$ & $\begin{array}{l}1.050 \\
1.315 \\
1.660 \\
1.900\end{array}$ & & $\begin{array}{l}14 \\
111 / 2 \\
111 / 2 \\
111 / 2\end{array}$ & $\begin{array}{l}3 / 4 \\
3 / 4 \\
3 / 4 \\
3 / 4\end{array}$ & & & & & & & & $\begin{array}{l}.302 \\
.244 \\
.470 \\
.453\end{array}$ & $\begin{array}{l}.000 \\
.000 \\
.000 \\
.000\end{array}$ & \\
\hline $\begin{array}{l}2 \\
21 / 2 \\
3 \\
31 / 2\end{array}$ & & & $\begin{array}{l}111 / 2 \\
8 \\
8 \\
8\end{array}$ & $\begin{array}{l}3 / 4 \\
3 / 4 \\
3 / 4 \\
3 / 4\end{array}$ & $\begin{array}{r}.93043 \\
.95000 \\
.95000 \\
1.17500\end{array}$ & & & & & & & $\begin{array}{l}.447 \\
.487 \\
.487 \\
.512\end{array}$ & $\begin{array}{l}.000 \\
.000 \\
.000 \\
.000\end{array}$ & \\
\hline $\begin{array}{l}4 \\
5 \\
6 \\
8\end{array}$ & & All. & $\begin{array}{l}8 \\
8 \\
8 \\
8\end{array}$ & $\begin{array}{l}3 / 4 \\
3 / 4 \\
8 / 4 \\
3 / 4 \\
\end{array}$ & $\begin{array}{l}1.17500 \\
1.40630 \\
1.51250 \\
1.71250\end{array}$ & & & & & & & $\begin{array}{l}.512 \\
.531 \\
.424 \\
.724\end{array}$ & $\begin{array}{l}.000 \\
.000 \\
.000 \\
.000\end{array}$ & \\
\hline $\begin{array}{l}10 \\
12 \\
114 \\
115\end{array}$ & & All. & $\begin{array}{l}8 \\
8 \\
8 \\
8\end{array}$ & $\begin{array}{c}3 / 4 \\
3 / 4 \\
3 / 4 \\
3 / 4 \\
\end{array}$ & & & & & & & & $\begin{array}{l}.762 \\
.562 \\
.687 \\
.587\end{array}$ & $\begin{array}{l}.000 \\
.000 \\
.000 \\
.000\end{array}$ & \\
\hline 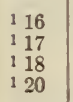 & $\begin{array}{l}16.000 \\
17.000 \\
18.000 \\
20.000\end{array}$ & & $\begin{array}{l}8 \\
8 \\
8 \\
8\end{array}$ & $\begin{array}{l}8 / 4 \\
8 / 4 \\
3 / 4 \\
3 / 4\end{array}$ & $\begin{array}{l}2.45000 \\
2.45000 \\
2.45000 \\
2.60000\end{array}$ & 20.80000 & $\begin{array}{l}2.70000 \\
2.70000 \\
2.70000 \\
2.85000\end{array}$ & $\begin{array}{l}\text { 3. } 07500 \\
\text { 3. } 22500\end{array}$ & .10000 & 19.63750 & $\begin{array}{l}17.84688 \\
19.83750\end{array}$ & $\begin{array}{l}.487 \\
.487 \\
.487 \\
.587\end{array}$ & $\begin{array}{l}.000 \\
.000 \\
.000 \\
.000\end{array}$ & \\
\hline
\end{tabular}

1 Outside diameter.

TABLE 6.-A. P. I. outside diameter plain-end line pipe

[All dimensions in inches at $68^{\circ} \mathrm{F}$.]

\begin{tabular}{|c|c|c|c|c|c|c|c|c|}
\hline \multirow{3}{*}{ 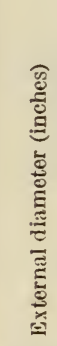 } & \multirow[b]{3}{*}{ 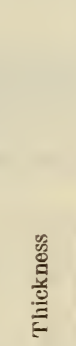 } & \multirow{3}{*}{ 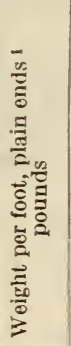 } & \multicolumn{3}{|c|}{$\begin{array}{l}\text { Test pressures } \\
\text { (poundspersquare } \\
\text { inch) }\end{array}$} & \multicolumn{3}{|c|}{$\begin{array}{l}\text { Working }{ }^{2} \text { pressure } \\
\text { steel pipe (pounds } \\
\text { per square inch) } \\
\text { safety factor of } 4\end{array}$} \\
\hline & & & \multicolumn{2}{|c|}{ Steel } & \multirow{2}{*}{ 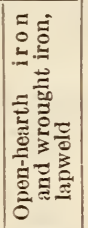 } & \multirow{2}{*}{ 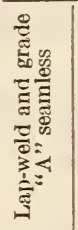 } & \multirow{2}{*}{ 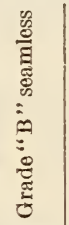 } & \multirow{2}{*}{ 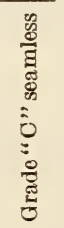 } \\
\hline & & & 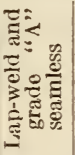 & 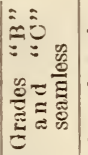 & & & & \\
\hline & $\left\{\begin{array}{l}0.216 \\
.241 \\
.254 \\
.289 \\
.300\end{array}\right.$ & $\begin{array}{r}7.575 \\
8.388 \\
8.805 \\
9,910 \\
10.252\end{array}$ & $\begin{array}{l}1,900 \\
2,000 \\
2,200 \\
2,500 \\
2,500\end{array}$ & $\begin{array}{l}2,300 \\
2,500 \\
2,500 \\
2,500 \\
2,500\end{array}$ & $\begin{array}{l}1,600 \\
1,800 \\
1,900 \\
2,200 \\
2,300\end{array}$ & $\begin{array}{l}1,542 \\
1,721 \\
1,814 \\
2,064 \\
2,142\end{array}$ & $\begin{array}{l}2,005 \\
2,237 \\
2,358 \\
2,683 \\
2,785\end{array}$ & $\begin{array}{l}2,314 \\
2,582 \\
2,721 \\
3,096 \\
3,214\end{array}$ \\
\hline & .226 & 9.109 & 1,700 & 2,100 & 1,500 & 1,412 & 1,836 & 2,118 \\
\hline & $\begin{array}{l}.237 \\
.250 \\
.271 \\
.3125\end{array}$ & $\begin{array}{l}10.790 \\
11.347 \\
12.240 \\
13.975\end{array}$ & $\begin{array}{l}1,600 \\
1,700 \\
1,800 \\
2,000\end{array}$ & $\begin{array}{l}2,000 \\
2,100 \\
2,300 \\
2,500\end{array}$ & $\begin{array}{l}1,400 \\
1,500 \\
1,600 \\
1,800\end{array}$ & $\begin{array}{l}1,316 \\
1,388 \\
1,505 \\
1,736\end{array}$ & $\begin{array}{l}1,711 \\
1,805 \\
1,957 \\
2,256\end{array}$ & $\begin{array}{l}1,974 \\
2,083 \\
2,258 \\
2,604\end{array}$ \\
\hline & $\left\{\begin{array}{l}.280 \\
.432\end{array}\right.$ & $\begin{array}{l}18.974 \\
28.573\end{array}$ & $\begin{array}{l}1,500 \\
2,000\end{array}$ & $\begin{array}{r}1,600 \\
2,500\end{array}$ & $\begin{array}{l}1,100 \\
1,800\end{array}$ & $\begin{array}{l}1,056 \\
1,630\end{array}$ & $\begin{array}{l}1,373 \\
2,119\end{array}$ & $\begin{array}{l}1,584 \\
2,445\end{array}$ \\
\hline $85 \%$ & $\left\{\begin{array}{l}.277 \\
.322 \\
.500\end{array}\right.$ & $\begin{array}{l}24.696 \\
28.554 \\
43.388\end{array}$ & $\begin{array}{l}1,000 \\
1,200 \\
1,700\end{array}$ & $\begin{array}{r}1,200 \\
1,400 \\
2,200\end{array}$ & $\begin{array}{r}850 \\
1,000 \\
1,500\end{array}$ & $\begin{array}{r}802 \\
933 \\
1,449\end{array}$ & $\begin{array}{l}1,043 \\
1,213 \\
1,884\end{array}$ & $\begin{array}{l}1,204 \\
1,399 \\
2,173\end{array}$ \\
\hline & $\left\{\begin{array}{l}.279 \\
.307 \\
.348\end{array}\right.$ & $\begin{array}{l}31.201 \\
34.240 \\
38.661\end{array}$ & $\begin{array}{r}800 \\
900 \\
1,000\end{array}$ & $\begin{array}{l}1,000 \\
1,100 \\
1,200\end{array}$ & $\begin{array}{l}700 \\
750 \\
850\end{array}$ & $\begin{array}{l}648 \\
713 \\
809\end{array}$ & $\begin{array}{r}843 \\
928 \\
1,052\end{array}$ & $\begin{array}{r}973 \\
1,070 \\
1,213\end{array}$ \\
\hline
\end{tabular}

${ }_{1}$ See par. $12(b)$

2 The indicated working pressures are given for reference purposes only. They apply for a safety factor of 4 throughout and do not take into account differences in pipe fabrication or in service requirements. To obtain the working pressures for other assumed factors of safety, multiply these pressures by 4 and divide by the safety factor desired.
TABLE 6.-A. P. I. outside diameter plain-enp line pipe-Continued

[All dimensions in inches at $68^{\circ} \mathrm{F}$.]

\begin{tabular}{|c|c|c|c|c|c|c|c|c|}
\hline \multirow{3}{*}{ 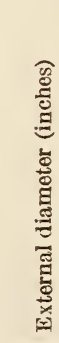 } & \multirow[b]{3}{*}{ 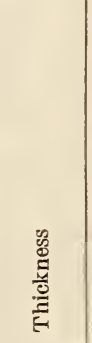 } & \multirow{3}{*}{ 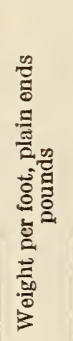 } & \multicolumn{3}{|c|}{$\begin{array}{l}\text { Test pressures } \\
\text { (pounds per square } \\
\text { inch) }\end{array}$} & \multicolumn{3}{|c|}{$\begin{array}{l}\text { Working pressure } \\
\text { steel pipe (pounds } \\
\text { per square inch) } \\
\text { safety factor of } 4\end{array}$} \\
\hline & & & \multicolumn{2}{|c|}{ Steel } & \multirow{2}{*}{ 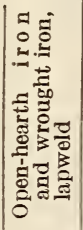 } & \multirow{2}{*}{ 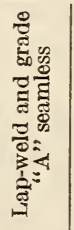 } & \multirow{2}{*}{ 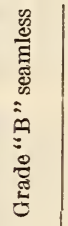 } & \multirow{2}{*}{ 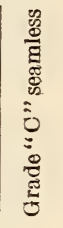 } \\
\hline & & & 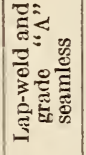 & 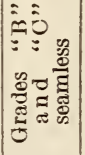 & & & & \\
\hline $10^{3}$ & $\left\{\begin{array}{r}0.365 \\
.500\end{array}\right.$ & $\begin{array}{l}40.483 \\
54.735 \\
\end{array}$ & $\begin{array}{l}1,000 \\
1,400\end{array}$ & $\begin{array}{l}1,300 \\
1,800\end{array}$ & $\begin{array}{r}900 \\
1,200\end{array}$ & $\begin{array}{r}848 \\
1,162\end{array}$ & $\begin{array}{l}1,103 \\
1,511\end{array}$ & $\begin{array}{l}1,273 \\
1,744\end{array}$ \\
\hline 123 & $\begin{array}{l}.330 \\
.34375 \\
.375 \\
.4375 \\
.500\end{array}$ & \begin{tabular}{|l|}
43.773 \\
45.547 \\
49.562 \\
57.531 \\
65.415
\end{tabular} & $\begin{array}{r}800 \\
800 \\
900 \\
1,000 \\
1,100\end{array}$ & $\begin{array}{l}1,000 \\
1,000 \\
1,100 \\
1,300 \\
1,500\end{array}$ & $\begin{array}{r}700 \\
700 \\
750 \\
900 \\
1,050\end{array}$ & $\begin{array}{l}647 \\
674 \\
735 \\
857 \\
980\end{array}$ & $\begin{array}{r}841 \\
876 \\
955 \\
1,115 \\
1,274\end{array}$ & $\begin{array}{r}970 \\
1,011 \\
1,102 \\
1,286 \\
1,470\end{array}$ \\
\hline 14 & $\begin{array}{l}.375 \\
.40625 \\
.4375 \\
.46875 \\
.500\end{array}$ & \begin{tabular}{|l|}
54.568 \\
58.980 \\
63.371 \\
67.741 \\
72.091
\end{tabular} & $\begin{array}{r}800 \\
900 \\
900 \\
1,000 \\
1,100\end{array}$ & $\begin{array}{l}1,000 \\
1,100 \\
1,200 \\
1,300 \\
1,400\end{array}$ & $\begin{array}{l}700 \\
750 \\
800 \\
900 \\
950\end{array}$ & $\begin{array}{l}669 \\
725 \\
781 \\
837 \\
892\end{array}$ & $\begin{array}{r}870 \\
943 \\
1,015 \\
1,088 \\
1,160\end{array}$ & $\begin{array}{l}1,004 \\
1,088 \\
1,171 \\
1,255 \\
1,339\end{array}$ \\
\hline 16 & $\begin{array}{l}.375 \\
.40625 \\
.4375 \\
.46875 \\
.500\end{array}$ & $\begin{array}{l}62.579 \\
67.658 \\
72.716 \\
77.754 \\
82.771\end{array}$ & $\begin{array}{r}700 \\
800 \\
900 \\
900 \\
1,000\end{array}$ & $\begin{array}{r}900 \\
950 \\
1,000 \\
1,100 \\
1,200\end{array}$ & $\begin{array}{l}600 \\
650 \\
800 \\
800 \\
900\end{array}$ & $\begin{array}{l}585 \\
634 \\
683 \\
732 \\
781\end{array}$ & $\begin{array}{r}761 \\
825 \\
888 \\
952 \\
1,015\end{array}$ & $\begin{array}{r}878 \\
952 \\
1,025 \\
1,098 \\
1,171\end{array}$ \\
\hline 18 & $\begin{array}{l}.40625 \\
.4375 \\
.46875 \\
.500\end{array}$ & $\begin{array}{l}76.336 \\
82.061 \\
87.767 \\
93.451\end{array}$ & $\begin{array}{l}700 \\
700 \\
750 \\
800\end{array}$ & $\begin{array}{r}850 \\
900 \\
1,000 \\
1,100\end{array}$ & $\begin{array}{l}600 \\
650 \\
700 \\
750\end{array}$ & $\begin{array}{l}564 \\
607 \\
651 \\
694\end{array}$ & $\begin{array}{l}733 \\
789 \\
846 \\
902\end{array}$ & $\begin{array}{r}846 \\
911 \\
976 \\
1,041\end{array}$ \\
\hline & $\begin{array}{l}.40625 \\
.4375 \\
.46875 \\
.500\end{array}$ & $\begin{array}{r}85.013 \\
91.407 \\
97.779 \\
104.131\end{array}$ & $\begin{array}{l}600 \\
650 \\
700 \\
750\end{array}$ & $\begin{array}{l}800 \\
850 \\
900 \\
950\end{array}$ & $\begin{array}{l}550 \\
550 \\
600 \\
650\end{array}$ & $\begin{array}{l}507 \\
546 \\
585 \\
625\end{array}$ & $\begin{array}{l}660 \\
710 \\
761 \\
812\end{array}$ & $\begin{array}{l}761 \\
820 \\
878 \\
937\end{array}$ \\
\hline
\end{tabular}


TABLE 7.-A. P. I. outside diameter plain-end $\mathrm{T}_{\mathrm{ABLE}}$ 7.-A. P. I. outside diameler plain-end line pipe

[(Light weight) or special diameters]

[All dimensions in inches at $68^{\circ} \mathrm{F}$.]

\begin{tabular}{|c|c|c|c|c|c|c|c|c|}
\hline \multirow{3}{*}{ 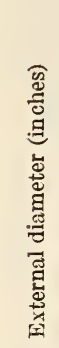 } & \multirow[b]{3}{*}{ 岕 } & \multirow{3}{*}{ 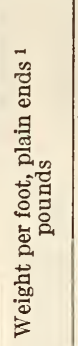 } & \multicolumn{3}{|c|}{$\begin{array}{l}\text { Test pressures } \\
\text { (pounds persquare } \\
\text { inch) }\end{array}$} & \multicolumn{3}{|c|}{$\begin{array}{l}\text { Working }{ }^{2} \text { pressure } \\
\text { steel pipe (pounds } \\
\text { per square inch) } \\
\text { safety factor of } 4\end{array}$} \\
\hline & & & \multicolumn{2}{|c|}{ Steel } & \multirow{2}{*}{ 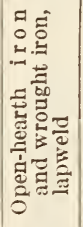 } & \multirow{2}{*}{ 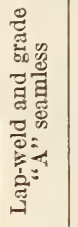 } & \multirow{2}{*}{ 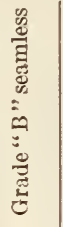 } & \multirow{2}{*}{ 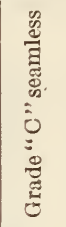 } \\
\hline & & & 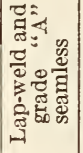 & 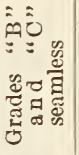 & & & & \\
\hline & 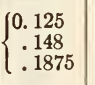 & $\begin{array}{l}\text { 4. } 505 \\
\text { 5. } 298 \\
6.633\end{array}$ & $\begin{array}{l}1,100 \\
1,300 \\
1,600\end{array}$ & $\begin{array}{l}1,400 \\
1,600 \\
2,000\end{array}$ & $\begin{array}{r}900 \\
1,100 \\
1,400\end{array}$ & $\begin{array}{r}892 \\
1,057 \\
1,339\end{array}$ & & $\begin{array}{l}1,33 \\
1,58 \\
2,00\end{array}$ \\
\hline & & $\begin{array}{l}5.293 \\
5.532 \\
6.038 \\
7.634\end{array}$ & $\begin{array}{l}1,000 \\
1,000 \\
1,100 \\
1,400\end{array}$ & $\begin{array}{l}1,200 \\
1,300 \\
1,400 \\
1,800\end{array}$ & $\begin{array}{r}850 \\
900 \\
1,000 \\
1,200\end{array}$ & $\begin{array}{r}800 \\
837 \\
925 \\
1,171\end{array}$ & & $\begin{array}{l}1,20 \\
1,25 \\
1,38 \\
1,75\end{array}$ \\
\hline & $\begin{array}{l}.1 \\
.18 \\
.18 \\
.1 \\
.1 \\
.2\end{array}$ & $\begin{array}{r}5.976 \\
6.609 \\
7.639 \\
8.635 \\
9.403 \\
11.652 \\
12.390 \\
12.891 \\
14.966 \\
15.906 \\
17.021\end{array}$ & $\begin{array}{r}850 \\
1,000 \\
1,100 \\
1,200 \\
1,400 \\
750 \\
800 \\
850 \\
1,000 \\
1,100 \\
1,100\end{array}$ & $\begin{array}{r}1,100 \\
1,200 \\
1,400 \\
1,600 \\
1,700 \\
900 \\
1,000 \\
1,100 \\
1,300 \\
1,300 \\
1,400\end{array}$ & $\begin{array}{r}750 \\
800 \\
1,000 \\
1,100 \\
1,200 \\
700 \\
700 \\
750 \\
900 \\
950 \\
1,000\end{array}$ & $\begin{array}{r}711 \\
788 \\
916 \\
1,041 \\
1,138 \\
637 \\
679 \\
707 \\
825 \\
879 \\
943\end{array}$ & $\begin{array}{r}924 \\
1,025 \\
1,191 \\
1,354 \\
1,480 \\
829 \\
883 \\
919 \\
1,073 \\
1,143 \\
1,226\end{array}$ & $\begin{array}{r}1,06 \\
1,18 \\
1,37 \\
1,56 \\
1,70 \\
95 \\
1,01 \\
1,06 \\
1,23 \\
1,31 \\
1,41\end{array}$ \\
\hline & & $\begin{array}{l}13.807 \\
15.644 \\
18.179 \\
20.692 \\
23.185\end{array}$ & $\begin{array}{r}600 \\
700 \\
800 \\
1,000 \\
1,100\end{array}$ & 800 & 900 & $\begin{array}{l}683 \\
781 \\
878\end{array}$ & $\begin{array}{r}670 \\
761 \\
888 \\
1,015 \\
1,142\end{array}$ & $\begin{array}{l}1,02 \\
1,17 \\
1,31\end{array}$ \\
\hline & & & $\begin{array}{l}550 \\
700 \\
800 \\
900 \\
900\end{array}$ & 1,200 & & & $\begin{array}{l}621 \\
706 \\
824 \\
942 \\
994\end{array}$ & $\begin{array}{r}95 \\
1,08 \\
1,14\end{array}$ \\
\hline & $\begin{array}{l}.1 \\
.2 \\
.2 \\
.2 \\
.2 \\
.3\end{array}$ & & $\begin{array}{r}550 \\
600 \\
700 \\
750 \\
850 \\
1,000\end{array}$ & 1,200 & & $\begin{array}{l}703 \\
781\end{array}$ & $\begin{array}{r}609 \\
676 \\
710 \\
812 \\
914 \\
1,015\end{array}$ & $\begin{array}{r}82 \\
93 \\
1,05 \\
1,17\end{array}$ \\
\hline 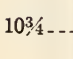 & .218 & $\begin{array}{l}24.6 \\
28.0\end{array}$ & $\begin{array}{l}600 \\
700\end{array}$ & $\begin{array}{l}75 \\
90\end{array}$ & $\begin{array}{l}55 \\
60\end{array}$ & $\begin{array}{l}50 \\
58\end{array}$ & & \\
\hline & & & 550 & 1,100 & & 716 & & $\begin{array}{r}96 \\
1,00 \\
1,07\end{array}$ \\
\hline & $\begin{array}{l}.250 \\
.28125 \\
.3125\end{array}$ & & & & & $\begin{array}{l}490 \\
551 \\
612\end{array}$ & & \\
\hline & $\left\{\begin{array}{l}.25 \\
.28 \\
.31\end{array}\right.$ & $\begin{array}{l}36.713 \\
41.208 \\
45.682 \\
50.136\end{array}$ & 550 & & & $\begin{array}{l}558 \\
613\end{array}$ & $\begin{array}{l}725 \\
797\end{array}$ & $\begin{array}{l}837 \\
920\end{array}$ \\
\hline & $\begin{array}{l}.250 \\
.28125 \\
.3125 \\
.34375\end{array}$ & $\begin{array}{l}42.053 \\
47.215 \\
52.357 \\
57.478\end{array}$ & $\begin{array}{l}450 \\
550 \\
600 \\
650\end{array}$ & $\begin{array}{l}60 \\
65\end{array}$ & $\begin{array}{l}40 \\
45\end{array}$ & $\begin{array}{l}390 \\
439 \\
488 \\
537\end{array}$ & $\begin{array}{l}507 \\
571 \\
634 \\
698\end{array}$ & 30 \\
\hline
\end{tabular}

1 See par. $12(b)$.

2 The indicated working pressures are given for reference purposes only. They apply for a safety factor of 4 throughout and do not take into account differences in pipe fabrication or in service requirements. To obtain the working pressures for other assumed factors of safety, multiply these pressures by 4 and divide by the safety factor desired.

\section{line pipe-Continued}

[(Light weight) or special diameters]

[All dimensions in inches at $68^{\circ} \mathrm{F}$.]

\begin{tabular}{|c|c|c|c|c|c|c|c|c|}
\hline \multirow{3}{*}{ 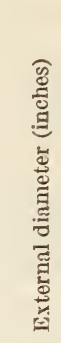 } & \multirow[b]{3}{*}{ 惫 } & \multirow{3}{*}{ 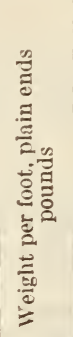 } & \multicolumn{3}{|c|}{$\begin{array}{l}\text { Test pressures } \\
\text { (pounds per square } \\
\text { inch) }\end{array}$} & \multicolumn{3}{|c|}{$\begin{array}{l}\text { Working yressure } \\
\text { steel pipe (pounds } \\
\text { per square inch) } \\
\text { safety tactor of } 4\end{array}$} \\
\hline & & & \multicolumn{2}{|c|}{ Steel } & \multirow{2}{*}{ 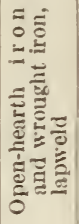 } & \multirow{2}{*}{ 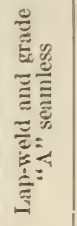 } & \multirow{2}{*}{ 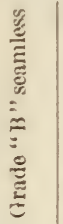 } & \multirow{2}{*}{ 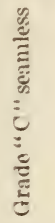 } \\
\hline & & & 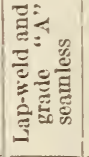 & 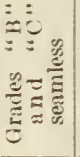 & & & & \\
\hline 18. & $\left\{\begin{array}{l}0.250 \\
.28125 \\
.3125 \\
.34375 \\
.375\end{array}\right.$ & $\begin{array}{l}47.393 \\
53.223 \\
59.032 \\
64.821 \\
70.589\end{array}$ & $\begin{array}{l}450 \\
450 \\
500 \\
550 \\
600\end{array}$ & $\begin{array}{l}550 \\
600 \\
650 \\
750 \\
800\end{array}$ & $\begin{array}{l}350 \\
400 \\
450 \\
500 \\
550\end{array}$ & $\begin{array}{l}347 \\
390 \\
434 \\
477 \\
520\end{array}$ & $\begin{array}{l}451 \\
507 \\
564 \\
620 \\
677\end{array}$ & $\begin{array}{l}520 \\
585 \\
651 \\
716 \\
781\end{array}$ \\
\hline 20. & $\begin{array}{l}.250 \\
.28125 \\
.3125 \\
.34375 \\
.375\end{array}$ & $\begin{array}{l}52.733 \\
59.231 \\
65.708 \\
72.164 \\
78.599\end{array}$ & $\begin{array}{l}400 \\
450 \\
450 \\
500 \\
600\end{array}$ & $\begin{array}{l}500 \\
550 \\
600 \\
650 \\
700\end{array}$ & $\begin{array}{l}350 \\
350 \\
400 \\
450 \\
500\end{array}$ & $\begin{array}{l}312 \\
351 \\
390 \\
429 \\
468\end{array}$ & $\begin{array}{l}406 \\
457 \\
507 \\
558 \\
609\end{array}$ & $\begin{array}{l}468 \\
527 \\
585 \\
644 \\
703\end{array}$ \\
\hline 22. & $\begin{array}{l}.3125 \\
.34375 \\
.375 \\
.40625 \\
.4375 \\
.46875 \\
.500\end{array}$ & $\begin{array}{r}72.383 \\
79.506 \\
86.609 \\
93.691 \\
100.752 \\
107.792 \\
114.811\end{array}$ & $\begin{array}{l}450 \\
450 \\
500 \\
550 \\
600 \\
600 \\
650\end{array}$ & $\begin{array}{l}550 \\
600 \\
650 \\
700 \\
750 \\
800 \\
850\end{array}$ & $\begin{array}{l}400 \\
400 \\
450 \\
500 \\
500 \\
550 \\
600\end{array}$ & $\begin{array}{l}355 \\
390 \\
426 \\
461 \\
497 \\
532 \\
568\end{array}$ & \begin{tabular}{l|}
461 \\
507 \\
553 \\
600 \\
646 \\
692 \\
738
\end{tabular} & $\begin{array}{l}532 \\
585 \\
639 \\
692 \\
745 \\
799 \\
852\end{array}$ \\
\hline & $\begin{array}{l}.3125 \\
.34375 \\
.375 \\
.40625 \\
.4375 \\
.46875 \\
.500\end{array}$ & $\begin{array}{r}79.058 \\
86.849 \\
94.619 \\
102.368 \\
110.097 \\
117.805 \\
125.491\end{array}$ & $\begin{array}{l}400 \\
450 \\
450 \\
500 \\
550 \\
550 \\
600\end{array}$ & $\begin{array}{l}500 \\
550 \\
600 \\
650 \\
700 \\
750 \\
800\end{array}$ & $\begin{array}{l}350 \\
400 \\
400 \\
450 \\
500 \\
500 \\
550\end{array}$ & $\begin{array}{l}325 \\
358 \\
390 \\
423 \\
455 \\
485 \\
520\end{array}$ & $\begin{array}{l}423 \\
465 \\
507 \\
550 \\
592 \\
634 \\
677\end{array}$ & $\begin{array}{l}488 \\
537 \\
585 \\
634 \\
683 \\
732 \\
781\end{array}$ \\
\hline
\end{tabular}

TABLE 8.-A. P. I. extra-strong pipe

[All dimensions in inches at $68^{\circ} \mathrm{F}$.]

\begin{tabular}{|c|c|c|c|c|c|c|}
\hline \multirow{2}{*}{$\begin{array}{l}\text { Nom- } \\
\text { inal } \\
\text { size }\end{array}$} & \multicolumn{2}{|c|}{ Diameters } & \multirow{2}{*}{$\begin{array}{l}\text { Thick- } \\
\text { ness }\end{array}$} & \multirow{2}{*}{$\begin{array}{l}\text { Weight } \\
\text { per foot } \\
\text { (pounds), } \\
\text { plain } \\
\text { ends } 1\end{array}$} & \multicolumn{2}{|c|}{$\begin{array}{l}\text { Test pressure } \\
\text { (pounds per } \\
\text { square inch) }\end{array}$} \\
\hline & $\begin{array}{l}\text { External, } \\
\text { "O. D." }\end{array}$ & $\begin{array}{l}\text { Internal, } \\
\text { "I. D." }\end{array}$ & & & $\begin{array}{l}\text { Butt- } \\
\text { welded }\end{array}$ & $\begin{array}{l}\text { Lap- } \\
\text { welded }\end{array}$ \\
\hline $\begin{array}{l}1 / 8 \\
1 / 4 \\
3 / 8 \\
1 / 2\end{array}$ & $\begin{array}{r}0.405 \\
.540 \\
.675 \\
.840\end{array}$ & $\begin{array}{r}0.215 \\
.302 \\
.423 \\
.546\end{array}$ & $\begin{array}{r}0.095 \\
.119 \\
.126 \\
.147\end{array}$ & $\begin{array}{r}0.314 \\
.535 \\
.738 \\
1.087\end{array}$ & $\begin{array}{l}700 \\
700 \\
700 \\
700\end{array}$ & \\
\hline $\begin{array}{l}3 / 4 \\
1 \\
11 / 4 \\
11 / 2\end{array}$ & $\begin{array}{l}1.050 \\
1.315 \\
1.660 \\
1.900\end{array}$ & $\begin{array}{r}.742 \\
.957 \\
1.278 \\
1.500\end{array}$ & $\begin{array}{l}.154 \\
.179 \\
.191 \\
.200\end{array}$ & $\begin{array}{l}1.473 \\
2.171 \\
2.996 \\
3.631\end{array}$ & $\begin{array}{r}700 \\
700 \\
1,500 \\
1,500\end{array}$ & $\begin{array}{l}2,500 \\
2,500\end{array}$ \\
\hline $\begin{array}{l}2 \\
21 / 2\end{array}$ & $\begin{array}{l}2.375 \\
2.875\end{array}$ & $\begin{array}{l}1.939 \\
2.323\end{array}$ & $\begin{array}{l}.218 \\
.276\end{array}$ & $\begin{array}{l}5.022 \\
7.661\end{array}$ & $\begin{array}{l}1,500 \\
1,500\end{array}$ & $\begin{array}{l}2,500 \\
2,500\end{array}$ \\
\hline $\begin{array}{l}3 \\
31 / 2\end{array}$ & $\begin{array}{l}3.500 \\
4.000\end{array}$ & $\begin{array}{l}\text { 2. } 900 \\
\text { 3. } 364\end{array}$ & $\begin{array}{l}.300 \\
.318\end{array}$ & $\begin{array}{l}10.252 \\
12.505\end{array}$ & 1,500 & $\begin{array}{l}2,500 \\
2,500\end{array}$ \\
\hline $\begin{array}{l}4 \\
5 \\
6 \\
8\end{array}$ & $\begin{array}{l}\text { 4. } 500 \\
\text { 5. } 563 \\
\text { 6. } 625 \\
8.625\end{array}$ & $\begin{array}{l}3.826 \\
4.813 \\
5.761 \\
7.625\end{array}$ & $\begin{array}{l}.337 \\
.375 \\
.432 \\
.500\end{array}$ & $\begin{array}{l}14.983 \\
20.778 \\
28.573 \\
43.388\end{array}$ & & $\begin{array}{l}2,200 \\
2,000 \\
2,000 \\
1,700\end{array}$ \\
\hline $\begin{array}{l}10 \\
12\end{array}$ & $\begin{array}{l}10.750 \\
12.750\end{array}$ & $\begin{array}{r}9.750 \\
11.750\end{array}$ & $\begin{array}{l}.500 \\
.500\end{array}$ & $\begin{array}{l}54.735 \\
65.415\end{array}$ & & $\begin{array}{l}1,400 \\
1,100\end{array}$ \\
\hline
\end{tabular}

1 See par. $12(b)$.

NoтE-For tests pressure on Grades A, B, and C seamless see corresponding sizes in Table 6 ; for test pressures not given in either
Tables 6 or 8 , see par. $6($ b $)$ in No. 5 -A, above. 
AMERICAN PETROLEUM I NST T T U T E, SPECIFICATIONS FOR OIL-WELI PUMPS, STANDARD NO. 11-A AND SUPPLEMENT NO. 1, JULY, 1931

\section{COLD-DRAWN WORKING BARRELS}

(These specifications include dimensional standards for cold-drawn working barrels as shown in Table 1 below. Working barrels are tubular and threaded at each end. The specifications do not include chemical or physical properties of materials.)

TABLE 1.-Cold-drawn working barrels

[All dimensions in inches at $68^{\circ} \mathrm{F}$.]

\begin{tabular}{|c|c|c|c|c|c|c|c|}
\hline \multirow{3}{*}{$\begin{array}{c}\text { Size } \\
\text { of } \\
\text { work- } \\
\text { ing } \\
\text { bar- } \\
\text { rel }\end{array}$} & \multicolumn{3}{|c|}{ Diameters } & \multicolumn{3}{|c|}{ Threads } & \multirow{3}{*}{$\begin{array}{l}\text { Length } \\
\text { of } \\
\text { barrel, } \\
\text { "D" }\end{array}$} \\
\hline & \multirow{2}{*}{$\begin{array}{l}\text { Nom- } \\
\text { inal } \\
\text { out- } \\
\text { side } \\
\text { "A" }\end{array}$} & \multicolumn{2}{|c|}{ Inside } & \multirow{2}{*}{$\begin{array}{l}\text { Num- } \\
\text { ber } \\
\text { threads } \\
\text { per } \\
\text { inch }\end{array}$} & \multirow{2}{*}{$\begin{array}{l}\text { Taper } \\
\text { inch } \\
\text { per } \\
\text { foot }\end{array}$} & \multirow{2}{*}{$\begin{array}{c}\text { Total } \\
\text { length } \\
\text { of } \\
\text { thread, "C" }\end{array}$} & \\
\hline & & "B" & $\begin{array}{l}\text { Toler- } \\
\text { ance }\end{array}$ & & & & \\
\hline & $\begin{array}{l}2.250 \\
2.75 \\
3.25 \\
4.25\end{array}$ & $\begin{array}{l}1.7810 \\
2.2500 \\
2.7500 \\
3.7500\end{array}$ & $\begin{array}{r} \pm 0.0082 \\
\pm .0082 \\
\pm .0082 \\
\pm .0102\end{array}$ & $\begin{array}{l}111 / 3 \\
111 / 2 \\
111 / 2 \\
11 \frac{1}{2}\end{array}$ & $\begin{array}{l}3 / 8 \\
3 / 4 \\
3 / 4 \\
3 / 4\end{array}$ & $\begin{array}{l}1.375 \\
1.625 \\
1.625 \\
1.625\end{array}$ & $\begin{array}{l}\text { See note } \\
\text { below. }\end{array}$ \\
\hline
\end{tabular}

Note.-For barrels of all diameters, lengths of 5, 6, 8, and 10 feet are considered standard; lengths of 4,7 , and 9 feet, and longer than 10 feet are considered special.

AMERICAN RAILWAY ASSOCIATION MECHANICAL DIVISION, 300-POUND SCREWED PIPE FITTINGS FOR STEAM LOCOMOTIVES, NO. D. V. $-741,1931$

\section{SCREWED PIPE FITTINGS}

(See 607.2, p. 422.)

\section{AMERICAN RAILWAY ASSOCIATION MECHANICAL DIVISION, SPECIFICA- TIONS FOR WELDED AND SEAMLESS STEEL PIPE, 1925}

\section{WELDED AND SEAMLESS STEEL PIPE}

(Substantially the same as A. S. T. M., A53-30, given below.)

\section{AMERICAN RAILWAY ASSOCIATION, PURCHASES AND STORES DIVISION, STANDARDIZATION AND SIMPLIFICA- TION OF STORES STOCKS, PROCEEDINGS, 1928}

\section{MECHANICAL STEEL TUBING}

(Recommended practice in stocking certain specified sizes of mechanical steel tubing. It is also recommended that in stating dimensions simplicity will be served by giving the outside diameter first, then the inside diameter.)

\section{HOSE CLAMPS}

(Recommended practice in stocking certain specified sizes of hose clamps, except clamps for tank hose.)
AMERICAN RAILWAY ASSOCIATION, SIGNAL SECTION, 1-INCH WELDED STEEL PIPE, SPECIFICATION 1222\%, JULY, $192 \%$

\section{WELDED STEEL PIPE}

PURPOSE

(a) The purpose of this specification is to provide welded steel pipe for the operation of mechanical units.

\section{CHEMIICAL PROPERTIES}

(a) The steel shall conform to the following requirements as to chemical composition:

$\begin{array}{ll}\text { Phosphorus, not more than } & \text { Per cent } \\ \text { Copper...11 }\end{array}$

PHYSICAL PROPERTIES AND TESTS

(a) The steel shall conform to the following minimum requirements as to tensile properties:

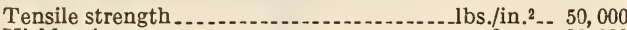

Yield point

Elongation in 8 inches......... per cent.- 18

(This is the same as for Bessemer in A. S. T. M., A53-30, given below.)

(b) The yield point shall be determined by the drop of the beam of the testing machine.

(c) Weights, dimensions, and hydrostatic pressure shall be as follows: ${ }^{78}$

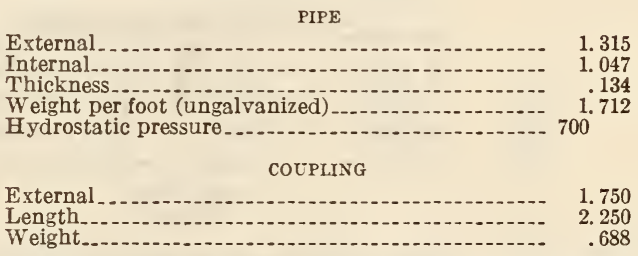

(d) The outside diameter at any point shall not vary more than one-sixty-fourth inch from the size specified.

(e) The inside diameter of all pipe must be large enough to receive a steel plug sixty-three-sixtyfourths inch in diameter for a distance of 6 inches.

$(f)$ The weight of the pipe and couplings shall not vary more than 5 per cent from that specified.

(g) The pipe shall be tested at the mill to the hydrostatic pressure specified above.

(h) A sufficient length of pipe shall stand being bent through $90^{\circ}$ around a cylindrical mandrel the diameter of which is twelve times the nominal diameter of the pipe, without developing cracks at any portion and without opening the weld.

(i) A piece of pipe 1 foot long will be selected at random and be subjected to a flattening test by hammering the piece until the opposite sides are within the thickness of the wall from each other. The piece shall show no cracks in the steel except at the weld.

(j) Specimens.-(1) Test specimens shall consist of sections cut from a pipe, (2) tension-test specimens shall be longitudinal, and (3) all specimens shall be tested cold.

(k) One of each of the tests specified above may be made on a length in each lot of 500 or less. Each length shall be subjected to the hydrostatic test.

(l) If the results of the tests of any lot do not conform to the requirements specified, retests of

${ }^{76}$ All weights are nominal and aregiven in pounds. All dimensions are nominal and are given in inches. 
two additional pipes shall be made, each of which shall conform to the requirements specified.

$(m)$ Contractor shall give the purchaser sufficient notice of time when material will be ready for testing.

$(n)$ Contractor shall provide, at point of production, apparatus and labor for making the required tests under supervision of the purchaser.
TEREADS

Each length of pipe shall have a thread $1 \frac{1}{8}$ inches long, three-eighths inch total taper per foot, $113 / 2$ slightly rounded top and bottom $v$ threads to the inch. The threaded portion of the pipe shall be of such diameter as to permit the coupling to be screwed on five turns by hand, with a permissible variation of one turn either way.
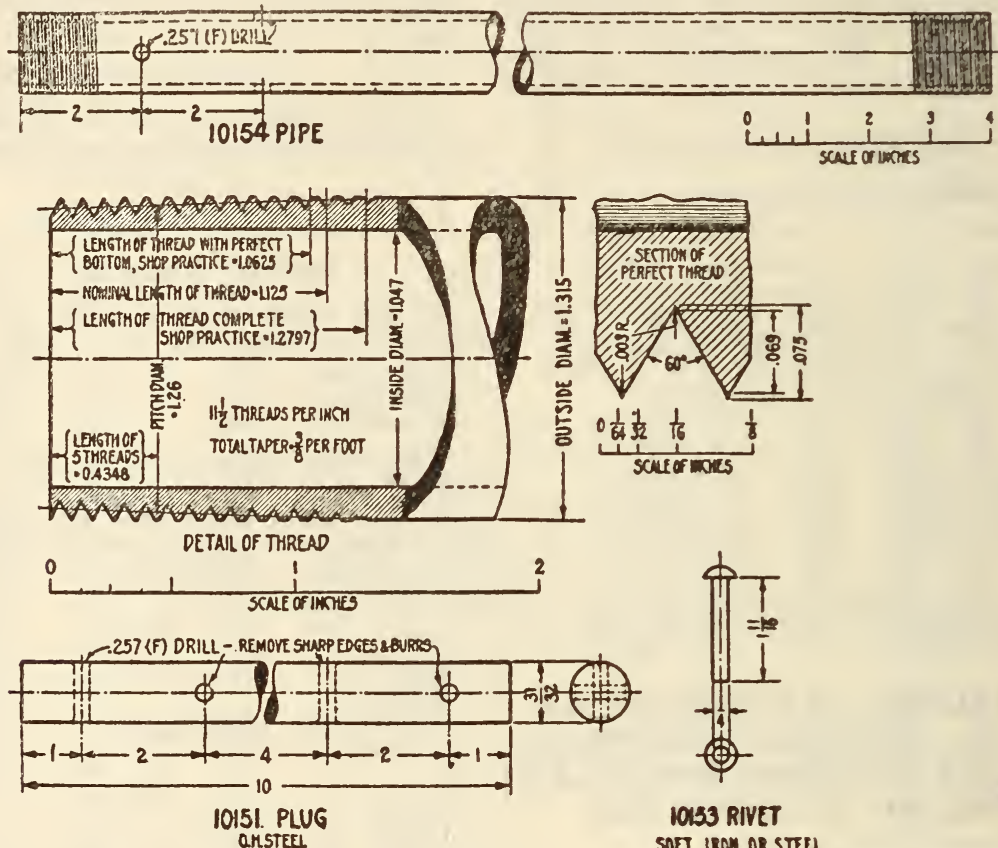

OMSTEE

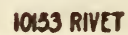

SOFT IRON OR STELL
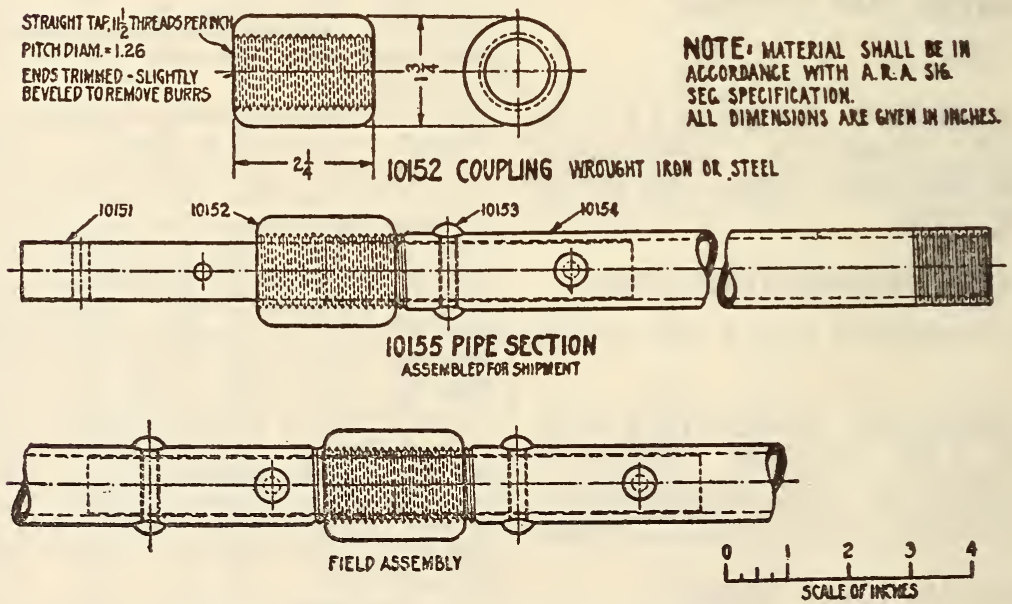

FIGURE 286.-One-inch signal pipe and coupling

(o) If tests are to be made at point of production, it shall be so stated. Purchaser will distinctly indicate which of the tests herein specified are to be made and what portion of the material shall be tested.

\section{ENDS}

(a) Ends of pipe must be cut square and two holes drilled 0.257 inch in diameter for two $1 / 4$-inch rivets on one end only; first rivet hole shall be drilled 2 inches from the end, and the second 2 inches from this and at right angles to it.

\section{COUPLINGS}

Each length of threaded pipe shall be provided with one coupling faced at ends, tapped straight through, having clear-cut threads, and of such pitch diameter as to make a tight joint. Couplings shall be made of wrought iron or steel.

\section{PLUGS}

Plugs shall be open-hearth steel, 10 inches long, thirty-one-thirty-seconds inch in diameter, drilled 0.257 inch in diameter, for four $1 / 4$-inch rivets, 
spacing to be 1 inch, 2 inches, 4 inches, 2 inches, and 1 inch, the first and third holes to be in the same plane, and the second and fourth holes at right angles thereto.

\section{RIVETS}

Rivets must be made of soft iron or steel, onefourth inch in diameter and $1^{11 / 16}$ inches long, and galvanized when specified.

\section{SALVANIZING}

Finished pipe and couplings, when specified, shall be hot galvanized inside and outside. The galvanizing shall be in accordance with $R$. S. A. specification 2912. The coating on the outside of the pipe shall weigh not less than 2 ounces per square foot of coated surface.

\section{LENGTHS}

Pipe shall be in random lengths of 16 to 22 feet. Jointers (which are two pieces coupled together) will be rejected.

\section{MATERIAL AND WORKMANSHIP}

Material and workmanship shall be first class in every respect.

\section{INSPECTION}

(a) Purchaser may inspect the material at all stages of manufacture.

(b) Purchaser may inspect the completed product to determine that the requirements of this specification have been met.

(c) If the material has not been accepted at point of production and if, upon arrival at destination, it does not meet the requirements of this specification, it may be rejected, and the contractor, upon request, shall advise the purchaser what disposition is to be made of the defective material. The contractor shall pay all freight charges.

(d) If purchaser is to make inspection at point of production, it shall be so stated.

\section{AMERICAN SOCIETY OF MECHANICAI ENGINEERS, STEEL PIPE FLANGES AND FLANGED FITTINGS, B16e-192\%}

[Approved as American Tentative Standard by the American Standards Association, ${ }^{77}$ June, 1927]

\section{STEEL PIPE FLANGES AND FLANGED FITTINGS}

For Maximum Working Steam Pressures of 250, 400, 600,900 , and 1,350 Pounds Per Square Inch (Gage) at a Temperature of $750^{\circ} \mathrm{F}$.

\section{INTRODUCTORY NOTES}

1. Pressure ratings and tests.-These standards shall be known as the American 250,400,600, 900 , and 1,350 pound steel flange standards, said pressure designation being the recommended rating at a temperature of $750^{\circ} \mathrm{F}$. Table 1 shows recommended ratings for various temperatures, together with hydrostatic shell test pressure

All tests shall be made with water at a temperature not to exceed $100^{\circ} \mathrm{F}$

2. Size.-The size of the fittings in the following tables is identified by the corresponding nominal pipe size. For pipe 14 inches and larger the corresponding outside diameter of the pipe is given.

3. Marking. - Each steel product (of a size permitting legible marking) produced with the intent

77 Sponsor organizations: American Society of Mechanical Engineers, Manufacturers' Standardization Society of Valve and Fittings Industry, and Heating and Piping Contractors National Association. of meeting the specifications of these American steel flange standards shall be marked for identification by means of, first, maker's name or trade-mark; second, size, and, third, maker's symbol, by which the service may be determined for which the maker recommends the article.

On products of small size or shape which will not permit all of the above markings, they may be omitted to the degree which conditions require, in the sequence of, first, size; second, maker's identification symbol; third, maker's name or trade-mark. Size marking shall be by means of the numeral or numerals involved in the nominal size designation.

Knowledge of the maker's identification symbol and the service recommendations which are signified by their application to products shall be made available to the public by means of the maker's catalogue, engineering data, or other comprehensive literature.

TABLE 1.-Pressure-temperature ratings and hydrostatic shell test pressures

[All pressures are in pounds per square inch (gauge)]

\begin{tabular}{|c|c|c|c|}
\hline $\begin{array}{l}\text { Maximum } \\
\text { working } \\
\text { steam-pres- } \\
\text { sure rating } \\
\text { at } 750^{\circ} \mathrm{F} \text {. }\end{array}$ & $\begin{array}{l}\text { Maximum } \\
\text { nonshock } \\
\text { working } \\
\text { hydraulic } \\
\text { rating at } \\
450^{\circ} \mathrm{F} \text {., such } \\
\text { as boiler } \\
\text { feed lines }\end{array}$ & $\begin{array}{l}\text { Maximum } \\
\text { nonshock } \\
\text { working } \\
\text { hydraulic } \\
\text { rating at } \\
\text { or near the } \\
\text { ordinary } \\
\text { range of } \\
\text { air tem- } \\
\text { peratures }\end{array}$ & $\begin{array}{l}\text { Hydrostatic } \\
\text { shell test, } \\
\text { maximum, } \\
100^{\circ} \mathrm{F} \text {. }\end{array}$ \\
\hline $\begin{array}{r}250 \\
400 \\
600 \\
900 \\
1,350\end{array}$ & $\begin{array}{r}325 \\
500 \\
720 \\
1,080 \\
1,625\end{array}$ & $\begin{array}{r}500 \\
750 \\
1,000 \\
1,500 \\
2,250\end{array}$ & $\begin{array}{r}750 \\
1,000 \\
1,500 \\
2,000 \\
3,000\end{array}$ \\
\hline
\end{tabular}

4. Materials-Steel castings.-The dimensional standards for steel castings, covered herein, are based on a high-grade product equal to that given in A. S. T. M. standard specifications for carbonsteel castings for valves, flanges, and fittings for high-temperature service, A95-29, suitable for the maximum temperatures and pressures for which the standards are designed. It is strongly recommended that the material for such maximum service be ordered and made in accordance with the latest revision of A. S. T. M. specification A95. In any event the castings shall be heat treated and the physical and chemical characteristics shall be in accordance with Table 2, which requirements are taken from A. S. T. M. standard specification A95-29. (See 611.41, p. 709.)

Bolting material. - The dimensional standards for bolting material, covered herein, including nuts and washers, are based on a high-grade product equal to that given in A. S. T. M. standard specifications for alloy-steel bolting material for high-temperature service, A96 (see 622.1, p. 1130), suitaole for the maximum temperatures and pressures for which the standards are designed. It is strongly recommended that the material for such maximum service be ordered and made in accordance with the latest revision of A. S. T. M. specification A96. The physical and chemical requirements shall be in accordance with Table 3 , which requirements are taken from A. S. T. M. standard specification A96. (See 622.1, p. 1130.)

While carbon-steel bolts and bolt studs purchased under a definite specification will resist the calculated stresses, it is strongly recommended that for the maximum service in each class, material be ordered and made in accordance with the latest revision of A. S. T. M. specification A96. 
It is also recommended that commercial steel bolts not be used at steam pressures over 160 pounds per square inch and/or temperatures over $450^{\circ} \mathrm{F}$.

Nuts shall be of carbon steel

TABLE 2.-Physical and chemical requirements of steel castings for flanges, fittings, and valves

Tensile strength, minimum. Yield point, minimum ............ 36, 000 Elongation in 2 inches, minimum.

Reduction of area, minimum

-

\section{TABLE 3.-Physical and chemical requirements of} alloy-steel bolting material

\begin{tabular}{|c|c|c|c|}
\hline & Class A & Class B & Class C \\
\hline $\begin{array}{l}\text { Tensile strength, minimum....lbs./in. }{ }^{2} \\
\text { Yield point, minimum } \\
\text { Elongation in } 2 \text { inches, minimum }\end{array}$ & $\begin{array}{l}95,000 \\
70,000\end{array}$ & $\begin{array}{r}105,000 \\
80,000\end{array}$ & $\begin{array}{l}125,000 \\
105,000\end{array}$ \\
\hline $\begin{array}{l}\text { Reduction of area, minimum } \\
\text { Phosphorus, maximum } \\
\text { Sulphur, maximum }\end{array}$ & $\begin{array}{r}20 \\
50 \\
10.05 \\
.05\end{array}$ & $\begin{array}{r}20 \\
50 \\
10.05 \\
.05\end{array}$ & $\begin{array}{r}16 \\
50 \\
10.05 \\
.05\end{array}$ \\
\hline
\end{tabular}

1 The phosphorous requirement of 0.05 per cent for alloy-steel bolting material agrees with the limits in A. S. T. M. specification A96-27 specified for check analysis on finished material. For the sake of uniformity in giving the phosphorus content for castings, bolting material, and forged steel flanges, the 0.05 per cent requirement is retained instead of the mill or ladle analysis requirement of 0.04 per cent as given in the A. S. T. M. specification.

TABLE 4.-Physical and chemical requirements, forged steel flanges

\begin{tabular}{|c|c|c|c|}
\hline & \multirow{2}{*}{$\begin{array}{l}\text { Flanges } \\
\text { for forge } \\
\text { weld- } \\
\text { ing- } \\
\text { class A }\end{array}$} & \multicolumn{2}{|c|}{$\begin{array}{l}\text { Flanges not for } \\
\text { forge welding }\end{array}$} \\
\hline & & $\begin{array}{l}\text { With } \\
\text { hubs- } \\
\text { class B }\end{array}$ & $\begin{array}{l}\text { With or } \\
\text { without } \\
\text { hubs- } \\
\text { class C }\end{array}$ \\
\hline Carbon, maximum & 0.17 & & \\
\hline Tensile strength, minimum & . - & 60,000 & 70,000 \\
\hline $\begin{array}{l}\text { Yield point, minimum } \\
\text { Elongation in } 2 \text { inches, minimum }\end{array}$ & & 30,000 & 36,000 \\
\hline Reduriont cent & & 25 & \\
\hline Reduction of area, minimum..... do & $--=-$ & 38 & 30 \\
\hline $\begin{array}{l}\text { Phosphorus, maximum } \\
\text { Sulphur, maximum }\end{array}$ & .05 & 0.05 & 0.05 \\
\hline Sulphur, maximum & & & \\
\hline
\end{tabular}

Washers when used under nuts shall be of forged or rolled carbon steel.

Forged steel flanges.-The dimensional standards for flanges covered herein are based on a high-grade product equal to class $\mathrm{C}$ steel given in A. S. T. M. standard specifications for forged or rolled steel pipe flanges for high-temperature service, A105-28, given below, suitable for the maximum temperatures and pressures for which the standards are designed. It is strongly recommended that the material for such maximum service be ordered and made in accordance with the latest revision of A. S. T. M. specification A105. The physical and chemical requirements shall be in accordance with Table 4 which requirements are taken from A. S. T. M. standard specification A105-28.

Where loose or unsupported flanges of classes A or B (Table 4) material are used they must be designed with adequate hubs. In the event that this can not be accomplished practically, the thickness of the flange shall be increased.

Where the yield point in steel flanges is below 36,000 pounds per square inch, hub or other reinforcement shall be provided to compensate for the lower efficiency in the strength of classes A and B materials used. Flanges forged integral with or forge welded to the walls of pipe or other structures shall be considered as being additionally reinforced.

5. Facings. - The raised face shall be the standard facing for fittings and valves. It shall be machined to the following dimensions:
Height of raised face-

250-pound standard. - 116 inch on all sizes. 400-pound standard 600 pound standard

900 pound standard.- $1 / 4$ inch on all sizes.

900 pound standard.

1,350-pound standard.

The raised face shall be provided in the following manner:

250-pound standard.- Included in the minimum flange thickness. 400-pound standard.

900-pound standard - - Added to the minimum flange thickness. 1,350-pound standard

Outside diameter of raised face.

utside diameter of raise

250-pound standard.

400-pound standard -- As given in the accompanying Table 5 in 600 -pound standard-- column headed "Outside diameter900 -pound standard

1,350-pound standard

Table 5 also gives dimensions for tongue-groove and male-female types of joints for the $250,400,600$, 900 , and 1,350 pound standards. The projecting contact surfaces, that is, the tongues and males, are, as described above, one-fourth inch beyond the edge of the flange. The bottom or contact surfaces of the groove and female facings shall be in the same plane as the edge of the flange.

The outside diameters of raised face, van stone, large tongue, and large male are the same, being the minimum diameter considered practical for van stone. The outside diameters of groove and female shall be one-sixteenth inch larger than the corresponding outside diameters of tongue and male, and the inside diameters of the groove shall be onesixteenth inch smaller than the corresponding inside diameters of the tongue.

A tolerance of plus or minus 0.016 inch (one sixty-fourth inch) is allowed on the inside and outside diameters of all facings. (See Table 5 for dimensions.)

The 1/16-inch raised face if applied to 400,600 , 900 , and 1,350 pound standard fittings shall not be cut from the minimum flange thicknesses specified in the respective standards but shall be provided by facing down the $1 / 4$-inch raised face.

Raised facings higher than one sixteenth-inch and tongue-groove and male-female facings may be specially required for 250-pound fittings, and when so required shall be furnished as follows: $(a)$ No metal shall be cut from the minimum flange thickness specified in the standard; $(b)$ the full flange thickness shall be first provided and then the raised - tongue or male-face shall be added thereto; (c) in the case of groove and female faces, the flange shall be built to full minimum thickness and sufficient metal added thereto so that the bottom or contact face of the groove or female is in the same plane with the face of flange edge of a full thickness flange.

6. Bolting.-Drilling templates are in multiples of four, so that fittings may be made to face in any quarter. Bolt holes straddle the center lines. Bolt holes are drilled one-eighth inch larger in diameter than the nominal size of bolt. Bolts or bolt studs threaded at both ends may be used and shall be equipped with cold-punched or cold-pressed semifinished nuts of American standard rough dimensions, chamfered and trimmed.

All bolts and bolt studs having diameters from one-half inch to and including 1 inch and the corresponding nuts shall be threaded with the American (national) standard screw thread,78 coarse thread series, medium fit (class 3). Bolts and bolt studs whose diameters are $1 \frac{1}{8}$ inches and larger shall have special threads of the American (national) form whose pitch is one-eighth inch ( 8 threads per inch). It is recommended that these special threads be allowed a pitch diameter tolerance of -0.006 inch and a lead tolerance of \pm 0.002 inch.

Bolt studs with a nut at each end are recommended for high-temperature service.

78 See Standards Pamphlet Bla-1924, "American Standard Screw Threads" published by the American Society of Mechanical Engineers, 29 West Thirty-ninth Street, New York, N. Y. 
The allowable working fiber stress, considering internal allowable working pressure only, in bolting material for valve-bonnet flanges, clean-out flanges, etc., shall not exceed 9,000 pounds per square inch assuming the pressure to act upon an area circumscribed by the periphery of the outside of the contact surface.

7. Spot facing.-All flanges shall be spot faced or back faced parallel to the flange face. Metal re-

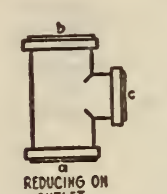

REDUCING ON
OUTLET

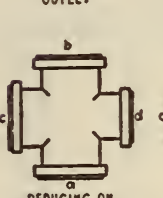

REDUCING ON
OHE OUTLET

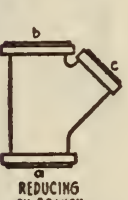

REDUCING
ON BRNCH

SIDE

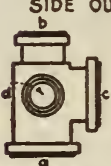

อ6แบ หมกอ

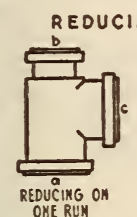

REDUCING

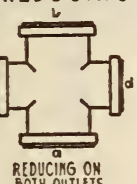

BOTH OUTLETS

REDUCING

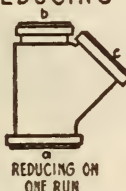

REDUCING ONI
OHE RUN

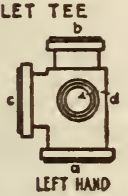

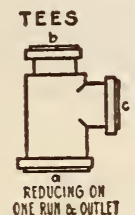

ONE RUH \& OUTLET
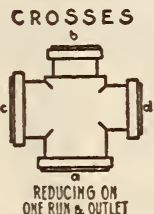

LATERALS
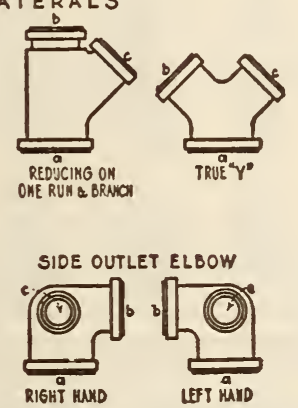

FIGURE 287.-Steel flanged fittings 250, 400, 600, 900 , and 1,350 pounds

Method of Designating Outlets of Reducing Fittings when Ordering

In designating the outlets of reducing fittings the openings should be read in the order indicated by the sequence of the letters $a, b$, c, and d. In designating the outlets of side outlet reducing fittings, the side outlet is named last.

moved in spot facing or back facing shall not reduce the thickness of the flange below the minimum given in the tables. Spot facing does not apply to forged steel flanges if the back of the flange is parallel to the flange face.

8. Metal thickness.-Minimum metal thicknesses specified in the tables are based on an allowable fiber stress of 7,000 pounds per square inch using the modified Barlow formula of the A. S. M. E. boiler construction code, section $\mathrm{P}-23$, for cylindrical sections and adding 50 per cent to the thickness thus determined to compensate for the shape of the fittings. The minimum commercial casting thickness is considered to be one-fourth inch, therefore the standards do not show thicknesses less than this. The minimum thickness in these standards means the minimum thickness in any part of the finished casting.

The modified Barlow formula as used in the A. S. M. E. boiler construction code is as follows:

For pipes having nominal diameters of from onefourth inch to 5 inches.

$$
P=\frac{2 S}{D}(t-0.065)-125
$$

For pipes of nominal diameters over 5 inches.

$$
P=\frac{2 S}{D}(t-0.1)
$$

where

$P=$ working pressure, pound per square inch.

$t=$ thickness of wall of pipe, inches.

$D=$ actual outside diameter of pipe, inches.

$S=7,000$ pounds per square inch.

9. Fitting dimensions.-One of the principles of design in this standard is the maintenance of a fixed position for the flange itself with reference to the body of the fitting and the addition of any facing is beyond the outside edge of the flange except for the 1/16-inch raised face in the 250-pound standard. (See note 4 in Table 5.) Tables $8,10,12$, and 14 show those dimensions which are made permanent to avoid pattern changes.

An inspection limit of $\pm 1 / 32$ inch shall be allowed on all center to contact surface dimensions for sizes up to and including 10 inches, and $\pm 1 / 16$ inch on sizes larger than 10 inches. An inspection limit of $\pm 1 / 16$ inch shall be allowed on all contact surface to contact surface dimensions for sizes up to and including 10 inches, and $\pm 1 / 8$ inch on sizes larger than 10 inches.

When elbows having longer radii than specified in the standards are required, the use of pipe bends is recommended.

10. Laterals. - The $45^{\circ}$ laterals of the larger sizes may require additional reinforcement to compensate for the inherent weakness in this shape of casting.
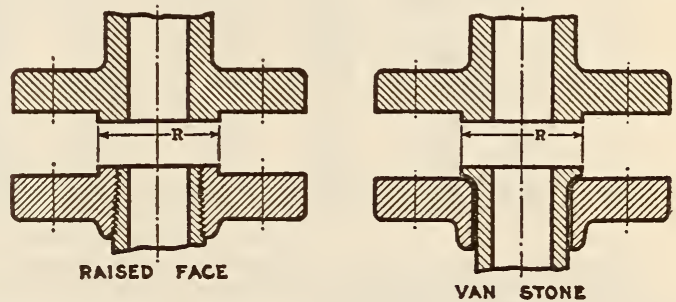
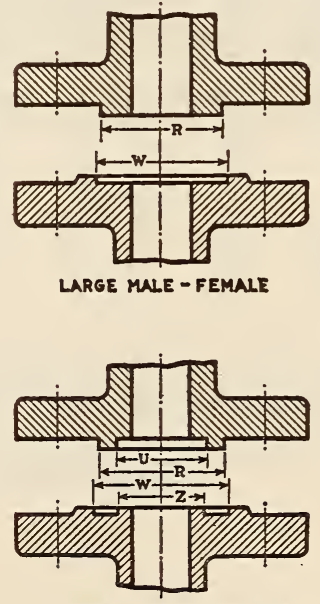

LARGE TONGUE - GROOVE
LARGE MULE - FEMALE

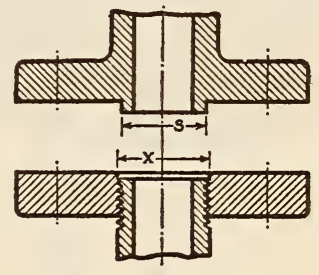

SMALL MALE-FEMALE

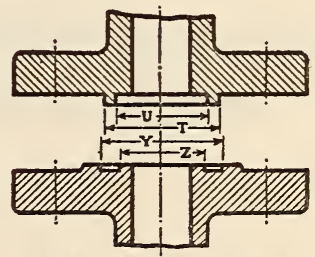

SMALL TONGUE-GROOVE
FIGURE 288

11. Valve dimensions.-The center to contact surface and contact surface to contact surface dimensions of 400,600, 900, and 1,350 pound American standard steel flange gate (both single and double disk), globe, angle, and check valves shall be the same as those prescribed for tees in the respective $400,600,900$, and 1,350 pound standards.

12. Reducing fittings.-Reducing fittings shall have same center to flange edge dimensions as those of straight size fittings of the largest opening.

13. Side-outlet fitings. - All side-outlet fittings shall have all openings on the intersecting center lines. 
STEEL FLANGED FITTINGS

TABLE 5.-Facing dimensions for the American 250,400,600,900, and 1,350 pound steel flanges

[All dimensions given in inches]

\begin{tabular}{|c|c|c|c|c|c|c|c|c|c|c|c|}
\hline \multirow[b]{2}{*}{$\begin{array}{l}\text { Nominal } \\
\text { pipe size }\end{array}$} & \multicolumn{3}{|c|}{ Outside diameter 1} & \multirow[b]{2}{*}{$\begin{array}{l}\text { Inside di- } \\
\text { ameter of } \\
\text { large and } \\
\text { small } \\
\text { tongue } 12\end{array}$} & \multicolumn{3}{|c|}{ Outside diameter 1} & \multirow[b]{2}{*}{$\begin{array}{l}\text { Inside di- } \\
\text { ameter of } \\
\text { large and } \\
\text { small } \\
\text { groove } 12\end{array}$} & \multicolumn{2}{|c|}{ Height } & \multirow[b]{2}{*}{$\begin{array}{l}\text { Depth of } \\
\text { groove or } \\
\text { female } \\
\text { compan- } \\
\text { ion } \\
\text { flanges }\end{array}$} \\
\hline & $\begin{array}{c}\text { Raised } \\
\text { face, } \\
\text { van stone, } \\
\text { large, } \\
\text { male, } \\
\text { and large } \\
\text { tongue? } \\
\\
\mathrm{R}\end{array}$ & $\underset{\text { male }^{23}}{\text { Small }}$ & $\begin{array}{c}\text { Small } \\
\text { tongue? }\end{array}$ & & $\begin{array}{l}\text { Large fe- } \\
\text { male and } \\
\text { large } \\
\text { groove? }\end{array}$ & $\underset{\text { female } 23}{\text { Small }}$ & $\begin{array}{c}\text { Small } \\
\text { groove }\end{array}$ & & $\begin{array}{l}\text { Raised } \\
\text { lace } \\
250- \\
\text { pound } \\
\text { stand- } \\
\text { ard }\end{array}$ & $\begin{array}{l}\text { Raised } \\
\text { face, } \\
\text { large and } \\
\text { small } \\
\text { male and } \\
\text { tongue } \\
400,600, \\
900, \text { and } \\
1,350 \\
\text { pound } \\
\text { stand- } \\
\text { ards }\end{array}$ & \\
\hline $\begin{array}{l}1 / 2 \\
3 / 4 \\
1 \\
11 / 4 \\
11 / 2\end{array}$ & $\begin{array}{l}13 / 8 \\
111 / 16 \\
2 \\
21 / 2 \\
27 / 8\end{array}$ & $\begin{array}{l}23 / 32 \\
15 / 16 \\
13 / 16 \\
11 / 2 \\
13 / 4\end{array}$ & $\begin{array}{l}13 / 8 \\
111 / 16 \\
17 / 8 \\
21 / 4 \\
21 / 2\end{array}$ & $\begin{array}{l}1 \\
15 / 16 \\
11 / 2 \\
17 / 8 \\
216\end{array}$ & $\begin{array}{l}17 / 16 \\
13 / 4 \\
23 / 16 \\
2 \% / 16 \\
215 / 16\end{array}$ & $\begin{array}{l}25 / 32 \\
1 \\
11 / 4 \\
19 / 16 \\
113 / 16\end{array}$ & $\begin{array}{l}17 / 16 \\
13 / 4 \\
115 / 10 \\
25 / 16 \\
2 \% 16\end{array}$ & $\begin{array}{l}15 / 16 \\
11 / 4 \\
17 / 16 \\
113 / 16 \\
21 / 16\end{array}$ & $\begin{array}{l}1 / 16 \\
1 / 16 \\
1 / 16 \\
1 / 10 \\
1 / 10\end{array}$ & $\begin{array}{l}1 / 4 \\
1 / 4 \\
1 / 4 \\
1\end{array}$ & $\begin{array}{l}3 / 16 \\
3 / 16 \\
3 / 16 \\
3 / 16 \\
3 / 10\end{array}$ \\
\hline $\begin{array}{l}2 \\
21 / 2 \\
3 \\
31 / 2 \\
4\end{array}$ & $\begin{array}{l}35 / 8 \\
41 / 8 \\
5 \\
51 / 2 \\
63 / 16\end{array}$ & $\begin{array}{l}21 / 4 \\
211 / 16 \\
35 / 16 \\
313 / 16 \\
45 / 16\end{array}$ & $\begin{array}{l}31 / 4 \\
33 / 4 \\
45 / 8 \\
51 / 8 \\
51116\end{array}$ & $\begin{array}{l}27 / 8 \\
33 / 8 \\
41 / 4 \\
43 / 4 \\
53 / 16\end{array}$ & $\begin{array}{l}311 / 16 \\
43 / 16 \\
51 / 16 \\
5 \% 16 \\
61 / 4\end{array}$ & $\begin{array}{l}25 / 16 \\
23 / 4 \\
33 / 8 \\
37 / 8 \\
43 / 8\end{array}$ & $\begin{array}{l}35 / 16 \\
313 / 16 \\
411 / 16 \\
53 / 16 \\
53 / 4\end{array}$ & $\begin{array}{l}213 / 16 \\
35 / 16 \\
43 / 16 \\
411 / 16 \\
518\end{array}$ & $\begin{array}{l}1 / 10 \\
1 / 10 \\
1 / 16 \\
1 / 16 \\
1 / 16\end{array}$ & $\begin{array}{l}1 / 4 \\
1 / 4 \\
1 / 4 \\
1\end{array}$ & $\begin{array}{l}3 / 16 \\
3 / 16 \\
3 / 16 \\
3 / 16 \\
3 / 16\end{array}$ \\
\hline $\begin{array}{r}5 \\
6 \\
8 \\
10 \\
12\end{array}$ & $\begin{array}{l}75 / 16 \\
81 / 2 \\
105 / 8 \\
123 / 4 \\
15\end{array}$ & $\begin{array}{r}53 / 8 \\
63 / 8 \\
83 / 8 \\
101 / 2 \\
121 / 2\end{array}$ & $\begin{array}{l}6^{13 / 10} \\
8 \\
10 \\
12 \\
141 / 4\end{array}$ & $\begin{array}{r}65 / 16 \\
71 / 2 \\
93 / 8 \\
111 / 4 \\
131 / 2\end{array}$ & $\begin{array}{c}73 / 8 \\
89 / 16 \\
10^{11} 116 \\
12^{13} / 16 \\
151 / 16\end{array}$ & $\begin{array}{r}57 / 16 \\
67 / 16 \\
87 / 16 \\
109 / 16 \\
12 \% 16\end{array}$ & $\begin{array}{c}67 / 8 \\
81 / 16 \\
101 / 16 \\
121 / 16 \\
145 / 16\end{array}$ & $\begin{array}{r}61 / 4 \\
77 / 16 \\
95 / 16 \\
113 / 16 \\
137 / 16\end{array}$ & $\begin{array}{l}1 / 16 \\
1 / 16 \\
1 / 16 \\
1 / 16 \\
1 / 16\end{array}$ & $\begin{array}{l}1 / 4 \\
1 / 4 \\
1 / 4 \\
1 / 4\end{array}$ & $\begin{array}{l}3 / 16 \\
3 / 16 \\
3 / 16 \\
3 / 16 \\
3 / 16\end{array}$ \\
\hline $\begin{array}{ll}6 & 14 \\
6 & 16 \\
6 & 18 \\
6 & 20 \\
6 & 24\end{array}$ & $\begin{array}{l}161 / 4 \\
181 / 2 \\
21 \\
23 \\
271 / 4\end{array}$ & $\begin{array}{l}133 / 4 \\
153 / 4 \\
173 / 4 \\
193 / 4 \\
233 / 4\end{array}$ & $\begin{array}{l}151 / 2 \\
175 / 8 \\
2018 \\
22 \\
261 / 4\end{array}$ & $\begin{array}{l}143 / 4 \\
163 / 4 \\
191 / 4 \\
21 \\
251 / 4\end{array}$ & $\begin{array}{l}165 / 16 \\
189 / 16 \\
211 / 16 \\
231 / 16 \\
275 / 16\end{array}$ & $\begin{array}{l}13^{13} / 16 \\
15^{13} / 16 \\
1713 / 16 \\
1913 / 16 \\
2313 / 16\end{array}$ & $\begin{array}{l}159 / 16 \\
1711 / 16 \\
203 / 16 \\
221 / 16 \\
265 / 16\end{array}$ & $\begin{array}{l}14^{11} / 16 \\
16^{1} 1 / 16 \\
19^{3} / 16 \\
20^{15} / 16 \\
25^{3} / 16\end{array}$ & $\begin{array}{l}1 / 16 \\
1 / 16 \\
1 / 16 \\
1 / 16 \\
1 / 16\end{array}$ & $\begin{array}{l}1 / 4 \\
1 / 4 \\
1 / 4 \\
1 / 4\end{array}$ & $\begin{array}{l}3 / 16 \\
3 / 16 \\
3 / 16 \\
3 / 16 \\
3 / 16\end{array}$ \\
\hline
\end{tabular}

1 A tolerance of plus or minus 0.016 inch ( 164 inch) is allowed on the inside and outside diameters of all facings.

2 Gaskets for male-female and tongue-groove joints shall cover the bottom of the recess with minimum clearances taking into account the tolerances prescribed in note 1.

(3) Care should be taken in the use of joints of these dimensions, as they apply particularly on lines where the joint is made on the end of pipe, to insure that pipe used is thick enough to permit sufficient bearing surface to prevent crushing the gasket.

4 Regular facing for 250-pound flange standard is a 1/16-inch raised face included in the minimum flange thickness dimensions giren in Table 6. A 1/16-inch raised face is also permitted on the $400,600,900$, and 1,350 pound flange standards, but it must be added to the miniTable 6. A 1/16-inch raised

m Regular facing for $400,600,900$, and 1,350 pound flange standards is a $1 / 4$-inch raised face not included in minimum flange thickness dimensions given in Tables 8 to 13, inclusive. See illustrations with tables showing application of various facings.

6 Outside diameter. 


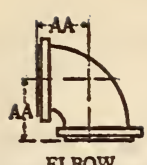

ELBOW

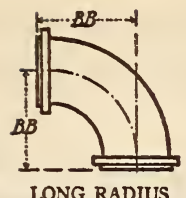

ELBOW

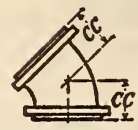

$45^{\circ}$ EIBOW

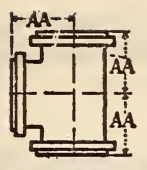

TEE

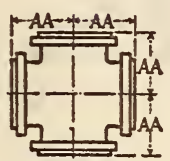

CROSS

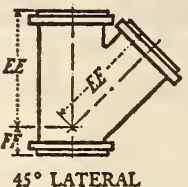

$45^{\circ}$ LATERAL

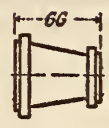

REDUCER

FIGURE 289.-250-pound steel flanged fittings

$\mathrm{T}_{\triangle \mathrm{BLE}}$ 6. - Dimensions of steel flanged fittings with projecting faces for maximum working steam pressure of .

[All dimensions given in inches]

\begin{tabular}{|c|c|c|c|c|c|c|c|c|c|c|c|c|c|}
\hline \multirow{3}{*}{$\begin{array}{l}\text { Nomina] } \\
\text { pipe size }\end{array}$} & & & & & & & & AA & BB & $\mathrm{CC}$ & $\mathrm{EE}$ & FF & $\mathrm{GG}$ \\
\hline & $\begin{array}{l}\text { Inside } \\
\text { diam- } \\
\text { eter of } \\
\text { fitting }\end{array}$ & $\begin{array}{l}\text { Mini- } \\
\text { mum } \\
\text { metal } \\
\text { thick- } \\
\text { ness of } \\
\text { fitting }\end{array}$ & $\begin{array}{l}\text { Out- } \\
\text { side } \\
\text { diam- } \\
\text { eter of } \\
\text { flange }\end{array}$ & $\begin{array}{l}\text { Mini- } \\
\text { mum } \\
\text { thick- } \\
\text { ness of } \\
\text { flange }\end{array}$ & $\begin{array}{l}\text { Diam- } \\
\text { eter of } \\
\text { bolt } \\
\text { circle }\end{array}$ & $\begin{array}{l}\text { Num- } \\
\text { ber of } \\
\text { bolts }\end{array}$ & $\begin{array}{c}\text { Size of } \\
\text { bolts }\end{array}$ & $\begin{array}{c}\text { Center } \\
\text { to } \\
\text { contact } \\
\text { surface } \\
\text { of } \\
\text { raised } \\
\text { face, } \\
\text { elbow, } \\
\text { tee, } \\
\text { and } \\
\text { cross } 234\end{array}$ & $\begin{array}{c}\text { Center } \\
\text { to } \\
\text { contact } \\
\text { surface } \\
\text { of } \\
\text { raised } \\
\text { face, } \\
\text { long } \\
\text { radius } \\
\text { ell } 234\end{array}$ & $\begin{array}{c}\text { Center } \\
\text { to } \\
\text { contact } \\
\text { surface } \\
\text { of } \\
\text { raised } \\
\text { face } \\
45^{\circ} \\
\text { ell }^{2} 3\end{array}$ & $\begin{array}{c}\text { Long } \\
\text { center } \\
\text { to } \\
\text { contact } \\
\text { surface } \\
\text { of } \\
\text { raised } \\
\text { face } \\
\text { lat- } \\
\text { eral } 234\end{array}$ & $\begin{array}{l}\text { Short } \\
\text { center } \\
\text { to } \\
\text { contact } \\
\text { surface } \\
\text { of } \\
\text { raised } \\
\text { face } \\
\text { lat- } \\
\text { eral } 234\end{array}$ & 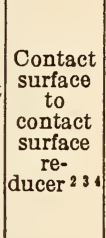 \\
\hline & \multicolumn{12}{|c|}{$\begin{array}{l}\text { For sizes below } 2 \text { inches, use the dimensions of } 600 \text {-pound fittings given in Tables } 10 \text { and } 11 \text {. When } \\
\text { a } 1 / 16 \text {-inch raised face is required, subtract } 31 / 1 \text { inch from center to contact surface dimensions given in } \\
\text { Table } 10 \text { and add } 1 / 16 \text { inch to center to flange edge dimensions given in Table } 11 .\end{array}$} & \\
\hline \begin{tabular}{l|}
2 \\
$21 / 2$ \\
3 \\
$31 / 2$ \\
4
\end{tabular} & \begin{tabular}{l|}
2 \\
$21 / 2$ \\
3 \\
$31 / 2$ \\
4
\end{tabular} & $\begin{array}{l}1 / 4 \\
1 / 4 \\
14 \\
2 / 32 \\
5 / 16\end{array}$ & $\begin{array}{c}61 / 2 \\
71 / 2 \\
81 / 4 \\
9 \\
10\end{array}$ & $\begin{array}{l}1^{7 / 8} \\
136 \\
13 / 16 \\
134\end{array}$ & \begin{tabular}{l|}
5 \\
$57 / 8$ \\
$65 / 8$ \\
$71 / 4$ \\
$77 / 8$
\end{tabular} & $\begin{array}{l}8 \\
8 \\
8 \\
8 \\
8\end{array}$ & $\begin{array}{l}5 / 8 \\
3 / 4 \\
3 / 4 \\
3 / 4 \\
3 / 4\end{array}$ & \begin{tabular}{l|}
5 \\
$51 / 2$ \\
6 \\
$61 / 2$ \\
7
\end{tabular} & $\begin{array}{l}61 / 2 \\
7 \\
73 / 4 \\
81 / 2 \\
9\end{array}$ & \begin{tabular}{l|}
3 \\
$31 / 2$ \\
$31 / 2$ \\
4 \\
$41 / 2$
\end{tabular} & $\begin{array}{l}9 \\
1016 \\
11 \\
1216 \\
131 / 2\end{array}$ & \begin{tabular}{l|}
$21 / 2$ \\
$21 / 2$ \\
3 \\
3 \\
3
\end{tabular} & $\begin{array}{l}5 \\
51 / 2 \\
6 \\
61 / 2 \\
7\end{array}$ \\
\hline $\begin{array}{r}5 \\
6 \\
8 \\
10 \\
12\end{array}$ & $\begin{array}{r}5 \\
6 \\
8 \\
10 \\
12\end{array}$ & $\begin{array}{l}36 \\
38 \\
7 / 16 \\
15 / 32 \\
15\end{array}$ & $\begin{array}{l}11 \\
121 / 2 \\
15 \\
171 / 2 \\
201 / 2\end{array}$ & $\begin{array}{l}136 \\
17 / 6 \\
15 / 8 \\
17 / 8 \\
2\end{array}$ & $\begin{array}{l}91 / 4 \\
105 / 8 \\
13 \\
151 / 4 \\
173 / 4\end{array}$ & $\begin{array}{r}8 \\
12 \\
12 \\
16 \\
16\end{array}$ & $\begin{array}{r}3 / 4 \\
3 / 4 \\
7 / 8 \\
1 \\
136\end{array}$ & $\begin{array}{l}8 \\
81 / 2 \\
10 \\
111 / 2 \\
13\end{array}$ & $\begin{array}{l}101 / 4 \\
111 / 2 \\
14 \\
161 / 2 \\
19\end{array}$ & \begin{tabular}{l|}
5 \\
$51 / 2$ \\
6 \\
7 \\
8
\end{tabular} & $\begin{array}{l}15 \\
171 / 2 \\
201 / 2 \\
24 \\
271 / 2\end{array}$ & $\begin{array}{l}31 / 2 \\
4 \\
5 \\
51 / 2 \\
6\end{array}$ & $\begin{array}{r}8 \\
9 \\
11 \\
12 \\
14\end{array}$ \\
\hline $\begin{array}{l}14 \\
16 \\
18 \\
120 \\
124\end{array}$ & $\begin{array}{l}131 / 4 \\
151 / 4 \\
17 \\
19 \\
23\end{array}$ & $\begin{array}{l}17 / 32 \\
19 / 32 \\
5 / 8 \\
11 / 16 \\
13 / 16\end{array}$ & $\begin{array}{l}23 \\
251 / 2 \\
28 \\
301 / 2 \\
36\end{array}$ & $\begin{array}{l}218 \\
21 / 4 \\
23 / 8 \\
21 / 2 \\
234\end{array}$ & $\begin{array}{l}201 / 4 \\
221 / 2 \\
243 / 4 \\
27 \\
32\end{array}$ & $\begin{array}{l}20 \\
20 \\
24 \\
24 \\
24\end{array}$ & $\begin{array}{l}138 \\
114 \\
11 / 4 \\
114 \\
11 / 2\end{array}$ & $\begin{array}{l}15 \\
161 / 2 \\
18 \\
191 / 2 \\
221 / 2\end{array}$ & $\begin{array}{l}211 / 2 \\
24 \\
261 / 2 \\
29 \\
34\end{array}$ & $\begin{array}{l}81 / 2 \\
91 / 2 \\
10 \\
101 / 2 \\
12\end{array}$ & $\begin{array}{l}31 \\
341 / 2 \\
371 / 2 \\
401 / 2 \\
471 / 2\end{array}$ & $\begin{array}{c}61 / 2 \\
715 \\
8 \\
81 / 2 \\
10\end{array}$ & $\begin{array}{l}16 \\
18 \\
19 \\
20 \\
24\end{array}$ \\
\hline
\end{tabular}

1 The fittings incorporated in this table are suitable also for other pressure and temperature ratings shown in Table 1.

A raised face of $1 / 16$ inch is included in $(a)$ minimum thickness of flanges, $(b)$ center to contact surface dimensions, and $(c)$ contact surface to contact surface dimensions. Inasmuch as the $1 / 16$-inch raised face is cut from the flange thickness, the center to contact surface dimen. sions are the same as the center to flange edge dimensions in this standard for this type of facing.

3 Where facings other than the 1 i 6 -inch raised face are used, the center to flange edge dimensions shall remain unchanged, and the new center to contact surface, or the new contact surface to contact surface dimensions shall be established to suit the facing used.

This standard provides for a 1/16-inch raised face on all openings of flanged fittings.

- Outside diameters. 

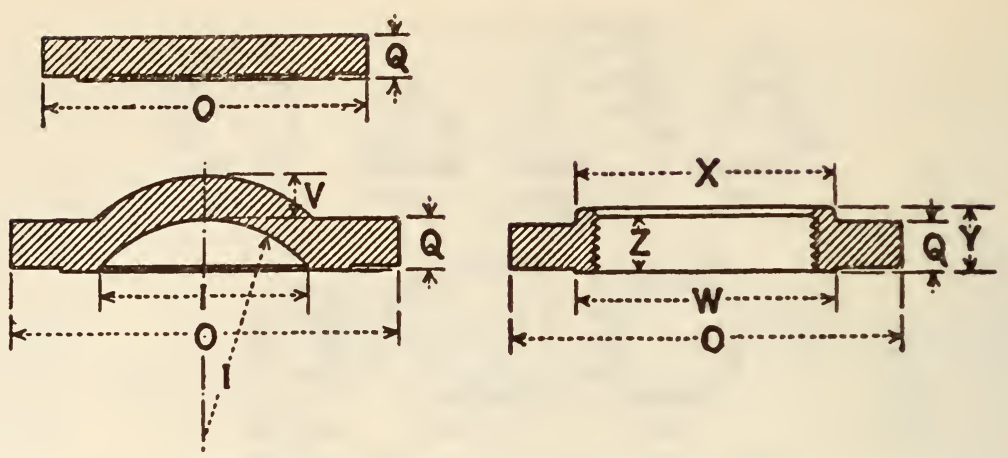

Figure 290.-250-pound steel flanges

TABLE 7.-Dimensions of steel screwed companion and blind flanges for maximum working steam pressure of 250 pounds per square inch at a temperature of $750^{\circ} \mathrm{F} .^{12}$

[All dimensions given in inches]

\begin{tabular}{|c|c|c|c|c|c|c|c|c|}
\hline \multirow[b]{2}{*}{$\begin{array}{l}\text { Nominal } \\
\text { pipe size }\end{array}$} & I & 0 & $Q^{8}$ & $\mathrm{~V}$ & $\mathbf{x}$ & $\mathbf{Y}$ & $\mathrm{Z}^{4}$ & W \\
\hline & $\begin{array}{c}\text { Diameter } \\
\text { of port }\end{array}$ & $\begin{array}{l}\text { Diameter } \\
\text { of flange }\end{array}$ & $\begin{array}{l}\text { Thick- } \\
\text { ness of } \\
\text { flange } \\
\text { mini- } \\
\text { mum }^{3}\end{array}$ & $\begin{array}{c}\text { Metal } \\
\text { thickness, } \\
\text { minimum }\end{array}$ & $\begin{array}{c}\text { Dismeter } \\
\text { hub }\end{array}$ & $\begin{array}{c}\text { Length } \\
\text { through } \\
\text { hub, } \\
\text { minimum }\end{array}$ & $\begin{array}{l}\text { Length } \\
\text { of } \\
\text { thresds, } \\
\text { minimum }\end{array}$ & $\begin{array}{l}\text { Diameter } \\
\text { of } \\
\text { raised } \\
\text { face }\end{array}$ \\
\hline \multirow{3}{*}{\multicolumn{4}{|c|}{ Dimensions same as in Table 6}} & & $\begin{array}{l}35 / 16 \\
315 / 16 \\
458 \\
51 / 4 \\
53 / 4\end{array}$ & $\begin{array}{l}11 / 4 \\
17 / 10 \\
19110 \\
158 \\
13 / 4\end{array}$ & $\begin{array}{l}1.00 \\
1.14 \\
1.20 \\
1.25 \\
1.30\end{array}$ & $\begin{array}{l}35,8 \\
41 / 8 \\
5 \\
51 / 2 \\
631 / 0\end{array}$ \\
\hline & & & & 1 & $\begin{array}{c}7 \\
81 / 8 \\
101 / 4 \\
1258 \\
143 / 4\end{array}$ & $\begin{array}{l}17 / 8 \\
1150 \\
2310 \\
238 \\
2910\end{array}$ & $\begin{array}{l}1.41 \\
1.51 \\
1.71 \\
1.92 \\
2.12\end{array}$ & $\begin{array}{l}73 / 16 \\
81 / 2 \\
1058 \\
123.4 \\
15\end{array}$ \\
\hline & & & & $\begin{array}{l}11 / 8 \\
11 / 4 \\
138 \\
11 / 2 \\
138\end{array}$ & $\begin{array}{l}161 / 4 \\
1838 \\
205 / 8 \\
223 / 4 \\
273 \%\end{array}$ & $\begin{array}{l}211 / 10 \\
27 / 8 \\
318 \\
3316 \\
311 / 16\end{array}$ & $\begin{array}{l}2.25 \\
2.45 \\
2.65 \\
2.85 \\
3.25\end{array}$ & $\begin{array}{l}161 / 4 \\
181 / 2 \\
21 \\
23 \\
2 \pi 1 / 4\end{array}$ \\
\hline
\end{tabular}

1 All blind flanges for sizes 10 inches (171/2 inches outside diameter) and larger must be dished with inside radius equal to the diameter of port.

2 These flanges are faced only and not drilled unless otherwise specified.

This column is the same as column for effective thread (E) in Table 1 of the American pipe thread standard, published by the' $A$ merican. Society of Mechanical Engineers, except the 2-inch size.

1 raised face of $1 / 10$ inch is included in the minimum thickness of flanges. 
400-Ib. STEEL FLATGED FITTINGS

(Elbow, $45^{\circ}$ elbow, tee, cross, $45^{\circ}$ lateral, and reducer illustrations are identical with those for 250pound steel flanged fittings in Table 6. .)

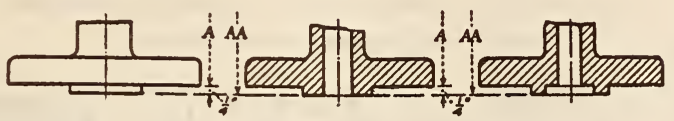

\section{FIGURE 291.-400-pound steel flanged fittings}

Raised face, male, and tongue facings for fittings

TABLE 8.-Center to contact surface dimensions for maximum working steam pressure of 400 pounds per square inch at a temperature of $750^{\circ} \mathrm{F}^{1}$

[All dimensions given in inches]

\begin{tabular}{|c|c|c|c|c|c|c|c|c|c|c|c|c|}
\hline \multirow{3}{*}{$\begin{array}{l}\text { Nom- } \\
\text { inal } \\
\text { pipe } \\
\text { size }\end{array}$} & & & & & & & & $\mathbf{A A}$ & $\mathrm{CC}$ & EE & $\mathrm{FF}$ & GG \\
\hline & $\begin{array}{l}\text { Inside } \\
\text { diam- } \\
\text { eter of } \\
\text { fitting }\end{array}$ & $\begin{array}{l}\text { Metal } \\
\text { thick- } \\
\text { ness of } \\
\text { fitting, } \\
\text { min- } \\
\text { imum }\end{array}$ & $\begin{array}{l}\text { Out- } \\
\text { side } \\
\text { diam- } \\
\text { eter of } \\
\text { flange }\end{array}$ & $\begin{array}{l}\text { Thick- } \\
\text { ness of } \\
\text { flange, } \\
\text { min- } \\
\text { imum }\end{array}$ & $\begin{array}{l}\text { Diam- } \\
\text { eter of } \\
\text { bolt } \\
\text { circle }\end{array}$ & $\begin{array}{l}\text { Num- } \\
\text { ber of } \\
\text { bolts }\end{array}$ & $\begin{array}{l}\text { Size of } \\
\text { bolts }\end{array}$ & $\begin{array}{l}\text { Center } \\
\text { to con- } \\
\text { tact sur- } \\
\text { face of } \\
\text { raised } \\
\text { face, } \\
\text { elbow, } \\
\text { tee, and } \\
\text { cross } 2 \text { a }^{2}\end{array}$ & $\begin{array}{l}\text { Center } \\
\text { to con- } \\
\text { tact sur- } \\
\text { face of } \\
\text { raised } \\
\text { face, } \\
45^{\circ} \\
\text { ell } 23\end{array}$ & $\begin{array}{c}\text { Long } \\
\text { center } \\
\text { to con- } \\
\text { tact sur- } \\
\text { face of } \\
\text { raised } \\
\text { face, } \\
\text { lat- } \\
\text { eral }\end{array}$ & $\begin{array}{c}\text { Short } \\
\text { center } \\
\text { to con- } \\
\text { tact sur- } \\
\text { face of } \\
\text { raised } \\
\text { face, } \\
\text { lat- } \\
\text { eral s }\end{array}$ & $\begin{array}{l}\text { Contact } \\
\text { surface } \\
\text { to con- } \\
\text { tact sur- } \\
\text { face re- } \\
\text { ducer } 28\end{array}$ \\
\hline & \multicolumn{11}{|c|}{ For sizes below 4 inches, use the dimensions of 600-pound fittings given in Table 10} & \\
\hline $\begin{array}{r}4 \\
5 \\
6 \\
8 \\
10\end{array}$ & $\begin{array}{r}4 \\
5 \\
6 \\
8 \\
10\end{array}$ & $\begin{array}{l}3 / 8 \\
7 / 16 \\
7 / 16 \\
9116 \\
11 / 16\end{array}$ & $\begin{array}{l}10 \\
11 \\
121 / 2 \\
15 \\
171 / 2\end{array}$ & $\begin{array}{l}13 / 8 \\
11 / 2 \\
1568 \\
17 / 8 \\
21 / 8\end{array}$ & $\begin{array}{l}77 / 8 \\
91 / 4 \\
105 / 8 \\
13 \\
151 / 4\end{array}$ & $\begin{array}{r}8 \\
8 \\
12 \\
12 \\
16\end{array}$ & $\begin{array}{l}7 / 8 \\
7 / 8 \\
7 / 8 \\
1 \\
136\end{array}$ & $\begin{array}{l}8 \\
9 \\
93 / 4 \\
113 / 4 \\
131 / 4\end{array}$ & $\begin{array}{l}51 / 2 \\
6 \\
61 / 4 \\
63 / 4 \\
73 / 4\end{array}$ & $\begin{array}{l}16 \\
163 / 4 \\
183 / 4 \\
221 / 4 \\
253 / 4\end{array}$ & $\begin{array}{l}41 / 2 \\
5 \\
51 / 4 \\
53 / 4 \\
61 / 4\end{array}$ & $\begin{array}{l}81 / 4 \\
91 / 4 \\
10 \\
12 \\
131 / 2\end{array}$ \\
\hline $\begin{array}{r}12 \\
114 \\
16 \\
18 \\
20 \\
124\end{array}$ & $\begin{array}{l}12 \\
1316 \\
15 \\
17 \\
187 / 8 \\
225 / 8\end{array}$ & $\begin{array}{l}3 / 4 \\
13 / 10 \\
7 / 8 \\
15 / 16 \\
11 / 16 \\
13 / 16\end{array}$ & $\begin{array}{l}201 / 2 \\
23 \\
251 / 2 \\
28 \\
301 / 2 \\
36\end{array}$ & $\begin{array}{l}21 / 4 \\
23 / 8 \\
21 / 2 \\
25 / 8 \\
23 / 4 \\
3\end{array}$ & $\begin{array}{l}173 / 4 \\
201 / 4 \\
221 / 2 \\
243 / 4 \\
27 \\
32\end{array}$ & $\begin{array}{l}16 \\
20 \\
20 \\
24 \\
24 \\
24\end{array}$ & $\begin{array}{l}11 / 4 \\
11 / 4 \\
13 / 8 \\
13 / 8 \\
11 / 2 \\
13 / 4\end{array}$ & $\begin{array}{l}15 \\
161 / 4 \\
173 / 4 \\
191 / 4 \\
203 / 4 \\
241 / 4\end{array}$ & $\begin{array}{r}83 / 4 \\
91 / 4 \\
101 / 4 \\
103 / 4 \\
111 / 4 \\
123 / 4\end{array}$ & $\begin{array}{l}293 / 4 \\
323 / 4 \\
361 / 4 \\
391 / 4 \\
423 / 4 \\
501 / 4\end{array}$ & $\begin{array}{l}61 / 2 \\
7 \\
8 \\
81 / 2 \\
9 \\
101 / 2\end{array}$ & $\begin{array}{l}151 / 4 \\
161 / 2 \\
181 / 2 \\
191 / 2 \\
21 \\
241 / 2\end{array}$ \\
\hline
\end{tabular}

The fittings incorporated in this table are suitable also for other pressure and temperature ratings shown in Table 1 .

1 raised face of $1 / 4$ inch is not included in the minimum thickness of flanges, but is included in center to contact surface of raised face dimensions, and contact surface to contact surface dimensions.

${ }^{3}$ Where facings other than the $1 / 4$-inch raised face are used, the center to flange edge dimensions shown in Table 9 shall remain unchanged, and the new center to contact surface, or the new contact surface to contact surface dimensions shall be established to suit the facing used.

Outside diameter. 


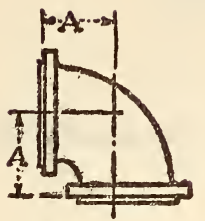

ELBOW

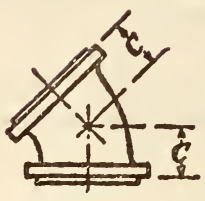

$45^{\circ}$ ELBOW

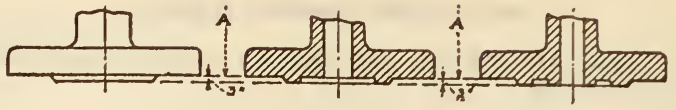

FIGURE 292.-400-pound steel flanged fittings

Groove and female facings for fittings

FIGURE 293.-400-pound steel flanged fittings

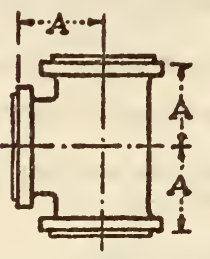

TEE

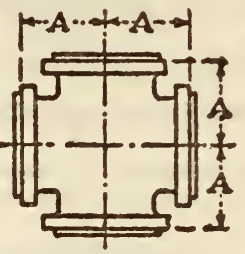

CROSS

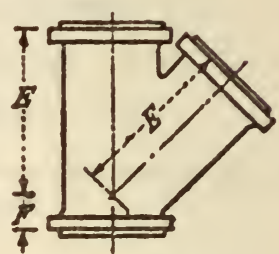

$45^{\circ}$ LATERAL

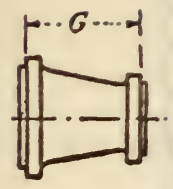

REDUCER

TABLE 9.-Center to flange edge dimensions for maximum working steam pressure of 400 pounds per square inch at a temperature of $750^{\circ} \mathrm{F} .{ }^{1}$

[All dimensions given in inches]

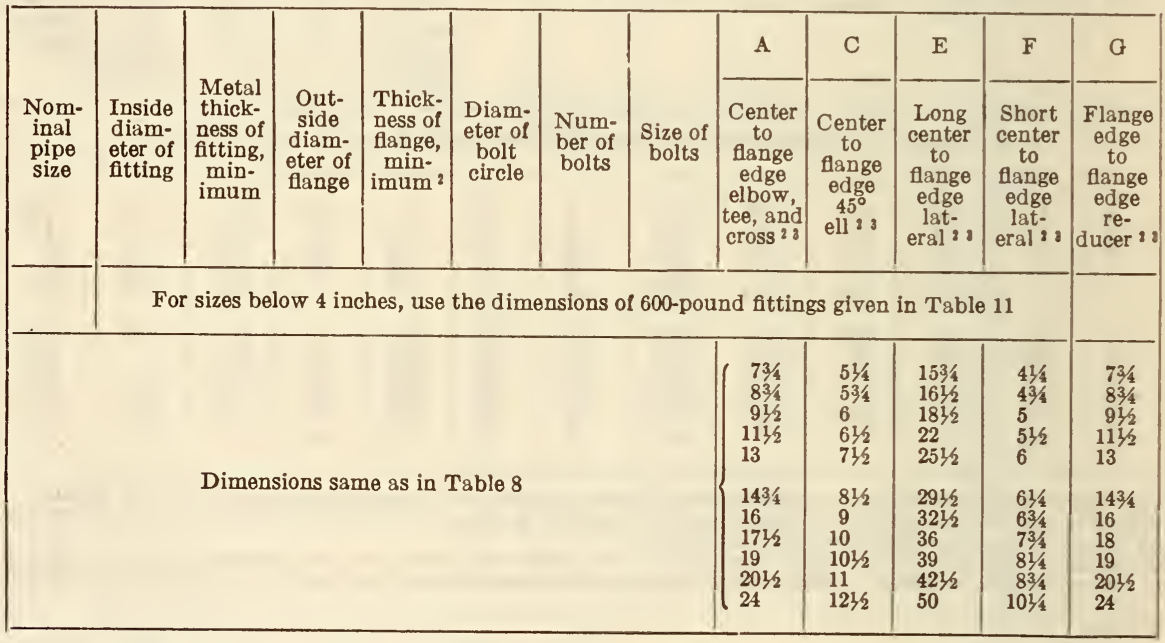

1 The fittings incorporated in this table are suitable also for other pressure and temperature ratings shown in Table 1.

2 A raised face of 14 inch is not included in (a) minimum thickness of flanges, $(b)$ center to flange edge dimensions, or $(c)$ flange edge to flange edge dimensions.

3. 3 Where facings other than the 1/4-inch raised face are used the center to flange edge dimensions shall remain unchanged, and the new center to contact surface dimensions shall be established to suit the facing used.

\section{0-POUND STEEL FLANGED FITTINGS}

(Elbow, $45^{\circ}$ elbow, tee, cross, $45^{\circ}$ lateral, and reducer illustrations are identical with those for 250-pound steel flanged fittings in Table 6.)
(Raised face, male, and tongue facings for fittings illustrations are identical with those for 400 -pound steel flanged fittings in Table 8.) 
TABLE 10.-Center to contact surface dimensions for maximum working steam pressure of 600 pounds per square inch at a temperature of $750^{\circ} \mathrm{F} .1$

[All dimensions given in inches]

\begin{tabular}{|c|c|c|c|c|c|c|c|c|c|c|c|c|}
\hline & & & & & & & & AA & $\mathrm{CC}$ & EE & FF & $\mathrm{GG}$ \\
\hline $\begin{array}{l}\text { Nominal } \\
\text { pipe size }\end{array}$ & $\begin{array}{l}\text { Inside } \\
\text { diame- } \\
\text { ter of } \\
\text { fittlng }\end{array}$ & $\begin{array}{l}\text { Metal } \\
\text { thick- } \\
\text { ness of } \\
\text { fitting, } \\
\text { mini- } \\
\text { mum }\end{array}$ & $\begin{array}{l}\text { Out- } \\
\text { side } \\
\text { diam- } \\
\text { eter of } \\
\text { flange }\end{array}$ & $\begin{array}{l}\text { Thick- } \\
\text { ness of } \\
\text { flange, } \\
\text { mini- } \\
\text { mum 2 }\end{array}$ & $\begin{array}{l}\text { Diam- } \\
\text { eter } \\
\text { of bolt } \\
\text { circle }\end{array}$ & $\begin{array}{l}\text { Num- } \\
\text { ber of } \\
\text { bolts }\end{array}$ & $\begin{array}{c}\text { Size of } \\
\text { bolts }\end{array}$ & $\begin{array}{l}\text { Center } \\
\text { to con- } \\
\text { tact } \\
\text { surface } \\
\text { of raised } \\
\text { face, } \\
\text { elbow } \\
\text { tee, and } \\
\text { cross }^{2}\end{array}$ & $\begin{array}{l}\text { Center } \\
\text { to con- } \\
\text { tact sur- } \\
\text { face of } \\
\text { raised } \\
\text { face, } \\
45^{\circ} \\
\text { ell } 3\end{array}$ & $\begin{array}{l}\text { Long } \\
\text { center } \\
\text { to con- } \\
\text { tact sur- } \\
\text { face of } \\
\text { raised } \\
\text { face, } \\
\text { lat- } \\
\text { eral :3 }\end{array}$ & $\begin{array}{l}\text { Short } \\
\text { center } \\
\text { to con- } \\
\text { tact sur- } \\
\text { face of } \\
\text { raised } \\
\text { face, } \\
\text { lat- } \\
\text { eral }:\end{array}$ & $\begin{array}{l}\text { Contact } \\
\text { surface } \\
\text { to con- } \\
\text { tact sur- } \\
\text { face } \\
\text { re- } \\
\text { ducer }\end{array}$ \\
\hline $\begin{array}{l}1 / 2 \\
3 / 4 \\
1 \\
11 / 4 \\
11 / 2\end{array}$ & $\begin{array}{l}1 / 2 \\
3 / 4 \\
1 \\
11 / 4 \\
11 / 2\end{array}$ & $\begin{array}{l}1 / 4 \\
1 / 4 \\
1 / 4 \\
1 / 4 \\
1 / 4\end{array}$ & $\begin{array}{l}33 / 4 \\
45 / 8 \\
47 / 8 \\
51 / 4 \\
616\end{array}$ & $\begin{array}{l}9 / 16 \\
5 / 8 \\
11 / 16 \\
13 / 16 \\
7 / 8\end{array}$ & $\begin{array}{l}25 / 8 \\
31 / 4 \\
31 / 2 \\
37 / 8 \\
41 / 2\end{array}$ & $\begin{array}{l}4 \\
4 \\
4 \\
4 \\
4\end{array}$ & $\begin{array}{l}1 / 2 \\
5 / 8 \\
5 / 8 \\
5 / 8 \\
3 / 4\end{array}$ & $\begin{array}{l}31 / 4 \\
33 / 4 \\
41 / 4 \\
41 / 2 \\
43 / 4\end{array}$ & $\begin{array}{l}2 \\
21 / 4 \\
21 / 4 \\
23 / 4 \\
3\end{array}$ & $\begin{array}{l}53 / 4 \\
63 / 4 \\
71 / 4 \\
8 \\
9\end{array}$ & $\begin{array}{l}13 / 4 \\
2 \\
21 / 4 \\
21 / 2 \\
23 / 4\end{array}$ & $\begin{array}{l}5 \\
5 \\
5 \\
5 \\
5\end{array}$ \\
\hline $\begin{array}{l}2 \\
21 / 2 \\
3 \\
31 / 2 \\
4\end{array}$ & $\begin{array}{l}2 \\
21 / 2 \\
3 \\
31 / 2 \\
4\end{array}$ & $\begin{array}{l}5 / 16 \\
368 \\
3 / 8 \\
7 / 16 \\
1 / 2\end{array}$ & $\begin{array}{c}61 / 2 \\
71 / 2 \\
81 / 4 \\
9 \\
103 / 4\end{array}$ & $\begin{array}{l}1 \\
11 / 8 \\
11 / 4 \\
13 / 8 \\
11 / 2\end{array}$ & $\begin{array}{l}5 \\
57 / 8 \\
65 / 8 \\
71 / 4 \\
81 / 2\end{array}$ & $\begin{array}{l}8 \\
8 \\
8 \\
8 \\
8\end{array}$ & $\begin{array}{l}5 / 8 \\
3 / 4 \\
3 / 4 \\
7 / 8 \\
7 / 8\end{array}$ & $\begin{array}{l}53 / 4 \\
61 / 2 \\
7 \\
71 / 2 \\
81 / 2\end{array}$ & $\begin{array}{l}41 / 4 \\
41 / 2 \\
5 \\
51 / 2 \\
6\end{array}$ & $\begin{array}{l}101 / 4 \\
111 / 2 \\
123 / 4 \\
14 \\
161 / 2\end{array}$ & $\begin{array}{l}31 / 2 \\
31 / 2 \\
4 \\
41 / 2 \\
41 / 2\end{array}$ & $\begin{array}{l}6 \\
63 / 4 \\
71 / 4 \\
73 / 4 \\
83 / 4\end{array}$ \\
\hline $\begin{array}{r}5 \\
6 \\
8 \\
10 \\
12\end{array}$ & $\begin{array}{l}5 \\
6 \\
77,8 \\
93 / 4 \\
113 / 4\end{array}$ & $\begin{array}{l}9 / 6 \\
5 / 8 \\
3 / 4 \\
7 / 8 \\
1\end{array}$ & $\begin{array}{l}13 \\
14 \\
161 / 2 \\
20 \\
22\end{array}$ & $\begin{array}{l}13 / 4 \\
178 \\
23 / 16 \\
21 / 2 \\
258\end{array}$ & $\begin{array}{l}101 / 2 \\
111 / 2 \\
133 / 4 \\
17 \\
191 / 4\end{array}$ & $\begin{array}{r}8 \\
12 \\
12 \\
16 \\
20\end{array}$ & $\begin{array}{l}1 \\
1 \\
11 / 8 \\
11 / 4 \\
11 / 4\end{array}$ & $\begin{array}{l}10 \\
11 \\
13 \\
151 / 2 \\
161 / 2\end{array}$ & $\begin{array}{l}7 \\
71 / 2 \\
81 / 2 \\
91 / 2 \\
10\end{array}$ & $\begin{array}{l}191 / 2 \\
21 \\
241 / 2 \\
291 / 2 \\
311 / 2\end{array}$ & $\begin{array}{l}6 \\
61 / 2 \\
7 \\
8 \\
81 / 2\end{array}$ & $\begin{array}{l}101 / 4 \\
111 / 4 \\
131 / 4 \\
153 / 4 \\
163 / 4\end{array}$ \\
\hline $\begin{array}{l}14 \\
+16 \\
118 \\
120 \\
+24\end{array}$ & $\begin{array}{l}127 / 8 \\
143 / 4 \\
161 / 2 \\
181 / 4 \\
22\end{array}$ & $\begin{array}{l}11 / 8 \\
11 / 4 \\
13 / 8 \\
11 / 2 \\
13 / 4\end{array}$ & $\begin{array}{l}23344 \\
27 \\
291 / 4 \\
32 \\
37\end{array}$ & $\begin{array}{l}2344 \\
3 \\
31 / 4 \\
31 / 2 \\
4\end{array}$ & $\begin{array}{l}203 / 4 \\
233 / 4 \\
253 / 4 \\
281 / 2 \\
33\end{array}$ & $\begin{array}{l}20 \\
20 \\
20 \\
24 \\
24\end{array}$ & $\begin{array}{l}13 / 8 \\
11 / 2 \\
158 \\
1568 \\
178\end{array}$ & $\begin{array}{l}171 / 2 \\
191 / 2 \\
211 / 2 \\
231 / 2 \\
271 / 2\end{array}$ & $\begin{array}{l}103 / 4 \\
113 / 4 \\
121 / 4 \\
13 \\
143 / 4\end{array}$ & $\begin{array}{l}341 / 4 \\
381 / 2 \\
42 \\
451 / 2 \\
53\end{array}$ & $\begin{array}{l}9 \\
10 \\
101 / 2 \\
11 \\
13\end{array}$ & $\begin{array}{l}173 / 4 \\
193 / 4 \\
213 / 4 \\
233 / 4 \\
273 / 4\end{array}$ \\
\hline
\end{tabular}

1 The fittings incorporated in this table are suitable also for other pressure and temperature ratings shown in Table 1.

2 A raised face of $1 / 4$ inch is not included in the minimum thickness of flanges, but is included in center to contact surface of raised.face dimensions, and contact surface to contact surface dimensions.

"Where facings other than the $1 / 4$-inch raised face are used, the "center to flange edge" dimensions shown in Table 11 shall remain unchanged, and the new "center to contact surface," or the new "contact surface to contact surface" dimensions shall be established to suit the facing used.

- Outside diameter.

(Elbow, $45^{\circ}$ elbow, tee, cross, $45^{\circ}$ lateral, and reducer illustrations are identical with those for 400-pound steel flanged fittings in Table 9.)

(Groove and female facings for fittings illustrations are identical with those for 400-pound steel flanged fittings in Table 9.)

$\mathrm{T}_{\mathrm{ABLE}}$ 11.-Center to flange edge dimensions for maximum working steam pressure of 600 pounds per square inch at a temperature of $750^{\circ} \mathrm{F} .1$

[All dimensions given in inches]

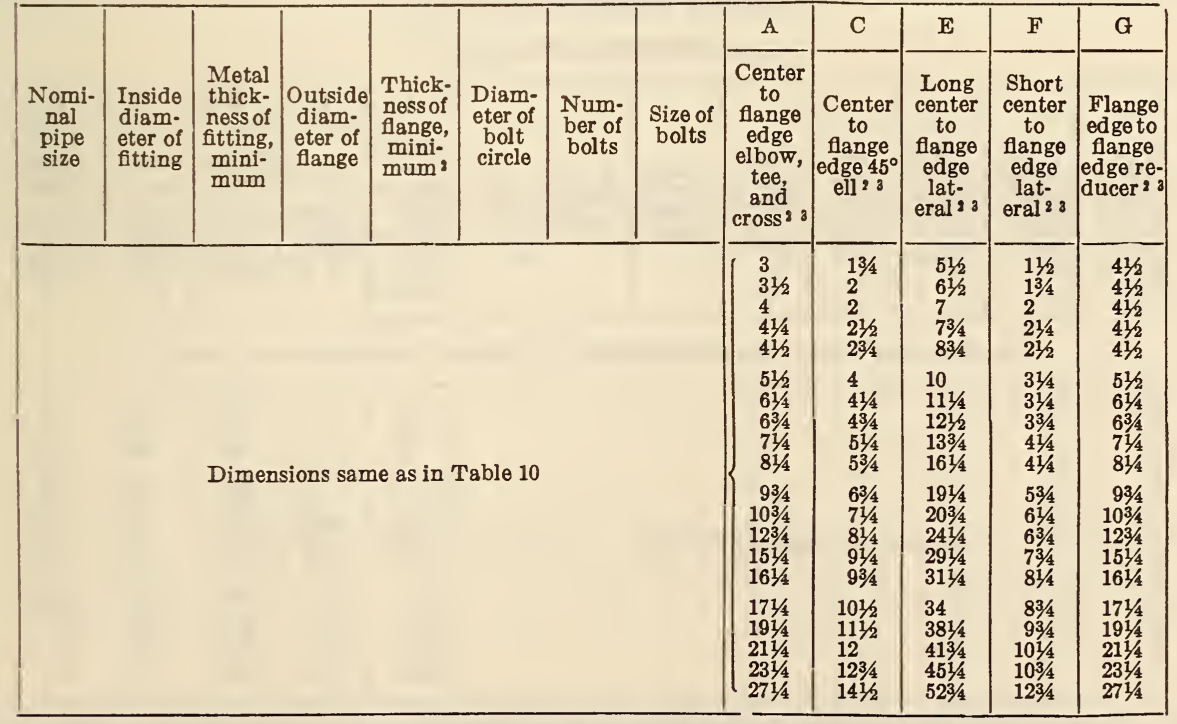

1 The fittings incorporated in this table are suitable also for other pressure and temperature ratings shown in Table 1.

A raised face of $1 / 4$ inch is not included in $(a)$ minimum thickness of flanges, $(b)$ center to flange edge dimensions, or $(c)$ flange edge to llange edge dimensions.

"Where facings other than the 1/4-inch raised face are used the "center to flange edge" dimensions shall remain unchanged, and the new "center to contact surface" dimensions shall be established to suit the facing used. 


\section{0-POUND STEEL FLANGED FITTINGS}

(Elbow, $45^{\circ}$ elbow, tee, cross, $45^{\circ}$ lateral, and reducer illustrations are identical with those for 250-pound steel flanged fittings in Table 6.)
(Raised face, male, and tongue facings for fittings illustrations are identical with those for 400 -pound steel flanged fittings in Table 8.)

TABLE 12.-Center to contact surface dimensions for maximum working steam pressure of 900 pounds per square inch at a temperature of $750^{\circ} \mathrm{F} .{ }^{1}$

[All dimensions given in inches]

\begin{tabular}{|c|c|c|c|c|c|c|c|c|c|c|c|c|}
\hline \multirow[b]{2}{*}{$\begin{array}{l}\text { Nominal } \\
\text { pipe size }\end{array}$} & \multirow[b]{2}{*}{$\begin{array}{l}\text { Inside } \\
\text { dism- } \\
\text { eter of } \\
\text { fitting }\end{array}$} & \multirow[b]{2}{*}{$\begin{array}{l}\text { Metal } \\
\text { thick- } \\
\text { ness of } \\
\text { fitting, } \\
\text { mini- } \\
\text { mum }\end{array}$} & \multirow[b]{2}{*}{$\begin{array}{l}\text { Outside } \\
\text { diam- } \\
\text { eter of } \\
\text { flange }\end{array}$} & \multirow[b]{2}{*}{$\begin{array}{l}\text { Thick- } \\
\text { ness of } \\
\text { flange, } \\
\text { mini- } \\
\text { mum I }\end{array}$} & \multirow[b]{2}{*}{$\begin{array}{l}\text { Diam- } \\
\text { eter of } \\
\text { bolt } \\
\text { circle }\end{array}$} & \multirow[b]{2}{*}{$\begin{array}{l}\text { Num- } \\
\text { ber of } \\
\text { bolts }\end{array}$} & \multirow[b]{2}{*}{$\begin{array}{c}\text { Size of } \\
\text { bolts }\end{array}$} & AA & $\mathrm{CC}$ & EE & FF & $\mathrm{GG}$ \\
\hline & & & & & & & & $\begin{array}{l}\text { Center } \\
\text { to con- } \\
\text { tact } \\
\text { surface } \\
\text { of } \\
\text { raised } \\
\text { face, } \\
\text { elbow, } \\
\text { tee, and } \\
\text { cross }^{2} \text { ? }\end{array}$ & $\begin{array}{l}\text { Center } \\
\text { to con- } \\
\text { tact } \\
\text { surface } \\
\text { of } \\
\text { raised } \\
\text { face, } 45^{\circ} \\
\text { ell } 2\end{array}$ & $\begin{array}{l}\text { Long } \\
\text { center } \\
\text { to con- } \\
\text { tact } \\
\text { surface } \\
\text { of } \\
\text { raised } \\
\text { face, } \\
\text { lat- } \\
\text { eral ? }\end{array}$ & $\begin{array}{l}\text { Short } \\
\text { center } \\
\text { to con- } \\
\text { tact } \\
\text { surface } \\
\text { of } \\
\text { raised } \\
\text { face, } \\
\text { lat- } \\
\text { eral } 8\end{array}$ & $\begin{array}{l}\text { Contact } \\
\text { surface } \\
\text { to con- } \\
\text { tact sur- } \\
\text { face re- } \\
\text { ducer'? }\end{array}$ \\
\hline & \multicolumn{11}{|c|}{ For sizes below 3 inches, use the dimensions of 1,350-pound fittings given in Table 14} & \\
\hline $\begin{array}{l}3 \\
31 / 2 \\
4\end{array}$ & $\begin{array}{l}278 \\
338 \\
37 / 8\end{array}$ & $\begin{array}{l}1 / 2 \\
9 / 16 \\
5 / 8\end{array}$ & $\begin{array}{r}91 / 2 \\
103 / 4 \\
111 / 2\end{array}$ & $\begin{array}{l}11 / 2 \\
15 \% 8 \\
13 / 4\end{array}$ & $\begin{array}{l}71 / 2 \\
81 / 2 \\
91 / 4\end{array}$ & $\begin{array}{l}8 \\
8 \\
8\end{array}$ & $\begin{array}{l}1^{7 / 8} \\
11 / 8\end{array}$ & $\begin{array}{l}71 / 2 \\
81 / 2 \\
9\end{array}$ & $\begin{array}{l}51 / 2 \\
6 \\
61 / 2\end{array}$ & $\begin{array}{l}141 / 2 \\
161 / 2 \\
171 / 2\end{array}$ & $\begin{array}{l}41 / 2 \\
41 / 2 \\
51 / 2\end{array}$ & $\begin{array}{l}73 / 4 \\
83 / 4 \\
61 / 4\end{array}$ \\
\hline $\begin{array}{r}5 \\
6 \\
8 \\
10 \\
12\end{array}$ & $\begin{array}{r}43 / 4 \\
53 / 4 \\
71 / 2 \\
93 / 8 \\
111 / 8\end{array}$ & $\begin{array}{l}3 / 4 \\
13 / 16 \\
11 / 16 \\
11 / 4 \\
17 / 16\end{array}$ & $\begin{array}{l}133 / 4 \\
15 \\
181 / 2 \\
211 / 2 \\
24\end{array}$ & $\begin{array}{l}2 \\
23 / 16 \\
21 / 2 \\
23 / 4 \\
31 / 8\end{array}$ & $\begin{array}{l}11 \\
121 / 2 \\
151 / 2 \\
181 / 2 \\
21\end{array}$ & $\begin{array}{r}8 \\
12 \\
12 \\
16 \\
20\end{array}$ & $\begin{array}{l}11 / 4 \\
11 / 8 \\
13 / 8 \\
13 / 8 \\
13 / 8\end{array}$ & $\begin{array}{l}11 \\
12 \\
141 / 2 \\
161 / 2 \\
19\end{array}$ & $\begin{array}{l}71 / 2 \\
8 \\
9 \\
10 \\
11\end{array}$ & $\begin{array}{l}21 \\
221 / 2 \\
271 / 2 \\
311 / 2 \\
341 / 2\end{array}$ & $\begin{array}{l}61 / 2 \\
61 / 2 \\
71 / 2 \\
81 / 2 \\
9\end{array}$ & $\begin{array}{l}111 / 4 \\
121 / 4 \\
143 / 4 \\
16^{3} / 4 \\
173 / 4\end{array}$ \\
\hline $\begin{array}{l}14 \\
16 \\
18 \\
20 \\
124\end{array}$ & $\begin{array}{l}121 / 4 \\
14 \\
153 / 4 \\
171 / 2 \\
21\end{array}$ & $\begin{array}{l}1 \% / 6 \\
113 / 16 \\
2 \\
21 / 4 \\
258\end{array}$ & $\begin{array}{l}251 / 4 \\
273 / 4 \\
31 \\
333 / 4 \\
41\end{array}$ & $\begin{array}{l}33 / 8 \\
31 / 2 \\
4 \\
41 / 4 \\
51 / 2\end{array}$ & $\begin{array}{l}22 \\
241 / 4 \\
27 \\
291 / 2 \\
351 / 2\end{array}$ & $\begin{array}{l}20 \\
20 \\
20 \\
20 \\
20\end{array}$ & $\begin{array}{l}11 / 2 \\
13 / 8 \\
17 / 8 \\
2 \\
21 / 2\end{array}$ & $\begin{array}{l}201 / 4 \\
221 / 4 \\
24 \\
26 \\
301 / 2\end{array}$ & $\begin{array}{l}111 / 2 \\
121 / 2 \\
131 / 4 \\
141 / 2 \\
18\end{array}$ & $\begin{array}{l}361 / 2 \\
403 / 4 \\
451 / 2 \\
501 / 4 \\
60\end{array}$ & $\begin{array}{l}91 / 2 \\
101 / 2 \\
12 \\
13 \\
151 / 2\end{array}$ & $\begin{array}{l}19 \\
21 \\
241 / 2 \\
261 / 2 \\
301 / 2\end{array}$ \\
\hline
\end{tabular}

1 The fittings incorporated in this table are suitable also for other pressure and temperature ratings shown in Table 1.

A raised face of $1 / 4$ inch is not, included in the minimum thickness of flanges, but is included in center to contact surface of raised face dimensions, and contact surface to contact surface dimensions.

"Where facings other than the $1 / 4$ inch raised face are used, the "center to flange edge" dimensions shown in Table 13 shall remain unchanged, and the new "center to contact surface," or the new "contact surface to contact surface" dimensions shall be established to suit the facing used.

Outside diameter.

(Elbow, $45^{\circ}$ elbow, tee, cross, $45^{\circ}$ lateral, and (Groove and female facings for fittings illustrareducer illustrations are identical with those for tions are identical with those for 400-pound steel 400 -pound steel flanged fittings in Table 9.)

flanged fittings in Table 9.)

TABLE]13.-Center to flange edge dimensions for maximum working steam pressure of 900 pounds per square inch at a temperature of $750^{\circ} \mathrm{F} .{ }^{1}$

[All dimensions given in inches]

\begin{tabular}{|c|c|c|c|c|c|c|c|c|c|c|c|c|}
\hline & & & & & & & & $\mathbf{A}$ & C & $\mathbf{E}$ & $F$ & $G$ \\
\hline $\begin{array}{c}\text { Nomi- } \\
\text { nal } \\
\text { pipe } \\
\text { size }\end{array}$ & $\begin{array}{l}\text { Inside } \\
\text { diam- } \\
\text { eter of } \\
\text { fitting }\end{array}$ & $\begin{array}{l}\text { Metal } \\
\text { thick- } \\
\text { ness of } \\
\text { fitting, } \\
\text { mini- } \\
\text { mum }\end{array}$ & $\begin{array}{l}\text { Out- } \\
\text { side } \\
\text { diam- } \\
\text { eter of } \\
\text { flange }\end{array}$ & $\begin{array}{l}\text { Thick- } \\
\text { ness of } \\
\text { flange, } \\
\text { mini- } \\
\text { mum }\end{array}$ & $\begin{array}{l}\text { Diam- } \\
\text { eter of } \\
\text { bolt } \\
\text { circle }\end{array}$ & $\begin{array}{l}\text { Num- } \\
\text { ber of } \\
\text { bolts }\end{array}$ & $\begin{array}{c}\text { Size } \\
\text { of } \\
\text { bolts }\end{array}$ & $\begin{array}{l}\text { Center } \\
\text { to } \\
\text { flange } \\
\text { edge } \\
\text { elbow, } \\
\text { teee, } \\
\text { and } \\
\text { cross' }^{2}\end{array}$ & 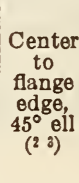 & $\begin{array}{c}\text { Long } \\
\text { center } \\
\text { to } \\
\text { flange } \\
\text { edge, } \\
\text { lateral } \\
\left(3^{3} 3\right)\end{array}$ & $\begin{array}{c}\text { Short } \\
\text { center } \\
\text { to } \\
\text { flange } \\
\text { edge, } \\
\text { lateral } \\
\text { (23) }\end{array}$ & $\begin{array}{l}\text { Flange } \\
\text { edge } \\
\text { to } \\
\text { flange } \\
\text { edge } \\
\text { reducer } \\
(23)\end{array}$ \\
\hline
\end{tabular}

For sizes below 3 inches, use the dimensions of 1,350-pound fittings given in Table 15

\begin{tabular}{|c|c|c|c|c|c|}
\hline \multirow{3}{*}{ Dimensions same as in Table 12} & $\left(\begin{array}{l}734 \\
814 \\
834\end{array}\right.$ & $\begin{array}{l}51 / 4 \\
53 / 4 \\
61 / 4\end{array}$ & $\mid$\begin{tabular}{c|}
1414 \\
1614 \\
$171 / 4$
\end{tabular} & $\begin{array}{l}41 / 4 \\
41 / 4 \\
51 / 4\end{array}$ & $\begin{array}{l}71 / 4 \\
81 / 4 \\
83 / 4\end{array}$ \\
\hline & $\begin{array}{l}1034 \\
1134 \\
141 / 4 \\
1614 \\
18^{3} / 4\end{array}$ & $\begin{array}{r}71 / 4 \\
73 / 4 \\
83 / 4 \\
934 \\
1034\end{array}$ & $\begin{array}{l}203 / 4 \\
221 / 4 \\
271 / 4 \\
3114 \\
341 / 4\end{array}$ & $\begin{array}{l}61 / 4 \\
61 / 4 \\
71 / 4 \\
81 / 4 \\
83 / 4\end{array}$ & $\begin{array}{l}10^{3} / 4 \\
113 / 4 \\
141 / 4 \\
161 / 4 \\
171 / 4\end{array}$ \\
\hline & \begin{tabular}{l|l}
20 \\
22 \\
$233 / 4$ \\
$253 / 4$ \\
$301 / 4$
\end{tabular} & $\begin{array}{l}111 / 4 \\
121 / 4 \\
13 \\
141 / 4 \\
173 / 4\end{array}$ & $\begin{array}{l}361 / 4 \\
401 / 2 \\
451 / 4 \\
50 \\
593 / 4\end{array}$ & $\begin{array}{r}91 / 4 \\
101 / 4 \\
113 / 4 \\
123 / 4 \\
151 / 4\end{array}$ & $\begin{array}{l}181 / 2 \\
201 / 2 \\
24 \\
26 \\
30\end{array}$ \\
\hline
\end{tabular}

1 Thesfittings incorporated in this table are suitable also for other pressure and temperature ratings shown in Table 1.

- A raised face of $1 / 4$ inch is not included in $(a)$ minimum thickness of flanges, $(b)$ center to flange edge dimensions, or $(c)$ flange edge to flange edge dimensions.

3 Where facings other than the $1 / 4$-inch raised face are used the "center to flange edge" dimensions shall remain unchanged, and the new "center to contact surface" dimensions shall be established to suit the facing used. 
1,350-POUND STEEL FLANGED FITTINGS

(Elbow, $45^{\circ}$ elbow, tee, cross, $45^{\circ}$ lateral, and reducer illustrations are identical with those for 250-pound steel flanged fittings in Table 6.)
(Raised face, male, and tongue facings for fittings illustrations are identical with those for 400-pound steel flanged fittings in Table 8.)

TABLE 14.-Center to contact surface dimensions for maximum working steam pressure of 1,350 pounds per square inch at a temperature of $750^{\circ} \mathrm{F} .{ }^{1}$

[All dimensions given in inches]

\begin{tabular}{|c|c|c|c|c|c|c|c|c|c|c|c|c|}
\hline & & & & & & & & AA & $\mathrm{CC}$ & EE & FF & $\mathrm{GG}$ \\
\hline $\begin{array}{c}\text { Nomi- } \\
\text { nal } \\
\text { pipe } \\
\text { size }\end{array}$ & $\begin{array}{l}\text { Inside } \\
\text { diam- } \\
\text { eter of } \\
\text { fltting }\end{array}$ & $\begin{array}{l}\text { Metal } \\
\text { thick- } \\
\text { ness of } \\
\text { fitting, } \\
\text { mini- } \\
\text { mum }\end{array}$ & $\begin{array}{l}\text { Out- } \\
\text { side } \\
\text { diam- } \\
\text { eter of } \\
\text { flange }\end{array}$ & $\begin{array}{l}\text { Thick- } \\
\text { ness of } \\
\text { flange, } \\
\text { mini- } \\
\text { mum }^{2}\end{array}$ & $\begin{array}{l}\text { Diam- } \\
\text { eter of } \\
\text { bolt } \\
\text { circle }\end{array}$ & $\begin{array}{l}\text { Num- } \\
\text { ber of } \\
\text { bolts }\end{array}$ & $\begin{array}{c}\text { Size } \\
\text { of } \\
\text { bolts }\end{array}$ & $\begin{array}{c}\text { Center } \\
\text { to } \\
\text { contact } \\
\text { surface } \\
\text { of } \\
\text { raised } \\
\text { face } \\
\text { elbow, } \\
\text { tee, and } \\
\text { cross }^{2} 4\end{array}$ & $\begin{array}{c}\text { Center } \\
\text { to } \\
\text { contact } \\
\text { surface } \\
\text { of } \\
\text { raised } \\
\text { face, } \\
45^{\circ} \text { ell } \\
(24)\end{array}$ & $\begin{array}{c}\text { Long } \\
\text { center } \\
\text { to } \\
\text { contact } \\
\text { surface } \\
\text { of } \\
\text { raised } \\
\text { face, } \\
\text { lateral } \\
(24)\end{array}$ & $\begin{array}{c}\text { Short } \\
\text { center } \\
\text { to } \\
\text { contact } \\
\text { surface } \\
\text { of } \\
\text { raised } \\
\text { face, } \\
\text { lateral } \\
(24)\end{array}$ & $\begin{array}{c}\text { Contact } \\
\text { surface } \\
\text { to } \\
\text { contact } \\
\text { surface } \\
\text { reducer } \\
(3)\end{array}$ \\
\hline $\begin{array}{l}1 \\
11 / 4 \\
11 / 2 \\
2\end{array}$ & $\begin{array}{r}7 / 8 \\
118 \\
138 \\
178\end{array}$ & $\begin{array}{r}3 / 8 \\
368 \\
7 / 16 \\
1 / 2\end{array}$ & $\begin{array}{l}57 / 8 \\
61 / 4 \\
7 \\
81 / 2\end{array}$ & $\begin{array}{l}118 \\
118 \\
114 \\
11 / 2\end{array}$ & $\begin{array}{l}4 \\
43 / 8 \\
47 / 8 \\
61 / 2\end{array}$ & $\begin{array}{l}4 \\
4 \\
4 \\
8\end{array}$ & $1_{7 / 8}^{7 / 8}$ & $\begin{array}{l}5 \\
51 / 2 \\
6 \\
71 / 4\end{array}$ & $\begin{array}{l}31 / 2 \\
4 \\
41 / 4 \\
43 / 4\end{array}$ & $\begin{array}{l}9 \\
10 \\
11 \\
1314\end{array}$ & $\begin{array}{l}21 / 2 \\
3 \\
31 / 2 \\
4\end{array}$ & $\begin{array}{l}5 \\
53 / 4 \\
61 / 4 \\
71 / 4\end{array}$ \\
\hline $\begin{array}{l}21 / 2 \\
3 \\
31 / 2 \\
4\end{array}$ & $\begin{array}{l}21 / 4 \\
23 / 4 \\
31 / 8 \\
358\end{array}$ & $\begin{array}{r}568 \\
11116 \\
13 / 16 \\
7 / 8\end{array}$ & $\begin{array}{l}95 / 8 \\
103 / 2 \\
11 \\
121 / 4\end{array}$ & $\begin{array}{l}1568 \\
17 / 8 \\
2 \\
21 / 8\end{array}$ & $\begin{array}{l}71 / 2 \\
8 \\
81 / 2 \\
91 / 2\end{array}$ & $\begin{array}{l}8 \\
8 \\
8 \\
8\end{array}$ & $\begin{array}{l}1 \\
11 / 8 \\
11 / 8 \\
11 / 4\end{array}$ & $\begin{array}{r}81 / 4 \\
91 / 4 \\
93 / 4 \\
103 / 4\end{array}$ & $\begin{array}{l}51 / 4 \\
534 \\
614 \\
71 / 4\end{array}$ & $\begin{array}{l}151 / 4 \\
171 / 4 \\
181 / 4 \\
191 / 4\end{array}$ & $\begin{array}{l}41 / 2 \\
5 \\
51 / 2 \\
6\end{array}$ & $\begin{array}{r}81 / 4 \\
91 / 4 \\
93 / 4 \\
1034\end{array}$ \\
\hline $\begin{array}{r}5 \\
6 \\
8 \\
10 \\
12\end{array}$ & $\begin{array}{l}436 \\
536 \\
7 \\
83 / 4 \\
1038\end{array}$ & $\begin{array}{r}11 / 16 \\
1316 \\
11 / 2 \\
11316 \\
21 / 8\end{array}$ & $\begin{array}{l}143 / 4 \\
151 / 2 \\
19 \\
23 \\
251 / 2\end{array}$ & $\begin{array}{l}23 / 8 \\
25 / 8 \\
3 \\
31 / 2 \\
41 / 8\end{array}$ & $\begin{array}{l}111 / 2 \\
121 / 2 \\
151 / 2 \\
19 \\
211 / 2\end{array}$ & $\begin{array}{r}8 \\
12 \\
12 \\
12 \\
16\end{array}$ & $\begin{array}{l}11 / 2 \\
13 / 8 \\
15 / 8 \\
17 / 8 \\
17 / 8\end{array}$ & $\begin{array}{l}123 / 4 \\
131 / 4 \\
153 / 4 \\
183 / 4 \\
201 / 4\end{array}$ & $\begin{array}{r}81 / 4 \\
83 / 4 \\
101 / 4 \\
111 / 4 \\
121 / 4\end{array}$ & $\begin{array}{l}223 / 4 \\
241 / 4 \\
291 / 4 \\
351 / 4 \\
381 / 4\end{array}$ & $\begin{array}{r}7 \\
71 / 2 \\
81 / 2 \\
91 / 2 \\
101 / 2\end{array}$ & $\begin{array}{l}123 / 4 \\
131 / 4 \\
153 / 4 \\
183 / 4 \\
201 / 4\end{array}$ \\
\hline
\end{tabular}

1 The fittings incorporated in this table are suitable also for other pressure and temperature ratings shown in Table 1.

- A raised face of 34 inch is not included in the minimum thickness of flanges, but included in center to contact surface of raised faced dimensions, and contact surface to contact surface dimensions.

"Class "C" bolt studs shall be used with these flanges. The physical and chemical requirements of class " $C$ " bolt studs is given in Table 3.

"Where facings other than the 34 inch raised face are used, the "center to flange edge" dimensions shown in Table 15 shall remain unchanged, and the new "center to contact surface" or the new "contact surface to contact surface" dimensions shall be established to suit the facing used.

(Elbow, $45^{\circ}$ elbow, tee, cross, $45^{\circ}$ lateral, and reducer illustrations are identical with those for 400 -pound steel flanged fittings in Table 9.)

(Groove and female facings for fittings illustrations are identical with those for 400-pound steel flanged fittings in Table 9.)

TABLE 15.-Center to flange edge dimensions for maximum working steam pressure of 1,350 pounds per square inch at a temperature of $750^{\circ} \mathrm{F}^{1}$

[All dimensions given in inches]

\begin{tabular}{|c|c|c|c|c|c|c|c|c|c|c|c|c|}
\hline $\begin{array}{l}\text { Nomi- } \\
\text { nal } \\
\text { pipe } \\
\text { size }\end{array}$ & $\begin{array}{l}\text { Inside } \\
\text { diam- } \\
\text { eter of } \\
\text { fitting }\end{array}$ & $\begin{array}{c}\text { Metal } \\
\text { thick- } \\
\text { ness of } \\
\text { fitting, } \\
\text { mini- } \\
\text { mum }\end{array}$ & $\begin{array}{l}\text { Out- } \\
\text { side } \\
\text { diam- } \\
\text { eter of } \\
\text { flange }\end{array}$ & $\begin{array}{l}\text { Thick- } \\
\text { ness of } \\
\text { flange, } \\
\text { mini- } \\
\text { mum }\end{array}$ & $\begin{array}{l}\text { Diam- } \\
\text { eter of } \\
\text { bolt } \\
\text { circle }\end{array}$ & $\begin{array}{l}\text { Num- } \\
\text { ber of } \\
\text { bolts }\end{array}$ & $\begin{array}{l}\text { Size of } \\
\text { bolts }\end{array}$ & $\begin{array}{c}\text { A } \\
\text { Center } \\
\text { to } \\
\text { flange } \\
\text { edge } \\
\text { elbow, } \\
\text { tee, and } \\
\text { cross ' }\end{array}$ & $\begin{array}{c}\text { Center } \\
\text { to } \\
\text { flange } \\
\text { edge, } \\
45^{\circ} \text { ell' }^{2}\end{array}$ & 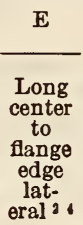 & 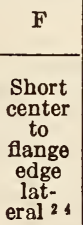 & $\begin{array}{c}G \\
\text { Flange } \\
\text { edge } \\
\text { to } \\
\text { flange } \\
\text { edge } \\
\text { reduc- } \\
\text { er } 24\end{array}$ \\
\hline & & Dimens & ions san & 10 as in 1 & able 14 & & & $\left\{\begin{array}{l}43 / 4 \\
51 / 4 \\
53 / 4 \\
7 \\
8 \\
9 \\
91 / 2 \\
101 / 2 \\
121 / 2 \\
13 \\
151 / 2 \\
1816 \\
20\end{array}\right.$ & $\begin{array}{l}31 / 4 \\
33 / 4 \\
4 \\
41 / 2 \\
5 \\
51 / 2 \\
6 \\
7 \\
8 \\
81 / 2 \\
10 \\
11 \\
12\end{array}$ & $\begin{array}{l}83 / 4 \\
93 / 4 \\
103 / 4 \\
13 \\
\\
15 \\
17 \\
18 \\
19 \\
\\
221 / 2 \\
24 \\
29 \\
35 \\
38\end{array}$ & $\begin{array}{r}21 / 4 \\
23 / 4 \\
31 / 4 \\
33 / 4 \\
\\
41 / 4 \\
434 \\
51 / 4 \\
534 \\
63 / 4 \\
61 / 4 \\
81 / 4 \\
91 / 4 \\
1014\end{array}$ & $\begin{array}{r}41 / 2 \\
51 / 4 \\
53 / 4 \\
63 / 4 \\
73 / 4 \\
83 / 4 \\
91 / 4 \\
101 / 4 \\
121 / 4 \\
123 / 4 \\
1514 \\
181 / 4 \\
193 / 4\end{array}$ \\
\hline
\end{tabular}

1 The fittings incorporated in this table are suitable also for other pressure and temperature ratings shown in Table 1.

2 A raised face of $1 / 4$-inch is not included in (a) minimum thickness of flanges, (b) center to flange edge dimensions, or (c) flange edge to flange edge dimensions.

Class C bolt studs shall be used with these flanges. The physical and chemical requirements of class $\mathrm{C}$ bolt studs are given in Table 3 . "Where facings other than the 14-inch raised face are used the "center of flange edge" dimensions shall remain unchanged and the new "center to contact surface" dimensions shall be established to suit the facing used. 
(Appendix A to this specification is attached for information only; it does not form part of the standard. It gives the dimensions and weight of pipe for $250,400,600,900$, and 1,350 pound steam pressures and high temperatures. The data are the same as in Table 1 in A. S. T. M., A106-29, given below.)

\section{AMERICAN SOCIETY FOR TESTING MA- TERIALS, STANDARD SPECIFICATIONS FOR WELDED AND SEAMLESS STEEL PIPE, DESIGNATION A53-30, 1930}

\section{WELDED AND SEAMLESS STEEL PIPE}

1. Scope.-These specifications cover "standard weight," "extra strong," and "double extra strong" welded and seamless steel pipe. Pipe ordered under these specifications is intended for coiling, bending, flanging, and other special purposes. Butt-welded pipe is not intended for flanging. The purposes for which the pipe is intended should be stated in the order. ${ }^{79}$ Where seamless pipe is ordered for coiling, bending, or welding, the low-carbon grade should be specified.

\section{MANUFACTORE}

2. (a) Process.-The steel for welded pipe shall he soft weldable quality made by the Bessemer or by the open-hearth process. The steel for seamless pipe shall be made by the open-hearth or electricfurnace process.

(b) Welded pipe 3 inches or under in nominal diameter may be butt-welded, unless otherwise specified. Welded pipe over 3 inches in nominal diameter shall be lap-welded.

\section{CHEMICAL PROPERTIES AND TESTS}

3. (a) Chemical composition.-Ladle tests of openhearth steel shall conform to the following requirement as to chemical composition:

\begin{tabular}{l|r|r}
\hline Phosphorus, not orer.......................... & $\begin{array}{r}\text { Ler ient } \\
0.06\end{array}$ & $\begin{array}{r}\text { Per ient } \\
0.045\end{array}$ \\
\hline
\end{tabular}

(b) Check analyses.-Analyses may be made by the purchaser from each lot of 500 lengths or less of finished pipe. The phosphorus content thus determined shall not exceed that specified in section 3 by more than 25 per cent. Drillings for analysis shall be taken from several points around each pipe selected for analysis.

\section{PHYSICAI PROPERTIES AND TESTS}

5. (a) Tension tests.-The material shall conform to the following minimum requirements as to tensile properties:

70 Double welding.-Unless double-welded pipe is specifically called for on tho order, single-welded pipe may be furnished. When double welded pipe is ordered the flattening test on crop ends specified in sec. 9 shall be made with the weld on the side.

\begin{tabular}{|c|c|c|c|c|}
\hline & \multicolumn{2}{|c|}{ Welded } & \multicolumn{2}{|c|}{ Seamless } \\
\hline & $\begin{array}{c}\text { Besse- } \\
\text { mer }\end{array}$ & $\begin{array}{l}\text { Open- } \\
\text { hearth }\end{array}$ & $\begin{array}{l}\text { Open. } \\
\text { hearth } \\
\text { low } \\
\text { carbon }\end{array}$ & $\begin{array}{l}\text { Open- } \\
\text { hearth } \\
\text { me- } \\
\text { dium } \\
\text { carbon }\end{array}$ \\
\hline $\begin{array}{l}\text { Tensile strength.........lbs./in.2.- } \\
\text { Yield point } \\
\text { Elongation in } 8 \text { inches....... doer cent.- } \\
\text { Elongation in } 2 \text { inches......do... }\end{array}$ & $\begin{array}{r}50,000 \\
30,000 \\
118\end{array}$ & $\begin{array}{r}45,000 \\
25,000 \\
20 \\
30\end{array}$ & $\begin{array}{r}48,000 \\
26,500 \\
\hdashline 40\end{array}$ & $\begin{array}{r}62,000 \\
35,000 \\
25\end{array}$ \\
\hline
\end{tabular}

1 Gage distances for measuring elongation on welded Bessemer pipe of nominal sizes $8 / 4$ inch and smaller shall be as follows:

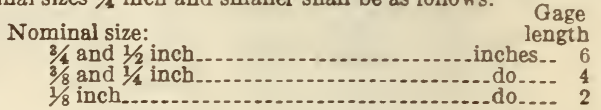

(b) The yield point shall be determined by the drop of the beam of the testing machine or other approved method.

6. Hydrostatic tests.-Each length of pipe shall be tested at the mill to the hydrostatic pressures specified in Table 1 . Welded pipe 2 inches and larger shall be jarred near one end while under test pressure.

7. (a) Flattening tests. - The flattening test shall be made on standard weight and extra strong pipe over 2 inches in nominal diameter. It shall not be required for double extra strong pipe.

(b) For lap-welded and butt-welded pipe the test section shall be 4 to 6 inches in length and the weld shall be located $45^{\circ}$ from the line of direction of the applied force.

(c) For seamless pipe the test section shall not be less than $2 \frac{1}{2}$ inches in length.

(d) The test shall consist in flattening a section of pipe, without developing cracks, between parallel plates until the distance between the plates is as follows:

For lap-welded pipe-One-third the outside diameter.

For butt-welded pipe-Sixty per cent of the outside diameter.

For low-carbon seamless pipe-One-third the outside diameter but in no case less than five times the thickness of the pipe wall.

For medium carbon seamless pipe-One-half the outside diameter but in no case less than seven times the thickness of the pipe wall.

8. Bend tests.-For pipe 2 inches or under in nominal diameter, a sufficient length of pipe shall stand being bent cold through $90^{\circ}$ around a cylindrical mandrel, the diameter of which is twelve times the nominal diameter of the pipe without developing cracks at any portion and without opening the weld. When ordered for close coiling, the pipe shall stand being bent cold through $180^{\circ}$ around a mandrel, the diameter of which is eight times the diameter of the pipe, without failure. Double extra strong pipe over $11 / 4$ inches in diameter need not be subjected to the bend test.

9. (a) Test specimens.-Tension-test specimens shall be cut longitudinally from the pipe and not flattened between gage marks. The sides of specimens shall be parallel between gage marks. If desired, the tension-test specimen may consist of a full section of the pipe. Where impracticable to pull a test specimen in full thickness the A. S. T. M. standard 2-inch gage length test specimen may be used. 
TABLE 1.-Hydrostatic pressures for welded and seamless steel pipe

[Pressures expressed in pounds per square inch]

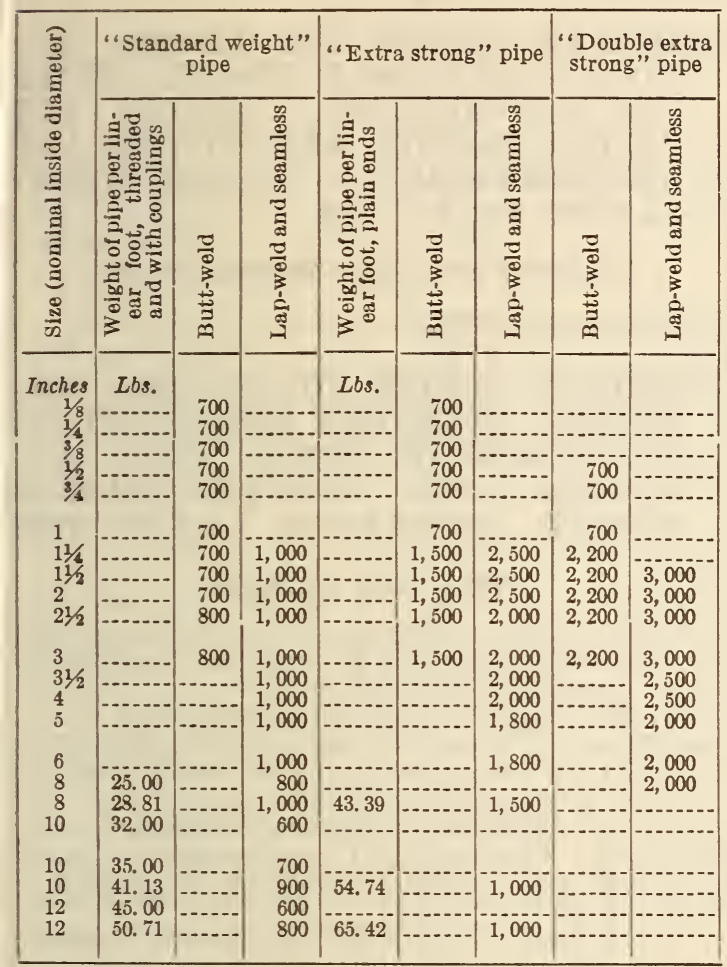

For pipes over 12 inches in inside diameter, the test pressures should be calculated by the formula $P=\frac{2 S t}{D}$ in which $P=$ pressure in pounds per square inch; $S=$ fiber stress, 12,000 pounds per square inch; $t=$ thickness of wall in inches; $D=$ outside diameter in inches.

(b) Test specimens for flattening and bend tests shall consist of sections cut from a pipe. Specimens for flattening tests shall be smooth on the ends and free from burrs, except when made on crop ends taken from welded pipe.

(c) All specimens shall be tested at room temperature.

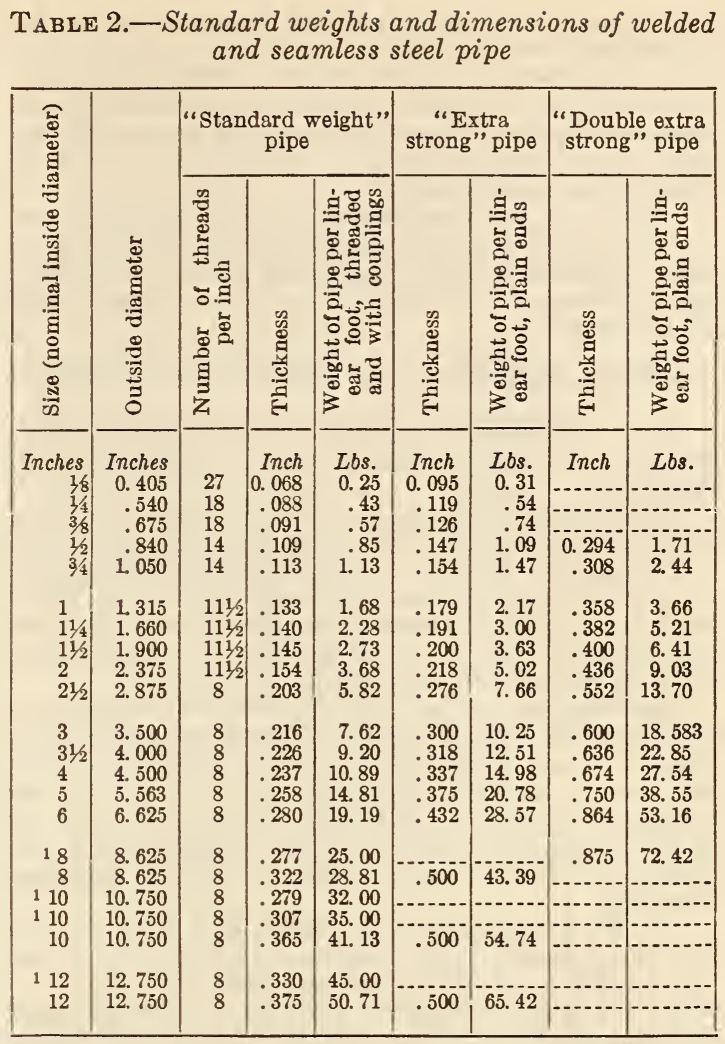

1 Unless specifically stated on the order the lighter weights will not be furnished. Weights given in the table are for pipes up to and in cluding 12 inches in nominal inside diameter, with threaded ends and couplings; sizes larger than those shown in the table are meas ured by the outside diameter and will be furnished with plain ends unless otherwise specified; for such sizes it will be necessary to accept manufacturers' weights or calculate the weights on the basis of 1 cubic inch of steel weighing 0.2833 pound.

10. (a) Number of tests.-One of each of the tests specified in sections 5,7 , and 8 shall be made on a length in each lot of 500 lengths or fraction thereof, of each size.

(b) In the case of welded pipe ordered for flanging, the crop ends cut from each length shall stand the flattening test specified in section 7 for lapwelded pipe. 
(c) Each length of pipe shall be subjected to the hydrostatic test specified in section 6 .

11. (a) Retests. - If the results of the physical test of any lot do not conform to the requirements specified in sections 5,7 , and 8 , retests of two additional pipes shall be made, each of which shall conform to the requirements specified.

(b) If any section fails when flattening tests are made on the crop ends of each length of welded pipe, other pieces from the length may be cut until satisfactory tests are obtained, otherwise the length shall be rejected.

\section{WEIGHTS, DIMENSIONS, AND PERMISSIBLE VARIATIONS}

12. (a) Standard weights.-The standard weights for pipe of various inside diameters are given in Table 2.

(b) Nipples shall be cut from pipe of the same weight and quality described in these specifications.

13. (a) Permissible variations-weight.-The weight of the pipe shall not vary more than 5 per cent for standard weight and extra strong pipe nor more than 10 per cent for double extra strong pipe from that specified in Table 2.

(b) Diameter.-For pipe $1 \frac{1}{2}$ inches or under in nominal diameter, the outside diameter at any point shall not vary more than one sixty-fourth inch over nor more than one thirty-second inch under the standard specified. For pipe 2 inches or over in nominal diameter, the outside diameter shall not vary more than 1 per cent over or under the standard specified.

(c) Thickness.-The minimum wall thickness at any point shall not be more than 12.5 per cent under the nominal wall thickness specified.

14. Lengths.-Unless otherwise specified, pipe lengths shall be in accordance with the following regular practice:

(a) Standard weight pipe shall be in random lengths of 16 to 22 feet, but not more than 5 per cent of the total number of lengths may be "jointers," which are two pieces coupled together. When ordered with plain ends, 5 per cent may be in lengths of 12 to 16 feet.

(b) Extra strong and double extra strong pipe shall be in random lengths of 12 to 22 feet. Five per cent may be in lengths of 6 to 12 feet.

\section{WORKMANSHIP AND FINISH}

15. Unless otherwise specified, pipe shall conform to the following regular practice:

(a) Ends.-Each end of standard weight welded pipe shall be threaded. Extra strong welded pipe and standard weight and extra strong seamless pipe and all double extra strong pipe shall be furnished with plain ends.

(b) Threads. - All threads shall be in accordance with the American standard pipe thread ${ }^{80}$ and cut so as to make a tight joint when the pipe is tested at the mill to the specified internal hydrostatic pressure. The variation from the standard, when tested with the standard working gage, shall not exceed a maximum of one and one-half turns either way.

(c) Couplings.-Each length of threaded pipe shall be provided with one coupling, having cleancut threads of such a pitch diameter as to make a tight joint. Couplings may be made of wrought iron or steel.

16. Finish.-The finished pipe shall be reasonably straight and free from injurious defects. All burrs at the ends of the pipe shall be removed.

80 A complete description of American standard pipe threads ap plicable to pipe, valves, and fittings is contained in the pipe thread bulletin published by the American Standards Association, B2-1929.
bulle

\section{INSPECTION AND REJECTION}

(Requirements covering inspection and rejection conform with those specified in A. S. T. M. standard specifications for steel. (See 605.0, p. 247.)

AMERICAN SOCIETY FOR TESTING MATERIALS, STANDARD SPECIFICATIONS FOR CARBON-STEEL CASTINGS FOR VALVES, FLANGES, AND FITTINGS FOR HIGH-TEMPERATURE SERVICE, DESIGNATION A95-29, 1929

\section{CASTINGS FOR HIGH TEMPERATORES}

(See 611.41, p. 709.)

\section{AMERICAN SOCIETY FOR TESTING MA-} TERIALS, STANDARD SPECIFICATIONS FOR FORGED OR ROLLED STEEL PIPE FLANGES FOR HIGH-TEMPERATURE SERVICE, DESIGNATION A105-28, 1928

\section{STEEL PIPE FLANGES}

1. (a) Scope.-These specifications cover forged or rolled steel flanges to be attached to piping for high-temperature service (note 1).

(b) Three classes of material are covered, designated class $\mathrm{A}, \mathrm{B}$, and $\mathrm{C}$, respectively. Class $\mathrm{A}$ is intended for forge welding and is classified by the chemical properties as specified in section $6(a)$ and (b) and by the physical properties as specified in section $9(a)$. Classes B and $\mathrm{C}$ are classified in accordance with their physical properties as specified in section $9(a)$.

2. Certification of test.-Unless otherwise specified on the order, a certification that the material conforms to the requirements of these specifications shall be the basis of acceptance of the material.

\section{MANOFACTURE}

3. Process.-The steel may be made by either or both of the following processes: Open-hearth or electric furnace.

4. Discard. - A sufficient discard shall be made from each ingot to secure freedom from injurious piping and undue segregation.

5. (a) Heat treatment.-Class A steel does not require heat treatment. (Note 2.)

(b) Classes B and C steels shall be heat treated. Heat treatment may consist of annealing or normalizing.

(c) Annealing.-The procedure for annealing shall consist in allowing the flanges, immediately after forging or rolling, to cool to a temperature below the critical range, under suitable conditions to prevent injuries by too rapid cooling. They shall then be uniformly reheated to the proper temperature to refine the grain (a group thus reheated being known as an "annealing charge") and allowed to cool uniformly in the furnace.

(d) Normalizing.-The procedure for normalizing shall consist in allowing the flanges, immediately after forging or rolling, to cool to a temperature below the critical range, under suitable conditions to prevent injuries by too rapid cooling. They shall then be uniformly reheated to the proper temperature to refine the grain (a group thus reheated being known as a "normalizing charge") and allowed to cool in still air.

(e) No flanges which have been quenched in any liquid medium shall be offered under these specifications. 
CREMICAL PROPERTIES AND TESTS

6. (a) Chemical composition.-The steel shall conform to the following requirements as to chemical composition:

\begin{tabular}{|c|c|c|}
\hline 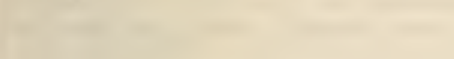 & Class A & $\begin{array}{l}\text { Classes B } \\
\text { and C }\end{array}$ \\
\hline Manganese & $\begin{array}{l}\text { Per cent } \\
0.35-0.60\end{array}$ & $\begin{array}{l}\text { Per cent } \\
0.40-0.80\end{array}$ \\
\hline $\begin{array}{l}\text { Phosphorous, not over } \\
\text { Sulphur, not over } \\
\text { Silicon, not over. }\end{array}$ & $\begin{array}{l}.05 \\
.05 \\
.40\end{array}$ & $\begin{array}{l}.05 \\
.05\end{array}$ \\
\hline
\end{tabular}

(b) The carbon content of class A material shall not exceed 0.20 per cent. The carbon content of class $\mathrm{B}$ and $\mathrm{C}$ material shall be a matter of agreement between the purchaser and the manufacturer.

7. Ladle analysis.-An analysis of each melt of steel shall be made by the manufacturer to determine the percentage of the elements specified in section 6 .

8. (a) Check analysis.-An analysis may be made by the purchaser from one forged or rolled flange representing each melt. The chemical composition thus determined shall conform to the requirements specified in section 6 , except that phosphorus and sulphur may not exceed 0.055 per cent.

(b) All drillings for analysis shall be taken at least one-fourth inch below the surface, or turnings may be taken from test specimens.

\section{PHYSICAL PROPERTIES AND TESTS}

9. (a) Tension tests.-Forged or rolled steel flanges shall conform to the following minimum requirements as to tensile properties:

\begin{tabular}{|c|c|c|c|}
\hline & $\begin{array}{l}\text { Flanges } \\
\text { for forge } \\
\text { welding }\end{array}$ & \multicolumn{2}{|c|}{$\begin{array}{l}\text { Flanges not for } \\
\text { forge welding }\end{array}$} \\
\hline & Class A & Class B & Class C \\
\hline 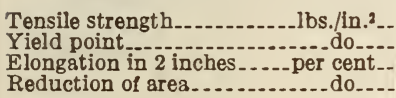 & $\begin{array}{r}54,000 \\
30,000 \\
25.0 \\
38.0\end{array}$ & $\begin{array}{r}60,000 \\
30,000 \\
25.0 \\
38.0\end{array}$ & $\begin{array}{r}70,000 \\
36,000 \\
22.0 \\
30.0\end{array}$ \\
\hline
\end{tabular}

(b) The yield point shall be determined by the drop of the beam of the testing machine, or by dividers, at a speed of head of the testing machine not to exceed one-eighth-inch per minute. The tensile strength shall be determined at a speed of head not to exceed $11 / 2$ inches per minute.

10. (a) Number of tests. - One tension test shall be made from each melt or for each annealing or normalizing charge. If more than one melt is represented in any annealing or normalizing charge, a test shall be made from each melt.

(b) If any test specimen shows defective machining or develops flaws, it may be discarded and another specimen substituted.

(c) If the percentage of elongation of any test specimen is less than that specified in section 9 (a) and any part of the fracture is more than threefourths inch from the center of the gage length, as indicated by scribe scratches marked on the specimen before testing, a retest shall be allowed.

(d) For purposes of testing, the necessary extra flanges shall be provided to furnish the specimens required in paragraph $(a)$. The test specimen shall be cut tangentially from the flange portion approximately midway between the inner and outer surfaces and approximately midway between the front and back faces.

11. (a) Tension-test specimens.-The tension-test specimens taken from the flanges shall conform to the dimensions shown in Figure 129 except as specified in paragraph $(b)$. The ends shall be of a form to fit the holders of the testing machine in such a way that the load shall be axial.

(b) In the case of small sections which will not permit of taking the standard-test specimen specified in paragraph $(a)$, the tension-test specimen shall be as large as feasible and its dimensions shall be proportional to those shown in Figure 129 in standard specifications for steel. (See 605.0, p. 247.) The gage length for measuring elongation shall be four times the diameter of the specimen. ${ }^{81}$

12. Retests.- If any of the results of the physical tests of any test lot do not conform to the requirements specified, the manufacturer may re-treat such lots one or more times and retests shall be made as specified in section $10(a)$.

\section{WORKMANSHIP AND FINISH}

13. (a) Workmanship.-The flanges shall conform to the sizes and shapes specified by the purchaser.

(b) The flanges shall be free from injurious defects and shall have a workmanlike finish.

\section{MARKING}

14. Identification.-Identification marks shall be stamped legibly on each flange.

\section{INSPECTION AND REJECTION}

(Requirements covering inspection, rejection, and rehearing conform with those specified in A. S. T. M. standard specifications for steel (see 605.0, p. 247), except that unless otherwise specified, any rejection based on tests shall be reported within 12 working days from the receipt of the samples.)

\section{EXPLANATORY NOTES}

(Note 1 is identical with that given in A. S. T. M. A95-29. See 611.41, p. 709.)

Note 2.-It is recommended that class A flanges and pipe be annealed or normalized following the forge-welding operation.

AMERICAN SOCIETY FOR TESTING MATERIALS, STANDARD SPECIFICATIONS FOR LAPWELDED AND SEAMLESS STEEL PIPE FOR HIGH-TEMPERATURE SERVICE, DESIGNATION A106-29, 1929

\section{PIPE FOR HIGH TEMPERATURES}

1. Scope.-These specifications cover lapwelded and seamless steel pipe for $250,400,600,900$, and 1,350 pounds per square inch pressure and high temperatures. (Note 1.) Pipe ordered under these specifications shall be suitable for bending, flanging, and similar forming operations. (Note 2.)

\section{MANUFACTURE}

2. (a) Process.-(Same as in A53, given above.)

(b) Welded pipe 2 inches or over in nominal diameter shall be made by the lap welded process. All pipe $11 / 2$ inches or under in nominal diameter shall be made by the seamless process, and may be either hot finished or cold drawn and annealed.

81 These requirements are in accordance with the provisions of sec. 8 of the Tentative Methods of Tension Testing of Metallic Materials (serial designation: E8-27T), see Proc. Am. Soc. Testing Mats., vol. 27, Pt. I, p. 1067, 1927; also 1928 Book of A. S. T. M. tentative standards. 
(c) Unless otherwise specified, seamless pipe 2 inches or over in nominal diameter shall be furnished hot finished. Upon agreement, cold drawn and annealed pipe may be furnished.

\section{CHEMICAL PROPERTIES AND TESTS}

3. Ladle analysis.-The steel for welded and seamless pipe shall conform to the following ladle analysis requirements as to chemical composition:

\begin{tabular}{l|r|r}
\hline & Welded & Seamless \\
\hline Phosphorus, not over... & $\begin{array}{r}\text { Per cent } \\
\text { (Note 3) 0.06 } \\
.05\end{array}$ & $\begin{array}{r}\text { Per cent } \\
0.04 \\
.05\end{array}$ \\
\hline
\end{tabular}

4. Check analyses.-Analyses may be made by the purchaser from each lot of 500 lengths or fraction thereof of finished pipe. The phosphorus and sulphur content thus determined shall not exceed that specified in section 3 by more than 25 per cent. Drillings for analysis shall be taken from several points around each pipe selected for analysis.

\section{PHYSICAL PROPERTIES AND TESTS}

5. (a) Tension tests.-(Requirements for welded pipe same as for welded open-hearth pipe; requirements for seamless, grade A pipe same as for seamless, open-hearth, low-carbon pipe; and requirements for seamless, grade B pipe same as for seamless, open-hearth, medium carbon pipe, all in A53, given above.)

(b) (Same as in A53, given above.)

6. Hydrostatic tests.-Unless otherwise agreed, each length of pipe ordered under these specifications shall be tested at the mill to the hydrostatic test pressures specified in the table below. Welded pipe shall be struck near both ends while under pressure, with a 2-pound hammer or equivalent.

(Working pressures and test pressures same as for carbon-steel castings for valves, flanges, and fittings for high-temperature service in A. S. T. M., A95. (See 611.41, p. 709.)

7. (a) Flattening tests.-For welded pipe, a section of pipe 6 inches long shall be flattened between parallel plates with the weld located at $90^{\circ}$ from the line of direction of the applied force, until opposite walls of the pipe meet. No opening in the weld shall take place until the distance between the plates is two-thirds of the original outside diameter of the pipe. No cracks or breaks in the metal elsewhere than in the lap weld shall occur until the distance between the plates is one-third of the original outside diameter of the pipe. Evidence of laminations or burnt material shall not develop during the entire flattening process. When doublewelded pipe is furnished on special order, the flattening test shall show no opening at the weld until the distance between the plates is one-third of the original outside diameter of the pipe.

(b) (For grade A seamless pipe over 2 inches in nominal diameter, same as for low-carbon seamless pipe in A53, given above.)

(c) (For grade B seamless pipe over 2 inches in nominal diameter, same as for medium-carbon seamless pipe in A53, given above.)

8. Bend tests.- (Same as for welded and seamless steel pipe in A53, given above.)

9. (a) Test specimens.-Tension-test specimens shall be cut longitudinally from the pipe and not flattened between gage marks. The width shall not be less than the wall thickness of the pipe nor more than three times the wall thickness. The sides of specimens shall be parallel between gage marks. If desired, the tension-test specimen may consist of a full section of the pipe.

Specimens from welded pipe shall be taken at a point approximately $90^{\circ}$ from the weld.

(b) and (c) (Same as for welded and seamless steel pipe in A53, given above.)

10. (a) Welded-Number of tests.-From each lot of 500 lengths or fraction thereof of each size of welded pipe, one length shall be selected for the tension test specified in section 5 .

The flattening test specified in section 7 (a) shall be made on both crop ends cut from each length of welded pipe.

Each length of welded pipe shall be subjected to the hydrostatic test specified in section 6 .

(b) Seamless.-From each lot of 500 lengths or fraction thereof of each size of seamless pipe, two lengths shall be selected for test. Upon one of these the tension test specified in section 5 shall be made. Upon both of them the test specified in sections $7(b),(c)$, or 8 shall be made.

Each length of seamless pipe shall be subjected to the hydrostatic test specified in section 6 . 
TABLE 1.-Dimensions and weights of lapwelded and seamless steel pipe ${ }^{1}$ for high-temperature service ${ }^{2}$

$[250,400,600,900$, and 1,350 pounds per square inch, working steam pressure ratings]

\begin{tabular}{|c|c|c|c|c|c|c|c|c|c|c|c|c|c|}
\hline \multicolumn{2}{|c|}{$\begin{array}{l}\text { Working steam } \\
\text { pressure rating }\end{array}$} & \multicolumn{2}{|c|}{250 pounds } & \multicolumn{2}{|c|}{400 pounds } & \multicolumn{2}{|c|}{400 pounds } & \multicolumn{2}{|c|}{600 pounds } & \multicolumn{2}{|c|}{900 pounds } & \multicolumn{2}{|c|}{1,350 pounds } \\
\hline \multicolumn{2}{|c|}{$\begin{array}{l}\text { Hydrostatic } \\
\text { test }\end{array}$} & \multicolumn{2}{|c|}{750 pounds } & \multicolumn{2}{|c|}{1,000 pounds } & \multicolumn{2}{|c|}{1,000 pounds } & \multicolumn{2}{|c|}{1,500 pounds } & \multicolumn{2}{|c|}{2,000 pounds } & \multicolumn{2}{|c|}{3,000 pounds } \\
\hline $\begin{array}{l}\text { Nomi- } \\
\text { nal pipe } \\
\text { size }\end{array}$ & $\begin{array}{c}\text { Outside } \\
\text { diam- } \\
\text { eter }\end{array}$ & $\begin{array}{l}\text { Wall } \\
\text { thick- } \\
\text { ness }\end{array}$ & $\begin{array}{l}\text { Weight } \\
\text { per } \\
\text { linear } \\
\text { foot, } \\
\text { plain } \\
\text { ends }\end{array}$ & $\begin{array}{l}\text { Wall } \\
\text { thick- } \\
\text { ness }\end{array}$ & $\begin{array}{l}\text { Weight } \\
\text { per } \\
\text { linear } \\
\text { foot, } \\
\text { plain } \\
\text { ends }\end{array}$ & $\begin{array}{l}\text { Wall } \\
\text { thick- } \\
\text { ness }\end{array}$ & $\begin{array}{l}\text { Weight } \\
\text { per } \\
\text { linear } \\
\text { foot, } \\
\text { plain } \\
\text { ends }\end{array}$ & $\begin{array}{l}\text { Wall } \\
\text { thick- } \\
\text { ness }\end{array}$ & $\begin{array}{l}\text { Weight } \\
\text { per } \\
\text { linear } \\
\text { foot, } \\
\text { plain } \\
\text { ends }\end{array}$ & $\begin{array}{l}\text { Wall } \\
\text { thick- } \\
\text { ness }\end{array}$ & $\begin{array}{l}\text { Weight } \\
\text { per } \\
\text { linear } \\
\text { foot, } \\
\text { plain } \\
\text { ends }\end{array}$ & $\begin{array}{l}\text { Wall } \\
\text { thick- } \\
\text { ness }\end{array}$ & $\begin{array}{l}\text { Weight } \\
\text { per linear } \\
\text { foot, plain } \\
\text { ends }\end{array}$ \\
\hline
\end{tabular}

SEAMLESS LOW OR MEDIUM CARBON PIPE

\begin{tabular}{|c|c|c|c|c|c|c|c|c|c|c|c|c|c|}
\hline $\begin{array}{r}\text { Inches } \\
1 / 2 \\
3 / 4 \\
11 \\
11 / 4 \\
11 / 2\end{array}$ & $\begin{array}{r}\text { Inches } \\
0.840 \\
1.050 \\
1.315 \\
1.660 \\
1.900\end{array}$ & $\begin{array}{c}\text { Inches } \\
0.109 \\
.113 \\
.133 \\
.140 \\
.145\end{array}$ & $\begin{array}{r}\text { Pounds } \\
0.850 \\
1.130 \\
1.678 \\
2.272 \\
2.717\end{array}$ & Inches & Pound & $\begin{array}{c}\text { Inches } \\
0.109 \\
.113 \\
.133 \\
.140 \\
.145\end{array}$ & $\begin{array}{r}\text { Pounds } \\
0.850 \\
1.130 \\
1.678 \\
2.272 \\
2.717\end{array}$ & $\begin{array}{r}\text { Inches } \\
0.147 \\
.154 \\
.179 \\
.191 \\
.200\end{array}$ & $\begin{array}{r}\text { Pounds } \\
1.087 \\
1.473 \\
2.171 \\
2.996 \\
3.631\end{array}$ & $\begin{array}{r}\text { Inches } \\
0.147 \\
.154 \\
.179 \\
.191 \\
.200\end{array}$ & $\begin{array}{r}\text { Pounds } \\
1.087 \\
1.473 \\
2.171 \\
2.996 \\
3.631\end{array}$ & $\begin{array}{l}\text { Inches } \\
0.147 \\
.1875 \\
.21875 \\
.250 \\
.28125\end{array}$ & $\begin{array}{c}\text { Pounds } \\
1.087 \\
1.727 \\
2.561 \\
3.764 \\
4.862\end{array}$ \\
\hline & & \multicolumn{4}{|c|}{$\begin{array}{l}\text { LAPWELDED OPEN-HEARTH } \\
\text { PIPE }\end{array}$} & \multicolumn{8}{|c|}{ SEAMLESS LOW OR MEDIUM CARBON PIPE } \\
\hline $\begin{array}{l}2 \\
21 / 2 \\
3 \\
31 / 2 \\
4\end{array}$ & $\begin{array}{l}2.375 \\
2.875 \\
3.500 \\
4.000 \\
4.500\end{array}$ & $\begin{array}{l}0.154 \\
.203 \\
.216 \\
.226 \\
.237\end{array}$ & $\begin{array}{c}3.652 \\
5.793 \\
7.575 \\
9.109 \\
10.79\end{array}$ & $\begin{array}{l}0.218 \\
.276 \\
.300 \\
.318 \\
.337\end{array}$ & $\begin{array}{r}5.022 \\
7.661 \\
10.25 \\
12.51 \\
14.98\end{array}$ & $\begin{array}{r}0.167 \\
.203 \\
.216 \\
.226 \\
.237\end{array}$ & $\begin{array}{r}3.938 \\
5.793 \\
7.575 \\
9.109 \\
10.79\end{array}$ & $\begin{array}{l}0.1875 \\
.217 \\
.241 \\
.28125 \\
.28125\end{array}$ & $\begin{array}{c}4.380 \\
6.160 \\
8.388 \\
11.17 \\
12.67\end{array}$ & $\begin{array}{l}0.250 \\
.276 \\
.3125 \\
.34375 \\
.375\end{array}$ & $\begin{array}{l}5.673 \\
7.661 \\
10.64 \\
13.42 \\
16.52\end{array}$ & $\begin{array}{l}0.3125 \\
.375 \\
.40625 \\
.46875 \\
.500\end{array}$ & $\begin{array}{c}6.883 \\
10.01 \\
13.42 \\
17.68 \\
21.36\end{array}$ \\
\hline $\begin{array}{r}5 \\
6 \\
8 \\
10 \\
12\end{array}$ & $\begin{array}{c}5.563 \\
6.625 \\
8.625 \\
10.75 \\
12.75\end{array}$ & $\begin{array}{l}.258 \\
.280 \\
.322 \\
.365 \\
.375\end{array}$ & $\begin{array}{l}14.62 \\
18.97 \\
28.55 \\
40.48 \\
49.56\end{array}$ & $\begin{array}{l}.375 \\
.432 \\
.500 \\
.500 \\
.500\end{array}$ & $\begin{array}{l}20.78 \\
28.57 \\
43.39 \\
54.74 \\
65.42\end{array}$ & $\begin{array}{l}.258 \\
.288 \\
.34375 \\
.395 \\
.500\end{array}$ & $\begin{array}{l}14.62 \\
19.49 \\
30.40 \\
43.68 \\
65.42\end{array}$ & $\begin{array}{l}.352 \\
.385 \\
.46875 \\
.53125 \\
.625\end{array}$ & $\begin{array}{l}19.59 \\
25.66 \\
40.83 \\
57.98 \\
80.94\end{array}$ & $\begin{array}{l}.4375 \\
.500 \\
.625 \\
.750 \\
.875\end{array}$ & \begin{tabular}{|c|}
23.95 \\
32.71 \\
53.40 \\
80.10 \\
111.0
\end{tabular} & $\begin{array}{c}.625 \\
.6875 \\
.875 \\
1.0625 \\
1.21875\end{array}$ & $\begin{array}{c}32.96 \\
43.60 \\
72.42 \\
109.9 \\
150.1\end{array}$ \\
\hline $\begin{array}{l}14 \\
16 \\
18 \\
20 \\
24\end{array}$ & $\begin{array}{l}14.0 \\
16.0 \\
18.0 \\
20.0 \\
24.0\end{array}$ & $\begin{array}{l}.40625 \\
.46875 \\
.500 \\
.5625 \\
.625\end{array}$ & $\begin{array}{r}58.98 \\
77.75 \\
93.45 \\
116.8 \\
156.0\end{array}$ & $\begin{array}{l}.625 \\
.6875 \\
.750 \\
.875 \\
.9375\end{array}$ & $\begin{array}{l}89.28 \\
112.4 \\
138.2 \\
178.7 \\
230.9\end{array}$ & $\begin{array}{l}.500 \\
.53125 \\
.59375 \\
.625 \\
.750\end{array}$ & $\begin{array}{c}72.09 \\
87.77 \\
110.4 \\
129.3 \\
186.2\end{array}$ & $\begin{array}{c}.65625 \\
.750 \\
.8125 \\
.90625 \\
1.03125\end{array}$ & $\begin{array}{l}93.52 \\
122.2 \\
149.1 \\
184.8 \\
253.0\end{array}$ & $\begin{array}{l}.9375 \\
1.03125 \\
1.15625 \\
1.28125 \\
1.500\end{array}$ & $\begin{array}{l}130.8 \\
164.9 \\
208.0 \\
256.1 \\
360.5\end{array}$ & $\begin{array}{l}1.34375 \\
1.500 \\
1.6875 \\
1.84375 \\
2.1875\end{array}$ & $\begin{array}{l}181.6 \\
232.3 \\
294.0 \\
357.5 \\
509.6\end{array}$ \\
\hline
\end{tabular}

1 While the thicknesses given in this table are theoretically ample to care for both bursting pressure and material removed in threading, it is recommended that where pipe is threaded, a weight at least equal to the 900-pound class be used to furnish added mechanical strength.

${ }^{2}$ This contemplates temperatures from $450^{\circ}$ to $750^{\circ} \mathrm{F}$. $\left(230^{\circ}\right.$ to $400^{\circ} \mathrm{C}$.), or at the discretion of the designing engineer, higher temperatures with appropriate pressures may be used.

8 All pressures in pound per square inch gage.

1 Wall thicknesses calculated from the modified Barlow formula given in par. P-23 of the 1927 edition of the A. S. M. E. Boiler Construction Code (as revised), increased to provide 12.5 per cent under thickness mill tolerance, then chosen as the next heavier commercial wall thickness.

11. (a) Retests.-If the results of any physical or chemical tests of any lot do not conform to the requirements specified in sections $3,4,5,7$ and 8 , retests of additional pipes of double the original number shall be made, each of which shall conform to the requirements specified.

(b) Should a crop end fail in the flattening test, one retest may be made from the failed end.

\section{DIMENSIONS, WEIGETS, AND PERMISSIBLE VARIATIONS}

12. (a) Weights and dimensions.-The nominal wall thicknesses and standard weights for pipe of various diameters are given in Table 1. (Note 4.)

(b) Nipples shall be cut from pipe of the same weight and quality as described in these specifications.

13. (a) Diameter-Permissible variations.-(Same as for diameter in A53, given above.)

(b) Thickness. -The minimum wall thickness at any point for welded and seamless pipe shall not be more than 12.5 per cent under the nominal wall thickness specified.

(c) Weight. - The weight of the pipe shall not exceed that specified in Table I by more than 5 per cent for the $250,400,600$, and 900 pound per square inch standards, nor more than 10 per cent for the 1,350-pound standard.

14. Lengths.-Pipe lengths shall be furnished in accordance with the following regular practice: (a) The lengths required shall be specified in the order and $(b)$ no jointers are permitted unless otherwise specified.

\section{WORKMANSEIP AND FINISH}

15. Ends.-Unless otherwise specified, pipe shall be furnished with plain ends.

16. Finish.-The finished pipe shall be reasonably straight and free from injurious defects. All burrs at the ends of the pipe shall be removed.

\section{MARKING}

17. Marking.-Each length of pipe shall be marked with appropriate symbols to show by whom manufactured and that it conforms to these specifications.

\section{INSPECTION AND REJECTION}

(Requirements covering inspection and rejection conform with those specified in A. S. T. M. standard specifications for steel. (See 605.0, p. 247.) 
EXPLAN ATORY NOTES

Nore 1.-This contemplates temperatures from $450^{\circ}$ to $750^{\circ} \mathrm{F}$. $\left(232^{\circ}\right.$ to $400^{\circ} \mathrm{C}$.), or at the discretion of the designing engineer, higher temperatures with appropriate pressures may be used.

NOTE 2- - rade A rather than grade $B$ seamless tubing should be used for cold bending or for fusion or forge welding. The purpose for which pipe is to be used should be stated on the face of the order.

Note 3.-The phosphorus limit for the finished pipe is set at 0.06 per cent maximum to permit rephosphorizing to facilitato welding.

Norf 4.-Table 1 is the recommendation of the sectional committee on standardization of dimensions and material of wrought iron and wrought steel pipe and tubing, functioning under the procedure of the American Standards Association, A. S. A. Project No. B-36.

AMERICAN SOCIETY FOR TESTING MATERIALS, TENTATIVE SPECIFICATIONS FOR BLACK AND HOT-DIPPED ZINCCOATED (GALVANIZED) WELDED AND SEAMLESS STEEL PIPE FOR ORDINARY USES, DESIGNATION A120-28T, 1928

\section{ORDINARY STEEL PIPE}

\section{SCOPE}

1. These specifications cover black and hotdipped galvanized standard weight, extra strong, and double extra strong welded and seamless steel pipe. Pipe ordered under these specifications is intended for ordinary uses such as low-pressure service in steam, water, and gas lines, and is not intended for close bending or coiling, or hightemperature service.

(In respect to manufacture; hydrostatic tests; hydrostatic pressures for welded and seamless steel pipe; weights, dimensions, and permissible variations; standard weights and dimensions of welded and seamless steel pipe; workmanship and finish, these specifications are identical with A53-30, given above, with the following exceptions:)

\section{MANUFACTURE}

2. Galvanized pipe shall be coated with zinc inside and outside by the hot-dip process.

\section{WORKMANSHIP AND FINISH}

3. All threads shall be in accordance with the American standard pipe thread and cut so as to make a tight joint when the pipe is tested at the mill to the specified internal hydrostatic pressure.

4. The zinc coating on galvanized pipe shall be free from injurious defects or excessive roughness.

\section{INSPECTION AND REJECTION}

(Requirements covering inspection and rejection conform with those specified in A. S. T. M. standard specifications for steel. See 605.0, p. 247.)
ASSOCIATION OF AMERICAN STEEL MAN UFACTURERS. STANDARD SPECIFICATIONS FOR SEAMLESS STEEL TUBES AND PIPE FOR GASOLINE CRACKING PLANTS, NO. 100, APRIL 1, 1932

\section{STEEL TUBES AND PIPE}

(This specification includes requirements on chemical composition, flattening test, flaring test, Brinell test, hydrostatic test, and permissible variations in dimensions, for oil-carrying tubes subject to furnace temperatures and for oil-transfer pipe.)

HEATING AND PIPING CONTRACTORS NATIONAL ASSOCIATION, WELDING NECK FLANGES FOR STANDARD AND EXTRAHEAVY PIPE, 1929

\section{PIPE FLANGES}

(See 607.14, p. 412.)

SOCIETY OF AUTOMOTIVE ENGINEERS, ELECTRICAL EQUIPMENT; CABLE, CONDUIT, AND TUBING CLIPS, S. A. E. STANDARD, AUGUST, 1928

CABLE, CONDUIT, AND TUBING CLIPS

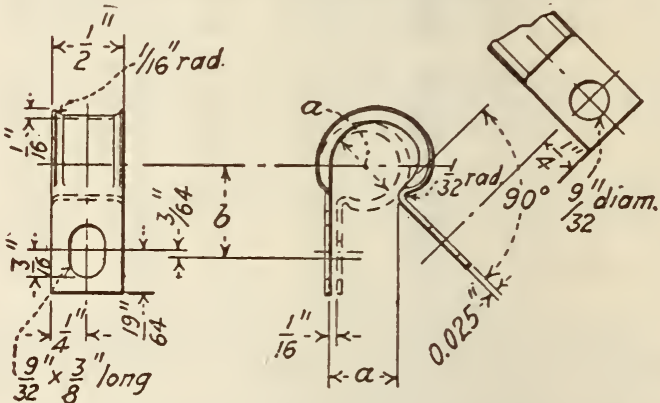

Figure 294.-Cable, conduit, and tubing clips

\begin{tabular}{l|l|r|r|r|r|r|r|r|r|r|r|r}
\hline$a$ & Diameter..... & $3 / 10$ & $1 / 4$ & $5 / 10$ & $3 / 8$ & $7 / 8$ & $1 / 2$ & $9 / 10$ & $5 / 8$ & $3 / 4$ & $7 / 8$ & 1 \\
$b$ & Length_...... & $15 / 32$ & $1 / 2$ & $17 / 32$ & $9 / 10$ & $18 / 32$ & $5 / 8$ & $21 / 32$ & $11 / 16$ & $3 / 4$ & $13 / 10$ & $7 / 8$ \\
\hline
\end{tabular}

Material.-S. A. E. No. 1010 steel or equivalent, cold rolled dead soft.

Clips shall be made to full lines, as shown above, and bent to dotted lines when assembling. They must be free from burrs and sharp edges.

\section{SOCIETY OF AUTOMOTIVE ENGINEERS, IRON AND STEEL; COLD-DRAWN SEAMLESS}

STEEL TUBING, S. A. E. STANDARD, JUNE, 1911

\section{COLD-DRAWN SEAMLESS STEEL TUBING}

Cold-drawn seamless steel tubing dimensions

\begin{tabular}{|c|c|c|c|c|c|c|c|c|c|c|c|c|c|c|c|c|c|c|}
\hline \multirow{2}{*}{\multicolumn{2}{|c|}{$\begin{array}{l}\text { Thickness, } \\
\text { B. w.g. \& } \\
\text { fractions }\end{array}$}} & \multicolumn{17}{|c|}{ Outside diameter (inches) } \\
\hline & & $1 / 2$ & 5 & $3 / 4$ & $7 / 8$ & 1 & $11 / 8$ & $11 / 4$ & $13 / 8$ & $11 \frac{1}{2}$ & 134 & 2 & $21 / 4$ & $23 \underline{6}$ & $23 / 4$ & 3 & 334 & $31 / 2$ \\
\hline $\begin{array}{l}20 \\
18 \\
16 \\
13 \\
11 \\
10 \\
5 / 32 \\
3 / 10 \\
1 / 4 \\
5 / 10 \\
3 / 8 \\
1 / 2 \\
5 / 8\end{array}$ & $\begin{array}{l}(0.035) \\
(.049) \\
(.065) \\
(.095) \\
(.120) \\
.134) \\
.156) \\
(.188) \\
(.250) \\
(.313) \\
(.375) \\
(.500) \\
(.625)\end{array}$ & * & : & * & $\begin{array}{l}* \\
* \\
* \\
* \\
* \\
* \\
*\end{array}$ & $\begin{array}{l}* \\
* \\
* \\
*\end{array}$ & $\begin{array}{l}* \\
* \\
* \\
* \\
* \\
* \\
* \\
*\end{array}$ & $\begin{array}{l}* \\
* \\
* \\
* \\
*\end{array}$ & $\begin{array}{l}* \\
* \\
* \\
* \\
* \\
* \\
* \\
*\end{array}$ & $\begin{array}{l}* \\
* \\
* \\
* \\
* \\
* \\
* \\
* \\
* \\
* \\
*\end{array}$ & $\begin{array}{l}* \\
* \\
* \\
* \\
* \\
* \\
* \\
*\end{array}$ & * & $\begin{array}{l}* \\
* \\
* \\
* \\
* \\
* \\
* \\
* \\
*\end{array}$ & $\begin{array}{l}* \\
* \\
* \\
* \\
* \\
* \\
* \\
* \\
*\end{array}$ & $\begin{array}{l}* \\
* \\
* \\
* \\
* \\
* \\
* \\
* \\
*\end{array}$ & * & $\begin{array}{l}* \\
* \\
* \\
* \\
* \\
* \\
* \\
* \\
*\end{array}$ & $\begin{array}{l}* \\
* \\
* \\
* \\
* \\
* \\
*\end{array}$ \\
\hline
\end{tabular}

Inside and outside diameter tolerances: From $1 / 2$ to $13 / 4$ inches outside diameters, plus or minus 0.005 inch; from 2 inches to $31 / 2$ inches outside diameters, plus or minus $0.010 \mathrm{inch}$.

Standard sizes are indicated by asterisks $\left({ }^{*}\right)$. 
SOCIETY OF AUTOMOTIVE ENGINEERS, POWER PLANT; CRANK-CASE DRAIN PLUGS, S. A. E. RECOMMENDED PRACTICE, MARCH, 1927

\section{CRANK-CASE DRAIN PLUGS}

\begin{tabular}{|c|c|c|c|}
\hline $\begin{array}{c}\text { Diam- } \\
\text { eter }\end{array}$ & Threads per inch & Head & $\begin{array}{l}\text { Width } \\
\text { across } \\
\text { flats }\end{array}$ \\
\hline $\begin{array}{c}\text { Inch } \\
36 \\
1 / 2 \\
7 / 6\end{array}$ & $\begin{array}{l}18 \text { (pipe) } \\
14 \text { (pipe) } \\
18\end{array}$ & $\begin{array}{l}\text { Square } \\
\text { Hexagon }\end{array}$ & $\begin{array}{r}\text { Inches } \\
7 / 16 \\
7 / 16 \\
11 / 8\end{array}$ \\
\hline
\end{tabular}

SOCIETY OF AUTOMOTIVE ENGINEERS, PARTS AND FITTINGS; FUEL AND LUBRICATION TUBE FITTINGS, S. A. E. STANDARD, JULY, 1925

\section{FUEL AND LUBRICATION FITTINGS}

(See 645.4, p. 1232.)

UNITED STATES GOVERNMENT, DEPARTMENT OF COMMERCE, STEEL PIPE NIP. PLES, COMMERCIAL STANDARD CS5-29, JANUARY, 1929

\section{STEEL PIPE NIPPLES}

Pipe nipples shall be made only from tested new, full, or short length and full weight steel pipe free from buckles, bends, kinks, cracks, laminations, imperfect welds, rust, injurious sand marks, or any other defects which might affect its strength or serviceability.

(In respect to manufacture; hydrostatic tests; hydrostatic pressures for welded and seamless steel pipe; weights, dimensions, and permissible variations standard weights and dimensions of welded and seamless steel pipe; workmanship and finish, this specification conforms to A. S. T. M., A53-30, given above, with the following exceptions:)

The weight of galvanized pipe shall not vary more than 3 per cent under that specified in Table 1 (class A, standard weight pipe), in F. S. B. WW-P431, given below; nor more than 3 per cent under that specified in A. S. T. M., A53-30, Table 11 (extra-strong pipe); nor more than 8 per cent under that specified in A. S. T. M., A53-30, Table 11 (double-extra-strong pipe), given above.

(Specifications covering taper-pipe-thread dimensions conform to those of the National Screw Thread Commission. See 607.0, p. 365.)

Pipe nipples shall be threaded on both ends in accordance with the latest issue of American standard for pipe thread B-2 of the American Standards Association. Gages used for inspection shall not vary from the basic dimensions more than one-half turn either way.

Inspection tolerances.-The maximum allowable variation in the commercial product is one turn plus or one turn minus from the gaging notch when using gages. This is equivalent to a maximum allowable variation of one and one-half turns from the basic dimensions on account of the tolerance of one-half turn on gages. Nipples shall be chamfered and reamed at an angle of $25^{\circ}$ to $40^{\circ}$ to the central axis. Nipples shall not exceed one-sixteenth inch under or over the specified length. Steel nipples are regularly made and stocked in the sizes shown in Tables 1, 2, 3, and 4 in Federal Specifications Board specification WW-N-351, given below. They are packed as shown in the accompanying table. Packing of standard-weight black or galvanized-steel
nipples

(Number contained in carton)

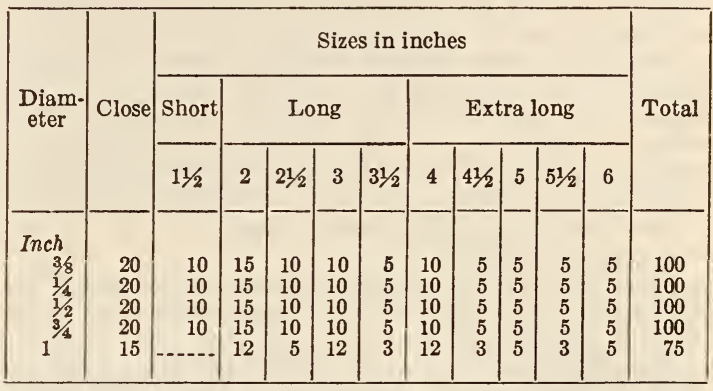

UNITED STATES GOVERNMENT, DEPARTMENT OF COMMERCE, STANDARD WEIGHT MALLEABLE IRON OR STEEL SCREWED UNIONS, COMMERCIAL STANDARD CS7-29, NOVEMBER 1, 1929

\author{
IRON OR STEEL SCREWED UNIONS
}

(See 607.2, p. 428.)

UNITED STATES GOVERNMENT, DEPARTMENT OF COMMERCE, BUREAU OF STANDARDS, WROUGHT-IRON AND WROUGHT-STEEL PIPE, VALVES, AND FITTINGS, SIMPLIFIED PRACTICE RECOMMENDATION NO. 57, 1926

\section{WROUGHT-IRON AND WROUGHT-STEEL PIPE, VALVES, AND FITTINGS}

(See 607.0, p. 365.)

UNITED STATES GOVERNMENT, DEPARTMENT OF COMMERCE, STEAMBOAT INSPECTION SERVICE, GENERAL RULES AND REGULATIONS PRESCRIBED BY THE BOARD OF SUPERVISING INSPECTORS, MAY 4, 1929

\section{WELDED STEAM AND WATER PIPES}

From one-eighth of an inch inside diameter up to and including 30 inches inside diameter.

The pipe shall be made of wrought iron or mild steel, smooth, straight, and free from defects.

Threaded pipe of standard thickness shall be avoided as far as possible. In steam pipes it is a very serious matter and shall not be allowed in any case on standard pipe over 5 inches in diameter. All pipe over 2 inches in diameter shall be lapwelded.

\section{TESTS}

The following tests shall be made before shipment by the manufacturer:

One-eighth inch inside diameter up to and including $31 / 2$ inches inside diameter shall be tested before shipment to 600 pounds per square inch hydrostatic pressure and not subject to any other test. 
For all diameters greater than $31 / 2$ inches inside: (a) For steel. - A test piece 2 inches in length cut from a pipe shall stand being flattened by hammering until the sides are brought parallel with the curve on the inside at the ends not greater than onethird the outside diameter of the pipe without showing cracks or flaws, the weld being $45^{\circ}$ from the curve.

For iron.-A test ring three times the thickness in length cut circumferentially from lapwelded wrought-iron pipe shall be crushed down to an inner diameter of one-third the outside diameter of the pipe. Wrought-iron pipe will not stand this test without fracture, but no lapwelded wrought-iron pipe shall be accepted where the break is short or crystalline or shows evidence of defective material or bad welding, and in every case the fiber of the iron shall be shown.

(b) Pulling tests shall be made from every 50 pieces furnished, or fraction thereof, and shall show the following results:

For Bessemer steel.-Tensile strength not less than 50,000 pounds per square inch. Elongation in 8-inch specimen, not less than 20 per cent.

For open-hearth steel. - Tensile strength not less than 45,000 pounds per square inch. Elongation in 8-inch specimen, not less than 20 per cent.

For iron.-Tensile strength, 40,000 to 48,000 pounds per square inch. Elastic limit, 22,000 to 30,000 pounds. Elongation in 8 -inch specimen, not less than 12 per cent.

All pipe from 4-inch diameter up shall be tested before shipment to not less than 500 pounds per square inch hydrostatic pressure.

\section{SEAMLESS STEEL STEAM AND WATER PIPES}

\section{MATERIAL}

The steel shall be made by the open-hearth process.

\section{SURFACE INSPECTION}

Pipe shall be free, inside and outside, from all surface defects that would materially weaken it or form starting points of corrosion. The defects to be especially avoided are snakes, checks, slivers, laps, pits, etc. Pipe shall be smooth and straight.

\section{TESTS}

The following tests shall be made before shipment by the manufacturer:

(a) A test piece, 2 inches in length, cut from a pipe, shall stand being flattened by hammering until the sides are brought parallel with the curve on the inside at the ends not greater than one-third the outside diameter of the pipe, without showing cracks or flaws.

(b) Pulling tests shall be made from every 50 pieces furnished, or fraction thereof, and shall show the following results:

Tensile strength, not less than 48,000 pounds per square inch.

Elongation in 8-inch specimen, not less than 12 per cent.

The name or brand of the manufacturer shall be legibly rolled in raised characters or stamped on the outer surface of each pipe.

\section{MAIN STEAM PIPE}

The thickness of and pressure allowed on main steam pipe constructed of riveted iron or steel plates that have been stamped and tested as required by section 4430, Revised Statutes, shall be determined in the same manner as required by section 4433 , Revised Statutes, to determine the pressure allowable on boilers.

The thickness of and steam pressure allowable on all lapwelded or solid-drawn steam pipe of wrought iron or steel shall be determined by the following formulas:

$$
\begin{gathered}
T=\frac{P \times D}{10,000}+0.125 \\
P=\frac{(T-0.125) \times 10,000}{D}
\end{gathered}
$$

Where

$P=$ pressure of steam allowable in pounds.

$T=$ thickness of pipe.

$D=$ diameter of pipe.

\section{Example}

Given $P=200$ pounds pressure. $D=5$ inches in diameter. Substituting and solving for $T$

$$
T=\frac{200 \times 5}{10,000}+0.125=0.225 \mathrm{inch}
$$

Substituting and solving for $P$

$$
P=\frac{(0.225-0.125) \times 10,000}{5}=200 \text { pounds. }
$$

\section{STATEMENT OR CERTIFICATE OF MANUFAC- TURER OF STEAM AND WATER PIPES}

When the manufacturers of steam and water pipes file with the supervising inspector general a certificate duly sworn to that all such pipes manufactured by them will stand all the tests and meet all the requirements of the foregoing rule, they shall be accepted by inspectors of the service and no other affidavit will be required.

Lapwelded or solid-drawn pipe of wrought iron or steel may be used for mud or steam drums not exceeding 24 inches in diameter for use on boilers when the ends of the pipe have been properly annealed before the holes are drilled or the heads are riveted in.

When pipe is used for steam lines where flanges are riveted on and calked, the ends of the pipe shall be properly annealed before drilling or riveting the flanges on.

UNITED STATES GOVERNMENT, FEDERAL SPECIFICATIONS BOARD, FEDERAL SPECIFICATIONS FOR UNIONS, MALLEABLE IRON OR STEEL, NO. 393, 1926. (CURRENT DESIGNATION WW-U-531)

\section{MALLEABLE IRON OR STEEL UNIONS}

(Conforms with Commercial Standard CS7-29 (see 607.2, p. 428), with the addition of tabulated dimensions on $2 \frac{1}{2}$ and 3 inch sizes.)

UNITED STATES GOVERNMENT, FEDERAL SPECIFICATIONS BOARD, FEDERAI SPECIFICATION FOR UNIONS, 300 POUND, MALLEABLE IRON OR STEEL, WW-U-536, 1932

\section{STEEL UNIONS, 300 POUNDS}

(See 607.2 p. 429.) 
UNITED STATES GOVERNMENT, FEDERAL SPECIFICATIONS BOARD, FEDERAL SPECIFICATION FOR NIPPLES, PIPE; BRASS, STEEL, AND WROUGHT IRON, WW-N-351, JUNE 24, 1930

\section{STEEL NIPPLES}

\section{APPLICABLE FEDERAL SPECIFICATIONS}

The following Federal specifications in so far as the requirements thereof are applicable, of the issue in effect on date of invitation for bids, shall form a part of this specification:

\begin{tabular}{|c|c|c|c|}
\hline $\begin{array}{l}\text { Federal spec- } \\
\text { ification No. }\end{array}$ & $\begin{array}{c}\text { Former } \\
\text { No. }\end{array}$ & Title & See \\
\hline$Q Q-M-151$ & 339 & $\begin{array}{l}\text { General specifications for } \\
\text { metals. }\end{array}$ & 600.0, p. 3 \\
\hline W W-P-351 & 342 & $\begin{array}{l}\text { Seamless brass pipe, iron- } \\
\text { pipe size, standard and } \\
\text { extra strong. }\end{array}$ & 645.23 p. 1217 \\
\hline W W-P-431 & 162 & $\begin{array}{l}\text { Welded steel pipe, black } \\
\text { and galvanized. }\end{array}$ & Below. \\
\hline $\mathrm{W} W-\mathrm{P}-441$ & 242 & $\begin{array}{l}\text { Welded wrought-iron pipe, } \\
\text { black and galvanized. }\end{array}$ & 607.3, p. 433 \\
\hline $\mathrm{GGG}-\mathrm{P}-351$ & 238 & Pipe threads & 607.0, p. 365. \\
\hline
\end{tabular}

GRADES, TYPES, AND SIZES

This specification covers wrought iron and steel nipples, coated and uncoated, in iron pipe sizes from $1 / 8$ to 12 inches, inclusive, of standard lengths and brass nipples in iron pipe sizes from $1 / 8$ to 6 inches, inclusive, of standard lengths.

Steel pipe nipples shall be of the following types, as required:

Type A. Standard weight (as defined by Federal specification WW-P-431).

Type B. Extra strong (as defined by Federal specification WW-P-431).

Type C. Double extra strong (as defined by Federal specification WW-P-431).

Wrought-iron pipe nipples shall be of the following types, as required:

Type A. Standard weight (as defined by Federal specification WW-P-441).

Type B. Extra strong (as defined by Federal specification WW-P-441).

Type C. Double extra strong (as defined by Federal specification WW-P-441).

Brass pipe nipples.

Brass pipe nipples shall be of the following types, as required:

Type A. Standard weight (as defined by Federal specification WW-P-351).

Type B. Extra strong (as defined by Federal specification WW-P-351).

Brass pipe nipples shall be of the following grades, as required:

Grade A (as defined by Federal specification $\mathrm{WW}-\mathrm{P}-351)$.

Grade B (as defined by Federal specification $\mathrm{WW}-\mathrm{P}-351$ ).

Grade C (as defined by Federal specification $\mathrm{WW}-\mathrm{P}-351$ ).

\section{MATERIAL AND WORKMANSHIP}

Steel pipe nipples shall be made of pipe conforming to the requirements of Federal specification $\mathrm{WW}-\mathrm{P}-431$.
Wrought-iron pipe nipples shall be made of pipe conforming to the requirements of Federal specification WW-P-441.

Brass pipe nipples shall be made of pipe conforming to the requirements of Federal specification WW-P-351.

\section{GENERAL REQUIREMENTS}

Pipe nipples shall be threaded on both ends with the standard taper pipe threads conforming to Federal specification GGG-P-351.

Nipples shall be chamfered on the outside at an angle of $25^{\circ}$ to $45^{\circ}$ to the central axis. All burrs on the inside shall be removed.

A tolerance of plus or minus one-sixteenth inch in length is permitted.

\section{DETAIL REQUIREMENTS}

Wrought iron and steel pipe nipples shall be uncoated or zinc coated as specified. Zinc-coated nipples shall be made of zinc-coated pipe.

Wrought iron and steel pipe nipples shall be of the respective dimensions given in Tables $1,2,3$, and 4 . Special sizes and lengths may be specified when required. Special lengths shall not vary more than one-sixteenth inch over or under the lengths required by the purchase order.

TABLE 1.-Wrought iron and steel pipe nipples, type $A$, standard weight, uncoated, iron pipe sizes and lengths

[All dimensions in inches]

\begin{tabular}{|c|c|c|c|c|c|}
\hline $\begin{array}{l}\text { Iron } \\
\text { pipe } \\
\text { size }\end{array}$ & Close & $\begin{array}{l}\text { Special } \\
\text { short }\end{array}$ & Short & Long & Extra long \\
\hline $\begin{array}{l}1 / 8 \\
1 / 4 \\
3 / 8 \\
1 / 2 \\
3 / 4 \\
1 \\
11 / 4 \\
11 / 2 \\
2 \\
21 / 2 \\
3 \\
31 / 2 \\
4 \\
5 \\
6 \\
8 \\
10 \\
12\end{array}$ & $\begin{array}{l}3 / 4 \\
7 / 8 \\
1 \\
11 / 8 \\
138 \\
11 / 2 \\
158 \\
13 / 4 \\
2 \\
21 / 2 \\
258 \\
23 / 4 \\
27 / 8 \\
3 \\
31 / 8 \\
31 / 2 \\
37 / 8 \\
41 / 2\end{array}$ & 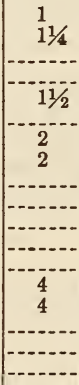 & $\begin{array}{l}11 / 2 \\
11 / 2 \\
11 / 2 \\
11 / 2 \\
2 \\
2 \\
21 / 2 \\
21 / 2 \\
21 / 2 \\
3 \\
3 \\
4 \\
4 \\
41 / 2 \\
41 / 2 \\
5 \\
5 \\
6\end{array}$ & $\begin{array}{r}2,21 / 2,3,31 / 2 \\
2,21 / 2,3,31 / 2 \\
2,21 / 2,3,31 / 2 \\
2,21 / 2,3,31 / 2 \\
21 / 2,3,31 / 2,4 \\
21 / 2,3,31 / 2,4 \\
3,31 / 2,4,41 / 2 \\
3,31 / 2,4,41 / 2 \\
3,31 / 2,4,41 / 2 \\
31 / 2,4,4112,5 \\
3112,4,41 / 2,5 \\
4112,5,51 / 2,6 \\
41 / 2,5,51 / 2,6 \\
5,51 / 2,6 \\
5,51 / 2,6 \\
6,7,8 \\
6,7,8 \\
8\end{array}$ & $\begin{array}{r}4,41 / 2,5,51 / 2,6,7,8,9,10,11,12 \\
4,41 / 2,5,51 / 2,6,7,8,9,10,11,12 \\
4,41 / 2,5,51 / 2,6,7,8,9,10,11,12 \\
4,412,5,51 / 2,6,7,8,9,10,11,12 \\
412,5,51 / 2,6,7,8,9,10,11,12 \\
41 / 2,5,512,6,7,8,9,10,11,12 \\
5,512,6,7,8,9,10,11,12 \\
5,51 / 2,6,7,8,9,10,11,12 \\
5,51 / 2,6,7,8,9,10,11,12 \\
51 / 2,6,7,8,9,10,11,12 \\
51 / 2,6,7,8,9,10,11,12 \\
7,8,9,10,11,12 \\
7,8,9,10,11,12 \\
7,8,9,10,11,12 \\
7,8,9,10,11,12 \\
10,12 \\
10,12 \\
10,12\end{array}$ \\
\hline
\end{tabular}

TABLE 2.-Wrought iron and steel pipe nipples, type $B$, extra strong, uncoated, iron pipe sizes and lengths

[All dimensions in inches]

\begin{tabular}{|c|c|c|c|c|c|}
\hline $\begin{array}{l}\text { Iron } \\
\text { pipe } \\
\text { size }\end{array}$ & Close & $\begin{array}{c}\text { Special } \\
\text { short }\end{array}$ & Short & Long & Extra long \\
\hline $\begin{array}{l}1 / 8 \\
1 / 4 \\
3 / 8 \\
1 / 2 \\
3 / 4 \\
1^{31} \\
11 / 4 \\
11 / 2 \\
2 \\
21 / 2 \\
3 \\
31 / 2 \\
4\end{array}$ & $\begin{array}{l}3 / 4 \\
7 / 8 \\
1 \\
11 / 8 \\
13 / 8 \\
11 / 2 \\
15 / 8 \\
13 / 4 \\
2 \\
21 / 2 \\
25 / 8 \\
28 / 4 \\
278\end{array}$ & 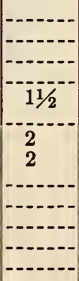 & $\begin{array}{l}11 / 2 \\
11 / 2 \\
11 / 2 \\
11 / 2 \\
2 \\
2 \\
21 / 2 \\
21 / 2 \\
21 / 2 \\
3 \\
3 \\
4 \\
4\end{array}$ & $\begin{array}{r}2,21 / 2,3,31 / 2 \\
2,21 / 2,3,31 / 2 \\
2,21 / 2,3,31 / 2 \\
2,21 / 2,3,31 / 2 \\
21 / 2,3,31 / 2,4 \\
21 / 2,3,31 / 2,4 \\
3,31 / 2,4,41 / 2 \\
3,31 / 2,4,41 / 2 \\
3,31 / 2,4,41 / 2 \\
31 / 2,4,41 / 2,5 \\
3112,4,4112,5 \\
5,6 \\
5,6\end{array}$ & $\begin{array}{r}4,5,6 \\
4,5,6 \\
4,4112,5,6 \\
4,412,5,6 \\
4112,5,6 \\
412,5,6 \\
5,6 \\
5,6 \\
5,6 \\
6 \\
6 \\
\end{array}$ \\
\hline
\end{tabular}


TABLE 3.-Wrought iron and steel pipe nipples, type $A$, standard weight, zinc coated, iron pipe sizes and lengths

[All dimensions in inches]

\begin{tabular}{|c|c|c|c|c|c|}
\hline $\begin{array}{l}\text { Iron } \\
\text { pipe } \\
\text { size }\end{array}$ & Close & $\begin{array}{l}\text { Spe- } \\
\text { cial } \\
\text { short }\end{array}$ & Short & Long & Extra long \\
\hline $\begin{array}{l}1 / 8 \\
1 / 4 \\
38 \\
1 / 2 \\
3 / 4 \\
1 \\
11 / 4 \\
11 / 2 \\
2 \\
21 / 2 \\
3 \\
31 / 2 \\
4\end{array}$ & $\begin{array}{l}3 / 4 \\
7 / 8 \\
1 \\
11 / 8 \\
13 / 8 \\
11 / 2 \\
158 \\
13 / 4 \\
2 \\
21 / 2 \\
258 \\
28 / 4 \\
27 / 8\end{array}$ & 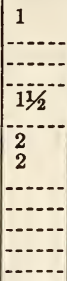 & $\begin{array}{l}11 / 2 \\
11 / 2 \\
11 / 2 \\
11 / 2 \\
2 \\
2 \\
21 / 2 \\
21 / 2 \\
21 / 2 \\
3 \\
3 \\
4 \\
4\end{array}$ & $\begin{array}{l}2,21 / 2,3,31 / 2 \\
2,21 / 2,3,31 / 2 \\
2,21 / 2,3,31 / 2 \\
2,21 / 2,3,31 / 2 \\
21 / 2,3,31 / 2,4 \\
21 / 2,3,31 / 2,4 \\
3,31 / 2,4,41 / 2 \\
3,31 / 2,4,41 / 2 \\
3,31 / 2,4,41 / 2 \\
31 / 2,4,5 \\
31 / 2,4,4112,5 \\
5,6 \\
41 / 2,5,51 / 2,6\end{array}$ & $\begin{array}{r}4,5,6 \\
4,5,6 \\
4,41 / 2,5,51 / 2,6,8,10,12 \\
4,41 / 2,5,5112,6,8,10,12 \\
41 / 2,5,51 / 2,6,8,10,12 \\
41 / 2,5,51 / 2,6,8,10,12 \\
5,51 / 2,6,8,10,12 \\
5,51 / 2,6,8,10,12 \\
5,51 / 2,6,8,10,12 \\
6,8,10,12\end{array}$ \\
\hline
\end{tabular}

TABLE 4.-Wrought iron and steel pipe nipples, right and left, type $A$ standard weight and type $B$ extra strong, uncoated, iron pipe sizes and lengths

[All dimensions in inches]

\begin{tabular}{|c|c|c|c|}
\hline $\begin{array}{l}\text { Iron } \\
\text { pipe } \\
\text { size }\end{array}$ & Short & Long & $\begin{array}{l}\text { Extra } \\
\text { long }\end{array}$ \\
\hline $\begin{array}{l}1 / 8 \\
1 / 4 \\
38 \\
1 / 2 \\
32 / 4 \\
1 \\
11 / 4 \\
11 / 2 \\
2\end{array}$ & $\begin{array}{l}2 \\
2 \\
21 / 2 \\
21 / 2 \\
21 / 2\end{array}$ & $\begin{array}{r}2,3 \\
2,21 / 2,3,31 / 2 \\
2112,3,31 / 2,4 \\
21 / 2,3,31 / 2,4 \\
3,31 / 2,4,41 / 2 \\
3,312,4,41 / 2 \\
3,312,4,41 / 2\end{array}$ & $\begin{array}{r}4,5,6 \\
4,5,6 \\
5,6 \\
5,6 \\
5,6 \\
5,6 \\
5,6\end{array}$ \\
\hline
\end{tabular}

Brass pipe nipples shall be of the respective dimensions given in Tables 5, 6, and 7. Special sizes and lengths may be specified when required. Special lengths shall not vary more than one-sixteenth-inch over or under the lengths required by the purchase order.

TABLE 5.-Brass pipe nipples, type A, standard weight, iron pipe sizes and lengths

[All dimensions in inches]

\begin{tabular}{|c|c|c|c|c|}
\hline $\begin{array}{l}\text { Iron } \\
\text { pipe } \\
\text { size }\end{array}$ & Close & $\begin{array}{l}\text { Special } \\
\text { short }\end{array}$ & Short & Long \\
\hline $\begin{array}{l}1 / 8 \\
1 / 2 \\
3 / 8 \\
1 / 2 \\
3 / 4 \\
1 \\
11 / 1 \\
11 / 2 \\
2 \\
21 / 2 \\
3 \\
4 \\
5 \\
6\end{array}$ & $\begin{array}{l}3 / 4 \\
7 / 8 \\
11 \\
11 / 8 \\
138 \\
11 / 2 \\
138 \\
13 / 4 \\
213 \\
21 / 2 \\
258 \\
278 \\
3 \\
31 / 8\end{array}$ & \begin{tabular}{c}
11 \\
$11 / 4$ \\
\hdashline $11 / 2$ \\
2 \\
2 \\
\hdashline-11 \\
-4 \\
4
\end{tabular} & $\begin{array}{l}11 / 2 \\
11 / 2 \\
11 / 2 \\
11 / 2 \\
2 \\
2 \\
21 / 2 \\
21 / 2 \\
21 / 2 \\
3 \\
3 \\
4 \\
41 / 2 \\
41 / 2\end{array}$ & 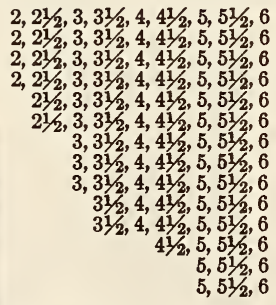 \\
\hline
\end{tabular}

TABLE 6.-Brass pipe nipples, type B, extra strong, iron pipe sizes and lengths

[All dimensions in inches]

\begin{tabular}{|c|c|c|c|}
\hline $\begin{array}{l}\text { Iron } \\
\text { pipe } \\
\text { size }\end{array}$ & Close & Short & Long \\
\hline $\begin{array}{l}1 / 8 \\
1 / 4 \\
3 / 8 \\
1 / 2 \\
33 / 4 \\
1 \\
11 / 4 \\
11 / 2 \\
2\end{array}$ & $\begin{array}{l}3 / 4 \\
7 / 8 \\
1 \\
11 / 8 \\
13 / 8 \\
11 / 2 \\
1568 \\
13 / 4 \\
2\end{array}$ & \begin{tabular}{r}
$r$ \\
2 \\
2 \\
\hdashline \\
-
\end{tabular} & $\begin{array}{r}2,3,4,5,6 \\
2,3,4,5,6 \\
2,3,4,5,6 \\
2,3,4,5,6 \\
3,4,5,6 \\
3,4,5,6 \\
3,4,5,6 \\
3,4,5,6 \\
3,4,5,6\end{array}$ \\
\hline
\end{tabular}

TABLE 7.-Brass pipe nipples, right and left, type $A$, standard weight, iron pipe sizes and lengths

[All dimensions in inches]

\begin{tabular}{|c|c|c|}
\hline $\begin{array}{l}\text { Iron } \\
\text { pipe } \\
\text { size }\end{array}$ & Short & Long \\
\hline $\begin{array}{l}3 / 8 \\
1 / 2 \\
3 / 4 \\
1 \\
11 / 4 \\
11 / 2 \\
2\end{array}$ & $\begin{array}{r}2 \\
2 \\
- \\
-\end{array}$ & $\begin{array}{r}2,3,4,5,6 \\
2,3,4,5,6 \\
3,4,5,6 \\
3,4,5,6 \\
3,4,5,6 \\
3,4,5,6 \\
3,4,5,6\end{array}$ \\
\hline
\end{tabular}

\section{METHOD OF SAMPIING, INSPECTION, AND TESTS}

Unless otherwise specified, the pipe from which nipples are to be made shall be inspected and tested in accordance with methods of inspection and tests covered by the respective specification listed in paragraph A-1.

When impracticable to inspect and test the pipe, the nipples shall be inspected and tested to insure that the material conforms to the respective specifications.

Nipples shall be inspected for dimensions, threading, and surface defects.

\section{PACKAGING, PACKING, AND MARKING}

Packaging.-Unless otherwise specified, commercial packages are acceptable under this specification.

Packing.-Unless otherwise specified, the subject commodity shall be delivered in substancial commercial containers, so constructed as to insure acceptance by common or other carriers for safe transportation, at the lowest rate, to the point of delivery.

Marking.-Unless otherwise specified, shipping containers shall be marked with the name of the material, the size, the type and the quantity contained therein, as defined by the contract or order under which the shipment is made, the name of the contractor, and the number of the contract or order.

\section{NOTES}

The dimensions for pipe nipples given herein are those in which they are regularly furnished. Nipples of special dimensions may be specified when required. 
Requisitions for pipe nipples should give iron pipe size, material (by reference to respective specification listed in section on "Material and Workmanship", type, length, number required, and, if of steel or iron, whether uncoated or zinc-coated nipples are required.

The dimensional requirements given herein correspond to those of United States Department of Commerce Commercial Standards CS5-29, CS6-29, and CS10-29.

It is the standard practice in some Government departments to use extra strong, right and left, wrought iron and steel nipples with standard weight wrought-iron and steel pipe. It is also the standard practice of some Government departments to use only grade A (Federal Specification WW-P-351) brass pipe nipples for installations of any grade of brass pipe.

It is believed that this specification is adequate and that normally no samples will be necessary to determine compliance with the requirements prior to award. If, for a particular purpose, samples with bids are necessary they should be specifically asked for in the invitaiion for bids.

\section{UNITED STATES GOVERNMENT, FEDERAI SPECIFICATIONS BOARD, FEDERAL SPECIFICATION FOR PIPE; WELDED- STEEL, BLACK AND ZINC-COATED, WW-P-431, OCTOBER 14, 1930}

\section{WELDED-STEEL PIPE}

\section{APPLICABLE FEDERAL SPECIFICATIONS}

The following Federal specifications, of the issue in effect on date of invitation for bids, shall form a part of this specification:

\begin{tabular}{|c|c|c|c|}
\hline $\begin{array}{l}\text { Federal speci- } \\
\text { fication } \\
\text { No. }\end{array}$ & $\begin{array}{c}\text { Former } \\
\text { No. }\end{array}$ & Title & See \\
\hline $\begin{array}{r}\text { GGG-P-351 } \\
Q Q-M-151\end{array}$ & $\begin{array}{l}238 \\
339\end{array}$ & $\begin{array}{l}\text { Pipe threads } \\
\text { General specifications for met- } \\
\text { als... }\end{array}$ & $\begin{array}{l}607.0, \text { p. } 365 \\
600.0, \text { p. } 3\end{array}$ \\
\hline
\end{tabular}

(In respect to classes of pipe this specification is identical with A. S. T. M., A120-28T, given above. In respect to manufacture, general requirements, and detail requirements it is substantially the same as A. S. T. M., A53-30, given above, with the following exceptions and additions:)

\section{MATERIAL AND WORKMANSHIP}

Pipe shall be straight and free from buckles, bends, kinks, cracks, laminations, imperfect welds, rust, injurious sand marks, or any other defects which might affect its strength or serviceability. Telescoped pipe shall not be furnished under this specification. All burrs at the ends of the pipe shall be removed. (Ordinarily liquor marks incident to manufacture of welded material shall not be considered cause for rejection.)

\section{GENERAL REQUIREMENTS}

Class B, extra-strong, and class C, double extrastrong pipe, shall conform to the following minimum requirements as to tensile properties:

\begin{tabular}{|c|c|c|c|}
\hline Classes & $\begin{array}{c}\text { Tensile } \\
\text { strength } \\
\text { per square } \\
\text { inch }\end{array}$ & $\begin{array}{c}\text { Yield } \\
\text { point per } \\
\text { square inch }\end{array}$ & $\begin{array}{c}\text { Elong8- } \\
\text { tion in 8 } \\
\text { inches }\end{array}$ \\
\hline B and C......... & $\begin{array}{c}\text { Pounds } \\
45,000\end{array}$ & $\begin{array}{c}\text { Pounds } \\
25,000\end{array}$ & $\begin{array}{c}\text { Per cent } \\
18\end{array}$ \\
\hline
\end{tabular}

The weight of zinc-coated (galvanized) pipe shall not vary more than 3 per cent under that specified in the accompanying table (class $A$, standard weight); nor more than 3 per cent under that specified in A. S. T. M., A53-30, Table 11 (extra-strong pipe); nor more than 8 per cent under that specified in A. S. T. M., A53-30, Table 11 (double extra-strong pipe).

TABLE 1.-Class $A$, standard-weight pipe

\begin{tabular}{|c|c|c|c|c|c|}
\hline \multirow[b]{2}{*}{$\begin{array}{l}\text { Iron } \\
\text { pipe } \\
\text { size }\end{array}$} & \multicolumn{2}{|c|}{ Weight per foot 1} & \multirow[b]{2}{*}{$\begin{array}{l}\text { Iron } \\
\text { pipe } \\
\text { size }\end{array}$} & \multicolumn{2}{|c|}{ Weight per foot 1} \\
\hline & $\begin{array}{l}\text { Plain } \\
\text { ends }\end{array}$ & $\begin{array}{l}\text { Ends } \\
\text { threaded } \\
\text { and cou- } \\
\text { pling on } \\
1 \text { end }\end{array}$ & & $\begin{array}{l}\text { Plain } \\
\text { ends }\end{array}$ & $\begin{array}{l}\text { Ends } \\
\text { threaded } \\
\text { and cou- } \\
\text { pling on } \\
1 \text { end }\end{array}$ \\
\hline $\begin{array}{c}\text { Inches } \\
1 / 8 \\
1 / 4 \\
38 \\
1 / 2 \\
3 / 4\end{array}$ & $\begin{array}{c}\text { Pounds } \\
0.244 \\
.424 \\
.567 \\
.850 \\
1.130\end{array}$ & $\begin{array}{c}\text { Pounds } \\
0.245 \\
.425 \\
.568 \\
.852 \\
1.134\end{array}$ & $\begin{array}{c}\text { Inches } \\
3 \\
31 / 2 \\
4 \\
5 \\
6\end{array}$ & $\begin{array}{r}\text { Pounds } \\
7.575 \\
9.109 \\
10.790 \\
14.617 \\
18.974\end{array}$ & $\begin{array}{c}\text { Pounds } \\
7.616 \\
9.202 \\
10.889 \\
14.810 \\
19.185\end{array}$ \\
\hline $\begin{array}{l}1 \\
11 / 1 / 2 \\
11 / 2 \\
2 \\
21 / 2\end{array}$ & $\begin{array}{l}1.678 \\
2.272 \\
2.717 \\
3.652 \\
5.793\end{array}$ & $\begin{array}{l}1.684 \\
2.281 \\
2.731 \\
3.678 \\
5.819\end{array}$ & $\begin{array}{r}8 \\
10 \\
12 \\
212\end{array}$ & $\begin{array}{l}28.554 \\
40.483 \\
49.562 \\
43.773\end{array}$ & $\begin{array}{l}28.809 \\
41.132 \\
50.706 \\
45.000\end{array}$ \\
\hline
\end{tabular}

1 Based on 20-foot lengths.

This is special-weight pipe which shall not be furnished unless specifled and shall conform to the requirements of class $A$ standardweight pipe.

Zinc coating (galvanizing) shall be done by the hot process, both inside and outside. The pipe shall be thoroughly and evenly coated and free from blisters and other defects. The increase in weight due to coating shall be not less than one-eighth pound per square foot of surface covered. The zinc bath shall contain not more than 2 per cent of elements other than zinc, as determined from samples taken at a point nalf the depth of the batin. Unless zinc coating is specified in the invitation for bids, black pipe shall be furnished.

All threads shall be adequately protected. Tapped rings or half-length couplings shall be provided on the end not protected by the coupling on all sizes of threaded pipe 2 inches iron pipe size or larger. Threads on smaller sizes shall be protected by having ends of bundles securely wrapped with burlap unless otherwise specified.

\section{DETAIL REQUIREMENTS}

Standard-weight pipe shall, unless otherwise specified, be threaded at both ends and have a standard pipe coupling of wrought iron or steel tightly screwed on one end. Extra-strong and double-extra-strong pipe shall, unless otherwise specified, be furnished with plain ends and without couplings. Zinc-coated (galvanized) couplings shall be furnished with zinc-coated pipe.

If impracticable to obtain samples from the bath or to weigh pipe immediately before coating, the quality of the zinc coating may be determined from the average of samples 6 inches long taken from both ends of finished pipe. In this event iron shall be disregarded as an impurity.

Hydrostatic tests shall be made in the presence of a Government inspector unless inspection is specifically waived, in which case the manufacturer shall furnish a certificate to the effect that the pipe has withstood satisfactorily the hydrostatic test required.

All pipe shall be surface inspected for defects by rolling on a set of wooden horses or other suitable supports, so that the entire surface of each pipe may be properly inspected. 
The number of flattening and bend tests for classes A, B, and C, and tension tests for classes B and $C$ shall be as follows for each size of pipe:

One test of each kind from each lot of 100 lengths of pipe 3 inches I. P. S. or less.

One test of each kind from each lot of 50 lengths of pipe over 3 inches and not over 6 inches I. P. S.

One test of each kind from each lot of 25 lengths of pipe over 6 inches and not over 8 inches I. P. S.

One test of each kind from each lot of 10 lengths of pipe over 8 inches I. P. S.

Note.-If the number of lengths is less than specified as lots in the above, one test of each kind shall be made.

\section{NOTES}

Pipe is regularly designated by iron-pipe size, class (standard weight, extra strong, or double extra strong), black, or zinc coated (galvanized).

Pipe under this specification should be ordered by number of feet required. In the case of pipe furnished with couplings, the length includes the coupling when tightly screwed on the pipe.

Pipe $11 / 4$ inches to 3 inches I. P. S., inclusive, is made by both the lap-welding and butt-welding processes. If either is specifically desired, the invitation for bids should so state, otherwise either lap-welded or butt-welded pipe between these sizes may be furnished and will be acceptable.

\section{0\%.5 Sheet Iron and Steel Pipe.}

\section{AMERICAN ASSOCIATION OF STATE HIGHWAY OFFICALS, TENTATIVE STANDARD SPECIFICATION FOR BASE METAL FOR CORRUGATED METAL SHEETS FOR CULVERTS, M-36, 1931}

\section{METAL-PIPE CULVERTS, CORRUGATED}

Corrugated metal-pipe culverts shall be fabricated from corrugated galvanized sheets, the base metal of which shall be made by the open-hearth process.

The base metal shall conform to the following chemical requirements:

\begin{tabular}{|c|c|c|c|c|c|c|}
\hline \multirow[b]{2}{*}{ Elements } & \multicolumn{5}{|c|}{$\begin{array}{l}\text { Chemical composition by ladle } \\
\text { analysis; position of base metals } \\
\text { does not indicate preference }\end{array}$} & \multirow{2}{*}{$\begin{array}{l}\text { Toler- } \\
\text { ance } \\
\text { by } \\
\text { check } \\
\text { analy- } \\
\text { sis } \\
\text { of } \\
\text { fin- } \\
\text { ished } \\
\text { sheets }\end{array}$} \\
\hline & $\begin{array}{l}\text { Pure } \\
\text { iron }\end{array}$ & $\begin{array}{l}\text { Cop- } \\
\text { per- } \\
\text { bear- } \\
\text { ing } \\
\text { pure } \\
\text { iron }\end{array}$ & $\begin{array}{l}\text { Cop- } \\
\text { per } \\
\text { iron }\end{array}$ & $\begin{array}{l}\text { Cop- } \\
\text { per- } \\
\text { molyb- } \\
\text { denum } \\
\text { steel }\end{array}$ & $\begin{array}{c}\text { Cop- } \\
\text { per } \\
\text { steel }\end{array}$ & \\
\hline Carbon, maximum & Per ct. & Perct. & Perct. & Per ct. & Perct. & Per ct. \\
\hline $\begin{array}{l}\text { Manganese, maxi- } \\
\text { mum }\end{array}$ & & & & & & \\
\hline Phosphorus, maxi- & 0.015 & 0.015 & 0.015 & 0.015 & & \\
\hline $\begin{array}{l}\text { Sulphur, maximum } \\
\text { Silicon, maximum... }\end{array}$ & .040 & .040 & .040 & .040 & 0.050 & 0.010 \\
\hline $\begin{array}{l}\text { Copper, minimum } \\
\text { Molybdenum, mini- } \\
\text { mum }\end{array}$ & 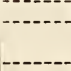 & .20 & .20 & .05 & .20 & .02 \\
\hline $\begin{array}{l}\text { Sum of first } 5 \text { ele- } \\
\text { ments, maximum -- } \\
\text { Sum of first } 6 \text { ele- } \\
\text { ments, maximum - }\end{array}$ & .10 & .10 & .25 & .25 & .70 & .04 \\
\hline
\end{tabular}

AMERICAN ASSOCIATION OF STATE HIGHWAY OFFICIALS, METHOD OF TEST AND INSPECTION OF CORRUGATED METAI CULVERT, TENTATIVE METHOD T-64, 1931

\section{TESTING CORRUGATED METAL CULVERT}

1. Corrugated metal culvert shall be subject to inspection at the factory, trench, or other point of delivery by the engineer or other authorized repre sentative.

2. The inspector shall determine the gage of the metal, inside diameter, depth and spacing of the corrugations, width of lap, rivet spacing, weight per linear foot of the finished pipe, condition of spelter coating, and other requirements as called for under the specifications. He shall also take a sample of the pipe material, approximately 6 inches wide and the full diameter of the pipe, for measurements, chemical analysis, and the determination of the amount of galvanizing or spelter coating.

3. Chemical analysis will be made of all metal culvert pipe, including rivets, to determine whether the requirements of the specifications as to quality of the material are met.

4. The amount of galvanizing or spelter coating will be determined in accordance with the methods for testing the amount of spelter coating on culvert metal.

5. Chemical analysis to be conducted in accordance with the standard method of chemical analysis of plain carbon steel in A. S. T. M., A33. (See 600.1, p. 10.)

\section{AMERICAN ELECTRIC RAILWAY ENGI- NEERING ASSOCIATION, DESIGN OF} SMALL BRIDGES, CULVERTS, AND TRESTLES, B209-26, 1926

\section{DESIGN OF PIPE CULVERTS}

In the design of any pipe culvert adequate head walls should be provided. This is especially true for the upstream side.

The maximum diameters of pipe recommended are:

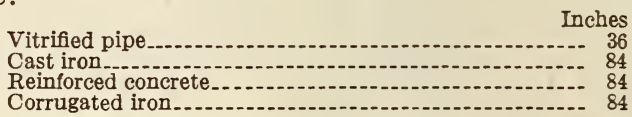

Figure 295 shows current practice in the design of head walls for pipe culverts.

AMERICAN RAILWAY ENGINEERING ASSOCIATION, SPECIFICATIONS FOR CORRUGATED METAL CULVERT PIPE FOR RAILROAD USE, 1930

(1) General.-Pipe shall be straight, of true circular form, and of lengths and net internal diameters indicated.

(2) Base metal.-Corrugated metal-pipe culverts shall be fabricated from corrugated galvanized sheets, the base metal of which shall be made by the open-hearth process, unless otherwise specified in the contract. 
(3) Analysis.-The base metal shall conform to one of the following chemical requirements. Bidder shall state classification he is bidding on.

Chemical composition.-(Identical with A. A. S. H. O., given above.)

(4) Gage.-U. S. standard gage for sheet-metal designation shall be used in specifying the thickness and weight of culvert-pipe sheets.

(5) Sheets. - All sheets shall be of first quality, prime stock, and free from injurious defects such as laminations, ragged edges, etc.

Corrugations.-Corrugations in sheets shall not exceed $2 \frac{3}{4}$ nor be less than $2 \frac{1}{2}$ inches in width, summit to summit, and not less than one-half inch nor more than three-fourths inch in depth.

(6) Weight and tolerance.-The average weight per square foot of the culvert sheets, as determined by weighing in lots of not less than 3,000 pounds and not exceeding 6,000 pounds, shall not vary
Longitudinal joints shall be riveted on not to exceed $23 / 4$-inch centers, with one row of rivets in pipe 21 inches or less in diameter and two rows of rivets in pipe of larger diameters.

Circumferential joints of pipe of all diameters shall be riveted on not to exceed 5 -inch centers.

The upsetting of the end of the rivets on the inside of the pipe shall give full hemispherical heads accomplished in such manner as not to remove or destroy the galvanizing, shall insure a tight joint, and shall present a neat and workmanlike job.

Rivets shall be driven without bending and must completely fill the holes.

(9) End reinforcement.-Culverts of 12-gage material and lighter shall have the ends reinforced by an iron rod of not less than one-half inch in diameter and a length equal to the circumference of the pipe, folded into the outer edge of the sheet forming the end section, or by use of a flat iron bar or other

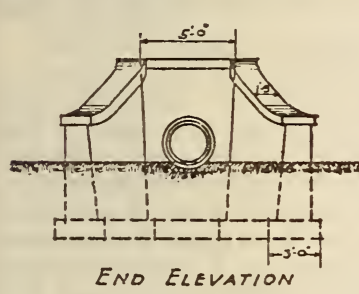

END ELEVATION

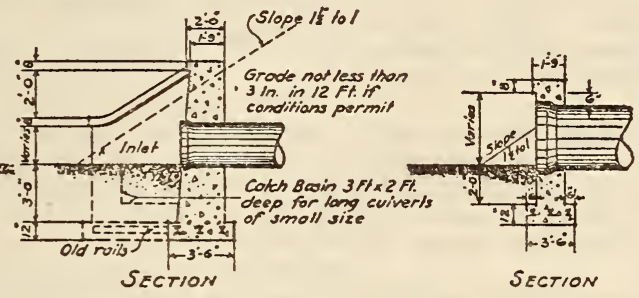

FIgURE 295.-Head walls for pipe culverts

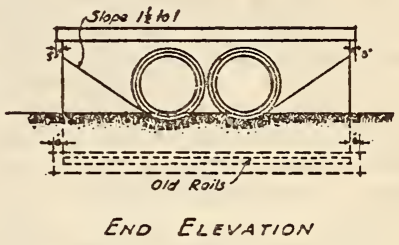

ENO ELEVATION from the theoretical weight by more than 5 per cent either way.

(7) Joints. - The various diameters of pipe shall have a lap of metal for longitudinal joints as follows:

\begin{tabular}{|c|c|}
\hline Diameter & Lap (for each longitudinal seam or joint) \\
\hline $\begin{array}{l}6 \text { to } 10 \text { inches } \\
11 \text { to } 15 \text { inches } \\
16 \text { to } 21 \text { inches } \\
22 \text { to } 27 \text { inches } \\
28 \text { to } 36 \text { inches } \\
37 \text { to } 45 \text { inches } \\
46 \text { inches and over... }\end{array}$ & $\begin{array}{l}\text { Not less than } 2.5 \text { inches. } \\
\text { Not less than } 3.0 \text { inches. } \\
\text { Not less than } 3.5 \text { inches. } \\
\text { Not less than } 4.0 \text { inches. } \\
\text { Not less than } 4.5 \text { inches. } \\
\text { Not less than } 5.0 \text { inches. } \\
\text { Not less than } 6.0 \text { inches. }\end{array}$ \\
\hline
\end{tabular}

(8) Riveting.-All rivets shall be manufactured of the same base metal as are the sheets. The rivets shall be of round head type, thoroughly galvanized with zinc spelter. The sizes of rivets to be used with different thicknesses of sheets shall be as follows:

\begin{tabular}{|c|c|c|c|c|}
\hline \multirow{2}{*}{ Sheets } & \multicolumn{2}{|c|}{2 thicknesses } & \multicolumn{2}{|c|}{3 thicknesses } \\
\hline & Diameter & Length & Diameter & Length \\
\hline $\begin{array}{l}16 \text { gage } \\
14 \text { gage } \\
12 \text { gage } \\
10 \text { gage } \\
8 \text { gage }\end{array}$ & $\begin{array}{r}\text { Inch } \\
5 / 16 \\
5 / 16 \\
3 / 8 \\
3 / 8 \\
3 / 8\end{array}$ & $\begin{aligned} & \text { Inch } \\
& 5 / 8 \\
& 5 / 8 \\
& 3 / 4 \\
& 3 / 4 \\
& 7 / 8\end{aligned}$ & \begin{tabular}{r|} 
Inch \\
$5 / 16$ \\
$5 / 16$ \\
38 \\
38 \\
$3 / 8$ \\
\end{tabular} & $\begin{array}{c}\text { Inch } \\
5 / 8 \\
3 / 4 \\
7 / 8 \\
1^{7 / 8}\end{array}$ \\
\hline
\end{tabular}

All rivets shall be placed with heads on outside of pipe in the recess or valley of corrugations. Where two lines of rivets are required, the minimum distance from center of rivet to edge of sheet shall not be less than two and one-half times and maximum distance not more than four times the diameter of the rivet.

Before placing the rivets the sheets shall be tightly and evenly clamped together to prevent the swelling of the rivets between the sheets. stiffener, riveted to or suitably attached to the end of the pipe, by folding back the end of the sheet or by other suitable means, and in such manner that the galvanizing will not be broken or flecked off, as may be specified by the purchaser.

(10) Galvanizing. - In all cases the sheets shall be corrugated and all shearing done before galvanizing, and in the case of perforated pipe the perforations also shall be punched before the galvanizing is done.

Sheets shall be uniformly coated with not less than 1 ounce of prime quality zinc spelter per square foot of surface of sheet, counting each surface of the sheet separately.

The galvanizing shall be free from cracks, peels, blisters, pinholes, uncoated spots, or imperfections of any kind, and, so far as is practicably possible, shall be of uniform thickness over all surfaces of the metal.

(11) Marking.-(a) All sheets shall be conspicuously branded or stenciled on the outside to show manufacturer's name, brand or quality of material, gage of sheet, and stock-card number (or heat number) of base metal so that complete data covering the manufacturing history of the sheet before galvanizing can be obtained.

(b) All culverts shall have a metal tag of durability equal to that of the culvert riveted to the inside of the pipe on the upstream end which will suitably identify the maker, kind of base metal, and year fabricated. Pipe ordered for use as drains shall have the identification tag attached on the outside of the pipe at least once each 300 feet.

(12) Field joints.-Unless otherwise specified, corrugated pipe may be furnished in as long sections as can be shipped economically, except that where the purchaser specifies a definite total length of pipe for any location, the lengths of joints shall be adjusted so that no section of less than 8 feet in length must be used.

Coupling bands or suitable devices for joining the pipes securely together shall be shipped with the pipe. 
(a) Coupling bands for pipe shall be of sufficient width to engage full corrugations on each side of the end of the pipe and be equipped with lugs or equivalent fastenings, as follows:

\begin{tabular}{|c|c|c|}
\hline Pipe diameters, inclusive & $\begin{array}{l}\text { Minimum number corruga- } \\
\text { tions to be engaged on each } \\
\text { section }\end{array}$ & $\begin{array}{l}\text { Num. } \\
\text { ber of } \\
\text { lugs }\end{array}$ \\
\hline $\begin{array}{l}6 \text { to } 15 \text { inches } \\
16 \text { to } 42 \text { inches } \\
43 \text { inches and larger. }\end{array}$ & $\begin{array}{l}11 / 2 \text { full corrugations } \\
2 \text { full corrugations } \\
3 \text { full corrugations }\end{array}$ & $\begin{array}{l}2 \\
3 \\
4\end{array}$ \\
\hline
\end{tabular}

Coupling bands shall be made of the same gage and quality of material used in the pipe.

(b) Lugs, or fastenings, on bands shall consist of forged wrought iron or such other material as may be specified by the purchaser and shall be fully galvanized to the same extent as required for the other material used in the culvert. Each half of the fastening lugs or other appliance shall be riveted to the band with not less than four rivets of threeeighths inch diameter or larger.

The draw bolts shall be of not less than one-half inch diameter, with $3 \frac{1}{2}$ inches or more, threaded, for pipe 30 inches or less in diameter, and of not less than three-fourths inch diameter with 4 inches or more threaded, for pipe of diameters above 30 inches.

Bolts and nuts must be galvanized after threading.

At the option of the purchaser, culverts of 54 inches diameter and larger shall be fabricated for field riveting, in which case necessary rivets, as specified under paragraph (8) "Riveting," must be furnished with the pipe. In such cases proper appliances for enlarging and tightening the sheets of end rings shall also be supplied.

(13) Workmanship.-Each section of pipe must be fabricated in a neat and workmanlike manner, of correct dimensions and true lines.

(14) Handling. - All material, during fabrication and in the loading and shipping of the finished pipe, must be so handled that the galvanizing or zinc coating shall not be broken or damaged.

(15) Inspections and rejections. - The failure of any section of pipe delivered to conform to these specifications in any particular shall constitute cause for rejection thereof.

\section{UNITED STATES GOVERNMENT, DEPART- MENT OF AGRICULTURE, BUREAU OF PUBLIC ROADS, SPECIFICATIONS FOR FOREST ROAD CONSTRUCTION, 1929}

\section{CORROGATED GALVANIZED METAL PIPE}

\section{DESCRIPTION}

This item shall consist of furnishing sheet-metal pipe conforming to these specifications and of the sizes and dimensions required on the plans, and installing such pipe where and as designated on the plans or by the engineer and in conformity with the lines and grades given. This item shall include the furnishing and construction of such joints, and such connections to existing pipes, catch basins, end walls, etc., as may be required to complete the work as shown on the plans.

\section{MATERIAL AND MANUFACTURE}

The pipe shall be fabricated from corrugated galvanized sheets, the base metal of which shall be made by the open-hearth process.

(The chemical requirements are identical with those for corrugated metal pipe culverts of the A. A. S. H. O., given above.)
Corrugated metal pipe culverts shall be fabricated from corrugated galvanized sheets, the base metal of which shall be made by the open-hearth process. The base metal in the finished sheets shall conform to the following chemical requirements:

The total amount of carbon, phosphorus, sulphur, manganese, and silicon shall not exceed 0.7 per cent. If the total of these five elements equals or exceeds 0.20 per cent, the metal shall contain not less than 0.17 per cent of copper and not more than 0.06 per cent of sulphur. If the total of these five elements is less than 0.20 per cent, the presence of copper is optional and sulphur shall not exceed 0.04 per cent.

All rivets shall be of the same material as the base metal specified for the corrugated sheets. They shall be thoroughly galvanized or sherardized.

The weight of the culvert sheets, as determined by weighing in lots not exceeding 6,000 pounds, shall not vary from the theoretical weight by more than 5 per cent either way for each lot of one gage and size.

\section{WEIGHT OF SPELTER COATING}

The base-metal sheets shall be uniformly galvanized on both sides by the hot process. A uniform coating of prime western spelter shall be applied at the rate of not less than 2 ounces per square foot of metal. If the average spelter coating as determined from samples shows less than 2 ounces of spelter per square foot of metal, or if any one sample shows less than 1.8 ounces of spelter per square foot of metal, the shipment shall be rejected. Sheets having blister spots, holes, or other imperfections in the galvanizing after corrugating shall be rejected.

No metal will be accepted under these specifications and no bids will be considered for the materials above described until after the sheet manufacturer's certified analysis and manufacturer's guarantee have been passed upon by the engineer and accepted.

Misbranding or other misrepresentation, and nonuniformity of product, will each be considered a sufficient reason to discontinue the acceptance of any brand under these specifications, and notice sent to the sheet manufacturer of the discontinuance of acceptance of any brand will be considered to be notice to any culvert companies which handle that particular brand.

The manufacturer of each brand shall file with the engineer a certificate setting forth the name or brand of metal to be furnished and a typical analysis showing the percentage of each of the five chemical elements. The certificate shall be sworn to for the manufacturing company by a person having legal authority to bind the company.

The manufacturer of the sheets shall submit with the certified analysis a guarantee providing that all metal furnished shall conform to the certified analysis filed, shall bear a suitable identification brand or mark, and shall be replaced without cost to the purchaser when not in conformity with the specified analysis, gage, or spelter coating; and the guarantee shall be so worded as to remain in effect so long as the manufacturer continues to furnish material.

No culverts will be accepted unless the metal is identified by a stamp on each section showing: First, name of sheet manufacturer; second, name of brand; third, the gage.

The identification brands shall be placed on the sheets by the manufacturer of the sheets in such a way that when rolled into culverts such identification shall appear on the outside of each section of each pipe. Pipe having any sections not so stamped shall be promptly rejected.

Laboratory tests shall follow the methods of the United States Department of Agriculture Bulletin No. 1216 , revised.

If the engineer so elects, he may have the material inspected at the rolling mill or the culverts inspected 
in the shop where they are fabricated. He may require a chemical analysis from the mill for any heat, also a physical test of the properties of the metal taken from any heat, to be made by the mill. The inclusion in any shipment of any material which has been rejected at the mill or shop will be considered sufficient cause for the rejection of the entire shipment.

\section{CONSTROCTION}

(Details of construction include shape of culverts, maximum and minimum diameters, other dimensions and weights, corrugations, rivets and riveting, coupling bands, workmanship and inspection, laying of pipe, and sampling.)

UNITED STATES GOVERNMENT, DEPARTMENT OF COMMERCE, BUREAU OF STANDARDS, SIMPLIFIED PRACTICE RECOMMENDATION FOR EAVES TROUGH, CONDUCTOR PIPE, CONDUCTOR ELBOWS, AND FITTINGS, R29, JULY 1, 1925

\section{EAVES TROUGH AND CONDUCTOR PIPE}

(Simplified practice recommended and accepted by industry establishing a limited list of standard sizes and weights.)

\section{CONDUCTOR PIPE SIZES}

Plain round conductor pipe, 2, 3, 4, 5, and 6 inches diameter. Round corrugated conductor pipe, $2,3,4,5$, and 6 inches diameter. Square corrugated conductor pipe, $2,3,4$, and 5 inches on side.

\section{EAVES TROUGH SIZES}

Eaves trough, 31/2, 4, 5, 6, 7, and 8 inches wide.

\section{CONDUCTOR PIPE ELBOWS}

No. $1,45^{\circ}$; No. $2,60^{\circ}$; No. $3,75^{\circ}$; No. $4,90^{\circ}$.

1. Along with the elimination of certain sizes of conductor pipe and eaves trough goes also the elimination of the fittings formerly used therewith.

2. No eaves trough or conductor pipe to be made lighter than 28 gage full weight; 27 gage is to be eliminated.

3. All elbows, shoes, miters, and all accessories, including ridge rolls, valleys, gutters, and so on, are to be of 28 gage full weight ( 0.0156 inch before galvanizing).

4. All eaves trough, conductor pipe, shoes, miters, and all accessories, including gutters, valleys, ridge rolls, and so on, when made of copper, to be not lighter than 16 ounces per square foot (equivalent to thickness of 0.0216 inch).

\section{0\%.6 VALVES, COCKS, AND HYDRANTS, FERROUS AND NONFERROUS.}

\section{AMERICAN BOTTLERS OF CARBONATED BEVERAGES, STANDARDS FOR BEV- ERAGE MANUFACTURING EQUIPMENT AND RECOMMENDED PRACTICES, EDU. CATIONAL BULLETIN NO. 4, 1930}

\section{CARBONATE WATER AND SIROP VALVES}

\section{A. B. C. B. RECOMMENDED PRACTICE}

Valves now used on carbonated water and sirup lines are of very great variety. While there are a few of them of notable construction, most are not suited to the service conditions encountered in carbonated water or sirup lines and are not in line with the sanitary trend of the bottling industry.

As a constructive suggestion for valves complying with the requirements of the beverage plant, a

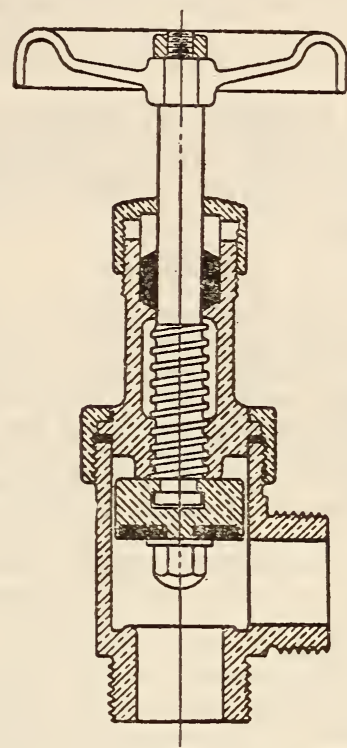

Figure 296.-Carbonated water valve

carbonated water valve and a sirup valve are shown in Figures 296 and 297.

Figure 296 shows a high-pressure carbonated water valve which offers an unobstructed flow to the carbonated water when wide open. The construction of the valve body is designed along very

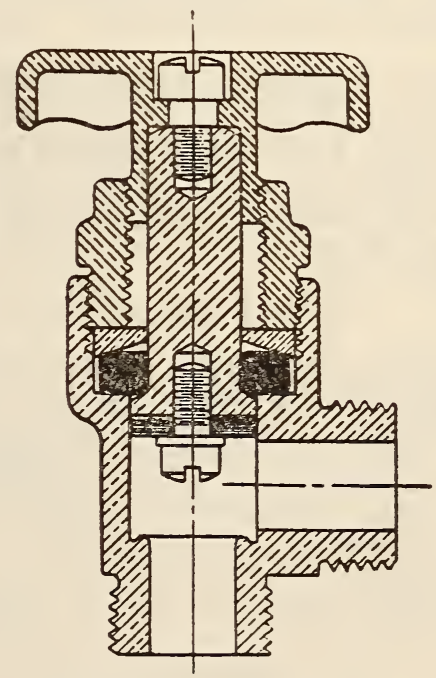

FIGURE 297.-Sirup valve

simple lines, therefore it is particularly suited for the manufacturing properties of the various materials which withstand corrosive action of carbonated water, many of which are difficult to obtain in the form of cored castings. The valve is easily taken apart for cleansing, inspection, or repair. 
Figure 297 illustrates low-pressure sirup valve. This valve was designed with the idea of reducing the possibility of bacterial contamination to a minimum. As will be noted, the valve has no pockets of any kind where bacteria can lodge, besides the liquid does not come into contact with the threads at any point. It can be readily taken apart for cleansing without removing it from the line.

\section{AMERICAN BUREAU OF SHIPPING, RULES FOR BUILDING AND CLASSING STEEI VESSELS; PUMPS AND PIPING SYS- TEMS, 1930}

\section{VALVES}

(For material of valves see 607.4, p. 435.)

1. All cast-iron and cast-steel valves to have flanged ends, bolted bonnets, and composition spindles, seats, and disks. Composition valves over 2 inches, for pressures over 100 pounds per square inch, and boiler stop and check valves are to have flanged ends and bolted bonnets. All valves intended for a working pressure exceeding 100 pounds per square inch shall bear the trademark of the manufacturer, legibily stamped or cast on the body of the valve, also the pressure in pounds per square inch that the manufacturer guarantees the valve to stand without distortion.

2. Valves 2 inches and over for working pressures exceeding 100 pounds per square inch, and valves below 2 inches for pressures of more than 300 pounds per square inch, are to be tested at the place of manufacture to not less than three times the working pressure.

\section{AMERICAN GAS ASSOCIATION, APPROVAI REQUIREMENTS FOR GAS WATER HEATERS, SEPTEMBER 1, 1931}

\section{GAS WATER HEATERS}

(See 614.2 , p. 855.)

AMERICAN RAILWAY ASSOCIATION, MECHANICAL DIVISION, STANDARD SPECI. FICATIONS FOR DETERMINING THE SIZE OF SAFETY VALVES, THEIR APPLICATION TO LOCOMOTIVES, AND THEIR REPAIR, 1912

\section{SAFETY VALVES}

1. Formula for determining the size of value required, assuming valves have $45^{\circ}$ seat.-

$D=$ the total of the actual diameters of the inner edge of the seats of valves required.

$H=$ total heating surface of boiler in square feet. (Superheating surface not to be included.)

$L=$ vertical lift of valve in inches.

$P=$ absolute boiler pressure in pounds per square inch.

$$
\begin{gathered}
D=0.036 \times \frac{H}{L \times P} \\
\text { Example } \\
\frac{0.036 \times 2,878}{0.1 \times 200}=5.2 \text { inch diameter }
\end{gathered}
$$

Which would require two 3 -inch valves.

2. Method of determining maximum capacity of safety valves. - The only accurate method of determining the capacity of safety valves is by actual test in a testing plant, with safety valves fully equipped with springs, as in actual road service. In order that it should be positively known that safety valves will prevent undue rise in pressure under extreme conditions, they should be subjected to a road test.

3. Number and size to be applied.-Every locomotive should be equipped with not less than two, and not more than three, safety valves, the size to be determined as per formula in paragraph 1. Safety valves to be set as follows: First, boiler pressure; second, 2 pounds in excess, and third, 3 pounds above second, or 5 pounds in excess of first.

4. Metal recommended for valves and valve seats.Bronze alloys should be used in the manufacture of valves and valve seats.

5. Stamping lift on valves.-Manufacturers should be required to stamp on the valve the lift in inches and the discharge capacity in pounds per minute, as determined by actual test, with valve in working condition, and set for a blow-back of not to exceed 3 pounds.

\begin{tabular}{|c|c|c|}
\hline $\begin{array}{l}\text { VALVE } \\
\text { Size }\end{array}$ & $\begin{array}{c}\text { SPacine } \\
\text { Deln } \\
\end{array}$ & RADIUS \\
\hline $4 \varepsilon^{\prime \prime}$ & $9^{\circ}$ & $50^{\circ}$ \\
\hline $4^{\circ}$ & $8 \frac{1}{2}^{\circ}$ & $47^{\circ}$ \\
\hline $32_{2}^{\circ}$ & $8^{\circ}$ & 45 \\
\hline $3^{\circ}$ & 74 & $4 \frac{3}{15}$ \\
\hline $26^{\circ}$ & $6 \frac{1}{2}$ & $39^{\circ}$ \\
\hline
\end{tabular}

6. Estimating steam discharge.-Steam discharge from safety valves of given size can be estimated

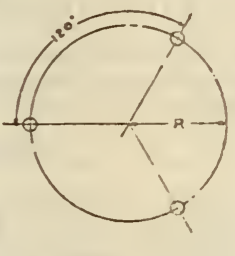

THAEE VALVES

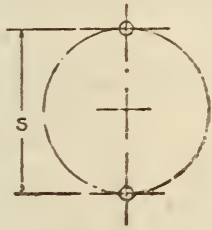

TES VALVES.

Figure 298.-Determining the size of safety valves, their application to locomotives, and their repairs

closely by the use of Napier's rule for flow of steam, as follows: Flow of steam per second-Absolute pressure in pounds per square inch, multiplied by area in square inches of discharge opening, divided by 70 . Multiplied by 3,600 gives flow in pounds per hour.

7. Location of valves on boiler.-Safety valves should be located at the highest point on the boiler, where clearance limits will permit, in vertical position, avoiding the use of piping, long nipples, and ells between safety valves and boiler.

8. Spacing in dome.-Where safety valves are located on an independent aome, they should be spaced as per Figure 298. The opening from boiler into dome and the area between supporting ribs in dome should not be less than inlet area of valves.

9. Hydrostatic and other tests.-The screwing down of the safety valve spring during a hydrostatic test of boiler should not be practiced. Valves with springs designed for certain pressures should not be subjected to extreme pressures. One safety valve should be removed and replaced with a special high-pressure valve, and the other valves should be removed and replaced with caps or plugs during test.

10. - Standard connections.-In order to make valves of different manufacture interchangeable, 
standard thread and diameter of valves at connection should be used as follows:

\begin{tabular}{|c|c|c|}
\hline $\begin{array}{c}\text { Safety } \\
\text { ralve }\end{array}$ & $\begin{array}{c}\text { U. S. } \\
\text { standard } \\
\text { pipe } \\
\text { thread }\end{array}$ & $\begin{array}{c}\text { Threads } \\
\text { to inch }\end{array}$ \\
\cline { 1 - 2 } Inches & Inchps & \\
$21 / 2$ & $21 / 2$ & 8 \\
3 & 3 & 8 \\
$31 / 2$ & $31 / 2$ & 8 \\
4 & 4 & 8 \\
$41 / 2$ & $41 / 2$ & 8 \\
\hline
\end{tabular}

Valves of above sizes to be made for U.S. standard wrench fit.

11. Inspection of old springs.-Old springs should be tested as to their deflection under load before being used in repaired valves.

12. Repairs to safety valves.- Safety valves should be thoroughly overhauled and put in good condition whenever the locomotive is in shop for general repairs. Standard gages should be used in order that important dimensions may be maintained as originally designed.

AMERICAN RAILWAY ASSOCIATION, MECHANICAL DIVISION, 5-INCH TANK CAR SAFETY VALVE, 1916 DESIGN, APPLICATION OF WASHER AND JAM NUTS TO VALVE STEM, CIR. NO. S-111-90

\section{TANK CAR SAFETY VALVE}

Figure 9 (a) shown in the tank car specifications, revised 1916, includes a design of spring follower with three lugs on the under side to engage tne single nut on the end of the valve stem. It was found in service that instead of the valve stem turning on its conical seat in the valve disk, when the disk was rotated to clear its face and seat of rust, etc., the stem turned in the nut and caused a change in the pressure setting of the valve. The design of the follower was therefore changed in 1918 to eliminate these lugs and double jam nuts applied to prevent the stem turning in the nut when the valve disk is rotated.

In the meantime a number of these valves were put into service. To avoid the frequent adjustment necessary to maintain the proper setting of the valves with this design of spring follower, it is desirable that when the valves are removed from the car for the adjustment of the spring setting a washer be introduced between the bottom of the follower and the nut, so that the lugs will not engage the nut; and that two nuts be applied, jammed against one another by the use of two wrenches, to prevent unscrewing when the valve disk is rotated. It may be found that the present stem is not long enough to permit the application of the washer and two nuts after the valve has been properly set, in which case a new stem of the dimensions shown should be used.

Figure 299 shows the application of the washer and nuts to the 1916 design.

NoTE. - Washer can be made of 3/4-inch pipe, or punched from 3/8-inch plate.

When the spring has been properly set for the required pressure the nuts on the valve stem should be jammed against one another by the use of two wrenches.

If the stem is not long enough to permit the application of the washer and double nuts when the spring has been properly set, it should be replaced by a new stem of the dimensions shown.
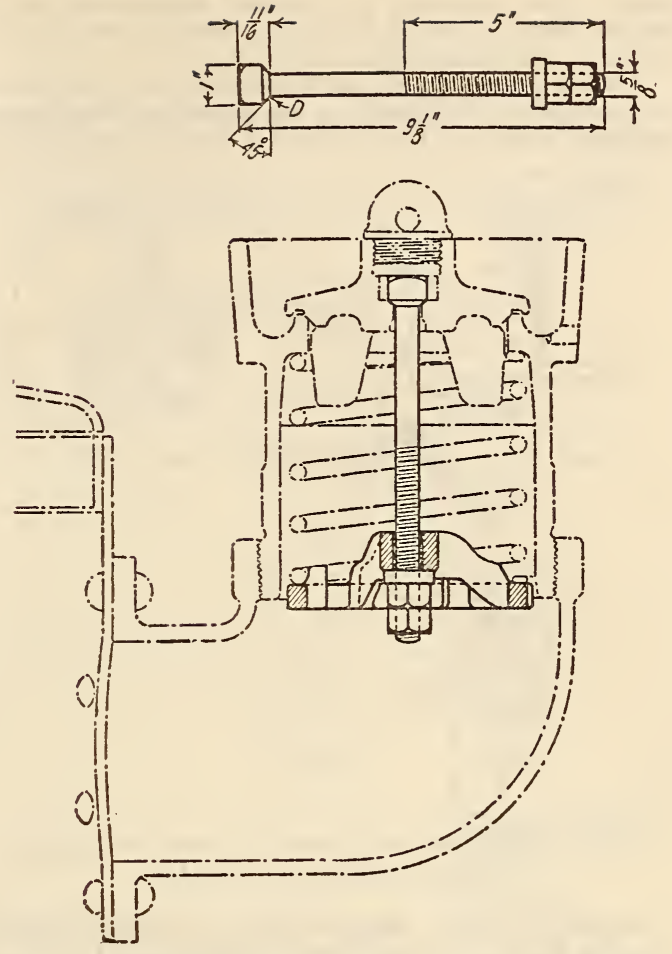

FIGURE 299.-Five-inch tank car safety valve, 1916 design

Application of washer and jam nuts to valve stem, $K-46-1931$

AMERICAN RAILWAY ASSOCIATION, MECHANICAL DIVISION, TRIPLE-VALVE, TEST REQUIREMENTS FOR FREIGHT SERVICE, RECOMMENDED PRACTICE, 1911

\section{TRIPLE-VALVE TESTS}

\section{CONDITION OF TESTS}

Construction of rack.-Triple valves on a rack representing the piping of a 100-car train. All cocks, angles, and connections will be as nearly as possible identical with those in train service. The rack shall conform to blue print No. C-11379 (rev. 3-9-09) in the hands of the committee, which gives the proper fittings, piping, cylinders, auxiliary reservoirs, main reservoirs, automatic brake valves, etc.

Reservoir capacity. - The main reservoir capacity shall be approximately 57,000 cubic inches.

The capacity of each auxiliary reservoir shall be such as will, with a pressure of 70 pounds, produce 50 pounds pressure in its brake cylinder when fully equalized in service application with 8 inches piston travel.

Air supply.-The air supply for the test rack shall be obtained from a locomotive type of air compressor having a capacity of from 80 to 120 cubic feet of free air per minute. The compressor to be controlled by a single top-pump governor adjusted to maintain 110 pounds main reservoir pressure.

Brake-pipe pressure.-Tests will be made with a brake-pipe pressure of 70 pounds, except when otherwise specified.

Brake-pipe leakage. With brake-pipe and auxiliary reservoirs charged to 70 pounds, the section of 
branch pipe between the cut-out cocks and triple valves, also the triple valves, should be tested with soapsuds and leakage eliminated.

Branch pipe cut-out cocks should then be closed and brake valve placed in lap position; brake-pipe leakage should then not exceed 2 pounds per minute.

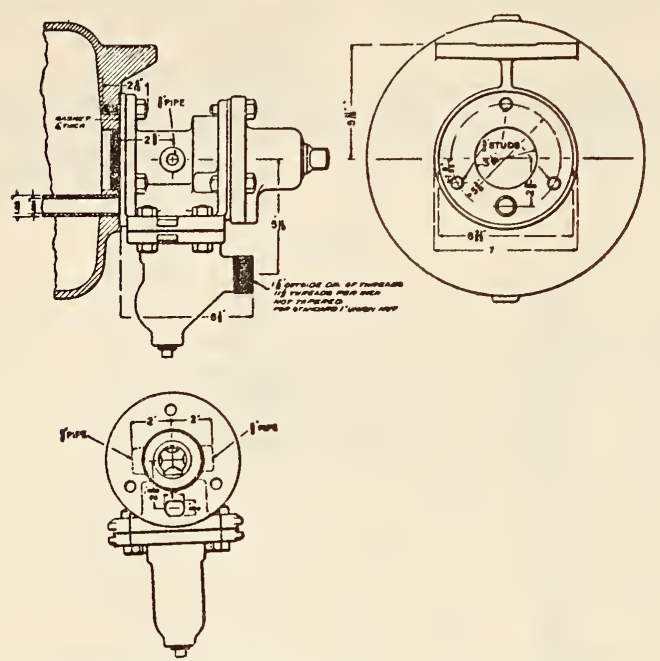

Figure 300.- $H^{2}$ triple valve on end of 10-inch freight reservoir, $D-15611$

Brake cylinders.-Brake-cylinder packing leathers must be maintained in good condition and free from leakage.

Piston travel. - All tests shall be made with 8-inch piston travel, except when otherwise specified.

Construction of triple valves.-Triples must be so constructed that they can be secured and operated on apparatus conforming to Figure 300 (which shows triple-valve end of auxiliary reservoir, branch-pipe union and location of bosses for retaining-valve pipe with detail dimensions of each, as well as detail dimensions between these parts when in the relative position they would occupy if triple valve were in place).

Gauges and recording instruments.-The auxiliary reservoirs, brake pipe and brake cylinder of the first, twenty-fifth, fiftieth, seventy-fifth, and one hundredth brakes shall be fitted with test gauges. All gauges must be calibrated and maintained in good condition.

Brake No. 1 shall be fitted with two recording pressure gauges, one to be connected to the brakepipe branch pipe, the other to the brake-cylinder, and brake No. 100 shall be fitted with a test gauge connected to the brake cylinder.

The attachments of electric circuit closers, also the general arrangement of the electric circuit wiring, shall be as shown on Figure 301.

Repetition of tests.-Tests shall be repeated three times under the same general condition, a record being taken of each test, also the average result of each three tests. The room temperalure at the time of the tests shall be recorded, also humidity.

Triple-valve essentials. - The essentials of a quickaction triple valve are: First, charging; second, service application; third, graduation; fourth, release; fifth, quick action.

\section{INDIVIDUAL TRIPLE-VALVE TESTS}

\section{No. 1.-Charging Tests}

(Time to charge auxiliary reservoir)

Not less than three triples, selected at random, shall be tested as follows:
With the triple valve cut out at the branch pipe cut-out cock, the auxiliary reservoir empty, and 90 -pound brake-pipe pressure maintained, the triple valve should be cut in.

A. Under these conditions the auxiliary reservoir should be charged from 0 to 70 pounds in not more than 90 seconds nor less than 70 seconds.

B. When triple is in normal release position the auxiliary reservoir should be charged from 0 to 70 pounds in not more than 60 seconds and not less than 40 seconds.

\section{No. 2.-Service Application Testa}

Section A.-(To determine sensitiveness to service application.)

1. Three valves, selected at random, shall be taken for this test and each tried separately. They will be tested on the first brake of the rack using the brake pipe only of the first car and locomotive, having the engine and tender brakes cut out.

2. These triple valves should apply in service when the brake-pipe pressure is reduced by direct discharge to the atmosphere through an orifice which will reduce brake-pipe pressure from 70 to 60 pounds, in 16 to 18 seconds, with brake valve and triple valves on locomotive and first brake cut out.

3. In preparing for this test insert the required disk in union shown on Figure 302 with all cocks closed, after which open cock $\mathrm{C}$ and start test by opening cock $\mathrm{B}$.

Section B.-(Graduating test.) 1. Three valves, selected at random, shall be taken for this test and each tried separately. They will be tested on the first brake of the rack, using the brake pipe only of
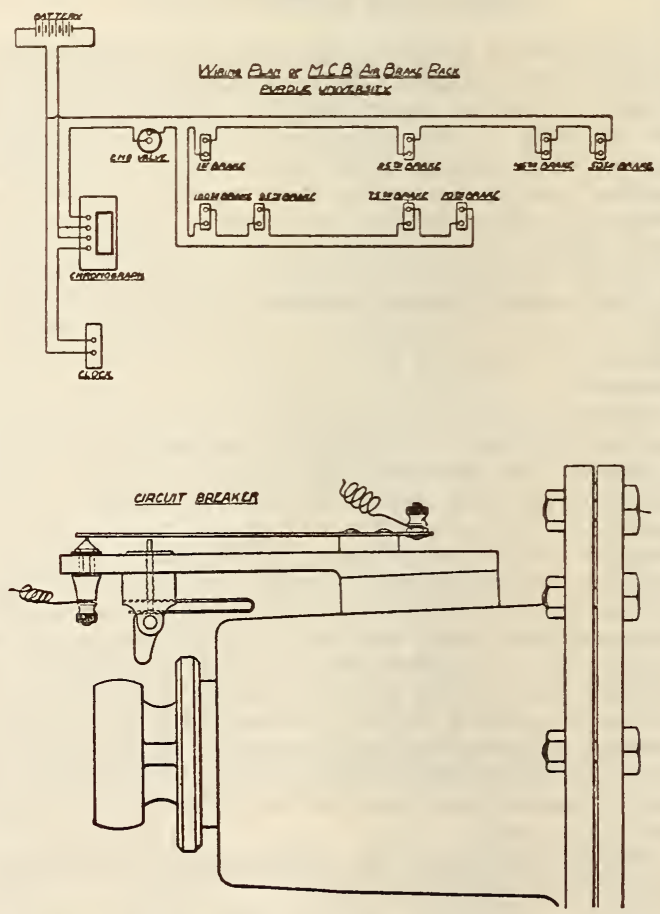

Figure 301.-Wiring plan of M. C. B. air brake rack

the first car and locomotive having the engine and tender brakes cut out.

2. The first admission to the cylinder should be made with a reduction of brake-pipe pressure not exceeding 5 pounds; each succeeding reduction 
should reduce the pressure in the auxiliary reservoir not to exceed 3 pounds, until equalization takes place. The pressure in the brake pipe should not be more than 3 pounds lower than the equalized pressure in the brake cylinder and reservoir at equalization.

Section C.-(Holding test.) Three valves, selected at random, will be taken for this test and each tried separately on the first brake on the rack, using the brake pipe only of the locomotive and the first car, having the triple valves cut out on engine and tender. The one brake will be applied, admitting as nearly as may be 15 pounds, into the brake clyinder following a service application. Record of pressures in the auxiliary reservoir clyinder and brake pipe will be taken as follows: First, at completion of application; second, in 5 minutes; third, in 10 minutes; and fourth, in 15 minutes.

In this test, when a constant brake-pipe pressure is maintained, the brake-cylinder pressure must not be increased more than 5 pounds in five minutes.

Section D.-(Release test.) Three triple valves, selected at random, shall be taken for this test and each tried separately. They will be tried on the first brake of the rack, using the brake pipe only of the first car and locomotive having the engine and tender brakes cut out. When the triple goes to normal release position it must exhaust the air from the brake cylinder from 50 to 0 pounds in not more than 15 seconds.

When the triple goes to retarded-release position it must exhaust the air from the brake cylinder from 50 pounds to 0 pounds in not more than 40 seconds.

No. 3.-Emergency Application Tests

(To determine sensitiveness to quick action)

Three triple valves, selected at random, shall be taken for this test and tried separately on the first brake of the rack. During this test the locomotive and tender triples are to be cut out.

Section $A$.- These triple valves must give a quickaction application when the brake-pipe pressure is reduced by direct discharge to the atmosphere through disk with a $14 / 64$-inch orifice.

Section B.-These triple valves must not give a quick action application when the brake-pipe pressure is reduced by direct discharge to the atmosphere through a disk with a $1 \%{ }_{64}$-inch orifice.

Section C.- (Holding test.) Three triple valves, selected at random, shall be taken for this test and tried separately on the first brake on the rack.

The brake will be applied in quick action by moving the brake-valve handle to emergency position, where it must remain until completion of test for the purpose of insuring the discharge of all brakepipe pressure. Record of pressure in auxiliary reservoir and brake cylinder will be taken as follows: First, at completion of application; second in 5 minutes; third, in 10 minutes; and fourth, in 15 minutes.

In this test, the auxiliary reservoir and brakecylinder pressure must not show a reduction of more than 5 pounds in five minutes.

\section{RACK TESTS}

No. 4.-Service Application Tests

Section A-(Service equalization.) With a service reduction of 25 pounds from brake-pipe pressure, a brake-cylinder pressure of not less than 48 pounds, nor more than 52 pounds, must be obtained.

Section B.-(Graduating test.) 1. A reduction of 5 pounds in brake-pipe pressure should apply lightly the 100 brakes. However, the brake-cylinder pressure may not be sufficient to show on all test gages.

2. A further reduction of 4 pounds to 6 pounds should increase the cylinder pressure of all brakes.
3. A further reduction, making a total of 25 pounds, should equalize the pressure between the auxiliary reservoirs and brake cylinders.

Section C.-(Service application time.) Brakes will be applied by reducing brake-pipe pressure 10 pounds.

There shall not be more than 25 seconds difference in the time of obtaining 10 pounds pressure in the cylinders of the first and one-hundredth brakes.

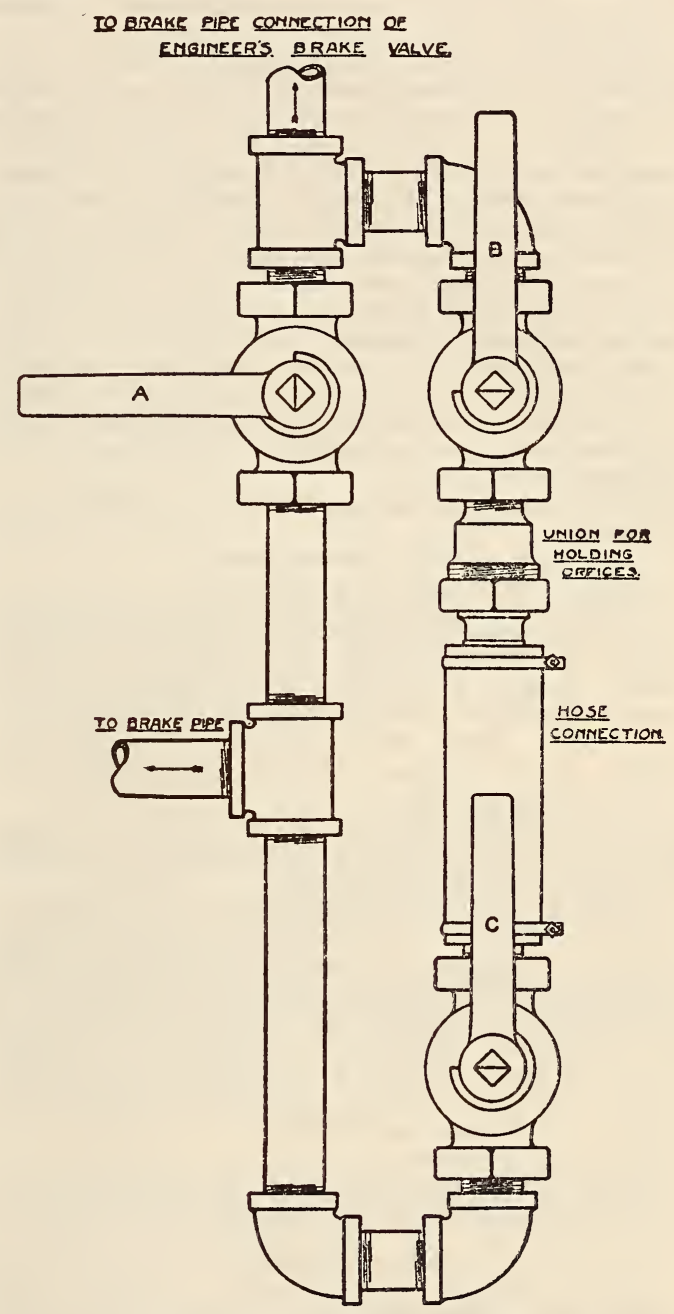

Figure 302.-Triple valve

\section{No. 5.-Emergency Application Tests}

Section A.-(Quick action, time, and pressure.) The one-hundredth brake must be applied with at least 45 pounds pressure in $61 / 4$ seconds from the movement of the brake-valve handle to emergency position and at least 55 pounds in seven seconds. The final maximum pressure in this test must not be less than 15 per cent nor more than 20 per cent above the pressure given by the same brake in full service application.

This test will also be made to determine that quick action is obtained with: First, 4 inches piston travel; and second, 12 inches piston travel.

NoтE.-The object of this test is to secure, as nearly as possible, uniformity of pressures in brake cylinders in an emergency application and uniformity of time required to obtain the pressures; to secure a minimum length of stop and a minimum of shock, and of trains parting. 
Section B.-(To determine whether quick action will follow a service application.) Using the 100 brakes, make a service reduction such as will give 20 pounds cylinder pressure on the first brake. Then place the brake-valve handle in emergency position, which should cause quick-action operation of all triple valves.

The pressure in the first cylinder will be increased or decreased by steps of about 5 pounds until the point at which quick action commences or ceases is determined.

Section C.-(Quick-action jumping test.) With brakes Nos. 1, 2, and 3 cut out, quick action should be obtained with the remainder of the brakes by an emergency reduction, and the time, from the movement of the brake-valve handle to emergency position to obtain 45 and 55 pounds cylinder pressure on the one-hundredth brake, should not be increased more than one second over that required to obtain the same pressures with all brakes cut in.

This test should be repeated with groups of three brakes cut out, consisting of Nos. 2-3-4, 3-4-5, $4-5-6$, and 5-6-7, and the time from the movement of the brake-valve handle to emergency position to obtain 45 and 55 pounds cylinder pressure in the one-hundredth brake should be the same as with all brakes cut in.

These tests will also be made with piston travel of 4 inches.

No. 6.-Folding Tests

Section A.-(Following a service application.) The 100 brakes will be applied, admitting, as nearly as may be, 15 pounds into the cylinder of the first brake. Record of pressures in the auxiliary reservoirs and cylinders will be taken at all record points as follows: First, at completion of application; second, in 5 minutes; third, in 10 minutes; and fourth, in 15 minutes.

In this test any increase of brake-cylinder pressure should be in proportion to the reduction in brakepipe pressure due to leakage.

Section B.- (Following a quick-action application.) The 100 brakes will be applied in quick action by placing the brake-valve handle in emergency position, where it will be left until completion of test, for the purpose of insuring the discharge of all brakepipe pressure. Record of pressures in auxiliary reservoirs and cylinders will be taken at all record points as follows: First, at completion of application; second, in 5 minutes; third, in 10 minutes; and fourth, in 15 minutes.

The results of this test must not indicate an excessive amount of back leakage into brake pipe.

\section{No. 7., Release Tests}

Section A.-(Release time.) The 100 brakes shall be applied with an 18-pound service reduction of brake-pipe pressure and brake valve, then placed in release position. Time will be taken from the movement of the brake valve into release position until pressure is reduced to 5 pounds in the cylinder of the first brake.

The pressure in the cylinder of the first brake should not reduce to 5 pounds in less than 18 seconds nor more than 25 seconds.

Note.-Main reservoir pressure must be 110 pounds at time of release.

\section{AMERICAN RAILWAY ASSOCIATION MECHANICAL DIVISION, AUXILIARY RESERVOIR RELEASE VALVE, RECOM- MENDED PRACTICE, 1929}

\section{RELEASE VALVE}
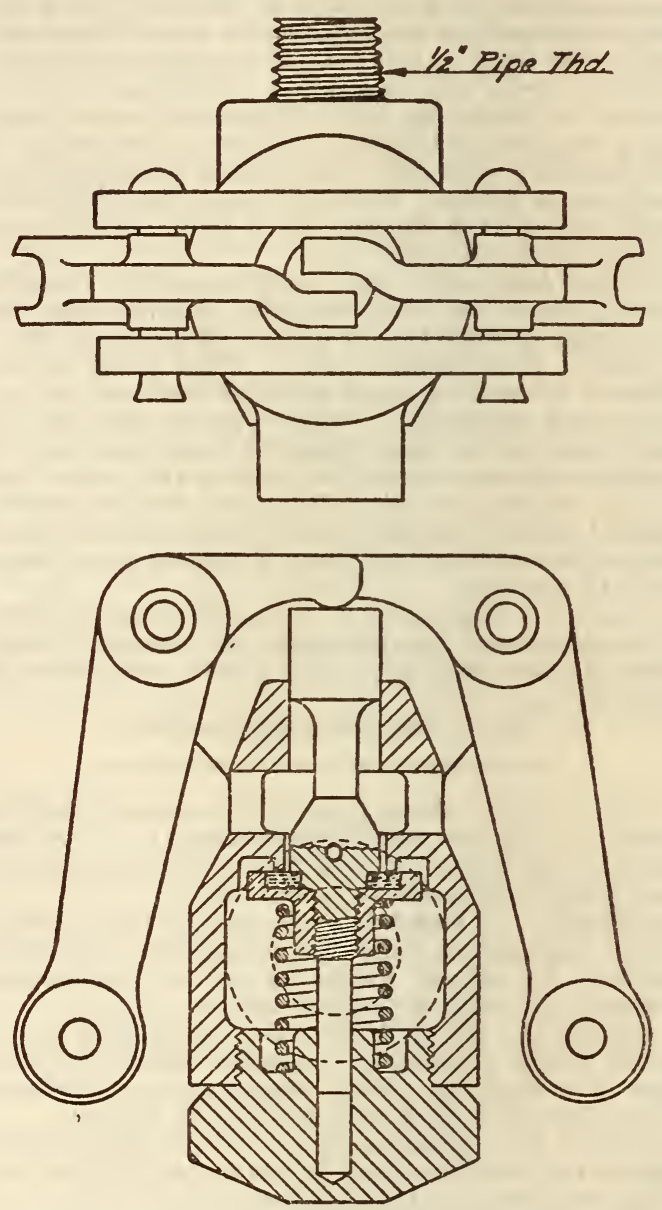

FIgURE 303. - Auxiliary reservoir release valve

AMERICAN RAILWAY ENGINEERING ASSOCIATION, WATER SERVICE AND SANITATION, 1929 MANUAL OF THE ASSOCIATION

\section{HYDRANTS AND VALVES}

(The 1916 specifications for hydrants and valves of the American Water Works Association have been adopted by this association and reprinted in its manual. For text of specifications see A. W. W. A. below.) 
AMERICAN SOCIETY FOR TESTING MATERIALS, STANDARD SPECIFICATIONS FOR CARBON-STEEL CASTINGS FOR VALVES, FLANGES, AND FITTINGS FOR HIGH-TEMPERATURE SERVICE, DESIGNATION A95-29, 1929

\section{CASTINGS FOR HIGH TEMPERATURES}

(See 611.41, p. 709.)

AMERICAN SOCIETY FOR TESTING MATERIALS, STANDARD SPECIFICATIONS FOR GRAY-IRON CASTINGS FOR VALVES, FLANGES, AND PIPE FITTINGS, DESIGNATION A126-30, 1930

\section{GRAY-IRON CASTINGS}

(See 611,11, p. 637.)

AMERICAN WATER WORKS ASSOCIATION, STANDARD SPECIFICATIONS FOR HYDRANTS AND VALVES, JUNE 9, 1916

\section{HYDRANTS}

1. Size.-The size of hydrant shall be designated by nominal diameter of the valve opening, which must be at least 4 inches for hydrants having two $2 \frac{1}{2}$-inch hose nozzles; 5 inches for hydrants having three $2 \frac{1}{2}$-inch nozzles; and 6 inches for hydrants having four 21/2-inch nozzles; and shall be classed as 1-way, 2-way, 3-way, or 4-way, etc., according to the number of $2 \frac{1}{2-i n c h}$ hose outlets for which they are designed.

The net area of the hydrant at the smallest part, when the valve is wide open, must not be less than 120 per cent that of the valve opening.

All hydrants must be fitted with bell ends to fit standard cast-iron pipe, or if flanged they must be fitted with flanges of the standard dimensions corresponding to the pressure under which they are to be used; connecting pipe or flange from main to hydrant in no case to be less in diameter than the valve opening. (The standards referred to are those adopted or that may be adopted by this association.)

2. General design.-(These design requirements are substantially the same as those of the Associated Factory Mutual Fire Insurance Companies' specifications for hydrants (see below, sec. 38 of A. F. M. $\mathrm{F}$. I. C. specifications) with the following exceptions:)

With a 5 -foot hydrant discharging 250 gallons per minute through each $21 / 2$-inch outlet, the total friction loss of the hydrant must not exceed 2 pounds for 2-way, 3 pounds for 3-way, and 4 pounds for 4-way hydrants.

Hydrants must be so designed, particularly as regards the pitch of the thread of the operating stem, so that when properly operated, the water hammer will not be caused which will give an increased pressure to exceed the working pressure when such pressure is over 60 pounds, nor increase the pressure more than 60 pounds when operated under less working pressure than 60 pounds.

(The A. W. W. A. specification contains no safety factor requirement on design for pressure as is given in the A. F. M. F. I. C. specification.)

3. Material.-(This specification contains the same requirements for material as given in the A. F. M. F. I. C. specification for hydrants given below, secs. 39 and 40 , with the following exceptions. This specification requires that the cast iron have a tonsile strength of 22,000 pounds per square inch instead of 21,000 ; that the specimen bars be loaded with 2,200 pounds and show a deflection of not less than 0.35 inch instead of the A. F. M. F. I. C. values of 1,900 and 0.30 , respectively; and that wrought iron have a tensile strength of 45,000 pounds per square inch instead of 48,000. Under composition metal the A. W. W. A. specification contains no requirements on elongation and no special strength requirements for inside hose valve stems.)

4. Hose nipples and valves.-Hose nipples must be of bronze or suitable noncorrodible metal, either threaded with a fine thread into the hydrants and securely pinned in place or carefully locked and calked in place.

Hose threads on all hydrants to be installed in any given community must of necessity be interchangeable with those already in service, but where practicable threads should conform to the national standard.

5. Hydrant seat and gate.-(This section is the same as the requirements under hydrant valve, sec. 42 , of the A. F. M. F. I. C. specification for hydrants given below.)

6. Drip valve.-(Requirements the same as in A. F. M. F. I. C. specification given below, sec. 43.)

7. Operating parts.-The operating threads of the hydrant must be so arranged as to do away with the working of any iron or steel. Either the operating screw or the operating nut must be made of noncorrodible metal and must be sufficiently strong to perform the work for which it is intended.

(The requirements for top nut are the same as in A. F. M. F. I. C. specifications below.)

8. Stuffing box and gland.-

9. Hydrant top.-

10. Hose cap.-

12. Testing.-

(The above four sections are the same as the equivalent sections in the A. F. M. F. I. C. specifications for hydrants given below except for the size of the lettering on hydrant tops which is required by A. W. W. A. to be one-half inch high and oneeighth inch in relief.)

11. Markings. - The hydrant must be marked with the name or particular mark of the manufacturer. All letters and figures must be cast on the hydrant barrel above the ground line.

\section{VALVES}

Castings.-(Requirements on general quality of castings and on dimensions and testing of test bars of cast iron are substantially the same as given in the specifications of the Associated Factory Mutual Fire Insurance Companies for outside-screw-andyoke valves, par. $3, b$, below, with the following exceptions. This specification requires that the cast iron have a tensile strength of 22,000 pounds per square inch instead of 21,000 , and that the specimen bars be loaded with 2,200 pounds and show a deflection of not less than 0.35 inch instead of the A. F. M. F. I. C. values of 1,900 and 0.30 , respectively.)

Name.-Each valve shall have the maker's name cast upon it.

Wrought iron.-All wrought iron used shall be of the best quality or refined iron of a tensile strength of at least 45,000 pounds per square inch.

Composition metals.-All composition metals to be of the best quality, and except for stems, to have a tensile strength of not less than 30,000 pounds per square inch, with 5 per cent elongation in eight diameters and 5 per cent reduction of area at breaking point.

Face joints. - All joints shall be faced true and smooth so as to make a perfectly water-tight joint when suitable gaskets are used. 
Fitting and interchangeable parts.-The fitting of all parts must be such as to make perfect joints and all parts of the valves of the same make and the same size shall be interchangeable. Valves to open as specified by the engineer.

Bolts and nuts.-All bolts and nuts in valves to be made from the best quality of double refined wrought iron or steel. Heads, nuts, and threads are to be of standard size.

Kind of valves.-Valves shall be fully mounted with bronze or suitable noncorrodible metal, and be either of the double disk or made-up gate type with bronze or suitable noncorrodible metal mounted wedging devices or have wedge-shaped gates with double faces and seats, designed to work equally well with pressure on either side of the gate. The gates (or disks) shall be of cast iron with bronze or suitable noncorrodible metal faces. These faces shall be machined, dovetailed, and driven into corresponding machined grooves in gates (or disks) or riveted on with bronze or suitable noncorrodible metal rivets. The seats for composition rings in body of valve shall be turned and threaded before rings are screwed in.

Seat and gate rings. - Both seat rings and gate (or disk) rings shall have smooth and true faces and make a perfectly water-tight joint. Valves shall have hub ends suitable for laying with classes $B$ and $\mathbf{C}$ of American Water Works Association standard pipe. All valves 24 inches in diameter and larger shall be geared.

$B y$-passes. - Where by-passes are required they shall, unless otherwise specified, be of the following sizes:

\begin{tabular}{|c|c||c|c|}
\hline $\begin{array}{c}\text { Size of } \\
\text { valve }\end{array}$ & By-pass & $\begin{array}{c}\text { Size of } \\
\text { valve }\end{array}$ & By-pass \\
\hline Inches & Inches & Inches & Inches \\
16 & 3 & 30 & 4 \\
18 & 3 & 36 & 6 \\
20 & 3 & 42 & 6 \\
24 & 4 & 48 & 8 \\
\hline
\end{tabular}

Weight.-Valves without by-passes shall be approximately not less than the following weights for the respective sizes:

\begin{tabular}{|c|c||c|c|}
\hline $\begin{array}{c}\text { Size of } \\
\text { valve }\end{array}$ & Weight & $\begin{array}{c}\text { Size of } \\
\text { valve }\end{array}$ & Weight \\
\hline Inches & Pounds & $\begin{array}{c}\text { Inches } \\
18\end{array}$ & Pounds \\
3 & 67 & 1,290 \\
4 & 85 & 20 & 1,700 \\
6 & 180 & 24 & 2,750 \\
8 & 255 & 30 & 5,200 \\
10 & 400 & 36 & 8,500 \\
12 & 500 & 42 & 12,000 \\
14 & 780 & 48 & 18,000 \\
16 & 900 & & \\
\hline
\end{tabular}

Valve stems.-Valve stems shall be made of solid brass or suitable noncorrodible metal, free from defects, and shall have a tensile strength of not less than 45,000 pounds per square inch.

Threads on the stems are to be square, acme, or $1 / 2 \mathrm{~V}$, and cut in most perfect manner, so as to work true and smooth and in perfect line throughout the lift of the valve.
Valve stems at the bottom or base of the thread shall not be less than the following sizes in diameter:

\begin{tabular}{|c|c|c|c|}
\hline $\begin{array}{c}\text { Size of } \\
\text { valve }\end{array}$ & $\begin{array}{c}\text { Diameter } \\
\text { stem }\end{array}$ & $\begin{array}{l}\text { Size of } \\
\text { valve }\end{array}$ & $\begin{array}{c}\text { Diameter } \\
\text { stem }\end{array}$ \\
\hline $\begin{array}{c}\text { Inches } \\
3 \\
4 \\
5 \\
6 \\
7 \\
8 \\
9 \\
10 \\
12\end{array}$ & $\begin{array}{l}\text { Inches } \\
4564 \\
8564 \\
55 \% 4 \\
1 \\
1 \\
1 \\
1864 \\
1864 \\
11364\end{array}$ & $\begin{array}{c}\text { Inches } \\
14 \\
16 \\
18 \\
20 \\
22 \\
24 \\
30 \\
36 \\
42 \\
48\end{array}$ & $\begin{array}{r}\text { Inches } \\
11264 \\
12864 \\
12864 \\
14864 \\
14864 \\
10364 \\
21364 \\
2^{3} 364 \\
2^{4864} \\
3^{3} 364\end{array}$ \\
\hline
\end{tabular}

The wrench nut on stem shall be 2 inches square with arrow cast on showing direction which valve is to turn to open.

Painting.-All iron work, after being thoroughly cleaned, to be painted throughout with asphaltum varnish, or suitable paint, or dipped in suitable coating material.

Testing. - Valves must be tested for leakage and distortion as follows: On double disk or made-up gate type, the body of the valve shall be drilled and tapped with a hole for pipe and a removable plug inserted, through this hole a hydraulic pressure of 300 pounds per square inch shall be applied; the wedge-shaped gate type by an hydraulic pressure of 300 pounds per square inch applied, first between one end and the gate, second between the opposite end and the gate, and third in the bonnet with gate open.

\section{ASSOCIATED FACTORY MUTUAL FIRE IN. SURANCE COMPANIES, RUBBER FAC- INGS FOR DRY PIPE VALVES AND CHECK VALVES, 1923}

\section{VALVE FACINGS}

\section{SERVICE REQUIREMENTS}

1. Rubber must not adhere to metal seat rings.

2. Rubber must be tough to withstand cutting and abrasion.

\section{CHEMTCAL TESTS}

1. Gum.-The facings shall be made from a compound containing not less than 40 per cent of washed and dried fine Para rubber or its equivalent and shall have all the characteristics of such a compound. They must not contain any kind of rubber substitute, old vulcanized or reclaimed rubber, or any substance injurious to rubber.

2. Acetone extract.-The organic acetone extract shall not exceed 5 per cent of the gum as found by chemical analysis.

3. Free sulphur.-The free sulphur shall not exceed 3 per cent of the gum as found by chemical analysis.

4. Alcoholic potash ether extract.-The saponifiable matter extracted by alcoholic potash after the acetone extraction has been made shall not exceed 3 per cent of the gum as found by chemical analysis. 
5. Total sulphur.-The total sulphur, exclusive of that which may be contained in the mineral matter as barytes, shall not exceed 10 per cent of the gum as found by chemical analysis.

\section{PHYSICAL TESTS}

All physical tests shall be performed upon samples one-half inch wide cut from the facings.

(The physical tests include plasticity on a sheet or section one-eighth inch thick, tensile strength not less than 1,800, and elongation at a uniform rate of 20 inches per minute.)

\section{MARKING}

The facings must be molded with the name of the manufacturer, the year of manufacture, and the identification mark in 1/4-inch letters raised on a depressed surface on one side of the facing and so placed that no portion of them shall extend more than three-eighths inch from the inside edge.

NoTE.-Manufacturers in submitting samples for approval should submit one lacing and a test sheet one-eighth inch thick. The test sheet should be at least 7 by 6 inches, from which test pieces for tensile-strength and permanent-set determinations may be cut.

\section{ASSOCIATED FACTORY MUTUAL FIRE} INSURANCE COMPANIES, VALVES, INDICATOR POSTS, AND HYDRANTS, JANUARY, 1930

\section{OUTSIDE-SCREW-AND-YOKE VALVES}

1. Size.-(a) The following will be recognized as standard sizes in fire protection work: $3 / 4,1,11 / 4$, $11 / 2,2,21 / 2,3,3 \frac{1}{2}, 4,5,6,7,8,10,12$, and 14 inches.

Larger sizes may occasionally be required, and the smaller ones of this type will probably have only limited use.

(b) Any given size of gate valve shall, when wide open, give an unobstructed waterway the nominal size of the pipe.

2. Type. $-(a)$ Shall be of the straightway type. Either solid or split wedge or built-up parallel seat gate is acceptable.

(b) Valves shall be designed to withstand safely \& working pressure of 150 pounds, with a factor of safety of at least 5 at the working pressure, for sizes less than 8 inches, and of at least 4 for sizes 8 inches and larger. Where the working pressure is greater than 150 pounds a heavier valve shall be used.

For example, a valve less than 8 inches in size whose working pressure is 150 pounds shall resist a pressure of 750 pounds before breaking and valves 8 inches or larger designed for the same pressure shall resist a pressure of 600 pounds. This test shall not be made in a press. In the average protective equipment it is expected that in time fire about 100 pounds will be carried, this pressure coming from pumps if gravity supplies do not furnish it. When pumps are runalways desirable to have some margin; therefore, it is required that the apparatus withstand a somewhat higher working pressure and 50 pounds is specified, as this standard practice.

Manufacturers shall determine that valves will withstand the high-pressure tests before submitting samples for approval. Valves so tested may have been dangerously stressed, and therefore should be destroyed, submitting for approval only untested samples.

3. Body and bonnet.-(a) The body and bonnet of valves 2 inches and smaller shall be made of bronze or other suitable noncorrodible metal.

(b) For larger sizes the body and bonnet shall be made of cast iron or some other hard, close metal so distributed as to insure sound castings and freedom from shrink cracks.

The metal for all iron castings must be of good quality and of such character as shall make the metal strong, tough, and of even grain, and soft enough satisfactorily to admit of drilling and machining. The metal must be made without any admixture of cinder iron or other inferior metal, and must be remelted in a cupola or air furnace. The castings must be smooth, free from scale, lumps, blisters, sand holes, and defects of every nature which unfit them for the use for which they are intended. No plugging or filling will be allowed.

Specimen bars of the metal used, each being 26 inches long by 2 inches wide and 1 inch thick must be made without charge as often as the inspection department may direct, and in default of definite instructions, the manufacturer must make and test at least one bar from each heat or run of metal. The bars when placed flatwise upon supports 24 inches apart, and loaded in the center, must support a load of 1,900 pounds, and show a deflection of not less than 0.30 of an inch before breaking; or if preferred, tensile bars may be made which must show a breaking point of not less than 21,000 pounds per square inch, bars to be cast as nearly as possible to the dimensions without finishing, but corrections may be made by the inspection department for variations in width and thickness, and the corrected result must conform to the requirements.

For floor-controlling valves a 11/4-inch drain outlet may be provided in the valve body, the nipple to be tapped into a boss cast on body.

(c) The guides shall be cast integral with the body and shall be of unequal widith if the gate can not be safely reversed.

All valves with built-up gates and the best solid wedge gates are reversible. In a few designs, however, the gates are not reversible, and the guides shall be of unequal width to prevent assembling the valve with the gate in the wrong position.

\section{TABLE 1}

[150 pounds per square inch]

\begin{tabular}{|c|c|c|c|c|c|}
\hline $\begin{array}{c}\text { Size of } \\
\text { pipe }\end{array}$ & $\begin{array}{c}\text { Dia- } \\
\text { meter } \\
\text { of } \\
\text { flange }\end{array}$ & $\begin{array}{c}\text { Flange } \\
\text { thick- } \\
\text { ness at } \\
\text { edge }\end{array}$ & $\begin{array}{c}\text { Dia- } \\
\text { meter } \\
\text { of bolt } \\
\text { circle }\end{array}$ & $\begin{array}{c}\text { Num- } \\
\text { ber of } \\
\text { bolts }\end{array}$ & $\begin{array}{c}\text { Size of } \\
\text { bolts }\end{array}$ \\
\hline Inches & Inches & Inch & Inches & & Inch \\
$3 / 4$ & $31 / 2$ & $11 / 32$ & $21 / 2$ & 4 & $3 / 8$ \\
1 & 4 & $3 / 8$ & 3 & 4 & $7 / 16$ \\
$11 / 4$ & $43 / 2$ & $13 / 32$ & $33 / 8$ & 4 & $7 / 16$ \\
$11 / 2$ & 5 & $7 / 16$ & 378 & 4 & 52 \\
2 & 6 & 112 & $43 / 4$ & 4 & $5 / 8$ \\
\hline
\end{tabular}

(For 21/2-inch size and larger refer to A. S. M. E. standard for cast-iron pipe flanges and flanged fittings, serial B16a-1928, Table No. 2. See 607.14, p. 385.)

(d) Valves may have threaded, flanged, or bell ends.

Flanges are preferred to screw ends for sizes $31 / 2$ inches and larger, as the valves can be more readily removed for repairs.

(e) Valves with threaded ends shall have American standard threads. (See United States Government National Screw Thread Commission, 607.0, p. 365.)

(f) For flanges the standard sizes and bolt layouts shall be used as per Table 1 . Where working pressure is from 151 to 250 pounds, the standard for heavy flanges shall be used as per Table 2 . Holes are not to be drilled on the center line, but symmetrically each side of it.

(g) Bell ends shall fit American Water Works Association standard pipe. (For dimensions, see A. W. W. A. under 607.12 , p. 379.) 
TABLE 2

(250 pounds per square inch)

\begin{tabular}{|c|c|c|c|c|c|}
\hline $\begin{array}{c}\text { Size of } \\
\text { pipe }\end{array}$ & $\begin{array}{l}\text { Diam- } \\
\text { eter of } \\
\text { flange }\end{array}$ & $\begin{array}{c}\text { Flange } \\
\text { thick- } \\
\text { ness at } \\
\text { edge }\end{array}$ & $\begin{array}{l}\text { Diam- } \\
\text { eter of } \\
\text { bolt } \\
\text { circle }\end{array}$ & $\begin{array}{l}\text { Num- } \\
\text { ber of } \\
\text { bolts }\end{array}$ & $\begin{array}{l}\text { Size of } \\
\text { bolts }\end{array}$ \\
\hline $\begin{array}{c}\text { Inches } \\
3 / 4 \\
1 \\
11 / 4 \\
11 / 2 \\
2\end{array}$ & $\begin{array}{c}\text { Inches } \\
4 \\
41 / 2 \\
5 \\
6 \\
61 / 2\end{array}$ & $\begin{array}{l}\text { Inch } \\
7 / 16 \\
1 / 2 \\
17 / 32 \\
916 \\
5 / 8\end{array}$ & $\begin{array}{c}\text { Inches } \\
27 / 8 \\
31 / 4 \\
33 / 4 \\
432 \\
5\end{array}$ & $\begin{array}{l}4 \\
4 \\
4 \\
4 \\
4 \\
4\end{array}$ & $\begin{array}{c}\text { Inch } \\
1 / 2 \\
1 / 2 \\
1 / 2 \\
5 / 8 \\
5 / 8\end{array}$ \\
\hline
\end{tabular}

(For $21 / 2$-inch size and larger refer to A. S. M. E. standard for cast-iron pipe flanges and flanged fittings, serial B16a-1928, Table No. 2. See 607.14, p. 385.)

(h) The body and bonnet shall be bolted together in $2 \frac{1}{2}$-inch valves and larger and the bolting of the bonnet shall be of such strength that the maximum stress at the bottom of the screw threads will not exceed 7,500 pounds per square inch for a water pressure of 150 pounds per square inch computed on an area out to center line of bolts.

(i) The yoke shall be of such proportions and secured to the bonnet in such a manner that it will be as strong relatively as other parts of the valve. The yoke shall be bolted on in valves larger than 4 inches. The design shall be such that the hand can

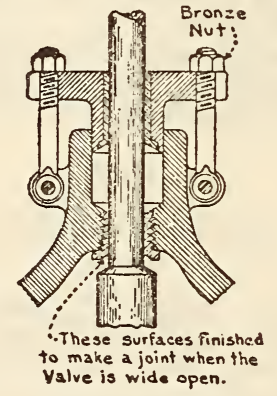

FIGURE 304

not be jammed between the yoke and the handwheel.

(j) Bolts, cap screws, or studs for attaching the yoke to the bonnet shall be at least one-half inch in diameter on valves larger than 4 inches.

Through bolts are preterred, as they are more readily replaced if broken off.

(k) Valves shall be plainly marked with the name or trade-mark of the manufacturer, the size of the valve, the year of manufacture, and the pressure for which it is designed. Valves designed for 125 pounds steam pressure shall be considered acceptable for 150 pounds working water pressure. Some identification mark satisfactory to the underwriters having jurisdiction shall be used only on approved valves so that these may be readily distinguished from those that are not approved. All letters and figures shall be placed upon the body or bonnet of the valve. They may be arranged part on body and part on bonnet if desired, with the exception that on iron valves the identification mark must be placed on the bonnet. The marking shall be easily readable and as large as possible, except that in no case need the letters or figures be higher than 1 inch, or raised more than one-eighth inch on the casting. Engraved letters may be used on bronze valves.

In Factory Mutual work this identification mark must consist of the letters FM between two triangles as follows: $4 \mathrm{FM}$

4. Gates.-(a) The gates for valves 2 inches and smaller shall be entirely of bronze or other suitable noncorrodible material. For larger sizes they may be of cast iron fitted with bronze rings.

(b) The surface of the iron gate inclosed by the bronze ring shall be so constructed as to clear the seat ring in all positions. The central part of the bronze gates larger than 1 inch shall be recessed.

(c) In built-up iron gates at least one wedge surface shall be of bronze or bronze mounted; the other may be of cast iron if the wedge parts fit loosely in the gate, and if not both shall be made of bronze or bronze mounted. In built-up gates for iron-bodied valves, guides or links must be provided to prevent the brass gate rings rubbing on the cast-iron body or bonnet during operation.

Experience has shown that bronze and cast iron work well together as wedge surfaces and do not cause trouble by rusting of the iron. Separate gate parts made of cast iron are liable to rust and become cemented together if not fitted loosely. Bronze is therefore required where close fitting is necessary.

5. Gate and seat rings.-(a) All iron bodies shall be fitted with bronze seat rings.

(b) All gate and seat rings shall have machined faces.

(c) The rings shall be held in place by a screw thread or by cold swaging, the former being preferred.

6. Stem.-(a) Stems shall be made of rolled bronze having a tensile strength of not less than 32,000 pounds per square inch, and preferably not less than 50,000 pounds.

(b) The threads shall be acme, modified acme, half $\mathbf{V}$, or square.

An acme standard thread is preferred. (For thread see United States Government National Screw Thread Commission., under 608.0, p. 529.)

(c) The stem and attached parts shall be so designed that all parts shall be equally strong. The connection between the stem and the gate shall be so made that they can not separate in use, and so that the stem will not be cramped when the gate seats.

(d) Valves $2 \frac{1}{2}$ inches and larger shall be so designed that when operated under severe conditions, such as forcing the gate against its seat, no part will break or the stem cripple before the failure of the yoke cap or stem nut.

It is of special importance that the stem be reliably connected to the gate, as any failure at this point would make it impossible to open the valve.1

(e) The twisting momen' in foot-pounds required on the handwheel to produce this failure shall not be less than that given in the following table:

\begin{tabular}{l|l|l|l|l|l|l|l|l|l|l|l|l|}
\hline Size of valve (inches) ........ & $21 / 2$ & 3 & $31 / 2$ & 4 & 5 & 6 & 7 & 8 & 10 & 12 & 14 \\
\hline Foot-pounds.............. & 150 & 150 & 200 & 250 & 300 & 300 & 400 & 400 & 600 & 900 & 900 \\
\hline
\end{tabular}

(f) The stem nut shall be of bronze.

(g) For valves 5 inches and larger a bronze washer shall be provided between the yoke and the handwheel, unless the design of the stem nut is such that the yoke and handwheel do not come in contact.

(h) The stem shall be so constructed as to make a joint with the underside of the bushing at the stem opening in the bonnet when the gate is wide open. (See fig. 304.)

This is required in order that the valve may be repacked with water on.

An acceptable construction is to provide a tightly fitted tapered collar on the stem.

In bronze-body valves, 2 inches and smaller, the required joint may be obtained by machining the underside of the bonnet at the stem opening.

(i) The diameters of the unthreaded sections of the stems and of the outside of the threads shall not be less than those given in the following table: 


\begin{tabular}{|c|c|c|c|c|c|c|c|c|c|c|c|c|c|c|c|c|}
\hline Size of valve (inches) & $3 / 4$ & 1 & $11 / 4$ & $11 / 2$ & 2 & $21 / 2$ & 3 & $31 / 2$ & 4 & 5 & 6 & 7 & 8 & 10 & 12 & 14 \\
\hline $\begin{array}{l}\text { Diameter of stem, working pres- } \\
\text { sure not over } 150 \text { pounds (inches)- } \\
\text { Diameter of stem, working pres- } \\
\text { sure } 151 \text { to } 250 \text { pounds (inches)... }\end{array}$ & $\begin{array}{l}716 \\
162\end{array}$ & $\begin{array}{l}7 / 16 \\
1 / 2\end{array}$ & $\begin{array}{l}16 \\
5 / 8\end{array}$ & $\begin{array}{l}1 / 2 \\
5 / 8\end{array}$ & $\begin{array}{l}5 / 8 \\
3 / 4\end{array}$ & $\begin{array}{l}3 / 4 \\
3 / 4\end{array}$ & $1^{3 / 4}$ & $1^{3 / 4}$ & $\begin{array}{l}1 \\
11 / 8\end{array}$ & $\begin{array}{l}11 / 8 \\
11 / 4\end{array}$ & $\begin{array}{l}138 \\
134\end{array}$ & $\begin{array}{l}11 / 4 \\
13 / 8\end{array}$ & $\begin{array}{l}11 / 4 \\
13 / 8\end{array}$ & $\begin{array}{l}13 / 8 \\
11 / 2\end{array}$ & $\begin{array}{l}11 / 2 \\
15 / 8\end{array}$ & \\
\hline
\end{tabular}

7. Stuffing box.-(a) The width of the stuffing boxes between stem and inner wall shall not be less than that given in the following table:

Size of $\nabla a l v e$

(inches)

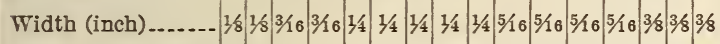

The depth of stuffing box shall be four times the width of the packing space.

The stuffing boxes shall be fully packed with a good packing such as long-fiber hemp saturated in hot tallow or a woven nonmetallic packing.

(b) With cast-iron valves, the opening through the bonnet for the stem shall be bushed with bronze, finished on the underside to make a joint with the stem when the gate is wide open, and the follower or gland shall be either of bronze or bushed with bronze. The bottom of the stuffing box and the end of the gland should be beveled, as per Figure 304 .

The construction shown in the sketch is satisfactory, and any other method of bushing equivalent to this will be acceptable.

(c) The gland shall be retained by bolts or studs and the diameters in $2 \frac{1}{2}$-inch valves and larger shall not be less than those given in the following table:

\begin{tabular}{|c|c|c|c|}
\hline Size of valves (inches) & $21 / 2$ & $3-4$ & $5-14$ \\
\hline Diameter gland bolts (inch). & $3 / 8$ & $1 / 2$ & $5 / 8$ \\
\hline
\end{tabular}

(d) Gland bolts or studs may be of bronze, iron, or steel. The nuts shall always be of bronze.

With bronze nuts such slight rusting of the iron bolts as is liable to occur will not cause the nuts to stick, as might be the case if iron nuts were used. Through bolts are preferred and $T$ bolts may be used if desired.

8. Handwheel.-(a) The handwheel shall be designed so as to furnish a ready grasp for the hands.

(b) Assuming the observer to be looking at the top of the handwheel, then the handwheel shall be turned counterclockwise to open.

(c) An arrow showing the direction to turn the handwheel to open the valve, with the word "OPEN" at the feather end, or in a break in the shaft, shall be cast on the rim of the handwheel, so as to be easily readable.

(d) On valves $2 \frac{1}{2}$ inches and smaller the arrow shall be $1 \frac{1}{4}$ inches long, on valves 3 inches to 5 inches the arrow shall be $2 \frac{1}{2}$ inches long, and on valves 6 inches and over the arrow shall be 4 inches long. If the shaft is broken to admit the word "OPEN" the sum of the parts of the arrow shall be at least three-fourths as long as specified for the unbroken arrow.

(e) The outside diameters of the handwheels shall be not less than those given in the following table:

\begin{tabular}{|c|c|c|c|c|c|c|c|c|c|c|c|c|c|}
\hline ze of valve (inches) ... & $3 / 4$ & & & 22 & $21 / 2$ & & $31 / 2$ & 4 & 5 & & & 8 & 10121 \\
\hline (i) & 3 & 3 & & & & & & & 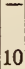 & & & & 16 \\
\hline
\end{tabular}

9. Tests.-(a) All valves shall be tested to twice the working pressure for leakage and distortion before leaving the factory: First, between one end and the gate; second, between the opposite end and the gate; third, in the bonnet with the gate open.

(b) Valves shall occasionally be taken at random from the regular output and tested by removing all of the packing from the stuffing box, opening the gate wide, applying the working pressure and noting the leakage through the stuffing box.

$V$ alves will be considered as passing the tests specified if they show no leakage through body or bonnet, no distortion, and only a slight weeping past the gates and through the stuffing boxes.

\section{INSIDE-SCREW VALVES}

\section{FOR ONDERGROOND WORK}

10. Size.-The following will be recognized as standard sizes in fire-protection work: $4,6,8,10$, 12 , and 14 inches.

Larger sizes may occasionally be required, in which case they should be designed to conform to these specifications as far as they apply.

11. General construction.-(a) Shall conform to the specifications for outside-screw-and-yoke valves as

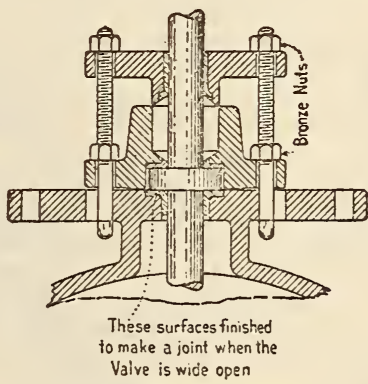

FIGURE 305

far as they apply as given above under sections $1(b) ; 2(a)$ and $(b) ; 3(b),(c),(d),(f),(g),(h)$, and $(k) ; 4(a),(b)$, and $(c) ; 5(a),(b)$, and $(c)$; $6(b),(c),(f)$, and $(i) ; 7(a),(c)$, and $(d) ; 9(a)$ and $(b)$.

(b) Stem shall be of bronze having a tensile strength of not less than 32,000 pounds per square inch and preferably not less than 50,000 pounds.

(c) The threaded stem nut shall be either a solid bronze casting or else bronze cast into a cast-iron member.

(d) When valve is closed the stem shall enter the stem nut a distance equal to at least one and onefourth times the outside diameter of the stem.

(e) The bearing of the stem collar on the bonnet bushing shall be finished to make a joint when the gate is wide open, thereby allowing the valve to be repacked with water on. (See fig. 305.)

(f) The diagonal of the base of the square tapered end of the stem on which the wrench nut fits shall be at least equal to the diameter of the stem.

(g) The valve shall be so designed that when operated under severe conditions, such as forcing the gate against its seat, no part will break before the stem fails. 
(h) The twisting moment in foot-pounds required on the wrench nut to cause the stem to fail shall not be less than that given in the following table:

\begin{tabular}{r|c|c|c|c|c|c|c}
\hline Size of valve (incles) ............... & 4 & 6 & 8 & 10 & 12 & 14 \\
\hline Foot-pounds.................. & 200 & 300 & 400 & 600 & 900 & 900 \\
\hline
\end{tabular}

The twisting moment at which failure occurs is the highest value obtained previous to the first halt or decrease in force required.

(i) The wrench nut shall be $115 / 16$ inches square at the top, 2 inches square at the base, and $13 / 4$ inches high, with a flange base upon which shall be

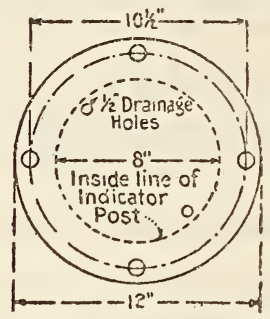

FigURE 306

cast an arrow at least 2 inches long showing the direction of opening and the word "OPEN" in distinct letters at least $1 / 2$ inch high.

(j) The lower part of the stuffing box and opening through the bonnet for the stem shall be bushed with bronze, and the follower or gland must be either of bronze or bronze bushed. The bottom of the stuffing box and the end of the gland should be beveled as per Figure 305.

The construction shown in the sketch is satisfactory, and any method of bushing equivalent to this will be acceptable.

12. Indicator post flange.-(a) All valves for indicator posts shall have a flange cast on the bonnet. For valves larger than 4 inches the flange shall be circular or square, of dimensions shown in Figure 306. The flange shall be at least five-eighths inch thick and be drilled for four 5/8-inch bolts. For smaller valves a smaller flange may be cast on bonnet, and an annular fiange of standard dimensions bolted to it.

(b) At least two holes one-half inch diameter shall be provided in the flange for drainage of the post.

13. Direction of opening.-(a) To open counterclockwise as specified for outside-screw-and-yoke valves, except where existing underground valves in the equipment open to the right, in which case the new valves should turn the same as the old ones for uniformity.

\section{STRAIGHTWAY HOSE VAIVES}

15. Size.-The size of valves for use on fire pumps and hydrants is $2 \frac{1}{2}$ inches, and for standpipes $1,1 \frac{1}{4}, 1 \frac{1}{2}, 2$, and $2 \frac{1}{2}$ inches.

16. General construction.- (a) Shall conform to the specifications given above, as far as they apply, as given in the following sections: $1(b) ; 2(a)$ and $(b) ; 3(a),(c),(d),(e),(f),(h)$, and $(k) ; 4(a)$ and $(b)$; $5(a),(b)$, and $(c) ; 6(b),(c),(f)$, and $(i) ; 8(a)$, (b), $(c),(d)$, and $(e) ; 9(a)$ and $11(b),(d)$, and $(f)$.

Item $3, h$ does not apply to valves having bonnets screwed to body.

(b) Valves for use on fire pumps and standpipes shall be made entirely of bronze or other suitable noncorrodible metal, with the exception of the hand- wheel. In this type of valve the bonnet may be threaded to the body.

(c) The bodies and bonnets of valves for use on hydrants may be made of cast iron bolted together following the requirements of $3, h$. The remainder of the valve with the exception of the handwheel should be of bronze.

(d) In pump and standpipe valves the guides shall be of unequal width so that the gate can not be reversed unless the design and construction are such that when gate is reversed the valve will be tight.

In hydrant valves a single-faced gate may be used.

(e) Pump and standpipe valves shall be fitted at inlet end with "American standard" female pipe thread. (See United States Government National Screw Thread Commission, 607.0, p. 365.)

Hydrant valves shall be designed for attachment to the hydrant barrel by two $3 / 4$-inch tap bolts spaced horizontally $5 \frac{5}{8}$ inches on centers.

$(f)$ The stuffing box shall be of sufficient size to contain at least four rings of packing $1 / 8$ inch square for 1 -inch valves and $3 / 16$ inch square for the other sizes. A follower nut may be used to retain the gland.

(g) In cast-iron valves the entire stuffing box shall be of bronze and the opening through the bonnet shall be bronze bushed.

17. Hose nipples.-(a) Nipples shall be of bronze cast in one piece with the body of bronze valves, and in cast-iron valves they shall be threaded into the body and pinned. A boss shall be provided in bronze valves at the bottom which may be tapped for a $1 / 8$-inch petcock.

(b) Hose threads for $2 \frac{1}{2}$-inch valves must of necessity be interchangcable with those already existing in the ity in each case, but where practicable and not otherwise specified shall conform to the national American standard adopted by the National Fire Protection Association. The essential features of the national American standard thread are a $60^{\circ} \mathrm{V}$ thread, outside diameter on male threads of $33 / 16$ inches, and $71 / 2$ threads per inch.

18. Hose caps.-(a) Hose caps are not necessary with pump and standpipe valves, but shall be provided on hydrant valves. The caps may be of cast iron.

(b) They shall be securely fastened to the hydrant body with a suitable chain made of stock not less than one-eighth inch diameter.

(c) A leather, rubber, or lead washer shall be provided in the hose cap set in a groove to prevent its falling out when the cap is remored.

(d) The hose cap nut shall be of the same size and shape as the stem nut of the hydrant.

\section{ANGLE HOSE VALVES}

19. Size.-The sizes of valves for use on standpipes are $1,11 / 4,1 \frac{1}{2}, 2$, and $21 / 2$ inches.

20. General construction.-(a) Shall conform to the specifications given above, as far as they apply, as given in the following sections: $2(b) ; 3(d),(e)$, and $(k) ; 6(a),(b),(c)$, and $(i) ; 8(a),(b),(c),(d)$, and $(e) ; 9(a)$ and $(b) ; 11(e) ; 16(b),(e),(f)$; $17(a)$ and $(b)$; and $18(a)$.

(b) Valves shall have outlet at right angles to inlet.

(c) The seat shall not extend into the valve beyond line of near side of hose nipple.

$(d)$ The width of the seat ( $A$, fig. 307 ) shall be that given in the following table:

\begin{tabular}{l|r|r|r|r|r}
\hline Size of valve (inches) ............. & 1 & $11 / 4$ & $11 / 2$ & 2 & $21 / 2$ \\
\hline Width of seat (inch) & & & \\
\hline
\end{tabular}


21. Disk.-(a) The lift of the disk shall be at least that given in the following table:

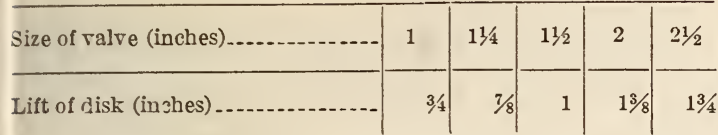

(b) The disk holder shall be free to turn on the stem.

This is necessary in order that the disk may find its seat without any rotary motion that would be liable to injure its face.

(c) When a lock nut is used to secure the disk holder to the stem it shall be pinned to the disk holder.

(d) The disk holder shall contain a suitable nonmetallic naterial having the same dimensic ns as the

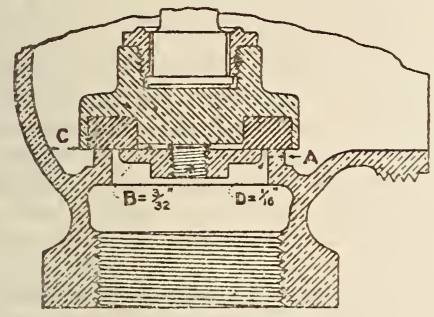

FIGURE 307

standard Jenkins disk (see Table 4), firmly secured and so arranged that it can be easily replaced. The disk holder shall extend over the outside edge of the disk until its edge is flush with the face of the disk. In order to prevent distortion, the support for the inside edge of the disk should extend to within three thirty-seconds inch of its face $(B$, fig. 307$)$.

TABLE 4

\begin{tabular}{|c|c|c|c|}
\hline $\begin{array}{c}\text { Size of } \\
\text { valve }\end{array}$ & $\begin{array}{c}\text { Outside } \\
\text { diameter }\end{array}$ & Inside diameter & $\begin{array}{c}\text { Thick- } \\
\text { ness }\end{array}$ \\
\cline { 2 - 4 } Inches & Inches & Inches & Inch \\
1 & $1^{25} / 64$ & $3 / 4$ by $5 / 8$ & $9 / 32$ \\
$11 / 4$ & $15 / 8$ & $15 / 16$ by $25 / 32$ & $11 / 32$ \\
$11 / 2$ & 2 & $11 / 4$ by $13 / 32$ & $11 / 32$ \\
2 & $219 / 32$ & 11932 by 11532 & $3 / 8$ \\
$21 / 2$ & $313 / 64$ & $23 / 32$ by $1^{3} 1 / 32$ & $15 / 32$ \\
\hline
\end{tabular}

(e) The disk nut or clamping ring shall be pinned in place.

(f) The clearance ( $C$, fig. 307) between the edge of the disk holder and the inside of the body shall not be less than that given in the following table:

\begin{tabular}{l|r|r|r|r|r}
\hline Size of valve (inches) .............. & 1 & $11 / 4$ & $11 / 2$ & 2 & $21 / 2$ \\
\hline $\begin{array}{l}\text { Clearance between disk and body } \\
\text { (inch)_.................... }\end{array}$ & $1 / 8$ & $3 / 16$ & $3 / 16$ & $1 / 4$ & $1 / 4$ \\
\hline
\end{tabular}

(g) The clearance ( $D$, fig. 307$)$ between the inside of the seat and the disk nut or clamping ring shall be one-sixteenth inch.

\section{SWING CHECK VALVES}

\section{REGULAR TYPE}

22. General construction.-(a) Shall conform to the specifications given above, as far as they apply, as given in the following sections: 1 (a) and $(b) ; 2$ $(b) ; 3(a),(b),(d),(e),(f),(g),(h)$, and $(k) ; 4(a)$ and $(b)$; and $5(a)$ and $(b)$.

The more important items of the specifications as applied to a valve are shown in Figure 308.

(b) Iron-body check valves when wide open shall have a waterway equal to the area of the pipe to permit the passing of large foreign bodies, and the toe of the clapper shall be free to raise above the waterway. In bronze valves the toe of the clapper shall raise at least as high as the upper edge of the seat opening. The body shall be as smooth inside as good foundry practice will permit and of such form as to minimize the danger of the clapper be-
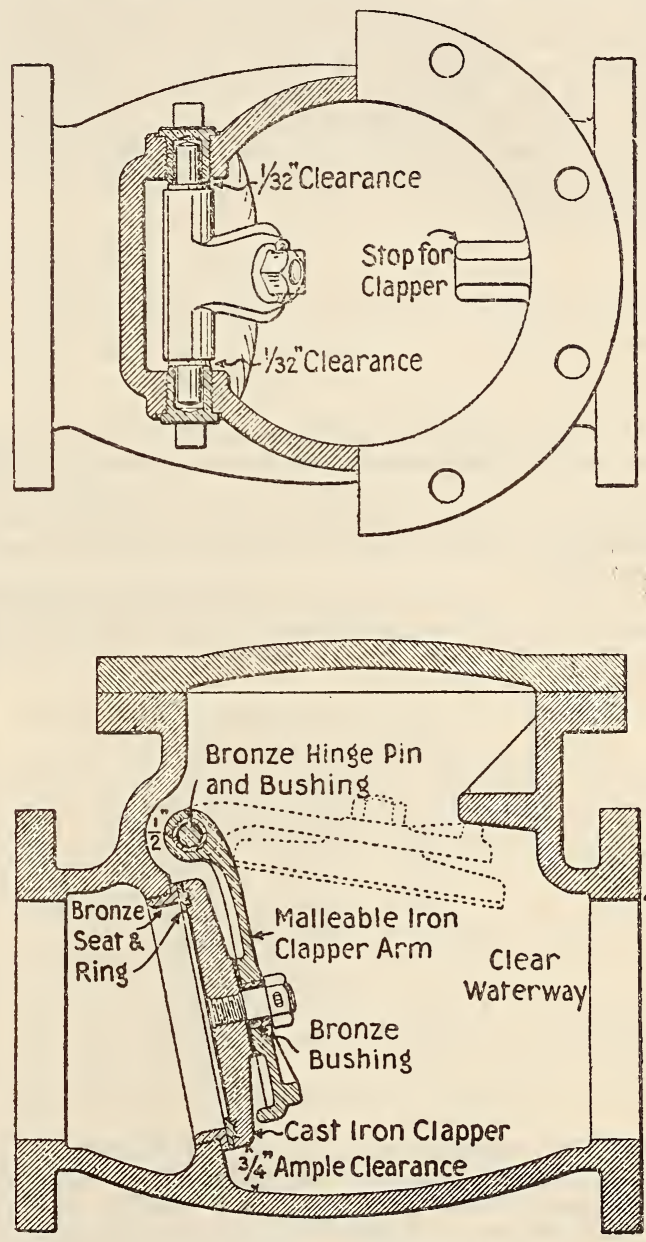

Figure 308.-Swing check valve

coming wedged when open and the chances of sediment lodging against the seat ring.

(c) The seat, clapper, and arm shall be so arranged that the clapper will close by gravity.

(d) The seat ring shall be screwed in position in iron-bodied valves.

(e) All working parts shall be removable through the bonnet opening.

23. Clapper and clapper arm.-(a) The clapper shall be of bronze for valves 2 inches and smaller. For larger valves it may be of cast iron, in which case it shall be provided with a bronze ring or a facing of leather or rubber.

(b) The facing for valves larger than 2 inches shall be protected for two-thirds of its thickness at its outer edge and clamped at least one-half inch at 
its inner edge. It shall be wide enough to overhang the seat at least one-eighth inch on the outside, one-fourth inch on the inside, and shall be backed up its full width by a finished surface on the clapper. The clamping ring shall be of bronze. It shall be turned concentric with clapper stud and come to a stop against a shoulder to prevent clamping too tightly.

(c) The clapper of valves 2 inches and smaller may be faced with a suitable nonmetallic material having the same dimerisions as the standard Jenkins disk (see Table 4) firmly attached, but so that it can be readily replaced. It shall be protected for twothirds its thickness at its outer edge and its inner edge shall be supported for a distance equal to three thirty-seconds inch less than its thickness to prevent distortion. The clearance between the inside of the seat and the clamping ring shall be one-sixteenth inch. The clamping ring shall be turned concentric with the clapper stud. The width of the seat for use with the above facing shall be that given in the following table:

\begin{tabular}{l|c|c|c|c|c}
\hline Size of valve (inches) ............. & $3 / 4$ & 1 & $11 / 4$ & $11 / 2$ & 2 \\
\hline Width of seat (inch) $\ldots$ & $3 / 32$ & $1 / 8$ & $1 / 8$ & $3 / 16$ & $7 / 32$ \\
\hline
\end{tabular}

(d) The clapper shall be secured to its arm by means of a bronze connection in such a manner that the parts can not separate in use, and so that there can be no leakage at this point. Sufficient play shall be allowed so that the clapper will find its seat properly.

(e) The diameter of the flat bearing between clapper and arm and the length of the bearing of the clapper stud in the arm must be not less than as follows:

\begin{tabular}{|c|c|c|}
\hline $\begin{array}{l}\text { Size of } \\
\text { valve }\end{array}$ & $\begin{array}{c}\text { Diameter } \\
\text { of flat }\end{array}$ & $\begin{array}{c}\text { Length of } \\
\text { bearing }\end{array}$ \\
\hline $\begin{array}{c}\text { Inches } \\
21 / 2 \\
3 \\
31 / 2 \\
4 \\
5\end{array}$ & $\begin{array}{r}\text { Inches } \\
11 / 4 \\
114 \\
11 / 2 \\
2 \\
21 / 4\end{array}$ & $\begin{array}{c}\text { Inch } \\
5 / 16 \\
5 / 16 \\
3 / 8 \\
38 \\
7 / 16\end{array}$ \\
\hline $\begin{array}{r}6 \\
7 \\
8 \\
10 \\
12\end{array}$ & $\begin{array}{l}21 / 2 \\
23 / 4 \\
3 \\
31 / 2 \\
4\end{array}$ & $\begin{array}{r}1 / 2 \\
9 / 16 \\
5 / 8 \\
11 / 16 \\
3 / 4\end{array}$ \\
\hline
\end{tabular}

(f) The clapper arm for valves 2 inches and smaller shall be bronze. For larger valves it may be of malleable iron, bronze busned where tne hinge pin and clapper stud pass through. The bushing for the hinge shall project one-eighth inch beyond the iron, or the side plug shall extend inside the body a like amount. All bushings snall be a tight fit.

(g) The clapper arm shall extend beyond the center of the clapper to a point near the rim in order to form a ston which will prevent the clapper tipping and catching under the seat ring in case the connection between the clapper and arm becomes badly worn.

(h) When the clapper is wide open the clapper arm shall bear on a definite stop. If this stop is on the cover, the latter shall be so designed that it will be impossible to assemble it incorrectly. The point of contact shall be located so that the reaction of the water will not tend to twist or bend the parts.

A stronger and mechanically better valve is obtained when the clapper arm and not the clapper comes against the stop. If the stop is not placed in the center line a twisting force will be exerted on the parts when the clapper is wide open.
24. Hinge-(a) The clapper arm shall be supported by a pin of rolled bronze whicn can not be thrown out of alignment.

(b) For valves larger tnan 2 inches, the diameters of the holes in the clapper arm and those in the body or side plugs used to support the hinge pin snall be from 0.03 inch to 0.05 inch larger than that of the pin.

(c) If side plugs are used to hold the pin, the hole shall be drilled concentric with the screw thread.

(d) The hinge pin shall be supported at each end for a distance at least equal to its diameter.

(e) The over-all length of bearing of the hinge pin in the hub of the clapper arm shall not be less than that given in the following table:

\begin{tabular}{l|c|c|c|c|c|c|c|c|c|c|c}
\hline Size of valve (inches) & $2 \frac{1}{2}$ & 3 & $31 / 2$ & 4 & 5 & 6 & 7 & 8 & 10 & 12 & 14 \\
\hline $\begin{array}{c}\text { Length of bearing } \\
\text { (inches) ............ }\end{array}$ & $13 / 4$ & $17 / 8$ & $21 / 8$ & $21 / 4$ & $23 / 4$ & 3 & $31 / 2$ & 4 & $43 / 4$ & $51 / 2$ & 7 \\
\hline
\end{tabular}

25. Clearances.-(a) The clearance between the rim of the clapper and the inside of the body in every position of the clapper from closed to wide open shall be at least three-fourths inch for iron-body valves, one-eighth inch for the $3 / 4$-inch and 1-inch, and onefourth inch tor the $1 \frac{1}{4}$-inch, 11/2-inch, and 2 -inch bronze valves.

(b) There snall be a clearance of at least one-half inch around the hub of the clapper arm for iron-body valves and one-eighth inch for the $3 / 4$-inch to 2 -inch bronze valves, inclusive.

(c) The length of the hub of the clapper arm in the iron valves shall be one-sixteenth inch less than the distance between the bearings.

26. Tests. - All valves shall be tested before leaving the factory to twice the working pressure for leakage and distortion, first, between the downstream end and the clapper, and, second, with the clapper blocked off its seat.

\section{TARGET TYPE OF INDICATOR POST} VERTICALIY MOVING TARGET

27. Barrel.-(a) The post barrel must be at least three-eighths of an inch thick and must have an inside diameter of not less than 4 inches at any part throughout its length, and for a length of 10 inches from bottom of lower part the inside diameter must be 8 inches; the length of the barrel must be such that the middle of the target windows will be at least 30 inches above the ground level; and when the post is made adjustable, the upper part must extend about 12 inches below the ground, and telescope over the lower part at least 12 inches.

(b) When the post is telescopic, the parts shall be clamped together by two $3 / 4$-inch set screws ilaced one above the other and so arranged as to prevent separation or rotation of the upper part. This may be accomplished by providing either, first, deeply slotted holes in the lower part for the ends of the set screws or, second, a series of horizontal ribs, spaced the same distance apart as the set screws, to prevent separation; and four vertical ribs cast on the lower part, to prevent rotation.

The upper part must be cylindrical or somewhat tapered, with a smooth outside surface, having no flange or other projection by which the action of frost may lift the part.

(c) The post must be provided with a flange of design and dimensions described in article 12, Figure 306 (specifications for gate and check valves), drilled for four $5 / 8$-inch bolts to secure the post to a similar indicator post flange on the valve bonnet.

(d) Two opposite window openings at least $11 / 4$ inches high and at least one-half inch wider than 
the words "OPEN" and "SHUT" and designed to show the targets clearly must be provided near the top of the post.

(e) The windows must be made of glass at least one-half inch thick and must be held in place by a suitable brass or malleable-iron ferrule or grating with brass screws.

Posts for use where the windows are especially likely to be broken should have a grating of bars one-eighth inch wide and one-fourth inch deep, arranged with one central horizontal bar and several vertical bars, the latter to come between the target letters. There should be a space of at least onefourth inch between the grating and the glass. The grating is to be furnished only on special order.

28. Extension rod.-(a) The extension rod must be made of the best quality wrought iron or mild steel having a tensile strength of at least 45,000 pounds per square inch and should have a sectional area at least 1 square inch throughout. It may be of other suitable material having a proper sectional area to give an equivalent torsional strength.

(b) The extension rod must be secured to the valve stem nut in such a manner as to permit the axis of the rod to swing in any direction at least $5^{\circ}$ off the vertical, thus allowing for the nonalignment of parts.

A cast-iron coupling, cored to fit loosely the turning members, with two bronze cotter pins at least one-fourth inch diameter, one through the coupling and stem nut and the other with axis at right angles to the former through the coupling and extension rod, both pins to be tight in the coupling but loose in other members, forms a satisfactory method of meeting the requirements. The strength of the joint must not be made dependent upon the shearing strength of the pins.

29. Post cap.-The cap must be weatherproof, must be secured by bolts at least one-half inch diameter, and must furnish a suitable bearing for the operating stem, with provision for lubrication.

30. Operating stem.-(a) The stem must be made of bronze, cored at least 4 inches deep to fit freely the extension rod, and its upper end must be finished in a solid nut $1 \frac{1}{4}$ inches square by 1 inch high except for post to be installed where existing posts have different shape or size of nut, in which case additional posts must have the same operating nut as the old ones for uniformity.

(b) The stem must turn freely in its bearings and must be so designed that it can not become clamped to its bearings by lock nut or be prevented from turning by the target nut in any position.

(c) Stems of same make must be interchangeable, regardless of number of turns or size of valve, the range of valves being from 4 to 12 inches, inclusive.

31. Wrench and handwheel.-(a) Either a wrench or handwheel must be provided with each post.

(b) The wrench must be of malleable iron 12 inches long from center of operating nut to end.

(c) The handwheel must be of heavy cast iron 14 inches in diameter.

32. Target.-(a) The target-nut threads must be made of bronze.

(b) The words "OPEN" and "SHUT" must be used on the target and each word must be on a separate plate of aluminum or other suitable noncorrodible white metal. The letters must be 1 inch high and raised one-eighth of an inch on the casting. The face of the letters should be finished smooth one-eighth inch wide and the background painted black. The words must be at least 3 inches wide. The proper word must be visible at both windows when the gate is open or shut, but they must not show when the target nut travels off the thread at top or bottom of stem.

(c) The target plates must be adjustable relative to each other to suit the number of turns required by the different sizes of the make of valve for which the post was designed. Plates shall also be interchangeable to suit either right or left hand valves.

(d) The target mechanism must be of such design and construction that it will not be liable to be wrongly assembled or become disarranged in operation.

33. Sealing and locking.-(a) Provision must be made for strapping or otherwise sealing the operating stem in both the open and shut positions.

(b) A secure locking arrangement must be furnished on special order.

34. Marking.-(a) The post must be plainly marked well above the ground line with the name or trade-mark of the manufacturer, the year of manufacture, and some identification mark satisfactory to the inspection department having jurisdiction, to be used only on approved posts. All letters and figures should be about three-fourth inch high, and raised one-eighth inch on the casting.

This identification mark may be a word, number, letter, or other character by means of which this line of posts may be easily distinguished from all others of the same make.

In Factory Mutual work this identification mark must consist of the letters FM inclosed in a diamond as follows: FM

These markings should be so placed as to allow ample room for stenciling or otherwise marking the post vertically with the service which the valve controls, as, for example, the name or number of a building.

(b) The post cap must be marked "OPEN" with letters 1 inch high and raised one-eighth inch on the casting, and also with an arrow 4 inches long raised one-eighth inch on the casting.

\section{ROTATING TARGET TYPE}

\section{One acceptable design shown in Figure 309.}

35. General construction--(a) This shall conform to the specifications above for vertically moving target indicator posts as far as they apply, as given in the following sections: $27,(a),(b),(c),(d)$, and $(e) ; 28,(a)$ and $(b) ; 29 ; 30,(a)$ and $(b) ; 31,(a)$ and (b); $34,(a)$ and $(b)$.

36. Target.-(a) Threads and gears shall be of bronze and where other moving parts are in contact at least one must be bronze.

(b) The words "OPEN" and "SHUT" shall be at least 3 inches wide and 1 inch high with letters raised one-eighth inch. The face of the letters shall be finished smooth and the background painted black. Letters shall be of aluminum or other suitable white metal, and if the post is adjustable for different sizes of valves, each word shall be on separate plates. The proper word shall be visible at both windows when the valve is open or shut.

(c) The target mechanism shall not limit the turning of the operating stem if the valve or extension rod is broken or disconnected. It shall be of such a design and construction that it will not be liable to be wrongfully assembled or become disarranged in operation.

\section{SPECIFICATIONS FOR HYDRANTS}

\section{FOR MILL YARDS}

These specifications are broad, covering only the more essential features of hydrants. The aim has been simply to require hydrants which are strong and reliable, and which as far as possible agree with present shop practices. The acceptable hydrant must, in addition to conforming with these specifications, be satisfactory in the construction of the parts, composition of the metals, and in workmanship. 
37. Size.-(a) Hydrants shall be designated by the number of $2 \frac{1}{2}$-inch hose outlets for which they are designed, the nominal diameter of the valve opening being at least 5 inches for 2 -way and 6 inches for 3 and 4 way hydrants.

For example, a hydrant having two $21 / 2$-inch hose outlets would be known as " 2 way," if fitted with three $21 / 2$-inch outlets, " 3 way," etc. If a steamer connection is also provided the hydrant would be designated, for example, "2 way and steamer connection."

(b) The net area of the waterway at the smallest part other than at the valve opening when the hydrant is wide open must not be less than 120 per cent that of the valve opening, and there must be
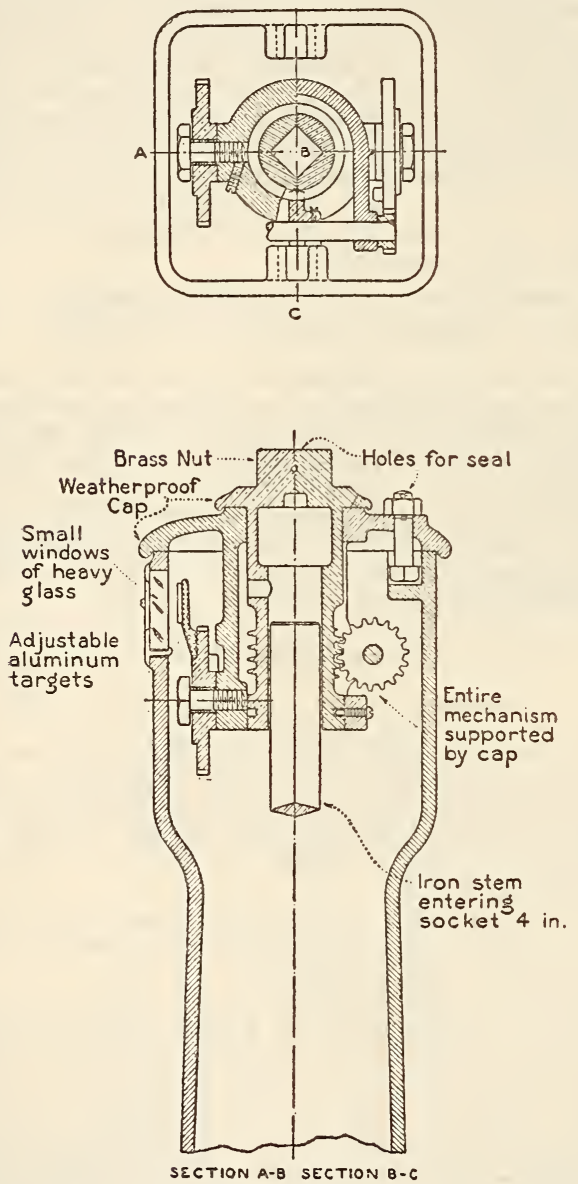

FigdRE 309.-Rotating target type indicator post

sufficient clear waterway through the hydrant when wide open to allow the passage of a ball at least $17 / 8$ inches in diameter for a 2 -way and $23 / 8$ inches for 3 and 4 way hydrants.

In new designs it is recommended that inside diameter of hydrants, especially at the outlets, be 7 inches for 2 -way and 8 inches for 3 and 4 way hydrants.

(c) Hydrants must be fitted with bell ends to fit American Water Works Association standard pipe (see 607.12 , p. 379 ) or with flanges of standard dimensions and having standard bolt layouts, as given in Table 2 of A. S. M. E. standard for castiron pipe flanges, and flanged fittings, serial B16a1928. (See 607.14, p. 385.) Holes are not to be drilled on the center line, but symmetrically each side of it.

(In Factory Mutual work the standard sizes for pipes as adopted by the New England Water Works Association September 10, 1902, are also acceptable.)
38. General design.-(a) Hydrants may be of compression or gate type.

(b) Hydrants must be designed to withstand safely a working pressure of 150 pounds, with a factor of safety of at least 5 at the working pressure. For example, a hydrant whose working pressure is 150 pounds must resist a pressure of 750 pounds before breaking. Where the working pressure is greater than 150 pounds a heavier hydrant must be used.

(c) Valve when shut must remain reasonably tight when upper portion of barrel is broken off.

(d) Any changes in diameter of the water passage through the hydrant must have easy curves, and all outlets must have rounded corners of good radius.

(e) With the hydrant discharging 250 gallons per minute through each $2 \frac{1}{2}$-inch hose outlet, the total friction loss of the hydrant must not exceed $13 / 4$ pounds for 2 -way, $2 \frac{1}{4}$ pounds for 3 -way, and 3 pounds for 4-way hydrants, except when fitted with inside hose gate valves, in which case the loss must not exceed $23 / 4$ pounds for 3 -way and $31 / 2$ pounds for 4-way hydrants.

(f) Hydrants must be so designed that with extraordinary usage they will not cause an increase of pressure in the system above normal of more than 60 pounds.

(g) Hydrants must be fitted with two lugs so that the leaded joint underground can be strapped.

(h) When hydrant barrel is made in two sections, the upper flange connection must be at least 2 inches above the ground line.

(i) All hydrant barrels must have a hole through the case, back of and level with the outlets, tapped for $1 / 4$-inch iron pipe thread, and plugged with a brass plug with large square head.

This tapped hole is desired for connecting a pressure gauge during field tests of pipe systems, etc., which may be made from time to time through the hydrants.

39. Material of body.-(a) The hydrant body must be made of cast iron.

40. Materials. - (a) (General quality requirements and strength-test requirements for cast iron are the same as specified above for outside-screw-and-yoke valves. See sec. $3(b)$ above.)

(b) All wrought iron or mild steel used must be of the best quality of refined iron, and have a tensile trength of at least 48,000 pounds per square inch.

(c) All composition or other noncorrodible metal used must be of the best quality, and have a tensile strength of not less than 32,000 pounds per square inch, with 5 per cent elongation in eight diameters and 5 per cent reduction of area at breaking point. Composition for inside hose valve stems must have a tensile strength of not less than 55,000 pounds per square inch and an elastic limit of not less than onehalf the tensile strength.

41. Hose valve and nipples.-(a) Hydrants must have at least two hose connections. If more than two hose connections are used each outlet must have a hose gate valve.

Steamer connections should never be on mill-yard hydrants when such hydrants are already connected with a fire pump.

(b) The hose gate valves may be of the outside detachable type or be built inside the barrel. The outside hose gate valves must be made in accordance with the specifications for this type of valve. Each valve must be bolted to the hydrant by two $3 / 4$-inch tap bolts, spaced horizontally $5 \% \frac{1}{8}$ inches on centers. The valves must not project further than necessary, and must be of the inside-screw type, placed in a vertical position, with the handwheel at least 3 inches below the base of the operating nut.

Inside hose gate valves must have composition metal working parts and be of rugged design, and must not introduce an unnecessary friction loss. There must be ample clearance between the gate and the hydrant body when the gate is in any 
position. The gate and parts should be interchangeable, and the valves should be located so as to be as accessible as possible for repairs. The gate must be designed so that it can not come off in use. The top of the stem must be below the level of the hydrant stem nut, so that the hydrant wrench can be freely operated.

(c) Hose nipples must be of composition metal threaded with a fine thread into the hydrant, and securely pinned in place. If desired, the nipple may be cast with two side lugs and be bolted to the hrdrant.

(d) Hose threads on all hydrants to be installed in any given mill yard must of necessity be interchangeable with those already in service, but, where practicable, threads should conform to the natonal standard adopted by the National Fire Protection Association. The essential features of the national standard thread in the $2 \frac{1}{2}$-inch size are a $60^{\circ} \mathrm{V}$ thread, outside diameter on male threads of $31 / 16$ inches, and $7 \frac{1}{2}$ threads per inch.

(e) The stems of hose valves must be not less than three-fourths inch in diameter for the $2 \frac{1}{2} 2$-inch valves, and not less than seven-eighths inch in diameter for the valves at the steamer connections.

( $f$ ) The stem nut of all inside hose and steamerconnection gate valves must be one-half inch square.

42. Hydrant valve.-(a) The valve seat must be made of composition metal securely fastened in place.

(b) The valve must be faced with a yielding material, such as rubber or leather, except that if of the gate type a bronze ring may be used. The valve must be designed so that it can be easily removed for repairs without digging up the hydrant.

(c) The clearance of parts must be such that corrosion will not make the parts inoperative.

43. Drip valve.-(a) A positively operating noncorrodible drip valve must be provided and arranged so as to drain the hydrant when the main valve is shut.

A drip ralre orerated by springs or gravity is not acceptable.

(b) The seat of the drip valve must be made of noncorrodible material and must be securely fastened in the hydrant. All other parts of the drip mechanism must be designed to be easily removed without digging up the hydrant.

44. Operating stem.- (a) The threaded section of the operating stem when located in the waterway must be of composition or other noncorrodible metal, but when above the stuffing box it may be of wrought iron. The operating stem where it passes through the stuffing box and gland, must be of composition metal or be fitted with a composition metal covering. The diameter of the operating stem at base of thread must be not less than $11 / 8$ inches for gate type of hydrant and 1 inch for compression and toggle types. The remaincer of stem may be of iron and the diameter must be not less than $13 / 8$ inches for gate type of hydrant and $11 / 4$ inches for compression and toggle types. The operating stem must be attached so that in operation it will be impossible for it to become detached.

If iron or steel is used, the threaded sections should receire the best of care and be kept oiled.

(b) The stem must terminate at the top in a nut of pentagonal shape, finished with slight taper to $1 \frac{1}{2}$ inches from point to flat, except for hydrants to be installed where existing hydrants have different shape or size of nut, in which case the additional hydrant must have the same operating nut as the old ones for uniformity. The nut socket in the wrench must be made without taper so as to be reversible.

(c) The thread which operates the valve must be acme, half $V$, or square.

The acme standard thread is considered preferable. (For thread see United States Gorernment National Screw Thread Commission, under 608.0 , p. 529 .)
45. Stuffing box and gland.-(a) The stuffing box and gland must be of composition metal or bushed with it. If a packing nut is used, it must be of composition metal. The bottom of the box and the end of the gland or packing nut must be slightly beveled.

(b) Gland bolts or studs must be of composition metal, iron, or steel, at least one-half inch in diameter. The nuts must always be of composition metal.

46. Hydrant top.-(a) The hydrant top must be designed so as to make the hydrant as weatherproof as possible, and thus overcome the danger of water getting in and freezing around the stem. Provision must be made for oiling both for lubrication and to prevent corrosion. A reasonably tight fit should be made around stems.

(b) There must be cast on the hydrant top, in characters raised one-eighth inch, an arrow at least $2 \frac{1}{2}$ inches long, showing direction to open, and the word "OPEN" in letters three-fourths inch high.

47. Hose caps.-(a) Hose caps must be provided for all hose outlets, and must be securely chained to the barrel with a welded chain of wire, not less than one-eighth inch in diameter.

(b) The hose cap nut must be of the same size and shape as the operating nut.

(c) A leather or rubber-or if requested, leadwasher or disk must be provided in the hose cap, set in a groove or otherw ise so retained as to prevent its falling out when the cap is removed.

48. Marking.-(a) Hydrants must be marked with the name or trade-mark of the manufacturer, the year of manufacture, the pressire for which they are designed, and some identification mark as agreed upon by the Underwriters having jurisdiction, to be used only on approved hydrants, so that these may be readily distinguished from those that are not approved. All letters and figures must be cast on the barrel well above the ground line. They must be 1 inch high, and raised one-eighth inch on the casting, except the date mark which may be smaller if legible.

This identification mark may be a number, letter, word, or other character by means of which this line of hydrants may be easily distinguished from all others of the same make.

In Factory Mutual work this identification mark must consist of the letters FM inclosed in a diamond as follows: $F M$

49. Testing.-(a) Hydrants, after being assembled, must be tested to at least 300 pounds per square inch before leaving the factory. If the working pressure is over 150 pounds per square inch, the hydrants must be tested to twice the working pressure. The test should be made with the valve open in order to test the whole barrel for porosity and strength of hydrant body. A second test should be made with the valve shut in order to test the strength and tightness of the valve.

(b) Hydrants must be fully opened and closed before shipping, in order to test the freedom and strength of the parts. The conditions of the test should be as severe as are liable to occur in service, when using a hydrant wrench at least 17 inches long.

50. Direction to open.- - (a) All hydrants must open to the left, except where existing hydrants open to the right, in which case additional hydrants should turn the same as the old ones for the sake of uniformity.

CHIORINE INSTITUTE (INC.), SPECIFICATIONS FOR THE CHLORINE INSTITUTE STANDARD VALVE FOR 100, 105, AND 150 POUND CONTAINERS, DECEMBER \%, 1928

\section{VALVE FOR 100 TO 150 POUND CONTAINERS}

I. General.-The valve shall be of the internal thread type, repackable under pressure, provided with outlet connection to accommodate present 
standard connections; that is, the $3 / 4$-inch straight pipe thread union and the standard Mathieson yoke and adapter. For assembly of valve see Figure 310. (Other detail dimension drawings of the valve parts may be obtained from the Chlorine Institute (Inc.), 30 East Forty-second Street, New York, N. Y.)

II. Body.-Brass forging consisting of 58 to 61 per cent copper, $1 \frac{1}{2}$ to $2 \frac{1}{2}$ per cent lead, and the remainder zinc.

(a) Outlet connection.-External 3/4-inch straight pipe thread, 14 pitch, V form; pitch diameter 0.9830 inch plus 0.000 inch, minus 0.0005 inch to minus 0.0035 inch limits. Inside of outlet boss to be counterbored; diameter of counterbore to be 0.5625 inch plus 0.003 inch minus 0.003 inch; depth
IV. Stem.-Monel metal with five-eighths inch by 8 Acme right-hand thread, rotating type. Top of stem to be three-eighths inch square. No handle required.

V. Packing nut.-Brass, external type, $11 \frac{1}{8}$ inches by 18 thread.

VI. Packing gland.-Brass, shaped to compress packing to stem. Clearance between follower and stem to be increased a short distance from the lower end to avoid freezing.

VII. Packing ring.-Brass, loose collar shaped to compress packing to stem.

VIII. Outlet cap gasket.-One-sixteenth-inch Garlock No. 902 or equivalent, diameter 0.960 inch.

IX. Outlet cap.-Brass, hexagonal in shape. Dimension from flat to flat of hexagon shall be same as

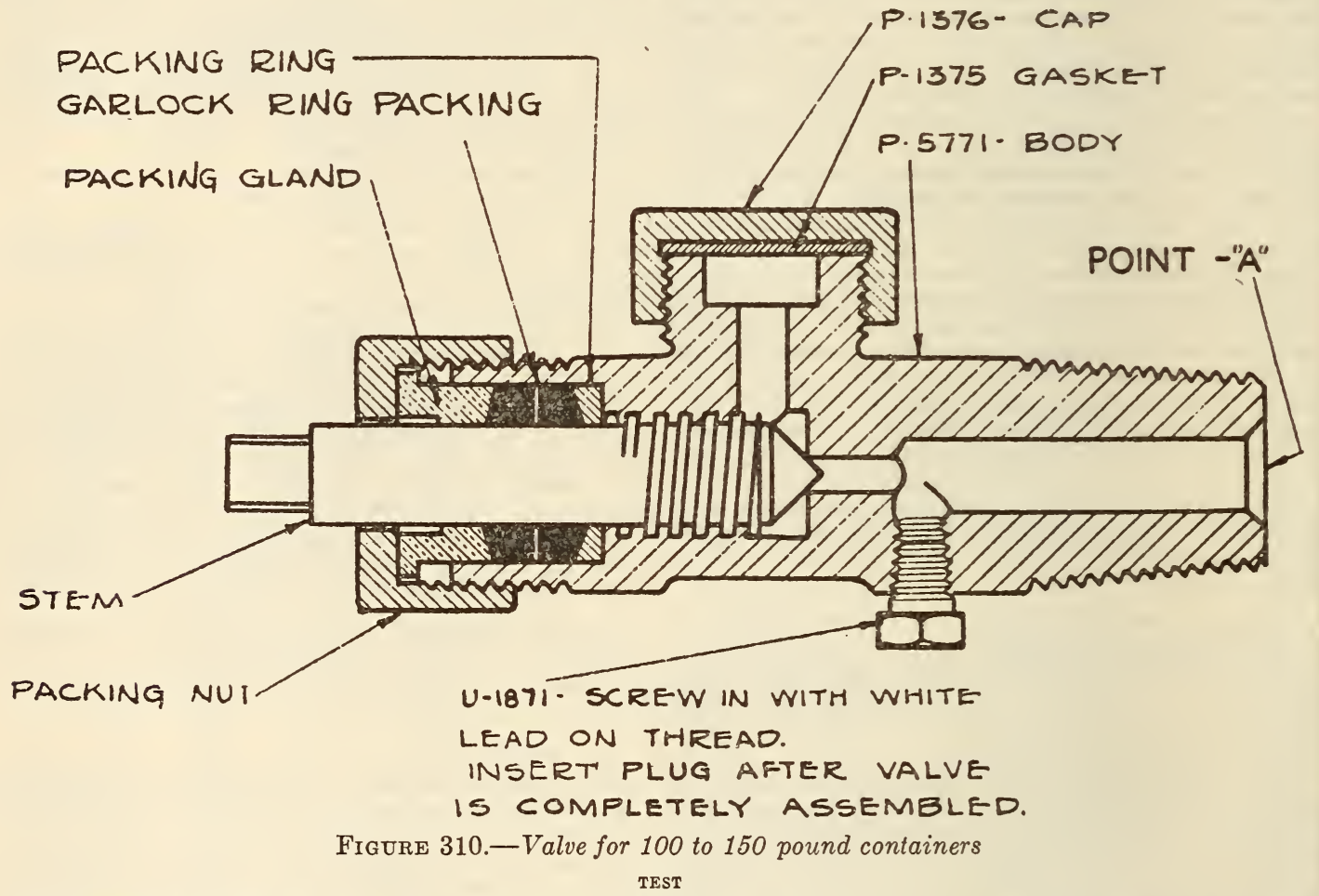

Apply 250 pound air pressure at point $A$ with valve stem closed and outlet cap removed, inspect for leak through valve seat and fusible metal plug

Place on outlet cap, open valve stem and inspect for leak through packing

fifteen sixty-fourths inch plus one sixty-fourth inch minus 0.000 inch; outer end of boss and bottom of counterbore to be machined smooth and flat to accommodate gaskets.

(b) Shank thread.- Standard Briggs pipe taper of such size as to enter a $3 / 4$-inch Briggs standard pipe gage one-half to one turn. Threads to be $11 / 8$ inches long; increased diameter to be in accordance with Briggs standard taper for overlength threads. Standard oversize for oversize cylinder threads shall be same as above with exception that the thread shall be one-sixteenth inch oversize in pitch diameter. In both cases the thread shall conform to the Briggs standard pipe thread without flats.

(c) Seat.- Integral with body, not renewable. Three-sixteenths-inch opening for 100,105 , and 150 pound containers.

III. Fusible plug and fusible metal.-The fusible plug shall consist of a threaded bronze container to screw into the valve body. The fusible metals shall be Niagara Alkali Co.- that is, Jaeger Salts formula. that of packing nut so that the same wrench may be used for both.

X. Packing.-Shall consist of two self-lubricating split ring type packing such as Garlock No. 343 or equivalent, free of oil, inside diameter one-half inch, outside diameter seven-eighths inch, thickness of each ring one-fourth inch.

CHLORINE INSTITUTE (INC.), SPECIFICATIONS FOR THE CHLORINE INSTITUTE STANDARD VALVE FOR 1-TON CONTAINERS, DECEMBER \%, 1928

\section{VALVE FOR 1-TON CONTAINER}

(This valve is the same as the standard valve for 100,105 , and 150 pound containers except that it has no fusible plug containing fusible metal and the opening through the valve seat is one-fourth inch instead of three-sixteenths inch.) 
HEATING AND PIPING CONTRACTORS NATIONAL ASSOCIATION, RADIATOR VALVES, ROUGHING-IN DIMENSIONS, 1929

\section{STANDARD ROUGHING-IN DIMENSIONS}
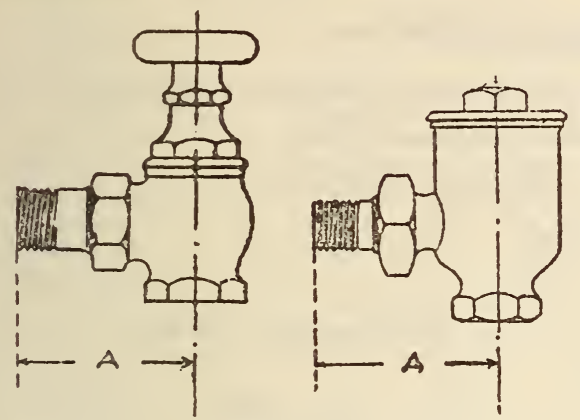

FIGURe 311.-Radiator valves, roughing-in dimensions

Angle-type valves

\begin{tabular}{|c|c|c|c|}
\hline Size of valre & $\begin{array}{c}\text { Dimension A, } \\
\text { steam and } \\
\text { hotwater } \\
\text { angle valves } \\
\text { and union } \\
\text { elbows, effec- } \\
\text { tive Jan. 1, } \\
1926\end{array}$ & $\begin{array}{c}\text { Dimension A, } \\
\text { modulating } \\
\text { valves, effec- } \\
\text { tive Jan. } 1 \text {, } \\
1926\end{array}$ & $\begin{array}{l}\text { Dimension A, } \\
\text { return line } \\
\text { vacuum } \\
\text { valves, effec- } \\
\text { tive Jan. 1, } \\
1925\end{array}$ \\
\hline $\begin{array}{c}\text { Inches } \\
1 / 2 \\
3 / 4 \\
11 \\
11 / 4 \\
11 / 2 \\
2 \\
\text { Tolerance }\end{array}$ & $\begin{array}{c}\text { Inches } \\
21 / 4 \\
23 / 4 \\
3 \\
31 / 2 \\
33 / 4 \\
41 / 4 \\
\pm 1 / 8\end{array}$ & $\begin{array}{c}\text { Inches } \\
23 / 4 \\
23 / 4 \\
3 \\
31 / 2 \\
33 / 4 \\
41 / 4 \\
\pm 1 / 8\end{array}$ & $\begin{array}{c}\text { Inches } \\
31 / 4\end{array}$ \\
\hline
\end{tabular}

Connecting ends shall be threaded and gaged as to threading according to the American (taper) pipe thread standard, A. S. A. No. B2-1919. (See United States Government, National Screw Thread Commission, Bureau of Standards miscellaneous publication No. 89, under 607.0 , p. 365.)

The standardization of the roughing-in dimensions of angle steam and hot water, and modulating radiator valves was made possible by the cooperation of the Manufacturers Standardization Society of the Valves and Fittings Industry.

\section{NATIONAL BOARD OF FIRE UNDER- WRITERS, INSTALLATION, MAINTE- NANCE, AND USE OF EMERGENCY GAS SHUT-OFF VALVES, 1924}

\section{GAS SHUT-OFF VALVES}

(This specification does not cover the design of emergency gas shut-off valves or the material of which they are made other than to require that such valves shall be simple and substantial in construction and so designed that the parts are not liable to stick or become clogged, or fail to close by the application of ordinary manual force. It is also required that valves on the operating stems shall be encased in suitable valve boxes, manholes, or vaults, and be adequately protected against water that might freeze. Automatic operation of such valves is not recommended, but provision is made for valves with positive mechanical connection to a control located at some readily accessible point.)
POWER PIPING SOCIETY, STANDARD SPECIFICATIONS FOR POWER PIPING, 1928

\author{
POWER PIPING
}

(See 607.0, p. 364.)

\section{REFRIGERATING MACHINERY ASSOCIA- TION, AMERICAN TENTATIVE STAND. ARD AMMONIA PIPE FLANGES AND FLANGED FITTINGS FOR MAXIMUM WORKING PRESSURES OF 300 POUNDS PER SQUARE INCH, 1931}

\section{AMMONIA PIPE FLANGES AND FLANGED FITTINGS}

(See 607.14, p. 413.)

UNDERWRITERS' LABORATORIES, STAND. ARD FOR CONSTRUCTION AND PERFORMANCE OF HAZARDOUS IIQUID VALVES, MAY, 1930

\section{HAZARDOUS LIQUID VALVES}

\section{GENERAL}

This standard covers the construction and performance of hazardous liquid valves that are intended to be incorporated in the assembly of discharge devices designed for dispensing motor fuels at automobile filling stations and intended for installation in accordance with the regulations of the National Board of Fire Underwriters for the installation of containers for storing and handling flammable liquids, or similarly employed in lines handling gasoline, kerosene, etc.

In this standard, the following words are used as defined below:

"Shall" is intended to indicate requirements.

"Should" is intended to indicate recommendations, or that which is advised but not required.

In this standard "valves" are divided into three groups: Shut-off valves (gate, globe, angle, needle), hose nozzle valves, and hose drain valves.

\section{SHOT-OFF VALVE}

Body

1. Bodies of valves shall be made of a good quality cast brass, or of other corrosion-resisting metal. They shall be free from sand holes and similar imperfections likely to permit leakage. The walls shall be not less than three thirty-seconds of an inch in thickness.

2. All joints between liquid-handling parts shall consist of metal-to-metal seating surfaces not requiring the use of gaskets to effect tightness against leakage.

3. (a) Plugs, caps, bonnets, spuds, etc., threaded into the valve body shall engage with at least five full threads of the body.

(b) Inlet and outlet openings of gate, angle, globe, and needle valves shall be threaded in accordance with the American pipe thread standard and the threads recessed or cored at the inner end, thereby providing sufficient clearance between the end of the thread and projections inside the body to reduce the possibility of the end of a pipe cutting into webs and projections or the pipe being kept from tightening sufficiently to prevent leakage.

\section{Stuffing Box}

4. (a) Stuffing boxes should be located on the open side of the valve so as not to be subjected to pressure when the valve is closed. 


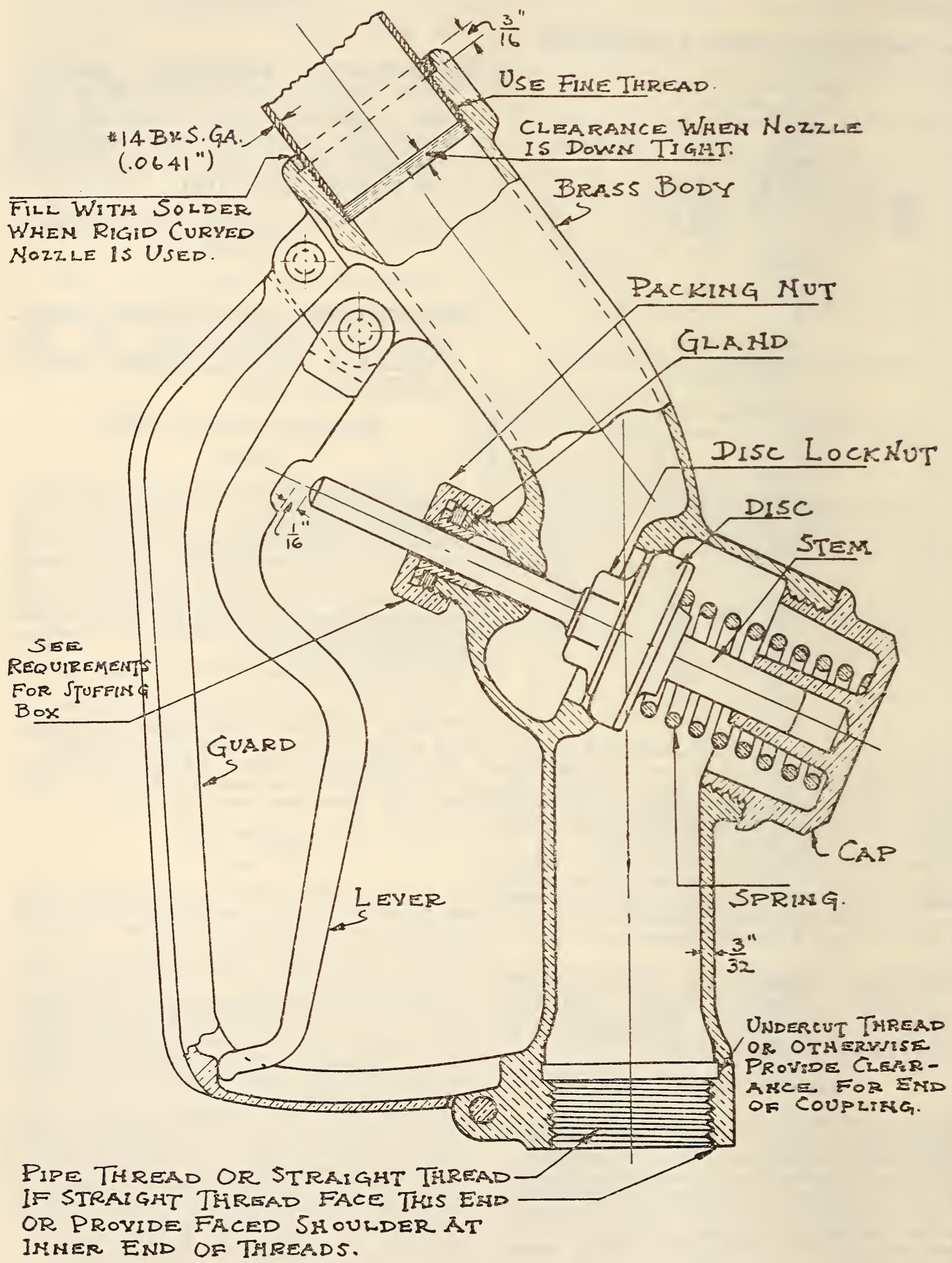

FIGURE 312.-Conventional design of hose nozzle valve 
(b) Acceptability of the construction submitted will be determined by the results of operating tests. (See secs. 27 and 28.)

5. (a) The stuffing box shall be provided with a removable, shouldered, unthreaded follower gland. The valve shall be designed with a nut or other acceptable means for adjusting the gland to maintain pressure on the packing as wear occurs.

(b) Packing nuts of hose nozzle and other selfclosing valves should be knurled to permit being adjusted by the fingers rather than requiring the application of a wrench.

6. The gland shall be made of corrosion-resisting material and shall be beveled at the lower end to conform to a corresponding angle of bevel that shall be provided in the bottom of the stuffing box recess. This construction is intended to assist in compressing the packing against the stem when force is applied to the gland. For rotating stems, the angle of bevel shall be not less than $25^{\circ}$, and for reciprocating between $35^{\circ}$ and $45^{\circ}$, measured from the perpendicular to the axis of the stem.

7. Automatic spring take-up for glands of valves, when supplied, shall employ a spring made of corrosion-resisting material or one acceptably coated to retard corrosion. Its fully compressed length and its strength shall be such that when the gland has entered the stuffing box only one-fourth inch the nut will be permitted to engage readily with at least one-fourth inch of the threaded section of the boss. The spring shall be of such length and strength that it will automatically advance the gland over onehalf the length of its travel without requiring further adjustment of the packing nut and still exert sufficient force to reduce leakage to a minimum when the device is subjected to the required test pressure. (See sec. 28.)

8. Before the valve is shipped, the stuffing box shall be fully packed with a suitable type of pliable packing material, the impregnation of which is not noticeably affected by contact with gasoline.

\section{Stem}

9. The valve stem should be of sufficient length to permit repacking the stuffing box without requiring the removal of the operating lever or handle. The stem shall be made from either cast or rod brass or other metal not readily affected by corrosion and shall possess sufficient strength to preclude the possibility of its being twisted off or distorted by normal operation.

10. Stems shall be so designed that they can not be backed out of the valve, and threads of stems shall not enter the stuffing box. Valve disks should be attached to the lower end of tine stem in such a manner as to permit swiveling, thus allowing the disk to seat properly.

\section{HOSE NOZZLE VALVE}

\section{Boỏy}

(See also secs. 1, 2, and 3.)

11. An illustration of a conventional type of hose nozzle valve is shown by Figure 312 . They shall be self-closing. If desired, the body may be of special shape or design to facilitate hanging or locking in place on the discharge device during intervals between operating periods.

12. Each valve shall incorporate an operating lever guard designed to guide the free end of the operating lever and to prevent opening of the valve and injury to the operating parts when the valve is dropped. The guard shall be of such strength as to permit operation of the valve subsequent to being subjected to rough usage tests. (See sec. 26.)

13. The valve seat shall be of ample width and if beveled shall be beveled at an angle not more acute than $45^{\circ}$, measured between the line of bevel and the axis of the valve stem.

14. The inlet opening shall be tapped either in accordance with the American pipe thread standard for taper pipe or with straight threads. When straight threads are used, either the outer edge of the body or a shoulder located at the inner end of the threads shall be faced for the seating of a gasket that is provided with the hose to secure a tight joint.

15. The discharge opening shall be tapped with straight fine threads for attachment of the nozzle, which shall be threaded and drawn tight in the opening before the inner end strikes webs or projections in the body. The outer end of the threaded opening of the body shall be recessed at least threesixteenths inch and the annular space shall be flooded with solder when a curved rigid nozzle is used.

Nozzles

16. Either flexible, or straight or curved nonflexible nozzles, not exceeding 12 inches in length,

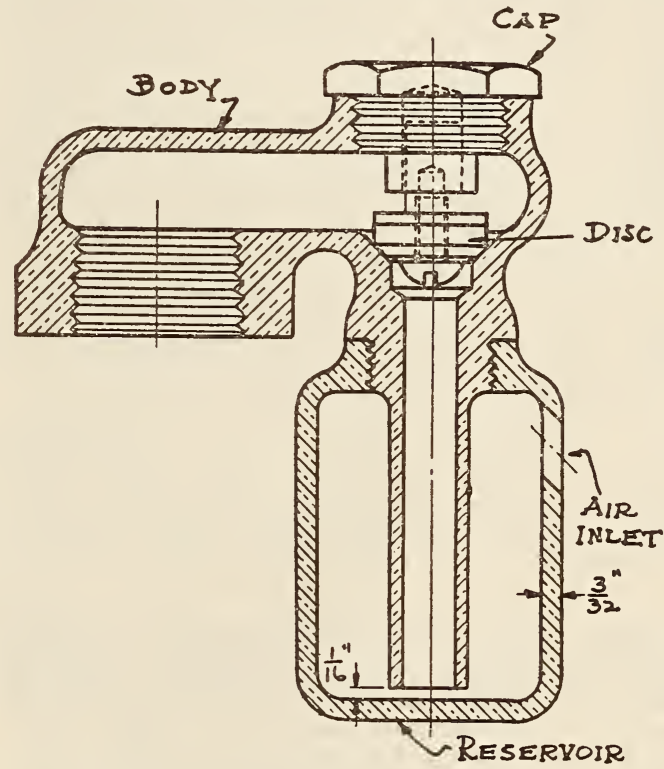

FIGURE 313.-Conventional design of hose drain valve

may be used. Nonflexible nozzles shall be made from hard drawn seamless brass tubing having a wall thickness of not less than No. 14 B. \& S: gage (0.0641 inch)

17. Flexible nozzles should be fitted with a securely attached threaded adapter that overlaps the end at least three-fourths inch for attachment to the body of the valve. The discharge end should terminate in a smooth ferrule. Tinning of ends of nozzles will assist materially in sweating the joints during assembly.

\section{Operating Parts}

18. Operating levers shall possess ample strength to resist bending or breaking in normal service and during operating tests, and shall be securely attached to the valve, the free end being guided by the lever guard. It shall be shaped and pivoted so that the contact of the valve stem with the lever is practically on the axis of the stem, thereby causing a minimum of side thrust on the stuffing box. A clearance of at least one-sixteenth inch shall be provided between the lever and the end of the valve stem to allow for wear of the disk, thus permitting it to seat though worn after continued use. 
19. Stuffing boxes and valve stems shall conform to the requirements of sections $4,5,6,8,9$, and 10 . The operating spring shall be made of corrosionresisting material or acceptably coated to retard corrosion.

20 . The valve shall close with the flow of liquid and the stem shall be guided to insure proper seating of the disk. The disk shall consist of acceptable resilient material not readily affected by the liquids handled and shall be securely attached to the valve stem. Care shall be exercised to prevent clamping washers or retaining cups from interfering with proper seating of the disk.

\section{HOSE DRAIN VALVE}

\section{Body}

(See also secs. 1, 2, and 3.)

21. A conventional type of hose drain valve is shown by Figure 313 . Valves of this type are designed to permit the entrance of air into a hose or other liquid-handling parts of discharge devices that are installed on the discharge side of shut-off valves. While thus assisting to secure ready drainage of parts following operation of the device, the hose drain valve is subjected to liquid pressure during operation. The conventional valve consists essentially of some form of check valve and a small cup designed to retain liquid which may leak past the check during operation. The retained liquid is intended to be drawn back into the discharge line with the incoming air when the hose is drained.

22. In view of the difficulty experienced in obtaining perfectly round balls for the ball check type of valve, manufacturers are urged to develop other types of valves more reliable in reducing, to an acceptable degree, leakage into the retention cup.

23. The retention cup shall be of sufficient capacity to contain the amount of liquid that passes the valve during discharge of liquid through the hose or other part to which it is attached. The relation of the depth and diameter of the cup and the location and size of the air inlet openings with respect to the diameter and length of the dip tube shall be such that the liquid collected in the cup during operation will be drawn into the discharge line when the hose is drained. The device shall be capable of being operated repeatedly in conjunction with the discharge device without permitting leakage or spilling of liquid through the air inlet openings.

24. (a) It is evident that the capacity of the retention cup that is required to prevent leakage and spilling of liquid during operation will depend on the tightness of the valve and to some extent on emptying the cup each time the hose is drained.

(b) To assist in obtaining the required results, the dip tube should extend to a point close to the bottom of the cup to aid in drawing collected liquid from the chamber. The air inlet openings should be placed at a high level and should be so proportioned and located as to permit a rapid inrush of air when the hose is drained.

\section{Tests}

25. (a) Each globe, angle, gate, needle, and hose nozzle valve body and stuffing box assembly shall be able to withstand without leakage or injury to operating parts, an internal hydrostatic pressure test, employing kerosene, of 300 pounds per square inch, held for one minute.

(b) The manufacturer shall test each globe, angle, gate, and needle valve body, valve seat, and stuffing box assembly, and each hose nozzle valve body and stuffing box to at least 25 pounds per square inch air pressure, with the valve immersed in water. Samples that show leakage at any point shall be rejected. (c) An air pressure test of 5 to 10 pounds per square inch shall be used by the manufacturer in testing the tightness of the seat of each hose nozzle valve.

26. The strength of the hose nozzle valve operating lever guard shall be sufficient to protect the lever and valve operating parts from injury when the valve is attached to a 10 -foot length of hose and dropped from a height of 6 feet onto a concrete floor at least 10 times.

27. Each hose nozzle, gate, angle, globe, and needle valve shall be capable of withstanding, without injury to the seat, spring, or stuffing box resulting in leakage, an operating test of 100,000 cycles of opening and closing. At the end of the test, the seat and stuffing box shall not show leakage under an air-pressure test of at least 50 pounds per square inch.

28. In the case of valves equipped with automatic spring take-ups, as described by section 7 , following the test specified by section 27 , the packing shall be removed from the stuffing box to permit the gland to advance over one-half the length of its travel. The remaining packing shall be firmly compressed and the valve shall again be operated in this condition for 1,000 cycles of operation subsequent to which the stuffing box shall not show leakage under a 50-pound air-pressure test.

29. Hose drain valves shall be capable of being operated repeatedly in conjunction with a dry hose type of discharge device without permitting escape of liquid through air inlet openings. The manufacturer shall test each seat and find it tight under a hydrostatic pressure of 5 pounds per square inch. Each retention cup shall be closely examined for pores or other imperfections likely to permit leakage.

\section{Marking}

30. Each valve shall be identified by a proper symbol agreed upon between the manufacturer and the laboratories which will be reserved for use only on such of his product as complies with the foregoing specifications.

\section{UNDERWRITERS' LABORATORIES, STAND- ARD FOR PRESSURE REGULATORS OR REDUCING VALVES, MARCH 13, 1928}

\section{PRESSURE REGULATORS OR REDUCING VALVES}

The following specifications apply to pressure regulators or reducing valves used in connection with oxygen, hydrogen, acetylene, and other gases employed in welding and cutting operations. They are designed to reduce the storage-cylinder pressures of these gases to the pressures required for blowpipes or torches.

\section{DESIGN AND CONSTRECTION}

(a) Devices of this type shall be of substantial construction and made of materials suitable for the purpose intended.

(b) Noninterchangeable union connections not requiring gaskets shall be provided for hose connections and for attachment to high-pressure cylinders.

NotE.-It is recommended that the International Acetylene Asso. ciation standard be followed. This provides for left-hand thread on fuel-gas fittings and right-hand thread on oxygen fittings.

It is also recommended that there be no shut-off or check valve on the outlet from the low-pressure compartment of oxygen welding regulators.

(c) These devices shall be marked with the name or registered trade-mark of the manufacturer. They shall also be marked with the proper type or style designation. 
(d) The workmanship shall be such as to insure a degree of uniformity suitable for devices of this character.

(e) Gauges reading over 1,000 pounds per square inch, if provided by the regulator manufacturer, shall conform with Underwriters' Laboratories' standard specifications for high-pressure gauges.

\section{PERFORMANCE TESTS}

Normal-operation test.-The regulator shall, when connected to a gas cylinder and to a proper blowpipe and subjected to normal operation, maintain the pressure within the limits required for proper operation of the blowpipe at any load within the rating of the regulator. Two samples of each type or style shall be tested.

Excess-pressure test.-The regulator shall be so constructed that if subjected to sudden application of full cylinder pressure to the low-pressure compartment, it shall either retain or safely release the pressure. Three samples of each type or style shall be tested. Each sample shall be connected to a full cylinder of oxygen and the cylinder valve opened manually as suddenly as possible.

UNDERWRITERS' LABORATORIES, STANDARD FOR OUTSIDE-SCREW-AND-YOKE GATE VALVES, 1921

(These specifications are the same as those of the Associated Factory Mutual Fire Insurance Companies' specifications for outside-screw-and-yoke valves given above.)

UNITED STATES GOVERNMENT, DEPARTMENT OF COMMERCE, BUREAU OF STANDARDS, WROUGHT-IRON AND WROUGHT-STEEL PIPE, VALVES, AND FITTINGS, SIMPLIFIED PRACTICE RECOMMENDATION NO. 57, 1926 WROUGHT-IRON AND WROUGHT-STEEL PIPE,
VALVES, AND FITTINGS

(See 607.0, p. 365.)

\section{UNITED STATES GOVERNMENT, DEPART-} MENT OF COMMERCE, STEAMBOAT INSPECTION SERVICE, GENERAI RULES AND REGULATIONS, MAY 4, 1929.

\section{LEVER SAFETY VALVES}

All common lever safety valves to be hereafter applied to the boilers of steam vessels shall be constructed in material, workmanship, and principle according to the requirements for a safety valve referred to in this section. When this construction of a safety valve is applied to the boilers of steamers navigating rough waters, the link may be connected direct with the spindle of the valve: Provided, always, that the fulcrum or points upon which the lever rests are made of steel, knife or sharp edged, and hardened; in this case, the short end of the lever shall be attached directly to the valve casing. In all cases the link requires but a slight movement not exceeding one-eighth of an inch.

\section{REQUIREMENTS IN CONSTRUCTION OF LEVER SAFETY VALVES}

All the points of bearing on lever shall be in the same plane.

The distance of the fulcrum shall in no case be less than the diameter of the valve opening.
The length of the lever shall not exceed the distance of the fulcrum multiplied by ten.

The width of the bearings of the fulcrum shall be not less than three-fourths of 1 inch.

The length of the fulcrum link shall be not less than 4 inches.

The lever and fulcrum link shall be made of wrought iron or steel and the knife-edged fulcrum points and bearings for the points shall be made of steel and hardened. But the chambers and saddle flanges of this and all other types of safety valves attached to boilers may be made of cast iron or other suitable material.

The valve, valve seat, and bushing for the stem or spindle shall be made of composition (gun metal) when the valve is intended to be attached to a boiler using salt water; but when the valve is to be attached to a boiler using fresh water and generating steam of a high pressure the parts named, with the exception of the bushings for the spindle, may be made of cast iron.

The valve shall be guided by its spindle, both above and below the ground seat and above the lever, through supports either made of composition (gun metal) or bushed with it.

The spindle shall fit loosely in the bearings or supports.

When the valve is intended to be applied to the boilers of steamers navigating rough waters, the fulcrum link may be connected directly with the spindle of the valve; providing always that the knife-edged fulcrum points are made of steel and hardened, and that the vertical movement of the valve is unobstructed by any lateral movement.

In all cases the weight shall be adjusted on the lever to the pressure of steam allowed in each case by a correct steam gauge attached to the boiler. The weight shall then be securely fastened in its position and the lever marked for the purpose of facilitating the replacing of the weight should it be necessary to remove the same, and in no case shall a line or any other device be attached to the lever or weight except in such a manner as will enable the engineer to raise the valve from its seat. (Sec. 4418, R. S.)

CAST STEEL, SEMISTEEL, FERROSTEEL, CAST IRON, MALLEA. BLE IRON, COMPOSITIONS MADE OF COPPER, TIN, AND ZINC, AND OTHER COMPOSITIONS PROPOSED TO BE USED IN THE MANUFACTURE OF VALVES, FITTINGS, AND OTHER APPLTANCES

Cast-steel fittings of any size or character and for any pressure and for any temperature may be used for any and all steam and feed pipe connections, and for valves, cocks, and all appliances subject to steam or water pressure when made by regular processes and by manufacturers who stamp such fittings and appliances with their trade-mark or identifying stamp and who guarantee the castings to possess the following physical characteristics: Tensile strength, minimum, 50,000; maximum, 78,000 pounds per square inch; elastic limit, minimum, not less than 45 per cent of tensile strength; elongation in 2 inches, minimum, 25 per cent. There shall be taken from each heat an annealed coupon or coupons, for the purpose of determining the physical tests, and the manufacturers shall furnish coupons to the local inspectors for tests when so required. All steel castings shall be thoroughly annealed.

The minimum thickness of steel fittings shall be determined by the following formula:

(where)

$$
T=\frac{P \times D}{7000}+0.188
$$

$P=$ working pressure in pounds.

$D=$ diameter in inches.

$T=$ thickness in inches. 
Cast iron, semisteel, or ferrosteel, possessing a tensile strength of not less than 22,000 pounds to the sectional square inch, may be used in the construction of valves and fittings for pressures not exceeding 300 pounds when such valves and fittings of 3 inches diameter or over are stamped with the trade-mark or identifying stamp of the manufacturer, and made in accordance with the following formula:

(where)

$$
T=\frac{P \times D}{3000}+0.25
$$

$T=$ thickness of casting in inches.

$P=$ pressure of steam allowable in pounds.

$D=$ internal diameter of the largest opening contained in the cylindrical part of the casting.

The above materials may also be used in the construction of manhole and handhole plates.

The headers of superheaters may be made of semisteel (so-called) in which the constituent part of high-grade uniform steel thereof shall be not less than 25 per cent and that the material shall have a tensile strength of not less than 28,000 pounds per square inch. The material must be soft and close grained, and the castings shall be tight under a hydrostatic pressure of 850 pounds per square inch.

Malleable iron possessing a tensile strength of not less than 30,000 pounds to the sectional square inch may be used in the construction of valves and fittings up to and including 6 inches in diameter, and for pressure not exceeding 300 pounds. Such valves and fittings of 3 inches diameter or over shall be extra heavy, the fittings beaded or banded, and the valves and fittings stamped with the trade-mark or identifying stamp of the manufacturer.

Hard brass, bronze, and other compositions, of which 95 per cent is copper, tin, and zinc, possessing a tensile strength of not less than 30,000 pounds to the square inch, may be used in the construction of all valves and fittings up to and including 12 inches in diameter, and for all pressures not exceeding 300 pounds per square inch. All such fittings of 3 inches in diameter or over shall be stamped with the trademark or identifying stamp of the manufacturer.

\section{VALVES}

Each and every valve for marine service shall have the trade-mark of the manufacturer cast or stamped on the chamber. All such valves of 2 inches diameter and over shall be subjected by the manufacturer before shipment to a hydrostatic pressure of three and one-half times the working pressure which the manufacturer guarantees them to stand without distortion, and shall also be stamped with such working pressure and also the letters " $\mathrm{M}$. S." which shall plainly indicate that the valve has met the test and requirements for marine service. All valves of over 2 inches diameter without regard for the service for which they are intended, installed after April 30, 1917, shall have bolted bonnets. Valves of 2 inches diameter or less connected directly to the boiler shall be of cast steel, hard brass, or bronze. Screwed bonnets on cast-iron valves of any size or for any character of service are positively prohibited. On all boilers built after July 1, 1896 , a stopcock or valve shall be placed between all check valves and boiler, and between all steam and water pipes and boiler: Provided, that this section shall not apply to safety valves that have been approved by the board of supervising inspectors.

\section{PIPE FITTINGS}

The term "pipe fittings" refers to all hollow cylindrical fittings, and does not apply to flanges or valves. All pipe fittings of more than 3 inches internal diameter shall be subjected by the manufacturer to a test of three and one-half times the pressure to which they will be subjected in service.

Where the thickness of the material in the boiler or drum, or the heads thereof, is not less than onehalf inch, or where such boiler, drum, or head thereof has been reinforced by having a pad or flange riveted on the same, to make the thickness not less than one-half inch, pipes or fittings of 2 inches in diameter or under may be screwed directly into the same. Where steam or feed pipes of 2 inches in diameter or under are screwed into the boiler, the stop valve shall be connected to the boiler by as short a nipple as it is possible to use, nipples to be of extra-heavy thickness.

All boiler connections of over 2 inches in diameter, except the connections for safety valves, shall be permanently flanged and attached directly to the boiler by bolts, studs, or rivets. Where the connecting point on the boiler is of circular form, distance pieces shall be allowed in order to square the point of attachment of the flanged fittings. Flanged distance pieces of not more than 8 inches between flanges may be allowed between stopcocks or valves and the boiler when necessary to care for and conveniently handle the valves, when such distance pieces are constructed of wrought material of sufficient thickness and strength to withstand any pressure to which they may be subjected and securely riveted to the boiler.

Cast-steel flanged fittings, when conforming strictly with the requirements for steel castings, may be used for the purpose of connecting main and auxiliary stop valves and other steam outlets, including safety valves, distance from axis of outlets of such fittings to point of connection with boiler to be as short as practicable.

Valves and fittings of 3 inches and under may be comnected by screw threads at their intermediate joints in pipe lines, but at point of connection with boiler all valves and fittings over 2 inches diameter shall be flanged and properly secured by bolts, studs, or rivets, and no fitting shall be of greater length than specified by the "Manufacturers' Standard."

Cast nozzles shall not be used when exposed to the direct action of the fire.

All sea valves or cocks secured to the skin of the vessel by bolts and connected to the engines or boilers by pipes shall be arranged so as to be acces sible at all times, so that if a leak or defect occurs it can be reached. All parts of said valves except the chamber shall be made of brass or bronze when used on wooden-hull vessels navigating salt water; but in the case of vessels of iron or steel the brass or bronze bolts may be dispensed with.

\section{AILOWABLE TEMPERATURES}

Cast iron, malleable iron, semisteel, and ferrosteel may be used in connection with temperatures up to and including $450^{\circ} \mathrm{F}$.

Hard brass, bronze, and other compositions, of which 95 per cent is copper, tin, and zinc, may be used in connection with temperatures up to and including $470^{\circ} \mathrm{F}$., but this temperature may be increased to $550^{\circ} \mathrm{F}$. when the manufacturer of the valves or fittings stamps them with the temperature they are guaranteed to stand without disintegration. This paragraph will also apply to any composition meeting the above physical requirements and stamped in accordance therewith.

For temperatures exceeding $550^{\circ} \mathrm{F}$. no valves or fittings other than steel or of a composition whose tensile strength has been demonstrated to exceed 50,000 pounds per sectional square inch with an elongation of 10 per cent in a length of 2 inches shall be allowed. Such valves and fittings shall be stamped as above required. 
The manufacturer of valves and fittings shall file with the supervising inspector general a certificate duly sworn to that all valves and fittings furnished by him for use on steam vessels comply with the requirements of the foregoing rule.

After July 1, 1911, local inspectors shall refuse to allow the use of any valves or fittings on steam vessels until notified by the supervising inspector genıral that such certificate is on file in his office. This shall not apply to valves and fittings installed previous to July 1, 1911.

UNITED STATES GOVERNMENT, FEDERAL SPECIFICATIONS BOARD, SPECIFICATION FOR PLUMBING FIXTURES (FOR LAND USE), NO. 448, NOVEMBER 22, 2926. (CURRENT DESIGNATION : FF$\mathrm{P}-451)$

\section{PLUMBING FIXTURES (FOR LAND USE)}

(See 617.73, p. 1062.)

\subsection{METALLIC HOSE, FERROUS AND NONFERROUS.}

AMERICAN MARINE STANDARDS COMMITTEE, SPECIFICATION FOR 3/4-INCH AND 2-INCH FLEXIBLE METALLIC HOSE, 0 NO. $2-1926$

\section{FLEXIBLE METALLIC HOSE}

1. Purpose.-The hose is intended to be used for steam, water, oil, or air, and it shall be suitable for working pressures up to 50 per cent of the straight test pressure specified herein.

2. Description.-Hose shall be made of copper, bronze, brass, or galvanized steel, as required. A sample showing the construction of hose offered must be submitted with bid. When galvanized steel hose is required, the coating shall be smooth and free from pinholes and must not interfere with the flexibility of the hose. Coverings of wire, wire and braid, rubber, and fabric shall be fitted when specifically required, and shall be of such a nature as not to be injurious to the operator's hands. The hose shall be so constructed as to be flexible and prevent excessive wear and breakage due to actual service.

3. Tests.-Each length or section of hose when lying straight shall be subjected to the hydrostatic test pressure siven in the table. Af er satisfact orily withstanding 1 his test, hose shall be coiled into a circle of the diameter specified in the table, and then the circle shall be rolled the entire length of hose while subjected to the hydrostatic test pressure given in the table. No leakage shall be in evidence during either of these tests. Oil shall be used for testing steel hose.

Test table

\begin{tabular}{|c|c|c|c|}
\hline $\begin{array}{l}\text { Inside } \\
\text { diameter } \\
\text { of hose }\end{array}$ & $\begin{array}{l}\text { Inside } \\
\text { diameter } \\
\text { of bend }\end{array}$ & $\begin{array}{c}\text { Test } \\
\text { pressure } \\
\text { per square } \\
\text { inch, } \\
\text { straight }\end{array}$ & $\begin{array}{c}\text { Test } \\
\text { pressure } \\
\text { per square } \\
\text { inch, } \\
\text { bent }\end{array}$ \\
\hline $\begin{array}{c}\text { Inches } \\
2^{3 / 4}\end{array}$ & $\begin{array}{r}\text { Inches } \\
13 \\
28\end{array}$ & $\begin{array}{r}\text { Pounds } \\
500 \\
300\end{array}$ & $\begin{array}{r}\text { Pounds } \\
400 \\
200\end{array}$ \\
\hline
\end{tabular}

4. Fittings, screw couplings.-All hose shall be provided, unless otherwise specified, with couplings of the following description:

Unless otherwise specified, couplings shall be made of composition in accordance with the latest issue of Navy Department specifications for valve bronze-composition "M." Couplings shall be of an approved type designed for use with an ordinary monkey wrench. Dimensions and threads shall be as specified in purchase order. The female coupling shall be of the swivel type, to permit of coupling without twisting the hose. The coupling shall withstand the pressure specified above and be substantial, of good workmanship, and designed so that it can readily be removed from or attached to the hose.

5. Exclusion of unsatisfactory brands.-Failure of any brand of hose to give satisfactory service, even though it meets the requirements outlined above, will operate to exclude the further purchase of the brand and type in question.

6. Hose lengths. - All hose shall be furnished in 50-foot, 25-foot, 20-foot, 10-foot, or other commercial lengths, as specified.

\section{SOCIETY OF AUTOMOTIVE ENGINEERS, ELECTRICAL EQUIPMENT; FLEXIBLE STEEL CONDUIT AND TUBING, S. A. E.} RECOMMENDED PRACTICE, JULY, 1926

\section{FLEXIBLE STEEL CONDUIT AND TUBING}

(Contains general specifications; tensile, breaking, and corrosion tests; dimensions, weights, stresses, and minimum bending radii for flexible steel conduit for insulated electric cables, for carburetor tubing, and for exhaust and heater tubing.)

608. NAILS, SCREWS, BOLTS, NUTS, AND RIVETS

\subsection{GENERAL ITEMS.}

\section{AMERICAN ELECTRIC RAILWAY ENGI- NEERING ASSOCIATION, OVERHEAD LINE HATERIAL FOR DIRECT AND CATENARY SUSPENSION, RECOMMEND- ED SPECIFICATION, D102-30, 1930}

\section{OVERHEAD LINE MATERIAL}

(D102-30 includes general requirements covering scope, materials, design, inspection, and rejection. For specification see 611.29, p. 682.)

\section{AMERICAN RAILWAY ASSOCIATION, MECHANICAI DIVISION, SCREW THREADS, STANDARD, 1926}

\section{SCREW THREADS}

The Sellers or Franklin Institute (United States standard) system of screw threads, boltheads, and nuts is the standard of the association, except for pipe work and special locomotive work, in which case either recommended practice or standards which have been adopted by the association should be used.

A set of gages for standard screw threads and a standard inch scale, 2 feet long, are held in the office of the secretary for reference.

Proportion of thread. - See Figure 314. Angle of thread, $60^{\circ}$. Divide the pitch, or side of thread, into eight equal parts; take off one part from the top $(c)$, and fill in one part in the bottom of the thread $(d)$, then the flat top and bottom will each equal one-eighth of the pitch; the wearing surface will be three-quarters of the pitch, and the diameter of the screw at the bottom of the thread will be expressed by the formula:

$$
\text { Diameter }=\frac{1.299}{\text { number of threads per inch }}
$$




\section{AMERICAN RAILWAY ASSOCIATION, TELEGRAPH AND TELEPHONE SECTION, SPECIFICATION FOR POLE IINE HARD- WARE, 1-A-20, NOVEMBER, 1927}

(See also 608.2 , p. 574 and 608.31 , p. 589.)

\section{POLE LINE HARDWARE}

PURPOSE

1. The purpose of this specification is to guide the seller or contractor in the manufacture of pole line hardware for use in the telegraph and telephone plant.

\section{GENERAI}

2. The quality of the material used and the method of manufacture shall be such as to insure

\section{GALVANIZING}

6. When galvanizing is specified on the drawing, it shall be done by the hot-dip process and shall conform to general specification for galvanizing for iron and steel.

\section{SAMPLES}

7. Samples for detailed examination and test may be selected at random by the inspector in such quantities as fairly to represent the shipment. Such samples as are rendered unfit for use by testing shall be furnished free of cost.

\section{PREPARATION FOR SHIPMENT}

8. The material shall be so prepared as to permit convenient handling and to protect against loss or damage during shipment. The quantities and the method of packing shall be as specified by the purchaser.
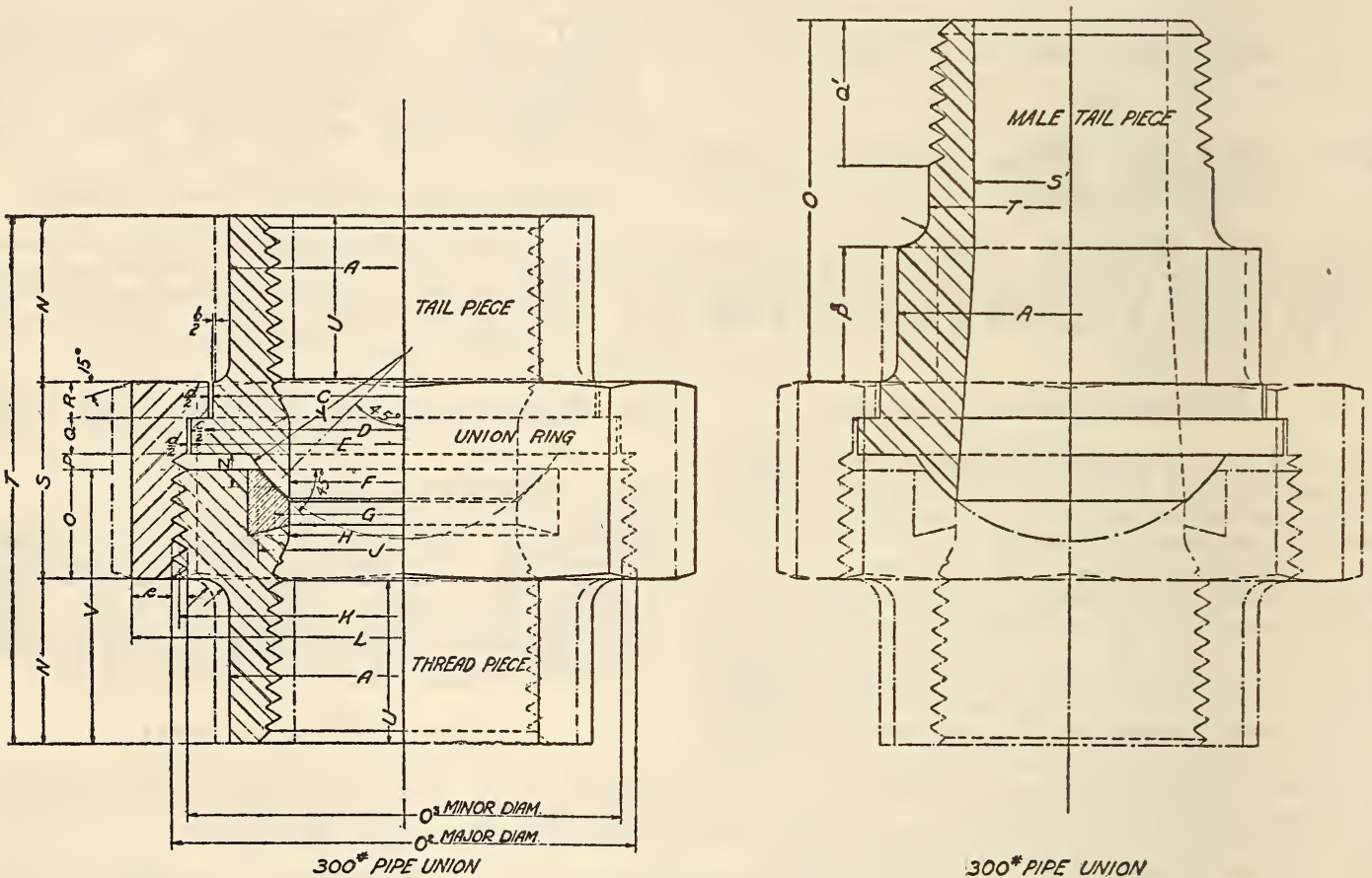

FIGURE 314.-Pipe unions of the nut and nipple type for locomotives, 300 pounds pressure

for the completed hardware the properties called for in this specification.

3. The specification and drawings are intended to cooperate with and supplement each other so that any details indicated in one and not in the other shall be executed the same as if indicated in both, unless exceptions are specifically mentioned within the body of this specification.

4. The seller or contractor must make sure that all material and work is in accordance with the specification before the hardware is offered.

\section{DESIGNS AND DIMENSIONS}

5. Designs and dimensions shall conform to the drawings. Allowable variations shall be within the limits specified; otherwise reasonable correspondence to the stated figures is required, consistent with good commercial practice.

\section{AMERICAN SOCIETY OF MECHANICAL ENGINEERS, AMERICAN STANDARD SCREW THREADS FOR BOLTS, MACHINE SCREWS, NUTS, AND COMMERCIALLY TAPPED HOLES, B1a-1924}

[Approved as American standard by the American Standards Assotion, 1924]

\section{STANDARD SCREW THREADS}

(Joint sponsor with the Society of Automotive Engineers under the auspices of the American Standards Association. Screw threads for bolts, machine screws, nuts, tapped holes, etc., for general use, prepared in collaboration with the National Screw Thread Commission. Substantially the same as Tables 1 to 14 in United States Department of Commerce, Bureau of Standards, Miscellaneous Publication No. 89, given below.) 
SOCIETY OF AUTOMOTIVE ENGINEERS, SCREW THREADS, S. A. E. (AMERICAN) STANDARD, FEBRUARY, 1926

[A pproved as American standard by the American Standards Association]

\section{STANDARD SCREW THREADS}

(Joint sponsor with the American Society of Mechanical Engineers under the auspices of the American Standards Association. Screw threads for bolts, machine screws, nuts, tapped holes, etc., for general use, prepared in collaboration with the National Screw Thread Commission. Substantially the same as corresponding tables in United States Department of Commerce, Bureau of Standards, Miscellaneous Publication No. 89, given below, with the following additional data on extrafine-thread series and fine-thread series over $1 \frac{1}{2}$ inches in diameter:)

The extra-fine-screw-thread series was adopted by the society in 1915 as the S. A. E. fine series for aeronautic and other applications where screwthreads finer than the present S. A. E. fine-screw thread series are necessary.

The extra-fine-screw-thread series is suited to parts (1) in which the thickness of the metal supporting the thread is not great, as in tubing, (2) which have a relatively light section, such as nuts, (3) which require fine adjustment, (4) where jar and vibration are very important factors, and (5) where assembly is made without the use of wrenches.
TABLE 14.-Basic diameters and thread data for the extra-fine-thread series

[Dimensions in inches]

\begin{tabular}{|c|c|r|r|r|r|r|}
\hline \hline Size & $\begin{array}{c}\text { Threads } \\
\text { per inch }\end{array}$ & $\begin{array}{c}\text { Basic } \\
\text { major } \\
\text { diameter }\end{array}$ & $\begin{array}{c}\text { Basic } \\
\text { pitch } \\
\text { diameter }\end{array}$ & $\begin{array}{c}\text { Basic } \\
\text { minor } \\
\text { diameter }\end{array}$ & Pitch 1 & $\begin{array}{c}\text { Basic } \\
\text { depth of } \\
\text { thread }\end{array}$ \\
\hline $1 / 4$ & 36 & 0.2500 & 0.2320 & 0.2139 & 0.0277778 & 0.01804 \\
$5 / 16$ & 32 & .3125 & .2922 & .2719 & .0312500 & .02030 \\
$3 / 8$ & 32 & .3750 & .3547 & .3344 & .0312500 & .02030 \\
$7 / 16$ & 28 & .4375 & .4143 & .3911 & .0357143 & .02319 \\
$1 / 2$ & 28 & .5000 & .4768 & .4536 & .0357143 & .02319 \\
$9 / 16$ & 24 & .5625 & .5354 & .5084 & .0416667 & .02706 \\
$5 / 8$ & 24 & .6250 & .5979 & .5709 & .0416667 & .02706 \\
$3 / 4$ & 20 & .7500 & .7175 & .6850 & .0500000 & .03248 \\
$7 / 8$ & 20 & .8750 & .8425 & .8100 & .0500000 & .03248 \\
1 & 20 & 1.0000 & .9675 & .9350 & .0500000 & .03248 \\
$11 / 8$ & 18 & 1.1250 & 1.0889 & 1.0528 & .0555556 & .03608 \\
$11 / 4$ & 18 & 1.2500 & 1.2139 & 1.1778 & .0555556 & .03608 \\
$11 / 2$ & 18 & 1.5000 & 1.4639 & 1.4278 & .0555556 & .03608 \\
$13 / 4$ & 16 & 1.7500 & 1.7094 & 1.6778 & .0625000 & .04060 \\
2 & 16 & 2.0000 & 1.9594 & 1.9278 & .0625000 & .04060 \\
$21 / 4$ & 16 & 2.2500 & 2.2094 & 2.1778 & .0625000 & .04060 \\
$21 / 2$ & 16 & 2.5000 & 2.4594 & 2.4278 & .0625000 & .04060 \\
$23 / 4$ & 16 & 2.7500 & 2.7094 & 2.6778 & .0625000 & .04060 \\
3 & 16 & 3.0000 & 2.9594 & 2.9278 & .0625000 & .04060 \\
$31 / 2$ & 16 & 3.5000 & 3.4594 & 3.4278 & .0625000 & .04060 \\
4 & 16 & 4.0000 & 3.9594 & 3.9278 & .0625000 & .04060 \\
\hline
\end{tabular}

1 This column is given to 7 decimal places for computation purposes only.

TABLEs 15 and 17.-Free fit (class $C$ ) and medium fit (class $D$ ) for screws in the extra-fine-thread series

[Dimensions in inches]

\begin{tabular}{|c|c|c|c|c|c|c|c|c|c|c|c|}
\hline \multirow{3}{*}{ Size } & \multirow{3}{*}{$\begin{array}{l}\text { Threads } \\
\text { per inch }\end{array}$} & \multicolumn{3}{|c|}{ Major diameter } & \multicolumn{6}{|c|}{ Pitch diameter } & \multirow{3}{*}{$\begin{array}{l}\text { Maxi- } \\
\text { mum } \\
\text { minor } \\
\text { diam- } \\
\text { eter }\end{array}$} \\
\hline & & \multirow{2}{*}{$\begin{array}{l}\text { Maxi- } \\
\text { mum } 1\end{array}$} & \multirow{2}{*}{$\begin{array}{l}\text { Toler- } \\
\text { ance }\end{array}$} & \multirow{2}{*}{$\begin{array}{l}\text { Mini- } \\
\text { mum }\end{array}$} & \multicolumn{3}{|c|}{ Class $\mathrm{C}$} & \multicolumn{3}{|c|}{ Class D } & \\
\hline & & & & & $\begin{array}{l}\text { Maxi- } \\
\text { mum }\end{array}$ & $\begin{array}{l}\text { Toler- } \\
\text { ance }^{2}\end{array}$ & $\begin{array}{l}\text { Mini- } \\
\text { mum }\end{array}$ & $\begin{array}{l}\text { Maxi- } \\
\text { mum } 1\end{array}$ & $\begin{array}{l}\text { Toler- } \\
\text { ance }^{2}\end{array}$ & $\begin{array}{l}\text { Mini- } \\
\text { mum }\end{array}$ & \\
\hline $\begin{array}{r}1 / 4 \\
5 / 16 \\
3 / 8 \\
7 / 16 \\
1 / 2\end{array}$ & $\begin{array}{l}36 \\
32 \\
32 \\
28 \\
28\end{array}$ & $\begin{array}{r}0.2500 \\
.3125 \\
.3750 \\
.4375 \\
.5000\end{array}$ & $\begin{array}{r}0.0050 \\
.0054 \\
.0054 \\
.0062 \\
.0062\end{array}$ & $\begin{array}{r}0.2450 \\
.3071 \\
.3696 \\
.4313 \\
.4938\end{array}$ & $\begin{array}{r}0.2320 \\
.2922 \\
.3547 \\
.4143 \\
.4768\end{array}$ & $\begin{array}{r}0.0027 \\
.0029 \\
.0030 \\
.0032 \\
.0033\end{array}$ & $\begin{array}{r}0.2293 \\
.2893 \\
.3517 \\
.4111 \\
.4735\end{array}$ & $\begin{array}{r}0.2320 \\
.2922 \\
.3547 \\
.4143 \\
.4768\end{array}$ & $\begin{array}{r}0.0018 \\
.0020 \\
.0021 \\
.0023 \\
.0024\end{array}$ & $\begin{array}{r}0.2302 \\
.2902 \\
.3526 \\
.4120 \\
.4744\end{array}$ & $\begin{array}{r}0.2159 \\
.2742 \\
.3367 \\
.3937 \\
.4562\end{array}$ \\
\hline $\begin{array}{l}9 / 16 \\
5 / 8 \\
3 / 4 \\
7 / 8\end{array}$ & $\begin{array}{l}24 \\
24 \\
20 \\
20\end{array}$ & $\begin{array}{l}.5625 \\
.6250 \\
.7500 \\
.8750\end{array}$ & $\begin{array}{l}.0066 \\
.0066 \\
.0072 \\
.0072\end{array}$ & $\begin{array}{l}.5559 \\
.6184 \\
.7428 \\
.8678\end{array}$ & $\begin{array}{l}.5354 \\
.5979 \\
.7175 \\
.8425\end{array}$ & $\begin{array}{l}.0035 \\
.0036 \\
.0040 \\
.0041\end{array}$ & $\begin{array}{l}.5319 \\
.5943 \\
.7135 \\
.8384\end{array}$ & $\begin{array}{r}.5354 \\
.5979 \\
.7175 \\
.8425\end{array}$ & $\begin{array}{l}.0025 \\
.0026 \\
.0029 \\
.0030\end{array}$ & $\begin{array}{l}.5329 \\
.5953 \\
.7146 \\
.8395\end{array}$ & $\begin{array}{l}.5114 \\
.5739 \\
.6887 \\
.8137\end{array}$ \\
\hline $\begin{array}{l}1 \\
118 \\
11 / 4 \\
11 / 2\end{array}$ & $\begin{array}{l}20 \\
18 \\
18 \\
18\end{array}$ & $\begin{array}{l}1.0000 \\
1.1250 \\
1.2500 \\
1.5000\end{array}$ & $\begin{array}{l}.0072 \\
.0082 \\
.0082 \\
.0082\end{array}$ & $\begin{array}{r}.9928 \\
1.1168 \\
1.2418 \\
1.4918\end{array}$ & $\begin{array}{r}.9675 \\
1.0889 \\
1.2139 \\
1.4639\end{array}$ & $\begin{array}{l}.0042 \\
.0045 \\
.0046 \\
.0048\end{array}$ & $\begin{array}{r}.9633 \\
1.0844 \\
1.2093 \\
1.4591\end{array}$ & $\begin{array}{r}.9675 \\
1.0889 \\
1.2139 \\
1.4639\end{array}$ & $\begin{array}{l}.0031 \\
.0033 \\
.0034 \\
.0036\end{array}$ & $\begin{array}{r}.9644 \\
1.0856 \\
1.2105 \\
1.4603\end{array}$ & $\begin{array}{r}.9387 \\
1.0568 \\
1.1818 \\
1.4318\end{array}$ \\
\hline $\begin{array}{l}13 / 4 \\
2 \\
21 / 4 \\
21 / 2\end{array}$ & $\begin{array}{l}16 \\
16 \\
16 \\
16\end{array}$ & $\begin{array}{l}1.7500 \\
2.0000 \\
2.2500 \\
2.5000\end{array}$ & $\begin{array}{l}.0090 \\
.0090 \\
.0090 \\
.0090\end{array}$ & $\begin{array}{l}1.7410 \\
1.9910 \\
2.2410 \\
2.4910\end{array}$ & $\begin{array}{l}\text { 1. } 7094 \\
\text { 1. } 9594 \\
\text { 2. } 2094 \\
\text { 2. } 4594\end{array}$ & $\begin{array}{l}.0051 \\
.0053 \\
.0055 \\
.0057\end{array}$ & $\begin{array}{l}1.7043 \\
1.9541 \\
2.2039 \\
2.4537\end{array}$ & $\begin{array}{l}\text { 1. } 7094 \\
\text { 1. } 9594 \\
\text { 2. } 2094 \\
\text { 2. } 4594\end{array}$ & $\begin{array}{l}.0039 \\
.0041 \\
.0042 \\
.0044\end{array}$ & $\begin{array}{l}\text { 1. } 7055 \\
1.9553 \\
2.2052 \\
2.4550\end{array}$ & $\begin{array}{l}1.6733 \\
1.9233 \\
2.1733 \\
2.4233\end{array}$ \\
\hline $\begin{array}{l}23 / 4 \\
3 \\
31 / 2 \\
4\end{array}$ & $\begin{array}{l}16 \\
16 \\
16 \\
16\end{array}$ & $\begin{array}{l}2.7500 \\
3.0000 \\
3.5000 \\
4.0000\end{array}$ & $\begin{array}{l}.0090 \\
.0090 \\
.0090 \\
.0090\end{array}$ & $\begin{array}{l}2.7410 \\
2.9910 \\
3.4910 \\
3.9910\end{array}$ & $\begin{array}{l}\text { 2. } 7094 \\
\text { 2. } 9594 \\
\text { 3. } 4594 \\
\text { 3. } 9594\end{array}$ & $\begin{array}{l}.0058 \\
.0060 \\
.0062 \\
.0065\end{array}$ & $\begin{array}{l}2.7036 \\
2.9534 \\
3.4532 \\
3.9529\end{array}$ & $\begin{array}{l}\text { 2. } 7094 \\
2.9594 \\
3.4594 \\
3.9594\end{array}$ & $\begin{array}{l}.0046 \\
.0047 \\
.0050 \\
.0053\end{array}$ & $\begin{array}{l}2.7048 \\
2.9547 \\
3.4544 \\
3.9541\end{array}$ & $\begin{array}{l}\text { 2. } 6733 \\
2.9233 \\
3.4233 \\
\text { 3. } 9233\end{array}$ \\
\hline
\end{tabular}

1 Basic diameter.

2 The tolerances specified for pitch diameter are cumulative and include errors of lead and angle.

Dimensions given are figured to the intersection of the worn tool arc with a center line through crest and root. Minimum flat at root equals $16 \times p$. 
TABLES 16 and 18.-Free fit (class $C$ ) and medium fit (class $D$ ) for nuts in the extra-fine-thread scries

[Dimensions in inches]

\begin{tabular}{|c|c|c|c|c|c|c|c|c|c|c|c|}
\hline \multirow{3}{*}{ Size } & \multirow{3}{*}{$\begin{array}{l}\text { Threads } \\
\text { per inch }\end{array}$} & \multirow{3}{*}{$\begin{array}{l}\text { Mini- } \\
\text { mum } \\
\text { major } \\
\text { diam- } \\
\text { eter } 1\end{array}$} & \multicolumn{6}{|c|}{ Pitch diameter } & \multicolumn{3}{|c|}{ Minor diameter } \\
\hline & & & \multicolumn{3}{|c|}{ Class C } & \multicolumn{3}{|c|}{ Class D } & \multirow{2}{*}{$\begin{array}{l}\text { Mini- } \\
\text { mum }\end{array}$} & \multirow{2}{*}{$\begin{array}{l}\text { Toler- } \\
\text { ance }\end{array}$} & \multirow{2}{*}{$\begin{array}{l}\text { Maxi- } \\
\text { mum }\end{array}$} \\
\hline & & & $\begin{array}{l}\text { Mini- } \\
\text { mum }{ }^{2}\end{array}$ & $\begin{array}{l}\text { Toler- } \\
\text { ance }^{3}\end{array}$ & $\begin{array}{l}\text { Maxi- } \\
\text { mum }\end{array}$ & $\begin{array}{l}\text { Mini- } \\
\text { mum } 2\end{array}$ & $\begin{array}{l}\text { Toler- } \\
\text { ance }^{3}\end{array}$ & $\begin{array}{l}\text { Maxi- } \\
\text { mum }\end{array}$ & & & \\
\hline $\begin{array}{r}1 / 4 \\
5 / 16 \\
3 / 8 \\
7 / 16 \\
1 / 2\end{array}$ & $\begin{array}{l}36 \\
32 \\
32 \\
28 \\
28\end{array}$ & $\begin{array}{r}0.2500 \\
.3125 \\
.3750 \\
.4375 \\
.5000\end{array}$ & $\begin{array}{r}0.2320 \\
.2922 \\
.3547 \\
.4143 \\
.4768\end{array}$ & $\begin{array}{r}0.0027 \\
.0029 \\
.0030 \\
.0032 \\
.0033\end{array}$ & $\begin{array}{r}0.2347 \\
.2951 \\
.3577 \\
.4175 \\
.4801\end{array}$ & $\begin{array}{r}0.2320 \\
.2922 \\
.3547 \\
.4143 \\
.4768\end{array}$ & $\begin{array}{r}0.0018 \\
.0020 \\
.0021 \\
.0023 \\
.0024\end{array}$ & $\begin{array}{r}0.2338 \\
.2942 \\
.3568 \\
.4166 \\
.4792\end{array}$ & $\begin{array}{r}0.2199 \\
.2787 \\
.3412 \\
.3988 \\
.4613\end{array}$ & $\begin{array}{r}0.0030 \\
.0034 \\
.0034 \\
.0039 \\
.0039\end{array}$ & $\begin{array}{r}0.2229 \\
.2821 \\
.3446 \\
.4027 \\
.4652\end{array}$ \\
\hline $\begin{array}{r}9 / 16 \\
5 / 8 \\
3 / 4 \\
7 / 8\end{array}$ & $\begin{array}{l}24 \\
24 \\
20 \\
20\end{array}$ & $\begin{array}{l}.5625 \\
.6250 \\
.7500 \\
.8750\end{array}$ & $\begin{array}{l}.5354 \\
.5979 \\
.7175 \\
.8425\end{array}$ & $\begin{array}{l}.0035 \\
.0036 \\
.0040 \\
.0041\end{array}$ & $\begin{array}{l}.5389 \\
.6015 \\
.7215 \\
.8466\end{array}$ & $\begin{array}{l}.5354 \\
.5979 \\
.7175 \\
.8425\end{array}$ & $\begin{array}{l}.0025 \\
.0026 \\
.0029 \\
.0030\end{array}$ & $\begin{array}{l}.5379 \\
.6005 \\
.7204 \\
.8455\end{array}$ & $\begin{array}{l}.5174 \\
.5799 \\
.6959 \\
.8209\end{array}$ & $\begin{array}{l}.0045 \\
.0045 \\
.0054 \\
.0054\end{array}$ & $\begin{array}{l}.5219 \\
.5844 \\
.7013 \\
.8263\end{array}$ \\
\hline $\begin{array}{l}1 \\
11 / 8 \\
11 / 4 \\
11 / 2\end{array}$ & $\begin{array}{l}20 \\
18 \\
18 \\
18\end{array}$ & $\begin{array}{l}1.0000 \\
1.1250 \\
1.2500 \\
1.5000\end{array}$ & $\begin{array}{r}.9675 \\
1.0889 \\
1.2139 \\
1.4639\end{array}$ & $\begin{array}{l}.0042 \\
.0045 \\
.0046 \\
.0048\end{array}$ & $\begin{array}{r}.9717 \\
1.0934 \\
1.2185 \\
1.4687\end{array}$ & $\begin{array}{r}.9675 \\
1.0889 \\
1.2139 \\
1.4639\end{array}$ & $\begin{array}{l}.0031 \\
.0033 \\
.0034 \\
.0036\end{array}$ & $\begin{array}{r}.9706 \\
1.0922 \\
1.2173 \\
1.4675\end{array}$ & $\begin{array}{r}.9459 \\
1.0649 \\
1.1899 \\
1.4399\end{array}$ & $\begin{array}{l}.0054 \\
.0060 \\
.0060 \\
.0060\end{array}$ & $\begin{array}{l}.9513 \\
\text { 1. } 0709 \\
1.1959 \\
1.4459\end{array}$ \\
\hline $\begin{array}{l}13 / 4 \\
2 \\
21 / 4 \\
21 / 2\end{array}$ & $\begin{array}{l}16 \\
16 \\
16 \\
16\end{array}$ & $\begin{array}{l}1.7500 \\
2.0000 \\
2.2500 \\
2.5000\end{array}$ & $\begin{array}{l}1.7094 \\
1.9594 \\
2.2094 \\
2.4594\end{array}$ & $\begin{array}{l}.0051 \\
.0053 \\
.0055 \\
.0057\end{array}$ & $\begin{array}{l}1.7145 \\
1.9647 \\
2.2149 \\
2.4651\end{array}$ & $\begin{array}{l}1.7094 \\
1.9594 \\
2.2094 \\
2.4594\end{array}$ & $\begin{array}{l}.0039 \\
.0041 \\
.0042 \\
.0044\end{array}$ & $\begin{array}{l}1.7133 \\
1.9635 \\
2.2136 \\
2.4638\end{array}$ & $\begin{array}{l}1.6523 \\
1.9323 \\
2.1823 \\
2.4323\end{array}$ & $\begin{array}{l}.0068 \\
.0068 \\
.0068 \\
.0068\end{array}$ & $\begin{array}{l}\text { 1. } 6591 \\
1.9391 \\
2.1891 \\
2.4391\end{array}$ \\
\hline $\begin{array}{l}23 / 4 \\
3 \\
31 / 2 \\
4\end{array}$ & $\begin{array}{l}16 \\
16 \\
16 \\
16\end{array}$ & $\begin{array}{l}\text { 2. } 7500 \\
\text { 3. } 0000 \\
\text { 3. } 5000 \\
\text { 4. } 0000\end{array}$ & $\begin{array}{l}\text { 2. } 7094 \\
2.9594 \\
3.4594 \\
3.9594\end{array}$ & $\begin{array}{l}.0053 \\
.00000 \\
.0062 \\
.0065\end{array}$ & $\begin{array}{l}\text { 2. } 7152 \\
\text { 2. } 9654 \\
\text { 3. } 4656 \\
\text { 3. } 9659\end{array}$ & $\begin{array}{l}\text { 2. } 7094 \\
2.9594 \\
3.4594 \\
3.9594\end{array}$ & $\begin{array}{l}.0046 \\
.0047 \\
.0050 \\
.0053\end{array}$ & $\begin{array}{l}2.7140 \\
2.9641 \\
3.4644 \\
3.9647\end{array}$ & $\begin{array}{l}\text { 2. } 6823 \\
2.9323 \\
3.4323 \\
3.9323\end{array}$ & $\begin{array}{l}.0068 \\
.0068 \\
.0068 \\
.0068\end{array}$ & $\begin{array}{l}\text { 2. } 6891 \\
2.9391 \\
3.4391 \\
3.9391\end{array}$ \\
\hline
\end{tabular}

1 Basic diameter. Dimensions given are allowable only with tap having theoretically sharp corners. Threaded hole must not reject correct basic "Go" gage by interference with rounded roots due to worn tap. Minimum flat at root equal $1 / 24 \times$.

2 Basic diameter.

3 The tolerances specified for pitch diameter are cumulative and include errors of lead and angle.

TABLE 19.-Basic diameter and thread data for finethread series over $1 \frac{1}{2}$ inches diameter

[Dimensions in inches]

\begin{tabular}{|l|r|r|r|r|r|r|}
\hline Size & $\begin{array}{c}\text { Threads } \\
\text { per inch }\end{array}$ & $\begin{array}{c}\text { Major } \\
\text { diam- } \\
\text { eter }\end{array}$ & $\begin{array}{c}\text { Pitch di- } \\
\text { ameter }\end{array}$ & $\begin{array}{c}\text { Minor } \\
\text { diam- } \\
\text { eter }\end{array}$ & Pitch 1 & $\begin{array}{c}\text { Basic depth } \\
\text { of thread }\end{array}$ \\
\cline { 1 - 2 } $13 / 4$ & 12 & 1.750 & 1.6959 & 1.6417 & 0.0833333 & 0.05413 \\
2 & 12 & 2.000 & 1.9459 & 1.8917 & .0333333 & .05413 \\
$21 / 4$ & 12 & 2.250 & 2.1959 & 2.1417 & .0833333 & .05413 \\
$21 / 2$ & 12 & 2.500 & 2.4459 & 2.3917 & .0833333 & .05413 \\
$23 / 4$ & 12 & 2.750 & 2.6959 & 2.6417 & .0833333 & .05413 \\
3 & 10 & 3.000 & 2.9350 & 2.8701 & .1000000 & .06495 \\
$31 / 2$ & 10 & 3.500 & 3.4350 & 3.3701 & .1000000 & .06495 \\
4 & 10 & 4.000 & 3.9350 & 3.8701 & .1000000 & .06495 \\
$41 / 2$ & 10 & 4.500 & 4.4350 & 4.3701 & .1000000 & .06495 \\
5 & 10 & 5.000 & 4.9350 & 4.8701 & .1000000 & .06495 \\
$51 / 2$ & 10 & 5.500 & 5.4350 & 5.3701 & .1000000 & .06495 \\
6 & 10 & 6.000 & 5.9350 & 5.8701 & .1000000 & .06495 \\
\hline
\end{tabular}
only. 
TABLes 20 and 22.-Free fit (class C) and medium fit (class D) for screws in the fine-thread series over $11 / 2$ inches diameter

[Dimensions in inches]

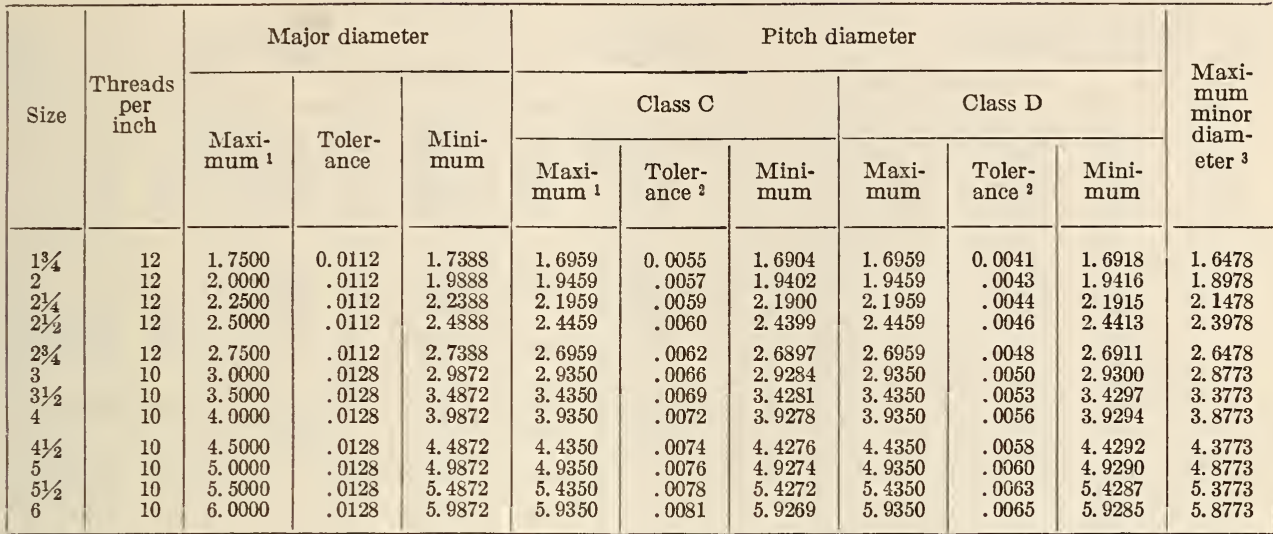

1 Basic diameter.

2 The tolerances specified for pitch diameter are cumulative and include errors of lead and angle.

3 Dimensions given are figured to the intersection of the worn tool are with a center line through crest and root. Minimum flat at root equals $1 / 8 \times p$.

TABLes 21 and 23.-Free fit (class C) and medium fit (class D) for nuts in the fine-thread series over 11/2 inches diameter

[Dimensions in inches]

\begin{tabular}{|c|c|c|c|c|c|c|c|c|c|c|c|}
\hline \multirow{3}{*}{ Size } & \multirow{3}{*}{$\begin{array}{c}\text { Threads } \\
\text { per } \\
\text { inch }\end{array}$} & \multirow{3}{*}{$\begin{array}{l}\text { Mini- } \\
\text { mum } \\
\text { major } \\
\text { diam- } \\
\text { eter }{ }^{2}\end{array}$} & \multicolumn{6}{|c|}{ Pitch diameter } & \multicolumn{3}{|c|}{ Minor diameter } \\
\hline & & & \multicolumn{3}{|c|}{ Class C } & \multicolumn{3}{|c|}{ Class D } & \multirow{2}{*}{$\begin{array}{l}\text { Mini- } \\
\text { mum }\end{array}$} & \multirow{2}{*}{$\begin{array}{l}\text { Toler- } \\
\text { ance }\end{array}$} & \multirow{2}{*}{$\begin{array}{l}\text { Maxi- } \\
\text { mum }\end{array}$} \\
\hline & & & $\begin{array}{l}\text { Mini- } \\
\text { mum } 2\end{array}$ & $\begin{array}{l}\text { Toler- } \\
\text { ance }^{3}\end{array}$ & $\begin{array}{l}\text { Maxi- } \\
\text { mum }\end{array}$ & $\begin{array}{l}\text { Mini- } \\
\text { mum }^{2}\end{array}$ & $\begin{array}{l}\text { Toler- } \\
\text { ance }{ }^{3}\end{array}$ & $\begin{array}{l}\text { Maxi- } \\
\text { mum }\end{array}$ & & & \\
\hline $\begin{array}{l}13 / 4 \\
2 \\
21 / 4 \\
21 / 2\end{array}$ & $\begin{array}{l}12 \\
12 \\
12 \\
12\end{array}$ & $\begin{array}{l}\text { 1. } 7500 \\
2.0000 \\
2.2500 \\
2.5000\end{array}$ & $\begin{array}{l}1.6959 \\
1.9459 \\
2.1959 \\
2.4459\end{array}$ & $\begin{array}{r}0.0055 \\
.0057 \\
.0059 \\
.0060\end{array}$ & $\begin{array}{l}\text { 1. } 7014 \\
\text { 1. } 9516 \\
\text { 2. } 2018 \\
\text { 2. } 4519\end{array}$ & $\begin{array}{l}1.6959 \\
1.9459 \\
2.1959 \\
2.4459\end{array}$ & $\begin{array}{r}0.0041 \\
.0043 \\
.0044 \\
.0046\end{array}$ & $\begin{array}{l}1.7000 \\
1.9502 \\
2.2003 \\
2.4505\end{array}$ & $\begin{array}{l}1.6598 \\
1.9098 \\
2.1598 \\
2.4098\end{array}$ & $\begin{array}{r}0.0090 \\
.0090 \\
.0090 \\
.0090\end{array}$ & $\begin{array}{l}1.6688 \\
1.9188 \\
2.1688 \\
2.4188\end{array}$ \\
\hline $\begin{array}{l}23 / 4 \\
3 \\
31 / 2 \\
4\end{array}$ & $\begin{array}{l}12 \\
10 \\
10 \\
10\end{array}$ & $\begin{array}{l}\text { 2. } 7500 \\
\text { 3. } 0000 \\
\text { 3. } 5000 \\
4.0000\end{array}$ & $\begin{array}{l}2.6959 \\
2.9350 \\
3.4350 \\
3.9350\end{array}$ & $\begin{array}{l}.0062 \\
.0066 \\
.0069 \\
.0072\end{array}$ & $\begin{array}{l}2.7021 \\
2.9416 \\
3.4419 \\
3.9422\end{array}$ & $\begin{array}{l}\text { 2. } 6959 \\
2.9350 \\
3.4350 \\
3.9350\end{array}$ & $\begin{array}{l}.0048 \\
.0050 \\
.0053 \\
.0056\end{array}$ & $\begin{array}{l}2.7007 \\
2.9400 \\
\text { 3. } 4403 \\
\text { 3. } 9406\end{array}$ & $\begin{array}{l}2.6598 \\
2.8917 \\
3.3917 \\
3.8917\end{array}$ & $\begin{array}{l}.0090 \\
.0108 \\
.0108 \\
.0108\end{array}$ & $\begin{array}{l}2.6688 \\
2.9025 \\
3.4025 \\
3.9025\end{array}$ \\
\hline $\begin{array}{l}41 / 2 \\
5 \\
51 / 2 \\
6\end{array}$ & $\begin{array}{l}10 \\
10 \\
10 \\
10\end{array}$ & $\begin{array}{l}4.5000 \\
5.0000 \\
5.5000 \\
6.0000\end{array}$ & $\begin{array}{l}4.4350 \\
4.9350 \\
5.4350 \\
5.9350\end{array}$ & $\begin{array}{l}.0074 \\
.0076 \\
.0078 \\
.0081\end{array}$ & $\begin{array}{l}\text { 4. } 4424 \\
\text { 4. } 9426 \\
\text { 5. } 4428 \\
\text { 5. } 9431\end{array}$ & $\begin{array}{l}4.4350 \\
4.9350 \\
5.4350 \\
5.9350\end{array}$ & $\begin{array}{l}.0058 \\
.0060 \\
.0063 \\
.0065\end{array}$ & $\begin{array}{l}4.4408 \\
4.9410 \\
5.4413 \\
5.9415\end{array}$ & $\begin{array}{l}4.3917 \\
4.8917 \\
5.3917 \\
5.8917\end{array}$ & $\begin{array}{l}.0108 \\
.0108 \\
.0108 \\
.0108\end{array}$ & $\begin{array}{l}4.4025 \\
4.9025 \\
5.4025 \\
5.9025\end{array}$ \\
\hline
\end{tabular}

71 Basic diameter. Dimensions given are allowable only with tap having theoretically sharp corners. Threaded hole must not reject correct basic "Go" gage by interference with rounded roots due to worn tap. Minimum flat at root equals $1 / 24 \times p$.

2 Basic diameter.

3 The tolerances specined for pitch diameter are cumulative and include errors of lead and angle.

SOCIETY OF AUTOMOTIVE ENGINEERS, SCREW THREADS; TAP-DRILL REFERENCE TABLES, MARCH, 1925

\section{TAP-DRILL REFERENCE TABLES}

\section{GENERAL INFORMATION}

The following reference tables give the nearest drill sizes for the basic, maximum, and minimum minor diameters specified in the American standard for screw threads. Where possible, drill sizes are specified that are within the minor-diameter limits, but drill sizes without the limits are so noted unless they are without the minor-diameter limits by less than one-half of 1 per cent of the major diameter. The tables serve to determine:

1. Dimensions of minor-diameter-limit gages.

2. The sizes of tap drills within the limits.

3 . The desired diameters of tap reamers.

The tap-drill tables give the tap drills recommended for general use. It should be noted that:
1. Sizes not marked by the superior letter " $a$ " or " $b$ " lie near the mean, favoring the high limit where the pitch is coarse relative to the screw size, and the low limit where the pitch is fine relative to the screw size; thus favoring the taps of small diameters and guarding against shallow threads in the large diameters. Used under normal conditions, these drills should produce holes that, when tapped, will be within the minor-diameter limits.

2. Sizes marked "a" are not recommended if the drill is allowed to cut larger than its nominal size.

3. Sizes marked "b" are preferable where the lengths of thread are less than three-fourths the major diameters in the smaller diameters, or are less than about two-thirds the major diameters in the larger diameters.

4. The size of drill best suited to a specific application is dependent upon the material, the nature of the cutting lubricant, the methods of drilling and tapping and the condition of the drill. To insure minor diameters acceptable to standard limit gages, the drills may have to be specially selected to meet these conditions. 
Minor diameters for extra-fine threads with nearest drill sizes

[Dimensions in inches]

\begin{tabular}{|c|c|c|c|c|c|c|c|c|c|c|}
\hline \multirow{2}{*}{$\begin{array}{c}\text { Diam- } \\
\text { eter }\end{array}$} & \multirow{2}{*}{$\begin{array}{l}\text { Threads } \\
\text { per inch }\end{array}$} & \multirow{2}{*}{$\begin{array}{c}\text { Bore, basic } \\
\text { thread } \\
\text { depth } 1\end{array}$} & \multicolumn{2}{|c|}{ Drill size } & \multirow{2}{*}{$\begin{array}{c}\text { Bore, } 5 / 6 \\
\text { thread } \\
\text { depth }{ }^{2}\end{array}$} & \multicolumn{2}{|c|}{ Drill size } & \multirow{2}{*}{$\begin{array}{c}\text { Bore, } 3 / 4 \\
\text { thread } \\
\text { depth }{ }^{3}\end{array}$} & \multicolumn{2}{|c|}{ Drill size } \\
\hline & & & Decimal & Nominal & & Decimal & Nominal & & Decimal & Nominal \\
\hline $\begin{array}{r}1 / 4 \\
5 / 16 \\
3 / 8 \\
7 / 16 \\
1 / 2\end{array}$ & $\begin{array}{l}36 \\
32 \\
32 \\
28 \\
28\end{array}$ & $\begin{array}{r}0.21392 \\
.27191 \\
.33441 \\
.39111 \\
.45361\end{array}$ & $\begin{array}{r}0.2130 \\
.2720 \\
.3346 \\
.3906 \\
.4531\end{array}$ & $\begin{array}{l}\text { No. } 3 \\
\text { I } \\
8.5 \mathrm{~mm} \\
25,64 \\
29 / 64\end{array}$ & $\begin{array}{r}0.21993 \\
.27867 \\
.34117 \\
.39884 \\
.46134\end{array}$ & $\begin{array}{r}0.2205 \\
.2795 \\
.3425 \\
.3970\end{array}$ & $\begin{array}{l}5.6 \mathrm{~mm} \\
7.1 \mathrm{~mm} \\
8.7 \mathrm{~mm} \\
\mathbf{X}\end{array}$ & $\begin{array}{r}0.22294 \\
.28205 \\
.34455 \\
.40270 \\
.46520\end{array}$ & $\begin{array}{r}0.2210 \\
.2812 \\
.3437 \\
.4040 \\
.4687\end{array}$ & $\begin{array}{l}\text { No. } 2 . \\
9 / 32 . \\
11 / 32 . \\
Y . \\
15 / 32 .{ }^{a}\end{array}$ \\
\hline $\begin{array}{l}9 / 16 \\
5 / 8 \\
3 / 4 \\
7 / 8 \\
1^{7 / 8}\end{array}$ & $\begin{array}{l}24 \\
24 \\
20 \\
20 \\
20\end{array}$ & $\begin{array}{l}.50837 \\
.57087 \\
.68505 \\
.81005 \\
.93505\end{array}$ & $\begin{array}{l}.5118 \\
.5709 \\
.6875 \\
.8125 \\
.9375\end{array}$ & $\begin{array}{l}13.0 \mathrm{~mm} \\
14.5 \mathrm{~mm} \\
11 / 16 \\
13 / 16 \\
15 / 16 \ldots\end{array}$ & $\begin{array}{l}.51739 \\
.57989 \\
.69587 \\
.82087 \\
.94587\end{array}$ & $\begin{array}{r}.5156 \\
.5781 \\
.9449\end{array}$ & 33/64 & $\begin{array}{l}.52191 \\
.58441 \\
.70129 \\
.82629 \\
.95129\end{array}$ & $\begin{array}{l}.7031 \\
.8268 \\
.9531\end{array}$ & $\begin{array}{l}45 / 64 . \\
21.0 \mathrm{~mm} . \\
61 / 64 .\end{array}$ \\
\hline $\begin{array}{l}11 / 8 \\
11 / 4 \\
11 / 2\end{array}$ & $\begin{array}{l}18 \\
18 \\
18\end{array}$ & $\begin{array}{l}1.05283 \\
1.17783 \\
1.42783\end{array}$ & $\begin{array}{l}1.0469 \\
1.1811 \\
\end{array}$ & $13 / 64^{a}$ & $\begin{array}{l}\text { 1. } 06486 \\
\text { 1. } 18986 \\
\text { 1. } 43986\end{array}$ & $\begin{array}{l}1.0630 \\
1.1875 \\
1.4375\end{array}$ & 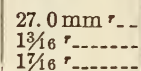 & $\begin{array}{l}\text { 1. } 07087 \\
1.19587 \\
1.44587\end{array}$ & 1.2008 & $30.5 \mathrm{~mm}$. \\
\hline $2^{3 / 4}$ & $\begin{array}{l}16 \\
16\end{array}$ & $\begin{array}{l}\text { 1. } 66881 \\
\text { 1. } 91881\end{array}$ & $\begin{array}{l}1.6718 \\
1.9218\end{array}$ & $\begin{array}{l}143 / 64 \ldots \\
159 \% 64 \ldots\end{array}$ & $\begin{array}{l}\text { 1. } 68234 \\
\text { 1. } 93234\end{array}$ & 1.9291 & $49.0 \mathrm{~mm}$ & $\begin{array}{l}\text { 1. } 68911 \\
\text { 1. } 93911\end{array}$ & $\begin{array}{l}1.6875 \\
1.9375\end{array}$ & $\begin{array}{l}111 / 16^{\circ} . \\
115 / 16^{\circ} .\end{array}$ \\
\hline $\begin{array}{l}21 / 4 \\
21 / 2 \\
23 / 4 \\
3\end{array}$ & $\begin{array}{l}16 \\
16 \\
16 \\
16\end{array}$ & $\begin{array}{l}\text { 2. } 16881 \\
2.41881 \\
\text { 2. } 66881 \\
2.91881\end{array}$ & $\begin{array}{l}2.1718 \\
2.4212 \\
2.6718 \\
2.9218\end{array}$ & $\begin{array}{l}211 / 64 \\
61.5 \mathrm{~mm} \\
2^{43} / 64 \\
259 / 64\end{array}$ & $\begin{array}{l}\text { 2. } 18234 \\
\text { 2. } 43234 \\
\text { 2. } 68234 \\
\text { 2. } 93234\end{array}$ & $\begin{array}{c}2.1851 \\
2.9330\end{array}$ & $55.5 \mathrm{~mm}$ & $\begin{array}{l}\text { 2. } 18911 \\
\text { 2. } 43911 \\
\text { 2. } 68911 \\
\text { 2. } 93911\end{array}$ & $\begin{array}{l}2.1875 \\
2.4375 \\
2.6875 \\
2.9375\end{array}$ & $\begin{array}{l}23 / 16^{\circ} . \\
27 / 16^{\circ} . \\
211 / 16^{\circ} . \\
215 / 16^{\circ} .\end{array}$ \\
\hline
\end{tabular}

1 Theoretical thread depth.

Minimum minor diameter.

3 Maximum minor diameter.

a Nearest size drill available is outside the minor-diameter limits by more than one-half of 1 per cent of the diameter.

- Reamer size.

Minor diameters for fine threads with nearest drill sizes

[Dimensions in inches]

\begin{tabular}{|c|c|c|c|c|c|c|c|c|c|c|}
\hline \multirow{2}{*}{$\begin{array}{l}\text { Diam- } \\
\text { eter }\end{array}$} & \multirow{2}{*}{$\begin{array}{l}\text { Threads } \\
\text { per inch }\end{array}$} & \multirow{2}{*}{$\begin{array}{l}\text { Bore, full } \\
\text { thread } \\
\text { depth } 1\end{array}$} & \multicolumn{2}{|c|}{ Drill size } & \multirow{2}{*}{$\begin{array}{l}\text { Bore, 5/6 } \\
\text { thread } \\
\text { depth }{ }^{2}\end{array}$} & \multicolumn{2}{|c|}{ Drill size } & \multirow{2}{*}{$\begin{array}{l}\text { Bore, } 3 / 4 \\
\text { thread } \\
\text { depth }\end{array}$} & \multicolumn{2}{|c|}{ Drill size } \\
\hline & & & Decimal & Nominal & & Decimal & Nominal & & Decimal & Nominal \\
\hline $\begin{array}{l}0 \\
1 \\
2 \\
3 \\
4\end{array}$ & $\begin{array}{l}80 \\
72 \\
64 \\
56 \\
48\end{array}$ & $\begin{array}{r}0.04376 \\
.05496 \\
.06570 \\
.07580 \\
.08494\end{array}$ & $\begin{array}{r}0.0433 \\
.0550 \\
.0650 \\
.0760 \\
.0846\end{array}$ & $\begin{array}{l}1.10 \mathrm{~mm} \\
\text { No. } 54 \\
1.65 \mathrm{~mm} \\
\text { No.... } 48 \\
2.15 \mathrm{~mm}\end{array}$ & $\begin{array}{r}0.04647 \\
.05796 \\
.06909 \\
.07967 \\
.08945\end{array}$ & $\begin{array}{r}0.04650 \\
.05710 \\
.06890 \\
.08070 \\
.08900\end{array}$ & $\begin{array}{l}\text { No. } 56 . . .- \\
1.45 \mathrm{~mm}{ }^{\circ}-- \\
1.75 \mathrm{~mm} . . .- \\
2.05 \mathrm{~mm} . . .- \\
\text { No. } 43 . . .-\end{array}$ & $\begin{array}{r}0.04782 \\
.05947 \\
.07078 \\
.08160 \\
.09170\end{array}$ & $\begin{array}{r}0.0472 \\
.0595 \\
.0709 \\
.0820 \\
.0905\end{array}$ & $\begin{array}{l}1.20 \mathrm{~mm} \text {. } \\
\text { No. } 53 . \\
1.80 \mathrm{~mm} \text {. } \\
\text { No. } 45 \\
2.30 \mathrm{~mm} \text {. }\end{array}$ \\
\hline $\begin{array}{r}5 \\
6 \\
8 \\
10 \\
12\end{array}$ & $\begin{array}{l}44 \\
40 \\
36 \\
32 \\
28\end{array}$ & $\begin{array}{l}.09548 \\
.10552 \\
.12792 \\
.14941 \\
.16961\end{array}$ & $\begin{array}{l}.0960 \\
.1063 \\
.1285 \\
.1495 \\
.1695\end{array}$ & $\begin{array}{l}\text { No. } 41 . . . \\
2.70 \mathrm{~mm} . \\
\text { No. } 30 \ldots . . \\
\text { No. } 25 \ldots \\
\text { No. } 18 . . .\end{array}$ & $\begin{array}{l}.10040 \\
.11094 \\
.13393 \\
.15617 \\
.17734\end{array}$ & $\begin{array}{l}.10150 \\
.11100 \\
.13390 \\
.15620 \\
.17720\end{array}$ & $\begin{array}{l}\text { No. } 38 \\
\text { No. } 34 \\
3.40 \mathrm{~mm} \\
5 \% 32 \\
4.50 \mathrm{~mm}\end{array}$ & $\begin{array}{l}.10286 \\
.11364 \\
.13694 \\
.15955 \\
.18120\end{array}$ & $\begin{array}{l}.1024 \\
.1130 \\
.1360 \\
.1590 \\
.1811\end{array}$ & $\begin{array}{l}2.60 \mathrm{~mm} \text {. } \\
\text { No. } 33 \text {. } \\
\text { No. } 29 . \\
\text { No. } 21 . \\
4.60 \mathrm{~mm} \text {. }\end{array}$ \\
\hline $\begin{array}{l}1 / 4 \\
5 / 16 \\
3 / 8 \\
7 / 16 \\
1 / 2\end{array}$ & $\begin{array}{l}28 \\
24 \\
24 \\
20 \\
20\end{array}$ & $\begin{array}{l}.20361 \\
.25837 \\
.32087 \\
.37255 \\
.43505\end{array}$ & $\begin{array}{l}.2040 \\
.2598 \\
.3228 \\
.3740 \\
.4375\end{array}$ & $\begin{array}{l}\text { No. } 6 \\
6.6 \mathrm{~mm} . . \\
8.2 \mathrm{~mm} \\
9.5 \mathrm{~mm} . \\
7 / 16\end{array}$ & $\begin{array}{l}.21134 \\
.26739 \\
.32989 \\
.38337 \\
.44587\end{array}$ & $\begin{array}{l}.21260 \\
.26770 \\
.33070 \\
.38580\end{array}$ & $\begin{array}{l}5.40 \mathrm{~mm} . \\
6.8 \mathrm{~mm} . . \\
8.4 \mathrm{~mm} . \\
9.8 \mathrm{~mm} . \\
\end{array}$ & $\begin{array}{l}.21520 \\
.27191 \\
.33441 \\
.38879 \\
.45129\end{array}$ & $\begin{array}{l}.2165 \\
.2720 \\
.3346 \\
.3898 \\
.4527\end{array}$ & $\begin{array}{l}5.5 \mathrm{~mm} .{ }^{a} \\
\mathrm{I} . \\
8.5 \mathrm{~mm} \text {. } \\
9.9 \mathrm{~mm} \text {. } \\
11.5 \mathrm{~mm} \text {. }\end{array}$ \\
\hline $\begin{array}{l}9 / 16 \\
5 / 8 \\
3 / 4 \\
7 / 8 \\
1^{7 / 8}\end{array}$ & $\begin{array}{l}18 \\
18 \\
16 \\
14 \\
14\end{array}$ & $\begin{array}{l}.49033 \\
.55283 \\
.66881 \\
.78221 \\
.90721\end{array}$ & $\begin{array}{l}.4921 \\
.5512 \\
.6693 \\
.78125 \\
.90625\end{array}$ & $\begin{array}{l}12.5 \mathrm{~mm} .- \\
14.0 \mathrm{~mm} \\
17.0 \mathrm{~mm}- \\
25 / 32 \\
29 / 32\end{array}$ & $\begin{array}{l}.50236 \\
.56486 \\
.68234 \\
.79768 \\
.92268\end{array}$ & $\begin{array}{l}.50000 \\
.56250 \\
.68750 \\
.79688 \\
.92188\end{array}$ & $\begin{array}{l}1 / 2 \ldots \ldots \\
916 \ldots \ldots \\
11116 \ldots \ldots \\
5164 \ldots \ldots \\
5964 \ldots \ldots \ldots\end{array}$ & $\begin{array}{l}.50837 \\
.57087 \\
.68911 \\
.80541 \\
.93041\end{array}$ & $\begin{array}{r}.5118 \\
.5709 \\
.6890 \\
.8071 \\
\end{array}$ & $\begin{array}{l}13.0 \mathrm{~mm} .^{a} \\
14.5 \mathrm{~mm} \text {. } \\
17.5 \mathrm{~mm} \text {. } \\
20.5 \mathrm{~mm} \text {. }\end{array}$ \\
\hline $\begin{array}{l}11 / 8 \\
11 / 4 \\
11 / 2 \\
13 / 4 \\
2\end{array}$ & $\begin{array}{l}12 \\
12 \\
12 \\
12 \\
12\end{array}$ & $\begin{array}{l}1.01675 \\
1.14175 \\
1.39175 \\
1.64175 \\
1,89175\end{array}$ & $\begin{array}{l}1.0156 \\
1.1417 \\
1.3906 \\
1.6406 \\
1.8906\end{array}$ & $\begin{array}{l}11 / 64 \ldots \\
29.0 \mathrm{~mm} \\
125 / 64 \ldots \\
14164 \ldots \\
157 / 64 \ldots\end{array}$ & $\begin{array}{l}1.03479 \\
1.15979 \\
1.40979 \\
1.65979 \\
1.90979\end{array}$ & $\begin{array}{l}1.03130 \\
1.15630 \\
1.4063 \\
1.6563 \\
1.9094\end{array}$ & $\begin{array}{l}11 / 32 \ldots \ldots \\
15 / 32 \ldots \ldots \\
113 / 32 \ldots \ldots \\
121 / 32 \ldots \\
48.5 \mathrm{~mm}\end{array}$ & $\begin{array}{l}1.04381 \\
1.16881 \\
1.41881 \\
1.66881 \\
1.91881\end{array}$ & $\begin{array}{l}1.0433 \\
1.1719 \\
1.4173 \\
1.6718 \\
1.9218\end{array}$ & $\begin{array}{l}26.5 \mathrm{~mm} . \\
11164 . \\
36.0 \mathrm{~mm} \text {. } \\
14364 . \\
15964 .\end{array}$ \\
\hline $\begin{array}{l}21 / 4 \\
21 / 2 \\
23 / 4 \\
3\end{array}$ & $\begin{array}{l}12 \\
12 \\
12 \\
10\end{array}$ & $\begin{array}{l}2.14175 \\
2.39175 \\
2.64175 \\
2.87010\end{array}$ & $\begin{array}{l}2.1406 \\
2.3906 \\
2.6406 \\
2.8740\end{array}$ & $\begin{array}{l}2964 \ldots \\
225 / 64 \ldots \\
241 / 64 \ldots \\
73.0 \mathrm{~mm}\end{array}$ & $\begin{array}{l}2.15979 \\
2.40979 \\
2.65979 \\
2.89175\end{array}$ & $\begin{array}{l}2.1563 \\
2.4063 \\
2.6574 \\
2.8906\end{array}$ & $\begin{array}{l}25 / 32 \ldots \ldots \\
213 / 32 \ldots \\
67.5 \mathrm{~mm} \\
2^{57} / 64 \ldots \ldots\end{array}$ & $\begin{array}{l}2.16881 \\
2.41881 \\
2.66881 \\
2.90257\end{array}$ & $\begin{array}{l}2.1718 \\
2.4212 \\
2.6718 \\
2.9063\end{array}$ & $\begin{array}{l}211 / 64 . \\
61.5 \mathrm{~mm} . \\
2^{43} 34 . \\
2^{29} / 32\end{array}$ \\
\hline
\end{tabular}

1 Theoretical thread depth. $\quad 2$ Minimum minor-diameter. $\quad{ }^{3}$ Maximum minor-diameter.

- The nearest drill size available is outside the minor-diameter limits by more than one-half of 1 per cent of the diameter. 
Minor diameters for coarse threads with nearest drill sizes

[Dimensions in inches]

\begin{tabular}{|c|c|c|c|c|c|c|c|c|c|c|}
\hline \multirow{2}{*}{$\begin{array}{l}\text { Diam. } \\
\text { eter }\end{array}$} & \multirow{2}{*}{$\begin{array}{l}\text { Threads } \\
\text { per inch }\end{array}$} & \multirow{2}{*}{$\begin{array}{l}\text { Bore, full } \\
\text { thread } \\
\text { depth } 1\end{array}$} & \multicolumn{2}{|c|}{ Drill size } & \multirow{2}{*}{$\begin{array}{l}\text { Bore, } 5 / 6 \\
\text { thread } \\
\text { depth }{ }^{2}\end{array}$} & \multicolumn{2}{|c|}{ Drill size } & \multirow{2}{*}{$\begin{array}{l}\text { Bore, 3/4 } \\
\text { thread } \\
\text { depth }{ }^{3}\end{array}$} & \multicolumn{2}{|c|}{ Drill size } \\
\hline & & & Decimal & Nominal & & Decimal & Nominal & & Decimal & Nominal \\
\hline $\begin{array}{l}1 \\
2 \\
2 \\
4 \\
5\end{array}$ & $\begin{array}{l}64 \\
56 \\
48 \\
40 \\
40\end{array}$ & $\begin{array}{r}0.05270 \\
.06280 \\
.07194 \\
.07952 \\
.09252\end{array}$ & $\begin{array}{r}0.0532 \\
.0630 \\
.0728 \\
.0807 \\
.0925\end{array}$ & $\begin{array}{l}1.35 \mathrm{~mm} \\
1.60 \mathrm{~mm} \\
1.85 \mathrm{~mm} \\
2.05 \mathrm{~mm} \\
2.35 \mathrm{~mm}\end{array}$ & $\begin{array}{r}0.05609 \\
.06667 \\
.07645 \\
.08494 \\
.09794\end{array}$ & $\begin{array}{r}0.0551 \\
.0669 \\
.0768 \\
.0846 \\
.0980\end{array}$ & $\begin{array}{l}1.40 \mathrm{~mm} \\
1.70 \mathrm{~mm} \\
1.95 \mathrm{~mm} \\
2.15 \mathrm{~mm} \\
\text { No. } 40\end{array}$ & $\begin{array}{r}0.05778 \\
.06860 \\
.07870 \\
.08764 \\
.10064\end{array}$ & $\begin{array}{r}0.0571 \\
.0889 \\
.0787 \\
.0866 \\
.0995\end{array}$ & $\begin{array}{l}1.45 \mathrm{~mm} . \\
1.75 \mathrm{~mm} . \\
2.0 \mathrm{~mm} . \\
2.20 \mathrm{~mm} \text {. } \\
\text { No. } 39 .\end{array}$ \\
\hline $\begin{array}{l}6 \\
8 \\
10 \\
12 \\
\quad 14\end{array}$ & $\begin{array}{l}32 \\
32 \\
24 \\
24 \\
20\end{array}$ & $\begin{array}{l}.09741 \\
.12341 \\
.13587 \\
.16187 \\
.18505\end{array}$ & $\begin{array}{l}.0980 \\
.1250 \\
.1360 \\
.1614 \\
.1850\end{array}$ & $\begin{array}{l}\text { No. } 40 \\
1 / 8 \\
\text { No. } 29 \\
4.10 \mathrm{~mm} \\
\text { No. } 13\end{array}$ & $\begin{array}{l}.10417 \\
.13017 \\
.14489 \\
.17089 \\
.19587\end{array}$ & $\begin{array}{l}.1040 \\
.1299 \\
.1457 \\
.1719 \\
.1960\end{array}$ & $\begin{array}{l}\text { No. } 37 \\
3.30 \mathrm{~mm} \\
3.70 \mathrm{~mm} \\
1164 \\
\text { No. } 9\end{array}$ & $\begin{array}{l}10755 \\
.13355 \\
.14941 \\
.17541 \\
.20129\end{array}$ & $\begin{array}{l}.1065 \\
.1339 \\
.1495 \\
.1732 \\
.2010\end{array}$ & $\begin{array}{l}\text { No. } 36 . \\
3.40 \mathrm{~mm} .{ }^{a} \\
\text { No. } 25 . \\
4.40 \mathrm{~mm} \text {. } \\
\text { No. } 7 \text {. }\end{array}$ \\
\hline $\begin{array}{l}5 / 16 \\
368 \\
7 / 16 \\
1 / 2 \\
9 / 16\end{array}$ & $\begin{array}{l}18 \\
16 \\
14 \\
13 \\
12\end{array}$ & $\begin{array}{l}.24033 \\
.29381 \\
.34471 \\
.40001 \\
.45425\end{array}$ & $\begin{array}{l}.2402 \\
.2950 \\
.3464 \\
.4040 \\
.4531\end{array}$ & $\begin{array}{l}6.1 \mathrm{~mm} \\
M \\
8.8 \mathrm{~mm} \\
Y \\
2964 \ldots\end{array}$ & $\begin{array}{r}.25236 \\
.30734 \\
.36018 \\
.41673 \\
.47229\end{array}$ & $\begin{array}{l}.2520 \\
.3071 \\
.3594 \\
.4134 \\
.4724\end{array}$ & $\begin{array}{l}6.4 \mathrm{~mm} \\
7.8 \mathrm{~mm} \\
23 / 64 \\
10.5 \mathrm{~mm} \\
12.0 \mathrm{~mm}\end{array}$ & $\begin{array}{l}.25837 \\
.31411 \\
.36791 \\
.42506 \\
.48131\end{array}$ & $\begin{array}{l}.2570 \\
.3125 \\
.3680 \\
.4219 \\
.4844\end{array}$ & $\begin{array}{l}F \\
5 / 16 . \\
U . \\
27 / 64 . \\
31 / 640^{\circ}\end{array}$ \\
\hline $\begin{array}{l}5 / 8 \\
3 / 4 \\
7 / 8 \\
1 \\
11 / 8\end{array}$ & $\begin{array}{r}11 \\
10 \\
9 \\
8 \\
7\end{array}$ & $\begin{array}{r}.50691 \\
.62010 \\
.73066 \\
.83762 \\
.93942\end{array}$ & $\begin{array}{l}.5118 \\
.6250 \\
.7344 \\
.8437 \\
.9375\end{array}$ & $\begin{array}{l}13.0 \mathrm{~mm} \\
568 \\
47 / 64 \\
27 / 32 \ldots \\
15 / 16 \ldots\end{array}$ & $\begin{array}{r}.52659 \\
.64175 \\
.75472 \\
.86468 \\
.97035\end{array}$ & $\begin{array}{l}.5312 \\
.6406 \\
.7500 \\
.8661 \\
.9687\end{array}$ & $\begin{array}{l}17 / 32 \ldots \\
4164 \ldots \\
3 / 4 a \\
22.0 \mathrm{~mm} \\
31 / 32 \ldots\end{array}$ & $\begin{array}{l}.53643 \\
.65257 \\
.76675 \\
.87822 \\
.98582\end{array}$ & $\begin{array}{l}.6496 \\
.7656 \\
.8750 \\
.9844\end{array}$ & $\begin{array}{l}16.5 \mathrm{~mm} . \\
49 \% 64 . \\
7 / 8 . \\
63 \% 64 .\end{array}$ \\
\hline $\begin{array}{l}11 / 4 \\
11 / 2 \\
13 / 4 \\
2\end{array}$ & $\begin{array}{l}7 \\
6 \\
5 \\
41 / 2\end{array}$ & $\begin{array}{l}1.06442 \\
1.28349 \\
1.49019 \\
1.71132\end{array}$ & $\begin{array}{l}1.0630 \\
1.2812 \\
1.4961 \\
1.7126\end{array}$ & $\begin{array}{l}27.0 \mathrm{~mm} \\
1 \% 32 \\
38.0 \mathrm{~mm} \\
43.5 \mathrm{~mm}\end{array}$ & $\begin{array}{l}\text { 1. } 09535 \\
\text { 1. } 31958 \\
\text { 1. } 53349 \\
\text { 1. } 75944\end{array}$ & $\begin{array}{l}1.0937 \\
1.3190 \\
1.5354 \\
1.7656\end{array}$ & $\begin{array}{l}13 / 32 \\
33.5 \mathrm{~mm} \\
39.0 \mathrm{~mm} \\
14964\end{array}$ & $\begin{array}{l}1.11082 \\
1.33762 \\
1.55514 \\
1.78349\end{array}$ & $\begin{array}{l}1.1093 \\
1.3386 \\
1.5551 \\
1.7812\end{array}$ & $\begin{array}{l}17 / 64 . \\
34.0 \mathrm{~mm} \text {. } \\
39.5 \mathrm{~mm} \text {. } \\
125 / 32 .\end{array}$ \\
\hline $\begin{array}{l}21 / 4 \\
21 / 2 \\
23 / 4 \\
3\end{array}$ & $\begin{array}{l}41 / 2 \\
4 \\
4 \\
4\end{array}$ & $\begin{array}{l}1.96132 \\
2.17524 \\
2.42524 \\
2.67524\end{array}$ & $\begin{array}{l}1.9687 \\
2.1719 \\
2.4219 \\
2.6772\end{array}$ & $\begin{array}{l}131 / 32 \ldots \\
2^{11} 164 \ldots \\
2^{27} / 64 \ldots \\
68.0 \mathrm{~mm}\end{array}$ & $\begin{array}{l}2.00944 \\
2.22940 \\
2.47940 \\
2.72940\end{array}$ & $\begin{array}{l}2.0079 \\
2.2244 \\
2.4803 \\
2.7344\end{array}$ & $\begin{array}{l}51.0 \mathrm{~mm} \\
56.5 \mathrm{~mm} \\
63.0 \mathrm{~mm} \\
247 / 64\end{array}$ & $\begin{array}{l}2.03349 \\
2.25643 \\
2.50643 \\
2.75643\end{array}$ & $\begin{array}{l}2.0312 \\
2.2500 \\
2.5000 \\
2.7559\end{array}$ & $\begin{array}{l}21 / 32 . \\
21 / 4 . \\
21 / 2 . \\
70.0 \mathrm{~mm} .\end{array}$ \\
\hline
\end{tabular}

1 Theoretical thread depth.

2 Minimum minor-diameter.

${ }^{3}$ Maximum minor-diameter.

a The nearest drill size available is outside the minor-diameter limits by more than one-half of 1 per cent of the diameter.

Tap drills for coarse and fine screw-thread series

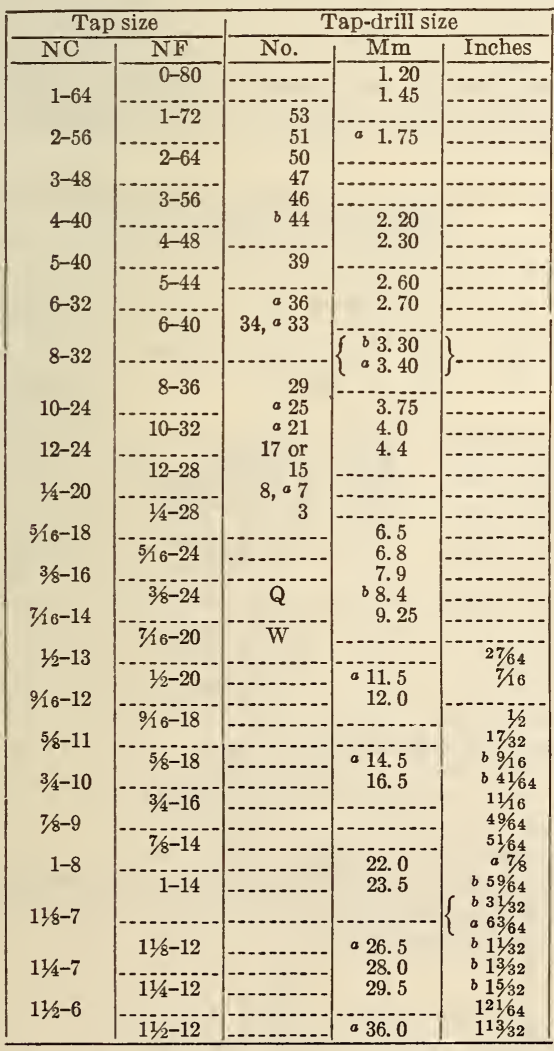

a Not recommended if drill is allowed to cut larger than its nominal size.

$b$ Preferable for thread lengths less than two-thirds of the nominal screw size.
Tap drills for extra-fine screw-thread series

\begin{tabular}{|c|c|c|c|}
\hline \multirow{2}{*}{$\begin{array}{c}\text { Screw } \\
\text { size }\end{array}$} & \multicolumn{3}{|c|}{ Tap-drill size } \\
\hline & No. & $\mathrm{Mm}$ & Inches \\
\hline $\begin{array}{r}1 / 4-36 \\
5 / 16-32 \\
3 / 8-32\end{array}$ & ${ }^{a} \mathrm{~K}$ & $\begin{array}{r}5.6 \\
6.1 \\
8.7\end{array}$ & a $11 / 32$ \\
\hline $7 / 16-28$ & $\bar{b}, a Y$ & & \\
\hline $1 / 2-28$ & & & $\begin{array}{l}\text { a } 15 / 32 \\
\text { b } 29 / 64\end{array}$ \\
\hline $\begin{array}{r}9 / 16-24 \\
5 / 8-24\end{array}$ & & ....... & $\begin{array}{r}3364 \\
37 / 64\end{array}$ \\
\hline $3 / 4-20$ & & $b 17.5$ & a $45 \% 64$ \\
\hline $\begin{array}{r}7 / 8-20 \\
1-20\end{array}$ & & $\begin{array}{l}a \text { r } 21.0 \\
b \quad 24.0\end{array}$ & a $61 / 34$ \\
\hline $11 / 8-18$ & (n) & $\begin{array}{r}24.0 \\
\text { r } 27.0\end{array}$ & $\begin{array}{l}+11 / 16 \\
.64\end{array}$ \\
\hline $11 / 4-18$ & & 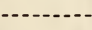 & ? $13 / 16$ \\
\hline
\end{tabular}

a Not recommended if drill is allowed to cut larger than its nominal size.
$b$ Preferable for thread lengths less than two-thirds of the nominal screw size.

$r$ Reamer size.

\section{UNITED STATES GOVERNMENT, DEPART- MENT OF COMMERCE, BUREAU OF STANDARDS, AMERICAN NATIONAI STANDARD SCREW THREADS (COARSE AND FINE THREAD SERIES) COMMER- CIAL STANDARD CS24-30, 1930}

\section{STANDARD SCREW THREADS}

(This commercial standard makes available for convenient use six of the most essential tables of dimensions of fastening screws as published in the 1928 report of the National Screw Thread Commission. The tables are identical with Tables 1, 2, $15,16,107$, and 108 in Miscellaneous Publication No. 89 , of the Bureau of Standards, given below.) 
UNITED STATES GOVERNMENT, DEPARTMENT OF COMIERCE, BUREAU OF STANDARDS, AIEERICAN NATIONAL SPECIAL SCREW THREADS, COMMERCIAL STANDARD CS25-30, 1930

\section{SPECIAL SCREW THREADS}

(This commercial standard presents in compact form the tables of dimensions of special screw threads having the American national form of thread $\left(60^{\circ}\right)$ as published in the 1928 report of the National Screw Thread Commission. The tables are identical with Tables 27, 28, 29, 30, 31, 87, and 88 in Miscellaneous Publication No. 89, of the Bureau of Standards, given below.)

UNITED STATES GOVERNMENT, FEDERAL SPECIFICATIONS BOARD, FEDERAI SPECIFICATION FOR BOLTS, NUTS, STUDS, AND TAP RIVETS (AND MATERIAL FOR SAME), FE-B-571, OCTOBER 13, 1931

\section{BOLTS, NUTS, STUDS, AND TAP RIVETS}

\section{A. APPLICABLE FEDIRAL SPECIFICATIONS}

Federal Specification QQ-M-151 (F. S. B. No. 339).-Metals; general specifications for, of the issue in effect on date of invitation for bids, forms a part of this specification.

\section{B. TYPES}

B-1. Bolts.- Shall be of the following types:

A. Squarehead bolts.

1. Rough machine bolts.

2. Semifinished machine bolts.

3 . Finished machine bolts.

B. Hexagonal-head bolts.

1. Rough machine bolts.

2. Semifinished machine bolts.

3. Finished machine bolts.

C. Round unslotted head bolts.

1. Machine bolts, button head

2. Carriage bolts, square neck.

B-2. Nuts. - Shall be of the following types:

A. Hexagonal nuts.

1. Rough and semifinished regular nuts.

2. Finished regular nuts.

3. Castellated nuts.

4. Finished and semifinished jam nuts.

5. Light nuts.

B. Square nuts.

1. Rough and semifinished regular nuts.

2. Finished regular nuts.

B-3. Tap rivets. - Shall be of but one type.

\section{MATERIAL AND WORKMANSHIP, ETC.}

C-1. Material.-

C-1a. Unless otherwise specified, all steel rods from which bolts, studs, and tap rivets are to be manufactured shall be of uniform quality.

$\mathrm{C}-1 \mathrm{~b}$. Unless otherwise specified, nuts for class A, B, or C steel bolts shall be of class $\mathrm{C}$ steel.

C-1c. Ferrous materials not covered in paragraph E-1a and nonferrous materials from which bolts, nuts, studs, and tap rivets are to be made, shall con- form, except as to dimensional requirements, to the issue, in effect at date of invitation for bids, of the applicable Federal specifications.

C-2. Workmanship.-

C-2a. The workmanship shall be compatible with the type and grade of product and class of fit and finish specified. The product shall be free from abnormal scale, fins, seams, or other defects. All bolts, nuts, studs, and tap rivets shall be free from any defects which might affect their serviceability.

$\mathrm{C}-2 \mathrm{~b}$. Unless the method of manufacture is specifically stated in the purchase order, the method of manufacture employed for the production of screw threads on bolts, nuts, studs, and tap rivets shall be by chasing, milling, tapping, die cutting, or rolling. Rolled threads on studs will not be permitted.

\section{GENERAL REQUIRZNENTS}

D-1. System of measurement.-All dimensions are stated in inches.

D-2. Screw threads.-Screw thread notation shall conform to Figure 315 under National Screw Thread Commission, subject matter, "screw threads," given below. The form of thread profile shall be the American national form, as covered by Figure 317 , also given below under National Screw Thread Commission, subject matter, screw threads.

D-3. Classes of fit and tolerances. - The class of fit of mating screw threads specified shall be class 1 , loose fit; class 2, free fit; class 3 , medium fit; class 4, close fit; or class 5, wrench fit-as established by the National Screw Thread Commission and determined by thread gages as specified below under National Screw Thread Commission, subject matter, screw threads.

D-4. Thread series.-Unless otherwise specified the number of threads per inch shall be that specified for the national coarse-thread series or the national fine-thread series, as covered by Table 1 . Threads shall be of the coarse-thread series, unless otherwise specified.

TABLE 1.-American national coarse and American national fine thread series

\begin{tabular}{|c|c|c|c|c|c|}
\hline \multirow{2}{*}{ Bolt size } & \multicolumn{2}{|c|}{$\begin{array}{l}\text { Threads per } \\
\text { inch }\end{array}$} & \multirow{2}{*}{ Bolt size } & \multicolumn{2}{|c|}{$\begin{array}{l}\text { Threads per } \\
\text { inch }\end{array}$} \\
\hline & Coarse & Fine & & Coarse & Fine \\
\hline $\begin{array}{l}\text { No. 10 } \\
\text { No. } 12 \\
114 \text { inch } \\
5 / 16 \text { inch } \\
3 / 8 \text { inch.... } \\
7 / 16 \text { inch... }\end{array}$ & $\begin{array}{l}24 \\
24 \\
20 \\
18 \\
16 \\
14\end{array}$ & $\begin{array}{l}32 \\
28 \\
28 \\
24 \\
24 \\
20\end{array}$ & $\begin{array}{l}1 \text { inch } \\
11 / 8 \text { inches....... } \\
11 / 4 \text { inches..... } \\
112 \text { inches...... } \\
13 / 4 \text { inches...... } \\
2 \text { inches........ }\end{array}$ & $\begin{array}{l}8 \\
7 \\
7 \\
6 \\
5 \\
41 / 2\end{array}$ & $\begin{array}{l}14 \\
12 \\
12 \\
12 \\
10 \\
10\end{array}$ \\
\hline $\begin{array}{l}1 / 2 \text { inch... } \\
9 / 16 \text { inch.- } \\
5 / 8 \text { inch... } \\
3 / 4 \text { inch... } \\
7 / 8 \text { inch... }\end{array}$ & $\begin{array}{r}13 \\
12 \\
11 \\
10 \\
9\end{array}$ & $\begin{array}{l}20 \\
18 \\
18 \\
16 \\
14\end{array}$ & $\begin{array}{l}21 / 4 \text { inches....... } \\
21 / 2 \text { inches...... } \\
23 / 4 \text { inches...... } \\
3 \text { inches......... } \\
3 \text { inches......... }\end{array}$ & $\begin{array}{l}41 / 2 \\
4 \\
4 \\
14 \\
31 / 2\end{array}$ & $\begin{array}{l}8 \\
8 \\
8 \\
8 \\
--\end{array}$ \\
\hline
\end{tabular}

1 Optional. Standard for marine work.

\section{E. DETAII REQUIREMENTS}

E-1. Bolts, studs, and tap rivets.-

E-1a. Unless otherwise specified, bolts, studs, and tap rivets shall be of class $\mathrm{A}, \mathrm{B}$, or $\mathrm{C}$ steel, as defined below. (See Par. I-6.) 
Physical and chemical requirements

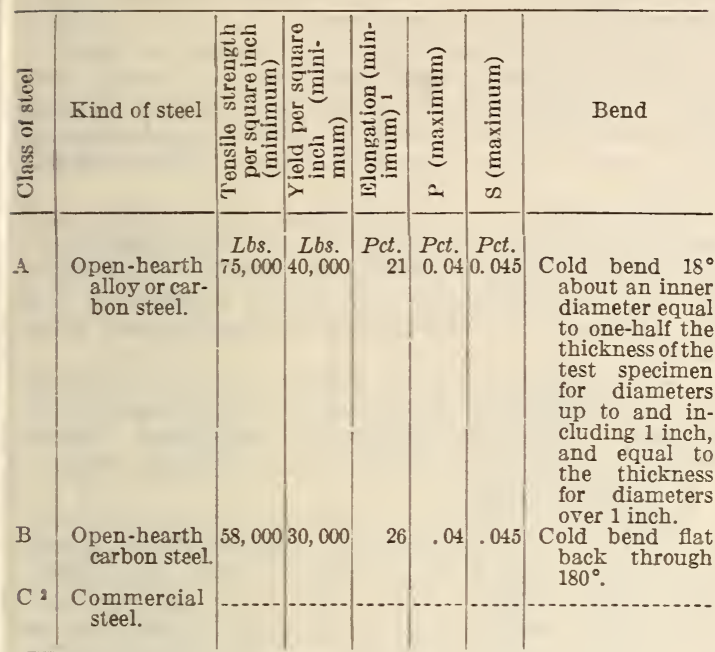

1 Elongation for rods 1/2-inch and less in diameter shall be measured in an original length equal to 12 times the diameter of the test specimen; for material over 36 -inch up to and including 1 inch in diameter the elongation shall be measured in a length of 8 inches; and for material over 1 inch in diameter up to and including 2 inches in diameter the required percentage of elongation, masured in a length of inces, shall be reduced by 1 for each increese in diameter of $1 \%$ inch or fraction theref above 1 inch. or full in full size when practicable. When the material is too large to so test and type 1 test specimen is used, an increase in elongation of 2 units of per cent over that specified in the table will be required.

2 Class C shall not be tested unless considered necessary by th

inspector to show that the material is equal to common steel of commercial quality. When tested the tensile strength shall be not less than 48,000 pounds.

\section{$\mathrm{E}-2$. Bolts and studs.-}

E-2a. The length of bolts and studs shall not vary from the specified length by more than the following:

Up to and including 6 inches, $\pm 1 / 32$ inch

Over 6 inches in length, $\pm 1 / 16$ inch.

$\mathrm{E}-2 \mathrm{~b}$. The dimensions and tolerances for body diameters of bolts and studs shall be as covered by Tables 64 and 65, given below, under National Screw Thread Commission, subject matter, wrench head bolts and nuts, and wrench head openings; introductory notes.

\section{E-3. Bolts -}

E-3a. The tops of heads of square and hexagonal bolts shall be flat and chamfered. The angle of chamfer with the top surface shall be $30^{\circ}$ for hexagonal bolts and $25^{\circ}$ for square bolts. The diameter of the top flat circle shall be from 85 to 100 per cent of the width across flats.

$\mathrm{E}-3 \mathrm{~b}$. The thickness of heads of square and hexagonal bolts shall be the distance from the top to the bearing surface.

E-3c. The width across flats of square and hexagonal boltheads shall be measured at the widest point of the heads. The total taper of any two opposite sides of boltheads shall not exceed $4^{\circ}$.

$\mathrm{E}-3 \mathrm{~d}$. The length of machine bolts shall be measured from the extreme point to the bearing surface of the hear. The length of the thread shall be measured from the extreme point to the last perfect thread.

$\mathrm{E}-3 \mathrm{e}$. Heads of all types of bolts shall be concentric with the body, within a tolerance of 3 per cent of the width across flats.

E-3f. The minimum length of thread of all types of bolts shall, unless otherwise specified, conform to Table 66, under National Screw Thread Commission, subject matter, wrench head bolts and nuts, and wrench openings; macnine bolts. (See 608.31, p. 605.) (Note that in FF-B-571, with diameter of bolt ranging from $5 / 8$ to $1 \frac{1}{4}$ inches and bolt length of 1 to $1 \frac{1}{2}$ inches, the bolt is threaded to the head. This is also true of bolts of diameter 1 to $1 \frac{1 / 4}{4}$ inches and $15 / 8$ to 2 inches long, of bolts of diameter $11 / 8$ and $1 \frac{1}{4}$ inches and $2 \frac{1}{8}$ to $2 \frac{1}{2}$ inches long, and of the $1 \frac{1}{4}$ inch bolt $2 \frac{5}{8}$ to 3 inches long. Note also that here the $7 / 8$-inch bolt $1 \frac{5}{8}$ to 2 inches long is threaded for $1 \frac{5}{8}$ inches instead of $13 / 4$ inches.)

$\mathrm{E}-3 \mathrm{~g}$. When bolts and nuts are ordered together, one nut shall be delivered on each bolt, which shall fit the bolt as specified. Bolts ordered separately shall be of the class of fit specified.

E-3h. Rough machine bolts, types $A-1$ and $B-1$, shall be threaded class 1 , loose fit. Head dimensions shall conform to the requirements covered by Table 67, under National Screw Thread Commission, subject matter, wrench head bolts and nuts, and wrench openings; machine bolts. (See 608.31, p. 605.) Heads shall be at right angles to the body, within $3^{\circ}$.

E-3i. Semifinished machine bolts, types A-2 and B-2, shall be threaded class 3 , medium fit, or class 2 , free fit, as specified. They need not be machined except that the bearing surfaces shall be finished smooth, the ends shall be chamfered, and heads shall conform to the requirements covered by N. S. T. C. Table 67 , referred to in the preceding paragraph. Heads shall be at right angles to the body, within $2^{\circ}$.

E-3j. Finished machine bolts, types $\mathrm{A}-3$ and $\mathrm{B}-3$, shall be threaded class 3 , medium fit, unless otherwise specified. They shall conform to the requirements covered by Table 68, under National Screw Thread Commission, subject matter, wrench head bolts and nuts, and wrench openings; machine bolts. (See 608.31, p. 605.) Heads shall be at right angles to the body, within $2^{\circ}$. All finished bolts shall be washer faced. The thickness of the washer face shall be about one-sixty-fourth inch. The diameter of the bearing surface of the washer face shall equal the width across flats, within plus or minus 5 per cent.

E-3k. Button-head machine bolts, type C-1, and carriage bolts, square neck, type C-2, shall be furnished in sizes from No. 10 to three-fourths inch, inclusive, as required. Unless otherwise specified, they shall be furnished in the coarse-thread series, class 2, free fit. Each bolt shall be furnished with one nut assembled on the bolt.

$\mathrm{E}-3 \mathrm{k}$ (1). The radius of fillet on types $\mathrm{C}-1$ and $\mathrm{C}-2$ bolts shall be as follows:

No. 10 to one-half inch, inclusive, $1 / 32$-inch fillet.

Nine-sixteenths to three-fourths inch, inclusive, 1/16-inch fillet.

E-3k (2). Head dimensions of button-head machine bolts, type $\mathrm{C}-1$, shall conform to Table 5 of American Society of Mechanical Engineers in B18e-1928. (See 608.31, p. 595.) Length of thread shall also conform to this Table 5 .

E-3k (3). Head dimensions of carriage bolts, round head, square neck, type $\mathrm{C}-2$, shall conform to Table 1 of American Society of Miechanical Engineers in B18e-1928. (See 608.31, p. 590.) Length of thread shall also conform to this Table 1 .

E-31. When zinc-coated (galvanized) bolts are required they shall conform to the dimensional requirements before coating. The coating shall be thoroughly and evenly applied by the hot process. The bolt shall be threaded class 1 , loose fit, before coating. After coating, a tolerance of 0.010 inch (maximum) will be permitted for the body diameter and height of head. The width across flats shall be less than the minimum wrench opening (National Screw Thread Commission). The nuts threaded to fits required shall assemble with the coated bolts.

E-3m. When cadmium-coated bolts are required they shall meet all dimensional requirements after 
coating. Coating shall be performed after threading, and the threads shall be completely coated.

E-4. Studs.-

$\mathrm{E}-4 \mathrm{a}$. Studs shall be furnished one-fourth inch and over in diameter and threaded on each end as specified.

$\mathrm{E}-4 \mathrm{~b}$. The length of the stud is measured from extreme end to extreme end. The length of threads is measured from extreme end to the last perfect thread.

E-4c. Studs shall be made to the thread series, class of fit, and finish specified.

$\mathrm{E}-4$ d. Unless otherwise specified, stud bolts shall be threaded as follows:

The stud end shall be threaded for a length equal to one and one-half times the diameter. The nut end shall be threaded for the respective length covered by Table 66 of the National Screw Thread Commission referred to above in paragraph $\mathrm{E}-3 \mathrm{f}$, with notations thereon. In case the stud is too short to permit such threading, the threading shall be equal length on each end to center.

E-5. Nuts.-

E-5a. Unless otherwise specified, semifinished or finished nuts shall be either cold punched or machined from bar stock. Rough nuts may be cold or hot punched or hot forged.

E-5b. When nuts are furnished with bolts the threads of the nuts shall, unless otherwise specified, be of the same thread series and class of fit as the threads of the bolts. When nuts are ordered separately the threads shall be of the thread series and class of fit specified.

E-5c. Unless otherwise specified, the washer face of finished nuts shall be at right angles to the axis of the threaded hole, within a tolerance of $1^{\circ}$. The bearing face of the rough and semifinished nuts shall be within $2^{\circ}$ of right angles of the axis of the holes.

E-5d. All finished and semifinished hexagonal and square regular nuts, castellated nuts, and hexagonal light nuts shall be washer faced. The thickness of the washer face, including that of jam nuts having a washer face, shall be about one sixty-fourth inch. The diameter of the bearing surface of the washer face shall equal the width across flats, within plus or minus 5 per cent. The thickness of the nut shall be the distance from the top to the bearing surface.

E-5e. The tops of regular square and hexagonal nuts when not castellated, of jam nuts, and of hexagonal light nuts when not castellated shall be flat and chamfered. The angle of chamfer with the top surface shall be $30^{\circ}$ for hexagonal nuts and $25^{\circ}$ for square nuts, and the diameter of the top flat circle shall be from 85 to 100 per cent of the width across flats.

E-5f. The width across flats of nuts shall be measured at the widest point. The total taper of any two opposite sides of nuts shall not exceed $4^{\circ}$.

E-5g. Rough and semifinished regular nuts, types $A-1$ and $B-1$, shall conform to Table 69 under National Screw Thread Commission, subject matter, wrench head bolts and nuts, and wrench openings; nuts. (See 608.32, p. 610.)

E-5h. Finished regular nuts, types $\mathrm{A}-2$ and $\mathrm{B}-2$, shall conform to Table 70 under National Screw Thread Commission, subject matter, wrench head bolts and nuts, and wrench openings; nuts. (See 608.32 , p. 610 .)

E-5i. Castellated nuts, type A-3, shall conform to Table 71 under National Screw Thread Commission, subject matter, wrench head bolts and nuts, and wrench openings; nuts. (See 608.32, p. 611.)

E-5j. Finished and semifinished jam nuts, type A-4, shall conform to Table 72 under National Screw Thread Commission, subject matter, wrench head bolts and nuts, and wrench openings; nuts. (See 608.32, p. 611.) Unless otherwise specified, jam nuts shall be double chamfered. If a single chamfer is specified, the opposite side shall be washer faced.

E-5k. Hexagonal light nuts, type $\mathrm{A}-5$, shall conform to Table 73 under National Screw Thread Commission, subject matter, wrench head bolts and nuts, and wrench openings; nuts. (See 608.32, p. 611.)

E-51. When zinc-coated (galvanized) nuts are required they shall conform to the dimensional requirements before threading or coating. The coating shall be thoroughly and evenly applied by the hot process. Threading shall be performed after coating.

$\mathrm{E}-5 \mathrm{~m}$. When cadmium-corted nuts are required they shall meet all dimensional requirements after coating. Threading shall be performed before coating, and the threads shall be completely coated.

E-6. Tap rivets.-

$\mathrm{E}-6 \mathrm{a}$. The dimensions of tap rivets shall conform to Table 1 under Federal Specifications Board specification $\mathrm{FF}-\mathrm{B}-571$, subject matter, tap rivets. (See 608.4, p. 615.) They shall have flat countersunk heads, except that square projections shall be provided on the tops of heads whereby they mav be screwed into place. The length of the rivet shall be measured from the extreme point to the maximum bearing surface of the head. Tap rivets shall be threaded class 3 , medium fit. The body of the rivet shall be threaded its entire length to the taper of the head.

\section{F. METHODS OF SAMPLING, INSPECTION, AND TESTS}

\section{$\mathrm{F}-1$. Bolts, studs, and tap rivets.-}

F-1a. Material from which bolts, studs, and tap rivets are to be manufactured shall, when so specified in invitation for bids, be tested by either melts or lots. When tested by melts, not less than two tension tests and two bend tests shall be selected from each size rod or bar from each melt. When the melt can not be identified the material shall be inspected and tested by lots. Each lot shall consist of 2,000 pounds or fraction thereof from each size submitted, from which two tension and two bend tests shall be selected. The physical properties shall conform to the requirements of the contract.

F-1b. The finished product shall pass the following physical tests: Bolts, studs, and tap rivets shall be tested whether or not the rod or bar stock has been tested before fabrication.

F-1b (1). Test specimens.-For purposes of inspection the finished product shall be divided into lots of not more than 1,000 pounds. There shall be selected not less than two specimens from each lot of the same material, having the same diameter, submitted for final inspection. One-half of the specimens so selected from each lot shall be used for bending tests and the other half for tension tests.

F-1b (2). Tension tests.-

F-1b (2) $a$. The tension-test specimens, selected as specified above, shall be subjected to a tension test, and the bolt, stud, or tap rivet shall meet the tensile strength specified for the material called for by the contract or order. The load shall be applied in an axial direction on the bearing surfaces of the head and a nut or threaded plate into which the bolt, stud, or tap rivet has been screwed a depth of one diameter. The test load shall be applied at a pulling speed of not more than 2 inches per minute.

F-1b (2) b. Test specimens for bolts, studs, or tap rivets larger than $1 \frac{1}{2}$ inches in diameter may be prepared by turning therefrom $11 / 4$-inch studs. The axis of the stud shall be halfway between the axis of the bolt and the outside diameter, or as near thereto as consistent. These studs shall be tested in a 
manner as specified for testing bolts, by fitting a $11 / 4$-inch nut on each end.

F-1b (2) c. In determining the tensile strength of bolts, studs, or tap rivets, the effective area will be the cross-sectional area based on pitch diameter for ferrous materials and on minor diameter for nonferrous materials. For this purpose the areas in Table 16 shall be used.

TABLE 16.-Cross-sectional areas of American national coarse and American national fine thread series

\section{NATIONAL COARSE THREAD SERIES}

\begin{tabular}{|c|c|c|c|}
\hline \multirow{2}{*}{ Sizes } & \multirow{2}{*}{$\begin{array}{l}\text { Threads } \\
\text { per inch }\end{array}$} & \multicolumn{2}{|c|}{$\begin{array}{l}\text { Cross-sectional } \\
\text { area based on- }\end{array}$} \\
\hline & & $\begin{array}{c}\text { Pitch } \\
\text { diameter }\end{array}$ & $\underset{\text { diameter }}{\text { Minor }}$ \\
\hline $\begin{array}{l}\text { No. } 10 \\
\text { No } 12 \\
1 / 4 \text { inch } \\
3 / 16 \text { inch- } \\
3 / 8 \text { inch }\end{array}$ & $\begin{array}{l}24 \\
24 \\
20 \\
18 \\
16\end{array}$ & $\begin{array}{r}\text { Sq. in. } \\
0.0208 \\
.0280 \\
.0372 \\
.0600 \\
.0878\end{array}$ & $\begin{array}{c}\text { Sq.in. } \\
0.0145 \\
.0206 \\
.0269 \\
.0454 \\
.0678\end{array}$ \\
\hline $\begin{array}{l}7 / 66 \\
1 / 2 \text { inch- } \\
9 / 6 \text { inch- } \\
58 \text { inch } \\
3 / 4 \text { inch }\end{array}$ & $\begin{array}{l}14 \\
13 \\
12 \\
11 \\
10\end{array}$ & $\begin{array}{l}.1201 \\
.1590 \\
.2030 \\
.2516 \\
.3685\end{array}$ & $\begin{array}{l}.0933 \\
.1257 \\
.1620 \\
.2018 \\
.3020\end{array}$ \\
\hline $\begin{array}{l}7 / 8 \text { inch } \\
1 \text { inch } \\
118 \text { inches-a. } \\
114 \text { inches-a } \\
112 \text { inches.... }\end{array}$ & $\begin{array}{l}9 \\
8 \\
7 \\
7 \\
6\end{array}$ & $\begin{array}{r}.5062 \\
.6630 \\
.8368 \\
1.0517 \\
1.5212\end{array}$ & $\begin{array}{r}.4193 \\
.5510 \\
.6931 \\
.8898 \\
1.2938\end{array}$ \\
\hline 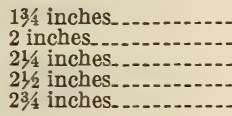 & $\begin{array}{l}5 \\
41 / 2 \\
41 / 2 \\
4 \\
4\end{array}$ & $\begin{array}{l}2.0615 \\
2.7046 \\
3.4824 \\
\text { 4. } 2917 \\
5.2588\end{array}$ & $\begin{array}{l}\text { 1. } 7333 \\
\text { 2. } 3001 \\
\text { 3. } 0212 \\
\text { 3. } 7161 \\
4.6194\end{array}$ \\
\hline $\begin{array}{l}3 \text { inches....... } \\
3 \text { inches... }\end{array}$ & $\begin{array}{l}4 \\
31 / 2\end{array}$ & $\begin{array}{l}\text { 6. } 3240 \\
6.2210\end{array}$ & $\begin{array}{l}5.6209 \\
5.4276\end{array}$ \\
\hline
\end{tabular}

\section{NATIONAL FINE THREAD SERIES}

\begin{tabular}{|c|c|c|c|}
\hline $\begin{array}{l}\text { No. } 8 \\
\text { No. } 10 \\
\text { No. } 12\end{array}$ & $\begin{array}{l}36 \\
32 \\
28\end{array}$ & $\begin{array}{r}0.0167 \\
.0226 \\
.0292\end{array}$ & $\begin{array}{r}0.0128 \\
.0175 \\
.0226\end{array}$ \\
\hline $\begin{array}{l}1 / 4 \text { inch } \\
5 / 16 \text { inch } \\
3 / 8 \text { inch } \\
7 / 16 \text { inch. } \\
1 / 2 \text { inch }\end{array}$ & $\begin{array}{l}28 \\
24 \\
24 \\
20 \\
20\end{array}$ & $\begin{array}{l}.0404 \\
.0640 \\
.0951 \\
.1288 \\
.1717\end{array}$ & $\begin{array}{l}.0326 \\
.0524 \\
.0809 \\
.1090 \\
.1486\end{array}$ \\
\hline $\begin{array}{l}9 / 16 \text { ineh... } \\
5 / 8 \text { inch } \\
3 / 4 \text { inch. } \\
7 / 8 \text { inch } \\
1 \text { inch }\end{array}$ & $\begin{array}{l}18 \\
18 \\
16 \\
14 \\
14\end{array}$ & $\begin{array}{l}.2176 \\
.2724 \\
.3953 \\
.5392 \\
.7142\end{array}$ & $\begin{array}{l}.1888 \\
.2400 \\
.3513 \\
.4805 \\
.6464\end{array}$ \\
\hline $\begin{array}{l}11 / 8 \text { inches. } \\
114 \text { inches. } \\
11 / 2 \text { inches. } \\
13 / 4 \text { inches. } \\
2 \text { inches. }\end{array}$ & $\begin{array}{l}12 \\
12 \\
12 \\
10 \\
10\end{array}$ & $\begin{array}{r}.9007 \\
1.1233 \\
1.6420 \\
2.2299 \\
2.9407\end{array}$ & $\begin{array}{l}.8118 \\
1.0238 \\
1.5212 \\
2.0615 \\
2.7468\end{array}$ \\
\hline $\begin{array}{l}21 / 4 \text { inches. } \\
21 / 2 \text { inches. } \\
23 / 4 \text { inches... } \\
3 \text { inches. }\end{array}$ & $\begin{array}{l}8 \\
8 \\
8 \\
8\end{array}$ & $\begin{array}{l}\text { 3. } 6943 \\
\text { 4. } 5950 \\
\text { 5. } 5940 \\
6.6911\end{array}$ & $\begin{array}{l}\text { 3. } 4228 \\
\text { 4. } 2917 \\
5.2588 \\
6.3240\end{array}$ \\
\hline
\end{tabular}

F-1b (2) $d$. Bolts of lengths and corresponding diameters shown below, and bolts longer than shown, shall be tested after fabrication:

\begin{tabular}{l|r|r|r|r|r|r|r|r|r|r|r}
\hline & In. & In. & In. & In. & In. & In. & In. & In & In. & In. & In. \\
Diameter_........ & $1 / 4$ & $5 / 16$ & $3 / 8$ & $7 / 16$ & $1 / 2$ & $9 / 16$ & $5 / 8$ & $3 / 4$ & $7 / 8$ & 1 & $11 / 8$ \\
Length_........ & 1 & 1 & $11 / 4$ & $11 / 4$ & $11 / 4$ & 116 & $13 / 4$ & 2 & $21 / 2$ & 3 & $31 / 2$
\end{tabular}

Materials for bolts shorter than provided for in the above table shall be tested before fabrication in accordance with paragraph $\mathrm{F}-1 \mathrm{a}$.

$\mathrm{F}-1 \mathrm{~b}(3)$. Bending tests.-

F-1b (3) $a$. Unless otherwise specified, no bending tests shall be made on nonferrous bolts, studs, or tap rivets.

F-1b (3) b. Ferrous bolts, studs, or tap rivets, unless of special material, shall be tested as follows: $\mathrm{F}-1 \mathrm{~b}$ (3) $b 1$. When the bolts are of sufficient length in the unthreaded portion to permit cold bending, the bolt selected for test shall withstand being bent $180^{\circ}$, without fracture, to a curve, the inner radius of which is equal to the radius of the bolt.

$\mathrm{F}-1 \mathrm{~b}$ (3) $b$ 2. When the bending test can not be applied, the test specimen shall withstand flattening cold to a thickness equal to one-half of its original diameter, without showing cracks. Casehardened bolts shall not be subjected to the above bending and flattening tests.

F-2. Nuts. -

F-2a. Unless otherwise specified, physical tests for nuts shall be made as follows:

F-2a (1). Tests specimens.-For purposes of inspection the product shall be divided into lots of not more than 1,000 pounds. There shall be selected not less than two specimens from each lot of the same material and of the same size ready for final inspection.

F-2a (2). Tests. - One-half of the specimens so selected shall be drifted cold until they break. The fracture must show normal uniform material.

F-2a (3). Unless otherwise specified, one-half of the specimens so selected shall withstand flattening axially, cold, to one-half of the original thickness without showing cracks. In the case of castellated nuts, the castellated portion shall be cut off before making this test. Casehardened nuts shall not be subjected to the above tests.

F-3. Gaging of dimensions. -

$\mathrm{F}-3 \mathrm{a}$. Inspection of dimensions.-Unless otherwise specified, the thread dimensions of bolts, nuts, studs, and tap rivets shall be gaged in order to insure that the manufactured product conforms to the specified dimensions within the limits of variations establishing the closest and loosest conditions of fit permissible for the class of fit specified. This requires the use of gages representing the limit of maximum metal, known as "go" gages, which control the minimum looseness or maximum tightness in the fit of mating parts and which accordingly control interchangeability, and the use of gages representing the limit of minimum metal, known as "not go" gages, which limit the amount of looseness between mating parts, and thus control in large measure the proper functioning of the parts. The body or head dimensions of bolts, nuts, and screws shall likewise be inspected by means of suitable gages or measuring instruments.

F-3b. Inspection gages.-Unless otherwise specified, the manufacturer shall make available to the inspector the necessary inspection gages, the accuracy of which shall be certified in a manner satisfactory to the inspector. In case of controversy the certification of the U. S. Bureau of Standards, based on the latest report of the National Screw Thread Commission, shall govern.

F-3c. Test specimens.-One specimen of each size shall be selected by the inspector from each lot of 100 or fraction thereof and shall be tested for accuracy of dimensions.

F-3d. Rejection.-The failure of any one test specimen to pass the dimensional requirements will result in the rejection of the lot from which the specimen was selected. If more than 10 per cent of the 
lots representing the entire submission or order fail, the entire submission or order shall be rejected.

F-4. Zinc coating (galvanizing) and cadmium coating.-Bolts, nuts, and studs shall be surface inspected to determine whether zinc coating (galvanizing) or cadmium coating is satisfactory.

\section{G. PACKAGING, PACKING, AND MARKING}

\section{G-1. Packing.-}

G-1a. Bolts and studs.-

$\mathrm{G}-1 \mathrm{a}(1)$. Bolts and studs when fitted with nuts, or ordered separately, shall be packaged as follows, as specified in the contract or order:

(a) In cardboard boxes, cartons, or paper wrappers.

(b) In bulk in accordance with best commercial practice.

G-1a (2). When packaged in accordance with paragraph G-1a (1) (a) the packages shall contain 25 units or multiples thereof not in excess of 100 units.

G-1a (3). Each package of bolts or studs shall contain one size only.

G-1a (4). No bolts or studs of sizes larger than those specified below shall be packaged in accordance with paragraph $\mathrm{G}-1 \mathrm{a}(1)(a)$ :

$1 / 4$-inch bolts up to 8 inches long.

$5 / 16$-inch bolts up to 10 inches long.

$3 / 8$-inch bolts up to 12 inches long.

$7 / 16$-inch bolts up to 12 inches long.

$1 / 2$-inch bolts up to 12 inches long.

$5 / 8$-inch bolts up to 8 inches long.

G-1a (5). When packaged in accordance with paragraph G-1a (1) (a), the bolts or studs shall be delivered in substantial commercial wooden cases or boxes so constructed as to insure acceptance by common or other carrier for safe transportation at the lowest rate to the point of delivery.

$\mathrm{G}-1 \mathrm{a}(6)$. When packed in accordance with paragraph G-1a (1) (b), in cases or kegs, the bolts or studs shall be delivered in substantial commercial wooden containers so constructed as to insure acceptance by common or other carrier for safe transportation at the lowest rate to the point of delivery, and each case or $\mathrm{keg}$ shall contain one size, only.

G-1b. Nuts.-

G-1b (1). When nuts are ordered separately they shall be packed in accordance with either paragraph G-1a (1) (a) or G-1a (1) (b), as specified in the contract or order.

$\mathrm{G}-1 \mathrm{~b}(2)$. When packaged in accordance with paragraph G-1a (1) (a), the packages shall contain 25 nuts, or multiples thereof, not in excess of 100 units.

G-1b (3). Each package of nuts shall contain one size only.

$\mathrm{G}-1 \mathrm{~b}(4)$. When packaged in accordance with paragraph G-1a (1) (a), the nuts shall be delivered in substantial commercial wooden cases, or boxes so constructed as to insure acceptance by common or other carrier for safe transportation at the lowest rate to the point of delivery.

$\mathrm{G}-1 \mathrm{~b}(5)$. When packed in accordance with paragraph G-1a (1) (b), in cases or kegs, the nuts shall be delivered in substantial commercial wooden containers so constructed as to insure acceptance by common or other carrier for safe transportation at the lowest rate to the point of delivery, and each case or keg shall contain one size only.

$\mathrm{G}-1$ c. Tap rivets. -

$\mathrm{G}-1 \mathrm{c}(1)$. Tap rivets shall be packed in substantial wooden cases or kegs so constructed as to insure acceptance by common or other carrier for safe transportation at the lowest rate to the point of delivery. Each case or $\mathrm{keg}$ shall contain one size only, unless the various sizes are properly packaged.

G-2. Marking.-

G-2a. Individual packages.-Each package of bolts, nuts, or studs shall bear a label stating the name of the manufacturer, the number of units, type, length of bolts or studs, type of nut, and also a mark designating the diameter, threads per inch, thread series, and class of fit; for example:

National coarse-thread series:

To specify a threaded part 1 inch diameter, 8 threads per inch, class 1 fit.

National fine-thread series:

To specify a threaded part 1 inch diameter, 14 threeds

per inch, class 4 fit..... $1^{\prime \prime}-14-\mathrm{NF}-4$

G-2b. Cases, boxes, or kegs.-Cases, boxes, or kegs containing bolts, nuts, or tap rivets shall be stenciled with a description of the contents, as required by paragraph $\mathrm{G}-2 \mathrm{a}$, for individual packages, and also with the name of the contractor, the number of the contract or order, the item or items contained in the case, box, or keg, and the gross weight in pounds. One end and one side and the top and bottom of the cases, boxes, or kegs shall be free from marks.

\section{NOTES}

I-1a. In ordering bolts, nuts, studis, or tap rivets, the following, as applicable, should be stated in the requisition or order:

I-1a. Quantity.-Bolts and nuts, when ordered together, or separately, should be ordered by the number of pieces.

I-1b. Type of bolt or nut.

I-1c. Nominal diameter.

I-1d. Thread series and class of fit.

I-1e. Length of bolt or stud.

I-1f. Kind and grade of material (referring to governing Federal specifications).

I-2. Square-head and hexagonal-head bolts shall be furnished $1 / 4$ inch in diameter and over.

I-3. Screw threads specified herein for bolts, nuts, and studs are in agreement with the report of the National Screw Thread Commission, revised 1928 (Bureau of Standards Miscellaneous Publication No. 89).

I-4. Boltheads and nuts herein specified are in agreement with the forms and dimensions specified in the standards issued by the American Standards Associations (report B18b-1927). In case oversize boltheads or nuts are manifestly necessary for a particular application the dimensions in the tables herein listed may be ignored and the dimensions actually required shall be specified by the purchaser.

I-5. In the purchase of material from which bolts, nuts, studs, and tap rivets are to be manufactured it is recommended that Table 19, which contains proper tolerances, be used. 
TABLE 19.-Limiting dimensions of round-bolt stock and hexagon bolt and nut stock for American national coarse and fine thread series, classes 2, 3, 4 , and 5 fits

\begin{tabular}{|c|c|c|c|c|c|c|}
\hline \multirow{4}{*}{$\begin{array}{l}\text { Bolt or screw } \\
\text { diameter }\end{array}$} & \multicolumn{4}{|c|}{ Bolt stock, round, diameter } & \multirow{3}{*}{\multicolumn{2}{|c|}{$\begin{array}{c}\text { Bolt and nut } \\
\text { stock, hexagon, } \\
\text { width across } \\
\text { flats }\end{array}$}} \\
\hline & \multirow{2}{*}{\multicolumn{2}{|c|}{$\begin{array}{c}\begin{array}{c}\text { Coarse-thread } \\
\text { series }\end{array} \\
\begin{array}{l}\text { Rough or semi- } \\
\text { finished, classes } \\
2 \text { and } 3 \text { fit }\end{array}\end{array}$}} & \multicolumn{2}{|c|}{$\begin{array}{l}\text { Coarse and fine } \\
\text { thread series }\end{array}$} & & \\
\hline & & & \multicolumn{2}{|c|}{ Finished } & & \\
\hline & $\begin{array}{l}\text { Maxi- } \\
\text { mum }\end{array}$ & $\begin{array}{l}\text { Mini- } \\
\text { mum }\end{array}$ & $\begin{array}{l}\text { Maxi- } \\
\text { mum }\end{array}$ & $\underset{\text { mum }}{\text { Mini- }}$ & $\begin{array}{l}\text { Maxi- } \\
\text { mum }\end{array}$ & $\begin{array}{l}\text { Mini- } \\
\text { mum }\end{array}$ \\
\hline & Inches & Inches & Inches & Inches & Inches & Inches \\
\hline $2(0.086)$ & $\begin{array}{r}0.0730 \\
.0860 \\
.0990 \\
.1120\end{array}$ & $\begin{array}{l}0.0678 \\
.0804 \\
.0928 \\
.1052\end{array}$ & $\begin{array}{l}0.1355 \\
.1485 \\
.1615 \\
.1745\end{array}$ & $\begin{array}{r}0.0730 \\
.0860 \\
.0990 \\
.1120\end{array}$ & & - \\
\hline o. $5(0.12$ & $\begin{array}{l}.1250 \\
.1380 \\
.1640 \\
.1900 \\
.2160\end{array}$ & $\begin{array}{l}.1182 \\
.1304 \\
.1564 \\
.1808 \\
.2068\end{array}$ & $\begin{array}{l}.1875 \\
.2005 \\
.2265 \\
.2525 \\
.2785\end{array}$ & $\begin{array}{l}.1250 \\
.1380 \\
.1640 \\
.1900 \\
.2160\end{array}$ & & \\
\hline $\begin{array}{l}1 / 4 \text { inc } \\
516 \text { in } \\
38 \text { inc } \\
76 \text { in }\end{array}$ & $\begin{array}{l}.2500 \\
.3125 \\
.3750 \\
.4375 \\
.5000\end{array}$ & $\begin{array}{l}.2399 \\
.3011 \\
.3624 \\
.4235 \\
.4852\end{array}$ & $\begin{array}{l}.3125 \\
.3750 \\
.4375 \\
.5000 \\
.5625\end{array}$ & $\begin{array}{l}.2500 \\
.3125 \\
.3750 \\
.4375 \\
.5000\end{array}$ & $\begin{array}{r}0.4375 \\
.5625 \\
.6250 \\
.7500 \\
.8125\end{array}$ & $\begin{array}{l}0.4280 \\
.5520 \\
.6130 \\
.7370 \\
.7990\end{array}$ \\
\hline $\begin{array}{l}9 / 16 \text { in } \\
5 / 8 \text { in } \\
3 / 4 \text { in } \\
7 / 8 \text { in } \\
1\end{array}$ & $\begin{array}{r}.5625 \\
.6250 \\
.7500 \\
.8750 \\
1.0000\end{array}$ & $\begin{array}{l}.5467 \\
.6080 \\
.7316 \\
.8550 \\
.9778\end{array}$ & $\begin{array}{r}.6350 \\
.6875 \\
.8125 \\
1.0000 \\
1.1250\end{array}$ & $\begin{array}{r}.5625 \\
.6250 \\
.7500 \\
.8750 \\
1.0000\end{array}$ & $\begin{array}{l}.8750 \\
1.0000 \\
1.1250 \\
1.3125 \\
1.5000\end{array}$ & $\begin{array}{r}.8610 \\
.9840 \\
1.1080 \\
1.2930 \\
1.4790\end{array}$ \\
\hline $\begin{array}{l}11 / 8 \text { inches.... } \\
11 / 4 \text { inches... } \\
11 / 2 \text { inches... } \\
13 / 4 \text { inches.... } \\
2 \text { inches..... }\end{array}$ & $\begin{array}{l}\text { 1. } 1250 \\
\text { 1. } 2500 \\
\text { 1. } 5000 \\
\text { 1. } 7500 \\
\text { 2. } 0000\end{array}$ & $\begin{array}{l}\text { 1. } 1002 \\
1.2252 \\
1.4710 \\
1.7162 \\
1.9632\end{array}$ & $\begin{array}{l}1.2500 \\
1.3750 \\
1.6250 \\
1.8750 \\
2.1250\end{array}$ & $\begin{array}{l}1.1250 \\
1.2500 \\
1.5000 \\
1.7500 \\
2.0000\end{array}$ & $\begin{array}{l}\text { 1. } 6875 \\
\text { 1. } 8750 \\
2.2500 \\
2.6250 \\
3.0000\end{array}$ & $\begin{array}{l}1.6650 \\
1.8500 \\
2.2220 \\
2.5930 \\
2.9640\end{array}$ \\
\hline $\begin{array}{l}21 / 4 \text { inches.. } \\
21,2 \text { inches.- } \\
23 \% 4 \text { inches.. } \\
3 \text { inches... }\end{array}$ & $\begin{array}{l}\text { 2. } 2500 \\
2.5000 \\
2.7500 \\
3.0000\end{array}$ & $\begin{array}{l}\text { 2. } 2132 \\
\text { 2. } 4592 \\
\text { 2. } 7092 \\
\text { 2. } 9592\end{array}$ & $\begin{array}{l}\text { 2. } 3750 \\
\text { 2. } 6250 \\
\text { 2. } 8750 \\
\text { 3. } 1250\end{array}$ & $\begin{array}{l}\text { 2. } 2500 \\
\text { 2. } 5000 \\
\text { 2. } 7500 \\
\text { 3. } 0000\end{array}$ & \begin{tabular}{|l|}
3.3750 \\
3.7500 \\
4.1250 \\
4.5000
\end{tabular} & $\begin{array}{l}\text { 3. } 3350 \\
\text { 3. } 7070 \\
\text { 4. } 0780 \\
4.4490\end{array}$ \\
\hline
\end{tabular}

I-6. In the purchase of bar stock for the manufacture of bolts complying with the requirements for class A steel and class B steel bolts, it is suggested that the minimum tensile strength per square inch be increased to 79,000 pounds and 62,000 pounds, respectively, in order that the finished bolts may meet the requirements of paragraph $\mathrm{E}-1$.

I-7. In accepting material before fabrication which complies with the requirements of the contract or order, no responsibility is assumed by the Government in the matter of tensile strength of finished bolts, studs, or tap rivets made therefrom.

I-12. Cap screws, set screws, machine screws, and stove bolts are covered by Federal Specification FF-S-91 (F. S. B. No. 548). (See below.)

\section{UNITED STATES GOVERNMENT, FEDERAL SPECIFICATIONS BOARD, FEDERAI SPECIFICATION FOR CAP SCREWS, SET SCREWS, MACHINE SCREWS, AND STOVE BOLTS, NO. 548, 1928. (CURRENT DES- IGNATION : FF-S-91)}

\section{MACHINE SCREWS AND STOVE BOLTS}

\section{MATERIAL AND WORKMANSHIP}

1. Material.-For special purposes, the material shall be in accordance with the requirements of the
United States Government master specification, or of the Government department concerned, covering the material desired.

2. When screws for general use are required, the composition of the material will not be specified.

3 . When screws are to be made of a special nonferrous metal, the materials recommended are rolled manganese bronze, rolled copper-nickel alloy, or rolled naval brass of the composition and properties specified in the applicable Government department specification.

4. Workmanship.-The workmanship shall be compatible with the type and grade of product, and class of fit and of finish specified. The product shall be free from abnormal scale, fins, seams, or other defects. All machine screws shall be finished and free from any defects which may affect their serviceability.

5. Unless otherwise specified, the method of manufacture employed for the production of screw threads on screws shall be by chasing, milling, tapping, die-cutting, or rolling.

\section{GENERAL REQUIREMENTS}

1. Physical requirements. - Screws shall meet the physical requirements specified by the Government department concerned.

2. Assembly requirements. - When screws are ordered with nuts, a nut shall be assembled on each.

3. Screw threads. - Screw threads shall be in accordance with the following specifications:

(a) Notation.-Screw-thread notation shall be in accordance with Figure 316. (See National Screw Thread Commission, below.)

(b) Thread form.-The form of thread profile shall be the national form, as shown in Figure 317 . (See National Screw Thread Commission, below.)

(c) Classes of fit and tolerances. - The class of fit of mating screw threads specified shall be class 1 , loose fit; class 2, free fit; class 3 , medium fit; or class 4, close fit; as established by the National Screw Thread Commission and determined by thread gages specified below.

\section{DETAIL REQUIREMENTS}

1. Thread series.-The diameters and threads per inch shall be those specified as the national coarse thread series, or the national fine thread series, as shown below in Tables Nos. 7 and 13 of the National Screw Thread Commission.

2. Body diameters.-The dimensions and tolerances for body diameter of screws shall be as in Tables 64 and 65 of the National Screw Thread Commission, given below.

3. Sysiem of measurements.-Unless otherwise specified, all dimensions are stated in inches.

4. Tolerances on length.-(Same as National Screw Thread Commission, given below.)

\section{METEODS OF INSPECTION AND TESTS}

In addition to such prior tests of the unfinished material as may have been specified in the order, the finished product shall, unless otherwise specified, pass the following tests, which shall be at the expense of the contractor.

1. Physical tests for screws.-Physical tests for screws shall be as follows:

(a) Test specimens. - For purposes of inspection the product shall be divided into lots of not more than 1,000 pounds. There shall be taken not less than two specimens from each lot of the same material having the same diameter and ready for final inspection. One-half of the specimens so selected from each lot shall be used for bending tests and the other half for tensile tests. 
(b) Tension tests.-The tension-test specimens selected as specified above shall be subjected to a tension test, and the screw shall meet the tensile strength specified for the material called for by the order. The force shall be applied in an axial direction on the bearing surfaces of the head and a nut or threaded plate into which the screw has been screwed to a depth of one diameter. The test load shall be applied at a pulling speed of not more than 2 inches per minute.

When the order calls for screws too short to apply the foregoing tension test, the number of tensiontest specimens specified above may be selected from the rods from which short screws are to be made. In determining the tensile strength of the screw, the effective area will be the cross-sectional area based on pitch diameter for ferrous metals and on minor diameter for nonferrous metals. For this purpose the areas given in Table 26 in $F$. S. B. FF-B-571, above, shall be used.

(c) Bending tests. - When specified, bending tests shall be made as required in the United States Government master specification covering the material, or specifications of the Government department concerned.

(1) Unless otherwise specified, no bending tests shall be made on nonferrous screws.

(2) Ferrous screws, unless of special material, shall be tested as follows:

When the screws are of sufficient length in the unthreaded portion to permit cold bending, the test specimen shall withstand being bent $180^{\circ}$, without fracture, to a curve the inner radius of which is equal to the radius of the bolt.

When the bending test can not be applied, the test specimen shall withstand flattening cold to a thickness equal to one-half of its original diameter, without showing cracks.

2. Gaging of dimensions-(a) Inspection of dimensions.-Unless otherwise specified, the thread dimensions of screws shall be gaged in order to insure that the manufactured product conforms to the specified dimensions within the limits of variation establishing the closest and loosest conditions of fit permissible for the class of fit specified. This requires the use of limit gages, namely "go" gages, representing the limit of maximum metal which must assemble with the product, and "not go" gages, representing the limit of minimum metal which must not assemble with the product. The body or head dimensions of screws shall likewise be inspected by means of suitable gages or measuring instruments.

Unless otherwise specified, the manufacturer shall make available to the inspector for the purchaser the necessary inspection gages, the accuracy of which shall be certified in a manner satisfactory to the purchaser. In case of controversy the certification of the Bureau of Standards, based on the latest report of the National Screw Thread Commission, shall govern.

(b) Test specimens.-When screws are made to class 1 , loose fit; or class 2 , free fit specifications; at least one specimen of each size shall be taken by the inspector from each lot of 100 , or fraction thereof, and shall be tested for the accuracy of dimensions.

When screws are made to class 3 , medium fit, or class 4, close fit specifications, at least five specimens of each size shall be taken by the inspector from each lot of 100, or fraction thereof, and shall be tested for the accuracy of dimensions.

3. Rejection. - The failure of any one test specimen to pass the foregoing requirements will result in the rejection of the lot from which the specimen was taken. If more than 10 per cent of the lots representing the entire submission or order fail, the entire submission or order shall be rejected. Rejected material shall not be resubmitted.

\section{PACKING AND MARKING OF SHIPMENTS}

1. Outside packing.-Unless otherwise required, screws shall be delivered packed in accordance with best commercial practice.

2. Inside packing.- - In addition to the above outside packing unless otherwise required, screws and stove bolts shall be packed in strong cartons, pasteboard boxes, or suitable paper wrappings. Each of these packages shall contain the same number; standard commercial packing may be either on the basis of a gross, a hundred, or fraction thereof. Each of such packages shall be marked with the name of the material and contents, type of head, length, diameter, threads per inch, quantity, the manufacturer's name, and description of contents as specified below. Packages containing one item only shall be neatly labeled on one end with the number and description of contents.

3. Marking of packages.-The description, which includes the type, style, finish, diameter, threads per inch, thread series, and class of fit, shall be in accordance with the system of marking specified by the National Screw Thread Commission, as follows:

The method of designating a screw thread by means of symbols is by the use of the initial letters of the thread series, preceded by the diameter in inches (or the screw number) and number of threads per inch, all in Arabic characters, followed by the classification of fit in Arabic numerals.

For example, to specify a bolt 5 inches long, 1 inch in diameter, 8 threads per inch, national coarse thread series, class 1 fit, writ To specify a cap screw 3 inches long, 1 inch in diameter, 14 threads per inch, national fine thread series, class 4 fit, write

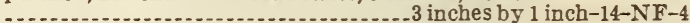

\section{NOTES}

1. Items to be stated in requisition.-In ordering screws, the following items should be stated in the requisition:

Kind of material and physical requirements. (Material should conform to the applicable United States Government master specification wherever possible.)

(a) Type of screw and shape of head or nut.

(b) Size, length, thread series, and class of fit.

Machine screws are not furnished with nuts unless specified.

2. Screw thread specifications.-Screw threads specified herein for screws are in agreement with the report of the National Screw Thread Commission, revised 1928. (Bureau of Standards miscellaneous publication No. 89; also with report B18c-1930, of the American Standards Association.)

3. Screw thread practice.-The usual commercial practice as to application of thread series and class of fit is indicated in Table 120 of the National Screw Thread Commission, given below.

4. Screw lengths. - Screws, except for special requirements, should be ordered in the following increments of length:

\begin{tabular}{|c|c|}
\hline Length (inches) & $\begin{array}{l}\text { Incre- } \\
\text { ment }\end{array}$ \\
\hline $\begin{array}{l}1 / 16 \text { to } 1 / 2 \\
\text { Over } 1 / 2 \text { to } 11 / 4 \\
\text { Over } 11 / 4 \text { to } 3 \\
\text { Over } 3\end{array}$ & $\begin{array}{c}\text { Inch } \\
1 / 16 \\
16 \\
1 / 4 \\
1 / 2\end{array}$ \\
\hline
\end{tabular}


UNITED STATES GOVERNMENT, NATIONAL SCREW THREAD COMMISSION, BUREAU OF STANDARDS MISCELIANEOUS PUB. IICATION NO. 89, JUNE 22, 1928

\section{SCREW THREADS}

(This report consists of 261 pages of text, tables, and illustrations. Included is a brief history of screw-thread standardization in the United States, beginning with the appointment of the special com

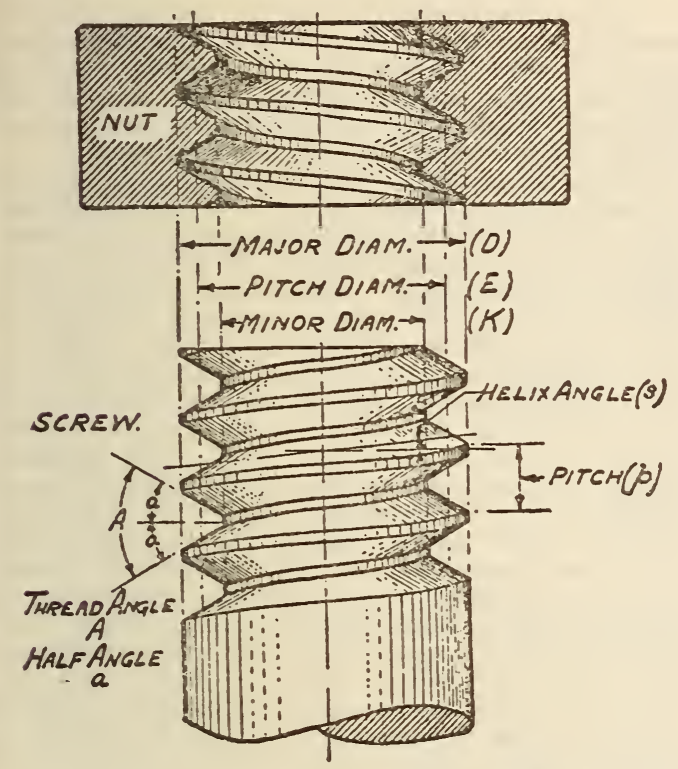

FIGURE 315.-Screw thread notation

mittee by the Franklin Institute on April 21, 1861, down to the act of Congress, on April 16, 1926, creating a self-perpetuating commission for the standardization of screw threads. In view of present-day manufacturing requirements the need of definite screw-thread specifications is set forth in some detail, extracts from which include the following):

\section{NEED OF DEFINITE SPECIFICATIONS}

A prerequisite of the present-day quantity production of machine parts is standardization of form and dimensions of parts, which involves also the specification of standard limiting dimensions of the manufactured product in order that interchangeability may be established. The economic advantages of producing interchangeable machined parts, particularly when made in different manufacturing plants located at a distance from each other, which will assemble without difficulty and in a dependable manner, are generally recognized.

The standardization of screw threads, involving as it does the specification of sizes which are necessary to industry, the elimination of unnecessary sizes, and the securing of interchangeability, is especially important because of their use in enormous quantities in all varieties of mechanisms. Such standardization is important to the user of a machine, as well as to the manufacturer, as the user should be able to buy locally a screw or nut for replacement in case of breakage or wear.

A screw-thread fit can not be accurately made with the same facility as the fit of a plain hole and shaft. In the fit of a plain hole and shaft only three elements are taken into account in securing a given class of fit, namely, rotundity, diameter, and length; whereas in a screw-thread fit it is necessary to consider rotundity, length, major diameter, pitch diameter, minor diameter, angle of thread, and pitch or lead. A variation in any one of these elements of a screw thread will prevent a good fit, so that it is much more difficult to make a good screwthread fit than it is to make a plain bearing fit.

\section{ARRANGEMENT OF REPORT}

There are included in the body of the report specifications for threaded products and gages, embodying sufficient information to permit the writing of definite and complete specifications for the purchase of screw-thread products. In the appendixes there is arranged supplementary information of both a general and technical nature, including such specifications as are not intended to be mandatory.

The specifications in the report have been arranged, as far as possible, by products. For example, one section deals with threads for bolts and nuts, etc., another with hose-coupling threads, another with pipe threads, etc. As far as practicable, each section is arranged in the following order: (1) Form of thread, (2) thread series, (3) classification and tolerances, (4) tables of dimensions, and (5) gages.

\section{TERMINOLOGY}

In this report there are utilized, as far as possible, nontechnical words and terms which best convey

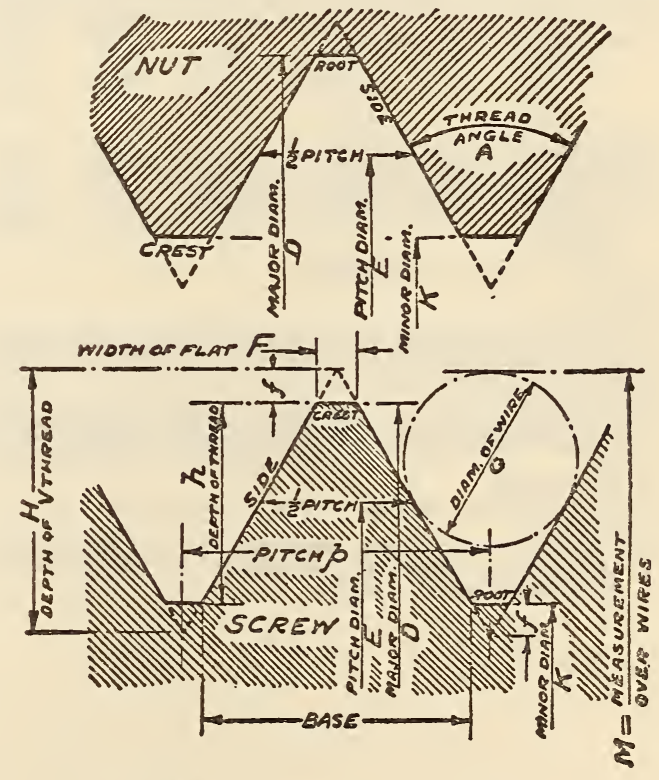

FIGURE 316.-Screw thread notation

alike to the producer and user of screw threads the information presented.

\section{AMERICAN NATIONAL FORM OF THREAD}

The form of thread profile specified, known previously as the United States standard or Sellers' profile, is adopted by the commission and shall hereafter be known as the American national form of thread.

The American national form of thread shall be used for all screw-thread work except when otherwise specified for special purposes. 


\section{THREAD SERIES}

It is the aim of the commission, in establishing thread systems for general use, to eliminate all unnecessary sizes and, in addition, to utilize as far as possible present predominating sizes. While from certain standpoints it would have been desirable to make simplifications in the thread systems and to establish more thoroughly consistent standards, it is believed that any radical change at the present time would be out of place and interfere with manufacturing conditions, and would involve great economic loss.

The testimony given at the various hearings held by the commission is very consistent in favoring the maintenance of the present coarse-thread and fine-

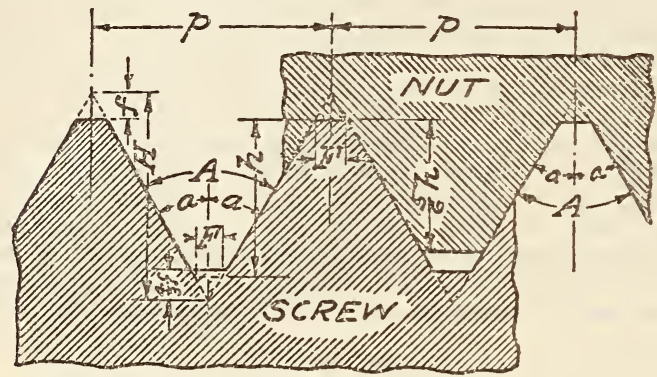

Figure 317.-American national form of thread

Note.-No allowance is shown. This condition exists in class 2 , free fit, and class 3 , medium fit, where both the minimum nut and the maximum screw are basic.

\section{NOTATION}

$n=$ number of threads per inch.

$H=0.866025 \mathrm{p}$ depth of $60^{\circ}$ sharp $\vee$ thread

$h=0.649519 p$ depth of American national form of thread.

$\begin{array}{rl}h=0.649519 & p \text { depth of American national form } \\ 5 / 6 h=0.541266 p \text { maximum depth of engagement. }\end{array}$

$F=0.125000 p$ width of fiat at crest and root of American national form

$f\left\{\begin{array}{l}=0.108253 p \\ =1 / 8 H \\ =1\end{array}\right\}$ depth of truncation.

Class 1 , loose fit:

Includes screw-thread work of rough commercial quality, where the threads must assemble readily, and a certain amount of shake or play is not objectionable.

Class 2 , free fit:

Includes the great bulk of screw-thread work of ordinary quality, of finished and semifinished bolts and nuts, machine screws, etc.

Class 3 , medium fit:

Includes the better grade of interchangeable screw-thread work. Class 4, close fit:

Includes screw-thread work requiring a fine snug fit, somewhat closer than the medium fit. In this class of fit selective assembly of parts may be necessary. thread series, the coarse-thread series being the United States standard threads, supplemented in the sizes below one-fourth inch by sizes taken from the standard established by the American Society of Mechanical Engineers. The fine-thread series is composed of standards that have been found necessary, and consists of sizes taken from the standards of the Society of Automotive Engineers and the finethread series of the American Society of Mechanical Engineers.

\section{CLASSIFICATION AND TOLERANCES}

There are established for general use four distinct classes of screw-thread fits as specified in the following brief outline. These four classes of fits, together with the accompanying specifications, are for the purpose of insuring the interchangeable manufacture of screw-thread parts throughout the country.

It is not the intention of the commission arbitrarily to place a general class or grade of work in a specific class of fit. Each manufacturer and user of screm threads is free to select the class of fit best adapted to his particular needs. The tolerances and dimensions for four classes of fit are given in Tables 3 to 14, inclusive, and summarized in Tables 15 and 16 .

An examination of the dimensional specifications for the various classes of fit shows that a screw made to one class of fit may be used with a nut or tapped hole made to some other class of fit. The resulting quality of fit may represent an intermediate class or may approximate one of the classes of fit adopted as standard. The use of different classes of fit on the screw and threaded hole may be justified when equipment available is such that one member can be economically produced to a higher accuracy than the other. For instance, common commercial machine screws are made to class 2 , free fit, while machine screw nuts are commonly supplied in class 1 , loose fit; or, ground taps may make it practicable to produce class 3 nuts for use with class 1 or class 2 screws.

\section{(a) AMERICAN NATIONAL COARSE-THREAD SERIES}

In Table 1 are specified the nominal sizes and basic dimensions of the American national coarsethread series.

The American national coarse-thread series is recommended for general use in engineering work, in machine construction where conditions are favorable to the use of bolts, screws, and other threaded components where quick and easy assembly of the parts is desired, and for all work where conditions do not require the use of fine-pitch threads. 
TABLE 1.-American national coarse-thread series

\begin{tabular}{|c|c|c|c|c|c|c|c|c|c|c|c|}
\hline \multicolumn{2}{|c|}{ Identification } & \multicolumn{3}{|c|}{ Basic diameter } & \multicolumn{7}{|c|}{ Thread data } \\
\hline Sizes & $\begin{array}{c}\text { Treads } \\
\text { per inch } \\
n\end{array}$ & $\begin{array}{c}\text { Major } \\
\text { diameter } \\
D\end{array}$ & $\begin{array}{c}\text { Pitch } \\
\underset{E}{\text { diameter }}\end{array}$ & $\underset{K}{\text { diameter }}$ & $\begin{array}{l}\text { Metric } \\
\text { equir- } \\
\text { alent } \\
\text { of major } \\
\text { diameter }\end{array}$ & $\begin{array}{c}\text { Pitch } \\
p\end{array}$ & $\begin{array}{c}\text { Depth of } \\
\text { thread } \\
h\end{array}$ & $\begin{array}{c}\text { Basic } \\
\text { width } \\
\text { of flat } \\
p / 8\end{array}$ & $\begin{array}{l}\text { Mini- } \\
\text { mum } \\
\text { width of } \\
\text { flat at } \\
\text { major } \\
\text { diameter } \\
\text { of nut } \\
p / 2 \pm\end{array}$ & $\begin{array}{c}\text { Helix } \\
\text { angle at } \\
\text { basic } \\
\text { pitch } \\
\text { diameter } \\
s\end{array}$ & $\begin{array}{c}\text { Basic } \\
\text { area of } \\
\text { section } \\
\text { at root } \\
\text { of thread } \\
\frac{\pi \pi^{2}}{4}\end{array}$ \\
\hline 1 & 2 & 3 & 4 & 5 & 6 & 7 & 8 & 9 & 10 & 11 & 12 \\
\hline $\begin{array}{l}1 \\
2 \\
3 \\
4\end{array}$ & $\begin{array}{l}64 \\
56 \\
48 \\
40 \\
40\end{array}$ & $\begin{array}{l}\text { Inches } \\
0.073 \\
.086 \\
.099 \\
.112 \\
.125\end{array}$ & $\begin{array}{r}\text { Inchcs } \\
0.0629 \\
.0744 \\
.0555 \\
.0958 \\
.1088\end{array}$ & $\begin{array}{r}\text { Inches } \\
0.0527 \\
.0628 \\
.0719 \\
.0795 \\
.0925\end{array}$ & $\begin{array}{l}m m \\
1.854 \\
2.184 \\
2.515 \\
2.845 \\
3.175\end{array}$ & $\begin{array}{c}\text { Inch } \\
0.01562 \\
.01786 \\
.02083 \\
.02500 \\
.02500\end{array}$ & $\begin{array}{c}\text { Inch } \\
0.01015 \\
.01160 \\
.01353 \\
.01624 \\
.01624\end{array}$ & $\begin{array}{c}\text { Inch } \\
0.00195 \\
.00223 \\
.00260 \\
.00312 \\
.00312\end{array}$ & $\begin{array}{c}\text { Inch } \\
0.00065 \\
.00074 \\
.00087 \\
.00104 \\
.00104\end{array}$ & $\begin{array}{lc}\circ & \prime \\
4 & 31 \\
4 & 22 \\
4 & 26 \\
4 & 45 \\
4 & 11\end{array}$ & $\begin{array}{c}\text { Square } \\
\text { inches } \\
0.0022 \\
.0031 \\
.0041 \\
.0050 \\
.0067\end{array}$ \\
\hline 10 & $\begin{array}{l}32 \\
32 \\
24 \\
24\end{array}$ & $\begin{array}{l}.138 \\
.164 \\
.190 \\
.216\end{array}$ & $\begin{array}{l}.1177 \\
.1437 \\
.1629 \\
.1889\end{array}$ & $\begin{array}{l}.0974 \\
.1234 \\
.1359 \\
.1619\end{array}$ & $\begin{array}{l}\text { 3. } 505 \\
4.166 \\
4.826 \\
5.486\end{array}$ & $\begin{array}{l}.0315 \\
.03125 \\
.04167 \\
.04167\end{array}$ & $\begin{array}{l}.02030 \\
.02030 \\
.02706 \\
.02706\end{array}$ & $\begin{array}{l}.00391 \\
.00391 \\
.00521 \\
.00521\end{array}$ & $\begin{array}{l}.00130 \\
.00130 \\
.00174 \\
.00174\end{array}$ & $\begin{array}{rr}4 & 50 \\
3 & 58 \\
4 & 39 \\
4 & 1\end{array}$ & $\begin{array}{l}.0075 \\
.0120 \\
.0145 \\
.0206\end{array}$ \\
\hline $\begin{array}{l}1 / 4 \ldots \ldots \\
5 / 16 \ldots \\
3 / 8 \ldots \ldots \\
5 / 16 \ldots \ldots \\
1 / 2 \ldots \ldots\end{array}$ & $\begin{array}{l}20 \\
18 \\
16 \\
14 \\
13\end{array}$ & $\begin{array}{l}.2500 \\
.3125 \\
.3750 \\
.4375 \\
.5000\end{array}$ & $\begin{array}{l}.2175 \\
.2764 \\
.3344 \\
.3911 \\
.4500\end{array}$ & $\begin{array}{l}.1850 \\
.2403 \\
.2938 \\
.3447 \\
.4001\end{array}$ & $\begin{array}{r}6.350 \\
7.933 \\
9.525 \\
11.113 \\
12.700\end{array}$ & $\begin{array}{l}.05000 \\
.05556 \\
.06250 \\
.07143 \\
.07692\end{array}$ & $\begin{array}{r}.03248 \\
.03608 \\
.04059 \\
.04639 \\
.04996\end{array}$ & $\begin{array}{l}.00625 \\
.00694 \\
.00781 \\
.00893 \\
.00962\end{array}$ & $\begin{array}{r}00208 \\
.00231 \\
.00260 \\
.00298 \\
.00321\end{array}$ & $\begin{array}{rr}4 & 11 \\
3 & 40 \\
3 & 24 \\
3 & 20 \\
3 & 7\end{array}$ & $\begin{array}{l}.0269 \\
.0454 \\
.0678 \\
.0933 \\
.1257\end{array}$ \\
\hline $\begin{array}{l}9 / 16- \\
58 \ldots \\
3,1 \ldots \\
7,8 \ldots \\
1 \ldots\end{array}$ & $\begin{array}{r}12 \\
11 \\
10 \\
9 \\
8\end{array}$ & $\begin{array}{r}.5625 \\
.6250 \\
.7500 \\
.8750 \\
1.0000\end{array}$ & $\begin{array}{l}.5084 \\
.5660 \\
.6850 \\
.8028 \\
.9188\end{array}$ & $\begin{array}{l}.4542 \\
.5069 \\
.6201 \\
.7307 \\
.8376\end{array}$ & $\begin{array}{l}14.258 \\
15.875 \\
19.050 \\
22.225 \\
25.400\end{array}$ & $\begin{array}{l}.08333 \\
.09091 \\
.10000 \\
.11111 \\
.12500\end{array}$ & $\begin{array}{r}.05413 \\
.05905 \\
.06495 \\
.07217 \\
.08119\end{array}$ & $\begin{array}{r}.01042 \\
.01136 \\
.01250 \\
.01389 \\
.01562\end{array}$ & $\begin{array}{l}.00347 \\
.00379 \\
.00417 \\
.00463 \\
.00521\end{array}$ & $\begin{array}{ll}2 & 59 \\
2 & 56 \\
2 & 40 \\
2 & 31 \\
2 & 29\end{array}$ & $\begin{array}{l}.1620 \\
.2018 \\
.3020 \\
.4193 \\
.5510\end{array}$ \\
\hline $\begin{array}{l}11 / 8 \ldots \\
11 / 4 \ldots \\
116 \ldots \\
13 / 4 \ldots \\
2 \ldots\end{array}$ & $\begin{array}{l}7 \\
7 \\
6 \\
5 \\
41 / 2\end{array}$ & $\begin{array}{l}\text { 1. } 1250 \\
\text { 1. } 2500 \\
1.5000 \\
1.7500 \\
\text { 2. } 0000\end{array}$ & $\begin{array}{l}1.0322 \\
1.1572 \\
1.3917 \\
1.6201 \\
1.8557\end{array}$ & $\begin{array}{l}.9394 \\
\text { 1. } 0644 \\
\text { 1. } 2835 \\
\text { 1. } 4902 \\
\text { 1. } 7113\end{array}$ & $\begin{array}{l}28.575 \\
31.750 \\
38.100 \\
44.450 \\
50.800\end{array}$ & $\begin{array}{l}.14286 \\
.14286 \\
.16667 \\
.20000 \\
.22222\end{array}$ & $\begin{array}{r}.09279 \\
.09279 \\
.10825 \\
.12990 \\
.14434\end{array}$ & $\begin{array}{r}.01786 \\
.01786 \\
.02083 \\
.02500 \\
.02778\end{array}$ & $\begin{array}{l}.00595 \\
.00595 \\
.00694 \\
.00833 \\
.00926\end{array}$ & $\begin{array}{ll}2 & 31 \\
2 & 15 \\
2 & 11 \\
2 & 15 \\
2 & 11\end{array}$ & $\begin{array}{r}.6931 \\
.8898 \\
\text { 1. } 2938 \\
\text { 1. } 7441 \\
\text { 2. } 3001\end{array}$ \\
\hline $\begin{array}{l}21 / 4 \ldots \\
21 / 2 \ldots \\
23 / 4 \ldots \\
311 \ldots \\
3 \ldots\end{array}$ & $\begin{array}{l}41 / 2 \\
4 \\
4 \\
4 \\
31 / 2\end{array}$ & $\begin{array}{l}2.2500 \\
2.5000 \\
2.7500 \\
3.0000 \\
3.0000\end{array}$ & $\begin{array}{l}2.1057 \\
2.3376 \\
2.5876 \\
2.8376 \\
2.8144\end{array}$ & $\begin{array}{l}\text { 1. } 9613 \\
\text { 2. } 1752 \\
\text { 2. } 4252 \\
\text { 2. } 6752 \\
\text { 2. } 6288\end{array}$ & $\begin{array}{l}57.150 \\
63.500 \\
69.850 \\
76.200 \\
76.200\end{array}$ & $\begin{array}{l}.22222 \\
.25000 \\
.25000 \\
.25000 \\
.28571\end{array}$ & $\begin{array}{r}.14434 \\
.16238 \\
.16238 \\
.16238 \\
.18558\end{array}$ & $\begin{array}{r}.02778 \\
.03125 \\
.03125 \\
.03125 \\
.03571\end{array}$ & $\begin{array}{r}.00926 \\
.01042 \\
.01042 \\
.01042 \\
.01190\end{array}$ & $\begin{array}{ll}1 & 55 \\
1 & 57 \\
1 & 46 \\
1 & 36 \\
1 & 51\end{array}$ & $\begin{array}{l}\text { 3. } 0212 \\
\text { 3. } 7161 \\
\text { 4. } 6194 \\
5.6209 \\
5.4276\end{array}$ \\
\hline
\end{tabular}

1 Optional. Standard for marine work.

(b) AMERICAN NATIONAL FINE-THREAD SERIES

In Table 2 are specified the nominal sizes and basic dimensions of the American national finethread series.
The American national fine-thread series is recommended for general use in automotive and aircraft work, for use where the design requires both strength and reduction in weight, and where special conditions require a fine thread. 
TABLE 2.-American national fine-thread series

\begin{tabular}{|c|c|c|c|c|c|c|c|c|c|c|c|}
\hline \multicolumn{2}{|c|}{ Identification } & \multicolumn{3}{|c|}{ Basic diameter } & \multicolumn{7}{|c|}{ Thread data } \\
\hline Sizes & $\begin{array}{l}\text { Threads } \\
\underset{n}{\text { per inch }}\end{array}$ & $\begin{array}{c}\text { Major } \\
\underset{D}{\text { diameter }}\end{array}$ & $\begin{array}{c}\text { Pitch } \\
\text { diameter } \\
E\end{array}$ & $\underset{K}{\text { Minor }}$ & $\begin{array}{l}\text { Metric } \\
\text { equiv- } \\
\text { alent } \\
\text { of major } \\
\text { diameter }\end{array}$ & $\begin{array}{c}\text { Pitch } \\
p\end{array}$ & $\begin{array}{c}\text { Depth of } \\
\text { thread } \\
h\end{array}$ & $\begin{array}{c}\text { Basic } \\
\text { width } \\
\text { of } f l a t \\
p / 8\end{array}$ & $\begin{array}{c}\text { Mini- } \\
\text { mum } \\
\text { width of } \\
\text { flat at } \\
\text { major } \\
\text { diameter } \\
\text { of nut } \\
\text { p } / 24 \\
\end{array}$ & $\begin{array}{c}\text { Helix } \\
\text { angle at } \\
\text { basic } \\
\text { pitch } \\
\text { diameter } \\
\mathbf{8}\end{array}$ & $\begin{array}{c}\text { Basic } \\
\text { area of } \\
\text { section } \\
\text { at root } \\
\text { of thread } \\
\frac{\pi K^{2}}{4}\end{array}$ \\
\hline 1 & 2 & 3 & 4 & 5 & 6 & 7 & 8 & 9 & 10 & 11 & 12 \\
\hline $\begin{array}{l}0 \ldots \\
1 \ldots \\
2 \ldots \\
3 \ldots \\
4 \ldots\end{array}$ & $\begin{array}{l}80 \\
72 \\
64 \\
56 \\
48\end{array}$ & $\begin{array}{l}\text { Inches } \\
0.060 \\
.073 \\
.086 \\
.099 \\
.112\end{array}$ & $\begin{array}{c}\text { Inches } \\
0.0519 \\
.0640 \\
.0759 \\
.0874 \\
.0985\end{array}$ & $\begin{array}{r}\text { Inches } \\
0.0438 \\
.0550 \\
.0657 \\
.0758 \\
.0849\end{array}$ & $\begin{array}{l}m m \\
1.524 \\
1.854 \\
2.184 \\
2.515 \\
2.845\end{array}$ & $\begin{array}{c}\text { Inch } \\
0.01250 \\
.01389 \\
.01562 \\
.01786 \\
.02083\end{array}$ & $\begin{array}{c}\text { Inch } \\
0.00812 \\
.00902 \\
.01015 \\
.01160 \\
.01353\end{array}$ & $\begin{array}{c}\text { Inch } \\
0.00156 \\
.00174 \\
.00195 \\
.00223 \\
.00260\end{array}$ & $\begin{array}{l}\text { Inch } \\
0.00052 \\
.00058 \\
.00065 \\
.00074 \\
.00087\end{array}$ & $\begin{array}{ll}\circ & 1 \\
4 & 23 \\
3 & 57 \\
3 & 45 \\
3 & 43 \\
3 & 51\end{array}$ & $\begin{array}{c}\text { Square } \\
\text { inches } \\
0.0015 \\
.0024 \\
.0034 \\
.0045 \\
.0057\end{array}$ \\
\hline $\begin{array}{l}5 \\
6 \\
8 \\
10 \\
12 \ldots\end{array}$ & $\begin{array}{l}44 \\
40 \\
36 \\
32 \\
28\end{array}$ & $\begin{array}{l}.125 \\
.138 \\
.164 \\
.190 \\
.216\end{array}$ & $\begin{array}{l}.1102 \\
.1218 \\
.1460 \\
.1697 \\
.1928\end{array}$ & $\begin{array}{l}.0955 \\
.1055 \\
.1279 \\
.1494 \\
.1696\end{array}$ & $\begin{array}{l}3.175 \\
3.505 \\
4.166 \\
4.826 \\
5.486\end{array}$ & $\begin{array}{l}.02273 \\
.02500 \\
.02778 \\
.03125 \\
.03571\end{array}$ & $\begin{array}{r}.01476 \\
.01624 \\
.01804 \\
.02030 \\
.02320\end{array}$ & $\begin{array}{r}.00284 \\
.00312 \\
.00347 \\
.00391 \\
.00446\end{array}$ & $\begin{array}{l}.00095 \\
.00104 \\
.00116 \\
.00130 \\
.00149\end{array}$ & $\begin{array}{ll}3 & 45 \\
3 & 44 \\
3 & 28 \\
3 & 21 \\
3 & 22\end{array}$ & $\begin{array}{l}.0072 \\
.0087 \\
.0128 \\
.0175 \\
.0226\end{array}$ \\
\hline $\begin{array}{l}1 / 4 \ldots \\
5 / 16 \ldots \\
36 \\
7 / 16 \ldots \\
1 / 2 \ldots\end{array}$ & $\begin{array}{l}28 \\
24 \\
24 \\
20 \\
20\end{array}$ & $\begin{array}{l}.2500 \\
.3125 \\
.3750 \\
.4375 \\
.5000\end{array}$ & $\begin{array}{l}.2268 \\
.2854 \\
.3479 \\
.4050 \\
.4675\end{array}$ & $\begin{array}{l}.2036 \\
.2584 \\
.3209 \\
.3725 \\
.4350\end{array}$ & $\begin{array}{r}6.350 \\
7.938 \\
9.525 \\
11.113 \\
12.700\end{array}$ & $\begin{array}{r}.03571 \\
.04167 \\
.04167 \\
.05000 \\
.05000\end{array}$ & $\begin{array}{l}.02320 \\
.02706 \\
.02706 \\
.03248 \\
.03248\end{array}$ & $\begin{array}{l}.00446 \\
.00521 \\
.00521 \\
.00625 \\
.00625\end{array}$ & $\begin{array}{r}.00149 \\
.00174 \\
.00174 \\
.00208 \\
.00208\end{array}$ & $\begin{array}{ll}2 & 52 \\
2 & 40 \\
2 & 11 \\
2 & 15 \\
1 & 57\end{array}$ & $\begin{array}{l}.0326 \\
.0524 \\
.0809 \\
.1090 \\
.1486\end{array}$ \\
\hline $\begin{array}{l}9 / 16 \ldots \\
5 / 8 \ldots- \\
3 / 4 \ldots \\
7 / 8 \ldots \\
1 \ldots\end{array}$ & $\begin{array}{l}18 \\
18 \\
16 \\
14 \\
14\end{array}$ & $\begin{array}{r}.5625 \\
.6250 \\
.7500 \\
.8750 \\
1.0000\end{array}$ & $\begin{array}{l}.5264 \\
.5889 \\
.7094 \\
.8286 \\
.9536\end{array}$ & $\begin{array}{l}.4903 \\
.5528 \\
.6688 \\
.7822 \\
.9072\end{array}$ & $\begin{array}{l}14.288 \\
15.875 \\
19.050 \\
22.225 \\
25.400\end{array}$ & $\begin{array}{r}.05556 \\
.05556 \\
.06250 \\
.07143 \\
.07143\end{array}$ & $\begin{array}{l}.03608 \\
.03608 \\
.04049 \\
.04639 \\
.04639\end{array}$ & $\begin{array}{l}.00694 \\
.00694 \\
.00781 \\
.00893 \\
.00893\end{array}$ & $\begin{array}{l}.00231 \\
.00231 \\
.00260 \\
.00298 \\
.00298\end{array}$ & $\begin{array}{ll}1 & 55 \\
1 & 43 \\
1 & 36 \\
1 & 34 \\
1 & 22\end{array}$ & $\begin{array}{l}.1888 \\
.2400 \\
.3513 \\
.4805 \\
.6464\end{array}$ \\
\hline $\begin{array}{l}11 / 8 \ldots \\
11 / 4 \ldots \\
11 / 2 \ldots \\
13 / 4 \ldots \\
2 \ldots\end{array}$ & $\begin{array}{l}12 \\
12 \\
12 \\
10 \\
10\end{array}$ & $\begin{array}{l}1.1250 \\
1.2500 \\
1.5000 \\
1.7500 \\
2.0000\end{array}$ & $\begin{array}{l}1.0709 \\
1.1959 \\
1.4459 \\
1.6850 \\
1.9350\end{array}$ & $\begin{array}{l}1.0167 \\
1.1417 \\
1.3917 \\
1.6201 \\
1.8701\end{array}$ & $\begin{array}{l}28.575 \\
31.750 \\
38.100 \\
44.450 \\
50.800\end{array}$ & $\begin{array}{l}.08333 \\
.08333 \\
.08333 \\
.10000 \\
.10000\end{array}$ & $\begin{array}{r}.05413 \\
.05413 \\
.05413 \\
.06495 \\
.06495\end{array}$ & $\begin{array}{r}.01042 \\
.01042 \\
.01042 \\
.01250 \\
.01250\end{array}$ & $\begin{array}{l}.00347 \\
.00347 \\
.00347 \\
.00417 \\
.00417\end{array}$ & $\begin{array}{rr}1 & 25 \\
1 & 16 \\
1 & 3 \\
1 & 5 \\
0 & 57\end{array}$ & $\begin{array}{r}.8118 \\
1.0238 \\
1.5212 \\
2.0615 \\
2.7468\end{array}$ \\
\hline $\begin{array}{l}21 / 4 \ldots \\
21 / 2 \ldots \\
23 / 4 \ldots \\
3 \ldots\end{array}$ & $\begin{array}{l}8 \\
8 \\
8 \\
8 \\
\end{array}$ & $\begin{array}{l}2.2500 \\
2.5000 \\
2.7500 \\
3.0000 \\
\end{array}$ & $\begin{array}{l}2.1688 \\
2.4188 \\
2.6688 \\
2.9188 \\
\end{array}$ & $\begin{array}{l}2.0876 \\
2.3376 \\
2.5876 \\
2.8376 \\
\end{array}$ & $\begin{array}{l}57.150 \\
63.500 \\
69.850 \\
76.200 \\
\end{array}$ & $\begin{array}{l}.12500 \\
.12500 \\
.12500 \\
.12500 \\
\end{array}$ & $\begin{array}{r}.08119 \\
.08119 \\
.08119 \\
.08119 \\
\end{array}$ & $\begin{array}{r}.01562 \\
.01562 \\
.01562 \\
.01562 \\
\end{array}$ & $\begin{array}{l}.00521 \\
.00521 \\
.00521 \\
.00521 \\
\end{array}$ & $\begin{array}{lr}1 & 3 \\
0 & 57 \\
0 & 51 \\
0 & 47 \\
\end{array}$ & $\begin{array}{l}3.4228 \\
4.2917 \\
5.2588 \\
6.3240 \\
\end{array}$ \\
\hline
\end{tabular}

TABLE 3.-Class 1, loose fit, allowances and tolerances for screws and nuts

\begin{tabular}{|c|c|c|c|c|c|}
\hline Threads per inch & $\begin{array}{l}\text { Allow- } \\
\text { ances }\end{array}$ & $\begin{array}{l}\text { Pitch } \\
\text { diame- } \\
\text { ter tol- } \\
\text { erances }\end{array}$ & \begin{tabular}{|} 
Lead \\
errors \\
con- \\
sum- \\
ing one- \\
half of \\
pitch- \\
diame- \\
ter tol- \\
erances
\end{tabular} & \multicolumn{2}{|c|}{$\begin{array}{l}\text { Errors } \\
\text { in half- } \\
\text { angle } \\
\text { con- } \\
\text { sum- } \\
\text { ing one- } \\
\text { half of } \\
\text { pitch- } \\
\text { diame- } \\
\text { ter tol- } \\
\text { erances }\end{array}$} \\
\hline 1 & 2 & 3 & 4 & \multicolumn{2}{|c|}{5} \\
\hline $\begin{array}{l}80 \\
64 \\
68\end{array}$ & $\begin{array}{l}\text { Inch } \\
0.0007 \\
.0007 \\
.0007 \\
.0008 \\
.0009\end{array}$ & $\begin{array}{l}\text { Inch } \\
0.0024 \\
.0025 \\
.0026 \\
.0028 \\
.0031\end{array}$ & $\begin{array}{l}\text { Inch } \\
0.0007 \\
.0007 \\
.0008 \\
.0008 \\
.0009\end{array}$ & $\begin{array}{l}\circ \\
3 \\
3 \\
3 \\
3 \\
2\end{array}$ & \\
\hline $\begin{array}{l}44 \\
40 \\
36 \\
32\end{array}$ & $\begin{array}{l}.0009 \\
.0010 \\
.0011 \\
.0011 \\
.0012\end{array}$ & $\begin{array}{l}.0032 \\
.0034 \\
.0036 \\
.0038 \\
.0043\end{array}$ & $\begin{array}{l}.0009 \\
.0010 \\
.0010 \\
.0011 \\
.0012\end{array}$ & $\begin{array}{l}2 \\
2 \\
2 \\
2 \\
2\end{array}$ & $\begin{array}{l}41 \\
36 \\
28 \\
19 \\
18\end{array}$ \\
\hline 18 & $\begin{array}{l}.0013 \\
.0015 \\
.0016 \\
.0018 \\
.0021\end{array}$ & $\begin{array}{l}.0046 \\
.0051 \\
.0057 \\
.0063 \\
.0070\end{array}$ & $\begin{array}{l}.0013 \\
.0015 \\
.0016 \\
.0018 \\
.0020\end{array}$ & $\begin{array}{l}2 \\
1 \\
1 \\
1 \\
1\end{array}$ & $\begin{array}{r}6 \\
57 \\
58 \\
55 \\
52\end{array}$ \\
\hline 10 (American national coarse) & $\begin{array}{l}.0022 \\
.0024 \\
.0026 \\
.0028 \\
.0028\end{array}$ & $\begin{array}{l}.0074 \\
.0079 \\
.0085 \\
.0092 \\
.0132\end{array}$ & $\begin{array}{l}.0021 \\
.0023 \\
.0025 \\
.0027 \\
.0038\end{array}$ & $\begin{array}{l}1 \\
1 \\
1 \\
1 \\
2\end{array}$ & $\begin{array}{l}50 \\
49 \\
47 \\
45 \\
31\end{array}$ \\
\hline 8 (American national coarse) & $\begin{array}{l}.0031 \\
.0034 \\
.0034 \\
.0039 \\
.0044\end{array}$ & $\begin{array}{l}.0100 \\
.0111 \\
.0145 \\
.0124 \\
.0145\end{array}$ & $\begin{array}{l}.0029 \\
.0032 \\
.0042 \\
.0036 \\
.0042\end{array}$ & $\begin{array}{l}1 \\
1 \\
2 \\
1 \\
1\end{array}$ & $\begin{array}{l}43 \\
42 \\
13 \\
39 \\
40\end{array}$ \\
\hline $\begin{array}{l}51 / 2-1 \\
41 / 2 \\
31 / 2\end{array}$ & $\begin{array}{l}.0052 \\
.0057 \\
.0064 \\
.0073\end{array}$ & $\begin{array}{l}.0169 \\
.0184 \\
.0204 \\
.0229\end{array}$ & $\begin{array}{l}.0049 \\
.0053 \\
.0059 \\
.0066\end{array}$ & $\begin{array}{l}1 \\
1 \\
1 \\
1\end{array}$ & $\begin{array}{l}37 \\
35 \\
33 \\
32\end{array}$ \\
\hline
\end{tabular}

12 See footnotes to Table 6.
TABLE 4.-Class 2, free fit, tolerances for screws and nuts (no allowances)

\begin{tabular}{|c|c|c|c|c|c|}
\hline \multirow[t]{2}{*}{ Threads per inch } & \multirow{2}{*}{$\begin{array}{l}\text { Allow- } \\
\text { ances } \\
\\
2\end{array}$} & $\begin{array}{l}\text { Pitch } \\
\text { diame- } \\
\text { ter tol- } \\
\text { erances }\end{array}$ & \begin{tabular}{|c|} 
Lead \\
errors \\
con- \\
sum- \\
ing one- \\
half of \\
pitch- \\
diame- \\
ter tol- \\
erances?
\end{tabular} & \multicolumn{2}{|c|}{$\begin{array}{l}\text { Errors } \\
\text { in half- } \\
\text { angle } \\
\text { con- } \\
\text { sum- } \\
\text { ing one- } \\
\text { half of } \\
\text { pitch- } \\
\text { diame- } \\
\text { ter tol- } \\
\text { erances } \\
\end{array}$} \\
\hline & & 3 & 4 & \multicolumn{2}{|c|}{5} \\
\hline 80 & $\begin{array}{l}\text { Inch } \\
0.0000 \\
.0000 \\
.0000 \\
.0000 \\
.0000\end{array}$ & $\begin{array}{l}\text { Inch } \\
0.0017 \\
.0018 \\
.0019 \\
.0020 \\
.0022\end{array}$ & $\begin{array}{l}\text { Inch } \\
0.0005 \\
.0005 \\
.0005 \\
.0006 \\
.0006\end{array}$ & $\begin{array}{l}\circ \\
2 \\
2 \\
2 \\
2 \\
2\end{array}$ & $\begin{array}{r}\prime \\
36 \\
28 \\
19 \\
8 \\
1\end{array}$ \\
\hline $\begin{array}{l}44 \\
40 \\
46 \\
36 \\
36 \\
28\end{array}$ & $\begin{array}{l}.0000 \\
.0000 \\
.0000 \\
.0000 \\
.0000\end{array}$ & $\begin{array}{l}.0023 \\
.0024 \\
.0025 \\
.0027 \\
.0031\end{array}$ & $\begin{array}{l}.0007 \\
.0007 \\
.0007 \\
.0008 \\
.0009\end{array}$ & $\begin{array}{l}1 \\
1 \\
1 \\
1 \\
1\end{array}$ & $\begin{array}{l}56 \\
50 \\
43 \\
39 \\
39\end{array}$ \\
\hline $\begin{array}{l}24 \\
20 \\
18 \\
16\end{array}$ & $\begin{array}{l}.0000 \\
.0000 \\
.0000 \\
.0000 \\
.0000\end{array}$ & $\begin{array}{l}.0033 \\
.0036 \\
.0041 \\
.0045 \\
.0049\end{array}$ & $\begin{array}{l}.0010 \\
.0010 \\
.0012 \\
.0013 \\
.0014\end{array}$ & $\begin{array}{l}1 \\
1 \\
1 \\
1 \\
1\end{array}$ & $\begin{array}{l}31 \\
22 \\
25 \\
22 \\
19\end{array}$ \\
\hline 10 (American national coarse) & $\begin{array}{l}.0000 \\
.0000 \\
.0000 \\
.0000 \\
.0000\end{array}$ & $\begin{array}{l}.0052 \\
.0056 \\
.0059 \\
.0064 \\
.0100\end{array}$ & $\begin{array}{l}.0015 \\
.0016 \\
.0017 \\
.0018 \\
.0029\end{array}$ & $\begin{array}{l}1 \\
1 \\
1 \\
1 \\
1\end{array}$ & $\begin{array}{l}17 \\
17 \\
14 \\
13 \\
55\end{array}$ \\
\hline $\begin{array}{l}8 \text { (American national coarse) } \\
8 \text { (American national fine) } \\
7\end{array}$ & $\begin{array}{l}.0000 \\
.0000 \\
.0000 \\
.0000 \\
.0000\end{array}$ & $\begin{array}{l}.0070 \\
.0076 \\
.0110 \\
.0085 \\
.0101\end{array}$ & $\begin{array}{l}.0020 \\
.0022 \\
.0032 \\
.0025 \\
.0029\end{array}$ & $\begin{array}{l}1 \\
1 \\
1 \\
1 \\
1\end{array}$ & $\begin{array}{r}12 \\
10 \\
41 \\
8 \\
9\end{array}$ \\
\hline 51/2 $41 / 2$ & $\begin{array}{l}.0000 \\
.0000 \\
.0000 \\
.0000 \\
\end{array}$ & $\begin{array}{l}.0116 \\
.0127 \\
.0140 \\
.0157 \\
\end{array}$ & $\begin{array}{l}.0033 \\
.0037 \\
.0040 \\
.0045 \\
\end{array}$ & $\begin{array}{l}1 \\
1 \\
1 \\
1 \\
\end{array}$ & $\begin{array}{l}6 \\
5 \\
4 \\
3 \\
\end{array}$ \\
\hline
\end{tabular}


TABLE 5.-Class 3, medium fit, tolerances for screws and nuts (no allowances)

\begin{tabular}{|c|c|c|c|c|}
\hline Threads per inch & $\begin{array}{l}\text { Allow- } \\
\text { ances }\end{array}$ & $\begin{array}{l}\text { Pitch } \\
\text { diame- } \\
\text { ter tol- } \\
\text { erances }^{1}\end{array}$ & $\begin{array}{c}\text { Lead } \\
\text { errors } \\
\text { con- } \\
\text { sum- } \\
\text { ing one- } \\
\text { half of } \\
\text { pitch- } \\
\text { diame- } \\
\text { ter tol- } \\
\text { erances }\end{array}$ & $\begin{array}{l}\text { Errors } \\
\text { in half- } \\
\text { angle } \\
\text { con- } \\
\text { sum- } \\
\text { ing one- } \\
\text { half of } \\
\text { pitch- } \\
\text { diame- } \\
\text { ter tol- } \\
\text { erances }\end{array}$ \\
\hline 1 & 2 & 3 & 4 & 5 \\
\hline $\begin{array}{l}80 \\
72 \\
64 \\
56 \\
48\end{array}$ & $\begin{array}{l}\text { Inch } \\
0.0000 \\
.0000 \\
.0000 \\
.0000 \\
.0000\end{array}$ & $\begin{array}{c}\text { Inch } \\
0.0013 \\
.0013 \\
.0014 \\
.0015 \\
.0016\end{array}$ & $\begin{array}{l}\text { Inch } \\
0.0004 \\
.0004 \\
.0004 \\
.0004 \\
.0005\end{array}$ & \begin{tabular}{ll}
$\circ$ & \multicolumn{1}{l}{} \\
1 & 59 \\
1 & 47 \\
1 & 43 \\
1 & 36 \\
1 & 28
\end{tabular} \\
\hline $\begin{array}{l}44 \\
40 \\
36 \\
32 \\
28\end{array}$ & $\begin{array}{l}.0000 \\
.0000 \\
.0000 \\
.0000 \\
.0000\end{array}$ & $\begin{array}{l}.0016 \\
.0017 \\
.0018 \\
.0019 \\
.0022\end{array}$ & $\begin{array}{l}.0005 \\
.0005 \\
.0005 \\
.0005 \\
.0006\end{array}$ & $\begin{array}{ll}1 & 21 \\
1 & 18 \\
1 & 14 \\
1 & 10 \\
1 & 11\end{array}$ \\
\hline $\begin{array}{l}24 \\
20 \\
18 \\
16\end{array}$ & $\begin{array}{l}.0000 \\
.0000 \\
.0000 \\
.0000 \\
.0000\end{array}$ & $\begin{array}{l}.0024 \\
.0026 \\
.0030 \\
.0032 \\
.0036\end{array}$ & $\begin{array}{l}.0007 \\
.0008 \\
.0009 \\
.0009 \\
.0010\end{array}$ & $\begin{array}{lr}1 & 6 \\
1 & 0 \\
1 & 2 \\
0 & 59 \\
0 & 58\end{array}$ \\
\hline 10 (American national coarse) & $\begin{array}{l}.0000 \\
.0000 \\
.0000 \\
.0000 \\
.0000\end{array}$ & $\begin{array}{l}.0037 \\
.0040 \\
.0042 \\
.0045 \\
.0084\end{array}$ & $\begin{array}{l}.0011 \\
.0012 \\
.0012 \\
.0013 \\
.0024\end{array}$ & $\begin{array}{ll}0 & 55 \\
0 & 55 \\
0 & 53 \\
0 & 52 \\
1 & 36\end{array}$ \\
\hline 8 (American national coarse) & $\begin{array}{l}.0000 \\
.0000 \\
.0000 \\
.0000 \\
.0000\end{array}$ & $\begin{array}{l}.0049 \\
.0054 \\
.0092 \\
.0059 \\
.0071\end{array}$ & $\begin{array}{l}.0014 \\
.0016 \\
.0027 \\
.0017 \\
.0020\end{array}$ & $\begin{array}{ll}0 & 51 \\
0 & 50 \\
1 & 24 \\
0 & 47 \\
0 & 49\end{array}$ \\
\hline $\begin{array}{l}51 / 2 \\
41 / 2 \\
4\end{array}$ & $\begin{array}{l}.0000 \\
.0000 \\
.0000 \\
.0000\end{array}$ & $\begin{array}{l}.0082 \\
.0089 \\
.0097 \\
.0107\end{array}$ & $\begin{array}{l}.0024 \\
.0026 \\
.0028 \\
.0031\end{array}$ & $\begin{array}{ll}0 & 47 \\
0 & 46 \\
0 & 44 \\
0 & 43\end{array}$ \\
\hline
\end{tabular}

12 See footnotes to Table 6.
TABLE 6.-Class 4, close fit, allowances and tolerances for screws and nuts

\begin{tabular}{|c|c|c|c|c|c|}
\hline Threads per inch & $\begin{array}{l}\text { Inter- } \\
\text { ferences } \\
\text { or neg- } \\
\text { ative } \\
\text { allow- } \\
\text { ances }\end{array}$ & $\begin{array}{c}\text { Pitch } \\
\text { diame- } \\
\text { ter tol- } \\
\text { erances } 1\end{array}$ & $\begin{array}{l}\text { Lead } \\
\text { errors } \\
\text { con- } \\
\text { sum- } \\
\text { ing one- } \\
\text { half of } \\
\text { pitch- } \\
\text { diame- } \\
\text { ter tol- } \\
\text { erances }{ }^{2}\end{array}$ & \multicolumn{2}{|c|}{\begin{tabular}{|} 
Errors \\
in half- \\
angle \\
con- \\
sum- \\
ing one- \\
half of \\
pitch- \\
diame- \\
ter tol- \\
erances
\end{tabular}} \\
\hline 1 & 2 & 3 & 4 & \multicolumn{2}{|l|}{5} \\
\hline S- & $\begin{array}{c}\text { Inch } \\
0.0001 \\
.0001 \\
.0001 \\
.0002 \\
.0002\end{array}$ & $\begin{array}{c}\text { Inch } \\
0.0006 \\
.0007 \\
.0007 \\
.0007 \\
.0008\end{array}$ & $\begin{array}{c}\text { Inch } \\
0.0002 \\
.0002 \\
.0002 \\
.0002 \\
.0002\end{array}$ & $\begin{array}{l}\circ \\
0 \\
0 \\
0 \\
0 \\
0\end{array}$ & \\
\hline 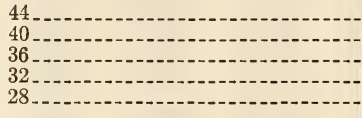 & $\begin{array}{l}.0002 \\
.0002 \\
.0002 \\
.0002 \\
.0002\end{array}$ & $\begin{array}{l}.0008 \\
.0009 \\
.0009 \\
.0010 \\
.0011\end{array}$ & $\begin{array}{l}.0002 \\
.0003 \\
.0003 \\
.0003 \\
.0003\end{array}$ & $\begin{array}{l}0 \\
0 \\
0 \\
0 \\
0\end{array}$ & $\begin{array}{l}40 \\
41 \\
37 \\
37 \\
35\end{array}$ \\
\hline 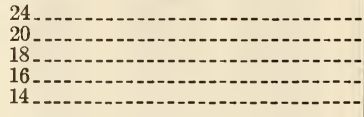 & $\begin{array}{l}.0003 \\
.0003 \\
.0003 \\
.0004 \\
.0004\end{array}$ & $\begin{array}{l}.0012 \\
.0013 \\
.0015 \\
.0016 \\
.0018\end{array}$ & $\begin{array}{l}.0003 \\
.0004 \\
.0004 \\
.0005 \\
.0005\end{array}$ & $\begin{array}{l}0 \\
0 \\
0 \\
0 \\
0\end{array}$ & $\begin{array}{l}33 \\
30 \\
31 \\
29 \\
29\end{array}$ \\
\hline 10 (American national coarse) & $\begin{array}{l}.0004 \\
.0005 \\
.0005 \\
.0006 \\
.0006\end{array}$ & $\begin{array}{l}.0019 \\
.0020 \\
.0021 \\
.0023 \\
.0042\end{array}$ & $\begin{array}{l}.0005 \\
.0006 \\
.0006 \\
.0007 \\
.0012\end{array}$ & $\begin{array}{l}0 \\
0 \\
0 \\
0 \\
0\end{array}$ & $\begin{array}{l}28 \\
28 \\
26 \\
26 \\
48\end{array}$ \\
\hline $\begin{array}{l}8 \text { (American national coarse) } \\
8 \text { (American national fine) } \\
6\end{array}$ & $\begin{array}{l}.0006 \\
.0007 \\
.0007 \\
.0008 \\
.0009\end{array}$ & $\begin{array}{l}.0024 \\
.0027 \\
.0046 \\
.0030 \\
.0036\end{array}$ & $\begin{array}{l}.0007 \\
.0008 \\
.0013 \\
.0009 \\
.0010\end{array}$ & $\begin{array}{l}0 \\
0 \\
0 \\
0 \\
0\end{array}$ & $\begin{array}{l}25 \\
25 \\
42 \\
24 \\
25\end{array}$ \\
\hline $31 / 2$ & $\begin{array}{l}.0010 \\
.0011 \\
.0013 \\
.0016\end{array}$ & $\begin{array}{l}.0041 \\
.0044 \\
.0048 \\
.0053\end{array}$ & $\begin{array}{l}.0012 \\
.0013 \\
.0014 \\
.0015\end{array}$ & $\begin{array}{l}0 \\
0 \\
0 \\
0\end{array}$ & $\begin{array}{l}23 \\
23 \\
22 \\
21\end{array}$ \\
\hline
\end{tabular}

1 The tolerances specified for pitch diameter include all errors of pitch diameter, lead, and angle. The full tolerance can not therefore be used on pitch diameter unless the lead and angle of the thread are perfect. Columns 4 and 5 give, for information, the errors in lead (per length of thread engaged) and in angle, each of which can be por for complon ulated, the pitch diameter of a bolt, for example, must be reduced by the full tolerance or it will not enter a basic nut or gage.

by the full tolerance or it will not enter a basic nut or gage.
2 Between any 2 threads not farther apart than the length of engagement. 
TABLE 7.-Class 1, loose fit, American national coarse-thread series

\begin{tabular}{|c|c|c|c|c|c|c|c|c|c|c|c|c|}
\hline \multirow{3}{*}{ Sizes } & \multirow{3}{*}{$\begin{array}{l}\text { Threads } \\
\text { per inch }\end{array}$} & \multicolumn{5}{|c|}{ Screw sizes } & \multicolumn{5}{|c|}{ Nut sizes } & \multirow{3}{*}{$\begin{array}{c}\text { Basic } \\
\text { major } \\
\text { diameter }\end{array}$} \\
\hline & & \multicolumn{2}{|c|}{ Major diameter } & \multicolumn{2}{|c|}{ Pitch diameter } & \multirow{2}{*}{$\begin{array}{c}\text { Minor } \\
\text { diameter, } \\
\text { maxi- } \\
\text { mum }\end{array}$} & \multicolumn{2}{|c|}{ Minor diameter } & \multicolumn{2}{|c|}{ Pitch diameter } & \multirow{2}{*}{$\begin{array}{l}\text { Major } \\
\text { diameter, } \\
\text { mini- } \\
\text { mum }\end{array}$} & \\
\hline & & $\begin{array}{l}\text { Maxi- } \\
\text { mum }\end{array}$ & $\begin{array}{l}\text { Mini- } \\
\text { mum }\end{array}$ & $\begin{array}{l}\text { Maxi- } \\
\text { mum }\end{array}$ & $\begin{array}{l}\text { Mini- } \\
\text { mum }\end{array}$ & & $\begin{array}{l}\text { Mini- } \\
\text { mum }\end{array}$ & $\begin{array}{l}\text { Maxi- } \\
\text { mum }\end{array}$ & $\begin{array}{l}\text { Mini- } \\
\text { mum }\end{array}$ & $\begin{array}{l}\text { Maxi- } \\
\text { mum }\end{array}$ & & \\
\hline \multirow[t]{2}{*}{1} & 2 & 3 & 4 & 5 & 6 & 7 & 8 & 9 & 10 & 11 & 12 & 13 \\
\hline & $\begin{array}{l}64 \\
56 \\
48 \\
40 \\
40\end{array}$ & $\begin{array}{c}\text { Inches } \\
0.0723 \\
.0852 \\
.0981 \\
.1110 \\
.1240\end{array}$ & $\begin{array}{c}\text { Inches } \\
0.0671 \\
.0796 \\
.0919 \\
.1042 \\
.1172\end{array}$ & $\begin{array}{c}\text { Inches } \\
0.0622 \\
.0736 \\
.0846 \\
.0948 \\
.1078\end{array}$ & $\begin{array}{c}\text { Inches } \\
0.0596 \\
.0708 \\
.0815 \\
.0914 \\
.1044\end{array}$ & $\begin{array}{c}\text { Inches } \\
0.0531 \\
.0633 \\
.0725 \\
.0803 \\
.0933\end{array}$ & $\begin{array}{c}\text { Inches } \\
0.0561 \\
.0667 \\
.0764 \\
.0849 \\
.0979\end{array}$ & $\begin{array}{c}\text { Inches } \\
0.0604 \\
.0715 \\
.0820 \\
.0913 \\
.1043\end{array}$ & $\begin{array}{c}\text { Inches } \\
0.0629 \\
.0744 \\
.0855 \\
.0958 \\
.1088\end{array}$ & $\begin{array}{c}\text { Inches } \\
0.0655 \\
.0772 \\
.0886 \\
.0992 \\
.1122\end{array}$ & $\begin{array}{c}\text { Inches } \\
0.0730 \\
.0860 \\
.0990 \\
.1120 \\
.1250\end{array}$ & $\begin{array}{c}\text { Inches } \\
0.073 \\
.086 \\
.099 \\
.112 \\
.125\end{array}$ \\
\hline $\begin{array}{l}6 \ldots \\
8 \ldots-. \\
10-. \\
12- \\
14 . .\end{array}$ & $\begin{array}{l}32 \\
32 \\
24 \\
24 \\
20\end{array}$ & $\begin{array}{l}.1369 \\
.1629 \\
.1887 \\
.2147 \\
.2485\end{array}$ & $\begin{array}{l}.1293 \\
.1553 \\
.1795 \\
.2055 \\
.2383\end{array}$ & $\begin{array}{l}.1166 \\
.1426 \\
.1616 \\
.1876 \\
.2160\end{array}$ & $\begin{array}{l}.1128 \\
.1388 \\
.1570 \\
.1830 \\
.2109\end{array}$ & $\begin{array}{l}.0986 \\
.1246 \\
.1376 \\
.1636 \\
.1872\end{array}$ & $\begin{array}{l}.1042 \\
.1302 \\
.1449 \\
.1709 \\
.1959\end{array}$ & $\begin{array}{l}.1118 \\
.1378 \\
.1541 \\
.1801 \\
.2060\end{array}$ & $\begin{array}{l}.1177 \\
.1437 \\
.1629 \\
.1889 \\
.2175\end{array}$ & $\begin{array}{l}.1215 \\
.1475 \\
.1675 \\
.1935 \\
.2228\end{array}$ & $\begin{array}{l}.1380 \\
.1640 \\
.1900 \\
.2160 \\
.2500\end{array}$ & $\begin{array}{l}.138 \\
.164 \\
.190 \\
.216 \\
.2500\end{array}$ \\
\hline $\begin{array}{l}5 / 16- \\
3 / 8- \\
7 / 16- \\
1 / 2 . \\
9 / 116 .\end{array}$ & $\begin{array}{l}18 \\
16 \\
14 \\
13 \\
12\end{array}$ & $\begin{array}{l}.3109 \\
.3732 \\
.4354 \\
.4978 \\
.5601\end{array}$ & $\begin{array}{l}.2995 \\
.3606 \\
.4214 \\
.4830 \\
.5443\end{array}$ & $\begin{array}{l}.2748 \\
.3326 \\
.3890 \\
.4478 \\
.5060\end{array}$ & $\begin{array}{l}.2691 \\
.3263 \\
.3820 \\
.4404 \\
.4981\end{array}$ & $\begin{array}{l}.2427 \\
.2965 \\
.3478 \\
.4034 \\
.4579\end{array}$ & $\begin{array}{l}.2524 \\
.3073 \\
.3602 \\
.4167 \\
.4723\end{array}$ & $\begin{array}{l}.2630 \\
.3184 \\
.3721 \\
.4290 \\
.4850\end{array}$ & $\begin{array}{l}.2764 \\
.3344 \\
.3911 \\
.4500 \\
.5084\end{array}$ & $\begin{array}{l}.2821 \\
.3407 \\
.3981 \\
.4574 \\
.5163\end{array}$ & $\begin{array}{l}.3125 \\
.3750 \\
.4375 \\
.5000 \\
.5625\end{array}$ & $\begin{array}{l}.3125 \\
.3750 \\
.4375 \\
.5000 \\
.5625\end{array}$ \\
\hline $\begin{array}{l}5 / 8 \ldots \\
3 / 4 \ldots \\
7 / 8 \\
17 . \\
11 / 8\end{array}$ & $\begin{array}{r}11 \\
10 \\
9 \\
8 \\
7\end{array}$ & $\begin{array}{r}.6224 \\
.7472 \\
.8719 \\
.9966 \\
1.1211\end{array}$ & $\begin{array}{r}.6054 \\
.7288 \\
.8519 \\
.9744 \\
1.0963\end{array}$ & $\begin{array}{r}.5634 \\
.6822 \\
.7997 \\
.9154 \\
1.0283\end{array}$ & $\begin{array}{r}.5549 \\
.6730 \\
.7897 \\
.9043 \\
1.0159\end{array}$ & $\begin{array}{l}.5109 \\
.6245 \\
.7356 \\
.8432 \\
.9458\end{array}$ & $\begin{array}{l}.5266 \\
.6417 \\
.7547 \\
.8647 \\
.9704\end{array}$ & $\begin{array}{l}.5397 \\
.6553 \\
.7689 \\
.8795 \\
.9858\end{array}$ & $\begin{array}{r}.5660 \\
.6850 \\
.8028 \\
.9188 \\
1.0322\end{array}$ & $\begin{array}{r}.5745 \\
.6942 \\
.8128 \\
.9299 \\
1.0446\end{array}$ & $\begin{array}{r}.6250 \\
.7500 \\
.8750 \\
1.0000 \\
1.1250\end{array}$ & $\begin{array}{r}.6250 \\
.7500 \\
.8750 \\
1.0000 \\
1.1250\end{array}$ \\
\hline $\begin{array}{l}114 \ldots \\
115 \ldots \\
13 / 4 \ldots \\
2 \\
213 \ldots \\
\end{array}$ & \begin{tabular}{l|}
7 \\
6 \\
5 \\
$41 / 2$ \\
412
\end{tabular} & $\begin{array}{l}1.2461 \\
1.4956 \\
1.7448 \\
1.9943 \\
2.2443\end{array}$ & $\begin{array}{l}1.2213 \\
1.4666 \\
1.7110 \\
1.9575 \\
2.2075\end{array}$ & $\begin{array}{l}1.1533 \\
1.3873 \\
1.6149 \\
1.8500 \\
2.1000\end{array}$ & $\begin{array}{l}\text { 1. } 1409 \\
\text { 1. } 3728 \\
1.5980 \\
1.8316 \\
2.0816\end{array}$ & $\begin{array}{l}1.0708 \\
1.2911 \\
1.4994 \\
1.7217 \\
1.9717\end{array}$ & $\begin{array}{l}1.0954 \\
1.3196 \\
1.5335 \\
1.7594 \\
\text { 2. } 0094\end{array}$ & $\begin{array}{l}1.1108 \\
1.3376 \\
1.5551 \\
1.7835 \\
2.0335\end{array}$ & $\begin{array}{l}1.1572 \\
1.3917 \\
1.6201 \\
1.8557 \\
2.1057\end{array}$ & $\begin{array}{l}1.1696 \\
1.4062 \\
1.6370 \\
1.8741 \\
2.1241\end{array}$ & $\begin{array}{l}1.2500 \\
1.5000 \\
1.7500 \\
2.0000 \\
2.2500\end{array}$ & $\begin{array}{l}\text { 1. } 2500 \\
\text { 1. } 5000 \\
\text { 1. } 7500 \\
\text { 2. } 0000 \\
\text { 2. } 2500\end{array}$ \\
\hline $\begin{array}{l}21 / 2 \ldots \\
23 / 4 \ldots \\
3 . \\
3 .\end{array}$ & $\begin{array}{l}4 \\
4 \\
4 \\
31 / 2\end{array}$ & $\begin{array}{l}2.4936 \\
2.7436 \\
2.9936 \\
2.9927\end{array}$ & $\begin{array}{l}2.4528 \\
2.7028 \\
2.9528 \\
2.9469\end{array}$ & $\begin{array}{l}\text { 2. } 3312 \\
2.5812 \\
2.8312 \\
2.8071\end{array}$ & $\begin{array}{l}2.3108 \\
2.5608 \\
2.8108 \\
2.7842\end{array}$ & $\begin{array}{l}\text { 2. } 1869 \\
\text { 2. } 4369 \\
\text { 2. } 6869 \\
\text { 2. } 6421\end{array}$ & $\begin{array}{l}\text { 2. } 2294 \\
\text { 2. } 4794 \\
\text { 2. } 7294 \\
\text { 2. } 6907\end{array}$ & $\begin{array}{l}2.2564 \\
2.5064 \\
2.7564 \\
2.7216\end{array}$ & $\begin{array}{l}2.3376 \\
2.5876 \\
2.8376 \\
2.8144\end{array}$ & $\begin{array}{l}2.3580 \\
2.6080 \\
2.8580 \\
2.8373\end{array}$ & $\begin{array}{l}2.5000 \\
2.7500 \\
3.0000 \\
3.0000\end{array}$ & $\begin{array}{l}2.5000 \\
2.7500 \\
3.0000 \\
3.0000\end{array}$ \\
\hline
\end{tabular}

1 : See footnotes to Table 14.

TABLE 8.-Class 2, free fit, American national coarse-thread series

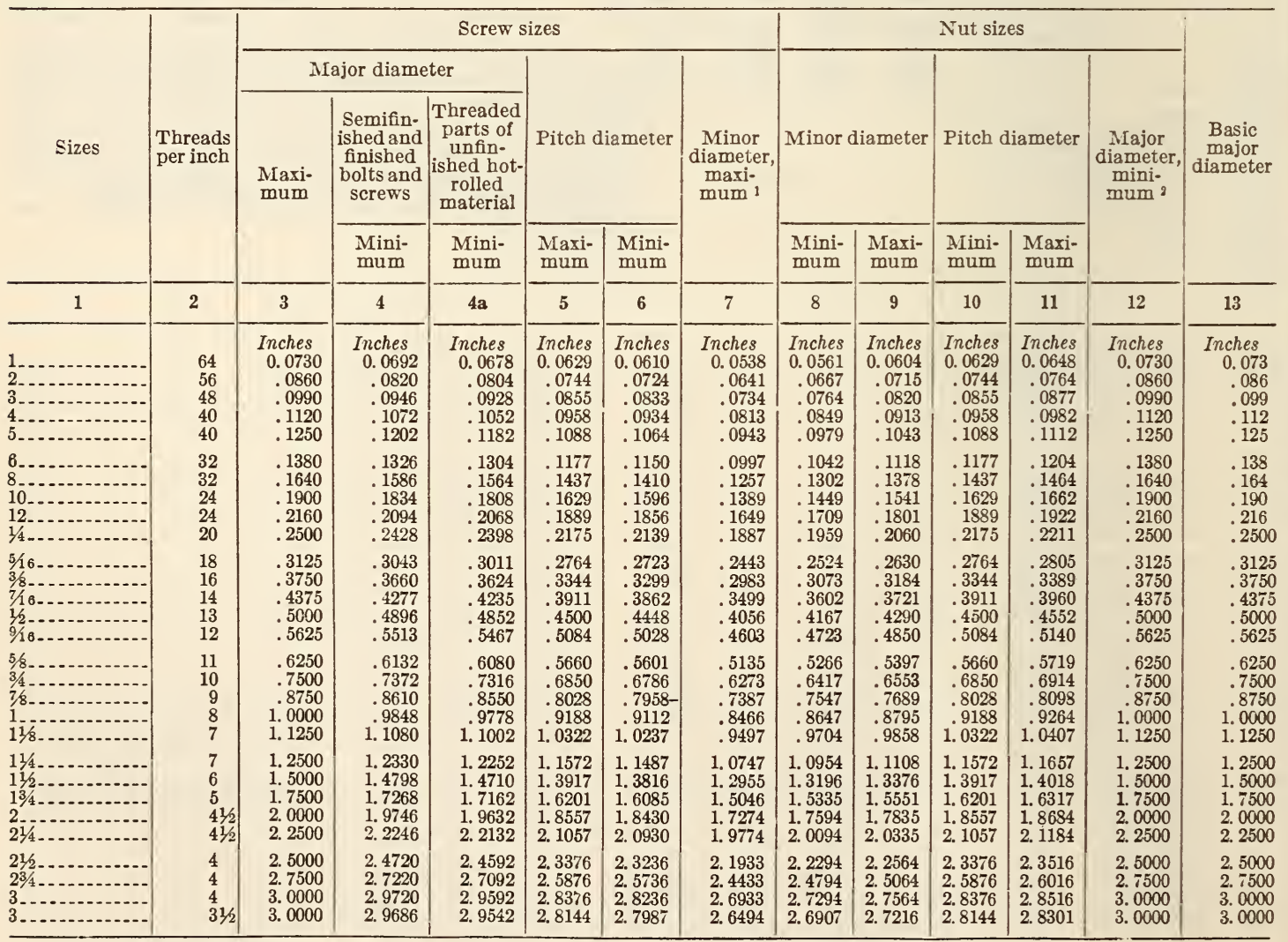

12 See footnotes to Table 14. 
TABLE 9.-Class 3, medium fit, American national coarse-thread series

\begin{tabular}{|c|c|c|c|c|c|c|c|c|c|c|c|c|}
\hline \multirow{3}{*}{ Sizes } & \multirow{3}{*}{$\begin{array}{l}\text { Threads } \\
\text { per inch }\end{array}$} & \multicolumn{5}{|c|}{ Screw sizes } & \multicolumn{5}{|c|}{ Nut sizes } & \multirow{3}{*}{$\begin{array}{c}\text { Basic } \\
\text { major } \\
\text { diameter }\end{array}$} \\
\hline & & \multicolumn{2}{|c|}{ Major diameter } & \multicolumn{2}{|c|}{ Pitch diameter } & \multirow{2}{*}{$\begin{array}{c}\text { Minor } \\
\text { diameter, } \\
\text { maxi- } \\
\text { mum }^{1}\end{array}$} & \multicolumn{2}{|c|}{ Minor diameter } & \multicolumn{2}{|c|}{ Pitch diameter } & \multirow{2}{*}{$\begin{array}{c}\text { Major } \\
\text { diameter, } \\
\text { mini- } \\
\text { mum }^{2}\end{array}$} & \\
\hline & & $\begin{array}{l}\text { Maxi- } \\
\text { mum }\end{array}$ & $\begin{array}{l}\text { Mini- } \\
\text { mum }\end{array}$ & $\begin{array}{l}\text { Maxi- } \\
\text { mum }\end{array}$ & $\begin{array}{l}\text { Mini- } \\
\text { mum }\end{array}$ & & $\begin{array}{l}\text { Mini- } \\
\text { mum }\end{array}$ & $\begin{array}{l}\text { Maxi- } \\
\text { mum }\end{array}$ & $\begin{array}{l}\text { Mini- } \\
\text { mum }\end{array}$ & $\begin{array}{l}\text { Maxi- } \\
\text { mum }\end{array}$ & & \\
\hline 1 & 2 & 3 & 4 & 5 & 6 & 7 & 8 & 9 & 10 & 11 & 12 & 13 \\
\hline & $\begin{array}{l}64 \\
56 \\
48 \\
40 \\
40\end{array}$ & $\begin{array}{r}\text { Inches } \\
0.0730 \\
.0860 \\
.0990 \\
.1120 \\
.1250\end{array}$ & $\begin{array}{c}\text { Inches } \\
0.0692 \\
.0820 \\
.0946 \\
.1072 \\
.1202\end{array}$ & $\begin{array}{r}\text { Inches } \\
0.0629 \\
.0744 \\
.0855 \\
.0958 \\
.1088\end{array}$ & $\begin{array}{c}\text { Inches } \\
0.0615 \\
.0729 \\
.0839 \\
.0941 \\
.1071\end{array}$ & $\begin{array}{c}\text { Inches } \\
0.0538 \\
.0641 \\
.0734 \\
.0813 \\
.0943\end{array}$ & $\begin{array}{c}\text { Inches } \\
0.0561 \\
.0667 \\
.0764 \\
.0849 \\
.0979\end{array}$ & $\begin{array}{r}\text { Inches } \\
0.0604 \\
.0715 \\
.0820 \\
.0913 \\
.1043\end{array}$ & $\begin{array}{c}\text { Inches } \\
0.0629 \\
.0744 \\
.0855 \\
.0958 \\
.0988\end{array}$ & $\begin{array}{r}\text { Inches } \\
0.0643 \\
.0759 \\
.0871 \\
.0975 \\
.1105\end{array}$ & $\begin{array}{c}\text { Inches } \\
0.0730 \\
.0860 \\
.0990 \\
.1120 \\
.1250\end{array}$ & $\begin{array}{r}\text { Inches } \\
0.073 \\
.086 \\
.099 \\
.112 \\
.125\end{array}$ \\
\hline & $\begin{array}{l}32 \\
32 \\
24 \\
24 \\
20\end{array}$ & $\begin{array}{r}.1380 \\
.1640 \\
.1900 \\
.2160 \\
.2500\end{array}$ & $\begin{array}{r}.1326 \\
.1586 \\
.1834 \\
.2094 \\
.2428\end{array}$ & $\begin{array}{r}.1177 \\
.1437 \\
.1629 \\
.1889 \\
.2175\end{array}$ & $\begin{array}{r}.1158 \\
.1418 \\
.1605 \\
.1865 \\
.2149\end{array}$ & $\begin{array}{l}.0997 \\
.1257 \\
.1389 \\
.1649 \\
.1887\end{array}$ & $\begin{array}{r}.1042 \\
.1302 \\
.1449 \\
.1709 \\
.1959\end{array}$ & $\begin{array}{l}.1118 \\
.1378 \\
.1541 \\
.1801 \\
.2060\end{array}$ & $\begin{array}{l}.1177 \\
.1437 \\
.1629 \\
.1889 \\
.2175\end{array}$ & $\begin{array}{r}.1196 \\
.1456 \\
.1653 \\
.1913 \\
.2201\end{array}$ & $\begin{array}{r}.1380 \\
.1640 \\
.1900 \\
.2160 \\
.2500\end{array}$ & $\begin{array}{l}.138 \\
.164 \\
.190 \\
.216 \\
.2500\end{array}$ \\
\hline & $\begin{array}{l}18 \\
16 \\
14 \\
13 \\
12\end{array}$ & $\begin{array}{r}.3125 \\
.3750 \\
.4375 \\
.5000 \\
.5625\end{array}$ & $\begin{array}{r}.3043 \\
.3660 \\
.4277 \\
.4896 \\
.5513\end{array}$ & $\begin{array}{l}.2764 \\
.3344 \\
.3911 \\
.4500 \\
.5084\end{array}$ & $\begin{array}{l}.2734 \\
.312 \\
.3875 \\
.4463 \\
.5044\end{array}$ & $\begin{array}{l}.2443 \\
.2983 \\
.3499 \\
.4056 \\
.4603\end{array}$ & $\begin{array}{l}.2524 \\
.3073 \\
.3602 \\
.4167 \\
.4723\end{array}$ & $\begin{array}{r}.2630 \\
.3184 \\
.3721 \\
.4290 \\
.4850\end{array}$ & $\begin{array}{l}.2764 \\
.3344 \\
.3911 \\
.4500 \\
.5084\end{array}$ & $\begin{array}{l}.2794 \\
.3376 \\
.3947 \\
.4537 \\
.5124\end{array}$ & $\begin{array}{r}.3125 \\
.3750 \\
.4375 \\
.5000 \\
.5625\end{array}$ & $\begin{array}{l}.3125 \\
.3750 \\
.4375 \\
.5000 \\
.5625\end{array}$ \\
\hline.- & $\begin{array}{r}11 \\
10 \\
9 \\
8 \\
7\end{array}$ & $\begin{array}{r}.6250 \\
.7500 \\
.8750 \\
1.0000 \\
1.1250\end{array}$ & $\begin{array}{r}.6132 \\
.7372 \\
.8610 \\
.9848 \\
1.1080\end{array}$ & $\begin{array}{r}.5660 \\
.6850 \\
.8028 \\
.9188 \\
1.0322\end{array}$ & $\begin{array}{r}.5618 \\
.6805 \\
.7979 \\
.9134 \\
1.0263\end{array}$ & $\begin{array}{l}.5135 \\
.6273 \\
.7387 \\
.8466 \\
.9497\end{array}$ & $\begin{array}{l}.5266 \\
.6417 \\
.7547 \\
.8647 \\
.9704\end{array}$ & $\begin{array}{l}.5397 \\
.6553 \\
.7689 \\
.8795 \\
.9858\end{array}$ & $\begin{array}{r}.5660 \\
.6850 \\
.8028 \\
.9188 \\
1.0322\end{array}$ & $\begin{array}{r}.5702 \\
.6895 \\
.8077 \\
.9242 \\
1.0381\end{array}$ & $\begin{array}{r}.6250 \\
.7500 \\
.8750 \\
1.0000 \\
1.1250\end{array}$ & $\begin{array}{r}.6250 \\
.7500 \\
.8750 \\
1.0000 \\
1.1250\end{array}$ \\
\hline & \begin{tabular}{l|}
7 \\
6 \\
5 \\
$41 / 2$ \\
$41 / 2$
\end{tabular} & $\begin{array}{l}1.2500 \\
1.5000 \\
1.7500 \\
2.0000 \\
2.2500\end{array}$ & $\begin{array}{l}1.2330 \\
1.4798 \\
1.7268 \\
1.9746 \\
\text { 2. } 2246\end{array}$ & $\begin{array}{l}1.1572 \\
\text { 1. } 3917 \\
1.6201 \\
1.8557 \\
2.1057\end{array}$ & $\begin{array}{l}\text { 1. } 1513 \\
\text { 1. } 3846 \\
1.6119 \\
1.8468 \\
2.0968\end{array}$ & $\begin{array}{l}1.0747 \\
1.2955 \\
1.5046 \\
1.7274 \\
1.9774\end{array}$ & $\begin{array}{l}1.0954 \\
1.3196 \\
1.5335 \\
1.7594 \\
2.0094\end{array}$ & $\begin{array}{l}1.1108 \\
1.3376 \\
1.5551 \\
1.7835 \\
2.0335\end{array}$ & $\begin{array}{l}\text { 1. } 1572 \\
\text { 1. } 3917 \\
1.6201 \\
1.8557 \\
2.1057\end{array}$ & $\begin{array}{l}1.1631 \\
1.3988 \\
1.6283 \\
1.8646 \\
2.1146\end{array}$ & $\begin{array}{l}\text { 1. } 2500 \\
1.5000 \\
1.7500 \\
2.0000 \\
2.2500\end{array}$ & $\begin{array}{l}1.2500 \\
1.5000 \\
1.7500 \\
2.0000 \\
2.2500\end{array}$ \\
\hline -- & $\begin{array}{l}4 \\
4 \\
4 \\
31 / 2\end{array}$ & $\begin{array}{l}2.5000 \\
2.7500 \\
3.0000 \\
3.0000\end{array}$ & 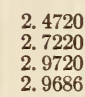 & $\begin{array}{l}2.3376 \\
2.5876 \\
2.8376 \\
2.8144\end{array}$ & $\begin{array}{l}2.3279 \\
2.5779 \\
2.8279 \\
2.8037\end{array}$ & $\begin{array}{l}2.1933 \\
2.4433 \\
2.6933 \\
2.6494\end{array}$ & $\begin{array}{l}2.2294 \\
2.4794 \\
2.7294 \\
2.6907\end{array}$ & $\begin{array}{l}2.2564 \\
2.5064 \\
2.7564 \\
2.7216\end{array}$ & $\begin{array}{l}2.3376 \\
2.5876 \\
2.8376 \\
2.8144\end{array}$ & $\begin{array}{l}2.3473 \\
2.5973 \\
2.8473 \\
2.8251\end{array}$ & $\begin{array}{l}2.5000 \\
2.7500 \\
3.0000 \\
3.0000\end{array}$ & $\begin{array}{l}\text { 2. } 5000 \\
\text { 2. } 7500 \\
3.0000 \\
\text { 3. } 0000\end{array}$ \\
\hline
\end{tabular}

12 See footnotes to Table 14.

TABLE 10.-Class 4, close fit, American national coarse-thread series

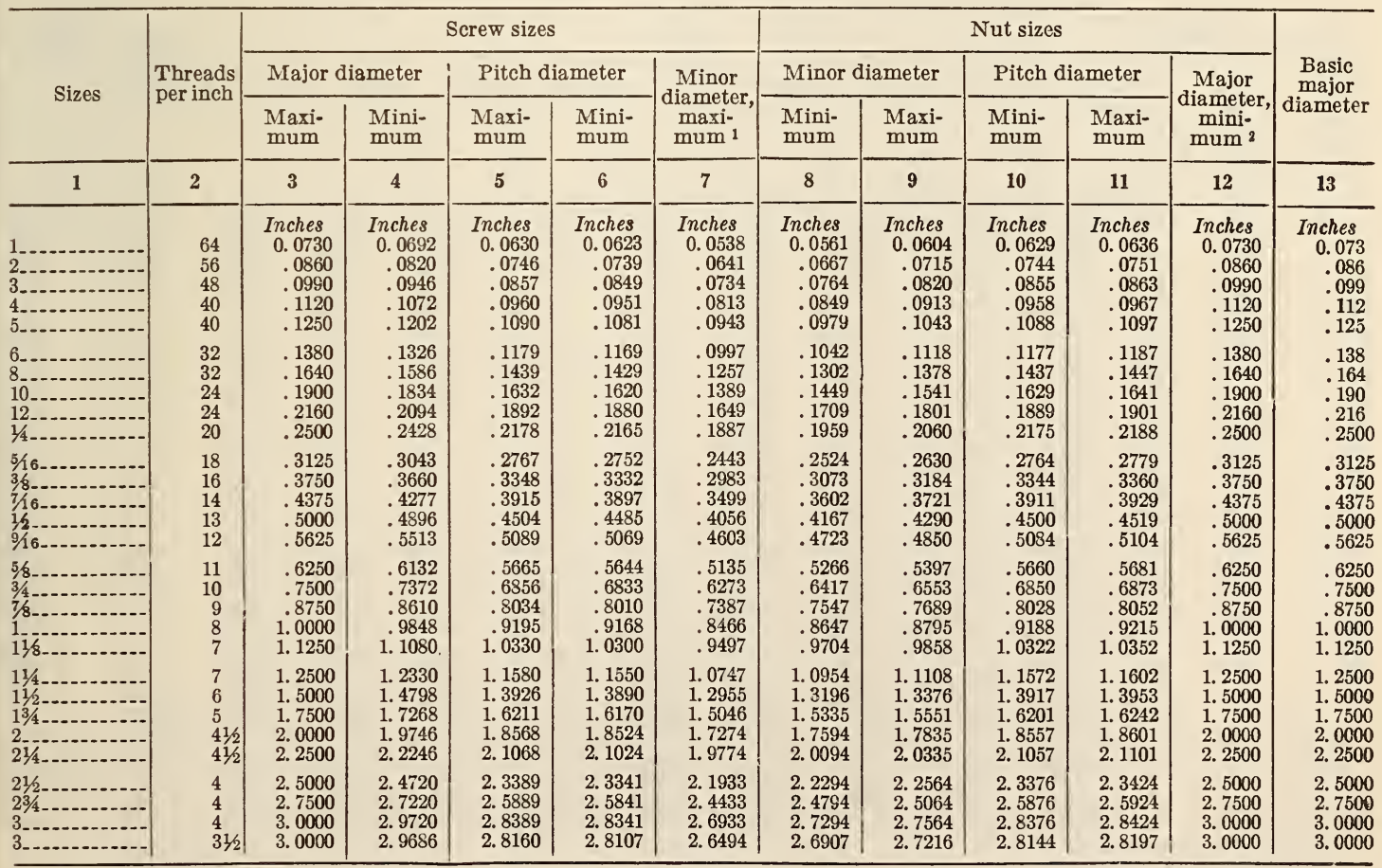

12 See footnotes to Table 14. 
Table 11.-Class 1, loose fit, American national fine-thread series

\begin{tabular}{|c|c|c|c|c|c|c|c|c|c|c|c|c|}
\hline \multirow{3}{*}{ Sizes } & \multirow{3}{*}{$\begin{array}{l}\text { Threads } \\
\text { per inch }\end{array}$} & \multicolumn{5}{|c|}{ Screw sizes } & \multicolumn{5}{|c|}{ Nut sizes } & \multirow{3}{*}{$\begin{array}{c}\text { Basic } \\
\text { major } \\
\text { diameter }\end{array}$} \\
\hline & & \multicolumn{2}{|c|}{ Major diameter } & \multicolumn{2}{|c|}{ Pitch diameter } & \multirow{2}{*}{$\begin{array}{l}\text { Minor } \\
\text { diameter, } \\
\text { maxi- } \\
\text { mum } 1\end{array}$} & \multicolumn{2}{|c|}{ Minor diameter } & \multicolumn{2}{|c|}{ Pitch diameter } & \multirow{2}{*}{$\begin{array}{l}\text { Major } \\
\text { diameter, } \\
\text { mini- } \\
\text { mum? }\end{array}$} & \\
\hline & & $\begin{array}{l}\text { Maxi- } \\
\text { mum }\end{array}$ & $\begin{array}{l}\text { Mini- } \\
\text { mum }\end{array}$ & $\begin{array}{l}\text { Maxi- } \\
\text { mum }\end{array}$ & $\begin{array}{l}\text { Mini- } \\
\text { mum }\end{array}$ & & $\begin{array}{l}\text { Mini- } \\
\text { mum }\end{array}$ & $\begin{array}{l}\text { Maxi- } \\
\text { mumn }\end{array}$ & $\begin{array}{l}\text { Mini- } \\
\text { mum }\end{array}$ & $\begin{array}{l}\text { Maxi- } \\
\text { mum }\end{array}$ & & \\
\hline 1 & 2 & 3 & 4 & 5 & 6 & 7 & 8 & 9 & 10 & 11 & 12 & 13 \\
\hline $4 \ldots$ & $\begin{array}{l}80 \\
72 \\
64 \\
56 \\
48\end{array}$ & $\begin{array}{c}\text { Inches } \\
0.0593 \\
.0723 \\
.0853 \\
.0982 \\
.1111\end{array}$ & $\begin{array}{c}\text { Inches } \\
0.0545 \\
.0673 \\
.0801 \\
.0926 \\
.1049\end{array}$ & $\begin{array}{c}\text { Inches } \\
0.0512 \\
.0633 \\
.0752 \\
.0866 \\
.0976\end{array}$ & $\begin{array}{c}\text { Inches } \\
0.0488 \\
.0608 \\
.0726 \\
.0838 \\
.0945\end{array}$ & $\begin{array}{c}\text { Inches } \\
0.0440 \\
.0553 \\
.0661 \\
.0763 \\
.0855\end{array}$ & $\begin{array}{c}\text { Inches } \\
0.0465 \\
.0580 \\
.0691 \\
.0797 \\
.0894\end{array}$ & $\begin{array}{c}\text { Inches } \\
0.0492 \\
.0610 \\
.0724 \\
.0834 \\
.0937\end{array}$ & $\begin{array}{c}\text { Inches } \\
0.0519 \\
.0640 \\
.0759 \\
.0574 \\
.0955\end{array}$ & $\begin{array}{c}\text { Inches } \\
0.0543 \\
.0665 \\
.0785 \\
.0902 \\
.1016\end{array}$ & $\begin{array}{c}\text { Inches } \\
0.0600 \\
.0730 \\
.0560 \\
.0990 \\
.1120\end{array}$ & $\begin{array}{c}\text { Inches } \\
0.060 \\
.073 \\
.056 \\
.099 \\
.112\end{array}$ \\
\hline $\begin{array}{l}5-\ldots \\
6--\cdot- \\
8-\ldots \\
10--. \\
12-.-\end{array}$ & $\begin{array}{l}44 \\
40 \\
36 \\
32 \\
28\end{array}$ & $\begin{array}{l}.1241 \\
.1370 \\
.1629 \\
.1889 \\
.2148\end{array}$ & $\begin{array}{l}.1177 \\
.1302 \\
.1557 \\
.1813 \\
.2062\end{array}$ & $\begin{array}{l}.1093 \\
.1208 \\
.1449 \\
.1686 \\
.1916\end{array}$ & $\begin{array}{l}.1061 \\
.1174 \\
.1413 \\
.1648 \\
.1873\end{array}$ & $\begin{array}{l}.0962 \\
.1063 \\
.1288 \\
.1506 \\
.1710\end{array}$ & $\begin{array}{l}.1004 \\
.1109 \\
.1339 \\
.1562 \\
.1773\end{array}$ & $\begin{array}{l}.1049 \\
.1158 \\
.1391 \\
.1619 \\
.1833\end{array}$ & $\begin{array}{l}.1102 \\
.1218 \\
.1460 \\
.1697 \\
.1923\end{array}$ & $\begin{array}{l}.1134 \\
.1252 \\
.1496 \\
.1735 \\
.1971\end{array}$ & $\begin{array}{l}.1250 \\
.1350 \\
.1640 \\
.1900 \\
.2160\end{array}$ & $\begin{array}{l}.125 \\
.138 \\
.164 \\
.190 \\
.216\end{array}$ \\
\hline $\begin{array}{l}1 / 4 \ldots . . \\
516 \ldots \\
3 / 8 \ldots \ldots \\
7 / 16 \ldots \\
1 / 2 \ldots . .\end{array}$ & $\begin{array}{l}28 \\
24 \\
24 \\
20 \\
20\end{array}$ & $\begin{array}{l}.2488 \\
.3112 \\
.3737 \\
.4360 \\
.4985\end{array}$ & $\begin{array}{l}.2402 \\
.3020 \\
.3645 \\
.4258 \\
.4883\end{array}$ & $\begin{array}{l}.2256 \\
.2841 \\
.3466 \\
.4035 \\
.4660\end{array}$ & $\begin{array}{l}.2213 \\
.2795 \\
.3420 \\
.3984 \\
.4609\end{array}$ & $\begin{array}{l}.2050 \\
.2601 \\
.3226 \\
.3717 \\
.4372\end{array}$ & $\begin{array}{l}.2113 \\
.2674 \\
.3299 \\
.3834 \\
.4459\end{array}$ & $\begin{array}{l}.2173 \\
.2739 \\
.3364 \\
.3906 \\
.4531\end{array}$ & $\begin{array}{l}.2269 \\
.2554 \\
.3479 \\
.4050 \\
.4675\end{array}$ & $\begin{array}{l}.2311 \\
.2900 \\
.3525 \\
.4101 \\
.4726\end{array}$ & $\begin{array}{l}.2500 \\
.3125 \\
.3750 \\
.4375 \\
.5000\end{array}$ & $\begin{array}{l}.2500 \\
.3125 \\
.3750 \\
.4375 \\
.5000\end{array}$ \\
\hline $\begin{array}{l}9 / 16 \\
5 / 8 \\
3 / 4 \\
7 / 8 \\
1 / 8\end{array}$ & $\begin{array}{l}18 \\
18 \\
16 \\
14 \\
14\end{array}$ & $\begin{array}{l}.5609 \\
.6234 \\
.7482 \\
.8729 \\
.9979\end{array}$ & $\begin{array}{l}.5495 \\
.6120 \\
.7356 \\
.8589 \\
.9839\end{array}$ & $\begin{array}{l}.5248 \\
.5873 \\
.7076 \\
.8265 \\
.9515\end{array}$ & $\begin{array}{l}.5191 \\
.5816 \\
.7013 \\
.8195 \\
.9145\end{array}$ & $\begin{array}{l}.4927 \\
.5552 \\
.6715 \\
.7853 \\
.9103\end{array}$ & $\begin{array}{l}.5024 \\
.5649 \\
.6823 \\
.7977 \\
.9227\end{array}$ & $\begin{array}{l}.5100 \\
.5725 \\
.6903 \\
.8062 \\
.9312\end{array}$ & $\begin{array}{l}.5264 \\
.5589 \\
.7094 \\
.8256 \\
.9536\end{array}$ & $\begin{array}{l}.5321 \\
.5946 \\
.7157 \\
.8356 \\
.9606\end{array}$ & $\begin{array}{r}.5625 \\
.6250 \\
.7500 \\
.8750 \\
1.0000\end{array}$ & $\begin{array}{r}.5625 \\
.6250 \\
.7500 \\
.8750 \\
1.0000\end{array}$ \\
\hline $\begin{array}{l}11 / 8 \\
114 \\
115 \\
13, \\
2\end{array}$ & $\begin{array}{l}12 \\
12 \\
12 \\
10 \\
10\end{array}$ & $\begin{array}{l}1.1226 \\
1.2476 \\
1.4976 \\
1.7472 \\
1.9972\end{array}$ & $\begin{array}{l}1.1068 \\
1.2318 \\
1.4818 \\
1.7288 \\
1.9788\end{array}$ & $\begin{array}{l}\text { 1. } 0685 \\
1.1935 \\
1.4435 \\
1.6822 \\
1.9322\end{array}$ & $\begin{array}{l}1.0606 \\
1.1856 \\
1.4356 \\
1.6690 \\
1.9190\end{array}$ & $\begin{array}{l}\text { 1. } 0204 \\
1.1454 \\
1.3954 \\
1.6245 \\
1.8745\end{array}$ & $\begin{array}{l}1.0348 \\
1.1598 \\
1.4098 \\
1.6417 \\
1.8917\end{array}$ & $\begin{array}{l}1.0438 \\
1.1658 \\
1.4188 \\
1.6525 \\
1.9025\end{array}$ & $\begin{array}{l}\text { 1. } 0709 \\
1.1959 \\
1.4459 \\
1.6550 \\
1.9350\end{array}$ & $\begin{array}{l}\text { 1. } 0788 \\
\text { 1. } 2038 \\
1.4535 \\
1.6982 \\
1.9482\end{array}$ & $\begin{array}{l}1.1250 \\
1.2500 \\
1.5000 \\
1.7500 \\
2.0000\end{array}$ & $\begin{array}{l}1.1250 \\
1.2500 \\
1.5000 \\
1.7500 \\
\text { 2. } 0000\end{array}$ \\
\hline $\begin{array}{l}21 / 4 \ldots \\
21 / 2 \ldots \\
23 / 4 \ldots \\
3\end{array}$ & $\begin{array}{l}8 \\
8 \\
8 \\
8\end{array}$ & $\begin{array}{l}\text { 2. } 2466 \\
\text { 2. } 4966 \\
\text { 2. } 7466 \\
2.9966\end{array}$ & $\begin{array}{l}\text { 2. } 2244 \\
\text { 2. } 4744 \\
\text { 2. } 7244 \\
\text { 2. } 9744\end{array}$ & $\begin{array}{l}\text { 2. } 1654 \\
\text { 2. } 4154 \\
\text { 2. } 6654 \\
\text { 2. } 9154\end{array}$ & $\begin{array}{l}2.1509 \\
2.4009 \\
2.6509 \\
2.9009\end{array}$ & $\begin{array}{l}\text { 2. } 0932 \\
\text { 2. } 3432 \\
\text { 2. } 532 \\
2.8432\end{array}$ & $\begin{array}{l}\text { 2. } 1147 \\
\text { 2. } 3647 \\
\text { 2. } 6147 \\
\text { 2. } 8647\end{array}$ & $\begin{array}{l}\text { 2. } 1282 \\
\text { 2. } 3782 \\
\text { 2. } 6282 \\
\text { 2. } 5782\end{array}$ & $\begin{array}{l}2.1683 \\
2.4183 \\
2.6685 \\
2.9185\end{array}$ & $\begin{array}{l}2.1533 \\
2.4333 \\
2.6333 \\
2.9333\end{array}$ & $\begin{array}{l}2.2500 \\
2.5000 \\
2.7500 \\
3.0000\end{array}$ & $\begin{array}{l}2.2500 \\
2.5000 \\
2.7500 \\
\text { 3. } 0000\end{array}$ \\
\hline
\end{tabular}

12 See footnotes to Table 14.

TABLe 12.-Class 2, free fit, American national fine-thread series

\begin{tabular}{|c|c|c|c|c|c|c|c|c|c|c|c|c|}
\hline \multirow{3}{*}{ Sizes } & \multirow{3}{*}{$\left|\begin{array}{l}\text { Threads } \\
\text { per inch }\end{array}\right|$} & \multicolumn{5}{|c|}{ Screw sizes } & \multicolumn{5}{|c|}{ Nut sizes } & \multirow{3}{*}{$\begin{array}{c}\text { Basic } \\
\text { major } \\
\text { diameter }\end{array}$} \\
\hline & & \multicolumn{2}{|c|}{ Major diameter } & \multicolumn{2}{|c|}{ Pitch diameter } & \multirow{2}{*}{$\begin{array}{c}\text { Minor } \\
\text { diameter, } \\
\text { maxi- } \\
\text { mum }{ }^{1}\end{array}$} & \multicolumn{2}{|c|}{ Minor diameter } & \multicolumn{2}{|c|}{ Pitch diameter } & \multirow{2}{*}{$\begin{array}{c}\text { Major } \\
\text { diameter, } \\
\text { mini-- } \\
\text { mum 2 }\end{array}$} & \\
\hline & & $\begin{array}{l}\text { Maxi- } \\
\text { mum }\end{array}$ & $\underset{\text { Mumi- }}{\text { Mini- }}$ & $\begin{array}{l}\text { Maxi- } \\
\text { mum }\end{array}$ & $\begin{array}{l}\text { Mrini- } \\
\text { mum }\end{array}$ & & $\underset{\text { mumi- }}{\text { Mini- }}$ & $\begin{array}{l}\text { Maxi- } \\
\text { mum }\end{array}$ & $\begin{array}{l}\text { Mini- } \\
\text { mum }\end{array}$ & $\begin{array}{l}\text { Mraxi- } \\
\text { mum }\end{array}$ & & \\
\hline 1 & 2 & 3 & 4 & 5 & 6 & 7 & 8 & 9 & 10 & 11 & 12 & 13 \\
\hline & $\begin{array}{l}80 \\
72 \\
64 \\
56 \\
48\end{array}$ & $\begin{array}{c}\text { Inches } \\
0.0600 \\
.0730 \\
.0860 \\
.0990 \\
.1120\end{array}$ & $\begin{array}{c}\text { Inches } \\
0.0566 \\
.0694 \\
.0822 \\
.0950 \\
.1076\end{array}$ & $\begin{array}{c}\text { Inches } \\
0.0519 \\
.0640 \\
.0759 \\
.0874 \\
.0985\end{array}$ & $\begin{array}{c}\text { Inches } \\
0.0502 \\
.0622 \\
.0740 \\
.0854 \\
0963\end{array}$ & $\begin{array}{c}\text { Inches } \\
0.0447 \\
.0560 \\
.0668 \\
.0771 \\
.0864\end{array}$ & $\begin{array}{c}\text { Inches } \\
0.0465 \\
.0580 \\
.0691 \\
.0797 \\
.0894\end{array}$ & $\begin{array}{c}\text { Inches } \\
0.0492 \\
.0610 \\
.0724 \\
.0834 \\
.0937\end{array}$ & $\begin{array}{c}\text { Inches } \\
0.0519 \\
.0640 \\
.0759 \\
.0574 \\
.0985\end{array}$ & $\begin{array}{c}\text { Inches } \\
0.0536 \\
.0658 \\
.0778 \\
.0594 \\
.1007\end{array}$ & $\begin{array}{c}\text { Inches } \\
0.0600 \\
.0730 \\
.0560 \\
.0990 \\
.1120\end{array}$ & $\begin{array}{r}\text { Inches } \\
0.060 \\
.073 \\
.086 \\
.099 \\
.112\end{array}$ \\
\hline & 44 & .1250 & .1204 & .1102 & .1079 & .0 & .1004 & .1049 & .1102 & .1 & & .125 \\
\hline & $\begin{array}{l}40 \\
36\end{array}$ & $\begin{array}{l}.1380 \\
.1640\end{array}$ & $\begin{array}{l}.13 \\
.13 \\
\end{array}$ & $\begin{array}{l}.12 \\
.14\end{array}$ & $: 1$ & & & $\begin{array}{r}11 \\
.13\end{array}$ & $: 1$ & 11 & $\begin{array}{r}.1380 \\
.1640\end{array}$ & 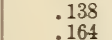 \\
\hline 0 & $\begin{array}{l}32 \\
28\end{array}$ & .19 & .18 & & & & & & & & & $: 1$ \\
\hline $34 \ldots$ & 28 & .21 & .2 & .1 & & & & & & & & .2 \\
\hline & 24 & .3125 & .30 & .28 & .2 & .2 & & & 23 & .2299 & & $\begin{array}{r}.2500 \\
.3125 \\
\end{array}$ \\
\hline 6 & $\begin{array}{l}24 \\
20\end{array}$ & .375 & .36 & .34 & .34 & & .32 & .33 & $.3=$ & .3 & .3750 & .3750 \\
\hline $32 \ldots$ & 20 & .5000 & .49 & $\begin{array}{l}.46 \\
.46\end{array}$ & .4 & .4 & .44 & .4 & .44 & & .43000 & .50 \\
\hline $9 / 16 \ldots$ & 18 & .5625 & .5543 & .526 & .52 & .49 & .5024 & .5100 & .5264 & .5305 & .5625 & .5625 \\
\hline & $\begin{array}{l}18 \\
16\end{array}$ & $\begin{array}{l}.6500 \\
.7500\end{array}$ & $: 61$ & $\begin{array}{r}.58 \\
.70\end{array}$ & .58 & .5 & .5 & & .58 & $\begin{array}{r}.5930 \\
.7139 \\
\end{array}$ & $\begin{array}{l}.6250 \\
.7500\end{array}$ & $\begin{array}{r}.6250 \\
.7500\end{array}$ \\
\hline 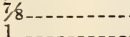 & ${ }_{14}^{14}$ & $\begin{array}{r}.8750 \\
1.0000\end{array}$ & $\begin{array}{l}.86 \\
99\end{array}$ & .82 & & & & & & & & .8750 \\
\hline & 12 & 1. 1250 & $\begin{array}{l}.002 \\
1.1138\end{array}$ & 1.07 (.) & 1.0 & & $1.0 \quad$ ( & & & & & 1.0000 \\
\hline & 12 & 1. 2500 & 1. 2388 & 1.19 & $\begin{array}{l}1.1903 \\
1.1902\end{array}$ & 1.1 & $\begin{array}{l}1.1598 \\
1.1598\end{array}$ & 1. 1688 & & & $\begin{array}{l}1.1500 \\
\text { 1. } 2500\end{array}$ & $\begin{array}{l}1.2500 \\
1.2500\end{array}$ \\
\hline$\frac{1}{4}-2$ & $\begin{array}{l}12 \\
10\end{array}$ & $\begin{array}{l}1.5 \\
1.7\end{array}$ & $\begin{array}{l}1.48 \\
1.73 \\
\text {. }\end{array}$ & $\begin{array}{l}1.4 \\
1.6\end{array}$ & & & & & & & & $\begin{array}{l}000 \\
500\end{array}$ \\
\hline 2 & 10 & 2.0000 & 1. 98 & 1.9350 & 1.92 & & 1.89 & & & & & 2. 0000 \\
\hline $234-\ldots . .$. & 8 & 2. 2500 & 2. 2348 & 2. 1688 & 2.1578 & 2.0 & 2. 1147 & 2. 1282 & 2. 1688 & 2. 1798 & 2. 2500 & 2. 2500 \\
\hline & 8 & $\begin{array}{l}2.5 \\
2.7\end{array}$ & 2.7 & $\begin{array}{l}2.4 \\
2,6\end{array}$ & 2, & & & & & & & $\begin{array}{l}2.5 \\
2.7\end{array}$ \\
\hline -.- & & 3. 0000 & 2. 9848 & 2. 9188 & 2. 9078 & 2. 8466 & 2. 8647 & 2. 8782 & 2.9188 & 2. 9298 & 3. 0000 & 3. 0000 \\
\hline
\end{tabular}

12 See footnotes to Table 14. 
TABLE 13.-Class 3, medium fit, American national fine-thread series

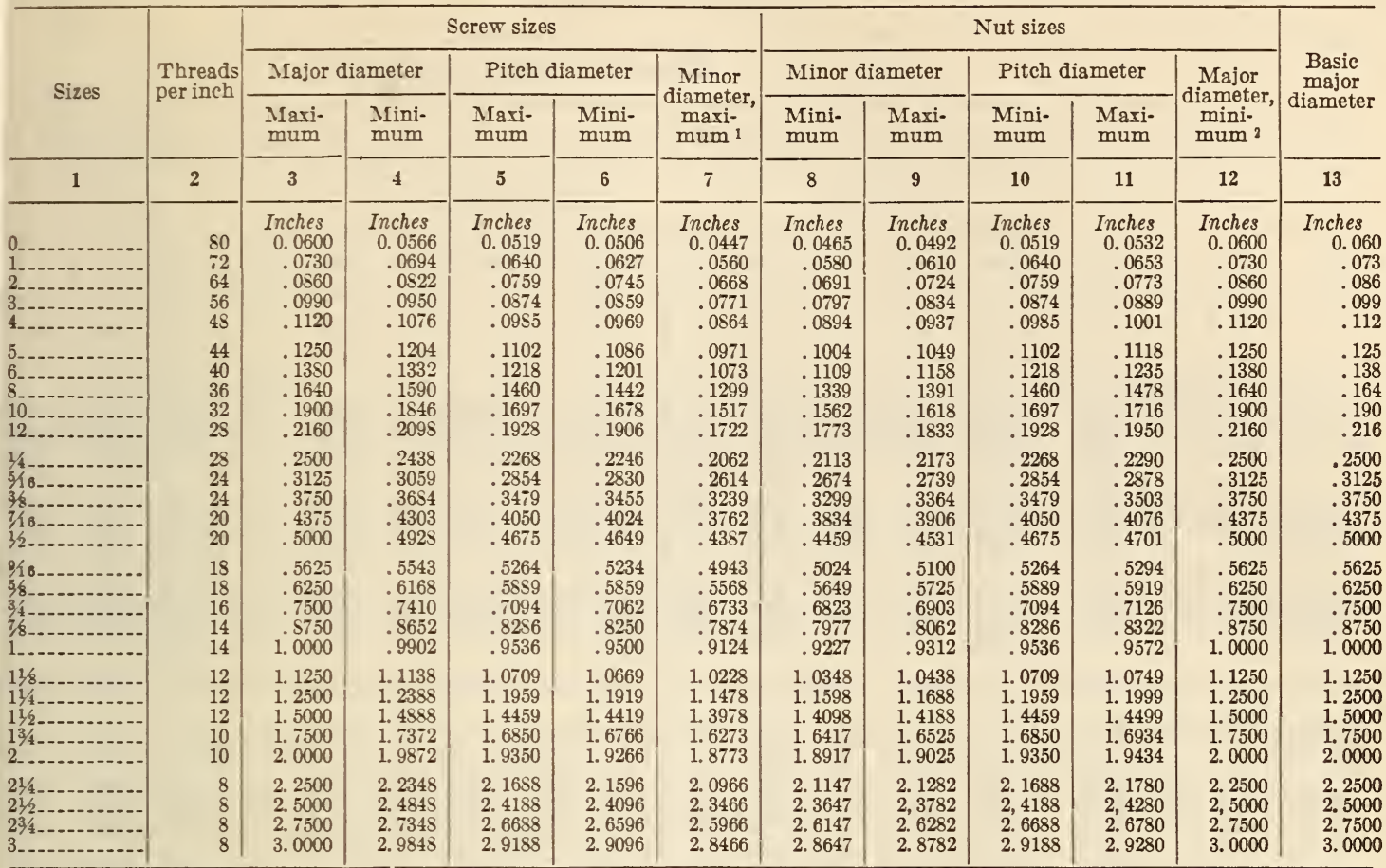

12 See footnotes to Table 14

TABle 14.-Class 4, close fit, American national fine-thread series

\begin{tabular}{|c|c|c|c|c|c|c|c|c|c|c|c|c|}
\hline \multirow{3}{*}{ Sizes } & \multirow{3}{*}{$\begin{array}{l}\text { Threads } \\
\text { per inch }\end{array}$} & \multicolumn{5}{|c|}{ Screw sizes } & \multicolumn{5}{|c|}{ Nut sizes } & \multirow{3}{*}{$\begin{array}{c}\text { Basic } \\
\text { major } \\
\text { diameter }\end{array}$} \\
\hline & & \multicolumn{2}{|c|}{ Major diameter } & \multicolumn{2}{|c|}{ Pitch diameter } & \multirow{2}{*}{$\begin{array}{l}\text { Minor } \\
\text { diameter, } \\
\text { maxi- } \\
\text { mum 1 }\end{array}$} & \multicolumn{2}{|c|}{ Minor diameter } & \multicolumn{2}{|c|}{ Pitch diameter } & \multirow{2}{*}{$\begin{array}{l}\text { Major } \\
\text { diameter, } \\
\text { mini- } \\
\text { mum }{ }^{2}\end{array}$} & \\
\hline & & $\begin{array}{l}\text { Maxi- } \\
\text { mum }\end{array}$ & $\begin{array}{l}\text { Mini- } \\
\text { mum }\end{array}$ & $\begin{array}{l}\text { Maxi- } \\
\text { mum }\end{array}$ & $\begin{array}{l}\text { Mini- } \\
\text { mum }\end{array}$ & & $\begin{array}{l}\text { Mini- } \\
\text { mum }\end{array}$ & $\begin{array}{l}\text { Maxi- } \\
\text { mum }\end{array}$ & $\begin{array}{l}\text { Mini- } \\
\text { mum }\end{array}$ & $\begin{array}{l}\text { Maxi- } \\
\text { mum }\end{array}$ & & \\
\hline 1 & 2 & 3 & 4 & 5 & 6 & 7 & 8 & 9 & 10 & 11 & 12 & 13 \\
\hline & $\begin{array}{l}80 \\
72 \\
64 \\
56 \\
48\end{array}$ & $\begin{array}{c}\text { Inches } \\
0.0600 \\
.0730 \\
.0860 \\
.0990 \\
.1120\end{array}$ & $\begin{array}{c}\text { Inches } \\
0.0566 \\
.0694 \\
.0822 \\
.0950 \\
.1076\end{array}$ & $\begin{array}{c}\text { Inches } \\
0.0520 \\
.0641 \\
.0760 \\
.0876 \\
.0987\end{array}$ & $\begin{array}{c}\text { Inches } \\
0.0514 \\
.0634 \\
.0753 \\
.0869 \\
.0979\end{array}$ & $\begin{array}{c}\text { Inches } \\
0.0447 \\
.0560 \\
.0668 \\
.0771 \\
.0864\end{array}$ & $\begin{array}{c}\text { Inches } \\
0.0465 \\
.0580 \\
.0691 \\
.0797 \\
.0894\end{array}$ & $\begin{array}{c}\text { Inches } \\
0.0492 \\
.0610 \\
.0724 \\
.0834 \\
.0937\end{array}$ & $\begin{array}{c}\text { Inches } \\
0.0519 \\
.0640 \\
.0759 \\
.0874 \\
.0985\end{array}$ & $\begin{array}{c}\text { Inches } \\
0.0525 \\
.0647 \\
.0766 \\
.0881 \\
.0993\end{array}$ & $\begin{array}{c}\text { Inches } \\
0.0600 \\
.0730 \\
.0860 \\
.0990 \\
.1120\end{array}$ & $\begin{array}{c}\text { Inches } \\
0.060 \\
.073 \\
.086 \\
.099 \\
.112\end{array}$ \\
\hline .... & $\begin{array}{l}44 \\
40 \\
36 \\
32 \\
28\end{array}$ & $\begin{array}{l}.1250 \\
1380 \\
.1640 \\
.1900 \\
2160\end{array}$ & $\begin{array}{l}.1204 \\
.1332 \\
.1590 \\
.1846 \\
.2098\end{array}$ & $\begin{array}{l}.1104 \\
.1220 \\
.1462 \\
.1699 \\
.1930\end{array}$ & $\begin{array}{l}.1096 \\
.1211 \\
.1453 \\
.1689 \\
1919\end{array}$ & $\begin{array}{l}.09 \\
.10 \\
.12 \\
.15 \\
.17\end{array}$ & $\begin{array}{l}.1004 \\
.1109 \\
.1339 \\
.1562 \\
.1773\end{array}$ & $\begin{array}{l}.1049 \\
.1158 \\
.1391 \\
.1618 \\
.1833\end{array}$ & $\begin{array}{l}.1102 \\
.1218 \\
.1460 \\
.1697 \\
.1928\end{array}$ & $\begin{array}{l}.1110 \\
.1227 \\
.1469 \\
.1707 \\
.1939\end{array}$ & $\begin{array}{l}.1250 \\
.1380 \\
.1640 \\
.1900 \\
.2160\end{array}$ & $\begin{array}{l}.125 \\
.138 \\
.164 \\
.190 \\
.216\end{array}$ \\
\hline 4 & $\begin{array}{l}28 \\
24 \\
24 \\
20 \\
20\end{array}$ & $\begin{array}{l}.2500 \\
.3125 \\
.3750 \\
.4375 \\
.5000\end{array}$ & $\begin{array}{l}.2438 \\
.3059 \\
.3684 \\
.4303 \\
.4928\end{array}$ & $\begin{array}{l}.2270 \\
.2857 \\
.3482 \\
.4053 \\
.4678\end{array}$ & $\begin{array}{l}.2259 \\
.2845 \\
.3470 \\
.4040 \\
.4665\end{array}$ & $\begin{array}{l}.2062 \\
.2614 \\
.3239 \\
.3762 \\
.4387\end{array}$ & $\begin{array}{l}.2113 \\
.2674 \\
.3299 \\
.3834 \\
.4459\end{array}$ & $\begin{array}{l}.2173 \\
.2739 \\
.3364 \\
.3906 \\
.4531\end{array}$ & $\begin{array}{l}.2268 \\
.2854 \\
.3479 \\
.4050 \\
.4675\end{array}$ & $\begin{array}{l}.2279 \\
.2866 \\
.3491 \\
.4063 \\
.4688\end{array}$ & $\begin{array}{l}.2500 \\
.3125 \\
.3750 \\
.4375 \\
.5000\end{array}$ & $\begin{array}{l}.2500 \\
.3125 \\
.3750 \\
.4375 \\
.5000\end{array}$ \\
\hline 6 & $\begin{array}{l}18 \\
18 \\
16 \\
14 \\
14\end{array}$ & $\begin{array}{r}.5625 \\
.6250 \\
.7500 \\
.8750 \\
1.0000\end{array}$ & $\begin{array}{l}.5543 \\
.6168 \\
.7410 \\
.8652 \\
.9902\end{array}$ & $\begin{array}{l}.5267 \\
.5892 \\
.7098 \\
.8290 \\
.9540\end{array}$ & $\begin{array}{l}.5252 \\
.5877 \\
.7082 \\
.8272 \\
.9522\end{array}$ & $\begin{array}{l}.4943 \\
.5568 \\
.6733 \\
.7874 \\
.9124\end{array}$ & $\begin{array}{l}.5024 \\
.5649 \\
.6823 \\
.7977 \\
.9227\end{array}$ & $\begin{array}{l}.5100 \\
.5725 \\
.6903 \\
.8062 \\
.9312\end{array}$ & $\begin{array}{l}.5264 \\
.5889 \\
.7094 \\
.8286 \\
.9536\end{array}$ & $\begin{array}{l}.5279 \\
.5904 \\
.7110 \\
.8304 \\
.9554\end{array}$ & $\begin{array}{r}.5625 \\
.6250 \\
.7500 \\
.8750 \\
1.0000\end{array}$ & $\begin{array}{r}.5625 \\
.6250 \\
.7500 \\
.8750 \\
1.0000\end{array}$ \\
\hline $118 \ldots$ & $\begin{array}{l}12 \\
12 \\
12 \\
10 \\
10\end{array}$ & $\begin{array}{l}1.1250 \\
1.2500 \\
1.5000 \\
1.7500 \\
2.0000\end{array}$ & $\begin{array}{l}1.1138 \\
1.2388 \\
1.4888 \\
1.7372 \\
1.9872\end{array}$ & $\begin{array}{l}\text { 1. } 0714 \\
1.1964 \\
1.4464 \\
1.6856 \\
1.9356\end{array}$ & $\begin{array}{l}1.0694 \\
\text { 1. } 1944 \\
\text { 1. } 4444 \\
1.6814 \\
1.9314\end{array}$ & $\begin{array}{l}1.0228 \\
1.1478 \\
1.3978 \\
1.6273 \\
1.8773\end{array}$ & $\begin{array}{l}1.0348 \\
1.1598 \\
1.4098 \\
1.6417 \\
1.8917\end{array}$ & $\begin{array}{l}1.0438 \\
1.1688 \\
1.4188 \\
1.6525 \\
1.9025\end{array}$ & $\begin{array}{l}1.0709 \\
1.1959 \\
1.4459 \\
1.6850 \\
1.9350\end{array}$ & $\begin{array}{l}1.0729 \\
1.1979 \\
1.4479 \\
1.6892 \\
1.9392\end{array}$ & $\begin{array}{l}1.1250 \\
1.2500 \\
1.5000 \\
1.7500 \\
2.0000\end{array}$ & $\begin{array}{l}1.1250 \\
1.2500 \\
1.5000 \\
1.7500 \\
2.0000\end{array}$ \\
\hline $\begin{array}{l}21 / 4- \\
212-.- \\
23 / 4- \\
3\end{array}$ & $\begin{array}{l}8 \\
8 \\
8 \\
8\end{array}$ & $\begin{array}{l}2.2500 \\
2.5000 \\
2.7500 \\
3.0000\end{array}$ & $\begin{array}{l}2.2348 \\
2.4848 \\
2.7348 \\
2.9848\end{array}$ & $\begin{array}{l}\text { 2. } 1695 \\
\text { 2. } 4195 \\
\text { 2. } 6695 \\
\text { 2. } 9195\end{array}$ & $\begin{array}{l}\text { 2. } 1649 \\
\text { 2. } 4149 \\
\text { 2. } 6649 \\
\text { 2. } 9149\end{array}$ & $\begin{array}{l}2.0966 \\
2.3466 \\
2.5966 \\
2.8466\end{array}$ & $\begin{array}{l}\text { 2. } 1147 \\
\text { 2. } 3647 \\
\text { 2. } 6147 \\
\text { 2. } 8647\end{array}$ & $\begin{array}{l}\text { 2. } 1282 \\
\text { 2. } 3782 \\
2.6282 \\
\text { 2. } 8782\end{array}$ & $\begin{array}{l}\text { 2. } 1688 \\
\text { 2. } 4188 \\
2.6688 \\
2.9188\end{array}$ & $\begin{array}{l}\text { 2. } 1734 \\
2.4234 \\
2.6734 \\
2.9234\end{array}$ & $\begin{array}{l}2.2500 \\
2.5000 \\
2.7500 \\
3.0000\end{array}$ & $\begin{array}{l}2.2500 \\
2.5000 \\
2.7500 \\
3.0000\end{array}$ \\
\hline
\end{tabular}

1 Dimensions given for the maximum minor diameter of the screw are figured to the intersection of the worn tool arc with a center line through crest and root. The minimum minor diameter of the screw shall be that corresponding to a flat at the minor diameter of the minimum screw equal to $1 / 8 \times p$, and may be determined by subtracting the basic thread depth, $h$ (or $0.6495 p$ ), from the minimum pitch diameter of the screw.

Dimensions for the minimum major diameter of the nut correspond to the basic flat $(1 / 8 \times p)$, and the profile at the major diameter produced by a worn tool must not fall below the basic outline. The maximum major diameter of the nut shall be that corresponding to a flat at the major diameter of the maximum nut equal to $1 / 24 \times p$, and may be determined by adding $126 \times h$ (or $0.7939 p$ ) to the maximum pitch diameter of the nut. 
TaBLe 15.-Limiting dimensions and tolerances, classes 1, 2, 3, and 4 fits, American national coarse-thread series

\begin{tabular}{|c|c|c|c|c|c|c|c|c|c|c|c|c|c|c|}
\hline & \multicolumn{14}{|c|}{ Machine screw number or nominal size } \\
\hline & 1 & 2 & 3 & 4 & 5 & 6 & 8 & 10 & 12 & $1 / 4$ & $5 / 16$ & $3 / 8$ & $7 / 10$ & $1 / 2$ \\
\hline & \multicolumn{14}{|c|}{ Threads per inch } \\
\hline & 64 & 56 & 48 & 40 & 40 & 32 & 32 & 24 & 24 & 20 & 18 & 16 & 14 & 13 \\
\hline 1 & 2 & 3 & 4 & 5 & 6 & 7 & 8 & 9 & 10 & 11 & 12 & 13 & 14 & 15 \\
\hline $\begin{array}{c}\text { BOLTS AND SCREwS } \\
\text { Class 1, major diameter }-\left\{\begin{array}{l}\text { Max..- } \\
\text { Min } \\
\text { Tol_.-.- }\end{array}\right.\end{array}$ & $\begin{array}{c}\text { Inch } \\
0.0723 \\
.0671 \\
.0052 \\
\end{array}$ & $\begin{array}{c}\text { Inch } \\
0.0852 \\
.0796 \\
.0056\end{array}$ & $\begin{array}{r}\text { Inch } \\
0.0981 \\
.0919 \\
.0062\end{array}$ & $\begin{array}{r}\text { Inch } \\
0.1110 \\
.1042 \\
.0068\end{array}$ & $\begin{array}{r}\text { Inch } \\
0.1240 \\
.1172 \\
.0068\end{array}$ & $\begin{array}{c}\text { Inch } \\
0.1369 \\
.1293 \\
.0076\end{array}$ & $\begin{array}{c}\text { Inch } \\
0.1629 \\
.1553 \\
.0076\end{array}$ & $\begin{array}{c}\text { Inch } \\
0.1887 \\
.1795 \\
.0092\end{array}$ & $\begin{array}{r}\text { Inch } \\
0.2147 \\
.2055 \\
.0092\end{array}$ & \begin{tabular}{|} 
Inch \\
0.2485 \\
.2383 \\
.0102
\end{tabular} & $\begin{array}{c}\text { Inch } \\
0.3109 \\
.2995 \\
.0114\end{array}$ & $\begin{array}{c}\text { Inch } \\
0.3732 \\
.3606 \\
.0126\end{array}$ & $\begin{array}{r}\text { Inch } \\
0.4354 \\
.4214 \\
.0140\end{array}$ & $\begin{array}{l}\text { Inch } \\
0.4978 \\
.4830 \\
.0148\end{array}$ \\
\hline $\begin{array}{l}\text { Classes 2, 3, and 4, major } \\
\text { diameter. }\end{array}\left\{\begin{array}{l}\text { Max.-. } \\
\text { Min } \\
\text { Tol...- }\end{array}\right.$ & $\begin{array}{l}.0730 \\
.0692 \\
.0038\end{array}$ & $\begin{array}{l}.0860 \\
.0820 \\
.0040\end{array}$ & $\begin{array}{l}.0990 \\
.0946 \\
.0044\end{array}$ & $\begin{array}{l}.1120 \\
.1072 \\
.0048\end{array}$ & $\begin{array}{l}.1250 \\
.1202 \\
.0048\end{array}$ & $\begin{array}{l}.1380 \\
.1326 \\
.0054\end{array}$ & $\begin{array}{l}.1640 \\
.1586 \\
.0054\end{array}$ & $\begin{array}{l}.1900 \\
.1834 \\
.0066\end{array}$ & $\begin{array}{l}.2160 \\
.2094 \\
.0066\end{array}$ & $\begin{array}{l}.2500 \\
.2428 \\
.0072\end{array}$ & $\begin{array}{l}.3125 \\
.3043 \\
.0082\end{array}$ & $\begin{array}{l}.3750 \\
.3660 \\
.0090\end{array}$ & $\begin{array}{l}.4375 \\
.4277 \\
.0098\end{array}$ & $\begin{array}{l}.5000 \\
.4896 \\
.0104\end{array}$ \\
\hline $\begin{array}{l}\text { Class 2, major diameter } \\
\text { (threaded parts of un- } \\
\text { finished, hot-rolled ma- } \\
\text { terial). }\end{array}$ & $\begin{array}{l}.0730 \\
.0678 \\
.0052\end{array}$ & $\begin{array}{l}.0860 \\
.0804 \\
.0056\end{array}$ & $\begin{array}{l}.0990 \\
.0928 \\
.0062\end{array}$ & $\begin{array}{l}.1120 \\
.1052 \\
.0068\end{array}$ & $\begin{array}{l}.1250 \\
.1182 \\
.0068\end{array}$ & $\begin{array}{l}.1380 \\
.1304 \\
.0076\end{array}$ & $\begin{array}{l}.1640 \\
.1564 \\
.0076\end{array}$ & $\begin{array}{l}.1900 \\
.1808 \\
.0092\end{array}$ & $\begin{array}{l}.2160 \\
.2068 \\
.0692\end{array}$ & $\begin{array}{l}.2500 \\
.2398 \\
.0102\end{array}$ & $\begin{array}{l}.3125 \\
.3011 \\
.0114\end{array}$ & $\begin{array}{l}.3750 \\
.3624 \\
.0126\end{array}$ & $\begin{array}{l}.4375 \\
.4235 \\
.0140\end{array}$ & $\begin{array}{l}.5000 \\
.4852 \\
.0148\end{array}$ \\
\hline Class 1, minor diameter... Miax.1.- & .0531 & .0633 & .0725 & .0803 & .0933 & .0986 & .1246 & .1376 & .1636 & .1872 & .2427 & .2965 & .3478 & .4034 \\
\hline $\begin{array}{l}\text { Classes 2, 3, and 4, minor }\} \text { Max.1 -- } \\
\text { diameter. }\end{array}$ & .0538 & .0641 & .0734 & .0813 & .0943 & .0997 & .1257 & .1389 & .1649 & .1887 & .2443 & .2983 & .3499 & .4056 \\
\hline 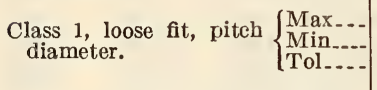 & $\begin{array}{l}.0622 \\
.0596 \\
.0026\end{array}$ & $\begin{array}{l}.0736 \\
.0708 \\
.0028\end{array}$ & $\begin{array}{l}.0846 \\
.0815 \\
.0031\end{array}$ & $\begin{array}{l}.0948 \\
.0914 \\
.0034\end{array}$ & $\begin{array}{l}.1078 \\
.1044 \\
.0034\end{array}$ & $\begin{array}{l}.1166 \\
.1128 \\
.0038\end{array}$ & $\begin{array}{l}.1426 \\
.1388 \\
.0038\end{array}$ & $\begin{array}{l}.1616 \\
.1570 \\
.0046\end{array}$ & $\begin{array}{l}.1876 \\
.1830 \\
.0046\end{array}$ & $\begin{array}{l}.2160 \\
.2109 \\
.0051\end{array}$ & $\begin{array}{l}.2748 \\
.2691 \\
.0057\end{array}$ & $\begin{array}{l}.3326 \\
.3263 \\
.0063\end{array}$ & $\begin{array}{l}.3890 \\
.3820 \\
.0070\end{array}$ & $\begin{array}{l}.4478 \\
.4404 \\
.0074\end{array}$ \\
\hline 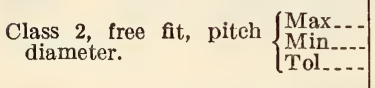 & $\begin{array}{l}.0629 \\
.0610 \\
.0019\end{array}$ & $\begin{array}{l}.0744 \\
.0724 \\
.0020\end{array}$ & $\begin{array}{l}.0855 \\
.0833 \\
.0022\end{array}$ & $\begin{array}{l}.0958 \\
.0934 \\
.0024\end{array}$ & $\begin{array}{l}.1088 \\
.1064 \\
.0024\end{array}$ & $\begin{array}{l}.1177 \\
.1150 \\
.0027\end{array}$ & $\begin{array}{l}.1437 \\
.1410 \\
.0027\end{array}$ & $\begin{array}{l}.1629 \\
.1596 \\
.0033\end{array}$ & $\begin{array}{l}.1889 \\
.1856 \\
.0033\end{array}$ & $\begin{array}{l}.2175 \\
.2139 \\
.0036\end{array}$ & $\begin{array}{l}.2764 \\
.2723 \\
.0041\end{array}$ & $\begin{array}{l}.3344 \\
.3299 \\
.0015\end{array}$ & $\begin{array}{l}.3911 \\
.3862 \\
.0049\end{array}$ & $\begin{array}{l}.4500 \\
.4448 \\
.0052\end{array}$ \\
\hline $\begin{array}{l}\text { Class 3, medium fit, pitch }\left\{\begin{array}{l}\text { Max...- } \\
\text { diameter. }\end{array} \text { Min }\right. \\
\text { Tol...-- }\end{array}$ & .0629 & $\begin{array}{l}.0744 \\
.0729 \\
.0015\end{array}$ & $\begin{array}{l}.0855 \\
.0839 \\
.0016\end{array}$ & $\begin{array}{l}.0958 \\
.0941 \\
.0017\end{array}$ & $\begin{array}{l}.1088 \\
.1071 \\
.0017\end{array}$ & $\begin{array}{l}.1177 \\
.1158 \\
.0019\end{array}$ & $\begin{array}{l}.1437 \\
.1418 \\
.0019\end{array}$ & $\begin{array}{l}.1629 \\
.1605 \\
.0024\end{array}$ & $\begin{array}{l}.1889 \\
.1865 \\
.0024\end{array}$ & $\begin{array}{l}.2175 \\
.2149 \\
.0026\end{array}$ & $\begin{array}{l}.2764 \\
.2734 \\
.0030\end{array}$ & $\begin{array}{l}.3344 \\
.3312 \\
.0032\end{array}$ & $\begin{array}{l}.3911 \\
.3875 \\
.0036\end{array}$ & $\begin{array}{l}.4500 \\
.4463 \\
.0037\end{array}$ \\
\hline 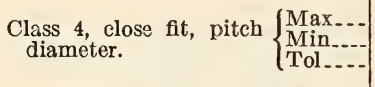 & .0630 & $\begin{array}{l}.0746 \\
.0739 \\
.0007\end{array}$ & $\begin{array}{l}.0857 \\
.0849 \\
.0008\end{array}$ & $\begin{array}{l}.0960 \\
.0951 \\
.0009\end{array}$ & $\begin{array}{l}.1090 \\
.1081 \\
.0009\end{array}$ & $\begin{array}{l}.1179 \\
.1169 \\
.0010\end{array}$ & $\begin{array}{l}.1439 \\
.1429 \\
.0010\end{array}$ & $\begin{array}{l}.1632 \\
.1620 \\
.0012\end{array}$ & $\begin{array}{l}.1892 \\
.1880 \\
.0012\end{array}$ & $\begin{array}{l}.2178 \\
.2165 \\
.0013\end{array}$ & $\begin{array}{l}.2767 \\
.2752 \\
.0015\end{array}$ & $\begin{array}{l}.3348 \\
.3332 \\
.0016 \\
\end{array}$ & $\begin{array}{l}.3915 \\
.3897 \\
.0018\end{array}$ & $\begin{array}{l}.4504 \\
.4485 \\
.0019\end{array}$ \\
\hline NUTS AND TAPPED HOLES & & & & & & & & & & & & & & \\
\hline $\begin{array}{l}\text { Classes } 1,2,3 \text {, and } 4,\} \text { Min. }{ }^{2}- \\
\text { major diameter. }\end{array}$ & .0730 & .0860 & .0990 & .1120 & .1250 & .1380 & .1640 & .1900 & .2160 & .2500 & .3125 & .3750 & .4375 & .5000 \\
\hline $\begin{array}{l}\text { Classes } 1,2,3, \text { and } 4, \\
\text { minor diameter. }\end{array}$ & .0604 & $\begin{array}{l}.0715 \\
.0667 \\
.0048\end{array}$ & $\begin{array}{l}.0820 \\
.0764 \\
.0056\end{array}$ & $\begin{array}{l}.0913 \\
.0849 \\
.0064\end{array}$ & $\begin{array}{l}.1043 \\
.0979 \\
.0064\end{array}$ & $\begin{array}{l}.1118 \\
.1042 \\
.0076\end{array}$ & $\begin{array}{l}.1378 \\
.1302 \\
.0076\end{array}$ & $\begin{array}{l}.1541 \\
.1449 \\
.0092\end{array}$ & $\begin{array}{l}.1801 \\
.1709 \\
.0092\end{array}$ & $\begin{array}{l}.2060 \\
.1959 \\
.0101\end{array}$ & $\begin{array}{l}.2630 \\
.2524 \\
.0106\end{array}$ & $\begin{array}{l}.3184 \\
.3073 \\
.0111\end{array}$ & $\begin{array}{l}.3721 \\
.3602 \\
.0119\end{array}$ & $\begin{array}{l}.4290 \\
.4167 \\
.0123\end{array}$ \\
\hline $\begin{array}{l}\text { Classes } 1,2,3 \text {, and } 4, \\
\text { pitch diameter. }\end{array}$ & .0629 & .0744 & .0855 & .0958 & .1088 & .1177 & .1437 & .1629 & .1889 & .2175 & .2764 & .3344 & .3911 & .4500 \\
\hline $\begin{array}{l}\text { Class 1, loose fit, pitch }\left\{\begin{array}{l}\text { Max... } \\
\text { diameter. }\end{array}\right. \\
\text { Tol... }\end{array}$ & .0655 & $\begin{array}{l}.0772 \\
.0028\end{array}$ & .0886 & .0992 & .1122 & .1215 & $\begin{array}{l}.1475 \\
.0038\end{array}$ & .1675 & $\begin{array}{l}.1935 \\
.0046\end{array}$ & .2226 & .2821 & $\begin{array}{l}.3407 \\
.0063\end{array}$ & .3981 & .4574 \\
\hline $\begin{array}{l}\text { Class 2, free fit, pitch }\left\{\begin{array}{l}\mathrm{Max}_{-.} \\
\text {diameter. }\end{array}\right.\end{array}$ & .0648 & $\begin{array}{l}.0764 \\
.0020\end{array}$ & .0877 & .0982 & .1112 & .1204 & .1464 & .1662 & .0022 & .2211 & .2805 & .3389 & .3960 &. .4552 \\
\hline $\begin{array}{l}\text { Class 3, medium fit, pitch }\left\{\begin{array}{l}\text { Max...- } \\
\text { diameter. }\end{array}\right. \\
\text { Tol.... }\end{array}$ & .0643 & $\begin{array}{l}.0759 \\
.0015\end{array}$ & .0871 & .0975 & .1105 & .1196 & $\begin{array}{l}.1456 \\
.0019\end{array}$ & $\begin{array}{l}.1653 \\
.0024\end{array}$ & .1913 & .2201 & .2794 & $\begin{array}{l}.3376 \\
.0032\end{array}$ & .3947 & .4537 \\
\hline $\begin{array}{l}\text { Class 4, close fit, pitch }\left\{\begin{array}{l}\text { Max... } \\
\text { diameter. }\end{array} \text { Tol.... }\right.\end{array}$ & .0636 & .0751 & $\begin{array}{l}.0863 \\
.0008\end{array}$ & $\begin{array}{l}.0967 \\
.0009\end{array}$ & $\begin{array}{l}.1097 \\
.0009\end{array}$ & $\begin{array}{l}.1187 \\
.0010\end{array}$ & $\begin{array}{l}.1447 \\
.0010\end{array}$ & .1641 & $\begin{array}{l}.1901 \\
.0012\end{array}$ & .2188 & $\begin{array}{l}.2779 \\
.0015\end{array}$ & $\begin{array}{l}.3360 \\
.0016\end{array}$ & $\begin{array}{l}.3929 \\
.0018\end{array}$ & $\begin{array}{l}.4519 \\
.0019\end{array}$ \\
\hline
\end{tabular}

12 See footnote to Table 16. 
TABLE 15.-Limiting dimensions and tolerances, classes 1, 2, 3, and 4, fits American national coarse-thread series-Continued

\begin{tabular}{|c|c|c|c|c|c|c|c|c|c|c|c|c|c|c|c|}
\hline & \multicolumn{15}{|c|}{ Sizes } \\
\hline & \%o & $5 / 8$ & $3 / 4$ & $7 / 8$ & 1 & $1 \frac{1}{8}$ & $11 / 4$ & $11 / 2$ & $18 / 4$ & 2 & $21 / 4$ & $21 / 2$ & $23 / 4$ & 3 & 3 \\
\hline & \multicolumn{15}{|c|}{ Threads per inch } \\
\hline & 12 & 11 & 10 & 9 & 8 & 7 & 7 & 6 & 5 & $4 \frac{1}{2}$ & $41 / 2$ & 4 & 4 & 4 & $31 / 2$ \\
\hline 1 & 16 & 17 & 18 & 19 & 20 & 21 & 22 & 23 & 24 & 25 & 26 & 27 & 28 & 29 & 30 \\
\hline BOLTS AND SCREWS $\left\{\begin{array}{l}\text { Max... } \\
\text { Class 1, major diameter......... } \\
\text { Tol } \ldots . . .\end{array}\right.$ & $\begin{array}{c}\text { Inch } \\
0.5601 \\
.5443 \\
.0158\end{array}$ & $\begin{array}{l}\text { Inch } \\
0.6224 \\
.6054 \\
.0170\end{array}$ & $\begin{array}{r}\text { Inch } \\
0.7472 \\
.7288 \\
.0184\end{array}$ & $\begin{array}{l}\text { Inch } \\
0.8719 \\
.8519 \\
.0200\end{array}$ & $\begin{array}{l}\text { Inch } \\
0.9966 \\
.9744 \\
.0222\end{array}$ & \begin{tabular}{|} 
Inches \\
1.1211 \\
1.0963 \\
.0248
\end{tabular} & $\begin{array}{r}\text { Inches } \\
1.2461 \\
1.2213 \\
.0248\end{array}$ & $\begin{array}{l}\text { Inches } \\
1.4956 \\
1.4666 \\
.0290\end{array}$ & $\mid \begin{array}{l}\text { Inches } \\
1.7448 \\
1.7110 \\
.0338\end{array}$ & \begin{tabular}{|} 
Inches \\
1.9943 \\
1.9575 \\
.0368
\end{tabular} & \begin{tabular}{|l|} 
Inches \\
2.2443 \\
2.2075 \\
.0368 \\
\end{tabular} & \begin{tabular}{|l|} 
Inches \\
2.4936 \\
2.4528 \\
.0408 \\
\end{tabular} & \begin{tabular}{|l|} 
Inches \\
2.7436 \\
2.7028 \\
.0408 \\
\end{tabular} & \begin{tabular}{|l|} 
Inches \\
2.9936 \\
2.9528 \\
.0408 \\
\end{tabular} & \begin{tabular}{l|l|l} 
& Inches \\
66 & 2.9927 \\
8 & 2.9469 \\
8 & .0458
\end{tabular} \\
\hline $\begin{array}{l}\text { Classes 2, 3, and 4, major diam- } \\
\text { eter.. }\end{array}$ & $\begin{array}{l}.5625 \\
.5513 \\
.0112\end{array}$ & $\begin{array}{l}.6250 \\
.6132 \\
.0118\end{array}$ & $\begin{array}{l}.7500 \\
.7372 \\
.0128\end{array}$ & $\begin{array}{l}.87501 \\
.8610 \\
.0140\end{array}$ & $\begin{array}{r}1.00001 \\
.98481 \\
.0152\end{array}$ & $\begin{array}{l}1.1250 \\
1.1080 \\
.0170\end{array}$ & $\begin{array}{r}1.2500 \\
1.2330 \\
.0107\end{array}$ & $\begin{array}{r}1.5000 \\
1.4798 \\
.0202\end{array}$ & $\begin{array}{r}1.7500 \\
1.7268 \\
.0232\end{array}$ & $\begin{array}{l}2.0000 \\
1.9746 \\
.0251\end{array}$ & $\begin{array}{r}2.2500 \\
2.2246 \\
.0254\end{array}$ & $\begin{array}{r}2.5000 \\
2.4720 \\
.0280\end{array}$ & $\begin{array}{r}2.7500 \\
2.7220 \\
.0280\end{array}$ & $\begin{array}{l}3.0000 \\
2.9720 \\
.0280\end{array}$ & \begin{tabular}{l|r}
0.0000 \\
0 \\
0.9686 \\
0 & .0314
\end{tabular} \\
\hline 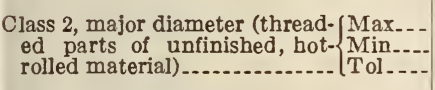 & $\begin{array}{l}.5625 \\
.5467 \\
.0158\end{array}$ & $\begin{array}{l}.6250 \\
.6080 \\
.0170\end{array}$ & $\begin{array}{l}.7500 \\
.7316 \\
.0184\end{array}$ & $\begin{array}{l}.87501 \\
.8550 \\
.0200\end{array}$ & $\begin{array}{r}1.00001 \\
.97781 \\
.0222\end{array}$ & $\begin{array}{l}1.2500 \\
1.1002 \\
.0248\end{array}$ & $\begin{array}{r}1.2500 \\
1.2252 \\
.0248\end{array}$ & $\begin{array}{r}1.5000 \\
1.4710 \\
.0290\end{array}$ & $\begin{array}{l}1.75002 \\
1.71621 \\
.0338\end{array}$ & $\begin{array}{l}2.0000 \\
1.9632 \\
.0368\end{array}$ & $\begin{array}{l}2.2500 \\
2.2132 \\
.0368\end{array}$ & $\begin{array}{l}2.5000 \\
2.4592 \\
.0408\end{array}$ & $\begin{array}{l}2.7500 \\
2.7092 \\
.0408\end{array}$ & $\begin{array}{l}3.0000 \\
2.9592 \\
.0408\end{array}$ & 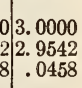 \\
\hline Class 1, minor diameter......... Max ${ }^{1}$ & .4579 & .5109 & .6245 & .7356 & .8432 & .9458 & 1.0708 & 1.2911 & 1.4994 & 1.7217 & 1.9717 & 2.1869 & 2.4369 & 2.6869 & 92.6421 \\
\hline $\begin{array}{l}\text { Classes } 2,3 \text {, and } 4 \text {, minor diam- }\} M_{\text {eter. }}{ }^{3} \text {.. }\end{array}$ & .4603 & .5135 & .6273 & .7387 & .8466 & .9497 & 1.0747 & 1.2955 & 1.50461 & 1.7274 & 1.9774 & 2.1933 & 2.4433 & 2.6933 & 2.6494 \\
\hline Class 1, loose fit, pitch diameter $\left\{\begin{array}{l}\text { Max... } \\
\text { Min } \\
\text { Tol } \ldots . .\end{array}\right.$ & $\begin{array}{l}.5060 \\
.4981 \\
.0079\end{array}$ & $\begin{array}{l}.5634 \\
.5549 \\
.0085\end{array}$ & $\begin{array}{l}.6822 \\
.6730 \\
.0092\end{array}$ & $\begin{array}{l}.7997 \\
.7897 \\
.0100\end{array}$ & $\begin{array}{l}.91541 \\
.90431 \\
.0111\end{array}$ & $\begin{array}{r}1.0283 \\
1.0159 \\
.0124\end{array}$ & $\begin{array}{l}1.1533 \\
1.1409 \\
.0124\end{array}$ & $\begin{array}{l}1.3873 \\
1.3728 \\
.0145\end{array}$ & $\begin{array}{l}1.6149 \\
1.59801 \\
.0169\end{array}$ & $\begin{array}{l}1.8500 \\
1.8316 \\
.0184\end{array}$ & $\begin{array}{r}2.1000 \\
2.0816 \\
.0184\end{array}$ & $\begin{array}{l}2.3312 \\
2.3108 \\
.0204\end{array}$ & $\begin{array}{r}2.5812 \\
2.5608 \\
.0204\end{array}$ & $\begin{array}{r}2.8312 \\
2.8108 \\
.0204\end{array}$ & $\begin{array}{l}22.8071 \\
82.7842 \\
.0229\end{array}$ \\
\hline Class 2, free fit, pitch diameter.- $\left\{\begin{array}{l}\text { Max. } \\
\text { Min } \\
\text { Tol.-. }\end{array}\right.$ & $\begin{array}{l}.5084 \\
.5028 \\
.0056\end{array}$ & $\begin{array}{l}.5660 \\
.5601 \\
.0059\end{array}$ & $\begin{array}{l}.6850 \\
.6786 \\
.0064\end{array}$ & $\begin{array}{l}.8028 \\
.7958 \\
.0070\end{array}$ & $\begin{array}{l}.9188 \\
.9112 \\
.0076\end{array}$ & $\begin{array}{r}1.0322 \\
1.0237 \\
.0085\end{array}$ & $\begin{array}{l}1.1572 \\
1.1487 \\
.0085\end{array}$ & $\begin{array}{l}1.3917 \\
1.3816 \\
.0101\end{array}$ & $\begin{array}{l}1.6201 \\
1.6085 \\
.0116\end{array}$ & $\begin{array}{l}1.8557 \\
1.8430 \\
.0127\end{array}$ & $\begin{array}{l}2.1057 \\
2.0930 \\
.0127\end{array}$ & $\begin{array}{r}2.3376 \\
2.3236 \\
.0140\end{array}$ & $\begin{array}{r}2.5876 \\
2.5736 \\
.0140\end{array}$ & $\begin{array}{r}2.8376 \\
2.8236 \\
.0140\end{array}$ & $\begin{array}{l}62.8144 \\
62.7987 \\
0.0157\end{array}$ \\
\hline 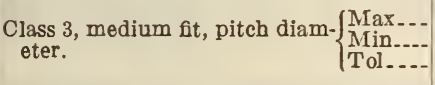 & $\begin{array}{l}.5084 \\
.5044 \\
.0040\end{array}$ & $\begin{array}{l}.5660 \\
.5618 \\
.0042\end{array}$ & $\begin{array}{l}.6850 \\
.6805 \\
.0045\end{array}$ & $\begin{array}{l}.8028 \\
.7979 \\
.0049\end{array}$ & $\begin{array}{l}.9188 \\
.9134 \\
.0054\end{array}$ & $\begin{array}{l}1.0322 \\
1.0263 \\
.0059\end{array}$ & $\begin{array}{r}1.1572 \\
1.1513 \\
.0059\end{array}$ & $\begin{array}{r}1.3917 \\
1.3846 \\
.0071\end{array}$ & $\begin{array}{l}1.62011 \\
1.61191 \\
.0082\end{array}$ & $\begin{array}{l}1.8557 \\
1.8468 \\
.0089\end{array}$ & $\begin{array}{l}2.1057 \\
2.0968 \\
.0089\end{array}$ & $\begin{array}{r}2.3376 \\
2.3279 \\
.0097\end{array}$ & $\begin{array}{r}2.5876 \\
2.5779 \\
.0097\end{array}$ & $\begin{array}{l}2.8376 \\
2.8279 \\
.0097\end{array}$ & $\begin{array}{l}62.8144 \\
92.8037 \\
70107\end{array}$ \\
\hline 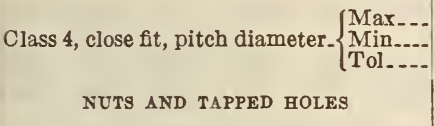 & $\begin{array}{l}.5089 \\
.5069 \\
.0020\end{array}$ & $\begin{array}{l}.5665 \\
.5644 \\
.0021\end{array}$ & $\begin{array}{l}.6856 \\
.6833 \\
.0023\end{array}$ & $\begin{array}{l}.8034 \\
.8010 \\
.0024\end{array}$ & $\begin{array}{l}.91951 \\
.91681 \\
.0027\end{array}$ & $\begin{array}{r}1.0330 \\
1.0300 \\
.0030\end{array}$ & $\begin{array}{r}1.1580 \\
1.1550 \\
.0030\end{array}$ & $\begin{array}{r}1.3926 \\
1.3890 \\
.0036\end{array}$ & $\begin{array}{l}1.62111 \\
1.61701 \\
.0041\end{array}$ & $\begin{array}{r}1.8568 \\
1.8524 \\
.0044\end{array}$ & $\begin{array}{r}2.1068 \\
2.1024 \\
.0044\end{array}$ & $\begin{array}{l}2.3389 \\
2.3341 \\
.0048\end{array}$ & $\begin{array}{r}2.5889 \\
2.5841 \\
.0048\end{array}$ & $\begin{array}{r}2.8389 \\
2.8341 \\
.0048\end{array}$ & $\begin{array}{l}92.8160 \\
2.8107 \\
.0053\end{array}$ \\
\hline $\begin{array}{l}\text { Classes } 1,2,3 \text {, and } 4 \text {, major diam- }\} \text { Min. } .^{2}-\text { - } \\
\text { eter. }\end{array}$ & .5625 & .6250 & .7500 & .87501 & 1.0000 & 1.1250 & 1.2500 & 1.5000 & 1.75002 & 2.0000 & 2.2500 & 2.5000 & 2.7500 & 3.0000 & 03.0000 \\
\hline 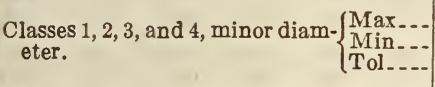 & $\begin{array}{l}.4850 \\
.4723 \\
.0127\end{array}$ & $\begin{array}{l}.5397 \\
.5266 \\
.0131\end{array}$ & $\begin{array}{l}.6553 \\
.6417 \\
.0136\end{array}$ & $\begin{array}{l}.7689 \\
.7547 \\
.0142\end{array}$ & $\begin{array}{l}.8795 \\
.8647 \\
.0148\end{array}$ & $\begin{array}{l}.9858 \\
.9704 \\
.0154\end{array}$ & $\begin{array}{l}1.1108 \\
1.0954 \\
.0154\end{array}$ & $\begin{array}{l}1.3376 \\
1.3196 \\
.0180\end{array}$ & $\begin{array}{l}1.5551 \\
1.5335 \\
.0216\end{array}$ & $\begin{array}{l}1.7835 \\
1.7594 \\
.0241\end{array}$ & $\begin{array}{r}2.0335 \\
2.0094 \\
.0241\end{array}$ & $\begin{array}{r}2.2564 \\
2.2294 \\
.0270\end{array}$ & $\begin{array}{r}2.5064 \\
2.4794 \\
.0270\end{array}$ & $\begin{array}{r}2.7564 \\
2.7294 \\
.0270\end{array}$ & \begin{tabular}{l|l}
4 & 2.7216 \\
4 & 2.6907 \\
0.0309
\end{tabular} \\
\hline Classes 1, 2, 3, and 4, pitch diameter_Min_- & .5084 & .5660 & .6850 & .8028 & .9188 & 1.0322 & 1.1572 & 1.3917 & 1.6201 & 1.8557 & 2.1057 & 2.3376 & 2.5876 & 2.8376 & 62.8144 \\
\hline Class 1, loose fit, pitch diameter $\left\{\begin{array}{l}\text { Max... } \\
\text { Tol...- }\end{array}\right.$ & $\begin{array}{l}.5163 \\
.0079\end{array}$ & $\begin{array}{l}.5745 \\
.0085\end{array}$ & .6942 & $\begin{array}{r}.8128 \\
.0100\end{array}$ & $\mid .92991$ & $\begin{array}{r}1.0446 \\
.0124\end{array}$ & $\begin{array}{r}1.1696 \\
.0124\end{array}$ & $\begin{array}{r}1.4062 \\
.0145\end{array}$ & $\begin{array}{r}1.6370 \\
.0169\end{array}$ & $\begin{array}{l}1.8741 \\
.0184\end{array}$ & $\begin{array}{r}2.1241 \\
.0184\end{array}$ & $\begin{array}{r}2.3580 \\
.0204\end{array}$ & $\begin{array}{r}2.6080 \\
.0204\end{array}$ & $\begin{array}{r}2.8580 \\
.0204\end{array}$ & \begin{tabular}{l|l}
0.8373 \\
0.0229
\end{tabular} \\
\hline 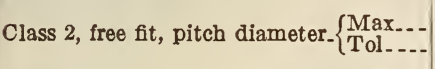 & .5140 & $\begin{array}{l}.5719 \\
.0059\end{array}$ & .6914 & $\begin{array}{r}.8098 \\
.0070\end{array}$ & .02641 & $\begin{array}{l}1.0407 \\
.0085\end{array}$ & $\begin{array}{l}1.1657 \\
.0085\end{array}$ & $\begin{array}{r}1.4018 \\
.0101 \\
\end{array}$ & $\begin{array}{r}1.6317 \\
.0116\end{array}$ & 1.8684 & 2.1184 & $\begin{array}{r}2.3516 \\
.0140\end{array}$ & $\begin{array}{r}2.6016 \\
.0140\end{array}$ & $\begin{array}{l}2.8516 \\
.0140\end{array}$ & $\begin{array}{ll}6 & 2.8301 \\
0.0157\end{array}$ \\
\hline $\begin{array}{l}\text { Class } 3 \text {, medium fit, pitch diam- }\left\{\begin{array}{l}\mathrm{Max}_{\mathrm{Ta}} . \\
\text { eter. }\end{array}\right.\end{array}$ & $\begin{array}{l}.5124 \\
.0040\end{array}$ & $\begin{array}{l}.5702 \\
.0042\end{array}$ & .6895 & $\begin{array}{l}.8077 \\
.0049\end{array}$ & .92421 & $\begin{array}{l}1.0381 \\
.0059\end{array}$ & $\begin{array}{r}1.1631 \\
.0059\end{array}$ & $\begin{array}{r}1.3988 \\
.0071\end{array}$ & $\begin{array}{l}1.62831 \\
.0082\end{array}$ & $\begin{array}{r}1.8646 \\
.0089\end{array}$ & $\begin{array}{r}2.1146 \\
.0089\end{array}$ & $\begin{array}{r}2.3473 \\
.0097\end{array}$ & $\begin{array}{r}2.5973 \\
.0097\end{array}$ & $\begin{array}{r}2.8473 \\
.0097\end{array}$ & $\begin{array}{l}3.8251 \\
.0107\end{array}$ \\
\hline Class 4, close fit, pitch diameter-\{$\left\{\begin{array}{l}\mathrm{Max}_{--} \\
\mathrm{Tol}_{---}\end{array}\right.$ & $\begin{array}{l}.5104 \\
.0020\end{array}$ & $\begin{array}{l}.5681 \\
.0021\end{array}$ & $\begin{array}{l}.6873 \\
.0023\end{array}$ & $\begin{array}{l}.8052 \\
.0024\end{array}$ & $\begin{array}{l}.9215 \\
.0027\end{array}$ & $\begin{array}{r}1.0352 \\
.0030\end{array}$ & $\begin{array}{r}1.1602 \\
.0030\end{array}$ & $\begin{array}{r}1.3953 \\
.0036\end{array}$ & $\begin{array}{r}1.62421 \\
.0041\end{array}$ & $\begin{array}{r}1.8601 \\
.0044\end{array}$ & $\begin{array}{l}2.1101 \\
.0044\end{array}$ & $\begin{array}{r}2.3424 \\
.0048\end{array}$ & $\begin{array}{r}2.5924 \\
.0048\end{array}$ & $\begin{array}{l}2.8424 \\
.0048\end{array}$ & $\begin{array}{l}4.8197 \\
8.0053\end{array}$ \\
\hline
\end{tabular}

12 See footnote to Table 16. 
TABLE 16.-Limiting dimensions and tolerances, classes 1, 2, 3, and 4 fits, American national fine-thread series

\begin{tabular}{|c|c|c|c|c|c|c|c|c|c|c|c|c|c|}
\hline & & \multicolumn{12}{|c|}{ Machine screw number or nominal size } \\
\hline & & 0 & 1 & 2 & 3 & 4 & 5 & 6 & 8 & 10 & 12 & $1 / 4$ & $5 / 10$ \\
\hline & & \multicolumn{12}{|c|}{ Threads per inch } \\
\hline & & 80 & 72 & 64 & 56 & 48 & 44 & 40 & 36 & 32 & 28 & 28 & 24 \\
\hline 1 & & 2 & 3 & 4 & 5 & 6 & 7 & 8 & 9 & 10 & 11 & 12 & 13 \\
\hline $\begin{array}{l}\text { BOLTS AND SCREWS } \\
\text { Class 1, major diameter.... }\end{array}$ & $\left\{\begin{array}{l}\text { Max... } \\
\text { Min...- } \\
\text { Tol.... }\end{array}\right.$ & $\begin{array}{c}\text { Inch } \\
0.0593 \\
.0545 \\
.0048\end{array}$ & $\begin{array}{c}\text { Inch } \\
0.0723 \\
.0673 \\
.0050\end{array}$ & $\begin{array}{l}\text { Inch } \\
0.0853 \\
.0801 \\
.0052\end{array}$ & $\begin{array}{c}\text { Inch } \\
0.0982 \\
.0926 \\
.0056\end{array}$ & $\begin{array}{r}\text { Inch } \\
0.1111 \\
.1049 \\
.0062\end{array}$ & $\begin{array}{l}\text { Inch } \\
0.1241 \\
.1177 \\
.0064\end{array}$ & $\begin{array}{r}\text { Inch } \\
0.1370 \\
.1302 \\
.0068\end{array}$ & $\begin{array}{r}\text { Inch } \\
0.1629 \\
.1557 \\
.0072\end{array}$ & $\begin{array}{r}\text { Inch } \\
0.1889 \\
.1813 \\
.0076\end{array}$ & $\begin{array}{r}\text { Inch } \\
0.2148 \\
.2062 \\
.0086\end{array}$ & $\begin{array}{r}\text { Inch } \\
0.2488 \\
.2402 \\
.0086\end{array}$ & $\begin{array}{l}\text { Inch } \\
0.3112 \\
.3020 \\
.0092\end{array}$ \\
\hline Classes 2, 3, and 4, major diameter. & $\left\{\begin{array}{l}\text { Max... } \\
\text { Min } \\
\text { Tol } \ldots . . .\end{array}\right.$ & $-\begin{array}{r}.0600 \\
-.0566 \\
.0034\end{array}$ & $\begin{array}{l}.0730 \\
.0694 \\
.0036\end{array}$ & $\begin{array}{l}.0860 \\
.0822 \\
.0038\end{array}$ & $\begin{array}{l}.0990 \\
.0950 \\
.0040\end{array}$ & $\begin{array}{l}.1120 \\
.1076 \\
.0044\end{array}$ & $\begin{array}{l}.1250 \\
.1204 \\
.0046\end{array}$ & $\begin{array}{l}.1380 \\
.1332 \\
.0048\end{array}$ & $\begin{array}{l}.1640 \\
.1590 \\
.0050\end{array}$ & $\begin{array}{l}.1900 \\
.1846 \\
.0054\end{array}$ & $\begin{array}{l}.2160 \\
.2098 \\
.0062\end{array}$ & $\begin{array}{l}.2500 \\
.2438 \\
.0062\end{array}$ & $\begin{array}{l}.3125 \\
.3059 \\
.0066\end{array}$ \\
\hline $\begin{array}{l}\text { Class } 1 \text {, minor diameter } \\
\text { Classes } 2,3 \text {, and } 4 \text {, minor diameter... }\end{array}$ & $\begin{array}{l}-\operatorname{Max}^{1}{ }^{1--} \\
\operatorname{Max} .^{1--}\end{array}$ & .0440 & $\begin{array}{l}.0553 \\
.0560\end{array}$ & .0661 & $\begin{array}{l}.0763 \\
.0771\end{array}$ & $\begin{array}{l}.0855 \\
.0864\end{array}$ & $\begin{array}{l}.0962 \\
.0971\end{array}$ & .1063 & $\begin{array}{l}.1288 \\
.1299\end{array}$ & $\begin{array}{l}.1506 \\
.1517\end{array}$ & $\begin{array}{l}.1710 \\
.1722\end{array}$ & $\begin{array}{l}.2050 \\
.2062\end{array}$ & $\begin{array}{l}.2601 \\
.2614\end{array}$ \\
\hline Class 1, pitch diameter... & $\left\{\begin{array}{l}\text { Max... } \\
\text { Min } \\
\text { Tol..... }\end{array}\right.$ & $\begin{array}{r}.0512 \\
-.0488 \\
.0024\end{array}$ & $\begin{array}{l}.0633 \\
.0608 \\
.0025\end{array}$ & $\begin{array}{l}.0752 \\
.0726 \\
.0026\end{array}$ & $\begin{array}{l}.0866 \\
.0838 \\
.0028\end{array}$ & $\begin{array}{l}.0976 \\
.0945 \\
.0031\end{array}$ & $\begin{array}{l}.1093 \\
.1061 \\
.0032\end{array}$ & $\begin{array}{l}.1208 \\
.1174 \\
.0034\end{array}$ & $\begin{array}{l}.1449 \\
.1413 \\
.0036\end{array}$ & $\begin{array}{l}.1686 \\
.1648 \\
.0038\end{array}$ & $\begin{array}{r}.1916 \\
.1873 \\
.0043\end{array}$ & $\begin{array}{l}.2256 \\
.2213 \\
.0043\end{array}$ & $\begin{array}{l}.2841 \\
.2795 \\
.0046\end{array}$ \\
\hline Class 2, pitch diameter... & $\left\{\begin{array}{l}\text { Max... } \\
\text { Min ... } \\
\text { Tol.... }\end{array}\right.$ & $\begin{array}{r}.0519 \\
-.0502 \\
-.0017\end{array}$ & $\begin{array}{l}.0640 \\
.0622 \\
.0018\end{array}$ & $\begin{array}{l}.0759 \\
.0740 \\
.0019\end{array}$ & $\begin{array}{l}.0874 \\
.0854 \\
.0020\end{array}$ & $\begin{array}{l}.0985 \\
.0963 \\
.0022\end{array}$ & $\begin{array}{l}.1102 \\
.1079 \\
.0023\end{array}$ & $\begin{array}{l}.1218 \\
.1194 \\
.0024\end{array}$ & $\begin{array}{l}.1460 \\
.1435 \\
.0025\end{array}$ & $\begin{array}{l}.1697 \\
.1670 \\
.0027\end{array}$ & $\begin{array}{l}.1928 \\
.1897 \\
.0031\end{array}$ & $\begin{array}{l}.2268 \\
.2237 \\
.0031\end{array}$ & $\begin{array}{l}.2854 \\
.2821 \\
.0033\end{array}$ \\
\hline Class 3, pitch diameter.-. & $\left\{\begin{array}{l}\text { Max... } \\
\text { Min.... } \\
\text { Tol .... }\end{array}\right.$ & $\begin{array}{r}.0519 \\
-.0506 \\
-.0013\end{array}$ & $\begin{array}{l}.0640 \\
.0627 \\
.0013\end{array}$ & $\begin{array}{l}.0759 \\
.0745 \\
.0014\end{array}$ & $\begin{array}{l}.0874 \\
.0859 \\
.0015\end{array}$ & $\begin{array}{l}.0985 \\
.0969 \\
.0016\end{array}$ & $\begin{array}{l}.1102 \\
.1086 \\
.0016\end{array}$ & $\begin{array}{l}.1218 \\
.1201 \\
.0017\end{array}$ & $\begin{array}{l}.1460 \\
.1442 \\
.0018\end{array}$ & $\begin{array}{l}.1697 \\
.1678 \\
.0019\end{array}$ & $\begin{array}{l}.1928 \\
.1906 \\
.0022\end{array}$ & $\begin{array}{l}.2268 \\
.2246 \\
.0022\end{array}$ & $\begin{array}{l}.2854 \\
.2830 \\
.0024\end{array}$ \\
\hline Class 4, pitch diameter.- & $\left\{\begin{array}{l}\text { Max... } \\
\text { Min.... } \\
\text { Tol_... }\end{array}\right.$ & $\begin{array}{r}.0520 \\
-.0514 \\
.0006\end{array}$ & $\begin{array}{l}.0641 \\
.0634 \\
.0007\end{array}$ & $\begin{array}{l}.0760 \\
.0753 \\
.0007\end{array}$ & $\begin{array}{l}.0876 \\
.0869 \\
.0007\end{array}$ & $\begin{array}{l}.0987 \\
.0979 \\
.0008\end{array}$ & $\begin{array}{l}.1104 \\
.1096 \\
.0008\end{array}$ & $\begin{array}{l}.1220 \\
.1211 \\
.0009\end{array}$ & $\begin{array}{l}.1462 \\
.1453 \\
.0009\end{array}$ & $\begin{array}{l}.1699 \\
.1689 \\
.0010\end{array}$ & $\begin{array}{l}.1930 \\
.1919 \\
.0011\end{array}$ & $\begin{array}{l}.2270 \\
.2259 \\
.0011\end{array}$ & $\begin{array}{l}.2857 \\
.2845 \\
.0012\end{array}$ \\
\hline NUTS AND TAPPED HOLES & & & & & & & & & & & & & \\
\hline Classes $1,2,3$, and 4 , major diameter.. & Min. ${ }^{2}-$ & .0600 & .0730 & .0860 & .0990 & .1120 & .1250 & .1380 & .1640 & .1900 & .2160 & .2500 & .3125 \\
\hline Classes $1,2,3$, and 4 , minor diameter.. & $\left\{\begin{array}{l}\text { Max... } \\
\text { Min.... } \\
\text { Tol.... }\end{array}\right.$ & $\begin{array}{r}.0492 \\
-.0465 \\
-.0027\end{array}$ & $\begin{array}{l}.0610 \\
.0580 \\
.0030\end{array}$ & $\begin{array}{l}.0724 \\
.0691 \\
.0033\end{array}$ & $\begin{array}{l}.0834 \\
.0797 \\
.0037\end{array}$ & $\begin{array}{l}.0937 \\
.0894 \\
.0043\end{array}$ & $\begin{array}{l}.1049 \\
.1004 \\
.0045\end{array}$ & $\begin{array}{l}.1158 \\
.1109 \\
.0049\end{array}$ & $\begin{array}{l}.1391 \\
.1339 \\
.0052\end{array}$ & $\begin{array}{l}.1618 \\
.1562 \\
.0056\end{array}$ & $\begin{array}{l}.1833 \\
.1773 \\
.0060\end{array}$ & $\begin{array}{l}.2173 \\
.2113 \\
.0060\end{array}$ & $\begin{array}{l}.2739 \\
.2674 \\
.0065\end{array}$ \\
\hline Classes $1,2,3$, and 4 , pitch diameter... & -Min & .0519 & .0640 & .0759 & .0874 & .0985 & .1102 & .1218 & .1460 & .1697 & .1928 & .2268 & .2854 \\
\hline Class 1, pitch diameter... & $\left\{\begin{array}{l}\text { Max... } \\
\text { Tol } \ldots\end{array}\right.$ & -.0543 & .0665 & $\begin{array}{l}.0785 \\
.0026\end{array}$ & $\begin{array}{l}.0902 \\
.0028\end{array}$ & $\begin{array}{l}.1016 \\
.0031\end{array}$ & $\begin{array}{l}.1134 \\
.0032\end{array}$ & $\begin{array}{l}.1252 \\
.0034\end{array}$ & $\begin{array}{l}.1496 \\
.0036\end{array}$ & $\begin{array}{l}.1735 \\
.0038\end{array}$ & $\begin{array}{l}.1971 \\
.0043\end{array}$ & .2311 & $\begin{array}{l}.2900 \\
.0046\end{array}$ \\
\hline Class 2, pitch diameter................. & $\left\{\begin{array}{l}\text { Max... } \\
\text { Tol } \ldots\end{array}\right.$ & -.0536 & $\begin{array}{l}.0658 \\
.0018\end{array}$ & $\begin{array}{l}.0778 \\
.0019\end{array}$ & $\begin{array}{l}.0894 \\
.0020\end{array}$ & $\begin{array}{l}.1007 \\
.0022\end{array}$ & $\begin{array}{l}.1125 \\
.0023\end{array}$ & $\begin{array}{l}.1242 \\
.0024\end{array}$ & .1485 & $\begin{array}{l}.1724 \\
.0027\end{array}$ & $\begin{array}{l}.1959 \\
.0031\end{array}$ & .2299 & $\begin{array}{l}.2887 \\
.0033\end{array}$ \\
\hline Class 3 , pitch diameter... & $\left\{\begin{array}{l}\text { Max... } \\
\text { Tol } \ldots\end{array}\right.$ & $\begin{array}{r}.0532 \\
.0013\end{array}$ & $\begin{array}{l}.0653 \\
.0013\end{array}$ & $\begin{array}{l}.0773 \\
.0014\end{array}$ & $\begin{array}{l}.0889 \\
.0015\end{array}$ & $\begin{array}{l}.1001 \\
.0016\end{array}$ & $\begin{array}{l}.1118 \\
.0016\end{array}$ & .1235 & $\begin{array}{l}.1478 \\
.0018\end{array}$ & $\begin{array}{l}.1716 \\
.0019\end{array}$ & $\begin{array}{l}.1950 \\
.0022\end{array}$ & $\begin{array}{l}.2290 \\
.0022\end{array}$ & $\begin{array}{l}.2878 \\
.0024\end{array}$ \\
\hline Class 4, pitch diameter................ & $\left\{\begin{array}{l}\text { Max... } \\
\text { Tol } \ldots\end{array}\right.$ & -.0525 & $\begin{array}{l}.0647 \\
.0007\end{array}$ & $\begin{array}{l}.0766 \\
.0007\end{array}$ & .0881 & $\begin{array}{l}.0993 \\
.0008\end{array}$ & $\begin{array}{l}.1110 \\
.0008\end{array}$ & $\begin{array}{l}.1227 \\
.0009\end{array}$ & $\begin{array}{l}.1469 \\
.0009\end{array}$ & $\begin{array}{r}.1707 \\
.0010\end{array}$ & $\begin{array}{r}.1939 \\
.0011\end{array}$ & $\begin{array}{r}.2279 \\
.0011\end{array}$ & $\begin{array}{l}.2866 \\
.0012\end{array}$ \\
\hline
\end{tabular}


TABLE 16.-Limiting dimensions and tolerances, classes 1, 2, 3, and 4 fits, American national fine-thread series-Continued

\begin{tabular}{|c|c|c|c|c|c|c|c|c|c|c|c|}
\hline & \multicolumn{11}{|c|}{ Sizes } \\
\hline \multirow[t]{3}{*}{ 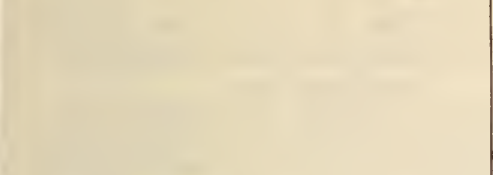 } & $3 / 8$ & 716 & $1 / 2$ & $9 / 16$ & $5 / 8$ & $3 / 4$ & $7 / 8$ & 1 & $11 / 8$ & 114 & $11 / 2$ \\
\hline & \multicolumn{11}{|c|}{ Threads per inch } \\
\hline & 24 & 20 & 20 & 18 & 18 & 16 & 14 & 14 & 12 & 12 & 12 \\
\hline 1 & 14 & 15 & 16 & 17 & 18 & 19 & 20 & 21 & 22 & 23 & 24 \\
\hline 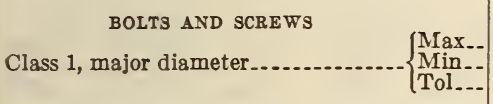 & $\begin{array}{l}\text { Inch } \\
0.3737 \\
.3645 \\
.0092\end{array}$ & \begin{tabular}{l|} 
Inch \\
0.4360 \\
.4258 \\
.0102
\end{tabular} & \begin{tabular}{l|} 
Inch \\
0.4985 \\
.4883 \\
.0102
\end{tabular} & \begin{tabular}{l|} 
Inch \\
0.5609 \\
.5495 \\
.0114
\end{tabular} & \begin{tabular}{l|} 
Inch \\
0.6234 \\
.6120 \\
.0114
\end{tabular} & \begin{tabular}{l|} 
Inch \\
0.7482 \\
.7356 \\
.0126
\end{tabular} & $\begin{array}{l}\text { Inch } \\
0.8729 \\
.8589 \\
.0140\end{array}$ & $\begin{array}{l}\text { Inch } \\
0.9979 \\
.9839 \\
.0140\end{array}$ & \begin{tabular}{c|} 
Inches \\
1.1226 \\
1.1068 \\
.0158
\end{tabular} & \begin{tabular}{c|} 
Inches \\
1.2476 \\
1.2318 \\
.0158
\end{tabular} & $\begin{array}{r}\text { Inches } \\
1.4976 \\
1.4818 \\
.0158\end{array}$ \\
\hline Classes 2, 3, and 4, major diameter...$\left\{\begin{array}{l}\text { Max.- } \\
\text { Min -- } \\
\text { Tol }-.-\end{array}\right.$ & $\begin{array}{l}.3750 \\
.3684 \\
.0066\end{array}$ & $\begin{array}{l}.4375 \\
.4303 \\
.0072\end{array}$ & $\begin{array}{l}.5000 \\
.4928 \\
.0072\end{array}$ & $\begin{array}{l}.5625 \\
.5543 \\
.0082\end{array}$ & $\begin{array}{l}.6250 \\
.6168 \\
.0082\end{array}$ & $\begin{array}{l}.7500 \\
.7410 \\
.0090\end{array}$ & $\begin{array}{l}.8750 \\
.8652 \\
.0098\end{array}$ & $\begin{array}{r}1.0000 \\
.9902 \\
.0098\end{array}$ & $\begin{array}{r}1.1250 \\
1.1138 \\
.0112\end{array}$ & $\begin{array}{r}1.2500 \\
1.2388 \\
.0112\end{array}$ & $\begin{array}{r}1.5000 \\
1.4888 \\
.0112\end{array}$ \\
\hline $\begin{array}{l}\text { Class } 1 \text {, minor diameter } \\
\text { Classes } 2,3 \text {, and } 4 \text {, minor diameter.... } \operatorname{Max}^{1}-\end{array}$ & $\begin{array}{l}.3226 \\
.3239\end{array}$ & $\begin{array}{l}.3747 \\
.3762\end{array}$ & .4372 & .4927 & .5552 & .6715 & .7853 & $\begin{array}{l}.9103 \\
.9124\end{array}$ & $\begin{array}{l}1.0204 \\
1.0228\end{array}$ & $\begin{array}{l}1.1454 \\
1.1478\end{array}$ & $\begin{array}{l}1.3954 \\
1.3978\end{array}$ \\
\hline Class 1 , pitch diameter & $\begin{array}{l}.3466 \\
.3420 \\
.0046\end{array}$ & $\begin{array}{l}.4035 \\
.3984 \\
.0051\end{array}$ & $\begin{array}{l}.4660 \\
.4609 \\
.0051\end{array}$ & $\begin{array}{l}.5248 \\
.5191 \\
.0057\end{array}$ & $\begin{array}{l}.5873 \\
.5816 \\
.0057\end{array}$ & $\begin{array}{l}.7076 \\
.7013 \\
.0063\end{array}$ & $\begin{array}{l}.8265 \\
.8195 \\
.0070\end{array}$ & $\begin{array}{l}.9515 \\
.9445 \\
.0070\end{array}$ & $\begin{array}{r}1.0685 \\
1.0606 \\
.0079\end{array}$ & $\begin{array}{r}1.1935 \\
1.1856 \\
.0079\end{array}$ & $\begin{array}{r}1.4435 \\
1.4356 \\
.0079\end{array}$ \\
\hline Class 2, pitch diameter $\ldots . . . . . . . . . . .-\left\{\begin{array}{l}\text { Max.- } \\
\text { Min }-- \\
\text { Tol }-.-\end{array}\right.$ & $\begin{array}{l}.3479 \\
.3446 \\
.0033\end{array}$ & $\begin{array}{l}.4050 \\
.4014 \\
.0036\end{array}$ & $\begin{array}{l}.4675 \\
.4639 \\
.0036\end{array}$ & $\begin{array}{l}.5264 \\
.5223 \\
.0041\end{array}$ & $\begin{array}{l}.5889 \\
.5848 \\
.0041\end{array}$ & $\begin{array}{l}.7094 \\
.7049 \\
.0045\end{array}$ & $\begin{array}{l}.8286 \\
.8237 \\
.0049\end{array}$ & $\begin{array}{l}.9536 \\
.9487 \\
.0049\end{array}$ & $\begin{array}{r}1.0709 \\
1.0653 \\
.0056\end{array}$ & $\begin{array}{r}1.1959 \\
1.1903 \\
.0056\end{array}$ & $\begin{array}{r}1.4459 \\
1.4403 \\
.0056\end{array}$ \\
\hline Class 3, pitch diameter............... $\left\{\begin{array}{l}\text { Max.- } \\
\text { Min }-. \\
\text { Tol.. }\end{array}\right.$ & $\begin{array}{l}.3479 \\
.3455 \\
.0024\end{array}$ & $\begin{array}{l}.4050 \\
.4024 \\
.0026\end{array}$ & $\begin{array}{l}.4675 \\
.4649 \\
.0026\end{array}$ & $\begin{array}{l}.5264 \\
.5234 \\
.0030\end{array}$ & $\begin{array}{l}.5889 \\
.5859 \\
.0030\end{array}$ & $\begin{array}{l}.7094 \\
.7062 \\
.0032\end{array}$ & $\begin{array}{l}.8286 \\
.8250 \\
.0036\end{array}$ & $\begin{array}{l}.9536 \\
.9500 \\
.0036\end{array}$ & $\begin{array}{r}1.0709 \\
1.0669 \\
.0040\end{array}$ & $\begin{array}{r}1.1959 \\
1.1919 \\
.0040\end{array}$ & $\begin{array}{r}1.4459 \\
1.4419 \\
.0040\end{array}$ \\
\hline Class 4, pitch diameter............... $\left\{\begin{array}{l}\text { Max.- } \\
\text { Min } \\
\text { Tol... } \\
\text { NUTS AND TAPPED HOLES }\end{array}\right.$ & $\begin{array}{l}.3482 \\
.3470 \\
.0012\end{array}$ & $\begin{array}{l}.4053 \\
.4040 \\
.0013\end{array}$ & $\begin{array}{l}.4678 \\
.4665 \\
.0013\end{array}$ & $\begin{array}{l}.5267 \\
.5252 \\
.0015\end{array}$ & $\begin{array}{l}.5892 \\
.5877 \\
.0015\end{array}$ & $\begin{array}{l}.7098 \\
.7082 \\
.0016\end{array}$ & $\begin{array}{l}.8290 \\
.8272 \\
.0018\end{array}$ & $\begin{array}{l}.9540 \\
.9522 \\
.0018\end{array}$ & $\begin{array}{r}1.0714 \\
1.0694 \\
.0020\end{array}$ & $\begin{array}{r}1.1964 \\
1.1944 \\
.0020\end{array}$ & $\begin{array}{r}1.4464 \\
1.4444 \\
.0020\end{array}$ \\
\hline Classes $1,2,3$, and 4, major diameter. Min ${ }^{2}-$ & .3750 & .4375 & .5000 & .5625 & .6250 & .7500 & .8750 & 1.0000 & 1.1250 & 1.2500 & 1.5000 \\
\hline 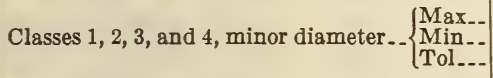 & $\begin{array}{l}.3364 \\
.3299 \\
.0065\end{array}$ & $\begin{array}{l}.3906 \\
.3834 \\
.0072\end{array}$ & $\begin{array}{l}.4531 \\
.4459 \\
.0072\end{array}$ & $\begin{array}{l}.5100 \\
.5024 \\
.0076\end{array}$ & $\begin{array}{l}.5725 \\
.5649 \\
.0076\end{array}$ & $\begin{array}{l}.6903 \\
.6823 \\
.0080\end{array}$ & $\begin{array}{l}.8062 \\
.7977 \\
.0085\end{array}$ & $\begin{array}{l}.9312 \\
.9227 \\
.0085\end{array}$ & $\begin{array}{r}1.0438 \\
1.0348 \\
.0090\end{array}$ & $\begin{array}{r}1.1688 \\
1.1598 \\
.0090\end{array}$ & $\begin{array}{r}1.4188 \\
1.4098 \\
.0090\end{array}$ \\
\hline Classes 1, 2, 3, and 4, pitch diameter.. Min..- & .3479 & .4050 & .4675 & .5264 & .5889 & .7094 & .8286 & .9536 & 1.0709 & 1. 1959 & 1.445 \\
\hline Class 1, pitch diameter................. $\left\{\begin{array}{l}\text { Max } \\
\text { Tol } . . .\end{array}\right.$ & .3525 & .4101 &. .4726 & .5321 & .5946 & .7157 & $\begin{array}{l}.8356 \\
.0070\end{array}$ & .9606 & $\begin{array}{r}1.0788 \\
.0079\end{array}$ & $\begin{array}{r}1.2038 \\
.0079\end{array}$ & $\begin{array}{r}1.4538 \\
.0079\end{array}$ \\
\hline Class 2, pitch diameter $\ldots . . . . . . . . . . .\left\{\begin{array}{l}\text { Max.- } \\
\text { Tol }-. .\end{array}\right.$ & $\begin{array}{l}.3512 \\
.0033\end{array}$ & $\begin{array}{l}.4086 \\
.0036\end{array}$ & .4711 & $\begin{array}{l}.5305 \\
.0041\end{array}$ & $\begin{array}{l}.5930 \\
.0041\end{array}$ & $\begin{array}{l}.7139 \\
.0045\end{array}$ & $\begin{array}{l}.8335 \\
.0049\end{array}$ & $\begin{array}{l}.9585 \\
.0049\end{array}$ & $\begin{array}{r}1.0765 \\
.0056\end{array}$ & $\begin{array}{r}1.2015 \\
.0056\end{array}$ & $\begin{array}{r}1.4515 \\
.0056\end{array}$ \\
\hline Class 3 , pitch diameter... & $\begin{array}{l}.3503 \\
.0024\end{array}$ & $\begin{array}{l}.4076 \\
.0026\end{array}$ & $\begin{array}{l}.4701 \\
.0026\end{array}$ & $\begin{array}{l}.5294 \\
.0030\end{array}$ & $\begin{array}{l}.5919 \\
.0030\end{array}$ & $\begin{array}{l}.7126 \\
.0032\end{array}$ & $\begin{array}{l}.8322 \\
.0036\end{array}$ & $\begin{array}{l}.9572 \\
.0036\end{array}$ & $\begin{array}{r}1.0749 \\
.0040\end{array}$ & $\begin{array}{r}1.1999 \\
.0040\end{array}$ & $\begin{array}{r}1.4499 \\
.0040\end{array}$ \\
\hline Class 4 , pitch diameter.... & $\begin{array}{l}.3491 \\
.0012\end{array}$ & $\begin{array}{l}.4063 \\
.0013\end{array}$ & $\begin{array}{r}.4688 \\
.0013\end{array}$ & $\begin{array}{l}.5279 \\
.0015\end{array}$ & $\begin{array}{l}.5904 \\
.0015\end{array}$ & $\begin{array}{l}.7110 \\
.0016\end{array}$ & $\begin{array}{l}.8304 \\
.0018\end{array}$ & $\begin{array}{l}.9554 \\
.0018\end{array}$ & $\begin{array}{r}1.0729 \\
.0020\end{array}$ & $\begin{array}{r}1.1979 \\
.0020\end{array}$ & $\begin{array}{r}1.4479 \\
.0020\end{array}$ \\
\hline
\end{tabular}


TABLE 16.-Limiting dimensions and tolerances, classes 1, 2, 3, and 4 fits, American national fine-thread series-Continued

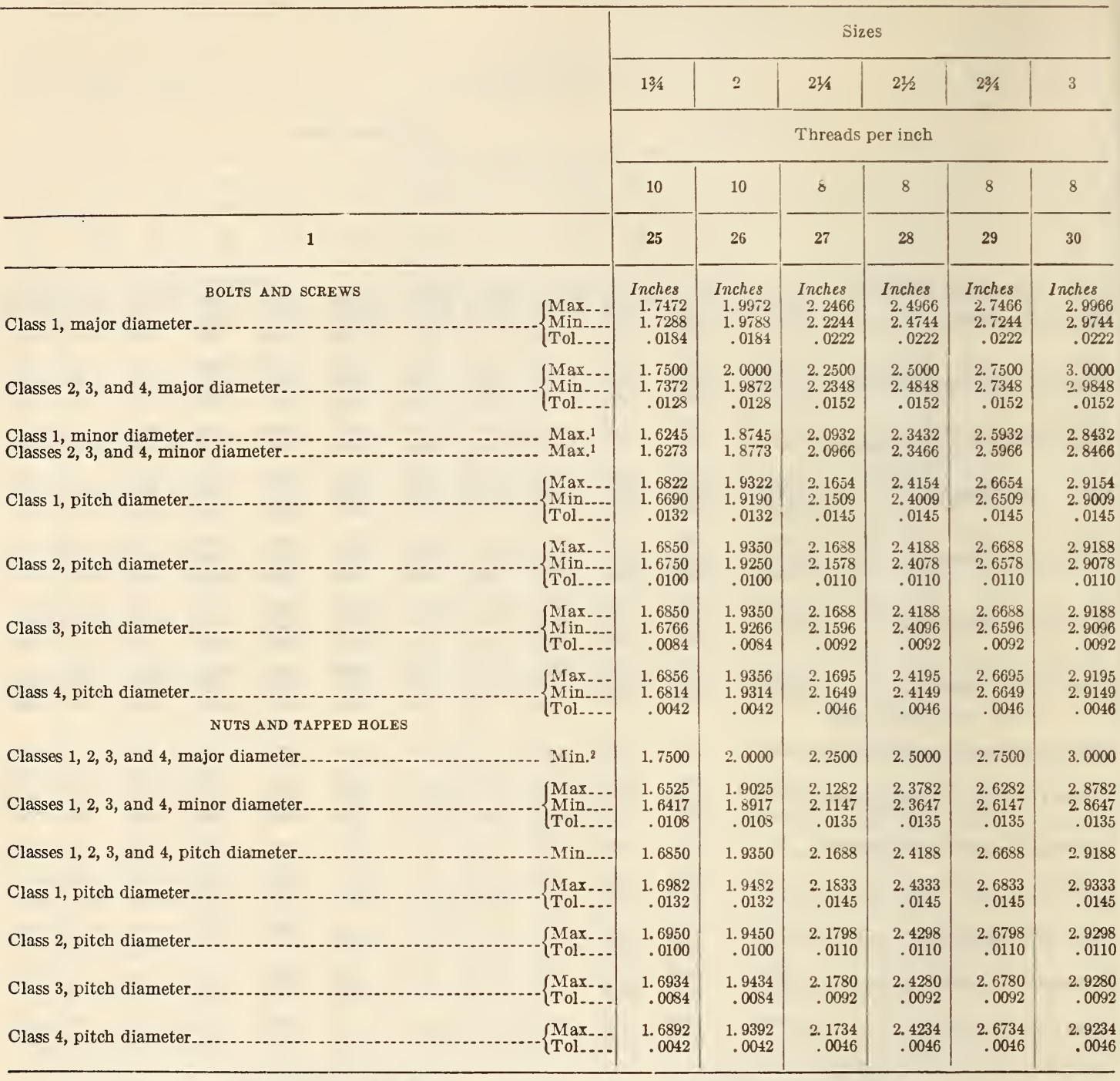

1 Dimensions given for the maximum minor diameter of the screw are figured to the intersection of the worn tool arc with a center line through crest and root. The minimum minor diameter of the screw shall be that corresponding to a flat at the minor diameter of the minimum screw equal to $1 / 8 \times p$, and may be determined by subtracting the basic thread depth, $h$ (or $0.6495 p)$, from the minimum pitch diameter mum screw equal to $18 \times p$, and may be determined by subtracting the basic thread depth, $h$ (or $0.6495 p$ ), from the minimum pitch diameter the screw.

2 Dimensions for the minimum major diameter of the nut correspond to the basic flat $(38 \times p)$, and the profile at the major diameter produced by a worn tool must not fall below the basic outline. The maximum major diameter of the nut shall be that corresponding to flat at the major diameter of the maximum nut equal to $1 / 24 \times p$, and may be determined by adding $126 \times h$ (or $0.7939 p$ ) to the maximum pitch diameter of the nut. 
UNITED STATES GOVERNMENT, NATIONAL SCREW THREAD COMMISSION, BUREAU OF STANDARDS MISCELLANEOUS PUBLICATION NO. 89, JUNE 22, 1928

\section{SCREW THREADS OF SPECIAL DIAMETERS, PITCHES, AND IENGTHS OF ENGAGEMENT}

The tolerances specified above in relation to standard threads apply in general to bolts, nuts, and tapped holes of standard pitches and diameters. They are based on the pitch of the thread and a length of engagement equal to the basic major diameter, but are used for lengths of engagement up to one and one-half diameters.

In addition to the foregoing threaded components, there are large quantities of threaded parts produced, such as hub and radiator caps in the automotive industry, threaded collars on machine tools, etc., where the diameters are larger, the pitches finer, and the lengths of engagement shorter than for bolt and nut practice.

\section{FORM OF THREAD}

The American national form of thread profile as specified above shall be used.

\section{THREAD SERIES}

It is recommended that one of the following pitches be selected whenever it is necessary to use a screw thread of special diameter and pitch: $4,6,8$, $10,12,14,16,18,20,24,28,32,36,40,48,56,64$ threads per inch.

Basic thread data for these pitches are given in Table 26. The use of 12 threads per inch, wherever possible, is recommended for all applications requiring special threads. This pitch is very convenient for a variety of miscellaneous work in machine construction, as for thin nuts on shafts and sleeves, on threaded studs, etc. It is the coarsest pitch, for a thread of basic depth, which will permit a threaded collar which screws onto a threaded shoulder to slip over a shaft, the difference in diameter between shoulder and shaft being one-eighth inch.

Below are given the limiting dimensions for 12 pitch threads of the following sizes: $1 / 2,9 / 16,5 / 8,11 / 16$, $3 / 4,13 / 16,7 / 8,15 / 16,1,11 / 16,11 / 8,13 / 16,11^{\prime}, 15 \% 16,13 / 8,11 / 2,13 \frac{1}{4}$, $2,2 \frac{1}{4}, 2 \frac{1}{2}, 2 \frac{3}{4}$, and 3 inches. Sizes up to and including $13 / 4$ inches are widely used in railroad practice, and die-head chasers for these sizes are stocked by manufacturers.

\section{CLASSIFICATION AND TOLERANCES}

There are established herein for general use four classes of screw-thread fits, which are named and numbered to correspond to the regular classification of fits given above. These four classes, together with the accompanying specifications, are intended to insure a uniform practice for screw threads not included in the American national coarse or fine thread series.
TABLE 26.-Thread data for recommended pitches for special threads

\begin{tabular}{|c|c|c|c|c|}
\hline $\begin{array}{c}\text { Threads per } \\
\text { inch } \\
n\end{array}$ & Pitch & $\begin{array}{c}\text { Depth of } \\
\text { thread } \\
\quad h\end{array}$ & $\begin{array}{c}\text { Basic width } \\
\text { of flat } \\
p / 8\end{array}$ & $\begin{array}{c}\text { Minimum } \\
\text { width of } \\
\text { Ilat at major } \\
\text { diameter } \\
\text { of nut } \\
p / 24\end{array}$ \\
\hline 1 & 2 & 3 & 4 & 5 \\
\hline $\begin{array}{l}64 \ldots \\
56 \\
48 \\
40 \\
36\end{array}$ & $\begin{array}{c}\text { Inch } \\
0.01562 \\
.01786 \\
.02083 \\
.02500 \\
.02778\end{array}$ & $\begin{array}{l}\text { Inch } \\
0.01015 \\
.01160 \\
.01353 \\
.01624 \\
.01804\end{array}$ & $\begin{array}{l}\text { Inch } \\
0.00195 \\
.00223 \\
.00260 \\
.00312 \\
.00347\end{array}$ & $\begin{array}{l}\text { Inch } \\
0.00065 \\
.00074 \\
.00087 \\
.00104 \\
.00116\end{array}$ \\
\hline $\begin{array}{l}32 \\
28 \\
24 \\
20\end{array}$ & $\begin{array}{l}.03125 \\
.03571 \\
.04167 \\
.05000\end{array}$ & $\begin{array}{l}.02030 \\
.02320 \\
.02706 \\
.03248\end{array}$ & $\begin{array}{l}.00391 \\
.00446 \\
.00521 \\
.00625\end{array}$ & $\begin{array}{l}.00130 \\
.00149 \\
.00174 \\
.00208\end{array}$ \\
\hline $\begin{array}{l}18 \\
16 \\
14 \\
12\end{array}$ & $\begin{array}{l}.05556 \\
.06250 \\
.07143 \\
.08333\end{array}$ & $\begin{array}{l}.03608 \\
.04059 \\
.04639 \\
.05413\end{array}$ & $\begin{array}{l}.00694 \\
.00781 \\
.00893 \\
.01042\end{array}$ & $\begin{array}{l}.00231 \\
.00260 \\
.00298 \\
.00347\end{array}$ \\
\hline $\begin{array}{l}10 \\
8 \\
6 \\
4\end{array}$ & $\begin{array}{l}.10000 \\
.12500 \\
.16667 \\
.25000\end{array}$ & $\begin{array}{l}.06495 \\
.08119 \\
.10825 \\
.16238\end{array}$ & $\begin{array}{l}.01250 \\
.01562 \\
.02083 \\
.03125\end{array}$ & $\begin{array}{l}.00417 \\
.00521 \\
.00694 \\
.01042\end{array}$ \\
\hline
\end{tabular}

It is not the intention of the commission arbitrarily to place a general class or grade of work in a specific class of fit. Each manufacturer and user of screw threads is free to select the class of fit best adapted to his particular needs.

(General specifications applying to all classes of fit specified for screw threads of special diameters, pitches, and length of engagement are included in this publication. These specifications cover tolerances, diameters, classification of fits, and rules for the use of the tables. The several classifications, with accompanying tables are as follows:)

Class 1, loose fit.-This class is intended to cover the manufacture of threaded parts where quick and easy assembly is necessary and a considerable amount of shake or play is not objectionable.

This class is made with an allowance on the screw, so as to permit ready assembly, even when the threads are slightly bruised or dirty.

Class 2, free fit.-This class is intended to cover the manufacture of threaded parts which are to assemble nearly or entirely with the fingers, and where a slight amount of shake or play between the assembled threaded members is not objectionable. It is the same in every particular as class 1 except that it has no allowance and the tolerances are smaller. Class 3, medium fit.-This class is intended to cover the manufacture of the higher grade of threaded parts which are to assemble nearly or entirely with the fingers, and must have the minimum amount of shake or play between the threaded members. It is the same as class 2 in every particular except that the tolerances are smaller.

Class 4 , close fit. - This class is intended to cover the manufacture of threaded parts of the finest commercial quality, where very little shake or play is desirable, and where a screw driver or wrench may be necessary for assembly. 
TABLE 27.-Thread dimensions of special screvo threads, classes 1, 2, 3, and if fits

\begin{tabular}{|c|c|c|c|c|c|c|c|c|c|c|c|c|c|}
\hline \multirow{4}{*}{$\begin{array}{l}\text { Threads per } \\
\text { inch }\end{array}$} & \multicolumn{9}{|c|}{ SCREW SIZES } & \multicolumn{4}{|c|}{ 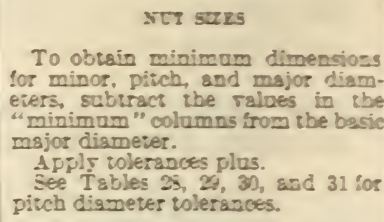 } \\
\hline & \multicolumn{4}{|c|}{ Major diameter } & \multirow{2}{*}{\multicolumn{3}{|c|}{ Pitch dismeter, marimum }} & \multirow{2}{*}{\multicolumn{2}{|c|}{$\begin{array}{l}\text { Minor dismeter, } \\
\text { marimum } 1\end{array}$}} & \multicolumn{2}{|c|}{ Minor diameter } & \multirow{2}{*}{$\begin{array}{l}\text { Pitch } \\
\text { dism- } \\
\text { eter, } \\
\text { min:- } \\
\text { mum }\end{array}$} & \multirow{2}{*}{$\begin{array}{l}\text { Mrior } \\
\text { dism- } \\
\text { eter, } \\
\text { mini- } \\
\text { mum2 }\end{array}$} \\
\hline & \multicolumn{2}{|c|}{ Meximum } & \multicolumn{2}{|c|}{ Tolerance } & & & & & & $\begin{array}{l}\text { Mini- } \\
\text { mum }\end{array}$ & $\begin{array}{l}\text { Toler- } \\
\text { ance }\end{array}$ & & \\
\hline & Class 1 & $\begin{array}{c}\text { Classes } \\
2,3 \text {, and } \\
4\end{array}$ & Class 1 & $\begin{array}{c}\text { Classes } \\
2,3 \text {, and } \\
4\end{array}$ & Class 1 & $\begin{array}{l}\text { Classes } \\
2 \text { end } 3\end{array}$ & Class 4 & Cless 1 & $\begin{array}{l}\text { Classes } \\
2,3.200 d \\
4\end{array}$ & \multicolumn{4}{|c|}{ Classes $1,2,3,80 d 4$} \\
\hline $\begin{array}{l}64 \ldots \ldots \\
56 \ldots \ldots \\
48 \ldots \ldots \\
40 \ldots \ldots \\
36 \ldots \ldots\end{array}$ & $\begin{array}{l}\text { Inch } \\
0.0007 \\
.0008 \\
.0009 \\
.0010 \\
.0011\end{array}$ & $\begin{array}{l}\text { Inch } \\
0.0000 \\
.0000 \\
.0000 \\
.0000 \\
.0000\end{array}$ & $\begin{array}{l}\text { Inch } \\
0.0052 \\
.0056 \\
.0052 \\
.0068 \\
.0072\end{array}$ & $\begin{array}{l}\text { Inch } \\
0.0535 \\
.0040 \\
.0044 \\
.0045 \\
.0050\end{array}$ & $\begin{array}{l}\text { Irch } \\
0.0105 \\
.0124 \\
.0114 \\
.0172 \\
.0191\end{array}$ & $\begin{array}{l}\text { Inch } \\
0.0101 \\
.0116 \\
.0135 \\
.0162 \\
.0130\end{array}$ & $\begin{array}{l}\text { Inch } \\
0.0100 \\
.0114 \\
.0133 \\
.0160 \\
.0178\end{array}$ & $\begin{array}{l}\text { Inch } \\
0.0199 \\
.0227 \\
.0255 \\
.0317 \\
.0352\end{array}$ & $\begin{array}{l}\text { Inch } \\
0.0192 \\
.0219 \\
.0259 \\
.0315 \\
.0341\end{array}$ & $\begin{array}{l}\text { Inch } \\
0.0169 \\
.0153 \\
.0225 \\
.0271 \\
.0351\end{array}$ & $\begin{array}{l}\text { Inck } \\
0.011 \\
.0019 \\
.0023 \\
.0028 \\
.0050\end{array}$ & $\begin{array}{l}\text { Irich } \\
0.0101 \\
.0115 \\
.0135 \\
.0162 \\
.0180\end{array}$ & $\begin{array}{l}\text { Inck } \\
0.0000 \\
.0000 \\
.0000 \\
.0000 \\
.0000\end{array}$ \\
\hline $\begin{array}{l}32 \ldots \ldots \ldots \\
28 \ldots \ldots \\
24 \ldots \ldots \\
20 \ldots \ldots\end{array}$ & $\begin{array}{l}.0011 \\
.0012 \\
.0013 \\
.0015\end{array}$ & $\begin{array}{l}.0000 \\
.0000 \\
.0090 \\
.0000\end{array}$ & $\begin{array}{l}.0076 \\
.0056 \\
.0982 \\
.0102\end{array}$ & $\begin{array}{l}.0054 \\
.0052 \\
.0596 \\
.0072\end{array}$ & $\begin{array}{l}.0214 \\
.0244 \\
.0234 \\
.0340\end{array}$ & $\begin{array}{l}.0303 \\
.0232 \\
.0271 \\
.0325\end{array}$ & $\begin{array}{l}.0201 \\
.0230 \\
.0263 \\
.0322\end{array}$ & $\begin{array}{l}.0324 \\
.0450 \\
.0524 \\
.0525\end{array}$ & $\begin{array}{l}.0353 \\
.0435 \\
.0511 \\
.0513\end{array}$ & $\begin{array}{l}.0335 \\
.0357 \\
.0451 \\
.0541\end{array}$ & $\begin{array}{l}.0034 \\
.0039 \\
.0045 \\
.0054\end{array}$ & $\begin{array}{l}.0233 \\
.0232 \\
.0271 \\
.0325\end{array}$ & $\begin{array}{l}\text { ogin } \\
.0000 \\
.0900 \\
.0000\end{array}$ \\
\hline $\begin{array}{l}18 \ldots \ldots \\
16 \ldots \ldots \\
12 \ldots \ldots\end{array}$ & $\begin{array}{l}.0016 \\
.0018 \\
.0021 \\
.0024\end{array}$ & $\begin{array}{l}.0000 \\
.0000 \\
.0000 \\
.0000\end{array}$ & $\begin{array}{l}.0114 \\
.0126 \\
.0140 \\
.0158\end{array}$ & $\begin{array}{l}.0052 \\
.0090 \\
.00959 \\
.0112\end{array}$ & $\begin{array}{l}.0377 \\
.0424 \\
.0455 \\
.0565\end{array}$ & $\begin{array}{l}.0361 \\
.0495 \\
.0454 \\
.0541\end{array}$ & $\begin{array}{l}.0335 \\
.0402 \\
.0460 \\
.0536\end{array}$ & $\begin{array}{l}.0095 \\
.0755 \\
.0395 \\
.1046\end{array}$ & $\begin{array}{l}.0592 \\
.0062 \\
.09-6 \\
.1022\end{array}$ & $\begin{array}{l}.0001 \\
.05 \pi \\
0 \div-3 \\
.0002\end{array}$ & $\begin{array}{l}.0000 \\
.0095 \\
0000 \\
.0900\end{array}$ & $\begin{array}{l}.0351 \\
.0455 \\
.0454 \\
.0541\end{array}$ & 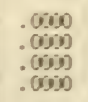 \\
\hline $\begin{array}{l}10 \ldots \ldots \\
8 \ldots \ldots \\
6 \ldots \ldots \\
4 \ldots\end{array}$ & $\begin{array}{l}.0028 \\
.0034 \\
.0044 \\
.0054\end{array}$ & $\begin{array}{l}.0000 \\
.0000 \\
.0000 \\
.00000\end{array}$ & $\begin{array}{l}.0184 \\
.0222 \\
.0290 \\
.0405\end{array}$ & $\begin{array}{l}.0125 \\
.0152 \\
.0302 \\
.0250\end{array}$ & $\begin{array}{l}.0575 \\
.0546 \\
.1127 \\
.1655\end{array}$ & $\begin{array}{l}.0650 \\
.0812 \\
.1053 \\
.1624\end{array}$ & $\begin{array}{l}.0644 \\
.0505 \\
.1074 \\
.1611\end{array}$ & $\begin{array}{l}.1255 \\
.1555 \\
.2559 \\
.3131\end{array}$ & $\begin{array}{l}129 \\
.1534 \\
.3045 \\
.365 \%\end{array}$ & $\begin{array}{l}.1053 \\
.13=3 \\
.1304 \\
.27(6)\end{array}$ & $\begin{array}{l}.0109 \\
.0135 \\
.0100 \\
.0270\end{array}$ & $\begin{array}{l}.0550 \\
.0512 \\
.10 \$ 3 \\
.1624\end{array}$ & $\begin{array}{l}.0000 \\
.0000 \\
.0000 \\
.0000\end{array}$ \\
\hline
\end{tabular}

1 Dimensions giren for the marimum minor dismeter of the screm are figued to the intersection of the worn tool grc with 8 cezier line through crest and root. The minimum minor diameter of the screw shall be that corresponding to a flst at the minor dismeter of the minimum screw equal to $38 \times p$, and may be determined by subtracting the basic thresd depth, $h$ (or $0.6495 p$ ) trom the minimum pitch dismeter of the screw.

2 Dimensions for the minimum major diameter of the nut correspond to the bssic flat (3/3 $p$ ), and the prosle st the major dismeter pro duced by a worn tool must not fall below the basic outline. The maximum msjor dismeter of the Iut shsill be thst correspondinz to 8 est

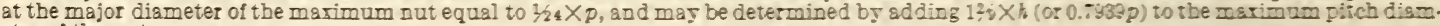
eter of the nut. 


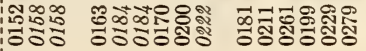
$\simeq \stackrel{\mathscr{\varrho}}{\rightleftarrows}$

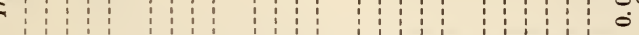

$$
\text { (2) }
$$

클

$\Xi$

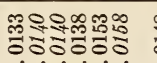
으.

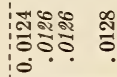

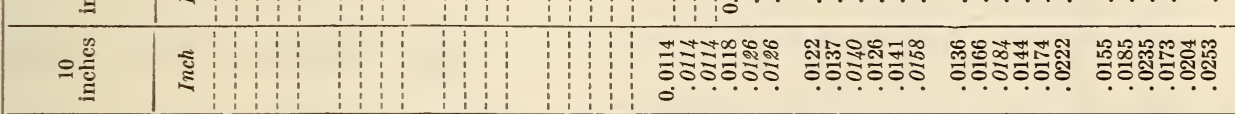

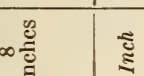

응ำสำสำ 0

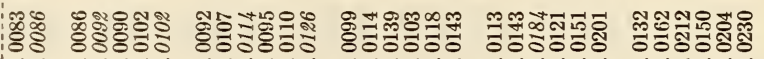

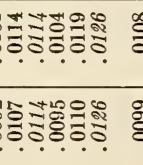

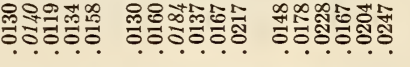

今

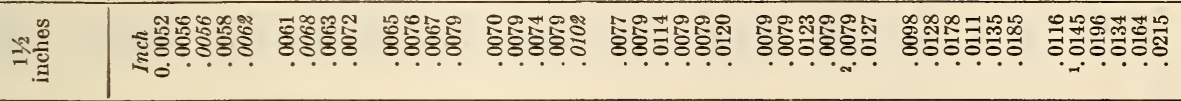

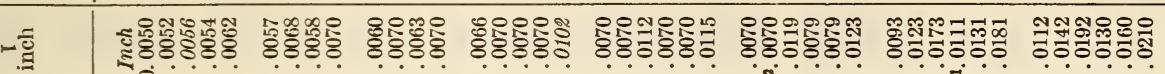

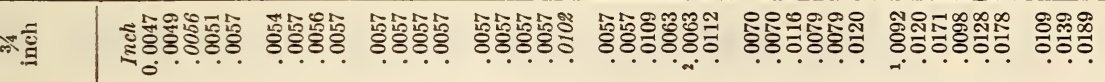

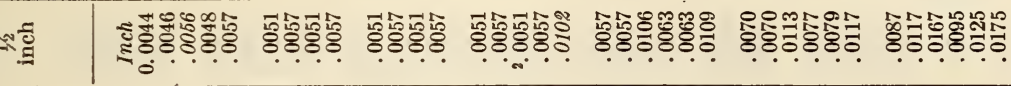

次音

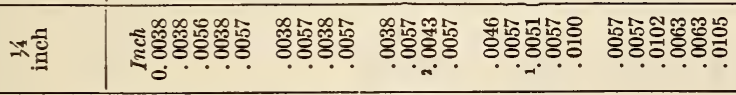

음

늡

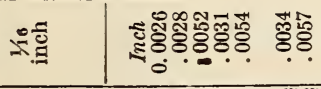

政

就结

प艹

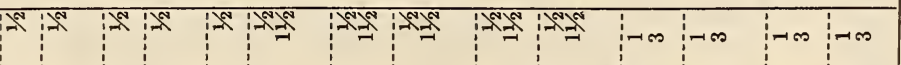
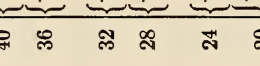


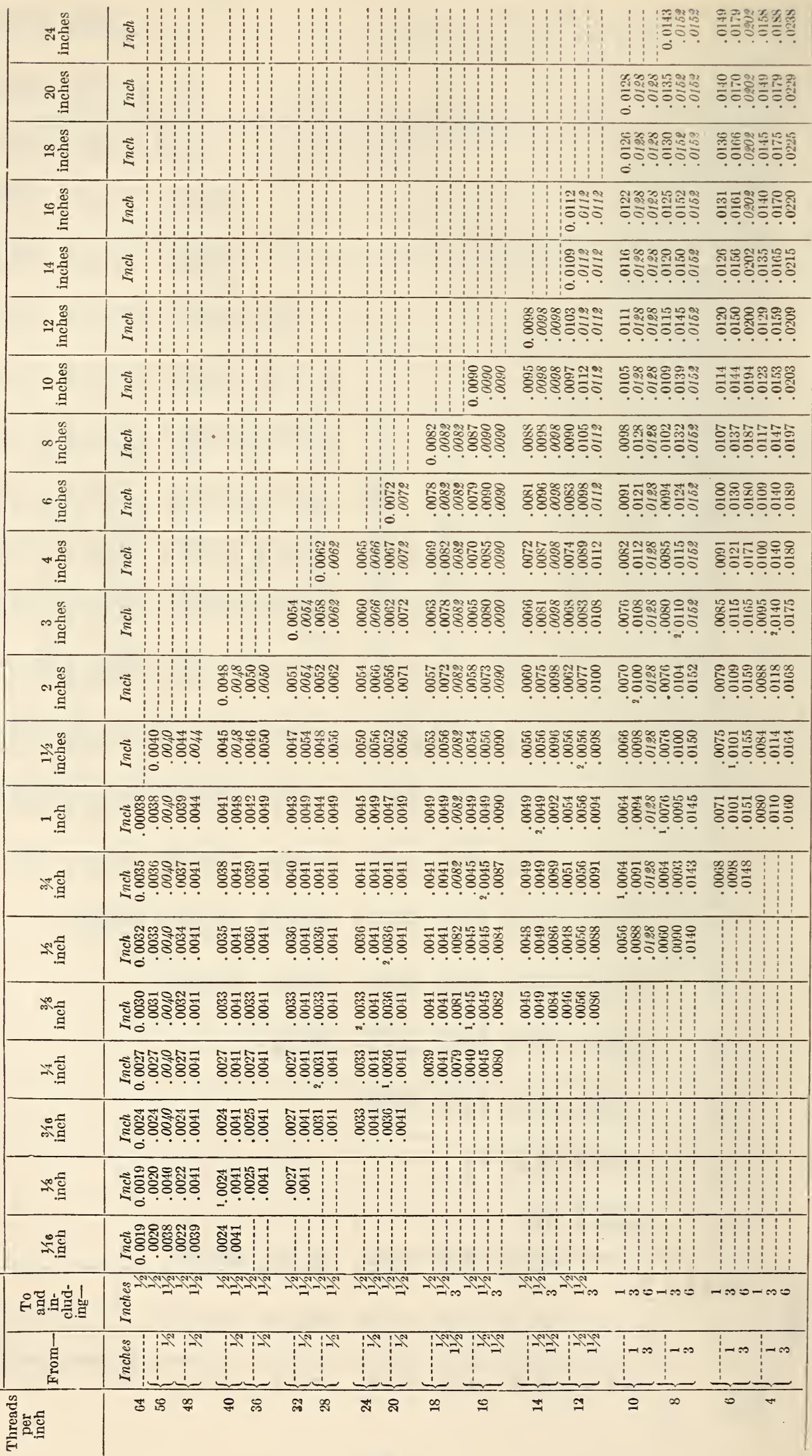




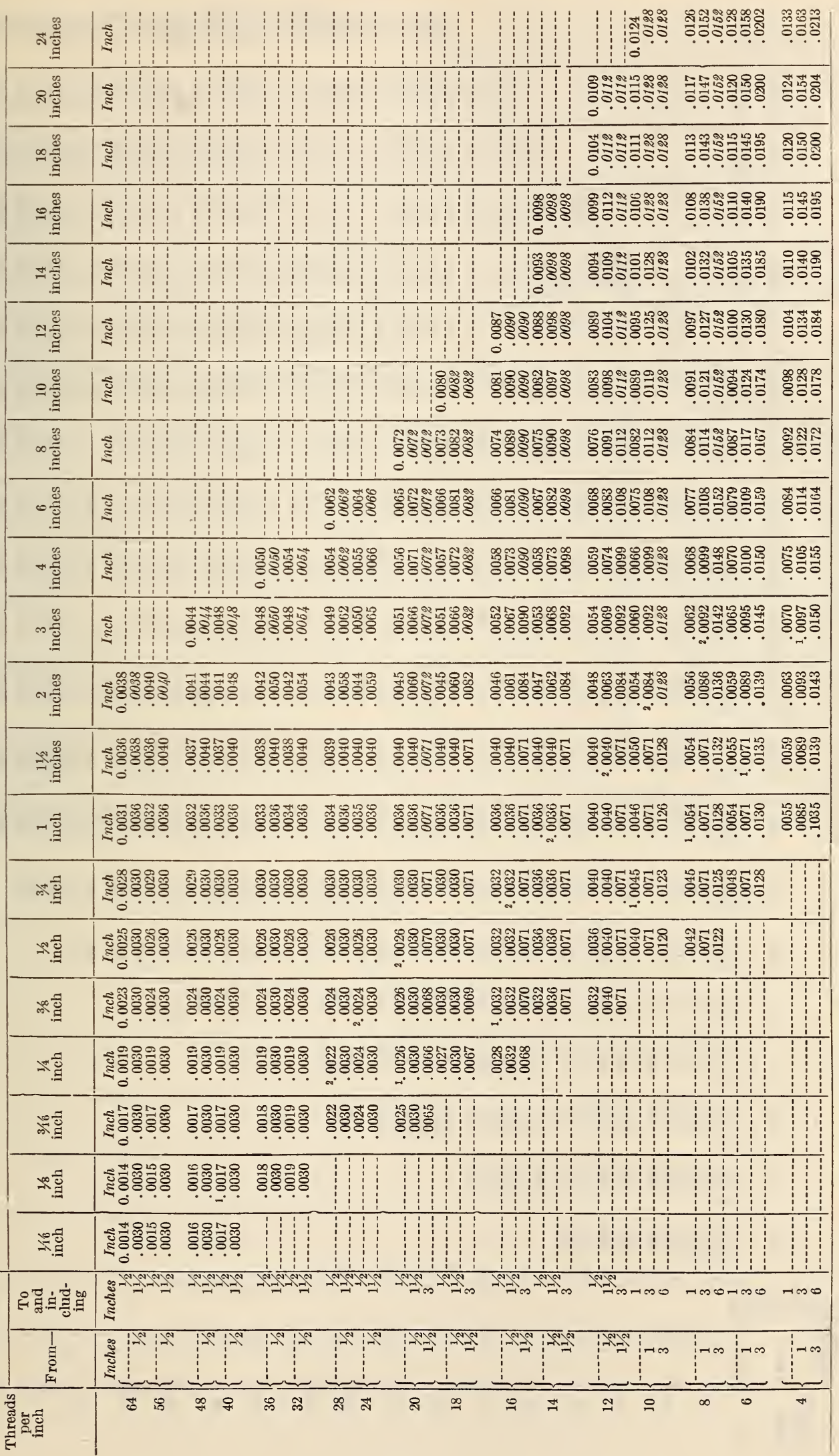




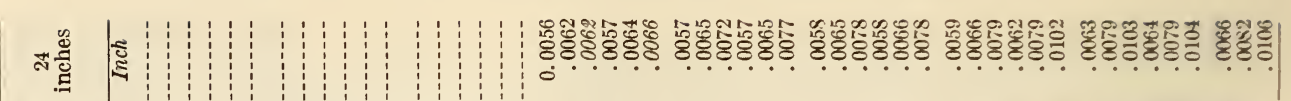

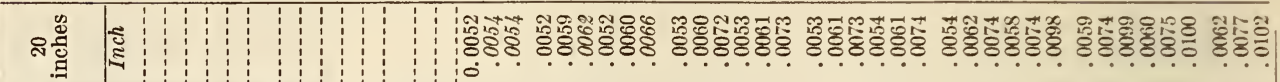
융 然 14000 7.대 ت

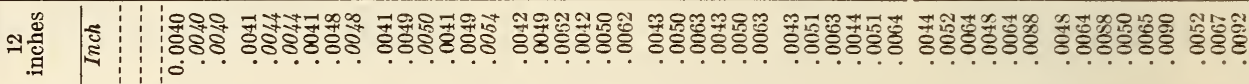

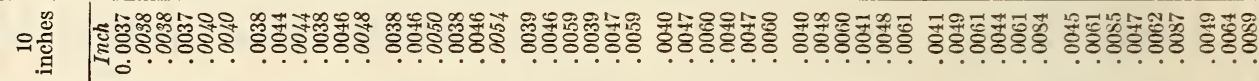

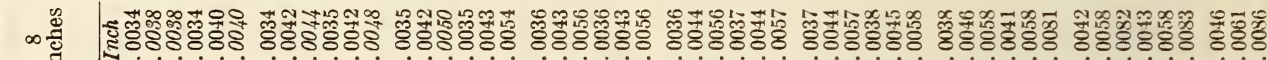
To.

○范

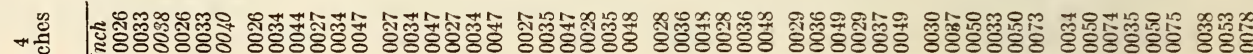

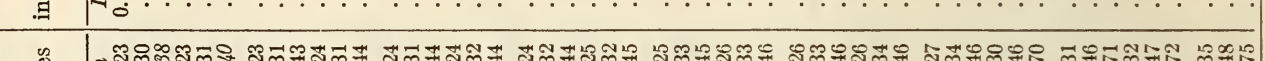

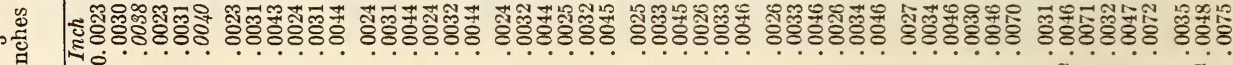

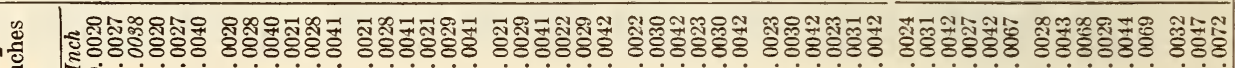

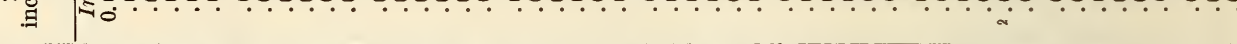

承 $\Rightarrow 1$

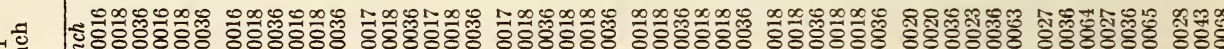
-

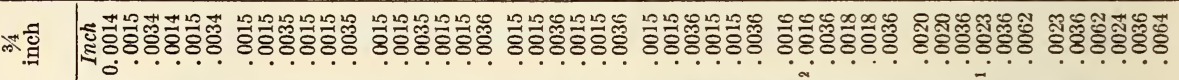

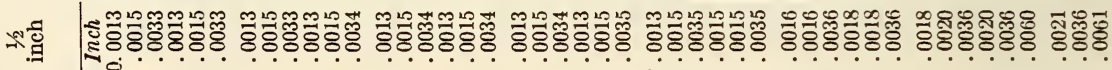

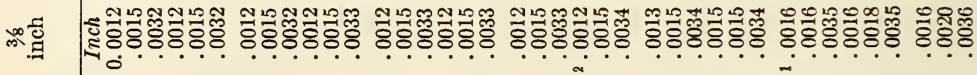

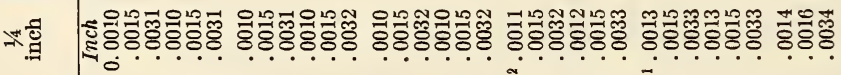

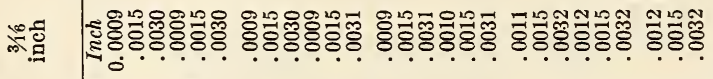

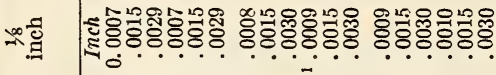

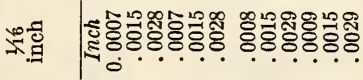

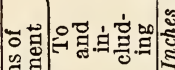

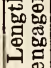

1

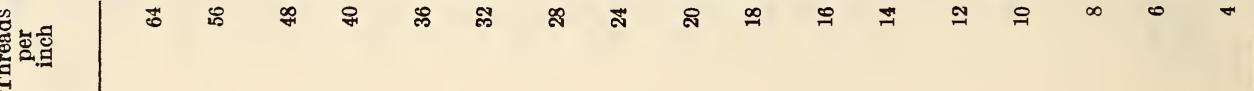




\section{SCREW THREADS OF SPECIAL DIAMETERS, 12 THREADS PER INCH (INCLUDING RAILWAY SIZES)}

Where special threads are required, it is sometimes essential to select a certain pitch as standard for a range of sizes. Also, in general practice, where the pitch of a special thread is optional, the uniform use of a selected pitch is advantageous. For such applications the 12 pitch is widely used, particularly for two distinct purposes as given below, but for different reasons.

Sizes of 12-pitch threads from one-half inch to and including $1 \frac{3}{4}$ inches are used in railroad practice, which require that worn stud holes be retapped with a tap of the next larger size, the increment being one-sixteenth inch throughout most of the range. ${ }^{82}$

The 12-pitch threads are also widely used in machine construction, as for thin nuts on shafts and sleeves. From the standpoints of good design and simplification of practice, it is desirable to maintain shoulder diameters to $1 / 8$-inch steps. The 12 pitch is the coarsest, for a thread of basic depth, which will permit a threaded collar which screws onto a threaded shoulder to slip over a shaft, the difference in diameter between shoulder and shaft being one-eighth inch.

There are, therefore, presented herein, supplementary to screw threads of special diameters, pitches, and lengths of engagement, given above, specifications and data covering a range of sizes of screw threads from $1 / 2$ to 3 inches, inclusive, having 12 threads per inch, and based on a length of engagement of 1 inch.

\section{FORM OF THREAD}

The American national form of thread profile as specified above shall be used.

\section{THREAD SERIES}

The nominal sizes and basic dimensions of the American national 12-pitch thread series are specified in Table 87.

82 See U. S. Department of Commerce Simplified Practice Recommendation No. 51, "Die Head Chasers."

\section{CLASSIFICATION AND TOLERANCES}

The general specifications and classification of fits referred to above are applicable to the American national 12-pitch thread series. The dimensions and tolerances for four classes of fit, based on a length of engagement of $1 \mathrm{inch}$, and derived from Tables 27 to 31 , given above, are given in Table 88 .

TABLE 87.-American national 12-pitch thread series

\begin{tabular}{|c|c|c|c|c|c|}
\hline \multicolumn{2}{|c|}{ Identification } & \multicolumn{3}{|c|}{ Basic diameters } & \multirow{2}{*}{$\begin{array}{l}\text { Metric } \\
\text { equiva- } \\
\text { lent of } \\
\text { major } \\
\text { diameter }\end{array}$} \\
\hline Sizes & $\begin{array}{l}\text { Threads } \\
\text { per inch }\end{array}$ & $\begin{array}{c}\text { Major } \\
\text { diameter } \\
D\end{array}$ & $\begin{array}{c}\text { Pitch } \\
\text { diameter } \\
E\end{array}$ & $\begin{array}{c}\text { Minor } \\
\text { diameter } \\
K\end{array}$ & \\
\hline 1 & 2 & 3 & 4 & 5 & 6 \\
\hline $\begin{array}{l}1 / 2 \\
9 / 10^{1} \\
5 / 8 \\
11 / 16 \\
3 / 4\end{array}$ & $\begin{array}{l}12 \\
12 \\
12 \\
12 \\
12\end{array}$ & $\begin{array}{l}\text { Inches } \\
0.5000 \\
.5625 \\
.6250 \\
.6875 \\
.7500\end{array}$ & $\begin{array}{l}\text { Inches } \\
0.4459 \\
.5084 \\
.5709 \\
.6334 \\
.6959\end{array}$ & $\begin{array}{l}\text { Inches } \\
0.3917 \\
.4542 \\
.5167 \\
.5792 \\
.6417\end{array}$ & $\begin{array}{c}m m \\
12.700 \\
14.288 \\
15.875 \\
17.463 \\
19.050\end{array}$ \\
\hline $\begin{array}{l}13 / 16 \\
7 / 8 \\
15 / 16 \\
11116\end{array}$ & $\begin{array}{l}12 \\
12 \\
12 \\
12 \\
12\end{array}$ & $\begin{array}{r}.8125 \\
.8750 \\
.9375 \\
1.0000 \\
1.0625\end{array}$ & $\begin{array}{r}.7584 \\
.8209 \\
.8834 \\
.9459 \\
1.0084\end{array}$ & $\begin{array}{l}.7042 \\
.7667 \\
.8292 \\
.8917 \\
.9542\end{array}$ & $\begin{array}{l}20.638 \\
22.225 \\
23.813 \\
25.400 \\
26.988\end{array}$ \\
\hline $\begin{array}{l}11 / 8^{2} \\
13310^{2} \\
1114^{2} \\
15 / 16\end{array}$ & $\begin{array}{l}12 \\
12 \\
12 \\
12\end{array}$ & $\begin{array}{l}1.1250 \\
1.1875 \\
1.2500 \\
1.3125\end{array}$ & $\begin{array}{l}1.0709 \\
1.1334 \\
1.1959 \\
1.2584\end{array}$ & $\begin{array}{l}1.0167 \\
1.0792 \\
1.1417 \\
1.2042\end{array}$ & $\begin{array}{l}28.575 \\
30.163 \\
31.750 \\
33.338\end{array}$ \\
\hline $\begin{array}{l}13,8 \\
112^{2} \\
13 / 4 \\
2\end{array}$ & $\begin{array}{l}12 \\
12 \\
12 \\
12\end{array}$ & $\begin{array}{l}1.3750 \\
1.5000 \\
1.7500 \\
2.0000\end{array}$ & $\begin{array}{l}1.3209 \\
1.4459 \\
1.6959 \\
1.9459\end{array}$ & $\begin{array}{l}1.2667 \\
1.3917 \\
1.6417 \\
1.8917\end{array}$ & $\begin{array}{l}34.925 \\
38.100 \\
44.450 \\
50.800\end{array}$ \\
\hline $\begin{array}{l}214 \ldots \\
21 / 2 \ldots-. \\
23 / 4 \ldots \\
3 .-. . \\
\end{array}$ & $\begin{array}{l}12 \\
12 \\
12 \\
12\end{array}$ & $\begin{array}{l}2.2500 \\
2.5000 \\
2.7500 \\
3.0000\end{array}$ & $\begin{array}{l}2.1959 \\
2.4459 \\
2.6959 \\
2.9459\end{array}$ & $\begin{array}{l}2.1417 \\
2.3917 \\
2.6417 \\
2.8917\end{array}$ & $\begin{array}{l}57.150 \\
63.500 \\
69.850 \\
76.200\end{array}$ \\
\hline
\end{tabular}

1 Standard size of the American national coarse-thread series. ${ }^{2}$ Standard size of the American national fine-thread series. 


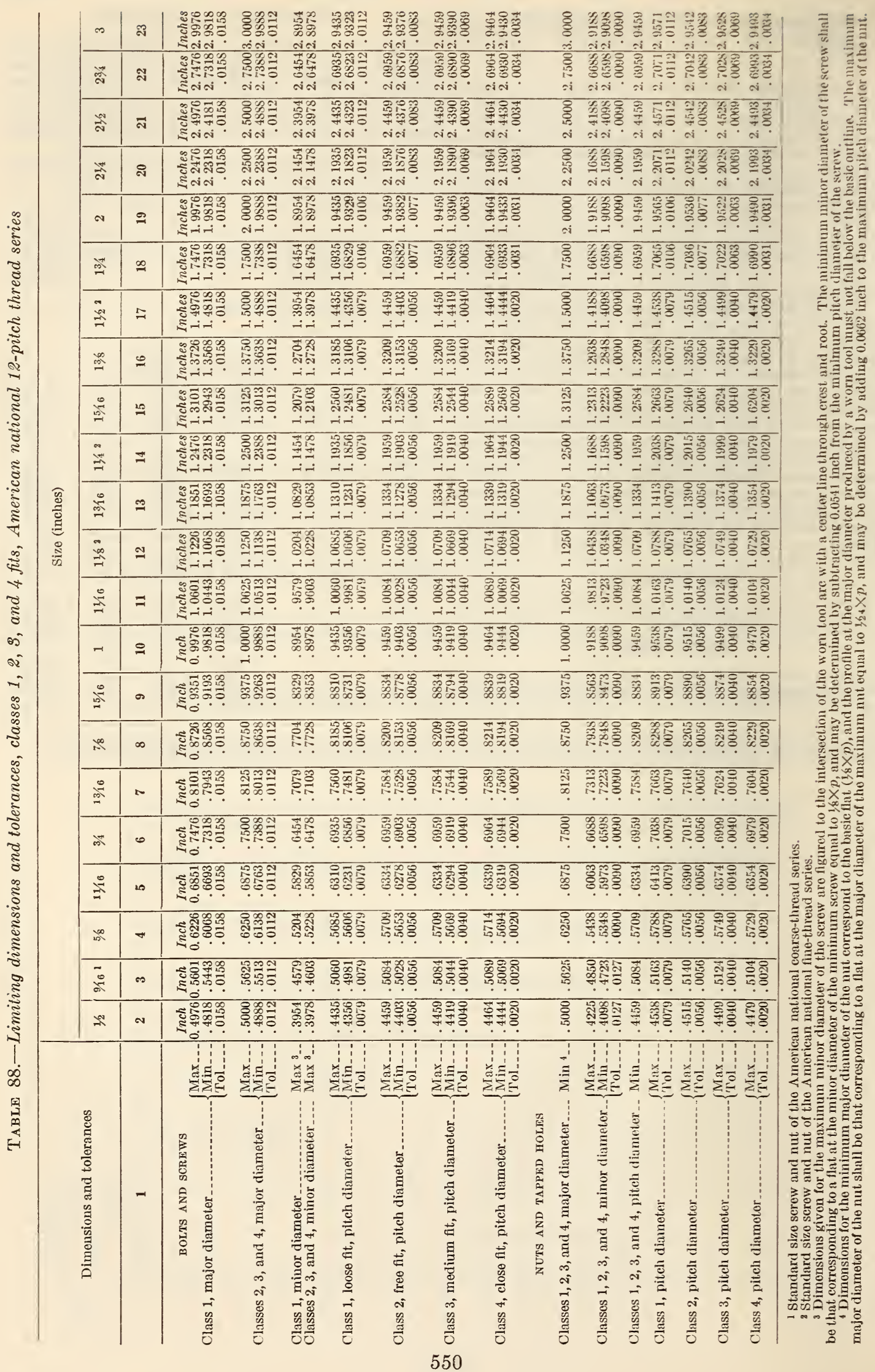


UNITED STATES GOVERNMENT, NATIONAL SCREW THREAD COMMISSION, AMERICAN NATIONAL SCREW, BOLT, AND NUT PROPORTIONS. BUREAU OF STANDARDS MISCELLANEOUS PUBIICATION NO. 89, JUNE 22, 1928

(See also 608.2 , p. $583 ; 608.31$, p. $604 ; 608.32$, p. $609 ; 615.42$, p. 883 .)

\section{WRENCH HEAD BOLTS AND NUTS, AND WRENCH OPENINGS}

\section{INTRODUCTORY NOTES}

These sizes of boltheads and nuts are intended to supersede all existing standards which have grown up for commercial standard boltheads and nuts. Through simplification of outside dimensions of boltheads and nuts by eliminating thirty-second inch sizes, and elimination of sizes little used, it has been possible to reduce the number of wrench openings required.

In all cases the basic widths across flats of boltheads and nuts are taken as maximum sizes and the tolerances on boltheads and nuts are minus only. The minimum wrench openings are made to provide a positive clearance between maximum nut and minimum wrench, and the tolerances on wrench openings are plus only. This insures a fit of the wrench to the bolthead and nut, whereas the tolerances allowed are as great as possible without causing the deformation of the corners of boltheads or nuts by the wrenches.

\section{GENERAI REQUIREMENTS}

(a) Workmanship.-It is recommended that workmanship be compatible with the type and grade of product, and class of fit and of finish specified; and that the product be free from abnormal scale, fins, seams, or other defects.

(b) Screw threads. - The form of thread profile is the American national form and the diameters and threads per inch are those specified as the American national coarse-thread series, or the American national fine-thread series.

(c) Body diameters.-The dimensions and tolerances for body diameter of bolts and screws are given in Tables 64 and 65 .

(d) Tolerance on length.-The length of all bolts and screws up to and including 6 inches in length does not vary from the nominal length by more than $\pm 1 / 32$ inch; and the length of all bolts and screws more than 6 inches in length does not vary from the nominal length by more than $\pm 1 / 16$ inch. (e) Bolt and screw lengths.-Bolts and screws, except for special requirements, should be ordered in the following increments of length:

\begin{tabular}{|c|c|}
\hline Length (inches) & Increment \\
\hline $\begin{array}{l}1 / 16 \text { to } 1 / 2 \\
\text { Over } 1 / 2 \text { to } 11 / 4 \\
\text { Over } 11 / 4 \text { to } 31 / 2 \\
\text { Over } 31 / 2 \text { to } 6 \\
\text { Over } 6 \text { to } 10 \\
\text { Over } 10\end{array}$ & $\begin{array}{c}\text { Inches } \\
1 / 16 \\
18 \\
1 / 4 \\
1 / 2 \\
1 \\
2\end{array}$ \\
\hline
\end{tabular}

TABLE 64.-Body diameter of bolts and screws, American national coarse-thread series

\begin{tabular}{|c|c|c|c|c|c|}
\hline \multirow[t]{2}{*}{ Sizes } & \multicolumn{2}{|c|}{ Class 1 , loose fit } & \multicolumn{2}{|c|}{$\begin{array}{l}\text { Class 2, free fit } 1 \text {; } \\
\text { class } 3 \text {, medium } \\
\text { fit; class } 4 \text {, close } \\
\text { fit }\end{array}$} & $\begin{array}{l}\text { Class } 2 \\
\text { free fit, } \\
\text { threaded } \\
\text { parts of } \\
\text { unfin- } \\
\text { ished, } \\
\text { hot- } \\
\text { rolled } \\
\text { material }\end{array}$ \\
\hline & $\begin{array}{l}\text { Maxi- } \\
\text { mum }\end{array}$ & $\begin{array}{l}\text { Mini- } \\
\text { mum }\end{array}$ & $\begin{array}{l}\text { Maxi- } \\
\text { mum }\end{array}$ & $\begin{array}{l}\text { Mini- } \\
\text { mum }\end{array}$ & $\begin{array}{l}\text { Mini- } \\
\text { mum }\end{array}$ \\
\hline 1 & 2 & 3 & 4 & 5 & 6 \\
\hline $\begin{array}{l}1 \ldots . . \\
2 \ldots \\
3 \ldots . \\
4 \ldots \\
5 \ldots\end{array}$ & $\begin{array}{r}\text { Inches } \\
0.0723 \\
.0352 \\
.0981 \\
.1110 \\
.1240\end{array}$ & $\begin{array}{c}\text { Inches } \\
0.0671 \\
.0796 \\
.0919 \\
.1042 \\
.1172\end{array}$ & $\begin{array}{r}\text { Inches } \\
0.0730 \\
.0860 \\
.0990 \\
.1120 \\
.1250\end{array}$ & $\begin{array}{r}\text { Inches } \\
0.0692 \\
.0820 \\
.0946 \\
.1072 \\
.1202\end{array}$ & $\begin{array}{r}\text { Inches } \\
0.0678 \\
.0804 \\
.0928 \\
.1052 \\
.1182\end{array}$ \\
\hline $\begin{array}{l}6 \ldots \ldots \\
8 \ldots \ldots \\
10 \ldots \ldots \\
12 \ldots \ldots \\
144 \ldots \ldots\end{array}$ & $\begin{array}{l}.1369 \\
.1629 \\
.1887 \\
.2147 \\
.2485\end{array}$ & $\begin{array}{l}.1293 \\
.1553 \\
.1795 \\
.2055 \\
.2383\end{array}$ & $\begin{array}{l}.1380 \\
.1640 \\
.1900 \\
.2160 \\
.2500\end{array}$ & $\begin{array}{r}.1326 \\
.1586 \\
.1834 \\
.2094 \\
.2428\end{array}$ & $\begin{array}{l}.1304 \\
.1564 \\
.1808 \\
.2068 \\
.2398\end{array}$ \\
\hline $\begin{array}{l}5 / 16 \ldots \\
3 / 8 \ldots \ldots \\
7 / 16 \ldots \\
1 / 2 \ldots \ldots \\
9 / 16 \ldots\end{array}$ & $\begin{array}{l}.3109 \\
.3732 \\
.4354 \\
.4978 \\
.5601\end{array}$ & $\begin{array}{l}.2995 \\
.3606 \\
.4214 \\
.4830 \\
.5443\end{array}$ & $\begin{array}{l}.3125 \\
.3750 \\
.4375 \\
.5000 \\
.5625\end{array}$ & $\begin{array}{r}.3043 \\
.3660 \\
.4277 \\
.4896 \\
.5513\end{array}$ & $\begin{array}{l}.3011 \\
.3624 \\
.4235 \\
.4852 \\
.5467\end{array}$ \\
\hline $\begin{array}{l}5 / 8 \\
3 / 4 \\
7 / 8 \\
1 \\
11 / 8\end{array}$ & $\begin{array}{r}.6224 \\
.7472 \\
.8719 \\
.9966 \\
1.1211\end{array}$ & $\begin{array}{r}.6054 \\
.7288 \\
.8519 \\
.9744 \\
1.0963\end{array}$ & $\begin{array}{r}.6250 \\
.7500 \\
.8750 \\
1.0000 \\
1.1250\end{array}$ & $\begin{array}{r}.6132 \\
.7372 \\
.8610 \\
.9848 \\
1.1080\end{array}$ & $\begin{array}{r}.6080 \\
.7316 \\
.8550 \\
.9778 \\
1.1002\end{array}$ \\
\hline $\begin{array}{l}1144 \ldots \\
11 / 2 \ldots \\
13 / 4 \ldots \\
2 \ldots\end{array}$ & $\begin{array}{l}\text { 1. } 2461 \\
\text { 1. } 4956 \\
\text { 1. } 7448 \\
\text { 1. } 9943\end{array}$ & $\begin{array}{l}1.2213 \\
1.4666 \\
1.7110 \\
1.9575\end{array}$ & $\begin{array}{l}1.2500 \\
1.5000 \\
1.7500 \\
2.0000\end{array}$ & $\begin{array}{l}1.2330 \\
1.4798 \\
1.7268 \\
1.9746\end{array}$ & $\begin{array}{l}1.2252 \\
1.4710 \\
1.7162 \\
1.9632\end{array}$ \\
\hline $\begin{array}{l}21 / 4- \\
21 / 2 \ldots \\
23 / 4- \\
3 \ldots\end{array}$ & $\begin{array}{l}2.2443 \\
2.4936 \\
2.7436 \\
2.9936\end{array}$ & $\begin{array}{l}2.2075 \\
2.4528 \\
2.7028 \\
2.9528\end{array}$ & $\begin{array}{l}2,2500 \\
2.5000 \\
2.7500 \\
3.0000\end{array}$ & $\begin{array}{l}2.2246 \\
2.4720 \\
2.7220 \\
2.9720\end{array}$ & $\begin{array}{l}2.2132 \\
2.4592 \\
2.7092 \\
2.9592\end{array}$ \\
\hline
\end{tabular}

1 For class 2, free fit, these minimum values apply only to semifinished and finished bolts and screws. 
TABLE 65.-Body diameters of bolts and screws, American national fine-thread series

\begin{tabular}{|c|c|c|c|c|}
\hline \multirow[t]{2}{*}{ Sizes } & \multicolumn{2}{|c|}{ Class 1 , loose fit } & \multicolumn{2}{|c|}{$\begin{array}{l}\text { Class 2, free fit; } \\
\text { class } 3 \text {, medium } \\
\text { fit; class } 4 \text {, close } \\
\text { fit }\end{array}$} \\
\hline & $\begin{array}{l}\text { Maxi- } \\
\text { mum }\end{array}$ & $\begin{array}{l}\text { Mini- } \\
\text { mum }\end{array}$ & $\begin{array}{l}\text { Maxi- } \\
\text { mum }\end{array}$ & $\begin{array}{l}\text { Mini- } \\
\text { mum }\end{array}$ \\
\hline 1 & 2 & 3 & 4 & 5 \\
\hline (12. & $\begin{array}{r}\text { Inches } \\
0.0593 \\
.0723 \\
.0853 \\
.0982 \\
.1111\end{array}$ & $\begin{array}{r}\text { Inches } \\
.0545 \\
.0673 \\
.0801 \\
.0926 \\
.1049\end{array}$ & $\begin{array}{l}\text { Inches } \\
0.0600 \\
.0730 \\
.0860 \\
.0990 \\
.1120\end{array}$ & $\begin{array}{c}\text { Inches } \\
0.0566 \\
.0694 \\
.0822 \\
.0950 \\
.1076\end{array}$ \\
\hline 8 & $\begin{array}{l}.1241 \\
.1370 \\
.1629 \\
.1889 \\
.2148\end{array}$ & $\begin{array}{l}.1177 \\
.1302 \\
.1557 \\
.1813 \\
.2062\end{array}$ & $\begin{array}{l}.1250 \\
.1380 \\
.1640 \\
.1900 \\
.2160\end{array}$ & $\begin{array}{l}.1204 \\
.1332 \\
.1590 \\
.1846 \\
.2098\end{array}$ \\
\hline $\begin{array}{l}1 / 4 \\
5 / 16 \ldots \ldots \\
3 / 8 \\
7 / 16 \\
1 / 2\end{array}$ & $\begin{array}{l}.2488 \\
.3112 \\
.3737 \\
.4360 \\
.4985\end{array}$ & $\begin{array}{l}.2402 \\
.3020 \\
.3645 \\
.4258 \\
.4883\end{array}$ & $\begin{array}{l}.2500 \\
.3125 \\
.3750 \\
.4375 \\
.5000\end{array}$ & $\begin{array}{l}.2438 \\
.3059 \\
.3684 \\
.4303 \\
.4928\end{array}$ \\
\hline $\begin{array}{l}9 / 16 \ldots \ldots \\
5 / 8 \ldots \ldots \\
3 / 4 \ldots \ldots \\
7 / 8 \ldots \ldots \\
1 \ldots \ldots\end{array}$ & $\begin{array}{l}.5609 \\
.6234 \\
.7482 \\
.8729 \\
.9979\end{array}$ & $\begin{array}{l}.5495 \\
.6120 \\
.7356 \\
.8589 \\
.9839\end{array}$ & $\begin{array}{r}.5625 \\
.6250 \\
.7500 \\
.8750 \\
1.0000\end{array}$ & $\begin{array}{l}.5543 \\
.6168 \\
.7410 \\
.8652 \\
.9902\end{array}$ \\
\hline $\begin{array}{l}11 / 8 \ldots \\
11 / 4 \\
11 / 2 \ldots \\
13 / 4 \ldots \\
2\end{array}$ & $\begin{array}{l}1.1226 \\
1.2476 \\
1.4976 \\
1.7472 \\
1.9972\end{array}$ & $\begin{array}{r}1.1068 \\
1.2318 \\
1.4818 \\
\cdot 1.7288 \\
1.9788\end{array}$ & $\begin{array}{l}1.1250 \\
1.2500 \\
1.5000 \\
1.7500 \\
2.0000\end{array}$ & $\begin{array}{l}1.1138 \\
1.2388 \\
1.4888 \\
1.7372 \\
1,9872\end{array}$ \\
\hline $\begin{array}{l}21 / 4 \ldots \ldots \\
21 / 2 \ldots \ldots \\
23 / 4 \ldots \ldots \\
3\end{array}$ & $\begin{array}{l}2.2466 \\
2.4966 \\
2.7466 \\
2.9966\end{array}$ & $\begin{array}{l}\text { 2. } 2244 \\
2.4744 \\
\text { 2. } 7244 \\
2.9744\end{array}$ & $\begin{array}{l}2.2500 \\
2.5000 \\
2.7500 \\
\text { 3. } 0000\end{array}$ & $\begin{array}{l}\text { 2. } 2348 \\
\text { 2. } 4848 \\
\text { 2. } 7348 \\
\text { 2. } 9848\end{array}$ \\
\hline
\end{tabular}

\section{UNITED STATES GOVERNMENT, NATIONAL SCREW THREAD COMMISSION, BUREAU OF STANDARDS MISCELLANEOUS PUB- IICATION NO. 89, JUNE 22, 1928}

\section{AMERICAN NATIONAL ACME SCREW THREADS STANDARD PRACTICE}

(a) Specifications

1. Ang'e of thread.-The angle between the sides of the thread measured in an axial plane shall be $29^{\circ}$. The line bisecting this $29^{\circ}$ angle shall be perpendicular to the axis of the screw thread.

2. Depth of thread. - The basic depth of the thread shall be equal to one-half of the pitch.

3. Thickness of thread.-The basic thickness of the thread at a diameter smaller by one-half the pitch than the basic major diameter shall be equal to onehalf of the pitch. ${ }^{83}$

4. Clearance at minor diameter.-A clearance shall be provided at the minor diameter for class $1 \mathrm{Acme}$ by making the minor diameter of the screw 0.020 $\sqrt{p}$ smaller than basic, and for classes 2,3 , and 4 Acme by making the minor diameter $0.005 \sqrt{p}$ smaller than basic.

5. Clearance at major diameter.-A clearance shall be provided at the major diameter for all classes by making the major diameter of the nut or threaded hole at least 0.020 inch larger than basic.

6. Fillets at minor diameter.-Fillets at the juncture of sides and root of the thread of the screw will develop on account of the rounding of the corners of the threading tool and the side cutting action of mill-

83 The diameter at which the thickness of thread is measured corresponds to the basic pitch diameter of $60^{\circ}$ screw threads used for bolts, nuts, etc. On threads whose included angle is equal to $45^{\circ}$ or more, nuts, etc. On threads whose included angle is equal to $45^{\circ}$ or more, diameter. On threads whose included angle is less than $45^{\circ}$, the thickness of the thread should be controlled directly. ing cutters when these threads are milled. It will be necessary, therefore, on tapped holes for all classes of fits, to provide a fillet or bevel at the minor diameter of the tap to remove the corner of the crest of the thread of the tapped hole. This fillet, or bevel, should be at least 0.010 inch for pitches of 3 threads per inch and finer, and at least 0.020 inch for pitches coarser than 3 threads per inch.

\section{(b) Ilustration} 318.

The basic form of this thread is shown in Figure

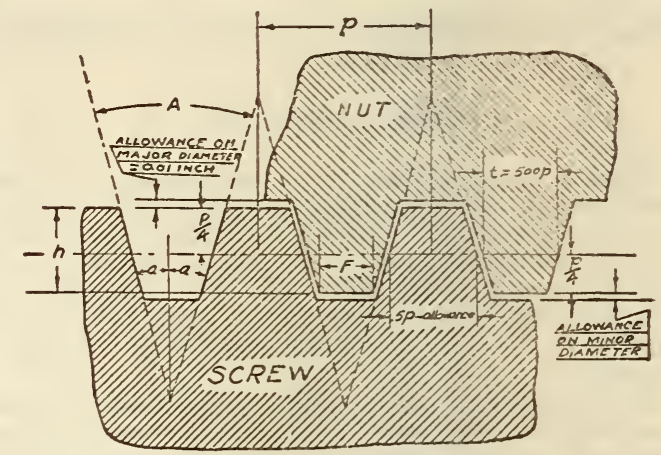

FIGURE 318.-American national acme form of thread

$$
\begin{aligned}
\text { Notation } \\
A=29^{\circ} 00^{\prime} . \\
a=14^{\circ} 30^{\circ} . \\
p=\text { pitch. } \\
n=\text { number of threads per inch. } \\
N=\text { number of turns per inch. } \\
h=0.5 p, \text { basic depth of thread. } \\
t=\text { thickness of thread. } \\
F=0.37069 p=\text { basic width of flat. }
\end{aligned}
$$

\section{THREAD SERIES}

As these threads are used for such a wide variety of purposes, it is not feasible arbitrarily to select and designate any given combinations of diameters and pitches as standard. It is recommended, however, that as far as practicable, some one of the pitches shown in Table 77 be used; also, that the diameter be not less than the minimum diameter

\begin{tabular}{|c|c|c|c|c|}
\hline $\begin{array}{l}\text { Number of threads } \\
\text { per inch, } n\end{array}$ & Pitch, $p$ & $\begin{array}{l}\text { Basic } \\
\text { depth of } \\
\text { thread, } \\
h=0.5 p\end{array}$ & $\begin{array}{l}\text { Recom- } \\
\text { mended } \\
\text { minimum } \\
\text { major di- } \\
\text { ameter }^{1}\end{array}$ & $\begin{array}{l}\text { Basic width } \\
\text { of flat, } \\
F=0.37069 p\end{array}$ \\
\hline 1 & 2 & 3 & 4 & 5 \\
\hline $\begin{array}{l}1 \\
1133 \\
11 / 2 \\
2\end{array}$ & $\begin{array}{c}\text { Inch } \\
1.00000 \\
.75000 \\
.66667 \\
.50000\end{array}$ & $\begin{array}{c}\text { Inch } \\
0.5000 \\
.3750 \\
.3333 \\
.2500\end{array}$ & $\begin{array}{l}\text { Inches } \\
4.5000 \\
\text { 3. } 5000 \\
3.0000 \\
\text { 2. } 2500\end{array}$ & $\begin{array}{l}\text { Inch } \\
0.3707 \\
.2780 \\
.2471 \\
.1853\end{array}$ \\
\hline $\begin{array}{l}21 / 2 \\
4 \\
4\end{array}$ & $\begin{array}{l}.40000 \\
.33333 \\
.25000 \\
.20000\end{array}$ & $\begin{array}{l}.2000 \\
.1667 \\
.1250 \\
.1000\end{array}$ & $\begin{array}{r}1.7500 \\
1.5000 \\
1.1250 \\
.8750\end{array}$ & $\begin{array}{l}.1483 \\
.1236 \\
.0927 \\
.0741\end{array}$ \\
\hline 10 & $\begin{array}{l}.16667 \\
.12500 \\
.10000 \\
.08333\end{array}$ & $\begin{array}{l}.0833 \\
.0625 \\
.0500 \\
.0417\end{array}$ & $\begin{array}{l}.7500 \\
.5625 \\
.4375 \\
.3750\end{array}$ & $\begin{array}{l}.0618 \\
.0463 \\
.0371 \\
.0309\end{array}$ \\
\hline
\end{tabular}
specified for each pitch. If a greater lead is required on a given diameter than that corresponding to the recommended maximum pitch, it is advisable to use a multiple thread of finer pitch rather than a single thread of coarser pitch.

TABLE 77.-Recommended pitches and corresponding minimum major diameters for American national acme threads

1 These recommended diameters correspond to a maximum helis angle (at the minor diameter) of approximately $5^{\circ}$. 
CLASSIFICATION AND TOLERANCES

There are established herein for general use four distinct classes of fit of American national acme screw threads. They are designated as follows:

Class 1. For general use.

Class 2. Adjusting screws with limited side play.

Class 3. Commercial lead screws.

Class 4. Precision lead screws.

In designating the various classes of fit in these specifications the class number is followed by the word "acme" in order to distinguish this classification from that applied to the various fits of fastening screws as given above.

(General specifications applying to all classes of fit specified for acme screw threads form part of this specification. These specifications cover tolerances, diameters, classification of fits, and examples of formula application. The several classifications are as follows:)

Class 1 acme.-This class is intended to cover the manufacture of threaded adjusting screws for gen- eral purposes where a considerable amount of shake or play, both side play and end play, is not objectionable.

This class is made with large allowances to permit ready assembly even when the threads are rough or dirty.

Class 2 acme.-This class is intended to cover the manufacture of threaded adjusting screws for general purposes, where the amount of side play must be kept to a minimum, such as valve stems, etc.

This class is made with allowances to permit ready assembly and lubrication.

Class 3 acme (commercial lead screw). -This class is intended to cover the manufacture of threaded lead screws used on machine tools, etc.

This class is made with allowances to permit ready assembly and lubrication.

Class 4 acme (precision lead screw). - This class is intended to cover the manufacture of precision threaded lead screws used in machine tools for tool room use, etc.

This class is made with allowances to permit ready assembly and lubrication.

TABLE 78.-Class 1 acme, allowances, thread proportions, and pitch and diameter increments

\begin{tabular}{|c|c|c|c|c|c|c|c|c|c|c|}
\hline \multirow[b]{2}{*}{$\begin{array}{l}\text { Number of } \\
\text { threads per } \\
\text { inch }\end{array}$} & \multirow[b]{2}{*}{ Pitch } & \multirow[b]{2}{*}{$\begin{array}{l}\text { Allowance } \\
\text { on thread } \\
\text { thickness }\end{array}$} & \multirow{2}{*}{\begin{tabular}{|c|} 
Combined \\
pitch and \\
diameter \\
increments \\
of thread \\
thickness \\
tolerance
\end{tabular}} & \multicolumn{3}{|c|}{ Screw } & \multicolumn{4}{|c|}{ Nut } \\
\hline & & & & $\begin{array}{c}\text { Tolerance } \\
\text { on major } \\
\text { diameters, } \\
\text { minus }\end{array}$ & $\begin{array}{l}\text { Maximum } \\
\text { minor di- } \\
\text { ameter= } \\
\text { basic major } \\
\text { diameter, } \\
\text { minus }\end{array}$ & $\begin{array}{c}\text { Maximum } \\
\text { thread } \\
\text { thickness }\end{array}$ & $\begin{array}{l}\text { Tolerance } \\
\text { on minor } \\
\text { diameter, } \\
\text { plus }\end{array}$ & $\begin{array}{c}\text { Minimum } \\
\text { minor di- } \\
\text { ameter = } \\
\text { basic major } \\
\text { diameter, } \\
\text { minus }\end{array}$ & $\begin{array}{c}\text { Maximum } \\
\text { thread } \\
\text { thickness }\end{array}$ & $\begin{array}{c}\text { Minimum } \\
\text { major di- } \\
\text { ameter = } \\
\text { basie major } \\
\text { diameter, } \\
\text { plus }\end{array}$ \\
\hline 1 & 2 & 3 & 4 & 5 & 6 & 7 & 8 & 9 & 10 & 11 \\
\hline $\begin{array}{l}13 .-. \\
1133-. \\
11 / 2 \ldots \\
2 \ldots\end{array}$ & $\begin{array}{l}\text { Inch } \\
1.00000 \\
.75000 \\
.66667 \\
.50000\end{array}$ & $\begin{array}{l}\text { Inch } \\
0.0050 \\
.0043 \\
.0041 \\
.0035\end{array}$ & $\begin{array}{l}\text { Inch } \\
0.00790 \\
.00689 \\
.00645 \\
.00559\end{array}$ & $\begin{array}{l}\text { Inch } \\
0.0500 \\
.0375 \\
.0333 \\
.0250\end{array}$ & $\begin{array}{c}\text { Inches } \\
1.0200 \\
.7673 \\
.6830 \\
.5141\end{array}$ & $\begin{array}{l}\text { Inch } \\
0.4950 \\
.3707 \\
.3293 \\
.2465\end{array}$ & $\begin{array}{l}\text { Inch } \\
0.0500 \\
.0375 \\
.0333 \\
.0250\end{array}$ & $\begin{array}{l}\text { Inch } \\
1.0000 \\
.7500 \\
.6667 \\
.5000\end{array}$ & $\begin{array}{l}\text { Inch } \\
0.5000 \\
.3750 \\
.3333 \\
.2500\end{array}$ & $\begin{array}{l}\text { Inch } \\
0.0200 \\
.0200 \\
.0200 \\
.0200\end{array}$ \\
\hline & $\begin{array}{l}.40000 \\
.33333 \\
.25000 \\
.20000\end{array}$ & $\begin{array}{l}.0032 \\
.0029 \\
.0025 \\
.0022\end{array}$ & $\begin{array}{l}.00497 \\
.00456 \\
.00395 \\
.00351\end{array}$ & $\begin{array}{l}.0200 \\
.0167 \\
.0125 \\
.0100\end{array}$ & $\begin{array}{l}.4126 \\
.3449 \\
.2600 \\
.2089\end{array}$ & $\begin{array}{l}.1968 \\
.1638 \\
.1225 \\
.0978\end{array}$ & $\begin{array}{l}.0200 \\
.0167 \\
.0125 \\
.0100\end{array}$ & $\begin{array}{l}.4000 \\
.3333 \\
.2500 \\
.2000\end{array}$ & $\begin{array}{l}.2000 \\
.1667 \\
.1250 \\
.1000\end{array}$ & $\begin{array}{l}.0200 \\
.0200 \\
.0200 \\
.0200\end{array}$ \\
\hline $\begin{array}{l}6 \ldots-. \\
8 \ldots-. \\
10-. . \\
12-. .\end{array}$ & $\begin{array}{l}.16667 \\
.12500 \\
.10000 \\
.08333\end{array}$ & $\begin{array}{l}.0020 \\
.0018 \\
.0016 \\
.0014\end{array}$ & $\begin{array}{l}.00322 \\
.00279 \\
.00248 \\
.00228\end{array}$ & $\begin{array}{l}.0083 \\
.0063 \\
.0050 \\
.0042\end{array}$ & $\begin{array}{l}.1748 \\
.1321 \\
.1063 \\
.0891\end{array}$ & $\begin{array}{l}.0813 \\
.0607 \\
.0484 \\
.0402\end{array}$ & $\begin{array}{l}.0083 \\
.0063 \\
.0050 \\
.0042\end{array}$ & $\begin{array}{l}.1667 \\
.1250 \\
.1000 \\
.0833\end{array}$ & $\begin{array}{l}.0833 \\
.0625 \\
.0500 \\
.0417\end{array}$ & $\begin{array}{l}.0200 \\
.0200 \\
.0200 \\
.0200\end{array}$ \\
\hline
\end{tabular}

TABLE 79.-Class 1 acme, length of engagement increments of thread thickness tolerances

\begin{tabular}{|c|c|c|c|}
\hline $\begin{array}{l}\text { Length } \\
\text { of en- } \\
\text { gage- } \\
\text { ment }\end{array}$ & $\begin{array}{l}\text { Length of } \\
\text { engage- } \\
\text { ment in- } \\
\text { crement }\end{array}$ & $\begin{array}{l}\text { Length } \\
\text { of en- } \\
\text { gage- } \\
\text { ment }\end{array}$ & $\begin{array}{l}\text { Length of } \\
\text { engage- } \\
\text { ment in- } \\
\text { crement }\end{array}$ \\
\hline 1 & 2 & 1 & 2 \\
\hline $\begin{array}{c}\text { Inches } \\
0.250 \\
.375 \\
.500 \\
.625 \\
.750\end{array}$ & $\begin{array}{c}\text { Inch } \\
0.00025 \\
.00038 \\
.00050 \\
.00063 \\
.00075\end{array}$ & $\begin{array}{r}\text { Inches } \\
4.000 \\
4.500 \\
5.000 \\
5.500 \\
6.000\end{array}$ & $\begin{array}{c}\text { Inch } \\
0.00400 \\
.00450 \\
.00500 \\
.00550 \\
.00600\end{array}$ \\
\hline $\begin{array}{l}1.000 \\
1.250 \\
1.500 \\
1.750\end{array}$ & $\begin{array}{l}.00100 \\
.00125 \\
.00150 \\
.00175\end{array}$ & $\begin{array}{l}6.500 \\
7.000 \\
7.500 \\
8.000\end{array}$ & $\begin{array}{l}.00650 \\
.00700 \\
.00750 \\
.00800\end{array}$ \\
\hline $\begin{array}{l}2.000 \\
2.500 \\
3.000 \\
3.500\end{array}$ & $\begin{array}{l}.00200 \\
.00250 \\
.00300 \\
.00350\end{array}$ & $\begin{array}{r}9.000 \\
10.000 \\
11.000 \\
12.000\end{array}$ & $\begin{array}{l}.00900 \\
.01000 \\
.01100 \\
.01200\end{array}$ \\
\hline
\end{tabular}


TABLE 80.-Classes 2, 3, and 4 acme, allowances, thread proportions, and pitch and diameter increment

\begin{tabular}{|c|c|c|c|c|c|c|c|c|c|c|c|c|}
\hline \multirow[b]{2}{*}{$\begin{array}{l}\text { Number of } \\
\text { threads per } \\
\text { inch }\end{array}$} & \multirow[b]{2}{*}{ Pitch } & \multirow[b]{2}{*}{$\begin{array}{c}\text { Allow- } \\
\text { ance on } \\
\text { thread } \\
\text { thickness }\end{array}$} & \multirow[b]{2}{*}{$\begin{array}{l}\text { Com- } \\
\text { bined } \\
\text { pitch and } \\
\text { diameter } \\
\text { incre- } \\
\text { ments of } \\
\text { thread } \\
\text { thickness } \\
\text { tolerance }\end{array}$} & \multirow[b]{2}{*}{$\begin{array}{c}\text { Radius of } \\
\text { fillet at } \\
\text { minor } \\
\text { diameter, } \\
\text { screw and } \\
\text { nut }\end{array}$} & \multicolumn{4}{|c|}{ Screw } & \multicolumn{4}{|c|}{ Nut } \\
\hline & & & & & $\begin{array}{l}\text { Tolerance } \\
\text { on major } \\
\text { diameter, } \\
\text { minus }\end{array}$ & $\begin{array}{l}\text { Maxi- } \\
\text { mum } \\
\text { minor } \\
\text { diameter } \\
\text { = basic } \\
\text { major } \\
\text { diameter } \\
\text { minus }\end{array}$ & $\begin{array}{l}\text { Tolerance } \\
\text { on minor } \\
\text { diameter, } \\
\text { minus }\end{array}$ & $\begin{array}{c}\text { Maxi- } \\
\text { mum } \\
\text { thread } \\
\text { thickness }\end{array}$ & $\begin{array}{c}\text { Tolerance } \\
\text { on minor } \\
\text { diameter, } \\
\text { plus }\end{array}$ & $\begin{array}{l}\text { Mini- } \\
\text { mum } \\
\text { minor } \\
\text { diameter } \\
\text { = basic } \\
\text { major } \\
\text { diameter } \\
\text { minus }\end{array}$ & $\begin{array}{c}\text { Maxi- } \\
\text { mum } \\
\text { thread } \\
\text { thickness }\end{array}$ & $\begin{array}{l}\text { Mini- } \\
\text { mum } \\
\text { major } \\
\text { diameter } \\
\text { = basic } \\
\text { major } \\
\text { diameter } \\
\text { plus }\end{array}$ \\
\hline 1 & 2 & 3 & 4 & 5 & 6 & 7 & 8 & 9 & 10 & 11 & 12 & 13 \\
\hline $\begin{array}{l}1 \\
113 \\
11 / 2 \\
2\end{array}$ & $\begin{array}{l}\text { Inch } \\
1.00000 \\
.75000 \\
.66667 \\
.50000\end{array}$ & $\begin{array}{l}\text { Inch } \\
0.0013 \\
.0011 \\
.0010 \\
.0009\end{array}$ & $\begin{array}{l}\text { Inch } \\
0.00790 \\
.00689 \\
.00645 \\
.00559\end{array}$ & $\begin{array}{r}\text { Inch } \\
0.02 \\
.02 \\
.02 \\
.02\end{array}$ & $\begin{array}{l}\text { Inch } \\
0.0500 \\
.0375 \\
.0333 \\
.0250\end{array}$ & $\begin{array}{c}\text { Inches } \\
1.0050 \\
.7543 \\
.6708 \\
.5035\end{array}$ & $\begin{array}{l}\text { Inch } \\
0.0100 \\
.0087 \\
.0082 \\
.0071\end{array}$ & $\begin{array}{l}\text { Inch } \\
0.4987 \\
.3739 \\
.3323 \\
.2491\end{array}$ & $\begin{array}{l}\text { Inch } \\
0.0100 \\
.0087 \\
.0082 \\
.0071\end{array}$ & $\begin{array}{l}\text { Inch } \\
1.0000 \\
.7500 \\
.6667 \\
.5000\end{array}$ & $\begin{array}{l}\text { Inch } \\
0.5000 \\
.3750 \\
.3333 \\
.2500\end{array}$ & $\begin{array}{c}\text { Inch } \\
0.0200 \\
.0200 \\
.0200 \\
.0200\end{array}$ \\
\hline $21 / 2$ & $\begin{array}{r}.40000 \\
.33333 \\
.25000 \\
.20000\end{array}$ & $\begin{array}{l}.0008 \\
.0007 \\
.0006 \\
.0006\end{array}$ & $\begin{array}{l}.00497 \\
.00456 \\
.00395 \\
.00351\end{array}$ & $\begin{array}{l}.02 \\
.01 \\
.01 \\
.01\end{array}$ & $\begin{array}{l}.0200 \\
.0167 \\
.0125 \\
.0100\end{array}$ & $\begin{array}{l}.4032 \\
.3362 \\
.2525 \\
.2022\end{array}$ & $\begin{array}{l}.0063 \\
.0058 \\
.0050 \\
.0045\end{array}$ & $\begin{array}{l}.1992 \\
.1660 \\
.1244 \\
.0994\end{array}$ & $\begin{array}{l}.0063 \\
.0058 \\
.0050 \\
.0045\end{array}$ & $\begin{array}{l}.4000 \\
.3333 \\
.2500 \\
.2000\end{array}$ & $\begin{array}{l}.2000 \\
.1667 \\
.1250 \\
.1000\end{array}$ & $\begin{array}{l}.0200 \\
.0200 \\
.0200 \\
.0200\end{array}$ \\
\hline $\begin{array}{l}6 \\
8 \\
10 \\
12\end{array}$ & $\begin{array}{r}.16667 \\
.12500 \\
.10000 \\
.08333\end{array}$ & $\begin{array}{l}.0005 \\
.0004 \\
.0004 \\
.0004\end{array}$ & $\begin{array}{l}.00322 \\
.00279 \\
.00248 \\
.00228\end{array}$ & $\begin{array}{l}.01 \\
.01 \\
.01 \\
.01\end{array}$ & $\begin{array}{l}.0083 \\
.0063 \\
.0050 \\
.0042\end{array}$ & $\begin{array}{l}.1687 \\
.1268 \\
.1016 \\
.0848\end{array}$ & $\begin{array}{l}.0041 \\
.0035 \\
.0032 \\
.0029\end{array}$ & $\begin{array}{l}.0828 \\
.0621 \\
.0496 \\
.0413\end{array}$ & $\begin{array}{l}.0041 \\
.0035 \\
.0032 \\
.0029\end{array}$ & $\begin{array}{l}.1667 \\
.1250 \\
.1000 \\
.0833\end{array}$ & $\begin{array}{l}.0833 \\
.0625 \\
.0500 \\
.0417\end{array}$ & $\begin{array}{l}.0200 \\
.0200 \\
.0200 \\
.0200\end{array}$ \\
\hline
\end{tabular}

TABLE 81.-Classes 2,3 , and 4 acme, length of engagement increments of thread thickness tolerances

\begin{tabular}{|c|c||c|c|}
\hline $\begin{array}{c}\text { Length } \\
\text { of en- } \\
\text { gage- } \\
\text { ment }\end{array}$ & $\begin{array}{c}\text { Length of } \\
\text { engagement } \\
\text { increment }\end{array}$ & $\begin{array}{c}\text { Length of } \\
\text { engage- } \\
\text { ment }\end{array}$ & $\begin{array}{c}\text { Length of } \\
\text { engagement } \\
\text { increment }\end{array}$ \\
\hline 1 & 2 & 1 & 2 \\
\hline Inches & Inch & Inches & Inch \\
0.250 & 0.00013 & 4.500 & 0.00225 \\
.375 & .00019 & 5.000 & .00250 \\
.500 & .00025 & 5.500 & .00275 \\
.625 & .00031 & 6.000 & .00300 \\
.750 & .00038 & 6.500 & .00325 \\
& & & \\
1.000 & .00050 & 7.000 & .00350 \\
1.250 & .00063 & 7.500 & .00375 \\
1.500 & .00075 & 8.000 & .00400 \\
1.750 & .00088 & 9.000 & .00450 \\
2.000 & .00100 & 10.000 & .00500 \\
2.500 & .00125 & 11.000 & .005500 \\
3.000 & .00150 & 12.000 & .006000 \\
3.500 & .00175 & & \\
4.000 & .00200 & & \\
& & & \\
\hline
\end{tabular}

\section{UNITED STATES GOVERNMENT, NATIONAL SCREW THREAD COMMISSION, BUREAU OF STANDARDS MISCELLANEOUS PUB- IICATION No. 89, JUNE 22, 1928}

\section{CLASS 5, WRENCH FIT FOR THREADED STUDS}

(Tentative specifications)

\section{FORM OF THREAD}

The American national form of thread profile shall be used. The thread form of the tapped hole is modified, however, by truncating the crest of the thread a greater amount than that specified for threads of strictly American national form. This truncation is such that the minimum depth of thread engagement is one-half of the basic thread depth. The maximum depth of engagement is governed by the tolerances specified for the major diameter of the stud and the minor diameter of the tapped hole.

\section{THREAD SERIES}

The range of sizes from $1 / 4$ to $11 / 2$ inches, inclusive, of the American national coarse-thread series and the American national fine-thread series of sizes and pitches, given above, are recommended for general use for class 5, wrench fit for threaded studs.

Definition.-The wrench-fit class is intended to cover the manufacture of threaded studs and holes

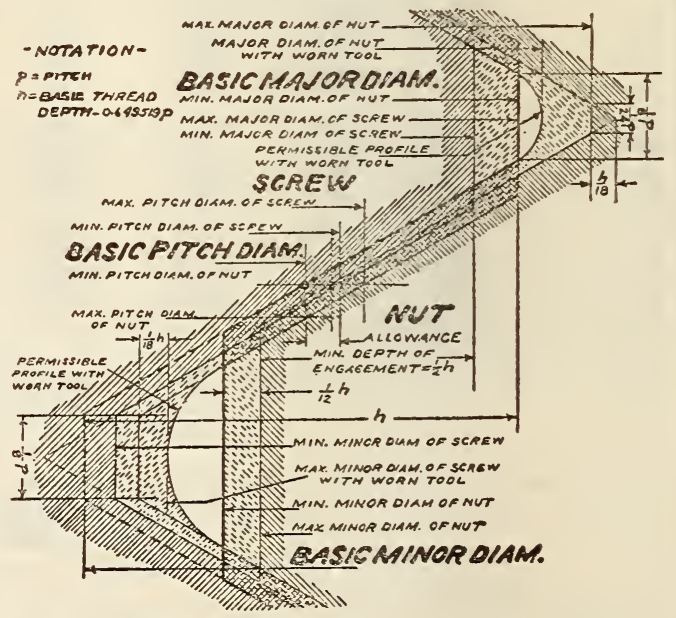

FIgURE 319.-Illustration of tolerances, allowance, and crest clearances for class 5 , wrench fit for threaded studs

which are to be assembled permanently by the application of power.

(General specifications applying to all materials for class 5, wrench fit for threaded studs, American national coarse-thread series and American national fine-thread series are included in this publication. These specifications cover pitch diameters, length of engagement, interferences, and tolerances. The relations of the maximum and minimum major, pitch, and minor diameters of stud and tapped hole are shown in Figures 319, 320, and 321.) 
CLASSIFICATION

Allowance and tolerance values.-Allowances and tolerances are specified in Tables 116 and 117, inclusive, for coarse-threaded and fine-threaded

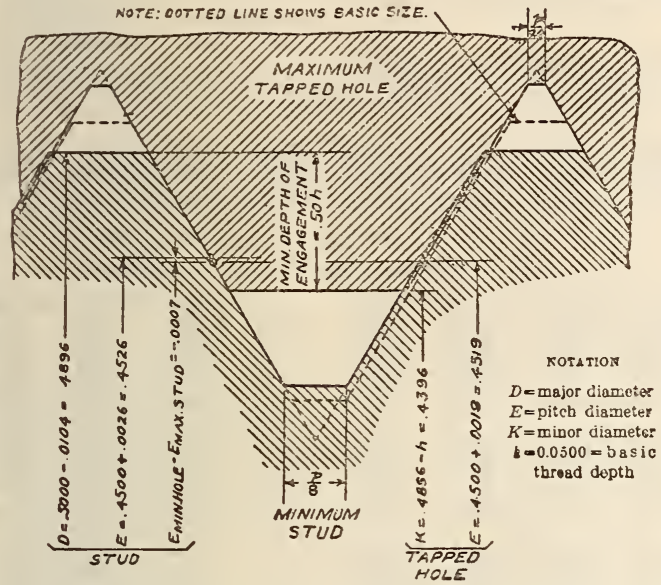

FIGURE 320.-Illustration of loosest condition for class 5, wrench fit for threaded studs, one-half inch, 13 threads, set in hard materials

studs set in hard materials, namely, cast iron, steel, and bronze. These are based upon data obtained in an experimental investigation and fulfill the conditions outlined in the above specifications.

\section{TABLES OF DIMENSIONS}

Tables 118 and 119 give recommended thread dimensions of studs and tapped holes which meet

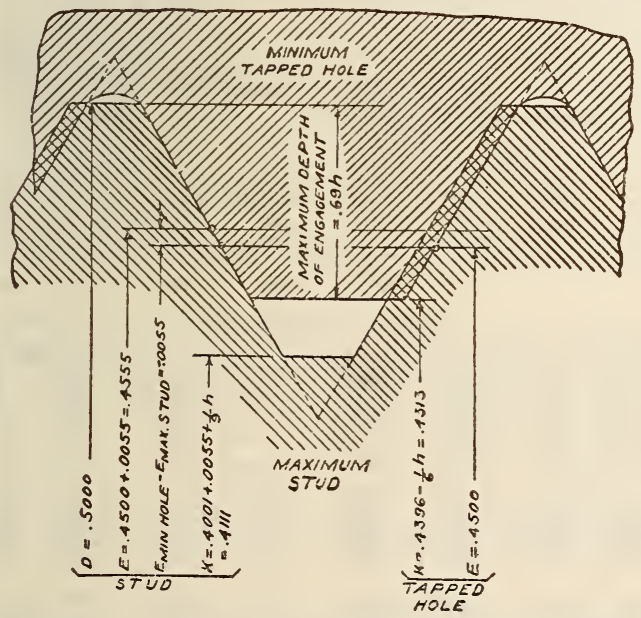

FIGURE 321.-Illustration of tightest condition for class 5, wrench fit for threaded studs, one-half inch, 13 threads, set in hard materials

\section{NOTATION}

$D=$ major diameter.

$E=$ pitch diameter.

$K=$ minor diameter.

$\begin{aligned} h & =0.0500=\text { basic thread depth. }\end{aligned}$

the above specifications for coarse-threaded and fine-threaded studs set in hard materials. Also the limiting values of the torques at full engagement (lever arm times force) which may be expected in the assembly of studs and tapped holes made to these dimensions are given.
TABLE 116.-Class 5, wrench fit for threaded studs, allowances and tolerances for studs and tapped holes, coarse-threaded studs in hard materials

\begin{tabular}{|c|c|c|c|c|c|c|c|c|}
\hline \multirow[t]{2}{*}{ Sizes } & \multirow{2}{*}{ 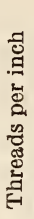 } & \multicolumn{2}{|c|}{$\begin{array}{l}\text { Interference on } \\
\text { pitch diameter }\end{array}$} & \multicolumn{2}{|c|}{$\begin{array}{l}\text { Pitch diameter } \\
\text { tolerances } 1\end{array}$} & \multicolumn{3}{|c|}{$\begin{array}{l}\text { Errors in half- } \\
\text { angle consuming } \\
\text { one-half of pitch } \\
\text { diameter toler- } \\
\text { ances }\end{array}$} \\
\hline & & $\begin{array}{l}\text { Mini- } \\
\text { mum }\end{array}$ & $\begin{array}{l}\text { Maxi- } \\
\text { mum }\end{array}$ & Stud & $\begin{array}{c}\text { Tapped } \\
\text { hole }{ }^{2}\end{array}$ & Stud & & $\begin{array}{l}\text { oped } \\
\text { ole }\end{array}$ \\
\hline 1 & 2 & 3 & 4 & 5 & 6 & 7 & & 8 \\
\hline $\begin{array}{l}1 / 4 \ldots \\
5116 \\
3 / 8 \\
7 / 16 \\
1 / 2 .\end{array}$ & $\begin{array}{l}20 \\
18 \\
16 \\
14 \\
13\end{array}$ & $\begin{array}{l}\text { Inch } \\
0.0003 \\
.0005 \\
.0005 \\
.0006 \\
.0007\end{array}$ & $\begin{array}{l}\text { Inch } \\
0.0018 \\
.0040 \\
.0045 \\
.0050 \\
.0055\end{array}$ & $\begin{array}{l}\text { Inch } \\
0.0007 \\
.0020 \\
.0024 \\
.0026 \\
.0029\end{array}$ & $\begin{array}{c}\text { Inch } \\
0.0008 \\
.0015 \\
.0016 \\
.0018 \\
.0019\end{array}$ & $\begin{array}{lc}\circ & , \\
0 & 16 \\
0 & 41 \\
0 & 44 \\
0 & 42 \\
0 & 44\end{array}$ & $\begin{array}{l}0 \\
0 \\
0\end{array}$ & $\begin{array}{l}\prime \\
25 \\
31 \\
29 \\
29 \\
28\end{array}$ \\
\hline $\begin{array}{l}9 / 16 \\
5 / 8 \\
3 / 4 \\
7 / 8\end{array}$ & $\begin{array}{r}12 \\
11 \\
10 \\
9\end{array}$ & $\begin{array}{l}.0008 \\
.0008 \\
.0009 \\
.0010\end{array}$ & $\begin{array}{l}.0060 \\
.0060 \\
.0065 \\
.0065\end{array}$ & $\begin{array}{l}.0032 \\
.0031 \\
.0033 \\
.0031\end{array}$ & $\begin{array}{l}.0020 \\
.0021 \\
.0023 \\
.0024\end{array}$ & $\begin{array}{ll}0 & 44 \\
0 & 39 \\
0 & 38 \\
0 & 32\end{array}$ & $\begin{array}{l}0 \\
0 \\
0 \\
0\end{array}$ & $\begin{array}{l}28 \\
26 \\
26 \\
25\end{array}$ \\
\hline $\begin{array}{l}1 . \\
11 / 8 . \\
11 / 4 . \\
11 / 2 .\end{array}$ & $\begin{array}{l}8 \\
7 \\
7 \\
6\end{array}$ & $\begin{array}{l}.0011 \\
.0011 \\
.0012 \\
.0013\end{array}$ & $\begin{array}{l}.0065 \\
.0065 \\
.0065 \\
.0070\end{array}$ & $\begin{array}{l}.0027 \\
.0024 \\
.0023 \\
.0021\end{array}$ & $\begin{array}{l}.0027 \\
.0030 \\
.0030 \\
.0036\end{array}$ & $\begin{array}{ll}0 & 25 \\
0 & 19 \\
0 & 18 \\
0 & 14\end{array}$ & $\begin{array}{l}0 \\
0 \\
0 \\
0\end{array}$ & $\begin{array}{l}25 \\
24 \\
24 \\
25\end{array}$ \\
\hline
\end{tabular}

1 Inasmuch as a moderate difference in lead between stud and tapped hole (about 0.005 inch per inch) has been shown to improve the quality of a stud fit having minimum pitch-diameter interference, no lead tolerance is specified. Therefore, the tolerances specified for pitch diameter include all errors of pitch diameter and angle but not of lead. Excessive lead errors, however, should be avoided, as they increase the tendency of the stud to loosen when subjected to load. Columns 7 and 8 give, for information, the errors in angle which can be compensated for by half the tolerance on pitch diameter given in columns 5 and 6 .

2 The tolerances on the tapped hole given in column 6 are the same as those specified for class 4 , close-fit screws and nuts, with the exception of the $1 / 4$-inch size.

TABLE 117.-Class 5, wrench fit for threaded studs, allowances and tolerances for studs and tapped holes, fine-threaded studs in hard materials

\begin{tabular}{|c|c|c|c|c|c|c|c|}
\hline \multirow[t]{2}{*}{ Sizes } & \multirow{2}{*}{ 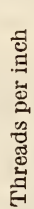 } & \multicolumn{2}{|c|}{$\begin{array}{l}\text { Interference on } \\
\text { pitch diameter }\end{array}$} & \multicolumn{2}{|c|}{$\begin{array}{c}\text { Pitch diameter } \\
\text { tolerances } 1\end{array}$} & \multicolumn{2}{|c|}{$\begin{array}{l}\text { Errors in half- } \\
\text { angle consuming } \\
\text { one-half of pitch } \\
\text { diameter toler- } \\
\text { ances }\end{array}$} \\
\hline & & $\begin{array}{l}\text { Mini- } \\
\text { mum }\end{array}$ & $\begin{array}{l}\text { Maxi- } \\
\text { mum }\end{array}$ & Stud & $\begin{array}{c}\text { Tapped } \\
\text { hole? }\end{array}$ & Stu & $\begin{array}{l}\text { pped } \\
\text { ole }\end{array}$ \\
\hline 1 & 2 & 3 & 4 & 5 & 6 & 7 & 8 \\
\hline $\begin{array}{l}1 / 4 . \\
51 / 10 \\
3 / 8 \\
7 / 10 \\
1 / 2\end{array}$ & $\begin{array}{l}28 \\
24 \\
24 \\
20 \\
20\end{array}$ & $\begin{array}{l}\text { Inch } \\
0.0005 \\
.0005 \\
.0006 \\
.0006 \\
.0007\end{array}$ & $\begin{array}{l}\text { Inch } \\
0.0034 \\
.0037 \\
.0044 \\
.0044 \\
.0050\end{array}$ & $\begin{array}{l}\text { Inch } \\
0.0018 \\
.0020 \\
.0026 \\
.0025 \\
.0030\end{array}$ & $\begin{array}{c}\text { Inch } \\
0.0011 \\
.0012 \\
.0012 \\
.0013 \\
.0013\end{array}$ & $\begin{array}{ll}\circ & , \\
0 & 5 \\
0 & 5 \\
1 & 1 \\
0 & 5 \\
1 & \end{array}$ & $\begin{array}{l}\prime \\
35 \\
33 \\
33 \\
30 \\
30\end{array}$ \\
\hline $\begin{array}{l}9 / 16 \ldots \\
5 / 8 \ldots \\
3 / 4 \ldots \\
7 / 8 \ldots\end{array}$ & $\begin{array}{l}18 \\
18 \\
16 \\
14\end{array}$ & $\begin{array}{l}.0007 \\
.0008 \\
.0008 \\
.0008\end{array}$ & $\begin{array}{l}.0050 \\
.0055 \\
.0059 \\
.0061\end{array}$ & $\begin{array}{l}.0028 \\
.0032 \\
.0035 \\
.0035\end{array}$ & $\begin{array}{l}.0015 \\
.0015 \\
.0016 \\
.0018\end{array}$ & $\begin{array}{ll}0 & 5 \\
1 & \\
1 & \\
0 & 5\end{array}$ & $\begin{array}{l}31 \\
31 \\
29 \\
29\end{array}$ \\
\hline $\begin{array}{l}1 \\
11 / 8- \\
11 / 4- \\
11 / 2-\end{array}$ & $\begin{array}{l}14 \\
12 \\
12 \\
12\end{array}$ & $\begin{array}{l}.0009 \\
.0009 \\
.0011 \\
.0012\end{array}$ & $\begin{array}{l}.0069 \\
.0067 \\
.0060 \\
.0050\end{array}$ & $\begin{array}{l}.0042 \\
.0038 \\
.0029 \\
.0018\end{array}$ & $\begin{array}{l}.0018 \\
.0020 \\
.0020 \\
.0020\end{array}$ & $\begin{array}{ll}1 & \\
0 & 5 \\
0 & 4 \\
0 & 2\end{array}$ & $\begin{array}{l}29 \\
28 \\
28 \\
28\end{array}$ \\
\hline
\end{tabular}

1 Inasmuch as a moderate difference in lead between stud and tapped hole (about 0.005 inch per inch) has been shown to improve the quality of a stud fit having minimum pitch diameter interference, no lead tolerance is specified. Therefore, the tolerances specified for pitch diameter include all errors of pitch diameter and angle but not of lead. Excessive lead errors, however, should be avoided as not they increase the tendency. load. Columns 7 and 8 give, for information, the errors in angle which can be compensated for by half the tolerance on pitch diameter given in columns 5 and 6 .

2 The tolerances on the tapped hole given in column 6 are the same as those specified for class 4 , close-fit screws and nuts. 
TABLE 118.-Class 5, wrench fit, American national coarse-thread series, steel studs set in hard materials (cast iron, semisteel, bronze, etc.)

\begin{tabular}{|c|c|c|c|c|c|c|c|c|c|c|c|c|c|c|c|}
\hline \multirow{3}{*}{ Sizes } & \multirow{3}{*}{ 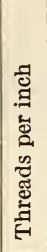 } & \multicolumn{5}{|c|}{ Stud sizes } & \multicolumn{5}{|c|}{ Tapped-hole sizes } & \multirow{2}{*}{\multicolumn{2}{|c|}{$\begin{array}{l}\text { Recommended } \\
\text { tap-drill size }\end{array}$}} & \multirow{2}{*}{\multicolumn{2}{|c|}{$\begin{array}{c}\text { A pproximate } \\
\text { torque at full } \\
\text { engagement of } \\
11 / 2 D\end{array}$}} \\
\hline & & \multicolumn{2}{|c|}{ Major diameter } & \multicolumn{2}{|c|}{ Pitch diameter } & \multirow{2}{*}{$\frac{\begin{array}{c}\text { Minor } \\
\text { diam- } \\
\text { eter }\end{array}}{\text { Maxi- }_{\text {mum 1 }}}$} & \multicolumn{2}{|c|}{ Minor diameter } & \multicolumn{2}{|c|}{ Pitch diameter } & \multirow{2}{*}{$\begin{array}{c}\begin{array}{c}\text { Major } \\
\text { diam- } \\
\text { eter }\end{array} \\
\begin{array}{c}\text { Mini- } \\
\text { mum 2 }\end{array}\end{array}$} & & & & \\
\hline & & $\begin{array}{l}\text { Maxi- } \\
\text { mum }\end{array}$ & $\begin{array}{l}\text { Mini- } \\
\text { mum }\end{array}$ & $\begin{array}{l}\text { Maxi- } \\
\text { mum }\end{array}$ & $\begin{array}{l}\text { Mini- } \\
\text { mum }\end{array}$ & & $\begin{array}{l}\text { Mini- } \\
\text { mum }\end{array}$ & $\begin{array}{l}\text { Maxi- } \\
\text { mum }\end{array}$ & $\begin{array}{l}\text { Mini- } \\
\text { mum }\end{array}$ & $\begin{array}{l}\text { Maxi- } \\
\text { mum }\end{array}$ & & $\begin{array}{c}\text { Nominal } \\
\text { size }\end{array}$ & $\begin{array}{l}\text { Diam- } \\
\text { eter }\end{array}$ & $\underset{\text { mumi- }}{\text { Maxi- }}$ & $\begin{array}{l}\text { Mini- } \\
\text { mum }\end{array}$ \\
\hline 1 & 2 & 3 & 4 & 5 & 6 & 7 & 8 & 9 & 10 & 11 & 12 & 13 & 14 & 15 & 16 \\
\hline $\begin{array}{l}1 / 4 \\
5 / 16 \\
3 / 8- \\
7 / 16 \\
1 / 2-\end{array}$ & $\begin{array}{l}20 \\
18 \\
16 \\
14 \\
13\end{array}$ & $\begin{array}{r}\text { Inches } \\
0.2500 \\
.3125 \\
.3750 \\
.4375 \\
.5000\end{array}$ & $\begin{array}{l}\text { Inches } \\
0.2428 \\
.3043 \\
.3660 \\
.4277 \\
.4896\end{array}$ & \begin{tabular}{|l|} 
Inches \\
0.2193 \\
.2804 \\
.3389 \\
.3961 \\
.4555 \\
\end{tabular} & $\begin{array}{c}\text { Inches } \\
0.2186 \\
.2784 \\
.3365 \\
.3935 \\
.4526\end{array}$ & $\begin{array}{l}\text { Inches } \\
0.1904 \\
.2483 \\
.3028 \\
.3549 \\
.4111\end{array}$ & $\begin{array}{l}\text { Inches } \\
0.2049 \\
.2622 \\
.3186 \\
.3736 \\
.4313\end{array}$ & $\begin{array}{c}\text { Inches } \\
0.2103 \\
.2682 \\
.3254 \\
.3813 \\
.4396\end{array}$ & $\begin{array}{l}\text { Inches } \\
0.2175 \\
.2764 \\
.3344 \\
.3911 \\
.4500\end{array}$ & $\begin{array}{l}\text { Inches } \\
0.2183 \\
.2779 \\
.3360 \\
.3929 \\
.4519\end{array}$ & $\begin{array}{l}\text { Inches } \\
0.2500 \\
.3125 \\
.3750 \\
.4375 \\
.5000\end{array}$ & $\begin{array}{r}\text { No. } 4 \\
H \\
\mathrm{P} \\
\mathrm{V} \\
7 / 16\end{array}$ & $\begin{array}{l}\text { Inches } \\
0.2090 \\
.2660 \\
.3230 \\
.3770 \\
.4375\end{array}$ & $\begin{array}{r}\text { In. }-l b s \\
105 \\
265 \\
420 \\
610 \\
850\end{array}$ & $\begin{array}{r}\text { In. }-1 b 8 \\
35 \\
80 \\
120 \\
180 \\
265\end{array}$ \\
\hline & $\begin{array}{r}12 \\
11 \\
10 \\
9\end{array}$ & $\begin{array}{l}.5625 \\
.6250 \\
.7500 \\
.8750\end{array}$ & $\begin{array}{l}.5513 \\
.6132 \\
.7372 \\
.8610\end{array}$ & $\begin{array}{l}.5144 \\
.5720 \\
.6915 \\
.8093\end{array}$ & $\begin{array}{l}.5112 \\
.5689 \\
.6882 \\
.8062\end{array}$ & $\begin{array}{l}.4663 \\
.5195 \\
.6338 \\
.7452\end{array}$ & $\begin{array}{l}.4882 \\
.5444 \\
.6614 \\
.7768\end{array}$ & $\begin{array}{l}.4972 \\
.5542 \\
.6722 \\
.7888\end{array}$ & $\begin{array}{l}.5084 \\
.5660 \\
.6850 \\
.8028\end{array}$ & $\begin{array}{l}.5104 \\
.5681 \\
.6873 \\
.8052\end{array}$ & $\begin{array}{l}.5625 \\
.6250 \\
.7500 \\
.8750\end{array}$ & $\begin{array}{r}12.5 \mathrm{~mm} \\
35 / 64 \\
43 / 64 \\
25 / 32\end{array}$ & $\begin{array}{l}.4921 \\
.5469 \\
.6719 \\
.7812\end{array}$ & $\begin{array}{l}1,170 \\
1,450 \\
2,300 \\
3,200\end{array}$ & $\begin{array}{r}360 \\
450 \\
730 \\
1,080\end{array}$ \\
\hline $\begin{array}{l}11-- \\
118- \\
114- \\
112=\end{array}$ & $\begin{array}{l}8 \\
7 \\
7 \\
6\end{array}$ & $\begin{array}{l}1.0000 \\
1.1250 \\
1.2500 \\
1.5000\end{array}$ & $\begin{array}{l}.9848 \\
1.1080 \\
1.2330 \\
1.4798\end{array}$ & $\begin{array}{l}.9253 \\
1.0387 \\
1.1637 \\
1.3987\end{array}$ & $\begin{array}{l}.9226 \\
1.0363 \\
1.1614 \\
1.3966\end{array}$ & $\begin{array}{r}.8531 \\
.9562 \\
1.0812 \\
1.3025\end{array}$ & $\begin{array}{r}.8901 \\
.9998 \\
1.1248 \\
1.3536\end{array}$ & $\begin{array}{l}.9036 \\
1.0152 \\
1.1402 \\
1.3716\end{array}$ & $\begin{array}{l}.9188 \\
\text { 1. } 0322 \\
1.1572 \\
1.3917\end{array}$ & $\begin{array}{l}.9215 \\
\text { 1. } 0352 \\
\text { 1. } 1602 \\
1.3953\end{array}$ & $\begin{array}{l}1.0000 \\
1.1250 \\
1.2500 \\
1.5000\end{array}$ & $\begin{array}{l}57 / 64 \\
11 / 8 \\
12364\end{array}$ & $\begin{array}{r}.8906 \\
1.0000 \\
1.1250 \\
1.3594\end{array}$ & $\begin{array}{r}4,250 \\
5,300 \\
6,950 \\
10,400\end{array}$ & $\begin{array}{l}1,500 \\
1,875 \\
2,535 \\
3,900\end{array}$ \\
\hline
\end{tabular}

1 Dimensions given for the maximum minor diameter of the screw are figured to the intersection of the worn tool arc with a center line through crest and root. The minimum minor diameter of the screw shall be that corresponding to a flat at the minor diameter of the screw equal to $1 / 8 \times p$, and may be determined by subtracting the basic thread depth, $h$ (or $0.6495 p)$, from the minimum pitch diameter of the screw. 2 Dimensions for the minimum major diameter of the tapped hole correspond to the basic flat $(1 / 8 \times p)$, and the profile at the major diameter produced by a worn tool must not fall below the basic outline. The maximum major diameter of the tapped hole shall be that corresponding to a flat at the major diameter of the tapped hole equal to $1 / 24 \times p$, and may be determined by adding $136 \times h$ (or $0.7939 p$ ) to the maxi-

mum pitch diameter of the nut. avoid breaking a mild-steel stud the maximum interference on pitch diameter of 0.0018 inch must not be exceeded.

TABLE 119.-Class 5, wrench fit, American national fine-thread series, steel studs set in hard materials (cast iron, semisteel, bronze, etc.)

\begin{tabular}{|c|c|c|c|c|c|c|c|c|c|c|c|c|c|c|c|}
\hline \multirow{3}{*}{ Sizes } & \multirow{3}{*}{ 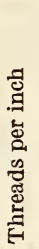 } & \multicolumn{5}{|c|}{ Stud sizes } & \multicolumn{5}{|c|}{ Tapped-hole sizes } & \multirow{2}{*}{\multicolumn{2}{|c|}{$\begin{array}{l}\text { Recommended } \\
\text { tap-drill size }\end{array}$}} & \multirow{2}{*}{\multicolumn{2}{|c|}{$\begin{array}{c}\text { Approximate } \\
\text { torque at full } \\
\text { engagement of } \\
11 / 2 D\end{array}$}} \\
\hline & & \multicolumn{2}{|c|}{ Major diameter } & \multicolumn{2}{|c|}{ Pitch diameter } & \multirow{2}{*}{$\frac{\begin{array}{c}\text { Minor } \\
\text { diam- } \\
\text { eter }\end{array}}{\begin{array}{c}\text { Maxi- } \\
\text { mum 1 }\end{array}}$} & \multicolumn{2}{|c|}{ Minor diameter } & \multicolumn{2}{|c|}{ Pitch diameter } & \multirow{2}{*}{$\begin{array}{c}\begin{array}{c}\text { Major } \\
\text { diam- } \\
\text { eter }\end{array} \\
\begin{array}{c}\text { Mini- } \\
\text { mum? }\end{array}\end{array}$} & & & & \\
\hline & & $\begin{array}{l}\text { Maxi- } \\
\text { mum }\end{array}$ & $\begin{array}{l}\text { Mini- } \\
\text { mum }\end{array}$ & $\begin{array}{l}\text { Maxi- } \\
\text { mum }\end{array}$ & $\begin{array}{l}\text { Mini- } \\
\text { mum }\end{array}$ & & $\begin{array}{l}\text { Mini- } \\
\text { mum }\end{array}$ & $\begin{array}{l}\text { Maxi- } \\
\text { mum }\end{array}$ & $\begin{array}{l}\text { Mini- } \\
\text { mum }\end{array}$ & $\begin{array}{l}\text { Maxi- } \\
\text { mum }\end{array}$ & & $\underset{\text { size }}{\text { Nominal }}$ & $\begin{array}{l}\text { Diam- } \\
\text { eter }\end{array}$ & $\begin{array}{l}\text { Maxi- } \\
\text { mum }\end{array}$ & $\begin{array}{l}\text { Mini- } \\
\text { mum }\end{array}$ \\
\hline 1 & 2 & 3 & 4 & 5 & 6 & 7 & 8 & 9 & 10 & 11 & 12 & 13 & 14 & 15 & 16 \\
\hline $\begin{array}{l}1 / 4 \ldots \\
5 / 16 \ldots \\
3 / 8 \\
7 / 16 \ldots \\
1 / 2 \ldots\end{array}$ & $\begin{array}{l}28 \\
24 \\
24 \\
20 \\
20\end{array}$ & $\begin{array}{l}\text { Inches } \\
0.2500 \\
.3125 \\
.3750 \\
.4375 \\
.5000\end{array}$ & $\begin{array}{l}\text { Inches } \\
0.2438 \\
.3059 \\
.3684 \\
.4303 \\
.4928\end{array}$ & $\begin{array}{l}\text { Inches } \\
0.2302 \\
.2891 \\
.3523 \\
.4094 \\
.4725\end{array}$ & $\begin{array}{l}\text { Inches } \\
0.2284 \\
.2871 \\
.3497 \\
.4069 \\
.4695\end{array}$ & $\begin{array}{l}\text { Inches } \\
0.2096 \\
.2650 \\
.3282 \\
.3805 \\
.4436\end{array}$ & $\begin{array}{l}\text { Inches } \\
0.2167 \\
.2743 \\
.3368 \\
.3924 \\
.4549\end{array}$ & $\begin{array}{l}\text { Inches } \\
0.2206 \\
.2788 \\
.3413 \\
.3978 \\
.4603\end{array}$ & $\begin{array}{l}\text { Inches } \\
0.2268 \\
.2854 \\
.3479 \\
.4050 \\
.4675\end{array}$ & $\begin{array}{l}\text { Inches } \\
0.2279 \\
.2866 \\
.3491 \\
.4063 \\
.4688\end{array}$ & $\begin{array}{l}\text { Inches } \\
0.2500 \\
.3125 \\
.3750 \\
.4375 \\
.5000\end{array}$ & $\begin{array}{r}7 / 32 \\
\mathrm{~J} \\
\mathrm{R} \\
\mathrm{X}\end{array}$ & $\begin{array}{l}\text { Inches } \\
0.2188 \\
.2770 \\
.3390 \\
.3970 \\
.4576\end{array}$ & $\begin{array}{r}\text { In - llbs. } \\
140 \\
230 \\
410 \\
540 \\
810\end{array}$ & $\begin{array}{c}\text { In. } 1 \text { lbs. } \\
45 \\
70 \\
125 \\
170 \\
260\end{array}$ \\
\hline $\begin{array}{l}9 / 16 \ldots \\
5 / 8 \ldots \\
34:- \\
7 / 8 \ldots\end{array}$ & $\begin{array}{l}18 \\
18 \\
16 \\
14\end{array}$ & $\begin{array}{l}.5625 \\
.6250 \\
.7500 \\
.8750\end{array}$ & $\begin{array}{l}.5543 \\
.6168 \\
.7410 \\
.8652\end{array}$ & $\begin{array}{l}.5314 \\
.5944 \\
.7153 \\
.8347\end{array}$ & $\begin{array}{l}.5286 \\
.5912 \\
.7118 \\
.8312\end{array}$ & $\begin{array}{l}.4993 \\
.5623 \\
.6792 \\
.7935\end{array}$ & $\begin{array}{l}.5122 \\
.5747 \\
.6936 \\
.8111\end{array}$ & $\begin{array}{l}.5182 \\
.5807 \\
.7004 \\
.8188\end{array}$ & $\begin{array}{l}.5264 \\
.5889 \\
.7094 \\
.8286\end{array}$ & $\begin{array}{l}.5279 \\
.5904 \\
.7110 \\
.8304\end{array}$ & $\begin{array}{l}.5625 \\
.6250 \\
.7500 \\
.8750\end{array}$ & $\begin{array}{l}33 / 64 \\
3764 \\
13 / 16\end{array}$ & & $\begin{array}{l}1,040 \\
1,430 \\
2,200 \\
3,070\end{array}$ & $\begin{array}{l}330 \\
460 \\
685 \\
945\end{array}$ \\
\hline $\begin{array}{l}117 \ldots \\
118 \ldots \\
114 \ldots \\
112 \ldots\end{array}$ & $\begin{array}{l}14 \\
12 \\
12 \\
12\end{array}$ & $\begin{array}{l}1.0000 \\
1.1250 \\
1.2500 \\
1.5000\end{array}$ & $\begin{array}{r}.9902 \\
1.1138 \\
1.2388 \\
1.4888\end{array}$ & $\begin{array}{r}.9605 \\
\text { 1. } 0776 \\
1.2019 \\
1.4509\end{array}$ & $\begin{array}{r}.9563 \\
1.0738 \\
1.1990 \\
1.4491\end{array}$ & $\begin{array}{r}.9193 \\
1.0295 \\
1.1538 \\
1.4028\end{array}$ & $\begin{array}{l}.9361 \\
1.0507 \\
1.1757 \\
1.4257\end{array}$ & $\begin{array}{r}.9438 \\
1.0597 \\
1.1847 \\
1.4347\end{array}$ & $\begin{array}{r}.9536 \\
1.0709 \\
1.1959 \\
1.4459\end{array}$ & $\begin{array}{r}.9554 \\
1.0729 \\
1.1979 \\
1.4479\end{array}$ & $\begin{array}{l}1.0000 \\
1.1250 \\
1.2500 \\
1.5000\end{array}$ & $\begin{array}{r}15 / 16 \\
30 \mathrm{~mm}\end{array}$ & $\begin{array}{r}.9375 \\
1.0552 \\
1.1811 \\
1.4302\end{array}$ & $\begin{array}{r}4,590 \\
5,620 \\
6,960 \\
10,070\end{array}$ & $\begin{array}{l}1,410 \\
1,750 \\
2,530 \\
4,215\end{array}$ \\
\hline
\end{tabular}

1 Dimensions given for the maximum minor diameter of the screw are figured to the intersection of the worn tool arc with a center line through crest and root. The minimum minor diameter of the screw shall be that corresponding to a flat at the minor diameter of the screw equal to $18 \times p$, and may be determined by subtracting the basic thread depth, $h$ (or $0.6495 p$ ), from the minimum pitch diameter of the screw.

2 Dimensions for the minimum major diameter of the tapped hole correspond to the basic flat $(1 / 8 \times p)$, and the profile at the major diameter produced by a worn tool must not fall below the basic outline. The maximum major diameier of the tapped hole shall be that corresponding to a flat at the major diameter of the tapped hole equal to $1 / 24 \times p$, and may be determined by adding $126 \times h$ (or $0.7939 p$ ) to the maximum pitch diameter of the nut. 
UNITED STATES GOVERNMENT, NATIONAL SCREW THREAD COMMISSION, APPENDIX NO. 6, BUREAU OF STANDARDS MISCELIANEOUS PUBLICATION NO. 89, JUNE 22, 1928

COMMON PRACTICE AS TO THREAD SERIES AND CLASS OF FIT FOR SCREWS, BOLTS, AND NUTS

The usual commercial practice as to application of thread series and class of fit to screws, bolts, and nuts is indicated in the accompanying table.

\begin{tabular}{|c|c|c|}
\hline Product & Thread series & Class of fit \\
\hline 1 & 2 & 3 \\
\hline 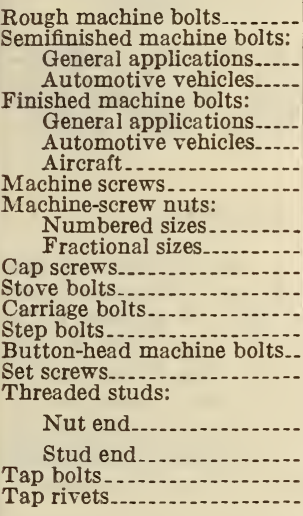 & $\begin{array}{l}\text { Fine } \\
\text { Coarse } \\
\text { Fine }\end{array}$ & $\begin{array}{l}\text { Class 1, loose fit. } \\
\text { Class 2, free fit. } \\
\text { Class 3, medium fit. } \\
\text { Do. } \\
\text { Do. } \\
\text { Do. } \\
\text { Class 2, free fit. } \\
\text { Class 1, loose fit. } \\
\text { Class 2, free fit. } \\
\text { Do. } \\
\text { Class 1, loose fit. } \\
\text { Class 2, free fit. } \\
\text { Do. } \\
\text { Do. } \\
\text { Class 3, medium fit. } \\
\text { Class 2, free fit. } \\
\text { Class 3, medium fit. } \\
\text { Class 5, wrench fit. } \\
\text { Class 2, free fit. } \\
\text { Class 3, medium fit. }\end{array}$ \\
\hline
\end{tabular}

UNITED STATES GOVERNMENT, NATIONAL SCREW THREAD COMMISSION, AMERICAN NATIONAL SCREW THREAD TABLES FOR SHOP USE, STANDARD THREADS (COARSE AND FINE THREAD SERIES), BUREAU OF STANDARDS MISCELIANEOUS PUBLICATION NO. 98, 1929

\section{STANDARD SCREW-THREAD TABLES FOR SHOP USE}

(This publication makes available for convenient use six of the most essential tables of dimensions of fastening screws as published in the 1928 report of the National Screw Thread Commission. These tables cover the basic sizes, limiting dimensions, tolerances, and tap-drill sizes for the American national coarse and fine thread series. They are identical with Tables $1,2,15,16,107$, and 108 in Miscellaneous Publication No. 89 of the Bureau of Standards, given above.)

UNITED STATES GOVERNMENT, NATIONAL SCREW THREAD COMMISSION, AMERICAN NATIONAL SCREW THREAD TABLES FOR SHOP USE, SPECIAL THREADS, BUREAU OF STANDARDS MISCELLANEOUS PUBLICATION NO. 99, 1929

\section{SPECIAL SCREW-THREAD TABLES FOR SHOP USE}

(This publication presents in compact form the tables of dimensions of special screw threads having the American national form of thread $\left(60^{\circ}\right)$ as published in the 1928 report of the National Screw Thread Commission. These tables cover the basic sizes, limiting dimensions, and tolerances for the American national 12-pitch thread series and other screw threads of special diameters, pitches, and lengths of engagement. They are identical with Tables 27, 28, 29, 30, 31, 87, and 88 in Miscellaneous Publication No. 89 of the Bureau of Standards, given above.)

608.1. NAIIS, BRADS, STAPLES, AND TACKS.

608.11. NAILS.

AMERICAN RAILWAY ENGINEERING ASSOCIATION, SPECIFICATIONS FOR DATING NAIIS, 1931

\section{DATING NAILS}

\section{MATERIAL}

The nails shall be made of iron or steel, galvanized with a coating of zinc (prime western, or equal)

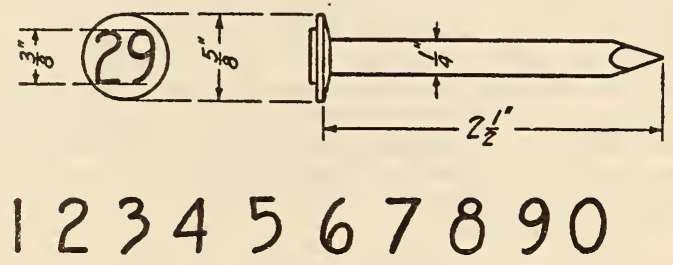

Figure 322

eveniy and uniformly applied by the hot-dip process so that it will adhere firmly to the surface of the iron or steel.

\section{CHEMICAL REQUIREMENTS}

(a) The sample shall be immersed in a standard solution of copper sulphate for one minute and then immediately washed in water thoroughly and wiped dry. This process shall be repeated. If after the fourth immersion there is a copper-colored deposit on the sample, or the zinc has been removed, the lot from which the sample was taken shall be rejected.

(b) The standard solution of copper sulphate is prepared by dissolving 36 parts of crystallized copper sulphate in 100 parts of water, then adding enough cupric oxide to neutralize any free acid. The solution is filtered or allowed to settle and decanted, then diluted with water until its specific gravity is 1.186 at $65^{\circ}$. F. While nails are being tested, the temperature shall be at no time less than $60^{\circ} \mathrm{F}$. nor more than $70^{\circ} \mathrm{F}$.

\section{DESIGN}

The shank of nail shall be one-fourth inch in diameter and $2 \frac{1}{2}$ inches long; the head of nail shall be five-eighths inch in diameter and one-sixteenth inch thick, and shall bear two raised figures designating the year, the figures to be three-eighths inch long and raised one-sixteenth inch, and of the following type.

(Inspection conforms to A. S. T. M. standard specifications for steel. See 605.0, p. 247.) 
AMERICAN SOCIETY FOR TESTING MATERIALS, STANDARD SPECIFICATIONS FOR HEAVY-WEIGHT SLATE-SURFACED ASPHALT ROLL ROOFING AND HEAVYWEIGHT SLATE-SURFACED ASPHALT SHINGLES, DESIGNATION D249-27, 1927

\section{ROOFING NAILS}

(For body of specification see Standards and Specifications for Nonmetallic Minerals and their Products, p. 156., publication M110 of Bureau of Standards.)

Nails. - The gage of the wire shall not be greater than A. S. \& W. No. 10 (0.135 inch) nor thinner than No. 12 ( 0.105 inch); the head shall be not less than three-eighths inch in diameter, and not less than 0.025 inch in thickness; the shank shall be between three-fourths and 1 inch long for slate roll roofing, and for shingles 1 inch long when laid on solid sheathing, or $1 \frac{1}{2}$ to $1 \frac{3}{4}$ inches when laid over old shingles. The lower end of the shank shall be pointed and either smooth or barbed, and, if the latter, then the barbings shall not increase the diameter of the lower two-thirds more than 0.01 inch. The nails shall be zinc coated with not less than one-half ounce nor more than 11/4 ounces per square foot of nail surface. Hot-galvanized, electro-galvanized, or sherardized coatings will be accepted. There shall be furnished per square of slate roll roofing not less than an average of 252 nails for 36-inch and not less than an average of 275 nails for 32-inch roofing. For shingles, the nails shall be purchased separately, and the number required per square will depend upon the size and form of the shingles and shall be agreed upon by the purchaser and seller.

\section{ASPHALT SHINGLE AND ROOFING IN- STITUTE, SPECIFICATIONS FOR NAILS TO BE USED FOR IAYING COMPOSITION SHINGLES, 1921}

\section{NAILS FOR COMPOSITION SHINGLES}

Gage of wire.-To be not thicker than 101/2 nor thinner than 12.

Length.-To be not less than 1 inch nor greater than $11 / 4$ inches.

Heads.-To be not less than three-eighths inch in diameter, nor less than 0.02 inch thick.

Shanks.-To be either smooth or barbed, and, if the latter, the barbings should not increase the diameter of the lower two-thirds of the shank more than 0.01 inch. The lower end of the shank to be pointed.

Count. - The count per pound for any given type of nail in different lots received from nail manufacturers shall not vary more than 2 per cent.

Protective coating.-Zinc-coated nails are recommended for composition shingles. The minimum amount of zinc shall be one-half ounce and the maximum $1 \frac{1}{4}$ ounces per square foot of nail surface.

ASPHALT SHINGLE AND ROOFING INSTITUTE, SPECIFICATIONS FOR NAILS TO BE USED FOR LAYING ROLL ROOFINGS, 1920

\section{NAILS FOR ROLI ROOFINGS}

Gage of wire.-To be not thicker than $10 \frac{1}{2}$ nor thinner than 12 .

Length.-To be not less than three-fourths inch nor greater than 1 inch.

Note. $-3 / 4$-inch nails are recommended for light-weight roll roofings and 1-inch nails for slate-surfaced roll roofings.
Heads.-To be not less than seven-sixteenths inch in diameter nor less than 0.025 inch thick.

Shanks. - To be either smooth or barbed, and, if the latter, then the barbings shall not increase the diameter of the lower two-thirds of the shank more than 0.01 inch. The lower end of shank to be pointed.

Count.-The count per pound for any given type of nail in different lots received from nail manufacturers shall not vary more than 2 per cent.

Note.-It is recommended that each manufacturer furnish not less than 288 nails for a square of 36 -inch roll roofing nor less than 307 nails for a square of 32 -inch roll roofing. This will be sufficient for 1 long seam and 2 end seams, when the nails are spaced 2 inches in 1 row along the long seam and 2 rows along each end seam.

Protective coating. - Nails to be zinc coated when so required. The minimum amount of zinc shall be one-half ounce and the maximum amount shall be $1 \frac{1}{4}$ ounces per square foot of nail surface.

UNDERWRITERS' IABORATORIES, SPECIFICATIONS FOR CLASS C ASPHALT RAG-FELT SHEET ROOFING AND SHINGLES, 1929

\section{NAILS FOR SHEET ROOFING}

Dimensions.-The diameter of wire shall not be greater than No. 10 (0.135 inch) nor thinner than No. 12 ( 0.105 inch); the heads shall not be less than three-eighths inch in diameter nor less than 0.025 inch thick; the shank shall be not less than threefourths inch long and shall be either smooth or barbed, and, if the latter, then the barbings shall not increase the diameter of the lower two-thirds more than 0.01 inch. The lower end of the shank shall be pointed.

Protective coating.-Nails shall be zinc coated with not less than one-half ounce nor more than $1 \frac{1}{4}$ ounces per square foot of nail surface. Hot-galvanized, electro-galvanized, or sherardized coating will be accepted.

Quantity.-There shall be furnished per square an average of not less than 252 nails for $36-i n c h$, 275 for 32-inch, 504 for 18-inch, and 550 for 16-inch roll roofings, respectively. Roofers' rolls shipped in carload lots need not contain nails.

UNITED STATES GOVERNMENT, DEPARTMENT OF COMMERCE, BUREAU OF STANDARDS, SIMPLIFIED PRACTICE RECOMMENDATION FOR CUT TACKS AND SMALL CUT NAIIS, R47-28, JULY 1, 1928

\section{CUT TACKS AND SMALL CUT NAILS}

(Simplified practice recommended and adopted by industry establishing a limited list of standard styles, sizes, and methods of packing.)

The following styles, sizes, and methods of packing will be considered a simplified list. Any sizes and styles not listed will be manufactured as special goods.

\section{HARDWARE IIST}

[This list is based on a redesignation from numbers to length in fractions of an inch and numbers]

Items in the simplified schedule shall be measured as follows:

(a) Nails with flat head or with square shoulder under head, and all tacks, are measured under head to end of point.

(b) Brad head, flat countersunk head, headless, and cement-coated nails are measured over all; that is, top of head to end of point. 
(c) Oval and round countersunk head nails are measured from the top of countersink (largest diameter) to end of point.

\section{BILL POSTER}

\begin{tabular}{|c|c|c|}
\hline Style and finishes & Designation & Sizes of packages \\
\hline Bright, blued, tinned... & 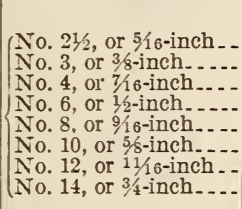 & $\begin{array}{l}\text { Pounds } \\
1 / 2,1,5,25,100\end{array}$ \\
\hline \multicolumn{3}{|c|}{ CARPET } \\
\hline $\begin{array}{l}\text { Bright, blued, tinned, } \\
\text { copper-plated.......... }\end{array}$ & 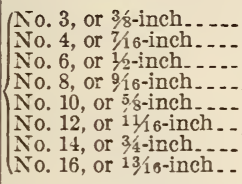 & $1 / 8,1 / 4,1 / 2,1,25,100$ \\
\hline
\end{tabular}

\section{UPHOLSTERER}

\begin{tabular}{|c|c|c|}
\hline Bright, blued, tinned.- & 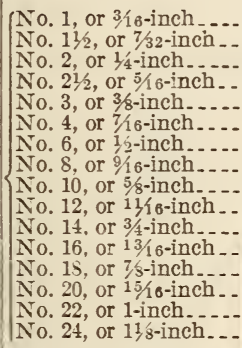 & $\begin{array}{l}1 / 8,1 / 4,1 / 2,1,5,10 \\
25,100 .\end{array}$ \\
\hline
\end{tabular}

TRIMMERS

(Formerly Swedes cut tacks)

\begin{tabular}{|c|c|c|}
\hline $\begin{array}{l}\text { Blued, tinned, copper- } \\
\text { plated, japanned.... }\end{array}$ & 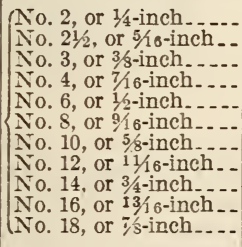 & $\left\{\begin{array}{c}1 / 8,1 / 4,1 / 2,1,5,10 \\
25,100 .\end{array}\right.$ \\
\hline \multicolumn{3}{|c|}{ BASKET } \\
\hline Bright, blued, tinned... & $\left\{\begin{array}{l}\text { No. } 3 \text {, or } 3 / 8 \text {-inch.... } \\
\text { No. } 4 \text {, or } 7 / 16 \text {-inch } \\
\text { No. } 6 \text {, or } 1 / 2 \text {-inch } \\
\text { No. } 8 \text {, or } 9 / 16 \text {-inch } \\
\text { No. } 10 \text {, or } 5 / 8 \text {-inch... } \\
\text { No. } 12 \text {, or } 11 / 16 \text {-inch. } \\
\text { No. } 14 \text {, or } 3 / 4 \text {-inch... }\end{array}\right.$ & $1,25,100$ \\
\hline 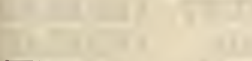 & TRUNK & \\
\hline $\begin{array}{l}\text { Blued, tinned, brass- } \\
\text { plated, coppered, } \\
\text { bright.............. }\end{array}$ & $\left\{\begin{array}{l}\text { No. } 6 \text {, or } 1 / 2 \text {-inch } \\
\text { No. } 8 \text {, or } 9 / 16 \text {-inch.-. } \\
\text { No. } 10 \text {, or } 5 / 8 \text {-inch } \\
\text { No. } 12 \text {, or } 11 / 16 \text {-inch.. } \\
\text { No. } 14 \text {, or } 3 / 4 \text {-inch } \\
\text { No. } 16 \text {, or } 13 / 16 \text {-inch.. } \\
\text { No. } 18 \text {, or } 7 / 8 \text {-inch. }\end{array}\right.$ & $1,25,100$ \\
\hline
\end{tabular}

SOLIJ HEAD LINING

\begin{tabular}{|c|c|c|}
\hline Style and finishes & Designation & Sizes of packages \\
\hline Japanned... & $\left\{\begin{array}{l}\text { No. } 6, \text { or } 1 / 2 \text {-inch } \\
\text { No. } 8 \text {, or } 9 / 16 \text {-inch } \\
\text { No. } 10 \text {, or } 5 / 8 \text {-inch } \\
\text { No. } 14 \text {, or } 3 / 4 \text {-inch... }\end{array}\right.$ & $\begin{array}{l}\text { Pounds } \\
\qquad 1 / 1,1,25,100\end{array}$ \\
\hline \multicolumn{3}{|c|}{ GIMP } \\
\hline $\begin{array}{l}\text { Blued, tinned, japan- } \\
\text { ned, copper-plated..- }\end{array}$ & $\left\{\begin{array}{l}\text { No. } 2 \text {, or } 5 / 16 \text {-inch } \\
\text { No. } 21 / 2 \text {, or } 3 / 6 \text {-inch... } \\
\text { No. } 3 \text {, or } 7 / 16 \text {-inch... } \\
\text { No. } 4 \text {, or } 1 / 2 \text {-inch } \\
\text { No. } 6 \text {, or } 9 / 16 \text {-inch... } \\
\text { No. } 8 \text {, or } 5 / 8 \text {-inch } \\
\text { No. } 12 \text {, or } 3 / 4 \text {-inch.... }\end{array}\right.$ & $\begin{array}{l}1 / 8,34,1,5,10,25, \\
100 .\end{array}$ \\
\hline
\end{tabular}

\section{LACE}

\begin{tabular}{|c|c|c|}
\hline $\begin{array}{l}\text { Bright, tinned, japan- } \\
\text { ned, blued, copper- } \\
\text { plated. }\end{array}$ & 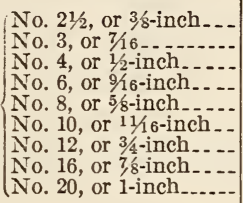 & $1 / 4,1 / 2,1,10,25,100$ \\
\hline
\end{tabular}

\section{HIDE}

\begin{tabular}{|c|c|}
\hline Bright, tinned.-- & $\left\{\begin{array}{l}\text { No. } 8, \text { or } 9 / 16 \text {-inch. } \\
\text { No. } 10 \text { or } 5 / 8 \text {-inch. } \\
\text { No. } 14 \text {, or } 3 / 4 \text {-inch } \\
\text { No. } 18 \text {, or } 78 \text {-inch.... } \\
\text { No. } 22 \text {, or } 1 \text {-inch } \\
\text { No. } 24 \text {, or } 11 / 8 \text {-inch... }\end{array}\right.$ \\
\hline
\end{tabular}

\section{CLOUT NAILS}

\begin{tabular}{|c|c|c|}
\hline $\begin{array}{l}\text { Bright, tinned, blued, } \\
\text { copper-plated.-... }\end{array}$ & $\left\{\begin{array}{l}13 / 4 \text {-inch } \\
11 / 2 \text {-inch } \\
11 / 4-\text { inch } \\
1 \text {-inch } \\
7 / 8 \text {-inch } \\
3 / 4 \text {-inch } \\
5 \text { /8-inch }\end{array}\right.$ & $\begin{array}{l}\text { Pounds } \\
\qquad 1 / 2,1,25,100\end{array}$ \\
\hline
\end{tabular}

TRUNK NAILS

\begin{tabular}{|c|c|c|}
\hline $\begin{array}{l}\text { Bright, tinned, cop- } \\
\text { pered, brass-plated, } \\
\text { blued. }\end{array}$ & $\left\{\begin{array}{l}11 / 2 \text {-inch } \\
11 \text {-inch } \\
1 \text {-inch } \\
\text { 7/8-inch } \\
3 / 4 \text {-inch } \\
5 / 8-i n c h\end{array}\right.$ & $32,1,25,10$ \\
\hline
\end{tabular}

BASKET NAILS

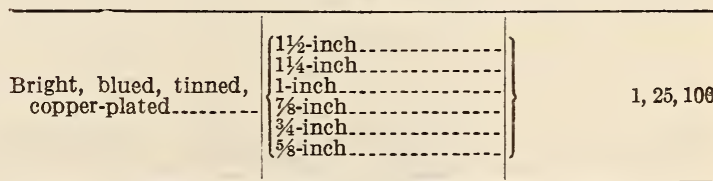

\section{COPPER TACKS}

No. 2 , or $1 / 4$-inch......

No. 3 , or $3 / 8$-inch.

No. 4 , or $7 / 16$-inch.

No. 6 , or $1 / 2$-inch

No. 8 , or $9 / 16$-inch.-

No. 10 or $5 / 8$-inch.....

No. 14 , or $3 / 4$-inch...

No. 18 , or 78 -inch

No. 22 , or 1 -inch. 
GALVANIZED TACKS

\begin{tabular}{|c|c|c|}
\hline Style and finishes & Designation & Sizes of packages \\
\hline & $\begin{array}{l}\text { No. } 3 \text {, or } 3 / 8 \text {-inch..... } \\
\text { No. } 6 \text {, or } 1 / 2 \text {-inch..... } \\
\text { No. } 8 \text {, or } 9 / 16 \text {-inch.... } \\
\text { No. } 10 \text {, or } 5 / 8 \text {-inch.... } \\
\text { No. } 12 \text {, or } 11 / 16 \text {-inch... } \\
\text { No. } 14 \text {, or } 3 / 4 \text {-inch.... } \\
\text { No. } 16 \text {, or } 13 / 16 \text {-inch... } \\
\text { No. } 18 \text {, or } 7 / 8 \text {-inch.... } \\
\text { No. } 22 \text {, or } 1 \text {-inch..... }\end{array}$ & $\begin{array}{l}\text { Pounds } \\
\qquad 1,25,100\end{array}$ \\
\hline
\end{tabular}

SHOE FINDERS' LIST

COBBLERS'

[16 gage]

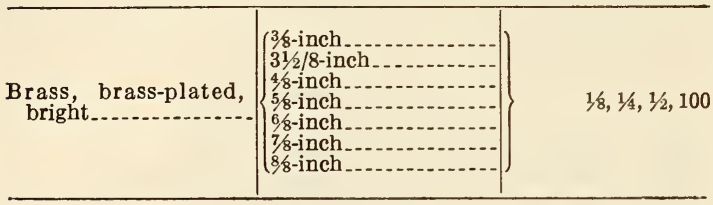

EXTRA IRON CLINCHING

\begin{tabular}{|c|c|c|}
\hline Bright.. & $\left\{\begin{array}{l}3 / 8-i n c h \\
31 / 2 / 8-i n c h \\
4 / 8-i n c h \\
41 / 2 / 8-i n c h . \\
5 / 8 \text {-inch } \\
51 / 2 / 8-i n c h . \\
6 / 8-i n c h \\
7 / 8-i n c h \\
8 / 8-i n c h\end{array}\right.$ & $\begin{array}{l}\text { Pounds } \\
1 / 8,1 / 4,1 / 2,1,100\end{array}$ \\
\hline
\end{tabular}

EXTRA CLINCHING

[Solid brass]

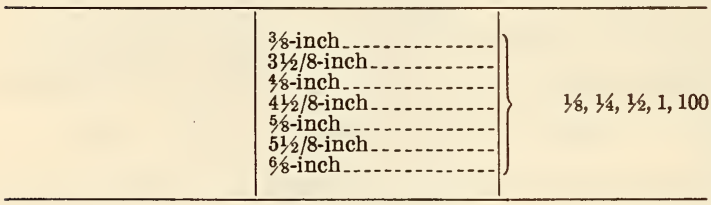

\section{HUNGARIAN NAILS}

Eliminate all styles and gages except-

ROUND HEAD NOS. 12 AND 13

\begin{tabular}{|c|c|c|}
\hline Bright.... & $\left\{\begin{array}{l}3 / 8 \text {-inch } \\
31 / 2 / 8-i n c h- \\
48-\text {-inch } \\
5 / 8 \text {-inch }\end{array}\right\}$ & $34,1 / 2,1,25,100$ \\
\hline
\end{tabular}

SHOT HEAD NO. 14

\begin{tabular}{|c|c|c|}
\hline Bright... & 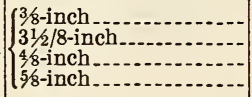 & $1 / 4,1 / 2,1,25,100$ \\
\hline
\end{tabular}

SHOT HEAD NO. 15

Bright_................ $\mid\left\{\begin{array}{l}3 / 8 \text {-inch } 31 / 2 / 8 \text {-inch................. } \\ \hline\end{array}\right.$

CONE HEAD NOS. 12 AND 13

\begin{tabular}{|c|c|c|}
\hline Bright.... & $\left|\begin{array}{l}3 / 8 \text {-inch } \\
31 / 2 / 8-\text { inch } \\
4 / 8-1 \text {-inch } \\
5 / 8 \text {-inch }\end{array}\right|$ & $1 / 4,1 / 2,1,25,100$ \\
\hline
\end{tabular}

GROOVED HEAD NO. 10

\begin{tabular}{|c|c|c|}
\hline Style and finishes & Designation & Sizes of packages \\
\hline Bright........ & $\left\{\begin{array}{l}3 / 8 \text {-inch } \\
31 / 2 / 8 \text {-inch } \\
4 / 8-i n c h \\
5 / 8-i n c h \\
6 / 8-i n c h . . .2 .\end{array}\right.$ & $\begin{array}{l}\text { Pounds } \\
1 / 4,1 / 2,1,25,100\end{array}$ \\
\hline \multicolumn{3}{|c|}{ SMOOTH HEAD NO. 10} \\
\hline Bright.. & $\left\{\begin{array}{l}3 / 8 \text {-inch } \\
31 / 2 / 8 \text {-inch...... } \\
4 / 8 \text {-inch }\end{array}\right.$ & $1 / 1,1 / 2,1,25,100$ \\
\hline
\end{tabular}

\section{CHANNEL NAILS}

(Regular and sharp points)

$[18,19$, and 20 gage $]$

\begin{tabular}{|c|c|c|}
\hline Bright, blued.-- & 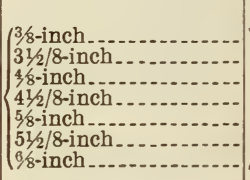 & $\begin{array}{l}\text { Pounds } \\
\qquad 1 / 4,1 / 2,100\end{array}$ \\
\hline
\end{tabular}

\section{HEEL NAILS}

[Gages Nos. 13, 14, 15, 16, and 17]

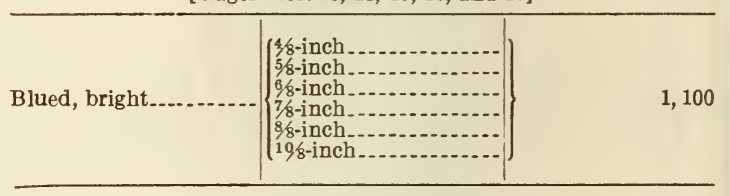

HAND SHOE TACKS

\begin{tabular}{|c|c|c|}
\hline Bright... & 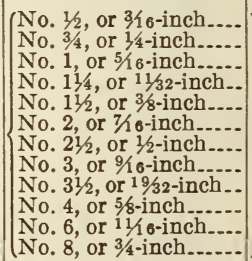 & $1 / 8,1 / 4,1 / 2,1,100$ \\
\hline
\end{tabular}

All special trade names for tacks, such as the following, have been eliminated: American cut, Swedes cut (substituting the name trimmers), Sweden carpet, baling, canvas, cheese-box, curtain, shade, broom, stamp, gagers, railroad, roofing, lookingglass, truckers, and berry-box tacks.

All tacks shall be labeled as designated in the above simplified schedule (by both number and size) on both outside and inside of package or individual container.

The conference recommends that the sale of tacks by count be discontinued, and that tacks shall be sold by weight only.

\section{UNITED STATES GOVERNMENT, FEDERAI SPECIFICATIONS BOARD, FEDERAL SPECIFICATION FOR NAILS, SPIKES, TACKS, AND STAPLES: FF-N-101, MAY 3, 1932}

\section{NAILS, SPIKES, TACKS, AND STAPLES}

\section{GENERAL SPECIFICATIONS}

There are no general specifications applicable to this specification. 


\section{TYPES AND CLASSES}

1. The types and classes of articles covered by this specification are limited to those named in the following list:

(a) Nails.-

(1) Barbed.

(1a) Barrel.

(2) Basket.

(3) Boat.

(4) Box.

(5) Broom.

(6) Car.

(7). Casing.

(8) Cement.

(9) Clinch.

(10) Clout.

(11a) Common brads.

(11b) Common nails.

(11c) Cut nails.

(11d) Copper-clad.

(11e) Copper and brass.

(11f) Cut copper.

(12) Double-head.

(13) Dowel (pins).

(14) Escutcheon (pins).

(15) Fence.

(16) Finishing.

(16a) Finishing, cut.

(17) Flooring.

(18) Foundry.

(19) Heel.

(20) Hinge.

(21) Hob.

(22) Horseshoe.

(23) Lath.

(24) Molding.

(25) Plaster-board.

(26) Purlin.

(27) Roofing.

(28) Saddle.

(29) Saddlery.

(30) Sheathing.

(31) Shingle.

(32) Shoe.

(33) Siding.

(34) Slating.

(35) Stem nails.

(36) Storm (rivets).

(37) Upholsterer's.

(b) Spikes.- Barge.

(1) Barge.

(3) Common (cut).

(4) Gutter.

(5) Round Wire.

(6) Track.

(c) Tacks.-
(1) Bill poster's.
(2) Carpet.
(2a) Copper.
(2b) Galvanized.
(3) Double pointed.
(4) Gimp.
(5) Saddle.
(6) Shoe.
(7) Upholsterer's.

(d) Staples.-
(1) Electrician's.
(2) Metal lath.
(3) Poultry netting.
(4) Wire fence.

\section{MATERIALS AND WORKMANSHIP}

1. Materials.-Articles included in this specification may be of any of the following materials, as designated by the purchaser: (a) Iron or steel, (b) copper, (c) zinc, $(d)$ brass, (e) muntz (yellow) metal, $(f)$ tin (coating only), and $(g)$ copper-bearing steel.

2. Quality of Materials-(a) Iron or steel.-(1) Iron or steel articles made from wire shall withstand cold bending $180^{\circ}$ flat on themselves without fracture.

(2) Iron or steel articles made from bars or plate, or made from rods (and not requiring to be hardened), shall withstand cold bending $180^{\circ}$ over a mandrel of the same diameter as the article to be tested, without fracture.

(3) Specially hardened steel articles shall break off square before bending $60^{\circ}$ and show a fine crystalline texture where broken.

(b) Copper.-(1) Copper shall show on analysis not less than 98 per cent pure copper.

(2) Copper-clad articles shall show on the normal section a thickness of copper not less than onetwelfth of the over-all diameter.

(3) Copper articles and copper-clad articles shall have the same ductility as required for articles of iron or steel wire. III, 2 (a) (1). Copper-clad articles shall not show any separation of the metals when tested for ductility.

(c) Zinc.-Zinc (except for coating) shall be equal to grade D of United States Government master specification for slab zinc (spelter), Federal Specifications Board specification No. 91 (current designations: QQ-Z-351). (See 683.1, p. 1311.)

(d) Brass-(1) Composition.-Copper, 59 to 63 per cent; tin, 0.5 to 1.5 per cent; iron, maximum, 0.06 per cent; lead, maximum, 0.2 per cent; and zinc, remainder.

(2) Ductility.- Same as required for articles of iron or steel wire. III, 2, (a), (1).

(e) Muntz metal-Composition-Copper, 59 per cent to 62 per cent; zinc, 38 per cent to 41 per cent; lead, maximum, 0.6 per cent; and iron, maximum, 0.2 per cent.

(f) Copper-bearing steel-(1) Composition.-Not less than 0.20 per cent of copper.

(2) Ductility.- Same as required for articles of iron or steel wire. III, 2, (a), (1).

3. Workmanship.-Articles of any type shall be true to shape, well finished, and be reasonably free from imperfectly formed or misshapen pieces and not vary in their principal dimensions more than the tolerances herein specified. All articles shall be clean and free from corrosion.

4. Finish-(a) Formation.-(1) Smooth and (2) barbed.

(b) Color.-(1) Black (annealed), (2) bright, (3) blued, and (4) coppered.

(c) Coatings.-(1) Zinc-coated articles shall be reasonably smooth and free from fins or lumps of zinc, and black or uncoated spots. 
(2) Zinc-coated articles shall carry a coating of not less than the following percentages of zinc to the weights of the uncoated articles for the diameters or thicknesses given.

(3) These percentages are equivalent to approximately 1 ounce of zinc per square foot of surface.

(4) Minimum percentages of zinc for coating articles of the diameters or thicknesses given:

\begin{tabular}{|c|c|c|c|c|c|}
\hline \multicolumn{3}{|c|}{$\begin{array}{l}\text { U. S. steel-wire gage, circular } \\
\text { sections }\end{array}$} & \multicolumn{3}{|c|}{$\begin{array}{l}\text { U. S. sheet-steel gage, square } \\
\text { sections }\end{array}$} \\
\hline Gage No. & Diameter & $\begin{array}{c}\text { Percent- } \\
\text { age } \\
\text { (zinc) }\end{array}$ & Gage No. & $\begin{array}{l}\text { Thick- } \\
\text { ness }\end{array}$ & $\begin{array}{l}\text { Percent- } \\
\text { age } \\
\text { (zinc) }\end{array}$ \\
\hline $\begin{array}{l}16 \\
15\end{array}$ & $\begin{array}{l}\text { Inch } \\
0.348 \\
.0475 \\
.054 \\
.0625 \\
.072\end{array}$ & $\begin{array}{l}21.5 \\
15.0 \\
13.25 \\
11.75 \\
10.2\end{array}$ & $\begin{array}{l}16 . .- \\
15-. \\
14-. \\
13-. \\
12 . .\end{array}$ & $\begin{array}{l}\text { Inch } \\
0.0625 \\
.0703 \\
.078 \\
.0937 \\
.1093\end{array}$ & $\begin{array}{r}11.5 \\
9.9 \\
9.0 \\
7.6 \\
6.7\end{array}$ \\
\hline $11 . .$. & $\begin{array}{l}.08 \\
.0915 \\
.1055 \\
.1205\end{array}$ & $\begin{array}{l}8.9 \\
7.85 \\
6.9 \\
6.15\end{array}$ & $\begin{array}{l}11 \ldots \\
10 . . . \\
9 \ldots \\
8 . . . . \\
\end{array}$ & $\begin{array}{l}.125 \\
.1406 \\
.1562 \\
.1718\end{array}$ & $\begin{array}{l}5.8 \\
5.15 \\
4.5 \\
4.1\end{array}$ \\
\hline $\begin{array}{l}10 \ldots \ldots \\
9 \\
9 \\
8 \\
7 \\
\end{array}$ & $\begin{array}{l}.135 \\
.1483 \\
.162 \\
.177\end{array}$ & $\begin{array}{l}5.6 \\
5.1 \\
4.7 \\
4.3\end{array}$ & $\begin{array}{l}7 \ldots \\
6-. \\
5-. \\
4 \ldots \\
-.-\end{array}$ & $\begin{array}{l}.1875 \\
.203 \\
.2187 \\
.2344\end{array}$ & $\begin{array}{l}3.7 \\
3.35 \\
3.15 \\
2.95\end{array}$ \\
\hline 6 & $\begin{array}{l}.192 \\
.207 \\
.225 \\
.2437\end{array}$ & $\begin{array}{l}\text { 3. } 95 \\
\text { 3. } 65 \\
3.3 \\
3.0\end{array}$ & 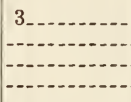 & $\begin{array}{l}.25 \\
5 / 16 \\
338 \\
7 / 16\end{array}$ & $\begin{array}{l}2.8 \\
2.10 \\
1.8 \\
1.55\end{array}$ \\
\hline $\begin{array}{l}2 \\
1 \\
0 \\
00\end{array}$ & $\begin{array}{l}.2625 \\
.283 \\
.3065 \\
.331\end{array}$ & $\begin{array}{l}2.7 \\
2.4 \\
2.15 \\
2.0\end{array}$ & \begin{tabular}{l}
$-\cdot-\cdot$ \\
\hdashline$-\cdot-$ \\
---
\end{tabular} & $\begin{array}{l}1 / 2 \\
9 / 16 \\
5 / 8\end{array}$ & $\begin{array}{l}1.4 \\
1.3 \\
1.2\end{array}$ \\
\hline
\end{tabular}

(5) Tin coating shall be smooth and bright.

(6) Brass plating shall be smooth and bright.

(7) Cement coating shall be evenly applied and shall not be sticky or gummy in any normal temperatures or atmospheric conditions.

(8) Cement-coated articles shall show an average increase in holding power, above that of identical uncoated articles, of not less than 50 per cent when tested as herein specified.

\section{GENERAL REQUIREMENTS}

1. Dimensions.-(a) Nails with flat head or with square shoulder under head, and all tacks, are measured under head to end of point.

(b) Brad head, flat countersunk head, headless, and cement-coated nails are measured over all, that is, top of head to end of point.

(c) Oval and round countersunk head nails are measured from top of countersink (largest diameter) to end of point.

Dimensions of staples shall be inside measurements.

Lengths and widths are given herein in inches and common fractions; diameters are given herein in common fractions when multiples of one-sixty-fourth inch; otherwise diameters are given in decimals of an inch. Diameter of pointed nails, tacks, etc., shall be measured near the gripper marks.

Gage numbers given herein for wire are U. S. steel-wire gage.

2. Tolerances.-Variation in diameter of articles made from wire shall not exceed one-quarter gage above or below the dimensions specified.

Variations in diameters of articles made from rods shall not exceed one-thirty-second inch above nor one-sixty-fourth inch below the dimension specified.
Measurements of diameters shall not include coatings.

Variation in length for articles $1 \frac{1}{2}$ inches long or less shall not exceed one-thirty-second inch and for articles over $1 \frac{1}{2}$ inches long shall not exceed onesixteenth inch from the length specified, except that variations in length for articles one-half inch or more in diameter shall not exceed one-eighth inch for articles up to 10 inches in length, and not more than one-fourth inch for articles 10 inches or over in length.

Articles intended for use in nail-driving machines shall be so sorted as to contain no pieces of different sizes or shapes.

\section{DETAIL REQUIREMENTS}

1. The articles herein listed shall conform to the descriptions for the classes named and to the dimensions given within the tolerances specified. The approximate number per pound when given, is for the purchaser's information and is not to be taken as accurate within any specified limits.

2. The finish, where more than one finish is specified, shall be as stated in the purchase order.

3 . In the tables included herein the various dimensions, etc., are indicated as follows:

$\mathrm{S}=$ nominal size or trade designation.

$\mathrm{L}=$ length in inches.

$\mathrm{D}=$ diameter in inches.

$\mathrm{G}=$ gage.

$\mathrm{H}=$ width of head.

No./lb.=Approximate number per pound.

(a) Nails-

(1) Barbed nails.-Flat head, diamond point, cement coated, brass and steel.

\begin{tabular}{|c|c|c|c|c|}
\hline \multirow{2}{*}{$\mathrm{L}$} & \multicolumn{4}{|c|}{ Diameter } \\
\hline & $20 \mathrm{G}$ & $18 \mathrm{G}$ & $16 \mathrm{G}$ & $14 \mathrm{G}$ \\
\hline $1 / 4$ & 0.035 & & & \\
\hline $3 / 8$ & .035 & 0.047 & & \\
\hline $\begin{array}{l}1 / 2 \\
5 / 8\end{array}$ & $\begin{array}{l}.035 \\
.035\end{array}$ & $\begin{array}{l}.047 \\
.047\end{array}$ & $\begin{array}{r}0.062 \\
.062\end{array}$ & \\
\hline $3 / 4$ & & .047 & .062 & 0.080 \\
\hline 1 & - & .047 & .062 & .080 \\
\hline $\begin{array}{l}114 \\
113\end{array}$ & ... & - n & .062 & .080 \\
\hline & & & 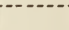 & \\
\hline
\end{tabular}

(1a) Barrel nails.-Flat head, diamond point.

\begin{tabular}{|c|c|c|c|c|c|c|}
\hline \multirow{2}{*}{ L } & \multicolumn{3}{|c|}{ Bright } & \multicolumn{3}{|c|}{ Cement coated } \\
\hline & G & $\mathrm{D}$ & No./lb. & G & D & No./lb. \\
\hline $\begin{array}{l}5 / 8 \\
3 / 4 \\
1^{7 / 8}\end{array}$ & $\begin{array}{l}151 / 2 \\
151 / 2 \\
141 / 2 \\
141 / 2\end{array}$ & $\begin{array}{r}0.067 \\
.067 \\
.076 \\
.076\end{array}$ & $\begin{array}{r}1,615 \\
1,346 \\
906 \\
755\end{array}$ & $\begin{array}{l}151 / 2 \\
141 / 2 \\
141 / 2\end{array}$ & $\begin{array}{r}0.067 \\
.076 \\
.076\end{array}$ & $\begin{array}{r}1,345 \\
905 \\
774\end{array}$ \\
\hline $\begin{array}{l}11 / 8 \\
11 / 4 \\
138 \\
11 / 2\end{array}$ & $\begin{array}{l}141 / 2 \\
14 \\
13 \\
13\end{array}$ & $\begin{array}{l}.076 \\
.080 \\
.092 \\
.092\end{array}$ & $\begin{array}{l}700 \\
568 \\
400 \\
357\end{array}$ & $\begin{array}{l}141 / 2 \\
14 \\
13 \\
13\end{array}$ & $\begin{array}{l}.076 \\
.080 \\
.092 \\
.092\end{array}$ & $\begin{array}{l}699 \\
567 \\
399 \\
366\end{array}$ \\
\hline
\end{tabular}

(2) Basket nails.-Flat head, needle point, bright

\begin{tabular}{|c|c|c|c|}
\hline $\mathrm{L}$ & $\mathrm{G}$ & $\mathrm{D}$ & No./lb. \\
\hline $\begin{array}{c}5 / 8 \\
3 / 4\end{array}$ & 18 & $\begin{array}{c}0.0475 \\
.0475\end{array}$ & 2,910 \\
\hline
\end{tabular}


(3) Boat nails.-Oval countersunk head, chisel point, bright (B) or zinc coated. (Z).

\begin{tabular}{|c|c|c|c|c|c|c|c|c|c|}
\hline \multicolumn{6}{|c|}{ Light } & \multicolumn{4}{|c|}{ Heavy } \\
\hline S & $\mathbf{L}$ & $\mathrm{D}$ & $\mathrm{H}$ & No./lb.B & No./lb.Z & $\mathrm{D}$ & $\mathrm{H}$ & No./lb.B & No./lb. \\
\hline $\begin{array}{l}4 \mathrm{~d} . . . \\
6 \mathrm{~d}-.- \\
8 \mathrm{~d}-.- \\
10 \mathrm{~d}-- \\
12 \mathrm{~d}-- \\
16 \mathrm{~d}-- \\
20 \mathrm{~d}-.\end{array}$ & $\begin{array}{l}11 / 2 \\
2 \\
21 / 2 \\
3 \\
31 / 4 \\
31 / 2 \\
4\end{array}$ & $\begin{array}{l}3 / 16 \\
3 / 16 \\
3 / 16 \\
1 / 4 \\
1 / 4 \\
1 / 4 \\
1 / 4\end{array}$ & $\begin{array}{l}13 / 32 \\
13 / 32 \\
13 / 32 \\
1 / 2 \\
1 / 2 \\
1 / 2 \\
1 / 2\end{array}$ & $\begin{array}{l}82 \\
62 \\
50 \\
22 \\
20 \\
18 \\
16\end{array}$ & $\begin{array}{l}79 \\
59 \\
48 \\
21 \\
19 \\
17.5 \\
15.5\end{array}$ & $\begin{array}{l}1 / 4 \\
1 / 4 \\
1 / 4 \\
3 / 8 \\
3 / 8 \\
3 / 8 \\
3 / 8\end{array}$ & $\begin{array}{l}1 / 2 \\
1 / 2 \\
1 / 2 \\
3 / 4 \\
3 / 4 \\
3 / 4 \\
3 / 4\end{array}$ & $\begin{array}{l}44 \\
32 \\
26 \\
14 \\
13 \\
12 \\
10\end{array}$ & $\begin{array}{l}43 \\
31 \\
25 \\
13 \\
12.8 \\
11.8 \\
9.8\end{array}$ \\
\hline
\end{tabular}

(4) Box nails.-Broad flat reinforced head, diamond point, smooth or barbed.

\begin{tabular}{|c|c|c|c|c|c|c|c|c|}
\hline \multirow{2}{*}{$\mathrm{s}$} & \multicolumn{4}{|c|}{ Bright } & \multicolumn{4}{|c|}{ Cement coated } \\
\hline & $\mathrm{L}$ & G & D & No./lb. & $\mathrm{L}$ & G & D & No./lb. \\
\hline $\begin{array}{l}2 \mathrm{~d} \\
3 \mathrm{~d} \\
4 \mathrm{~d} \\
5 \mathrm{~d} \\
6 \mathrm{~d}\end{array}$ & \begin{tabular}{l|}
1 \\
$11 / 4$ \\
$11 / 2$ \\
$13 / 4$ \\
2
\end{tabular} & $\begin{array}{l}151 / 2 \\
141 / 2 \\
14 \\
14 \\
121 / 2\end{array}$ & $\begin{array}{r}0.067 \\
.076 \\
.080 \\
.080 \\
.098\end{array}$ & $\begin{array}{r}1,010 \\
635 \\
473 \\
406 \\
236\end{array}$ & $\begin{array}{l}1 \\
11 / 8 \\
13 / 8 \\
15 / 8 \\
17 / 8\end{array}$ & $\begin{array}{l}161 / 2 \\
16 \\
151 / 2 \\
15 \\
131 / 2\end{array}$ & $\begin{array}{r}0.058 \\
.063 \\
.067 \\
.072 \\
.086\end{array}$ & $\begin{array}{r}1,300 \\
950 \\
710 \\
537 \\
306\end{array}$ \\
\hline $\begin{array}{r}7 \mathrm{~d} \\
8 \mathrm{~d} \\
9 \mathrm{~d} \\
10 \mathrm{~d} \\
12 \mathrm{~d}\end{array}$ & $\begin{array}{l}21 / 4 \\
21 / 2 \\
23 / 4 \\
3 \\
31 / 4\end{array}$ & $\begin{array}{l}121 / 2 \\
111 / 2 \\
111 / 2 \\
101 / 2 \\
101 / 2\end{array}$ & $\begin{array}{r}.098 \\
.113 \\
.113 \\
.128 \\
.128\end{array}$ & $\begin{array}{r}210 \\
145 \\
132 \\
94 \\
88\end{array}$ & $\begin{array}{l}21 / 8 \\
238 \\
25 / 8 \\
27 / 8 \\
31 / 8\end{array}$ & $\begin{array}{l}131 / 2 \\
121 \\
121 / 2 \\
111 / 2 \\
111 / 2\end{array}$ & $\begin{array}{l}.086 \\
.098 \\
.098 \\
.113 \\
.113\end{array}$ & $\begin{array}{l}268 \\
186 \\
168 \\
118 \\
108\end{array}$ \\
\hline $\begin{array}{l}16 \mathrm{~d} \\
20 \mathrm{~d} \\
30 \mathrm{~d} \\
40 \mathrm{~d}\end{array}$ & $\begin{array}{l}31 / 2 \\
41 / 2 \\
5\end{array}$ & $\begin{array}{r}10 \\
9 \\
9 \\
8\end{array}$ & $\begin{array}{r}.135 \\
.148 \\
.148 \\
.162\end{array}$ & $\begin{array}{l}71 \\
52 \\
46 \\
35\end{array}$ & $\begin{array}{l}33 / 8 \\
37 / 8 \\
4^{3} / 8 \\
4^{7} / 8\end{array}$ & $\begin{array}{r}11 \\
10 \\
10 \\
9\end{array}$ & $\begin{array}{l}.120 \\
.135 \\
.135 \\
.148\end{array}$ & $\begin{array}{l}91 \\
62 \\
52 \\
41\end{array}$ \\
\hline
\end{tabular}

(4a) Box nails.-Coolers, flat head, cement coated; sinkers, countersunk head, cement coated.

\begin{tabular}{|c|c|c|c|c|}
\hline S & $\mathrm{L}$ & G & D & No./lb. \\
\hline $\begin{array}{l}2 d \\
3 d \\
4 d \\
5 d \\
6 d\end{array}$ & $\begin{array}{l}1 \\
11 / 8 \\
13 / 8 \\
15 / 8 \\
17 / 8\end{array}$ & $\begin{array}{l}16 \\
151 / 2 \\
14 \\
131 / 2 \\
13\end{array}$ & $\begin{array}{l}0.0625 \\
.0673 \\
.08 \\
.0858 \\
.0915\end{array}$ & $\begin{array}{r}1,084 \\
848 \\
488 \\
364 \\
275\end{array}$ \\
\hline $\begin{array}{r}7 \mathrm{~d} \\
8 \mathrm{~d} \\
9 \mathrm{~d} \\
10 \mathrm{~d}\end{array}$ & $\begin{array}{l}218 \\
23 / 8 \\
25 / 8 \\
27 \% 8\end{array}$ & $\begin{array}{l}121 / 2 \\
11 / 2 \\
111 / 2 \\
11\end{array}$ & $\begin{array}{l}.0985 \\
.113 \\
.113 \\
.120\end{array}$ & $\begin{array}{l}212 \\
144 \\
127 \\
104\end{array}$ \\
\hline
\end{tabular}

(5) Broom nails.-Flat head or star head, diamond point, smooth, bright.

\begin{tabular}{|c|c|c|c|}
\hline L & G & D & No./lb. \\
\hline $\begin{array}{l}5 / 8 \\
5 / 8 \\
3 / 4 \\
3 / 4\end{array}$ & $\begin{array}{l}15 \\
14 \\
15 \\
14\end{array}$ & $\begin{array}{r}0.072 \\
.080 \\
.072 \\
.080\end{array}$ & $\begin{array}{r}1,256 \\
988 \\
1,056 \\
863\end{array}$ \\
\hline
\end{tabular}

(6) Car nails.-Countersunk heads, either flat or oval; diamond point; barbed.

BRIGHT OR ANNEALED

\begin{tabular}{|c|c|c|c|c|c|c|c|}
\hline \multirow{2}{*}{$\mathrm{S}$} & \multirow{2}{*}{ L } & \multicolumn{3}{|c|}{ Geavy } & \multicolumn{3}{|c|}{ Light } \\
\hline & & G & $\mathrm{D}$ & No./lb. & $\mathrm{G}$ & $\mathrm{D}$ & No./lb. \\
\hline $\begin{array}{l}4 d \\
5 d \\
6 d \\
7 d \\
8 d\end{array}$ & $\begin{array}{l}11 / 2 \\
13 / 4 \\
2 \\
21 / 4 \\
21 / 2\end{array}$ & $\begin{array}{r}10 \\
9 \\
9 \\
8 \\
8\end{array}$ & $\begin{array}{r}0.135 \\
.148 \\
.148 \\
.162 \\
.162\end{array}$ & $\begin{array}{r}165 \\
118 \\
103 \\
76 \\
69\end{array}$ & $\begin{array}{r}12 \\
10 \\
10 \\
9 \\
9\end{array}$ & $\begin{array}{c}0.1055 \\
.135 \\
.135 \\
.148 \\
.148\end{array}$ & $\begin{array}{r}274 \\
142 \\
124 \\
92 \\
82\end{array}$ \\
\hline $\begin{array}{r}9 \mathrm{~d} \\
10 \mathrm{~d} \\
12 \mathrm{~d} \\
16 \mathrm{~d} \\
20 \mathrm{~d}\end{array}$ & $\begin{array}{l}23 / 4 \\
3 \\
31 / 4 \\
31 / 2 \\
4\end{array}$ & $\begin{array}{l}7 \\
7 \\
6 \\
6 \\
5\end{array}$ & $\begin{array}{r}.177 \\
.177 \\
.192 \\
.192 \\
.207\end{array}$ & $\begin{array}{l}54 \\
50 \\
42 \\
35 \\
26\end{array}$ & $\begin{array}{l}8 \\
8 \\
7 \\
7 \\
6\end{array}$ & $\begin{array}{l}.162 \\
.162 \\
.177 \\
.177 \\
.192\end{array}$ & $\begin{array}{l}62 \\
57 \\
50 \\
43 \\
31\end{array}$ \\
\hline $\begin{array}{l}30 \mathrm{~d} \\
40 \mathrm{~d} \\
50 \mathrm{~d} \\
60 \mathrm{~d}\end{array}$ & $\begin{array}{l}41 / 2 \\
5 \\
51 / 2 \\
6\end{array}$ & $\begin{array}{l}5 \\
4 \\
3 \\
3\end{array}$ & $\begin{array}{l}.207 \\
.225 \\
.244 \\
.244\end{array}$ & $\begin{array}{l}24 \\
18 \\
15 \\
13\end{array}$ & $\begin{array}{l}6 \\
5 \\
4 \\
4\end{array}$ & $\begin{array}{r}.192 \\
.207 \\
.225 \\
.225\end{array}$ & $\begin{array}{l}28 \\
21 \\
17 \\
15\end{array}$ \\
\hline
\end{tabular}

CEMENT COATED

\begin{tabular}{|c|c|c|c|c|c|c|c|}
\hline \multirow{2}{*}{$\mathrm{s}$} & \multirow{2}{*}{ L } & \multicolumn{3}{|c|}{ Heavy } & \multicolumn{3}{|c|}{ Light } \\
\hline & & G & D & No./lb. & $G$ & D & No./lb. \\
\hline $\begin{array}{l}4 d \\
5 d \\
6 d \\
7 d \\
8 d\end{array}$ & $\begin{array}{l}11 / 2 \\
13 / 4 \\
2 \\
21 / 4 \\
21 / 2\end{array}$ & $\begin{array}{r}11 \\
10 \\
10 \\
9 \\
9\end{array}$ & $\begin{array}{r}0.120 \\
.135 \\
.135 \\
.148 \\
.148\end{array}$ & $\begin{array}{r}197 \\
138 \\
117 \\
86 \\
78\end{array}$ & $\begin{array}{l}13 \\
11 \\
11 \\
10 \\
10\end{array}$ & $\begin{array}{r}0.091 \\
.120 \\
.120 \\
.135 \\
.135\end{array}$ & $\begin{array}{r}335 \\
177 \\
149 \\
103 \\
96\end{array}$ \\
\hline $\begin{array}{r}9 \mathrm{~d} \\
10 \mathrm{~d} \\
12 \mathrm{~d} \\
16 \mathrm{~d} \\
20 \mathrm{~d}\end{array}$ & $\begin{array}{l}23 / 4 \\
3 \\
31 / 4 \\
31 / 2 \\
4\end{array}$ & $\begin{array}{l}8 \\
8 \\
7 \\
7 \\
6\end{array}$ & $\begin{array}{r}.162 \\
.162 \\
.177 \\
.177 \\
.192\end{array}$ & $\begin{array}{l}62 \\
56 \\
45 \\
39 \\
30\end{array}$ & $\begin{array}{l}9 \\
9 \\
8 \\
8 \\
7\end{array}$ & $\begin{array}{l}.148 \\
.148 \\
.162 \\
.162 \\
.177\end{array}$ & $\begin{array}{l}74 \\
65 \\
52 \\
48 \\
37\end{array}$ \\
\hline $\begin{array}{l}30 \mathrm{~d} \\
40 \mathrm{~d} \\
50 \mathrm{~d} \\
60 \mathrm{~d}\end{array}$ & $\begin{array}{l}41 / 2 \\
5 \\
51 / 2 \\
6\end{array}$ & $\begin{array}{l}6 \\
5 \\
4 \\
4\end{array}$ & $\begin{array}{r}.192 \\
.207 \\
.225 \\
.225\end{array}$ & $\begin{array}{l}27 \\
21 \\
16 \\
14\end{array}$ & $\begin{array}{l}7 \\
6 \\
5 \\
5\end{array}$ & $\begin{array}{l}.177 \\
.192 \\
.207 \\
.207\end{array}$ & $\begin{array}{l}31 \\
24 \\
18 \\
16\end{array}$ \\
\hline
\end{tabular}

(7) Casing nails.-Long countersunk narrow head, diamond point, smooth.

\begin{tabular}{|c|c|c|c|c|c|c|c|}
\hline \multirow{2}{*}{$\mathrm{s}$} & \multirow{2}{*}{$\mathrm{L}$} & \multicolumn{3}{|c|}{ Bright } & \multicolumn{3}{|c|}{ Cement coated } \\
\hline & & G & D & No./lb. & G & D & No./lb. \\
\hline $\begin{array}{l}2 \mathrm{~d} \\
3 \mathrm{~d} \\
4 \mathrm{~d} \\
5 \mathrm{~d} \\
6 \mathrm{~d}\end{array}$ & $\begin{array}{l}1 \\
11 / 4 \\
11 / 2 \\
13 / 4 \\
2\end{array}$ & $\begin{array}{l}151 / 2 \\
141 / 2 \\
14 \\
14 \\
121 / 2\end{array}$ & $\begin{array}{l}0.057 \\
.076 \\
.080 \\
.080 \\
.098\end{array}$ & $\begin{array}{r}1,010 \\
635 \\
473 \\
406 \\
236\end{array}$ & $\begin{array}{l}16 \\
15 \\
15 \\
15 \\
13\end{array}$ & $\begin{array}{r}0.062 \\
.072 \\
.072 \\
.072 \\
.091\end{array}$ & $\begin{array}{r}1,170 \\
686 \\
606 \\
514 \\
272\end{array}$ \\
\hline $\begin{array}{r}7 \mathrm{~d} \\
8 \mathrm{~d} \\
9 \mathrm{~d} \\
10 \mathrm{~d} \\
12 \mathrm{~d}\end{array}$ & $\begin{array}{l}21 / 4 \\
21 / 2 \\
23 / 4 \\
3 \\
31 / 4\end{array}$ & $\begin{array}{l}121 / 2 \\
111 / 2 \\
111 / 2 \\
101 / 2 \\
101 / 2\end{array}$ & $\begin{array}{r}.098 \\
.113 \\
.113 \\
.128 \\
.128\end{array}$ & $\begin{array}{r}210 \\
145 \\
132 \\
94 \\
87\end{array}$ & $\begin{array}{l}13 \\
12 \\
12 \\
11 \\
11\end{array}$ & $\begin{array}{l}.091 \\
.105 \\
.105 \\
.120 \\
.120\end{array}$ & $\begin{array}{r}236 \\
168 \\
146 \\
110 \\
99\end{array}$ \\
\hline $\begin{array}{l}16 \mathrm{~d} \\
20 \mathrm{~d} \\
30 \mathrm{~d} \\
40 \mathrm{~d}\end{array}$ & $\begin{array}{l}31 / 2 \\
4 \\
41 / 2 \\
5\end{array}$ & $\begin{array}{r}10 \\
9 \\
9 \\
8\end{array}$ & $\begin{array}{r}.135 \\
.148 \\
.148 \\
.162\end{array}$ & $\begin{array}{l}71 \\
52 \\
46 \\
35\end{array}$ & $\begin{array}{r}11 \\
10 \\
10 \\
9\end{array}$ & $\begin{array}{r}.120 \\
.135 \\
.135 \\
.148\end{array}$ & $\begin{array}{l}88 \\
63 \\
56 \\
41\end{array}$ \\
\hline
\end{tabular}


(8) Cement nails.-Oval head, diamond point, smooth, bright.

\begin{tabular}{|c|c|c|c|}
\hline $\mathrm{L}$ & $\mathrm{G}$ & $\mathrm{D}$ & No./lb. \\
\hline $1^{5 / 8}$ & $\mathbf{9}$ & $\begin{array}{c}0.148 \\
.162\end{array}$ & $\begin{array}{c}286 \\
153\end{array}$ \\
\hline
\end{tabular}

(9) Clinch nails.-Oval head or flat head; duckbill point; smooth, bright, or annealed.

\begin{tabular}{|c|c|c|c|c|c|c|c|c|c|}
\hline S & L & G & D & No./lb. & $\mathrm{s}$ & L & $G$ & D & No./lb. \\
\hline $\begin{array}{l}2 \mathrm{~d} \\
3 \mathrm{~d} \\
4 \mathrm{~d} \\
5 \mathrm{~d} \\
6 \mathrm{~d} \\
7 \mathrm{~d}\end{array}$ & $\begin{array}{l}1 \\
11 / 4 \\
11 / 2 \\
13 / 4 \\
2 \\
21 / 4\end{array}$ & $\begin{array}{l}14 \\
13 \\
12 \\
12 \\
11 \\
11\end{array}$ & $\begin{array}{r}0.080 \\
.091 \\
.05 \\
.105 \\
.120 \\
.120\end{array}$ & $\begin{array}{l}710 \\
429 \\
274 \\
235 \\
157 \\
139\end{array}$ & $\begin{array}{r}8 \mathrm{~d} \\
9 \mathrm{~d} \\
10 \mathrm{~d} \\
12 \mathrm{~d} \\
16 \mathrm{~d} \\
20 \mathrm{~d}\end{array}$ & $\begin{array}{l}21 / 2 \\
23 / 4 \\
3 \\
31 / 4 \\
31 / 2 \\
4\end{array}$ & $\begin{array}{r}10 \\
10 \\
9 \\
9 \\
8 \\
7\end{array}$ & $\begin{array}{r}0.135 \\
.135 \\
.148 \\
.148 \\
.162 \\
.177\end{array}$ & $\begin{array}{l}99 \\
90 \\
69 \\
62 \\
49 \\
37\end{array}$ \\
\hline
\end{tabular}

(10) Clout nails.-Flat head; duck-bill point; smooth, bright, or tinned.

\begin{tabular}{|c|c|c|c|}
\hline L & G & D & No./lb. \\
\hline $\begin{array}{l}108 \\
3 / 4 \\
7\end{array}$ & 15 & 0.072 & 1,160 \\
\hline $1^{1 / 8}$ & $\begin{array}{l}14 \\
14\end{array}$ & $\begin{array}{l}.080 \\
.080\end{array}$ & $\begin{array}{l}808 \\
705\end{array}$ \\
\hline $11 / 4$ & 13 & .091 & 423 \\
\hline $\begin{array}{l}118 \\
11 / 2\end{array}$ & $\begin{array}{l}13 \\
13\end{array}$ & .091 & $\begin{array}{l}390 \\
350\end{array}$ \\
\hline
\end{tabular}

(11) Common brads.-Long, narrow flat head, diamond point, smooth, bright.

LIGHT GAGE

\begin{tabular}{|c|c|c|c|c|c|}
\hline L & D & No./lb. & L & D & No./lb. \\
\hline Inch & Inch & & Inches & Inch & \\
\hline $\begin{array}{l}3 / 8 \\
1 / 2 \\
\end{array}$ & $\begin{array}{l}0.0348 \\
.0348\end{array}$ & $\begin{array}{l}9,500 \\
7,060\end{array}$ & $\begin{array}{l}11 / 4 \\
11 / 2\end{array}$ & $\begin{array}{l}0.0475 \\
.0625\end{array}$ & 1,550 \\
\hline $5 / 8$ & .0348 & 5,680 & $13 / 4$ & .0625 & 672 \\
\hline $\begin{array}{l}3 / 4 \\
7 / 8\end{array}$ & $\begin{array}{r}.0348 \\
0.0348\end{array}$ & $\begin{array}{r}4,800 \\
4,202\end{array}$ & ${ }_{21}^{2}$ & $\begin{array}{l}.0625 \\
0\end{array}$ & $\begin{array}{l}580 \\
520 \\
320\end{array}$ \\
\hline 18 & .0475 & 2,040 & $21 / 2$ & .080 & 290 \\
\hline
\end{tabular}

MEDIUM GAGE

\begin{tabular}{|c|c|c|c|c|c|}
\hline L & $\mathrm{D}$ & No./lb. & L & $\mathrm{D}$ & No./lb. \\
\hline $\begin{array}{c}\text { Inch } \\
1 / 2 \\
5 / 8 \\
3 / 4 \\
7 / 8 \\
1\end{array}$ & $\begin{array}{c}\text { Inch } \\
0.0475 \\
.0475 \\
.0475 \\
.0475 \\
.0625\end{array}$ & $\begin{array}{l}3,990 \\
3,200 \\
2,620 \\
2,220 \\
1,120\end{array}$ & $\begin{array}{c}\text { Inches } \\
11 / 4 \\
11 / 2 \\
13 / 4 \\
2\end{array}$ & $\begin{array}{c}\text { Inch } \\
0.0625 \\
.080 \\
.080 \\
.080\end{array}$ & $\begin{array}{l}940 \\
470 \\
400 \\
350\end{array}$ \\
\hline
\end{tabular}

(11a) Common nails.-Heavy gages, flat head, diamond point, smooth.

\begin{tabular}{|c|c|c|c|c|c|c|c|c|}
\hline \multirow{2}{*}{$\mathrm{S}$} & \multicolumn{4}{|c|}{ Bright } & \multicolumn{4}{|c|}{ Cement coated } \\
\hline & L & $G$ & $\mathrm{D}$ & No./lb. & $\mathrm{L}$ & G & $\mathrm{D}$ & No./lb. \\
\hline $\begin{array}{l}2 d \\
3 d \\
4 d \\
5 d\end{array}$ & $\begin{array}{l}1 \\
11 / 4 \\
11 / 2 \\
13 / 4\end{array}$ & $\begin{array}{l}15 \\
14 \\
121 / 2 \\
121 / 2\end{array}$ & $\begin{array}{r}0.072 \\
.080 \\
.098 \\
.098\end{array}$ & $\begin{array}{l}876 \\
568 \\
316 \\
271\end{array}$ & \begin{tabular}{l|}
1 \\
$11 / 8$ \\
$13 / 8$ \\
$15 / 8$
\end{tabular} & $\begin{array}{l}16 \\
15 \\
131 / 2 \\
131 / 2\end{array}$ & $\begin{array}{r}0.062 \\
.072 \\
.086 \\
.086\end{array}$ & $\begin{array}{r}1,176 \\
758 \\
427 \\
372\end{array}$ \\
\hline $\begin{array}{l}6 \mathrm{~d} \\
7 \mathrm{~d} \\
8 \mathrm{~d} \\
9 \mathrm{~d}\end{array}$ & $\begin{array}{l}2 \\
21 / 4 \\
21 / 2 \\
23 / 4\end{array}$ & $\begin{array}{l}111 / 2 \\
111 / 2 \\
101 / 4 \\
101 / 4\end{array}$ & $\begin{array}{r}.113 \\
.113 \\
.131 \\
.131\end{array}$ & $\begin{array}{r}181 \\
161 \\
106 \\
96\end{array}$ & $\begin{array}{l}17 / 8 \\
21 / 8 \\
23 / 8 \\
25 / 8\end{array}$ & $\begin{array}{l}121 / 2 \\
121 / 2 \\
11 \\
11\end{array}$ & $\begin{array}{r}.098 \\
.098 \\
.120 \\
.120\end{array}$ & $\begin{array}{l}245 \\
221 \\
134 \\
121\end{array}$ \\
\hline $\begin{array}{l}10 \mathrm{~d} \\
12 \mathrm{~d} \\
16 \mathrm{~d} \\
20 \mathrm{~d}\end{array}$ & $\begin{array}{l}3 \\
31 / 4 \\
31 / 2 \\
4\end{array}$ & $\begin{array}{l}9 \\
9 \\
8 \\
6\end{array}$ & $\begin{array}{l}.148 \\
.148 \\
.162 \\
.192\end{array}$ & $\begin{array}{l}69 \\
64 \\
49 \\
31\end{array}$ & $\begin{array}{l}27 / 8 \\
31 / 8 \\
33 / 8 \\
37 / 8\end{array}$ & $\begin{array}{r}10 \\
10 \\
9 \\
7\end{array}$ & $\begin{array}{r}.135 \\
.135 \\
.148 \\
.177\end{array}$ & $\begin{array}{l}89 \\
80\end{array}$ \\
\hline $\begin{array}{l}30 \mathrm{~d} \\
40 \mathrm{~d} \\
50 \mathrm{~d} \\
60 \mathrm{~d}\end{array}$ & $\begin{array}{l}41 / 2 \\
5 \\
51 / 2 \\
6\end{array}$ & $\begin{array}{l}5 \\
4 \\
3 \\
2\end{array}$ & $\begin{array}{l}.207 \\
.225 \\
.244 \\
.262\end{array}$ & $\begin{array}{l}24 \\
18 \\
16 \\
11\end{array}$ & $\begin{array}{l}43 / 8 \\
47 / 8 \\
53 / 8 \\
57 / 8\end{array}$ & $\begin{array}{l}6 \\
5 \\
4 \\
3\end{array}$ & $\begin{array}{l}.192 \\
.207 \\
.225 \\
.244\end{array}$ & $\begin{array}{l}29 \\
22 \\
17 \\
13\end{array}$ \\
\hline
\end{tabular}

(11b) Common nails.-Flat head, diamond point, smooth.

\begin{tabular}{|c|c|c|c|c|c|c|c|c|}
\hline \multirow{2}{*}{$\mathrm{S}$} & \multicolumn{4}{|c|}{ Bright } & \multicolumn{4}{|c|}{ Cement coated } \\
\hline & L & $\mathrm{G}$ & $\mathrm{D}$ & No./lb. & L & G & $\mathrm{D}$ & No./lb. \\
\hline $\begin{array}{l}2 \mathrm{~d} . . \\
3 \mathrm{~d}-. . \\
4 \mathrm{~d}-. . \\
5 \mathrm{~d} . .\end{array}$ & $\begin{array}{l}1 \\
11 / 4 \\
11 / 2 \\
13 / 4\end{array}$ & $\begin{array}{l}15 \\
14 \\
121 / 2 \\
121 / 2\end{array}$ & $\begin{array}{r}0.072 \\
.080 \\
.098 \\
.098\end{array}$ & $\begin{array}{l}876 \\
568 \\
316 \\
271\end{array}$ & $\begin{array}{l}1 \\
11 / 8 \\
13 / 8 \\
15 / 8\end{array}$ & $\begin{array}{l}16 \\
151 / 2 \\
14 \\
131 / 2\end{array}$ & $\begin{array}{r}0.062 \\
.067 \\
.080 \\
.086\end{array}$ & $\begin{array}{r}1,085 \\
850 \\
490 \\
364\end{array}$ \\
\hline $\begin{array}{l}\text { 6d.... } \\
\text { 7d... } \\
\text { 8d.-. } \\
\text { 9d.... }\end{array}$ & $\begin{array}{l}2 \\
21 / 4 \\
21 / 2 \\
23 / 4\end{array}$ & $\begin{array}{l}111 / 2 \\
111 / 2 \\
101 / 4 \\
101 / 4\end{array}$ & $\begin{array}{r}.113 \\
.113 \\
.131 \\
.131\end{array}$ & $\begin{array}{r}181 \\
161 \\
106 \\
96\end{array}$ & $\begin{array}{l}17 / 8 \\
21 / 8 \\
23 / 8 \\
25 / 8\end{array}$ & $\begin{array}{l}13 \\
121 / 2 \\
111 / 2 \\
111 / 2\end{array}$ & $\begin{array}{l}.091 \\
.098 \\
.113 \\
.113\end{array}$ & $\begin{array}{l}275 \\
212 \\
142 \\
130\end{array}$ \\
\hline $\begin{array}{l}\text { 10d.- } \\
12 \mathrm{~d} . \\
16 \mathrm{~d}-. \\
20 \mathrm{~d} .\end{array}$ & $\begin{array}{l}3 \\
31 / 4 \\
31 / 2 \\
4\end{array}$ & $\begin{array}{l}9 \\
9 \\
8 \\
6\end{array}$ & $\begin{array}{r}148 \\
.148 \\
.162 \\
.192\end{array}$ & $\begin{array}{l}69 \\
63 \\
49 \\
31\end{array}$ & $\begin{array}{l}27 / 8 \\
31 / 8 \\
31 / 4 \\
33 / 4\end{array}$ & $\begin{array}{r}11 \\
10 \\
9 \\
7\end{array}$ & $\begin{array}{l}.120 \\
.135 \\
.148 \\
.177\end{array}$ & $\begin{array}{r}105 \\
77 \\
61 \\
37\end{array}$ \\
\hline $\begin{array}{l}30 \mathrm{~d} . . \\
40 \mathrm{~d} . . \\
50 \mathrm{~d}- \\
60 \mathrm{~d}-\end{array}$ & $\begin{array}{l}41 / 2 \\
5 \\
51 / 2 \\
6\end{array}$ & $\begin{array}{l}5 \\
4 \\
3 \\
2\end{array}$ & $\begin{array}{l}.207 \\
.225 \\
.244 \\
.262\end{array}$ & $\begin{array}{l}24 \\
18 \\
14 \\
11\end{array}$ & $\begin{array}{l}41 / 4 \\
43 \\
51 / 4 \\
53 / 4\end{array}$ & $\begin{array}{l}6 \\
5 \\
4 \\
3\end{array}$ & $\begin{array}{l}.192 \\
.207 \\
.225 \\
.244\end{array}$ & $\begin{array}{l}16 \\
13\end{array}$ \\
\hline
\end{tabular}

Values for zinc-coated nails are the same as for bright above except the number per pound which are as follows: $788,517,293,251,171,153,100,90$, $65,60,47,30,23,17.5,13.5,10.5$.

(11c) Cut iron or steel common nails.-

\begin{tabular}{|c|c|c|c|}
\hline \multirow{2}{*}{$\mathrm{S}$} & \multirow{2}{*}{ L } & \multicolumn{2}{|c|}{$\begin{array}{l}\text { Approximate } \\
\text { number per } \\
\text { pound }\end{array}$} \\
\hline & & Plain & $\begin{array}{l}\text { Zinc, } \\
\text { coated }\end{array}$ \\
\hline $\begin{array}{r}2 \mathrm{~d} \\
3 \mathrm{~d} \\
31 / 2 \mathrm{~d} \\
4 \mathrm{~d} \\
5 \mathrm{~d}\end{array}$ & $\begin{array}{l}1 \\
11 / 4 \\
13 / 8 \\
11 / 2 \\
13 / 4\end{array}$ & $\begin{array}{l}667 \\
448 \\
372 \\
300 \\
200\end{array}$ & $\begin{array}{l}580 \\
390 \\
340 \\
275 \\
185\end{array}$ \\
\hline $\begin{array}{l}6 \mathrm{~d} \\
7 \mathrm{~d} \\
8 \mathrm{~d} \\
9 \mathrm{~d}\end{array}$ & $\begin{array}{l}2 \\
21 / 4 \\
21 / 2 \\
23 / 4\end{array}$ & $\begin{array}{r}150 \\
120 \\
96 \\
72\end{array}$ & $\begin{array}{r}141 \\
113 \\
92 \\
69\end{array}$ \\
\hline $\begin{array}{l}10 \mathrm{~d} \\
12 \mathrm{~d} \\
16 \mathrm{~d} \\
20 \mathrm{~d}\end{array}$ & $\begin{array}{l}3 \\
31 / 4 \\
31 / 2 \\
4\end{array}$ & $\begin{array}{l}56 \\
44 \\
30 \\
25\end{array}$ & $\begin{array}{l}54 \\
42 \\
29 \\
24\end{array}$ \\
\hline $\begin{array}{l}30 \mathrm{~d} \\
40 \mathrm{~d} \\
50 \mathrm{~d} \\
60 \mathrm{~d}\end{array}$ & $\begin{array}{l}41 / 2 \\
5 \\
51 / 2 \\
6\end{array}$ & $\begin{array}{l}18 \\
16 \\
12 \\
81 / 2\end{array}$ & $\begin{array}{r}17.5 \\
15.6 \\
11.7 \\
8.3\end{array}$ \\
\hline
\end{tabular}

(11d) Copper-clad common nails.-

\begin{tabular}{l|l|l|r||l|l|l|r}
\hline $\mathrm{S}$ & $\mathrm{L}$ & $\mathrm{D}$ & No./1b. & $\mathrm{S}$ & $\mathrm{L}$ & \multicolumn{1}{c}{$\mathrm{D}$} & No./1b. \\
\hline 2d & 1 & 0.072 & 823 & $10 \mathrm{~d}$ & 3 & 0.144 & 65 \\
3d & $11 / 4$ & .081 & 534 & $12 \mathrm{~d}$ & $31 / 4$ & .144 & 59 \\
$4 \mathrm{~d}$ & $11 / 2$ & .091 & 297 & $16 \mathrm{~d}$ & $31 / 2$ & .162 & 46 \\
$5 \mathrm{~d}$ & $13 / 4$ & .091 & 255 & $20 \mathrm{~d}$ & 4 & .182 & 29 \\
$6 \mathrm{~d}$ & 2 & .102 & 170 & $30 \mathrm{~d}$ & $41 / 2$ & .204 & 22 \\
$7 \mathrm{~d}$ & $21 / 4$ & .102 & 151 & $40 \mathrm{~d}$ & 5 & .204 & 17 \\
$8 \mathrm{~d}$ & $21 / 2$ & .128 & 100 & $50 \mathrm{~d}$ & $51 / 2$ & .229 & 13 \\
$9 \mathrm{~d}$ & $23 / 4$ & .128 & 90 & $60 \mathrm{~d}$ & 6 & .258 & 10 \\
\hline
\end{tabular}

(11e) Common copper or brass wire nails.-

\begin{tabular}{|c|c|c|c|c|c|}
\hline L & D & No./lb. & L & D & No./lb. \\
\hline $3 / 4$ & 0.083 & 752 & $21 / 2$ & 0.120 & 107 \\
\hline $1 / 8$ & .083 & 675 & $\begin{array}{l}21 / 2 \\
032\end{array}$ & $\begin{array}{r}.165 \\
\end{array}$ & 58 \\
\hline $\begin{array}{l}1 \\
11 / 4\end{array}$ & $\begin{array}{l}.072 \\
.083\end{array}$ & $\begin{array}{l}767 \\
473\end{array}$ & 33 & $\begin{array}{l}.134 \\
.134\end{array}$ & $\begin{array}{l}80 \\
74\end{array}$ \\
\hline $11 / 4$ & . 120 & 206 & 3 & .165 & 45 \\
\hline $\begin{array}{l}11 / 2 \\
11 / 2\end{array}$ & $\begin{array}{r}.095 \\
120\end{array}$ & $\begin{array}{l}307 \\
176\end{array}$ & $\begin{array}{l}31 / 4 \\
31 / 2\end{array}$ & .148 & $\begin{array}{l}54 \\
42\end{array}$ \\
\hline $\begin{array}{l}11 / 2 \\
13 / 4\end{array}$ & $\begin{array}{r}120 \\
.109\end{array}$ & $\begin{array}{l}176 \\
202\end{array}$ & $\begin{array}{l}13 / 2 \\
31 / 2\end{array}$ & $\begin{array}{l}.165 \\
.180\end{array}$ & $\begin{array}{l}42 \\
35\end{array}$ \\
\hline 2 & .109 & 179 & 4 & .238 & 21 \\
\hline${ }_{21 / 4}^{2}$ & $\begin{array}{l}.134 \\
.120\end{array}$ & $\begin{array}{l}109 \\
120\end{array}$ & $\begin{array}{l}41 / 2 \\
5\end{array}$ & .238 & $\begin{array}{l}17 \\
13.2\end{array}$ \\
\hline $21 / 4$ & .148 & 76 & 6 & .284 & 8.2 \\
\hline
\end{tabular}


(11f) Standard cut nails.-Copper, Muntz metal, or zinc.

\begin{tabular}{|c|c|c|c|c|c|c|c|}
\hline \multirow{2}{*}{ S } & \multirow{2}{*}{ L } & \multicolumn{2}{|c|}{$\begin{array}{l}\text { Approximate } \\
\text { number per } \\
\text { pound }\end{array}$} & \multirow{2}{*}{$\mathrm{S}$} & \multirow{2}{*}{ L } & \multicolumn{2}{|c|}{$\begin{array}{l}\text { Approximate } \\
\text { number per } \\
\text { pound }\end{array}$} \\
\hline & & Copper & $\begin{array}{l}\text { Muntz } \\
\text { metal }\end{array}$ & & & Copper & $\begin{array}{l}\text { Muntz } \\
\text { metal }\end{array}$ \\
\hline $\begin{array}{l}2 \mathrm{~d} \\
3 \mathrm{~d}\end{array}$ & $\begin{array}{c}5 / 8 \\
3 / 4 \\
7 / 8 \\
1 \\
11 / 4\end{array}$ & $\begin{array}{l}930 \\
760 \\
640 \\
400 \\
310\end{array}$ & $\begin{array}{r}1,400 \\
700 \\
600 \\
368 \\
288\end{array}$ & $\begin{array}{r}9 \mathrm{~d} \\
10 \mathrm{~d} \\
12 \mathrm{~d} \\
16 \mathrm{~d} \\
20 \mathrm{~d}\end{array}$ & $\begin{array}{l}23 / 4 \\
3 \\
31 / 4 \\
31 / 2 \\
4\end{array}$ & $\begin{array}{l}60 \\
55 \\
45 \\
35 \\
30\end{array}$ & $\begin{array}{l}80 \\
75 \\
65 \\
55 \\
29\end{array}$ \\
\hline $\begin{array}{l}4 \mathrm{~d} \\
5 \mathrm{~d} \\
6 \mathrm{~d} \\
7 \mathrm{~d} \\
8 \mathrm{~d}\end{array}$ & $\begin{array}{l}11 / 2 \\
13 / 4 \\
2 \\
21 / 4 \\
21 / 2\end{array}$ & $\begin{array}{r}235 \\
150 \\
130 \\
100 \\
70\end{array}$ & $\begin{array}{r}245 \\
170 \\
150 \\
115 \\
90\end{array}$ & $\begin{array}{l}30 \mathrm{~d} \\
40 \mathrm{~d} \\
50 \mathrm{~d} \\
60 \mathrm{~d}\end{array}$ & $\begin{array}{l}41 / 2 \\
5 \\
51 / 2 \\
6\end{array}$ & $\begin{array}{r}20 \\
15 \\
10 \\
8\end{array}$ & $\begin{array}{l}21 \\
18 \\
14 \\
10\end{array}$ \\
\hline
\end{tabular}

(12) Double-head wire nails.-Thick, flat head, with flange or collar below the head to prevent the nail from sinking into the material into which it is driven.

\begin{tabular}{|c|c|c|r|}
\hline $\mathrm{L}$ & $\mathrm{G}$ & $\mathrm{D}$ & No./lb. \\
\hline 2 & $111 / 2$ & 0.113 & 170 \\
$21 / 2$ & $101 / 4$ & .131 & 100 \\
3 & 9 & .148 & 65 \\
$31 / 2$ & 8 & .162 & 46 \\
\hline
\end{tabular}

(13) Dowel pins.-Headless, cut off square, diamond point, barbed.

\begin{tabular}{|c|c|c|c|c|}
\hline \multirow{2}{*}{$\mathrm{L}$} & \multicolumn{3}{|c|}{$\begin{array}{c}\text { Approximate number } \\
\text { per pound }\end{array}$} \\
\cline { 2 - 5 } & $8 \mathrm{G}$ & $9 \mathrm{G}$ & $10 \mathrm{G}$ & $11 \mathrm{G}$ \\
\hline $1 / 2$ & 395 & 467 & 568 & 704 \\
$5 / 8$ & 290 & 363 & 440 & 549 \\
$\frac{5}{3 / 4}$ & 250 & 297 & 390 & 450 \\
$7 / 8$ & 210 & 252 & 330 & 380 \\
$11 / 8$ & 190 & 218 & 277 & 329 \\
$11 / 8$ & 165 & 192 & 251 & 290 \\
$11 / 4$ & 150 & 172 & 221 & 260 \\
$13 / 8$ & 130 & 156 & 200 & 235 \\
$11 / 2$ & 120 & 141 & 181 & 215 \\
$13 / 4$ & 100 & 121 & 146 & 183 \\
2 & 90 & 105 & 127 & 159 \\
\hline
\end{tabular}

(14) Escutcheon pins.-Oval head, needle point, smooth bright steel, brass plated, or brass.

LIGHT GAGE

\begin{tabular}{|c|c|c||c|c|r|}
\hline $\mathrm{L}$ & $\mathrm{D}$ & $\mathrm{No} / \mathrm{lb}$ & $\mathrm{L}$ & $\mathrm{D}$ & No./lb. \\
\cline { 2 - 5 } Inch & Inch & & Inches & Inch & \\
$1 / 4$ & 0.0475 & 6,590 & 1 & 0.0625 & 1,050 \\
$3 / 8$ & .0475 & 4,430 & $11 / 4$ & .0625 & 865 \\
$1 / 2$ & .0475 & 3,680 & $11 / 2$ & .08 & 445 \\
$5 / 8$ & .0475 & 3,490 & $13 / 4$ & .08 & 375 \\
$3 / 4$ & .0625 & 1,440 & 2 & .08 & 345 \\
$7 / 8$ & .0625 & 1,150 & & & \\
\hline
\end{tabular}

\section{MEDIUM GAGE}

\begin{tabular}{|c|c|c|c|c|c|c|c|}
\hline $\mathrm{L}$ & $\begin{array}{l}\text { No./lb. } \\
\text { (0.08 di- } \\
\text { ameter) }\end{array}$ & $\begin{array}{c}\text { No./lb. } \\
(0.1055 \\
\text { diame- } \\
\text { ter })\end{array}$ & $\begin{array}{l}\text { No./lb. } \\
\text { (0.0625 } \\
\text { diame- } \\
\text { ter) }\end{array}$ & L & $\begin{array}{l}\text { No./lb. } \\
\text { (0.08 di- } \\
\text { ameter) }\end{array}$ & $\begin{array}{c}\text { No. } / \mathrm{b} . \\
(0.1055 \\
\text { diame- } \\
\text { ter })\end{array}$ & $\begin{array}{c}\text { No./lb. } \\
(0.0625 \\
\text { diame- } \\
\text { ter })\end{array}$ \\
\hline $\begin{array}{r}\text { Inch } \\
1 / 4 \\
3 / 8 \\
1 / 2 \\
5 / 8 \\
3 / 4 \\
7 / 8\end{array}$ & $\begin{array}{l}815 \\
770\end{array}$ & 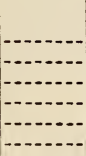 & $\begin{array}{l}3,700 \\
2,780 \\
2,020 \\
1,640 \\
\end{array}$ & $\begin{array}{c}\text { Inches } \\
1 \\
11 / 4 \\
11 / 2 \\
13 / 4 \\
2\end{array}$ & $\begin{array}{c}660 \\
530 \\
-\end{array}$ & $\begin{array}{r}255 \\
225 \\
190\end{array}$ & 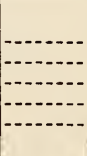 \\
\hline
\end{tabular}

HEAVY GAGE

\begin{tabular}{|c|c|c|c|c|c|c|c|}
\hline $\mathbf{L}$ & $\begin{array}{c}\text { No./lb. } \\
(0.185 \\
\text { diame- } \\
\text { ter })\end{array}$ & $\begin{array}{l}\text { No./lb. } \\
\text { (0.08 di- } \\
\text { ameter) }\end{array}$ & $\begin{array}{l}\text { No./lb. } \\
(0.1055 \\
\text { diame- } \\
\text { ter })\end{array}$ & L & $\begin{array}{c}\text { No./lb. } \\
(0.185 \\
\text { diame- } \\
\text { ter })\end{array}$ & $\begin{array}{l}\text { No./lb. } \\
\text { (0.08 di- } \\
\text { ameter) }\end{array}$ & $\begin{array}{c}\text { No./lb. } \\
(0.1055 \\
\text { diame- } \\
\text { ter })\end{array}$ \\
\hline $\begin{array}{r}\text { Inch } \\
1 / 4 \\
3 / 8 \\
1 / 2 \\
5 / 8 \\
3 / 4 \\
7 / 8\end{array}$ & $\begin{array}{l}-\cdots \\
-\cdots \\
\cdots \\
\cdots\end{array}$ & $\begin{array}{l}2,180 \\
1,660 \\
1,380 \\
1,010 \\
\end{array}$ & $\begin{array}{r}465 \\
405\end{array}$ & $\begin{array}{c}\text { Inches } \\
1 \\
11 / 4 \\
11 / 2 \\
13 / 4 \\
2\end{array}$ & $\begin{array}{c}160 \\
130 \\
115\end{array}$ & $\begin{array}{l}--- \\
-- \\
-- \\
-- \\
--\end{array}$ & $\begin{array}{c}375 \\
285 \\
- \\
\\
\end{array}$ \\
\hline
\end{tabular}

(15) Fence nails.-Flat head, diamond point, smooth.

\begin{tabular}{|c|c|c|c|c|c|c|c|}
\hline \multirow{2}{*}{$\mathbf{S}$} & \multirow{2}{*}{ L } & \multicolumn{3}{|c|}{ Bright } & \multicolumn{3}{|c|}{ Cement coated } \\
\hline & & G & D & No./lb. & $G$ & $\mathrm{D}$ & No./lb. \\
\hline $\begin{array}{l}5 \mathrm{~d} \\
6 \mathrm{~d} \\
7 \mathrm{~d} \\
8 \mathrm{~d} \\
9 \mathrm{~d}\end{array}$ & $\begin{array}{l}13 / 4 \\
2 \\
21 / 4 \\
21 / 2 \\
23 / 4\end{array}$ & $\begin{array}{r}10 \\
10 \\
9 \\
9 \\
8\end{array}$ & $\begin{array}{r}0.135 \\
.135 \\
.148 \\
.148 \\
.163\end{array}$ & $\begin{array}{r}142 \\
124 \\
92 \\
82 \\
62\end{array}$ & $\begin{array}{r}11 \\
11 \\
10 \\
10 \\
9\end{array}$ & $\begin{array}{r}0.120 \\
.120 \\
.135 \\
.135 \\
.148\end{array}$ & $\begin{array}{r}174 \\
150 \\
110 \\
100 \\
71\end{array}$ \\
\hline $\begin{array}{l}10 \mathrm{~d} \\
12 \mathrm{~d} \\
16 \mathrm{~d} \\
20 \mathrm{~d}\end{array}$ & $\begin{array}{l}3 \\
31 / 4 \\
31 / 2 \\
4\end{array}$ & $\begin{array}{l}7 \\
6 \\
5 \\
4\end{array}$ & $\begin{array}{l}.177 \\
.192 \\
.207 \\
.225\end{array}$ & $\begin{array}{l}50 \\
40 \\
30 \\
23\end{array}$ & $\begin{array}{l}8 \\
7 \\
6 \\
5\end{array}$ & $\begin{array}{l}.162 \\
.177 \\
.192 \\
.207\end{array}$ & $\begin{array}{l}50 \\
41 \\
34 \\
25\end{array}$ \\
\hline
\end{tabular}

(15a) Cut iron or steel fence nails.-

\begin{tabular}{|c|c|c|c|}
\hline \multirow{2}{*}{$\mathrm{S}$} & \multirow{2}{*}{ L } & \multicolumn{2}{|c|}{$\begin{array}{l}\text { A pproximate num } \\
\text { ber per pound }\end{array}$} \\
\hline & & Plain & $\begin{array}{c}\text { Zinc } \\
\text { coated }\end{array}$ \\
\hline $\begin{array}{l}5 \mathrm{~d} \\
6 \mathrm{~d} \\
7 \mathrm{~d} \\
8 \mathrm{~d} \\
9 \mathrm{~d}\end{array}$ & $\begin{array}{l}13 / 4 \\
2 \\
21 / 4 \\
21 / 2 \\
23 / 4\end{array}$ & $\begin{array}{r}119 \\
84 \\
62 \\
48 \\
38\end{array}$ & $\begin{array}{r}113 \\
80 \\
59 \\
45 \\
36\end{array}$ \\
\hline $\begin{array}{l}10 \mathrm{~d} \\
12 \mathrm{~d} \\
16 \mathrm{~d} \\
20 \mathrm{~d}\end{array}$ & $\begin{array}{l}3 \\
31 / 4 \\
31 / 2 \\
4\end{array}$ & $\begin{array}{l}30 \\
24 \\
20 \\
18\end{array}$ & $\begin{array}{l}28 \\
23 \\
19 \\
17\end{array}$ \\
\hline
\end{tabular}

(16) Finishing nails.-Long, narrow, flat head, diamond point, smooth.

\begin{tabular}{|c|c|c|c|c|c|c|c|c|}
\hline \multirow{2}{*}{$S$} & \multicolumn{4}{|c|}{ Bright } & \multicolumn{4}{|c|}{ Cement coated } \\
\hline & $\mathrm{L}$ & $G$ & $\mathrm{D}$ & No./lb. & $\mathrm{L}$ & $G$ & $\mathrm{D}$ & No./lb. \\
\hline $\begin{array}{l}2 \mathrm{~d} \\
3 \mathrm{~d} \\
4 \mathrm{~d} \\
5 \mathrm{~d}\end{array}$ & \begin{tabular}{l|}
1 \\
$11 / 4$ \\
$11 / 2$ \\
$18 / 4$
\end{tabular} & $\begin{array}{l}161 / 2 \\
151 / 2 \\
15 \\
15\end{array}$ & $\begin{array}{r}0.058 \\
.067 \\
.072 \\
.072\end{array}$ & $\begin{array}{r}1,351 \\
807 \\
584 \\
500\end{array}$ & \begin{tabular}{l|}
1 \\
$11 / 4$ \\
$11 / 2$ \\
$13 / 4$
\end{tabular} & $\begin{array}{l}161 / 2 \\
151 / 2 \\
15 \\
15\end{array}$ & $\begin{array}{r}0.058 \\
.067 \\
.072 \\
.072\end{array}$ & $\begin{array}{r}1,285 \\
786 \\
600 \\
500\end{array}$ \\
\hline $\begin{array}{l}6 \mathrm{~d} \\
7 \mathrm{~d} \\
8 \mathrm{~d} \\
9 \mathrm{~d}\end{array}$ & \begin{tabular}{l|}
2 \\
$21 / 4$ \\
$21 / 2$ \\
$28 / 4$
\end{tabular} & $\begin{array}{l}13 \\
13 \\
121 / 2 \\
121 / 2\end{array}$ & $\begin{array}{l}.092 \\
.092 \\
.098 \\
.098\end{array}$ & $\begin{array}{l}309 \\
238 \\
189 \\
172\end{array}$ & \begin{tabular}{l|}
2 \\
$21 / 4$ \\
$21 / 2$ \\
$23 / 4$
\end{tabular} & $\begin{array}{l}131 / 2 \\
13 \\
121 / 2 \\
121 / 2\end{array}$ & $\begin{array}{r}.086 \\
.092 \\
.098 \\
.098\end{array}$ & $\begin{array}{l}286 \\
235 \\
180 \\
169\end{array}$ \\
\hline $\begin{array}{l}10 \mathrm{~d} \\
12 \mathrm{~d} \\
16 \mathrm{~d} \\
20 \mathrm{~d}\end{array}$ & $\begin{array}{l}3 \\
31 / 4 \\
31 / 2 \\
4\end{array}$ & $\begin{array}{l}111 / 2 \\
111 / 2 \\
11 \\
10\end{array}$ & $\begin{array}{r}.113 \\
.113 \\
.120 \\
.135\end{array}$ & $\begin{array}{r}121 \\
113 \\
90 \\
62\end{array}$ & $\begin{array}{l}3 \\
31 / 1 \\
31 / 2 \\
37 / 8\end{array}$ & $\begin{array}{l}111 / 2 \\
111 / 2 \\
11 \\
10\end{array}$ & $\begin{array}{r}.113 \\
.113 \\
.120 \\
.135\end{array}$ & $\begin{array}{r}119 \\
110 \\
87 \\
64\end{array}$ \\
\hline
\end{tabular}


(16a) Cut iron or steel finishing nails.

\begin{tabular}{|c|c|c|c|}
\hline \multirow{2}{*}{ S } & \multirow{2}{*}{ L } & \multicolumn{2}{|c|}{$\begin{array}{c}\text { Approximate } \\
\text { number per } \\
\text { pound }\end{array}$} \\
\hline & & $\begin{array}{l}\text { Stand- } \\
\text { ard }\end{array}$ & Fine \\
\hline $3 d$ & $11 / 4$ & 660 & \\
\hline $4 d$ & $11 / 2$ & 432 & \\
\hline $5 d$ & $13 / 4$ & 256 & \\
\hline $6 \mathrm{~d}$ & 2 & 172 & 208 \\
\hline $7 d$ & $21 / 4$ & 124 & 176 \\
\hline $8 d$ & $21 / 2$ & 110 & 144 \\
\hline $\begin{array}{r}9 \mathrm{~d} \\
10 \mathrm{~d}\end{array}$ & $\begin{array}{l}23 / 4 \\
3\end{array}$ & 82 & 92 \\
\hline $12 \mathrm{~d}$ & $31 / 4$ & 54 & - \\
\hline $16 d$ & $31 / 2$ & 38 & 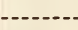 \\
\hline $20 d$ & 4 & 29 & \\
\hline
\end{tabular}

(17) Flooring nails.-Pointing cone head, blunt diamond point.

\begin{tabular}{|r|c|c|c|c|}
\hline \multicolumn{5}{|c|}{ Cement coated } \\
\hline $\mathrm{S}$ & $\mathrm{L}$ & $\mathrm{G}$ & $\mathrm{D}$ & No./1b. \\
\hline 6d & $13 / 4$ & $111 / 2$ & 0.113 & 178 \\
$7 \mathrm{~d}$ & 2 & 11 & .120 & 138 \\
8d & $21 / 4$ & $101 / 2$ & .128 & 116 \\
9d & $21 / 2$ & 10 & .135 & 94 \\
10d & $233 / 4$ & $91 / 2$ & .142 & 77 \\
12d & 3 & 9 & .148 & 65 \\
16d & $31 / 4$ & 8 & .162 & 47 \\
20d & $33 / 4$ & 7 & .177 & 32 \\
\hline
\end{tabular}

(17a) Flooring brads.-Long, narrow, countersunk head, blunt diamond point, smooth.

\begin{tabular}{|c|c|c|c|c|c|c|c|c|}
\hline \multirow{2}{*}{$\mathrm{S}$} & \multicolumn{4}{|c|}{ Bright } & \multicolumn{4}{|c|}{ Cement coated } \\
\hline & $\mathrm{L}$ & G & D & No./lb. & L & G & D & No./lb. \\
\hline $\begin{array}{l}6 d \\
7 d \\
8 d \\
9 d\end{array}$ & $\begin{array}{l}2 \\
21 / 4 \\
21 / 2 \\
23 / 4\end{array}$ & $\begin{array}{l}11 \\
11 \\
10 \\
10\end{array}$ & $\begin{array}{r}0.120 \\
.120 \\
.135 \\
.135\end{array}$ & $\begin{array}{r}157 \\
139 \\
99 \\
90\end{array}$ & $\begin{array}{l}2 \\
21 / 4 \\
23,8 \\
23 / 4\end{array}$ & $\begin{array}{l}12 \\
12 \\
11 \\
11\end{array}$ & $\begin{array}{r}0.105 \\
.105 \\
.120 \\
.120\end{array}$ & $\begin{array}{l}207 \\
179 \\
128 \\
111\end{array}$ \\
\hline $\begin{array}{l}10 \mathrm{~d} \\
12 \mathrm{~d} \\
16 \mathrm{~d} \\
20 \mathrm{~d}\end{array}$ & $\begin{array}{l}3 \\
31 / 4 \\
31 / 2 \\
4\end{array}$ & $\begin{array}{l}9 \\
8 \\
7 \\
6\end{array}$ & $\begin{array}{r}.148 \\
.162 \\
.177 \\
.192\end{array}$ & $\begin{array}{l}69 \\
54 \\
43 \\
31\end{array}$ & $\begin{array}{l}3 \\
314 \\
3112 \\
4\end{array}$ & $\begin{array}{r}10 \\
9 \\
8 \\
7\end{array}$ & $\begin{array}{l}.135 \\
.148 \\
.162 \\
.177\end{array}$ & $\begin{array}{l}89 \\
61 \\
49 \\
37\end{array}$ \\
\hline
\end{tabular}

(17b) Parquet flooring nails (brads).-Narrow countersunk flat head, needle point, smooth or barbed.

\begin{tabular}{|c|c|c|c|}
\hline L & G & D & No./1b. \\
\hline 1 & 15 & 0.072 & 888 \\
$11 / 8$ & 15 & .072 & 704 \\
$11 / 4$ & 15 & .072 & 592 \\
\hline
\end{tabular}

(17c) Cut iron or steel flooring nails.

\begin{tabular}{|c|c|c|c|c|r|}
\hline $\mathrm{S}$ & $\mathrm{L}$ & No./lb. & $\mathrm{S}$ & $\mathrm{L}$ & No./lb. \\
\cline { 2 - 6 } 4d & $11 / 2$ & 314 & 9d & $23 / 4$ & 85 \\
5d & $13 / 4$ & 221 & $10 \mathrm{~d}$ & 3 & 68 \\
6d & 2 & 176 & $12 \mathrm{~d}$ & 314 & 49 \\
7d & $21 / 4$ & 138 & $16 \mathrm{~d}$ & $31 / 2$ & 36 \\
8d & $21 / 2$ & 114 & 20d & 4 & 32 \\
\hline
\end{tabular}

(18) Foundry nails.-Large, flat, thin head, diamond point, smooth bright.

\begin{tabular}{|c|c|c|c|c|}
\hline \multirow[b]{2}{*}{ L } & \multicolumn{4}{|c|}{ Approximate number per pound } \\
\hline & $\begin{array}{c}0.162 \\
\text { inch } \\
\text { diameter, } \\
1 / 2 \text {-inch } \\
\text { head }\end{array}$ & $\begin{array}{c}0.148 \\
\text { inch } \\
\text { diameter, } \\
\begin{array}{c}1 / 2 \text {-inch } \\
\text { bead }\end{array}\end{array}$ & $\begin{array}{c}0.135 \\
\text { inch } \\
\text { diameter, } \\
1 / 2 \text {-inch } \\
\text { head }\end{array}$ & $\begin{array}{l}0.1205 \\
\text { inch } \\
\text { diameter, } \\
\text { 7/16-inch } \\
\text { head }\end{array}$ \\
\hline $\begin{array}{l}8 / 4 \\
7 / 8 \\
1 \\
11 / 8 \\
11 / 4 \\
11 / 2 \\
13 / 4 \\
2 \\
21 / 4 \\
21 / 2 \\
23 / 4 \\
3 \\
31 / 4 \\
31 / 2\end{array}$ & $\begin{array}{r}194 \\
170 \\
150 \\
135 \\
122 \\
103 \\
90 \\
79 \\
71 \\
64 \\
59 \\
54 \\
51 \\
47\end{array}$ & $\begin{array}{r}226 \\
201 \\
176 \\
160 \\
144 \\
122 \\
106 \\
94 \\
83 \\
76 \\
69 \\
64 \\
59 \\
55\end{array}$ & $\begin{array}{r}288 \\
252 \\
220 \\
197 \\
178 \\
153 \\
131 \\
117 \\
111 \\
94 \\
85 \\
79 \\
73 \\
68\end{array}$ & $\begin{array}{r}348 \\
307 \\
275 \\
241 \\
221 \\
190 \\
162 \\
142 \\
129 \\
116 \\
106 \\
98 \\
90 \\
83\end{array}$ \\
\hline
\end{tabular}

(19) Heel nails.-For rubber heels. Steel-wire nail, flat countersunk head or brad head, flat thin clinching point, bright.

\begin{tabular}{|l|r|r|r|}
\hline L & G & \multicolumn{1}{|l|}{ D } & No./lb. \\
\hline & & & \\
\hline $3 / 4$ & 14 & 0.08 & 995 \\
$13 / 16$ & 14 & .08 & 910 \\
$7 / 8$ & 14 & .08 & 850 \\
$15 / 16$ & 14 & .08 & 782 \\
1 & 14 & .08 & 739 \\
\hline
\end{tabular}

(19a) Heel nails.-Steel-wire nail, flat countersunk head, needle point or diamond point, bright.

\begin{tabular}{|c|c|c|c||c|c|c|c|}
\hline L & G & D & No./lb. & L & G & D & No./lb. \\
\hline & & & & & & \\
\hline $3 / 4$ & 14 & 0.08 & 863 & $13 / 8$ & 14 & 0.08 & 495 \\
$7 / 8$ & 14 & .08 & 765 & $11 / 2$ & 14 & .08 & 459 \\
$111 / 8$ & 14 & .08 & 667 & $15 / 8$ & 14 & .08 & 428 \\
$11 / 4$ & 14 & .08 & 586 & $13 / 4$ & 14 & .08 & 398 \\
\hline
\end{tabular}

(19b) Heel nails.-Cut steel or iron, square tapering shank, flat point, no head, blued or bright.

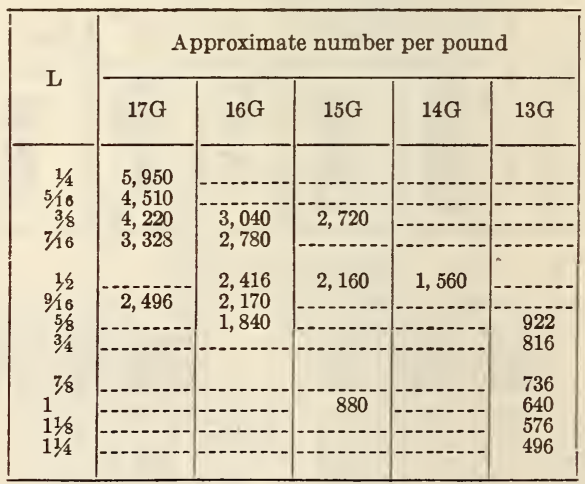


(20) Hinge nails. - Oval head, or countersunk flat head; chisel point; smooth, bright, or annealed.

\begin{tabular}{|c|c|c|c|c|}
\hline \multirow{2}{*}{$\mathrm{L}$} & \multicolumn{2}{|c|}{ Heavy } & \multicolumn{2}{|c|}{ Light } \\
\hline & $\mathrm{D}$ & No./lb. & $\mathrm{D}$ & No./lb \\
\hline $11 / 2$ & $1 / 4$ & 50 & $3 / 16$ & 82 \\
\hline 2 & $1 / 4$ & 38 & $\begin{array}{l}310 \\
3 / 16\end{array}$ & 62 \\
\hline $21 / 2$ & $\begin{array}{l}1 / 4 \\
336\end{array}$ & 30 & $3 / 16$ & 50 \\
\hline $\begin{array}{l}31 / 4 \\
3\end{array}$ & $3 / 8$ & 11 & $\begin{array}{l}14 \\
1 / 4\end{array}$ & $\begin{array}{l}20 \\
23\end{array}$ \\
\hline $31 / 2$ & $3 / 8$ & 10 & 14 & 22 \\
\hline 4 & $3 / 8$ & 9 & $1 / 4$ & 19 \\
\hline
\end{tabular}

(20a) Hinge nails.-Oval head; long diamond point; smooth, bright, or annealed.

\begin{tabular}{|c|c|c|c|c|}
\hline \multirow{2}{*}{ L } & \multicolumn{2}{|c|}{ Light } & \multicolumn{2}{|c|}{ Heavy } \\
\hline & $\mathrm{D}$ & No./lb. & $\mathrm{D}$ & No./lb. \\
\hline $11 / 2$ & $3 / 16$ & 81 & $1 / 4$ & 47 \\
\hline $13 / 4$ & $3 / 16$ & 68 & $1 / 4$ & 41 \\
\hline${ }_{21 / 4}^{2}$ & $\begin{array}{l}3 / 16 \\
3 / 1\end{array}$ & 61 & 1/4 & 33 \\
\hline $\begin{array}{l}274 \\
21 / 2\end{array}$ & $\begin{array}{l}8 / 16 \\
3 / 16\end{array}$ & $\begin{array}{l}54 \\
48\end{array}$ & $\begin{array}{l}1 / 4 \\
1 / 4\end{array}$ & $\begin{array}{l}11 \\
28\end{array}$ \\
\hline $23 / 4$ & $3 / 16$ & 45 & $1 / 4$ & 26 \\
\hline 3 & $3 / 16$ & 41 & $1 / 4$ & 24 \\
\hline
\end{tabular}

(21) Hob nails.-Steel or iron, No. 10 gage; high oval smooth head or square grooved head; approximately five-sixteenths inch wide; long sharp point; bright finish.

\begin{tabular}{|r|r|r|}
\hline L & $\begin{array}{r}\text { Grooved } \\
\text { No./lb. }\end{array}$ & $\begin{array}{r}\text { Smooth } \\
\text { No./1b. }\end{array}$ \\
\cline { 1 - 2 } $3 / 8$ & 384 & 336 \\
$3 \frac{1}{31 / 8}$ & 320 & 304 \\
$4 / 8$ & 288 & 272 \\
$5 / 8$ & 256 & 240 \\
$6 / 8$ & 224 & $\ldots$ \\
\hline
\end{tabular}

(22) Horseshoe nails.-Regular head.

\begin{tabular}{|c|c|r|}
\hline S & L & No./lb. \\
\hline 5 & 2 & 182 \\
\hline 6 & $21 / 8$ & 137 \\
7 & $21 / 4$ & 114 \\
8 & $21 / 2$ & 88 \\
9 & $25 / 8$ & 70 \\
\hline
\end{tabular}

(23) Lath nails.-For wood lath; flat head; diamond point.

\begin{tabular}{|c|c|c|c|c|c|c|c|}
\hline \multirow{2}{*}{$\mathrm{S}$} & \multicolumn{4}{|c|}{ Bright or blued } & \multicolumn{3}{|c|}{ Cement coated } \\
\hline & L & G & $\mathrm{D}$ & No./lb. & G & $\mathrm{D}$ & No./lb. \\
\hline \multirow{4}{*}{$\begin{array}{l}2 \mathrm{~d} \\
2 \mathrm{~d} \\
3 \mathrm{~d} \\
3 \mathrm{~d} \\
3 \mathrm{~d} \\
4 \mathrm{~d}\end{array}$} & \multirow{4}{*}{$\begin{array}{l}1 \\
1 \\
118 \\
11 / 8 \\
114 \\
11 / 2\end{array}$} & \multirow{4}{*}{$\begin{array}{l}17 \\
161 / 2 \\
16 \\
15 \\
14 \\
14\end{array}$} & \multirow{4}{*}{$\begin{array}{l}0.054 \\
.058 \\
.062 \\
.072 \\
.08 \\
.080\end{array}$} & \multirow{4}{*}{$\begin{array}{r}1,560 \\
1,351 \\
1,015 \\
778 \\
568 \\
473\end{array}$} & $171 / 2$ & 0.051 & 1,715 \\
\hline & & & & & 16 & .0625 & 1,015 \\
\hline & & & & & & & \\
\hline & & & & & -- &.-- & \\
\hline & & & & & & & \\
\hline
\end{tabular}

(23a) Lath nails.-For metal lath; thin, flat hook head; diamond point.

\begin{tabular}{|l|r|r|r|r|}
\hline L & G & D & \multicolumn{2}{|r|}{ Approximate number per pound } \\
\hline 138 & 12 & 0.105 & Bright or blued & Zinc coated \\
\hline
\end{tabular}

(24) Molding nails (brads).-Narrow countersunk flat head, needle point, smooth, bright, or cement coated.

\begin{tabular}{|c|c|c|c|}
\hline L & G & D & No./lb. \\
\hline $\begin{array}{l}1^{7 / 8} \\
11 / 8 \\
11 / 4\end{array}$ & $\begin{array}{l}14 \\
14 \\
14 \\
14\end{array}$ & $\begin{array}{r}0.08 \\
.08 \\
.08 \\
.08\end{array}$ & $\begin{array}{l}816 \\
760 \\
670 \\
580\end{array}$ \\
\hline
\end{tabular}

(25) Plaster-board nails.-Broad flat head, diamond point, smooth, bright, or cement coated.

\begin{tabular}{|c|r|r|r|r||c|c|c|c|c|}
\hline L & G & D & E & No./lb. & L & G & D & H & No./lb. \\
\hline 1 & 9 & 0.148 & $1 / 2$ & 193 & $11 / 4$ & 10 & 0.135 & $1 / 2$ & 183 \\
$11 / 4$ & 9 & .148 & 112 & 156 & $11 / 2$ & 10 & .135 & $1 / 2$ & 153 \\
$11 / 2$ & 9 & .148 & 112 & 131 & $13 / 4$ & 10 & .135 & $1 / 2$ & 133 \\
$13 / 4$ & 9 & .148 & $1 / 2$ & 113 & $11 / 4$ & 11 & .120 & $7 / 6$ & 251 \\
\hline
\end{tabular}

(26) Purlin nails.-Flat head or curved head, point cut off square, annealed or zinc coated.

\begin{tabular}{|r|r|r|r|}
\hline & & \multicolumn{2}{|c|}{$\begin{array}{c}\text { Approximate } \\
\text { number per } \\
\text { pound }\end{array}$} \\
L & D & \multicolumn{2}{|c|}{} \\
\cline { 3 - 4 } & & Plain & $\begin{array}{c}\text { Zinc } \\
\text { coated }\end{array}$ \\
\hline & & & \\
\hline 6 & $1 / 8$ & 46 & 44 \\
7 & $1 / 8$ & 40 & 38 \\
8 & $1 / 8$ & 34 & 32 \\
9 & $1 / 8$ & 31 & 30 \\
10 & $1 / 8$ & 28 & 27 \\
12 & $1 / 8$ & 23 & 22 \\
14 & $1 / 8$ & 20 & 19 \\
\hline
\end{tabular}

(27) Roofing nails, standard.-Flat head, diamond point, barbed.

\begin{tabular}{|c|c|c|c|c|c|c|}
\hline & \multicolumn{3}{|c|}{ Bright } & \multicolumn{3}{c|}{ Cement coated } \\
\cline { 2 - 7 } & G & D & No./lb. & G & D & No./lb. \\
\hline & & & & & & \\
$3 / 4$ & 13 & 0.091 & 714 & 14 & 0.080 & 870 \\
$7 / 8$ & 12 & .105 & 469 & $121 / 2$ & .098 & 487 \\
1 & 12 & .105 & 411 & $121 / 2$ & .098 & 429 \\
$11 / 8$ & 12 & .105 & 365 & $121 / 2$ & .098 & 400 \\
$11 / 4$ & 11 & .120 & 251 & 12 & .105 & 307 \\
$13 / 8$ & 11 & .120 & 230 & 12 & .105 & 280 \\
$11 / 2$ & 10 & .135 & 176 & 12 & .105 & 260 \\
$13 / 4$ & 10 & .135 & 151 & 12 & .105 & 228 \\
2 & 9 & .148 & 103 & 11 & .120 & 156 \\
\hline
\end{tabular}


(27a) Roofing nails.-Large head, barbed, diamond point, bright (B) or zinc coated (Z).

\begin{tabular}{|c|c|c|c|c|c|c|c|c|c|c|c|c|c|c|c|c|}
\hline \multirow{3}{*}{$G$} & \multirow{3}{*}{$\mathrm{D}$} & \multirow{3}{*}{$\mathbf{H}$} & \multicolumn{14}{|c|}{ Approximate number per pound } \\
\hline & & & \multicolumn{2}{|c|}{$\mathrm{L} 3 / 4$} & \multicolumn{2}{|c|}{ L 7/8 } & \multicolumn{2}{|c|}{ L 1} & \multicolumn{2}{|c|}{$\mathrm{L} 11 / 8$} & \multicolumn{2}{|c|}{ L $11 / 4$} & \multicolumn{2}{|c|}{$\mathrm{L} 11 / 2$} & \multicolumn{2}{|c|}{$\mathrm{L} 13 / 4$} \\
\hline & & & $\mathbf{B}$ & $\mathbf{Z}$ & $\mathbf{B}$ & $\mathbf{Z}$ & B & $\mathbf{Z}$ & B & $\mathbf{Z}$ & $\mathbf{B}$ & $\mathbf{Z}$ & $\mathbf{B}$ & $\mathbf{Z}$ & B & $\mathbf{Z}$ \\
\hline $\begin{array}{l}12 \\
11 \\
10 \\
91 / 2 \\
9 \\
8\end{array}$ & $\begin{array}{r}0.1055 \\
.1205 \\
.1350 \\
.1417 \\
.1483 \\
.1620\end{array}$ & $\begin{array}{l}3 / 8 \\
7 / 16 \\
7 / 16 \\
1 / 2 \\
1 / 2 \\
1 / 2\end{array}$ & $\begin{array}{l}510 \\
345 \\
275 \\
255 \\
240 \\
200\end{array}$ & $\begin{array}{l}464 \\
315 \\
255 \\
235 \\
214 \\
177\end{array}$ & $\begin{array}{l}435 \\
305 \\
240 \\
220 \\
210 \\
175\end{array}$ & $\begin{array}{l}396 \\
280 \\
225 \\
200 \\
184 \\
160\end{array}$ & $\begin{array}{l}397 \\
280 \\
215 \\
210 \\
185 \\
155\end{array}$ & $\begin{array}{l}361 \\
255 \\
210 \\
190 \\
165 \\
140\end{array}$ & $\begin{array}{l}346 \\
255 \\
196 \\
184 \\
173 \\
142\end{array}$ & $\begin{array}{l}315 \\
232 \\
185 \\
177 \\
157 \\
132\end{array}$ & $\begin{array}{l}314 \\
230 \\
190 \\
175 \\
160 \\
130\end{array}$ & $\begin{array}{l}286 \\
210 \\
170 \\
165 \\
148 \\
125\end{array}$ & $\begin{array}{l}273 \\
195 \\
165 \\
150 \\
135 \\
110\end{array}$ & $\begin{array}{l}249 \\
180 \\
155 \\
138 \\
125 \\
102\end{array}$ & $\begin{array}{r}230 \\
165 \\
140 \\
130 \\
115 \\
95\end{array}$ & $\begin{array}{r}210 \\
150 \\
135 \\
120 \\
107 \\
88\end{array}$ \\
\hline
\end{tabular}

(27b) Roofing nails.-For prepared felt roofing, | point or diamond point, bright (B) or zinc coated $5 / 8$-inch diameter, flat, reinforced head, needle $(Z)$.

\begin{tabular}{|c|c|c|c|c|c|c|c|c|c|c|c|c|}
\hline \multirow{3}{*}{$G$} & \multirow{3}{*}{$\mathrm{D}$} & \multirow{3}{*}{ H } & \multicolumn{10}{|c|}{ Approximate number per pound } \\
\hline & & & \multicolumn{2}{|c|}{$\mathrm{L} 3 / 4$} & \multicolumn{2}{|c|}{ L $7 / 8$} & \multicolumn{2}{|c|}{ L 1} & \multicolumn{2}{|c|}{ L 138} & \multicolumn{2}{|c|}{ L $11 / 4$} \\
\hline & & & B & $\mathrm{z}$ & $\mathbf{B}$ & Z & B & $\mathrm{Z}$ & $\mathbf{B}$ & $\mathrm{Z}$ & B & $\mathrm{Z}$ \\
\hline $\begin{array}{l}11 \\
12\end{array}$ & $\begin{array}{r}0.1205 \\
.1055\end{array}$ & $\begin{array}{l}9 / 16 \\
1 / 2\end{array}$ & $\begin{array}{l}184 \\
205\end{array}$ & $\begin{array}{l}164 \\
180\end{array}$ & $\begin{array}{l}175 \\
195\end{array}$ & $\begin{array}{l}157 \\
172\end{array}$ & $\begin{array}{l}155 \\
180\end{array}$ & $\begin{array}{l}145 \\
160\end{array}$ & $\begin{array}{l}149 \\
170\end{array}$ & $\begin{array}{l}135 \\
152\end{array}$ & $\begin{array}{l}136 \\
165\end{array}$ & $\begin{array}{l}125 \\
148\end{array}$ \\
\hline
\end{tabular}

(28) Saddle nails.-Oval head, plain bright, japanned black, or colors, brass capped and copper capped, tapering shanks, square point.

\begin{tabular}{|c|c|c|c|}
\hline \multirow{2}{*}{$\mathrm{L}$} & \multicolumn{3}{|c|}{$\begin{array}{c}\text { Approximate number } \\
\text { per pound }\end{array}$} \\
\cline { 2 - 4 } & $\mathrm{H} 3 / 8$ & $\mathrm{H} 1 / 2$ & $\mathrm{H} 5 / 8$ \\
\hline & & & \\
$3 / 4$ & 462 & 300 & 208 \\
$7 / 8$ & 430 & 287 & 198 \\
$11 / 8$ & 400 & 273 & 189 \\
$11 / 4$ & $-\cdots$ & 249 & 185 \\
\hline
\end{tabular}

(29) Saddlery nails.-Oval head, long diamond point, smooth, annealed.

\begin{tabular}{|c|c|c|c|}
\hline L & $G$ & $D$ & No./lb. \\
\hline $\begin{array}{c}11 / 2 \\
11 / 2\end{array}$ & $\begin{array}{c}7 \\
71 / 2\end{array}$ & $\begin{array}{c}0.177 \\
.169\end{array}$ & $\begin{array}{r}90 \\
99\end{array}$ \\
\hline
\end{tabular}

(30) Sheathing nails.-Cut copper or Muntz metal.

\begin{tabular}{|c|c|c|c|c|c|}
\hline \multirow{2}{*}{ L } & \multicolumn{2}{|c|}{$\begin{array}{l}\text { Approximate } \\
\text { number per } \\
\text { pound }\end{array}$} & \multirow{2}{*}{ L } & \multicolumn{2}{|c|}{$\begin{array}{l}\text { Approximate } \\
\text { number per } \\
\text { pound }\end{array}$} \\
\hline & Copper & $\begin{array}{c}\text { Muntz } \\
\text { metal }\end{array}$ & & Copper & $\begin{array}{l}\text { Muntz } \\
\text { mutal }\end{array}$ \\
\hline $\begin{array}{l}3 / 4 \\
1 / 8 \\
11 / 8 \\
11 / 8 \\
13 / 8 \\
11 / 2\end{array}$ & $\begin{array}{c}316 \\
308 \\
236 \\
228 \\
184 \\
116 \\
96\end{array}$ & $\begin{array}{r}160 \\
152 \\
140 \\
130 \\
124 \\
88 \\
84\end{array}$ & $\begin{array}{l}13 / 4 \\
21 / 4 \\
21 / 4 \\
21 / 2 \\
23 / 4 \\
3\end{array}$ & $\begin{array}{l}84 \\
60 \\
52 \\
44\end{array}$ & $\begin{array}{l}72 \\
64 \\
60 \\
56 \\
46 \\
36\end{array}$ \\
\hline
\end{tabular}

(31) Shingle nails.-Flat head; diamond point; smooth, bright, or zinc coated.

\begin{tabular}{|c|c|c|c|c|c|c|c|c|}
\hline \multirow{2}{*}{$\mathrm{S}$} & \multicolumn{4}{|c|}{ Bright } & \multicolumn{4}{|c|}{ Zinc coated } \\
\hline & L & $G$ & $\mathrm{D}$ & No./lb. & $\mathbf{L}$ & $G$ & $\mathrm{D}$ & No./lb. \\
\hline \multirow[t]{2}{*}{$\begin{array}{r}3 \mathrm{~d} \\
31 / 2 \mathrm{~d} \\
4 \mathrm{~d} \\
5 \mathrm{~d} \\
6 \mathrm{~d}\end{array}$} & $\begin{array}{l}11 / 4 \\
13 / 8 \\
11 / 2 \\
13 / 4 \\
2\end{array}$ & $\begin{array}{l}13 \\
121 / 2 \\
12 \\
12 \\
12\end{array}$ & $\begin{array}{r}0.091 \\
.098 \\
.105 \\
.105 \\
.105\end{array}$ & $\begin{array}{l}429 \\
305 \\
274 \\
235 \\
204\end{array}$ & $\begin{array}{l}11 / 4 \\
13 / 8 \\
11 / 2 \\
13 / 4 \\
2\end{array}$ & $\begin{array}{l}13 \\
121 / 2 \\
12 \\
12 \\
12\end{array}$ & $\begin{array}{r}0.091 \\
.098 \\
.105 \\
.105 \\
.105\end{array}$ & $\begin{array}{l}337 \\
266 \\
227 \\
186 \\
158\end{array}$ \\
\hline & \multicolumn{4}{|c|}{ Cement coated (heavy) } & \multicolumn{4}{|c|}{ Cement coated (light) } \\
\hline $\begin{array}{l}3 d \\
4 d \\
5 d \\
6 d\end{array}$ & $\begin{array}{l}11 / 4 \\
13 / 8 \\
13 / 4 \\
2\end{array}$ & $\begin{array}{l}131 / 2 \\
121 / 2 \\
121 / 2 \\
121 / 2\end{array}$ & $\begin{array}{r}0.086 \\
.098 \\
.098 \\
.098\end{array}$ & $\begin{array}{l}464 \\
321 \\
254 \\
220\end{array}$ & $\begin{array}{l}11 / 4 \\
13 / 8 \\
15 / 8 \\
17 / 8\end{array}$ & $\begin{array}{l}14 \\
13 \\
13 \\
13\end{array}$ & $\begin{array}{r}0.080 \\
.091 \\
.091 \\
.091\end{array}$ & $\begin{array}{l}520 \\
372 \\
310 \\
267\end{array}$ \\
\hline
\end{tabular}

(31a) Cut iron or steel shingle nails.-

\begin{tabular}{|c|c|c|c|}
\hline \multirow{2}{*}{$\mathrm{S}$} & \multirow{2}{*}{ L } & \multicolumn{2}{|c|}{$\begin{array}{l}\text { Approximate } \\
\text { number per } \\
\text { pound }\end{array}$} \\
\hline & & Plain & $\begin{array}{l}\text { Zinc } \\
\text { coated }\end{array}$ \\
\hline $\begin{array}{l}2 \mathrm{~d} \\
3 \mathrm{~d} \\
31 / 2 \mathrm{~d} \\
4 \mathrm{~d} \\
5 \mathrm{~d} \\
6 \mathrm{~d}\end{array}$ & $\begin{array}{l}1 \\
11 / 4 \\
13 / 8 \\
11 / 2 \\
13 / 4 \\
2\end{array}$ & $\begin{array}{l}558 \\
390 \\
338 \\
273 \\
192 \\
141\end{array}$ & $\begin{array}{l}475 \\
345 \\
320 \\
250 \\
175 \\
132\end{array}$ \\
\hline
\end{tabular}

(31b) Copper-wire shingle nails.-(See common copper-wire nails $(11 e)$.)

(31c) Copper-clad shingle nails.-(See copperclad common nails (11d).) 
(32) Shoe nails (cobbler's). - Iron or steel wire nails; narrow, flat countersunk head; long sharp point; bright finish; brass plated.

\begin{tabular}{|r|r||r|r|}
\hline $\mathrm{L}$ & $\begin{array}{c}\text { Approxi- } \\
\text { mate } \\
\text { number } \\
\text { per pound } \\
(16 \mathrm{G})\end{array}$ & $\mathrm{L}$ & $\begin{array}{c}\text { Approxi- } \\
\text { mate } \\
\text { number } \\
\text { per pound } \\
(16 \mathrm{G})\end{array}$ \\
\hline $3 / 8$ & 3,290 & $51 / 2 / 8$ & 1,670 \\
$33 / 8 / 8$ & 2,810 & $6 / 8$ & 1,550 \\
$3 / 8$ & 2,320 & $61 / 2 / 8$ & 1,420 \\
$43 / 8$ & 2,060 & $7 / 8$ & 1,290 \\
$5 / 8$ & 1,790 & $8 / 8$ & $\ldots$ \\
\hline
\end{tabular}

(33) Siding nails.-Flat head, diamond point, smooth, bright.

\begin{tabular}{|c|c|c|c|c|c|c|c|c|c|}
\hline $\mathrm{S}$ & L & $\mathrm{G}$ & D & No./lb. & $\mathrm{S}$ & L & $G$ & D & No./lb. \\
\hline $2 \mathrm{~d}$ & & & 0671 & 1.010 & $9 \mathrm{~d}$ & & $11^{1 / 6}$ & 0.113 & \\
\hline $3 \mathrm{~d}$ & 114 & $141 / 2$ & .0 & 35 & 10 & 3 & $101 / 2$ & .1 & 94 \\
\hline $4 \mathrm{~d}$ & $11 / 2$ & & .0 & 3 & 11 & $31 / 4$ & 10 & .1 & 88 \\
\hline $5 d$ & $13 / 4$ & 14 & .0 & 406 & 16 & $3 i$ & 10 & .1 & $=$ \\
\hline 6 & 2 & 12 & 0 & 236 & 20 & 4 & 9 & .1 & 5 \\
\hline & $21 / 4$ & 121 & .0 & 210 & 30 & 4132 & 0 & & 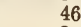 \\
\hline $8 c$ & $2 \frac{1}{2}$ & $11 \frac{1}{2}$ & .113 & 145 & $40 c$ & & 8 & .162 & 35 \\
\hline
\end{tabular}

(34) Slating nails.-Flat head, diamond point, smooth bright.

\begin{tabular}{|c|c|c|c|c|c|c|c|c|c|}
\hline \multirow{2}{*}{$\mathrm{S}$} & \multirow{2}{*}{ L } & \multicolumn{4}{|c|}{ Bright } & \multicolumn{4}{|c|}{ Cement Coated } \\
\hline & & G & $\mathrm{D}$ & $\mathrm{H}$ & No./lb. & G & D & H & No./lb. \\
\hline $\begin{array}{l}2 d \\
3 d \\
4 d \\
5 d \\
6 d\end{array}$ & $\begin{array}{l}1 \\
11 / 4 \\
11 / 2 \\
13 / 4 \\
2\end{array}$ & $\begin{array}{l}12 \\
101 / 2 \\
101 / 2 \\
10 \\
9\end{array}$ & $\begin{array}{r}0.105 \\
.128 \\
.128 \\
.135 \\
.148\end{array}$ & $\begin{array}{r}5 / 16 \\
3 / 8 \\
36 \\
13 / 32 \\
7 / 16\end{array}$ & $\begin{array}{l}411 \\
225 \\
187 \\
142 \\
103\end{array}$ & $\begin{array}{l}13 \\
12 \\
12 \\
11 \\
10\end{array}$ & $\begin{array}{r}0.091 \\
.105 \\
.105 \\
.120 \\
.135\end{array}$ & $\begin{array}{r}5 / 16 \\
3 / 8 \\
3 / 8 \\
13 / 32 \\
7 / 16\end{array}$ & $\begin{array}{l}500 \\
315 \\
271 \\
174 \\
120\end{array}$ \\
\hline
\end{tabular}

(34a) Slating nails.-3/8-inch head; zinc coated.

\begin{tabular}{|l|l|l|r|}
\hline $\mathrm{L}$ & $\mathrm{G}$ & $\mathrm{D}$ & No./lb. \\
\hline 1 & 12 & 0.105 & 370 \\
$11 / 8$ & $111 / 2$ & .113 & 330 \\
$11 / 4$ & 11 & .120 & 201 \\
$11 / 2$ & 11 & .120 & 160 \\
$13 / 4$ & $101 / 2$ & .128 & 113 \\
2 & 10 & .135 & 100 \\
\hline
\end{tabular}

(34b) Copper-wire slating nails-Large flat head, five-sixteenths inch to three-eighths inch; diamond point.

\begin{tabular}{|c|c|c|c|}
\hline L & G & $\mathrm{D}$ & No./lb. \\
\hline $7 / 8$ & 12 & 0.105 & 390 \\
\hline 1 & 12 & .105 & 350 \\
\hline $11 / 4$ & 12 & .105 & 289 \\
\hline $11 / 4$ & 11 & .120 & 206 \\
\hline $11 / 4$ & 10 & .135 & 173 \\
\hline $11 / 2$ & 11 & .120 & 176 \\
\hline $11 / 2$ & 10 &. .135 & 145 \\
\hline
\end{tabular}

(34c) Copper-clad slating nails.-Flat head five-sixteenths inch to three-eighth inch; diamond point.

\begin{tabular}{|l|r|r|r|}
\hline L & \multicolumn{1}{|c|}{ G } & D & No./lb. \\
\cline { 1 - 2 } & 10 & 0.135 & 386 \\
1134 & 9 & .148 & 211 \\
$11 / 2$ & 8 & .162 & 176 \\
$13 / 4$ & 8 & .162 & 133 \\
2 & 7 & .177 & 87 \\
\hline
\end{tabular}

(34d) Cut slating nails.-Copper or Muntz metal,

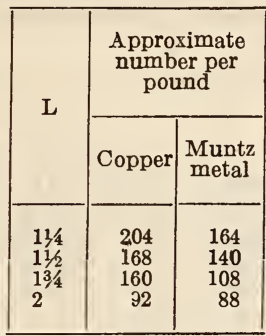

(34e) Cut iron or steel slating nails.-

\begin{tabular}{|c|c|c|c|}
\hline & & \multicolumn{2}{|c|}{$\begin{array}{c}\text { Approximate } \\
\text { number per } \\
\text { pound }\end{array}$} \\
S & L & \multicolumn{2}{|c|}{} \\
\cline { 1 - 2 } & & Plain & $\begin{array}{c}\text { Zinc } \\
\text { coated }\end{array}$ \\
\hline 21/2d & $11 / 8$ & 465 & 405 \\
3d & $11 / 4$ & 373 & 324 \\
31/2d & 138 & 323 & 296 \\
4d & $11 / 2$ & 260 & 238 \\
5d & $13 / 4$ & 180 & 175 \\
6d & 2 & 109 & 105 \\
\hline
\end{tabular}

(35) Stem nails.-Cut Muntz metal.

\begin{tabular}{|c|c||c|c|}
\hline $\mathrm{L}$ & No./lb. & $\mathrm{L}$ & No./lb. \\
\hline & & & \\
\hline 114 & 88 & $21 / 2$ & 30 \\
1132 & 64 & $23 / 4$ & 24 \\
134 & 52 & 3 & 22 \\
2 & 48 & 4 & 16 \\
234 & 32 & $-\cdots$ &..$--\cdot$ \\
\hline
\end{tabular}

(36) Copper storm nails (rivets).-Broad fla1, round, square, or octagon heads; soft-wire shanks.

\begin{tabular}{|c|c|c|c|c|c|}
\hline & \multirow{2}{*}{$\mathrm{L}$} & $\mathrm{G}$ & $\mathrm{D}$ & \multicolumn{3}{|c|}{$\begin{array}{c}\text { Approximate number } \\
\text { per pound }\end{array}$} \\
\cline { 4 - 6 } & & & $\mathrm{H} 5 / 8$ & $\mathrm{H} 13 / 16$ & $\mathrm{H} 1$ \\
\hline & & & & & \\
\hline $5 / 8$ & 14 & 0.080 & 741 & 665 & 628 \\
$11 / 16$ & 14 & .080 & 691 & 615 & 518 \\
$3 / 4$ & 14 & .080 & 648 & 572 & 535 \\
$7 / 8$ & 14 & .080 & 582 & 506 & 469 \\
1 & 14 & .080 & 533 & 457 & 420 \\
$5 / 8$ & 13 & .092 & 610 & 534 & 497 \\
$11 / 16$ & 13 & .092 & 572 & 496 & 459 \\
$3 / 4$ & 13 & .092 & 540 & 464 & 427 \\
$7 / 8$ & 13 & .092 & 489 & 413 & 376 \\
1 & 13 & .092 & 451 & 375 & 338 \\
\hline
\end{tabular}

(37) Upholsterer's nails.-Oval head, brass or japanned to imitate leather in various shades of red, brown, maroon, green, and black; steel shanks; needle point.

\begin{tabular}{|c|c|}
\hline $\mathrm{L}$ & $\mathrm{H}$ \\
\hline 38 & $1 / 4$ \\
$31 / 2$ & $5 / 16$ \\
538 & $7 / 16$ \\
$3 / 4$ & 112 \\
\hdashline$-11 / 6$ \\
\hdashline & $13 / 16$ \\
\hline
\end{tabular}


(b) Spikes-(1) Barge spikes.-Oval or square head; chisel point; plain or zinc coated. Number per keg of 200 pounds approximately the same as for boat spikes (2).

\begin{tabular}{|c|c|}
\hline $\mathrm{D}$ & L \\
\hline 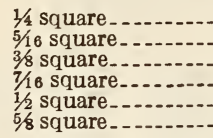 & $\begin{array}{l}3 \text { to } 8 . \\
3 \text { to } 8 . \\
3 \text { to } 12 . \\
6 \text { to } 12 . \\
6 \text { to } 12 . \\
8 \text { to } 12 .\end{array}$ \\
\hline
\end{tabular}

(2) Boat spikes.-Square or diamond shaped head, chisel point; plain or zinc coated.

\begin{tabular}{|c|c|c|c|c|c|c|}
\hline \multirow{2}{*}{ D } & \multicolumn{6}{|c|}{$\begin{array}{l}\text { Approximate number per keg of } 200 \\
\text { pounds }\end{array}$} \\
\hline & L 3 & L $31 / 2$ & $\mathbf{L}$ & & L 5 & L 6 \\
\hline $\begin{array}{l}5 / 8 \\
1 / 2 \\
7 / 16 \\
3 / 16 \\
1 / 4\end{array}$ & $\begin{array}{r}2,176 \\
3,400\end{array}$ & $\begin{array}{r}-1,265 \\
1,824 \\
2,942\end{array}$ & $\begin{array}{l}-1 \\
1,6 \\
2,6\end{array}$ & $\begin{array}{l}10 \\
346\end{array}$ & $\begin{array}{r}920 \\
1,380 \\
2,040\end{array}$ & $\begin{array}{r}434 \\
572 \\
748 \\
1,138 \\
1,748\end{array}$ \\
\hline \multirow{2}{*}{ D } & \multicolumn{6}{|c|}{$\begin{array}{l}\text { Approximate number per } \mathrm{keg} \text { of } 200 \\
\text { pounds }\end{array}$} \\
\hline & L 7 & L 8 & L 9 & L 10 & L 11 & L 12 \\
\hline 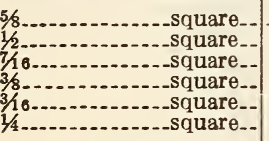 & $\begin{array}{r}384 \\
484 \\
634 \\
974 \\
1,456\end{array}$ & $\begin{array}{r}260 \\
338 \\
414 \\
554 \\
858 \\
1,294\end{array}$ & $\begin{array}{c}234 \\
294 \\
380 \\
500 \\
----\end{array}$ & $\begin{array}{r}210 \\
262 \\
348 \\
458 \\
- \\
- \\
-\end{array}$ & $\begin{array}{r}194 \\
236 \\
318 \\
416 \\
\hdashline--\end{array}$ & $\begin{array}{r}178 \\
212 \\
392 \\
378 \\
\hdashline- \\
\hdashline-\end{array}$ \\
\hline
\end{tabular}

(3) Common cut iron or steel spikes.-Plain or zinc coated.

\begin{tabular}{|c|c|c||c|c|c|}
\hline S & L & No./lb. & S & L & No./lb. \\
\hline 20d & 4 & 21 & $70 d$ & $61 / 2$ & 7 \\
30d & $41 / 2$ & 15 & $80 d$ & 7 & 634 \\
50d & 5 & 12 & $90 d$ & $71 / 2$ & $61 / 2$ \\
50d & $51 / 2$ & 10 & $100 d$ & 8 & $61 / 4$ \\
60d & 6 & $71 / 2$ & & & \\
\hline
\end{tabular}

(4) Gutter spikes.-Oval head, chisel point, or flat head, diamond point; one-fourth inch diameter; length $5 \frac{1}{2}$ inches to $10 \frac{1}{2}$ inches; bright or zinc coated.

(5) Round wire spikes.-Oval countersunk head, chisel point, or flat head, diamond point; smooth bright or zinc coated.

\begin{tabular}{|c|c|c|c|c|c|}
\hline \multirow{2}{*}{$\mathrm{s}$} & \multirow{2}{*}{ L } & \multirow{2}{*}{$G$} & \multirow{2}{*}{ D } & \multicolumn{2}{|c|}{$\begin{array}{l}\text { Approximate } \\
\text { number per } \\
\text { pound }\end{array}$} \\
\hline & & & & Bright & $\begin{array}{l}\text { Zine } \\
\text { coated }\end{array}$ \\
\hline $\begin{array}{l}10 \mathrm{~d} \\
12 \mathrm{~d} \\
16 \mathrm{~d} \\
20 \mathrm{~d}\end{array}$ & $\begin{array}{l}3 \\
31 / 4 \\
31 / 2 \\
4\end{array}$ & $\begin{array}{l}6 \\
6 \\
5 \\
4\end{array}$ & $\begin{array}{r}0.192 \\
.192 \\
.207 \\
.225\end{array}$ & $\begin{array}{l}41 \\
38 \\
30 \\
23\end{array}$ & $\begin{array}{l}39 \\
36 \\
28 \\
22\end{array}$ \\
\hline $\begin{array}{l}30 \mathrm{~d} \\
40 \mathrm{~d} \\
50 \mathrm{~d} \\
60 \mathrm{~d}\end{array}$ & $\begin{array}{l}41 / 2 \\
5 \\
51 / 2 \\
6\end{array}$ & $\begin{array}{l}3 \\
2 \\
1 \\
1\end{array}$ & $\begin{array}{l}.244 \\
.2625 \\
.283 \\
.283\end{array}$ & $\begin{array}{r}17 \\
13 \\
10 \\
9\end{array}$ & $\begin{array}{l}16 \\
12 \\
11 \\
8.5\end{array}$ \\
\hline $\begin{array}{r}7 \\
8 \\
9 \\
10 \\
12\end{array}$ & $\begin{array}{r}7 \\
8 \\
9 \\
10 \\
12\end{array}$ & $\begin{array}{l}5 / 16 \\
338 \\
338 \\
38 \\
3 / 8\end{array}$ & & $\begin{array}{l}7 \\
4 \\
31 / 2 \\
3 \\
21 / 4\end{array}$ & $\begin{array}{l}6.5 \\
3.8 \\
3.4 \\
2.9 \\
2.0\end{array}$ \\
\hline
\end{tabular}

(6) Track spikes.-

\begin{tabular}{|c|c|c|c|}
\hline Size & $\begin{array}{l}\text { Number } \\
\text { per keg } \\
\text { of } 200 \\
\text { pounds }\end{array}$ & Size & $\begin{array}{l}\text { Number } \\
\text { per keg } \\
\text { of } 200 \\
\text { pounds }\end{array}$ \\
\hline $\begin{array}{l}51 / 2 \text { by } 9 / 16 \\
5 \text { by } 1 / 2, \ldots \\
41 / 2 \text { by } 1 / 2 \ldots \\
4 \text { by } 1 / 2 \\
31 / 2 \text { by } 1 / 2 \ldots\end{array}$ & $\begin{array}{l}340 \\
460 \\
528 \\
592 \\
660\end{array}$ & $\begin{array}{l}3 \text { by } 3 / 8 \\
21 / 2 \text { by } 3 / 8 \\
21 / 4 \text { by } 3 / 8 \\
2 \text { by } 3 / 8 \\
4 \text { by } 5 / 16\end{array}$ & $\begin{array}{l}1,342 \\
1,600 \\
1,750 \\
1,902 \\
1,630\end{array}$ \\
\hline $\begin{array}{l}3 \text { by } 1 / 2=- \\
21 / 2 \text { by } 1 / 2 . \\
5 \text { by } 7 / 16= \\
41 / 2 \text { by } 76-\end{array}$ & $\begin{array}{l}732 \\
814 \\
664 \\
712\end{array}$ & $\begin{array}{l}31 / 2 \text { by } 5 / 16 \ldots \\
3 \text { by } 516 \\
21 / 2 \text { by } 5 / 16 \\
21 / 4 \text { by } 5 / 16 \ldots\end{array}$ & $\begin{array}{l}1,810 \\
2,066 \\
2,380 \\
2,760\end{array}$ \\
\hline $\begin{array}{l}4 \text { by } 7 / 16 \\
31 / 2 \text { by } 7 / 16 \ldots \\
3 \text { by } 7 / 16 . \\
21 / 2 \text { by } 7 / 16 \ldots\end{array}$ & $\begin{array}{r}764 \\
854 \\
1,032 \\
1,210\end{array}$ & $\begin{array}{l}2 \text { by } 5 / 16 \ldots \ldots \\
11 / 2 \text { by } 5 / 16 \ldots \\
3 \text { by } 1 / 4-\ldots \\
21 / 2 \text { by } 1 / 4 \ldots\end{array}$ & $\begin{array}{l}2,912 \\
4,200 \\
3,266 \\
4,120\end{array}$ \\
\hline $\begin{array}{l}5 \text { by } 3 / 8 \\
41 / 2 \text { by } 3 / 8 \ldots \\
4 \text { by } 3 / 8 \ldots \\
31 / 2 \text { by } 3 / 8 \ldots\end{array}$ & $\begin{array}{r}908 \\
1,000 \\
1,092 \\
1,200\end{array}$ & $\begin{array}{l}21 / 4 \text { by } 1 / 4 \ldots \ldots \\
2 \text { by } 11 / 4 . \ldots \\
11 / 2 \text { by } 114 \ldots \\
11 / 4 \text { by } 1 / 4 \ldots\end{array}$ & $\begin{array}{l}4,600 \\
4,778 \\
6,000 \\
7,920\end{array}$ \\
\hline
\end{tabular}

(c) Tacks-(1) Bill posters' tacks.-Flat head; bright, blued, or tinned.

\begin{tabular}{|r|r|r|}
\hline $\mathrm{S}$ & $\mathrm{L}$ & No./lb. \\
\hline & & \\
\cline { 1 - 2 } 3 & $3 / 8$ & 1,570 \\
6 & $1 / 2$ & 873 \\
10 & 58 & 561 \\
14 & $3 / 4$ & 398 \\
\hline
\end{tabular}

(2) Carpet tacks.-Bright, blued, or tinned; flat

\begin{tabular}{|c|c|c|}
\hline S & L & No./b. \\
\hline $\begin{array}{r}6 \\
10\end{array}$ & $\begin{array}{l}3 / 2 \\
588\end{array}$ & $\begin{array}{l}1,570 \\
1,280\end{array}$ \\
\hline 14 & & \\
\hline
\end{tabular}

(2a) Copper or brass tacks.-

\begin{tabular}{|c|c|c|c|}
\hline \multirow{2}{*}{$\mathrm{S}$} & \multirow{2}{*}{ L } & \multicolumn{2}{|c|}{$\begin{array}{l}\text { Approximate } \\
\text { number per } \\
\text { pound }\end{array}$} \\
\hline & & $\begin{array}{l}\text { Flat } \\
\text { head }\end{array}$ & $\begin{array}{l}\text { Oval } \\
\text { head }\end{array}$ \\
\hline 3 & $3 / 6$ & 3,245 & 2,700 \\
\hline $\begin{array}{r}6 \\
10\end{array}$ & $\begin{array}{l}1 / 2 \\
5 / 8\end{array}$ & $\begin{array}{l}1,490 \\
1,145\end{array}$ & $\begin{array}{r}1,500 \\
900\end{array}$ \\
\hline $\begin{array}{l}14 \\
18\end{array}$ & $\begin{array}{l}3 / 4 \\
7 / 8\end{array}$ & $\begin{array}{l}875 \\
660\end{array}$ & $\begin{array}{r}660 \\
55\end{array}$ \\
\hline $\begin{array}{l}18 \\
22\end{array}$ & $1^{1 / 8}$ & $\begin{array}{l}0.00 \\
585\end{array}$ & $\begin{array}{l}530 \\
400\end{array}$ \\
\hline
\end{tabular}

(2b) Galvanized tacks.-

\begin{tabular}{|c|c|c|}
\hline No. & $\mathbf{L}$ & No./1b. \\
\hline 10 & $\begin{array}{l}3 / 8 \\
1 / 2 \\
5 / 8 \\
3 / 4 \\
7 / 8 \\
1^{7 / 8}\end{array}$ & $\begin{array}{r}2,400 \\
1,250 \\
850 \\
650 \\
500 \\
400\end{array}$ \\
\hline
\end{tabular}

(3) Double-pointed tacks.-Bright or blued.

\begin{tabular}{|c|c|c|c|}
\hline No. & L & Width & $G$ \\
\hline 8. & $\begin{array}{r}13 / 32 \\
7 / 6\end{array}$ & $3 / 16$ & 16 \\
\hline 10 & $\begin{array}{r}1,16 \\
1532 \\
1\end{array}$ & $\begin{array}{l}1 / 32 \\
1 / 4 \\
0\end{array}$ & $\begin{array}{l}10 \\
16\end{array}$ \\
\hline & 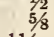 & $\begin{array}{l}3 / 322 \\
5 / 16\end{array}$ & $\begin{array}{l}15 \\
15\end{array}$ \\
\hline $\begin{array}{ll}14-- \\
16-\end{array}$ & $\begin{array}{r}13 / 16 \\
3 / 4\end{array}$ & $\begin{array}{r}5 / 16 \\
3 / 8\end{array}$ & $\begin{array}{l}14 \\
14\end{array}$ \\
\hline
\end{tabular}


(4) Gimp tacks.-Oval head; blued, tinned, or japanned.

\begin{tabular}{|l|r||r|r|}
\hline $\mathrm{S}$ & $\mathrm{L}$ & $\mathrm{S}$ & $\mathrm{L}$ \\
& & & \\
\hline $21 / 2$ & $3 / 8$ & 6 & $9 / 16$ \\
3 & $7 / 6$ & 8 & $5 / 8$ \\
4 & $1 / 2$ & 12 & $3 / 4$ \\
\hline
\end{tabular}

(5) Saddle tacks.-Oval head; plain bright, japanned black, and colors; brass capped and copper capped; long sharp point.

\begin{tabular}{|r|c|c|}
\hline $\mathrm{I}$ & $\mathrm{H}$ & $\mathrm{H}$ \\
\cline { 1 - 2 } $1 / 2$ & $3 / 8$ & $1 / 2$ \\
$9 / 16$ & $3 / 8$ & $1 / 2$ \\
$5 / 8$ & $3 / 8$ & $1 / 2$ \\
$11 / 16$ & $3 / 8$ & $1 / 2$ \\
$3 / 4$ & $3 / 8$ & $1 / 2$ \\
$13 / 16$ & $3 / 8$ & $1 / 2$ \\
\hline
\end{tabular}

(6) Shoe tacks.-Flat head, sharp point, bright.

\begin{tabular}{|c|c||c|r|}
\hline$S$ & L & $S$ & \multicolumn{1}{|c|}{ L } \\
\cline { 1 - 1 } & & & \\
\cline { 1 - 2 } $1 / 2$ & $3 / 16$ & 3 & $9 / 16$ \\
$3 / 4$ & $1 / 4$ & 4 & $5 / 8$ \\
$111 / 2$ & $5 / 16$ & 6 & $11 / 16$ \\
$11 / 2$ & 8 & $3 / 4$ \\
$21 / 2$ & $1 / 2$ & & \\
\hline
\end{tabular}

(7) Upholsterer's tacks.-Flat head, blued or tinned.

\begin{tabular}{|l|c||c|c|}
\hline \multicolumn{1}{|c|}{$S$} & $\mathrm{~L}$ & $\mathrm{~S}$ & $\mathrm{I}$ \\
\cline { 1 - 2 } 1 & $7 / 32$ & 10 & $5 / 8$ \\
2 & 932 & 14 & $3 / 4$ \\
$21 / 2$ & $5 / 16$ & 18 & $7 / 8$ \\
3 & $3 / 8$ & 22 & 118 \\
4 & $7 / 16$ & 24 & 118 \\
6 & $1 / 2$ & & \\
\hline
\end{tabular}

(d) Staples-(1) Electrician's staples.-Bright or coppered.

\begin{tabular}{|c|c|c|c|}
\hline S & G & D & No./1b. \\
\cline { 1 - 1 } 3 & & & \\
\cline { 1 - 1 } $1 / 8$ & 15 & 0.072 & 980 \\
$1 / 2$ & 15 & .072 & 700 \\
$5 / 8$ & 15 & .072 & 590 \\
$3 / 4$ & 15 & .072 & 500 \\
\hline
\end{tabular}

(2) Metal-lath staples.-Bright, blued, or zinc coated.

\begin{tabular}{|c|c|c|c|c|}
\hline S & G & D & $\begin{array}{c}\text { Bright } \\
\text { No./lb. }\end{array}$ & $\begin{array}{c}\text { Zinc } \\
\text { coated } \\
\text { No./lb. }\end{array}$ \\
\hline 1 & 14 & 0.08 & 390 & 352 \\
$11 / 8$ & 14 & .08 & 350 & 300 \\
$11 / 4$ & 14 & .08 & 315 & 255 \\
$11 / 2$ & 14 & .08 & 225 & 185 \\
\hline
\end{tabular}

(3) Poultry-netting staples.-Zinc coated.

\begin{tabular}{|c|c|c|c|}
\hline$S$ & $G$ & $D$ & No./lb. \\
\hline $3 / 4$ & 14 & 0.08 & 480 \\
$7 / 8$ & 14 & .08 & 416 \\
1 & 14 & .08 & 352 \\
\hline
\end{tabular}

(4) Wire-fence staples.-

\begin{tabular}{|c|c|r|r|r|}
\hline $\mathrm{S}$ & $\mathrm{G}$ & $\mathrm{D}$ & $\begin{array}{r}\text { Bright } \\
\text { No./lb. }\end{array}$ & $\begin{array}{c}\text { Zinc } \\
\text { coated } \\
\text { No./lb. }\end{array}$ \\
\hline 3 & & & & \\
\hline 34 & 9 & 0.148 & 152 & 147 \\
$7 / 8$ & 9 & .148 & 120 & 116 \\
1118 & 9 & .148 & 108 & 105 \\
$11 / 4$ & 9 & .148 & 96 & 93 \\
$11 / 2$ & 9 & .148 & 87 & 84 \\
$13 / 4$ & 9 & .148 & 72 & 70 \\
21 & 9 & .148 & 65 & 63 \\
214 & 9 & .148 & 58 & 56 \\
$21 / 2$ & 9 & .148 & 40 & 38 \\
\hline
\end{tabular}

4. Packing and marking.-Containers shall be substantial and so constructed as to preserve the contents in good condition; they shall be so made that the contents may be removed without destroying the containers.

Containers shall be marked with the type, class, material, finish, and length (in inches) of the article contained; and shall also be marked with the net weight of the contents or with the count number, and with the name of the manufacturer.

Articles intended for use in automatic driving machines shall be so labeled.

\section{METHOD OF INSPECTION AND TESTS}

1. Inspection.-Inspection shall be made by the purchaser after delivery (unless otherwise agreed upon) by examining not less than one package in every 25 packages, or portion thereof, of each type and class included in the order. The articles will be examined for type, class, material, size, finish, workmanship, and net weight. The count numbers will be verified when orders are based on count numbers.

2. Tests.-Test specimens in such numbers as required shall be taken from the packages examined.

(a) Tests for ductility.-Articles tested for ductility shall be bent $180^{\circ}$ and flattened on themselves as required in III $2(a)(1)$, or bent $180^{\circ}$ over a mandrel as required in III $2(a)(2)$, by any suitable means available.

(b) Tests for hardness.-Articles tested for hardness shall be held rigidly in a vice and bent by means of a clamp or similar device, so attached to the free end of the specimen that the bend shall be confined to a length not exceeding two times the diameter of the specimen, and suitable means be provided for measuring the angle through which the specimen is bent before breaking.

(c) Tests of zinc coating.-The articles to be tested shall be weighed on scales sensitive to 0.05 per cent, and then immersed in concentrated hydrochloric acid (specific gravity 1.20) to each $100 \mathrm{c}$ c of which has been added $5 \mathrm{c}$ c of a solution made by dissolving $20 \mathrm{~g}$ of antimony trioxide in $1,000 \mathrm{c}$ c of concentrated hydrochloric acid.

The specimens shall be immersed for one minute, then washed and scrubbed in running water to remove deposited antimony, and dried and reweighed. The loss in weight represents the weight of the zinc coating.

(d) Tests of cement coating shall be made by removing the coating from one-half of the number of test specimens. An equal number of the cementcoated and uncoated specimens, not less than 10 of each, shall be driven into sound, dry, shortleaf yellow pine, hemlock, fir, or larch, perpendicular to the grain, with heads protruding one-fourth inch. The specimens shall be withdrawn by apparatus that will exert a direct pull and indicate the force 
applied. The force required to start the withdrawal shall be taken as the resistance. This shall be expressed in percentages of the average resistance of the cement-coated above that of the uncoated for each group of identical specimens tested. Thus, if the average resistance of the uncoated is 100 and that of the cement-coated 160, the increased holding capacity would be 60 per cent.

3. Methods of analysis for copper, zinc, brass, yellow metals, and copper steel.-In all cases the sample must be clean and free from foreign substances.

(a) Copper.-Dissolve $5 \mathrm{~g}$ of the sample in a mixture of $25 \mathrm{c}$ c of water, $10 \mathrm{c}$ c of concentrated sulphuric acid, and $7 \mathrm{c} \mathrm{c}$ of concentrated nitric acid. Boil gently to remove nitrous oxides, dilute to 225 c c and electrolyze for about 18 hours with a current of 0.5 ampere and 2.0 to 2.5 volts, using gauze electrodes. Remove the cathode without interrupting the current; wash with water, then with alcohol; dry for a few minutes at $100^{\circ} \mathrm{C}$.; and weigh.

If tin and antimony are present, decompose a $5 \mathrm{~g}$ sample with $50 \mathrm{c} \mathrm{c}$ of nitric acid $(1: 1)$ and boil to remove nitrous oxides. Dilute to $150 \mathrm{c}$ c and digest on the steam bath for several hours or over night. Filter and wash with a hot 1 per cent solution of nitric acid. Add $10 \mathrm{c}$ c concentrated sulphuric acid to the filtrate and evaporate to expel nitric acid. Dilute to about $225 \mathrm{c} \mathrm{c}$ with distilled water, add $7 \mathrm{c}$ c of concentrated nitric acid, and electrolyze as described above.

(b) Zinc.-Dissolve $1 \mathrm{~g}$ of the sample in $20 \mathrm{c} \mathrm{c}$ of nitric acid $(1: 1)$ and boil to remove nitrous fumes. If a precipitate forms, filter it off. Add $10 \mathrm{c} c$ of concentrated sulphuric acid and evaporate to expel all nitric acid. Dilute to 300 c c with distilled water and saturate with hydrogen sulphide. Filter and boil the filtrate to remove the hydrogen sulphide.

Neutralize the acid with ammonia, using methyl orange as indicator, dilute to about $900 \mathrm{c}$ c with distilled water, and make 0.008 to $0.009 N$ with sulphuric acid. Cool to room temperature and pass a rapid stream of hydrogen sulphide through the solution for about 40 minutes. Allow the precipitate to settle for one-half hour to two hours, filter, and wash with cool distilled water. Ignite at as low a temperature as possible until the paper is consumed. Increase the heat to $750^{\circ}$ to $800^{\circ} \mathrm{C}$., complete the conversion of sulphide to oxide, and then heat at a temperature of not over $900^{\circ} \mathrm{C}$. for about five minutes. Cool; ignite, and weigh as zinc oxide.

(c) Brass and muntz metal.-Dissolve $1 \mathrm{~g}$ of the sample in $20 \mathrm{c} \mathrm{c}$ of nitric acid $(1: 1)$ boil to expel nitrous oxides, and, if a precipitate forms, dilute to $150 \mathrm{c} \mathrm{c}$ with hot water, digest on the steam bath for several hours or overnight. Filter and wash with a hot 1 per cent solution of nitric acid. Ignite the filter at as low a temperature as possible until carbon is consumed, then blast in an oxidizing flame for three to five minutes. Cool, and weigh as stannic oxide. Neutralize the filtrate with ammonia, add an excess of $7 \mathrm{c} \mathrm{c}$ of concentrated nitric acid, dilute to $225 \mathrm{c} \mathrm{c}$, and electrolyze for about 18 hours with a current of 0.5 ampere and 2.0 to 2.5 volts. Remove the solution from the electrodes without interrupting the current, and wash the electrodes with water as fast as they are exposed. Finally rinse thoroughly in water and then in alcohol. Dry the cathode for a few minutes at $100^{\circ} \mathrm{C}$. and weigh. Dry the anode at $210^{\circ} \mathrm{C}$. for one-half hour, weigh as $\mathrm{PbO}_{2}$, and use the factor 0.866 to calculate the amount of lead. Add a slight excess of ammonia to the electrolyte, boil, filter, and wash the precipitate with hot water. Ignite and weigh as $\mathrm{Fe}_{2} \mathrm{O}_{3}$, or dissolve the precipitate and determine the iron by volumetric procedure. Pass hydrogen sulphide into the ammoniacal filtrate. A white precipitate shows the presence of zinc, which is then reported by difference.

(d) Determination of copper in copper steel by precipitation with hydrogen sulphide.-Dissolve $10.00 \mathrm{~g}$ of steel in $100 \mathrm{c}$ c of dilute sulphuric acid (1:1) and, when solution is complete, dilute to at least 500 c c with water. Heat to boiling and saturate with hydrogen sulphide. Digest a while, filter on paper or paper pulp, and wash with acidulated hydrogen sulphide water. Ignite the residue and paper in a porcelain crucible and fuse with a small amount of alkali pyrosulphate. Dissolve the cooled melt in the crucible in 1 to $2 \mathrm{c}$ c of hydrochloric acid (specific gravity 1.19) and a few c c of water, transfer to a $200 \mathrm{c} \mathrm{c}$ beaker, dilute to $100 \mathrm{c} \mathrm{c}$, and add sodium hydroxide ( 5 per cent) solution in slight excess. Boil, digest, and filter, in order to separate such elements as vanadium, tungsten, and molybdenum. Dissolve the precipitate in hot dilute nitric acid (1:1), add $5 \mathrm{c}$ c of sulphuric acid (specific gravity 1.84), evaporate to the appearance of fumes of sulphuric acid, cool, dilute to $40 \mathrm{c}$ c, filter, and, add $10 \mathrm{c}$ c of ammonium hydroxide (specific gravity 0.90 ). Heat the feebly acid solution to boiling and saturate with hydrogen sulphide. Digest a while, filter, wash thoroughly with acidulated hydrogen sulphide water, and proceed according to method 1 or 2 below.

(1) Electrolytic method.-Dissolve the sulphides obtained as above in hot dilute nitric acid $(1: 1)$, and electrolyze after the addition of sulphuric acid according to usual procedures.

(2) Gravimetric method.-Ignite the sulphide obtained as above and weigh as copper oxide. As this compound is somewhat hygroscopic, care must be observed to prevent the absorption of moisture.

\section{PACKING AND MARKING OF SHIPMENTS}

Shall be in accordance with best commercial practice unless otherwise specified.

\section{NOTES}

Requisitions or orders should give lengths in inches.

Copper should be used in work exposed to salt or brackish water.

Muntz metal should not be used in work exposed to salt water or brackish water.

Copper or Muntz metal or zinc, or zinc-coated articles, should be used in work of the best grade when exposed to salt air, and should be used in connection with roofing work of the best grade.

\section{UNITED STATES GOVERNMENT, FEDERAL SPECIFICATIONS BOARD, FEDERAL SPECIFICATION FOR BOXES; WOOD, NAILED AND IOCKED CORNER, NN-B- 621, DECEMBER 9, 1930}

\section{BOX NAILS}

(Contains tables showing sizes of cement-coated steel wire nails, and sizes of cement-coated, cooler, sinker, and box nails for unstrapped boxes.

\subsection{BRADS.}

AMERICAN RAILWAY ASSOCIATION, PURCHASES AND STORES DIVISION, STAND. ARDIZATION AND SIMPLIFICATION OF STORES STOCKS, PROCEEDINGS 1928

\section{WIRE BRADS}

(Recommended practice in stocking certain specified sizes of wire brads, ranging from one-half inch No. 20 to 2 inch No. 14.) 
UNITED STATES GOVERNMENT, FEDERAL SPECIFICATIONS BOARD, FEDERAL SPECIFICATIONS FOR NAILS, SPIKES, TACKS, AND STAPLES, FF-N-101, MAY 3, 1932.

\section{COMMON BRADS}

(Common brads are included in above Federal specification No. 534 (FF-N-101) for nails, etc. See 608.11, p. 560.)

608.13 STAPLES.

AMERICAN RAILWAY ENGINEERING ASSOCIATION, SPECIFICATIONS FOR STANDARD RIGHT-OF-WAY FENCES, 1926

\section{STAPLES FOR RIGHT-OF-WAY FENCES}

The staples used for fastening the longitudinal wires to the posts shall be made of No. 9 galvanized steel wire. They shall be 1 inch long for hardwood and $1 \frac{1}{2}$ inches long for softwood.

(For A. R. E. A. specifications for right-of-way fences see 603.43, p. 191.)

UNITED STATES GOVERNMENT, FEDERAL SPECIFICATIONS BOARD, FEDERAL SPECIFICATION FOR NAILS, SPIKES, TACKS, AND STAPLES, FF-N-101, MAY 3, 1932.

ELECTRICIAN'S STAPLES, METAL LATH STAPLES, POULTRY NETTING STAPLES, WIRE FENCE STAPLES, DOUBLE-POINTED TACKS

(Above items are included in Federal specification FF-N-101 for nails, etc. See 608.11, p. 560.) 608.14 TACKS.

UNITED STATES GOVERNMENT, DEPARTMENT OF COMMERCE, BUREAU OF STANDARDS, CUT TACKS AND SMALL CUT NAILS, SIMPLIFIED PRACTICE RECOMMENDATION R47-28, JULY 1, 1928

\section{CUT TACKS AND SMALI CUT NAILS}

(See 608.11, p. 558.)

UNITED STATES GOVERNMENT, FEDERAL SPECIFICATIONS BOARD, FEDERAL SPECIFICATION FOR NAILS, SPIKES, TACKS, AND STAPLES, FFN-101, MAY 3, 1932.

\section{TACKS}

(Bill posters' tacks, carpet tacks, copper or brass tacks, galvanized tacks, double-pointed tacks, gimp tacks, saddle tacks, shoe tacks, upholsterers' tacks, are included in Federal specification No. 534 (FF-N-101) for nails, etc. See 608.11, p. 560.)

UNITED STATES GOVERNMENT, FEDERAL SPECIFICATIONS BOARD, FEDERAL SPECIFICATION FOR THUMB TACKS, FF-T-311, DECEMBER 8, 1931

\section{THUMB TACKS}

\section{A. APPLICABLE FEDERAL SPECIFICATIONS}

A-1. There are no other Federal specifications applicable to this specification.

\section{B. TYPES AND GRADE}

B-1. Thumb tacks shall be of the highest grade and of the following types:

Type I. Nickel-silver heads.

Type II. Brass or steel heads.

Type III. Stamped steel.

Type IV. Center pull.

Type V. Colored heads.

\section{MATERIAL AND WORKMANSHIP}

C-1. Material.

C-1a. Type I heads to be made from the best rolled stock.

C-1b. Type II heads to be of the best commercial stock.

C-1c. Type III to be of the best material suitable for the purpose.

C-1d. Type IV heads may be of nickel-silver or steel.

C-1e. Type V heads may be enameled or celluloid.

C-2. Workmanship. - Shall be of the best and points of pins shall register accurately with centers of heads.

\section{GENERAL REQUIREMENTS}

D-1. Sizes.-Sizes shall be as specified in the invitation for bids.

D-2. Samples.-In all respects not specifically covered by this specification the thumb tacks shall be equal to sample submitted. (See Sec. I.)

\section{E. DETAIL REQUIREMENTS}

E-1. Type I heads.-Heads shall be as thin as practicable, top surface convex in section or flat with bevel edges. The under edges of heads shall be slightly beveled or rounded to permit the ready insertion of extracting tool without injury to paper. The steel pins shall be shouldered, screwed, and riveted into heads. Pins to be tool steel properly tempered and to have at least two complete threads and to be turned to a needle point.

E-2. Type II heads.-Heads and pins shall be swaged together but in such manner that pins do not extend through the heads. The pins are to be needle pointed and the swaged end to be headed. Steel heads shall be nickel plated.

E-3. Type III.-These tacks shall be nickel plated and shall be made from one piece of metal having the pins stamped out of the center of the head but not disconnected therefrom. The pins shall be turned to a needle point.

E-4. Type IV heads.-Heads of center-pull thumb tacks shall have holes or slots for the insertion of the pulling instrument. Thumb tacks may be turned from a solid piece of steel, or the pins may be screwed into nickel-silver or steel heads with at least two complete threads and riveted thereto, or the pin may have a shoulder integral with the pin to prevent it being forced through head, the top of the pin being solidly riveted in place. Pins shall be of steel, needle pointed.

E-5. Type $V$ heads.-Heads shall be of metal and shall be covered with colored celluloid or enameled in colors. Pins shall be of steel, needle pointed, and swaged to the heads.

E-6. Finish.-Metal heads shall be highly polished.

\section{F. METHOD OF INSPECTION AND TESTS}

F-2. Thumb tacks will be cut open so as to expose the heads of the pins to show the manner of construction.

\section{G. PACKAGING, PACKING, AND MARKING}

G-2. Packaging.

G-2a. Type I thumb tacks shall be mounted on cork board in sets of 1 dozen and packed in cardboard boxes containing 12 dozen. 
G-2b. Type II thumb tacks shall be mounted on blocks in sets of 1 dozen or packed in boxes of 100 each, as required.

G-2c. Type III thumb tacks shall be packed in cardboard boxes containing 100 each.

G-2d. Type IV thumb tacks shall be packed in cardboard boxes containing 1 dozen, or shall be mounted on cork board in sets of 1 dozen. One extracting tool for each 1 dozen center-pull thumb tacks shall be furnished for the type requiring same.

G-2e. Type V thumb tacks shall be mounted on blocks in sets of 1 dozen of one color and packed in boxes containing 12 dozen, or packed loose in boxes containing 100 in assorted colors, as required.

G-3. Packing.-Unless otherwise specified, the subject commodity shall be delivered in standard commercial containers, so constructed as to insure acceptance by common or other carriers, for safe transportation, at the lowest rate, to the point of delivery.

G-4. Marking.-Each box shall be plainly marked on the outside to indicate size, brand, manufacturer's name, and type of thumb tacks contained therein. Shipping containers shall be marked with the name of the material, the type, size, and the quantity contained therein, as defined by the contract or order, the name of the contractor, and the number of contract or order.

\section{NOTES}

I-1. Purchasers should exercise all options offered herein and should specify type, grade, class, etc., required.

I-2. Unless otherwise specified, samples are required with bid for the purpose of determining compliance with this specification as to composition of material, character of construction, and finish.

I-2a. Samples.-One dozen of each of the smallest and largest sizes of each type of metal thumb tacks and one of each color of the colored thumb tacks shall be submitted with the bids.

$\mathrm{I}-2 \mathrm{~b}$. The name of the manufacturer shall be indicated on the samples.

I-3. This specification governs all United States Government purchases of this commodity.

608.15 SPIKES.

(Railroad track spikes. See 606.5, p. 357.)

UNITED STATES GOVERNMENT, FEDERAL SPECIFICATIONS BOARD, FEDERAL SPECIFICATION FOR NAILS, SPIKES, TACKS, AND STAPLES, FF-N-101, MAY 3,1932 .

\section{SPIKES}

(Barge and boat spikes, common cut iron or steel spikes, gutter spikes, round wire spikes, and track spikes are included in Federal specification No. 534 (FF-N-101) for nails, etc. See 608.11, p. 560.)

608.2 SCREWS.

\section{AMERICAN ELECTRIC RAILWAY ENGI- NEERING ASSOCIATION, OVERHEAD LINE MATERIAL FOR DIRECT AND CAT- ENARY SUSPENSION, RECOMMENDED SPECIFICATION, D102-30, 1930}

\section{OVERHEAD IINE MATERIAL}

(D102-30, paragraph 311, includes requirements for lag screws. For specification see 611.29 , p. 682.)
AMERICAN MARINE STANDARDS COMMITTEE, SPECIFICATION E NO. 11 , PROPELLER HUB STUDS, NUTS, AND LOCK SCREWS, A. M. S. C. 30, SEPTEMBER 20, 1927

(See also 608.31 , p. 586 ; 608.32, p. 605 ; and 646.59 , p. 1257.)

\section{IOCK SCREWS}

(Contains specifications, dimensions, and illustrations of propeller hub studs, nuts, and lock screws.)

\section{AMERICAN RAILWAY ASSOCIATION, PURCHASES AND STORES DIVISION, STANDARDIZATION AND SIMPLIFI- CATION OF STORES STOCKS, D. V1- 238, MAY 20, 1930}

\section{SCREWS}

(Recommended practice in stocking certain specified sizes and types of screws-wood and machine screws of brass with flat, oval, and round heads; wood and machine screws of iron with flat and round heads; and machine screws of iron with fillister heads.)

AMERICAN RAILWAY ASSOCIATION, TELEGRAPH AND TELEPHONE SECTION, SPECIFICATION FOR POLE LINE HARDWARE, 1-A-20, NOVEMBER, 1927

\section{FETTER DRIVE SCREWS}
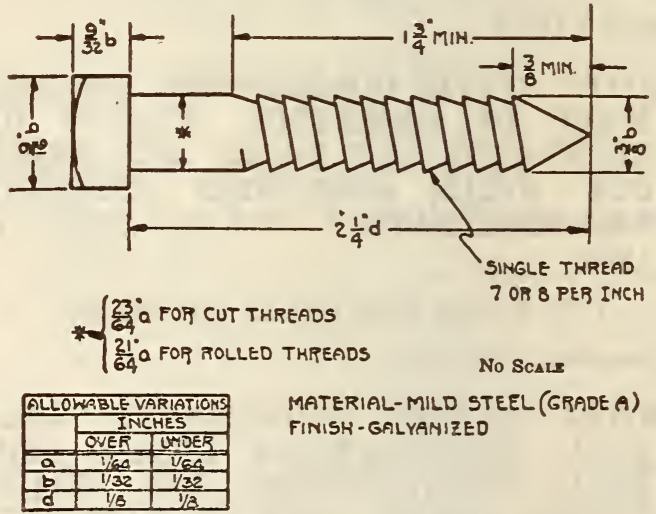

FIGURE 323.- $3 / 8$ inch fetter drive screw

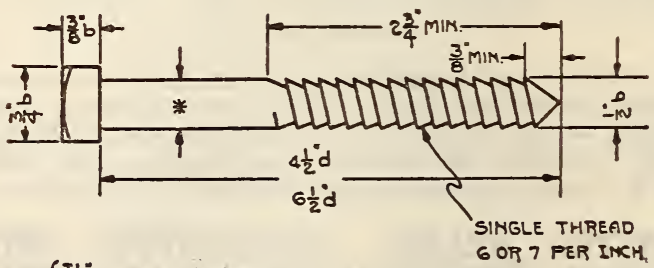

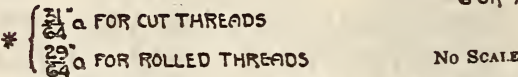

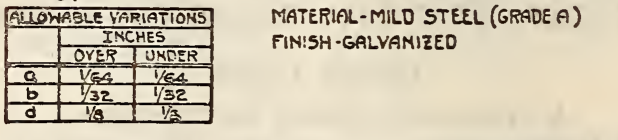

FIgURE 324,-One-half inch fetter drive screw 
(For general specifications see 608.0 , p. 516; for grades, tests, and workmanship see 608.31 , p. 589; requirements covering inspection and rejection conform in general to those specified in A. S. T. M., standard specifications for steel, see 605.0, p. 247.

\section{AMERICAN SOCIETY OF MECHANICAL EN- GINEERS, WRENCH HEAD BOLTS AND NUTS AND WRENCH OPENINGS, B18b- 1927}

[A pproved as American tentative standard by the American Standards Association, February, 1927]

(See also 608.31, p. 590; 608.32, p. 606; and 615.42, p.883.)

\section{FINISHED HEXAGONAL CAP SCREW HEADS}

(Dimensions of finished hexagonal cap screw heads are identical with those given below in National Screw Thread Commission, Table 75. The footnotes conform to the N. S. T. C. specifications for hexagon head cap screws, given below. Formulas for computing dimensions are appended.)

\section{SET SCREW HEADS}

(Dimensions of set screw heads are identical with those given below in Federal Specifications Board, No. 548 (current designation: FF-S-91), Table 24. The footnotes conform to the F. S. B. specifications for set screws, given below. Formulas for computing dimensions are appended.)

\section{AMERICAN SOCIETY OF MECHANICAL EN- GINEERS, SLOTTED-HEAD PROPOR- TIONS, MACHINE SCREWS, CAP SCREWS, AND WOOD SCREWS, B18c-1930}

[Approved by American Standards Association, April, 1930]

\section{MACHINE SCREWS, CAP SCREWS, AND WOOD}

\section{FLATHEAD MACHINE SCREWS}

1. The flathead screw has a flat surface for the top of the head and a countersink angle of $82^{\circ}$. This type of head is standard for machine screws, cap screws, and wood screws.

(Dimensions of flathead machine screws are identical with those given below in Federal Specifications Board No. 548 (FF-S-91), Table 14. Formulas for computing dimensions are appended as footnotes to the A. S. M. E. table.)

Note 1.-The unthreaded body diameter of machine screws will have approximately the same tolerances as the pitch diameter of the threads shown in the American national standard for screw threads, class 2 , free fit, where the number of threads per inch is the same.

\section{ROUND-HEAD MACHINE SCREWS}

1. The round-head screw has a semiellipticalshaped head. This type of head is standard for machine screws, cap screws, and wood screws, but for the cap screw it is called "button head."

(Dimensions of round-head machine screws are identical with those given below in Federal Specifications Board, No. 548 (FF-S-91), Table 15. Formulas for computing dimensisns are appended
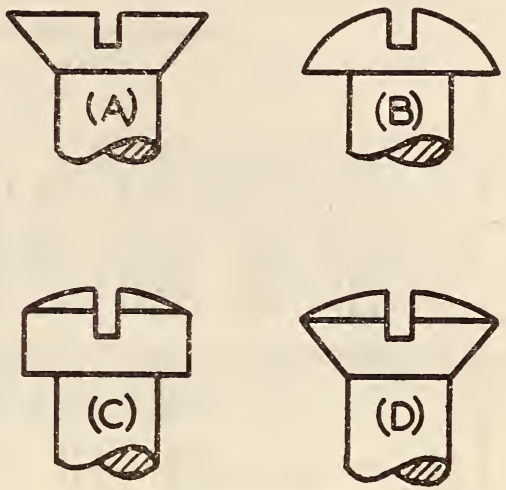

Figure 325 $(A)$, flat-head screw. $(B)$, round-head screw. $(C)$, flllister-head
screw. $(D)$, oval-head screw

as footnotes to the A. S. M. E. table. Note 1 is similar to note 1 under flathead machine screws.)

\section{OVAL-HEAD MACHINE SCREWS}

1. The oval-head screw has a rounded surface for the top of the head and a countersink angle of $82^{\circ}$. This type of head is standard for machine screws and wood screws.

(Dimensions of oval-head machine screws are identical with those given below in Federal Specifications Board, No. 548 (FF-S-91), Table 17, except that the latter carries two additional sizesseven-sixteenths inch and one-half inch. Formulas for computing dimensions are appended to the A. S. M. E. table. Note 1 is similar to note 1 under flathead machine screws.)

\section{FILIISTER-HEAD MACHINE SCREWS}

1. The fillister-head screw has a rounded surface for the top of the head, the remainder of the head being cylindrical. This type of head is standard for machine screws and cap screws.

(Dimensions of fillister-head machine screws are identical with those given below in Federal Specifications Board, No. 548 (FF-S-91), Table 16, except for slightly lower values for maximum total height of head in the F. S. B. table. Formulas for computing dimensions are appended to the A. S. M. E. table. Note 1 is similar to note 1 under flathead machine screws.) 
TABLE 5.-Preferred screw lengths and heads, machine screws

\begin{tabular}{|c|c|c|c|c|c|c|c|c|c|c|c|}
\hline \multirow{4}{*}{$\begin{array}{c}\text { Length } \\
\text { in } \\
\text { inches } \\
\text { L }\end{array}$} & \multicolumn{11}{|c|}{ Nominal size } \\
\hline & 2 & 3 & 4 & 5 & 6 & 8 & 10 & 12 & 34 & $5 / 16$ & $3 / 8$ \\
\hline & \multicolumn{11}{|c|}{ Coarse-thread series, free fit (class 2) (common pitches) (threads per inch) } \\
\hline & 56 & 48 & 40 & 40 & 32 & 32 & 24 & 24 & 20 & 18 & 16 \\
\hline $\begin{array}{l}1 / 8 \\
3 / 16 \\
1 / 4\end{array}$ & $\begin{array}{l}\text { FRP } \\
\text { FROP } \\
\text { FROP }\end{array}$ & $\begin{array}{l}\text { FRP } \\
\text { FROP } \\
\text { FROP }\end{array}$ & $\begin{array}{l}\text { FRP } \\
\text { FROP } \\
\text { FROP }\end{array}$ & $\begin{array}{l}\text { RP } \\
\text { FRP } \\
\text { FRP }\end{array}$ & $\begin{array}{l}\text { RP } \\
\text { FROP } \\
\text { FROP }\end{array}$ & $\begin{array}{l}\text { RP } \\
\text { FRP } \\
\text { FROP }\end{array}$ & $\begin{array}{l}\text { RP } \\
\text { ROP }\end{array}$ & $\mathrm{RP}$ & & & \\
\hline $\begin{array}{l}5 / 16 \\
3 / 8 \\
7 / 16 \\
1 / 2\end{array}$ & $\begin{array}{l}\text { FRP } \\
\text { FRP } \\
\text { FRP } \\
\text { FRP }\end{array}$ & $\begin{array}{l}\text { FRP } \\
\text { FRP } \\
\text { FRP } \\
\text { FRP }\end{array}$ & $\begin{array}{l}\text { FROP } \\
\text { FROP } \\
\text { FROP } \\
\text { FROP }\end{array}$ & $\begin{array}{l}\text { FRP } \\
\text { FRP } \\
\text { FRP } \\
\text { FRP }\end{array}$ & $\begin{array}{l}\text { FROP } \\
\text { FROP } \\
\text { FROP } \\
\text { FROP }\end{array}$ & $\begin{array}{l}\text { FROP } \\
\text { FROP } \\
\text { FROP } \\
\text { FROP }\end{array}$ & $\begin{array}{l}\text { FROP } \\
\text { FROP } \\
\text { FROP } \\
\text { FROP }\end{array}$ & $\begin{array}{l}\text { FRP } \\
\text { FROP } \\
\text { FROP } \\
\text { FROP }\end{array}$ & $\begin{array}{l}\text { FRP } \\
\text { FRP } \\
\text { FRP } \\
\text { FROP }\end{array}$ & FROP & FRP \\
\hline $\begin{array}{r}5 / 8 \\
3 / 4 \\
7 / 8 \\
1^{7 / 8}\end{array}$ & $\begin{array}{l}\text { FRP } \\
\text { FR } \\
\text { R }\end{array}$ & $\begin{array}{l}\text { FRP } \\
\text { FR } \\
\text { R }\end{array}$ & $\begin{array}{l}\text { FROP } \\
\text { FRP } \\
\text { FRP } \\
\text { FRP }\end{array}$ & $\begin{array}{l}\text { FRP } \\
\text { FRP } \\
\text { FRP } \\
\text { FRP }\end{array}$ & $\begin{array}{l}\text { FROP } \\
\text { FROP } \\
\text { FROP } \\
\text { FROP }\end{array}$ & $\begin{array}{l}\text { FROP } \\
\text { FROP } \\
\text { FROP } \\
\text { FROP }\end{array}$ & $\begin{array}{l}\text { FROP } \\
\text { FROP } \\
\text { FROP } \\
\text { FROP }\end{array}$ & $\begin{array}{l}\text { FROP } \\
\text { FROP } \\
\text { FROP } \\
\text { FROP }\end{array}$ & $\begin{array}{l}\text { FROP } \\
\text { FROP } \\
\text { FROP } \\
\text { FROP }\end{array}$ & $\begin{array}{l}\text { FROP } \\
\text { FROP } \\
\text { FROP } \\
\text { FRP }\end{array}$ & $\begin{array}{l}\text { FRP } \\
\text { FROP } \\
\text { FROP } \\
\text { FRP }\end{array}$ \\
\hline $\begin{array}{l}11 / 4 \\
11 / 2 \\
13 / 4 \\
2\end{array}$ & & & $\begin{array}{l}\mathrm{R} \\
\mathrm{R}\end{array}$ & $\begin{array}{l}\text { FR } \\
\text { FR } \\
\text { F }\end{array}$ & $\begin{array}{l}\text { FROP } \\
\text { FROP } \\
\text { FROP } \\
\text { FROP }\end{array}$ & $\begin{array}{l}\text { FROP } \\
\text { FROP } \\
\text { FROP } \\
\text { FROP }\end{array}$ & $\begin{array}{l}\text { FROP } \\
\text { FROP } \\
\text { FROP } \\
\text { FROP }\end{array}$ & $\begin{array}{l}\text { FROP } \\
\text { FROP } \\
\text { FROP } \\
\text { FROP }\end{array}$ & $\begin{array}{l}\text { FROP } \\
\text { FROP } \\
\text { FROP } \\
\text { FROP }\end{array}$ & $\begin{array}{l}\text { FRP } \\
\text { FRP } \\
\text { FRP } \\
\text { FRP }\end{array}$ & $\begin{array}{l}\text { FRP } \\
\text { FRP } \\
\text { FRP } \\
\text { FRP }\end{array}$ \\
\hline $\begin{array}{l}21 / 4 \\
21 / 2 \\
23 / 4 \\
3\end{array}$ & & & & & $\mathrm{R}$ & $\begin{array}{l}\text { FRP } \\
\text { FR } \\
\text { FR } \\
\text { FR }\end{array}$ & $\begin{array}{l}\text { FRP } \\
\text { FRP } \\
\text { FR } \\
\text { FR }\end{array}$ & $\begin{array}{l}\text { FRP } \\
\text { FRP } \\
\text { FR } \\
\text { FR }\end{array}$ & $\begin{array}{l}\text { FROP } \\
\text { FRP } \\
\text { FRP } \\
\text { FRP }\end{array}$ & $\begin{array}{l}\text { FRP } \\
\text { FRP } \\
\text { FRP } \\
\text { FRP }\end{array}$ & $\begin{array}{l}\text { FRP } \\
\text { FRP } \\
\text { FRP } \\
\text { FRP }\end{array}$ \\
\hline
\end{tabular}

\begin{tabular}{|c|c|c|c|c|c|c|c|c|c|c|}
\hline \multirow{4}{*}{\begin{tabular}{|c}
$\begin{array}{c}\text { Length } \\
\text { in } \\
\text { inches }\end{array}$ \\
$\mathrm{L}$
\end{tabular}} & \multicolumn{10}{|c|}{ Nominal size } \\
\hline & 2 & 3 & 4 & 6 & 8 & 10 & 12 & $1 / 4$ & $5 / 16$ & $3 / 8$ \\
\hline & \multicolumn{10}{|c|}{ Fine-thread series, free fit (class 2) (less common pitches) (threads per inch) } \\
\hline & 64 & 56 & 48 & 40 & 36 & 32 & 28 & 28 & 24 & 24 \\
\hline $\begin{array}{l}1 / 8 \\
3116 \\
1 / 4\end{array}$ & $\begin{array}{l}\mathrm{FR} \\
\mathrm{FR} \\
\mathrm{FR}\end{array}$ & $\begin{array}{l}\text { FRP } \\
\text { FRP } \\
\text { FRP }\end{array}$ & $\begin{array}{l}\text { FRP } \\
\text { FRP } \\
\text { FRP }\end{array}$ & $\begin{array}{l}\text { RP } \\
\text { RP } \\
\text { FRP }\end{array}$ & $\begin{array}{l}\mathrm{RP} \\
\mathrm{FRP}\end{array}$ & FROP & & & & \\
\hline $\begin{array}{l}5 / 16 \\
3 / 8 \\
7 / 16 \\
1 / 2\end{array}$ & $\begin{array}{l}\mathrm{FR} \\
\mathrm{F} \\
\mathrm{F} \\
\mathrm{F}\end{array}$ & $\begin{array}{l}\text { FR } \\
\text { FR } \\
\text { FR } \\
\text { FR }\end{array}$ & $\begin{array}{l}\text { FRP } \\
\text { FRP } \\
\text { FRP } \\
\text { FRP }\end{array}$ & $\begin{array}{l}\text { FRP } \\
\text { FROP } \\
\text { FROP } \\
\text { FROP }\end{array}$ & $\begin{array}{l}\text { FRP } \\
\text { FROP } \\
\text { FROP } \\
\text { FROP }\end{array}$ & $\begin{array}{l}\text { FROP } \\
\text { FROP } \\
\text { FROP } \\
\text { FROP }\end{array}$ & $\begin{array}{l}\text { F } \\
\text { FROP } \\
\text { FROP } \\
\text { FROP }\end{array}$ & $\begin{array}{l}\text { F } \\
\text { FR } \\
\text { FR } \\
\text { FR }\end{array}$ & $\mathrm{R}$ & \\
\hline $\begin{array}{r}5 / 8 \\
34 \\
7 / 8 \\
1^{7 / 8}\end{array}$ & & $\stackrel{\mathrm{R}}{\mathrm{R}}$ & $\begin{array}{l}\mathrm{FR} \\
\mathrm{FR}\end{array}$ & $\begin{array}{l}\text { FRP } \\
\text { FRP } \\
\text { FROP } \\
\text { FR }\end{array}$ & $\begin{array}{l}\text { FROP } \\
\text { FROP } \\
\text { FROP } \\
\text { FROP }\end{array}$ & $\begin{array}{l}\text { FROP } \\
\text { FROP } \\
\text { FROP } \\
\text { FROP }\end{array}$ & $\begin{array}{l}\text { FROP } \\
\text { FROP } \\
\text { FRP } \\
\text { FRP }\end{array}$ & $\begin{array}{l}\text { FRP } \\
\text { FRP } \\
\text { FRP } \\
\text { FRP }\end{array}$ & $\begin{array}{l}\text { FRP } \\
\text { FRP } \\
\text { FRP } \\
\text { FR }\end{array}$ & $\begin{array}{l}\text { FR } \\
\text { FR } \\
\text { FR } \\
\text { FR }\end{array}$ \\
\hline $\begin{array}{l}11 / 4 \\
11 / 2 \\
13 / 4 \\
2\end{array}$ & & & & $\underset{\mathrm{R}}{\mathrm{FR}}$ & $\begin{array}{l}\text { FRP } \\
\text { FR } \\
\text { F } \\
\text { F }\end{array}$ & $\begin{array}{l}\text { FROP } \\
\text { FROP } \\
\text { FROP } \\
\text { FROP }\end{array}$ & $\begin{array}{l}\text { FRP } \\
\text { FRP } \\
\text { FRP } \\
\text { FROP }\end{array}$ & $\begin{array}{l}\text { FRP } \\
\text { FRP } \\
\text { FRP } \\
\text { FRP }\end{array}$ & $\begin{array}{l}\mathrm{FR} \\
\mathrm{F} \\
\mathrm{F} \\
\mathrm{F}\end{array}$ & $\begin{array}{l}F \\
F \\
F \\
F\end{array}$ \\
\hline $\begin{array}{l}21 / 4 \\
21 / 2 \\
23 / 4 \\
3\end{array}$ & & & & & $\begin{array}{l}F \\
F \\
F \\
F\end{array}$ & $\begin{array}{l}\text { FRP } \\
\text { FRP } \\
\text { FRP } \\
\text { FRP }\end{array}$ & $\begin{array}{l}\mathrm{FR} \\
\mathrm{F} \\
\mathrm{F}\end{array}$ & $\begin{array}{l}\mathrm{FR} \\
\mathrm{F} \\
\mathrm{F} \\
\mathrm{F}\end{array}$ & $\begin{array}{l}F \\
F \\
F \\
F\end{array}$ & $\begin{array}{l}F \\
F \\
F \\
F\end{array}$ \\
\hline
\end{tabular}

Note 1.-The table of screw lengths reproduced above is intended only as a guide to the users of these screws. A number of the listed sizes and lengths will not be regularly stocked by the manufacturers but will be available on order of a sufficient quantity. Letters in the vertical column under the nominal screw sizes indicate the style of head for a particular length of screw; hence, $F=$ flat head, $R=$ round head, $O=0$ val head, $P=$ fillister head.

NOTE 2.-Tolerance in length $(L)=-3$ per cent; but not less than -0.025 inch.

NOTE 3.-Where length of screw $(L)$ is $1 \frac{1}{4}$ inches or less, the length of thread $(l)$ will extend to as near the head as is practicable. Where the length of screw is over 134 inches, the thread length shall not be less than $11 / 4$ inches.

\section{FLAT-HEAD CAP SCREWS}

1. Cap screw lengths.-The length shall be measured from the largest diameter of the bearing surface of the head to the extreme point, on a line parallel to the axis of the screw.

2. The difference between consecutive lengths of screw:

For screw lengths $1 / 4$ to 1 inch shall be $1 / 8$ inch.

For screw lengths 1 to 4 inches shall be 14 inch.
For screw lengths 4 to 6 inches shall be $1 / 2$ inch.
3. The tolerance in screw length shall be 3 per cent of the nominal length, with a minimum tolerance of 0.030 inch; one-third of the tolerance to be applied minus, and two-thirds plus.

4. Thread lengths.-Slotted-head cap screws shall be regularly threaded coarse pitch, and when so threaded shall have a length of thread equal to $2 \mathrm{D}+1 / 4$ inch. Screws too short to allow the formula length of thread may be threaded as close to the head as practicable. 
5. Screw points.-The points of all cap screws shall be flat, the flat being normal to the axis of the screw; and shall be chamfered at an angle of $35^{\circ}$ with the surface of the flat, $+5^{\circ},-0^{\circ}$; the the chamfer to extend to the bottom of the thread. The edge of the chamfer is to be slightly rounded.

(Dimensions of flat-head cap screws are substantially the same as those given below in Federal Specifications Board, No. 548 (FF-S-91), Table 14, for screws ranging in size from $7 / 16$ to $3 / 4$ inch. Dimensions of other sizes not covered by F. S. B., No. 548 (FF-S-91), Table 14, are as follows:)

[All dimensions are in inches]

\begin{tabular}{|c|c|c|c|c|c|c|c|c|}
\hline \multirow{2}{*}{$\begin{array}{c}\text { Nomi- } \\
\text { nal } \\
\text { size }\end{array}$} & \multirow{2}{*}{$\begin{array}{l}\text { Maxi- } \\
\text { mum } \\
\text { diame- } \\
\text { ter }\end{array}$} & \multicolumn{2}{|c|}{$\begin{array}{c}\text { Head diame- } \\
\text { ter }\end{array}$} & \multirow{2}{*}{$\begin{array}{c}\text { Height } \\
\text { of } \\
\text { head, } \\
\text { nomi- } \\
\text { nal }\end{array}$} & \multicolumn{2}{|c|}{ Width of slot } & \multicolumn{2}{|c|}{ Depth of slot } \\
\hline & & $\begin{array}{l}\text { Maxi- } \\
\text { mum }\end{array}$ & $\begin{array}{l}\text { Mini- } \\
\text { mum }\end{array}$ & & $\begin{array}{l}\text { Maxi- } \\
\text { mum }\end{array}$ & $\begin{array}{l}\text { Mini- } \\
\text { mum }\end{array}$ & $\begin{array}{l}\text { Maxi- } \\
\text { mum }\end{array}$ & $\begin{array}{l}\text { Mini- } \\
\text { mum }\end{array}$ \\
\hline $\begin{array}{l}1 / 4 \\
5 / 16 \\
3 / 8\end{array}$ & $\begin{array}{c}0.250 \\
.3125 \\
.375\end{array}$ & $\begin{array}{l}1 / 2 \\
5 / 8 \\
3 / 4\end{array}$ & $\begin{array}{r}0.477 \\
.598 \\
.719\end{array}$ & $\begin{array}{r}0.146 \\
.183 \\
.220\end{array}$ & $\begin{array}{r}0.070 \\
.079 \\
.088\end{array}$ & $\begin{array}{r}0.058 \\
.065 \\
.074\end{array}$ & $\begin{array}{r}0.073 \\
.091 \\
.110\end{array}$ & $\begin{array}{r}0.053 \\
.066 \\
.080\end{array}$ \\
\hline
\end{tabular}

(Formulas for computing dimensions are appended to the A. S. M. E. table. Note 1 is similar to note 1 under flat-head machine screws.)

\section{BUTTON-HEAD CAP SCREWS}

(Dimensions of button-head cap screws are substantially the same as those given below in Federal
Specifications Board, No. 548 (FF-S-91), Table 15, for screws ranging in size from seven-sixteenths to three-fourths inch. Dimensions of other sizes not covered by F. S. B., No. 548 (FF-S-91), Table 15, are as follows:)

[All dimensions are in inches]

\begin{tabular}{|c|c|c|c|c|c|c|c|c|c|}
\hline \multirow{2}{*}{$\begin{array}{l}\text { Nom- } \\
\text { inal } \\
\text { size }\end{array}$} & \multirow{2}{*}{$\begin{array}{l}\text { Maxi- } \\
\text { mum } \\
\text { diam- } \\
\text { eter }\end{array}$} & \multicolumn{2}{|c|}{$\begin{array}{c}\text { Head diam. } \\
\text { eter }\end{array}$} & \multicolumn{2}{|c|}{$\begin{array}{c}\text { Height of } \\
\text { head }\end{array}$} & \multicolumn{2}{|c|}{$\begin{array}{l}\text { Width of } \\
\text { slot }\end{array}$} & \multicolumn{2}{|c|}{$\begin{array}{l}\text { Depth of } \\
\text { slot }\end{array}$} \\
\hline & & $\begin{array}{l}\text { Maxi- } \\
\text { mum }\end{array}$ & $\begin{array}{l}\text { Mini- } \\
\text { mum }\end{array}$ & $\begin{array}{l}\text { Maxi- } \\
\text { mum }\end{array}$ & $\begin{array}{l}\text { Mini- } \\
\text { mum }\end{array}$ & $\begin{array}{l}\text { Maxi- } \\
\text { mum }\end{array}$ & $\begin{array}{l}\text { Mini- } \\
\text { mum }\end{array}$ & $\begin{array}{l}\text { Maxi- } \\
\text { mum }\end{array}$ & $\begin{array}{l}\text { Mini- } \\
\text { mum }\end{array}$ \\
\hline $\begin{array}{l}1 / 4 \\
5 / 6 \\
3 / 8\end{array}$ & $\begin{array}{l}0.250 \\
.3125 \\
.375\end{array}$ & $\begin{array}{r}7 / 16 \\
9 / 16 \\
5 / 8\end{array}$ & $\begin{array}{c}0.418 \\
.541 \\
.602\end{array}$ & $\begin{array}{l}0.191 \\
.246 \\
.273\end{array}$ & $\begin{array}{r}0.175 \\
.226 \\
.251\end{array}$ & $\begin{array}{r}0.070 \\
.079 \\
.088\end{array}$ & $\begin{array}{l}0.058 \\
.065 \\
.074\end{array}$ & $\begin{array}{l}0.117 \\
.151 \\
.167\end{array}$ & $\begin{array}{r}0.097 \\
.126 \\
.137\end{array}$ \\
\hline
\end{tabular}

(Formulas for co mputing dimensions are appended to the A. S. M. E. table. Note 1 is similar to note 1 under flat-head machine screws.)

\section{FILIISTER-HEAD CAP SCREWS}

(Dimenions of fillister-head cap screws are substantially the same as those given below in Federal Specifications Board, No. 548 (FF-S-91), Table 16 , for screws ranging in size from $7 / 16$ to 1 inch. Dimensions of other sizes not covered by F. S. B., No. 548 (FF-S-91), Table 16, are as follows:)

[All dimensions are in inches]

\begin{tabular}{|c|c|c|c|c|c|c|c|c|c|c|c|c|c|}
\hline \multirow{2}{*}{ Nominal size } & \multirow{2}{*}{$\begin{array}{l}\text { Maxi- } \\
\text { mum } \\
\text { diam- } \\
\text { eter }\end{array}$} & \multicolumn{2}{|c|}{ Head diameter } & \multicolumn{2}{|c|}{ Height of head } & \multicolumn{2}{|c|}{ Width of slot } & \multicolumn{2}{|c|}{ Depth of slot } & \multicolumn{2}{|c|}{ Height of oval } & \multicolumn{2}{|c|}{$\begin{array}{l}\text { Total height } \\
\text { of head }\end{array}$} \\
\hline & & $\begin{array}{l}\text { Maxi- } \\
\text { mum }\end{array}$ & $\begin{array}{l}\text { Mini- } \\
\text { mum }\end{array}$ & $\begin{array}{l}\text { Maxi- } \\
\text { mum }\end{array}$ & $\begin{array}{l}\text { Mini- } \\
\text { mum }\end{array}$ & $\begin{array}{l}\text { Maxi- } \\
\text { mum }\end{array}$ & $\begin{array}{l}\text { Mini- } \\
\text { mum }\end{array}$ & $\begin{array}{l}\text { Maxi- } \\
\text { mum }\end{array}$ & $\begin{array}{l}\text { Mini- } \\
\text { mum }\end{array}$ & $\begin{array}{l}\text { Maxi- } \\
\text { mum }\end{array}$ & $\begin{array}{l}\text { Mini- } \\
\text { mum }\end{array}$ & $\begin{array}{l}\text { Maxi- } \\
\text { mum }\end{array}$ & $\begin{array}{l}\text { Mini- } \\
\text { mum }\end{array}$ \\
\hline $36-$ & $\begin{array}{l}0.250 \\
.3125 \\
.375\end{array}$ & $\begin{array}{l}3 / 8 \\
7 / 16 \\
916\end{array}$ & $\begin{array}{r}0.363 \\
.424 \\
.547\end{array}$ & $\begin{array}{r}1364 \\
1364 \\
1 / 4\end{array}$ & $\begin{array}{l}0.157 \\
.186 \\
.229\end{array}$ & $\begin{array}{r}0.070 \\
.079 \\
.088\end{array}$ & $\begin{array}{r}0.058 \\
.065 \\
.074\end{array}$ & $\begin{array}{r}0.097 \\
.115 \\
.142\end{array}$ & $\begin{array}{r}0.077 \\
.090 \\
.112\end{array}$ & $\begin{array}{r}0.044 \\
.050 \\
.064\end{array}$ & $\begin{array}{r}0.038 \\
.044 \\
.056\end{array}$ & $\begin{array}{r}0.216 \\
.253 \\
.314\end{array}$ & $\begin{array}{r}0.195 \\
.230 \\
.285\end{array}$ \\
\hline
\end{tabular}

(Formulas for computing dimensions are appended to the A. S. M. E. table. Note 1 is similar to note 1 under flat-head machine screws.)

\section{WOOD SCREWS}

(Dimensions of round-head, flat-head, and ovalhead wood screws are identical with those given below in National Screw Thread Commission, wood screws, Tables 56, 57, and 58, respectively. Formulas for computing dimensions are also identical with those of the N. S. T. C.)

(Length tolerances of round-head wood screws are in agreement with those of the National Screw Thread Commission, wood screws, given below in Table 61. Lengths of flat-head and oval-head wood screws are substantially the same as those of the N. S. T. C., wood screws, given below in Table 60.)

\section{BRASS WOOD SCREWS}

(Standard sizes of brass wood screws are identical with those of the National Screw Thread Commission, wood screws, given below in Table 63.)

\section{STEEL WOOD SCREWS}

(Standard sizes of steel wood screws are identical with those of the National Screw Thread Commission, wood screws, given below in Table 62.)
SOCIETY OF AUTOMOTIVE ENGINEERS, CAP SCREWS, BOLTS AND NUTS, AND WRENCH OPENINGS, S. A. E. STANDARDS, JANUARY, 1930

[Approved by American Standards Association, April, 1930]

(See also 608.31 , p. $600 ; 608.32$, p. 606 ; and 615.42 , p. 883.$)$

\section{CAP SCREWS, BOLTS AND NUTS, AND WRENCH OPENINGS}

1. The following specifications have been adopted as standard for all cap screws, bolts, and nuts for general use.

2. Cap screws, bolts, and nuts not conforming to these specifications will be considered special.

\section{CAP SCREWS}

1. Cap screws when threaded coarse pitch shall have a length of thread equal to $2 \mathrm{D}+1 / 4$ inch. When threaded fine pitch the length shall be $1 \frac{1}{2} \mathrm{D}+1 / 4$ inch. (D equals body diameter of screw.) Screws too short to allow the formula length thread may be threaded as close to the head as practicable.

2. Body lengths of all cap screws shall be measured from the largest diameter of the bearing under the 
head to the extreme point in a line parallel to the axis of the screw.

3. Points of all cap screws shall be flat, the flat being normal to the axis of the screw; the point shall be chamfered at an angle of $35^{\circ}$ to the surface of the flat, with a tolerance of $+5^{\circ}$ and $-0^{\circ}$, the chamfer to extend to the bottom of the thread.

4. Material.-All cap screws unless otherwise specified shall be made of steel.

5. Slots, cotter-pin holes, heat treatment.-Hexagonhead cap screws are made regularly without slots or cotter-pin holes. If slots, cotter-pin holes, or special heat treatments are desired, these should be specified by the purchaser.

\section{FINISHED HEXAGONAL CAP SCREWS}

(Dimensions of finished hexagonal cap screws are identical with those given below in National Screw Thread Commission, hexagon-head cap screws, Table 75, except that the S. A. E. table includes threads per inch. The footnotes conform to the N. S. T. C. specifications for hexagon-head cap screws, given below.)

\section{SET SCREWS}

(Dimensions of set screws are identical with those given below in Federal Specifications Board, No. 548 (FF-S-91), Table 24, except that the S. A. E. table includes threads per inch. The footnotes conform to the F. S. B. specifications for set screws, given below.)

\section{FLAT-HEAD MACHINE SCREWS}

(Dimensions of flat-head machine screws are identical with those given below in Federal Specifications Board, No. 548 (FF-S-91), Table 14. Note 1 is identical with note 1 under flat-head machine screws, American Society of Mechanical Engineers, given above. Formulas for computing dimensions are appended.)

\section{OVAL-HEAD MACHINE SCREWS}

(Dimensions of oval-head machine screws are identical with those given below in Federal Specifications Board, No. 548 (FF-S-91), Table 17, except that the latter carries two additional sizes - sevensixteenths inch and one-half inch. Note 1 is similar to note 1 under flat-head machine screws. Formulas for computing dimensions are appended.)

\section{FILLISTER-HEAD MACHINE SCREWS}

(Dimensions of fillister-head machine screws are identical with those given below in Federal Specifications Board, No. 548 (FF-S-91), Table 16, except for slightly lower values for maximum total height of head in the F. B. S. table. Note 1 is similar to note 1 under flat-head machine screws. Formulas for computing dimensions are appended.)

\section{ROUND-HEAD MACHINE SCREWS}

(Dimensions of round-head machine screws are identical with those given below in Federal Specifications Board, No. 548 (FF-S-91), Table 15. Note 1 is similar to note 1 under flat-head machine screws. Formulas for computing dimensions are appended.)

\section{SLOTTED-HEAD CAP SCREW LENGTHS}

(Specifications covering cap screw lengths, thread lengths, and screw points are identical with those of the American Society of Mechanical Engineers, given above.)

\section{FLAT-HEAD CAP SCREWS}

(Dimensions of flat-head cap screws are substantially the same as those given below in Federal Speci- fications Board, No. 548 (FF-S-91), Table 14, for screws ranging in size from seven-sixteenths to threefourths inch. Dimensions of one-fourth, five-sixteenths, and three-eighths inch flat-head cap screws are identical with those of the American Society of Mechanical Engineers, given above. Note 1 is similar to note 1 under flat-head machine screws. Formulas for computing dimensions are appended.)

\section{FILLISTER-HEAD CAP SCREWS}

(Dimensions of fillister-head cap screws are substantially the same of those given below in Federal Specifications Board, No. 548 (FF-S-91), Table 16 , for screws ranging in size from $7 / 16$ to 1 inch. Dimensions of one-fourth, five-sixteenths, and threeeighths inch fillister-head cap screws are identical with those of the American Society of Mechanical Engineers, given above. Note 1 is similar to note 1 under flat-head machine screws. Formulas for computing dimensions are appended.)

\section{BUTTON-HEAD CAP SCREWS}

(Dimensions of button-head cap screws are substantially the same as those given below in Federal Specifications Board, No. 548 (FF-S-91), Table 15, for screws ranging in size from seven-sixteenths to three-fourths inch. Dimensions of one-fourth, five-sixteenths, and three-eighths inch are identical with those of the American Society of Mechanical Engineers given above. Note 1 is similar to note 1 under flat-head machine screws. Formulas for computing dimensions are appended.)

\section{WOOD SCREWS}

(Dimensions of round-head, flat-head, and ovalhead wood screws are identical with those given below in National Screw Thread Commission, wood screws, Tables 57, 56, and 58, respectively. Formulas for computing dimensions are also identical with those of the N. S. T. C.)

(Length tolerances of round-head wood screws are in agreement with those of the National Screw Thread Commission, wood screws given below in Table 61. Lengths of flat-head and oval-head wood screws are substantially the same as those of the N. S. T. C., given below in Table 60.)

\section{SOCIETY OF AUTOMOTIVE ENGINEERS,} CAP SCREWS, BOLTS, AND NUTS; SLOT AND COTTER-PIN HOLE DIMENSIONS, JANUARY, 1927

\section{SLOT AND COTTER-PIN HOLE DIMENSIONS}

Cap screws and bolts

\begin{tabular}{|c|c|c|c|c|}
\hline \multirow{2}{*}{$\begin{array}{l}\text { Nominal } \\
\text { cap screw } \\
\text { and bolt } \\
\text { diameter }\end{array}$} & \multirow{2}{*}{$\begin{array}{l}\text { Cotter-pin } \\
\text { hole di- } \\
\text { ameter }\end{array}$} & \multicolumn{2}{|c|}{ Head slots } & \multirow{2}{*}{$\begin{array}{l}\text { Center of } \\
\text { cotter-pin } \\
\text { hole to ex- } \\
\text { treme poin } \\
\text { of screw } 1\end{array}$} \\
\hline & & Width & Depth & \\
\hline $\begin{array}{c}\text { Inches } \\
1 / 4 \\
5 / 16 \\
3 / 8 \\
7 / 16 \\
1 / 2\end{array}$ & $\begin{array}{l}\text { Inch } \\
564 \\
5 \% 64 \\
18 \\
18 \\
18\end{array}$ & $\begin{array}{l}\text { Inch } \\
1 / 16 \\
1 / 16 \\
3 / 32 \\
3 / 32 \\
3 / 32\end{array}$ & $\begin{array}{l}\text { Inch } \\
3 / 32 \\
7 / 64 \\
118 \\
18 \\
1 / 8\end{array}$ & $\begin{array}{r}\text { Inch } \\
7 / 64 \\
118 \\
5 / 32 \\
5 / 32 \\
11 / 64\end{array}$ \\
\hline $\begin{array}{l}9 / 16 \\
5 / 8 \\
3 / 4 \\
7 / 8\end{array}$ & $\begin{array}{l}5 / 32 \\
5 / 32 \\
5 / 32 \\
5 / 32\end{array}$ & $\begin{array}{l}3 / 32 \\
3 / 32 \\
3 / 32 \\
3 / 32\end{array}$ & $\begin{array}{l}18 \\
18 \\
118 \\
18\end{array}$ & $\begin{array}{l}3 / 16 \\
3 / 16 \\
7 / 32 \\
134\end{array}$ \\
\hline $\begin{array}{l}1 \\
11 / 8 \\
11 / 4 \\
11 / 2\end{array}$ & $\begin{array}{l}5 / 32 \\
7 / 32 \\
7 / 32 \\
1 / 4\end{array}$ & $\begin{array}{l}3 / 32 \\
5532 \\
5 / 32 \\
3 / 16\end{array}$ & $\begin{array}{l}1 / 8 \\
7 / 32 \\
7 / 32 \\
1 / 4\end{array}$ & $\begin{array}{l}1 / 4 \\
9 / 32 \\
9 / 32 \\
5 / 16\end{array}$ \\
\hline
\end{tabular}

1 It is suggested that this dimension be used to determine the distance from the cotter-pin hole to the under face of the head of the screw or bolt. 
SOCIETY OF AUTOMOTIVE ENGINEERS, SLOTTED-HEAD WOOD SCREWS, 1930

\section{WOOD SCREWS}

(Same as the standards of the National Screw Thread Commission for wood screws, see U. S. Gov., N. S. T. C., below.)

UNITED STATES GOVERNMENT, DEPARTMENT OF COMMERCE, BUREAU OF STANDARDS, COMMERCIAL STANDARD FOR STEEL BONE PLATES AND SCREWS, CS37-31, 1931

\section{SCREWS FOR BONE PLATES}

(See 622.9, p. 1138.)

\section{UNITED STATES GOVERNMENT, FEDERAL SPECIFICATIONS BOARD, FEDERAL SPECIFICATION FOR SCREWS; WOOD,} FF-S-111, APRIL 28, 1931

\section{WOOD SCREWS}

\section{A. APPLICABLE FEDERAL SPECIFICATIONS}

A-1. There are no other Federal specifications applicable to this specification.

\section{B. TYPES}

B-1. Wood screws shall be furnished in three types, namely, flat, oval, or round head, as specified in the invitation for bids.

B-2. Wood screws shall be made of steel or brass as specified in the invitation for bids.

(General requirements, standard size numbers, diameters, and pitches of screws, standard head proportions, tolerances on length, and standard stock sizes of steel screws and of brass screws are the same as given in the standards of the National Screw Thread Commission for wood screws (see 608.2, p. 584), with the exceptions noted below.)

Minimum height of head for flat-head and for oval-head wood screws

\begin{tabular}{|c|c|c|c|}
\hline Nominal size & $\begin{array}{l}\text { Height of } \\
\text { head, min- } \\
\text { imum }\end{array}$ & Nominal size & $\begin{array}{l}\text { Height of } \\
\text { head, min- } \\
\text { imum }\end{array}$ \\
\hline $\begin{array}{l}0 \\
0 \\
1 \\
3 \\
3\end{array}$ & $\begin{array}{r}\text { Inches } \\
0.026 \\
.033 \\
.040 \\
.048 \\
.055\end{array}$ & $\begin{array}{l}9 \\
10 \\
11 \\
14 \\
14\end{array}$ & $\begin{array}{l}\text { Inch } \\
0.091 \\
.098 \\
.105 \\
.112 \\
.127\end{array}$ \\
\hline $\begin{array}{l}5-1-1-1 \\
6 \\
7 \\
8 \\
8-\end{array}$ & $\begin{array}{l}.062 \\
.069 \\
.076 \\
.084\end{array}$ & $\begin{array}{l}16- \\
18 \\
20 \\
24\end{array}$ & $\begin{array}{l}.141 \\
.155 \\
.170 \\
.198\end{array}$ \\
\hline
\end{tabular}

TABLE 6.-Tolerances on length of round-head screws

[Dimensions in inches]

\begin{tabular}{|r|r||r|r||r|r|}
\hline $\begin{array}{r}\text { Screw } \\
\text { No. }\end{array}$ & $\begin{array}{c}\text { Tolerance 1 } \\
\text { minus }\end{array}$ & $\begin{array}{r}\text { Screw } \\
\text { No. }\end{array}$ & $\begin{array}{c}\text { Tolerance 1 } \\
\text { minus }\end{array}$ & $\begin{array}{c}\text { Screw } \\
\text { No. }\end{array}$ & $\begin{array}{c}\text { Tolerance 1 } \\
\text { minus }\end{array}$ \\
\hline 0 & 0.06 & 6 & 0.10 & 12 & 0.15 \\
1 & .07 & 7 & .11 & 14 & .16 \\
2 & .08 & 8 & .12 & 16 & .18 \\
3 & .08 & 9 & .13 & 18 & .20 \\
4 & .09 & 10 & .13 & 20 & .22 \\
5 & .10 & 11 & .14 & 24 & .27 \\
\hline
\end{tabular}

1 Plus tolerance $=0$.
F. METHODS OF SAMPLING INSPECTION AND TESTS

F-1. Approximately 2 per cent of each size of screws in a given shipment may be taken at random and measured for compliance with the detail dimensions and number of threads.

\section{G. PACKAGING, PACKING, AND MARKING OF SHIPMENTS}

G-2. Packaging.-Unless otherwise specified, commercial packages are acceptable under this specification.

G-3. Packing.-Unless otherwise specified, the subject commodity shall be delivered in substantial commercial containers so constructed as to insure acceptance by common or other carriers, for safe transportation, at the lowest rate, to the point of delivery.

$\mathrm{G}-4$. Unless otherwise specified, shipping containers shall be marked with the name of the actual manufacturer, the length, the numbered size, the material, and the quantity contained therein, as defined by the contract or order under which shipment is made, the name of the contractor and the number of the contract or order.

\section{NOTES}

I-1. Wood screws are designated commercially by the length and the numbered size. The length is given first, for example: $1 \frac{1}{2}$ inch, No. 12.

I-2. It is believed that this specification adequately describes the characteristics necessary to secure the desired material, and that normally no samples will be necessary prior to award to determine compliance with this specification. If, for any particular purpose, samples with bids are necessary, they should be specifically asked for in the invitation for bids and the particular purpose to be served by the bid samples should be definitely stated, the specification to apply in all other respects.

\section{UNITED STATES GOVERNMENT, FEDERAI SPECIFICATIONS BOARD, FEDERAL SPECIFICATION FOR CAP SCREWS, SET SCREWS, MACHINE SCREWS, AND STOVE BOLTS, NO. 548 (CURRENT DESIGNA- TION : FF-S-91), MARCH 5, 1928}

\section{MACHINE SCREWS AND STOVE BOLTS}

(For material and workmanship, general requirements, detail requirements, methods of inspection and tests, packing and marking of shipments, and notes, see F. S. B. No. 548 (FF-S-91), under commodity designation 608.0 , p. 527.)

\section{CAP SCREWS}

(Specifications for cap screws conform to National Screw Thread Commission specifications for hexagon-head cap screws, given below, except in the following particulars:)

1. (a) Cap screws.-Cap screws, one-fourth to one-half inch, are identical with machine bolts and machine screws when supplied with the same style of head, and they may be used interchangeably.

(b) Flat-head cap screws.-The head dimensions of flat-head cap screws shall be in accordance with Table 14.

Flat-head cap screws shall be threaded the full length under the head.

(c) Button-head cap screws.-The head dimensions of button-head cap screws shall be in accordance with Table 15

The profile of the head shall be a semiellipse.

(d) Oval fillister-head cap screws. -The dimensions of oval fillister-head cap screws shall be in accordance with Table 16. 
(e) Finished hexagon-head cap screws.-(Dimensions are identical with those given below in National Screw Thread Commission, hexagon-head cap screws, Table 75.)

\section{MACHINE SCREWS}

(Specifications for machine screws conform to National Screw Thread Commission specifications for hexagon-head cap screws, given below, except in the following particulars:)

1. Machine screws.-Machine screws are regularly furnished in standard numbered sizes to No. 12, and in fractions of an inch from one-fourth to onehalf inch, inclusive. They are furnished with various styles of slotted heads. Machine screws, sizes one-fourth to one-half inch, are identical with cap screws when supplied with the same style of head, and they may be used interchangeably. They are furnished with or without nuts as required.

2. The length of machine screws is measured from the largest diameter of the bearing surface of the head to the extreme point. The length of thread is measured from the extreme point to the last perfect thread.

3. Machine screws having flat, round, oval, or fillister heads shall have the dimensions as specified in Tables $14,15,16$, or 17 .

\section{SET SCREWS}

1. Set screws.-Set screws are regularly furnished in coarse-thread series unless otherwise specified, with square heads, $1 / 4$ to $1 \frac{1}{2}$ inches, inclusive, in diameter, with recessed (safety) heads, and headless slotted No. 5 to three-fourth-inch, inclusive.

2 . They are provided with round point, cup point, conical point, and pivot (dog) point, as required. Set screws are hardened and tempered or casehardened, as required.

3. Headless set screws.-Threaded the entire length. Set screws with heads are threaded from the under side of the head to the largest diameter of the point. The length of headless set screws is the over-all length. The length of set screws with heads is measured from under the head to the extreme end.

4. Headed set screws.-The head dimensions of headed set screws shall be in accordance with Table 24 .

5 . Headed set screws may be necked unless otherwise specified, and when necked set screws are furnished the neck shall not be longer than twice the pitch of the thread. When not necked the head shall be beveled not more than $40^{\circ}$ on the under side.

6 . The radius of the crown of the head shall be approximately two and one-half times the diameter of the screw.

7. The threads of headed set screws shall extend from the under side of the head to the extreme point. When necked the threads shall extend from the neck to the extreme point.

8. Headless slotted set screws.-Headless slotted set screws shall have the slot dimensions as given in Table 25.

9. Headless slotted set screws shall be threaded their entire length.

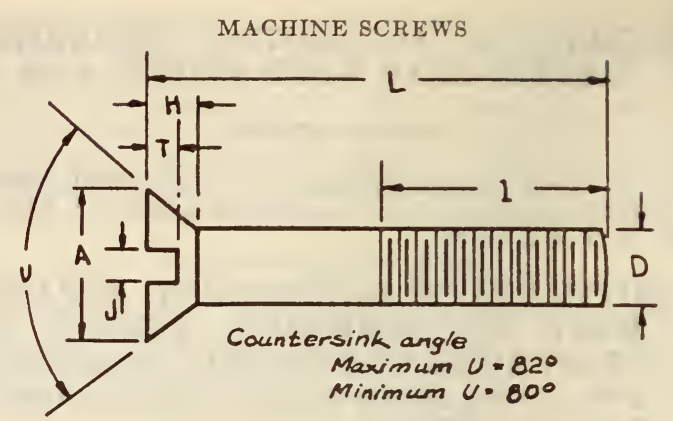

Figure 326.-Flat-head cap screw

TABLE 14.-Dimensions of flat-head cap-screw, machine-screw, and stove-bolt heads

[All dimensions in inches]

\begin{tabular}{|c|c|c|c|c|c|c|c|c|}
\hline \multirow{3}{*}{$\underset{\operatorname{size}}{\text { Nominal }}$} & $\mathrm{D}$ & \multicolumn{2}{|c|}{ A } & $\mathrm{H}$ & \multicolumn{2}{|c|}{$\mathbf{J}$} & \multicolumn{2}{|c|}{$\mathrm{T}$} \\
\hline & \multirow{2}{*}{$\begin{array}{l}\text { Uiam- } \\
\text { eter }\end{array}$} & \multicolumn{2}{|c|}{$\begin{array}{l}\text { Head diam- } \\
\text { eter }\end{array}$} & \multirow{2}{*}{$\begin{array}{c}\text { Nomi- } \\
\text { nal } \\
\text { height } 1 \\
\text { of head }\end{array}$} & \multicolumn{2}{|c|}{$\begin{array}{l}\text { Width of } \\
\text { slot }\end{array}$} & \multicolumn{2}{|c|}{$\begin{array}{l}\text { Depth of } \\
\text { slot }\end{array}$} \\
\hline & & $\begin{array}{l}\text { Maxi- } \\
\text { mum }\end{array}$ & $\begin{array}{l}\text { Mini- } \\
\text { mum }\end{array}$ & & $\begin{array}{l}\text { Max- } \\
\text { mum }\end{array}$ & $\begin{array}{l}\text { Mini- } \\
\text { mum }\end{array}$ & $\begin{array}{l}\text { Maxi- } \\
\text { mum }\end{array}$ & $\begin{array}{l}\text { Mini- } \\
\text { mum }\end{array}$ \\
\hline $\begin{array}{l}\text { No. } 2 \\
\text { No. } 3 \\
\text { No. } 4 \\
\text { No. } 5{ }^{2} \\
\text { No. } 6 \\
\text { No. } 8^{2}\end{array}$ & $\begin{array}{l}0.086 \\
.099 \\
.112 \\
.125 \\
.138 \\
.164\end{array}$ & $\begin{array}{r}0.172 \\
.199 \\
.225 \\
.252 \\
.279 \\
.332\end{array}$ & $\begin{array}{r}0.156 \\
.181 \\
.207 \\
.232 \\
.257 \\
.308\end{array}$ & $\begin{array}{l}0.045 \\
.054 \\
.060 \\
.068 \\
.076 \\
.092\end{array}$ & $\begin{array}{l}0.036 \\
.038 \\
.040 \\
.043 \\
.045 \\
.050\end{array}$ & $\begin{array}{l}0.024 \\
.026 \\
.028 \\
.031 \\
.033 \\
.037\end{array}$ & $\begin{array}{l}0.023 \\
.027 \\
.030 \\
.034 \\
.038 \\
.045\end{array}$ & $\begin{array}{l}0.015 \\
.017 \\
.020 \\
.022 \\
.024 \\
.029\end{array}$ \\
\hline $\begin{array}{l}\text { No. } 10^{2} \\
\text { No. } 12^{2} \\
1 / 4{ }^{2} \\
5 / 16^{2} \\
3 / 8\end{array}$ & $\begin{array}{l}.190 \\
.216 \\
.250 \\
.3125 \\
.375\end{array}$ & $\begin{array}{l}.385 \\
.438 \\
.507 \\
.636 \\
.762\end{array}$ & $\begin{array}{l}.359 \\
.410 \\
.477 \\
.600 \\
.722\end{array}$ & $\begin{array}{l}.108 \\
.122 \\
.142 \\
.180 \\
.215\end{array}$ & $\begin{array}{l}.055 \\
.059 \\
.066 \\
.077 \\
.088\end{array}$ & $\begin{array}{l}.041 \\
.045 \\
.051 \\
.061 \\
.072\end{array}$ & $\begin{array}{l}.053 \\
.060 \\
.070 \\
.088 \\
.106\end{array}$ & $\begin{array}{l}.034 \\
.039 \\
.046 \\
.058 \\
.070\end{array}$ \\
\hline
\end{tabular}

\section{CAP SCREWS}

\begin{tabular}{l|l|r|r|r|r|r|r|r}
\hline $7 / 16 \ldots \ldots \ldots$ & 0.4375 & 0.812 & 0.780 & 0.220 & 0.098 & 0.084 & 0.110 & 0.075 \\
$1 / 2 \ldots \ldots \ldots$ & .500 & .875 & .841 & .220 & .109 & .094 & .110 & .070 \\
$9 / 16 \ldots \ldots \ldots$ & .5625 & 1.000 & .962 & .256 & .120 & .104 & .128 & .083 \\
$5 / 8 \ldots \ldots \ldots$ & .625 & 1.125 & 1.083 & .293 & .132 & .115 & .146 & .116 \\
$3 / 4 \ldots \ldots \ldots$ & .750 & 1.375 & 1.326 & .366 & .155 & .135 & .183 & .123 \\
\hline
\end{tabular}

${ }_{1}$ Nominal $H$ subject to variations in $A, D$, and angle $U$.

2 These sizes of screws in the coarse-thread series when furnished with nuts are interchangeable with stove bolt sizes as listed below:

\begin{tabular}{|c|c|}
\hline Machine screw & $\begin{array}{l}\text { Stove } \\
\text { bolt }\end{array}$ \\
\hline $\begin{array}{l}\text { No. } 5 \\
\text { No. } 8 \\
\text { No. } 10 \\
\text { No. } 12 \\
1 / 4 \text { inch } \\
5 / 16 \text { inch } \\
3 / 8 \text { inch }\end{array}$ & $\begin{array}{l}\text { Inch } \\
1 / 8 \\
5 / 32 \\
3 / 16 \\
7 / 32 \\
1 / 4 \\
5 / 16 \\
3 / 8\end{array}$ \\
\hline
\end{tabular}


IRON AND STEEL

MACHINE SCREWS

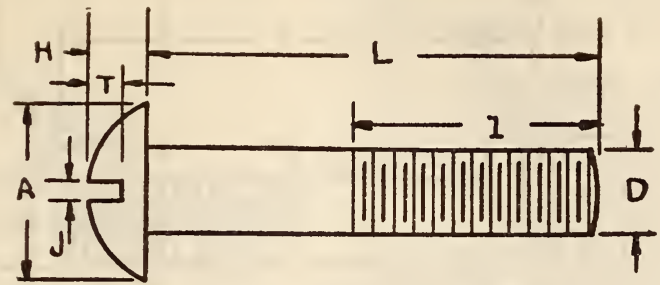

FigdRE 327.-Button-head cap screw

TABLE 15.-Dimensions of button-head cap-screw, round-head machine-screw, and stove-bolt heads

[All dimensions in inches]

\begin{tabular}{|c|c|c|c|c|c|c|c|c|c|}
\hline \multirow{3}{*}{$\begin{array}{l}\text { Nomi- } \\
\text { nal size }\end{array}$} & D & \multicolumn{2}{|c|}{ A } & \multicolumn{2}{|c|}{ H } & \multicolumn{2}{|c|}{$\mathbf{J}$} & \multicolumn{2}{|c|}{$\mathrm{T}$} \\
\hline & & \multicolumn{2}{|c|}{$\underset{\text { diameter }}{\text { Head }}$} & \multicolumn{2}{|c|}{$\begin{array}{l}\text { Height of } \\
\text { head }\end{array}$} & \multicolumn{2}{|c|}{$\begin{array}{l}\text { Width of } \\
\text { slot }\end{array}$} & \multicolumn{2}{|c|}{$\begin{array}{l}\text { Depth of } \\
\text { slot }\end{array}$} \\
\hline & & Max- & Min- & Max- & imum & Max- & Min- & Max- & $\begin{array}{l}\text { Min- } \\
\text { imum }\end{array}$ \\
\hline 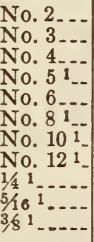 & $\begin{array}{l}0.086 \\
.099 \\
.112 \\
.125 \\
.138 \\
.164 \\
.190 \\
.216 \\
.250 \\
.3125 \\
.375\end{array}$ & $\begin{array}{r}0.162 \\
.187 \\
.211 \\
.236 \\
.260 \\
.309 \\
.359 \\
.408 \\
.472 \\
.591 \\
.708\end{array}$ & $\begin{array}{r}0.146 \\
.169 \\
.193 \\
.217 \\
.240 \\
.287 \\
.334 \\
.382 \\
.443 \\
.557 \\
.670\end{array}$ & $\begin{array}{l}0.070 \\
.078 \\
.086 \\
.095 \\
.103 \\
.119 \\
.136 \\
.152 \\
.174 \\
.214 \\
.254\end{array}$ & $\begin{array}{r}0.059 \\
.067 \\
.075 \\
.083 \\
.091 \\
.107 \\
.124 \\
.140 \\
.161 \\
.200 \\
.239\end{array}$ & $\begin{array}{l}0.036 \\
.038 \\
.040 \\
.043 \\
.045 \\
.050 \\
.055 \\
.059 \\
.066 \\
.077 \\
.088\end{array}$ & $\begin{array}{l}0.024 \\
.026 \\
.028 \\
.031 \\
.033 \\
.037 \\
.041 \\
.045 \\
.051 \\
.061 \\
.072\end{array}$ & $\begin{array}{l}0.048 \\
.053 \\
.058 \\
.062 \\
.067 \\
.076 \\
.086 \\
.095 \\
.108 \\
.130 \\
.153\end{array}$ & $\begin{array}{l}0.036 \\
.040 \\
.043 \\
.047 \\
.050 \\
.057 \\
.064 \\
.071 \\
.080 \\
.097 \\
.114\end{array}$ \\
\hline
\end{tabular}

CAP SCREWS

\begin{tabular}{|c|c|c|c|c|c|c|c|c|c|}
\hline $\begin{array}{l}7 / 16 \ldots \ldots \\
1 / 2 \\
916 \\
58 \\
3 / 4\end{array}$ & $\begin{array}{l}0.4375 \\
.500 \\
.5625 \\
.625 \\
.750\end{array}$ & $\begin{array}{r}0.750 \\
.812 \\
.938 \\
1.000 \\
1.250\end{array}$ & $\begin{array}{r}0.725 \\
.786 \\
.908 \\
.970 \\
1.215\end{array}$ & $\begin{array}{r}0.328 \\
.355 \\
.410 \\
.438 \\
.547\end{array}$ & $\begin{array}{r}0.302 \\
.328 \\
.379 \\
.405 \\
.506\end{array}$ & $\begin{array}{l}0.098 \\
.109 \\
.120 \\
.132 \\
.155\end{array}$ & $\begin{array}{r}0.084 \\
.084 \\
.104 \\
.115 \\
.135\end{array}$ & $\begin{array}{r}0.202 \\
.219 \\
.254 \\
.270 \\
.337\end{array}$ & $\begin{array}{r}0.167 \\
.179 \\
.209 \\
.220 \\
.277\end{array}$ \\
\hline
\end{tabular}

1 These sizes of screws in the coarse-thread series when furnished with nuts are interchangeable with stove bolt sizes as listed below:

\begin{tabular}{|c|c|}
\hline Machine screw & $\begin{array}{c}\text { Stove } \\
\text { bolt }\end{array}$ \\
\hline $\begin{array}{l}\text { No. } 5 \\
\text { No. } 8 \\
\text { No. } 10 \\
\text { No. } 12 \\
1 / 4 \text { inch } \\
5 / 6 \text { inch }\end{array}$ & $\begin{array}{c}\text { Inch } \\
1 / 8 \\
5 / 32 \\
3 / 16 \\
7 / 32 \\
1 / 4 \\
5 / 16 \\
3 / 8\end{array}$ \\
\hline
\end{tabular}




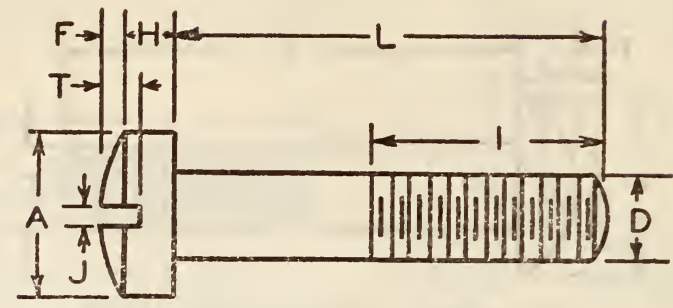

FrgURE 328.- Oval fillister-head cap screw

$T_{\triangle B L E}$ 16.-Dimensions of oval fillister-head cap-screw and machine-screw heads

[All dimensions in inches]

\begin{tabular}{|c|c|c|c|c|c|c|c|c|c|c|c|c|c|}
\hline \multirow{3}{*}{ Nominal size } & $\mathrm{D}$ & & & & & $\mathrm{J}$ & & & & & & $\mathrm{F}+$ & H \\
\hline & \multirow{2}{*}{$\begin{array}{l}\text { Diam- } \\
\text { eter }\end{array}$} & \multicolumn{2}{|c|}{ Head diameter } & \multicolumn{2}{|c|}{ Height of head } & \multicolumn{2}{|c|}{ Width of slot } & \multicolumn{2}{|c|}{ Depth of slot } & \multicolumn{2}{|c|}{ Height of oval } & \multicolumn{2}{|c|}{$\begin{array}{l}\text { Total height } \\
\text { of head }\end{array}$} \\
\hline & & $\begin{array}{l}\text { Maxi- } \\
\text { mum }\end{array}$ & $\begin{array}{l}\text { Mini- } \\
\text { mum }\end{array}$ & $\begin{array}{l}\text { Maxi- } \\
\text { mum }\end{array}$ & $\begin{array}{l}\text { Mini- } \\
\text { mum }\end{array}$ & $\begin{array}{l}\text { Maxi- } \\
\text { mum }\end{array}$ & $\begin{array}{l}\text { Mini- } \\
\text { mum }\end{array}$ & $\begin{array}{l}\text { Maxi- } \\
\text { mum }\end{array}$ & $\begin{array}{l}\text { Mini- } \\
\text { mum }\end{array}$ & $\begin{array}{l}\text { Maxi- } \\
\text { mum }\end{array}$ & $\begin{array}{l}\text { Mini- } \\
\text { mum }\end{array}$ & $\begin{array}{l}\text { Maxi- } \\
\text { mum }\end{array}$ & $\begin{array}{l}\text { Mini- } \\
\text { mum }\end{array}$ \\
\hline $\begin{array}{l}\text { No. } 2 \\
\text { No. } 3 \\
\text { No. } 4 \\
\text { No. } 5 \\
\text { No. } 6 \\
\text { No. } 8\end{array}$ & $\begin{array}{l}0.086 \\
.099 \\
.112 \\
.125 \\
.133 \\
.164\end{array}$ & $\begin{array}{l}0.140 \\
.161 \\
.183 \\
.205 \\
.226 \\
.270\end{array}$ & $\begin{array}{l}0.124 \\
.145 \\
.166 \\
.187 \\
.208 \\
.250\end{array}$ & $\begin{array}{l}0.055 \\
.063 \\
.072 \\
.081 \\
.089 \\
.106\end{array}$ & $\begin{array}{l}0.045 \\
.052 \\
.060 \\
.068 \\
.076 \\
.091\end{array}$ & $\begin{array}{r}0.036 \\
.038 \\
.040 \\
.043 \\
.045 \\
.050\end{array}$ & $\begin{array}{l}0.024 \\
.026 \\
.028 \\
.031 \\
.033 \\
.037\end{array}$ & $\begin{array}{l}0.037 \\
.043 \\
.048 \\
.054 \\
.060 \\
.071\end{array}$ & $\begin{array}{l}0.021 \\
.026 \\
.031 \\
.036 \\
.041 \\
.050\end{array}$ & $\begin{array}{l}0.028 \\
.032 \\
.035 \\
.039 \\
.043 \\
.050\end{array}$ & $\begin{array}{l}0.018 \\
.021 \\
.024 \\
.027 \\
.029 \\
.035\end{array}$ & $\begin{array}{l}0.073 \\
.084 \\
.096 \\
.107 \\
.118 \\
.141\end{array}$ & $\begin{array}{l}0.063 \\
.073 \\
.084 \\
.095 \\
.105 \\
.126\end{array}$ \\
\hline $\begin{array}{l}\text { No. } 10 \\
\text { No. } 12 \\
1 / 4 \\
5 / 16\end{array}$ & $\begin{array}{l}.190 \\
.216 \\
.250 \\
.3125 \\
.375\end{array}$ & $\begin{array}{l}.313 \\
.357 \\
.414 \\
.519 \\
.622\end{array}$ & $\begin{array}{l}.292 \\
.334 \\
.389 \\
.490 \\
.590\end{array}$ & $\begin{array}{l}.123 \\
.141 \\
.163 \\
.205 \\
.246\end{array}$ & $\begin{array}{l}.107 \\
.123 \\
.143 \\
.181 \\
.218\end{array}$ & $\begin{array}{l}.055 \\
.059 \\
.066 \\
.077 \\
.088\end{array}$ & $\begin{array}{l}.041 \\
.045 \\
.051 \\
.061 \\
.072\end{array}$ & $\begin{array}{l}.083 \\
.094 \\
.109 \\
.137 \\
.164\end{array}$ & $\begin{array}{l}.060 \\
.070 \\
.083 \\
.106 \\
.129\end{array}$ & $\begin{array}{l}.057 \\
.064 \\
.074 \\
.092 \\
.109\end{array}$ & $\begin{array}{l}.041 \\
.047 \\
.054 \\
.068 \\
.082\end{array}$ & $\begin{array}{l}.164 \\
.187 \\
.217 \\
.272 \\
.327\end{array}$ & $\begin{array}{r}.148 \\
.169 \\
.197 \\
.249 \\
.300\end{array}$ \\
\hline $\begin{array}{l}7 / 10 \\
1 / 2 \\
9 / 10\end{array}$ & $\begin{array}{c}0.4375 \\
.500 \\
.5625 \\
.625 \\
.750 \\
.875 \\
1.000\end{array}$ & $\begin{array}{r}0.625 \\
.750 \\
.812 \\
.875 \\
1.000 \\
1.125 \\
1.312\end{array}$ & $\begin{array}{r}0.608 \\
.731 \\
.792 \\
.853 \\
.976 \\
1.098 \\
1.282\end{array}$ & $\begin{array}{l}0.299 \\
.328 \\
.375 \\
.422 \\
.500 \\
.594 \\
.656\end{array}$ & $\begin{array}{r}0.274 \\
.301 \\
.347 \\
.392 \\
.466 \\
.556 \\
.613\end{array}$ & $\begin{array}{l}0.098 \\
.109 \\
.120 \\
.132 \\
.155 \\
.177 \\
.200\end{array}$ & $\begin{array}{l}0.084 \\
.094 \\
.104 \\
.115 \\
.135 \\
.156 \\
.176\end{array}$ & $\begin{array}{l}0.168 \\
.188 \\
.214 \\
.240 \\
.283 \\
.334 \\
.372\end{array}$ & $\begin{array}{l}0.133 \\
.148 \\
.169 \\
.190 \\
.223 \\
.264 \\
.292\end{array}$ & $\begin{array}{l}0.071 \\
.084 \\
.091 \\
.099 \\
.112 \\
.126 \\
.146\end{array}$ & $\begin{array}{l}0.063 \\
.075 \\
.081 \\
.088 \\
.100 \\
.113 \\
.131\end{array}$ & $\begin{array}{l}0.368 \\
.412 \\
.466 \\
.521 \\
.612 \\
.720 \\
.802\end{array}$ & $\begin{array}{r}0.337 \\
.376 \\
.428 \\
.480 \\
.566 \\
.669 \\
.744\end{array}$ \\
\hline
\end{tabular}

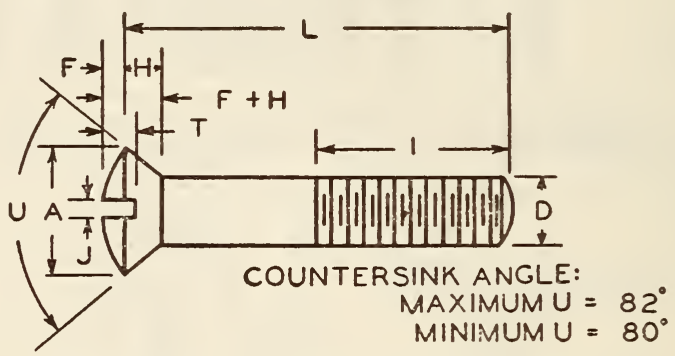

Frgure 329.-Oval-head machine screw head

TABLE 17.-Dimensions of oval-head machine-screw heads

[All dimensions in inches]

\begin{tabular}{|c|c|c|c|c|c|c|c|c|c|c|c|c|}
\hline \multirow{3}{*}{ Nominal size } & $\mathrm{D}$ & & & H & & & & & & & & \\
\hline & \multirow{2}{*}{$\begin{array}{l}\text { Diam- } \\
\text { eter }\end{array}$} & \multicolumn{2}{|c|}{ Head diameter } & \multirow{2}{*}{$\begin{array}{c}\text { Nomi- } \\
\text { nal } \\
\text { height1 } \\
\text { of head }\end{array}$} & \multicolumn{2}{|c|}{ Width of slot } & \multicolumn{2}{|c|}{ Depth of slot } & \multicolumn{2}{|c|}{ Height of oval } & \multicolumn{2}{|c|}{$\begin{array}{c}\text { Total height of } \\
\text { head }\end{array}$} \\
\hline & & $\begin{array}{l}\text { Maxi- } \\
\text { mum }\end{array}$ & $\begin{array}{l}\text { Mini- } \\
\text { mum }\end{array}$ & & $\begin{array}{l}\text { Maxi- } \\
\text { mum }\end{array}$ & $\begin{array}{l}\text { Mini- } \\
\text { mum }\end{array}$ & $\begin{array}{l}\text { Maxi- } \\
\text { mum }\end{array}$ & $\begin{array}{l}\text { Mini- } \\
\text { mum }\end{array}$ & $\begin{array}{l}\text { Maxi- } \\
\text { mum }\end{array}$ & $\begin{array}{l}\text { Mini- } \\
\text { mum }\end{array}$ & $\begin{array}{l}\text { Maxi- } \\
\text { mum }\end{array}$ & $\begin{array}{l}\text { Mini- } \\
\text { mum }\end{array}$ \\
\hline $\begin{array}{l}\text { No. } 2 \\
\text { No. } 3 \\
\text { No. } 4 \\
\text { No. } 5\end{array}$ & $\begin{array}{r}0.086 \\
.099 \\
.112 \\
.125\end{array}$ & $\begin{array}{r}0.172 \\
.199 \\
.225 \\
.252\end{array}$ & $\begin{array}{r}0.156 \\
.181 \\
.207 \\
.232\end{array}$ & $\begin{array}{r}0.045 \\
.055 \\
.060 \\
.068\end{array}$ & $\begin{array}{r}0.036 \\
.038 \\
.040 \\
.043\end{array}$ & $\begin{array}{r}0.024 \\
.026 \\
.028 \\
.031\end{array}$ & $\begin{array}{r}0.045 \\
.052 \\
.059 \\
.067\end{array}$ & $\begin{array}{r}0.037 \\
.043 \\
.049 \\
.055\end{array}$ & $\begin{array}{r}0.029 \\
.033 \\
.037 \\
.041\end{array}$ & $\begin{array}{l}0.022 \\
.026 \\
.029 \\
.033\end{array}$ & $\begin{array}{r}0.080 \\
.092 \\
.104 \\
.116\end{array}$ & $\begin{array}{r}0.063 \\
.073 \\
.084 \\
.095\end{array}$ \\
\hline $\begin{array}{l}\text { No. } 6 \\
\text { No. } 8 \\
\text { No. } 10 \\
\text { No. } 12\end{array}$ & $\begin{array}{l}.138 \\
.164 \\
.190 \\
.216\end{array}$ & $\begin{array}{l}.279 \\
.332 \\
.385 \\
.438\end{array}$ & $\begin{array}{l}.257 \\
.308 \\
.359 \\
.410\end{array}$ & $\begin{array}{l}.075 \\
.092 \\
.107 \\
.120\end{array}$ & $\begin{array}{l}.045 \\
.050 \\
.055 \\
.059\end{array}$ & $\begin{array}{l}.033 \\
.037 \\
.041 \\
.045\end{array}$ & $\begin{array}{l}.074 \\
.088 \\
.103 \\
.117\end{array}$ & $\begin{array}{l}.060 \\
.072 \\
.084 \\
.096\end{array}$ & $\begin{array}{l}.045 \\
.053 \\
.061 \\
.069\end{array}$ & $\begin{array}{l}.036 \\
.043 \\
.050 \\
.057\end{array}$ & $\begin{array}{l}.128 \\
.152 \\
.176 \\
.200\end{array}$ & $\begin{array}{l}.105 \\
.126 \\
.148 \\
.169\end{array}$ \\
\hline $\begin{array}{l}1 / 4 \ldots \\
5 / 16 \ldots \\
3 / 8 \\
7 / 16 \\
1 / 2\end{array}$ & $\begin{array}{l}.250 \\
.3125 \\
.375 \\
.4375 \\
.500\end{array}$ & $\begin{array}{r}.507 \\
.636 \\
.762 \\
.891 \\
1.017\end{array}$ & $\begin{array}{l}.477 \\
.600 \\
.722 \\
.845 \\
.967\end{array}$ & $\begin{array}{l}.142 \\
.180 \\
.215 \\
.250 \\
.285\end{array}$ & $\begin{array}{l}.066 \\
.077 \\
.088 \\
.100 \\
.111\end{array}$ & $\begin{array}{l}.051 \\
.061 \\
.072 \\
.082 \\
.092\end{array}$ & $\begin{array}{l}.136 \\
.171 \\
.206 \\
.241 \\
.275\end{array}$ & $\begin{array}{l}.112 \\
.141 \\
.170 \\
.198 \\
.227\end{array}$ & $\begin{array}{l}.079 \\
.098 \\
.117 \\
.136 \\
.155\end{array}$ & $\begin{array}{l}.066 \\
.083 \\
.100 \\
.116 \\
.133\end{array}$ & $\begin{array}{l}.232 \\
.290 \\
.347 \\
.405 \\
.463\end{array}$ & $\begin{array}{l}.197 \\
.249 \\
.300 \\
.351 \\
.402\end{array}$ \\
\hline
\end{tabular}

1 Nominal $\mathrm{H}$ subject to variations in $\mathrm{A}, \mathrm{D}$, and angle $\mathrm{U}$. 

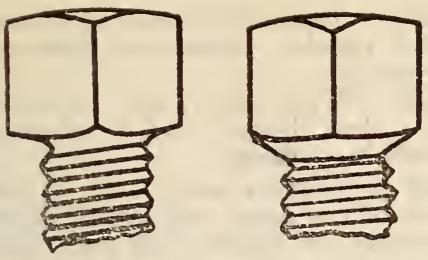

Figure 330.- Set screw heads

TABLE 24.-Dimensions of set-screw heads

[All dimensions in inches]

\begin{tabular}{|c|c|c|c|c|c|}
\hline \multirow{2}{*}{$\begin{array}{l}\text { Nominal diaweter of } \\
\text { screw }\end{array}$} & \multicolumn{2}{|c|}{$\begin{array}{l}\text { Width across } \\
\text { flats }\end{array}$} & \multicolumn{3}{|c|}{ Height } \\
\hline & $\begin{array}{l}\text { Maxi- } \\
\text { mum }\end{array}$ & $\begin{array}{l}\text { Mini- } \\
\text { mum }\end{array}$ & $\begin{array}{l}\text { Nomi- } \\
\text { nal }\end{array}$ & $\begin{array}{l}\text { Maxi- } \\
\text { mum }\end{array}$ & $\begin{array}{l}\text { Mini- } \\
\text { mum }\end{array}$ \\
\hline 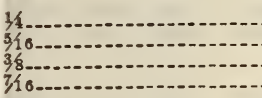 & $\begin{array}{r}0.2500 \\
.3125 \\
.3750 \\
.4375\end{array}$ & $\begin{array}{l}0.241 \\
.302 \\
.362 \\
.423\end{array}$ & $\begin{array}{l}3 / 16 \\
15 / 64 \\
9 \% 32 \\
21 \% 64\end{array}$ & $\begin{array}{l}0.197 \\
.245 \\
.293 \\
.341\end{array}$ & $\begin{array}{l}0.179 \\
.224 \\
.270 \\
.315\end{array}$ \\
\hline 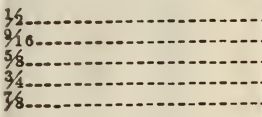 & $\begin{array}{l}.5000 \\
.5625 \\
.6250 \\
.7500 \\
.8750\end{array}$ & $\begin{array}{l}.484 \\
.545 \\
.607 \\
.729 \\
.852\end{array}$ & $\begin{array}{l}3 / 8 \\
27 / 64 \\
15 / 32 \\
9 / 16 \\
21 / 32\end{array}$ & $\begin{array}{l}.389 \\
.437 \\
.485 \\
.582 \\
.678\end{array}$ & $\begin{array}{l}.361 \\
.407 \\
.452 \\
.544 \\
.635\end{array}$ \\
\hline $\begin{array}{l}1 \\
138 \\
134 \\
132\end{array}$ & $\begin{array}{l}1.0000 \\
1.1250 \\
1.2500 \\
1.5000\end{array}$ & $\begin{array}{l}1.974 \\
1.097 \\
1.219 \\
1.464\end{array}$ & $\begin{array}{l}3 / 4 \\
27 / 32 \\
15 / 16 \\
138\end{array}$ & $\begin{array}{r}.774 \\
.870 \\
.967 \\
1.159\end{array}$ & $\begin{array}{r}.726 \\
.817 \\
.909 \\
1.091\end{array}$ \\
\hline
\end{tabular}

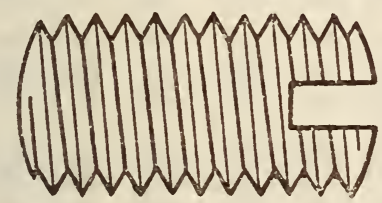

FIgURE 331.-Slotted headless set screw

TABLE 25.-Dimensions of slots of slotted headless set screws

[All dimensions in inches]

\begin{tabular}{|c|c|c|c|c|}
\hline \multirow{2}{*}{$\begin{array}{c}\text { Nominal diameter } \\
\text { of screw }\end{array}$} & \multicolumn{2}{|c|}{ Depth of slot } & \multicolumn{2}{|c|}{ Width of slot } \\
\hline & Maximum & Minimum & Maximum & Minimum \\
\hline $\begin{array}{l}\text { No. } 5 \\
\text { No. } 10 \\
1 / 4 \\
5 / 16\end{array}$ & $\begin{array}{l}0.034 \\
.053 \\
.070 \\
.088 \\
.106\end{array}$ & $\begin{array}{l}0.022 \\
.034 \\
.046 \\
.058 \\
.070\end{array}$ & $\begin{array}{l}0.027 \\
.035 \\
.042 \\
.050 \\
.057\end{array}$ & $\begin{array}{r}0.022 \\
.029 \\
.035 \\
.042 \\
.049\end{array}$ \\
\hline $\begin{array}{l}7 / 16 \ldots \\
162 \\
616 \\
56 \\
3 / 4\end{array}$ & $\begin{array}{l}.124 \\
.142 \\
.160 \\
.178 \\
.214\end{array}$ & $\begin{array}{l}.082 \\
.094 \\
.106 \\
.118 \\
.142\end{array}$ & $\begin{array}{l}.065 \\
.072 \\
.080 \\
.088 \\
.103\end{array}$ & $\begin{array}{l}.056 \\
.062 \\
.069 \\
.076 \\
.090\end{array}$ \\
\hline
\end{tabular}

UNITED STATES GOVERNMENT, NATIONAL SCREW THREAD COMMISSION, AMERICAN NATIONAI SCREW, BOLT, AND NUT PROPORTIONS. BUREAU OF STANDARDS MISCELLANEOUS PUBLICATION NO. 89, JUNE 22, 1928

(See also 608.0, p. 551; 608.31, p. 604; 608.32, p. $609 ; 615.42$, p. 883.$)$

\section{HEXAGON-HEAD CAP SCREWS}

Cap screws are usually manufactured by upsetting or by machining. They are usually threaded definite lengths for each diameter irrespective of length of screw. They are furnished without nuts. The smallest diameter regularly furnished is onefourth inch.

The length of cap screws is measured from the bearing surface of the head to the extreme point. The length of thread is measured from the extreme point to the last perfect thread.

The head is at right angles to the body within $2^{\circ}$, and concentric with the body within a tolerance of 3 per cent of the width across flats.

Cap screws 1 inch in length and under are threaded from the extreme point to the head. For cap screws longer than 1 inch, the minimum length of thread is one and one-half times the diameter plus one-fourth inch.

The dimensions of finished hexagon-head cap screws are given in Table 75.

The tops are smooth, flat, and chamfered, the maximum angle of chamfer with the top surface is $30^{\circ}$, and the diameter of the top flat circle is from 85 to 100 per cent of the width across flats.

All finished hexagon cap-screw heads are washer faced. The thickness of the washer face is about one sixty-fourth inch. The diameter of the bearing surface of the washer face is equal to the width across flats within plus or minus 5 per cent. The thickness of the head is the distance from the top to the bearing surface.

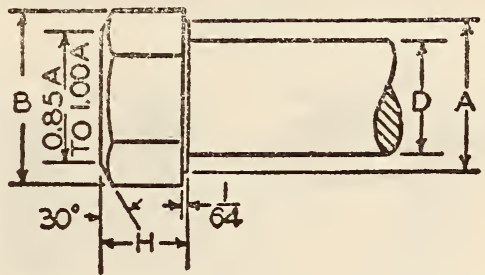

Figure 332.-Hexagon cap screw heads

TABLE 75.-Dimensions of finished hexagon cap-screw heads

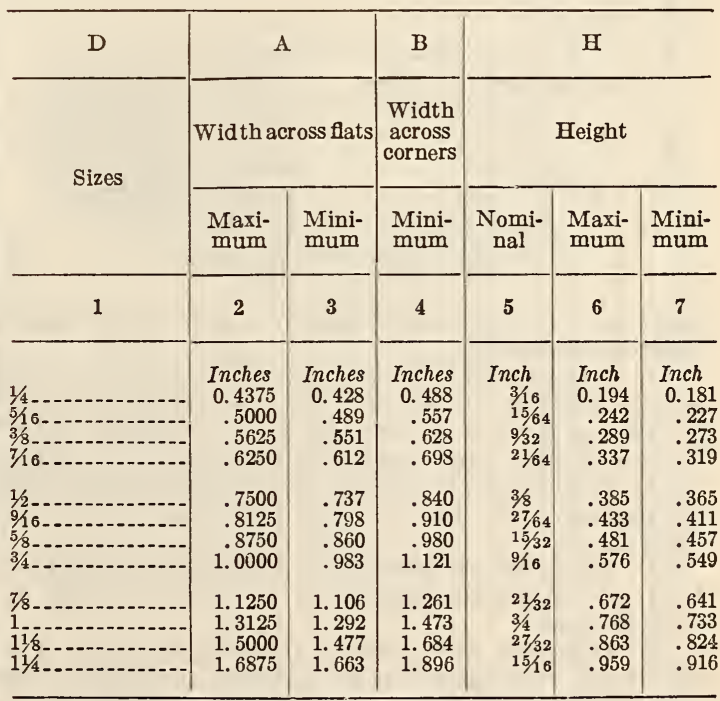

Note.-Cap screws with hexagon heads are similar to machine bolts with hexagon heads, Table 68 , and they may be used interchangeably. (See 608.31, p. 605.) 
UNITED STATES GOVERNMENT, NATIONAL SCREW THREAD COMMISSION, AMERICAN NATIONAL WOOD-SCREW SIZES, BUREAU OF STANDARDS MISCELLANEOUS PUBLICATION NO. 89, JUNE 22, 1928

\section{WOOD SCREWS}

\section{GENERAL SPECIFICATIONS}

(a) Types.-Wood screws shall be furnished in three types, namely, flat, round, or oval head, as specified.

(b) Material and workmanship.-Wood screws shall be made of steel or brass, as specified, and
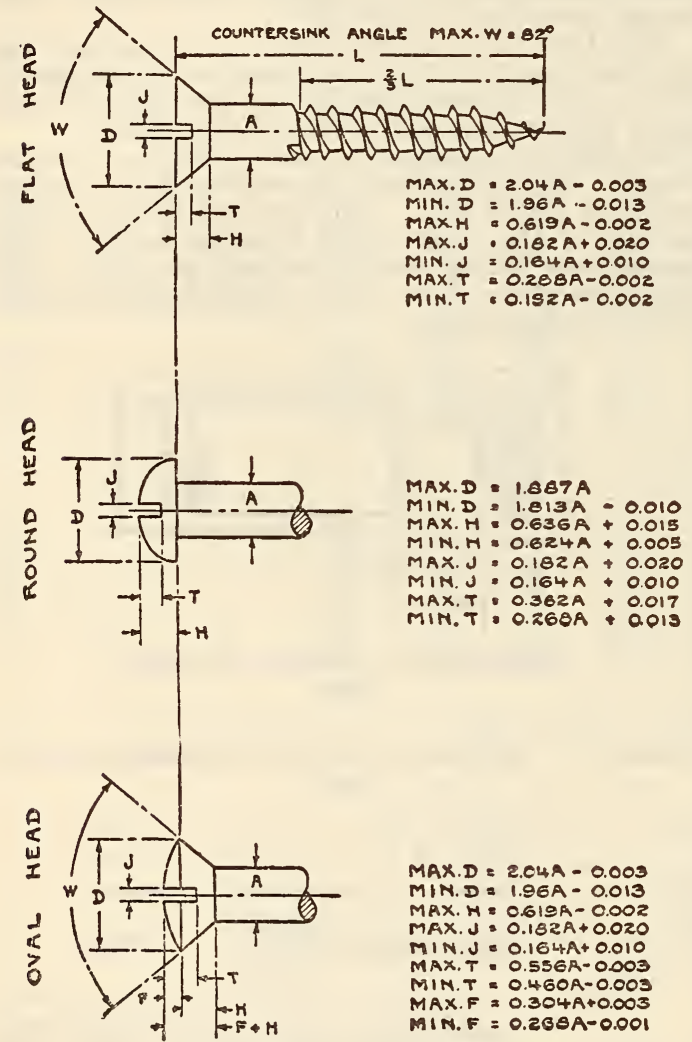

MAX.D $=2.04 A-0.003$ MIN.D $=1.96 A-0.013$ MAX. H $=0.619 A-0.002$ MAX. J = 0.182A+0.020 MIN.J $=0.164 A+0.010$ MAX.T $0.556 R-0.003$ MIN. $=0.460 \mathrm{~A}-0.003$ MIN.F $=0.258 A-0.001$

FigURE 333.-Wood screws

shall be free from any defects which would affect their serviceability.

(c) Measurement of lengths. - The length of all screws shall be measured from the largest diameter of bearing surface of the head to the extreme end of the point measured parallel to the axis of the screw.

(d) Measurement of diameters. - The diameter shall be measured on the body of the screw under the head.

(e) Tolerance on diameter.-The maximum variation on diameter permitted is from +0.004 to -0.007 inch.

(f) Tolerance on number of threads per inch.-The maximum variations in the number of threads per inch permittedpl are us or minus 10 per cent. (g) Points.-Standard screws shall be furnished with gimlet points. Cone and diamond pointed screws are special.

(h) Finish.-Wood screws shall be furnished plain, uncoated, unless blued, nickel plated, or other special finish is specified.

(i) Head proportions and length of thread.-The proportions of the head and length of thread shall be as indicated in Figure 333 and Tables 56, 57 and 58.

TABLE 56.-Head proportions of flat-head wood screws

\begin{tabular}{|c|c|c|c|c|c|c|c|c|}
\hline \multirow{3}{*}{$\underset{\text { size }}{\text { Nominal }}$} & A & \multicolumn{2}{|c|}{ D } & H & \multicolumn{2}{|c|}{$\mathrm{J}$} & \multicolumn{2}{|c|}{$\mathrm{T}$} \\
\hline & \multirow{2}{*}{$\begin{array}{l}\text { Diam- } \\
\text { eter }\end{array}$} & \multicolumn{2}{|c|}{ Head diameter } & \multirow{2}{*}{$\begin{array}{l}\text { Height } \\
\text { of } \\
\text { head, } \\
\text { maxi- } \\
\text { mum }\end{array}$} & \multicolumn{2}{|c|}{ Width of slot } & \multicolumn{2}{|c|}{ Depth of slot } \\
\hline & & $\begin{array}{l}\text { Maxi- } \\
\text { mum }\end{array}$ & $\begin{array}{l}\text { Mini- } \\
\text { mum }\end{array}$ & & $\begin{array}{l}\text { Maxi- } \\
\text { mum }\end{array}$ & $\begin{array}{l}\text { Mini- } \\
\text { mum }\end{array}$ & $\begin{array}{l}\text { Maxi- } \\
\text { mum }\end{array}$ & $\begin{array}{l}\text { Mini- } \\
\text { mum }\end{array}$ \\
\hline & $\begin{array}{c}\text { Inch } \\
0.060 \\
.073 \\
.086 \\
.099 \\
.112\end{array}$ & $\begin{array}{c}\text { Inch } \\
0.119 \\
.146 \\
.172 \\
.199 \\
.225\end{array}$ & $\begin{array}{c}\text { Inch } \\
0.105 \\
.130 \\
.156 \\
.181 \\
.207\end{array}$ & $\begin{array}{c}\text { Inch } \\
0.035 \\
.043 \\
.051 \\
.059 \\
.067\end{array}$ & $\begin{array}{c}\text { Inch } \\
0.031 \\
.033 \\
.036 \\
.038 \\
.040\end{array}$ & $\begin{array}{l}\text { Inch } \\
0.020 \\
.022 \\
.024 \\
.026 \\
.028\end{array}$ & $\begin{array}{l}\text { Inch } \\
0.015 \\
.019 \\
.023 \\
.027 \\
.030\end{array}$ & $\begin{array}{l}\text { Inch } \\
0.010 \\
.012 \\
.015 \\
.017 \\
.020\end{array}$ \\
\hline & $\begin{array}{l}.125 \\
.138 \\
.151 \\
.164 \\
.177\end{array}$ & $\begin{array}{r}.252 \\
.279 \\
.305 \\
.332 \\
.358\end{array}$ & $\begin{array}{l}.232 \\
.257 \\
.283 \\
.308 \\
.334\end{array}$ & $\begin{array}{l}.075 \\
.083 \\
.091 \\
.000 \\
.108\end{array}$ & $\begin{array}{l}.043 \\
.045 \\
.047 \\
.050 \\
.052\end{array}$ & $\begin{array}{l}.031 \\
.033 \\
.035 \\
.037 \\
.039\end{array}$ & $\begin{array}{l}.034 \\
.038 \\
.041 \\
.045 \\
.049\end{array}$ & $\begin{array}{l}.022 \\
.024 \\
.027 \\
.029 \\
.032\end{array}$ \\
\hline & $\begin{array}{l}.190 \\
.203 \\
.216 \\
.242\end{array}$ & $\begin{array}{l}.385 \\
.411 \\
.438 \\
.491\end{array}$ & $\begin{array}{l}.359 \\
.385 \\
.410 \\
.461\end{array}$ & $\begin{array}{l}.116 \\
.124 \\
.132 \\
.148\end{array}$ & $\begin{array}{l}.055 \\
.057 \\
.059 \\
.064\end{array}$ & $\begin{array}{l}.041 \\
.043 \\
.045 \\
.050\end{array}$ & $\begin{array}{l}.053 \\
.056 \\
.060 \\
.068\end{array}$ & $\begin{array}{l}.034 \\
.037 \\
.039 \\
.044\end{array}$ \\
\hline 20 & $\begin{array}{l}.268 \\
.294 \\
.320 \\
.372\end{array}$ & $\begin{array}{l}.544 \\
.597 \\
.650 \\
.756\end{array}$ & $\begin{array}{l}.512 \\
.563 \\
.614 \\
.716\end{array}$ & $\begin{array}{r}.164 \\
.180 \\
.196 \\
.228\end{array}$ & $\begin{array}{l}.069 \\
.074 \\
.078 \\
.088\end{array}$ & $\begin{array}{l}.054 \\
.058 \\
.062 \\
.071\end{array}$ & $\begin{array}{l}.075 \\
.083 \\
.090 \\
.105\end{array}$ & $\begin{array}{l}.049 \\
.054 \\
.059 \\
.069\end{array}$ \\
\hline
\end{tabular}

TABLE 57.-Head proportions of round-head wood screws

\begin{tabular}{|c|c|c|c|c|c|c|c|c|c|}
\hline \multirow{3}{*}{$\underset{\text { size }}{\text { Nominal }}$} & A & \multicolumn{2}{|c|}{ D } & \multicolumn{2}{|c|}{ H } & \multicolumn{2}{|l|}{ J } & \multicolumn{2}{|c|}{$\mathrm{T}$} \\
\hline & & $\begin{array}{r}\text { Head } \\
\text { et }\end{array}$ & $\begin{array}{l}\text { diam- } \\
\text { er }\end{array}$ & $\begin{array}{r}\text { Heig } \\
\text { he }\end{array}$ & $\begin{array}{l}\text { ht of } \\
\text { ad }\end{array}$ & $\begin{array}{l}\text { Widt } \\
\text { slc }\end{array}$ & $\begin{array}{l}\text { th of } \\
\text { ot }\end{array}$ & $\begin{array}{r}\text { Dep } \\
\text { sl }\end{array}$ & $\begin{array}{l}\text { th of } \\
\text { ot }\end{array}$ \\
\hline & & $\begin{array}{l}\text { Maxi- } \\
\text { mum }\end{array}$ & $\begin{array}{c}\text { Mini- } \\
\text { mum }\end{array}$ & $\begin{array}{l}\text { Maxi- } \\
\text { mum }\end{array}$ & $\underset{\text { Mumi- }}{\text { Mini- }}$ & $\begin{array}{c}\text { Maxi- } \\
\text { mum }\end{array}$ & $\underset{\text { mumi- }}{\text { Mini- }}$ & $\begin{array}{l}\text { Maxi- } \\
\text { mum }\end{array}$ & $\begin{array}{l}\text { Mini- } \\
\text { mum }\end{array}$ \\
\hline & $\begin{array}{r}\text { Inch } \\
0.060 \\
.073 \\
.086 \\
.099 \\
.112\end{array}$ & $\begin{array}{r}\text { Inch } \\
0.113 \\
.138 \\
.162 \\
.187 \\
.211\end{array}$ & $\begin{array}{c}\text { Inch } \\
0.099 \\
.122 \\
.146 \\
.169 \\
.193\end{array}$ & $\begin{array}{r}\text { Inch } \\
0.053 \\
.061 \\
.070 \\
.078 \\
.086\end{array}$ & $\begin{array}{l}\text { Inch } \\
0.042 \\
.051 \\
.059 \\
.067 \\
.075\end{array}$ & $\begin{array}{c}\text { Inch } \\
0.031 \\
.033 \\
.036 \\
.038 \\
.040\end{array}$ & $\begin{array}{l}\text { Inch } \\
0.020 \\
.022 \\
.024 \\
.026 \\
.028\end{array}$ & $\begin{array}{r}\text { Inch } \\
0.039 \\
.043 \\
.048 \\
.053 \\
.058\end{array}$ & $\begin{array}{l}\text { Inch } \\
0.029 \\
.033 \\
.036 \\
.040 \\
.043\end{array}$ \\
\hline $\begin{array}{l}5 \\
6 \\
7 \\
8 \\
9\end{array}$ & $\begin{array}{l}.125 \\
.138 \\
.151 \\
.164 \\
.177\end{array}$ & $\begin{array}{l}.236 \\
.260 \\
.285 \\
.309 \\
.334\end{array}$ & $\begin{array}{l}.217 \\
.240 \\
.264 \\
.287 \\
.311\end{array}$ & $\begin{array}{l}.095 \\
.103 \\
.111 \\
.119 \\
.128\end{array}$ & $\begin{array}{l}.083 \\
.091 \\
.099 \\
.107 \\
.115\end{array}$ & $\begin{array}{l}.043 \\
.045 \\
.047 \\
.050 \\
.052\end{array}$ & $\begin{array}{l}.031 \\
.033 \\
.035 \\
.037 \\
.039\end{array}$ & $\begin{array}{l}.062 \\
.067 \\
.072 \\
.076 \\
.081\end{array}$ & $\begin{array}{l}.047 \\
.050 \\
.053 \\
.057 \\
.060\end{array}$ \\
\hline $\begin{array}{l}10 \\
11 \\
12 \\
14\end{array}$ & $\begin{array}{r}.190 \\
.203 \\
.216 \\
.242\end{array}$ & $\begin{array}{l}.359 \\
.383 \\
.408 \\
.457\end{array}$ & $\begin{array}{l}.334 \\
.358 \\
.382 \\
.429\end{array}$ & $\begin{array}{l}136 \\
.144 \\
.152 \\
.169\end{array}$ & $\begin{array}{l}.124 \\
.132 \\
.140 \\
.156\end{array}$ & $\begin{array}{l}.055 \\
.057 \\
.059 \\
.064\end{array}$ & $\begin{array}{l}.041 \\
.043 \\
.045 \\
.050\end{array}$ & $\begin{array}{l}.086 \\
.090 \\
.095 \\
.105\end{array}$ & $\begin{array}{l}.064 \\
.067 \\
.071 \\
.078\end{array}$ \\
\hline $\begin{array}{l}16 \\
18 \\
20 \\
24\end{array}$ & $\begin{array}{l}.268 \\
.294 \\
.320 \\
.372\end{array}$ & $\begin{array}{l}.506 \\
.555 \\
.604 \\
.702\end{array}$ & $\begin{array}{l}.476 \\
.523 \\
.570 \\
.664\end{array}$ & $\begin{array}{l}.185 \\
.202 \\
.219 \\
.252\end{array}$ & $\begin{array}{l}.172 \\
.188 \\
.205 \\
.237\end{array}$ & $\begin{array}{l}.069 \\
.074 \\
.078 \\
.088\end{array}$ & $\begin{array}{l}.054 \\
.058 \\
.062 \\
.071\end{array}$ & $\begin{array}{l}.114 \\
.123 \\
.133 \\
.152\end{array}$ & $\begin{array}{l}.085 \\
.092 \\
.099 \\
.113\end{array}$ \\
\hline
\end{tabular}


TABLE 58.- Head proportions of oval-head wood screws

\begin{tabular}{|c|c|c|c|c|c|c|}
\hline \multirow{3}{*}{$\underset{\text { size }}{\text { Nominal }}$} & $\mathbf{A}$ & & & H & & \\
\hline & \multirow{2}{*}{$\begin{array}{l}\text { Diam- } \\
\text { eter }\end{array}$} & \multicolumn{2}{|c|}{$\begin{array}{l}\text { Head diam- } \\
\text { eter }\end{array}$} & $\begin{array}{l}\text { Height } \\
\text { of head }\end{array}$ & \multicolumn{2}{|c|}{$\begin{array}{l}\text { Width of } \\
\text { slot }\end{array}$} \\
\hline & & $\begin{array}{l}\text { Maxi- } \\
\text { mum }\end{array}$ & $\begin{array}{l}\text { Mini- } \\
\text { mum }\end{array}$ & $\begin{array}{l}\text { Maxi- } \\
\text { mum }\end{array}$ & $\begin{array}{l}\text { Maxi- } \\
\text { mum }\end{array}$ & $\begin{array}{l}\text { Mini- } \\
\text { mum }\end{array}$ \\
\hline 4 & $\begin{array}{c}\text { Inch } \\
0.060 \\
.073 \\
.086 \\
.099 \\
.112\end{array}$ & $\begin{array}{c}\text { Inch } \\
0.119 \\
.146 \\
.172 \\
.199 \\
.225\end{array}$ & $\begin{array}{c}\text { Inch } \\
0.105 \\
.130 \\
.156 \\
.181 \\
.207\end{array}$ & $\begin{array}{c}\text { Inch } \\
0.035 \\
.043 \\
.051 \\
.059 \\
.067\end{array}$ & $\begin{array}{c}\text { Inch } \\
0.031 \\
.033 \\
.036 \\
.038 \\
.040\end{array}$ & $\begin{array}{c}\text { Inch } \\
0.020 \\
.022 \\
.024 \\
.026 \\
.028\end{array}$ \\
\hline $\begin{array}{l}5 .- \\
6- \\
7- \\
8 .- \\
9 .\end{array}$ & $\begin{array}{l}.125 \\
.138 \\
.151 \\
.164 \\
.177\end{array}$ & $\begin{array}{l}.252 \\
.279 \\
.305 \\
.332 \\
.358\end{array}$ & $\begin{array}{l}.232 \\
.257 \\
.283 \\
.308 \\
.334\end{array}$ & $\begin{array}{l}.075 \\
.083 \\
.091 \\
.100 \\
.108\end{array}$ & $\begin{array}{l}.043 \\
.045 \\
.047 \\
.050 \\
.052\end{array}$ & $\begin{array}{l}.031 \\
.033 \\
.035 \\
.037 \\
.039\end{array}$ \\
\hline $\begin{array}{l}10 . \\
11 . \\
12 . \\
14 .\end{array}$ & $\begin{array}{l}.190 \\
.203 \\
.216 \\
.242\end{array}$ & $\begin{array}{l}.385 \\
.411 \\
.438 \\
.491\end{array}$ & $\begin{array}{l}.359 \\
.385 \\
.410 \\
.461\end{array}$ & $\begin{array}{l}.116 \\
.124 \\
.132 \\
.148\end{array}$ & $\begin{array}{l}.055 \\
.057 \\
.059 \\
.064\end{array}$ & $\begin{array}{l}.041 \\
.043 \\
.045 \\
.050\end{array}$ \\
\hline $\begin{array}{l}16 \\
18 \\
20 \\
24\end{array}$ & $\begin{array}{l}.268 \\
.294 \\
.320 \\
.372\end{array}$ & $\begin{array}{l}.544 \\
.597 \\
.650 \\
.756\end{array}$ & $\begin{array}{l}.512 \\
.563 \\
.614 \\
.716\end{array}$ & $\begin{array}{l}.164 \\
.180 \\
.196 \\
.228\end{array}$ & $\begin{array}{l}.069 \\
.074 \\
.078 \\
.088\end{array}$ & $\begin{array}{l}.054 \\
.058 \\
.062 \\
.071\end{array}$ \\
\hline \multirow{3}{*}{$\underset{\text { size }}{\text { Nominal }}$} & \multicolumn{2}{|c|}{$\mathrm{T}$} & \multicolumn{2}{|c|}{$F$} & \multicolumn{2}{|c|}{$\mathrm{F}+\mathrm{H}$} \\
\hline & \multicolumn{2}{|c|}{$\begin{array}{l}\text { Depth of } \\
\text { slot }\end{array}$} & \multicolumn{2}{|c|}{$\begin{array}{l}\text { Height of } \\
\text { oval }\end{array}$} & \multirow{2}{*}{$\begin{array}{l}\text { Maxi- } \\
\text { mum }\end{array}$} & \multirow{2}{*}{$\begin{array}{l}\text { Mini- } \\
\text { mum }\end{array}$} \\
\hline & $\begin{array}{l}\text { Maxi- } \\
\text { mum }\end{array}$ & $\begin{array}{l}\text { Mini- } \\
\text { mum }\end{array}$ & $\begin{array}{l}\text { Maxi- } \\
\text { mum }\end{array}$ & $\begin{array}{l}\text { Mini- } \\
\text { mum }\end{array}$ & & \\
\hline 4 & $\begin{array}{c}\text { Inch } \\
0.030 \\
.038 \\
.045 \\
.052 \\
.059\end{array}$ & $\begin{array}{c}\text { Inch } \\
0.025 \\
.031 \\
.037 \\
.043 \\
.049\end{array}$ & $\begin{array}{l}\text { Inch } \\
0.021 \\
.025 \\
.029 \\
.033 \\
.037\end{array}$ & $\begin{array}{l}\text { Inch } \\
0.015 \\
.019 \\
.022 \\
.026 \\
.029\end{array}$ & $\begin{array}{l}\text { Inch } \\
0.056 \\
.068 \\
.080 \\
.092 \\
.104\end{array}$ & $\begin{array}{c}\text { Inch } \\
0.041 \\
.052 \\
.062 \\
.074 \\
.084\end{array}$ \\
\hline $\begin{array}{l}5 \ldots \ldots . \\
6 \ldots \ldots \\
7 \\
8 \\
9 \\
9\end{array}$ & $\begin{array}{l}.067 \\
.074 \\
.081 \\
.088 \\
.095\end{array}$ & $\begin{array}{l}.055 \\
.060 \\
.066 \\
.072 \\
.078\end{array}$ & $\begin{array}{l}.041 \\
.045 \\
.049 \\
.053 \\
.057\end{array}$ & $\begin{array}{l}.033 \\
.036 \\
.039 \\
.043 \\
.046\end{array}$ & $\begin{array}{l}.116 \\
.128 \\
.140 \\
.153 \\
.165\end{array}$ & $\begin{array}{l}.095 \\
.105 \\
.115 \\
.127 \\
.137\end{array}$ \\
\hline $\begin{array}{l}10 \\
11 \\
12 \\
14\end{array}$ & $\begin{array}{r}.103 \\
.110 \\
.117 \\
.132\end{array}$ & $\begin{array}{l}.084 \\
.090 \\
.096 \\
.108\end{array}$ & $\begin{array}{l}.061 \\
.065 \\
.069 \\
.077\end{array}$ & $\begin{array}{l}.050 \\
.053 \\
.057 \\
.064\end{array}$ & $\begin{array}{l}.177 \\
.189 \\
.201 \\
.225\end{array}$ & $\begin{array}{l}.148 \\
.158 \\
.169 \\
.191\end{array}$ \\
\hline $\begin{array}{l}16 \ldots- \\
18 \ldots- \\
20 \ldots \\
24 \ldots\end{array}$ & $\begin{array}{l}.146 \\
.160 \\
.175 \\
.204\end{array}$ & $\begin{array}{r}.120 \\
.132 \\
.144 \\
.168\end{array}$ & $\begin{array}{l}.084 \\
.092 \\
.100 \\
.116\end{array}$ & $\begin{array}{l}.071 \\
.078 \\
.085 \\
.099\end{array}$ & $\begin{array}{l}.248 \\
.272 \\
.296 \\
.344\end{array}$ & $\begin{array}{l}.212 \\
.233 \\
.254 \\
.297\end{array}$ \\
\hline
\end{tabular}

(j) Included angle.-The included angle of the head on flat and oval head screws shall be $82^{\circ}$, with permissible variations of $+0^{\circ}$ and $-2^{\circ}$.

\section{TEREAD SERIES}

The numbered screw sizes, basic and limiting diameters, and threads per inch given in Table 59 are standard.
TABLE 59.-American national wood screw standard size numbers, diameters, and pitches

\begin{tabular}{|c|c|c|c|c|c|c|c|c|c|}
\hline \multirow[b]{2}{*}{$\begin{array}{l}\text { Number of } \\
\text { screw }\end{array}$} & \multirow{2}{*}{ 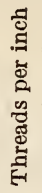 } & \multicolumn{3}{|c|}{ Diameter } & \multirow[b]{2}{*}{$\begin{array}{c}\text { Number of } \\
\text { screw }\end{array}$} & \multirow{2}{*}{ 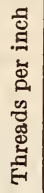 } & \multicolumn{3}{|c|}{ Diameter } \\
\hline & & 怘 & 胥 & 昜 & & & 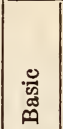 & 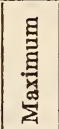 & 悬 \\
\hline & $\begin{array}{l}32 \\
28 \\
26 \\
24 \\
22\end{array}$ & $\begin{array}{r}\text { Inch } \\
0.060 \\
.073 \\
.086 \\
.099 \\
.112\end{array}$ & $\begin{array}{c}\text { Inch } \\
0.064 \\
.077 \\
.090 \\
.103 \\
.116\end{array}$ & $\begin{array}{r}\text { Inch } \\
0.053 \\
.066 \\
.079 \\
.092 \\
.105\end{array}$ & $\begin{array}{l}10 \\
11 \\
12 \\
14\end{array}$ & $\begin{array}{r}13 \\
12 \\
11 \\
10 \\
9\end{array}$ & $\begin{array}{r}\text { Inch } \\
0.190 \\
.203 \\
.216 \\
.242 \\
.268\end{array}$ & $\begin{array}{l}\text { Inch } \\
0.194 \\
.207 \\
.220 \\
.246 \\
.272\end{array}$ & $\begin{array}{c}\text { Inch } \\
0.185 \\
.196 \\
.209 \\
.235 \\
.261\end{array}$ \\
\hline 5 & $\begin{array}{l}20 \\
18 \\
16 \\
15 \\
14\end{array}$ & $\begin{array}{r}.125 \\
.138 \\
.151 \\
.164 \\
.177\end{array}$ & $\begin{array}{r}.129 \\
.142 \\
.155 \\
.168 \\
.181\end{array}$ & $\begin{array}{l}.118 \\
.131 \\
.144 \\
.157 \\
.170\end{array}$ & 18 & $\begin{array}{l}8 \\
8 \\
7\end{array}$ & $\begin{array}{l}.294 \\
.320 \\
.372\end{array}$ & $\begin{array}{l}.298 \\
.324 \\
.376\end{array}$ & $\begin{array}{l}.287 \\
.313 \\
.365\end{array}$ \\
\hline
\end{tabular}

\section{TOLERANCES ON LENGTH}

(a) Flat and oval head screws.-The maximum variations permitted in the length of flat and oval head screws are given in Table 60.

(b) Round-head screws. - The maximum variations permitted in the length of round-head screws are given in Table 61.

TABLE 60.-Tolerances on length of flat and oval head screws ${ }^{1}$

\begin{tabular}{|c|c|c|c|}
\hline $\begin{array}{l}\text { Nominal length in } \\
\text { inches }\end{array}$ & $\begin{array}{l}\text { Minus } \\
\text { tolerance }\end{array}$ & $\begin{array}{l}\text { Nominal length in } \\
\text { inches }\end{array}$ & $\begin{array}{l}\text { Minus } \\
\text { tolerance }\end{array}$ \\
\hline $\begin{array}{l}1 / 4 \\
3 / 8 \\
1 / 8 \\
6 / 8 \\
3 / 4 \\
3 / 4\end{array}$ & $\begin{array}{r}\text { Inch } \\
0.031 \\
.033 \\
.035 \\
.037 \\
.039\end{array}$ & $\begin{array}{l}2 \\
21 / 4 \\
21 / 2 \\
23 / 4\end{array}$ & $\begin{array}{r}\text { Inch } \\
0.060 \\
.064 \\
.068 \\
.072 \\
.076\end{array}$ \\
\hline $\begin{array}{l}7 / 8 \\
111 / 4 \\
11 / 2 \\
13 / 4\end{array}$ & $\begin{array}{l}.041 \\
.043 \\
.048 \\
.052 \\
.056\end{array}$ & $31 / 2$ & $\begin{array}{l}.084 \\
.092 \\
.101 \\
.109\end{array}$ \\
\hline
\end{tabular}

1 Plus tolerance $=0$.

TABLE 61.-Tolerances on length of round-head screws ${ }^{1}$

\begin{tabular}{|c|c|c|c|c|c|c|c|c|c|}
\hline \multirow{2}{*}{$\begin{array}{l}\text { Nominal } \\
\text { length in } \\
\text { inches }\end{array}$} & \multicolumn{9}{|c|}{ Screw numbers } \\
\hline & 0 & 1 & 2 & 3 & 4 & 5 & 6 & 7 & 8 \\
\hline \multirow{13}{*}{$\begin{array}{l}1 / 4 \\
3 / 8 \\
1 / 2 \\
5 / 8 \\
3 / 4 \\
7 / 8 \\
1 \\
113 / 4 \\
11 / 2 \\
\end{array}$} & \multirow{13}{*}{$\begin{array}{r}\text { Inch } \\
0.064 \\
.065\end{array}$} & \multirow{13}{*}{$\begin{array}{r}\text { Inch } \\
0.071 \\
.073 \\
.075 \\
\\
\end{array}$} & \multirow{13}{*}{$\begin{array}{r}\text { Inch } \\
0.077 \\
.079 \\
.081 \\
.083 \\
.085\end{array}$} & \multirow{5}{*}{$\begin{array}{r}\text { Inch } \\
0.084 \\
.086 \\
.088 \\
.090 \\
.092\end{array}$} & \multirow{5}{*}{$\begin{array}{l}\text { Inch } \\
.090 \\
.092 \\
.094 \\
.096 \\
.098\end{array}$} & Inch & Inch & Inch & Inch \\
\hline & & & & & & 0.099 & $0 . \overline{105}$ & $0 . \overline{112}$ & $0.1 \overline{18}$ \\
\hline & & & & & & .101 & .107 & .114 & .120 \\
\hline & & & & & & .103 & .109 & .116 & .12 \\
\hline & & & & & & .1 & .111 & .11 & .12 \\
\hline & & & & .094 & .100 & . 107 & .113 & .120 & .12 \\
\hline & & & & .0 & .102 & .109 & .115 & .122 & .1 \\
\hline & & & & - & .106 & .113 & .119 & .126 & - 12 \\
\hline & & & & & & & .127 & .134 & .14 \\
\hline & & & & & & & .131 & .138 & \\
\hline & & & & & & & .135 & .142 & .1 \\
\hline & & & & & & & .1 & & \\
\hline & & & & & & & & & \\
\hline
\end{tabular}

1 Plus tolerance $=0$. Minus tolerances as given in body of table. 
TABLE 61.-Tolerances on length of round-head screws ${ }^{1-}$ Continued

\begin{tabular}{|c|c|c|c|c|c|c|c|c|c|}
\hline \multirow{2}{*}{$\begin{array}{l}\text { Nomina } \\
\text { length in } \\
\text { inches }\end{array}$} & \multicolumn{9}{|c|}{ Screw numbers } \\
\hline & 9 & 10 & 11 & 12 & 14 & 16 & 18 & 20 & 24 \\
\hline & $\begin{array}{l}\text { Inch } \\
0.127\end{array}$ & $\begin{array}{l}\text { Inch } \\
0.133\end{array}$ & Inch & Inch & Inch & Inch & Inch & Inch & Inch \\
\hline & .129 & .135 & 0.142 & 0.148 & & & 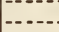 & . & - \\
\hline & .131 & .137 & .144 & .150 & 0.163 & 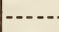 & 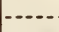 & 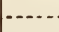 & $\cdots$ \\
\hline & $\begin{array}{l}.133 \\
.135\end{array}$ & $\begin{array}{l}.139 \\
.141\end{array}$ & $\begin{array}{l}.146 \\
.148\end{array}$ & $\begin{array}{l}.152 \\
.154\end{array}$ & $\begin{array}{l}.165 \\
.167\end{array}$ & 0.180 & & & $-\cdots$ \\
\hline & .139 & .145 & .152 & .158 & .171 & .184 & 0.19 & & \\
\hline & .143 & .149 & .156 & .162 & .175 & .188 & .202 & 0.215 & \\
\hline & .147 & .153 & .160 & .166 & .179 & .192 & .206 & .219 & \\
\hline & .151 & .157 & .164 & .170 & .183 & .196 & .210 & .223 & \\
\hline & .155 & .16 & .168 & .174 & .187 & .200 & .214 & .227 & \\
\hline & & 1 & & & .1 & .204 & .218 & .231 & -1 \\
\hline & .163 & .169 & .1 & .1 & .195 & .208 & .222 & .235 & \\
\hline & .167 & .173 & .180 & 186 & 199 & .212 & .226 & .239 & 0.26 \\
\hline & 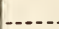 & .181 & .188 & .194 & .207 & .220 & .234 & .247 & .273 \\
\hline & & & & .202 & .215 & .228 & .242 & .255 & .281 \\
\hline & & & & & & .236 & .250 & .263 & .2 \\
\hline & & & & & .231 & .244 & .258 & .271 & .29 \\
\hline
\end{tabular}

1 Plus tolerance $=0$. Minus tolerances as given in body of table.

AMERICAN NATIONAI STANDARD SIZES OF WOOD SCREWS

(a) Steel screws.-The standard sizes of steel screws are given in Table 62.

(b) Brass screws.-The standard sizes of brass screws are given in Table 63.

TABLE 62.-Standard sizes of steel screws

[F, flat head; $R$, round head; $O$, oval head]

\begin{tabular}{|c|c|c|c|c|c|c|c|c|c|}
\hline $\begin{array}{l}\text { eng } \\
\text { nch }\end{array}$ & $\begin{array}{l}\text { No. } 0 \\
\text { diam } \\
\text { eter, } \\
0.060 \\
\text { inch }\end{array}$ & $\begin{array}{l}\text { No. } 1 \\
\text { diam- } \\
\text { eter, } \\
0.073 \\
\text { inch }\end{array}$ & $\begin{array}{l}\text { No. } 2 \\
\text { diam- } \\
\text { eter, } \\
0.086 \\
\text { inch }\end{array}$ & $\begin{array}{l}\text { No. } 3 \\
\text { diam- } \\
\text { eter, } \\
0.099 \\
\text { inch }\end{array}$ & $\begin{array}{l}\text { No. } 4 \\
\text { diam- } \\
\text { eter, } \\
0.112 \\
\text { inch }\end{array}$ & $\begin{array}{l}\text { No. } 5 \\
\text { diam- } \\
\text { eter, } \\
0.125 \\
\text { inch }\end{array}$ & $\begin{array}{l}\text { No. } 6 \\
\text { diam- } \\
\text { eter, } \\
0.138 \\
\text { inch }\end{array}$ & $\begin{array}{l}\text { No. } 7 \\
\text { diam } \\
\text { eter, } \\
0.151 \\
\text { inch }\end{array}$ & $\begin{array}{l}\text { No. } 8 \\
\text { diam- } \\
\text { eter, } \\
0.164 \\
\text { inch }\end{array}$ \\
\hline & & $\mathrm{FR}$ & \multirow{5}{*}{$\begin{array}{l}\text { FR } \\
\text { FR } \\
\text { FR } \\
\text { FR } \\
\text { FR }\end{array}$} & $\mathrm{FR}$ & $\mathrm{FR}$ & & & & \\
\hline & $\mathrm{FR}$ & $\mathrm{F}$ & & FRO & FRO & $\mathrm{FR}$ & $\mathrm{FR}$ & $\mathrm{FR}$ & $\mathrm{FR}$ \\
\hline & & & & $\mathrm{FR}$ & $\mathrm{FRO}$ & $\mathrm{FRO}$ & $\mathrm{FRO}$ & FRO & FRO \\
\hline & & & & $\mathrm{FR}$ & $\mathrm{FRO}$ & FRO & FRO & FRO & $\mathrm{FR}$ \\
\hline & & & & $\mathrm{FR}$ & FRO & $\mathrm{FRO}$ & FRO & $\mathrm{FRO}$ & FRO \\
\hline & & & & $\mathrm{Fl}$ & $\mathrm{FR}$ & $\mathrm{FR}$ & $\mathrm{FR}$ & FRO & FRO \\
\hline & & & & F & & FRO & FRO & & \\
\hline & & & & & & $\mathrm{FR}$ & FRO & $\mathrm{FRO}$ & $\mathrm{FR}$ \\
\hline & & & & & $\mathrm{FR}$ & $\mathrm{FR}$ & & & \\
\hline & & & & & & & FRO & FRO & FRO \\
\hline & & & & & & & $\mathrm{F}$ & $\mathrm{FR}$ & $\mathrm{FR}$ \\
\hline & & & & & & & FR & FR & FR \\
\hline & & & & & & & $\mathrm{FR}$ & FR & \\
\hline & & & & & & & & & \\
\hline
\end{tabular}

No. $9 \mid$ No.10|No.11 $\mid$ No.12 $\mid$ No. 14 $\mid$ No.16 $\mid$ No.18 $\mid$ No.20|No. 24 Lengths diam-diam-diam-diam-diam- diam- diam- diam- diam(inches) eter, eter, eter, eter, eter, eter, eter, eter, eter,

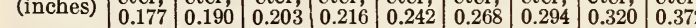
inch inch inch inch inch inch inch inch inch

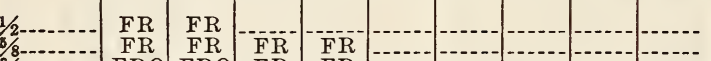

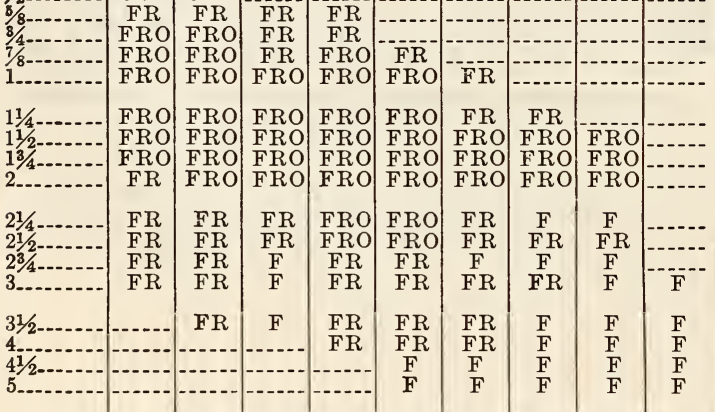

TABLE 63.- Standard sizes of brass screws

[F, flat head; $R$, round head; $O$, oval head]

\begin{tabular}{|c|c|c|c|c|c|c|c|c|}
\hline $\begin{array}{l}\text { Lengths } \\
\text { (inches) }\end{array}$ & $\begin{array}{l}\text { No. } 0 \\
\text { diam- } \\
\text { eter, } \\
0.060 \\
\text { inch }\end{array}$ & $\begin{array}{l}\text { No. } 1 \\
\text { diam- } \\
\text { eter, } \\
0.073 \\
\text { inch }\end{array}$ & $\begin{array}{l}\text { No. } 2 \\
\text { diam- } \\
\text { eter, } \\
0.086 \\
\text { inch }\end{array}$ & $\begin{array}{l}\text { No. } 3 \\
\text { diam- } \\
\text { eter, } \\
0.099 \\
\text { inch }\end{array}$ & $\begin{array}{c}\text { No. } 4 \\
\text { diam- } \\
\text { eter, } \\
0.112 \\
\text { inch }\end{array}$ & $\begin{array}{l}\text { No. } 5 \\
\text { diam- } \\
\text { eter, } \\
0.125 \\
\text { inch }\end{array}$ & $\begin{array}{l}\text { No. } 6 \\
\text { diam- } \\
\text { eter, } \\
0.138 \\
\text { inch }\end{array}$ & $\begin{array}{l}\text { No. } 7 \\
\text { diam- } \\
\text { eter, } \\
0.151 \\
\text { inch }\end{array}$ \\
\hline & FRO & FRO & FRO & FRO & FRO & & & \\
\hline & FRO & $\begin{array}{l}\text { FRO } \\
\text { FRO }\end{array}$ & $\begin{array}{l}\text { FRO } \\
\text { FRO }\end{array}$ & $\begin{array}{l}\text { FRO } \\
\text { FRO }\end{array}$ & $\begin{array}{l}\text { FRO } \\
\text { FRO }\end{array}$ & $\begin{array}{l}\text { FRO } \\
\text { FRO }\end{array}$ & FRO & $\mathrm{FPO}$ \\
\hline & & & FRO & FRO & FRO & FRO & FRO & FRO \\
\hline & & & FRO & FRO & FRO & FRO & FRO & FRO \\
\hline & & & & & FRO & FRO & FRO & FRO \\
\hline & & & & & FRO & FRO & FRO & RO \\
\hline & & & & & & & FRO & FRO \\
\hline & & & & & & & & \\
\hline & No. 8 & No. 9 & No. 10 & No. 11 & No. 12 & No. 14 & No. 16 & No. 18 \\
\hline & eter, & eter, & eter, & eter, & eter, & eter, & eter, & $\begin{array}{l}\text { lam- } \\
\text { eter, }\end{array}$ \\
\hline & $\begin{array}{l}0.164 \\
\text { inch }\end{array}$ & $\begin{array}{l}0.177 \\
\text { inch }\end{array}$ & $\begin{array}{l}0.190 \\
\text { inch }\end{array}$ & $\begin{array}{l}0.203 \\
\text { inch }\end{array}$ & $\begin{array}{l}0.216 \\
\text { inch }\end{array}$ & $\begin{array}{l}0.242 \\
\text { inch }\end{array}$ & $\begin{array}{l}0.268 \\
\text { inch }\end{array}$ & $\begin{array}{l}0.294 \\
\text { inch }\end{array}$ \\
\hline & & & & & & & & \\
\hline & FRO & & & & & & & \\
\hline & FRO & FRO & FRO & & & & & \\
\hline & FRO & FRO & FRO & $\mathrm{FR}$ & $\begin{array}{l}\text { FRO } \\
\text { FR }\end{array}$ & & & \\
\hline & FRO & FRO & $\mathrm{FRC}$ & $\mathrm{FR}$ & FRO & FRO & & \\
\hline & FRO & FRO & $\mathrm{FP}$ & FR & $\mathrm{FP}$ & FRO & & \\
\hline & FRO & & & & & FRO & & ..... \\
\hline & FRO & FRO & FRO & FR & FRO & FRO & $\mathrm{F}$ & F \\
\hline & & & $\mathrm{FP}$ & $\mathrm{FR}$ & FR & FR & F & F \\
\hline & & & $\mathrm{F}$ & & FR & FR & $F$ & $\mathrm{~F}$ \\
\hline & & & & & $\mathrm{F}$ & $\mathrm{F}$ & F & $\mathrm{F}$ \\
\hline
\end{tabular}

608.3 BOLTS AND NUTS, EXCEPT RAILWAY TRACK BOLTS.

608.31 BOLTS.

AMERICAN ELECTRIC RAILWAY ENGINEERING ASSOCIATION, OVERHEAD LINE MATERIAL FOR DIRECT AND CATENARY SUSPENSION, RECOMMENDED SPECIFICATION, D102-30, 1930

\section{OVERHEAD LINE MATERIAL}

(D102-30, paragraph 304, includes requirements for bolts and nuts. For specification see 611.29 , p. 682.)

\section{AMERICAN MARINE STANDARDS COM-} MITTEE, SPECIFICATION E NO. 11 , PROPELLER HUB STUDS, NUTS, AND LOCK SCREWS, A. M. S. C. 30, SEPTEMBER 20, 1927

(See also 608.2, p. 574; 608.32, p. 605; and 646.59, p. 1257.)

\section{нов STUDS}

(Contains specifications, dimensions, and illustrations of propeller hub studs, nuts, and lock screws.)

\section{AMERICAN RAILWAY ASSOCIATION, ME- CHANICAL DIVISION ; SPECIFICATIONS FOR MATERIALS, BOLTS AND NUTS, 1916}

\section{BOLTS AND NUTS}

1. Scope.-These specifications shall apply to all bolts and nuts. 
MANOFACTORE

2. Process.-The bolts and nuts may be wrought iron or steel, made in accordance with A. R. A. specifications for refined wrought-iron bars or mildsteel bars (see 603.1, p. 137).

\section{CHEMICAL PROPERTIES AND TESTS}

3. Chemical composition.-The steel for the bolts shall conform to the requirements of the A. R. A. specifications for mild steel.

\section{PHYSICAL PROPERTIES AND TESTS}

4. Tension tests.-The wrought iron or steel conform to the physical properties as specified in the A. R. A. specifications for refined wrought-iron bars or mild-steel bars.

\section{PERMISSIBLE VARIATIONS}

5. Gage of bolts.-Rods shall conform to the limits as specified in A. R. A. specifications for refined wrought-iron bar or mild-steel bars.

\section{SPECIFICATIONS FOR BOLTS AND NUTS}

6. Threads.-Nuts shall be so tapped that a U. S. standard plug gage of normal size will pass through the nut, and in that position be snug and not show an appreciable shake. Bolts to be threaded so as to fit the nut in such a manner that it may be turned to within one of the threads of the threaded portion of the bolt. When in this position there shall be no appreciable shake.

7. Dimensions of boltheads and nuts.-Finished bolts and nuts shall conform to dimensions in Table No. 1. Short diameter of rough boltheads and nuts may vary from this table as follows: For nuts under one-half inch, one-sixteenth inch under and one sixty-fourth inch over; for nuts one-half inch and larger, one-sixteenth inch under and three sixtyfourths inch over. All other dimensions of nuts and heads of bolts may vary one-sixteenth inch either way, except that for bolts and nuts under one-half inch, only $1 / 32$-inch variation either way will be permitted.

8. Tapping of nuts.-The nuts shall be tapped so that, when applied, the angle between the bearing surfaces shall not exceed $2^{\circ}$, as shown by the cut

9. Length. - The finished length of the bolt shall not vary more than one-eighth inch from the ordered dimension and unless otherwise specified, the threads shall be cut two and one-half times the diameter of the bolt.

\section{WORKMANSHIP AND FINISH}

10. Workmanship.-The heads of all bolts shall be neatly formed, firmly joined to the body of the bolt, free from large projecting fins, and no nicking or reduction of the size of the body of the bolt by dies.

11. Threads.-Bolts and nuts shall be cut with a U. S. and A. R. A. standard thread, the threads being cut full and clean. Unless otherwise specified, each bolt shall be furnished with a nut screwed on.

12. Finish.-Where the keyways are cut in the ends of bolts, they shall be full and clean, and the metal around them shall show that it has not been injured by cutting the keyways more than is necessary.

\section{INSPECTION AND REJECTION}

(Substantially the same as American Society for Testing Materials, specifications for steel. (See 605.0 , p. 247).)

TABLE 1

[All dimensions in inches]

\begin{tabular}{|c|c|c|c|c|c|c|c|c|c|c|c|}
\hline \multirow{3}{*}{ Diameter of screw } & \multicolumn{3}{|c|}{ Screw threads } & \multicolumn{4}{|c|}{ Nuts, hexagon and square } & \multicolumn{4}{|c|}{ Heads, hexagon and square } \\
\hline & \multirow{2}{*}{$\begin{array}{l}\text { Threads } \\
\text { per inch }\end{array}$} & \multirow{2}{*}{$\begin{array}{c}\text { Diameter } \\
\text { at root } \\
\text { of thread }\end{array}$} & \multirow{2}{*}{$\begin{array}{l}\text { Width of } \\
\text { flat }\end{array}$} & \multicolumn{2}{|c|}{ Short diameter } & \multicolumn{2}{|c|}{ Thickness } & \multicolumn{2}{|c|}{ Short diameter } & \multicolumn{2}{|c|}{ Thickness } \\
\hline & & & & Rough & Finish & Rough & Finish & Rough & Finish & Rough & Finish \\
\hline $\begin{array}{l}1 / 4 \ldots \ldots \\
5 / 16 \ldots . . \\
3 / 8 \ldots \ldots \\
7 / 16 \ldots \\
1 / 2 \ldots \ldots\end{array}$ & $\begin{array}{l}20 \\
18 \\
16 \\
14 \\
13\end{array}$ & $\begin{array}{l}0.185 \\
.240 \\
.294 \\
.344 \\
.400\end{array}$ & $\begin{array}{l}0.0062 \\
.0074 \\
.0078 \\
.0089 \\
.0096\end{array}$ & $\begin{array}{l}1 / 2 \\
19 / 32 \\
11 / 16 \\
25 / 32 \\
7 / 8\end{array}$ & $\begin{array}{l}7 / 16 \\
17 / 32 \\
5 / 8 \\
23 / 32 \\
13 / 16\end{array}$ & $\begin{array}{l}1 / 4 \\
5 / 16 \\
3 / 8 \\
7 / 16 \\
1 / 2\end{array}$ & $\begin{array}{l}3 / 16 \\
1 / 4 \\
5 / 16 \\
3 / 8 \\
7 / 10\end{array}$ & $\begin{array}{l}3 / 8 \\
15 / 32 \\
9 / 16 \\
21 / 32 \\
3 / 4\end{array}$ & $\begin{array}{l}5 / 16 \\
13 / 32 \\
1 / 2 \\
19 / 32 \\
11 / 16\end{array}$ & $\begin{array}{l}3 / 16 \\
15 / 64 \\
9 / 32 \\
21 / 64 \\
3 / 8\end{array}$ & $\begin{array}{l}1 / 8 \\
11 / 6 \\
7 / 32 \\
176 \\
5 / 16\end{array}$ \\
\hline $\begin{array}{l}9 / 6 \ldots \\
5 / 6 \ldots \\
3 / 4 \ldots \\
7 / 8 \ldots \\
1 \ldots . .\end{array}$ & $\begin{array}{r}12 \\
11 \\
10 \\
9 \\
8\end{array}$ & $\begin{array}{l}.454 \\
.507 \\
.620 \\
.731 \\
.837\end{array}$ & $\begin{array}{l}.0104 \\
.0113 \\
.0125 \\
.0138 \\
.0156\end{array}$ & $\begin{array}{l}31 / 32 \\
11 / 16 \\
11 / 4 \\
17 / 16 \\
15 / 8\end{array}$ & $\begin{array}{l}1^{29 / 32} \\
13 / 16 \\
13 / 8 \\
19 / 16\end{array}$ & $\begin{array}{l}9 / 16 \\
5 / 8 \\
3 / 4 \\
7 / 8 \\
1\end{array}$ & $\begin{array}{l}1 / 2 \\
916 \\
11 / 16 \\
1316 \\
15 / 16\end{array}$ & $\begin{array}{l}27 / 32 \\
15 / 16 \\
11 / 8 \\
15 / 16 \\
17 / 16\end{array}$ & $\begin{array}{c}25 / 32 \\
7 / 8 \\
11 / 16 \\
11 / 4 \\
11 / 2\end{array}$ & $\begin{array}{l}27 / 64 \\
15 / 32 \\
9 / 16 \\
21 / 32 \\
3 / 4\end{array}$ & $\begin{array}{l}23 / 6 \\
13 / 32 \\
1 / 2 \\
19 / 32 \\
11 / 10\end{array}$ \\
\hline & $\begin{array}{l}7 \\
7 \\
6 \\
6 \\
51 / 2\end{array}$ & $\begin{array}{l}.940 \\
1.065 \\
1.160 \\
1.284 \\
1.389\end{array}$ & $\begin{array}{l}.0178 \\
.0178 \\
.0208 \\
.0208 \\
.0227\end{array}$ & $\begin{array}{l}1^{13 / 16} \\
2 \\
23 / 16 \\
238 \\
29 / 16\end{array}$ & $\begin{array}{l}13 / 4 \\
115 / 16 \\
21 / 8 \\
25 / 16 \\
21 / 2\end{array}$ & $\begin{array}{l}11 / 8 \\
114 \\
13 / 8 \\
11 / 2 \\
15 / 8\end{array}$ & $\begin{array}{l}11 / 16 \\
13 / 16 \\
15 / 16 \\
17 / 16 \\
19 / 16\end{array}$ & $\begin{array}{l}111 / 16 \\
17 / 8 \\
21 / 16 \\
21 / 4 \\
27 / 16\end{array}$ & $\begin{array}{l}15 / 8 \\
1^{13} / 16 \\
2 \\
23 / 16 \\
23 / 8\end{array}$ & $\begin{array}{c}27 / 32 \\
15 / 16 \\
11 / 32 \\
118 \\
17 / 32\end{array}$ & $\begin{array}{l}25 / 32 \\
7 / 8 \\
31 / 32 \\
11 / 1 \\
15 / 32\end{array}$ \\
\hline $\begin{array}{l}134 \\
178 \\
2 \\
2144 \\
21 / 2\end{array}$ & \begin{tabular}{l|}
5 \\
5 \\
$41 / 2$ \\
$41 / 2$ \\
4
\end{tabular} & $\begin{array}{l}1.491 \\
1.616 \\
1.712 \\
1.961 \\
2.175\end{array}$ & $\begin{array}{l}.0250 \\
.0250 \\
.0277 \\
.0277 \\
.0312\end{array}$ & $\begin{array}{l}23 / 4 \\
215 / 16 \\
318 \\
31 / 2 \\
37 \%\end{array}$ & $\begin{array}{l}211 / 16 \\
27 / 8 \\
31 / 16 \\
37 / 16 \\
3^{13} / 16\end{array}$ & $\begin{array}{l}13 / 4 \\
178 \\
2 \\
21 / 4 \\
21 / 2\end{array}$ & $\begin{array}{l}111 / 16 \\
113 / 16 \\
115 / 16 \\
23 / 16 \\
27 / 16\end{array}$ & $\begin{array}{l}25 / 8 \\
2^{13} / 16 \\
3 \\
238 \\
3^{3} / 4\end{array}$ & $\begin{array}{l}29 / 16 \\
23 / 4 \\
2^{15} 156 \\
35 / 16 \\
3^{11 / 16}\end{array}$ & $\begin{array}{l}15 / 16 \\
113 / 32 \\
11 / 2 \\
111 / 16 \\
17 / 8\end{array}$ & $\begin{array}{l}11 / 4 \\
111 / 32 \\
17 / 16 \\
15 / 8 \\
113 / 16\end{array}$ \\
\hline $\begin{array}{l}23 / 4 \ldots \\
3 \\
31 / 4 \\
31 / 2 \\
334\end{array}$ & $\begin{array}{l}4 \\
31 / 2 \\
31 / 2 \\
31 / 4 \\
3\end{array}$ & $\begin{array}{l}2.425 \\
2.629 \\
2.879 \\
3.100 \\
3.317\end{array}$ & $\begin{array}{l}.0312 \\
.0357 \\
.0357 \\
.0384 \\
.0416\end{array}$ & $\begin{array}{l}41 / 4 \\
45 / 8 \\
5 \\
53 / 8 \\
53 / 4\end{array}$ & $\begin{array}{l}43 / 16 \\
49116 \\
415 / 16 \\
55 / 16 \\
5^{11} 116\end{array}$ & $\begin{array}{l}23 / 4 \\
3 \\
31 / 4 \\
31 / 2 \\
33 / 4\end{array}$ & $\begin{array}{l}211 / 16 \\
215 / 16 \\
33 / 16 \\
37 / 16 \\
311 / 16\end{array}$ & $\begin{array}{l}41 / 8 \\
41 / 2 \\
47 / 8 \\
51 / 4 \\
55 / 8\end{array}$ & $\begin{array}{l}41 / 16 \\
47 / 16 \\
413116 \\
53 / 16 \\
5916\end{array}$ & $\begin{array}{l}21 / 16 \\
21 / 4 \\
27 / 16 \\
25 / 8 \\
213 / 16\end{array}$ & $\begin{array}{l}2 \\
23 / 16 \\
23 / 8 \\
29116 \\
23 / 4\end{array}$ \\
\hline $4 \begin{array}{l}4144 \\
412 \\
43 / 4\end{array}$ & \begin{tabular}{l|}
3 \\
$27 / 8$ \\
$23 / 4$ \\
$25 / 8$
\end{tabular} & $\begin{array}{l}\text { 3. } 567 \\
3.798 \\
4.028 \\
4.255\end{array}$ & $\begin{array}{l}.0416 \\
.0434 \\
.0454 \\
.0475\end{array}$ & $\begin{array}{l}61 / 8 \\
61 / 2 \\
67 / 8 \\
71 / 4\end{array}$ & $\begin{array}{l}61 / 16 \\
67 / 16 \\
6^{13} / 16 \\
73 / 16\end{array}$ & $\begin{array}{l}4 \\
41 / 4 \\
41 / 2 \\
43 / 4\end{array}$ & $\begin{array}{l}3^{15} / 16 \\
4^{3} / 16 \\
4^{7} / 16 \\
4^{11} 1 / 16\end{array}$ & $\begin{array}{l}6 \\
63 / 8 \\
63 / 4 \\
71 / 8\end{array}$ & $\begin{array}{l}5^{15} / 16 \\
65 / 16 \\
6^{11} 1 / 16 \\
71 / 16\end{array}$ & \begin{tabular}{l|l|}
3 \\
$33 / 16$ \\
$33 / 8$ \\
$3 \% 16$
\end{tabular} & $\begin{array}{l}215 / 16 \\
31 / 8 \\
35 / 16 \\
31 / 2\end{array}$ \\
\hline $\begin{array}{l}5 \\
51 / 4 \\
512 \\
534 \\
6\end{array}$ & $\begin{array}{l}21 / 2 \\
21 / 2 \\
23 / 8 \\
23 / 8 \\
21 / 4\end{array}$ & $\begin{array}{l}4.480 \\
4.730 \\
4.953 \\
5.203 \\
5.423\end{array}$ & $\begin{array}{l}.0500 \\
.0500 \\
.0526 \\
.0526 \\
.0555\end{array}$ & $\begin{array}{l}75 / 8 \\
8 \\
83 / 8 \\
83 / 4 \\
91 / 8\end{array}$ & $\begin{array}{l}79 / 16 \\
713 / 16 \\
85 / 16 \\
811 / 16 \\
91 / 16\end{array}$ & $\begin{array}{l}5 \\
51 / 4 \\
51 / 2 \\
53 / 4 \\
6\end{array}$ & $\begin{array}{l}415 / 16 \\
53 / 16 \\
57 / 16 \\
5^{11} 1 / 16 \\
5^{15 / 16}\end{array}$ & $\begin{array}{l}71 / 2 \\
778 \\
81 / 4 \\
85 / 8 \\
9\end{array}$ & $\begin{array}{l}77 / 16 \\
713 / 16 \\
83 / 16 \\
89 / 16 \\
813 / 16\end{array}$ & $\begin{array}{l}33 / 4 \\
315 / 16 \\
4118 \\
45 / 16 \\
41 / 2\end{array}$ & $\begin{array}{l}311 / 16 \\
37 / 8 \\
41 / 16 \\
41 / 4 \\
47 / 16\end{array}$ \\
\hline
\end{tabular}


AMERICAN RAILWAY ASSOCIATION, MECHANICAL DIVISION; MISCELLANEOUS STANDARDS AND RECOMMENDED PRACTICE, 1930

CASTLE NUTS, COTTER PINS, AND LOCATION OF COTTER-PIN HOLES IN PROJECTING BOLT ENDS

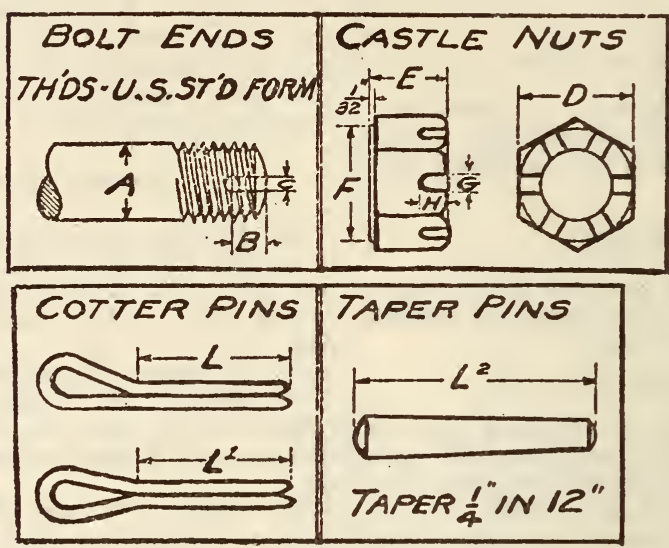

TABLF 1

[All dimensions in inches]

\begin{tabular}{|c|c|c|c|c|c|c|c|c|c|c|c|c|c|c|c|}
\hline \multirow{2}{*}{$\mathbf{A}$} & \multirow{2}{*}{ B } & \multirow{2}{*}{ C } & \multicolumn{6}{|c|}{ Standard } & \multicolumn{2}{|c|}{ Thin } & \multirow{2}{*}{$\begin{array}{l}\text { Nom- } \\
\text { inal } \\
\text { diam- } \\
\text { eter }\end{array}$} & \multirow{2}{*}{ L } & \multirow{2}{*}{$\mathrm{L}^{1}$} & \multirow{2}{*}{$\underset{\text { ber }}{\text { Num- }}$} & \multirow{2}{*}{$\mathrm{L}^{\mathrm{g}}$} \\
\hline & & & $D$ & $\begin{array}{c}\text { No. } \\
\text { threads }\end{array}$ & E & $\mathbf{F}$ & G & $\mathbf{H}$ & $\mathbf{E}$ & $\begin{array}{c}\text { No. } \\
\text { threads }\end{array}$ & & & & & \\
\hline $\begin{array}{l}1 / 2 \\
5 / 8 \\
3 / 4 \\
7 / 8 \\
1 \\
11 / 8 \\
11 / 4 \\
13 / 8 \\
11 / 2 \\
15 / 8 \\
13 / 4 \\
17 / 8 \\
2 \\
21 / 4 \\
21 / 2 \\
23 / 4 \\
3 \\
31 / 4 \\
31 / 2\end{array}$ & $\begin{array}{r}3 / 8 \\
3 / 8 \\
3 / 8 \\
7 / 16 \\
7 / 16 \\
1 / 2 \\
1 / 2 \\
1 / 2 \\
1 / 2 \\
1 / 2 \\
9116 \\
9 / 16 \\
5 / 8 \\
5 / 8 \\
11 / 16 \\
11 / 16 \\
13 / 16 \\
13 / 16 \\
13 / 16\end{array}$ & $\begin{array}{r}3 / 16 \\
3 / 16 \\
316 \\
1 / 4 \\
1 / 4 \\
5 / 16 \\
5 / 16 \\
5 / 16 \\
5 / 16 \\
5 / 16 \\
3 / 8 \\
38 \\
3 / 8 \\
3 / 8 \\
13 \\
1 / 2 \\
1 / 2 \\
12 \\
1 / 2\end{array}$ & $\begin{array}{l}7 / 8 \\
11 / 16 \\
11 / 4 \\
17 / 16 \\
15 / 8 \\
113 / 16 \\
2 \\
23 / 16 \\
25 / 8 \\
29 / 16 \\
23 / 4 \\
215 / 16 \\
31 / 8 \\
31 / 2 \\
37 / 8 \\
414 \\
45 / 8 \\
5 \\
53 / 8\end{array}$ & $\begin{array}{l}13 \\
11 \\
10 \\
9 \\
8 \\
7 \\
7 \\
6 \\
6 \\
51 / 2 \\
5 \\
5 \\
41 / 2 \\
41 / 2 \\
4 \\
4 \\
315 \\
312 \\
314\end{array}$ & $\begin{array}{l}3 / 4 \\
13 / 16 \\
7 / 8 \\
15 / 16 \\
1 \\
11 / 8 \\
11 / 4 \\
1318 \\
11 / 2 \\
158 \\
13 / 4 \\
178 \\
2 \\
21 / 4 \\
21 / 2 \\
23 / 4 \\
3 \\
314 \\
31 / 2\end{array}$ & $\begin{array}{l}13 / 16 \\
1 \\
13 / 16 \\
13 / 8 \\
19116 \\
13 / 4 \\
115 / 16 \\
2138 \\
2516 \\
21 / 2 \\
211 / 16 \\
27 / 8 \\
31116 \\
37 / 16 \\
313 / 16 \\
43 / 16 \\
43 / 16 \\
415 / 16 \\
55 / 16\end{array}$ & $\begin{array}{r}1 / 4 \\
1 / 4 \\
1 / 4 \\
5 / 16 \\
5 / 16 \\
38 \\
38 \\
36 \\
38 \\
38 \\
368 \\
7 / 16 \\
7 / 16 \\
7 / 16 \\
7 / 16 \\
9116 \\
916 \\
568 \\
5,8 \\
56\end{array}$ & $\begin{array}{r}3 / 8 \\
3 / 8 \\
3 / 8 \\
7 / 16 \\
7 / 16 \\
132 \\
1 / 2 \\
1 / 2 \\
1 / 2 \\
132 \\
9116 \\
9116 \\
9116 \\
9116 \\
1116 \\
1116 \\
3 / 4 \\
3 / 4 \\
3 / 4\end{array}$ & $\begin{array}{l}116 \\
116 \\
118 \\
13116 \\
114 \\
15 / 16 \\
15 / 16 \\
13 / 8 \\
13 / 8 \\
17 / 16 \\
115 \\
19116 \\
15 \% 8 \\
111 / 16 \\
13 / 4\end{array}$ & $\begin{array}{l}8 \\
8 \\
8 \\
8 \\
8 \\
8 \\
8 \\
8 \\
8 \\
8 \\
8 \\
8 \\
8 \\
8\end{array}$ & $\begin{array}{r}3 / 16 \\
3 / 16 \\
3 / 16 \\
1 / 4 \\
1 / 1 \\
5 / 16 \\
5116 \\
5 / 16 \\
5 / 16 \\
5 / 16 \\
3 / 8 \\
3 / 8 \\
3 / 8 \\
368 \\
112 \\
1 / 2 \\
1 / 2 \\
112 \\
1 / 2\end{array}$ & $\begin{array}{l}7 / 8 \\
1 \\
11 / 4 \\
11 / 2 \\
11 / 2 \\
13 / 4 \\
13 / 4 \\
2 \\
2 \\
2 \\
21 / 2 \\
21 / 2 \\
3 \\
3 \\
31 / 2 \\
31 / 2 \\
4 \\
5 \\
5\end{array}$ & $\begin{array}{l}11 / 4 \\
11 / 2 \\
13 / 4 \\
2 \\
2 \\
21 / 4 \\
21 / 2 \\
21 / 2 \\
3 \\
3 \\
31 / 2 \\
31 / 2 \\
31 / 2 \\
4 \\
5 \\
5 \\
5 \\
6 \\
6\end{array}$ & $\begin{array}{l}2 \\
2 \\
2 \\
4 \\
4 \\
6 \\
6 \\
6 \\
6 \\
6 \\
6 \\
6 \\
7 \\
7 \\
8 \\
8 \\
9 \\
9 \\
9\end{array}$ & $\begin{array}{l}11 / 4 \\
11 / 4 \\
11 / 2 \\
13 / 4 \\
2 \\
21 / 4 \\
21 / 4 \\
21 / 2 \\
23 / 4 \\
3 \\
314 \\
31 / 4 \\
31 / 2 \\
33 / 4 \\
41 / 2 \\
41 / 2 \\
5 \\
514 \\
5314\end{array}$ \\
\hline
\end{tabular}

AMERICAN RAILWAY ASSOCIATION, PURCHASES AND STORES DIVISION; STAND. ARDIZATION AND SIMPLIFICATION OF STORES STOCK, NO. D V1, 135, 1926

\section{BOLTS AND RIVETS}

(Contains tables showing the standard sizes of boiler lagging, flat and curved; carriage bolts; square-head machine bolts; locomotive-boiler rivets; and structural rivets that should be carried in stock by railway companies.)
AMERICAN RAILWAY ASSOCIATION, PURCHASES AND STORES DIVISION ; STAND. ARDIZATION AND SIMPLIFICATION OF STORES STOCK, PROCEEDINGS, 1928

\section{FLEXIBLE STAY BOLTS}

1. As a means of reducing the number of sizes carried, the committee recommends 1-inch steps in lengths of the most generally used sizes; and, in the longer lengths, the listing of lengths in 2-inch steps. Most roads confine diameters to $1,11 \frac{1}{16}, 11 / 8$, $13 / 16$, and $1 \frac{1}{4}$ inches. 


\section{STOVE BOLTS}

(The stocking of 20 different sizes of flat-head stove bolts, and 24 different sizes of round-head stove bolts, is recommended. The flat-head stove bolts range in size from $3 / 16$ by $1 / 2$ inch to $3 / 8$ by 3 inches. The round-head stove bolts range in size from $1 / 8$ by 1 inch to $3 / 8$ by 3 inches.)

\section{AMERICAN RAILWAY ASSOCIATION, TELE- GRAPH AND TELEPHONE SECTION ; SPEC- IFICATION FOR POLE LINE HARDWARE, 1-A-20, NOVEMBER, 1927}

\section{BOITS}

(For general specifications see 608.0, p. 516.)

GRADE OF STEEL

1. The hardware shall be made from grade $\mathrm{A}$ or grade B steel, as designated, unless a special grade of steel is specifically called for in the drawings.

2. Grade A steel used in the manufacture of pole line hardware, herein specified, shall be a mild steel and shall conform to the physical properties and tests as shown below.

\section{PHYSICAL PROPERTIES AND TESTS}

3. Tension tests. - The material shall conform to the following requirements as to tensile properties:

Tensile strength

Yield point, minimum, 0.5 tensile strength,

but in no case less than.......lbs./in.2 --

Elongation in 8 inches, minimum_per cent-- $\frac{1,400,000}{\text { Tensile strength }}$

Elongation in 2 inches, minimum... do .... Tensile strength

4. The yield point shall be determined by the drop of the beam of the testing machine.

\section{MODIFICATION OF ELONGATION FOR STRUCTURAL STEEL}

5. The percentage of elongation in 8 inches specified above is dependent upon the diameter and thickness and shall be reduced as follows: (a) By one for each 1/8-inch increase in thickness or diameter above three-fourths inch to a minimum of 18 per cent; and (b) two and one-half for each $1 / 16$-inch decrease in thickness or diameter below five-sixteenths inch.

\section{BEND TESTS}

6. The test specimen for plates, shapes, and bars shall bend cold through $180^{\circ}$ without cracking the steel on the outside of the bent portion, as follows:

\begin{tabular}{l|l}
\hline $\begin{array}{c}\text { Thickness of material } \\
\text { (in inches) }\end{array}$ & \multicolumn{1}{c}{ Specimen to bend- } \\
\hline $\begin{array}{l}34 \text { or under } \\
\text { Over } 3 / 4 \text { to and including } \\
11 / 4 \\
\text { Over } 13 / 4\end{array}$ & $\begin{array}{l}\text { Flat on itself. } \\
\text { Around a pin the diameter of which is } \\
\text { equal to the thickness of the specimen. } \\
\text { Around a pin the diameter of which is } \\
\text { equal to twice the thickness of the } \\
\text { specimen. }\end{array}$ \\
\hline
\end{tabular}

7. Test specimens.-Test specimens shall be prepared from the material in its rolled or forged condition, except where it is to be annealed.

8. Test specimens for annealed material shall be prepared from the material as annealed for use, or from a short length of a full section similarly treated.

9. Grade B.-Grade B shall be a good grade of mild steel.

\section{WORKMANSHIP}

10. Workmanship shall be of a good commercial grade.
ANCHOR RODS, BOLTS, AND SPINDLES

11. Bolt and anchor-rod eyes shall be drop-forged. Heads and nuts shall have one face rounded or chamfered, unless otherwise noted. Threads shall conform in size, pitch, and number per inch to the U. S. standard, except for steel pins with wood cobs.

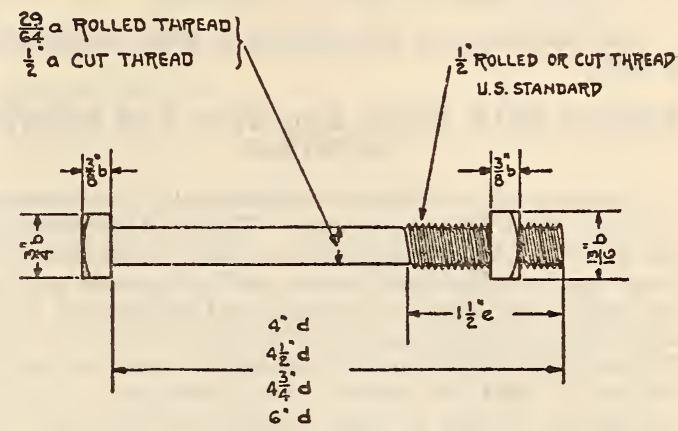

FigURE 334.-One-half inch machine bolt

Material.-Mild steel (grade A).

Finish.-Galvanized.

Assembly.-Before packing nut to be run down to at least two threads beyond end of bolt.

\begin{tabular}{|c|c|c}
\hline & \multicolumn{2}{|c}{$\begin{array}{c}\text { Allowable } \\
\text { variations }\end{array}$} \\
\cline { 1 - 3 } & Over & Under \\
& & \\
\cline { 2 - 3 }$a$ & Inch & Inch \\
$a$ & $1 / 64$ & $1 / 64$ \\
$b$ & $1 / 32$ & $1 / 32$ \\
$d$ & $1 / 8$ & $1 / 8$ \\
$e$ & $1 / 4$ & $1 / 4$ \\
\hline
\end{tabular}

No scale.

12. Threads shall be full, clean, and concentric and formed so that, unless otherwise specified on the drawings, the nut can be run for one-half the length of the thread (in no case less than the thickness of the nut) without undue forcing with the fingers, and the remaining length with a 6-inch wrench without undue forcing.

13. Zinc coating on the threaded portion of nuts may be omitted.

\section{INSPECTION AND REJECTION}

(Requirements covering inspection and rejection conform in general to those specified in A. S. T. M. standard specifications for steel. See 605.0, p. 247. The illustrations for cross-arm bolt, double-arming bolt, and carriage bolt are identical with the illustrations for through bolt, double-arming bolt, and carriage bolt, respectively, in A. E. R. E. A. D102-30. See 611.29, p. 682. Figure 334, shown herewith, illustrates the $1 / 2$-inch machine bolt.)

\section{AMERICAN SOCIETY OF MECHANICAL EN- GINEERS, T SLOTS, THEIR BOLTS, NUTS, TONGUES, AND CUTTERS, B5a-1927}

[Approved by the American Standards Association, March, 1927.]

\section{T SLOTS, THEIR BOLTS, NUTS, TONGUES, AND CUTTERS}

(Contains dimensions of $\mathrm{T}$ slots, $\mathrm{T}$ bolts, $\mathrm{T}$ nuts, $T$ slot cutters, tongues and tongue seats for singlewidth $T$ slots, and reversible tongues and tongue seats for slots for two sizes of $\mathrm{T}$ bolts.) 


\section{AMERICAN SOCIETY OF MECHANICAL EN- GINEERS, WRENCH HEAD BOLTS AND NUTS AND WRENCH OPENINGS, B18b- 1927}

[Approved as American tentative standard by the American Standards Association, February, 1927]

(See also 608.2 , p. $575 ; 608.32$, p. 606 ; and 615.42 , p. 883.)

\section{WRENCH HEAD BOLTS AND NOTS AND WRENCH OPENINGS}

(Introductory notes are substantially the same as those of the National Screw Thread Commission. See 608.0, p. 551. Specifications and dimensions for rough and semifinished square and hexagonal regular boltheads and finished square and hexagonal boltheads are the same as those given below under the National Screw Thread Commission, machine bolts, Tables 67 and 68, except that formulas for computing the several tabular dimensions are appended to the A. S. M. E. tables. These formulas are, however, not of general application.)

\section{AMERICAN SOCIETY OF MECHANICAL EN- GINEERS, ROUND UNSLOTTED-HEAD BOLTS; CARRIAGE, STEP, AND MA. CHINE BOLTS, B18e-1928}

[Approved by the American Standards Association, February, 1928]

\section{ROUND ONSLOTTED-HEAD BOLTS}

1. All screw threads are to be American standard coarse-thread series, free fit, class 2 , with special major diameter tolerances provided for unfinished hot-rolled material. (See Table 8, National Screw Thread Commission, 608.0, p. 534.)

2. The threads on these bolts shall be produced by cutting or rolling. When rolled, the shank diameter will necessarily be smaller than the shank diameter for corresponding cut threads.

3 . The tables of bolt and thread lengths reproduced below are intended as a guide to the users of these bolts. A number of the listed sizes and lengths will not be regularly stocked by the manufacturers but will be available on order of a sufficient quantity.

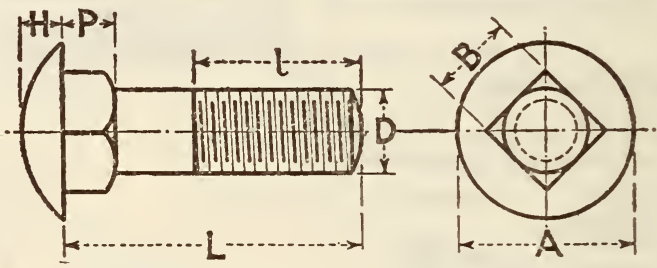

FIgURE 335.-Square neck carriage bolt

TABLE 1.-Square-neck carriage bolt

[All dimensions given in inches]

\begin{tabular}{|c|c|c|c|c|c|c|c|c|c|c|c|}
\hline \multirow{3}{*}{$\underset{\text { size }}{\text { Nominal }}$} & \multicolumn{2}{|c|}{ D } & \multirow{3}{*}{$\begin{array}{c}\text { Threads } \\
\text { per } \\
\text { inch }\end{array}$} & \multicolumn{2}{|l|}{ A } & \multicolumn{2}{|c|}{ H } & \multicolumn{2}{|c|}{$\mathbf{P}$} & \multicolumn{2}{|c|}{ B } \\
\hline & \multicolumn{2}{|c|}{$\begin{array}{l}\text { Major diameter } \\
\text { of thread }\end{array}$} & & \multicolumn{2}{|c|}{ Diameter of head } & \multicolumn{2}{|c|}{ Height of head } & \multicolumn{2}{|c|}{ Depth of square } & \multicolumn{2}{|c|}{ Width of square } \\
\hline & $\begin{array}{l}\text { Maxi- } \\
\text { mum, } \\
\text { basic }\end{array}$ & $\begin{array}{l}\text { Toler- } \\
\text { ance } \\
(-)\end{array}$ & & Basic & $\begin{array}{l}\text { Toler- } \\
\text { ance }(+) \\
\text { or }(-)\end{array}$ & Basic & $\begin{array}{c}\text { Toler- } \\
\text { ance }(+) \\
\text { or }(-)\end{array}$ & Basic & $\begin{array}{l}\text { Toler- } \\
\text { ance } \\
(t)\end{array}$ & Basic & $\begin{array}{l}\text { Toler- } \\
\text { ance } \\
(-)\end{array}$ \\
\hline $\begin{array}{c}\text { No. } 10 \\
1 / 4 \\
5 / 16 \\
3 / 8 \\
7 / 16 \\
1 / 2 \\
9 / 10 \\
5 / 8 \\
3 / 4\end{array}$ & $\begin{array}{l}0.190 \\
.250 \\
.313 \\
.375 \\
.438 \\
.500 \\
.563 \\
.625 \\
.750\end{array}$ & $\begin{array}{l}0.009 \\
.010 \\
.013 \\
.015 \\
.015 \\
.015 \\
.016 \\
.017 \\
.020\end{array}$ & $\begin{array}{l}24 \\
20 \\
18 \\
16 \\
14 \\
13 \\
12 \\
11 \\
10\end{array}$ & $\begin{array}{r}0.438(7 / 16) \\
.563(916) \\
.688(1116) \\
.813(1316) \\
.938(1516) \\
1.063(11 / 16) \\
1.188(1316) \\
1.313(15116) \\
1.563(196)\end{array}$ & $\begin{array}{l}0.010 \\
.010 \\
.010 \\
.010 \\
.010 \\
.010 \\
.015 \\
.015 \\
.015\end{array}$ & $\begin{array}{l}0.094 \\
.125 \\
.156 \\
.188 \\
.219 \\
.250 \\
.281 \\
.313 \\
.375\end{array}$ & $\begin{array}{l}0.010 \\
.010 \\
.010 \\
.010 \\
.010 \\
.010 \\
.015 \\
.015 \\
.015\end{array}$ & $\begin{array}{l}0.188 \\
.219 \\
.250 \\
.281 \\
.313 \\
.344 \\
.375 \\
.406 \\
.469\end{array}$ & $\begin{array}{l}0.031 \\
.031 \\
.031 \\
.031 \\
.031 \\
.031 \\
.031 \\
.031 \\
.031\end{array}$ & $\begin{array}{r}0.190 \\
.250 \\
.313 \\
.375 \\
.438 \\
.500 \\
.563 \\
.625 \\
.750\end{array}$ & $\begin{array}{l}0.009 \\
.010 \\
.013 \\
.015 \\
.015 \\
.015 \\
.016 \\
.017 \\
.020\end{array}$ \\
\hline
\end{tabular}


TABLE 1.-Square-neck carriage bolt-Continued

\begin{tabular}{|c|c|c|c|c|c|c|c|c|c|c|}
\hline$\underset{\text { size }}{\text { Nominal }}$ & No. 10 & 34 & $5 / 16$ & $3 / 8$ & $7 / 16$ & $1 / 2$ & $\% 16$ & $5 / 8$ & $3 / 4$ & \multirow{3}{*}{$\begin{array}{l}\text { Length } \\
\text { tolerance } \\
(+) \text { or }(-)\end{array}$} \\
\hline $\begin{array}{l}\text { Threads } \\
\text { per inch }\end{array}$ & 24 & 20 & 18 & 16 & 14 & 13 & 12 & 11 & 10 & \\
\hline $\begin{array}{l}\text { Length of } \\
\text { bolt (L) }\end{array}$ & \multicolumn{9}{|c|}{ Minimum length of thread $(l)$ including point } & \\
\hline $\begin{array}{l}1 / 2 \\
3 / 4 \\
1^{31 / 4} \\
11 / 4 \\
11 / 2\end{array}$ & $\begin{array}{r}\mathrm{T} \text { to } \cdot \mathrm{S} \\
3 / 8 \\
1 / 2 \\
1 / 2 \\
1 / 2\end{array}$ & $\begin{array}{r}\mathrm{T} \text { to } \mathrm{S} \\
3 / 8 \\
1 / 2 \\
5 / 8 \\
3 / 4\end{array}$ & $\begin{array}{l}35 \\
12 \\
5 / 8 \\
3 / 4\end{array}$ & $\begin{array}{l}3 / 8 \\
5 / 8 \\
5 / 8 \\
3 / 4\end{array}$ & $\begin{array}{l}5 / 8 \\
3 / 4 \\
7 / 8\end{array}$ & $\begin{array}{r}\mathrm{T} \text { to } \mathrm{S} \\
3 / 4 \\
7 / 8\end{array}$ & $\begin{array}{l}\mathrm{T} \text { to } \\
\mathrm{T} \text { to } \mathrm{S}\end{array}$ & $\begin{array}{l}\mathrm{T} \text { to } \\
\mathrm{T} \text { to } \mathrm{S}\end{array}$ & $\mathrm{T}$ to $\mathrm{S}$ & $\begin{array}{l}1 / 64 \\
1 / 64 \\
1664 \\
1 / 64 \\
1 / 64\end{array}$ \\
\hline $\begin{array}{l}13 / 4 \\
2 \\
21 / 4 \\
21 / 2 \\
23 / 4\end{array}$ & $\begin{array}{l}5 / 8 \\
58 \\
5 / 8 \\
5 / 8 \\
3 / 4\end{array}$ & $\begin{array}{l}3 / 4 \\
3 / 4 \\
3 / 4 \\
3 / 4 \\
3 / 2\end{array}$ & $\begin{array}{l}3 / 4 \\
3 / 4 \\
3 / 4 \\
3 / 4 \\
7 / 8\end{array}$ & $\begin{array}{l}3 / 4 \\
3 / 4 \\
3 / 4 \\
7 / 8 \\
7 / 8\end{array}$ & $\begin{array}{l}7 / 8 \\
7 / 8 \\
7 / 8 \\
7 / 8 \\
7 / 8\end{array}$ & $\begin{array}{l}1 \\
1 \\
1 \\
1 \\
1\end{array}$ & $11 / 4$ & $11 / 4$ & 1134 & $\begin{array}{l}1 / 64 \\
164 \\
164 \\
164 \\
164\end{array}$ \\
\hline $\begin{array}{l}3 \\
31 / 4 \\
31 / 2 \\
33 / 4 \\
4\end{array}$ & $\begin{array}{l}3 / 4 \\
3 / 4 \\
3 / 4\end{array}$ & $\begin{array}{l}3 / 4 \\
3 / 4 \\
3 / 4 \\
3 / 4 \\
7 / 8\end{array}$ & $\begin{array}{l}7 / 8 \\
7 / 8 \\
7 / 8 \\
7 / 8 \\
7 / 8\end{array}$ & $\begin{array}{l}7 / 8 \\
78 \\
7 / 8 \\
7 / 8 \\
7 / 8\end{array}$ & $\begin{array}{l}1^{7 / 8} \\
1 \\
1 \\
1\end{array}$ & $\begin{array}{l}118 \\
118 \\
118 \\
118 \\
118\end{array}$ & $13 / 8$ & $\begin{array}{l}13 / 8 \\
-13 / 8 \\
-13 / 2\end{array}$ & $\begin{array}{l}1 \frac{1 / 2}{2} \\
-1 \overline{1} / 2 \\
11 \frac{1}{2}\end{array}$ & $\begin{array}{l}1 / 64 \\
1 / 32 \\
1 / 32 \\
1 / 32 \\
1 / 32\end{array}$ \\
\hline $\begin{array}{l}41 / 2 \\
5 \\
51 / 2 \\
6\end{array}$ & $\begin{array}{l}-\cdot- \\
-- \\
--\cdot \\
-\cdot\end{array}$ & $7 / 8$ & $\begin{array}{l}1 \\
1 \\
11 / 8 \\
118\end{array}$ & $\begin{array}{l}1 \\
1 \\
11 / 8 \\
118\end{array}$ & $\begin{array}{l}11 / 8 \\
118 \\
11 / 8 \\
11 / 8\end{array}$ & $\begin{array}{l}11 / 4 \\
11 / 4 \\
114 \\
114\end{array}$ & $\begin{array}{l}11 / 2 \\
112 \\
11 / 2 \\
11 / 2\end{array}$ & $\begin{array}{l}11 / 2 \\
112 \\
11 / 2 \\
11 / 2\end{array}$ & $\begin{array}{l}11 / 2 \\
11 / 2 \\
11 / 2 \\
11 / 2\end{array}$ & $\begin{array}{l}1 / 32 \\
1 / 32 \\
1 / 32 \\
1 / 32\end{array}$ \\
\hline $\begin{array}{l}61 / 2 \\
7 \\
71 / 2 \\
8\end{array}$ & $-\cdot-$ & & 118 & $\begin{array}{l}11 / 8 \\
118 \\
118 \\
114\end{array}$ & $\begin{array}{l}11 / 8 \\
11 / 4 \\
11 / 4 \\
114\end{array}$ & $\begin{array}{l}11 / 4 \\
11 / 4 \\
11 / 4 \\
13 / 8\end{array}$ & $\begin{array}{l}15 / 8 \\
15 / 8 \\
15 / 8 \\
15 / 8\end{array}$ & $\begin{array}{l}15 / 8 \\
15 \% 8 \\
158 \\
15 \%\end{array}$ & $\begin{array}{l}13 / 4 \\
13 / 4 \\
13 / 4 \\
17 / 8\end{array}$ & $\begin{array}{l}1116 \\
116 \\
1 / 16 \\
1 / 16\end{array}$ \\
\hline $\begin{array}{c}81 / 2 \\
9 \\
91 / 2 \\
10\end{array}$ & & & & & $\begin{array}{l}11 / 4 \\
11 / 4 \\
11 / 4 \\
11 / 4\end{array}$ & $\begin{array}{l}13 / 8 \\
13 / 8 \\
138 \\
13 / 8\end{array}$ & $\begin{array}{l}13 / 4 \\
13 / 4 \\
13 / 4 \\
13 / 4\end{array}$ & $\begin{array}{l}13 / 4 \\
13 / 4 \\
13 / 4 \\
13 / 4\end{array}$ & $\begin{array}{l}17 / 8 \\
2 \\
2 \\
2\end{array}$ & $\begin{array}{l}1 / 16 \\
1 / 16 \\
1 / 16 \\
1 / 16\end{array}$ \\
\hline
\end{tabular}

$\mathrm{T}$ to $\mathrm{S}$ means threaded to square as near as is practicable. Radius of fllet between body and head 1/32 inch on sizes No. 10 to $1 / 2$ inch, inclusive, and $1 / 16$ inch on sizes $\% / 16,5 / 8$, and $3 / 4$ inch. 


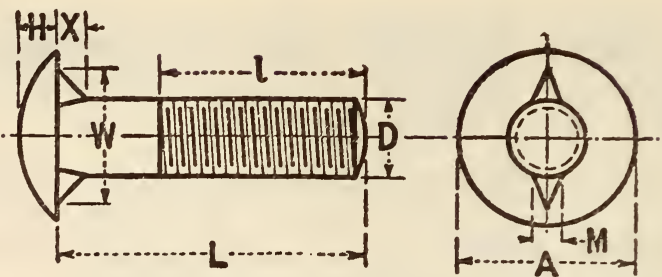

FigURE 336.-Fin neck carriage bolt

TABLE 2.-Fin-neck carriage bolt

[All dimensions in inches]

\begin{tabular}{|c|c|c|c|c|c|c|c|c|c|c|c|c|c|}
\hline \multirow{3}{*}{$\begin{array}{l}\text { Nom- } \\
\text { inal } \\
\text { size }\end{array}$} & \multicolumn{2}{|c|}{$\mathrm{D}$} & \multirow{3}{*}{$\begin{array}{c}\text { Threads } \\
\text { per } \\
\text { inch }\end{array}$} & \multicolumn{2}{|c|}{ A } & \multicolumn{2}{|r|}{$\mathbf{H}$} & \multicolumn{2}{|c|}{$\mathrm{X}$} & \multicolumn{2}{|r|}{ W } & \multicolumn{2}{|r|}{$\mathbf{M}$} \\
\hline & \multicolumn{2}{|c|}{$\begin{array}{l}\text { Major diam- } \\
\text { eter of } \\
\text { thread }\end{array}$} & & \multicolumn{2}{|c|}{ Diameter of head } & \multicolumn{2}{|c|}{ Height of head } & \multicolumn{2}{|c|}{$\begin{array}{l}\text { Depth of } \\
\text { fins }\end{array}$} & \multicolumn{2}{|c|}{$\begin{array}{l}\text { Distance across } \\
\text { fins }\end{array}$} & \multicolumn{2}{|c|}{$\begin{array}{l}\text { Maximum } \\
\text { thickness of } \\
\text { fins }\end{array}$} \\
\hline & $\mid \begin{array}{l}\text { Maxi- } \\
\text { mum } \\
\text { basic }\end{array}$ & $\begin{array}{c}\text { Toler- } \\
\text { ance } \\
(-)\end{array}$ & & Basic & $\begin{array}{l}\text { Toler- } \\
\text { ance }(+) \\
\text { or }(-)\end{array}$ & Basic & $\begin{array}{l}\text { Toler- } \\
\text { ance (t) } \\
\text { or (-) }\end{array}$ & Basic & $\begin{array}{c}\text { Toler- } \\
\text { ance } \\
(+)\end{array}$ & Basic & $\begin{array}{l}\text { Toler- } \\
\text { ance (+) } \\
\text { or }(-)\end{array}$ & Basic & $\begin{array}{l}\text { Toler- } \\
\text { ance (+) } \\
\text { or (-) }\end{array}$ \\
\hline $\begin{array}{c}\text { No. } 10 \\
1 / 4 \\
5 / 16 \\
3 / 8 \\
7 / 16 \\
1 / 2\end{array}$ & $\begin{array}{r}0.190 \\
.250 \\
.313 \\
.375 \\
.438 \\
.500\end{array}$ & $\begin{array}{r}0.009 \\
.010 \\
.013 \\
.015 \\
.015 \\
.015\end{array}$ & $\begin{array}{l}24 \\
20 \\
18 \\
16 \\
14 \\
13\end{array}$ & $\begin{array}{r}0.469(15 / 32) \\
.594(19 / 32) \\
.719(23 / 32) \\
.844(27 / 32) \\
.969(31 / 32) \\
1.094(13 / 32)\end{array}$ & $\begin{array}{l}0.010 \\
.010 \\
.010 \\
.010 \\
.010 \\
.010\end{array}$ & $\begin{array}{r}0.078 \\
.109 \\
.141 \\
.172 \\
.203 \\
.234\end{array}$ & $\begin{array}{l}0.010 \\
.010 \\
.010 \\
.010 \\
.010 \\
.010\end{array}$ & $\begin{array}{l}0.078 \\
.094 \\
.125 \\
.141 \\
.172 \\
.188\end{array}$ & $\begin{array}{r}0.010 \\
.010 \\
.010 \\
.010 \\
.010 \\
.010\end{array}$ & $\begin{array}{r}0.375 \\
.438 \\
.531 \\
.625 \\
.719 \\
.813\end{array}$ & $\begin{array}{l}0.010 \\
.010 \\
.010 \\
.010 \\
.010 \\
.010\end{array}$ & $\begin{array}{l}0.078 \\
.094 \\
.125 \\
.141 \\
.172 \\
.188\end{array}$ & $\begin{array}{r}0.010 \\
.010 \\
.010 \\
.010 \\
.010 \\
.010\end{array}$ \\
\hline
\end{tabular}

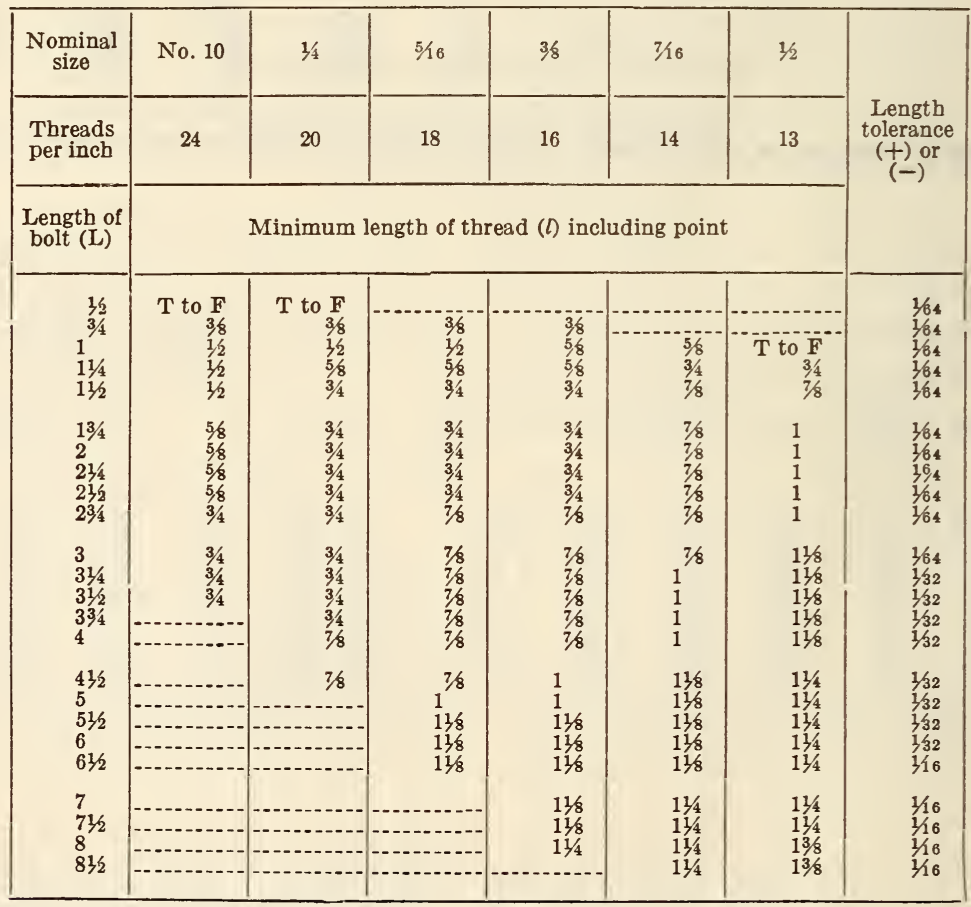

$\mathrm{T}$ to $\mathrm{F}$ means threaded to fin as near as is practicable. Radius of fillet between body and bead $1 / 32$ inch. 


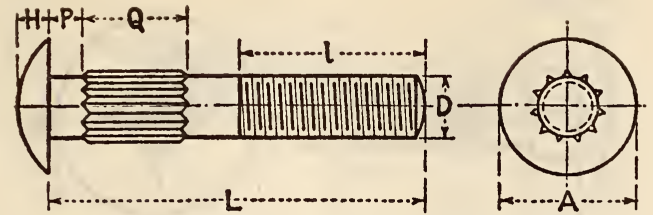

FIGURE 337.-Ribbed carriage bolt

TABLE 3.-Ribbed carriage bolt

[All dimensions in inches]

\begin{tabular}{|c|c|c|c|c|c|c|c|c|c|c|c|c|c|}
\hline \multirow{3}{*}{$\begin{array}{l}\text { Nomi- } \\
\text { nal } \\
\text { size }\end{array}$} & \multicolumn{2}{|c|}{ D } & \multirow{3}{*}{$\begin{array}{l}\text { Threads } \\
\text { per inch }\end{array}$} & \multicolumn{2}{|c|}{ A } & \multicolumn{2}{|r|}{ H } & \multicolumn{2}{|r|}{$\mathbf{P}$} & \multicolumn{2}{|c|}{$Q$} & \multirow{3}{*}{$\begin{array}{l}\text { Max- } \\
\text { imum } \\
\text { num- } \\
\text { ber } \\
\text { of } \\
\text { ribs }\end{array}$} & \multirow{3}{*}{$\begin{array}{c}\text { Included } \\
\text { angle of } \\
\text { rib }\end{array}$} \\
\hline & \multicolumn{2}{|c|}{$\begin{array}{l}\text { Major diam. } \\
\text { eter of thread }\end{array}$} & & \multicolumn{2}{|c|}{ Diameter of head } & \multicolumn{2}{|c|}{ Height of head } & \multicolumn{2}{|c|}{$\begin{array}{l}\text { Distance of rib } \\
\text { below head }\end{array}$} & \multicolumn{2}{|c|}{ Length of rib } & & \\
\hline & $\begin{array}{l}\text { Maxi- } \\
\text { mum } \\
\text { basic }\end{array}$ & $\begin{array}{c}\text { Toler- } \\
\text { ance } \\
(-)\end{array}$ & & Basic & $\begin{array}{c}\text { Tolerance } \\
(+) \text { or } \\
(-)\end{array}$ & Basic & $\begin{array}{c}\text { Tolerance } \\
(+) \text { or } \\
(-)\end{array}$ & Basic & $\begin{array}{c}\text { Tolerance } \\
(+) \text { or } \\
(-)\end{array}$ & $\begin{array}{l}\text { When } \\
\mathrm{L} \text { is } \\
11 / 8 \\
\text { inch } \\
\text { and } \\
\text { under }\end{array}$ & $\begin{array}{l}\text { When } \\
\mathrm{L} \text { is } \\
11 / 4 \\
\text { inch } \\
\text { and } \\
\text { over }\end{array}$ & & \\
\hline $\begin{array}{c}\text { No.10 } \\
1 / 4 \\
5 / 16 \\
3 / 8 \\
7 / 16 \\
1 / 2\end{array}$ & $\begin{array}{r}0.190 \\
.250 \\
.313 \\
.375 \\
.438 \\
.500\end{array}$ & $\begin{array}{l}0.009 \\
.010 \\
.013 \\
.015 \\
.015 \\
.015\end{array}$ & $\begin{array}{l}24 \\
20 \\
18 \\
16 \\
14 \\
13\end{array}$ & $\begin{array}{r}0.438(7 / 16) \\
.563(9 / 16) \\
.688(11 / 16) \\
.813(13 / 16) \\
.938(15 / 16) \\
1.063(1116)\end{array}$ & $\begin{array}{l}0.010 \\
.010 \\
.010 \\
.010 \\
.010 \\
.010\end{array}$ & $\begin{array}{r}0.094 \\
.125 \\
.156 \\
.188 \\
.219 \\
.250\end{array}$ & $\begin{array}{r}0.010 \\
.010 \\
.010 \\
.010 \\
.010 \\
.010\end{array}$ & $\begin{array}{r}0.094 \\
.094 \\
.094 \\
.094 \\
.094 \\
.094\end{array}$ & $\begin{array}{r}0.031 \\
.031 \\
.031 \\
.031 \\
.031 \\
.031\end{array}$ & $\begin{array}{r}0.375 \\
.375 \\
.375 \\
.375 \\
.375 \\
.375\end{array}$ & $\begin{array}{r}0.500 \\
.500 \\
.500 \\
.500 \\
.500 \\
.500\end{array}$ & $\begin{array}{r}9 \\
10 \\
12 \\
12 \\
14 \\
16\end{array}$ & $\begin{array}{c}\text { Approxi- } \\
\text { mately } \\
90^{\circ}\end{array}$ \\
\hline
\end{tabular}

\begin{tabular}{|c|c|c|c|c|c|c|c|}
\hline$\underset{\text { size }}{\text { Nominal }}$ & No. 10 & $1 / 4$ & $5 / 16$ & $3 / 8$ & $7 / 16$ & 12 & \\
\hline $\begin{array}{l}\text { Threads } \\
\text { per inch }\end{array}$ & 24 & 20 & 18 & 16 & 14 & 13 & tolerance \\
\hline $\begin{array}{l}\text { Length of } \\
\text { bolt (L) }\end{array}$ & \multicolumn{6}{|c|}{ Minimum length of thread $(l)$ including point } & $(+)$ or $(-)$ \\
\hline $\begin{array}{l}1 \\
11 / 4 \\
11 / 2 \\
13 / 4 \\
2\end{array}$ & $\begin{array}{c}\mathrm{T} \text { to } \mathrm{R} \\
1 / 2 \\
1 / 2 \\
5 / 8 \\
5 / 8\end{array}$ & $\begin{array}{c}\text { T to } R \\
5 / 8 \\
3 / 4 \\
3 / 4 \\
3 / 4\end{array}$ & $\begin{array}{c}T \text { to } R \\
5 / 8 \\
3 / 4 \\
3 / 4 \\
3 / 4\end{array}$ & $\begin{array}{c}\mathrm{T} \text { to } \mathrm{R} \\
\mathrm{T} \text { to } \mathrm{R} \\
3 / 4 \\
3 / 4 \\
3 / 4\end{array}$ & $\begin{array}{c}\mathrm{T} \text { to } \mathrm{R} \\
\mathrm{T} \text { to } \mathrm{R} \\
\mathrm{T} \text { to } \mathrm{R} \\
7 / 8 \\
7 / 8\end{array}$ & $\begin{array}{c}\mathrm{T} \text { to } \mathrm{R} \\
\mathrm{T} \text { to } \mathrm{R} \\
\mathrm{T} \text { to } \mathrm{R} \\
1 \\
1\end{array}$ & $\begin{array}{l}1 / 64 \\
1 / 64 \\
1 / 64 \\
164 \\
1 / 64\end{array}$ \\
\hline $\begin{array}{l}21 / 4 \\
21 / 2 \\
23 / 4 \\
3 \\
31 / 4\end{array}$ & $\begin{array}{l}5 / 8 \\
5 / 8 \\
3 / 4 \\
3 / 4 \\
3 / 4\end{array}$ & $\begin{array}{l}3 / 4 \\
3 / 4 \\
3 / 4 \\
3 / 4 \\
3 / 4\end{array}$ & $\begin{array}{l}3 / 4 \\
3 / 4 \\
7 / 8 \\
7 / 8 \\
7 / 8\end{array}$ & $\begin{array}{l}3 / 4 \\
3 / 4 \\
7 / 8 \\
7 / 8 \\
7 / 8\end{array}$ & $\begin{array}{l}7 / 8 \\
7 / 8 \\
7 / 8 \\
7 / 8 \\
1^{7 / 8}\end{array}$ & $\begin{array}{l}1 \\
1 \\
1 \\
11 / 8 \\
11 / 8\end{array}$ & $\begin{array}{l}164 \\
164 \\
1 / 64 \\
164 \\
1 / 32\end{array}$ \\
\hline $\begin{array}{l}31 / 2 \\
33 / 4 \\
4 \\
41 / 2 \\
5\end{array}$ & $3 / 4$ & $\begin{array}{l}3 / 4 \\
3 / 4 \\
7 / 8 \\
7 / 8\end{array}$ & $\begin{array}{l}7 / 8 \\
7 / 8 \\
7 / 8 \\
1^{7}\end{array}$ & $\begin{array}{l}7 / 8 \\
7 / 8 \\
7 / 8 \\
1\end{array}$ & $\begin{array}{l}1 \\
1 \\
1 \\
11 / 8 \\
11 / 8\end{array}$ & $\begin{array}{l}11 / 8 \\
11 / 8 \\
118 \\
11 / 4 \\
11 / 4\end{array}$ & $\begin{array}{l}1 / 32 \\
1 / 32 \\
1 / 32 \\
1 / 32 \\
1 / 32\end{array}$ \\
\hline $\begin{array}{l}51 / 2 \\
6 \\
61 / 2 \\
7 \\
71 / 2\end{array}$ & & & $\begin{array}{l}11 / 8 \\
11 / 8 \\
11 / 8\end{array}$ & $\begin{array}{l}11 / 8 \\
11 / 8 \\
11 / 8 \\
11 / 8 \\
11 / 8\end{array}$ & $\begin{array}{l}11 / 8 \\
11 / 8 \\
11 / 8 \\
11 / 4 \\
11 / 4\end{array}$ & $\begin{array}{l}11 / 4 \\
11 / 4 \\
11 / 4 \\
11 / 4 \\
11 / 4\end{array}$ & $\begin{array}{l}1 / 32 \\
1 / 32 \\
1 / 16 \\
1 / 16 \\
1 / 16\end{array}$ \\
\hline $\begin{array}{l}8 \\
81 / 6 \\
9 \\
91 / 2 \\
10\end{array}$ & & & & $11 / 4$ & $\begin{array}{l}11 / 4 \\
11 / 4 \\
11 / 4 \\
11 / 4 \\
11 / 4\end{array}$ & $\begin{array}{l}13 / 8 \\
13 / 8 \\
13 / 8 \\
13 / 8 \\
13 / 8\end{array}$ & $\begin{array}{l}1 / 16 \\
1 / 16 \\
1 / 16 \\
1 / 16 \\
1 / 16\end{array}$ \\
\hline
\end{tabular}

$\mathrm{T}$ to $\mathrm{R}$ means threaded to rib as near as is practicable. Radius of fillet between body and head $1 / 32$ inch. 


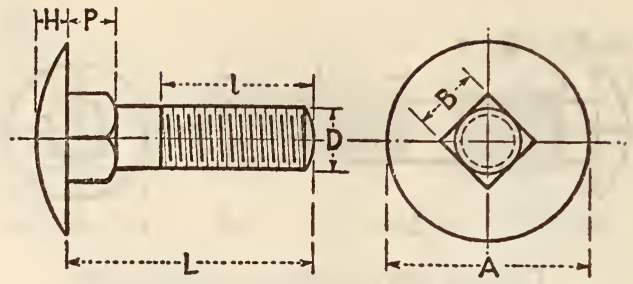

FIGURE 338. - Step bolt

TABLE 4.-Step bolt

[All dimensions in inches]

\begin{tabular}{|c|c|c|c|c|c|c|c|c|c|c|c|}
\hline \multirow{3}{*}{$\begin{array}{l}\text { Nominal } \\
\text { size }\end{array}$} & \multicolumn{2}{|c|}{$\mathbf{D}$} & \multirow{3}{*}{$\begin{array}{l}\text { Threads } \\
\text { per inch }\end{array}$} & \multicolumn{2}{|c|}{$A$. } & \multicolumn{2}{|r|}{$\mathrm{H}$} & \multicolumn{2}{|c|}{$\mathrm{P}$} & \multicolumn{2}{|c|}{ B } \\
\hline & \multicolumn{2}{|c|}{$\begin{array}{l}\text { Major diameter } \\
\text { of thread }\end{array}$} & & \multicolumn{2}{|c|}{ Diameter of head } & \multicolumn{2}{|c|}{ Height of head } & \multicolumn{2}{|c|}{ Depth of square } & \multicolumn{2}{|c|}{ Width of square } \\
\hline & $\begin{array}{l}\text { Maxi- } \\
\text { mum } \\
\text { basic }\end{array}$ & $\begin{array}{l}\text { Toler- } \\
\text { ance } \\
(-)\end{array}$ & & Basic & $\begin{array}{c}\text { Toler- } \\
\text { ance } \\
(+) \text { or }(-)\end{array}$ & Basic & $\begin{array}{c}\text { Toler- } \\
\text { ance } \\
(+) \text { or }(-)\end{array}$ & Basic & $\begin{array}{l}\text { Toler- } \\
\text { ance } \\
(+)\end{array}$ & Basic & $\begin{array}{l}\text { Toler- } \\
\text { ance } \\
(-)\end{array}$ \\
\hline $\begin{array}{c}\text { No. } 10 \\
1 / 4 \\
5 / 16 \\
3 / 8 \\
7 / 16 \\
1 / 2\end{array}$ & $\begin{array}{r}0.190 \\
.250 \\
.313 \\
.375 \\
.438 \\
.500\end{array}$ & $\begin{array}{r}0.009 \\
.010 \\
.013 \\
.015 \\
.015 \\
.015\end{array}$ & $\begin{array}{l}24 \\
20 \\
18 \\
16 \\
14 \\
13\end{array}$ & $\begin{array}{l}0.625(5 / 8) \\
.813(13 / 16) \\
1.000(1) \\
1.188(13 / 16) \\
1.375(13 / 8) \\
1.563(1 \% 16)\end{array}$ & $\begin{array}{l}0.010 \\
.010 \\
.010 \\
.100 \\
.010 \\
.010\end{array}$ & $\begin{array}{r}0.094 \\
.125 \\
.156 \\
.188 \\
.219 \\
.250\end{array}$ & $\begin{array}{r}0.010 \\
.010 \\
.010 \\
.010 \\
.010 \\
.010\end{array}$ & $\begin{array}{r}0.188 \\
.219 \\
.250 \\
.281 \\
.313 \\
.344\end{array}$ & $\begin{array}{r}0.031 \\
.031 \\
.031 \\
.031 \\
.031 \\
.031\end{array}$ & $\begin{array}{l}0.190 \\
.250 \\
.313 \\
.375 \\
.438 \\
.500\end{array}$ & $\begin{array}{r}0.009 \\
.010 \\
.013 \\
.015 \\
.015 \\
.015\end{array}$ \\
\hline
\end{tabular}

\begin{tabular}{|c|c|c|c|c|c|c|c|}
\hline$\underset{\text { size }}{\text { Nominal }}$ & No. 10 & $1 / 4$ & $5 / 16$ & $3 / 8$ & $7 / 16$ & $1 / 2$ & \\
\hline $\begin{array}{l}\text { Threads } \\
\text { per inch }\end{array}$ & 24 & 20 & 18 & 16 & 14 & 13 & tolerance \\
\hline $\begin{array}{l}\text { Iength of } \\
\text { bolt (L) }\end{array}$ & \multicolumn{6}{|c|}{ Minimum length of thread $(l)$ including point } & $(+)$ or $(-)$ \\
\hline $\begin{array}{l}1 / 2 \\
3 / 4 \\
1 \\
11 / 4 \\
11 / 2\end{array}$ & $\begin{array}{c}\mathrm{T} \text { to } \mathrm{S} \\
\mathrm{T} \text { to } \mathrm{S} \\
1 / 2 \\
1 / 2 \\
1 / 2 \\
\end{array}$ & $\begin{array}{c}\mathrm{T} \text { to } \mathrm{S} \\
\mathrm{T} \text { to } \mathrm{S} \\
1 / 2 \\
5 / 8 \\
3 / 4\end{array}$ & $\begin{array}{c}\mathrm{T} \text { to } \mathrm{S} \\
\mathrm{T} \text { to } \mathrm{S} \\
1 / 2 \\
5 / 8 \\
3 / 4\end{array}$ & $\begin{array}{c}\mathrm{T} \text { to } \mathrm{S} \\
5 / 8 \\
5 / 8 \\
3 / 4\end{array}$ & $\begin{array}{c}\text { T to } \mathrm{S} \\
5 / 8 \\
3 / 4 \\
7 / 8\end{array}$ & $\begin{array}{c}\mathrm{T} \text { to } \mathrm{S} \\
3 / 4 \\
7 / 8\end{array}$ & $\begin{array}{l}1 / 64 \\
1 / 64 \\
1 / 64 \\
164 \\
1 / 64\end{array}$ \\
\hline $\begin{array}{l}13 / 4 \\
2 \\
21 / 4 \\
21 / 2 \\
23 / 4\end{array}$ & $\begin{array}{l}5 / 8 \\
5 / 8 \\
5 / 8 \\
5 / 8 \\
3 / 4\end{array}$ & $\begin{array}{l}3 / 4 \\
3 / 4 \\
3 / 4 \\
3 / 4 \\
3 / 4\end{array}$ & $\begin{array}{l}3 / 4 \\
3 / 4 \\
3 / 4 \\
3 / 4 \\
7 / 8\end{array}$ & $\begin{array}{l}3 / 4 \\
3 / 4 \\
3 / 4 \\
3 / 4 \\
7 / 8\end{array}$ & $\begin{array}{l}7 / 8 \\
7 / 8 \\
7 / 8 \\
7 / 8 \\
7 / 8\end{array}$ & $\begin{array}{l}1 \\
1 \\
1 \\
1 \\
1\end{array}$ & $\begin{array}{l}1 / 64 \\
164 \\
164 \\
164 \\
1 / 64\end{array}$ \\
\hline $\begin{array}{l}3 \\
31 / 4 \\
31 / 2 \\
33 / 4 \\
4\end{array}$ & $\begin{array}{l}3 / 4 \\
3 / 4 \\
3 / 4\end{array}$ & $\begin{array}{l}3 / 4 \\
3 / 4 \\
3 / 4 \\
3 / 4 \\
7 / 8\end{array}$ & $\begin{array}{l}7 / 8 \\
7 / 8 \\
7 / 8 \\
7 / 8 \\
7 / 8\end{array}$ & $\begin{array}{l}7 / 8 \\
7 / 8 \\
7 / 8 \\
7 / 8 \\
7 / 8\end{array}$ & $\begin{array}{l}1^{7 / 8} \\
1 \\
1\end{array}$ & $\begin{array}{l}11 / 8 \\
11 / 8 \\
11 / 8 \\
11 / 8 \\
11 / 8\end{array}$ & $\begin{array}{l}1 / 64 \\
1 / 32 \\
1 / 32 \\
1 / 32 \\
1 / 32\end{array}$ \\
\hline $\begin{array}{l}41 / 2 \\
5 \\
51 / 2 \\
6 \\
61 / 2\end{array}$ & & $7 / 8$ & $\begin{array}{l}1 \\
1 \\
11 / 8 \\
11 / 8 \\
11 / 8\end{array}$ & $\begin{array}{l}1 \\
1 \\
11 / 8 \\
11 / 8 \\
11 / 8\end{array}$ & $\begin{array}{l}11 / 8 \\
11 / 8 \\
11 / 8 \\
1118 \\
11 / 8\end{array}$ & $\begin{array}{l}11 / 4 \\
11 / 4 \\
11 / 4 \\
11 / 4 \\
11 / 4\end{array}$ & $\begin{array}{l}1 / 32 \\
1 / 32 \\
1 / 32 \\
1 / 32 \\
1 / 16\end{array}$ \\
\hline $\begin{array}{l}7 \\
71 / 2 \\
8 \\
81 / 2\end{array}$ & - & & 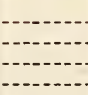 & $\begin{array}{l}11 / 8 \\
11 / 8 \\
11 / 4\end{array}$ & $\begin{array}{l}11 / 4 \\
11 / 4 \\
11 / 4 \\
11 / 4\end{array}$ & $\begin{array}{l}11 / 4 \\
11 / 4 \\
13 / 8 \\
13 / 8\end{array}$ & $\begin{array}{l}1 / 16 \\
1 / 16 \\
1 / 16 \\
1 / 16\end{array}$ \\
\hline
\end{tabular}

T to $\mathrm{S}$ means threaded to square as near as is practicable. Radius of fillet between body and head 132 inch. 


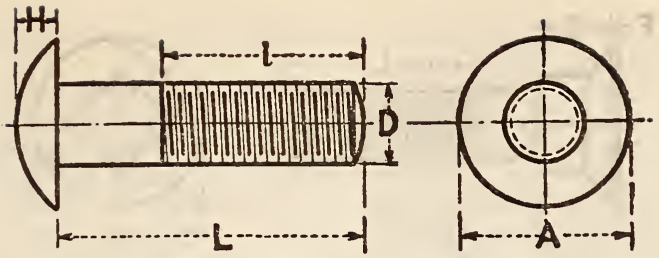

FIGURE 339.-Button head machine bolt

TABLE 5.-Button-head machine bolt

[All dimensions in inches]

\begin{tabular}{|c|c|c|c|c|c|c|c|}
\hline \multirow{3}{*}{$\underset{\text { size }}{\text { Nominal }}$} & \multicolumn{2}{|c|}{$\mathrm{D}$} & \multirow{3}{*}{$\begin{array}{l}\text { Threads } \\
\text { per inch }\end{array}$} & \multicolumn{2}{|c|}{ A } & \multicolumn{2}{|c|}{$\mathbf{H}$} \\
\hline & \multicolumn{2}{|c|}{$\begin{array}{c}\text { Major diameter of } \\
\text { thread }\end{array}$} & & \multicolumn{2}{|c|}{ Diameter of head } & \multicolumn{2}{|c|}{ Height of head } \\
\hline & $\begin{array}{l}\text { Maxi- } \\
\text { mum } \\
\text { basic }\end{array}$ & $\begin{array}{c}\text { Tolerance } \\
(-)\end{array}$ & & Basic & $\begin{array}{l}\text { Tolerance } \\
(+) \text { or }(-)\end{array}$ & Basic & $\begin{array}{l}\text { Tolerance } \\
(+) \text { or }(-)\end{array}$ \\
\hline $\begin{array}{c}\text { No. } 10 \\
1 / 4 \\
5 / 16 \\
3 / 8 \\
7 / 16\end{array}$ & $\begin{array}{l}0.190 \\
.250 \\
.313 \\
.375 \\
.438\end{array}$ & $\begin{array}{l}0.009 \\
.010 \\
.013 \\
.015 \\
.015\end{array}$ & $\begin{array}{l}24 \\
20 \\
18 \\
16 \\
14\end{array}$ & $\begin{array}{cc}0.438(7 / 16) \\
.563(9 / 16) \\
.688(11 / 16) \\
.813(13 / 16) \\
.938(15 / 16)\end{array}$ & $\begin{array}{r}0.010 \\
.010 \\
.010 \\
.010 \\
.010\end{array}$ & $\begin{array}{r}0.094 \\
.125 \\
.156 \\
.188 \\
.219\end{array}$ & $\begin{array}{r}0.010 \\
.010 \\
.010 \\
.010 \\
.010\end{array}$ \\
\hline $\begin{array}{l}1 / 2 \\
9 / 16 \\
5 / 8 \\
3 / 4\end{array}$ & $\begin{array}{l}.500 \\
.563 \\
.625 \\
.750\end{array}$ & $\begin{array}{l}.015 \\
.016 \\
.017 \\
.020\end{array}$ & $\begin{array}{l}13 \\
12 \\
11 \\
10\end{array}$ & $\begin{array}{l}1.063(11 / 16) \\
1.188(13 / 16) \\
1.313(1516) \\
1.563(19 / 16)\end{array}$ & $\begin{array}{l}.010 \\
.015 \\
.015 \\
.015\end{array}$ & $\begin{array}{r}.250 \\
.281 \\
.313 \\
.375\end{array}$ & $\begin{array}{l}.010 \\
.015 \\
.015 \\
.015\end{array}$ \\
\hline
\end{tabular}

\begin{tabular}{|c|c|c|c|c|c|c|c|c|c|c|}
\hline $\begin{array}{c}\text { Nominal } \\
\text { size }\end{array}$ & No. 10 & $1 / 4$ & $5 / 16$ & $3 / 8$ & $7 / 16$ & $1 / 2$ & $\% 16$ & $5 / 8$ & $3 / 4$ & \multirow{3}{*}{$\begin{array}{c}\text { Length } \\
\text { tolerance } \\
(+) \text { or }(-)\end{array}$} \\
\hline $\begin{array}{l}\text { Threads } \\
\text { per inch }\end{array}$ & 24 & 20 & 18 & 16 & 14 & 13 & 12 & 11 & 10 & \\
\hline $\begin{array}{l}\text { Length of } \\
\text { bolt (L) }\end{array}$ & \multicolumn{9}{|c|}{ Minimum length of thread $(l)$ including point } & \\
\hline $\begin{array}{l}1 / 2 \\
3 / 4 \\
11 / 4 \\
11 / 4 \\
11 / 2\end{array}$ & $\begin{array}{l}3 / 8 \\
3 / 8 \\
1 / 2 \\
1 / 2 \\
1 / 2\end{array}$ & $\begin{array}{l}3 / 8 \\
3 / 8 \\
1 / 2 \\
5 / 8 \\
3 / 4\end{array}$ & $\begin{array}{l}3 / 8 \\
3 / 8 \\
1 / 2 \\
5 / 8 \\
3 / 4\end{array}$ & $\begin{array}{l}38 \\
58 \\
58 \\
3 / 4\end{array}$ & $\begin{array}{l}1 / 2 \\
5 / 8 \\
3 / 4 \\
7 / 8\end{array}$ & $\begin{array}{l}3 / 4 \\
3 / 4 \\
7 / 8\end{array}$ & $11 / 8$ & $11 / 8$ & 118 & $\begin{array}{l}1 / 64 \\
1 / 64 \\
1 / 64 \\
164 \\
164\end{array}$ \\
\hline $\begin{array}{l}13 / 4 \\
2 \\
21 / 4 \\
21 / 2 \\
23 / 4\end{array}$ & $\begin{array}{l}5 / 8 \\
5 / 8 \\
5 / 8 \\
5 / 8 \\
3 / 4\end{array}$ & $\begin{array}{l}3 / 4 \\
3 / 4 \\
3 / 4 \\
3 / 4 \\
3 / 4\end{array}$ & $\begin{array}{l}3 / 4 \\
3 / 4 \\
3 / 4 \\
3 / 4 \\
7 / 8\end{array}$ & $\begin{array}{l}3 / 4 \\
3 / 4 \\
3 / 4 \\
7 / 8 \\
7 / 8\end{array}$ & $\begin{array}{l}7 / 8 \\
7 / 8 \\
7 / 8 \\
7 / 8 \\
7 / 8\end{array}$ & $\begin{array}{l}1 \\
1 \\
1 \\
1 \\
1\end{array}$ & $11 / 4$ & $11 / 4$ & $11 / 4$ & $\begin{array}{l}164 \\
164 \\
164 \\
164 \\
164\end{array}$ \\
\hline $\begin{array}{l}3 \\
31 / 4 \\
31 / 2 \\
33 / 4 \\
4\end{array}$ & $\begin{array}{r}3 / 4 \\
3 / 4 \\
3 / 4 \\
\end{array}$ & $\begin{array}{l}3 / 4 \\
3 / 4 \\
3 / 4 \\
3 / 4 \\
7 / 8\end{array}$ & $\begin{array}{l}7 / 8 \\
788 \\
7 / 8 \\
78 \\
78\end{array}$ & $\begin{array}{l}7 / 8 \\
7 / 8 \\
7 / 8 \\
78 \\
7 / 8\end{array}$ & $\begin{array}{l}1^{7 / 8} \\
1 \\
1 \\
1\end{array}$ & $\begin{array}{l}11 / 8 \\
11 / 8 \\
11 / 8 \\
11 / 8 \\
11 / 8\end{array}$ & $\begin{array}{c}13 / 8 \\
13 / 8 \\
11 / 2\end{array}$ & $\begin{array}{r}13 / 8 \\
13 / 8 \\
11 / 2\end{array}$ & $\begin{array}{c}11 / 2 \\
-11 / 2 \\
11 / 2\end{array}$ & $\begin{array}{l}1 / 64 \\
1 / 32 \\
1 / 32 \\
1 / 32 \\
1 / 32\end{array}$ \\
\hline $\begin{array}{l}41 / 2 \\
5 \\
51 / 2 \\
6\end{array}$ & & $7 / 8$ & $\begin{array}{l}1 \\
1 \\
11 / 8 \\
11 / 8\end{array}$ & $\begin{array}{l}1 \\
1 \\
11 / 8 \\
11 / 8\end{array}$ & $\begin{array}{l}118 \\
11 / 8 \\
11 / 8 \\
11 / 8\end{array}$ & $\begin{array}{l}11 / 4 \\
11 / 4 \\
11 / 4 \\
11 / 4\end{array}$ & $\begin{array}{l}11 / 2 \\
11 / 2 \\
1112 \\
11 / 2\end{array}$ & $\begin{array}{l}112 \\
11 / 2 \\
11 / 2 \\
11 / 2\end{array}$ & $\begin{array}{l}11 / 2 \\
11 / 2 \\
11 / 2 \\
11 / 2\end{array}$ & $\begin{array}{l}1 / 32 \\
1 / 32 \\
1 / 32 \\
1 / 32\end{array}$ \\
\hline $\begin{array}{l}61 / 2 \\
7 \\
71 / 2 \\
8\end{array}$ & & & $11 / 8$ & $\begin{array}{l}11 / 8 \\
118 \\
118 \\
11 / 4\end{array}$ & $\begin{array}{l}11 / 8 \\
11 / 4 \\
11 / 4 \\
11 / 4\end{array}$ & $\begin{array}{l}114 \\
11 / 4 \\
114 \\
13 / 8\end{array}$ & $\begin{array}{l}15 / 8 \\
158 \\
15 \% \\
158\end{array}$ & $\begin{array}{l}158 \\
15 / 8 \\
15 \% \\
158\end{array}$ & $\begin{array}{l}13 / 4 \\
13 / 4 \\
13 / 4 \\
17 / 8\end{array}$ & $\begin{array}{l}1 / 16 \\
1 / 16 \\
1 / 16 \\
1 / 16\end{array}$ \\
\hline $\begin{array}{c}81 / 2 \\
9 \\
91 / 2 \\
10^{1 / 2}\end{array}$ & & & & & $\begin{array}{l}11 / 4 \\
11 / 4 \\
11 / 4 \\
11 / 4\end{array}$ & $\begin{array}{l}13 / 8 \\
13 / 8 \\
13 / 8 \\
13 / 8\end{array}$ & $\begin{array}{l}13 / 4 \\
134 \\
134 \\
134 \\
134\end{array}$ & $\begin{array}{l}13 / 4 \\
13 / 4 \\
13 / 4 \\
13 / 4\end{array}$ & $\begin{array}{l}17 / 8 \\
2 \\
2 \\
2\end{array}$ & $\begin{array}{l}1 / 16 \\
1 / 16 \\
1 / 16 \\
1 / 16\end{array}$ \\
\hline
\end{tabular}

Radius of fillet between body and head 1/32 inch on sizes No. 10 to 1/2 inch, inclusive, and 1/16 inch on sizes $9 / 16,5 / 8$, and $3 / 4$ inch; 


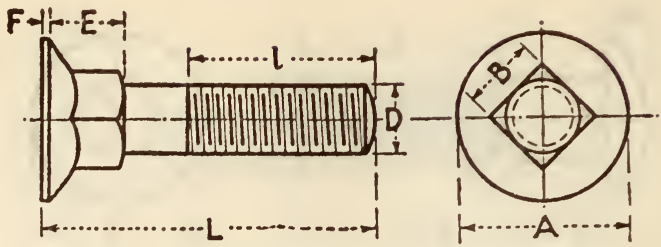

Figdre 340.-Countersunk carriage bolt

TABLE 6.-Countersunk carriage bolt

[All dimensions in inches]

\begin{tabular}{|c|c|c|c|c|c|c|c|c|c|c|c|}
\hline \multirow{3}{*}{$\begin{array}{c}\text { Nominal } \\
\text { size }\end{array}$} & \multicolumn{2}{|c|}{$\mathrm{D}$} & \multirow{3}{*}{$\begin{array}{c}\text { Threads } \\
\text { per } \\
\text { inch }\end{array}$} & \multicolumn{2}{|l|}{ A } & \multirow[t]{3}{*}{$\mathbf{F}$} & \multirow{3}{*}{$\begin{array}{l}\text { In- } \\
\text { cluded } \\
\text { angle } \\
\text { (degs.) }\end{array}$} & \multicolumn{2}{|c|}{ E } & \multicolumn{2}{|c|}{ B } \\
\hline & \multicolumn{2}{|c|}{$\begin{array}{l}\text { Major diameter } \\
\text { of thread }\end{array}$} & & \multicolumn{2}{|c|}{ Diameter of head } & & & \multicolumn{2}{|c|}{ Depth of square } & \multicolumn{2}{|c|}{ Width of square } \\
\hline & $\begin{array}{l}\text { Maxi- } \\
\text { mum } \\
\text { basic }\end{array}$ & $\begin{array}{l}\text { Toler- } \\
\text { ance } \\
(-)\end{array}$ & & Basic & $\begin{array}{l}\text { Tolerance } \\
(+) \text { or }(-)\end{array}$ & & & Basic & $\begin{array}{l}\text { Toler- } \\
\text { ance } \\
(+)\end{array}$ & Basic & $\begin{array}{l}\text { Toler- } \\
\text { ance } \\
(-)\end{array}$ \\
\hline $\begin{array}{l}1 / 4 \\
5 / 16 \\
3 / 8 \\
7 / 16\end{array}$ & $\begin{array}{r}0.250 \\
.313 \\
.375 \\
.438\end{array}$ & $\begin{array}{r}0.010 \\
.013 \\
.015 \\
.015\end{array}$ & $\begin{array}{l}20 \\
18 \\
16 \\
14\end{array}$ & $\begin{array}{r}0.625(5 / 8) \\
.750(3 / 4) \\
.875(7 / 8) \\
1.000(1)\end{array}$ & $\begin{array}{r}0.010 \\
.010 \\
.010 \\
.010\end{array}$ & $\begin{array}{r}0.016 \\
.031 \\
.031 \\
.031\end{array}$ & $\begin{array}{l}114 \\
114 \\
114 \\
114\end{array}$ & $\begin{array}{r}0.281 \\
.344 \\
.406 \\
.469\end{array}$ & $\begin{array}{r}0.031 \\
.031 \\
.031 \\
.031\end{array}$ & $\begin{array}{r}0.250 \\
.313 \\
.375 \\
.438\end{array}$ & $\begin{array}{r}0.010 \\
.013 \\
.015 \\
.015\end{array}$ \\
\hline $\begin{array}{l}1 / 2 \\
9 / 16 \\
5 / 8 \\
3 / 4\end{array}$ & $\begin{array}{l}.500 \\
.563 \\
.625 \\
.750\end{array}$ & $\begin{array}{l}.015 \\
.016 \\
.017 \\
.020\end{array}$ & $\begin{array}{l}13 \\
12 \\
11 \\
10\end{array}$ & $\begin{array}{l}1.125(11 / 8) \\
1.250(11 / 4) \\
1.375(13 / 8) \\
1.625(15 / 8)\end{array}$ & $\begin{array}{l}.010 \\
.015 \\
.015 \\
.015\end{array}$ & $\begin{array}{l}.031 \\
.031 \\
.031 \\
.047\end{array}$ & $\begin{array}{l}114 \\
114 \\
114 \\
114\end{array}$ & $\begin{array}{l}.531 \\
.594 \\
.656 \\
.781\end{array}$ & $\begin{array}{l}.031 \\
.031 \\
.031 \\
.031\end{array}$ & $\begin{array}{l}.500 \\
.563 \\
.625 \\
.750\end{array}$ & $\begin{array}{l}.015 \\
.016 \\
.017 \\
.020\end{array}$ \\
\hline
\end{tabular}

\begin{tabular}{|c|c|c|c|c|c|c|c|c|c|c|}
\hline$\underset{\text { size }}{\text { Nominal }}$ & No. 10 & 144 & $5 / 16$ & $3 / 8$ & $7 / 10$ & $1 / 2$ & \%०o & 58 & $3 / 4$ & \\
\hline $\begin{array}{l}\text { Threads } \\
\text { per inch }\end{array}$ & 24 & 20 & 18 & 16 & 14 & 13 & 12 & 11 & 10 & $\begin{array}{c}\text { Length } \\
\text { tolerance } \\
(+) \text { or }(-)\end{array}$ \\
\hline $\begin{array}{l}\text { Length of } \\
\text { bolt (L) }\end{array}$ & \multicolumn{9}{|c|}{ Minimum length of thread $(l)$ including point } & \\
\hline $\begin{array}{l}3 / 4 \\
1^{314} \\
11 / 2 \\
13 / 4\end{array}$ & 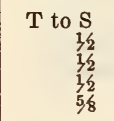 & $\begin{array}{r}\mathrm{T} \text { to } \mathrm{S} \\
1 / 2 \\
5 / 8 \\
3 / 4 \\
3 / 4\end{array}$ & 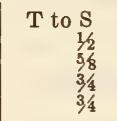 & $\begin{array}{r}\mathrm{T} \text { to } \mathrm{S} \\
\mathrm{T} \text { to } \mathrm{S} \\
5 / 8 \\
3 / 4 \\
3 / 4\end{array}$ & $\begin{array}{l}\mathrm{T} \text { to } \mathrm{S} \\
\mathrm{T} \text { to } \mathrm{S} \\
\mathrm{T} \text { to } \mathrm{S} \\
7 / 8 \\
7 / 8\end{array}$ & $\begin{array}{l}\mathrm{T} \text { to } \mathrm{S} \\
\mathrm{T} \text { to } \mathrm{S} \\
\mathrm{T} \text { to } \mathrm{S} \\
1\end{array}$ & $\begin{array}{l}\mathrm{T} \text { to } \mathrm{S} \\
\mathrm{T} \text { to } \mathrm{S}\end{array}$ & $\begin{array}{l}\mathrm{T} \text { to } \mathrm{S} \\
\mathrm{T} \text { to } \mathrm{S}\end{array}$ & T to $\mathrm{S}$ & $\begin{array}{l}164 \\
164 \\
164 \\
164 \\
164\end{array}$ \\
\hline $\begin{array}{l}2 \\
21 / 4 \\
21 / 2 \\
23 / 4 \\
3\end{array}$ & $\begin{array}{l}5 / 8 \\
5 / 8 \\
5 / 8 \\
3 / 4 \\
3 / 4\end{array}$ & $\begin{array}{l}3 / 4 \\
3 / 4 \\
3 / 4 \\
3 / 4 \\
3 / 4\end{array}$ & $\begin{array}{l}3 / 4 \\
3 / 4 \\
3 / 4 \\
7 / 8 \\
7 / 8\end{array}$ & $\begin{array}{l}3 / 4 \\
7 / 8 \\
7 / 8 \\
7 / 8 \\
7 / 8\end{array}$ & $\begin{array}{l}7 / 8 \\
7 / 8 \\
7 / 8 \\
7 / 8 \\
7 / 8\end{array}$ & $\begin{array}{l}1 \\
1 \\
1 \\
1 \\
1168\end{array}$ & $\begin{array}{c}1144 \\
11 / 4 \\
136^{1 / 4}\end{array}$ & T to $\mathrm{S}$ & $\begin{array}{c}\mathrm{T} \text { to } \mathrm{S} \\
11 / 4 \\
11 / 2\end{array}$ & $\begin{array}{l}164 \\
164 \\
164 \\
164 \\
164\end{array}$ \\
\hline $\begin{array}{l}31 / 4 \\
31 / 2 \\
33 / 4 \\
4\end{array}$ & $\begin{array}{r}3 / 4 \\
3 / 4 \\
\end{array}$ & $\begin{array}{l}3 / 4 \\
34 \\
3 / 4 \\
7 / 8\end{array}$ & $\begin{array}{l}7 / 8 \\
7 / 8 \\
7 / 8 \\
7 / 8\end{array}$ & $\begin{array}{l}7 / 8 \\
7 / 8 \\
7 / 8 \\
7 / 8\end{array}$ & $\begin{array}{l}1 \\
1 \\
1 \\
1\end{array}$ & $\begin{array}{l}118 \\
118 \\
11 / 8 \\
118\end{array}$ & $\frac{138^{--}}{11 / 2}$ & 1138 & $11 / 2$ & $\begin{array}{l}1 / 32 \\
1 / 32 \\
1 / 32 \\
1 / 32\end{array}$ \\
\hline $\begin{array}{l}41 / 2 \\
5 \\
51 / 2 \\
6\end{array}$ & 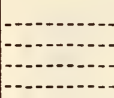 & $\begin{array}{c}7 / 8 \\
\end{array}$ & $\begin{array}{l}1 \\
1 \\
11 / 8 \\
118\end{array}$ & $\begin{array}{l}1 \\
1 \\
118 \\
11 / 8\end{array}$ & $\begin{array}{l}118 \\
11 / 8 \\
11 / 8 \\
118\end{array}$ & $\begin{array}{l}11 / 4 \\
11 / 4 \\
11 / 4 \\
11 / 4\end{array}$ & $\begin{array}{l}11 / 2 \\
11 / 2 \\
15 / 8 \\
158\end{array}$ & $\begin{array}{l}11 / 2 \\
11 / 2 \\
158 \\
15 / 8\end{array}$ & $\begin{array}{l}15 / 8 \\
15 / 8 \\
13 / 4 \\
13 / 4\end{array}$ & $\begin{array}{l}1 / 32 \\
1 / 32 \\
1 / 32 \\
1 / 32\end{array}$ \\
\hline $\begin{array}{l}61 / 2 \\
7 \\
71 / 2 \\
8\end{array}$ & $\mid-1-1$ & 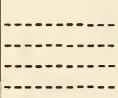 & $\begin{array}{c}116 \\
\end{array}$ & $\begin{array}{l}118 \\
118 \\
118 \\
114\end{array}$ & $\begin{array}{l}116 \\
11 / 4 \\
11 / 4 \\
114\end{array}$ & $\begin{array}{l}11 / 4 \\
11 / 4 \\
11 / 4 \\
1318\end{array}$ & $\begin{array}{l}15 / 8 \\
15 / 8 \\
158 \\
15 / 8\end{array}$ & $\begin{array}{l}15 / 8 \\
15 \% 8 \\
15 / 8 \\
15 / 8\end{array}$ & $\begin{array}{l}13 / 4 \\
13 / 4 \\
13 / 4 \\
17 / 8\end{array}$ & $\begin{array}{l}1 / 16 \\
1 / 16 \\
1 / 16 \\
116\end{array}$ \\
\hline $\begin{array}{l}81 / 2 \\
9 \\
91 / 2 \\
10\end{array}$ & 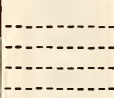 & & 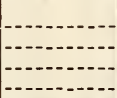 & 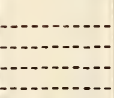 & $\begin{array}{l}11 / 4 \\
114 \\
11 / 4 \\
11 / 4\end{array}$ & $\begin{array}{l}138 \\
138 \\
138 \\
138\end{array}$ & $\begin{array}{l}13 / 4 \\
13 / 4 \\
13 / 4 \\
13 / 4\end{array}$ & $\begin{array}{l}15 / 8 \\
13 / 4 \\
13 / 4 \\
13 / 4\end{array}$ & $\begin{array}{l}17 / 8 \\
2 \\
2 \\
2\end{array}$ & $\begin{array}{l}1 / 16 \\
1 / 16 \\
1 / 16 \\
1 / 16\end{array}$ \\
\hline
\end{tabular}

'T to $\mathrm{S}$ means threaded to square as near as is practicable.

Radius of fillet between body and head 1/32 inch on sizes No. 10 to 1/2 inch, inclusive, and 1/10 inch on sizes $\% / 6,5 / 8$, and $3 / 4$ inch.

\section{AMERICAN SOCIETY OF MECHANICAL ENGINEERS, PLOW BOLTS, B18f-1928, APRIL, 1928}

[Approved as American tentative standard by the American Standards Association, ${ }^{84}$ April, 1928]

\section{PLOW BOLTS}

INTRODUCTORY NOTES

Round head, square neck, countersunk type.-This is generally used in steel parts where countersink- ing the holes is necessary. It is also used to some extent where the hole can be either dry sand or green sand cored. With an included angle of the head of $80^{\circ}$, the hole can be countersunk conveniently leaving a sufficiently strong head without too much top surface which might be objectionable in soft center moldboards where scouring is essential.

Square head, countersunk type.-This bolt is used only in cast iron or malleable parts, where the hole is green sand cored. The head with the $80^{\circ}$ angle is rather flat and, therefore, is desirable for use in cast shares that are very often quite thin. The $80^{\circ}$ angle was adopted for this bolt as a fair compromise

a Sponsor organizations: American Society of Mechanical Engineers and Society of Automotive Engineers. 
and because it is now used by two of the largest plow manufacturers.

Round head, heavy key, countersunk type.-This bolt is used in chilled and cast moldboards where the holes are always dry sand cored. This bolt with its $40^{\circ}$ angle of head was adopted for the reason that three of the largest plow manufacturers now use it.

Round head, reverse key, countersunk type.-This bolt is used in steel parts. The key on this bolt being larger on the bottom makes it very desirable for parts of plows such as the moldboard for the reason that the head can wear down with the surface of the board without wearing away the entire key. The key does not extend outside of the top portion of the bolt, and therefore, if the key on the bolthead does not fill the entire slot in the hole, this is not noticeable. The $60^{\circ}$ angle on this bolt makes it very desirable for plow bottom work as the head is not any larger than necessary. It is very important that the soft head of a bolt in a highly tempered and polished moldboard should be no larger than necessary on account of difficult conditions of scouring. The $60^{\circ}$ angle of head was adopted for this bolt because it is now used by several of the large plow manufacturers.

Screw thread. - The threaded parts of these four standard plow bolts are to conform to the American National Standard Coarse-Thread Series-Free Fit (class 2).

\section{ROUND HEAD, SQUARE NECK, COUNTERSUNK PLOW BOLT}

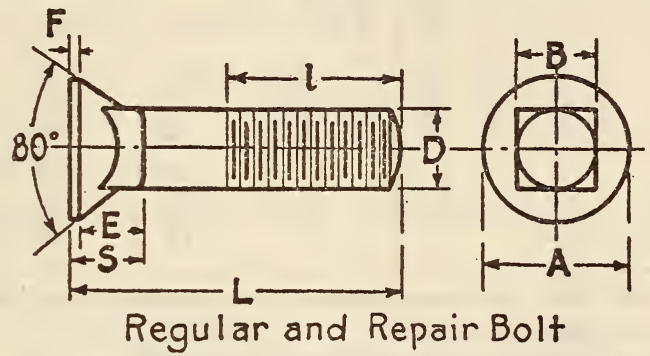

FIGURE 341.-Round head, square neck, countersunk plow bolt

\section{Regular bolt}

[All dimensions given in inches]

\begin{tabular}{|c|c|c|c|c|c|c|c|c|c|}
\hline $\mathrm{D}$ & \multirow{3}{*}{$\begin{array}{l}\text { Threads } \\
\text { per inch }\end{array}$} & A & & & & \multirow{3}{*}{$\begin{array}{l}\text { Included } \\
\text { angle of } \\
\text { head }{ }^{1} \\
\text { (degrees) }\end{array}$} & s & $\mathbf{F}$ & $\mathbf{E}$ \\
\hline \multirow{2}{*}{$\begin{array}{l}\text { Nominal } \\
\text { diameter }\end{array}$} & & \multicolumn{2}{|c|}{ Head diameter } & \multicolumn{2}{|c|}{ Square neck } & & \multirow{2}{*}{$\begin{array}{l}\text { Mini- } \\
\text { mum }\end{array}$} & \multirow{2}{*}{$\begin{array}{c}\text { Feed } \\
\text { thickness, } \\
\text { maxi- } \\
\text { mum }\end{array}$} & \multirow{2}{*}{$\begin{array}{l}\text { Mini- } \\
\text { mum }\end{array}$} \\
\hline & & Minimum & $\begin{array}{l}\text { Toler- } \\
\text { ance } \\
(+)\end{array}$ & $\begin{array}{l}\text { Maxi- } \\
\text { mum }\end{array}$ & $\begin{array}{l}\text { Toler- } \\
\text { ance } \\
(-)\end{array}$ & & & & \\
\hline $\begin{array}{l}5 / 10 \\
38 \\
7 / 10 \\
1 / 2 \\
9 / 10 \\
58 \\
34\end{array}$ & $\begin{array}{l}18 \\
16 \\
14 \\
13 \\
12 \\
11 \\
10\end{array}$ & $\begin{array}{r}0.562(9 / 10) \\
.656(21 / 32) \\
.765(4964) \\
.875(7 / 8) \\
.968(31 / 32) \\
1.062(11 / 16) \\
1.219(17 / 32)\end{array}$ & $\begin{array}{l}0.015 \\
.015 \\
.015 \\
.015 \\
.031 \\
.031 \\
.031\end{array}$ & $\begin{array}{r}0.312 \\
.375 \\
.437 \\
.500 \\
.562 \\
.625 \\
.750\end{array}$ & $\begin{array}{l}0.012 \\
.012 \\
.012 \\
.015 \\
.015 \\
.015 \\
.015\end{array}$ & $\begin{array}{l}80 \\
80 \\
80 \\
80 \\
80 \\
80 \\
80\end{array}$ & $\begin{array}{l}0.243 \\
.281 \\
.328 \\
.375 \\
.416 \\
.456 \\
.491\end{array}$ & $\begin{array}{l}0.025 \\
.031 \\
.036 \\
.042 \\
.045 \\
.050 \\
.050\end{array}$ & $\begin{array}{l}0.218 \\
.250 \\
.292 \\
.333 \\
.371 \\
.406 \\
.441\end{array}$ \\
\hline \multicolumn{10}{|c|}{ Repair bolt 1} \\
\hline $\mathrm{D}$ & \multirow{3}{*}{$\begin{array}{l}\text { Threads } \\
\text { per inch }\end{array}$} & \multicolumn{2}{|l|}{ a } & \multicolumn{2}{|c|}{ b } & \multirow{3}{*}{$\begin{array}{l}\text { Included } \\
\text { angle of } \\
\text { head } 3 \\
\text { (degrees) }\end{array}$} & s & f & e \\
\hline \multirow[b]{2}{*}{$\begin{array}{l}\text { Nominal } \\
\text { diameter }\end{array}$} & & \multicolumn{2}{|c|}{ Head diameter ${ }^{2}$} & \multicolumn{2}{|c|}{ Square neck } & & \multirow[b]{2}{*}{$\begin{array}{l}\text { Mini- } \\
\text { mum }\end{array}$} & \multirow{2}{*}{$\begin{array}{c}\text { Feed } \\
\text { thickness, } \\
\text { maxi- } \\
\text { mum }\end{array}$} & \multirow[b]{2}{*}{$\begin{array}{l}\text { Mini- } \\
\text { mum }\end{array}$} \\
\hline & & Minimum & $\begin{array}{l}\text { Toler- } \\
\text { ance } \\
(+)\end{array}$ & $\begin{array}{l}\text { Maxi- } \\
\text { mum }\end{array}$ & $\begin{array}{l}\text { Toler- } \\
\text { ance } \\
(-)\end{array}$ & & & & \\
\hline \multicolumn{2}{|c|}{$\begin{array}{l}\text { Dimensions same } \\
\text { as for regular } \\
\text { bolt. }\end{array}$} & $\begin{array}{r}0.516(33 / 64) \\
.609(39 / 64) \\
.719(23 / 32) \\
.828(53 / 64) \\
.922(59 / 64) \\
1.016(11 / 64) \\
1.172(111 / 64)\end{array}$ & \multicolumn{4}{|c|}{ Dimensions same as for regular bolt... } & $\begin{array}{r}0.212 \\
.247 \\
.294 \\
.340 \\
.383 \\
.418 \\
.453\end{array}$ & $\begin{array}{l}0.020 \\
.025 \\
.030 \\
.035 \\
.040 \\
.040 \\
.040\end{array}$ & $\begin{array}{l}0.192 \\
.222 \\
.264 \\
.305 \\
.343 \\
.378 \\
.413\end{array}$ \\
\hline
\end{tabular}

1 The reference letters corresponding to the dimensions of the repair bolts are identical with those of the regular bolts but are printed in lower-case type.

"To distinguish the repair bolts from regular bolts of the same type, a large " $R$ " shall be stamped on the top of its head or this top surface shall be painted red

The tolerance on the included angle shall be plus $2^{\circ}$.

(The dimensions given above for square neck are for cold upset wire.) 
Bolt and thread lengths, etc.

[All dimensions given in inches]

\begin{tabular}{|c|c|c|c|c|c|c|c|c|}
\hline $\begin{array}{l}\text { Nominal } \\
\text { diameter } \\
\text { of bolt }\end{array}$ & $5 / 16$ & $3 / 8$ & $7 / 16$ & $1 / 2$ & $8 / 10$ & $5 / 8$ & $3 / 4$ & \multirow{3}{*}{ 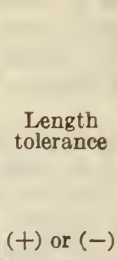 } \\
\hline $\begin{array}{l}\text { Threads } \\
\text { per inch }\end{array}$ & 18 & 16 & 14 & 13 & 12 & 11 & 10 & \\
\hline $\begin{array}{l}\text { Length of } \\
\text { bolt (L) }\end{array}$ & \multicolumn{7}{|c|}{ Minimum length of thread ${ }^{1}(l)$} & \\
\hline $1^{3 / 4}$ & $\begin{array}{l}\mathrm{T} \text { to } \mathrm{H} \\
\mathrm{T} \text { to } \mathrm{H} \\
\mathrm{T} \text { to } \mathrm{H}\end{array}$ & $\begin{array}{l}\mathrm{T} \text { to } \mathrm{H} \\
\mathrm{T} \text { to } \mathrm{H} \\
\mathrm{T} \text { to } \mathrm{H}\end{array}$ & $\begin{array}{l}\mathrm{T} \text { to } \mathrm{H} \\
\mathrm{T} \text { to } \mathrm{H} \\
\mathrm{T} \text { to } \mathrm{H}\end{array}$ & $\begin{array}{l}\mathrm{T} \text { to } \mathrm{H} \\
\mathrm{T} \text { to } \mathrm{H}\end{array}$ & & & & $\begin{array}{l}1 / 32 \\
1 / 32 \\
1 / 32\end{array}$ \\
\hline $\begin{array}{l}11 / 8 \\
11 / 4\end{array}$ & $\begin{array}{r}11 / 16 \\
3 / 4\end{array}$ & $\begin{array}{l}3 / 4 \\
3 / 4\end{array}$ & $\begin{array}{l}5 / 8 \\
5 / 8\end{array}$ & $\begin{array}{l}\mathrm{T} \text { to } \mathrm{H} \\
\mathrm{T} \text { to } \mathrm{H}\end{array}$ & $\mathrm{T}$ to $\mathrm{H}$ & $\mathrm{T}$ to $\mathrm{H}$ & & $\begin{array}{l}1 / 32 \\
1 / 32\end{array}$ \\
\hline $\begin{array}{l}13 / 8 \\
11 / 2 \\
13 / 4 \\
2\end{array}$ & $\begin{array}{l}1 / 8 \\
7 / 8 \\
7 / 8\end{array}$ & $\begin{array}{l}3 / 4 \\
7 / 8 \\
7 / 8 \\
7 / 8\end{array}$ & $\begin{array}{l}3 / 4 \\
7 / 8 \\
7 / 8 \\
7 / 8\end{array}$ & $\begin{array}{l}3 / 4 \\
7 / 8 \\
7 / 8 \\
7 / 8\end{array}$ & T to $\begin{array}{r}\mathrm{H} \\
3 / 4 \\
7 / 8 \\
11 / 8\end{array}$ & T to $\begin{array}{c}\mathrm{H} \\
7 / 8 \\
11 / 8 \\
11 / 8\end{array}$ & 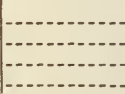 & $\begin{array}{l}1 / 32 \\
1 / 32 \\
1 / 16 \\
1 / 16\end{array}$ \\
\hline $\begin{array}{l}21 / 4 \\
21 / 2 \\
23 / 4 \\
3\end{array}$ & 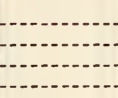 & $1^{7 / 8}$ & $\begin{array}{l}1 \\
1 \\
1 \\
1\end{array}$ & $\begin{array}{l}1 \\
1 \\
1 \\
1\end{array}$ & $\begin{array}{l}11 / 8 \\
11 / 4 \\
11 / 4 \\
11 / 4\end{array}$ & $\begin{array}{l}11 / 4 \\
11 / 4 \\
11 / 4 \\
114\end{array}$ & $\begin{array}{l}11 / 4 \\
11 / 4 \\
11 / 4 \\
11 / 4\end{array}$ & $\begin{array}{l}\text { Yo } \\
110 \\
110 \\
1 / 10\end{array}$ \\
\hline
\end{tabular}

"As stated in the "Introductory notes" these threads are to conform to the American Standard B1a-1924.

"T to $\mathrm{H}$ " means threaded to head as near as practicable.

The table of bolt and thread lengths reproduced above is intended as a guide to the users of these bolts. A number of the listed sizes and lengths will not be regularly stocked by the manufacturers but will be available on order of a sufficient quantity.

SQUARE HEAD COUNTERSUNK PLOW BOLT

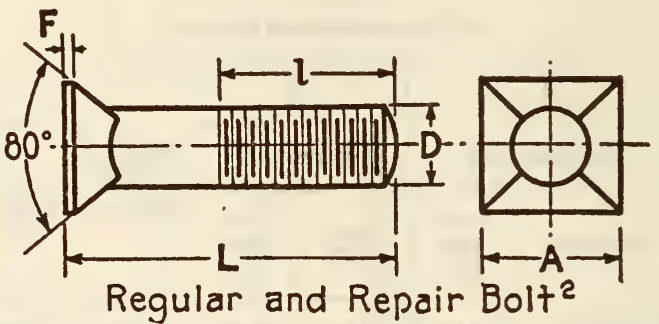

FigdRE 342.-Square head, countersunk plow bolt

Regular bolt

[All dimensions in inches]

\begin{tabular}{|c|c|c|c|c|c|}
\hline D & \multirow{3}{*}{$\begin{array}{c}\text { Threads } \\
\text { per } \\
\text { inch }\end{array}$} & \multicolumn{2}{|l|}{ A } & $\mathbf{F}$ & \multirow{3}{*}{$\begin{array}{l}\text { In- } \\
\text { cluded } \\
\text { angle } \\
\text { of } \\
\text { head } \\
\text { (de- } \\
\text { grees) }\end{array}$} \\
\hline \multirow{2}{*}{$\begin{array}{l}\text { Nomi- } \\
\text { nal di- } \\
\text { ameter }\end{array}$} & & \multicolumn{2}{|c|}{ Head diameter } & \multirow{2}{*}{$\begin{array}{l}\text { Feed } \\
\text { thick- } \\
\text { ness, } \\
\text { maxi- } \\
\text { mum }\end{array}$} & \\
\hline & & Minimum & $\begin{array}{c}\text { Tolerance } \\
(+)\end{array}$ & & \\
\hline $\begin{array}{l}5 / 16 \\
3 / 8 \\
7 / 16 \\
1 / 2 \\
8 / 16\end{array}$ & $\begin{array}{l}18 \\
16 \\
14 \\
13 \\
12\end{array}$ & $\begin{array}{l}0.562(8 / 16) \\
.687(11 / 16) \\
.750(3 / 4) \\
.812(13 / 16) \\
.937(15 / 16)\end{array}$ & $\begin{array}{r}0.015(1 / 64) \\
.015(1 / 64) \\
.015(1 / 64) \\
.015(1 / 64) \\
.031(1 / 32)\end{array}$ & $\begin{array}{l}0.025 \\
.031 \\
.036 \\
.042 \\
.045\end{array}$ & $\begin{array}{l}80 \\
80 \\
80 \\
80 \\
80\end{array}$ \\
\hline $\begin{array}{r}5 / 8 \\
3 / 4 \\
7 / 8 \\
1^{7 / 8}\end{array}$ & $\begin{array}{r}11 \\
10 \\
9 \\
8\end{array}$ & $\begin{array}{l}1.000(1) \\
1.125(11 / 8) \\
1.312(1516) \\
1.500(11 / 2)\end{array}$ & $\begin{array}{l}.031(1 / 32) \\
.031(1 / 32) \\
.031(1 / 32) \\
.031(1 / 32)\end{array}$ & $\begin{array}{l}.050 \\
.050 \\
.050 \\
.050\end{array}$ & $\begin{array}{l}80 \\
80 \\
80 \\
80\end{array}$ \\
\hline
\end{tabular}

Repair bolt

\begin{tabular}{|c|c|c|c|c|c|}
\hline \multirow{3}{*}{$\begin{array}{c}\mathrm{D} \\
\\
\text { Nomi- } \\
\text { nal di- } \\
\text { ameter }\end{array}$} & \multirow{3}{*}{$\begin{array}{c}\text { Threads } \\
\text { per } \\
\text { inch }\end{array}$} & \multicolumn{2}{|c|}{ a } & f & \multirow{3}{*}{$\begin{array}{l}\text { In- } \\
\text { cluded } \\
\text { angle } \\
\text { of } \\
\text { head } \\
\text { (de- } \\
\text { grees) }\end{array}$} \\
\hline & & \multicolumn{2}{|c|}{ Head diameter } & \multirow{2}{*}{$\begin{array}{l}\text { Feed } \\
\text { thick- } \\
\text { ness, } \\
\text { maxi- } \\
\text { mum }\end{array}$} & \\
\hline & & Minimum & $\begin{array}{c}\text { Tolerance } \\
(+)\end{array}$ & & \\
\hline & & $\begin{array}{l}0.515(33 / 64) \\
.640(4164) \\
.703(4564)\end{array}$ & \multirow{3}{*}{$\begin{array}{l}\text { Dimensions } \\
\text { s a me as } \\
\text { for regular } \\
\text { bolt. }\end{array}$} & $\begin{array}{r}0.020 \\
.025 \\
.030\end{array}$ & \multirow{3}{*}{$\begin{array}{l}\text { Same } \\
\text { as for } \\
\text { regu- } \\
\text { lar } \\
\text { bolt }\end{array}$} \\
\hline \multirow{2}{*}{\multicolumn{2}{|c|}{$\begin{array}{l}\text { Dimensions same } \\
\text { as for regular } \\
\text { bolt. }\end{array}$}} & $\begin{array}{l}.765(4964) \\
.890(5764) \\
.953(6164)\end{array}$ & & $\begin{array}{l}.035 \\
.040 \\
.040\end{array}$ & \\
\hline & & $\begin{array}{l}1.078(1564) \\
1.265(11764) \\
1.453(12964)\end{array}$ & & $\begin{array}{l}.040 \\
.040 \\
.040\end{array}$ & \\
\hline
\end{tabular}

(Footnotes same as for round head, square neck bolts.) 
IRON AND STEEL

Bolt and thread lengths, etc.

[All dimensions in inches]

\begin{tabular}{|c|c|c|c|c|c|c|c|c|c|c|}
\hline $\begin{array}{l}\text { Nominal } \\
\text { diameter }\end{array}$ & $5 / 16$ & $3 / 8$ & 716 & $1 / 2$ & 916 & $5 / 8$ & $3 / 4$ & $7 / 8$ & 1 & \multirow{3}{*}{$\begin{array}{c}\text { Length } \\
\text { tolerance } \\
(+) \text { or } \\
(-)\end{array}$} \\
\hline $\begin{array}{l}\text { Threads } \\
\text { per inch }\end{array}$ & 18 & 16 & 14 & 13 & 12 & 11 & 10 & 9 & 8 & \\
\hline $\begin{array}{l}\text { Length } \\
\text { of bolt } \\
\text { (L) }\end{array}$ & \multicolumn{9}{|c|}{ Minimum length of thread $(l)$} & \\
\hline $1^{3 / 4}$ & $\begin{array}{l}\mathrm{T} \text { to } \mathrm{H} \\
\mathrm{T} \text { to } \mathrm{H} \\
\mathrm{T} \text { to } \mathrm{H}\end{array}$ & $\begin{array}{l}\mathrm{T} \text { to } \underset{\mathrm{H}}{\mathrm{T}} \\
\mathrm{T} \text { to } \underset{\mathrm{H}}{\mathrm{H}}\end{array}$ & $\begin{array}{l}\mathrm{T} \text { to } \mathrm{H} \\
\mathrm{T} \text { to } \mathrm{H} \\
\mathrm{T} \text { to } \mathrm{H}\end{array}$ & T to $\overline{\mathrm{H}}$ & $\cdots$ & & & - & - & $\begin{array}{l}1 / 32 \\
1 / 32 \\
1 / 32\end{array}$ \\
\hline $\begin{array}{l}11 / 8 \\
11 / 4 \\
13 / 8\end{array}$ & $\begin{array}{c}11 / 16 \\
3 / 4\end{array}$ & $\begin{array}{l}3 / 4 \\
3 / 4 \\
3 / 4\end{array}$ & $\begin{array}{l}5 / 8 \\
5,8 \\
3 / 4\end{array}$ & $\begin{array}{l}\mathrm{T} \text { to } \underset{3 / 4}{\mathrm{H}} \\
\mathrm{T} \text { to } \\
\mathrm{H}\end{array}$ & T to $\mathrm{H}$ & $\begin{array}{l}\mathrm{T} \text { to } \mathrm{H} \\
\mathrm{H}\end{array}$ & & & -- & $\begin{array}{l}1 / 32 \\
1 / 32 \\
1 / 32\end{array}$ \\
\hline $\begin{array}{l}13 / 2 \\
13 / 4 \\
2\end{array}$ & 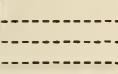 & $\begin{array}{l}7 / 8 \\
7 / 8 \\
7 / 8\end{array}$ & $\begin{array}{l}7 / 8 \\
7 / 8 \\
7 / 8\end{array}$ & $\begin{array}{l}7 / 8 \\
7 / 8 \\
7 / 8\end{array}$ & $\begin{array}{r}7 / 8 \\
7 / 8 \\
118\end{array}$ & $1_{118}^{7 / 8}$ & 118 & $--\cdot$ & -- & $\begin{array}{l}1 / 32 \\
1 / 16 \\
1 / 16\end{array}$ \\
\hline $\begin{array}{l}21 / 4 \\
21 / 2 \\
23 / 4 \\
3\end{array}$ & 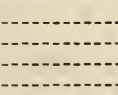 & $1^{7 / 8}$ & $\begin{array}{l}1 \\
1 \\
1 \\
1\end{array}$ & $\begin{array}{l}1 \\
1 \\
1 \\
1\end{array}$ & $\begin{array}{l}138 \\
11 / 4 \\
114 \\
11 / 4\end{array}$ & $\begin{array}{l}11 / 4 \\
11 / 4 \\
11 / 4 \\
11 / 4\end{array}$ & $\begin{array}{l}11 / 4 \\
11 / 4 \\
11 / 4 \\
11 / 4\end{array}$ & $\begin{array}{l}11 / 4 \\
11 / 4 \\
11 / 4 \\
11 / 4\end{array}$ & $\begin{array}{l}11 / 4 \\
11 / 4 \\
11 / 4 \\
11 / 4\end{array}$ & $\begin{array}{l}116 \\
116 \\
1 / 16 \\
1 / 16\end{array}$ \\
\hline
\end{tabular}

(Footnotes same as for round head, square neck bolts.)

ROUND HEAD, HEAVY KEY, COUNTERSUNK PLOW BOLT

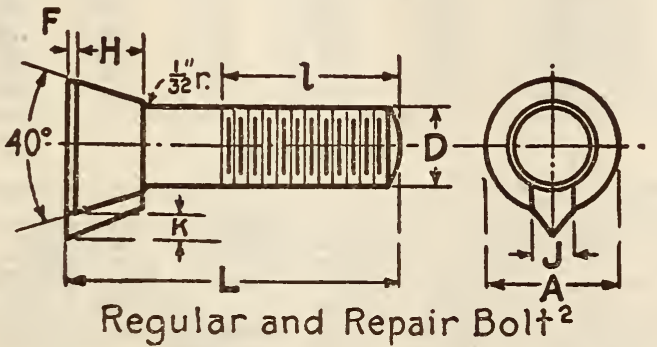

FigURE 343.-Round head, heavy key, countersunk plow bolt

Regular bolt

[All dimensions in inches]

\begin{tabular}{|c|c|c|c|c|c|c|c|c|}
\hline D & \multirow{3}{*}{$\begin{array}{l}\text { Threads } \\
\text { per inch }\end{array}$} & I & & $\mathbf{F}$ & \multirow{3}{*}{$\begin{array}{c}\text { Included } \\
\text { angle of } \\
\text { head, } \\
\text { (degrees) }\end{array}$} & $\mathbf{H}$ & $\mathrm{J}$ & $\mathrm{K}$ \\
\hline \multirow{2}{*}{$\begin{array}{l}\text { Nominal } \\
\text { diameter }\end{array}$} & & Head d & ameter & \multirow{2}{*}{$\begin{array}{l}\text { Feed } \\
\text { thickness, } \\
\text { maximum }\end{array}$} & & \multirow{2}{*}{$\begin{array}{l}\text { Height of } \\
\text { head } \\
\text { maximum }\end{array}$} & \multirow{2}{*}{$\begin{array}{c}\text { Key } \\
\text { width, } \\
\text { maximum }\end{array}$} & \multirow{2}{*}{$\begin{array}{c}\text { Key } \\
\text { project, } \\
\text { maximum }\end{array}$} \\
\hline & & Minimum & $\begin{array}{l}\text { Tolerance } \\
(+)\end{array}$ & & & & & \\
\hline $\begin{array}{l}3 / 8 \\
7 / 16 \\
1 / 2\end{array}$ & $\begin{array}{l}16 \\
14 \\
13\end{array}$ & $\begin{array}{r}0.725 \\
.796 \\
.867\end{array}$ & $\begin{array}{r}0.015 \\
.015 \\
.015\end{array}$ & $\begin{array}{r}0.050 \\
.050 \\
.050\end{array}$ & $\begin{array}{l}40 \\
40 \\
40\end{array}$ & $\begin{array}{r}0.310 \\
.367 \\
.430\end{array}$ & $\begin{array}{r}0.156 \\
.172 \\
.188\end{array}$ & $\begin{array}{r}0.100 \\
.100 \\
.100\end{array}$ \\
\hline
\end{tabular}

(Footnotes same as for round head, square neck bolts.)

Repair bolt

\begin{tabular}{|c|c|c|c|c|}
\hline $\mathrm{D}$ & $\mathrm{a}$ & $\mathrm{f}$ & $\mathrm{b}$ \\
\cline { 1 - 1 } $\begin{array}{c}\text { Nominal } \\
\text { diameter }\end{array}$ & $\begin{array}{c}\text { Threads } \\
\text { per inch }\end{array}$ & $\begin{array}{c}\text { Head } \\
\text { diameter, } \\
\text { maxi- } \\
\text { mum }\end{array}$ & $\begin{array}{c}\text { Feed } \\
\text { thickness, } \\
\text { maxi- } \\
\text { mum }\end{array}$ & $\begin{array}{c}\text { Height } \\
\text { of head, } \\
\text { maxi- } \\
\text { mum }\end{array}$ \\
\hline $\begin{array}{c}3 / 8 \\
7 / 16\end{array}$ & 16 & $\begin{array}{r}0.708 \\
.770 \\
1 / 2\end{array}$ & $\begin{array}{r}0.025 \\
.030 \\
.836\end{array}$ & $\begin{array}{r}0.286 \\
.339 \\
.402\end{array}$ \\
\hline
\end{tabular}

(Footnotes same as for round head, square neck bolts.)
Bolt and thread lengths, etc.

[All dimensions in inches]

\begin{tabular}{|c|c|c|c|c|}
\hline $\begin{array}{l}\text { Nominal } \\
\text { diameter } \\
\text { of bolt }\end{array}$ & $3 / 8$ & $7 / 16$ & $1 / 2$ & \multirow{2}{*}{$\begin{array}{l}\text { Length } \\
\text { tolerance }\end{array}$} \\
\hline $\begin{array}{l}\text { Threads } \\
\text { per inch }\end{array}$ & 16 & 14 & 13 & \\
\hline $\begin{array}{l}\text { Length of } \\
\text { bolt (L) }\end{array}$ & \multicolumn{3}{|c|}{ Minimum length of thread ( $l$ ) } & $(+)$ or $(-)$ \\
\hline $\begin{array}{l}3 / 4 \\
7 / 8\end{array}$ & $\begin{array}{l}\mathrm{T} \text { to } \mathrm{H} \\
\mathrm{T} \text { to } \mathrm{H} \\
\mathrm{T} \text { to } \mathrm{H}\end{array}$ & $\begin{array}{l}\mathrm{T} \text { to } \mathrm{H} \\
\mathrm{T} \text { to } \mathrm{H} \\
\mathrm{T} \text { to } \mathrm{H}\end{array}$ & $\begin{array}{l}\mathrm{T} \text { to } \mathrm{H} \\
\mathrm{T} \text { to } \mathrm{H} \\
\mathrm{T} \text { to } \mathrm{H}\end{array}$ & $\begin{array}{l}1 / 32 \\
1 / 32 \\
1 / 32\end{array}$ \\
\hline $\begin{array}{l}11 / 8 \\
11 / 4\end{array}$ & $\begin{array}{l}\mathrm{T} \text { to } \mathrm{H} \\
\mathrm{T} \text { to } \mathrm{H}\end{array}$ & $\begin{array}{l}\mathrm{T} \text { to } \mathrm{H} \\
\mathrm{T} \text { to } \mathrm{H}\end{array}$ & $\begin{array}{l}\mathrm{T} \text { to } \mathrm{H} \\
\mathrm{T} \text { to } \mathrm{H}\end{array}$ & $\begin{array}{l}1 / 32 \\
1 / 32\end{array}$ \\
\hline $\begin{array}{l}138 \\
11 / 2 \\
13 / 4 \\
2\end{array}$ & $\begin{array}{c}\mathrm{T} \text { to } \mathrm{H} \\
15 / 16 \\
7 / 8 \\
7 / 8\end{array}$ & $\begin{array}{c}\mathrm{T} \text { to } \mathrm{H} \\
7 / 8 \\
7 / 8 \\
7 / 8\end{array}$ & $\begin{array}{c}\mathrm{T} \text { to } \mathrm{H} \\
7 / 8 \\
7 / 8 \\
7 / 8\end{array}$ & $\begin{array}{l}1 / 32 \\
1 / 32 \\
1 / 16 \\
1 / 16\end{array}$ \\
\hline $\begin{array}{l}21 / 4 \\
21 / 2 \\
23 / 4 \\
3\end{array}$ & $\begin{array}{l}7 / 8 \\
7 / 8 \\
1 \\
1\end{array}$ & $\begin{array}{l}1 \\
1 \\
1 \\
1\end{array}$ & $\begin{array}{l}1 \\
1 \\
1 \\
1\end{array}$ & $\begin{array}{l}1 / 16 \\
1 / 16 \\
1 / 16 \\
1 / 16\end{array}$ \\
\hline
\end{tabular}

(Footnotes same as for round head, square neck bolts.) 
ROUND HEAD, REVERSE KEY, COONTERSONK PLOW BOLT

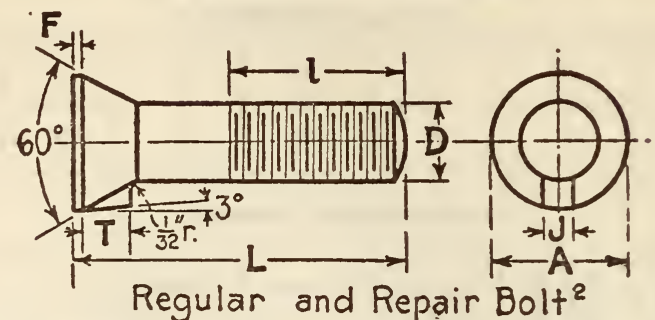

Figure 344.-Round head, reverse key, countersunk plow bolt

Regular bolt

[All dimensions in inches]

\begin{tabular}{|c|c|c|c|c|c|c|c|c|c|}
\hline $\mathrm{D}$ & \multirow{3}{*}{$\begin{array}{l}\text { Threads } \\
\text { per inch }\end{array}$} & A & & $\mathrm{F}$ & & & J & & $\Gamma$ \\
\hline \multirow{2}{*}{$\begin{array}{l}\text { Nominal } \\
\text { diameter }\end{array}$} & & \multicolumn{2}{|c|}{ Head diameter } & \multirow{2}{*}{$\begin{array}{l}\text { Feed } \\
\text { thickness } \\
\text { maxi- } \\
\text { mum }\end{array}$} & \multirow{2}{*}{$\begin{array}{l}\text { angle of } \\
\text { head (de- } \\
\text { grees) }\end{array}$} & \multicolumn{2}{|c|}{ Key width } & \multicolumn{2}{|c|}{ Key length } \\
\hline & & Minimum & $\underset{(+)}{\text { Tolerance }}$ & & & $\begin{array}{l}\text { Maxi- } \\
\text { mum }\end{array}$ & $\underset{(-)}{\text { Tolerance }}$ & $\begin{array}{l}\text { Mini- } \\
\text { mum }\end{array}$ & $\begin{array}{l}\text { Tolerance } \\
(t)\end{array}$ \\
\hline $\begin{array}{r}5 / 16 \\
3 / 8 \\
7 / 16 \\
1 / 2 \\
9 / 16 \\
5 / 8 \\
3 / 4\end{array}$ & $\begin{array}{l}18 \\
16 \\
14 \\
13 \\
12 \\
11 \\
10\end{array}$ & $\begin{aligned} & 0.562(9 / 16) \\
& .625(5 / 8) \\
& .734(47 / 64) \\
& .843(27 / 32) \\
& .968(31 / 32) \\
& 1.063(11 / 16) \\
& 1.218(17 / 32)\end{aligned}$ & $\begin{array}{l}0.015 \\
.015 \\
.015 \\
.015 \\
.015 \\
.015 \\
.015\end{array}$ & $\begin{array}{l}0.025 \\
.031 \\
.036 \\
.042 \\
.045 \\
.050 \\
.050\end{array}$ & $\begin{array}{l}60 \\
60 \\
60 \\
60 \\
60 \\
60 \\
60\end{array}$ & $\begin{array}{l}0.156 \\
.156 \\
.156 \\
.156 \\
.156 \\
.156 \\
.156\end{array}$ & $\begin{array}{r}0.005 \\
.005 \\
.005 \\
.005 \\
.005 \\
.005 \\
.005\end{array}$ & $\begin{array}{r}0.185 \\
.187 \\
.227 \\
.267 \\
.321 \\
.348 \\
.375\end{array}$ & $\begin{array}{l}0.015 \\
.015 \\
.015 \\
.015 \\
.015 \\
.015 \\
.015\end{array}$ \\
\hline
\end{tabular}

Repair bolt

\begin{tabular}{|c|c|c|c|c|c|c|c|c|c|}
\hline $\mathrm{D}$ & & a & & f & & & j & & $t$ \\
\hline & Threads & Head dia & neter & Feed & angle of & $\mathrm{Key}$ & width & Key & length \\
\hline $\begin{array}{l}\text { Nominal } \\
\text { diameter }\end{array}$ & $1 \mathrm{ch}$ & Minimum & $\begin{array}{c}\text { Tolerance } \\
(+)\end{array}$ & $\begin{array}{l}\text { thlckness } \\
\text { maxi- } \\
\text { mum }\end{array}$ & $\begin{array}{l}\text { head (de- } \\
\text { grees) }\end{array}$ & $\begin{array}{l}\text { Maxi- } \\
\text { mum }\end{array}$ & $\begin{array}{c}\text { Tolerance } \\
(-)\end{array}$ & $\begin{array}{l}\text { Mini- } \\
\text { mum }\end{array}$ & $\begin{array}{c}\text { Tolerance } \\
(+)\end{array}$ \\
\hline $\begin{array}{l}\text { Dimensio } \\
\text { as for } \\
\text { bolt. }\end{array}$ & $\begin{array}{l}\text { ns same } \\
\text { regular }\end{array}$ & $\begin{array}{r}0.531(17 / 32) \\
.593(19 / 32) \\
.703(45 / 64) \\
.812(13 / 16) \\
.937(15 / 16) \\
1.031(11 / 32) \\
1.187(13 / 16)\end{array}$ & $\begin{array}{c}\text { Same as } \\
\text { for reg- } \\
\text { u l a r } \\
\text { bolt. }\end{array}$ & $\begin{array}{l}0.020 \\
.025 \\
.030 \\
.035 \\
.040 \\
.040 \\
.040\end{array}$ & $\begin{array}{l}\text { Dimensi } \\
\text { ular b }\end{array}$ & same & as for reg- & $\begin{array}{l}0.158 \\
.158 \\
.199 \\
.239 \\
.293 \\
.321 \\
.347\end{array}$ & $\begin{array}{c}\text { Same as } \\
\text { for reg- } \\
\text { u } 18 \text { r } \\
\text { bolt. }\end{array}$ \\
\hline
\end{tabular}

(Footnotes same as for round head, square neck bolts.)

Bolt and thread lengths, etc.

[All dimensions in inches]

\begin{tabular}{|c|c|c|c|c|c|c|c|c|}
\hline $\begin{array}{l}\text { Nominal } \\
\text { diam. } \\
\text { e } t \text { e } r \\
\text { of bolt }\end{array}$ & $5 / 16$ & $3 / 8$ & $7 / 16$ & $1 / 2$ & $9 / 16$ & 58 & $3 / 4$ & \multirow{2}{*}{$\begin{array}{c}\text { Length } \\
\text { tolerance } \\
(+) \text { or }(-)\end{array}$} \\
\hline $\begin{array}{l}\text { Threads } \\
\text { per inch }\end{array}$ & 18 & 16 & 14 & 13 & 12 & 11 & 10 & \\
\hline $\begin{array}{l}\text { Length of } \\
\text { bolt (L) }\end{array}$ & \multicolumn{7}{|c|}{ Minimum length of thread $(l)$} & \\
\hline $\begin{array}{l}3 / 4 \\
7 / 8 \\
1 \\
11 / 8 \\
11 / 4 \\
13 / 8 \\
11 / 2 \\
13 / 4 \\
2 \\
21 / 4 \\
21 / 2 \\
23 / 4 \\
3 \\
\end{array}$ & $\begin{array}{l}\mathrm{T} \\
\mathrm{T} \text { to } \mathrm{T} \\
\mathrm{T} \text { to }{ }_{1} \mathrm{H} \\
\\
\end{array}$ & $\begin{array}{c}\mathrm{T} \text { to } \mathrm{H} \\
\mathrm{T} \text { to } \mathrm{H} \\
\mathrm{T} \text { to } \frac{\mathrm{H}}{3 / 4} \\
3 / 4 \\
3 / 4 \\
7 / 8 \\
7 / 8 \\
7 / 8 \\
7 / 8 \\
1 \\
1 \\
1 \\
\end{array}$ & $\begin{array}{c}\mathrm{T} \text { to } \mathrm{H} \\
\mathrm{T} \text { to } \mathrm{H} \\
\mathrm{T} \text { to } \underset{5}{\mathrm{H}} \\
5 / 8 \\
5 / 8 \\
3 / 4 \\
7 / 8 \\
7 / 8 \\
7 / 8 \\
1 \\
1 \\
1 \\
1\end{array}$ & $\begin{array}{c}\mathrm{T} \text { to } \mathrm{H} \\
\mathrm{T} \text { to } \mathrm{H} \\
\mathrm{H} \\
\frac{3 / 4}{7 / 8} \\
7 / 8 \\
1^{7 / 8} \\
1 \\
1 \\
1\end{array}$ & \begin{tabular}{|c|} 
T to $\mathrm{H}$ \\
T to $\frac{\mathrm{H}}{7 / 8}$ \\
\\
\\
\\
$11 / 8$ \\
$11 / 8$ \\
$11 / 4$ \\
$11 / 4$ \\
$11 / 4$
\end{tabular} & 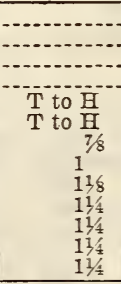 & $\begin{array}{r}116 \\
1114 \\
114 \\
11 / 4 \\
114 \\
114 \\
\end{array}$ & $\begin{array}{l}1 / 32 \\
1 / 32 \\
1 / 32 \\
132 \\
1 / 32 \\
1 / 32 \\
1 / 32 \\
1 / 16 \\
1116 \\
1116 \\
1 / 16 \\
1 / 16 \\
1 / 16 \\
\end{array}$ \\
\hline
\end{tabular}

(Footnotes same as for round head, square neck bolts.)

\section{SOCIETY OF AUTOMOTIVE ENGINEERS, /CAP SCREWS, BOLTS AND NOTS, AND WRENCH CAP SCREWS, BOLTS AND NUTS, AND WRENCH OPENINGS, S. A. E. STAND- \\ OPENINGS
OPA AND WRENCH FINISHED AND SEMUINISHED BOITS} ARDS, JANUARY, 1930

[Approved by American Standards Association, April, 1930]

(See also 608.2, p. 577; 608.32, p. 606; and 615.42, p. 883 .)

(In respect to general requirements, body lengths, points, material, slots, cotter-pin holes, and heat treatment, this specification is identical with that for cap screws. (See 608.2, p. 577.) Dimensions of rough and semifinished square and hexagonal regu- 
lar boltheads, and finished square and hexagonal boltheads, are identical with those of the National Screw Thread Commission, machine bolts, in Tables 67 and 68 , given below.)

SOCIETY OF AUTOMOTIVE ENGINEERS,
CAP SCREWS, BOLTS, AND NUTS; CONNECTING-ROD BOLTS, S. A. E. STAND. ARD, AUGUST, 1920

\section{CONNECTING-ROD BOLTS}

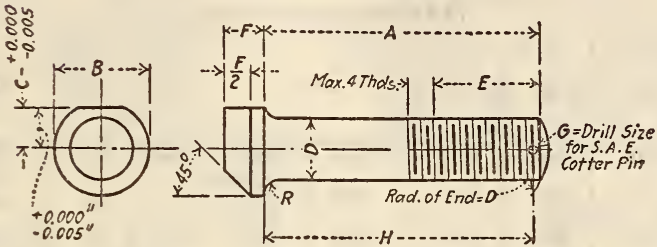

FIGURE 345.-Connecting-rod bolt

[All dimensions in inches]

\begin{tabular}{|c|c|c|c|c|c|c|c|c|c|c|}
\hline 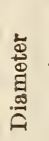 & A & B & C & D & 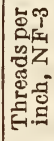 & E 1 & $\mathrm{~F}$ & $\begin{array}{c}G \\
\text { No. }\end{array}$ & 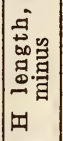 & $\mathrm{R}$ \\
\hline 5/. & & $3 / 16$ & $3 / 16$ & $\left\{\begin{array}{r}0.3125 \\
3105\end{array}\right.$ & 24 & $1 / 2$ & $3 / 16$ & 48 & $3 / 32$ & \\
\hline $3 / 8$ & छ & $5 / 8$ & $7 / 32$ & $\left\{\begin{array}{l}.3750 \\
.3730\end{array}\right.$ & 24 & $5 / 8$ & $7 / 32$ & 36 & $\%$ & $\begin{array}{r}0.01 \\
\text { to. } 1 / 32\end{array}$ \\
\hline $7 / 10$ & 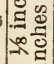 & $11 / 16$ & $1 / 4$ & $\left\{\begin{array}{l}.4375 \\
.4355\end{array}\right.$ & 20 & $11 / 16$ & $1 / 4$ & 36 & $9 \% 4$ & \\
\hline $1 / 2$ & D. & $3 / 4$ & $9 / 32$ & $\left\{\begin{array}{l}.5000 \\
.4975\end{array}\right.$ & 20 & $3 / 4$ & $9 / 32$ & 36 & $\%$ & \\
\hline$\%$ & $\vec{a}$ & $7 / 8$ & $5 / 16$ & $\left\{\begin{array}{l}.5625 \\
.5600\end{array}\right.$ & 18 & $7 / 8$ & $5 / 10$ & 28 & $3 / 16$ & to 3 \\
\hline $5 / 8$ & 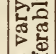 & & $3 / 8$ & $\left\{\begin{array}{l}.6250 \\
.6220\end{array}\right.$ & 18 & 1 & $3 / 8$ & 28 & $3 / 16$ & \\
\hline $3 / 4$ & 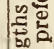 & $11 / 8$ & $7 / 16$ & $\left\{\begin{array}{l}.7500 \\
.7470\end{array}\right.$ & 16 & $11 / 8$ & $7 / 16$ & 28 & $3 / 16$ & $1 / 32$ \\
\hline $7 / 8$ & $\Phi$ & $13 / 8$ & $3 / 2$ & $\left\{\begin{array}{l}.8750 \\
.8715\end{array}\right.$ & 14 & $11 / 4$ & $1 / 2$ & 28 & $3 / 16$ & \\
\hline 1 & & $11 / 2$ & $9 / 16$ & $\left\{\begin{array}{r}1.0000 \\
.9965\end{array}\right.$ & 14 & $11 / 2$ & $5 / 8$ & 28 & $3 / 18$ & \\
\hline
\end{tabular}

1 Minimum length of usable threads.

Recommended practice for material is S. A. E. Steel No. 2330 or 3130 . Heat treatment should give a Brinell test of from 223 to 285 .

\section{SOCIETY OF AUTOMOTIVE ENGINEERS, AERONAUTIC; PLAIN HEXAGON-HEAD BOLTS, S. A. E. STANDARD, AUGUST, 1928}

\section{PLAIN HEXAGON-HEAD BOLTS} PHYSICAL PROPERTIES

Assembled bolts shall show required minimum tensile strength when tested in tension between the head and the nut.

Bolts shall stand cold-bending in the plain part without fracture through an angle of $180^{\circ}$ over a diameter equal to the diameter of the bolt. In short bolts the test may be made in threaded part, the angle of the bend to be through $35^{\circ}$.

\section{MARKING}

Bolts conforming to these specifications shall be marked in some distinctive manner where shown.

Bolts conforming entirely to Army-Navy material specifications shall be marked with $\mathrm{X}$.

Bolts not marked in either manner will be understood to be made from low-strength material. 


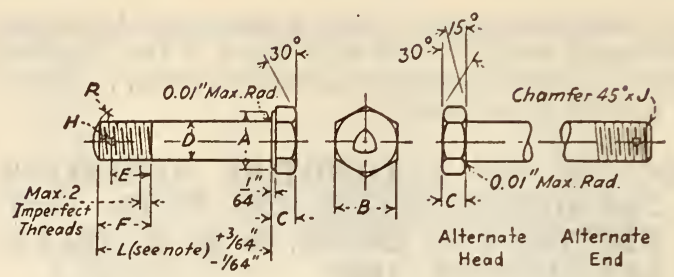

Figure 346.-Plain hexagon head bolts

Plain hexagon-head bolt dimensions

[All dimensions in inches]

\begin{tabular}{|c|c|c|c|c|c|c|c|c|c|c|c|}
\hline Nominal bolt size & $\begin{array}{l}\text { Threads } \\
\text { per } \\
\text { inch } 1\end{array}$ & $A$ & $B$ & $C$ & $D^{2}$ & $E$ & F & $H$ & $J$ & $R$ & $\begin{array}{c}\text { Tensile } \\
\text { strength, } \\
\text { mini- } \\
\text { mum, } \\
\text { pounds }\end{array}$ \\
\hline $0.1640(\mathrm{No} .8) \ldots$ & 36 & $5 / 10$ & $5 / 10$ & $1 / 8$ & $0.164+0.0000$ & $15 / 64$ & $3 / 8$ & 0.070 & $1 / 32$ & $1 / 8$ & - \\
\hline .1900 (No. 10) ... & 32 & $3 / 8$ & $3 / 8$ & $1 / 8$ & $.190+.0000$ & $1 / 4$ & $3 / 8$ & .070 & $1 / 32$ & $3 / 10$ & 2,190 \\
\hline $.2500(1 / 4) \ldots$ & 28 & $7 / 10$ & $7 / 10$ & $5 / 32$ & $\begin{array}{r}.250 \mp .0054 \\
\mp .0062\end{array}$ & $\% / 32$ & $7 / 10$ & .076 & $1 / 32$ & $1 / 4$ & 4,070 \\
\hline $.3125(5 / 10) \ldots$ & 24 & $1 / 2$ & $1 / 2$ & $3 / 10$ & $.3125+.0000$ & $21 / 64$ & $7 / 10$ & .076 & $3 / 64$ & $5 / 20$ & 6,550 \\
\hline $.3750(3 / 8) \ldots$ & 24 & $9 / 10$ & $9 / 10$ & $7 / 32$ & $\begin{array}{r}.375+.0000 \\
-.0066\end{array}$ & $13 / 32$ & $9 / 10$ & .106 & $3 / 64$ & 36 & 10,100 \\
\hline $4375(7 / 10) \ldots$ & 20 & $5 / 8$ & $5 / 8$ & $1 / 4$ & $.4375+.0000$ & $2 \% 4$ & $\% 10$ & .106 & 364 & $7 / 10$ & 13,620 \\
\hline $.5000(1 / 2) \ldots$ & 20 & $3 / 4$ & $3 / 4$ & $9 / 32$ & $\begin{array}{r}.500+.0000 \\
-.0072\end{array}$ & $9 / 10$ & $11 / 10$ & .108 & 364 & $1 / 2$ & 18,580 \\
\hline $.5625(9 / 10)$ & 18 & $7 / 8$ & $7 / 8$ & $5 / 10$ & $.5625+.0000$ & $3 \% 64$ & $3 / 4$ & .141 & 364 & $\% / 10$ & 23,600 \\
\hline $.6250(5 / 8) \ldots$ & 18 & $15 / 10$ & $15 / 10$ & $11 / 32$ & $\begin{array}{r}.625+.0000 \\
-.0082\end{array}$ & 1316 & $13 / 10$ & .141 & $3 / 64$ & $5 / 8$ & 30,000 \\
\hline
\end{tabular}

1 Threads are American standard (NF), with class 3 tolerances. NF indicates national fine pitch.

3 Finished sizes include plating or protective coating.

Bolt lengths 14 to 8 inches by 18 -inch increments.

Where $L$ is shorter than $F$, bolt is to be threaded entire length.

SOCIETY OF AUTOMOTIVE ENGINEERS, AERONAUTIC; EYEBOLTS, S. A. E. STANDARD JANUARY, 1931

EYEBOLTS

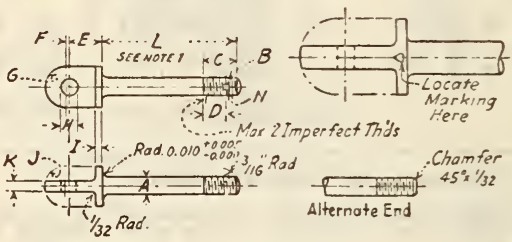

Figure 347.-Eyebolts

Eyebolt dimensions

[All dimensions in inches]

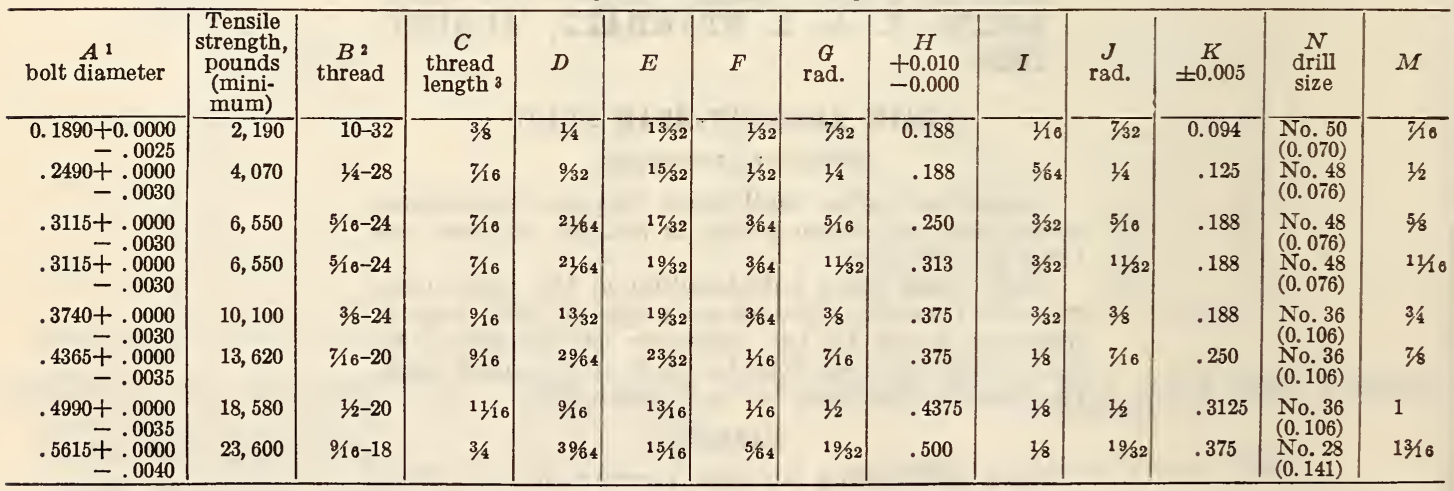

1 Finished sizes include plating or protective coating.

2 Threads are American standard (NF), with class 3 tolerances. NF indicates national fine pitch.

8 Where length of bolt $(L)$ is shorter than $C$ (thread length), bolt is to be threaded entire length. (See illustration.)

- Standard bolt lengths $(L) 34$ to 8 inches by 1/8-inch increments. Tolerances on length, $+3 / 64,-164$. (See illustration.)

Tolerances are \pm 0.010 except where otherwise indicated.

(Physical properties and marking are identical with those specifled for plain hexagon-head bolts, given above.) 
SOCIETY OF AUTOMOTIVE ENGINEERS, AER ONAUTIC; PROPELLER-BLADE CLAMP RINGS, BOLTS, AND NUTS, S. A. E. RECOMMENDED PRACTICE, JUNE, 1929

PROPELLER-BLADE CLAMP RINGS, BOLTS, AND NUTS
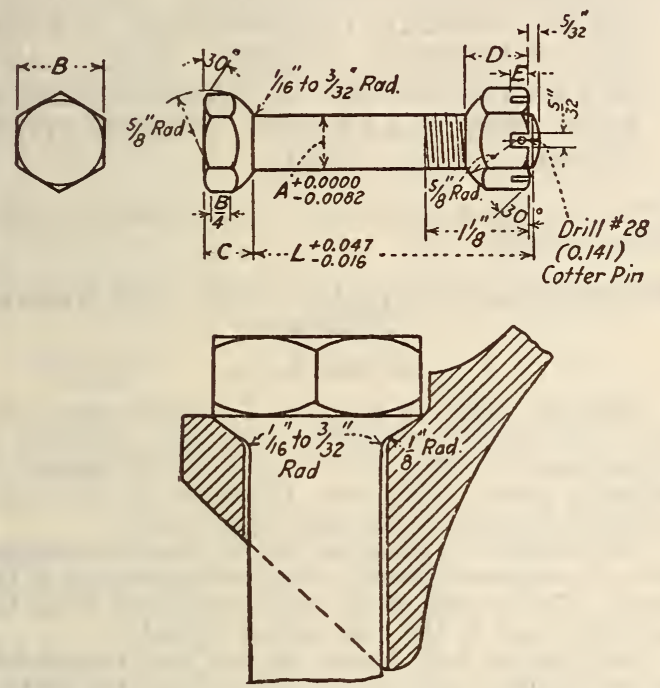

Frgure 348.-Propeller blade clamp, ring bolts, and nuts

Clamp-ring bolts and nuts

[All dimensions in inches]

\begin{tabular}{|c|c|c|c|c|c|c|c|c|}
\hline Size & A & B & C & D & E & F & G & $\begin{array}{c}\text { Clamp } \\
\text { ring }\end{array}$ \\
\hline $\begin{array}{r}9 / 16-18 \\
5 / 8-18\end{array}$ & 0.5625 & $\begin{array}{r}7 / 6 \\
.6250\end{array}$ & $\begin{array}{c}15 / 132 \\
33 / 64\end{array}$ & $\begin{array}{c}21 / 32 \\
4 \% 64\end{array}$ & $\begin{array}{c}3 / 16 \\
1 / 4\end{array}$ & $\begin{array}{c}27 / 8 \\
3\end{array}$ & $\begin{array}{c}0.5264 \\
.5889\end{array}$ & $\begin{array}{l}41 / 4-43 / 8 \\
45 / 8-5\end{array}$ \\
\hline
\end{tabular}

Threads.-American standard fine pitch, class 3 fit.

Bolts.-S. A. E. Steel 2330, 6130, or 6135 H. T. to 125,000 lbs. per sq. in T. S.

Nuts.-S. A. E. Steel 1025.

Scleroscope hardness for bolts 35-40.

Finish.-Cadmium plate.

\section{SOCIETY OF AUTOMOTIVE ENGINEERS,} ROUND UNSLOTTED-HEAD BOLTS, S. A. E. STANDARDS, FEBRUARY, 1929

\section{ROUND UNSLOTTED-HEAD BOLTS}

(Under this heading are included: Square-neck carriage bolts, fin-neck carriage bolts, ribbed carriage bolts, step bolts, button-head machine bolts, and countersunk carriage bolts. The dimensions of these bolts are identical with those given above under the American Society of Mechanical Engi- neers, B18e-1928, except that the S. A. E. tables omit minimum lengths of thread and corresponding tolerances.)

SOCIETY OF AUTOMOTIVE ENGINEERS, FRAMES AND SPRINGS; SPRING REBOUND CLIPS, SPACERS, AND BOLTS, S. A. E. RECOMMENDED PRACTICE, JANUARY, 1931

SPRING REBOUND CLIPS, SPACERS, AND BOLTS

(See 611.54, p. 810.)

SOCIETY OF AUTOMOTIVE ENGINEERS, FRAMES AND SPRINGS; SPRING CENTER BOLTS, S. A. E. STANDARD, JANUARY, 1931

\section{SPRING CENTER BOITS}

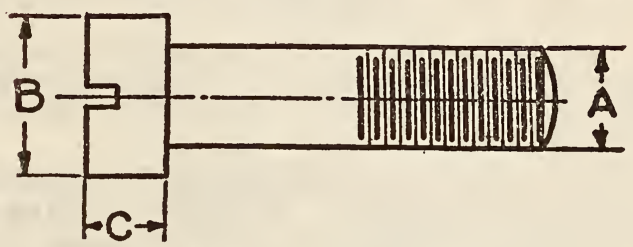

Figdre 349.-Spring center-bolt

TABLE 1.-Center-bolt application

[All dimensions in inches]

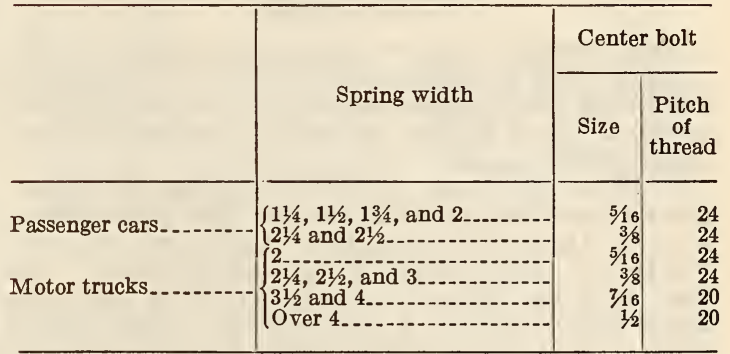

TABLE 2.-Center-bolt dimensions

[All dimensions in inches]

\begin{tabular}{|c|c|c|c|c|c|c|c|}
\hline \multirow{2}{*}{$\begin{array}{l}\text { Body } \\
\text { diam- } \\
\text { eter A }\end{array}$} & \multirow{2}{*}{$\begin{array}{l}\text { Pitch } \\
\text { of } \\
\text { thread }\end{array}$} & \multirow{2}{*}{$\begin{array}{l}\text { Head } \\
\text { diam- } \\
\text { eter B }\end{array}$} & \multirow{2}{*}{$\underset{\text { Ceight }}{\text { Head }}$} & \multicolumn{2}{|c|}{ Slot width } & \multicolumn{2}{|c|}{ Slot depth } \\
\hline & & & & $\begin{array}{l}\text { Maxi- } \\
\text { mum }\end{array}$ & $\begin{array}{l}\text { Mini- } \\
\text { mum }\end{array}$ & $\begin{array}{l}\text { Maxi- } \\
\text { mum }\end{array}$ & $\begin{array}{l}\text { Mini- } \\
\text { mum }\end{array}$ \\
\hline $\begin{array}{r}5 / 16 \\
3 / 8 \\
7 / 16 \\
1 / 2\end{array}$ & $\begin{array}{l}24 \\
24 \\
20 \\
20\end{array}$ & $\begin{array}{r}1 / 2 \\
9 / 16 \\
5 / 8 \\
3 / 4\end{array}$ & $\begin{array}{l}3 / 8 \\
3 / 8 \\
1 / 2 \\
1 / 2\end{array}$ & $\begin{array}{r}0.079 \\
.088 \\
.098 \\
.110\end{array}$ & $\begin{array}{r}0.065 \\
.074 \\
.083 \\
.094\end{array}$ & $\begin{array}{r}0.115 \\
.142 \\
.168 \\
.188\end{array}$ & $\begin{array}{r}0.090 \\
.112 \\
.133 \\
.148\end{array}$ \\
\hline
\end{tabular}

All threads American standard (NF), class 2 fit. Thread length to be $11 / 2 \mathrm{~A}+1 / 4$ inch. The edge of the bolthead shall have a maximum chamfer or rounding of $1 / 32$ inch. Where passenger-car springs are more than $21 / 2$ inches wide, center bolts specified for motor-truck springs of similar width shall be used.

Center-bolt nuts.--Standard cold-pressed hexagon blanks shall be used for spring center-bolt nuts. 
UNITED STATES GOVERNMENT, DEPARTMENT OF COMMERCE, BUREAU OF STANDARDS, PLOW BOLTS, SIMPLIFIED PRACTICE RECOMMENDATION NO. 23, 1925

\section{PLOW BOLTS}

(This recommendation confines itself to nominal diameters and threads per inch of the four standard types of plow bolts, the data on which are in agreement with the American Society of Mechanical Engineers, given above.)

UNITED STATES GOVERNMENT, DEPARTIMENT OF COMMERCE, BUREAU OF STANDARDS, PACKING OF CARRIAGE, MACHINE, AND LAG BOLTS, SIMPLIFIED PRACTICE RECOMMENDATION NO. 60 , MARCH 15, 1930

\section{PACKING OF CARRIAGE, MACHINE, AND LAG BOLTS}

(Contains tabular matter covering dimensions, quantity, and weights of cut or roll thread carriage, machine, and lag bolts in containers accepted as standard by industry.)

UNITED STATES GOVERNMENT, FEDERAL SPECIFICATIONS BOARD, FEDERAL SPECIFICATION FOR CAP SCREWS, SET SCREWS, MACHINE SCREWS, AND STOVE BOLTS, NO. 548 (CURRENT DESIGNATION : FF-S-91), MARCH 5, 1928

\section{STOVE BOLTS}

(For material and workmanship, general requirements, detail requirements, methods of inspection and tests, packing and marking of shipments, and notes, see F. S. B. No. 548 under commodity designation 608.0 , p. 527.)

1. Stove bolts are regularly furnished in coarsethread series standard sizes of one-eighth, five thirtyseconds, three-sixteenths, seven thirty-seconds, onefourth, five-sixteenths and three-eighths inch. The first four are interchangeable with the machinescrew sizes Nos. 5, 8, 10, and 12, coarse-thread series. They are furnished with flat or round heads, slotted. Stove bolts are furnished with square nuts. (See National Screw Thread Commission, Table 74, under commodity designation 608.32, p. 611.)

2 . Stove bolts 2 inches and under in length are usually threaded from the extreme point to the least diameter of the bearing surface of the head. Stove bolts over 2 inches in length are usually threaded about two-thirds of their length. The length of stove bolts is measured from the largest diameter of the bearing surface of the head to the extreme point.

3 . Stove bolts having flat and round heads shall have the dimensions specified in Federal Specifications Board specification No. 548 (FF-S-91), Tables 14 and 15. (See 608.2, p. 579.)

4. The head shall be at right angles to the body within $2^{\circ}$ and concentric with the body within a tolerance of 3 per cent of the diameter of the head.

UNITED STATES GOVERNMENT, FEDERAL SPECIFICATIONS BOARD; FEDERAI SPECIFICATION FOR BOLTS, NUTS, STUDS, AND TAP RIVETS (AND MATERIAL FOR SAME), FF-B-571, OCTOBER 13,1931

\section{BOLTS AND STUDS}

(For detail specifications covering applicable Federal specifications, types, material and work- manship, general requirements, detail requirements-including references to tabular matter in other specifications, such as National Screw Thread Commission and the American Society of Mechanical Engineers - methods of sampling, inspection, and tests; packaging, packing, and marking; and notes, see FF-B-571 under commodity classification 608.0, p. 522.)

UNITED STATES GOVERNMENT, NATIONAL SCREW THREAD COMMISSION, AMERICAN NATIONAL SCREW, BOLT, AND NUT PROPORTIONS. BUREAU OFSTAND. ARDS MISCELIANEOUS PUBLICATION NO. 89, JUNE 22, 1928

(See also 608.0 , p. 551 ; 608.2, p. 583 ; 608.32, p. 609; 615.42, p. 883.) WRENCH-HEAD BOLTS AND NUTS, AND WRENCH
OPENINGS

\section{MACHINE BOLTS}

Machine bolts are regularly furnished one-fourth inch and over in diameter and with hexagon or squareheads. They are furnished with square or hexagon nuts. They are threaded for a length dependent upon the diameter and length.

The length of a machine bolt is the measurement from the extreme point to the bearing surface of the head. The length of thread is measured from the extreme point to the last perfect thread.

The tops of bolt heads are flat and chamfered; the maximum angle of chamfer with the top surface is $30^{\circ}$, and the diameter of the top flat circle is from 85 to 100 per cent of the width across flats.

The width across flats of bolt heads is measured at the widest point. The taper of the sides of bolt heads does not exceed $4^{\circ}$.

Bolt heads are concentric with the body within a tolerance of 3 per cent of the width across flats.

The minimum lengths of thread, unless otherwise specified, are given in Table 66 .

The thickness of the head is the distance from the top to the bearing surface.

(a) Rough and semifinished bolts.-Rough bolts are made by cold or hot pressing, punching, or forging. They are threaded but not machined.

Semifinished bolts are those which meet the tolerances prescribed without regard to the amount of machining needed to meet such tolerances, except that the bearing surfaces are finished smooth.

The dimensions of rough and semifinished bolts are given in Table 67.

Rough and semifinished boltheads are at right angles to the body within $3^{\circ}$.

(b) Finished bolts.-Finished bolts and nuts are machined all over, and threaded.

The dimensions of finished bolts are given in Table 68 .

Finished boltheads are at right angles to the body within $2^{\circ}$.

All finished bolts are washer faced. The thickness of the washer face is about one sixty-fourth inch. The diameter of the bearing surface of the washer face is equal to the width across flats within plus or minus 5 per cent.

\section{TAP BOLTS}

Tap bolts are the same as machine bolts, except that they are threaded to the head, and they may be used interchangeably. Tap bolts are regularly furnished in the coarse-thread series one-fourth inch and over in diameter, without nuts, and with square or hexagon heads. The length of bolt is measured from the extreme point to the bearing surface of the head. 
TABLE 66.-Minimum length of threaded portion of machine and tap bolts

\begin{tabular}{|c|c|c|c|c|c|c|}
\hline \multirow{3}{*}{$\begin{array}{l}\text { Length of bolt, } L \\
\text { (inches) }\end{array}$} & \multicolumn{6}{|c|}{ Diameter of bolt, $D$ (inches) } \\
\hline & $1 / 4$ & $5 / 16$ & $3 / 8$ & $7 / 10$ & $1 / 2$ & $9 / 16$ \\
\hline & \multicolumn{6}{|c|}{ Length of threaded part, $l$} \\
\hline 1 & 2 & 3 & 4 & $\mathbf{5}$ & 6 & 7 \\
\hline $\begin{array}{l}1 \text { to } 11 / 2 \\
13 / 8 \text { to } 2 \\
21 / 8 \text { to } 21 / 2 \\
258 / 8 \text { to } 3 \\
31 / 8 \text { to } 4 \\
418 \text { to } 8 \\
81 / 8 \text { to } 12 \\
121 / 8 \text { to } 20\end{array}$ & $\begin{array}{c}\text { Inch } \\
3 / 4 \\
7 / 8 \\
1 \\
1 \\
1 \\
1 \\
1 \\
1\end{array}$ & $\begin{array}{c}\text { Inch } \\
3 / 4 \\
7 / 8 \\
1 \\
1 \\
1 \\
1 \\
1 \\
1\end{array}$ & $\begin{array}{c}\text { Inches } \\
7 / 8 \\
1 \\
1 \\
1 \\
11 / 4 \\
11 / 4 \\
11 / 4 \\
11 / 2\end{array}$ & \begin{tabular}{|c|} 
Inches \\
$7 / 8$ \\
1 \\
1 \\
1 \\
$11 / 4$ \\
$11 / 4$ \\
$11 / 4$ \\
$11 / 2$
\end{tabular} & $\begin{array}{c}\text { Inches } \\
1 \\
1 \\
1 \\
1 \\
11 / 4 \\
11 / 4 \\
11 / 2 \\
2\end{array}$ & \begin{tabular}{|c} 
Inches \\
1 \\
1 \\
1 \\
1 \\
$11 / 4$ \\
$11 / 4$ \\
$11 / 2$ \\
2
\end{tabular} \\
\hline \multirow{3}{*}{$\begin{array}{l}\text { Length of bolt, } L \\
\text { (inches) }\end{array}$} & \multicolumn{6}{|c|}{ Diameter of bolt, $D$ (inches) } \\
\hline & $5 / 8$ & $3 / 4$ & $7 / 8$ & 1 & $11 / 8$ & $11 / 4$ \\
\hline & \multicolumn{6}{|c|}{ Length of threaded part, $l$} \\
\hline 1 & 8 & 9 & 10 & 11 & 12 & 13 \\
\hline $\begin{array}{l}1 \text { to } 11 / 2- \\
15 / 8 \text { to } 2 \\
21 / 8 \text { to } 21 / 2- \\
25 / 8 \text { to } 3\end{array}$ & $\begin{array}{r}\text { Inches } \\
11 / 4 \\
11 / 4 \\
11 / 4 \\
11 / 4\end{array}$ & $\begin{array}{r}\text { Inches } \\
111 / 2 \\
11 / 2 \\
11 / 2\end{array}$ & $\begin{array}{r}\text { Inches } \\
13 / 4 \\
13 / 4 \\
13 / 4\end{array}$ & \begin{tabular}{c} 
Inches \\
\hdashline $1^{3} / 4$ \\
2
\end{tabular} & \begin{tabular}{l} 
Inches \\
\hdashline \\
\hdashline $21 / 4$
\end{tabular} & Inches \\
\hline $\begin{array}{l}31 / 8 \text { to } 4 \\
41 / 8 \text { to } 8 \\
81 / 8 \text { to } 12 \\
121 / 8 \text { to } 20\end{array}$ & $\begin{array}{l}11 / 2 \\
11 / 2 \\
13 / 4 \\
2\end{array}$ & $\begin{array}{l}11 / 2 \\
13 / 4 \\
2 \\
2\end{array}$ & $\begin{array}{l}13 / 4 \\
21 \\
21 / 4 \\
21 / 2\end{array}$ & $\begin{array}{l}2 \\
21 / 4 \\
21 / 2 \\
3\end{array}$ & $\begin{array}{l}21 / 2 \\
23 / 4 \\
3 \\
31 / 4\end{array}$ & $\begin{array}{l}21 / 2 \\
31 / 2 \\
31 / 4 \\
31 / 2\end{array}$ \\
\hline
\end{tabular}

Note.-Machine bolts under 1 inch in length are threaded to the head. Machine bolts of any diameter, over 20 inches in length, and bolts over $11 / 4$ inches in diameter, if length permits, are threaded a length equal to three times the diameter unless otherwise specified.
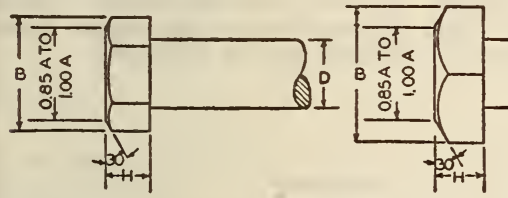

FIgURE 350.-Machine and tap bolt heads

TABLE 67.-Dimensions of rough and semifinished, square and hexagon, machine and tap bolt heads

\begin{tabular}{|c|c|c|c|c|c|c|c|}
\hline D & & & I & 3 & & H & \\
\hline \multirow{2}{*}{ Sizes } & \multicolumn{2}{|c|}{$\begin{array}{l}\text { Width across } \\
\text { flats }\end{array}$} & \multicolumn{2}{|c|}{$\begin{array}{l}\text { Minimum } \\
\text { width across } \\
\text { corners }\end{array}$} & \multicolumn{3}{|c|}{ Height } \\
\hline & $\begin{array}{l}\text { Maxi- } \\
\text { mum }\end{array}$ & $\begin{array}{l}\text { Mini- } \\
\text { mum }\end{array}$ & $\begin{array}{l}\text { Hexa- } \\
\text { gon }\end{array}$ & Square & $\begin{array}{c}\text { Nom- } \\
\text { inal }\end{array}$ & $\begin{array}{l}\text { Maxi- } \\
\text { mum }\end{array}$ & $\begin{array}{l}\text { Mini- } \\
\text { mum }\end{array}$ \\
\hline 1 & 2 & 3 & 4 & 5 & 6 & 7 & 8 \\
\hline & $\begin{array}{r}\text { Inches } \\
0.3750 \\
.5000 \\
.5625 \\
.6250 \\
.7500\end{array}$ & $\begin{array}{c}\text { Inches } \\
0.363 \\
484 \\
.544 \\
.603 \\
.725\end{array}$ & $\begin{array}{c}\text { Inches } \\
0.414 \\
552 \\
.620 \\
.687 \\
.827\end{array}$ & $\begin{array}{c}\text { Inches } \\
0.498 \\
.665 \\
.747 \\
.828 \\
.995\end{array}$ & $\begin{array}{c}\text { Inches } \\
1164 \\
1364 \\
1 / 4 \\
1964 \\
21 / 64\end{array}$ & \begin{tabular}{|c|} 
Inches \\
0.188 \\
.220 \\
.268 \\
.316 \\
.348
\end{tabular} & $\begin{array}{r}\text { Inches } \\
0.156 \\
.186 \\
.232 \\
.278 \\
.308\end{array}$ \\
\hline & $\begin{array}{r}.8750 \\
.9375 \\
1.1250 \\
1.3125 \\
1.5000\end{array}$ & $\begin{array}{r}.847 \\
.906 \\
1.088 \\
1.269 \\
1.450\end{array}$ & $\begin{array}{r}.966 \\
\text { 1. } 033 \\
1.240 \\
1.447 \\
1.653\end{array}$ & $\begin{array}{l}1.163 \\
1.244 \\
1.494 \\
1.742 \\
1.991\end{array}$ & $\begin{array}{l}3 / 8 \\
27 / 64 \\
1 / 2 \\
19 / 32 \\
21 / 32\end{array}$ & $\begin{array}{l}.396 \\
.444 \\
.524 \\
.620 \\
.684\end{array}$ & $\begin{array}{l}.35 \\
.40 \\
.47 \\
.56 \\
.62\end{array}$ \\
\hline & $\begin{array}{l}1.6875 \\
1.8750 \\
2.2500 \\
2.6250 \\
3.0000\end{array}$ & $\begin{array}{l}1.631 \\
1.813 \\
2.175 \\
2.538 \\
2.900\end{array}$ & $\begin{array}{l}1.859 \\
2.067 \\
2.480 \\
2.893 \\
3.306\end{array}$ & $\begin{array}{l}2.239 \\
2.489 \\
2.986 \\
3.485 \\
3.982\end{array}$ & $\begin{array}{l}3 / 4 \\
27 / 32 \\
1 \\
15 / 32 \\
111 / 32\end{array}$ & $\begin{array}{r}.780 \\
.876 \\
1.036 \\
1.196 \\
1.388\end{array}$ & $\begin{array}{r}.72 \\
.81 \\
.96 \\
1.11 \\
1.30\end{array}$ \\
\hline $\begin{array}{l}21 / 4 \ldots \\
21 / 2 \ldots \\
23 / 4 \ldots \\
3 \ldots\end{array}$ & $\begin{array}{l}3.3750 \\
3.7500 \\
4.1250 \\
4.5000\end{array}$ & $\begin{array}{l}3.263 \\
3.625 \\
3.988 \\
4.350\end{array}$ & $\begin{array}{l}\text { 3. } 720 \\
\text { 4. } 133 \\
\text { 4. } 546 \\
\text { 4. } 959\end{array}$ & $\begin{array}{l}4.480 \\
4.977 \\
5.476 \\
5.973\end{array}$ & $\begin{array}{l}11 / 2 \\
121 / 32 \\
153 \% 4 \\
2\end{array}$ & $\begin{array}{l}1.548 \\
1.708 \\
1.885 \\
2.060\end{array}$ & $\begin{array}{l}1.452 \\
1.604 \\
1.773 \\
1.940\end{array}$ \\
\hline
\end{tabular}
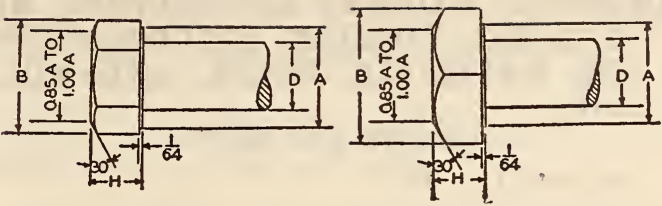

FigURE 351.-Machine and tap bolt heads

TABLE 68.-Dimensions of finished square and hexagon, machine and tap bolt heads

\begin{tabular}{|c|c|c|c|c|c|c|c|}
\hline \multirow{3}{*}{ Sizes } & \multicolumn{2}{|c|}{ A } & \multicolumn{2}{|c|}{13} & \multicolumn{3}{|c|}{$\overline{\mathrm{H}}$} \\
\hline & \multicolumn{2}{|c|}{$\begin{array}{l}\text { Width across } \\
\text { flats }\end{array}$} & \multicolumn{2}{|c|}{$\begin{array}{l}\text { Minimum } \\
\text { width across } \\
\text { corners }\end{array}$} & \multicolumn{3}{|c|}{ Height } \\
\hline & $\begin{array}{l}\text { Maxi- } \\
\text { mum }\end{array}$ & $\begin{array}{l}\text { Mini- } \\
\text { mum }\end{array}$ & $\begin{array}{c}\text { Hexa- } \\
\text { gon }\end{array}$ & Square & $\begin{array}{l}\text { Nom- } \\
\text { inal }\end{array}$ & $\begin{array}{l}\text { Maxi- } \\
\text { mum }\end{array}$ & $\begin{array}{l}\text { Mini- } \\
\text { mum }\end{array}$ \\
\hline 1 & 2 & 3 & 4 & 5 & 6 & 7 & 8 \\
\hline & $\begin{array}{l}\text { Inches } \\
0.4375 \\
.5625 \\
.6250 \\
.7500 \\
.8125\end{array}$ & $\begin{array}{c}\text { Inches } \\
0.428 \\
.552 \\
.613 \\
.737 \\
.799\end{array}$ & $\begin{array}{c}\text { Inches } \\
0.488 \\
.629 \\
.699 \\
.840 \\
.911\end{array}$ & $\begin{array}{c}\text { Inches } \\
0.588 \\
.758 \\
.842 \\
1.012 \\
1.097\end{array}$ & $\begin{array}{c}\text { Inches } \\
3 / 16 \\
15 / 64 \\
9 / 32 \\
2164 \\
3 / 8\end{array}$ & $\begin{array}{l}\text { Inches } \\
0.194 \\
.242 \\
.289 \\
.337 \\
.385\end{array}$ & $\begin{array}{c}\text { nches } \\
0.180 \\
.227 \\
.273 \\
.319 \\
.365\end{array}$ \\
\hline & $\begin{array}{r}.8750 \\
.9375 \\
1.1250 \\
1.3125 \\
1.5000\end{array}$ & $\begin{array}{r}.861 \\
.922 \\
1.108 \\
1.293 \\
1.479\end{array}$ & $\begin{array}{l}.982 \\
1.051 \\
1.263 \\
1.474 \\
1.686\end{array}$ & $\begin{array}{l}1.7 \\
2.0\end{array}$ & $\begin{array}{l}27 / 64 \\
15 / 32 \\
9 / 16 \\
21 / 32 \\
3 / 4\end{array}$ & .672 & $\begin{array}{l}.41 \\
.45 \\
.54 \\
.64 \\
.73\end{array}$ \\
\hline & $\begin{array}{l}1.6875 \\
1.8750 \\
2.2500 \\
2.6250 \\
3.0000\end{array}$ & $\begin{array}{l}2.222 \\
2.593 \\
2.964\end{array}$ & $\begin{array}{l}1.898 \\
2.109 \\
2.533 \\
2.956 \\
3.379\end{array}$ & 3.5 & $\begin{array}{c}27 / 32 \\
15 / 16 \\
11 / 8 \\
15 / 16 \\
11 / 2\end{array}$ & $\begin{array}{r}.863 \\
.959 \\
1.150 \\
1.341 \\
1.533\end{array}$ & $\begin{array}{l}.82 \\
.91 \\
1.10 \\
1.28 \\
1.46\end{array}$ \\
\hline 4 & $\begin{array}{l}3.3750 \\
3.7500 \\
4.1250 \\
4.5000\end{array}$ & $\begin{array}{l}\text { 3. } 335 \\
\text { 3. } 707 \\
4.078 \\
4.449\end{array}$ & $\begin{array}{l}\text { 3. } 802 \\
\text { 4. } 226 \\
4.649 \\
\text { 5. } 072\end{array}$ & & $\begin{array}{l}111 / 16 \\
17 / 8 \\
21 / 16 \\
21 / 4\end{array}$ & $\begin{array}{l}\text { 1. } 724 \\
\text { 1. } 915 \\
\text { 2. } 106 \\
\text { 2. } 298\end{array}$ & $\begin{array}{l}1.651 \\
\text { 1. } 835 \\
2.019 \\
2.203\end{array}$ \\
\hline
\end{tabular}

608.32 NUTS.

AMERICAN ELECTRIC RAILWAY ENGI.

NEERING ASSOCIATION, OVERHEAD

LINE MATERIAL FOR DIRECT AND CAT-

ENARY SUSPENSION, RECOMMENDED

SPECIFICATION, D102-30, 1930

\section{OVERHEAD LINE MATERIAI}

(D102-30, paragraph 304, includes requirements for nuts. For specification see 611.29, p. 682.)

\section{AMERICAN MARINE STANDARDS COM-} MITTEE, SPECIFICATION E NO. \%, PROPELLER SHAFT DETAILS, A. M. S. C. 28, JUNE 10, 1927

(See also 608.52, p. 616.)

\section{PROPELLER NUTS}

(Contains specifications, dimensions, and illustrations of tail shafts and stern tube bearings, propeller keys, propeller nuts, and stern tube stuffing boxes.)

AMERICAN MARINE STANDARDS COMMITTEE, SPECIFICATION E NO. 11, PROPELLER HUB STUDS, NUTS, AND LOCK SCREWS, A. M. S. C. 30, SEPTEMBER 20, 1927

(See also 608.2 , p. $574 ; 608.31$, p. 586 ; and 646.59 , p. 1257.)

\section{NUTS}

(Contains specifications, dimensions, and illustrations of propeller hub studs, nuts, and lock screws.) 
AMERICAN RAILWAY ASSOCIATION, MECHANICAL DIVISION, SPECIFICATIONS FOR MATERIALS, BOLTS, AND NUTS, 1916

BOLTS AND NUTS

(See 608.31, p. 586.)

AMERICAN RAILWAY ASSOCIATION, MECHANICAL DIVISION, MISCELLANEOUS STANDARDS AND RECOMMENDED PRACTICE, 1930

CASTLE NUTS, COTTER PINS, AND LOCATION OF COTTER-PIN HOLES IN PROJECTING BOLT ENDS

(See 608.31, p. 588.)

\section{AMERICAN SOCIETY OF MECHANICAI} ENGINEERS, T SLOTS, THEIR BOLTS, NUTS, TONGUES, AND CUTTERS, B5a-1927

[Approved by the American Standards Association, March, 1927] T SLOTS, THEIR BOLTS, NUTS, TONGUES, AND CUTTERS

(Contains dimensions of $T$ slots, $T$ bolts, $T$ nuts, $T$ slot cutters, tongues and tongue seats for singlewidth $T$ slots, and reversible tongues and tongue seats for slots for two sizes of $T$ bolts.)

\section{AMERICAN SOCIETY OF MECHANICAI ENGINEERS, WRENCH HEAD BOLTS AND NUTS, AND WRENCH OPENINGS, B18b-1927}

[Approved as American tentative standard by the American Standards Association, February, 1927]

(See also 608.0 , p. $551 ; 608.2$, p. $575 ; 608.31$, p. 590 ; $615.42,883$.
WRENCH HEAD BOLTS AND NUTS, AND WRENCH OPENINGS

(Introductory notes are identical with those of the National Screw Thread Commission (see 608.0, p. 551). Specifications and dimensions for rough and semifinished square and hexagonal regular nuts, finished square and hexagonal regular nuts, finished and semifinished hexagonal jam nuts; hexagonal plain nuts (fine threads), hexagonal and square machine screw and stove bolt nuts, and hexagonal castellated nuts-all are identical with those given below for the National Screw Thread Commission. Formulas for computing dimensions are appended to the A. S. M. E. tables, but these formulas are not general in their application.)

\section{SOCIETY OF AUTOMOTIVE ENGINEERS,} CAP SCREWS, BOLTS AND NUTS, AND WRENCH OPENINGS, S. A. E. STAND. ARDS, JANUARY, 1930

[Approved by the American Standards Association, April, 1930]

(See also 608.2, p. 577; 608.31, p. 600; and 615.42, p. 833.)

CAP SCREWS, BOLTS AND NUTS, AND WRENCH OPENINGS

(For general specifications see 608.2 , p. 577 . Specifications and dimensions for rough and semifinished square and hexagonal regular nuts, finished square and hexagonal regular nuts, finished and semifinished hexagonal jam nuts, hexagonal plain nuts (one-fourth to one-half inch), hexagonal and square machine screw and stove bolt nuts, and hexagonal castellated nuts-all are identical with those given below for the National Screw Thread Commission. The dimensions of hexagonal plain nuts (fine thread) regular, are as follows:)

[All dimensions in inches]

\begin{tabular}{|c|c|c|c|c|c|c|c|c|c|}
\hline \multicolumn{2}{|c|}{$\begin{array}{c}\text { Diameter of } \\
\text { bolt }\end{array}$} & \multirow{2}{*}{$\begin{array}{c}\begin{array}{c}\text { Threads } \\
\text { per inch } 1\end{array} \\
\begin{array}{c}\text { Fine } \\
(\mathrm{NF})\end{array}\end{array}$} & \multicolumn{3}{|c|}{ Width across flats } & \multirow{2}{*}{$\begin{array}{c}\text { Mini- } \\
\text { mum } \\
\text { width } \\
\text { across cor- } \\
\text { ners of } \\
\text { hexagon }\end{array}$} & \multicolumn{3}{|c|}{ Thickness } \\
\hline $\begin{array}{c}\text { Nomi- } \\
\text { nal }\end{array}$ & $\begin{array}{l}\text { Maxi- } \\
\text { mum }\end{array}$ & & $\underset{\text { nal }}{\text { Nomi- }}$ & $\begin{array}{l}\text { Maxi- } \\
\text { mum }\end{array}$ & $\begin{array}{l}\text { Mini- } \\
\text { mum }\end{array}$ & & $\begin{array}{l}\text { Nomi- } \\
\text { nal }\end{array}$ & $\begin{array}{l}\text { Maxi- } \\
\text { mum }\end{array}$ & $\begin{array}{l}\text { Mini- } \\
\text { mum }\end{array}$ \\
\hline $\begin{array}{l}9 / 16 \\
5 / 8 \\
3 / 4 \\
7 / 8\end{array}$ & $\begin{array}{r}0.5625 \\
.6250 \\
.7500 \\
.8750\end{array}$ & $\begin{array}{l}18 \\
18 \\
16 \\
14\end{array}$ & $\begin{array}{l}7 / 8 \\
11 / 8 \\
15 / 16\end{array}$ & $\begin{array}{l}0.8750 \\
1.0000 \\
1.1250 \\
1.3125\end{array}$ & $\begin{array}{r}0.861 \\
.985 \\
1.108 \\
1.293\end{array}$ & $\begin{array}{l}0.981 \\
1.123 \\
1.263 \\
1.474\end{array}$ & $\begin{array}{l}1 / 2 \\
35 / 64 \\
21 / 32 \\
4964\end{array}$ & $\begin{array}{r}0.511 \\
.559 \\
.670 \\
.781\end{array}$ & $\begin{array}{r}0.489 \\
.535 \\
.642 \\
.750\end{array}$ \\
\hline $\begin{array}{l}1 \\
11 / 8 \\
11 / 4 \\
11 / 2\end{array}$ & $\begin{array}{l}1.0000 \\
1.1250 \\
1.2500 \\
1.5000\end{array}$ & $\begin{array}{l}14 \\
12 \\
12 \\
12\end{array}$ & $\begin{array}{l}11 / 2 \\
111 / 16 \\
17 / 8 \\
21 / 4\end{array}$ & $\begin{array}{l}1.5000 \\
1.6875 \\
1.8750 \\
2.2500\end{array}$ & $\begin{array}{l}1.479 \\
1.665 \\
1.850 \\
2.222\end{array}$ & $\begin{array}{l}1.686 \\
1.898 \\
2.109 \\
2.533\end{array}$ & $\begin{array}{l}1^{7 / 8} \\
13 / 32 \\
15 / 16\end{array}$ & $\begin{array}{l}.893 \\
1.019 \\
1.115 \\
1.338\end{array}$ & $\begin{array}{r}.858 \\
.981 \\
1.072 \\
1.228\end{array}$ \\
\hline
\end{tabular}

1 Not included in American tentative standard. 
SOCIETY OF AUTOMOTIVE ENGINEERS, CAP SCREWS, BOLTS, AND NUTS; GAGING OF CASTLE-NUT SLOTS, FEBRUARY, 1925

\section{GAGING OF CASTLE-NOT SLOTS}

\section{GENERAL INFORMATION}

1. The method of gaging castle-nut slots that is indicated in the accompanying table permits as mnch variation in the location of the cotter-pin hole in the screw as in the location of the cotter-pin slot in the nut. Some approximations will have to be made in the application of this method, owing to differences of bore due to the drilling and to the tapping of the nut. The errors can be reduced by the nut manufacturer by making the diameter of the plug suitable for the minimum drilled hole, and by the user by making the plug suitable for the minimum minor-diameter of the threaded hole.
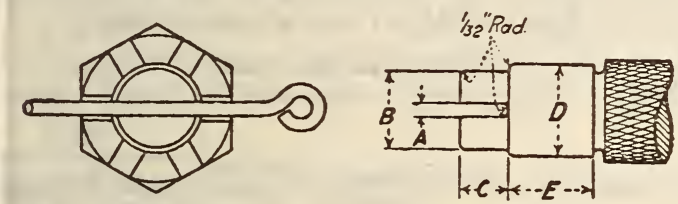

FIGURE 352.-Gaging castle-nut slots

[All dimensions in inches]

\begin{tabular}{|c|c|c|c|c|c|c|c|c|c|}
\hline \multirow{2}{*}{ Nut } & \multirow{2}{*}{$\begin{array}{l}\text { Slot } \\
\text { width }\end{array}$} & \multirow{2}{*}{$\begin{array}{l}\text { Cotter- } \\
\text { pin } \\
\text { diam- } \\
\text { eter? }\end{array}$} & \multicolumn{2}{|c|}{ Drill rod } & \multirow{2}{*}{$A$} & \multirow{2}{*}{$B$} & \multirow{2}{*}{$C$} & \multirow{2}{*}{$D^{1}$} & \multirow{2}{*}{$E$} \\
\hline & & & No. & Inch & & & & & \\
\hline $\begin{array}{r}1 / 4 \\
5 / 16 \\
38 \\
7 / 16 \\
3 / 2\end{array}$ & $\begin{array}{r}5 / 64 \\
5 \% 64 \\
1 / 8 \\
1 / 8 \\
18\end{array}$ & $\begin{array}{l}1 / 16 \\
1 / 16 \\
3 / 32 \\
3 / 32 \\
3 / 32\end{array}$ & $\begin{array}{l}49 \\
49 \\
34 \\
34 \\
34\end{array}$ & $\begin{array}{r}0.072 \\
.072 \\
.110 \\
.110 \\
.110\end{array}$ & $\begin{array}{r}0.072 \\
.072 \\
.110 \\
.110 \\
.110\end{array}$ & $\begin{array}{r}0.182 \\
15 / 64 \\
194 \\
11 / 32 \\
13 / 32\end{array}$ & $\begin{array}{r}5 / 32 \\
5 / 32 \\
3 / 16 \\
3 / 16 \\
1 / 4\end{array}$ & $\begin{array}{r}0.2113 \\
.2674 \\
.3299 \\
.3834 \\
.4459\end{array}$ & $\begin{array}{r}1 / 4 \\
19 \% 64 \\
11 / 32 \\
25 \% 64 \\
7 / 16\end{array}$ \\
\hline
\end{tabular}

The drill rod shall preferably be tempered and should fit in the slot of the gage without shake.

1 Minimum minor-diameter for free fit (class 2) nuts. If the nuts are not threaded in accordance with the American standard for screw threads (see 608.0, p. 529 ), the diameter shall be the same as the diameter of the "go" plug gage for the bore.

S. A. E. standard dimensions.
2. The diameter of the gaging pin is intended to be the mean of the width of the slot in the gage and the diameter of the cotter pin, the drill-rod size nearest this dimension, but larger, being used. The diameter of the slotted part of the gage plug is made smaller so as to clear the burrs resulting from the blanking and the slotting of the nut.

\section{SOCIETY OF AUTOMOTIVE ENGINEERS,} CAP SCREWS, BOLTS, AND NUTS; HIGH NUTS, S. A. E. STANDARD, JUIY, 1924

\section{HIGH NOTS}

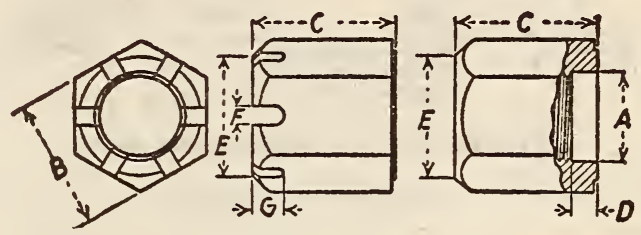

FIGURE 353.-High nuts

[All dimensions in inches]

\begin{tabular}{|c|c|c|c|c|c|c|}
\hline $\operatorname{Size} A$ & $B$ & $C$ & $D^{1}$ & $E$ & $F$ & $G$ \\
\hline $\begin{array}{r}1 / 4 \\
5 / 16 \\
3 / 8 \\
7 / 16 \\
1 / 2\end{array}$ & $\begin{array}{r}7 / 16 \\
1 / 2 \\
9 / 16 \\
5 / 8 \\
3 / 4\end{array}$ & $\begin{array}{r}3 / 8 \\
29 / 64 \\
17 / 32 \\
39 / 64 \\
11 / 16\end{array}$ & $\begin{array}{r}1 / 16 \\
5 / 64 \\
3 / 32 \\
7 / 64 \\
1 / 8\end{array}$ & $\begin{array}{l}25 / 64 \\
29 / 64 \\
33 / 64 \\
37 / 64 \\
11 / 16\end{array}$ & $\begin{array}{l}5 / 64 \\
5 / 64 \\
1 / 8 \\
1 / 8 \\
1 / 8\end{array}$ & $\begin{array}{r}1 / 8 \\
9 / 64 \\
5 / 32 \\
11 / 64 \\
3 / 16\end{array}$ \\
\hline $\begin{array}{r}9 / 16 \\
5 / 8 \\
3 / 4 \\
7 / 8 \\
1\end{array}$ & $\begin{array}{l}178 \\
1 \\
11 / 8 \\
15 / 16 \\
11 / 2\end{array}$ & $\begin{array}{l}49 / 64 \\
27 / 32 \\
1 \\
15 / 32 \\
15 / 16\end{array}$ & $\begin{array}{r}9 / 64 \\
5 / 32 \\
3 / 16 \\
7 / 32 \\
1 / 4\end{array}$ & $\begin{array}{c}51 / 64 \\
55 / 64 \\
63 / 64 \\
15 / 32 \\
121 / 64\end{array}$ & $\begin{array}{l}5 / 32 \\
5 / 32 \\
5 / 32 \\
5 / 32 \\
5 / 32\end{array}$ & $\begin{array}{r}13 / 64 \\
7 / 32 \\
1 / 4 \\
9 / 32 \\
5 / 16\end{array}$ \\
\hline
\end{tabular}

1 The counterbore indicated is optional.

All screw threads shall be in accordance with the free (class 2) fit as given in the American standard for screw threads. (See 608.0, p. 529.)

The angle of chamfer shall not exceed $40^{\circ}$ with the top face of the nut. 


\section{SOCIETY OF AUTOMOTIVE ENGINEERS, AERONAUTIC; CASTLE-HEXAGON NUTS, S. A. E. STANDARD, AUGUST, 1928}

\section{CASTLE-HEXAGON NUTS}
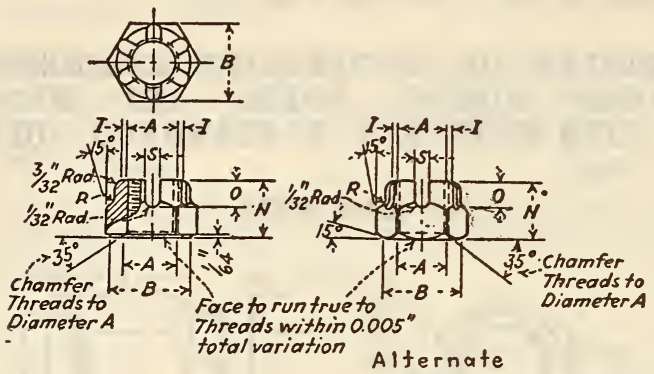

Figure 354.-Castle-hexagon nuts

[All dimensions in inches]

\begin{tabular}{|c|c|c|c|c|c|c|c|}
\hline Bolt size $A^{1}$ & $\begin{array}{l}\text { Threads } \\
\text { per inch }\end{array}$ & $B^{2}$ & $N$ & $\begin{array}{c}S \\
+0.010 \\
-0.000\end{array}$ & $0^{3}$ & $R$ & $I^{4}$ \\
\hline $\begin{array}{l}0.1640-(N 0.8) \\
0.1900-(N 0.10) \\
0.2500-(1 / 4) \\
0.3125-(5 / 16) \\
0.3750-(3 / 8)\end{array}$ & $\begin{array}{l}36 \\
32 \\
28 \\
24 \\
24\end{array}$ & $\begin{array}{r}3 / 8 \\
3 / 8 \\
7 / 16 \\
112 \\
9 / 16\end{array}$ & $\begin{array}{r}15 / 64 \\
1 / 4 \\
9 / 32 \\
21 / 64 \\
13 / 32\end{array}$ & $\begin{array}{l}5 / 64 \\
5 / 64 \\
5 / 64 \\
5 / 64 \\
1 / 8\end{array}$ & $\begin{array}{l}9 / 64 \\
964 \\
5 / 32 \\
5 / 32 \\
3 / 16\end{array}$ & $\begin{array}{l}3 / 32 \\
3 / 32 \\
3 / 32 \\
3 / 32 \\
3 / 32\end{array}$ & $\begin{array}{l}1 / 64 \\
164 \\
1 / 32 \\
1 / 32 \\
1 / 32\end{array}$ \\
\hline $\begin{array}{l}0.4375-(7 / 16) \\
0.5000-(1 / 2)- \\
0.5625-(8 / 16) . \\
0.6250-(5 / 8) \ldots\end{array}$ & $\begin{array}{l}20 \\
20 \\
18 \\
18\end{array}$ & $\begin{array}{r}5 / 8 \\
3 / 4 \\
7 / 8 \\
15 / 16\end{array}$ & $\begin{array}{r}29 / 64 \\
9616 \\
39 / 64 \\
11 / 16\end{array}$ & $\begin{array}{r}168 \\
1 / 8 \\
5 / 32 \\
5 / 32\end{array}$ & $\begin{array}{r}3 / 16 \\
13 / 64 \\
7 / 32 \\
7 / 32\end{array}$ & $\begin{array}{r}3 / 32 \\
18 \\
5 / 32 \\
5 / 32\end{array}$ & $\begin{array}{l}3 / 64 \\
3 / 64 \\
3 / 64 \\
3 / 64\end{array}$ \\
\hline
\end{tabular}

1 Finished sizes include plating or protective coating.

2 Also size of hexagon.

Also depth of slot.

Distance from $A$ to beginning of $3 / 32$-inch radius, whose center is floating to satisfy curve.

(Threads, tolerances, and physical properties are identical with those specified for plain hexagonal nuts, given below.)
SOCIETY OF AUTOMOTIVE ENGINEERS, AERONAUTIC; PLAIN HEXAGONAI NUTS, S. A. E. STANDARD, AUGUST, 1928

\section{PLAIN HEXAGONAL NUTS}

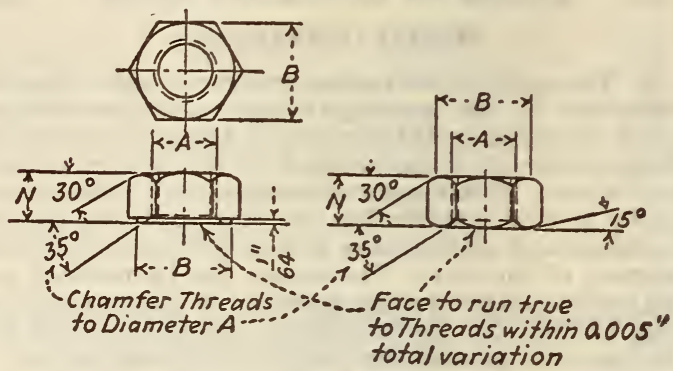

Alternate

Figure 355.-Plain hexagonal nuts [All dimensions in inches]

\begin{tabular}{|c|c|c|c|c|}
\hline \multirow[b]{2}{*}{ Nominal size $A$} & \multirow[b]{2}{*}{$\begin{array}{l}\text { Threads } \\
\text { per inch }{ }^{2}\end{array}$} & \multirow[b]{2}{*}{$\begin{array}{c}B \text { Width } \\
\text { across } \\
\text { flats }\end{array}$} & \multicolumn{2}{|c|}{$N^{8}$} \\
\hline & & & $\begin{array}{l}\text { Full- } \\
\text { strength } \\
\text { nuts } \\
\pm 0.005\end{array}$ & $\begin{array}{l}\text { Thin and } \\
\text { check } \\
\text { nuts } \\
\pm 0.005\end{array}$ \\
\hline $\begin{array}{l}0.1640-(N 0.8) \\
0.1900-(N 0.10) \\
0.2500-1 / 4\end{array}$ & $\begin{array}{l}36 \\
32 \\
28\end{array}$ & $\begin{array}{r}36 \\
38 \\
7 / 16\end{array}$ & $\begin{array}{r}1 / 8 \\
964 \\
3 / 16\end{array}$ & $\begin{array}{l}1 / 8 \\
964 \\
1 / 8\end{array}$ \\
\hline $\begin{array}{l}0.3125-5 / 16 \ldots-1 \\
0.3750-3 / 8 \\
0.4375-7 / 16 \ldots-\end{array}$ & $\begin{array}{l}24 \\
24 \\
20\end{array}$ & $\begin{array}{r}1 / 2 \\
9 / 16 \\
5 / 8\end{array}$ & $\begin{array}{r}15 / 64 \\
9 / 32 \\
21 / 64\end{array}$ & $\begin{array}{l}5 / 32 \\
3 / 16 \\
7 / 32\end{array}$ \\
\hline $\begin{array}{l}0.5000-1 / 2 \\
0.5625-9 / 16 \\
0.6250-5 / 8\end{array}$ & $\begin{array}{l}20 \\
18 \\
18\end{array}$ & $\begin{array}{r}3 / 4 \\
7 / 8 \\
15 / 16\end{array}$ & $\begin{array}{r}3 / 8 \\
27 / 64 \\
15 / 32\end{array}$ & $\begin{array}{l}1 / 4 \\
9 / 32 \\
5 / 10\end{array}$ \\
\hline
\end{tabular}

Finished sizes include plating or protective coating.

2 Threads are American standard (NF), with class 3 tolerances. $\mathrm{NF}$ indicates national fine pitch

${ }^{8}$ For full-strength nuts $\mathrm{N}=3 A / 4$. For check nuts and thin nut for shear bolts $1 / 4$ inch diameter and larger $\mathrm{N}=\mathrm{A} / 2$.

Tolerances are \pm 0.010 except where otherwise indicated. 
Nuts shall be manufactured from bar steel having the following physical properties:

Tensile strength (minimum) _..._._._._._bs./in.2. 70,000 Yield point (minimum) .......... 50,000 Yeld point (minimum) in 2-inch $\begin{array}{ll}\text { Elongation (minimum), in 2-inch......... per cent.- } & 10 \\ \text { Reduction of area (minimum) } & 40\end{array}$

Nuts shall withstand being increased 25 per cent of their original internal diameter without fracture and decreased 25 per cent of their internal diameter without failure.

SOCIETY OF AUTOMOTIVE ENGINEERS, PARTS AND FITTINGS; FUEL AND LUBRICATION TUBE FITTINGS, S. A. E. STANDARD, JULY, 1924

FUEL AND LUBRICATION TUBE FITTINGS

(See 645.4, p. 1232.)

SOCIETY OF AUTOMOTIVE ENGINEERS, AER O N A T I C; PROPELLER-BLADE CLAMP RINGS, BOLTS, AND NUTS, S. A. E. RECOMMENDED PRACTICE, JUNE, 1929

PROPELLER-BLADE CLAMP RINGS, BOLTS, AND NUTS

(See 608.31, p. 603.)

UNITED STATES GOVERNMENT, FEDERAL SPECIFICATIONS BOARD; FEDERAL SPECIFICATION FOR BOLTS, NUTS, STUDS, AND TAP RIVETS (AND MATERIAL FOR SAME), FF-B-5\%1, OCTOBER 13,1931

\section{NUTS}

(For detail specifications covering applicable Federal specifications, types, material and workmanship, general requirements, detail requirements (including references to tabular matter in other specifications, such as National Screw Thread Commission and the American Society of Mechanical Engineers), methods of sampling, inspection, and tests; packaging, packing, and marking; and notes, see FF-B-571 under commodity classification 608.0 p. 522.)

UNITED STATES GOVERNMENT, NATIONAL SCREW THREAD COMMISSION, AMERICAN NATIONAL SCREW, BOLT, AND NUT PROPORTIONS. BUREAU OF STANDARDS MISCELIANEOUS PUBLICA. TION NO. 89, JUNE 22, 1928

(See also 608.0 , p. $551 ; 608.2$, p. $583 ; 608.31$, p. $604 ; 615.42$, p. 883.)
WRENCH-HEAD BOLTS AND NUTS, AND WRENCH OPENINGS

\section{NUTS}

Nuts are commonly either hot forged, cold punched, or machined from bar stock.

The tops of regular square and hexagon nuts when not castellated, of jam nuts, square and hexagon machine screw nuts, and of hexagon light nuts when not castellated are flat and chamfered; the maximum angle of chamfer with the top surface is $25^{\circ}$; and the diameter of the top flat circle is from 85 to 100 per cent of the width across flats.

The width across flats of nuts is measured at the widest point. The taper of sides of nuts does not exceed $4^{\circ}$.

All finished hexagon and square regular nuts, castellated nuts, and hexagon light nuts are washer faced. The thickness of the washer face, including that of jam nuts having a washer face, is about one sixty-fourth inch. The diameter of the bearing surface of the washer face is equal to the width across flats within plus or minus 5 per cent. The thickness of the nut is the distance from the top to the bearing surface.

(a) Rough and semifinished regular nuts.-Rough nuts are made by cold or hot pressing, punching, or forging. They are threaded but not machined.

Semifinished nuts are those which meet the tolerances prescribed without regard to the amount of machining needed to meet such tolerances, except that the bearing surfaces are finished smooth.

The dimensions of rough and semifinished square and hexagon regular nuts are given in Table 69.

Semifinished nuts are faced on the bearing surface and are at right angles to the axis of the threaded hole within $3^{\circ}$.

(b) Finished regular nuts.-The dimensions of finished square and hexagon regular nuts are given in Table 70.

The axis of the threaded hole is at right angles to the washer face within a tolerance of $2^{\circ}$.

(c) Castellated nuts.-The dimensions of castellated nuts are given in Table 71.

The axis of the threaded hole is at right angles to the washer face within a tolerance of $2^{\circ}$

(d) Finished and semifinished jam nuts.-The dimensions of finished and semifinished jam nuts are given in Table 72. Jam nuts are either single or double chamfered as specified. If a single chamfer is specified, the opposite side is washer faced.

The axis of the threaded hole is at right angles to the washer or finished face within a tolerance of $2^{\circ}$.

(e) Hexagon light nuts.-The dimensions of hexagon light nuts are given in Table 73.

$(f)$ Hexagon and square machine screw nuts and square stove-bolt nuts. - The dimensions of hexagon and square machine screw nuts and square stovebolt nuts are given in Table 74 .

Machine screw nuts, when made from hexagon bar stock, are faced on the bearing surface and this surface is at right angles to the axis of the threaded hole within $5^{\circ}$. 

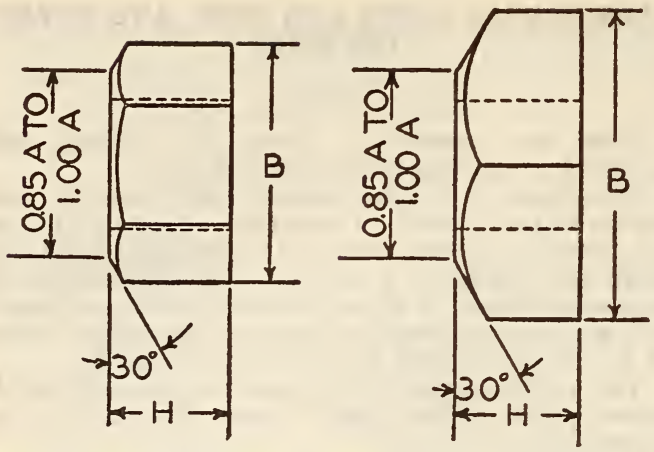

FIgURE 356.-Rough and semifinished square and hexagon regular nuts

TABLE 69.-Dimensions of rough and semifinished square and hexagon regular nuts

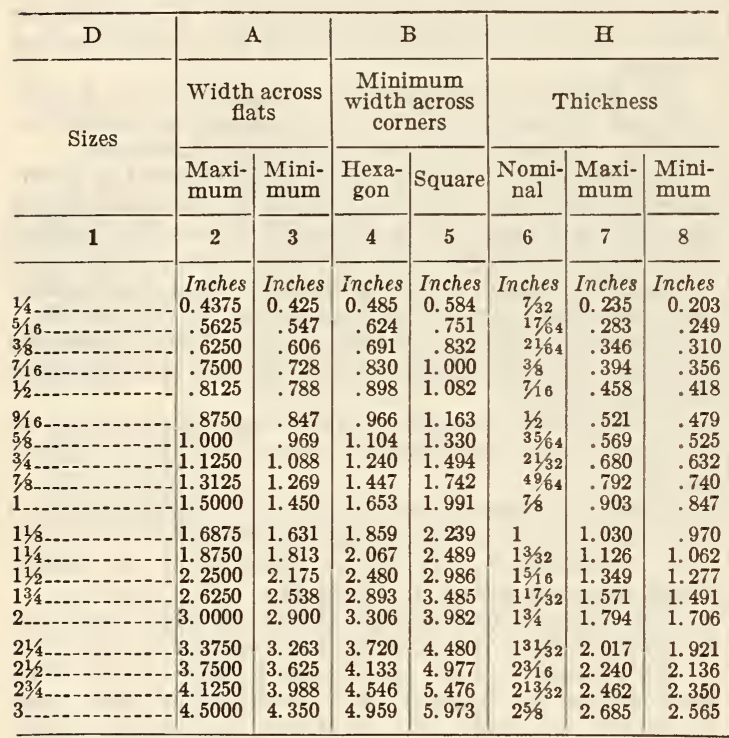
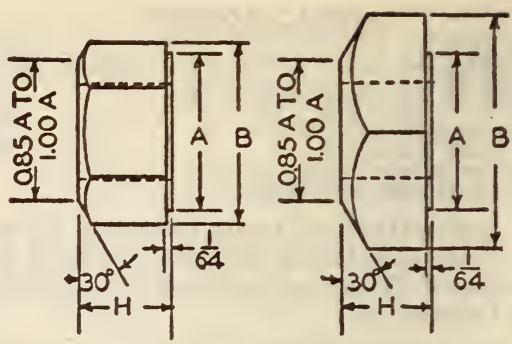

FIgURE 357.-Finished square and hexagon regular nuts

TABLE 70.-Dimensions of finished square and hexagon regular nuts

\begin{tabular}{|c|c|c|c|c|c|c|c|}
\hline $\mathrm{D}$ & \multicolumn{2}{|c|}{ A } & \multicolumn{2}{|c|}{ B } & \multicolumn{3}{|c|}{ H } \\
\hline \multirow{2}{*}{ Sizes } & \multicolumn{2}{|c|}{$\begin{array}{l}\text { Width across } \\
\text { flats }\end{array}$} & \multicolumn{2}{|c|}{$\begin{array}{c}\text { Minimum } \\
\text { width across } \\
\text { corners }\end{array}$} & \multicolumn{3}{|c|}{ Thickness } \\
\hline & $\begin{array}{l}\text { Maxi- } \\
\text { mum }\end{array}$ & $\begin{array}{l}\text { Mini- } \\
\text { mum }\end{array}$ & $\begin{array}{l}\text { Hexa- } \\
\text { gon }\end{array}$ & Square & $\begin{array}{c}\text { Nomi- } \\
\text { nal }\end{array}$ & $\begin{array}{l}\text { Maxi- } \\
\text { mum }\end{array}$ & $\begin{array}{l}\text { Mini- } \\
\text { mum }\end{array}$ \\
\hline 1 & 2 & 3 & 4 & 5 & 6 & 7 & 8 \\
\hline $\begin{array}{l}1 / 4 \ldots \\
5 / 16 \ldots \\
3 / 8 \ldots \\
7 / 16 \ldots \\
1 / 2 \ldots\end{array}$ & $\begin{array}{l}\text { Inches } \\
0.4375 \\
.5625 \\
.6250 \\
.7500 \\
.8125\end{array}$ & $\begin{array}{c}\text { Inches } \\
0.428 \\
.552 \\
.613 \\
.737 \\
.799\end{array}$ & $\begin{array}{c}\text { Inches } \\
0.488 \\
.629 \\
.699 \\
.840 \\
.911\end{array}$ & $\begin{array}{c}\text { Inches } \\
0.588 \\
.758 \\
.842 \\
1.012 \\
1.097\end{array}$ & $\begin{array}{c}\text { Inches } \\
7 / 32 \\
17 / 64 \\
2164 \\
3 / 8 \\
7 / 16\end{array}$ & $\begin{array}{c}\text { Inches } \\
0.225 \\
.273 \\
.336 \\
.384 \\
.448\end{array}$ & $\begin{array}{r}\text { Inches } \\
0.212 \\
.258 \\
.320 \\
.366 \\
.428\end{array}$ \\
\hline $\begin{array}{l}9 / 16 \ldots \\
5 / 8 \ldots \\
3 / 4 \ldots \\
7 / 8 \ldots \\
1 \ldots \ldots\end{array}$ & $\begin{array}{l}.8750 \\
1.000 \\
1.1250 \\
1.3125 \\
1.5000\end{array}$ & $\begin{array}{r}.861 \\
.985 \\
1.108 \\
1.293 \\
1.479\end{array}$ & $\begin{array}{r}.982 \\
1.123 \\
1.263 \\
1.474 \\
1.686\end{array}$ & $\begin{array}{l}1.182 \\
1.352 \\
1.521 \\
1.775 \\
2.031\end{array}$ & $\begin{array}{l}1 / 2 \\
35 / 64 \\
21 / 32 \\
1964 \\
7 / 8\end{array}$ & $\begin{array}{r}.511 \\
.559 \\
.670 \\
.781 \\
.893\end{array}$ & $\begin{array}{l}.489 \\
.535 \\
.642 \\
.750 \\
.858\end{array}$ \\
\hline $\begin{array}{l}116 . \\
11 / 4- \\
112 . \\
13 / 4 . \\
2 \ldots\end{array}$ & $\begin{array}{l}1.6875 \\
1.8750 \\
2.2500 \\
2.6250 \\
3.0000\end{array}$ & $\begin{array}{l}1.665 \\
1.850 \\
2.222 \\
2.593 \\
2.964\end{array}$ & $\begin{array}{l}1.898 \\
2.109 \\
2.533 \\
2.956 \\
3.379\end{array}$ & $\begin{array}{l}\text { 2. } 286 \\
2.540 \\
\text { 3. } 051 \\
\text { 3. } 560 \\
4.070\end{array}$ & $\begin{array}{l}1 \\
13 / 32 \\
15 / 16 \\
117 / 32 \\
13 / 4\end{array}$ & $\begin{array}{l}1.019 \\
1.115 \\
1.338 \\
1.560 \\
1.783\end{array}$ & $\begin{array}{l}.981 \\
1.072 \\
1.288 \\
1.503 \\
1.718\end{array}$ \\
\hline $\begin{array}{l}214 \ldots \\
21 / 2 \ldots \\
2344 \ldots \\
3 \ldots \ldots\end{array}$ & $\begin{array}{l}\text { 3. } 3750 \\
3.7500 \\
4.1250 \\
4.5000\end{array}$ & $\begin{array}{l}3.335 \\
3.707 \\
4.078 \\
4.449\end{array}$ & $\begin{array}{l}3.802 \\
4.226 \\
4.649 \\
5.072\end{array}$ & $\begin{array}{l}4.579 \\
5.090 \\
5.599 \\
6.108\end{array}$ & $\begin{array}{l}131 / 32 \\
23 / 16 \\
213 / 32 \\
25 \% 8\end{array}$ & $\begin{array}{l}2.005 \\
2.228 \\
2.450 \\
2.673\end{array}$ & $\begin{array}{l}1.932 \\
2.148 \\
2.363 \\
2.578\end{array}$ \\
\hline
\end{tabular}



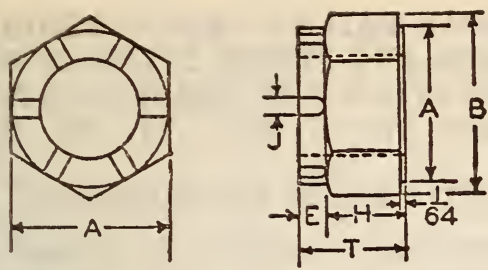

FIGURE 358.-Castellated nuts

$\mathrm{T}_{\mathrm{ABLE}}$ 71.-Dimensions of castellated nuts

\begin{tabular}{|c|c|c|c|c|c|c|c|c|c|}
\hline $\mathrm{D}$ & \multicolumn{2}{|c|}{ A } & $\mathrm{B}$ & \multicolumn{3}{|c|}{$\mathrm{T}$} & H & \multicolumn{2}{|c|}{$\mathrm{J}$} \\
\hline \multirow{2}{*}{ Sizes } & \multicolumn{2}{|c|}{$\begin{array}{c}\text { Width } \\
\text { across flats }\end{array}$} & \multirow{2}{*}{$\begin{array}{l}\text { Mini- } \\
\text { mum } \\
\text { width } \\
\text { across } \\
\text { cor- } \\
\text { ners } \\
\end{array}$} & \multicolumn{3}{|c|}{ Over-all thickness } & \multirow{2}{*}{$\begin{array}{l}\text { Thick- } \\
\text { ness, } \\
\text { nom- } \\
\text { inal }\end{array}$} & \multicolumn{2}{|c|}{ Slot } \\
\hline & $\begin{array}{c}\text { Maxi- } \\
\text { mum }\end{array}$ & $\left|\begin{array}{c}\text { Mini- } \\
\text { mum }\end{array}\right|$ & & $\begin{array}{l}\text { Nom- } \\
\text { inal }\end{array}$ & $\begin{array}{l}\text { Maxi- } \\
\text { mum }\end{array}$ & $\begin{array}{l}\text { Mini- } \\
\text { mum }\end{array}$ & & Width & Depth \\
\hline 1 & 2 & 3 & 4 & 5 & 6 & 7 & 8 & 9 & 10 \\
\hline 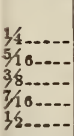 & $\begin{array}{r}\text { Inches } \\
0.4375 \\
.5000 \\
.5625 \\
.6250 \\
.7500\end{array}$ & $\begin{array}{r}\text { Inches } \\
0.422 \\
.489 \\
.551 \\
.612 \\
.737\end{array}$ & $\begin{array}{r}\text { Inches } \\
0.488 \\
.557 \\
.628 \\
.699 \\
.840\end{array}$ & $\begin{array}{c}\text { Inches } \\
9 / 32 \\
21,64 \\
13,32 \\
2964 \\
9116\end{array}$ & $\begin{array}{r}\text { Inches } \\
0.288 \\
.335 \\
.414 \\
.462 \\
.573\end{array}$ & $\begin{array}{r}\text { Inches } \\
0.275 \\
.321 \\
.398 \\
.444 \\
.553\end{array}$ & $\begin{array}{c}\text { Inches } \\
3 / 16 \\
15 \% 4 \\
9 / 32 \\
2164 \\
3 / 8\end{array}$ & $\begin{array}{r}\text { Inch } \\
564 \\
564 \\
18 \\
18 \\
18 \\
18\end{array}$ & \begin{tabular}{|c} 
Inch \\
$3 / 32$ \\
$3 / 32$ \\
$1 / 8$ \\
$1 / 8$ \\
$3 / 16$
\end{tabular} \\
\hline 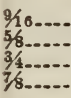 & $\begin{array}{l}.8750 \\
1.000 \\
1.1250 \\
1.3125\end{array}$ & $\begin{array}{r}.860 \\
.985 \\
1.108 \\
1.293\end{array}$ & $\begin{array}{r}.980 \\
1.123 \\
1.263 \\
1.474\end{array}$ & $\begin{array}{l}39,64 \\
23 / 32 \\
13 / 16 \\
29 / 32\end{array}$ & $\begin{array}{l}.620 \\
.731 \\
.826 \\
.921\end{array}$ & $\begin{array}{l}.598 \\
.707 \\
.799 \\
.891\end{array}$ & $\begin{array}{l}27 / 64 \\
15 / 32 \\
9 / 16 \\
21 / 32\end{array}$ & $\begin{array}{l}5 / 32 \\
5 / 32 \\
5 / 32 \\
5 / 32\end{array}$ & $\begin{array}{l}3 / 16 \\
1 / 4 \\
14 \\
1 / 4\end{array}$ \\
\hline $\begin{array}{l}134 \ldots \\
11 / 2 \ldots\end{array}$ & $\begin{array}{l}1.5000 \\
1.6875 \\
1.8750 \\
2.2500\end{array}$ & $\begin{array}{l}1.479 \\
1.665 \\
1.850 \\
2.222\end{array}$ & $\begin{array}{l}1.686 \\
1.898 \\
2.109 \\
2.533\end{array}$ & $\begin{array}{l}1 \\
15 / 32 \\
11 / 4 \\
11 / 2\end{array}$ & $\begin{array}{l}1.017 \\
1.173 \\
1.272 \\
1.525\end{array}$ & $\begin{array}{r}.983 \\
1.139 \\
1.229 \\
1.475\end{array}$ & $\begin{array}{c}3 / 4 \\
27 / 32 \\
15 / 16 \\
138\end{array}$ & $\begin{array}{l}5 / 32 \\
7 / 32 \\
7 / 32 \\
1 / 4\end{array}$ & $\begin{array}{l}1 / 4 \\
5 / 1 \\
5 / 16 \\
3 / 86\end{array}$ \\
\hline
\end{tabular}
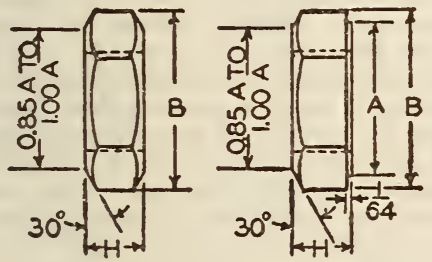

FIGURE 359.-Finished and semifinished jam nuts

TABLE 72.-Dimensions of finished and semifinished jam nuts

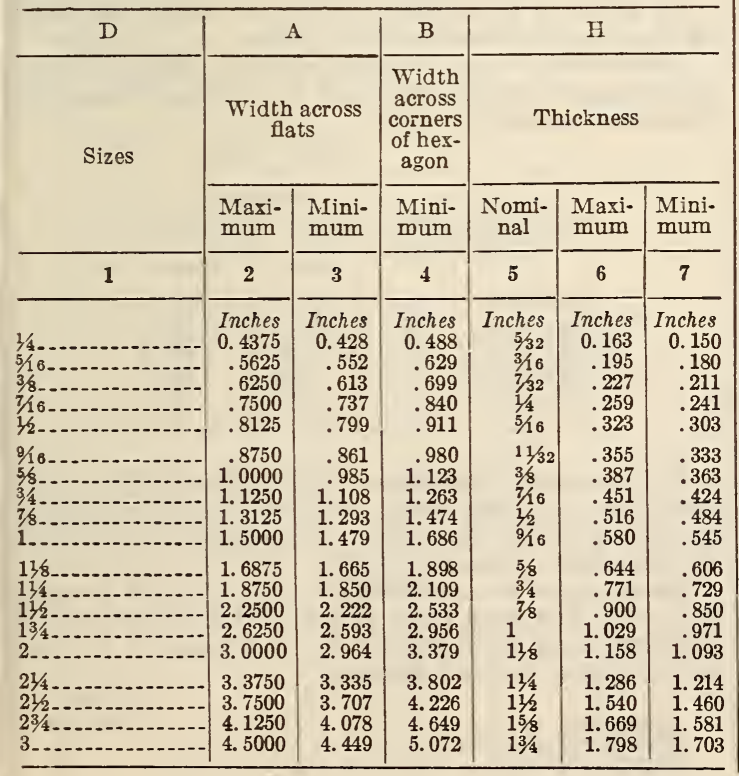

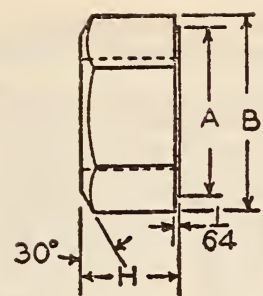

FIGURE 360.-Hexagon light nuts

TABLE 73.-Dimensions of hexagon light nuts

\begin{tabular}{|c|c|c|c|c|c|c|}
\hline $\mathrm{D}$ & \multicolumn{2}{|c|}{ A } & B & \multicolumn{3}{|c|}{ H } \\
\hline \multirow{2}{*}{ Sizes } & \multicolumn{2}{|c|}{$\begin{array}{l}\text { Width across } \\
\text { flats }\end{array}$} & $\begin{array}{l}\text { Width } \\
\text { across } \\
\text { corners } \\
\text { of hex- }\end{array}$ & \multicolumn{3}{|c|}{ Thickness } \\
\hline & $\begin{array}{l}\text { Maxi- } \\
\text { mum }\end{array}$ & $\begin{array}{l}\text { Mini- } \\
\text { mum }\end{array}$ & $\begin{array}{l}\text { Mini- } \\
\text { mum }\end{array}$ & $\begin{array}{c}\text { Nomi- } \\
\text { nal }\end{array}$ & $\begin{array}{l}\text { Maxi- } \\
\text { mum }\end{array}$ & $\begin{array}{l}\text { Mini- } \\
\text { mum }\end{array}$ \\
\hline 1 & 2 & 3 & 4 & 5 & 6 & 7 \\
\hline - & $\begin{array}{c}\text { Inch } \\
0.4375 \\
.5000 \\
.5625 \\
.6250 \\
.7500 \\
\end{array}$ & $\begin{array}{l}\text { Inch } \\
0.428 \\
.489 \\
.551 \\
.612 \\
.737 \\
\end{array}$ & $\begin{array}{l}\text { Inch } \\
0.488 \\
.557 \\
.628 \\
.698 \\
.840 \\
\end{array}$ & $\begin{array}{c}\text { Inch } \\
7 / 32 \\
17 / 64 \\
21 / 64 \\
3 / 8 \\
7 / 16 \\
\end{array}$ & $\begin{array}{l}\text { Inch } \\
0.225 \\
.273 \\
.336 \\
.384 \\
.448 \\
\end{array}$ & $\begin{array}{c}\text { Inch } \\
0.212 \\
.259 \\
.320 \\
.366 \\
.428 \\
\end{array}$ \\
\hline
\end{tabular}
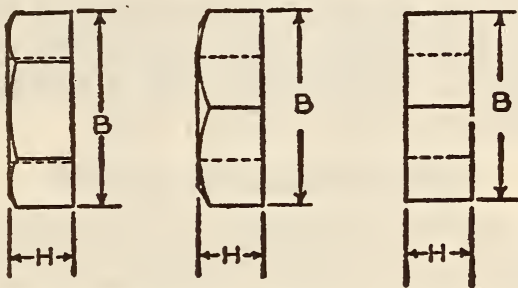

FIGURE 361.- Hexagon and square machine screw nuts and square stove bolt nuts

TABLE 74.-Dimensions of hexagon and square machine screw nuts and square stove bolt nuts

\begin{tabular}{|c|c|c|c|c|c|c|}
\hline $\mathrm{D}$ & \multicolumn{2}{|c|}{$\mathbf{A}$} & B & \multicolumn{3}{|c|}{ H } \\
\hline \multirow{2}{*}{ Sizes } & \multicolumn{2}{|c|}{$\begin{array}{l}\text { Width across } \\
\text { flats }\end{array}$} & $\begin{array}{l}\text { Width } \\
\text { across } \\
\text { corners }\end{array}$ & \multicolumn{3}{|c|}{ Thickness } \\
\hline & $\begin{array}{l}\text { Maxi- } \\
\text { mum }\end{array}$ & $\begin{array}{l}\text { Mini- } \\
\text { mum }\end{array}$ & $\begin{array}{l}\text { Mini- } \\
\text { mum }\end{array}$ & $\underset{\text { nal }}{\text { Nomi- }}$ & $\begin{array}{l}\text { Maxi- } \\
\text { mum }\end{array}$ & $\begin{array}{l}\text { Mini- } \\
\text { mum }\end{array}$ \\
\hline 1 & 2 & 3 & 4 & 5 & 6 & 7 \\
\hline $\begin{array}{l}\text { No. } 0-0.0600 \\
\text { No. } 1-0.0730 \\
\text { No. } 2-0.0860 \\
\text { No. } 3-0.0990 \\
\text { No. } 4-0.1120\end{array}$ & $\begin{array}{l}\text { Inch } \\
0.1562 \\
.1562 \\
.1875 \\
.1875 \\
.2500\end{array}$ & $\begin{array}{l}\text { Inch } \\
0.150 \\
.150 \\
.180 \\
.180 \\
.241\end{array}$ & $\begin{array}{l}\text { Inch } \\
0.171 \\
.171 \\
.205 \\
.205 \\
.275\end{array}$ & \begin{tabular}{r|} 
Inch \\
$3 / 64$ \\
$3 / 64$ \\
$1 / 16$ \\
$1 / 16$ \\
$3 / 32$
\end{tabular} & $\begin{array}{l}\text { Inch } \\
0.050 \\
.050 \\
.066 \\
.066 \\
.098\end{array}$ & $\begin{array}{l}\text { Inch } \\
0.043 \\
.043 \\
.057 \\
.057 \\
.087\end{array}$ \\
\hline $\begin{array}{l}\text { No. } 5^{1}-0.1250 \ldots \\
\text { No. } 6-0.1380 \ldots \\
\text { No. } 81-0.1640 \ldots \\
\text { No. } 101-0.1900 \\
\text { No. } 121-0.2160\end{array}$ & $\begin{array}{l}.3125 \\
.3125 \\
.3437 \\
.3750 \\
.4375\end{array}$ & $\begin{array}{l}.302 \\
.302 \\
.332 \\
.362 \\
.423\end{array}$ & $\begin{array}{l}.344 \\
.344 \\
.378 \\
.413 \\
.482\end{array}$ & $\begin{array}{l}7 / 64 \\
7 / 64 \\
1 / 8 \\
1 / 8 \\
5 / 32\end{array}$ & $\begin{array}{l}.114 \\
.114 \\
.130 \\
.130 \\
.161\end{array}$ & $\begin{array}{l}.102 \\
.102 \\
.117 \\
.117 \\
.148\end{array}$ \\
\hline $\begin{array}{l}1 / 4^{1}-0.2500 \\
5 / 16^{1}-0.3125 \\
3 / 81-0.3750 \\
7 / 16-0.4375 \\
1 / 2-0.5000\end{array}$ & $\begin{array}{l}.4375 \\
.5625 \\
.6250 \\
.7500 \\
.8125\end{array}$ & $\begin{array}{l}.423 \\
.545 \\
.607 \\
.729 \\
.790\end{array}$ & $\begin{array}{l}.482 \\
.621 \\
.692 \\
.831 \\
.901\end{array}$ & $\begin{array}{l}3 / 16 \\
7 / 32 \\
1 / 4 \\
9 / 32 \\
5 / 16\end{array}$ & $\begin{array}{l}.193 \\
.225 \\
.257 \\
.289 \\
.321\end{array}$ & $\begin{array}{l}.178 \\
.208 \\
.239 \\
.269 \\
.299\end{array}$ \\
\hline
\end{tabular}


TABLE 74.-Dimensions of hexagon and square machine screw nuts and square stove bolt nuts-Con.

\begin{tabular}{|c|c|}
\hline Machine screw & $\begin{array}{c}\text { Stove } \\
\text { bolt }\end{array}$ \\
\hline $\begin{array}{l}\text { No. } 5 \\
\text { No. } 8 \\
\text { No. } 10 \\
\text { No. } 12\end{array}$ & $\begin{array}{r}\text { Inch } \\
1 / 8 \\
5 / 32 \\
3 / 16 \\
7 / 32\end{array}$ \\
\hline $\begin{array}{l}\text { 1/4-inch } \\
5 / 6 \text {-inch-... } \\
3 / 8 \text {-inch }\end{array}$ & $\begin{array}{l}1 / 4 \\
5 / 16 \\
3 / 8\end{array}$ \\
\hline
\end{tabular}

608.4 RIVETS, IRON OR STEEL.

AMERICAN BUREAU OF SHIPPING, RULES FOR BUILDING AND CLASSING STEEL VESSELS; QUALITY AND TESTING OF MATERIALS-SHIP CONSTRUCTION, 1930

\section{STEEL AND IRON RIVETS}

(For general information and requirements see 600.0, p. 2.)

A sufficient number of sample steel or iron rivets are to be selected by the surveyor from each diameter of rivet and are to be tested hot and cold by bending, crushing, or in such other way as may be required. The shank must stand being doubled together cold without fracture, and the rivet head must stand being flattened hot until its diameter is two and one-half times the diameter of the shank.

AMERICAN BUREAU OF SHIPPING, RULES FOR BUILDING AND CLASSING STEEL VESSELS; MATERIALS FOR MACHINERY, BOILERS, AND PIPING, 1930

\section{STEEL RIVETS FOR BOILERS}

(For general information and requirements see 600.0 , p. 1 . For test requirements see 600.1 , p. 7 .

(Specifications identical with those given above in ship construction.)

AMERICAN RAILWAY ASSOCIATION, MECHANICAI DIVISION, HIGH CHROME STEEL RIVET MATERIAL AND RIVETS FOR NITRIC ACID TANK CARS, RECOMMENDED PRACTICE, 1929

HIGH CHROME STEEL RIVETS

(See 621.31, p. 1122.)

AMERICAN RAILWAY ASSOCIATION, MECHANICAL DIVISION, RIVET STEEL AND RIVETS FOR LOCOMOTIVE TENDERS, PASSENGER AND FREIGHT EQUIPMENT CARS, 1925

\section{RIVET STEEL AND CAR RIVETS}

(See 603.21, p. 141.)

AMERICAN RAILWAY ASSOCIATION, MECHANICAL DIVISION, RIVET STEEL AND RIVETS FOR STEAM BOILERS AND OTHER PRESSURE VESSELS, 1923

\section{RIVET STEEL FOR BOILERS}

(See 603.21, p. 142.)
AMERICAN RAILWAY ASSOCIATION, PURCHASES AND STORES DIVISION, STAND. ARDIZATION AND SIMPLIFICATION OF STORES STOCK, NO. DV1. 135, 1928

\section{LOCOMOTIVE BOILER AND STROCTURAL RIVETS}

(Contains tables showing the standard sizes of boiler lagging, flat and curved; carriage bolts; squarehead machine bolts; locomotive boiler rivets; and structural rivets that should be carried in stock by railway companies.)

AMERICAN SOCIETY OF MECHANICAL ENGINEERS, AMERICAN STANDARD SMALL RIVETS, $7 / 16$ INCH NOMINAL DIAMETER AND UNDER, B-18a-1927

[Approved by American Standards Association, July 1927]

\section{SMALL RIVETS}

\section{INTRODUCTORY NOTES}

The standard types of small rivets covered by this report are designated, respectively, as flathead, countersunk head, button head, pan head, and truss or wagon-box head. All other types of small rivets within the limits of diameters given are to be considered special.

Tabular diameters. - The diameters of rivets in fractions of an inch as given for the respective types of rivets on Tables $1,2,3,4$, and 5 shall be considered standard. Other values for diameters of rivets, as in Birmingham wire gage numbers, may be used in catalogues in conjunction with the standard diameters, it being recommended, however, that the data be in such form as will make clear which diameters are standard and which are not standard.

Proportions and dimensions. - The proportions and dimensions indicated in the tahles for the heads of the respective rivets shall be standard; other proportions or dimensions are to be considered special. Where nonstandard diameters are given for rivets, the proportions of heads will follow the diagrammatic proportions given in the respective diagrams, in terms of the diameter of body.

Fillets.-Rivets, other than of the countersunk type, shall be acceptable with fillets under the head up to one thirty-second inch radii.

Tolerances.-The tolerances on the nominal diameters of the shanks shall be those given in the following table:

\begin{tabular}{|c|c|c|}
\hline \multirow{2}{*}{$\begin{array}{l}\text { Body diameters (in- } \\
\text { clusive) }\end{array}$} & \multicolumn{2}{|c|}{ Tolerances } \\
\hline & Plus & Minus \\
\hline $\begin{array}{l}3 / 32 \text { to } 5 / 32 \ldots \\
3 / 16 \text { to } 1 / 4 \ldots \\
9 / 32 \text { to } 11 / 32 \ldots \ldots \\
3 / 8 \text { to } 7 / 16 \ldots \ldots\end{array}$ & $\begin{array}{l}0.002 \\
.003 \\
.004 \\
.005\end{array}$ & $\begin{array}{r}0.004 \\
.006 \\
.008 \\
.010\end{array}$ \\
\hline
\end{tabular}

No standard tolerances for the dimensions of the heads are contemplated. For work where restrictions as to head tolerances are necessary these shall be considered special.

Rivet material-composition and physical properties.-(a) The steel from which the rivets are manufactured shall be made by the open-hearth process and conform to the following:

Per cent

Manganese................ $0.30-0.50$

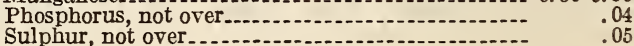


(b) The material, when tested, shall conform to the following:

Tensile strength, $1 \mathrm{bs} . / \mathrm{in} .{ }^{2}, 45,000$ to 55,000

Yield point, lbs./in. ${ }^{2}, 0.5$ tensile strength.

Elongation in 8 inches (minimum per cent) $=1,500,000$ The elongation need not, however, exceed 30 per cent.

The preceding requirements are not applicable to tests on finished rivets. They are for general information only in regard to the production of suitable rivet material.

Physical tests.--Rivets selected at random from each size shall comply with the following:

(a) Cold test for ductility.-One-half of the rivets selected for each test shall be flattened to one-fourth of their original diameter and then bent through $180^{\circ}$ flat on themselves and shall show no signs of cracks, flaws, or any other defects.

(b) Hot test for ductility. - The remaining rivets shall be heated to a red heat and flattened to onefourth of their original diameter, then reheated and bent through $180^{\circ}$ flat on themselves and shall show no signs of cracks, flaws, or any other defects.

(c) Hardness test.- The hardness of rivets shall register between No. 20 and No. 26, inclusive, when tested by the Shore scleroscope. Any other degrees of hardness shall be considered special.

Finish.-The finished rivets shall be free from injurious defects.

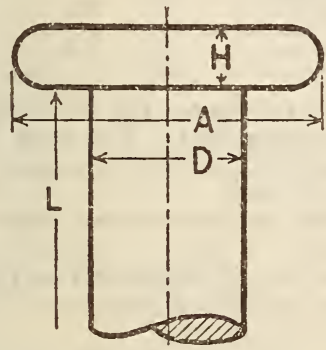

FigURE 362 rivet - Flat head

TABLE 1.-Flat head rivets

[All dimensions given in inches.]

\begin{tabular}{|c|c|c|c|c|c|}
\hline \multicolumn{3}{|c|}{$D$} & \multirow{3}{*}{$\begin{array}{l}\text { Diam- } \\
\text { eter of } \\
\text { head }\end{array}$} & $H$ & $L$ \\
\hline \multicolumn{3}{|c|}{ Diameter of body } & & \multirow{2}{*}{$\begin{array}{l}\text { Height } \\
\text { of head }\end{array}$} & \multirow{2}{*}{$\begin{array}{c}\text { Length } \\
\text { under head }\end{array}$} \\
\hline Nominal & $\begin{array}{l}\text { Maxi- } \\
\text { mum }\end{array}$ & $\begin{array}{l}\text { Mini- } \\
\text { mum }\end{array}$ & & & \\
\hline $\begin{array}{r}3 / 32-0.094 \\
1 / 8-.125 \\
5 / 32-.156\end{array}$ & $\begin{array}{r}0.096 \\
.127 \\
.158\end{array}$ & $\begin{array}{r}0.090 \\
.121 \\
.152\end{array}$ & $\begin{array}{r}0.190 \\
.250 \\
.312\end{array}$ & $\begin{array}{r}0.032 \\
.042 \\
.052\end{array}$ & \\
\hline $\begin{array}{r}3 / 16-.188 \\
7 / 32-.219 \\
1 / 4-.250\end{array}$ & $\begin{array}{l}.191 \\
.222 \\
.253\end{array}$ & $\begin{array}{l}.182 \\
.213 \\
.244\end{array}$ & $\begin{array}{l}.374 \\
.440 \\
.500\end{array}$ & $\begin{array}{l}.062 \\
.073 \\
.083\end{array}$ & $\begin{array}{l}\text { Ordered } \\
\text { length } \\
\text { under }\end{array}$ \\
\hline $\begin{array}{r}9 / 32-.281 \\
5 / 16-.313 \\
11 / 32-.344\end{array}$ & $\begin{array}{l}.285 \\
.317 \\
.348\end{array}$ & $\begin{array}{l}.273 \\
.305 \\
.336\end{array}$ & $\begin{array}{l}.562 \\
.624 \\
.686\end{array}$ & $\begin{array}{l}.094 \\
.104 \\
.114\end{array}$ & $\begin{array}{l}\text { head to } \\
\text { end. }\end{array}$ \\
\hline $\begin{array}{r}3 / 8-.375 \\
7 / 16-.438\end{array}$ & $\begin{array}{l}.380 \\
.443\end{array}$ & $\begin{array}{l}.365 \\
.428\end{array}$ & $\begin{array}{l}.750 \\
.874\end{array}$ & $\begin{array}{l}.125 \\
.146\end{array}$ & \\
\hline
\end{tabular}

Approximate proportions- $A=2.00 \times D$

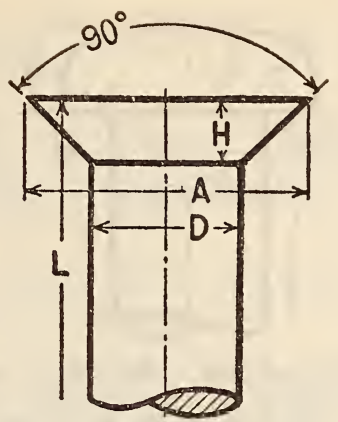

Figdre 363.-Countersunk head rivet

TABLE 2.-Countersunk head rivets

[All dimensions given in inches]

\begin{tabular}{|c|c|c|c|c|c|}
\hline$D$ & & $A$ & $H$ & \multirow{3}{*}{$\begin{array}{c}\text { In- } \\
\text { cluded } \\
\text { angle } \\
\text { (de- } \\
\text { grees) }\end{array}$} & $L$ \\
\hline Diameter of $b$ & $d y$ & \multirow{2}{*}{$\begin{array}{l}\text { Diam- } \\
\text { eter of } \\
\text { head }\end{array}$} & \multirow{2}{*}{$\begin{array}{c}\text { Depth } \\
\text { of } \\
\text { head }\end{array}$} & & \multirow{2}{*}{$\begin{array}{l}\text { Length } \\
\text { overall }\end{array}$} \\
\hline Nominal / Maximum & Minimum & & & & \\
\hline \multirow{4}{*}{ Same as for flat head $r$} & & $\begin{array}{r}0.176 \\
.231 \\
.289\end{array}$ & $\begin{array}{r}0.040 \\
.053 \\
.066\end{array}$ & $\begin{array}{l}90 \\
90 \\
90\end{array}$ & \multirow{4}{*}{$\begin{array}{l}\text { Ordered } \\
\text { length. }\end{array}$} \\
\hline & vets.. & $\begin{array}{l}.346 \\
.407 \\
.463\end{array}$ & $\begin{array}{l}.079 \\
.094 \\
.106\end{array}$ & $\begin{array}{l}90 \\
90 \\
90\end{array}$ & \\
\hline & & $\begin{array}{l}.520 \\
.577 \\
.635\end{array}$ & $\begin{array}{l}.119 \\
.133 \\
.146\end{array}$ & $\begin{array}{l}90 \\
90 \\
90\end{array}$ & \\
\hline & & $\begin{array}{l}.694 \\
.808\end{array}$ & $\begin{array}{l}.159 \\
.186\end{array}$ & $\begin{array}{l}90 \\
90\end{array}$ & \\
\hline
\end{tabular}

A pproximate proportions- $A=1.850 \times D$

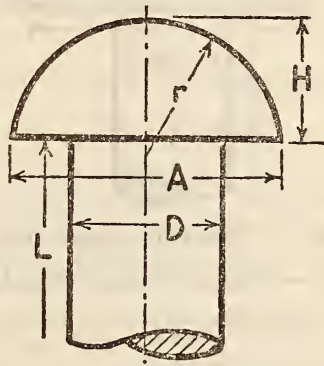

Figure 364.-Button head rivet

TABLE 3.-Button head rivets

[All dimensions given in inches]

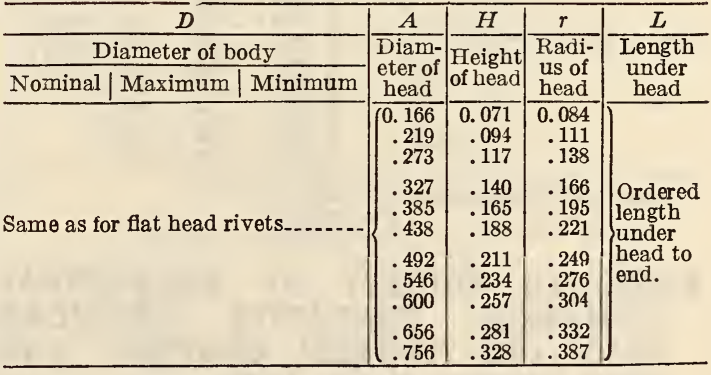

Approximate proportions $-A=1.750 \times D$

$H=0.750 \times D$ 


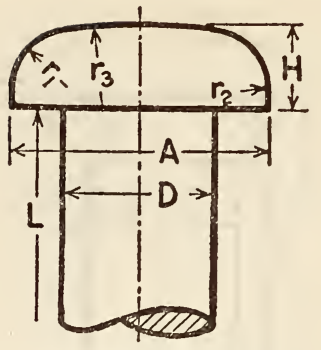

FiguRe 365.-Pan head rivet

TABLE 4.-Pan head rivets

[All dimensions given in inches]

\begin{tabular}{|c|c|c|c|c|c|c|c|c|}
\hline \multicolumn{3}{|c|}{$D$} & $A$ & $H$ & \multicolumn{3}{|c|}{$r$} & $L$ \\
\hline \multicolumn{3}{|c|}{ Diameter of body } & \multirow{2}{*}{$\begin{array}{l}\text { Diam- } \\
\text { eter of } \\
\text { head } \\
\end{array}$} & \multirow[b]{2}{*}{$\begin{array}{l}\text { Height } \\
\text { of head }\end{array}$} & \multicolumn{3}{|c|}{ Radii of head } & \multirow{2}{*}{$\begin{array}{l}\text { Length } \\
\text { under } \\
\text { head }\end{array}$} \\
\hline $\begin{array}{l}\text { Nom- } \\
\text { inal }\end{array}$ & $\begin{array}{l}\text { Maxi- } \\
\text { mum }\end{array}$ & $\begin{array}{l}\text { Mini- } \\
\text { mum }\end{array}$ & & & $r_{1}$ & $r_{2}$ & $r_{3}$ & \\
\hline \multirow{4}{*}{\multicolumn{3}{|c|}{$\begin{array}{l}\text { Same as for fllat head } \\
\text { rivets. }\end{array}$}} & $\begin{array}{r}0.163 \\
.215 \\
.268\end{array}$ & $\begin{array}{l}0.054 \\
.072 \\
.089\end{array}$ & $\begin{array}{r}0.030 \\
.039 \\
.049\end{array}$ & $\begin{array}{l}0.080 \\
.106 \\
.133\end{array}$ & $\begin{array}{r}0.326 \\
.429 \\
.535\end{array}$ & \multirow{4}{*}{$\begin{array}{l}\text { Ordered } \\
\text { length } \\
\text { under } \\
\text { head to } \\
\text { end. }\end{array}$} \\
\hline & & & $\begin{array}{l}.321 \\
.378 \\
.429\end{array}$ & $\begin{array}{r}.107 \\
.126 \\
.143\end{array}$ & $\begin{array}{l}.059 \\
.069 \\
.079\end{array}$ & $\begin{array}{l}.159 \\
.186 \\
.213\end{array}$ & $\begin{array}{l}.641 \\
.754 \\
.858\end{array}$ & \\
\hline & & & $\begin{array}{l}.482 \\
.535 \\
.589\end{array}$ & $\begin{array}{l}.161 \\
.178 \\
.196\end{array}$ & $\begin{array}{l}.088 \\
.098 \\
.108\end{array}$ & $\begin{array}{l}.239 \\
.266 \\
.292\end{array}$ & $\begin{array}{r}.963 \\
1.070 \\
1.176\end{array}$ & \\
\hline & & & $\begin{array}{l}.644 \\
.750\end{array}$ & $\begin{array}{l}.215 \\
.250\end{array}$ & $\begin{array}{r}118 \\
.137\end{array}$ & $\begin{array}{r}319 \\
.372\end{array}$ & $\begin{array}{l}1.286 \\
1.500\end{array}$ & \\
\hline
\end{tabular}

Approximate proportions $-A=1.720 \times D$

$H=.570 \times D$

$r_{1}=.314 \times D$

$r_{2}=.850 \times D$

$r_{3}=3.430 \times D$

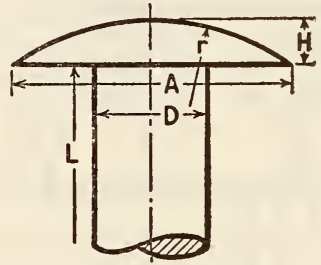

FIguRE 366.-Truss or wagon box head rivet

TABLE 5.-Truss or wagon box head rivets [All dimensions given in inches]

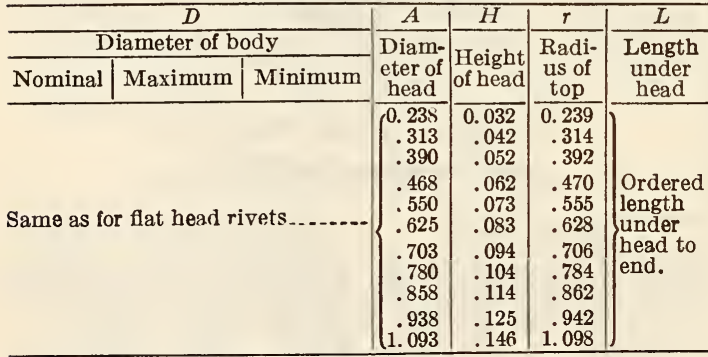

Approximate proportions $-A=2.500 \times D$ $\begin{aligned} H & =2.500 \times D \\ r & =2.512 \times D\end{aligned}$

\section{AMERICAN SOCIETY OF MECHANICAL ENGINEERS, TENTATIVE AMERICAN STANDARD TINIERS', COOPERS', AND BELT RIVETS, B-18g-1928}

[Approved as American tentative standard by the American Standards Association, May, 1928]

\section{TINNERS', COOPERS', AND BELT RIVETS INTRODUCTORY NOTES}

The standard types of small rivets covered by this report are designated, respectively, as tinners', coopers', belt. All other types of rivets for this class shall be considered special.

Tabular sizes. - The sizes of rivets as given for the respective types of rivets on Tables 1,2 , and 3 shall be considered standard. Other values for sizes of rivets may be used in catalogs in conjunction with the standard sizes; it being recommended, however, that the data be in such form as will make clear which are standard and which are not standard.

Proportions. - The proportions indicated below, in Tables 1, 2, and 3, for the heads and points of the respective sizes of rivets shall be standard; other proportions are to be considered special. In nonstandard sizes of rivets, the heads and points shall be of the same proportions; the lengths of rivets covered by Tables 1 and 2 being determined from the table by interpolation.

Tolerances. - The tolerance on the nominal diameter of belt rivets shall be plus 0.002 inch and minus 0.004 inch.

The tolerances on the body diameters of coopers' and tinners' rivets shall be those given in the following table:

\begin{tabular}{|c|c|c|}
\hline \multirow{2}{*}{$\begin{array}{c}\text { Size } \\
\text { (inclusive) }\end{array}$} & \multicolumn{2}{|c|}{ Tolerances } \\
\hline & Plus & Minus \\
\hline 8 ounces to $2 \frac{1}{2}$ & 0.002 & 0.004 \\
\hline 3 pounds to 10 & & \\
\hline${ }_{12}^{\text {pounds }}$ pounds to 16 & .003 & .006 \\
\hline pounds & .004 & .008 \\
\hline
\end{tabular}

No standard tolerances for the dimensions of the heads are contemplated. For work where restrictions as to head tolerances are necessary, these shall be considered special.

Finish.-The finished rivets shall be free from injurious defects.

(Rivet material: Composition and physical properties, physical tests, and finish are the same as for small rivets, given above.)

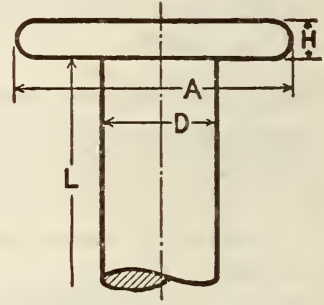

Figure 367.-Tinners' rivet

TABLE 1.-Tinners' rivets

[All dimensions given in inches]

\begin{tabular}{|c|c|c|c|c|}
\hline \multirow[b]{2}{*}{ Size No.1 } & $D$ & $A$ & $H$ & $L$ \\
\hline & $\begin{array}{l}\text { Diameter } \\
\text { of body }\end{array}$ & $\begin{array}{l}\text { Diameter } \\
\text { of head }\end{array}$ & $\begin{array}{l}\text { Height } \\
\text { of head }\end{array}$ & $\begin{array}{l}\text { Length } \\
\text { under } \\
\text { head }\end{array}$ \\
\hline $\begin{array}{l}8 \text { ounces... } \\
12 \text { ounces... } \\
11 \text { pound } \\
21 / 2 \text { pounds. }\end{array}$ & $\begin{array}{l}0.089 \\
.105 \\
.111 \\
.130 \\
.144\end{array}$ & $\begin{array}{l}0.207 \\
.236 \\
.249 \\
.292 \\
.324\end{array}$ & $\begin{array}{l}0.027 \\
.031 \\
.033 \\
.039 \\
.043\end{array}$ & $\begin{array}{l}0.16 \\
.19 \\
.20 \\
.23 \\
.27\end{array}$ \\
\hline $\begin{array}{l}21 / 2 \text { pounds } \\
3 \text { pounds } \\
6 \text { pounds } \\
8 \text { pounds }\end{array}$ & $\begin{array}{l}.148 \\
.160 \\
.176 \\
.203 \\
.224\end{array}$ & $\begin{array}{l}.333 \\
.360 \\
.396 \\
.456 \\
.504\end{array}$ & $\begin{array}{l}.044 \\
.048 \\
.052 \\
.060 \\
.067\end{array}$ & $\begin{array}{l}.28 \\
.31 \\
.34 \\
.39 \\
.44\end{array}$ \\
\hline $\begin{array}{l}10 \text { pounds } \\
12 \text { pounds } \\
14 \text { pounds } \\
16 \text { pounds }\end{array}$ & $\begin{array}{l}.238 \\
.259 \\
.284 \\
.300\end{array}$ & $\begin{array}{l}.535 \\
.582 \\
.639 \\
.675 \\
\end{array}$ & $\begin{array}{l}.071 \\
.077 \\
.085 \\
.090 \\
\end{array}$ & $\begin{array}{l}.47 \\
.50 \\
.52 \\
.53 \\
\end{array}$ \\
\hline
\end{tabular}

1 Size numbers refer to the trade name or weight of 1,000 rirets.

Approximate proportions $-A=2.25 \times D$

$H=.30 \times D$ 


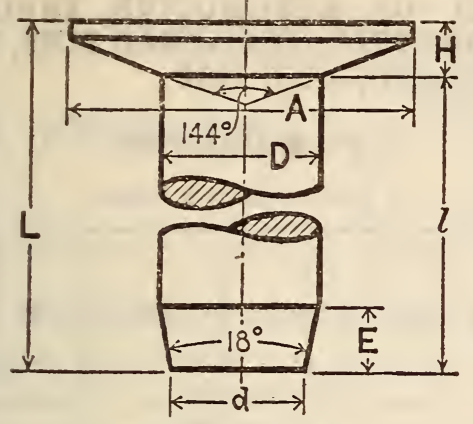

FigURe 368.-Coopers' rivet

TABLE 2.-Coopers' rivets [All dimensions given in inches]

\begin{tabular}{|c|c|c|c|c|c|c|c|c|}
\hline . & $D$ & $\bar{A}$ & $H$ & & $l$ & $L$ & $E$ & $d$ \\
\hline Size No.\$ & 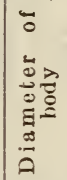 & 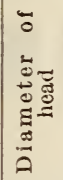 & 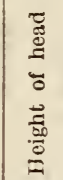 & 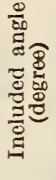 & 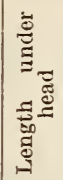 & 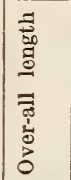 & 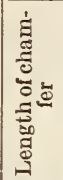 & 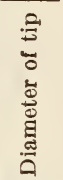 \\
\hline $\begin{array}{l}1 \text { pound } \\
11 / 2 \text { pounds. } \\
2 \text { pounds } \\
21 / 2 \text { pounds }\end{array}$ & $\begin{array}{l}0.109 \\
.127 \\
.141 \\
.148\end{array}$ & $\begin{array}{r}0.245 \\
.285 \\
.317 \\
.333\end{array}$ & $\begin{array}{r}0.032 \\
.038 \\
.042 \\
.044\end{array}$ & $\begin{array}{l}144 \\
144 \\
144 \\
144\end{array}$ & $\begin{array}{r}0.187 \\
.218 \\
.250 \\
.281\end{array}$ & $\begin{array}{r}0.219 \\
.256 \\
.292 \\
.325\end{array}$ & $\begin{array}{r}0.043 \\
.050 \\
.056 \\
.059\end{array}$ & $\begin{array}{r}0.098 \\
.114 \\
.126 \\
.133\end{array}$ \\
\hline $\begin{array}{l}3 \text { pounds } \\
4 \text { pounds } \\
6 \text { pounds } \\
8 \text { pounds...... }\end{array}$ & $\begin{array}{l}.156 \\
.165 \\
.203 \\
.238\end{array}$ & $\begin{array}{l}.351 \\
.371 \\
.455 \\
.535\end{array}$ & $\begin{array}{l}.046 \\
.049 \\
.060 \\
.071\end{array}$ & $\begin{array}{l}144 \\
144 \\
144 \\
144\end{array}$ & $\begin{array}{l}.312 \\
.343 \\
.406 \\
.500\end{array}$ & $\begin{array}{l}.358 \\
.392 \\
.466 \\
.571\end{array}$ & $\begin{array}{l}.062 \\
.066 \\
.081 \\
.095\end{array}$ & $\begin{array}{l}.140 \\
.148 \\
.182 \\
.214\end{array}$ \\
\hline $\begin{array}{l}10 \text { pounds } \\
12 \text { pounds } \\
14 \text { pounds } \\
16 \text { pounds }\end{array}$ & $\begin{array}{l}.250 \\
.259 \\
.271 \\
.281\end{array}$ & $\begin{array}{l}.562 \\
.582 \\
.609 \\
.632 \\
\end{array}$ & $\begin{array}{l}.075 \\
.077 \\
.081 \\
.084\end{array}$ & $\begin{array}{l}144 \\
144 \\
144 \\
144\end{array}$ & $\begin{array}{l}.531 \\
.531 \\
.562 \\
.593\end{array}$ & $\begin{array}{l}.606 \\
.608 \\
.643 \\
.677 \\
\end{array}$ & $\begin{array}{l}.100 \\
.103 \\
.108 \\
.112 \\
\end{array}$ & $\begin{array}{l}.225 \\
.233 \\
.243 \\
.252 \\
\end{array}$ \\
\hline
\end{tabular}

1 Size numbers refer to the trade name or weight of 1,000 rivets Approximate proportions $-A=2.25 \times D$ $\begin{aligned} d= & =.90 \times D \\ E & =.40 \times D\end{aligned}$ $E=.40 \times D$
$H=.30 \times D$

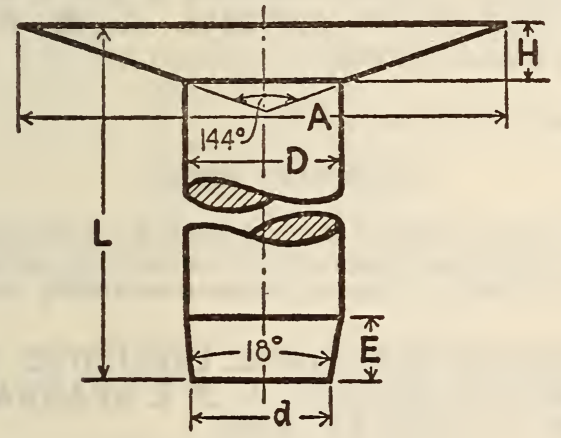

FIGURE 369.-Belt rivet

TABLE 3.-Belt rivets [All dimensions given in inches.]

\begin{tabular}{|c|c|c|c|c|c|c|c|}
\hline \multirow[b]{2}{*}{ Size No. } & $D$ & $A$ & $H$ & \multirow[b]{2}{*}{$\begin{array}{l}\text { In- } \\
\text { cluded } \\
\text { angle } \\
\text { (de- } \\
\text { grees) }\end{array}$} & $L$ & $E$ & $d$ \\
\hline & $\begin{array}{c}\text { Diam- } \\
\text { eter } \\
\text { of } \\
\text { body }\end{array}$ & $\begin{array}{l}\text { Diam- } \\
\text { eter } \\
\text { of } \\
\text { head }\end{array}$ & $\begin{array}{c}\text { Depth } \\
\text { of } \\
\text { head }\end{array}$ & & $\begin{array}{l}\text { Length } \\
\text { overall }\end{array}$ & $\begin{array}{l}\text { Length } \\
\text { of } \\
\text { chamfer }\end{array}$ & $\begin{array}{l}\text { Diam- } \\
\text { eter } \\
\text { of tip }\end{array}$ \\
\hline 12. & $\begin{array}{l}0.180 \\
.165 \\
.148 \\
.134 \\
.120 \\
.109 \\
.095\end{array}$ & $\begin{array}{r}0.504 \\
.462 \\
.414 \\
.375 \\
.336 \\
.305 \\
.266\end{array}$ & $\begin{array}{l}0.054 \\
.049 \\
.044 \\
.040 \\
.036 \\
.032 \\
.028\end{array}$ & $\begin{array}{l}144 \\
144 \\
144 \\
144 \\
144 \\
144 \\
144\end{array}$ & $\left\{\begin{array}{l}\text { Lengths } \\
\text { to be } \\
\text { from } 3 / 8 \\
\text { to } 3 / 4 \\
\text { inches } \\
\text { by } 1 / 8 \\
\text { inch } \\
\text { incre- } \\
\text { ments. }\end{array}\right.$ & $\begin{array}{r}0.072 \\
.066 \\
.059 \\
.053 \\
.048 \\
.043 \\
.038\end{array}$ & $\begin{array}{r}0.162 \\
.148 \\
.133 \\
.120 \\
.108 \\
.098 \\
.085\end{array}$ \\
\hline
\end{tabular}

1 Size number refers to the stubs iron wire gage number of the stock used in the body of the rivet.

Approximate proportions. $-A=2.8 \times D$ $\begin{aligned} d & =.9 \times D \\ E & =.4 \times D\end{aligned}$ $E=.4 \times D$
$H=.3 \times D$
Tolerances.-The tolerances on the nominal diameter of belt rivets shall be plus 0.002 inch and minus 0.004 inch.

AMERICAN SOCIETY FOR TESTING MATERIALS, STANDARD SPECIFICATIONS FOR RIVET STEEL FOR SHIPS, DESIGNATION A13-24, 1924

RIVET STEEL FOR SHIPS

(See 605.15, p. 269.)

AMERICAN SOCIETY FOR TESTING MATERIALS, STANDARD SPECIFICATIONS FOR BOILER RIVET STEEL, DESIGNATION A31-24, 1924

\section{BOILER RIVET STEEL}

(See 603.21, p. 144.)

SOCIETY OF AUTOMOTIVE ENGINEERS, PARTS AND FITTINGS, SPLIT AND TUBULAR RIVETS, S. A. E. RECOMMENDED PRACTICE, JUNE, 1929

\section{SPLIT AND TUBULAR RIVETS}

(See 645.9, p. 1240.)

\section{UNITED STATES GOVERNMENT, FEDERAL} SPECIFICATIONS BOARD, FEDERAL SPECIFICATION FOR BOLTS, NUTS, STUDS, AND TAP RIVETS (AND MATERIAL FOR SAME), FF-B-5\%1, OCTOBER 13,1931

(See also 608.0, p.522; 608.31,p.604; 608.32, p.609.)

\section{TAP RIVETS}

(For applicable Federal specifications, types, material, and workmanship, general requirements, detail requirements, methods of sampling, inspection, and tests; packaging, packing, and marking; and notes, - see 608.0, p. 522.)

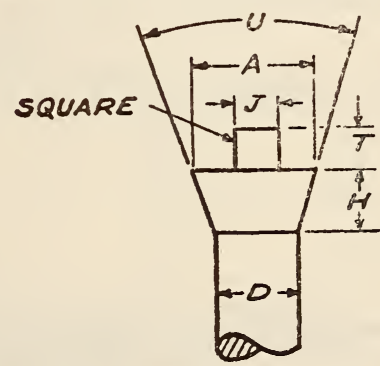

FIGURE 370.-Tap rivet head

TABLE 1.-Dimensions of tap rivet heads

\begin{tabular}{|c|c|c|c|c|c|}
\hline $\begin{array}{l}\text { Nom- } \\
\text { inal } \\
\text { diam- } \\
\text { eter } \\
\text { of } \\
\text { screw } \\
(D)\end{array}$ & $A$ & $H$ & $J$ & $T$ & $U$ \\
\hline $\begin{array}{c}\text { Inches } \\
1 / 2 \\
5 / 8 \\
3 / 4 \\
7 / 8 \\
11 \\
11 / 8 \\
11 / 4\end{array}$ & $\begin{array}{c}\text { Inches } \\
0.788 \\
.985 \\
1.183 \\
1.380 \\
1.577 \\
1.774 \\
1.971\end{array}$ & $\begin{array}{c}\text { Inch } \\
1 / 4 \\
5516 \\
3 / 8 \\
7 / 16 \\
1 / 2 \\
9 / 16 \\
5 / 8\end{array}$ & $\begin{array}{c}\text { Inch } \\
3 / 8 \\
7716 \\
112 \\
9116 \\
588 \\
5 / 8 \\
11 / 16\end{array}$ & $\begin{array}{c}\text { Inch } \\
1 / 2 \\
5 / 8 \\
58 \\
23 / 32 \\
23 / 32 \\
23 / 32 \\
23 / 32\end{array}$ & $\begin{array}{l}\circ \\
60 \\
60 \\
60 \\
60 \\
60 \\
60 \\
60\end{array}$ \\
\hline
\end{tabular}


608.5 PINS AND SHAFT KEYS.

608.51 PINS, COTTER, DOWEL, ROD END PINS, ETC.

AMERICAN RAILWAY ASSOCIATION, MECHANICAL DIVISION, MISCELLANEOUS STANDARDS AND RECOMMENDED PRACTICE, 1930

CASTLE NUTS, COTTER PINS, AND LOCATION OF COTTER-PIN HOLES IN PROJECTING BOLT ENDS

(See 608.31, p. 588.)

AMERICAN RAILWAY ASSOCIATION, PURCHASES AND STORES DIVISION, STANDARDIZATION AND SIMPLIFICATION OF

STORES STOCK, PROCEEDINGS, 1928

\section{COTTER KEYS}

(The stocking of 21 different sizes of cotter keys is recommended. These keys range in size from $1 / 16$ inch by $1 \frac{1}{2}$ inches to $3 / 4$ inch by 4 inches.)

SOCIETY OF AUTOMOTIVE ENGINEERS, PARTS AND FITTINGS; COTTER PINS, S. A. E. STANDARD, JULY, 1928

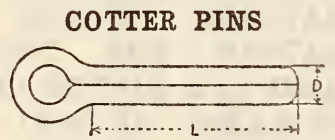

Figure 371.-Cotter pin

[All dimensions in inches]

\begin{tabular}{|c|c|c|c|c|c|c|c|c|}
\hline \multirow{2}{*}{ Length $(L)$} & \multicolumn{8}{|c|}{ Nominal trade diameter $(D)$} \\
\hline & $1 / 16$ & $3 / 32$ & $1 / 8$ & $5 / 32$ & $3 / 16$ & $7 / 32$ & $1 / 4$ & $5 / 16$ \\
\hline $\begin{array}{l}5 / 16 \\
7 / 16 \\
1 / 2 \\
5 / 8 \\
3 / 4 \\
7 / 8 \\
11 \\
1138 \\
114 \\
13 / 8 \\
11 / 2 \\
158 \\
13 / 4 \\
2 \\
21 / 4 \\
21 / 2 \\
23 / 4 \\
3\end{array}$ & $\begin{array}{l}* \\
* \\
* \\
* \\
* \\
*\end{array}$ & $\begin{array}{l}* \\
* \\
* \\
* \\
* \\
*\end{array}$ & $\begin{array}{l}* \\
* \\
* \\
* \\
* \\
* \\
* \\
*\end{array}$ & $\begin{array}{l}* \\
* \\
* \\
* \\
* \\
* \\
*\end{array}$ & $\begin{array}{l}* \\
* \\
* \\
* \\
*\end{array}$ & $\begin{array}{l}* \\
* \\
* \\
* \\
*\end{array}$ & $\begin{array}{l}* \\
* \\
* \\
*\end{array}$ & $\begin{array}{l}* \\
* \\
*\end{array}$ \\
\hline $\begin{array}{l}\text { Diameter } \\
\text { Limits } 1\end{array}$ & 0.061 & $\begin{array}{r}0.090 \\
.086\end{array}$ & 0.122 & $\begin{array}{r}0.150 \\
.146\end{array}$ & $\begin{array}{r}0.176 \\
.172\end{array}$ & $\begin{array}{l}0.207 \\
.202\end{array}$ & $\begin{array}{l}0.225 \\
.220\end{array}$ & $\begin{array}{r}0.280 \\
.275\end{array}$ \\
\hline
\end{tabular}

1 Actual cotter-pin or wire diameter limits.
SOCIETY OF AUTOMOTIVE ENGINEERS, AERONAUTIC; FLAT-HEAD PINS, S. A. E. STANDARD, MAY, 1930

\section{FLAT-HEAD PINS}

[All dimensions in inches]

\begin{tabular}{|c|c|c|c|c|c|c|c|}
\hline $\begin{array}{l}\text { Nom- } \\
\text { inal } \\
\text { pin } \\
\text { size }\end{array}$ & $A$ & $B$ & $C$ & $D$ & Drill $H$ & $X$ & $\begin{array}{l}\text { Mini- } \\
\text { mum } \\
\text { single } \\
\text { shear } \\
\text { strength } \\
\text { in pounds }\end{array}$ \\
\hline $\begin{array}{l}1 / 8 \\
3 / 16 \\
1 / 4 \\
5 / 16 \\
3 / 8\end{array}$ & $\begin{array}{l}1 / 4 \\
5 / 16 \\
3 / 8 \\
7 / 16 \\
1 / 2\end{array}$ & $\begin{array}{l}3 / 64 \\
3 / 64 \\
1 / 16 \\
1 / 16 \\
1 / 16\end{array}$ & $\begin{array}{l}3 / 64 \\
3 / 64 \\
1 / 16 \\
5 / 64 \\
5 / 64\end{array}$ & $\begin{array}{l}0.124 \\
.186 \\
.248 \\
.311 \\
.373\end{array}$ & $\begin{array}{ll}\text { No. } & \\
50 & (0.070) \\
48 & (.076) \\
48 & (.076) \\
36 & (.1065) \\
36 & (.1065)\end{array}$ & $\begin{array}{r}0.035 \\
.038 \\
.038 \\
.053 \\
.053\end{array}$ & $\begin{array}{r}940 \\
2,130 \\
3,770 \\
6,000 \\
8,650\end{array}$ \\
\hline $\begin{array}{l}7 / 16 \\
1 / 2 \\
9 / 16 \\
5 / 8\end{array}$ & $\begin{array}{c}9 / 16 \\
5 / 8 \\
11 / 16 \\
3 / 4\end{array}$ & $\begin{array}{l}3 / 32 \\
3 / 32 \\
3 / 32 \\
3 / 32\end{array}$ & $\begin{array}{l}5 / 64 \\
5 / 64 \\
5 / 64 \\
5 / 64\end{array}$ & $\begin{array}{r}.436 \\
.497 \\
.560 \\
.622\end{array}$ & $\begin{array}{ll}36 & (.1065) \\
36 & (.1065) \\
28 & (.141) \\
28 & (.141)\end{array}$ & $\begin{array}{l}.053 \\
.053 \\
.070 \\
.070\end{array}$ & $\begin{array}{l}11,840 \\
15,400 \\
19,550 \\
24,150\end{array}$ \\
\hline
\end{tabular}

It is recommended that pins of three-eighths to seven-sixteenths inch in diameter be carried up to 3 inches in length only, and that pins one-half to five-eighths inch in diameter be carried up to 4 inches in length, as there is no known demand for greater lengths.

On pins longer than 2 inches the tolerance should be changed from $+0.000-0.002$ to $+0.000-0.003$.

Pins to be ordered in increments of one-sixteenth inch only.

608.52 SHAFT KEYS.

AMERICAN MARINE STANDARDS COMMITTEE, SPECIFICATION E NO. 6, PROPELLER-SHAFT DETAILS, A. M. S. C. 28, JUNE 10, 1927

(See also 608.32, p. 605.)

\section{PROPELLER KEYS}

(Contains specifications, dimensions, and illustrations of tail shafts ana stern-tube bearings, propeller keys, propeller nuts, and stern-tube stuffing boxes.)

AMERICAN PETROLEUM INSTITUTE, RIGIRON STANDARDS, A. P. I. STANDARDS NO. 6, JANUARY, 1930

\section{SHAFT KEYS AND KEY WAYS}

(For general specifications see American Petroleum Institute, cranks and shafts, 611.52 , p. 760.$)$ 
GIB-HEAD TAPER KEYS

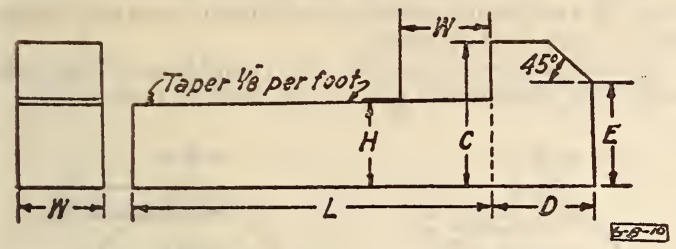

FIGURE 372.-Gib head taper key

TABLE 1.-Keys for A. P. I. Standard rig irons and sand reels

[See fig. 372]

[All dimensions in inches at $68^{\circ} \mathrm{F}$.]

\begin{tabular}{|c|c|c|c|c|c|c|}
\hline & \multirow{2}{*}{ 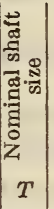 } & \multirow{2}{*}{ 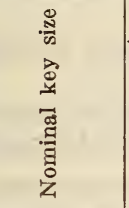 } & Width & Height & \multirow{2}{*}{ 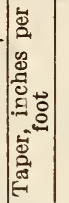 } & \multirow[b]{2}{*}{ 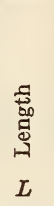 } \\
\hline & & & 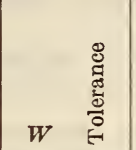 & 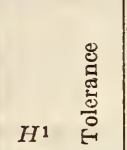 & & \\
\hline Sand reel shaft. & 4 & 1 by $3 / 4$ & $\overline{1.000+0.000}$ & $0.750+0.003$ & $1 / 8$ & \\
\hline Crank shaft... & $41 / 2$ & 1 by $3 / 4$ - & $1.000+.000$ & $.750+.003$ & $1 / 8$ & \\
\hline Sand reel shaft. & 5 & 134 by $7 / 8$. & $1.250+.000$ & $.875+.003$ & $1 / 8$ & \\
\hline Crank shaft... & 5 & $11 / 4$ by $7 / 8$. & $1.250+.000$ & $.875 \bar{t} .003$ & $1 / 8$ & \\
\hline Crank shaft... & 2 & $13 / 4$ by $7 / 8$. & $1.250 \bar{\tau} .000$ & $.875+.00$ & $1 / 8$ & \\
\hline and reel shaft. & 6 & $11 / 2$ & $1.500+.000$ & & $1 / 8$ & \\
\hline
\end{tabular}

1 The height $H$ is measured at the distance $W$ from gib.

2 The 6-inch crank shaft uses the same size key as the 5-inch, as shown.

Note.-The dimensions indicated in Figure 372 and not shown in the above table are not essential to the fit; they can be obtained from A. P. I. Standards 7. Transmission Standards.

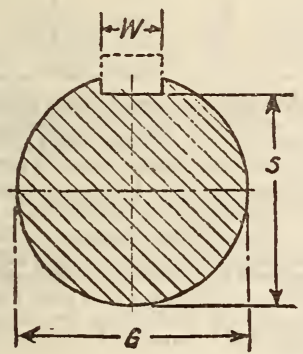

FIGURE 373.- Shaft details

TABLE 2.-Shaft details

[See fig. 373]

[All dimensions in inches at $68^{\circ} \mathrm{F}$.]

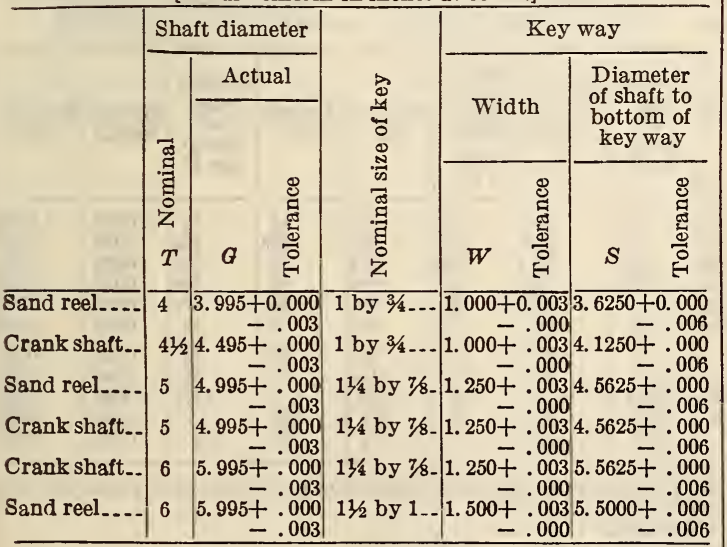

Note.-All key ways shall be cut on a diametral center line.

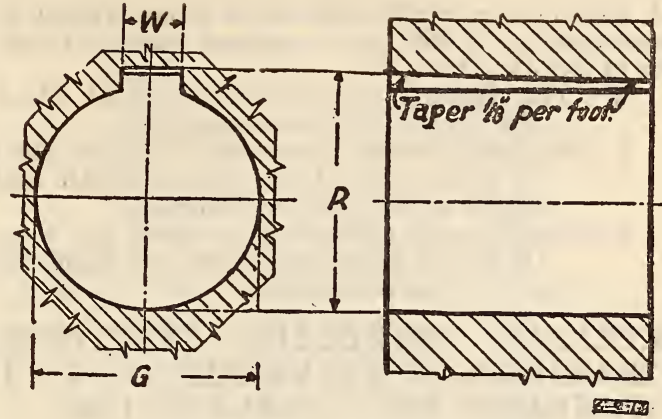

FigURE 374.-Bored member

TABLE 3.-Bored member

[See fig. 374]

[All dimensions in inches at $68^{\circ} \mathrm{F}$.]

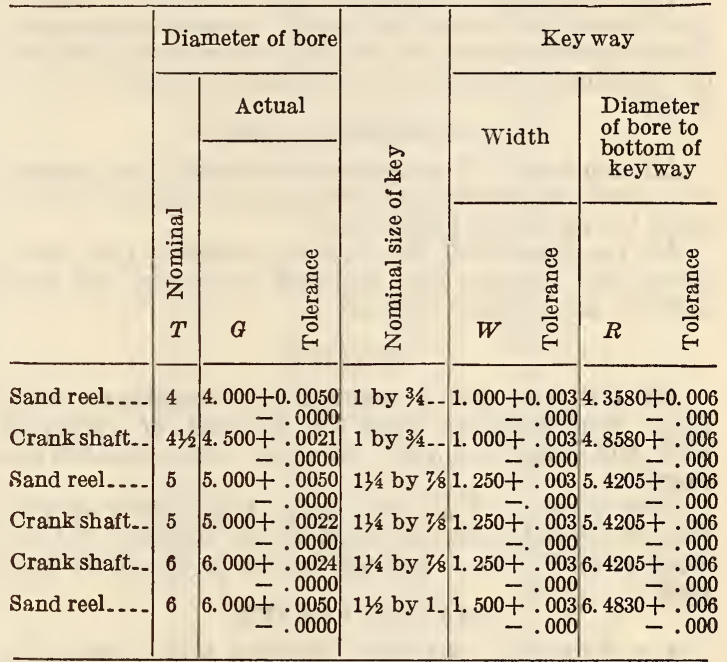

Note.-All key ways shall be cut on a diametral center line.

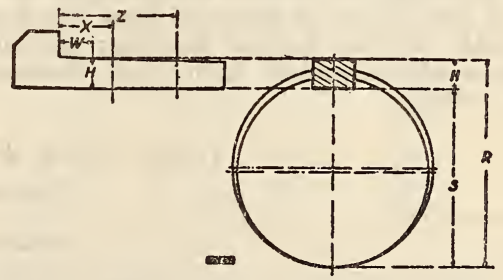

FIGURE 3741/2.-Projection of taper key under maximum and minimum tolerances

The numerical values of $R$ and $S$ are computed as follows:

$$
\begin{gathered}
R=(T+H / 2-0.017)+0.006^{\prime \prime} \\
S=(T-H / 2)+0.000^{\prime \prime} \\
-0.000^{\prime \prime}
\end{gathered}
$$

Where

$R=$ The diameter of the bore to the bottom of key way, in inches.

$S=$ The diameter of shaft to the bottom of key way, in inches.

$T=$ Nominal diameter of shaft, in inches.

The reason for deducting 0.017 inch from the nominal depth of the key way in the bored member is to establish the minimum projection of the key greater than $W$. 
A difference of 0.001 inch in $H$ is equivalent to a movement of 0.096 inch measured parallel to the base of the key from $W$.

$W=$ The distance from the gib head at which the values for $H$ are computed.

$X=$ Minimum projection of key with key ways $(R$ minus $S)$ at maximum depth and height of key $(H)$ at minimum.

$Z=$ Maximum projection of key with key ways $(R$ minus $S)$ at minimum and height of key $(H)$ at maximum.

\section{AMERICAN PETROLEUM INSTITUTE, TRANSMISSION STANDARDS, A. P. I.} STANDARDS NO. \%, JANUARY, 1930

\section{SHAFTING AND KEYS}

\section{MATERIAL COVERED}

These specifications apply to the following material: Shafting and keys.

The standards contained and illustrated herein are dimensional only, and apply to new equipment. These specifications do not include either chemical or physical properties of material, except as to finish.

\section{WORKMANSHIP AND FINISH}

All machined or mating surfaces shall be finished in a good and workmanlike manner and shall be free from burrs, rough edges, etc.

All castings shall be of good quality, free from blowholes, rough edges chipped or ground off, and shall be thoroughly cleaned.

\section{MARKING}

All material manufactured in accordance with these specifications shall be marked or stamped with the manufacturer's name or other identifying mark.

Where the A. P. I. monogram is specified on mill roll or forged material it shall be applied with a steel stencil, size of monogram to be one-half inch high.

\section{SHAFTING AND KEYS}

4-a. Shafting and keys selected from Tables 1, 2 , and 3 shall be standard.

4-b. In order to reduce the number of sizes of shafting, the following range of sizes was adopted:

One-half to four inches diameter (inclusive) in variations of one-eighth inch; this eliminates the 1116-inch sizes.
Four and one-fourth to six inches diameter (inclusive) in variations of one-fourth inch; this eliminates the $1 / 16$ and $1 / 8$ inch sizes.

Over 6 inches diameter, in variations of one-half inch; this eliminates the $1 / 16,1 / 8$, and $1 / 4$ inch sizes.
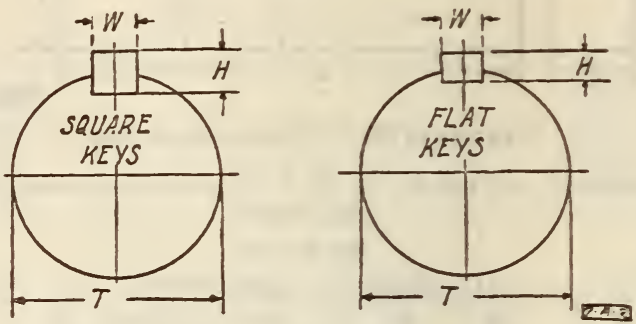

FIGURE 375.-Shafting and plain keys

TABLE 1.-A. P. I. standard shafting, square and flat keys, and key tolerances

[See fig. 375. All dimensions in inches at $68^{\circ}$ ]

\begin{tabular}{|c|c|c|c|c|c|}
\hline \multirow{2}{*}{$\begin{array}{c}\text { Shait diameter } \\
\text { (nominal) } \\
T\end{array}$} & \multicolumn{2}{|c|}{$\begin{array}{c}\text { Square stock } \\
\text { keys }\end{array}$} & \multicolumn{2}{|c|}{ Flat stock keys } & \multirow{2}{*}{$\begin{array}{l}\text { Toler- } \\
\text { ances } \\
\text { on stock } \\
\text { keys } 1 \\
\text { (minus) }\end{array}$} \\
\hline & $\underset{W}{\text { Width }}$ & Heigth & $\underset{W}{W i d t h}$ & $\stackrel{\text { Height }}{H}$ & \\
\hline $\begin{array}{l}58,3 \\
1,118,1144 \\
138,113,158,134 \\
13,8,2,218,21 / 4 \\
238,21 / 2,258,234\end{array}$ & $\begin{array}{l}18 \\
3 / 16 \\
1 / 4 \\
38 \\
1 / 2 \\
5,8\end{array}$ & $\begin{array}{l}1 / 8 \\
3 / 16 \\
134 \\
38 \\
1 / 2 \\
5 / 8\end{array}$ & $\begin{array}{l}38 \\
319 \\
1,6 \\
38 \\
132 \\
58\end{array}$ & $\begin{array}{l}3 / 32 \\
138 \\
3 / 16 \\
14 \\
38 \\
716\end{array}$ & $\begin{array}{l}0.0020 \\
.0020 \\
.0020 \\
.0020 \\
.0025 \\
.0025\end{array}$ \\
\hline 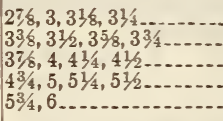 & $\begin{array}{l}3,1 \\
378 \\
178 \\
134 \\
112\end{array}$ & $\begin{array}{l}3 / 4 \\
7 / 8 \\
11 / 4 \\
11 / 2\end{array}$ & $\begin{array}{l}3 / 4 \\
7 / 8 \\
11 / 4 \\
11 / 4 \\
112\end{array}$ & $\begin{array}{l}1 / 2 \\
5,8 \\
3 / 4 \\
7 / 8 \\
1\end{array}$ & $\begin{array}{l}.0025 \\
.0030 \\
.0030 \\
.0030 \\
.0030\end{array}$ \\
\hline
\end{tabular}

${ }_{1}$ Keys. - These tolerences are negative and represent the maximum allowable variation below the exact decimal size. For instance, the standard stock square key for a 2-inch shaft has a maximum size of 0.500 by 0.500 inch, and a minimum size of 0.4975 by 0.4975 inch. It is understood that these kevs are to be cut from cold-finished stock and are to be used without further machining.

Note-Shafting.-The shafting diameters contained in Table 1 are standard. No tolerences are specified, due to different methods used by various manufacturers in manufacturing shafting; that is, machine turned or ground from hot fished and cold fnished. Then specified items requiring the use of shafting are standardized, the tolerances on the shafting must be specified to insure interchangeability.

TABLE 2.-A. P. I. Standard gib-head taper keys

[All dimensions in inches at $68^{\circ} \mathrm{F}$.]

\begin{tabular}{|c|c|c|c|c|c|c|c|c|c|c|c|c|}
\hline \multirow[b]{3}{*}{ Shaft diameter (nominal) $T$} & \multicolumn{5}{|c|}{ Square type 1} & \multicolumn{5}{|c|}{ Flat type 1} & \multirow{2}{*}{\multicolumn{2}{|c|}{$\begin{array}{c}\text { Tolerances on } \\
\text { keys }\end{array}$}} \\
\hline & \multicolumn{2}{|c|}{ Key } & \multicolumn{3}{|c|}{ Gib head } & \multicolumn{2}{|c|}{ Кey } & \multicolumn{3}{|c|}{ Gib head } & & \\
\hline & $\begin{array}{l}\text { Maxi- } \\
\text { mum } \\
\text { width } \\
W\end{array}$ & $\begin{array}{l}\text { Mini- } \\
\text { mum } \\
\text { height } \\
\text { large } \\
\text { end } \\
H^{2}\end{array}$ & Height & $\underset{D}{\text { Length }}$ & $\begin{array}{c}\text { Height } \\
\text { edge } \\
\text { of } \\
\text { cham- } \\
\text { fer } E\end{array}$ & $\begin{array}{c}\text { Maxi- } \\
\text { mum } \\
\text { width } \\
W\end{array}$ & \begin{tabular}{|c|} 
Mini- \\
mum \\
height \\
large \\
end \\
$H^{2}$
\end{tabular} & $\underset{C}{\text { Height }}$ & $\underset{D}{\text { Length }}$ & $\begin{array}{l}\text { Height } \\
\text { edge } \\
\text { of } \\
\text { cham- } \\
\text { fer } E\end{array}$ & $\begin{array}{l}\text { Width, } \\
\text { minus }\end{array}$ & $\begin{array}{l}\text { Height, } \\
\text { plus }\end{array}$ \\
\hline $\begin{array}{l}1 / 2, \\
5 / 8,3 / 4,7 / 8 \\
1,118,114 \\
138,11 / 2,15 \%, 13 / 4 \\
178,2,21 / 8,21 / 4 \\
23 / 8,21 / 2,258,23 / 4\end{array}$ & $\begin{array}{l}18 \\
3 / 16 \\
1 / 4 \\
3 / 8 \\
1 / 2 \\
5 / 8\end{array}$ & $\begin{array}{l}18 \\
3 / 16 \\
14 \\
38 \\
1 / 2 \\
5 / 8\end{array}$ & $\begin{array}{l}14 \\
5 / 16 \\
716 \\
11 / 16 \\
7 / 8 \\
11 / 16\end{array}$ & $\begin{array}{r}7 / 32 \\
932 \\
1132 \\
1532 \\
1932 \\
23 / 32\end{array}$ & \begin{tabular}{r|}
$5 / 32$ \\
$7 / 32$ \\
1132 \\
$15 / 32$ \\
$5 / 8$ \\
$3 / 4$
\end{tabular} & $\begin{array}{l}1 / 8 \\
3 / 16 \\
1 / 4 \\
3 / 8 \\
1 / 2 \\
58\end{array}$ & $\begin{array}{l}3 / 32 \\
18 \\
3 / 16 \\
14 \\
38 \\
7 / 16\end{array}$ & $\begin{array}{l}3 / 16 \\
114 \\
5316 \\
716 \\
58 \\
3 / 4\end{array}$ & $\begin{array}{l}1 / 8 \\
3 / 16 \\
1 / 4 \\
3 / 8 \\
1 / 2 \\
58\end{array}$ & $\begin{array}{l}1 / 8 \\
532 \\
3 / 16 \\
5116 \\
7116 \\
1 / 2\end{array}$ & $\begin{array}{l}0.0020 \\
0020 \\
.0020 \\
.0020 \\
.0025 \\
.0025\end{array}$ & $\begin{array}{l}0.0020 \\
.0020 \\
.0020 \\
.0020 \\
.0025 \\
.0025\end{array}$ \\
\hline $\begin{array}{l}27 / 8,3,31 / 8,314 \\
33 / 8,31 / 2,358,33 / 4 \\
37 / 8,4,414,412 \\
43 / 4,5,514,512 \\
53 / 4,6\end{array}$ & $\begin{array}{l}3 / 4 \\
7 / 8 \\
1 \\
134 \\
11 / 2\end{array}$ & $\begin{array}{l}3 / 4 \\
7 / 8 \\
1 \\
11 / 4 \\
11 / 2\end{array}$ & $\begin{array}{l}13 / 4 \\
11 / 2 \\
13 / 4 \\
2 \\
21 / 2\end{array}$ & $\begin{array}{l}7 / 8 \\
1 \\
13 / 16 \\
17 / 16 \\
13 / 4\end{array}$ & $\begin{array}{l}7 / 8 \\
13 / 16 \\
13 / 16 \\
13 / 4\end{array}$ & $\begin{array}{l}3 / 4 \\
7 / 8 \\
1 \\
114 \\
11 / 2\end{array}$ & $\begin{array}{l}1 / 2 \\
5,8 \\
3 / 4 \\
1 / 8 \\
1\end{array}$ & $\begin{array}{l}7 / 8 \\
1116 \\
114 \\
112 \\
13 / 4\end{array}$ & $\begin{array}{l}3 / 4 \\
7 / 8 \\
1 \\
11 / 4 \\
11 / 2\end{array}$ & $\begin{array}{l}5 / 8 \\
3 / 4 \\
13 / 6 \\
114 \\
114\end{array}$ & $\begin{array}{l}.0025 \\
.0030 \\
.0030 \\
.0030 \\
.0030\end{array}$ & $\begin{array}{l}.0025 \\
.0030 \\
.0030 \\
.0030 \\
.0030\end{array}$ \\
\hline
\end{tabular}

1 The minimum stock length of keys is equal to 4 times the key width, and the maximum stock length of keys is equal to 16 times the key width. The increments of increase in length are equal to 2 times the width.

2 The height $H$ of the key is measured at the distance $W$ from the large end.

Letters indicating dimensions are the same as in Figure 372 in shaft keys and key ways, given abore. 

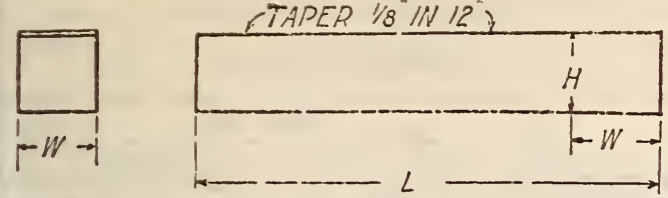

FIGURE 3751/2.-Plain head taper key

TABLE 3.-A. P. I. standard plain-head taper keys [See fig. 3751/2]

[All dimensions in inches at $68^{\circ} \mathrm{F}$.]

\begin{tabular}{|c|c|c|c|c|c|c|}
\hline \multirow[b]{2}{*}{$\begin{array}{l}\text { Shaft diameter (nominal) } \\
T\end{array}$} & \multicolumn{2}{|c|}{ Square type } & \multicolumn{2}{|c|}{ Flat type } & \multicolumn{2}{|c|}{ Tolerances } \\
\hline & 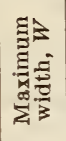 & 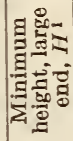 & 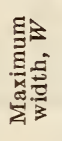 & 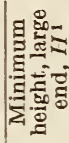 & 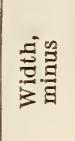 & 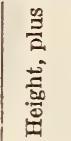 \\
\hline 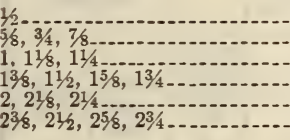 & $\begin{array}{l}1 / 8 \\
3 / 16 \\
1 / 4 \\
3 / 8 \\
1 / 2 \\
5 / 8\end{array}$ & $\begin{array}{l}1 / 8 \\
3 / 16 \\
1 / 4 \\
3 / 8 \\
1 / 2 \\
5 / 8\end{array}$ & $\begin{array}{l}1 / 8 \\
3 / 16 \\
1 / 4 \\
3 / 8 \\
1 / 2 \\
5 / 8\end{array}$ & $\begin{array}{l}3 / 32 \\
1 / 8 \\
3 / 10 \\
1 / 4 \\
3 / 8 \\
7 / 10\end{array}$ & $\begin{array}{l}0.0020 \\
.0020 \\
.0020 \\
.0020 \\
.0025 \\
.0025\end{array}$ & $\begin{array}{l}0.0020 \\
.0020 \\
.0020 \\
.0020 \\
.0025 \\
.0025\end{array}$ \\
\hline $\begin{array}{l}27 / 8,3,31 / 8,31 / 4 \\
338,31 / 2,35 \%, 3 \\
37 / 8,4,41 / 4,41 / 2 \\
43 / 4,5,51 / 4,51 / 2\end{array}$ & $\begin{array}{l}5 / 4 \\
7 / 8 \\
1 \\
11 / 4 \\
11 / 2\end{array}$ & $\begin{array}{l}3 / 4 \\
7 / 8 \\
11 / 4 \\
11 / 2\end{array}$ & $\begin{array}{l}3 / 4 \\
7 / 8 \\
1 \\
11 / 4 \\
11 / 2\end{array}$ & $\begin{array}{l}1 / 2 \\
5 / 8 \\
3 / 4 \\
1^{7 / 8}\end{array}$ & $\begin{array}{l}.0025 \\
.0030 \\
.0030 \\
.0030 \\
.0030\end{array}$ & $\begin{array}{r}.0025 \\
.0030 \\
.0030 \\
.0030 \\
.0030\end{array}$ \\
\hline
\end{tabular}

3 The height $H$ of the key is measured at the distance $W$ from the large end.

The minimum stock length of keys is equal to 4 times the key width, and the maximum stock length of keys is equal to 16 times the

key width. The increments of increase in length are equal to 2 times the width.

\section{AMERICAN SOCIETY OF MECHANICAL ENGINEERS, SQUARE AND FLAT STOCK KEYS, STANDARD WIDTHS AND HEIGHTS, B17b-1925}

[Approved as American Tentative Standard by the American Standards Association, June, 1925]

SQUARE AND FLAT STOCK KEYS

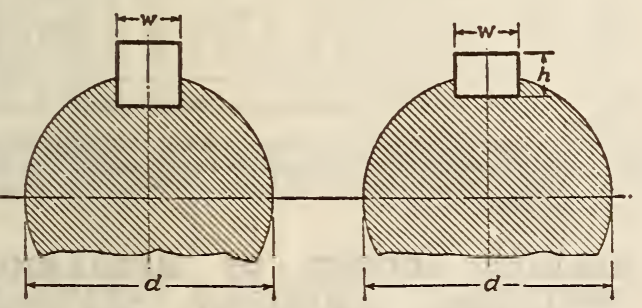

FiguRe 376.-Square and flat stock keys
Dimensions and tolerances

\begin{tabular}{|c|c|c|c|}
\hline $\begin{array}{c}\text { Shaft } \\
\text { diame- } \\
\text { ters } d \\
\text { (inches) }\end{array}$ & $\begin{array}{l}\text { Square } \\
\text { stock } \\
\text { keys } w \\
\text { (inches) }\end{array}$ & $\begin{array}{l}\text { Flat stock } \\
\text { keys } w \times h \\
\text { (inches) }\end{array}$ & $\begin{array}{c}\text { Toler- } \\
\text { ances } 1 \text { on } \\
\text { stock } \\
\text { keys (-) } \\
\text { (inches) }\end{array}$ \\
\hline $\begin{array}{c}1 / 2 \\
9 / 16 \\
5 / 8 \\
11 / 16 \\
3 / 4\end{array}$ & $\begin{array}{l}1 / 8 \\
1 / 8 \\
3 / 16 \\
3 / 16 \\
3 / 16\end{array}$ & $\begin{array}{l}1 / 8 \text { by } 3 / 32 \\
1 / 8 \text { by } 3 / 32 \\
3 / 16 \text { by } 1 / 8 \\
3 / 16 \text { by } 1 / 8 \\
3 / 16 \text { by } 1 / 8\end{array}$ & $\begin{array}{l}0.0020 \\
.0020 \\
.0020 \\
.0020 \\
.0020\end{array}$ \\
\hline $\begin{array}{l}13 / 10 \\
7 / 8 \\
15 / 10 \\
11 / 10\end{array}$ & $\begin{array}{l}3 / 16 \\
3 / 16 \\
1 / 4 \\
1 / 4 \\
1 / 4\end{array}$ & $\begin{array}{l}3 / 16 \text { by } 1 / 8 \\
3 / 16 \text { by } 1 / 8 \\
1 / 4 \text { by } 3 / 16 \\
1 / 4 \text { by } 3 / 16 \\
1 / 4 \text { by } 3 / 16\end{array}$ & $\begin{array}{l}.0020 \\
.0020 \\
.0020 \\
.0020 \\
.0020\end{array}$ \\
\hline $\begin{array}{l}11 / 8 \\
13 / 16 \\
114 \\
15 / 16 \\
138\end{array}$ & $\begin{array}{l}1 / 4 \\
1 / 4 \\
1 / 4 \\
3 / 8 \\
3 / 8\end{array}$ & $\begin{array}{ll}1 / 4 & \text { by } 3 / 16 \\
1 / 4 & \text { by } 3 / 16 \\
1 / 4 & \text { by } 3 / 16 \\
3 / 8 & \text { by } 1 / 4 \\
3 / 8 & \text { by } 1 / 4\end{array}$ & $\begin{array}{l}.0020 \\
.0020 \\
.0020 \\
.0020 \\
.0020\end{array}$ \\
\hline $\begin{array}{l}17 / 16 \\
11 / 2 \\
191 / 6 \\
15 / 8 \\
111 / 16\end{array}$ & $\begin{array}{l}3 / 8 \\
3 / 8 \\
3 / 8 \\
3 / 8 \\
3 / 8\end{array}$ & $\begin{array}{ll}3 / 8 & \text { by } 1 / 4 \\
3 / 8 & \text { by } 1 / 4 \\
3 / 8 & \text { by } 1 / 4 \\
3 / 8 & \text { by } 1 / 4 \\
3 / 8 & \text { by } 1 / 4\end{array}$ & $\begin{array}{l}.0020 \\
.0020 \\
.0020 \\
.0020 \\
.0020\end{array}$ \\
\hline $\begin{array}{l}13 / 4 \\
1^{13 / 16} \\
17 / 8 \\
1^{15 / 16} \\
2^{15}\end{array}$ & $\begin{array}{l}3 / 8 \\
1 / 2 \\
1 / 2 \\
1 / 2 \\
1 / 2\end{array}$ & $\begin{array}{ll}3 / 8 & \text { by } 1 / 4 \\
1 / 2 & \text { by } 3 / 8 \\
1 / 2 & \text { by } 3 / 8 \\
1 / 2 & \text { by } 3 / 8 \\
1 / 2 & \text { by } 3 / 8\end{array}$ & $\begin{array}{l}.0020 \\
.0025 \\
.0025 \\
.0025 \\
.0025\end{array}$ \\
\hline $\begin{array}{l}21 / 16 \\
21 / 8 \\
23 / 16 \\
214 \\
25 / 16\end{array}$ & $\begin{array}{l}1 / 2 \\
1 / 2 \\
1 / 2 \\
1 / 2 \\
5 / 8\end{array}$ & $\begin{array}{ll}1 / 2 & \text { by } 3 / 8 \\
1 / 2 & \text { by } 3 / 8 \\
1 / 2 & \text { by } 3 / 8 \\
1 / 2 & \text { by } 3 / 8 \\
5 / 8 & \text { by } 7 / 16\end{array}$ & $\begin{array}{l}.0025 \\
.0025 \\
.0025 \\
.0025 \\
.0025\end{array}$ \\
\hline $\begin{array}{l}23 / 8 \\
27 / 16 \\
21 / 2 \\
25 / 8 \\
23 / 4\end{array}$ & $\begin{array}{l}5 / 8 \\
5 / 8 \\
5 / 8 \\
5 / 8 \\
5 / 8\end{array}$ & $\begin{array}{ll}5 / 8 & \text { by } 7 / 16 \\
5 / 8 & \text { by } 7 / 16 \\
5 / 8 & \text { by } 7 / 16 \\
5 / 8 & \text { by } 7 / 16 \\
5 / 8 & \text { by } 7 / 16\end{array}$ & $\begin{array}{l}.0025 \\
.0025 \\
.0025 \\
.0025 \\
.0025\end{array}$ \\
\hline $\begin{array}{l}27 / 8 \\
215 / 16 \\
3 \\
31 / 8 \\
31 / 4\end{array}$ & $\begin{array}{l}3 / 4 \\
3 / 4 \\
3 / 4 \\
3 / 4 \\
3 / 4\end{array}$ & $\begin{array}{ll}3 / 4 & \text { by } 1 / 2 \\
3 / 4 & \text { by } 1 / 2 \\
3 / 4 & \text { by } 1 / 2 \\
3 / 4 & \text { by } 1 / 2 \\
3 / 4 & \text { by } 1 / 2\end{array}$ & $\begin{array}{l}.0025 \\
.0025 \\
.0025 \\
.0025 \\
.0025\end{array}$ \\
\hline $\begin{array}{l}33 / 8 \\
37 / 16 \\
31 / 2 \\
35 / 8 \\
33 / 4\end{array}$ & $\begin{array}{l}7 / 8 \\
7 / 8 \\
7 / 8 \\
7 / 8 \\
7 / 8\end{array}$ & $\begin{array}{ll}7 / 8 & \text { by } 5 / 8 \\
7 / 8 & \text { by } 5 / 8 \\
7 / 8 & \text { by } 5 / 8 \\
7 / 8 & \text { by } 5 / 8 \\
7 / 8 & \text { by } 5 / 8\end{array}$ & $\begin{array}{l}.0030 \\
.0030 \\
.0030 \\
.0030 \\
.0030\end{array}$ \\
\hline $\begin{array}{l}37 / 8 \\
315 / 10 \\
4 \\
41 / 4 \\
47 / 10\end{array}$ & $\begin{array}{l}1 \\
1 \\
1 \\
1 \\
1\end{array}$ & $\begin{array}{ll}1 & \text { by } 3 / 4 \\
1 & \text { by } 3 / 4 \\
1 & \text { by } 3 / 4 \\
1 & \text { by } 3 / 4 \\
1 & \text { by } 3 / 4\end{array}$ & $\begin{array}{l}.0030 \\
.0030 \\
.0030 \\
.0030 \\
.0030\end{array}$ \\
\hline $\begin{array}{l}41 / 2 \\
43 / 4 \\
4^{15 / 16} \\
5 \\
51 / 4\end{array}$ & $\begin{array}{l}1 \\
11 / 4 \\
11 / 4 \\
11 / 4 \\
11 / 4\end{array}$ & $\begin{array}{ll}1 & \text { by } 3 / 4 \\
11 / 4 & \text { by } 7 / 8 \\
11 / 4 & \text { by } 7 / 8 \\
11 / 4 & \text { by } 7 / 8 \\
11 / 4 & \text { by } 7 / 8\end{array}$ & $\begin{array}{l}.0030 \\
.0030 \\
.0030 \\
.0030 \\
.0030\end{array}$ \\
\hline $\begin{array}{l}57 / 16 \\
51 / 2 \\
53 / 4 \\
515 / 16 \\
6\end{array}$ & $\begin{array}{l}11 / 4 \\
11 / 4 \\
11 / 2 \\
11 / 2 \\
11 / 2\end{array}$ & $\begin{array}{ll}11 / 4 & \text { by } 7 / 8 \\
11 / 4 & \text { by } 7 / 8 \\
11 / 2 & \text { by } 1 \\
11 / 2 & \text { by } 1 \\
11 / 2 & \text { by } 1\end{array}$ & $\begin{array}{l}.0030 \\
.0030 \\
.0030 \\
.0030 \\
.0030\end{array}$ \\
\hline
\end{tabular}

1 These tolerances are negative and represent the maximum allowable variation below the exact nominal size. For instance, the standard stock square key for a 2-inch shaft has a maximum size of 0.500 by 0.500 inches and a minimum size of 0.4975 by 0.4975 inches.

It is understood that these keys are to be cut from cold-finished stock and are to be used without machining. 


\section{AMERICAN SOCIETY OF MECHANICAL ENGINEERS, PLAIN TAPER STOCK KEYS, SQUARE AND FLAT, B17d-1927}

[Approved as American tentative standard by the American Standards Association, February, 1927]

PLAIN TAPER STOCK KEYS
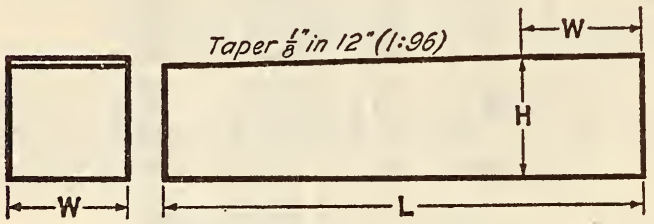

FIgURE 376 $1 / 2$.- Plain taper stock key

\section{Dimensions and tolerances}

[All dimensions in inches]

\begin{tabular}{|c|c|c|c|c|c|c|}
\hline \multirow[b]{2}{*}{$\begin{array}{l}\text { Diameters of } \\
\text { shafts } \\
\text { (inclusive) }\end{array}$} & \multicolumn{2}{|c|}{ Square type } & \multicolumn{2}{|c|}{ Flat type } & \multicolumn{2}{|c|}{ Tolerances on keys } \\
\hline & $\begin{array}{c}\text { Maxi- } \\
\text { mum } \\
\text { width } \\
W \\
W\end{array}$ & $\begin{array}{c}\begin{array}{c}\text { Height } \\
\text { at large } \\
\text { end } 1 \\
H\end{array} \\
H\end{array}$ & $\begin{array}{c}\underset{\substack{\text { Maxi- } \\
\text { width }}}{ } \\
W\end{array}$ & $\begin{array}{c}\text { Height } \\
\text { at large } \\
\text { end } 1 \\
H\end{array}$ & $\begin{array}{c}\text { Width } \\
\text { (minus) }\end{array}$ & $\begin{array}{l}\text { Height } \\
\text { (plus) }\end{array}$ \\
\hline $\begin{array}{c}1 / 2-9 / 16 \\
5 / 8-7 / 8 \\
15 / 16-11 / 4\end{array}$ & $\begin{array}{l}1 / 8 \\
3 / 16 \\
1 / 4\end{array}$ & $\begin{array}{l}1 / 8 \\
3 / 16 \\
1 / 4\end{array}$ & $\begin{array}{l}1 / 8 \\
3 / 16 \\
1 / 4\end{array}$ & $\begin{array}{l}3 / 32 \\
1 / 8 \\
3 / 16\end{array}$ & $\begin{array}{l}0.0020 \\
.0020 \\
.0020\end{array}$ & $\begin{array}{r}0.0020 \\
.0020 \\
.0020\end{array}$ \\
\hline $\begin{array}{l}15 / 16-13 / 4 \\
113 / 16-21 / 4 \\
25 / 16-23 / 4 \\
27 / 8 \quad-31 / 4\end{array}$ & $\begin{array}{l}3 / 8 \\
1 / 2 \\
5 / 8 \\
3 / 4\end{array}$ & $\begin{array}{l}3 / 8 \\
1 / 2 \\
5 / 8 \\
3 / 4\end{array}$ & $\begin{array}{l}3 / 8 \\
1 / 2 \\
5 / 8 \\
3 / 4\end{array}$ & $\begin{array}{l}1 / 4 \\
3 / 8 \\
7 / 16 \\
1 / 2\end{array}$ & $\begin{array}{l}.0020 \\
.0025 \\
.0025 \\
.0025\end{array}$ & $\begin{array}{l}.0020 \\
.0025 \\
.0025 \\
.0025\end{array}$ \\
\hline $\begin{array}{ll}33 / 8 & -33 / 4 \\
37 / 8 & -41 / 2 \\
43 / 4 & -51 / 2 \\
53 / 4 & -6\end{array}$ & $\begin{array}{l}1^{7 / 8} \\
11 / 4 \\
11 / 2\end{array}$ & $\begin{array}{l}7 / 8 \\
11 \\
11 / 4 \\
11 / 2\end{array}$ & $\begin{array}{l}7 / 8 \\
1 \\
114 \\
11 / 2\end{array}$ & $\begin{array}{r}5 / 8 \\
3 / 4 \\
7 / 8 \\
1\end{array}$ & $\begin{array}{l}.0030 \\
.0030 \\
.0030 \\
.0030\end{array}$ & $\begin{array}{l}.0030 \\
.0030 \\
.0030 \\
.0030\end{array}$ \\
\hline
\end{tabular}

\section{Diameters of}

shafts

(inclusive)

\begin{tabular}{|c|c|c|c|c|c|c|c|}
\hline $\begin{array}{c}1 / 2-9 / 16 \\
5 / 8-7 / 8 \\
15 / 16-11 / 4\end{array}$ & $\begin{array}{l}1 / 2 \\
1^{3 / 4}\end{array}$ & $\begin{array}{r}3 / 4 \\
11 / 8 \\
11 / 2\end{array}$ & $\begin{array}{l}1 \\
11 / 2 \\
2\end{array}$ & $\begin{array}{l}11 / 4 \\
17 / 8 \\
21 / 2\end{array}$ & $\begin{array}{l}11 / 2 \\
21 / 4 \\
3\end{array}$ & $\begin{array}{l}13 / 4 \\
25 / 8 \\
31 / 2\end{array}$ & $\begin{array}{l}2 \\
3 \\
4\end{array}$ \\
\hline $\begin{array}{l}15 / 16-13 / 4 \\
113 / 16-21 / 4 \\
25 / 16-23 / 4\end{array}$ & $\begin{array}{l}11 / 2 \\
2 \\
21 / 2\end{array}$ & $\begin{array}{l}21 / 4 \\
3 \\
33 / 4\end{array}$ & $\begin{array}{l}3 \\
4 \\
5\end{array}$ & $\begin{array}{l}33 / 4 \\
5 \\
61 / 4\end{array}$ & $\begin{array}{l}41 / 2 \\
6 \\
71 / 2\end{array}$ & $\begin{array}{l}51 / 4 \\
7 \\
83 / 4\end{array}$ & $\begin{array}{r}6 \\
8 \\
10\end{array}$ \\
\hline $\begin{array}{ll}27 / 8 & -31 / 4 \\
33 / 8 & -33 / 4 \\
37 / 8 & -41 / 2 \\
43 / 4 & -51 / 2 \\
53 / 4 & -6\end{array}$ & $\begin{array}{l}3 \\
31 / 2 \\
4 \\
5 \\
6\end{array}$ & $\begin{array}{l}41 / 2 \\
51 / 4 \\
6 \\
71 / 2 \\
9\end{array}$ & $\begin{array}{r}6 \\
7 \\
8 \\
10 \\
12\end{array}$ & $\begin{array}{l}71 / 2 \\
83 / 4 \\
10 \\
121 / 2 \\
15\end{array}$ & $\begin{array}{l}9 \\
101 / 2 \\
12 \\
15 \\
18\end{array}$ & $\begin{array}{l}101 / 2 \\
121 / 4 \\
14 \\
171 / 2 \\
21\end{array}$ & $\begin{array}{l}12 \\
14 \\
16 \\
20 \\
24\end{array}$ \\
\hline
\end{tabular}

1 This height of the key is measured at the distance $W$ from the large end.

${ }^{2}$ The minimum stock length of keys is equal to 4 times the key width, and the maximum stock length of keys is equal to 16 times the key width. The increments of increase of length are equal to 2 times the width.

\section{AMERICAN SOCIETY OF MECHANICAL ENGINEERS, GIB-HEAD TAPER STOCK KEYS, SQUARE AND FLAT, B1\%e-192\%}

[Approved as American tentative standard by the American Standard Association, February, 1927.]

\section{GIB-HEAD TAPER STOCK KEYS}

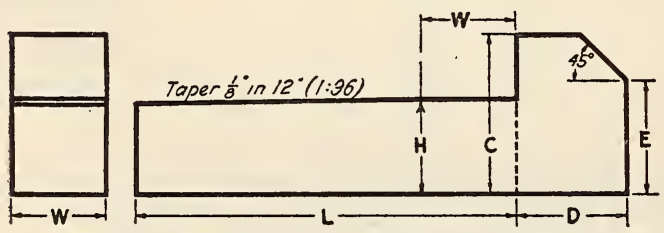

FIGURE 377.-Gib head taper stock key
Dimensions and tolerances

[All dimensions in inches]

\begin{tabular}{|c|c|c|c|c|c|}
\hline \multirow{3}{*}{$\begin{array}{c}\text { Diameters of } \\
\text { shafts } \\
\text { (inclusive) }\end{array}$} & \multicolumn{5}{|c|}{ Square type } \\
\hline & \multicolumn{2}{|c|}{ Кey } & \multicolumn{3}{|c|}{ Gib head } \\
\hline & $\begin{array}{l}\text { Maxi- } \\
\text { mum } \\
\text { width } \\
W\end{array}$ & $\begin{array}{c}\text { Height } \\
\text { at large } \\
\text { end } 1 \\
H\end{array}$ & $\begin{array}{c}\text { Height } \\
\qquad C\end{array}$ & $\begin{array}{c}\text { Length } \\
\quad D\end{array}$ & $\begin{array}{c}\text { Height } \\
\text { edge of } \\
\text { chamfer } \\
E\end{array}$ \\
\hline $\begin{array}{c}1 / 2-9 / 16 \\
5 / 8-7 / 8 \\
15 / 16-11 / 4 \\
15 / 16-134 \\
113 / 16-214 \\
25 / 16-23 / 4\end{array}$ & $\begin{array}{l}1 / 8 \\
3 / 16 \\
1 / 4 \\
3 / 8 \\
1 / 2 \\
5 / 8\end{array}$ & $\begin{array}{l}1 / 8 \\
3 / 16 \\
1 / 4 \\
3 / 8 \\
1 / 2 \\
5 / 8\end{array}$ & $\begin{array}{l}1 / 4 \\
5 / 10 \\
7 / 16 \\
11 / 10 \\
7 / 8 \\
11 / 10\end{array}$ & $\begin{array}{l}7 / 32 \\
9 / 32 \\
11 / 32 \\
15 / 32 \\
19 / 22 \\
23 / 32\end{array}$ & $\begin{array}{l}5 / 32 \\
7 / 32 \\
11 / 32 \\
15 / 32 \\
5 / 8 \\
3 / 4\end{array}$ \\
\hline $\begin{array}{ll}27 / 8 & -31 / 4 \\
33 / 8 & -33 / 4 \\
37 / 8 & -41 / 2 \\
43 / 4 & -51 / 2 \\
53 / 4 & -6\end{array}$ & $\begin{array}{l}3 / 4 \\
7 / 8 \\
1 \\
11 / 4 \\
11 / 2\end{array}$ & $\begin{array}{l}3 / 4 \\
7 / 8 \\
11 \\
11 / 4 \\
11 / 2\end{array}$ & $\begin{array}{l}11 / 4 \\
11 / 2 \\
13 / 4 \\
2 \\
21 / 2\end{array}$ & $\begin{array}{l}7 / 8 \\
13 / 16 \\
13 / 16 \\
13 / 4\end{array}$ & $\begin{array}{l}7 / 8 \\
13 / 16 \\
13 / 16 \\
17 / 4\end{array}$ \\
\hline
\end{tabular}

\begin{tabular}{|c|c|c|c|c|c|c|c|}
\hline \multirow[b]{3}{*}{$\begin{array}{l}\text { Diameters } \\
\text { of shafts } \\
\text { (inclusive) }\end{array}$} & \multicolumn{5}{|c|}{ Flat type } & \multirow{2}{*}{\multicolumn{2}{|c|}{$\begin{array}{c}\text { Tolerances } \\
\text { on keys }\end{array}$}} \\
\hline & \multicolumn{2}{|c|}{ Key } & \multicolumn{3}{|c|}{ Gib head } & & \\
\hline & $\begin{array}{c}\text { Maxi- } \\
\text { mum } \\
\text { width } \\
W \\
W\end{array}$ & $\begin{array}{c}\text { Height } \\
\text { at } \\
\text { large } \\
\text { end 1 } \\
H\end{array}$ & $\begin{array}{c}\text { Height } \\
C\end{array}$ & $\begin{array}{c}\text { Length } \\
D\end{array}$ & \begin{tabular}{|c|} 
Height \\
edge of \\
cham- \\
fer \\
$E$
\end{tabular} \mid & $\begin{array}{l}\text { Width } \\
\text { (mi- } \\
\text { nus) }\end{array}$ & $\begin{array}{l}\text { Height } \\
\text { (plus) }\end{array}$ \\
\hline $\begin{array}{l}1 / 2-9 / 16 \\
5 / 8-7 / 8 \\
15 / 16-11 / 4\end{array}$ & $\begin{array}{l}11 / 8 \\
3 / 16 \\
1 / 4\end{array}$ & $\begin{array}{l}3 / 32 \\
1 / 8 \\
3 / 16\end{array}$ & $\begin{array}{l}3 / 16 \\
1 / 4 \\
5 / 16\end{array}$ & $\begin{array}{l}1 / 8 \\
3 / 16 \\
1 / 4\end{array}$ & $\begin{array}{l}1 / 8 \\
5 / 32 \\
3 / 10\end{array}$ & $\begin{array}{l}0.0020 \\
.0020 \\
.0020\end{array}$ & $\begin{array}{l}0.0020 \\
.0020 \\
.0020\end{array}$ \\
\hline $\begin{array}{l}15 / 16-13 / 4 \\
113 / 16-21 / 4 \\
25 / 16-23 / 4\end{array}$ & $\begin{array}{l}3 / 8 \\
1 / 2 \\
5 / 8\end{array}$ & $\begin{array}{l}1 / 4 \\
3 / 8 \\
7 / 16\end{array}$ & $\begin{array}{l}7 / 16 \\
5 / 8 \\
3 / 4\end{array}$ & $\begin{array}{l}3 / 8 \\
1 / 2 \\
5 / 8\end{array}$ & $\begin{array}{l}5 / 16 \\
7 / 16 \\
1 / 2\end{array}$ & $\begin{array}{l}.0020 \\
.0025 \\
.0025\end{array}$ & $\begin{array}{l}.0020 \\
.0025 \\
.0025\end{array}$ \\
\hline $\begin{array}{ll}27 / 8 & -31 / 4 \\
33 / 8 & -33 / 4 \\
37 / 8 & -41 / 2 \\
43 / 4 & -51 / 2 \\
53 / 4 & -6\end{array}$ & $\begin{array}{l}3 / 4 \\
1^{7 / 8} \\
11 / 4 \\
11 / 2\end{array}$ & $\begin{array}{c}1 / 2 \\
5 / 8 \\
3 / 4 \\
1^{7 / 8}\end{array}$ & $\begin{array}{l}7 / 8 \\
11 / 16 \\
11 / 4 \\
11 / 2 \\
13 / 4\end{array}$ & $\begin{array}{l}3 / 4 \\
7 / 8 \\
1^{7 / 8} \\
11 / 4 \\
11 / 2\end{array}$ & $\begin{array}{l}5 / 8 \\
3 / 4 \\
13 / 16 \\
1 \\
11 / 4\end{array}$ & $\begin{array}{l}.0025 \\
.0030 \\
.0030 \\
.0030 \\
.0030\end{array}$ & $\begin{array}{l}.0025 \\
.0030 \\
.0030 \\
.0030 \\
.0030\end{array}$ \\
\hline
\end{tabular}

\begin{tabular}{|c|c|c|c|c|c|c|c|}
\hline $\begin{array}{l}\text { Diameters } \\
\text { of shafts } \\
\text { (inclusive) }\end{array}$ & \multicolumn{7}{|c|}{ Stock length of key ${ }^{2}(L)$} \\
\hline $\begin{array}{l}1 / 2-9 / 16 \\
5 / 8-7 / 8 \\
15 / 16-11 / 4\end{array}$ & $1^{1 / 2}$ & $\begin{array}{r}3 / 4 \\
11 / 8 \\
11 / 2\end{array}$ & $\begin{array}{l}1 \\
11 / 2 \\
2\end{array}$ & $\begin{array}{l}11 / 4 \\
378 \\
21 / 2\end{array}$ & $\begin{array}{l}11 / 2 \\
21 / 4 \\
3\end{array}$ & $\begin{array}{l}13 / 4 \\
25 / 8 \\
31 / 2\end{array}$ & $\begin{array}{l}2 \\
3 \\
4\end{array}$ \\
\hline $\begin{array}{l}15 / 16-13 / 4 \\
113 / 16-21 / 4 \\
251 / 16-23 / 4 \\
27 / 8-314\end{array}$ & $\begin{array}{l}11 / 2 \\
2 \\
21 / 2 \\
3\end{array}$ & $\begin{array}{l}21 / 4 \\
3 \\
33 / 4 \\
41 / 2\end{array}$ & $\begin{array}{l}3 \\
4 \\
5 \\
6\end{array}$ & $\begin{array}{l}33 / 4 \\
5 \\
61 / 4 \\
71 / 2\end{array}$ & $\begin{array}{l}41 / 2 \\
6 \\
71 / 2 \\
9\end{array}$ & $\begin{array}{r}51 / 4 \\
7 \\
83 / 4 \\
101 / 2\end{array}$ & $\begin{array}{r}6 \\
8 \\
10 \\
12\end{array}$ \\
\hline $\begin{array}{ll}33 / 8 & -33 / 4 \\
37 / 8 & -41 / 2 \\
43 / 4 & -51 / 2 \\
53 / 4 & -6\end{array}$ & $\begin{array}{l}31 / 2 \\
4 \\
5 \\
6\end{array}$ & $\begin{array}{l}51 / 4 \\
6 \\
71 / 2 \\
9\end{array}$ & $\begin{array}{r}7 \\
8 \\
10 \\
12\end{array}$ & $\begin{array}{l}83 / 4 \\
10 \\
121 / 2 \\
15\end{array}$ & $\begin{array}{l}101 / 2 \\
12 \\
15 \\
18\end{array}$ & $\begin{array}{l}121 / 4 \\
14 \\
171 / 2 \\
21\end{array}$ & $\begin{array}{l}14 \\
16 \\
20 \\
24\end{array}$ \\
\hline
\end{tabular}

${ }_{1}^{1}$ This height of the key is measured at the distance $W$ from the gib head.

2 The minimum stock length of keys is equal to 4 times the key width, and the maximum stock length of keys is equal to 16 times the key width. The increments of increase of length are equal to 2 times the width.

\section{AMERICAN SOCIETY OF MECHANICAL ENGINEERS, AMERICAN STANDARD WOODRUFF KEYS, KEY SLOTS, AND CUTTERS, B17f-1930}

[Approved as American Standard by the American Standards Association, December, 1930]

\section{WOODRUFF KEYS, KEY SLOTS, AND CUTTERS}

(Dimensions of keys and key slots conform to those of the Society of Automotive Engineers, given below. In addition, this specification contains the Woodruff key-slot cutter dimensions shown in the accompanying table.)

Key-slot cutters. - Two series of key-slot cutters, fine and coarse teeth, both of the shank type and having a shank diameter of one-half inch for all sizes have been adopted as best suited to the re- 
quirements. The key numbers established to designate the size of key apply also to the corresponding cutters. The over-all dimensions, number of teeth for both fine and coarse series, tolerances, and general design features are given in Table 3.

It should be noted that a back clearance of 0.006 to 0.010 inch per inch (20 to 40 minutes of arc) shall be ground on each side of the cutter and that each side face may be recessed to a depth of not more than one-quarter of the cutter width.

The accuracy in grinding shall be such that the cutting edges of the teeth will be concentric within 0.002 inch and both sides will be parallel within 0.0005 inch indicator reading.

In order that the cutters may be centered accurately for regrinding, provision for which has been made in the maximum dimensions (note 2, name or trade-mark.
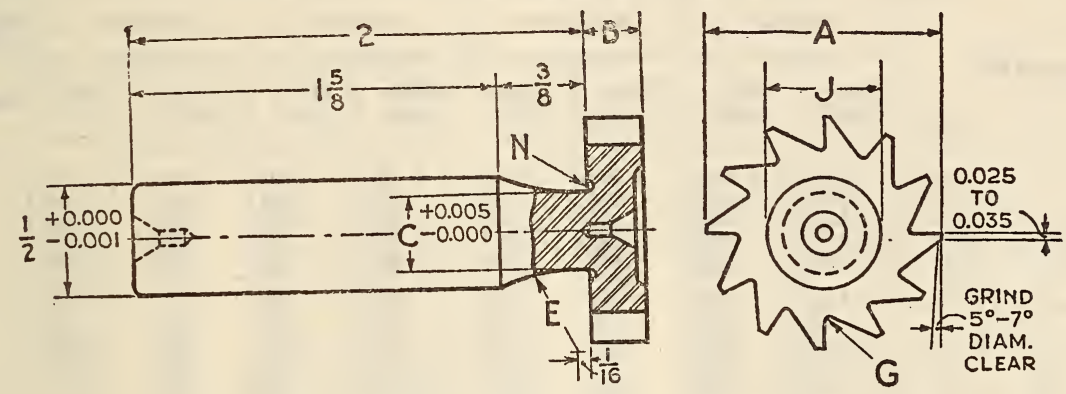

FIGURE 378.-Woodruff key-slot cutter

TABLE 3.-Woodruff key-slot cutter dimensions

[All dimensions given in inches]

\begin{tabular}{|c|c|c|c|c|c|c|c|c|c|c|c|c|c|c|}
\hline \multirow{2}{*}{$\begin{array}{c}\text { Key }{ }^{1} \\
\text { No. }\end{array}$} & \multirow{2}{*}{$\underset{\text { size }}{\text { Nominal key }}$} & \multicolumn{2}{|c|}{$\begin{array}{c}\text { Number of } \\
\text { teeth }\end{array}$} & \multicolumn{2}{|c|}{$\begin{array}{c}\text { Cutter }{ }^{2} \text { diameter } \\
\text { A }\end{array}$} & \multicolumn{2}{|c|}{$\begin{array}{c}\text { Cutter }{ }^{3} \text { width } \\
\text { B }\end{array}$} & \multicolumn{2}{|c|}{ Neck diameter } & \multicolumn{4}{|c|}{ Radii } & \multirow{2}{*}{$\begin{array}{c}\begin{array}{c}\text { Recess } \\
\text { diam- } \\
\text { eter }\end{array} \\
J\end{array}$} \\
\hline & & Fine & Coarse & $\begin{array}{l}\text { Maxi- } \\
\text { mum }\end{array}$ & $\begin{array}{l}\text { Mini- } \\
\text { mum }\end{array}$ & $\begin{array}{l}\text { Maxi- } \\
\text { mum }\end{array}$ & $\begin{array}{l}\text { Mini- } \\
\text { mum }\end{array}$ & $C$ & $C$ & $E$ & $E^{4}$ & $G$ & $N$ & \\
\hline $\begin{array}{l}204 \\
304 \\
305 \\
404 \\
405\end{array}$ & 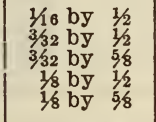 & $\begin{array}{l}10 \\
10 \\
10 \\
10 \\
10\end{array}$ & $\begin{array}{l}8 \\
8 \\
8 \\
8 \\
8\end{array}$ & $\begin{array}{l}0.515 \\
.515 \\
.640 \\
.515 \\
.640\end{array}$ & $\begin{array}{l}0.510 \\
.510 \\
.635 \\
.510 \\
.635\end{array}$ & $\begin{array}{r}0.0625 \\
.0938 \\
.0938 \\
.1250 \\
.1250\end{array}$ & $\begin{array}{l}0.0620 \\
.0933 \\
.0933 \\
.1245 \\
.1245\end{array}$ & $\begin{array}{r}0.130 \\
.160 \\
.191 \\
.191 \\
.223\end{array}$ & & $\begin{array}{l}23 / 64 \\
3 / 8 \\
13 / 32 \\
13 / 32 \\
7 / 16\end{array}$ & & $\begin{array}{l}1 / 64 \\
1 / 64 \\
1 / 32 \\
1 / 32 \\
1 / 32\end{array}$ & $\begin{array}{l}1 / 64 \\
1 / 64 \\
1 / 64 \\
1 / 64 \\
1 / 64\end{array}$ & $\begin{array}{l}1 / 4 \\
1 / 4 \\
5 / 16 \\
1 / 4 \\
5 / 16\end{array}$ \\
\hline $\begin{array}{l}406 \\
505 \\
506 \\
507 \\
606\end{array}$ & $\begin{array}{r}1 / 8 \text { by } 3 / 4 \\
5 / 32 \text { by } 5 / 8 \\
5 / 32 \text { by } 3 / 4 \\
5 / 32 \text { by } 7 / 8 \\
3 / 16 \text { by } 3 / 4\end{array}$ & $\begin{array}{l}10 \\
10 \\
10 \\
12 \\
10\end{array}$ & $\begin{array}{l}8 \\
8 \\
8 \\
9 \\
8\end{array}$ & $\begin{array}{l}.765 \\
.640 \\
.765 \\
.892 \\
.765\end{array}$ & $\begin{array}{l}.760 \\
.635 \\
.760 \\
.887 \\
.760\end{array}$ & $\begin{array}{l}.1250 \\
.1563 \\
.1563 \\
.1563 \\
.1873\end{array}$ & $\begin{array}{l}.1245 \\
.1558 \\
.1558 \\
.1558 \\
.1868\end{array}$ & $\begin{array}{l}.217 \\
.253 \\
.247 \\
.247 \\
.279\end{array}$ & & $\begin{array}{l}13 / 32 \\
15 / 32 \\
7 / 16 \\
7 / 16 \\
1 / 2\end{array}$ & & $\begin{array}{l}1 / 32 \\
1 / 32 \\
1 / 32 \\
1 / 32 \\
1 / 32\end{array}$ & $\begin{array}{l}1 / 64 \\
1 / 64 \\
1 / 64 \\
1 / 64 \\
1 / 64\end{array}$ & $\begin{array}{l}3 / 8 \\
5 / 16 \\
3 / 8 \\
7 / 16 \\
3 / 8\end{array}$ \\
\hline $\begin{array}{l}607 \\
608 \\
609 \\
807 \\
808\end{array}$ & $\begin{array}{l}3 / 16 \text { by } 7 / 8 \\
3 / 16 \text { by } 1 \\
3 / 16 \text { by } 118 \\
3 / 4 \text { by } 7 / 8 \\
1 / 4 \text { by } 1\end{array}$ & $\begin{array}{l}12 \\
12 \\
12 \\
12 \\
12\end{array}$ & $\begin{array}{r}9 \\
10 \\
10 \\
9 \\
10\end{array}$ & $\begin{array}{r}.892 \\
1.017 \\
1.142 \\
.892 \\
1.017\end{array}$ & $\begin{array}{r}.887 \\
1.012 \\
1.137 \\
.887 \\
1.012\end{array}$ & $\begin{array}{l}.1873 \\
.1873 \\
.1873 \\
.2497 \\
.2497\end{array}$ & $\begin{array}{l}.1868 \\
.1868 \\
.1868 \\
.2492 \\
.2492\end{array}$ & $\begin{array}{l}.279 \\
.279 \\
.312 \\
.342 \\
.342\end{array}$ & & $\begin{array}{l}1 / 2 \\
1 / 2 \\
9 / 16 \\
2132 \\
21 / 32\end{array}$ & & $\begin{array}{l}1 / 32 \\
1 / 32 \\
1 / 32 \\
1 / 32 \\
1 / 32\end{array}$ & $\begin{array}{l}1 / 64 \\
1 / 64 \\
1 / 64 \\
164 \\
1 / 64\end{array}$ & $\begin{array}{l}7 / 10 \\
1 / 2 \\
91 / 6 \\
71 / 6 \\
1 / 2\end{array}$ \\
\hline $\begin{array}{l}809 \\
810 \\
811 \\
812\end{array}$ & $\begin{array}{l}1 / 4 \text { by } 11 / 8 \\
1 / 4 \text { by } 11 / 4 \\
1 / 4 \text { by } 13 / 8 \\
1 / 4 \text { by } 11 / 2\end{array}$ & $\begin{array}{l}12 \\
14 \\
14 \\
16\end{array}$ & $\begin{array}{l}10 \\
10 \\
10 \\
12\end{array}$ & $\begin{array}{l}1.142 \\
1.270 \\
1.395 \\
1.520\end{array}$ & $\begin{array}{l}1.137 \\
1.265 \\
1.390 \\
1.515\end{array}$ & $\begin{array}{l}.2497 \\
.2497 \\
.2497 \\
.2497\end{array}$ & $\begin{array}{l}.2492 \\
.2492 \\
.2492 \\
.2492\end{array}$ & $\begin{array}{l}.373 \\
.373\end{array}$ & $\begin{array}{r}0.401 \\
.435\end{array}$ & $3 / 4$ & $\begin{array}{l}3 / 64 \\
1 / 32\end{array}$ & $\begin{array}{l}1 / 32 \\
1 / 32 \\
1 / 32 \\
1 / 32\end{array}$ & $\begin{array}{l}1 / 64 \\
1 / 64 \\
1 / 32 \\
1 / 32\end{array}$ & $\begin{array}{l}9 / 10 \\
5 / 8 \\
1316 \\
3 / 4\end{array}$ \\
\hline $\begin{array}{l}1008 \\
1009 \\
1010 \\
1011\end{array}$ & $\begin{array}{l}5 / 16 \text { by } 1 \\
5 / 16 \text { by } 11 / 8 \\
5 / 16 \text { by } 11 / 4 \\
5 / 16 \text { by } 13 / 8\end{array}$ & $\begin{array}{l}12 \\
12 \\
14 \\
14\end{array}$ & $\begin{array}{l}10 \\
10 \\
10 \\
10\end{array}$ & $\begin{array}{l}1.017 \\
1.142 \\
1.270 \\
1.395\end{array}$ & $\begin{array}{l}1.012 \\
1.137 \\
1.265 \\
1.390\end{array}$ & $\begin{array}{l}.3121 \\
.3121 \\
.3121 \\
.3121\end{array}$ & $\begin{array}{l}.3116 \\
.3116 \\
.3116 \\
.3116\end{array}$ & & $\begin{array}{l}.401 \\
.435 \\
.435 \\
.467\end{array}$ & & $\begin{array}{l}3 / 64 \\
1 / 32 \\
1 / 32 \\
1 / 64\end{array}$ & $\begin{array}{l}1 / 32 \\
1 / 32 \\
1 / 32 \\
1 / 32\end{array}$ & $\begin{array}{l}1 / 32 \\
1 / 32 \\
1 / 32 \\
1 / 32\end{array}$ & $\begin{array}{l}1 / 2 \\
9 / 16 \\
518 \\
11 / 16\end{array}$ \\
\hline $\begin{array}{l}1012 \\
1210 \\
1211 \\
1212\end{array}$ & $\begin{array}{l}5 / 16 \text { by } 11 / 2 \\
3 / 8 \text { by } 11 / 4 \\
3 / 8 \text { by } 13 / 8 \\
3 / 8 \text { by } 11 / 2\end{array}$ & $\begin{array}{l}16 \\
14 \\
14 \\
16\end{array}$ & $\begin{array}{l}12 \\
10 \\
10 \\
12\end{array}$ & $\begin{array}{l}1.520 \\
1.270 \\
1.395 \\
1.520\end{array}$ & $\begin{array}{l}1.515 \\
1.265 \\
1.390 \\
1.515\end{array}$ & $\begin{array}{l}.3121 \\
.3745 \\
.3745 \\
.3745\end{array}$ & $\begin{array}{l}.3116 \\
.3740 \\
.3740 \\
.3740\end{array}$ & & $\begin{array}{l}.467 \\
.467 \\
.467 \\
.467\end{array}$ & & $\begin{array}{l}1 / 64 \\
164 \\
1164 \\
364\end{array}$ & $\begin{array}{l}1 / 32 \\
1 / 32 \\
1 / 32 \\
1 / 32\end{array}$ & $\begin{array}{l}1 / 32 \\
1 / 32 \\
1 / 32 \\
1332\end{array}$ & $\begin{array}{l}3 / 4 \\
5 / 8 \\
11 / 16 \\
3 / 4\end{array}$ \\
\hline
\end{tabular}

$1 \mathrm{Key}$ numbers indicate the nominal key dimensions. The last 2 digits give the nominal diameter $(B)$ in eighths of an inch and the digits preceding the last 2 give the nominal width $(A)$ in thirty-seconds of an inch. Thus, 204 indicates a key $3 / 32$ by $4 / 8$ or $1 / 16$ by $1 / 2$ inch; 1210 indicates a key $12 / 32$ by $10 \% 8$ or $3 / 8$ by $11 / 4$ inches.

2 The maximum diameters of cutters given in Table 3 will permit regrinding once or twice, depending on the widths.

3 The minimum cutter width is equal to the minimum key-slot width plus 0.0005 inch. The width of the key slot cut by the maximum cutter will be found in the majority of cases to be slightly less than the maximum key-slot width given in Table 2.

4 For keys larger than No. 810, cutters with cylindrical necks are specified, in which case $\mathrm{E}$ is the small radius between the cylindrical neck and shank. 
SOCIETY OF AUTOMOTIVE ENGINEERS, PARTS AND FITTINGS; WOODRUFF KEYS, S. A. E. STANDARD, JUNE, 1929

WOODRUFF KEYS
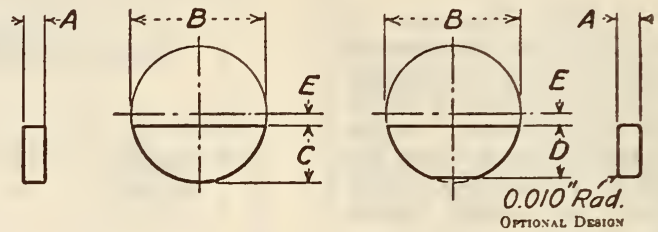

FIGURE 379.-Woodruff keys

[All dimensions in inches]

\begin{tabular}{|c|c|c|c|c|c|c|c|c|c|c|}
\hline \multirow{2}{*}{ S. A. E. nominal size } & \multicolumn{2}{|c|}{$A$ width } & \multicolumn{2}{|c|}{$B$ diameter } & \multicolumn{2}{|c|}{$C$ height } & \multicolumn{2}{|c|}{$D$ height } & \multirow{2}{*}{$\frac{E \text { height }}{\text { Nonimal }}$} & \multirow{2}{*}{$\begin{array}{l}\text { Approxi- } \\
\text { mate } \\
\text { weight } \\
\text { per } 1,000 \\
\text { pounds }\end{array}$} \\
\hline & $\begin{array}{l}\text { Mini- } \\
\text { mum }\end{array}$ & $\begin{array}{l}\text { Maxi- } \\
\text { mum }\end{array}$ & $\begin{array}{l}\text { Mini- } \\
\text { mum }\end{array}$ & $\begin{array}{l}\text { Maxi- } \\
\text { mum }\end{array}$ & $\begin{array}{l}\text { Mini- } \\
\text { mum }\end{array}$ & $\begin{array}{l}\text { Maxi- } \\
\text { mum }\end{array}$ & $\begin{array}{l}\text { Mini- } \\
\text { mum }\end{array}$ & $\begin{array}{l}\text { Maxi- } \\
\text { mum }\end{array}$ & & \\
\hline $\begin{array}{l}1 / 16 \text { by } 1 / 2 \\
3 / 32 \text { by } 1 / 2 \ldots \\
1 / 8 \text { by } 1 / 2 \\
3 / 32 \text { by } 5 / 8 \\
1 / 8 \text { by } 5 / 8\end{array}$ & $\begin{array}{r}0.0625 \\
.0938 \\
.1250 \\
.0938 \\
.1250\end{array}$ & $\begin{array}{l}0.0635 \\
.0948 \\
.1260 \\
.0948 \\
.1260\end{array}$ & $\begin{array}{r}0.490 \\
.490 \\
.490 \\
.615 \\
.615\end{array}$ & $\begin{array}{r}0.500 \\
.500 \\
.500 \\
.625 \\
.625\end{array}$ & $\begin{array}{r}0.198 \\
.198 \\
.198 \\
.245 \\
.245\end{array}$ & $\begin{array}{r}0.203 \\
.203 \\
.203 \\
.250 \\
.250\end{array}$ & $\begin{array}{r}0.188 \\
.188 \\
.188 \\
.234 \\
.234\end{array}$ & $\begin{array}{r}0.194 \\
.194 \\
.194 \\
.240 \\
.240\end{array}$ & $\begin{array}{l}364 \\
364 \\
364 \\
1 / 16 \\
1 / 16\end{array}$ & $\begin{array}{l}1.3 \\
1.9 \\
2.5 \\
3.0 \\
3.9\end{array}$ \\
\hline 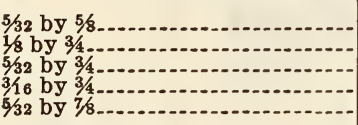 & $\begin{array}{l}.1563 \\
.1250 \\
.1563 \\
.1875 \\
.1563\end{array}$ & $\begin{array}{l}.1573 \\
.1260 \\
.1573 \\
.1885 \\
.1573\end{array}$ & $\begin{array}{l}.615 \\
.740 \\
.740 \\
.740 \\
.865\end{array}$ & $\begin{array}{l}.625 \\
.750 \\
.750 \\
.750 \\
.875\end{array}$ & $\begin{array}{l}.245 \\
.308 \\
.308 \\
.308 \\
.370\end{array}$ & $\begin{array}{r}.250 \\
.313 \\
.313 \\
.313 \\
.375\end{array}$ & $\begin{array}{l}.234 \\
.297 \\
.297 \\
.297 \\
.359\end{array}$ & $\begin{array}{l}.240 \\
.303 \\
.303 \\
.303 \\
.365\end{array}$ & $\begin{array}{l}1 / 16 \\
116 \\
116 \\
1 / 16 \\
1 / 16\end{array}$ & $\begin{array}{r}4.9 \\
6.1 \\
7.5 \\
9.0 \\
11\end{array}$ \\
\hline $\begin{array}{l}3 / 16 \text { by } 7 / 8 . \\
1 / 4 \text { by } 7 / 8 . \\
3 / 16 \text { by } 1 .- \\
1 / 4 \text { by } 1 . . \\
5 / 16 \text { by } 1 .-\end{array}$ & $\begin{array}{l}.1875 \\
.2500 \\
.1875 \\
.2500 \\
.3125\end{array}$ & $\begin{array}{l}.1885 \\
.2510 \\
.1885 \\
.2510 \\
.3135\end{array}$ & $\begin{array}{l}.865 \\
.865 \\
.990 \\
.990 \\
.990\end{array}$ & $\begin{array}{r}.875 \\
.875 \\
1.000 \\
1.000 \\
1.000\end{array}$ & $\begin{array}{l}.370 \\
.370 \\
.433 \\
.433 \\
.433\end{array}$ & $\begin{array}{l}.375 \\
.375 \\
.438 \\
.438 \\
.438\end{array}$ & $\begin{array}{l}.359 \\
.359 \\
.422 \\
.422 \\
.422\end{array}$ & $\begin{array}{l}.365 \\
.365 \\
.428 \\
.428 \\
.428\end{array}$ & $\begin{array}{l}1 / 16 \\
1 / 16 \\
1 / 16 \\
116 \\
1 / 16\end{array}$ & $\begin{array}{l}13 \\
17 \\
17 \\
23 \\
29\end{array}$ \\
\hline $\begin{array}{l}3 / 16 \text { by } 11 / 8 \\
1 / 4 \text { by } 11 / 8 \\
5 / 16 \text { by } 11 / 8 \\
1 / 4 \text { by } 11 / 4\end{array}$ & $\begin{array}{l}.1875 \\
.2500 \\
.3125 \\
.2500\end{array}$ & $\begin{array}{l}.1885 \\
.2510 \\
.3135 \\
.2510\end{array}$ & $\begin{array}{l}1.115 \\
1.115 \\
1.115 \\
1.240\end{array}$ & $\begin{array}{l}1.125 \\
1.125 \\
1.125 \\
1.250\end{array}$ & $\begin{array}{l}.479 \\
.479 \\
.479 \\
.542\end{array}$ & $\begin{array}{l}.484 \\
.484 \\
.484 \\
.547\end{array}$ & $\begin{array}{l}.469 \\
.469 \\
.469 \\
.531\end{array}$ & $\begin{array}{l}.475 \\
.475 \\
.475 \\
.537\end{array}$ & $\begin{array}{l}5 / 64 \\
564 \\
5 / 64 \\
564\end{array}$ & $\begin{array}{l}22 \\
29 \\
36 \\
36\end{array}$ \\
\hline $\begin{array}{l}5 / 16 \text { by } 11 / 4 \\
3 / 8 \text { by } 11 / 4,4 \\
1 / 4 \text { by } 13 / 8 \\
5 / 16 \text { by } 138\end{array}$ & $\begin{array}{l}.3125 \\
.3750 \\
.2500 \\
.3125\end{array}$ & $\begin{array}{l}.3135 \\
.3760 \\
.2510 \\
.3135\end{array}$ & $\begin{array}{l}\text { 1. } 240 \\
1.240 \\
1.365 \\
1.365\end{array}$ & $\begin{array}{l}1.250 \\
1.250 \\
1.375 \\
1.375\end{array}$ & $\begin{array}{l}.542 \\
.542 \\
.589 \\
.589\end{array}$ & $\begin{array}{l}.547 \\
.547 \\
.594 \\
.594\end{array}$ & $\begin{array}{l}.531 \\
.531 \\
.578 \\
.578\end{array}$ & $\begin{array}{l}.537 \\
.537 \\
.584 \\
.584\end{array}$ & $\begin{array}{l}5 / 64 \\
5 / 64 \\
3 / 32 \\
3 / 32\end{array}$ & $\begin{array}{l}45 \\
54 \\
43 \\
54\end{array}$ \\
\hline $\begin{array}{l}3 / 8 \text { by } 13 / 8 \\
1 / 4 \text { by } 11 / 2 \ldots \\
5 / 16 \text { by } 11 / 2 \ldots \\
3 / 8 \text { by } 11 / 2 \ldots \ldots\end{array}$ & $\begin{array}{l}.3750 \\
.2500 \\
.3125 \\
.3750\end{array}$ & $\begin{array}{l}.3760 \\
.2510 \\
.3135 \\
.3760\end{array}$ & $\begin{array}{l}1.365 \\
1.490 \\
1.490 \\
1.490\end{array}$ & $\begin{array}{l}1.375 \\
1.500 \\
1.500 \\
1.500\end{array}$ & $\begin{array}{l}.589 \\
.636 \\
.636 \\
.636\end{array}$ & $\begin{array}{l}.594 \\
.641 \\
.641 \\
.641\end{array}$ & $\begin{array}{l}.578 \\
.625 \\
.625 \\
.625\end{array}$ & $\begin{array}{l}.584 \\
.631 \\
.631 \\
.631\end{array}$ & $\begin{array}{l}3 / 32 \\
7 / 64 \\
7 / 64 \\
7 / 64\end{array}$ & $\begin{array}{l}65 \\
50 \\
63 \\
75\end{array}$ \\
\hline
\end{tabular}

Width A.-Dimensions shown are set with the minimum key width as basic.

Tolerance on all key widths to be 0.001 .

Maximum key width is basic plus tolerance.

Diameter B.-Dimensions shown are set with maximum diameter as basic.

Tolerance on all key diameters to be 0.010 .

Minimum key diameter is basic minus tolerance.

Height C.-Dimensions shown are set with maximum height as

Tolerance on all key heights to be 0.005 .

Minimum key height is basic minus tolerance.

Height $D$.-Keys of this type are standard with some manufacturers Dimensions shown have been furnished by those manufacturers. Height E.-This dimension is given for convenience of draftsmen. Weights.-Weights shown are approximate.

Material.-S. A. E. Steel 1035 or 2330 as specified. Keys of nickel teel are marked with depressions on the edges to distinguish them from carbon-steel keys. Nickel-steel keys are heat treated. basic. 


\section{KEY SLOTS AND KEY WAYS}
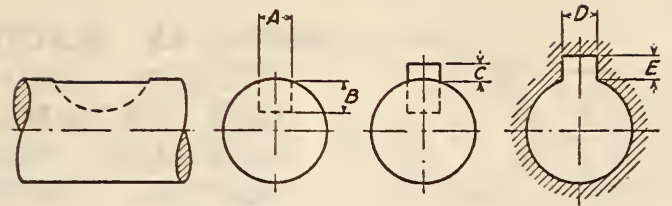

FIGURE 380.-Key slots and key ways

Woodruff key slot, key above shaft, and key way dimensions

[All dimensions in inches]

\begin{tabular}{|c|c|c|c|c|c|c|c|c|c|c|c|c|c|c|}
\hline \multirow{4}{*}{ Woodruff key size } & \multirow{2}{*}{\multicolumn{5}{|c|}{ Key slot }} & \multirow{2}{*}{\multicolumn{3}{|c|}{ Key above shaft }} & \multicolumn{6}{|c|}{ Key way } \\
\hline & & & & & & & & & \multicolumn{4}{|c|}{ Width $D$} & \multirow{2}{*}{\multicolumn{2}{|c|}{$\frac{\text { Depth } E}{\text { All classes }}$}} \\
\hline & \multicolumn{3}{|c|}{ Width $A$} & \multicolumn{2}{|c|}{ Depth $B$} & \multicolumn{3}{|c|}{ Height $C$} & \multicolumn{2}{|c|}{ Class 2} & \multicolumn{2}{|c|}{ Class 1} & & \\
\hline & Basic & $\begin{array}{l}\text { Mini- } \\
\text { mum }\end{array}$ & $\underset{\text { mum }}{\text { Maxi- }}$ & $\begin{array}{l}\text { Mini- } \\
\text { mum }\end{array}$ & $\begin{array}{l}\text { Maxi- } \\
\text { mum }\end{array}$ & Basic & $\begin{array}{l}\text { Mini- } \\
\text { mum }\end{array}$ & $\begin{array}{l}\text { Maxi- } \\
\text { mum }\end{array}$ & $\begin{array}{l}\text { Mini- } \\
\text { mum }\end{array}$ & $\underset{\text { mum }}{\text { Maxi- }}$ & $\begin{array}{l}\text { Mini- } \\
\text { mum }\end{array}$ & $\begin{array}{l}\text { Maxi- } \\
\text { mum }\end{array}$ & $\begin{array}{l}\text { Mini- } \\
\text { mum }\end{array}$ & $\begin{array}{l}\text { Maxi- } \\
\text { mum }\end{array}$ \\
\hline $\begin{array}{l}316 \text { by } 132 \\
3 / 32 \text { by } 132 \\
18 \text { by } 1 / 2 \\
3,32 \text { by } 5 / 8 \\
18 \text { by } 58 .\end{array}$ & $\begin{array}{l}0.0625 \\
.0938 \\
.1250 \\
.0938 \\
.1250\end{array}$ & $\begin{array}{r}0.0615 \\
.0928 \\
.1240 \\
.0928 \\
.1240\end{array}$ & $\begin{array}{r}0.0630 \\
.0943 \\
.1255 \\
.0943 \\
.1255\end{array}$ & $\begin{array}{l}0.1668 \\
.1511 \\
.1355 \\
.1981 \\
.1825\end{array}$ & $\begin{array}{l}0.1718 \\
.1561 \\
.1405 \\
.2031 \\
.1875\end{array}$ & $\begin{array}{l}0.312 \\
.0469 \\
.0625 \\
.0469 \\
.0625\end{array}$ & $\begin{array}{l}0.0262 \\
.0419 \\
.0575 \\
.0419 \\
.0575\end{array}$ & $\begin{array}{l}0.0362 \\
.0519 \\
.0675 \\
.0519 \\
.0675\end{array}$ & $\begin{array}{l}0.0635 \\
.0948 \\
.1260 \\
.0948 \\
.1260\end{array}$ & $\begin{array}{l}0.0655 \\
.0968 \\
.1280 \\
.0968 \\
.1280\end{array}$ & $\begin{array}{l}0.0635 \\
.0948 \\
.1260 \\
.0948 \\
.1260\end{array}$ & $\begin{array}{l}0.0685 \\
.0998 \\
.1310 \\
.0998 \\
.1310\end{array}$ & $\begin{array}{l}0.0372 \\
.0529 \\
.0685 \\
.0529 \\
.0685\end{array}$ & $\begin{array}{l}0.0422 \\
.0579 \\
.0735 \\
.0579 \\
.0735\end{array}$ \\
\hline $\begin{array}{l}5 / 32 \text { by } \\
18 \text { by } 3 \\
432 \text { by } \\
316 \text { by } \\
932 \text { by }\end{array}$ & $\begin{array}{l}.1563 \\
.1250 \\
.1563 \\
.1375 \\
.1563\end{array}$ & $\begin{array}{l}.1553 \\
.1240 \\
.1553 \\
.1863 \\
.1553\end{array}$ & $\begin{array}{l}.1568 \\
.1255 \\
.1568 \\
.1880 \\
.1568\end{array}$ & $\begin{array}{l}.1669 \\
.2455 \\
.2299 \\
.2143 \\
.2919\end{array}$ & $\begin{array}{l}.1719 \\
.2505 \\
.2349 \\
.2193 \\
.2969\end{array}$ & $\begin{array}{l}.0781 \\
.0625 \\
.0781 \\
.0937 \\
.0781\end{array}$ & $\begin{array}{l}.0731 \\
.0575 \\
.0731 \\
.0887 \\
.0731\end{array}$ & $\begin{array}{l}.0831 \\
.0675 \\
.0831 \\
.0987 \\
.0831\end{array}$ & $\begin{array}{l}.1573 \\
.1260 \\
.1573 \\
.1885 \\
.1573\end{array}$ & $\begin{array}{l}.1593 \\
.1280 \\
.1593 \\
.1905 \\
.1593\end{array}$ & $\begin{array}{l}.1573 \\
.1260 \\
.1573 \\
.1885 \\
.1573\end{array}$ & $\begin{array}{l}.1623 \\
.1310 \\
.1623 \\
.1935 \\
.1623\end{array}$ & $\begin{array}{l}.0841 \\
.0685 \\
.0841 \\
.0997 \\
.0841\end{array}$ & $\begin{array}{l}.0891 \\
.0735 \\
.0891 \\
.1047 \\
.0891\end{array}$ \\
\hline $\begin{array}{l}3 / 16 \text { by } 7 \\
1 / 4 \text { by } 7 / 8 \\
3 / 16 \text { by } 1 \\
1 / 4 \text { by } 1 \\
1 / 16 \text { by } 1\end{array}$ & $\begin{array}{l}.1875 \\
.2500 \\
.1875 \\
.2500 \\
.3125\end{array}$ & $\begin{array}{l}.1863 \\
.2487 \\
.1863 \\
.2487 \\
.3111\end{array}$ & $\begin{array}{l}.1880 \\
.2505 \\
.1880 \\
.2505 \\
.3130\end{array}$ & $\begin{array}{l}.2763 \\
.2450 \\
.3393 \\
.3080 \\
.2768\end{array}$ & $\begin{array}{l}.2813 \\
.2500 \\
.3443 \\
.3130 \\
.2818\end{array}$ & $\begin{array}{l}.0937 \\
.1250 \\
.0937 \\
.1250 \\
.1562\end{array}$ & $\begin{array}{l}.0887 \\
.1200 \\
.0887 \\
.1200 \\
.1512\end{array}$ & $\begin{array}{l}.0987 \\
.1300 \\
.0987 \\
.1300 \\
.1612\end{array}$ & $\begin{array}{l}.1885 \\
.2510 \\
.1885 \\
.2510 \\
.3135\end{array}$ & $\begin{array}{l}.1905 \\
.2530 \\
.1905 \\
.2530 \\
.3155\end{array}$ & $\begin{array}{l}.1885 \\
.2510 \\
.1885 \\
.2510 \\
.3135\end{array}$ & $\begin{array}{l}.1935 \\
.2560 \\
.1935 \\
.2560 \\
.3185\end{array}$ & $\begin{array}{l}.0997 \\
.1310 \\
.0997 \\
.1310 \\
.1622\end{array}$ & $\begin{array}{l}.1047 \\
.1360 \\
.1047 \\
.1360 \\
.1672\end{array}$ \\
\hline $\begin{array}{l}316 \text { by } 138 \\
34 \text { by } 138 \\
316 \text { by } 118 \\
14 \text { by } 114 .\end{array}$ & $\begin{array}{l}.1875 \\
.2500 \\
.3125 \\
.2500\end{array}$ & $\begin{array}{l}.1863 \\
.2487 \\
.3111 \\
.2487\end{array}$ & $\begin{array}{l}.1830 \\
.2505 \\
.3130 \\
.2505\end{array}$ & $\begin{array}{l}.3853 \\
.3540 \\
.3228 \\
.4170\end{array}$ & $\begin{array}{l}.3903 \\
.3590 \\
.3278 \\
.4220\end{array}$ & $\begin{array}{l}.0937 \\
.1250 \\
.1562 \\
.1250\end{array}$ & $\begin{array}{l}.0887 \\
.1200 \\
.1512 \\
.1200\end{array}$ & $\begin{array}{l}.0987 \\
.1300 \\
.1612 \\
.1300\end{array}$ & $\begin{array}{l}.1885 \\
.2510 \\
.3135 \\
.2510\end{array}$ & $\begin{array}{l}.1905 \\
.2530 \\
.3155 \\
.2530\end{array}$ & $\begin{array}{l}.1885 \\
.2510 \\
.3135 \\
.2510\end{array}$ & $\begin{array}{l}.1935 \\
.2560 \\
.3185 \\
.2560\end{array}$ & $\begin{array}{l}.0997 \\
.1310 \\
.1622 \\
.1310\end{array}$ & $\begin{array}{l}.1047 \\
.1360 \\
.1672 \\
.1360\end{array}$ \\
\hline $\begin{array}{l}\$ 16 \text { by } 134 \ldots \\
38 \text { by } 134 \ldots \\
14 \text { by } 136 \ldots \\
916 \text { by } 136 \ldots\end{array}$ & $\begin{array}{l}.3125 \\
.3750 \\
.2500 \\
.3125\end{array}$ & $\begin{array}{l}.3111 \\
.3735 \\
.2487 \\
.3111\end{array}$ & $\begin{array}{l}.3130 \\
.3755 \\
.2505 \\
.3130\end{array}$ & $\begin{array}{l}.3858 \\
.3545 \\
.4640 \\
.4328\end{array}$ & $\begin{array}{l}.3908 \\
.3595 \\
.4690 \\
.4378\end{array}$ & $\begin{array}{l}.1562 \\
.1875 \\
.1250 \\
.1562\end{array}$ & $\begin{array}{l}.1512 \\
.1825 \\
.1200 \\
.1512\end{array}$ & $\begin{array}{l}.1612 \\
.1925 \\
.1300 \\
.1612\end{array}$ & $\begin{array}{l}.3135 \\
.3760 \\
.2510 \\
.3135\end{array}$ & $\begin{array}{l}.3155 \\
.3780 \\
.2530 \\
.3155\end{array}$ & $\begin{array}{l}.3135 \\
.3760 \\
.2510 \\
.3135\end{array}$ & $\begin{array}{l}.3185 \\
.3810 \\
.2560 \\
.3185\end{array}$ & $\begin{array}{l}.1622 \\
.1935 \\
.1310 \\
.1622\end{array}$ & $\begin{array}{l}.1672 \\
.1985 \\
.1360 \\
.1672\end{array}$ \\
\hline $\begin{array}{l}3 / 8 \text { by } 13 / 2 \ldots \\
1 / 4 \text { by } 112 \ldots \\
5 / 16 \text { by } 112 \ldots \\
38 \text { by } 112 \ldots\end{array}$ & $\begin{array}{l}.3750 \\
.2500 \\
.3125 \\
.3750\end{array}$ & $\begin{array}{l}.3735 \\
.2487 \\
.3111 \\
.3735\end{array}$ & $\begin{array}{l}.3755 \\
.2505 \\
.3130 \\
.3755\end{array}$ & $\begin{array}{l}.4015 \\
.5110 \\
.4798 \\
.4485\end{array}$ & $\begin{array}{l}.4065 \\
.5160 \\
.4848 \\
.4535\end{array}$ & $\begin{array}{l}.1875 \\
.1250 \\
.1562 \\
.1875\end{array}$ & $\begin{array}{l}.1825 \\
.1200 \\
.1512 \\
.1825\end{array}$ & $\begin{array}{l}.1925 \\
.1300 \\
.1612 \\
.1925\end{array}$ & $\begin{array}{l}.3760 \\
.2510 \\
.3135 \\
.3760\end{array}$ & $\begin{array}{l}.3780 \\
.2530 \\
.3155 \\
.3780\end{array}$ & $\begin{array}{l}.3760 \\
.2510 \\
.3135 \\
.3760\end{array}$ & $\begin{array}{l}.3810 \\
.2560 \\
.3185 \\
.3810\end{array}$ & $\begin{array}{l}.1935 \\
.1310 \\
.1622 \\
.1935\end{array}$ & $\begin{array}{l}.1985 \\
.1360 \\
.1672 \\
.1985\end{array}$ \\
\hline
\end{tabular}

KEY SLOT

Width A.-Dimensions shown are set with the maximum key-slot width as that figure which will receive a key with the greatest amount of looseness permissible to assure the key sticking in the slot.

Minimum key-slot width is that figure permitting the largest shaft distortion accoptable when assembling maximum key in minimum key slot.

The largest shaft distortion acceptable in all calculations was 0.0003 not more than 16 inches from side of assembled key.

Depth $B$.-Dimensions shown are set with the maximum as that figure which receives a key of maximum height and permits the key to project above the shaft a distance equal to one-hali the basic width. Tolerance on all key-slot depths 0.005 .

Minimum key-slot depth is maximum minus tolerance.

NOTE.-The word "depth" as applied to "key slot" should be construed to mean the depth of the key slot taken at the side.
KEY ABOVE SHAFT

Height $C .-$ Dimensions shown are set with one-half the basic key as basic height. Tolerance on key height above shaft plus or minus 0.005 .

NотE.-The wording "key above shaft" and "height" should be construed to mean the amount of key exposed after being fitted into place.

\section{KEY WAY}

Width $D$.-Dimensions shown are determined by using for width of key way the maximum width of key minus 0.000 plus 0.002 for class 2 and minus 0.000 plus 0.005 for class 1 .

Depth E.-Dimensions shown are determined by using for depth of key way the maximum key in the minimum key slot minus 0.001 plus 0.006 . 
Details of Woodruff key slots, keys above shaft, and key ways

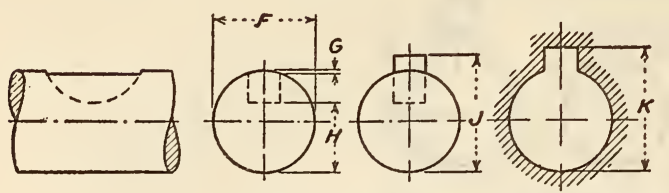

FIGURE 381

[All dimensions in inches]

\begin{tabular}{|c|c|c|c|c|c|c|c|c|c|}
\hline \multirow{3}{*}{$\begin{array}{l}\text { Wood- } \\
\text { ruff } \\
\text { size }\end{array}$} & $\begin{array}{r}\text { Shaft } \\
\text { et }\end{array}$ & $\begin{array}{l}\text { diam- } \\
\text { er }\end{array}$ & \multirow[t]{2}{*}{$\begin{array}{l}\text { Versed } \\
\text { sine }\end{array}$} & \multirow{2}{*}{\multicolumn{2}{|c|}{$\frac{\begin{array}{c}\text { Bottom of } \\
\text { key slot to } \\
\text { opposite } \\
\text { side }\end{array}}{H}$}} & \multicolumn{2}{|c|}{$\begin{array}{l}\text { Top of key } \\
\text { to opposite } \\
\text { side }\end{array}$} & \multicolumn{2}{|c|}{$\begin{array}{l}\text { Bottom of } \\
\text { key way to } \\
\text { opposite } \\
\text { side }\end{array}$} \\
\hline & \multicolumn{2}{|c|}{$F$} & & & & & $\boldsymbol{J}$ & \multicolumn{2}{|c|}{$K$} \\
\hline & $\begin{array}{l}\text { Frac- } \\
\text { tion }\end{array}$ & $\begin{array}{l}\text { Deci- } \\
\text { mal }\end{array}$ & $G$ & $\begin{array}{l}\text { Min- } \\
\text { imum }\end{array}$ & $\begin{array}{l}\text { Max- } \\
\text { imum }\end{array}$ & $\begin{array}{l}\text { Min- } \\
\text { imum }\end{array}$ & $\begin{array}{l}\text { Max- } \\
\text { imum }\end{array}$ & $\underset{\text { imum }}{\text { Min- }}$ & $\begin{array}{l}\text { Max- } \\
\text { imum }\end{array}$ \\
\hline
\end{tabular}

(General information covering machined dimensions are appended to this specification. Included are data on key slots and key pended to this secter mays maximum diameter, as machined to accommodate the 27 different shown above. The dimensions are obtained as shown below:)

Shaft diameter F.-Decimal equivalents are given to four places and all figures in the remaining columns are calculated from these basic dimensions. Any change in the shaft diameter from basic will necessarily change all other figures and, in this case, should accurate dimensions be required, the formula in the next paragraph should be used.

Versed sine $G$.- The versed sines specified are determined from the following formula.

$$
G=\frac{F}{2}-\sqrt{\frac{F^{2}-A^{2}}{4}}
$$

$A$ being the basic width of the key slot.

Bottom of key slot to opposite side of shaft $H$.-These figures are obtained by subtracting the versed sine $G$ and the depth of the key slot $B$, from the shaft diameter $F$.

Top of key to opposite side of shaft.-These figures are obtained by adding to the dimension, $H$, the heighth of the key $C$, these figures being as follows:

\begin{tabular}{|c|c|c|}
\hline \multirow{2}{*}{$\begin{array}{l}\text { Key di- } \\
\text { ameter }\end{array}$} & \multicolumn{2}{|c|}{ Key height } \\
\hline & $\begin{array}{l}\text { Mini- } \\
\text { mum }\end{array}$ & $\begin{array}{l}\text { Maxi- } \\
\text { mum }\end{array}$ \\
\hline $\begin{array}{r}\text { Inches } \\
1 / 2 \\
5 / 8 \\
3 / 4 \\
7 / 8 \\
1 \\
11 / 8 \\
11 / 4 \\
13 / 8 \\
11 / 2\end{array}$ & $\begin{array}{l}\text { Inch } \\
0.198 \\
.245 \\
.308 \\
.370 \\
.433 \\
.479 \\
.542 \\
.589 \\
.636\end{array}$ & $\begin{array}{l}\text { Inch } \\
0.203 \\
.250 \\
.313 \\
.375 \\
.438 \\
.484 \\
.547 \\
.594 \\
.641\end{array}$ \\
\hline
\end{tabular}

Bottom of key way to opposite side of bore.-These figures are obtained by first subtracting the versed sine $G$ from the shaft diameter $F$ and then adding to this figure the depth of the key way $E$.
608.6 WASHERS.

AMERICAN ELECTRIC RAILWAY ENGINEERING ASSOCIATION, OVERHEAD LINE MATERIAL FOR DIRECT AND CATENARY CONSTRUCTION, RECOMMENDED SPECIFICATION, D102-30, 1930

\section{OVERHEAD LINE MATERIAI}

(D102-30, paragraph 315, includes requirements for steel washers. For specification see 611.29, p. 682.)

\section{SOCIETY OF AUTOMOTIVE ENGINEERS,} AERONAUTIC ; FLAT WASHERS, S. A. E. STANDARD, AUGUST, 1928

\section{FLAT WASHERS}

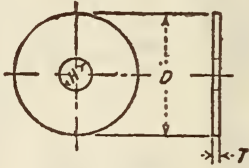

IARGE

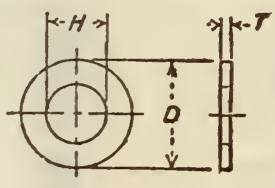

SMALL
FIGURE 382.-Flat washers

[All dimensions in inches]

\begin{tabular}{|c|c|c|c|c|c|}
\hline \multirow{2}{*}{ Nominal bolt size } & \multirow{2}{*}{$H$} & \multicolumn{2}{|c|}{ Large } & \multicolumn{2}{|c|}{ Small } \\
\hline & & $D$ & $T$ & $D$ & $T$ \\
\hline $\begin{array}{l}\text { No. } 8 \\
\text { No. } 10 \\
14 \\
516 \\
36 \\
76 \\
16\end{array}$ & $\begin{array}{l}11 / 64 \\
13 / 64 \\
17 / 64 \\
21 / 64 \\
25 / 64 \\
2964 \\
3364 \\
37 / 64 \\
4164\end{array}$ & $\begin{array}{l}7 / 8 \\
11 / 8 \\
13 / 8 \\
15 / 8 \\
113 / 16 \\
2 \\
23 / 16 \\
238\end{array}$ & $\begin{array}{l}1 / 16 \\
116 \\
116 \\
116 \\
110 \\
110 \\
118 \\
168\end{array}$ & $\begin{array}{r}5 / 16 \\
3 / 8 \\
1 / 2 \\
\% 16 \\
11 / 16 \\
13 / 16 \\
15 / 16 \\
11 / 16 \\
11 / 8\end{array}$ & $\begin{array}{l}1 / 32 \\
1 / 32 \\
1 / 16 \\
1 / 16 \\
1 / 16 \\
1 / 16 \\
1 / 16 \\
1 / 16 \\
1 / 16\end{array}$ \\
\hline
\end{tabular}

SOCIETY OF AUTOMOTIVE ENGINEERS, PARTS AND FITTINGS; LOCK WASHERS, S. A. E. STANDARD, AUGUST, 1922

\section{LOCK WASHERS}

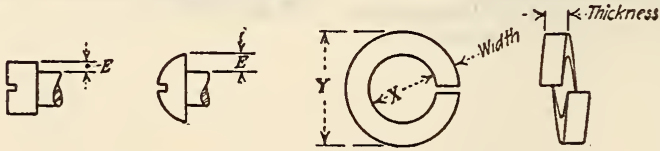

FIGURE 383.-Machine screw lock washer 
Dimensions of machine-screw lock washers

[All dimensions in inches]

\begin{tabular}{|c|c|c|c|c|c|c|c|c|}
\hline \multirow{2}{*}{ Туре } & \multirow{2}{*}{$\begin{array}{l}\text { Screw } \\
\text { No. }\end{array}$} & \multicolumn{2}{|c|}{ Diameter } & \multirow{2}{*}{$\begin{array}{l}\text { Clear- } \\
\text { ance } 1\end{array}$} & \multirow{2}{*}{$\mathrm{E}$} & \multirow{2}{*}{$\mathbf{X}$} & \multirow{2}{*}{ Section ${ }^{2}$} & \multirow{2}{*}{$Y$} \\
\hline & & Screw & Head & & & & & \\
\hline Round head. & $\begin{array}{r}2 \\
4 \\
6 \\
8 \\
10 \\
12 \\
2 \\
4 \\
6 \\
8 \\
10 \\
12\end{array}$ & $\begin{array}{l}0.086 \\
.112 \\
.138 \\
.164 \\
.190 \\
.216 \\
.086 \\
.112 \\
.138 \\
.164 \\
.190 \\
.216\end{array}$ & $\begin{array}{l}0.132 \\
.175 \\
.217 \\
.260 \\
.303 \\
.345 \\
.154 \\
.202 \\
.250 \\
.298 \\
.346 \\
.394\end{array}$ & $\begin{array}{l}0.003 \\
.003 \\
.005 \\
.005 \\
.010 \\
.010 \\
.003 \\
.003 \\
.005 \\
.005 \\
.010 \\
.010\end{array}$ & $\begin{array}{l}0.023 \\
.031 \\
.040 \\
.048 \\
.056 \\
.065 \\
.034 \\
.045 \\
.056 \\
.067 \\
.078 \\
.089\end{array}$ & $\begin{array}{l}0.089 \\
.115 \\
.143 \\
.169 \\
.200 \\
.226 \\
.089 \\
.115 \\
.143 \\
.169 \\
.200 \\
.226\end{array}$ & $\begin{array}{r}1 / 32 \text { by } 0.022 \\
1 / 32 \text { by } 1 / 32 \\
3 / 64 \text { by } 1 / 32 \\
3 / 64 \text { by } 3 / 64 \\
1 / 16 \text { by } 3 / 64 \\
1 / 16 \text { by } 1 / 16 \\
1 / 32 \text { by } 1 / 32 \\
1 / 16 \text { by } 1 / 32 \\
5 / 64 \text { by } 1 / 32 \\
5 / 64 \text { by } 3 / 64 \\
3 / 32 \text { by } 3 / 64 \\
3 / 32 \text { by } 1 / 16\end{array}$ & $\begin{array}{l}0.1515 \\
.1775 \\
.2367 \\
.2627 \\
.3250 \\
.3510 \\
.1515 \\
.2400 \\
.2992 \\
.3252 \\
.3875 \\
.4135\end{array}$ \\
\hline
\end{tabular}

1 Inside diameter of washer less diameter of screw.

2 Lock-washer sizes should be designated by giving the machine-screw diameter, the lock-washer width, and the thickness in the order given; as $1 / 2$ by 1164 by $1 / 8$.
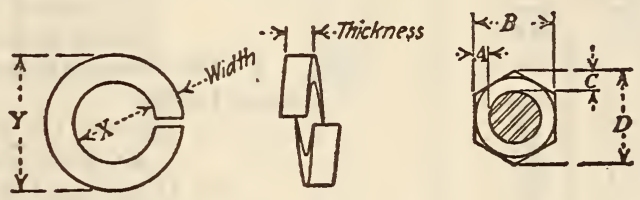

FiguRe 384.- Standard bolt lock washer

Dimensions of S. A. E. standard bolt lock washers

[All dimensions in inches]

\begin{tabular}{|c|c|c|c|c|c|c|c|c|}
\hline $\begin{array}{c}\text { Bolt } \\
\text { diameter }\end{array}$ & $\begin{array}{l}\text { Clear- } \\
\text { ance } 1\end{array}$ & $\mathbf{A}$ & B & C & D & $\mathrm{X}$ & Section ${ }^{2}$ & $\mathrm{Y}$ \\
\hline $\begin{array}{l}1 / 4 \\
5 / 16 \\
368 \\
7 / 16 \\
1 / 2\end{array}$ & $\begin{array}{l}1 / 32 \\
1 / 32 \\
1 / 32 \\
1 / 32 \\
1 / 32\end{array}$ & $\begin{array}{l}3 / 32 \\
3 / 32 \\
3 / 32 \\
3 / 32 \\
1 / 8\end{array}$ & $\begin{array}{l}7 / 16 \\
1 / 2 \\
9 / 16 \\
5 / 8 \\
3 / 4\end{array}$ & $\begin{array}{r}0.128 \\
.133 \\
.137 \\
.173 \\
.183\end{array}$ & $\begin{array}{r}0.505 \\
.578 \\
.650 \\
.722 \\
.866\end{array}$ & $\begin{array}{l}9 / 32 \\
11 / 32 \\
13 / 32 \\
15 / 32 \\
17 / 32\end{array}$ & $\begin{array}{r}3 / 32 \text { by } 1 / 16 \\
1 / 8 \text { by } 1 / 16 \\
1 / 8 \text { by } 3 / 32 \\
5 / 32 \text { by } 1 / 8 \\
1164 \text { by } 1 / 8\end{array}$ & $\begin{array}{l}15 / 32 \\
19 / 32 \\
21 / 32 \\
25 / 32 \\
7 / 8\end{array}$ \\
\hline $\begin{array}{l}9 / 16 \\
5 / 8 \\
11 / 16 \\
3 / 4 \\
7 / 8\end{array}$ & $\begin{array}{l}1 / 32 \\
1 / 32 \\
1 / 32 \\
1 / 16 \\
1 / 16\end{array}$ & $\begin{array}{l}5 / 32 \\
5 / 32 \\
5 / 32 \\
5 / 32 \\
3 / 16\end{array}$ & $\begin{array}{l}7 / 8 \\
15 / 16 \\
1 \\
11 / 16 \\
11 / 4\end{array}$ & $\begin{array}{l}.224 \\
.229 \\
.234 \\
.239 \\
.284\end{array}$ & $\begin{array}{l}1.011 \\
1.083 \\
1.156 \\
1.228 \\
1.444\end{array}$ & $\begin{array}{l}19 / 32 \\
21 / 32 \\
23 / 32 \\
13 / 16 \\
15 / 16\end{array}$ & $\begin{array}{r}3 / 16 \text { by } 1 / 8 \\
13 / 64 \text { by } 5 / 32 \\
7 / 32 \text { by } 3 / 16 \\
1 / 4 \text { by } 3 / 16 \\
17 / 64 \text { by } 3 / 16\end{array}$ & $\begin{array}{l}31 / 32 \\
11 / 16 \\
15 / 32 \\
15 / 16 \\
115 / 32\end{array}$ \\
\hline $\begin{array}{l}1 \\
11 / 8 \\
11 / 4 \\
138 \\
11 / 2\end{array}$ & $\begin{array}{l}1 / 16 \\
1 / 16 \\
1 / 16 \\
1 / 16 \\
1 / 16\end{array}$ & $\begin{array}{l}7 / 32 \\
1 / 4 \\
9 / 32 \\
5 / 16 \\
11 / 32\end{array}$ & $\begin{array}{l}17 / 16 \\
15 / 8 \\
113 / 16 \\
2 \\
23 / 16\end{array}$ & $\begin{array}{l}.330 \\
.376 \\
.422 \\
.468 \\
.513\end{array}$ & $\begin{array}{l}1.660 \\
1.877 \\
2.093 \\
2.310 \\
2.527\end{array}$ & $\begin{array}{l}11 / 16 \\
13 / 16 \\
15 / 16 \\
17 / 16 \\
1 \% 16\end{array}$ & $\begin{array}{r}5 / 16 \text { by } 1 / 4 \\
3 / 8 \text { by } 1 / 4 \\
7 / 16 \text { by } 1 / 4 \\
7 / 16 \text { by } 5 / 16 \\
1 / 2 \text { by } 5 / 16\end{array}$ & $\begin{array}{l}111 / 16 \\
115 / 16 \\
23 / 16 \\
25 / 16 \\
29 / 16\end{array}$ \\
\hline
\end{tabular}

1 Inside diameter of washer less diameter of bolt.

2 Lock-washer sizes should be designated by giving the bolt diameter, the lock-washer width, and the thickness in the order given; as $1 / 2$ by $11 \% 64$ by $1 / 8$.

Special machine-screw lock washers

[All dimensions in inches]

\begin{tabular}{|c|c|c|c|c|c|}
\hline \multirow{2}{*}{ Туре } & \multirow{2}{*}{$\begin{array}{l}\text { Screw } \\
\text { No. }\end{array}$} & \multicolumn{2}{|c|}{ Special light } & \multicolumn{2}{|c|}{ Special heavy } \\
\hline & & Section & $\mathrm{Y}$ & Section & $\mathrm{Y}$ \\
\hline Fillister head & $\left\{\begin{array}{r}2 \\
4 \\
6 \\
8 \\
10 \\
12\end{array}\right.$ & $\begin{array}{l}0.022 \text { by } 0.022 \\
1 / 32 \text { by } 0.022 \\
1 / 32 \text { by } 1 / 32 \\
3 / 64 \text { by } 1 / 32 \\
3 / 64 \text { by } 3 / 64 \\
1 / 16 \text { by } 3 / 64\end{array}$ & $\begin{array}{r}0.1330 \\
.1775 \\
.2055 \\
.2627 \\
.2937 \\
.3510\end{array}$ & $\begin{array}{l}1 / 32 \text { by } 1 / 32 \\
3 / 64 \text { by } 1 / 32 \\
3 / 64 \text { by } 364 \\
1 / 16 \text { by } 3 / 64 \\
1 / 16 \text { by } 1 / 16 \\
5 / 64 \text { by } 1 / 16\end{array}$ & $\begin{array}{r}0.1515 \\
.2087 \\
.2367 \\
.2940 \\
.3250 \\
.3822\end{array}$ \\
\hline Round head. & $\left\{\begin{array}{r}2 \\
4 \\
6 \\
8 \\
10 \\
12\end{array}\right.$ & $\begin{array}{l}1 / 32 \text { by } 0.022 \\
3 / 64 \text { by } 1 / 32 \\
1 / 16 \text { by } 1 / 32 \\
564 \text { by } 1 / 32 \\
564 \text { by } 3 / 64 \\
3 / 32 \text { by } 3 / 64\end{array}$ & $\begin{array}{l}.1515 \\
.2087 \\
.2680 \\
.3252 \\
.3562 \\
.4135\end{array}$ & $\begin{array}{l}3 / 64 \text { by } 1 / 32 \\
5 / 64 \text { by } 1 / 32 \\
5 / 64 \text { by } 3 / 64 \\
3 / 32 \text { by } 3 / 64 \\
3 / 32 \text { by } 1 / 16 \\
1 / 6 \text { by } 3 / 64\end{array}$ & $\begin{array}{l}.1827 \\
.2712 \\
.2992 \\
.3565 \\
.3875 \\
.4760\end{array}$ \\
\hline
\end{tabular}

Special S. A. E. standard bolt lock washers [All dimensions in inches]

\begin{tabular}{|c|c|c|c|c|}
\hline \multirow{2}{*}{$\begin{array}{l}\text { Bolt di- } \\
\text { ameter }\end{array}$} & \multicolumn{2}{|c|}{ Special light } & \multicolumn{2}{|c|}{ Special heavy } \\
\hline & Section & $Y$ & Section & $\mathrm{Y}$ \\
\hline $\begin{array}{l}1 / 4 \\
5 / 16 \\
3 / 8 \\
7 / 16\end{array}$ & $\begin{array}{r}3 / 32 \text { by } 3 / 64 \\
1 / 8 \text { by } 3 / 64 \\
1 / 8 \text { by } 1 / 16 \\
5 / 32 \text { by } 1 / 16\end{array}$ & $\begin{array}{l}15 / 32 \\
19 / 32 \\
21 / 32 \\
25 / 32\end{array}$ & $\begin{array}{c}3 / 32 \text { by } 5 / 64 \\
1 / 8 \text { by } 3 / 32 \\
1 / 8 \text { by } 1 / 8 \\
5 / 32 \text { by } 5 / 32\end{array}$ & $\begin{array}{l}15 / 32 \\
19 / 32 \\
21 / 32 \\
25 / 32\end{array}$ \\
\hline $1 / 2$ & $11 / 64$ by $1 / 16$ & $7 / 8$ & $11 / 64$ by $11 / 64$ & $7 / 8$ \\
\hline $\begin{array}{c}9 / 16 \\
5 / 8 \\
11 / 16 \\
3 / 4 \\
7 / 8\end{array}$ & $\begin{array}{r}3 / 16 \text { by } 3 / 32 \\
13 / 64 \text { by } 3 / 32 \\
7 / 32 \text { by } 1 / 8 \\
1 / 4 \text { by } 18 \\
17 / 64 \text { by } 5 / 32\end{array}$ & $\begin{array}{l}31 / 32 \\
11 / 16 \\
15 / 32 \\
15 / 16 \\
115 / 32\end{array}$ & $\begin{aligned} 3 / 16 & \text { by } 3 / 16 \\
13 / 64 & \text { by } 13 / 64 \\
7 / 32 & \text { by } 7 / 32 \\
1 / 4 & \text { by } 1 / 4 \\
17 / 64 & \text { by } 17 / 64\end{aligned}$ & $\begin{array}{l}31 / 32 \\
11 / 16 \\
15 / 32 \\
15 / 16 \\
115 / 32\end{array}$ \\
\hline $\begin{array}{l}1 \\
11 / 8 \\
11 / 4 \\
138 \\
11 / 2\end{array}$ & $\begin{array}{l}5 / 16 \text { by } 3 / 10 \\
3 / 8 \text { by } 3 / 16 \\
7 / 16 \text { by } 3 / 10 \\
7 / 10 \text { by } 1 / 4 \\
1 / 2 \text { by } 1 / 4\end{array}$ & $\begin{array}{l}11116 \\
115 / 16 \\
23 / 16 \\
25 / 16 \\
29116\end{array}$ & $\begin{array}{l}5 / 16 \text { by } 5 / 16 \\
3 / 8 \text { by } 3 / 8 \\
7 / 16 \text { by } 5 / 16 \\
7 / 16 \text { by } 388 \\
1 / 2 \text { by } 3 / 8\end{array}$ & $\begin{array}{l}111 / 10 \\
115 / 16 \\
23 / 16 \\
25 / 16 \\
2 \% 16\end{array}$ \\
\hline
\end{tabular}


Temper test.-After compressing the lock washer flat, the reaction shall be sufficient to indicate necessary spring power, and on a subsequent compression to flat, the lock washer shall manifest no appreciable loss in reaction.

Toughness test.-Forty-five per cent of the lock washer, including one end, shall be secured firmly in a vise, and forty-five per cent, including the other end, shall be secured firmly between parallel jaws of a wrench. Movement of wrench at right angles to the heilcal curve shall twist the lock washer through $45^{\circ}$ without it showing signs of fracturing, and shall twist the lock washer entirely apart within $135^{\circ}$.

SOCIETY OF AUTOMOTIVE ENGINEERS, PARTS AND FITTINGS; PLAIN WASHERS, S. A. E. STANDARD, MARCH, 1923

\section{PLAIN WASHERS}

[All dimensions in inches]

\begin{tabular}{|c|c|c|c|}
\hline $\begin{array}{c}\text { Bolt and } \\
\text { screw } \\
\text { sizes }\end{array}$ & $\begin{array}{c}\text { Inside } \\
\text { diame- } \\
\text { ter }\end{array}$ & $\begin{array}{c}\text { Outside } \\
\text { diame- } \\
\text { ter }\end{array}$ & $\begin{array}{c}\text { Thick- } \\
\text { ness } \\
\pm 0.010\end{array}$ \\
\hline $\begin{array}{r}2 \\
4 \\
6 \\
8 \\
10\end{array}$ & $\begin{array}{r}3 / 32 \\
1 / 8 \\
5 / 32 \\
3 / 16 \\
7 / 32\end{array}$ & $\begin{array}{r}1 / 4 \\
5 / 16 \\
3 / 8 \\
7 / 16 \\
1 / 2\end{array}$ & $\begin{array}{l}1 / 32 \\
1 / 32 \\
3 / 64 \\
3 / 64 \\
1 / 16\end{array}$ \\
\hline $12 \begin{array}{r}1 / 4 \\
5 / 16 \\
3 / 8\end{array}$ & $\begin{array}{r}1 / 4 \\
9 / 32 \\
11 / 32 \\
13 / 32\end{array}$ & $\begin{array}{r}9 / 16 \\
5 / 8 \\
11 / 16 \\
13 / 16\end{array}$ & $\begin{array}{l}1 / 10 \\
1 / 16 \\
1 / 10 \\
1 / 10\end{array}$ \\
\hline $\begin{array}{r}7 / 16 \\
1 / 2 \\
9 / 16 \\
5 / 8\end{array}$ & $\begin{array}{l}15 / 32 \\
17 / 32 \\
19 / 32 \\
21 / 32\end{array}$ & $\begin{array}{l}15 / 16 \\
11 / 16 \\
13 / 16 \\
15 / 16\end{array}$ & $\begin{array}{l}1 / 10 \\
3 / 32 \\
3 / 32 \\
3 / 32\end{array}$ \\
\hline $\begin{array}{r}11 / 16 \\
3 / 4 \\
7 / 8 \\
1^{7 / 8}\end{array}$ & $\begin{array}{l}23 / 32 \\
13 / 16 \\
15 / 16 \\
11 / 16\end{array}$ & $\begin{array}{l}13 / 8 \\
11 / 2 \\
13 / 4 \\
2\end{array}$ & $\begin{array}{r}3 / 32 \\
1 / 8 \\
1 / 8 \\
1 / 8\end{array}$ \\
\hline $\begin{array}{l}11 / 8 \\
11 / 4 \\
13 / 8 \\
11 / 2\end{array}$ & $\begin{array}{l}13 / 16 \\
15 / 16 \\
17 / 16 \\
1 \% 16\end{array}$ & $\begin{array}{l}21 / 4 \\
21 / 2 \\
23 / 4 \\
3\end{array}$ & $\begin{array}{l}1 / 8 \\
5 / 32 \\
5 / 32 \\
5 / 32\end{array}$ \\
\hline
\end{tabular}

Washers shall be flat and free from burrs.

\subsection{TURNBUCKLES.}

\section{AMERICAN MARINE STANDARDS COM- MITTEE, RIGGING FITTINGS FOR SHIPS, H. No. 6-1928 AND H No. 7-1928}

\section{RIGGING SCREWS}

\section{GENERAL NOTES}

Materials and Workmanship

1. The turnbuckles, screws, and pins are to be of mild steel of tensile strength of 55,000 to 65,000 pounds per square inch. It is intended that the materials and workmanship shall conform to the requirements of the American Bureau of Shipping, but inspection and tests shall be required only when specified in the purchase order.

2. The dimensions tabulated correspond to sizes and strengths of ropes given in the rules of the American Bureau of Shipping; if ropes of lesser or greater strengths are used, rigging screws of corresponding strengths should be selected from the table and their end connections adapted to fittings required to suit the rope actually used.

3. Mast shrouds and stays should be served for a length of 6 feet from the lower end. Other wire ropes should be finished as follows:

(a) Those under five-eighths inch nominal diameter should be parceled and single served only in way of splices and thimbles.

(b) Those of five-eighths inch nominal diameter and over should be single served in way of splices, and wormed, parceled, and single served in way of thimbles.

4. The materials used to serve wire ropes should be as follows:

(a) Marline for wire ropes up to and including seven-sixteenths inch nominal diameter.

(b) Houseline for wire ropes from one-half inch to $1 \frac{1}{2}$ inches nominal diameter, inclusive.

(c) Hambroline for wire ropes from $1 \frac{5}{8}$ to $23 / 4$ inches nominal diamter, inclusive.

(The nominal diameter of wire rope is the overall diameter.)

5. Details and dimensions of open and closed sockets for various sizes of wire rope are shown by American Marine Standard H No. 9-1926. The size of the pin required in the socket must in every instance be specified when ordering these fittings from manufacturers, as the sizes required might not be commercial standards.

6. Socketing of rope should be done according to the best practice. Zinc (not lead or babbitt) should be used for filling the socket.

7. All screw threads are to be of medium fit and conform to the American (national) standard, sponsored by the Society of Automotive Engineers and the American Society of Mechanical Engineers, and approved by the American Standards Association in May, 1924, as standard B1a-1924. This standard is in complete accordance with the report of the National Screw Thread Commission (revised, 1928). (Miscellaneous Publication No. 89, Bureau of Standards.)

8. Pins of diameters not conforming to standard screw-thread sizes shall have their threaded end of the standard screw-thread size next below the pin size. 


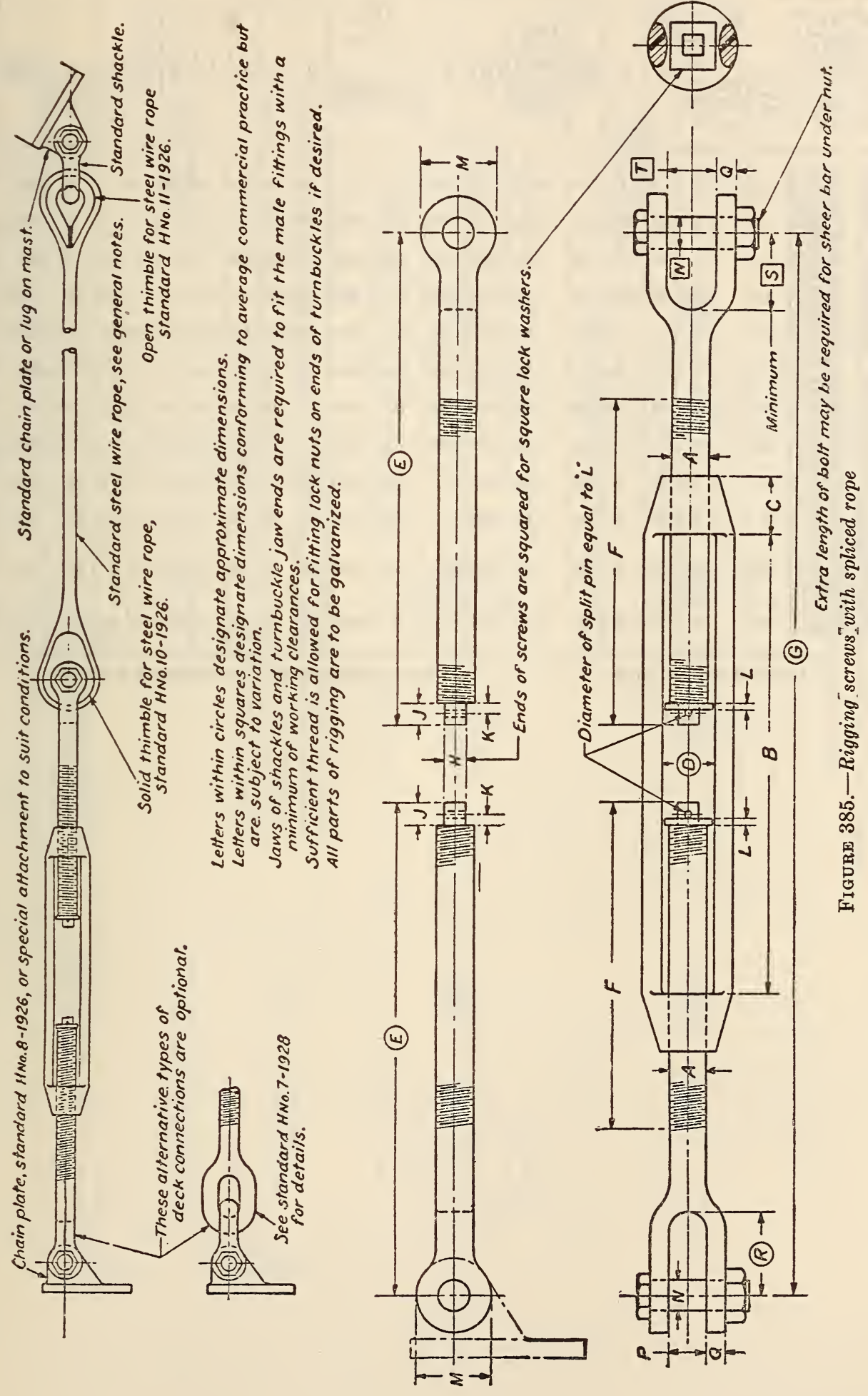


Rigging screws with spliced rope

[All dimensions in inches]

\begin{tabular}{|c|c|c|c|c|c|c|c|c|c|c|c|c|c|c|c|c|c|c|c|c|c|c|c|}
\hline \multirow{2}{*}{$\begin{array}{l}\text { Size } \\
\text { No. } \\
\text { of } \\
\text { rig- } \\
\text { ging }\end{array}$} & \multirow{2}{*}{$\begin{array}{c}\text { Rope } \\
\text { sizes } \\
\text { (cir- } \\
\text { cum- } \\
\text { fer- } \\
\text { ences) } \\
\text { in } \\
\text { inches }\end{array}$} & \multirow{2}{*}{$\begin{array}{l}\text { As- } \\
\text { sumed } \\
\text { load in } \\
\text { pounds } \\
\text { (factor } \\
\text { of } \\
\text { safety } \\
=5 \text { ) }\end{array}$} & \multicolumn{2}{|r|}{ A } & \multirow[b]{2}{*}{ B } & \multirow[b]{2}{*}{ C } & \multirow[b]{2}{*}{ (D) } & \multirow{2}{*}{$\begin{array}{l}\text { Mini- } \\
\text { mum } \\
\text { area of } \\
1 \text { rein } \\
\text { (square } \\
\text { inches) }\end{array}$} & \multirow[b]{2}{*}{ (E) } & \multirow[b]{2}{*}{$\mathbf{F}$} & \multicolumn{2}{|c|}{ (G) } & \multirow[b]{2}{*}{ H } & \multirow[b]{2}{*}{$\mathrm{J}$} & \multirow[b]{2}{*}{ K } & \multirow[b]{2}{*}{$\mathrm{L}$} & \multirow[b]{2}{*}{$\mathbf{M}$} & \multirow[b]{2}{*}{$\mathrm{N}$} & \multirow[b]{2}{*}{$P$} & \multirow[b]{2}{*}{ Q } & \multirow[b]{2}{*}{ (B) } & \multirow[b]{2}{*}{$\mathrm{S}$} & \multirow[b]{2}{*}{$\mathbf{T}$} \\
\hline & & & $\begin{array}{c}\text { Di- } \\
\text { ame- } \\
\text { ter }\end{array}$ & $\left|\begin{array}{l}\text { Threads } \\
\text { per inch }\end{array}\right|$ & & & & & & & $\begin{array}{l}\text { Mini- } \\
\text { mum }\end{array}$ & $\begin{array}{l}\text { Maxi- } \\
\text { mum }\end{array}$ & & & & & & & & & & & \\
\hline 1 & $\begin{array}{l}13 / 8 \\
11 / 2\end{array}$ & $\begin{array}{l}1,612 \\
1,926\end{array}$ & $3 / 4$ & 10 & 9 & $11 / 8$ & $11 / 8$ & 0.21 & $91 / 2$ & $61 / 2$ & 19 & 27 & $1 / 2$ & $1 / 2$ & $3 / 16$ & $1 / 8$ & $11 / 2$ & $5 / 8$ & $3 / 4$ & $3 / 8$ & $15 / 8$ & $15 / 8$ & 1 \\
\hline 2 & $\begin{array}{l}13 / 4 \\
17 / 8\end{array}$ & $\begin{array}{l}2,600 \\
3,000\end{array}$ & $7 / 8$ & 9 & 12 & $15 / 16$ & $11 / 4$ & .29 & $113 / 4$ & 814 & $231 / 2$ & $311 / 2$ & $1 / 2$ & $1 / 2$ & $3 / 18$ & $1 / 8$ & $13 / 4$ & $3 / 4$ & $7 / 8$ & $1 / 2$ & $13 / 4$ & $17 / 8$ & $11 / 1$ \\
\hline 3 & $\begin{array}{l}2 \\
21 / 8 \\
21 / 4\end{array}$ & $\begin{array}{l}3,400 \\
3,900 \\
4,340\end{array}$ & 1 & 8 & 12 & $11 / 2$ & $13 / 8$ & .39 & $12^{3} / 4$ & $81 / 2$ & $251 / 2$ & $351 / 2$ & 58 & $3 / 2$ & $3 / 16$ & $1 / 8$ & 2 & $7 / 8$ & 1 & $5 / 8$ & 2 & $21 / 8$ & 13 \\
\hline 4 & $\begin{array}{l}23 / 8 \\
21 / 2 \\
25 / 8\end{array}$ & $\begin{array}{l}4,840 \\
5,320 \\
5,900\end{array}$ & 138 & 7 & 12 & $111 / 16$ & $11 / 2$ & .49 & $133 / 4$ & $87 / 8$ & $271 / 2$ & $391 / 8$ & $5 / 8$ & $11 / 16$ & $9 / 32$ & $3 / 16$ & $21 / 4$ & 1 & $11 / 8$ & $3 / 4$ & $25 / 16$ & 23,8 & $15 / 8$ \\
\hline 5 & $\begin{array}{l}2^{3}, 8 \\
2^{7 / 8}\end{array}$ & $\begin{array}{l}6,440 \\
7,080\end{array}$ & $11 / 4$ & 7 & 12 & $17 / 8$ & $13 / 4$ & .62 & 15 & 914 & 30 & $405 / 8$ & $3 / 4$ & $11 / 16$ & $9 / 32$ & $3 / 16$ & $21 / 2$ & $11 / 8$ & $11 / 4$ & $13 / 16$ & $2^{11} 1 / 16$ & $25 \%$ & $15 / 8$ \\
\hline 6 & 3 & $\begin{array}{l}7,700 \\
8,380\end{array}$ & $11 / 2$ & 6 & 18 & $21 / 4$ & 2 & .91 & $191 / 4$ & $123 / 4$ & $381 / 2$ & $543 / 4$ & $7 / 8$ & $7 / 8$ & $3 / 8$ & $1 / 4$ & 3 & $11 / 4$ & $13 / 8$ & $13 / 16$ & $31 / 4$ & $31 / 8$ & $17 / 8$ \\
\hline 17 & $\begin{array}{l}3,4 \\
33 / 8 \\
31 / 2\end{array}$ & $\begin{array}{r}9,000 \\
9,720 \\
10,580\end{array}$ & $13 / 4$ & 5 & 18 & $25 / 8$ & $21 / 2$ & 1. 22 & $203 / 4$ & $133 / 8$ & $411 / 2$ & $573 / 4$ & 1 & $7 / 8$ & $3 / 8$ & $1 / 4$ & $31 / 2$ & $13 / 2$ & $15 / 8$ & $15 / 16$ & $43 / 16$ & $37 / 8$ & $25 / 10$ \\
\hline 8 & $\begin{array}{l}35,8 \\
334 \\
378\end{array}$ & $\begin{array}{l}11,240 \\
12,040 \\
12,900\end{array}$ & $13 / 4$ & 5 & 18 & 25,8 & $21 / 2$ & 1.22 & $203 / 4$ & $133 / 8$ & $411 / 2$ & $573 / 4$ & 1 & $7 / 8$ & $3 / 8$ & $1 / 4$ & $31 / 2$ & $11 / 2$ & $15 \%$ & $15 / 16$ & $43 / 16$ & 378 & 25 \\
\hline 29 & $\begin{array}{l}4 / 8 \\
41 / 8\end{array}$ & $\begin{array}{l}13,700 \\
14,560\end{array}$ & 2 & $41 / 2$ & 24 & 3 & 234 & 1. 61 & $251 / 4$ & 17 & $501 / 2$ & $72^{33} / 4$ & 138 & $7 / 8$ & $3 / 8$ & 34 & 4 & $13 / 4$ & $17 / 8$ & $11 / 4$ & $17 / 8$ & 458 & $27 / 10$ \\
\hline 10 & $\begin{array}{l}41 / 4 \\
43 / 8 \\
41 / 2 \\
45 / 8\end{array}$ & $\begin{array}{l}15,460 \\
16,400 \\
17,340 \\
18,280\end{array}$ & 2 & $41 / 2$ & 24 & 3 & $23 / 4$ & 1.61 & $251 / 4$ & 17 & $501 / 2$ & $72^{3 / 4}$ & 136 & $7 / 8$ & $3 / 8$ & 144 & 4 & $13 / 4$ & $17 / 8$ & 134 & $47 / 8$ & $45 / 8$ & $27 / 1$ \\
\hline 11 & $\begin{array}{l}438 \\
434 \\
47 / 8 \\
5\end{array}$ & $\begin{array}{l}18,280 \\
19,300 \\
20,340 \\
21,360\end{array}$ & $21 / 4$ & 432 & 24 & $33 / 8$ & $31 / 4$ & 2.11 & 27118 & $175 / 8$ & $541 / 4$ & $761 / 4$ & $13 / 4$ & $7 / 8$ & $3 / 8$ & 34 & $41 / 2$ & 178 & 2 & $11 / 2$ & $53 / 4$ & 538 & 236 \\
\hline 12 & $\begin{array}{l}514 \\
51 / 2\end{array}$ & $\begin{array}{l}23,560 \\
25,840\end{array}$ & $21 / 2$ & 4 & 24 & $33 / 4$ & $31 / 4$ & 2.60 & 29 & 1814 & 58 & 8014 & $13 / 8$ & $7 / 8$ & $3 / 8$ & $1 / 4$ & 5 & $21 / 8$ & 234 & 158 & $67 / 10$ & 634 & 25,8 \\
\hline
\end{tabular}

1 Use chain plate size No. 8.

2 Use chain plate size No. 10. See standard H No. 8-1926. 


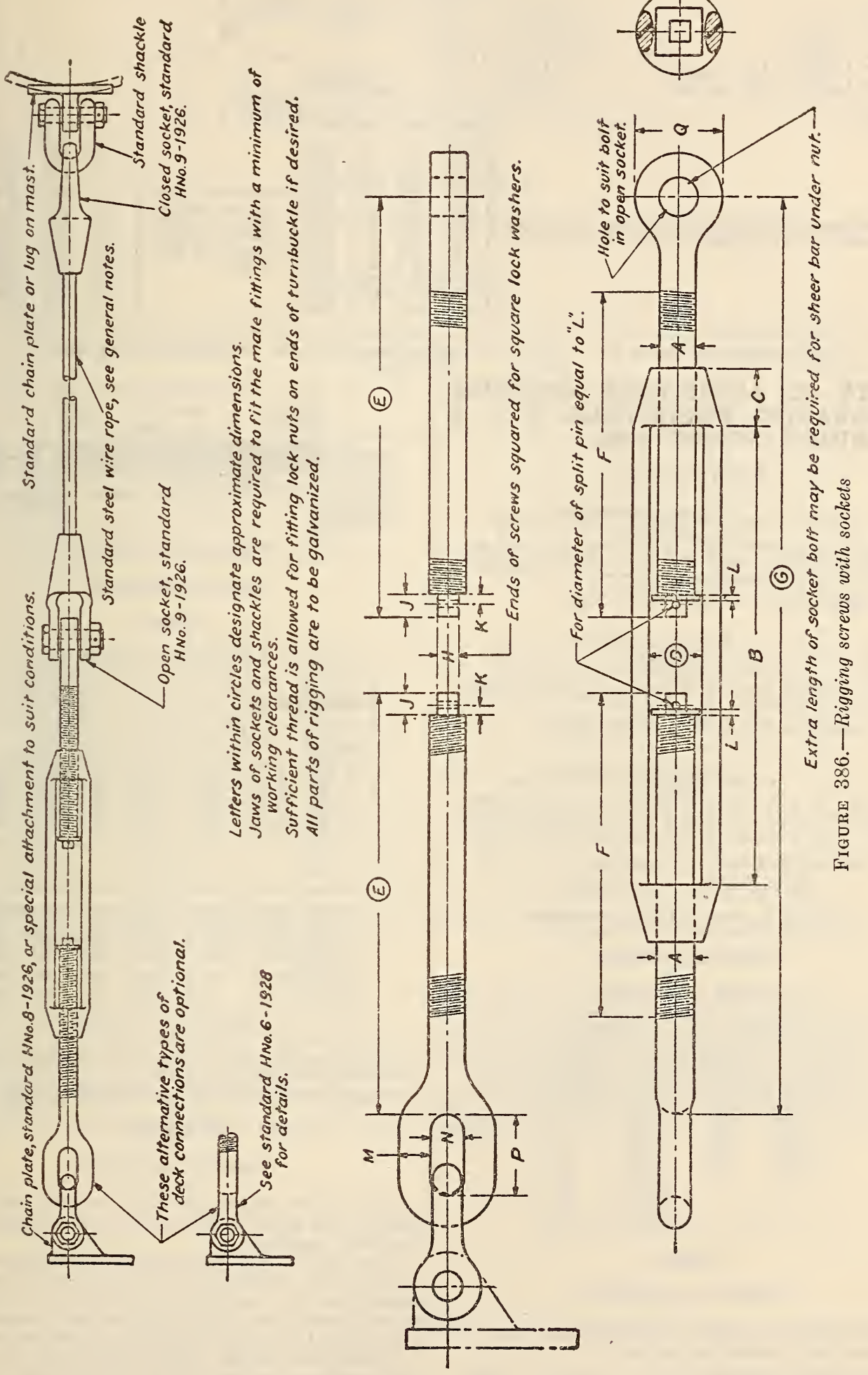


Rigging screws with sockets

[All dimensions in inches]

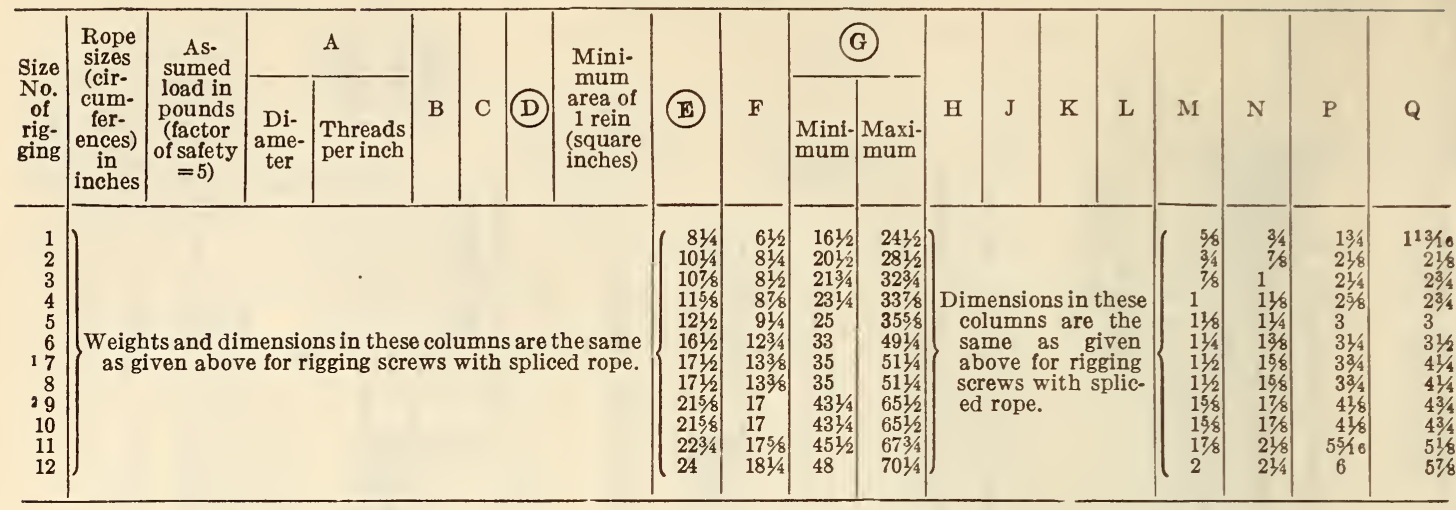

1 Use chain plate size No. 8.

2 Use chain plate size No. 10. See standard H No. 8-1926.

SOCIETY OF AUTOMOTIVE ENGINEERS, AERONAUTIC; TURNBUCKLES, S. A. E. STANDARD, AUGUST, 1928

\section{TURNBUCKLES}

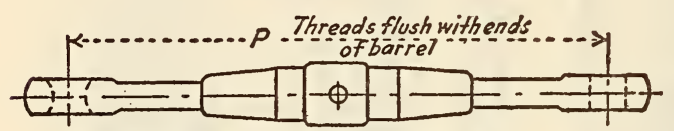

Assembly No.1 Cable-Eye and Pin-Eye

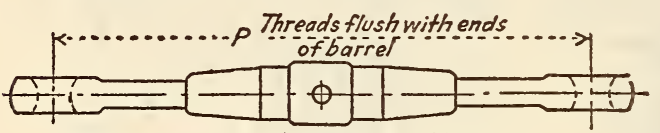

Assembly No.2. Cable-Eye and Cable-Eye

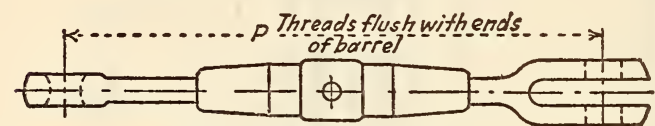

Assembly No.3 Cable-Eye and Fork

Frgure 387.-Turnbuckles

$P=4 \frac{1}{2}$ inches, assembled length-short type $P=8$ inches, assembled length-long type

TURNBUCKLE WEIGHTS

(General information)

\begin{tabular}{|c|c|c|c|}
\hline \multicolumn{2}{|c|}{ Short type } & \multicolumn{2}{c|}{ Long type } \\
\hline Strength & $\begin{array}{c}\text { Approxi- } \\
\text { mate } \\
\text { weight }\end{array}$ & Strength & $\begin{array}{c}\text { Approxi- } \\
\text { mate } \\
\text { weight }\end{array}$ \\
\cline { 2 - 3 } Pounds & Pound & Pounds & Pound \\
800 & 0.030 & 1,600 & 0.094 \\
1,600 & .065 & 2,100 & .116 \\
2,100 & .084 & 3.200 & .172 \\
3,200 & .112 & 4,600 & .250 \\
4,600 & .165 & 6,100 & .368 \\
& & 8,000 & .420 \\
& & 12,500 & .630 \\
& & 17,500 & .935 \\
\hline
\end{tabular}

BARREI

PHYSICAL PROPERTIES

Barrels shall be made of rolled brass to provide the following physical properties:

Tensile strength (minimum) ..............lbs./in. ${ }^{2}$.. $\quad 67,000$ Yield point (minimum) .................... do ... 45, 000 Elongation (minimum) in 2 inches........-per cent.- 22

Reduction of area (minimum) .................. do....

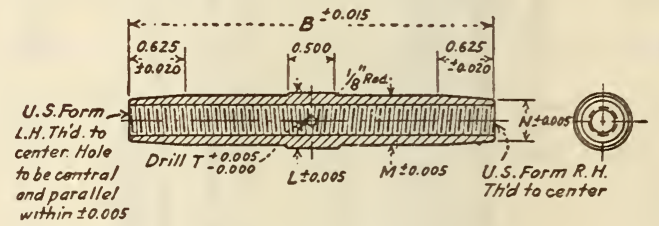

FIGURE 388.-Barrel

Barrel dimensions

SHORT

[All dimensions in inches]

\begin{tabular}{|r|r|r|r|r|r|r|}
\hline Strength & Thread $^{1}$ & \multicolumn{1}{|c|}{$L$} & \multicolumn{1}{|c|}{$M$} & \multicolumn{1}{|c|}{$N$} & $B$ & $T$ \\
\cline { 1 - 3 } Pounds & & & & & & \\
800 & $6-40$ & 0.250 & 0.219 & 0.188 & 2.25 & 0.125 \\
1,600 & $10-32$ & .375 & .281 & .250 & 2.25 & .125 \\
2,100 & $12-28$ & .375 & .328 & .281 & 2.25 & .125 \\
3,200 & $14-28$ & .438 & .391 & .328 & 2.25 & .125 \\
4,600 & $516-24$ & .500 & .438 & .406 & 2.25 & .125 \\
\hline
\end{tabular}

LONG

\begin{tabular}{|r|r|r|r|r|r|r|}
\hline 1,600 & $10-32$ & 0.375 & 0.281 & 0.250 & 4.00 & 0.125 \\
2,100 & $12-28$ & .375 & .328 & .281 & 4.00 & .125 \\
3,200 & $1 / 4-28$ & .438 & .391 & .328 & 4.00 & .125 \\
4,600 & $5 / 16-24$ & .500 & .438 & .406 & 4.00 & .125 \\
6,100 & $3 / 8-24$ & .625 & .594 & .469 & 4.00 & .125 \\
8,000 & $7 / 16-20$ & .750 & .688 & .563 & 4.25 & .188 \\
12,500 & 7625 & .875 & .813 & .625 & 4.25 & 1.88 \\
17,500 & $1 / 2-20$ & .875 & & & & \\
\hline
\end{tabular}

1 Threads are American standard (NF), with class 3 tolerances. $\mathrm{NF}$ indicates national fine pitch.

Tolerances are \pm 0.010 except where otherwise indicated.

\section{CABLE EYE}

\section{PHYSICAL PROPERTIES}

Turnbuckle cable eyes shall be subjected to a tensile test after plating and shall withstand the test load specified.

The shanks shall be machined, preferably from heat-treated cold-drawn or cold-rolled bars. If shanks are not made from heat-treated cold-drawn or cold-rolled bars, they shall be heat treated after machining to give the physical properties specified.

Turnbuckle shanks meeting the tensile test without breaking shall be held in a square-nose vise and bent through an angle of $90^{\circ}$ without failing or cracking. 


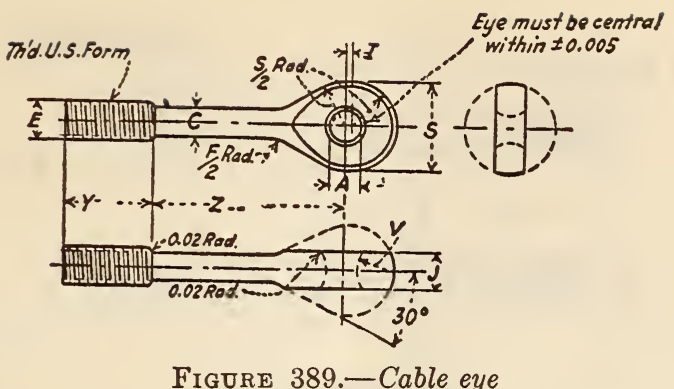

FIGURE 389.-Cable eye

Cable-eye dimensions

SHORT

[All dimensions in inches]

\begin{tabular}{|c|c|c|c|c|c|c|c|c|c|c|c|c|}
\hline Hand & Strength & Thread ${ }^{1}$ & $E$ & $\begin{array}{c}A \\
+0.010 \\
-0.000\end{array}$ & $\begin{array}{c}C \\
+0.006 \\
-0.000\end{array}$ & $F / 2$ & $\begin{array}{c}I \\
+0.010 \\
-0.000\end{array}$ & $J$ & $S$ & $V$ & $\begin{array}{c}Y \\
\pm 0.047\end{array}$ & $\begin{array}{c}Z \\
+0.031 \\
-0.015\end{array}$ \\
\hline L. H. & $\left\{\begin{array}{r}\text { Pounds } \\
800\end{array}\right.$ & $6-40$ & $\{0.138+0.0000$ & 0.188 & 0.094 & 0.125 & 0.031 & 0.125 & 0.375 & 0.094 & 0.375 & 1.125 \\
\hline L. H. & 1,600 & $10-32$ & $\{.190+.0000$ & .219 & .133 & .156 & .031 & . 188 & .500 & .172 & .500 & 1.125 \\
\hline R. H. & 2,100 & $12-28$ & $\begin{array}{r}.216 \pm .0000 \\
-.0062\end{array}$ & .219 & .155 & .156 & .031 & .188 & .500 & .172 & .563 & 1. 125 \\
\hline L. H. & 3,200 & $1 / 4-28$ & $\begin{array}{r}.250+.0000 \\
-.0062\end{array}$ & .281 & .189 & .219 & .047 & .219 & .625 & .203 & .625 & 1.125 \\
\hline L. H. & 4,600 & $5 / 16-24$ & $\begin{array}{r}.3125+.0000 \\
-.0066\end{array}$ & .313 & .243 & .250 & .047 & .281 & .688 & .250 & .750 & 1. 125 \\
\hline
\end{tabular}

LONG

\begin{tabular}{|c|c|c|c|c|c|c|c|c|c|c|c|c|}
\hline $\begin{array}{l}\text { L. H. } \\
\text { R. H. }\end{array}$ & 1,600 & $10-32$ & $\left\{\begin{array}{r}0.190+0.0000 \\
-.0054\end{array}\right.$ & 0.219 & 0.133 & 0.156 & 0.031 & 0.188 & 0.500 & 0.172 & 0.500 & 2.000 \\
\hline R. & 2,100 & $12-28$ & $\left\{\begin{array}{r}.216+.0000 \\
-.0062\end{array}\right.$ & .219 & .155 & .156 & .031 & .188 & .500 & .172 & .563 & 2. 000 \\
\hline $\begin{array}{l}\text { L. H. } \\
\text { R. H. }\end{array}$ & 3,200 & $1 / 4-28$ & $\begin{array}{r}.250+.0000 \\
-.0062\end{array}$ & .281 & .189 & .219 & .047 & .219 & .625 & .203 & .625 & 2.000 \\
\hline R. $\mathrm{H}$. & 4,600 & $5 / 16-24$ & $\begin{array}{r}.3125+.0000 \\
-.0066\end{array}$ & .313 & .243 & .250 & .047 & .281 & .688 & .250 & .750 & 2.000 \\
\hline L. & 6,100 & $3 / 8-24$ & $\left\{\begin{array}{r}.375+.0000 \\
\div .0066\end{array}\right.$ & .344 & .256 & .281 & .063 & .281 & .750 & .313 & .875 & 2.000 \\
\hline $\begin{array}{l}\text { L. H. } \\
\text { R. H. }\end{array}$ & 8,000 & $3 / 8-24$ & $\left\{\begin{array}{r}.375+.0000 \\
-.0066\end{array}\right.$ & .375 & .306 & .281 & .063 & .328 & .875 & .375 & .875 & 2.000 \\
\hline $\begin{array}{l}\text { L. H. } \\
\text { R. H. }\end{array}$ & 12,500 & $7 / 16-20$ & $\left\{\begin{array}{r}.4375+.0000 \\
-.0072\end{array}\right.$ & .469 & .362 & .359 & .078 & .375 & 1. 063 & .453 & 1.000 & 2. 375 \\
\hline $\begin{array}{l}\text { L. H. } \\
\text { R. H. }\end{array}$ & 17,500 & $1 / 2-20$ & $\left\{\begin{array}{r}.500+.0000 \\
-.0072\end{array}\right.$ & .563 & .425 & .406 & .078 & .469 & 1. 188 & .500 & 1.000 & 2. 625 \\
\hline
\end{tabular}

1 Threads are American standard (NF) with class 3 tolerances. NF indicates national fine pitch.

Finished sizes include plating or protective coating.

Tolerances are \pm 0.010 except where otherwise indicated.

\section{PIN EYE}

\section{PHYSICAL PROPERTIES}

Turnbuckle pin eyes shall be subjected to a tensile test after plating and shall withstand the test load specified.

The pin eye shall be machined preferably from heat-treated cold-drawn or cold-rolled bars. If pin eyes are not made from heat-treated cold-drawn or cold-rolled bars, they shall be heat-treated after machining to give physical properties specified.

Turnbuckle pin eyes meeting the tensile test without breaking shall be held in a square-nose vise and bent through an angle of $90^{\circ}$ without failing or cracking. 


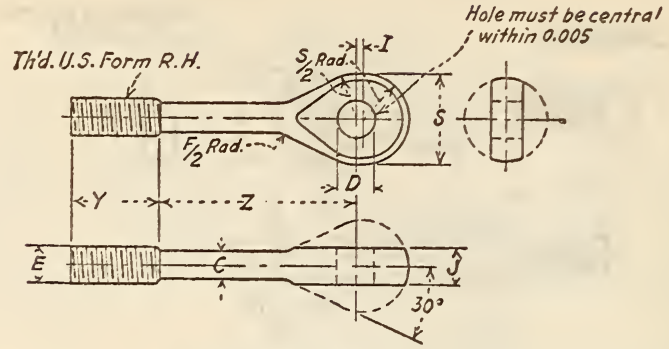

Figure 390.-Pin eye

Pin-eye dimensions

SHORT

[All dimensions in inches]

\begin{tabular}{|c|c|c|c|c|c|c|c|c|c|c|}
\hline Strength & Thread 1 & $E$ & $\begin{array}{c}D \\
+0.010 \\
-0.000\end{array}$ & $\begin{array}{c}C \\
+0.006 \\
-0.000\end{array}$ & $F / 2$ & $\begin{array}{c}I \\
+0.010 \\
-0.000\end{array}$ & $J$ & $S$ & $\begin{array}{c}Y \\
\pm 0.047\end{array}$ & $\begin{array}{c}Z \\
+0.031 \\
-0.015\end{array}$ \\
\hline $\begin{array}{c}\text { Pounds } \\
800\end{array}$ & $6-40$ & $\begin{array}{r}0.1218+0.0900 \\
-.0017\end{array}$ & 0.188 & 0.094 & 0.125 & 0.031 & 0.125 & 0.375 & 0.375 & 1.125 \\
\hline 1,600 & $10-32$ & $\begin{array}{r}.1697+.0000 \\
-.0019\end{array}$ & .188 & .133 & .156 & .031 & .188 & .500 & .500 & 1.125 \\
\hline 2,100 & $12-28$ & $\begin{array}{r}.1928+.0000 \\
-.0022\end{array}$ & .188 & .155 & .156 & .031 & .188 & .500 & .563 & 1.125 \\
\hline 3,200 & $1 / 4-28$ & $\begin{array}{r}.2268+.0000 \\
-.0022\end{array}$ & .250 & .189 & .219 & .047 & .219 & .625 & .625 & 1.125 \\
\hline 4,600 & $5 / 16-24$ & $\begin{array}{r}.2854+.0000 \\
-.0024\end{array}$ & .313 & .243 & .250 & .047 & .281 & .658 & .750 & 1.125 \\
\hline
\end{tabular}

LONG

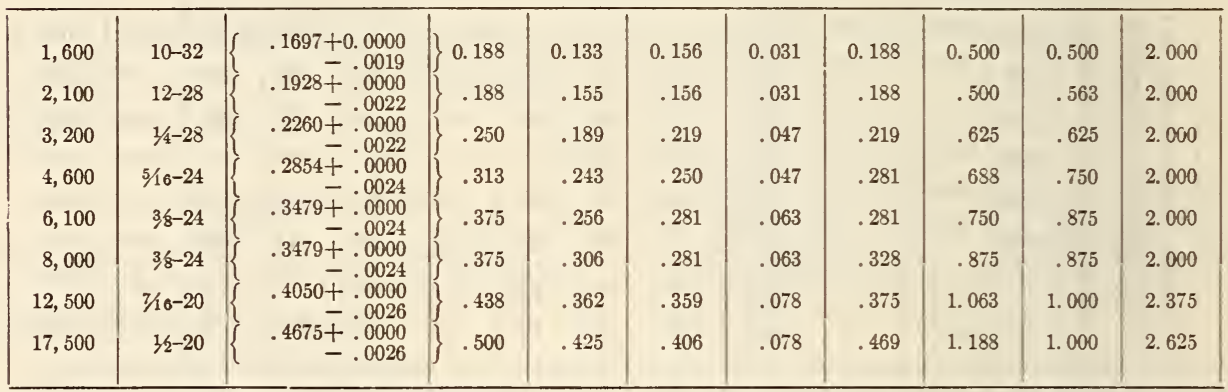

1 Threads are American Standard (NF), with class 3 tolerances. NF indicates national fine pitch.

Finished sizes include plating or protective coating.

Tolerances are \pm 0.010 except where otherwise indicated.

\section{FORK}

\section{PHYSICAL PROPERTIES}

Turnbuckle forks shall be subjected to a tensile test after plating and shall withstand the test load specified.

The forks shall be machined, preferably from heat- treated cold-drawn or cold-rolled bars. If forks are not made from heat-treated cold-drawn or cold-rolled bars, they shall be heat treated after machining to give the physical properties specified.

Turnbuckle forks meeting the tensile test without breaking shall be held in a square-nose vise and bent through an angle of $90^{\circ}$ without failing or cracking. 


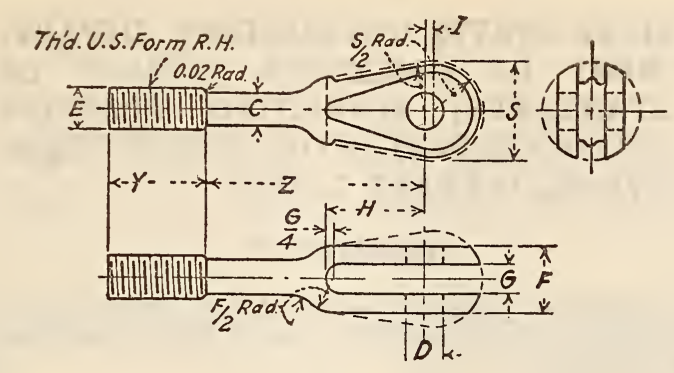

Figure 391.-Fork

Slot, jaw, and hole to be central and parallel within \pm 0.005 on fork

\section{Fork dimensions}

SHORT

[All dimensions in inches]

\begin{tabular}{|c|c|c|c|c|c|c|c|c|c|c|c|}
\hline Strength & Thread 1 & $E$ & $\begin{array}{c}C \\
+0006 \\
-0.000\end{array}$ & $\begin{array}{c}D \\
+0.010 \\
-0.000\end{array}$ & $\begin{array}{c}F \\
+0.010 \\
-0.005\end{array}$ & $\begin{array}{c}G \\
+0.010 \\
-0.000\end{array}$ & $H$ & $\begin{array}{c}1 \\
+0.010 \\
-0.000\end{array}$ & $S$ & $\begin{array}{c}Y \\
\pm 0.047\end{array}$ & $\begin{array}{c}Z \\
+0.031 \\
-0.015\end{array}$ \\
\hline $\begin{array}{c}\text { Pounds } \\
800\end{array}$ & $6-40$ & $\begin{array}{r}0.138+0.0000 \\
-.0048\end{array}$ & 0.094 & 0.188 & 0.250 & 0.109 & 0.375 & 0.031 & 0.375 & 0.375 & 1. 125 \\
\hline 1,600 & $10-32$ & $\begin{array}{r}.190 \pm .0000 \\
-.0054\end{array}$ & .133 & .188 & .313 & .150 & .469 & .031 & .500 & .500 & 1. 125 \\
\hline 2,100 & $12-28$ & $.216 \pm .0000$ & .155 & .188 & .313 & .150 & .562 & .031 & .500 & .563 & 1. 125 \\
\hline 3,200 & $34-28$ & $\begin{array}{r}.250+.0000 \\
-.0062\end{array}$ & .189 & .250 & .438 & .203 & .625 & .047 & .625 & .625 & 1.125 \\
\hline 4,600 & $5 / 10-24$ & $\begin{array}{r}.3125 \pm .0000 \\
-.0066\end{array}$ & .243 & .313 & .500 & .203 & .656 & .047 & .688 & .750 & 1. 125 \\
\hline
\end{tabular}

\section{LONG}

\begin{tabular}{|c|c|c|c|c|c|c|c|c|c|c|c|}
\hline 1,600 & $10-32$ & $\begin{array}{r}0.190+0.0000 \\
-.0054\end{array}$ & 0.133 & 0.188 & 0.313 & 0.150 & 0.469 & 0.031 & 0.500 & 0.500 & 2. 000 \\
\hline 2,100 & $12-28$ & $.216 \pm .0000$ & . 155 & . 188 & . 313 & .150 & .562 & . 031 & .500 & .563 & 2. 000 \\
\hline 3,200 & $14-28$ & $\begin{array}{r}.250+.0000 \\
-.0062\end{array}$ & .189 & .250 & .438 & .203 & .625 & .047 & .625 & .625 & 2.000 \\
\hline 4,600 & $5 / 16-24$ & $\begin{array}{r}.3125+.0000 \\
-.0066\end{array}$ & .243 & .313 & .500 & .203 & .656 & .047 & .688 & .750 & 2. 000 \\
\hline 6,100 & $3 / 8-24$ & $.375 \pm .0000$ & .256 & .375 & .563 & .203 & .843 & .063 & .750 & .875 & 2. 000 \\
\hline 8,000 & $3 / 8-24$ & $\begin{array}{r}.375 \pm .0000 \\
-.0066\end{array}$ & .306 & .375 & .563 & .266 & .875 & .063 & .875 & .875 & 2.000 \\
\hline 12,500 & $7 / 16-20$ & $\begin{array}{r}.4375+.0000 \\
-.0072\end{array}$ & .362 & .438 & .719 & .344 & 1.000 & .078 & 1.063 & 1.000 & 2. 375 \\
\hline 17,500 & $1 / 2-20$ & $\begin{array}{r}.500 \pm .0000 \\
-.0072\end{array}$ & .425 & .500 & .813 & .406 & 1. 188 & .078 & 1. 188 & 1.000 & 2.625 \\
\hline
\end{tabular}

1 Threads are American Standard (NF), with class 3 tolerances. NF indicates national fine pitch.

Finished sizes include plating or protective coating.

Tolerances are \pm 0.010 except where otherwise indicated. 
UNITED STATES GOVERNMENT, DEPARTMENT OF COMMERCE, BUREAU OF STANDARDS; SIMPIIFIED PRACTICE RECOMMENDATION FOR TURNBUCKLES, R71-28, JANUARY 1, 1928

\section{TURNBUCKLES}

(Simplified practice recommended and accepted by industry establishing a limited list of standard stock sizes.)

TABLE 1.-Turnbuckles with and without stubs

\begin{tabular}{|c|c|c|c|c|c|c|c|c|c|}
\hline \multirow{2}{*}{$\begin{array}{l}\text { Diam- } \\
\text { eter } \\
\text { (inches) }\end{array}$} & \multicolumn{9}{|c|}{ Opening (in inches) } \\
\hline & 4 & $41 / 2$ & 6 & 9 & 12 & 18 & 24 & 36 & 48 \\
\hline 13 & $x$ & & & & & & & & \\
\hline & & $\mathbf{x}$ & $x$ & $\cdots$ & - & --- & $-\cdots$ & (n...... & $-\cdots$ \\
\hline & & $\ldots$ & $x$ & $x$ & $x$ & & - & & $\ldots$ \\
\hline & & $\mid-\cdots$ & $x$ & $x$ & $x$ & $x$ & & 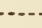 & $-\cdots$ \\
\hline & & & $x$ & $x$ & x & $\mathrm{x}$ & $\mathbf{x}$ & 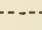 & $\cdots$ \\
\hline $7 / 8$ & & $\cdots$ & $x$ & $\cdots$ & $x$ & $\mathbf{x}$ & $x$ & & .... \\
\hline & 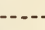 & $\mid-\ldots$. & $x$ & 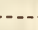 & $\mathrm{x}$ & $x$ & $\mathrm{x}$ & $\mathbf{x}$ & .... \\
\hline $11 / 8 \ldots$ & $\cdots$ & $\mid \cdots-$ & $x$ & $\cdots$ & $x$ & $x$ & $\mathrm{x}$ & $\mathbf{x}$ & $\cdots$ \\
\hline $\begin{array}{l}1344 \ldots \\
138\end{array}$ & $\cdots$ & $-\cdots$ & $\frac{x}{x}$ & - & $\mathrm{x}$ & $\mathrm{x}$ & $\mathrm{x}$ & $x$ & $\cdots$ \\
\hline $11 / 2$ & & | & $x$ & & $x$ & $x$ & $x$ & $x$ & $\mathbf{I}$ \\
\hline $15 / 8$ & .... & $\mid \ldots .$. & $x$ & 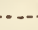 & 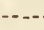 & 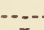 & 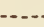 & & \\
\hline $\begin{array}{l}13, \\
2\end{array}$ & & {$[-\cdots$} & $\begin{array}{l}x \\
x\end{array}$ & -- & - & $x$ & $\begin{array}{l}x \\
x\end{array}$ & $\begin{array}{l}x \\
x\end{array}$ & $\frac{x}{x}$ \\
\hline & & {$[\cdots$} & $\mathrm{x}$ & $\cdots$ & $\ldots$. & $\cdots$ & $\mathrm{x}$ & $\mathrm{x}$ & $x$ \\
\hline 23 & & & $\mathrm{x}$ & & & & $x$ & $x$ & $x$ \\
\hline $23 / 4$ & -3 & $\cdots$ & & $\mathrm{x}$ & 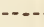 & 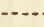 & & & $x$ \\
\hline & & & & $\begin{array}{l}x \\
x\end{array}$ & -- & & & & $\begin{array}{l}x \\
x\end{array}$ \\
\hline & & & & $x$ & & & & & $x$ \\
\hline
\end{tabular}

TABLE 2.-Turnbuckles with hook, eye, and jaw ends

\begin{tabular}{|c|c|c|c|c|c|c|c|c|c|}
\hline \multirow{2}{*}{$\begin{array}{c}\text { Diam- } \\
\text { eter } \\
\text { (inches) }\end{array}$} & \multicolumn{9}{|c|}{ Opening (in inches) } \\
\hline & 4 & $41 / 2$ & 6 & 9 & 12 & 18 & 24 & 36 & 48 \\
\hline $\begin{array}{l}14 . \\
5 / 16\end{array}$ & $x$ & $\mathbf{x}$ & & & & - & ..... & 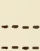 & 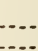 \\
\hline & & & $\frac{x}{x}$ & $x$ & $x$ & & 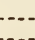 & 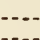 & $\cdots$ \\
\hline & & & $\vec{x}$ & $\mathrm{x}$ & $\mathbf{x}$ & $x$ & (n) & (n. & (n- \\
\hline $3 / 4$ & & & $x$ & $x$ & $x$ & $x$ & $x$ & -- & $\cdots$ \\
\hline 3. & 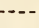 & 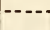 & & & $\mathrm{x}$ & $x$ & $x$ & $\cdots$ & \\
\hline 12 & & & & & $\frac{x}{x}$ & ${ }_{x}^{x}$ & $\frac{x}{x}$ & $\begin{array}{l}x \\
x\end{array}$ & 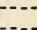 \\
\hline $114 \ldots$ & & & & & $\mathrm{x}$ & $x$ & $\vec{x}$ & $x$ & $\cdots$ \\
\hline $11 / 2$ & & & & & $x$ & $x$ & $x$ & $x$ & $x$ \\
\hline & - & $\cdots$ & . & & & $x$ & $x$ & $x$ & $x$ \\
\hline & & 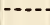 & $\ldots$ & & & & $x$ & $x$ & $x$ \\
\hline 2 & & & & & & & $\frac{x}{x}$ & $\frac{x}{x}$ & $\begin{array}{l}x \\
x\end{array}$ \\
\hline & & & & & & & & & \\
\hline
\end{tabular}


611. CASTINGS AND FORGINGS

611.0 GENERAL ITEMS.

\section{UNITED STATES GOVERNMENT, DEPART- MENT OF COMMERCE, BUREAU OF STANDARDS; STANDARD SAMPLES, CIRCULAR NO. 25 AND SUPPLEMENT, 1927}

\section{SAMPLES OF CAST IRON}

(The Bureau of Standards prepares and sells standard samples of cast iron and other materials for use by industrial organizations and others as comparison standards for checking the accuracy of analysis of cast iron, etc. Each sample is accompanied by a certificate giving the analysis of that particular sample. Samples number $4 \mathrm{~d}, 5 \mathrm{f}, 6 \mathrm{~d}, 7 \mathrm{~b}$, and 74 are cast iron, sample No. 55 is ingot iron, and sample No. 82 is nickel-chromium cast iron.)

\subsection{GRAY-IRON CASTINGS.}

\subsection{GRAY CAST IRON.}

AMERICAN BUREAU OF SHIPPING, RULES FOR BUILDING AND CLASSING STEEL VESSELS; MATERIALS FOR MACHINERY, BOILERS, AND PIPING, 1930

\section{IRON CASTINGS}

(For general information and requirements see 600.0, p. 1 . For test requirements see 600.1, p. 7.)

1. Iron castings subject to test and inspection shall be made by the cupola or other approved process. The material must be of uniform grain, free from blowholes, porous spots, or other defects. The physical characteristics of the material shall be determined by a tension-test piece (similar in outline to test piece shown in A. B. S. 600.1, p. 7.) With a sectional area of 0.5 square inches, or by arbitration bar $1 \frac{11}{4}$ inches diameter and 15 inches long.

2. The minimum tensile strength of the material as shown by the tension test shall not be less than 20,000 pounds per square inch. The minimum breaking strength of the material under transverse load as shown by the arbitration bar shall not be less than 2,500 pounds with the points of support 12 inches apart. Satisfactory evidence must be furnished the surveyor to identify the arbitration bar as representing the material to be tested.

3 . Semisteel castings subject to test and inspection shall comply with the general requirements for cast iron. The materials must have a minimum tensile strength of 28,000 pounds per square inch.

4. The number of tests for iron and semisteel castings is to be in accordance with the requirements for steel castings. (See 611.41, p.706, par. 2.)
AMERICAN ELECTRIC RAILWAY ENGINEERING ASSOCIATION, SPECIFICATION FOR MATERIALS FOR USE IN THE MANUFACTURE OF SPECIAL TRACK WORK, W104-28, 1928

(Approved as American tentative standard by the American Standards Association, January 23, 1929)

\section{SPECIAL TRACK-WORK MATERIALS}

(For gray-iron castings see W104-28, under 606.3, p. 342.)

AMERICAN RAILWAY ASSOCIATION, SIGNAL SECTION, GRAY-IRON CASTINGS, SPECIFICATION NO. 1630, JULY, 1930

\section{GRAY-IRON CASTINGS}

(Same as A. S. T. M. A48-29, given below, except for the following items:)

\section{DRA WIITGS}

Contractor shall furnish, with his tender, drawings forming an essential part thereof.

\section{TENDER}

The tender shall be for apparatus meeting the requirements of this specification. If the contractor wishes to vary from the specification, a tender may be submitted covering the apparatus he desires to furnish. This tender shall be accompanied by full information showing wherein the requirements of the specification are varied from.

\section{MATERIAL AND WORKMANSHIP}

The manufacturer's pattern number must appear on each casting and be so located that it will not wear off or interfere with the fitting of other parts to the casting.

\section{TESTS}

Tests may be made at point of production, or on samples submitted, and may also be made at destination.

Contractor shall give the purchaser sufficient notice of time when material will be ready for testing.

Contractor shall provide, at point of production, apparatus and labor for making the required tests under supervision of the purchaser.

The arbitration test bar shall conform to that shown in A. S. T. M. A124-29. (See 600.1, p. 18.)

\section{PACKING}

Material shall be so prepared as to permit convenient handling and to protect against loss or damage during shipment. 


\section{MARKIHG}

Purchaser's order, requisition, and package numbers; name of consignor; and name and address of consignee shall be plainly marked on outside of package.

Detail list of loose pieces, containers, and their contents shall be furnished for each shipment. Where carload shipments are made, routing and car identification shall be shown.

Where carload shipments are made, name and address of consignee may be omitted.

\section{WARRANTY}

Contractor shall warrant the material covered by this specification to be free from defects in material and workmanship under ordinary use and service, his obligation under this warranty being limited to making, at point of production, any part or parts to replace those which shall be found defective within one year after shipment to the purchaser. This warranty shall not apply to any apparatus which shall have been repaired or altered in any way by anyone other than the manufacturer thereof, so as to affect, in the contractor's judgment, its proper functioning or reliability, or which has been subject to misuse, negligence, or accident.

\section{AMERICAN RAILWAY ENGINEERING AS- SOCIATION, SPECIFICATIONS FOR BUILDINGS FOR RAILWAY PURPOSES}

\section{CAST IRON}

(The association indorses American Society for Testing Materials specification A48, for high-test gray-iron castings (below).)

\section{AMERICAN RAILWAY ENGINEERING AS- SOCIATION, SPECIFICATIONS FOR STEEL RAILWAY BRIDGES, 1924}

(See also 605.11, p. 249; 611.41, p. 707; and 611.51 p. 753.)

\section{CAST IRON}

(In respect to process, finish, chemical composition, classification, test bar, tension tests, number of tests, and deflection at rupture, this specification is identical with A. S. T. M., A48-29, given below. In respect to transverse tests it provides that:)

Transverse tests. - A transverse test of each bar cast shall be made. The supports shall be spaced 12 inches apart and the load applied at the middle. The load on the test bar at rupture shall be not less than the following:

$\begin{array}{ll} & \text { Pound } \\ \text { Might castings... } & \end{array}$

Heavy castings....... 3, 300

\section{AMERICAN SOCIETY FOR TESTING MATE- RIALS, STANDARD SPECIFICATIONS FOR GRAY-IRON CASTINGS, DESIGNATION A48-29, 1929}

(A48-32T has recently been issued)

\section{GRAY-IRON CASTINGS SCOPE}

1. These specifications cover three classes of grayiron castings, as follows:

(a) Light castings, those having any section less than one-half inch in thickness;

(b) Heavy castings, those in which no section is less than 2 inches in thickness;

(c) Medium castings, those not included in either of the above two classes.
BASIS OF PORCHASE

2. The tension test will be made only when specified by the purchaser and at his expense. ${ }^{84}$

\section{MANUFACTURE}

3. The castings shall be made by the cupola process, unless furnace iron is specified.

\section{CHEMICAL PROPERTIES AND TESTS}

4. (a) Drillings taken from the fractured end of the transverse test bars shall conform to the following requirements as to sulphur:

Light castings, not over. Per cent

Medium castings, not over 0.10

Heavy castings, not over....

(b) One sulphur determination shall be made for each mold of test bars cast in accordance with the standard methods of sampling and chemical analysis of pig and cast iron in A. S. T. M., A64-27. (See 601.20, p. 119). In case of dispute, the standards of the Bureau of Standards shall be used for comparison.

\section{PHYSICAL PROPERTIES AND TESTS}

5. (a) The transverse test specimens (arbitration test bars) specified in section $7(a)$, when placed horizontally upon supports 18 inches apart and tested under a centrally applied load, shall conform to the following minimum requirements, interpreted in accordance with section 9:

\begin{tabular}{l|r|r|r}
\hline & \multicolumn{3}{|c}{ Class of casting } \\
\cline { 2 - 4 } & Light & Medium & Heary \\
\hline $\begin{array}{l}\text { Load at center..........-pounds.- } \\
\text { Deflection at center........inches.- }\end{array}$ & $\begin{array}{r}1,500 \\
0.20\end{array}$ & $\begin{array}{r}1,750 \\
0.20\end{array}$ & $\begin{array}{r}2,000 \\
0.20\end{array}$ \\
\hline
\end{tabular}

(b) The rate of application of the load shall be such that a central deflection of 0.10 inch is produced in from 20 to 40 seconds.

6 . When tension tests are specified, the tensiontest specimen shall conform to the following minimum requirements as to tensile strength:

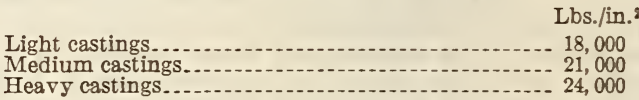

7. (a) Arbitration test bar.-The arbitration test bar shall be in accordance with the standard specifications for the arbitration test bar and tensiontest specimen for cast iron in A. S. T. M., A124-29. (See 600.1, p. 18.)

(b) Tension-test specimen.-When tension tests are specified, the tension-test specimen shall conform to the requirements specified in the standard specifications A124-29.

8. (a) Two sets of two arbitration test bars each shall be cast from each melt, one set from the first and the other set from the last iron going into the castings. Where the melt exceeds 20 tons, an additional set of two bars shall be cast for each additional 20 tons or fraction thereof. In case of a change of mixture during the melt, one set of two bars shall also be cast for every mixture other than the regular one. Each set of two bars shall be cast in a single mold.

(b) All arbitration test bars cast shall be tested as specified in section $5(a)$.

81 It is recommended by the committee that the tension test shall not be made, for the reason that cast iron is almost devoid of elasticity and hence any deviation from an absolutely straight pull in com. mercial testing machines yields inaccurate results. 
9. One arbitration test bar of each set cast shall conform to the requirements specified in section 5 (a); otherwise the castings represented by such bars shall be rejected.

\section{WORKMANSHIP AND FINISH}

10. The castings shall be true to pattern, and free from cracks, flaws, and excessive shrinkage. In other respects they shall conform to whatever points may be specially agreed upon between the manufacturer and the purchaser.

\section{INSPECTION}

(Conforms to paragraph 7 of A. S. T. M. standard specifications for steel. See 605.0, p. 247.)

\section{AMERICAN SOCIETY FOR TESTING MA- TERIALS, STANDARD METHODS OF SAMPLING AND CHEMICAL ANALYSIS OF PIG AND CAST IRON, DESIGNATION A64-27, 1927}

\section{CHEMICAL ANALYSIS OF CAST IRON}

(See 601.20, p. 119.)

AMERICAN SOCIETY FOR TESTING MATERIALS, STANDARD SPECIFICATIONS FOR HIGH-TEST GRAY-IRON CASTINGS, DESIGNATION A88-31, 1931

\section{HIGH-TEST GRAY-IRON CASTINGS}

(In respect to rate of application of transverse test load, arbitration test bar, tension-test specimen, number of tests, test requirements, and workmanship and finish, this specification is identical with A. S. T. M., A48, given above, including the references in A48 to A. S. T. M., A124. Otherwise it provides as follows:)

\section{SCOPE}

These specifications cover all classes (note) of gray-iron castings (free from massive cementite) which conform to the minimum physical requirements specified herein.

Note.-These specifications cover the so-called semisteels, pearlitic irons, special alloy irons, and any other gray irons which conform to the requirements for physical properties.

\section{MANUFACTURE}

The castings may be made by the cupola, airfurnace, electric-furnace, or any other generally approved process. Where heat treatment, heated molds, or other expedients are utilized for modification of the properties of the casting, the equivalent of these shall be used in the preparation of test bars

\section{PHYSICAL PROPERTIES AND TESTS}

(a) The transverse test specimens (arbitration test bars, see A48, given above) when placed horizontally upon supports 18 inches apart and tested under a centrally applied load, shall withstand a minimum load of 2,500 pounds and deflect under this load at least 0.24 inch at the center.

When tension tests are specified, the tension-test specimen shall show a minimum tensile strength of 33,000 pounds per square inch.

When a large casting is made for which test bars are required, the test bar shall be cast from the same ladle of iron used to pour the casting. If two ladles are used for pouring the casting, the iron for the test bars shall be poured from a ladle of iron caught between the pouring of these two ladles. either case the test bars shall be poured at as near as practicable the same temperature at which the casting is poured.

(Conforms to paragraph 7 of A. S. T. M. standard specifications for steel. See 605.0, p. 247.)

\section{AMERICAN SOCIETY FOR TESTING MA- TERIAIS, STANDARD SPECIFICATIONS FOR THE ARBITRATION TEST BAR AND TENSION-TEST SPECIMEN FOR CAST IRON, DESIGNATION A124-29, 1929}

\section{CAST-IRON TEST SPECIMEN}

(See 600.1, p. 18.)

\section{AMERICAN SOCIETY FOR TESTING MA. TERIALS, STANDARD SPECIFICATIONS FOR GRAY-IRON CASTINGS FOR VALVES, FLANGES, AND PIPE FITTINGS, DESIGNA- TION A126-30, 1930}

\section{GRAY-IRON CASTINGS FOR VALVES \\ SCOPE}

1. These specifications cover gray iron for castings such as valve bodies, fittings, flanges, including parts to be assembled into valves, manufactured in advance and supplied for sale from stock by the manufacturer, jobber, or other dealer.

Two classes of castings are included as follows: Class A.-Regular gray iron.

Class B.-Higher-strength gray iron including the so-called semisteels used in valves and fittings.

\section{MANUFACTURE}

2. The iron shall be made by the cupola, airfurnace, electric-furnace, or other approved process. The iron shall be produced under regular chemical and physical control and shall be gray iron of high quality.

\section{CHEMICAL PROPERTIES AND TESTS}

3. Drillings taken from test ingots, broken test specimens, or from castings shall conform to the following requirements as to chemical composition:

Per cent

Sulphur, not over 0.12

Phosphorus, not over

\section{PHYSICAL PROPERTIES AND TESTS}

4. (a) The tension-test specimen shall conform to the following requirements as to tensile strength:

Class A, regular gray iron.....Lbs./in.2... Not less than 21,000 Class $B$, higher-strength gray iron...do... Not less than 30,000

(b) After reaching a stress of 15,000 pounds per square inch, the speed of the crosshead of the testing machine shall not exceed one-eighth inch per minute.

5. (a) The transverse test shall be optional. When transverse tests are made, the transversetest specimen, when placed horizontally upon supports 12 inches apart and tested under a centrally applied load, shall conform to the following requirements:

\begin{tabular}{|c|c|c|}
\hline & $\begin{array}{c}\text { Regular gray } \\
\text { iron }\end{array}$ & $\begin{array}{l}\text { Higher-strength } \\
\text { gray iron }\end{array}$ \\
\hline $\begin{array}{l}\text { Load at center......... pounds.. } \\
\text { Deflection at center.......inches.- }\end{array}$ & $\begin{array}{l}\text { Not less than } \\
2,500 . \\
\text { Not less than } \\
0.10 \text {. }\end{array}$ & $\begin{array}{l}\text { Not less than } \\
3,300 \text {. } \\
\text { Not less than } \\
0.12 \text {. }\end{array}$ \\
\hline
\end{tabular}


(b) The rate of application of the load shall be such that a central deflection of 0.10 inch is produced in from 20 to 40 seconds.

(c) In case the transverse-test specimen varies from the specified diameter of 1.20 inches, a correction factor conforming to the following shall be applied:

(For table of diameters of transverse-test specimens and correction factors see A. S. T. M., A12429. See 600.1, p. 18.)

6 . One mold containing one or more tension-test specimens and, when desired, one transverse-test specimen, made as indicated in Figure 393 shall be poured at least twice a day from each heat from which castings are made under these specifications. The molds for these test specimens shall be made from plate pattern, on a standard molding machine if possible, from the same lot of sand and in the same general way as that in which molds for the castings are made. Each specimen shall be marked to show cupola or furnace number and date and hour when

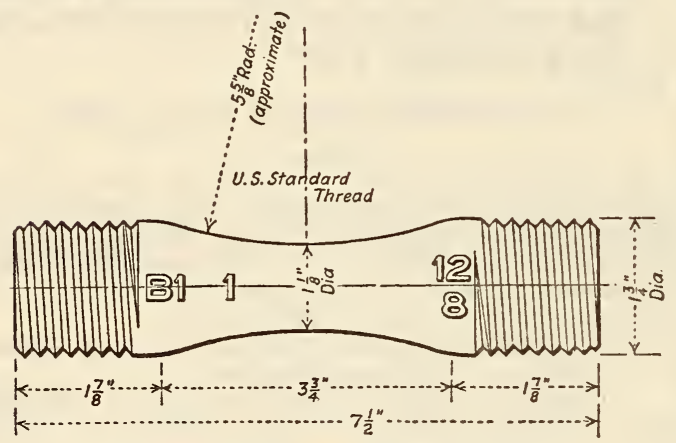

Figure 393.-Tension test specimen

the mold is poured by either casting in the mold or stamping the specimens as soon as possible after shaking out.

Note.-The numbering shown on the test specimens in Figures 392 and 393 is intended simply to illustrate a method of designation. In the particular method shown 128 refers to December $8, B 1$ is the cupola number, and the numeral 1 which follows shows the hour cast (1 p. m.). (For fig. 392 see insert facing p. 69.)

7. Tension-test specimens, cast as illustrated in Figure 392, shall conform to the dimensions shown in Figure 393, shall be reasonably smooth and round at breaking section, and shall be tested without machining except that the enlarged ends shall be threaded (13/4-inch. U.S. standard threads are recommended). The cross-sectional area at the breaking section shall be within \pm 5 per cent of 1 square inch and the ultimate stress shall be calculated on the minimum cross section.

8. Ball-and-socket specimen holders or sphericalseated bearings or other device which will insure that the specimen, when under load, will be as nearly as possible in pure axial tension without transverse stress shall be used in making the tension test.

NotE.-Suitable ball-and-socket specimen holders and sphericalseated bearing devices are shown in Figures 30 and 32 and described in section 13 of the tentative methods of tension testing of metallic materials in A. S. T. M., E8-27T (see 600.1, p. 34).

9. Records of the chemical composition of the iron and physical properties of the test specimens shall be systematically made and maintained.

\section{WORKMANSHIP AND FINISE}

10. The castings shall be sound, clean, free from sand, of workmanlike finish, and soft enough to machine well.

\section{CERTIFICATION}

11. Upon request of the purchaser, the manufacturer shall be prepared to certify that his product conforms to the requirements of these specifications.

\section{EXPLANATORY NOTE}

NotE.-Reference may be made to the dimensional standards for pressures and temperatures formulated by the sectional committee on standardization of pipe flanges and fittings functioning under the procedure of the American Standards Association, A. S. A. Project No. B16, and more specifically to American Standards B1fia-1928 B16b-1928, and B16b1, referring respectively to the 125,250 , and 800 pounds per square inch cast-iron pipe flanges and flanged-fittings standards (see 607.14 , pp. $385,395,401$ ).

\section{UNITED STATES GOVERNMENT, FEDERAI SPECIFICATIONS BOARD, FEDERAL SPE- CIFICATION FOR HIGH-TEST GRAY-IRON CASTINGS (SEMISTEEL), QQ-I-656, FEB- RUARY 2, 1932}

\section{HIGH-TEST GRAY-IRON CASTINGS}

(This specification is the same as Federal specification QQ-I-651 given below, with the exceptions noted herewith.)

(Under material and workmanship, specifiication QQ-I-656 includes the following:) Steel scrap may be added to the melt.

(Detail requirements in Federal specification QQ-I-656 are as follows:)

\section{DETAIL REQUIREMENTS}

4-A. The chemical requirements shall be as shown in Table 1.

B. When not otherwise stated in the contract or purchase order, the requirements for transverse strength shown in Table 1 shall be complied with.

TABLE 1

\begin{tabular}{c|r|r|r|r}
\hline Class & Chemical & \multicolumn{2}{|c|}{ Transverse test } & $\begin{array}{c}\text { Tension } \\
\text { test }\end{array}$ \\
\cline { 2 - 3 } & $\begin{array}{c}\text { Sulphur } \\
\text { maximum }\end{array}$ & $\begin{array}{c}\text { Load at } \\
\text { center }\end{array}$ & $\begin{array}{r}\text { Deflec- } \\
\text { tion at } \\
\text { center }\end{array}$ & $\begin{array}{r}\text { Minimum } \\
\text { pounds per } \\
\text { square inch }\end{array}$ \\
\cline { 2 - 5 } & Per cent & Pounds & Inches & \\
Light castings.......... & 0.11 & 2,100 & 0.24 & 28,000 \\
Medium castings...... & .11 & 2,250 & .24 & 30,000 \\
Heavy castings....... & .11 & 2,400 & .24 & 35,000 \\
\hline
\end{tabular}

(Under Method of Inspection and Tests, Federal specification QQ-I-656 contains the following requirement in addition to those given in Federal specification QQ-I-651.)

When required by the contract or purchase order a sample of the iron shall be taken from the first ladle of metal poured and shall be chilled in a castiron mold as shown in Figure 394. The sample shall be allowed to cool in the mold until it is dark red or almost black, when it shall be knocked out and quenched in water. This chill-test specimen, on being broken, shall show a close-grain gray iron, with a well-defined border of white iron at the bottom of the fracture. The depth of the white iron shall not be less than one-sixteenth inch as measured at the center line. 

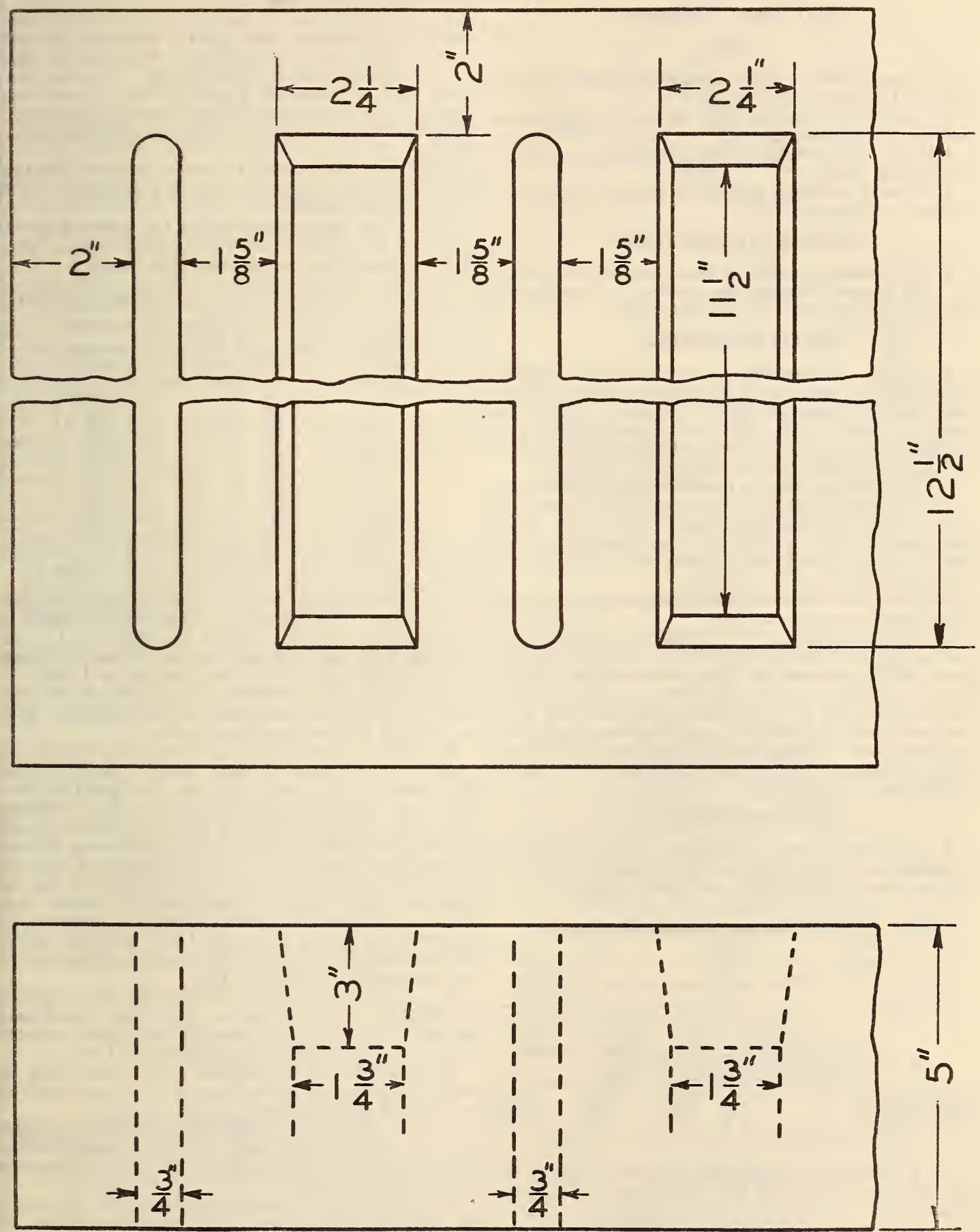

FigURE 394.-Mold for chill test specimen 
UNITED STATES GOVERNMENT, FEDERAL SPECIFICATIONS BOARD, FEDERAL SPECIFICATION FOR GRAY-IRON CASTINGS, QQ-I-651, FEBRUARY 2, 1932

\section{GRAY-IRON CASTINGS}

\section{CLASSES}

1. There shall be three classes of gray-iron castings, as follows:

A. Light castings, having a section less than onehalf inch in thickness.

B. Medium castings, falling outside the remaining classes, and

C. Heavy castings, having no section less than 2 inches in thickness.

\section{MIATERIAL AND WORKMANSHIP}

2. The castings shall be made from iron melted by any process, unless the process is specifically stated in the contract or purchase order.

\section{GENERAL REQUIREMENTS}

3-A. The castings shall be of uniform quality and condition, free from blowholes, porous places, hard spots, shrinkage defects, cracks, or other injurious defects, and shall be smooth and well cleaned before inspection by sand blasting, tumbling, pickling, or other approved process.

B. All castings shall be commercially machinable.

C. Castings shall not be repaired, plugged, or welded without permission from the inspector. Such permission will be given only when the defects are small and do not adversely affect the strength, use, or machinability of the casting.

D. Distinctive marks shall be placed around welds prior to shipment of castings.

E. The responsibility of furnishing castings that can be laid out and machined to the finished dimensions within the specified tolerances as shown on the drawings, and that will conform to such gages as may be specified in individual cases, shall rest with the contractor. Sufficient stock shall be allowed for shrinkage and, where required, for finishing. Castings unreasonably in excess of the specified size or weight may be rejected.

\section{DETAIL REQUIREMENTS}

4-A. Unless otherwise specified in the contract or purchase order, the chemical and physical properties of gray iron castings shall conform to Table 1.

TABLE 1.-Chemical and physical requirements

\begin{tabular}{|c|c|c|c|c|}
\hline \multirow[b]{2}{*}{ Class } & \multirow{2}{*}{$\frac{\text { Chemical }}{\substack{\text { Sulphur } \\
\text { maxi- } \\
\text { mum) }}}$} & \multicolumn{2}{|c|}{ Transverse test } & \multirow{2}{*}{ 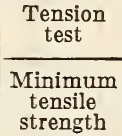 } \\
\hline & & $\begin{array}{l}\text { Load at } \\
\text { center } \\
\text { min. }\end{array}$ & $\begin{array}{c}\text { Deflection } \\
\text { at center } \\
\text { min. }\end{array}$ & \\
\hline $\begin{array}{l}\text { Light castings......... } \\
\text { Medium castings } \\
\text { Heavy castings }\end{array}$ & $\begin{array}{r}\text { Per cent } \\
0.11 \\
.11 \\
.11\end{array}$ & $\begin{array}{r}\text { Pounds } \\
1,5000 \\
1,750 \\
2,000\end{array}$ & $\begin{array}{c}\text { Inch } \\
0.20 \\
.20 \\
.20\end{array}$ & $\begin{array}{r}\text { Lbs./in. }{ }^{2} \\
18,000 \\
21,000 \\
24,000\end{array}$ \\
\hline
\end{tabular}

C. An analysis of each melt shall be furnished by the contractor showing the percentage of sulphur present.

\section{METHOD OF INSPECTION AND TESTS}

5-A. Transverse test bars shall have the form and dimensions of the arbitration test bar for cast iron as shown in Figure 13. The mold may be made in cores or in dry sand, but in either case it shall be made in accordance with Figure 13 as to the amount of sand as well as the dimension of the bars.
When the mold is made in dry sand the flask shall be rammed up with green molding sand-a little damper than usual, well mixed and put through a No. 8 sieve, with a mixture of 1 to 12 bituminous facing. The mold shall be rammed evenly and fairly hard, thoroughly dried, and not cast until it is cold. The bottom of the bar shall be 0.05 inch smaller in diameter than the top to allow for draft and for the strain of pouring. The pattern shall not be rapped before withdrawing. The test bars shall not be removed from the mold until cold enough to be handled. It shall not be rumbled or otherwise treated, being simply brushed off before testing.

B. The pattern shall be made of such size that the arbitration test bar will have a diameter of 1.20 inches at the center.

C. In case the transverse-test bar varies from the specified diameter of 1.20 inches, a correction factor shall be applied in accordance with Table 2 .

\section{TABLE 2.-Arbitration test bar correction factors}

\begin{tabular}{|c|c||c|c|}
\hline $\begin{array}{c}\text { Diameter } \\
\text { of arbitra- } \\
\text { tion test } \\
\text { bar (inches) }\end{array}$ & $\begin{array}{c}\text { Correction } \\
\text { factor }\end{array}$ & $\begin{array}{c}\text { Diameter } \\
\text { of arbitra- } \\
\text { tion test } \\
\text { bar (inches) }\end{array}$ & $\begin{array}{c}\text { Correction } \\
\text { factor }\end{array}$ \\
\cline { 1 - 2 } 1.10 & 0.770 & 1.21 & 1.025 \\
1.11 & 791 & 1.22 & 1.051 \\
1.12 & .813 & 1.23 & 1.077 \\
1.13 & .835 & 1.24 & 1.103 \\
1.14 & .857 & 1.25 & 1.130 \\
1.15 & .880 & 1.26 & 1.158 \\
1.16 & .903 & 1.27 & 1.185 \\
1.17 & .927 & 1.28 & 1.214 \\
1.18 & .951 & 1.29 & 1.242 \\
1.19 & .975 & 1.30 & 1.271 \\
1.20 & 1.000 & & \\
\hline
\end{tabular}

D. If the test bar is not round the correction factor can not be obtained from Table 2 and shall be calculated as follows: Square the depth of the bar measured at point of application of load, multiply by the width, and divide the product by 1.728 .

E. In order to correct to a diameter of 1.20 inch the breaking load obtained in testing, the bar shall be divided by the correction factor.

F. When physical tests are required two sets of transverse-test bars ( 2 bars per set) shall be cast from each melt, one set from the first and the other set from the last iron going into the castings. Where the melt exceeds 20 tons, an additional set of two bars shall be cast for each additional 20 tons or fraction thereof. In case of a change of mixture during the melt, one set of two bars shall also be cast for every mixture other than the regular one. Each set of two bars shall be cast in a single mold as shown in Figure 13. One transverse-test bar of each cast shall conform to the requirements specified in paragraphs $5-\mathrm{G}$ and $5-\mathrm{H}$.

$\mathrm{G}$. The transverse-test specimens, when placed horizontally upon supports 18 inches apart and tested under a centrally applied load, shall conform to the minimum requirements shown in Table 1 .

$H$. The rate of application of the load shall be such that a central deflection of 0.10 inch is produced in from 20 to 40 seconds.

I. The tension-test specimen may be turned from any of the broken pieces of the transverse-test specimens and shall conform to the dimensions shown in Figure 14.

$K$. Check analyses may be made by the inspector, or through him by any Government laboratory or other designated representative, and without cost to the contractor. The chemical composition thus determined shall conform to the requirements specified.

L. Umpire method of analysis.- Suitable methods for umpire analysis are being developed and will be added in a later revision. 


\section{PACKING AND MARKING}

6-A. Large castings shall be stamped with the melt or lot number and, where practicable, with the pattern number.

B. Small castings shall be properly separated by melts or lots when presented for inspection and when shipped and shall be so marked that their identity will be clear at the point of delivery.

C. A lot shall consist of castings poured from the same melt, unless otherwise mutually agreed upon by the contractor and the inspector.

D. If special packing be necessary to secure the acceptance of castings by common or other carriers for safe transportation at the lowest rate to the point of delivery, such packing shall be provided by the contractor without additional expense to the purchaser.

\section{ADDITIONAL INFORMATION}

7-A. All patterns furnished by the purchaser shall be properly cared for, and when being returned to the purchaser shall be suitably boxed or crated by the contractor to prevent damage or breakage. Before shipment from the foundry, patterns shall be inspected by the representative of the purchaser and report made by him if found to be in unsatisfactory condition.

B. All purchase orders must state the physical test desired; it is intended to test the material for physical properties and to reject castings for failure to comply with the physical requirements.

611.12 MANHOLE AND CONDUIT CASTINGS.

AMERICAN RAILWAY ASSOCIATION, TELEGRAPH AND TELEPHONE SECTION, COMMUNICATION UNDERGROUND CONDUIT CONSTRUCTION, 1-C-1, 1927

\section{MANHOLE AND HANDHOLE FRAMES AND COVERS}

Manhole and handhole frames and covers should be of cast iron or other approved material.

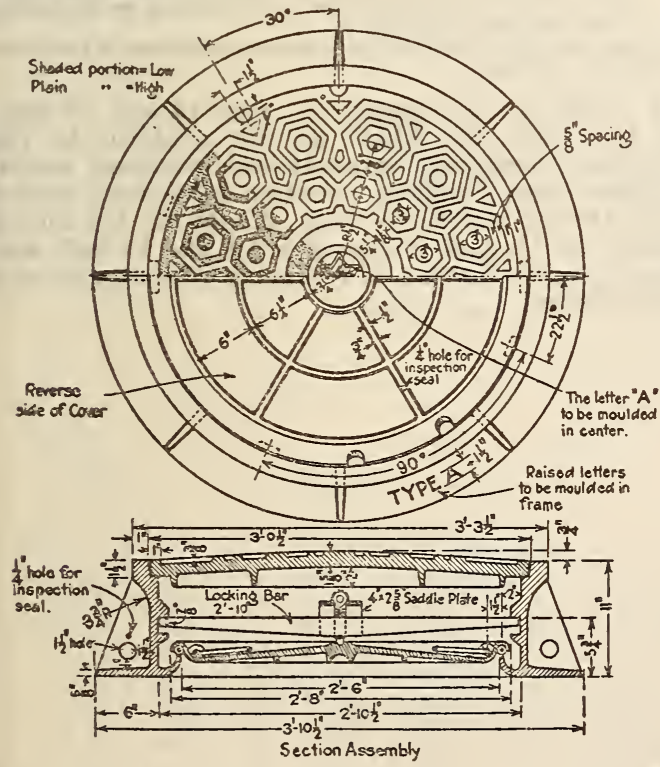

FIgUREs 395 and 396.-Manhole frame and cover, 30inch opening, type $A$

Three standard types of manhole frames and covers are shown in the accompanying illustrations. (See figs. 395, 399, and 400)
Type A frames, which are equipped with double covers, should only be used in special situations
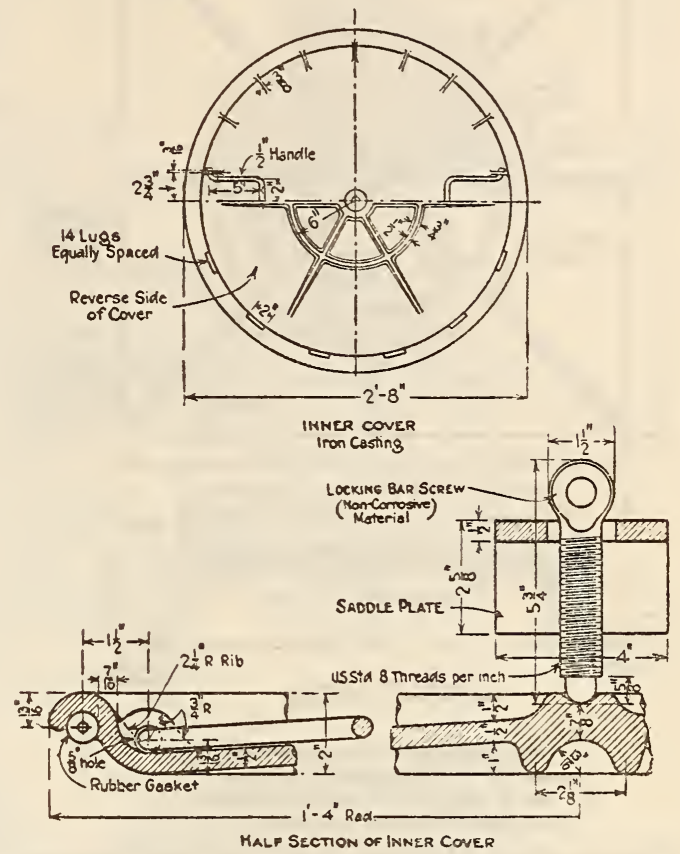

Figures 397 and 398.-Manhole inner cover, type $A$

where required by local regulations or municipal ordinances.

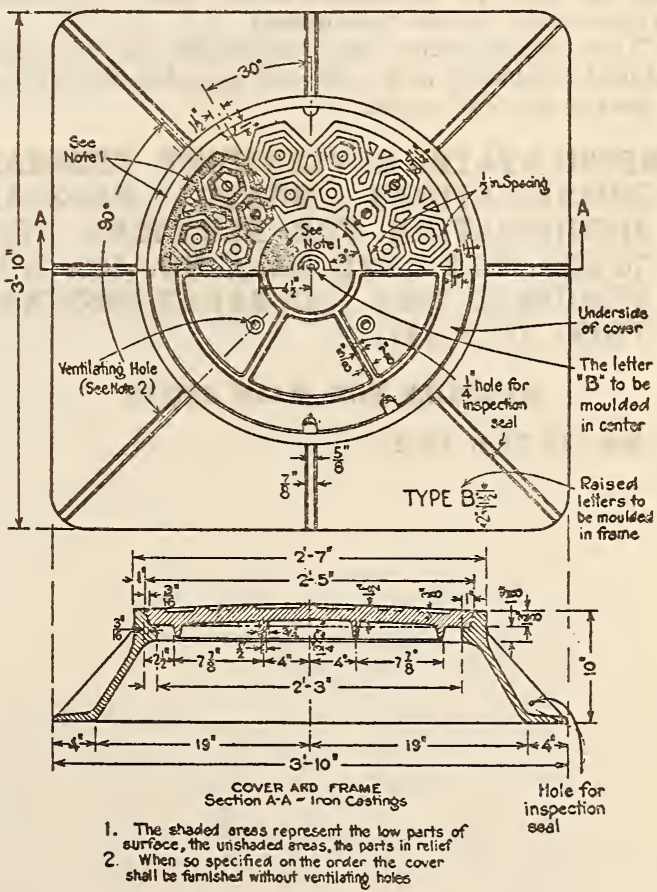

FiguRE 399.-Manhole frame and cover, 27-inch opening, type $B$

Type B frames and covers should be used in city construction or in other cases where the manhole covers will be subjected to heavy loads. 


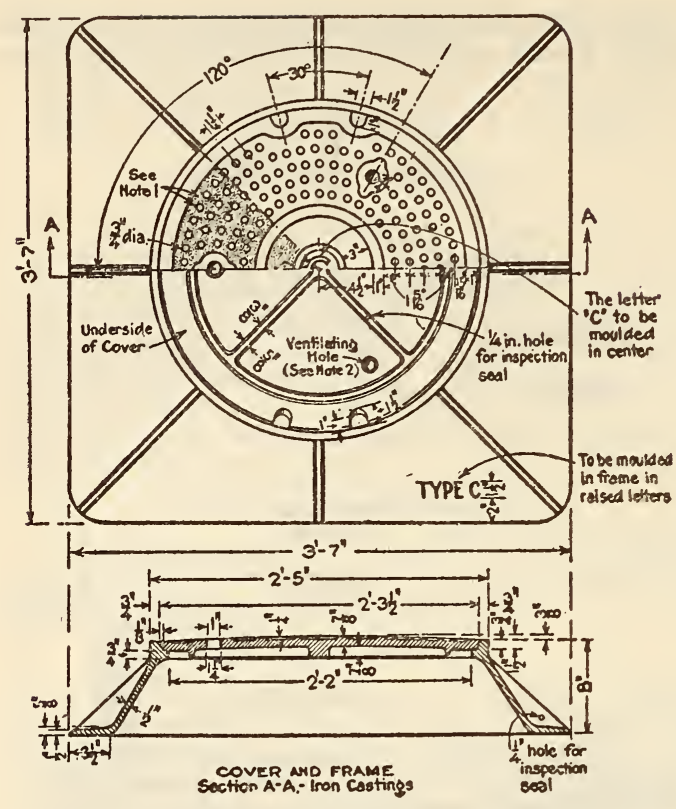

1 The shoded oreas reprecent the low ports of surface, the unshadod ereas the parts in relief.

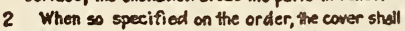
be furnished without ventilating holes.

Figure 400.- Manhole frame and cover, 26-inch opening, type $C$

Type $\mathbf{C}$ frames and covers, or type $\mathrm{C}$ covers resting upon special cast-iron rings set in concrete collars, may be used in situations where only very light service loads will be experienced.

(This specification also provides for cast-iron reduced couplings and cast-iron caps for connecting $\mathbf{U}$ guards to pipe bends.)

UNITED STATES GOVERNMENT, FEDERAL
SPECIFICATIONS BOARD, FEDERAL SPECIFICATION FOR PLUMBING FIXTURES (FOR LAND USE), NO. 448, NOVEMBER 22, 1926. (CURRENT DESIGNATION ; FF-P-451)

\section{MANHOLE FOR MAIN SEWER}

(See 617.73, p. 1062.)
611.13 SLUICE GATES AND MOORING BITTS. UNITED STATES GOVERNMENT, DEPARTMENT OF COMMERCE, AMERICAN MARINE STANDARDS COMMITTEE, MOORING BITTS, CAST IRON, H NO. 21925

\section{MOORING BITTS}

\section{GENERAL NOTES}

1. Where this type of bitt, in excess of the 7-inch size, is intended for towing, the next size larger than required for mooring should be installed.

2. The material is to be gray-iron casting, in accordance with the specifications of the American Bureau of Shipping. (Sec. 40, par. 9, of the bureau's rules.) Inspection by the bureau shall be required only when specified in the purchase order.

3. Holes for fastenings may be cored and reamed to size, or they may be drilled as preferred.

4. The countersink for the heads of the fastenings is to have an angle of $221^{\circ}$ ( $45^{\circ}$ center angle) and a depth equal to half the diameter of the fastening. 5. The bottom faying surfaces of castings shall be rough planed for flat surfaces, or chipped to fit uneven surfaces.

6. The wearing surfaces where rough are to be chipped or ground smooth.

7. The numbers and diameters of rivets or bolts are in accordance with the British standard No. 3005-1922, from which the following rules in regard thereto are quoted:

Number of rivets or bolts $=\frac{A}{2}+3$ :

Where the result is an odd number, the nearest lower even num ber to be used.

Diameter of iron rivets in eighths of an inch $=\frac{\mathrm{A}}{2}+4$ :

Where the result is not a whole number the nearest one-eighth below to be used, but no rivet to be less than seven-eighths, except for 4 -inch bollards.

If bolts are used they should be of the same diameter as the rivets but should be of steel.

8. There are two cases, viz, sizes 14 and 18 inch, the bolt sizes for which do not conform to the American (national) screw-thread standard series. In those cases if bolts are desired to conform to the screw-thread standards they should be $1 \frac{1}{2}$ inches instead of $13 / 8$ inches diameter for the 14 -inch size and $13 / 4$ inches instead of $15 \%$ inches diameter for the 18-inch size. 

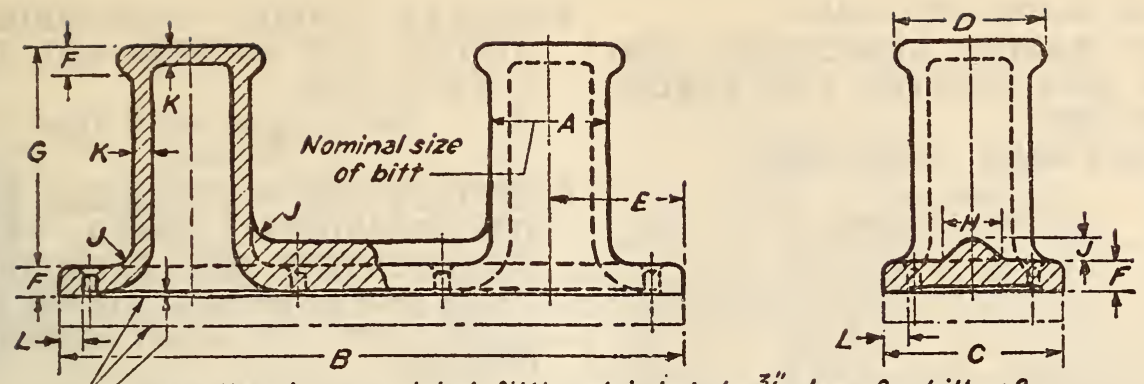

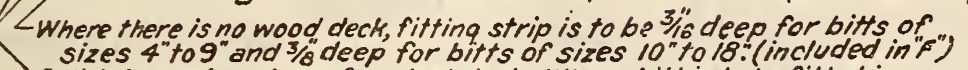

solid lines show base for steel deck. Where bitt is to be filted in way of wood deck, the depth of base shall equal at least the thickness of deck plus/:.
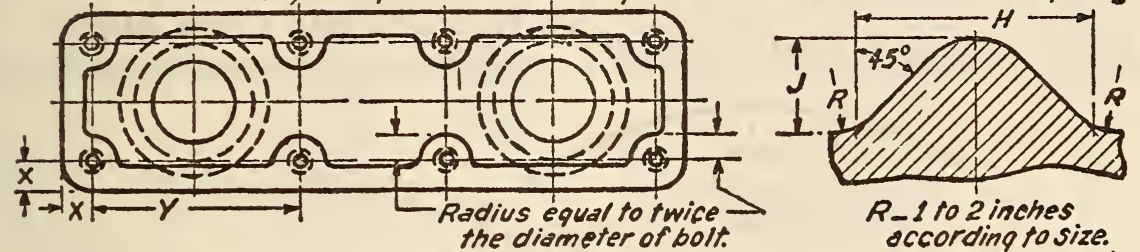

FIGURE 401.-Mooring bitts, cast-iron
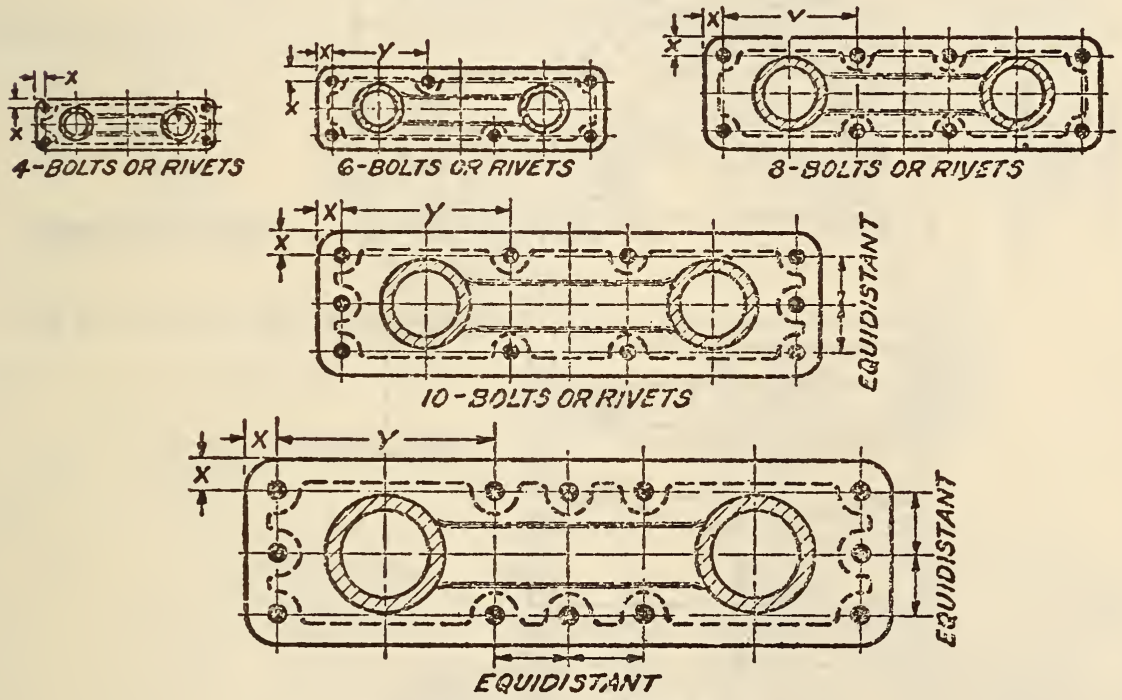

12-BOLTS OR AIVETS

Pivets may be of Iron but bolrs should be of steel.

Figure 402.-Mooring bitts, cast-iron

[All dimensions in inches]

\begin{tabular}{|c|c|c|c|c|c|c|c|c|c|c|c|c|c|c|}
\hline \multirow{3}{*}{$\begin{array}{c}\text { Size } \\
\text { A }\end{array}$} & \multirow{3}{*}{ B } & \multirow{3}{*}{ C } & \multirow{3}{*}{ D } & \multirow{3}{*}{$\mathrm{E}$} & \multirow{3}{*}{ F } & \multirow{3}{*}{ G } & \multirow{3}{*}{ H } & \multirow{3}{*}{$\mathbf{J}$} & \multirow{3}{*}{ K } & \multirow{3}{*}{$\mathbf{L}$} & \multicolumn{4}{|c|}{ Bolts or rivets } \\
\hline & & & & & & & & & & & \multirow{2}{*}{$\underset{\text { ber }}{\text { Num }}$} & \multirow{2}{*}{$\begin{array}{c}\text { Diam- } \\
\text { eter }\end{array}$} & \multicolumn{2}{|c|}{ Spacing } \\
\hline & & & & & & & & & & & & & $\mathrm{X}$ & $\mathrm{Y}$ \\
\hline $\begin{array}{l}4 \\
5 \\
6 \\
7\end{array}$ & $\begin{array}{l}21 \\
261 / 4 \\
311 / 2 \\
37\end{array}$ & $\begin{array}{l}6 \\
71 / 2 \\
9 \\
101 / 2\end{array}$ & $\begin{array}{l}5 \\
61 / 4 \\
71 / 2 \\
83 / 4\end{array}$ & $\begin{array}{l}41 / 2 \\
5 \% 8 \\
63 / 4 \\
77 / 8\end{array}$ & $\begin{array}{l}1 \\
11 / 4 \\
11 / 2 \\
13 / 4\end{array}$ & $\begin{array}{r}71 / 2 \\
93 / 8 \\
111 / 4 \\
131 / 8\end{array}$ & $\begin{array}{l}2 \\
21 / 2 \\
3 \\
31 / 2\end{array}$ & $\begin{array}{l}13 / 16 \\
1^{13 / 16} \\
138\end{array}$ & $\begin{array}{c}5 / 8 \\
3 / 4 \\
15 / 16 \\
11 / 16\end{array}$ & $\begin{array}{r}3 / 4 \\
7 / 8 \\
118 \\
11 / 4\end{array}$ & $\begin{array}{l}4 \\
4 \\
6 \\
6\end{array}$ & $\begin{array}{l}3 / 4 \\
7 / 8 \\
7 / 8 \\
7 / 8\end{array}$ & $\begin{array}{l}1 \\
11 / 4 \\
11 / 2 \\
13 / 4\end{array}$ & $\begin{array}{l}101 / 2 \\
121 / 4\end{array}$ \\
\hline $\begin{array}{r}8 \\
9 \\
10 \\
11\end{array}$ & $\begin{array}{l}42 \\
471 / 4 \\
521 / 2 \\
573 / 4\end{array}$ & $\begin{array}{l}12 \\
131 / 2 \\
15 \\
161 / 2\end{array}$ & $\begin{array}{l}10 \\
111 / 4 \\
121 / 2 \\
133 / 4\end{array}$ & $\begin{array}{c}9 \\
1018 \\
1134 \\
123 \%\end{array}$ & $\begin{array}{l}2 \\
21 / 4 \\
21 / 2 \\
23 / 4\end{array}$ & $\begin{array}{l}15 \\
16^{7 / 8} \\
183 / 4 \\
205 / 8\end{array}$ & $\begin{array}{l}4 \\
41 / 2 \\
5 \\
51 / 2\end{array}$ & $\begin{array}{l}15 / 8 \\
1^{13 / 16} \\
2 \\
21 / 4\end{array}$ & $\begin{array}{l}13 / 16 \\
13 / 8 \\
11 / 2 \\
15 / 8\end{array}$ & $\begin{array}{l}11 / 2 \\
15 / 8 \\
13 / 4 \\
2\end{array}$ & $\begin{array}{l}6 \\
6 \\
8 \\
8\end{array}$ & $\begin{array}{l}1 \\
1 \\
118 \\
11 / 8\end{array}$ & $\begin{array}{l}2 \\
21 / 4 \\
21 / 2 \\
23 / 4\end{array}$ & $\begin{array}{l}14 \\
1534 \\
171 / 2 \\
191 / 4\end{array}$ \\
\hline $\begin{array}{l}12 \\
14 \\
16 \\
18\end{array}$ & $\begin{array}{l}63 \\
731 / 2 \\
84 \\
941 / 2\end{array}$ & $\begin{array}{l}18 \\
21 \\
24 \\
27\end{array}$ & $\begin{array}{l}15 \\
171 / 2 \\
20 \\
221 / 2\end{array}$ & $\begin{array}{l}131 / 2 \\
153 / 4 \\
18 \\
201 / 4\end{array}$ & $\begin{array}{l}3 \\
31 / 2 \\
4 \\
41 / 2\end{array}$ & $\begin{array}{l}221 / 2 \\
2614 \\
30 \\
333 / 4\end{array}$ & $\begin{array}{l}6 \\
7 \\
8 \\
9\end{array}$ & $\begin{array}{l}23 / 8 \\
2^{13 / 16} \\
33 / 16 \\
35 / 8\end{array}$ & $\begin{array}{l}113 / 16 \\
21 / 8 \\
23 / 8 \\
2^{11} 1 / 16\end{array}$ & $\begin{array}{l}23 / 16 \\
21 / 2 \\
27 / 8 \\
31 / 4\end{array}$ & $\begin{array}{r}8 \\
10 \\
10 \\
12\end{array}$ & $\begin{array}{r}114 \\
113 / 8 \\
11 / 2 \\
1158\end{array}$ & $\begin{array}{l}3 \\
31 / 2 \\
4 \\
41 / 2\end{array}$ & $\begin{array}{l}21 \\
241 / 2 \\
28 \\
311 / 2\end{array}$ \\
\hline
\end{tabular}

See Figure 402 for arrangement of fastenings.

1 See General Note 8. 
611.14 FLOOR DRAINS AND TRAPS.

AMERICAN MARINE STANDARDS COMMITTEE, SHIP SCUPPER AND DRAINS, H NO. 36-1928

\section{DECK DRAIN, PLAIN TYPES}

(See fig. 403)

[All dimensions in inches]

\begin{tabular}{|c|c|c|c|c|c|c|c|c|c|c|c|c|c|}
\hline $\begin{array}{c}\text { Size } \\
\text { A }\end{array}$ & B & C & D & $\mathrm{E}^{1}$ & $\mathrm{~F}$ & $G$ & H & $\mathbf{J}$ & $\mathrm{K}$ & L & $\mathrm{M}$ & $\mathrm{N}$ & $P$ \\
\hline $2^{11 / 2}$ & $\begin{array}{l}6 \\
7\end{array}$ & $\begin{array}{l}43 / 4 \\
51 / 2\end{array}$ & $\begin{array}{l}13 / 8 \\
13 / 8\end{array}$ & $\begin{array}{l}5 / 8 \\
11 / 16\end{array}$ & $\begin{array}{l}31 / 4 \\
4\end{array}$ & $\begin{array}{l}31 / 8 \\
37 / 8\end{array}$ & $\begin{array}{l}25 / 8 \\
33 / 8\end{array}$ & $\begin{array}{l}5 / 16 \\
11 / 32\end{array}$ & $\begin{array}{l}1118 \\
11 / 2\end{array}$ & $\begin{array}{l}111 / 16 \\
27 / 16\end{array}$ & $\begin{array}{l}25 / 8 \\
33 / 8\end{array}$ & $\begin{array}{l}2 \\
21 / 2\end{array}$ & $\begin{array}{c}21 / 2 \\
3\end{array}$ \\
\hline${ }_{3}^{21 / 2}$ & $\begin{array}{l}71 / 2 \\
81 / 2\end{array}$ & $\begin{array}{l}6 \\
7\end{array}$ & $\begin{array}{l}17 / 8 \\
178\end{array}$ & $\begin{array}{l}3 / 4 \\
13 / 16\end{array}$ & $\begin{array}{l}41 / 2 \\
51 / 2\end{array}$ & $\begin{array}{l}438 \\
53 / 8\end{array}$ & $\begin{array}{l}37 / 8 \\
478\end{array}$ & $\begin{array}{l}3 / 8 \\
13 / 32\end{array}$ & $\begin{array}{l}13 / 4 \\
21 / 4\end{array}$ & $\begin{array}{l}215 / 16 \\
3^{15 / 16}\end{array}$ & $\begin{array}{l}37 / 8 \\
47 / 8\end{array}$ & \begin{tabular}{|l|}
3 \\
$35 / 8$
\end{tabular} & $\begin{array}{l}35 / 8 \\
43 / 8\end{array}$ \\
\hline
\end{tabular}

1 Composition may be substituted for galvanized cast iron with thicknesses of flanges reduced to the following: E 1/2, 9/16, 5/8, 11/16.
AMERICAN MARINE STANDARDS COMMITTEE, SHIP SCUPPER AND DRAINS, H. NO. 37-1928

\section{DECK DRAIN, TRAP TYPE}

(See fig. 404)

UNITED STATES GOVERNMENT, FEDERAL SPECIFICATIONS BOARD, FEDERAL SPECIFICATION FOR PLUMBING FIXTURES (FOR IAND USE), NO.448, NOVEMBER 22, 1926. (CURRENT DESIGNATION : FF-P-451)

\section{DRAINS AND TRAPS}

(See 617.73 , o. 1062.)

611.15 GRATES AND GRATE BARS.

(No nationally recognized specifications available.)
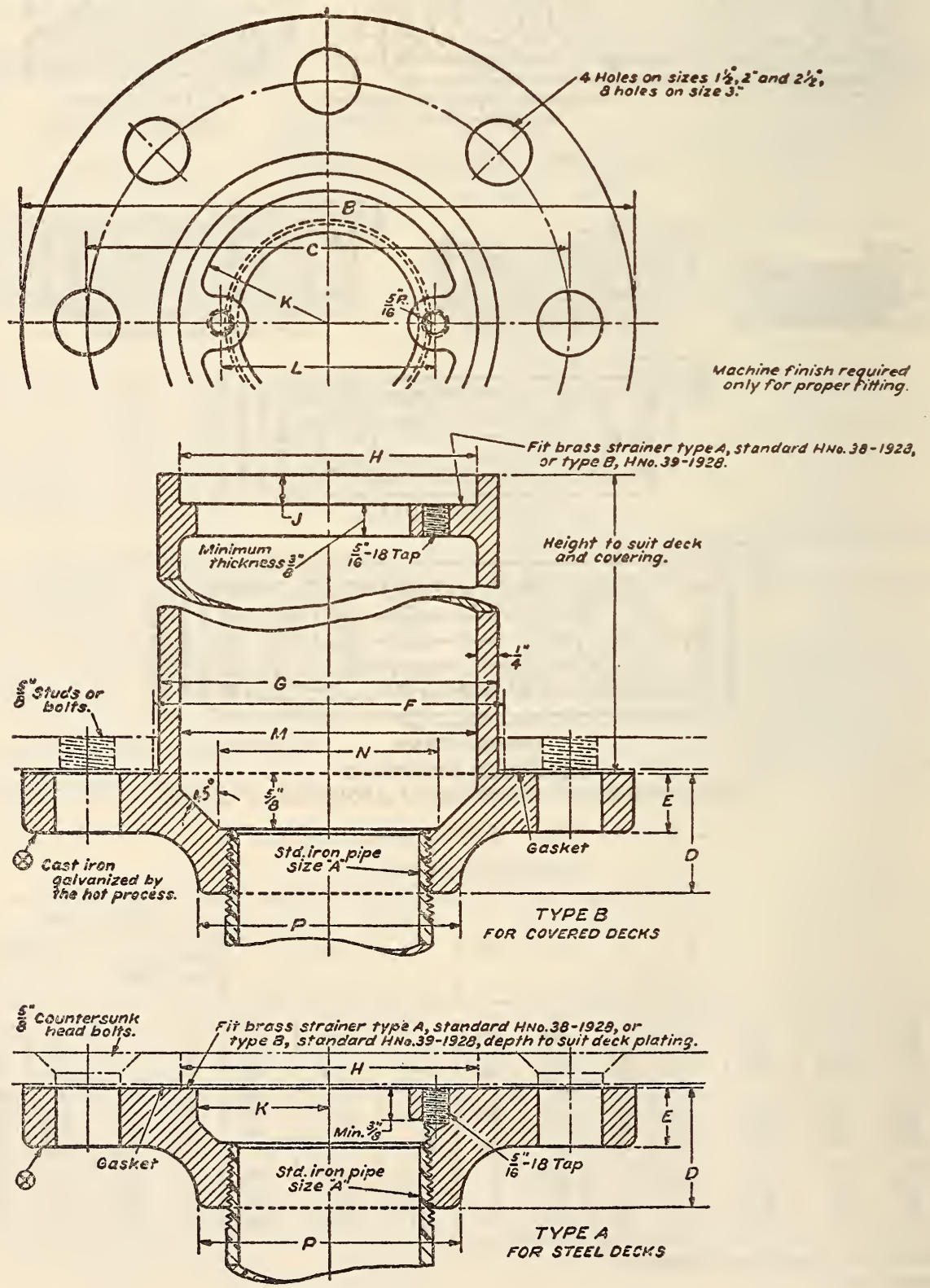

Figure 403.-Deck drain, plain type 


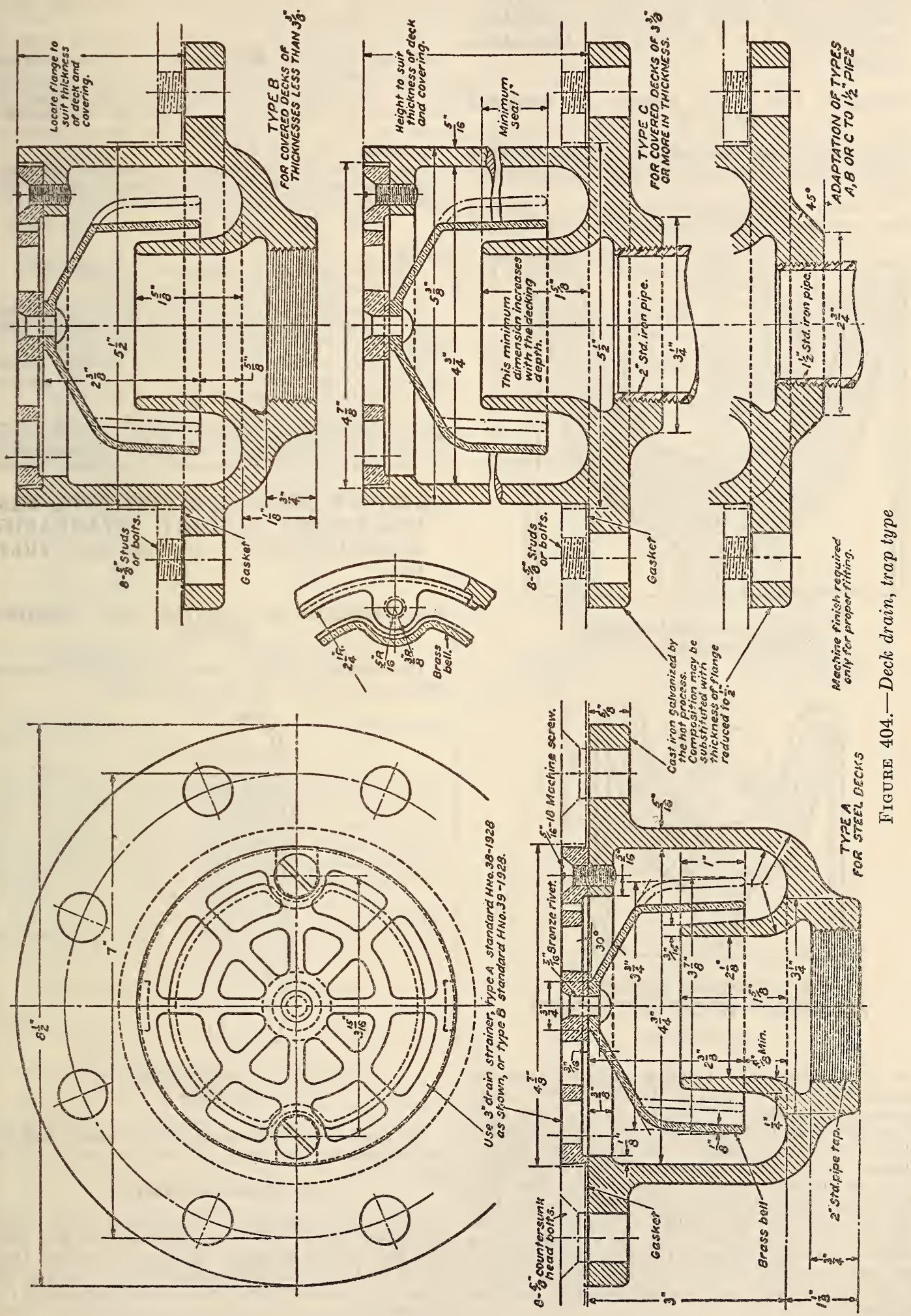


611.16 PULLEYS.

AMERICAN PETROLEUM INSTITUTE, SPECIFICATIONS FOR MANILA CORDAGE, STANDARD NO. 9-B, 4TH ED., JANUARY, 1930

\section{STANDARD SIZE AND FORMS OF CORDAGE GROOVES}

No standard shall be approved for a crown pulley having a combination groove for both manila and wire lines.

The bull rope grooves in the tug rims of the bull wheel and band wheel shall be made for $2 \frac{1}{2}$-inch

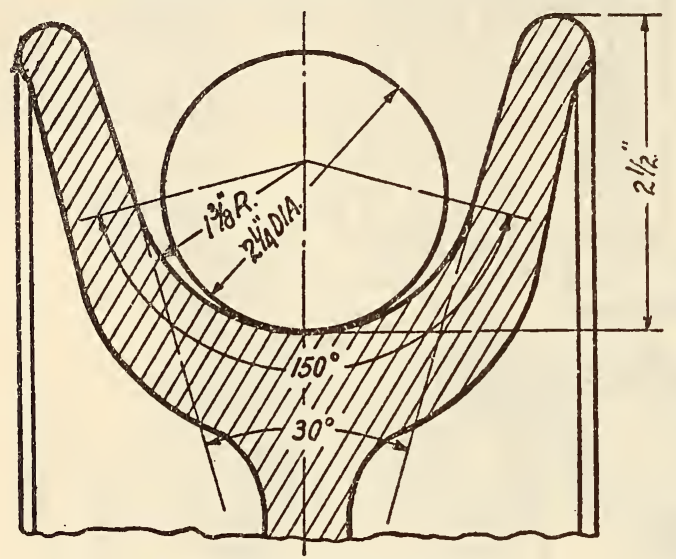

\section{A.P.I. STANDARD GROOVE FOR CROWN SHEAVES}

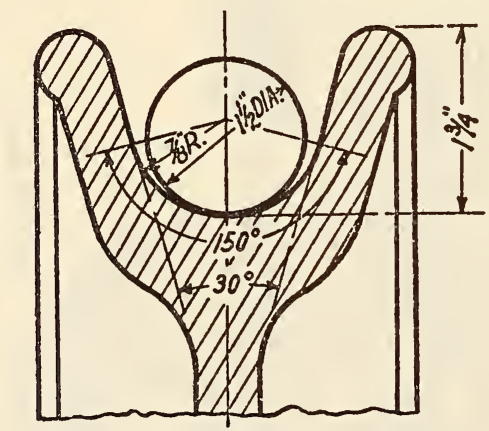

\section{A. P.I. STANDARD GROOVE FOR DERRICK OR CAT LINE SHEAVES}

Figure 405

rope as standard; the included angle between the sides of each groove shall be preferably $60^{\circ}$, but in no case greater than $80^{\circ}$; the depth of groove shall be not less than 17/8 inches nor more than $2 \% / 8$ inches (allowances must be made in the die to produce the minimum depth of $17 / 8$ inches specified on the product. It is recommended, where practicable, that the groove be rolled slightly deeper so that the bull rope will not ride the bottom of the groove); the bottoms of the grooves shall be rounded to a radius of one-half inch with a tolerance of minus 0 inch plus one-eighth inch; the top edges of the grooves shall be rounded to a radius of not less than one-half inch.

The standard groove for manila drilling cable shall be made for the $23_{4}^{\prime}$-inch cable. The bottom of the groove shall be an arc with a radius of $1 \frac{3 / 8}{}$ inches. The sides of the groove shall be tangent to the ends of this arc, and shall have an included angle of $30^{\circ}$. The denth of the groove shall be $21 / 2$ inches. (See fig. 405.)

The groove in the derrick line sheave shall be made for 11/2-inch manila rope. The bottom of the groove shall be an arc with a radius of seven-eighths inch. The sides of the groove shall be tangent to the ends of this arc, and shall have an included angle of $30^{\circ}$. The depth of the groove shall bs $13 / 4$ inches. (See fig. 405.)

For sheaves for any other size manilla drill lines, and other manila lines, the bottom of the groove shall be an arc having a radius equal to

where

$$
R=(D+t) / 2+1 / 8^{\prime \prime}
$$

$R=$ radius of bottom of groove, inches.

$D=$ nominal diameter of line, inches.

$t=$ specified tolerance in line diameter, inches.

The depth of the groove shall be one and oneeighth times the nominal diameter of the line.

The sides of the groove shall be tangent to the ends of the arc forming the bottom of the groove and shall have an included angle of $30^{\circ}$.

Note.-The radius " $R$ " should bo expressed in values not less than sixteenths of an inch. Should the solution of the above equation result in a fractional part of an inch smaller than one-sixteenthinch, the value found should be increased to the next higher sixteenth.

AMERICAN PETROLEUM INSTITUTE, MISCELLANEOUS ROTARY STANDARDS, STANDARD NO. 7-A, 4TH ED., JULY, 1931

\section{MEASUREMENT OF SHEAVES - AND HOISTING BLOCKS}

The diameter of all sheaves shall be the sutside diameter, as shown by $A$ in Figure 406.

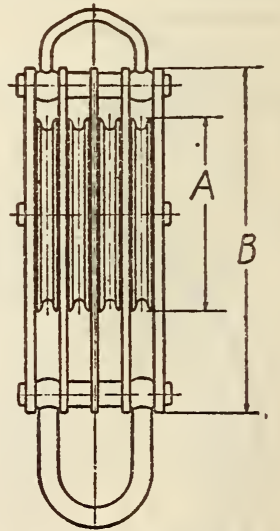

Frgure 406.-Hoisting blocks

The size of hoisting or traveling blocks shall be determined by the length of the side bar or $B$ in Figure 406.

\section{GROOVE FORM IN SHEAVES}

The form of groove in all sheaves shall be as stipulated in the A. P. I. specifications for wire rope and manila cordage. It is recommended that the A. P. I. standard form of groove be used on all sheaves in crown blocks, or any other equipment involving the use of sheaves not specifically mentioned herein. See A. P. I. Standards No. 9-A: "Wire Rope"; and A. P. I. Stds. No. 9-B: "Manila Cordage." (See below.) 
MARKING

The size of the A. P. I. groove form and the diameter of the sheave shall be stamped or cast on the outer rim of the groove of the sheave, and this information shall also be stamped on the name plates of complete crown and hoisting blocks. For example, a 30 -inch sheave with $1 \frac{1}{8}$-inch A. P. I. groove should be marked as follows:

$$
11 / 8 \text {-A. P. I. }-30
$$

\section{AMERICAN PETROLEUM INSTITUTE, SPEC- IFICATIONS FOR WIRE ROPE, STAND- ARD NO. 9-A, 8TH ED., JULY, 1931}

\section{GROOVES FOR SHEAVES FOR WIRE ROPE}

(Appendix B of Standard No. 9-A includes the following requirements on sheave grooves.)

B-5. The grooves of drilling and casing line sheaves shall be mada for 1 -inch rope as standard.

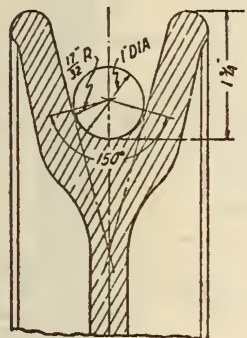

A.PL STANDARO GROOVE FOP SHEAVES

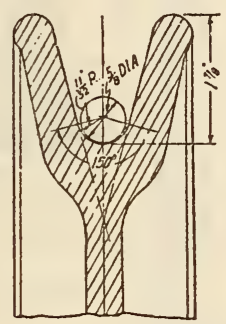

API STANOARO GROOVE TOR SAND LINE SHEAVES
Figure 407

To the above grove radius may be added a manufacturing tolerance $=+164^{\circ}-0^{\circ}$.

The bottom of the groove shall have $150^{\circ}$ arc of support with a radius of seventeen-thirty-seconds inch with a sheave-manufacturing tolerance of plus one-sixty-fourth minus 0 . The depth of the groove shall be one and three-fourths times the diameter of the rope. The sides of the groove shall be tangent to the ends of the bottom arc. See paragraph B-6 for size of rope other than 1 inch.

B-6. The groove for any other size of drilling and casing line is to be specified by the purchaser, the radius of the bottom of the groove to be:

$$
R=\frac{D+t}{2}+\text { Sheave manufacturing tolerance of }+364^{\prime \prime}-0^{\prime \prime} .
$$
where

$R=$ radius of the bottom of the groove, in inches.

$D=$ nominal diameter of line, in inches.

$t=$ specified plus tolerance of line, in inches.

The depth of the groove to be one and threefourths times the nominal diameter of the rope, and to have a $150^{\circ}$ arc of support.

B-7. The groove for sand-line sheaves shall be made for $5 / 8$-inch rope as standard. The bottom of the groove shall have a $150^{\circ}$ arc of support, with a radius of eleven thirty-seconds inch with a sheave manufacturing tolerance of plus one sixty-fourthinch minus 0 . The depth of the groove shall be three times the diameter of the rope. The sides of the groove shall be tangent to the ends of the b ttom arc.

B-8. Sheaves having grooves made according to the foregoing specifications should be marked plainly with the size of line for which the sheave is designed.
B-9. It is recommended that the hole in the rope socket and sockets for swivels be five thirty-secondsinch larger than the line to be inserted.

B-10. Combination sheaves for manila and wire lines are not recommended.

B-11. Grooves on rollers of oil savers should be made to the same tolerances as the grooves on the sheaves.

\section{AMERICAN RAILWAY ASSOCIATION, ME- CHANICAI DIVISION, CAR LIGHTING, RECOMMENDED PRACTICE, 1927}

\section{FLANGED PULLEYS}

(This specification covers the design and application of electric-light equipment to passenger equip-

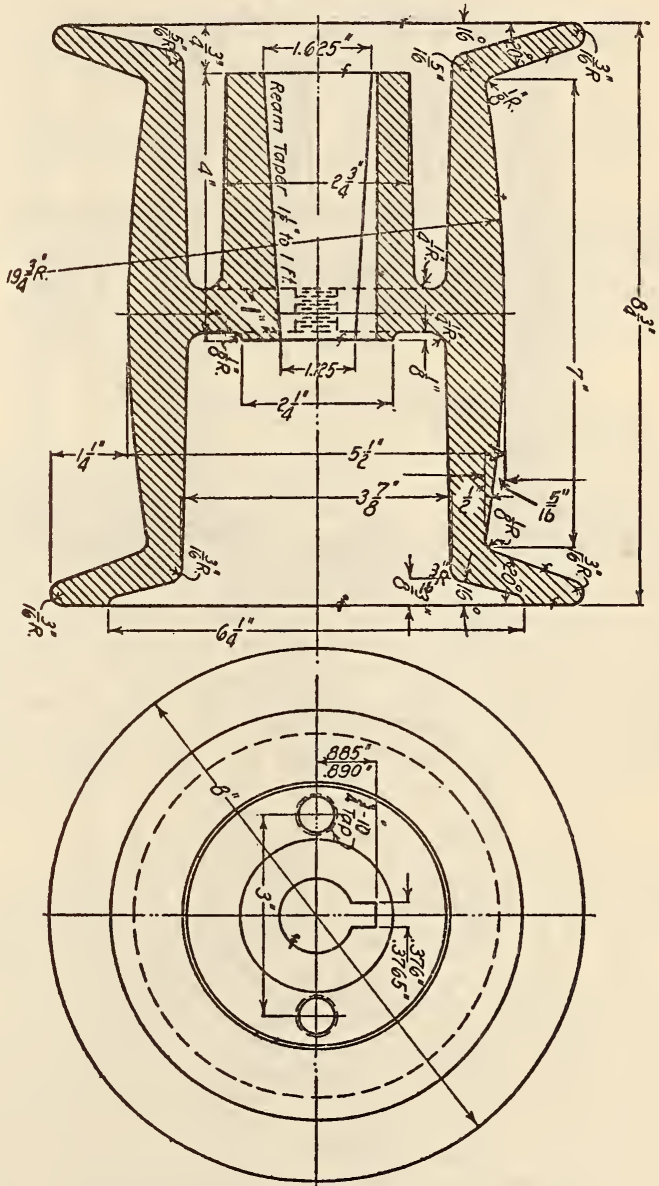

Figure 408.-Generator pulley, 51/2-inch

ment cars. A component part of this lighting equipment consists of various kinds of pulleys, the specifications for which are as follows:)

Axle pulley.-(a) The diameter of the axle pulley shall be as large as the construction of the car will permit with proper clearance, preferably 21 inches.

(b) The hub of the axle pulley shall have a uniform internal diameter of $71 / 2$ inches; the length of the hub shall not be less than $61 / 2$ inches.

Axle pulley bushing.-The bushing, when used, shall preferably be secured to the axle by the clamping action of the pulley; shall have an external diameter throughout its length of $71 / 2$ inches, and shall be not less than $8 \frac{1}{2}$ inches in length. 


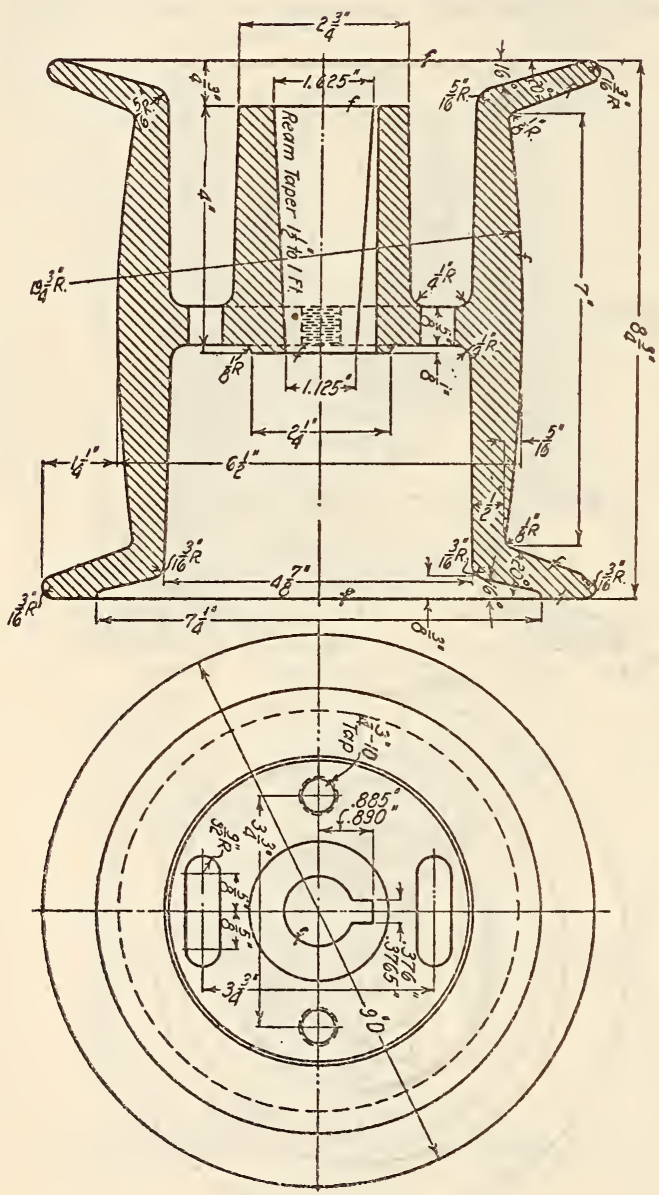

FigurE 409.-Generator pulley, 61/2-inch

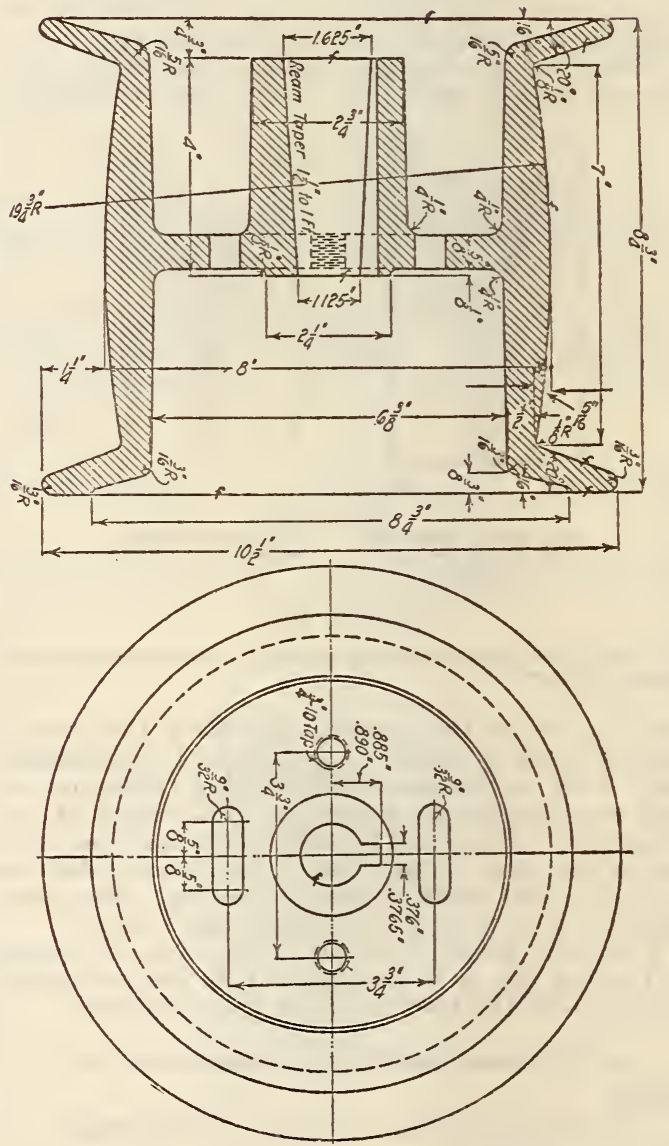

Figure 410.-Generator pulley, 8-inch. 


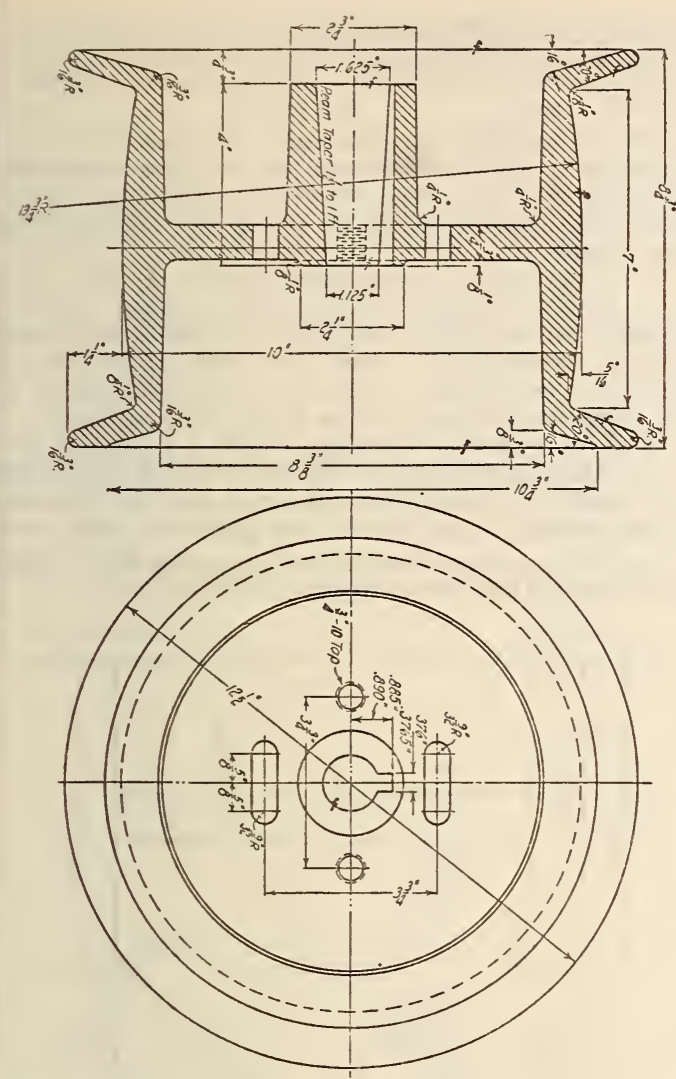

Figure 411.-Generator pulley, 10-inch

Generator pulley.-(a) The generator pulley shall be flanged and crowned and shall be in accord with the dimensions shown in the accompanying illustrations.

(b) The generator pulley shall be in perfect mechanical balance.

\section{NATIONAL MACHINE TOOL BUILDERS'} ASSOCIATION, DRIVING SPEEDS, STANDARD NO. T-1, OCTOBER 12, 1927

\section{MACHINE PULIEYS}

(Among this association's recommended standards for drive speeds for machine tools and speeds for constant-speed motors for machine-tool drives are the following recommended pulley sizes.)

As standard sizes for machine pulleys, the following diameters are recommended: $8,10,12,14,16$, 18,20 , and 24 inches.

611.17 HEATING AND COOKING VESSELS.

(No nationally recognized specifications available.)

611.18 CAST IRON WHEELS.

AMERICAN ELECTRIC RAILWAY ENGINEERING ASSOCIATION, STANDARD DESIGN FOR TREAD AND FLANGE OF WHEEL, NO. E13-26, 1926

\section{TREAD AND FLANGE OF WHEEL}

(See 611.53, p. 775.)
AMERICAN RAILWAY ASSOCIATION, MECHANICAL DIVISION, STANDARD WHEEL TREAD AND FLANGE FOR CAST-IRON WHEELS, 1930

TREAD AND FLANGE CONTOUR FOR CAST-IRON WHEELS

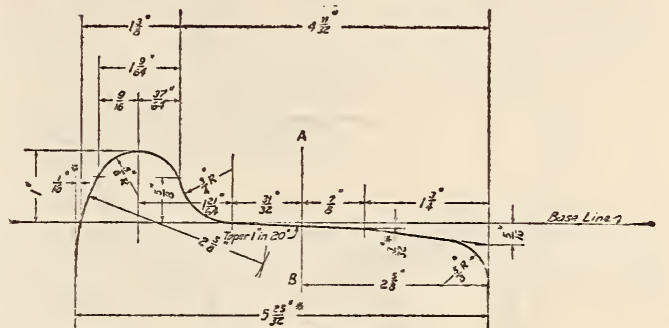

Figure 412.-Tread and flange contour for castiron wheels

These dimensions should not be used in layout of contour. They are approximate and for information purposes only.

\section{AMERICAN RAILWAY ASSOCIATION, ME- CHANICAL DIVISION; WHEELS, CAST IRON, FOR LOCOMOTIVES, TENDER, AND CARS, RECOMMENDED PRACTICE, 1928}

\section{CAST-IRON WHEELS}

(In respect to scope, material, chemical composition, sample and check analyses, shipment, patterns, dimensions, drop test, chill test, thermal test, taping, weights, test specimens, additional tests, marking, and rejection limits, this specification conforms to A. S. T. M., A46, given below, except that in chemical composition the total carbon desired, minimum, is in this case 3.35 per cent, and the phosphorus desired, maximum, is 0.35 per cent. In respect to inspection this specification conforms to paragraph 7 of A. S. T. M. standard specifications for steel. (See 605.0, p. 247.) Otherwise this specification provides as follows:)

\section{WORKMANSHIP AND FINISH}

1. Workmanship.-(a) Chill shall have an inside profile that, in the finished wheel, will produce the exact form of flange and tread contour as shown by A. R. A. drawings.

(b) The depth of clear white iron shall not exceed seven-eighths of an inch at the throat and 1 inch at the center line of tread and must not be less than one-half of an inch at the center line of tread and seven-sixteenths inch at the throat. These limits apply to all weights of wheels. The blending of clear white iron with the gray behind it shall be without any distinct line of demarcation, and the iron shall not bave a mottled appearance in any part of the wheel at a greater distance than $13 / 4$ inches from the throat and the tread. The depth of clear white iron shall not vary more than onefourth inch around the tread in any one plane in the same wheel.

2. Finish.-(b) All wheels shall be thoroughly cleaned by removing all sand and foreign substances. Wheels offered for inspection shall not be covered with any substance which will hide defects. The body of the wheel shall be smooth and free from slag, shrinkage or blow holes, swollen rims, or other defects. The tread and throat shall be smooth and free from deep and irregular wrinkles, slag, sand wash, or chill cracks and sweat. The 
hub core shall not be more than one-sixteenth inch out of center.

3. Rejection at destination.-Wheels which show injurious defects while being finished by the purchaser, or which crack while being pressed on axle,

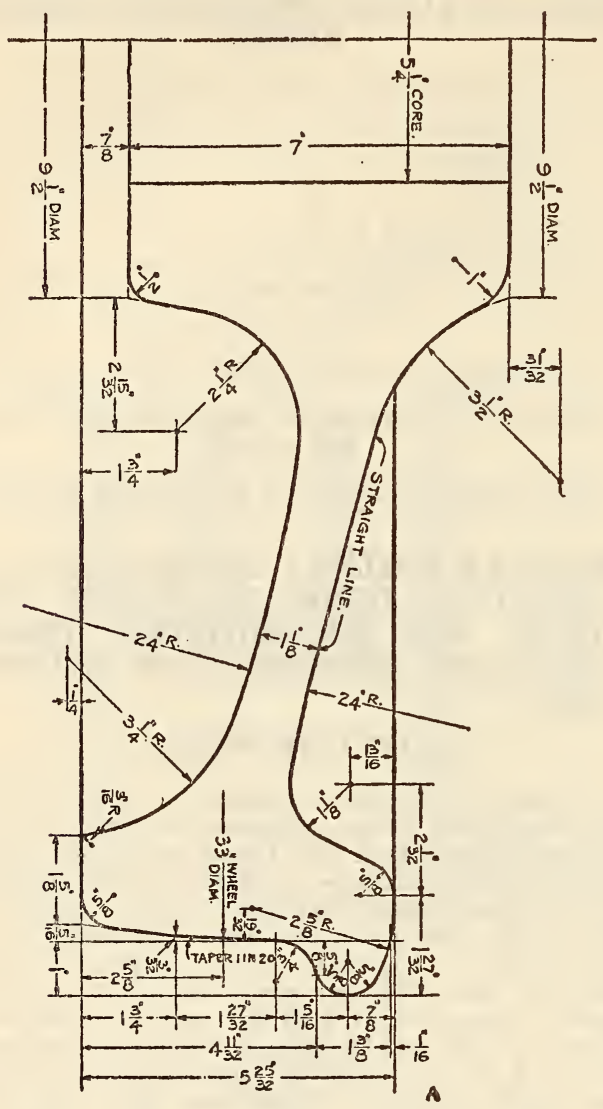

Figure 413.-Wheels, cast-iron, 33-inch diameter, for cars of maximum gross weight not to exceed 103,000 pounds

650 -pound wheel for 414 by 8 inch axle.

shall be rejected provided the axle pressure is not excessive and the bore of the wheel shows no evidence of faulty machine work or gouging.

4. Rehearing.-Samples for chemical analysis which represent rejected material shall be preserved for 14 days from date of test report, so that, in case of dissatisfaction with the results of the test, the manufacturer may make claim for rehearing within that time.

\section{FOUNDRY-PRACTICE REQUIREMENTS}

5. Composition.-The composition of the mixture shall be such that it will produce the analysis specified and an adequate depth of chill in the wheel.

6. Chill-test block.-A chill-test block must be taken at least once out of every 10 wheels poured. The size of the test bar shall be $1 \frac{1}{2}$ by 3 by $61 / 2$ inches, the $1 \frac{1}{2}$-inch face to be placed against the chiller.

Note.-The depth of chill measurements shall be recorded in sixteenths.

7. Analysis-test block.-(a) A test block 3 by 3 inches minimum shall be cast, preferably in dry sand, to represent each 40 wheels.
Note.-The drillings shall be obtained from the end of the test block, the first drillings shall be discarded, the drilling continued, and the resultant chips, etc., dumped from the hole and taken for sample, or the block may be broken and the drillings taken at the point of fracture.

(b) An analysis may be made for each block and recorded or, at the option of the manufacturer, the samples may be mixed of equal quantities, but at least one analysis shall be made to represent the day's heat.

8. Irregularities.-The irregularities in the foundry, which produce failure from seams, broken flanges, broken rims, and broken treads are almost entirely traceable to care and preparation of the ladles for service and failure to pour the metal at the proper speed and at the proper temperature.

9. Temperature.-The iron shall be melted and poured at a temperature sufficiently high to eliminate any possibility of cold-iron defects, such as wrinkles in the tread, blowholes in the plates or rims, etc.

Note.- It is recommended that the temperature of the molten metal as poured into wheel molds be not less than $2,400^{\circ} \mathrm{F}$.

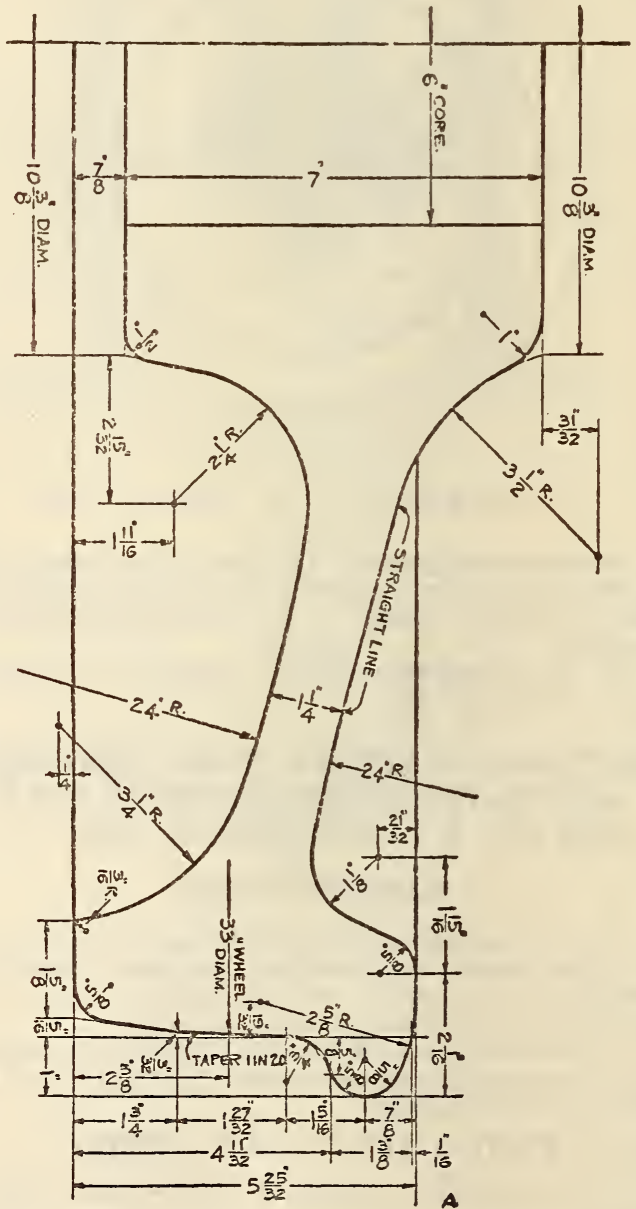

Frgure 414.-Wheels, cast-iron, 33-inch'diameter, for cars of maximum gross weight not to exceed 136,000 pounds

700-pound wheel for 5 by 9 inch axle.

10. Ladles.-(a) During the heat, the bull ladle, which shall be of the teapot type, shall be kept at least half full of molten metal, for better mixing of the metal and for preserving a uniform temperature.

(b) The bull ladle and small ladles shall be thoroughly heated prior to use. 
(c) Ladles shall be heated by the use of an oil burner, in which the heat is applied for a sufficient length of time to dry the lining thoroughly all the way through to the metal shell. It is preferable to heat the small ladles in specially designed ovens with closed doors, in which the ladles can be thoroughly baked throughout. Before any ladle is put into service, it shall have been two-thirds filled with molten iron which shall be discarded after 2 minutes.

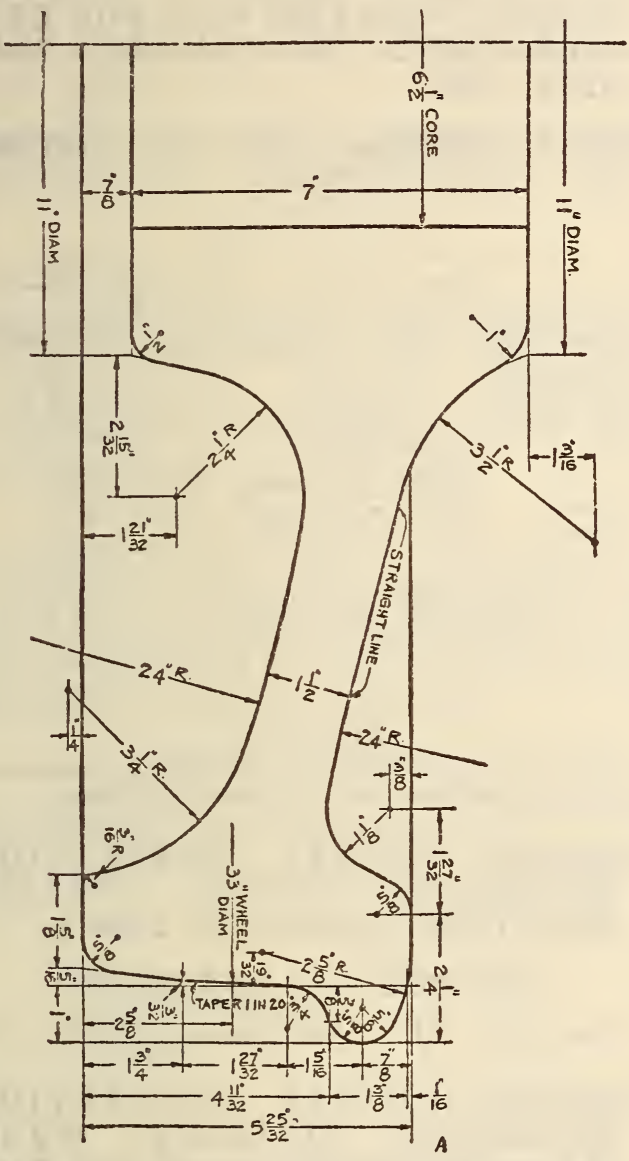

FIGURE 415.-Wheels, cast-iron, 39-inch diameter, for cars of maximum gross weight not to exceed 169,000 pounds

750 -pound wheel for $5 \frac{1}{2}$ by 10 inch axle.

(d) An alternative method is to give the ladles a preliminary heating with wood, coke, oil, or gas fire, then fill the ladle at least two-thirds full of molten iron. After 2 minutes the metal shall be discarded and the ladle immediately placed in service.

11. Last iron.- Under no conditions shall the last two ladles of iron in the reservoir or bull ladle be poured into wheels, nor shall iron which has been transferred from one small ladle to another be poured into wheel molds.

12. Pouring.-In ordinary pouring, the metal must be transferred from the bull ladle to the mold as quickly as possible; not more than 2 minutes shall elapse between the time the small ladles are filled and the wheel poured. Under no circumstances shall any material be placed in the bull ladle or pouring ladle, nor any other artificial means used, for cooling purposes. When starting to pour a mold, no let-up or intermission is permissible. This is most important, as it is essential that the metal be poured in a fast and steady stream. The time of pouring wheels of various weights shall not exceed the following:

650-pound wheels, 11 seconds.

700 -pound wheels, 12 seconds.

750 -pound wheels, 13 seconds.

850 -pound wheels, 15 seconds.

13. Shaking out.-Wheels shall be shaken out from the mold as quickly as permissible without bleeding. The interval of time between pouring and shaking out must not exceed the following limits:

650 -pound wheels, 40 minutes.

700 -pound wheels, 45 minutes.

750-pound wheels, 55 minutes.

850-pound wheels, 65 minutes.

After the wheels are shaken out they must be transferred to the annealing pit and pitted, not to exceed 4 minutes for the entire operation; the average time should not exceed 2 minutes. The time shall start with the lifting of the cope.

14. Pits.-(a) At the time of pitting, the temperature of the air in the middle of the pit, with

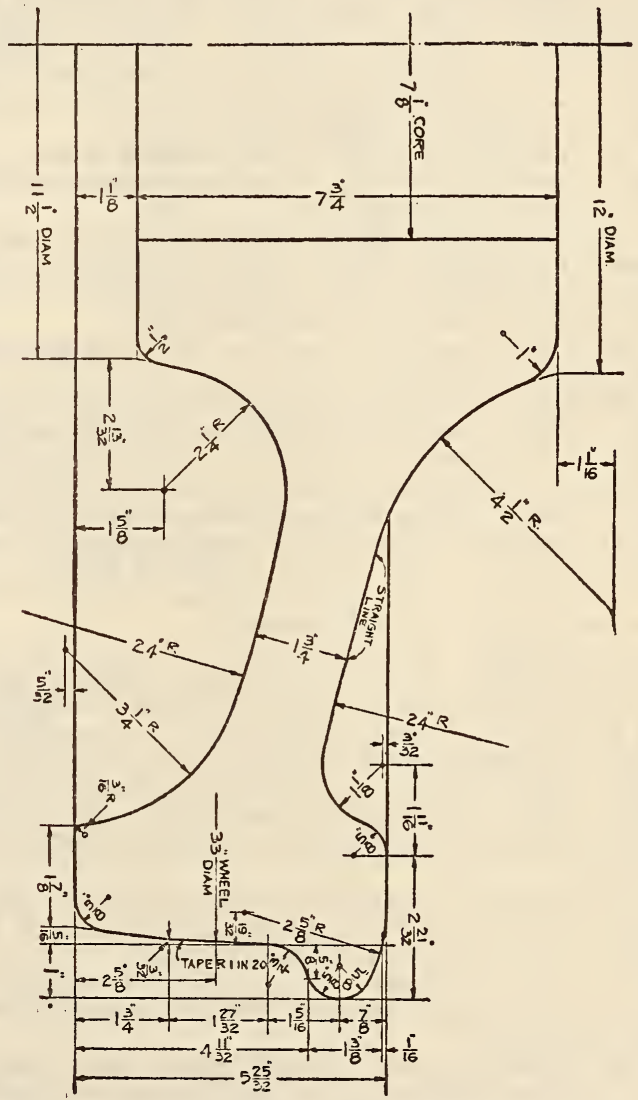

FIGURE 416.-Wheels, cast-iron, 39-inch diameter, for cars of maximum gross weight not to exceed 210,000 pounds

850-pound wheel for 6 by 11 inch axle.

cover closed, shall be not less than $600^{\circ} \mathrm{F}$., preferably $800^{\circ} \mathrm{F}$. or over.

The pits shall preferably be as deep as practical in order to reduce the number of top-pit wheels. The maximum depth is limited by the ability to remove the wheels and maintain the temperature specified.

(b) Pits which have not been in daily use must be fired a sufficient length of time not only to heat and 
dry the pit but also to heat the surrounding inclosure. The pit temperature shall be ascertained and recorded before the wheels are placed in the pits.

15. Rate of cooling. - The treads of wheels when placed in the pits must be of a cherry-red color, indicating a minimum surface temperature of the plates of $1,350^{\circ} \mathrm{F}$. or more.

The rate of cooling shall be such that 24 hours after the pit is closed the temperature of the top wheel shall be not less than $900^{\circ} \mathrm{F}$.

The top and second wheels shall be repitted. This shall be done before the temperature of the wheels in question has dropped below $900^{\circ} \mathrm{F}$.

16. Pit covers.-(a) The cover shall be so insulated as to prevent as far as possible rapid transfer of heat from the top wheel. A space equal to at least two wheels shall exist between the bottom of the cover and the top wheel.

(b) The pit covers must be placed as quickly as possible after the pit is filled with wheels.

(c) The primary pit covers must remain undisturbed until time for removal of the wheels, and must be replaced as soon as the wheels are removed.

17. Pyrometers. - (a) In order to determine the temperature of the pits and hot wheels, a portable pyrometer must be provided. Thermal couples must be provided for obtaining temperature of the pits and of hot wheels from the foundry, and as removed from the primary pit.

(b) The handle of the thermal couple must never be lowered into the pit. The temperature readings will be obtained as follows:

The pit cover shifted to one side just enough to admit the thermal couple, which is lowered to the middle portion of the pit; care being taken to keep the tip from contact with the brick lining.

(c) The temperature readings of the plates shall be obtained as follows:

The tip of the thermal couple is applied to the face of the wheel midway between the hub and the rim, and contact made with the metal surface, tip of thermal couple being protected from air currents.

(d) When taking the temperature of newly fired pits, the tip of the thermal couple must be kept well away from the glowing coals and flame.

(e) Pyrometer suitable for taking temperature of molten metal should be provided.

18. Selection of test wheels.-In selecting the wheels for test, careful consideration must be given to the following items, which may affect the strength of the wheel. Position in pit, top pit, second top pit, or any other position which has proved questionable, high shrinkage, lightest design, chill on test piece, last wheels poured, new ladles, any indication of cold iron, interruption of service, ladle treatment.

At least one manufacturer's test shall be taken from each cupola each operating day, and 50 per cent of the tests shall be thermal tests.

19. Chill on wheels. - The combined depth of the chill and mottled metal shall be not less than threequarters inch.

20. Plant operation. - When plants are operated less than six days per week, care should be taken to avoid so far as possible consecutive "shut-down days."

21. Records.-A permanent record shall be maintained of the following: (a) Wheel number, (b) name of railroad, $(c)$ type of wheel, $(d)$ diameter of wheel, (e) actual weight of wheel, $(f)$ date made, $(g)$ tape size of wheel, $(h)$ order of pouring, $(i)$ location of wheels in primary pit (top and second top), ( $j)$ chill on test piece, $(k)$ irregulatities or interruption in service, $(l)$ details of shop tests and rejections, $(m)$ condition of iron during heat, $(n)$ bull ladle treatment, (o) individual ladle treatment $(p)$ temperature of iron from pouring ladle, (q) wheels pitted at improper temperatures, $(r)$ all temperatures taken, $(s)$ first wheel poured from any ladle, and $(t)$ chemical analysis.

AMERICAN RAILWAY ASSOCIATION, MECHANICAL DIVISION; MAXIMUM FLANGE THICKNESS GAGE FOR CASTIRON WHEELS, RECOMMENDED PRACTICE, 1930

\section{FLANGE THICKNESS GAGE FOR CAST-IRON WHEELS}

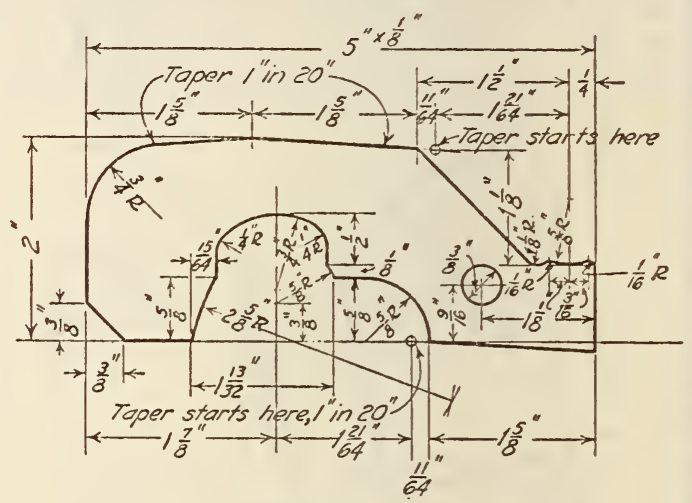

Figure 417

Note.-This gage is used only by inspectors at the foundries, and provides for a maximum flange thickness of $113 / 32$ inches.

\section{AMERICAN RAILWAY ASSOCIATION, MECHANICAL DIVISION; WHEELS, MOUNTING, STANDARD, 1926}

\section{MOUNTING CAST-IRON WHEELS}

(See A. R. A.-M., mounting wheels, 611.53, p. 780.)

AMERICAN RAILWAY ASSOCIATION, MECHANICAL DIVISION; WHEEL FLANGE THICKNESS GAGES FOR CASTIRON WHEELS, HEIGHT AND THROAT RADIUS GAGES FOR SOLID-STEEI AND STEEL-TIRED WHEELS, STANDARD, 1930

WHEEL FLANGE THICKNESS GAGES

(See A. R. A.-M., solid wrought carbon-steel wheels, 611.53 , p. 783.$)$

\section{AMERICAN RAILWAY ASSOCIATION, MECHANICAL DIVISION ; WHEEI MOUNTING AND CHECK GAGE FOR CAST-IRON AND CAST-STEEL WHEELS, STANDARD, 1930}

\section{WHEEL MOUNTING AND CHECK GAGE}

(See A. R. A.-M., solid wrought carbon-steel wheels, 611.53, p 783.) 
AMERICAN RAILWAY ASSOCIATION, MECHANICAL DIVISION ; WHEEL CIRCUMFERENCE MEASURE FOR CASTIRON, CAST-STEEL, WROUGHT-STEEL, AND STEEL-TIRED WHEELS, STANDARD, 1919

WHEEL CIRCUMFERENCE MEASURE FOR CAST

(See A. R. A.-M., solid wrought carbon steel wheels, 611.53, p. 783. )

AMERICAN RAILWAY ENGINEERING ASSOCIATION, AXLE ASSEMBLY, 1929

\section{AXLE ASSEMBLY}

(See 611.52, p. 770.)

\section{AMERICAN SOCIETY FOR TESTING MATERIALS, STANDARD SPECIFI. CATIONS FOR CHILIED CAST-IRON WHEELS, DESIGNATION A 46-24, 1924}

\section{CAST-IRON WHEELS}

\section{MATERIAL}

1. Wheels shall be made of a mixture of such composition that will produce the required chill in the tread and not show any mottled iron in the plates except around the chaplets, where it shall not extend more than one-half inch $(12.7 \mathrm{~mm})$. (The use of steel chaplets is preferred.)

\section{CHEMICAL REQUIREMENTS}

2. (a) The wheels shall conform to the following requirements as to chemical composition:

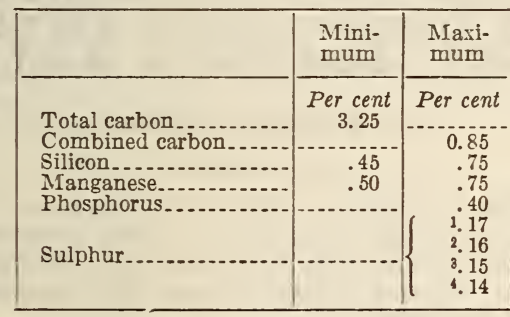

\footnotetext{
1 For 1923 to 1924 , inclusive.

2 For 1925 to 1926 , inclusive.

- For 1927 to 1928 , inclusive.

- For 1929 and thereafter.
}

(b) The minimum percentage of manganese shall be not less than three times the sulphur content.

3. Analysis sball be made by the manufacturer from test blocks poured during the day's melt from each cupola from which wheels purchased under these specifications are poured to determine the percentages of total carbon, manganese, phosphorus, sulphur, and silicon. The chemical composition thus determined shall be reported to the purchaser or his representative upon request.

4. Drillings for check chemical analysis shall be taken from drill hole bored entirely through the back double plate of the wheel midway between the core leg holes.

\section{DIMENSIONS, WEIGHT, AND DESIGN OF WHEELS}

5. Patterns and chillers shall be such that they will produce wheels according to the dimensions shown on drawings furnished by the purchaser.

6. The normal circumference conforming to the specified diameter shall be measured at a point $1 \frac{23 / 32}{3}$ inches $(43.65 \mathrm{~mm})$ from the gaging point on the throat of the flange. Wheels shall not vary more than seven-sixteenths inch $(11.11 \mathrm{~mm})$ above or below normal size, measured on the circumference, but only five-sixteenths inch $(7.94 \mathrm{~mm})$ variation above or below normal size shall be allowed for any day's cast from one cupola.

The thickness of the flange shall be regulated by a maximum and minimum flange thickness gage which shall be not more than one-sixteenth inch $(1.59 \mathrm{~mm})$ over nor more than one-sixteenth inch $(1.59 \mathrm{~mm})$ under the normal flange.

7. All wheels shall be taped with a standard design of wheel circumference tape and have the numbers $1,2,3,4$, and 5 stamped one-eighth inch $(3.18 \mathrm{~mm})$ apart, the figure " 3 " to represent normal tape size limited by one-sixteenth inch $(1.59 \mathrm{~mm})$ over or under the standard circumference, which for 33 -inch $(838.2 \mathrm{~mm})$ wheels will be 103.67 inches $(2,633.2 \mathrm{~mm})$. The figure " 1 " shall represent the smallest diameter, and the figure " 5 " the largest diameter. Tape sizes shall be stenciled in plain figures on the plate of the wheel or otherwise designated, as may be required by the purchaser.

8. (a) If wheels are ordered in accordance with A. R. A. standard drawings the weights shall be as follows:

\begin{tabular}{|c|c|c|c|}
\hline \multicolumn{2}{|c|}{$\begin{array}{l}\text { Maximum gross } \\
\text { weight of car }\end{array}$} & $\begin{array}{c}\begin{array}{c}\text { Normal } \\
\text { weight of } \\
\text { wheel }\end{array} \\
\text { Lbs. } k q\end{array}$ & $\begin{array}{c}\text { Minimum } \\
\text { weight of } \\
\text { wheel }\end{array}$ \\
\hline $\begin{array}{r}\text { Lbs. } \\
95,000 \\
132,000 \\
169,000 \\
210,000\end{array}$ & $\begin{array}{c}k g \\
43,000 \\
59,750 \\
76,500 \\
95,250\end{array}$ & $\begin{array}{ll}\text { Lbs. } & \mathrm{kg} \\
650 & 295 \\
700 & 317 \\
750 & 340 \\
850 & 385\end{array}$ & $\begin{array}{ll}\text { Lbs. } & \mathrm{kg} \\
640 & 290 \\
690 & 313 \\
740 & 335 \\
835 & 380\end{array}$ \\
\hline
\end{tabular}

(b) In the case of wheels ordered with cores smaller in diameter than the standard, the additional weight shall be considered as additional to the normal weigh given in the above table and be paid for by the purchaser.

(c) In any shipment where the average weight of wheels is above the normal weight given in the above table the excess weight shall be at the expense of the manufacturer.

\section{INSPECTION}

(Inspection conforms to paragraph 7 of A. S. T. M. standard specifications for steel. See 605.0, p. 247.)

10. The body of the wheel shall be free from slag, shrinkage, or blowholes. The tread and throat shall be free from irregular wrinkles, slag, sand wash, chill cracks, or sweat. Wheels will not be rejected on account of cracks around the circumference of the center core commonly known as "drawn hubs" or slight shrinkage holes on the face of the hub which will not interfere with the proper mounting of the wheels.

11. All wheels shall be marked and numbered consecutively in accordance with instructions issued by the purchaser. All wheels shall have the initials of the purchaser, wheel number, weight of wheel, month, day, and year when made plainly cast on the inside plate of the wheel. No wheels shall have duplicate numbers and all indistinct or duplicate numbers may be corrected only after having received the approval of the inspector. All wheels shall have the name of the manufacturer and place of manufacture cast on the outside plate of the wheel.

12. When ready for inspection, the wheels shall be arranged in groups, all wheels of the same date being grouped together, and for each 102 wheels which pass inspection and are ready for shipment, two representative wheels shall be taken, one of which shall be subjected to the thermal test and the other shall be used for the drop test. 
13. (a) In making the thermal test the wheels shall be laid with the flange downward in the sand and a channel way $13 / 4$-inches $(44 \mathrm{~mm})$ in width at the center of the tread for all wheels up to and including 700 pounds in weight and 2 inches $(51 \mathrm{~mm})$ in width at the center of the tread for all wheels over 700 pounds in weight and 4 inches $(102 \mathrm{~mm})$ deep shall be molded in green sand around the wheel, the tread of the wheel to form one side of the channel way and the clean flange forming as much of the bottom as its width will cover. This channel way shall be filled with molten cast iron which shall be hot enough when poured, so that the ring cast, when the metal is cold, will be solid and free from wrinkles. The time when pouring ceases shall be noted and after two minutes has elapsed an examination of the wheel shall be made. If any cracks develop in any part of the wheel, all wheels bearing the same tape size as represented by the sample shall be rejected.

(b) In order to prevent spicting while pouring, the tread and flange may be covered with a coat of shellac. Wheels selected for test which are wet or which have been exposed to snow or frost may be warmed sufficiently to dry them or remove the frost before testing, but under no circumstances shall the thermal test be applied to a wheel that in any part feels warm to the hand.

14. (a) The anvil of the drop-testing machine shall be supported on rubble masonry or a concrete foundation at least 2 feet $(610 \mathrm{~mm})$ deep and shall weigh not less than 1,700 pounds $(770 \mathrm{~kg})$. The striking face of the tup shall be 9 inches $(229 \mathrm{~mm})$ in diameter and flat. The face of each of the three bosses supporting the wheel under test shall be flat and measure 5 inches $(127 \mathrm{~mm})$ in width.

(b) The test wheel shall be so placed on the three supports with the flange down, that the tup will strike centrally on the hub. The test wheel shall pass the requirements given in Table 1 without breaking in two or more pieces. If the test wheel fails, all wheels bearing the same tape size shall be rejected.

TABLE 1

\begin{tabular}{|c|c|c|c|}
\hline Weight of wheel & $\begin{array}{l}\text { Weight of } \\
\text { tup }\end{array}$ & $\begin{array}{l}\text { Height of } \\
\text { drop }\end{array}$ & $\begin{array}{l}\text { Num- } \\
\text { ber of } \\
\text { blows }\end{array}$ \\
\hline $\begin{array}{ll}\text { Lbs. } & k g \\
650 & 295 \\
700 & 317 \\
750 & 340 \\
850 & 385\end{array}$ & $\begin{array}{lr}\text { Lbs. } & \mathrm{kg} \\
250 & 113 \\
250 & 113 \\
250 & 113 \\
250 & 113\end{array}$ & $\begin{array}{cc}F t . & m \\
9 & 2.75 \\
101 / 2 & 3.20 \\
12 & 3.65 \\
15 & 4.60\end{array}$ & $\begin{array}{l}12 \\
12 \\
12 \\
12\end{array}$ \\
\hline
\end{tabular}

(c) If the test wheel cracks before the twelfth blow it shall be required to stand 15 blows before breaking in two or more pieces.

15. The wheel selected for the drop and therma! test shall, following such test, be broken so that the chill may be examined at at least four different portions of the wheel. The depth of pure white iron shall conform to the following requirements:

\begin{tabular}{|c|c|c|c|c|c|}
\hline \multirow[b]{2}{*}{$\begin{array}{c}\text { Weight of } \\
\text { wheel }\end{array}$} & \multicolumn{3}{|c|}{ Maximum depth } & \multirow{2}{*}{\multicolumn{2}{|c|}{$\begin{array}{c}\text { Minimum } \\
\text { depth at } \\
\text { throat }\end{array}$}} \\
\hline & $\begin{array}{l}\text { At mid- } \\
\text { dle of } \\
\text { tread }\end{array}$ & At throat & $\begin{array}{l}\text { At mid- } \\
\text { dle of } \\
\text { tread }\end{array}$ & & \\
\hline $\begin{array}{lr}\text { Lbs. } & \text { kg } \\
650 & 295 \\
700 & 317 \\
750 & 340 \\
850 & 385\end{array}$ & $\begin{array}{l}\text { Inst. } m m \\
1 \quad 25.5 \\
111627 \\
11 / 828.6 \\
11828.6\end{array}$ & $\begin{array}{ll}\text { In. } & m m \\
7 / 8 & 22.2 \\
7 / 8 & 22.2 \\
7 / 8 & 22.2 \\
7 / 8 & 22.2\end{array}$ & $\begin{array}{cc}\text { In } & m m \\
1 / 2 & 12.7 \\
1 / 2 & 12.7 \\
1 / 2 & 12.7 \\
1 / 2 & 12.7\end{array}$ & $\begin{array}{l}\text { In. } \\
3 / 8 \\
7 / 16 \\
136 \\
1 / 2\end{array}$ & $\begin{array}{r}m m \\
9.5 \\
11.1 \\
12.7 \\
12.7\end{array}$ \\
\hline
\end{tabular}

The depth of the chill shall not vary more than one-quarter inch $(6.35 \mathrm{~mm})$ in the middle of the tread on the different portions measured. If the sample does not conform to the requirements stated, all wheels of the tape size represented by the sample shall be rejected.

Note.-The chill and physical tests specified only cover 33-inch wheels of the weights listed. Requirements for wheels of other sizes are to be supplied by the committee.

\section{RETEST}

16. Should the test wheel selected for the drop test, thermal test, or chill test fail to meet the requirements specified, all wheels bearing the same tape size shall be rejected. (Secs. 13, 14, and 15.) The inspector shall then select another wheel of \& different tape size, which shall be submitted to the same test as the wheel which failed and if it passes the requirements of the test the remainder of the lot shall be accepted, provided the requirements of all other tests have been met.

\section{REJECTION}

17. The numbers of all wheels which have been rejected because of failure to meet the requirements of the drop test, thermal test, or chill test shall be noted and such wheels shall not be submitted for test at any future time.

18. Individual wheels shall be rejected if: (a) They are under the minimum weight specified, (b) they have surface defects mentioned in section 10 , and $(c)$ they are over or under the specified dimensions.

(The appendix is identical with that in A46-30T, given below, except that in A46-24 the first 33-inch wheel weighs 650 pounds with a maximum load per wheel of 11,875 pounds; the second 33-inch wheel weighs 700 pounds, with a maximum load per wheel of 16,500 pounds; and the first 36 -inch wheel weighs 740 pounds, with a maximum load per wheel of 12,000 pounds.)

\section{AMERICAN SOCIETY FOR TESTING MA- TERIALS, TENATATIVE SPECIFICATIONS FOR CHILLED-TREAD CAST-IRON WHEELS, DESIGNATION A 46-30T, 1930}

\section{CAST-IRON WHEELS}

\section{MANUFACTURE}

1. Wheels shall be made of a mixture of such composition that, with good foundry practice and proper annealing, they will have the required chill in the tread, and otherwise meet the requirements of these specifications.

2. The fracture shall show a soft, clean, gray iron free from defects such as holes more than one-fourth inch in diameter containing slag or dirt, or clusters of holes honeycombing the hub, or white iron in the plate or hub.

\section{CHEMICAL PROPERTIES AND TESTS}

3. The wheels shall conform to the following requirements as to chemical composition:

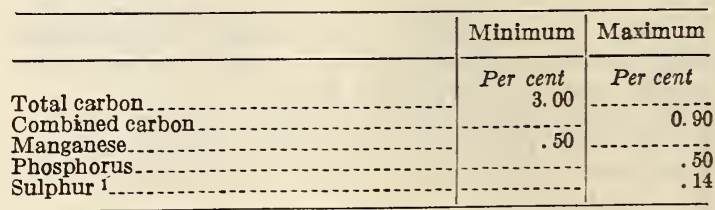

1 Sulphur content.-The essential condition of chemical composition of chilled tread wheels is a balance between sulphur, carbon, and manganese, and variation in these constituents must not disturb this relation. If, on check analysis, the sulphur is found to be not more than 0.02 per cent greater than that specified, it shall not be considered sufficient cause for the rejection of the wheels, provided const that the wheels conform to all physical and is at least three and oneand provided that the manganese content is at least three and onehalf times the sulphur content. Such failure to conform to the specifications should be called to the attention of the manufacturer,
subsequent shipments or lots shall conform to the specifications. 
4. Analyses shall be made by the manufacturer from test blocks poured during the day's melt from each cupola from which wheels purchased to these specifications are poured. The percentages of manganese, phosphorus, and sulphur shall be determined. The determination of carbon shall also be furnished merely for information. A copy of the analysis shall be furnished the purchaser or his representative on request.

5. An analysis may be made by the purchaser from one of the test wheels representing each 100 wheels. The sample for analysis shall be taken from drillings obtained by drilling a hole into the back of the plate of the wheel midway between the flange and the hub. The chemical composition thus determined shall conform to the requirements for manganese, phosphorus, and sulphur specified in section 3 .

6. Chemical analyses shall be determined in accordance with the standard methods of sampling and chemical analysis of pig and cast iron A. S. T. M. A64. (See 601.20, p. 119.)

7. The purchaser shall not require the manufacturer to hold all lots of wheels until check analysis can be obtained unless it has been found that the manufacturer's product has not been conforming to these specifications.

\section{PHYSICAL PROPERTIES AND TESTS}

8. (a) In making the thermal test, the wheel shall be laid with the flange downward in the sand with the channel way molded in green sand around the wheel, 4 inches deep and of a width as shown in the following table. The tread of the wheel shall form one side of the channel way, the clean flange forming as much of the bottom as its width will cover. This channel way shall be filled with molten cast iron, which shall be hot enough when poured so that the cast ring, when cold, will be solid and free from wrinkles. The time when pouring ceases shall be noted, and after the time specified in the following table has elapsed, the wheel shall be examined. If a crack develops in the wheel within the time limit specified in the table all wheels bearing that same tape size shall be rejected.

\begin{tabular}{|c|c|c|}
\hline $\begin{array}{c}\text { Weight } \\
\text { of } \\
\text { wheel }\end{array}$ & $\begin{array}{c}\text { Cooling } \\
\text { time }\end{array}$ & $\begin{array}{c}\text { Width } \\
\text { of chan- } \\
\text { nelway }\end{array}$ \\
\hline Pounds & $\begin{array}{c}\text { Minutes } \\
650\end{array}$ & $\begin{array}{c}\text { Inches } \\
700\end{array}$ \\
750 & 5 & 2 \\
850 & 5 & 2 \\
& 7 & 3 \\
\hline
\end{tabular}

(b) The use of a permanent mold for making the thermal test in place of the sand mold described in paragraph $(a)$ is permissible. The permanent mold shall consist of an iron ring and bottom board of such dimensions that the wheel to be tested can be immediately placed in the mold and centered to the required channel way.

(c) In order to prevent spitting while pouring, the tread and flange may be covered with a coat of chiller paste. Wheels selected for test which are wet or which have been exposed to snow or frost may be warmed sufficiently to dry them or remove the frost before testing, but under no circumstances shall the thermal test be applied to a wheel that in any part feels warm to the hand.

9. (a) The test wheel shall conform to the droptest requirements given in the following table without breaking in two or more pieces. If the test wheel fails all wheels bearing the same tape size shall be rejected:

\begin{tabular}{|c|c|c|c|}
\hline $\begin{array}{c}\text { Weight of } \\
\text { wheel }\end{array}$ & $\begin{array}{c}\text { Weight of } \\
\text { tup }\end{array}$ & $\begin{array}{l}\text { Height of } \\
\text { drop }\end{array}$ & $\begin{array}{l}\text { Number } \\
\text { of blows }\end{array}$ \\
\hline $\begin{array}{cc}\text { Lbs. } & \mathrm{kg} \\
650 & 295 \\
700 & 317 \\
750 & 340 \\
850 & 385\end{array}$ & $\begin{array}{cc}\text { Lbs. } & \mathrm{kg} \\
250 & 113 \\
250 & 113 \\
250 & 113 \\
250 & 113\end{array}$ & $\begin{array}{lc}F t . & m \\
9 & 2.75 \\
101 / 2 & 3.20 \\
12 & 3.65 \\
15 & 4.57\end{array}$ & $\begin{array}{l}12 \\
12 \\
12 \\
12\end{array}$ \\
\hline
\end{tabular}

(b) If the test wheels crack in the plate with three blows or less, they shall be considered as having failed.

(c) Apparatus.-The anvil of the drop-testing machine shall weigh not less than 1,700 pounds $(770$ $\mathrm{kg}$ ) and shall be supported on a foundation of rubble masonry or concrete at least 2 feet $(61 \mathrm{~cm})$ in depth. The striking face of the tup shall be flat and 9 inches $(229 \mathrm{~cm})$ in diameter. The face of each of the three bosses supporting the wheel under test shall be flat and 5 inches $(12.7 \mathrm{~cm})$ in width. The test wheel shall be so placed on the three supports, with the flange down, that the tup will strike centrally on the hub.

10. (a) After having been subjected to the thermal and drop tests, the test wheels shall be broken so that the chill may be examined at at least four different portions of the wheel. The depth of pure white iron shall conform to the following requirements and the depth of the chill shall not vary more than one-fourth inch $(6.35 \mathrm{~mm})$ in the middle of the tread on the different portions measured:

\begin{tabular}{|c|c|c|}
\hline $\begin{array}{c}\text { Weight of } \\
\text { wheel }\end{array}$ & $\begin{array}{l}\text { Maximum } \\
\text { depth }\end{array}$ & $\underset{\text { depth }}{\text { Minimum }}$ \\
\hline $\begin{array}{cc}\text { Lbs. } & k g \\
650 & 295 \\
700 & 317 \\
750 & 340 \\
850 & 385\end{array}$ & $\begin{array}{ll}\text { Ins. } & m m \\
1 & 25.5 \\
11116 & 27 \\
138 & 28.6 \\
118 & 28.6\end{array}$ & $\begin{array}{rr}\text { In. } & m m \\
3 / 8 & 9.5 \\
7 / 16 & 11.1 \\
1 / 2 & 12.7 \\
1 / 2 & 12.7\end{array}$ \\
\hline
\end{tabular}

(b) If the test wheel does not conform to the requirements specified, all wheels of the tape size represented by the test wheel shall be rejected.

Notr.-The physical properties and tests specified cover only 33-inch wheels of the weights listed. Requirements for wheels of other sizes are to be supplied by the committee.

11. (a) Sampling.-When ready for inspection, the wheels shall be arranged in groups, all wheels of the same weight being grouped together, and for each 102 wheels which pass inspection and are ready for shipment, two representative wheels shall be taken, one of which shall be subjected to the thermal test, and the other shall be used for the drop test.

(b) When ordered in lots of 100 or more of each weight, the manufacturer shall arrange to cast them in sufficient quantity so that at least one test wheel may be taken from the wheels cast each day.

\section{DIMENSIONS, WEIGHT, AND DESIGN OF WHEELS}

12. Patterns and chillers shall be such that they will produce wheels in accordance with the dimensions shown on drawings furnished by the purchaser.

13. The normal circumference conforming to the specified diameter shall be measured at a point $123 / 32$ inches $(43.65 \mathrm{~mm})$ from the gaging point on 
the throat of the flange. When measured on the circumference, wheels shall not vary more than fivesixteenths inch above or below the normal size. Each wheel shall be so nearly circular that a true metallic ring, placed on its tread and bearing somewhere on the cone, shall at no point, be more than one thirty-second inch from the tread. The thickness of the flange shall be determined by a minimum and maximum flange thickness gage which shall be regulated to not more than one thirty-second inch $(0.794 \mathrm{~mm})$ over nor more than one-sixteenth inch $(1.59 \mathrm{~mm})$ under the normal flange thickness.

14. All wheels shall be taped with a standard design of wheel circumference tape. The wheels shall have the figures $1,2,3,4$, and 5 cast on the back plate in three-sixteenths inch $(4.76 \mathrm{~mm})$ raised figures, three-fourths inch in height, and oneeighth inch apart. The figure " 3 " shall represent normal tape size limited by one-sixteenth inch $(1.59 \mathrm{~mm})$ over or under the standard circumference (which for 33 -inch $(838.2 \mathrm{~mm})$ wheels will be 103.67 inches \pm 1116 inch $(2,633.2 \mathrm{~mm} \pm 1.59 \mathrm{~mm}))$. The figure " 1 " shall represent the smallest diameter, and the figure " 5 " the largest diameter. When taping a wheel the figure representing the tape size shall be chipped off. Tape sizes shall either be stenciled in plain figures on the plate of the wheel or as may be otherwise designed by the purchaser.

15. (a) If wheels are ordered in accordance with A. R. A. standard drawings the weights shall be as follows:

\begin{tabular}{|c|c|c|c|}
\hline $\begin{array}{l}\text { Maximu } \\
\text { weight }\end{array}$ & of car & $\begin{array}{c}\text { Normal } \\
\text { weight of } \\
\text { wheel }\end{array}$ & $\begin{array}{c}\text { Minimum } \\
\text { weight of } \\
\text { wheel }\end{array}$ \\
\hline $\begin{array}{r}\text { Lbs. } \\
103,000 \\
136,000 \\
169,000 \\
210,000\end{array}$ & $\begin{array}{c}\mathrm{kg} \\
46,700 \\
61,000 \\
76,500 \\
95,250 \ldots\end{array}$ & $\begin{array}{lc}\mathrm{Lbs} . & \mathrm{kg} \\
650 & 295 \\
700 & 317 \\
750 & 340 \\
850 & 385\end{array}$ & $\begin{array}{lc}\text { Lbs. } & \mathrm{kg} \\
640 & 290 \\
690 & 313 \\
740 & 335 \\
835 & 380\end{array}$ \\
\hline
\end{tabular}

(b) In the case of wheels ordered with cores smaller in diameter than the standard, the additional weight shall be considered as additional to the normal weight given in the above table and shall be paid for by the purchaser.

(c) In any shipment where the average weight of wheels is above the normal weight given in the above table the excess weight shall be at the expense of the manufacturer.

\section{FINISH}

16. The body of the wheel shall be free from slag, shrinkage, or blowholes. The tread and throat shall be free from irregular wrinkles, slag, sand wash, chill cracks, or sweat. Wheels will not be rejected on account of cracks around the circumference of the center core commonly known as "drawn hubs" or slight shrinkage holes on the face of the hub which will not interfere with the proper mounting of the wheels.

\section{MARKING}

17. All wheels shall be marked and numbered consecutively in accordance with instructions issued by the purchaser. All wheels shall have plainly cast on the inside plate of the wheel the initials of the purchaser, the wheel number, weight of the wheel, and the month, day, and year when cast. No wheels shall have duplicate numbers and all indistinct or duplicate numbers may be corrected only after having received the approval of the inspector. All wheels shall have the name of the manufacturer and the place of manufacture cast on the outside plate of the wheel.

\section{INSPECTION AND REJECTION}

(Inspection conforms to paragraph 7 of A. S. T. M. standard specifications for steel. See 605.0 , p. 247.)

19. (a) Should the test wheel selected for the drop test, thermal test, or chill test fail to conform to the requirements specified, all wheels bearing the same tape size shall be rejected. (See secs. 8, 9, and 10.) The inspector shall then select another wheel of a different tape size, which shall be submitted to the same test as the wheel which failed, and if it passes the requirements of the test the remainder of the lot shall be accepted, provided the requirements of all other tests have been met.

(b) The numbers of all wheels which have been rejected because of failure to meet the requirements of the drop test, thermal test, or chill test shall be noted, and such wheels shall not be submitted for test at any future time.

(c) Individual rejection.-Individual wheels shall be rejected in accordance with the following: (1) If they are under the minimum weight specified, (2) if they have surface defects described in section 16 , and (3) if they are under the specified dimensions.

\section{APPENDIX}

As information of possible value to the users of these specifications, committee $\mathrm{A}-3$ on cast iron wishes to state that the Association of Manufacturers of Chilled Car Wheels has adopted recommended designs of chilled-tread car wheels of 24,26 , $28,30,33,36,39$, and 42 inch diameter. The designs for the 33 -inch diameter single plate wheels have been approved by the American Pailway Association. The wheels for which designs have been made, the standard for maximum load and estimated weight of the wheels are given below:

\begin{tabular}{|c|c|c|c|c|c|}
\hline \multirow{2}{*}{$\begin{array}{l}\text { Diam- } \\
\text { oter of } \\
\text { wheel }\end{array}$} & \multicolumn{2}{|c|}{ Standard for- } & \multirow{2}{*}{$\begin{array}{l}\text { Diam- } \\
\text { eter of } \\
\text { wheel }\end{array}$} & \multicolumn{2}{|c|}{ Standard for- } \\
\hline & $\begin{array}{l}\text { Maximum } \\
\text { load per } \\
\text { wheel }\end{array}$ & $\begin{array}{l}\text { Esti- } \\
\text { mated } \\
\text { weight }\end{array}$ & & $\begin{array}{c}\text { Maximum } \\
\text { load per } \\
\text { wheel }\end{array}$ & $\begin{array}{l}\text { Esti- } \\
\text { mated } \\
\text { weight }\end{array}$ \\
\hline $\begin{array}{c}\text { Inches } \\
24 \\
24 \\
24 \\
24 \\
26 \\
26 \\
26 \\
26 \\
28 \\
28 \\
28 \\
28 \\
30 \\
30 \\
30 \\
30\end{array}$ & $\begin{array}{r}\text { Pounds } \\
6,000 \\
9,000 \\
12,000 \\
16,000 \\
6,000 \\
9,000 \\
12,000 \\
16,000 \\
6,000 \\
9,000 \\
12,000 \\
16,000 \\
6,000 \\
9,000 \\
12,000 \\
16,000\end{array}$ & $\begin{array}{c}\text { Pounds } \\
320 \\
360 \\
400 \\
440 \\
360 \\
410 \\
460 \\
510 \\
410 \\
460 \\
510 \\
560 \\
455 \\
530 \\
575 \\
615\end{array}$ & $\begin{array}{c}\text { Inches } \\
33 \\
33 \\
33 \\
33 \\
36 \\
36 \\
36 \\
39 \\
39 \\
39 \\
42 \\
42 \\
42\end{array}$ & $\begin{array}{r}\text { Pounds } \\
12,875 \\
17,000 \\
21,125 \\
26,250 \\
16,000 \\
20,000 \\
25,000 \\
16,000 \\
20,000 \\
25,000 \\
16,000 \\
20,000 \\
25,000\end{array}$ & $\begin{array}{c}\text { Pounds } \\
650 \\
700 \\
750 \\
850 \\
800 \\
860 \\
980 \\
875 \\
950 \\
1,100 \\
1,000 \\
1,075 \\
1,225\end{array}$ \\
\hline
\end{tabular}

\section{ASSOCIATION OF MANUFACTURERS OF CHILLED CAR WHEELS, CHILIED-TREAD WHEEIS FOR LOCOMOTIVES, TENDERS, AND CARS, 1930}

\section{CHILIED-TREAD WHEELS}

(In respect to surface and rejections at destination this specification conforms to A. R. A.-M. specification for cast-iron wheels, given above. In respect to illustration of tread and flange contour it is identical with A. R. A.-M. standard wheel tread and flange for cast-iron wheels, also given above. In respect to analysis, check analyses, patterns and 
chillers, dimensions, taping, cores, marking, sampling, thermal test, drop test, chill test, and individual rejection, this specification conforms to A. S. T. M., A46-24, likewise given above. It differs from the above-referred-to specifications in the following particulars:)

\section{Section I. WHEELS FOR STANDARD RAIIWAY USES}

Scope.-These specifications cover all 33-inch $(838.20 \mathrm{~mm})$ wheels and over in diameter for rail way service for loads of maximum gross weights not exceeding $12,000,16,000,20,000$, and 25,000 pounds per wheel.

\section{MANUFACTURE}

Composition.-The composition of the mixture shall be such that it will produce the analysis specified and an adequate depth of chill in the wheel.

Chill.-(c) The depth of chill shall not be less than one-half inch and not more than $1 \frac{11}{4}$ inches at the center line of tread. The blending of the chill with the gray iron shall be without any distinct line of demarcation. The depth of chill shall not vary more than one-fourth inch around the tread in any one plane in the same wheel. These limits apply to all weights of wheels.

Chemical composition.-Wheels shall conform to the following requirements as to chemical composition:

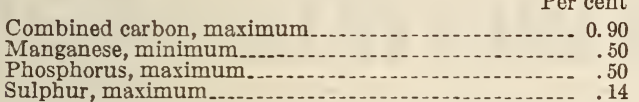

Sulphur content.-The essential condition of chemical composition of chilled-tread wheels is a balance between sulphur, carbon, and manganese, and variation in these constituents must not disturb this relation. If, on check analysis, the sulphur is found to be not more than 0.02 per cent greater than that specified, it shall not be considered sufficient cause for the rejection of the wheels provided that the wheels conform to all physical and inspection requirements and provided that the manganese content is at least three and one-half times the sulphur content. Such failure to conform to the specifications should be called to the atten- tion of the manufacturer and subsequent shipments or lots shall conform to the specifications.

Chemical analysis procedure.-Chemical analyses shall be determined in accordance with the standard methods of sampling and chemical analysis of pig and cast iron in A. S. T. M., A64. (See 601.20, p. 119.)

Dimensions.-39-inch wheels shall not vary more than seven-sixteenths inch $(11.11 \mathrm{~mm})$ and 42 -inch wheels seven-sixteenths inch $(11.11 \mathrm{~mm})$ above or below normal size, measured on the circumference.

Taping.-All 39-inch $(990.6 \mathrm{~mm})$ and all 42 -inch $(1066.8 \mathrm{~mm})$ wheels shall be taped in a like manner (to the taping of 33-inch and 36-inch wheels) except that the tape shall be marked with numbers from 0 to 6 , inclusive, each of which shall be stamped oneeighth inch $(3.18 \mathrm{~mm})$ apart. The figure 0 shall represent the smallest circumference and the figure 6 the largest circumference.

\begin{tabular}{|c|c|c|}
\hline Diameter & $\begin{array}{l}\text { Normal circum- } \\
\text { ference }\end{array}$ & $\begin{array}{l}\text { Maximum } \\
\text { allowable } \\
\text { variation in } \\
\text { cil cumfer- } \\
\text { ence above } \\
\text { or below the } \\
\text { normals }\end{array}$ \\
\hline \begin{tabular}{cr} 
Ins. & \multicolumn{1}{c}{ cm } \\
33 & 83.82 \\
36 & 91.44 \\
39 & 99.06 \\
42 & 106.68
\end{tabular} & $\begin{array}{lc}\text { Ins. } & c m \\
103.67 & 263.3 \\
113.10 & 287.3 \\
122.52 & 311.2 \\
131.95 & 335.2\end{array}$ & $\begin{array}{rr}I n . & m m \\
5 / 16 & 7.9 \\
5 / 16 & 7.9 \\
7 / 16 & 11.1 \\
7 / 16 & 11.1\end{array}$ \\
\hline
\end{tabular}

A tape number plate shall be cast on the back plate of the wheel. When taping a wheel the number of this plate representing the tape size shall be chipped off. Under no circumstances are these numbers to be removed after the wheel is received from the foundry.

At the request of the purchaser, tape sizes may in addition be stenciled in plain figures with white paint on the front plate of the wheel, or as otherwise designated, without extra cost to the purchaser.

Weights. - Wheels shall be furnished in accordance with the weights shown in the accompanying table, based on standard drawing:

\begin{tabular}{|c|c|c|c|c|c|c|c|c|c|c|c|}
\hline \multicolumn{2}{|c|}{ Diameter } & \multicolumn{2}{|c|}{$\begin{array}{l}\text { Maximum gross } \\
\text { load on wheel }\end{array}$} & \multicolumn{2}{|c|}{ Core size } & \multicolumn{2}{|c|}{ To bore } & \multicolumn{2}{|c|}{$\begin{array}{l}\text { Nominal } \\
\text { weight }\end{array}$} & \multicolumn{2}{|c|}{$\underset{\text { weight }}{\text { Minimum }}$} \\
\hline $\begin{array}{c}\text { Ins. } \\
33 \\
33 \\
33 \\
33 \\
36\end{array}$ & $\begin{array}{c}c m \\
83.83 \\
83.82 \\
83.82 \\
83.82 \\
91.44\end{array}$ & $\begin{array}{l}L b s . \\
12,000 \\
16,000 \\
20,000 \\
25,000 \\
12,000\end{array}$ & $\begin{array}{r}k g \\
5,443 \\
7,257 \\
9,072 \\
11,340 \\
5,443\end{array}$ & $\begin{array}{l}\text { Ins. } \\
51 / 4 \\
6 \\
61 / 2 \\
7 \\
534\end{array}$ & \begin{tabular}{l}
\multicolumn{1}{c}{$m m$} \\
133.35 \\
152.4 \\
165.1 \\
177.8 \\
146.05
\end{tabular} & $\begin{array}{l}\text { Ins. } \\
53 / 4 \\
61 / 2 \\
7 \\
71 / 2 \\
61 / 4\end{array}$ & $\begin{array}{l}\quad m m \\
146.05 \\
165.1 \\
177.8 \\
190.5 \\
158.75\end{array}$ & $\begin{array}{l}L b s . \\
650 \\
700 \\
750 \\
850 \\
740\end{array}$ & $\begin{array}{c}\mathrm{kg} \\
295 \\
318 \\
340 \\
386 \\
336\end{array}$ & $\begin{array}{l}L b s . \\
640 \\
690 \\
740 \\
835 \\
730\end{array}$ & $\begin{array}{c}\mathrm{kg} \\
290 \\
313 \\
336 \\
379 \\
331\end{array}$ \\
\hline $\begin{array}{l}36 \\
36 \\
36 \\
39 \\
39\end{array}$ & $\begin{array}{l}91.44 \\
91.44 \\
91.44 \\
99.06 \\
99.06\end{array}$ & $\begin{array}{l}16,000 \\
20,000 \\
25,000 \\
16,000 \\
20,000\end{array}$ & $\begin{array}{r}7,257 \\
9,072 \\
11,340 \\
7,257 \\
9,072\end{array}$ & $\begin{array}{l}61 / 4 \\
63 / 4 \\
71 / 4 \\
61 / 4 \\
63 / 4\end{array}$ & $\begin{array}{l}158.75 \\
171.45 \\
184.15 \\
158.75 \\
171.45\end{array}$ & $\begin{array}{l}63 / 4 \\
71 / 4 \\
73 / 4 \\
63 / 4 \\
71 / 4\end{array}$ & $\begin{array}{l}171.45 \\
184.15 \\
196.85 \\
171.45 \\
184.15\end{array}$ & $\begin{array}{l}800 \\
860 \\
980 \\
875 \\
950\end{array}$ & $\begin{array}{l}363 \\
390 \\
445 \\
395 \\
430\end{array}$ & $\begin{array}{l}785 \\
845 \\
960 \\
860 \\
930\end{array}$ & $\begin{array}{l}356 \\
383 \\
435 \\
390 \\
422\end{array}$ \\
\hline $\begin{array}{l}39 \\
42 \\
42 \\
42\end{array}$ & $\begin{array}{r}99.06 \\
106.68 \\
106.68 \\
106.68\end{array}$ & $\begin{array}{l}25,000 \\
16,000 \\
20,000 \\
25,000\end{array}$ & $\begin{array}{r}11,340 \\
7,257 \\
9,072 \\
11,340\end{array}$ & $\begin{array}{l}71 / 4 \\
61 / 2 \\
7 \\
71 / 2\end{array}$ & $\begin{array}{l}184.15 \\
165.1 \\
177.8 \\
190.5\end{array}$ & $\begin{array}{l}73 / 4 \\
7 \\
71 / 2 \\
8\end{array}$ & $\begin{array}{l}196.85 \\
177.8 \\
190.5 \\
203.2\end{array}$ & $\begin{array}{l}1,100 \\
1,000 \\
1,075 \\
1,225\end{array}$ & $\begin{array}{l}458 \\
454 \\
488 \\
566\end{array}$ & $\begin{array}{r}1,075 \\
975 \\
1,050 \\
1,190\end{array}$ & $\begin{array}{l}488 \\
442 \\
477 \\
540\end{array}$ \\
\hline
\end{tabular}


Rejection limits. - If any lot of wheels submitted for test fails to meet the requirements of drop, chill, fracture, thermal, or chemical test as regards combined carbon contents, all wheels of the same tape size shall be rejected. If any lot of wheels fails to meet chemical requirements, all wheels of that lot shall be rejected.

TABLE 5-A.-Dimensions of channel way in making thermal test

\begin{tabular}{|c|c|c|c|c|c|c|c|}
\hline \multirow{2}{*}{\multicolumn{2}{|c|}{ Diameter }} & \multirow{2}{*}{\multicolumn{2}{|c|}{$\begin{array}{l}\text { Weight of } \\
\text { wheel }\end{array}$}} & \multicolumn{3}{|c|}{ Channel way } & \multirow[b]{2}{*}{ Time } \\
\hline & & & & \multicolumn{2}{|c|}{$\begin{array}{l}\text { Width at } \\
\text { center of } \\
\text { tread }\end{array}$} & Depth & \\
\hline $\begin{array}{l}\text { Ins. } \\
33 \\
33 \\
33 \\
33 \\
36\end{array}$ & $\begin{array}{c}m m \\
838.2 \\
838.2 \\
838.2 \\
838.2 \\
914.4\end{array}$ & $\begin{array}{l}\text { Lbs. } \\
650 \\
700 \\
750 \\
850 \\
740\end{array}$ & $\begin{array}{c}k g \\
295 \\
318 \\
340 \\
386 \\
336\end{array}$ & $\begin{array}{l}\text { Ins. } \\
2 \\
2 \\
3 \\
3 \\
13 / 4\end{array}$ & $\begin{array}{l}m m \\
50.8 \\
50.8 \\
76.2 \\
76.2 \\
44.5\end{array}$ & $\begin{array}{cc}\text { Ins. } & m m \\
4 & 101.6 \\
4 & 101.6 \\
4 & 101.6 \\
4 & 101.6 \\
4 & 101.6\end{array}$ & $\begin{array}{c}\text { Min. } \\
3 \\
5 \\
5 \\
7 \\
2\end{array}$ \\
\hline $\begin{array}{l}36 \\
36 \\
36 \\
39 \\
39\end{array}$ & $\begin{array}{l}914.4 \\
914.4 \\
914.4 \\
990.6 \\
990.6\end{array}$ & $\begin{array}{l}800 \\
860 \\
980 \\
875 \\
950\end{array}$ & $\begin{array}{l}363 \\
390 \\
445 \\
395 \\
430\end{array}$ & $\begin{array}{l}13 / 4 \\
2 \\
2 \\
13 / 4 \\
2\end{array}$ & $\begin{array}{l}44.5 \\
50.8 \\
50.8 \\
44.5 \\
50.8\end{array}$ & $\begin{array}{ll}4 & 101.6 \\
4 & 101.6 \\
4 & 101.6 \\
4 & 101.6 \\
4 & 101.6\end{array}$ & $\begin{array}{l}2 \\
2 \\
2 \\
2 \\
2\end{array}$ \\
\hline $\begin{array}{l}39 \\
42 \\
42 \\
42\end{array}$ & $\begin{array}{r}990.6 \\
1,066.8 \\
1,066.8 \\
1,066.8\end{array}$ & $\begin{array}{l}1,100 \\
1,000 \\
1,225 \\
1,075\end{array}$ & $\begin{array}{l}488 \\
454 \\
566 \\
458\end{array}$ & $\begin{array}{l}2 \\
13 / 4 \\
2 \\
2\end{array}$ & $\begin{array}{l}50.8 \\
44.5 \\
50.8 \\
50.8\end{array}$ & $\begin{array}{ll}4 & 101.6 \\
4 & 101.6 \\
4 & 101.6 \\
4 & 101.6\end{array}$ & $\begin{array}{l}2 \\
2 \\
2 \\
2\end{array}$ \\
\hline
\end{tabular}

TABLE 5-B

\begin{tabular}{|c|c|c|c|c|c|}
\hline Diameter & $\begin{array}{l}\text { Weight of } \\
\text { wheel }\end{array}$ & $\begin{array}{l}\text { Weight of } \\
\text { tup }\end{array}$ & \multicolumn{2}{|c|}{$\begin{array}{l}\text { Height of } \\
\text { drop }\end{array}$} & $\begin{array}{c}\text { Num- } \\
\text { ber } \\
\text { of }\end{array}$ \\
\hline $\begin{array}{l}\text { Diamete } \\
\text { weig } \\
\text { wheel } \\
\text { in the } \\
\text { ing tal }\end{array}$ & $\begin{array}{l}\text { er and } \\
\text { ht of } \\
\text { same as } \\
\text { preced- } \\
\text { ble. }\end{array}$ & $\begin{array}{cc}L b s & k g \\
250 & 113 \\
250 & 113 \\
250 & 113 \\
250 & 113 \\
250 & 113 \\
& \\
250 & 113 \\
250 & 133 \\
250 & 113 \\
250 & 113 \\
250 & 113 \\
& \\
250 & 113 \\
250 & 113 \\
250 & 113 \\
250 & 113\end{array}$ & $\begin{array}{l}F t . \\
9 \\
101 / 2 \\
12 \\
15 \\
9 \\
\\
101 / 2 \\
12 \\
13 \\
101 / 2 \\
12 \\
\\
13 \\
101 / 2 \\
13 \\
12\end{array}$ & $\begin{array}{l}m \\
2.75 \\
3.20 \\
3.65 \\
4.57 \\
2.75 \\
\\
3.20 \\
3.65 \\
3.95 \\
3.20 \\
3.65 \\
3.95 \\
3.20 \\
3.95 \\
3.65\end{array}$ & $\begin{array}{l}12 \\
12 \\
12 \\
12 \\
12 \\
12 \\
12 \\
12 \\
12 \\
12 \\
12 \\
12 \\
12 \\
12\end{array}$ \\
\hline
\end{tabular}

\section{REQUIREMENTS FOR MAKING DROP TEST}

If the test wheel cracks in the plate with three blows or less, it will be considered as having failed.

Retest.- Should the test wheel selected for the drop test, thermal test, or chill test fail to meet the requirements specified, all wheels bearing the same tape size shall be rejected. The inspector shall then select another wheel of a different tape size, which shall be submitted to the same test as the wheel which failed and if it passes the requirements of the test the remainder of the lot shall be accepted, provided the requirements of all other tests have been met.

Rehearing on check analyses.-Samples for chemical analysis which represent wheels rejected for failure to meet chemical specifications shall be preserved for 30 days from the date of test report, so that in case of dissatisfaction with the results of the test, the manufacturer may have a rehearing. In the event the purchaser and the manufacturer shall not agree, the purchaser and the manufacturer shall have their respective chemists select a third chemist by mutual agreement, a joint analysis shall be made and the result so obtained shall be binding both on the purchaser and the manufacturer. The cost of such arbitration analysis shall be borne by the party in error.

(Inspection conforms to paragraph 7 of A. S. T. M. standard specification for steel. See 605.0, p. 247.)

\section{Section II. INDUSTRIAL WHEELS}

Wheels for industrial and steam-railway uses ranging in diameters from 10 inches $(25.40 \mathrm{~cm})$ to 30 inches $(76.20 \mathrm{~cm})$, including the standard designs of the Association of Manufacturers of Chilled Car Wheels, both of single-plate and double-plate design, which designs are recommended for adoption as standard, shall conform to the following requirements:

Material.-(Same as for locomotive, tender, and car wheels.)

Chill.-Depth of clean white iron in tread and throat sections shall conform to the following table:

TABLE 6

\begin{tabular}{|c|c|c|c|c|c|c|}
\hline \multirow{2}{*}{\multicolumn{2}{|c|}{ Wheel diameters }} & \multicolumn{3}{|c|}{ Maximum } & \multicolumn{2}{|c|}{ Minimum } \\
\hline & & $\mathrm{Mic}_{\mathrm{tr}}$ & $\begin{array}{l}\text { dle of } \\
\text { ead }\end{array}$ & $\begin{array}{c}\text { Middle of } \\
\text { throat }\end{array}$ & $\begin{array}{c}\text { Middle of } \\
\text { tread }\end{array}$ & $\begin{array}{l}\text { Middle of } \\
\text { throat }\end{array}$ \\
\hline $\begin{array}{l}\text { Ins. } \\
10-16 \\
18-24 \\
26-30\end{array}$ & $\begin{array}{c}c m \\
\text { 25. } 40-40.64 \\
\text { 45. } 72-60.96 \\
66.04-76.20\end{array}$ & $\begin{array}{l}\text { In. } \\
3 / 4 \\
7 / 8 \\
1^{7 / 8}\end{array}$ & $\begin{array}{r}m m \\
19.05 \\
22.23 \\
25.40\end{array}$ & $\begin{array}{lc}\text { In. } & m m \\
5 / 8 & 15.88 \\
3 / 4 & 19.05 \\
7 / 8 & 22.23\end{array}$ & $\begin{array}{cl}\text { In. } & m m \\
3 / 16 & 4.8 \\
1 / 4 & 6.35 \\
5 / 16 & 7.9\end{array}$ & $\begin{array}{rl}I_{\text {i }} & m m \\
\text { 1/8 } & 3.17 \\
3 / 16 & 4.8 \\
3 / 16 & 4.8\end{array}$ \\
\hline
\end{tabular}

This specification covers so wide a range of weights within the range of diameters given, it must be recognized that a deeper chill can safely be given in the wheels of the heavier weights, but that the limits shown in the above table should be followed with respect to the lighter-weight wheels of the diameters shown.

The blending of the white iron with the gray iron shall be in conformity with that for locomotive, tender, and car wheels.

Chemical composition.-Wheels shall conform to the following requirements as to chemical composition:

TABLe 7

\begin{tabular}{|c|c|c|}
\hline & $\begin{array}{l}\text { Mini- } \\
\text { mum }\end{array}$ & $\underset{\text { mumi- }}{\text { Maxi- }}$ \\
\hline & $\begin{array}{l}\text { Per } \\
\text { cent }\end{array}$ & $\begin{array}{l}\text { Per } \\
\text { cent }\end{array}$ \\
\hline $\begin{array}{l}\text { Combined carbon } \\
\text { Silicon }\end{array}$ & 0.45 & 0.90 \\
\hline Manganese. & .50 & .85 \\
\hline Phosphorus . . . . . . . . & - n & .40 \\
\hline 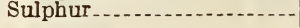 & $-\cdots$ & .18 \\
\hline
\end{tabular}

The minimum percentage of manganese shall under no condition be less than three times the sulphur content.

Analyses.- (Same as for locomotive, tender, and car wheels. See A. S. T. M., A46, given above.)

\section{Dimensions, Weights, and Designs}

Patterns and chillers shall be such that they will produce wheels according to dimensions shown on drawings.

Dimensions. - The normal circumference of the wheels produced by the chiller must be measured at a point $123 / 32$ inches $(43.65 \mathrm{~mm})$ from the gaging point on the throat of the flange. The gaging point is determined by a line drawn parallel to the flange and forming a tangent to the circle forming the throat.

The maximum allowable variation in the circumference above or below the normal circumference shall be governed by the following table: 
TABLE 8

\begin{tabular}{|c|c|c|c|c|}
\hline \multicolumn{2}{|c|}{ Diameter } & \multicolumn{2}{|c|}{$\begin{array}{c}\text { Normal } \\
\text { circumference }\end{array}$} & $\begin{array}{l}\text { Maximum } \\
\text { allowable } \\
\text { variation in } \\
\text { circumference } \\
\text { above or } \\
\text { below the } \\
\text { normal }\end{array}$ \\
\hline $\begin{array}{r}\text { Ins. } \\
10 \\
12 \\
14 \\
16 \\
18 \\
20\end{array}$ & $\begin{array}{c}c m \\
25.40 \\
30.48 \\
35.36 \\
40.64 \\
45.72 \\
50.80\end{array}$ & $\begin{array}{l}\text { Ins. } \\
31.42 \\
37.70 \\
43.95 \\
50.27 \\
56.55 \\
62.83\end{array}$ & $\begin{array}{c}c m \\
79.80 \\
95.76 \\
111.72 \\
127.68 \\
143.64 \\
159.60\end{array}$ & $\begin{array}{cc}\text { In. } & m m \\
1 / 8 & 3.2 \\
1 / 8 & 3.2 \\
3 / 16 & 4.8 \\
3 / 16 & 4.8 \\
3 / 16 & 4.8 \\
1 / 4 & 6.3\end{array}$ \\
\hline $\begin{array}{l}22 \\
24 \\
26 \\
28 \\
30\end{array}$ & $\begin{array}{l}55.88 \\
60.96 \\
66.04 \\
71.12 \\
76.20\end{array}$ & $\begin{array}{l}69.12 \\
75.40 \\
81.68 \\
87.86 \\
94.25\end{array}$ & $\begin{array}{l}175.56 \\
191.52 \\
207.4 \\
223.4 \\
239.4\end{array}$ & $\begin{array}{rr}1 / 4 & 6.3 \\
5 / 16 & 7.9 \\
5 / 16 & 7.9 \\
5 / 16 & 7.9 \\
5 / 16 & 7.9\end{array}$ \\
\hline
\end{tabular}

The thickness of the flange shall be regulated by a maximum and minimum flange gage, which thickness shall not vary in an individual wheel more than one-sixteenth inch $(1.59 \mathrm{~mm})$ over or more than one-sixteenth inch $(1.59 \mathrm{~mm})$ under the normal flange.

Taping.-Manufacturers shall not be required to tape wheels under 24 inches $(609.6 \mathrm{~mm})$ in diameter. Wheels 24 inches $(609.6 \mathrm{~mm})$ to 30 inches $(762.0 \mathrm{~mm})$ in diameter shall be taped in accordance with the requirements for locomotive, tender, and car wheels.

Weights.-(1) The maximum permissible loads under normal conditions for wheels of various weights and diameters are shown in Table 9 (which table is based on association standards). Wheels will be furnished in accordance with these weights.

TABLE 9

\begin{tabular}{|c|c|c|c|c|c|}
\hline \multicolumn{2}{|c|}{$\begin{array}{l}\text { Single plate } \\
\text { diameter }\end{array}$} & \multicolumn{2}{|c|}{$\begin{array}{l}\text { Maximum per- } \\
\text { missible load } \\
\text { per wheel }\end{array}$} & \multicolumn{2}{|c|}{$\begin{array}{c}\text { Nominal } \\
\text { weight }\end{array}$} \\
\hline $\begin{array}{c}\text { Ins. } \\
10 \\
10 \\
10 \\
10 \\
12\end{array}$ & $\begin{array}{r}c m \\
25.40 \\
25.40 \\
25.40 \\
25.40 \\
30.48\end{array}$ & $\begin{array}{r}L b s . \\
500 \\
1,000 \\
1,800 \\
2,200 \\
600\end{array}$ & $\begin{array}{c}\mathrm{kg} \\
227 \\
454 \\
726 \\
998 \\
272\end{array}$ & $\begin{array}{c}L b s \\
35 \\
45 \\
65 \\
70 \\
47\end{array}$ & $\begin{array}{l}k g \\
16 \\
20 \\
25 \\
32 \\
21\end{array}$ \\
\hline $\begin{array}{l}12 \\
12 \\
12 \\
14 \\
14\end{array}$ & $\begin{array}{l}30.48 \\
30.48 \\
30.48 \\
35.56 \\
35.56\end{array}$ & $\begin{array}{r}1,300 \\
2,100 \\
2,900 \\
700 \\
1,000\end{array}$ & $\begin{array}{r}590 \\
954 \\
1,315 \\
318 \\
454\end{array}$ & $\begin{array}{l}60 \\
78 \\
95 \\
57 \\
75\end{array}$ & $\begin{array}{l}27 \\
35 \\
43 \\
26 \\
34\end{array}$ \\
\hline $\begin{array}{l}14 \\
14 \\
14\end{array}$ & $\begin{array}{l}35.56 \\
35.56 \\
35.56\end{array}$ & $\begin{array}{l}2,000 \\
3,000 \\
4,000\end{array}$ & $\begin{array}{r}909 \\
1,361 \\
1,814\end{array}$ & $\begin{array}{r}90 \\
115 \\
140\end{array}$ & $\begin{array}{l}41 \\
52 \\
64\end{array}$ \\
\hline $\begin{array}{l}16 \\
16\end{array}$ & $\begin{array}{l}40.64 \\
40.64\end{array}$ & $\begin{array}{r}800 \\
1.600\end{array}$ & $\begin{array}{l}363 \\
726\end{array}$ & $\begin{array}{r}80 \\
105\end{array}$ & $\begin{array}{l}36 \\
48\end{array}$ \\
\hline $\begin{array}{l}16 \\
16 \\
16 \\
18 \\
18 \\
18 \\
18 \\
18 \\
20 \\
20\end{array}$ & $\begin{array}{l}40.64 \\
40.64 \\
40.64 \\
45.72 \\
45.72 \\
45.72 \\
45.73 \\
45.72 \\
50.80 \\
50.80\end{array}$ & $\begin{array}{r}2,800 \\
4,000 \\
5,200 \\
900 \\
2,200 \\
3,600 \\
5,000 \\
6,400 \\
1,000 \\
2,800\end{array}$ & $\begin{array}{r}1,270 \\
1,814 \\
2,359 \\
408 \\
998 \\
1,633 \\
2,268 \\
2,903 \\
454 \\
1,270\end{array}$ & $\begin{array}{l}130 \\
155 \\
180 \\
100 \\
120 \\
163 \\
210 \\
245 \\
140 \\
180\end{array}$ & $\begin{array}{r}59 \\
70 \\
82 \\
45 \\
54 \\
75 \\
95 \\
111 \\
64 \\
82\end{array}$ \\
\hline $\begin{array}{l}20 \\
20 \\
20 \\
22 \\
22\end{array}$ & $\begin{array}{l}50.80 \\
50.80 \\
50.80 \\
55.88 \\
55.88\end{array}$ & $\begin{array}{l}4,400 \\
6,000 \\
7,600 \\
1,100 \\
3,400\end{array}$ & $\begin{array}{r}1,995 \\
2,722 \\
3,447 \\
499 \\
1,542\end{array}$ & $\begin{array}{l}215 \\
265 \\
315 \\
160 \\
215\end{array}$ & $\begin{array}{r}98 \\
120 \\
143 \\
72 \\
98\end{array}$ \\
\hline $\begin{array}{l}22 \\
22 \\
22 \\
24\end{array}$ & $\begin{array}{l}55.88 \\
55.88 \\
55.88 \\
60.96\end{array}$ & $\begin{array}{l}5,200 \\
7,000 \\
8,800 \\
1,200\end{array}$ & $\begin{array}{r}2,359 \\
3,175 \\
3,992 \\
544\end{array}$ & $\begin{array}{l}280 \\
325 \\
385 \\
185\end{array}$ & $\begin{array}{r}127 \\
147 \\
175 \\
84\end{array}$ \\
\hline $\begin{array}{l}24 \\
22 \\
24 \\
24\end{array}$ & $\begin{array}{l}60.96 \\
60.96 \\
60.96 \\
60.96\end{array}$ & $\begin{array}{r}4,000 \\
6,000 \\
8,000 \\
10,000\end{array}$ & $\begin{array}{l}1,814 \\
2,722 \\
3,629 \\
4,536\end{array}$ & $\begin{array}{l}245 \\
330 \\
395 \\
450\end{array}$ & $\begin{array}{l}111 \\
150 \\
179 \\
204\end{array}$ \\
\hline
\end{tabular}

TABLE 9-Continued

DOUBLE PLATE

\begin{tabular}{|c|c|c|c|c|c|}
\hline \multicolumn{2}{|c|}{$\begin{array}{l}\text { Single plate } \\
\text { diameter }\end{array}$} & \multicolumn{2}{|c|}{$\begin{array}{l}\text { Maximum per- } \\
\text { missible load } \\
\text { per wheel }\end{array}$} & \multicolumn{2}{|c|}{$\begin{array}{c}\text { Nominal } \\
\text { weight }\end{array}$} \\
\hline $\begin{array}{c}\text { Ins. } \\
24 \\
24 \\
24 \\
24\end{array}$ & $\begin{array}{c}c m \\
60.96 \\
60.96 \\
60.96 \\
60.96\end{array}$ & $\begin{array}{r}\text { Lbs. } \\
6,000 \\
9,000 \\
12,000 \\
16,000\end{array}$ & $\begin{array}{c}\mathrm{kg} \\
2,722 \\
4,082 \\
5,443 \\
7,257\end{array}$ & $\begin{array}{l}L b s . \\
320 \\
360 \\
400 \\
440\end{array}$ & $\begin{array}{l}k g \\
145 \\
163 \\
181 \\
200\end{array}$ \\
\hline $\begin{array}{l}26 \\
26 \\
26 \\
26\end{array}$ & $\begin{array}{l}66.04 \\
66.04 \\
66.04 \\
66.04\end{array}$ & $\begin{array}{r}6,000 \\
9,000 \\
12,000 \\
16,000\end{array}$ & $\begin{array}{l}2,722 \\
4,082 \\
5,443 \\
7,257\end{array}$ & $\begin{array}{l}360 \\
410 \\
460 \\
510\end{array}$ & $\begin{array}{l}163 \\
186 \\
209 \\
231\end{array}$ \\
\hline $\begin{array}{l}28 \\
28 \\
28 \\
28\end{array}$ & $\begin{array}{l}71.12 \\
71.12 \\
71.12 \\
71.12\end{array}$ & $\begin{array}{r}6,000 \\
9,000 \\
12,000 \\
16,000\end{array}$ & $\begin{array}{l}2,722 \\
4,082 \\
5,443 \\
7,257\end{array}$ & $\begin{array}{l}410 \\
460 \\
510 \\
560\end{array}$ & $\begin{array}{l}186 \\
209 \\
231 \\
254\end{array}$ \\
\hline $\begin{array}{l}30 \\
30 \\
30 \\
30\end{array}$ & $\begin{array}{l}76.20 \\
76.20 \\
76.20 \\
76.20\end{array}$ & $\begin{array}{r}6,000 \\
9,000 \\
12,000 \\
16,000\end{array}$ & $\begin{array}{l}2,722 \\
4,082 \\
5,443 \\
7,257\end{array}$ & $\begin{array}{l}485 \\
530 \\
575 \\
615\end{array}$ & $\begin{array}{l}220 \\
240 \\
259 \\
277\end{array}$ \\
\hline
\end{tabular}

In the case of either single or double plate wheels the width of tread or length of hub may be increased, or the diameter of the core reduced as required, but the additional weight shall be considered as addi-

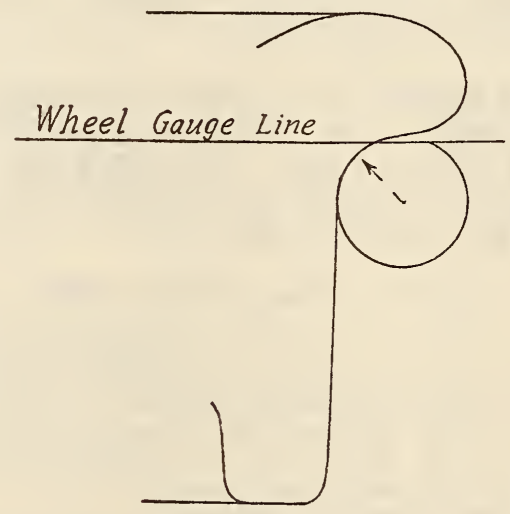

FIgure 418

tional to the normal weight given in the table above and be paid for by the purchaser.

In the case of double-plate wheels ordered with cores smaller in diameter than the standard, the additional weight shall be considered as additional to the normal weights given in the table above and shall be paid for by the purchaser.

In any shipment of wheels ordered with standard core sizes, where the average weight of the wheels is above the normal weight for such shipment, the excess weight shall be at the expense of the manufacturer.

\section{Inspection}

General conditions and surface shall be governed by requirements referred to above under car wheels.

Marking.-Shall be as provided for in A. S. T. M., A 46, given above under car wheels, except that no tape-number plate shall be required on wheels under 24 inches $(609.6 \mathrm{~mm})$ in diameter on account of lack of space on the inside or back plate of these wheels.

Sampling.-Wheels, when ready for inspection, shall be arranged in groups and where purchaser requires tests for each 51 wheels which pass surface inspection and are ready for shipment, one repre- 
sentation shall be furnished by the manufacturer without expense, to be broken for chill test. Where there are less than 50 wheels to be inspected the test wheel or wheels taken for chill test shall be paid for by the purchaser unless by special agreement with the manufacturer.

Drop and thermal tests.-No drop or thermal test shall be required on wheels of 10 inches $(254.0 \mathrm{~mm})$ to 30 inches $(762.0 \mathrm{~mm})$ diameter inclusive.

Chill test.-The wheel selected for chill test shall be broken so that the chill may be examined. The depth of pure white iron shall conform to requirements give above under "chill." If the sample does not conform to requirements stated, all wheels submitted shall then be taped and the inspector shall select another wheel. If this wheel does not meet the requirements, all wheels of the same tape size shall be rejected and the test continued on other tape sizes. The inspector shall then select another wheel of a different tape size, which shall be broken for chill test as specified, and if it passes the requirements the remainder of the lot shall be accepted provided the requirements of all other tests have been met.

Rejection.- If any lot of wheels fails to meet requirements of chill test or fails to meet chemical requirements, such wheels shall be rejected. Individual wheels may be rejected as provided for above, and wheels may be rejected at destination as provided for above under "car wheels."

Rehearing on check analyses.- Shall be as provided for above under "car wheels."

\section{ASSOCIATION OF MANUFACTURERS OF CHILLED CAR WHEELS, STANDARD PRACTICE FOR FOUNDRIES AND SPECIFICATIONS FOR TESTS, CHILLED TREAD WHEELS, 1930}

\section{CHILLED TREAD WHEEL TESTS}

(In respect to composition, chill-test block, temperature of molten metal, ladles, last iron, pouring, pits, pit covers, pyrometers, selection of test wheels, plant operation, and records, this specification conforms to A. R. A.-M. cast-iron wheels for locomotives, tenders, and cars, given above, with the following exceptions and additions:)

Chill-test block. - The size of the test bar shall be $1 \frac{1}{2}$ inches wide, 3 to 4 inches thick, and $6 \frac{1}{2}$ to 8 inches long.

Analysis-test block.-(a) A test block 3 by 3 inches minimum section and $4 \frac{1}{2}$ inches minimum in length shall be cast, preferably in dry sand, one test bar poured from the first and one from the last tap, and at least two bars from intermediate parts of the heat.

(b) The drillings shall be obtainec from the enci of the test block. The first drillings shall be discarded, the drilling continued, and the resultant chips, etc., dumped from the hole and taken for sample, or the block may be broken and the drillings taken at the point of fracture.

(c) An analysis may be made for each block and recorded, or, at the option of the manufacturer, the samples may be mixed of equal quantities, but at least one analysis shall be made to represent the day's heat.

Ladles.-Ladles may be heated in specially designed ovens in which they can be thoroughly baked throughout, or they may be heated by the use of a forced-blast fire using coke, oil, or gas as fuel; in which case, before using, the ladles shall be filled at least two-thirds full of molten iron. After two minutes the metal shall be discarded and the ladle immediately placed in service.
Pouring.-The rate of pouring off the heat should be held constant with the melting rate of the cupola. The number of ladles used shall be so regulated that there will be no long wait between taps. Ladles held 4 minutes shall be drained, and if not used for 10 minutes or more shall be treated as a green ladle and reheated or washed before being used.

Shaking out.-(a) Wheels shall be shaken out from the mold as quickly as permissible without bleeding. The interval of time between pouring and shaking out must not exceed the following limits:

\begin{tabular}{|c|c|c|}
\hline $\begin{array}{c}\text { Weight } \\
\text { of wheel }\end{array}$ & $\begin{array}{c}\text { Single } \\
\text { plate }\end{array}$ & $\begin{array}{c}\text { Double } \\
\text { plate }\end{array}$ \\
\hline Pounds & Minutes & Minutes \\
650 & 45 & 35 \\
700 & 50 & 40 \\
750 & 55 & 45 \\
850 & 60 & 50 \\
\hline
\end{tabular}

(b) After the wheels are sbaken out they must be transferred to the annealing pit and pitted, not to exceed six minutes for the entire operation; the average time should not exceed four minutes. The time shall start with the lifting of the cope.

(c) The surface of the plate of the wheel shall not be less than $1,350^{\circ} \mathrm{F}$. when pitted.

Pits.-(a) At the time of pitting, the temperature of the air in the middle of the primary pit, with the over closed, shall be not less than $700^{\circ} \mathrm{F}$., preferably $900^{\circ} \mathrm{F}$. or over.

(b) When the temperature of the pit drops below the minimum, artificial means shall be used for raising the temperature.

(c) Temperatures of not less than five representative primary pits shall be taken and recorded before refilling.

Annealing temperatures.-(a) The rate of cooling shall be such that approximately 24 hours after the pit is closed the temperature of the top wheel shall be not less than $900^{\circ} \mathrm{F}$., after two days not less than $750^{\circ} \mathrm{F}$, and after three days not less than $650^{\circ} \mathrm{F}$.

(b) Temperatures of not less than five representative top-pit wheels shall be taken and recorded at time of removal.

(c) When secondary pits are not used, the toppit wheel must either be repitted or cover wheels used.

Pit covers.-(a) The cover shall be so insulated as to prevent, as far as possible, rapid transfer of heat from the top wheel. A space equal to at least one wheel shall exist between the bottom of the cover and the top of the wheel, but if by this method the temperature of the plate of the top wheel at the end of approximately 20 hours is less than $900^{\circ} \mathrm{F}$., a space equal to two wheels shall exist between the bottom of the cover and the top wheel.

(In respect to drop test and thermal test, this specification conforms to A. S. T. M., A46, given above, with the following exceptions and additions:)

Drop test.- (a) In addition to the requirements of paragraph $9(\mathrm{c})$ of A. S. T. M., A46, given above, care should be taken to see that the tup falls free in the guides and that the supporting bosses are solidly anchored to the large base plate, and so located that only the back of the flange rests on them. The space betweeen the hub of the wheel and the base plate shall be kept free from sand and broken pieces of wheel.

(b) The tup shall weigh 250 pounds when first placed in service, and shall be removed when found to weigh less than 247 pounds.

(c) If test wheel cracks in the plate with nine blows or less, it will be considered as having failed. 
(d) The drop tests shall be to destruction. (After 36 blows the height of drop may be increased or big drop used.)

Thermal test.-

\begin{tabular}{|c|c|c|}
\hline $\begin{array}{c}\text { Weight of } \\
\text { wheel }\end{array}$ & \multicolumn{2}{|c|}{ Double plate } \\
\cline { 2 - 3 } & $\begin{array}{c}\text { Cooling } \\
\text { time }\end{array}$ & $\begin{array}{c}\text { Width of } \\
\text { channel- } \\
\text { way }\end{array}$ \\
\hline Pounds & Minutes & Inches \\
650 & 2 & 134 \\
700 & 2 & 134 \\
750 & 2 & 2 \\
850 & 2 & 2 \\
\hline
\end{tabular}

The wheel shall be observed for at least 10 minutes after the ring is poured to note and record any structural failure.

Chill on wheels. - The depth of chill shall be not less than one-half inch and not more than $11 / 4$ inches at the center line of tread. The blending of the chill with the gray iron shall be without any distinct line of demarcation. The depth of chill shall not vary more than one-fourth inch around the tread in any one plane in the same wheel. These limits apply to all weights of wheels.

Tape lines.-Tape lines should be checked daily on a car wheel used as a master. This wheel shall be an exact tape 3 as checked by association master tape line. The exact weight of the master wheel should also be determined and the correct weight stamped on same, so that it may be used for checking the scales used for weighing the new wheels.

NATIONAL ELECTRICAL MANUFACTURERS ASSOCIATION, WHEEL CONTOUR, RECOMMENDED PRACTICE, 1923

\section{WHEEL CONTOUR}

The standard contour of locomotive wheels shall be as in Figure 419.

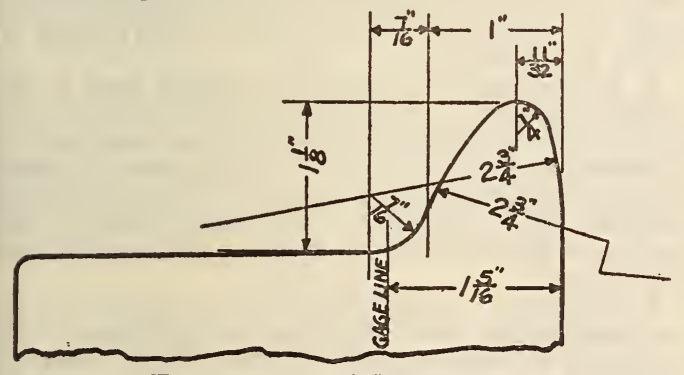

FIgURE 419.-Wheel contour

611.19 MISCELLANEOUS MANUFACTURES OF GRAY CAST IRON.

AMERICAN MARINE STANDARDS COMMITTEE, SPECIFICATION FOR BUILTUP PROPELLERS, E NO. 23-1928

\section{BUILT-UP PROPELLERS}

(A) GENERAL CONDITIONS

1. This specification is issued only for general guidance. It defines a complete propeller but is intended to apply only to the items actually included in the contract or purchase order. Such details as may be omitted in whole or in part in this specifica- tion and which are included in the purchase order in form of either material or workmanship shall be supplied in accordance with the best modern practice.

2. In all cases where propellers are to be subject to inspection and approval by ship-classification societies, the requirements of such societies shall govern as to compositions, grades, and tests of the materials, also thicknesses of blades.

\section{(B) COMPONENT PARTS}

1. For the purpose of this specification, a complete built-up propeller shall be understood to consist of the following parts unless exceptions are made in the purchase order: $(a)$ hub; $(b)$ blades, including spares, if ordered; $(c)$ blade studs, including spares, if ordered; $(d)$ stud nuts, including spares, if ordered; (e) lock screws for stud nuts, including spares, if ordered; $(f)$ studs, nuts, and split pins for fair-water cap; and $(g)$ plugs fitted in filling holes.

2. The following additional parts, or either of them, shall be furnished with the propeller wheel if specifically ordered by the purchaser: (a) A fairwater cap fitted and secured on the hub and (b) A packing gland conforming to American Marine Standard E No. 12-1927 (publication A. M. S. C. 31), complete, with studs, nuts, and split pins.

\section{(C) MATERIALS}

1. The purchaser should specify the characteristics of the materials desired in asking for quotations and in purchasing. When the characteristics of the materials are not definitely specified, the minimum requirements for grade and tests of any of the materials hereinafter mentioned shall be those specified by the American Bureau of Shipping. (See A. B. S. rules for building and classing steel vessels, secs. 33 and 40.) The standard specifications of the American Society for Testing Materials may be used in conjunction herewith to define the characteristics of the materials to be furnished, provided that their requirements are not less than those of the American Bureau of Shipping.

2. Hubs.-These may be of either cast iron, semisteel, or cast steel, as specified by the purchaser in asking for quotations and in ordering.

(a) Cast-iron hubs. - These shall be high-grade gray-iron castings of uniform grain, without blowholes, porous spots, or other defects that might impair strength or lasting quality of the propeller in service.

(b) Semisteel hubs.-These shall be high-grade gray-iron castings, containing a proper proportion of good steel scrap. The steel scrap shall be introduced into the iron in the cupola and not in the pouring ladle. The castings shall be of close grain, without blowholes, porous spots, or other defects that might impair strength or lasting quality of the propeller in service.

(c) Cast-steel hubs.-The castings shall be of the kind generally referred to in trade as soft steel. They shall be sound, without blowholes, porous spots, or other defects that might impair strength or lasting quality of the propeller in service, and they shall be annealed.

3. Blades. - These shall be either of cast steel or of manganese bronze, as specified by the purchaser in asking for quotations and in ordering.

(a) Cast-steel blades.-(1) The castings shall be of the kind generally referred to in trade as soft steel. They shall be sound, without blowholes, porous spots, or other defects that might impair strength or lasting quality of the propeller in service. They shall be annealed in a suitable furnace of ample proportions to bring the whole casting to a uniform annealing temperature. They must not be removed 
from the annealing furnace until the temperature has dropped to $700^{\circ} \mathrm{F}$. or lower.

(2) Local heating, bending, or straightening of annealed blade castings shall be permitted only if approved by representatives of the purchaser and the inspection authorities.

(b) Bronze blades. - (1) The actual manganesebronze composition of the castings shall be left to the manufacturer unless the purchaser should specify his own requirements, in which case the latter shall govern.

(2) The castings shall be sound, without blowholes, sponge, dross, cold shuts, seams, cracks, and all other defects which might impair the strength or lasting quality of the propeller in service. All surfaces shall be smooth.

(3) Scrap shall not be used in the castings, but metal of identical composition remaining from propeller castings made by the same manufacturer may be used to a proportion not exceeding 50 per cent.

(4) Only virgin copper pigs 99.8 per cent pure and spelter 99.7 per cent pure shall be used in the manganese-bronze ingots which shall form at least 50 per cent of each heat.

4. Blade studs. - The studs shall be of high-grade forged steel. Cold-rolled or cold-drawn steel shall in no case be used.

5. Stud nuts.-The nuts shall be of high-grade bronze having physical characteristics equivalent to those required for bronze blades.

6. Lock screws for stud nuts. - These shall be of high-grade rolled bronze having characteristics equivalent to those required for bronze blades.

7. Fair-water cap.-This shall be of cast iron, semisteel, cast steel, or bronze as may be specified by the purchaser in asking for quotations and in ordering. In any case the castings shall be of the same grade as hereinbefore specified for castings of the respective materials, and they shall be equally free of defects.

8. Studs, nuts, and splut pins for fair-water cap.The studs shall be of steel for cast-iron, semisteel, or cast-steel propellers, and of high-grade bronze for bronze propellers. The nuts shall be of highgrade bronze in all cases, but of different composition from bronze studs. The split pins shall be of commercial standard bronze.

9. Plugs for filling holes.-These shall be commercial standard cast-brass pipe plugs.

10. Packing gland. - If included in the order, the gland shall be cast of high-grade bronze possessing physical characteristics equivalent to those required for bronze propellers. The studs shall be of highgrade steel for cast-iron, semisteel, or cast-steel propellers, and of high-grade rolled bronze for bronze propellers. The nuts shall be of high-grade bronze in all cases, but of different composition from bronze studs. The split pins shall be of commercial standard bronze.

\section{(D) TESTS AND INSPECTION OF MATERIAL}

1. When the conditions of purchase call for physical tests and chemical analyses of any of the materials, the manufacturer shall furnish to the purchaser the requisite certificates of tests and analyses, identified with the test coupons or samples and the materials they represent. The tests and analyses shall be made and certified by a recognized testing laboratory, and the cost of the testing shall be borne by the manufacturer.

2 . For vessels classing with any of the ship-classification societies, test coupons shall be marked by the surveyor for identification before they are detached from the castings. Corresponding marks shall be placed on the castings. On blade castings this mark shall be placed in line with the point of attachment of the coupon.
3. All physical tests snall be made in the presence of representatives of the purchaser and the inspection authorities. This, however, may be waived by agreement if the said representatives are willing to accept matching of fractures.

4. Each blade casting shall be hung free and hammered for detection of possible shrinkage cracks and other flaws.

5. Any casting or other material developing defects in the process of machining shall be replaced without cost to the purchaser, notwithstanding any prior acceptance of the unfinished casting or material.

\section{(E) MACHINING}

(Included in this specification are details of machine work on hubs, blades, blade studs, nuts, lock screws, screw threads, boltheads, and nuts.)

\section{(F) ACCURACY OF DIMENSIONS AND PITCH}

1. Castings shall be true to drawings or patterrs furnished by the purchaser. Variations from the designed dimensions when the pattern is furnished by the manufacturer or from the pattern dimensions when the pattern is furnished by the purchaser must not exceed the proper tolerances generally allowed in modern foundry practice.

2. All necessary facilities shall be furnished by the manufacturer for measuring the pitch of the blades. The pitch shall be carefully set and verified in the presence of the representative of the purchaser.

3. When each blade is mounted on the hub with the pitch accurately set to that called for by the design at a radius between 66 to 75 per cent (preferably 70 per cent) of the radius to the blade tip, the variation from the designed pitch at any other radius shall not, unless otherwise specified in the purchase order, exceed 2 per cent plus or minus if the pattern is made by the manufacturer, or 2 per cent plus or minus from the pitch of the pattern when the latter is furnished by the purchaser.

\section{(G) ASSEMBLING AND BALANCING}

1. All blades shall be interchangeable on all faces of the hub, and in case of doubt on this point they shall be assembled to the hub to verify their fit before shipment.

2 . In the case of 4-bladed propellers each of the blades, including spares, snall be weighed. The two nearest the same weight shall be mounted on opposite sides of the hub. The pair next nearest the same weight shall be mounted on the remaining opposite sides of the hub. The blades and the hub shall be marked to identify the location of each blade thus placed.

3. Assembled propellers shall be balanced as may be specified in the conditions of purchase. The purchaser's requirements should in all cases be ascertained, as the degree of accuracy of balance required depends upon the service conditions for the propeller.

Note.-It is recommended that wheels for high-speed work be brought to a perfect static balance. For low or medium speed work, the final difference in weight to be allowed between blades should not tip of the blade:
Propeller diameter:
7 to 9.5 feet
9.5 to 12 feet
12 to 15 feet.
18.5 to 21 feet. Weight (pounds)
(1.00

4. If the propeller is to be permanently assembled at the place of manufacture before shipment, the spaces in the blade flanges surrounding the studs when the blades are finally set shall be solidly filled with hardwood properly fitted in natural dry con- 
dition. White metal shall be used for filling instead of wood if specified in the conditions of purchase.

(H) MAIUFACTURER'S GUARANTY

When the conditions of purchase do not require tests of the materials to determine their physical characteristics, the manufacturer shall, if required by the purchaser, guarantee that the materials furnished by him will not break due to defects within one year from the date of shipment when the ship is operated under normal conditions in free water. Should disagreement arise between the manufacturer and purchaser in case of breakage apparently due to defective material, the question shall be determined by a representative of ship-classification societies to be designated by the purchaser and a representative of the manufacturer designated by him. Should these two fail to agree, they shall appoint a third party, satisfactory to the manufacturer and purchaser, whose decision shall be final.

\section{(J) MARKINGS}

1. When the blades are assembled on the hub at the pitch called for by the purchase order or drawing, coinciding index lines shall be marked on each flange and the hub, for guidance in reassembling the propeller and setting the blades at the proper pitch. These index lines shall also be put on the spare blades. If included in the conditions of purchase, brass pins shall be tapped into the flanges of cast-steel blades to receive these lines. The lines shall be identified on 3-bladed propellers by stamping the blades with the numbers 1, 2, and 3, respectively, and on 4-bladed propellers by stamping the blades with the numbers $1,2,3$, and 4 , respectively. Corresponding numbers shall be stamped near the lines on the hubs.

2. Nuts, studs, and lock screws shall, in general, be interchangeable, but, in order to provide for the contingency of slight differences which might cause difficulty in reassembling, they shall be marked by stamping and corresponding marks shall be stamped at their respective locations on the various parts for individual identification as fitted.

3. The manufacturer's name and location of his plant shall either be cast in raised letters not less than one-eighth inch high, or stamped on the fillet on the back of each blade. Under this shall be stamped the name of the ship if known, also the manufacturer's order number, weight of blade, and date of manufacture. Spare blades shall be stamped "Spare," in addition to the other markings, in a conspicuous manner on both sides of the blade. All markings shall be put on before shipment.

\section{(K) SHIPMENT}

1. The parts shall be completely assembled for shipment or shipped separately as directed by the purchaser. If shipped separately, all shall be marked for proper assembling as hereinafter specified.

2. In preparing assembled propellers or separate parts for shipment the manufacturer shall place adequate protection over all finished parts to safeguard them against damage or deterioration under normal conditions in handling or in transit. Hubs may be shipped with studs, nuts, and lock screws in place, provided that they are properly protected.

3. The manufacturer shall be responsible for proper loading and blocking of assembled propellers or parts so as to comply with transportation regulations.

4. If spare blades or studs are included in the order, they shall accompany the shipment unless otherwise directed by the purchaser.

\section{AMERICAN MARINE STANDARDS COM- MITTEE, SPECIFICATION FOR PROPEL- IERS CAST IN ONE PIECE, E NO. 25- 1928}

\section{CAST PROPELLERS}

(In respect to general conditions, characteristics of materials, tests and inspection of material, accuracy of dimensions and pitch, balancing, manufacturer's guaranty, and shipment, this specification is like A. M. S. C., E23, given above. It differs from E23 in the following particulars:)

\section{(B) COMPONEIT PARTS}

1. Unless otherwise specified, the order for a solid propeller shall be understood to cover the propeller wheel only, fully machined, with plugs fitted in filling holes.

2. The following additional parts or either of them shall be furnished with the propeller wheel if specifically ordered by the purchaser:

(a) A fair-water cap fitted and secured on the hub.

(b) A packing gland conforming to American Marine Standard E No. 12-1927 (publication A. M. S. C., 31), complete with studs, nuts, and split pins.

\section{(C) MATERIALS}

1. Solid propellers may be required of cast iron, semisteel, cast steel, or bronze. The purchaser shall specify which one of the materials is to be furnished.

2. Cast-iron propellers.-These shall be high-grade gray-iron castings of uniform grain, without blowholes, porous spots, or other defects that might impair strength or lasting quality of the propeller in service.

3. Semisteel propellers.-These shall be high-grade gray-iron castings, containing a proper proportion of good steel scrap. The steel scrap shall be introduced into the iron in the cupola and not in the pouring ladle. The castings shall be of close grain, without blowholes, porous spots, or other defects that might impair strength or lasting quality of the propeller in service.

4. Cast-steel propellers.-(a) The castings shall be of the kind generally referred to in trade as soft steel. They shall be sound, without blowholes, porous spots, or other defects that might impair strength or lasting quality of the propeller in service. They shall be annealed in a suitable furnace of ample proportions to bring the whole casting to a uniform annealing temperature. They must not be removed from the annealing furnace until the temperature has dropped to $700^{\circ} \mathrm{F}$. or lower.

(b) Local heating, bending, or straightening of annealed castings shall be permitted only if approved by representatives of the purchaser and the inspection authorities.

5. Bronze propellers.-(a) The actual manganesebronze composition of the castings shall be left to the manufacturer unless the purchaser should specify his own requirements, in which case the latter shall govern.

(b) The castings shall be sound, without blow. holes, sponge, dross, cold shuts, seams, cracks, and all other defects which might impair the strength or lasting quality of the propeller in service. All surfaces shall be smooth.

(c) Scrap shall not be used in the castings, but metal of identical composition remaining from propeller castings made by the same manufacturer may be used to a proportion not exceeding 50 per cent. 
(d) Only virgin copper pigs 99.8 per cent pure and spelter 99.7 per cent pure shall be used in the manganese-bronze ingots which shall form at least 50 per cent of each heat.

6. Fair-water cap.-If included in the order and unless otherwise specified, the fair-water cap shall be cast of the same material as the propeller and shall be equally free of defects.

7. Studs, nuts, and split pins for fair-water cap.The studs shall be of steel for cast-iron, semisteel, or cast-steel propellers, and of high-grade bronze for bronze propellers. The nuts shall be of high-grade bronze in all cases, but of different composition from bronze studs. The split pins shall be of commercial standard bronze.

8. Plugs for filling holes.-These shall be commercial standard cast-brass pipe plugs.

9. Packing gland.- If included in the order, the gland shall be cast of high-grade bronze possessing physical characteristics equivalent to those required for bronze propellers. The studs shall be of highgrade steel for cast-iron, semisteel, or cast-steel propellers, and of high-grade rolled bronze for bronze propellers. The nuts shall be of high-grade bronze in all cases, but of different composition from bronze studs. The split pins shall be of commercial standard bronze.

\section{(E) MACHINING}

(Included in this specification are details of machine work on hubs, blades, screw threads, boltheads, and nuts.)

\section{(J) MARKINGS}

Each propeller shall be marked with the manufacturer's name and location of his plant and the name of the ship if known; also with the manufacturer's order number and date of manufacture. These markings shall either be cast on in raised letters not less than one-eighth inch high, or stamped in a conspicuous place other than on the face of the blades.

\section{AMERICAN MARINE STANDARDS COM- MITTEE, BOLLARDS AND CLEATS FOR DOCKS, $P$ NO. 1 TO $P$ NO. 4, 1931}

\section{BOLLARDS AND CLEATS}

(This specification covers five sizes of single bollards, cleats, and bollard cleats, and three sizes of double bollards for both cast-iron and cast-steel construction. The external forms and dimensions are identical for both metals, the variations being in the thicknesses and cores. Iron castings conform to A. S. T. M., A48, steel castings conform to class B steel castings in A. S. T. M., A27, steel for bolts and nuts conforms to A. S. T. M., A9. Screw threads in bolts and nuts conform to A. S. A., B1a-1924, while the dimensions of boltheads and nuts conform to A. S. A., B18b-1927.)

AMERICAN RAILWAY ASSOCIATION, MECHANICAL DIVISION; WELDING WIRE AND RODS, RECOMMENDED PRACTICE, 1924

\section{WELDING WIRE AND RODS}

(See 603.41, p. 156; 631.31, p. 1157; and 645.11, p. 1203.)
AMERICAN RAILWAY ENGINEERING ASSOCIATION, SPECIFICATIONS FOR RAILWAY BUILDINGS, ORNAMENTAL AND MISCELLANEOUS METAL WORK, 1929

\section{CASTINGS}

Ornamental castings shall be made in stove plate molding sand. Cast metal shall be uniform in thickness throughout, free from sand, holes, or other defects. Ornamental castings shall not exceed three-eighths inch thickness unless shown on drawings or otherwise permitted. Ornamental details shall be strictly adhered to and profiles made in accordance with full-size drawings. Surface lines and straight-line moldings shall be hand filed, ground, and soft wheeled. Ornamental detail shall be modeled in clay and then cast directly from the plaster reproduction of the clay model. No ornamental detail shall be cast from wood patterns or molds.

The exposed surfaces of ornamental castings shall be perfectly clean and sharp with the ornamental detail brought out clear and strong, well modeled in good relief and undercut when necessary, and with all lines, arrises, profiles, and ornaments true and in accordance with details and approved models. Pierced work shall have openings and outlines free from irregularities. Castings shall be neatly filed and chased to remove any superficial imperfections, and shall be altered if necessary until they are satisfactory to the engineer in all respects.

(For bronze castings, see 646.41, p. 1250.)

\section{AMERICAN RAILWAY ASSOCIATION, MECHANICAL DIVISION, STANDARD BRAKE SHOES, 1910}

\section{BRAKE SHOES}

(See 611.43, p. 726.)

\section{NATIONAL BOARD OF FIRE UNDER-} WRITERS, BUILDING CODE, CONSTRUCTION, STEEL AND IRON, 1931

\section{STEEL AND IRON CONSTRUCTION}

General.-Except as otherwise specifically provided in this ordinance or in rules duly promulgated by the building official, the standard specification for structural steel for buildings, and the coce of standard practice as adopted and amended from time to time by the American Institute of Steel Construction, shall be deemed to be the generally accepted good practice in steel construction. (The A. I. S. C. indorses specification A9 of the American Society for Testing Materials. (See 605.12, p. 252.) (In addition, this specification contains structural details, such as thicknesses of cast-iron and steel columns, cast-iron bases and lintels, lintel spans, bolting of beams, thickness of web in riveted girders, riveting, bolting, welding, gas cutting, and protection against corrosion.)

\section{UNITED STATES GOVERNMENT, DE- PARTMENT OF COMMERCE; BUILDERS' HARDWARE (NONTEMPLATE), COM- MERCIAL STANDARD CS22-30, DE- CEMBER 11, 1930}

\section{BUILDERS' HARDWARE (NONTEMPLATE)}

(See 617.0, p. 977.) 
UNITED STATES GOVERNMENT, FEDERAL SPECIFICATIONS BOARD; FEDERAL SPECIFICATION FOR PLUMBING FIXTURES (FOR LAND USE), NO. 448, NOVEMBER 22, 1926. (CURRENT DESIGNATION : FF-P-451)

ROOF DRAINS

(See 617.73, p. 1062.)

611.2 MALLEABLE-IRON CASTINGS.

611.21 MALLEABLE CAST IRON.

AMERICAN ELECTRIC RAILWAY ENGINEERING ASSOCIATION, SPECIFICATION FOR MATERIALS FOR USE IN THE MANUFACTURE OF SPECIAL TRACK WORK, W104-28, 1928

Approved as American tentative standard by the American Stand ards Association, January 23, 1929]

SPECIAL TRACK WORK MATERIALS

(See 606.3, p. 342.)

\section{AMERICAN RAILWAY ASSOCIATION, MECHANICAL DIVISION ; MALLEABLE- IRON CASTINGS, RECOMMENDED PRAC- TICE, 1928}

\section{MALLEABLE-IRON CASTINGS}

(Identical with A. S. T. M., A47, given below, except that the yield point is 30,000 pounds per square inch and the percentage of elongation in 2 inches is 15 instead of 10 . Inspection and rejection conform to A. R. A. galvanized sheets. See 604.32, p. 243.)

\section{AMERICAN RAILWAY ASSOCIATION,} SIGNAL SECTION; MALLEABLE-IRON CASTINGS, 1930

\section{MALLEABLE-IRON CASTINGS}

(Conforms to A. S. T. M., A47, given below, except that yield point is 30,000 instead of 32,500 pounds per square inch.)

\section{AMERICAN SOCIETY FOR TESTING MATERIALS, STANDARD SPECIFICA- TIONS FOR MALLEABLE CASTINGS, DESIGNATION A47-30, 1930}

\section{MALLEABLE CASTINGS}

1. These specifications cover malleable castings for railroad, motor vehicle, agricultural implement, and general machinery purposes.

\section{MANUFACTURE}

2. The castings shall be produced by either the air-furnace, open-hearth, or electric-furnace process.

\section{PHYSICAL PROPERTIES AND TESTS}

3. (a) The tension-test specimens specified in section 5 shall conform to the following minimum requirements as to tensile properties: (b) The yield point may be determined by the drop of the beam of the testing machine or by the divider method.

4. (a) All castings, if of sufficient size, shall have cast thereon test lugs of a size proportional to the thickness of the casting, but not exceeding fiveeighths by three-fourths inch in cross section. On castings which are 24 inches or over in length, a test lug shall be cast near each end. These test lugs shall be attached to the casting at such a point that they will not interfere with the assembling of the castings, and may be broken off by the inspector.

(b) If the purchaser or his representative so desires, a casting may be tested to destruction. Such a casting shall show good, tough malleable iron.

5. (a) Tension-test specimens shall be of the form and dimensions shown in Figure 420. Specimens whose mean diameter at the smallest section is less than nineteen thirty-seconds inch will not be accepted for test.

(b) A set of three tension-test specimens shall be cast from each melt, without chills, using heavy risers of sufficient height to secure sound bars. The specimens shall be suitably marked for identification with the melt. Each set of specimens so cast shall be placed in some one oven containing castings to be annealed.

6. (a) After annealing, three tension-test specimens shall be selected by the inspector as represent-

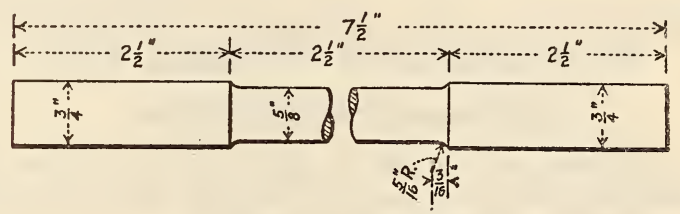

Figure $420 .-T e s t$ specimen

ing the castings in the oven from which these specimens are taken.

(b) If the first specimen conforms to the specified requirements, or if, in the event of failure of the first specimen, the second and third specimens conform to the requirements, the castings in that oven shall be accepted, except that any casting may be rejected if its test lug shows that it has not been properly annealed. If either the second or third specimen fails to conform to the requirements, the entire contents of that oven shall be rejected. In case one of the retest specimens contains a flaw which results in the failure of the bar to meet the specifications, at the discretion of the inspector additional test specimens from the same oven may be tested, or test specimens may be cut from castings.

7. Any castings rejected for insufficient annealing may be reannealed once. The reannealed castings shall be inspected and if the remaining test lugs, or castings broken as specimens, show the castings to be thoroughly annealed, they shall be accepted; if not, they shall be finally rejected.

\section{WORKMANSHIP AND FINISH}

8. The castings shall conform substantially to the patterns or drawings furnished by the purchaser, and also to gages which may be specified in individual cases. The castings shall be made in a workmanlike manner. A variation of one-eighth inch per foot will be permitted.

9. The castings shall be free from injurious defects.

10. The manufacturer's identification mark and the pattern numbers assigned by the purchaser shall 
be cast on all castings of sufficient size in such positions that they will not interfere with the service of the castings.

\section{INSPECTION AND REJECTION}

11. (a) The inspector representing the purchaser shall have free entry, at all times while work on the contract of the purchaser is being performed, to all parts of the manufacturer's works which concern the manufacture of the castings ordered. The manufacturer shall afford the inspector, without charge, all reasonable facilities to satisfy him that the castings are being furnished in accordance with these specifications. All tests and inspection shall be made at the place of manufacture prior to shipment, unless otherwise specified, and shall be so conducted as not to interfere unnecessarily with the operation of the works.

(b) The manufacturer shall be required to keep a record of each melt from which castings are produced, showing tensile strength and elongation of test specimens cast from such melts. These records shall be available and shown to the inspector whenever required.

12. Castings which show injurious defects subsequent to their acceptance at the manufacturer's works may be rejected and, if rejected, shall be replaced by the manufacturer free of cost to the purchaser.

\section{MALLEABLE IRON RESEARCH INSTITUTE, MALLEABLE-IRON CASTINGS, 1929}

\section{MALLEABLE-IRON CASTINGS}

(Identical with A. R. A., given above, including references therein to A. S. T. M., A47, and A. R. A. specifications for galvanized sheets.)

\section{MALLEABLE IRON RESEARCH INSTITUTE, MALLEABLE-IRON CASTINGS FOR RAIL- ROADS, MOTOR VEHICLES, AGRICUI- TURAL IMPLENENTS, AND GENERAL MACHINERY, 1922}

\section{MALLEABLE-IRON CASTINGS}

(Identical with A. S. T. M., A47, given above.)

\section{SOCIETY OF AUTOMOTIVE ENGINEERS, IRON AND STEEL SPECIFICATIONS, MARCH, 1922}

\section{MALLEABLE-IRON CASTINGS}

(For prefatory remarks, see 600.0 , p. 2. This specification is substantially the same as A. S. T. M., A47-30, given above. It differs only in respect to tensile strength and percentage of elongation in 2 inches, and in omitting the minimum yield point. It provides that:)

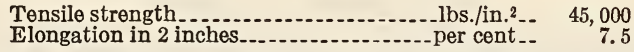

\section{UNITED STATES GOVERNMENT, FEDERAI SPECIFICATIONS BOARD; FEDERAL SPECIFICATION FOR IRON, MALLEA- BLE; CASTINGS, QQ-I-666, FEBRUARY 2, 1932}

\section{MALLEABLE-IRON CASTINGS}

(This specification includes F. S. B. No. 339a (current designation: QQ-M-151). (See 600.0, p.
3.) In many respects it is similar to A. S. T. M. A47, given above, including diagram of test specimen. It differs from $\mathrm{A} 47$ in the following particulars:)

$$
\text { GRADE AND TYPES }
$$

B-1. Grade.-Malleable-iron castings shall be of but one grade.

B-2. Types.-Malleable-iron castings shall be of two types, as follows:

Type A.-Black (ungalvanized).

Type B.-Zinc coated (galvanized).

C-5. Zinc coating (galvanizing) shall be accomplished by the hot-dipping process.

\section{GENERAL REQUIREMENTS}

D-1. The responsibility of furnishing castings that can be laid out and machined to the finished

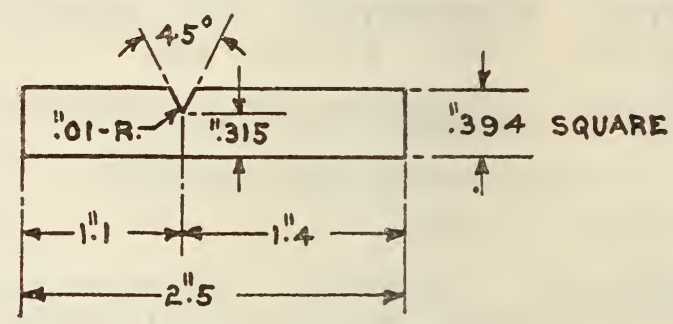

Figure 421.-I Iod

dimensions within the specified tolerances as shown on the drawings, and that will conform to such gages as may be specified in individual cases, shall rest with the contractor. Sufficient stock shall be allowed for shrinkage and, where required, for finish-

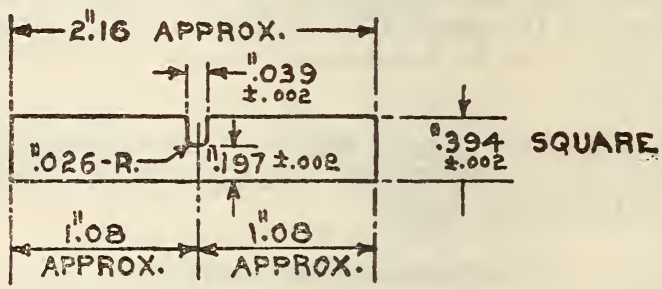

Figure 422.-Charpy

ing. Castings unreasonably in excess of the specified size or weight may be rejected.

\section{E. DETAIL REQUIREMENTS}

E-1. The physical properties shall conform to the respective requirements of Table 1 .

TABLE 1

\begin{tabular}{|c|c|c|c|c|}
\hline \multirow{2}{*}{ Type } & \multirow{2}{*}{$\begin{array}{l}\text { Tensile } \\
\text { strength, } \\
\text { pounds per } \\
\text { square } \\
\text { inch }\end{array}$} & \multirow{2}{*}{$\begin{array}{c}\text { Elonga- } \\
\text { tion, per } \\
\text { cent in } 2 \\
\text { inches, } \\
\text { minimum }\end{array}$} & \multicolumn{2}{|c|}{$\begin{array}{l}\text { Impact strength, foot- } \\
\text { pounds }\end{array}$} \\
\hline & & & $\begin{array}{l}\text { Izod, min- } \\
\text { imum }\end{array}$ & $\begin{array}{l}\text { Charpy, } \\
\text { minimum }\end{array}$ \\
\hline $\begin{array}{l}\text { Ungalvanized.... } \\
\text { Galvanized }\end{array}$ & $\begin{array}{l}50,000 \\
50,000\end{array}$ & $\begin{array}{l}10 \\
10\end{array}$ & 5 & -- \\
\hline
\end{tabular}

F-4. Impact-test specimens shall be cast to the form and dimensions shown in the annexed diagrams (according to impact-test method employed) except that the notch shall not be cast in the specimen but shall be machined in either case. 
F-6. When annealed, test specimens shall be so located as to receive the least favorable annealing of any of the castings.

F-7. Tension and impact test specimens representing zinc-coated (galvanized) malleable iron shall be zinc coated (galvanized) with the castings they represent and the impact specimens machined for test after having been galvanized.

F-8. Three tension and three impact test specimens (the latter for zinc-coated (galvanized) castings only) representing each melt in each annealing charge will be tested. If more than one of each group of three fail to conform to the requirements, the charge represented by that group shall be rejected.

F-9. Any black (ungalvanized) malleable-iron castings rejected for insufficient annealing may be reannealed once.

F-10. Malleable-iron castings shall not be reannealed or reheated after zinc coating (galvanizing), to above $350^{\circ} \mathrm{F}$.

\section{G. PACKAGING, PACKING, AND MAREING}

\section{G-1. Packing-}

G-1a. Small castings shall be properly separated by melts or lots when shipped. A lot shall consist of castings poured from the same melt, unless otherwise mutually agreed upon by the contractor and the inspector.
G-1b. If special packing be necessary to secure the acceptance of castings by common or other carrier for safe transportation at the lowest rate to the point of delivery, such packing shall be provided by the contractor without additional expense to the Government.

G-2. Marking-

G-2a. Small castings shall be so marked that their identity will be clear at the point of delivery.

G-2b. Large castings shall be stamped with the melt or lot number and, where practicable, with the pattern number.

G-2c. Shipping containers shall be marked with the name of the material, the size, the type, and the quantity contained therein as defined by the contract or order under which shipment is made; the name of the contractor; and the number of the contract or order.

\subsection{JOURNAL BOXES.}

\section{AMERICAN ELECTRIC RAILWAY ENGI- NEERING ASSOCIATION, STANDARD JOURNAL BOXES AND CONTAINED PARTS, E2-31, 1931}

\section{JOURNAL BOXES}

For journals 3 by 6 inches, $3 \frac{1}{4}$ by 6 inches, $33 / 4$ by 7 inches, $4 \frac{1}{4}$ by 8 inches, 5 by 9 inches, and $51 / 2$ by 10 inches.

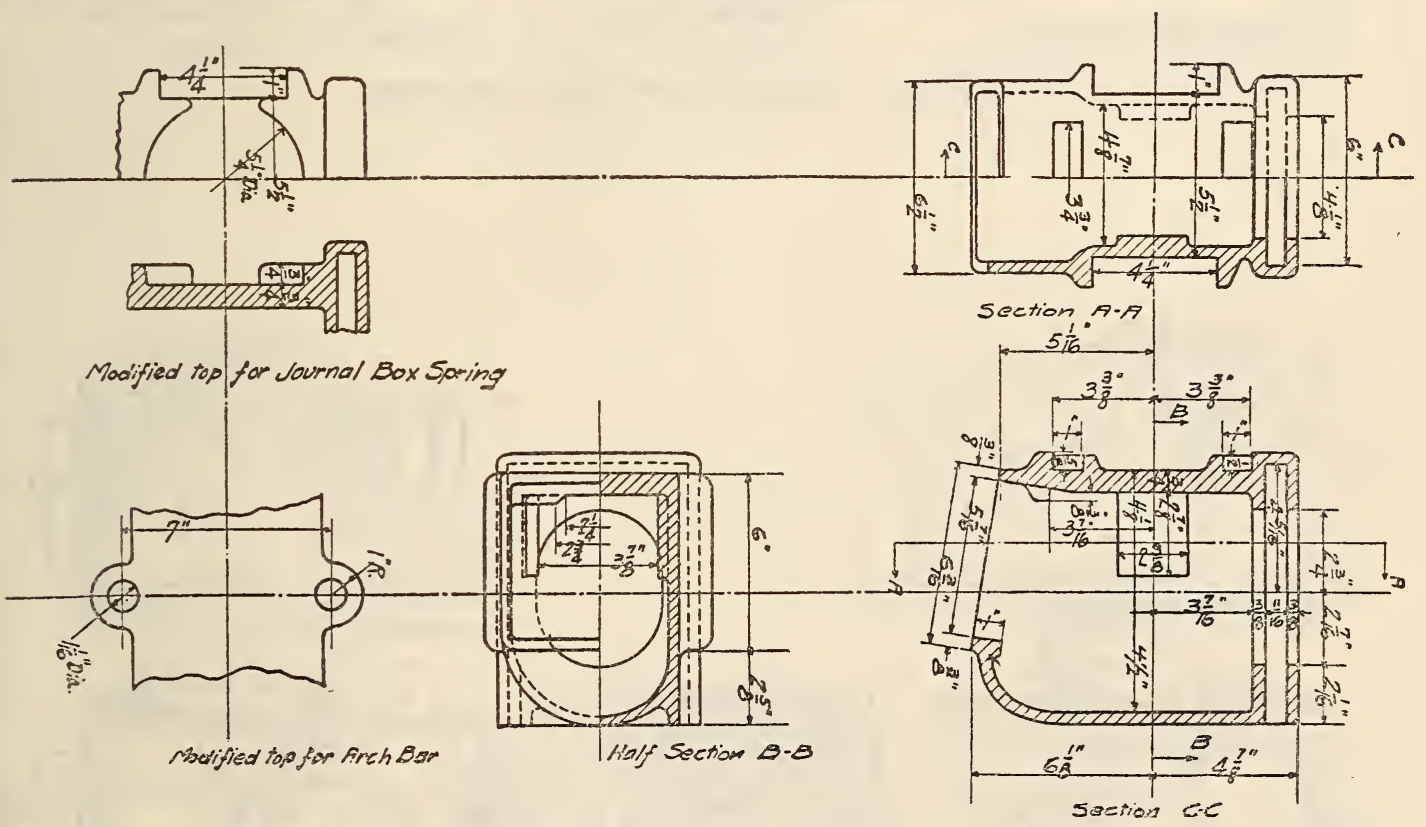

Figure 423.-Journal box for 3 by 6 inch and 31/4 by 6 inch journal 


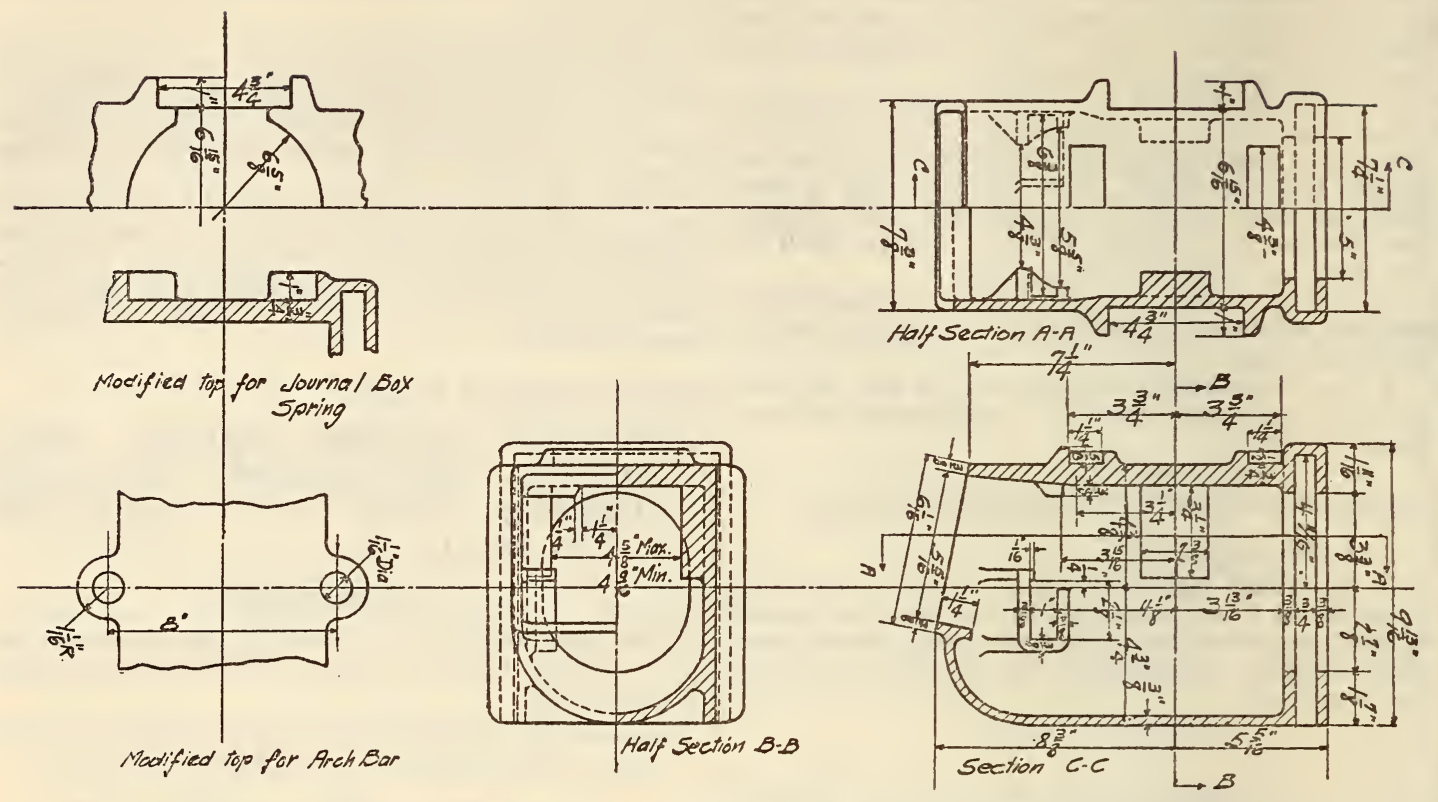

FIGURE 424.-Journal box for $31 / 4$ by 7 inch journal
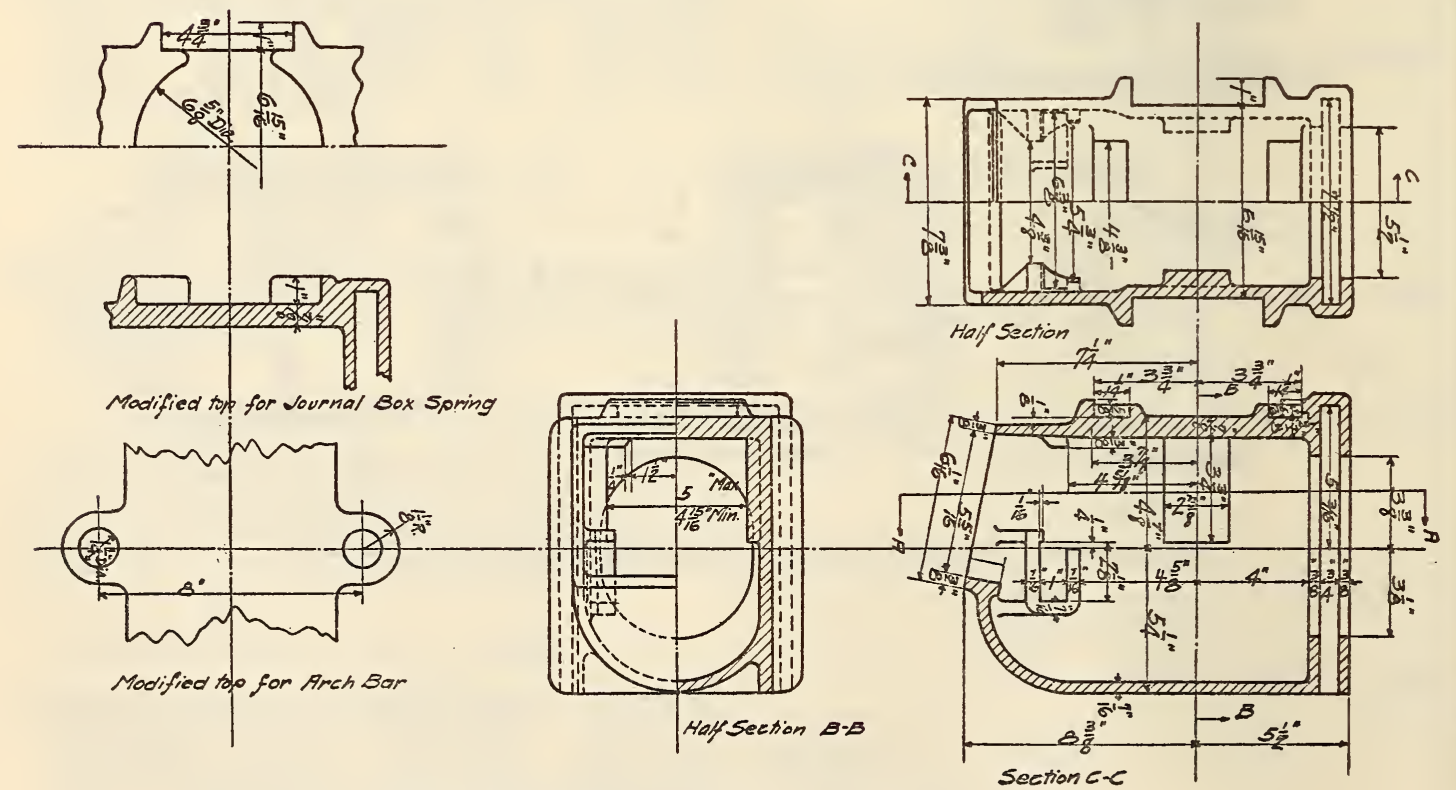

FIGURE 425.-Journal box for $4 \frac{1}{4}$ by 8 inch journal 


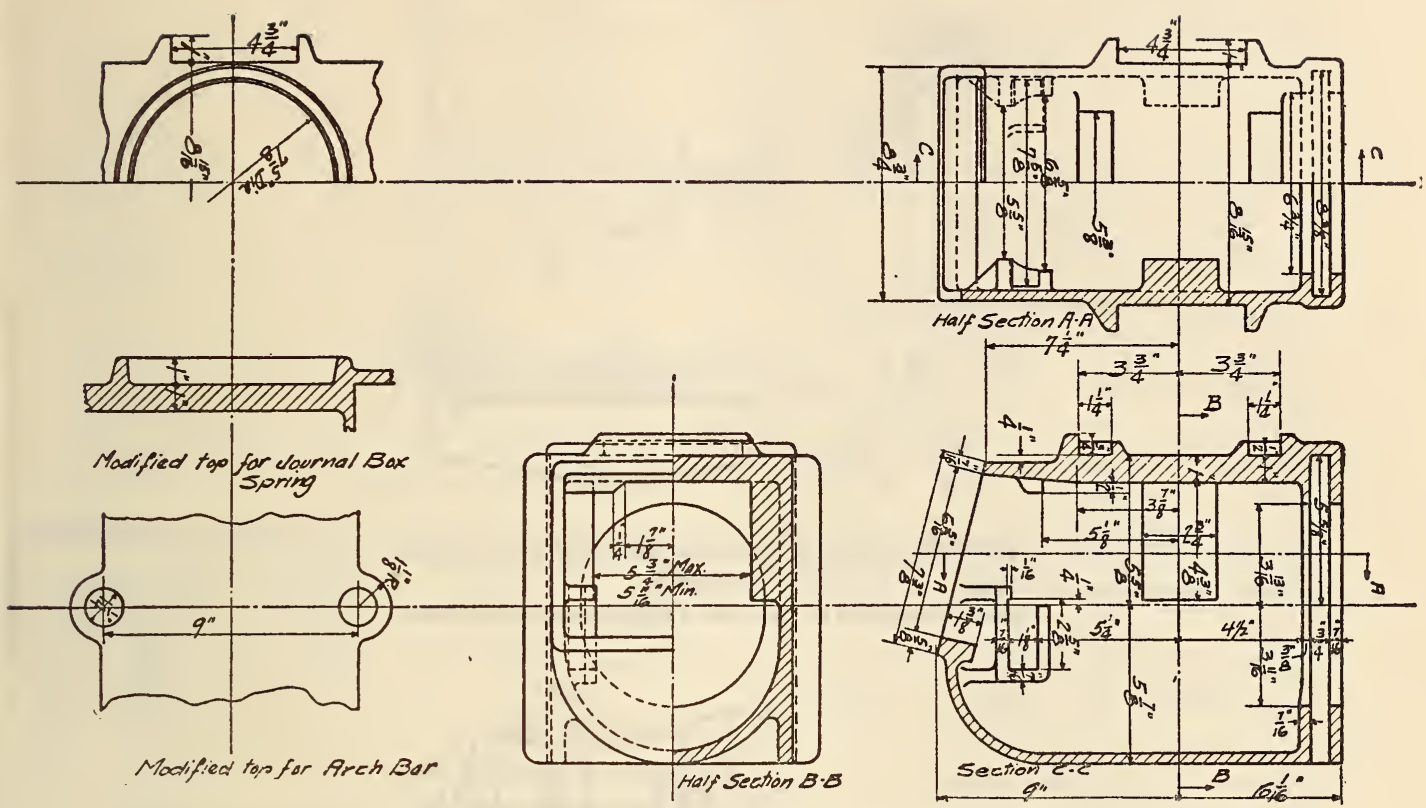

FigURe 426.-Journal box for 5 by 9 inch journal
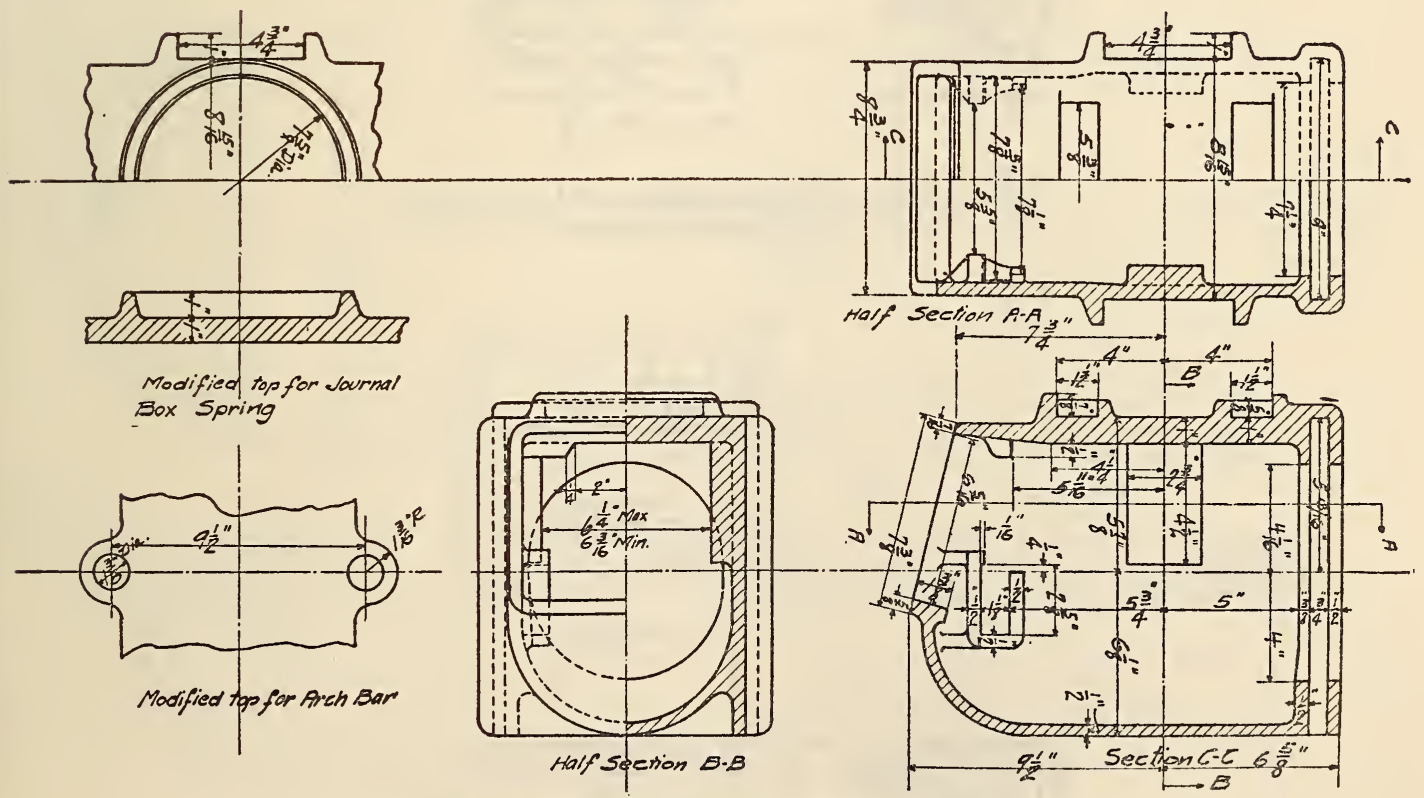

FIG URE 427.-Journal box for $5 \frac{1}{2}$ by 10 inch journal 

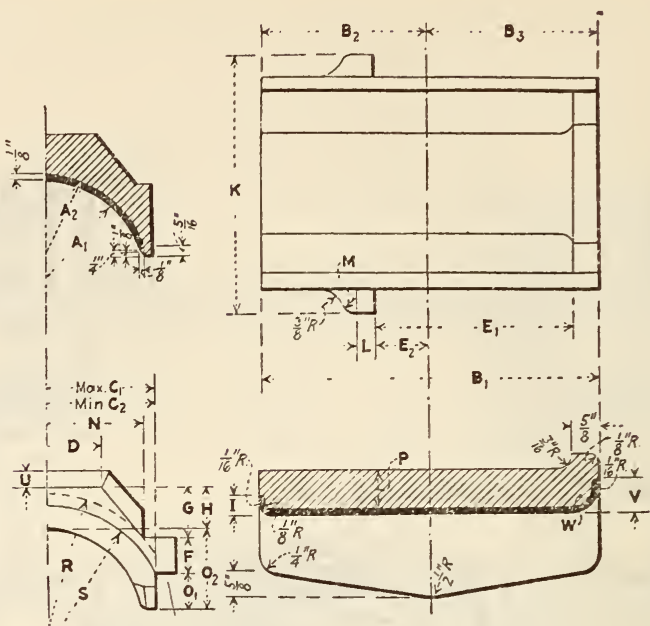

Figure 428.-Dimension drawing for journal bearings

Detail dimensions for each size of bearing are given in Table

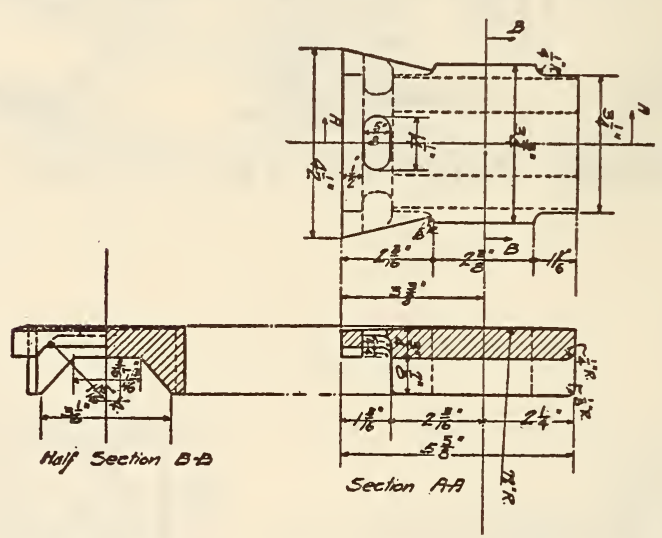

Figure 429.-Journal wedge for 3 by 6 inch and $31 / 4$ by 6 -inch journals

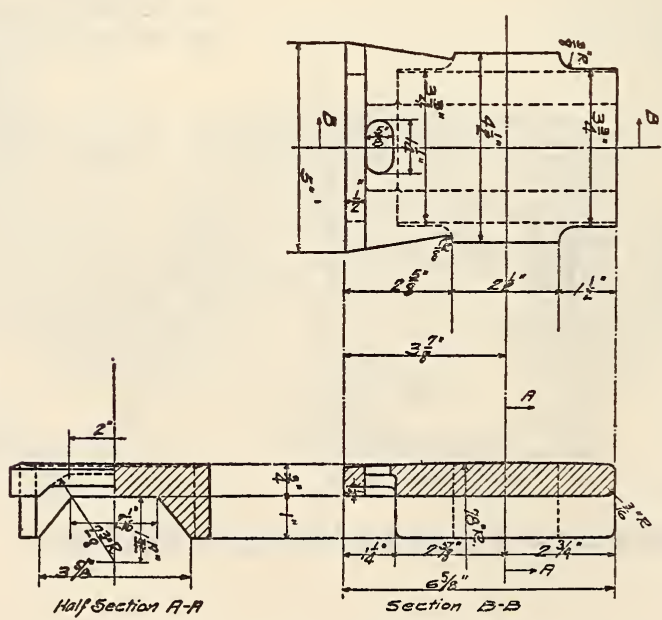

FIGURE 430.-Journal wedge for $33 / 4$ by 7 inch journal 


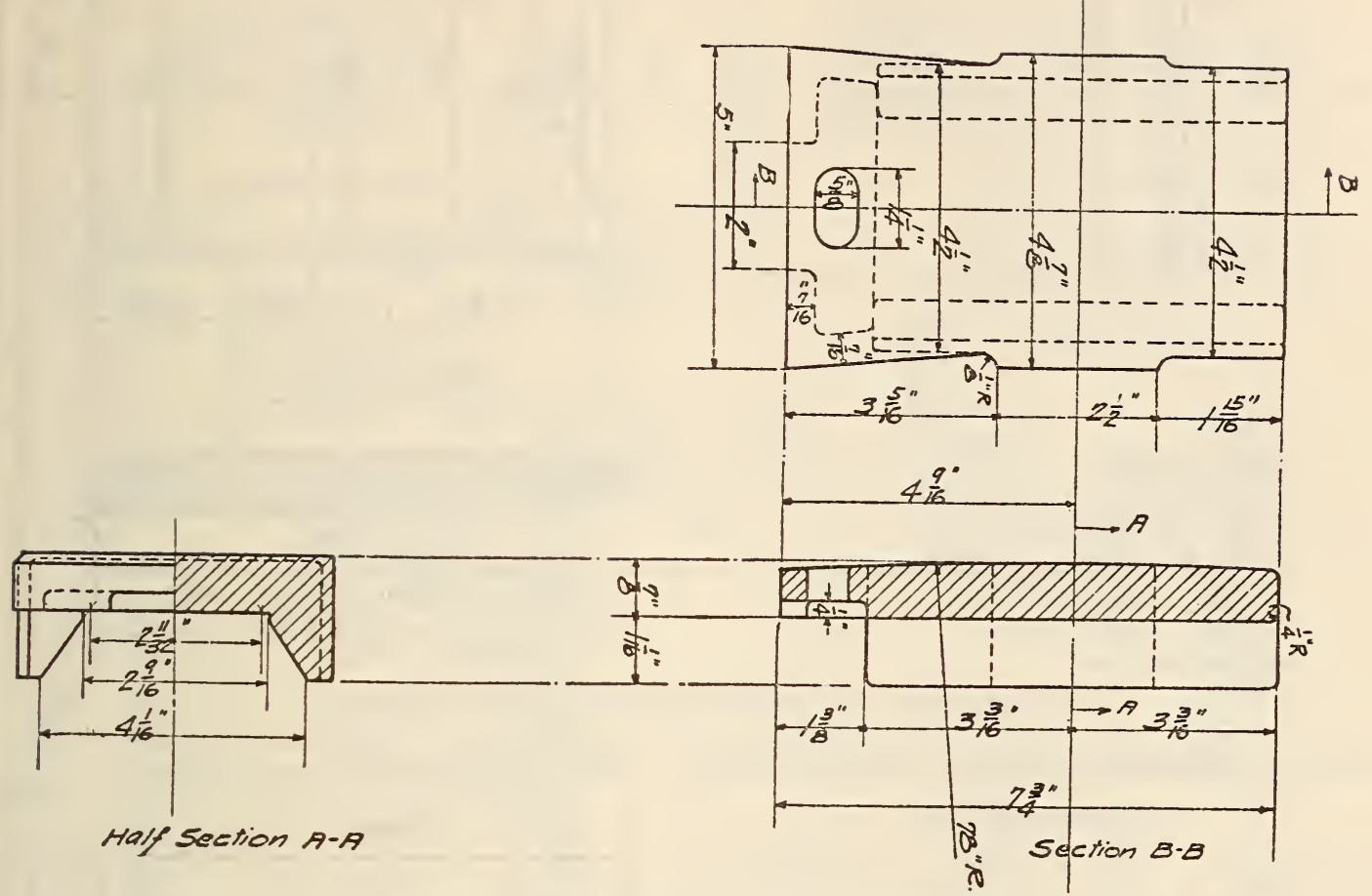

FIGURE 431.-Journal wedge for $4 \frac{1}{2}$ by 8 inch journal

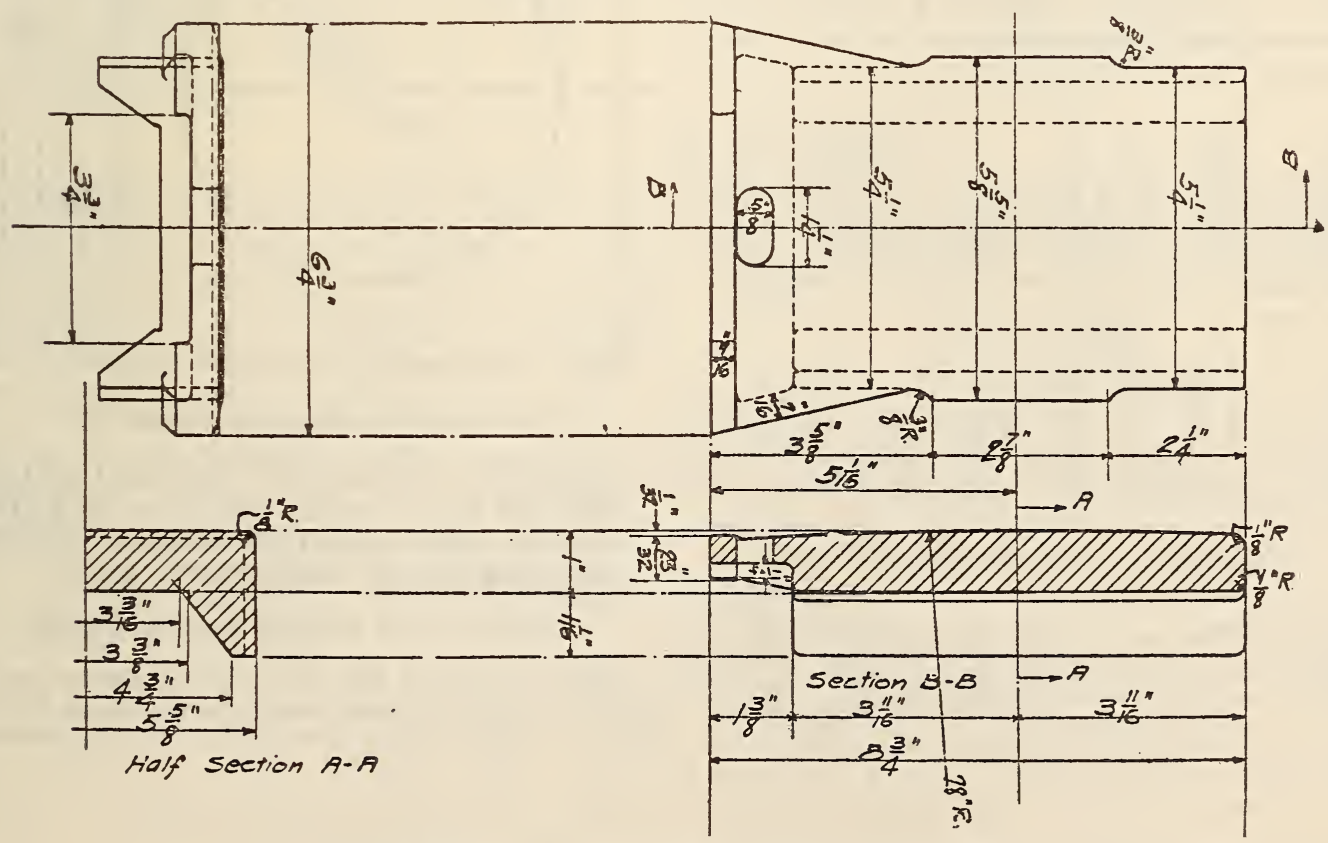

FIGURE 432.-Journal wedge for 5 by 9 inch journal 


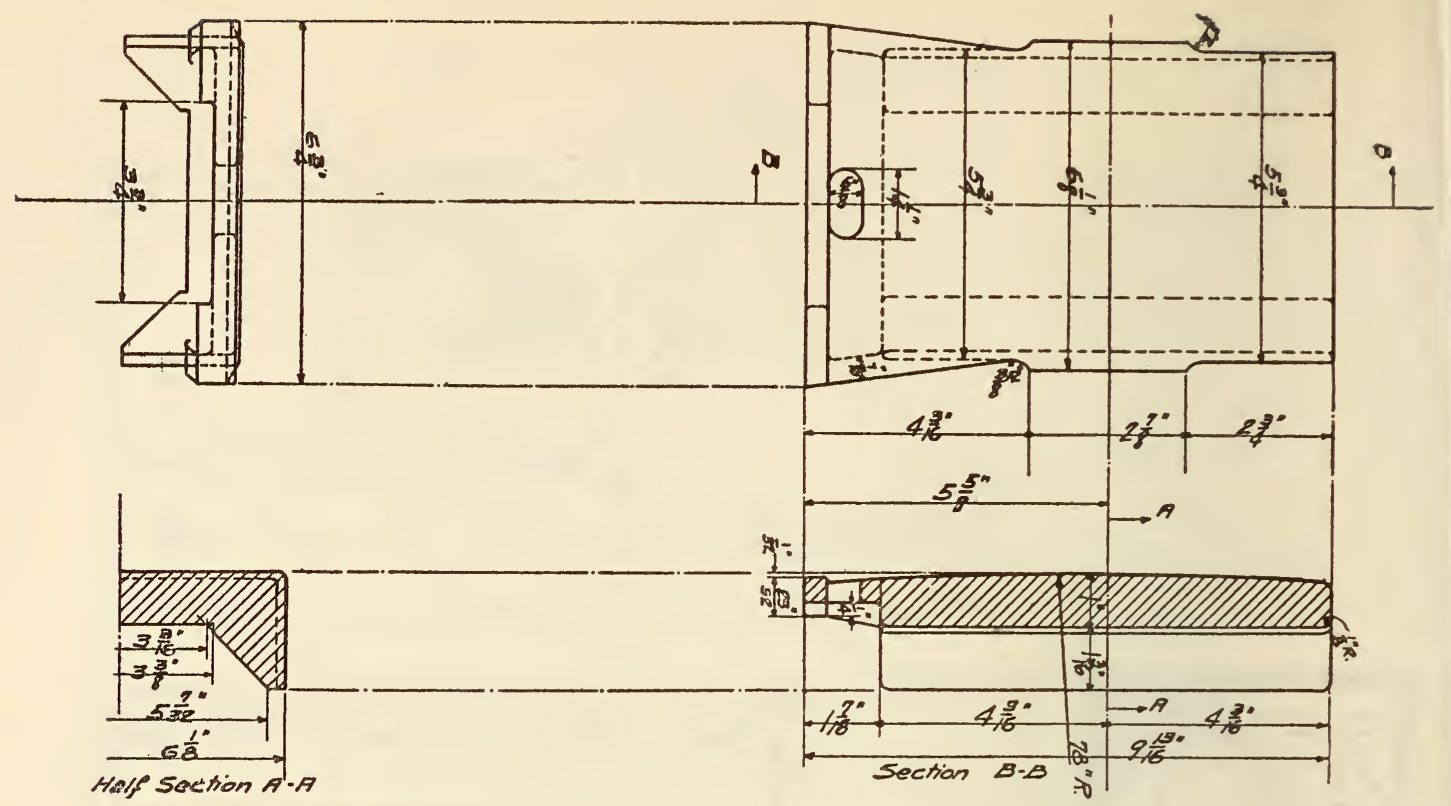

FIGURE 433.- Journal wedge for $5 \frac{1}{2}$ by 10 inch journal

TABLE 1.-Dimensions of standard journal bearings [All dimensions in inches]

\begin{tabular}{|c|c|c|c|c|c|c|c|c|c|}
\hline Size journal & A1 & A2 & B1 & & B2 & B3 & $\mathrm{C} 1$ & C2 & D \\
\hline $\begin{array}{l}3 \text { by } 6 \\
31 / 4 \text { by } 6 \\
334 \text { by } 7 \\
414 \text { by } 8 \\
5 \text { by } 9 \\
51 / 2 \text { by } 10\end{array}$ & $\begin{array}{l}117 / 32 \\
-12132 \\
1^{29} / 32 \\
2^{5} / 32 \\
2^{17 / 32} \\
2^{25 / 32}\end{array}$ & $\begin{array}{l}117 / 32 \\
1^{213} / 32 \\
1^{29} / 32 \\
25 / 32 \\
2^{17 / 32} \\
2^{25 / 32}\end{array}$ & $\begin{array}{l}57 \\
57 \\
67 \\
73 \\
83 \\
93\end{array}$ & & \begin{tabular}{l|l}
$215 / 16$ & 2 \\
$17 / 16$ & 2 \\
$77 / 8$ & 3 \\
378 & 3 \\
378 & 4 \\
7178 & 4
\end{tabular} & $\begin{array}{l}215 / 16 \\
21516 \\
37 / 16 \\
37 / 8 \\
43 / 8 \\
47 / 8\end{array}$ & $\begin{array}{l}327 / 32 \\
322 / 32 \\
4^{217 / 32} \\
4^{29 / 32} \\
521 / 32 \\
65 / 32\end{array}$ & $\begin{array}{l}313 / 16 \\
313 / 16 \\
41 / 2 \\
47 / 8 \\
55 / 8 \\
618\end{array}$ & $\begin{array}{l}134 \\
13 / 4 \\
2 \\
25 / 16 \\
31 / 8 \\
31 / 8\end{array}$ \\
\hline Size journal & E1 & E2 & F & G & & H & $\mathrm{K}$ & L & M \\
\hline 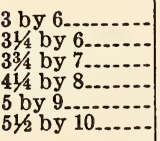 & $\begin{array}{l}39 / 16 \\
3916 \\
41 / 16 \\
413 \\
53 / 16 \\
51116\end{array}$ & $\begin{array}{l}114 \\
134 \\
114 \\
114 \\
1716 \\
17 / 16\end{array}$ & $\begin{array}{c}3 / 4 \\
7 / 8 \\
7 / 8 \\
13116 \\
1116 \\
11 / 16\end{array}$ & & \begin{tabular}{l|l} 
& 1 \\
.18 & 1 \\
3.16 & 1 \\
3.16 & 1 \\
$5 / 16$ & 1
\end{tabular} & \begin{tabular}{l|l}
1 & $3 / 6$ \\
1 & $3 /$ \\
1 & $9 / 1$ \\
1 & $9 / 1$ \\
$11 / 8$ & $5 / 8$ \\
$11 / 8$ & $5 / 8$
\end{tabular} & 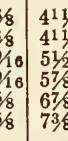 & $\begin{array}{l}1 / 2 \\
1 / 2 \\
1 / 2 \\
1 / 2 \\
5 / 8 \\
5 / 8\end{array}$ & \begin{tabular}{l|l}
2 & 38 \\
2 & $3 / 8$ \\
2 & $3 / 8$ \\
2 & $3 / 8$ \\
8 & $3 / 8$ \\
8 & $1 / 2$
\end{tabular} \\
\hline Size journal & $\mathrm{N}$ & 01 & $\mathrm{O} 2$ & $\mathrm{P}$ & $\mathrm{R}$ & $\mathrm{s}$ & U & $\mathrm{V}$ & W \\
\hline $\begin{array}{l}37 \text { by } 6 \\
314 \text { by } 6 \\
334 \text { by } 7 \\
41 / 4 \text { by } 8 \\
5 \text { by } 9 \\
51 / 2 \text { by } 10 \\
\end{array}$ & \begin{tabular}{l|}
314 \\
314 \\
$33 / 4$ \\
4118 \\
434 \\
$53 / 8$
\end{tabular} & \begin{tabular}{r|}
$3 / 4$ \\
$5 / 8$ \\
$1 / 2$ \\
718 \\
118 \\
118
\end{tabular} & $\begin{array}{l}11 / 2 \\
11 / 2 \\
11 / 2 \\
17 / 8 \\
21 / 4 \\
23 / 8\end{array}$ & $\begin{array}{l}7 / 8 \\
7 / 8 \\
7 / 8 \\
7 / 8 \\
1 \\
1\end{array}$ & $\begin{array}{l}2 \\
2 \\
2^{7 / 16} \\
2^{11} 1 / 16 \\
31 / 8 \\
3^{3} / 8\end{array}$ & \begin{tabular}{|l|l}
2 \\
2 \\
616 \\
$2^{7 / 16}$ \\
$2^{11} / 1$ \\
$31 / 8$ \\
$3 \% 8$
\end{tabular} & $\begin{array}{l}1 / 4 \\
1 / 4 \\
1 / 4 \\
3 / 8 \\
3 \\
3 \\
3\end{array}$ & \begin{tabular}{|l|l|}
4 & $3 / 4$ \\
4 & 3 \\
4 & 34 \\
8 & $15 / 16$ \\
8 & $15 / 16$ \\
8 & 1 \\
8 & 1
\end{tabular} & $\begin{array}{l}1 / 2 \\
1 / 2 \\
3 / 4 \\
3 / 4 \\
34 \\
3 / 4\end{array}$ \\
\hline \multicolumn{3}{|c|}{ Size of journal } & A & & B & C & D & $\mathrm{E}$ & F \\
\hline \multicolumn{3}{|c|}{$\begin{array}{l}3 \text { by } 6 \text { and } 3144 \text { by } 6 \\
33 / 4 \text { by } 7 \\
41 / 4 \text { by } 8 \\
5 \text { by } 9 \text { by } 10\end{array}$} & - & 1 & \begin{tabular}{r|}
$77 / 8$ \\
858 \\
994 \\
$103 / 8$ \\
$107 / 8$
\end{tabular} & $\begin{array}{l}41 / 2 \\
43 / 4 \\
554 \\
57 / 8 \\
61 / 8\end{array}$ & \begin{tabular}{|l|}
31516 \\
$413 / 16$ \\
5516 \\
6316 \\
61116
\end{tabular} & $\begin{array}{l}33 / 8 \\
37 / 8 \\
4 \\
41 / 2 \\
43 / 4\end{array}$ & $\begin{array}{l}9 / 16 \\
58 \\
58 \\
58 \\
58\end{array}$ \\
\hline
\end{tabular}

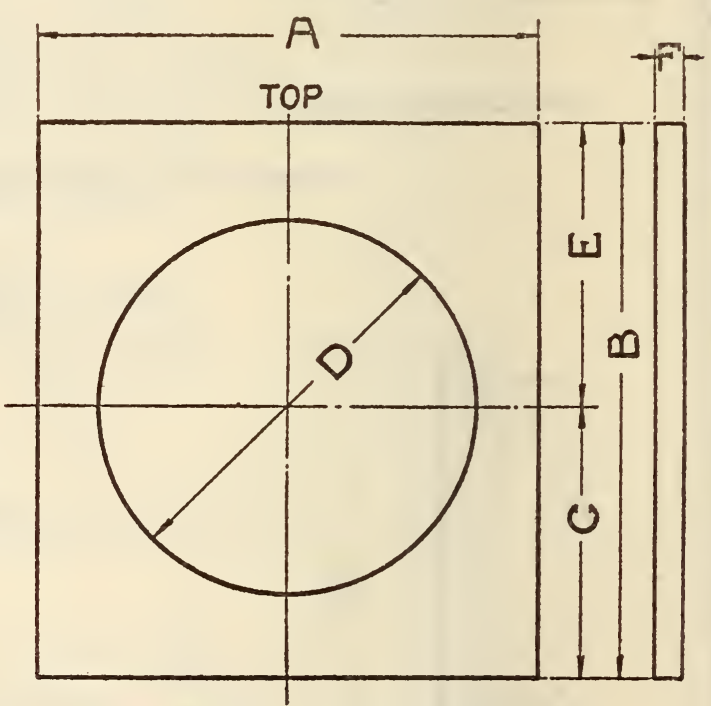

FIGURE 434.-Standard dust guards

AMERICAN RAILWAY ASSOCIATION, MECHANICAL DIVISION ; DRIVING BOXES AND ENGINE-TRUCK BOXES, RECOMMENDED PRACTICE, 1928

DRIVING AND ENGINE-TRUCK BOXES

Figures 435 and 436 show the practices recommended for driving and engine-truck boxes, respectively. Figure 435 is practically self-explanatory. 


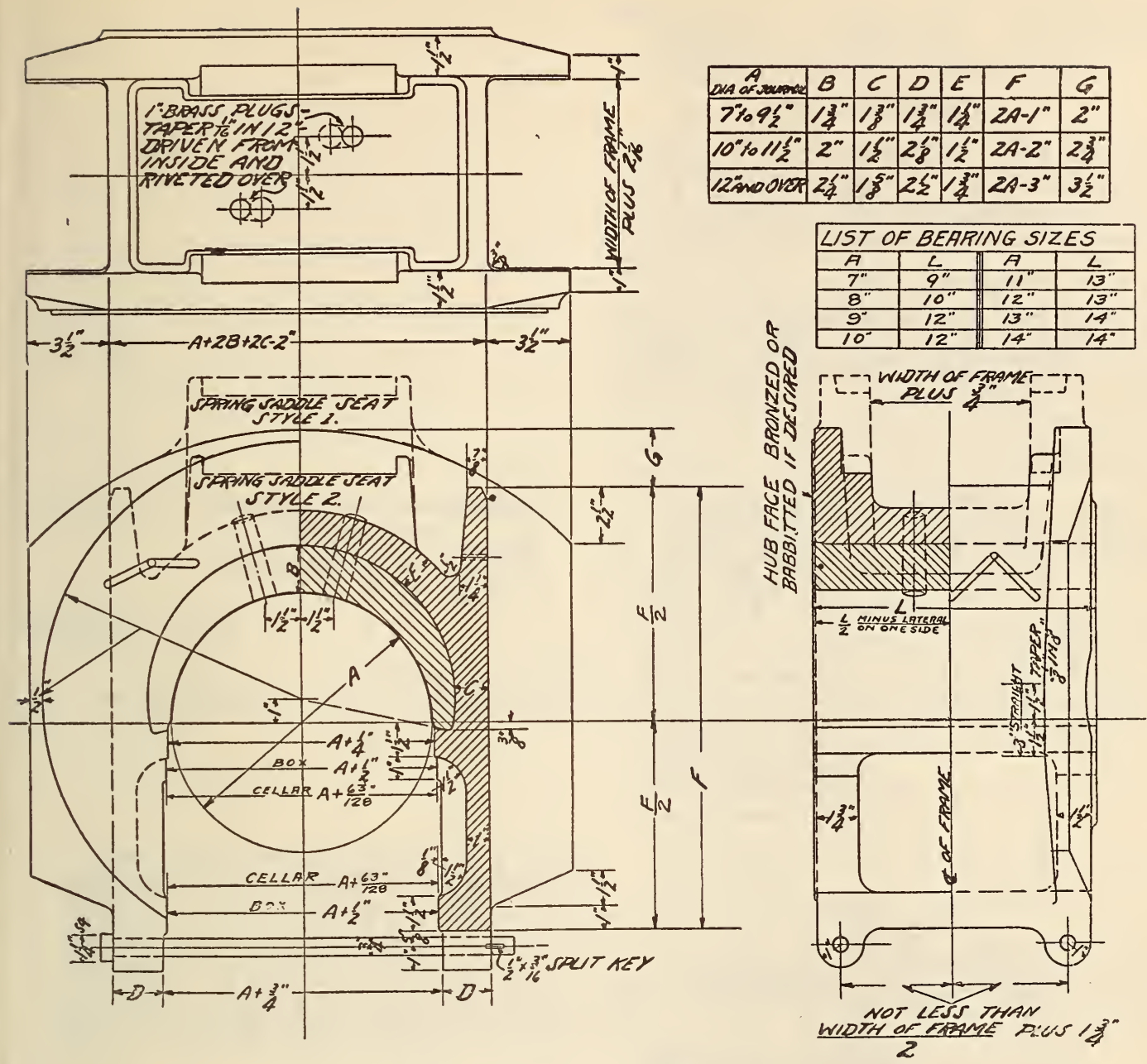

Figure 435.-Driving and engine truck boxes

In its treatment of driving-box design, the committee has not attempted to cover the design of cellars, nor has it laken up the matter of fitting and grooving brasses, as the arrangement of these details depends very largely upon the kind of lubricant used, and there is an extremely wide variation in the practices of the different railroads. Practically all enginetruck boxes are lubricated with oil that is fed to the journal from waste-packed cellars. Believing that the amount of packing carried in engine-truck cellars of most modern locomotives is not as large as it should be, the committee has provided for the greatest depth of cellar which is felt could be applied in connection with the 30 -inch diameter enginetruck wheel while maintaining $1 \frac{1}{2}$ inches of clearance between the pedestal binder and the bottom of the engine-truck box and cellar, as well as a clearance of not less than $2 \frac{1}{2}$ inches between the top of rail and the lowest part of the engine-truck frame. The several diagrams in Figure 436 show the construction recommended for boxes of 2-wheel engine trucks using plate springs of the semielliptical type. With suitable modifications at the top this box becomes applicable to four-wheel front trucks of the type generally used on passenger locomotives.

Material recommended for both driving and engine-truck boxes is cast steel as per A. R. A. specifications for carbon-steel castings adopted as recommended practice, 1923 . (See 611.41, p. 707.)
AMERICAN RAILWAY ASSOCIATION MECHANICAL DIVISION ; STANDARD SPECIFICATIONS FOR DUST GUARDS, 1930

\section{DUST GUARDS}

(See figs. 437 and 438 )

1. Purpose.-To exclude dust from the journal box and to retain the oil.

2. Requirement.-To serve its purpose when new guard is applied, it must fit the axle with not more than one thirty-second of an inch clearance all around.

3. Material.-Material in contact with axle must be of such composition that it will not cut the axle in service.

The inside dimension of guard must be such that it can be easily applied to the axle and of such outside dimension that it can easily enter the box.

The guard must not bind when applied.

4. A wedge or plug shall be applied to close the top of the guard well in the box, or provision made in the construction of the guard to close this opening in order to exclude dirt.

5. Service.-The service life of the guard shall be equal to at least the service life of a pair of new wheels.

A wood dust guard to meet these specifications must not be less than three-ply laminated and secured by use of water-resisting glue. 


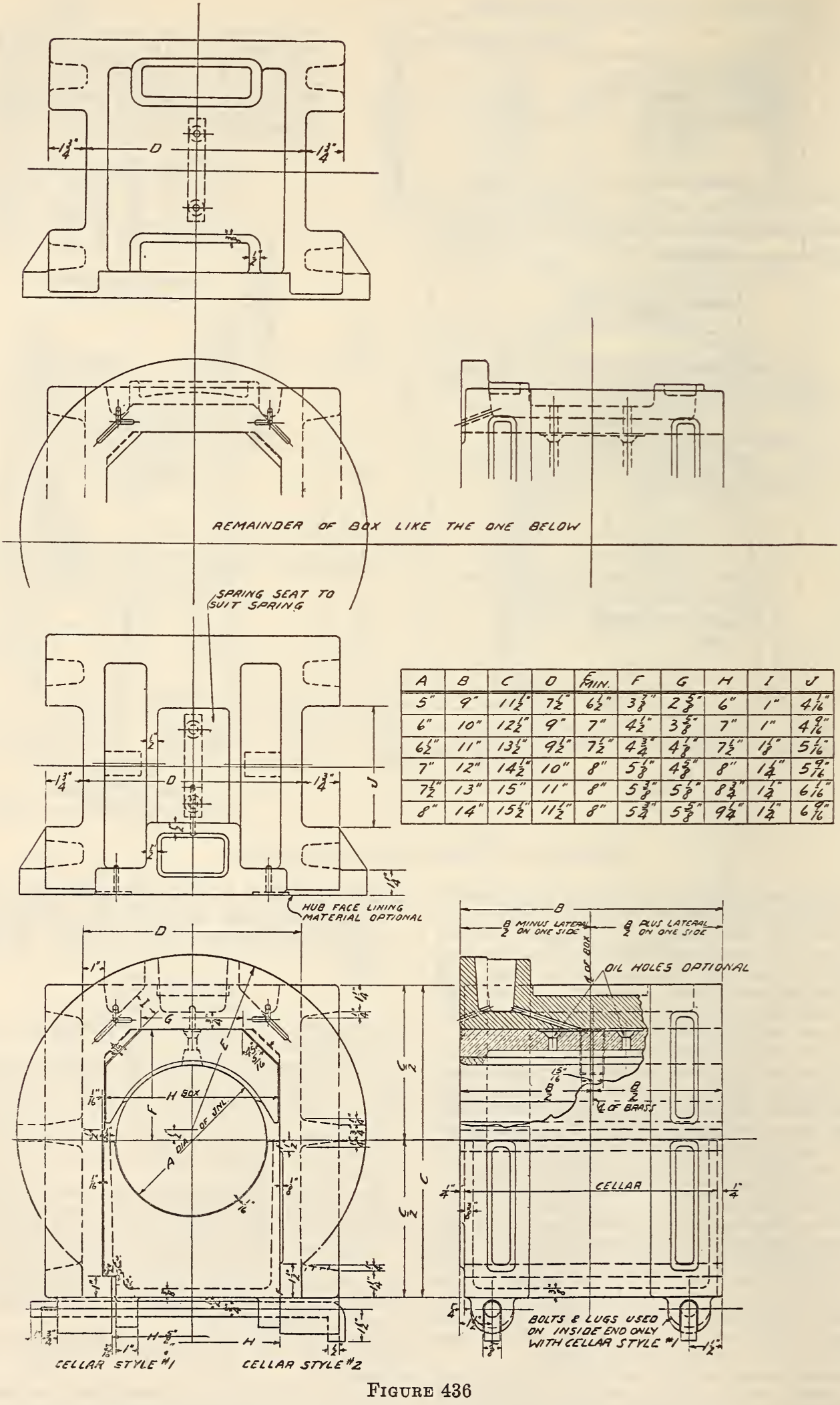




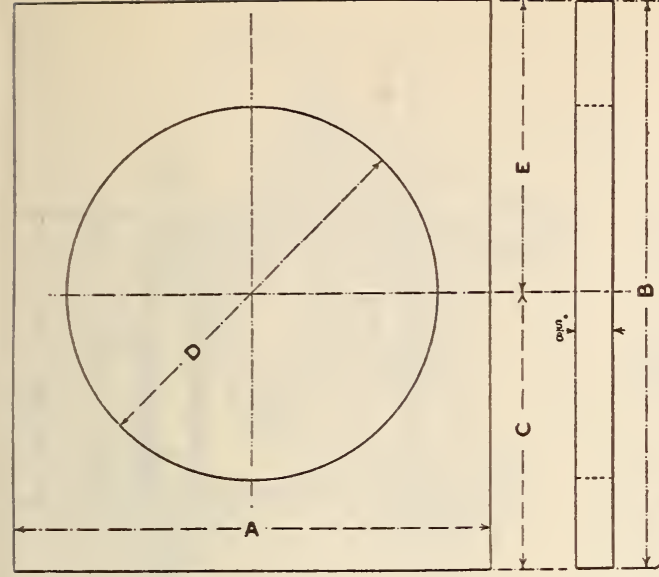

FIgURE 437.-Dust guards for journal boxes $A$ and $B$

[All dimensions in inches]

\begin{tabular}{|c|c|c|c|c|c|c|}
\hline $\begin{array}{l}\text { Classification of } \\
\text { guard }\end{array}$ & $\begin{array}{l}\text { Size of } \\
\text { journal }\end{array}$ & $A$ & B & C & D & $\mathrm{E}$ \\
\hline $\begin{array}{l}\mathrm{A} . . . \\
\mathrm{B} . . .\end{array}$ & $\begin{array}{l}334 \text { by } 7 .-- \\
414 \text { by } 8 . .-\end{array}$ & $\frac{6}{71 / 4}$ & $\begin{array}{l}71 / 2 \\
83 / 4\end{array}$ & $\begin{array}{l}33 / 4 \\
438\end{array}$ & $\begin{array}{l}413 / 16 \\
55 / 16\end{array}$ & $\begin{array}{l}334 \\
438\end{array}$ \\
\hline
\end{tabular}

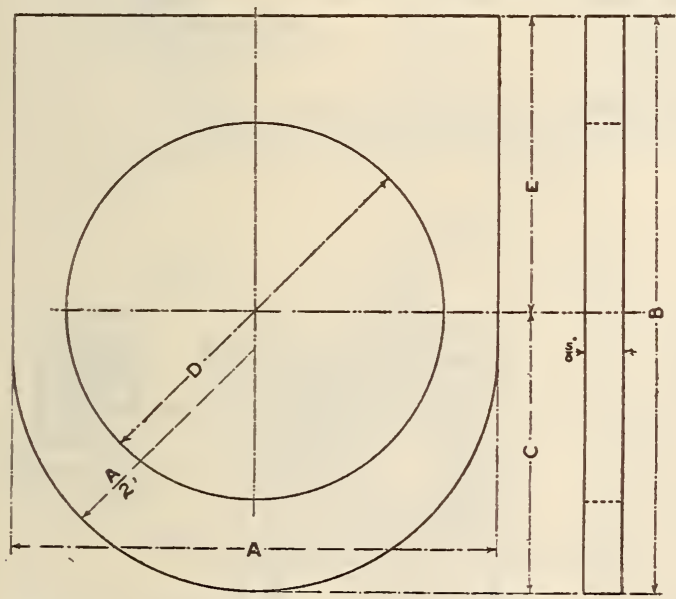

FIgORE 438.-Dust guards for journal boxes $C$, $D, E$, and $F$

[All dimensions in inches]

\begin{tabular}{|c|c|c|c|c|c|c|}
\hline $\begin{array}{l}\text { Classification of } \\
\text { guard }\end{array}$ & $\begin{array}{c}\text { Size of } \\
\text { journal }\end{array}$ & A & B & C & D & $\mathrm{E}$ \\
\hline $\begin{array}{l}\mathrm{C} . . . . \\
\mathrm{D} \\
\mathrm{E} \\
\mathrm{E} \\
\mathrm{F}\end{array}$ & $\begin{array}{l}5 \text { by } 9 \\
51 / 2 \text { by } 10 . \\
6 \text { by } 11 \\
61 / 2 \text { by } 12 .\end{array}$ & $\begin{array}{l}8 \\
87 / 8 \\
105 / 8 \\
11\end{array}$ & $\begin{array}{l}91 / 2 \\
10 \\
103 / 4 \\
1158\end{array}$ & $\begin{array}{l}45 / 8 \\
478 \\
518 \\
53 \%\end{array}$ & $\begin{array}{l}63 / 16 \\
611 / 16 \\
7516 \\
713 / 16\end{array}$ & $\begin{array}{l}47 / 8 \\
518 \\
55 / 8 \\
61 / 4\end{array}$ \\
\hline
\end{tabular}

AMERICAN RAILWAY ASSOCIATION, MECHANICAL DIVISION; STANDARD JOURNAL BOXES A AND B, 1930

\section{JOURNAL BOXES}

Section of box may be made either circular or square below the center line and material may be cast iron, when alloyed for proper strength and wearing qualities, malleable iron, pressed steel, or cast steel, provided all the essential dimensions are adhered to.

When journal box is made of material other than cast iron, reduction in thickness of metal and coring to lighten weight is permissible provided all the essential dimensions which affect interchangeability and the proper fitting of contained parts are adhered to

If the method of manufacture does not permit of placing the letters "A. R. A." on the side of the journal box, they may be placed on the top between the hinge lug and seat of truck sides.

When used with side frames of such design that the bottom tie bar is omitted, or is not an essential strength member for carrying the load, the bottom box lugs may be omitted.

When used in connection with other side frames, the bottom box lugs must be equivalent, both in location and strength, to those shown.

The lid face of bar should be reasonably straight and smooth. For journal boxes used on passengertrain equipment, a machine fit is recommended.

Figure 439 shows details for journal box A for journal $33 / 4$ by 7 inches.

Figure 440 shows details for journal box B for journal $4 \frac{1}{4}$ by 8 inches.

\section{SPECIFICATIONS FOR JOURNAL BOXES, C, D, E, AND F}

Arrow heads, without figures, indicating radii, represent one-fourth inch radius.

Dimensions for tolerances underscored are plus or minus one-sixteenth inch; lesser tolerances are given with the dimensions. Other dimensions of lesser importance may vary, plus or minus, one-eighth inch.

Metal thickness given in table under " $\mathrm{T}$ " is minimum.

Section of any box may be made either circular or square, below center line.

The metal may be cast iron, malleable iron, pressed steel, or cast steel, provided all of the essential dimensions, which affect interchangeability and the proper fitting of contained parts, are adhered to.

When used with side frames of such design that the bottom tie bar is omitted, or is not an essential strength member for carrying the load, the bottom box lugs may be omitted.

When used in connection with other side frames, the bottom box lugs must be equivalent, both in location and strength, to those shown.

If the method of manufacture does not permit the placing of letters "A. R. A." on the side of the journal box, they may be placed on the top, between the hinge lugs and the seat of truck sides.

The lid face of box should be reasonably straight and smooth. For journal boxes used on passengertrain equipment, a machine fit is recommended. 

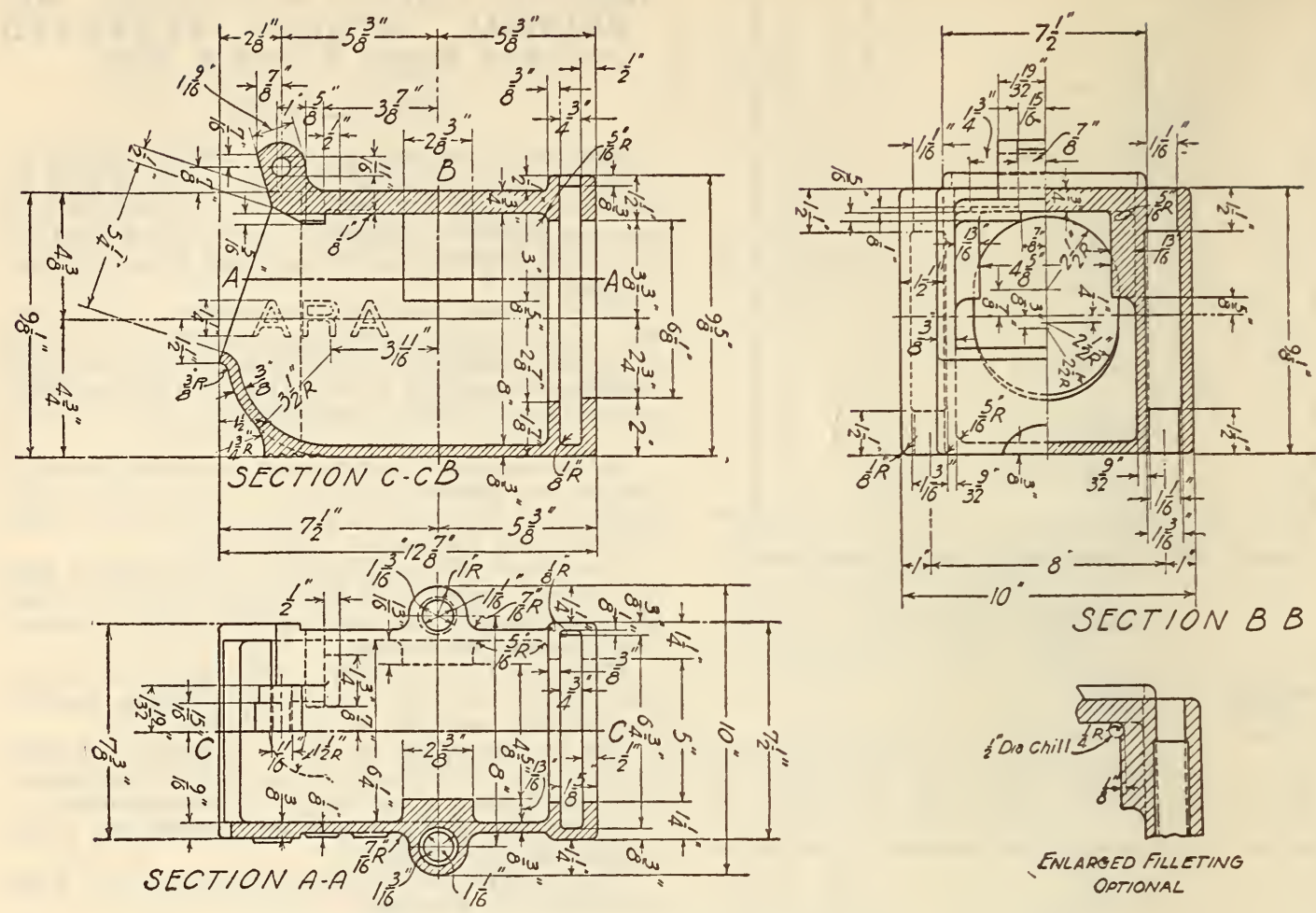

FIGURE 439.-Journal box for freight cars for $33 / 4$ by 7 inch journal
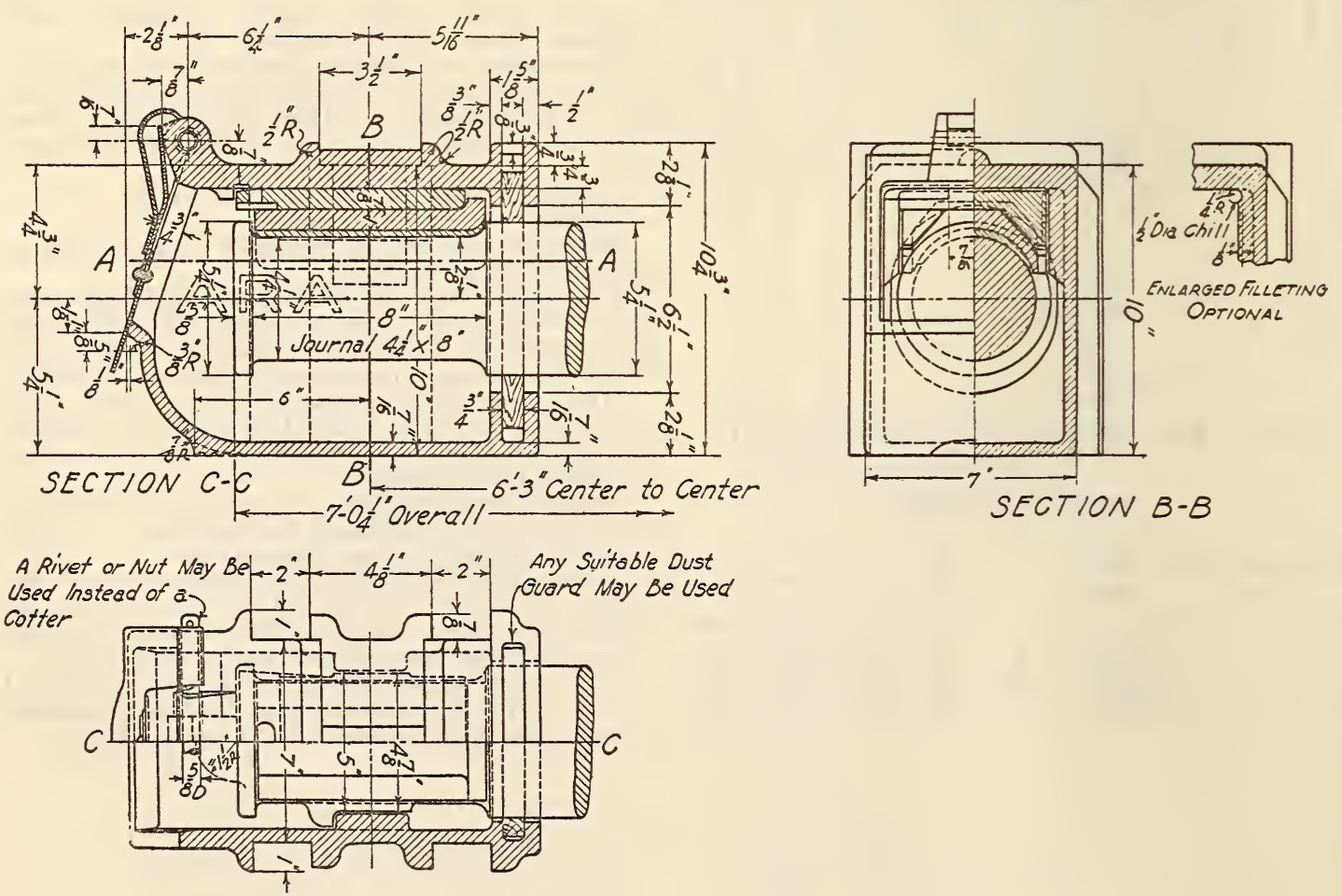

$$
\text { SECTION A-A }
$$

FIGURE 440.- Journal box and contained parts for freight cars for $41 / 4$ by 8 inch journal 


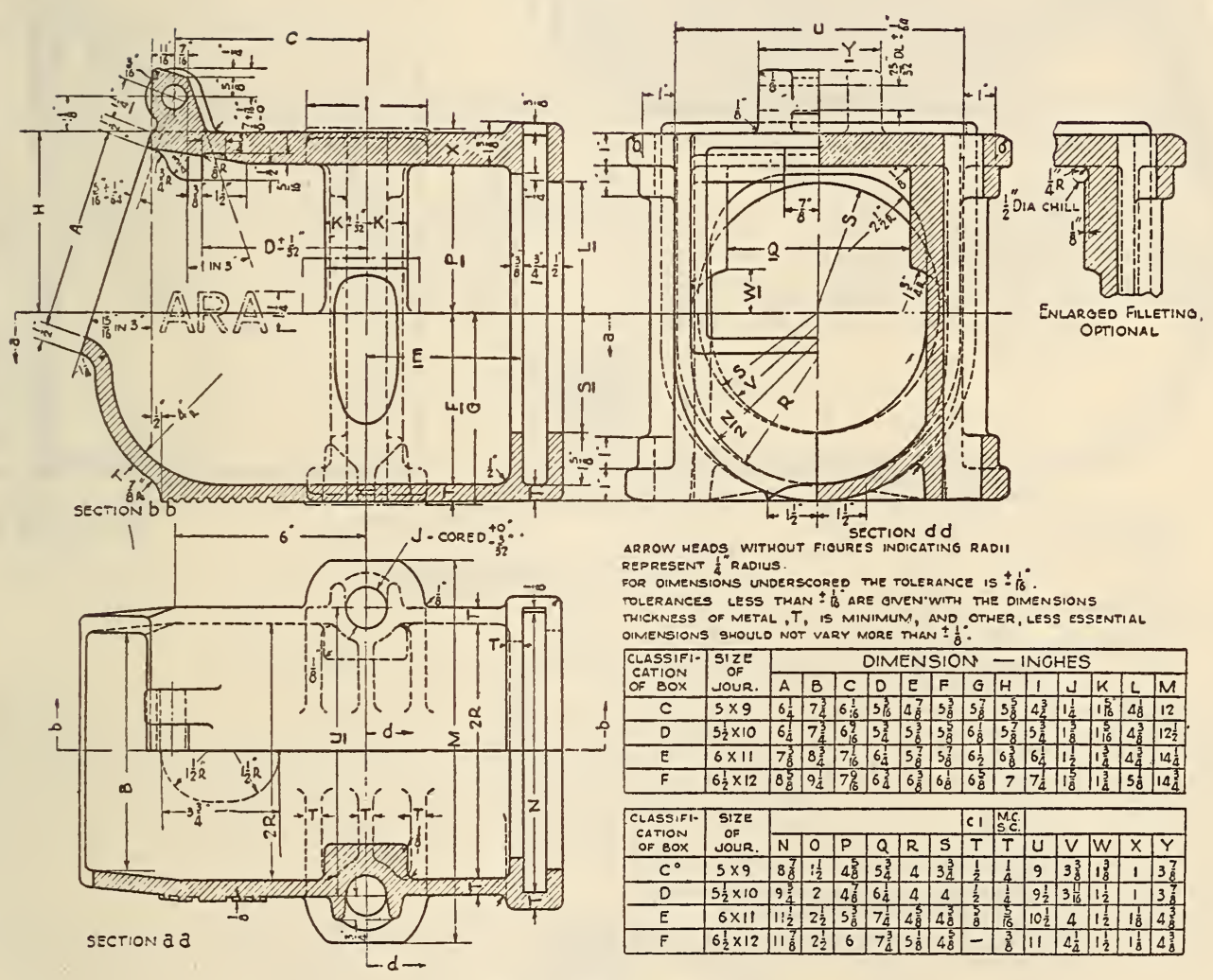

Figdre 441.-Journal boxes $C, D, E$, and $F$ 


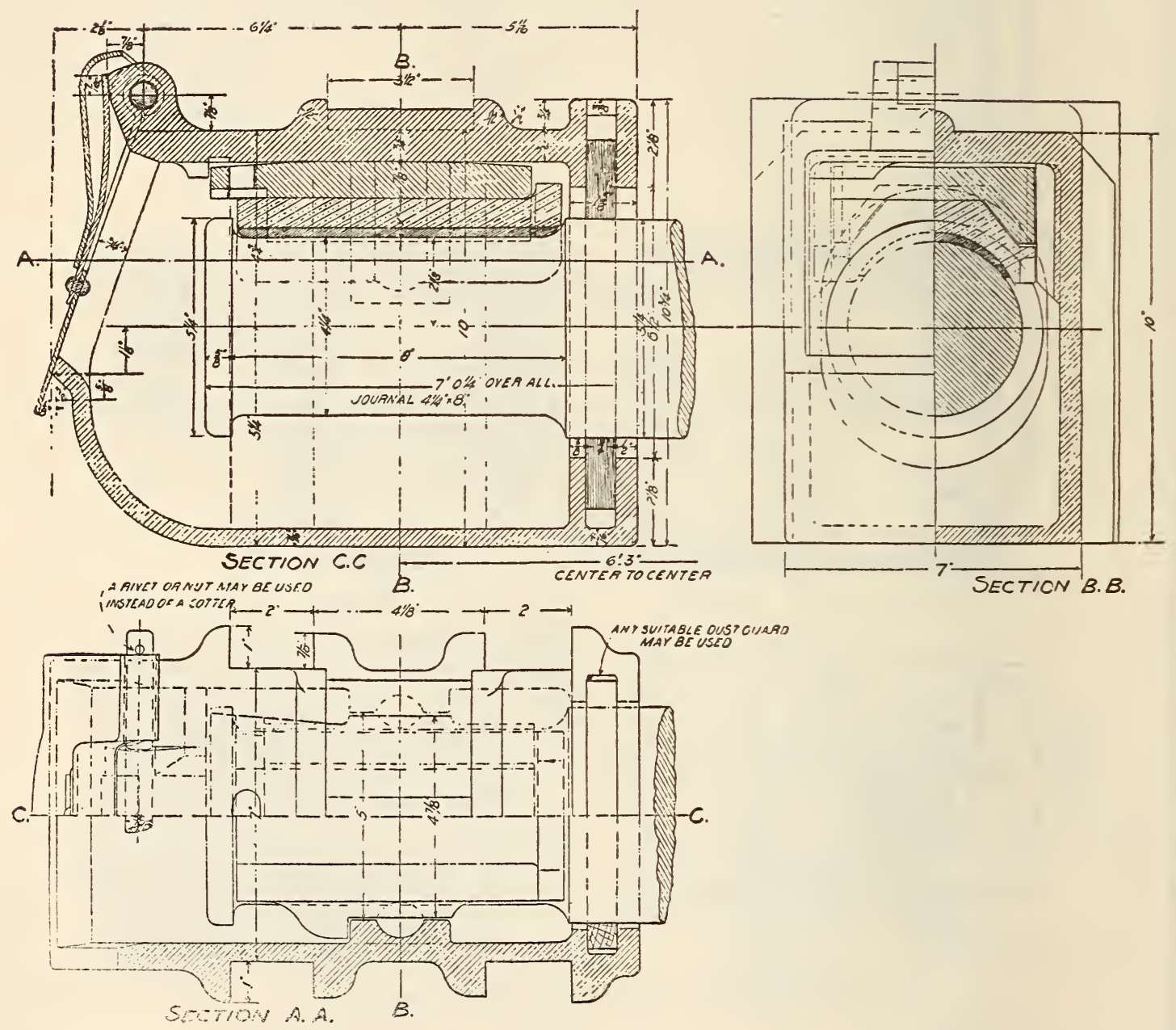

Figdre 442

Note.-Section of box may be made either circular or square below the center line, provided all the essential dimensions are adhered to. When journal box is made of malleable iron, reduction in thickness of metal and coring to lighten weight is permissible, provided all the essential dimensions which affect interchangeability and the proper fitting of contained parts are adhered to. 


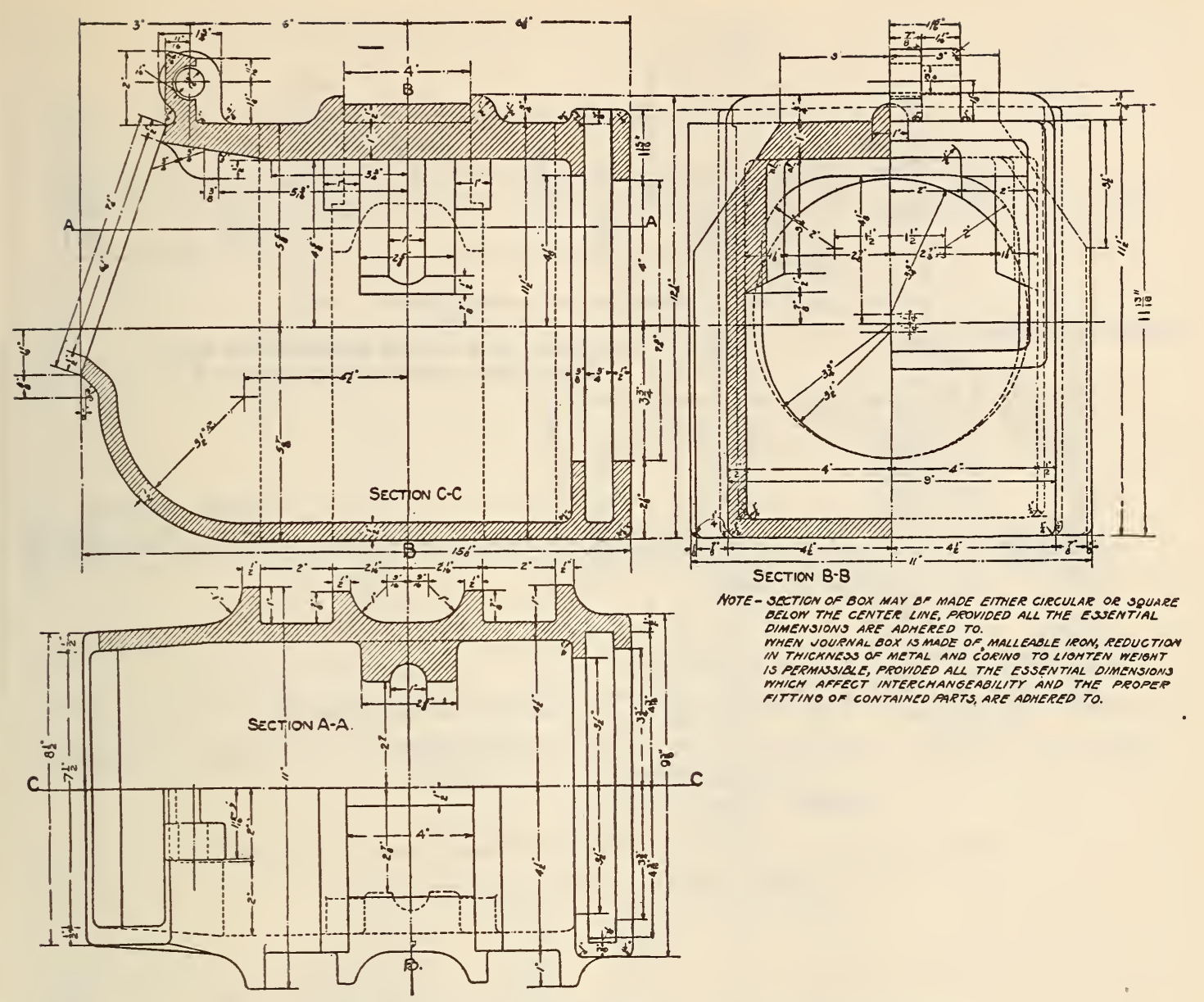

FIGURE 443.-Journal box for passenger cars for 5 by 9 inch journals

Hole for lid pin may be cored thirteenth-sixteenths inch diameter, provided the amount that the edge of the hcle projects outside of the plane of box lid face does not exceed nine-sixty-fourths inch, and is not less than three-thirty-seconds inch. Hole for lid pin must be horizontal, and also parallel with the lid face of the box.

\section{AMERICAN RAILWAY ASSOCIATION, ME- CHANICAL DIVISION; STANDARD JOURNAL-BOX GAGES FOR JOURNAL BOXES, CLASSES C, D, E, AND F, 1923 JOURNAL-BOX GAGES}

The table of dimensions for journal-box gages for journals $\mathrm{C}, \mathrm{D}, \mathrm{E}$, and $\mathrm{F}$ is as follows:

(See figs. 444, 445, 446, and 447)
[All dimensions in inches]

\begin{tabular}{|c|c|c|c|c|c|c|c|c|c|c|c|}
\hline $\begin{array}{c}\text { Class of } \\
\text { box }\end{array}$ & $\begin{array}{c}\text { Size of } \\
\text { journal }\end{array}$ & A & B & C & $\mathrm{D}$ & $\mathbf{E}$ & $\mathbf{F}$ & G & H & I & $\mathbf{J}$ \\
\hline $\begin{array}{l}\mathrm{C} \\
\mathrm{D} \\
\mathrm{E} \\
\mathrm{F}\end{array}$ & $\begin{array}{l}5 \text { by } 9 \\
51 / 2 \text { by } 10 \\
6 \text { by } 11 \\
61 / 2 \text { by } 12\end{array}$ & $=\mid \begin{array}{c}9 \\
91 / 2 \\
101 / 2 \\
11\end{array}$ & \begin{tabular}{|l|}
6 \\
$61 / 4$ \\
$63 / 4$ \\
$71 / 8$
\end{tabular} & $\begin{array}{l}121 / 8 \\
125 / 8 \\
143 / 8 \\
147 / 8\end{array}$ & \begin{tabular}{l|c}
8 & $15 / 3$ \\
8 & $1 \% / 3$ \\
8 & $113 / 3$ \\
8 & $117 / 3$
\end{tabular} & \begin{tabular}{l|l}
32 & $11 / 2$ \\
32 & 2 \\
32 & $21 / 2$ \\
32 & $21 / 2$
\end{tabular} & $\begin{array}{r}83 / 4 \\
95 / 8 \\
113 / 8 \\
113 / 4\end{array}$ & \begin{tabular}{l|l}
$3 / 4$ & $65 / 8$ \\
$5 / 8$ & $71 / 4$ \\
$3 / 8$ & $77 / 8$ \\
$3 / 4$ & $83 / 8$
\end{tabular} & $\begin{array}{l}73 / 4 \\
81 / 4 \\
9 \\
95 / 8\end{array}$ & \begin{tabular}{l|l}
4 & $11 / 2$ \\
4 & $11 / 2$ \\
$11 / 2$ \\
$11 / 2$
\end{tabular} & $\left\{\begin{array}{l}5 / 32 \\
523 / 32 \\
67 / 33 \\
623 / 32\end{array}\right.$ \\
\hline $\begin{array}{c}\text { Class } \\
\text { of } \\
\text { box }\end{array}$ & $\begin{array}{c}\text { Size of } \\
\text { journal }\end{array}$ & $\mathrm{K}$ & L & $\mathrm{M}$ & $\mathrm{N}$ & 0 & $\mathrm{P}$ & $Q$ & R & $\mathrm{S}$ & $\mathrm{T}$ \\
\hline $\begin{array}{l}\text { C.- } \\
\text { D.- } \\
\text { E.- } \\
\text { F.- }\end{array}$ & $\begin{array}{l}5 \text { by } 9 \\
51 / 2 \text { by } 10 \\
6 \text { by } 11- \\
61 / 2 \text { by } 12\end{array}$ & $\begin{array}{l}611 / 16 \\
73 / 16 \\
71116 \\
83 / 16\end{array} \mid$ & \begin{tabular}{l|}
$43 / 8$ \\
$47 / 8$ \\
$53 / 8$ \\
$57 / 8$
\end{tabular} & \begin{tabular}{l|l}
$67 / 8$ & 5 \\
$67 / 8$ & 6 \\
778 & 7 \\
838 & 7
\end{tabular} & $\begin{array}{l}13 / 16 \\
55 / 16 \\
55 / 16 \\
13 / 16\end{array}$ & $\left|\begin{array}{l}5^{11} 1 / 16 \\
63 / 16 \\
73 / 16 \\
711 / 16\end{array}\right|$ & \begin{tabular}{l|l}
7 & 1 \\
$71 / 2$ & 1 \\
$81 / 2$ & 1 \\
9 & 1
\end{tabular} & $\begin{array}{l}111 / 32 \\
111 / 32 \\
125 / 32 \\
125 / 32\end{array}$ & \begin{tabular}{l|l}
$3 / 8$ \\
$3 / 8$ \\
$1 / 2$ \\
$3 / 4$
\end{tabular} & \begin{tabular}{|c|}
$29 / 32$ \\
$11 / 32$ \\
$13 / 32$ \\
$17 / 32$
\end{tabular} & $\begin{array}{l}115 / 32 \\
119 / 32 \\
121 / 32 \\
125 / 33\end{array}$ \\
\hline
\end{tabular}




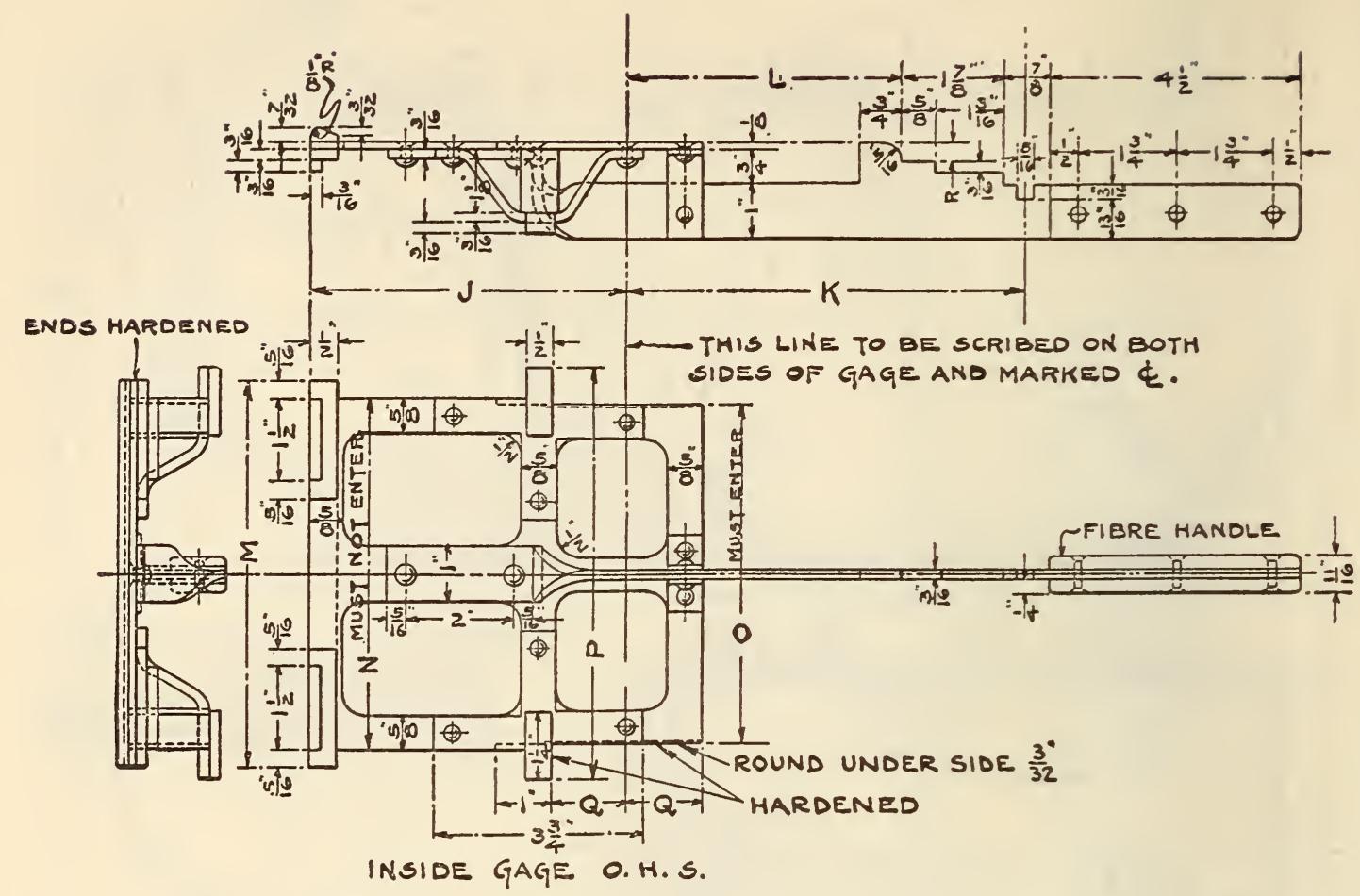

Figure 444.-Journal box gages for journal boxes, class $C, D, E$, and $F$ Gage No. 1.-Inside gage. (For dimensions see p. 679.)

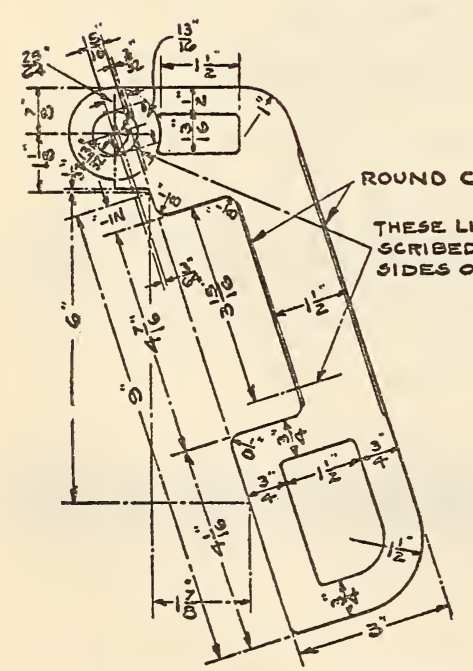

FRONT OPENING ANO LUG GAGE. Б̈O.H.S. HARDENEO.

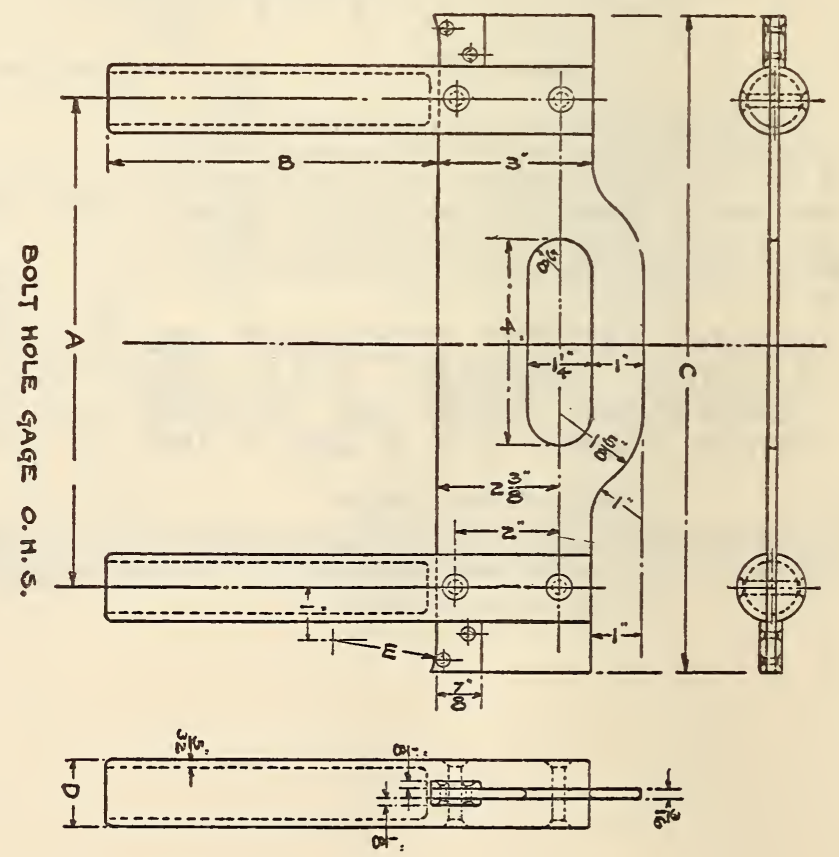

Figure 445.-Journal box gages for journal boxes, class $C, D, E$, and $F$ Gage No. 2.-Journal box bolt hole gage. (For dimensions see p. 679.) 


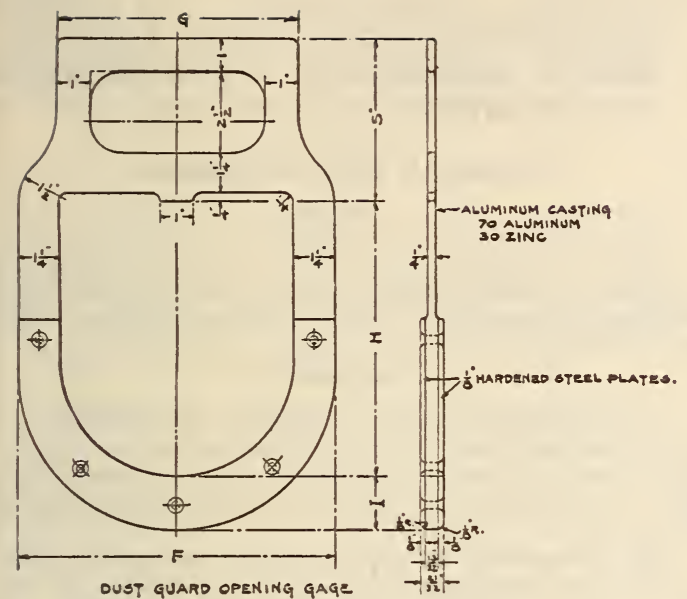

FigURE 446.-Journal box gages for journal boxes, class $C, D, E$, and $F$

Gage No. 3.-Dust guard gage. (For dimensions see p. 679.)

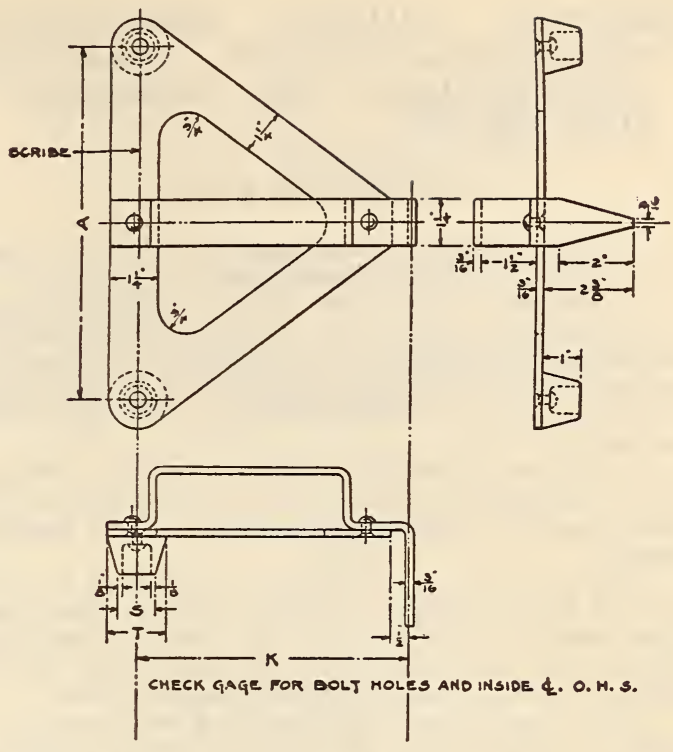

Figure 447.-Journal box gage for journal boxes, class $C, D, E$, and $F$.

Gage No.5.-Bolt hole hinge lug gage. (For dimensions see p. 679.)

\section{AMERICAN RAILWAY ASSOCIATION, MECHANICAL DIVISION, JOURNAL-BEARING WEDGES, STANDARD, 1931}

JOURNAL-BEARING WEDGES
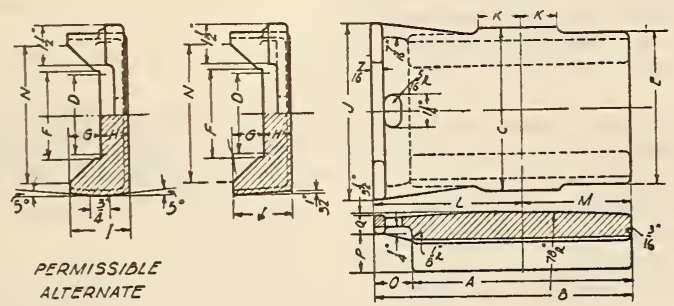

FIGURE 448.-Journal bearing wedges

[All dimensions in inches]

\begin{tabular}{|c|c|c|c|c|c|c|c|c|c|c|c|c|c|c|c|c|c|c|}
\hline $\begin{array}{l}\text { Classification of } \\
\text { wedge }\end{array}$ & $\begin{array}{l}\text { Size of } \\
\text { journal }\end{array}$ & A & B & $\mathrm{C}$ & D & $\mathrm{E}$ & F & $G$ & H & I & $\mathrm{J}$ & K & L & M & $\mathbf{N}$ & 0 & $\mathbf{P}$ & Q \\
\hline $\begin{array}{l}\mathrm{C} . . . \\
\mathrm{D} \\
\mathrm{E} \\
\mathrm{F} \\
\mathrm{F}\end{array}$ & $\begin{array}{l}33 / 4 \text { by } 7 \\
4145 \text { by } 8 \\
5 \text { by } 9 \\
51 / 2 \text { by } 10 \\
6 \text { by } 11 \\
61 / 2 \text { by } 12\end{array}$ & \begin{tabular}{r|}
$53 / 8$ \\
638 \\
$73 / 8$ \\
838 \\
938 \\
1038
\end{tabular} & $\begin{array}{c}6 \% 16 \\
73,4 \\
834 \\
913 / 16 \\
101 / 16 \\
111316\end{array}$ & $\begin{array}{l}41 / 2 \\
47 / 8 \\
55,8 \\
618 \\
718 \\
75 \%\end{array}$ & $\begin{array}{l}21 / 16 \\
21132 \\
33 / 13 \\
33116 \\
3916 \\
41 / 16\end{array}$ & \begin{tabular}{l|l|}
4 \\
$41 / 2$ \\
$51 / 4$ \\
534 \\
$67 / 8$ \\
$73 / 8$
\end{tabular} & $\begin{array}{l}21 / 4 \\
2916 \\
3316 \\
338 \\
334 \\
414\end{array}$ & $\begin{array}{l}1 \\
11 / 16 \\
11 / 16 \\
1316 \\
138 \\
11 / 2\end{array}$ & $\begin{array}{l}3 / 4 \\
1^{7 / 8} \\
1 \\
11 / 8 \\
13 / 8\end{array}$ & $\begin{array}{l}13 / 4 \\
115 / 16 \\
21 / 16 \\
23 / 16 \\
21 / 2 \\
27 / 8\end{array}$ & \begin{tabular}{l|}
5 \\
5 \\
$63 / 4$ \\
$6^{33}$ \\
$73 / 4$ \\
$81 / 4$
\end{tabular} & $\begin{array}{l}114 \\
11 / 4 \\
17 / 16 \\
17 / 6 \\
17 / 8 \\
17 / 8\end{array}$ & $\begin{array}{l}37 / 8 \\
49 / 6 \\
51 / 16 \\
558 \\
618 \\
65 / 8\end{array}$ & $\begin{array}{l}211 / 16 \\
33 / 16 \\
311 / 16 \\
4316 \\
411 / 16 \\
53 / 16\end{array}$ & \begin{tabular}{l|}
$35 / 8$ \\
$41 / 16$ \\
434 \\
$57 / 32$ \\
618 \\
$67 / 16$
\end{tabular} & \begin{tabular}{l|}
$13 / 16$ \\
138 \\
138 \\
$17 / 16$ \\
$17 / 16$ \\
$17 / 16$
\end{tabular} & $\begin{array}{c}31 / 32 \\
1532 \\
1932 \\
1132 \\
1232 \\
23 / 32 \\
232\end{array}$ & (1) \\
\hline
\end{tabular}

PERMISSIBLE VARIATIONS

\begin{tabular}{|c|c|c|c|c|c|c|c|c|c|c|c|c|c|c|c|c|c|}
\hline $\begin{array}{l}\text { May be greater by } \\
\text { May be less by }\end{array}$ & $\begin{array}{l}1 / 16 \\
1 / 16\end{array}$ & $\begin{array}{l}1 / 32 \\
1 / 32\end{array}$ & $\begin{array}{l}1 / 32 \\
1 / 32\end{array}$ & $0^{1 / 32}$ & & & $0_{164}$ & $\begin{array}{l}1 / 32 \\
1 / 16\end{array}$ & - & $\begin{array}{l}1 / 8 \\
1 / 8\end{array}$ & $\begin{array}{l}1 / 8 \\
1 / 8\end{array}$ & $\begin{array}{l}1 / 32 \\
1 / 16\end{array}$ & $\begin{array}{l}1 / 32 \\
1 / 16\end{array}$ & $0^{1 / 32}$ & & & $-\int_{1 / 16}^{0}$ \\
\hline
\end{tabular}

All wedges revised, 1927 and 1930.

Nоте.-Wedge shall be of malleable iron, forged or cast steel. 


\section{AMERICAN RAILWAY ASSOCIATION, MECHANICAL DIVISION, STANDARD SPECIFICATIONS FOR JOURNAL-BOX LIDS, 1929}

\section{JOURNAL-BOX LIDS}

1. Scope.-This specification covers all lids for use on A. R. A. standard journal boxes.

2. Material.-Lids when made of malleable iron or cast steel to be not less than one-eighth inch in thickness. When made of pressed steel, thickness to be not less than five thirty-seconds of an inch and material to have a carbon content of at least 0.25 per cent.

3. Functions.-(a) Lid must protect the packing by preventing the entrance into the journal box of dust, sand, fine coal, snow, water, or other foreign matter.

4. Construction. - $(a)$ Lid to be attached to the journal box by means of a pin, bolt, or rivet one thirty-second of an inch less in diameter than the size of the hinge lug hole in the bcx. The use of separable cotter pins or split keys is prohibited.

(b) Springs of the coiled ty pe to be used, and preferably so located that the force on the lid in closed position shall be at or near the center of the lid.

(c) The above lid fastenings to be so arranged that the lid can be easily opened and closed and retain itself in a fully opened position without danger of closing.

(d) When closed, the tension between the lid and fastening must be sufficient to prevent vibration of lid or any parts thereof.

(e) Lids of the hinged type to have the hinge located at the top of the journal box so arranged that the lid will open outward and upward to at least an angle of $90^{\circ}$ with the lid face of the journal box. Lids of other types should provide an equivalent opening.

(f) A tight contact between the lid and the face of the journal box must be maintained in order to meet the requirements as stated under item No. 3.

For journal boxes used on passenger-train equipment a machined fit is recommended for the lid as well as the journal box. Die forming of lids, after pressings or casting to insure a smooth, flat surface where lid bears against face of box, will be considered equivalent to machining.

(g) A ledge, flange, or other suitable arrangement must be provided on the inside of the lid, particularly along the lower part, so that oil thrown against the inside face will drain back into the box.

(h) Top and side lips at least one-fourth of an inch in projection to be located around the outside edge of the lid, except that side lips may be omitted where the lid itself is ground or machined.

(i) The hinge lug must be housed.

(j) The eyes of cast lids must be integrally closed their full length.

Pressed-steel lids to have the eyes carefully welded their full length, or to have each eye secured by not less than two $3 / 8$-inch rivets.

The eyes of the lid must be parallel with the contact face and gaged so as to insure a tight lid fit.

(k) The inspection of lids that will insure complying with the above requirements is recommended.

\subsection{ROWLOCKS.}

(No nationally recognized specifications available.)
611.29 MISCELLANEOUS MANUFACTURES MALLEABLE CAST IRON.

\section{AMERICAN ELECTRIC RAILWAY ENGI- NEERING ASSOCIATION, OVERHEAD LINE MATERIAL FOR DIRECT AND CAT. ENARY SUSPENSION, RECOMMENDED SPECIFICATION, D102-30, 1930}

\section{OVERHEAD LINE MATERIAIS}

\section{SCOPE}

101. This specification covers material used in overhead trolley construction, both direct and catenary suspension, and overhead-feeder cable construction.

\section{MATERIALS}

201. Nonferrous alloy castings.-(a) Nonferrous alloy castings shall be of such proportions of copper, tin, zinc, lead, and/or other metals as to produce an alloy suitable for the duty for which the casting is designed.

(b) Alloys shall be highly resistive to the corrosive action of severe atmospheric pollution due to the products of combustion, steam, and to salt fogs.

(c) Alloys used in current-carrying parts shall be of the highest electrical conductivity consistent with the strength requirements, and the size and design of such parts shall be such as to provide the necessary conductivity with the alloy used.

(d) Alloys shall have tensile strength not less than those shown in table below.

\begin{tabular}{|c|c|}
\hline Use & $\begin{array}{c}\text { Minimum } \\
\text { tensile } \\
\text { strength }\end{array}$ \\
\hline $\begin{array}{l}\text { For main tension members, such as splicers, sockets, etc- } \\
\text { For parts where stiffness is a prime requisite, such as } \\
\text { frogs, crossings, clips, jaws, etc } \\
\text { For parts which must be bent cold, such as clinch ears, } \\
\text { approaches, etc-. } \\
\text { (Yield point not in excess of } 40 \text { per cent of tensile } \\
\text { strength.) }\end{array}$ & $\begin{array}{r}L b 8 . / i n .^{2} \\
30,000 \\
28,500 \\
26,000\end{array}$ \\
\hline
\end{tabular}

202. Copper.-Copper shall confrom to the requirements for low-resistance lake of current specification designation B-4 for lake copper, or to the current specification designation $\mathrm{B}-5$ for electrolvtic copper, both of the A.S. T. M. (See 641.11, p. 1173.)

203. Malleable iron.-(a) Malleable-iron castings shall conform to the current specification designation A-47 for malleable castings of the A. S. T. M. (See 611.21, p. 665.)

(b) Such castings shall not show brittleness after galvanizing nor shall their tensile strength or ductility be reduced to values less than those given in the above specification after any heat treatment to remove embrittlement.

204. Steel.-Steel shall conform to the current specification designation A-7 for structural steel for bridges of the A. S. T. M. (See 605.11, p. 249.)

209. Wrought iron.-Wrought iron shall conform to the current specification designation $A-41$ for refined wrought-iron bars of the A. S. T. M. (See 603.1, p. 139.)

210. Protective coatings. - (a) Zinc protective coatings shall conform to the current specification manual section D6 for galvanizing or sherardizing 
on iron and steel of the American Electric Railway Engineering Association. (See 600.3, p. 60.)

(b) Other protective coatings may be used provided they furnish a degree of protection not less than that of zinc.

211. Nonferrous materials.-Nonferrous materials may be used, notwithstanding the specification herein of iron or steel, provided adequate strength and satisfactory dimensions are realized.

\section{DESIGN}

301. Drawings.-Drawings which accompany these specifications shall be considered a part thereof.

302. Protection.-All ferrous materials and fittings shall have a zinc or other approved protective coating, firmly adherent, uniform, and smooth.

\section{A. GENERAL OVER-HEAD IINE MATERIAL}

303. Scope.-This specification covers material used in overhead line construction of all kinds, including feeder and trolley wire construction.

304. Bolts and nuts, general.-(a) Bolts and nuts shall be of steel or of nonferrous materials as specified. Heads and nuts shall be finished with one face rounded or chamfered.

(b) Bolt threads may be cut or rolled as specified and shall be full, clean, and concentric with shank. They shall conform in size, pitch and number per inch to the U. S. standard given below:

\begin{tabular}{|c|c|}
\hline $\begin{array}{c}\text { Nominal } \\
\text { diameter } \\
\text { of bolt }\end{array}$ & $\begin{array}{c}\text { Threads } \\
\text { per inch }\end{array}$ \\
\hline Inch & \\
$1 / 4$ & 20 \\
$3 / 8$ & 16 \\
$1 / 2$ & 13 \\
$5 / 8$ & 11 \\
$3 / 4$ & 10 \\
$7 / 8$ & 9 \\
1 & 8 \\
\hline
\end{tabular}

When rolled thread is used, the diameter specified applies to the threaded portion and the shank may be slightly less in size.

(c) Nuts shall be square or hexagonal as specified and must develop the full strength of the bolt. They shall be symmetrically formed, with hole central and at right angles to face, tapped to fit corresponding thread so that they can be run the entire length of the thread by the fingers without undue forcing or without noticeable play or rocking. When hot galvanized, the galvanizing may be omitted upon the threaded portion of the nut.

(d) Various types of bolts and nuts shall be of dimensions shown in the following figures:

Carriage bolt_._._._._. . Figure 449.

Eyebolt_....... Figure 450.

Double arming (spacing) bolt. Figure 451 .

Machine (through) bolt_..... Figure 452.

It shall be understood that they are to be furnished complete with nuts as shown in figures.
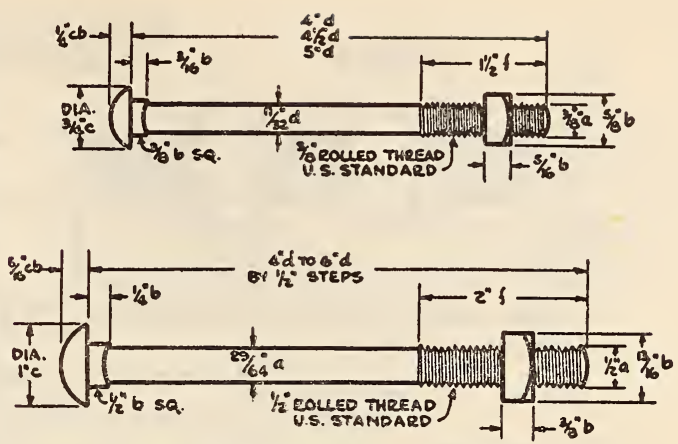

Figure 449.-Carriage bolt

\begin{tabular}{|c|c|c|}
\hline & \multicolumn{2}{|c|}{$\begin{array}{c}\text { Allowable va- } \\
\text { riations }\end{array}$} \\
\hline & Over & Under \\
\hline & Inch & Inch \\
$a$ & $1 / 64$ & $1 / 64$ \\
$b$ & $1 / 32$ & $1 / 32$ \\
$c$ & $1 / 16$ & $1 / 16$ \\
$d$ & $1 / 8$ & $1 / 8$ \\
$f$ & $1 / 4$ & 114 \\
$c b$ & $1 / 16$ & $1 / 32$ \\
\end{tabular}

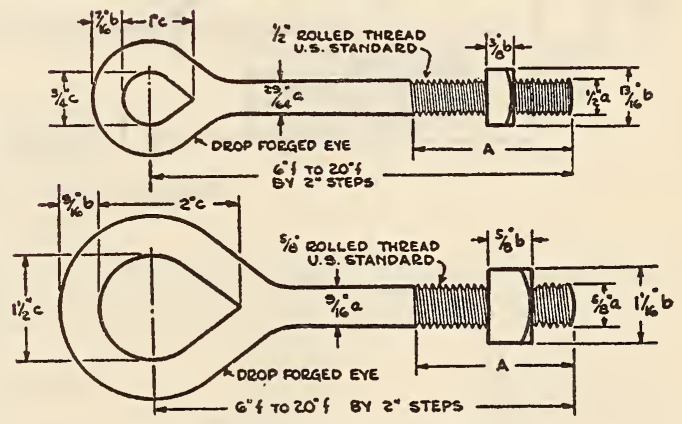

Figure 450.-Eyebolt

\begin{tabular}{|l|}
\hline A \\
\hline 3 inches $(f)$ for 6 -inch bolt. \\
4 inches $(f)$ for 8,10 , and 12 inch bolt. \\
6 inches $(f)$ for 14 to 20 inch bolt.
\end{tabular}

\begin{tabular}{|c|c|c|}
\hline & \multicolumn{2}{|c|}{$\begin{array}{l}\text { Allowable va- } \\
\text { riations }\end{array}$} \\
\hline & Over & Under \\
\hline $\begin{array}{l}a \\
b \\
c \\
d \\
f\end{array}$ & $\begin{array}{c}\text { Inch } \\
1 / 64 \\
1 / 32 \\
1 / 16 \\
1 / 8 \\
1 / 4\end{array}$ & $\begin{array}{c}\text { Inch } \\
1164 \\
1 / 32 \\
1116 \\
1 / 8 \\
1 / 4\end{array}$ \\
\hline
\end{tabular}




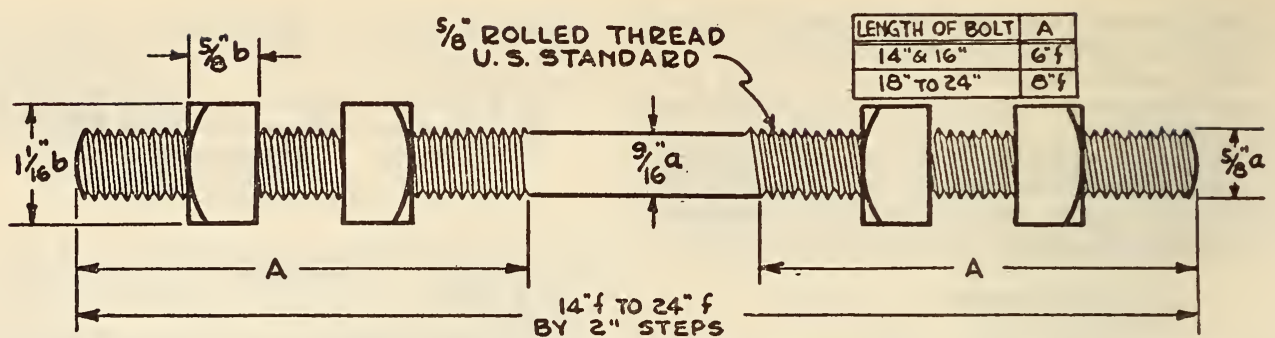

FIGURE 451.-Double arming (spacing) bolt

\begin{tabular}{|c|c|c|}
\hline & \multicolumn{2}{|c|}{$\begin{array}{l}\text { Allowable va- } \\
\text { riations }\end{array}$} \\
\hline & Over & Under \\
\hline $\begin{array}{l}a \\
b \\
f\end{array}$ & $\begin{array}{c}\text { Inch } \\
1 / 64 \\
1 / 32 \\
1 / 4\end{array}$ & $\begin{array}{c}\text { Inch } \\
1 / 64 \\
1 / 32 \\
1 / 4\end{array}$ \\
\hline
\end{tabular}

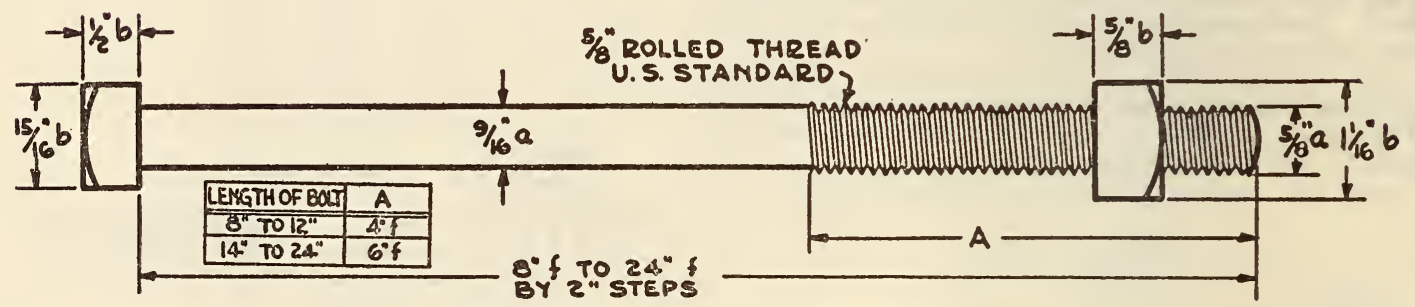

FIGURE 452.-Machine (through) bolt

\begin{tabular}{|c|c|c|}
\hline & \multicolumn{2}{|c|}{$\begin{array}{l}\text { Allowable va- } \\
\text { riations }\end{array}$} \\
\hline & Over & Under \\
\hline $\begin{array}{l}a \\
b \\
f\end{array}$ & $\begin{array}{c}\text { Inch } \\
1 / 64 \\
1 / 32 \\
1 / 4\end{array}$ & $\begin{array}{c}\text { Inch } \\
1 / 64 \\
1 / 32 \\
1 / 4\end{array}$ \\
\hline
\end{tabular}

305. Clamps, guy.-(a) Guy clamps shall be of steel of dimensions shown in Figure 453. The ends of the groove shall be rounded and the clamping surface may be either smooth or with rounded diagonal ridges to fit the lay of any strand size from $5 / 10$ inch to $1 / 2$ inch. 

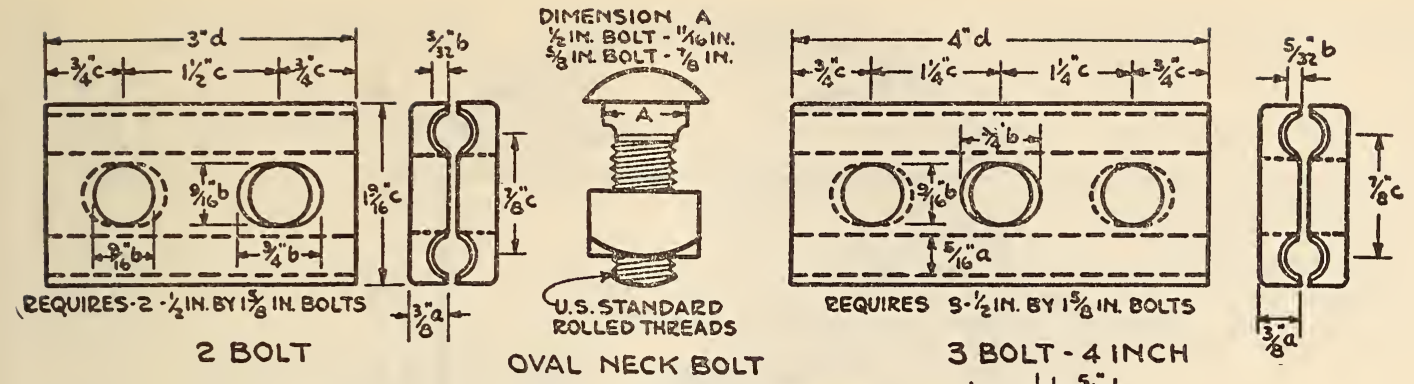

REQUIRES-

3-5/6IN. BY IT/4IN. BOLTS
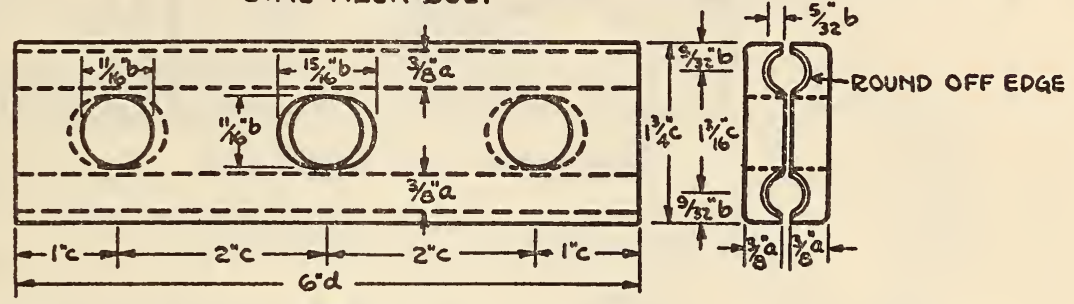

3 BOLT - 6 INCH

Figure 453.-Guy clamp

\begin{tabular}{|c|c|c|}
\hline \multicolumn{1}{|c|}{} & \multicolumn{2}{|c|}{$\begin{array}{c}\text { Allowable va- } \\
\text { riations }\end{array}$} \\
\hline & Over & Under \\
\hline & & \\
\hline & Inch & Inch \\
$a$ & $1 / 64$ & $1 / 64$ \\
$b$ & $1 / 32$ & $1 / 32$ \\
$c$ & $1 / 16$ & $1 / 16$ \\
$d$ & $1 / 8$ & $1 / 8$ \\
$f$ & $1 / 4$ & $1 / 4$ \\
\hline
\end{tabular}

(b) Bolts shall have square or oval neck to prevent turning, and shall be of special high-carbon or heat-treated stock to give extra strength. Alternate bolts shall be inverted.
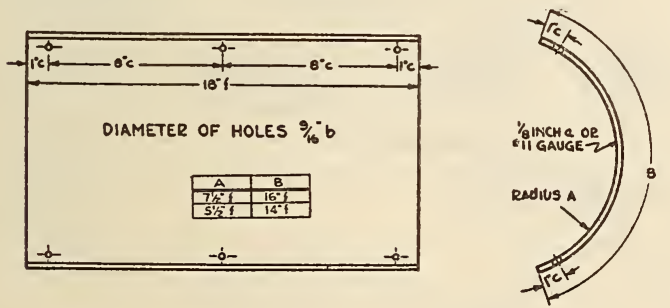

FIgURE 454.-Hub (pole) guard

\begin{tabular}{|l|r|r|}
\hline & \multicolumn{2}{|c|}{$\begin{array}{c}\text { Allowable va- } \\
\text { riations }\end{array}$} \\
\hline & Over & Under \\
\hline & & \\
\hline & Inch & Inch \\
$a$ & $1 / 64$ & $1 / 64$ \\
$b$ & $1 / 32$ & $1 / 32$ \\
$c$ & $1 / 16$ & $1 / 16$ \\
$f$ & $1 / 4$ & $1 / 4$ \\
\hline
\end{tabular}

306. Guards, hub (pole).-Hub guards shall be of steel of dimensions shown in Figure 454.

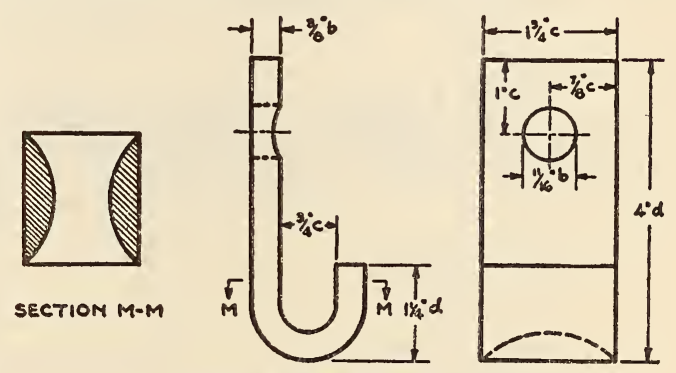

FIGURE 455.-Guy hook

\begin{tabular}{|c|c|c|}
\hline & \multicolumn{2}{|c|}{$\begin{array}{c}\text { Allowable va- } \\
\text { riations }\end{array}$} \\
\hline & Over & Under \\
\hline & Inch & Inch \\
$b$ & $1 / 32$ & $1 / 32$ \\
$c$ & $1 / 16$ & $1 / 16$ \\
$d$ & $1 / 8$ & $1 / 8$ \\
\hline
\end{tabular}

307. Hooks, guy.-Guy hooks shall be of steel of dimensions shown in Figure 455. 


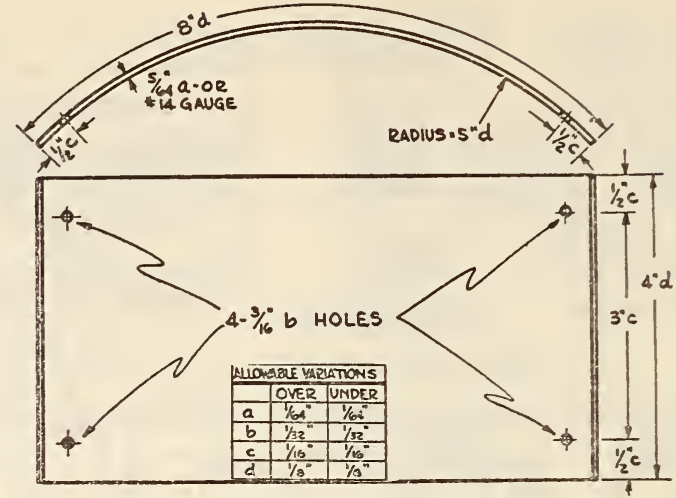

FigURE 456.-Guy (strain) plate

309. Plates, guy (strain).-Guy plates shall be of steel of dimensions shown in Figure 456.

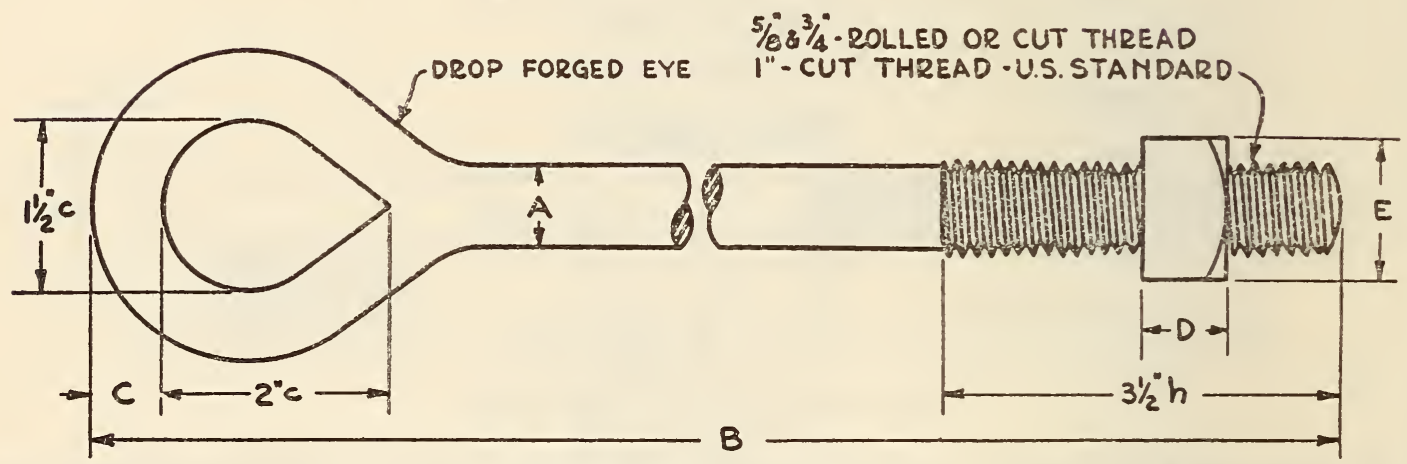

FIgURe 457.-Anchor rod

\begin{tabular}{|c|c|c|c|c|c|}
\hline Size & A & B & $\mathrm{C}$ & $\mathrm{D}$ & $\mathrm{E}$ \\
\hline $\begin{array}{c}\text { Inch } \\
5 / 8 \\
1^{3 / 4}\end{array}$ & $\begin{array}{l}5 / 8 \text { inch }(a) . \\
3 / 4 \text { inch }(a) \text {. } \\
1 \text { inch }(a) .\end{array}$ & $\begin{array}{l}6 \text { feet }(k) \text { and } 8 \text { feet }(k) \text {. } \\
8 \text { feet }(k) \text { and } 10 \text { feet }(k) \text {. } \\
10 \text { feet }(k) \text { and } 12 \text { feet }(k) \text {. }\end{array}$ & $\begin{array}{l}9 / 16 \text { inch }(b) . \\
5 / 8 \text { inch }(b) . \\
7 / 8 \text { inch }(b) .\end{array}$ & $\begin{array}{l}5 / 8 \text { inch }(b) \text {. } \\
3 / 4 \text { inch }(b) \text {. } \\
1 \text { inch }(b) \text {. }\end{array}$ & $\begin{array}{l}11 / 16 \text { inches }(b) . \\
11 / 4 \text { inches }(b) . \\
15 / 8 \text { inches }(b) .\end{array}$ \\
\hline
\end{tabular}

\begin{tabular}{|c|c|c|}
\hline & \multicolumn{2}{|c|}{$\begin{array}{c}\text { Allowable va. } \\
\text { riations }\end{array}$} \\
\cline { 2 - 3 } & Over & Under \\
\hline & & \\
\hline$a$ & & Inch \\
$b$ & $1 / 64$ & 164 \\
$c$ & $1 / 32$ & $1 / 32$ \\
$h$ & $1 / 16$ & $1 / 16$ \\
$k$ & $1 / 2$ & $1 / 2$ \\
\hline
\end{tabular}

310. Rods, anchor.-Anchor rods shall be of steel of dimensions shown in Figure 457. 


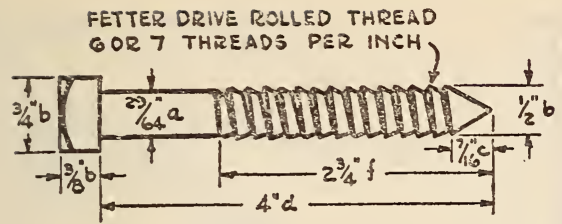

$1 / 3$ INCH LAG SCREW

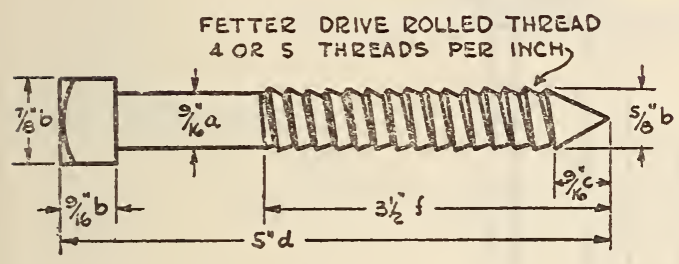

$\%$ INCH LAG SCREW

FIgURE 458.- Lag screw

Note.-Gimlet point instead of fetter drive may be specified.

\begin{tabular}{|c|c|c|}
\hline & \multicolumn{2}{|c|}{$\begin{array}{c}\text { Allowable va- } \\
\text { riations }\end{array}$} \\
\cline { 2 - 3 } & Over & Under \\
\hline & Inch & Inch \\
$a$ & $1 / 64$ & $1 / 64$ \\
$b$ & $1 / 32$ & $1 / 32$ \\
$c$ & $1 / 16$ & $1 / 16$ \\
$d$ & $1 / 8$ & $1 / 8$ \\
$f$ & $1 / 4$ & $1 / 4$ \\
\hline
\end{tabular}

311. Screws, lag.-Lag screws shall be of steel of dimensions shown in Figure 458. They shall have the fetter drive thread unless gimlet point is specified.

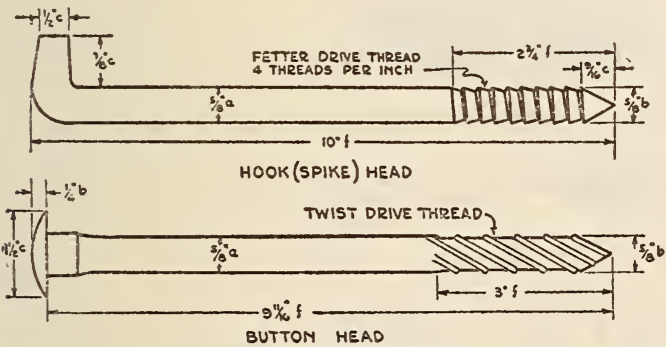

FigURe 459.-Pole steps

\begin{tabular}{|c|c|c|}
\hline & \multicolumn{2}{|c|}{$\begin{array}{c}\text { Allowable va- } \\
\text { riations }\end{array}$} \\
\cline { 2 - 3 } & Over & Under \\
\hline & Inch & Inch \\
$a$ & $1 / 64$ & $1 / 64$ \\
$b$ & $1 / 32$ & $1 / 32$ \\
$c$ & $1 / 16$ & $1 / 16$ \\
$f$ & $1 / 4$ & $1 / 4$ \\
\hline
\end{tabular}

312. Steps, pole.-Pole steps shall be of steel of dimensions shown in Figure 459. They may be either the hook-head type with fetter drive, or the button-head type with twist drive.

313. Strand (guy wire) (messenger).-(a) Strand for guys, backbones, messengers, and similar purposes shall consist of concentrically stranded wires. (Steel strand is usually left-hand lay, and copper and bronze right-hand lay. The direction of lay desired should be specified for both strand and fittings.)

(b) Galvanized-steel strand cable shall conform to the current specification designation A-122 for zinccoated (galvanized) steel wire strand of the A. S. T. M. (See 603.42, p. 181.)
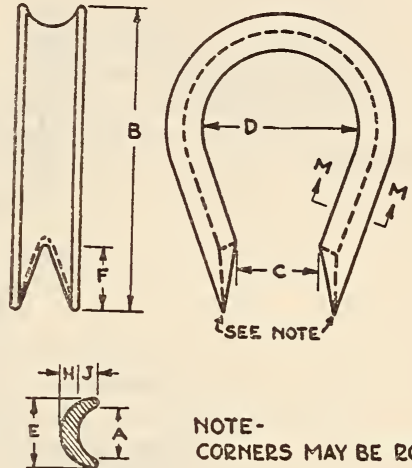

NOTE-

CORNERS MAY BE ROUNDED

SECTION M-M

FIgURE 460.-Thimble

\begin{tabular}{|c|c|c|c|c|}
\hline Size & A & B & C & D \\
\hline $\begin{array}{c}\text { Inch } \\
3 / 8 \\
1 / 2 \\
5 / 8\end{array}$ & $\begin{array}{l}3 / 8 \text { inch }(b) . \\
1 / 2 \text { inch }(b) \text {. } \\
5 / 8 \text { inch }(b) \text {. }\end{array}$ & $\begin{array}{l}23 / 8 \text { inches }(f) . \\
3 \text { inches }(f) \text {. } \\
35 / 8 \text { inches }(f) .\end{array}$ & $\begin{array}{l}1 / 2 \text { inch }(d x) . \\
3 / 4 \text { inch }(d x) \text {. } \\
1 \text { inch }(d x) .\end{array}$ & $\begin{array}{l}\text { 7/8 inch }(c) \text {. } \\
11 / 4 \text { inches }(c) \text {. } \\
17 / 8 \text { inches }(c) \text {. }\end{array}$ \\
\hline Size & $\mathrm{E}$ & $\mathrm{F}$ & $\mathrm{H}$ & $\mathrm{J}$ \\
\hline $\begin{array}{c}\text { Inch } \\
3 / 8 \\
1 / 2 \\
5 / 8\end{array}$ & $\begin{array}{l}1 / 2 \text { inch }(b) . \\
11 / 16 \text { inch }(b) . \\
13 / 16 \text { inch }(b) .\end{array}$ & $\begin{array}{l}\text { 3/8 inch }(c) \text {. } \\
5 / 8 \text { inch }(c) \text {. } \\
3 / 4 \text { inch }(c) \text {. }\end{array}$ & $\begin{array}{l}3 / 32 \text { inch }(a) . \\
5 / 32 \text { inch }(a) . \\
7 / 32 \text { inch }(a) .\end{array}$ & $\begin{array}{l}3 / 32 \text { inch }(a) . \\
1 / 4 \text { inch }(a) \text {. } \\
7 / 32 \text { inch }(a) .\end{array}$ \\
\hline
\end{tabular}

\begin{tabular}{|c|c|c|}
\hline & \multicolumn{2}{|c|}{$\begin{array}{l}\text { Allowable va- } \\
\text { riations }\end{array}$} \\
\hline & Over & Under \\
\hline $\begin{array}{c}a \\
b \\
c \\
f \\
d x\end{array}$ & $\begin{array}{c}\text { Inch } \\
1 / 64 \\
1 / 32 \\
1 / 16 \\
1 / 4 \\
1 / 8\end{array}$ & $\begin{array}{l}\text { Inch } \\
1 / 64 \\
1 / 32 \\
1 / 16 \\
1 / 4 \\
0^{1 / 4}\end{array}$ \\
\hline
\end{tabular}

314. Thimbles.-Thimbles shall be of steel of dimensions shown in Figure 460. 

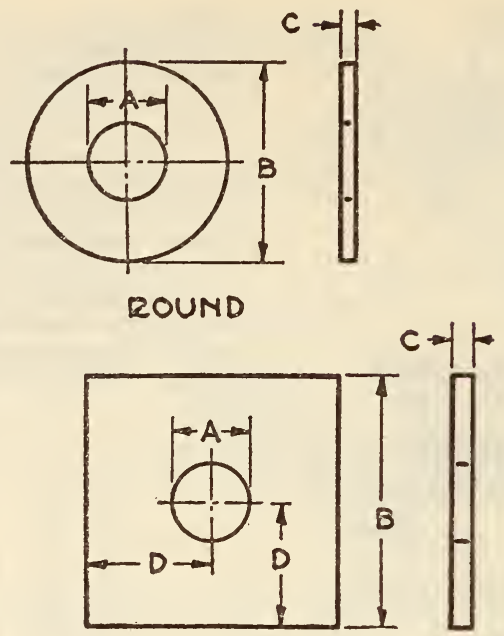

SQUARE

Figure 461.-Washers

\begin{tabular}{|c|c|c|c|c|}
\hline \multicolumn{2}{|c|}{ Size of bolt } & A & B & $\mathrm{C}$ \\
\hline \multicolumn{2}{|c|}{$\begin{array}{l}3 / 8 \text {-inch carriage } \\
\text { bolt nut. } \\
3 / 8 \text { inch carriage } \\
\text { bolt head. } \\
1 / 2 \text { inch carriage } \\
\text { bolt nut or lag. } \\
1 / 2 \text { inch carriage } \\
\text { bolt head or } 5 / 8- \\
\text { inch lag. }\end{array}$} & $\begin{array}{l}7 / 16 \text { inch }(b) . \\
1 / 2 \text { inch }(b) . \\
9 / 16 \text { inch }(b) . \\
11 / 16 \text { inch }(b) .\end{array}$ & $\begin{array}{l}1 \text { inch }(c) \\
11 / 4 \text { inches }(c) \\
13 / 8 \text { inches }(c) \\
13 / 4 \text { inches }(c)\end{array}$ & $\begin{array}{l}5 / 64 \text { inch }(a)- \\
\text { No. } 14 \text { gage. } \\
5 / 64 \text { inch }(a)- \\
\text { No. } 14 \text { gage. } \\
764 \text { inch }(a)- \\
\text { No. } 12 \text { gage. } \\
1 / 8 \text { inch }(a)- \\
\text { No. } 10 \text { gage. }\end{array}$ \\
\hline Size & A & B & $\mathrm{C}$ & $\mathrm{D}$ \\
\hline $\begin{array}{l}\text { Inch } \\
5 / 8 \\
3 / 4 \\
1^{3 / 4}\end{array}$ & $\begin{array}{l}13 / 16 \text { inch }(b) \\
7 / 8 \text { inch }(b) . \\
11 / 8 \text { inches } \\
(b) .\end{array}$ & $\begin{array}{l}21 / 4 \text { inches }(d) \\
3 \text { inches }(d) \\
4 \text { inches }(d)\end{array}$ & $\begin{array}{l}3 / 16 \text { inch }(b) . \\
1 / 4 \text { inch }(b) . \\
1 / 2 \text { inch }(b) .\end{array}$ & $\begin{array}{l}11 / 8 \text { inches }(d) . \\
11 / 2 \text { inches }(d) \\
2 \text { inches }(d)\end{array}$ \\
\hline
\end{tabular}

\begin{tabular}{|c|c|c|}
\hline & $\begin{array}{c}\text { Allowable va- } \\
\text { riations }\end{array}$ \\
\hline & Over & Under \\
\hline & Inch & Inch \\
& $1 / 64$ & $1 / 64$ \\
$b$ & $1 / 32$ & $1 / 32$ \\
$c$ & $1 / 16$ & $1 / 16$ \\
$d$ & $1 / 8$ & $1 / 8$ \\
\hline
\end{tabular}

315. Washers. - Washers shall be of steel of dimensions shown in Figure 461.

\section{B. MATERIAL FOR FEEDER CONSTRUCTION}

316. Scope.-This specification covers material used in feeder cable construction for voltages not exceeding 750 volts. 


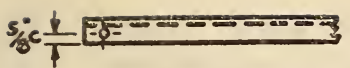

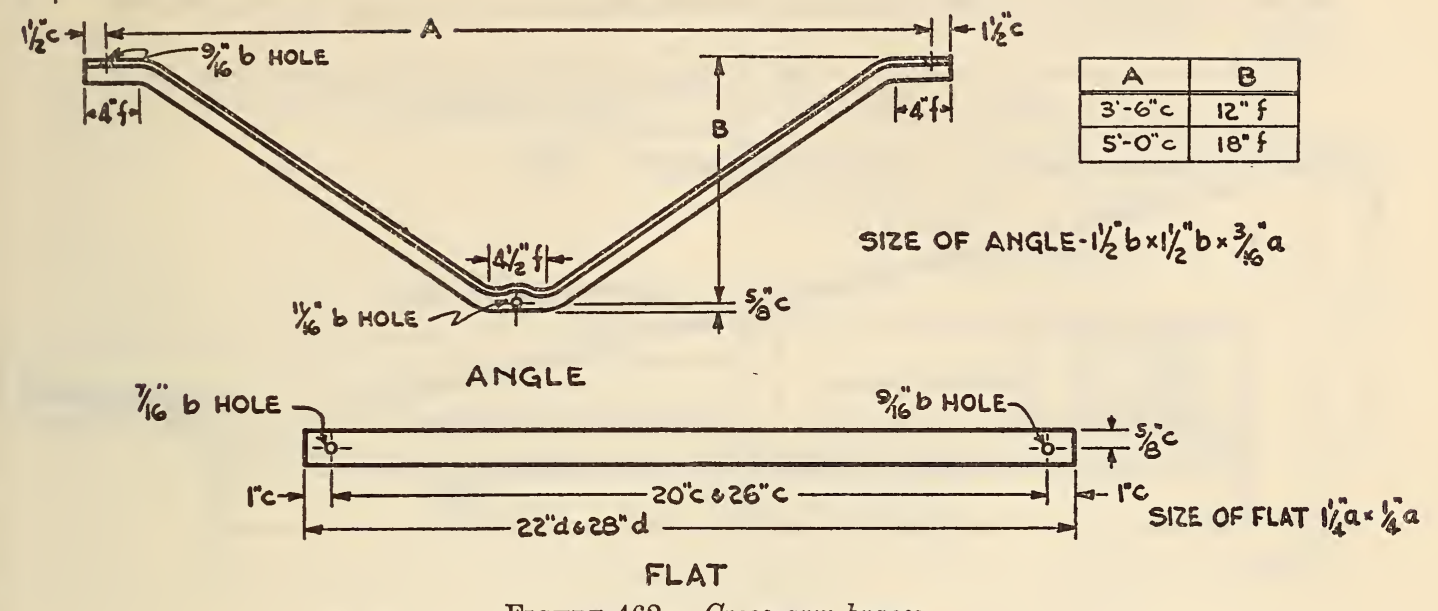

Figdre 462.-Cross-arm braces

\begin{tabular}{|c|c|c|}
\hline & \multicolumn{2}{|c|}{$\begin{array}{l}\text { Allowable va- } \\
\text { riations }\end{array}$} \\
\hline & Over & Under \\
\hline $\begin{array}{l}a \\
b \\
c \\
d \\
f\end{array}$ & $\begin{array}{c}\text { Inch } \\
1 / 64 \\
1 / 32 \\
1 / 16 \\
118 \\
1 / 4\end{array}$ & $\begin{array}{c}\text { Inch } \\
1 / 64 \\
1 / 32 \\
1 / 16 \\
1 / 8 \\
1 / 4\end{array}$ \\
\hline
\end{tabular}

317. Braces, cross arm.-Cross-arm braces shall be of steel of dimensions shown in Figure 462. The horizontal leg of the angle brace shall be so formed to a 3 -inch radius at the apex as to give clearance for the bolthead or nut and wrench.

322. Pins, insulator. - (a) Insulator pins shall be of wood or malleable iron or steel.
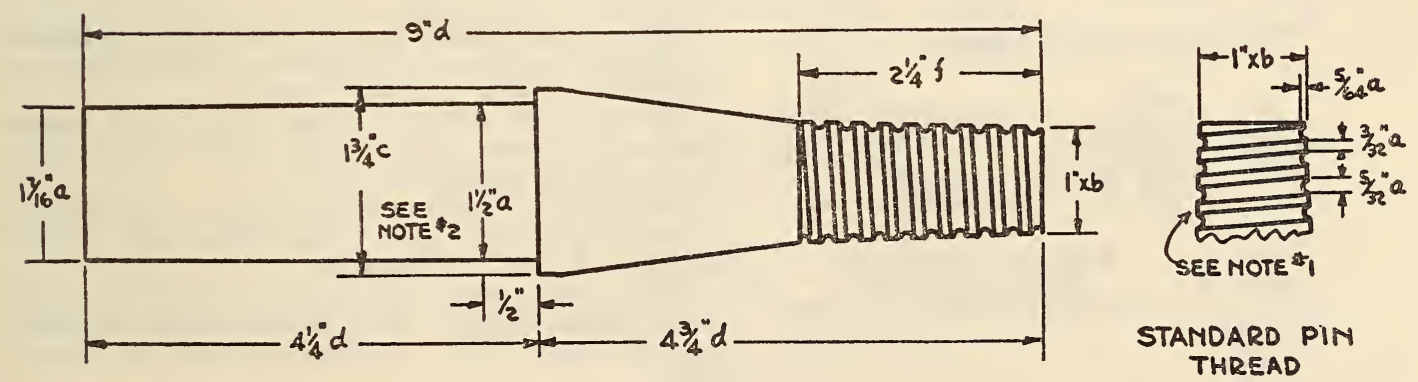

-2 PINTO BE I\%/2INGH IN OIA. YूINCH BELOW SHOULDER.

* - 4 THREADS PER INCH TAPERING Y INCH IN DIATO IINSH INLENGTH.

Figure 463.-Feeder insulator pin

\begin{tabular}{|c|c|c|}
\hline & \multicolumn{2}{|c|}{$\begin{array}{l}\text { Allowable va- } \\
\text { riations }\end{array}$} \\
\hline & Over & Under \\
\hline $\begin{array}{c}a \\
c \\
d \\
f \\
x b\end{array}$ & $\begin{array}{c}\text { Inch } \\
1 / 64 \\
116 \\
118 \\
1 / 4 \\
0^{1 / 4}\end{array}$ & $\begin{array}{c}\text { Inch } \\
1 / 64 \\
1 / 16 \\
1 / 8 \\
1 / 4 \\
1 / 32\end{array}$ \\
\hline
\end{tabular}




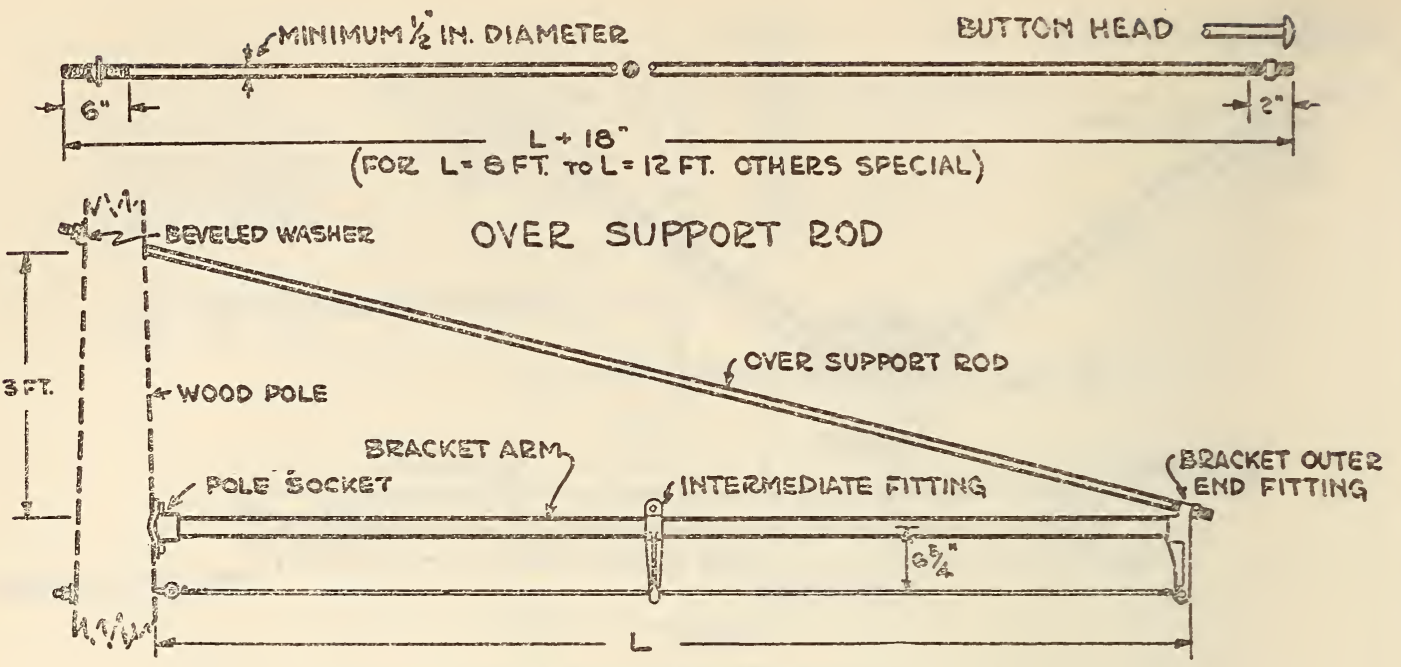

FIGURB 464.-Pole bracket arm assembly, direct suspension
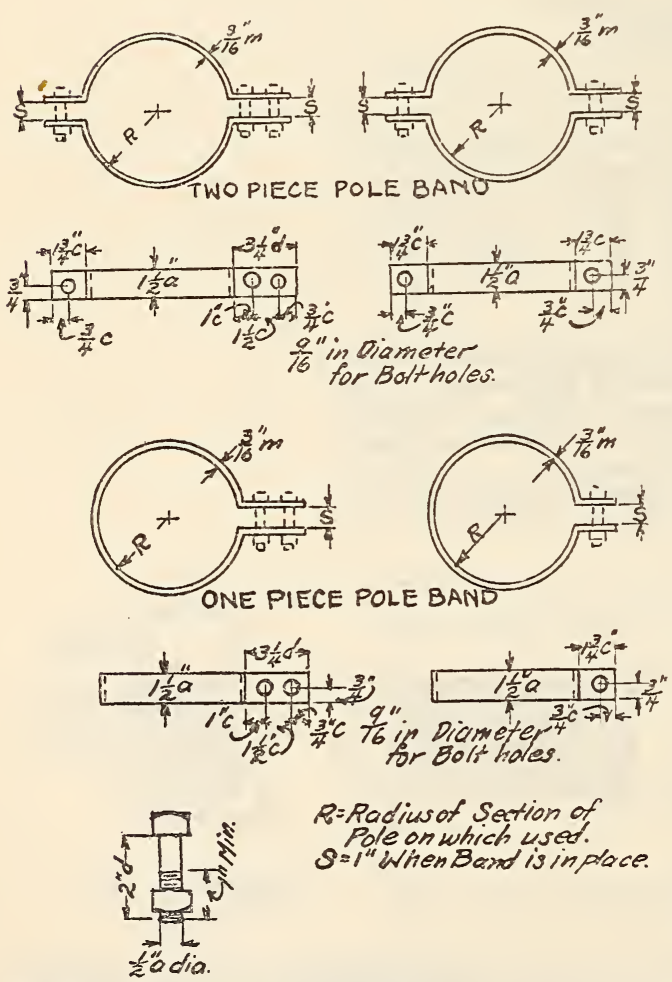

$P=$ Radius of Section of $s=1$ "When Bard is in place.

FigURE 465.-Pole bands

\begin{tabular}{|c|c|c|}
\hline & \multicolumn{2}{|c|}{$\begin{array}{c}\text { Permissible } \\
\text { variations }\end{array}$} \\
\cline { 2 - 3 } & Over & Under \\
\hline & Inch & Inch \\
$a$ & $1 / 64$ & 164 \\
$c$ & $1 / 16$ & $1 / 16$ \\
$d$ & $1 / 8$ & $1 / 8$ \\
$m$ & $1 / 16$ & 0 \\
\hline
\end{tabular}

(b) Wood insulator pins and standard malleableiron or forged-steel corner pins shall be of dimensions shown in Figure 463. They shall have standard 1-inch pin threads.

(c) Various types of metallic pins using $5 / 8$-inch or $3 / 4$-inch bolt through arm, having forged or malleable body or spacer, and having integral or separate lead tips with pin thread, or steel, malleable or zinc separable thimbles with machine screw or pin threads, are available and give greater strength than wood pins. If not cemented into insulator they shall have standard 1 -inch pin threads. The body or spacer shall have a base of such shape as to fit the arm or surface to which attached.

\section{IIATERIAL FOR DIRECT TROLIEY SUSPENSION}

324. Scope.-This specification covers material used principally in direct suspension over-head trolley construction, for voltages not exceeding 750 volts.

325. Arms, bracket (see fig. 464).-(a) Bracket arms shall be of grade $\mathrm{C}$ structural steel tubing, or of steel $\mathrm{T}$ or other structural shapes.

(b) For light service, tubing shall be 1.9 inches external diameter with walls 0.145 inch thick, weighing 2.68 pounds per foot.

(c) For heavy service, tubing shall be 2.375 inches external diameter, with walls 0.154 inch thick, weighing 3.6 pounds per foot.

326. Bands, pole.-Pole bands for tubular steel pole shall be of steel. They may be either one piece or two piece with dimensions shown in Figure 465 .

327. Bolts.-All stud bolts or machine (tap) bolts of any type for the suspension of line-material devices shall be of steel, five-eighths inch diamater with U. S. standard thread, 11 threads per inch threaded at least thirteenth-sixteenths inch and of proper length to project five-eighths inch to eleven. sixteenths inch below shoulder or seat of suspensior device.

328. Bracket intermediate fittings (see fig. 464).(a) Bracket intermediate fittings for tubular arm. shall be of malleable iron. 


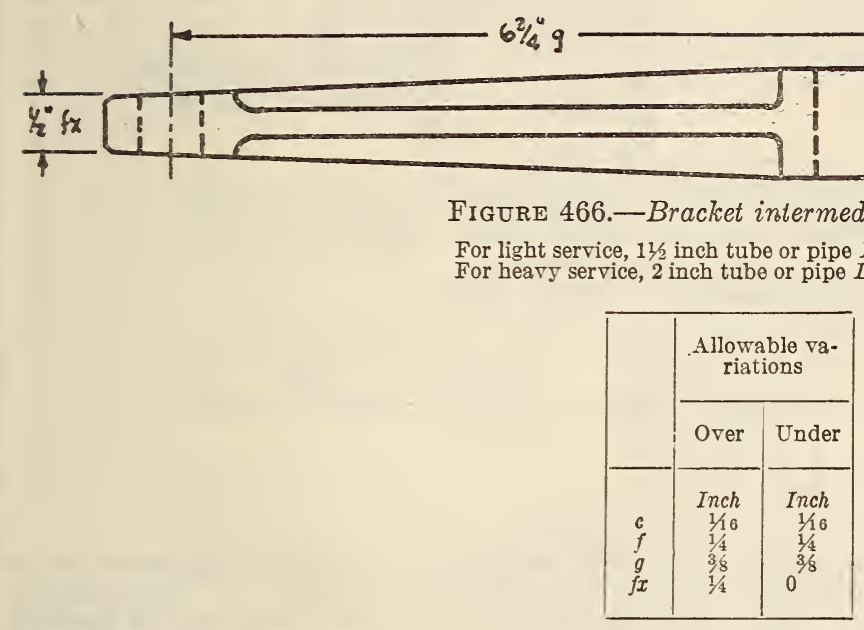

(b) Uninsulated type shall be of dimensions shown in Figure 466.
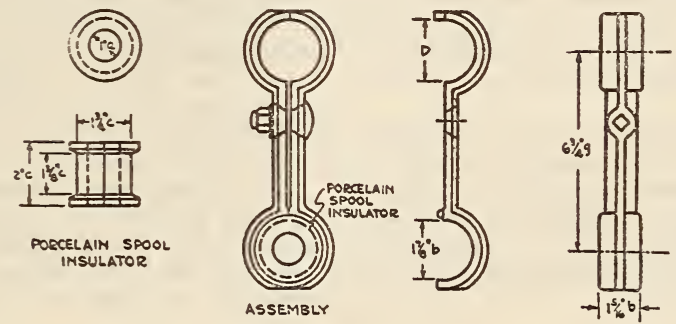

FIGURE 467.-Bracket intermediate fitting, feed tap type

For light service, $1 \frac{1}{2}$ inch tube or pipe $D=2$ inches. For heavy service, 2 inch tube or pipe $D=27 / 16$ inches.

\begin{tabular}{|c|c|c|}
\hline \multicolumn{2}{|c|}{} & \multicolumn{2}{|c|}{$\begin{array}{c}\text { Allowable va- } \\
\text { riations }\end{array}$} \\
\cline { 2 - 3 } & Over & Under \\
\hline & Inch & Inch \\
$a$ & $1 / 64$ & $1 / 64$ \\
$b$ & $1 / 32$ & $1 / 32$ \\
$c$ & $1 / 16$ & $1 / 16$ \\
$g$ & $3 / 8$ & $3 / 8$ \\
\hline
\end{tabular}

(c) Feed-tap type shall be furnished with porcelain-spool insulator and of dimensions shown in Figure 467.

329. Bracket outer-end fittings (see fig. 464).Bracket outer-end fittings for tubular arms shall be of malleable iron of dimensions shown in Figure 468. 


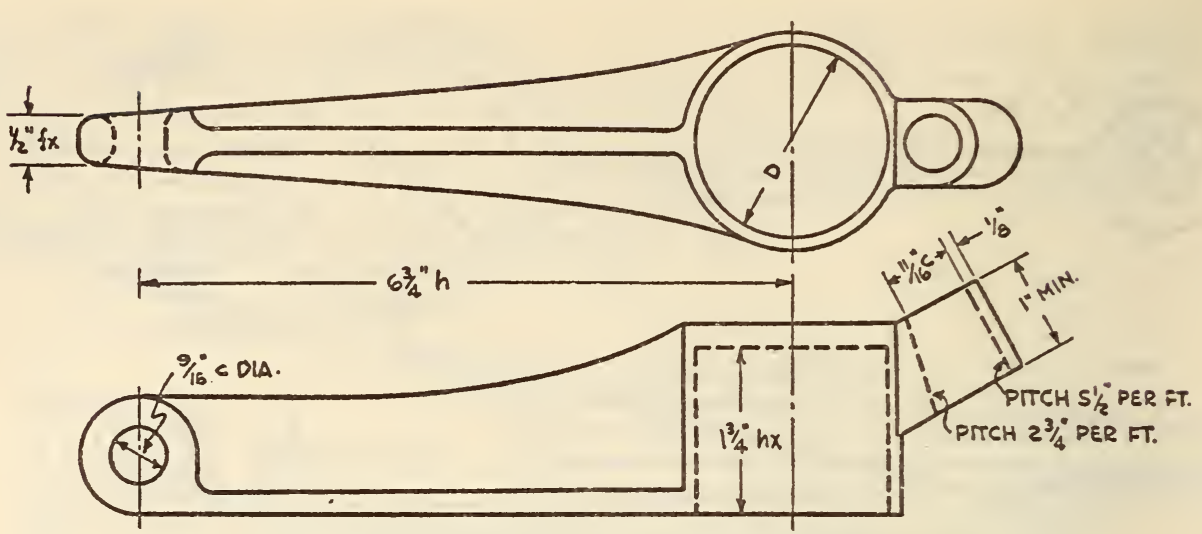

FIgURE 468.-Bracket outer end fitting, tubular arm

For light service, $1 \frac{1}{2}$ inch tube or pipe $D=2$ inches.

For heavy service, 2 inch tube or pipe $D=2^{7} / 16$ inches.

\begin{tabular}{|c|c|c|}
\hline & \multicolumn{2}{|c|}{$\begin{array}{c}\text { Allowable va- } \\
\text { riations }\end{array}$} \\
\cline { 2 - 4 } & Over & Under \\
\cline { 2 - 4 }$c$ & Inch & Inch \\
$h$ & $1 / 16$ & $1 / 16$ \\
$f x$ & $1 / 2$ & $1 / 2$ \\
$h x$ & $1 / 4$ & 0 \\
\hline
\end{tabular}

330. Crossings.- (a) Crossings shall consist of a malleable-iron or bronze body or pan, with separable bronze approaches, and may be either rigid, with fixed angularity, or adjustable.

(b) Crossings shall be of such design that both trolley wires of any standard size can be carried through without cutting or excessive bending and that adjustment along the wires can be made after installation. The clamping devices shall hold the wires securely without slipping and without injury to the wires.

(c) Body design shall be such as to give sufficient rigidity against bending due to maximum trolley tensions, and such as to prevent wheel or harp of dewired pole from catching. Rigid-type crossings shall have at least two pull-off eyes. All separable parts shall be cotter pinned or otherwise suitably locked to prevent working loose. (d) Runners shall be of such cross section as to permit satisfactory operation of the currentcollecting device, and of such design and so guarded as to cause collector to track without dewirement and to reduce arcing to a minimum. For wheel operation the design of the runners and the pan shall be such that either a standard new wheel or an old wheel with groove worn to a depth of $11 / 8$ inches shall pass with minimum abruptness.

(e) The bronze approaches shall be tapered, easily applied, and securely retained in position and shall be adapted for any of the standard round or grooved trolley-mire sizes.

331. Crossings, insulated.-(a) Insulated crossings, in addition to general requirements for crossings, shall provide suitable wood or other insulation between the two wires in such fashion that dust, snow, or sleet can not bridge the insulation.
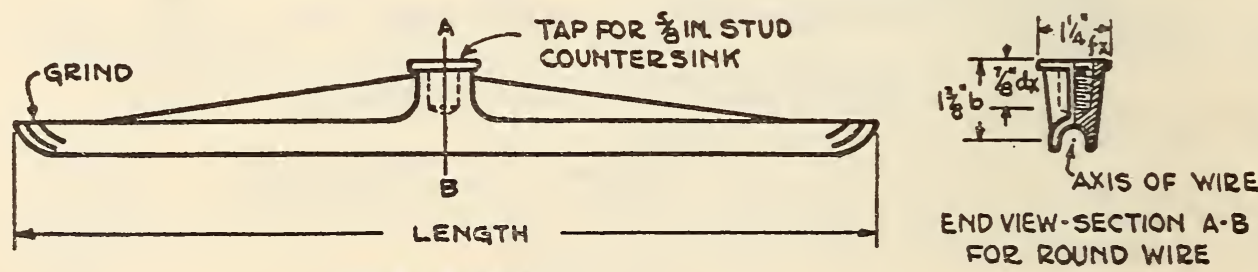

SIDE ELEVATION

\section{TOP VIEW}

FIGURe 469.-Clinch ear

\begin{tabular}{|c|c|c|}
\hline & \multicolumn{2}{|c|}{$\begin{array}{c}\text { Allowable va- } \\
\text { riations }\end{array}$} \\
\cline { 2 - 3 } & Over & Under \\
\hline & Inch & Inch \\
$b$ & $1 / 32$ & $1 / 32$ \\
$f$ & $1 / 4$ & $1 / 4$ \\
$d x$ & 118 & 0 \\
$f x$ & $1 / 4$ & 0 \\
\hline
\end{tabular}


(b) The runner for one wire shall be of wood or fiber for at least 4 inches on either side of gap, and shall be readily removable for replacement or repair.

332. Ears, clinch.- (a) Clinch ears shall be of bronze of dimensions shown in Figure 469. The standard lengths shall be 9 inches, 12 inches, and 15 inches. The central boss shall be slightly countersunk and tapped for five-eighths inch U. S. standard thread, 11 threads per inch.

(b) Groove in ear for round wire shall be milled smooth and shall be of such dimensions as to fit size of wire used, and hold wire with axis $13 / 8$ inches below top of boss. Lips shall be ground thin and smooth at edges and ends. They shall be of such shape and length as to form properly about the wire, meeting for at least the center two-thirds of the length, and of such thickness as to offer the least resistance to current collector.

(c) Groove in ear for grooved or figure 8 wire shall have lips designed to spring into the groove of the wire by hand. They shall be of such shape, length, and thickness that, after crimping, they will hold the wire securely with its axis $13 / 8$ inches below top of boss, and will not interfere with current collector.

(d) Ears having separate boss or rib of bronze or other metal, or having copper lips, or of the underslung or clip type are approved if they fulfill the general requirements above.

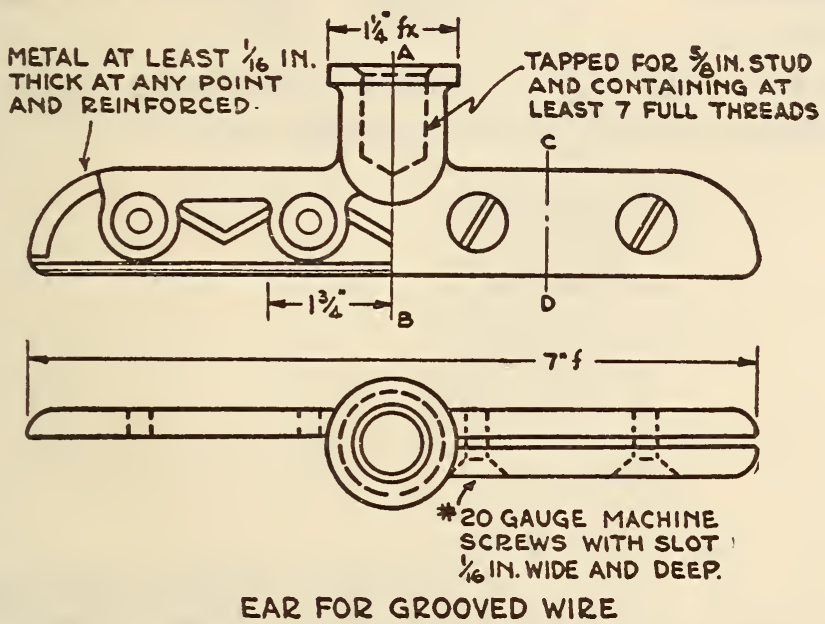

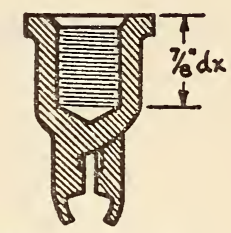

SECTION A-B

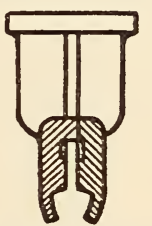

SECTION C.D

Figure 470.-Screw clamp ear

\begin{tabular}{|c|c|c|}
\hline & \multicolumn{2}{|c|}{$\begin{array}{l}\text { Allowable va- } \\
\text { riations }\end{array}$} \\
\hline & Over & Under \\
\hline$\underset{d x}{f x}$ & $\begin{array}{r}\text { Inch } \\
1 / 4 \\
118 \\
1 / 4\end{array}$ & $\begin{array}{c}\text { Inch } \\
1 / 4 \\
0 \\
0\end{array}$ \\
\hline
\end{tabular}

333. Ears, screw ciamp.-(a) Screw clamp ears shall be of malleable iron or bronze, with four No. 20 steel or bronze machine screws. They shall conform to the requirements for boss threading, height dimension, and clearance in paragraph 332 and shall have dimensions shown in Figure 470 .

(b) Cross section shall be such as to permit internal stiffening ribs such that when installed ear can not be buckled sufficiently to offset its grip on the wire.

(c) Lips shall be of such size and shape as to take desired size of round, grooved, or figure 8 wire as specified.

334. Frogs.- (a) Frogs shall be of malleable iron or bronze, with separable bronze approaches.

(b) Frogs shall be of such design that trolley wires of any standard size can be carried through without cutting or excessive bending and that adjustment along the wires can be made after installation. The clamping devices shall hold the wires securely without slipping and without injury to the wires.

(c) Body design shall be such as to give sufficient rigidity against bending due to maximum trolley tensions, and such as to prevent the wheel or harp of a dewired pole from catching. Frogs shall have at least four pull-off eyes. All separable parts shall be cotter pinned or otherwise suitably locked to prevent working loose.

(d) Runners shall be of such cross section as to permit satisfactory operation of the current-collecting device, and of such design and so guarded as to cause collector to track without dewirement, and to reduce arcing to a minimum. For wheel operation the design of the runners shall be such that either a standard new wheel or an old wheel with groove worn to depth of $1 \frac{1}{8}$ inches shall pass with minimum abruptness.

(e) The bronze approaches shall be tapered, easily applied, and securely retained in position, and shall be adapted for any of the standard round or grooved trolley-wire sizes.

338. Rods, over-support. (See fig. 464.)-(a) Over-support rod shall be of round steel, not less than one-half inch diameter. The end next the pole shall be threaded for 6 inches and the end next the bracket shall either be threaded for 2 inches or shall have a button head. 


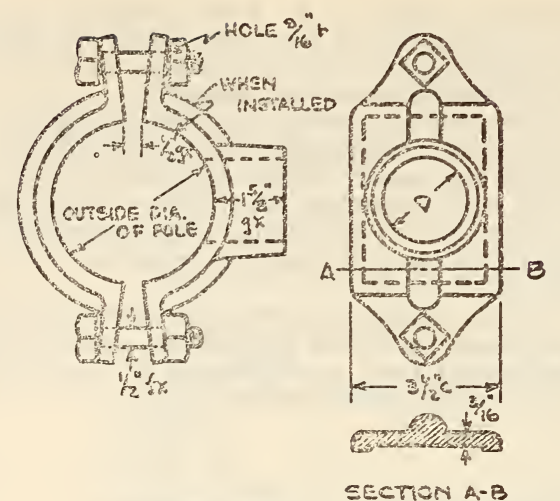

FOR STEEL POLE
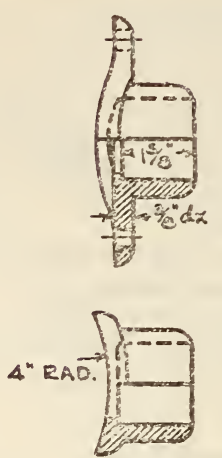

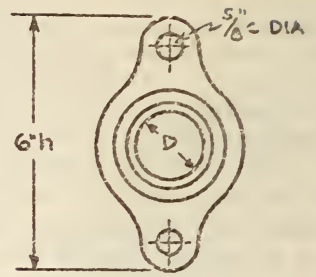

FOE LIGHT EERYICE $1 \% "$ TUEE CE PIPE D $02 \mathrm{IN}$ FOP HEAY SERVICE. 2 "TUEE OR PIPE D=2\% $1 \mathrm{H}$.

Figure 471.-Pole socket for tubular arm

\begin{tabular}{|c|c|c|}
\hline & \multicolumn{2}{|c|}{$\begin{array}{l}\text { Allowable va- } \\
\text { riations }\end{array}$} \\
\hline & Over & Under \\
\hline $\begin{array}{c}b \\
c \\
f \\
h \\
d x \\
f x \\
h x\end{array}$ & $\begin{array}{c}\text { Inch } \\
1 / 32 \\
1 / 16 \\
1 / 4 \\
1 / 2 \\
1 / 8 \\
1 / 4 \\
1 / 2\end{array}$ & $\begin{array}{l}\text { Inch } \\
1 / 32 \\
1 / 16 \\
1 / 4 \\
1 / 2 \\
0 \\
0 \\
0\end{array}$ \\
\hline
\end{tabular}

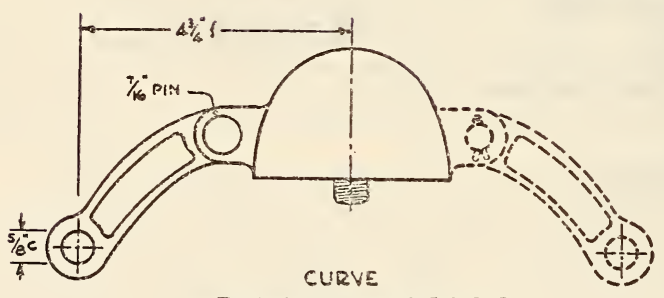

WITH ONE A.RM-SINGLE CURVE WITH TWO ARMS-DQUBLE CURVE

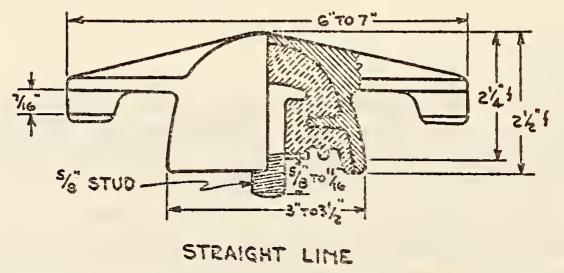

FIGURE 472.-Suspension (hanger) round top type

\begin{tabular}{|c|c|c|}
\hline \multicolumn{1}{|c|}{} & \multicolumn{2}{|c|}{$\begin{array}{c}\text { Allowable va- } \\
\text { riations }\end{array}$} \\
\hline Over & Under \\
\hline & Inch & $\begin{array}{c}\text { Inch } \\
1 / 16 \\
c\end{array}$ \\
$1 / 4$ & $1 / 4$ \\
\hline
\end{tabular}

(b) The length of the over-support rod for bracket arms from 9 feet to 12 feet in length shall be 18 inches greater than the length of the arm.

(c) Rod shall be furnished with one nut for each threaded end and one beveled cast-iron washer.

339. Sockets, pole, for bracket arms. (See fig. 464.)-Pole sockets for bracket arms shall be of malleable iron of dimensions shown in Figure 471 . They shall have internal socket diameters as follows: For light service (11/2-inch) bracket arms, 2 inches; for heavy service (2-inch) bracket arms, 2716 inches.

340. Splicers, trolley wire.-(a) Splicers shall be of bronze, steel, or other suitable material, the whole device to have a strength and conductivity at least equal to that of the wire on which it is used.

(b) The center of gravity shall be $10 \%$ and the shape and design such that the current collector will pass satisfactorily without shock or blow and with a minimum of arcing.

(c) The wire passages shall be of such size that the trolley wire can be easily threaded through, and the clamping devices shall be capable of securely holding either new or worn wire without reducing the strength of the wire.

341. Suspensions (hangers).--(a) Suspensions, as to form of insulation, shall be of four general types:

1. Round top type (fig. 472).

2. Cap and cone type (figs. 473 and 474 ).

3. Insulated bolt (west end) type (fig. 475).

4. Yoke (uninsulated) type (fig. 476).

They shall be of malleable iron, with steel studs or bolts, and (except the yoke type) with molded composition insulation of suitable shape andimensions for their use. 

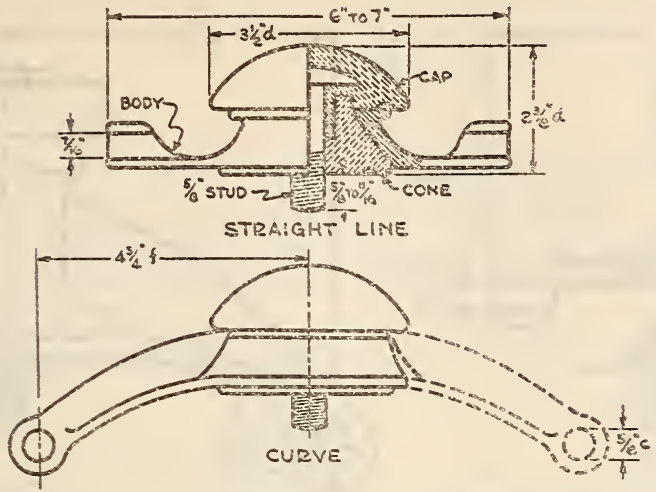

WITH ONE ARM-SINGLE CURVE WITHTWO ARMS-COUBLE CUEVE

FIgURE 473.-Suspension (hanger) cap and cone type

\begin{tabular}{|c|c|c|}
\hline & \multicolumn{2}{|c|}{$\begin{array}{c}\text { Allowable va- } \\
\text { riations }\end{array}$} \\
\hline & Over & Under \\
\hline & Inch & Inch \\
& $1 / 16$ \\
$c$ & 116 & $1 / 8$ \\
$d$ & $1 / 8$ & $1 / 4$ \\
$f$ & $1 / 4$ & $1 / 8$ \\
\hline
\end{tabular}

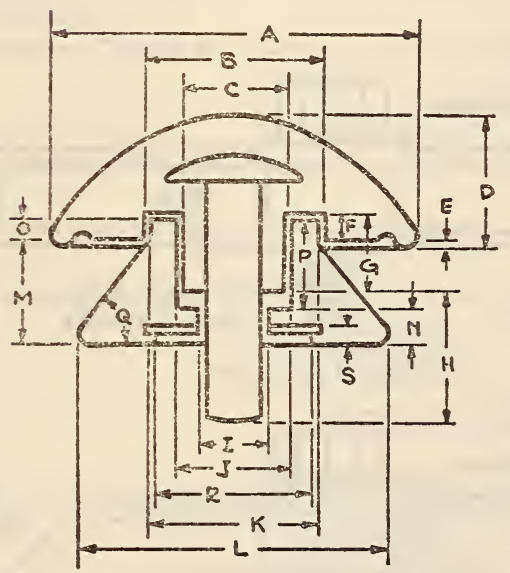

FiguRE 474.-Cap and cone insulators

\begin{tabular}{|c|c|c|}
\hline Letter & Dimension & Allowable variations \\
\hline$A$ & $31 / 2$ inches. & Not over $18 \mathrm{sinch}$. \\
\hline$\stackrel{B}{C}$ & $\begin{array}{l}121 / 32 \text { inches } \\
1 \text { inch }\end{array}$ & $\begin{array}{l}\text { Not over } 1_{64} \text { inch. } \\
\text { Not over. }\end{array}$ \\
\hline$D$ & $\begin{array}{l}\text { Minimum } \\
18 \% 4 \text { inch- }\end{array}$ & $\begin{array}{l}\text { Maximum } 12464 \text { in- } \\
\text { ches. }\end{array}$ \\
\hline$E$ & es. & Not over 16 inch \\
\hline$F$ & $1 / 4$ inch. & Not less. \\
\hline $\begin{array}{l}G \\
H \\
I \\
J\end{array}$ & $\begin{array}{l}3 / 4 \text { inch } \\
11 / 4 \text { inches.-- } \\
21 / 32 \text { inch } \\
11 / 32 \text { inches }\end{array}$ & $\begin{array}{l}\text { Not over. } \\
\text { Not over } 164 \text { inch. } \\
\text { Not over } 118 \text { inch. } \\
\text { Not less. }\end{array}$ \\
\hline$K$ & $15 \%$ inches & Not over. \\
\hline$L$ & $\begin{array}{l}\text { Minimum } \\
27,8 \text { inches. }\end{array}$ & Maximum 3 inches. \\
\hline $\begin{array}{c}M \\
N \\
O\end{array}$ & $\begin{array}{l}1 \text { inch } \\
3 / 8 \text { inch } \\
1 / 4 \text { inch }\end{array}$ & $\begin{array}{l}\text { Not over } 164 \text { inch. } \\
\text { Not over. } \\
\text { Not over. }\end{array}$ \\
\hline $\begin{array}{l}P \\
Q \\
R \\
S\end{array}$ & $\begin{array}{l}\text { 7/6 inch } \\
50 \text { degrees.... } \\
11 / 2 \text { inches..- } \\
1 / 8 \text { inch }\end{array}$ & $\begin{array}{l}\text { None. } \\
\text { None. } \\
\text { Not over } 18 \text { inch. } \\
\text { Not over } 18 \text { inch. }\end{array}$ \\
\hline
\end{tabular}




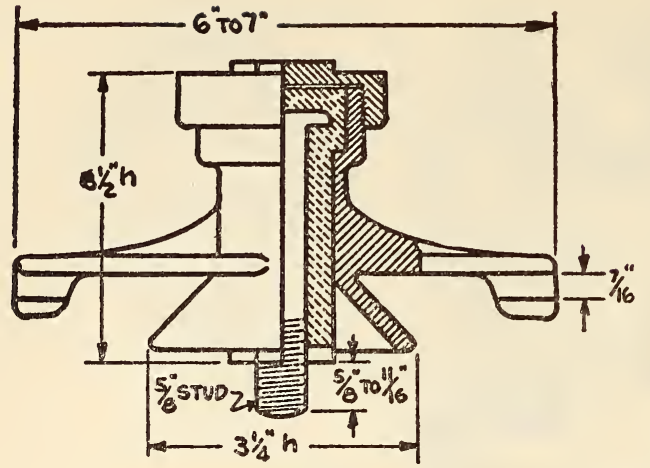

STRAIGHT L.ANE

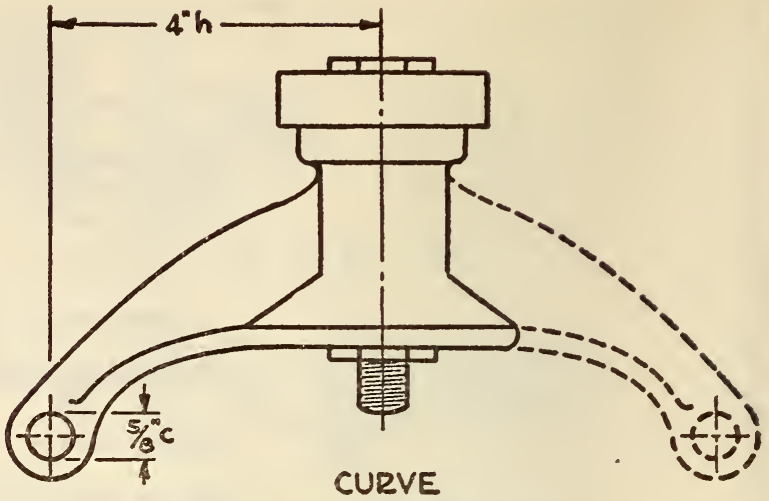

WITH ONE ARM-SINGLE CURVE WITH TWO ARMS-DOUBLE CURVE

FIGURE 475.-Suspension (hanger) insulated bolt type

\begin{tabular}{|c|c|c|}
\hline \multicolumn{1}{|c|}{} & \multicolumn{2}{|c|}{$\begin{array}{c}\text { Allowable va- } \\
\text { riations }\end{array}$} \\
\hline & Over & Under \\
\hline & Inch & $\begin{array}{c}\text { Inch } \\
1 / 16 \\
c\end{array}$ \\
$1 / 16$ & $1 / 2$ \\
$h$ & $1 / 2$ & \\
\hline
\end{tabular}

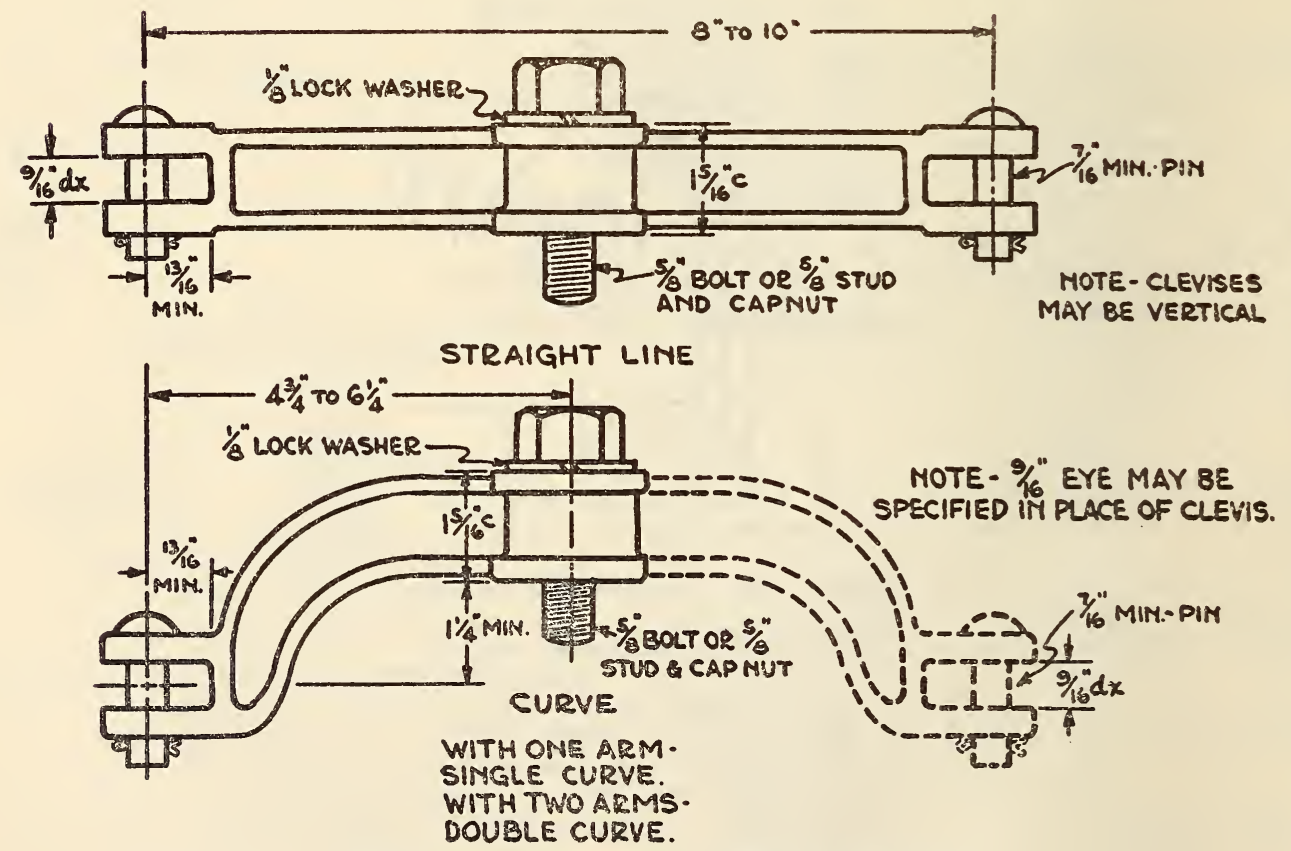

FIGURE 476.-Suspension (hanger) yoke type

\begin{tabular}{|c|c|c|}
\hline & \multicolumn{2}{|c|}{$\begin{array}{c}\text { Allowable va- } \\
\text { riations }\end{array}$} \\
\hline & Over & Under \\
\hline & $\begin{array}{c}\text { Inch } \\
1 / 16 \\
1 / 8\end{array}$ & $\begin{array}{c}\text { Inch } \\
1 / 16\end{array}$ \\
\hline$d x$ & 0 \\
\hline
\end{tabular}


(b) Suspensions shall be classified as to use, as:

1. Straight line.

2. Single curve (pullover).

3. Double curve (pullover).

4. Barn, bridge, ceiling, or trough.

5. Bracket arm.

and shall have suitable size and shape of body and facilities for attachment for such use.

(c) All studs and bolts for ear boss shall be fiveeignths inch diameter, U. S. standard thread, projecting five-eighths inch to eleven-sixteenths inch below shoulder or seat of suspension.

(d) Suspensions with molded or composition insulation for service on 750 volts shall have the following minimum characteristics:

\begin{tabular}{|c|c|c|c|}
\hline & $\begin{array}{c}\text { Round } \\
\text { top }\end{array}$ & $\begin{array}{l}\text { Cap } \\
\text { and } \\
\text { cone }\end{array}$ & $\begin{array}{l}\text { Insu- } \\
\text { lated } \\
\text { bolt }\end{array}$ \\
\hline $\begin{array}{l}\text { Breaking strength } \\
\text { Potential test }(5 \text { seconds) } \\
\text { Flash-over voltage }\end{array}$ & $\begin{array}{r}7,000 \\
5,000 \\
10,000\end{array}$ & $\begin{array}{r}8,000 \\
5,000 \\
10,000\end{array}$ & $\begin{array}{r}6,000 \\
5,000 \\
10,000\end{array}$ \\
\hline
\end{tabular}
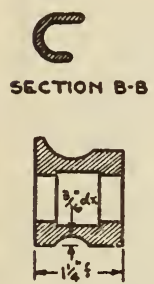

SECTIONA-A

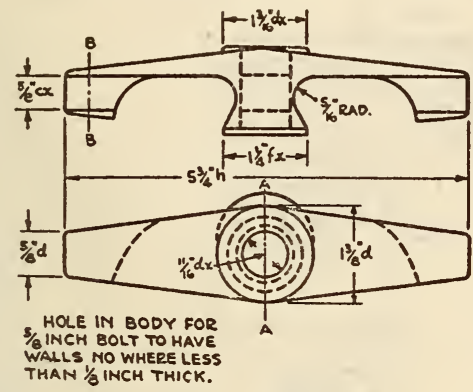

Figure 477.-Feed in suspension (hanger)

\begin{tabular}{|c|c|c|}
\hline & \multicolumn{2}{|c|}{$\begin{array}{c}\text { Allowable va- } \\
\text { riations }\end{array}$} \\
\cline { 2 - 3 } & Over & Under \\
\hline & Inch & Inch \\
$d$ & $1 / 8$ & $1 / 8$ \\
$f$ & $1 / 4$ & $1 / 4$ \\
$h$ & $1 / 2$ & $1 / 2$ \\
$c x$ & $1 / 16$ & 0 \\
$d x$ & 18 & 0 \\
$f x$ & $1 / 4$ & 0 \\
\hline
\end{tabular}

342. Suspensions, feed-in (feeder hangers or yokes).(a) Feed-in suspensions shall be of bronze of dimensions shown in Figure 477. (b) They shall be furnished with five-eighths-inch steel machine bolt and one-eighth-inch steel lock washer, or with five-eighths-inch steel stud and cap nut.
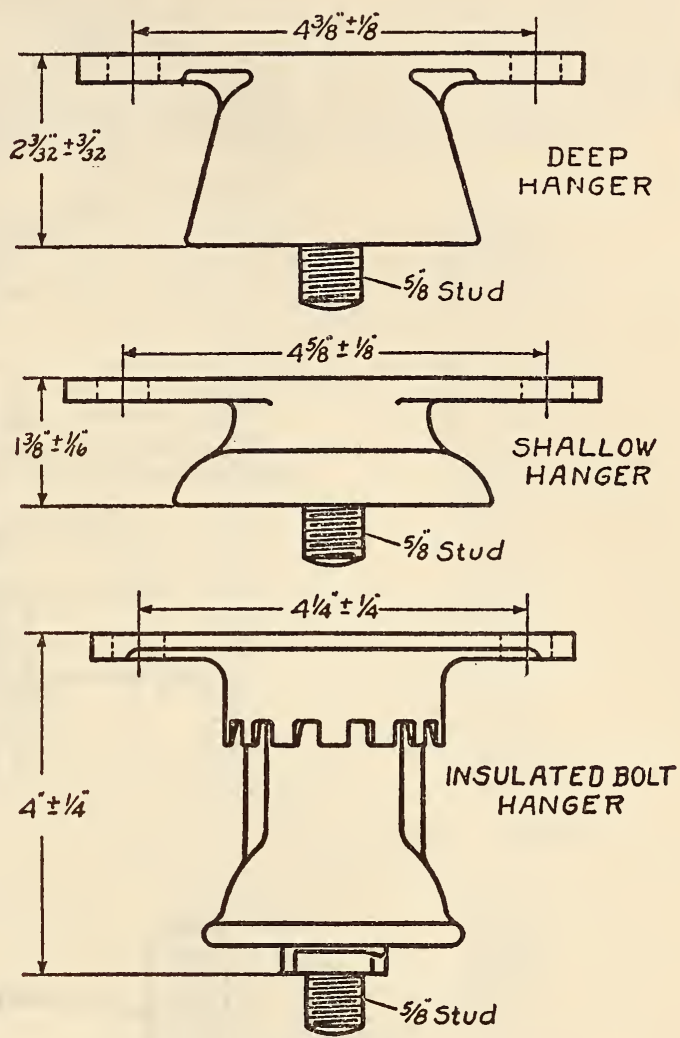

Figure 478.-Trough suspension with stud

343. Suspensions, trough.-Trough suspensions shall comply with the general requirements of paragraph 341. Types with stud are shown in Figure 478 and type for contact bar in Figure 479.

\section{MATERIAL FOR CATENARY SUSPENSION}

344. Scope.-This specification covers material used in catenary construction, simple suspension, utilizing poles with pole spacing of not more than 150 feet and for voltages not exceeding 1,200 volts. 

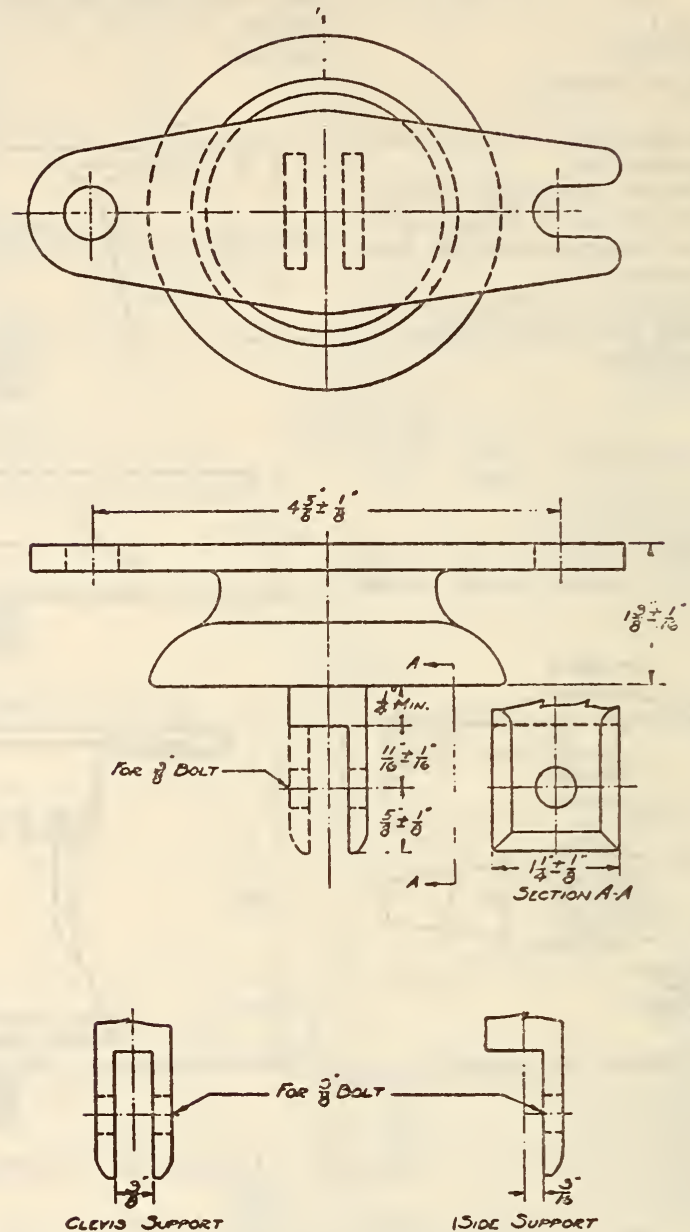

FIGURE 479.-Trough suspension for contact bar
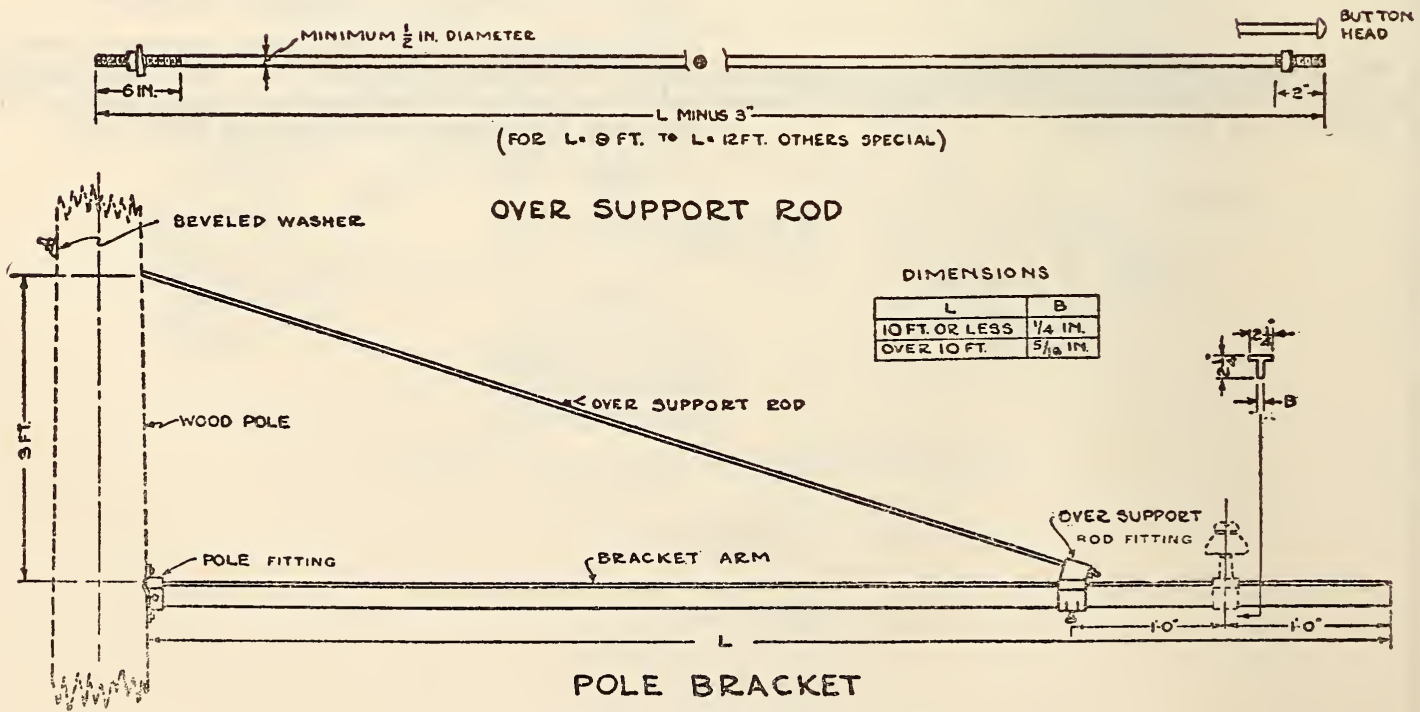

FIGURE 480.-Bracket arm assembly 
345. Arms, bracket. (See figs. 480, 481, and 482.) - (a) Pole bracket arms and over-support rod shall be made of steel and the fittings of malleable iron.

(b) Bracket arm, when 10 feet total length or less, shall consist of $2 \frac{1}{4}$ by $2 \frac{1}{4}$ by one-fourth inch T sec-
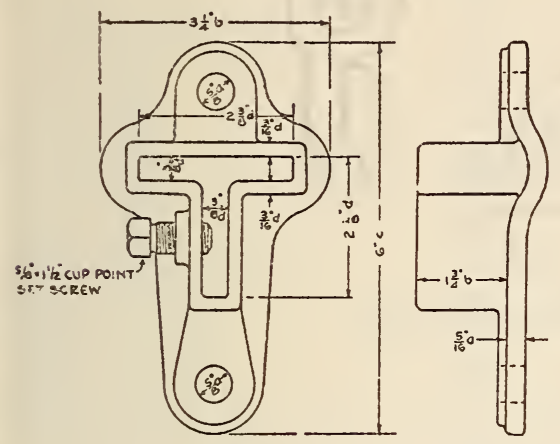

POLE CASTING
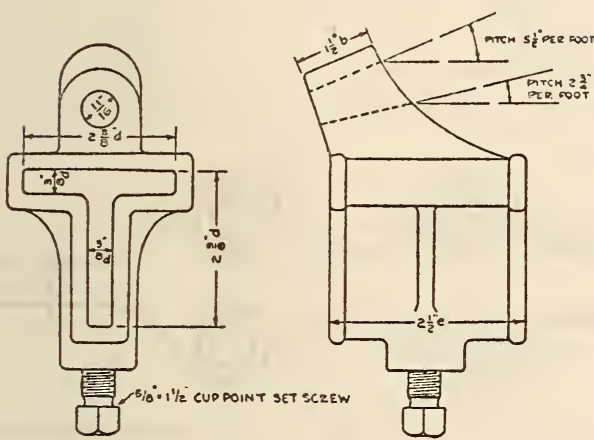

OVER SUPPORT ROD CASTIMG

FIGURE 481.-Pole socket and rod fittings, T-iron arm

\begin{tabular}{|c|c|c|}
\hline & \multicolumn{2}{|c|}{$\begin{array}{l}\text { Allowable } \nabla a- \\
\text { riations }\end{array}$} \\
\hline & Over & Under \\
\hline $\begin{array}{l}a \\
b \\
c \\
d \\
e\end{array}$ & $\begin{array}{c}\text { Inch } \\
1 / 16 \\
1 / 4 \\
1 / 2 \\
1 / 16 \\
1 / 2\end{array}$ & $\begin{array}{c}\text { Inch } \\
1 / 16 \\
1 / 4 \\
1 / 2 \\
0 \\
0\end{array}$ \\
\hline
\end{tabular}

tion. Bracket arm when more than 10 feet in total length shall consist of $2 \frac{1}{4}$ by $2 \frac{1}{4}$ by five-sixteenths inch $T$ section.

(c) Bracket arm shall be not less than 12 inches longer than distance from pole face to center line of track. When used on curved track where it is necessary to attach pull-over span to outer end of bracket arm, the length of bracket arm shall be increased as necessary.

(d) Over-support rod shall be not less than onehalf inch in diameter. The end next the pole shall be threaded for 6 inches and the end next the bracket shall either be threaded for 2 inches or shall have a button head. Rod shall be provided with one nut on each threaded end and one beveled cast-iron washer.

(e) Pole fitting for attachment of bracket to pole shall be of socket type. Provision shall be made for attaching to pole by two or more lag screws and means shall be provided for locking bracket arm into fitting.

$(f)$ Outer end and intermediate fittings for use with steady span or pull off construction shall be of such length that contact wire will be held at the prevailing height, and shall have provision for locking fittings in place. Outer end fitting shall slip over arm, intermediate fitting may slip over arm or be hinged or clamped to facilitate attachment.
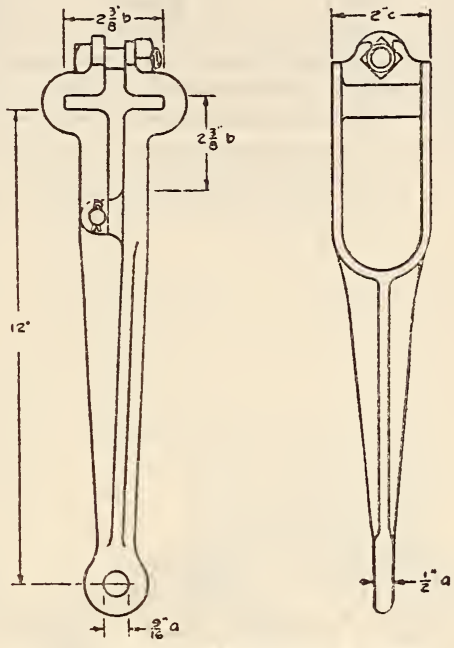

CLAMP TYPE INTERMEDIATE CASTING

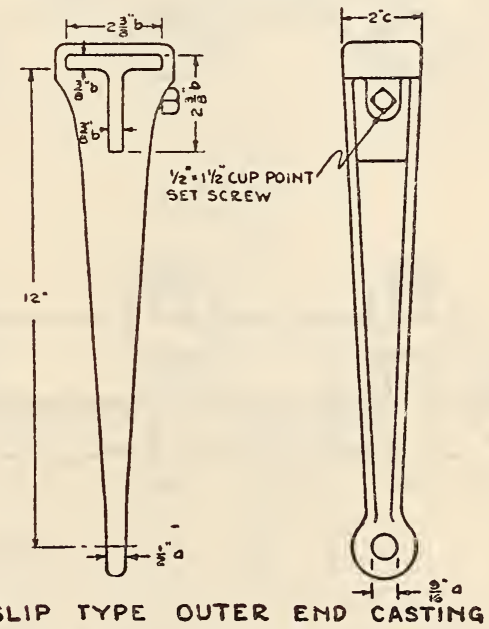

FIGURE 482.-Bracket fittings, T-iron arm

\begin{tabular}{|c|c|c|}
\hline & \multicolumn{2}{|c|}{$\begin{array}{c}\text { Allowable va- } \\
\text { riations }\end{array}$} \\
\hline & Over & Under \\
\hline & & \\
\hline & Inch & Inch \\
$a$ & $1 / 16$ & $1 / 16$ \\
$b$ & $1 / 16$ & 0 \\
$c$ & $1 / 2$ & 0 \\
\hline
\end{tabular}

(g) Over-support rod fitting shall slip over bracket arm and have provision for locking in place at desired location. Fitting shall have suitable provision for attachment of over-support rod. 

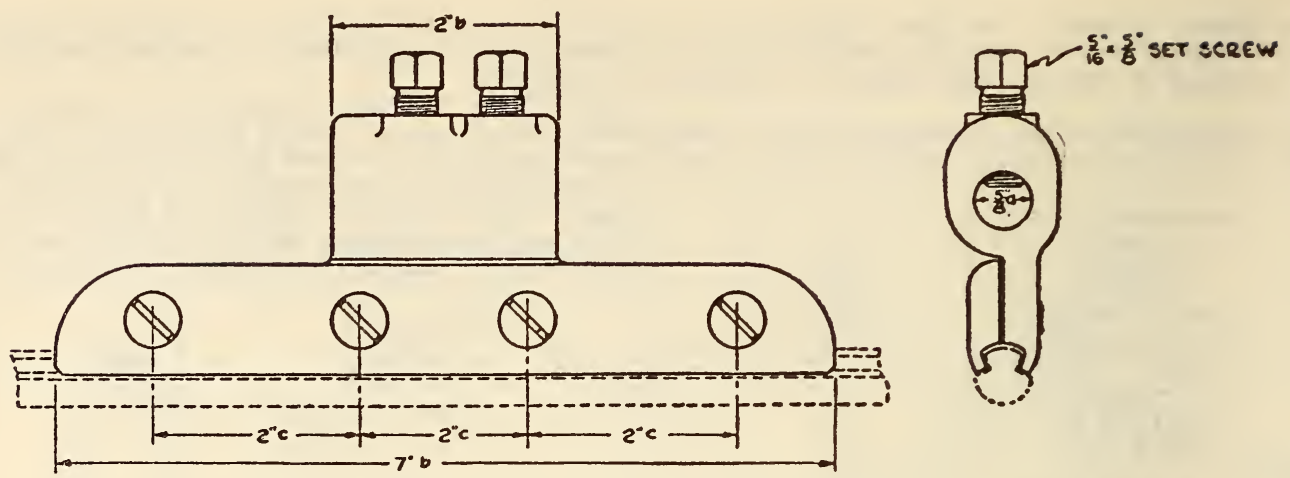

Figure 483.-Contact wire feed clamp, grooved wire

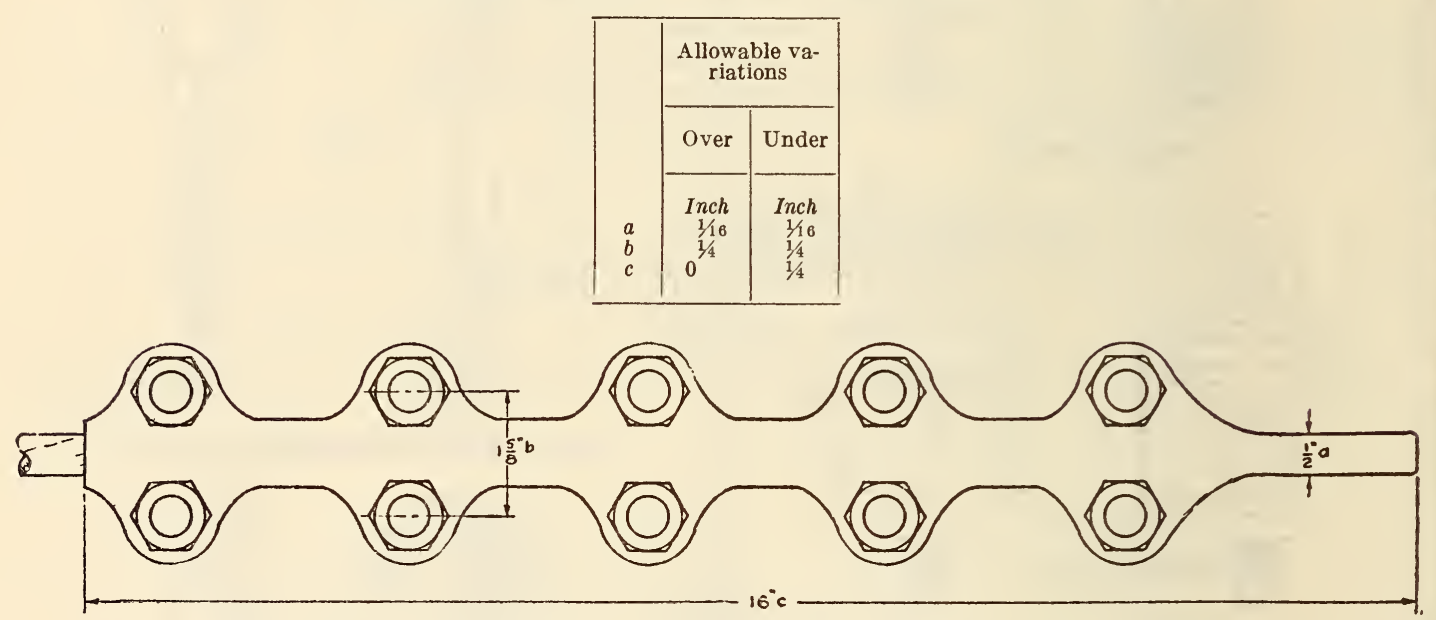

$16^{\circ} \mathrm{c}$

EITHER EYE OR
CLEVIS MAY BE USED

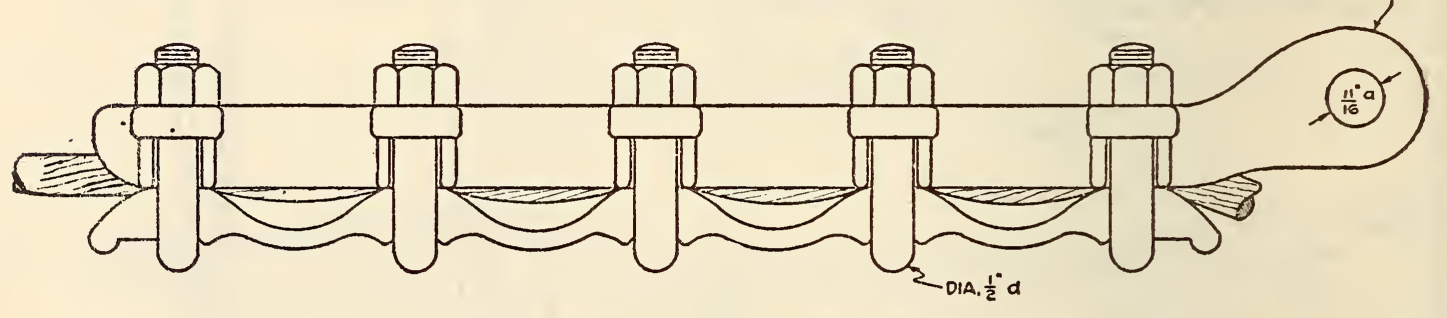

Figure 484.-Dead end clamp

\begin{tabular}{|c|c|c|}
\hline & \multicolumn{2}{|c|}{$\begin{array}{l}\text { Allowable va- } \\
\text { riations }\end{array}$} \\
\hline & Over & Under \\
\hline $\begin{array}{l}a \\
b \\
c \\
d\end{array}$ & $\begin{array}{c}\text { Inch } \\
1 / 16 \\
1 / 8 \\
11 / 8 \\
1 / 8\end{array}$ & $\begin{array}{l}\text { Inch } \\
1 / 16 \\
1^{1 / 8} \\
0^{1 / 8}\end{array}$ \\
\hline
\end{tabular}

346. Clamp feed. (See fig. 483.)-(a) Contact wire feed clamp shall be of bronze.

(b) Clamp shall be so designed that it grips contact wire and has suitable provision for attaching a flexible top of $0000 \mathrm{AWG}$ size. (c) Clamp shall be of such design as to avoid interference with satisfactory operation of current collector, and shall conform to the shape of contact wire so as to form a good gripping surface and prevent slipping along contact wire. 
347. Clamp, messenger dead end. (See fig. 484.)(a) Messenger dead end clamp shall be of wrought iron, steel, malleable iron, or bronze.

(b) Clamp shall be so designed as to grip messenger without damage to messenger strands.
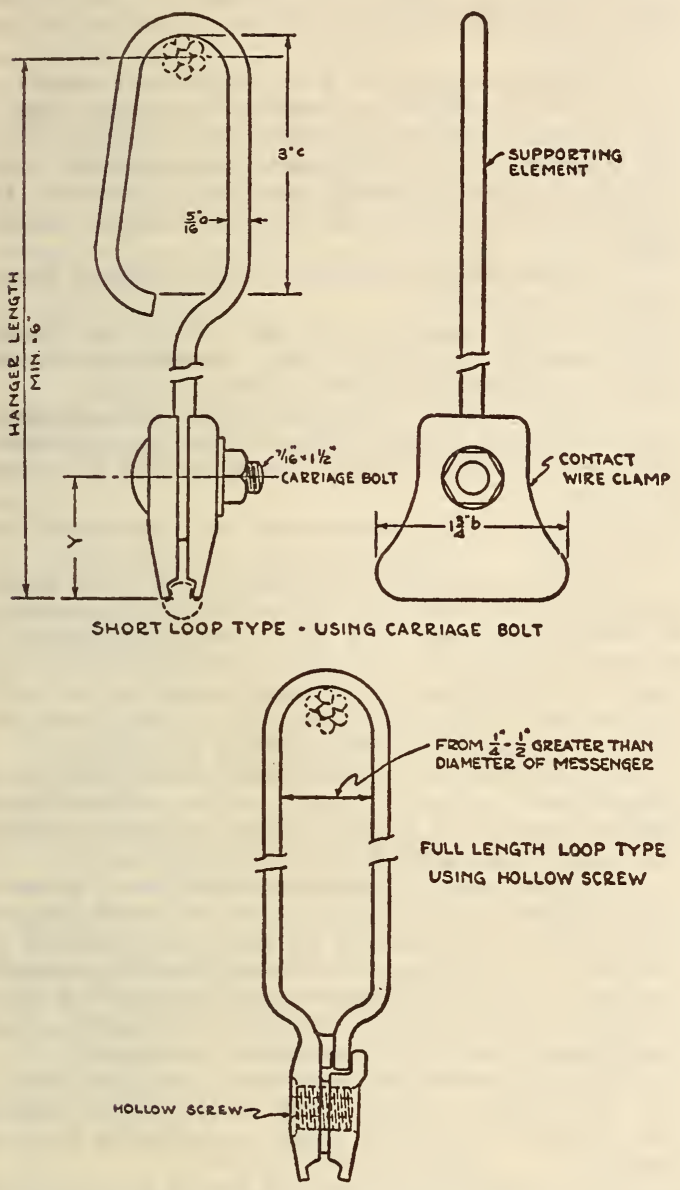

Figure 485.-Catenary hangers

\begin{tabular}{|c|c|c|}
\hline $\begin{array}{c}\text { Di- } \\
\text { men- } \\
\text { sion }\end{array}$ & Wheel & $\begin{array}{l}\text { Panto- } \\
\text { graph }\end{array}$ \\
\hline \multirow[t]{3}{*}{ Y } & $\begin{array}{c}\text { Inches } \\
15 \%\end{array}$ & $\begin{array}{l}\text { Inch } \\
1\end{array}$ \\
\hline & \multicolumn{2}{|c|}{$\begin{array}{l}\text { Allowable va- } \\
\text { riations }\end{array}$} \\
\hline & Over & Under \\
\hline $\begin{array}{l}a \\
b \\
c\end{array}$ & $\begin{array}{c}\text { Inch } \\
1 / 16 \\
1 / 2 \\
1 / 2\end{array}$ & $\begin{array}{c}\text { Inch } \\
1 / 16 \\
1 / 2 \\
0^{1 / 2}\end{array}$ \\
\hline
\end{tabular}

(c) Clamp shall be so designed that when properly installed on messenger, it shall be capable of withstanding a tension not less than 85 per cent of the breaking strength of messenger, without slippage.

(d) Clamp shall have a suitable eye or clevis for the dead ending guy.
348. Crossings.-Crossings for pantograph operation shall conform to the specifications of similar devices used in direct suspension, so far as they apply, and shall be of such design as to provide sufficient clearance for pantograph on both wires.

349. Frogs.-Frogs shall conform to the specifications of similar devices used in direct suspension. When both pole and pantograph current collectors are used, the design of frog shall be such that both types of collectors will pass satisfactorily.

350. Hanger, catenary. (See figs. 485 and 486.)(a) Catenary hanger shall be of such design that it grips the contact wire securely and suspends it a fixed distance from the messenger.

(b) Hangers for use where contact wire is maintained in the same vertical plane as the messenger

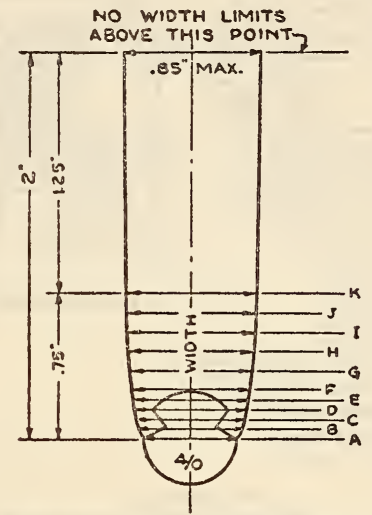

Figure 486.-Cross section of contact wire clamp

Width dimensions must not exceed values shown below.

\begin{tabular}{|c|c|c|}
\hline $\begin{array}{l}\text { Sec- } \\
\text { tion }\end{array}$ & $\begin{array}{l}\text { Dis- } \\
\text { tance } \\
\text { from } \\
\text { bottom } \\
\text { of } \\
\text { clamp }\end{array}$ & Width \\
\hline $\begin{array}{l}\mathrm{A} \\
\mathrm{B} \\
\mathrm{C} \\
\mathrm{D} \\
\mathrm{E} \\
\mathrm{F}\end{array}$ & $\begin{array}{l}\text { Inch } \\
0.0 \\
.05 \\
.1 \\
.15 \\
.2 \\
.25\end{array}$ & $\begin{array}{c}\text { Inch } \\
0.482 \\
.53 \\
.56 \\
.575 \\
.585 \\
.60\end{array}$ \\
\hline $\begin{array}{c}\mathrm{G} \\
\mathrm{H} \\
\mathrm{I} \\
\mathrm{J} \\
\mathrm{K}\end{array}$ & $\begin{array}{l}.35 \\
.45 \\
.55 \\
.65 \\
.75\end{array}$ & $\begin{array}{l}.63 \\
.65 \\
.66 \\
.67 \\
.68\end{array}$ \\
\hline
\end{tabular}

(as on tangent track or on curves with pull-over construction) shall be of the flexible type, so arranged that hanger may be lifted up from messenger and permit the distance between contact wire and messenger at hanger to be less than hanger length.

(c) Hangers for use on curves with "inclined catenary" where contact wire is not in the same vertical plane as messenger may be either of the flexible type with a loose fitting loop around messenger or of the rigid type arranged to clamp the messenger so that the distance between contact wire and messenger at hangers can not change.

(d) Contact wire clamp of hanger shall be of steel, malleable iron, or bronze, as specified.

(e) Contact wire clamp shall be of such design as to avoid interference with satisfactory operation of current collector (see fig. 486) and shall conform to the shape of contact wire so as to form a good 
gripping surface and prevent clamp from slipping along contact wire, and shall have the necessary strength to support the weight.

$(f)$ Messenger clamp of rigid hanger shall be of steel, malleable iron, or bronze.

(g) Messenger clamp of rigid hanger shall be of such design as to clamp securely in place on messenger without injury to messenger strands.
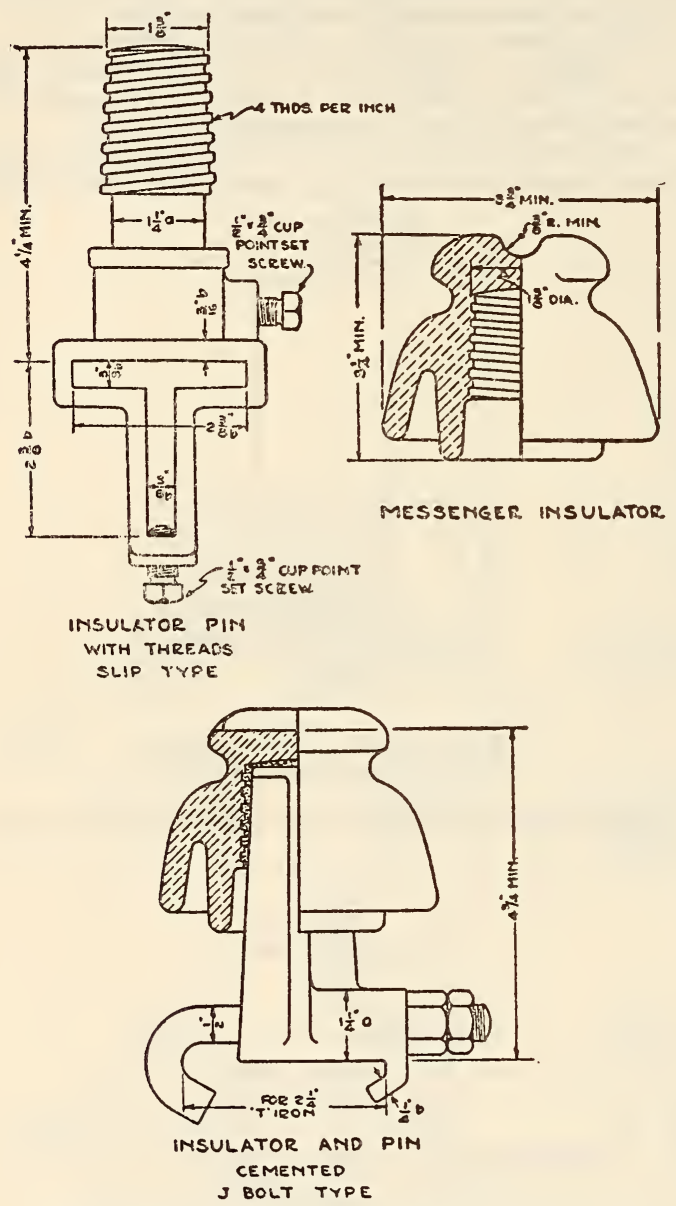

FIgURE 487.-Messenger insulator and pin

\begin{tabular}{|c|c|c|}
\hline & \multicolumn{1}{|c|}{$\begin{array}{c}\text { Allowable va- } \\
\text { riations }\end{array}$} \\
\hline & Over & Under \\
\hline & $\begin{array}{c}\text { Inch } \\
1 / 8\end{array}$ & $\begin{array}{c}\text { Inch } \\
1 / 8 \\
a\end{array}$ \\
\hline $1 / 16$ & 0 \\
\hline
\end{tabular}

(h) Supporting element of hanger shall be of steel, bronze, copper, or other suitable material.

(i) Supporting element of flexible hanger shall be made with a loop, which will permit a vertical movement of hanger and shall be designed to prevent hanger from becoming detached from messenger.

(j) Hangers for "inclined catenary" shall have the assembly of supporting element and contact wire clamp such as to provide the desired angle between a vertical line through contact wire and the axis of supporting element. (k) The length of a catenary hanger shall be the distance from the center line of messenger to the lower edge of contact wire clamp. (In ordering hangers, the diameter of messenger must be specified.)

351. Hanger, cross-span messenger.-( (i) Crossspan messenger hanger shall be of malleable iron or bronze.

(b) Messenger hanger shall be of such design as to provide for attaching messenger to cross span or steady span. It may be either of two types-one for attachment to simple cross span, and the other for attachment to steady span with provision for suspension from cross span located above steady span.

(c) Messenger hanger shall prevent lateral movement of messenger.

354. Links, spacer. (See fig. 48\%.)-(a) Spacer link assembly shall hold two paralleling contact wires at a desired distance between centers.

(b) Spacer links may be either nonadjustable (fixed center between contact wires) or adjustable. Link shall consist of two or more clamps connected by a bar.

(c) Contact wire clamps shall be of malleable iron or bronze.

(d) Contact wire clamp shall be of such design as to avoid interference with satisfactory operation of current collector and shall conform to shape of contact wire, so as to form a good gripping surface and prevent clamp from slipping along contact wire. In the case of the adjustable link, it shall have suitable provision for securely locking to link bar.

(e) Link bar for nonadjustable link shall be of formed steel, bronze, copper, or other suitable material, either solid or tubing. Link bar for adjustable link shall be a straight pipe of suitable length.

355. Messenger.-Messenger shall be of a material suitable for the condition to be met as regards conductivity, mechanical strength, and liability of corrosion. In all cases the desired breaking strength and yield point shall be given in addition to the other physical characteristics necessary. Stranding shall be in accordance with commercial standards.

356. Pin, messenger insulator. (See fig. 488.)(a) Messenger insulator pin shall be of standard wrought iron, mild steel, or malleable iron, as specified.

(b) Pin shall be so designed that it can be either slipped over bracket arm and securely locked or clamped in place.

(c) Pin shall be furnished with $13 / 8$-inches standard pin threads or be of such design that messenger insulator may be cemented on.

(d) Pins provided with pin threads shall be so designed that when insulator is tightened pin may be rotated to bring wire groove in insulator in alignment with messenger, and then locked to prevent further rotation.

(e) Pin shall withstand, without fracture or serious deflection, a bending moment of not less than 5,000 inch-pounds.

357. Pull over, flexible. (See fig. 489.)-(a) Flexible pull over shall be of iron, steel, or bronze of such size and design as to withstand without permanent distortion a direct pull of 150 pounds.

(b) Pull over shall be of such design that when properly installed it will pull the contact wire into alignment without decreasing the vertical movement or flexibility of the overhead system.

(c) Pull over shall be designed to give a vertical clearance of at least 6 inches at a distance of 30 inches from contact wire.

(d) Pull over shall have provision for a suitable clamping arrangement for attachment to contact wire and shall also have provision for attachment of pull-off wire. 
358. Steady, cross span--(a) Cross-span steady shall be of steel, malleable iron, or bronze.

(b) Steady shall be so designed that when attached to cross-span wire, it will restrain lateral movement of contact wire.

(c) Steady shall have provision for rigid attachment to cross-span wire and for suitable attachment to contact wire, ear, or clamp.

(d) Steady shall have sufficient clearance for current collector.

359. Steady, pole bracket. (See fig. 490).-(a) Pole bracket steady assembly shall be so designed that when attached to pole bracket it will restrain lateral movement but will not retard moderate vertical or longitudinal movement of contact wire.

(b) Assembly shall provide sufficient clearance for current collector.

(c) Bracket arm clamp shall be of steel, malleable iron, or bronze.

(d) Bracket arm clamp shall clamp securely to bracket arm at desired location.

(e) Insulation shall be provided between contact wire and bracket arm suitable for trolley voltage used.

(f) Rod shall be of steel or bronze not less than five-eighths inch in diameter if solid.

(g) Assembly shall have sufficient strength to prevent distortion by wind load on, or by side sway of contact wire.

\section{WORKMANSHIP AND FINISH}

401. General.-The general workmanship shall be appropriate to the service for which the article is designed.

402. Finish. (a) Surfaces not in contact shall have such finish as results from good commercial practice in manufacture.

(b) Surfaces to be given a protective coating shall be of such character as properly to receive and retain the protective coat.

(c) Surfaces in fixed contact shall be so finished as to give good true bearing.

(d) Surfaces in moving contact shall be finished by machining or other process to inake them suitable for the particular service.

\section{PACKING AND SEIPPING}

501. Packing.-All material shall be so packed for shipment that it will be suitably protected from injury. Larger material should be bundled, baled, barreled, or crated. Smaller material should be packed in layers in cartons or boxes so that check of quantity is easily made.

502. Marking.-Each package shall be plainly marked, giving the description and quantity of material and the purchaser's order number.

\section{INSPECTION AND REJECTION}

601. Facilities for inspection.-Inspection shall, unless otherwise agreed, be made at the point of

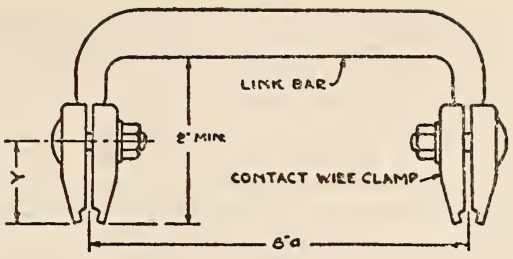

MON-ADJUSTABLE SPACER LINK

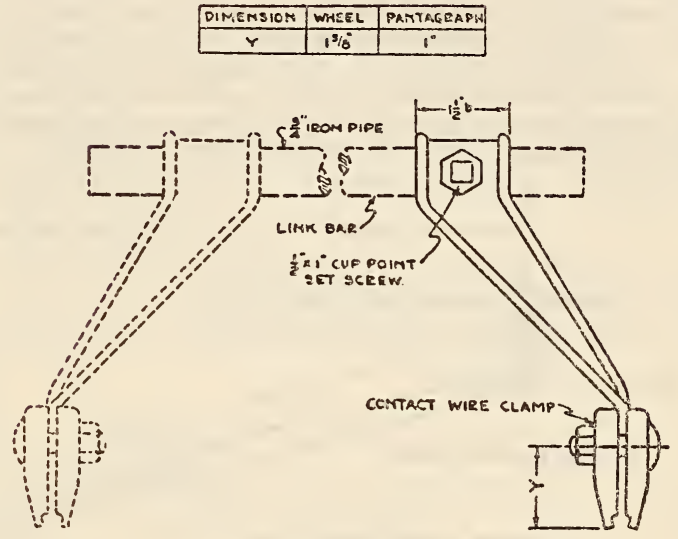

ADJUSTABLE SPACER LINK

FIgure 488.-Spacer link

\begin{tabular}{|c|c|c|}
\hline & \multicolumn{2}{|c|}{$\begin{array}{l}\text { Allowable va- } \\
\text { riations }\end{array}$} \\
\hline & Over & Under \\
\hline $\begin{array}{l}a \\
b\end{array}$ & $\begin{array}{c}\text { Inch } \\
1 / 4 \\
1 / 4\end{array}$ & $\begin{array}{l}\text { Inch } \\
0^{1 / 4}\end{array}$ \\
\hline
\end{tabular}

manufacture. The accredited representative of the purchaser shall be afforded every reasonable facility to satisfy himself that the material and workmanship conforms to this specification.

602. Waiving inspection.-If inspection of material at the point of manufacture is waived or is impractical, the manufacturer shall, at the demand of the purchaser, furnish certified copies of the manufacturer's inspection report for the approval of the purchaser prior to shipment of material.

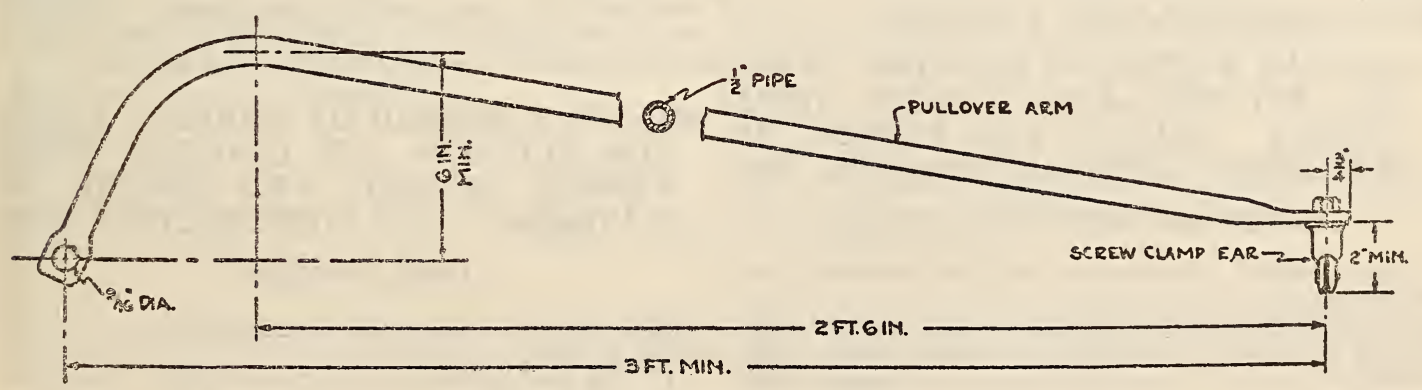


603. Method of inspection.-Representative samples for inspection shall be selected at random, totaling not more than 10 per cent of shipments up to 500 pieces and not more than 5 per cent of larger quantities. In case of the failure of more than 20 per cent of the samples so selected to pass inspection, the manufacturer shall provide for the inspection of an additional number of samples equal to those in the first lot.

604. Testing to destruction.-The testing of material to destruction is liable to entail considerable expense and possible delay to shipments, and such tests shall be required only when specifically agreed by the purchaser and manufacturer.

605. Rejection. - All samples which fail to conform to the requirements of this specification shall be rejected, and the failure of 20 or more per cent of the samples in each of the two sample lots taken as above shall be cause for the rejection of the entire lot.

606. Failure in service.-Any material which fails in service due to inherent defects in either material or workmanship shall be replaced by the manufacturer free of charge, including transportation charges.
2. Anchors may be of either the ordinary or stockless type. The weight of the stock of an ordinary anchor is to be equal to one-fourth that of the anchor. The weight of the head of a stockless anchor is not to be less than three-fifths of the total weight of the anchor.

3. All anchors are to be tested under the inspection of a surveyor of the bureau, in a machine recognized for such purposes by the committee of the American Bureau of Shipping.

4. Prior to testing, the actual weight of the anchor is to be ascertained. The proof tests provide for anchors weighing from 56 pounds to 18,088 pounds in 28-pound gradations. The proof tests range from 6,400 pounds for the 56-pound anchor to 195,160 pounds for the 18,088-pound anchor. Anchors of the ordinarr type are weighed without the stock.

5. After testing, all anchors shall be carefully examined-disassembled where necessary for this purposeand if any evidence is found of flaw or material weakness the anchors shall be rejected. Any anchor which after being tested is found to have more than three-

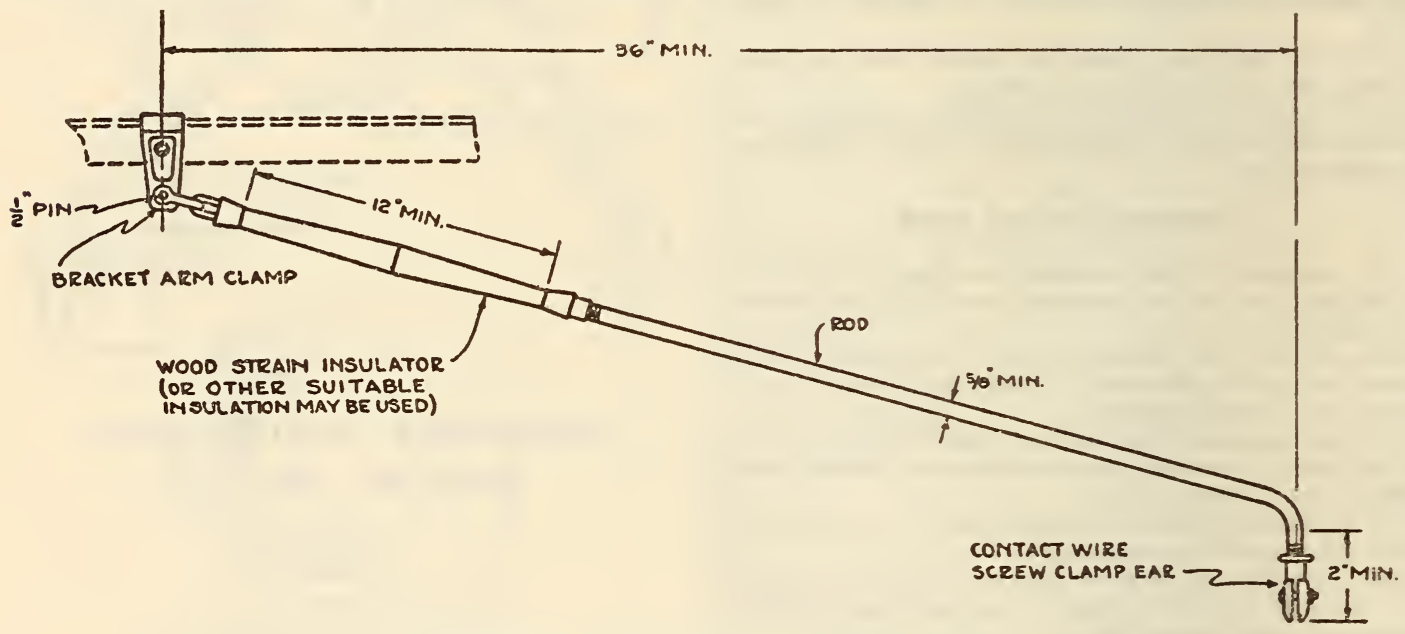

Figure 490.-Pole bracket steady assembly

(Appendixes to this specification cover studies of alloy and composite messenger cables, of pin and suspension type insulators, and data on catenary systems for railway electrification with copper, alloy, and composite messengers.)

UNITED STATES GOVERNMENT, DEPARTMENT OF COMMERCE, BUILDERS' HARDWARE (NONTEMPLATE), COMMERCIAI STANDARD CS22-30, DECEMBER 11, 1930

BUILDERS' HARDWARE (NONTEMPLATE)

(See 617.0, p. 977.)

\subsection{WROUGHT-IRON FORGINGS.}

\section{AMERICAN BUREAU OF SHIPPING, RULES} FOR BUILDING AND CLASSING STEEL VESSELS; QUALITY AND TESTING OF MATERIALS-SHIP CONSTRUCTION, 1930

\section{FORGED WROUGHT-IRON ANCHORS}

(For general information and requirements see 600.0, p. 2.)

1. Anchors are to be made of forged wrought iron, forged open-hearth ingot steel, or cast steel. The shackles may be of wrought iron or of forged steel, unwelded. fourths inch permanent set shall be rejected. When an anchor which has already been in use is submitted for testing or retesting the certificate issued in regard to same will be marked in red: "This anchor is not new but has been previously used."

6 . When anchors have satisfactorily passed the above requirements they are to be clearly stamped by the manufacturer. The stampings include identifying number, initials of surveyor, date of test, proof test, weight, and the bureau's mark of approval, A. B.

7. One side of the anchor should be reserved solely for these marks, and the other side be solely used for the maker's name or other trade-marks that may be desired. If the design of the anchor does not admit of the above marks being placed or grouped as indicated, a suitable boss should be cast on each arm, on which the marks should be stamped.

AMERICAN BUREAU OF SHIPPING, RULES FOR BUILDING AND CLASSING STEEL VESSELS; QUALITY AND TESTING OF MATERIALS-SHIP CONSTRUCTION, 1930

\section{IRON FORGINGS}

(For general information and requirements see 600.0, p. 2.)

1. The test specimens are to be taken from the body of the forging. The material is to have a 
tensile strength of 49,000 pounds per square inch, with an elongation of 20 per cent on a gage length corresponding to three and one-half times the diameter of the test specimen. Specimens 1 inch square are to stand being bent cold to $90^{\circ}$ over a radius of $11 / 2$ inches without fracture.

\section{AMERICAN BUREAU OF SHIPPING, RULES FOR BUILDING AND CLASSING STEEI VESSELS; MATERIALS FOR MACHINERY, BOILERS, AND PIPING, 1930}

\section{IRON FORGINGS}

(For general information and requirements see 600.0 , p. 1. For test requirements see 600.1, p. 7 .)

1. The number of tests is the same as that prescribed for steel forgings. (See 611.51, p. 750, par. 3.) The physical characteristics of the material as shown by standard test pieces must meet with the following requirements:

Tensile strength............... 44,000-54,000 pounds per square inch Yield point .......................... 50 per cent of tensile strength.

Minimum elongation. $1,100,000 ; 25$ to 20 per cent.

Bend without fracture. Tensile strength $120^{\circ}$; radius of bend $11 / 2$ inches.

AMERICAN SOCIETY FOR TESTING MATERIALS, STANDARD SPECIFICATIONS FOR WROUGHT-IRON ROLLED OR FORGED BLOOMS AND FORGINGS FOR LOCOMOTIVES AND CARS, DESIGNATION A73-30, 1930

\section{WROUGHT-IRON FORGINGS} SCOPE

1. These specifications cover two classes of wrought-iron blooms and forgings for locomotives and cars, as determined by the kind of material used in their manufacture: namely, class $A$, as defined in section $2(a)$; class $\mathrm{B}$, as defined in section $2(b)$.

\section{MANOFACTORE}

2. (a) Class A.-Blooms and forgings of class A shall be made wholly from puddled pig iron. The puddle mixture and the component parts of the piles from which the blooms and forgings are made shall be free from any admixture of iron scrap or steel. The term "iron scrap" applies only to foreign or bought scrap, and does not include the manufacturer's own scrap from the same product and of equal quality.

(b) Class B.-Blooms and forgings of class B shall be made from selected No. 1 wrought-iron scrap, or a mixture of such scrap and the puddled iron described in paragraph $(a)$.

3 . Cross piling will not be permitted in either class of forging. In the final pile for the bloom or forging, all bars and slabs shall be of the full length of the pile.

\section{CHEMICAL PROPERTIES AND TESTS}

4. The iron shall conform to the following requirement as to chemical composition:

\begin{tabular}{l|r|r}
\hline & Class A & Class B \\
\hline Manganese, maximum ....................... & $\begin{array}{r}\text { Per cent } \\
0.07\end{array}$ & $\begin{array}{r}\text { Per cent } \\
0.15\end{array}$ \\
\hline
\end{tabular}

5. (a) An analysis may be made by the purchaser from each specimen used for the tension test. The chemical composition thus determined shall conform to the requirement specified in section 4 .

(b) Drillings or chips for analysis shall be so taken as to represent the full cross section of the specimen.

\section{PHYSICAL PROPERTIES AND TESTS}

6. (a) The iron shall conform to the following minimum requirements as to tensile properties:

\begin{tabular}{|c|c|c|}
\hline & Blooms & $\begin{array}{c}\text { Forgings, } \\
\text { classes A } \\
\text { and B }\end{array}$ \\
\hline Tensile strength & 45,000 & 45,000 \\
\hline Yield point & 0.5 tensile & $\begin{array}{l}0.5 \text { tensile } \\
\text { strength. }\end{array}$ \\
\hline $\begin{array}{l}\text { Elongation in } 4 \text { inches } \\
\text { Reduction of area }\end{array}$ & $\begin{array}{r}22 \\
30\end{array}$ & 24 \\
\hline
\end{tabular}

(b) The fracture shall be free from crystallization.

(c) The yield point shall be determined by the: drop of the beam of the testing machine. The speed of the crosshead of the machine shall not exceed three-eighths inch per minute.

7. The entire cross section of the prolongation of the bloom or forging shall be ground or polished, and shall show no seams or flaws of injurious character. It shall then be etched ${ }^{85}$ and, in class A blooms or

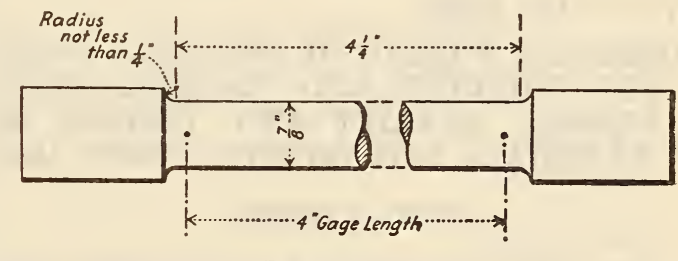

Figure 491

NoTE.-The gage length, parallel portions, and fillets shall be as shown, but the ends may be of any form which will fit the holders of the testing meahine.

forgings, shall show freedom from steel.

8. (a) Tension-test specimens shall be taken from a full-size prolongation of the bloom or forging. The axis of the specimen shall be located at any point midway between the center and surface of the bloom or forging, and shall be parallel to the axis of the bloom or forging.

(b) The specimens shall conform to the dimensions shown in Figure 491. The ends shall be of a form to fit the holders of the testing machine in such a way that the load shall be axial.

9. Unless otherwise specified, one test shall be made from each lot of 10 blooms or less and from each lot of 4,000 pounds or less of forgings. These tests shall be made on prolongations the full size of the blooms or the largest section of the forging and, for test purposes, prolongations shall be furnished on at least 20 per cent of the blooms or forgings. In the case of small forgings, one forging may be used for test purposes, in which case one test will represent not more than 100 forgings.

\section{MARKING}

12. Identification marks shall be legibly stamped on each bloom or forging and on each test specimen. The purchaser shall indicate the location of such identification marks.

85 A solution of 10 per cent hydrochloric acid (specific gravity 1.19), 30 per cent sulphuric acid (specific gravity 1.84), and 60 per cent water; or 25 per cent nitric acid (specific gravity 1.42 ), and 75 per cent water, is recommended for the etch test. 
(Requirements covering workmanship and finish conform with those in A. S. T. M. A18-30. (See 611.51, p. 754.) Requirements covering inspection and rejection and rehearing conform with those in A. S. T. M. standard specifications for steel, paragraphs 7,8 , and 9 . (See 605.0, p. 247.))

\section{UNITED STATES GOVERNMENT, FEDERAL SPECIFICATIONS BOARD, FEDERAL SPEC- IFICATION FOR LADLES, PLUMBERS' GGG-I-51 MARCH 1, 1932}

\section{PLUMBERS' LADLES}

(See 615.9, p. 940.)

\subsection{STEEL CASTINGS.}

\author{
611.40 GENERAL ITEMS.
}

\section{AMERICAN RAILWAY ASSOCIATION, ME- CHANICAL DIVISION, STANDARD DROP. TEST MACHINE FOR A. R. A. COUPLERS AND AXLES, 1911}

\section{DROP-TEST MACHINE}

(See 611.50, p. 750.)

611.41 CAST STEEL.

AMERICAN BUREAU OF SHIPPING, RULES FOR BUILDING AND CLASSING STEEL VESSELS; QUALITY AND TESTING OF MATERIALS, SHIP CONSTRUCTION, 1930

\section{STEEL CASTINGS}

(For general information and requirements, see 600.0, p. 2.)

1. Where steel castings are to be used instead of forgings, every care is to be taken to avoid abrupt changes in sectional area. Each casting must be thoroughly annealed and subjected to percussive and hammering tests, as well as to tests for tensile strength and ductility. The castings are to be made by the open-hearth process, and are to be accurately molded and free from defects. The material is to have a tensile strength of not less than 58,000 pounds, and not exceeding 78,400 pounds per square inch, with a corresponding elongation varying from 20 per cent with 58,000 pounds to 15 per cent with 78,400 pounds, measured on the standard test specimens of the American Bureau of Shipping. (See fig. 5, 600.1, p. 8.)

2. The specimens for testing must have formed part of the actual casting submitted for approval, subjected to similar and simultaneous annealing, and are not to be detached from the casting until after the annealing is completed nor until they have been stamped by the surveyor. The piece for ductility test should not be less than 1 inch by three-fourths inch with edges rounded to a radius of one-sixteenth inch, and must stand being bent cold through an angle of $120^{\circ}$ over a radius of 1 inch without any appearance of fracture.

3. Castings of stern frames, posts, single-plate rudder frames, brackets, and quadrants are to be raised to a height of 6 to 10 feet, according to the character and form of the castings, and dropped bodily on to a hard surface, holes being prepared to receive bosses or similar projections. When large stern frames are cast in one piece, they may be dropped through an angle of $45^{\circ}$ instead of being lifted bodily. Castings of a complex design may, at the discretion of the surveyor, be exempted from the drop test; and where the material is thin the limits of elongation may be reduced by 5 per cent. All castings are to be slung clear of the ground, and well hammered all over with a heavy sledge hammer to test the soundness of the material. All steel castings which have satisfactorily passed the requirements are to be clearly stamped A.B. and with the identification marks furnished by the surveyor.

4. In addition to the foregoing tests, large castings are to be drilled, as required by the surveyors for the purpose of examination, at locations where porosity, cavities, and contraction cracks usually occur. Such small holes are to be subsequently filled up with screwed plugs or the equivalent.

5 . In the event of any casting proving unsatisfactory or defective in the course of preparation or fitting in the ship, such casting shall be rejected irrespective of any previous certificate of satisfactory testing.

\section{AMERICAN BUREAU OF SHIPPING, RULES FOR BUILDING AND CLASSING STEEL VESSELS; MATERIALS FOR MACHINERY, BOILERS, AND PIPING, 1930}

\section{STEEL CASTINGS}

(For general information and requirements, see 600.0 , p. 1. For test requirements, see 600.1 , p. 7.)

1. Steel castings subject to test and inspection must be made by the open hearth or by such other process as may be approved by the committee. All castings shall be thoroughly cleaned and must be annealed in an annealing furnace of ample proportions to bring the whole casting to a uniform annealing temperature. They must not be removed from the annealing furnace until the temperature has dropped to about $700^{\circ} \mathrm{F}$. Local heating or cooling, also bending and straightening, of annealed castings is only permitted with the approval of the surveyor. Where the use of steel castings has been approved where ordinarily forgings are required, the castings must be submitted for the surveror's inspection in the green. In the case of failure of the physical tests, castings may be reannealed and retested. The material shall comply with the following requirements:

\begin{tabular}{l|c|c|c|c|c}
\hline Grade & $\begin{array}{c}\text { Limits of ten- } \\
\text { sile strength }\end{array}$ & Yield point & $\begin{array}{c}\text { Mini- } \\
\text { mum } \\
\text { elonga- } \\
\text { tion }\end{array}$ & $\begin{array}{c}\text { Bend without } \\
\text { fracture }\end{array}$ & Stamp \\
\hline & $5 S, 000-68,000$ & $\begin{array}{c}45 \text { per cent } \\
\text { of tensile } \\
\text { strength. }\end{array}$ & $\begin{array}{c}\text { Per cent } \\
23-20\end{array}$ & $\begin{array}{c}120^{\circ} \text { to } 1 \text { inch } \\
\text { radius. }\end{array}$ & A.B. \\
\hline
\end{tabular}

Grade 1 required for castings to take the place of engine forgings and for propellers.

2. At least one tension and one bend test shall be made from each heat, and the test specimens must have formed part of the actual castings submitted for approval. Where a number of small castings are made from one heat and have been simultaneously annealed, test coupons should be cast on a sufficient number of the castings for the selection of the surveyor, and two tests shall be made from each heat. All castings are to be slung clear of the ground and hammered all over with a heavy sledge hammer to test the soundness of the material. Casting showing defects during this test shall be rejected, irrespective of satisfactory tension and bending tests. 
AMERICAN ELECTRIC RAILWAY ENGINEERING ASSOCIATION, SPECIFICATION FOR MATERIALS FOR USE IN THE MANUFACTURE OF SPECIAL TRACK WORK, W104-28, 1928

Approred as American tentatice standard by the American Standards Association, January 23, 1929]

SPECIAL TRACK WORK MATERIALS

(See 606.3, p. 342.)

AMERICAN RAILWAY ASSOCIATION, MECHANICAL DIVISION, SPECIFICATIONS FOR STEEL CASTINGS, CARBON, 1923

\section{CARBON-STEEL CASTINGS}

(Identical with A. S. T. M., A87-27, given below, including references in $A S 7-27$ to $A$. S. T. M., A95-29, and A. S. T. M. standard specifications for steel in 605.0.)

\section{AMERICAN RAILWAY ENGINEERING SOCIATION, SPECIFICATIONS STEEL RAILWAY BRIDGES, 1924}

AS-

(See also 605.11, p. 249; 611.11, p. 636; and 611.51$, p. 753.$)$

\section{CAST STEEI}

(In respect to process, heat treatment, chemical and phrical properties, ladle analyses, check analyses, rield point, bend tests, test specimens, number of tests, workmanship and finish, and rejection this specification conforms with A. S. T. M., A27-24, given below, including references in A27 to A. S. T. M., $195-29$, and to A. S. T. M. standard specifications for steel in 605.0.)

AMERICAN SOCIETY OF MECHANICAL ENGINEERS, SPECIFICATIONS FOR STEEL CASTINGS, S-11, BOILER CONSTRUCTION CODE, SECTION II, 1930

\section{STEEL CASTINGS}

(Class A castings based on class A castings of A. S. T. Mi. specification A27-24, given below.)

(Class B castings based on grade A annealed requirements of $A$. S. T. M. specification A87-27, given below.)

AMERICAN SOCIETY OF MECHANICAI ENGINEERS, SPECIFICATIONS FOR CARBON-STEEL CASTINGS FOR VALVES, FLANGES, AND FITTINGS FOR HIGHTEMPERATURE SERVICE, BOILER CONSTRUCTION CODE, 1930

\section{CARBON-STEEL CASTINGS}

(This specification conforms with A. S. T. M., A95-29, given below.)
AMERICAN SOCIETY FOR TESTING MATERIALS, STANDARD SPECIFICATIONS FOR STEEL CASTINGS, DESIGNATION A27-24, 1924

\section{STEEL CASTINGS}

\section{SCOPE}

These specifications cover steel castings for general service and for ships. Two classes of castings are covered, namely, class A, castings for which no physical requirements are specified; class B, castings for which physical requirements are specified. These are of three grades; hard, medium, and soft.

\section{MANUFACTURE}

The steel shall be made by one or more of the following processes: Open-hearth, electric-furnace, converter, or crucible. Class A castings need not receive any heat treatment unless so specified, or unless the carbon content exceeds 0.30 per cent. Class A castings, in which the carbon content is over 0.30 per cent, and all class B castings, shall receive a heat treatment proper to the design and chemical composition of the castings.

(Details covering heat treatment, annealing, and normalizing are identical with those in A. S. T. M., A95-29, given below.)

\section{CHEMICAL PROPERTIES AND TESTS}

The steel shall conform to the following requirements as to chemical composition:

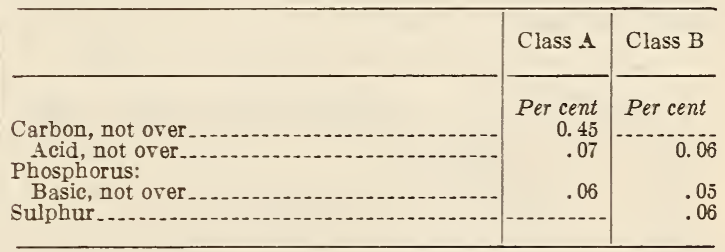

(Details covering ladle analyses and check analyses of class A castings are identical with those in A. S. T. M., A 95-29, given below.)

Analyses of class B castings may be made by the purchaser from a broken tension-test specimen. The phosphorus and sulphur content thus determined shall not exceed that specified above by more than 20 per cent.

\section{PHYSICAL PROPERTIES AND TESTS}

(For Class B Castings Only)

The castings shall conform to the following minimum requirements as to tensile properties:

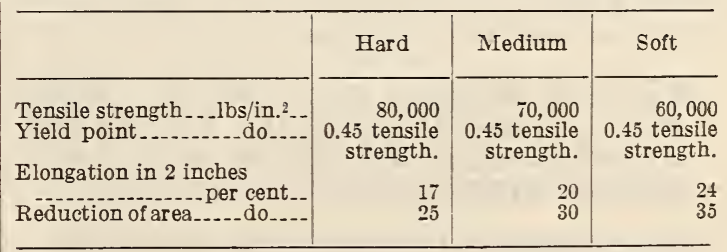

The yield point shall be determined by the drop of the beam of the testing machine. 
Bend tests.-Bend tests shall not be required for castings of the hard grade, and shall be required for castings of the medium and soft grades only when so specified in the order.

When a bend test is specified, the test specimen shall stand being bent cold without cracking on the outside of the bent portion, around a pin 1 inch in diameter through the following angles:

\begin{tabular}{l|c|c|c}
\hline & Hard & $\begin{array}{c}\text { Medi- } \\
\text { um }\end{array}$ & Soft \\
\hline & No bend test_........ & ${ }_{90}$ & $\circ$ \\
\hline Angle of bend & & \\
\hline
\end{tabular}

Alternative tests to destruction.-The tensile properties of the castings shall be determined from test bars, unless in the case of castings weighing not over 150 pounds the manufacturer desires to offer, as an alternative, tests to destruction. Upon agreement with the inspector, the castings shall be grouped in lots, each lot containing castings from the same melt and from the same heat-treatment charge, and the inspector shall select from each lot one casting to represent the lot. The representative casting shall be tested to destruction as a substitute for the tension test. This test shall show the material to be ductile and free from injurious defects.

(Details covering test specimens, number of tests, retests, workmanship and finish, inspection, rejection, and rehearing - all are indentical with those in A. S. T. M., A95-29, given below. Tension-test specimens shall conform to the dimensions shown in Figure 129 in A. S. T. M. standard specifications for steel. See 605.0, p. 247.)

\section{SPECIAL REQJIREMENTS FOR CASTINGS FOR SHIPS}

In addition to the preceding requirements, castings for ships, when so specified, shall conform to the following requirements:

All castings shall be of class B, medium or soft grade, and shall receive a proper heat treatment.

One tension and one bend test shall be made from each of the following castings: Stern frames, stern posts, twin-screw spectacle frames, propellershaft brackets, rudders, steering quadrants, tillers, stems, anchors, and other castings when specified.

When a casting is made from more than one melt, four tension and four bend tests shall be made from each casting.

A percussion test shall be made on each of the following castings: Stern frames, stern posts, twinscrew spectacle frames, propeller-shaft brackets, rudders, steering quadrants, tillers, stems, anchors, and other castings when specified.

For this test the casting shall be suspended by chains and hammered all over with a hammer of a weight approved by the purchaser or his representative. If cracks, flaws, defects, or weakness appear after such test, the casting may be rejected.

\section{AMERICAN SOCIETY FOR TESTING MATE- RIALS, STANDARD SPECIFICATIONS FOR CARBON-STEEL CASTINGS FOR RAIL- ROADS, DESIGNATION A87-27, $192 \%$}

\section{CARBON-STEEL CASTINGS FOR RAILROADS SCOPE}

1. (a) These specifications cover all carbon-steel castings for locomotive and car equipment, and for miscellaneous use, classified as grade $\mathrm{A}$ and grade $\mathrm{B}$.

(b) The purposes for which the two grades are generally used are:

Grade A, unannealed, covers only such special castings as may be approved by the purchaser.
Grade A, annealed, covers such castings not covered by grade $\mathrm{A}$, unannealed.

Grade B covers all castings for high stresses such as truck side frames, bolsters, couplers and coupler parts, locomotive frames, and locomotive driving and trailer wheel centers.

2. Grade A castings shall be furnished annealed unless otherwise specified by the purchaser.

Grade B castings shall be furnished annealed.

\section{MANOFACTURE}

3. The steel may be made bv one or more of the following processes: Open-hearth, electric-furnace, crucible, or converter.

4. (a) The castings shall be allowed to become cool. They shall then be uniformly heated to the proper temperature to refine the grain, and allowed to cool uniformly.

(b) For the purpose of determining the quality of annealing of all castings weighing 150 pounds or over, and of such lighter castings as shall be required by the purchaser or his representative, there shall be provided for removal by the inspector after annealing and also after reannealing, if necessary, at least one and not more than two annealing lugs. The location of the annealing lugs shall be such that when removed by the inspector they shall be indicative of the character of annealing. The standard annealing lug shall be 1 inch in height and 1 inch in width and five-eighths inch in thickness where it joins the casting.

(c) If, in the opinion of the inspector, a casting is not properly annealed, he may at his option require the casting to be reannealed.

\section{CHEMICAL PROPERTIES AND TESTS}

5. The steel shall conform to the following requirements as to chemical composition:

\begin{tabular}{|c|c|c|}
\hline & Grade $\mathbf{A}$ & Grade B \\
\hline $\begin{array}{l}\text { Manganese, not over } \\
\text { Phosphorus, not over } \\
\text { Sulphur, not over }\end{array}$ & $\begin{array}{r}\text { Per cent } \\
0.85 \\
.05 \\
.05\end{array}$ & $\begin{array}{r}\text { Per cent } \\
0.85 \\
.05 \\
.05\end{array}$ \\
\hline
\end{tabular}

(Ladle analyses and check analyses are the same as in A. S. T. M., A95-29, given below.)

\section{PHYSICAL PROPERTIES AND TESTS}

8. (a) The steel shall conform to the following minimum requirements as to tensile properties:

\begin{tabular}{|c|c|c|c|}
\hline & \multicolumn{2}{|c|}{ Grade A } & \multirow{2}{*}{$\begin{array}{l}\text { Grade B } \\
\text { annealed }\end{array}$} \\
\hline f & $\begin{array}{c}\text { Un- } \\
\text { annealed }\end{array}$ & Annealed & \\
\hline Yield point........... & 29,250 & 29,250 & 36,000 \\
\hline \multirow{2}{*}{ Elongation in 2 inches.. per cent } & $1,450,000$ & $1,600,000$ & $1,600,000$ \\
\hline & $\begin{array}{l}\text { Tensile } \\
\text { strength }\end{array}$ & $\begin{array}{l}\text { Tensile } \\
\text { strength }\end{array}$ & $\begin{array}{l}\text { Tensile } \\
\text { strength }\end{array}$ \\
\hline But in no case less than... & 22 & 24 & 22 \\
\hline \multirow[t]{2}{*}{ Reduction of area......-per cent.. } & $2,250,000$ & $2,600,000$ & $2,500,000$ \\
\hline & $\begin{array}{l}\text { Tensile } \\
\text { strength }\end{array}$ & $\begin{array}{l}\text { Tensile } \\
\text { strength }\end{array}$ & $\begin{array}{l}\text { Tensile } \\
\text { strength }\end{array}$ \\
\hline But in no case less than..-. & 30 & 35 & 30 \\
\hline
\end{tabular}

(b) The tensile strength shall be reported as information.

(The yield point determination is the same as in A. S. T. M., A95-29, given below.) 
9. (a) A sufficient number of test coupons from which the required test specimen may be prepared shall be cast, attached in the neighborhood of each end of each locomotive frame, attached to each locomotive cylinder, to each wheel center, and to miscellaneous castings weighing over 150 pounds. These test coupons shall remain attached to the castings throughout the annealing and until the castings are presented for inspection. If the design of the casting is such that the test coupons can not be attached, they shall be cast in runners outside of the casting, but attached to it, to represent each melt. The location of the test coupons, as well as the method of casting such coupons, shall be subject to mutual agreement between the inspector and the manufacturer. In the case of any orders for castings weighing under 150 pounds, the physical properties as required in section 8 , may be determined from an extra or spare test bar cast with and attached to some other casting from the same melt.

(b) When sufficient coupons have not been cast, a test specimen may be cut from a finished casting at a location mutually agreed upon by the inspector and the manufacturer.

(c) Tension-test specimens shall conform to dimensions shown in Figure 129 in A. S. T. M. standard specifications for steel in 605.0, p. 247. The ends shall be not less than seven-eighths inch in diameter and of a length and form to fit the holders of the testing machine in such a way that the load shall be axial.

10. (a) One tension test shall be made from the neighborhood of each end of each locomotive frame and both tests shall meet the requirements of the specifications. One tension test may be made from each wheel center and each locomotive cylinder casting, but of such castings at least one of each kind in each melt shall be tested. For miscellaneous castings from melts which do not include frames, wheel centers, or cylinders, one tension test shall be made from each melt, except as provided in section $11(a)$.

(b) If the test specimen shows defective machining or develops flaws, it may be discarded and another specimen substituted.

(c) If the percentage of elongation of any tensiontest specimen is less than that specified in section 8 and any part of the fracture is more than threefourths inch from the center of the gage length, as indicated by scribe scratches marked on the specimen before testing, a retest shall be allowed.

(d) No part of these specifications shall operate to cause any one tension test to apply to more than 40 tons of castings as offered for inspection.

11. (a) After 15 consecutive melts, which may contain any or all classes of castings (except frames, wheel centers, and cylinders) covered by these specifications, on one or more orders, have been tested and accepted in accordance with the above requirements, the manufacturer may group the succeeding melts in lots of five melts each, but each lot not to exceed 40 tons, the entire group to be accepted if the test specimen selected from the lot fulfills the chemical and physical requirements herein specified. If this test fails, a rehearing will be granted on the melt that the failed bar represents, and the other four melts of the group shall be tested individually.

(b) If there is a period of more than six months between shipments of the class of castings covered by these specifications, then each melt shall be tested individually until 15 consectuive melts have been accepted, after which the melts may again be grouped as in paragraph $(a)$.

(c) If one or more melts are rejected, each succeeding melt shall be tested individually until 15 consecutive melts have been accepted, after which melts may again be grouped as in paragraph $(a)$.
12. In case of small orders for bolsters, truck sides, draft arms, yokes, or castings weighing over 150 pounds where the size of the order and the available pattern and foundry equipment are such that not more than five castings can be cast in any one melt, the physical properties, as required in section 8 , will be determined from an extra or spare test coupon cast with and attached to some other casting of the same melt.

13. If the results of the physical test lot do not conform to the requirements specified, the manufacturer may reanneal such lot not more than twice and retest shall be as specified in section 8 .

14. In the case of orders including only castings not exceeding 150 pounds in weight, a test to destruction of one casting from each 100 castings or smaller lot may be substituted for the tension tests, at the option of the inspector. This test shall show the material to be ductile, free from injurious defects, and suitable for the purpose intended.

\section{WORKMANSHIP AND FINISH}

(Substantially the same as in A. S. T. M., A95-29, given below.)

\section{MARIING}

17. The manufacturer's name or identification mark and the specified pattern number shall be cast on all castings. In addition, the month and year when made shall be cast on all bolsters, truck sides, frames, wheel centers, cylinders, and similar castings. The location and size of numbers shall be agreed upon by the manufacturer and the inspector. In accordance with the standard practice of the individual foundry to identify individual castings, a serial number may be cast or the melt number may be stamped on bolsters, truck sides, frames, wheel centers, cylinders, and similar castings as agreed upon by the manufacturer and the inspector. The melt number shall be legibly stamped on all other castings weighing over 150 pounds.

\section{INSPECTION AND REJECTION}

(Inspection, rejection, and rehearing conform with A. S. T. M. standard specifications for steel. (See 605.0 , p. 247.)

\section{AMERICAN SOCIETY FOR TESTING MATE- RIALS, STANDARD SPECIFICATIONS FOR CARBON-STEEL CASTINGS FOR VALVES, FLANGES, AND FITTINGS FOR HIGH- TEMPERATURE SERVICE, DESIGNATION A95-29, 1929}

\section{CASTINGS FOR HIGH TEMPERATURES SCOPE}

1. These specifications cover carbon-steel castings for valves, flanges, and fittings for high-temperature service. (See explanatory note.)

\section{MANUFACTURE}

2. The steel shall be made by the electric-furnace, open-hearth or other process approved by the purchaser.

3. (a) All castings shall receive a heat treatment proper to the design and chemical composition of the castings.

(b) Heat treatment, unless otherwise specified by the purchaser, may consist of annealing, or of normalizing, or of normalizing followed by annealing, or of normalizing followed by a draw-back to a temperature below the critical range. No castings which have been quenched in any liquid medium shall be offered under these specifications. 
(c) Annealing.-The procedure for annealing shall consist in allowing the castings to cool, after pouring, to a temperature below the critical range. They shall then be uniformly reheated to the proper temperature to refine the grain and allowed to cool uniformly in the furnace.

(d) Normalizing. - The procedure for normalizing shall consist in allowing the castings to cool, after pouring, to a temperature below the critical range. They shall then be reheated to the proper temperature to refine the grain and allowed to cool in still air.

\section{CHEMICAL PROPERTIES AND TESTS}

4. The steel shall conform to the following requirements as to chemical composition:

$\begin{array}{ll}\text { Carbon } & \text { Per cent } \\ \text { Manganese, not under } & \\ \text { Silicon, not under } & \\ \text { Phosphorus, not over.15 to } 0.45 \\ \text { Sulphur, not over }\end{array}$

5. An analysis of each melt of steel shall be made by the manufacturer to determine the percentages of the elements specified in section 4. This analysis shall be made from drillings taken at least one-fourth inch beneath the surface of a test ingot obtained during the pouring of the melt. The chemical composition thus determined shall be reported to the purchaser or his representative and shall conform to the requirements specified in section 4 .

6. Analyses may be made by the purchaser from the broken tension-test specimen or from a casting representing each melt. The chemical composition thus determined shall conform to the requirements specified in section 4 . Samples for analysis shall be taken not less than one-fourth inch beneath the surface, except in the case of castings which are too thin, and shall be taken in such a manner as not to impair the usefulness of the casting.

\section{PHYSICAL PROPERTIES AND TESTS}

7. (a) The castings shall conform to the following minimum requirements as to tensile properties:

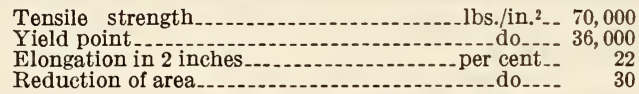

(b) The yield point shall be determined by the drop of the beam of the testing machine at a crosshead speed not to exceed one-eighth inch per minute. The tensile strength shall be determined at a speed not to exceed $1 \frac{1}{2}$ inches per minute.

8. (a) Bend tests shall be required for castings only when so specified in the order.

(b) When a bend test is specified, the test specimens shall stand being bent cold through an angle of $90^{\circ}$ around a pin 1 inch in diameter, without cracking on the outside of the bent portion.

9. Each casting shall be tested after machining to the hydrostatic pressures specified in the table below (note 1), and shall show no leaks. Castings ordered under these specifications, for working pressures other than those listed below, shall be tested to such pressures as may be agreed upon.

\begin{tabular}{|c|c|}
\hline $\begin{array}{c}\text { Class of } \\
\text { castings, } \\
\text { working } \\
\text { steam } \\
\text { pressure }\end{array}$ & $\begin{array}{c}\text { Standard } \\
\text { test } \\
\text { pressure }\end{array}$ \\
\cline { 1 - 2 } \cline { 1 - 2 } Lbs./in.2 & Lbs./in..$^{2}$ \\
250 & 750 \\
400 & 1,000 \\
600 & 1,500 \\
900 & 2,000 \\
1,350 & 3,000 \\
\hline
\end{tabular}

10. (a) Tension-test specimens and, when a bend test is specified, bend-test specimens shall be taken from test bars cast attached to the eastings where practicable. If the design of the castings is such that test bars should not be attached to the castings, the test bars shall be cast attached to special blocks, a sufficient number of which shall be provided for each lot of castings. Test bars from which tension and bend test specimens are to be taken shall remain attached to the castings or blocks they represent through heat treatment and until presented for inspection. Test bars shall be provided in sufficient numbers to furnish the tests required in section 11.

(b) If satisfactory to the manufacturer and inspector, tension-test specimens may be cut frcm heat-treated castings instead of from test bars.

(c) Tension-test specimens shall conform to the dimensions shown in Figure 129 in A. S. T. M. standard specifications for steel. (See 605.0, p. 247.) The ends shall be of a form to fit the holders of the testirg machine in such a way that the load shall be axial.

(d) Bend-test specimens shall be machined to 1 by one-half inch in section with the corners rounded to a radius of not over one-sixteenth inch.

11. (a) One tension test and, when specified, one bend test shall be made from each melt in each heattreatment charge and, when specified, from each casting weighing 500 pounds or over.

(b) If any test specimen shows defective machining or develops flaws, it may be discarded, in which case another specimen from the same lot shall be substituted.

(c) If the percentage of elongation of any tensiontest specimen is less than that specified in section 7 (a) and any part of the fracture is more than threefourths inch from the center of the gage length, as indicated by scribe scratches marked on the specimen before testing, a retest shall be allowed.

12. If the results of the physical tests for any lot do not conform to the requirements specified, such lot may be retreated, but not more than twice. Retests shall be made as specified in sections 7 and 8 .

\section{WORKMANSHIP AND FINISH}

13. The castings shall conform substantially to the shapes and sizes indicated by the patterns and drawings submitted by the purchaser.

14. (a) The castings shall be clean and free from injurious defects.

(b) The castings shall not be peened to stop leaks.

(c) Defects which do not impair the strength of the castings may be welded by an approved process. The defects shall be cleaned out to solid metal, before welding, and when so required by the inspector, shall be submitted to him in this condition for his approval. All castings shall be heat treated after welding in accordance with the requirements in section 3, except that, with permission of the inspector, castings with minor welds need not be re-heat-treated.

\section{INSPECTION AND REJECTION}

(Requirements covering inspection and rejection conform with those specified in A. S. T. M. standard specifications for steel (see 605.0, p. 247) with the following exceptions:)

1. If, in the case of important castings for special purposes, surface inspection in the green state is required, this shall be so specified in the order.

2 . Unless otherwise specified, any rejection based on tests made in accordance with section 6 shall be reported within 12 working days from the receipt of samples. 


\section{EXPLANATORY NOTE}

This contemplates temperatures up to $750^{\circ} \mathrm{F}$. $\left(400^{\circ} \mathrm{C}_{\text {. })}\right.$; or, at the discretion of the designing engineer, higher temperatures with appropriate pressures may be used. Reference may be made to the dimensional standards for pressures and temperatures formulated by the sectional committee on standardization of pipe flanges and fittings under the procedure of the American Standards Association, A. S. A. project No. B16, and, more specifically, to the American steel pipe flanges and flanged fittings standard B16e-1927. (See 607.4, p. 465.)

\section{SOCIETY OF AUTOMOTIVE ENGINEERS, IRON AND STEEL SPECIFICATIONS,} S. A. E. STANDARD, 1928

\section{STEEL CASTINGS}

(For prefatory remarks, see 600.0 , p. 2. This specification is substantially the same as that for class B castings in A. S. T. M., A 27-24, given above.)

Chemical composition

\begin{tabular}{l|c|c|c}
\hline $\begin{array}{c}\text { S. A. E. steel } \\
\text { No.- }\end{array}$ & Carbon & $\begin{array}{c}\text { Phos- } \\
\text { phorus, } \\
\text { maxi- } \\
\text { mum }\end{array}$ & $\begin{array}{c}\text { Sul- } \\
\text { phur, } \\
\text { maxi- } \\
\text { mum }\end{array}$ \\
\hline & As required by physical properties__ & $\begin{array}{r}\text { Per cent } \\
0.05\end{array}$ & $\begin{array}{c}\text { Per cent } \\
0.055\end{array}$ \\
\hline
\end{tabular}

UNITED STATES GOVERNMENT, FEDERAL SPECIFICATIONS BOARD, FEDERAL SPECIFICATION FOR STEEL CASTINGS, QQ-S-681, MAY 3, 1932

\section{STEEL CASTINGS}

GRADES

1. Stee] castings shall be furnished in five gradesspecial, hard, medium, soft, and common.

\section{MATERIAI AND WORKMANSHIP}

2. Unless otherwise required in detailed specifications, contract, or purchase order, steel castings may be made by one or more of the following processes: Electric, open hearth, converter, and crucible.

\section{GENERAL REGUIREMENTS}

3. (a) The responsibility for furnishing castings that can be laid out and machined to the finished dimensions within the tolerances given, without further straightening after final heat treatment, shall rest with the contractor. Castings of excessive size or weight shall not be furnished.

(b) Castings shall be well cleaned, with heads and gates removed, for inspection in the green. They shall be sound and free from injurious defects. When heads and gates are removed by burning, this burning shall be done at least one-half inch from the body of the casting, the remaining metal to be removed by grinding, cutting, or machining.

(c) Castings shall not be repaired, plugged, or welded without permission from the inspector. Such permission will be given only for welding to be completed prior to final heat treatment and when the defects, after being thoroughly cleaned out to sound metal, are judged not to affect the strength, use, or machinability of the castings. Distinctive marks shall be placed around welds prior to shipment of the casting. No welding will be permitted on steam piping or any casting subjected to steam pressure. Shrinkage cracks shall not be welded without specific authority of the purchaser.

(d) Castings shall be annealed in a properly constructed pit or furnace, and they shall be held at the treatment temperature at least long enough for each casting to be uniformly heated throughout its mass. They shall not be removed from the furnace until they have been cooled down to a temperature of about $700^{\circ} \mathrm{F}$. Rapid cooling of castings, or any further heat treatment other than reannealing, shall not be undertaken without specific authority from the purchaser.

(e) All castings shall be annealed so that the fracture of any part shall show to the eye a fine grain structure. If, in the opinion of the inspector, a casting has been improperly heat treated, he may require it to be retreated.

$(f)$ Castings shall be well cleaned for final inspection by sand blasting, tumbling, pickling, or other approved process.

\section{DETAIL REQUIREMENTS}

4. (a) Castings shall conform to the chemical and physical requirements shown in Table 1.

TABLE 1

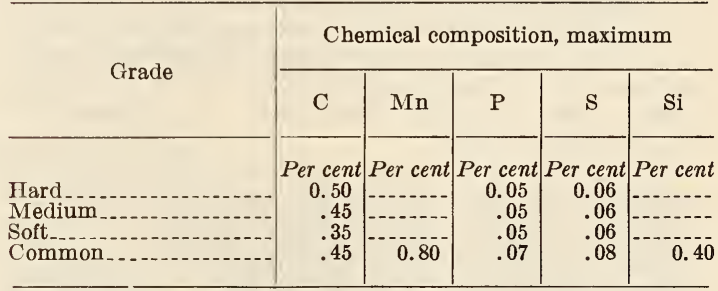

(Tensile properties for hard, medium, and soft grades are the same as given in A. S. T. M. specification A27-24. See 611.41, p. 707.)

For the special grade of castings chemical composition and physical requirements shall be as specified in detail specifications, contract, or purchase order.

For the common grade of castings physical requirements are not specified.

(The following cold bend-test requirements apply.)

Cold bend test

\begin{tabular}{|c|l|}
\hline \multicolumn{1}{|c|}{ Grade } & \multicolumn{1}{|c|}{ Angle of bend } \\
\hline Hard & No bend test. \\
\hline Medium & $90^{\circ}$ \\
\hline Soft & $120^{\circ}$.
\end{tabular}

(b) An analysis of each melt shall be furnished by the contractor, showing the percentage of the elements specified and, in addition, any alloying metal present in an amount greater than two-tenths of 1 per cent. This analysis shall be made from drillings taken at least one-quarter inch beneath the surface of the metal.

\section{METHOD OF INSPECTION AND TESTS}

5. (a) The inspector shall have free entry at all times to all parts of the contractor's works which concern the manufacture of the castings ordered. The contractor shall afford the inspector free of cost all necessary facilities to determine if all steps in the manufacture, such as melting, pouring, annealing, 
etc., have been properly performed, either by exhibiting records, supplying data, or both, as requested.

(b) All castings shall be submitted and inspected in the green.

(c) The physical properties of common castings will not be determined unless the inspector has reason to doubt that the castings are of a quality suitable for the purpose for which intended.

(d) Large castings, when required by the inspector shall be suspended and hammered all over with a hammer weighing not less than $7 \frac{1}{2}$ pounds. If cracks, defects, or unsoundness are indicated which may render the castings unfit for service, such castings shall be rejected.

(e) Whenever practicable, coupons from which tension and bend test specimens may be machined shall be cast on the body of a casting. Their number and location shall be such as thoroughly to exhibit the character of the metal throughout the casting, but the responsibility for furnishing sufficient test, specimens shall rest with the contractor. Coupons shall not be detached from the casting until it has received its final heat treatment and until properly stamped and authorized by the inspector. The unauthorized chilling of test coupons shall cause rejection of the material represented.

$(f)$ When cast-on coupons are not used, sufficient coupons shall be cast with and gated to castings to represent the lot, or the particular casting when necessary. When this is impracticable, the inspector may authorize: (1) Separate coupons to be cast from the same ladle as the castings they represent, and (2) additional castings to be made from which test specimens may be machined. In all cases separately cast coupons shall bear the inspector's identification stamp.

(g) At least one tension and one bend test will be required from each casting the estimated weight of which is 500 pounds or more, unless otherwise specified by the department concerned.

(h) At least two tension tests and one bend test will be taken from each lot of castings estimated to weigh less than 500 pounds, unless otherwise specified by the department concerned. A lot shall consist of: (1) Castings made from the same openhearth, converter, or electric melt treated in the same furnace charge, and (2) castings made from the same pouring of a ladle containing a number of crucible heats, and heat treated in the same furnace charge.

(i) Tension-test specimens shall conform to the dimensions of type 1 specimen shown in Figure 1 of Federal specifications for metals No. 339a. (See 600.0 , p. 3.) The ends shall be of a form to fit holders of the testing machine in such a way that the load shall be axial.

(j) Bend-test specimens shall be about 6 inches long and have a section 1 inch by one-half inch with long edges rounded to a radius of not over onesixteenth of an inch. The specimen shall be bent cold around a pin 1 inch in diameter to the angle specified in the table above without cracking on the outside of the bent portion.

(k) When any test specimen shows defective machining or obvious lack of continuity of metal, it may be discarded and replaced by another specimen selected by the inspector. The nature of the flaw or lack of continuity of the metal, when deemed characteristic of the material under inspection, shall be taken into consideration when determining the acceptability of the material. (l) If the percentage of elongation of a tensiontest specimen is less than that specified and any part of the fracture is within one-quarter inch of the scribe marks made on the specimen before testing or outside the scribe marks, the inspector shall select another test specimen to replace it.

(m) Castings in which injurious defects are revealed subsequent to acceptance shall be rejected.

( $n$ ) Check analyses may be made by the inspector, or through him, by any Government laboratory or other designated representative and without cost to the contractor. The chemical composition thus determined shall conform to the requirements specified and be in substantial agreement with the contractor's analysis regarding the presence and amount of alloys. Drilling for analysis may be taken from broken test specimens or from a casting representing a melt. Castings shall be drilled in such a manner as not to impair their usefulness and drillings taken within one-fourth inch from the surface shall be discarded.

(o) Any casting may be subjected to macroscopic and microscopic examination.

\section{PACKING AND MARKING}

6. (a) Castings shall be marked with the pattern or mark number, and where possible with the melt or lot number, in a position on the casting where they will not be machined off.

(b) If special packing be necessary to secure the acceptance of castings for safe transportation at the lowest rate to the point of delivery, such packing shall be provided by the contractor without additional expense to the purchaser.

(c) All shipments shall be marked with the contract or order number, name of manufacturer, and contents.

\section{ADDITIONAL INFORMATION}

7. (a) Common-grade castings are intended for commercial fittings where structural strength is not involved. They ordinarily would have the following minimum values: Tensile strength, 55,000 pounds per square inch; yield point, 25,000 pounds per square inch; elongation in 2 inches, 15 per cent; reduction of area, 25 per cent.

(b) Soft-grade castings are intended for parts subject to tensile, vibratory, or shock stresses.

(c) Medium-grade castings are intended for the same general purpose as the soft grade, but where greater strength is required.

(d) Hard-grade castings are intended for all inportant parts subject to crushing stresses or surface wear only.

(e) Special-grade castings are intended for cases where specific properties not covered by the other grades are required.

$(f)$ All patterns furnished by the purchaser shall be properly cared for and when baing returned shall be suitably boxed or crated by the contractor to prevent damage or breakage. The patterns shall be examined by the inspector before shipment and report made if found in unsatisfactory condition.

\section{GENERAL SPECIFICATIONS}

8. General specifications for metals, Federal Specification No. 339a (current designation: QQ-M-151), in effect on date of issuance of proposals, shall form a part of this specification. (See 600.0, p. 3.) 


\section{AMERICAN RAILWAY ASSOCIATION, MECHANICAL DIVISION, STANDARD BRAKE- BEAM GAGES, 1930}

BRAKE-BEAMS GAGES

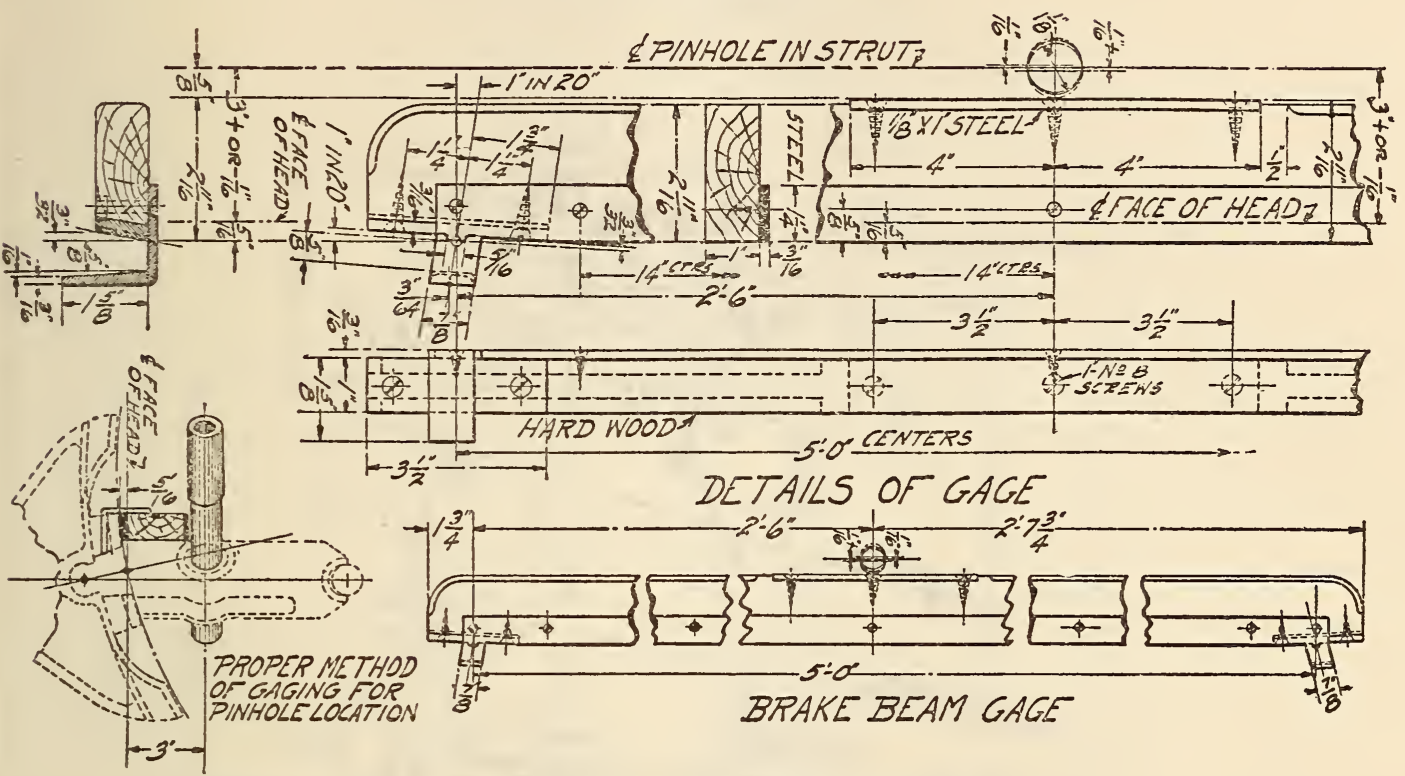

Figure 492.-Brake beam gage

AMERICAN RAILWAY ASSOCIATION, MECHANICAL DIVISION, STANDARD BRAKE-BEAM SPECIFICATIONS AND TESTS, 1917

\section{BRAKE BEAMS}

1. Scope.-This specification covers all complete brake beams for M. C. B. equipment cars.

\section{MANUFACTURE}

2. Material.-The tension member shall conform to the M. C. B. specifications for mild steel.

3. Heads. - The heads and struts, where struts are of malleable iron, shall conform to M. C. B. specifications for malleable iron.

\section{PHYSICAL PROPERTIES AND TESTS}

4. Proof tests. - The manufacturer shall give each beam a proof-test load equal to the deflection load, as shown in Table 1, before offering it for inspection and test.

5. Deflection-measurement tests.-The test bedm will be mounted in the testing machine in accordance with section 8 and a load applied equal to deflection loan as shown by Table 1 and then reduced to 500 pounds and the deflection instrument set at zero. The deflection load will then be reapplied, and the deflection shall not be more than 0.07 inch; in event this deflection is exceeded, no further tests will be made, but the lot will be rejected and held in accordance with paragraphs $15(b)$ and 17.

6. Set test.-After the deflection measurements have been completed, the beam will be loaded to the set load shown in Table 1 and then released to 500 pounds, when the beam shall not show a set greater than 0.01 inch.
7. Total-deflection test.-The brake beam shall stand a total movement of the head of the test machine of not less than 2 inches without failure at any point. The speed of the head of the machine shall be 1 inch per minute.

Table 1

\begin{tabular}{|c|c|c|c|}
\hline M. C. B. beam No.- & $\begin{array}{c}\text { Deflection } \\
\text { load }\end{array}$ & Set load & Ratio \\
\hline $\begin{array}{l}1 \\
2 \\
2 \\
2 \\
4\end{array}$ & $\begin{array}{r}\text { Pounds } \\
6,500 \\
12,000 \\
15,000 \\
18,000 \\
24,000 \\
28,000 \\
36,000\end{array}$ & $\begin{array}{r}\text { Pounds } \\
14,000 \\
24,000 \\
27,000 \\
30,000 \\
36,000 \\
39,200 \\
48,000\end{array}$ & $\begin{array}{l}47 \\
50 \\
55 \\
60 \\
66.7 \\
71.4 \\
75\end{array}$ \\
\hline
\end{tabular}

8. Machine.-The brake-beam testing machine shall have suitable castings to represent the tread of the car wheels so that the beam can be mounted with the load applied at the pinhole in strut.

9. Test specimen.-The test specimen shall consist of a complete brake beam and may, at the option of the inspector, be either right or left hand.

10. Number of tests. - The manufacturer shall have the brake beams filed according to size, regardless of requisition numbers, and sampled as follows: If the beams in a pile are either right or left hand, the lot shall consist of 501 beams or less, and if the pile consists of both right and left hand beams the lot shall consist of 502 beams; but in either case only one beam will be selected for test. The extra beam in the lot of 502 will be returned to stock. If the pile contains more than 501 of either right or left hand beams, or 502 of both right and left hand beams, and not more than 1,002 or 1,004 , two samples will be taken, and so on for each additional 500 beams. 


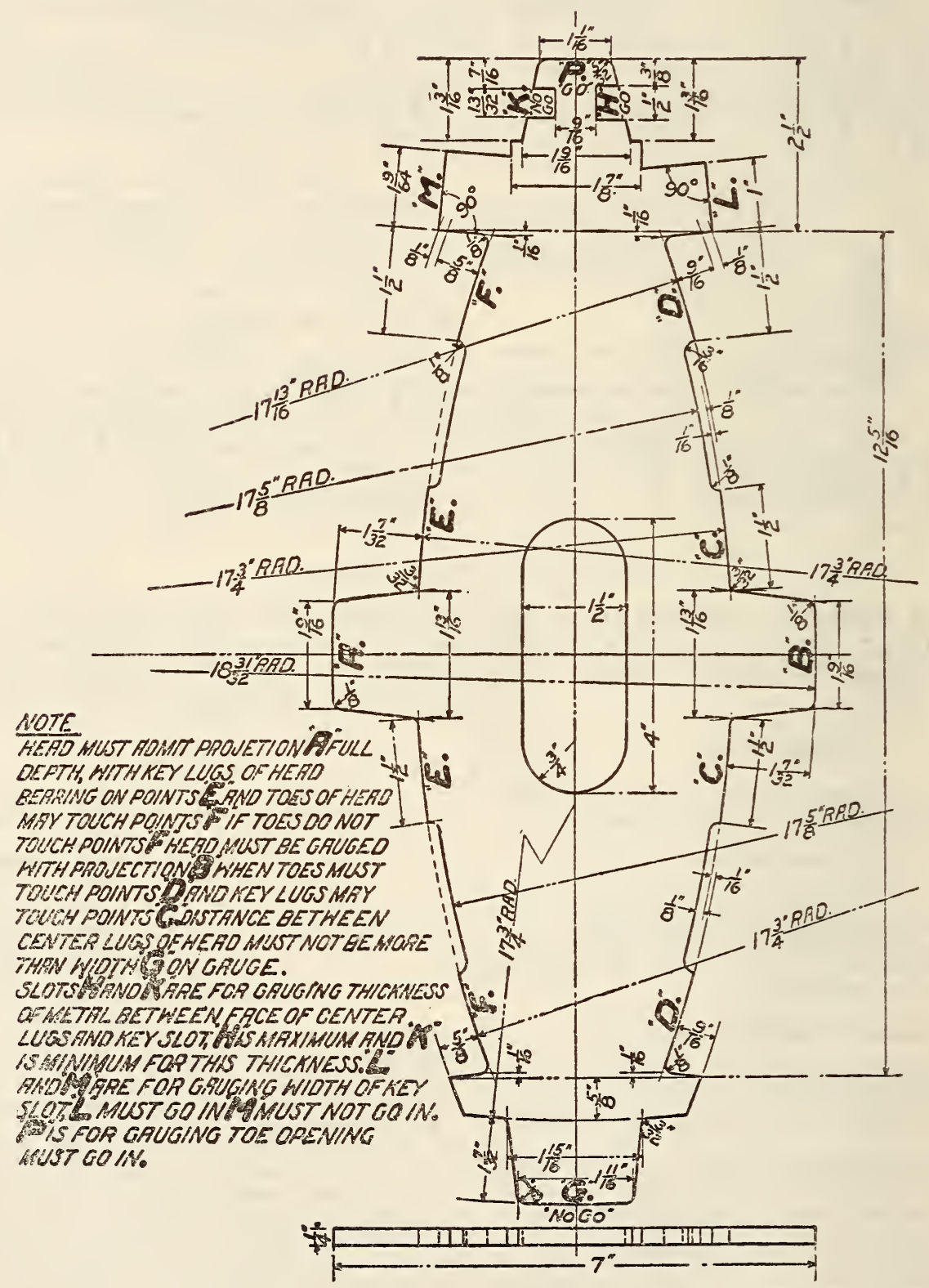

Figure 493.-Brake beam gages-brake head gage 


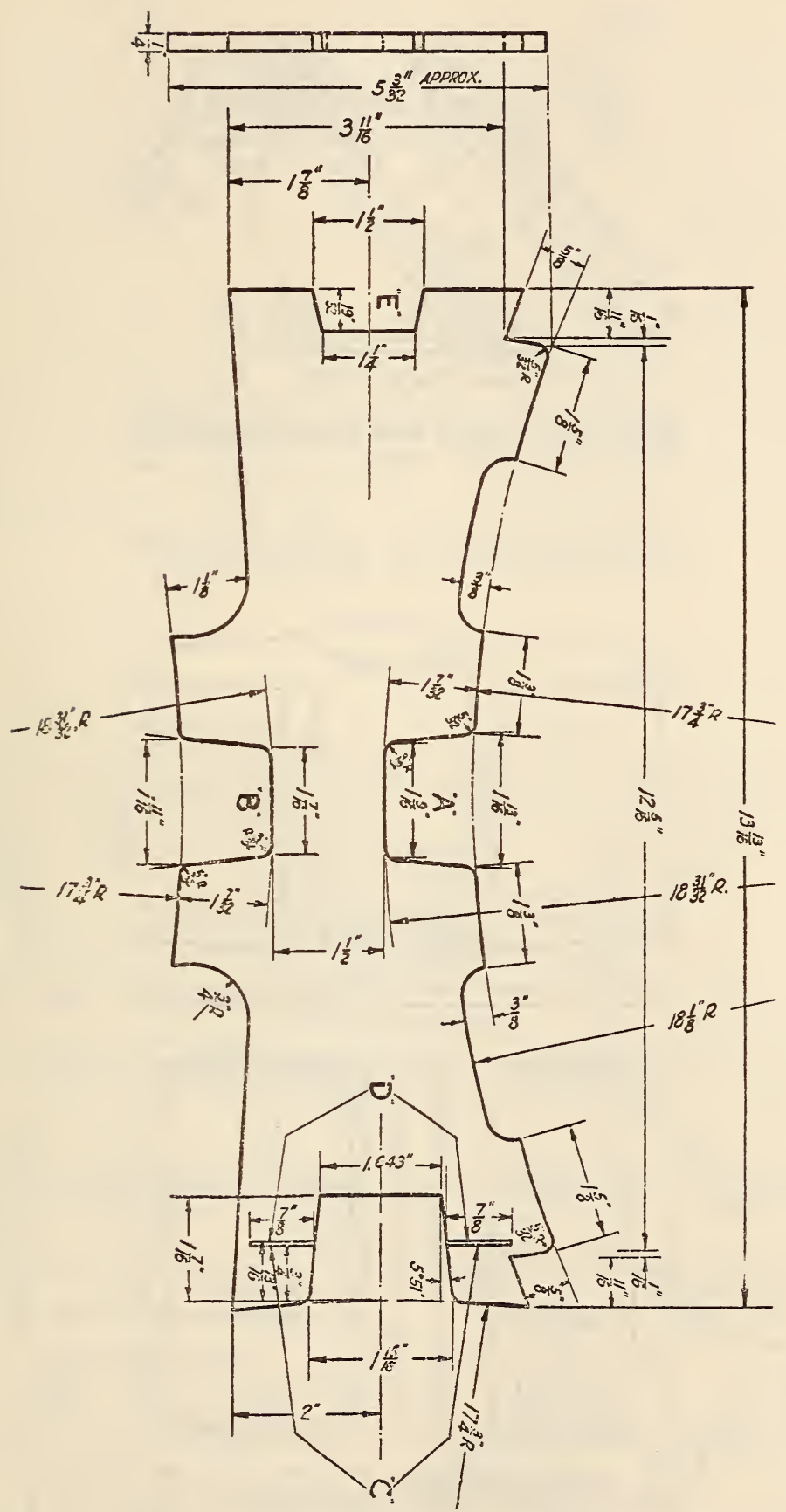

FIgURE 494.-Brake shoe gage

" $A$ " - $A$ " is maximum and " $B$ " is minimum width for center lug. " $A$ " " $A$ " is minimum maximum af slot in center lug, measured from Line " $C$ is minimum height of slot in center lug, measured from back of " $D$ " $D$ " is maximum height of slot in center of lug, measured from back of shoe.

" $E$ " is maximum thickness of end lugs on back of shoe. 


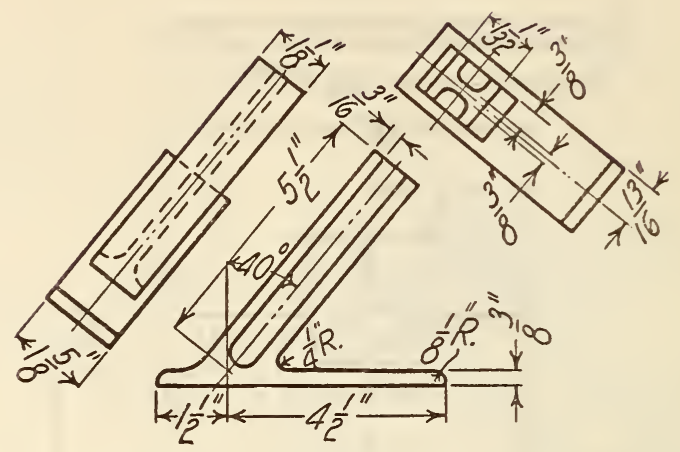

FiguRe 495.-Brake beam gages-angle gage for strut
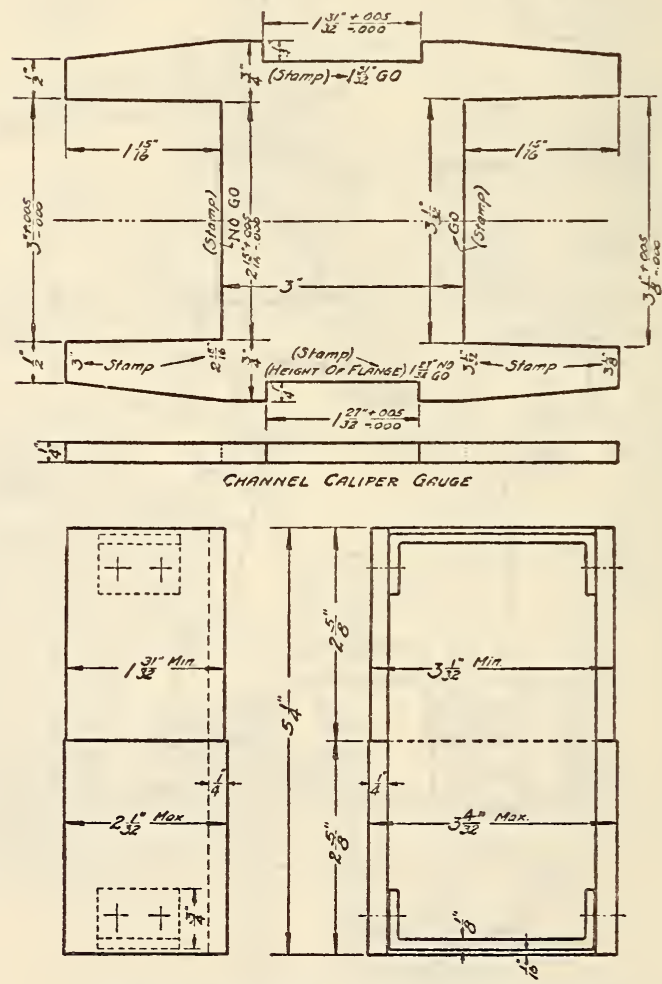

CanNeL OPENING GavGe FOR HEAD \& STRUT

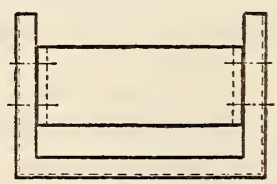

FIGURE 496.-Brake beam gages-channel caliper gage, and channel opening gage for head and strut 

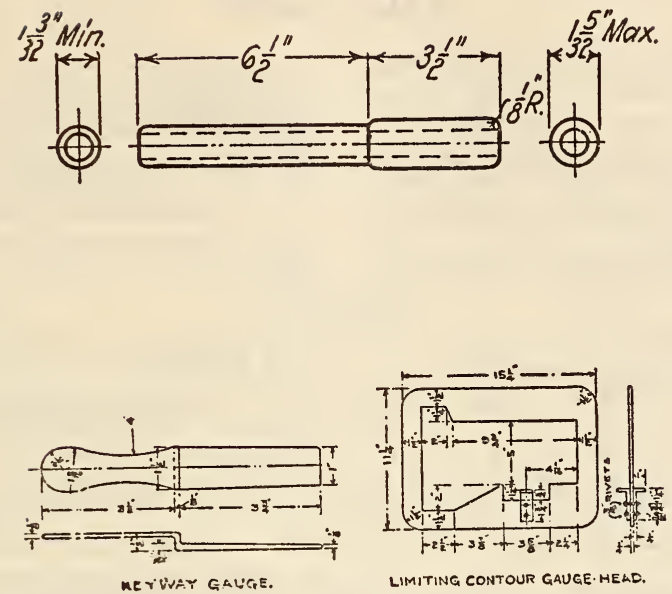

Figure 497.-Brake beam gages-lever pin hole gage, keyway gage, and limiting contour gage head

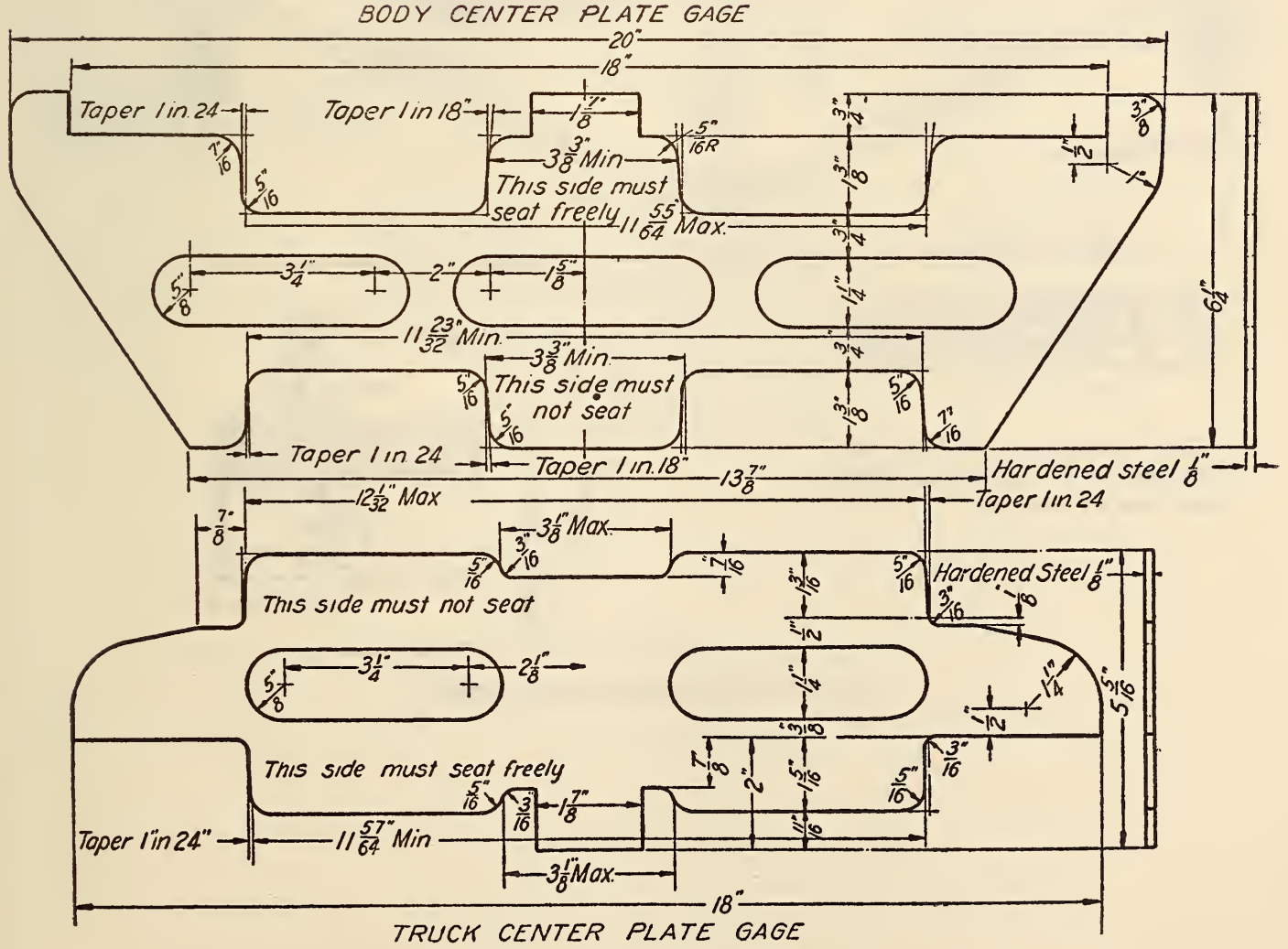

Figure 498.-Center plate gages 
AMERICAN RAILWAY ASSOCIATION, MECHANICAL DIVISION, STANDARD BRAKE BEAMS, LIMITING OUTLINE FOR, 1920; BRAKE BEAMS, COMPLETE, 1928

\section{BRAKE BEAMS}

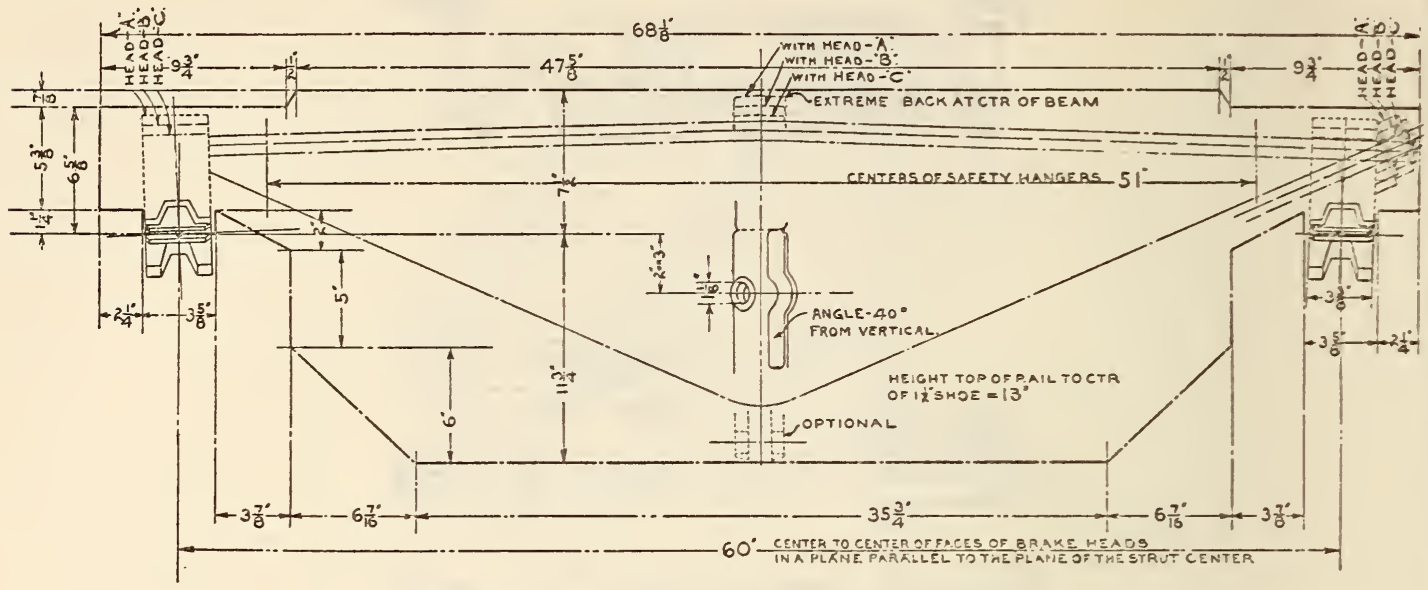

Figdre 499.-Brake beams, limiting outline for

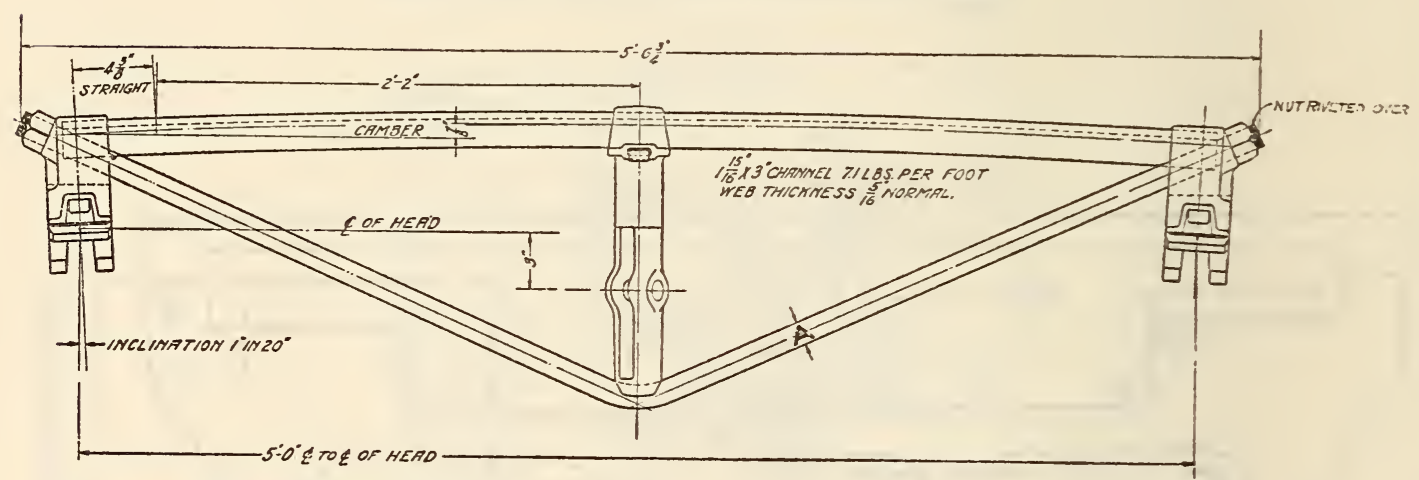

\begin{tabular}{|c|c|c|c|}
\hline $\begin{array}{l}\text { BEAM } \\
\text { NUMBER }\end{array}$ & Rog & $\begin{array}{l}\text { BEAM } \\
\text { CAPACIT }\end{array}$ & THREAOING OF ROD \\
\hline 2 & 16 & $12000^{2}$ & LS.5TD If -7 THDS. $2 \xi \angle O N G$ \\
\hline $2+$ & 18 & $15000^{\prime \prime}$ & 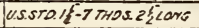 \\
\hline
\end{tabular}

Note.-A tolerance of $1 / 8$ in. plus or minus in the camber of brake beam may be allowed.

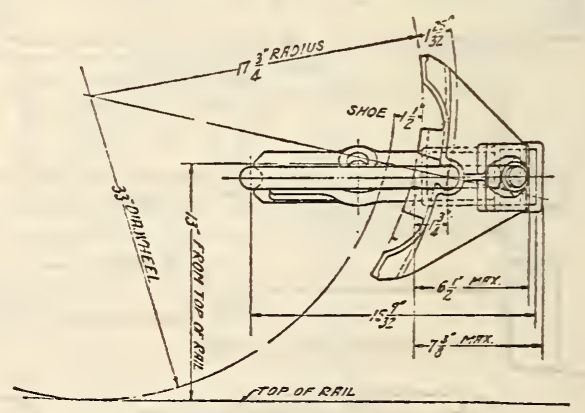

FigURe 500.-Brake beams, complete 
III. PERMISSIBLE VARIATIONS

11. Gage.-Brake beams shall conform to the dimensions shown below in A. R. A. brake-beam gages.

\section{WORKMANSHIP AND FINISH}

12. Workmanship. - The beam shall be made in a workmanlike manner and be free from injurious defects.

13. Finish.-(a) Brake beams shall be free from rust, scale, paint, or any covering that will hide defects.

(b) After the beams are inspected and marked, they shall be given cine coat of a quick-drying, black, protective paint.

\section{MARKING}

14. Marking.-The designation "A. R. A." and the capacity of the beam as "No. 2 ," "No. $2+$," etc., shall be marked on the center strut where they can readily be seen when the beam is in place on the car. The letters and figures shall be not less than five-eighths inch high, being one-eighth inch in relief on a cast strut and plainly stamped on a forged strut.

\section{INSPECTION AND REJECTION}

(Inspection and rejection conform to A. R. A. specifications for galvanized sheets (see 604.32, p. 243) except in the following instances:)

15. Inspection.-(b) When the manufacturer's testing facilities are, in the judgment of the purchaser, adequate, tests shall preferably be made at the manufacturer's laboratory. The purchaser may, however, have tests made at his own or other laboratories, providing the facilities are adequate, and if the manufacturer desires he may witness such tests.

16. Rejection.-Material or completed beams which, subsequent to above tests at the manufacturer's plant or elsewhere, and their acceptance, develop weak spots or imperfections, or fail to pass any one of the tests herein required, such tests to be made within 30 days of the reccipt of the material by the purchaser, will be rejected and shall be replaced by the manufacturer at his own expense.

\section{AMERICAN RAILWAY ASSOCIATION, MECHANICAL DIVISION, STANDARD BRAKE BEAMS, STRUT, 1908}

BRAKE BEAMS, STRUT

sioring of Srrut; Letters ans

Numbers to be Not less Than $5^{\circ}$

High Mark Strut No. I or No.2 Etc.

According, to its Casacity.

For Malleable Iron Providing Sare

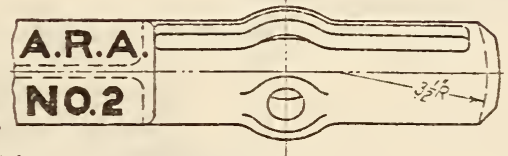

Strength Strut and Fin Hole Beariry

Area is 'Furnisher'

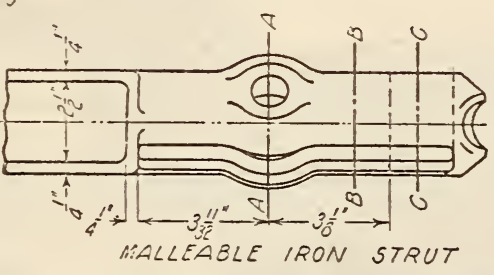

FigURE 501.-Brake beams, strut

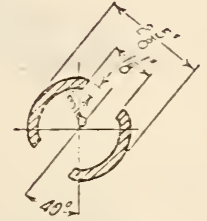

SECTION B-E

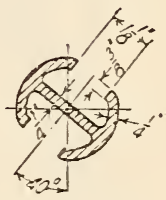

SECTION C-C

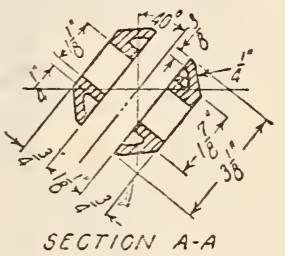


611.43 RAILWAY BRAKE SHOES, CAST STEEL AND CAST IRON.

AMERICAN ELECTRIC RAILWAY ENGINEERING ASSOCIATION, STANDARD DESIGN OF BRAKE SHOES, BRAKE-SHOE HEADS, BRAKE-SHOE KEYS, AND GAGES, E1-27, 1927

BRAKE SHOES, HEADS, KEYS, AND GAGES

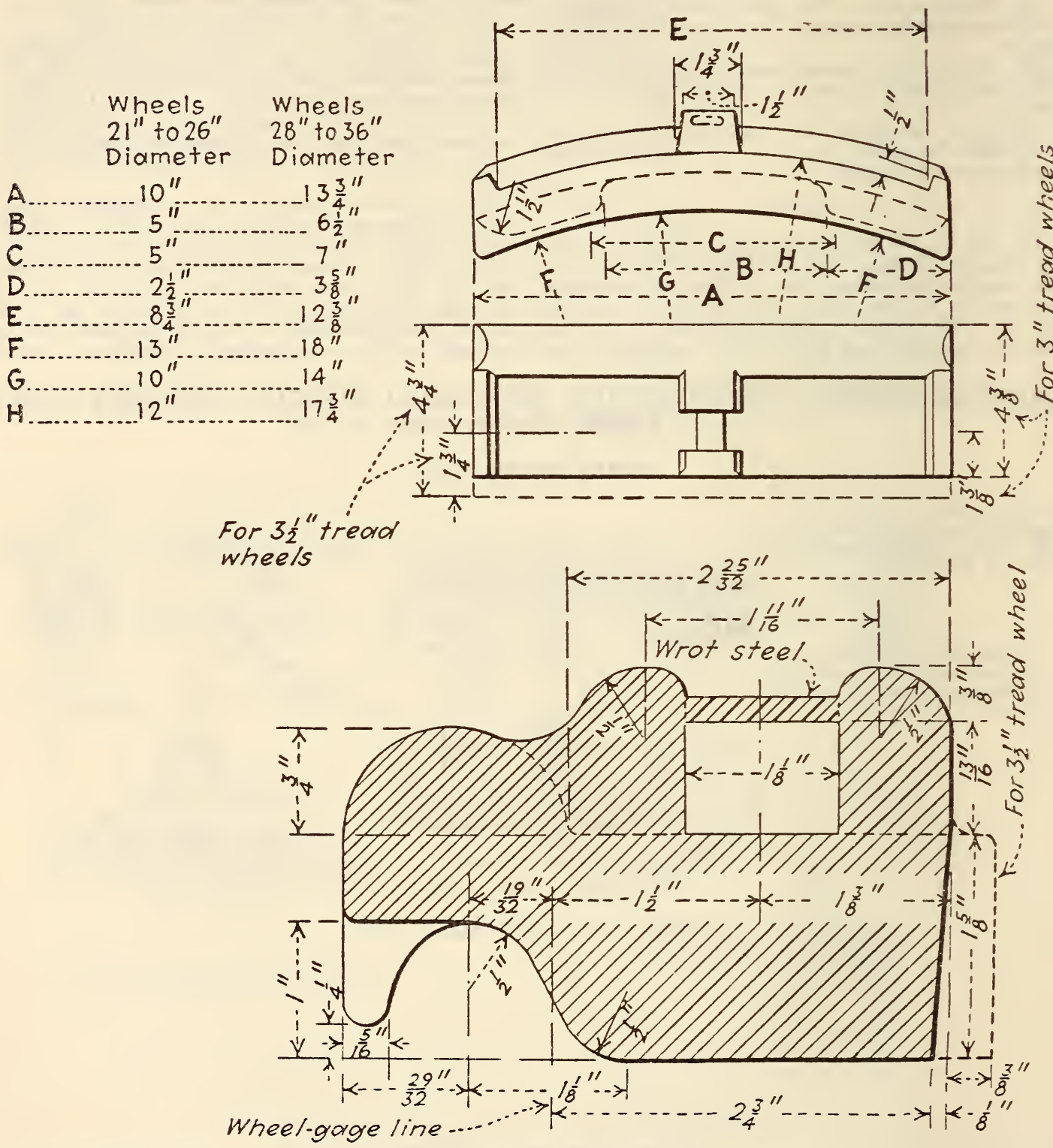

Figure 502.- Standard brake shoes for 3 and $3 \frac{1}{2-i n c h}$ tread wheels with contours $A 3, A 31 / 2, B S$, and DS and diameters 21 to 36 inches 

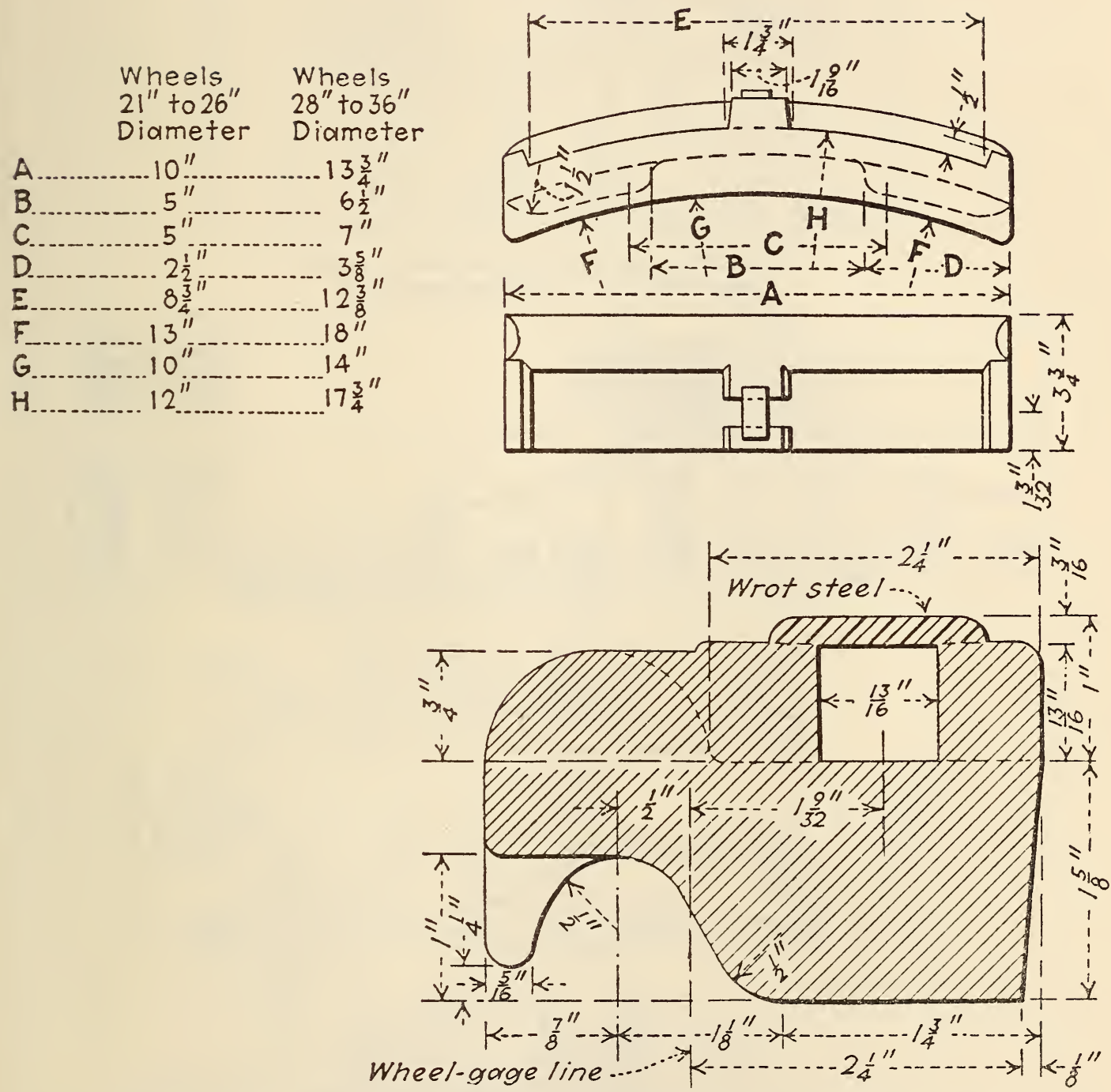

FIGURE 503.-Standard brake shoes for 21/2-inch tread wheels with contours B21/2, C21/2 and D21/2 and diameters 21 to 36 inches 


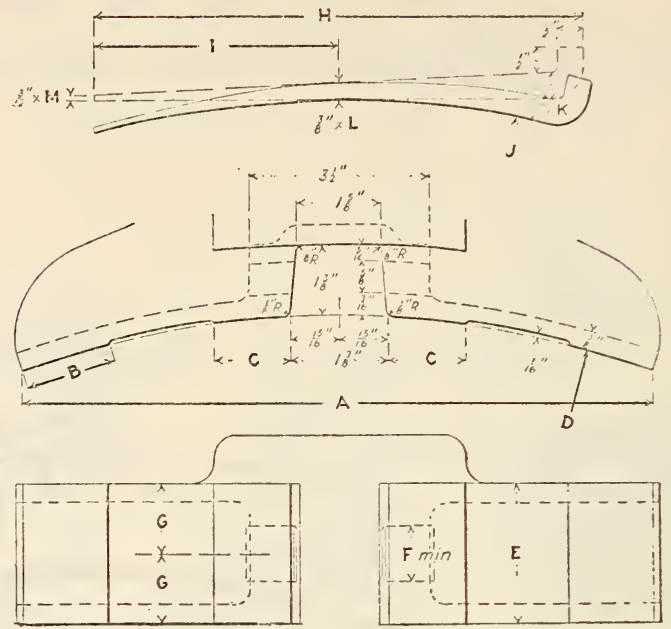

FIgURE 504.- Standard brake shoe heads and keys for A. E. R.E. A. standard brake shoes

\begin{tabular}{|c|c|c|c|c|c|c|}
\hline 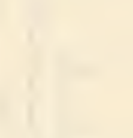 & 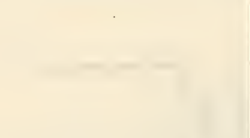 & $\begin{array}{l}\text { For } \\
\text { wheels } \\
28 \text { to } 36 \\
\text { inches } \\
\text { diameter }\end{array}$ & $\begin{array}{l}\text { For } \\
\text { wheels } \\
21 \text { to } 26 \\
\text { inches } \\
\text { diameter }\end{array}$ & & $\begin{array}{l}\text { For } \\
\text { wheels } \\
\text { with } 3 \\
\text { and } 31 / 2 \text {. } \\
\text { inch } \\
\text { tread }\end{array}$ & $\begin{array}{l}\text { For } \\
\text { wheels } \\
\text { with } 21 / 2- \\
\text { inch } \\
\text { tread }\end{array}$ \\
\hline Shoe head.. & $\left\{\begin{array}{l}\mathrm{A} \\
\mathrm{B} \\
\mathrm{C} \\
\mathrm{D}\end{array}\right.$ & $\begin{array}{r}\text { Inches } \\
121 / 4 \\
13 / 4 \\
11 / 2 \\
173 / 4 \\
91 / 2 \\
43 / 4 \\
181 / 2 \\
\end{array}$ & $\begin{array}{c}\text { Inches } \\
85 / 8 \\
11 / 4 \\
1 \\
12 \\
7 \\
31 / 2 \\
121 / 2 \\
\end{array}$ & 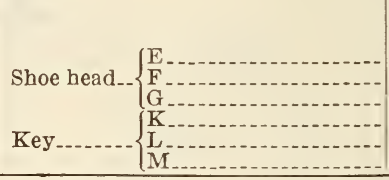 & $\begin{array}{c}\text { Inches } \\
211 / 10 \\
11 / 16 \\
111 / 32 \\
115 / 16 \\
7 / 8\end{array}$ & $\begin{array}{c}\text { Inches } \\
23 / 16 \\
13 / 16 \\
13 / 32 \\
3 / 4 \\
11 / 16 \\
5 / 8\end{array}$ \\
\hline
\end{tabular}

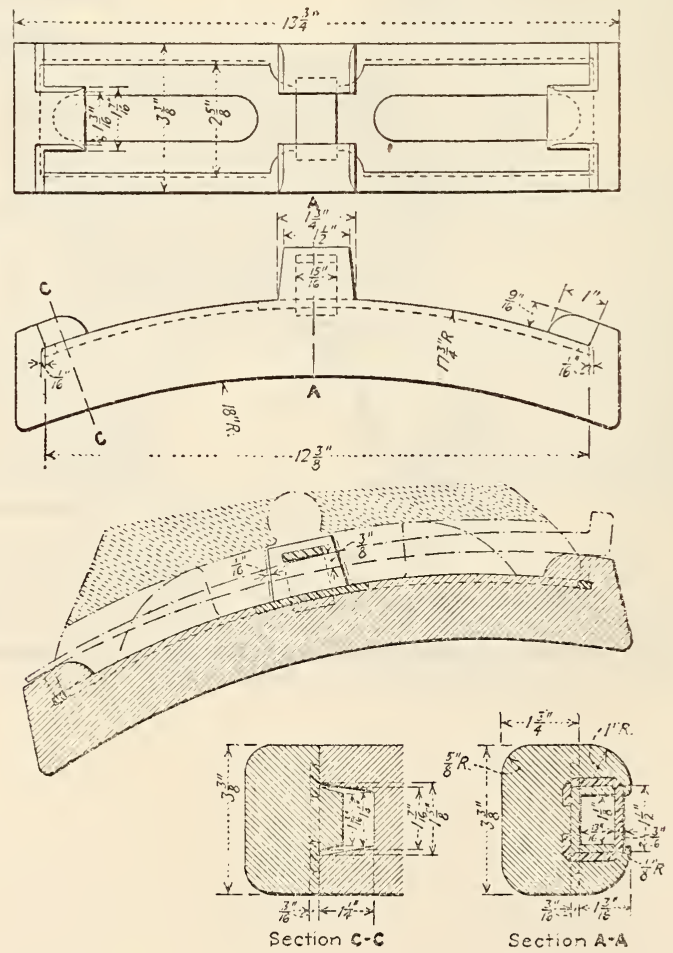

Figure 505.-Design of unflanged brake shoe for use in A. R. A. standard wheel contours adopted standard of the American Electric Railway Engineering Association in 1923 

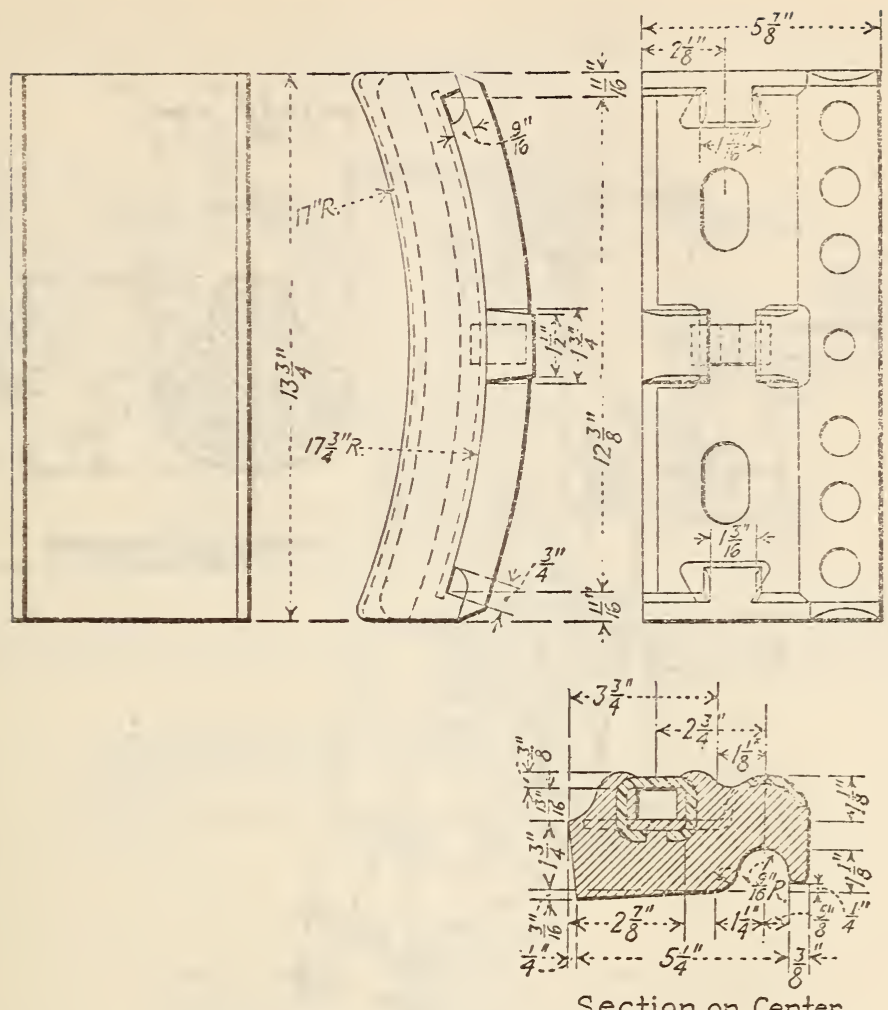

Section on Center

FIGUPi 506.-Design of flanged brake shoe for A. R. A. standard wheel contours adopted as standard of the American Electric Railway Engineering Association in 1913

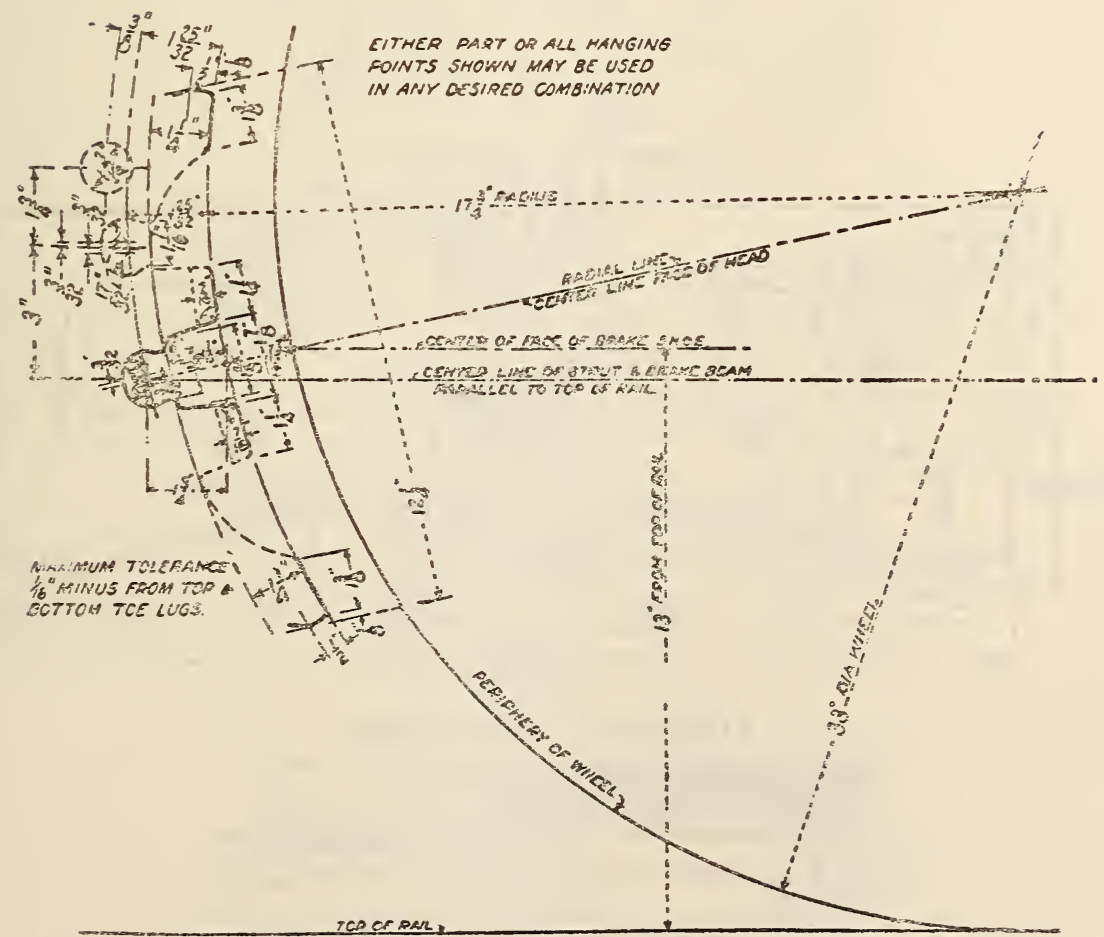

FIGURE 507.-Procedure for laying out drawing of brake head to fit brakeshoes shown in Figures 505 and 506 


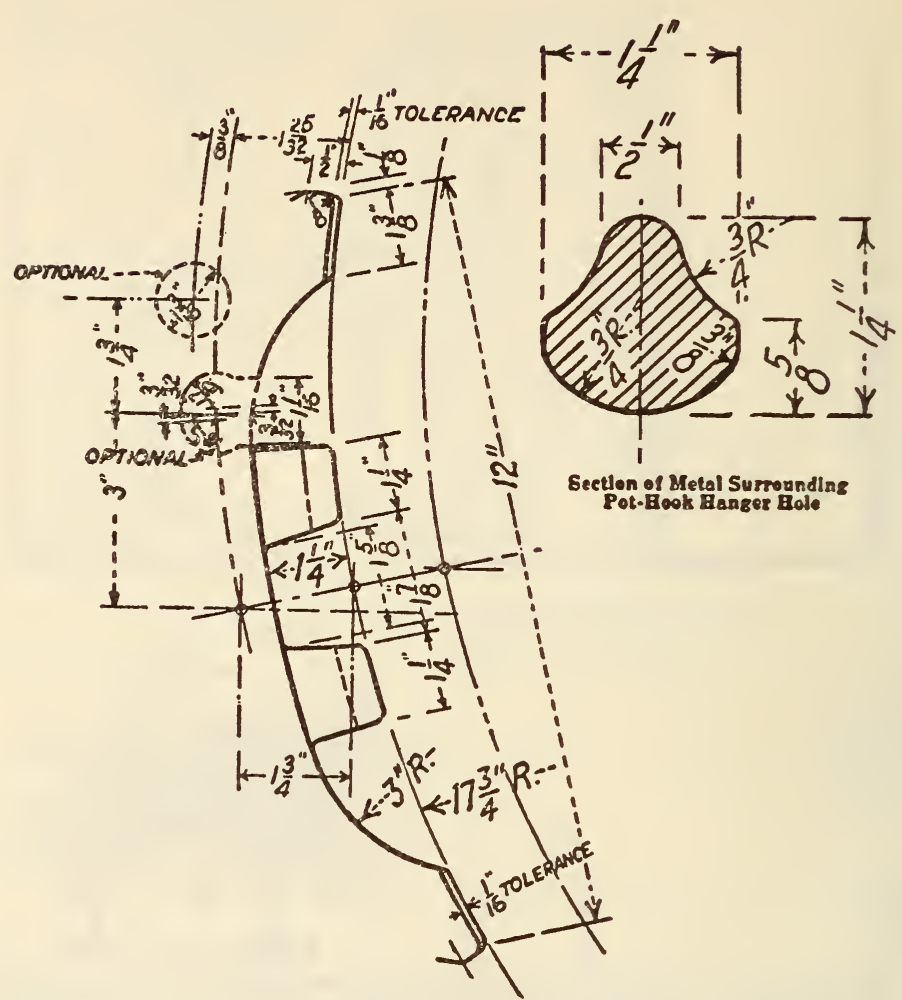

Frgure 508.-Dimensions of brake head to show attachment to brake shoes

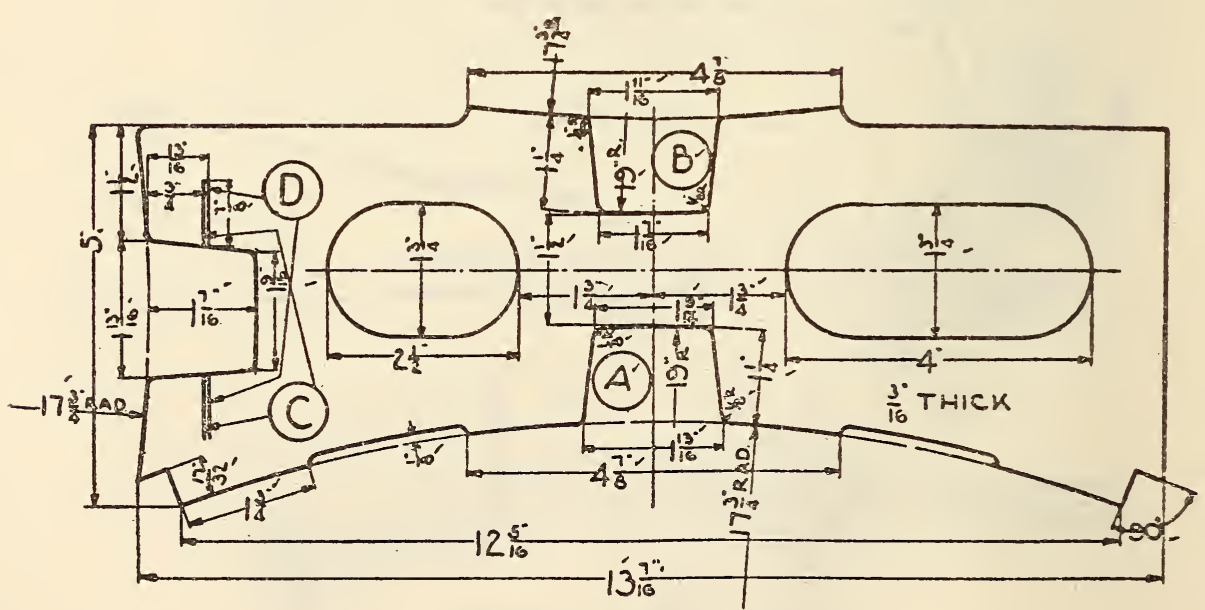

Figure 509.-Brake shoe gage

Side of gage marked $A$ must fit back of shoe.

$A$, is maximum width for center lugk.

$B$, is minimum width for center lug.

Line $C$ is minimum height of slot in center of lug measured from back of shoe.

Line $D$ is .

Line $D$ is maximum height of slot in center of lug measured from
back of shoe. 


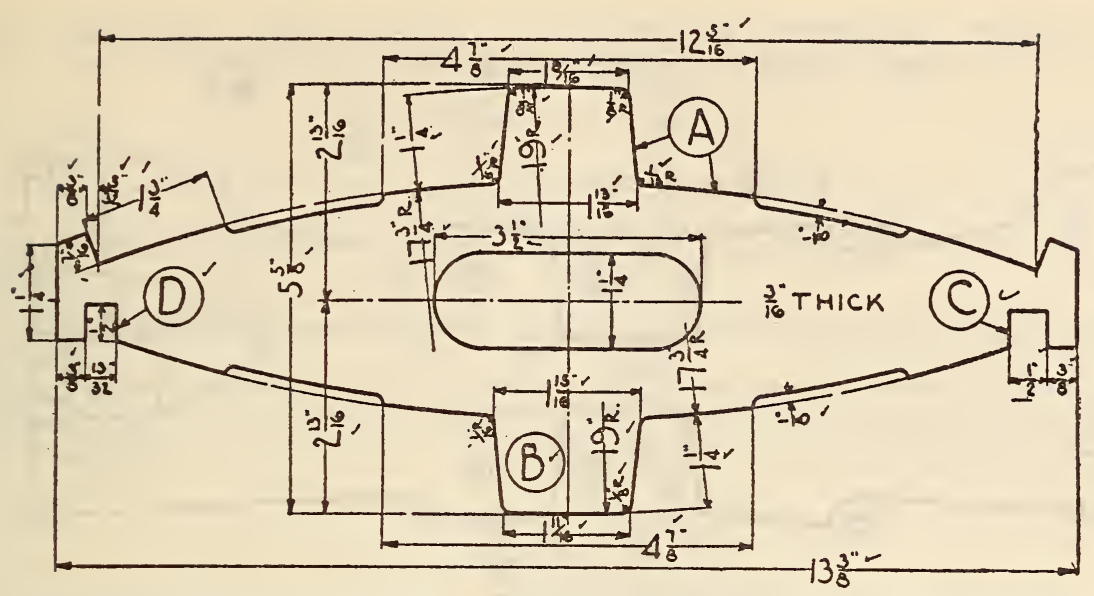

FIGURE 510.-Gages for brake heads and brake shoes for 3-inch tread wheels 33-inch diameter and over, and for 21/2-inch tread wheels 28-inch diameter and over

\section{Brake head gage}

$A$, head must admit side of gage to full depth and must fit radius. $B$, is maximum distance between lugs of head.

$C$, is maximum thickness of metal between face of center lugs and key slot.

$D$, is minimum thickness of metal between face of center lugs and key slot.

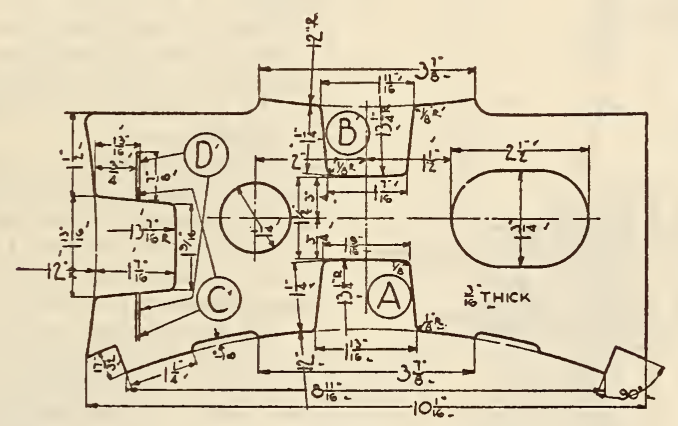

FigURe 511.-Brake shoe gage

Side of gage marked $A$, must fit back of shoe.

$A$ is maximum width for center lug.

Line $C$ is minimum height of slot in center of lug measured from

back of shoe.
Line $D$ is maximum height of slot in center of lug measured from back of shoe.

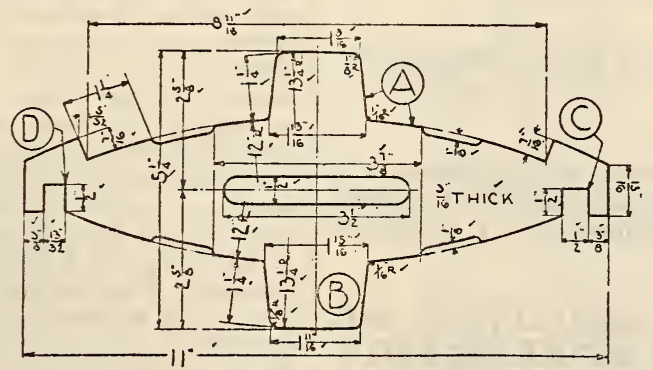

Figure 512.-Gages for brake heads and brake shoes for 3-inch tread wheels 26 -inch diameter and under, and for 21/2-inch tread wheels 26 inch diameter and under

\section{Brake head gage}

$A$, head must admit side of gage to full depth and must fit radius. $B$, is maximum distance between center lugs of head.

$C$, is maximum thickness of metal between face of center lugs and key slot.

$D$, is minimum thickness of metal between face of center lugs and key slot. 


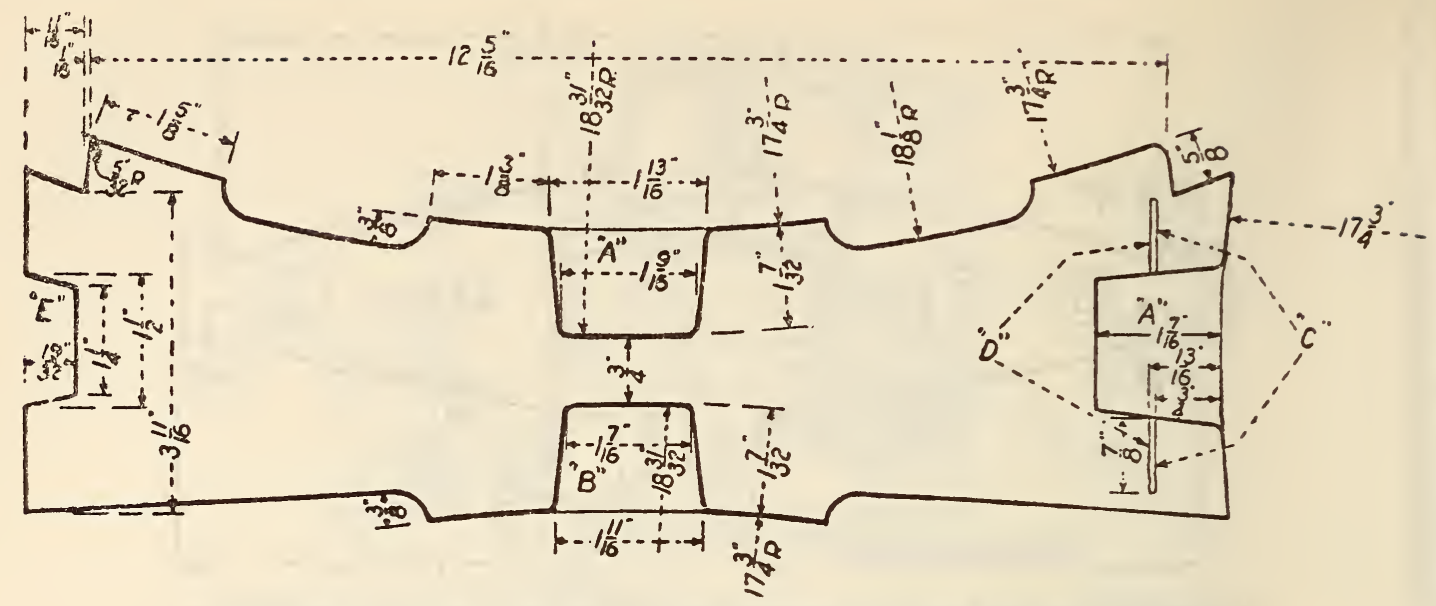

Figure 513.-Brake shoe gage for brake shoes shown in Figures 505 and 506

$A$ is maximum and $B$ is minimum width for center lug. Line $C$ is minimum height of slot in center lug, measured from back of shoe.

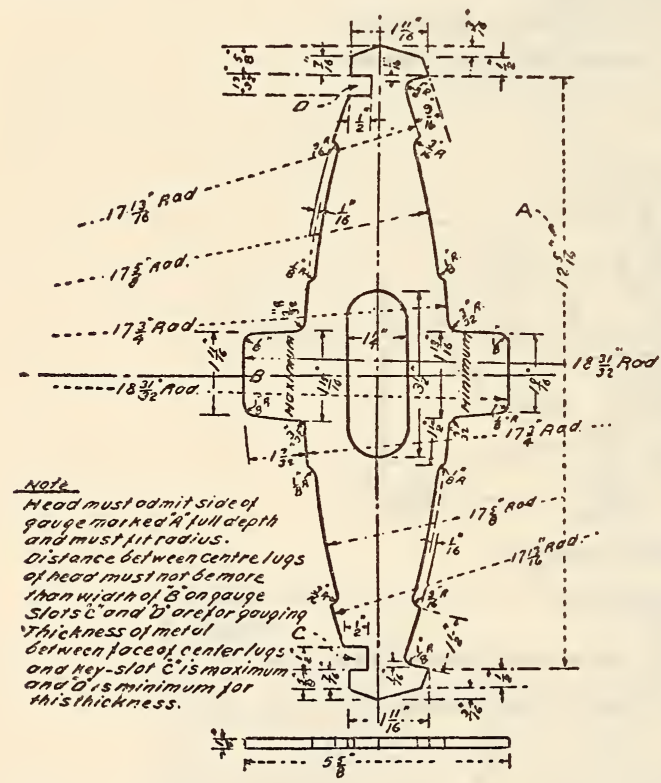

FIGURE 514.-Dimensions of brake head gage for brake head shown in Figures $50 \%$ and 508

(Demensions and illustration of brake-shoe key for attaching brake shoes to head are identical with those in the A. R. A. specification for reinforced brake shoes, given below.)

\section{AMERICAN RAILWAY ASSOCIATION, ME- CHANICAL DIVISION, STANDARD SPECI- FICATIONS FOR BRAKE SHOES, 1910}

\section{BRAKE SHOES}

\section{TESTS}

1. Kinds of test.-Shoes shall be tested for coefficient of friction and for wear upon the American Railroad Association testing machine, or upon a machine with equivalent characteristics.
Line $D$ is maximum height of slot in center of lug, measured from back of shoe.

Line $E$ is maximum thickness of end lug on back of shoe.

\section{COEFFICIENT OF FRICTION TEST}

2. Cast-iron wheel.-Shoes shall developupon the cast-iron wheel, in effecting stops from an initial speed of 40 miles per hour, a mean coefficient范 of friction of not less than:

(a) Twenty-two per cent when the brake-shoe pressure is 2,808 pounds.

(b) Sixteen per cent when the brake-shoe pressure is 6,840 pounds.

3. Steel-tired wheel.-Shoes shall develop upon the steel or steel-tired wheel, in effecting stops from an initial speed of 65 miles per hour, a mean coefficient of friction of not less than:

(a) Twelve and one-half per cent when the brakeshoe pressure is 6,840 pounds.

(b) Eleven per cent when the brake-shoe pressure is 12,000 pounds.

(c) No limitation is placed upon the rise in coefficient of friction at the end of the stop.

\section{SHOE-WEAR TEST}

4. Cast-iron wheel.-Shoe wear shall be determined upon the cast-iron wheel by making not less than 100 applications of the shoe to the wheel, under pressure of 2,808 pounds, and at a constant peripheral speed of the wheel of 20 miles per hour. At each application, the shoe shall remain in contact with the wheel during 190 revolutions of the latter and between applications the shoe shall remain out of contact during 610 revolutions of the wheel. Under these conditions, the shoe shall lose in weight not more than 0.8 of a pound for each $100,000,000$ foot-pounds of work done.

5. Steel-tired wheel.-Shoe wear shall be determined upon the steel or steel-tired wheel by making not less than 10 stops from initial speed of 65 miles per hour and under pressure of 12,000 pounds. Ten minutes shall intervene between successive applications of the shoe. Under these conditions the shoes shall lose in weight not more than 4.0 pounds from each $100,000,000$ foot-pounds of work:

Note.-When a shoe, not entirely metallic in its composition, is tested for wear, its actual loss in weight shall be increased in the ratio which the density of the cast iron bears to the mean density of the abraded parts of the shoe, in order to determine the weight which is to be compared with the specifications.

\section{GAGING AND DRAWING}

6. Gaging.-That the back of the shoe be made to conform to the gage shown in the Manual of Standard and Recommended Practice-Gages. 
AMERICAN RAILWAY ASSOCIATION, MECHANICAL DIVISION, BRAKE SHOE, REINFORCED, RECOMMENDED PRACTICE, 1922

\section{REINFORCED BRAKE SHOE}
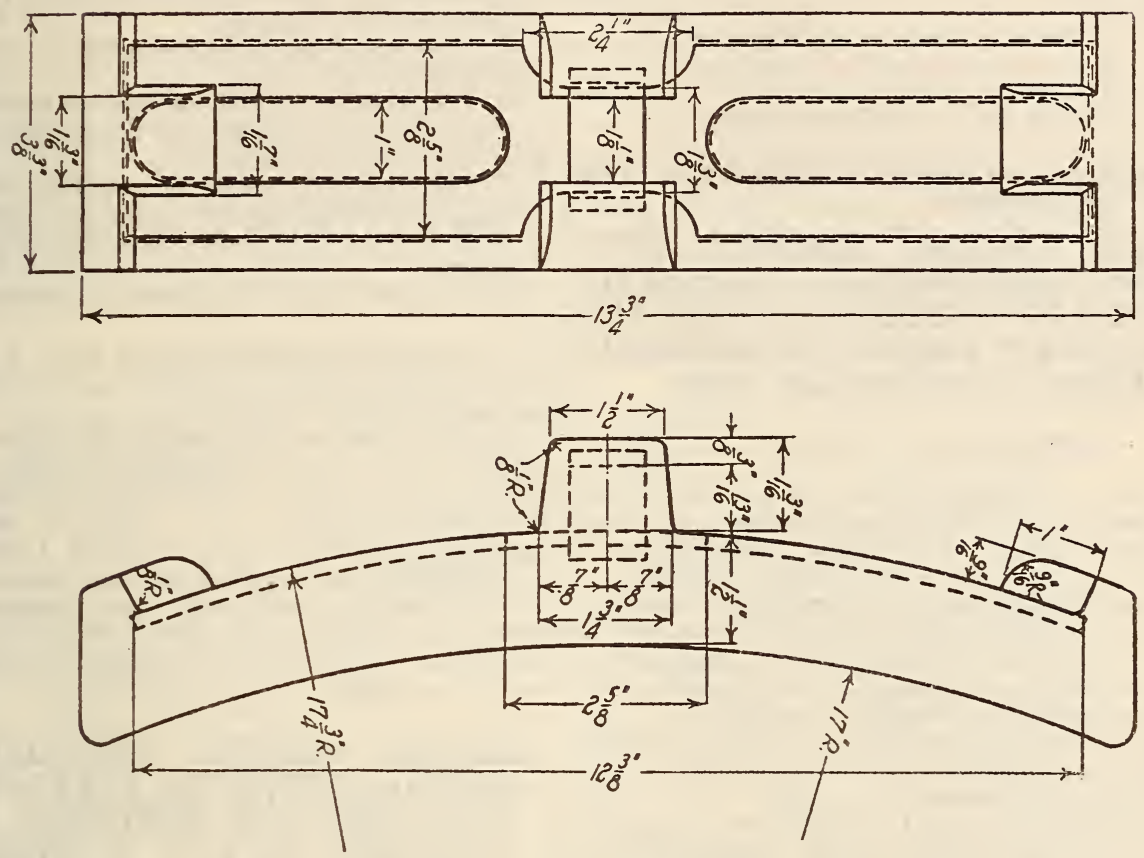

Figure 515.-Brake shoe, reinforced

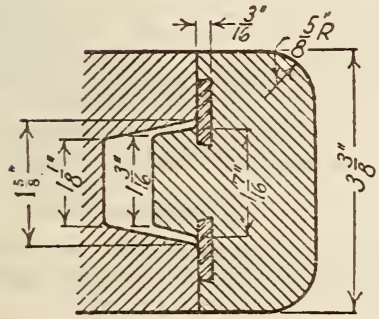

SECTION C-C

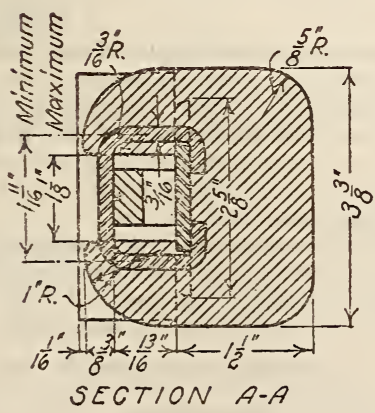

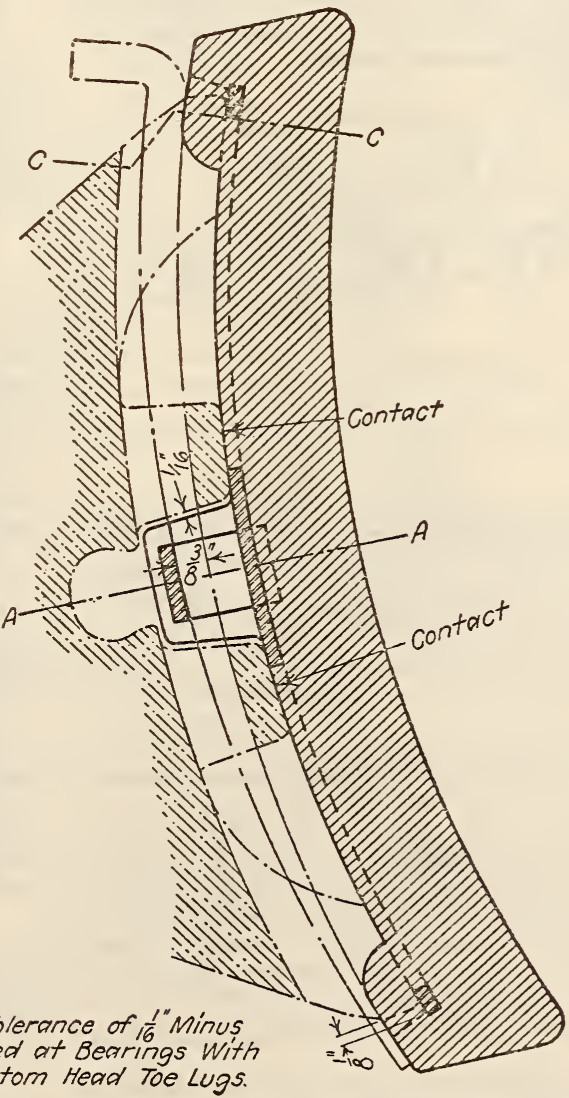

Maximum Tolerance of $\frac{1}{6}$ Minus to be Allowed at Bearings With

Top and Bottom Head Toe Lugs.

FIGURE 516.-Brake shoe, reinforced back 


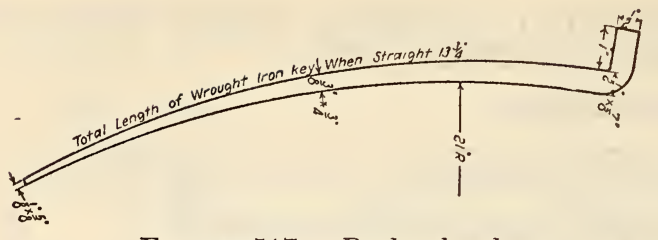

Figure 517.-Brake shoe key

\subsection{RAILWAY COUPLERS, CAST STEEL AND FORGED STEEL}

\section{AMERICAN ELECTRIC RAILWAY ENGI- NEERING ASSOCIATION, AUTOMATIC COUPLERS FOR INTERURBAN CARS AND RADIAL DRAFT RIGGING, RECOMMEND- ED SPECIFICATION E100-29, 1929}

\section{AUTOMATIC COUPLERS}

1. For use on interurban cars, a coupler of the vertical-plane type is recommended which has contour lines of knuckle and guard arm automatically to couple with standard steam railroad couplers.

2. The carrier supports for couplers when mounted on cars of standard height should be located as closely to the coupler head as possible, and should not allow vertical movement sufficient to cause un. coupling due to sudden changes of grade.

3. The length of the radial coupler measured from the center of the pocket pin to the pulling face must not be less than 58 inches.

It is advisable at present to maintain a $1 \frac{1}{2}$-inch link slot and coupling pin hole in the knuckle of automatic couplers.

4. All couplers must be made to couple automatically by impact and to uncouple without the necessity of going between the cars.

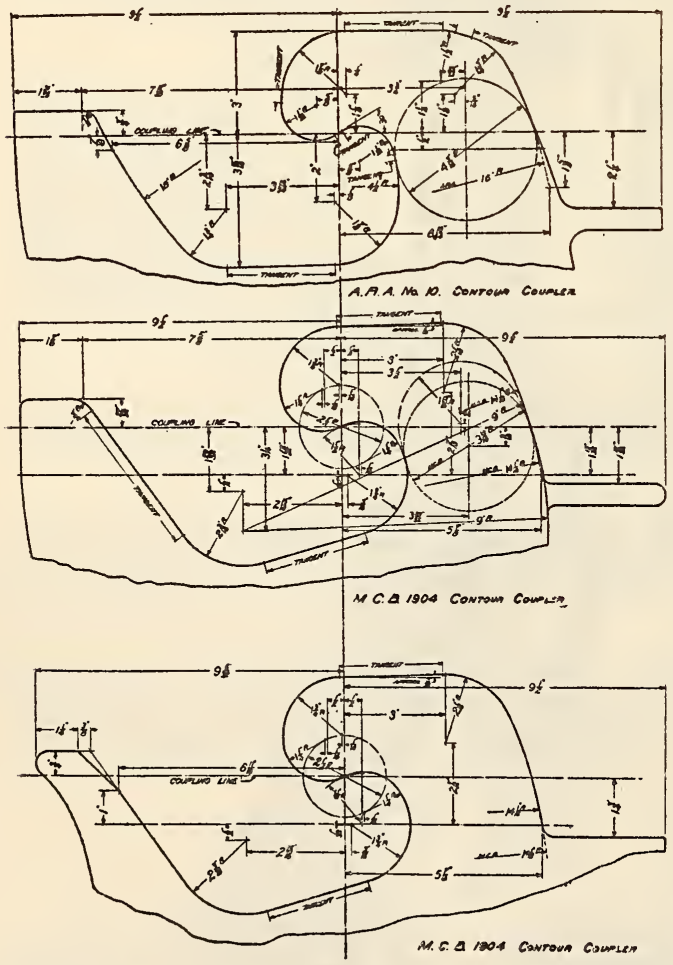

FigURE 518.-Details of guard arm and abutting wall for automcitic couplers
5. All couplers should be mounted to operate over 35 feet center radius track curves broken from the point of tangency.

6. There should be an arrangement to release and open the knuckle without requiring the operator to pass between the cars.

7. The face of the knuckle vertically should be 16 inches maximum.

8. The height of drawbar center should be $311 / 2$ inches minimum loaded, and $341 / 2$ inches maximum above the head of the rail.

9. The coupling center of couplers must have a minimum projection of 6 inches beyond buffer faces at any point between the working limits of the counlers.

10. Couplers should be placed on both ends of the car.

11. The coupler head should have a guard arm on one side, and an abutting wall on the other in accordance with Figure 518.

12. The condemning point for worn knuckles shall be $5 \frac{1}{8} 8$ inches as measured by A. R. A. gages shown on page 500 of 1926 Manual of the A. R. A. Mechanical Division, or C. E. R. M. M. A. defect gage, page 12, Figure 6, C. E. R. A. Code of Rules.

(Requirements covering process, annealing, chemical composition, ladle and check analyses, tension tests, and yield point are identical with those specified below for A. R. A. couplers, knuckles, locks, and other parts.)

AMERICAN RAILWAY ASSOCIATION, MECHANICAL DIVISION, STANDARD SPECIFICATIONS FOR PURCHASE AND ACCEPTANCE OF A. R. A. STANDARD D COUPLERS, KNUCKLES, LOCKS, AND OTHER PARTS, 1925

\section{COUPLERS, KNUCKLES, LOCKS}

NotE.-These specifications replace specifications for M. C. B. automatic couplers adopted in 1899 . When couplers other than type $\mathrm{D}$ are ordered under these specifications, they shall conform to the requirements for quality of steel, workmanship, and details of inspection. No tests of the couplers as a whole will be required.

1. Scope.-These specifications cover all cast steel for complete couplers and for repair parts.

\section{MANUFACTURE}

2. Process.-The steel shall be made by the openhearth or electric-furnace process and in accordance with the best foundry methods.

3. Annealing.-(a) Castings shall be allowed to become cool. They shall then be uniformly heated to the proper temperature to refine the grain and allowed to cool uniformly.

(b) Annealing lugs.-The location of the annealing lugs shall be as shown on Figures 519 and 520. These annealing lugs shall be cast with and attached to each casting when presented for inspection, and shall be removed by the inspector for the purpose of determining the character of the annealing.

(c) If, in the opinion of the inspector, a casting is not properly annealed, he may at his option require the casting to be reannealed.

(d) If, after annealing or reannealing, any casting is so much out of gage as to require heating in order to bring it within the gage limits, it shall be reannealed before it shall be accepted.

\section{CHEMICAL PROPERTIES AND TESTS}

4. Chemical composition.-The steel shall conform to the following requirements as to chemical composition:

Manganese, not over.

Per cent

Sulphur, not over 
5. Ladle analysis.-An analysis of each melt of steel shall be made by the manufacturer to deterinine the percentage of carbon, manganese, phosphorus, sulphur, and silicon. This analysis shall be made from drillings taken at least one-fourth inch beneath the surface of a test ingot obtained during the pouring of the melt. The chemical composition thus determined shall be reported to the purchaser or his representative when requested, and shall conform to the requirements specified in section 4 .

6. Check analysis. - An analysis may be made by the purchaser from the broken tension-test specimen or from a casting representing each melt. The chemical composition thus determined shall conform to the requirements specified in section 4 . Drillings for analysis shall be taken not less than minute. The tensile strength shall be determined at a speed not to exceed $1 \frac{1}{2}$ inches per minute.

8. Test specimens.-(a) A sufficient number of test coupons to provide a test for each melt shall be cast with and attached to the coupler bodies or knuckles when presented for inspection. The size of coupon shall be sufficient to provide for a machined test piece as required in paragraph $(d)$, and shall be cast on the coupler bodies or knuckles at a location optional with the manufacturer.

(b) For the purpose of identifying each casting in a melt, the manufacturer shall keep a record by serial or heat numbers of the coupler bodies and knuckles which have been poured from that melt.

(c) When sufficient coupons have not been cast, a test specimen may be cut from the finished casting:at

Markings for Standard A.R.A. D. Coupler and Fittings.

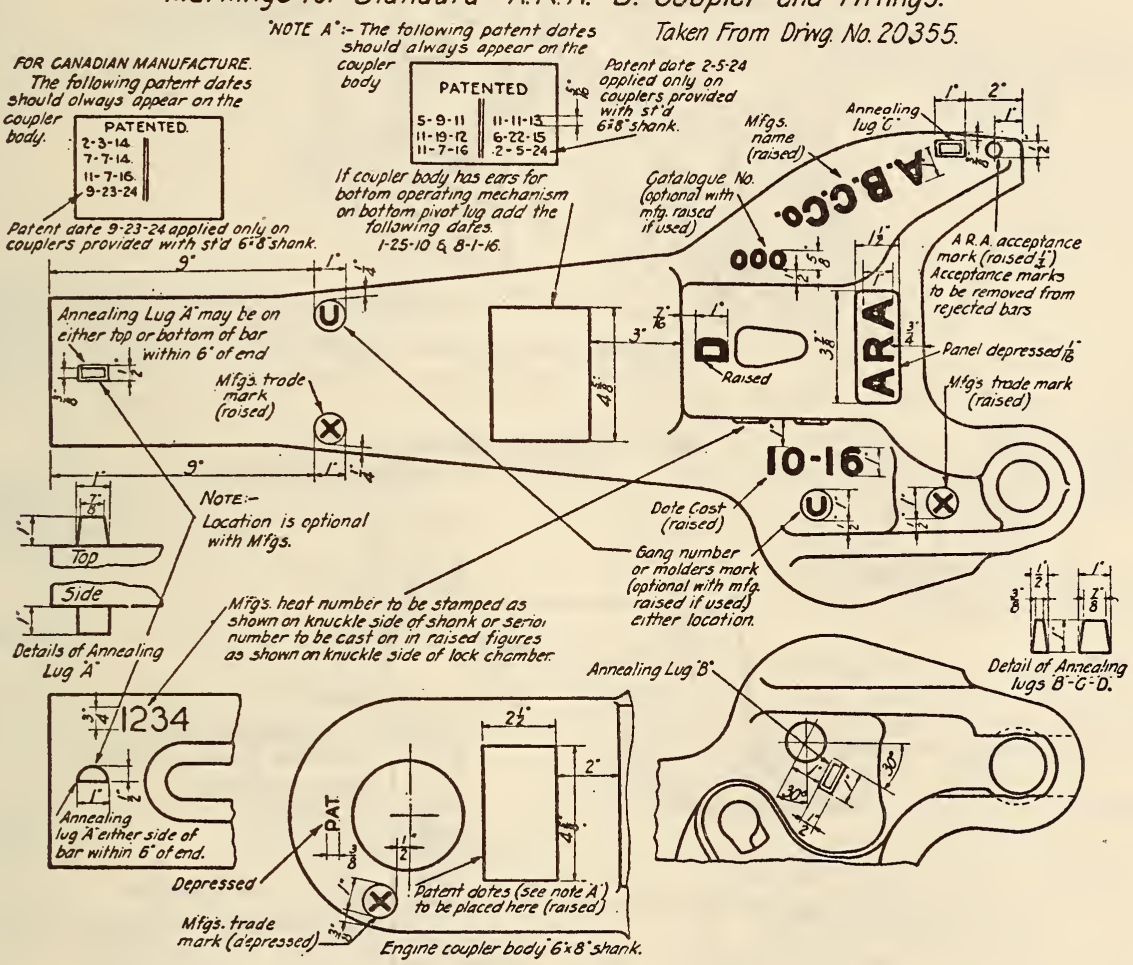

Figure 519

one-fourth inch beneath the surface, and shall be taken in such a manner as not to impair the usefulness of a casting.

\section{PHYSICAL PROPERTIES AND TESTS}

7. Tension tests.-(a) The steel shall conform to the following minimum requirements as to tensile properties:

Yield point............................

Elongation in 2 inches $\quad 1,600,000$

Elongation in 2 inches............. per cent.-- $\frac{1,600,000}{\text { Tensile strength }}$

Elongation, not under..............do.... $\quad 2,500,00022$

Reduction of area................... $\frac{2,500,000}{\text { Tensile strength }}$

Reduction of area not under...............

(b) The tensile strength shall be reported.

(c) The yield point shall be determined by the drop of the beam or by the dividers, the method being optional with the purchaser, and at a crosshead speed not to exceed one-eighth inch per a location mutually agreed upon by the inspector and the manufacturer, and this casting replaced in the lot.

(d) Tension-test specimens shall be machined to 0.505 inch in diameter with a 2-inch gage length. The ends shall be not less than seven-eighths inch in diameter and of a length and form to fit the holders of the test machine in such a manner that the load shall be axial.

9. Number of tests. - (a) At least one chemical and physical test shall be made from each melt of steel except as provided in section $10(a)$.

(b) If the test specimen shows defective machining or develops flaws, it may be discarded and another specimen substituted.

(c) If the percentage of elongation of any, tensiontest specimen is less than that specified in section 7 and any part of the fracture is more than threefourths inch from the center of the gage length, as indicated by scribe scratches marked on the specimen before testing, a retest shall be allowed. 
(d) No part of these specifications shall operate to cause any one tension test to apply to more tha.] 40 tons of castings as offered for inspection.

10. Number of tests, consecutive melts.-(a) After 15 consecutive melts have been tested and accepted in accordance with the above requirements, the manufacturer may group the succeeding melts in lots of five melts each, but each lot not to exceed 40 tons, the entire group to be accepted if the test specimen selected from the lot fulfills the chemical and physical requirements herein specified. If this test fails, a rehearing will be granted on the melt that the failed bar represents, and the other four melts of the group shall be tested individually. shall preferably be from the same melt, and the manufacturer shall furnish the purchaser a copy of his record showing satisfactory chemical analysis and physical properties obtained from the melt of steel in question, and the annealing lug showing his proof of the appearance of the fracture of the annealing lugs.

13. Grouping.-The manufacturer shall have the material grouped in lots of 100 complete couplers or 100 detail parts, and this will be an inspection lot. Where possible, care should be taken to put all couplers of the same melt number in the same lot. The manufacturer should endeavor to cast as many parts from one melt on a given order as is possible.
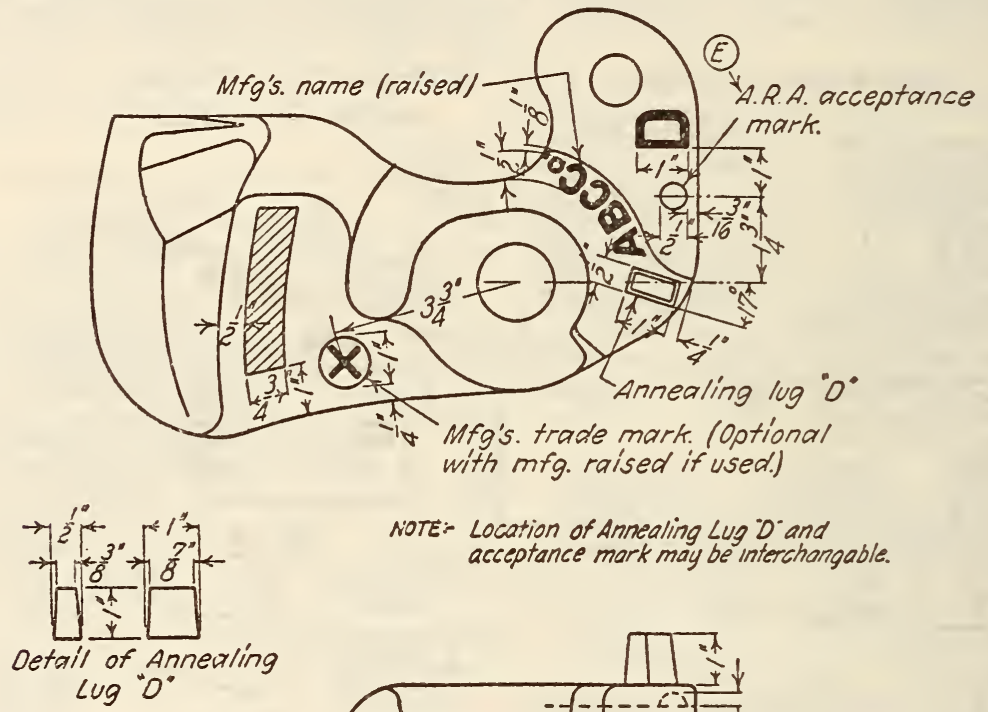

NOTE:- Location of Annealing LUg D and acceptance mark may be interchangable.

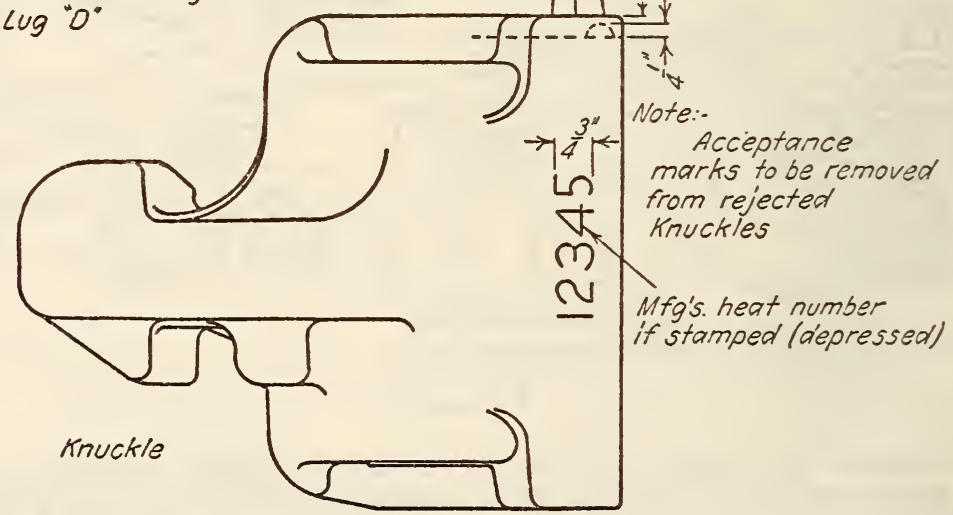

FigURE 520

(b) If there is a period of more than six months between shipments of couplers or parts, then each melt shall be tested individually until 15 consecutive melts have been accepted, after which the melts may again be grouped as in paragraph $(a)$.

(c) If one or more melts are rejected, each succeeding melt shall be tested individually until 15 consecutive melts have been accepted, after which melts may again be grouped as in paragraph $(a)$.

11. Retests. - If the results of the physical test lot do not conform to the requirements specified, the manufacturer may reanneal such lot not more than twice and retest shall be made as specified in section 7 .

12. Waiving tests. - Tests will be waived on orders for less than 10 complete couplers or less than 100 parts other than bars, in which event all castings
14. Tests of knuckle pivot pins.-For each two lots of 100 couplers or less, otherwise accepted, one pivot pin shall be removed from a complete coupler and subjected to the requirements of the A. R. A. specifications for heat-treated knuckle pivot pins. If this pin fails to pass the specifications, all pins in this lot of couplers will be rejected. Pins thus removed from the assembled couplers shall be replaced by the manufacturer by an additional pin after the test in order to complete the coupler.

\section{VARIATION IN WEIGHT AND GAGE}

15. Weight.-(a) One detail part of the lots as established by section 13 shall be weighed and come within the limits as shown below. Failure of any part to come within the minimum weight shall reject that detail and each such detail in the entire 
lot so represented shall then be weighed and every piece less than the minimum weight shall be rejected.

(b) When couplers are purchased complete and assembled, 10 per cent of the couplers in each lot of 100 or less shall be individually weighed and shall come within the limits as shown below. Upon the failure of a coupler to come within the limits as shown below, the weighing shall be extended to the entire lot. Failure of any coupler to come within the limits shall be sufficient cause to reject the coupler unless the weight can be adjusted by the manufacturer. Detail parts shall come within the limits as shown below, one coupler being dismantled in each 100 and weighed.

(c) When castings are more than the allowable maximum weight as shown below, and all other

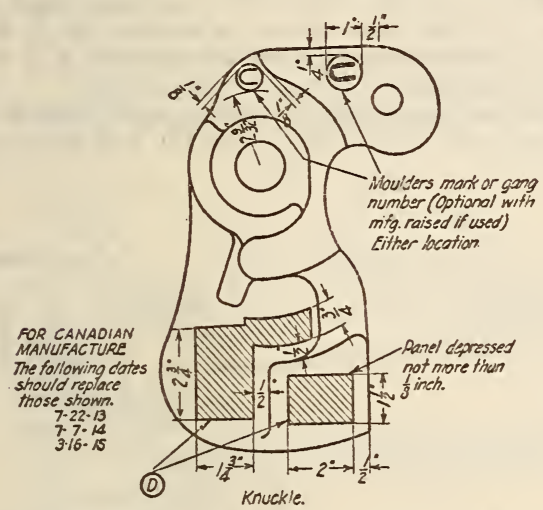

Markings indicated on knuckle to be located as shown. The follawing markings are to be placed upon the upper and lower surfaces of the kinuckle tail within shaded areas indicated, to be raised unless othenwise specified.

Dates cast

10-16 I-Not kess than $5^{\circ}$ -

Patent dates

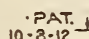$$
\begin{aligned}
& 10 \cdot 8 \cdot 12 \\
& 4 \cdot 13 \cdot 15
\end{aligned}
$$

12345 Rat less thon $5^{\circ}$ Raised not more than $3^{\circ}$

Mifgs. serial Na if cast

When mfos haat number is used in place of serial number it is to be stamped on tack of knuckle as indicated.

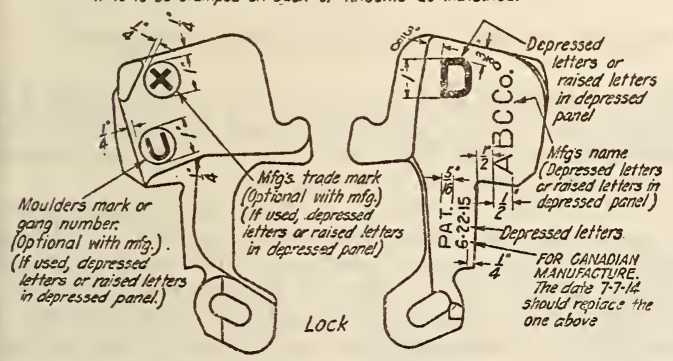

Frgore 521

requirements are satisfactory, castings may be accepted at the maximum allowable weight, excess weight being at the expense of the manufacturer.

16. Gage.-(a) Castings shall conform to the prescribed limits and gages. Five per cent of each lot of 100 or less, but in all cases at least one of the complete couplers or separate parts in each, shall be completely gaged. Assembled couplers shall be dismantled before gaging.

(b) Failure of any part in the lot to come within the gages or limits shall be sufficent cause to reject

the lot so represented. Failure of a lot to meet the gages will not be sufficient cause to reject a reoffering of the same lot on the same order after the manufacturer has adjusted these parts to the gages. After this adjustment by the manufacturer, the inspector will then gage 10 couplers or pieces selected at random in the lot, and failure of any to meet the

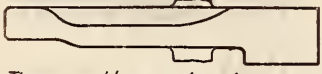

These markings apoly, only

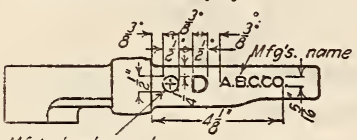

info's. trade mark.

lootional with mfg. raised if used
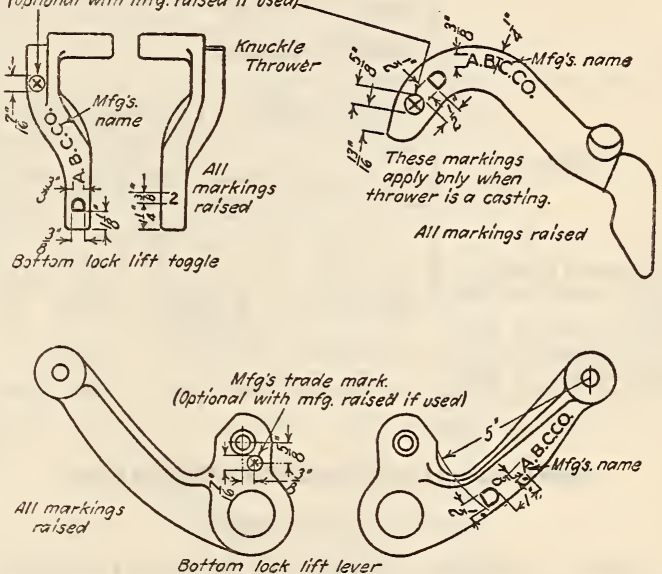

FIGURE 522

gages will reject the entire lot, which shall not again be offered.

(c) The inspector shall inspect and gage each coupler in a lot in order to determine if they comply to the contour gage.

(d) Gages referred to above are listed below.

$$
\text { Limiting weights-" } D \text { " coupler }
$$

[All weights in pounds]

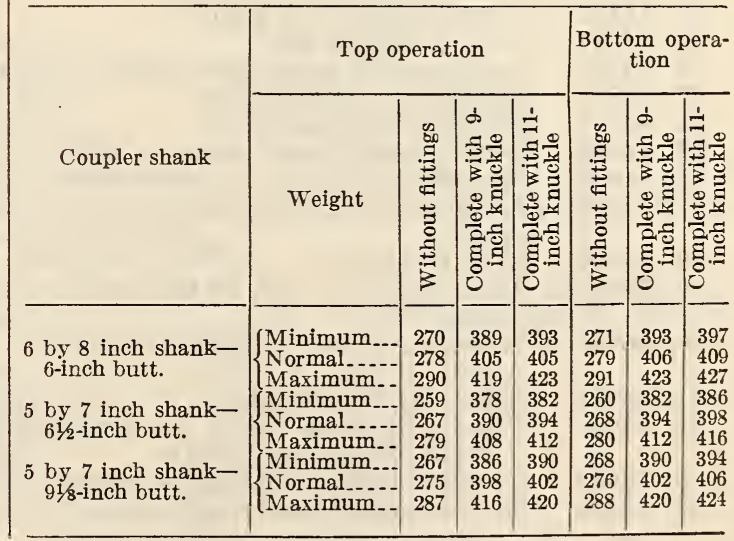




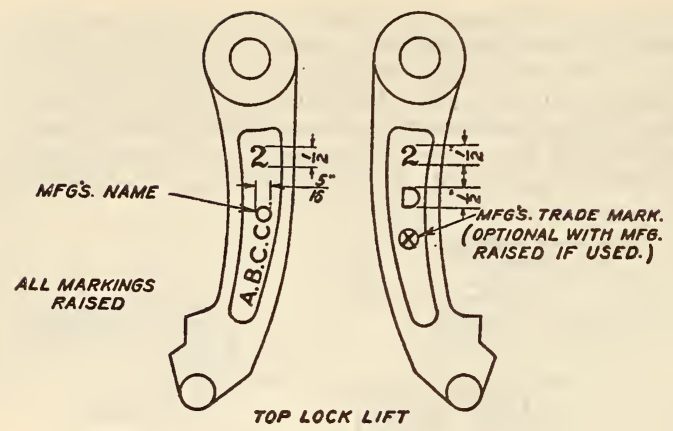

FIGURE 523

Weight of fittings

[All weight in pounds]

\begin{tabular}{|c|c|c|c|c|c|c|}
\hline \multirow{2}{*}{ Fittings } & \multicolumn{3}{|c|}{ Top operation } & \multicolumn{3}{|c|}{ Bottom operation } \\
\hline & $\begin{array}{l}\text { Mini- } \\
\text { mum }\end{array}$ & $\begin{array}{l}\text { Nor- } \\
\text { mal }\end{array}$ & $\begin{array}{l}\text { Maxi- } \\
\text { mum }\end{array}$ & $\begin{array}{l}\text { Mini- } \\
\text { mum }\end{array}$ & $\begin{array}{l}\text { Nor- } \\
\text { mal }\end{array}$ & $\begin{array}{l}\text { Maxi- } \\
\text { mum }\end{array}$ \\
\hline $\begin{array}{l}\text { 9-inch cored knuckle--. } \\
\text { 11-inch cored knuckle.- } \\
\text { Lock }\end{array}$ & $\begin{array}{l}90 \\
94 \\
13.5\end{array}$ & $\begin{array}{l}93 \\
97 \\
14.5\end{array}$ & $\begin{array}{c}97 \\
101 \\
15.5\end{array}$ & $\begin{array}{l}90 \\
94 \\
13.5\end{array}$ & $\begin{array}{l}93 \\
97 \\
14.5\end{array}$ & $\begin{array}{c}97 \\
101 \\
15.5\end{array}$ \\
\hline $\begin{array}{l}\text { Total weight of parts } \\
\text { (with 9-inch knuckle) } \\
\text { Total weight of parts } \\
\text { (with 11-inch knuckle) }\end{array}$ & $\begin{array}{l}119 \\
123\end{array}$ & $\begin{array}{l}123 \\
127\end{array}$ & $\begin{array}{l}129 \\
133\end{array}$ & $\begin{array}{l}122 \\
126\end{array}$ & 126 & 132 \\
\hline
\end{tabular}

\section{Gages to Insure Interchangeability of Coupler Parts}

(Standard "D" coupler)

Class I, inspectors' gages. - To be used by inspectors for rejection of couplers and parts not conforming to same.

Gage No.

1. 13242 Knuckle thrower gage.

2. 13815 Movable point knuckle gage.

3. 13818 Inspectors' contour gage, No. 10 contour.

4. 13906 Knuckle pin protector gage.

5. 14220 Knuckle hub gage.

6. 14413 Drawbar pulling lug gage.

7. 14414 Drawbar pin protector gage.

8. 14437 Knuckle tail height gage.

9. 14480-1 Drawbar lock chamber gage.

10. 14487 Drawbar lock hole gage.

11. 14491 Lock thickness gage.

12. 14492

13. 14513

14. 14514

15. 14604

16. 14628

17. 14643

Lock contour gage-guard arm side.

Drawbar pivot lug gage.

Top lock lift gage.

Lock contour gage-knuckle side.

Knuckle pulling lug gage for top half.

Knuckle pulling lug gage for bottom half.

18. 14690 Lock slot gage.

19. 20843-1 Minimum gage- $15 / 8$ by $6 \frac{5}{8}$ inch key slot.

20. 20843-2 Maximum gage- $1 \frac{5}{8}$ by $6 \frac{5}{8}$ inch key slot.

21. 15014 Lock toggle hole gage.

22. 15037 Pivot pin used with gages Nos. 14413, 14414, and 14511.

23. 16652 Gage for hole in butt end of coupler.

24. 18334 Bottom lock lift and toggle assembly.
Class II, checking gages.-To be used by inspectors periodically, but not as rejection gages.

Gage No.

1. 13167 Top lock lift hole gage.

2. 14480-2 Maximum gage, lock chamber and lock hole.

3. 14511 Drawbar knuckle thrower hole gage.

4. 15077 Top operating anticreep gage.

5. 16259-1 Bottom lock lift lever gage.

6. 16259-2 Bottom lock lift toggle gage.

7. 16259-3 Bottom lock lift lever gage pin.

NotE.-Drawings of the above gages are held by the sectetary of the American Railway Association.

\section{WORKMANSHIP AND FINISH}

17. Workmanship.-(a) The castings shall conform to the size and shape as shown on A. R. A. standard drawings and shall be finished in a workmanlike manner.

(b) When assembled, knuckles and locking pins or blocks must work freely, but the lost motion be-

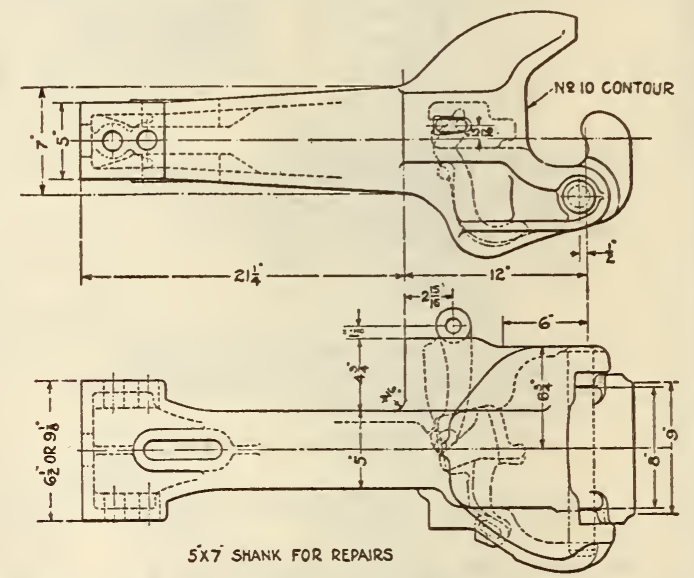

Figure 524.-Standard " $D$ " coupler, 5 by 7 inch shank

For details of shank, butt end, and key-slot, see Figure 527 .

tween knuckles and locks must be such that the knuckle can not be pulled forward by hand beyond the proper contour line, but $1 / 8$ to $1 / 4$ inch lost motion in opposite direction is desirable.

(c) In order to determine that the requirements of paragraph (b) have been complied with, not less than 10 couplers out of each lot of 100 shall be tried. Failure of any one to operate properly will be sufficient cause for rejection of the lot, but this does not prohibit the manufacturer reoffering the lot on the same order for inspection after adjustment, providing it is shown that all other requirements of the specifications have been complied with.

18. Finish. - The castings shall be free from blowholes, sand pockets, shrinkage cracks, and other injurious defects.

19. Sand or shot blast.-All castings shall be properly cleaned by sand or shot blast or other approved process. The inspector may require that any or all castings again be subjected to sand or shot blast or cleaning in order to better examine for checks and shrinkage cracks which in his opinion would be detrimental to the strength of the castings. 


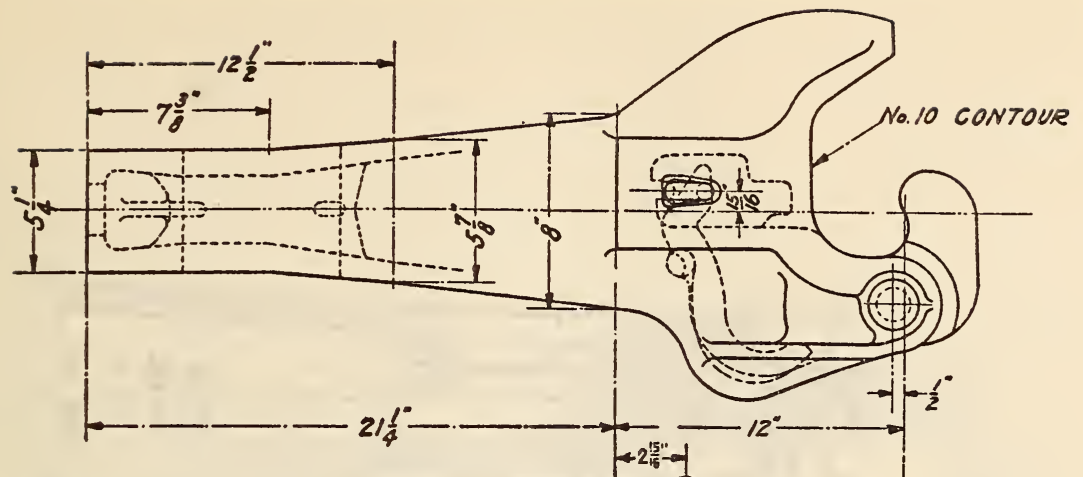

TOP OPERATION:- USEO WITH EITHER SIZE SHANK. TOTAL LIFT TO LOCK SET 3/1". TOTAL LIFT TO THROW KNUCKLE 5:

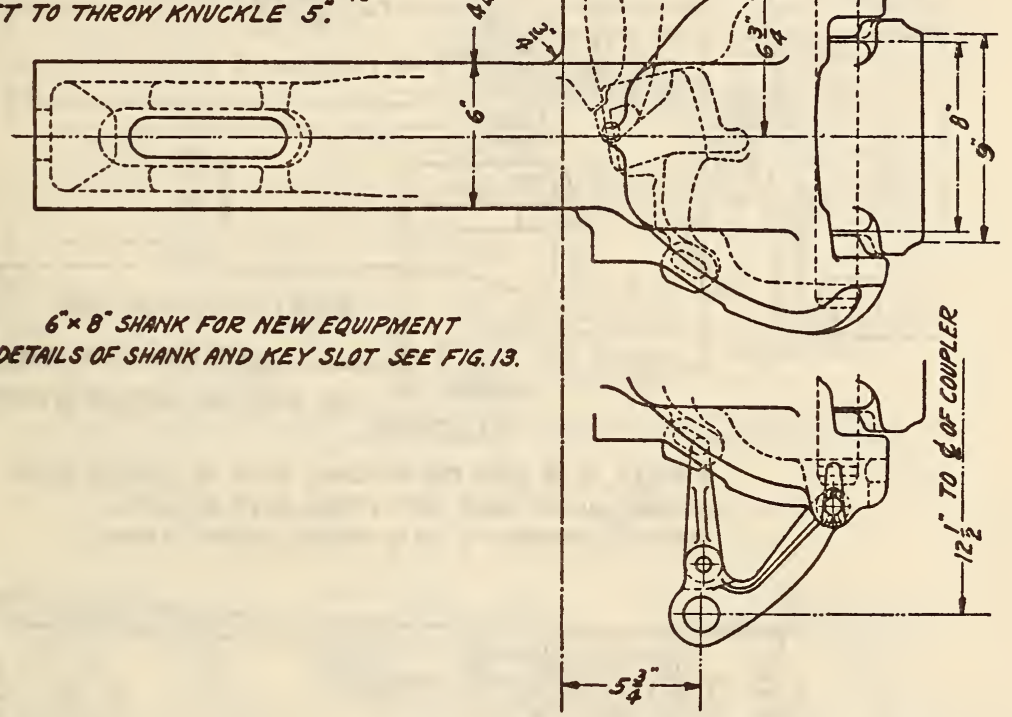

BOTTOM OPERATION:- USED WITH EITHER SIZE SHANK. TOTAL LIFT TO LOCK SET 3\%: TOTAL LIFT TO THEOW KNUCKLE 5 SIS" $^{\circ}$

FigURE 525.-Standard " $D$ " coupler, 6 by 8 inch shank
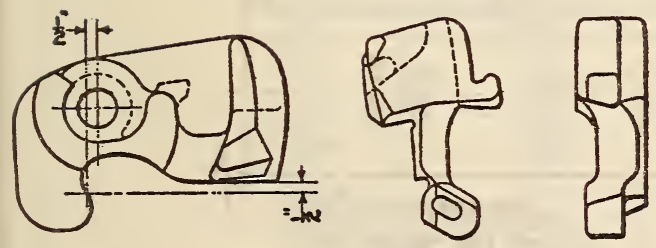

LOCK
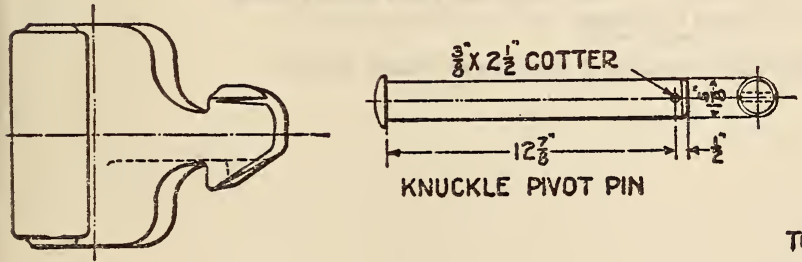

TOP LOCK LIFT

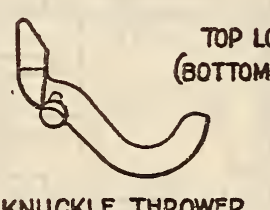

KNUCKLE THROWER

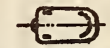

LIOCK LIFT HOLE CAP BOTTOM OPERATING COUPLER)
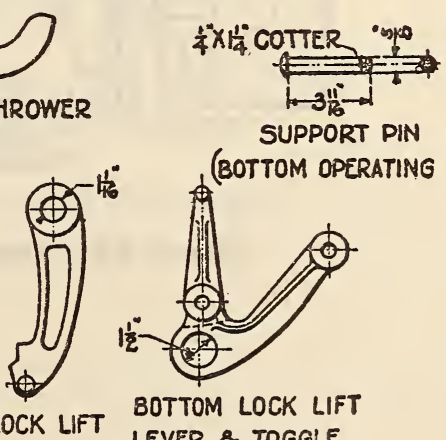

KNUCKLE 


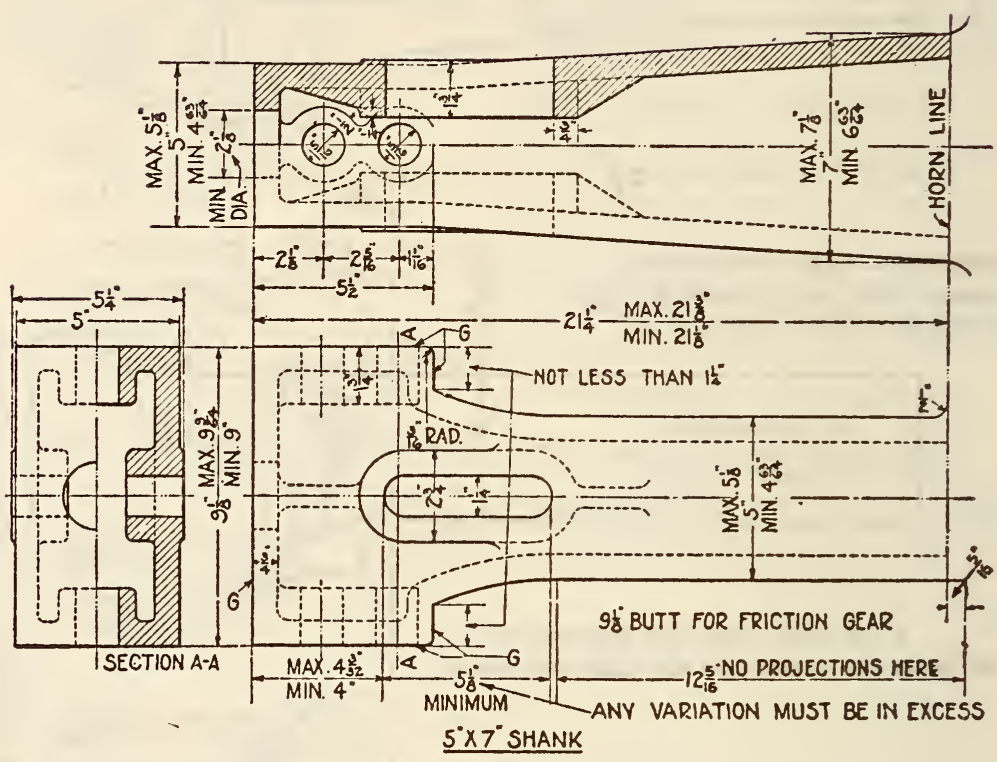

$11^{\circ}$ RIVETS TO BE USED FOR ATTACHING YOKES TO COUPLER BUTTS. COUPLER MAY BE MADE WITH EITHER 9:8 OR 61. BUTTS. SURFACES MARKED " $G$ " TO BE GROUND SQUARE TO GAUGE.

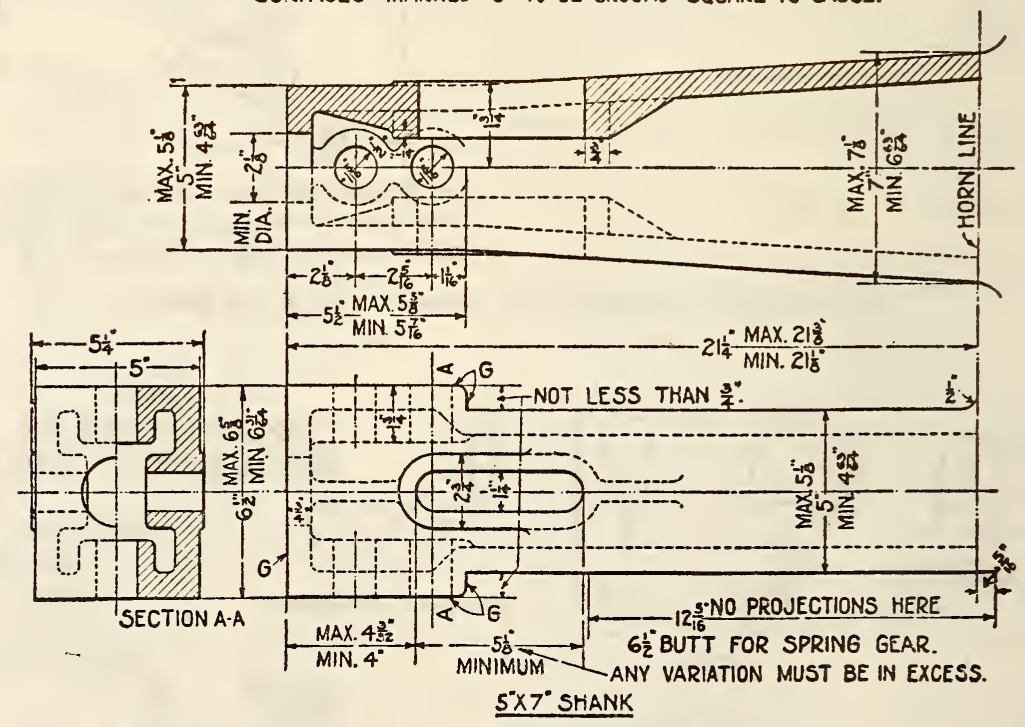

F1GURe 527.-Standard " $D$ " coupler, 5 by 7 inch shank details 


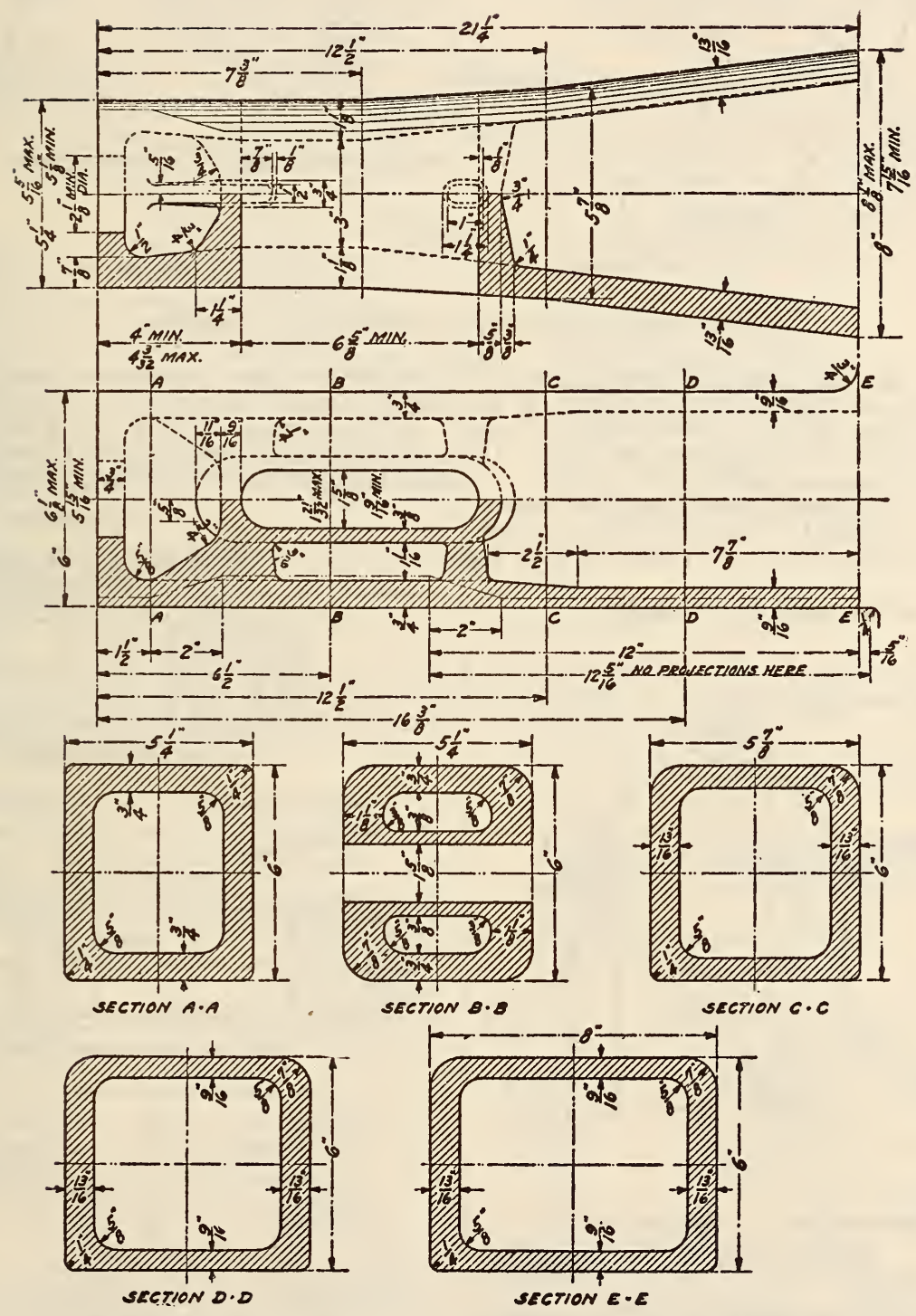

Figure 528.-Standard " $D$ " coupler, 6 by 8 inch shank details 


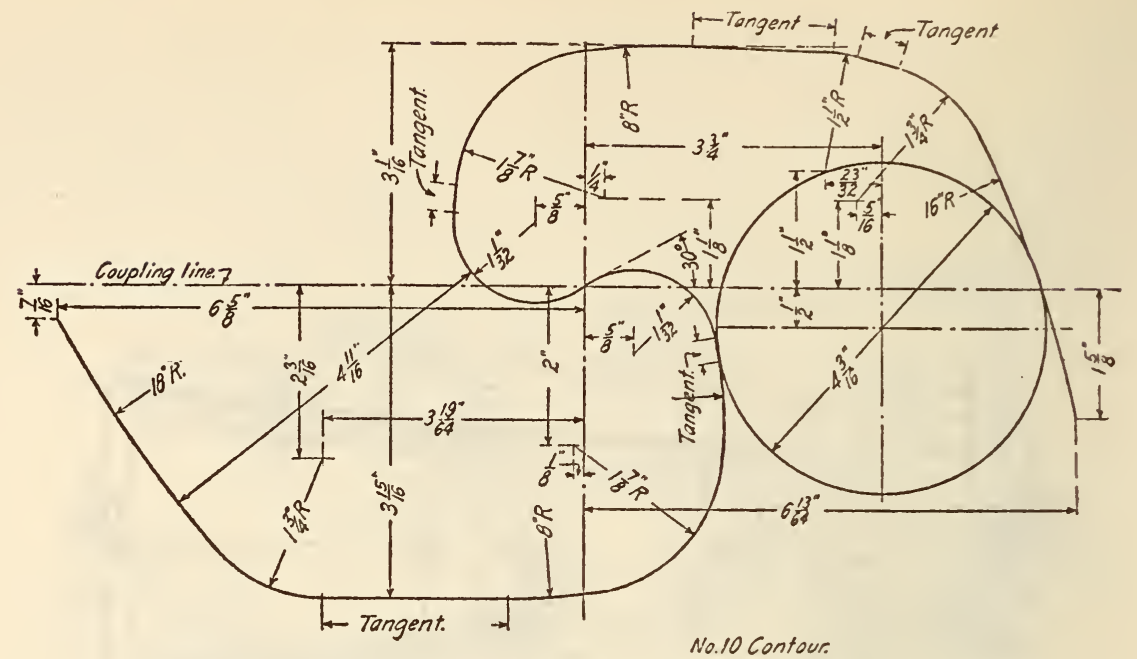

Figure 529.-Standard "D" coupler, No. 10 contour

20. Welding.-Welding minor imperfections which do not impair the strength of the castings will be permitted when done under the jurisdiction of the inspector and by a practice approved by him. All castings shall be reannealed after such welding.

21. Painting.-The castings as offered for inspection shall: not be painted or covered with any substance that will hide defects, nor rusted to such an

STANDARD "D" COUOLER

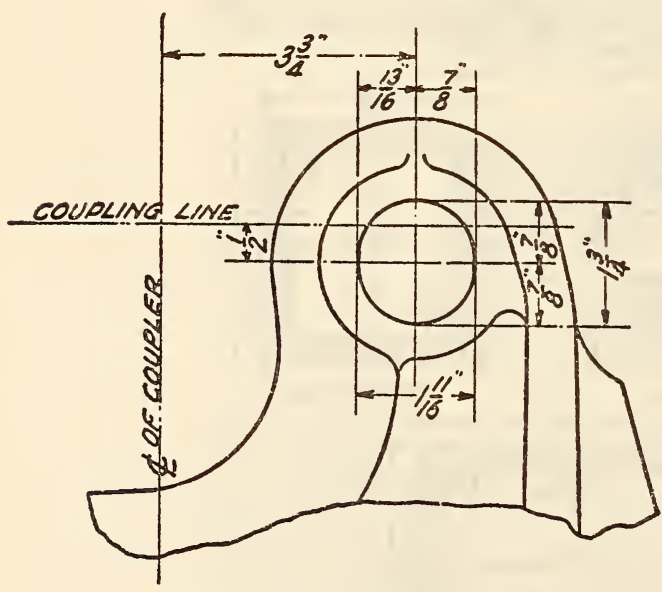

DETAIL OF MNUCHLE PIVOT PIN MOLE

Figure 530

extent as to make defects invisible. If the castings are to be painted, it shall be so specified on the order, but this shall only be done after complete inspection and acceptance of the material by the inspector, and then with a quick-drying paint.

22. Lubrication.-Couplers when assembled shall be properly lubricated.

23. Marking.-(a) All parts shall have the manufacturer's name or identification mark, and when required, serial numbers, and such additional marking as shown on Figures 519, 520, 521, 522, and 523, cast legibly in letters or figures of size, manner, and location as specified. Manufacturer to give purchaser list of serials in each melt. When melt numbers are used in place of serial numbers they shall be legibly stamped on each piece in the melt.

(b) The serial number for each manufacturer, when used, shall begin with " 1 " and continue consecutively throughout the month, when a new series
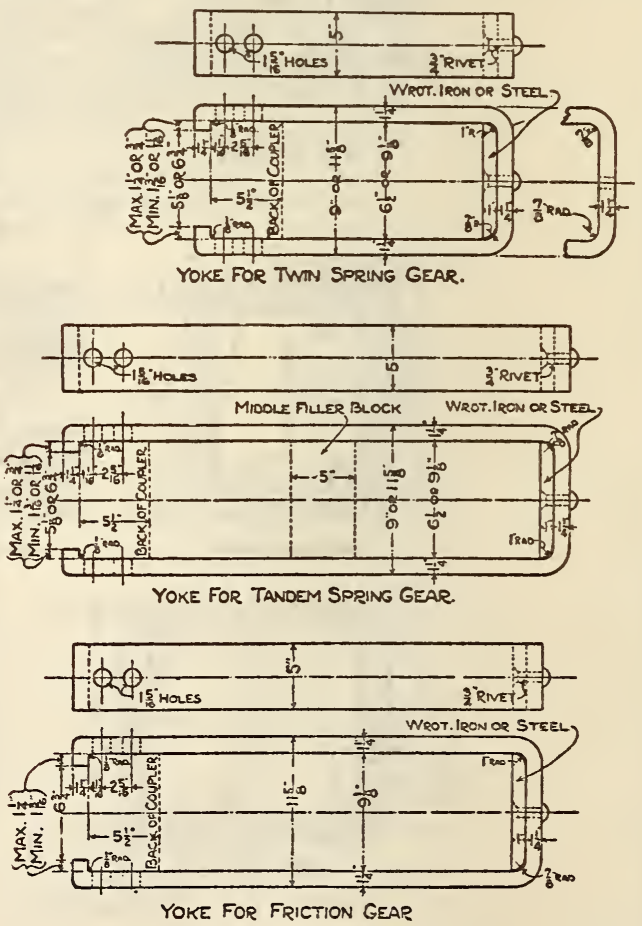

Figure 531.-Coupler yokes

shall be started. In all cases, however, a new serial number shall start at the beginning of each month.

(c) After the material has been inspected and is ready to be shipped, the inspector shall stamp each casting with his private mark.

\section{INSPECTION AND REJECTION}

24. Inspection.-(a) The inspector representing the purchaser shall have free entry at all times while 


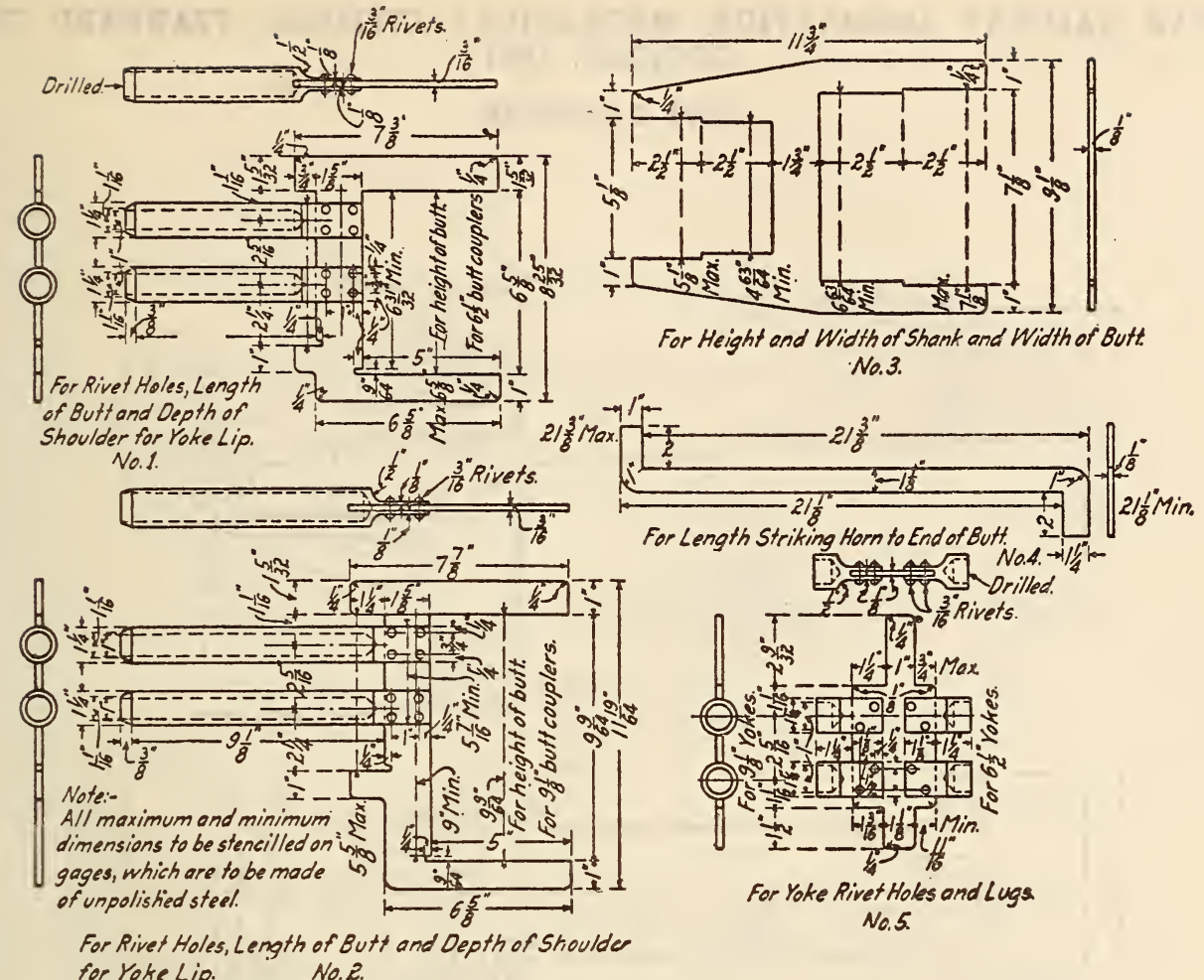

Figure 532.-Gages 1, 2, 3, 4, and 5 for coupler and yoke

work on the contract of the purchaser is being performed, to all parts of the manufacturer's works which concern the manufacture, test, and inspection of the material ordered. The manufacturer

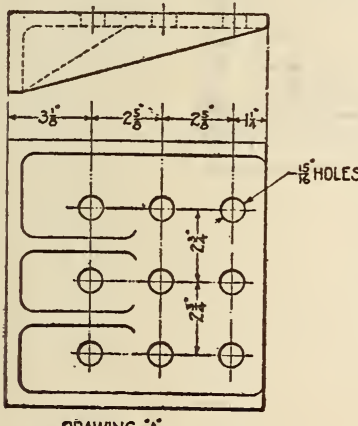

DRAWING "Ä:

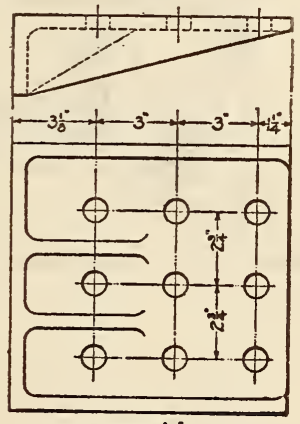

DRAWING ${ }^{\circ} B^{\circ}$
FIGURe 533.-Front and back stop

shall afford the inspector, free of charge, all reasonable facilities to satisfy himself that the material is being furnished in accordance with the specifications.

(b) The purchaser may make the chemical tests to govern the acceptance or rejection of the material in his own laboratory or elsewhere. Such chemical tests, however, shall be made at the expense of the purchaser.

(c) The physical tests may be made at the plant of the manufacturer, providing the purchaser is satisfied with the accuracy of the test machine.

(d) All tests and inspection shall be so conducted as not to interfere unnecessarily with the operation of the works.

(e) After the inspection and tests have been completed and before castings are loaded, at the option of the purchaser, all remaining annealing lugs shall be removed and surface where located put in a workmanlike condition.

25. Rejections.- (a) If any of the test coupons or annealing lugs selected to represent the melt do not

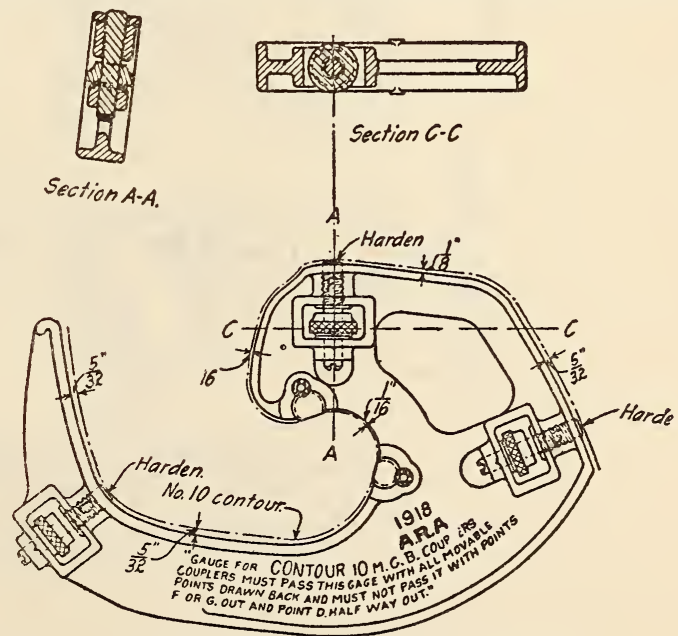

FIGURE 534.-Coupler limit gage

conform to the requirements specified in sections 3 , 6 , and 7 , the lot will be rejected.

(b) All castings which show any injurious defects or do not conform to the weights or gages shall be rejected.

(c) From each bar or knuckle rejected by the inspector under these specifications, he shall cause to be chipped the A. R. A. acceptance mark shown on Figures 519 and 520 . 
AMERICAN RAILWAY ASSOCIATION, MECHANICAL DIVISION, STANDARD TYPE E COUPLER, 1931

TYPE E COUPLER

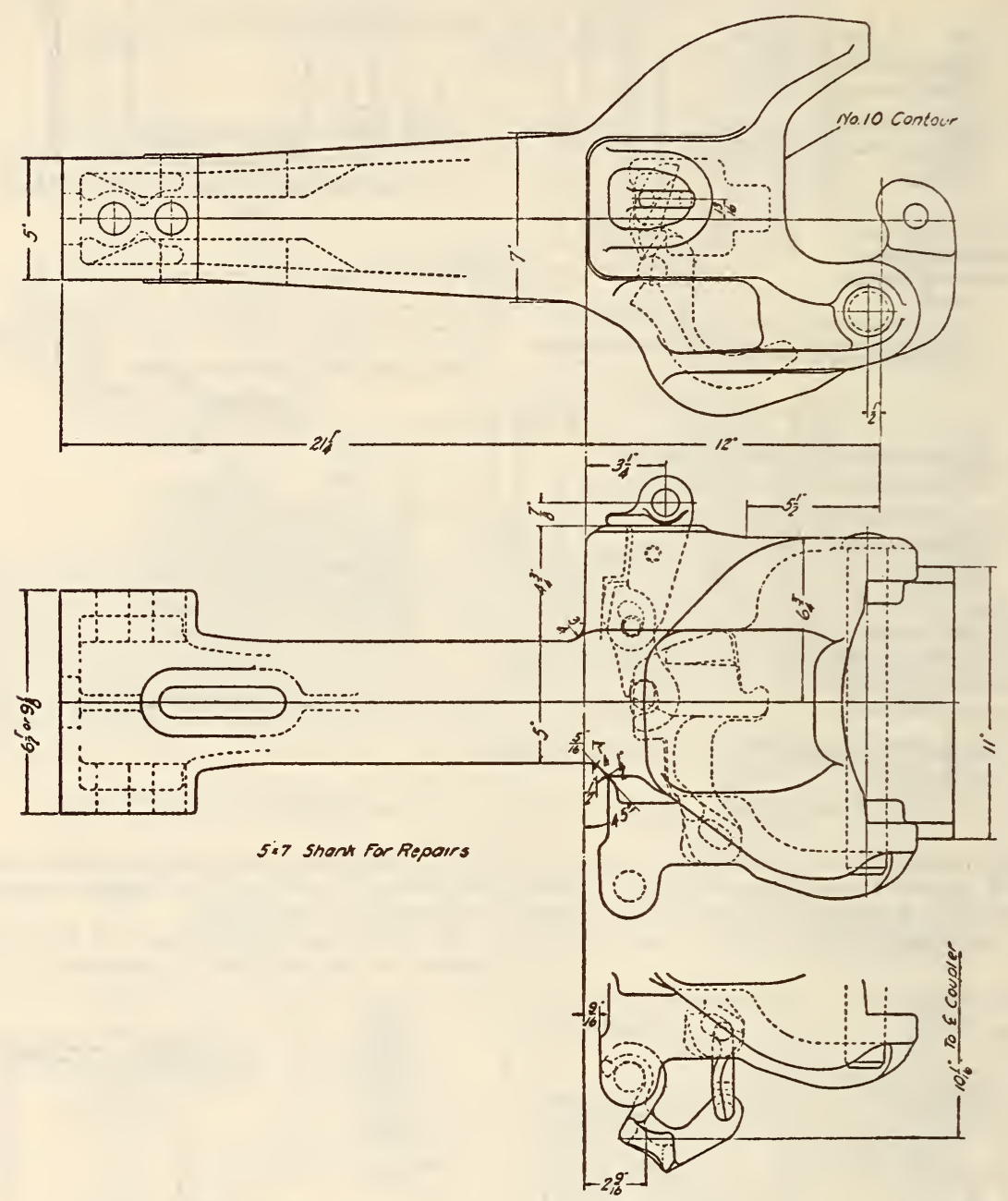

Figure 535.-Type $E$ coupler, 5 by $\gamma$ inch shank 


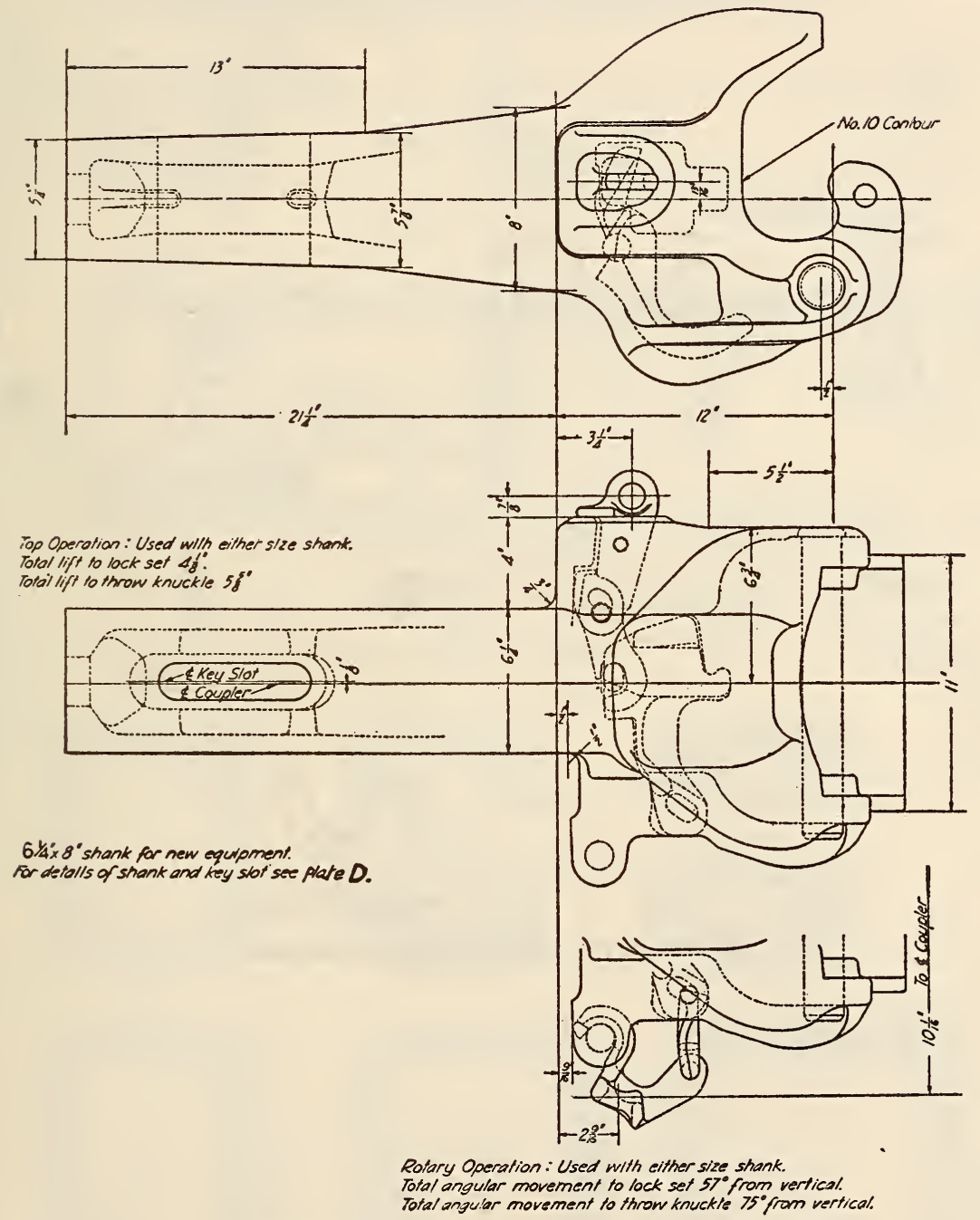

Figure 536.-Type E coupler, $61 / 4$ by 8 inch shank 

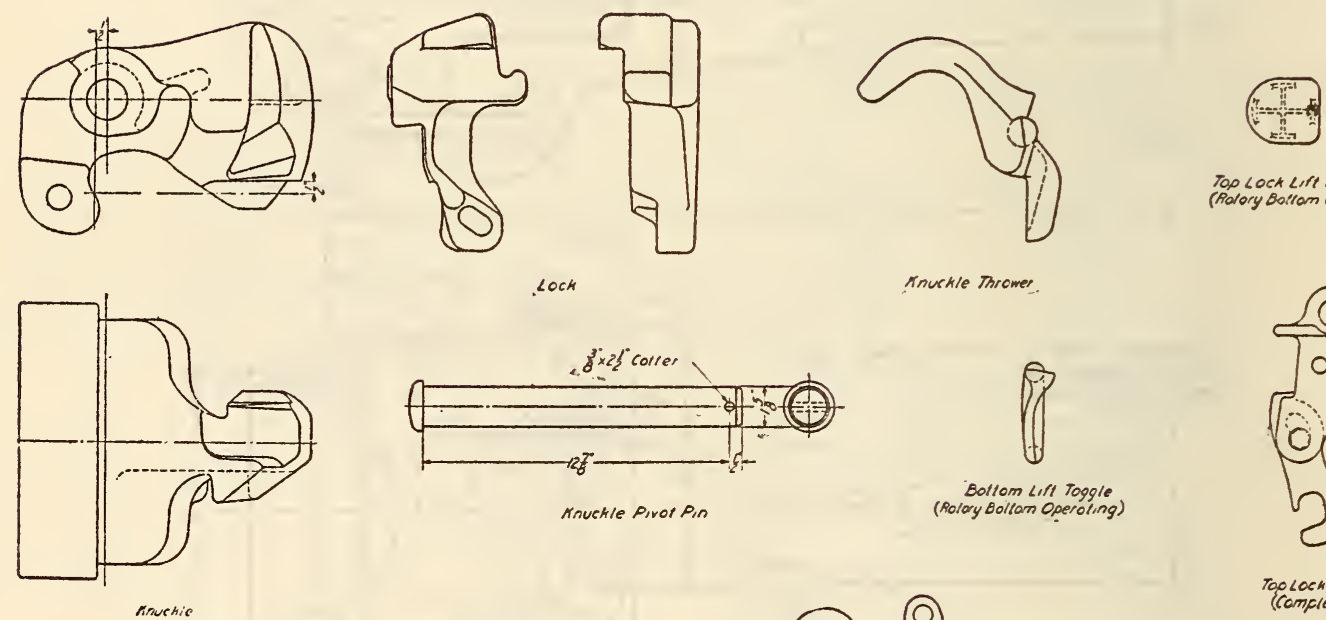

Anuckle Thrower.
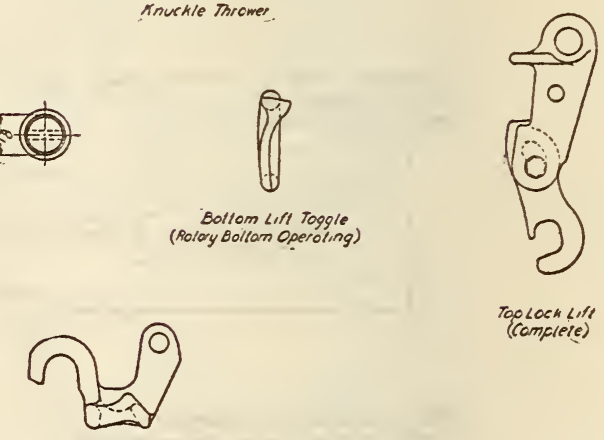

Too LOCA Lift
(Complete)

$$
\begin{aligned}
& \text { Lock Liff Lever } \\
& \text { (Polory Bollom operoting) }
\end{aligned}
$$

Figdre 537.-Type E coupler, detail parts 

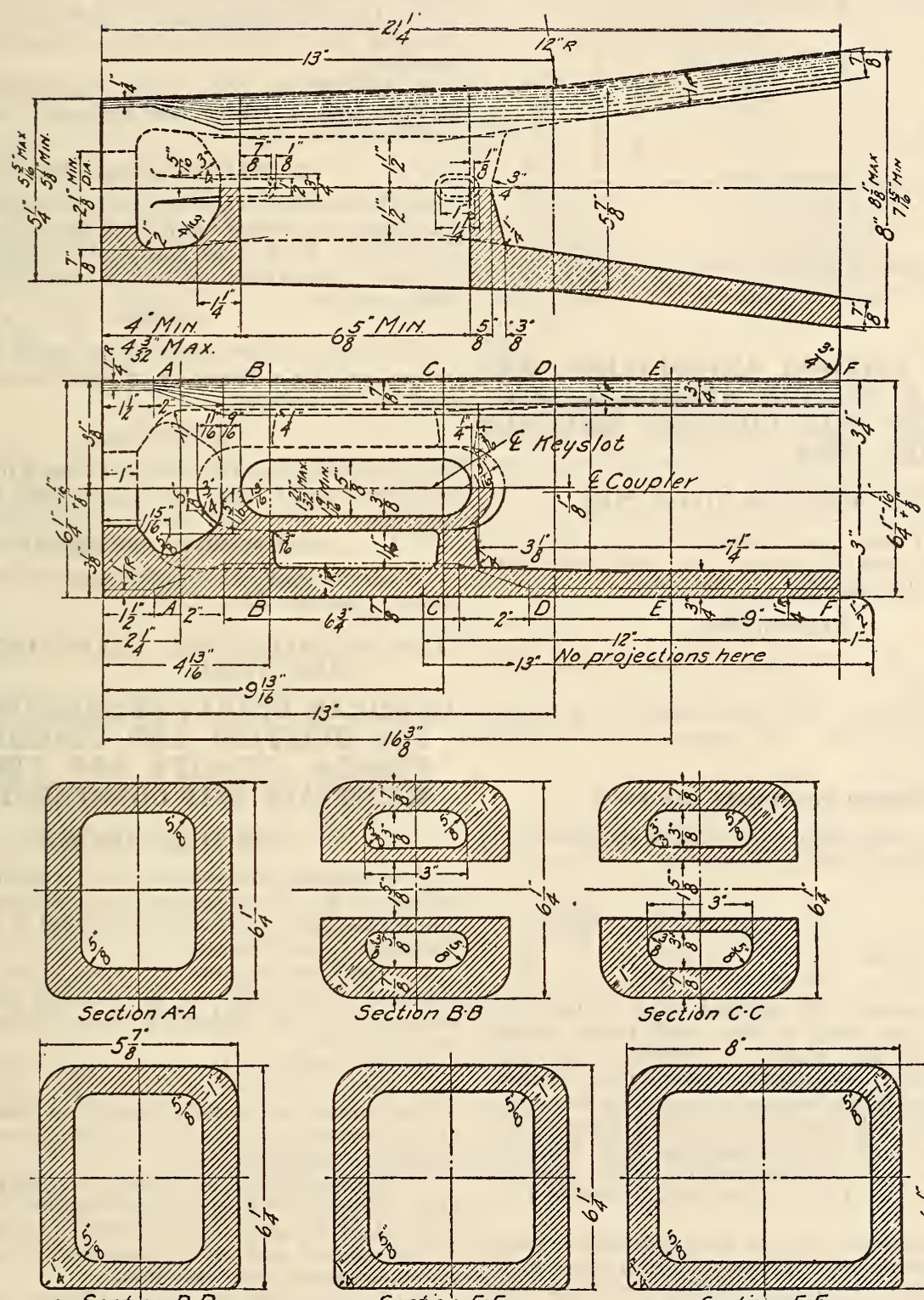

Section E-E

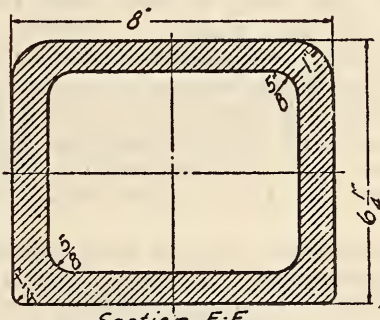

FIGURE 538.-Type E coupler, $61 \frac{1}{4}$ by 8 inch shank details 


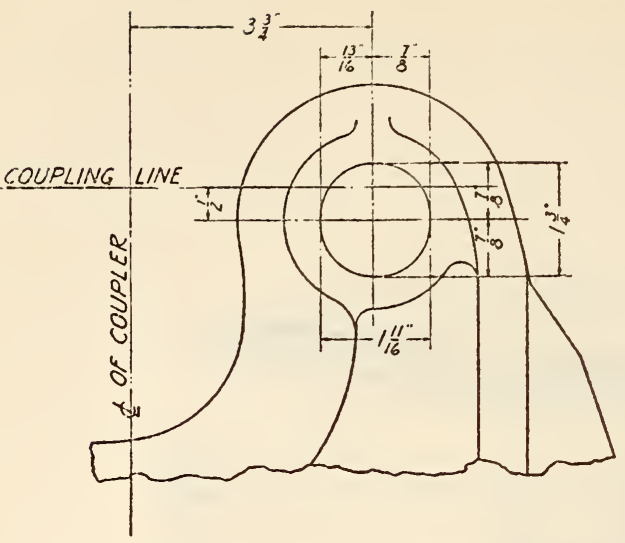

DETAIL OF KNUCKLE PIVOT PIN HOLE

Figure 539.-Type E coupler, detail of knuckle pivot pinhole

\section{AMERICAN RAILWAY ASSOCIATION, ME- CHANICAL DIVISION, STANDARD SPEC- IFICATIONS FOR COUPLER KNUCKLE PIVOT PINS, 1928}

\section{COUPLER KNUCKLE PIVOT PINS}

1. Scope.-These specifications cover all heat treated carbon steel knuckle pivot pins and other coupler pins; also bars for making pins.

\section{MANUFACTURE}

2. Process. - The steel shall be made by the openhearth process.

3. Heat treatment.-The pins shall be properly heat treated to meet the requirements of the physical tests.

\section{CHEMICAL PROPERTIES AND TESTS}

4. Chemical composition.-The steel shall conform to the following requirements as to chemical composition:

\begin{tabular}{lr} 
Carbon & Per cent \\
Manganese & \\
Phosphorus, not over & $.55-0.75$ \\
Sulphur, not over & .90 \\
\hline
\end{tabular}

5. Ladle analyses.-An analysis shall be made by the manufacturer from a test ingot taken during the pouring of each melt, to determine the percentage of carbon, manganese, phosphorus, sulphur and silicon. Drillings for analysis shall be taken not less than one-fourth inch beneath the surface of the test ingot. A copy of this analysis shall be given the purchaser or his representative. This analysis shall conform to the requirements specified in section 4.

6. Check analyses.-A check analysis shall be made from the finished material representing each melt, by the purchaser or his representative, and shall meet the requirements specified in section 4 .

\section{PHYSICAI PROPERTIES AND TESTS}

7. Drop test.-(a) This test shall be made on a standard A. R. A. drop-test machine, or a drop-test machine that will give equivalent results, the pin resting on rounded supports held rigidly 10 inches center to center. If made on a standard A. R. A. machine, the pin shall be struck one blow by a tup weighing 1,640 or 2,240 pounds falling from a height determined from the following formula:

$$
\text { For } 1,640 \text { pounds tup: } \mathrm{H}=1.16 \mathrm{~d}^{2}
$$

$i_{n}$ which $\mathrm{H}$ is the height of the drop in feet and $d$ $i_{s}$ the diameter of the pin in inches. The height of drop for the standard $15 / 8$-inch pin shall be 3 feet (36 inches), for 1,640-pound tup and 2.25 feet ( 27 inches), for 2,240-pound tup.

(b) The pin shall show a deflection of not less than $15^{\circ}$, nor more than $30^{\circ}$, without cracking or breaking.

8. Number of tests.-The manufacturers shall furnish, free of charge, one extra pin with each lot of 200 or less.

\section{PERMISSIBLE VARIATIONS}

9. Permissible variations.-The diameter of the pin may vary not more than plus or minus one-sixtyfourth inch from that specified. The length shall not vary more than one-eighth inch below or above that specified.

10. Finish.-The finished pin shall be straight, have a smooth surface, be uniform in diameter and shall not be painted.

\section{MAREINGS}

11. Markings. - The manufacturer's name or identification marks shall be stamped on the head of each pin.

\section{INSPECTION AND REJECTION}

(Conform to A. R. A. specifications for galvanized sheets. See 604.32 , p. 243.)

\subsection{MISCELLANEOUS MANUFACTURES OF CAST STEEL.}

\section{AMERICAN BUREAU OF SHIPPING, RULES FOR BUILTING AND CLASSING STEEL VESSELS; QUALITY AND TESTING OF MATERIALS, SHIP CONSTRUCTION, 1930}

\section{CAST-STEEL ANCHORS}

(For general information and requirements see 600.0 , p. 2. Specifications are the same as for forged wrought-iron anchors (see 611.3, p. 704) with the following additions):

1. Cast-steel anchors, or when such anchors are made of more than one piece, each piece shall be subjected to the following drop, hammering, and bending tests:

2. Each piece is to be thoroughly annealed, the annealing to extend over three days for castings of 1,700 pounds and under, and the time increased with increased weight. The castings are then to be stamped "annealed steel."

3. The anchor or piece shall be raised to a height of 12 feet and dropped on an iron slab. If this test is satisfactory the anchor or piece is to be slung clear of the ground and well hammered all over to test the soundness of the material.

4. A piece sufficient to provide two or three test specimens must be cast on each casting submitted for approval, and is not to be detached from the casting until after the annealing is complete nor until it has been stamped by the surveyor.

5. A specimen which should not be less than 1 by $3 / 4$ inch, with edges rounded to a radius of one-sixteenth inch, must stand being bent cold through an angle of $120^{\circ}$ over a radius of 1 inch without any appearance of fracture. 
AMERICAN MARINE STANDARDS COM- AMERICAN MARINE STANDARDS COMMITTEE, SPECIFICATION FOR BUILT- MITTEE, SPECIFICATION FOR PROPELUP PROPELLERS, E NO. 23-1928

BUILT-UP PROPELLERS

(See 611.19, p. 661.) LERS CAST IN ONE PIECE, E NO. 25, 1928 PROPELLERS CAST IN ONE PIECE

(See 611.19, p. 663.)

\section{AMERICAN MARINE STANDARDS COMMITTEE, SHIP FITTINGS FOR DECKS AND RIGGING, H NO. 8-1926; H NO. 13-1926}

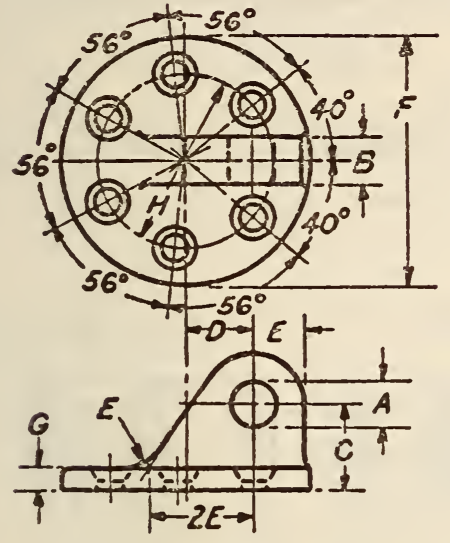

FIgURE 540.-Chain plates, American Marine standard H No. 8-1926

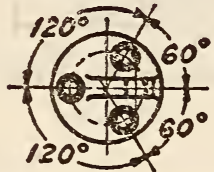

1- All holes for countersunk head rivets.

2. Material:-Cast steel, in accordance with requirements of the American Bureau of Shipping for ship construction steel castings. - Inspection and tests ito be required only when specified in the purchase order.

[All dimensions in inches]

\begin{tabular}{|c|c|c|c|c|c|c|c|c|c|c|c|c|}
\hline \multirow{2}{*}{$\begin{array}{l}\text { Size } \\
\text { No. }\end{array}$} & \multirow{2}{*}{$\begin{array}{l}\text { Working } \\
\text { load (in } \\
\text { pounds) }\end{array}$} & \multirow{2}{*}{$\begin{array}{c}\text { Diam- } \\
\text { eter of } \\
\text { pin }\end{array}$} & \multirow{2}{*}{ A } & \multirow{2}{*}{ B } & \multirow{2}{*}{ C } & \multirow{2}{*}{$\mathrm{D}$} & \multirow{2}{*}{$\mathrm{E}$} & \multirow{2}{*}{$\mathrm{F}$} & \multirow{2}{*}{$\mathrm{G}$} & \multirow{2}{*}{$\mathrm{H}$} & \multicolumn{2}{|c|}{ Rivets } \\
\hline & & & & & & & & & & & Num- & $\begin{array}{l}\text { Diam- } \\
\text { eter }\end{array}$ \\
\hline $\begin{array}{l}1 \\
2 \\
3 \\
4\end{array}$ & $\begin{array}{l}2,240 \\
3,000 \\
4,340 \\
5,320\end{array}$ & $\begin{array}{r}5 / 8 \\
3 / 4 \\
7 / 8 \\
1^{7 / 8}\end{array}$ & $\begin{array}{l}11 / 16 \\
13 / 16 \\
15 / 16 \\
13 / 16\end{array}$ & $\begin{array}{l}5 / 8 \\
3 / 4 \\
7 / 8 \\
1^{7 / 8}\end{array}$ & $\begin{array}{l}15 / 16 \\
17 / 16 \\
111 / 16 \\
115 / 16\end{array}$ & $\begin{array}{l}1^{7 / 8} \\
11 / 4 \\
17 / 16\end{array}$ & $\begin{array}{l}11 / 16 \\
13 / 16 \\
15 / 16 \\
11 / 8\end{array}$ & $\begin{array}{l}35 / 8 \\
41 / 8 \\
47 / 8 \\
51 / 2\end{array}$ & $\begin{array}{l}3 / 8 \\
3 / 8 \\
1 / 2 \\
1 / 2\end{array}$ & $\begin{array}{l}21 / 4 \\
23 / 4 \\
31 / 8 \\
3^{11 / 16}\end{array}$ & $\begin{array}{l}3 \\
3 \\
3 \\
4\end{array}$ & $\begin{array}{l}1 / 2 \\
1 / 2 \\
58 \\
58\end{array}$ \\
\hline $\begin{array}{l}5 \\
6 \\
7 \\
8\end{array}$ & $\begin{array}{r}7,080 \\
8,380 \\
10,480 \\
12,040\end{array}$ & $\begin{array}{l}11 / 8 \\
11 / 4 \\
13 / 8 \\
11 / 2\end{array}$ & $\begin{array}{l}13 / 16 \\
15 / 16 \\
17 / 16 \\
1 \% 16\end{array}$ & $\begin{array}{l}118 \\
11 / 4 \\
136 \\
116\end{array}$ & $\begin{array}{l}23 / 16 \\
27 / 16 \\
29 / 16 \\
2^{15} / 16\end{array}$ & $\begin{array}{l}19 / 16 \\
113 / 16 \\
17 / 8 \\
23 / 16\end{array}$ & $\begin{array}{l}11 / 4 \\
138 \\
11 / 2 \\
15 / 8\end{array}$ & $\begin{array}{l}6 \\
67 / 8 \\
716 \\
8\end{array}$ & $\begin{array}{r}58 \\
3 / 4 \\
1^{3 / 4}\end{array}$ & $\begin{array}{l}4 \\
49 / 16 \\
43 / 4 \\
51 / 4\end{array}$ & $\begin{array}{l}4 \\
4 \\
4 \\
4\end{array}$ & $\begin{array}{r}3 / 4 \\
7 / 8 \\
7 / 8 \\
1^{7 / 8}\end{array}$ \\
\hline $\begin{array}{r}9 \\
10 \\
11 \\
12\end{array}$ & $\begin{array}{l}14,560 \\
16,400 \\
21,360 \\
25,840\end{array}$ & $\begin{array}{l}15 / 8 \\
13 / 4 \\
17 / 8 \\
218\end{array}$ & $\begin{array}{l}111 / 16 \\
113 / 16 \\
115 / 16 \\
23 / 16\end{array}$ & $\begin{array}{l}158 \\
13 / 4 \\
178 \\
218\end{array}$ & $\begin{array}{l}33 / 16 \\
336 \\
31 / 2 \\
33 / 4\end{array}$ & $\begin{array}{l}27 / 16 \\
238 \\
31 / 16 \\
2^{13 / 16}\end{array}$ & $\begin{array}{l}13 / 4 \\
17 / 8 \\
2 \\
214\end{array}$ & $\begin{array}{c}83 / 4 \\
9 \\
105 / 8 \\
105 / 8\end{array}$ & $\begin{array}{l}11 / 8 \\
11 / 8 \\
11 / 8 \\
11 / 8\end{array}$ & $\begin{array}{l}511 / 16 \\
57 / 8 \\
71 / 2 \\
71 / 2\end{array}$ & $\begin{array}{l}4 \\
4 \\
6 \\
6\end{array}$ & $\begin{array}{l}118 \\
11 / 8 \\
11 / 8 \\
11 / 8\end{array}$ \\
\hline
\end{tabular}

PAD EYES AND LINKS, H NO. 13-1926

Material.-Pads to be of cast steel in accordance with requirements of the American Bureau of Shipping for ship-construction steel castings. Links to be of wrought steel in accordance with the requirements of the American Bureau of Shipping for chains. Inspection and tests to be required only when specified in the purchase order. 


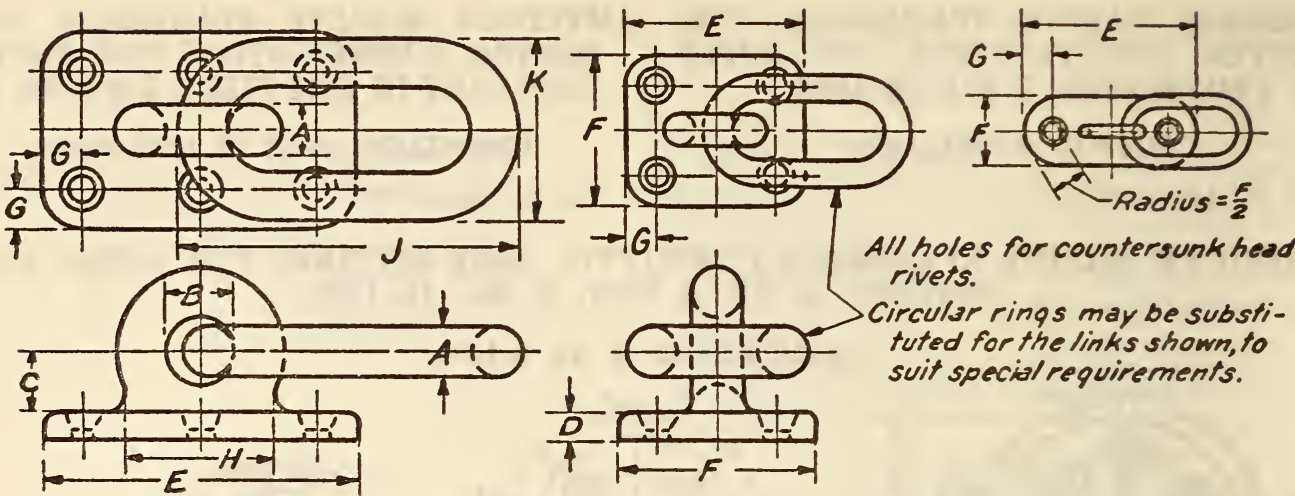

Figdre 541.-Pad eyes and links, American Marine standard H No. 13-1926

[All dimensions in inches]

\begin{tabular}{|c|c|c|c|c|c|c|c|c|c|c|c|c|c|}
\hline \multirow{2}{*}{$\begin{array}{l}\text { Size } \\
\text { No. }\end{array}$} & \multirow{2}{*}{$\begin{array}{l}\text { Working } \\
\text { load (in } \\
\text { pounds) }\end{array}$} & \multirow[b]{2}{*}{ A } & \multirow[b]{2}{*}{ B } & \multirow[b]{2}{*}{ C } & \multirow[b]{2}{*}{ D } & \multirow[b]{2}{*}{$\mathbf{E}$} & \multirow[b]{2}{*}{$F$} & \multirow[b]{2}{*}{$G$} & \multirow[b]{2}{*}{ H } & \multirow[b]{2}{*}{$\mathrm{J}$} & \multirow[b]{2}{*}{$\mathrm{K}$} & \multicolumn{2}{|c|}{ Rivets } \\
\hline & & & & & & & & & & & & Num- & $\begin{array}{l}\text { Diam- } \\
\text { eter }\end{array}$ \\
\hline $\begin{array}{l}1 \\
2 \\
3 \\
4 \\
5\end{array}$ & $\begin{array}{l}2,400 \\
4,000 \\
5,800 \\
7,800 \\
9,800\end{array}$ & $\begin{array}{l}1 / 2 \\
5 / 8 \\
3 / 4 \\
7 / 8 \\
1^{7 / 8}\end{array}$ & $\begin{array}{l}3 / 4 \\
7 / 8 \\
1 \\
118 \\
13 / 8\end{array}$ & $\begin{array}{c}5 / 8 \\
3 / 4 \\
7 / 8 \\
1 \\
13 / 16\end{array}$ & $\begin{array}{l}3 / 8 \\
1 / 2 \\
5 / 8 \\
3 / 4 \\
3 / 4\end{array}$ & $\begin{array}{l}41 / 2 \\
51 / 2 \\
61 / 2 \\
71 / 2 \\
6\end{array}$ & $\begin{array}{l}13 / 4 \\
23 / 16 \\
25 / 8 \\
3 \\
51 / 2\end{array}$ & $\begin{array}{c}3 / 4 \\
7 / 8 \\
11 / 16 \\
11 / 4 \\
11 / 4\end{array}$ & $\begin{array}{l}15 / 8 \\
115 / 16 \\
234 \\
258 \\
3\end{array}$ & $\begin{array}{l}31 / 4 \\
41 / 16 \\
478 \\
511 / 16 \\
612\end{array}$ & $\begin{array}{l}13 / 4 \\
23 / 16 \\
258 \\
3116 \\
312\end{array}$ & $\begin{array}{l}2 \\
2 \\
2 \\
2 \\
4\end{array}$ & $\begin{array}{l}1 / 2 \\
5 / 8 \\
3 / 4 \\
7 / 8 \\
7 / 8\end{array}$ \\
\hline $\begin{array}{r}6 \\
7 \\
8 \\
9 \\
10\end{array}$ & $\begin{array}{l}12,200 \\
14,600 \\
17,400 \\
20,400 \\
23,800\end{array}$ & $\begin{array}{l}118 \\
11 / 4 \\
138 \\
11 / 2 \\
158\end{array}$ & $\begin{array}{l}11 / 2 \\
158 \\
13 / 4 \\
2 \\
218\end{array}$ & $\begin{array}{l}15 / 16 \\
17 / 16 \\
1916 \\
13 / 4 \\
17 / 8\end{array}$ & $\begin{array}{l}1^{3 / 4} \\
1 \\
1 \\
1\end{array}$ & $\begin{array}{l}61 / 4 \\
63 / 4 \\
73,4 \\
93 / 4 \\
93 / 4\end{array}$ & $\begin{array}{l}51 / 2 \\
61 / 4 \\
61 / 2 \\
63 / 4 \\
67 / 8\end{array}$ & $\begin{array}{l}1134 \\
1388 \\
138 \\
138 \\
138\end{array}$ & $\begin{array}{l}33 / 8 \\
33 / 4 \\
41 / 8 \\
41 / 2 \\
47 / 8\end{array}$ & $\begin{array}{c}7516 \\
818 \\
815 / 16 \\
93 / 4 \\
10 \% 16\end{array}$ & $\begin{array}{l}315 / 16 \\
4^{33} 8 \\
4^{13 / 16} \\
51 / 4 \\
5^{111 / 16}\end{array}$ & $\begin{array}{l}4 \\
4 \\
4 \\
6 \\
6\end{array}$ & $\begin{array}{l}1^{7 / 8} \\
1 \\
1 \\
1\end{array}$ \\
\hline $\begin{array}{l}11 \\
12 \\
13 \\
14 \\
15\end{array}$ & $\begin{array}{l}27,400 \\
31,400 \\
35,800 \\
40,400 \\
45,400\end{array}$ & $\begin{array}{l}13 / 4 \\
17 / 8 \\
2 \\
21 / 8 \\
214\end{array}$ & $\begin{array}{l}21 / 4 \\
23,8 \\
21 / 2 \\
25,8 \\
23 / 4\end{array}$ & $\begin{array}{l}2 \\
2118 \\
214 \\
2318 \\
21 / 2\end{array}$ & $\begin{array}{l}1 \\
1118 \\
118 \\
114 \\
11 / 4\end{array}$ & $\begin{array}{r}934 \\
1018 \\
1118 \\
111 / 2 \\
131 / 2\end{array}$ & $\begin{array}{l}7 \\
73 / 4 \\
73 / 4 \\
81 / 2 \\
81 / 2\end{array}$ & $\begin{array}{l}13 / 8 \\
1 \% 16 \\
1916 \\
13 / 4 \\
13 / 4\end{array}$ & $\begin{array}{l}51 / 8 \\
51 / 2 \\
57 / 8 \\
61 / 4 \\
65 \% 8\end{array}$ & $\begin{array}{l}113 / 8 \\
123 / 16 \\
13 \\
13^{13} / 16 \\
145 / 8\end{array}$ & $\begin{array}{l}61 / 8 \\
6916 \\
7 \\
77 / 16 \\
77 / 8\end{array}$ & $\begin{array}{l}6 \\
6 \\
6 \\
6 \\
6\end{array}$ & $\begin{array}{l}1 \\
11 / 8 \\
11 / 8 \\
11 / 4 \\
11 / 4\end{array}$ \\
\hline
\end{tabular}

AMERICAN MARINE STANDARDS COM-| bitts (see 611.13,p.642) except that the material is MITTEE, MOORING BITTS, CAST STEEL, to be grade 1 steel, annealed, in accordance with H NO. $17-1926$

\section{MOORING BITTS}

(Specifications for cast-steel mooring bits are substantially the same as those for cast-iron mooring American Bureau of Shipping specifications (sec: 40 , par. 8 , of the bureau's rules), and the thickness of metal of the top and base of the bitt is, in general, less than the dimensions for cast-iron bitts of the same size.) 


\section{AMERICAN MARINE STANDARDS COMMITTEE, SHIP SCUPPER AND DRAINS, H No. $35-1928$}

DECK SCUPPER

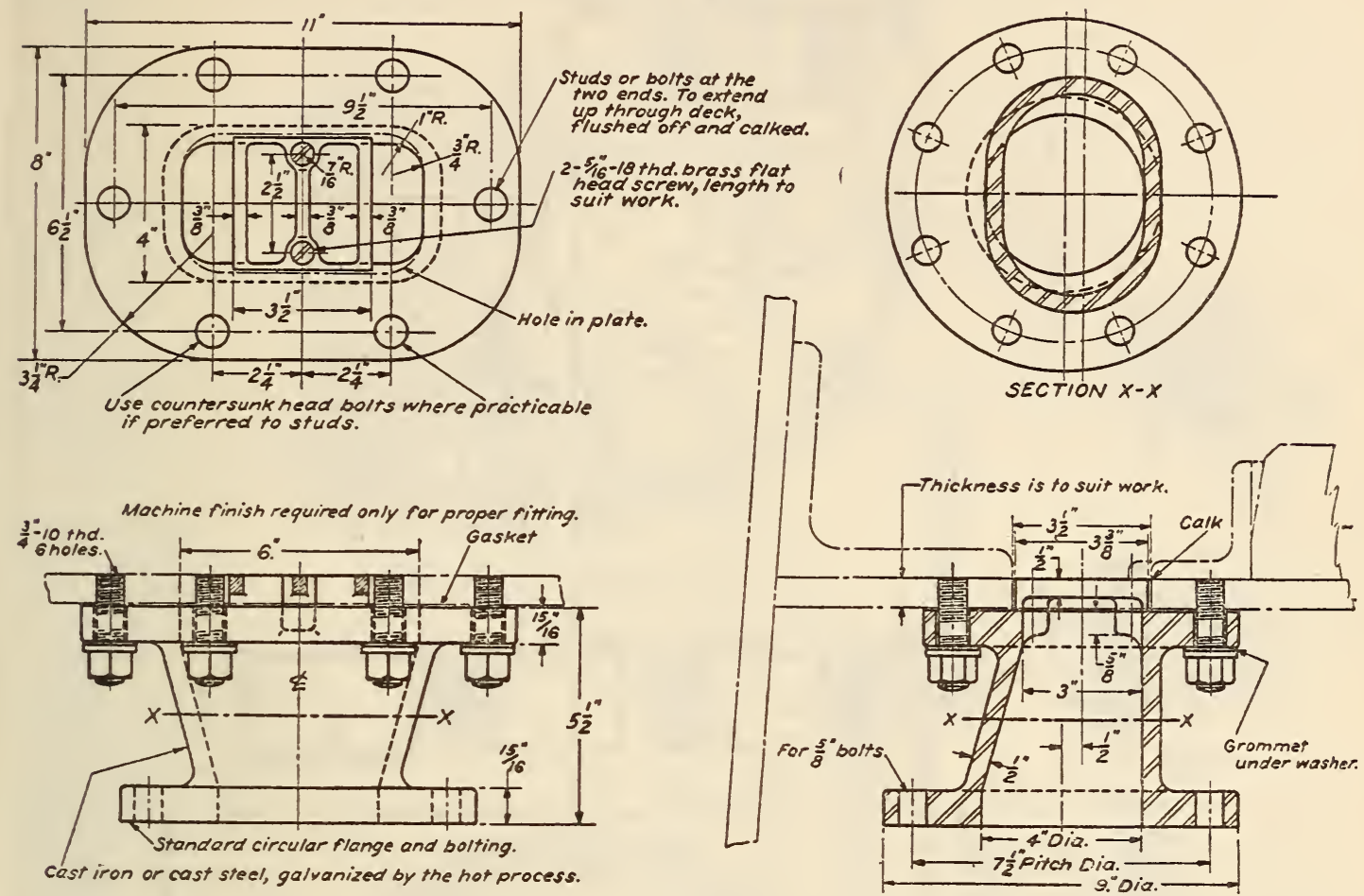

FIGURE 542.-4-inch weather deck scupper

AMERICAN MARINE STANDARDS COMMITTEE, BOLLARDS AND CLEATS FOR DOCKS, P No. 1 TO P No. 4, 1931

\section{BOLLARDS AND CLEATS}

(See 611.19, p. 664.)

AMERICAN RAILWAY ASSOCIATION, MECHANICAI DIVISION, COUPIER YOKES, RECOMMENDED PRACTICE, 1929

\section{COUPLER YOKES}

(See 605.13, p. 259.)

AMERICAN RAILWAY ASSOCIATION, MECHANICAI DIVISION, DRIVING AND TRAILER WHEELS FOR LOCOMOTIVES, RECOMMENDED PRACTICE, 1928

\section{LOCOMOTIVE WHEELS}

Cast-steel driving-wheel centers should preferably be uncut and shrinkage slots omitted; if cut, slots should be machined out and closed with solid castiron liners driven in. No lead or white metal should be used.
For wheel centers, when the permissible total weight of the locomotive will allow, the rims should preferably be cast solid without cores, so as to obtain the maximum section and have full bearing of tires; the section in square inches should be approximately 0.45 of the sectional area of the tire when new.

In presenting Figure 543, the committee recommends hubs and solid rim sections as shown in diagrams $5-b$ and 5-c for all locomotive designs in which limitations of weight and counterbalance will permit their use. These features, together with the spokes of diagrams $5-d$ and $5-e$, greatly simplify pattern and foundry work in the manufacture of driving wheels. The solid rim also provides a better support for tires than a cored rim, and solid rims are therefore recommended in all cases where they can be used.

The demands for locomotives of increased power which must be built without exceeding certain very definite limits of weight and counterbalance effects have made it desirable to recognize and approve any feature of design that will reduce weight and for this reason the committee has included as an alternative practice the cored rim section of diagrams $5-f, 5-g$, and $5-h$, and the elliptical spoke section of diagrams $5-i$ ana $5-j$, which conform to the foundry practice usually followed in producing driving wheels with cored rims. Diagrams 5- $j$ and $5-k$ show an arrangement of reinforcing webs between spokes, which is recommended as an optional feature on account of its extensive use on a number of roads. 


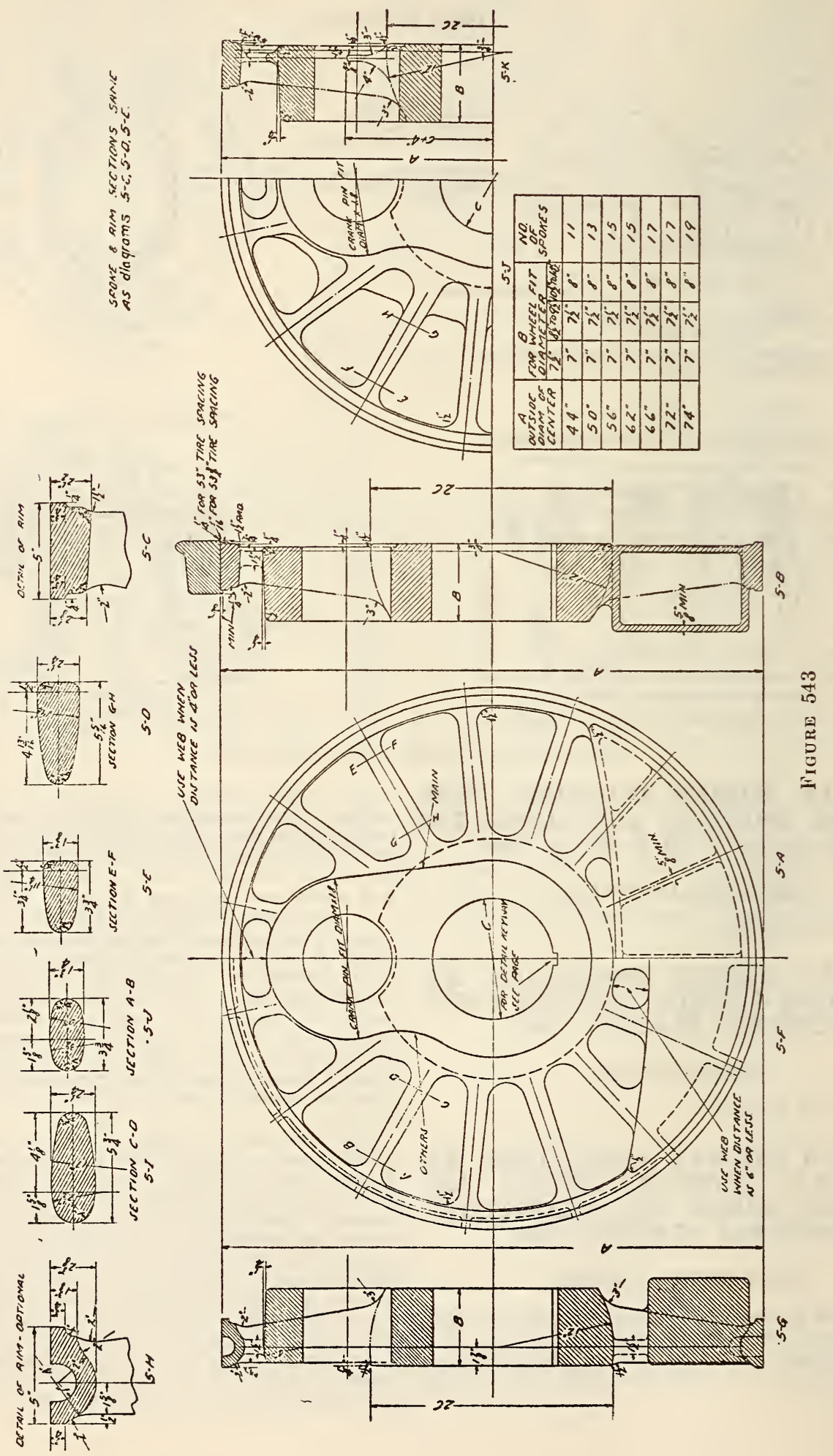


The committee recommends solid counterweights $\mid$ weights, the committee recommends the pocket concast as an integral part of the driving-wheel center in all cases in which the desired counterbalance can \begin{tabular}{l|l|l} 
be secured with solid cast counterweights. Small & the rims of the wheels, these \\
lead pockets as shown by diagram 5-f are recom- & covered when tires are in place.
\end{tabular}

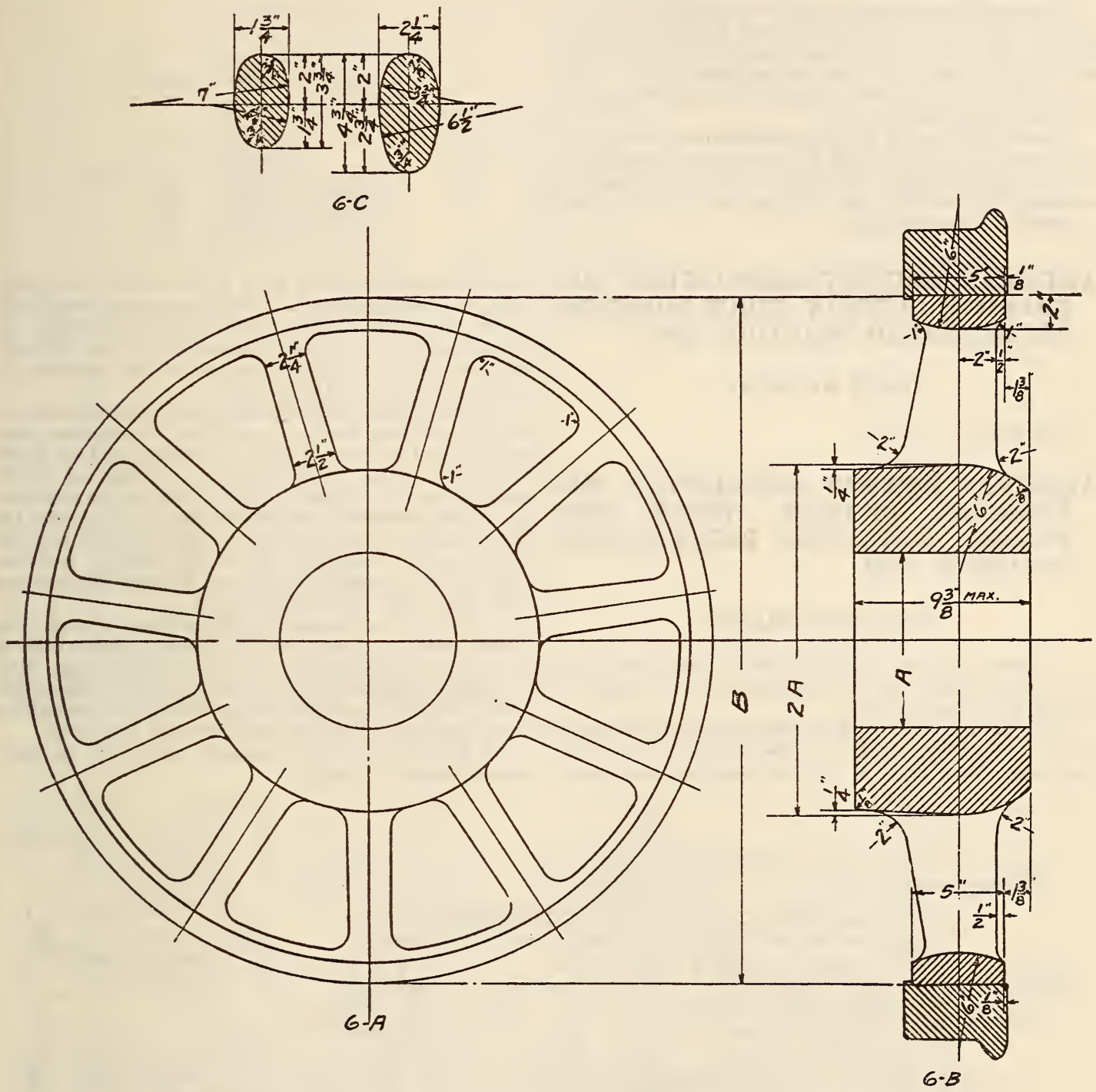

FigURE 544.-Inside and outside hub faces to be machined to suit wheel seat on axle

\begin{tabular}{|c|c|}
\hline$A$ & Journal \\
\hline $\begin{array}{r}\text { Inches } \\
83 / 8 \\
93 / 8 \\
10^{3} / 8\end{array}$ & $\begin{array}{l}\text { Inches } \\
7 \text { by } 14 \\
8 \text { by } 14 \\
9 \text { by } 14\end{array}$ \\
\hline
\end{tabular}

\begin{tabular}{|c|c|}
\hline $\begin{array}{c}\mathrm{B} \\
\text { diameter }\end{array}$ & $\begin{array}{c}\text { Num- } \\
\text { ber of } \\
\text { spokes }\end{array}$ \\
\cline { 1 - 2 } & \\
$\begin{array}{c}\text { Inches } \\
333^{3}\end{array}$ & 9 \\
37 & 11 \\
40 & 11 \\
43 & 13 \\
\hline
\end{tabular}

mended for obtaining closer adjustments than can be had with solid counterweights. Solid cast counterweights can usually be used with entire satisfaction on all driving wheels other than main, and on ome locomotives, particularly those having drivings wheels of relatively large diameters, solid counterweights can be used on main driving wheels. For driving wheels which require lead-filled counterstruction shown by diagram 5-a, in which the pockets are arranged to be filled through cored holes in the rims of the wheels, these holes being entirely covered when tires are in place. 
steel wheels that will be entirely satisfactory for use on trailer trucks requiring wheel centers $33 \frac{3}{4}$ inches in diameter or smaller. Some rolled-steel trailer wheels of this size are now in use, but their performance indicates that further development and improvement is desirable.

The experience of some of the roads has been that more trouble has been experienced with breakage of tires of the bolted type than has been experienced with plain shrinkage tires of the same sizes. The committee therefore feels that shrinkage tires should be used in all cases.

Cast steel as per A. R. A. specifications for carbonsteel castings, adopted as recommended practice in 1913 and revised in 1919, is recommended as the material to be used for driving and trailer wheel centers of these designs.

AMERICAN RAILWAY ASSOCIATION, MECHANICAL DIVISION, TRUCK BOLSTERS, RECOMMENDED PRACTICE, 1929

\section{TRUCK BOLSTERS}

(See 605.13, p. 255.)

\section{AMERICAN RAILWAY ASSOCIATION, ME- CHANICAL DIVISION, TRUCK SIDE FRAMES, CAST STEEL, RECOMMENDED PRACTICE, 1929}

\section{TRUCK SIDE FRAMES}

1. Scope.-These specifications cover cast-steel side frames for freight equipment with integral or separable journal boxes.

2. Material.--Shall be of cast steel in accordance with grade B of A. R. A. specifications for steel castings, which form a part of these specifications. (b) $T$ sections, angles, and vertical web I beam sections are prohibited under these specifications.

4. Test requirements. - (a) The basis of test for side frames shall be the axle capacity (C) in accordance with the values given in Table 1 .

TABLE 1

\begin{tabular}{|c|c|c|}
\hline $\begin{array}{c}\text { Size of } \\
\text { journal }\end{array}$ & $\begin{array}{c}\text { Capacity } \\
\text { of axle }\end{array}$ & $\begin{array}{c}\text { Type of } \\
\text { truck }\end{array}$ \\
\hline & $\mathrm{C}$ & \\
& $\begin{array}{c}\text { Pounds } \\
5 \text { by } 9-\ldots\end{array}$ & 32,000 \\
$51 / 2$ by $10 .-$ & 40,000 & $2 \mathrm{C}$. \\
6 by $11 .-$ & 50,000 & $2 \mathrm{D}$. \\
$61 / 2$ by $12 .-$ & 60,000 & $2 \mathrm{~F}$. \\
\hline
\end{tabular}

(b) The vertical-test load shall act on the spring base, or its equivalent. The transverse load shall act on the bolster guides, one-half on each guide, on a line located above the nominal center line through the two axles, an amount equal to the journal diameter of axle less $1 \frac{1}{2}$ inches.

(c) The deflection measuring instruments shall be located midway between supports of specimen and shall be set at zero under an initial load of 5,000 pounds. In the vertical test only, a load of 20,000 pounds shall be applied to the frame for five minutes, and then released to 5,000 pounds. The load for the deflection measurement shall be 5,000 pounds additional to the load shown in Table 2, but the load for the permanent set and minimum breaking load shall be as given in Table 2 .

5. Tests.-(a) At least two specimens of each new design shall be tested on a suitable static testing machine. The vertical and transverse tests shall be made separately. In the case of separable journal boxes, the boxes and parts shall be assembled and bolted into place for the vertical test only.

(b) Side frames shall comply with the requirements shown in Table 2.

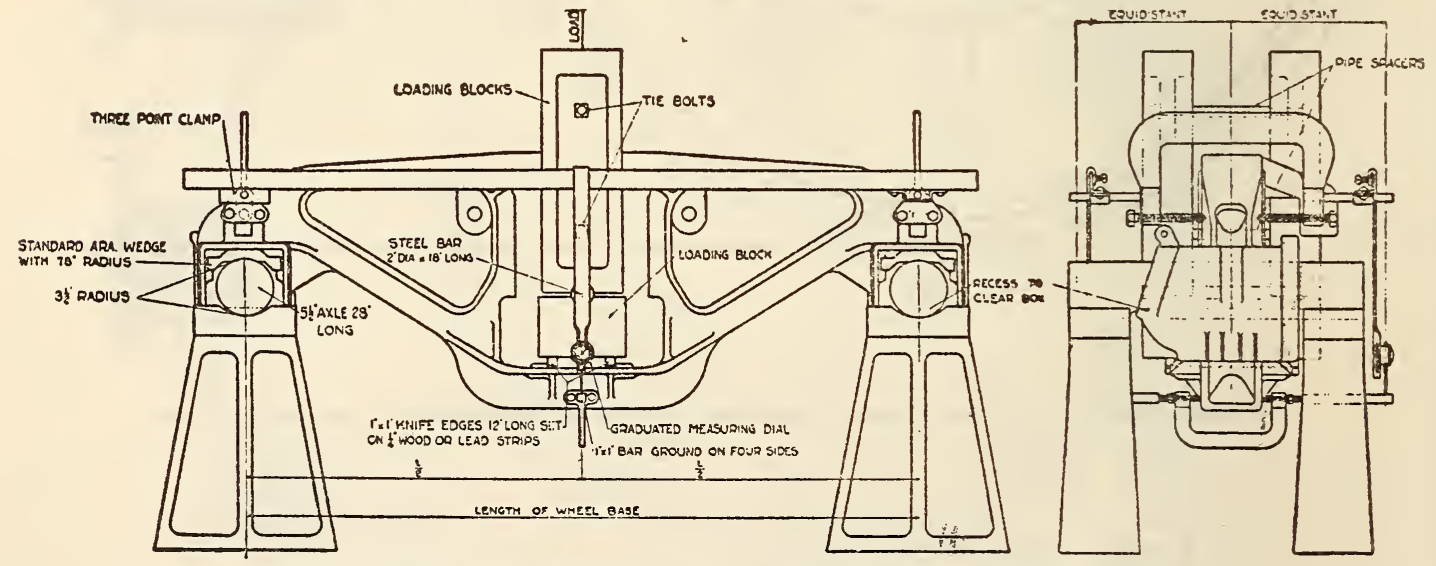

FIGURE. 545.-Truck side frames, cast steel design test requirements

3. Design.-(a) The basis of design shall be the axle capacity $\mathrm{C}$. The maximum combined unit stress in the design shall not exceed 16,000 pounds per square inch. The vertical design load shall be taken as acting on the spring base (or its equivalent, for test) and shall be $1 \frac{1}{2}$ C. The transverse load shall be taken as acting on the bolster guides, onehalf on each guide-on a line located above the nominal center line through the two axles, an amount equal to the journal diameter of axle less $1 \frac{11}{2}$ inches, and shall be $0.4 \mathrm{C}$.
Table 2

\begin{tabular}{|c|c|c|}
\hline & \multicolumn{2}{|c|}{ Side frames } \\
\hline & Vertical & $\begin{array}{c}\text { Trans- } \\
\text { verse }\end{array}$ \\
\hline $\begin{array}{l}\text { Load for zero setting of instrument.-....-pounds. } \\
\text { Load at } 0.04 \text { inch maximum deflection.-. }\end{array}$ & $\begin{array}{r}5,000 \\
2,25 \mathrm{C}\end{array}$ & 5,000 \\
\hline Load at 0.07 inch maximum deflection & & $0.60 \mathrm{C}$ \\
\hline $\begin{array}{l}\text { Load at } 0.01 \text { inch maximum permanent set } \\
\text { Minimum breaking load }\end{array}$ & $\begin{array}{r}4.50 \mathrm{C} \\
12 \mathrm{C}\end{array}$ & $1.20 \mathrm{C}$ \\
\hline
\end{tabular}



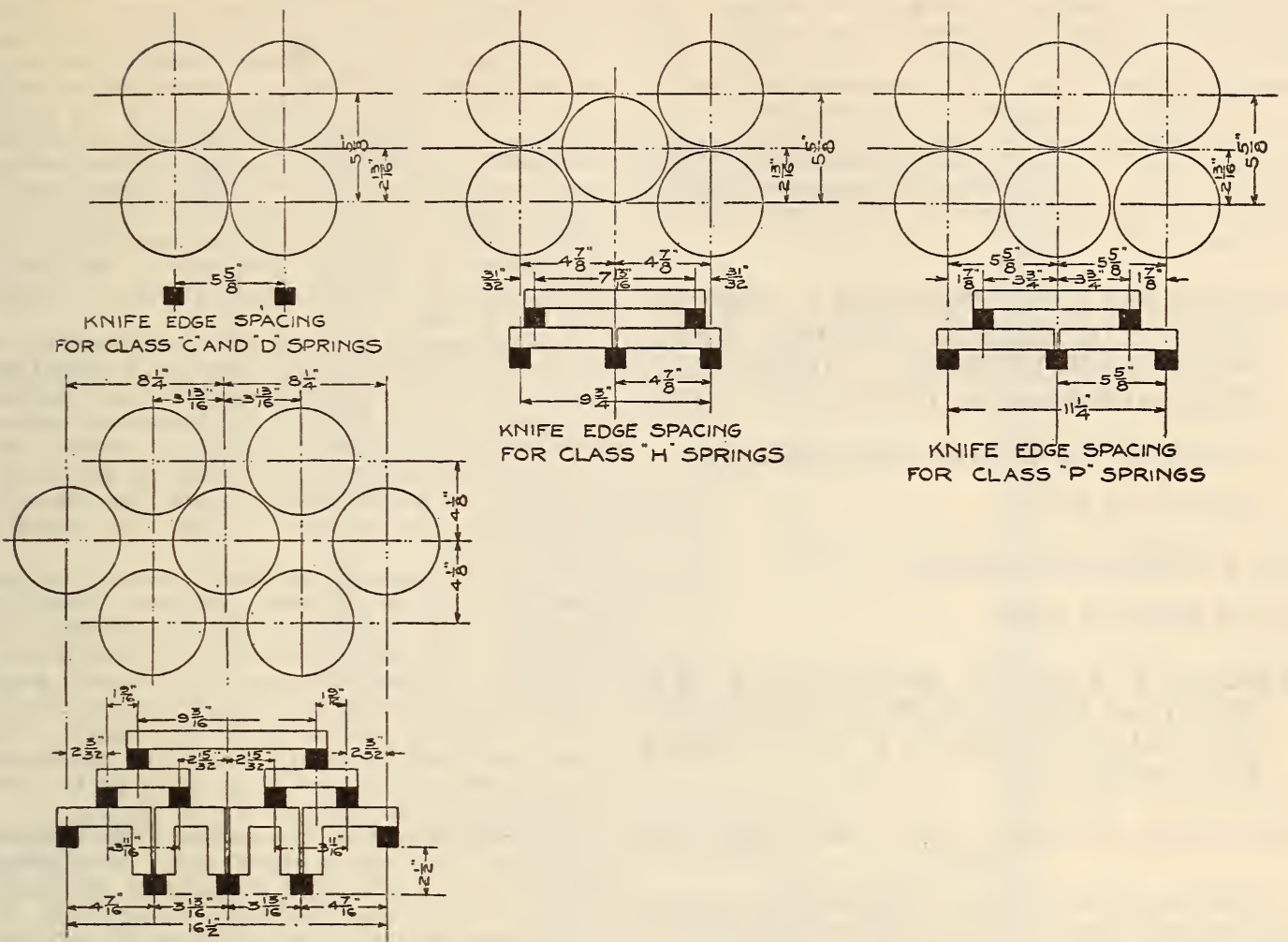

KNIFE EDGE SPACING FOR T-SPRING NEST

WITH 2 END-SPRINGS SEATED ABOVE MIDDLE GROUP

FIGURE 546.-Truck side frames, cast-steel knife-edge spacings for test loading
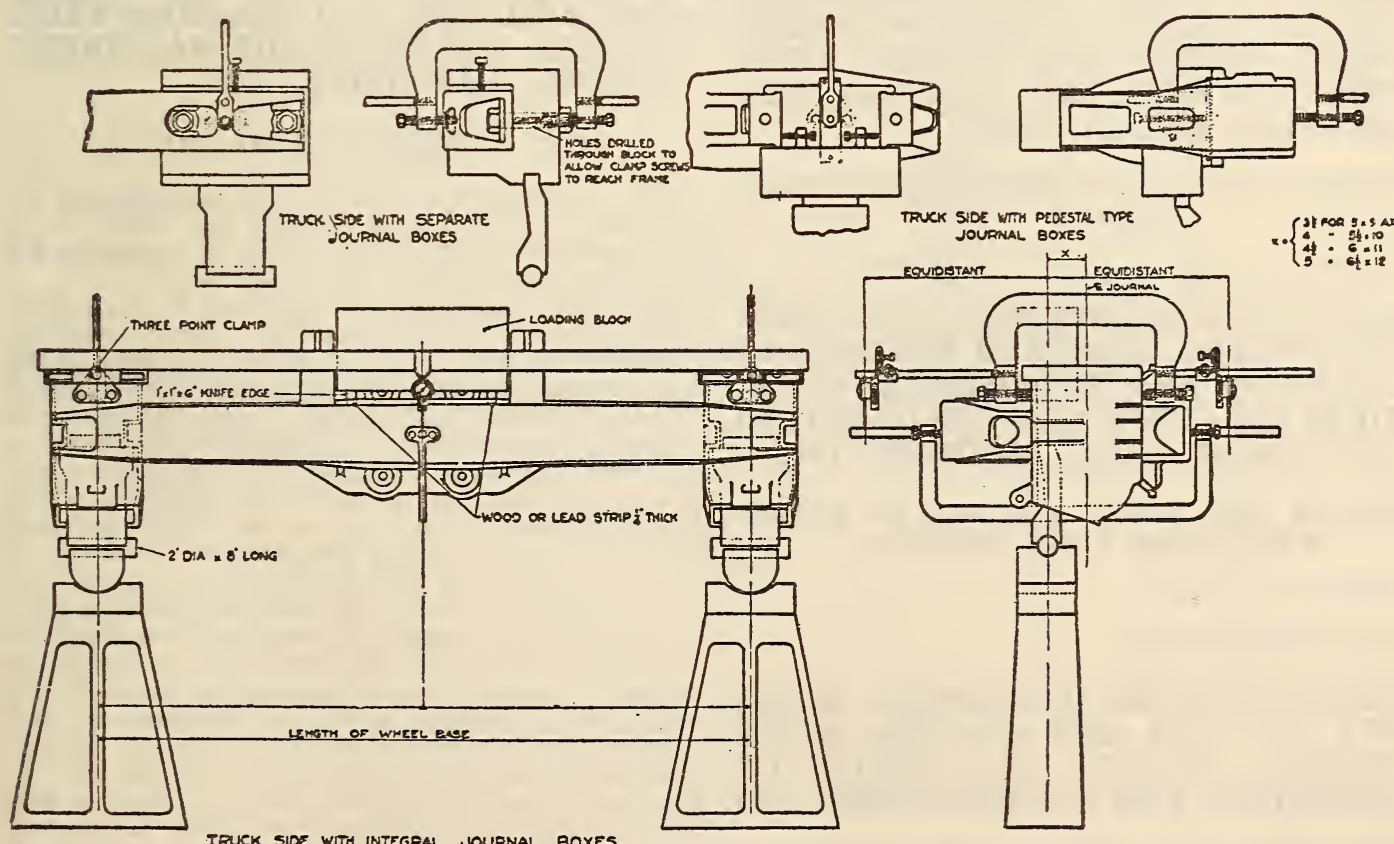

FigdRe 547.-Frames, cast-steel, design test requirements 
6. Loading diagrams.-Figure 545 shows the recommended method of vertical-test loading. The knife-edges should be located in accordance with the spring grouping as shown in Figure 546. Figure 547 shows the method of transverse test loading.

7. Marking.-In addition to the requirements for marking given in A. R. A. specifications for steel castings, all truck side frames shall bear the cast letters A. R. A., followed by the date of adoption or revision of this specification to which they conform, such as "A. R. A.-29."

UNITED STATES GOVERNMENT, DEPARTMENT OF COMMERCE, BUILDERS' HARDWARE (NONTEMPLATE), COMMERCIAL STANDARD CS22-30, DECEMBER 11, 1930

BUILDERS' HARDWARE (NONTEMPLATE)

(See 617.0 , p. 977.)

\subsection{STEEL FORGINGS.}

\subsection{GENERAL ITEMS.}

AMERICAN RAILWAY ASSOCIATION, MECHANICAL DIVISION, STANDARD DROP. TEST MACHINE FOR A. R. A. COUPLERS AND AXLES, 1911

\section{DROP-TEST MACHINE FOR COUPLERS AND} AXLES

(The accompanying diagram shows the standard drop-test machine for couplers and axles adopted by the American Railway Association. The manual of the association shows this machine set up for standard jerk, striking, face, knuckle pin, and separate knuckle tests. See fig. 548.)

AMERICAN SOCIETY FOR TESTING MATERIALS, STANDARD METHODS OF SAMPLING ROLLED AND FORGED STEEL PRODUCTS FOR CHECK ANALYSIS, DESIGNATION A130-30, 1930

SAMPLING ROLLED AND FORGED STEEL PROD-

(See 600.1, p. 20.)

ASSOCIATION OF AMERICAN STEEL MANUFACTURERS, STANDARD METHODS OF SAMPLING AND PERMISSIBLE VARIATIONS FOR CHECK ANALYSIS OF ROLLED AND FORGED STEEL PRODUCTS, 1928

SAMPLING AND CHECK ANALYSIS OF ROLLED AND FORGED STEEL PRODUCTS

(See 600.1, p. 47.)

\subsection{FORGED STEEL.}

AMERICAN BUREAU OF SHIPPING, RULES FOR BUILDING AND CLASSING STEEL VESSELS; QUALITY AND TESTING OF MATERIAIS, SHIP CONSTRUCTION, 1930

\section{INGOT STEEL AND WELDED STEEL FORGINGS}

(For general information and requirements, see 600.0, p. 2 .)
1. Ingot steel forgings are to be forged from sourd ingots made by the open-hearth process. The sectional area of the body of the forging when it leaves the hammer should not exceed one-fifth and no part of the forging should exceed three-fourths the area of the original ingot. Forgings are to be properly annealed in an annealing furnace, and must be free from defect. The tensile strength of the forgings is to be from 60,000 to 72,000 pounds per square inch. The elongations are not to be less than 30 per cent with 60,000 pounds nor 25 per cent with 72,000 pounds tensile strength measured on the standard test specimens of the American Bureau of Shipping. (See fig. 5, 600.1, p. 8.)

2. Specimens 1 inch by three-fourths inch with edges rounded to $1 / 16$-inch radius are to stand being bent cold to $180^{\circ}$ without fracture over a radius of one-fourth inch. The test specimens are to be cut lengthwise from a part of the forging which is not of less sectional area than the body of the forgings. They are not to be detached until they have been stamped by the surveyors nor until the forging has been annealed.

3. One tension and one bend test are to be taken from each forging. Where a number of small forgings are made from one ingot, specimens which represent each end of the ingot will be sufficient.

4. All ingot steel forgings are to be legibly marked in such a way that they can be traced to the heat from which the material has been made. Those which have satisfactorily passed the requirements are to be clearly stamped A. B., and with the identification marks furnished by the surveyor.

5. Welded steel forgings may be made from especially soft open-hearth ingot steel. Such forgings are to have a tensile strength between the limits of 49,000 and 56,000 pounds per square inch. The elongations are not to be less than 35 per cent at 49,000 pounds, nor 32 per cent at 56,000 pounds. The remaining tests and requirements are to be as prescribed above for ingot steel forgings.

AMERICAN BUREAU OF SHIPPING, RULES FOR BUILDING AND CLASSING STEEL VESSELS; MATERIALS FOR MACHINERY, BOILERS, AND PIPING, 1930

\section{STEEL FORGINGS FOR MACHINERY}

(For general requirements covering materials for machinery, boilers, and piping, see 600.0 , p. 1 . For requirements covering tests of materials for machirery, boilers, and piping, see 600.1, p. 7.)

1. Steel forgings for machinery shall be made from ingots manufactured by the open hearth, crucible, or electric process. The cross-sectional area of the unmachined finished forging shall not exceed 20 per cent of the area of the ingot. Palms, flanges, and similar enlargements on forgings shall be reduced so as not to exceed 70 per cent of the area of the ingot.

2. Unless especially approved by the surveyor, the discard by weight shall not be less than 5 per cent from the bottom and 25 per cent from the top of poured ingots; or 3 per cent from the bottom, and 10 per cent from the top of fiuid-compressed ingots.

3. All forgings must be sound and free from defects; they are also to be annealed in an annealing furnace of ample proportions to bring the whole forging to a uniform annealing temperature, and must not be removed from the annealing furnace until the temperature has dropped to about $700^{\circ} \mathrm{F}$. Forgings for special purposes may be quenched and drawn in place of annealing if previously sanctioned by the surveyor or approved on the plans submitted. The test specimens are to be cut longitudinally in direction of forgings, and where practicable taken at one-half the outside radius of the finished forging 


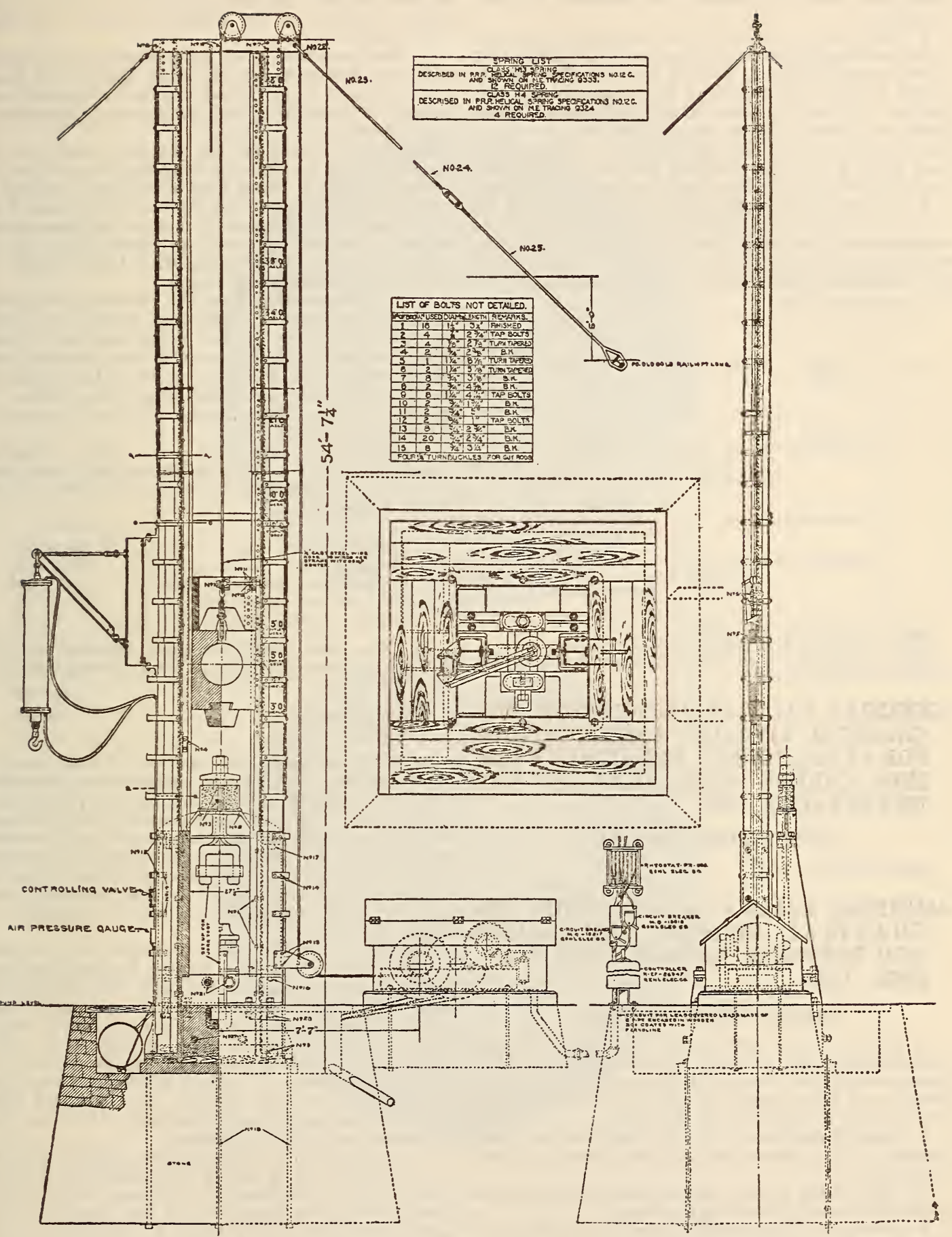

Figure 548.-Drop test machine for A. R. A. couplers and axles 
from a part having a sectional area of not less than the body of the forging; and they are not to be detached until they have been stamped by the surveyor and until the forging has received its final treatment.

4. One tension and one bend test are to be taken from each forging, except in the case of forgings over 7,000 pounds weight, and of all propeller shafts where tests will be required from each end of the forging. Where a number of bolts or pins are made from one larger forging, one tension and one bend test from the latter will be sufficient. Where one long forging is to be cut into two or more shafts, tests should be made from both ends of the forging.

5. All important forgings subject to test and inspection are to be examined during the process of manufacture when machined, or, in the case of shafts, when rough turned and when finished. Material of the following characteristics will be approved for the purposes noted:

\begin{tabular}{|c|c|c|c|c|}
\hline Grade & $\begin{array}{l}\text { Tensile } \\
\text { strength }\end{array}$ & Yield point & Minimum elonga- & $\begin{array}{l}\text { Bend of } \\
180^{\circ} \\
\text { radius } \\
\text { of bend }\end{array}$ \\
\hline \multirow[t]{2}{*}{$1 \ldots \ldots$} & \multirow[t]{2}{*}{$\begin{array}{c}\text { Lbs./in. }{ }^{2} \\
60,000-70,000\end{array}$} & \multirow{3}{*}{$\begin{array}{l}50 \text { per cent } \\
\text { of tensile } \\
\text { strength. }\end{array}$} & $\begin{array}{l}\text { Per cent } \\
180,000\end{array}$ & \multirow{2}{*}{$\begin{array}{l}\text { Inch } \\
\qquad 1 / 4\end{array}$} \\
\hline & & & $\begin{array}{l}\text { Tensile strength. } \\
\qquad 180,000\end{array}$ & \\
\hline $2 \ldots \ldots$ & $70,000-80,000$ & & $\overline{\text { Tensile strength. }}$ & \multirow{3}{*}{$3 / 8$} \\
\hline \multirow[t]{2}{*}{$3 \ldots \ldots$} & \multirow[t]{2}{*}{$80,000-90,000$} & \multirow{2}{*}{$\begin{array}{l}60 \text { per cent } \\
\text { of tensile } \\
\text { strength. }\end{array}$} & 180,000 & \\
\hline & & & Tensile strength. & \\
\hline
\end{tabular}

Grade 1: Approved for all purposes.

Grade 2: Approved for all purposes, excepting propeller shafts.

Grade 3: A pproved for all engine forgings, excepting main shafting, pistons, and connecting rods.

\section{AMERICAN RAILWAY ASSOCIATION, ME- CHANICAI DIVISION; SPECIFICATIONS FOR AXLES, SHAFTS, AND OTHER FORG- INGS, CARBON STEEL, ANNEALED AND UNANNEALED, 1926}

\section{CARBON-STEEL FORGINGS}

(See 611.52, p. 765.)

\section{AMERICAN RAILWAY ASSOCIATION, ME- CHANICAL DIVISION; NORMALIZED AND TEMPERED CARBON-STEEL FORG- INGS, 1929}

\section{CARBON-STEEL FORGINGS}

1. Scope.-These specifications cover normalized carbon-steel shafts; driving, engine-truck, and trailer axles; crank pins; main and side rods; piston rods; straps; and other steel forgings for locomotives and cars as may be specified on the order.

2. Basis of purchase.-(a) The manufacturer shall employ the normalized and tempered method of heat treatment.

(b) Quenching in any liquid medium will not be permitted under these specifications.

3. Process.-The steel shall be made by the openhearth or other process approved by the purchaser.

4. Discard. - A sufficient discard shall be made from each ingot to insure freedom from piping and segregation.

5. Forging practice.-The forgings may be made direct from the ingot or from billets, the total reduction from ingot to forging being not less than three to one.
6. Prolongation for test.-Full-sized prolongation shall be left on at least 20 per cent of the forgings for test purposes. For forgings with large ends or collars the prolongation may be of the same cross section as that of the forgings back of the large end or collar.

7. Heat treatment.-(a) After forging, the forgings shall be allowed to cool to below the critical range and shall then be subjected to a heat treatment consisting of normalizing and tempering as specified in this section.

(b) For normalizing, the forgings shall be uniformly heated to a proper temperature to refine the grain and, after being held a sufficient length of time at this temperature, shall be withdrawn from the furnace and allowed to cool in the atmosphere to below the critical range, protected from rain or snow. A group of forgings thus treated shall be known as a normalizing charge.

(c) For tempering, after the previous treatment, the forgings shall be uniformly heated to a temperature below the critical range and, after being held a proper length of time at this temperature, shall be allowed to cool uniformly either in the furnace or in the atmosphere, protected from rain or snow. A group of forgings thus treated shall be known as a tempering charge.

8. Chemical composition.-(a) The chemical composition of the forgings determined from samples taken as described in section 10 shall conform to the following:

Per cent

Carbon

$0.40-0.55$

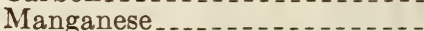

Phosphorus, not more than.... 60

Sulphur, not more than...... 045

Silicon, not less than

(b) The steel shall not show residual alloying metal impurities in excess of the following limits:

Per cent

Chromium_.................. 0.18

Nickel

9. Ladle analyses.-An analysis of each melt of steel shall be made by the manufacturer to determine the percentage of carbon, manganese, phosphorus, sulphur, and silicon. This analysis shall be made on drillings taken at least one-fourth inch beneath the surface of the test ingot taken during the pouring of the melt. The chemical composition thus determined shall be reported to the purchaser, and shall conform to the requirements specified in section 8 .

10. Check analysis.-(a) An analysis may be made by the purchaser from a forging representing each melt. The chemical composition thus determined shall conform to the requirements specified in section 8. Drillings for analysis may be taken with a $5 / 8$-inch drill from the forging or from a full-sized prolongation of the same, at any point midway between the center and surface of solid forgings, and at any point midway between the inner and outer surface of the wall of bored forgings; or turnings may be taken from a test specimen.

(b) In addition to the complete analysis specified in paragraph $(a)$, a carbon determination may be made by the purchaser on drillings taken with a $5 / 8$-inch drill from the center of the forging or fullsized prolongation of the same, to determine by the variation in carbon the amount of segregation. This determination shall show the carbon content to be within 12 per cent of the amount found at any point midway between the center and surface. This requirement does not apply to bored forgings.

11. Tension tests. - (a) The forgings shall conform to the following minimum requirements as to tensile properties: 


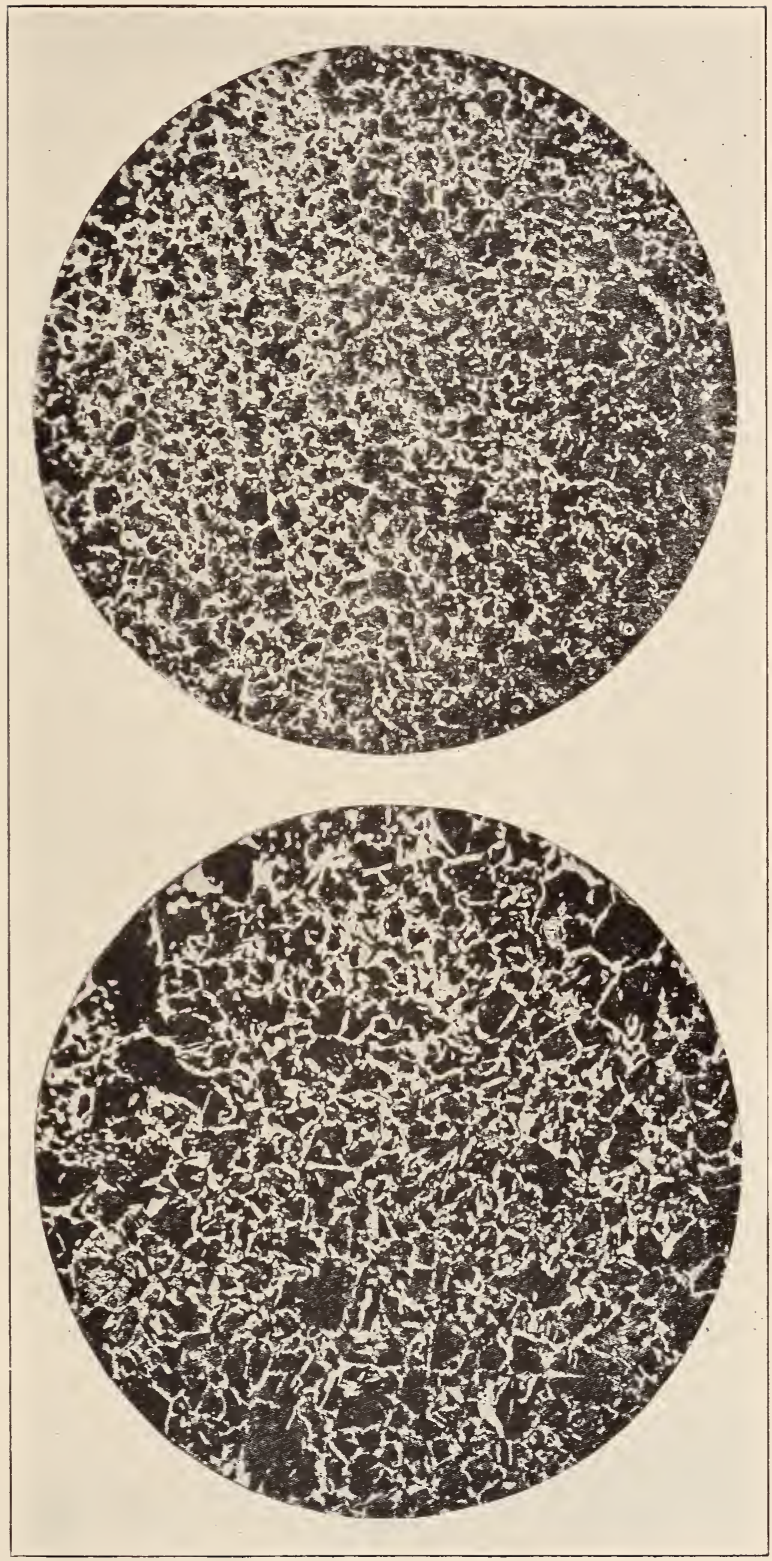

Figure 549.-Upper, fine-grained uniform structure; desired. Lower, maximum grain structure; acceptable (For specification see p. 752.) 



\begin{tabular}{|c|c|c|c|c|c|c|}
\hline \multirow{2}{*}{ Size, outside diameter or over-all thickness } & \multirow{2}{*}{$\begin{array}{l}\text { Tensile } \\
\text { strength }\end{array}$} & \multirow{2}{*}{$\begin{array}{l}\text { Yield point, pounds } \\
\text { per square inch }\end{array}$} & \multicolumn{2}{|c|}{$\begin{array}{l}\text { Elongation in } 2 \text { inches, } \\
\text { per cent }\end{array}$} & \multicolumn{2}{|c|}{$\begin{array}{l}\text { Reduction of area, per } \\
\text { cent }\end{array}$} \\
\hline & & & Inverse ratio & $\begin{array}{l}\text { Not } \\
\text { under }\end{array}$ & Inverse ratio & $\begin{array}{l}\text { Not } \\
\text { under }\end{array}$ \\
\hline $\begin{array}{l}\text { Not over } 8 \text { inch diameter or thickness, } 4 \\
\text { inch maximum wall. }\end{array}$ & $\begin{array}{l}\text { Lbs./in. }{ }^{2} \\
85,000\end{array}$ & 0.52 tensile strength & $\frac{2,300,000}{\text { Tensile strength }}$ & 25 & $\frac{3,650,000}{\text { Tensile strength }}$ & 40 \\
\hline $\begin{array}{l}\text { Over } 8 \text { to } 12 \text { inches outside diameter or thick- } \\
\text { ness, } 6 \text { inch maximum wall. }\end{array}$ & 83,000 & ..... do....... & $\frac{2,100,000}{\text { Tensile strength }}$ & 24 & $\frac{3,320,000}{\text { Tensile strength }}$ & 38 \\
\hline $\begin{array}{l}\text { Over } 12 \text { to } 20 \text { inches outside diameter or thick- } \\
\text { ness, } 10 \text { inch maximum wall. }\end{array}$ & 83,000 & -..... do & $\frac{2,000,000}{\text { Tensile strength }}$ & 23 & $\frac{3,100,000}{\text { Tensile strength }}$ & 36 \\
\hline
\end{tabular}

(b) The classification by size of forgings shall be determined by the specified finished diameter or thickness which governs the size of the prolongation from which the test specimen is taken.

(c) The yield point shall be determined by the drop of the beam or by the dividers; the method being optional with the purchaser, and at a crosshead speed of from one-sixteenth to one-fourth inch per minute. The tensile strength shall be determined at a speed not to exceed $1 \frac{1}{2}$ inches per minute.

12. Tension-test specimens.-(a) Tension-test specimens shall be taken from the full-sized prolongation of any forging or, at the option of the manufacturer, from an extra forging furnished for the purpose. The axis of the specimen shall be located at any point midway between the center and surface of the solid forging or full-sized prolongation, and at any point midway between the inner and outer surface of wall of bored forgings, and shall be parallel to the axis of the forging in the direction in which the metal is most drawn out.

(Tension-test specimens shall conform to the dimensions shown in Figure 129 in A. S. T. M. standard specifications for steel under commodity designation 605.0 , p. 247.)

13. Micrographic tests.-(a) A specimen representing each class by size of each melt of each heattreated lot shall be taken for microscopic tests from the tension-test specimen. The sections for microscopic tests shall be cut from the large undistorted portion of the test specimen in such a way as will give one face normal and one face parallel to the axis of the specimen.

(b) Both faces shall be polished practically free from scratches. The transverse face shall be etched with 4 per cent solution of nitric acid in alcohol. The longitudinal face to be left unetched. The specimen shall be examined under a magnification of 100 diameters.

(c) The whole of the transverse section shall show uniform, well-broken-up, fine-grained structure, and shall conform to the requirements illustrated in photomicrographs, Figure 549. Only one irregular mesh as large as one-half inch in diameter shall be permitted in a field 3 inches in diameter, as shown on the screen or photomicrograph.

(d) For information only, the longitudinal unetched face will be examined for solid nonmetallic impurities and should show such impurities well scattered over the field.

14. Macroscopic test.-For information only, a coupon taken with a core drill or sawed normal to the axis of the forging shall be cut from the prolongation from which drillings for chemical analysis are obtained. This coupon representing one-half of the diameter of the prolongation shall be marked before detaching to indicate direction of forging and shall be used for macroscopic examination.

15. Number of tests.-(a) One tension and one microscopic etch test and, for information only, one microscopic unetched and one microscopic etch test shall be made from each class by size of each melt of each heat-treated lot.

(b) If the test specimen shows defective machining or develops flaws, it may be discarded and another specimen substituted.

(c) If the percentage of elongation of any tensiontest specimen is less than that specified in section 11, and any part of the fracture is more than threefourths inch from the center of the gage length, as indicated by scribe scratches marked on the specimen, a retest shall be allowed.

(d) If the results of the tests of any lot do not conform to the requirements specified, the manufacturer may re-treat such lot, but not more than three times, and retests shall be made as specified in sections 11 and 13 .

16. Workmanship.-The forgings shall conform to the sizes and shapes specified by the purchaser. When centered, $60^{\circ}$ centers with clearance drilled for points shall be used. When forgings are drilled from both ends, and the hole thus drilled is out of line, the diameter of the hole may be increased by boring or reaming in order to make the hole smooth and continuous, but the diameter of the hole shall not be increased more than one-eighth inch.

17. Finish.-The forgings shall be free from in jurious defects and shall have a workmanlike finish.

18. Marking.-(a) The forgings shall have the manufacturer's identification marks, melt number, forging number, and date legibly stamped on each forging with steel stencils at such location as shall be designated by the purchaser with letters and figures not less than three-eighths inch high. In case it is necessary to move the above markings, due to machining, it may be done only after permission has been obtained from the inspector.

(b) After the material has been inspected the inspector shall stamp each accepted forging with his private mark.

(Inspection, rejection, and rehearing conform to A. S. T. M. standard specifications for steel. See 605.0 , p. 247.$)$

\section{AMERICAN RAILWAY ENGINEERING ASSO- CIATION, SPECIFICATIONS FOR MOVA- BLE RAILWAY BRIDGES, FORGED STEEL, 1922}

(See also 603.32, p. $151 ; 611.41$, p. 707; 647.31, p. $1268 ; 692.1$, p. 1320 .)

\section{FORGED STEEL}

(With respect to workmanship, chemical analyses, and annealing requirements, this specification is similar to A. S. T. M., A18, given below. Its requirements for tensile tests of forgings whose maximum outside diameter or over-all thickness is not over 20 inches are identical with "E annealed" in 
A18. With respect to test specimens, bend tests, retests, and marking, its requirements are similar to A20-29T, also given below. Otherwise it provides that:)

1. Steel for forging shall be made by the openhearth or the crucible process and shall conform to the following chemical requirements:

Per cent

Phosphorus, maximum 0.04

Sulphur, maximum

2. Check analysis may be made by the engineer from a broken tension or bend test specimen. The phosphorus and sulphur content thus determined shall not exceed that specified by more than 20 per cent.

\section{AMERICAN SOCIETY OF MECHANICAL EN- GINEERS, BOILER CONSTRUCTION CODE, MATERIAL SPECIFICATIONS FOR SEAM- LESS-STEEL DRUM FORGINGS, 1930}

\section{SEAMLESS-STEEL DRUM FORGINGS}

(With respect to process, discard, ladle and check analyses, heat treatment, tests, finish, and marking, this specification is substantially the same as A. S. T. M., A20-29T, given below, with the following exceptions and additional requirements:)

1. Forgings. - The forgings shall be made from solid cast ingots, punched, bored, or hot trephined. The walls of the hollowed ingot shall be reduced in thickness at least one-half by forging on mandrels.

2. Chemical composition. - The steel shall conform to the following requirements as to chemical composition:

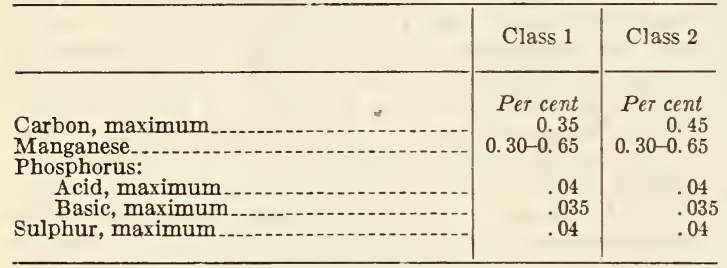

3. Tension tests.--The forging shall conform to the following requirements as to tensile properties:

\begin{tabular}{|c|c|c|}
\hline & Class 1 & Class 2 \\
\hline Tensile strength, minimum _._._lbs./in. ${ }^{2}{ }^{-}$ & 60,000 & 75,000 \\
\hline Yield point, minimum & $\begin{array}{l}0.5 \text { tensile } \\
\text { strength. }\end{array}$ & $\begin{array}{l}0.5 \text { tensile } \\
\text { strength. }\end{array}$ \\
\hline Elongation in 2 inches, minimum & & \\
\hline Reduction of area........ & $\begin{array}{l}26 \\
42\end{array}$ & $\begin{array}{l}24 \\
38\end{array}$ \\
\hline
\end{tabular}

4. One bend-test specimen shall be taken from the end of the forging corresponding to the top of the ingot. The axis of the specimen shall be in a diametral plane perpendicular to the axis of the forging. The bend-test specimen shall be 1 inch by one-half inch in section, with edges rounded to $1 / 16$-inch radius.

5. Two tension tests and one bend test shall be made from each forging.

6. Permissible variation.-The thickness of the parallel wall of each forging shall not be less than that specified. If the thickness of a portion of the wall of the forging is less than that specified, due to accidental or unavoidable irregularity of bore, the forgings may be accepted by the purchaser, provided such irregularity will not require lowering of the allowable working pressure below that for which the drum is designed.

7. The overweight limits are considered a matter of contract between the manufacturer and the purchaser.

\section{AMERICAN SOCIETY FOR TESTING MATE- RIAIS, STANDARD SPECIFICATIONS FOR CARBON-STEEL AND ALLOY-STEEL FORGINGS, DESIGNATION A18-30, 1930}

\section{CARBON-STEEL AND ALLOY-STEEL FORGINGS}

\section{$\mathrm{SCOPE}$}

1. (a) These specifications cover the various classes of carbon-steel and alloy-steel forgings now commonly used and not covered by other existing specifications of the American Society for Testing Materials.

(b) The purposes for which these classes are frequently used are as follows:

Class $A$, for forgings which may be welded or casehardened.

Class $B$, for mild-steel forgings for structural purposes, for minor ship fittings, etc.

Class $C$, for mild-steel forgings for structural purposes, for ships, etc.

Classes $D, E, F, G, H$, and $I$, for various machinery forgings, choice depending upon design and upon the stresses and service to be imposed.

Classes $K, L$, and $M$, for various machinery forgings, choice depending upon design and upon the stresses and services to be imposed, and upon the character of machining operations to be done.

\section{MANUFACTORE}

2. The steel shall be made by either or both the following processes: Open hearth or electric furnace.

3. A sufficient discard shall be made from each ingot to secure freedom from injurious piping and undue segregation.

4. Unless otherwise specified, for test purposes at least 20 per cent of the forgings shall be provided with prolongations or, at the manufacturer's option, a forging may be selected.

5. If boring is specified, the diameter of the hole shall be at least 20 per cent of the maximum outside diameter or thickness of the forging, exclusive of collars and flanges.

6. Heat treatment, if required, shall consist of either annealing or quenching and tempering, as specified.

(a) The procedure to be followed in annealing shall consist in allowing the objects, immediately after forging, to cool to a temperature below the critical range, under suitable conditions to prevent injury by too rapid cooling. They shall then be uniformly reheated to the proper temperature to refine the grain (a group thus heated being known as an annealing charge), and allowed to cool uniformly.

(b) The procedure to be followed in quenching and tempering shall consist in allowing the objects, immediately after forging, to cool to a temperature below the critical range, under suitable conditions to prevent injury by too rapid cooling. They shall then be uniformly reheated to the proper temperature to refine the grain (a group thus reheated being known as quenching charge), and quenched in some medium under substantially uniform conditions for each quenching charge. Finally, they shall be uniformly reheated to the proper temperature for tempering or "drawing back" (a group thus reheated being known as a tempering charge), and allowed to cool uniformly. 
CHEMICAL PROPERTIES AND TESTS

7. (a) The steel shall conform to the following requirements as to chemical composition:

TABLE 1

\begin{tabular}{|c|c|c|c|c|c|}
\hline \multirow{2}{*}{ Elements considered } & \multicolumn{5}{|c|}{ Classes } \\
\hline & A & $\begin{array}{l}\mathrm{B}, \mathrm{C}, \mathrm{D}, \\
\mathrm{E}, \mathrm{F}, \mathrm{G}\end{array}$ & $\mathrm{H}, \mathrm{I}$ & $\mathrm{K}, \mathrm{L}$ & M \\
\hline Manganese. . & $\begin{array}{r}\text { Per cent } \\
0.30-0.60\end{array}$ & $\begin{array}{r}\text { Per cent } \\
0.40-0.80\end{array}$ & $\begin{array}{r}\text { Per cent } \\
0.40-0.80\end{array}$ & $\begin{array}{l}\text { Per } \\
\text { cent }\end{array}$ & $\begin{array}{l}\text { Per } \\
\text { cent }\end{array}$ \\
\hline $\begin{array}{l}\text { Phosphorus: } \\
\text { Acid, not over... } \\
\text { Basic, not over.... } \\
\text { Sulphur, not over.... } \\
\text { Nickel, not under..... }\end{array}$ & $\begin{array}{r}.05 \\
.05 \\
.05 \\
\end{array}$ & $\begin{array}{r}.05 \\
.05 \\
.05 \\
-0 .--\end{array}$ & $\begin{array}{r}.04 \\
.04 \\
.05 \\
3.00\end{array}$ & $\begin{array}{r}0.05 \\
.04 \\
.05\end{array}$ & $\begin{array}{r}0.04 \\
.04 \\
.05\end{array}$ \\
\hline
\end{tabular}

(b) The composition of alloy steel, other than phosphorus and sulphur, to be used in forgings of classes $K, L$, and $M$, shall be agreed upon by the manufacturer and the purchaser.
8. An analysis of each melt of steel shall be made by the manufacturer to determine the percentage of carbon, manganese, and the elements specified in section 7. This analysis shall be madef rom a test ingot taken during the pouring of the melt. The chemical composition thus determined shall be reported to the purchaser or his representative, and shall conform to the requirements specified in section 7 .

9. An analysis may be made by the purchaser from a forging representing each melt. The chemical composition thus determined shall conform to the requirements specified in section 7. Drillings for analysis may be taken from the forging or from a full-size prolongation of the same, at any point midway between the center and surface of solid forgings, and at any point midway between the inner and outer surfaces of the wall of bored forgings; or turnings may be taken from a test specimen.

\section{PHYSICAL PROPERTIES AND TESTS}

10. (a) The forgings shall conform to the requirements as to tensile properties specified in Tables 1,2 , and 3 .

\section{TABLe 1.-Tensile proxerties (classes $A$ to $F$, inclusive)}

[For forgings whose maximum outside diameter or over-all thickness is not orer 20 inches]

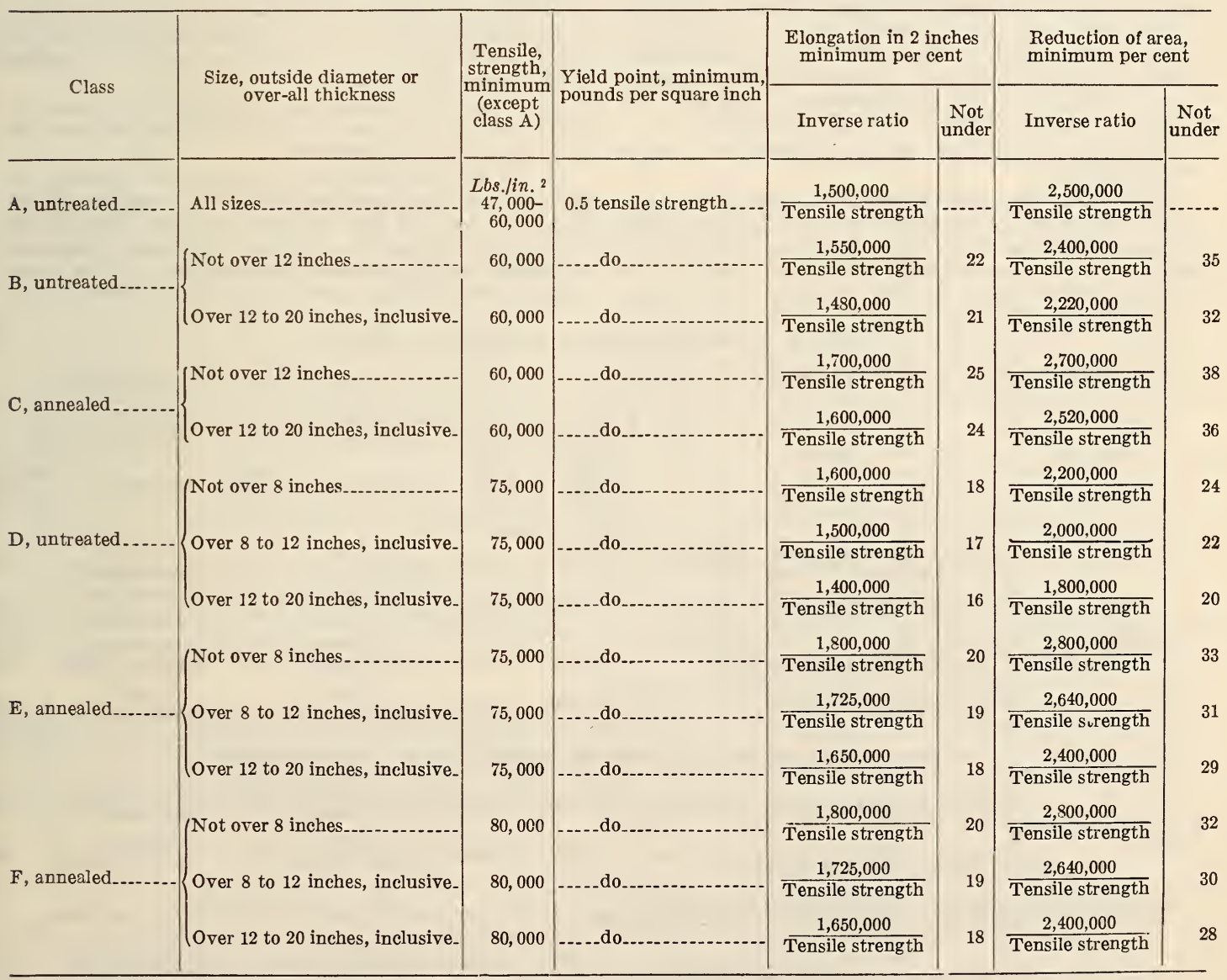


(b) The classification by size of the forging shall be determined by the specified diameter or thickness which governs the size of the prolongation from which the test specimen is taken.

(c) The yield point shall be determined by the drop of the beam of the testing machine.

$(d)$ The elastic limit called for by these specifications shall be determined by an extensometer reading to 0.0002 inch. The extensometer shall be attached to the specimen at the gage marks and not to the shoulders of the specimen nor to any part of the testing machine. When the specimen is in place and the extensometer attached, the testing

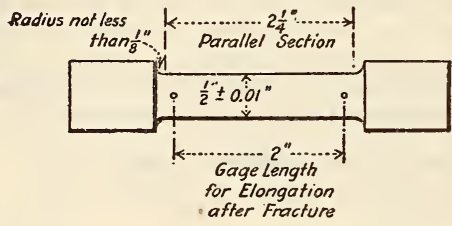

Note:- The Gage Length Parallel Section, and Fillets shall be as Shown, but the Ends may be of any Shape to fit the Holders of - the Testina Machine in such a Way that the

Figure 550.-Standard test piece

(This figure is identical with fig. 129.)

machine shall be operated so as to increase the load on the specimen at a uniform rate. The observer shall watch the elongation of the specimen as shown by the extensometer and shall note, for this determination, the load at which the rate of elongation shows a sudden increase. The extensometer shall then be removed from the specimen, and the test continued to determine the tensile strength.

(e) Tests of forgings shall be made only after final treatment.

11. (a) Tension-test specimens shall be taken from a full-size prolongation of any forging. For forgings with large ends or collars, the prolongation may be of the same cross section as that of the forging back of the large end or collar. Specimens may be taken from the forging itself with a hollow drill, if approved by the purchaser.

(b) The axis of the specimen shall be located at any point midway between the center and surface of solid forgings, and at any point midway between the inner and outer surfaces of the wall of bored forgings, and shall be parallel to the axis of the forging in the direction in which the metal is most drawn out.

(c) The specimens shall conform to the dimensions shown in Figure 550. The ends shall be of a form to fit the holders of the testing machine in such a way that the load shall be axial. (This figure is identical with Figure 129 in standard specifications for steel. See 605.0, p. 247.)

12. Unless otherwise specified by the purchaser, tests shall be made as follows:

(a) For untreated forgings, one tension test shall be made from each melt.

(b) For annealed forgings, one tension test shall be made from each annealing charge. If more than one melt is represented in an annealing charge, one tension test shall be made from each melt.

(c) For quenched and tempered forgings, one tension test shall be made from each tempering charge. If more than one quenching charge is represented in a tempering charge, one tension test shall be made from each quenching charge. If more than one melt is represented in a quenching charge, one tension test shall be made from each melt.

$(d)$ If more than one class of forgings by size is represented in any lot, one tension test from a forging of each class by size shall be made as specified in sections 10 and 11 .

(e) If any test specimen shows defective machining or develops flaws, it may be discarded and another specimen substituted.

$(f)$. If the percentage of elongation of any test specimen is less than that specified in section $10(a)$, and any part of the fracture is more than threefourths inch from the center of the gage length, as indicated by scribe scratches marked on the specimen before testing, a retest shall be allowed.

\section{TABle 2.-Tensile properties (classes $G, H$, and $I$ )}

[For forgings whose maximum outside diameter or thickness is not over 10 inches when solid, and not over 20 inches when bored]

\begin{tabular}{|c|c|c|c|c|c|c|c|}
\hline \multirow{2}{*}{ Class } & \multirow{2}{*}{ Size } & \multirow{2}{*}{$\begin{array}{l}\text { Tensile } \\
\text { strength, } \\
\text { mini- } \\
\text { mum }\end{array}$} & \multirow{2}{*}{$\begin{array}{l}\text { Elastic } \\
\text { limit, } \\
\text { mini- } \\
\text { mum }\end{array}$} & \multicolumn{2}{|c|}{$\begin{array}{l}\text { Elongation in } 2 \text { inches, } \\
\text { minimum, per cent }\end{array}$} & \multicolumn{2}{|c|}{$\begin{array}{l}\text { Reduction of area, } \\
\text { minimum, per cent }\end{array}$} \\
\hline & & & & Inverse ratio & $\left|\begin{array}{c}\text { Not } \\
\text { under }\end{array}\right|$ & Inverse ratio & $\begin{array}{c}\text { Not } \\
\text { under }\end{array}$ \\
\hline \multirow{4}{*}{$\begin{array}{l}\text { G, quenched and } \\
\text { tempered. }\end{array}$} & $\left(\begin{array}{l}\mathrm{Up} \text { to } 4 \text { inches in outside diameter or } \\
\text { thickness, } 2 \text {-inch maximum wall. }\end{array}\right.$ & $\begin{array}{r}\text { Lbs./in. }{ }^{2} \\
90,000\end{array}$ & $\begin{array}{r}L b s . / i n .^{2} \\
55,000\end{array}$ & $\frac{2,100,000}{\text { Tensile strength. }}$ & 20.5 & $\frac{4,000,000}{\text { Tensile strength. }}$ & \\
\hline & $\begin{array}{l}\text { Over } 4 \text { to } 7 \text { inches in outside diameter or } \\
\text { thickness, } 31 / 2 \text {-inch maximum wall. }\end{array}$ & 85,000 & 50,000 & $\frac{2.000,000}{\text { Tensile strength. }}$ & 20.5 & $\frac{3.800,000}{\text { Tensile strength. }}$ & \\
\hline & $\begin{array}{l}\text { Over } 7 \text { to } 10 \text { inches in outside diameter or } \\
\text { thickness, } 5 \text {-inch maximum wall. }\end{array}$ & 85,000 & 50,000 & $\frac{1,900,000}{\text { Tensile strength. }}$ & 19.5 & $\frac{3,600,000}{\text { Tensile strength. }}$ & \\
\hline & $\begin{array}{l}\text { Outside diameter or thickness not over } 20 \\
\text { inches, } 5 \text { to 8-inch wall. }\end{array}$ & 82,500 & 48,000 & $\frac{1,800,000}{\text { Tensile strength. }}$ & 19 & $\frac{3,400,000}{\text { Tensile strength. }}$ & 36 \\
\hline
\end{tabular}

For forgings whose maximum outside diameter or over-all thickness is not over 20 inches

\begin{tabular}{|c|c|c|c|c|c|c|c|}
\hline $\mathrm{H}$, nickel steel, an- & $\left\{\begin{array}{l}\text { Outside diameter of over-all thickness not } \\
\text { over } 12 \text { inches. }\end{array}\right.$ & 80,000 & 50,000 & $\frac{2,000,000}{\text { Tensile strength. }}$ & 22 & $\frac{3,600,000}{\text { Tensile strength. }}$ & 40 \\
\hline & $\begin{array}{l}\text { Outside diameter or over-all thickness } \\
\text { over } 12 \text { to } 20 \text { inches, inclusive. }\end{array}$ & 80,000 & 50,000 & $\frac{1, y 00,000}{\text { Tensile strength. }}$ & 21 & $\frac{0,4 v 0,000}{\text { Tensile strength. }}$ & 38 \\
\hline
\end{tabular}

For forgings whose maximum outside diameter or thickness is not over 10 inches when solid, and not over 20 inches when bored

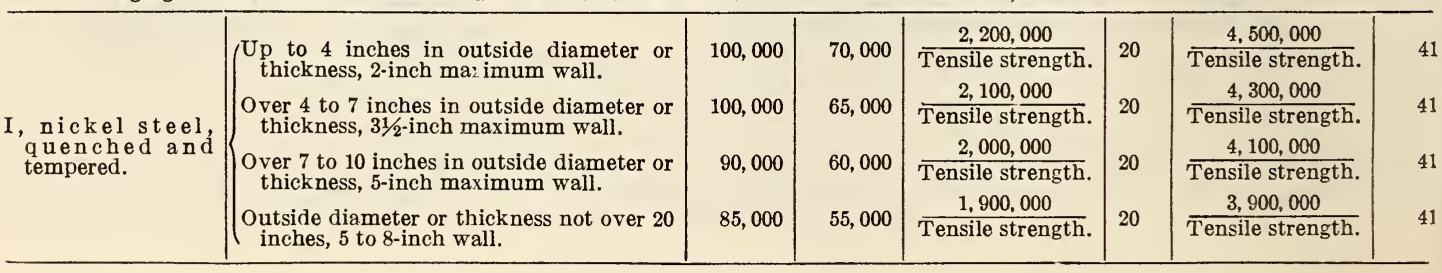


TABLE 3.-Tensile properties (classes $K, L$, and $M$ )

[For forgings whose maximum outside diameter or thickness is not over 10 inches when solid, and not over 20 inches when bored]

\begin{tabular}{|c|c|c|c|c|c|}
\hline Class & Size & Tensile strength & $\begin{array}{l}\text { Elastic } \\
\text { limit, } \\
\text { minimum }\end{array}$ & $\begin{array}{l}\text { Elonga- } \\
\text { tion in } \\
2 \text { inches, } \\
\text { mini- } \\
\text { mum }\end{array}$ & $\begin{array}{l}\text { Reduc- } \\
\text { tion of } \\
\text { area, } \\
\text { mini- } \\
\text { mum }\end{array}$ \\
\hline \multirow{5}{*}{$\begin{array}{l}\text { K, alloy steel, } \\
\text { quenched and } \\
\text { tempered. }\end{array}$} & $\begin{array}{l}\text { Up to } 2 \text { inches in outside diameter or thickness, } 1 \text {-inch maxi- } \\
\text { mum wall. }\end{array}$ & $\begin{array}{l}\text { Lbs./in. }{ }^{2} \\
95,000-115,000\end{array}$ & $\begin{array}{l}\text { Lbs./in. }{ }^{2} \\
\quad 70,000\end{array}$ & $\begin{array}{r}\text { Per cent } \\
20\end{array}$ & $\begin{array}{l}\text { Per cent } \\
50\end{array}$ \\
\hline & $\begin{array}{l}\text { Over } 2 \text { to } 4 \text { inches in outside diameter or thickness, } 2 \text {-inch maxi- } \\
\text { mum wall. }\end{array}$ & $90,000-110,000$ & 65,000 & 20 & \\
\hline & $\begin{array}{l}\text { Over } 4 \text { to } 7 \text { inches in outside diameter or thickness, } 31 / 2 \text {-inch } \\
\text { maximum wall. }\end{array}$ & $90,000-110,000$ & 65,000 & 20 & 50 \\
\hline & $\begin{array}{l}\text { Over } 7 \text { to } 10 \text { inches in outside diameter or thickness, } 5 \text {-inch } \\
\text { maximum wall. }\end{array}$ & $90,000-110,000$ & 65,000 & 20 & 50 \\
\hline & $\begin{array}{l}\text { Outside diameter or thickness not over } 20 \text { inches, } 5 \text { to } 8 \text { inch } \\
\text { wall. }\end{array}$ & $85,000-105,000$ & 60,000 & 20 & 50 \\
\hline \multirow{5}{*}{$\begin{array}{l}\text { L, alloy steel, } \\
\text { quenched and } \\
\text { tempered. }\end{array}$} & $\begin{array}{l}\text { Up to } 2 \text { inches in outside diameter or thickness, } 1 \text {-inch maxi- } \\
\text { mum wall. }\end{array}$ & $105,000-125,000$ & 80,000 & 20 & 50 \\
\hline & $\begin{array}{l}\text { O ver } 2 \text { to } 4 \text { inches in outside diameter or thickness, } 2 \text {-inch maxi- } \\
\text { mum wall. }\end{array}$ & $100,000-120,000$ & 75,000 & 20 & 50 \\
\hline & $\left\{\begin{array}{l}\text { Over } 4 \text { to } 7 \text { inches in outside diameter or thickness, } 3 \frac{1}{2} \text {-inch } \\
\text { maximum wall. }\end{array}\right.$ & $100,000-120,000$ & 75,000 & 20 & 50 \\
\hline & $\begin{array}{l}\text { Over } 7 \text { to } 10 \text { inches in outside diameter or thickness, } 5 \text {-inch } \\
\text { maximum wall. }\end{array}$ & $100,000-120,000$ & 75,000 & 18 & 4 \\
\hline & $\begin{array}{l}\text { Outside diameter or thickness not over } 20 \text { inches, } 5 \text { to } 8 \text { inch } \\
\text { wall. }\end{array}$ & $95,000-115,000$ & 70,000 & 18 & 4 \\
\hline \multirow{5}{*}{$\begin{array}{l}\text { M, alloy steel, } \\
\text { quenched and } \\
\text { tempered. }\end{array}$} & $\begin{array}{l}\text { Up to } 2 \text { inches in outside diameter or thickness, } 1 \text {-inch maxi- } \\
\text { mum wall. }\end{array}$ & $\underset{125,000}{\text { Minimum }}$ & 105,000 & 16 & 50 \\
\hline & $\begin{array}{l}\text { Orer } 2 \text { to } 4 \text { inches in outside diameter or thickness, } 2 \text {-inch maxi- } \\
\text { mum wall. }\end{array}$ & 00 & 000 & 16 & \\
\hline & $\left\{\begin{array}{l}\text { Over } 4 \text { to } 7 \text { inches in outside diameter or thickness, } 31 / 2 \text {-inch } \\
\text { maximum wall. }\end{array}\right.$ & 110,000 & 85,000 & 16 & 45 \\
\hline & $\begin{array}{l}\text { Over } 7 \text { to } 10 \text { inches outside diameter or thickness, } 5 \text {-inch maxi- } \\
\text { mum wall. }\end{array}$ & 100,000 & $7 \tilde{5}, 000$ & 18 & \\
\hline & $\begin{array}{l}\text { Outside diameter or thickness not orer } 20 \text { inches, } 5 \text { to } 8 \text {-inch } \\
\text { wall. }\end{array}$ & 100,000 & 70,000 & 18 & \\
\hline
\end{tabular}

13. (a) When annealed or quenched-and-tempered forgings are specified, if the results of the physical tests of any test lot of forgings do not conform to the requirements specified, the manufacturer may re-treat such lot one or more times, and retests shall be made as specified in section 12 .

(b) When untreated forgings (classes A, B, and D) are specified, if the results of the physical tests of any lot of forgings do not conform to the requirements specified, the manufacturer may anneal such lot one or more times, and retests shall be made as specified in section 12 .

14. The forgings shall conform to the sizes and shapes specified by the purchaser. When centered $60^{\circ}$ centers with clearance drilled for points shall be used.

(Requirements covering workmanship and finish, marking, inspection and rejection, and rehearing conform with those specified in A. S. T. M. standard specifications for steel, paragraphs $5,6,7,8$, and 9 . (See 605.0, p. 247.)

\section{AMERICAN SOCIETY FOR TESTING MATE- RIALS, TENTATIVE SPECIFICATIONS TOR CARBON-STEEL FORGINGS FOR IOCO- MOTIVES, DESIGNATION A20-31T, 1931}

\section{CARBON-STEEL FORGINGS FOR LOCOMOTIVES}

\section{SCOPE}

1. These specifications cover untreated, annealed, and normalized-and-tempered carbon-steel driving axles, engine and trailing-truck axles, main and side rods, straps, crank pins, piston rods, and annealed car and tender axles.

2. The manufacturer may at his option furnish annealed forgings when untreated forgings are speci- fied by the purchaser, provided they conform to the requirements specified for untreated forgings.

\section{MANUFACTERE}

(Requirements covering manufacture are practically the same as for carbon-steel and alloy-steel forgings in A18, given above, except that boring requirements differ, and the process of normalizing replaces that of quenching, as indicated in the following paragraphs.)

3. Boring.- If boring is required in the case of axles, shafts, and similar forgings, it shall be performed before heat treatment, and the diameter of the hole, unless otherwise specified, shall be at least 20 per cent of the maximum outside diameter or thickness of the forging, exclusive of collars and fianges.

3. (a) Normalizing.-For normalizing, the forgings shall be uniformly reheated to a proper temperature to refine the grain and, after being held a sufficient length of time at this temperature, shall be withdrawn from the furnace and allowed to cool uniformly in the atmosphere, protected from rain or snow, to a temperature below the critical range. A group of forgings thus heated shall be known as a normalizing charge. Normalizing, when specified, shall be followed either by annealing or by tempering.

\section{CHEMTCAL PROPERTIES AND TESTS}

4. The steel shall conform to the following requirements as to chemical composition:

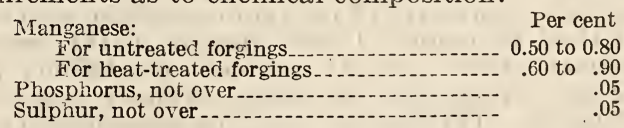

(Chemical analysis is identical with that in A18, given above.) 


\section{PHYSICAL PROPERTIES AND TESTS}

(The requirements covering tension tests are substantially the same as those for carbon-steel and alloy-steel forgings in A18, given above, except that no reference is made to elastic-limit tests by means of the extensometer, and the speed of the machine for testing yield point is specified as to be not less than one-sixteenth inch nor more than oneeighth inch per minute. The minimum tensile requirements for untreated forgings not over 8 inches and over 8 to 12 inches, inclusive, are the same as for D untreated in A18, given above. Otherwise the minimum tensile requirements are as follows.)

\section{TABLE 1.-Minimum tensile requirements}

[For forgings whose maximum outside diameter or over-al] thickness is not over 12 inches when untreated, and not over 20 inches when treated]

ANNEALED

\begin{tabular}{|c|c|c|c|c|c|c|}
\hline \multirow{2}{*}{$\begin{array}{l}\text { Size, outside diameter or over-al] thickness, in } \\
\text { inches }\end{array}$} & \multirow{2}{*}{$\begin{array}{l}\text { Tensile } \\
\text { strength } \\
\text { Lbs./in.2 }\end{array}$} & \multirow{2}{*}{$\begin{array}{l}\text { Yield point } \\
\text { Lbs./in.2 }\end{array}$} & \multicolumn{2}{|c|}{$\begin{array}{l}\text { Elongation in } 2 \text { inches, } \\
\text { per cent }\end{array}$} & \multicolumn{2}{|c|}{$\begin{array}{l}\text { Reduction of area, per } \\
\text { cent }\end{array}$} \\
\hline & & & Inverse ratio & $\begin{array}{l}\text { Not } \\
\text { under }\end{array}$ & Inverse ratio & $\begin{array}{l}\text { Not } \\
\text { under }\end{array}$ \\
\hline Not over 8, 4-inch maximum wall.......... & 80,000 & 0.48 tensile strength... & $\frac{1,980,000}{\text { Tensile strength. }}$ & 22 & $\frac{3.100,000}{\text { Tensile strength. }}$ & 35 \\
\hline Over 8 to 12,6 -inch maximum wall.......... & 80,000 & ..... do & $\frac{1,900,000}{\text { Tensile strength. }}$ & 21 & $\frac{2,900,000}{\text { Tensile strength }}$ & 33 \\
\hline Over 12 to 20,10 -inch maximum wall.......... & 80,000 & ......do... & $\frac{1,820,000}{\text { Tensile strength. }}$ & 20 & $\frac{2,640,000}{\text { Tensile strength. }}$ & 31 \\
\hline
\end{tabular}

NORMALIZED AND TEMPERED

\begin{tabular}{|c|c|c|c|c|c|c|}
\hline Not over 8, 4-inch maximum wall.............. & 85,000 & 0.52 tensile strength... & $\frac{2,300,000}{\text { Tensile strength. }}$ & 25 & $\frac{3.650,000}{\text { Tensile strength. }}$ & 40 \\
\hline Over 8 to 12,6 -inch maximum wall .............. & 83,000 & 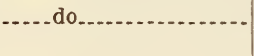 & $\frac{2,100,000}{1 \text { ensile strength. }}$ & 23 & $\frac{3,320,000}{\text { Tensile strength. }}$ & 37 \\
\hline Over 12 to 20,10 -inch maximum wall & 83,000 & -..... do... & $\frac{2,000,000}{\text { Tensile strength. }}$ & 22 & $\frac{3,100,000}{\text { Tensile strength. }}$ & 35 \\
\hline
\end{tabular}

5. Tests of forgings shall be made only after final heat treatment.

6 . If specified by the purchaser, bend tests shall be made on annealed and normalized-and-tempered forgings as follows: The test specimen shall stand being bent cold through $180^{\circ}$ around a pin one-half inch in diameter without cracking on the outside of the bent portion.

(Bend-test specimens are subjected to the same requirements as tension-test specimens in paragraph 11 (a) in A18, given above.)

7. Bend-test specimens shall be one-half inch square in section with corners rounded to a radius of not over one-sixteenth inch, and the length need not exceed 6 inches.

(Requirements covering tests on treated and untreated forgings are the same as given above in A18, except that bend tests are included here, when specified, and normalized forgings replace the quenched forgings specified in A18. In the matter of retests, this specification provides that:)

8. If the results of the physical tests of any test lot do not conform to the requirements specified, the manufacturer may re-treat such lot, but not more than three additional times unless authorized by the purchaser.

9. When annealed or normalized-and-tempered forgings are specified, if the fracture of any test specimen shows over 15 per cent crystalline a second test shall be made. If the fracture of the second specimen shows over 15 per cent crystalline, the forgings represented by such specimen shall be re-treated. The fracture shall be considered crys- talline if the crystals which it contains are so large that the cleavage planes or sides of these crystals are easily visible to the naked eye.

\section{WORKMANSHIP AND FINISH}

10. The forgings shall conform to the sizes and shapes specified by the purchaser. Axles, shafts, and similar forgings, unless otherwise specified, shall be rough-turned all over with an allowance of one-eighth inch on the surface for finishing. In centering, $60^{\circ}$ centers with clearance drilled for points shall be used.

(Requirements covering marking, inspection and rejection, and rehearing conform to those specified in A. S. T. M. standard specifications for steel, paragraphs $6,7,8$, and 9 . See 605.0, p. 247.)

\subsection{AXLES, SHAFTS, AND SIMILAR FORGINGS.}

\section{AMERICAN ELECTRIC RAILWAY ENGI- NEERING ASSOCIATION, STANDARD DESIGN OF AXLES FOR ELECTRIC RAII- WAY MOTORS, E3-30, 1930}

\section{RAIIWAY MOTOR AXIES}

(Axle, wheel, and gear data are summarized in tabular form in this specification, also axle stresses and journal loadings. Form, dimensions, and tolerances for axles of various capacities are shown in the diagrams accompanying the tabulated data.) 


\section{AMERICAN ELECTRIC RAILWAY ENGI- NEERING ASSOCIATION, STANDARD SPECIFICATION FOR QUENCHED AND TER PERED CARBON-STEEL AXLES, SHAFTS, AND SIMILAR FORGINGS, E5-24, 1924}

\section{CARBON-STEEL FORGINGS}

(In respect to manufacture, chemical properties and tests, physical properties and tests, workmanship and finish, inspection, rejection, and rehearing, this specification conforms to the American Railway Association specification for axles, shafts, and other forgings, carbon steel, quenched and tempered, given below, including the references in the latter specification to A. S. T. M., A18-30, to A. S. T. M. standard specifications for steel in 605.0, and to the American Railway Association specification for alloy-steel forgings, quenched and tempered. This specification differs from the American Railway Association specification for axles, shafts, and other forgings, carbon steel, quenched and tempered, in the following particulars:)

(Boring requirements are omitted.)

The variation in thickness or diameter of forging, as determined by the specified diameter or thickness which governs the size of prolongation from which the test specimens are taken, shall not exceed 2 inches for forgings included in the same quenching or tempering charge.

\section{TURNING}

The forgings shall conform to sizes and shapes specified by the purchaser.

Unless otherwise specified by the purchaser, axles, shafts, and similar round forgings shall have a collar approximately 2 inches in width left rough forged on each forging, and the remainder of forging shall be rough turned, with an allowance of one-eighth inch on the surface for finishing.

Unless otherwise specified, all axles, shafts, and similar forgings shall have ends faced, with an allowance of one-eighth inch on each end for finishing, and shall be centered for $60^{\circ}$ lathe centers, with clearance for points.

\section{PHYSICAL PROPERTIES AND TESTS}

Tensile tests.-After final treatment the forgings shall conform to the following minimum tensile properties:

\begin{tabular}{|c|c|c|c|c|}
\hline Size & $\begin{array}{c}\text { Tensile } \\
\text { strength }\end{array}$ & $\begin{array}{c}\text { Elastic } \\
\text { limit }\end{array}$ & $\begin{array}{c}\text { Elonga- } \\
\text { tion in } 2 \\
\text { inches, } \\
\text { not under }\end{array}$ & $\begin{array}{l}\text { Reduc- } \\
\text { tion of } \\
\text { area }\end{array}$ \\
\hline $\begin{array}{l}\text { Up to } 4 \text { inches in diameter } \\
\text { or thickness. } \\
\text { Over } 4 \text { inches up to } 7 \\
\text { inches diameter or } \\
\text { thickness.............. }\end{array}$ & $\begin{array}{c}\text { Lbs./in. }{ }^{2} \\
90,000 \\
85,000\end{array}$ & $\begin{array}{r}\text { Lbs./in. }{ }^{2} \\
55,000 \\
50,000\end{array}$ & $\begin{array}{r}\text { Per cent } \\
20.5\end{array}$ & Per cent \\
\hline
\end{tabular}

For forgings with large ends or collars (except axles), the prolongation may be of the same crosssection as that of the forging back of the large end or collar. For axles, the nominal diameter governing the size of the prolongation shall be the finished diameter of the motor-bearing seat.:

\section{MAREING}

Each billet shall be legibly stamped with an identification number representing melt, which shall be transferred to the final forging immediately after reduction of billet has been completed.

Before treatment, each forging shall be stamped with a shop identification number. These shop numbers are to permit of identifying the forgings in each quenching and tempering charge to determine the number of required physical tests.

The name or trade-mark of the manufacturer, identification number, and inspector's mark shall be legibly stamped on each finished forging in location indicated by the purchaser.

The manufacturer shall report to the purchaser upon each shipment, the number of quenching and tempering charges, together with the melt numbers and identification numbers of forgings included in each quenching and tempering charge.

\section{REJECTION}

Unless otherwise agreed upon between the purchaser and the manufacturer, any rejection based on test made in accordance with specified requirements shall be reported within 10 working days from the receipt of samples (instead of within five working days as specified in A. S. T. M. standard specifications for steel in 605.0 , p. 247).

\section{AMERICAN ELECTRIC RAILWAY ENGI- NEERING ASSOCIATION, STANDARD SPECIFICATION FOR ANNEALED CARBON-STEEL AXLES, SHAFTS, AND SIMILAR FORGINGS, E6-23, 1923}

\section{CARBON-STEEL FORGINGS}

\section{MANUFACTURE}

(Requirements covering manufacture are practically the same as for carbon-steel and alloy-steel forgings in A.S. T. M., A18-30 (see 611.51, p. 754), including the heat treatment specified in paragraph $6(a)$, but omitting the boring.)

\section{TORNING}

(Same as in American Electric Railway Engineering Association specification E5-24, given above.)

\section{CHEMICAL PROPERTIES AND TESTS}

Chemical composition.-(Same for maximum limit as for medium forgings, class B, in American Railway Association specification for axles, shafts, and other forgings, carbon steel, annealed and unannealed, given below, while ladle and check analyses are the same as in A. S. T. M., A18-30, with the addition of the following paragraph:)

In addition to the complete analysis, a phosphorus determination may be made by the purchaser from turnings from each tensile-test specimen, and this determination shall conform to the requirements for phosphorus specified.

\section{PHYSICAL PROPERTIES AND TESTS}

Tensile tests.-After annealing, the forgings shall conform to the following minimum tensile properties:

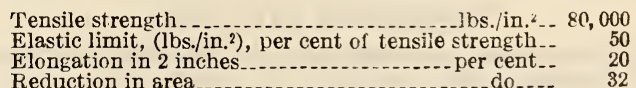

Bend tests. The bend-test specimen shall bend cold through $180^{\circ}$ around a 1-inch flat mandrel having a rounded edge of $1 / 2$-inch radius, without cracking on the outside of the bent portion. 
Bend-test specimens shall be one-half inch square in section with corners rounded to a radius of not over one thirty-second inch, and need not exceed 6 inches in length.

(Requirements covering determination of elastic limit by means of the extensometer, tension and bend test specimens (including illustration), are the same as for carbon-steel and alloy-steel forgings in A. S. T. M., A18-30. See 611.51, p. 754.)

(Requirements covering number of tests and re-treating are the same as for axles, shafts, and other forgings, alloy steel, quenched and tempered, American Railway Association specification (see $622.6, p .1136$ ) except that annealing is substituted in E6 for tempering and quenching in the A. R. A. specification. Marking is the same as in E5-24, given above, while finish, inspection and rejection, and rehearing conform to A. S. T. M. standard specifications for steel in 605.0, p. 247.)

\section{AMERICAN PETROLEUM INSTITUTE, RIG- IRON STANDARDS, A. P. I. STANDARDS NO. 6, JANUARY, 1930}

\section{CRANKS AND SHAFTS MATERIAL COVERED}

These specifications apply to rig irons for oilcountry purposes. Three sizes-41/2 inch, 5 inch,
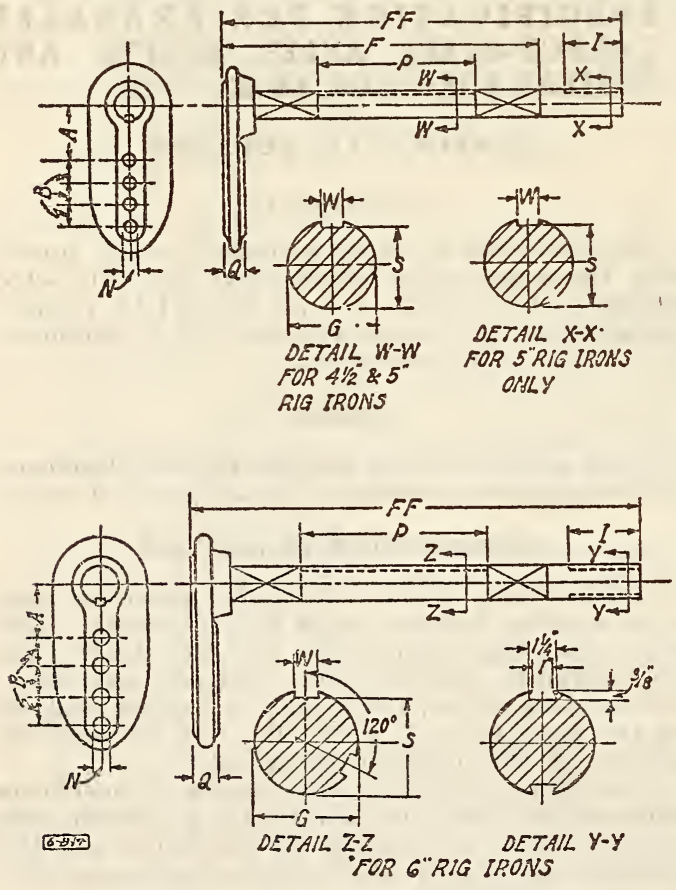

FIgTre 551.-Cranks and shafts

and 6 inch are standard. The standards are dimensional only and apply to new material. These specifications do not include chemical or physical properties of materials.

\section{WORKMANSHIP AND FINISH}

All machined surfaces shall be finished in a good and workmanlike manner and shall be free from burrs, rough edges, etc.
All castings shall have rough edges chipped or ground off, shall be commercially clean, and shall be free from injurious defects. All cored bolt holes shall be drifted to insure free passage of proper sized bolts.

All forgings shall be free from injurious defects, and shall have a workmanlike finish.

\section{MARKING}

Rig irons manufactured in accordance with these dimensional standards shall be stamped or marked with the official A. P. I. monogram as stipulated in these specifications. No rig iron material shall be stamped with the A. P. I. monogram that does not comply with these specifications: Authority will be granted to use the A. P. I. monogram in accordanc with the rules and conditions contained in the appendix.

All rig iron material manufactured in accordance with these specifications shall also be marked with the manufacturer's name or other identifying mark.

Where the A. P. I. monogram is specified on forged metal it shall be applied with a steel stencil, size of monogram to be one-half inch high as follows:

When the A. P. I. monogram is specified on cast material the design of the monogram shall be affixed to the pattern, letters to be $1 \frac{1}{2}$ inches long, in a 2-inch circle; height of letters and circle to be not less than one-eighth inch, substantially as follows:

Cranks and shafts for A. P. I. standard rig irons shall be dimensioned as shown in Figure 551 and Table 1.

The crank and shaft shall be considered as one unit. They shall be so assembled that the crank is permanently attached to the shaft. Each shaft shall be the same diameter, within the tolerance specified herein, throughout its length, disregarding that part of the shaft in the crank.

All shaft diameters, keys, and keyways shall be as specified (for keys and keyways see 608.52, p. 616), except clutch keyways at end of 6-inch crank shaft, which shall be as shown in the accompanying figure.

All flange keyways, $P$, shall be cut the full length of that part of the shaft between the front and back jack-post boxes; other keyways as shown.

Shafts of $4 \frac{1}{2}$ and 5 inches shall have one flange keyway.

The 6-inch shaft shall have two flange keyways, spaced $120^{\circ}$, and located on the upper side of the shaft at $60^{\circ}$ on either side of a vertical center line when the crank is in a downward vertical position.

One accessory keyway shall be provided for the 5 -inch shaft in the 6-foot 6-inch length.

The 6-inch shaft shall have two clutch keyways, spaced $180^{\circ}$. The crank and shaft for $4 \frac{1}{2}$-inch rig irons shall be made in one length of shaft only.

The crank and shaft for 5-inch rig irons may be made in two lengths of shafts, namely, 5-foot 6-inch length with keyways for flanges only, 6-foot 6-inch length with keyways for flanges and for accessory equipment.

The crank and shaft for 6-inch rig irons may be made in two lengths of shafts -7 feet 6 inches and 8 feet.

The A. P. I. monogram shall be cast on the same side of the crank as the manufacturer's name or trade-mark, and shall be stamped with a steel die on the end of the shaft. 
TABLE 1.-Keys for A. P. I. standard rotary line shafts

[See fig. $552 a$ and fig. 552b]

[All dimensions in inches at $68^{\circ} \mathrm{F}$.]

\begin{tabular}{|c|c|c|c|c|c|c|c|c|c|}
\hline \multirow{3}{*}{ Member } & \multirow{3}{*}{$\begin{array}{c}\text { Nomi- } \\
\text { nal } \\
\text { shaft } \\
\text { size } \\
\text { "T" }\end{array}$} & \multicolumn{8}{|c|}{ Keys } \\
\hline & & \multirow{2}{*}{ Type } & \multirow{2}{*}{ Nominal size } & \multirow{2}{*}{$\begin{array}{c}\text { Taper, } \\
\text { inches } \\
\text { per } \\
\text { foot }\end{array}$} & \multirow{2}{*}{ Length } & \multicolumn{2}{|c|}{ Width } & \multicolumn{2}{|c|}{ Height 1} \\
\hline & & & & & & "W" & Tolerance & "G" & Tolerance \\
\hline Tight members, keyed... & 4 & Taper-- & 1 by $3 / 4 \ldots$ & $1 / 8$ & Optional & 1.000 & $\left\{\begin{array}{r}+0.000 \\
-.003\end{array}\right.$ & 0.750 & $\left\{\begin{array}{r}+0.003 \\
-.000\end{array}\right.$ \\
\hline Sliding members, keyed & 4 & Feather... & 1 by $1 \ldots \ldots$ & -..... & ..... do do.. & 1.000 & $\left\{\begin{array}{l}+.000 \\
-.003\end{array}\right.$ & 1.000 & $\left\{\begin{array}{l}+.000 \\
-.003\end{array}\right.$ \\
\hline Tight members, keyed.......... & 5 & Taper. & $11 / 4$ by $7 / 8$ & $1 / 8$ & .... do. & 1. 250 & $\left\{\begin{array}{l} \pm .000 \\
-.003\end{array}\right.$ & .875 & $\begin{array}{l}+.003 \\
-.000\end{array}$ \\
\hline Sliding members, keyed... & 5 & Feather. & $11 / 4$ by $11 / 4$ & & -..... do.. & 1. 250 & $\left\{\begin{array}{l}+.000 \\
-.003\end{array}\right.$ & 1. 250 & $\begin{array}{l}+.000 \\
-.003\end{array}$ \\
\hline Tight members, keyed.......... & 6 & Taper. & $11 / 2$ by 1 & $1 / 8$ & ..... do... & 1.500 & $\left\{\begin{array}{l}+.000 \\
-.003\end{array}\right.$ & 1.000 & $\begin{array}{l}+.003 \\
-.000\end{array}$ \\
\hline Sliding members, keyed & 6 & Feather. & $11 / 2$ by $11 / 2 \ldots$ & 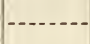 & .....do do & 1.500 & $\left\{\begin{array}{l}+.000 \\
-.003\end{array}\right.$ & 1.500 & $\left\{\begin{array}{l} \pm .000 \\
-.003\end{array}\right.$ \\
\hline
\end{tabular}

1 The height " $\mathrm{H}$ " is measured at the distance "W" from gib on taper keys.

The dimensions indicated in fig. $552 a$ and not shown in the above table are not essential to the fit; they can be obtained from A. P. I. Standards 7: "Transmission Standards."

\section{TABLE 2.-Shaft details for A. P. I. standard rotary line shafts}

[See fig. 552c]

[All dimensions in inches at $68^{\circ} \mathrm{F}$.]

\begin{tabular}{|c|c|c|c|c|c|c|c|c|c|}
\hline \multirow{3}{*}{ Membar } & \multicolumn{3}{|c|}{ Shaft diameter } & \multicolumn{2}{|c|}{ Key } & \multicolumn{4}{|c|}{ Kejway 1} \\
\hline & \multirow{2}{*}{ 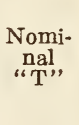 } & \multirow{2}{*}{ "G" } & \multirow{2}{*}{ Tolerance } & \multirow{2}{*}{ Tyре } & \multirow{2}{*}{ Nominal size } & \multicolumn{2}{|c|}{ Width } & \multicolumn{2}{|c|}{$\begin{array}{l}\text { Diameter of shaft to } \\
\text { bottom of keyway }\end{array}$} \\
\hline & & & & & & "W" & Tolerance & "S" & Tolerance \\
\hline Tight"members, keyed_-.-- & 4 & 3.995 & $\left\{\begin{array}{r}+0.000 \\
-.003\end{array}\right.$ & Taper.- & 1 by $3 / 4 \ldots$ & 1.000 & $\left\{\begin{array}{r}+0.003 \\
-.000\end{array}\right.$ & 3.6250 & $\left\{\begin{array}{r}+0.000 \\
-.005\end{array}\right.$ \\
\hline Slidíng members, keyed & 4 & 3.995 & $\left\{\begin{array}{l}+.000 \\
-.003\end{array}\right.$ & Feather... & 1 by $1 \ldots$ & 1.000 & $\left\{\begin{array}{l}+.003 \\
-.000\end{array}\right.$ & 3.4380 & \\
\hline Tight members, keyed.-- & 5 & 4.995 & $\left\{\begin{array}{l}+.000 \\
-.003\end{array}\right.$ & \} Taper.. & $11 / 4$ by 7/8 & 1. 250 & $\left\{\begin{array}{l}+.003 \\
-.000\end{array}\right.$ & 4. 5625 & $\begin{array}{l}+.000 \\
-.006\end{array}$ \\
\hline Sliding members, keyed. & 5 & 4. 995 & $\left\{\begin{array}{l}+.000 \\
-.003\end{array}\right.$ & Feather. & $11 / 4$ by $11 / 4 \ldots$ & 1. 250 & $\left\{\begin{array}{l}+.003 \\
-.000\end{array}\right.$ & 4. 2500 & $\begin{array}{l}+.000 \\
-.006\end{array}$ \\
\hline Tight members, keyed.............. & 6 & 5.995 & $\left\{\begin{array}{l}+.000 \\
-.003\end{array}\right.$ & Taper-- & 132 by $1 \ldots$ & 1.500 & $\left\{\begin{array}{l}+.003 \\
-.000\end{array}\right.$ & 5.5000 & $\begin{array}{l}+.000 \\
-.006\end{array}$ \\
\hline Sliding members, keyed. & 6 & 5.995 & $\left\{\begin{array}{r}+.000 \\
-.003\end{array}\right.$ & Feather. & $11 / 2$ by $11 / 2 \ldots$ & 1.500 & $\left\{\begin{array}{l}+.003 \\
-.000\end{array}\right.$ & 1) 5.1250 & $\left\{\begin{array}{l}+.000 \\
-.000\end{array}\right.$ \\
\hline
\end{tabular}

1 All keyways shall be cut on a diametral center line; see fig. 37412 in A. P. I. specification for shaft keys and keyways (608.52, p. 617 ) for explanation of tolerances on taper keys. 

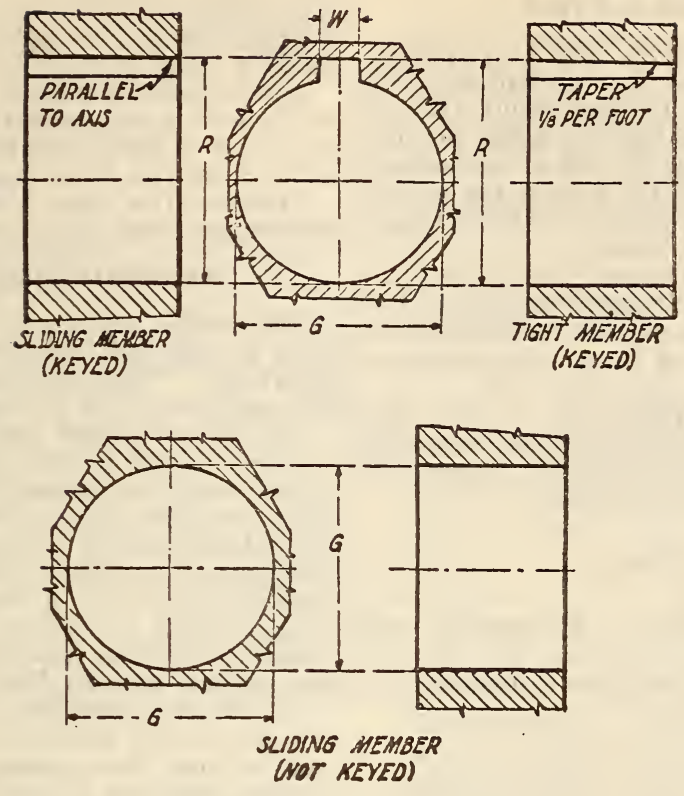

FIGURE 553.-Bored members

TABLE 3.-Details of bored members for A. P. I. standard rotary line shafts

[See fig. 553]

[All dimensions in inches at $68^{\circ} \mathrm{F}$.]

\begin{tabular}{|c|c|c|c|c|c|c|c|c|c|}
\hline \multirow{3}{*}{ Member } & \multicolumn{3}{|c|}{ Diameter at bore } & \multirow{3}{*}{ Type of key } & \multirow{3}{*}{$\begin{array}{c}\text { Nominal size } \\
\text { of key }\end{array}$} & \multicolumn{4}{|c|}{ Keyway 1} \\
\hline & \multirow{2}{*}{$\begin{array}{l}\text { Nomi- } \\
\text { nal }\end{array}$} & \multicolumn{2}{|c|}{ Actual } & & & \multicolumn{2}{|c|}{ Width } & \multicolumn{2}{|c|}{$\begin{array}{l}\text { Diameter of bore } t \\
\text { bottom of keyway }\end{array}$} \\
\hline & & "G" & Tolerance & & & “W" & Tolerance & " $\mathrm{R} "$ & Tolerance \\
\hline Tight members, keyed.... & 4 & 4. 000 & $\left\{\begin{array}{r}+0.002 \\
-.000\end{array}\right.$ & Taper-- & 1 by $3 / 4 \ldots$ & 1.000 & $\left\{\begin{array}{r}+0.003 \\
-.000\end{array}\right.$ & 4. 3580 & $\left\{\begin{array}{r}+0.006 \\
-.000\end{array}\right.$ \\
\hline Sliding members, keyed.. & 4 & 4.015 & $\left\{\begin{array}{l}+.005 \\
-.000\end{array}\right.$ & Feather. & 1 by $1 \ldots$ & 1.015 & $\left\{\begin{array}{l}+.010 \\
-.000\end{array}\right.$ & 4. 4680 & $\left\{\begin{array}{l}+.006 \\
-.000\end{array}\right.$ \\
\hline Sliding memb $\mathrm{e}_{\mathrm{e} s}$, not keyed.. & 4 & 4.005 & $\begin{array}{l}+.003 \\
-.000\end{array}$ & & & & & & -.................. \\
\hline Tight members, keyed.... & 5 & 5.000 & $\begin{array}{l}+.002 \\
+.000\end{array}$ & Taper.. & $11 / 4$ by $7 / 8 \ldots$ & 1. 250 & $\left\{\begin{array}{l} \pm .003 \\
-.000\end{array}\right.$ & 5.4205 & $\left\{\begin{array}{l}+.006 \\
-.000\end{array}\right.$ \\
\hline Sliding $\mathrm{m}_{\mathrm{e}}$ mbers, keyed.. & 5 & 5.015 & $\begin{array}{l}+.005 \\
-.000\end{array}$ & Feather. & 1144 by $11 / 4 \ldots$ & 1.265 & $\left\{\begin{array}{l}+.010 \\
-.000\end{array}\right.$ & 5. 5310 & $\begin{array}{l}+.006 \\
-.000\end{array}$ \\
\hline Sliding members, not keyed.. & 5 & 5.005 & $\begin{array}{l}+.003 \\
-.000\end{array}$ & & & & & & $\cdots$ \\
\hline Tight members, keyed.... & 6 & 6.000 & $\begin{array}{l}+.002 \\
-.000\end{array}$ & Taper. & $11 / 2$ by $1 \ldots$ & 1.500 & $\left\{\begin{array}{l}+.003 \\
-.000\end{array}\right.$ & 6.4830 & $\left\{\begin{array}{l}+.006 \\
-.000\end{array}\right.$ \\
\hline sliding members, keyed... & 6 & 6.015 & $\begin{array}{l}+.005 \\
-.000\end{array}$ & Feather.. & $11 / 2$ by $11 / 2 \ldots$ & 1.515 & $\left\{\begin{array}{r}+.010 \\
-.000\end{array}\right.$ & 6.6560 & $\left\{\begin{array}{l}+.006 \\
-.000\end{array}\right.$ \\
\hline Sliding members, not keyed. & 6 & 6.005 & $\begin{array}{l} \pm .003 \\
-.000\end{array}$ & & & & & & \\
\hline
\end{tabular}

1 All keyways shall be cut on a diametral center line; see fig. $3741 / 2$ for explanation of tolerances on taper keys.

(Explanation of tolerances on taper keys is identical with that given in the A. P. I. specification for shaft keys and keyways. See 608.52, p. 616.)

AMERICAN RAILWAY ASSOCIATION, MECHANICAL DIVISION, AXLES, CARBON STEEL, FOR CARS AND LOCOMOTIVE TENDERS, 1926

\section{CARBON-STEEL AXLES SCOPE}

These specifications cover tapered axles up to and including those $6 \frac{1}{2}$ inches in nominal diameter at the center. Axles over $61 / 2$ inches in diameter at the center shall be purchased under the latest issue of specifications for annealed carbon steel axles. (See below.)

\section{MANUFACTURE}

Process.-The steel shall be made by the openhearth or electric process.

\section{CHEMICAL PROPERTIES AND TESTS}

(The chemical composition is identical with that for medium forgings class $\mathrm{A}$ in the American Railway Association specification for annealed and unannealed carbon steel forgings, given below, while the ladle and check analyses conform to the requirements specified in A. S. T. M. A18-30. See 611.51, p. 754.) 


\section{PHYSICAL PROPERTIES AND TESTS}

Drop tests.-(a) The test axles shall be so placed on supports 3 feet apart that the tup will strike it midway between the ends. It shall stand without fracture five blows from a tup of 2,240 pounds falling from a height $H$, such that $H$ in feet equals the square of the diameter of the axle at the center in inches, $H=d^{2}$. The axle shall be rotated through $180^{\circ}$ after the first and third blows.

(b) Before an axle is drop tested, the center shall be calipered to the nearest one-eighth inch, and the height of drop in feet, to the nearest half foot, shall not be less than the square of the actual diameter, at the center, in inches.

(c) The permanent set produced by the first blow shall not exceed that given by the following formula, in which $L=$ length of axle in inches and $d=$ diameter of axle at center in inches:

$$
\frac{L}{1.9 d}-\frac{d}{2}+1 / 2 \text { inch. }
$$

(d) The American Railway Association has adopted five standard sizes of axles, the requirements for which, based on the above formula, are given in the following table:

\begin{tabular}{|c|c|c|c|c|c|c|}
\hline $\begin{array}{c}\text { Classification } \\
\text { of axles }\end{array}$ & $\begin{array}{l}\text { Size of } \\
\text { journal }\end{array}$ & $\begin{array}{l}\text { Diam- } \\
\text { eter of } \\
\text { axle } \\
\text { at } \\
\text { center }\end{array}$ & $\begin{array}{c}\text { Length } \\
\text { of } \\
\text { axle }\end{array}$ & $\begin{array}{c}\text { Height } \\
\text { of } \\
\text { drop }\end{array}$ & $\begin{array}{l}\text { Num- } \\
\text { ber of } \\
\text { blows }\end{array}$ & $\begin{array}{l}\text { Maxi- } \\
\text { mum } \\
\text { perma- } \\
\text { nent } \\
\text { set }\end{array}$ \\
\hline & Inches & Inches & Inches & Feet & & Inches \\
\hline A. & $33 / 4$ by 7 & $\left\{\begin{array}{l}41 / 4 \\
43 / 8 \\
41 / 2 \\
43 / 4\end{array}\right.$ & $831 / 4$ & $\begin{array}{l}18 \\
19 \\
20 \\
221\end{array}$ & 5 & $\begin{array}{l}834 \\
83 / 8 \\
8 \\
71 / 2\end{array}$ \\
\hline B. & $41 / 4$ by 8 & $\begin{array}{l}47 / 8 \\
5 \\
53\end{array}$ & $841 / 4$ & $\begin{array}{l}231 / 2 \\
25\end{array}$ & 5 & $\begin{array}{l}71 / 4 \\
7 \\
61\end{array}$ \\
\hline & 5 by 9 & $51 / 2$ & $861 / 2$ & 30 & 5 & $\begin{array}{l}6 \\
53 / 4\end{array}$ \\
\hline D. & $51 / 2$ by 10 & $\begin{array}{l}07 / 8 \\
61 / 8\end{array}$ & $881 / 2$ & $\begin{array}{l}341 / 2 \\
36 \\
371 / 2\end{array}$ & 5 & $51 / 4$ \\
\hline $\mathrm{E}_{\text {. }}$ & 6 by 11 & $\begin{array}{r}6716 \\
6916 \\
6^{11} 116\end{array}$ & $90^{3} / 4$ & $\begin{array}{l}411 / 2 \\
43 \\
441 / 2\end{array}$ & 5 & $\begin{array}{l}41 / 2 \\
41 / 4\end{array}$ \\
\hline
\end{tabular}

(e) The permanent set is the difference between the distance from a straightedge to the middle point of the axle, measured before the first blow, and the distance measured in the same manner after the blow. The straightedge shall rest only on the end collars or the ends of the axle.

(f) The temperature of the test axle shall be between $40^{\circ}$ and $120^{\circ} \mathrm{F}$.

Drop-test machine.-The anvil of the drop-test machine shall be supported on 12 springs, as shown on the A. R. A. drawings, and shall be free to move in a vertical direction, and shall weigh 17,500 pounds. The radii of the striking face of the tup and of the supports shall be 5 inches.

Number of tests. - One drop test shall be made from each melt. The weight of axles offered from any one melt shall not be less than the equivalent of the weight of thirty 6 by 11 inch axles.

\section{WORKMANSHIP AND FINISH}

Workmanship.-(a) All axles shall be made and finished in a workmanlike manner, and all journals and wheel seats shall be rough turned. In centering, unless otherwise specified, $60^{\circ}$ centers shall be used, with large diameter of countersink not less than seven-eighths inch and with clearance drilled onehalf inch deep. (b) Unless otherwise specified, axles shall be smooth forged, except the wheel seats and journals shall be rough turned in a workmanlike manner.

(c) Any straightening of axles, if required, shall be done at a temperature not below $1,100^{\circ} \mathrm{F}$. (low red), and in such a manner as to leave the surface of the axles free from unnecessary scars.

Finish.-The axles shall be free from injurious defects and shall have a workmanlike finish.

\section{PERMISSIBLE VARIATIONS AND WEIGHTS}

Permissible variation.-(a) The axles shall conform in size and shape to the standard A. R. A. drawings. The length shall not be less than shown, and not more than one-eighth inch over.

(b) Smooth forged axles. - The diameters of the straight center portion of the axle and the black collars shall not be less than specified, and shall not be more than one-fourth inch over. The intermediate portion of the axles shall not be under the dimensions specified, and shall be forged to a straight taper in a workmanlike manner. The rough turned diameters of the journals and wheel seats shall be from one-eigth to one-quarter inch over the finished sizes to provide for proper finishing.

(c) Rough-turned axles.-The diameters of the straight center portion and the black collars shall not be less than specified. The straight center portion shall not be more than one-eighth inch over and the black collars not more than one-fourth inch over the diameters specified. The intermediate portion of the axle shall not be under the dimensions specified, and shall be turned to a straight taper in a workmanlike manner.

Weights.-The maximum weights of smoothforged and rough-turned axles in any shipment shall not exceed those given below:

\begin{tabular}{|c|c|c|c|}
\hline \multirow[b]{2}{*}{$\begin{array}{c}\text { Classification } \\
\text { of axles }\end{array}$} & \multirow[b]{2}{*}{$\begin{array}{c}\text { Size of } \\
\text { journal }\end{array}$} & \multicolumn{2}{|c|}{ Maximum weight } \\
\hline & & $\begin{array}{l}\text { Smooth forged } \\
\text { with rough- } \\
\text { turned journals } \\
\text { and wheel } \\
\text { seats }\end{array}$ & $\begin{array}{l}\text { Rough } \\
\text { turned } \\
\text { all over }\end{array}$ \\
\hline $\begin{array}{l}\mathrm{A} \\
\mathrm{B} \\
\mathrm{C} \\
\mathrm{D} \\
\mathrm{E}\end{array}$ & $\begin{array}{l}\text { Inches } \\
33 / 4 \text { by } 7 \\
41 / 4 \text { by } 8 \\
5 \text { by } 9 \\
51 / 2 \text { by } 10 \\
6 \text { by } 11\end{array}$ & $\begin{array}{c}\text { Pounds } \\
435 \\
530 \\
705 \\
835 \\
1,015\end{array}$ & $\begin{array}{c}\text { Pounds } \\
425 \\
520 \\
695 \\
825 \\
1,005\end{array}$ \\
\hline
\end{tabular}

\section{MARKING AND STORING}

Marking.-Each axle shall have the manufacturer's name or brand legibly stamped on one of the black collars, and the melt number, the month and year when made stamped on the other.

Storing.-If, as a result of the inspection and tests, more axles are accepted than the order calls for, such accepted axles in excess shall be stamped by the inspector with his own name, and will then be piled and allowed to remain in stock at the works, subject to further orders from the purchasing agent. On receipt of further orders, axles once accepted will not be subject to further test. In all cases the inspector will keep an accurate record of the melt numbers and the number of axles in each melt which are stored and will transmit this information with each report.

\section{INSPECTION AND REJECTION}

(Requirements covering inspection, rejection, and rehearing conform with those specified in A. S. T. M. standard specifications for steel, paragraphs 7,8 , and 9 . See 605.0, p. 247.) 


\section{AMERICAN RAILWAY ASSOCIATION, ME- CHANICAL DIVISION, AXLES, SHAFTS, AND OTHER FORGINGS, CARBON STEEL, ANNEALED AND UNANNEALED, 1926}

\section{CARBON-STEEL FORGINGS}

\section{SCOPE}

(a) These specifications cover two grades of forgings, distinguished, respectively, as "medium" and "mild," with the "medium" grade divided into class A for unannealed and class B for annealed forgings. Medium forgings are intended for the important large forged parts of locomotives, such as axles, rods, rod straps, pins, guides, etc. Mild forgings are to be used only where so ordered, for parts of minor importance, and those which are to be casehardened, etc.

(b) The manufacturer may, at his option, furnish annealed forgings when unannealed forgings are specified by the purchaser, provided they conform to the requirements specified for unannealed forgings.

(c) Unless otherwise specified, annealed material shall be furnished for medium forgings. Mild forgings may be furnished unannealed unless otherwise specified.

\section{MANOFACTORE}

Process.-The steel may be made by the openhearth or electric process.

Prolongation for test.-The manufacturer and the purchaser shall agree upon forging on which a prolongation for test purposes shall be provided.

Annealing.-(a) For annealing, the forgings shall be allowed to become cold after forging. They shall then be uniformly reheated to the proper temperature to refine the grain (a group thus reheated being known as an annealing charge) and allowed to cool uniformly.

(b) Material ordered to these specifications must under no circumstances be quenched.

\section{CHEMICAL PROPERTIES AND TESTS}

Chemical composition.-The steel shall conform to the following requirements as to chemical composition:

\begin{tabular}{|c|c|c|c|}
\hline & \multicolumn{2}{|c|}{ Medium forgings } & \multirow{2}{*}{$\begin{array}{c}\text { Mild } \\
\text { forgings }\end{array}$} \\
\hline & Class A & Class B & \\
\hline $\begin{array}{l}\text { Carbon } \\
\text { Manganese- } \\
\text { Phosphorus, maximum } \\
\text { Sulphur, maximum. }\end{array}$ & $\begin{array}{r}\text { Per cent } \\
0.40-0.55 \\
.04-.70 \\
.05 \\
.05\end{array}$ & $\begin{array}{r}\text { Per cent } \\
0.45-0.60 \\
.40-.70 \\
.05 \\
.05\end{array}$ & $\begin{array}{r}\text { Percent } \\
0.05-0.15 \\
.30-.60 \\
.05 \\
.05\end{array}$ \\
\hline
\end{tabular}

(Ladle and check analyses, classification by size, time of testing forgings, tension-test specimens (including illustration), number of tests-all conform to the requirements specified in A. S. T. M. A18-30 (see 611.51, p. 754) except that in this specification there is no reference to quenching, since it specifically provides that:)

Material ordered to these specifications must under no circumstances be quenched.

\section{PHYSICAL PROPERTIES}

(Tensile properites of forgings whose maximum outside diameter or over-all thickness is not over 12 inches when unannealed and not over 20 inches when annealed are identical, respectively, with class $\mathrm{D}$ untreated and class $\mathrm{F}$ annealed in A. S. T. M. A18-30.) Mild forgings of all sizes shall conform to the following minimum requirements as to tensile properties:

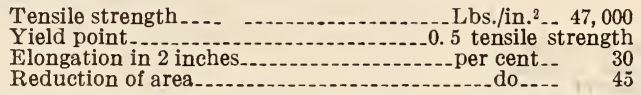

The yield point shall be determined by the drop of the beam of the testing machine, the speed of the crosshead of the machine not exceeding one-half inch per minute. For the determination of tensile strength the speed shall not exceed 2 inches per minute.

(Retests are the same as in A. S. T. M. A20 (see 611.51 , p. 757), while workmanship and finish, marking, inspection and rejection, and rehearing conform to A. S. T. M. standard specifications for steel, pars. $5,6,7,8$, and 9 (see 605.0 , p. 247).)

AMERICAN RAILWAY ASSOCIATION, MECHAIICAL DIVISION, AXLES, SHAFTS, AND OTHER FORGINGS, CARBON STEEL, QUENCHED AND TEMPERED, 1925

\section{CARBON-STEEL FORGINGS}

SCOPE

These specifications are to cover the various classes of carbon steel forgings now commonly used in locomotive construction, classified as class A and class $B$ on the basis of size.

\section{MANUFACTURE}

(Requirements covering manufacture are practially the same as for carbon-steel and alloy-steel forgings in A. S. T. M. A18-30 (see 611.51, p. 754) including the heat-treatment specified in par. $6(b)$.)

\section{CHEMICAL PROPERTIES AND TESTS}

Chemical composition.-The steel shall conform to the following requirements as to chemical composition:

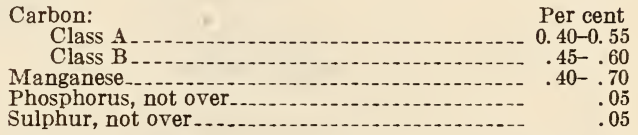

(Ladle and check analyses are the same as in A. S. T. M. A18-30, and include the additional paragraph in regard to phosphorus determination given in the American Railway Association specification for alloy-steel forgings, quenched and tempered. See 622.6, p. 1136.)

\section{PHYSICAL PROPERTIES AND TESTS}

Tension tests.-The forgings shall conform to the requirements as to tensile properties specified in Table 1. 
TABLE 1

For forgings whose diameter or thickness is not over 10 inches when solid.

\begin{tabular}{|c|c|c|c|c|c|c|}
\hline \multirow{2}{*}{ Size } & \multirow{2}{*}{$\begin{array}{l}\text { Tensile } \\
\text { strength }\end{array}$} & \multirow{2}{*}{$\begin{array}{l}\text { Elastic } \\
\text { limit }\end{array}$} & \multicolumn{2}{|c|}{$\begin{array}{l}\text { Elongation in } \\
2 \text { inches, per } \\
\text { cent }\end{array}$} & \multicolumn{2}{|c|}{$\begin{array}{l}\text { Reduction of } \\
\text { area, per cent }\end{array}$} \\
\hline & & & $\begin{array}{l}\text { Inverse } \\
\text { ratio }\end{array}$ & $\begin{array}{l}\text { Not } \\
\text { un- } \\
\text { der }\end{array}$ & $\begin{array}{l}\text { Inverse } \\
\text { ratio }\end{array}$ & $\begin{array}{l}\text { Not } \\
\text { un- } \\
\text { der }\end{array}$ \\
\hline $\begin{array}{l}\text { Class A, up to } 7 \\
\text { inches outside } \\
\text { diameter or } \\
\text { thickness when } \\
\text { solid, or } 31 / 2- \\
\text { inch maximum } \\
\text { w all w h en } \\
\text { bored........ }\end{array}$ & $\begin{array}{c}\text { Lbs./in }{ }^{2} \\
85,000\end{array}$ & $\begin{array}{c}\text { Lbs./in.2 } \\
50,000\end{array}$ & $\frac{2,000,000}{\text { Tens. str. }}$ & 20.5 & $\frac{3,800,000}{\text { Tens. str. }}$ & 39 \\
\hline $\begin{array}{l}\text { Class B, over } 7 \text { to } \\
10 \text { inches, in- } \\
\text { clusive, outside } \\
\text { diameter or } \\
\text { thickness when } \\
\text { solid, or 5-inch } \\
\text { maximum wall } \\
\text { when bored...- }\end{array}$ & 85,000 & 50,000 & $\frac{1,900,000}{\text { Tens.str. }}$ & 19.5 & $\frac{3,600,000}{\text { Tens. str. }}$ & 37 \\
\hline
\end{tabular}

(Requirements covering classification of forgings by size, determination of elastic limit by means of the extensometer, time of testing forgings, tension and bends test specimens (including illustration), are practically the same as for carbon-steel and alloy-steel forgings in A. S. T. M. A18-30. See 651.11, p. 754.)

The speed of the test machine shall not exceed one-fourth inch per minute until the elastic limit has been reached.

(Requirements covering bends tests, proof test, number of tests, and retests are identical with those in the American Railway Association specification for alloy-steel forgings, quenched and tempered. See 622.6 , p. 1136.)

(Requirements covering workmanship and finish, marking, inspection and rejection, and rehearing conform with those specified in A. S. T. M. standard specifications for steel, pars. $5,6,7,8$, and 9 . See 605.0 , p. 247.)

Freight charges.-All rejected forgings will be returned to the manufacturer who shall pay freight charges both ways.
AMERICAN RAILWAY ASSOCIATION, MECHANICAL DIVISION, AXLES FOR LOCOMOTIVES, RECOMMENDED PRACTICE, 1927

\section{AXLES FOR LOCOMOTIVES}

(See figs. 554 to 558 )

AMERICAN RAILWAY ASSOCIATION, MECHANICAL DIVISION, JOURNAL LENGTH GAGE, RECOMMENDED PRACTICE, 1930

\section{JOURNAL LENGTH GAGE}

(See fig. 559)

This gage is to be used for measuring length of journals on classes $\mathrm{A}$ to $\mathrm{F}$ axles, inclusive. Classes $\mathrm{A}$ to $\mathrm{F}$ axles are defined under A. R. A. standard axles, given below.

\section{AMERICAN RAILWAY ASSOCIATION, ME- CHANICAL DIVISION, STANDARD AXLES, 1928}

\section{STANDARD AXIES}

In 1899 it was decided that the standard axles should be known by letters. In 1920 the same classification was extended to journal boxes and contained parts, trucks, and cars; 2 -axle trucks to be designated as $2 \mathrm{~A}, 2 \mathrm{~B}, 2 \mathrm{C}$, etc.; 3 -axle trucks to be designated as $3 \mathrm{~A}, 3 \mathrm{~B}, 3 \mathrm{C}$, etc.; 4-axle cars to be designated as $4 \mathrm{~A}, 4 \mathrm{~B}, 4 \mathrm{C}$, etc.; 6-axle cars to be designated as $6 \mathrm{~A}, 6 \mathrm{~B}, 6 \mathrm{C}$, etc.

Axle A, with journals, $3 \frac{3}{4}$ by 7 inches, is designed to carry 15,000 pounds.

Axle B, with journals, $4 \frac{1}{4}$ by 8 inches, is designed to carry 24,000 pounds.

Axle $\mathrm{C}$, with journals, 5 by 9 inches, is designed to carry 32,000 pounds.

Axle $\mathrm{D}$ with journals, $5 \frac{1}{2}$ by 10 inches, is designed to carry 40,000 pounds.

Axle E, with journals, 6 by 11 inches, is designed to carry 50,000 pounds.

Axle F, with journals, $61 / 2$ by 12 inches, is designed to carry 60,000 pounds.

In 1928 the standard axle drawing was revised to shown an increase in the length of wheel seat of three-eighths inch. 


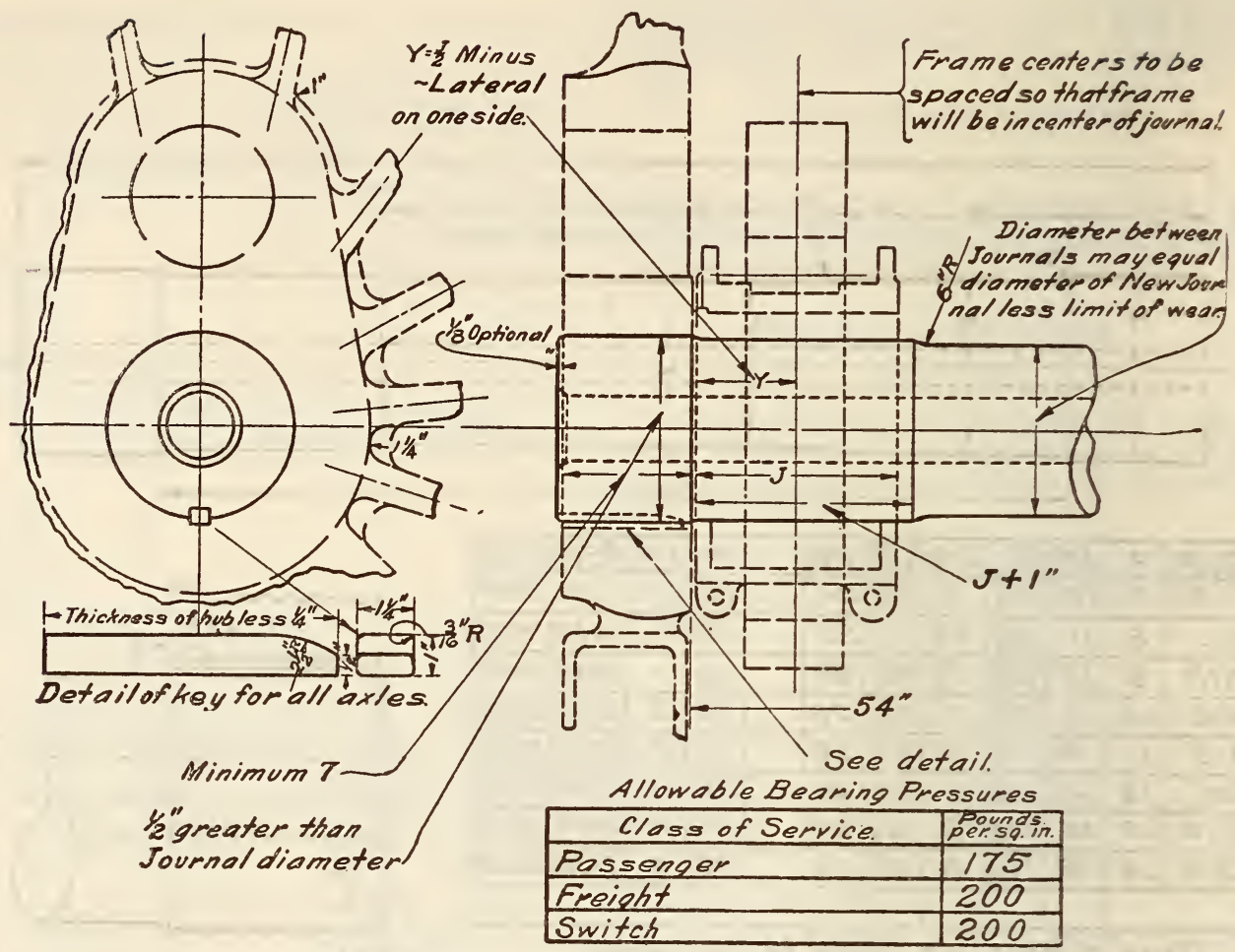

FIGURE 555.-Driving axles for locomotives

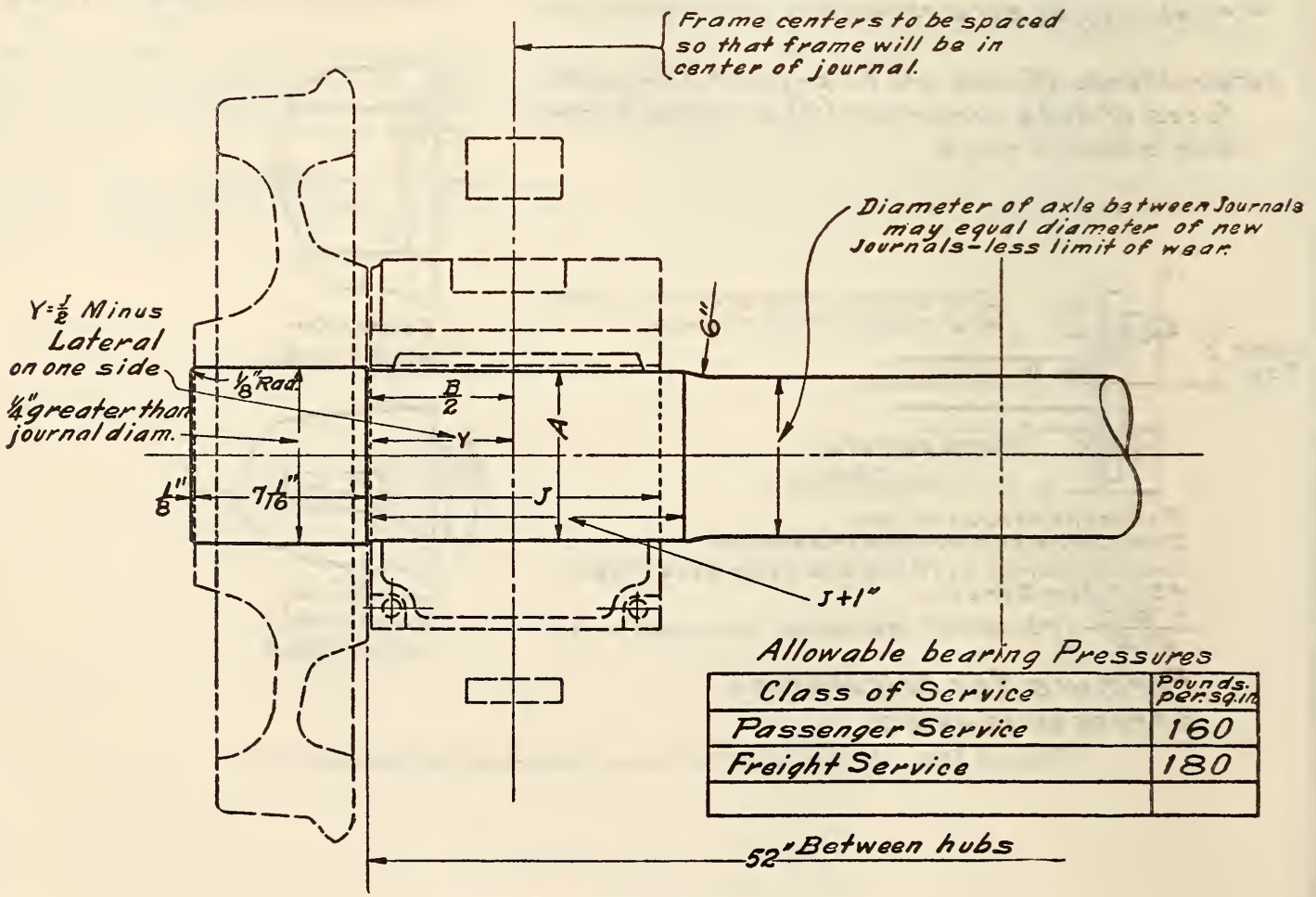

FigURE 556.-Engine truck axles 


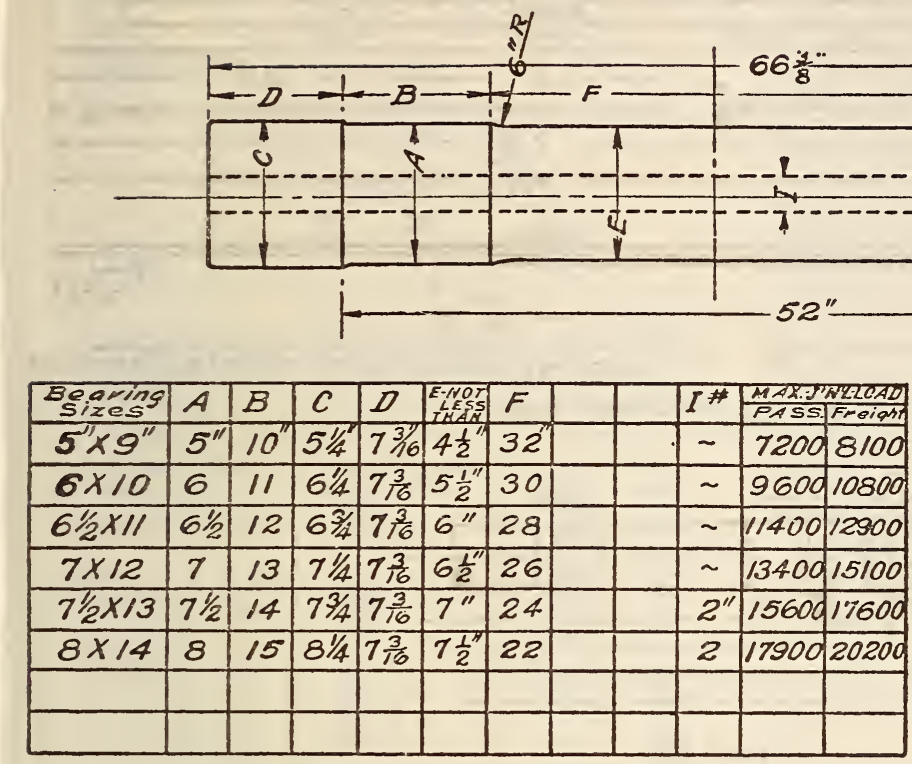

\#Axles may be solidor bored, at option of purchaser.

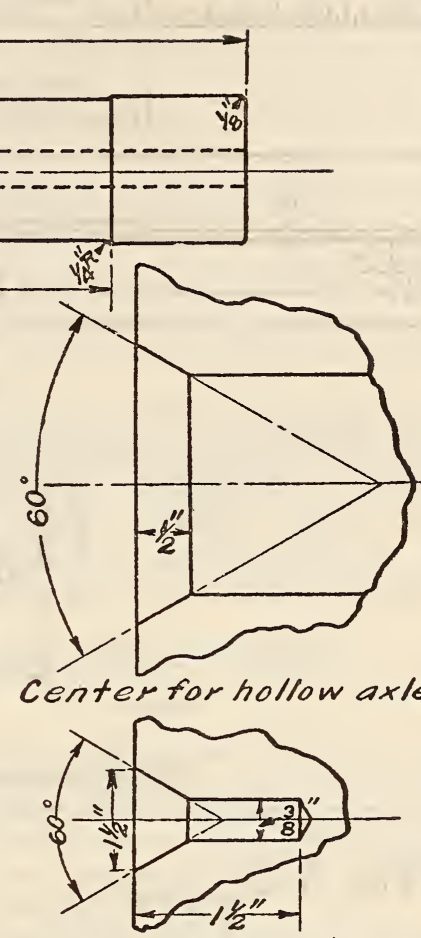

FIGURE 557.-Engine truck axles, sizes and dimensions of

centerforsolid axle.

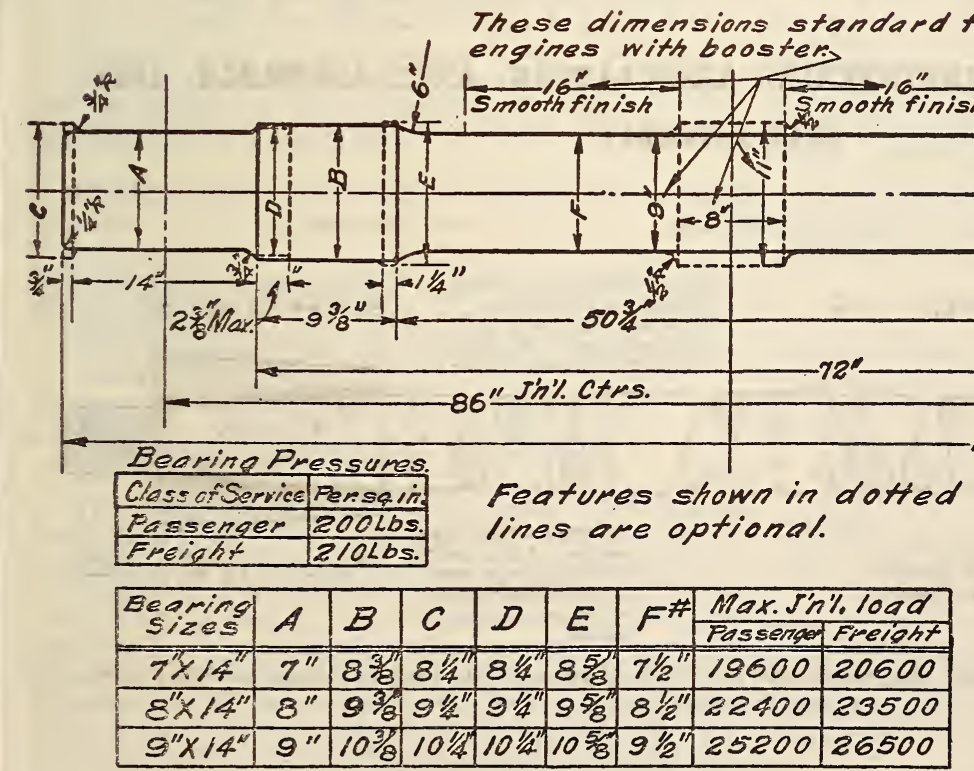

* This dimension applies to Non Buoster axles only. Non Boostor axles tobe Roughturnedbetween wheel fir's.

FIGURE 558.- Trailer truck aales, sizes and dimensions of, for trailer trucks of the 2-wheel type 


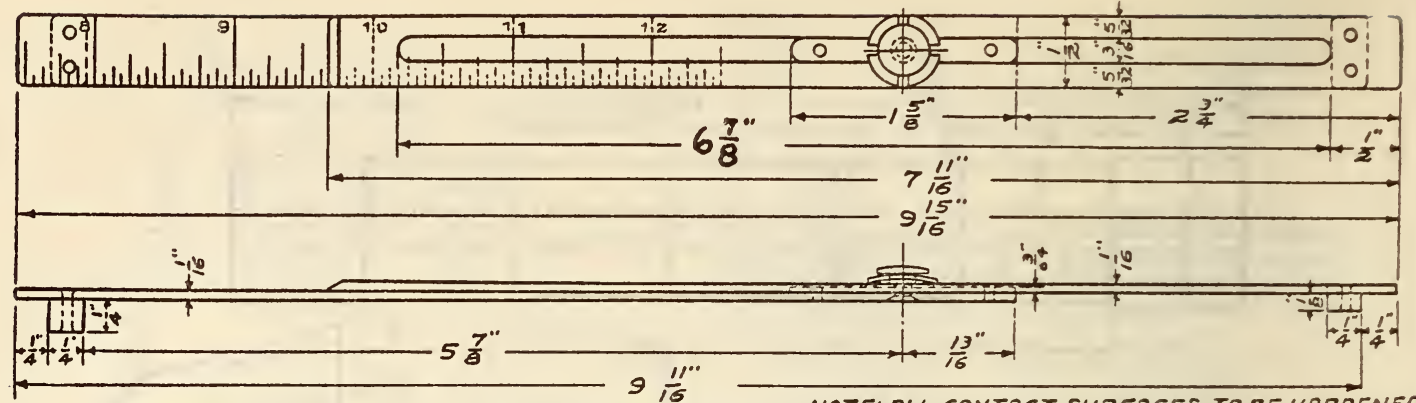

Frgure 559.-Journal length gage

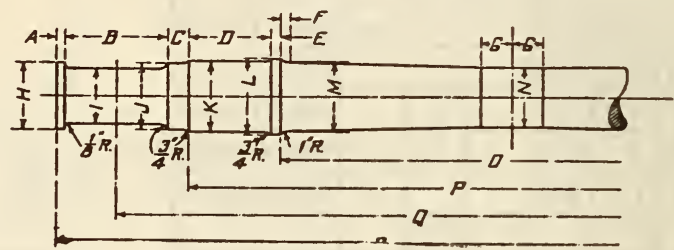

FIGURE 560

(For text accompanying fig. 560 and this table, see A. R. A. standard axles, p. 766)

\begin{tabular}{|c|c|c|c|c|c|c|c|c|c|c|c|c|c|c|c|c|c|c|c|}
\hline \multirow{2}{*}{$\begin{array}{l}\text { Classifica- } \\
\text { tion of axle }\end{array}$} & \multirow{2}{*}{$\begin{array}{l}\text { Size of } \\
\text { journal }\end{array}$} & \multicolumn{18}{|c|}{ Dimensions } \\
\hline & & A & B & $\mathrm{C}$ & D & $\mathbf{E}$ & F & $G$ & H & I & $J$ & $\mathrm{~K}$ & L & $M$ & $\mathrm{~N}$ & 0 & $P$ & Q & $\mathrm{R}$ \\
\hline $\begin{array}{l}- \\
\cdots\end{array}$ & $\begin{array}{l}\text { Inches } \\
33 / 4 \text { by } 7 \\
41 / 4 \text { by } 8 \\
5 \text { by } 9 \\
51 / 2 \text { by } 10 \\
6 \\
61 / 2 \text { by } 11 \\
6\end{array}$ & \begin{tabular}{r|}
$I n$. \\
$5 / 8$ \\
$5 / 8$ \\
$3 / 4$ \\
$3 / 4$ \\
$7 / 8$ \\
$7 / 8$
\end{tabular} & $\begin{array}{r}I n . \\
7 \\
8 \\
9 \\
10 \\
11 \\
12\end{array}$ & $\begin{array}{l}I n . \\
21 / 2 \\
2 \\
2 \\
2 \\
214 \\
21 / 4\end{array}$ & $\begin{array}{l}\text { In } \\
81 i_{6} \\
81 / 16 \\
8116 \\
81 / 16 \\
858 \\
85 \%\end{array}$ & \begin{tabular}{r|}
$I n$. \\
$13 / 16$ \\
$13 / 16$ \\
$13 / 16$ \\
1316 \\
$13 / 4$ \\
134 \\
13
\end{tabular} & \begin{tabular}{c|} 
In. \\
$23 / 32$ \\
$23 / 32$ \\
$23 / 32$ \\
$23 / 32$ \\
$3 / 4$ \\
$3 / 4$
\end{tabular} & $\begin{array}{c}I n . \\
11 / 2 \\
11 / 2 \\
11 / 2 \\
3 \\
3 \\
3\end{array}$ & \begin{tabular}{r|}
$I n$. \\
$43 / 4$ \\
$51 / 4$ \\
$61 / 8$ \\
$65 / 8$ \\
$71 / 4$ \\
$73 / 4$
\end{tabular} & $\begin{array}{c}\text { In. } \\
33 / 4 \\
41 / 4 \\
5 \\
51 / 2 \\
6 \\
61 / 2\end{array}$ & $\begin{array}{r}I n . \\
43 / 4 \\
51 / 4 \\
618 \\
65 / 8 \\
714 \\
73 / 4\end{array}$ & \begin{tabular}{c|}
$I n$. \\
518 \\
$53 / 4$ \\
$61 / 2$ \\
7 \\
$75 / 8$ \\
818
\end{tabular} & $\begin{array}{c}\text { In. } \\
51 / 2 \\
638 \\
67 / 8 \\
73 / 8 \\
8 \\
81 / 2\end{array}$ & 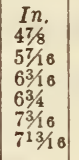 & $\begin{array}{l}I n . \\
414 \\
434 \\
538 \\
578 \\
67 / 16 \\
678\end{array}$ & \begin{tabular}{cc|}
$F t$. & in \\
3 & $91 / 4$ \\
3 & $91 / 4$ \\
3 & $91 / 4$ \\
3 & $91 / 4$ \\
3 & 534 \\
3 & $53 / 4$
\end{tabular} & $\begin{array}{cc}\text { Ft. in. } \\
5 & 3 \\
5 & 3 \\
5 & 3 \\
5 & 3 \\
5 & 21 / 2 \\
5 & 21 / 2\end{array}$ & $\begin{array}{cc}\text { Ft. in. } \\
6 & 3 \\
6 & 3 \\
6 & 4 \\
6 & 5 \\
5 & 6 \\
6 & 7\end{array}$ & $\begin{array}{lr}\text { Ft. } & \text { in } \\
6 & 1144 \\
7 & 34 \\
7 & 21 / 2 \\
7 & 412 \\
7 & 63 / 7 \\
7 & 83 / 4\end{array}$ \\
\hline
\end{tabular}

AMERICAN RAILWAY ENGINEERING ASSOCIATION, AXLE ASSEMBLY, 1929 AXLE ASSEMBLY

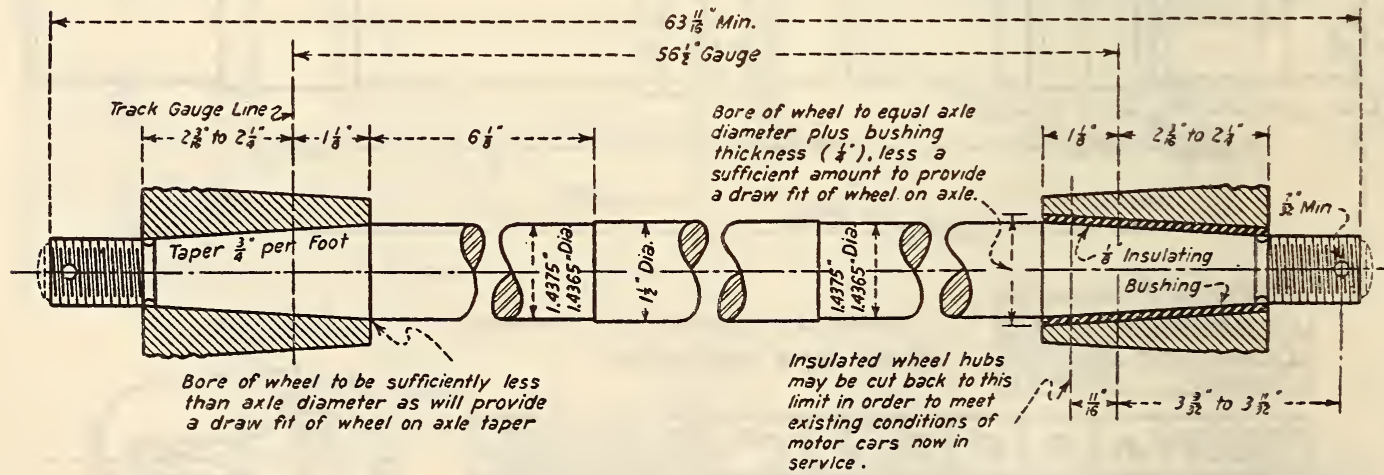

Fradre 561.-One and one-haif inch diameter axle assembly showing tight ana insulated wheels for maintenance motor cars 
AMERICAN SOCIETY OF MECHANICAL ENGINEERS, COLD-FINISHED SHAFTING, STANDARD DIAMETERS AND LENGTHS, B17a-1924

[A pproved as American Tentative Standard by American Standards Association, December, 1924]

\section{COLD-FINISHED SHAFTING}

Diameters and tolerances

\begin{tabular}{|c|c|c|c|c|c|}
\hline $\begin{array}{l}\text { Trans- } \\
\text { mission } \\
\text { shafting } \\
\text { sizes }\end{array}$ & $\begin{array}{l}\text { Machin- } \\
\text { ery shaft- } \\
\text { ing sizes }\end{array}$ & $\begin{array}{c}\text { Toler- } \\
\text { ances on } \\
\text { diame- } \\
\text { ters } 1 \\
(-)\end{array}$ & $\begin{array}{c}\text { Trans- } \\
\text { mission } \\
\text { shafting } \\
\text { sizes }\end{array}$ & $\begin{array}{l}\text { Machin- } \\
\text { ery shaft- } \\
\text { ing sizes }\end{array}$ & $\begin{array}{c}\text { Toler- } \\
\text { ances on } \\
\text { diame- } \\
\text { ters } 1 \\
(-)\end{array}$ \\
\hline Inches & $\begin{array}{c}\text { Inches } \\
16 \\
9 / 16 \\
5 / 8\end{array}$ & $\begin{array}{l}\text { Inch } \\
0.002 \\
.002 \\
.002\end{array}$ & $\begin{array}{l}\text { Inches } \\
23 / 16\end{array}$ & $\begin{array}{c}\text { Inches } \\
23 / 16 \\
21 / 4 \\
2316\end{array}$ & $\begin{array}{l}\text { Inch } \\
0.004 \\
.004 \\
.004\end{array}$ \\
\hline - & $\begin{array}{l}11 / 16 \\
314 \\
13 / 16 \\
7 / 8\end{array}$ & $\begin{array}{l}.002 \\
.002 \\
.002 \\
.002\end{array}$ & $27 / 16$ & $\begin{array}{l}23 / 8 \\
27 / 16 \\
21 / 2 \\
25 / 8\end{array}$ & $\begin{array}{l}.004 \\
.004 \\
.004 \\
.004\end{array}$ \\
\hline $15 / 16$ & $\begin{array}{l}15 / 16 \\
1 \\
11 / 16 \\
118\end{array}$ & $\begin{array}{l}.002 \\
.002 \\
.003 \\
.003\end{array}$ & $215 / 16$ & $\begin{array}{l}23 / 4 \\
27 / 8 \\
3 \\
31 / 8\end{array}$ & $\begin{array}{l}.004 \\
.004 \\
.004 \\
.004\end{array}$ \\
\hline $13 / 16$ & $\begin{array}{l}13 / 16 \\
11 / 4 \\
15 / 16 \\
13 / 8\end{array}$ & $\begin{array}{l}.003 \\
.003 \\
.003 \\
.003\end{array}$ & $37 / 16$ & $\begin{array}{l}31 / 4 \\
33 / 8 \\
31 / 2 \\
35 / 8\end{array}$ & $\begin{array}{l}.004 \\
.004 \\
.004 \\
.004\end{array}$ \\
\hline $17 / 16$ & $\begin{array}{l}17 / 16 \\
11 / 2 \\
1 \% / 16 \\
15 / 8\end{array}$ & $\begin{array}{l}.003 \\
.003 \\
.003 \\
.003\end{array}$ & $3^{15 / 16}$ & $\begin{array}{l}33 / 4 \\
37 / 8 \\
4 \\
41 / 4\end{array}$ & $\begin{array}{l}.004 \\
.004 \\
.004 \\
.005\end{array}$ \\
\hline $111 / 16$ & $\begin{array}{l}111 / 16 \\
13 / 4 \\
113 / 16 \\
17 / 8\end{array}$ & $\begin{array}{l}.003 \\
.003 \\
.003 \\
.003\end{array}$ & $\begin{array}{l}47 / 16 \\
415 / 16\end{array}$ & $\begin{array}{l}41 / 2 \\
43 / 4 \\
5 \\
514\end{array}$ & $\begin{array}{l}.005 \\
.005 \\
.005 \\
.005\end{array}$ \\
\hline $115 / 16$ & $\begin{array}{l}115 / 16 \\
2 \\
21 / 16 \\
21 / 8\end{array}$ & $\begin{array}{l}.003 \\
.003 \\
.004 \\
.004\end{array}$ & $\begin{array}{l}57 / 16 \\
5^{15} / 16\end{array}$ & $\begin{array}{l}51 / 2 \\
53 / 4 \\
6\end{array}$ & $\begin{array}{l}.005 \\
.005 \\
.005\end{array}$ \\
\hline
\end{tabular}

1 These tolerances are negative and represent the maximum allowable variation below the exact nominal size. For instance, the maximum diamater of the $115 / 16$-inch shaft is 1.938 inches and its minimum allowable diameter is 1.935 inches.

Standard stock lengths for cold-finished shafting shall be: 16,20 , and 24 feet.

\section{AMERICAN SOCIETY OF MECHANICAI ENGINEERS, CODE FOR DESIGN OF TRANSMISSION SHAFTING, B17c-1927}

[Approved by American Standards Association, November, 1927]

\section{TRANSMISSION SHAFT DESIGN}

This code covers designing formulas for the cases most frequently met in the design of transmission shafting, also diagrams for use in designing shafting.

Appendix A covers the various stress conditions that may be set up in a transmission shaft; the various theories of elastic failure evolved from time to time leading to the many formulas found in engineering literature; the application and limitation of the various theories of elastic failure as applied to ductile ferrous material used in shafts; and the elastic shearing strength of ductile ferrous materials.

Appendix B covers designing stresses, shafting steels, effect of straining action upon working stresses, and the use of constant or base stress with shock and fatigue factors.
The formulas recommended in this report for the design of shafts subjected to torsion or bending only, are based upon the well recognized common theories of torsion and flexure. For such cases standardization is confined to the adoption of proper unit stresses suited to the material of which the shaft is made and to the operating conditions. For the case of a shaft subjected to torsion combined with flexure or end thrust, or both, no rational method of design has become recognized as standard in the literature of machine design. The formulas are perfectly general and apply to all cases of shaft design, even to shafts in special machines. The only limitation imposed is that these formulas provide only for strength and do not deal with the rigidity or deformation of the shafting system.

\section{AMERICAN SOCIETY FOR TESTING MATE- RIALS, STANDARD SPECIFICATIONS FOR QUENCHED-AND-TEMPERED CARBON- STEEL AXLES, SHAFTS, AND OTHER FORGINGS FOR LOCOMOTIVES AND CARS, DESIGNATION A19-27, 1927}

\section{CARBON-STEEL FORGINGS}

\section{SCOPE}

When used for forgings for locomotives, these specifications cover quenched-and-tempered carbonsteel driving axles, engine and trailing truck axles, main and side rods, straps, crank pins, and piston rods.

\section{MANUFACTURE}

(Requirements covering manufacture are practically the same as for carbon-steel and alloy-steel forgings in A. S. T. M. specification A18-30 (see 611.51 , p. 754 ), including the heat treatment specified in paragraph $6(b)$.

\section{BORING}

Unless otherwise specified by the purchaser, all forgings over 7 inches in diamater shall be bored, and all axles, shafts, and similar forgings shall be rough turned all over. The boring shall be done before quenching.

If boring is specified, the diamater of the hole shall be at least 20 per cent of the maximum outside diamater or thickness of the forging, exclusive of collars and flanges.

\section{CHEMICAL PROPERTLES AND TESTS}

7. The steel shall conform to the following requirements as to chemical composition:

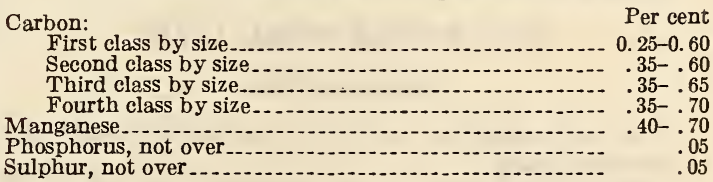

(Ladle and check analyses are the same as in A. S. T. M. A18-30, and include the additional paragraph in regard to phosphorus determination given in the American Railway Association specification for alloy-steel forgings, quenched and tempered. See 622.6, p. 1136.)

\section{PHYSICAL PROPERTIES AND TESTS}

(Requirements covering classification of forgings by size, determination of elastic limit by means of the extensometer, time of testing forgings, tension and bend test specimens (including illustration), are 
practically the same as for carbon-steel and alloysteel forgings in A. S. T. M. A18-30. See 611.51, p. 754. The tensile properties are identical with those for grade $\mathrm{G}$, quenched and tempered, in A18-30.)

(Requirements covering bend tests, proof tests, number of tests, and retests conform to those specified by the American Railway Association for quenched and tempered alloy-steel forgings. See 622.6, p. 1136.)

(Requirements covering workmanship and finish, marking, inspection and rejection, and rehearing conform to those specified in A. S. T. M. standard specifications for steel, pars. 5, 6, 7, 8, and 9 . See 605.0, p. 247.)

\section{AMERICAN SOCEITY FOR TESTING MA- TERIALS, TENTATIVE SPECIFICATIONS FOR CARBON-STEEL FORGINGS FOR LOCOMOTIVES, DESIGNATION A20-31T, 1931}

\section{LOCOMOTIVE FORGINGS}

(See 611.51, p. 757.)

\section{ANERICAN SOCIETY FOR TESTING MA- TERIALS, STANDARD SPECIFICATIONS FOR CARBON-STEEL CAR AND TENDER AXLES, DESIGNATION A21-2\%, 192\%}

\section{CARBON-STEEL AXIES}

(Same as American Railway Association specification for axles, carbon steel, for cars and locomotive tenders, given above, including the references given in the latter to the A. R. A. specification for annealed and unannealed carbon-steel forgings, and additional references to A. S. T. M. specification A18-30, and standard specifications for steel in 605.0. In addition A21-27 provides that for axles 65 inches or under in length the permanent set produced by the first blow shall not exceed that given by the formula:)

$$
\left(\frac{L}{1.9 d}-\frac{d}{2}\right)+1 \text { inch }
$$

\section{NOMBER OF TESTS}

One drop test shall be made from each melt. Not less than 15 tons shall be offered from any one melt unless otherwise specified.

\section{AMERICAN SOCIETY FOR TESTING MA- TERIALS, STANDARD SPECIFICATIONS FOR COLD-ROLLED STEEL AXLES, DES- IGNATION A22-21, 1921}

\section{COLD-ROLLED STEEL AXLES}

\section{MANUFACTURE}

The axles shall be cold-rolled to finished size from hot-rolled bars.

(Process and discard are the same as in A. S. T. M. A18-30. See 611.51, p. 754.)

\section{CHEMICAL PROPERTIES AND TESTS}

Chemical composition.-The steel shall conform to the following requirements as to chemical composition:

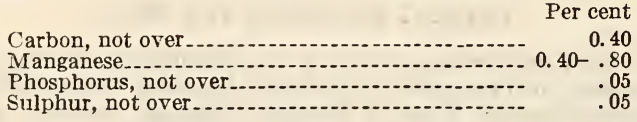

(Ladle and check analyses are the same as in A. S. T. M. A18-30.)
PHXSICAL PROPERTIES AND TESTS

The axles shall conform to the following minimum requirements as to tensile properties:

$\begin{array}{ll}\text { Tensile strength } & \text { Elastic limit } \\ \text { Elongation in } 2 \text { inches. } & \end{array}$

(Requirements covering determination of elastic limit by means of the extensometer, tension and bend test specimens (including illustration) are practically the same as for carbon-steel and alloy-steel forgings in A. S. T. M. A18-30. See 611.51, p. 754.)

Bend test.-The test specimen shall bend cold through $180^{\circ}$ around a pin 1 inch in diameter, without cracking on the outside of the bent portion.

Bend test specimens shall be one-half inch square in section with corners rounded to a radius not over one-sixteenth inch, and need not exceed 6 inches in length.

Number of tests.-One tension and one bend test shall be made from each lot of 50 axles or less from each melt.

If any test specimen shows defective machining or develops flaws it may be discarded and another specimen substituted.

If the percentage of elongation of any tension-test specimen is less than that specified above, and any part of the fracture is more than three-fourths inch from the center of the gage length, as indicated by scribe scratches marked on the specimen before testing, a retest shall be allowed.

\section{WORKMANSHIP AND FINISH}

The axles shall conform to the sizes and shapes specified by the purchaser and shall not vary more than 0.002 inch from the diameter specified. When centered, $60^{\circ}$ centers with clearance drilled for points shall be used.

The axles, either finished or plain, shall be straight and free from injurious defects and shall have a workmanlike finish.

\section{MARKING}

Identification marks shall be legibly stamped on each axle, and on each test specimen. The purchaser shall indicate the location of such identification marks.

(Requirements covering inspection and rejection and rehearing, conform to those specified in A. S. T. M. standard specifications for steel, pars. 7, 8, and 9 . See 605.0, p. 247.)

\section{NATIONAL MACHINE TOOL BUILDERS ASSOCIATION, SHAFT DIAMETERS USED IN MACHINE-TOOL CONSTRUCTION, MAY 22, 1928}

\section{MACHINE TOOL SHAFT DIAMETERS}

Diameters of shafts under 1 inch to be in sixteenths of an inch or even multiples thereof.

Diameters of shafts from 1 inch to $23 / 4$ inches to be in eighths of an inch or even multiples thereof.

Diameters of shafts from $23 / 4$ inches to 6 inches to be in fourths of an inch or even multiples thereof.

611.53 WHEELS AND TIRES, STEEL.

AMERICAN ELECTRIC RAILWAY ENGINEERING ASSOCIATION, STANDARD SPECIFICATION FOR SOLID WROUGHT CARBON STEEL WHEELS FOR ELECTRIC RAILWAY SERVICE, E7-21, 1921

\section{SOLID WROUGHT CARBON STEEL WHEELS}

(Identical with A. S. T. M. A25, given below.) 
AMERICAN ELECTRIC RAILWAY ENGINEERING ASSOCIATION, STANDARD IIMIT-OF-WEAR GAGES FOR A. E. R. E. A. AND A. R. A. STANDARD FLANGE CONTOURS, E8-26, 1926

\section{LIMIT-OF-WEAR GAGES}

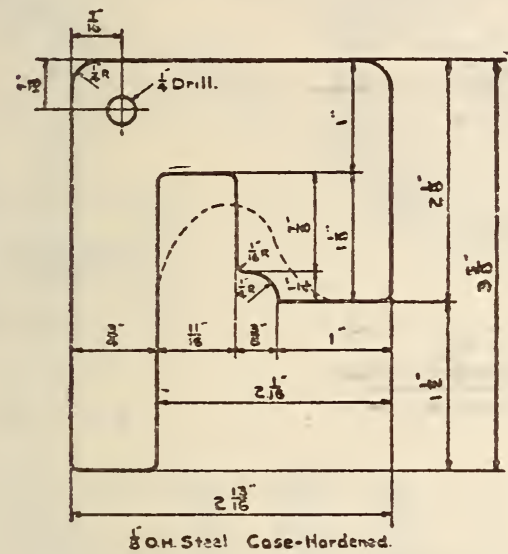

FIGURE 562-Limit-of-wear gage for $1 \frac{1}{8}$ and $13 / 16$ inch flanges

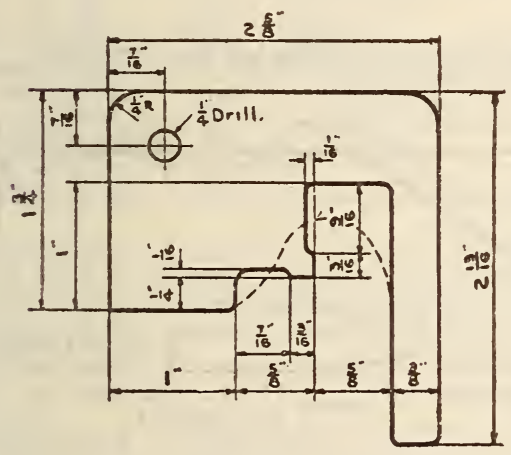

\$Q.H.Steel Cose-Hordened

FIGURE 563-Limit-of-wear gage for 1-inch flange
Gages as shown are for use with rolled-steel wheels only. The same general dimensions apply to gages for use with cast-iron wheels except that the part which projects down the back of the wheel should be cut off in line with the tread face.

These gages are to be used only for testing wheels and not to determine their suitability for remounting.
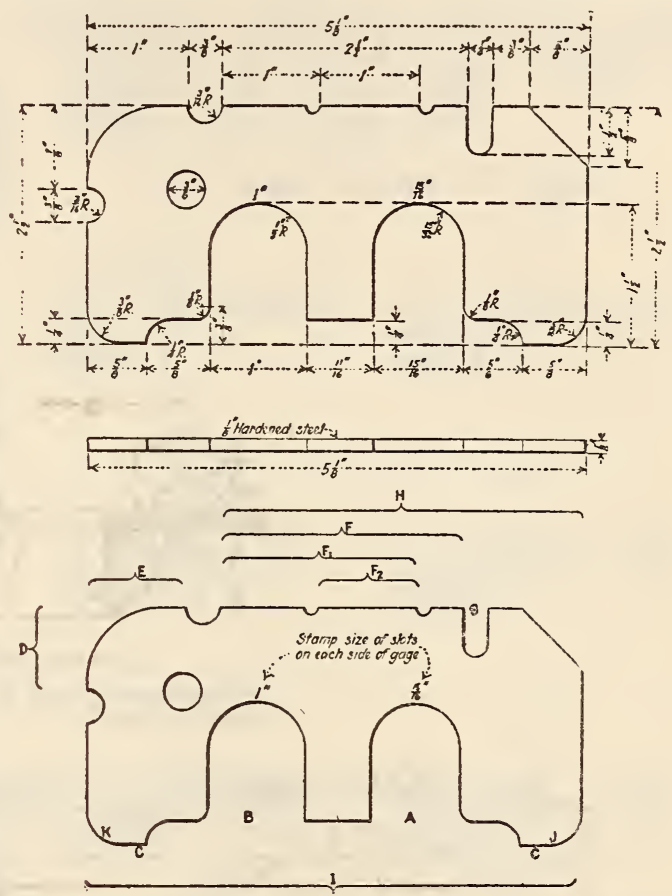

FIGURE 564.- Limit-of-wear gage for standard A. R. A. wheel contours

Slot $G$, one-fourth inch wide, is for gaging the thickness of worn journal collars.

Length $H, 31 / 4$ inch, is for gaging chipped rims.

Overall length $I, 51 / 8$ inch, is for gaging distance between point of knuckle and guard arm of couplers, measured perpendicularly to the guard arm.

Radii $J$ and $K$, five-sixteenth inch and one-eighth inch, respectively, are for checking the fillets of journals.

Slots $A$ and $B$, fifteen-sixteenth inch and 1 inch, respectively, are for gaging flanges worn thin; gage is to be used with heel $C$ on tread of wheel.

Corners $D$ and $E$, seven-eighthinch and 1 inch, respectively, are for measuring height of vertical wheel flange wear above tread.

Lengths $F, F_{1}$, and $F_{2}\left(2 \frac{1}{2}, 2\right.$, and 1 inch, respectively) are for gaging the length of shelled out or flat spots on wheel tread. 


\section{AMERICAN ELECTRIC RAILWAY ENGINEERING ASSOCIATION, STANDARD PLANE GAGE FOR SOLID STEEL WHEELS, E9-19, 1919}

\section{PLANE GAGE FOR SOLID STEEL WHEELS}
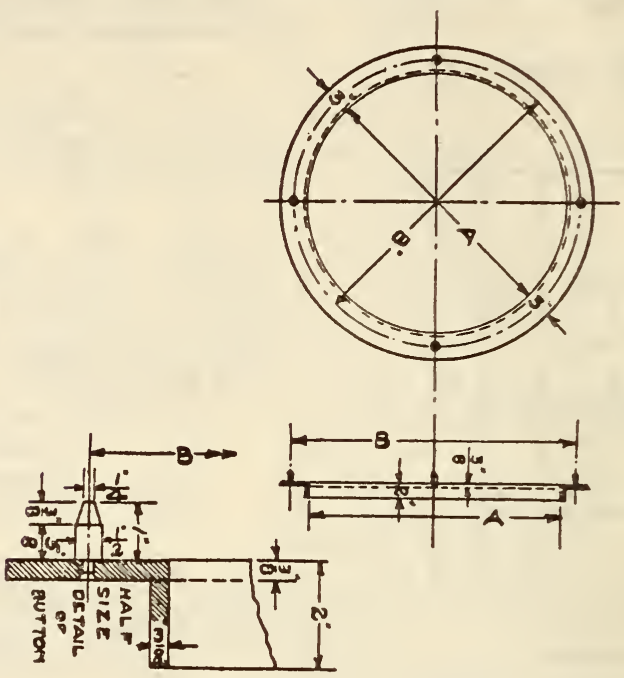

Figure 565.-Plane gage

Measurements $A$ and $B$ vary, depending on diameter of wheel to be gaged.

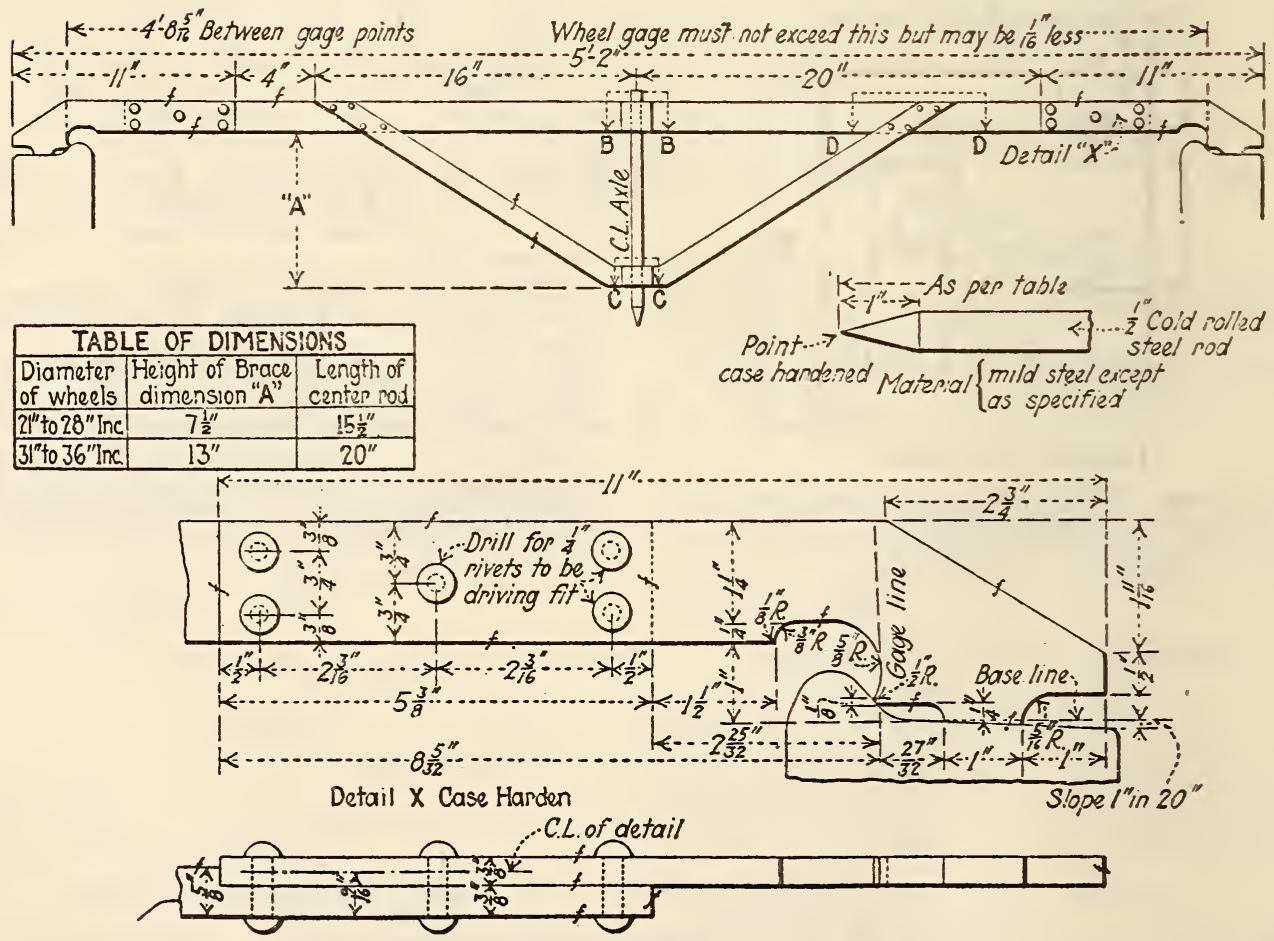

Figure 566.-Wheel mounting gage

This design of gage is for mounting wheels for contours: A-3, A-316, B-21/2, B-3, C-21/2, C-3, D-21/2, D-3 only. Wheel gage is shown applied to $13 / 16$ inch flange for wheel contours $A-3$ and $A-31 \frac{1}{2}$. (See E10-25, p. 775)

There has been adopted as standard the plane by placing it on the back of the rim. The tolergage for rolled-steel wheels shown in the figure. ances allowed will be governed by the wheel This is used in checking warped condition of wheels $/$ specifications. 
AMERICAN ELECTRIC RAILWAY ENGINEERING ASSOCIATION, STANDARD WHEEL-MOUNTING GAGE, E10-25, 1925 WHEEL-MOUNTING GAGE

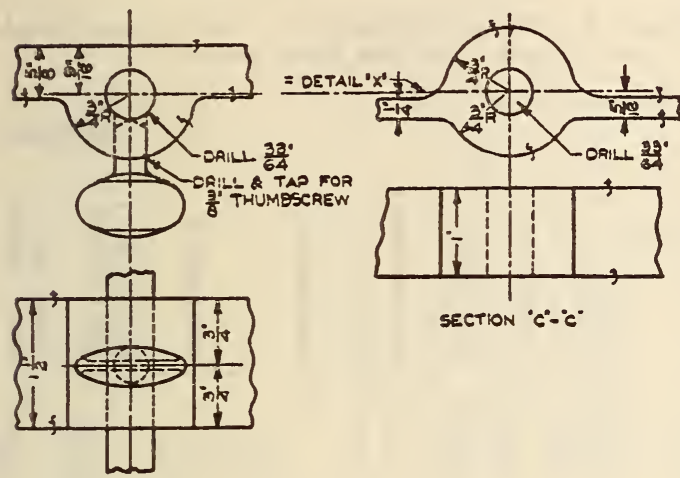

SECTION 'B'-'8

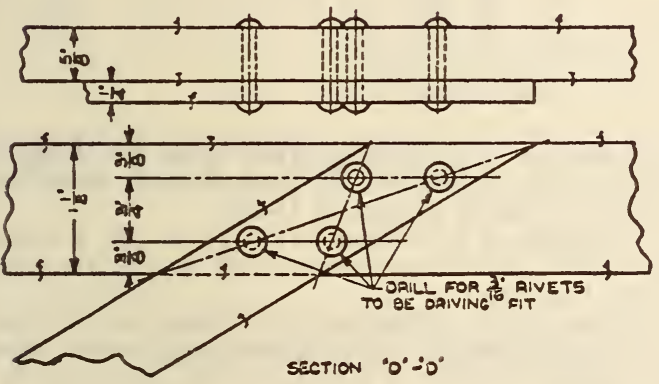

FigURE 567.-Detail of wheel mounting gage

This gage is made with a pointer for the center line of the axle so that the first wheel will be pressed on from the center line of the axle, thereby insuring that the wheels will not only be located the proper distance from each other but will be located centrally on the axle.

\section{AMERICAN ELECTRIC RAILWAY ENGI- NEERING ASSOCIATION, STANDARD ROTUNDITY GAGE FOR SOLID-STEEL WHEELS, E11-19, 1919 \\ ROTUNDITY GAGE}

There has been adopted as standard the rotundity gage for steel wheels shown in Figure 589. (See A.
R. A. solid wrought carbon steel wheels, given below.) As all standard wheels have a taper of 1 inch in 20 inches in checking the wheel, the gage will be used that will become tight on the wheel about the center of the tread. The tolerances governing this are included in the wheel specifications.

AMERICAN ELECTRIC RAILWAY ENGI. NEERING ASSOCIATION, STANDARD DESIGH FOR TREAD AND FLANGE OF WHEEL, NO. E13-26, 1926

\section{TREAD AND FLANGE OF WHEEL}
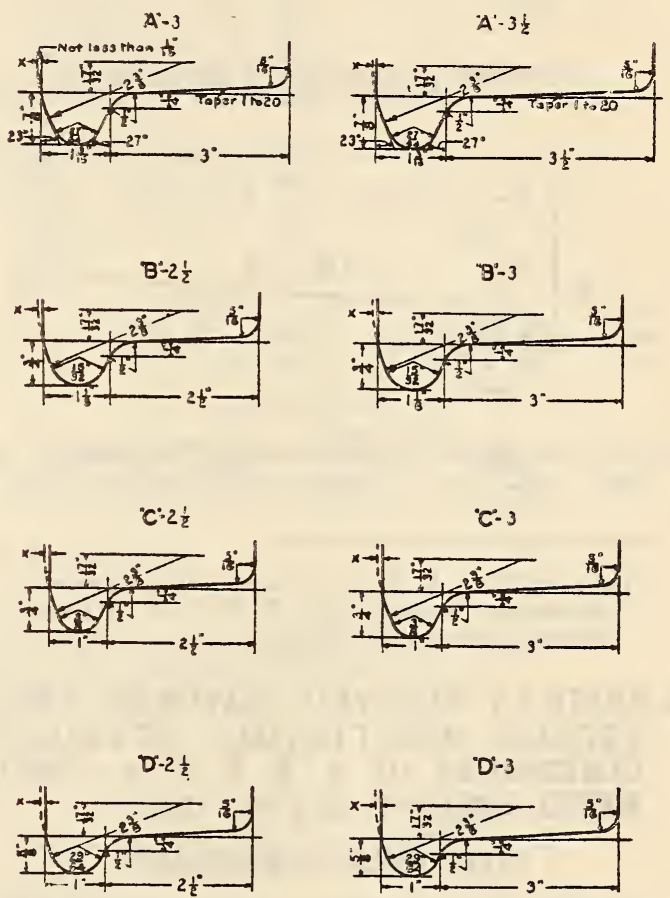

FigURE 568.-A.E.R.E. A. standard tread and flange contours for steel and chilled tron wheels

Full lines. steel wheels Dotted lines Dimension $X=$ not less than $1 / 16$ inch.

\section{Tapers}

Wheel tread 1 to 20

Flange-tread side $-27^{\circ}$

Flange-guard side All as shown on flange $A-3$. 
CONTOUR E FOR CHILLED IRON WHEELS (A.R.A. 1909)

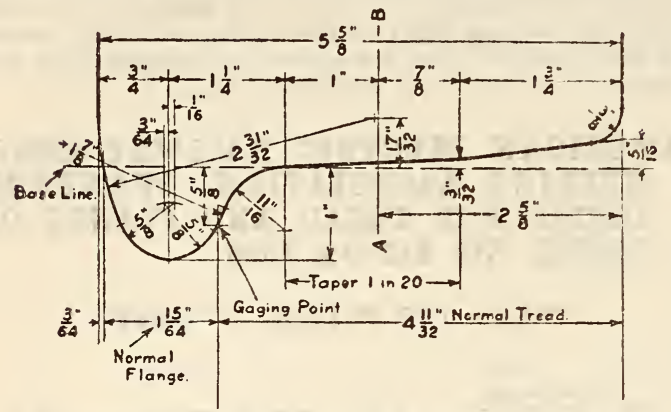

CONTOUR F FOR STEEL \& STEEL TIRED WHEELS (A.R.A. 1920)

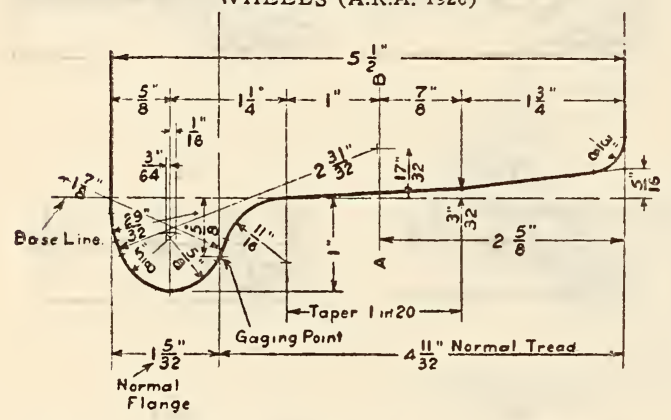

Diometer of Wheel is to te measured on line $A-B$

Figure 569.-A. R. A. standard wheel contours adopted as standards of A.E. R. E. A. for service requiring wheels of this type

\section{AMERICAN ELECTRIC RAILWAY ENGI- NEERING ASSOCIATION, STANDARD DIMENSIONS OF A. E. R. E. A. STEEL- WHEEL DESIGNS, E14-29, 1929}

\section{STEEL-WHEEL DIMENSIONS}

The hub diameters given in the tables of steelwheel designs should be looked upon merely as

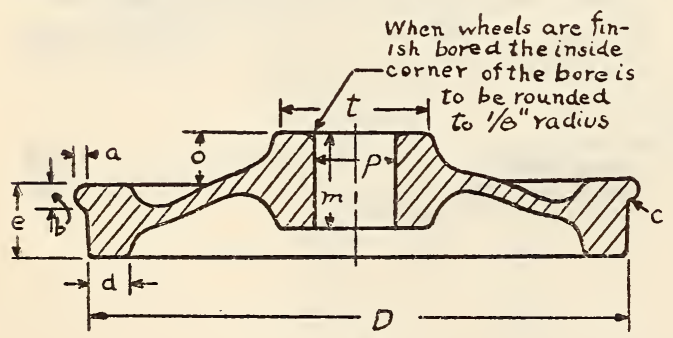

Figure 570

maximum limits to take care of eccentric bores. In the inspection and acceptance of wheels, A. E. R. E. A. Manual, section E7 (same as A. S. T. M., A25, given below) should govern in all cases, and wheels accepted or rejected on hub wall thickness within the tolerances given in the specifications.

Four holes should be included in standard wheel designs for electric railway service in order to pro- vide for the bolting on of pads to reduce noise in operation. These holes should be at least 1 inch in diameter and should be located on a radius approximately 2 inches in from the inside diameter of the rim.

TABLE 1.-Flange thickness (b), 1 inch; flange height (a), $5 / 8$ inch; tread and flange contour $D-21 / 2$

\begin{tabular}{|c|c|c|c|c|c|c|c|c|}
\hline \multirow[b]{2}{*}{ Wheel } & \multirow[b]{2}{*}{ 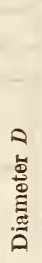 } & \multirow[b]{2}{*}{ 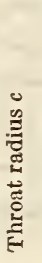 } & \multirow[b]{2}{*}{ 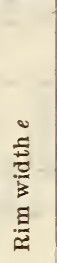 } & \multirow[b]{2}{*}{ 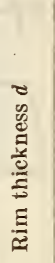 } & \multirow[b]{2}{*}{ 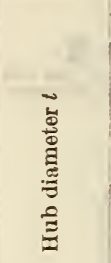 } & \multirow[b]{2}{*}{ 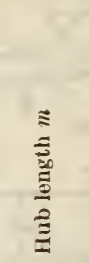 } & \multicolumn{2}{|c|}{$\begin{array}{c}\text { Hub projec- } \\
\text { tion }\end{array}$} \\
\hline & & & & & & & 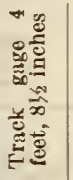 & 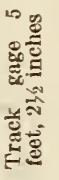 \\
\hline & $\begin{array}{r}I n \\
21 \\
22 \\
24 \\
26\end{array}$ & $\begin{array}{l}\text { In. } \\
1 / 2 \\
1 / 2 \\
1 / 2 \\
1 / 2\end{array}$ & $\begin{array}{l}\text { In. } \\
31 / 2 \\
31 / 2 \\
31 / 2 \\
31 / 2\end{array}$ & $\begin{array}{r}\text { In. } \\
2 \\
2 \\
2 \\
2\end{array}$ & $\begin{array}{ll}\text { In. } & \\
& \frac{7}{71 / 2} \\
& \frac{71}{112} \\
81 / 4 & \text { or } 91 / 4\end{array}$ & $\begin{array}{l}I n \text {. } \\
41_{2} \text { or } 5 \\
41 / 2 \text { or } 5 \\
41 / 2 \text { or } 5 \\
\frac{1}{2} / 2 \text { or } 5\end{array}$ & $\begin{array}{r}\text { In. } \\
31 / 8 \\
318 \\
318 \\
318\end{array}$ & In. \\
\hline & $\begin{array}{l}28 \\
31 \\
33 \\
34\end{array}$ & $\begin{array}{l}1 / 2 \\
1 / 2 \\
1 / 2 \\
1 / 2\end{array}$ & $\begin{array}{l}31 / 2 \\
31 / 2 \\
31 / 2 \\
31 / 2\end{array}$ & $\begin{array}{l}2 \\
2 \\
21 / 2 \\
21 / 2\end{array}$ & $\begin{array}{l}81 / 4 \text { or } 91 / 4 \\
81 / 4 \text { or } 91 / 4 \\
81 / 4 \text { or } 914 \\
81 / 4 \text { or } 91 / 4\end{array}$ & $\begin{array}{l}41 / 2 \text { or } 5 \\
41 / 2 \text { or } 5 \\
41 / 2 \text { or } 5 \\
41 / 2 \text { or } 5\end{array}$ & $\begin{array}{l}31 / 8 \\
31 / 8 \\
31,8 \\
31,8\end{array}$ & 41 \\
\hline
\end{tabular}

Note.-Minimum rough bore (p) $3 \frac{3}{4}$ inches.

Table No. 2 is identical with Table No. 1 except that flange height $(a)$ is three-fourths inch, and tread and fiange contour is $\mathrm{C}-2 \frac{1}{2}$.

Table No. 3 is identical with Table No. 1 except that tread and flange contour is $\mathrm{D}-3$, and rim width (e) is 4 inches.

Table No. 4 is identical with Table No. 1 except that flange height $(a)$ is three-fourths inch, tread and flange contour is $\mathrm{C}-3$, and rim width $(e)$ is 4 $i^{\text {nches. }}$

TABLE 5.-Flange thickness (b), 11/8 inches; flange height (a), three-fourths inch; tread and flange contour $B-21 \%$

\begin{tabular}{|c|c|c|c|c|c|c|c|c|}
\hline 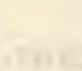 & & & - & & the & & \multicolumn{2}{|c|}{$\begin{array}{l}\text { Hub projec- } \\
\text { tion }\end{array}$} \\
\hline Wheel & 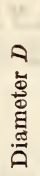 & 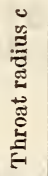 & 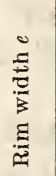 & 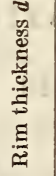 & 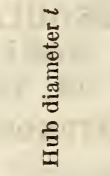 & 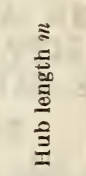 & 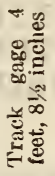 & 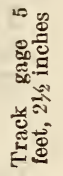 \\
\hline & $\begin{array}{r}\text { In. } \\
21 \\
22 \\
24 \\
26 \\
28\end{array}$ & $\begin{array}{r}\text { In. } \\
1 / 2 \\
1 / 2 \\
1 / 2 \\
1 / 2 \\
1 / 2\end{array}$ & $\begin{array}{r}\text { In. } \\
35 \% \\
358 \\
358 \\
35 \% \\
35 \%\end{array}$ & $\begin{array}{r}\text { In. } \\
2 \\
2 \\
2 \\
2 \\
2\end{array}$ & $\begin{array}{c}\text { In. } \\
\\
\\
\begin{array}{c}\tau_{11 / 2} \\
71 / 4\end{array} \text { or } 91 / 4 \\
81 / 4 \text { or } 91 / 4\end{array}$ & $\begin{array}{c}\text { In. } \\
41 / 2 \text { or } 5 \\
41 / 2 \text { or } 5 \\
41 / 2 \text { or } 5 \\
41 / 2 \text { or } 5 \\
41 / 2 \text { or } 5\end{array}$ & $\begin{array}{r}\text { In. } \\
3 \\
3 \\
3 \\
3 \\
3\end{array}$ & In \\
\hline & $\begin{array}{l}31 \\
33 \\
34 \\
36\end{array}$ & $\begin{array}{l}1 / 2 \\
1 / 2 \\
1 / 2 \\
1 / 2\end{array}$ & $\begin{array}{l}35 \% \\
35 \% \\
35 \% \\
35 \%\end{array}$ & $\begin{array}{l}2 \\
21 / 2 \\
21 / 2 \\
21 / 2\end{array}$ & $\begin{array}{l}81 / 4 \text { or } 91 / 4 \\
91 / 4 \text { or } 111 / 2 \\
91 / 4 \text { or } 111 / 2 \\
91 / 4 \text { or } 111 / 2\end{array}$ & $\begin{array}{r}41 / 2 \text { or } 5 \\
6 \\
6 \\
6\end{array}$ & 3 & \\
\hline
\end{tabular}

NoтE.-Minimum rough bore (p) 33\% inches.

Table No. 6 is identical with Table No. 5 except that tread and flange contour is $\mathrm{B}-3$. and rim width (e) is $4 \frac{1}{8}$ inches. 
TABLE 7.-Flange thickness (b), $13 \% 16$ inches; flange height $(a)$, seven-eighths inch; tread and flange contour, A-3.

\begin{tabular}{|c|c|c|c|c|c|c|c|c|}
\hline \multirow[b]{2}{*}{ Wheel } & \multirow[b]{2}{*}{ 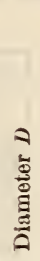 } & \multirow[b]{2}{*}{ 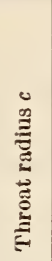 } & \multirow[b]{2}{*}{ 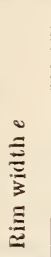 } & \multirow[b]{2}{*}{ 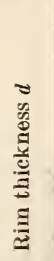 } & \multirow[b]{2}{*}{ 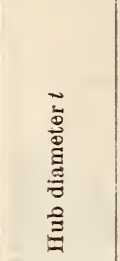 } & \multirow[b]{2}{*}{ 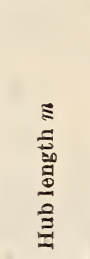 } & \multicolumn{2}{|c|}{$\begin{array}{c}\text { Hub projec- } \\
\text { tion }\end{array}$} \\
\hline & & & & & & & 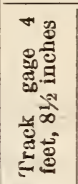 & 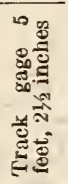 \\
\hline & $\begin{array}{r}\text { In. } \\
21 \\
22 \\
24 \\
26 \\
28\end{array}$ & $\begin{array}{r}I n . \\
1 / 2 \\
1 / 2 \\
1 / 2 \\
1 / 2 \\
1 / 2\end{array}$ & $\begin{array}{l}\text { In. } \\
4316 \\
4316 \\
43116 \\
4316 \\
43116\end{array}$ & $\begin{array}{r}\text { In. } \\
2 \\
2 \\
2 \\
2 \\
2\end{array}$ & $\begin{aligned} & \text { In. } \\
& 71 / 2 \\
& 71 / 2 \\
& 71 / 2 \\
& 81 / 4 \text { or } 91 / 4 \\
& 81 / 4 \text { or } 91 / 4\end{aligned}$ & $\begin{array}{l}I n \text {. } \\
41 / 2 \text { or } 5 \\
412 \text { or } 5 \\
412 \text { or } 5 \\
4112 \text { or } 5 \\
412 \text { or } 5\end{array}$ & $\begin{array}{l}I n . \\
2^{15} / 16 \\
21516 \\
21516 \\
2^{11516} \\
2^{1} 516\end{array}$ & $\begin{array}{l}I n . \\
3^{15} / 16 \\
3^{15 / 16} \\
3^{15 / 16} \\
3^{15 / 16} \\
3^{15} / 16\end{array}$ \\
\hline & $\begin{array}{l}31 \\
33 \\
34 \\
36\end{array}$ & $\begin{array}{l}1,2 \\
1 / 2 \\
1 / 2 \\
1 / 2\end{array}$ & $\begin{array}{l}43 / 16 \\
4316 \\
43116 \\
43116\end{array}$ & $\begin{array}{l}2 \\
2135 \\
21 / 2 \\
21 / 2\end{array}$ & $\begin{array}{l}81 / 4 \text { or } 91 / 4 \\
91 / 4 \text { or } 111 / 2 \\
91 / 4 \text { or } 111 / 2 \\
914 \text { or } 111 / 2\end{array}$ & $\begin{array}{r}41 / 2 \text { or } 5 \\
6 \\
6 \\
6\end{array}$ & $\left\{\begin{array}{l}2^{15 / 16} \\
2^{15 / 16}\end{array}\right.$ & $\begin{array}{r}315 / 16 \\
115 / 16\end{array}$ \\
\hline
\end{tabular}

Nots.-Minimum rough bore (p) $33 \frac{3}{4}$ inches.

Table No. 8 is identical with Table No. 7 excep that tread and flange contour is $\mathrm{A}-3 \frac{1}{2}$, and rim width $(e)$ is $4^{11} 116$ inches.

\section{AMERICAN ELECTRIC RAILWAY ENGINEERING ASSOCIATION, WHEEL CHECKING GAGE FOR A. E. R. E. A. CONTOURS A, B, C, AND D, RECOMMENDED DESIGN, E112-26, 1926}

\section{WHEEL CHECKING GAGE}
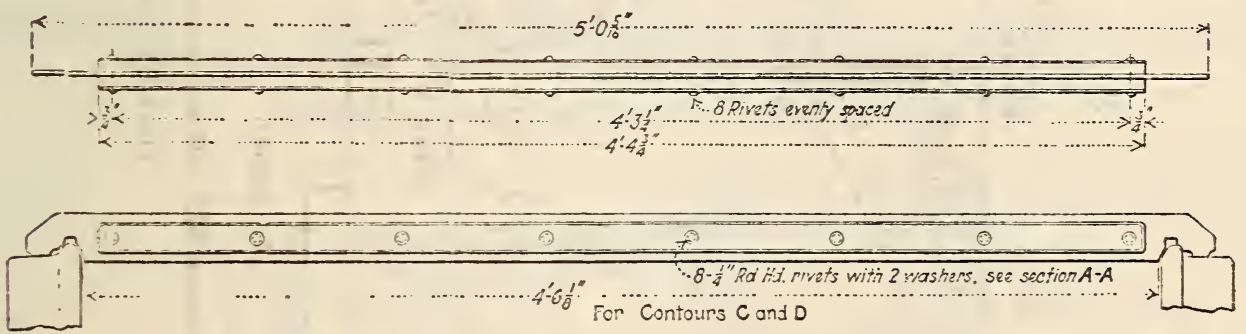

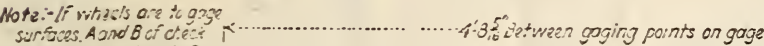

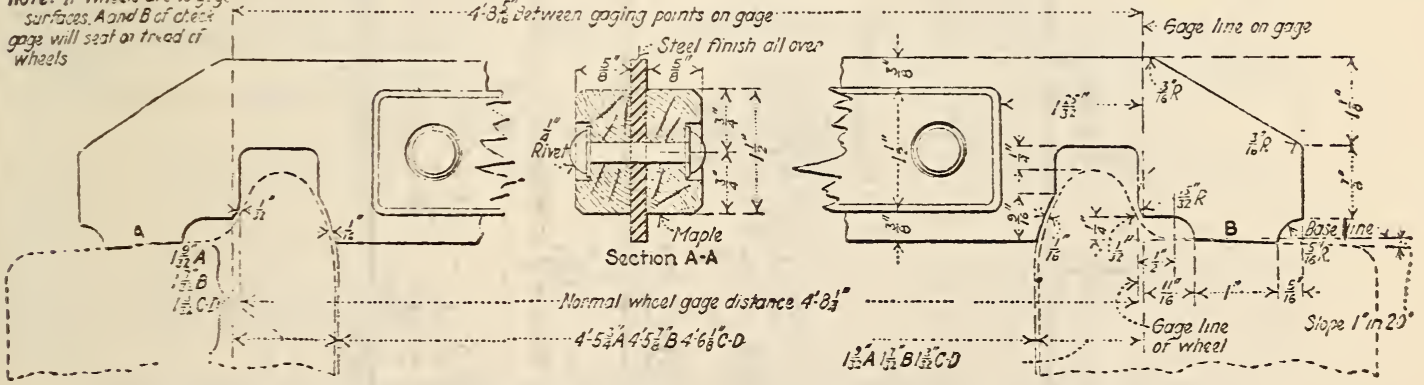

FigURE 571.-Wheel checking gage for contours $A, B, C$, and $D$

The dimensions followed by letters A, B, C, and D apply to the gage itself, and not to the wheels which are shown dotted in the illustration. This gage is for use only in checking wheels in service and is not to be used for mounting wheels. Letters $\mathrm{A}$, B, C, D are for corresponding wheel contours. 


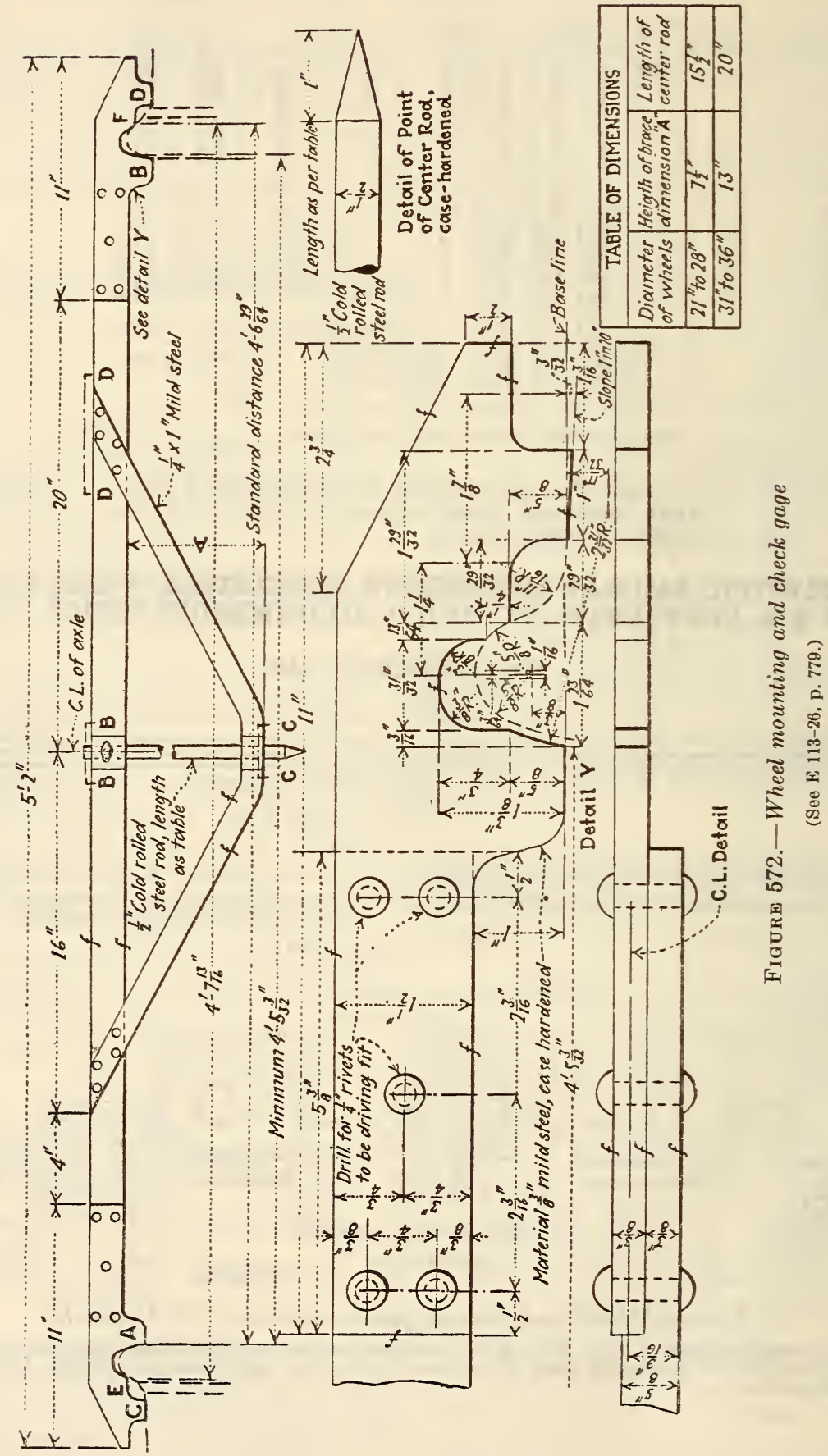




\section{AMERICAN ELECTRIC RAILWAY ENGINEER- ING ASSOCIATION, WHEEL MOUNTING AND CHECK GAGE FOR A. R. A. CON- TOURS E AND F, RECOMMENDED DE- SIGN, E113-26, 1926}

\section{WHEEL MOUNTING AND CHECK GAGE}

In the mounting of wheels, new or second hand, the standard wheel mounting and check gage should be used in the following manner:

After one wheel is pressed into position, place the stop $A$ or $B$ of the check gage against the inside of the flange of the wheel with the thinner flange, with the corresponding tread stop $C$ or $D$ against the tread of the wheel. Press the other wheel on the axle until the opposite tread stop comes in contact with the tread and the corresponding gage point $E$ or $F$ is in contact with the outside of the thicker flange.

Wheels should be mounted centrally on the axle. All axles should be center punched and the center point of the gage should be used to locate the first wheel pressed on the axle from this center punch mark.

This gage is to be used for mounting and checking wheels with A. R. A. contours $E$ and $F$ only. It is the same as standardized by the American Railway Association in 1911 (for illustration of latter see A. R. A. M. solid wrought carbon-steel wheels, given below), except that a center point has been added. The wheel mounting and check gage as shown is designed for and applied to contour $E$. When used with contour $F$ the clearance from the gage to the back of the flange will be increased to a maximum of five-sixty-fourth inch at the tread level.

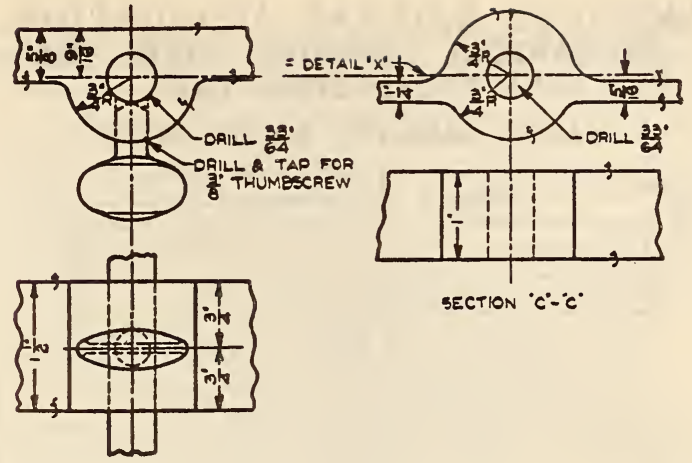

SECTION $A \cdot-8$

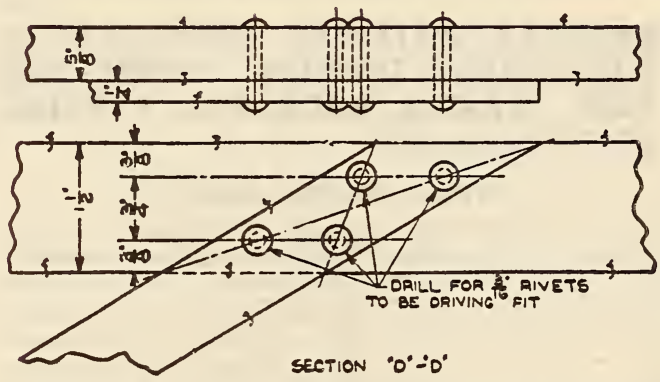

FigURE 573.-Detail of wheel mounting and check gage

AMERICAN RAILWAY ASSOCIATION, MECHANICAL DIVISION; MOUNTING GAGE FOR WROUGHT-STEEL WHEELS, RECOMMENDED PRACTICE, 1927

\section{MOUNTING GAGE FOR WROUGHT-STEEL WHEELS}

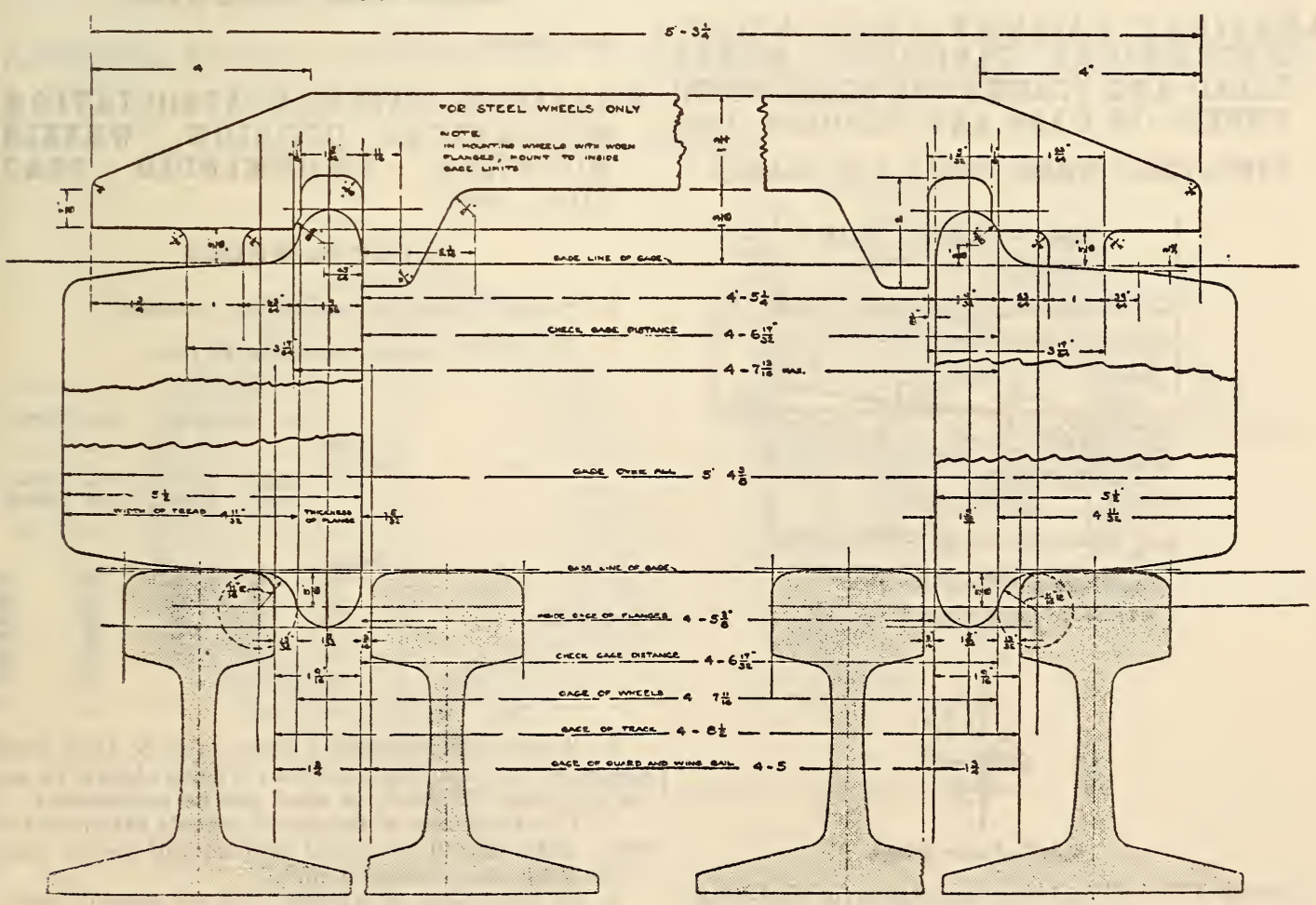

FIGURE 574.-Mounting gage for wrought-steel wheels 
AMERICAN RAILWAY ASSOCIATION, MECHANICAL DIVISION; TIRES, MASTER GAGE FOR BORING, 1908

MASTER GAGE FOR BORING TIRES

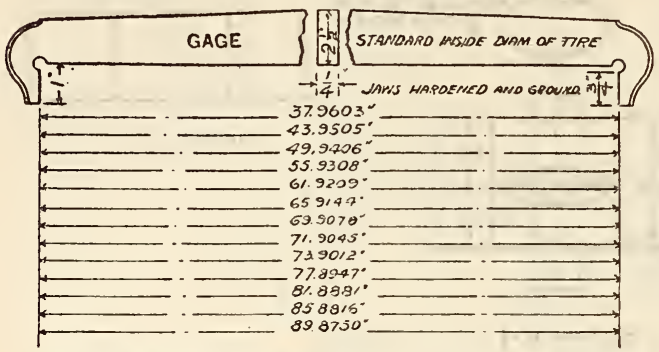

Figure 575.-Tires, master gage for boring

AMERICAN RAILWAY ASSOCIATION, MECHANICAL DIVISION; WHEEL CENTERS, IMASTER GAGE FOR TURNING, STANDARD, 1908

WHEEL-CENTER GAGE

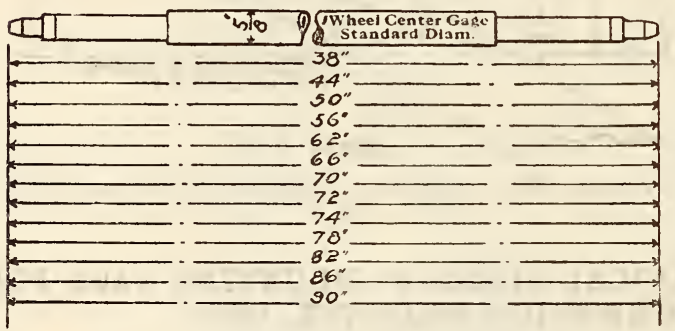

Figure 576.-Wheel centers, master gage for turning

AMERICAN RAILWAY ASSOCIATION, MECHANICAL DIVISION ; WHEEL TREAD AND FLANGE FOR STEEL-TIRED WHEELS ON CARS AND TENDERS, 1930 STEEL-TIRED WHEEL TREAD AND FLANGE
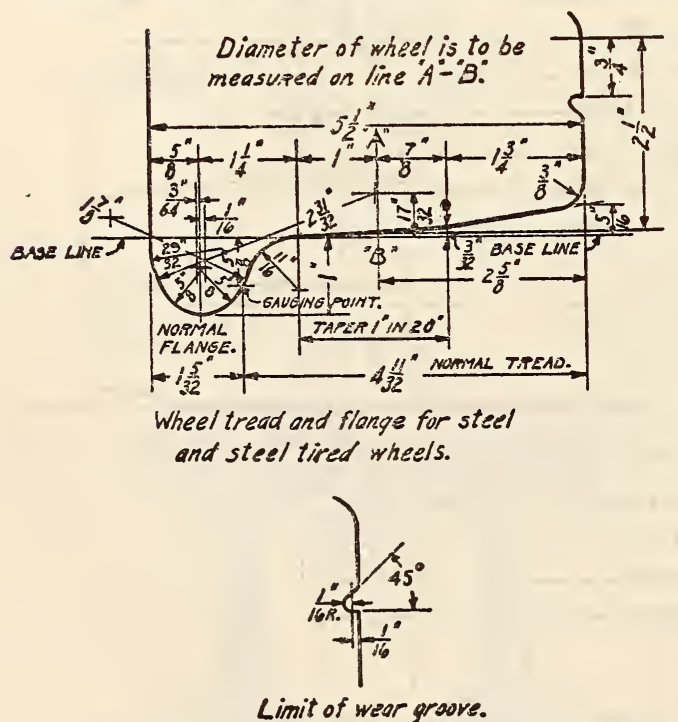

FigURE 577.- Wheel tread and flange for steel-tired wheels on cars and tenders
AMERICAN RAILWAY ASSOCIATION, MECHANICAL DIVISION ; WHEEL TREAD AND FLANGE FOR WROUGHT. STEEL WHEELS ON CARS AND TEN. DERS, 1923

\section{STEEL-WHEEL TREAD AND FLANGE}

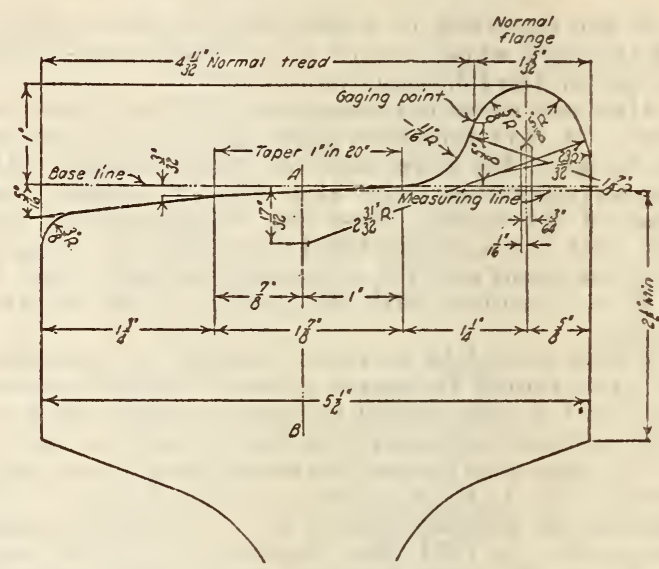

FIGURE 578. - Wheel tread and flange for wroughtsteel wheels on cars and tenders

Nore.-See above for section of tire for steel-tired engine and tender-truck wheels.

AMERICAN RAILWAY ASSOCIATION, MECHANICAL DIVISION; WHEELS AND TRACK, TERMS AND GAGING POINTS FOR, 1894

\section{WHEELS AND TRACK GAGE}

(See 606.0, p. 310.)

AMERICAN RAILWAY ASSOCIATION, MECHANICAL DIVISION ; WHEELS, MOUNTING, RECOMMENDED PRAC. TICE, 1926

\section{MOUNTING WHEELS}

1. Standard table of mounting pressure:

Mounting pressures in tons

\begin{tabular}{|c|c|c|c|c|c|}
\hline \multirow{2}{*}{ Axle } & \multirow{2}{*}{$\begin{array}{l}\text { Wheel } \\
\text { seat di- } \\
\text { ameter }\end{array}$} & \multicolumn{2}{|c|}{ Cast iron wheels } & \multicolumn{2}{|c|}{ Steel wheels } \\
\hline & & $\begin{array}{l}\text { Mini- } \\
\text { mum }\end{array}$ & $\begin{array}{l}\text { Maxi- } \\
\text { mum }\end{array}$ & $\begin{array}{l}\text { Mini- } \\
\text { mum }\end{array}$ & $\underset{\text { Maxi- }}{\text { Mum }}$ \\
\hline $\begin{array}{l}\mathrm{A} \\
\mathrm{B} \\
\mathrm{C} \\
\mathrm{D} \\
\mathrm{D} \\
\mathrm{E} \\
\mathrm{F}\end{array}$ & $\begin{array}{r}\text { Inches } \\
51 / 8 \\
53 / 4 \\
61 / 2 \\
7 \\
751 \\
81 / 8\end{array}$ & $\begin{array}{l}30 \\
35 \\
40 \\
45 \\
50\end{array}$ & $\begin{array}{l}55 \\
60 \\
65 \\
70 \\
75\end{array}$ & $\begin{array}{l}50 \\
55 \\
70 \\
75 \\
80 \\
85\end{array}$ & $\begin{array}{r}70 \\
80 \\
100 \\
110 \\
120 \\
130\end{array}$ \\
\hline
\end{tabular}

2. Wheels having flanges worn so as to take limit gages for remounting cast-iron wheels shown in accompanying illustrations shall not be remounted.

3. The thickness of flanges of wheels fitted on the same axle should be equal and should never vary more than one-sixteenth inch.

4. In mounting of wheels, new or second hand, the standard wheel mounting and check gage should 
be used in the following manner (for illustration of this gage see A. R. A.-M, solid wrought carbon-steel wheels, given above):

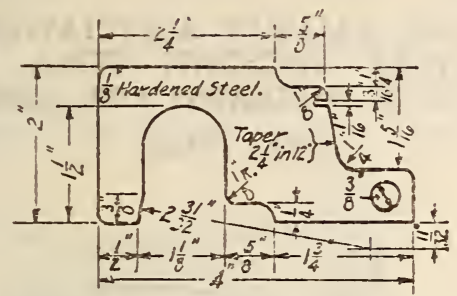

FIGURE 579.-For cast-iron wheels less than 80,000-pounds capacity; one-wear wrought-steel wheels and cast-steel wheels

\section{WHEELS CAST IRON, LIMIT GAGES FOR REMOUNTING}

After one wheel is pressed into position, place the stop $A$ or $B$ of the check gage against the inside of

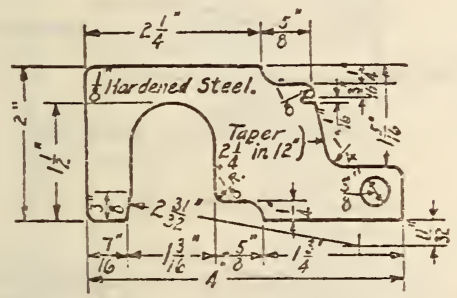

Figure 580.-For cast-iron wheels 80,000-pounds capacity and over

the flange of the wheel with the thinner flange with the corresponding tread stop $C$ or $D$ against the tread of the wheel. Press the other wheel on the axle until the opposite tread stop comes in contact with the tread of the corresponding gage point $E$ or $F$ in contact with the outside of the thicker flange.

5. The wheel seats on all axles must be turned to uniform diameter throughout the entire length of each wheel seat and must be smooth and free from ridges so as to provide even bearing for the wheel fit throughout. The mounting of wheels on axles having the wheel fit tapered is not permissible. The wheels must also have a straight bore with the exception of the chamfering for not more than threeeighths inch at the back hub face which may be allowed to facilitate application to axles.

6 . Wheels should be mounted centrally on the axle. All axles should be center punched and some form of gage be used to measure the location of the wheel from this center-punch mark. The central mounting of wheels is necessary in order to secure proper running of the wheels and to prevent hot boxes.

7. Wheels should ordinarily be fitted to axles and not axles fitted to the wheels. It is usually unnecessary to turn the wheel seat of second-hand axles unless they are found a tapered fit.

8. In mounting either new or second-hand wheels, care should be used to see that the wheels are of the same diameter. In the case of new wheels, the wheels should be taped in order to check the tapesize marking, and in no case should wheels of different tape sizes be applied to the same axle. In the case of new wrought-steel wheels, those mounted on the same axle should not differ in the carbon content by more than 0.05 per cent.

9. The alignment of axle lathe, the trueness of the centers, and the jaws on boring mills should be checked frequently in order to insure proper mounting of the wheels.

10. While the wheels are being bored the high spots on the flange should be marked, and in mating the high spots should be put opposite low spots on the mate wheels.

\section{AMERICAN RAILWAY ASSOCIATION, MECHANICAL DIVISION; WHEELS, STEEL TIRED, TIRE FASTENING FOR, RECOMMENDED PRACTICE, 1930}

\section{TIRE FASTENING}
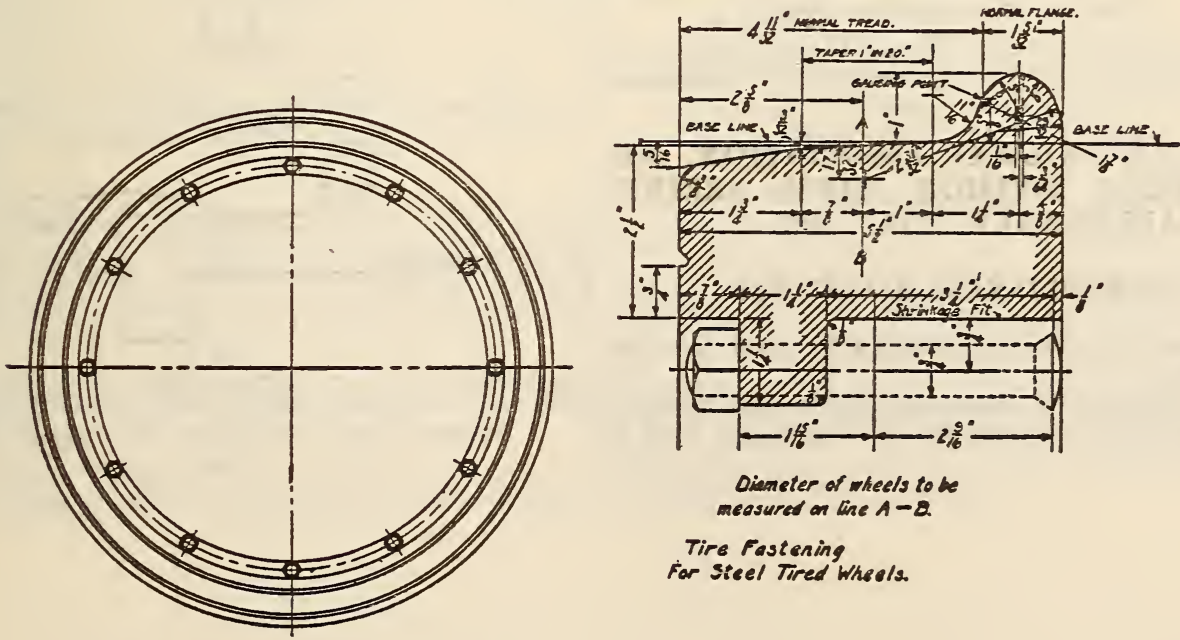

FIGURE 581.-Wheels, steel-tired, tire fastening for 
AMERICAN RAILWAY ASSOCIATION, MECHANICAL DIVISION ; SECTION OF TIRE, 1930 SECTION OF TIRE

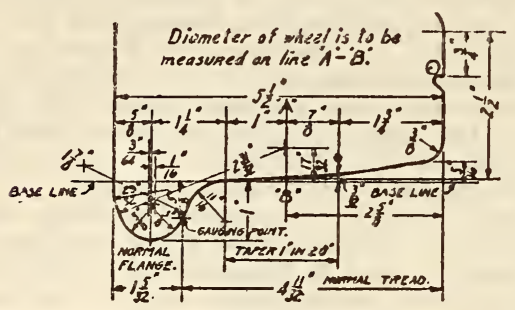

FIG.1-- FOR STEEL TIRED

EngINE AND TENDER TRUCK WheELS AND FLANGED DRIVING TIRES IN SWITCHING SERVKE.

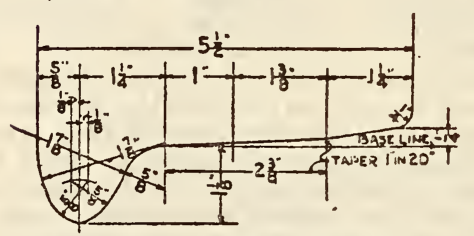

FIG.2- SECTION OF STEEL TIRE FOR Flanged Driving and Tranling Wheel OF LOCOMOTIVES IN ROAD SERVICE.

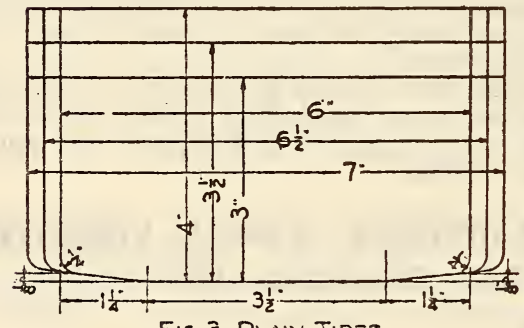

FIG 3-PLAIN TIRES

FIGURE 582.- Section of tire

Note. See below for wheel tread and flange'for wrought-steel wheels and steel-tired wheels on cars and tenders.f

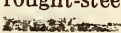

\section{AMERICAN RAILWAY ASSOCIATION, ME- CHANICAL DIVISION ; TIRES, SHRINK- AGE ALIOWANCE FOR, 1907}

\section{TIRE-SHRINKAGE ALLOWANCE}

Standard shrinkage allowances for steel tires are as follows:

For cast-iron and cast-steel centers less than 66 inches in diameter, one-eightieth inch per foot in diameter.
For cast-iron and cast-steel centers 66 inches and over in diameter, one-sixtieth inch per foot in diameter.

AMERICAN RAILWAY ASSOCIATION, MECHANICAL DIVISION; TIRES, STEEL, MINIMUM THICKNESS FOR, 1923

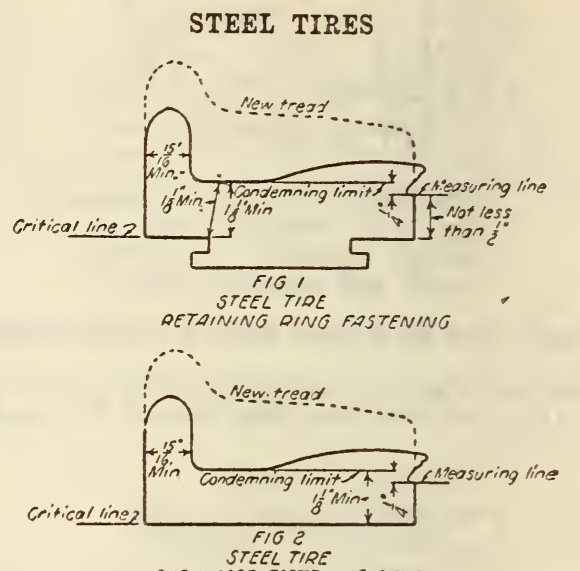

SHRINKAGE FASTENING ONLY
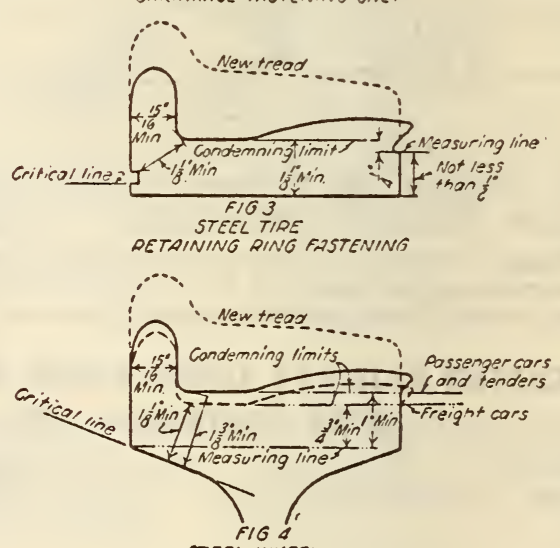

STEEL WHEEL

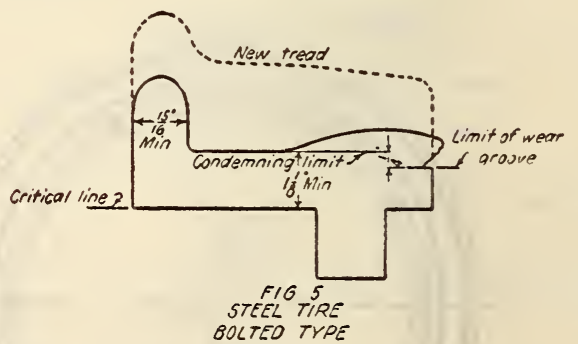

Figure 583.-Minimum thickness for steel tires 
AMERICAN RAILWAY ASSOCIATION, MECHANICAL DIVISION; WHEELS, SOLID WROUGHT CARBON STEEL, 1926

\section{CARBON-STEEL WHEELS}

F (Substantially the same as A. S. T. M., A57, given'below, including references in A57 to A.S.T.M.',

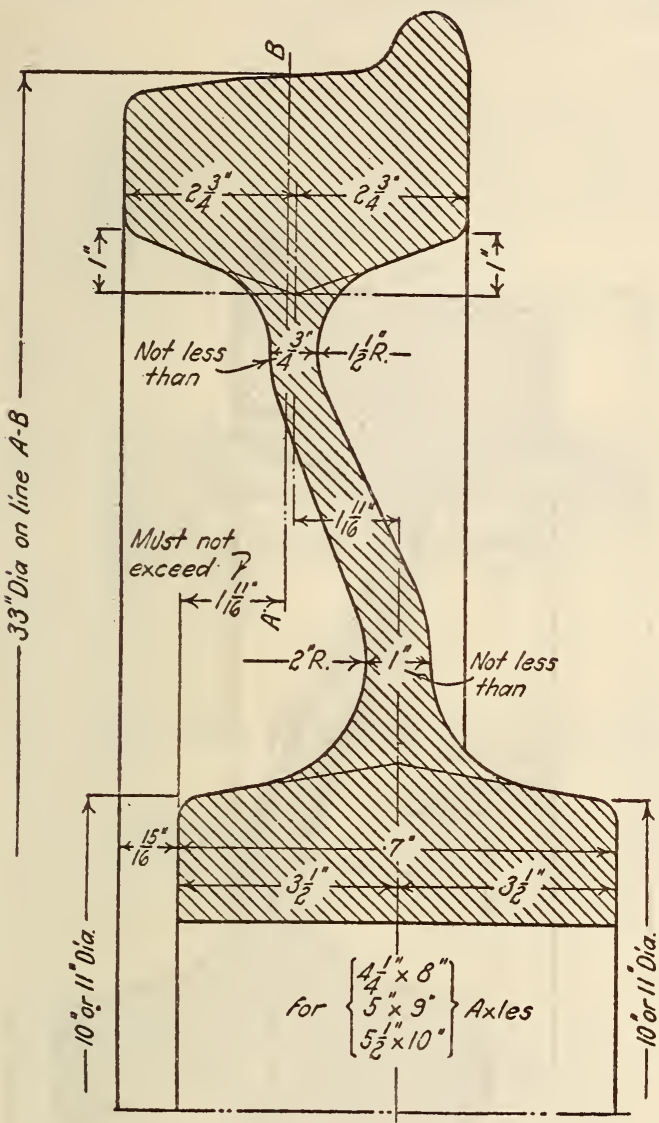

FIGURE 584.-Wheels, wrought-steel, 33 inches diameter, for $4 \frac{1}{4}$ by 8,5 by 9 , and $51 / 2$ by 10 inch axles

A25, to standard specifications for steel under 605.0 , and to A. R. A. specification for ladle analysis under 621.32 , with the addition of the following cuts showing contour of tread and flange of wheels and various kinds of gages:)

\section{INSTRUCTIONS FOR USING STANDARD WHEEL CIRCDMFER-} ENCE MEASURE FOR STEEL, STEEL-TIRED, AND CAST-IRON WHEELS

The wheel circumference measure is used for taping wheels in order to insure that wheels mounted on the same axle shall be of the same diameter and that new wheels are within the specification limits. It is calibrated by eighths of an inch of circumference with tape 157 representing a diameter of exactly 33 inches and a circumference of 103.67 inches For new cast-iron wheels, tape 3 represents a wheel of exact nominal diameter. The tape size of a wheel is considered to be that of the lower graduation until the next graduation is reached. The four lugs which contact on the face of the flange are to hold the tape in the center of the tread; these lugs should prefer-

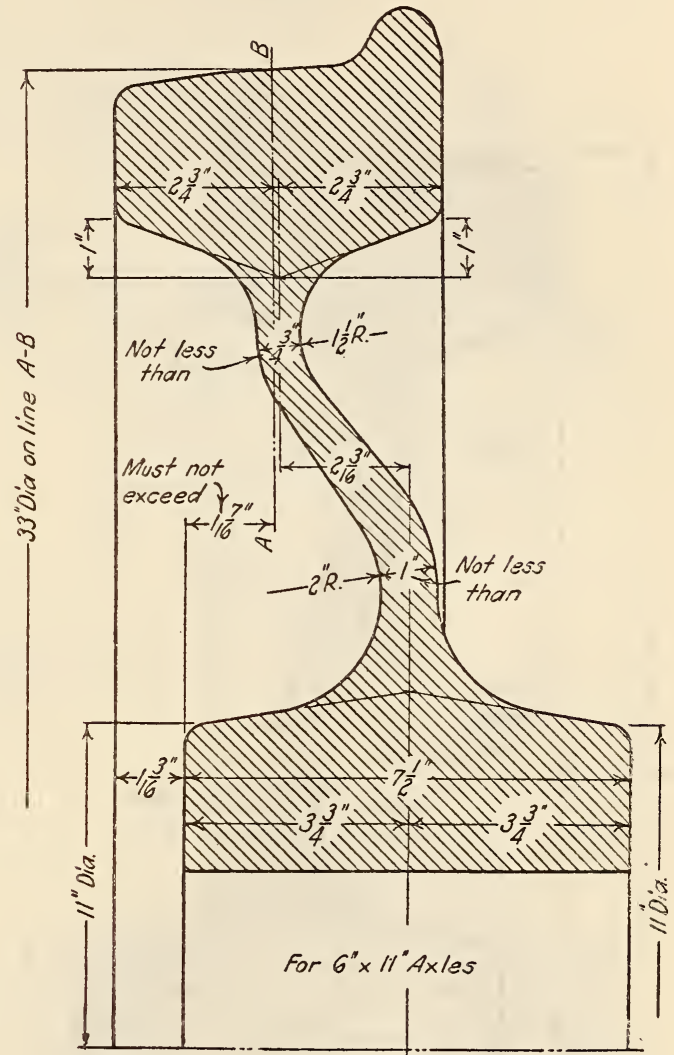

FIGURE 585.-Wheels, wrought-steel, 33 inches diameter, for 6 by 11 inch axles

ably have ribs pressed into them to reduce a tendency to bend out of shape. In use, the tape should lie flat on the tread all the way around including the handle and lugs, so that kinks in the tape should not be tolerated; such kinks are a manufacturing problem except in cases where the tape has been abused. For taping cast-iron wheels exclusively, the tape tires may be put on the handle only and satisfactory tapes of this type are available from some of the wheel foundries for taping new cast-iron wheels. All wheel tapes should be checked periodically on a machine of known accuracy. 


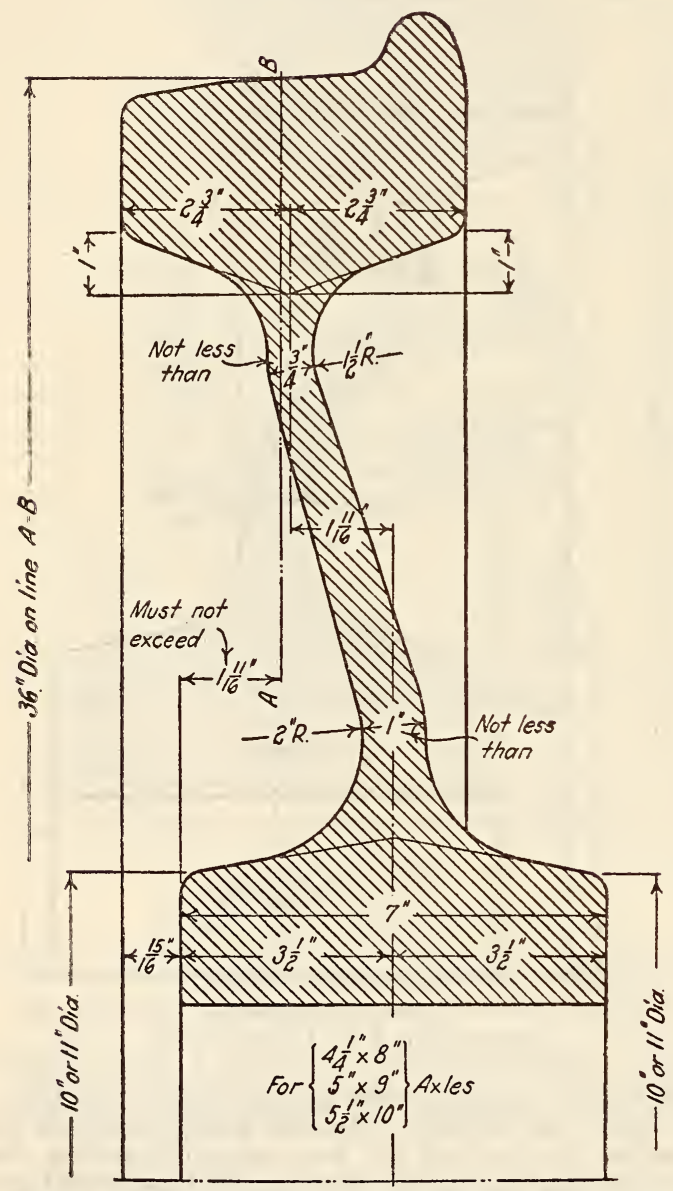

FigURE 586.-Wheels, wrought-steel, $\$ 6$ inches diameter, for $4 \frac{1}{4}$ by 8,5 by 9 , and $51 / 2$ by 10 inch axles

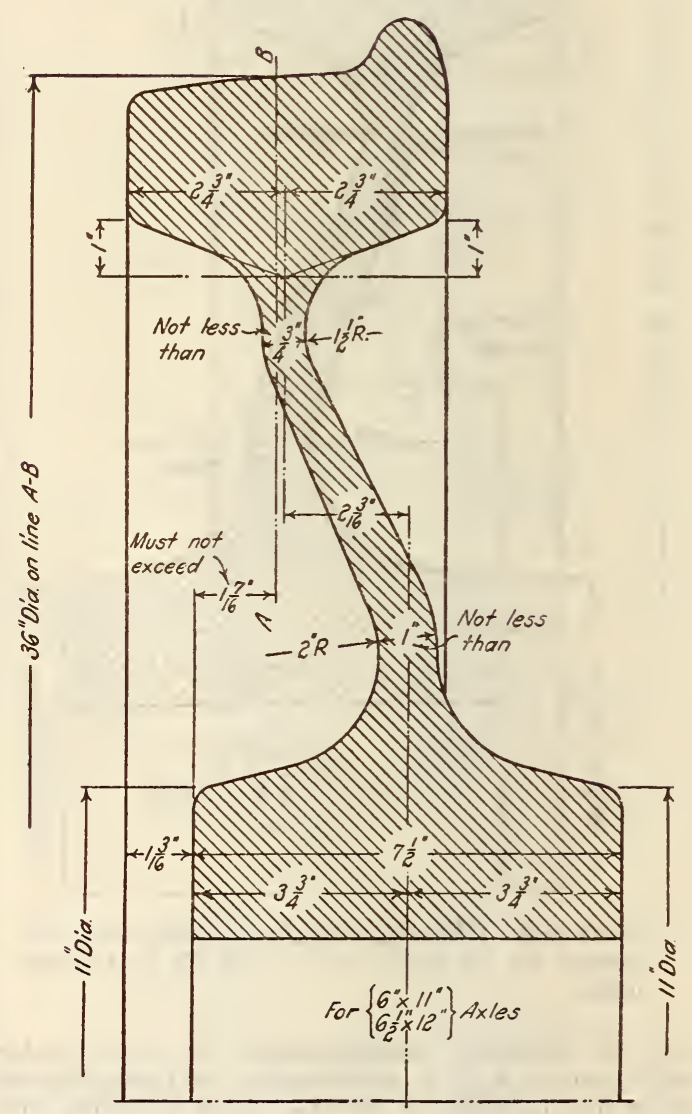

FIGURE 587.-Wheels, wrought-steel, 36 inches diameter, for 6 by 11 and $6 \frac{1}{2}$ by 12 inch axles 


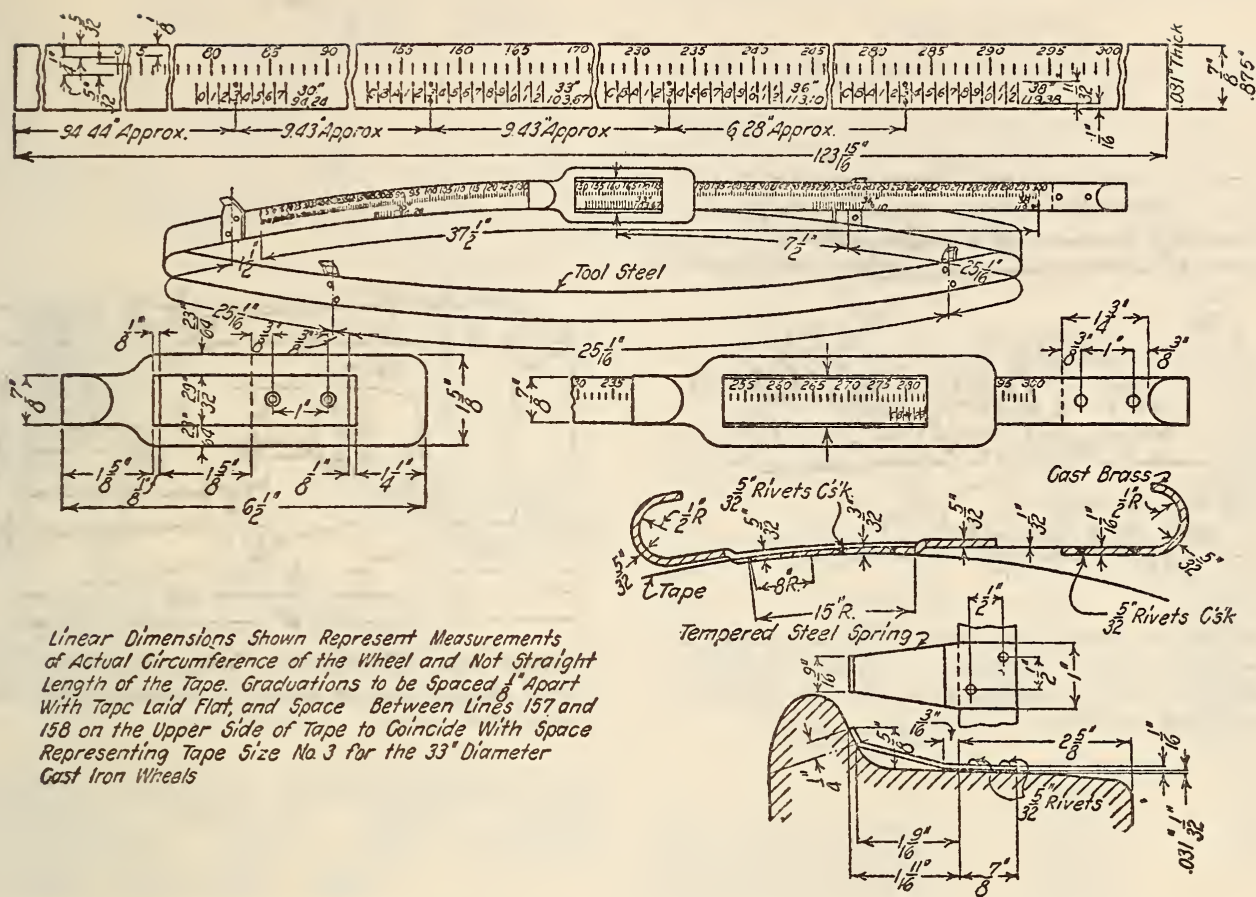

FIGURE 588.- Wheel circumference measure for cast-iron, cast-steel, wrought-steel, and steeltired wheels

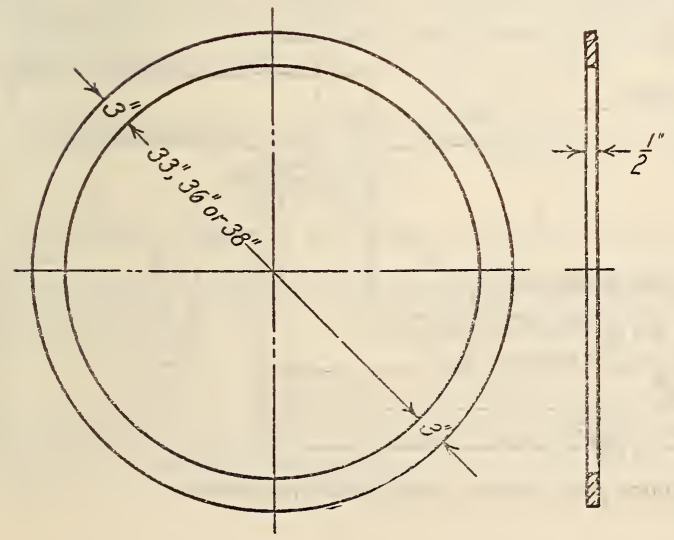

ROTUNDITY GAGE

FOR 33,"36" OR 38" SOLID STEEL WHEELS

Frgure 589.-Wheels, steel, rotundity gage for

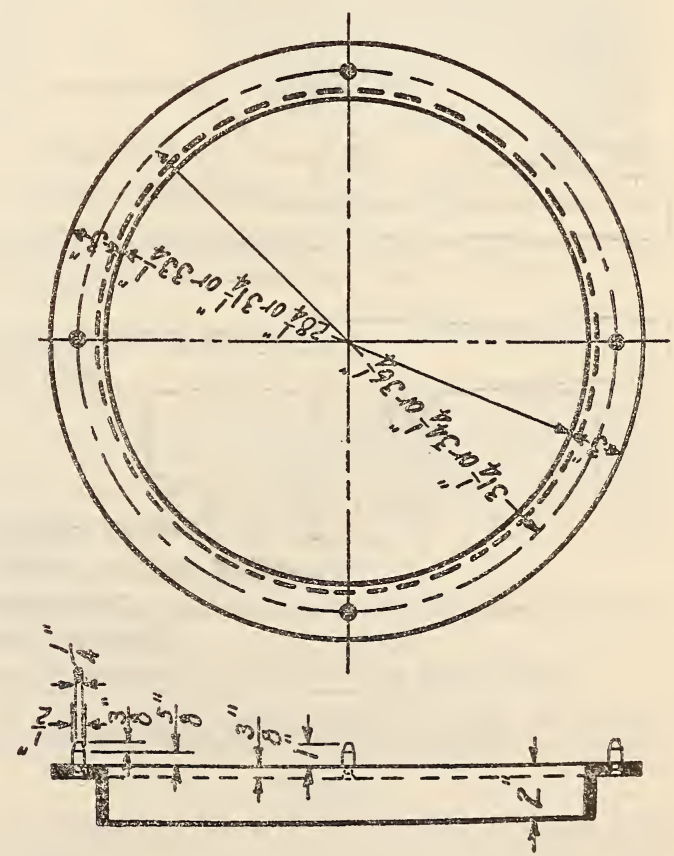

FIGURE 590.-Wheels, wrought-steel, plane gage for 


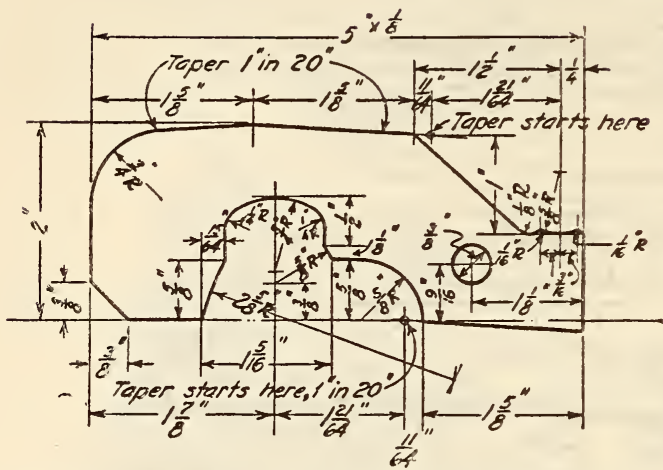

Figdre 591.-Minimum flange thickness gage for cast-iron wheels. Maximum flange thickness, height, and throat radius gage for wrought-steel wheels and steel tires

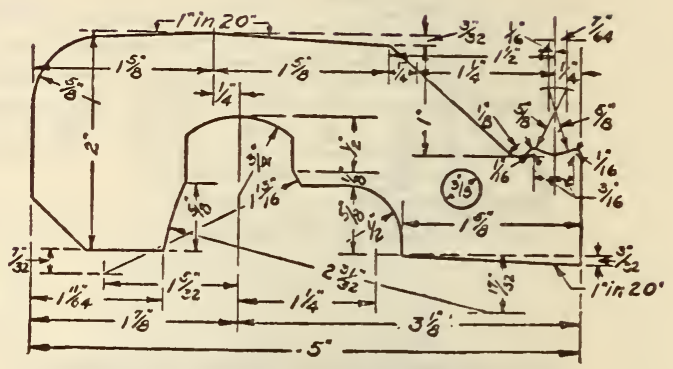

Frgure 592.-Minimum flange thickness, height, and throat radius gage for wrought-steel wheels and steel tires

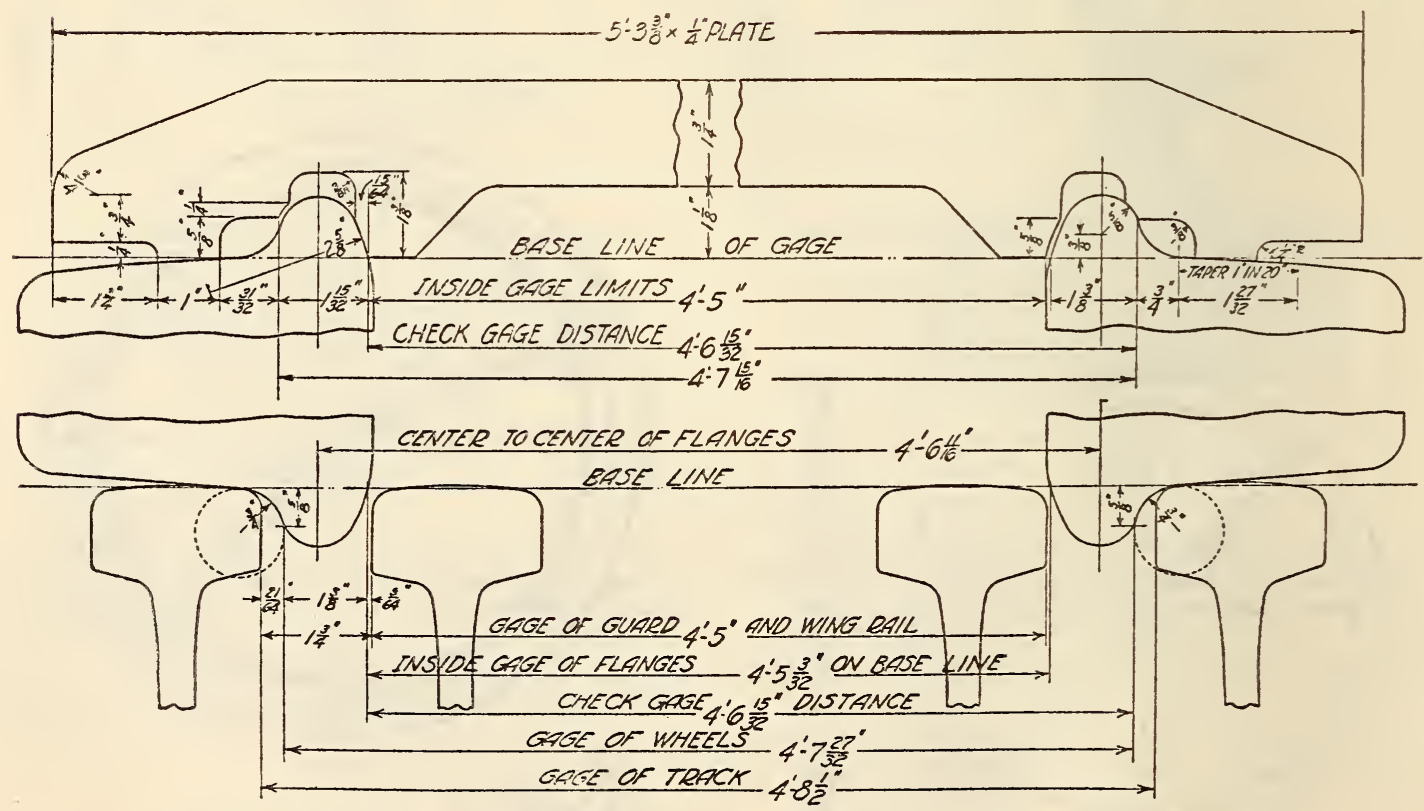

FigURE 593.-Wheel mounting and check gage for cast-iron and cast-steel wheels 
Acvised Sept 111029

70 calioratio

70 se calioretco

0 to $2-.056$

2 to. $3=.049$

$3104-.049$
$4105-.051$

5 to $6=.037$

$6+07=.041$
$1+08$
$1+00$

8 to $\left.9-.058^{\circ}\right)$

Calibration of nange

height scale or aiogram Ne-

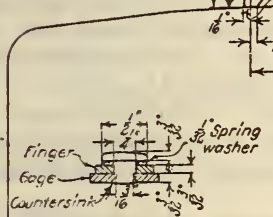

SECTION THROUEH UOINT

PECTION THROUGH NOINT

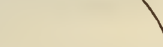

STEEL WHEEL GAGE

For use in measuring tread thickness, amount of metel to be turned off tread to restore flange contour and limit of dispith and locotion of witress groove in flomge. slid flat spets.
vertical nanges, chipped rims and axle collar thickness.

FIGURE 594.-Gage for measuring steel wheels to restore contour

AMERICAN RAILWAY ASSOCIATION, MECHANICAL DIVISION; TIRES, STEEL, IOCOMOTIVES AND CARS, RECOMMENDED PRACTICE, 1920

\section{STEEL TIRES FOR LOCOMOTIVES AND CARS}

(Identical with A. S. T. M., A26, given below, including references in A26 to A. R. A. specification for steel tank plates in 621.32, and A. S. T. M. standard specifications for steel in 605.0 , except that in this case the steel shall be made by either the open-hearth or electric process, and the height of flange shall not be less but may be one-sixteenth inch more than that specified.)

\section{AMERICAN SOCIETY FOR TESTING MA- TERIALS, STANDARD SPECIFICATIONS FOR WROUGHT SOLID CARBON-STEEL WHEELS FOR ELECTRIC RAILWAY SERV- ICE, DESIGNATION A25-24, 1924}

\section{WHEELS FOR ELECTRIC RAILWAYS}

\section{MANUFACTURE}

1. The steel shall be made by the open-hearth process.

2. A sufficient discard shall be made from each ingot to secure freedom from injurious piping and undue segregation.

\section{CHEMICAL PROPERTIES AND TESTS}

3. The steel shall conform to the following requirements as to chemical composition:

\begin{tabular}{|c|c|c|}
\hline & Acid & Basic \\
\hline Carbon & $\begin{array}{l}\text { Per cent } \\
0.60-0.80\end{array}$ & $\begin{array}{l}\text { Per cent } \\
0.65-0.85\end{array}$ \\
\hline Manganese & $.55-.80$ & $.55-.80$ \\
\hline $\begin{array}{l}\text { Phosphorus, not over } \\
\text { Sulphur, not over }\end{array}$ & .05 & $\begin{array}{l}.05 \\
.05\end{array}$ \\
\hline Silicon. & $.15-.35$ & $.10-.30$ \\
\hline
\end{tabular}

\section{IADIE ANALYSIS}

(Same as for steel tank plates in American Railway Association specification. See 621.32, p. 1124.)

\section{CHECK ANAIYSIS}

5. An analysis may be made by the purchaser from a wheel representing each melt. The chemical composition thus determined shall conform to the requirements specified in section 3 . A sample may be taken from any one point in the plate; or two samples may be taken, in which case they shall be on radii at right angles to each other. Samples shall not be taken in such a way as to impair the usefulness
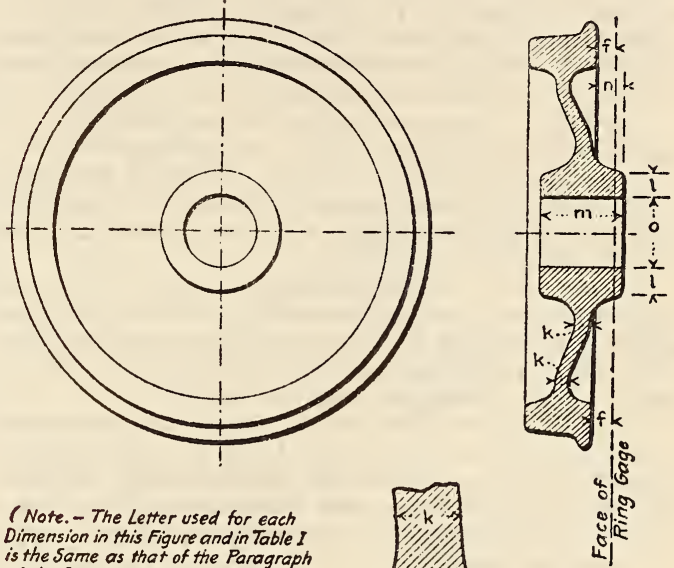

Dimension in this Figure for each is the Same as that of the Paragraph is the Same as that of the Paragraph Covering that Dimension.)

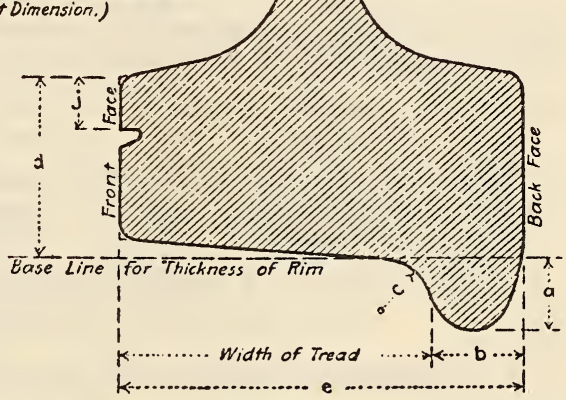

Figure 595.-Diagram showing points at which the dimensions covered by specifications are measured

For the permissible variations in these dimensions, see Table 1 or section 7 .

of the wheel. Drillings for analysis shall be taken by boring entirely through the sample parallel to the axis of the wheel; they shall be clean and free from scale, oil, and other foreign substances. All drillings from any one wheel shall be thoroughly mixed together.

\section{MATING}

6. The wheels shall be mated as to tape sizes and shipped in pairs.

\section{PERMISSIBLE VARIATIONS IN DIMENSIONS 80}

7. The wheels shall conform to the dimensions specified within the following permissible variations:

\section{FLANGE}

(a) Height of flange.-The height of flange shall not vary more than one-sixteenth inch from that specified.

${ }_{86}$ To facilitate the use of these specifications, the various dimensions are illustrated in fig. 595, and the permissible variations in those dimensions are also given in tabular form in Table 1. 
(b) Thickness of flange.-The thickness of flange shall not vary more than one-sixteenth inch from that specified.

(c) Radius of throat.-The radius of throat shall not vary more than one-sixteenth inch from that specified.

(d) Thickness of rim.-The thickness of rim shall not vary more than one-fourth inch over nor more than one-eighth inch under that specified. The thickness of rim shall be measured radially of the wheel on the front face and is the distance from the inner edge of the rim to the point at which a base line, drawn parallel to the axis of the wheel from the point of tangency of the throat and tread, meets the front face.

(e) Width of rim. - The width of rim shall not vary more than one-eighth inch from that specified.

(f) Plane.-The wheels shall be gaged with a ring gage placed concentric with and perpendicular to the axis of the wheel. For all points on the back of the rim equidistant from the center, the variation from the plane of the gage when so placed shall not exceed one-sixteenth inch.

(g) Rotundity.- The tread shall be gaged with a ring gage, and the opening between the tread and this gage at any point shall not exceed one-sixteenth inch.

(h) Block marks on tread.-Block marks shall not exceed one sixty-fourth inch in height.

(i) Tape sizes. - Wheels with treads under 3 inches in width shall not vary more than 6 tapes over nor more than 4 tapes under the size specified. Wheels with treads 3 inches or over in width shall not vary more than 9 tapes over nor more than 5 tapes under the size specified.

(j) Limit-of-wear groove.-When a limit-of-wear groove is specified, its location shall not vary more than one-sixteenth inch from that specified.

TABLE 1.-Permissible variations in dimensions of wrought-steel wheels for electric railway service

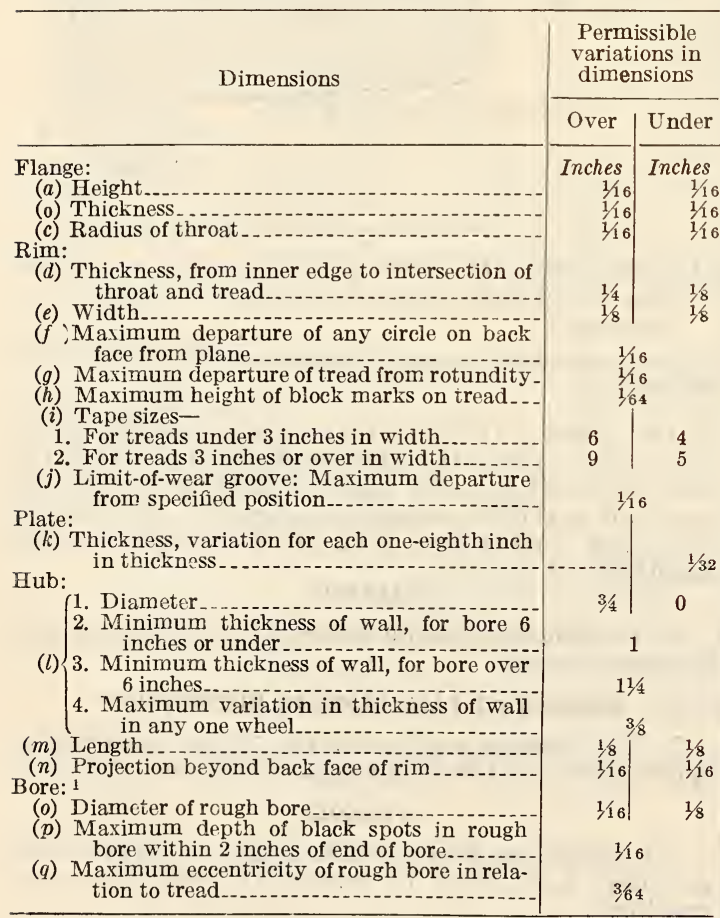

1 (If not specified, rough bore shall be one-fourth inch less than finished bore.)

NoTE.-The letter used for each dimension in this table and in fig. 595 is the same as that of the paragraph of sec. 7 covering that dimension.
PLATE

(k) Thickness of plate.-The plate may vary in thickness, but the variation less than that specified shall not exceed one thirty-second inch for each one-eighth inch in the thickness of the plate.

\section{нО В}

(l) Diameter of hub.-The diameter of hub shall not be less but may be three-fourths inch more than that specified. The thickness of wall of the finished bored hub, unless otherwise specified, shall not be less than 1 inch at any point for bores 6 inches or under in diameter, nor less than $11 / 4$ inches for bores over 6 inches in diameter. The thickness of the hub wall in any wheel shall not vary more than three-eighths inch at any two points equidistant from the face of the hub.

(m) Length of hub.-The length of hub shall not vary more than one-eighth inch from that specified.

(n) Projection of hub.-The projection of the hub beyond the back face of the rim shall not vary more than one-sixteenth inch from that specified.

\section{BORE}

(o) Diameter of rough bore.-The diameter of rough bore shall not vary more than one-sixteenth inch over nor more than one-eighth inch under that specified. When finished-bore diameter only is specified, the rough-bore diameter shall be made one-fourth inch less with the permissible variations specified above.

(p) Black spots in bore.-Black spots deeper than one-sixteenth inch will not be permitted in rough bore within 2 inches of either face of the hub.

(q) Eccentricity of bore.-The eccentricity between the tread at its center line and the rough bore shall not exceed three-sixty-fourths inch.

\section{FINISH}

8. (a) The wheel shall be free from injurious defects and shall have a workmanlike finish.

(b) Wheels shall not be offered for inspection if covered with paint, rust, or any other substance to such an extent as to hide defects.

\section{MARKING}

9. (a) The name or brand of the manufacturer, date, and serial number shall be legibly stamped on each wheel in such a way that the wheel may be readily identified.

(b) The tape size shall be legibly marked on each wheel.

\section{INSPECTION AND REJECTION}

10. (a) The manufacturer shall provide suitable gages and tapes which shall conform to the contour and dimensions specified.

(Otherwise inspection and rejection conform to A. S. T. M. standard specifications for steel, paragraphs 7,8 , and 9 (see 605.0, p. 247) except that rejections shall be reported within 10 working days, and the rejected samples shall be preserved for one month from the date of the test report.)

\section{AMERICAN SOCIETY FOR TESTING MATE- RIALS, STANDARD SPECIFICATIONS FOR STEEI TIRES, DESIGNATION A26-16, 1916}

\section{STEEL TIRES \\ SCOPE}

1. (a) These specifications cover three classes of tires.

(b) The purposes for which these classes are frequently used are as follows:

Class $A$, for driving tires for passenger locomotives. 
Class $B$, for driving tires for freight locomotives and tires for locomotive-truck, tender-truck, trailer, and car wheels, and miscellaneous service.

Class $C$, for driving tires for switching locomotives.

\section{MAN UFACTURE}

2. The steel shall be made by the open-hearth process.

3. A sufficient discard shall be made from each ingot to secure freedom from injurious piping and undue segregation.

\section{CHEMICAL PROPERTIES AND TESTS}

4. The steel shall conform to the following requirements as to chemical composition:

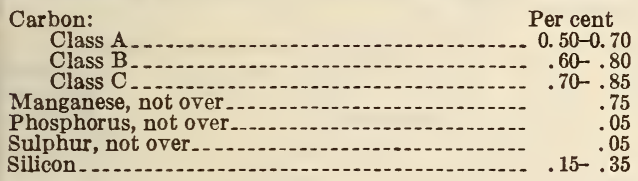

LADLE AND CHECK ANALYSES

(Same as for steel tank plates in American Railway Association specification. See 621.32, p. 1124.)

\section{PHYSICAL PROPERTIES AND TESTS}

7. If tension tests from representative bars in accordance with section 8 are specified by the purchaser, the tensile properties shown shall conform to the following minimum requirements:

\begin{tabular}{l|r|r|r}
\hline & Class A & Class B & Class C \\
\cline { 2 - 4 } & & \\
\hline Tensile strength & & \\
Elongation in 2 inches.........per cent.. & 105,000 & 115,000 & 125,000 \\
Reduction of area............do.... & 10 & 14 & 8 \\
\hline
\end{tabular}

8. (a) The tension-test specimen representing each melt shall be taken from a test ingot taken during the pouring of the melt, and shall have received approximately the same amount of work sa the tires which it represents.

(Tension-test specimens shall conform to the dimensions shown in Figure 129 in A. S. T. M. standard specifications for steel. See 605.0, p. 247.)

9. (a) If specified by the purchaser, one tension test shall be made from each melt.

(b) If any test specimen shows defective machining or develops flaws, it may be discarded and another specimen substituted.

(c) If the percentage of elongation of any test specimen is less than that specified in section 7 and any part of the fracture is more than three-fourths inch from the center of the gage length, as indicated by scribe scratches marked on the specimen before testing, a retest shall be allowed.

10. If the results of the tension test for any melt do not conform to the requirements of section 7 , a retest may be made on a specimen cut from a tire of the same melt furnished at the expense of the manufacturer. This retest shall give results conforming to the requirements of section 7 .

\section{MATING}

11. The tires shall be grouped as to outside diameters and shipped in sets.

\section{PERMISSIBLE VARIATIONS IN DIMENSIONS}

12. Tires may be furnished with all surfaces as rolled, and shall conform to the dimensions specified within the following permissible variations: (a) Height of flange.-The height of flange shall not vary more than three thirty-seconds inch from that specified.

(b) Thickness of flange.-The thickness of flange shall not vary more than one-sixteenth inch from that specified.

(c) Radius of throat. - The radius of throat shall not vary more than one-eighth inch over nor more than one-sixteenth inch under that specified.

(d) Width of tire.-The width of tire shall not vary more than one-eighth inch over nor more than onesixteenth inch under that specified.

(e) Inside diameter.-The rough inside diameter shall not be more, but may be one-fourth inch less than that specified. When finished inside diameter only is specified, the rough diameter shall be from three-sixteenths to seven-sixteenths inch less than this diameter.

(f) Outside diameter.-Unless otherwise specified the outside diameter when 54 inches or under shall not be less but may be one-half inch more than that specified; and when over 54 inches shall not vary more than one-eighth inch under nor more than three-eighths inch over that specified.

(g) Set diameters.-The tires shall be furnished in sets and the variation in outside diameters in each set shall not exceed one-sixteenth inch for tires 33 inches or under in outside diameter nor three thirty-seconds inch for tires over 33 inches in outside diameter.

(h) Rotundity. - Tires shall not be out of round more than one-sixteenth inch for tires 33 inches or under in outside diameter nor more than three thirty-seconds inch for tires over 33 inches in outside diameter.

(Finish, marking, inspection, rejection, and rehearing conform to A. S. T. M. standard specifications for steel, pars. 5, 6, 7, 8, and 9. See 605.0, p. 247.)

AMERICAN SOCIETY FOR TESTING MATERIALS, STANDARD SPECIFICATIONS FOR WROUGHT SOIID C AR B ON-STEEI WHEELS FOR STEAM-RAILWAY SERVICE, DESIGNATION A57-29, 1929

\section{WHEELS FOR STEAM RAILWAYS}

\section{MANUFACTURE}

1. The steel shall be made by either or both the following processes: Open hestrh or electric furnace.

2. A sufficient discard shall be made from each ingot to secure freedom from injurious piping and undue segregation.

\section{CHEMICAL PROPERTIES AND TESTS}

3. The steel shall conform to the following requirements as to chemical composition:

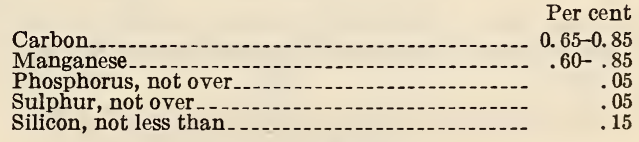

\section{LADIE ANALYSIS}

(Same as for steel tank plates in American Railway Association specification. See 621.32, p. 1124.)

\section{CHECK ANALYSIS}

(Same as in A. S. T. M., A25, given above, except that drillings for check analysis shall be taken direct from the plate, or from a slug hot punched or drilled out of the plate, and shall be taken so as not to impair the usefulness of the wheel.) 


\section{MATING}

6. (a) The wheels shall be mated as to tape sizes and shipped in pairs.

(b) When so specified in the purchaser's order, wheels of each type and rough-bore diameter in each carload shall be so selected as to permit of mating so that the ladle analysis for any pair shall not show a difference in carbon content exceeding 5 points.

\section{PERMISSIBLE VARIATIONS IN DIMENSIONS 87}

7. The wheels shall conform to the dimensions specified within the following permissible variations.

\section{FLANGE}

(Same as in A. S. T. M., A25, given above, except that the variation in the height of the flange may be one-eight inch more than that specified.)

\section{RIM}

(d) Thickness of rim.-The thickness of the rim shall be measured radially of the wheel on the back
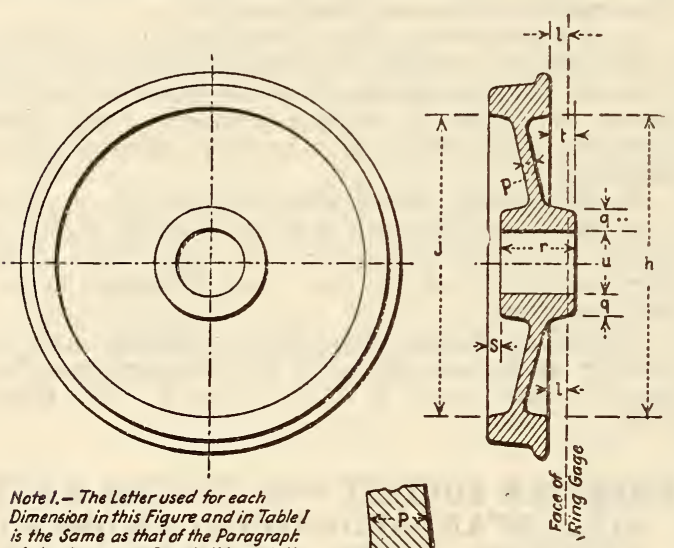

Note 1. - The Letter used for each is the Same as that of the Paragrapt of the Section on Permissible Variation covering that Dimension.

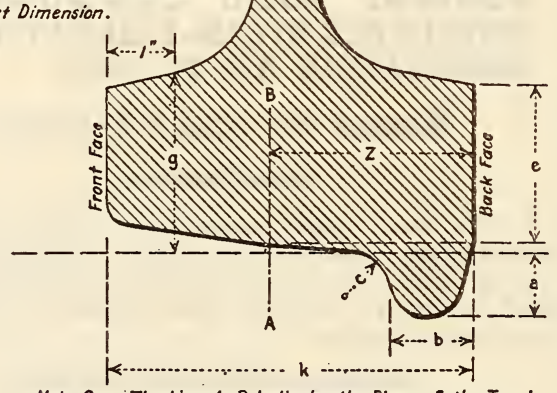

Note 2. - The Line $A-B$ indicates the Plane of the Troad Circle. In the Standard Wheels with Rims $5 \frac{1}{2}$ "wide the Distance $Z$ from the Line $A-B$ to the Back Face of the Rim is $2 \frac{7}{8}$.

FIGURE 596.-Diagram showing points at which the dimensions covered by specifications are measured

For the permissible variations in these dimensions see Table 1 or section 7 .

face of the rim, and is the distance between the inside diameter of the rim and the point at which the back rim of the wheel is cut by a line drawn parallel to the axis of the wheel through the intersection of the line $A-B$ (fig. 596), and the tread of the wheel.

Note.-The line $A-B$ (fig. 596), indicates the plane of the tread circle. In the standard American wheels with rims $51 / 2$ inches wide the distance from the line $A-B$ to the back face of the rim is $27 \% 8$ inches. (e) Thickness of rim thus measured shall be not less than that specified, and in any one wheel shall not vary more than one-eighth inch.

(f) To facilitate measurement of rim thickness, the radius of the inside edge of the back face of rim shall not exceed one-eighth inch. A sharp corner is preferable.

(g) The rim thickness measured radially on a plane 1 inch from the front face of the wheel shall not vary more than one-eighth inch in any one wheel. The contour of the inner surface of the rim shall be practically symmetrical on both sides of the plate.

TABLE 1.-Permissible variations in dimensions of wrought solid carbon-steel wheels for steum railway service

\begin{tabular}{|c|c|c|}
\hline \multirow[t]{2}{*}{ Dimensions } & \multicolumn{2}{|c|}{$\begin{array}{l}\text { Permissible } \\
\text { variations in } \\
\text { dimensions }\end{array}$} \\
\hline & Over & Under \\
\hline $\begin{array}{l}\text { Flange: } \\
\text { (a) Height } \\
\text { (b) Thickness } \\
\text { (c) Radius of throat.... }\end{array}$ & $\begin{array}{c}\text { Inches } \\
1 / 8 \\
1 / 16 \\
1 / 16\end{array}$ & $\begin{array}{l}\text { Inch } \\
0 \\
1 / 16 \\
1 / 10\end{array}$ \\
\hline $\begin{array}{l}\text { Rim: }\left\{\begin{array}{l}\text { 1. Thickness, at back face of wheel } \\
2 \text {. Maximum variation in rim thickness at } \\
\text { back face in any one wheel }\end{array}\right. \\
\text { (g) Maximum } \nabla \text { ariation in rim thickness measured } \\
\text { 1 inch from front face of wheel }\end{array}$ & (1) $\left.\right|_{18}$ & 720 \\
\hline $\begin{array}{l}\text { (h) Inside diameter of rim at back face of wheel } \\
(j) \text { Inside diameter of rim at front face of wheel in } \\
\text { relation to inside diameter at back face of }\end{array}$ & (1) & 36 \\
\hline (k) Width of rim & & $\begin{array}{l}1 / 4 \\
1 / 8\end{array}$ \\
\hline $\begin{array}{l}\text { (l) Maximum departure of any circle on back face } \\
\text { from plane }\end{array}$ & & \\
\hline $\begin{array}{l}(m) \text { Maximum departure of tread from rotundity.. } \\
(n) \text { Maximum height of block marks on tread... }\end{array}$ & & \\
\hline $\begin{array}{l}\text { (o) Tape sizes. } \\
\text { Plate: }\end{array}$ & 14 & 0 \\
\hline 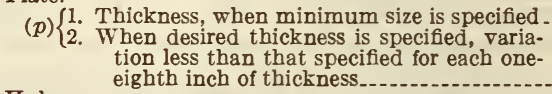 & & 0 \\
\hline (q) $\left\{\begin{array}{l}\text { 1. Diameter } \\
\text { 2. Minimum thickness of wall } \\
\text { 3. Maximum variation in thickness of wall in } \\
\text { any one wheel }\end{array}\right.$ & $11 / 4$ & $4^{(2)}$ \\
\hline (r) Length & $\begin{array}{l}1 / 8 \\
1 / 8\end{array}$ & $0^{1 / 8}$ \\
\hline $\begin{array}{l}\text { (t) Projection beyond back face of rim: } \\
\text { 1. If finished machined } \\
\text { 2. If not finished machined, allowance for } \\
\text { finish to be not less than one-sixteenth } \\
\text { inch nor more than three-sixteenth inch. }\end{array}$ & 0 & 0 \\
\hline $\begin{array}{l}\text { Bore: } \\
(u) \text { Diameter of rough bore. } \\
(w) \text { Maximum eccentricity of rough bore in rela- } \\
\text { tion to tread. }\end{array}$ & $1 / 26$ & $1 / 8$ \\
\hline
\end{tabular}

1 Limited by tape size. $\quad{ }^{2}$ Limited by wall thickness.

Note.-The letter used for each dimension in this table and in Figure 596 is the same as that of the paragraph of section 7 covering that dimension.

(h) The inside diameter of the rim at the back face of the wheel shall not vary more than threeeighths inch under that specified.

(j) Diameter of rim. - The inside diameter of the rim at the front face of the wheel shall not exceed the inside diameter at the back face nor vary under that dimension by more than one-fourth inch.

(Width of rim, plane, rotundity, and block marks on tread all are the same as in A. S. T. M., A25, given above.)

(o) Tape sizes. - The tape sizes of the wheels shall not be less than that specified, and shall not exceed this by more than 14 tapes.

87 To facilitate the use of the specifications, the various dimensions are illustrated in fig. 596, and the permissible variations in those dimensions are also given in tabular form in Table 1. 


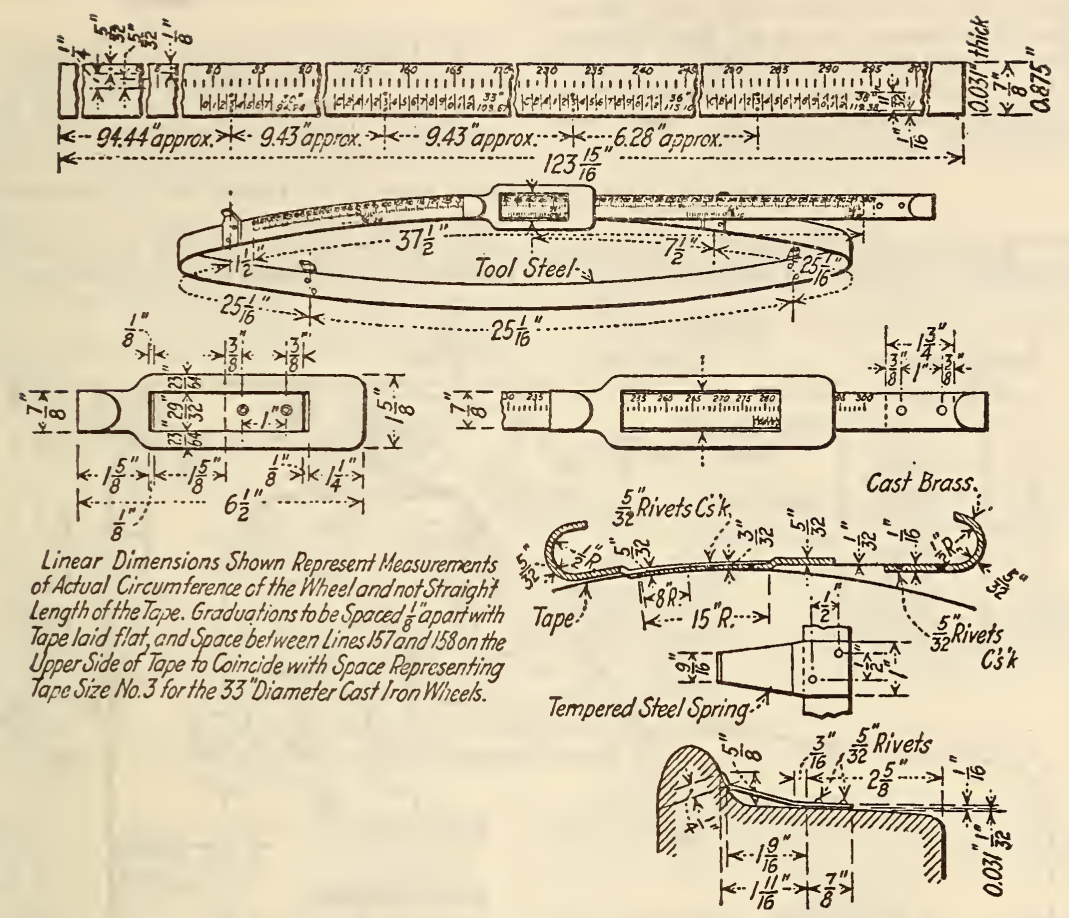

(a). Wheel Circumference Gage.

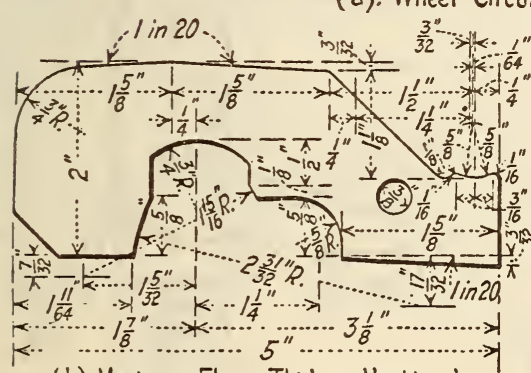

(b). Maximum Flange Thickness, Height and

Throat Radius Gage for Solid Steel Wheels.

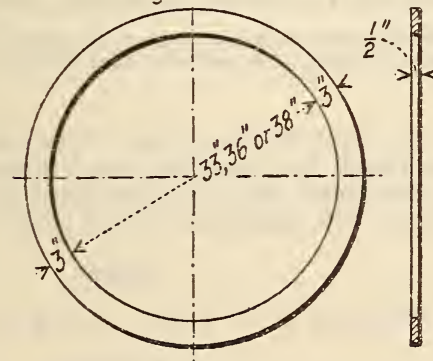

(d). Rotundity Gage for $33^{\prime \prime}, 36^{\prime \prime}$ or $38^{\prime \prime}$ Solid Steel Wheels.

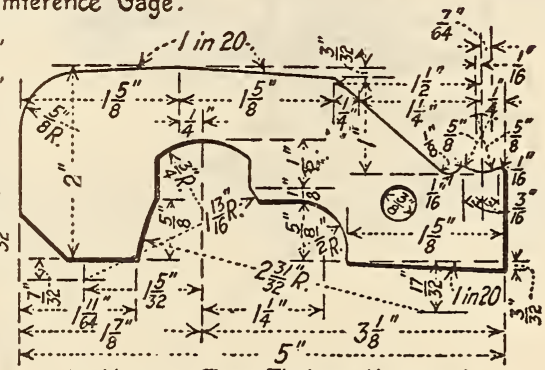

(c). Minimum Flange Thickness, Height and Throat Radius Gage for Solid Steel Wheels.

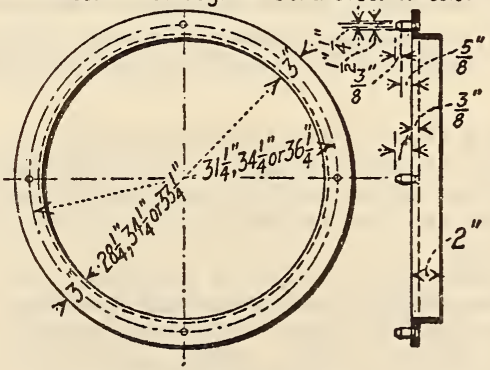

(e). Plane Gage for Wrought Steel Wheels.

FIgURE 597.-Standards of American Railway Association, mechanical division 
PLATE

(p) Thickness of plate.-The plate may vary in thickness. When the drawings specified with an order show minimum thicknesses for the plate, the plate thickness shall not be less than that shown. When the plate thicknesses shown by the drawings are not specified as minimum, the variation in plate thickness less than that shown by the drawings shall not exceed one thirty-second inch for each oneeighth inch in the thickness of the plate.

\section{HО B}

(q) Diameter of hub.-The diameter of the hub may vary, but unless otherwise specified the thick- rim, or there shall be left on the back face of the hub not more than three-sixteenth inch nor less than one-sixteenth inch stock for finishing by the purchaser.

\section{BORE}

(u) Diameter of bore.-The diameter of rough bore shall not vary more than one-sixteenth inch over nor more than one-eighth inch under the dimensions specified. When not specified, the rough bore shall be one-fourth inch less in diameter than the finished bore, subject to the above limitations.

(v) Black spots in bore.-No wheels shall have black spots in the rough bore which will not be completely removed in finish boring.

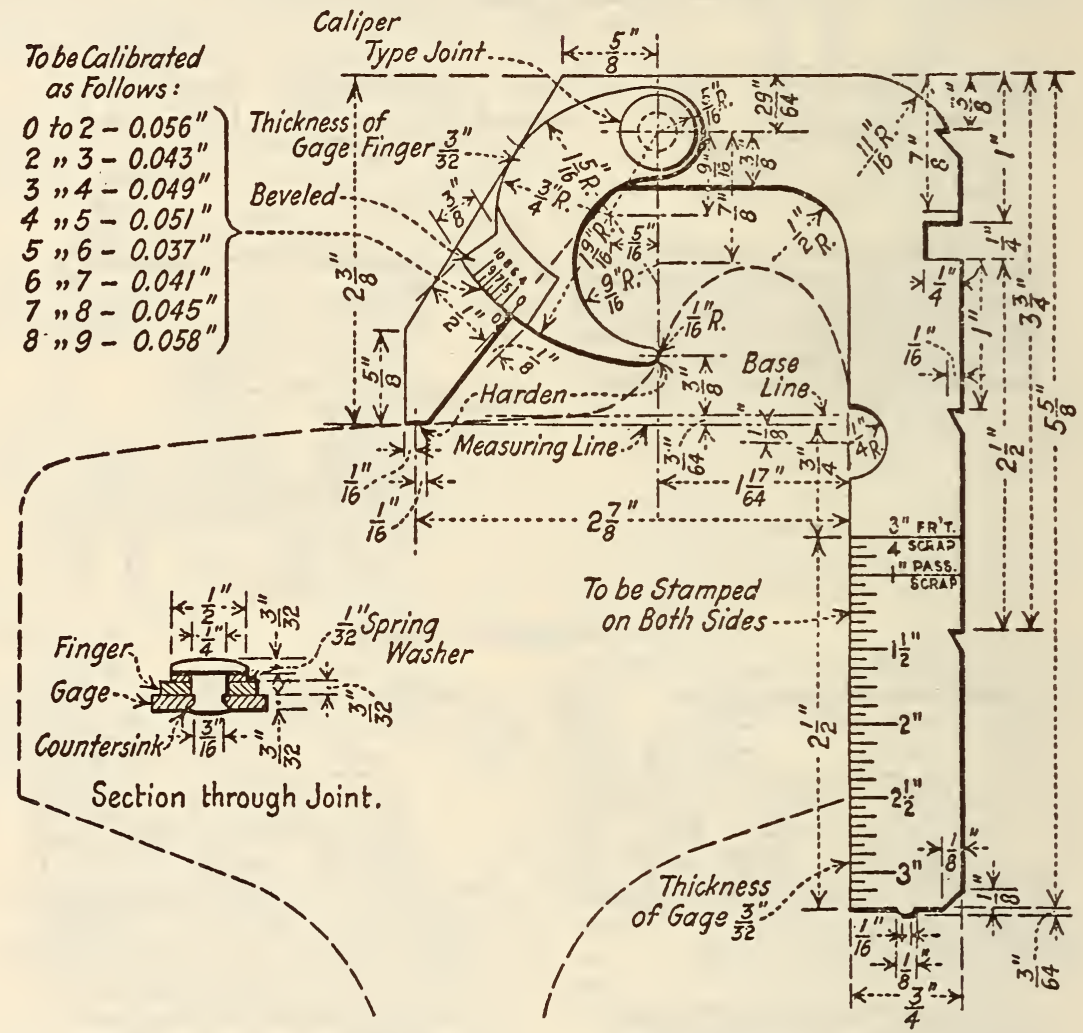

Figure 598.- Steel wire gage of American Railway Association, mechanical division

For use in measuring tread thickness, amount of metal to be turned off tread to restore flange ccntour, and limit of depth and location of witness groove in flange, slid flat spots, vertical flanges, chipped rims, and axle collar thickness.

ness of the wall of the finished bored hub shall not be less than $11 / 4$ inches at any point. The thickness of the hub wall in any wheel shall not vary more than one-fourth inch at any two points equidistant from the face of the hub.

(r) Length of hub.-The length of hub shall not vary more than one-eighth inch from that specified.

(s) Depression of hub.-For car and tender wheels and wheels of similar design, with journal-box bearing on front face of the hub, the depression of the hub below the front face of the rim shall not be less but may be one-eighth inch more than that specified.

( $t$ ) Projection of hub.-For locomotive-truck wheels and wheels of similar design with journal-box bearing on back face of the hub, the wheels may be furnished with the back face of the hub smooth machined to the exact specified dimension for the projection of the hub beyond the back face of the (w) Eccentricity of bore.-The eccentricity between the rough bore and the tread measured in the plane of the tread circle, as indicated by the line $A-B$ in Figure 596, shall not exceed three sixty-fourth inch.

\section{FINISH}

(Same as A. S. T. M., A25, given above.)

\section{MARKING}

9. (a) The date (month and year), brand of manufacturer, manufacturer's serial number, and heat number shall be legibly stamped on the back face of the rim approximately one-fourth inch from the inner edge of the rim. The height of characters shall not be less than five-eighths inch for hot stamping or one-half inch for cold stampins.

(b) The tape size shall be plainly marked on the plate in figures at least 1 inch in height. 
(c) When so specified in the purchaser's order, the wheels shall be stamped so as to indicate the carbon content shown by the ladle analysis.

\section{INSPECTION AND REJECTION}

10. (a) The gages and tapes used shall be based on American Railway Association standards, as illustrated in figures 597, and 598.

(Otherwise inspection and rejection conform to A. S. T. M. standard specifications for steel, paragraphs 7, 8, and 9 . (See 605.0, p. 247.)

\section{ASSOCIATION OF IRON AND STEEL ELEC- TRICAL ENGINEERS, GENERAL SPECIFI- CATIONS FOR ELECTRIC OVERHEAD TRAVELING CRANES, HEAVY-DUTY MILL SERVICE, 1921}

\section{BRIDGE AND CRANE TRACK WHEELS \\ TRACK WHEELS}

(a) Track wheels shall preferably be of rolled or forged steel. Cast-steel wheels may be used if specified.

(b) The standard tread diameters of bridge track wheels shall be 18,24,27, and 30 inches, and tread shall be made to suit a standard rail not smaller than 85 pounds section. Treads for heavier rails to be made to suit.

(c) The standard bores of these wheels shall be as follows:

18-inch diameter.
24 -inch diameter.
30-inch diameter.

(d) A standard increase in diameter of oneeighth inch for all bridge track wheel axles in the wheel hubs is recommended.

(e) All bridge track wheels on same crane to be interchangeable.

(f) The A. I. \& S. E. E. standard for bridge runway rails shall be an 85-pound open-hearth rail for ordinary cranes, and for special cranes requiring over an 85-pound rail, purchaser shall specify type and size of rail. (g) The standard tread diameters of trolley track wheels shall be $12,15,18,21$, and 24 inches.

(h) The standard bores of these wheels shall be as follows:

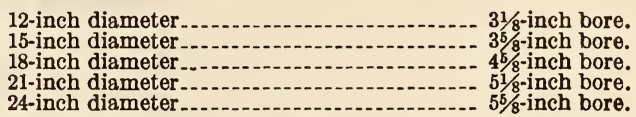

(i) With maximum load on crane, track wheel bearing pressures must not exceed 750 pounds per square inch projected area.

NATIONAL ELECTRICAL MANUFACTURERS ASSOCIATION, WHEEL CONTOUR, RECOMMENDED PRACTICE, 1923

WHEEL CONTOUR

(See 611.18, p. 661.)

611.54 SPRINGS, HELICAL AND ELLIPTICAL, AND PARTS.

\section{AMERICAN RAILWAY ASSOCIATION, MECHANICAL DIVISION; SPRINGS, EL- LIPTICAL, RECOMMENDED PRACTICE, 1917}

\section{ELLIPTICAL SPRINGS}

(This specification is substantially the same as A. S. T. M., A62, given below, including the references in A62 to other A. S. T. M. specifications.)

\section{AMERICAN RAILWAY ASSOCIATION, MECHANICAL DIVISION; STANDARD SPRINGS, HELICAL, 1930}

\section{HELICAL SPRINGS}

(This specification is substantially the same as A. S. T. M., A61, given below, including the references in A61 to other A. S. T. M. specifications.) 
AMERICAN RAILWAY ASSOCIATION, MECHANICAL DIVISION; STANDARD SPRINGS AND SPRING CAPS FOR FREIGHT-CAR TRUCKS, 1924

SPRINGS AND SPRING CAPS

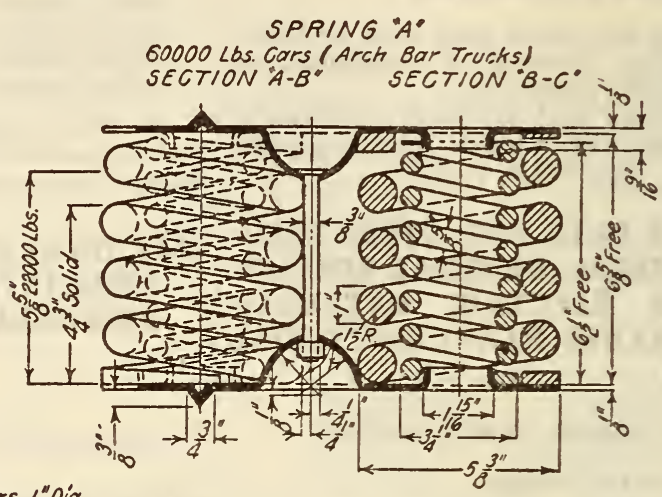

Eight Bars, Four Bars I"Dia. $65 \frac{1}{4}^{\circ}$ Long, Tapered to $72 \frac{1}{4}$ Four Bars, $\frac{5}{8}$ "Oí, $62 \frac{1}{2} "$ Long. Tapered $4066 \frac{3^{\circ}}{4}$

Normal Wt. of Each ist four Bars 14 Lbs. 12 Oz., Minimum WH. 14 Lbs. $50 z$

Normal Wt. of Each 2nd four Bars 5 Lbs. 6 Oz., Minimum WH. 5 lbs $30 z$.

outside oiam /st four coils $5 \frac{3}{8}$, and four coils $3 \bar{f}^{\prime \prime}$ Heights 1 st Four coils $6_{\frac{5}{8}}^{5^{3}}$ Free, $4 \frac{3^{\prime}}{4}$ solid, 5 ह" 3970 Lbs, Copy. 7440 Lbs. Heights 2 nd Four Coils $6 \frac{1}{2}$ Free, $44^{\circ}$ solid, $58^{5} 1530^{\circ}$ Lbs., Cap'y. 3060 Lbs. Cluster or Springs Heights Exclusive of caps $6 \frac{5}{8}$ "Free, $4 \frac{3{ }^{\prime}}{8}$ solid, $5 \frac{5}{8}$ 22000 Lbs., Cap'y. 42000 Lbs.
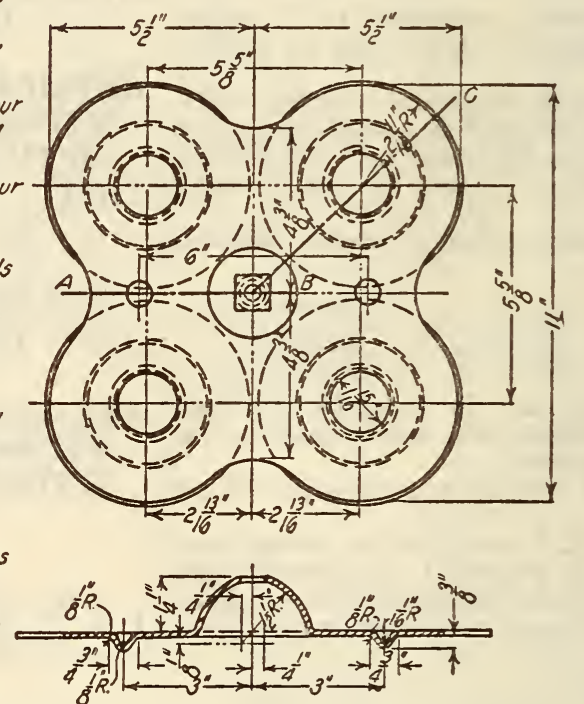

FIGURE ¿599.-Springs and spring caps for freight-car trucks, spring $A$ 


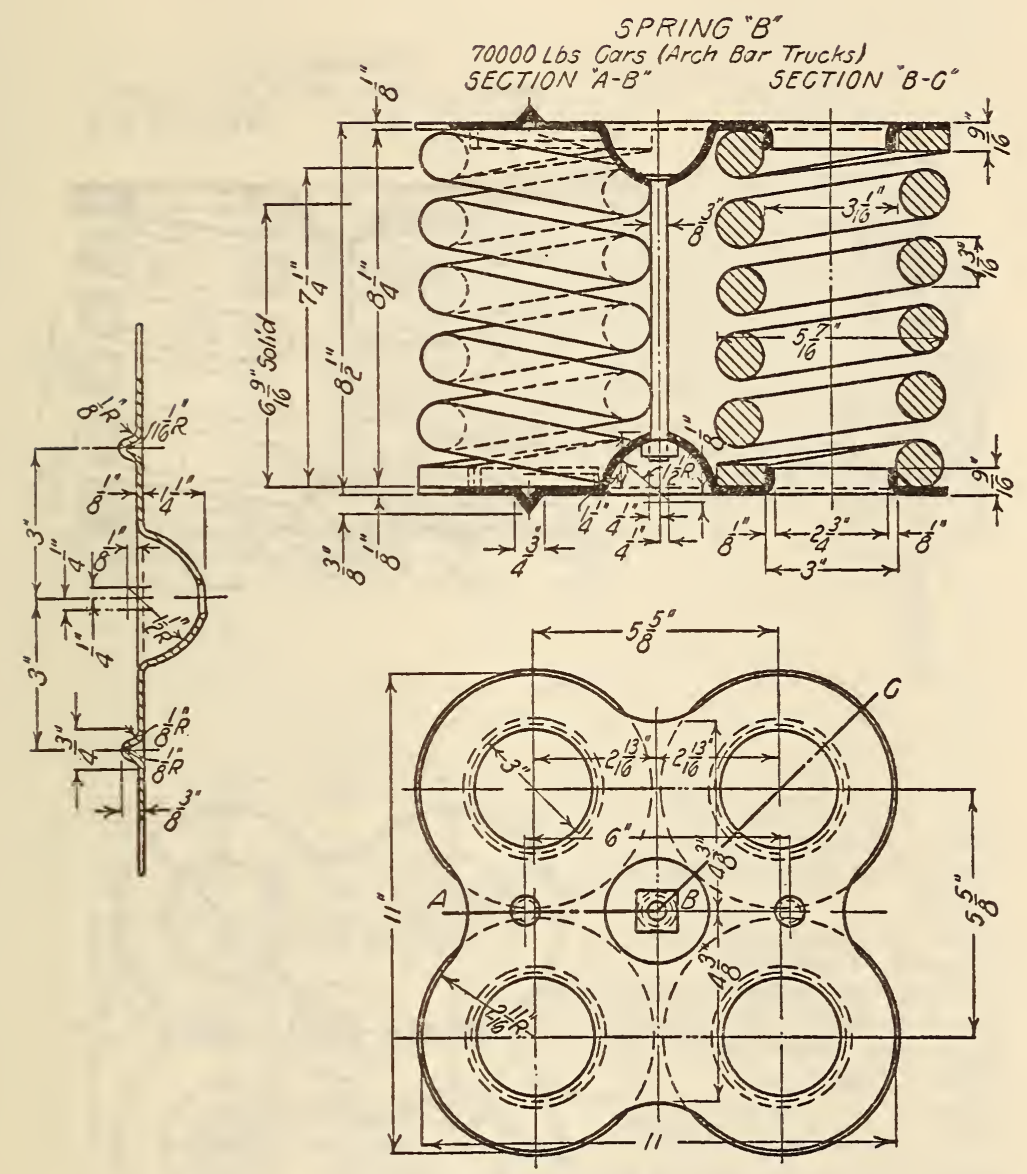

4 Bars 1/3" Dia $733^{3}$ "Long Tapered to $80 \frac{3}{8}^{\prime \prime}$

Normal Wt. of Each Bar 23 Lbs, Minimun Wt 22 LbS $50 z$.

outside Diam $5,7^{4}$

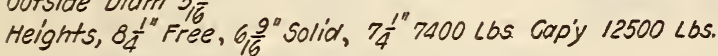

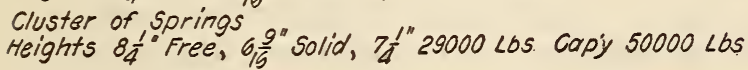

FIGURE 600.-Springs and spring caps for freight-car trucks, spring $B$ 

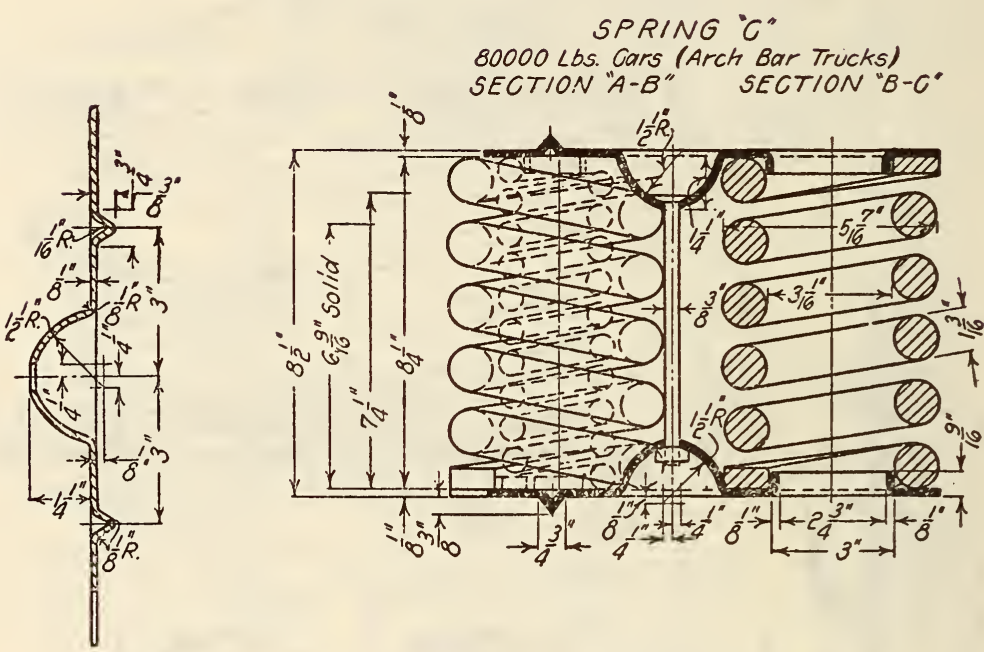
6 Bars. 4 Bars $1 / 3^{\prime \prime}$ Día. $73 \frac{3}{4} "$ Long
Tapered to $803^{\prime \prime}$

2 Bars ${ }^{5} \overline{8}^{\circ}$ Día. $744^{\prime \prime}$ Lorig Tapered to $77 \frac{3}{4}$.

Normal Wt. of Each Ist. Four Bars 23 Lbs. Min. Wt. 22 Lbs. $50 z$

Normal Wt. of Each 2nd. Two Bars 6 Lbs. 7 Oz. Minimum Wt 6Lbs. $40 z$. outside Diam. of I st. Four Coils 5/6" 2ind. Two Coils $2 \frac{7 "}{8}$

Heights ist Four Goils $8 \frac{1}{4}$ " Free $69^{\prime \prime}$ Solid: $7 \frac{1}{4} " 7400$ Lbs. Cap'y 12500 Lbs. Heights 2nd. Two Coils $8 \frac{1}{4} "$ Free $6 \frac{9}{16}$ solid: $7 \frac{1}{4} " 2100$ Lbs. Gap'y. 3500 Lbs. cluster of Springs Heights Without Caps; $8 \frac{1}{4} "$ Free $6 \frac{9}{6}$ " solid: $7 \overline{4}^{10} 33800$ Lbs. Cap'y. $57000^{16}$ Lbs

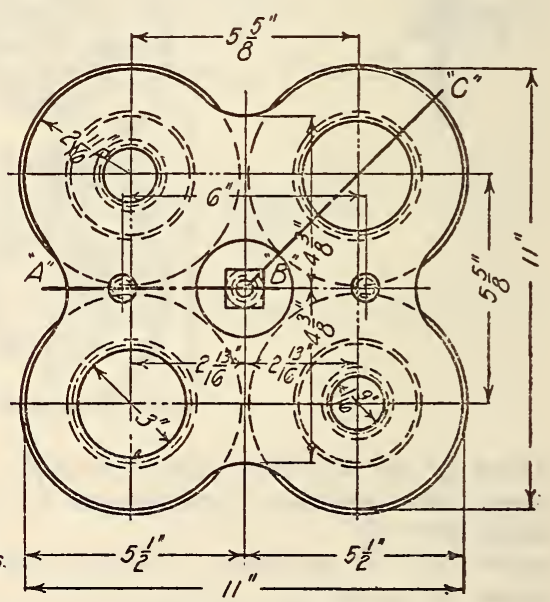

FigURE 601.-Springs and spring caps for freight-car trucks, spring $C$ 


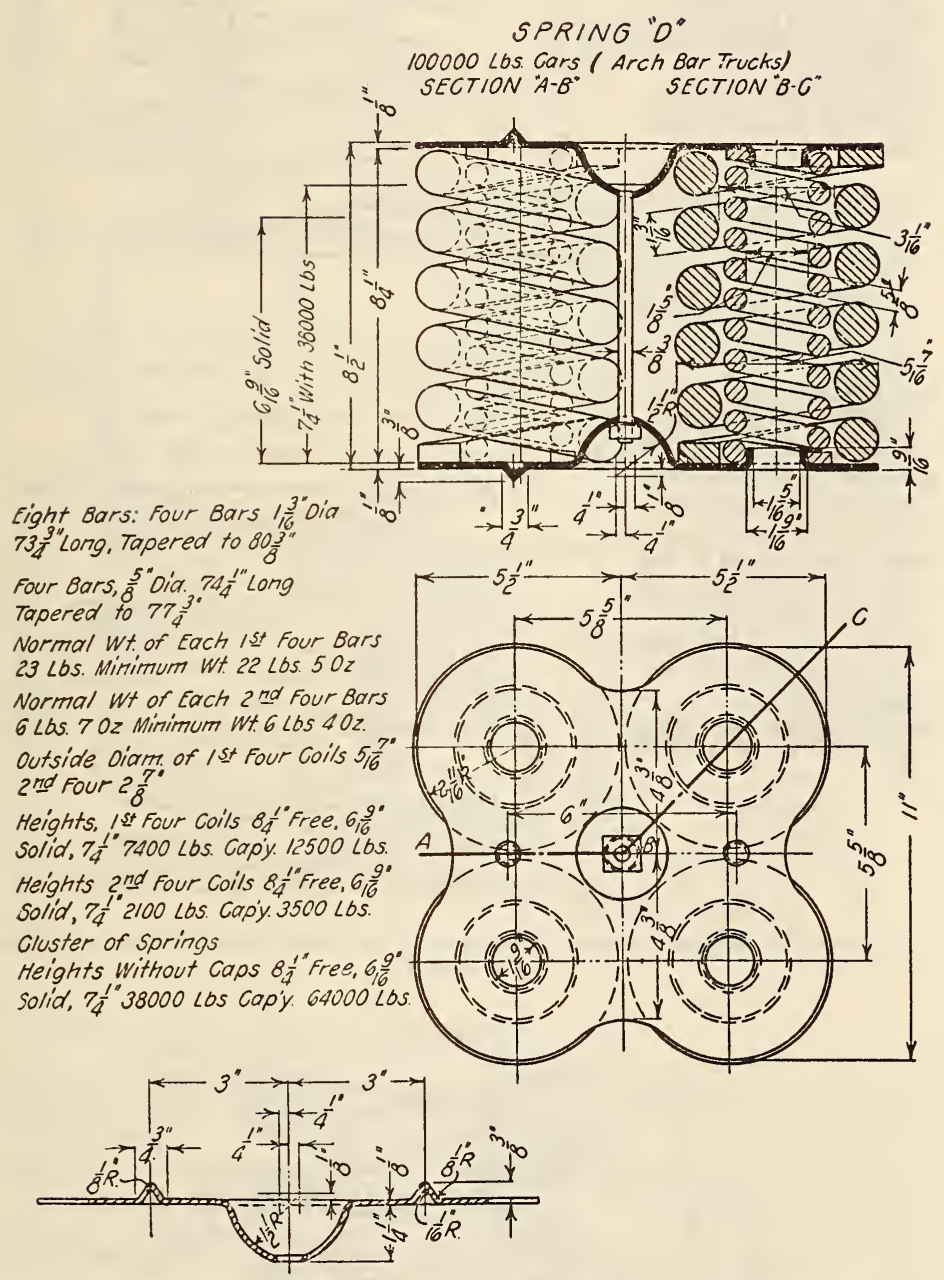

FigURE 602.-Springs and spring caps for freight-car trucks, spring $D$ 
Two bars, 1st bar $18 / 8^{\prime \prime}$ diam., $6712^{n}$ long, tapered to $763 / 8$ : 2nd bar $7 / 8$ " diam., $611 / 4$ " long, tapered to $66^{3} / 8^{\prime \prime}$.

Normal wt. 1st bar 28 ibs. 802 ., minimum wt. $27 \mathrm{lbs} .10 \mathrm{oz}$.

Normal wt. 2 nd bar 10 ibs. 8 oz. Normal wt. 2nd bar $10 \mathrm{lbs} .802$

Ouinimum wt. 10 lbs. 2 oz. Outside diam. 1st coil 7", 2nd coll 41/"; heights 1st coll $718^{\prime \prime}$ free, $51 /^{n}$ solid, $61 / 8^{n}$ 8270 ibsen cap $y 15,530$ ibs. solid, $618^{n} 3080^{n}$ lbs., cap'y solid, 61/8 3080 clustors.

Cluster of springs. $618^{\prime \prime} 11,350$ lbs., cap'y 22,530 ibs.

Two bars, Ist bar $11 / 2^{\prime \prime}$ dia., $761 / 2^{\prime \prime}$ long, tapered to $86^{3} / \mathrm{f}^{\prime \prime}$; 2 2nd bar 2 s" $^{\prime \prime}$ diam. $74^{\prime \prime}$ long, tapered to $80^{n}$.

Normal wt. 1 st bar 38 lbs. 6 oz., minimum wt. 37 lbs. 4 0z. Normal wt. 2 nd bar $14 \mathrm{lbs} .60 \mathrm{oz}$. minimum wt. 13 lbs. $150 z$.

Outside diam. 1st coil $8^{\prime \prime}, 2$ nd coll $47 / 8^{n}$; heights, 1st coil $737^{\prime \prime}$ free, $5 \%^{\prime \prime}$ solid, $65 \%^{\prime \prime}$ $9200 \mathrm{lbs}$., cap'y 19,400 Ibs. Heights, 2 nd coil $737^{m}$ free, Heights, 2nd coil $73 / 4^{\prime \prime}$ free,
$5 \% 8^{\prime \prime}$ solid, $65 / 8^{\prime \prime} 3780$ lbs., $55 / 8^{\prime \prime}$ solid, $65 / 8^{\prime \prime} 3780$ lbs., cap'y 7100 ibs.

Cluster of springs.

Heights $734^{\prime \prime}$ free, $55 / 8^{\prime \prime}$ solld, $658^{\prime \prime} 12,950$ lbs., cap'y 26,500 ibs.

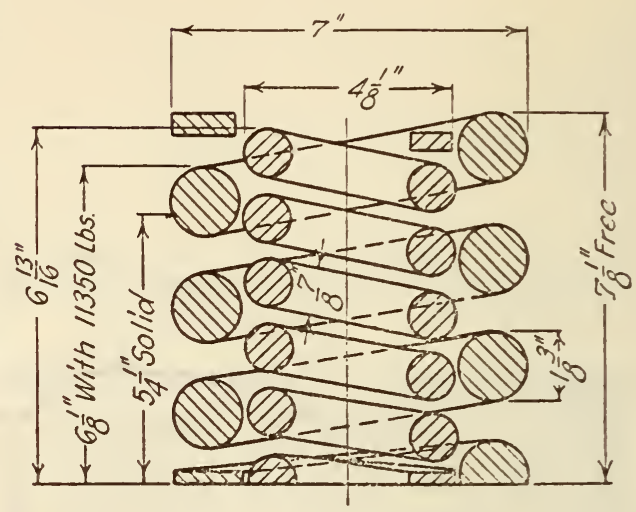

SPRING "F"

80000 Lbs. Cars (Pedestal Trucks)

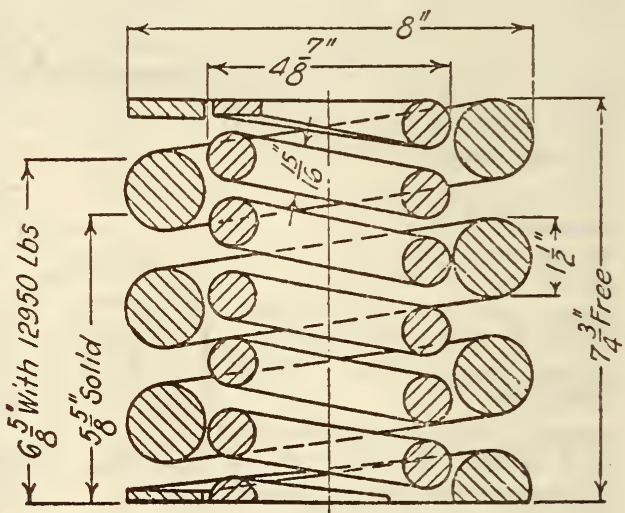

FIGURE 603.-Springs and spring caps for freight-car trucks, springs $E$ and $F$

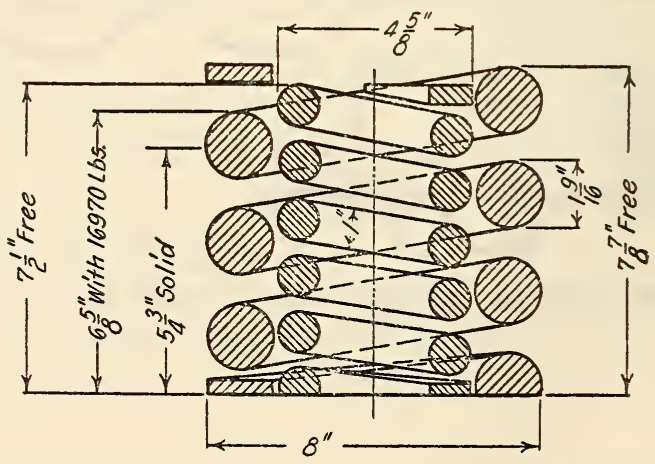

FIGURE 604.-Spring $G, 100,000$ pounds cars (pedestal trucks)

Two bars, first bar, $1 \% 16$ inches diameter, $743 / 8$ inches long, tapered to $841 / 2$ inches; second bar, 1 inch diameter, $651 / 2$ inches long, tapered to 7114 inches. Normal weight, first bar, 40 pounds 5 ounces; minimum weight, 39 pounds 116 ounces. Normal weight, second bar, 14 pounds, 8 ounces; minimum weight, 14 pounds 1 ounce. Outside diameter, first coil, 8 inches; second pounds 1 ounce. Outside diameter, first coil, 8 inches; second coil, $45 / 8$ inches. Heights, first coil, $77 / 8$ inches, free; $53 / 4$ inches,
solid; $65 \% 8$ inches, 11,940 pounds; capacity, 20,300 pounds. Second coil, $71 / 2$ inches, free; $53 / 4$ inches, solid; $65 / 8$ inches, 5,030 pounds; capacity, 10,060 pounds. Cluster of springs, heights, 778 inches, free; $5 \frac{3}{4}$ inches, solid; $65 / 8$ inches, 16,970 pounds; capacity, 30,360 pounds. 

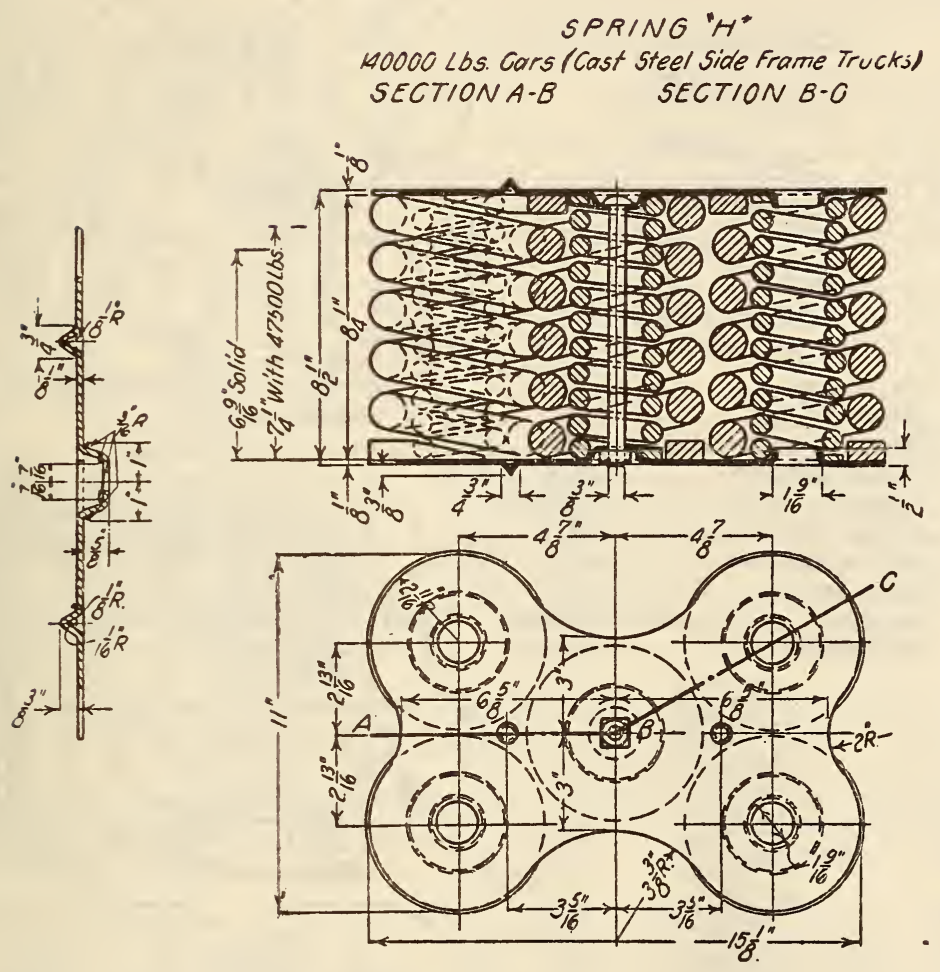

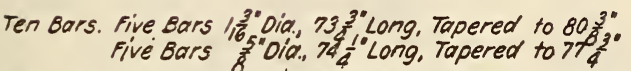

Normal wt of Each Ist Five Bars 23 Lbs. Minimum 22 Lbs $50 z$.

Normal Wt. of Each 2nd Five Bars 6 Lbs. $70 z$. Mimimum $6 \mathrm{lbs} .40 \mathrm{z}$.

outside Diam. of First five Coils 5, $\frac{7}{6}$, Second five $2 \frac{7}{8}$

Heights: First five coils $8 \frac{1}{4}$. Free; $6 \frac{16}{6}$ ' Solid; $7 \frac{1{ }^{\prime \prime}}{17400}$ Lbs. Cop'y. 12500 Lbs.

Heights: Second Five Coils $8 \frac{1}{4}$ "Free $6 \frac{9}{16}$ "Solid $7 \frac{1 "}{4} 2100$ Los. Cap'y. 3500 Lbs.

Cluster of springs

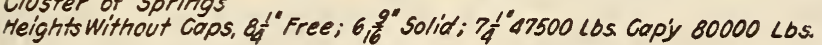

FIGURE 605.-Springs and spring caps for freight-car trucks, spring $H$ 


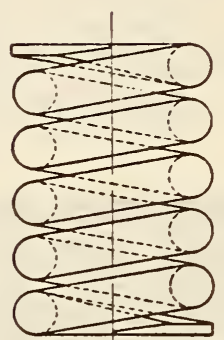

COLL NOI

DIA. OF BAR -..............

LENGTH OF BAR

TAPERED LENGTH OF BAR -..76\%"

NORMAL WEIGHT-..-.---22ZBS.

MINUMUM WEIGHT-........-21-1BS.60Z

OUTSIOE DIA. OF COIL_-....-.-516

FREE HEIGHT---:-.-----84"

SOLID HEIGHT -..........- 6án

MIN. HEIGHT 7द्स WITH LOAD OF T737LBS

LOAD SOLID
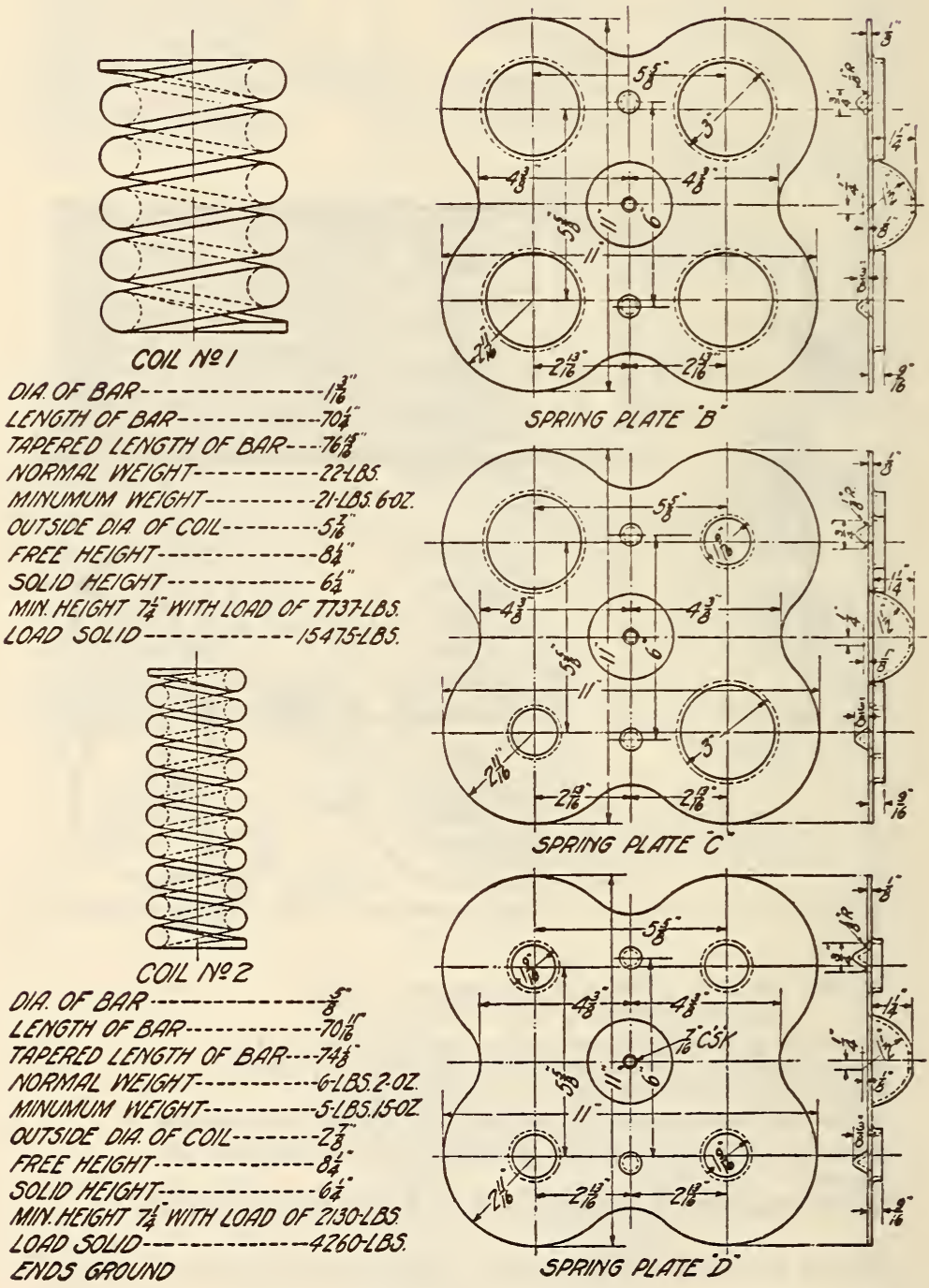

FigURE 606.-Springs and spring caps for freight cars, classes $L, M, N$, $O$, and $P$ 

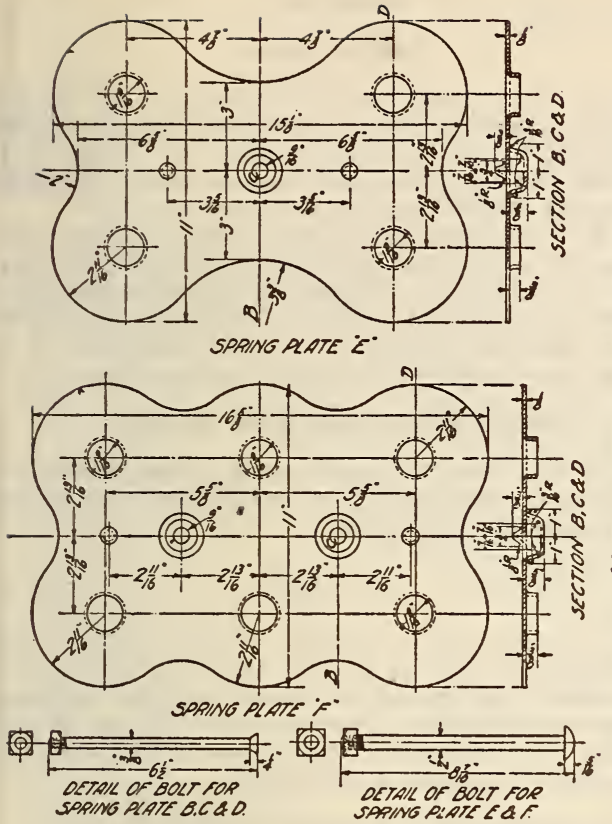

TABLE OF SPRING GPOUPS

\begin{tabular}{|c|c|c|c|c|c|c|c|c|}
\hline \multicolumn{2}{|c|}{ CIASS of } & \multicolumn{2}{|c|}{ me ar colls } & \multirow{2}{*}{$\begin{array}{l}\text { SPAING } \\
\text { RLATE }\end{array}$} & \multicolumn{2}{|c|}{ WESAHT ALESES } & \multicolumn{2}{|c|}{ CAPACITY } \\
\hline TRUCA & $\operatorname{sen} 1 \pi$ & $M O$ & M?? & & TREE & 50210 & 50210 & Faes'-72 \\
\hline $2 B$ & $\bar{L}$ & 4 & 0 & $B$ & $85^{\circ}$ & $67^{\circ}$ & 61900 & 30950 \\
\hline $2 C$ & $M$ & 4 & 2 & C & $81^{\circ}$ & $66^{\circ}$ & 70420 & 35210 \\
\hline 20 & $N$ & 4 & 4 & $D$ & Bt $2^{\circ}$ & $65^{\circ}$ & 78940 & 39470 \\
\hline $2 E$ & 0 & 5 & 5 & $E$ & $65^{\circ}$ & $6 i^{\circ}$ & 98675 & 49338 \\
\hline $2 F$ & $P$ & 6 & 6 & $F$ & ff & $65^{\circ}$ & 118410 & 59205 \\
\hline
\end{tabular}

FIGURE 607.-Springs and spring caps for freight cars, classes $L, M, N, O$, and $P$

\section{AMERICAN SOCIETY OF MECHANICAL ENGINEERS, BIBLIOGRAPHY ON ME- CHANICAI SPRINGS, 1930}

\section{Mechanical Springs}

(A pamphlet of 72 pages containing 600-odd references to publications relating to mechanical springs. The items are arranged both alphabetically with respect to author's name and chronologically with respect to date of issue of the publication, beginning with Hooke in 1678 . A comprehensive subject index associates each author with the particular aspect of spring treatment with which his work is identified.)

\section{AMERICAN SOCIETY FOR TESTING MA- TERIALS, STANDARD SPECIFICATIONS FOR HELICAL STEEL SPRINGS FOR RAIL- WAYS, DESIGNATION A61-16, 1916}

\section{HELICAL RAILWAY SPRINGS} SCOPE

1. These specifications cover all helical steel springs for suspension, draft, and buffer purposes for locomotives, tenders, and cars.

2. (a) The springs shall be made of carbon-steel bars conforming to the requirements of the standard specifications for carbon-steel bars for railway springs, A. S. T. M., A14 (see 603.33, p. 152), unless otherwise specified.

(b) If carbon-steel bars with special silicon requirements or alloy-steel bars are specified, the manufacturer and the purchaser shall agree upon the type and grade of the bars to be used, in which case the bars shall conform, for the respective types, to the standard specifications for carbon-steel bars for railway springs with special silicon requirements, A. S. T. M., A68 (see 603.33, p. 153); the standard specifications for silico-manganese steel bars for automobile and railway springs, A. S. T. M., A59 (See 621.34, p. 1128); or the standard specifications for chrome-vanadium-steel bars for automobile and railway springs, A.S.T.M., A60 (see 621.34, p. 1128).

(c) Drillings for chemical analysis shall be taken from pieces sheared from the bars during the process of manufacture, and not from a finished spring, unless otherwise agreed upon.

\section{CHEMICAL PROPERTIES AND TESTS}

3. (a) An analysis may be made by the purchaser from a sample representing each size of spring steel involved. The chemical composition thus determined shall conform to the requirements specified in section $2(a)$ or $(b)$.

(b) Drillings for analysis shall be taken from the unworked portion of the bars and shall represent the full cross section after rejecting any decarburized material.

(c) In case of dispute, check and arbitration analyses of carbon steel shall be made in accordance with the standard methods of chemical analysis of plain carbon steel, A. S. T. M., A33 (see 600.1, p. 10), the carbon being determined by the directcombustion method; and of alloy steel, in accordance with the standard methods of chemical analysis of alloy steel, A. S. T. M., A55 (see 621.30, p. 1107).

\section{WORKMANSHIP}

4. The purchaser or his representative may examine all springs in each lot for workmanship and general dimensions.

5. (a) The springs shall be submitted for inspection complete in the condition required by the drawings, and shall conform to these drawings with the permissible variations specified in this section and section 7.

(b) The springs shall be of uniform pitch with ends taperfd to give a reasonably square, firm bearing. The points of bars shall not protrude beyond the outside diameter of the springs.

(c) The outside dimensions of the springs, excepting the height, shall not vary more than one-sixteenth inch from those specified.

\section{PHYSICAL PROPERTIES AND TESTS}

6. (a) From each lot of springs which has met the requirements of section 5 , the purchaser or his representative may select for physical tests at least 10 per cent, to be tested in accordance with the requirements of $s$ ction 7 and the appenaix.

(b) If any of the springs representing a lot fail to meet the requirements as to physical properties specified in section 7 , but at least balf of the springs representing a lot do meet these requirements, each spring of the lot shall be tested and those which meet the requirements shall be accepted. If more than half of the springs representing a lot fail to meet the requirements specified in section 7 , the lot represented will be rejected.

7. The properties specified in paragraphs $(a),(b)$, $(c)$, and $(d)$, modified if necessary to conform to the requirements of the appendix, shall be determined in the order specified. The spring shall not be rapped or otherwise disturbed during the test.

(a) Solid height.-The solid height is the per pendicular distance between the plates of the testing machine when the spring is compressed solid with a 
test load at least one and one-fourth times that necessary to bring all the coils in contact. The solid height shall not vary more than one-eighth inch from that specified.

(b) Free height.-The free height is the height of the spring when the load specified in paragraph $(a)$ has been released, and is determined by placing a straight edge across the top of the spring and measuring the perpendicular distance from the plate on which the spring stands to the straight edge, at the approximate center of the spring. The free height shall not vary more than one-eighth inch from that specified.

(c) Loaded height.-The loaded height is the distance between the plates of the testing machine when the specified working load is applied. The loaded height shall not vary more than one-eighth inch over nor more than one-sixteenth inch under that specified.

(d) Permanent set.-The permanent set is the difference, if any, between the free height and the height (measured at the same point and in a similar manner) after the spring has been compressed solid three times in rapid succession with the test load specified in paragraph $(a)$. The permanent set shall not exceed one thirty-second inch.

\section{MARKING}

8. (a) The name or brand of the manufacturer, the year and month of manufacture, and, if specified, the purchaser's class number shall be legibly stamped on each spring at a place not detrimental to the life or service of the spring.

(b) Any stamping by the inspector shall be so placed as not to be detrimental to the life or service of the spring.

\section{INSPECTION AND REJECTION}

(Conform to A. S. T. M. standard specifications for steel. See 605.0, p. 247.)

\section{REWORKING}

12. Any springs which fail to meet the requirements as to dimensions or physical tests may be again submitted after being worked.

${ }^{88}$ This stress is given solely as a limiting stress not to be exceeded in testing by the methods covered by the specifications. It is not intended as a guide in the design of springs, as the proper working fiber stress will depend on the class and design of the spring and on the service for which it is intended.

\section{APPENDIX}

\section{Test Fiber Stress}

1. (a) The properties and methods of testing specified in section 7 of the specifications have been established for carbon-steel springs on the assumption that the maximum fiber stress under test shall not exceed 90,000 pounds per square inch. ${ }^{88}$

(b) For alloy-steel springs practice has not been sufficiently well established to enable definite fiber stresses to be given. Unless otherwise agreed upon by the manufacturer and the purchaser, alloy-steel springs shall be tested under the conditions as to fiber stress specified for carbon-steel springs.

\section{Relation Between Fiber Stress and Load}

2. To find the maximum load under which the conditions of paragraph $1(a)$ are fulfilled, the following formula shall be used:

$$
P=\frac{3.1416 S d^{3}}{8 M}
$$

where $P=$ the load in pounds; $S=$ the stress of the most strained fiber in pounds per square inch; $d=$ the diameter of the bar in inches; and $M=$ the mean diameter of the helix in inches-this is found by subtracting the diameter or thickness of the bar from the outside diameter of the spring.

\section{Test of Springs Not in Accordance with Preceding Rule}

3 . If it is desired to purchase under these specifications springs in which the maximum fiber stress exceeds that specified in paragraph $1(a)$, the tests shall be made as specified in section 7 except that the maximum test load to be applied in determining the properties specified in sections $7(a)$ and $(d)$ shall not be sufficient to compress the spring solid, but shall be the load which corresponds to a fiber stress of 90,000 pounds per square inch, as determined by equation (1).

\section{Table to Facilitate Calculations}

4. Table 1 is arranged to facilitate the calculations necessary to determine whether a spring ean be tested in accordance with the specifications without exceeding the stress given in paragraph $1(a)$. The table shows the loads at which springs of various sizes are stressed to 90,000 pounds per square inch. These are the test loads to be used for springs tested in accordance with section 15 . In testing in accordance with section $7(a)$ the load given in Table 1 for the given size of spring shall not be exceeded, unless with this load the spring is compressed solid. 
TABLE 1.-Test loads for helical steel springs, in pounds, which will correspond to a maximum fiber stress of 90,000 pounds per square inch

\begin{tabular}{|c|c|c|c|c|c|c|c|c|c|c|c|c|c|c|c|c|c|c|c|c|c|}
\hline \multirow{2}{*}{$\begin{array}{l}\text { Outside } \\
\text { diam. } \\
\text { eter of } \\
\text { spring } \\
\text { (inches) }\end{array}$} & \multicolumn{21}{|c|}{ Diameter of bar (inches) } \\
\hline & $5 / 16$ & $3 / 8$ & $7 / 16$ & $1 / 2$ & $9 / 16$ & $5 / 8$ & $11 / 16$ & $3 / 4$ & $13 / 16$ & $7 / 8$ & $15 / 16$ & 1 & $11 / 16$ & 138 & $13 / 16$ & $11 / 4$ & $15 / 16$ & $13 / 8$ & $17 / 16$ & $11 / 2$ & $1 \% 16$ \\
\hline 2 & $\begin{array}{r}639 \\
557\end{array}$ & 1,147 & 895 & & & & & & & & & & & & & & & & & & \\
\hline $21 / 2$ & 493 & & $\begin{array}{l}1,633 \\
1,435\end{array}$ & $\begin{array}{l}2,525 \\
2,210\end{array}$ & $\begin{array}{l}3,730 \\
3,245\end{array}$ & 4,600 & & & & & & & & & & & & & & & 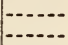 \\
\hline 3 & 442 & 785 & 1,280 & 1,964 & 2,875 & 4,060 & 5,570 & 6.625 & & 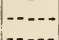 & - & &.- & $-\cdots$ & & & & & & & $\cdots$ \\
\hline $31 / 4$ & & & 1005 & 1607 & 2,000 & & 4 & 5. 95 & 77 & & & & & & & & & & & & \\
\hline $31 / 2$ & & & $\begin{array}{l}1,052 \\
966\end{array}$ & 1,473 & 2,140 & & & 5,420 & & 9,020 & & & & & & & & & & & \\
\hline $\begin{array}{l}334 \\
4\end{array}$ & $\ldots$ & & (n..... & $\begin{array}{l}1,359 \\
1,262\end{array}$ & $\begin{array}{l}1,973 \\
1,830\end{array}$ & $\begin{array}{l}2,760 \\
2,555\end{array}$ & $\begin{array}{l}3,750 \\
3,465\end{array}$ & $\begin{array}{l}4,970 \\
4,590\end{array}$ & 6,455 & 8,235 & $\begin{array}{r}10,350 \\
9\end{array}$ & 11780 & & & & & & & & & \\
\hline $41 / 4$ & & & & & 1,706 & 2,3 & 3,225 & 4,260 & 5,515 & 7,015 & 8,790 & 10,880 & 13,300 & & & & & & & & \\
\hline $\begin{array}{l}41 / 2 \\
43 / 4\end{array}$ & & & & & 1,598 & 2,225 & 3,010 & $\begin{array}{l}3,975 \\
3,725\end{array}$ & 5,140 & 6,530 & 8,175 & $\begin{array}{r}10,100 \\
9\end{array}$ & $\begin{array}{l}12,330 \\
11500\end{array}$ & 14,910 & 10.60 & & & & & & \\
\hline 5 & $\cdots$ & & & 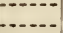 & (n).... & 1,972 & 2,665 & 3,510 & 4,52 & 5,740 & 7,170 & 8,835 & 10,770 & 12,990 & 15,520 & 18,410 & & & & & \\
\hline $\begin{array}{l}514 \\
512\end{array}$ & $\ldots$ & & & & & & {$\left[\begin{array}{l}2,5 \\
2,38\end{array}\right.$} & $\begin{array}{l}3,3 \\
3,1\end{array}$ & $\begin{array}{l}4,270 \\
4,045\end{array}$ & $\begin{array}{l}5,410 \\
5,120\end{array}$ & $\begin{array}{l}6,755 \\
6,385\end{array}$ & 8,315 & $\begin{array}{r}10,120 \\
9\end{array}$ & 12,200 & $\begin{array}{l}14,570 \\
13,720\end{array}$ & 17 & 20,300 & $\cdots$ & & & \\
\hline $53 / 4$ & & & & & & & 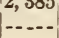 & 2,98 & 3,84 & $\mid \begin{array}{l}4 \\
4,855\end{array}$ & $\begin{array}{l}0,385 \\
6,050\end{array}$ & 7,440 & $\begin{array}{l}9,000 \\
9,045\end{array}$ & 10,880 & 12,970 & 10, & 19, & 21 , & 24,340 & & \\
\hline 6 & & & & & & & & 2,840 & 3,655 & 4,620 & 5,750 & 7,070 & 8,585 & 10,320 & 12,300 & 14,530 & 17,050 & 19,870 & 23,010 & 26,510 & 30,380 \\
\hline 634 & & & & & & & & & $\begin{array}{l}3,485 \\
3\end{array}$ & 4,405 & 5,490 & 6,730 & 8,170 & 9,820 & 11, & $\begin{array}{l}13,810 \\
13\end{array}$ & 16 & 18, & 21,820 & 238 & $\begin{array}{l}3,760 \\
710\end{array}$ \\
\hline $63 / 4$ & & & & & & & & & & 4,030 & $\begin{array}{l}5,250 \\
5,010\end{array}$ & $\begin{array}{l}0,425 \\
6,145\end{array}$ & 7,455 & 8,945 & 11, & 12,550 & 14 & 17,090 & $\begin{array}{l}20, \\
19,760\end{array}$ & $\begin{array}{l}\mid \begin{array}{l}25,800 \\
22,720\end{array} \\
2\end{array}$ & , 990 \\
\hline 7 & & & & & & & $\cdots$ & & $\ldots$ & 3,865 & 4,805 & 5,890 & 7,140 & 8,565 & 10,180 & 12,010 & & 16,330 & 18,870 & 21,690 & 24,800 \\
\hline $71 / 2$ & & & & & & & & & & & 4,440 & 5,435 & 6,585 & 7,895 & 9,375 & 11,050 & 12,920 & 15,000 & 17,320 & 19,8 & 22,710 \\
\hline $\begin{array}{l}8 \\
81 / 2\end{array}$ & & & & & & & & & & & - & 5,050 & 6,110 & 7,320 & & 10 , & & & 15,600 & & 20,940 \\
\hline & & & & & & & & & & & & & 0,100 & 6,390 & & & & & 14 & & $\begin{array}{l}450 \\
130\end{array}$ \\
\hline $1 / 2$ & & & & & & & & & & & & & & & 7,120 & 8,365 & 9,760 & 11,310 & 13,020 & 14, & 16,990 \\
\hline & & & & & & & & & & & & & & & & 7,890 & 9,200 & 10,650 & 12,260 & 14, & 15,980 \\
\hline & & & & & & & & & & & & & & & & & & $\begin{array}{r}10,010 \\
9,545\end{array}$ & & 12,560 & \\
\hline & & & & & & & & & & & & & & & & & & & 10,430 & & \\
\hline & & & & & & & & & & & & & & & & & & & & & \\
\hline
\end{tabular}

Nоте.-All values under 2,000 pounds have been shown to the nearest pound; those between 2,000 and 10,000 pounds, to the nearest 5 pounds and those over 10,000 pounds to the nearest 10 pounds.

\section{AMERICAN SOCIETY FOR TESTING MATE- RIALS, STANDARD SPECIFICATIONS FOR ELLIPTICAL STEEL SPRINGS FOR RAIL. WAYS, DESIGNATION A62-16, 1916}

\section{ELLIPTICAL RAILWAY SPRINGS SCOPE}

1. These specifications cover all elliptic steel springs for suspension, draft, and buffer purposes for locomotives, tenders, and cars.

\section{BASIS OF PURCHASE}

(Requirements covering basis of purchase are identical with those in A. S. T. M., A61, given above, with the following additions:)

(c) The bands of the springs shall be made of wrought iron conforming to the requirements of the standard specifications for refined wrought-iron bars, A. S. T. M., A41 (see 603.1 , p. 139), or if agreed upon, they may be made of "dead-soft" open-hearth steel, the carbon content of which shall not exceed 0.15 per cent. Bands of special design, subject to agreement between the manufacturer and the purchaser, may be made of steel castings conforming to the requirements of the standard specifications for steel castings, Class B, soft grade, A. S. T. M., A27. (See 611.41, p. 707.)

(e) In determining the loaded height and loaded length of the springs, the "compression" method of section 7 shall be used, unless the "release" method of section 8 is specified.

\section{CHEMICAL PROPERTIES AND TESTS}

(Same as A. S. T. M., A61, given above.)

\section{WORKMANSHIP}

(Same as A. S. T. M., A61, given above, paragraphs 4 and $5(a)$, with the following additions and variations:)

(a) Dimensions which affect the contour only and do not affect the interchange or service of the springs need only be approximated.

(b) The springs shall have the leaves properly graduated in length, properly bent, and fitted to reasonably true circular arcs.

(c) The bands of the springs shall not vary from the specified dimensions more than one-sixteenth inch in width and one-thirty-second inch in thickness of straps, nor more than one-eighth inch in width across the spring.

\section{PHYSICAL PROPERTIES AND TESTS}

6. (a) From each lot of springs which has met the requirements of section 5 , the purchaser or his representative may select for physical tests at least 25 per cent, to be tested in accordance with the "compression" method of section 7, or, if specified, in accordance with the "release" method of section 8 , and the appendix.

(b) If any of the springs representing a lot fail to meet the requirements as to physical properties specified in section 7 or section 8 as required, but at least half of the springs representing a lot do meet these requirements, each spring of the lot shall be 
tested and those which meet the requirements shall be accepted. If more than half of the springs representing a lot fail to meet the requirements specified in section 7 or section 8 as required, the lot represented will be rejected.

7. When the "compression" method is to be used, the properties specified in paragraphs $(a),(b)$, and (c), modified if necessary to conform to the requirements of the appendix, shall be determined, in the order specified. The spring shall not be rapped or otherwise distributed during the test. The ends of half-elliptic springs shall be supported on roller or swing bearings.

(a) Free height.-The free height is the height of the spring after a test load of one and one-half times the specified working load has been applied and fully released.

(b) Loaded height and loaded length.-The loaded height and loaded length are, respectively, the height and length when the specified working load is applied. The load shall be applied gradually and in such a way that the specified working load shall not be exceeded. If it is exceeded, the load shall be released to not more than one-half the specified working load and then increased to the specified working load. The loaded height shall not be less but may be three eighths inch more than that specified. The loaded length shall not vary more than one-fourth inch from that specified.

(c) Permanent set.-The permanent set is the difference, if any, between the free height and the height after the test load of one and one-half times the specified working load has again been applied and fully released. The following two requirements shall be met:

(1) The permanent set shall not exceed one thirty-second inch.

(2) If there is any permanent set not exceeding one thirty-second inch the difference between the free height and the height after the test load of one and one-half times the specified working load has been applied and fully released two additional times, shall not be greater than the permanent set first measured.

8. When the "release" method is specified, the properties specified in paragraphs $(a),(b)$, and $(c)$, modified if necessary to conform to the requirements of the appendix, shall be determined in the order specified. The spring shall not be rapped or otherwise disturbed during the test. The ends of halfelliptic springs shall be supported on roller or swing bearings.

(a) Free height.-The free height is the height of the spring after a test load of one and one-half times the specified working load has been applied and fully released.

(b) Loaded height and loaded length.-The loaded height and loaded length are, respectively, the height and length when a test load of one and onehalf times the specified working load has been applied and is slowly released to the specified working load. If released to less than the specified working load, the load shall again be raised to one and one-half times the specified working load and then released to the specified working load. The loaded height shall not be more, but may be three-eighths inch less than that specified. The loaded length shall not vary more than one-fourth inch from that specified.

(c) Permanent set.-The permanent set is the difference, if any, between the free height and the height after the test load of one and one-half times the specified working load has again been applied and fully released. The following two requirements shall be met:

(1) The permanent set shall not exceed onethirty-second inch.
(2) If there is any permanent set not exceeding one thirty-second inch, the difference between the free height and the height after the test load of one and one-half times the specified working load has been applied and fully released two additional times, shall not be greater than the permanent set first measured.

\section{MARKING}

9. (a) The name or brand of the manufacturer, the year and month of manufacture, and, if specified the purchaser's class number shall be legibly stamped on each spring at a place not detrimental to the life or service of the spring.

(b) Any stamping by the inspector shall be so placed as not to be detrimental to the life or service of the spring.

\section{INSPECTION AND REJECTION}

(Inspection, rejection, and rehearing conform to A. S. T. M. standard specifications for steel. See 605.0 , p. 247. Reworking is identical with A.S. T. M., A61, given above.)

\section{APPENDIX}

\section{Test Fiber Stress}

1. (a) The properties and methods of testing specified in sections 7 and 8 , have been established for carbon-steel springs on the assumption that the maximum fiber stress under test shall not exceed 127,500 pounds per square inch. ${ }^{88}$

(b) For alloy-steel springs, practice has not been sufficiently well established to enable definite fiber stresses to be given. Unless otherwise agreed upon by the manufacturer and the purchaser, alloy-steel springs shall be tested under the conditions as to fiber stress specified for carbon-steel springs.

\section{Relation Between Fiber Stress and Load}

2. To find the maximum loads under which the conditions of section 1 (a) in appendix are fulfilled, the following formula shall be used:

$$
P=\frac{2 S n b h^{2}}{3 L}
$$

where $P=$ the load in pounds; $S=$ the stress of the most strained fiber in pounds per square inch; $n=$ the number of plates in half-elliptic springs, or half the number of plates in full-elliptic springs; $b=$ the width of each plate in inches; $h=$ the thickness of each plate in inches; and $L=$ the distance between supports, in inches, when the spring is loaded.

\section{Test of Springs Not in Accordance with Preceding Rules}

3. If it is desired to pruchase under these specifications springs in which the maximum fiber stress exceeds that specified in section $1(a)$ in appendix the tests shall be made as specified in section 7 or 8 except that the test load to be applied shall not be one and one-half times the specified working load but shall be the load which corresponds to a fiber stress of 127,500 pounds per square inch, as determined by equation (1).

\section{Test of Springs for Which No Working Load is Specified}

4. If springs are ordered to free height and length only, and no working load and no loaded dimensions are specified, the tests specified in sections 7 or 8

89 This stress is given solely as a limiting stress not to be exceeded in testing by the methods covered by the specifications. It is no intended as a guide in the design of springs, as the proper wortin fiber stress will depend on the class and design of the spring and on the service for which it is intended. 
shall be made using instead of the specified working load the load which corresponds to a fiber stress of 75,000 pounds per square inch, as determined by equation (1).

\section{Table to Facilitate Calculations}

5. Table 1 is arranged to facilitate the calculations necessary to determine whether a spring can be tested in accordance with the specifications without exceeding the stress given in section $1(a)$ in appendix. The table shows, for various lengths and for various thicknesses of plate, the loads per inch of effective width of spring corresponding to a fiber stress of 127,500 pounds per square inch. These are the maximum allowable test loads, and shall not b? exceeded in any tests.

The loads are given per inch of effective width of spring. The effective width of spring is understood to mean the width of the individual plate multiplied by the number of plates as defined in section 2 in appendix. Consequently the total load in pounds for any spring is found by multiplying the value from Table 1 by the product of the width of plate into the number of plates.

TABLE 1.-Test loads for elliptical springs, in pounds per inch of effective width, which will correspond to a maximum fiber stress of $12 \%, 500$ pounds per square inch

\begin{tabular}{|c|c|c|c|c|c|c|c|}
\hline \multirow{2}{*}{ 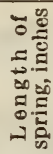 } & \multicolumn{7}{|c|}{ Thickness of plates, (inches) } \\
\hline & $3 / 16$ & $1 / 4$ & $5 / 16$ & $3 / 8$ & $7 / 16$ & $1 / 2$ & 916 \\
\hline 20 & 150 & 265 & & & & & \\
\hline 22 & 136 & 241 & 377 & & & & \\
\hline 24 & 125 & 221 & 345 & 497 & & & \\
\hline 26 & 115 & 204 & 319 & 460 & 625 & & \\
\hline 28 & 107 & 190 & 296 & 427 & 581 & 760 & \\
\hline 30 & 100 & 177 & 277 & 398 & 543 & 707 & 896 \\
\hline 32 & -...... & 166 & 259 & 374 & 509 & 665 & 840 \\
\hline 34 & ...... & 156 & 244 & 351 & 479 & 625 & 791 \\
\hline 36 & & 148 & 230 & 332 & 452 & 590 & 748 \\
\hline 38 & & 140 & 218 & 314 & 429 & 560 & 708 \\
\hline 40 & & 133 & 208 & 298 & 407 & 531 & 673 \\
\hline 42 & .. & 126 & 198 & 284 & 388 & 506 & 640 \\
\hline 44 & & & 189 & 272 & 370 & 483 & 612 \\
\hline 46 & & & 180 & 260 & 354 & 462 & 585 \\
\hline 48 & & & 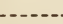 & 249 & 339 & 443 & 561 \\
\hline 50 & & & & 239 & 325 & 425 & 538 \\
\hline 52 & -- & & & $-\cdots$ & 313 & 409 & 518 \\
\hline 54 & & & & & 302 & 394 & 499 \\
\hline 56 & - & & & & -- & 380 & 481 \\
\hline $\begin{array}{l}58 \\
60\end{array}$ & & & & & & $\begin{array}{l}366 \\
354\end{array}$ & $\begin{array}{l}465 \\
450\end{array}$ \\
\hline
\end{tabular}

\section{AMERICAN SOCIETY FOR TESTING MATE- RIALS, TENTATIVE SPECIFICATIONS FOR HEAT-TREATED CARBON-STEEL HELICAL SPRINGS, DESIGNATION A125-31T, 1931}

\section{HEAT-TREATEN HELICAL SPRINGS SCOPE}

1. These specifications cover hot-coiled, helical compression springs made of round bars one-half inch and larger in diameter and suitable for use on railway equipment.

\section{MANUFACTORE}

2. The steel may be made by one or more of the following processes: Open hearth, crucible, or electric urnace. The rolled bars shall be practically straight; free from surface cracks, rolïing seams, folds and flat areas, or other injurious defects.

3. The bars after being suitably tapered or otherwise prepared shall be heated slowly and uniformly to a temperature of approximately $1,700^{\circ} \mathrm{F} .\left(930^{\circ}\right.$ C. $)$ and coiled on a preheated mandrel. No water shall be allowed to come in contact with the heated bar at any time.

4. After coiling, the springs shall be allowed to cool uniformly to a distinct black heat. They shall then be reheated uniformly to a temperature sufficient to refine the grain and quenched in an ample volume of oil. After quenching the springs shall be allowed to cool to at least $400^{\circ} \mathrm{F}$. $\left(200^{\circ} \mathrm{C}\right.$.) and shall then be reheated uniformly to a temperature below the critical, but sufficiently high to give a proper drawback or tempering effect.

\section{SAMPLING}

5. The springs offered for inspection shall be arranged in groups with 500 or fraction thereof of a size in a group. The inspector shall select one spring from each group to represent that group and shall suitably mark the sample so selected for identification.

\section{CHEMICAL PROPERTIES AND TESTS}

6. (a) Unless otherwise specified, the steel shall conform to the following requirements as to chemical composition:

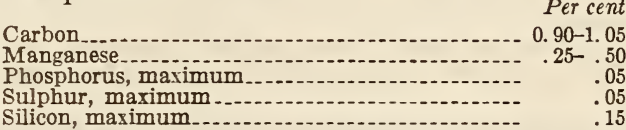

(b) Other grades of steel may be used when mutually agreed upon by the purchaser and the manufacturer.

7. One check analysis may be made by the purchaser from each 500 springs or fraction thereof of each size of bar.

8. For chemical analysis, a spring if small shall be taken or if large a piece weighing about one-half pound shall be cut off. If the sample is cut off hot it shall be cooled in such a way as not to permit it to harden. Drillings for check analysis shall be made from the samples so selected and the drill used shall be approximately one-half the diameter of the bar. The drillings for analysis shall be selected from the thoroughly mixed total drillings obtained by pass. ing nearly through the section of the bar after discarding the surface drillings.

\section{PHYSICAL PROPERTIES AND TESTS}

9. From each group of springs described in section 5 which conform to the requirements of sections 15 and $16(a)$ the inspector may select 10 per cent to be tested in accordance with the requirements of sections 10 and 11 .

10. (a) The properties specified in paragraphs (b) to $(f)$ shall be determinea in the order enumerated The springs shall not be rapped or otherwise disturbed during the test.

(b) Solid height.-The solid height is the perpendicular distance between the plates of the testing machine when the spring is compressed solid with a test load of approximately one and one-quarter times that necessary to bring all coils in contact.

(c) Free height. - The free height is the height of the spring when the load specified in paragraph (b) has been released and is determined by placing a straight-edge across the top of the spring and measuring the perpendicular distance from the plate on which the spring stands to the straight-edge at the approximate center of the spring. 
(d) Loaded height.-The loaded height is the perpendicular distance between the plates of the testing machine when the specified working load has been applied.

(e) Uniformity of pitch. - The pitch of the coils shall be sufficiently uniform that when the springs are compressed to a point where any two coils are in contact there shall be no space exceeding onethirty-second inch between the other coils, excluding the tapered ends.

(f) Permanent set.-The permanent set is the difference, if any, between the free height and the height after the spring has been compressed solid three times with the test load specified in paragraph (b), measured at the same point and in the same manner.

11. (a) Springs having a free height of 10 inches and under shall conform to the following tolerances when tested in accordance with section 10:

Diameter.-The diameter of the springs up to 6 inches in outside diameter shall not vary more than one-sixteenth inch from that specified and for springs over 6 inches in outside diameter the diameter shall not vary more than one-eighth inch from that specified.

Solid height.-Not more than one-sixteenth inch over that specified.

Free height. - Not more than one-eighth inch over or under that specified.

Loaded height.- Not more than one-eighth inch over nor more than one-sixteenth inch under that specified.

Uniformity of pitch.-As specified in section $10(e)$.

Permanent set.- Not more than one-thirty-second inch.

(b) For springs having a free height over 10 inches but not over 15 inches the tolerances specified in paragraph (a) shall be increased 50 per cent.

(c) For springs having a free height over 15 inches but not over 20 inches the tolerances specified

in Paragraph (a) shall be increased 100 per cent.

(d) For springs having a free height over 20 inches the tolerances shall be mutually agreed upon by the manufacturer and the purchaser.

12. (a) The properties and methods of testing specified in sections 10 and 11 have been established on the assumption that the maximum fiber stress at the solid height shall not exceed 100,000 pounds per square inch and that the several heights specified are commensurate. The stress of 100,000 pounds per square inch referred to is given solely as a limiting stress not to be exceeded in testing. This stress is not intended as a guide in the design of springs, as the proper working fiber stress will depend upon the class of the spring and upon the service for which it is intended.

(b) Unless otherwise mutually agreed upon by the manufacturer and purchaser, springs which do not meet the conditions of paragraph $(a)$ shall not be subject to the requirements of sections 10 and 11 , but shall be submitted to a test load which corresponds to a maximum fiber stress of 100,000 pounds per square inch as determined in section 13 . The free height as measured after application and release of such test load shall conform to the requirements of section 11 .

13. The test load corresponding to a maximum fiber stress of 100,000 pounds per square inch shall be calculated from the following equation:

$$
C=\frac{3.1416 S d^{3}}{8 M}
$$

where

$C=$ the capacity in pounds.

$S=$ the stress at the most strained fiber $=$ 100,000 pounds per square inch.

$d=$ the diameter of the bar in inches.

$M=$ the mean diameter of the helix in inches, found by subtracting the diameter of the bar from the outside diameter of the spring.

Table 1 shows test loads in pounds and deflection per turn in inches for helical springs, corresponding to a maximum fiber stress of 100,000 pounds per square inch for springs 2 to 12 inches in outside diameter.

TABLE 1.-Test loads in pounds and deflection per turn in inches for helical springs, corresponding to maximum fiber stress of 100,000 pounds per square inch

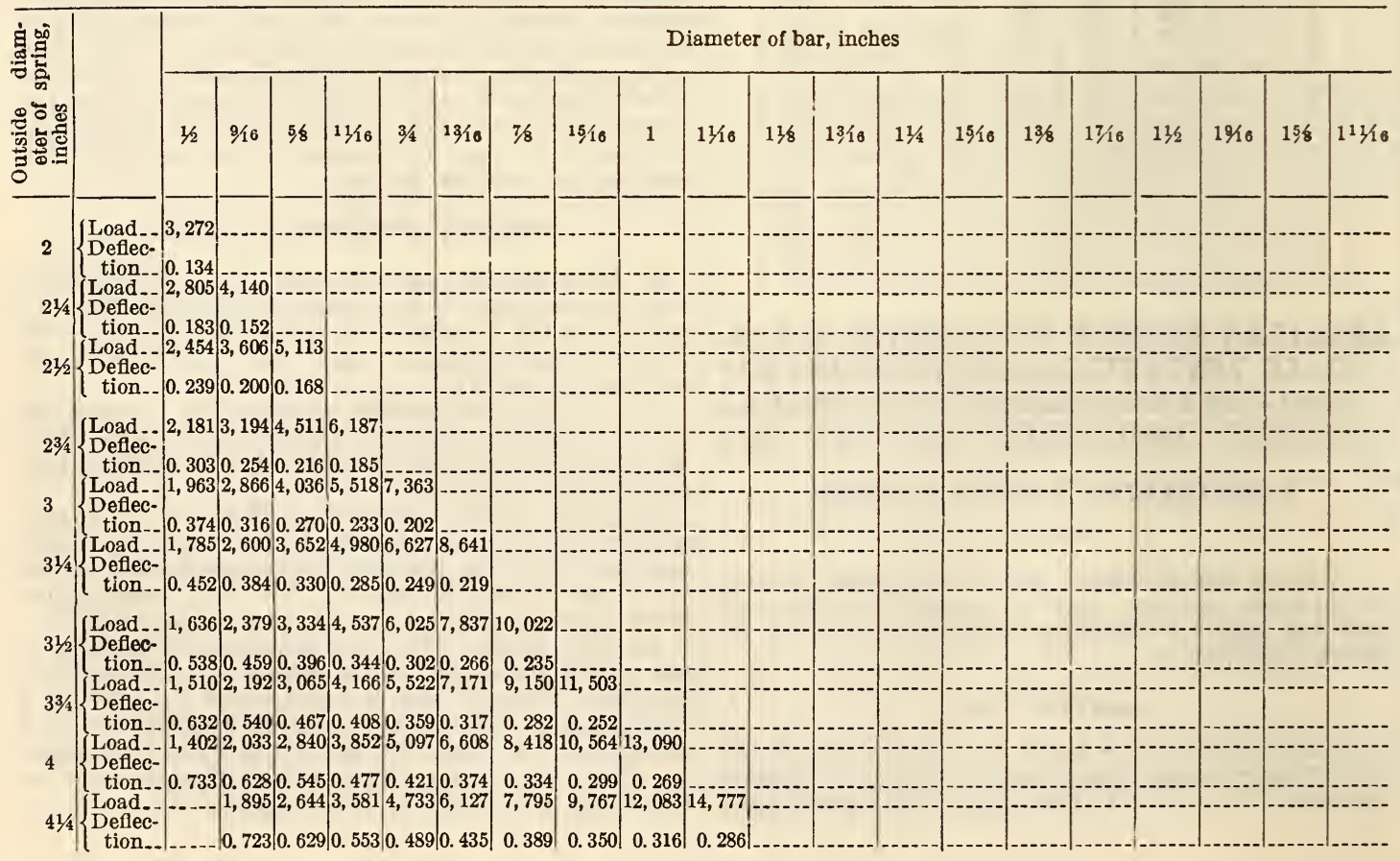


TABLE 1.-Test loads in pounds and deflection per turn in inches for helical springs, corresponding to a maximum fiber stress of 100,000 pounds per square inch-Continued

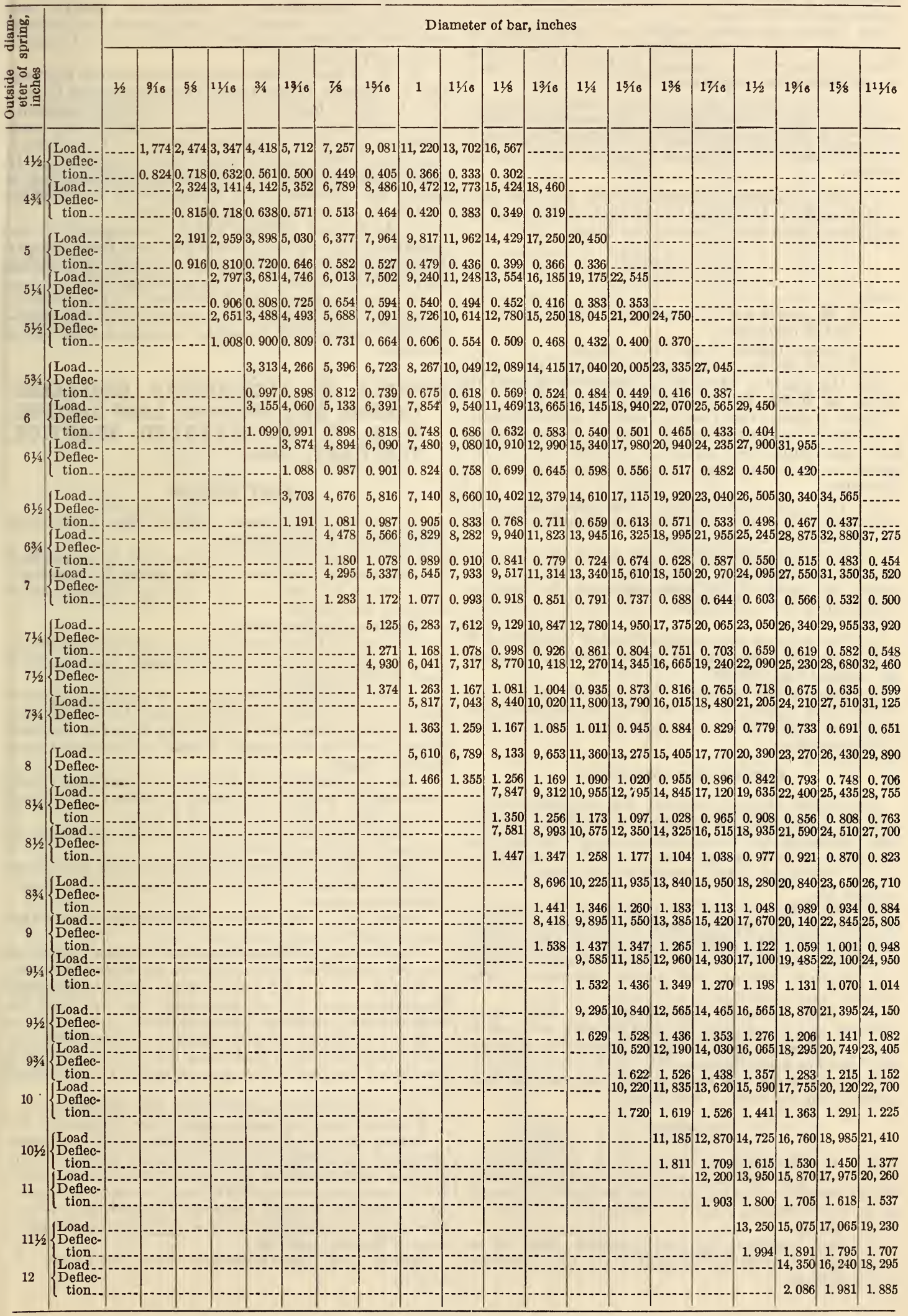


14. To determine whether or not the specified heights fulfill the conditions of section $12(a)$ the calculations described in paragraphs $(a)$ to $(f)$, inclusive, shall be made.

(a) Divide the solid height by the diameter of the bar to obtain the number of turns in the spring. From the figure thus obtained subtract $1 \frac{1}{2}$ to obtain the number of acting turns.

NotE-There is one turn of inactive material at each end of a spring counting from the tip of the tapered bar. In measurements parallel to the axis of the spring, this is equal to one and one-half times the the diameter of the bar, being made up of the two tips of the bar, each one-quarter of the bar diameter in thickness and one full bar diameter.

(b) The deflection corresponding to the load determined by the equation in section 13 shall be calculated from the following equation:

$$
F=\frac{3.1416 S M^{2} N}{G d}
$$

where

$S, M$, and $d$ are the same as in equation (1);

$F=$ the deflection;

$N=$ the number of active turns; and

$G=$ the effective torsional modulus of elasticity.

For the grade of steel herein specified $G$ shall be taken at 10,500,000 pounds per square inch.

Table 1 shows test loads in pounds and deflection per turn in inches for helical springs, corresponding to a maximum fiber stress of 100,000 pounds per square inch for springs 2 to 12 inches in outside diameter.

(c) The sum of the solid height and the total deflection $F$ is the normal free height and should not be exceeded by the free height.

(d) In helical springs the loads and deflections are directly proportional. Therefore, the deflection, $X$, under any load, $P$, less than the capacity shall be determined from the ratio: $C: F:: P: X$ by solving for $X$.

(e) The sum of the specified loaded height and $X$ is the required free height which shall not exceed the normal free height.

(f) Should the specified free height exceed the normal free height or be less thain the required free height or both, the several specified heights are not commensurate.

\section{WOREMANSHIP AND FINISH}

15. Before offering springs for inspection they shall be compressed solid three times. The springs shall be submitted for inspection singly or grouped as may be shown by the drawings and shall conform to the drawings within the tolerances specified in section 11 .

16. (a) The ends of the bars shall be properly tapered to give when coiled a reasonably square, firm bearing. The points of the bars shall be in approximate contact with the adjacent coil and shall not protrude beyond the outside diameter of the springs.

(b) When mutually agreed upon by the manufacturer and purchaser, the end bearing surfaces of the springs shall be ground to produce a uniform bearing of at least three-fourths of the circumference and square with the axis of the helix within oneeighth inch per foot of free length.

\section{MAREING}

17. (a) The name or brand of the manufacturer, the year and month of manufacture and, if specified, the purchaser's class number shall be legibly stamped on each spring at a place not detrimental to the life of the spring.

(b) Any stamping by the inspector shall be so placed as not to be detrimental to the life or service of the spring.

\section{INSPECTION AND REJECTION}

(Conform to the requirements of the American Railway Association for galvanized sheets (see 604.32 , p. 243) in respect to inspection and rehearing, with the following original requirements as to rejection and reworking:)

18 (a) If any of the springs representing a group fail to conform to the requirements specified in section 11, but at least one-half of the springs representing a group do conform to these requirements, each spring of the group shall be tested, and those which conform to the requirements shall be accepted. If more than one-half of the springs representing a group fail to conform to requirements specified in section 11, the group shall be rejected.

(b) Individual springs which, subsequent to inspection at the mills or elsewhere and their acceptance, show defects or imperfections will be rejected and shall be replaced by the manufacturer.

19. Springs which fail to conform to the requirements of the physical tests or conform to the specified dimensions may be again submitted after being reworked.

\section{SOCIETY OF AUTOMOTIVE ENGINEERS,} FRAMES AND SPRINGS; LEAF SPRINGS, S. A. E. RECOMMENDED PRACTICE, FEBRUARY, 1929

\section{LEAF SPRINGS}

All letter dimensions refer to the leaf-spring specification form herewith.

Load.-Load is the force in pounds to be applied by the testing machine to the spring in question. Load is to be read on the testing machine after rapping the spring.

Height.-See dimension $E$.

Clearance.-Clearance is the greatest distance in inches the spring may deflect after the specified load has been applied.

Opening.-See dimension $F$. Where the exposed surface of the main leaf comes below the center line of the spring eyes, when the spring is in the position illustrated, the dimension $F$ shall be expressed as a minus quantity.

Rate (or flexibility).-Rate is one-half the force, measured in pounds, necessary to deflect the spring from $F$ plus 1 inch to $F$ minus 1 inch.

Finish.-Finish refers to the surface condition of the steel after heat treatments.

Finish No. 1.-Leaves shall be left in the same condition as that in which they come from the heattreating process.

Finish No. 2.-All burrs on the bearing sides of the leaves shall be removed.

Finish No. 3. - The sheared and trimmed edges of the leaves shall be chamfered on their bearing sides.

Finish No. 4.-All loose scale shall be removed. Combinations of these numbers may be used to indicate the complete finish desired.

Leaf numbers.-Leaves shall be designated by their numbers, No. 1 leaf being the main leaf; No. 2 leaf being the leaf above and adjacent to it; and so on.

Nominal width of spring steel.-See dimension $G$.

Finished width of spring steel.-See dimension $H$.

Spring ends shall be finished to a width of onesixteenth inch less than the nominal width of the springs, with a plus or minus tolerance of 0.005 inch for passenger cars and 0.010 inch for motor trucks, to a point far enough back on the spring to allow free shackle movement or free sliding movement in case of flat-end springs.

Spring eyes and bushings.--See dimensions $K$ and $L$. 
TOLERANCES ON SPRING EYES AND BUSHINGS

\begin{tabular}{|c|c|c|}
\hline \multirow{2}{*}{ Part } & \multicolumn{2}{|c|}{ Diameter tolerance } \\
\hline & Plus & Minus \\
\hline 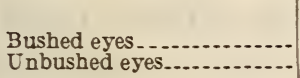 & $\begin{array}{l}\text { Inch } \\
0.001 \\
.001\end{array}$ & $\begin{array}{l}\text { Inch } \\
0.003 \\
.004\end{array}$ \\
\hline
\end{tabular}

The nominal wall thickness of spring-eye bushings shall be one-eighth inch for all sizes of bushing.

\section{LEAF-SPRING STEEI}

Rolling tolerances for automobile concave spring steel.-The finished bars shall be of double-concave section with round edges.
Leaf-spring steel bars shall not have more than 1 inch curvature in 20 feet, or $1 \frac{11}{4}$ inches in 25 feet, or $1 \frac{1}{2}$ inches in 30 feet.

The concavity, or difference between the thickness at the edges and at the center of the bar, shall be as specified in Table 3.

TABLE 3.-Allowable variations in concavity

\begin{tabular}{|c|c|c|c|}
\hline Width & $\begin{array}{l}\text { Nominal } \\
\text { concavity }\end{array}$ & $\begin{array}{l}\text { Maximum } \\
\text { concavity }\end{array}$ & $\begin{array}{l}\text { Minimum } \\
\text { concavity }\end{array}$ \\
\hline $\begin{array}{c}\text { Inches } \\
11 / 2 \\
13 / 4 \\
2 \\
21 / 4 \\
21 / 2\end{array}$ & $\begin{array}{l}\text { Inch } \\
0.007 \\
.008 \\
.010 \\
.011 \\
.013\end{array}$ & $\begin{array}{l}\text { Inch } \\
0.009 \\
.010 \\
.012 \\
.013 \\
.015\end{array}$ & $\begin{array}{l}\text { Inch } \\
0.004 \\
.005 \\
.006 \\
.007 \\
.009\end{array}$ \\
\hline $\begin{array}{l}3 \\
31 / 2 \\
4 \\
5\end{array}$ & $\begin{array}{l}.016 \\
.018 \\
.021 \\
.029\end{array}$ & $\begin{array}{l}.018 \\
.020 \\
.023 \\
.031\end{array}$ & $\begin{array}{l}.012 \\
.013 \\
.016 \\
.023\end{array}$ \\
\hline
\end{tabular}

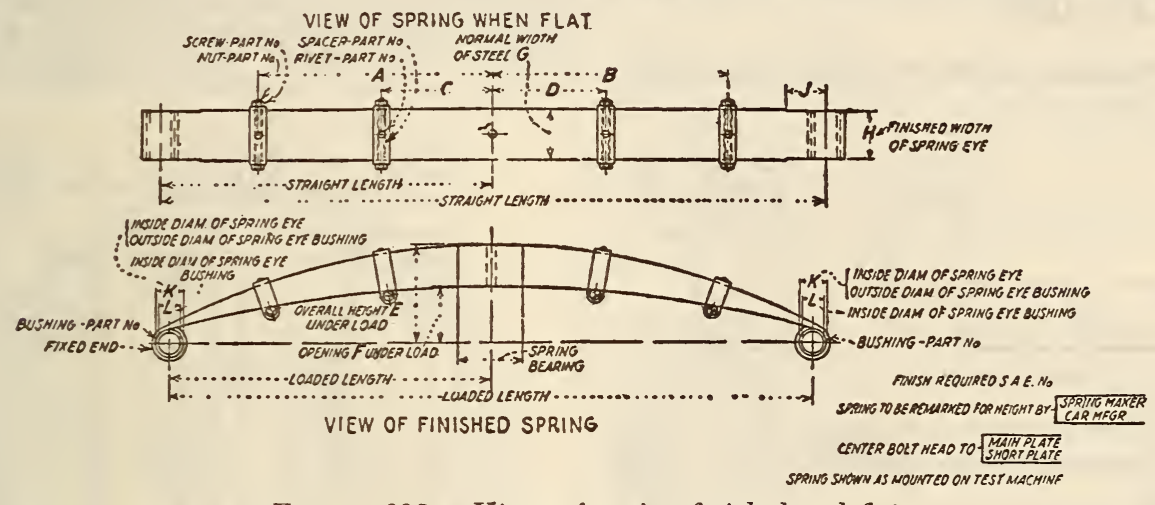

FIGURE 608.-Views of spring finished and flat

TABLE 1.-Width and thickness tolerances

\begin{tabular}{|c|c|c|c|c|c|}
\hline \multicolumn{2}{|c|}{ Width of flat } & \multicolumn{2}{|c|}{ Width } & \multicolumn{2}{|c|}{ Thickness 1} \\
\hline Over- & $\begin{array}{l}\text { To, in- } \\
\text { clus- } \\
\text { ive- }\end{array}$ & Plus & Minus & Plus & Minus \\
\hline $\begin{array}{c}\text { Inches } \\
0 \\
21 / 4 \\
3\end{array}$ & $\begin{array}{c}\text { Inches } \\
21 / 4 \\
3 \\
5\end{array}$ & $\begin{array}{r}\text { Inch } \\
1 / 32 \\
364 \\
1 / 16\end{array}$ & $\begin{array}{r}\text { Inch } \\
0 \\
0 \\
0\end{array}$ & $\begin{array}{l}\text { Inch } \\
0.005 \\
.006 \\
.007\end{array}$ & $\begin{array}{l}\text { Inch } \\
0.005 \\
.006 \\
.007\end{array}$ \\
\hline
\end{tabular}

1 Thickness measurements shall be taken at the edge of the bar where the concave surface intersects the rounded edge.

The radii of the arcs of the two concave surfaces shall be of equal length.

Rolls to produce the round edges shall be turned to a radius equal to two-thirds the thickness of the bar.

All bars ordered to gage shall be rolled to the Birmingham wire gage.

All bars must meet the width and thickness tolerances specified in Table 1 .

The difference in thickness between the two edges of each bar shall not be greater than those given in Table 2.

TABLE 2.-Differences in thickness

\begin{tabular}{|c|c|c|}
\hline \multicolumn{2}{|c|}{ Width of flat } & Difference \\
\cline { 1 - 2 } Over- & $\begin{array}{c}\text { To, inclu- } \\
\text { sive- }\end{array}$ & \\
\hline in thickness \\
\hline Inches & $\begin{array}{c}\text { Inches } \\
0\end{array}$ & $\begin{array}{c}\text { Inch } \\
2\end{array}$ \\
3 & 3 & .002 \\
3 & 5 & .003 \\
\hline
\end{tabular}

LEAF-SPRING TESTS

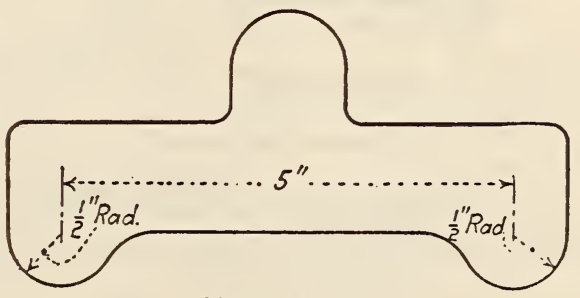

Figure 609

All leaf springs shall be tested in an upright position and supported so as to permit free movement.

All tests or inspection measurements shall be made after rapping the leaf spring in the test or inspection position.

TESTS FOR PARALLELISM OF SPRING EYES

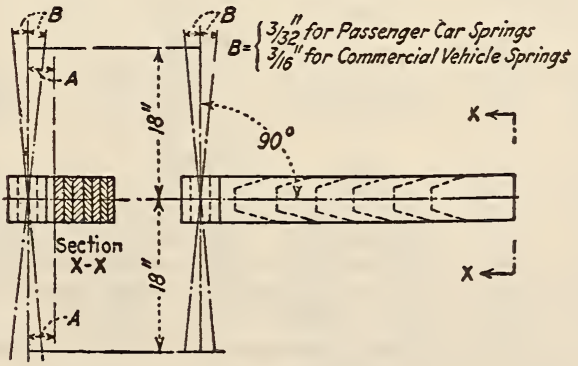

Figure 610 
Eyes of main leaves shall be parallel and square (within specified limits) to the main leaf and parallel to each other.

The test shall be made by inserting two 3-foot bars in the eyes as shown.

SOCIETY OF AUTOMOTIVE ENGINEERS, FRAMES AND SPRINGS ; SPRING CENTERBOLTS, S. A. E. STANDARD; JANUARY, 1931

SPRING CENTER BOLTS

(See 608.31, p. 603.)

SOCIETY OF AUTOMOTIVE ENGINEERS, FRAMES AND SPRINGS; SPRING REBOUND CLIPS, SPACERS, AND BOLTS, S. A. E. RECOMMENDED PRACTICE. JANUARY, 1931

SPRING REBOUND CLIPS, SPACERS, AND BOLTS

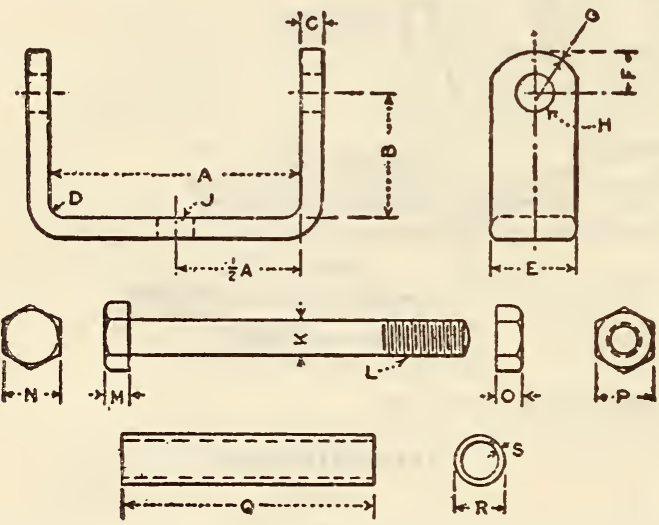

FIGURE 611.-Spring rebound clips, spacers, and

[Dimensions in inches]

\begin{tabular}{|c|c|c|c|}
\hline \multirow{2}{*}{ Dimensions } & \multirow{2}{*}{$\begin{array}{l}\text { Passenger-car } \\
\text { springs (except } \\
\text { cantilevers or } \\
\text { when wider than } \\
21 / 2 \text { inches) }\end{array}$} & \multicolumn{2}{|c|}{$\begin{array}{l}\text { Motor-truck springs (also } \\
\text { cantilevers on passenger cars) }\end{array}$} \\
\hline & & $\begin{array}{l}21 / 1,21 / 2,3 \text { inches } \\
\text { wide }\end{array}$ & $\begin{array}{c}31 / 2 \text { inches wide } \\
\text { and over }\end{array}$ \\
\hline & $\begin{array}{l}3 / 16 \\
1 / 8 \\
3 / 4 \\
3 / 8\end{array}$ & $1_{1 / 2}^{1 / 4}$ & $\begin{array}{c}1 / 4 \\
3 / 16 \\
11 / 4 \\
5 / 8\end{array}$ \\
\hline & $\begin{array}{l}7 / 16 \\
11 / 32\end{array}$ & $\begin{array}{l}5 / 8 \\
13 / 32\end{array}$ & $\begin{array}{l}3 / 4 \\
15 / 32\end{array}$ \\
\hline $\begin{array}{l}\text { I (diameter } \\
\text { rivet) } \\
\mathrm{J} \text { (omit with } \\
\text { inverted } \\
\text { clins) }\end{array}$ & $5 / 16$ & $3 / 8$ & $3 / 8$ \\
\hline $\begin{array}{l}\mathrm{L}_{1}^{1} \\
\mathrm{M}-\end{array}$ & $\begin{array}{l}5 / 16 \\
5 / 16-18 \\
7 / 32 \\
1 / 2\end{array}$ & $\begin{array}{l}3 / 8 \\
3 / 8 \\
7,32 \\
9 / 16\end{array}$ & $\begin{array}{l}7 / 16 \\
7 / 16-14 \\
25 / 64 \\
25 / 32\end{array}$ \\
\hline S $(\mathrm{B} . \mathrm{w} . \mathrm{g})$. & $\begin{array}{c}7 / 32 \\
1 / 2 \\
7 / 6 \\
\text { Not less than } 20 \\
\text { (0.035 inch). }\end{array}$ & $\begin{array}{c}7 / 32 \\
9 / 16 \\
1 / 2 \\
18 \text { to } 20(0.049 \text { to } \\
0.035 \text { inch). }\end{array}$ & $\begin{array}{c}7 / 16 \\
35 / 32 \\
5 / 8 \\
15 \text { to } 17^{5 / 8}(0.072 \\
\text { to } 0.058 \text { inch). }\end{array}$ \\
\hline
\end{tabular}

1 Threads to be American standard (NC) coarse, class 2 fit, length $11 / 2 \mathrm{~K}+1 / 4$ inch, with points rounded.

A To be $1 / 32$ to $1 / 16$ inch greater than nominal width of spring. B To be made to suit spring.

$Q$ To be $1 / 32$ inch greater than nominal width of spring.

Clearance between the rebound clip bolts (for spacer tubes if used) and spring plates shall not exceed $3 / 32$ inch for passenger cars or $3 / 16$ inch for motor trucks.
611.55 PINIONS AND GEARS.

AMERICAN ELECTRIC RAILWAY ENGINEERING ASSOCIATION, STANDARD WEB HOLES FOR RAILWAY GEARS, E15-24, 1924

\section{WEB HOLES FOR RAILWAY GEARS}

The number of web holes in railway gears shall be four.

(In addition to the above statement this specification consists of an extended table which contains the outside diameter in inches-the diameters ranging in size from 15.75 to 31.20 inches-supplemented by data on number of teeth, diametral pitch, width of face, thickness of rim, and hub diameter. Also diameters of holes and distance between hole centers, and the identifying pattern numbers assigned to these gears by the manufacturing concerns in the Pittsburgh district engaged in gear manufacturing. The following table contains a summary showing the number of railway-gear blanks for various web-hole diameters and centers:)

\begin{tabular}{|c|c|c|c|c|c|}
\hline $\begin{array}{l}\text { Diam- } \\
\text { eter of } \\
\text { holes }\end{array}$ & $\begin{array}{c}\text { Hole } \\
\text { centers }\end{array}$ & $\begin{array}{l}\text { Num- } \\
\text { ber of } \\
\text { blanks }\end{array}$ & $\begin{array}{l}\text { Diam- } \\
\text { eter of } \\
\text { holes }\end{array}$ & $\begin{array}{l}\text { Hole } \\
\text { centers }\end{array}$ & $\begin{array}{l}\text { Num- } \\
\text { ber of } \\
\text { blenks }\end{array}$ \\
\hline $\begin{array}{l}\text { Inches } \\
1 \\
11 / 2 \\
11 / 2 \\
11 / 2 \\
2 \\
2 \\
2 \\
2 \\
2 \\
2 \\
2 \\
2 \\
2 \\
21 / 2 \\
21 / 2 \\
21 / 2 \\
21 / 2 \\
21 / 2 \\
21 / 2\end{array}$ & $\begin{array}{l}\text { Inches } \\
17 \\
10 \\
111 / 2 \\
171 / 2 \\
10^{3 / 4} \\
111 / 2 \\
121 / 2 \\
13 \\
141 / 2 \\
151 / 2 \\
16 \\
17 \\
18 \\
111 / 2 \\
12 \\
121 / 2 \\
131 / 2 \\
14 \\
141 / 2\end{array}$ & $\begin{array}{l}1 \\
2 \\
1 \\
1 \\
2 \\
1 \\
2 \\
2 \\
7 \\
1 \\
1 \\
1 \\
1 \\
2 \\
1 \\
1 \\
3 \\
1 \\
3\end{array}$ & $\begin{array}{c}\text { Inches } \\
21 / 2 \\
21 / 2 \\
21 / 2 \\
21 / 2 \\
21 / 2 \\
3 \\
3 \\
3 \\
31 / 2 \\
31 / 2 \\
31 / 2 \\
31 / 2 \\
31 / 2 \\
31 / 2 \\
31 / 2 \\
31 / 2 \\
31 / 2 \\
31 / 2 \\
31 / 2\end{array}$ & $\begin{array}{l}\text { Inches } \\
15 \\
16 \\
161 / 2 \\
17 \\
18 \\
12 \\
141 / 2 \\
16 \\
111 / 2 \\
13 \\
14 \\
141 / 2 \\
15 \\
16 \\
17 \\
171 / 2 \\
18 \\
181 / 2 \\
19\end{array}$ & $\begin{array}{r}1 \\
5 \\
1 \\
3 \\
1 \\
1 \\
1 \\
1 \\
1 \\
2 \\
4 \\
28 \\
9 \\
7 \\
4 \\
2 \\
2 \\
2 \\
1\end{array}$ \\
\hline
\end{tabular}

\section{AMERICAN ELECTRIC RAILWAY ENGI- NEERING ASSOCIATION, RECOMMENDED SPECIFICATION FOR CASEHARDENED FORGED STEEL AND QUENCHED AND TEMPERED FORGED CARBON-STEEL GEARS, E117-29, 1929}

\section{CARBON-STEEL GEARS}

\section{MANUFACTORE}

101. Material.-All blanks for gears shall be made from open-hearth steel which has been worked thoroughly to secure a homogeneous dense material, free from all injurious defects.

\section{CHEMICAL PROPERTLES AND TESTS}

201. Chemical properties.-The steel shall conform to the following requirements as to chemical composition:

\begin{tabular}{|c|c|c|}
\hline Element & $\begin{array}{l}\text { Quenched and tem. } \\
\text { pered forged carbon } \\
\text { steel }\end{array}$ & $\begin{array}{l}\text { Casehardened forged } \\
\text { steel }\end{array}$ \\
\hline $\begin{array}{l}\text { Phosphorus..... } \\
\text { Sulphur.......-. } \\
\text { Carbon-...-. } \\
\text { Manganese...... }\end{array}$ & $\begin{array}{l}\text { Not over } 0.05 \text { per cent. } \\
\text { do }\end{array}$ & $\begin{array}{l}\text { Not over } 0.05 \text { per cent. } \\
\text { Do. } \\
0.20 \text { per cent-not less } \\
\text { than } 0.12 \text { per cent nor } \\
\text { more than } 0.28 \text { per cent. } \\
0.50 \text { per cent - not less } \\
\text { than } 0.40 \text { per cent nor } \\
\text { more than } 0.60 \text { per cent. }\end{array}$ \\
\hline
\end{tabular}


These specifications cover a medium carbon steel, heat treated. It is not thought desirable to bind manufacturers specifically as to chemical composition for the reason that different heat treatments can be used for different analyses.

202. Check analysis. - A check analysis may be made by the purchaser or his representative from one or more gear blanks from each lot of 100 or fraction thereof ordered, and this analysis shall conform to the requirements specified above. The sample for check analysis is to be taken at the pitch line so that the blank will not be destroyed.

\section{PHYSICAL PROPERTIES AND TESTS (FOR CASE HARDENED FORGED STEEL GEARS ONLY)}

301. Hardness. - The hardness of casehardened forged-steel gears as shown by the scleroscope shall not be less than 80 , taken at the center of the top of the tooth after treatment.

302. Treatment.-All casehardened forged-steel gears, after the teeth are cut, shall be carbonized to a depth approximately one-sixth of the thickness of the teeth on the pitch line.

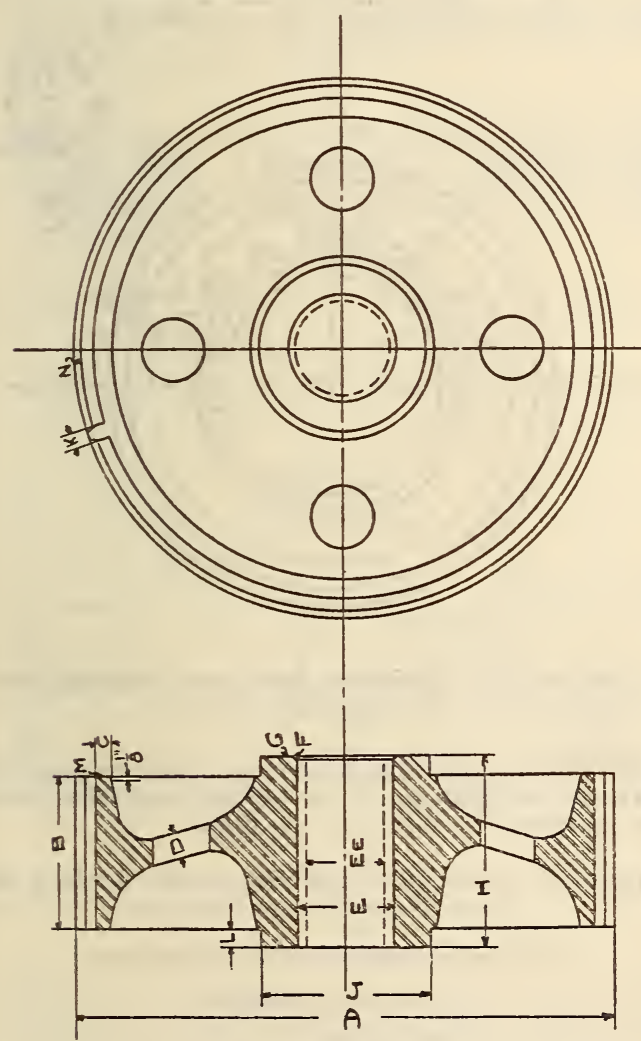

FigURE 612.-Reference letters for gear dimensions

FINISH

401. Dimensions.-(a) The outside diameter $(A)$ over the teeth as machined must not vary from that specified by more than plus 0 inch nor minus one thirty-second inch.

$(b)$ The face $(B)$ of the gears must not vary from the specified width by more than plus one thirtysecond inch nor minus one thirty-second inch. (c) The minimum thickness of the rim (C) under the teeth shall be as follows, measured one-eighth

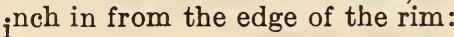

\begin{tabular}{|c|c|}
\hline Pitch & $\begin{array}{c}\text { Thickness } \\
\text { of rim }\end{array}$ \\
\hline & Inch \\
2 & $1 / 2$ \\
$21 / 4$ & $15 / 32$ \\
$21 / 2$ & $7 / 6$ \\
3 & 386 \\
$31 / 2$ & $3 / 8$ \\
4 & $31 / 8$ \\
$41 / 4$ & $3 / 8$ \\
\hline
\end{tabular}

402. Web.-The web $(D)$ of all gears shall have four holes. The diameter and radius on which they are spaced shall be in accordance with the requirements of E15, referred to above.

403. Bore.-(a) The diameter for the bore of gears with hard centers shall be 0.001 inch per inch of diameter under that specified, and 0.0015 inch per inch of diameter for gears with soft centers to allow for press fit.

(b) The permissible variation from the finish bore shall be plus 0.001 inch and minus 0.001 inch.

(c) The diameter of rough bore $(E e)$ shall not vary more than one-sixteenth inch over nor one-eighth inch under that specified.

(d) The ends of finished bores shall be chamfered $(F)$ one-sixteenth inch on motor side to avoid injury to shaft when mounting.

(e) Bores shall be measured with pin gage or inside micrometer.

404. Hub.- (a) The face $(G)$ of hub $(H)$ next to the lining is to have a smooth bearing finish and run true with the bore.

(b) The variation from the specified dimensions of hub $(H)$ and hub extension $(L)$ shall not exceed the following:

Length of hub $(H)$ over all, plus 0 inch to minus 0.02 inch.

Length of hub extension $(L)$, plus one thirtysecond inch to minus one thirty-second inch.

Diameter of hub extension $(J)$, plus 0 inch to minus 0.03 inch

405. Teeth.- (a) The thickness $(K)$ of the teeth at the pitch line must be to specified dimensions as a maximum or to specified dimensions minus 0.010 inch as a minimum.

The teeth, unless otherwise specified, shall be of one of the following three forms: (a) Brown \& Sharpe standard $1412^{\circ}$ involute; $(b)$ long and short addendum spur as shown in Table $\mathrm{A}$, and (c) long and short addendum helical as shown in Table $B$. For new equipment, the use of tooth form $(b)$ or (c) is recommended.

TABLE A.-Tooth data and dimensions for long and short addendum spur gearing

[Gears (short addendum), $20^{\circ}$ pressure angle]

[Dimensions in inches]

\begin{tabular}{|c|c|c|c|c|c|c|}
\hline $\begin{array}{l}\text { Diametrical } \\
\text { pitch }\end{array}$ & $\begin{array}{l}\text { Circu- } \\
\text { lar } \\
\text { pitch }\end{array}$ & $\begin{array}{l}\text { Thick- } \\
\text { ness of } \\
\text { tooth on } \\
\text { pitch } \\
\text { line, } 42 \\
\text { per cent } \\
\text { circular } \\
\text { pitch }\end{array}$ & $\begin{array}{l}\text { Adden- } \\
\text { dum } \\
=\frac{0.654}{D . P .}\end{array}$ & $\begin{array}{l}\text { Deden- } \\
\text { dum } \\
=\frac{1.5031}{D . P .}\end{array}$ & $\begin{array}{l}\text { Working } \\
\text { depth of } \\
\text { tooth } \\
=\frac{2}{D . P .}\end{array}$ & $\begin{array}{l}\text { Whole } \\
\text { depth of } \\
\text { tooth } \\
=\frac{2.1571}{D \cdot P .}\end{array}$ \\
\hline $\begin{array}{l}2 \\
21 / 4 \ldots \\
21 / 2 \ldots \\
3 \\
31 / 2 \ldots \\
4 \\
41 / 2\end{array}$ & $\begin{array}{r}1.5708 \\
1.3963 \\
1.2566 \\
1.0472 \\
.8976 \\
.7854 \\
.7392\end{array}$ & $\begin{array}{r}0.6597 \\
.5864 \\
.5278 \\
.4398 \\
.3770 \\
.3299 \\
.3105\end{array}$ & $\begin{array}{r}0.3270 \\
.2906 \\
.2616 \\
.2180 \\
.1868 \\
.1635 \\
.1539\end{array}$ & $\begin{array}{r}0.7515 \\
.6681 \\
.6012 \\
.5010 \\
.4295 \\
.3758 \\
.3537\end{array}$ & $\begin{array}{r}1.0000 \\
.8888 \\
.8000 \\
.6667 \\
.5714 \\
.5000 \\
.4706\end{array}$ & $\begin{array}{r}1.0785 \\
.9587 \\
.8628 \\
.7190 \\
.6163 \\
.5393 \\
.5076\end{array}$ \\
\hline
\end{tabular}


TABLE B.-Tooth data and dimensions for long and short addendum helical gearing

Gear (short addendum)]

[Dimensions in inches]

\begin{tabular}{|c|c|c|c|c|c|c|c|}
\hline $\begin{array}{l}\text { Diametrical } \\
\text { pitch }\end{array}$ & $\begin{array}{l}\text { Circu- } \\
\text { lar } \\
\text { pitch }\end{array}$ & $\begin{array}{l}\text { Tooth } \\
\text { thick- } \\
\text { ness on } \\
\text { pitch } \\
\text { line arc }\end{array}$ & $\begin{array}{l}\text { Adden- } \\
\text { dum }\end{array}$ & $\begin{array}{l}\text { Deden- } \\
\text { dum }\end{array}$ & $\begin{array}{l}\text { Work- } \\
\text { ing } \\
\text { depth } \\
\text { of } \\
\text { tooth }\end{array}$ & $\begin{array}{l}\text { Whole } \\
\text { depth } \\
\text { of } \\
\text { tooth }\end{array}$ & $\begin{array}{l}\text { Pres- } \\
\text { sure } \\
\text { angle } \\
\text { (de- } \\
\text { grees) }\end{array}$ \\
\hline $1 / 4 \ldots$ & $\begin{array}{r}1.5708 \\
1.3963 \\
1.2566 \\
1.0472 \\
.8976 \\
.7854 \\
.7392\end{array}$ & $\begin{array}{r}0.6854 \\
.5864 \\
.5446 \\
.4561 \\
.3876 \\
.3354 \\
.3146\end{array}$ & $\begin{array}{r}0.3110 \\
.2906 \\
.2420 \\
.1750 \\
.1643 \\
.1700 \\
.1264\end{array}$ & $\begin{array}{r}0.6540 \\
.6681 \\
.5560 \\
.4530 \\
.4000 \\
.3750 \\
.3300\end{array}$ & $\begin{array}{r}0.8400 \\
.8887 \\
.6980 \\
.5280 \\
.4786 \\
.4700 \\
.3858\end{array}$ & $\begin{array}{r}0.9650 \\
.9587 \\
.7980 \\
.6280 \\
.5643 \\
.5450 \\
.4564\end{array}$ & $\begin{array}{l}20 \\
20 \\
20 \\
201 / 2 \\
201 / 2 \\
21 \\
211 / 2\end{array}$ \\
\hline
\end{tabular}

NoTE.-Theoretical dimensions do not allow for backlash.

\section{MARKING}

501. Marking.-All gears shall be plainly stamped on motor side of $\operatorname{rim}(M)$ as follows:

Grade

Month, year, serial number of manufacturer consecutive for each month.

Name, initials, or trade-mark of manufacturer. Long and short addendum spur gearing shall be distinguished from standard addendum by two grooves around the periphery of each gear and pinion.

\section{INSPECTION AND REJECTION}

601. Inspection.-(a) All gears shall be tested for smooth running. The teeth must be equally spaced so that the gear will run smoothly in both directions with a master pinion.

(b) Records of all chemical analyses and physical tests shall be kept by the manufacturer and shall be available to the purchaser for a period of one year.

602. Rejection.- The purchaser reserves the right to reject any portion of or all the material which does not conform to the above specifications in every particular.

\section{AMERICAN ELECTRIC RAILWAY ENGI- NEERING ASSOCIATION, RECOMMENDED SPECIFICATION FOR CASEHARDENED FORGED STEEL AND QUENCHED AND TEMPERED FORGED CARBON-STEEL PINIONS, E118-29, 1929}

\section{CARBON-STEEL PINIONS}

(This specification conforms to A. E. R. E. A., E117, given above, except in the following particulars:)

403. Bore.-(a) The taper for the bore of pinions shall be $1 \frac{1}{4}$ inches on the diameter per 1 foot in length.

(b) All bores $(C-E)$ must be finished after treatment. The diameter of the bore must be such that the standard plug gage (see section $601(b)$ ) will not project less than one-thirty-second inch nor more than one-sixteenth inch, measured at the large end of the bore $(C)$ and have bearing the full length $(D)$ of bore $(C-E)$.

404. Counterbore.- $(a)$ The depth $(H)$ of the counterbore must not vary from that specified by more than plus 0 inch nor minus one-thirty-second inch.

(b) The diameter $(F)$ of the counterbore must not vary from that specified by more than plus onethirty-second inch nor minus 0 inch.

405. Keyway.- (a) The sides $(G)$ of the keyway must be cut parallel with the center line of the pinion.

(b) The width $(L)$ of the keyway must not vary from that specified by more than plus 0.003 inch nor minus 0 inch. (c) The depth $(P)$ of the keyway must not vary from that specified by more than plus one-sixtyfourth inch nor minus 0 inch.

(d) The fillet $(J)$ at the bottom of the keyway may be a minimum of one-sixty-fourth inch radius.

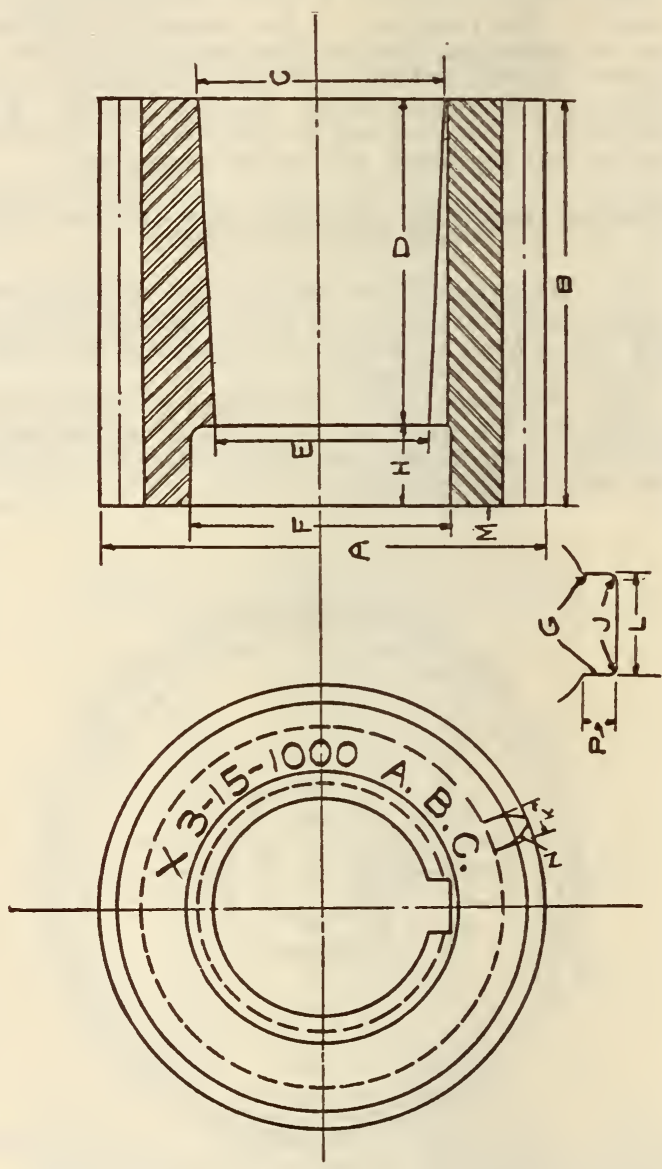

FIGURE 613.--Reference letters and marking for pinions

With this specification, a clearance shall be provided between the bottom of the keyway and the pinion key equal to the radius of the fillet.

TABLE A.-Tooth data and dimensions for long and short addendum spur gearing

[Pinions (long addendum) $20^{\circ}$ pressure angle]

[Dimensions in inches]

\begin{tabular}{|c|c|c|c|c|c|c|}
\hline $\begin{array}{l}\text { Diametrical } \\
\text { pitch }\end{array}$ & $\begin{array}{c}\text { Circular } \\
\text { pitch }\end{array}$ & $\begin{array}{l}\text { Thick- } \\
\text { ness of } \\
\text { tooth } \\
\text { on } \\
\text { pitch } \\
\text { line, } \\
58 \text { per } \\
\text { cent } \\
\text { circular } \\
\text { pitch }\end{array}$ & $\begin{array}{c}\begin{array}{c}\text { Adden- } \\
\text { dum } \\
1.346\end{array} \\
=\frac{P . P}{}\end{array}$ & $\begin{array}{l}\begin{array}{c}\text { Deden- } \\
\text { dum } \\
0.8111\end{array} \\
=\frac{P . P .}{D .}\end{array}$ & $\begin{array}{l}\text { Work- } \\
\text { ing } \\
\text { depth } \\
\text { of } \\
\text { tooth } \\
=\frac{2}{D \cdot P .}\end{array}$ & $\begin{array}{l}\text { Whole } \\
\text { depth } \\
\text { of } \\
\text { tooth } \\
=\frac{2.1571}{D . P .}\end{array}$ \\
\hline $\begin{array}{l}\text { Same as for gea } \\
\text { given above. }\end{array}$ & n E117, & $\left\{\begin{array}{l}0.9111 \\
.8099 \\
.7288 \\
.6074 \\
.5206 \\
.4555 \\
.4287\end{array}\right.$ & $\begin{array}{r}0.6730 \\
.5982 \\
.5384 \\
.4487 \\
.3846 \\
.3365 \\
.3167\end{array}$ & $\begin{array}{r}0.4055 \\
.3605 \\
.3244 \\
.2703 \\
.2317 \\
.2028 \\
.1909\end{array}$ & \multicolumn{2}{|c|}{$\begin{array}{l}\text { Same as for } \\
\text { gears in E117, } \\
\text { given above. }\end{array}$} \\
\hline
\end{tabular}


TABLE B.-Tooth data and dimensions for long and short addendum helical gearing

[Pinion (long addendum)]

[Dimensions in inches]

\begin{tabular}{|c|c|c|c|c|c|c|c|}
\hline $\begin{array}{l}\text { Diametrical } \\
\text { pitch }\end{array}$ & $\begin{array}{l}\text { Circu- } \\
\text { lar } \\
\text { pitch }\end{array}$ & $\begin{array}{l}\text { Tooth } \\
\text { thick- } \\
\text { ness on } \\
\text { pitch } \\
\text { line } \\
\text { arc }\end{array}$ & $\begin{array}{l}\text { Ad- } \\
\text { den- } \\
\text { dum }\end{array}$ & $\begin{array}{l}\text { De- } \\
\text { den- } \\
\text { dum }\end{array}$ & $\begin{array}{l}\text { Work- } \\
\text { ing } \\
\text { depth } \\
\text { of } \\
\text { tooth }\end{array}$ & $\begin{array}{c}\text { Whole } \\
\text { depth } \\
\text { of } \\
\text { tooth }\end{array}$ & $\begin{array}{l}\text { Pres- } \\
\text { sure } \\
\text { angle }\end{array}$ \\
\hline $\begin{array}{l}\text { Same as for } \\
\text { E117, given }\end{array}$ & $\begin{array}{l}\text { ears in } \\
\text { above. }\end{array}$ & $\left\{\begin{array}{r}0.8854 \\
.8099 \\
.7120 \\
.5911 \\
.5100 \\
.4500 \\
.4246\end{array}\right.$ & $\begin{array}{r}0.5290 \\
.5982 \\
.4560 \\
.3530 \\
.3143 \\
.3000 \\
.2594\end{array}$ & $\begin{array}{r}0.4360 \\
.3605 \\
.3420 \\
.2750 \\
.2500 \\
.2450 \\
.1970\end{array}$ & $\begin{array}{r}0.8400 \\
.8887 \\
.6980 \\
.5280 \\
.4786 \\
.4700 \\
.3858\end{array}$ & \multicolumn{2}{|c|}{$\begin{array}{l}\text { Same as for } \\
\text { gears in } \\
\text { E117, given } \\
\text { above. }\end{array}$} \\
\hline
\end{tabular}

NotE.-Theoretical dimensions do not allow for backlash.

601. Finish.-(b) All pinions shall be gaged with a standard taper plug gage which shall be the same size as the nominal size of the bore at the large end.

\section{INSPECTION AND REJECTION}

701. Inspection.-All pinions shall be free from any seams, cracks, or other defects that would in any way affect their service.

\section{AMERICAN SOCIETY OF MECHANICAL EN- GINEERS, AMERICAN TENTATIVE STANDARD SPUR-GEAR TOOTH FORM, $\mathrm{B} 6 \mathrm{~b}-1927$}

[Approved by American Standards Association, January, 1927.]

\section{SPUR-GEAR TOOTH FORM}

FO URTEEN AND ONE-HALF DEGREE COMPOSITE SYSTEM

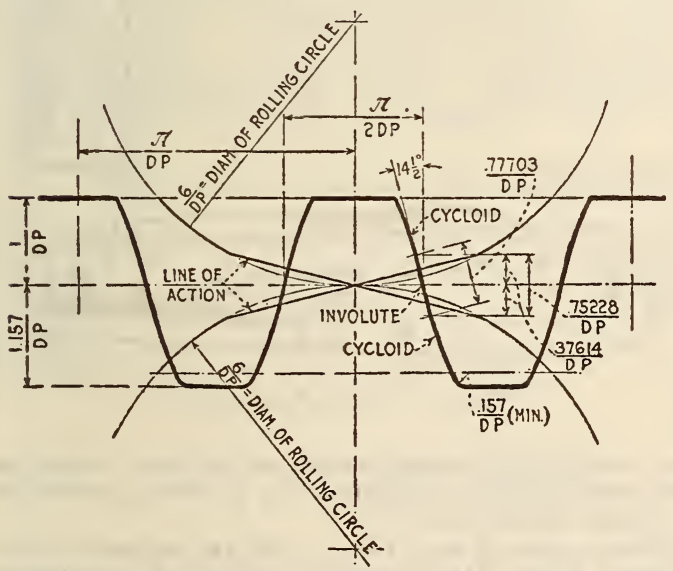

Figure 614.-Basic rack for $14^{1 / 2^{\circ}}$ composite system (full depth tooth)

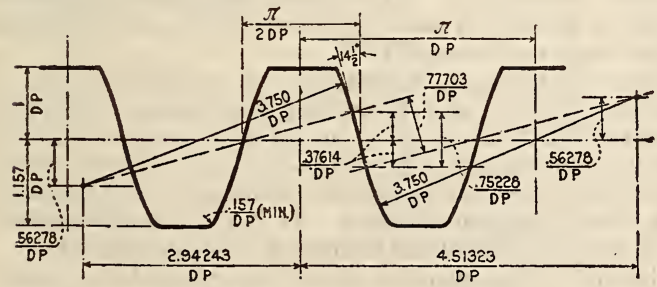

FIGURE 615.-Approximation to basic rack for $14^{1 / 2^{\circ}}$ composite system (full depth tooth)

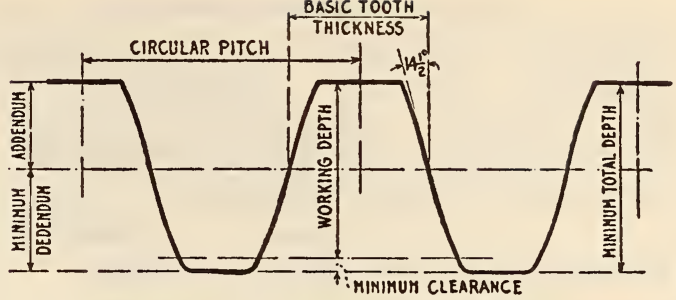

FigURE 616.-Full depth tooth proportions for spur gears

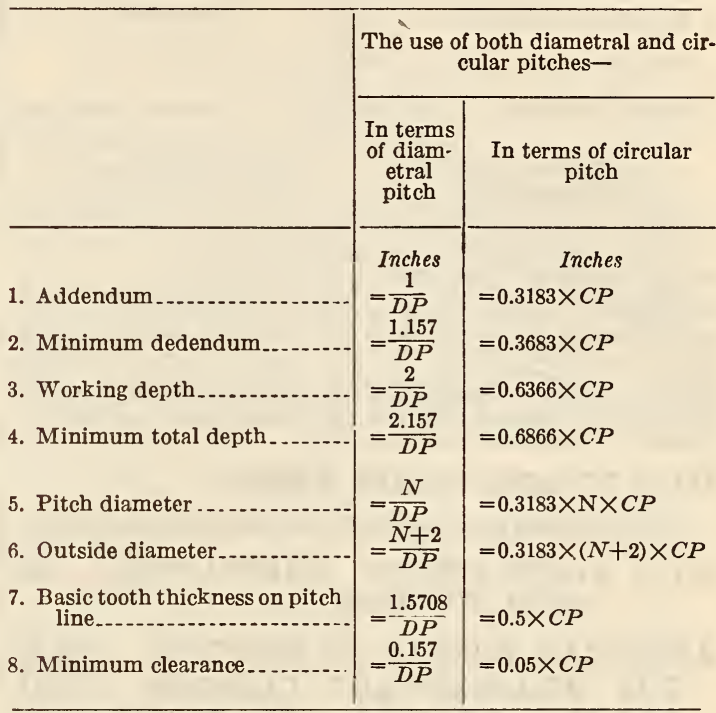

$N=$ Number of teeth.

$D P=$ Diametral pitch.

$C P=$ Circular pitch.

NOTE. - The term diametral pitch is used up to $1 D P$ inclusive and the term circular pitch is used for 3 inches $C P$ and over.

NoTs.-A suitable working tolerance should be considered in connection with all minimum recommendations.

note - Minimum clearance refers to the clearance between the top of the gear tooth and the bottom of the mating gear space, and is specified as "minimum" so as to allow for necessary cutter clearance for all methods of producing gears. At the present time this value can not be standardized.

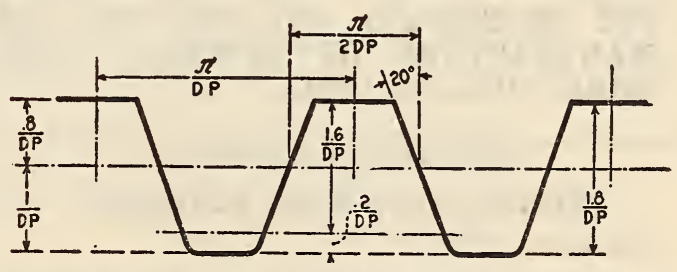

Figure 617.-Basic rack for $20^{\circ}$ stub involute system for spur gears

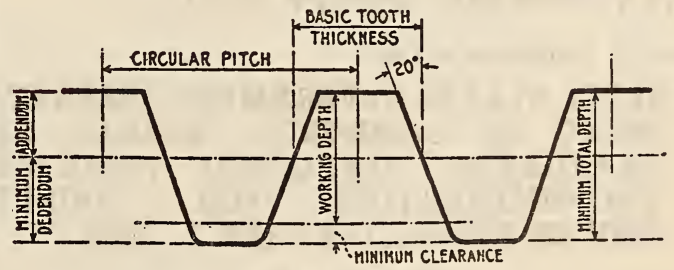

Figure 618.-Proportions for spur gears 
Proportions for spur gears

\begin{tabular}{|c|c|c|}
\hline \multirow[t]{2}{*}{-} & \multicolumn{2}{|c|}{$\begin{array}{l}\text { The use of both diametral and cir- } \\
\text { cular pitches- }\end{array}$} \\
\hline & $\begin{array}{l}\text { In terms } \\
\text { of diam- } \\
\text { etral } \\
\text { pitch }\end{array}$ & $\begin{array}{l}\text { In terms of circular } \\
\text { pitch }\end{array}$ \\
\hline & Inches & Inches \\
\hline 1. Addendum ....... & $=\frac{0.8}{D P}$ & $=0.2546 \times C P$ \\
\hline 2. Minimum dedendum... & $=\frac{1}{D P}$ & $=0.3183 \times C P$ \\
\hline 3. Working depth... & $=\frac{1.6}{D P}$ & $=0.5092 \times C P$ \\
\hline 4. Minimum total depth. & $=\frac{1.8}{D P}$ & $=0.5729 \times C P$ \\
\hline 5. Pitch diameter.. & $=\frac{N}{D P}$ & $=0.3183 \times N \times C P$ \\
\hline 6. Outside diameter.- & $=\frac{N+1.6}{D P}$ & $=P D+(2$ addendums $)$ \\
\hline
\end{tabular}

$N=$ Number of teeth.

$D P=$ Diametral pitch

$P D=$ Pitch diameter.

$C P=$ Circular pitch.

Note.-The term diametral pitch is used up to $1 D P$ inclusive and the term circular pitch is used for 3 inches $C P$ and over.

Note.-These proportions are identical with those of the A. G. M. A. recommended practice for herringbone gears.

Note.-A minimum root clearance of 0.2 inch/diametral pitch is recommended for new cutters and gears. There is correct tooth action, however, between gears cut to this standard system and those cut to the Nuttall system, the only dimension affected being the clearance. Where the proposed gear tooth meshes with a Nuttall gear space there is a clearance of $0.1425 \mathrm{inch} /$ diametral pitch, and where the Nuttall tooth runs with the proposed gear space there is a clearance of 0.2146 inch/diametral pitch.

\subsection{TURBINE BUCKET WHEELS.}

(No nationally recognized specifications available.)

611.59 MISCELLANEOUS MANUFACTURES OF STEEL FORGINGS.

AMERICAN BUREAU OF SHIPPING, RULES FOR BUILDING AND CLASSING STEEL VESSELS; QUALITY AND TESTING OF MATERIAIS-SHIP CONSTRUCTION, 1930

\section{INGOT-STEEL ANCHORS}

(For genelal iniormation and requirements see 600.0, p. 2 . Specifications are the same as for forged wrought-iron anchors. (See 611.3, p. 704.)

\section{AMERICAN ELECTRIC RAILWAY ENGIN- EERING ASSOCIATION, SPECIFICATION FOR MATERIALS FOR USE IN THE MANUFACTURE OF SPECIAL TRACK WORK, W104-28, 1928}

[Approved as American tentatice standard by the American Standards Association, January 23, 1929]

\section{SPECIAL TRACK-WORK MATERIALS}

(See 606.3, p. 342.)

612. CUTLERY, HOLIOW WARE, AND OTHER HOUSEHOLD UTENSILS.

612.1 CUTLERY, EXCEPT TOOLS.

612.11 POCKETKNIVES.

UNITED STATES GOVERNMENT, DEPARTMENT OF COMMERCE, BUREAU OF STANDARDS; SIMPLIFIED PRACTICE RECOMMENDATION FOR POCKET KNIVES, R99-30, JANUARY 1, 1930

\section{POCKETKNIVES}

On November 13,1928, a general conference of representative manufacturers, distributors, and users of pocketknives drafted a simplified-practice recommendation. The industry has since adopted, and approved for promulgation by the Department of Commerce, this recommendation which limits the varieties of pocketknives to those described herein.

The basis of this simplification is the shape and size of the haft or handle which are governed by the basic die. To aid in identification, 140 illustrations referred to are shown as complete knives in the Pocket Knife Manufacturers Standard Catalogue. The haft of these knives represent the shape and size of 140 basic dies.

Special attention is drawn to the fact that the covers, as well as the style of blade, size of blade, and number of blades, are shown only to aid in identifying the hafts. The dimension indicated beneath each illustration represents the length of the haft. It should be remembered that the shape and size of the haft is to be considered only as a means of identifying the pertinent approved basic die.

It is permissible for a pocketknife manufacturer to fit any standard haft, any required type of blade, or

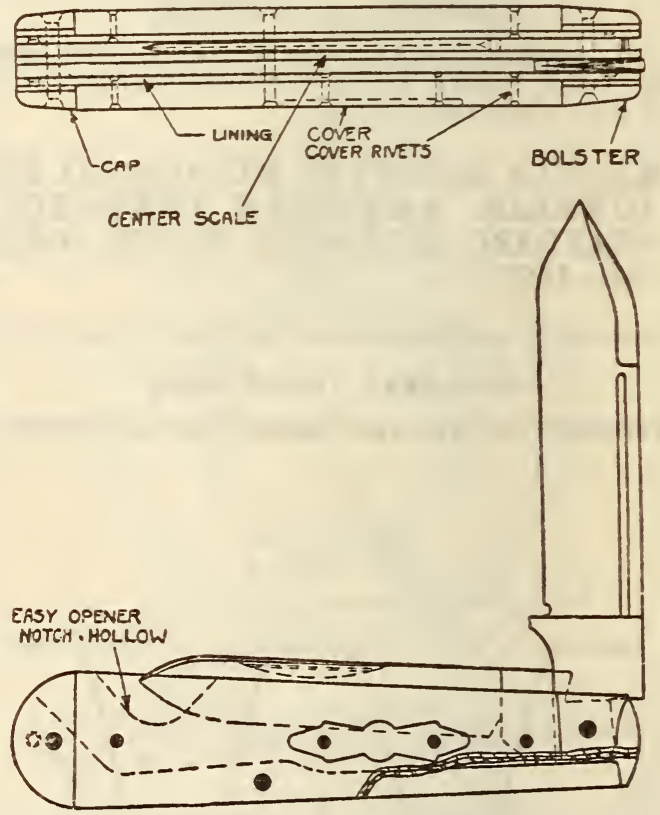

FiguRe 619.-Pocketknife

any number of blades, so long as the total variety in the line of knives produced by that manufacturer does not exceed 500 .

One hundred and forty cuts are shown in the Pocket Knife Manufacturers Standard Catalogue, but it should be noted that any one manufacturer is limited to 107 basic dies, selected from the abovenamed catalogue. Moreover, any one manufacturer is allowed 5 basic dies not shown, provided his total does not exceed 107.

The haft-length tolerances or deviations are as follows: Up to and including $3 \frac{5}{8}$ inches in length, one-sixteenth inch, plus or minus; longer than $3 \%$ inches, one-eighth inch, plus or minus. These deviations are allowed in order to accommodate the existing dies of manufacturers. It is further recognized that proportionate deviations in widths will occur.

Item 1. The number of styles or numbers should not exceed 500 knives in any one line; different colors of celluloid to constitute separate numbers.

Item 2. One hundred and seven basic dies shall not be exceeded in any one manufacturer's line. 
Item 3. Fifteen standard colors of celluloid with 10 additional optional shall not be exceeded, these colors to be revised as necessary.

Item 4. The number of celluloid-handled knives in any manufacturer's line shall not exceed 200 .

Item 5. The number of pearl knives in any manufacturer's line shall not exceed 75 .

Item 6. Nickel-silver bolsters not sanctioned on pruners, barlows, and stabbers.

Item 7. Steel linings only sanctioned on pruners, barlows, and stabbers.

Item 8. Brass or nickel-silver linings sanctioned on all numbers except pruners, barlows, and stabbers.

Item 9. Black insides sanctioned only on pruners, barlows, stabbers, and such other patterns as may be developed from the stabber category; that is, basic dies Nos. 16 and 33 of the Pocket Knife Manufacturers Standard Catalogue.

Item 10. Pruners, barlows, and stabbers should have glaze-finished blades only.

Item 11. The catalogue shows illustrations of hafts or handles with or without easy-opener notch hollows, and these handles may be made either way without being considered as a deviation from the basic dies, each constituting a separate number.

Item 12. The 15 standard colors of celluloid are as follows:

White.

Red.

Black.

Shell.

Candy stripe.

Red horn.

Buffalo horn.

Gray horn.

Tinsel.

Ultra pearl.

Golden pearl.

Abalone pearl.

Green pearl.

Blue pearl.

Smoke pearl.

\subsection{RAZORS.}

UNITED STATES GOVERNMENT, DEPARTMENT OF COMMERCE, BUREAU OF STANDARDS, SIMPLIFIED PRACTICE RECOMMENDATION FOR PACKAGING OF RAZOR BLADES, R69, JULY 1, 1928

\section{PACKAGING OF RAZOR BLADES}

In accordance with the unanimous action on March 31, 1927, of a general conference of representatives of manufacturers and distributors, the Department of Commerce, through the Bureau of Standards, recommends the establishment of the following schedule for packing razor blades:

\begin{tabular}{|c|c|c|}
\hline 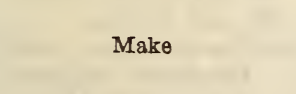 & $\begin{array}{l}\text { Pack- } \\
\text { ages } \\
\text { per } \\
\text { carton }\end{array}$ & $\begin{array}{l}\text { Blades per } \\
\text { package }\end{array}$ \\
\hline $\begin{array}{l}\text { Arrow } \\
\text { Auto-Strop } \\
\text { Christy } \\
\text { Darwin. } \\
\text { Durham Duplex. }\end{array}$ & $\begin{array}{l}20 \\
20 \\
20 \\
10 \\
20\end{array}$ & 5 and $10-10$ \\
\hline $\begin{array}{l}\text { Enders. } \\
\text { Ever-Ready } \\
\text { Gem } \\
\text { Gillette } \\
\text { Keen }\end{array}$ & $\begin{array}{l}20 \\
20 \\
20 \\
20 \\
10\end{array}$ & $\begin{array}{r}5 \\
5 \\
6 \\
5 \text { and } 10-10 \\
10\end{array}$ \\
\hline $\begin{array}{l}\text { Keen Kutter } \\
\text { Radium } \\
\text { Schick } \\
\text { Sexto } \\
\text { Vibro Shave }\end{array}$ & $\begin{array}{l}20 \\
20 \\
20 \\
20 \\
10\end{array}$ & $\begin{array}{r}5 \text { and } 10-10 \\
5 \\
3 \text { and } 10-5 \\
10 \text { and } 10-5\end{array}$ \\
\hline
\end{tabular}

All cataloguing, listing, billing, or invoicing is to be by the decimal system.
612.13 SCISSORS AND SHEARS.

UNITED STATES GOVERNMENT, FEDERAL SPECIFICATIONS BOARD, FEDERAL SPECIFICATIONS FOR SHEARS AND SCISSORS, OFFICE, NO. 361A, MAY 14, 1929. (CURRENT DESIGNATION: GGGS-101)

\section{OFFICE SHEARS AND SCISSORS}

\section{GENERAL SPECIFICATIONS}

There are no general specifications applicable to this specification.

\section{TYPES AND GRADES}

1. The shears furnished under this specification shall be of two types, as follows:

Type $A$, Bankers, double pointed.

Type B, Straight trimmers, one point sharp and one blunt.

2. They shall be furnished in two grades as follows:

Grade 1, Mild steel frames with high carbon steel blades welded to the frame to provide the cutting edges.

Grade 2, Solid forged steel.

3. Both types and grades shall be furnished with nickel-plated blades and japanned handles, or full nickel plated, as specified.

4. The scissors furnished under this specification shall be double pointed, one point sharp, or both points blunt, as ordered, in solid forged steel, full nickel plated.

5. Shears and scissors to be furnished in the various sizes listed in recognized manufacturers' catalogues.

\section{MATERIAI AND WORKMANSHIP}

1. Blades of grade 1 shears shall be of 0.9 per cent to 1.1 per cent carbon steel.

2. Grade 2 shears and scissors shall be of 0.65 per cent to 0.75 per cent carbon steel.

3. Blades of all shears and scissors shall be joined together with a hardened, threaded steel screw except for straight trimmers 9 inches or larger, a steel bolt and nut shall be used. The latter method may also be employed for straight trimmers less than 9 inches in length, if specified.

4. All shears and scissors shall be properly hardened, tempered, adjusted, and set in accordance with the recognized practices of the trade. They shall be smoothly finished, free from defects or blemishes that may affect appearance and service.

\section{GENRRAI REQUIREMENTS}

See Section III, Material and workmanship.

\section{จ. DETAILED REQUIREMENTS}

See Section III, Material and workmanship.

\section{METHOD OF INSPECTION AND TESTS}

1. At least six pairs of shears and scissors shall be taken at random from each delivery. For large deliveries, tests shall be made at the rate of three pairs for each gross. These samples will be carefully examined for defects in workmanship or appearance, and shall be tested for proper operation of the blades. Chemical analysis to determine conformance to the requirements for grade of steel will be made at the option of the purchaser.

2. Each sample shall be tested by cutting the following materials:

(a) Tissue paper, weight 25 by 40,$500 ; 11.6$ pounds ( 24 by 36,$500 ; 10$ pounds). 
(b) Cotton sheeting, weight 4.3 ounces per square yard.

3. The paper shall be thoroughly moistened with water before the test cuts are made.

(a) Cut one thickness of moistened paper as described above.

(b) Cut one thickness of cotton sheeting as described above.

(c) Cut multiple ${ }^{-*}$ thicknesses of cotton sheeting using the number of thicknesses specified in the following table:

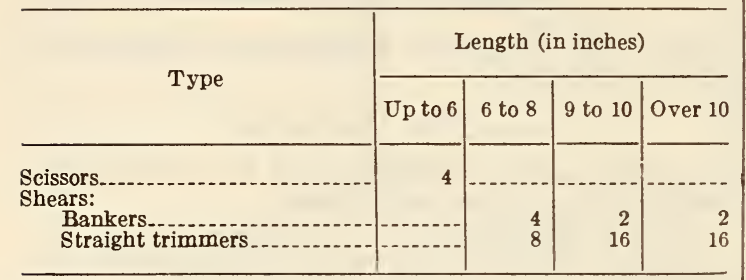

(d) Cut one thickness of cotton sheeting.

(e) Cut one thickness of moistened tissue paper.

(f) All test cuts specified to be made three times as follows: 1 inch from point to point; from heel clear to point; 1 inch from point to point.

4. Failure to cleanly and accurately cut the test material specified shall cause rejection. The cut material shall fall away from the blades and any tendency of the shears or scissors to hold the material after the blades are completely closed shall be accepted as evidence of imperfect adjustment and shall cause rejection.

\section{PACKING AND MARKING OF SHIPMENTS}

Each pair of shears or scissors shall be enclosed in a paper sleeve or sheath and packed not more than one dozen to a box. The box shall be properly labeled on one end to indicate size and description of the various articles contained.

\subsection{TABLE AND KITCHEN CUTLERY.}

(No nationally recognized specifications available.)

\subsection{HOLLOW AND STAMPED WARE, IRON AND STEEL.}

\subsection{GENERAI ITEMS.}

UNITED STATES GOVERNMENT, DEPARTMENT OF COMMERCE, BUREAU OF STANDARDS, COMMERCIAL STANDARD FOR COLORS FOR COLORED SANITARY WARE CS30-31, JULY 1, 1931

\section{COLORS FOR SANITARY WARE}

On January 23, 1931, a general conference of representative manufacturers, distributors, and users of colored sanitary ware adopted six commercial standard colors for products in this field. The industry has since accepted and approved for promulgation by the Department of Commerce, through the Bureau of Standards, the commercial standard as described herein.

SCOPE

This standard designates colors to be used as a guide in production of sanitary ware, plumbing fixtures, and allied products made of vitreous china, porcelain (all-clay) enameled iron, metals, wood, or glass.
The development of individual colors for special pieces-for instance, drinking fountains-and the matching of colors for special work will not come within the scope of this commercial standard.

\section{PURPOSE}

Manufacturers of colored sanitary ware have encountered problems due to the multiplicity of colors that are expensive to manufacture, impracticable to stock, and unsatisfactory to sell. The increasing variety of colors has resulted in a lack of harmony, especially when one manufacturer supplies the vitreous china lavatory, another, the enameled iron bathtub, and still another the seat for the watercloset bowl. This standard is offered as a solution of these problems.

It is not the purpose of this standard to retard initiative on the part of the individual producers or to limit the introduction of new colors, but it is intended to provide an authoritative record of the recommendation of the industry as a whole based on composite experience.

\section{METHOD OF MAIING COLOR COMPARISONS}

When comparing colors rf colored sanitary ware the line of observation shall be at right angles to the surface of the sample, which shall be so placed under the diffused light of a northern sky that minute shadows affecting the appearance of the colored surface will be reduced to a minimum, and the light source, reflections of lights, or differently cclored surfaces should be prevented from being mirrored in the gross of the sample.

The two surfaces to be compared shall be covered by a mask of neutral gray paper at least 8 inches square, with a rectangular opening $1 \frac{1}{2}$ by 4 inches cut in its center. The exposed area of each surface shall be approximately $1 \frac{1}{2}$ by 2 inches. The purpose of the mask is to conceal the surrounding colors, to bring into immediate focus the single color to be examined, and to restrict the area of the fixture to that of the color sample with which it is compared.

The normal recovery of the eye from the natural fatigue occurring by examining colors is distinctly rapid. Nevertheless, some time should be permitted to elapse between making successive comparisons.

No attempt should be made to match colors from memory.

\section{DETAIL REQUIREMENTS}

The six colors recommended as standard colors for sanitary ware shall be designated as follows:

$$
\begin{array}{ll}
\text { SC-11, green. } & \text { SC-40, blue. } \\
\text { SC-20, orchid. } & \text { SC-51, light brown. } \\
\text { SC-30, ivory. } & \text { SC-60, black. }
\end{array}
$$

The standard color samples shall be retained at the Bureau of Standards. Duplicate reference color samples may be obtained at a cost of $\$ 1.50$ per set from the secretary ${ }^{90}$ of the Manufacturers' Advisory Committee on Colors for Sanitary Ware as a basis for production control. The manufacturers' advisory committee has fixed light and dark limits for each color, and samples are to be retained at the Bureau of Standards. Such samples will be available only for the settlement of disputes. While any color within these limits will no doubt conform with the requirements, it is understood that every effort will be made to conform as nearly as possible to the reference color samples.

The color of sanitary ware shall be considered to match the standard color sample when, on comparison of the ware with a duplicate reference color sample under the specified lighting conditions and by the specified method, there is no appreciable difference in color.

${ }^{80} \mathrm{G}$. W. Wray, Bureau cf Standards, Washington, D. C. 
The advisory committee recommends that secrecy in connection with the subject of colored glazes and $\epsilon$ namels be eliminated. In order that manufacturers may have a thorough understanding concerning colored glazes the formulas, firing temperatures, and other pertinent information have been filed with the Bureau of Standards, and are available upon request.

It is recommended that all colored sanitary ware shall be considered as special rather than staple owing to the number of different types and shapes and the cost involved in stocking such a variety.

\section{UNITED STATES GOVERNMENT, DEPART- MENT OF COMMERCE, BUREAU OF STANDARDS, SIMPLIFIED PRACTICE RECOMMENDATION FOR TINWARE, GAL- VANIZED, AND JAPANNED WARE, R55, JULY 1, 1926}

\section{TINWARE, GALVANIZED WARE, AND JAPANNED WARE}

(Simplified practice recommended and adopted by industry establishing a limited list of standard stock sizes and varieties.)

Descriptions contained in the following recommendations refer to catalogue numbers from catalogue No. 35 of the National Enameling \& Stamping Co. and have been used as a matter of converience, as they are well known to all sheet-metal ware manufacturers and to the trade.

In these descriptions the gages, sizes, and trade capacities are considered to be approximate and are intended to apply to those articles, produced by other manufacturers, which most nearly approach thereto or articles substantially similar in the described particulars and for the same use.

It is recommended that manufacturers work toward japanning bread boxes, flour boxes, round sugar boxes, and cake closets in white and gray or some other one color only.

The so-called "specialties" or articles made by one manufacturer only, which are not comparable with anything made by anyone else, have been omitted from consideration in the simplification program.

TABLE 1.-Stamped ware

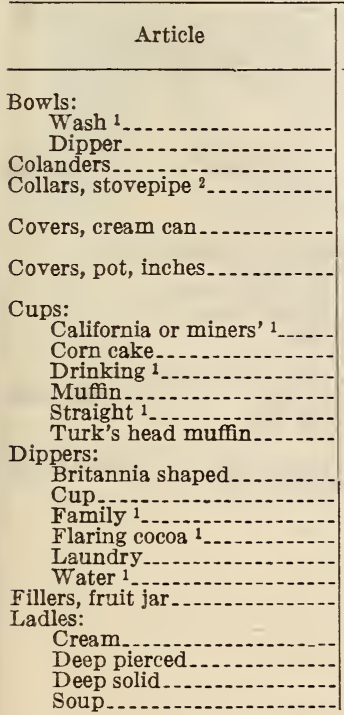

Catalogue number

$06,07,071 / 2,08$

$12,13,14$.

$104,205,306$. $4,41 / 2,5,51 / 2,6,7 ; 03,04,041 / 2,05$ $051 / 2,06,07$ $81 / 2$ inches, shallow; $81 / 2$ inches, extra deep. $1 / 2,7,71 / 2,8,81 / 2,9,91 / 2,10,101 / 2,11$
$11 \frac{1}{2}, 12,121 \frac{1}{2}, 13,13 \frac{1}{2}, 14,141 / 2,15$ 02.

\section{2.}

010 .

60.

023,26

064 .

455,450 .

10.

$040,041,042$.

051.

(63.

$0,2,4$.

$8,9,10,11$

$08,09,010,011$

$38,229$.
TABLE 1.-Stamped ware-Continued

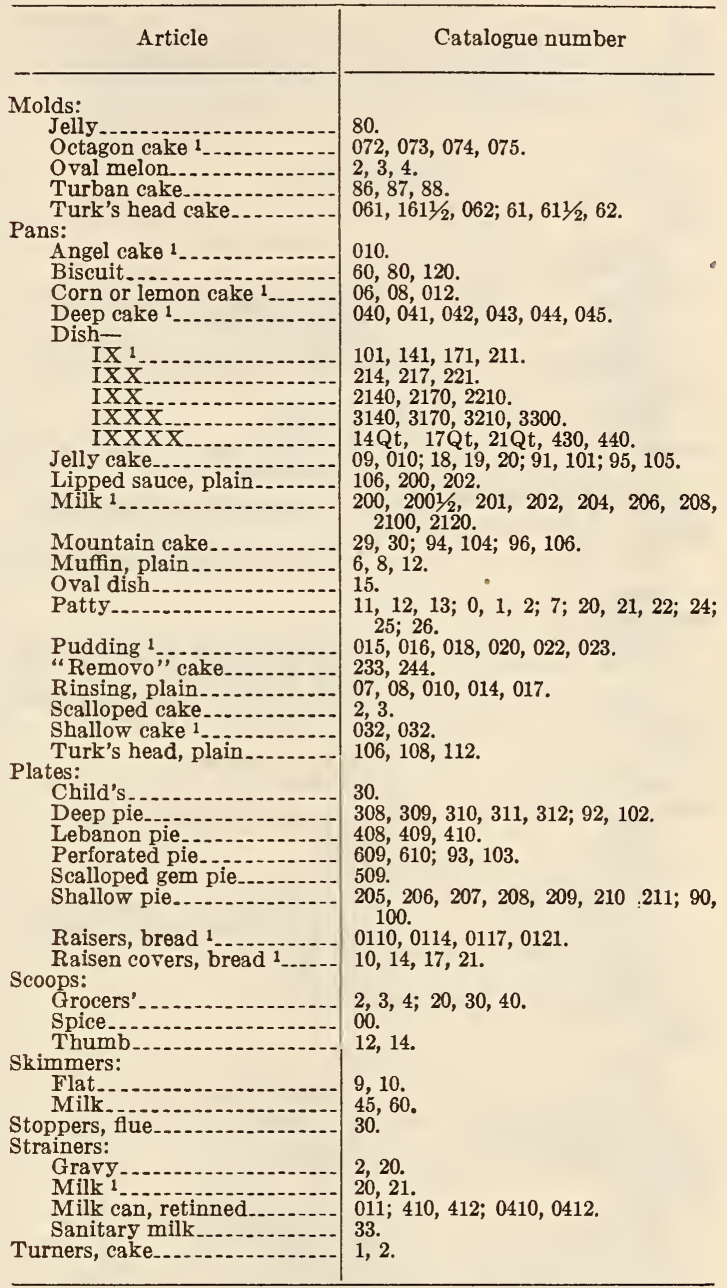

1 Both plain and retinned.

? Plain, lacquered, or japanned.

TABLE 2.-Metallic sieves

\begin{tabular}{|c|c|}
\hline Article & Catalogue number \\
\hline $\begin{array}{l}\text { Sieves: } \\
\text { Champion } \\
\text { Flour............. }\end{array}$ & $\begin{array}{l}1,11 / 2,2 . \\
116 ; 316,318 ; 1316,1318 .\end{array}$ \\
\hline
\end{tabular}

Table 3.-Pieced tinware

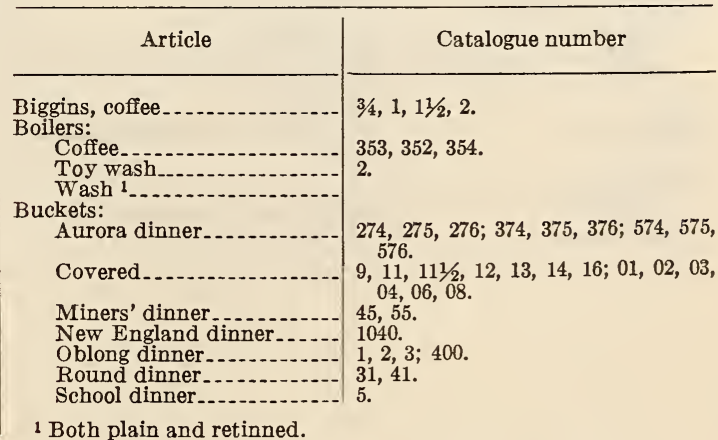


TABLE 3.-Pieced tinware-Continued

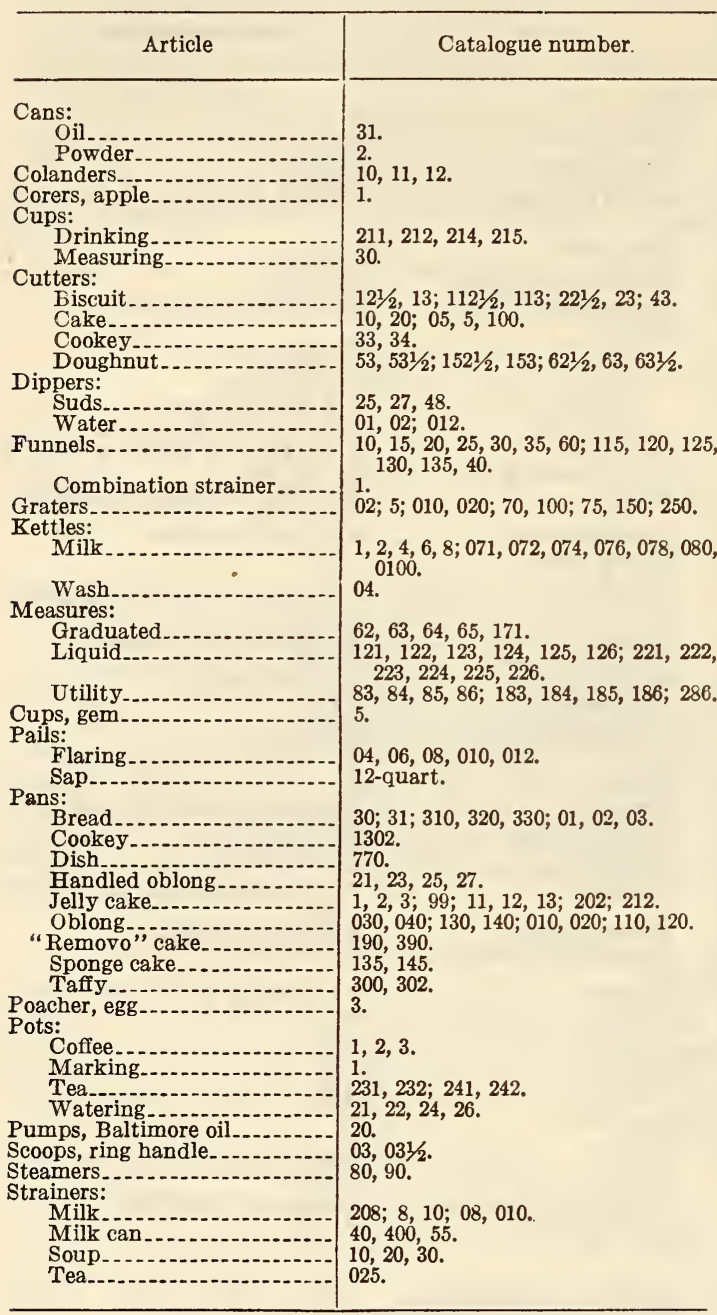

TABLE 4.-Heavy tinware

\begin{tabular}{|c|c|}
\hline Article & Catalogue number \\
\hline 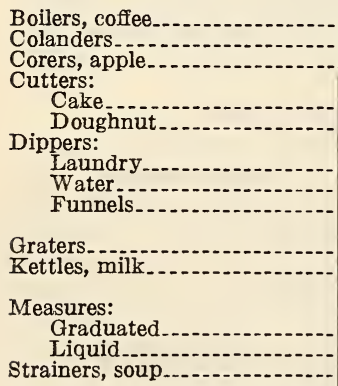 & $\begin{array}{l}612,616,620 . \\
113 \frac{1}{2} . \\
11 . \\
821 \frac{1}{2}, 83 . \\
9212,23 . \\
223,224 . \\
404 . \\
215,220,225,230 ; 235 ; 315,320,325, \\
330,335 . \\
40 . \\
171,172,174,176 ; 178,210,212,216, \\
220 . \\
191 . \\
043,044,045,046,143,144,145,146 . \\
110,120 .\end{array}$ \\
\hline
\end{tabular}

TABLE 5.-Japanned ware

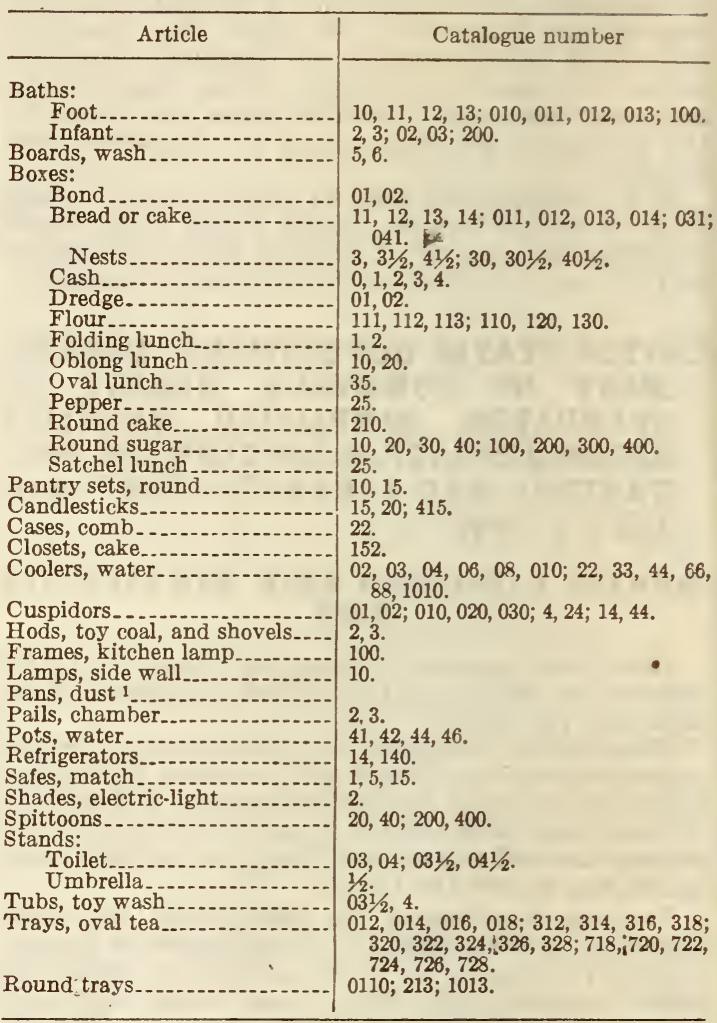

1 It was decided that no limitations be imposed on the varieties of this item, consideration being reserved for a regular annual revision meeting of the standing committee.

\section{TABLE 6.-Stock items of galvanized ware}

[Descriptions refer to catalogue numbers in section 12 of the National Enameling \& Stamping Co.'s catalogue No. 35, with the addition of items as listed from Wheeling Corrugating $\mathrm{Co}$. and Central Stamping Co. catalogues]

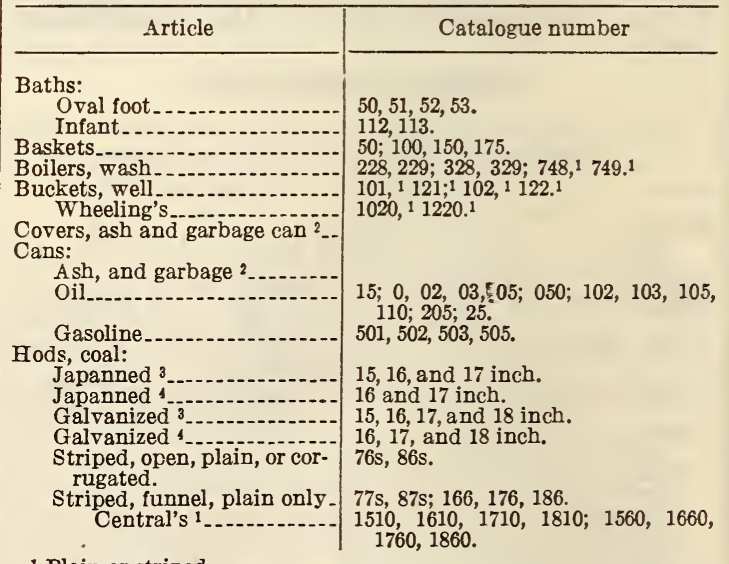

1 Plain or striped.

2 All varieties of these items were retained for the present, except wood-stave ash cans and japanned garbage cans.

3 Open, plain, or corrugated.

- Funnel, plain, or corrugated. 
TABLE 6.-Stock items of galvanized ware-Con.

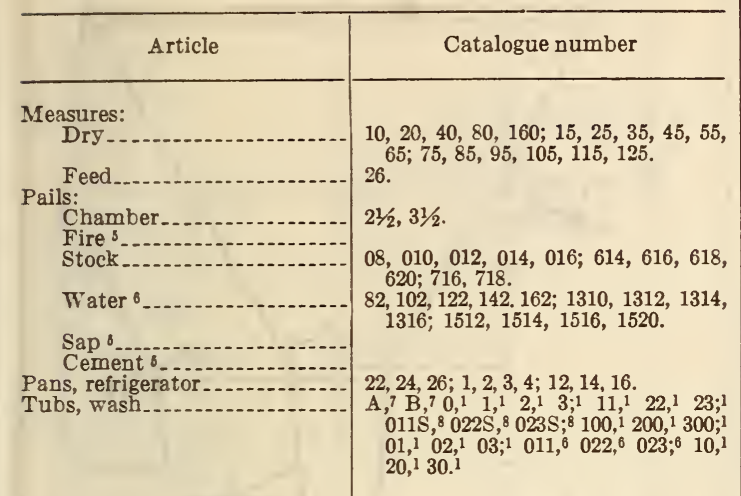

1 Plain or striped.

5 It was decided that no limitations be imposed on the varieties of this item, consideration being reserved for a regular annual revision meeting of the standing committee.

6 All water pails furnished plain or striped with or without wood roll.

7 Plain only.

Striped only.

\subsection{BATH TUBS.}

UNITED STATES GOVERNMENT, FEDERAL SPECIFICATIONS BOARD, SPECIFICATION FOR PLUMBING FIXTURES (FOR IAND USE), NO. 448, NOVEMBER 22, 1926. (CURRENT DESIGNATION : FF-P-451)

PLUMBING FIXTURES (FOR LAND USE)

\section{BATH TUBS}

Enameled Iron Ware

(See 617.73, p. 1062.)

612.22 HOTEL AND HOUSEHOLD HOLLOW AND STAMPED WARE.

(See 612.20, p. 817 for standard sizes.)

612.23 WATER-CLOSETS AND SINKS.

UNITED STATES GOVERNMENT, FEDERAL SPECIFICATIONS BOARD, SPECIFICATION FOR PLUMBING FIXTURES (FOR LAND USE), NO. 448, NOVEMBER 22, 1926. (CURRENT DESIGNATION : FF-P451)

\section{PLUMBING FIXTURES (FOR LANDESE)}

(See 617.73, p. 1062.)
612.29 MISCELLANEOUS HOLLOW AND STAMPED WARE.

AMERICAN RAILWAY ASSOCIATION, MECHANICAL DIVISION, TINWARE, STANDARDIZATION OF, RECOMMENDED PRACTICE, 1915

\section{TINWARE}

\section{Lap Seam}

Double folded Seam L

Riveted Lap Seam

Lap Jornt

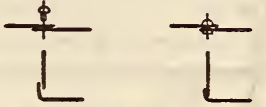

Sountersunk Lap Joint

Inserted Botfom

Double folded Joint J
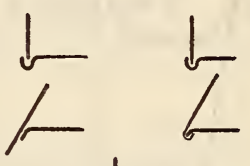

Double Folded Countersunk Joint

Wire inserted Botfom

Riveted Joint

Groove Joint

FIGURE 620.-Typical seams and joints
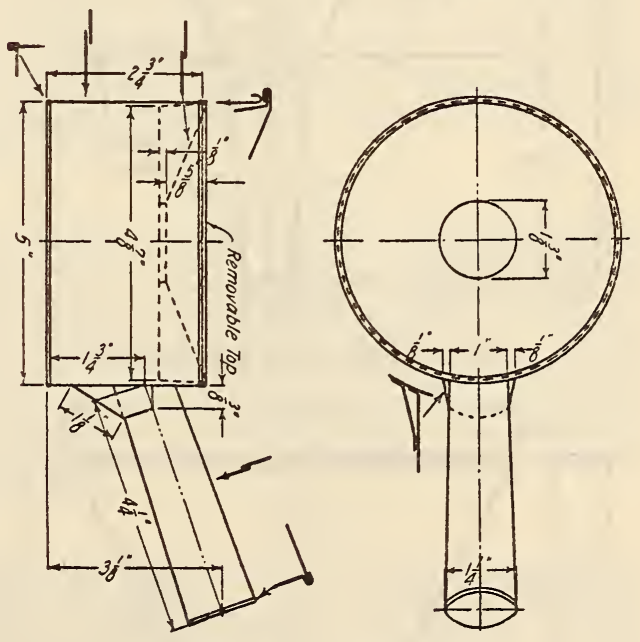

FIGURE 621.-Marking pot 


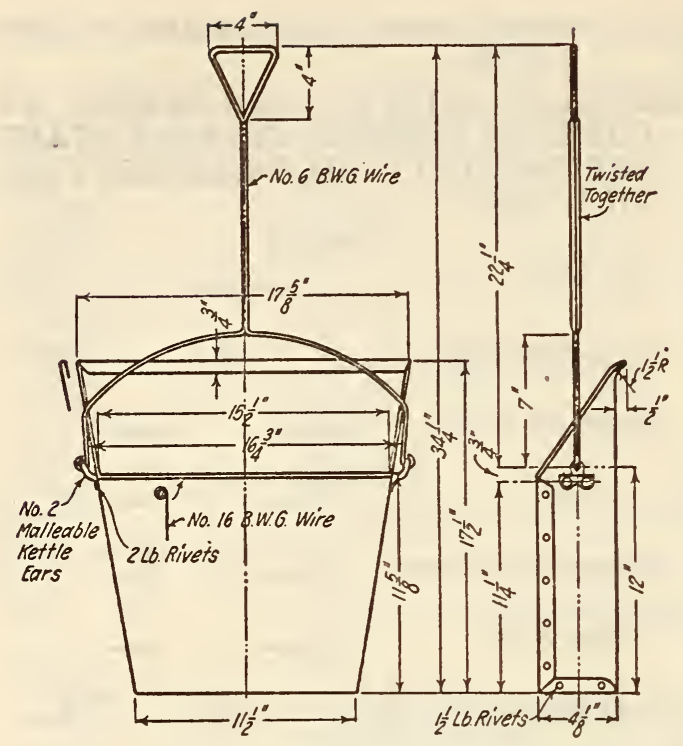

Figure 622.-Dust pan

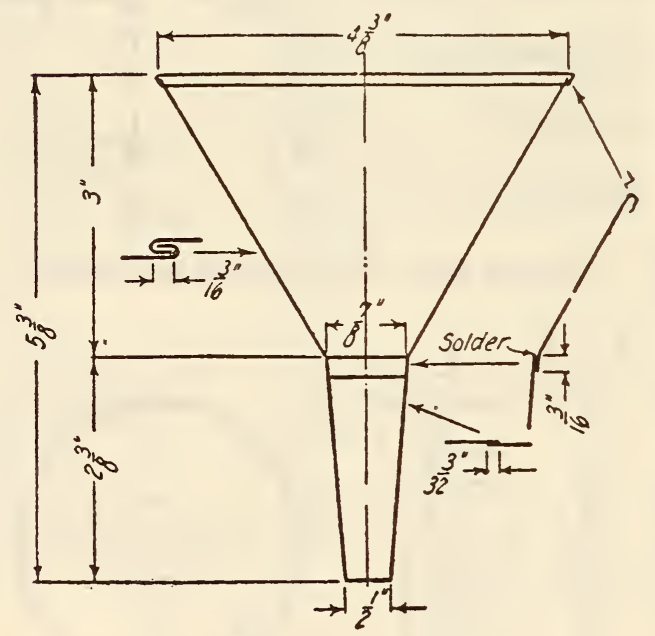

Figure 623.-One pint funnel

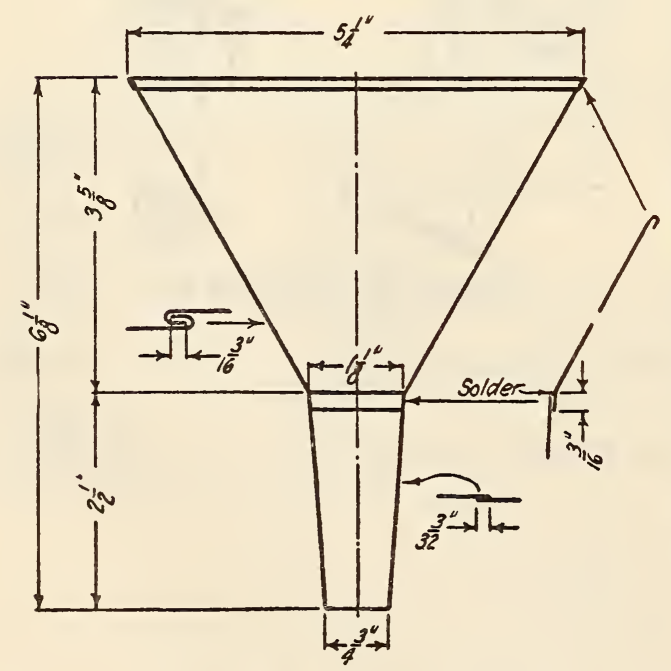

Figure 624.-One quart funnel

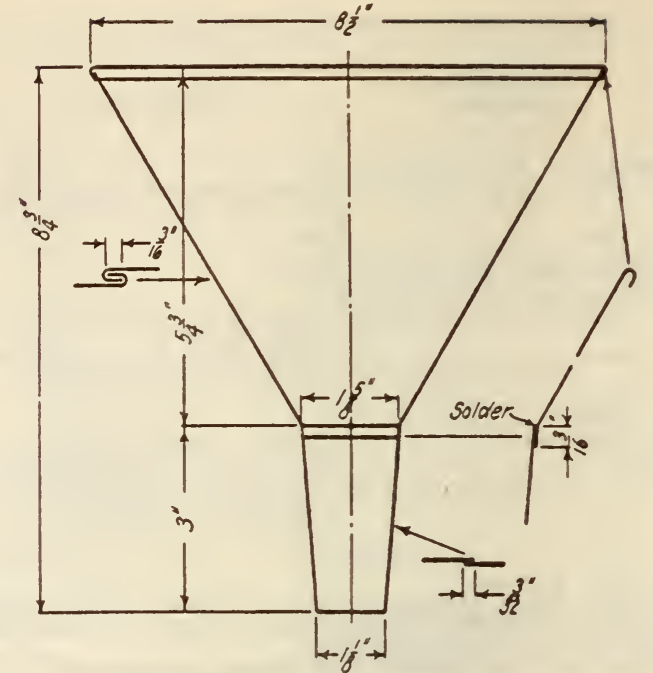

Figdre 625.-One gallon funnel

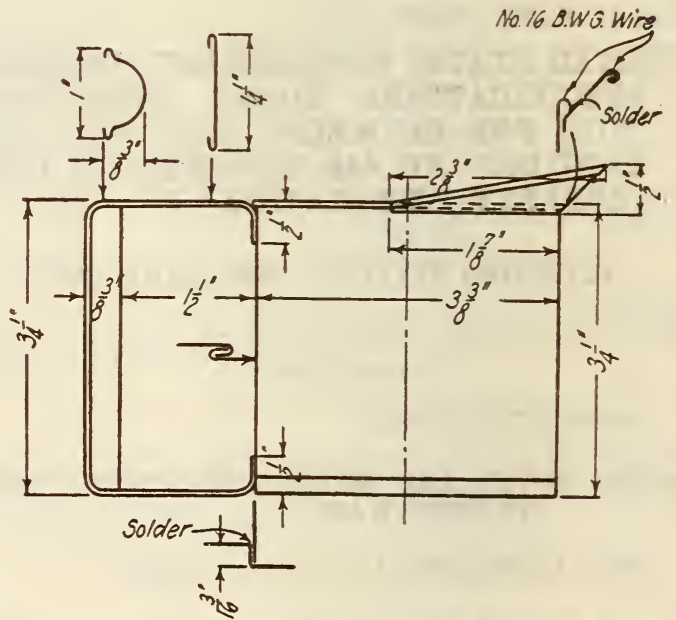

Figure 626.-One pint measure

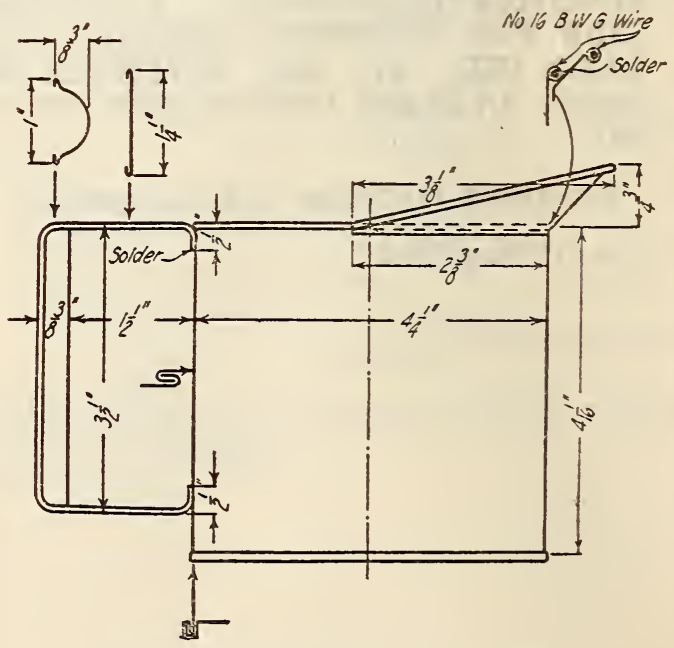

FigdRE 627.-One quart measure 


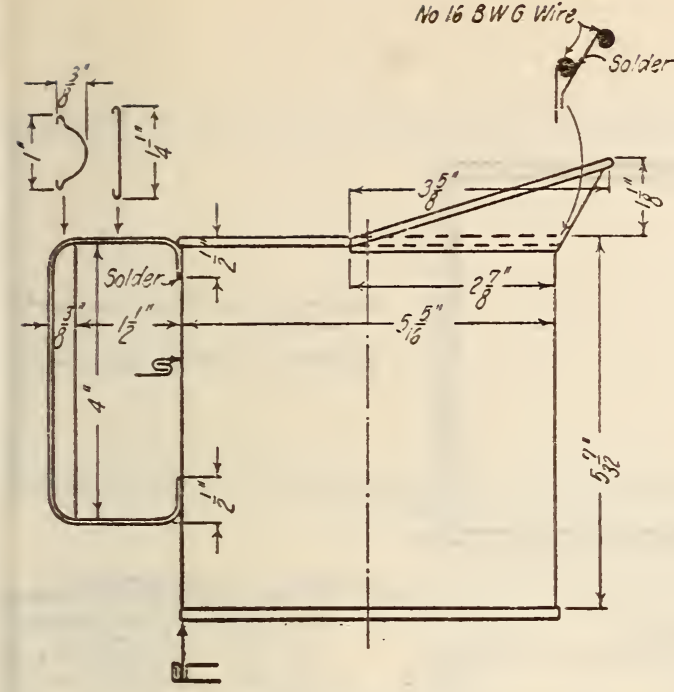

FIGURE 628.-Two quart measure

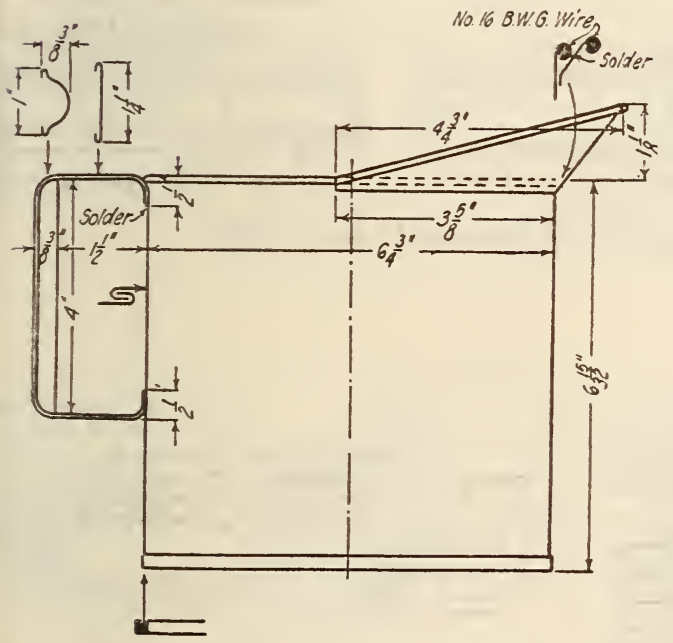

FIGURE 629.-One gallon measure

A universal form of marking pot.

A convenient and sanitary form of dust pan.

Three sizes of funnels which should take care of all requirements in the mechanical department.

One-pint, 1-quart, 2-quart, and 1-gallon measures. These measures are made with parallel sides to economize in labor and material.

All to be made of IX bright tin No. 28 B. W. G. or cold-rolled steel of suitable thickness.

\section{METAL FURNITURE AND FIXTURES}

613.1 BEDS, BED SPRINGS, COTS.

AMERICAN MARINE STANDARDS COM-

MITTEE, METAL FRAME BERTHS AND ACCESSORIES FOR SHIPS, H NOS. 53, 54, $55,56,59$, AND 60, 1929

\section{SHIP BERTHS}

\section{MATERIALS}

(a) Tubular parts of berth frames.-To be commercial grade high-carbon brazed steel tubing of the following sizes and minimum thicknesses: Upper rail, 1.05 inches outside diameter No. 17 B. w. g. (0.058 inch). Lower rail, 1.25 inches outside diameter No. 15 B. w. g. (0.072 inch). Tubing of other metal, and of equivalent strength, may be substituted at the option of the purchaser.

(b) Angle end rails of berth frames.-To be commercial grade soft-steel rolled shape $1 \frac{1}{2}$ by $1 \frac{1}{2}$ by $3 / 16$ inches.

(c) Castings for berth frames and accessories.-All castings shall be of malleable iron or equally suitable material. They shall be sound and free from defects that would impair their serviceability or good appearance. Should any part of the tubular frames be ordered of brass or other material to be brightmetal finished, the cast parts connecting them shall be of corresponding metal and their exposed surfaces shall be polished.

(d) Stanchions for fixed berths.-These shall be tubes $1 \frac{1}{2}$ inches outside diameter. They may be of commercial grade high-carbon brazed steel tubing of minimum thickness equivalent to No. 14 B. w. g. (0.083 inch) or of other equally suitable material.

(e) Stanchions for hinged berths. - These shall be of ordinary $1 \frac{1}{2}$-inch size commercial standard weight or extra strong pipe according to conditions.

(f) Chains and hooks for hinged berths.-To be of bright-steel wire unless specifically ordered of other material. The chains may be of any suitable commercial type having a breaking strength of at least 1,200 pounds.

\section{WORKMANSHIP}

All surfaces of berth frames and accessories shall be smooth and free of defects. Joints of assembled parts shall be closely fitted. Machining shall be required only as necessary for assembly and installation. Fastenings shall be well fitted and smoothly finished. Welding may be substituted for riveting at the option of the purchaser to fasten the parts of berth frames or to attach fittings to stanchions or to the ship structure. All welds shall be smoothly finished.

\section{FINISH}

The character of finish or coating and color desired shall be specified by the purchaser in asking for quotations and in ordering. If no finish or coating is thus specified, none shall be applied by the berth manufacturer. 


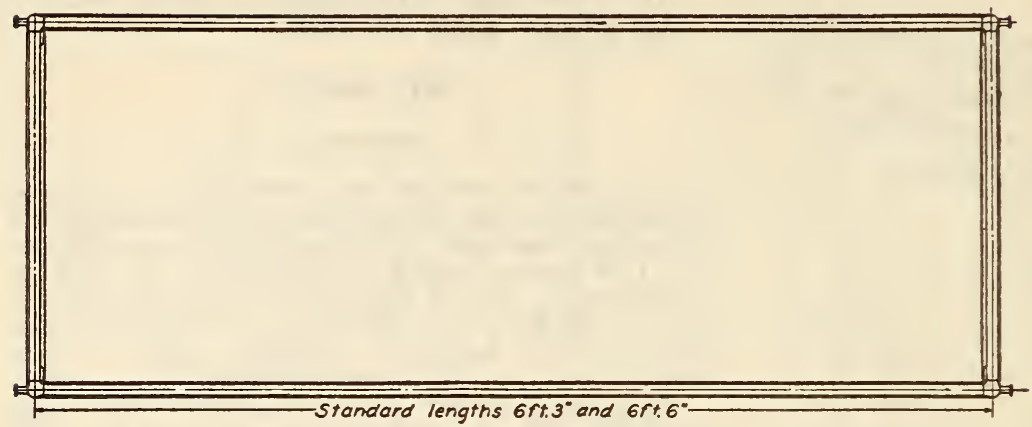
See general notes for porticulars of
materials.

QStandard lengths of tubes or angles.

Erazed steel tube. $1.05^{\circ}$ cutside diameter, minimum thickness No. 17 B.W.G. $\left(0.058^{\circ}\right)$-7)

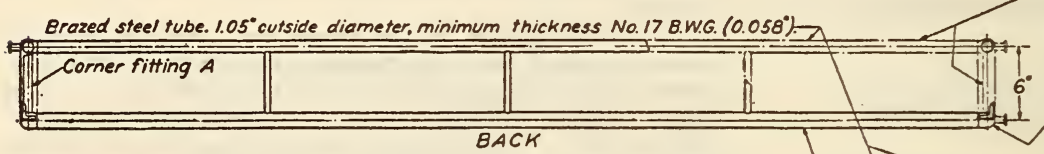

Upper rail and spills may be omitted at back and ends when berth is to be installed against partitions or bulkheads.

Use corner fitting $B$ where both back and end top rails are omitted.

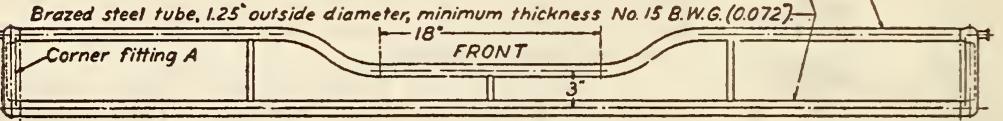

- $06 \mathrm{rt} 1 \frac{1}{2}$ for $6 \mathrm{ft} 3^{\circ}$ berth and 6 frt $4 \frac{1}{2}$ for 6 frt $6^{\circ}$ berth-

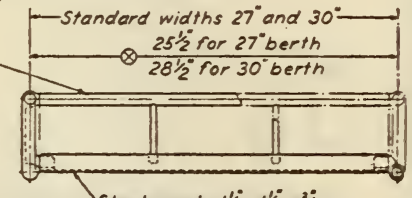

Figure 630.-Shallow berths, American Marine standard H No. 59-1929
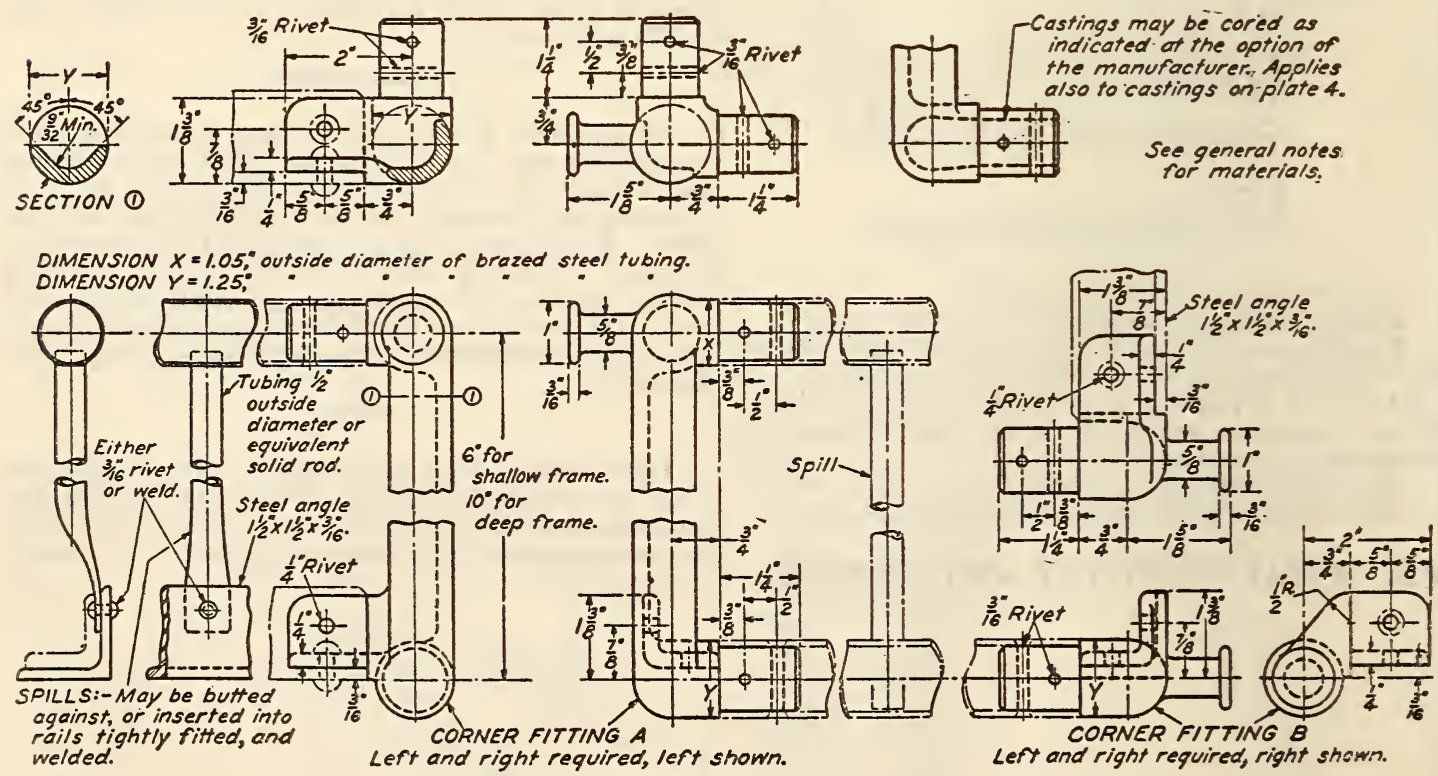

FIGURE 631.- Shallow berth details 

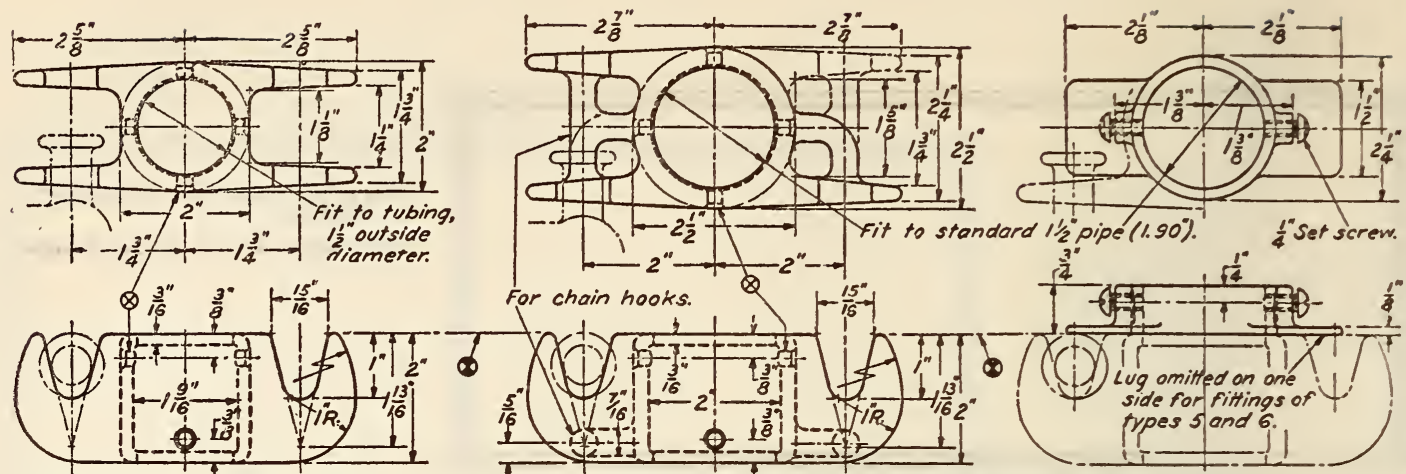

BRACKET FOR FIXED EERTHS STANDARD HNO.55-1929.

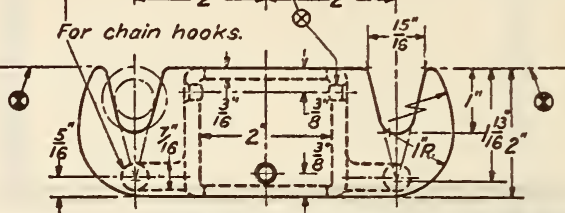
BRACKET AND OPTIONAL RETAINER FOR HINGEO BERTHS
STANDARD HNO.56-1929.

QHoles for $\frac{1}{4}$ rivet, see specification for option to weld.

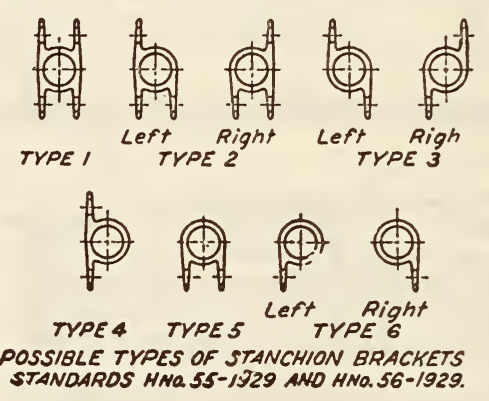

Figure 634.-Stanchion fittings for American Marine standard berths

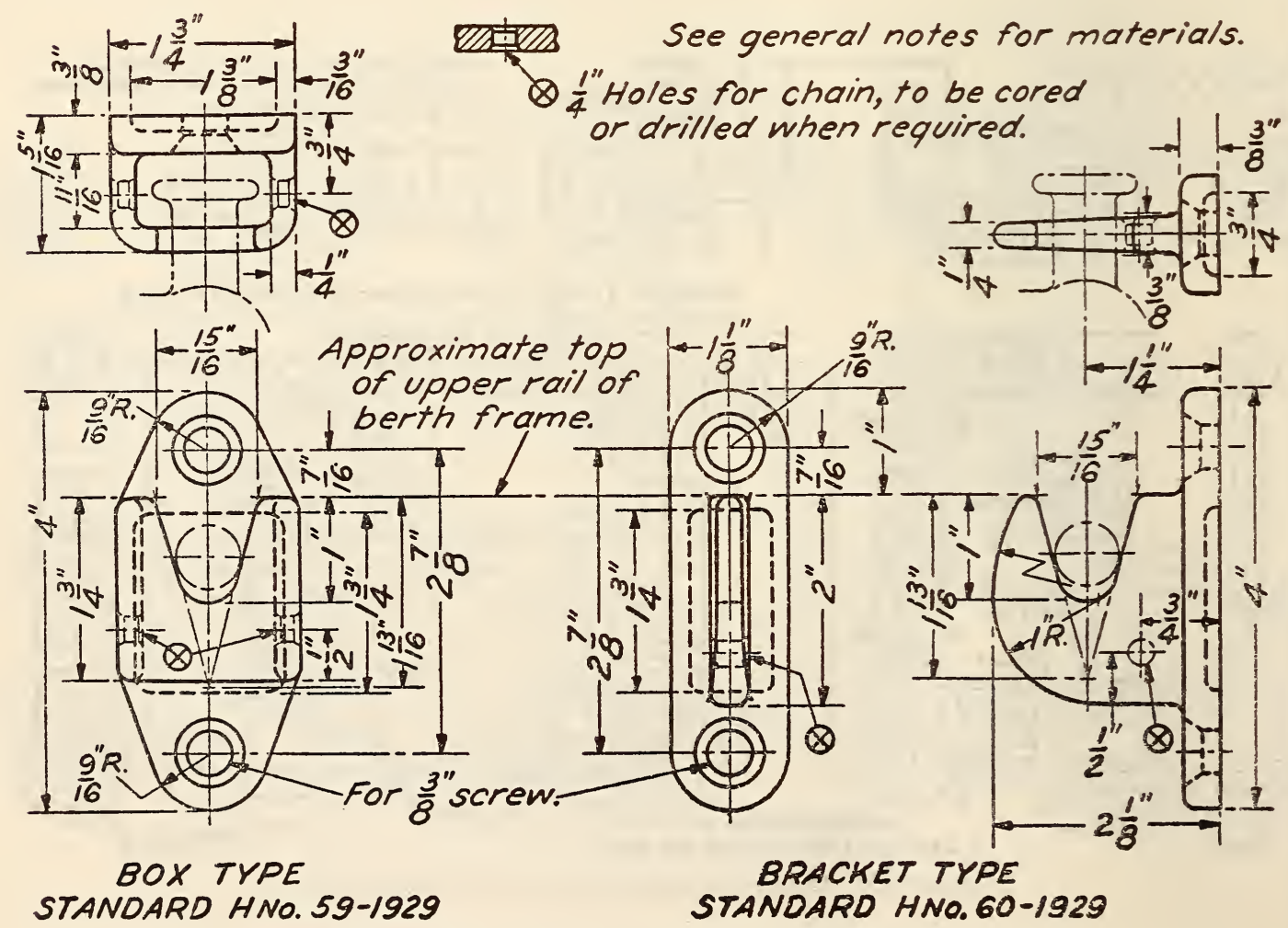

Figure 635.-Wall supports for American Marine standard berths 
PACKING AND MARKING FOR SHIPMENT

Berth frames and fittings shall be tagged or otherwide marked for convenient identification with the items in the purchaser's order. They shall be packed for shipment in such manner as to insure safe and economical handling and transportation. Containers shall be marked to identify them with the number or date of the purchaser's order.

\section{GUARANTY}

Berth frames, stanchions, or fittings furnished on the basis of these standards shall be guaranteed by the manufacturer against failure due to faulty material or workmanship. In case of any failure from such causes, the manufacturer shall furnish replacements without charge to the purchaser or shall reimburse the latter for the costs of necessary repairs.

\section{UNITED STATES GOVERNMENT, DEPARTMENT OF COMMERCE, BUREAU OF STAND. ARDS, SIMPLIFIED PRACTICE RECOMMENDATION FOR BEDSTEADS, SPRINGS, AND MATTRESSES, R2-30, SEPTEMBER 1, 1922}

\section{BEDSTEADS, SPRINGS, AND MATTRESSES}

(Simplified practice recommended and accepted by industry establishing a limited schedule of standard sizes and types.)

TABLE 1.-Wooden beds

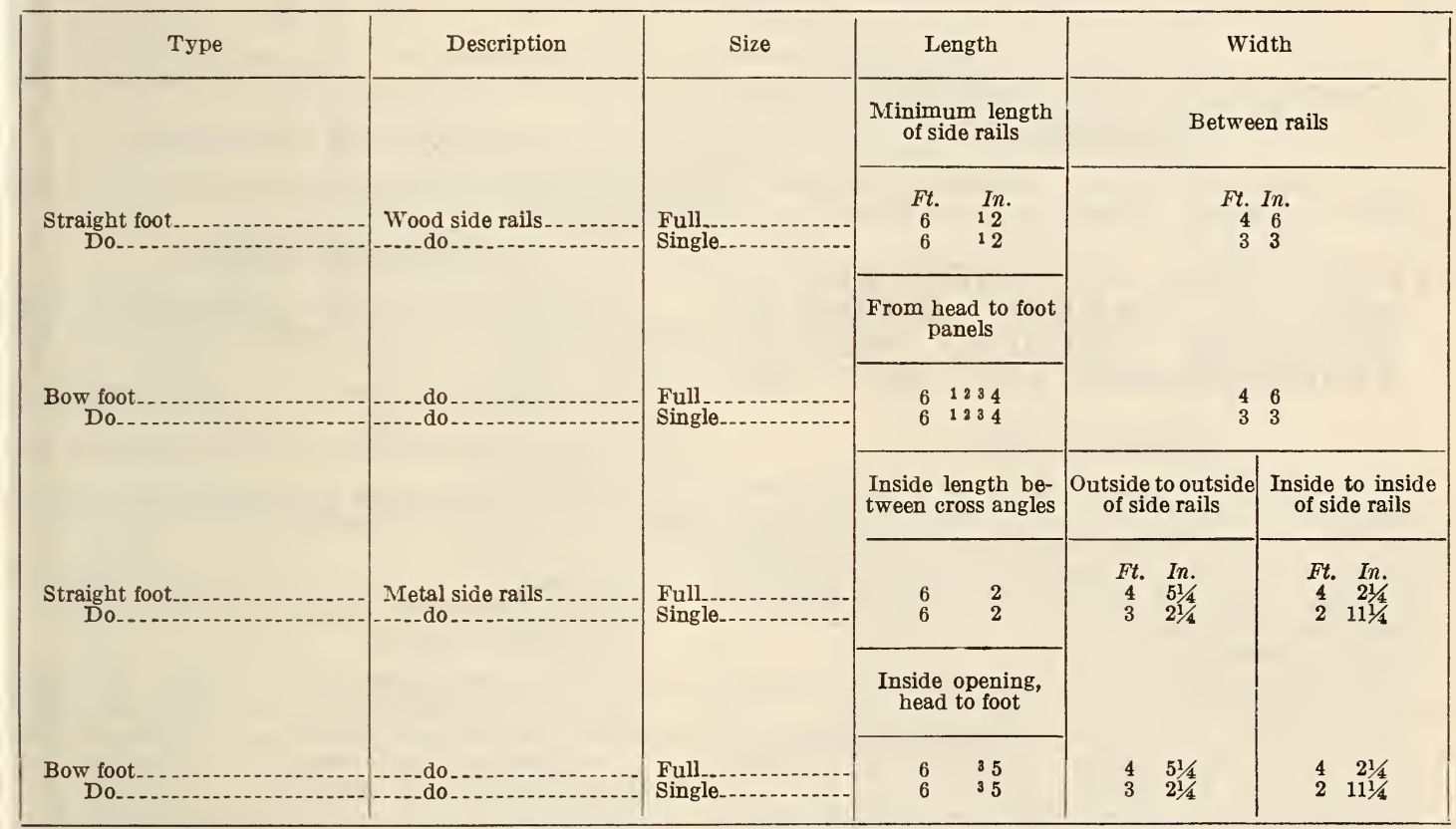

1 Where necessary to use blocks to hold bed rail hook pins, blocks must not extend over $3 / 8$ inch inside rails.

2 Crossbars or braces where necessary in bow ends shall be set even with, or below, the top of slat bearer. It is preferred these crossbars be eliminated if possible.

3 All bow-foot beds are to be made with radius not exceeding 7 inches.

TABLE 2.-Iron, steel, or brass beds

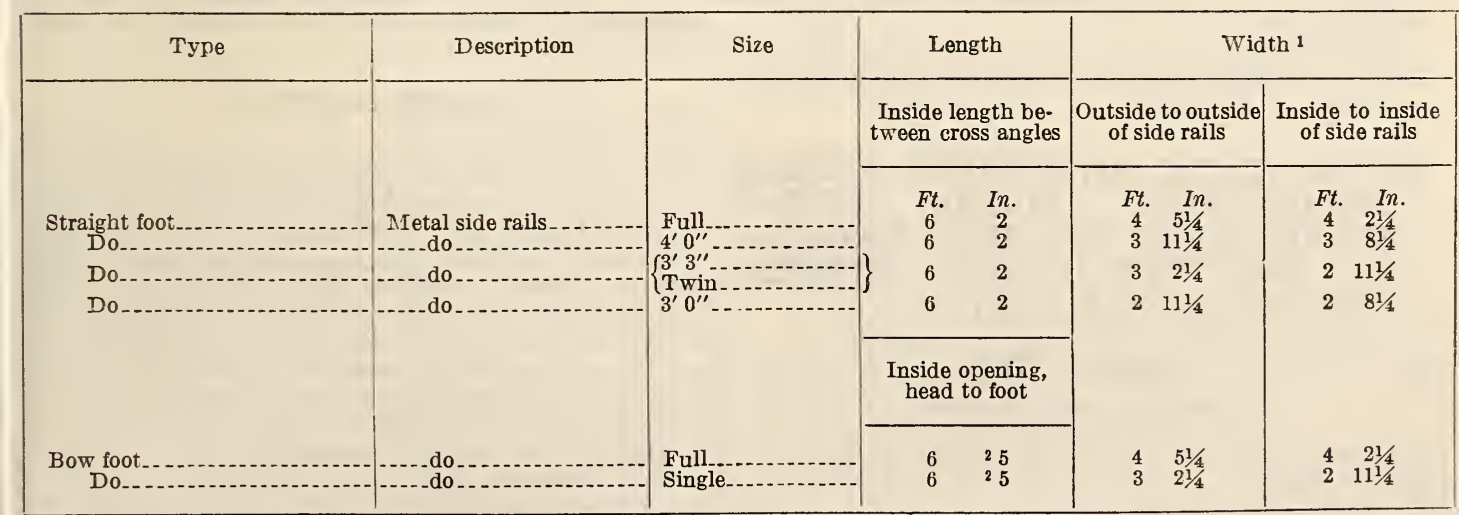

$1 \mathrm{It}$ is recommended that the top flange of side rails measure $1 \frac{1}{2}$ inches in order that the inside openings of the established sizes of beds will conform to the recommended dimensions for the inside widths.

2 All bow-foot beảs are to be made with radius not exceeding 7 inches. 
TABLE 3.-Springs

\begin{tabular}{|c|c|c|c|c|c|c|}
\hline \multirow{2}{*}{ Class } & \multirow{2}{*}{ Type } & \multirow{2}{*}{ Size } & \multirow{2}{*}{\multicolumn{2}{|c|}{ Length }} & \multicolumn{2}{|c|}{ Width } \\
\hline & & & & & Top & Bottom \\
\hline \multirow[t]{2}{*}{ Spiral or coil wire. } & \multirow[t]{2}{*}{ With metal bases...... } & \multirow[t]{3}{*}{$\begin{array}{l}\text { Full } \\
4^{\prime}, 0^{\prime \prime}, \ldots \\
3^{\prime} 3^{\prime \prime} \\
\text { Twin } \\
3^{\prime} 0^{\prime \prime}, \ldots\end{array}$} & \multicolumn{2}{|c|}{$\begin{array}{cc}\text { Ft. } & \text { In. } \\
6 & 1 \\
6 & 1 \\
6 & 1 \\
6 & 1\end{array}$} & $\begin{array}{cc}\text { Ft. } & \text { In. } \\
4 & 4 \\
3 & 10 \\
3 & 1 \\
2 & 10\end{array}$ & $\begin{array}{cr}\text { Ft. } & \text { In } \\
4 & 48 / 4 \\
3 & 108 / 4 \\
3 & 18 / 4 \\
2 & 108 / 4\end{array}$ \\
\hline & & & & & $\begin{array}{l}\text { Length of } \\
\text { end rail }\end{array}$ & $\begin{array}{l}\text { Width of } \\
\text { hangers }\end{array}$ \\
\hline Metal frame... & $\left\{\begin{array}{l}\text { With link, slat, cable, or } \\
\text { woven-wire fabric. }\end{array}\right.$ & & $\begin{array}{l}6 \\
6 \\
6 \\
6\end{array}$ & $\begin{array}{l}1 \\
1 \\
1 \\
1\end{array}$ & $\begin{array}{rr}4 & 43 / 4 \\
3 & 10^{3} / 4 \\
3 & 13 / 4 \\
2 & 10^{8} / 4\end{array}$ & $\begin{array}{rr}4 & 48 / 4 \\
3 & 103 / 4 \\
3 & 18 / 4 \\
2 & 103 / 4\end{array}$ \\
\hline
\end{tabular}

NorE.-It is recommended that springs for bow-foot beds be provided with a 7-inch radius corner at the foot end to conform to similar radius in bow-foot beds.

\section{MATTRESSES}

It is recommended that mattresses be made to conform to spring dimensions given above.

UNITED STATES GOVERNMENT, DEPARTMENT OF COMMERCE, BUREAU OF STANDARDS, SIMPLIFIED PRACTICE RECOMMENDATION R24, JUNE 3, 1924

\section{HOSPITAL BEDS}

In accordance with the action of a general conference of representatives of various hospital interests, the United States Department of Commerce, through its Bureau of Standards, recommends that simplified sizes and varieties of hospital beds be established as follows:

1. For general hospital use:

(a) Length, inside distance between head and foot posts, 78 inches.

(b) Width of end angles of springs, 36 inches.

(c) Height from floor to top of springs, inclusive of casters, etc., 27 inches.

2. For certain institutional use the need for a narrower bed is recognized. In these cases the recommended width is 33 inches, with other dimensions the same as in $(a)$ and $(c)$ above.

3 . For private-room use, where a wider than standard bed is desired, the recommended width is 39 inches, with other dimensions the same as in $(a)$ and $(c)$ above.

These recommendations are to become effective January 1,1925 , subject to regular annual revision by similar conference.

\section{UNITED STATES GOVERNMENT, DESIGNATION : AA-B-201)} SPECIFICATION FOR BEDS, HOSPITAL, NO. 186a, JULY 11, 192\%. (CURRENT

\section{HOSPITAL BEDS}

\section{GENERAL SPECIFICATIONS}

There are no general specifications applicable to this specification.

II. TYPE

Hospital beds shall be of one type but subject to alternatives in connection with certain detail requirements.
III. MATERIAL AND WORKMANSHIP

Hospital beds shall be of first-class material and workmanship.

\section{GENERAL REQUIREMENTS}

1. Hospital beds shall be constructed in three pieces, as follows: (a) Headpiece, (b) foot piece, and (c) spring bottom.

2. The general dimensions of the beds shall be as follows:

(a) Length, inside distance between head and foot posts, 78 inches.

(b) Width of end angles of spring bottom, dimension X (as specified by purchaser).

Generally

For certain institutional uses ......... 33

For private-room use............. 39

(c) Height of spring bottom from floor, including wood shoes, casters, etc. (all widths), 27 inches.

(d) Height above floor, including wood shoes, casters, etc., to top of headpiece, 52 inches.

(e) Height above floor, including wood shoes, casters, etc., to top of foot piece, 42 inches.

$(2-a, 2-b, 2-c$, above, are in conformity with simplified practice recommendation R24 of Bureau of Standards above.)

3. The finish of the bed shall be as specified in the contract or order.

4. A tolerance of 5 per cent above or below the dimensions specified will be allowed for buttwelded steel tubing.

\section{DETAIL REQUIREMENTS}

1. Headpiece-(a) Continuous post.-For the bed 36 inches in width this part of the headpiece shall be of hot-rolled butt-welded steel tubing 1.25 inches outside diameter by 0.089 inch thick, bent to form a continuous post having approximately 4-inch radius at bends and measuring approximately $34^{3} / 4$ inches center to center of the vertical legs. The width distance between centers of vertical legs of the continuous posts for beds 33 inches and 39 inches in width shall be appropriate for those widths, and suitable for the dimensions of the widths of the end angles of the spring bottom as stated in the foregoing.

(b) Cross rods.- The cross rods shall be of buttwelded steel tubing 0.625 inch outside diameter by 0.069 inch thick connected to the vertical legs of the continuous post with cast chills located in the lower portion of and forming a part of the cast-chill connection securing the female hook parts of the corner 
locks to the legs of the continuous post or at about 6 inches to center of cross rod below top of end angles of spring bottom.

Note- This aroids the necessity for additional chills on the headpiece and at the same time admits of ample room for making beds without injury to the fingers of attendants.

- (c) Upright rods or fillers. - The upright rods or fillers shall be three in number spaced at equidistant (e) Welded connections.-Unless otherwise specified by the purchaser, welded connections may be used instead of the cast chills wherever specified herein; in which case the connections for the cross rods, fillers, etc., shall be made by inserting them into the connecting tubing to the full depth admissible by maans of a driving fit and then welding completely around the entrance opening or joint.

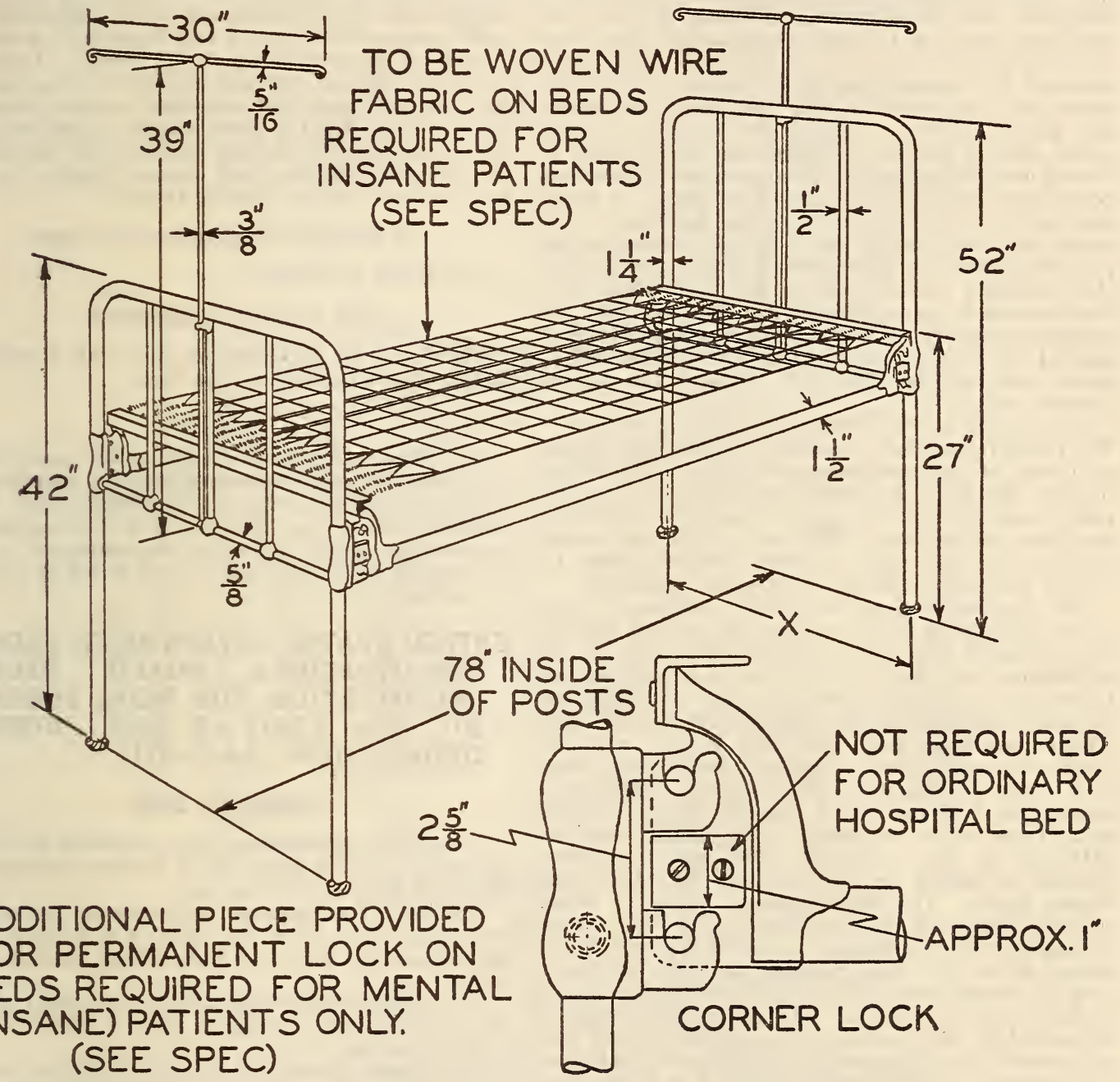

\section{HOSPITAL BED}

FIGURe 636

intervals between the vertical legs of the continuous post. They shall be of butt-welded steel tubing 0.500 inch outside diameter by 0.065 inch thick connected to the top rail of post by insertion and welding and to the cross rod by cast chills. A square socket shall be provided in the lower cast chill connecting the center upright rod to the cross rod for the purpose of taking the lower end of the mosquito rod, and an eye casting shall be provided on the center upright at about 1 inch below the under side of the top rail of the continuous post for use as an upper socket for the mosquito rod.

(d) All cast chills shall be smooti and manufactured from pig iron and machinery scrap only.
The attachments for the mosquito rod may be by welded connections or other means as approved by the purchaser.

2. Foot piece. - The foot piece in all its parts shall be of the sama design and construction as specified above for the headpiece except that the height to top of continuous post from floor, including wooden shoes, casters, etc., shall be 42 inches.

3. Corner lock. - The corner lock shall be a doublesocket connection, as shown in the accompanying sketch which forms a part of this specification. The

Note.-A flat fracture bar will not be required, as the cross rod appears to be suitably located for that purpose. 
female hook parts shall be chill cast to the upright posts. The risers of the spring bottom part shall be substantially constructed of malleable iron with nine-sixteenths inch diameter steel pins, so located as to accurately mesh with the female parts on center of $25 \% 8$ inches as shown in the accompanying sketch. The malleable-iron risers shall have a socket io receive 1.500 inches outside diameier by 0.095 inch thick butt-welded straight tubular side rails. The height of the riser shall be such that the height of the fabric (spring botiom) shall be not less than $41 / 2$ inches above the top of the side rail. On beds as required for mental patients (insane), the corner locks shall be provided with the additional pieces and screws shown in sketch for fastening to the center web of the riser between the hook pieces to form a lock to prevent the haad and foot pieces from being detached from the spring bottom. Or as an alternative, if approved by the purchaser, the corner lock may be of the contractor's own design, provided after investigation it is demonstrated that the proposed corner lock is the equivalent of a double-socket connection in rigidity and can be suitably arranged, if desired, for the special use of mental patients (insane) by an interlocking device which can not be detached without the use of suitable tools.

4. (a) Fabric and fabric frame (spring bottom).The fabric frame shall consist of two straight tubular side rails of butt-welded steel tubing 1.500 inches outside diameter by 0.095 inch thick. The end rails shall consist of angles made of hot-rolled medium carbon steel. The size of the end angles shall be not less than $1 \frac{1}{2}$-inch vertical flange by 2 -inch horizontal flange by $3 / 16$-inch thickness.

(b) The fabric shall be of the "national" type, about 16 squares long, and the size of the link for the square of the width shall be such that by the addition or subtraction of a line of squares of the fabric for the 36-inch bad the width of fabric can be made suitable for the 39-inch and 33-inch beds. The cencer links and links and $V$ links shall be made of tinned drawn wire about 0.117 inch diameter. The 3-hole and 4-hole plates shall be of steel about 0.05 inch thick, flat and free from burrs and tinned after stamping. The fabric shall be attached to the end angles with an appropriate number of helical springs (not less than 19), black Japan finish. The helical springs shall be about three-fourths inch outside diameter, of high carbon spring wire, finely tempered, 0.1055 inch diameter, about 20 to 21 convolutions, and $35 / 16$ inches in length over all, not stretched (total stretch in length of bed about $1 \frac{1}{2}$ inches). Or as an alternative, if approved by the purchaser, the spring boitom may be made of any single-wire or double-wire fabric of the contractor's own design, and of a guaranteed rustless or electro galvanized finish, provided the proposed fabric is of a scrength and tension equivalent to that described in the foregoing for the "national" type of fabric; and provided further that the spring bottom of the bed intended for the use of mental patients (insane) shall, in all cases, be of the best commercial quality, strong and durable woven-wire construction, fastened to the end angles securely, and in a manner to be approved by the purchaser.

5. Mosquito bar rods. - T mosquito bar rods of not less than three-eighths inch round steel or iron shall be furnished with the bed. They shall be 39 inches high with the cross rod 30 inches long. The cross rod shall be five-sixteenths inch round steel or iron and shall be connected to the upright portion with a chill or by weld. The lower end of the vertical portion of the rod shall be pointed and of squared cross section in order to resc in the square hole provided in the rod socket on the centar upright rod. The extreme ends of the cross rod shall be bent downward into the form of eyes. The holes so formed shall be one-half inch inside diameter.

6. Wooden shoes or casters.-The material of the shoes shall be of clear rock maple, or beech, well seasoned and sound. The ball shall be $1 \frac{7 / 8}{8}$ inche。 in diameter and $13 / 8$ inches long, slightly rounded at the top and bottom. The pin shall be $21 / 2$ inches high and shall taper so as to make a snug fit into the tubular legs of the continuous post. The entire pin shall be smoothly finished and thoroughly soaked in linseed oil for approximately 10 hours. If required by the purchaser, all four legs of the hospital bed shall be equipped with noiseless casters instead of wooden shoes, and the lower lengths of legs shortened so as to compensate for the height of the casters and yet maintain the specified over-all height from the floor to top of spring bottom fabric.

\section{METHOD OF INSPECTION AND TESTS}

No details specified.

\section{PACKING OF SHIPMENTS}

Packing shall be in accordance with commercial practice unless otherwise specified.

\section{NOTES}

The illustration accompanying this specification shows the general characteristics of a hospital bed, with cast-chill connections, "national" type fabric double-socket corner locks, and with an adaptable interlocking device for use in connection with beds for mental patients (insane) and is for general information only.

\section{UNITED STATES GOVERNMENT, FEDERAI SPECIFICATIONS BOARD, FEDERAL SPECIFICATION FOR BEDS, SURGICAL, NO. 187a, APRIL 25, 1927. (CURRENT DESIGNATION : AA-B-211)}

\section{SURGICAI BEDS}

(General specifications, type, material and workmanship, are the same as in Federal specification No. 186a (AA-B-201) above.)

(Section IV on general requirements is the same as Section IV of F. S. No. 186a (AA-B-201) except for paragraphs $2(b)$ and $2(d)$, which read as given below.)

(b) Width of end angles of mechanically adjust. able spring bottom exclusive of riser plates of corner locks, 35 inches.

(c) Height of spring in mechanically adjustable bottom from floor, including wood shoes, casters, etc., 30 inches.

(Detail requirements for headpiece and foot piece are the same as given in F. S. 186a (AA-B-201) above for 36 -inch beds, with the additional requirements given below.)

Height to center of cross rod above floor shall be about 22 inches.

\section{CORNER IOCK}

8. The corner lock shall be a double-socket connection as shown in the accompanying sketch which forms a part of this specification. The female hook parts shall be chill cast to the upright posts. The riser of the adjustable spring bottom part shall be a

NotE.-The distance between the centers of vertical legs of headpiece (and foot piece) shall not vary more than plus or minus oneeighth inch from the approximate dimensions given above. The distance between inside surfaces of the vertical bracket plates on the outer angle frame of the adjustable spring bottom shall not be the outer angle frame
less than $351 / 4$ inches. 
plate of wrought iron or of malleable iron with ninesixteenths inch diameter steel pins, securely fastened thereto, and so located as to accurately mesh with the female hook parts on posts on centers of $2 \frac{5}{8}$ inches as shown in the accompanying sketch. This plate riser shall be thoroughly riveted to the outer frame of the adjustable spring bottom. As an alternative, if approved by the purchaser, the corner lock may be of the contractor's own design provided after investigation it is demonstrated that the pro-
Lifting handles shall be omitted as unnecessary with the mechanical adjusting device. The fabric of the movable portions shall be the "national" type, made of tinned drawn wire about 0.117 inch diameter. The plates shall be of steel about 0.05 inch thick, flat and free from burrs and tinned after stamping. The fabric shall be suspended to sides and ends of frame with a sufficient number of black Japanned helical springs, not less than 18 of No. 12 ste-l wire gage 0.105 inch diameter each side, and

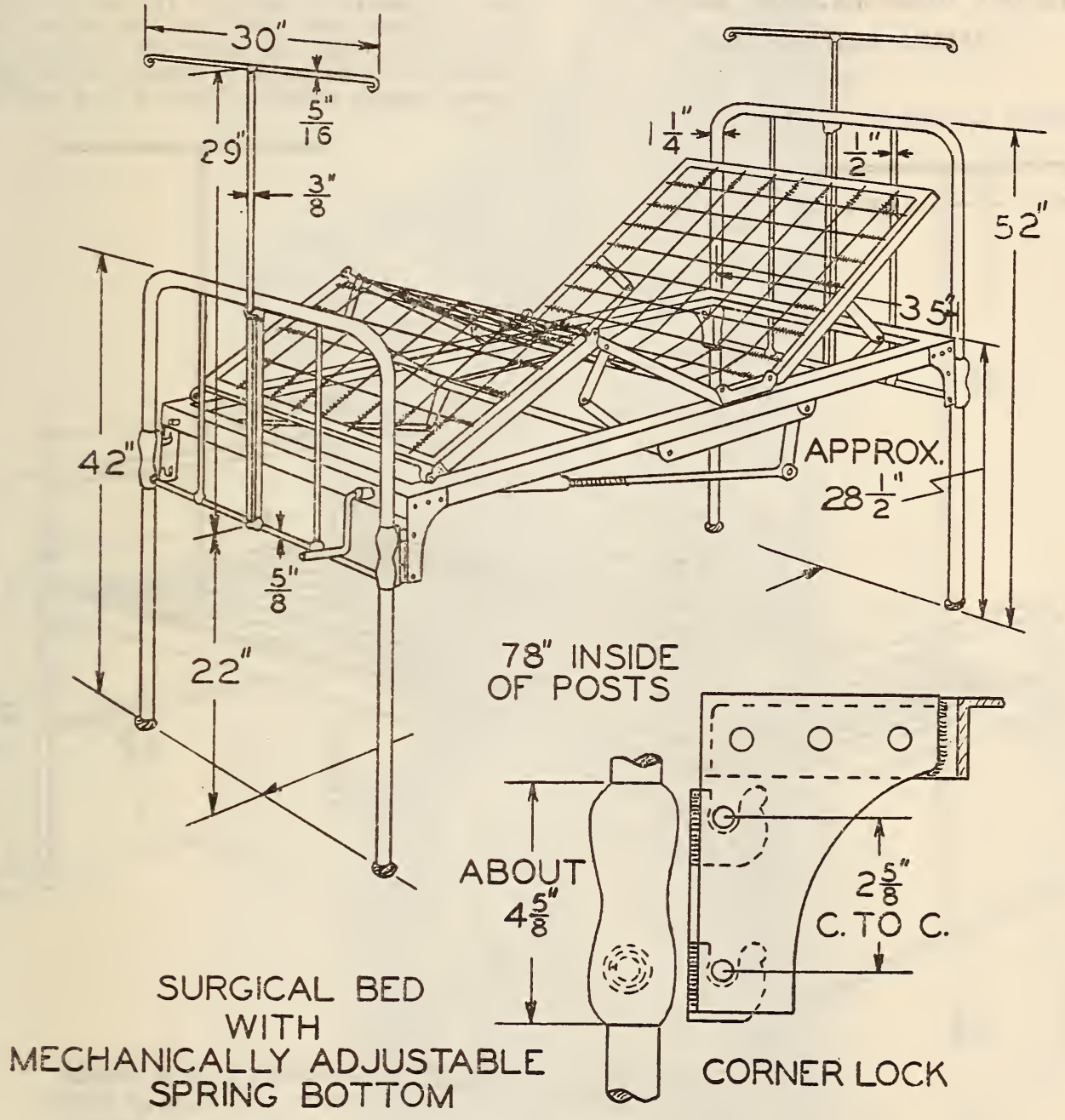

Figure 637

posed corner lock is the equivalent of a doublesocket connection in rigidity.

\section{FABRIC AND FABRIC FRAME (MECHANICALLY ADJUSTABLE: SPRING BOTTOM)}

9. The outer frame shall consist of $1 \frac{3}{4}$ by $13 / 4$ by $1 / 8$ inch angles. The inner frame shall consist of four sections made of suitably sized angles for strength and rigidity - three of the sections to ba movable and one section stationary. The movable sections shall be capable of being adjusted to a variety of heights and angles by means of mechanical levers operated by a removable handle located at the foot of the bed. The cross brace of the frame shall be bowed to allow sufficient space for sag of fabric with a heavy patient in bed, approximately 3 inches. not less than 2 and not more than 4 of No. 11 steel wire gage 0.12 inch diameter at each end.

(Detail requirements for mosquito bar rods and for wooden shoes or casters are the same as given in F. S. 186a (AA-B-201) above.)

\section{PACKING AND MARKING OF SHIPMENTS}

The fillers and cross rods shall be wrapped in paper and the posts shall be wrapped in excelsior pads, then boin head and foot pieces complete to be protected by fiber board of a width not less than 6 inches narrower than the heights of these end pieces covering both sides. This covering shall be tied laterally and longitudinally, and then the end pieces securely mounted on the under side of the spring frame. The ends and sides of spring frame shall be wrapped in excelsior pads. 
VII. NOTES

The sketch (fig. 637) showing the adjustable spring bottom is illustrative only and is not intended to show exact construction.

\section{UNITED STATES GOVERNMENT, FEDERAL SPECIFICATIONS BOARD, FEDERAL SPECIFICATION FOR FOLDING HOS- PITAL COT, NO. 188, JULY 30, 1924. (CURRENT DESIGNATION: AA-C-581) \\ FOLDING HOSPITAL COT TYPE} 1. Folding hospital cots shall be of but one type: $\mid$ rivet.

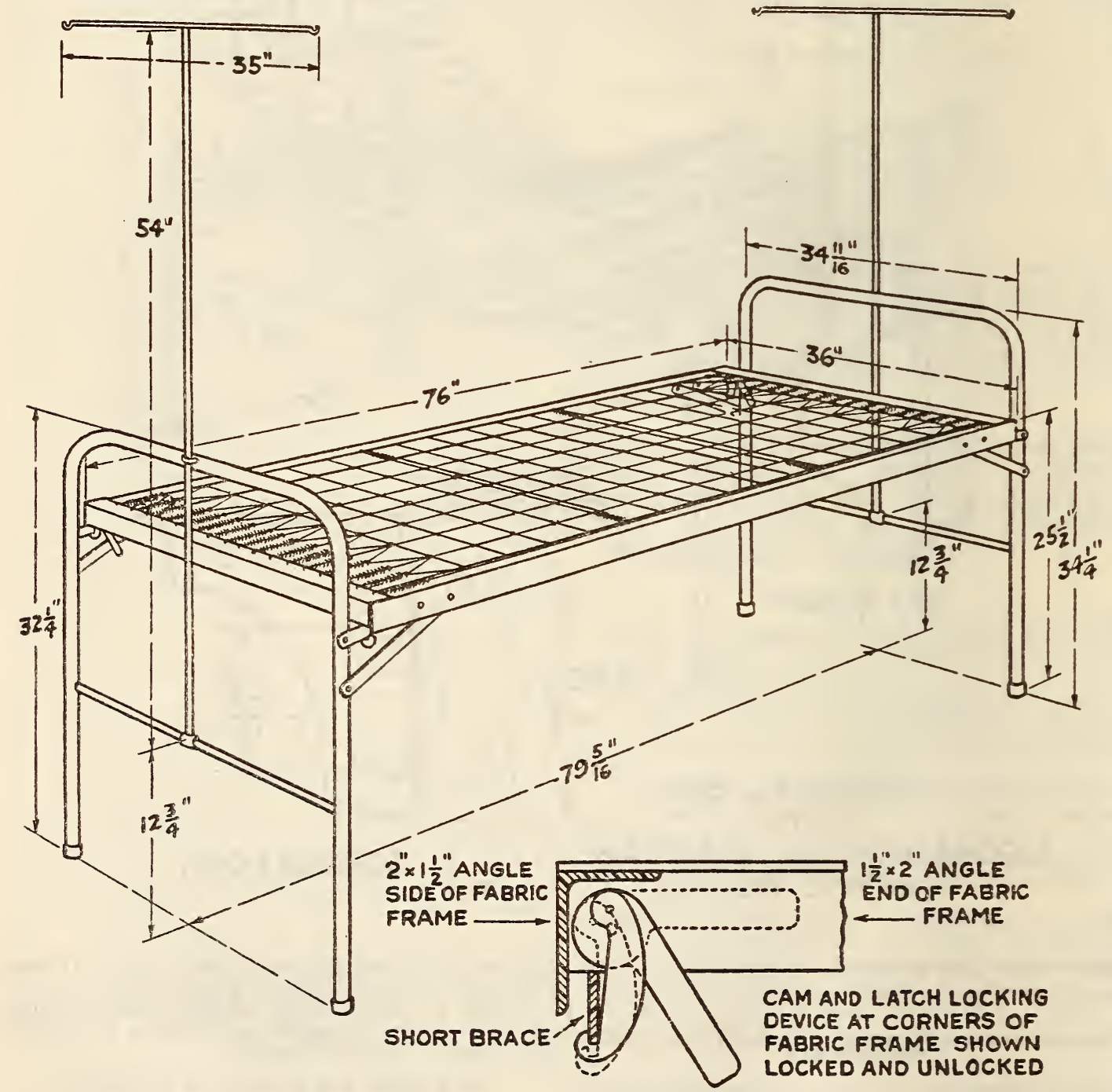

FIgURE 638.-Folding hospital cot

4. (a) Ends.-Ends made of butt welded or high carbon brazed tube (that will withstand bending without opening seams), outside diameter 1.05 inches with corners bent to a radius of not more than 6 inches so as to form continuous posts measuring $34^{11} 1 / 16$ inches wide outside, head end to stand $341 / 4$ inches high from the floor, foot end $321 / 4$ inches from the floor.

(b) Cross rods. - There shall be one cross rod $7 / 16$ inch in diameter in each end $123 / 4$ inches above the floor. These cross rods shall be carried at full thickness through the inside wall of pipe and be shouldered on the inside of outside wall of pipe and riveted against outside of pipe with a well-headed
MATERIAI AND WOREMANSHIP, ETC.

2. Folding hospital cots shall be of first class material and workmanship.

\section{GENERAI REQUIREMENTS}

3. Each cot shall be plainly marked with the manufacturer's name in such a manner that marking can not be made illegible. (c) Steel caps.-Steel caps shall be made of No. 20 gage steel or heavier, $3 / 4$ inch high crowned on end; to be firmly driven on ends of posts and center punched to hold same firmly in position.

(d) Fabric frame.-Fabric frame shall have side angles 76 inches long by 2 by $1 \frac{1 / 2}{\text { by }} 1 / 8$ inch and end angles 36 inches long, 2 by $1 \frac{1}{2}$ by $1 / 8$ inch high carbon steel, securely riveted at corners and braced in two corners of one end by 1 by $3 / 16$ inch flat steel braces 


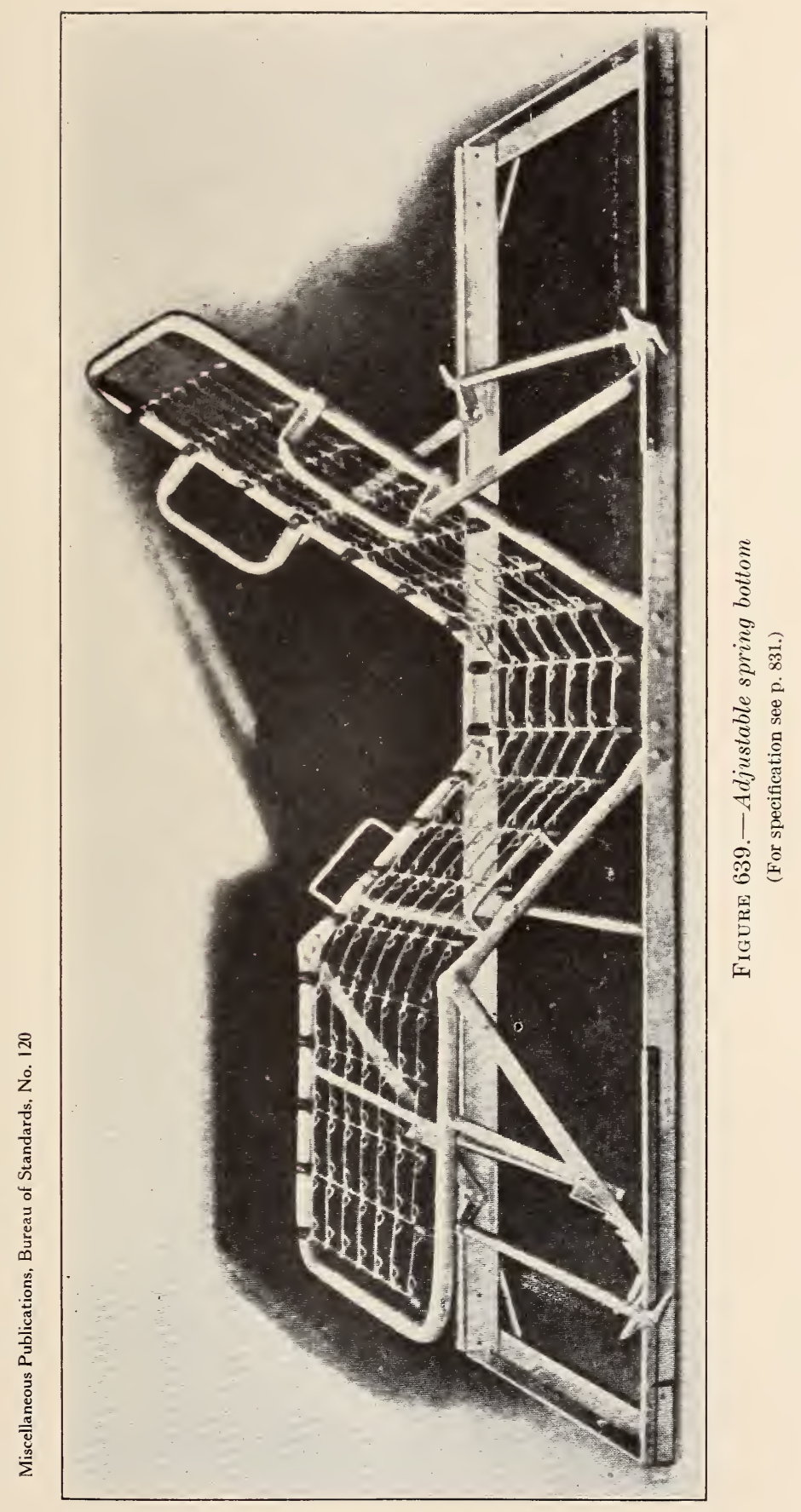



$73 / 4$ inches long, holes punched $63 / 4$ inches on center, riveted to frame diagonally across corners; holes in frame to be punched $6^{11} 116$ inches on center to prevent frame from twisting. These corner braces shall have offset of one-fourth inch to allow proper alignment of end helicals.

Ends shall fold under and within frame and be attached to frame at each corner by two 1 by 118 inch flat steel braces. Short brace shall have rivet holes 6 inches on center; holes in long brace 10 inches on center; the short brace to be offset so as to clear in folding. In riveting braces which connect the head and foot ends to fabric frame, the heads of rivets shall be placed against the movable piece; height from floor to top of fabric to be $25 \frac{1}{2}$ inches.

(e) Locking device.-There shall be placed at the four corners of the cot a locking device consisting of a cam latch riveted to the end angles of the cot. When the cot is set up the latch shall engage into the 1 by $1 / 8$ inch short brace, holding the cot firmly in position. The latch and lock bar shall be made in accordance with detail drawing forming a part of these specifications. Manufacturers are required to attach a tag to each bed showing action of locking device.

(f) Fabric.-Any double-wire link fabric made of not less than No. 14 steel wire gage galvanized wire 17 strands wide and not less than 15 links long, or any single link fabric of equal strength made of not less than No. 13 steel wire gage galvanized wire which can be interchangeable with a 17-strand double wire fabric, can be used subject to approval; fabric to be of a length that will insure proper tension when mounted on frame with helical springs as specified; fabric to be attached to frame at each end by 17 standard No. 12 steel wire gage 16-coil helical springs, high carbon steel, which are to be finished in black japan. No single continuous strand or wire fabric will be permitted.

(g) Support.-The support shall be made of fabric reinforced by two supporting bands made of 1 -inch No. 22 gage hoop steel, attached to side rails by four No. 12 steel wire gage 16 coil high carbon steel helical springs. These shall be located 26 inches from each end of the frame.

(h) $T$ canopy.-The canopy shall be made of seven sixteenth inch round iron, or soft steel; upright rod to be 4 feet 6 inches in length, extending through staple in the middle of head and foot ends on the outside as shown on detail drawing, forming a part of these specifications, and to rest in socket in the center of cross rod. Socket shall be made of $11 / 4$ by $1 / 8$ inch flat bar iron pressed on center of cross rod. Top cross arms of canopy shall be connected at top of upright with $\mathrm{T}$ ball connection and shall extend $17 \frac{1}{2}$ inches on opposite side of upright.

\section{METHOD OF INSPECTION, TESTS, ETC.}

5. Folding hospital cots shall be inspected as to conformity with these specifications.

\section{ADDITIONAL INFORMATION}

7. The sketch (fig. 638) accompanying these specifications is to be considered as forming a part thereof.

\section{UNITED STATES GOVERNMENT, FEDERAL SPECIFICATIONS BOARD, FEDERAL SPECIFICATION FOR ADJUSTABLE SPRING BOTTOM (GATCH FRAME), NO. 189 , JULY 30, 1924 (CURRENT DESIGNATION : AA-B-571)}

\section{ADJUSTABLE SPRING BOTTOM}

(Gatch Frame)

TYPE

1. Adjustable spring bottoms shall be of but one type.

\section{MATERIAI AND WORKMANSHIP}

2. Adjustable spring bottoms shall be of first-class material and workmanship.

\section{GENERAL REQUIREMENTS}

3. The frames of the adjustable spring bottom shall be finished in black or in aluminum as required.

\section{DETAIL REQUIREMENTS}

4. Fabric and fabric frame.-The outer frame shall consist of $13 / 4$ by $13 / 4$ by $1 / 8$ inch angles. The inner frame shall consist of four sections made from 1.05 inches diameter butt-welded tubing (national tube standard). Three of these sections shall be movable and one stationary. (See sketch). The movable sections shall be capable of being adjusted to a variety of heights and angles. Head and foot sections shall operate independently of each other from either side of the bed. Catches controlling the movement of head and foot sections shall be of ratchet type. The cross braces of inner Gatch frame shall be made of butt-welded steel tubing 1.05 inches diameter (national tube standard), and be so shaped as to allow sufficient space between it and the fabric with a heavy patient in the bed. Lifting handles on inner Gatch frame shall be of five-eighths inch butt-welded steel bedstead tubing. The fabric of the movable bottom shall be of the "national" type, thoroughly coated with pure tin applied by the molten bath process after assembling the fabric and before attaching to the frame. The fabric shall be suspended on each side with 18 No. 12 steel wire gage ( 0.1055 inch diameter) black-japanned helical springs and on each end with 2 No. 11 steel wire gage (0.1205 inch diameter) black-japanned helical springs.

METHOD OF INSPECTION, TESTS, ETC.

5. Adjustable spring bottoms shall be inspected as to conformity with these specifications.

\section{PACKING AND MARKING}

6. Adjustable spring bottoms shall be first papered and then carefully packed in excelsior pads.

\section{ADDITIONAL INFORMATION}

7. The adjustable spring bottom described in Figure 639 is intended for use on the standard folding hospital cot. The outer frame of the adjustable bottom shall fit the dimensions of the folding hospital cot which is covered in separate specifications. 
UNITED STATES GOVERNMENT, FEDERAL SPECIFICATIONS BOARD, FEDERAL SPECIFICATION FOR LIBERTY STEEL COT, NO. 190, JULY 30, 1924. (CURRENT DESIGNATION : AA-C-591)

\section{LIBERTY STEEL COT}

\section{TYPE}

1. Liberty steel cots shall be of but one type.

MATERIAL AND WORKMANSHIP, ETC.

2. Liberty steel cots shall be of first-class material and workmanship. (b) Cross rods. - There shall be one cross rod $7 / 16$ inch in diameter in each end 7 inches above the floor; these cross rods to be carried at full thickness through the inside wall of pipe and to be shouldered on the inside of outside wall of pipe and riveted against outside of pipe with a well-headed rivet.

(c) Steel caps.-Steel caps shall be made of No. 20 U. S. standard gage steel or heavier, three-fourths inch high crowned on end; to be firmly driven on ends of posts and center punched to hold same firmly in position.

(d) Fabric frame.-Fabric frame shall have side angles 76 inches long by 2 by $1 \frac{11}{2}$ by $1 / 8$ inch and end angles 30 inches long, 2 by $1 \frac{1}{2}$ by $1 / 8$ inch high carbon steel, securely riveted at corners and braced at two corners of one end by 1 by $3 / 16$ inch flat steel braces $73 / 4$ inches long, holes punched $63 / 4$ inches on

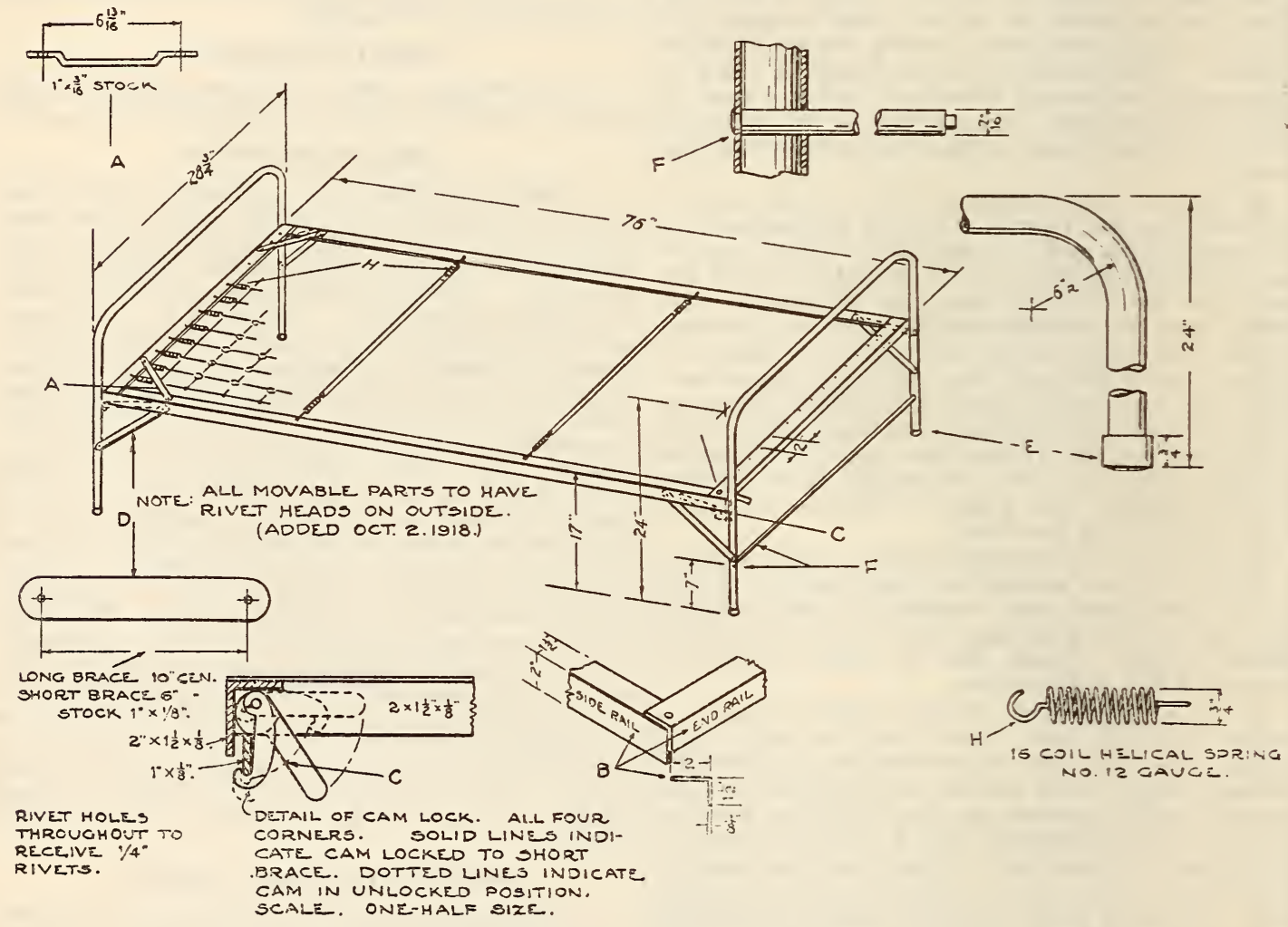

FiguRe 640.-Liberty steel cot

\section{GENERAL REQUIREMENTS}

3. (a) Entire cot shall be finished in gray or black japan or gray or black metallic paint, except fabric, which shall be made of galvanized wire. Support shall be finished in gray or black japan or gray or black metallic paint.

(b) Each cot shall be plainly marked with the manufacturer's name in such a manner that marking can not be made illegible.

\section{DETAIL REQUIREMENTS}

4. (a) Ends.-Ends shall be made of butt welded or high carbon brazed tube (that will withstand bending without opening seams) outside diameter 1.05 inches with corners bent to a radius of not more than 6 inches so as to form continuous posts, measuring $283 / 4$ inches wide outside and to stand 24 inches high from the floor. center, riveted to frame diagonally across corners; holes in frame to be punched $6^{11 / 16}$ inches on center, to prevent frame from twisting. These corner braces to have ofiset of $1 / 4$ inch to allow proper alignment of end helicals.

Ends shall fold under and within frame and be attached to frame at each corner by two $1-b r-1 / 8$-inch flat steel braces. Short brace to have rivet holes 6 inches on center; holes in long brace 10 inches on center; the short brace to be offset so as to clear in folding. In riveting braces which connect the head and foot ends to fabric frame the heads of rivets shall be placed against the movable piece; height from floor to top of fabric to be 17 inches.

(e) Locking device.-There shall be placed at two corners of the cot diagonally opposite a locking device consisting of a cam latch riveted to the end angle of the cot. When the cot is set up the latch shall engage into the 1 by $1 / 8$ inch short brace, holding the cot firmly in position. The latch and lock bar shall 
be made in accordance with detail drawing. Manufacturers are required to attach a tag to each bed showing action o locking device.

(f) Fabric. - Any double-wire link fabric made of not less than 14 gage galvanized wire 14 strands wide and not less than 15 links long, or any single-link fabric of equal strength made of not less than No. 13 gage galvanized wire, which can be interchangeable with a 14-strand double-wire fabric can be used subject to approval; fabric to be of a length that will insure proper tension when mounted on frame with helical springs as specified; fabric to be attached to frame at each end by 14 standard No. 12 gage, 16-coil helical springs, high-carbon steel, which shall be finished in black japan. No single continuous strand or wire fabric shall be permitted.

(g) Support.-The support shall be made of fabric reinforced by two supporting bands made of $3 / 4$-inch No. 22 gage hoop steel, attached to side rails by four No. 12 gage 16-coil high-carbon steel helical springs. These shall be located 26 inches from each end of the frame.

\section{METEOD OF INSPECTION, TESTS, ETC.}

5 Liberty steel cots shall be inspected as to con. formity with these specifications|

\section{ADDITIONAL INFORMATION}

7. Sketch (fig. 640) accompanying these specifications is to be considered a part thereof.

\subsection{BOOK CASES.}

UNITED STATES GOVERNMENT, FEDERAL SPECIFICATIONS BOARD, FEDERAI SPECIFICATION FOR FURNITURE AND CABINETS; OFFICE, SECTIONAL, STEEL, AA-F-791, NOVEMBER 11, 1930

\section{BOOKCASE SECTIONS WITH DISAPPEARING GLASS DOORS}

(Specifications for these sections will be found in the complete text of the specification $\mathrm{AA}-\mathrm{F}-791$. See 613.7, p. 850 .)

\subsection{CHAIRS AND STOOLS.}

(No nationally recognized specifications available for metal chairs and stools.)

\subsection{DESKS.}

UNITED STATES GOVERNMENT, FEDERAL SPECIFICATIONS BOARD, FEDERAL SPECIFICATION FOR DESKS, STEEL, AAD-191, MARCH 31, 1931

\section{STEEL DESKS}

\section{A. APPLICABLE FEDERAL SPECIFICATIONS.}

A-1. There are no Federal specifications applicable to this specification.

\section{B, TYPE}

B-1. This specification includes the following types of desks:

B-1a. Flat top, single pedestal desks.

B-1b. Flat top, double pedestal desks.

B-1c. Flat top, double desks.

B-1d. Typewriter desks, drop center, single pedestal, flat top.
B-1e. Typewriter desks, drop center, double pedestal, flat top.

B-1f. Typewriter desks, double pedestal, pedestal device, flat top.

B-1g. Roll top, double pedestal desks.

\section{MATERIAL AND WORKMANSHIP, ETC.}

C-1. All exposed sheet steel used for exposed surfaces to be open hearth steel, full pickled, full cold rolled, stretcher leveled, resquared, deoxidized, and oiled. All other sheets to be single pickled, full cold rolled, deoxidized, and oiled.

C-2. All workmanship shall be the best known to the trade.

\section{GENERAL REQUIREMENTS}

D-1. Sizes of the various desks to be approximate commercial dimensions, but minor variations will be permitted except in height, which shall be $301 / 2$ inches from floor to top of writing bed.

\section{E. DETAIL REQUIREMENTS}

E-1. All desks shall be of the sanitary type; the distance from floor to bottom of pedestal shall be approximately $8 \frac{1}{2}$ inches.

$\mathrm{E}-2$. Construction shall be knock-down, permitting quick assembly or the replacement of any section that might become damaged or broken.

E-3. General construction shall be such as to insure rigidity.

E-4. All exposed edges of steel sheets, if not folded, beaded, or flanged, shall be so filed or otherwise smoothed as to avoid sharp cutting edges.

E-5. Finish.-

E-5a. All metal surfaces shall be thoroughly cleaned and free from dirt, oil, grease, and moisture before applying the finish.

E-5b. Exposed surfaces.-After a thoroughly reliable priming coat and filler, where necessary, the color coats shall be sufficient when properly dried so that a surface guaranteed not to crack or spall under severe usage will be produced. The final coat shall be a dull egg shell or satin finish. The final colors shall harmonize with the standard colors adopted by the General Supply Committee, Washington, D. C.

E-5c. All unexposed surfaces or component parts to be carefully coated to harmonize with outside color.

$\mathrm{E}-5 \mathrm{~d}$. In addition to the above requirements, whenever especially called for in the purchase specifications to take care of tropical climatic conditions, all bolts, nuts, screws, small levers, or similar parts, shall be made of brass or galvanized steel. All other parts shall be rust resistant metal or they shall be treated with a rustproof coating. Bidder shall give a complete description of the methods he proposes to use in applying the rust resisting coating and finish coat, in order to prevent the coating from chipping off.

E-6. Hardware.-

E-6a. Drawer pulls of hocded type similar in appearance to those supplied on wood desks, or bar pulls of brass or bronze brush finish may be used. Pulls shall be securely attached by concealed fasteners but in such a manner as to be easily replaced.

E-6b. Drawer locks shall be brass case cabinet locks, two flat keys each. Curtain locks shall have pin tumblers and corrugated flat keys. Shall lock into sweep arms, not into bed of desk, center drawer to have separate lock and to automatically lock all drawers on the sides. Drawer locking device shall be so built as to permit a drawer left open to be closed after center drawer is locked. 


\section{E-7. Drawers.-}

E-7a. Double-wall construction shall be used for drawer fronts. Outside drawer heads shall be constructed of 20-gage steel, inner head of at least 24gage steel, body of at least 24-gage steel, back of at least 22-gage steel. The top edges of sides shall be either folded, flanged, $r$ beaded.

E-7b. Assembly shall be by electric spot welding or lock joints reinforced where necessary by acetylene or arc welding, to insure strength and rigidity. Box drawers shall be mounted in channel suspension slides, but progressive suspension slides will be preferred for letter file or similar drawers. All drawer suspensions shall be so constructed as to reduce friction and noise in operating the drawers.

E-8. Fittings.-

E-8a. Lower drawers in right side of flat and roll top desks, except typewriter desks, shall be double height and be fitted with vertical filing device fitted with compressor block or follower plates to support the drawer contents in its proper reference position. Double desks shall have both sides so fitted. Rolltop desks shall have pigeonhole case fitted with document box drawers and private compartment with door fitted with lock.

E-9. Tops.- Shall be of at least 18-gage steel, rigidly reinforced and covered with $1 / 8$-inch battleship linoleum or equal, as approved. Linoleum to be uniformly cemented to steel tops in a manner that will insure permanent adhesion. Edges of linoleum to be protected and secured by rounded bronze strip, closely fitting to the linoleum, the strips to be attached with concealed screws or by other secure methods. If binding strip is not a sufficient width, the edge of top beneath the bronze strip shall be finished off by steel binding strip which shall be securely fastened by screws, rivets, or adequate means to the top, making the over-all thickness of top, including the linoleum not less than $1 \frac{1}{2}$ inches. This strip may be an integral part of the top itself.

E-10. Sliding shelves.-Shall be of at least 18-gage steel, and pan-type construction. Linoleum top covering need not be furnished unless especially asked for in the purchase specifications. Suitable bumpers or coil springs shall be provided to stop shelf when shelf is extended to limit or forced into the pedestal.

E-11. Legs.-Shall be of at least 18-gage steel, approximately 2 by 2 inches square, closed on all sides below pedestal and securely attached to panel and pedestal by means of bolts, welding, or other secure methods or they may be designed so that by means of suitable slots, the panels may be fitted into them so as to be retained in the legs without use of bolts or other fastenings. When legs are not tubular their entire length a 16-gage angular or curved reinforcing member not less than 10 inches long, and extending not less than 5 inches above and below lower edge of pedestal, shall be welded to inside of each leg. Brass or bronze shoes at least $1 \frac{1}{2}$ inches high, securely fastened to the legs shall be provided. The interior legs may be omitted.

E-12. Panels.-Outside panels shall be of at least 20-gage steel and inside panels shall be of at least 22-gage steel and all panels shall be flanged and reinforced or other suitable means shall be used to insure rigidity and prevent vibrations.

E-13. Curtains or rolls. - Shall be made of slats of at least 24-gage strip to provide interlocking of adjacent slats. Interlocking methods to provide flexibility of operation and security from pulling apart.

E-14: Typewriter beds.-Shall be rigid, give clearance over the desk bed for all standard ma- chines, and shall be constructed of at least 16-gage sheet steel flanged on all sides and reinforced to prevent vibration. Device on which bed operates shall be substantial in construction, easily operated, and shall be securely attached to a rigid part of the desk.

\section{F. METHOD OF INSPECTION AND TESTS}

F-1. All desks may be inspected at the factory by a representative of the purchaser at any time during process of manufacture, the purpose of this inspection being to determine compliance with the terms of the specification as to workmanship, material, details of construction, hardware, and finish. Final inspection will be made at the point of delivery.

\section{G. PACKING AND MIARING}

$\mathrm{G}-1$. Unless otherwise specified, the subject commodity shall be delivered in standard commercial containers, for safe transportation, at the lowest rate, to the point of delivery.

G-2. Each desk shall be clearly marked in an inconspicuous place showing the name of the manufacturer. Unless otherwise specified shipping containers shall be marked with the name of the material, the size, type, and the quantity contained therein, as defined by the contract or order under which the shipment is made, the name of the contractor, and the number of the contract or order.

\section{NOTES}

I-1. All gages mentioned are United States standard gages.

I-2. Purchasers shall exercise all options offered herein and should specify type, grade, class, etc., required.

I-3. It is believed that this specification adequately describes the characteristics necessary to secure the desired material, and that normally no samples will be necessary prior to award to determine compliance with this specification. If, for any particular purpose, samples with bids are necessary, they should be specifically asked for in the invitation for bids, and the particular purpose to be served by the bid sample should be definitely stated, the specification to apply in all other respects.

\subsection{LOCKERS AND SAFES.}

\section{UNDERWRITERS' LABORATORIES, PRO- POSED STANDARD ON MINIMUM BURGLARY RESISTANCE OF KEY- LOCKED SAFES (CLASS K L), 1927}

\section{KEY-LOCKED SAFES}

(Specification covers practicability and durability of safe and locking mechanism, time limit on resistance to burglar attack, mounting requirements, tests for lock picking, drifting and drilling, door sledging and wedging, dismounting and fishing test.)

\section{UNDER WRITERS' LABORATORIES, STAND- ARD FOR RELOCKING DEVICES FOR SAFE AND VAULT DOORS, 1927}

\section{RELOCKING DEVICES}

(Applies to relocking devices for safes and chests, light vault doors, and heavy vault doors. Requirements cover design and construction, practicability, durability, reliability of operation, and tests for relocking when combination is tampered with.) 
UNDERWRITERS' LABORATORIES, STANDARD FOR SAFES AND INSULATED CABINETS, 1918

\section{SAFES AND INSULATED CABINETS}

(Safes and insulated cabinets are classified according to their fire retardent and impact resistive properties. Safes are separated into three classesclass A, class B, and class C. All three classes are effective against fairly heavy impacts. As fire retardents, class $\mathrm{A}$ is effective against severe fires for at least four hours, class B for at least two hours, class $\mathrm{C}$ for at least one hour. Insulated cabinets are effective against fires of moderate severity for at least 45 minutes, but are not subject to impacts from falling or from falling bodies. Specification also includes general design, strength requirements, and fire stream tests.)

UNITED STATES GOVERNMENT, DEPARTMENT OF COMMERCE, BUREAU OF STANDARDS, SIMPLIFIED PRACTICE RECOMMENDATION FOR STEEL LOCKERS, R35-28, FEBRUARY 1, 1928

\section{STEEL IOCKERS}

(Simplified practice recommended and accepted by industry establishing a limited list of standard stock sizes for single, double, and multiple tier steel lockers.)

TABLE 1.-Single tier

\begin{tabular}{|c|c|c|c|}
\hline Width 1 & Depth & Height & $\begin{array}{c}\text { Maximum } \\
\text { over-all height, } \\
\text { including leg }\end{array}$ \\
\hline Inches & Inches & Inches & Inches \\
12 & 12 & 60 & 66 \\
12 & 15 & 60 & 66 \\
12 & 18 & 60 & 66 \\
15 & 15 & 60 & 66 \\
15 & 18 & 60 & 66 \\
18 & 21 & 60 & 66 \\
12 & 12 & 72 & 78 \\
12 & 15 & 72 & 78 \\
12 & 18 & 72 & 78 \\
15 & 15 & 72 & 78 \\
15 & 18 & 72 & 78 \\
18 & 18 & 72 & 78 \\
18 & 21 & 72 & \\
\hline
\end{tabular}

1 This dimension does not include the projecting boltheads. When filling fixed spaces with rows of lockers allowance should be made for suchiprojections.

TABLE 2.-Double tier

\begin{tabular}{|c|c|c|c|}
\hline Width 1 & Depth & Height & $\begin{array}{c}\text { Maximum } \\
\text { over-all height, } \\
\text { including leg }\end{array}$ \\
\hline Inches & Inches & Inches & Inches \\
12 & 12 & 30 & 66 \\
12 & 12 & 36 & 78 \\
12 & 15 & 36 & 78 \\
15 & 15 & 36 & 78 \\
12 & 15 & 42 & 290 \\
15 & 15 & 42 & 290 \\
\hline
\end{tabular}

1 This dimension does not include the projecting boltheads. When filling fixed spaces with rows of lockers allowance should be made for such projetcions.

When 3 -inch legs are used the over-all height will be 87 inches.
TABLE 3.-Multiple tier (box)

\begin{tabular}{|c|c|c|c|c|}
\hline Lockers per stack & Width 1 & Depth & Height & $\begin{array}{c}\text { Maximum } \\
\text { over-all } \\
\text { height, } \\
\text { including } \\
\text { leg }\end{array}$ \\
\hline $\begin{array}{l}5 \text { or } 6 \\
5 \text { or } 6 \\
5\end{array}$ & $\begin{array}{r}\text { Inches } \\
12 \\
12 \\
15 \\
15\end{array}$ & $\begin{array}{r}\text { Inches } \\
12 \\
15 \\
15 \\
15\end{array}$ & $\begin{array}{c}\text { Inches } \\
12 \\
12 \\
15 \\
1425\end{array}$ & $\begin{array}{l}\text { Inches } \\
66 \text { or } 78 \\
66 \text { or } 78 \\
66 \\
78\end{array}$ \\
\hline
\end{tabular}

1 This dimension does not include the projecting boltheads. When filling fixed spaces with rows of lockers allowance should be made for such projections.

\section{Lockers purchased as samples}

Single tier. 12 by 15 by 60 inches.

Double tier

Multiple tier.................... 12 by 15 by 12 inches ( 5 high).

Where recess installation is to be made, the recess height should be $41 / 2$ inches less than the maximum over-all height of corresponding leg lockers.

The recommended sizes for single, double, and multiple tier lockers are supplied in single row construction (wall type) and in double row construction (back to back type).

UNITED STATES GOVERNMENT, FEDERAI
SPECIFICATIONS BOARD, FEDERAI SPECIFICATION FOR CABINETS; STATIONARY, STORAGE, AND CLOTHING (STEEL), AA-C-31, FEBRUARY 2, 1932

\section{STEEL STORAGE CABINETS}

\section{B. TYPES}

Type I. Single-door clothing cabinet.

Type II. Double-door clothing cabinet.

Type III. Single-door stationery and storage cabinet.

Type IV. Double-door stationery and storage cabinet.

Type V. Janitors' single-door storage cabinet.

Type VI. Janitors' double-door storage cabinet.

\section{MATERIAL AND WORKMANSHIP}

C-1. General.- Material shall be the best quality of the kind specified. It shall be free from defects and imperfections which impair the appearance or usefulness of the products.

C-2. Steel sheets. - Shall be open-hearth steel, cold rolled for tops, bases, sides, backs, shelves, and structural parts generally.

C-3. Hardware.-Hinges shall be of good quality commercial steel. Door handles shall be of formed or solid bronze or brass, malleable iron, cast steel, or formed steel suitable for the purpose.

C-4. Workmanship.-The workmanship on all parts shall be first class in every respect and of a type to insure rigidity of the assembled articles.

\section{GENERAL REQUIREMENTS}

D-1. Strength and rigidity of parts shall be secured by flanging or reinforcing so they will properly perform the function for which they are designed. Special precautions shall be used to insure proper alignment of doors with door openings or frames so that doors may be freely operated and latched. All bases shall be closed. 
D-2. All cabinets shall have the maker's name in full or a trade-mark appearing in an inconspicuous place on same. The name and marks on cabinets for foreign shipment shall comply with existing export regulations.

D-3. Locks on equipment furnished on any order shall be different or alike, as ordered, and shall be master-keyed only if so ordered. Two keys shall be supplied with each cabinet and two master keys with each order when master keys are ordered.

$\mathrm{D}-4$. The general assembly of parts shall be by electric spot-welding or oxy-acetylene welds or bolted together with rustproof bolts. No spot weld shall exceed three-sixteenths inch in diameter. If bolted, no exposed bolts shall show on front or ends. All bolt holes in adjoining parts shall be true and even.

D-5. Finish.

D-5a. All parts shall be thoroughly cleaned and dried before finishing materials are applied. The finish shall consist of not less than two coats of baked enamel or a baked primer and two coats of pyroxylin lacquer. The standard color to be olive green. When specifically ordered, cabinets shall be furnished in mahogany or walnut.

D-5b. Handles shall be finished in baked enamel or to resemble brass or bronze or nickel plated. The hinges shall harmonize with the finish of the cabinet or locker.

D-6. Patent features shall be owned or licensed by the manufacturer, who shall assume full responsibility and protect the purchaser.

\section{E. DETAIL REQUIREMENTS}

E-1. Sizes of cabinets.-Approximate outside dimensions:

E-1a. 36 inches wide (18 and 24 inches) deep and 72 inches high.

E-1b. 24 inches wide (18 and 24 inches) deep and 72 inches high.

$\mathrm{E}-2$. Interior equipment.

E-2a. Clothing cabinets, Types I and II, shall be equipped with a hat shelf and one coat rod extending the full width of shelf.

E-2b. Stationery or storage cabinets, Types III and IV, shall be equipped with or without adjustable shelves as called for in the invitation for bids.

E-2c. Janitors' storage cabinets, Types V and VI, shall be equipped with one top shelf, underside fitted with not less than two ceiling hooks.

E-3. Shelves. - All cabinets shall provide for adjustment of shelves on not more than 2 -inch centers. Extra shelves may be ordered for any cabinet if required.

E-4. Doors.-Single doors shall be used for widths up to 24 inches, and double doors for widths greater than 24 inches. Substantial hinges shall be provided, not less than three for each door; if of butt type, they shall have tight pins. Single doors or swinging doors of those in pairs shall have $T$ or lever handles for operating the latching mechanism. The standing door of those in pairs shall be provided with a rigid $T$ or lever handle, and a suitable means for interlocking with the other door. Each door shall be reinforced with flanged edges and a stiffening panel.

E-5. Latching mechanism.-Single doors and swinging doors of those in pairs shall be provided with a latching device that makes engagement at three points. The latching mechanism shall be controlled by a suitable flat key lock, master-keyed when so specified.

E-6. Gages of parts.-The following are minimum gages of parts-U. S. standard gage:

E-6a. Tops, No. 22 gage, 0.0306 inch.

E-6b. Backs, No. 22 gage, 0.0306 inch.
E-6c. Twenty-four inch bottoms, No. 20 gage, 0.0368 inch.

E-6d. Thirty-six inch bottoms, No. 18 gage, 0.0490 inch.

E-6e. Doors, No. 20 gage, 0.0368 inch.

E-6f. Shelves (36 inches), No. 18 gage, 0.0490 inch:

E-6g. Shelves (24 inches), No. 20 gage, 0.0368 inch.

E-6h. Sides, No. 22 gage, 0.0306 inch.

\section{F. METHODS OF SAMPLING, INSPECTION, AND TESTS}

F-1. The manufacturer shall permit a representative of the Government, at any time, to make inspections of all products during process of manufacture and such tests as may be necessary to ascertain that proper materials are being used. Final inspection will be made at point of delivery. All defective parts or articles shall be replaced by the manufacturer without cost to the purchaser.

\section{G. PACKING AND MARKING}

G-1. Articles when shipped from factory shall be so crated, packed or wrapped, as to insure delivery in first-class condition. Packages shall be clearly marked. When intended for foreign delivery, packing shall be in accordance with rules of the Department of Commerce.

G-2. Shipping containers or packages shall be marked with the name of the material, the type and quantity contained therein as defined by the contract or order under which shipment is made, the name of the contractor, and the number of the contract or order.

\section{UNITED STATES GOVERNMENT, FEDERAL SPECIFICATIONS BOARD, FEDERAL SPECIFICATION FOR CASES; TRANSFER, STEEL, AA-C-121, FEBRUARY 2, 1932}

\section{STEEL TRANSFER CASES}

$$
\text { B. TYPES }
$$

B-1. Type I.-For invoice-size papers; outide depth, 25 inches; inside dimensions of drawer to be not less than 10 inches wide by 22 inches deep by $8 \frac{1}{2}$ inches high.

B-2. Type II.-For letter-size papers; outside depth, 25 inches; inside dimensions of drawer to be not less than 12 inches wide by 22 inches deep by $10 \frac{1}{2}$ inches high.

B-3. Type III.-For cap-size papers; outside depth, 25 inches; inside dimensions of drawer to be not less than $15 \frac{1}{4}$ inches wide by 22 inches deep by $10 \frac{1}{2}$ inches high.

B-4. Type IV.-For sheets 10 by 16 inches; outside depth, 25 inches; inside dimensions of drawer to be not less than $16 \frac{1}{2}$ inches wide by 22 inches deep by $11 \frac{1}{4}$ inches high.

\section{MATERIAL AND WORKMANSHIP}

C-1. All sheet steel shall be roller leveled and all workmanship shall be the best known to the trade.

\section{GENERAI REQUIREMENTS}

D-1. Finish.

D-1a. All metal surfaces shall be subjected to cleaning and shall be thoroughly dried before they are treated further.

D-1b. Parts and their members shall have a priming coat and all exposed surfaces, two coats of enamel baked on the finish coat to be an egg-shell gloss to match the general supply committee's 
standard green, or other color designated by the contracting officer.

D-2. All sections shall intermember with and match in size the standard sections adopted by the general supply committee.

D-3. All exposed edges of steel sheets shall be so rounded or smoothed as to avoid sharp-cutting edges.

\section{E. DETAIL REQUIREMENTS}

E-1. Case body.

E-1a. The case body shall be construcied of sheet steel not lighter than 24-gage (0.0245 inch), shall have closed sides and back, and shall have front and back edges reinforced with a band in the body or back. The band shall be not less than one-eighth inch thick and not less than $1 \frac{1}{4}$ inches wide.

E-2. Drawers.

E-2a. Shall have closed backs and shall operate on steel-roller bearings provided at the bottom front of the case body. These rollers shall be at least onehalf inch in diameter and three-eighths inch wide. Drawer bottoms shall be so constructed or reinforced as to prevent these rollers from indenting or corrugating the bottom of the drawer when loaded to capacity.

E-2b. Drawer fronts shall be provided with suitabla label holders and drawer pulls securely fastened thereto.

E-2c. Compressors shall be of durable construction, shall have strong positive grips and shall be capable of easy adjustment. They shall be readily removable and shall not operate upon a guide rod.

E-2d. Assembly of drawers and case body shall be by spot weld spaced not more than 4 inches apart.

$\mathrm{E}-2 \mathrm{e}$. Front head of drawers shall be at least 22gage ( 0.0306 inch), and sides and rear head shall be at least 24-gaga steel (0.0245 inch).

E-2f. Bolts and holes shall be provided for interlocking the cases, horizontally at the front and back. A device shall be provided at the back to prevent the tilting of the case when ihe drawer is extended. This device shall operate independent of the interlocking bolts.

$\mathrm{E}-3$. Leg bases.

E-3a. Shall be $81 / 2$ inches high, 25 inches deep, and 6 inches under the rail. At least 20 gaga (0.0368 inch), sheet steel shall be used in the base construction.

E-3b. Legs shall be securely fastened to the rail, shall be approximately 2 inches square and so formed as to be rigid and give a sufficiently smooth bearing surface to prevent undue marring of floors.

\section{F. METHOD OF INSPECTION AND TESTS}

F-1. All sections may be inspected at the factory by a representative of the Government at any time during the process of manufacture. Final inspection will also be made at point of delivery.

\section{G. PACKAGING, PACKING, AND MARKING}

G-1. Packaging.-Unless otherwise specified, commercial packages are acceptable under this specificavion.

G-2. Packing.-Unless otherwise specified, the subject commodity shall be delivered in standard commercial containers so constructed as to insure acceptance by common or other carrier for safe transportation at the lowest rate to the point of delivery.

G-3. Marking.-Shipping containers or packages shall be marked with the name of the material, the type, and quantity contained therein, as defined by the contractor or order under which shipment is made, the name of the contractor, and the number of the contract or order.

\section{UNITED STATES GOVERNMENT, FEDERAL SPECIFICATIONS BOARD, FEDERAL SPECIFICATION FOR SAFES, INSULATED, AA-S-81, DECEMBER 8, 1931}

\section{INSULATED SAFES}

\section{A. APPIICABLE FEDERAL SPECIFICATIONS}

A-1. There are no other Federal specifications applicable to this specification.

\section{B. CLASSES}

B-1. This specification covers three classes of safes as determined by fire resistance as follows:

Class A. Having 4-hour firer esistance as defined in section E-2.
Class B. Having 2-hour fire resistance as defined in section E-2.

Class C. Having 1-hour fire resistance as defined in section E-2.

\section{MATERIAL AND WORKMANSHIP}

C-1. Material.-Structural shapes shall be of wrought iron or open-hearth steel and plates and sheets of open-hearth or Bessemer sheet stock, or other material of at least equal tensile strength.

C-2. Workmanship. - All joints between sheets, plates, and shapes shall be made to develop the required strength of the assembly, and to prevent undue openings when exposed to fire or impacts.

\section{GENERAL REQUIREMENTS}

D-1. Insulation.-The insulation and design of the safe shall be such as to prevent sweating and swelling from the insulation, appreciable loss of insulating value with age, or disruption from explosion of gases generated within it when exposed to fire. The insulation shall be so molded, jointed, or reinforced as to preserve its insulating function when safe is subjected to fire and impacts.

D-2. Rollers and casters. - The safes shall be supported on heavy swivel casters with antifriction bearings or on wheels properly proportioned for the weight of the safe. They shall be secured to the safe by means of screws or bolts in such a manner as not to unduly strain any portion thereof and make possible replacement from the outside. Steel housings may be welded to the supporting plate.

D-3. Door details.

D-3a. The outer surface of the doors shall be made to fit each other and the opening, with no greater face clearance than one-sixteenth inch. The maximum clearance within the door joint on at least one line shall not be more than one-sixteenth inch. A gasket of asbestos not more than oneeighth inch thick suitably filled and attached, a flexible steel strip not more than one-thirty-second inch thick, or equivalent of either of these, may be used to assist in meeting this requirement. The hinges shall be of steel, semisteel, or malleable iron, securely attached to the door and doorframe, reinforcing plates being used where necessary. Centers of hinges are to be placed not more than 9 inches from top and bottom edges of doors. When the distance between those centers exceeds 30 inches a third similar hinge shall be placed at the middle of the height. One hinge shall be added for each additional 18 inches in height. These maximum spacings apply to the heavier hinges. The lighter hinges must be given closer spacings.

D-3b. For doors weighing over 220 pounds, ball race bearings and hardened steel balls shall be provided between knuckles or other means to prevent undue wear and friction. Where the conditions of use require it, such as location of safe against a pier between windows or close to side walls, purchasing officers may specify a $250^{\circ}$ swing for the door, or 
may specify the maximum distance beyond the end faces of the safe that any portions of the door or doors must be swung in order to make the whole interior equipment of the safe removable. Doors shall be provided at the back or hinge side with not less than two fixed or hand-operated bolts, engaging suitably cupped holes, in addition to the regular locking bolts, or the hinge side of the door shall have one or more projecting heels fitting into continuous recesses in the jamb. The joint between double doors shall have one or more tongue and groove interlocks.

D-4. Locking requirements. - The safes shall be designed and equipped to provide such lock protection as is consistent with the general construction. All parts of the locking mechanism are to be suitably protected against corrosion and be metal inclosed or otherwise protected from dust, corrosion, or other effects from the insulation, and be protected against contact with the safe contents. The inside doorplate shall be removable to give access to lock and bolt work.

D-4a. Locking bolts.-All single doors or swinging doors of those in pairs shall have not less than two locking bolts and safes of 30 -inch or greater inside height shall have not less than three bolts. For single doors over 30 inches high and for double door safes at least one bolt shall be a horizontal bolt engaging opening in the opposing door or jamb framing. The standing door of those in pairs shall have not less than two vertical bolts, one at the top and one at the bottom.

D-4b. Modification for doorplate thickness.- Safes having doorplate thickness accepted under section $\mathrm{E}-4 \mathrm{~b}$ shall have not less than three locking bolts on separate drawbars or on middle or opposite ends of drawbars, that must be withdrawn before safe can be opened, not less than one bolt and adjacent portion of bar assembly of which must be protected on the outside by metal of thickness not less than that specified in section E-4a for the outer doorplate, or, such safes must have not less than four locking bolts on separate drawbars or on middle or opposite ends of drawbars that must be withdrawn before safe can be opened. Bolts between doors in double-door safes are not to be considered as effective in preventing opening of safe after all bolts in both doors engaging the jambs have been withdrawn.

D-4c. Details of bolts. - Locking bolts are to be of cold-rolled steel properly polished and finished to prevent corrosion, having well-cupped openings in doorframe and doors, cups to be tight and flush with the doorframe or jamb. A step may be provided to engage the bolts in lieu of cupped holes. The diameter of bolts shall not be less than fiveeighths inch. They are to be yoked together for simultaneous response to levers or cams operated by lever or $T$ handles. With doors in pairs provision shall be made to prevent locking of bolts in the swinging door while the bolts of the standing door are in unlocked position, and for preventing unlocking the bolts of the standing door while the bolts of the swinging door are in locked position.

D-4d. Locks. - The bolt-work system shall be checked by one combination lock having not less than three changeable tumblers in addition to the driving wheel; the tumblers shall be key changeable or hand changeable as will be specified. The handchangeable lock must be made so that all numbers stamped on tumblers can be used. The instructions for changing combinations must be supplied with each safe. A device must be provided that will check the bolt-work automatically in case the lock tumblers are driven out of position, or that will securely maintain the door in closed position in case of burglarious attack. Unless the lock case is of material and is so mounted that it can not be driven out of position with reference to the bolt-work, this device must be located outside of the lock case.

A guard plate made of drill-proof steel and sufficient in size to guard the lock and lock connection must be provided. This plate shall be not less than three-sixteenths inch thick. Two plates of drillproof steel each not less than one-eighth inch thick, may be substituted for the single three-sixteenths inch thick plate, or a single one-eighth inch thick drill-proof plate may be used if backed with soft steel plates aggregating not less than three-sixteenths inch in thickness.

D-5. Finish.-The interior finish of the safes shall be of rich olive green and the exterior finish the Government standard olive green, unless otherwise specified. The unexposed surface of sheets and plates shall be suitably protected against corrosion unless the insulation forms a protective covering.

D-6. Sizes.-The sizes requisitioned shall be the commercial sizes, but preference will be given those safes the dimensions of which will most adequately accommodate the Government sectional furniture, the dimensions of which are as follows:

Small and horizontal sections, $16 \frac{1}{2}$ and 33 inches wide by 17 and 25 inches deep. These sections advance in height in multiples of $65 / 8$ inches.

\section{E. DETAII REQUIREMENTS}

E-1. Structural strength. - Safes shall withstand an applied load maintained constant one week and equal to the weight of the safe without equipment plus three times the weight of contents, disposed and computed as given in section F-5, without deformation or sagging that interferes with easy operation of doors and locking bolts.

E-2. Fire resistance.

E-2a. Class A safes shall be able to withstand a standard fire test as described in section F-6 for four hours without attainment of temperatures within the safe at any thermocouple location higher than $350^{\circ}$ F. $\left(177^{\circ}\right.$ C.) either during or after the fire exposure.

E-2b. Class B safes shall be able to withstand a similar test for two hours without exceeding the same inside temperature limits.

E-2c. Class C safes shall be able to withstand a similar test for one hour without exceeding the same inside temperature limits.

E-2d. All safes shall withstand the explosion hazard test, in cases where this test is required, without development of explosive pressures sufficient to open forcibly the doors or distort the safe structure.

$\mathrm{E}-3$. Impact resistance.

E-3a. Safes of classes A and B must preserve their contents in legible condition during a reheat test of one hour following a clear drop of safe of 30 feet while in heated condition from an initial fire exposure of one hour under the testing procedure described in section F-7.

$\mathrm{E}-3 \mathrm{~b}$. Safes of class $\mathrm{C}$ must meet the same requirements with the exception that the initial and reheat tests are to be of one-half hour duration.

E-4. Shell thickness.

E-4a. The metal thickness of the outer shell shall be not less than 0.05 inch (No. 18 gage) for class $A$ and class B safes and not less than 0.0375 inch (No. 20 gage) for class $\mathrm{C}$ safes. The thickness of the outer doorplate shall be not less than 0.109 inch (No. 12 gage) for class $A$ and class B safes and not less than 0.078 inch (No. 14 gage) for class $\mathrm{C}$ safes. The thickness of the inner shell and inner doorplate shall be not less than 0.0375 inch (No. 20 gage). The outer doorplate may be made of two sheets having combined thickness of not less than 0.109 
inch, welded together at points not more than 6 inches apart.

If the outer doorplate is not continuous with the jambs it shall be welded to the jamb metal at intervals not exceeding 3 inches. The inner shell shall be suitably reinforced where necessary to safely support the safe contents without distortion.

E-4b. Modification for locking bolts. - The outer doorplate may be of the same minimum thickness as that specified above for the outer shell provided the number and distribution of the locking bolts conform with the requirements of section D-4b.

E-5. Weight.- The weight of safes without interior equipment or contents shall not exceed 325 pounds per square foot of floor area occupied for class A safes, 275 pounds per square foot for class B safes, and 225 pounds per square foot for class C safes. These weight limitations apply to safes not over 6 feet in body height.

\section{F. METHODS OF SAMPLING, INSPECTION, AND TESTS}

F-1. Information required.-When requested, the manufacturer shall supply with his proposal shop drawings showing and noting every detail of structural, heat insulating, and thief-resisting features for the different classes and sizes. Catalogues shall be supplied showing equipment items, finished appearance, weight of each safe in the lines proposed upon, both total and per square foot of floor area covered; and attested statements as to results of tests if available. These will be considered confidential and used only for the information of the Government in determining the acceptability of the safes.

F-2. Inspection. - The manufacturer shall provide facilities for such inspection of materials and process at the factory as may be deemed necessary. Purchasing officers should ascertain whether the given manufacturer's product is being regularly inspected by any Government department in order to avoid unnecessary duplication of inspection work. The safes will be finally inspected for acceptance at the point of destination.

F-3. Samples for test.-If requested two safes of each class proposed upon, one of medium size and one of the largest size it is proposed to furnish, with the interior equipped with open shelves and accompanied by shop drawings, shall be delivered by the successful bidder or contractor, free of expense to the Government, at the time and place designated by the purchasing officer. If the explosion hazard test is required an additional safe of medium or large size will be required for this test. These safes will be submitted to such examinations and tests as may be deemed necessary to determine their properties and performance. Two samples of insulation, 18 by 18 inches in area, of the same thickness and contained in the same manner as in the safes proposed upon shall be submitted with the sample safes.

F-4. Service tests. - Examination and tests of the unloaded safe will be made to determine ease of operations of doors, bolts, and lock under conditions of uniform support and support only at two or three corners. Determination will be made of the degrea of swing of the door that is required to make possible removal of interior equipment and of the force required to move the safe on its base supports over a level floor.

F-5. Strength tests.

F-5a. The safe to be tested shall be weighed with all interior equipment that is not fixed in place removed. The inside volume of the safe shall then be determined, the depth to be taken as the distance between the inner doorplate and the back of the inner shell. In case fixed interior equipment is present, this shall be included in the weight of the safe and the inside volume computed so as not to include the space occupied by such equipment. The weight of contents shall be taken as 25 pounds per cubic foot of inside volume or thrice the weight of contents as 75 pounds per cubic foot of inside volume.

$\mathrm{F}-5 \mathrm{~b}$. During the loading tests a plate not less than one-fourth inch thick shall be placed under one corner support so as to place the weight mainly on this and the diagonally opposite corner support. In case of single-door safes, the plate shall be placed under the corner adjacent to the lock side of the door.

F-5c. The load required under section E-1 shall be distributed as follows: One-half of the load shall be placed on the front central part of the upper door jamb with no portion of the load nearer than onefourth of the outside width from the sides of the safe or one-third of the outside depth from the back.

F-5d. One-fourth of the load shall be placed on the front central part of the lower door jamb with no portion of the load nearer than one-fifth of the inside width from the sides of the inner shell or onethird of the inside depth from the back of the inner shell.

F-5e. One-fourth of the load shall be hung on the door of 1-door safes or symmetrically on the two doors of 2-door safes with center of support 2 inches from the door edge. During the test the doors shall be kept open to their maximum swing, but not more than $180^{\circ}$ from the closed position.

$\mathrm{F}-5 \mathrm{f}$. After the loads have been in place for one week, the whole door load and two-thirds of each of the top and bottom door-jamb loads shall be removed. With the plate remaining under the corner support there shall be no indications of binding or lack of easy closure or operation of doors and bolt work.

F-6. Fire endurance tests.-The sample used in this test shall be of medium size, and lightly loaded with loose papers and records. The time-temperature curve followed shall conform to that defined in American Tentative Standard A2-1926 of the American Standards Association. The flames and hot gases shall bathe the sample on not less than five sides without undue impingements, the door side to be always fully exposed. It is placed in the furnace on incombustible foundations with suitable equipment for measuring temperature on the inside, installed so as not to affect the insulating efficiency of the safe. Temperatures shall be measured at several representative points within the safe, including not less than two points 6 inches from the top, 1 inch from the sides opposite joints between door and jamb or between doors, and 2 inches from inside door or doorplate.

After exposure to the fire test for the duration specified for the pertaining class, the sample shall be allowed to cool in the furnace, with furnace doors closed, the inside safe temperature being measured until the maximum is reached.

F-7. Impact test. - The sample used in this test shall be the largest size on which proposal is made, loaded with a content of papers and records of 25 pounds per cubic foot of safe capacity, supported oi shelves. The sample shall be subjected to the fire test defined in section F-6, for on and class $B$ rating and for one-half hour for class $C$ rating, except that it shall be dropped base downward a clear distance of 30 feet while in the highly heated condition on a riprap of broken bricks. After cooling to normal temperature it shall be replaced in the furnace, bottom upward, and subjected to the standard fire test for one hour for class A and class B rating and for one-half hour for class $\mathrm{C}$ rating. 
Safes are to withstand the fire, impact, and reheat test above specified without destroying the legibility of the records inside.

F-8. Explosion hazard test.-The sample used in this test shall be of medium or large size and lightly loaded with loose papers and records. It shall be subjected by any means to a very quick hot fire for about one-half hour, the intensity of which is to be considerably higher than that of the fireendurance test described under section F-6.

\section{G. PACKAGING, PACKING, AND MARKING}

G-2. Packing.-Unless otherwise specified, the subject commodity shall be delivered in standard commercial containers, so constructed as to insure acceptance by common or other carriers, for safe transportation, at the lowest rate, to the point of delivery.

G-3. Marking.-Unless otherwise specified, shipping containers shall be marked with the name of the material and class as defined by the contract or order under which the shipment is made, the name of the contractor, and the number of the contract or order.

\section{NOTES}

I-1. Items to be specified.-Purchasers should exercise all options specified herein and should specify class, finish, and size, the last being usually best stated in terms of ininimum width, height, and depth inside of inner shell and inner doorplate, and whether hand-changeable or key-changeable lock combination is wanted. If a limit on the swing of the door or doors required to make the interior equipment removable is needed, or a $250^{\circ}$ swing required, this should be specified. If the explosion hazard test is required, this should be stated.

On account of cost of samples and their transportation it is suggested that sample safes be not requested from all bidders, but that such requests be made only of the successful bidder or contractor per section F-3. For small orders it may be possible to base acceptance on examination of detail drawings and safe details and subjection of the sample or samples to the nondestructive loading tests outlined in section F-5. In such cases, fire endurance and fire and impact resistance of the safe might be judged from the information the manufacturer can supply as to results of such tests with the class of safe concerned. Where tests are required, the Bureau of Standards at Washington, D. C., is equipped to conduct for Government departments and establishments the examination and tests required by this specification.

I-2. Scope of protection afforded.

I-2a. The safes covered by this specification are intended mainly to afford protection against fire and theft and such resistance against forcible entry as is consistent with the general construction. They should not be considered as giving burglar protection approaching that afforded by burglar-resistive safes meeting requirements such as those of Federal Specification AA-S-71.

I-2b. For buildings of fire-resistive interior and exterior construction that can withstand a complete burning-out of contents without collapse of floors and supports, the maximum equivalent severity of the fire is fairly determinate and can be stated as not exceeding the fire resistance of class $\mathrm{C}$ safes where combustibles, such as wood and paper contained in building contents, finish floors, and trim, do not exceed 10 pounds per square foot, that of class B safes where not exceeding 20 pounds per square foot, nor that of class A safes where not exceeding 35 or 40 pounds per square foot. The weight of combustibles per unit of floor area can be obtained by dividing the total weight within rooms or areas not exceeding approximately 25 by 30 feet, by the floor area involved. Extra high cabinets should not be placed closely adjacent to safes as the hot débris from them in contact with the safe may give a more severe exposure than the general room temperatures. For locations involving ordinary combustibles in excess of the amounts given above, safes of 4 -hour or less fire resistance would afford temporary protection, and the contents would be saved provided the fire is extinguished at a sufficiently early stage or its severity diminished by this means or by other conditions present, that prevent development of a severity in excess of the protection afforded by the safe. Full protection against these severe fire conditions can be obtained by recourse to vaults having walls and supports of suitable material and thickness and well-insulated doors.

I-2c. For fire-resistive buildings the severity of the exposure is largely independent of the size, height, or location of the buildings.

I-2d. As concerns buildings with combustible or nonfire-resistive interior construction less definite estimates of the severity of the fire exposure to be expected can be made. While the duration of the fire before building collapse takes place may be relatively brief, a prolonged exposure from contact with hot ruins may follow. It is recommended that in such buildings, safes, of class B or higher fire resistance be used generally, although class $\mathrm{C}$ safes may be adequate in 1-story buildings not adjoining higher nonfire-resistive buildings and that house the lighter occupancies from the fire-hazard standpoint. The hazard increases with the height of the building and weight of combustible material present on account of possibility of greater volume of hot débris and severer impacts. Hot débris covering safes should be cooled with water or the safes removed as soon as conditions permit.

I-2e. Generally, considering possible changes in location and ownership, it is preferable to provide containers having fire resistance not less than that of class B safes. The safes with the higher fire resistance also afford better theft protection on account of thicker insulation and body plates.

I-3. This specification governs all United States Government purchases of this commodity.

UNITED STATES GOVERNMENT, FEDERAL SPECIFICATIONS BOARD, FEDERAL SPECIFICATION FOR STERILIZERS, DRESSING CONTAINERS AND STANDS, PORTABLE LOCKERS FOR USE IN CONJUNCTION WITH STERLIZIERS, ELECTRIC HEATERS, AND STEAM STERILIZING APPARATUS, NO. 68A, OCTOBER 25, 1923. (CURRENT DESIGNATION: GG-S-751)

\section{PORTABLE LOCKERS}

(The following requirements from the above specification are applicable to portable lockers for use in conjunction with sterilizers.)

\section{TYPE}

Portable lockers for use in conjunction with sterilizers shall be of but one type, as hereinafter covered in detail.

\section{MATERIAL AND WORKMANSHIP}

The material used in the manufacture of portable lockers for use in conjunction with sterilizers shall be as hereinafter covered. 
All details of outfit, its design, material, workmanship, finish, and operation under service conditions shall be first class in every respect.

\section{GENERAL REQUIREMENTS}

Each complete apparatus shall be fitted with a single brass name plate in a conspicuous location in order that it may be read after installation. The name plate shall contain the following data:

\section{Name of manufacturer.}

Shop number.

Year of manufacture.

\section{DETAIL REQUIREMENTS}

1. When ordered, portable lockers about 12 inches high, constructed of 0.05 inch terneplate and of about the depth of the tubular stand, with bottom not less than 4 inches above the deck, shall be located under the combination sterilizing outfits. Top, bottom, back, and sides of lockers shall be watertight, and each locker shall be fitted with a tightclosing door or doors provided with hinges and fastenings of heavily nickeled brass.

2. Doors shall be suitably stiffened and door frames shall be fitted.

3. Lockers shall be finished with two undercoats and two finishing coats of high-grade white enamel baked on.

\section{UNITED STATES GOVERNMENT, FEDERAL SPECIFICATIONS BOARD, FEDERAL SPECIFICATION FOR LOCKERS; CLOTHES, STEEL, AA-I-486, FEBRUARY 2, 1932}

\section{STEEL LOCKERS}

\section{A. APPIICABLE FEDERAL SPECIFICATIONS}

A-1. There are no Federal specifications applicable to this specification:

\section{B. TYPES AND CLASSES}

B-1. Type 1.--Single-tier lockers. Class (a). Semilouvered door.

Class (b). Full-louvered door.

Class (c). Full-perforated door.

Class (d). Open-mesh "Tropical."

B-2. Type II.-Double-tier lockers.

Class $(a)$. Semilouvered door.

Class (b). Full-louvered door.

Class (c). Full-perforated door.

Class $(d)$. Open-mesh "Tropical."

\section{MATERIAL AND WORZMANSHIP}

C-1. General.-Material shall be the best quality of the kind specified. It shall be free from defects and imperfections which impair the appearance or usefulness of the products.

C-2. Steel sheets. - Shall be open-hearth steel, voldrolled for tops, bottoms, sides, backs, shelves, and structural parts generally.

$\mathrm{C}-3$. Hardware.-Hinges shall be of good quality commercial steel. Door handles shall be of malleable iron, cast steel, or formed steel suitable for the purpose.

C-4. Workmanship.-The workmanship on all parts shall be first class in every respect and of a type to insure rigidity of the assembled article.

\section{GENERAL REQUIREMENTS}

D-1. Lockers shall be furnished in single units or in groups of two or more units, but not exceeding 10 in one group for single-tier lockers, or 20 for double- tier lockers, as ordered. Groups may be double, two rows back to back when so ordered, and so constructed as to provide for mutual back for such double rows or groups when so ordered.

D-2. Strength and rigidity of parts shall be secured by flanging or reinforcing so they will properly perform the function for which they are designed. Special precautions shall be used to insure proper alignment of doors with door openings or frames so that doors may be freely operated and latched.

D-3. All lockers shall have brass or aluminum number plates on the doors, the numbers to be consecutive, or otherwise to meet requirements. The scheme of numbering shall be supplied by purchaser. The manufacturer's name and location of factory shall also be placed on such number plates.

D-4. Locks on equipment furnished on any order shall be different and shall be master-keyed. Two keys shall be supplied with each locker and two master keys with each order. Built-in keyless or combination locks, self-locking type, shall be furnished in lieu of flat-key locks when specified by the purchasing officer. Provision shall be made for fastening by means of padlock, when specified by the purchasing officer, padlocks to be furnished by user.

D-5. The general assembly of parts shall be by bolts. Bolt holes in adjoining parts shall be true and even so that knock-down lockers may be set up without difficulty. All assembly bolts shall be rustproof.

D-6. Finish.

D-6a. All parts shall be thoroughly cleaned and dried before finishing materials are applied. The finish shall consist of not less than two coats of baked enamel or a primer and two coats of pyroxylin lacquer. The standard color to be olive green.

D-6b. Hardware shall be of rust-resisting finish, manufacturer's standard.

D-7. Patented features shall be owned or licensed by the manufacturer, who shall assume full responsibility and protect the purchaser.

\section{E. DETAIL RIQUIREMENTS}

E-1. Sizes of lockers.-Approximate outside dimensions:

E-1a. Type I.- Single tier.

\begin{tabular}{|r|r|r|r|}
\hline Width & Depth & Height & $\begin{array}{c}\text { Over-all height, } \\
\text { including legs }\end{array}$ \\
\cline { 2 - 3 } Inches & \multicolumn{1}{|c|}{ Inches } & Inches & \multicolumn{2}{|c|}{ Inches } \\
12 & 12 & 60 & 66 \\
12 & 15 & 60 & 66 \\
12 & 18 & 60 & 66 \\
15 & 15 & 60 & 66 \\
15 & 18 & 60 & 66 \\
12 & 12 & 72 & 78 \\
12 & 15 & 72 & 78 \\
12 & 18 & 72 & 78 \\
15 & 15 & 72 & 78 \\
18 & 18 & 72 & 78 \\
18 & 21 & 72 & 78 \\
\hline
\end{tabular}

E-1b. Type II.-Double tier.

\begin{tabular}{|c|c|c|c|}
\hline Width & Depth & Height & $\begin{array}{c}\text { Over-all } \\
\text { height, includ- } \\
\text { ing legs }\end{array}$ \\
\hline Inches & Inches & Inches & Inches \\
12 & 12 & 36 & 78 \\
12 & 15 & 36 & 78 \\
15 & 15 & 36 & 78 \\
12 & 12 & 42 & 90 \\
12 & 15 & 42 & 90 \\
\hline
\end{tabular}


Width dimension does not include the projecting bolt heads. When filling fixed spaces with sections of lockers, allowance shall be made for such projections.

E-2. Interior equipment.

E-2a. Shelves. - Hat shelves shall be furnished for single-tier lockers only.

E-2b. Hooks. - A double-prong ceiling hook shall be furnished for each locker. Lockers 12 inches wide shall be provided with at least one hook on the back and one on each side. Lockers over 12 inches wide shall be provided with two hooks on the back and one on each side. Hooks shall be of malleable iron or cast steel and of rustproof finish.

E-3. Legs.- Each locker shall have four legs 6 inches long, with feet that may be secured to the floor. Legs may be separate members or extensions of framing parts. Vertical adjustment shall be provided either with the legs or foot.

E-4. Doors.

E-4a. Locker doors shall be reinforced with steel angle members or equal, along the vertical edges, with top and bottom edges flanged. Doors not over 36 inches high which are flanged only shall have oxyacetylene or electric welded corners. For "Tropical" lockers, class 4, hard steel angle frames shall be used for doors, which shall be assembled by oxyacetylene or electric welding. A reinforcing plate shall be provided, on which the handle and lock shall be mounted. Expanded metal not less than No. 14 gage, of three-fourths-inch diamond mesh, shall be welded to the angle door frame of "Tropical" lockers.

E-4b. Semilouvered doors shall have six louvers near top and six louvers near bottom of door. Fulllouvered doors and full-perforated doors shall have as many louvers or perforations as may conveniently be placed without weakening structural strength.

E-5. Hinges.-Heavy steel butt hinges with tight pins shall be mounted, if possible, with ends concealed. Other types of approved standard hinges will be considered. Hinges to be bolted or riveted to door and riveted to door frames.

E-6. Latching mechanism.-The latching mechanism for doors up to 42 inches high shall make engagement at not less than two points, doors of greater size shall have a 3-point device. The latching-mechanism shall be controlled by a suitable flat key lock that is master-keyed, or built-in keyless lock, as specified in the invitation for bids.

E-7. Gages of parts. - The following are minimum gages of parts-United States standard gage:

E-7a. Tops, backs, bottoms, sides, and partitions, shelves No. 24 gage-0.0245 inch.

E-7b. Doors, when reinforced-No. 20 gage, 0.0368 inch. inch.

E-7c. Doors, when flanged-No. 16 gage, 0.0613

\section{F. METHOD OF SAMPLING, INSPECTION, AND TESTS}

F-1. The manufacturer shall permit a representative of the Government at any time, to make inspections of all products during process of manufacture and such tests as may be necessary to ascertain that proper materials are being used. Final inspection will be made at point of delivery. All defective parts or articles shall be replaced by the manufacturer without cost to the purchaser.

\section{G. PACKING AND MARKING}

G-1. Articles when shipped from factory shall be so crated, packed, or wrapped, as to insure delivery in first-class condition. Packages shall be clearly marked. When intended for foreign delivery, packing shall be in accordance with rules of the Department of Commerce.
G-2. Shipping containers or packages shall be marked with the name of the material, the type and quantity contained therein, as defined by the contract or order under which shipment is made, the name of the contractor, and the number of the contract or order.

$$
\text { I. NOTES }
$$

Lockers, which are usually simple to erect, are purchased more economically knocked down. The requirements as to assembling shall be stated by the purchaser.

\section{UNITED STATES GOVERNMENT, FEDERAL SPECIFICATIONS BOARD, FEDERAL SPECIFICATION FOR SAFES; BURGLAR RESISTING, AA-S-71, NOVEMBER 11, 1930}

\section{BURGLAR-RESISTING SAFES}

\section{A. APPLICABLE FEDERAI SPECIFICATIONS}

A-1. The following Federal specifications, or parts thereof that are below indicated, of the issue in effect on date of invitation for bids, shall form a part of this specification:

\begin{tabular}{c|r|c|c}
\hline $\begin{array}{c}\text { Federal } \\
\text { specifica- } \\
\text { tion }\end{array}$ & $\begin{array}{c}\text { Former } \\
\text { No. }\end{array}$ & Title & . See \\
\hline $\begin{array}{c}\text { QQ-S-681 } \\
\text { QQ-B-691 } \\
\text { QQ-S-711 }\end{array}$ & $\begin{array}{r}170 \\
\text { 172a }\end{array}$ & $\begin{array}{l}\text { Steel castings (soft grade) } \\
\text { Bronze castings (grade No. 5) } \\
\text { Structural steel for bridges_-- }\end{array}$ & $\begin{array}{l}611.41, \text { p. } 711 . \\
640.41, \text { p. 1252. } \\
605.11, \text { p. 251. }\end{array}$ \\
\hline
\end{tabular}

\section{B. TYPES}

B-1. The burglar-resisting safes covered by this specification shall be of the following types:

Type A.- Safes of this type shall offer resistance to edge-cutting tools, explosives, and heat destruction by the oxyacetylene torch or other heat devices.

Type B.- Safes of this type shall be the same as those of type A but having a higher degree of resistance to heat destruction by the oxyacetylene torch or other heat devices than that of type A.

\section{MATERIAL AND WORKMANSHIP}

C-1. General. - The material shall be of the best quality of the material specified. It shall be free from defects and imperfections which impair the appearance, burglar resistance, or usefulness of the safe.

C-2. Iron castings. - The iron castings shall be commercial cast iron.

C-3. Steel castings.-The steel castings shall comply with the requirements of Federal Specification QQ-S-681 (former No. 170) for steel castings, soft grade.

C-4. Structural steel.-The structural steel shall comply with Federal Specification QQ-S-711 (former No. 351a) for structural steel for bridges.

C-5. Machine steel. - The machine steel shall be commercial open-hearth steel.

C-6. Tool steel.-The tool steel shall be finegrained, high-carbon crucible steel containing sufficient chromium to cause it to become glass hard, i. e., can not be penetrated with edge-cutting tools after it has been quenched from a high temperature in a large body of water at a temperature below $60^{\circ} \mathrm{F}$.

C-7. Five-ply steel.-The 5-ply steel shall consist of two layers of tool steel and three layers of structural steel, arranged alternately. Each layer shall be uniform in thickness and the five layers welded 
together, over their entire contact surfaces, by rolling under high pressure at a temperature sufficiently elevated for that purpose. Five-ply plates shall be uniform in thickness.

C-8. Bronze.-Bronze may be either bronze castings or rolled, drawn, or extruded bronze. The bronze castings shall comply with Federal Specification QQ-B-691 (former No. 172a) for bronze castings, grade No. 5. Rolled, drawn, or extruded bronze shall be of good commercial grade.

C-9. Packing.-The packing shall be nonabsorbent, acid proof, and fireproof.

C-10. Workmanship.-The workmanship on all parts of the safe shall be of the highest grade.

\section{GENERAL REQUIREMENTS}

D-1. General.-The construction of the walls and the door of the safe shall be what is known com-
D-3. Intermediate layer.-The intermediate layer, except on the back wall of the safe, shall consist of four splice plates to secure the boxes to each other and a frame to reinforce the door jamb, made from rolled structural steel angles. The corners of the frame shall be solidly welded. Each splice plate shall be one piece, full width, between the frame and the inside of the rear box, and used over the joint between the two boxes. These plates, madesfom structural steel, shall be fitted closely into the space at the top and bottom and on both sides between the frame and the inside back of the safe.

D-4. Inner layer. - For type $\mathrm{A}$ the inner layer (plates and angles) of the safe body and of the outer door shall be cast iron. For type B the inner layer (plates and angles) of the safe body and of the outer door shall be cast-metal alloy or any material having a resistance to heat destruction by the oxyacetylene torch or other heat devices of a greater

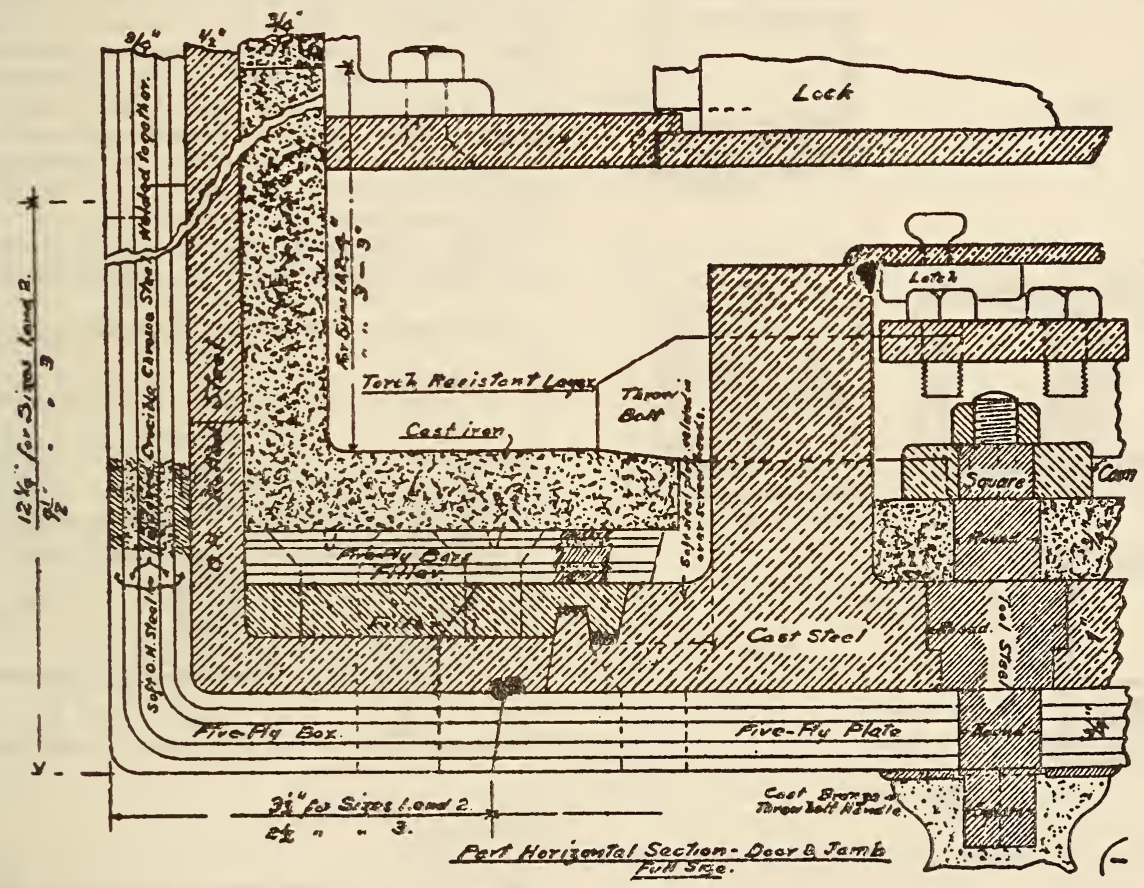

Figure 641

mercially as laminated. In this construction two or more separate layers of plates lie in close contact and are secured from within by countersunk flathead machine screws. To prevent the introduction of liquid explosives into any space which may unavoidably be left between the layers, these spaces shall be filled completely with a self-hardening metallic cement.

D-2. Outer layer.-The outer layer of the body of the safe shall consist of six pieces only. The back and half of each side shall be formed from one piece. The ends of this U-shaped section shall be closed by fitting and welding in two plates, each of which will form half of the top and half of the bottom outer layer. The front and half of each side, top, and bottom shall be formed in the same manner. Each box section shall be made from 5-ply steel and have the ends welded in place before tempering. Each of the boxes formed shall have the edges of each side rebated and have the necessary holes for assembling screws drilled and tapped. The opening for the outer door of the safe shall be cutfrom the solid face of the box used as the front of the safe. degree than that afforded by the material specified for type $A$, as measured by the tests hereinafter specified under section F-3.

$\mathrm{D}-4 \mathrm{a}$. The inner layer shall consist of a frame, forming part of the door jamb, and machined-edge plates closely fitted on both sides, top, bottom, and back of the body of the safe. Each plate to be a single piece, full size, between the frame and inside face of back or sides and top and bottom of safe. The frame shall be made in the form of cast angles. The corners of the frame shall be mitered.

D-5. Door jamb. - The door jamb on the body of the safe shall be built up with fillers to the thickness necessary to form the stepping. These fillers shall be made from 5-ply and structural steel bars or plates.

D-6. Safe door.-The outer door of the safe shall be a steel casting forming the intermediate layer, with an outer and inner layer fastened to it. The intermediate layer casting shall have a rib integral with the casting around the top, bottom, and sides to form a continuous bolt frame. The width of this rib parallel to the face of the door shall not be less 
than the diameter of the throw bolts, and the depth of the rib at right angles to the face shall not be less than two and one-half times the diameter of the throw bolts. The outer layer shall consist of a single plate of 5-ply steel. The inner layer shall consist of a single plate of material, as described under section C-4. The door may have rounded corners,
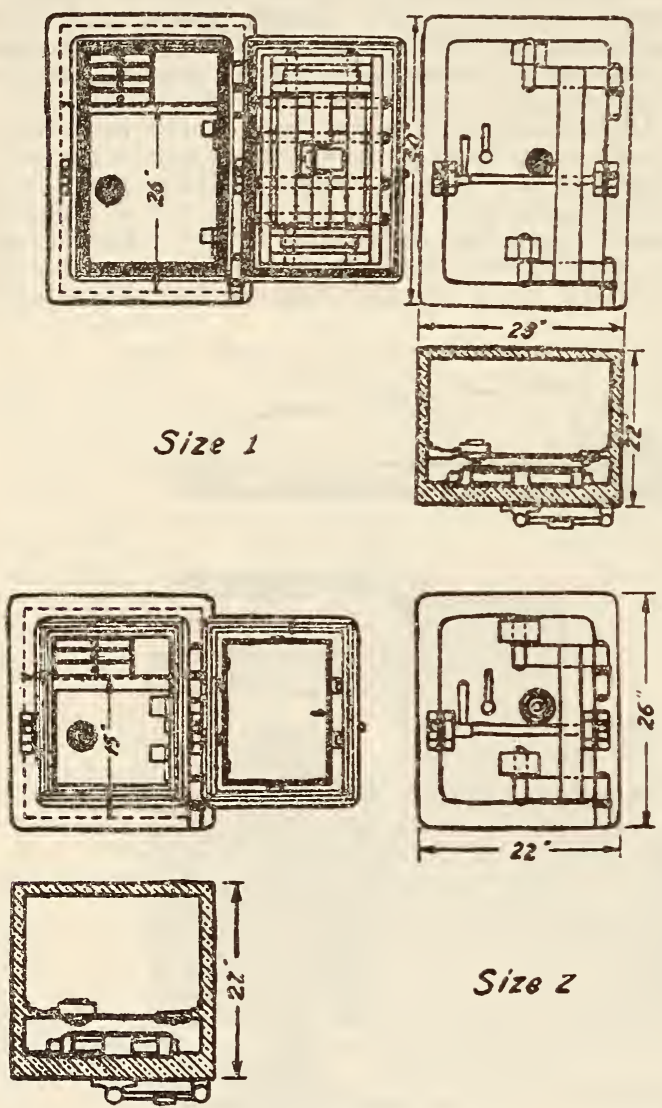

Size $z$

Figure 642

but they must fit snugly into the fillets in the door frame.

D-7. Thickness of walls and of door.-The top, bottom, and side walls of the body of the safe shall be not less than 2 inches thick. The back of the safe shall be not less than $1 \frac{1 / 2}{}$ inches thick. The door jamb shall be not less than 3 inches thick. The outer door of the safe shall be not less than $2 \frac{1}{2}$ inches thick.

D-8. Screus for body and door.-The distance between screws shall be measured along lines perpendicular to the corners of the safe. The screws for securing one layer of the body of the safe to the next layer shall be spaced not less than 2 inches nor more than 6 inches from center to center of the screws, except that the screws fastening the intermediate layer (splice plates) at the top, bottom, and sides shall be 2 inches center to center of the screws. The distance from the center of a screw to the edge of a plate or of an angle shall not be less than 1 inch.

$\mathrm{D}-8 \mathrm{a}$. The screws fastening the intermediate layer to the outer layer shall be of hardened 7-ply steel, three-fourths inch in diameter, except as shown in Figure 641. They shall be driven from within, and the tapped holes in the outer layer shall be through holes except in the back wall of the safe, where there is no intermediate layer.
D-8b. The screws fastening the inner laver to the intermediate layer or to the outer layer at the back of the safe shall be one-half inch in diameter. The screws fastening the bars or plates (sec. C-5) used to "build up" the door jamb shall be one-half inch in diameter. They shall be driven from within, and the tapped holes in the intermediate laver shall extend through the plate or angle. The tapped holes in the back of the safe (outer laver) shall be "blind," i. e., they shall extend into the plate for three-fifths of its thickness.

D-9. Screws for hinges and pressure bar.-Each hinge bracket and each pressure-bar bracket shall be attached, at the proper location, to the front face of the safe body by two $1 / 2$-inch screws through the intermediate layer and the outer layer, while the door brackets shall be attached by $1 / 2$-inch studs, blind tapped into the outer layer of the door. The heads of all these screws shall be keved to prevent back turnings, and their shanks shall extend through the outer laver, the hinge bracket, and into cylindrical nuts supplied with depressions for engaging a spanner wrench or screw driver. These depressions shall be filled, and the face of these blocks shall be smooth. Each hinge bracket and each pressurebar bracket shall be counterbored for the cylindrical nuts, so that the outer surface of the nut is flush with the surface of the bracket.

D-10. Stepping around door.-The edge of the door and the jamb shall be stepped (or tongue and
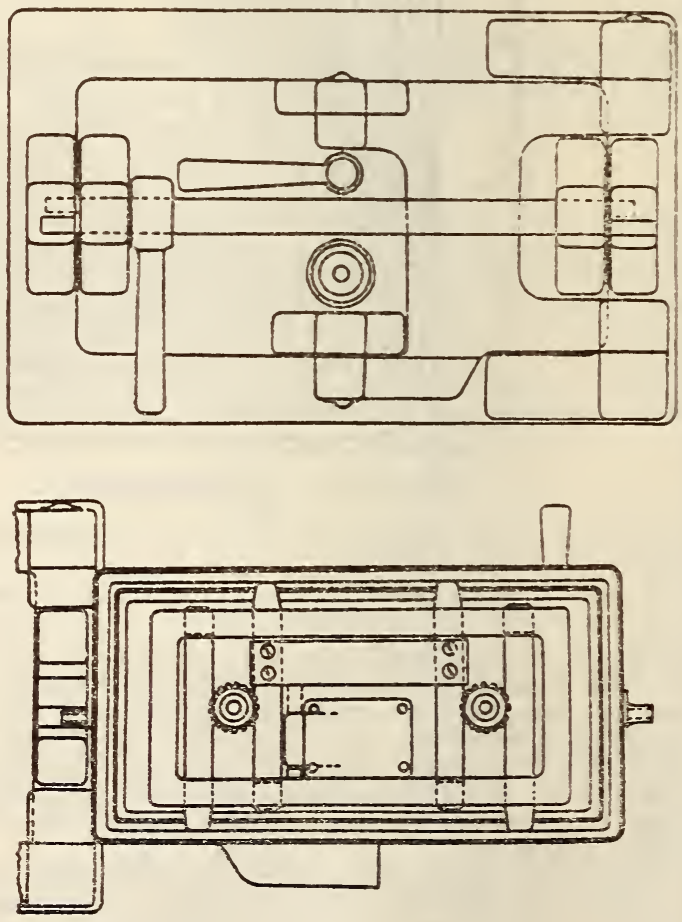

Size 3

\section{Figere 643}

grooved), as shown in detail in Figure 641. The stepping on the door shall be machined from the steel casting and outer layer plate and be continuous around the edges of the door and jamb. Each step shall have the face (parallel to the face of the door) fitted so that the step on the door is in continuous surface contact with the step on the jamb.

$\mathrm{D}-10 \mathrm{a}$. The beveled edges (surfaces at an angle to the face of the door) shall have no more clearance 
than is necessary for operating the door, except that the tongue on the door shall bear, metal to metal, on the tongue on the jamb to prevent opening of the joint between the door and the jamb if wedges are used to introduce liquid explosive. The end of the tongue on the door and on the jamb shall be serrated.

D-11. Recesses for packing. - The recess in the door and in the jamb into which the serrated end of the tongue extends shall be suitable for packing. A recess shall also extend around the door at the inner angle of the first step on the jamb into which the inner corner of the first step on the door projects when the door is closed.

D-12. Packing. - In the completed safe the packing recesses shall be filled with a flat strip of packing which fits the recess tightly and is securely attached to the sides and bottom of the recess. When the door is fully closed the serrated tongues shall press heavily into the packing for its entire length.

D-12a. The recess in the first step shall have a round strip of packing calked into it which shall project so that the corner of the door, which shall be slightly chamfered, shall bear uniformly on the packing for its entire length at the same time that the face on the step on the door is in contact with the step on the jamb.

D-13. Hardening of outer layer.-After all machine work has been performed on the outer layer of the safe body and of the door, also the door jamb filler bars, these parts of the safe shall, before assembling, be hardened so that the tool-steel element therein becomes glass hard and can not be penetrated with edge-cutting tools. The tapped holes in these parts shall be filled with fire clay before hardening, and every care shall be taken to prevent injury to the threads while the parts are heated.

D-14. Hinges. - The door shall swing to the right of the entrance. The hinge shall be of the singlepiece, gooseneck pattern, crane type. Each part of the hinge shall be large enough and have sufficient strength to carry the door without spring or sag. The hinge shall be made of cast steel. The hinge pins shall be tool steel, hardened and ground to size. They shall be of sufficient size to carry the weight of the door and shall fit their bearings closely. The end of each pin shall be covered by a suitable tip.

D-14a. Roller thrust bearings shall be provided on each bearing between the hinge arms and the jamb. These bearings shall consist of two hardened tool steel collars between which hardened tool steel rollers are fitted. Screws for adjusting the height of the door shall be provided in the arms at the hinge bearings on the door.

D-15. Lock-bolt work. - The safe outer door shall be fitted with a system of heavy, round lock-bolt work. This system shall consist of throw bolts, $11 / 4$ inches in diameter, operating in the bolt frame, which is an integral part of the door casting and arranged in double-acting system. The throw bolts shall be cross connected and shall be operated from a common point. The ends of the bolts which project from the bolt frame shall be tapered. The number and the positions of the bolts are shown in Figures 642 and 643 . The locking-bolt work shall be covered by a structural steel plate. This cover plate shall be attached to the door on one side by hinges and be provided with a thumb latch on the other side to hold it in the closed position. The bolt work shall be checked by a combination lock.

D-15a. The bolts shall seat in recesses on the inner face of the door jamb. These recesses shall be hand fitted to the taper of the locking throw bolts so that the bolts can only be thrown to their full travel when the door is fully seated; that is, the step on the door is in contact, metal to metal, with the step on the jamb. If the recesses are correctly fitted, the edge of the door will remain in contact with the jamb after the pressure system is thrown off.

D-16. Combination lock. -The size of the combination lock shall not be less than $37 / 8$ by $2 \frac{7 / 8}{\text { by }}$ $13 \frac{1}{8}$ inches. It shall have four adjustable circular tumblers operated with a graduated dial handle in extension of the lock spindle. The dial shall rotate in a stop-mark ring and shall have its periphery uniformly graduated with short radial marks spaced not more than $3.6^{\circ}$ apart, at least every tenth mark being numbered on the face of the dial (graduations from 0 to 20 may be omitted). The stop-mark ring shall be rigidly attached to the face of the door and shall bear upon its face two stop marks, one an upper mark at the top upon which to set up the combination for unlocking the safe door and another mark either to the right or left of the upper mark upon which to set up the combination for releasing the lock-case cover and also for inserting the changing key for the purpose of changing the combination numbers. The tumbler pin shall be a stud, shouldered and riveted into a hole, countersunk on the opposite side of the tumbler center. The sliding bolt shall be operated by a catch or drop lever, which is dependent only on the force of gravity for connection to the spindle. The combination lock shall be so placed in relation to the bolt work that the lock mechanism shall not be strained by the forces exerted by the throw bolts. The tumblers shall be of the key-changing type, and the inner and outer sections shall be held together by means of interlocking teeth.

D-16a. The cover of the lock case shall be locked on by the tumblers so that the combination must be set to the side mark on the dial ring before the cover can be removed. The escutcheon for the insertion of the key for changing the combination numbers must be located under the lock-case cover and can not be inserted into the tumblers except when combination has been set upon the side mark on the dial. This prevents changing or ascertaining of the numbers of the combination by an unauthorized person.

D-16b. The case of the combination lock with its gears, tumblers, and all internal parts shall be made of bronze, except the screws for attaching lock to door, which shall be of steel.

D-17. Dials and bolt-throwing handle.-The dials of the combination lock and the handle for throwing the bolts shall be made from bronze and be so connected with the mechanism on the inside of the door by shouldered spindles of such design and so placed in the door that they can neither be pulled out nor driven in. A heavy bar-bolt frame or bridge on the inner face of the door may be provided to prevent driving in of any spindle. This frame shall be fastened to the door by machine screws one-half inch in diameter. All spindles shall be hardened so that they can not be cut or penetrated with edge-cutting tcols, and shall be fitted into the door so as to give a liquid-proof joint.

D-18. Pressure bar. - Each safe shall be provided with a pressure-bar system for forcing the safe door into contact with the jamb. The pressure bar, cams, and pins shall be one piece and shall be made from machinery steel. The pressure-bar brackets shall be steel. The sleeves shall be made from machinery steel and case-hardened. The pressure-bar handle shall be made from bronze.

D-19. Finish of safe body and door.-The entire interior metal surface of the safe, including the bolt frame and the plate on the door, but excluding steel contact surfaces, polished steel, and bronze parts shall be painted white.

D-19a. The exterior, including the crane hinge and the pressure-bar system with its brackets, shall be filled, rubbed to a smooth surface, finished in 
black lacquer, and varnished. Striping shall be the only decoration permitted. Contact surfaces shall not be painted but shall be coated with petrole um jelly.

$\mathrm{D}-19 \mathrm{~b}$. In the center of the upper part of the door there shall be, in appropriate colors, the name of the bureau and the initials and emblem of the United States department for which the safe is ordered. Another inscription or emblem shall be substituted for the one described above, at the request of the purchaser.

D-20. Finish of fittings.-All bolt work on the interior of the door and the stepping on the safe (outer) door and the jamb shall be highly polished. The hinge tips, pressure bar, and handle, throw-bolt handle, and lock dial knob on the exterior of the door shall be triple nickel plated on copper ground. The face of the dial and rim shall be black lacquer, with the marks and figures incised in luminous (radium) paint.

D-21. Lock-operating instruction.-A copy of the manufacturer's instructions for operating and changing the lock combinations bearing the name and address of the manufacturer and the date of the contract shall be pasted to the inside face of the cover plate on the inside of the safe door so that the paper will not become detached. These instructions shall be printed on heavy white paper. They shall be so coated with waterproof varnish that the reading matter will remaio perfectly legible.

\section{E. DETAIL REQUIREMENTS}

E-1. Dimensions.-The dimensions of the safe shall not be less than those given in Table 1 for the size ordered.

TABLE 1.-Minimum dimensions of burglarresisting safes

\begin{tabular}{|c|c|c|c|c|c|c|}
\hline \multirow{2}{*}{ Drawing No. } & \multicolumn{2}{|c|}{ Height } & \multicolumn{2}{|c|}{ Width } & \multicolumn{2}{|c|}{ Depth } \\
\hline & $\begin{array}{l}\text { Out- } \\
\text { side }\end{array}$ & Inside & $\begin{array}{l}\text { Out- } \\
\text { side }\end{array}$ & Inside & $\begin{array}{l}\text { Out- } \\
\text { side }\end{array}$ & Inside \\
\hline $\begin{array}{l}\text { Size 1, I and II....... } \\
\text { Size 2, I and II } \\
\text { Size 3, III. }\end{array}$ & \begin{tabular}{|r} 
Inches \\
40 \\
26 \\
15
\end{tabular} & \begin{tabular}{|c} 
Inches \\
36 \\
22 \\
11
\end{tabular} & $\begin{array}{r}\text { Inches } \\
28 \\
22 \\
26\end{array}$ & $\begin{array}{r}\text { Inches } \\
24 \\
18 \\
22\end{array}$ & $\begin{array}{r}\text { Inches } \\
22 \\
22 \\
17\end{array}$ & $\begin{array}{r}\text { Inches } \\
171 / 2 \\
171 / 2 \\
121 / 2\end{array}$ \\
\hline
\end{tabular}

Safes of size 1 and size 2 shall be fitted with sneak-proof compartment and drawer cabinet. Safes of size 3 shall not be fitted with sneak-proof compartment and drawer cabinet.

E-2. Sneak-proof compartments.-

E-2a. The sneak-proof compartment shall be formed by a front frame and a top of structuralsteel plate. The top, which shall be put in place before the front frame, shall be one-fourth inch thick. The front frame may be formed of two or more pieces welded together after the front is in place in the safe. The door and front shall be one-half inch thick.

$\mathrm{E}-2 \mathrm{~b}$. The top and front shall fit snugly against the inside surface of the safe. They shall be attached to the safe by either lugs or ribs $1 / 4$ inch thick by $1 \frac{1}{2}$ inches wide. The top edge of the front shall fit snugly against the bottom surface of the top, and the joint shall be welded its full length.

E-2c. The inner door to the compartment shall be a single plate of structural steel. The edges shall be square (without stepping). The door-jamb edge of the front frame shall be machined around the opening to form one step, which shall have a depth equal to the thickness of the compartment door.
The edges of the door shall seat on this step without packing. The clearance between the edge of the door and the frame shall be no more than is necessary for the door to swing freely, allowing for the paint on the door and frame.

E-2d. This door shall swing to a position of $90^{\circ}$ with the front frame and shall be as wide and as high as will permit it to take this position. On the edge of the door to which the hinges are attached there shall be two stationary back bolts, which shall prevent the door from being opened if the hinges are removed. These bolts shall be made from 3 by 3 by $5 / 16$ inch structural-steel plate. They shall be offset for the thickness of the frame and shall be attached to the door by rivets having countersunk heads flush with the outside surface of the door.

E-2e. The door shall be hung on center-pin, 5knuckle, shutter-flap hinges. The hinges shall be made from bronze, except the pins, which shall be tool steel, hardened. The countersunk head machine screws shall be inserted from the inside of the door so that the heads are in the hinge flaps and the shanks are in tapped holes in the door and in the frame.

E-2f. The inner compartment door shall be secured with a combination lock having a size of not less than $3 \frac{7}{8}$ by $2 \frac{7}{8}$ by $11 / 4$ inches. This lock shall be of the same pattern and material as hereinbefore described for the outer door, except that the lock shall have three key-changing tumblers instead of four.

E-3. Cabinet.-The space above the sneak-proof compartment shall be provided on the left-hand side with a cabinet of drawers made either from wood or metal, which shall be securely fastened to the safe. If the drawer case is made of wood, the wood shall be one-fourth inch thick and the drawer sides shall be dovetailed into front and back and bottom grooved into sides and nailed to back. Each drawer shall be furnished with a suitable type of drawer lock having duplicate keys. Each drawer shall be partitioned into compartments from side to side for United States paper money. These compartments shall have handholes. The drawers shall be fitted into a case and shall clear the outside door of the safe when withdrawn. There shall also be furnished with each safe a light, removable, wooden change tray which shall contain six cup depressions, one each for 1-cent, 5-cent, 10-cent, 25-cent, 50-cent, and $\$ 1$ silver pieces. This tray shall be of such size as to fit inside any of the drawers when partitions are removed. The cabinet, drawers, and change tray shall be neatly finished in clear varnish, rubbed down to a smooth surface.

\section{F. METHOD OF SAMPLING, INSPECTION, AND TESTS}

F-1. Fit of safe (outer) door.-White-lead paint shall be thinned with turpentine until it gives a thin continuous coating of white when spread on a metal surface. This liquid shall be used to coat all the edges of the safe (outer) door as thinly as possible. The door shall then be closed and locked. When opened the door jamb shall be examined, and if the white liquid has not been transferred to every portion of the jamb, the door and jamb have not come into continuous metal-to-metal contact. It is therefore not air-tight, and liquid explosive can be introduced if the packing is defective.

F-2. Inspection. - Unless otherwise stipulated in the invitation for proposals, inspection and tests will be made during the progress of the work at the shops of the manufacturer, who shall furnish all labor, tools, and material (test pieces) required for proof tests free of expense to the purchaser and conduct such tests in the manner to be directed by the duly authorized representative of the purchaser. 
Tests shall be for the purpose of determining the quality of the material and the workmanship, as follows:

F-2a. Quality of material.-In addition to the drill tests for hardness of parts which the manufacturer should make for his own satisfaction, and oxyacetylene torch resisting property of the inner layer, tensile and ductility tests of cast steel, hardness of tempered tool steel and casehardened machine steel and fire and acid-proof quality of packing for grooves in door and jamb are to be made unless specifically waived in writing by the purchaser.

F-2b. Workmanship. - Workmanship shall cover in a careful manner the fitting of doors to jambs, lock and throw-bolt operating handle spindles, hinge pins, ball and roller bearings, throw-bolt bearings in frame and on jamb, connection of lock to throw bolt work, bearings of pressure-bar system, and general finish of machine work.

F-3. Torch test.-Type B only.

F-3a. The inspector shall select at random not fewer than 10 per cent of the plates for the inner layer of the safe before they are fastened into place in the safe. Each of these plates shall be tested to determine the resistance to the oxyacetylene cutting torch test and oxygen lance test (either one or both of the tests in the discretion of the inspector) in the following manner:

$\mathrm{F}-3 \mathrm{~b}$. The plate shall be placed in a vertical position, with its back at least 1 inch from the supports. The supports shall be fire brick or some similar nonconducting material. The supports may touch the back of the plate at not more than two places, and the area of the plate in contact with each of the supports at the back shall not be greater than 1 square inch.

F-3c. Oxygen lance test.-Each plate shall be attacked separately at any place on its surface selected by the inspector which is not less than 2 inches from the edge of the plate or holes therein. The attack shall be made by an oxygen lance consisting of a $1 / 8$-inch steel pipe and a $3 / 8$-inch-diameter steel rod. The pipe and the rod shall be held parallel and in contact, so that one end of the rod and one end of the pipe can be brought in contact with the plate. The other end of the pipe shall be connected to a tank containing not less than 100 cubic feet of oxygen. The pressure in the tank at the beginning of the test of any plate shall be not less than 1,100 pounds, and the pressure after the test is completed shall be not less than 200 pounds. The free end of the lance shall be placed close to the plate in a "well" formed by a 11/2-inch-diameter perforation in the center of a block of fire clay, cast iron, or graphite. This block shall be not less than 6 by 6 by $1 \frac{1}{2}$ inches and shall be clamped to the test plate.

F-3d. The free end of the lance for about $11 / 2$ inches shall be brought to fusion temperature by an oxvacetylene welding torch. Oxygen shall then immediately be passed through the pipe under a pressure at the regulator on the oxygen tank of 50 pounds per square inch. The hose connecting the pipe and the oxygen tank shall not be longer than 25 feet, and the internal diameter shall not be less than one-fourth inch. The lance shall be kept in contact with the plate with a force not to exceed 5 pounds after the end of the lance has been heated by the welding torch. The end of the lance may be moved across the face of the plate as far as the "well" will permit. The end of the lance shall remain in contact with the portion of the plate within the "well" during this test until the plate has been perforated or until 50 cubic feet of oxygen has been consumed or the test has continued without interruption for five minutes. In any case the test shall then be discontinued and the plate examined. If the plate is penetrated to a depth of one-half inch from the original surface where the attack was made, the plate shall be considered to have failed and no plates represented by the test specimen shall be used in the safe.

F-3e. Cutting-torch test.-Each plate shall be attacked separately at any place on its surface selected by the inspector which is not less than 2 inches from the edge of the plate or holes therein. The attack shall be made by an oxyacetylene cutting torch having at least four but not more than six preheating flames and having an oxygen outlet of approximately a No. 40 drill (0.098 inch in diameter).

F-3f. The torch shall be lighted and the pressure of the oxygen and of the acetylene shall be increased until the largest practicable neutral preheating flame is obtained. This flame shall be maintained as nearly as practicable throughout the test.

F-3g. The cutting torch, held with the longitudinal axis of the tip at a convenient angle with the plate, shall be used to melt the surface of the plate at the place selected by the inspector. The end of a fluxing rod shall be kept in contact or close proximity with the portion of the plate which is being tested. The fluxing rod shall be a steel rod three-eighths inch in diameter, 12 feet long. The amount of carbon in the fluxing rod shall not exceed 0.40 per cent.

$\mathrm{F}-3 \mathrm{~h}$. The test shall continue without interruption and without shifting to another place on the plate until the plate has been penetrated or until 9 feet of the fluxing rod have been consumed, when the torch and rod shall be removed and the plate be examined. If the plate is penetrated to a depth of one-half inch below the original surface of the plate, the plate shall be considered to have failed, and no plates represented by this test specimen shall be used in the safe.

\section{G. PACKAGING, PACKING, AND MARKING}

G-1. Packaging and packing.-Each safe with the combinations set in the unlocked positions shall be delivered at the destination named in the invitation for proposals in a substantial wooden crate, securely packed for reshipment.

G-2. Marking.- Shall be as follows:

G-2a. Each crate shall be marked with the number of the safe, size, type, contract number and date, and the name of the contractor.

$\mathrm{G}-2 \mathrm{~b}$. A copy of the manufacturer's instructions, inclosed in a substantial envelope appropriately marked, shall be securely attached to the inside of the crate in a conspicuous position.

\section{NOTES}

I-1. Type A safes are intended for use in places where the exposure to and likelihood of attack is a minimum, such as in United States Government department buildings, or other United States public buildings where a continuous (24-hour) watch service is provided, and on board of naval vessels in active commission.

I-2. Type B safes are intended for use in places where the exposure is maximum, such as United States public buildings remotely situated and without continuous watch service, at home or in foreign countries, navy yards, storage supply warehouses of the Army, Coast Guard Service, and the like.

I-3. This specification is not mandatory in the purchase of burglar-resisting safes of the special sizes and types required for use in vaults installed in Federal buildings erected under the supervision of the Supervising Architect and which are Federal property, nor to chests contained in insulated (fireproof) safes for use in leased post-office quarters. 
I-4. It is believed that this specification adequately describes the characteristics necessary to secure the desired material and that normally no samples will be necessary prior to award to determine compliance with this specification. If, for any particular purpose, samples, with bids are necessary, they should be specifically asked for in the invitation for bids, and the particular purpose to be served by the bid sample should be definitely stated, the specification to apply in all other respects.

I-5. This specification governs all United States Government purchases of this commodity.

\subsection{TABLES.}

\section{AMERICAN RAILWAY ASSOCIATION, TELE- GRAPH AND TELEPHONE SECTION, SPECIFICATION FOR TELEGRAPH TABLE, 2-G-38, 1928}

\section{TELEGRAPH TABLE}

PURPOSE

The purpose of this specification is to guide the seller or contractor in the manufacture of telegraph tables ARA-1-A for use in telegraph offices.

\section{FINISH}

Metal parts that are to be framed shall be ground smooth. All metal parts shall be well cleaned and given a coat of durable mineral filler and painted, then given a coat of air drying olive $F$ enamel. Finish is to be eggshell without gloss.

\section{PACKING}

Metal details shall be carefully wrapped in paper and so packed in substantial wood crates as to prevent damage to table during transit. Small details and screws shall be enclosed in a canvas sack securely nailed to the inside of the crate.

A detail list of loose pieces, containers and their contents shall be furnished for each shipment.

AMERICAN RAILWAY ASSOCIATION, TELEGRAPH AND TELEPHONE SECTION, SPECIFICATION FOR DISPATCHERS TABLES ARA-1-A AND ARA-2-A, 2-G-39, 1928

\section{DISPATCHERS' TABLES}

PURPOSE

The purpose of this specification is to guide the seller or contractor in the manufacture of single

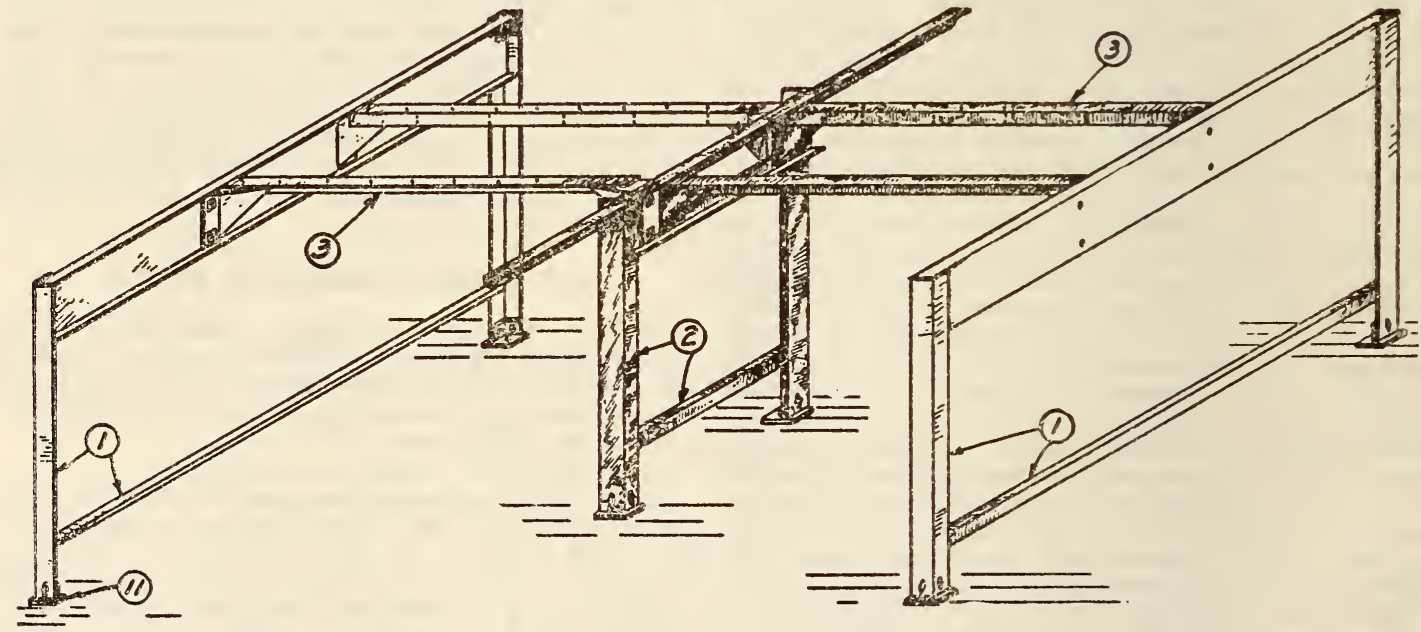

NOTE:-

INTERMEOIATE GECTION SHPOED.

Figure 644.-Telegraph table ARA-1-A, assembly of steel framework

(The specification covers general items, number of units required for different lengths of table, material and workmanship, dimensions, finish, assembly, inspection, tests, packing, marking, and warranty. It is accompanied by 11 drawings, one of which is reproduced below, showing the assembly of steel framework. The metal requirements are as follows:)

\section{MATERIAL AND WORKMATSTIP}

Sheet steel. - The sheet-metal parts shall be mild steel, cold rolled and double annealed, free from buckle, and shall be roller leveled and pickled.

Angles.-The angles, bars, etc., used shall be Carnegie standard or equivalents.

Castings.-Castings shall be good quality gray cast iron.

Similar parts shall be identical in size so as to be strictly interchangeable. position dispatcher's table ARA-1-A and double position dispatcher's table ARA-2-A.

(This specification covers general items, material, manufacture, dimensions, finish, inspection, tests, packing, marking, and warranty. The metal requirements are as follows:)

\section{MATERIAI}

Except where otherwise stated, all steel is to be the best mild cold-rolled, pickeled, double annealed, and patent leveled, free from scales and buckle. The plates used on exposed parts to be the particular special grade known as metallic furniture stock. Framing parts are to be built of rolled or formed shapes of mild steel. Drawer pulls, door handles and binding for linoleum top shall be of bronze. Footings for legs shall be bronze plated. Yale (or equivalent) paracentric locks shall be fitted in all drawer heads. 


\section{MANUFACTURE}

Pedestals shall be formed of No. 18 gage steel with 2 -inch square legs and panels sunk three-eightinch. Pedestals shall be flanged at bottom for stiffness and to hold bottom plates. Dividing shelves between drawers are to be of No. 22 gage. Legs shall be formed of No. 18 gage steel and welded to pedestals. Legs shall be equipped with flush bronze-plated footings. Foot rest shall be made of $1 \frac{1}{4}$ inch pipe secured to pedestals by wrought flanges.

Cupboard pedestal shall have an inside cupboard case. Uprights of this case are to be made of No. 13 gage steel reinforced by means of $3 / 16$ by 1 -inch strike bars electrowelded thereto. Bottom plate shall be No. 18 gage steel flanged on all edges and formed at front with recess to form door strike. Back of this case shall be No. 22 gage steel flanged on all edges and bolted or riveted to uprights. Doors shall be No. 8 gage steel and shall be fitted with solid castbronze door knobs arranged to operate a steel latch and up-and-down bolts which shall hold the doors securely at top and bottom when latched. Doors shall be hung on heavy wrought hinges.

Sliding shelf boxes shall be No. 18 gage steel flanged on three edges and with front formed to a flat face channel section with $\% / 16$-inch flat face. Sliding shelves shall be made of No. 14 gage steel and shall have sides and front formed into a channel section. Front corners shall be rounded to a $3 / 8$ inch radius. The table top shall be of No. 14 gage steel and shall be made in two parts securely spliced in center. All edges shall be formed into a channel shape. This top shall be reinforced by channelshaped corrugations formed of steel on both sides just back of apron. Steel straps $1 / 8$ by $1 / 2$ inch shall be hung in channel shape in center of top to carry wiring. A rigid top shelf made of No. 14 gage japanned steel with edges formed into channel shape, shall be placed $16^{3}{ }_{4}^{\prime}$ inches above main top and supported by 2 -inch square tubing posts with steel panels between. These panels are to be framed with neat steel molding. Subshelves made of No. 16 gage steel shall be placed at each end with an opening $3 y^{\prime \prime}$ inches between top side of same and bottom side of top shelf. Two upright plates of No. 13 gage steel shall run from main top to underside of shelf just inside the 2-inch posts with No. 16 gage steel panel between.

Outside heads of box drawers shall be flat and made of No. 24 gage steel with edges formed into a 5/16-inch box channel section slightly beveled from front to rear to insure a perfect fit in the compartments. All corners shall be oxyacetylene welded to prevent opening of joints and ground to a slight radius to eliminate sharp corners. Intermediate heads shall be made of No. 22 gage steel flanged back at side and bottom, placed tightly against outside head and electrowelded through flanges to body. Inside heads shall be made of No. 24 gage steel, formed or flanged on all edges. Each shall be made of No. 18 gage steel full height of sides. Body shall be made with one piece of No. 24 gage steel with sides to be bent up at right angles to bottom with top edges formed into a $5 \% 16$-inch square tubing section and rear edges flanged and electrowelded to back. Channel suspension slides shall be provided consisting of No. 16 gage steel channels electrowelded to sides of drawers engaging larger channels, mortised to the pedestals. Head of drawer shall be fitted with bronze piece of suitable design securely fastened through outside and intermediate heads. Yale (or equivalent) locks and duplicate keys.
All gages specified shall be United States standard, except where otherwise stated.

\section{FINISH}

The finished color of table shall be olive enamel without stripes. All steel parts shall be thoroughly cleaned before finishing, framing parts made smooth, plate surfaces well sandpapered, and the entire product treated to a benzine dip to remove oil and dirt, followed by a heavy coat of well-baked mineral filler applied by dipping or spraying the product to insure covering all surfaces. This shall be thoroughly rubbed down to a smooth even surface, coated with best baking japan, and baked to a temperature of from 150 to $300^{\circ}$, exact temperature and number of coats to depend on the color. Final finish is to be

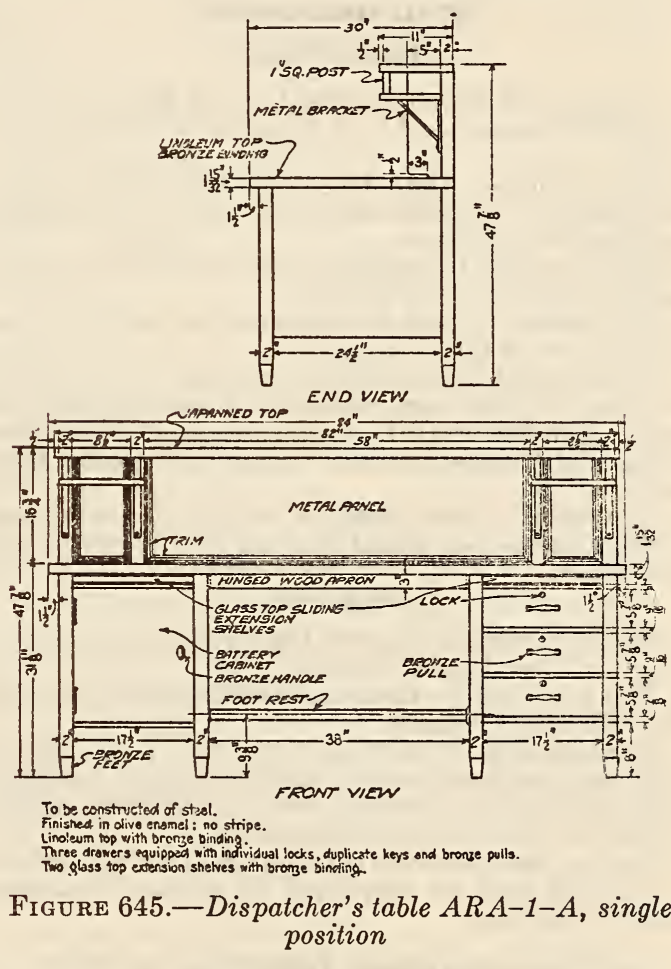

rubbed to an eggshell gloss. All brass or bronze hard ware, ornaments, and moldings are to be thoroughly buffed to a high polish and given the required finish by hand brushing, oxidizing, etc. Work shall be thoroughly cleaned between each operation and finally dried and given a heavy spray coat of best grade lacquer. All brass or bronze castings must be free from sand holes, pit marks, and other imperfections.

\section{PACKING}

Table shall be knocked down into four pieces, consisting of top shelf, main top, cupboard section, and drawer section. Each piece shall be securely wrapped with heavy wrapping paper and so crated in substantial wood crates as to prevent damage to table during transit. Small details, screws, etc., shall be inclosed in a canvas sack and securely nailed on inside of crate. A detail list of loose pieces, containers, and their contents shall be furnished for each shipment. 


\section{UNITED STATES GOVERNMENT, FEDERAL SPECIFICATIONS BOARD, FEDERAL SPECIFICATION FOR LAUNDRY APPLI- ANCES, 00-I-131, APRIL 28, 1931}

(Various types of laundry appliances are covered in above specification, of which the following requirements are applicable to laundry tables and to starch tables.)

\section{IAUNDRY TABLES}

There are no other Federal specifications applicable to this specification.

Household types of laundry appliances are not covered by this specification.

\section{DETAIL REQUIREMENTS}

Laundry Tables

Laundry tables shall have tops of approximately the following sizes- 3 by 8 feet, 3 by 10 feet, 3 by 12 feet.

The table top shall be of not less than $1 \frac{3}{8} 8$-inch nonresinous, nonwarping white wood, thoroughly seasoned and dressed.

The top shall be smooth and constructed so as to prevent opening of the seams.

The table top shall be supported on cast-iron legs about 2 feet 9 inches high.

Tables shall have at least three legs, except those less than 10 feet long, which shall have at least two legs. The legs shall be shaped so as to insure stability of the table and be well secured to the table top.

If required, tables shall be suitable for mounting a seam dampener, collar shaper, and collar former and edger.

$$
\text { Starch Table }
$$

Starch tables shall have a top approximately 4 feet long by 2 feet 8 inches wide.

The top shall be made in accordance with one of the following methods:

Nonresinous, nonwarping white wood, thoroughly seasoned, not less than $1 \frac{3}{8}$ inches thick, and covered with zinc, tinned copper or an approved nickelcopper or other noncorrodible alloy.

Iron or steel coated with white porcelain fused on.

The top shall be supported by at least two rigid iron or steel legs.

METHODS OF SAMPLING, INSPECTION, AND TESTS

Laundry appliances shall be inspected and tested to determine compliance with this specification.

\subsection{FILING FURNITURE.}

UNITED STATES GOVERNMENT, FEDERAL SPECIFICATIONS BOARD, FEDERAL SPECIFICATION FOR FURNITURE AND CABINETS ; OFFICE, SECTIONAL, STEEL, AA-F-791, NOVEMBER 11, 1930

\section{SECTIONAL FURNITURE AND CABINETS}

\section{A. APPLICABLE FEDERAL SPECIFICATIONS}

A-1. There are no other Federal specifications applicable to this specification.

\section{B. TYPE}

B-1. This specification includes steel sectional office furniture, including small sections, horizontal sections, upright sections and cabinets, card filing.

\section{MATERIAL AND WORKMANSBIP}

C-1. All sheet steel shall be of the quality and grade known as first-grade metallic furniture steel. All workmanship shall be the best known to the trade; all materials shall be the best of the kind specified.

\section{GENERAL REQUIREMENTS}

D-1. The technical terms herein employed shall be interpreted in accordance with the highest and best known standards of actual commercial practice in the art of high-grade furniture construction and design.

D-2. All sections shall intermember with, and match in size, exterior appearance and design, the standard sections adopted by the General Supply Committee.

D-3. Finish.-

D-3a. All metal surfaces shall be thoroughly cleaned and free from dirt, oil, grease, and moisture, before applying the finish.

D-3b. After a thoroughly reliable priming coat and filler, where necessary, the color coats shall be sufficient when properly baked so that in connection with the varnish, a surface guarantee not to crack or spall under severe usage will be produced. Each coat to be baked and rubbed down separately. All exposed surfaces to receive a final varnish rubbing to a satin finish. Final colors shall match the standard colors adopted by the General Supply Committee.

D-3c. In addition to the above requirements, whenever especially called for in the purchase specifications to take care of tropical climatic conditions, all small bolts, nuts, screws, small levers, rods, guides or similar parts, shall be made of brass or galvanized steel. All other parts shall be treated with a rustresistant alloy or tern coating. The tern coating shall be done after the fabrication. Bidder shall give a complete description of the methods he proposes to use in applying the rust-resistant coating and enamel in order to prevent the coating and enamel from flaking or chipping off.

D-4. Hardware.-

D-4a. Pulls, label holders, rod heads, and door knobs are to be solid cast bronze, brush finish. The mixture of metals for the bronze shall consist of 88 per cent copper, 10 per cent tin, and 2 per cent zinc.

D-5. Drawer suspensions.-

All vertical file drawers such as letter, legal and bill sizes and similar drawers with subdivisions, space permitting, shall be fitted with side-arm suspensions slides. Where the width of the cabinet will not permit the use of side-arm slides, bottom extension slides shall be used of the type known as progressive drawer slides. The steel used for the side-arm slides, for the housing in which the slides operate and for the runner attached to the drawers, shall be made rust resistant by electric galvanizing, nickelplating, or by other equally effective process and not lighter than No. 16 gage. The slides shall travel easily and smoothly with the drawer and the mechanism shall be sufficiently strong to sustain the weight of the drawer when loaded to capacity, and fully open. All slide rollers shall have at least $1 / 4$-inch bearing surface and be accurately latheturned from drawn screw stock, no stamped or formed rollers will be permitted. Slides with traveling rollers or fixed rollers with ball bearings are preferred, but other methods will be considered. Special note will be made of the method of assembling the rollers and roller housings or retainers to the slide arm. Assembling of these parts shall be by solid or tubular riveting. Vertical file drawers more than 16 inches in width and 10 inches in height shall be fitted with two pairs of extension slides. 
D-6. Vertical file drawers shall be equipped with suitable stops to relieve the shock and deaden the noise of opening and closing the drawers and to prevent the drawers from dropping out when opened, but the stops must be so constructed that the drawers may be readily removed and replaced. Drawers shall not be permitted to stop against the back of the files.

D-7. All card index and vertical file drawers shall be fitted with compressors of approved type. The compressors shall have strong, positive grips and shall be capable of easy adjustment. Vertical file compressors shall be removable and shall not operate upon the countersunk guide rod.

\section{E. DETAIL REQUIREMENTS}

$\mathrm{E}-1$. Small sections and horizontal sections.-

E-1a. Outside dimensions. - Sixteen and one-half and thirty-three inches wide by 17 and 25 inches deep. These sections to advance in height in multiples of $6 \%$ inches. The steel used in the manufacture of the various parts shall not be less than the gage specified below:

\begin{tabular}{|c|c|c|}
\hline & No. & Inch \\
\hline $\begin{array}{l}\text { End } \\
\text { Back } \\
\text { Top } \\
\text { Bottom } \\
\text { Partition, front } \\
\text { Partition, side } \\
\text { End lining } \\
\text { Card index drawer front } \\
\text { Vertical file drawer front } \\
\text { Card index drawer body } \\
\text { Vertical file drawer body } \\
\text { Shelf } \\
\text { Top section } \\
\text { Base frame. } \\
\text { Base leg }\end{array}$ & $\begin{array}{l}18 \\
20 \\
20 \\
20 \\
18 \\
24 \\
24 \\
20 \\
18 \\
24 \\
20 \\
20 \\
18 \\
18 \\
18\end{array}$ & $\begin{array}{l}0.0490 \\
.0368 \\
.0368 \\
.0368 \\
.0490 \\
.0245 \\
.0245 \\
.0368 \\
.0490 \\
.0245 \\
.0368 \\
.0368 \\
.0490 \\
.0490 \\
.0490\end{array}$ \\
\hline
\end{tabular}

E-1b. Construction.-Assembling shall be by electric spot welding. Tops and bottoms shall be welded to the flanges of the ends and back with spots not more than 3 inches apart. All sections shall have vertical and horizontal partitions between drawers. Partitions, where space permits, and ends and drawer fronts, shall be double-wall construction. The four front corners as well as the four back corners of the sections shall be joined by acetylene or arc welding for the purpose of strengthening and giving the sections a finished appearance. All surplus metal shall be filed off and sanded smooth. Top and bottom of section shall be tied together by wire brace rods or a similar device to prevent spreading. Sections shall be assembled so that spot welds shall not show on exposed portions. All drawer fronts shall be assembled by welding directly to the drawer body. Sections shall be fitted with interlocking device which shall prevent tilting of the sections when the loaded drawers are extended. Ends and backs of sections shall be smooth and flush and the raw edges of steel shall not protrude.

E-1c. All top sections shall be $2 \frac{1}{4}$ inches high and base sections 9 inches or 16 inches high.

E-1d. All card index and box drawers shall be made with bail suspension, so that the drawers can be readily removed.

E-2. Bookcase sections with disappearing glass panel doors.-

E-2a. Outside dimensions shall be 33 inches wide by 13 inches deep by $123 / 4,143 / 4,163 / 4$, or $18 \%$ inches high. Inside dimensions in the clear, when door is opened, shall be not less than 31 inches wide by $101 / 2$ inches deep by $9 \frac{1}{2}, 11 \frac{1}{2}, 14$, or 16 inches high. Bookcase sections without doors shall have same outside dimenions and inside dimensions except that the inside heights shall be $11,13,15$, or 17 inches and the depth 12 inches.

$\mathrm{E}-2 \mathrm{~b}$. Construction shall be the same as for small and horizontal sections.

E-3. Upright sections.-

E-3a. Outside dimensions $52 \%$ inches high by 25 inches deep by such width as may be required. These sections shall be so constructed that the detachable leg bases $45 \%$ inches high may be attached, bringing the total height to 57 inches.

$\mathrm{E}-3 \mathrm{~b}$. The steel used in the manufacture of upright sections shall not be less than the gage specified below:

\begin{tabular}{|c|c|c|c|c|c|}
\hline & No. & Inch & & No. & Inch \\
\hline $\begin{array}{l}\text { End } \\
\text { Back... } \\
\text { Top. } \\
\text { Bottom }\end{array}$ & $\begin{array}{l}18 \\
18 \\
18 \\
18\end{array}$ & $\begin{array}{r}0.0490 \\
.0490 \\
.0490 \\
.0490\end{array}$ & $\begin{array}{l}\text { Shelf } \\
\text { Drawer body } \\
\text { Drawer front... }\end{array}$ & $\begin{array}{l}18 \\
20 \\
18\end{array}$ & $\begin{array}{r}0.0490 \\
.0368 \\
.0490\end{array}$ \\
\hline
\end{tabular}

E-3c. Assembly shall be by electric spot welding or lock joints reinforced where necessary by acetylene or arc welding to insure strength and rigidity. Sections shall have shelves between drawers. Flanges of shelves shall be spot welded to the ends of the cabinets. Drawer fronts shall be spot welded directly to the drawer bodies. No spot welds are to appear on exposed portions of cabinet. Distance between spot welds shall not be more than 4 inches. Double-wall construction shall be used for drawer fronts. The four front and four back outside corners shall be acetylene or arc welded to prevent opening of joints. The two front top corners shall be reinforced by steel angles running from front to rear welded into position and reinforced by acetylene or are welding.

E-3d. Double compartment card index drawers in upright sections shall have side slide suspensions, single drawers, bail suspension.

$\mathrm{E}-4$. Card index cabinets.-

E-4a. Outside depths shall be either 12 inches or 16 inches.

E-4b. The steel used in the manufacture of the various parts shall not be less than the gage listed below:

\begin{tabular}{|c|c|c|c|c|c|}
\hline & No. & Inch & & No. & Inch \\
\hline $\begin{array}{l}\text { End } \\
\text { Top } \\
\text { Bottom } \\
\text { Back } \\
\text { Partition front }\end{array}$ & $\begin{array}{l}20 \\
20 \\
20 \\
20 \\
20\end{array}$ & $\begin{array}{r}0.0368 \\
.0368 \\
.0368 \\
.0368 \\
.0368\end{array}$ & $\begin{array}{l}\text { Partition side.... } \\
\text { Drawer front... } \\
\text { Drawer body. } \\
\text { End lining } \\
\text { False bottom..... }\end{array}$ & $\begin{array}{l}24 \\
20 \\
24 \\
24 \\
20\end{array}$ & $\begin{array}{l}0.0245 \\
.0368 \\
.0245 \\
.0245 \\
.0368\end{array}$ \\
\hline
\end{tabular}

E-4c. Assembly shall be by electric spot welding reinforced where necessary by acetylene or arc welding to insure strength and rigidity. The distance between spot welding shall not be more than 4 inches. Cabinets shall have shelves between drawers. Flanges of shelves shall be spot welded to the ends of the cabinets. Drawer fronts shall be spot welded direct to the drawer bodies. No spot welds shall appear on exposed portions of cabinets. All four corners, both front and rear, shall be acetylene or arc welded to prevent opening of joints. Doublewall construction shall be used for drawer fronts.

E-4d. Double compartment card index drawers in the upright sections shall have side slide suspensions; single drawers, bail suspensions.

\section{F. METHOD OF INSPECTION AND TESTS}

F-1. The articles may be inspected at the factory, by a representative of the purchaser, at any time 
during process of manufacture. The purpose of this factory inspection being to determine compliance with the terms of the specification as to workmanship, material, details of construction, hardware, and finish. Final inspection will also be made at point of delivery.

\section{G. PACKING AND MARKING}

G-1. Packing.-Unless otherwise specified, the subject commodity shall be delivered in standard commercial containers, for safe transportation, at the lowest rate, to the point of delivery.

G-2. Marking.-Each article shall be clearly marked in an inconspicuous place with the name of the manufacturer.

G-2a. Unless otherwise specified, shipping containers shall be marked with the name of the material, the size, type, and the quantity contained therein, as defined by the contract or order under which the shipment is made, the name of the contractor, and the number of the contract or order.

\section{NOTES}

I-1. Purchasers should exercise all options offered herein and should specify type, grade, class, etc., required.

I-2. It is believed that this specification adequately describes the characteristics necessary to secure the desired material and that normally no samples will be necessary prior to award to determine compliance with this specification. If, for any particular purpose, samples with bids are necessary, they should be specifically asked for in the invitation for bids, and the particular purpose to be served by the bid sample should be definitely stated, the specification to apply in all other respects.

I-3. This specification governs all United States Government purchases of this commodity.

\subsection{MISCELLANEOUS METAL FURNI- TURE AND FIXTURES.}

\section{NATIONAL BOARD OF FIRE UNDER- WRITERS, CONTAINERS FOR STORING AND HANDLING FLAMMABLE LIQUIDS, 1927}

\section{STORAGE CABINETS}

Storage cabinets may be used where it is desired to keep more than 10 gallons of oils, varnishes, lacquers, or similar materials inside buildings, no individual container to exceed 5 gallons capacity. Not over 50 gallons should be stored in any one cabinet. Such cabinets shall be of construction conforming with or equivalent in fire resistance to the following requirements:

Bottom, top, and sides of cabinet shall be made of sheet iron at least No. 18 gage in thickness and double walled with $1 \frac{1}{2}$-inch air space. Joints shall be riveted or made tight by some equally efficient means. Doors shall be of construction equivalent to walls, provided with 3-point lock, and kept closed when not in use; door sill shall be raised at least 2 inches above bottom of cabinet. Cabinet shall be conspicuously labeled in red letters, "Flammable, Keep Fire Away."

Note.-When deemed necessary, cabinets shall be vented. Volatile flammable liquids shall be kept in metal safety cans, or other approved metal containers.
UNITED STATES GOVERNMENT, FEDERAL SPECIFICATIONS BOARD, FEDERAL SPECIFICATIONS FOR CUPBOARDS AND LOCKERS ; STEEL, AND EQUIPMENT, NO. 471, MARCH 5, 1927. (CURRENT DESIGNATION : AA-C-791)

\section{COPBOARDS AND LOCKERS}

(Superseded by AA-C-31, p. 835, and AA-L -486, p. 841)

614. STOVES

\section{AND FURNACES, EXCEPT ELECTRIC}

614.0 GENERAL ITEMS.

AMERICAN GAS ASSOCIATION, REQUIREMENTS FOR HOUSE PIPING AND APPLIANCE INSTALLATION, 1928

GAS PIPING

(See 607.0, p. 361.)

AMERICAN GAS ASSOCIATION, AMERICAN STANDARD GAS SAFETY CODE FOR INSTALLATIONS AND WORK IN BUILD. INGS, K2-1927

\section{GAS SAFETY CODE}

[Approved by the American Standards Association] (See 607.0, p. 361.)

ASSOCIATED FACTORY MUTUAL FIRE INSURANCE COMPANIES, FUEL-OIL IN. STALLATIONS FOR FURNACES AND ENGINES, 1928

\section{FUEL OIL INSTALLATIONS}

(Requirements covering capacities of tanks, material, location, and construction details conform to those specified under 605.23, p. 293 , for containers for storing and handling flammable liquids by the National Board of Fire Underwriters.)

NATIONAL BOARD OF FIRE UNDERWRITERS, INSTALLATION, MAINTENANCE, AND USE OF PIPING AND FITTINGS FOR CITY GAS, 1920

\author{
PIPING FOR CITY GAS
}

(See 607.0, p. 364.)

RAILWAY FIRE PROTECTION ASSOCIATION, GENERAL RECOMMENDATIONS IN CONNECTION WITH CHIMNEYS, FLUES, STOVES, AND FURNACES, 1925

\section{HEATING EQUIPMENT}

(This specification contains detail recommendations covering material, location, and structural features of chimneys, smoke pipes, stoves, gas stoves, furnaces, hot-air pipes, hot-air registers, and steam and hot water pipes.) 
614.1 COAL STOVES AND RANGE BOILERS. UNITED STATES GOVERNMENT, DEPARTMENT OF COMMERCE, BUREAU OF STANDARDS, SIMPLIFIED PRACTICE RECOMMENDATION FOR RANGE BOILERS AND EXPANSION TANKS, R8-29, NOVEMBER 1, 1929

\section{RANGE BOILERS AND EXPANSION TANKS}

(Simplified practice recommended and adopted by industry establishing a limited list of standard sizes and capacities.)

TABLE 1.-Range boilers

\begin{tabular}{|c|c|c|c|c|c|}
\hline \multicolumn{2}{|c|}{ Size } & \multirow{2}{*}{ Capacity } & \multicolumn{2}{|c|}{ Size } & \multirow{2}{*}{ Capacity } \\
\hline $\begin{array}{l}\text { Diam- } \\
\text { eter } 1\end{array}$ & Length ${ }^{2}$ & & $\underset{\text { eter }^{1}}{\text { Diam- }}$ & Length ${ }^{2}$ & \\
\hline $\begin{array}{r}\text { Inches } \\
12 \\
12 \\
12 \\
14 \\
14 \\
16 \\
16\end{array}$ & $\begin{array}{r}\text { Inches } \\
36 \\
48 \\
60 \\
48 \\
60 \\
48 \\
60\end{array}$ & $\begin{array}{c}\text { Gallons } \\
18 \\
24 \\
30 \\
32 \\
40 \\
42 \\
52\end{array}$ & $\begin{array}{r}\text { Inches } \\
18 \\
20 \\
22 \\
24 \\
24 \\
24\end{array}$ & $\begin{array}{r}\text { Inches } \\
60 \\
60 \\
60 \\
60 \\
72 \\
96\end{array}$ & $\begin{array}{c}\text { Gallons } \\
66 \\
82 \\
100 \\
120 \\
144 \\
192\end{array}$ \\
\hline
\end{tabular}

1 Diameters refer to inside measurements.

${ }^{2}$ Lengths mean lengths of sheet, not over-all lengths of boilers.

Notes.-Working pressure.-Range boilers may be made in two classes-" "standard" 85 pounds working pressure and "extra heavy" 150 pounds working pressure.

Four side tappings. - The revision conference of April 18, 1928, felt that at this time the demand for range boilers with four side tappings is not sufficient to warrant inclusion in the simplified-practice reco is not sucied -practice recommendation. However, in deference to a resolution of the National Association of Master Plumbers, it is recommended that when orders be placed in line in the side at 6,18 , and 26 inches from the bottom and 6 inches from the top.

It is further recommended that-

1. Range boilers have one side tapping 6 inches from the top and one 6 inches up from the bottom (measurements to be made from the edge of the shell plate), and two tappings in the top and one in the bottom. All tappings to be 1 inch $(1 / 2$-inch top and the $3 / 4$-inch side and bottom tappings on vertical boilers to be eliminated)

2. Five 1-inch openings to be considered as standard for horizontal boilers.

3. The 16 by 48 inch boiler be designated by its actual capacity (viz, 42 gallons), in lieu of "No. 18," to avoid confusion with the 18-gallon boiler.

4. All range boilers and combination boiler and gas water heaters be rated by their actual water capacities in gallons.

TABLE 2.-Expansion tanks

\begin{tabular}{|c|c|c|c|c|c|}
\hline \multicolumn{2}{|c|}{ Size } & \multirow{2}{*}{ Capacity } & \multicolumn{2}{|c|}{ Size } & \multirow{2}{*}{ Capacity } \\
\hline$\underset{\text { eter }}{\text { Diam- }}$ & Length & & $\begin{array}{c}\text { Diam- } \\
\text { eter }\end{array}$ & Length & \\
\hline $\begin{array}{r}\text { Inches } \\
12 \\
12 \\
14\end{array}$ & $\begin{array}{r}\text { Inches } \\
20 \\
30 \\
30\end{array}$ & $\begin{array}{c}\text { Gallons } \\
10 \\
15 \\
20\end{array}$ & $\begin{array}{r}\text { Inches } \\
16 \\
16 \\
16\end{array}$ & $\begin{array}{r}\text { Inches } \\
30 \\
36 \\
48\end{array}$ & $\begin{array}{c}\text { Gallons } \\
26 \\
32 \\
42\end{array}$ \\
\hline
\end{tabular}

NOTE-Gage glass tappings to be $131 / 2$ inches between centers to provide for exposure of 12 inches of vertical gage glass.
614.2 GAS STOVES, RANGES, AND WATER HEATERS.

AMERICAN GAS ASSOCIATION, APPROVAL REQUIREMENTS FOR GAS RANGES, MARCH 1,1930

\section{GAS RANGES}

\section{CONSTRUCTION REQUIREMENTS}

Notes

1. Unless otherwise specified, all gages are U. S. standard.

2. The terms steel and iron are used interchangeably in these requirements. Either steel or iron is considered satisfactory.

\section{Section 1. Sheet Metal}

(a) No sheet-metal parts shall be less than No. 24 gage excepting rack supports, top back strip, and finishing strips, which may be No. 26 gage.

(b) Gage of enameled parts shall be not less than that herein specified for such parts.

\section{Section 2. Front Frame and Top Frame}

(a) All ranges shall be equipped with front and top frames. The top frame shall extend on all four sides of the cooking top.

(b) If made of cast iron, no part of frame must have a cross-sectional area less than one-fourth square inch.

(c) If made of angle iron, no part of frame must have a cross-sectional area less than one-eighth square inch.

$(d)$ If made of sheet steel, frame must not be less than No. 18 gage and no part must have a crosssectional area less than one-eighth square inch.

(e) When any other material is used, the strength of the part shall be at least equivalent to that specified for cast iron in $(b)$, or for structural iron in (c) and $(d)$ above.

(f) Band iron or other structural steel shapes shall have joints securely riveted or welded or bolted.

(g) Cast frames, when not cast in one piece, shall have joints securely fastened to give effectively a 1 -piece construction.

Section 3. Base

(a) All ranges shall be equipped with a base.

(b) Bases extending around only the two sides and back of a range shall be of 1-piece construction with ends securely bolted to front frame, but in such cases no side shall be longer than 24 inches.

(c) Band iron, angle iron, or other structural steel shapes shall have joints securely riveted or welded.

(d) Sheet iron, when used, shall be not less than No. 18 gage and edges shall be reinforced, as by folding.

(e) Bases shall be strong enough to withstand without permanent vertical or horizontal deflection, as measured at the center of the long sides, an added weight of 300 pounds when applied for a period of five minutes. Application of load shall be made over a width of 8 inches and a depth of approximately that of cooking top. For cabinet ranges, the load is to be placed on inside half of cooking top, alongside of ovens; for other types of ranges it is to be placed in the middle of the cooking top, or in special cases as close to the middle as conditions permit. 
Section 4. Cven and Broiler Tops

(a) The outside part of the tops of ovens on elevated and cabinet types of ranges, if of sheet metal, shall be not less than No. 20 gage.

\section{Section 5. Oven and Broiler Linings}

(a) Ovens and broilers shall be furnished with linings at sides and back to either (1) provide airspace insulation, or (2) inclose solid insulation.

(b) Where linings are used for air-space insulation solely there shall be not less than one-fourth inch noncirculating air space.

(c) Where the back space is used for flue purposes, there is to be not less than one-half inch space.

(d) Oven shall be furnished with a top lining, providing not less than one-half inch space.

(The full text of these approval requirements on construction of ovens includes requirements on removability and gage thickness of linings, structural requirements and thickness requirements for some of the sheet-metal parts for burner box and burner box lining, oven bottoms, extreme bottoms and broiler bottoms, rack supports, flue collars, doors, legs, bolts and screw sizes, open top grates, closed (solid) tops, end shelves, top and simmering burners, oven and broiler burners, oven-burner supports, lighters, mixer heads, air shutters, cocks, manifolds, burner trays, oven racks and broiler pans, solid insulation, air supply to elevated ovens, removal of piping on cabinet ranges, oven thermostats and their pilots, and assembly and name-plate requirements.)

\section{PERFORMANCE REQUIREMENTS}

\section{Test Gases}

In conducting the performance tests specified herein, gases with characteristics approximately as follows shall be used:

\begin{tabular}{|c|c|c|}
\hline & $\begin{array}{l}\text { Heating } \\
\text { value (B. } \\
\text { t.u. per } \\
\text { cubic foot) }\end{array}$ & $\begin{array}{l}\text { Specific } \\
\text { gravity } \\
(\text { air }=1.0)\end{array}$ \\
\hline $\begin{array}{l}\text { Gas A (natural gas) } \\
\text { Gas B (manufactured gas) } \\
\text { Gas C (manufactured gas) }\end{array}$ & $\begin{array}{r}1,135 \\
535 \\
400\end{array}$ & $\begin{array}{r}0.65 \\
.38 \\
.70\end{array}$ \\
\hline
\end{tabular}

\section{Pressures}

(a) Normal pressures specified herein shall be 3.5 inches equivalent water column for gases B and C, and 7.0 inches equivalent water column for gas $\mathrm{A}$.

(b) Unless otherwise stated, each test specified herein shall consist of a series of three tests: One made at normal pressure, on which the adjustment of air shutters shall be made without change in adjustment, one at 0.5 normal gas pressure, and one at 1.25 normal gas pressure.

\section{Burner Capacities}

(a) The gas-burning capacities of range burners shall be as follows:

Separate simmering burner, not less than 1,800 B. t. u. per hour.

Regular top burner, not less than 9,000 B. t. u. per hour. hour

Giant top burner, not less than 12,000 B. t. u. per

Oven burner, not less than 8,000 B. t. u. per hour per cubic foot of oven space, when oven space does not exceed 2.4 cubic feet. For ovens larger than 2.4 cubic feet oven space, the manufacturer to furnish B. t. u. rating.

Broiler burner, not less than 140 B. t. u. per hour per square inch of broiling area.

\section{Oven Heating Capacity}

The capacity of all oven-burner controls shall be such that the oven temperature will reach $500^{\circ} \mathrm{F}$. within 20 minutes.

\section{Capacities at Low Pressure}

All burners shall have sufficient capacity to burn gas at rates specified above when using gas $\mathrm{A}$ at 4 inches pressure and gas $\mathrm{B}$ at 2 inches pressure.

\section{Burner Operating Characteristics}

All burners shall fulfill the following requirements throughout a range of gas pressures from 1.25 normal to 0.5 normal with the same adjustment of the air shutter as provided for the normal pressure.

(a) Burners must not flash back upon immediate ignition, 2 to 20 seconds delayed ignition, or turning the gas cock until the gas supply is one fifth of the full supply at the test pressure.

(b) Burners shall ignite completely and without hesitation. In the case of top burners, this observation to be made with $7 \frac{1}{2}$-inch diameter (bottom) utensils over the burners.

(c) Burners shall operate without carbonizing flames. This observation to be made with $7 \frac{1}{2}$-inch diameter (bottom) utensils over the top burners.

(d) Burners shall extinguish without undue noise.

(e) Burners must not produce a back pressure in the mixers, causing a leakage of gas through the air inlets when consuming gas at a pressure 1.25 normal, nor at a pressure only high enough to support combustion at the ports.

\section{Fire Hazard}

The maximum temperature at points 6 inches from the back and sides of a gas range and on the floor under the range shall be not more than $90^{\circ} \mathrm{F}$. in excess of the room temperature.

\section{Flue Gas Analysis}

A gas range shall produce no carbon monoxide. This requirement shall be deemed met when a concentration not in excess of 0.01 per cent is produced in a room of 1,000 cubic feet content with 4 air changes occurring during the combustion of an amount of gas liberating 60,000 B. t. u.

\section{Oven Heat Distribution}

The heat distribution in the oven shall be so uniform that biscuits distributed in the oven, heated to $450^{\circ} \mathrm{F}$., will be evenly browned in not more than 20 minutes.

\section{Broiler Heat Distribution}

The heat distribution in the broiler shall be uniform. This requirement shall be deemed met when bread, covering entire broiling area, will be toasted to an even brown in not more than 10 minutes.

\section{Relief for Explosion}

The oven doors shall open when a pulling force of 50 pounds is applied at each door handle. $n$ a direction which is normal to the plane of the door.

\section{Top Burner Thermal Efficiency}

The thermal efficiency of gas range top burners shall be not less than 30 per cent.

\section{Air Shutters}

The air-gas ratio of the mixture in any gas range burner shall be not greater than 1.1, with the air shutter in the completely closed position when operating on gas B at the rated burner capacity. 
(Other performance requirements given in the full text of these approval requirements for ranges using natural and manufactured gas, include performance requirements for lighters, adjustable orifices, oven thermostats, absence of leakage, rustresisting oven interiors, test requirements for coated sheet steel, safety devices to prevent escape of unburned gas, temperature rise limits for gas-cock bodies, time clocks, and oven and broiler tops.)

(The full text of these approval requirements includes methods of test for determining if the performance requirements are met.)

(In addition to the above requirements for ranges using natural and manufactured gases, the full text of these approval requirements includes performance requirements for ranges using propane gas and for ranges using butane-air gas.)

\section{AMERICAN GAS ASSOCIATION, APPROVAL REQUIREMENTS FOR GAS WATER HEAT- ERS, SEPTEMBER 1, 1931}

\section{GAS WATER HEATERS}

(The full text of these approval requirements contains construction requirements for gas water heaters including security and removability of burners, protection of pilot flames, construction of gas cocks, flue collars, draft hoods, and relief valves, provision of means independent of automatic devices for shutting off pilot and main gas supply, and a recommendation that automatic water heaters be equipped with a safety device to prevent escape of unburned gas.)

\section{PERFORMANCE REQUIREMENTS}

(Performance requirements are specified under the same test gas characteristics and pressures as given in the A. G. A. performance requirements for gas ranges, above.)

\section{BURNER OPERATION}

A water heater shall produce no carbon monoxide. This requirement shall be deemed met when a concentration of carbon monoxide not in excess of 0.02 per cent is present in the air-free products of combustion, when tested in a room with approximately a normal oxygen supply at gas pressures of normal, 50 per cent below normal, and 25 per cent above normal, and not in excess of 0.04 per cent when tested at a gas pressure of 50 per cent above normal.

\section{GAS COCKS}

Gas cocks shall show no evidence of leakage when subjected to an air pressure of 6.0 inches mercury column for one minute.

Gas cocks shall pass sufficient quantities of 500 B. t. u., 0.6 specific gravity gas to supply the manufacturer's B. t. u. input rating with a pressure drop through the cock not in excess of 0.5 inch water column.

\section{THERMAL EFFICIENCY}

The corrected thermal efficiency of all gas water heaters shall be not less than 65 per cent.

\section{FIRE HAZARD}

The maximum rise above room temperature, when the heater has been operated for one hour from a cold start at a gas pressure of 1.5 normal, shall not exceed $90^{\circ} \mathrm{F}$. at points 6 inches from the nearest part of the heater jacket or flue and on the floor under the heater.
The maximum temperature of the products of combustion at the flue collar shall be not more than $550^{\circ} \mathrm{F}$. during any test.

\section{OTHER REQUIREMENTS}

(The full text of the approval requirements includes performance requirements for pilot flame, water pressure relief valves, water temperature limiting devices, safety devices to prevent escape of unburned gas, thermostatic control, gas pressure regulators, draft hoods, and accuracy of capacity rating.)

(Prescribed methods of test are also included in the full text of these requirements for determining whether the device meets the above performance requirements. Methods are also prescribed for determining heat losses from storage vessels, and stand-by loss for automatic combination heaters and water storage vessels, Type 4-A-2, insulated.)

\section{AMERICAN GAS ASSOCIATION, APPROVAL REQUIREMENTS FOR HOT PLATES AND LAUNDRY STOVES, 1929}

\section{HOT PLATES AND LAUNDRY STOVES}

\section{CONSTRUCTION REQUIREMENTS}

(These approval requirements include structural requirements for burners, mixer heads, air shutters, cocks, manifolds, leg sheets and burner trays, and hose end nozzles. Materials and construction requirements for the body are given below.)

Unless otherwise specified, all gages are U. S. standard.

The terms steel and iron are used interchangeably in these requirements, either steel or iron is considered satisfactory.

\section{TOP FRAMES}

One-burner hot plates.-(a) If made of sheet steel, frames must not be less than No. 18 gage.

Two or more burner hot plates and laundry stoves.(b) If made of cast iron, no part of frame must have a cross-sectional area less than one-fourth square inch

(c) If made of angle iron, no part of frame must have a cross-sectional area less than one-eighth square inch.

(d) If made of sheet steel, frame must not be less than No. 18 gage, and no part must nave a cross-sectional area less than one-eignth square inch.

(e) When any other material is used, the strength of the part shall be at least equivalent to that specified for cast iron in (b) or for structural iron in $(c)$ and $(d)$ above.

All hot plates and laundry stoves.-( $f$ ) Band iron or other structural steel shapes shall have joints securely riveted, welded, or bolted.

(g) Cast frames, when not cast in one piece, shall have joints securely fastened to give effectively a 1-piece construction.

\section{BURNER BOXES}

Sheet steel burner boxes, when used, shall have a rust-resisting finish. Painted, jappanned, and enameled burner boxes shall meet the tests prescribed under performance requirements.

\section{LEGS}

(a) Legs, if detachable, shall be interchangeable and rigidly held in place.

(b) Sheet steel legs shall be of at least No. 18 gage. 


\section{BOITS}

(a) No bolts or screws shall be used in the general assembly of not plates or laundry stoves excepting those having stove bolt threads. It is strongly urged that the following thread dimensions be used:

\begin{tabular}{|c|c|c|}
\hline $\begin{array}{c}\text { Nominal } \\
\text { diameter }\end{array}$ & $\begin{array}{c}\text { Actual } \\
\text { diameter }\end{array}$ & $\begin{array}{r}\text { Threads } \\
\text { per inch }\end{array}$ \\
\cline { 1 - 2 } Inch & $\begin{array}{l}\text { Inch } \\
3 / 16\end{array}$ & \\
$1 / 4$ & 0.195 & 24 \\
$5 / 16$ & .250 & 18 \\
$3 / 8$ & .312 & 18 \\
& .375 & 16 \\
\hline
\end{tabular}

(b) No bolts smaller than three-sixteenths inch shall be used (bolts for attaching name and number plates excepted).

(c) In general, through bolts with nuts shall be used throughout. Where tap bolts are used to attach air shutters and manifold supports, they are to be of rust-resisting material, or if of steel, to have a metallic rust-resisting coating.

(d) Where tap screws are used for attaching manifold supports, they are to be not less than one-fourth inch size.

\section{OPEN GRATES}

(Includes requirements on beveling of edges, interchangeability of grates, detachability, prevention of inversion of grates, and the following requirements:)

The top surface shall be raised at least five thirtyseconds inch above the level of the top frame.

Design of grate arms shall be such that they will support a utensil of $3 \frac{1}{2}$-inch diameter or greater when placed centrally over a burner.

\section{PERFORMANCE REQUIREMENTS}

General.-Normal pressure hereinafter specified shall be $3 \frac{1}{2}$ inches for manufactured gas and 7 inches for natural gas.

Burner capacities.-The gas-burning capacities of hot plates and laundry stove burners shall be as follows:

B.t.u.
per hour

Regular burner, not less than_................ 9, 000

Giant burner, not less than

Capacities at low pressure.-All burners shall have sufficient capacity to burn gas at the rates specified above when using natural gas at 4 inches pressure and coke-oven gas at 2 inches pressure.

Burner operating characteristics. - (These requirements are the same as given above in the A. G. A. approval requirements for gas ovens with the exception of the requirement for operation without carbonizing flames, which is as given herewith.)

Burners shall operate without carbonizing flames. This observation is to be made with a $71 / 2$-inch diameter (bottom) utensil over the burner in the case of a single-burner hot plate and with a No. 9 roundend wash boiler ( 22 by 12 inches on bottom) placed centrally over any two adjacent burners of a hot plate having two or more burners, or of a laundry stove.

Fire hazard, flue gas analysis, burner thermal effciency.-(Requirements are the same as those given under similar headings in the performance requirements of the A. G. A. for gas ovens given above.)

(Other performance requirements included in the full text of these approval requirements cover lighters, adjustable orifices and cocks, absence of leakage, test requirements for coated sheet steel. Methods of test are also given for determining if performance requirements are met.)
AMERICAN GAS ASSOCIATION, APPROVAL REQUIREMENTS FOR SPACE HEATERS, DECEMBER 1, 1931

\section{GAS-BURNING ROOM HEATER}

\section{CONSTRUCTION REQUIREMENTS}

(These approval requirements include structural requirements for burners, air shutters, pilots, sizes and construction of cocks, manifolds, gas connections, flue outlets, and hose and nozzles. Meterials and construction of body are as given below.)

\section{MATERIALS AND CONSTRUCTION}

(a) The construction of the body shall be substantial to the extent that it will support without damage the following weight applied uniformly over the top:

150 pounds for heaters weighing up to 50 pounds.

300 pounds for heaters weighing 50 pounds and over.

(b) A heater shall be solidly supported so that it can not be tipped by any reasonable pressure or pull.

\section{METHOD OF TEST}

For all heaters weighing up to 100 pounds, the heater shall be tipped in any direction at an angle of $15^{\circ}$ from the vertical and shall not tip over when released.

For heaters weighing over 100 pounds, the heater shall not tip over when a horizontal pull, in any direction, equal to one-tenth of the heater weight is applied at the center of the top of the heater.

(c) Sheet metal heating elements and baffles on circulating type heaters shall be of not less than No. 20 gage.

(d) The outer casing or jacket and all other sheet metal parts, except heating elements, of circulating type heaters shall be of not less than 22 gage, except finish strips and ornamental trimmings.

(e) The construction of every part of the heater shall be such that it will not show undue signs of becoming so warped, bent, or broken as to negate the heater's compliance with any of these requirements.

$(f)$ Materials shall be of such composition that they will not disintegrate to the extent that particles will separate and drop upon the burner, and affect combustion.

(g) The body finish shall be durable and uniformly applied. After the termination of all tests specified herein, it shall not show undue discoloration or deterioration.

(Among assembly requirements specified in these approval requirements are the requirements that joints of heating surfaces in vented types of heaters shall not depend primarily on cement for tightness.)

\section{PERFORMANCE REQUIREMENTS}

(Performance requirements are specified under the same test gas characteristics and pressures as given in the A. G. A. performance requirements for gas ranges, above.)

\section{BURNER OPERATION}

(a) A space heater shall produce no carbon monoxide. This requirement shall be deemed met when:

1. A concentration of carbon monoxide, not in excess of 0.02 per cent, is present in an air-free sample of the products of combustion, when tested in a room with approximately a normal oxygen supply.

2. A concentration of carbon monoxide, not in excess of 0.05 per cent, is produced in a 1,000 cubic 
foot air-tight room with no air changes occurring during the combustion of an amount of gas liberating 20,000 B. t. u.

\section{FIRE HAZARD}

(a) The maximum temperature at points 6 inches from the back and sides of a space heater and on the floor under the heater and within 3 feet of it, shall be not more than $90^{\circ} \mathrm{F}$. in excess of the room temperature except wall heaters and those designed for use only in fireproof fireplaces suitable for the combustion of solid fuels.

(b) The maximum temperature at points of a wall heater which comes into contact with walls and at points 6 inches below the bottom of the heater shall be not more than $90^{\circ} \mathrm{F}$. in excess of the room temperature.

(c) The maximum temperature of the flue products, from a circulating type space heater, shall not exceed $550^{\circ} \mathrm{F}$.

\section{EXPLOSION HAZARD}

The construction of the heater shall be such that, should sufficient gas accumulate before ignition so as to cause an explosion, the explosive force will vent itself without damage.

\section{HEATING EFFICIENCY}

(a) Flue connections shall not carry from the heater more than 35 per cent of the total heat input when operated at the manufacturer's B. t. u. rating.

(b) An appliance represented as a radiant heater shall have a radiant efficiency of not less than 28 per cent.

(In addition to the above, the full text of these approval requirements includes performance requirements as regards pilot operation, effect of orifice on air injection, absence of leakage of gas, permissible pressure drop through gas cocks, provision of safety guards and screens, operating features of draft hoods, venting requirements, permissible corrosion or oxidation of burner parts.)

\section{GAS-FIRED STEAM AND HOT WATER RADIATORS}

All gas-fired steam and hot water radiators shall meet the following requirements:

(a) They shall withstand, without leakage, a hydrostatic pressure test of 100 pounds per square inch.

(b) A relief valve shall be provided which shall operate at a pressure not more than 40 pounds per square inch.

(c) Double diaphrams or equivalent construction shall be provided in pressure or thermostatic controls to prevent steam or water from entering the gas lines.

(d) All radiators up to 33 inches in height shall be constructed or provided with a shield, screen, or other means, so that when installed under a window the curtains, drapes, or other inflammable materials hanging directly above and close to the top of a radiator will not be scorched or ignited from a delayed ignition of 10 seconds, at normal pressure, with the gas flowing at the manufacturer's hourly B. t. u. starting rating.

(e) The water-filling cup on all steam and hot water radiators shall be so constructed that when the cap is removed, it will break any vacuum that might be formed within the radiator.

(f) The average heat content of the flue gases of a gas-fired steam or hot water radiator, when measured at a point one-fourth inch below the escapement into the air, shall not be more than 35 per cent of the total B. t. u. input in the fuel gas for vented radiators nor more than 50 per cent of the total B. $t$. $u$. input in the fuel gas for unvented radiators, with the gas burning at the manufacturer's hourly B. t. u. input rate.

(g) The ratings of gas-fired steam and hot water radiators shall be given in square feet of standard cast-iron column radiation. (Rules are included in the full text of these requirements for expressing the rating for vented and for unvented radiators.)

\section{METHODS OF TEST}

(The full text of these approval requirements includes methods of test for determining if the performance requirements are met for both gas-burning room heaters and gas-fired steam and hot water radiators.)

\section{UNDERWRITERS' LABORATORIES, STAN- DARDS FOR CONSTRUCTION AND PER- FORMANCE OF COMPRESSED GAS SYS- TEMS OTHER THAN ACETYLENE FOR LIGHTING AND HEATING, FEBRUARY, 1928}

\section{COMPRESSED GAS SYSTEMS}

(For class A systems introducing only gas into buildings and for class B systems introducing liquid into buildings, these standards include requirements on general design and thickness of metal for cabinets, permissible types of storage cylinders, permissible gas or liquid pressures, types and performance requirements of pressure regulators and of valves, sizes of connecting tubing and piping, types of connectors and fittings, working pressure capacity of expansion tanks, and the following requirements for stoves and ranges.)

\section{STOVES OR RANGES}

(For use with compressed gas other than acetylene)

The frame shall consist of noncombustible material rigidly assembled to provide ample strength for supporting the several parts of the stove and the materials likely to be placed on it. It shall be made to inclose the burners on the bottom and three sides.

Sheet metal supporting parts and braces used in the construction of the frame shall be of material at least No. 26 U. S. gage $(0.01875 \mathrm{inch})$ and corrugated or otherwise stiffened where strength is needed.

(The full text of this standard includes general requirements on the provision of legs of proper height and the provision of drip pan or base that is removable or readily accessible for cleaning.)

Legs of stoves of class B systems, for stoves that weigh less than approximately 100 pounds, shall be provided with means for attachment to the floor to prevent the stove being moved and breaking the fuel line. Legs may consist of castings or sheet metal suitably designed and stiffened to carry the weight likely to be placed on them under ordinary service conditions. These legs shall be attached to the frame in a manner which will insure rigidity.

Shelves and cabinets located above the burners shall be constructed in a substantial manner, and shall be securely fastened to the stove. Valves of oven burners of class B systems shall be provided with interferences which make it necessaty to open the broiler door before the valve can be opened.

\section{BURNERS}

Individual or utility burners for use under coffee urns, etc., shall be constructed with a drip pan or base, designed to serve as a heat deflector to protect wocden or other combustible materials on which it is installed.

(Requirements on the provision of operating instructions for stoves and gas systems are included in the full text of this standard.) 
UNITED STATES GOVERNMENT, DEPARTMENT OF COMMERCE, BUREAU OF STANDARDS, METHOD FOR TESTING GAS APPLIANCES TO DETERMINE THEIR SAFETY FROM PRODUCING CARBON MONOXIDE, PUBLICATION T304, 1926

\section{TESTING GAS APPLIANCES}

(This technologic paper covers the description of tests developed at the Bureau of Standards for testing gas appliances under forced operation and under normal operation conditions. It includes tests for the determination of flash-back, blow-off, and yellow-tip curves, determination of limit of complete combustion, and of normal injection curve. Typical test results are shown.)

614.3 OIL AND GASOLINE STOVES, RANGES, AND WATER HEATERS.

UNDERWRITERS' LABORATORIES, STANDARDS FOR CONSTRUCTION AND PERFORMANCE OF COMPRESSED GAS SYSTEMS OTHER THAN ACETYLENE FOR LIGHTING AND HEATING, FEBRUARY, 1928

(This standard includes requirements for stoves and ranges. See 614.2 , p. 857.)

UNDERWRITERS' LABORATORIES, STAND. ARDS FOR CONSTRUCTION AND PERFORMANCE OF GASOLINE STOVES FOR COOKING AND HEATING, FEBRUARY, 1928

\section{STATIONARY GASOLINE STOVES}

These specifications apply to devices of the wickless type, intended for heating or cooking, that use gasoline either for securing generation or as a fuel.

\section{FRAME}

This shall consist of noncombustible material rigidly assembled to provide ample strength for supporting the several parts of the stove and the materials likely to be placed on it and shall have the ends and backs inclosed.

Sheet-metal supporting parts and braces used in the construction of the frame shall be of material at least No. 26 U. S. gage $(0.01875$ inch) and corrugated or otherwise stiffened where strength is needed.

(The full text of these standards includes general requirements on provision of legs of proper strength and length, provision of metal drip pan, rigid connection of shelves and cabinets that are located above burners, and provision for attachment to floor for those stoves equipped with outside storage tanks.)

\section{FUEL SUPPLY}

The fuel may be supplied to the burners either by gravity or by air pressure from a suitably designed pressure tank.

Liquid capacity of tanks located at the stove shall not exceed approximately one gallon and shall be rigidly supported at the stove. When made removable, an automatic valve shall be provided in the end of the fuel line to prevent leakage of fuel when the tank is removed.

Liquid capacity of pressure tanks installed outside of buildings shall not exceed approximately 6 gallons.
(The full text of this standard includes general requirements on permissible materials, shapes, types of joints, and structural features of fuel tanks, including the requirements given herewith.)

Material for lift-out tanks and founts to be as follows: Copper sheets shall be at least No. 24 B. \& S. gage $(0.0201$ inch), brass sheets of at least No. 26 B. \& S. gage (0.0159 inch), and steel sheets of at least No. 27 U. S. gage (0.0172 inch), measured under their coating. Founts shall be made with dished bottoms.

Pressure tanks shall be designed for a working pressure of not less than 30 pounds and shall possess a factor of safety of at least 6 . The stove design shall be such that pressures in excess of 50 pounds per square inch will not be required for proper operation and the pumps supplied with the equipment should be of such capacity as to make it difficult to obtain pressures in excess of that for which the tank is designed. Two pressure tanks picked at random from each day's production shall be subjected to an intermittent hydrostatic pressure test lasting one-half hour with a pressure of 100 pounds per square inch, on for 5-minute periods and off for 1-minute periods during the test, and shall not show leakage or any permanent distortion. All pressure tanks shall be tested to at least twice the working pressure and proven tight.

Pressure tanks mounted on stove shall be cylindrical or elliptical in shape and constructed of at least No. 22 U. S. gage (0.0312 inch) steel sheet suitably protected from corrosion, or of brass or copper of a thickness which will provide necessary strength to withstand the pressure tests. (Other requirements included in the full text of the standard include the use of filler caps which will relieve tank pressure before opening tank, provision of pressure and fuel level gages and a positive shut-off valve in the fuel line.)

(Requirements on construction of tanks to be installed outside of buildings are also included in full text of this standard.)

\section{GENERATORS AND BURNERS}

Generators shall be initially heated by means which will cbviate the use of an open gasoline drip cup or other open handling and exposure of gasoline.

(This standard also includes requirements on structural features and installation of generators, burners, valves, piping and fittings, material and thickness of fuel piping.)

\section{PORTABLE HEATERS}

In general, heaters of this class shall conform to the foregoing requirements but shall be provided with antiflooding devices in all cases and may not be designed for connection with tanks of larger capacity than approximately 1 gallon.

A portable heater shall be so constructed that its center of gravity is low to enable it to regain its stability on being tipped. It shall return to the upright position on being tipped forward or sideways to an angle of $30^{\circ}$ from the vertical and likewise from a backward tip of $20^{\circ}$ from the vertical when the fuel tank is practically empty.

UNDERWRITERS' IABORATORIES, STANDARDS FOR CONSTRUCTION AND PERFORMANCE OF KEROSENE STOVES FOR COOKING AND HEATING, AUGUST, 1927

\section{KEROSENE STOVES}

These specifications cover the construction and performance of the nonportable type of kerosene stoves. 
FRAME

(Requirements on the construction of the frame are the same as for gasoline stoves given above except that there is no requirement that the ends and back be enclosed. It contains the additional requirement that burner flames shall be protected by a suitable enclosure around each burner or the frame of the stove shall be sufficiently inclosed on at least five sides.)

\section{FUEL SUPPLY}

Fuel may be supplied to the burner either by gravity or by a suitably designed pressure tank. If the feed mechanism is not designed to maintain the fuel level below the top edge of the fuel containing part of the burners, an antiflooding device shall be incorporated in the stove to prevent flooding of the burners.

Fuel tanks shall not exceed 2 gallons capacity and shall be rigidly supported at the stove. When made removable, means shall be provided to prevent leakage of fuel.

(With the exception that glass is permitted in the construction of lift-out tanks, the requirements for lift-out tanks and founts and for pressure tanks mounted at the stove are the same as given in the Underwriters' Laboratories standard for gasoline stoves, see above.)

\section{GENERATORS AND BURNERS}

(In addition to general requirements on construction and installation, type of joint permitted, etc., are the following requirements:)

Generators shall be initially heated by a means which will obviate the use of any fuel other than kerosene and may consist of either the individual or common type.

Burners may consist of either the wick, lighting ring, or wickless types. Wick type burners shall be provided either with a wick stop or with means to control the wick travel.

Burners of the lighting ring or wick types shall have the fuel level in the burners sufficiently far below the tops of the burner bowls or wick tubes to prevent danger of flooding when not set level, or leveling means shall be provided.

(The full text of this standard also includes structural requirements and operating features for valves, permissible materials and minimum sizes and wall thicknesses of piping, and permissible types of fittings.)

\section{UNDERWRITERS' LABORATORIES, STAND- ARD REQUIREMENTS FOR CONSTRUC- TION AND PERFORMANCE OF PORT- ABLE KEROSENE HEATERS, JUNE 2, 1924}

\section{PORTABLE KEROSENE HEATERS}

\section{INTRODUCTORY}

These requirements are intended as minimum requirements which must be met by devices of this class under consideration in order to be judged acceptable.

\section{DEFINITION}

These standard requirements apply to portable oil heaters using kerosene as fuel, which is contained in a small reservoir and burned with a wick. These heaters consist commonly of a lower drum supported on legs and containing the reservoir on which the burner is mounted. An upper section or drum serves as a radiator and is usually attached to the lower section by a hinge and catch.
3. UPPER DROM

The side walls of the upper drum may be of sheet iron or steel not less than No. 28 U. S. gage $(0.016$ inch). The vertical seam may be a hook seam or a riveted lap joint, or may be welded. The drum shall be stiffened by having corrugations rolled into it circumferentially, or by an equivalent means. A sufficient number of permanent openings shall be provided to prevent smoking when the top ventilator is closed.

A suitable window shall be provided in the side of the drum through which the height and condition of the flame may readily be observed. It should be covered with fine wire mesh screen or with a good grade of mica which should preferably be protected by a metal guard or framework.

\section{TOP}

The top may be of sheet iron or steel not less than No. 24 U. S. gage ( 0.025 inch) or of a suitable casting. The edge shall be flanged to provide stiffening and in some cases to provide for attachment to the drum. It may or may not be provided with an adjustable shutter. When the carrying handle is attached to it the top may be fastened to the drum by not less than four $1 / 8$-inch rivets or two or more $3 / 16$-inch tie bolts may be used to clamp the drum between the top and center section. If tie bolts are used, the ends of the drum should rest in suitable grooves in the top and center section. If the carrying handle is not attached to it the top may be fastened to the upper drum by at least three $3 / 16$-inch bolts or the equivalent.

\section{CENTER SECTION}

The center section shall be of sheet iron or steel not less than No. $24 \mathrm{U}$. S. gage $(0.025 \mathrm{inch})$ or of a suitable casting. It may be attached to the drum by not less than four $1 / 8$-inch rivets or three $3 / 16$-inch bolts or by tie rods, as mentioned under section 4 . The flame collar should be securely attached to the center section and be of sheet iron or steel not less than No. 24 U. S. gage. It should have a suitable flange or shoulder on the lower edge for stiffening and for seating against the upper edge of the gallery.

\section{CARRYING HANDIE}

The bail should be of tinned or nickeled steel wire not less than No. 8 W. \& M. gage (0.162 inch). (Other recommendations include method of attachment of handle, that it be not closer than 1 inch to stove when in position of rest and not closer than 6 inches when in raised position, that it does not get uncomfortably hot, etc.)

\section{LOWER DRUM}

The walls of the lower drum should be of sheet iron or steel not less than No. 28 U. S. gage (0.016 inch). It should be perforated with a sufficient number of openings or otherwise arranged to permit adequate circulation of air and cooling of the reservoir. The upper edge shall be reinforced by being rolled over a wire or by means of a band or the equivalent. The lower edge should be rivted to the base ring by not less than four $3 / 16$-inch rivets well headed over or by an equivalent method. A substantial ring or lugs attached securely to the drum or a part of the base ring shall be provided to support the reservoir. The base ring should be flanged or otherwise suitably stiffened and should be of sheet iron or steel not less than No. 20 U. S. gage (0.0375 inch).

\section{LEGS}

The heater should be supported by not less than four substantial legs curved or otherwise sufficiently 
stiffened. They should be of sheet iron or steel not less than No. 20 U. S. gage $(0.0375$ inch) or of suitable castings and should be fastened to the base ring by at least two $3 / 16$-inch rivets well headed over. If the legs are extensions of the base ring, they shall be not less than No. 19 U.S. gage $(0.043$ inch), and the legs should be shaped to provide sufficient stiffness.

$$
\text { 9. HINGE-10. IATCE }
$$

(The full text of this standard includes requirements on material, minimum thicknesses of parts, and methods of attachment of hinge, general performance and ease of operation of latch.)

\section{RESERVOIR}

The reservoir may be of brass, terneplate, or the equivalent. If made of brass the bowl should be not thinner than No. 24 B. \& S. gage $(0.020$ inch) and if of terneplate, not less than No. 26 U. S. gage $(0.018 \mathrm{inch})$. The cover of the reservoir, if made of terneplate shall be at least No. 26 U. S. gage (0.018 inch) or if of brass, at least No. 26 B. \& S. gage $(0.0159 \mathrm{inch})$. The lower section shall be joined to the cover by a standing seam or the equivalent.

The inner wick tube, or draft tube may be of the same material as the fount. If the lower section of the fount does not include the wick tube as a part of itself, this tube should be seamed to the bottom and additionally soldered for tightness. Reinforcement of the bottom to prevent movement is desirable.

(Other requirements and recommendations in this standard deal with the provision, size, and attachment of handle to reservoir, structural features of pinion shaft for adjusting wick and method of attaching to reservoir, provision and performance requirements of fill plug for reservoir, gage for reservoir, wick stop, wich carrier, floor pan, and the following requirements.)

\section{STABILITY}

These heaters shall be so constructed that their centers of gravity are low and that they have a minimum tipping angle of $33^{\circ}$ with the reservoir empty.

\section{OPERATION}

These heaters shall be capable of operating satisfactorily for a reasonably long time, requiring a minimum of care and attention.

The maximum flame obtainable shall be so limited and the heater so constructed that, when placed as close to a vertical wall as the legs will permit, it will not cause ignition of combustible materials on the wall during continued operation as long as the heater will burn on one filling of oil.

The maximum heat developed during continued operation shall not be such as to overheat and ignite the oil in the reservoir or to induce an abnormally high and smoky flame.

20. TEST OF RESERVOIR

Each reservoir shall be tested and proven tight against leakage under a pressure of 5 pounds per square inch.

614.4 HOUSE HEATING BOILERS, RADIATORS, AND HOT-WATER TANKS.

\section{AMERICAN GAS ASSOCIATION, APPROVAL REQUIREMENTS FOR CENTRAL HOUSE HEATING GAS APPLIANCES, MAY 1, 1930}

\section{GAS BOILERS}

(This specification includes requirements on the construction of gas burners; size and type of main burner cock; method of connection, minimum size of supply pipe, and nonflash back requirements for pilot burners; structural and operating features of air mixers and electric-control valves; requirements on the provision of main control valve, limiting devices for steam pressures and hot-water temperatures, and gas pressure regulators.)

(The requirements for the construction of castiron boilers including the requirements for fittings and appliances are identical in nearly all provisions with the requirements for cast-iron low-pressure heating boilers given in the boiler construction code of the American Society of Mechanical Engineers. See p. 864.)

\section{PERFORMANCE REQUIREMENTS}

\section{Section 1. Test Gases}

In conducting performance tests for approval"of gas boilers, two kinds of test gases shall be used:

(a) Natural gas, approximately 1,125 B. t. u. per cubic foot, 0.65 specific gravity.

(b) Manufactured gas, approximately 550 B. t. u. per cubic foot, 0.40 specific gravity.

Section 2. Normal Pressures

For the purpose of these requirements normal gas test pressures are defined as $31 \frac{1}{2}$-inch water column for manufactured gas and 7 -inch water column for natural gas. Pressures shall be measured at the regulator inlet.

\section{Section 3. Thermal Efficiency}

Steam and hot-water boilers shall have a thermal efficiency of at least 75 per cent based on the total heating value of the gas.

Section 4. Burrer Operation

(a) A gas boiler shall produce no carbon monoxide. This requirement shall be deemed met when a concentration not in excess of 0.04 per cent is present in an air-free sample of the products of combustion when the boiler is tested in an atmosphere with approximately a normal oxygen supply.

\section{Section 5. Pilct Operation}

(a) Pilot flames shall effectively ignite the gas at the burners and shall be adequately protected from drafts. A draft hood which meets the requirements of the American Gas Association shall be interpreted as fulfilling the second part of this requirement as far as chimney drafts are concerned.

(b) Luminous flame pilots shall not show carbon deposits during the period of test when adjusted according to the manufacturer's instructions.

(c) Where escapement pilots are used they shall ignite freely from a constant burning pilot.

\section{Section 6. Steam Pressure Iimiting Devices}

(a) Boilers shall be provided with a device which will limit the maximum steam pressure to 10 per cent below that for which the safety valve is set.

(b) The limiting device shall be capable of maintaining a uniform steam pressure.

\section{Section $\%$ Gas Pressure Regulatcrs}

Gas pressure regulators shall limit the manifold pressure to not more than one-half inch above that occurring with normal pressure at the regulator inlet.

Section 8. Allowable Pressure Drop

When the boiler is operated at the manufacturer's hourly B. t. u. rating with a gas of 0.6 specific 
gravity and 500 B. t. u. per cubic foot, the pressure drop through all controls shall not exceed 1.0 inch water column.

\section{Section 9. Fire Hazard}

The maximum temperature rise at points 6 inches from the back and sides of the boiler shall not exceed $90^{\circ} \mathrm{F}$. above room temperature when the boiler has been operating for one hour at the manufacturer's hourly B. t. u. rating.

(In addition to the above performance requirements, the full text of this specification includes detailed methods of test for determining whether the performance requirements are met.)

\section{AMERICAN GAS ASSOCIATION, APPROVAI REQUIREMENTS FOR CENTRAL HOUSE HEATING GAS APPLIANCES, MAY 1, 1930}

\section{GAS WARM AIR FURNACES}

(This specification includes requirements on the construction of gas burners; size and type of main burner cock, etc.; the same as for gas boilers above, first paragraph, excepting the requirements for limiting devices for steam pressures and hot water temperatures. In addition the following structural requirements apply to gas warm air furnaces.)

\section{Section 8. Primary and Secondary Air Intake}

All primary and secondary air for burners must be drawn from the outside of the furnace casing or from furnace openings which extend through the outside casing.

\section{Section 9. Accessibility for Cleaning}

The flue gas passage-way through furnaces shall be accessible for cleaning where:

1. The products of combustion are drawn below the level of the burner.

2 . The temperature of the combustion products is less than $250^{\circ} \mathrm{F}$. when the furnace is operated at normal pressure with the gas rate within \pm 2 per cent of the manufacturer's hourly B. t. u. rating.

3. The inside walls of any heating surfaces are closer than $1 \frac{1}{2}$ inches.

\section{Saction 10. Air Circulation in Floor Furnaces}

(a) Floor furnaces shall be so constructed and designed that they will provide a constant circulation of heated air at all times during their operation. The difference in temperature between the heated air and that in the cold air return duct shall in no case be less than $90^{\circ} \mathrm{F}$.

(b) The ratio of the net free area of the cold air returns to the net free area through the grating of the warm air section of floor furnaces shall in no case be less than 100 per cent.

\section{Section 11. Draft Hoods}

A suitably designed draft hood shall be furnished with each furnace. Such devices shall meet the approval requirements of the American Gas Association. No flue damper shall be used.

\section{Section 12. Joints in Eeating Surface}

All joints of heating surfaces shall be of welded, brazed, screwed, lock seamed, machined and bolted, or of formed, slip joint, or flanged construction tightly bolted together and enclosing gaskets or other similar packing materials.

\section{Section 13. Disposal of Combustion Products}

The construction of the furnace shall be such that no part of the products of combustion becomes mixed with the warm air discharged by the furnace.

Section 14. Vent Connections

Vent connections shall extend beyond the furnace casing a sufficient distance to insure secure fastening of the vent pipe.

\section{Section 15. Furnace Openings}

Furnace openings other than vent connections, such as secondary air supplies, doors, etc., shall extend through the outside casing a sufficient distance to permit secure fastening of outside frames and to prevent possible leakage of combustion products into the warm air ducts.

\section{Section 16. Thickness of Material}

(a) Where outside casings are constructed of sheet iron or steel, they shall not be less than $26 \mathrm{U}$. S. gage for furnaces of less than $100,000 \mathrm{~B}$. $t$. $u$. per hour input capacity. For furnaces with a capacity in excess of this amount, the thickness of the furnace casing shall not be less than 24 gage. Where reinforcing or a double construction is employed, strength equivalent to that afforded by weights of metals mentioned above shall be regarded as acceptable.

(b) Where sheet steel or iron is used in the construction of furnace heating surfaces, the thickness shall not be less than $22 \mathrm{U}$. S. standard gage.

\section{Section 17. Fan Location}

Furnaces which include fans as integral parts of their construction shall have the fans so placed that they discharge into the furnace casings.

\section{Section 18. Fan Control}

Furnaces which include fans as integral parts of their construction shall include means to prevent the temperature of the air in the warm air ducts exceeding $250^{\circ} \mathrm{F}$. in the event of fan stoppage.

\section{Section 19. Observation Doors}

Observation doors of floor furnaces shall fit tightly and shall be so machined or of such weight and construction that they will not be displaced by any normal jar or pressure created during normal operation of the furnace.

\section{Section 20. Ratings}

All gas furnaces shall bear a permanent name plate on which shall appear in permanent form:

(a) The manufacturer's hourly B. t. u. input rating.

(b) The allowable warm air duct area in square inches, which shall be computed on the basis of not more than 100 per cent of the effective free area through the furnace.

\section{PERFORMANCE REQUIREMENTS}

(Performance requirements for warm air furnaces are the same as given above for gas boilers as given in sections $1,2,4,5,7,8$, and 9 . In addition the following performance requirements are specified.)

Heating efficiency. - Warm air furnaces, except floor furnaces, shall have a heating efficiency of at least 70 per cent based on the total heating value of the gas.

Pilot operation.-Pilot flames shall not become extinguished when the main burners are turned on or off in a normal manner during the laboratory tests. 
Allowable air temperature.-When tested at normal gas pressure, the furnace shall not discharge air at a temperature in excess of $220^{\circ} \mathrm{F}$.

The temperature of the air discharged through any single warm-air opening, when the furnace is tested at normal pressure, shall not vary more than $30^{\circ} \mathrm{F}$. from the average of the discharge temperature observed at all of the warm-air openings. In case the furnace is designed for discharge into a single warm-air duct, the test prescribed shall be carried out by dividing the outlet into four equal areas and observing the temperature at the center of each.

(In addition to the above performance requirements, the full text of this specification includes detailed methods of test for the various performance requirements.)

\section{AMERICAN SOCIETY OF HEATING AND VENTILATING ENGINEERS, CODE FOR TESTING RADIATORS, JANUARY, 1927}

\section{HOUSE HEATING RADIATORS}

\section{THE CONDENSATION METHOD FOR THE TESTING OF
DIRECT RADIATORS}

(This standard method of test includes requirements on size of test room, permissible window size, maintenance of uniform temperature and humidity, pressure and superheat of steam used in test, measurement of condensate, painting of radiator, standard formula for computing heating capacity.)

\section{AMERICAN SOCIETY OF HEATING AND VENTILATING ENGINEERS, PROPOSED CODE FOR TESTING STEAM HEATING BOILERS BURNING OIL FUEL, MARCH, 1931}

\section{BOILERS BURNING OIL FUEL}

(This code provides standard methods for conducting and reporting tests to determine heat efficiencies and performance characteristics of steam heating boilers using oil fuel. It includes standard set-up of oil burner and boiler to be tested, types of instruments and set-up of test apparatus, sampling of fuel, duration of tests, starting and stopping continuous tests, starting and stopping of intermittent tests, test procedure, and standard data and test forms.)

\section{AMERICAN SOCIETY OF HEATING AND VENTILATING ENGINEERS, PIPE SIZES, 1930}

\section{PIPE SIZES FOR HEATING SYSTEMS}

(The pipe sizes recommended by this society are the same as those recommended for steam heating by the Heating and Piping Contractors National Association. See H. \& P. C. N. A., below.)

\section{AMERICAN SOCIETY OF HEATING AND VENTILATING ENGINEERS, COMMITTEE REPORT ON CODE FOR RATING OF HEATING BOILERS BURNING SOLID FUELS, JANUARY, 1929}

\section{RATING OF HEATING BOILERS}

(The report of the committee presented a code by which the rating of a solid fuel boiler is determined either from the performance in relation to flue gas temperature or the performance in relation to the coal burned per unit area of grate, the lower of the two outputs plus 30 per cent of their difference being chosen as the rating. The boiler to be rated is tested in accordance with the code for testing lowpressure steam heating boilers of the American Society of Heating and Ventilating Engineers. Performance curves plotted from the data obtained from the test are used in conjunction with a standard rating chart to determine the rating of the particular boiler. The code provides for an anthracite rating and a bituminous rating.)

\section{AMERICAN SOCIETY OF HEATING AND} VENTILATING ENGINEERS, PERFORMANCE TEST CODE FOR STEAM HEATING SOLID FUEL BOILERS, CODE NO. 3, JUNE, 1929

\section{PERFORMANCE TEST CODE FOR HEATING BOILERS}

(This code specifies the tests to be conducted and standard methods for conducting and reporting tests to determine the efficiencies and performance of heating boilers. It includes types and arrangement of apparatus and instruments, connecting up of apparatus and boiler, methods of starting and stopping test, standard log sheets and test forms.)

AMERICAN SOCIETY OF HEATING AND VENTILATING ENGINEERS, STANDARD AND SHORT FORM HEAT BALANCE CODES FOR TESTING LOW-PRESSURE STEAM HEATING SOLID FUEL BOILERS, CODES 1 AND 2, JUNE, 1929

\section{TESTING LOW PRESSURE HEATING BOILERS}

(These standard test methods include definitions of grate area and combustion rate for solid fuel boilers, set-up of boiler to be tested, types of instruments and measuring apparatus required, sampling procedure for the fuel, duration of tests, new fire method of starting and stopping tests and the continuous firing method of starting and stopping tests, method of conducting tests, and standard form for recording test data and test results.)

\section{AMERICAN SOCIETY OF MECHANICAL ENGINEERS, BOILER CONSTRUCTION CODE; BOILERS USED EXCLUSIVELY FOR LOW-PRESSURE STEAM HEATING, HOT- WATER HEATING, AND HOT-WATER SUPPLY, 1927}

\section{STEEL-PLATE BOILERS}

H-1. These rules for steel-plate boilers shall apply:

(a) To all steam boilers for operation at pressures not exceeding 15 pounds per square inch.

(b) To hot water boilers to be operated at pressures not exceeding 160 pounds per square inch, or temperatures not exceeding $250^{\circ} \mathrm{F}$.

(c) For conditions exceeding those specified above, the rules for construction and setting of power boilers shall apply.

H-2. Wherever the term maximum allowable working pressure is used herein, it refers to gauge pressure or the pressure above the atmosphere in pounds per square inch. 
H-3. The maximum allowable working pressure shall not exceed 15 pounds per square inch on a steelplate boiler built under these rules to be used for low-pressure steam heating.

The maximum allowable working temperature at or near the outlet of a hot-water steel-plate boiler shall not exceed $250^{\circ} \mathrm{F}$, or the maximum allowable working pressure 160 pounds per square inch.

$\mathrm{H}-4$. The maximum allowable working pressure on the shell or drum of steel plate steam heating and hot-water boilers shall be determined by the strength of the weakest course, computed from the thickness of the plate, the tensile strength stamped thereon, as provided for in paragraph S-17 of Section II of the code, the efficiency of the longitudinal joint, or of the ligament between the tube holes in shell or drum (whichever is the least), the inside diameter of the course, and the factor of safety, but in no case shall the pressure on which the factor of safety is based be considered less than 30 pounds per square inch.

$\frac{T S \times t \times E}{R \times F S}=\underset{\text { pounds per square inch. }}{\operatorname{maximum}}$ allowable working pressure, where

$T S=$ ultimate tensile strength stamped on shell plates, as provided for in paragraph S-17 of Section II of the code, pounds per square inch.

$t=$ minimum thickness of shell plates in weakest course, inch.

$E=$ efficiency of longitudinal joint or of ligaments between tube holes (whichever is the least).

$R=$ inside radius of the weakest course of the shell or drum, inches.

$F S=$ factor of safety, or the ratio of the ultimate strength of the material to the allowable stress.

5 .

For new constructions, FS in the above formulac

\section{MATERIALS}

$\mathrm{H}-5$. Specifications are given in paragraphs $\mathrm{S}-1$ to S-285 of Section II of the code, for the important materials used in the construction of boilers, and where so given the materials herein mentioned for boiler parts required to resist internal pressure shall conform thereto except as specified herein for autogenously welded boilers.

$\mathrm{H}-6$. Steel plates for any part of a boiler where under pressure, also manhole and handhole covers and other parts subjected to pressure, and braces and lugs when made of steel plate, shall be of firebox or flange quality as designated in the specifications for steel boiler plate, except for base metal as specified for autogenous welding.

$\mathrm{H}-7$. Braces when made of parts welded together shall be of wrought iron of the quality designated in the specifications for extra-refined bar iron.

\section{ULTIMATE STRENGTH OF MATERIAL}

H-8. Tensile strength of steel plate.-In determining the maximum allowable working pressure, the tensile strength used in the computations for steel plates shall be that stamped on the plates as herein provided, which is the minimum of the stipulated range, or 55,000 pounds per square inch for all steel plates except for special grades having a lower tensile strength.

H-9. Crushing strength of steel plate.-The resistance to crushing of steel plate shall be taken at 95,000 pounds per square inch of cross-sectional area.

$\mathrm{H}-10$. Strength of rivets in shear.-In computing the ultimate strength of rivets in shear, the following values, in pounds per square inch of the crosssectional area of the rivet shank, shall be used:

Iron rivets in single shear

Iron rivets in double shear

Steel rivets in single shear.............................. 44, 000

Steel rivets in double shear.................. 88, 000

The cross-sectional area used in the computations shall be that of the rivet shank after driving.

\section{MINIMUM THICKNESS OF PLATES AND TUBES}

H-11. The minimum thickness of any boiler plate under pressure shall be one-fourth inch.

$\mathrm{H}-12$. The minimum thickness of shell plates, heads and tube sheets for various shell diameters of steel-plate heating boilers shall be as shown in Table $\mathrm{H}-1$.

TABLE H-1.-Minimum allowable thickness of shell
plates

\begin{tabular}{|c|c|c|}
\hline \multirow{2}{*}{ Diameter of shell, tube sheet or head } & \multicolumn{2}{|c|}{$\begin{array}{l}\text { Minimum thickness } \\
\text { allowable under rules }\end{array}$} \\
\hline & Shell & $\begin{array}{l}\text { Tube sheet } \\
\text { or head }\end{array}$ \\
\hline 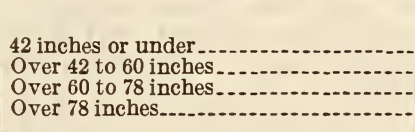 & \begin{tabular}{c|} 
Inch \\
$1 / 4$ \\
$5 / 16$ \\
$3 / 8$ \\
$7 / 16$
\end{tabular} & $\begin{array}{r}\text { Inch } \\
5 / 16 \\
3 / 8 \\
7 / 16 \\
1 / 2\end{array}$ \\
\hline
\end{tabular}

H-13. The minimum thickness of butt straps for double-strap joints shall be as given in Table $\mathrm{H}-2$. For plate thickness exceeding three-fourths inch, the thickness of butt straps shall be not less than two-thirds of the thickness of the plate.

TABLE H-2.-Minimum thickness of butt straps

\begin{tabular}{|c|c||c|c|}
\hline $\begin{array}{c}\text { Thickness } \\
\text { of shell, } \\
\text { plates }\end{array}$ & $\begin{array}{c}\text { Minimum } \\
\text { thickness } \\
\text { of butt } \\
\text { straps }\end{array}$ & $\begin{array}{c}\text { Thickness } \\
\text { of shell } \\
\text { plates, }\end{array}$ & $\begin{array}{c}\text { Minimum } \\
\text { thickness } \\
\text { of butt } \\
\text { straps }\end{array}$ \\
\cline { 1 - 2 } Inch & Inch & Inch & Inch \\
$1 / 4$ & 34 & $15 / 32$ & $3 / 8$ \\
$9 / 32$ & 114 & $1 / 2$ & $7 / 16$ \\
5116 & 114 & 1732 & 716 \\
$3 / 32$ & $1 / 4$ & $9 / 16$ & $7 / 16$ \\
$13 / 32$ & $5 / 16$ & $5 / 8$ & $1 / 2$ \\
$7 / 16$ & $3 / 16$ & $3 / 4$ & $1 / 2$ \\
\hline
\end{tabular}

H-14. (a) The minimum thickness of tubes used in water-tube or fire-tube boilers measured by Birmingham wire gage, shall be as given in Table $\mathrm{H}-3$. TABLE H-3.-Tubes for water-tube and fire-tube
boilers

\begin{tabular}{|c|c|}
\hline & $\begin{array}{l}\text { Minimum thick- } \\
\text { ness of tubes }\end{array}$ \\
\hline $\begin{array}{l}\text { Diameters } 1 \text { inch or over, but less than } 21 / 2 \text { inches } \\
\text { Diameters } 21 / 2 \text { inches or over, but less than } 31 / 4\end{array}$ & $\begin{array}{l}\text { No. } 13 \text { B. W. G. } \\
\text { No. } 12 \text { B. W. G. }\end{array}$ \\
\hline $\begin{array}{l}\text { inches. } \\
\text { Diameters } 31 / 4 \text { inches or over, but less than } 4 \\
\text { inches. }\end{array}$ & No. 11 B. W. G. \\
\hline $\begin{array}{l}\text { Diameters } 4 \text { inches or over, but less than } 5 \text { inches } \\
\text { Diameters } 5 \text { inches. }\end{array}$ & $\begin{array}{l}\text { No. } 10 \text { B.W. G. } \\
\text { No. } 9 \text { B. W. G. }\end{array}$ \\
\hline
\end{tabular}


(b) The minimum thickness of tubes, if of copper, either straight, bent, or coiled, for use in watertube or fire-tube boilers, shall be as follows:

In water boilers where working pressures of over 30 pounds and not to exceed 160 pounds per square inch may be used:

$$
t=\frac{D}{30}+0.03
$$

where

$t=$ thickness of tube wall in inches, and

$D=$ outside diameter of tube in inches.

For steam boilers to be used at pressures not exceeding 15 pounds per square inch, and water boilers where the maximum allowable working pressure does not exceed 30 pounds per square inch:

$$
t-\frac{D}{45}+0.03
$$

In no case shall a tube thinner than No. 16 gage (B. W. G.) be used.

Par. $\mathrm{H}-15$ to $\mathrm{H}-20$ cover joint efficiency, lap riveting, length of longitudinal joints in butt-anddouble-strap construction, and shaping of shell plate ends.

Par. H-21 to H-26 include a formula for calculating the maximum allowable working pressure in pounds per square inch, the shielding of the ends of stays fitted with nuts from direct radiant fire heat, the area of heads to be stayed, and a table giving the maximum allowable stresses for staybolts and stays or braces.

Boiler openings, including threaded openings, a table giving the minimum thickness of material for tappings, and requirements for flanged connections are covered in paragraphs $\mathrm{H}-27$ to $\mathrm{H}-31$.

Supports for horizontal-return tubular boilers 54 inches and up to and including 72 inches in diameter; also such boilers over 72 inches in diameter are covered in paragraphs $\mathrm{H}-32$ to $\mathrm{H}-34$.

Details of setting and installation, including connections for safety and water-relief valves are covered in paragraphs $\mathrm{H}-35$ to $\mathrm{H}-42$.

Fittings and appliances-in which are included safety valves, water-relief valves, minimum allowable sizes of safety valves for steam-heating boilers, also minimum allowable sizes of water-relief valves for water-heating boilers and for water-supply boilers, steam-gauges, pressure and altitude gauges, thermometers, temperature-combustion regulators, pressure-combustion regulators, bottom-blow-off, watergage glasses, gauge cocks, water-column pipes, and fusible plugs-are covered in paragraphs $\mathrm{H}-43$ to H-64.

\section{MYUROSTATIC TEST}

H-65. All hot-water boilers, the maximum allowable working pressure of which is not in excess of 30 pounds per square inch, and steam-heating boilers, shall be subjected to a hydrostatic test of 60 pounds per square inch at the shop where constructed. Hot-water boilers, the maximum allowable working pressure of which exceeds 30 pounds per square inch, shall be subjected to a hydrostatic test of one and one-half times the maximum allowable working pressure both at the shop where constructed and in the field when erected and ready for service.

Any hydrostatic-pressure test to be made on either a steam-heating boiler or hot-water boiler, after the boiler has been in service, shall be at a pressure one and one-half times the maximum allowable working pressure.

In making hydrostatic-pressure tests the pressure shall be under such control that in no case shall the required test pressure be exceeded by more than 6 per cent.
$\mathrm{H}-66$. Individual shop inspection shall not be required for boilers which come under the rules of this exception, except for boilers constructed by autogenous welding. (See par. H-81.)

H-67. Each plate of a completed boiler shall bear the plate maker's name with brand and tensile strength, except that these marks need not appear on the butt straps after completion of boiler.

H-68. All boilers built according to these rules and no other boilers shall be marked A. S. M. E. Std.- Heat, and with the manufacturer's name and maximum allowable working pressure. These markings shall be stamped with letters and figures at least five-sixteenths inch high on some conspicuous portion of the boiler proper, preferably over or near the fire door. Boilers suitable for use for both steam and water shall have the stamps arranged substantially as follows:

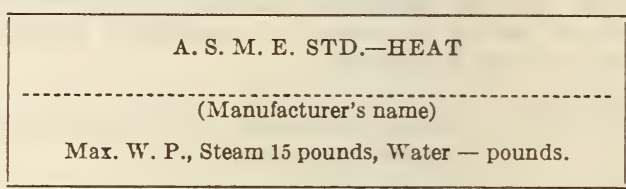

Boilers suitable for use for water only shall have the stamps arranged substantially as follows:

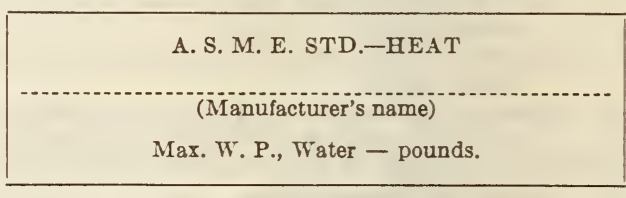

These stamps shall not be covered with insulating or other material. The symbol authorized for use on power boilers shall not be used on heating boilers, nor shall any accessory or part of the boiler be marked A.S. M. E. or A.S. M. E. standard unless so specified in the code.

(The design, construction, and stamping of autogeneously welded boilers, including autogeneous welds, base-metal material, filling material, method of welding, longitudinal joint provisions, and welding of stay bolts into stayed plates are covered in paragraphs $\mathrm{H}-69$ to $\mathrm{H}-83$.)

\section{CAST-IRON BOILERS}

$\mathrm{H}-84$. These rules for cast-iron boilers shall apply:

(a) To all steam boilers for operation at pressures not exceeding 15 pounds per square inch.

(b) To hot-water boilers to be operaced at pressure noc exceeding 160 pounds per square inch, or temperatures not exceeding $250^{\circ} \mathrm{F}$.

(c) For conditions exceeding those specified above, cast-iron construction is not permitted.

$\mathrm{H}-85$. Wherever the term maximum allowable working pressure is usad herein, it refers to gauge pressure or the pressure above the atmosphere in pounds per square inch.

H-86. The maximum allowable working pressure shall not exceed 15 pounds per square inch on a castiron boiler built under these rules to be used exclusively for low-pressure steam heating.

The maximum allowable working temperature at or near the outlet of a hot-water cast-iron boiler shall not exceed $250^{\circ} \mathrm{F}$.

\section{BOIIER OPENINGS}

H-87. Washout openings.-All cast-iron steam and hot-water boilers shall be provided with suitable washout openings to permit the removal of any sediment that may accumulate therein. Washout open- 
ings may be used for return pipe connactions and the washout plug placed in a tee so that the plug is directly opposite and as close as possible to the opening in the boiler.

H-88. Flanged connections.-Flanged pipe connection openings in boilers shall conform to the American standard given in Tables A-5 or A-6 of the appendix to the code, for the corresponding pipe size, and shall have the corresponding drilling for bolts or studs.

H-89. Threaded openings.-Pipe connections if threaded shall be tapped into material having a minimum thickness as specified in Table $\mathrm{H}-9$.

TABLE H-9.-Minimum thickness of material for threaded connections to boilers

\begin{tabular}{|c|c|}
\hline Size of pipe connection (in inches) & $\begin{array}{c}\text { Minimum } \\
\text { thickness } \\
\text { of material } \\
\text { required }\end{array}$ \\
\hline $\begin{array}{l}3 / 4 \text { and under } \\
1 \text { to } 21,2 \text {, inclusive } \\
3 \text { to } 312 \text {, inclusive } \\
4 \text { to } 5 \text {, inclusive. } \\
6 \text { to } 8 \text {, inclusive } \\
9 \text { to } 12 \text {, inclusive }\end{array}$ & $\begin{array}{c}\text { Inches } \\
5 / 16 \\
7 / 16 \\
5 / 8 \\
7 / 8 \\
1 \\
11 / 4\end{array}$ \\
\hline
\end{tabular}

(Details of installation, including connections for safety and water-relief valves, are provided for in paragraphs $\mathrm{H}-90$ to $\mathrm{H}-95$.)

(Under fittings and appliances will be found substantially the same requirements as specified for steel place boilers.)

\section{HYDROSTATIC TESTS}

H-118. All hot-water boilers, the maximum allowable working pressure of which is not in excess of 30 pounds per square inch, and steam-heating boilers, shall be subjected to a hydrostatic test of 60 pounds per square inch at the shop where made, on each individual section. Hot-water boilers, the maximum allowable working pressure of which exceeds 30 pounds per square inch, shall be subjected to a hydrostatic test of two and one-half times the maximum allowable working pressure both at the shop where made, on each individual section, and in the field when erected and ready for service.

Any hydrostatic pressure test to be made on either a steam-heating boiler or hot-water boiler, after the boiler has been in service, shall be at a pressure of one and one-half times the maximum allowable working pressure.

In making hydrostatic pressure tests the pressure shall be under such control that in no case shall the required test pressure be exceeded by more than 6 per cent.

$\mathrm{H}-119$. Individual shop inspection shall not be required for boilers which come under the rules of this section.

H-120. All boilers shall be plainly and permanently marked with the manufacturer's or distributor's name and the maximum allowable working pressure. All letters and figures shall be at least five-sixteenths inch high.

The maximum allowable working pressure shall be stamped, cast, or irremovably attached to the front and rear cored sections of vertical sectional cast-iron boilers and on the dome section of horizontal sectional cast-iron boilers. The marking of maximum allowable working pressure on cast-iron boilers suicable for use for steam or water shall be as follows:

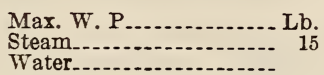

Boilers suitable for use as water boilers only shall be marked as follows:

Max. W. P............. Lb.

Water
All boilers built according to these rules, and no other boilers, shall be marked A. S. M. E. Standard, as follows:

$$
\text { A. S. M. E. STD. }
$$

The symbol authorized for use on power boilers shall not be used on heating boilers.

When an insulating or other form of covering is used that portion of the front cored section of vertical sectional cast-iron boilers, and the dome cored section of horizontal sectional cast-iron boilers bearing the foregoing marking, shall either be provided with a removable cover plate or be left uncovered.

(Pars. $\mathrm{H}-121$ to $\mathrm{H}-136$ have in this edition been placed in Sec. II as pars. S-264 to S-279.)

\section{AMERICAN SOCIETY OF MECHANICAI ENGINEERS, RULES FOR CONSTRUC- TION OF UNFIRED PRESSURE VESSELS,
A. S. M. E. BOILER CONSTRUCTION CODE, 1931

UNFIRED PRESSURE VESSELS

(See 605.23, p. 288.)

\section{HEATING AND PIPING CONTRACTORS NATIONAL ASSOCIATION, ING STANDARDS, 1930}

\section{FIGURING RADIATION}

(Standard rules are given for determining the required amount of radiation area of radiators required to properly heat a given space, based on the heat required to offset the losses due to the transfer of heat through the walls, windows, floors, ceilings, and roofs, and the additional heat required to bring the air that leaks into that space up to the required temperature. Tables of data are included for amount of heat transmitted through various types of wall, ceiling, and floor constructions, for heat transmitted through windows and doors, and allowances are made in figuring the radiation such that various multiplication factors are applied dependent on the relative location of the room being heated as regards exposure to wind and the prevailing winter temperatures.)

\section{NET SQUARE FEET RADIATION LOADS IN $70^{\circ} \mathrm{F}$. RECOMMENDED FOR LOW PRESSURE HEATIYG BOILERS}

(These recommendations are based on the use of direct cast-iron radiation and include allowances for heat loss in piping system, morning peak load, and attention factor. The maximum recommended net radiation area of radiators attached to a given boiler are given for various grate sizes and types of assemblies of round boilers and of cast-iron square boilers of straight draft or smokeless type. Recommendations are based on the use of bituminous coal of a given heat value and rules are given for making corrections to take care of fuel of other heat values. Rules are included for computing loads for other than cast-iron column radiation. Radiation loads are given for both hot water and steam radiation.)

\section{PIPE SIZES}

(These standards are recommended pipe sizes for various maximum capacities as measured in square feet of equivalent radiation for small and for large 1-pipe and 2-pipe steam systems, for 2-pipe vapor systems, for vacuum pump systems, and for gravity circulation hot water systems.) 


\section{INSTITUTE OF BOILER AND RADIATOR MANUFACTURERS, STEAM HEATING BOILER TESTING CODE, JANUARY, 1929}

\section{HEATING BOILERS}

(This code specifies the tests to be conducted and provides a standard method for conducting and reporting tests to determine the efficiencies and performance of solid fuel burning, low pressure steam heating boilers. It is identical with code No. 3, performance test code for steam heating solid fuel boilers, of the American Society of Heating and Ventilating Engineers, briefly described above, except that a latent heat of evaporation of 970.4 is used in the I. B. \& R. M. code as contrasted with the latent heat value of 971.7 used in the A. S. H. \& V. E. Code.)

\section{NATIONAL DISTRICT HEATING ASSOCIA- TION, STANDARD RULES FOR COMPUT- ING REQUIRED RADIATION, 1919}

\section{RADIATOR REQUIREMENTS FOR BUILDINGS}

(These standard rules are applied to the computation of the required amount of radiating area for either steam or hot-water heating systems and take into consideration the heat required to warm up the air in the room assuming a certain number of air changes per hour and the heat lost through walls, ceiling, floor, doors, and windows. Data are included for the rate of heat transmission through various building materials and types of construction for walls, ceilings, etc. Correction factors are given for high ceilings, for room exposure other than southern, and for coefficient of heat transmission for various types and sizes of radiators.)

\section{NATIONAL WARM AIR HEATING ASSOCIA-} TION, THE AMERICAN SOCIETY OF HEATING AND VENTILATING ENGINEERS, AND NATIONAL ASSOCIATION SHEET METAL CONTRACTORS, STANDARD CODE REGULATING THE INSTALLATION OF GRAVITY WARM-AIR HEATING SYSTEMS IN RESIDENCES, SEPTEMBER 1,1930

\section{GRAVITY WARM-AIR IEATING SYSTEMS}

(This code includes standard methods and formulas for determining sizes of warm-air pipes, wall stacks, and furnaces for use in residences, taking into consideration the type of wall, degree of exposure, number of windows and doors, etc. Regulations are given for the location and installation of furnace, casings, warm-air pipes, wall stacks, registers, smoke pipes, etc. The furnace rating formula is as given below.)

\section{FURNACE RATING FORMOLA}

$$
L=1.75 G(1+0.02(R-20))
$$

$L=$ square inches of warm-air pipe connected to the furnace as calculated.

$G=$ grate area in square inches; the area of the fire pot at the grate level, its most restricted area.

$R=$ ratio of heating surface area to grate area.

$1.75=$ a constant based upon the results obtained in the association research on a furnace having 20 square feet of heating surfáce for each square foot of grate.
The above equation is based on the following: Efficiency of heater, 0.55 ; combustion rate of 7.5 pounds of coal per square foot of grate per hour; calorific value of fuel, 12,790 B. t. u. per pound; percentage of heat available at register, 0.75 ; average $B$. t. $u$. delivering value of 1 square inch of leader pipe area, assuming half of the heat is sent to each floor, 136; and on an operating temperature of $175^{\circ}$ F. at the register.

The formula allows 1.75 square inckes of warm-air pipe area for each square inch of grate area, for the furnace having a ratio of heating surface to grate surface of 20 to 1 . For furnaces having other ratios of heating surfaces to grate surface, it adds 2 per cent or deducts 2 per cent for each unit above or below a ratio of 20 .

\section{UNITED STATES GOVERNMENT, DEPART-} MENT OF COMMERCE, BUREAU OF STANDARDS, SIMPLIFIED-PRACTICE RECOMMENDATION FOR HOT-WATER STORAGE TANKS, R25, JANUARY 1, 1925

\section{HOT-WATER STORAGE TANKS}

(Simplified practice recommended and accepted by industry establishing a limited list of standard dimensions and capacities.)

\section{SIZES}

\begin{tabular}{|c|c|c|c|c|c|}
\hline $\begin{array}{l}\text { Diam- } \\
\text { eter }{ }^{1}\end{array}$ & Length 1 & $\begin{array}{c}\text { Actual } \\
\text { capacity }\end{array}$ & $\begin{array}{l}\text { Diam- } \\
\text { eter }{ }^{1}\end{array}$ & Length 1 & $\begin{array}{c}\text { Actual } \\
\text { capacity }\end{array}$ \\
\hline $\begin{array}{c}\text { Inches } \\
20 \\
24 \\
24 \\
30 \\
30 \\
36 \\
36\end{array}$ & $\begin{array}{c}\text { Feet } \\
5 \\
5 \\
6 \\
6 \\
8 \\
6 \\
8\end{array}$ & $\begin{array}{c}U . S . \\
\text { gallons } \\
82 \\
118 \\
141 \\
220 \\
294 \\
318 \\
423\end{array}$ & $\begin{array}{c}\text { Inches } \\
42 \\
42 \\
42 \\
42 \\
48 \\
43 \\
48\end{array}$ & $\begin{array}{c}\text { Feet } \\
7 \\
\delta \\
10 \\
14 \\
10 \\
16 \\
20\end{array}$ & $\begin{array}{r}U . S . \\
\text { gallons } \\
504 \\
576 \\
720 \\
1,008 \\
940 \\
1,504 \\
1,880\end{array}$ \\
\hline
\end{tabular}

$1 \mathrm{By}$ diameter is meant inside diameter, length means length of sheet, not orer-all length of tank.

\section{WORKING PRESSURES, CLASSIFICATION, AND MAREING}

(a) These sizes to be made in two working pressures, viz, 65 pounds per square inch, and 100 pounds per square inch.

(b) Those made for 65 pounds working pressure are to be classified as "STANDARD," and those for 100 pounds working pressure as "ExTRA HEATY."

(c) Each tank is to be stenciled with its proper classification, working pressure, also the name and address of its manufacturer, as follows:

\section{STANDARD \\ Guaranteed for 65 pounds working pressure Manufacturer's name and address \\ ExTRA HEATY \\ Guaranteed for 100 pounds working pressure Manufacturer's name and address}

3. FACTORS OF SAFETY, THICKNESSES, ETC.

These are to be in accord with the A. S. M. E. code for "Unfired pressure vessels." (See 605.23 p. 288.)

\section{INTERCHANGEABILITY}

The tanks above listed are to be made interchangeable for either horizontal or vertical installation.

\section{TAPPINGS}

There are to be six tappings in each tank, placed as follows: One in center of convex head; two in the 
shell, in line (parallel with axis of tank), each to be centered 12 inches from the edge of the sheet; one 12 inches from the conver end and one 12 inches from the concave end; 3 in the shell, in line, said line to be diametrically opposite from the line of the 2 just mentioned. Of these three, one is to be centered 12 inches in from the convex end; another 24 inches in from that end; and the third is to be centered 12 inches in from the concave end. All measurements are taken from the edge of the sheet. There is no tapping in the concave, or bottom, end. All tappings in tanks up to but not including 30 inches in diameter are to be $1 \frac{1}{2}$ inches; from 30 inches up to but not including 48 inches are to be 2 inches; and from 48 inches upward, the tappings are to be 3 inches. All threads are to be American standard taper pipe threads. These tappings apply to either horizontal or vertical installations.

\section{MANHOLES}

These are to be standard size, 11 by 15 inches, and may be placed either in the shell or the head.

\section{HANDHOLES}

These are to be 4 by 6 inches, located as desired.

\section{HEATING COIIS}

Coils for either horizontal or vertical installation of these tanks must not be less in size or total length than appears in the following table:

\begin{tabular}{|c|c|c|c|c|c|c|c|}
\hline \multicolumn{2}{|c|}{$\begin{array}{l}\text { Tank dimen- } \\
\text { sions }\end{array}$} & \multirow{2}{*}{$\begin{array}{l}\text { Size of } \\
\text { pipe }\end{array}$} & \multirow{2}{*}{$\begin{array}{l}\text { Mini- } \\
\text { mum } \\
\text { length } \\
\text { of heat- } \\
\text { ing coil }\end{array}$} & \multicolumn{2}{|c|}{$\begin{array}{l}\text { Tank dimen- } \\
\text { sions }\end{array}$} & \multirow{2}{*}{$\begin{array}{l}\text { Size of } \\
\text { pipe }\end{array}$} & \multirow{2}{*}{$\begin{array}{l}\text { Mini- } \\
\text { mum } \\
\text { length } \\
\text { of heat } \\
\text { ing coil }\end{array}$} \\
\hline $\begin{array}{l}\text { Diam- } \\
\text { eter }\end{array}$ & Length & & & $\begin{array}{c}\text { Diam- } \\
\text { eter }\end{array}$ & Length & & \\
\hline $\begin{array}{c}\text { Inches } \\
20 \\
24 \\
24 \\
30 \\
30 \\
36 \\
36\end{array}$ & $\begin{array}{r}\text { Feet } \\
5 \\
5 \\
6 \\
6 \\
8 \\
6 \\
8\end{array}$ & $\begin{array}{c}\text { Inches } \\
1 \\
11 / 4 \\
11 / 4 \\
11 / 4 \\
11 / 4 \\
11 / 2 \\
11 / 2\end{array}$ & $\begin{array}{r}\text { Feet } \\
14 \\
14 \\
18 \\
18 \\
26 \\
18 \\
26\end{array}$ & $\begin{array}{c}\text { Inches } \\
42 \\
42 \\
42 \\
42 \\
48 \\
48 \\
48\end{array}$ & $\begin{array}{r}\text { Feet } \\
7 \\
8 \\
10 \\
14 \\
10 \\
16 \\
20\end{array}$ & $\begin{array}{c}\text { Inches } \\
11 / 2 \\
11 / 2 \\
11 / 2 \\
11 / 2 \\
2 \\
2 \\
2\end{array}$ & $\begin{array}{r}\text { Feet } \\
22 \\
26 \\
34 \\
50 \\
34 \\
58 \\
74\end{array}$ \\
\hline
\end{tabular}

UNITED STATES GOVERNMENT, FEDERAL SPECIFICATIONS BOARD, SPECIFICATION FOR PIUMBING FIXTURES (FOR LAND USE), NO. 448, NOVEMBER 22, 1926. (CURRENT DESIGNATION : FF-P451)

\author{
WATER HEATERS AND STORAGE TANKS
}

(See 617.73, p. 1062.)

\subsection{MISCELLANEOUS HEATING COOKING EQUIPMENT.}

AMERICAN OIL CHEMISTS' SOCIETY, OFFICIAL METHODS OF CHEMICAL ANALYSIS, AUGUST 1, 1930

\section{JACKETED OVEN}

(This society has adopted a standard design for a constant temperature oven for maintaining a temperature of $101^{\circ} \mathrm{C}$. for use in testing cottonseed and cottonseed products. The oven is jacketed with glycerine and water and made of 48-ounce copper sheet with all seams brazed. Outside dimensions are $201 / 8$ inches long by $13 \frac{1}{4}$ inches wide by 14 inches high, inside dimensions are 18 inches long, $113 / 4$ inches wide, and 10 inches high. It is supported from the floor by angle iron supports and legs at a height of 9 inches. Heat is supplied beneath the oven by electrical elements of approximately 2,000 watts capacity.)

\section{VACUUM OVEN}

This oven has been developed as the standard vacuum oven of the society with the idea of affording a simple and compact vacuum oven which will give as uniform temperature as possible on the shelf. It consists of a piece of 10-inch extra heavy iron pipe, with back and attachments welded on and turned down to dimensions. The front door can be made air tight by grinding into position with emery flour, or a gasket may be used. The single shelf is heated by means of a brass coil soldered on to the shelf, heat being supplied by vapor from boiling carbon tetrachloride which is circulated through the coil. (The boiling point of carbon tetrachloride varies from $76.1^{\circ} \mathrm{C}$. at 743 millimeters of mercury pressure to $25^{\circ}$ C. at 116 millimeters of mercury pressure.) The carbon tetrachloride is contained in a flask fitted with a reflux condenser and heated by means of a small electric hot plate. This flask and heater are outside the oven, and connection is made to the brass coil inside the oven by means of glass tubing connecting to the outside terminals of the coil. The advantage of heating by means of a constant boiling liquid, rather than by electric coils entailing the difficulties of electrical control, is considerable. Insulation of the oven is recommended, also attachment of a small pet cock at a point low down on the door to regulate circulation within the oven.

(Assembly drawings of both the official jacketed oven and the standard vacuum oven are shown in the Official Methods of Chemical Analysis of the American Oil Chemists Society.)

\section{ASSOCIATED FACTORY MUTUAL FIRE IN- SURANCE COMPANIES, GRAVITY WATER TANKS AND STEEL TOWERS, VOLUME II, PIPING, FITTINGS, AND HEATING SYSTEMS, 1929}

\section{TANK HEATING EQUIPMENTS}

(These rules and recommendations cover recommended construction and design of steam heaters, using steam coils, the water from the tank circulating through the heater and around the steam pipes by gravity; recommended makes of coal-burning heaters where the tank water circulates through chambers or pipe coils around or above the fire; special cases were steam may be led directly into the tank itself; and methods of determining necessary heater capacities.)

NATIONAL BOARD OF FIRE UNDERWRITERS, REGULATIONS FOR THE INSTALLATION AND OPERATION OF ACETYIENE EQUIPMENT FOR IIGHTING, HEATING, AND COOKING, MARCH 1, 1930

\section{ACETYLENE FOR LIGHTING, HEATING, AND COOKING}

(These regulations cover six classes of equipmentstationary automatic apparatus permitted for outside installation only, stationary automatic apparatus permitted for inside or outside installation, stationary nonautomatic apparatus for outside installation only, semiportable automatic apparatus permitted for inside installation, dissolved acetylene 
under pressure, and portable generators for lighting. They include requirements on the location of generator; on the construction, ventilation, and heating of outside generator houses; on sizes and capacities of pipe; requirements for conductor and escape piping; operation of generators; storage and installation of cylinders; storage of calcium carbide.)

NATIONAL BOARD OF FIRE UNDERWRITERS, REGULATIONS FOR OVENS FOR JAPAN, ENAMEL, AND OTHER FLAMMABLE FINISHES, MARCH 15, 1931

\section{OVENS}

(These regulations cover the location and arrangement of ovens used in enameling and japanning, construction of oven as regards safety from explosion damage, adequacy of openings for bringing in fire hose in case of fire, constructed of brick, concrete, or of steel and approved noncombustible materials, regulations on type and amount of ventilation, permissible types and installation requirements for heating equipment for ovens, regulations on amount and permissible types of fire-extinguishing equipment.)

UNDERWRITERS' LABORATORIES, STANDARDS FOR CONSTRUCTION AND PERFORMANCE OF FUEL OIL BURNERS FOR DOMESTIC USE, JUNE, 1928

\section{DOMESTIC OIL BURNERS}

(These standards apply to burners designed for operation without an attendant constantly on the premises. These standards require that the burners be reliable and uniform in operation and free from hazardous carbonization; that they shall contain no chambers which allow the dangerous accumulation of gases; they shall have no oil conveying pipes or parts which are subject to intense heat. Minimum sizes of oil line connections and tubing are specified; strainers are to be accessible for cleaning with at least 4 square inches of effective screening area per gallon per hour of liquid flow; general regulations on the provision, performance requirements, and permissible types of draft regulators, pumps, packing, unions, valves, antiflooding devices, automatic valves, floats, overflow cups, overflow pipe, base, fire pots, gas pilot lights, electric ignition equipment oil pilot lights, etc. Structural features and safety provisions for steam generators used for atomizing, operating features of preheating and ignition devices, location of vacuum tanks, electrical wiring and equipment installation regulations are also covered.)

\section{UNDERWRITERS' LABORATORIES, STAND- ARD SPECIFICATIONS FOR GAS GA- RAGE HEATERS, DECEMBER, 1917}

\section{GAS GARAGE HEATERS}

(These standards relate to gas heaters with protected combusion chambers and include the following construction requirements.)

\section{HEAT RADIATING DROM}

It should be of sufficient size to permit of ready passing of heat into the circulating air so as to prevent heating the drum to an unsafe temperature. It should be made of rust-resisting metal not less than No. 24 U. S. gage (0.025 inch thick). All joints should be double seamed or made in an equivalent manner. The bottom of the drum should be at least 6 inches above the floor to permit of proper circulation of heat. Screens for air inlet openings should be mechanically secured in place without the use of solder. Perforated sheets should be used in this connection and the sheets should measure not less than 0.025 inch in thickness and the openings should not be larger than 0.027 inch and should aggregate 400 per square inch. Connections for flue and inlet pipes should not depend upon solder for security. The lowest portion of the drum should be connected with an accessible plugged or capped drain so as to permit of the removal of condensation.

(Other general provisions of these regulations deal with area and accessibility for cleaning of air inlet screens, provision of burners adaptable to both artificial and natural gas, installation of burners and air regulators, location and control of pilot light, and the following requirements for outside casing.)

\section{OUTSIDE CASING}

The heat radiating drum should be inclosed at the sides and ends by a casing placed at such distance from the drum that it can not become unduly heated (not less than 2 inches for unlined casing and 1 inch or more for casing lined with asbestos). The material used for such a casing should be galvanized iron and should measure not less than No. 24 U. S. gage $(0.025 \mathrm{inch})$ in thickness over the galvanizing. The edges and corners of this casing should be reinforced and substantial plates provided for the attachment of legs and braces. A screen made of $1 / 4$-inch mesh galvanized iron wire should be substantially mounted and mechanically fastened at the top of the outer casing, and should be located at least 6 inches above the top of the heat radiating drum. The bottom of this casing may be open but should not extend more than 3 inches below the plane of the bottom of the drum.

\section{FLUE AND AIR INLET PIPES}

Flue and air inlet pipes should be made of not less than No. 26 U. S. gage (0.0187 inch) galvanized iron and should be mechanically secured to the drum. Joints should be made in the form of a hook joint having an offset. These pipes should be not less than 3 inches in diameter. Dampers should be of such design that they will not interfere with the operation of the burner when entirely closed.

(Other requirements in this standard deal with the provision of ventilators or rain caps for flue pipes, structural and operating features of doors, ignition devices, piping and valves.)

\section{UNDERWRITERS' LABORATORIES, PRO- POSED STANDARDS FOR OIL BURNER HIGH TENSION ELECTRIC IGNITION SYSTEMS, MAY, 1928}

(These standards are the same as given for electric ignition under the Underwriters' Laboratories standards for construction and performance of fuel-oil burners for domestic use. See above.)

UNDERWRITERS' LABORATORIES, STANDARDS FOR CONSTRUCTION AND PERFORMANCE OF OIL-HEATED INCUBATORS (CLASS B), OCTOBER, 1927

\section{OIL HEATERS FOR INCUBATORS}

(This standard includes raquirements on structural features of the incubator, protection of the end 
of incubator case next to heater by heat-insulating material and sheet metal or having a lamp inclosure providing equivalent protection, prevention of direct heat from the oil flame from entering incubator case, insulating of hot-air pipe entering case, stability of case and attachment of legs, and the following raquirements for the heater.)

The heater shall be constructed wholly of noncombustible material and installed at the end of the incubator case. It shall be so located that all parts are at least 1 inch from the case and that the center of the burners shall not be lass than 5 inches from the end of the case. (Other provisions in the full text of the standard cover sizes of screws to be used and installation requirements of heater and provision and performance of inclosure for lamp.)

Lamps shall be made with seamless bottoms of not less than No. 28 U. S. gage metal suitably protected to retard corrosion. If galvanized, they shall be galvanized after forming, and if of terneplate, deepdrawing metal carrying at least an 8-pound coating shall be employed. Joints between the tops and bottoms shall be made mechanically secure in addition to soldering and so formed that the top will not provide a receptacle for dirt, oil, etc. Braces shall be provided between the tops and bottoms of bowls which will effectually prevent any bellows action.

The handle of the bowl shall be of such size and so located with respect to the filling opening that it will be impossible to replenish the fuel supply while the lamp is installed in the inclosure. (Other provisions include requirements on the sweating-in of bushings, provision of and general requirements for drip pan for each lamp.)

Burners shall be of substantial construction with wick tube and flame slot in proper alignment and must be provided with a guide ring to insure proper engagement with and positive seating of the bottom of the chimney. Chimney shall be made of metal.

\section{HAND TOOLS, METAL-WORKING}

\subsection{GENERAL ITEMS.}

UNITED STATES GOVERNMENT, DEPARTMENT OF COMMERCE, BUREAU OF ST ANDARDS, SIMPLIFIED-PRACTICE RECOMMENDATION FOR FORGED TOOLS, R17-31, JULY 1, 1931

FORGED TOOLS

(See 616.60, p. 960.)

615.1 CHISELS, DRILLS, PUNCHES, REAMERS, TAPS, AND DIES.

615.11 CHISELS.

UNITED STAGES GOVERNMENT, DEPARTMENT OF COMMERCE, BUREAU OF STANDARDS, SIMPLIFIED-PRACTICE RECOMMENDATION FOR FORGED TO0LS, R17-31, JULY 1, 1931

\section{CHISELS}

(A list of the standard sizes and varieties of track chisels will be found in Table 7 of the simplifiedpractice recommendation R17-31 for forged tools. Standard sizes and dimensions of blacksmith chisels will be found in Table 8 , and standard sizes of rivet buster or side chisel will be found in Table 10 of R17-31. See 616.60 , p. 960.)
615.12 DRILLS.

UNITED STATES GOVERNMENT, FEDERAL SPECIFICATIONS BOARD, FEDERAI SPECIFICATION FOR DRILLS, BREAST, NO. 417, JULY 9, 1926. (CURRENT DESIGNATION : GGG-D-651.)

\section{BREAST DRILLS}

I. GENERAL SPECIFICATIONS

There are no general specifications applicable to this specification.

\section{TYPE}

Shall be the single-speed type of breast drills.

\section{MATERIAL AND WORKMANSHIP}

These drills shall be made of high-grade material and in accordance with the best commercial practice. They shall be free from all defects or blemishes which may affect their serviceability or appearance.

\section{GENERAL REQUIREMENTS}

Breast drills shall have the manufacturer's name or trade-mark stamped or otherwise permanently marked on them.

\section{DETAIL REQUIREMENTS}

1. Breast drills shall consist of a stock, a spirit level, a breastplate, a chuck, a spindle, two or three gears, a crank, and two wooden handles.

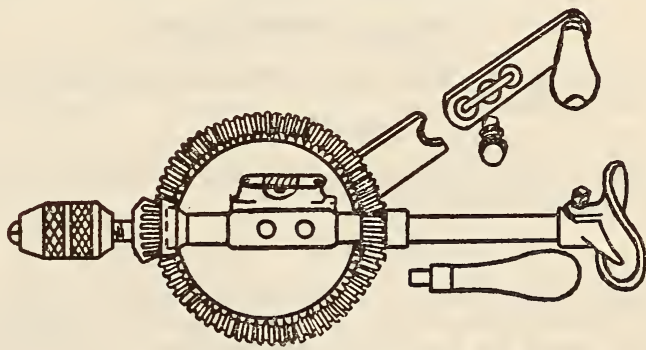

FIgURE 646.-Breast drill

2. The stóck shall be made of steel, and shall have mounted thereon a spirit level within a metal case, secured by screws to the stock.

3. The breastplate shall be made of malleable iron, and shall be adjustable and secured to the stock by means of a set screw. It shall be polished on the bearing surface and painted on the underside.

4. The chuck shall be internally threaded to screw on the spindle, and shall be provided with steel jaws capable of securely gripping straight shanks or tapered square shanks of drills from one-eighth inch up to and including one-half inch diameter.

5 . The spindle shall be made of one piece of steel and suitably shaped and threaded at one end to accommodate the chuck; the other end shall be provided with a bevel pinion. The spindle shall run on hardened steel balls bearing or hardenedsteel ball races. It shall be operated by means of a driving plate gear which shall engage with the bevel pinion on the spindle. The ratio of the géars shall not be less than $4 \frac{1}{2}$ to 1 .

6. The driving plate gear shall be provided with a steel crank which shall be adjustable to lengths of $5 \frac{1}{2}$ to 7 inches. There shall be provided either an idler pinion diametrically opposite the driving pinion or a guard near the driving pinion to counteract the outward thrust of the plate gear. 
7. All gears shall be machine cut and shall operate freely. The plate gear shall be neatly finished with a smooth coat of paint, and all exposed metal parts except bearing surfaces shall be nickel plated and polished unless otherwise stated.

8. Two detachable handles shall be provided, one for a crank handle and the other for a grip handle, which shall be threaded to screw in the stock opposite the gear plate. The handles shall be made of hardwood and smoothly finished. The wood portion of the crank handle shall be free to rotate. The wood portion of the grip handle shall be rigidly secured to its shank.

9. These drills shall be similar to the following sketch: Length over all, about 15 inches.

Representative samples shall be taken from each lot delivered and inspected for compliance with this specification.

\section{PACKING AND MARKING}

1. Breast drills shall be packed in accordance with the manufacturer's trade practice, unless otherwise specified.

\section{UNITED STATES GOVERNMENT, FEDERAL SPECIFICATIONS BOARD, FEDERAL SPECIFICATION FOR DRILIS, HAND, WITH HOLLOW HANDLES, NO. 509, JULY 11, 192\%. (CURRENT DESIGNA- TION GGG-D-671.)}

\section{HAND DRILLS}

\section{GENERAL SPECIFICATIONS}

There are no general specifications applicable to this specification.

\section{TYPE}

The drills shall be of the hollow-handle type, 0 to $3 / 8$ inch capacity.

\section{MATERIAL AND WORKMANSHIP}

The drills shall be made of high-grade material and in accordance with the best commercial practice. They shall be free from all defects or blemishes affecting their appearance or that may affect their serviceability.

\section{GENERAL REQUIREMENTS}

See Section V.

\section{DETAIL REQUIREMENTS}

1. Hand drills shall be composed essentially of a frame, a spindle either two or three gears, a crank, a chuck, and two handles exclusive of the crank handle. Provision shall be made so that all bearing surfaces of these drills may be readily oiled. Each hand drill shall be marked in a permanent manner with the manufacturer's name or known trademark.

2 . The frame shall be made of malleable iron or other material equally as suitable for the purpose. The frame shall be so designed as to withstand without injury the maximum strain to which it may be subjected under ordinary service conditions. It shall have a protective coating of paint or enamel.

3 . The spindle shall be made of a good grade of steel and the lower portion shall be threaded so as to be a neat fit when screwed into the chuck. The upper portion of the spindle shall be provided with a bevel pinion made of a good grade of steel. The bevel pinion shall have machine-cut teeth. Ball or roller bearings shall be provided to facilitate ease with which the spindle may be operated. The spindle shall be operated by means of a driving gear which shall engage with the bevel pinion on the spindle. The ratio of the gears shall be not less than 4 to 1 .

4. The driving gear shall be made of high-grade iron or steel and shall be provided with a removable crank made of steel, nickel plated. The crank shall be securely held to the driving gear and shall be provided with a well-made hardwood handle. The teeth of the driving gear shall be machine cut and shall operate freely with the teeth of the pinion gear. There shall be provided either an idler pinion gear diametrically opposite the driving pinion gear or a guard near the driving pinion gear to counteract outward thrust of the driving gear. The driving gear shall have a coating of paint or enamel on all exposed surfaces except the teeth and rim. The rim of the gear shall be nickel plated.

5 . The chuck shall be made of a good grade of steel or malleable iron, nickel plated on the outside, and shall be approximately $11 / 8$-inch diameter and 2 to $2 \frac{1}{2}$ inches long. It shall be threaded internally to screw on the spindle. The chuck shall be knurled on the outside diameter so as to afford a good hand grip. The chuck shall be provided with three hardened tool steel jaws, so designed that the jaws will not become separated when the chuck is removed from spindle. Suitable springs shall be provided to hold the jaws firmly against the tapered bearing on the inside of the chuck. The jaws shall open and close as the chuck is revolved and they shall close evenly and center accurately round shanks from 0 to $3 / 8$-inch diameter.

6 . The handles of the drills shall be made of cocobola, rosewood, or other hardwood equally as suitable for the purpose. The handles shall be neatly finished and so shaped as to afford a good hand grip. A side handle or knob shall be provided with each drill. This handle shall have a suitable ferrule on the small end and it shall be removable from the frame of the drill. The large or main handle shall be securely fastened to the frame of the drill and a suitable ferrule shall be provided on the small end where it joins the frame. This handle shall be hollowed for holding wood-boring bit or drill points. A removable screw cap of suitable shape to fit the handle shall be a part of each handle. Eight woodboring drill points ranging in size from one-sixteenth to eleven sixty-fourths inch in diameter shall be furnished with each drill.

7. The drills shall be not less than 13 inches nor more than 16 inches long, and shall weigh approximately 2 pounds.

\section{METHOD OF INSPECTION AND TESTS}

1. Representative samples, at least three, shall be taken from each lot and inspected to determine whether the drills comply with this specification.

2 . When so requested, bidders under requisitions or schedules shall submit, free of expense to the Government, for examination and test, sample hand drills similar to those they propose to furnish. Samples shall remain the property of the exhibitors, but a sample may be accepted as part of a delivery in the case of a successful bidder. However, the right is reserved to destroy the sample submitted if such action is considered necessary to determine the quality of material and method of manufacture, in which case no expense to the Government shall accrue. If award is made on samples submitted, it will be with the understanding that the hand drills furnished shall be equal in all respects to samples.

\section{PACKING OF SHIPMENTS}

Hand drills shall be packed in accordance with the manufacturer's trade practice unless otherwise specified. 
615.13 PUNCHES.

UNITED STATES GOVERNMENT, DEPARTMENT OF COMMERCE, BUREAU OF STANDARDS, SIMPLIFIED PRACTICE RECOMMENDATION FOR FORGED TOOLS, R17-31, JULY, 1931

\section{PUNCHES}

(Standard sizes and varieties of tie plug punches and of track punches will be found in Table 7 of simplified practice recommendation R17-31 for forged tools. Standard sizes of blacksmith punches will be found in Table 8 and standard sizes of backing-out punches will be found in Table 10 of R17-31. See 616.60 , p. 960.)

\subsection{REAMERS.}

(No nationally recognized specifications available.)

615.15. TAPS AND DIES.

\section{AMERICAN SOCIETY OF MECHANICAL ENGINEERS, TAPS, CUT AND GROUND THREADS, B5e-1930}

[Approved by American Standards Association, 1930]

\section{TAPS}

(Same as National Screw Thread Commission, given below, except for tapper-tap and nut-tap cut threads, the maximum and minimum pitch diameters and major diameters of which are slightly smaller for coarse threads in the A. S. M. E. specification than in the N. S. T. C. report.)

\section{UNITED STATES GOVERNMENT, DEPART- MENT OF COMMERCE, DIE-HEAD CHAS- ERS. BUREAU OF STANDARDS PUBLI- CATION SIMPLIFIED PRACTICE RECOM- MENDATION R51-29, 1929}

\section{DIE-HEAD CHASERS}

(For self-opening and Adjustable Die Heads)

A joint conference of manufacturers, distributors, and users of chasers for self-opening and adjustable die heads, held on December 4, 1925, drafted a simplified practice recommendation which was later accepted by the industry. This recommendatior: as revised by subsequent meetings of the standing committee on December 20, 1927, and May 20, 1929 , and approved by the industry for promulgation by the Department of Commerce, establishes the following simplified list of stock sizes and varieties:

NotE.-While self-opening die heads have a diameter adjustment, all chasers not stamped as indicated in the following tables will be regarded as specials.

The stockable sizes shown are for right-hand threads only.

TABLE 1

\begin{tabular}{|c|c|c|c|c|c|c|c|}
\hline \multicolumn{4}{|c|}{$\begin{array}{c}\text { American national coarse-thread } \\
\text { series }\end{array}$} & \multicolumn{4}{|c|}{$\begin{array}{c}\text { American national fine-thread } \\
\text { series }\end{array}$} \\
\hline Size & 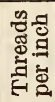 & Size & 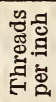 & Size & 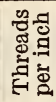 & Size & 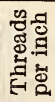 \\
\hline $\begin{array}{l}5^{1} \\
6 \\
8 \\
8 \\
10^{2}=\end{array}$ & $\begin{array}{l}40 \\
32 \\
32 \\
24\end{array}$ & $\begin{array}{l}3 / 4 \ldots \\
7 / 8 \\
1 \\
11 / 8 \ldots\end{array}$ & $\begin{array}{r}10 \\
9 \\
8 \\
7\end{array}$ & $\begin{array}{l}6 \\
8 \\
10^{4} \\
12\end{array}$ & $\begin{array}{l}40 \\
36 \\
32 \\
28\end{array}$ & $\begin{array}{l}7 / 8 \ldots \\
1118 \ldots \\
11 / 4 \ldots\end{array}$ & $\begin{array}{l}14 \\
14 \\
12 \\
12\end{array}$ \\
\hline $\begin{array}{l}12 \\
1 / 4{ }^{3} \\
5 / 16 \\
3 / 8\end{array}$ & $\begin{array}{l}24 \\
20 \\
18 \\
16\end{array}$ & $\begin{array}{l}11 / 4 \\
11 / 2 \\
13 / 4 \\
2 .\end{array}$ & $\begin{array}{l}7 \\
6 \\
5 \\
41 / 2\end{array}$ & $\begin{array}{l}1 / 4{ }^{5} \\
5 / 16 \ldots \\
3 / 8 \\
7 / 16 \ldots\end{array}$ & $\begin{array}{l}28 \\
24 \\
24 \\
20\end{array}$ & $\begin{array}{l}11 / 2 \\
13 \% \\
2 \\
214\end{array}$ & $\begin{array}{r}12 \\
10 \\
10 \\
8\end{array}$ \\
\hline $\begin{array}{l}7 / 16 . \\
1 / 2 \ldots \\
9 / 16- \\
5 / 8 \ldots\end{array}$ & $\begin{array}{l}14 \\
13 \\
12 \\
11\end{array}$ & $\begin{array}{l}21 / 4 \\
21 / 2 \\
23 / 4 \\
3 .\end{array}$ & $\begin{array}{l}41 / 2 \\
4 \\
4 \\
4\end{array}$ & & $\begin{array}{l}20 \\
18 \\
18 \\
16\end{array}$ & $\begin{array}{l}21 / 2 \\
23 / 4 \\
3 .\end{array}$ & $\begin{array}{l}8 \\
8 \\
8\end{array}$ \\
\hline
\end{tabular}

1 Identical with size $1 / 8-40$.

2 This size should be used in place of size $3 / 16-24$.

3 This size should be used in place of size 14-20.

4 This size should be used in place of size $3 / 16-32$.

$\checkmark$ This size should be used in place of size 14-28.

The American national coarse-thread series is the "U. S. standard" threads, supplemented in the sizes below one-fourth inch by sizes taken from the standard established by the American Society of Mechanical Engineers. The fine-thread series is composed of standards that have been found necessary and consists of sizes taken from the standards of the Society of Automotive Engineers and the finethread series of the American Society of Mechanical Engineers.

(For both coarse and fine thread series, see National Screw Thread Commission, screw threads, 608.0, p. 529.)

TABLE 1A

\begin{tabular}{|c|c|c|c|}
\hline \multicolumn{2}{|c|}{ S. A. E. standard spark plug } & \multicolumn{2}{|c|}{$\begin{array}{l}\text { Regular bushing thread for } \\
\text { spark plugs }\end{array}$} \\
\hline Size & $\begin{array}{l}\text { Threads } \\
\text { per inch }\end{array}$ & Size & $\begin{array}{l}\text { Threads } \\
\text { per inch }\end{array}$ \\
\hline 7/8 $\ldots$ & 18 & $13 / 16$ & 24 \\
\hline
\end{tabular}

REFERENCES.-For detailed dimensions of bolt threads and threaded holes, see the 1928 Report of the National Screw Thread Commission, which is No. 89 of the Miscellaneous Publications of the National Bureau of Standards, or Publication B-1-a (1924) of the American Standards Association.

For detailed information concerning S. A. E. standards, see the published tables of the Society of Automotive Engineers. 
TABLE 3.-Threads for lighting fixtures and fittings ${ }^{1}$

[American National, or United States form]

\begin{tabular}{|c|c|c|c|}
\hline Size & $\begin{array}{l}\text { Threads } \\
\text { per inch }\end{array}$ & Size & $\begin{array}{l}\text { Threads } \\
\text { per inch }\end{array}$ \\
\hline $\begin{array}{l}1 / 46-16 \\
5 / 16 \\
38 \\
7 / 16 \\
1 / 2\end{array}$ & $\begin{array}{l}27 \\
27 \\
27 \\
27 \\
27\end{array}$ & 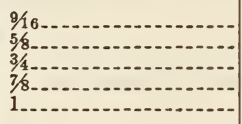 & $\begin{array}{l}27 \\
27 \\
27 \\
27 \\
27\end{array}$ \\
\hline
\end{tabular}

1 For detailed dimensions, see Tables VII- 6 and VII-7 of the National Screw Thread Commission's Communication No. 81, dated Apr. 16, 1924. The sizes shown above are identical with those in National Screw Thread Commission's Communication No. 81, except that the size $25 \% 64-27$ is omitted because it has only special application.

TABLE 4.-Railway sizes 1

[American National, or United States form]

Straight and taper of $3 / 4$ inch per foot

\begin{tabular}{|c|c|c|c|c|c|}
\hline Size & $\begin{array}{l}\text { Threads } \\
\text { per inch }\end{array}$ & Size & $\begin{array}{l}\text { Threads } \\
\text { per inch }\end{array}$ & Size & $\begin{array}{l}\text { Threads } \\
\text { per inch }\end{array}$ \\
\hline 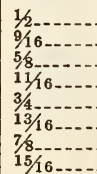 & $\begin{array}{l}12 \\
12 \\
12 \\
12 \\
12 \\
12 \\
12 \\
12\end{array}$ & $\begin{array}{l}11 \\
1116 \\
118 \\
13116 \\
114 \\
15116 \\
138\end{array}$ & $\begin{array}{l}12 \\
12 \\
12 \\
12 \\
12 \\
12 \\
12\end{array}$ & $\begin{array}{l}11 / 2 \ldots \\
13 / 4 \ldots \\
2 \\
21 / 4 \\
21 / 2 \ldots \\
234 \\
3\end{array}$ & $\begin{array}{l}12 \\
12 \\
12 \\
12 \\
12 \\
12 \\
12\end{array}$ \\
\hline
\end{tabular}

1 The sizes in Table 4 can be used for special thin adjusting or clamping nuts and, in general, machine design.

TABLE 5.-American national (Briggs) pipe thread Straight and taper

\begin{tabular}{|c|c|c|c|}
\hline Size & $\begin{array}{l}\text { Threads } \\
\text { per inch }\end{array}$ & Size & $\begin{array}{l}\text { Threads } \\
\text { per inch }\end{array}$ \\
\hline $\begin{array}{l}18 \\
144 \\
3 / 8 \\
112\end{array}$ & $\begin{array}{l}27 \\
18 \\
18 \\
14\end{array}$ & $\begin{array}{l}3 / 4 \ldots \ldots \\
113 \ldots \\
11 / 2 \ldots\end{array}$ & $\begin{array}{l}14 \\
111 / 2 \\
111 / 2 \\
111 / 2\end{array}$ \\
\hline
\end{tabular}

REFERENCES.-For detailed dimensions of pipe threads, see the 1928 Report of the National Screw Thread Commission, which is No. 89 of the Miscellaneous Publications of the National Bureau of Standards, or the 1919 Publication No. 3 of the American Standards Association.

\section{RIGHT-HAND AND LEFT-HAND THREADING}

Fi? While the question of right-hand and left-hand threading was not made the subject of a definite recommendation by the general conference, it developed during the period of acceptance of the proposed simplified practice that the industry is defi- nitely of the op nion that in addition to the desirability of concentrating on thread sizes shown in the tables, $i$; is important to make sure that all threads are "right-hand" except in very special cases where a left-hand thread is desirable. Left-hand threads are usually made to order by die-head manufacturers, and the demand is very small. A thread is always to be considered "right-hand" unless otherwise specifically stated.

\section{UNITED STATES GOVERNMENT, NA- TIONAL SCREW THREAD COMMISSION ; TAP SPECIFICATIONS, BUREAU OF STANDARDS MISCELLANEOUS PUBLI- CATION NO. 89, JUNE 22, 1928}

\section{TAPS}

\section{TAP DIMENSIONS AND TOLERANCES}

The dimensions and tolerances for cut and ground thread machine-screw, hand, tapper, nut, and pulley taps given in Tables 99 to 103 , inclusive, were prepared with the cooperation of a comm ttee of representative tap manufacturers and users, and represent present practice for the production of tapped holes of the classes indicated below. These standards may, therefore, be regarded as representing the best present commercial practice. They should be regarded as suggestive only and in no way mandatory.

The use, however, of taps that are in conformity with these tables should not be taken as a guaranty that the tapped holes produced thereby will necessarily fall with $n$ any given class of fit. Taps may cut oversize an amount depending on the cutting speed, condition of the tapping machine, lubrication or cooling agent, relief and chamfer of tap, and other factors. In general, cut-thread taps made to these specifications, when used under normal conditions, should in the majority of cases produce holes within class 2 free-fit tolerances; ground-thread taps, within class 3 , medium-fit tolerances.

The general dimensions contained in these tables do not pertain to screw threads, but are essential to the complete standardization of taps.

The point of measurement for the pitch diameter for all taps shall be at the first full thread.

The form of thread at the minor diameter is the American national form of thread, or sharper.

The maximum lead errors permitted are \pm 0.0030 inch in 1 inch of thread for cut-thread taps, and \pm 0.0005 inch in 1 inch of thread for ground-thread taps.

All taps have center holes at both ends, with the exception that cut thread taps up to and including five-sixteenths inch are pointed on the thread end.

Clearance on a ground thread tap may be provided by a slight rad al relief or a back taper of 0.0005 to 0.0010 inch per inch. 


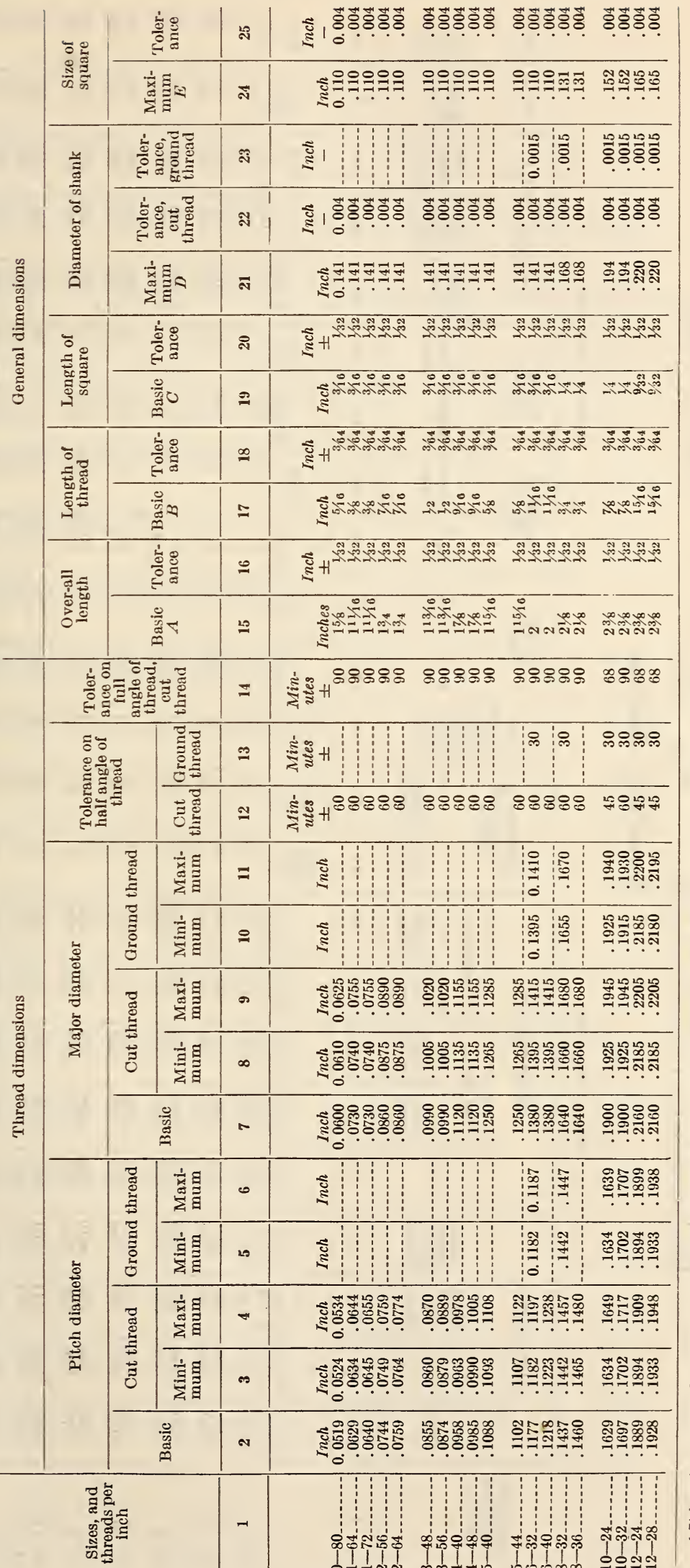




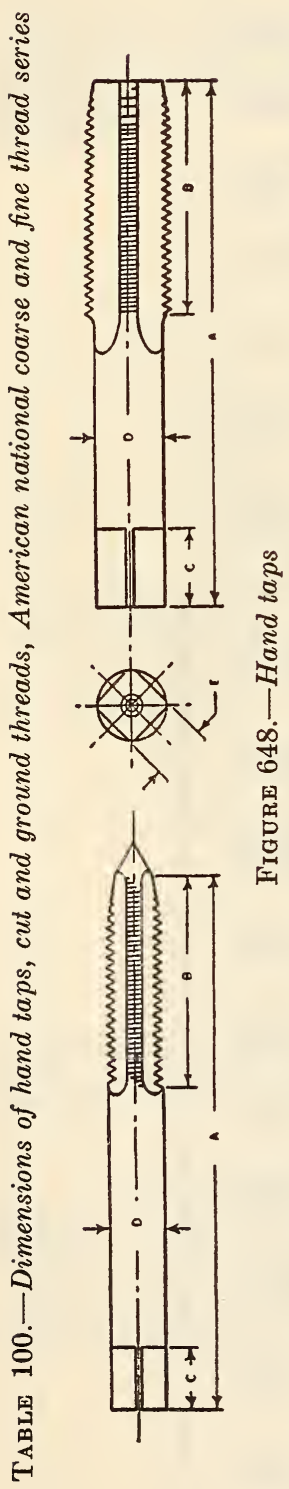

\begin{tabular}{|c|c|c|c|}
\hline \multirow{2}{*}{$\frac{\frac{2}{\frac{2}{2}}}{\frac{2}{4}}$} & & & \\
\hline & 离細四 & \& & 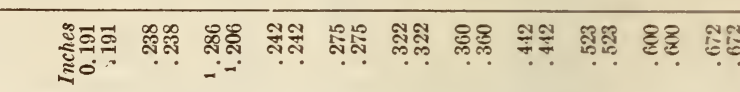 \\
\hline \multirow[t]{3}{*}{ (1) } & 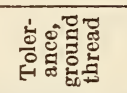 & $\approx$ & 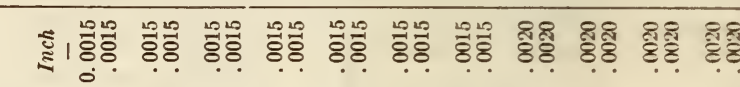 \\
\hline & 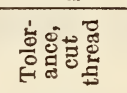 & न & 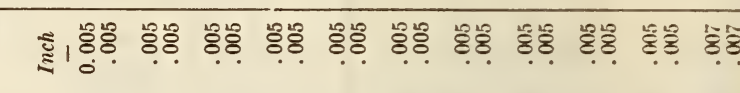 \\
\hline & 离羄 & $\approx$ & 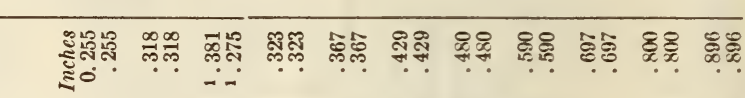 \\
\hline & 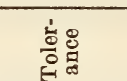 & $=$ & 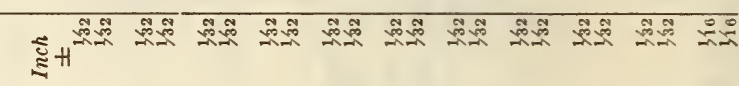 \\
\hline & 递 & 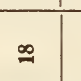 & 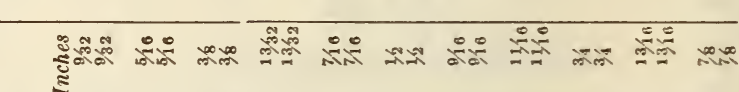 \\
\hline & 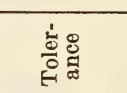 & $=$ & 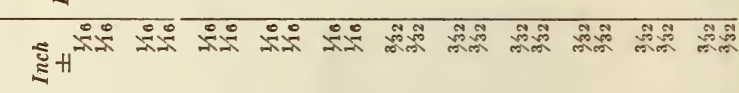 \\
\hline & 总 & $\cong$ & 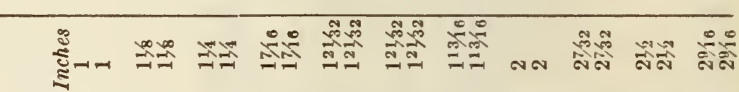 \\
\hline \multirow{2}{*}{$\bar{c}$} & 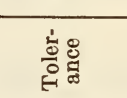 & $\stackrel{2}{2}$ & हnt \\
\hline & 总恼 & $=$ & 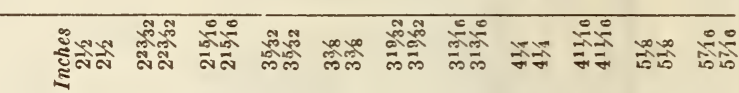 \\
\hline \multicolumn{2}{|c|}{ 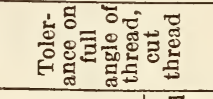 } & $\cong$ & 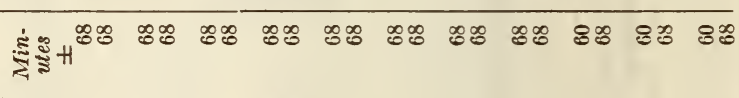 \\
\hline \multirow{2}{*}{ 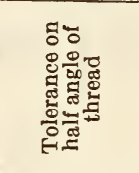 } & 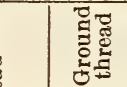 & $\cong$ & 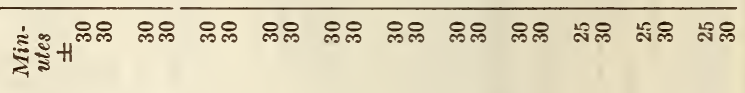 \\
\hline & 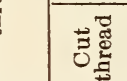 & $=1$ & 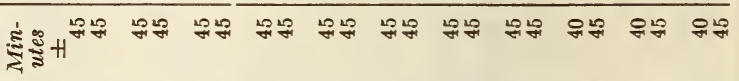 \\
\hline & 害㫷 & $=$ & 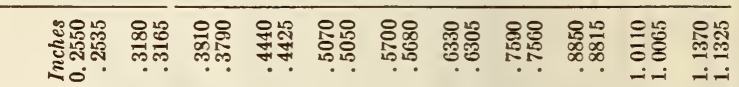 \\
\hline & 高悬 & . & 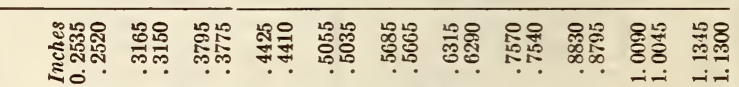 \\
\hline & 䇏 & $\infty$ & 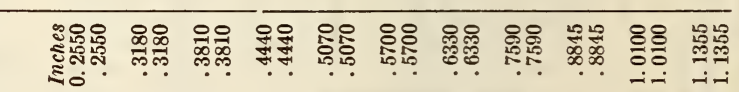 \\
\hline & 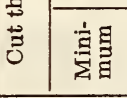 & - & 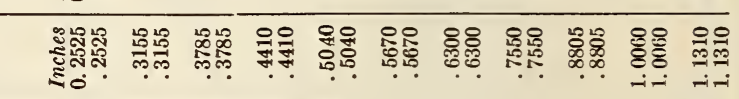 \\
\hline & 鄫 & . & 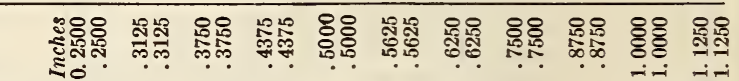 \\
\hline \multirow{4}{*}{$\frac{5}{\underline{\underline{z}}}$} & 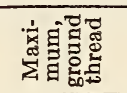 & 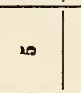 & 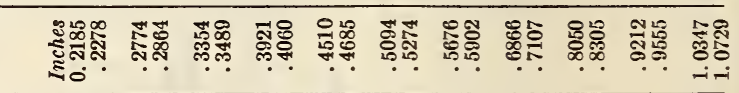 \\
\hline & 高稿譆 & $*$ & 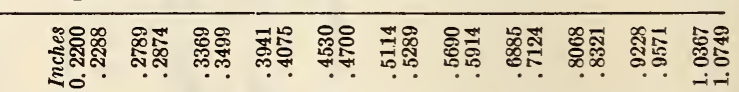 \\
\hline & 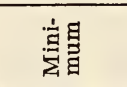 & $\infty$ & 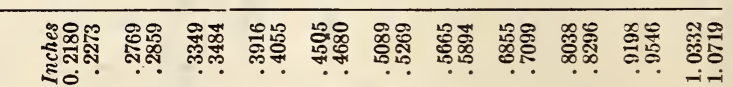 \\
\hline & 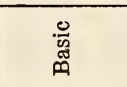 & " & 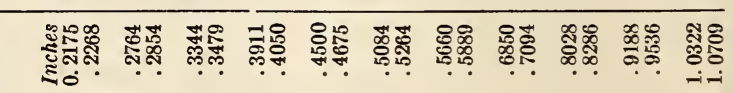 \\
\hline \multicolumn{2}{|c|}{ 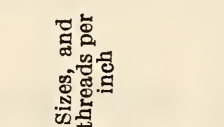 } & & \\
\hline
\end{tabular}




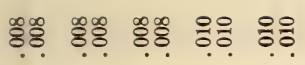

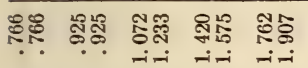

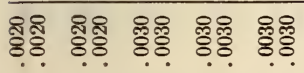

影

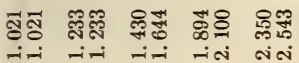

ฮำ

$\stackrel{\infty}{2}=$

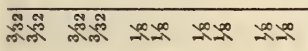

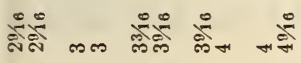

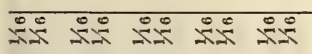

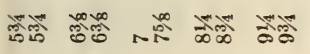

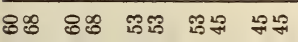

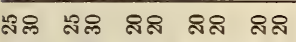

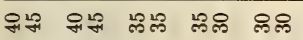

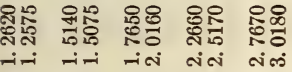

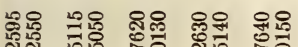

in ti-i min nin

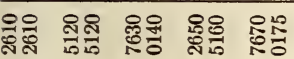

-i-i -i-i ni nim

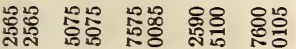

I-

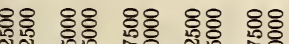

i- i-i in vin

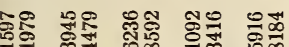

I- -i-i ri vin

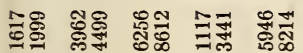

¿-

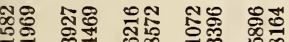

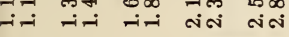

싱여

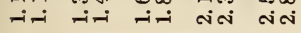

II 


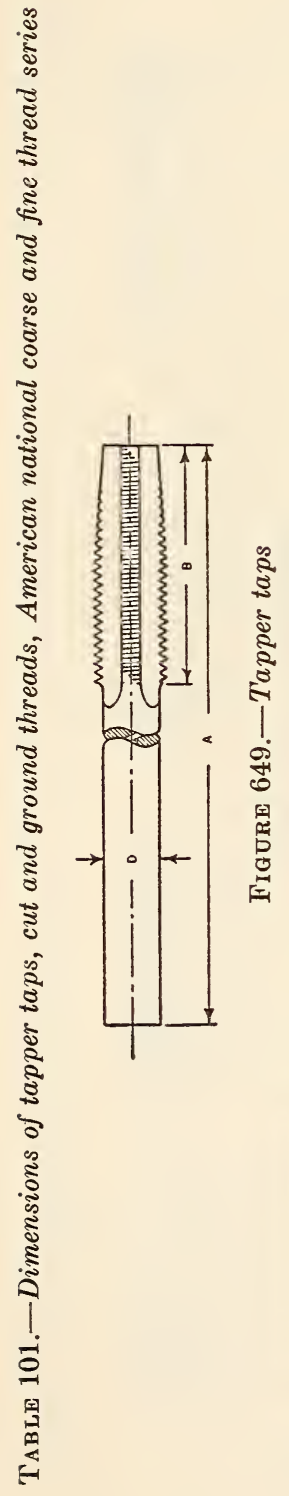

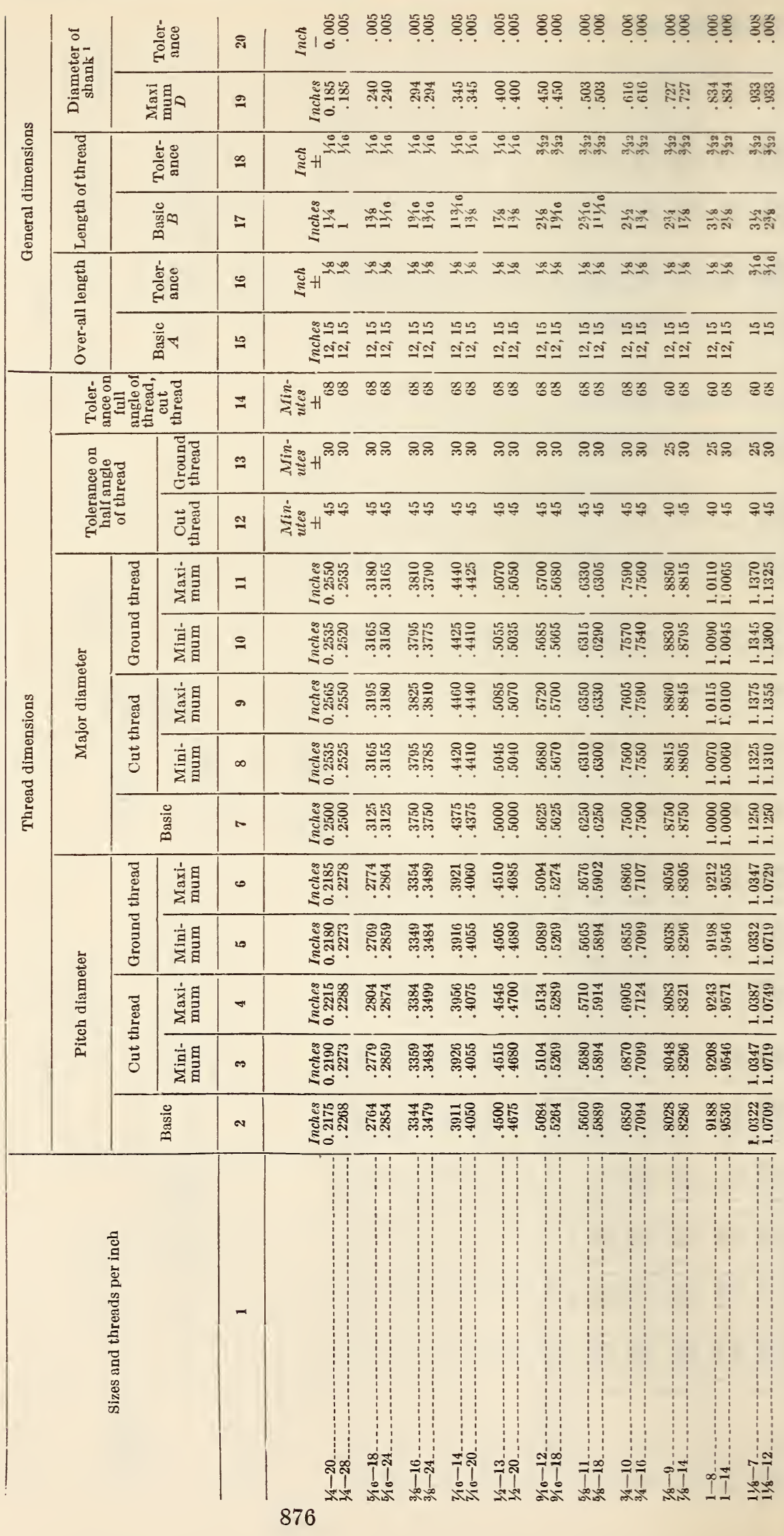


:

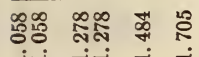

א్

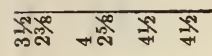

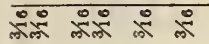

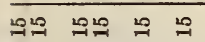

$8 \% 8 \%$ เึ

ผำ ผำ สิ สิ

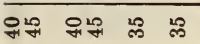

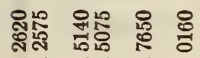

-i-i-i

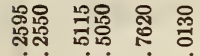

-i -i-i $-i$

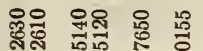

iٓi $i-i$ i

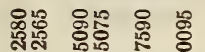

i-i $-i-i$ i

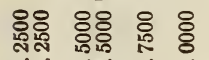

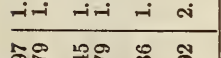

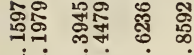

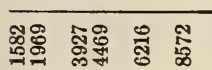

i- $\rightarrow-T_{-1}$

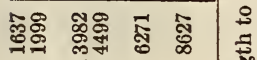

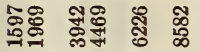

1 1 


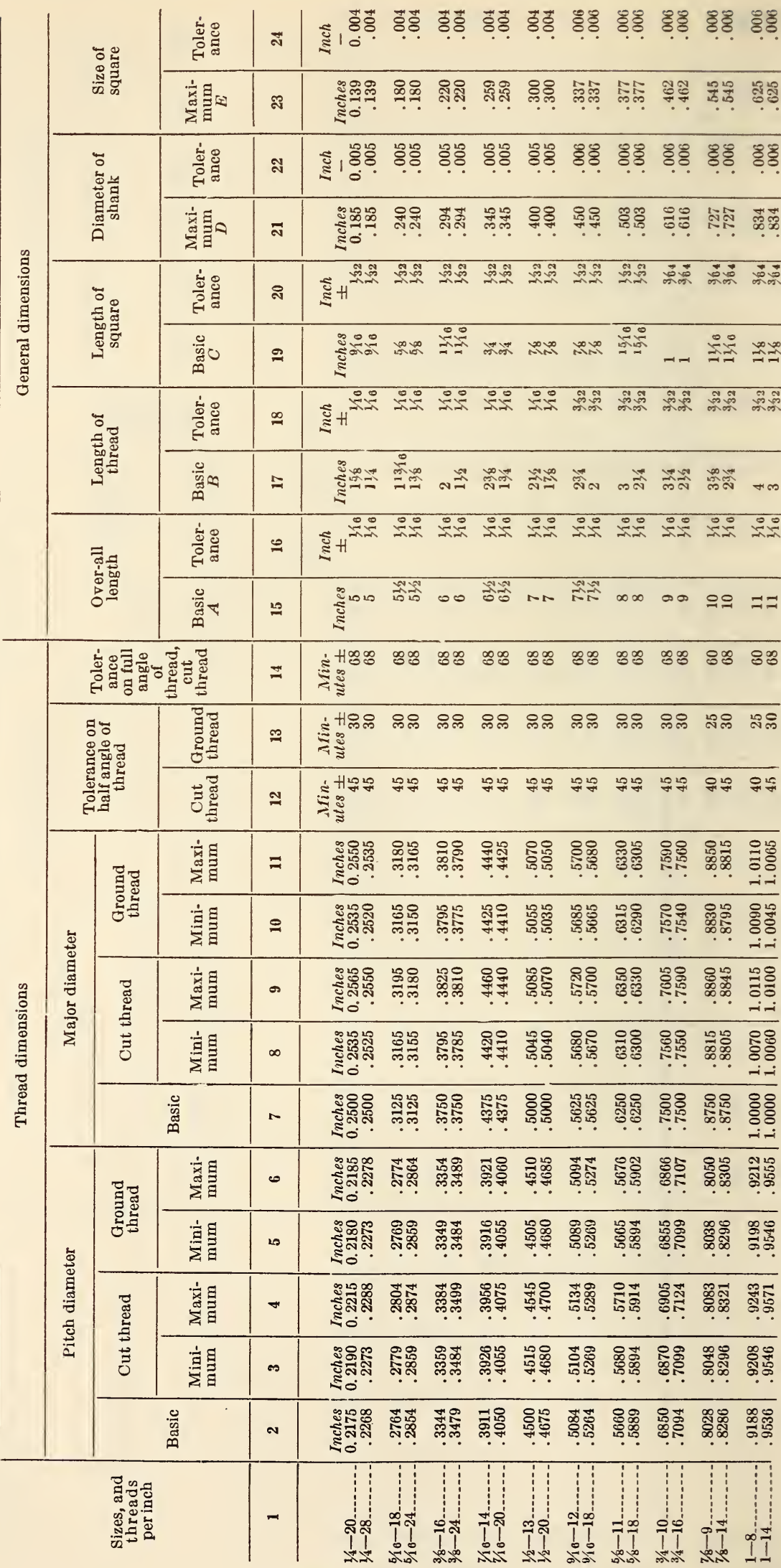




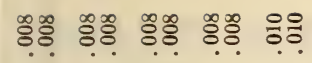

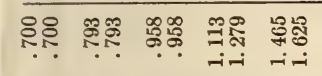

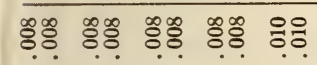

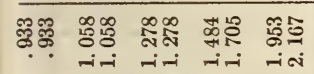

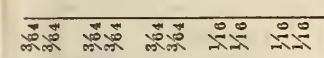

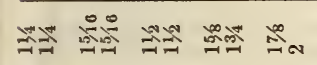

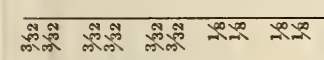

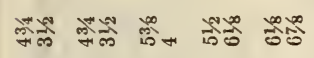

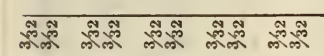

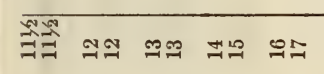
용 8\% $8 \%$ :

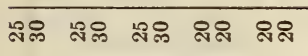

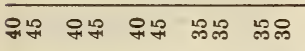

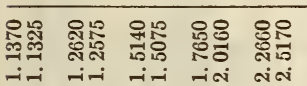

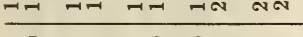

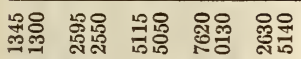

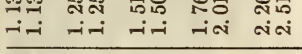

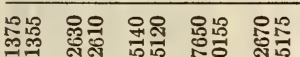

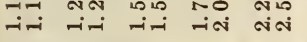

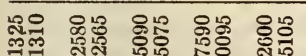
-i -i -i-i nioi vioi

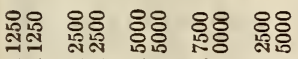
-i-

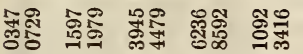

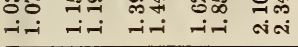

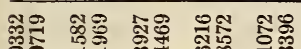

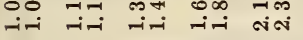

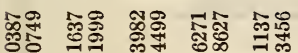

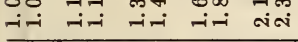

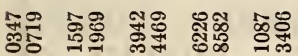

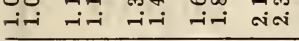

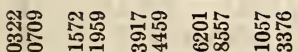
隹 $\lim _{x \rightarrow \infty}$ 
TABLE 103.-Dimensions of pulley taps, cut threads, American national coarse and fine thread series

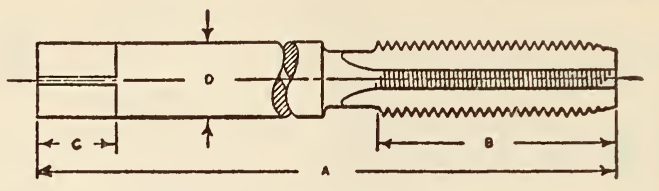

Figure 651.-Pulley taps

\begin{tabular}{|c|c|c|c|c|c|c|c|c|c|c|c|c|c|c|c|c|c|c|}
\hline \multirow{3}{*}{$\begin{array}{l}\text { Sizes, and } \\
\text { threads per } \\
\text { inch }\end{array}$} & \multicolumn{8}{|c|}{ Thread dimensions } & \multicolumn{10}{|c|}{ General dimensions } \\
\hline & \multicolumn{3}{|c|}{ Pitch diameter } & \multicolumn{3}{|c|}{ Major diameter } & \multirow{2}{*}{ 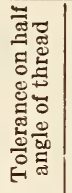 } & \multirow{2}{*}{ 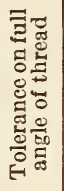 } & \multicolumn{2}{|c|}{ Over-all length } & \multicolumn{2}{|c|}{$\begin{array}{l}\text { Length of } \\
\text { thread }\end{array}$} & \multicolumn{2}{|c|}{$\begin{array}{l}\text { Length of } \\
\text { square }\end{array}$} & \multicolumn{2}{|c|}{$\begin{array}{l}\text { Diameter } \\
\text { shank }\end{array}$} & \multicolumn{2}{|c|}{$\begin{array}{l}\text { Size of } \\
\text { square }\end{array}$} \\
\hline & Basic & $\begin{array}{l}\text { Mini- } \\
\text { mum }\end{array}$ & $\begin{array}{l}\text { Maxi- } \\
\text { mum }\end{array}$ & Basic & $\begin{array}{l}\text { Mini- } 1 \\
\text { mum }\end{array}$ & $\begin{array}{l}\text { Maxi- } \\
\text { mum }\end{array}$ & & & Basic $A$ & $\begin{array}{l}\text { Toler- } \\
\text { ance }\end{array}$ & $\left|\begin{array}{c}\text { Basic } \\
B\end{array}\right|$ & $\begin{array}{l}\text { Toler- } \\
\text { ance }\end{array}$ & $\stackrel{\text { Basic }}{C}$ & $\begin{array}{l}\text { Toler- } \\
\text { ance }\end{array}$ & $\underset{D}{\operatorname{Maxi}-}$ & $\begin{array}{l}\text { Toler- } \\
\text { ance }\end{array}$ & $\underset{E}{\operatorname{maxi}-}$ & $\begin{array}{l}\text { Toler- } \\
\text { ance }\end{array}$ \\
\hline 1 & 2 & 3 & 4 & $\mathbf{5}$ & 6 & 7 & 8 & 9 & 10 & 11 & 12 & 13 & 14 & 15 & 16 & 17 & 18 & 19 \\
\hline $\begin{array}{l}1 / 4-20 \\
5 / 16-18 \ldots \\
3 / 8-16 \\
7 / 16-14\end{array}$ & $\begin{array}{r}\text { Inch } \\
0.2175 \\
.2764 \\
.3344 \\
.3911\end{array}$ & \begin{tabular}{r|} 
Inch \\
0.2180 \\
.2769 \\
.3349 \\
.3916
\end{tabular} & $\begin{array}{l}\text { Inch } \\
.2200 \\
.2789 \\
.3369 \\
.3941\end{array}$ & $\begin{array}{l}\text { Inch } \\
0.2500 \\
.3125 \\
.3750 \\
.4375\end{array}$ & \begin{tabular}{|l|} 
Inch \\
0.2525 \\
.3155 \\
.3785 \\
.4410
\end{tabular} & $\begin{array}{l}\text { Inch } \\
0.2550 \\
.3180 \\
.3810 \\
.4440\end{array}$ & $\begin{array}{c}\text { Min- } \\
\text { utes } \\
\pm \\
\quad 45 \\
45 \\
45 \\
45\end{array}$ & \begin{tabular}{|c|} 
Min- \\
utes \\
\pm \\
68 \\
68 \\
68 \\
68
\end{tabular} & $\begin{array}{l}\text { Inches } \\
6,8 \\
6,8 \\
6,8,10,12,14 \\
6,8,10,12,14\end{array}$ & \begin{tabular}{|c|} 
Inch \\
\pm \\
$1 / 16$ \\
$11 / 6$ \\
$1 / 16$ \\
$1 / 16$
\end{tabular} & $\begin{array}{l}\text { Inches } \\
1 \\
11 / 8 \\
11 / 4 \\
17 / 16\end{array}$ & $\mid \begin{array}{c}\text { Inch } \\
\pm \\
1 / 16 \\
1116 \\
1 / 16 \\
1 / 16\end{array}$ & $\begin{array}{c}\text { Inch } \\
5 / 16 \\
3 / 8 \\
7 / 16 \\
1 / 2\end{array}$ & $\begin{array}{l}\text { Inch } \\
\pm \\
13 / 32 \\
132 \\
1 / 32 \\
1 / 32\end{array}$ & $\begin{array}{l}\text { Inch } \\
0.255 \\
.318 \\
.381 \\
.444\end{array}$ & $\begin{array}{l}\text { Inch } \\
0.005 \\
.005 \\
.005 \\
.005\end{array}$ & $\begin{array}{l}\text { Inch } \\
0.191 \\
.238 \\
.286 \\
.333\end{array}$ & $\begin{array}{l}\text { Inch } \\
0.004 \\
.004 \\
.004 \\
.004\end{array}$ \\
\hline $\begin{array}{l}1 / 2-13 \\
9 / 16-12 \\
5 / 8-11 \\
3 / 4-10\end{array}$ & $\begin{array}{l}.4500 \\
.5084 \\
.5660 \\
.6850 \\
\end{array}$ & $\begin{array}{l}.4505 \\
.5089 \\
.5665 \\
.6855 \\
\end{array}$ & $\begin{array}{l}.4530 \\
.5114 \\
.5690 \\
.6885 \\
\end{array}$ & $\begin{array}{l}.5000 \\
.5625 \\
.6250 \\
.7500 \\
\end{array}$ & $\begin{array}{l}.5040 \\
.5670 \\
.6300 \\
.7550 \\
\end{array}$ & $\begin{array}{l}.5070 \\
.5700 \\
.6330 \\
.7590 \\
\end{array}$ & $\begin{array}{l}45 \\
45 \\
45 \\
45 \\
\end{array}$ & $\begin{array}{l}68 \\
68 \\
68 \\
68 \\
\end{array}$ & $\begin{array}{l}6,8,10,12,14 \\
6,8,10,12,14 \\
6,8,10,12,14 \\
10,12,14 \ldots\end{array}$ & $\begin{array}{l}1 / 16 \\
1 / 16 \\
1 / 16 \\
1 / 16 \\
\end{array}$ & $\begin{array}{l}121 / 32 \\
121 / 32 \\
113 / 16 \\
2\end{array}$ & $\begin{array}{l}1 / 16 \\
1 / 16 \\
1 / 16 \\
1 / 16\end{array}$ & $\begin{array}{l}9 / 16 \\
5 / 8 \\
11 / 16 \\
3 / 4 \\
\end{array}$ & $\begin{array}{l}1 / 32 \\
1 / 32 \\
1 / 32 \\
1 / 32 \\
\end{array}$ & $\begin{array}{l}.507 \\
.570 \\
.633 \\
.759 \\
\end{array}$ & $\begin{array}{l}.005 \\
.005 \\
.005 \\
.005 \\
\end{array}$ & $\begin{array}{l}.380 \\
.427 \\
.475 \\
.569 \\
\end{array}$ & $\begin{array}{l}.004 \\
.006 \\
.006 \\
.006 \\
\end{array}$ \\
\hline
\end{tabular}

\section{TOLERANCES FOR TAPS FOR SPECIAL THREADS}

Tables of tolerances for two classes of taps for threads of special diameter and pitch are given, namely, cut-thread taps and ground-thread taps. (See Tables 104 and 105.)

The selection of the tap will depend upon the pitch-diameter tolerance on the tapped hole. A tap should be selected whose tolerance doas not exceed 75 per cent of the pitch-diameter tolerance on the tapped hole.

TABLE 104.-Tolerances for cut-thread taps, special diameters and pitches

\begin{tabular}{|c|c|c|c|c|c|c|c|c|c|}
\hline \multirow{2}{*}{ Sizes } & & \multicolumn{4}{|c|}{$\begin{array}{l}\text { Coarser pitches than } \\
\text { American national } \\
\text { fine-thread series }\end{array}$} & \multicolumn{4}{|c|}{$\begin{array}{l}\text { Finer pitches than } \\
\text { American national } \\
\text { fine-thread series }\end{array}$} \\
\hline & & $\begin{array}{l}\text { Ma- } \\
\text { jor } \\
\text { diam- } \\
\text { eter }\end{array}$ & Pitch & $\mathrm{h}$ diam & aeter & $\begin{array}{c}\text { Ma- } \\
\text { jor } \\
\text { diam- } \\
\text { eter }\end{array}$ & Pitch & $\mathrm{h}$ diam & neter \\
\hline ! & ! & 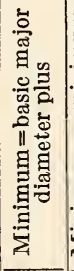 & 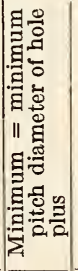 & 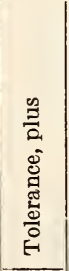 & 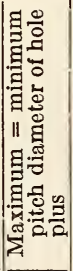 & 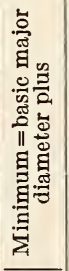 & 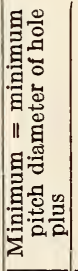 & 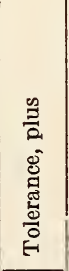 & 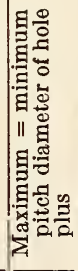 \\
\hline \multicolumn{2}{|l|}{1} & 2 & 3 & 4 & 5 & 6 & 7 & 8 & 9 \\
\hline $\begin{array}{r}1 / 4 \\
7 / 16 \\
3 / 4 \\
11 / 8\end{array}$ & $\begin{array}{r}3 / 8 \\
5 / 8 \\
11 \\
11 / 2\end{array}$ & $\begin{array}{l}\text { Inch } \\
0.0030 \\
.0040 \\
.0055 \\
.0070\end{array}$ & $\begin{array}{c}\text { Inch } \\
0.0005 \\
.0005 \\
.0010 \\
.0010\end{array}$ & $\begin{array}{r}\text { Inch } \\
0.0020 \\
.0025 \\
.0030 \\
.0035\end{array}$ & $\begin{array}{c}\text { Inch } \\
0.0025 \\
.0030 \\
.0040 \\
.0045\end{array}$ & $\begin{array}{c}\text { Inch } \\
0.0020 \\
.0025 \\
.0025 \\
.0035\end{array}$ & $\begin{array}{l}\text { Inch } \\
0.0005 \\
.0005 \\
.0005 \\
.0010\end{array}$ & $\begin{array}{l}\text { Inch } \\
0.0015 \\
.0020 \\
.0025 \\
.0030\end{array}$ & $\begin{array}{c}\text { Inch } \\
0.0020 \\
.0025 \\
.0030 \\
.0040\end{array}$ \\
\hline $\begin{array}{l}15 / 8 \\
21 / 8 \\
25 / 8 \\
31 / 8\end{array}$ & $\begin{array}{l}2 \\
21 / 2 \\
3 \\
4\end{array}$ & $\begin{array}{l}.0085 \\
.010 \\
.0100 \\
.0100\end{array}$ & $\begin{array}{l}.0015 \\
.0015 \\
.0020 \\
.0020\end{array}$ & $\begin{array}{l}.0040 \\
.0045 \\
.0050 \\
.0055\end{array}$ & $\begin{array}{l}.0055 \\
.0060 \\
.0070 \\
.0075\end{array}$ & $\begin{array}{l}.0035 \\
.0045 \\
.0045 \\
.0055\end{array}$ & $\begin{array}{l}.0010 \\
.0010 \\
.0010 \\
.0010\end{array}$ & $\begin{array}{l}.0030 \\
.0035 \\
.0035 \\
.0045\end{array}$ & $\begin{array}{l}.0040 \\
.0045 \\
.0045 \\
.0055\end{array}$ \\
\hline
\end{tabular}

Note.-Maximum lead error $= \pm 0.003$ inch in 1 inch of thread. Tolerance on half angle of thread $= \pm 40$ minutes.
TABLE 105.-Tolerances for ground-thread taps, special diameters and pitches

\begin{tabular}{|c|c|c|c|c|c|c|c|c|c|}
\hline & & \multicolumn{4}{|c|}{$\begin{array}{l}\text { Coarser pitches than } \\
\text { American national } \\
\text { fine-thread series }\end{array}$} & \multicolumn{4}{|c|}{$\begin{array}{l}\text { Finer pitches than } \\
\text { American national } \\
\text { fine-thread series }\end{array}$} \\
\hline \multicolumn{2}{|c|}{ Sizes } & $\begin{array}{l}\text { Major } \\
\text { diam- } \\
\text { eter }\end{array}$ & \multicolumn{3}{|c|}{ Pitch diameter } & Major & \multicolumn{3}{|c|}{ Pitch diameter } \\
\hline g & b & 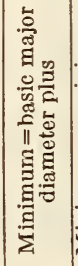 & 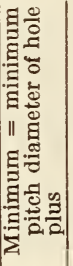 & 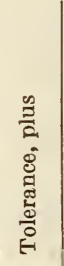 & 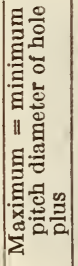 & 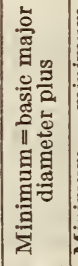 & 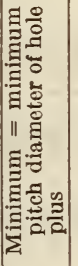 & 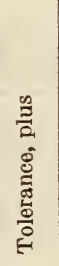 & 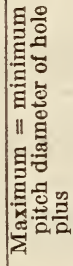 \\
\hline \multicolumn{2}{|c|}{1} & 2 & 3 & 4 & 5 & 6 & 7 & 8 & 9 \\
\hline & $\begin{array}{l}1 / 2 \\
1 / 8 \\
11 / 2\end{array}$ & $\begin{array}{c}\text { Inch } \\
0.0025 \\
.0030 \\
.0035 \\
.0040 \\
.0050\end{array}$ & $\begin{array}{c}\text { Inch } \\
0.0006 \\
.0007 \\
.0008 \\
.0001 \\
.0012\end{array}$ & $\begin{array}{c}\text { Inch } \\
0.0005 \\
.0005 \\
.0010 \\
.0010 \\
.0015\end{array}$ & $\begin{array}{c}\text { Inch } \\
0.0011 \\
.0012 \\
.0018 \\
.0020 \\
.0027\end{array}$ & $\begin{array}{c}\text { Inch } \\
0.0015 \\
.0020 \\
.0020 \\
.0025 \\
.0025\end{array}$ & $\begin{array}{c}\text { Inch } \\
0.0005 \\
.0005 \\
.0006 \\
.0007 \\
.0008\end{array}$ & $\begin{array}{l}\text { Inch } \\
0.0005 \\
.0005 \\
.0008 \\
.0008 \\
.0010\end{array}$ & \begin{tabular}{l|l} 
Inch \\
5 \\
5.0010 \\
5 & .0010 \\
8.0014 \\
0.0015 \\
0.0018
\end{tabular} \\
\hline & $\begin{array}{l}2 \\
21 / 2 \\
3 \\
4\end{array}$ & $\begin{array}{l}.0065 \\
.0080 \\
.0080 \\
.0080\end{array}$ & $\begin{array}{l}.0015 \\
.0020 \\
.0020 \\
.0020\end{array}$ & $\begin{array}{l}.0020 \\
.0020 \\
.0020 \\
.0025\end{array}$ & $\begin{array}{l}.0035 \\
.0040 \\
.0040 \\
.0045\end{array}$ & $\begin{array}{l}.0030 \\
.0030 \\
.0035 \\
.0035\end{array}$ & $\begin{array}{l}.0010 \\
.0010 \\
.0015 \\
.0015\end{array}$ & $\begin{array}{l}.0015 \\
.0015 \\
.0015 \\
.0020\end{array}$ & \begin{tabular}{l|l}
5 & .0025 \\
5 & .0025 \\
5 & .0030 \\
0 & .0035 \\
\end{tabular} \\
\hline
\end{tabular}

Note.-Maximum lead error $= \pm 0.0005$ inch in 1 inch of thread. Tolerance of half angle of thread $= \pm 20$ minutes.

\subsection{HAMMERS AND SLEDGES.}

\subsection{GENERAL ITEMS.}

UNITED STATES GOVERNMENT, DEPARTMENT OF COMMERCE, BUREAU OF STANDARDS, SIMPLIFIED PRACTICE RECOMMENDATION FOR FORGED TOOLS, R17-31, JULY 1, 1931

\section{HAMMERS AND SLEDGES}

(Standard sizes and varieties of sledges, hammers, and mauls will be found in Table 6 of simpli- 
fied practice recommendation $\mathrm{R} 17-31$ for forged tools. Standard sizes and varieties of spike mauls and track sledges will be found in Table 7 of R17-31. See 616.60, p. 960.)

\subsection{HAMMERS.}

(For standard sizes and varieties see United Government, Department of Commerce, Bureau of Standards; simplified practice recommendation R17-31 for forged tools. See 616.60 , p. 960.)

\subsection{SLEDGE HAMMERS.}

\section{AMERICAN RAILWAY ENGINEERING AS- SOCIATION, PLANS FOR TRACK TOOLS, 1930}

\section{TRACK TOOLS}
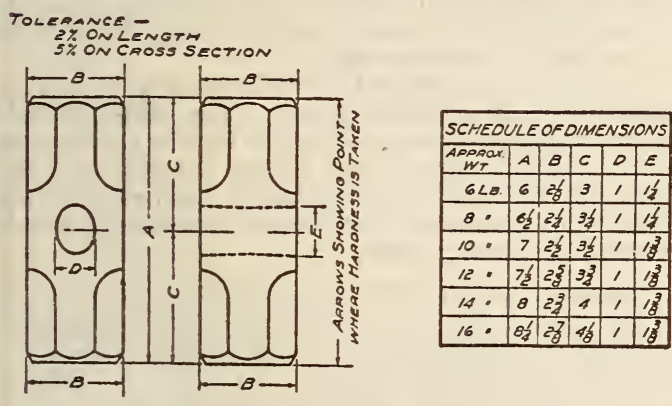

BRINELL

ROCMWELL
SCLEAOSCODE $=425-500$
$49-55$
$64-75$

FIgURE 652.-Double-faced sledge

Eye tapered to center
$4^{\circ}$ on long diameter
$3^{\circ}$ on short diameter

615.3 PINCERS, PLIERS, PLIER CUTTERS, AND SHEARS.

\subsection{PINCERS.}

(No nationally recognized specifications available.)

\subsection{PLIERS AND PLIER CUTTERS.}

(No nationally recognized specifications available.)

\author{
615.33 SHEARS.
}

UNITED STATES GOVERNMENT, FEDERAL SPECIFICATIONS BOARD; FEDERAL SPECIFICATION FOR SHEARS, TINNERS', HAND (SNIPS), NO. 451, NOVEMBER 22, 1926. (CURRENT DESIGNATION: GGGS-291.)

\section{TINNERS' HAND SHEARS}

\section{GENERAL SPECIFICATIONS}

There are no general specifications applicable to this specification.

\section{TYPES}

The shears shall be of the following types: A, straight cut; B, circular cut.

\section{MATERIAL AND WORKMANSHIP}

The shears shall be of high-grade material and workmanship. They shall be smoothly finished and free from all defects or blemishes affecting their appearance or that may affect their serviceability.

\section{GENERAI REQUIREMENTS}

Shears shall have the maker's name or known trade-mark stamped or permanently marked thereon and shall be furnished left-hand cut unless otherwise specified.

\section{DETAIL REQUIREMENTS}

1. Tinner's hand shears, straight cut, type A.These shears shall be made entirely of steel, with a durable cutting edge. The blades may be bimetallic with high-grade tool-steel cutting edge and mildsteel body. If bimetallic form of blade is furnished, the tool-steel cutting edge shall be such as to permit any number of grindings which may be required during the life of the shears. The cutting edges of all shears shall be suitably beveled and properly hardened, tempered, and ground. The blades shall be firmly held together with a finished steel bolt and nut, and adjustable in such manner as to insure easy and smooth cutting when the shears are in use. The bolt shall be flattened on the nut end or otherwise designed to prevent turning or working loose when the shears are operated. The bearing between the blades shall be accurately finished and of ample width to prevent the blades from springing open when cutting the maximum thickness of sheet steel or iron for which the shears are designed.

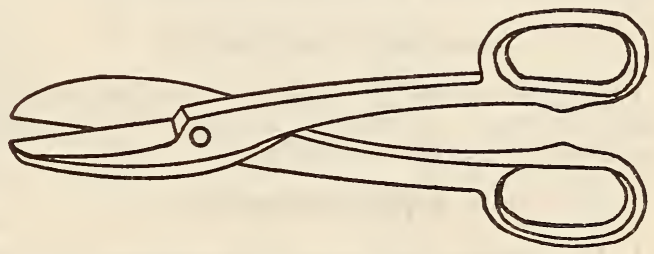

FIgURE 653.-Tinners' hand shears

2. The handles shall be bow shaped and shall provide a comfortable grip for the hand. The handles shall have a japan or enamel black finish.

3. The shears shall be similar to Figure 653 and of the weight and dimensions stated below:

\section{Minimum weight_._... 1 pound 14 ounces. \\ Length of cut..... \\ Minimum length over all..........}

4. Tinners' hand shears, circular cut, type B.These shears shall be similar, in all respects, to straight-cut shears, type A, except that the cutting edges shall be curved to approximately a $3 \frac{3}{8}$-inch radius.

\section{METHOD OF INSPECTION AND TESTS}

Representative samples, at least three, shall be taken from each lot submitted for inspection and tested as follows:

Each shear shall be used in cutting a dozen strips, about one-half inch wide by 12 inches long, from low-carbon sheet steel of a minimum thickness of 0.0201 inch. In this test the shears shall cut along the entire length of the blade. Two of the abovementioned strips shall then be cut into one-half inch lengths or squares by placing them (one at a time) at the ends of the blades about 3 inches from the bolt. All cuts shall be made without unnecessary pressure being applied to the shear handles and without the blades springing apart or allowing the sheet steel to bend or wedge between the cutting surfaces of the shears. As a further evidence that the cutting edges are properly hardened and tempered, each pair of shears shall be required to sever a com- 
mercial (common type) sixpenny wire nail $(0.113$ inch in diameter) without marring the cutting edges.

\section{PACKING AND MARKING}

Packing and marking shall be in accordance with best commercial practice unless otherwise spehified.

\subsection{MISCELLANEOUS CUTTING TOOLS.}

UNITED STATES GOVERNMENT, FEDERAL SPECIFICATIONS BOARD, FEDERAL SPECIFICATION FOR CUTTERS, PIPE, NO. 43\%, SEPTEMBER 25, 1926. (CURRENT DESIGNATION : GGG-C-771)

\section{PIPE CUTTERS}

\section{GENERAL SPECIFICATIONS}

There are no general specifications applicable to this specification.

\section{TYPE}

Pipe cutters shall be of the 3-wheel cutter type.

\section{MATERIAL AND WORKMANSHIP}

Pipe cutters shall be made of good quality material and shall be of good workmanship and in accordance with best commerical practice.

\section{GENERAL REQUIREMENTS}

Each pipe cutter shall have the manufacturer's name or known trade-mark stamped or otherwise permanently marked on the frame.

\section{DETAIL REQUIREMENTS}

1. Pipe cutters shall consist of a frame, a movable jaw, a threaded feed rod with handle, and three

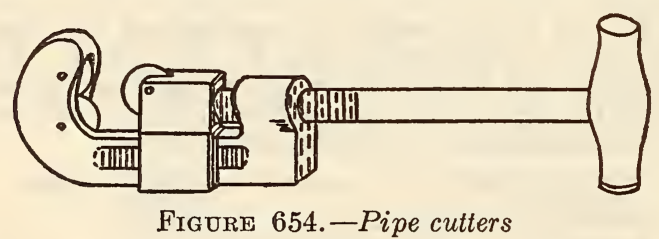

cutter wheels. All parts of pipe cutters shall be fastened together so that no part may become detached while in use under ordinary service conditions.

2. The frame shall be made of malleable iron or steel and shall be finished where the movable jaw fits over the frame so that alignment of the cutters will be insured. The frame shall carry either one or two of the three cutter wheels, and shall be either tapped for insertion of the threaded feed rod or there shall be a hardened nut fitted to engage with the threads of the feed rod, and held firmly in the frame so that it is immovable when adjusment is made.

3. The cutter wheels shall be made of tool steel, hardened and tempered; the hubs shall have ample bearing surface on the pins, or by other suitable means provision shall be made to hold the cutter wheels in alignment.

4. The movable jaw shall consist of one piece of steel or malleable iron and shall be equipped with one or two cutter wheels. The jaw shall be finished where sliding contact is made with the frame, and the jaw and frame shall not bind when adjustment is made.

5 . The threaded feed rod shall be made of steel and shall have suitable threads to engage with the tapped hole in the frame or nut as required. This rod shall be fitted to the movable jaw so as to operate without possibility of becoming detached. A handle of malleable iron or steel shall be firmly fixed on the cutter end of the feed rod.

6. Pipe cutters shall be similar to Figure 654 and conform to the table below. The illustration is incorporated in this specification for the convenience of the supply officers and others interested. It is not intended that the illustration shall restrict purchase of pipe cutters that are otherwise in accordance with specifications.

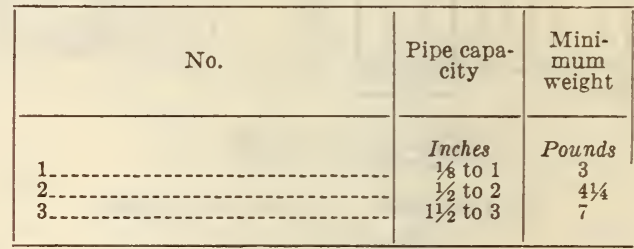

VI. TESTS

A sufficient number of pipe cutters of each size shall be selected and each shall operate satisfactorily when cutting pipe of the respective sizes indicated above. The adjustment of each pipe cutter shall operate smoothly in both directions between the maximum and minimum limits.

\section{PACKING AND MARKING}

1. Pipe cutters shall be packed in accordance with the best commercial practice unless otherwise specified.

\subsection{VISES, WRENCHES, AND VISE CLAMPS.}

\subsection{VISES.}

(No nationally recognized specifications available.) 
615.42 WRENCHES.

AMERICAN RAILWAY ENGINEERING ASSOCIATION, PLANS FOR TRACK TOOIS, 1930 TRACK TOOLS

TOLERANCE-

E\% ON LENGTH

$5 \%$ ON GROSS SECTION

ALL WRENCH JAWS SMALL EE MILLEO

TOEXACT DIMENSIONS SHOWN

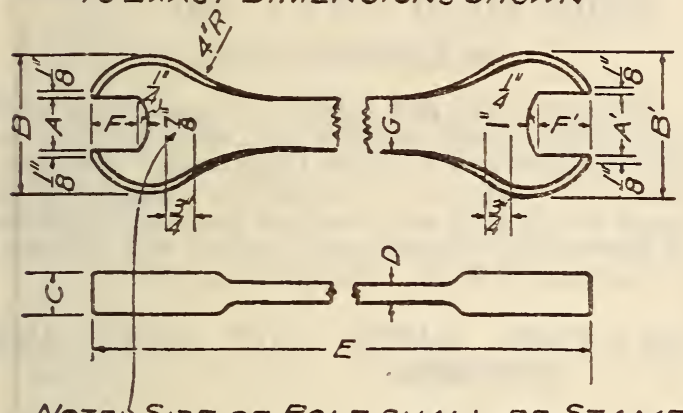

NOTE:- SIZE OF EOLT SHALL EE STAMPED PLAINLY ON UNE SIDE OF HEAD IN

POSITION AS SHOWN:

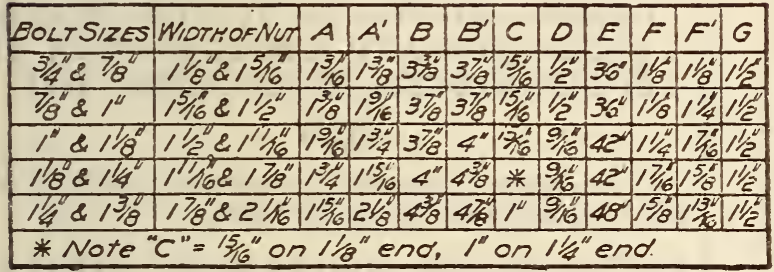

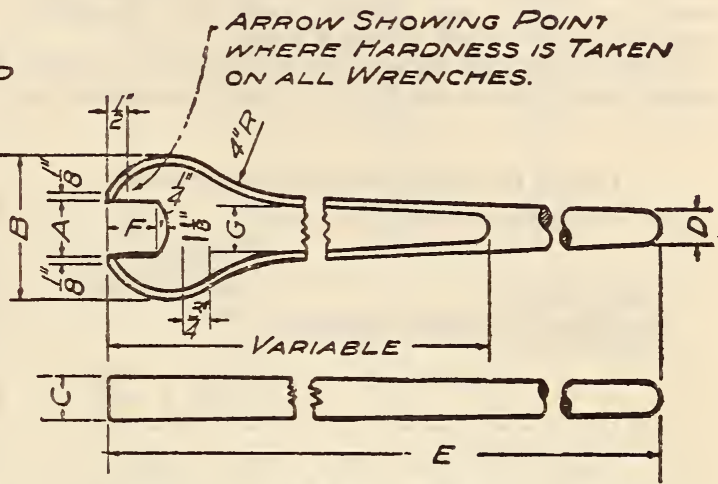

Note:- SINGLE END WRENCM SHALL EE FURNISHEO WITH END TAPERED FOR LAST 6"TOZ"DIA AT END NHEN SO SPECIFIEO

\begin{tabular}{|c|c|c|c|c|c|c|c|c|}
\hline $\begin{array}{l}8 O L \\
\text { SIZE }\end{array}$ & $\begin{array}{l}\text { Wiorh } \\
\text { oENuT }\end{array}$ & $A$ & $\theta$ & C & $D$ & $E$ & $F$ & \\
\hline $3 / 4$ & $1 / 80$ & $13 / 6$ & $3 \%$ & $13 / 10^{2}$ & $34^{\circ}$ & $30^{\prime \prime}$ & $1 / 8$ & \\
\hline$\frac{1}{0}$ & $15 / 6$ & $13 / 8$ & $3 \%$ & 15,16 & $3 / 4$ & $30^{\prime \prime}-36^{\prime \prime}$ & $1 / 8$ & $1 \%$ \\
\hline 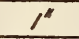 & $1 / 2^{21}$ & $19 / 10$ & $3 \%$ & $151 / 6$ & 788 & $366^{\prime \prime}$ & $11 / 4$ & \\
\hline $1 / 8$ & $11 / 36$ & $13 / 4$ & $4^{\prime \prime}$ & 15,11 & 78 & $36^{2}-42^{2}$ & $17 / 6$ & $1 / 2$ \\
\hline $11 / 4$ & $17 / 8^{11}$ & $15 / 16$ & $4 \%$ & $1^{\prime \prime}$ & $\geqslant 8$ & $42^{\prime}$ & $15 \%$ & $1 / 2$ \\
\hline $13 / 8$ & 2116 & $2 \%$ & 478 & $\gamma^{\prime \prime}$ & $\geqslant 8$ & $42 \div 48$ & $1^{13} y_{1 / 2}^{\prime \prime}$ & \\
\hline $11 / 2$ & $21 / 4$ & $25 / 6$ & $5 \%$ & $1^{\prime \prime}$ & $1 \% 8$ & $48^{\prime \prime}$ & $17 / 8$ & \\
\hline
\end{tabular}

$$
\begin{aligned}
& \text { BRINELL } \\
& \text { POCMWELL }-375-450 \\
& \text { SCLEPOSCOPE - } 45-51 \\
& \hline 5-66
\end{aligned}
$$

GenERAL NOTE. ABOVE JAW OPENINGS DESIGNED TO FIT NEN A.SA. NUTS - JON 1930.

FiguRE 655.-Track wrenches

AMERICAN SOCIETY OF MECHANICAL ENGINEERS, WRENCH-HEAD BOLTS AND NUTS, AND WRENCH OPENINGS, B18b, 1927

[Approved as American tentative standard by the American Standards Association, February, 1927]

\section{WRENCH OPENINGS}

(Identical with those of the National Screw Thread Commission, given below.)

SOCIETY OF AUTOMOTIVE ENGINEERS,

CAP SCREWS, BOLTS AND NUTS, AND

WRENCH OPENINGS, S. A. E. STANDARDS, JANUARY, 1930

[Approved as American tentative standard by the American Standards Association, February, 1927]

\section{WRENCH OPENINGS}

(Identical with those of the National Screw Thread Commission, given below.)
UNITED STATES GOVERNMENT, DEPARTMENT OF COMMERCE, BUREAU OF STANDARDS, SIMPLIFIED PRACTICE RECOMMENDATION FOR FORGED TOOLS R17-31, JULY 1, 1931

\section{TRACK WRENCHES}

(See 616.60 , p. 960.)

UNITED STATES GOVERNMENT, NATIONAI SCREW THREAD COMMISSION, AMERICAN NATIONAL SCREW, BOLT, AND NUT PROPORTIONS. BUREAU OF STANDARDS MISCELLANEOUS PUBLICATION NO. 89, JUNE 22, 1928

(See also 608.0 , p. $551 ; 608.2$, p. $583 ; 608.31$, p. $604 ; 608.32$, p. 609 .) 


\section{WRENCH HEAD BOLTS AND NUTS AND WRENCH OPENINGS}

\section{WRENCH OPENINGS}

Wrench openings for open-end wrenches are given in Table 76. The sizes given in the table for the maximum and minimum columns are sizes of "go" and "not go" gage blocks used for inspecting wrenches and are not product sizes.

Wrenches should be marked with the basic width across flats (maximum width of nut), as shown in column 1.

TABLE 76.-Open-end wrench openings

\begin{tabular}{|c|c|c|c|c|}
\hline \multirow{2}{*}{$\begin{array}{l}\text { Basic width } \\
\text { across flats, bolt- } \\
\text { heads and nuts }\end{array}$} & \multirow[t]{2}{*}{ Clearance } & \multirow[t]{2}{*}{ Tolerance } & \multicolumn{2}{|c|}{$\begin{array}{l}\text { Dimensions of } \\
\text { measuring blocks } \\
\text { for wrench open- } \\
\text { ings }\end{array}$} \\
\hline & & & $\begin{array}{l}\text { Maxi- } \\
\text { mum }\end{array}$ & $\begin{array}{l}\text { Mini- } \\
\text { mum }\end{array}$ \\
\hline 1 & 2 & 3 & 4 & 5 \\
\hline $\begin{array}{c}\text { Inches } \\
5 / 32-0.1562 \\
3 / 16-0.1875 \\
1 / 4-0.2500 \\
5 / 16-0.3125 \\
11 / 32-0.3437\end{array}$ & $\begin{array}{c}\text { Inch } \\
0.0014 \\
.0016 \\
.0018 \\
.0019 \\
.0022\end{array}$ & $\begin{array}{l}\text { Inch } \\
0.005 \\
.005 \\
.005 \\
.007 \\
.007\end{array}$ & $\begin{array}{r}\text { Inches } \\
0.163 \\
.194 \\
.257 \\
.321 \\
.353\end{array}$ & $\begin{array}{r}\text { Inches } \\
0.158 \\
.189 \\
.252 \\
.314 \\
.346\end{array}$ \\
\hline $\begin{array}{r}3 / 3-0.3750 \\
7 / 16-0.4375 \\
1 / 2-0.5000 \\
9 / 16-0.5625 \\
5 / 8-0.6250\end{array}$ & $\begin{array}{l}.0024 \\
.0025 \\
.0030 \\
.0035 \\
.0040\end{array}$ & $\begin{array}{l}.007 \\
.007 \\
.007 \\
.007 \\
.007\end{array}$ & $\begin{array}{l}.384 \\
.447 \\
.510 \\
.573 \\
.636\end{array}$ & $\begin{array}{l}.377 \\
.440 \\
.503 \\
.566 \\
.629\end{array}$ \\
\hline $\begin{array}{r}3 / 4-0.7500 \\
13 / 16-0.8125 \\
7 / 8-0.8750 \\
15 / 16-0.9375 \\
1-1.0000\end{array}$ & $\begin{array}{l}.0050 \\
.0050 \\
.0050 \\
.0055 \\
.0060\end{array}$ & $\begin{array}{l}.008 \\
.008 \\
.008 \\
.009 \\
.009\end{array}$ & $\begin{array}{r}.763 \\
.826 \\
.888 \\
.952 \\
1.015\end{array}$ & $\begin{array}{r}.755 \\
.818 \\
.880 \\
.943 \\
1.006\end{array}$ \\
\hline $\begin{array}{r}11 / 8-1.1250 \\
11 / 4-1.2500 \\
15 / 16-1.3125 \\
11 / 2-1.5000 \\
11116-1.6875\end{array}$ & $\begin{array}{l}.0070 \\
.0070 \\
.0075 \\
.0090 \\
.0095\end{array}$ & $\begin{array}{l}.010 \\
.010 \\
.011 \\
.012 \\
.013\end{array}$ & $\begin{array}{l}1.142 \\
1.267 \\
1.331 \\
1.521 \\
1.710\end{array}$ & $\begin{array}{l}1.132 \\
1.257 \\
1.320 \\
1.509 \\
1.697\end{array}$ \\
\hline $\begin{array}{r}17 / 8-1.8750 \\
21 / 4-2.2500 \\
258-2.6250 \\
3-3.0000\end{array}$ & $\begin{array}{l}.0100 \\
.0120 \\
.0140 \\
.0160\end{array}$ & $\begin{array}{l}.013 \\
.015 \\
.017 \\
.019\end{array}$ & $\begin{array}{l}\text { 1. } 898 \\
2.277 \\
2.656 \\
\text { 3. } 035\end{array}$ & $\begin{array}{l}1.885 \\
2.262 \\
2.639 \\
3.016\end{array}$ \\
\hline $\begin{array}{l}33 / 8-3.3750 \\
33 / 4-3.7500 \\
41 / 8-4.1250 \\
41 / 2-4.5000\end{array}$ & $\begin{array}{l}.0180 \\
.0200 \\
.0220 \\
.0230\end{array}$ & $\begin{array}{l}.021 \\
.023 \\
.024 \\
.026\end{array}$ & $\begin{array}{l}\text { 3. } 414 \\
\text { 3. } 793 \\
4,171 \\
\text { 4. } 549\end{array}$ & $\begin{array}{l}3.393 \\
3.770 \\
4,147 \\
4.523\end{array}$ \\
\hline
\end{tabular}

\subsection{VISE CLAMPS.} able.)

(No nationally recognized specifications avail-

615.5 ANVILS, BELLOWS, HARDIES, AND FORGES.

\subsection{ANVIIS.} able.)

(No nationally recognized specifications avail-

\subsection{BELLOWS.} able.)

(No nationally recognized specifications avail-

\subsection{HARDIES.}

(For standard sizes of hardies see United States Government, Department of Commerce, Bureau of Standards; simplified practice recommendation R17-31 for forged tools. See 616.60, p. 960.)

\subsection{FORGES.}

(No nationally recognized specifications available.)

\subsection{MISCELLANEOUS BLACKSMITH TOOLS.}

UNITED STATES GOVERNMENT, DEPART-

MENT OF COMMERCE, BUREAU OF

STABDARDS; SIMPLIFIED PRACTICE

RECOMMENDATION FOR FORGED

TOOLS, R17-31, JULY 1, 1931

\section{BLACKSMITH TOOLS}

(Standard sizes and varieties of blacksmith anvil tools will be found in Table 8 of simplified practice recommendation R17-31 for forged tools. Standard sizes and varieties of horseshoers' tongs will be found in Table 9 and standard sizes and varieties of blacksmith hammers and sledges will be found in Table 6 of R17-31. See 616.60, p. 960.)

615.6 FILES, RASPS, HACK SAWS, AND SCRIBERS.

\subsection{FILES.}

AMERICAN RAILWAY ASSOCIATION, PURCHASES AND STORES DIVISION ; STANDARDIZATION AND SIMPLIFICATION OF STORES STOCKS, PROCEEDINGS, 1928

\section{FILES}

(Recommended practice in stocking certain specified types and sizes of standard files.)

\section{UNITED STATES GOVERNMENT, DEPART-}

MENT OF COMMERCE, BUREAU OF STANDARDS, SIMPLIFIED PRACTICE RECOMMENDATION FOR FILES AND RASPS, R6, NOVEMBER 1, 1923 Ne

\section{FILES}

(Simplified practice recommended and accepted by industry establishing a limited list of standard sizes and types.)

Mill:

Bastard, 3, 4, 5, 6, 7, 8, 9, 10, 12, 14, 16, and 18 inch.

Second cut, 4, 5, 6, 7, 8, 9, 10, 12, 14, and 16 inch.

Smooth, 6, 8, 10, 12, 14, and 16 inch.

Mill (1 R. E.):

Bastard, 6, 7, 8, 9, 10, 12, and 14 inch.

Second cut, 6, 7, 8, 9, 10, and 12 inch.

Mill (2 R. E.):

Bastard, 6, 7, 8, 9, 10, and 12 inch.

Second cut, $6,8,10$, and 12 inch.

Mill (blunt):

Bastard _._- $\} 6,7,8$, and 10 inch.

Tapers:

Single cut, $3,3 \frac{1}{2}, 4,4 \frac{1}{2}, 5,5 \frac{1}{2}, 6,7,8,10$, and 12 inch.

Double cut, $4,41 \frac{1}{2}, 5,6,7$, and 8 inch.

Slim, 3, 31/2, 4, 41/2, 5, 51/2, 6, 7, 8, 10, and 12 inch.

Extra slim, 4, 41/2, 5, 51/2, 6, 7, and 8 inch.

Double extra slim, 4, 41/2, 5, 51/2, 6, 7, and 8 inch.

Handsaw:

Blunt, 4, 41/2, 5, 6, 7, and 8 inch.

Blunt slim, 4, 5, 6, and 8 inch.

Bandsaw (blunt and taper):

Regular, 4, 41/2, 5, 6, 7, 8, and 10 inch.

Slim, 6 and 8 inch. 
Pit saw, 4, 41/2, 5, 51/2, 6, 7, and 8 inch.

Double ender, $7,8,9,10$, and 12 inch.

Cant saw, 5, 6, 7, 8, 10, and 12 inch.

Crosscut, 6, 8, 9, 10, and 12 inch.

Planer knife, 8 and 10 inch.

Flat:

Bastard, 4, 5, 6, 7, 8, 9, 10, 12, 14, 16, 18, and 20 inch.

Second cut_- $\} 4,5,6,7,8,9,10,12,14,16$, and 18 Smooth_..._ inch.

Hand:

Bastard, 4, 5, 6, 7, 8, 9, 10, 12, 14, 16, 18, and 20 inch.

Second cut_- $\} 4,5,6,7,8,9,10,12,14,16$, and Smooth_...- 18 inch.

Square:

Bastard, 4, 5, 6, 7, 8, 10, 12, 14, 16, 18, and 20 inch.

Second cut.-- $\} 4,5,6,7,8,10,12,14,16$, and 18 Smooth....- $\}^{2}$ inch.

Three square:

Bastard__-_- 4 4, 5, 6, 7, 8, 10,12, 14, 16, and 18

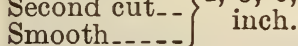

Half round:

Bastard .....-

Second cut_- $\} 4,5,6,7,8,10,12,14,16$, and 18 Smooth_..._ inch.

Round:

Bastard, 4, 5, 6, 7, 8, 9, 10, 12, 14, 16, 18, and 20 inch. Second cut_- $\} 4,5,6,7,8,9,10,12,14,16$, and 18 Smooth...._ inch.

Warding:

Bastard ....-

Second cut-- $\} 4,5,6,8$, and 10 inch.

Smo

Bastard ...-

Second cut_- $\} 4,5,6,8,10$, and 12 inch.

Smo

Bastard, 6, 8, 10, 12, 14, and 16 inch.

Second cut_- $\} 6,8,10$, and 12 inch.

Square blunt bastard, $8,10,12,14,16$, and 18 inch. Round blunt bastard, 8, 10, 12, and 14 inch.

Lead float and wood files:

Flat_-_-_-- $6,8,10,12,14$, and 16 inch.

Hand finishing:

Second cut_- $\} 12$ and 14 inch.

Cabinet files, 6, 8, 10, 12, and 14 inch.

\subsection{HACK SAWS.}

UNITED STATES GOVERNMENT, DEPARTMENT OF COMMERCE, BUREAU OF STANDARDS, SIMPLIFIED PRACTICE RECOMMENDATION FOR HACK-SAW BLADES, R90-29, JULY 1, 1929

\section{HACK-SAW BLADES}

(Simplified practice recommended and accepted by industry establishing a limited list of standard sizes.)

STANDARD TUNGSTEN AND CARBON BLADES

TABLE 1.-Hand hack-saw blades (all hard and flexible)

\begin{tabular}{|c|c|c|c|}
\hline Length & Width & Thickness & $\begin{array}{c}\text { Number of teeth } \\
\text { per inch }\end{array}$ \\
\hline Inches & Inch & Inch & \\
8 & $7 / 16$ & 0.025 & $18,24,32$ \\
10 & $1 / 2$ & .025 & $14,18,24,32$ \\
12 & $1 / 2$ & .025 & $14,18,24,32$ \\
12 & $9 / 16$ & .025 & $14,18,24$ \\
\hline
\end{tabular}

TABLE 2.-Power hack-saw blades (all hard)

\begin{tabular}{|c|c|c|c|}
\hline Length & Width & Thickness & $\begin{array}{c}\text { Number of teeth } \\
\text { per inch }\end{array}$ \\
\hline $\begin{array}{c}\text { Inches } \\
10\end{array}$ & $\begin{array}{c}\text { Inches } \\
3 / 4\end{array}$ & $\begin{array}{c}\text { Inch } \\
0.049\end{array}$ & \\
12 & 55 & .032 & 10 \\
12 & $3 / 4$ & .032 & 14,18 \\
12 & $3 / 4$ & .049 & 14,18 \\
12 & 1 & .049 & 10,14 \\
14 & $3 / 4$ & .049 & 10,14 \\
14 & 1114 & .049 & 10,14 \\
14 & $11 / 4$ & .065 & 10,14 \\
17 & 1 & .049 & 4,10 \\
17 & $11 / 4$ & .065 & 10,14 \\
18 & $11 / 4$ & .065 & $4,6,10$ \\
21 & $11 / 2$ & .065 & 6,10 \\
24 & $11 / 2$ & .065 & 6,10 \\
\hline
\end{tabular}

\section{HIGH-SPEED BLADES}

TABLE 3.-Hand blades

\begin{tabular}{|c|c|c|c|}
\hline Length & Width & Thickness & $\begin{array}{c}\text { Number of teeth } \\
\text { per inch }\end{array}$ \\
\hline $\begin{array}{c}\text { Inches } \\
10\end{array}$ & $\begin{array}{c}\text { Inch } \\
9 / 16\end{array}$ & $\begin{array}{c}\text { Inch } \\
0.025\end{array}$ & $18,24,32$ \\
12 & $9 / 16$ & .025 & $14,18,24,32$ \\
\hline
\end{tabular}

TABLE 4.- Power blades

\begin{tabular}{|c|c|c|c|}
\hline Length & Width & Thickness & $\begin{array}{c}\text { Number of teeth } \\
\text { per inch }\end{array}$ \\
\cline { 1 - 2 } $\begin{array}{c}\text { Inches } \\
12\end{array}$ & $\begin{array}{c}\text { Inches } \\
1\end{array}$ & $\begin{array}{c}\text { Inch } \\
0.049\end{array}$ & \\
12 & 1 & .065 & 14 \\
14 & 1 & .049 & 6,10 \\
14 & 1 & .065 & 14 \\
14 & $11 / 4$ & .065 & 6,10 \\
17 & 1 & .049 & $4,6,10$ \\
17 & 1 & .065 & 14 \\
17 & 114 & .065 & 6,10 \\
18 & 1 & .065 & $4,6,10$ \\
18 & $11 / 4$ & .065 & 10 \\
21 & $11 / 2$ & .065 & $4,6,10$ \\
24 & $11 / 2$ & .065 & 6,10 \\
\hline
\end{tabular}

\section{UNITED STATES GOVERNMENT, FEDERAL} SPECIFICATIONS BOARD, FEDERAL SPECIFICATION FOR BLADES, HACK SAW, NO. 415A, OCTOBER 1, 1928. (CURRENT DESIGNATION: GGG-B-451)

\section{HACK-SAW BLADES}

\section{GENERAL SPECIFICATIONS}

There are no general specifications applicable to this specification.

$$
\text { II. TYPES }
$$

Hack-saw blades shall be of the following types: $A$, hand frame blades, all hard; $B$, hand frame blades, flexible; C, machine blades, all hard; D, machine blades, all hard, for cutting corrosionresisting steel.

\section{MATERIAL AND WORKMANSHIP}

Hack-saw blades shall be made of high-grade material and be free from all defects affecting their appearance or serviceability. They shall be of highgrade workmanship and made in accordance with the best commercial practice. 


\section{GENERAL REQUIREMENTS}

The teeth shall be accurately cut, evenly spaced, of uniform height, and properly set. The maximum set shall be not greater than is necessary for free, smooth, and rapid cutting. The length of blades shall be the distance from center to center of holes The ends of all blades shall be in the annealed state in order to preclude probability of holes pulling out or the ends breaking when the saw is in tension between holders. Each hack-saw blade shall be stamped or otherwise permanently marked with the maker's name, brand, or trade-mark.

\section{DETAIL REQUIREMENTS}

1. Hand-frame blades, all hard, type A.-These blades shall be uniformly hardened throughout except the ends. They shall be made of alloy tool steel. They shall conform to dimensions in the following table:

Hand-frame blades, all hard

\begin{tabular}{|c|c|c|c|}
\hline Length & Width & Thickness & $\begin{array}{c}\text { Teeth per } \\
\text { inch }\end{array}$ \\
\hline Inches & Inch & Inch & \\
8 & $7 / 16$ & 0.025 & 18 \\
10 & $1 / 2$ & .025 & 18 \\
10 & $1 / 2$ & .025 & 24 \\
12 & $1 / 2$ or $9 / 16$ & .025 & 14 \\
12 & $1 / 2$ or $9 / 16$ & .025 & 24 \\
\hline
\end{tabular}

NoTE.-A plus or minus tolerance of $3 / 16$ inch in distance between holes and a plus or minus tolerance of 0.003 inch in thickness will be allowed.

2. Hand-frame blades, flexible, type B.-These blades shall have soft backs and shall be hardened on the cutting edge only. They shall be identical otherwise to the type A all hard hand-frame blades as described under Section V 1 above. They shall conform to dimensions in the following table:

Hand-frame blades, flexible

\begin{tabular}{|c|c|c|c|}
\hline Length & Width & Thickness & $\begin{array}{c}\text { Teeth per } \\
\text { inch }\end{array}$ \\
\cline { 1 - 1 } Inches & Inch & Inch & \\
8 & $7 / 16$ & 0.025 & 18 \\
8 & $7 / 16$ & .025 & 32 \\
10 & $11 / 2$ & .025 & 18 \\
10 & $1 / 2$ or $9 / 2$ & .025 & 32 \\
12 & $1 / 2$ or 916 & .025 & 14 \\
12 & .025 & 32 \\
\hline
\end{tabular}

Note.-Flexible blades with soít centers will be accepted under this type, provided they are otherwise in accordance with specifications.

3. Machine blades, all hard type C.-These blades shall be uniformly hardened throughout except the ends. They shall be made of alloy tool steel. They shall conform to dimensions in the following table:

Machine blades, all hard

\begin{tabular}{|c|c|c|c|c|}
\hline \multirow{3}{*}{ Length } & \multirow{2}{*}{ Width } & \multicolumn{2}{|c|}{ Thickness } & Teeth per \\
\cline { 3 - 5 } & & Minimum & Maximum & \\
\cline { 3 - 5 } & & & & \\
\cline { 3 - 5 } Inches & Inch & Inch & Inch & \\
12 & $5 / 8$ & 0.029 & 0.035 & 14 \\
14 & $3 / 4$ & .046 & .068 & 14 \\
14 & 1 & .046 & .068 & 10 \\
17 & 1 & .046 & .068 & 10 \\
\hline
\end{tabular}

NoTE.-A plus or minus tolerance of $1 / 2$ inch in distance between holes will be allowed.
4. Machine blades, all hard, for cutting corrosionresisting steel, type D.-These blades shall be similar to type C blades as described under Section V 3 above except that they shall be suitable for cutting corrosion-resisting steel and shall conform to dimensions in the following table:

Machine blades, all hard, for cutting corrosion-resis
steel
\begin{tabular}{|c|c|c|c|c|}
\hline Length & Width & \multicolumn{2}{|c|}{ Thickness } & $\begin{array}{c}\text { Teeth per } \\
\text { inch }\end{array}$ \\
\cline { 2 - 5 } & Minimum & Maximum & \\
\hline $\begin{array}{c}\text { Inches } \\
14 \\
17\end{array}$ & $\begin{array}{c}\text { Inch } \\
1\end{array}$ & $\begin{array}{c}\text { Inch } \\
0.058\end{array}$ & $\begin{array}{c}\text { Inch } \\
0.068\end{array}$ & 10 \\
.068 & 10 \\
\hline
\end{tabular}

Nоте.-A plus or minus tolerance of $1 / 2$ inch in distance between holes will be allowed.

\section{METHOD OF INSPECTION AND TEST}

1. Tests.-Selective tests, as described herein, will be conducted to determine the work values of hacksaw blades submitted by the various manufacturers. The results obtained in these tests will be entered on the lists of work values maintained by the department concerned. Separate lists will be maintained, respectively, of the average work values for types $A$ and $B$ hand-frame blades, the work values for type $C$ machine blades, and the work values for type D blades for cutting corrosion-resisting steel. Each list will also contain the name of the manufacturer, brand name, and the type or types of hack-saw blades represented by the respective work values.

Work value tests will be conducted on the following representive types and sizes of hack-saw blades conforming to this specification:

\begin{tabular}{|c|c|c|}
\hline Type & Length & $\begin{array}{c}\text { Teeth per } \\
\text { inch }\end{array}$ \\
\hline $\begin{array}{l}\mathrm{B} \\
\mathrm{B} \\
\mathrm{C} \\
\mathrm{D}\end{array}$ & $\begin{array}{c}\text { Inches } \\
12 \\
12 \\
17 \\
17\end{array}$ & $\begin{array}{l}14 \\
14 \\
10 \\
10\end{array}$ \\
\hline
\end{tabular}

2. Hack-saw blades will be tested for work values in machines operated under uniform conditions for the respective type of blades, as follows:

\begin{tabular}{|c|c|c|c|}
\hline Type & $\begin{array}{c}\text { Tension on } \\
\text { blade }\end{array}$ & $\begin{array}{c}\text { Maximum } \\
\text { feed pres- } \\
\text { sure during } \\
\text { stroke }\end{array}$ & $\begin{array}{c}\text { Strokes } \\
\text { per } \\
\text { minute }\end{array}$ \\
\hline & Pounds & Pounds & \\
A and B...- & 275 & 12 & 60 \\
C_.... & 550 & 60 & 60 \\
D.-. & 550 & 125 & 60 \\
\hline
\end{tabular}

The types A, B, and C blades will be used to cut disks from annealed tool steel 3 inches in diameter of a grade specified by the department concerned. The type D blades will be used to cut disks from 3inch diameter high nickel corrosion-resisting steel of a grade specified by the department concerned. All cutting shall be done without a lubricant.

3. Work values will be determined by testing three blades of each of the above types and sizes. Each blade will be tested to failure. Except when a blade is damaged accidentally, failure will be considered to have occurred when the blade cuts at an 
angle, cuts at an excessively slow rate, suffers breakage of body or teeth, or becomes otherwise unfit for useful service in the machine used for testing.

4. Records of the tests will contain data on the number of strokes required to cut off each disk from the bar and the number of disks removed by each blade before failure occurs.

5. The work value for any sat of three blades of the same type and discription will be computed from the test data, as follows:

\begin{tabular}{|c|c|c|}
\hline $\begin{array}{c}\text { Blade } \\
\text { No. }\end{array}$ & $\begin{array}{c}\text { Number } \\
\text { of disks } \\
\text { removed } \\
\text { before } \\
\text { failure }\end{array}$ & $\begin{array}{c}\text { A verage } \\
\text { strokes } \\
\text { per disk }\end{array}$ \\
\hline 1 & $\mathrm{~A}$ & $\mathrm{D}$ \\
2 & $\mathrm{~B}$ & $\mathrm{E}$ \\
3 & $\mathrm{C}$ & $\mathrm{F}$ \\
\hline
\end{tabular}

$\frac{\text { Total number of strokes made by blade No. } 1 \text { before failure }}{A}=D$

$\frac{\text { Total number of strokes made by blade No. } 2}{B}=E$

$\frac{\text { Total number of strokes made by blade No. } 3}{C}=F$

$\frac{A+B+C}{3}=$ A verage disks per blade, $G$

$\frac{D+E+F}{3}=$ general average strokes per disk, $H$

Work value $=\frac{G \times 10,000}{H}$.

6. The method of averaging the work value of hand-frame blades types A and B is as follows:

$\begin{aligned} & \text { A verage work value } \\ & \text { for hand frame }\end{aligned}=\frac{\text { Work value, type A+work value, type B }}{2}$

7. Inspection.-From every four gross of blades under inspection or from the total number if less than four gross, three blades of each type and size shall be taken at random for test. Blades having 18 teeth per inch or less shall, under conditions of the original test, show a work value equal to that of blades of like description originally tested.

Hand-frame blades having more than 18 teeth per inch shall be tested as follows:

\begin{tabular}{|c|c|}
\hline $\begin{array}{c}\text { Teeth } \\
\text { per } \\
\text { inch } \\
\end{array}$ & Test \\
\hline $\begin{array}{l}24 \\
32\end{array}$ & $\begin{array}{l}6 \text { cuts through commercial brass pipe, } 3 / 4 \text {-inch iron pipe size } \\
6 \text { cuts through commercial hard drawn brass tubing, wall } \\
\text { thickness } 0.041 \text { to } 0.057 \text { inch. }\end{array}$ \\
\hline
\end{tabular}

Blades shall complete the six cuts without breakage of body or teeth.

If blades with 18 teeth or less fail under test to equal the original work value of blades of like description, or if 10 per cent of the number of sample blades with more than 18 teeth per inch, taken at random for test, fail to meet the tests specified, the entire shipment shall be rejected.

8. The right is reserved to reject bids on brands of handsaw blades which have not been subjected to the required tests and on those of which tests have been authorized but not completed by opening date of the bids. The attention of manufacturers is called to this requirement, and they are urged to forward as early as practicable samples of hack-saw blades which they propose to offer to the department concerned in the future in order that tests may be made. These tests will be conducted at the expense of the manufacturers. Information with reference to the expense involved and as to where the sample should be sent will be supplied upon application to the department of the Governmenc issuing the specification and invitation for bids. Hack-saw blades not marked in a permanent manner with the brand name, name, or trade-mark of the manufacturer will not be tested.

\section{PACKING AND MARKING OF SHIPMENTS}

Hack-saw blades shall be packed and marked in accordance with best commercial practice, unless otherwise specified.

\section{NOTES}

1. Award of contract will be made to the bidder offering hack-saw blades having the highest selective factor and purchase of which it is considered would be to the best interest to the department concerned. Selective factors for hand-frame blades will be based on the average work values for hand-frame blades and the average price per dozen quoted for the respective makes of hand-frame blades. Selective factors for machine blades will be based on the work value for machine blades and the average price per dozen quoted for the respective makes of machine blades. Selective factors are computed by dividing the work value attained by each manufacturer's product in tests described in Section VI hereunder $b y$ the average price per dozen as quoted in his bid.

2. Hack-saw blades described in this specification are intended to cover the general needs of the Government. It is possible that other types and sizes may be needed from time to time. In such cases, requisitions should state the reasons for requiring the other types and sizes and wherein the hack-saw blades described in specification will not be suitable.

3. Requisitions should state type of blades, length, and number of teeth desired.

4. Typical uses of blades covered by this specification are as follows:

(a) Type A hand-frame blades, all hard are suitable for general purposes.

(b) Type B hand-frame blades, flexible, are suitable where awkward cutting positions are encountered and for use by unskilled workers, because of their flexibility and ability to withstand abuse.

(c) For hand-blades types A and B, recommended uses for the different teeth per inch are as follows:

14 teeth: For cutting soit steel, bronze, copper, and aluminum.

18 teeth: For cutting tool steel, cast iron, and brass.

24 teeth: For cutting iron-pipe, heavy tubing, and electrical conduit.

32 teeth: For cutting thin tubing and metal sheets of 0.050 inch thickness and under.

NOTE.-Ail hard blades preferable for cutting tool steel, cast iron, or brass.

(d) Type C machine blades are suitable for general purposes.

(e) Type D machine blades are suitable for cutting corrosion-resisting steel having a high nickel content and to meet other severe conditions that type $\mathrm{C}$ blades are not capable of withstanding.

\section{UNITED STATES GOVERNMENT, FEDERAL SPECIFICATIONS BOARD, FEDERAL SPECIFICATION FOR FRAMES, HACK SAW, GGG-F-671, NOVEMBER 10, 1931}

\section{HACK-SAW FRAMES}

\section{A. APPLICABLE FEDERAL SPECIFICATIONS}

A-1. There are no other Federal specifications applicable to this specification. 


\section{B. TYPES}

B-1. Hack-saw frames shall be furnished in the following types, as specified: Type A, straighthandle, adjustable; type $\mathrm{B}$, pistol-grip handle, adjustable; and type $\mathrm{C}$, heavy, deep throat, solid.

\section{MATERIAL AND WORKMANSHIP, ETC.}

C-1. Material.-Hack-saw frames shall be made throughout of high-grade material suitable for the purpose.

C-2. Workmanship.- Shall be first class.

\section{GENERAL REQUIREMENTS}

D-1. Marking of frames.-All hack-saw frames shall be marked in a plain and permanent manner with the manufacturer's name or with a trade-mark of such known character that the source of manufacture may be readily determined.

D-2. Handles.-All handles shall be suitably shaped so as to provide a comfortable grip to the hand.

D-3. Finish.-All frames shall be smoothly finished. Except where made of stainless or corrosion-resisting material each frame shall be heavily plated in accordance with the best commercial practice with nickel, cadmium, chromium, or other equally suitable material which will resist corrosion and tarnishing.

D-4. Threaded parts.-A full thread shall be provided for all threaded parts. Such parts shall fit neatly together without undue looseness.

D-5. Saw grips. - Each frame shall have two saw grips, one at either end, for rigidly holding the blade in alignment under all service conditions. Means shall be provided for allowing the grips to be turned to any of four positions $90^{\circ}$ apart, and for retaining them in these positions when the saw is tightened. While under a tenson of 50 pounds between the saw grips, they shall retain the position to which they may be set without turning or damage to any part of the frame when a torsion strain of 35 inch-pounds is applied to either or both of the grips.

Each grip shall be fitted with a pin approximately one-eighth inch (0.124 to 0.130 inch) in diameter, driven through and rigidly secured to the grip to hold the blade, and inclined at a slight angle so as to force the blade against the grip when tightened. Both grips shall be machined to provide a flat bearing surface for the blade.

\section{E. DETAIL REQUIREMENTS}

E-1. Type A.-Straight handle, adjustable, hacksaw frames.

E-1a. Design.-These frames shall have a handle in normal alignment with the blade. The handle shall be made of hardwood of a suitable quality that will resist splitting. The handle shall be smoothly finished and protected from the absorption of moisture by a coating of varnish, paint, or enamel. The frames shall be adjustable to accommodate hacksaw blades of 8-inch, 10-inch, and 12-inch lengths and so designed that all parts of the frame shall be held together when the blade is not in place. Tension shall be applied to the blade either by means of a threaded sleeve within the handle engaging a threaded section of the rear saw grip or by a thumb nut engaging a threaded section of the forward saw grip.

E-1b. Handle-tension style.-In the case of the handle-tension style of frame, the sleeve within the handle shall be made of steel and secured to prevent it from turning or being withdrawn from the handle under service conditions. The end of the sleeve shall be flush with the end of the handle or bear upon a heavy ferrule secured in such manner that the tension of the blade shall not tend to withdraw sleeve from handle. A friction washer shall be provided between the handle and the frame. The length of the threaded section of the sleeve shall not be less than the nominal diameter of the threaded section of the saw grip.

E-1c. Thumb-nut tension style.-If the thumb. nut-tension style of frame is provided, the rear saw grip shall be rigidly secured to the handle. The rear saw grip shall be held in position either by a shoulder or by a ferrule on the handle bearing on the frame. If the grip is held in position by a ferrule bearing on the frame, a pin shall pass through the ferrule and the grip to prevent the grip from being withdrawn from the handle. The length of the threaded section of the thumb nut shall be not less than the nominal diameter of the threaded section of the saw grip on which it operates. A friction washer shall be provided between the thumb nut and the frame.

E-1d. Dimensions, weight, strength, etc.-Type A hack-saw frames shall be similar to illustration Figure 656. They shall conform to the dimensions and weights and withstand without indications of weakness of any nature the proof test outlined in Table 1.

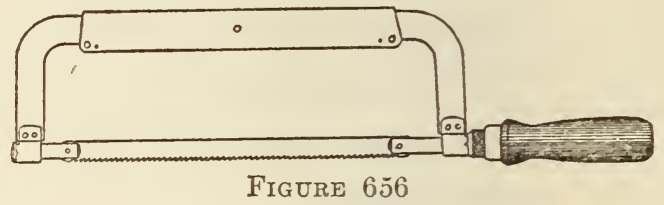

TABLE 1

\begin{tabular}{|c|c|c|c|c|c|}
\hline \multirow[t]{2}{*}{ Capacity (blade length) } & \multicolumn{2}{|c|}{$\begin{array}{l}\text { Depth from } \\
\text { teeth of blade } \\
\text { to nearest in- } \\
\text { side edge or } \\
\text { projection of } \\
\text { frame }\end{array}$} & \multicolumn{2}{|c|}{$\begin{array}{l}\text { Weight com- } \\
\text { plete }\end{array}$} & \multirow{2}{*}{$\begin{array}{l}\text { Tension } \\
\text { be- } \\
\text { tween } \\
\text { saw } \\
\text { grips } \\
\text { (mini- } \\
\text { mum) }\end{array}$} \\
\hline & $\begin{array}{l}\text { Mini- } \\
\text { mum }\end{array}$ & $\begin{array}{l}\text { Maxi- } \\
\text { mum }\end{array}$ & $\begin{array}{l}\text { Mini- } \\
\text { mum }\end{array}$ & $\begin{array}{l}\text { Maxi- } \\
\text { mum }\end{array}$ & \\
\hline 8,10 & $\begin{array}{r}\text { Inches } \\
2 \% 16\end{array}$ & $\begin{array}{l}\text { Inches } \\
35 / 8\end{array}$ & $\begin{array}{c}\text { Ounces } \\
15\end{array}$ & $\begin{array}{c}\text { Ounces } \\
18\end{array}$ & $\begin{array}{r}\text { Pounds } \\
275\end{array}$ \\
\hline
\end{tabular}

E-2. Type B.-Pistol grip, adjustable, hack-saw frames.

E-2a. Design.-These frames shall have a pistolgrip type of handle set at an angle of not less than $25^{\circ}$ or more than $35^{\circ}$ with relation to a perpendicular line drawn at right angles to the longitudinal axis of the frame and blade. Unless otherwise specified the handles shall be made of steel, malleable iron, or of a suitable composition which will withstand, without flaking off or breakage, a drop of 5 feet on a concrete floor. When specifically required the handles shall be of steel or malleable iron or of a nonconducting material which will withstand the above-mentioned drop test. Nonconducting styles of handles shall be designed so that no metal will be exposed at the handle end. Composition handles shall be supported by a steel shank securely fastened to the frame and extending into the handle a distance of not less than 2 inches. All handles of this type of frame shall be knurled or impressed to provide a secure grip. These frames shall be adjustable to accommodate hack-saw blades of 8-inch, 10-inch, and 12-inch lengths and so designed that all parts of the frame shall be held together when the blade is not in place. Tension shall be applied to the blade by means of a thumbscrew engaging a threaded section of one of the saw grips. If thumb nut is located on the rear saw grip sufficient clearance shall be provided between the handle and the thumb nut or 
the end of the saw grip to preclude the operator's hand or fingers coming in contact with the thumb nut or the end of the saw grip under normal service conditions. The length of the threaded section of the thumb nut shall be not less than the nominal diameter of the threaded section of the saw grip on which it operates. A friction washer shall be provided between the thumb nut and the frame.

E-2b. Dimensions, weight, etc.-Type B hack-saw frames shall be similar to illustration Figure 657. They shall conform to the dimensions and weights and withstand without indications of weakness of any nature the proof test outlined in Table 2.

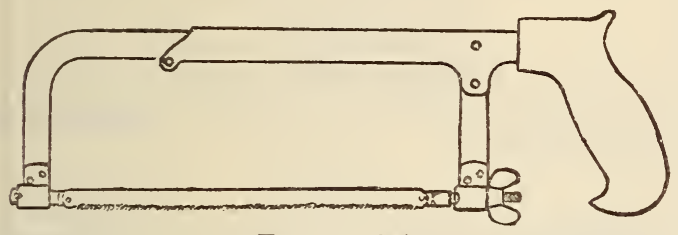

,Figure 657

TABLE 2

\begin{tabular}{|c|c|c|c|c|c|}
\hline \multirow[t]{2}{*}{ Capacity (blade length) } & \multicolumn{2}{|c|}{$\begin{array}{l}\text { Depth from } \\
\text { teeth of blade } \\
\text { to nearest in- } \\
\text { side edge or } \\
\text { projection of } \\
\text { frame }\end{array}$} & \multicolumn{2}{|c|}{$\begin{array}{l}\text { Weight com- } \\
\text { plete }\end{array}$} & \multirow{2}{*}{$\begin{array}{l}\text { Tension } \\
\text { be- } \\
\text { tween } \\
\text { saw } \\
\text { grips } \\
\text { (mini- } \\
\text { mum) }\end{array}$} \\
\hline & $\begin{array}{l}\text { Mini- } \\
\text { mum }\end{array}$ & $\begin{array}{l}\text { Maxi- } \\
\text { mum }\end{array}$ & $\begin{array}{l}\text { Mini- } \\
\text { mum }\end{array}$ & $\begin{array}{l}\text { Maxi- } \\
\text { mum }\end{array}$ & \\
\hline $8,10,12$ inch & $\begin{array}{r}\text { Inches } \\
29 / 16\end{array}$ & $\begin{array}{r}\text { Inches } \\
35,8\end{array}$ & $\begin{array}{c}\text { Ounces } \\
18\end{array}$ & $\begin{array}{c}\text { Ounces } \\
26\end{array}$ & $\begin{array}{r}\text { Pounds } \\
275\end{array}$ \\
\hline
\end{tabular}

E-3. Type C.-Heavy, deep-throat, fixed, hack-saw frame.

E-3a. Design.-These frames shall be fitted with the conventional style of carpenters handsaw handle secured to the frame by means of three telescopic oval head screws, the body diameters of which shall

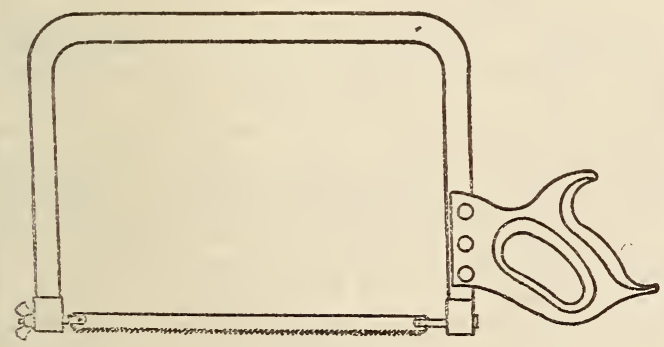

FIgURE 658

be not less than three-sixteenths inch. The handles shall be made of beech or other tough hardwood equally suited for the purpose. The grip portion of the handle shall set at an angle of not less than $40^{\circ}$ or more than $50^{\circ}$ with relation to the vertical portion of the frame to which the handle is secured. These frames shall be of the solid style to accommodate hack-saw blades of 12-inch length. Tension shall be applied to the blade by means of a thumbscrew engaging a threaded section of the forward saw grip. The length of the threaded section of the thumb nut shall be not less than the nominal diameter of the threaded section of the saw grips on which it operates. A friction washer shall be provided between the thumb nut and the frame.
E-3b. Dimensions, weight, etc.-Type C hack-saw frames shall be similar to illustration Figure 658 . They shall conform to the dimensions and weights, and withstand without indications of weakness of any nature the proof test outlined in Table 3.

\section{TABLE 3}

\begin{tabular}{|c|c|c|c|c|c|}
\hline \multirow{2}{*}{$\begin{array}{c}\text { Capacity (blade } \\
\text { length) }\end{array}$} & \multicolumn{2}{|c|}{$\begin{array}{l}\text { Depth from } \\
\text { teetn of blade } \\
\text { to inside edge } \\
\text { of frame }\end{array}$} & \multicolumn{2}{|c|}{ Weight complete } & \multirow{2}{*}{$\begin{array}{c}\text { Tension } \\
\text { be- } \\
\text { tween } \\
\text { saw } \\
\text { grips } \\
\text { (mini- } \\
\text { mum) }\end{array}$} \\
\hline & $\begin{array}{l}\text { Mini- } \\
\text { mum }\end{array}$ & $\begin{array}{l}\text { Maxi- } \\
\text { mum }\end{array}$ & $\begin{array}{l}\text { Mini- } \\
\text { mum }\end{array}$ & $\begin{array}{l}\text { Maxi- } \\
\text { mum }\end{array}$ & \\
\hline 12 inches... & $\begin{array}{r}\text { Inches } \\
10\end{array}$ & $\begin{array}{r}\text { Inrhes } \\
10 \frac{1}{2}\end{array}$ & $\underset{2}{\text { Lhs. Ozs. }}$ & $\begin{array}{cc}\text { Lbs. } & \text { Ozs } \\
3 & 4\end{array}$ & $\begin{array}{r}\text { Pounds } \\
275\end{array}$ \\
\hline
\end{tabular}

\section{F. METHODS OF SAMPLING, INSPECTION, AND TESTS}

F-1. All hack-saw frames shall be inspected to determine whether they conform to this specification. In addition to a careful surface inspection at least three samples (in no instance less than 2 per cent of the entire quantity under inspection) shall be taken at random for those under inspection and

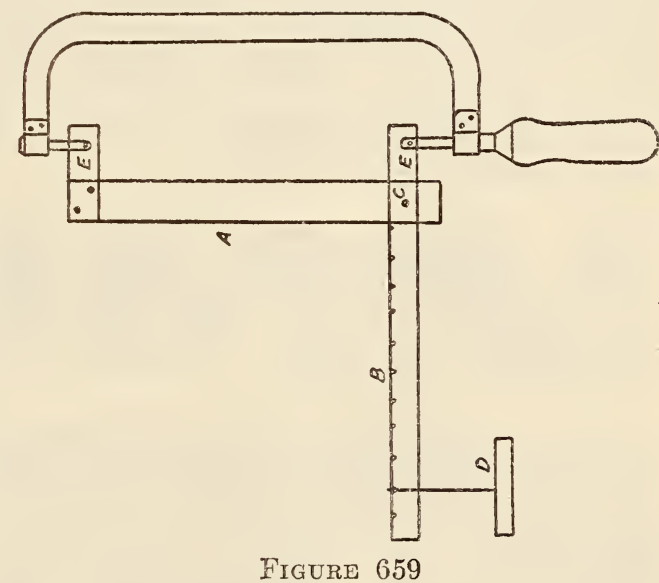

subjected to the test outlined hereunder to determine whether the frames meet the minimum strength requirements of this specification.

Center punch marks shall be applied near either end of the frame, preferably between the rivets securing the grip holders in place. The distance between these two punch marks shall be measured while the frame is subjected to a tension of 20 pounds between the grips. The contraction of the frame shall be not more than one-fourth inch when this tension is increased from 20 to 275 pounds and there shall be no permanent set when the load is decreased from 275 pounds to 20 pounds as indicated by a difference in the distance between punch marks at 20 pounds tension before and after the application of the 275 pounds tension. The adjustable frames types $A$ and $B$ shall be set to maximum capacity (for 12-inch blade) when subjected to the above strength test, and care shall be exercised to see that the load is applied to the saw grips.

Note.-The apparatus as shown in Figure 659 may be used and is approved for conducting the tension test on these frames.

\section{G. PACKAGING, PACKING, AND MARKING}

G-1. Packaging.-Unless otherwise specified, commercial packages are acceptable, provided that 
not more than six frames shall be contained in any one box or package.

G-2. Packing.-Unless otherwise specified the subject commodity shall be delivered in standard commercial containers so constructed as to insure acceptance by common or other carrier, for safe transportation, at the lowest rate, to the point of delivery.

G-3. Marking.-Each box or package shall be plainly marked with the name of the article, the size, the quantity contained therein, and the name of the manufacturer.

G-3a. Unless otherwise specified, shipping containers shall be marked with the name of the article, the type, the size, and the quantity contained therein, as defined by the contract or order under which shipment is made, the name of the contractor, and the number of the contract or order.

\section{NOTES}

I-1. The illustration is included for the convenience of those interested, and is not intended to preclude the purchase of hack-saw frames which are otherwise in accordance with this specification.

I-2. It is believed that this specification adequately describes the characteristics necessary to secure the desired material, and that normally no samples will be necessary prior to award to determine compliance with this specification. If, for any particular purpose, samples with bids are necessary, they should be specifically asked for in the invitation for bids, and the particular purpose to be served by the bid sample should be definitely stated, the specification to apply in all other respects.

\section{UNITED STATES GOVERNMENT, FED-}

ERAL SPECIFICATIONS BOARD, FEDERAL SPECIFICATIONS FOR SAWS, NO. 480, APRIL 25, 192\%. (CURRENT DESIGNATION : GGG-S-61)

(In this specification are included requirements for metal cutting band saws and for hand hack saws, wood cutting. See 616.17, p. 951.)

615.63 RASPS.

UNITED STATES GOVERNMENT, DEPARTMENT OF COMMERCE, BUREAU OF STANDARDS, SIMPLIFIED PRAC. TICE RECOMMENDATION FOR FILES AND RASPS, R6, NOVEMBER 1, 1923.

\section{RASPS}

(Simplified practice recommended and accepted by industry establishing a limited list of standard sizes and types.)

Cabinet rasps:

Second cut_- $\} 6,8,10,12$, and 14 inch.

Half-round wood rasps:

Bastard_._-_ $\} 6,8,10,12,14$, and 16 inch.

Flat-wood rasps:

Bastard_...- $\} 8,10,12,14$, and 16 inch.

Shoe rasps:

Flat_-round__ $\} 8,9$, and 10 inch.

Horse rasps, plain:

$1 / 2$ file, $12,14,15,16$, and 18 inch.

Slim ( $1 / 2$ file), 16,18 , and 20 inch.
Horse rasps, tanged:

Regular, 12, 13, 13, 15, and 16 inch.

Slim, 14, 15, and 16 inch.

Thin, 12, 13, 14, 15, and 16 inch.

\subsection{SCRIBERS.}

UNITED STATES GOVERNMENT, FEDERAL SPECIFICATIONS BOARD, FEDERAL SPECIFICATION FOR SCRIBERS, MA. CHINISTS', NO. 441, OCTOBER 28, 1926. (CURRENT DESIGNATION: GGG-S-111)

\section{SCRIBERS}

\section{GENERAL SPECIFICATIONS}

There are no general specifications applicable to this specification.

\section{TYPE}

Machinists' scribers shall be of the doublepoint type.

\section{MATERIAL AND WORKMANSHIP}

Machinists' scribers shall be of the best material and workmanship and made in accordance with the best commercial practice. They shall be free from all defects or blemishes affecting their appearance or that may affect their serviceability.

\section{GENERAL REQUIREMIENTS}

See Section V.

\section{DETAIL REQUIREMENTS}

1. Machinists' scribers shall be marked in a permanent manner with the manufacturer's name or known trade-mark. They shall be made from a good grade of tool steel suitable for the purpose, and so designed as to be easily held, when in service, without cramping or turning in the fingers. The points of these scribers shall be properly hardened and tempered in such manner that they will not break off or bend under ordinary service conditions. One end of the scriber shall be straight and ground to a long, smooth, tapered sharp point not less than 1 inch long. The opposite end shall be bent at right angles to the scriber and ground to a smooth tapered, sharp point not less than three-eights inch long, measured from the axis of the scriber proper to the end of the bent point. The total length of these scribers shall be approximately 9 inches. The round steel used in the manufacture of the scribers shall be not less than one-eighth inch in diameter. The knurled grip sleeve shall be not less than one-fourth inch diameter and not less than 21/2 inches long. The grip sleeve may be adjustable along the scriber or may be fixed. If adjustable, the sleeve shall be provided with a locking nut so designed as to securely hold the sleeve to any desired location on the scriber. If fixed grip sleeves are provided, they shall be located at the center of the scriber and so designed as to firmly hold each point in position.

2. A tolerance of plus or minus 1 inch will be allowed in the over-all length.

\section{METHOD OF INSPECTION}

Representative samples, at least three, shall be taken from each lot and inspected for compliance with this specification.

\section{PACKING AND MARKING}

Packing and marking shall be in accordance with best commercial practice unless otherwise specified. 


\subsection{TINNERS' AND COPPERSMITHS' TOOLS.}

615.71 SOLDERING COPPERS.

UNITED STATES GOVERNMENT, FEDERAL SPECIFICATIONS BOARD, FEDERAL SPECIFICATION FOR COPPERS, SOLDERING, NO. 439, SEPTEMBER 25, 1926. (CURRENT DESIGNATION : GGG-C-571)

\section{SOLDERING COPPERS}

\section{GENERAL SPECIFICATIONS}

There are no general specifications applicable to this specification.

\section{TYPES}

Soldering coppers shall be of the following types, as required: $\mathrm{A}$, regular tinner's point (pyramid), and $\mathrm{B}$, flat point (wedge).

\section{MATERIAL AND WORKMANSHIP}

The entire copper, exclusive of handle and handle rod, shall be made of commercially pure wrought copper, free from cracks, seams, or imperfections. Workmanship shall be in accordance with the best commercial practice.

\section{GENERAL REQUIREMENTS}

1. The cross section of the copper shall be approximately square, with chamfered edges. Weight per pair, including the iron handle, shall be not less than specified in contract or order.

2. Each soldering copper shall be provided with an iron or steel handle rod. The hanale rod shall be suitably pointed for insertion in a wooden handle.

3. The handle rod shall be securely fastened to the copper by bending so as to form a loop, which shall be formed by passing the iron through the copper at right angles to its axis and then closing the loop by welding. The loop shall be so flattened

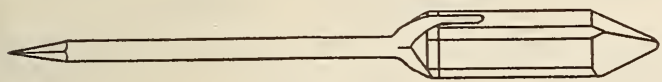

FIGURE 660

that its sides are imbedded in the copper and do not project beyond its lateral surfaces.

4. Soldering coppers shall be furnished without wooden handles.

5. The size (weight per pair) shall be deeply stamped on each copper.

6. Soldering coppers shall be of the dimensions given below:

\begin{tabular}{|r|c|c|c|c|}
\hline $\begin{array}{c}\text { Size } \\
\text { (weight } \\
\text { per pair) }\end{array}$ & $\begin{array}{c}\text { Length of } \\
\text { copper }\end{array}$ & $\begin{array}{c}\text { Thickness } \\
\text { of copper }\end{array}$ & $\begin{array}{c}\text { Diameter } \\
\text { of handle } \\
\text { rod }\end{array}$ & $\begin{array}{c}\text { Length of } \\
\text { handle } \\
\text { rod 1 }\end{array}$ \\
\cline { 1 - 3 } Pounds & Inches & Inches & Inch & Inches \\
2 & $33 / 4-41 / 4$ & $3 / 4-1$ & $3 / 8-1 / 2$ & $8-81 / 2$ \\
3 & $41 / 2-43 / 4$ & $7 / 8-11 / 8$ & $3 / 8-1 / 2$ & $8-91 / 2$ \\
4 & $45 / 8-5$ & $1-11 / 2$ & $112-5 / 8$ & $8-10$ \\
5 & $5-51 / 2$ & $1-11 / 4$ & $1 / 2-5 / 8$ & $8-10$ \\
6 & $51 / 2-6$ & $11 / 4-11 / 2$ & $1 / 2-5 / 8$ & $9-11$ \\
8 & $6-65 / 8$ & $13 / 8-15 / 8$ & $1 / 2-5 / 8$ & $10-12$ \\
10 & $6-63 / 4$ & $11 / 2-13 / 4$ & $1 / 2-5 / 8$ & $10-12$ \\
12 & $63 / 4-71 / 4$ & $15 / 8-11 / 8$ & $1 / 2-5 / 8$ & $10-12$ \\
14 & $7-71 / 2$ & $13 / 4-2$ & $5 / 8-7 / 8$ & $10-12$ \\
\hline
\end{tabular}

1 Length of handle rod is distance from point to first point of contact with the copper.

\section{DETAIL REQUIREMENTS}

1. Type A copper shall be pointed in the shape of a square pyramid, with sharp point removed. (See fig. 660.) The length of the pointed part shall be from one and one-fourth to one and one-half times the thickness of copper given in the table above.

2. Type B copper shall have two sides uniformly sloped to give a wedge-shaped point, with sharp edge removed (see fig. 661), of the same width as the thickness given in above table. Length of

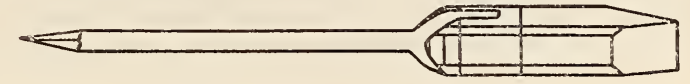

\section{FIgURE 661}

the pointed portion shall be from one and one-fourth to one and one-half times the thickness given in the table.

\section{METHOD OF INSPECTION}

Representative samples shall be taken from each lot delivered and inspected to determine whether they comply with this specification.

\section{PACKING AND MARKING}

Soldering coppers shall be packed and marked in accordance with the manufacturer's trade practice unless otherwise specified.

\subsection{BLOW TORCHES AND SOLDERING POTS.}

(No nationally recognized specifications available.)

\subsection{BENCH PLATES AND STAKES.}

(No nationally recognized specifications available.)

\subsection{MEASURING TOOLS.}

\subsection{CALIPERS AND DIVIDERS.}

\section{UNITED STATES GOVERNMENT, FEDERAL SPECIFICATIONS BOARD, FEDERAL SPECIFICATION FOR DIVIDERS GGG-D- 351, MARCH 1, 1932}

\section{DIVIDERS}

\section{GENERAL SPECIFICATIONS}

There are no general specifications applicable to this specification.

\section{TYPES}

Dividers shall be of the following types: A, spring; $\mathrm{B}$, firm joint; and $\mathrm{C}$, wing.

\section{MATERIAL AND WORKMANSHIP}

Dividers shall be made of high-grade steel suitable for the purpose and in accordance with the best commercial practice. They shall be free from all defects or blemishes affecting their appearance or that may affect their serviceability.

\section{GENERAL REQUIREMENTS}

1. Each divider shall have the maker's name or known trade-mark stamped or otherwise permanently marked thereon.

2. The points of all dividers shall be such as to avoid breakage, undue wear, or bending under ordinary service conditions. 


\section{จ. DETAIL REQUIREMENTS}

1. Spring dividers, type A.-These dividers shall be provided with a circular band spring at the hinged end which shall cause the dividers to open and be held in a rigid manner. The dividers shall be adjustable by means of a nut traveling over a finely threaded steel rod, which shall be hinged to one leg of the divider and pass through an attachment fastened to the other leg and terminating in a small ball end to prevent the nut from coming off the rod. The bearing surfaces where the nut or friction washer bears on the attachment fastened to one of the legs shall be smoothly and neatly finished to reduce friction when the nut is operated.

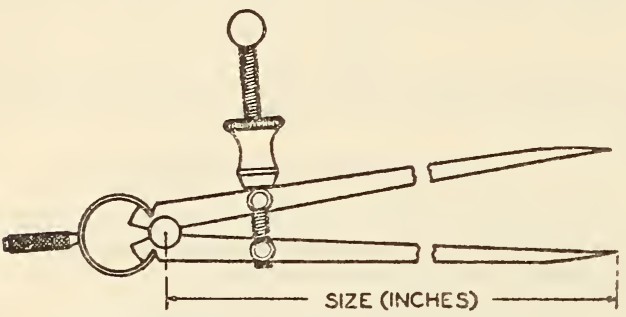

FIgURE 662.-Type A, spring dividers

The legs of these dividers shall be of either rectangular or round cross section. The legs shall be polished and straight, with the ends ground to a sharp point. The ends shall come evenly together when the dividers are in a closed position. A knurled finger and thunb attachment shall be provided and securely fastened to the circular band spring for convenience in handling the dividers when in use. The fulcrum stud shall be suitably hardened to reduce wear. These dividers shall be furnished in $3,4,6$, and 8 inch sizes and shall be similar to the sketch incorporated herein. Size refers to the distance from the center of the fulcrum stud to the points.

2. Firm-joint dividers, type B.-These dividers shall be so designed as to give any degree of friction desired in the joint. The legs shall be polished, approximately parallel on the sides, tapered on the edges, and shall terminate in sharply ground points at

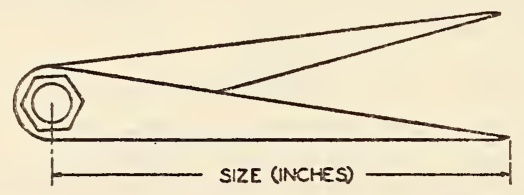

Frgure 663.-Type $B$, firm joint

the ends. The ends shall come evenly together when the dividers are in a closed position. These dividers shall be furnished in 6 and 12 inch sizes, and shall be similar to the sketch incorporated herein. Size refers to distance from the center of the joint to the points.

3. Wing dividers, type C.-These divider's shall be provided with an adjusting arc or wing, which shall pass through a slot in one leg of the divider. The threaded end of the adjusting arc shall pass through a hole in the other leg of the divider, after passing through an adjusting spring which shall be secured on inside of the leg. The adjusting spring shall be of a good grade of spring steel. The adjusting nut shall have a round head, which shall be knurled. The slotted leg of the dividers shall be provided with a thumbscrew for securing that leg to the adjusting arc. All ares shall be of sufficient length to allow opening and adjusting of dividers to a minimum of $70^{\circ}$. The upper part of the legs of these dividers shall be square, the lower part round, having a long taper terminating in sharply ground points at the ends. The ends shall come evenly together when the dividers are in a closed position. These dividers shall be furnished in 8 , 12 , and 24 inch sizes, and shall be similar to the sketch incorporated herein. Size refers to the distance from the center of the hinged joint to the points.

\section{METHOD OF INSPECTION}

From each lot under inspection at least three dividers of each type and size shall be taken at random for inspection with a view of determining whether the dividers being furnished are in accordance with specifications. Results of inspection shall be considered as representative of the lot, and acceptance or rejection will be based thereon.

In addition to a careful surface inspection for size, design, finish, workmanship, marking requirements, etc., each sample shall also be subjected to the following practical test:

With the divider set to maximum capacity, at least 12 circles shall be scribed on metal which shall have a hardness equal to 45 to 50 scleroscope. Under this test there shall be no perceptible in-

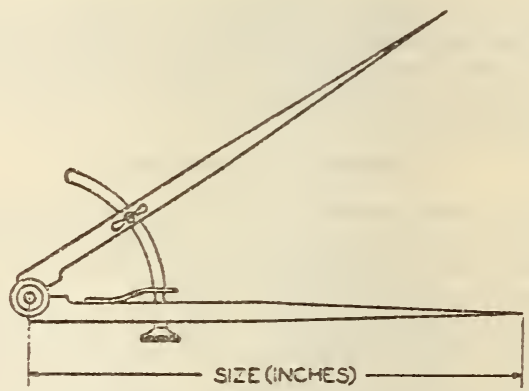

FIGURE 664.-Type $C$, wing dividers

crease in the width of the lines scribed nor shall there be any apparent damage to the points of the divider.

\section{PACKING AND MARKING}

They shall be packed and marked in accordance with best commercial practice unless otherwise specified.

\section{NOTES}

1. The illustrations (figs. 662, 663, and 664) are included for the convenience of those interested and are not intended to prevent the purchase of dividers which are otherwise in accordance with specifications.

\subsection{GAGES, DIMENSION.}

\section{GAGES}

NotE.-Gages for use with a particular item only will be found under the same commodity designation as that item.

\section{AMERICAN PETROLEUM INSTITUTE, LINE. PIPE SPECIFICATIONS, NO. 5-I, 1931}

\section{TUBULAR GAGES (LINE PIPE)}

Each manufacturer of A. P. I. threaded line pipe shall provide himself with plug and ring reference gages which have been manufactured in accordance with the gage tables appended, except that gages may be used which have been manufactured in accordance with the American engineering standards for pipe threads, in which case the additional length of thread specified for line pipe will project through the American standards ring gage; provided that 
such existing gages are certified by the National Bureau of Standards to be in accordance with the gage tables appended before their use is authorized under this specification.

\section{PLUG}

The certified master plug shall be the control and shall be a physical reproduction of the thread on the pipe. A plug gage, within the following limits, shall be considered satisfactory The lead and taper shall be measured over a length of axis beginning and ending not less than one full thread in from the ends of the threaded portion on gages up to 2 inches nominal size, inclusive, and not less than two full threads in from the ends of the threaded portion on gages over 2 inches nominal size.

\begin{tabular}{|c|c|c|c|c|}
\hline Pitch & Lead & Taper & $\begin{array}{l}\text { Pitch di- } \\
\text { ameter }\end{array}$ & $\begin{array}{c}1 / 2 \text { thread } \\
\text { angle }\end{array}$ \\
\hline 27 & $\begin{aligned} & \text { Inch } \\
\pm & 0.0002\end{aligned}$ & $\begin{array}{c}\text { Inch } \\
+0.0003\end{array}$ & $\begin{array}{c}\text { Inch } \\
\pm 0.0002\end{array}$ & $\begin{array}{c}\text { Minutes } \\
\pm 15\end{array}$ \\
\hline 18 & \pm .0002 & $\begin{array}{r}-.0004 \\
+.0004\end{array}$ & \pm .0004 & \pm 15 \\
\hline 14 & \pm .0003 & $\begin{array}{l}+.0006 \\
+.000\end{array}$ & \pm .0006 & \pm 10 \\
\hline 1113 & \pm .0004 & $\begin{array}{r}7.0000 \\
+.0008\end{array}$ & \pm .0007 & \pm 10 \\
\hline 8 & \pm .0005 & $\begin{array}{l}-.0000 \\
+.0010 \\
-.0000\end{array}$ & \pm .0010 & \pm 10 \\
\hline
\end{tabular}

The above tolerances are subject to the additional condition that the cumulative error must not exceed one-third turn for gages of $3 / 4$-inch taper per foot. This means that if the error in any element exceeds the specified unit tolerance some other element or elements must be a sufficien amount inside of the specified tolerance to compensate for the excess error.

\section{RING}

The ring is not standard and its definition of size is obtained by calibration from the certified master plug. To be a reliable transfer medium it must be certified for lead, taper, and form of thread. A ring gage, within the following limits, shall be considered satisfactory. The lead and taper shall be measured over a length of axis beginning and ending not less than one full thread in from the ends of the threaded portion on gages up to 2 inches nominal size, inclusive, and not less than two full threads in from the ends of the threaded portion on gages over 2 inches nominal size.

\begin{tabular}{|c|c|c|c|}
\hline Pitch & Lead & Taper & $\begin{array}{l}16 \text { thread } \\
\text { angle }\end{array}$ \\
\hline 27 & $\begin{array}{c}\text { Inch } \\
\pm 0.0004\end{array}$ & $\begin{array}{l}\text { Inch } \\
-0.0002\end{array}$ & $\begin{array}{c}\text { Minutes } \\
\quad \pm 20\end{array}$ \\
\hline 18 & \pm .0004 & $\begin{array}{l}-.0000 \\
-.0002\end{array}$ & \pm 20 \\
\hline 14 & \pm .0006 & $\begin{array}{l}-.0002 \\
-.0002\end{array}$ & \pm 15 \\
\hline $111 / 2$ & \pm .0008 & $\begin{array}{l}-.0009 \\
-.0002\end{array}$ & \pm 15 \\
\hline 8 & \pm .0010 & $\begin{array}{l}-.0012 \\
-.0002 \\
-.0014\end{array}$ & \pm 15 \\
\hline
\end{tabular}

The above tolerances are subject to the additional condition that the cumulative error must not exceed one-third turn for gages of $3 / 4$-inch taper per foot. This means that if the error in any element exceeds the specified unit tolerance, some other element or elements must be a sufficient amount inside of the specified tolerance to compensate for the excess error.

\section{GENERAL}

Certified master plugs should preferably be of hardened and ground al oy steel properly heat treated and aged to prevent secular change of size.

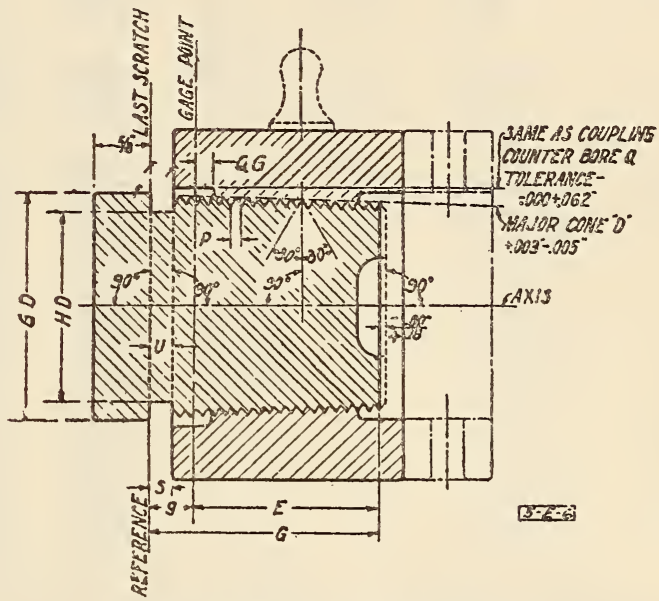

Figure 665. - Line pipe gage

$a$, All measurements referred to shoulder marked "last scratch;" $b$, the small ends of gages are not measuring planes, but are finished square with axis to assist in certification; $c$, the length of the collar on plug gages, also the dimensions $G D, Q G, U$, and $H D$ are unimportant within the tolerances shown, and gages shall not be rejected for noncompliance, unless interfering with the proper use of the gage; $d$, the length of the threads in the ring gage should not be less than $E$ minus $11 / 2$ pitches; $e$, the ring gage may be extended as shown to provide sockets for make-up bar; $f$, the outside diameter $G D$ of the plug collar to be equal to the outside diameter $O D$ of the pipe plus or minu 0.010 in minus $U$ to be .060 inch less than the minor (root) diameter of the product thread at the gage point plus or minus 0.020 inch. $h$, the depth of counterbore $Q G$ on ring gages to be one-half pitch minimum; $i$, the crests of the threads on gages shall be truncated as indicated in column $h$ in gage tables. The roots of the threads on gages shall be cut to a sharp $V$ or, when ground, shall be undercut to prevent the gage from bearing on crest or root of the thread on the product.

Transfer ring gages up to 7 inches pitch diameter may be of soft steel with chased thread.

Transfer ring gages over 7 inches pitch diameter should preferably be of hardened and ground alloy steel. If the transfer ring is solid (not built up), a fitting plate may be attached to the large end face. If the transfer ring is solid and no fitting plate is attached the dimension $S$ need not be held closely to a defined size. When fitting plate is used, the value $S$ may be specified as an absolute amount at the option of the purchaser. In any case, the value $S$ after calibration with the master plug, shall be stamped on the ring gage. 
TABLE 1-B-A. P. I. Line-pipe gages

[See fig. 665]

[All dimensions in inches at $68^{\circ} \mathrm{F}$ ]

\begin{tabular}{|c|c|c|c|c|c|c|c|c|c|c|}
\hline$\underset{\text { size }}{\text { Nominal }}$ & $\begin{array}{c}\text { Outside } \\
\text { diameter } \\
O . D \text {. }\end{array}$ & $\begin{array}{c}\text { Number of } \\
\text { threads } \\
\text { per inch } \\
B\end{array}$ & $\begin{array}{c}\text { Included } \\
\text { taper, } \\
\text { inches } \\
\text { per foot } \\
b\end{array}$ & $\begin{array}{l}\text { Pitch } \\
\text { diameter } \\
\text { at gage } \\
\text { point } \\
C\end{array}$ & $\begin{array}{l}\text { Major } \\
\text { diameter } \\
\text { at gage } \\
\text { point } \\
D\end{array}$ & $\begin{array}{c}\text { Distance } \\
\text { from gage } \\
\text { point to last } \\
\text { scratch } \\
g\end{array}$ & $\begin{array}{l}\text { Width } \\
\text { of notch } \\
U\end{array}$ & $\begin{array}{c}\text { Height } \\
\text { of thread } \\
\text { (truncated) } \\
h\end{array}$ & $\underset{P}{\text { Pitch }}$ & $\underset{S^{1}}{\text { Stand-off }}$ \\
\hline $\begin{array}{l}1 / 8-. \\
1 / 4-. \\
3 / 8- \\
1 / 2-\end{array}$ & $\begin{array}{r}0.405 \\
.540 \\
.675 \\
.840\end{array}$ & $\begin{array}{l}27 \\
18 \\
18 \\
14\end{array}$ & $\begin{array}{l}3 / 4 \\
3 / 4 \\
3 / 4 \\
3 / 4\end{array}$ & $\begin{array}{r}0.37537 \\
.49556 \\
.63056 \\
.78286\end{array}$ & $\begin{array}{r}0.40004 \\
.53256 \\
.66756 \\
.83043\end{array}$ & $\begin{array}{l}0.185 \\
.278 \\
.278 \\
.357\end{array}$ & $\begin{array}{r}0.111 \pm 0.100 \\
.167 \pm .100 \\
.167 \pm .100 \\
.214 \pm .100\end{array}$ & $\begin{array}{r}0.02467 \\
.03700 \\
.03700 \\
.04757\end{array}$ & $\begin{array}{r}0.0370370 \\
.0555555 \\
.0555555 \\
.0714285\end{array}$ & $\begin{array}{r}0.111 \pm 0.10 \\
.167 \pm .10 \\
.167 \pm .10 \\
.214 \pm .10\end{array}$ \\
\hline $\begin{array}{l}3 / 4 \ldots \\
11 \\
114 . \\
11 / 2-.\end{array}$ & $\begin{array}{l}1.050 \\
1.315 \\
1.660 \\
1.900\end{array}$ & $\begin{array}{l}14 \\
111 / 2 \\
111 / 2 \\
111 / 2\end{array}$ & $\begin{array}{l}3 / 4 \\
3 / 4 \\
3 / 4 \\
3 / 4\end{array}$ & $\begin{array}{l}.99286 \\
1.24543 \\
1.59043 \\
1.83043\end{array}$ & $\begin{array}{l}\text { 1. } 04043 \\
\text { 1. } 30335 \\
\text { 1. } 64835 \\
1.88835\end{array}$ & $\begin{array}{l}.357 \\
.435 \\
.435 \\
.435\end{array}$ & $\begin{array}{l}.214 \pm .100 \\
.261 \pm .100 \\
.261 \pm .100 \\
.261 \pm .100\end{array}$ & $\begin{array}{l}.04757 \\
.05792 \\
.055792 \\
.05792\end{array}$ & $\begin{array}{l}.0714285 \\
.0869565 \\
.0869565 \\
.0869565\end{array}$ & $\begin{array}{l}.214 \pm .10 \\
.261 \pm .10 \\
.261 \pm .10 \\
.261 \pm .10\end{array}$ \\
\hline $\begin{array}{l}2 \\
21 / 2 \\
3 \\
31 / 2\end{array}$ & $\begin{array}{l}2.375 \\
2.875 \\
3.500 \\
4.000\end{array}$ & $\begin{array}{l}111 / 2 \\
8 \\
8 \\
8\end{array}$ & $\begin{array}{l}3 / 4 \\
3 / 4 \\
3 / 4 \\
3 / 4\end{array}$ & $\begin{array}{l}\text { 2. } 30543 \\
2.77500 \\
3.40000 \\
3.90000\end{array}$ & $\begin{array}{l}2.36335 \\
2.85825 \\
3.48325 \\
3.98325\end{array}$ & $\begin{array}{l}.435 \\
.625 \\
.625 \\
.625\end{array}$ & $\begin{array}{l}.261 \pm .100 \\
.375 \pm .100 \\
.375 \pm .100 \\
.375 \pm .100\end{array}$ & $\begin{array}{l}.05792 \\
.08325 \\
.08325 \\
.08325\end{array}$ & $\begin{array}{l}.0869565 \\
.1250000 \\
.1250000 \\
.1250000\end{array}$ & $\begin{array}{l}.261 \pm .10 \\
.375 \pm .10 \\
.375 \pm .10 \\
.375 \pm .10\end{array}$ \\
\hline $\begin{array}{l}4-. \\
5-- \\
6-- \\
8--\end{array}$ & $\begin{array}{l}4.500 \\
5.563 \\
6.625 \\
8.625\end{array}$ & $\begin{array}{l}8 \\
8 \\
8 \\
8\end{array}$ & $\begin{array}{l}3 / 4 \\
3 / 4 \\
3 / 4 \\
3 / 4\end{array}$ & $\begin{array}{l}4.40000 \\
5.46300 \\
6.52500 \\
8.52500\end{array}$ & $\begin{array}{l}4.48325 \\
5.54625 \\
6.60825 \\
8.60825\end{array}$ & $\begin{array}{l}.625 \\
.625 \\
.625 \\
.625\end{array}$ & $\begin{array}{l}.375 \pm .100 \\
.375 \pm .100 \\
.375 \pm .100 \\
.375 \pm .100\end{array}$ & $\begin{array}{l}.08325 \\
.08325 \\
.08325 \\
.08325\end{array}$ & $\begin{array}{l}.1250000 \\
.1250000 \\
.1250000 \\
.1250000\end{array}$ & $\begin{array}{l}.375 \pm .10 \\
.375 \pm .10 \\
.375 \pm .10 \\
.375 \pm .10\end{array}$ \\
\hline $\begin{array}{l}10 \\
12 \\
14 \\
15\end{array}$ & $\begin{array}{l}10.750 \\
12.750 \\
14.000 \\
15.000\end{array}$ & $\begin{array}{l}8 \\
8 \\
8 \\
8\end{array}$ & $\begin{array}{l}3 / 4 \\
3 / 4 \\
3 / 4 \\
3 / 4\end{array}$ & $\begin{array}{l}10.65000 \\
12.65000 \\
13.90000 \\
14.90000\end{array}$ & $\begin{array}{l}10.73325 \\
12.73325 \\
13.98325 \\
14.98325\end{array}$ & $\begin{array}{l}.625 \\
.625 \\
.625 \\
.625\end{array}$ & $\begin{array}{l}.375 \pm .100 \\
.375 \pm .100 \\
.375 \pm .100 \\
.375 \pm .100\end{array}$ & $\begin{array}{l}.08325 \\
.08325 \\
.08325 \\
.08325\end{array}$ & $\begin{array}{l}.1250000 \\
.1250000 \\
.1250000 \\
.1250000\end{array}$ & $\begin{array}{l}.375 \pm .10 \\
.375 \pm .10 \\
.375 \pm .10 \\
.375 \pm .10\end{array}$ \\
\hline 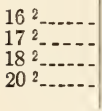 & $\begin{array}{l}16.000 \\
17.000 \\
18.000 \\
20.000\end{array}$ & $\begin{array}{l}8 \\
8 \\
8 \\
8\end{array}$ & $\begin{array}{l}3 / 4 \\
3 / 4 \\
3 / 4 \\
3 / 4\end{array}$ & $\begin{array}{l}15.90000 \\
16.90000 \\
17.90000 \\
19.90000\end{array}$ & $\begin{array}{l}15.98325 \\
16.98325 \\
17.98325 \\
19.98325\end{array}$ & $\begin{array}{l}.625 \\
.625 \\
.625 \\
.625\end{array}$ & $\begin{array}{l}.375 \pm .100 \\
.375 \pm .100 \\
.375 \pm .100 \\
.375 \pm .100\end{array}$ & $\begin{array}{l}.0 \$ 325 \\
.08325 \\
.80325 \\
.08325\end{array}$ & $\begin{array}{l}.1250000 \\
.1250000 \\
.1250000 \\
.1250000\end{array}$ & $\begin{array}{l}.375 \pm .10 \\
.375 \pm .10 \\
.375 \pm .10 \\
.375 \pm .10\end{array}$ \\
\hline
\end{tabular}

1 The purchaser may specify the $S$ dimension to suit his requirements, ignoring the values giren. In the absence of definite specifications. for $S$ from the purchaser, the values giren in the table will be used. The calibrated ralue of $S$ in an $y$ case must be stamped on the ring gage Fitting plates may be added to gages if desired.

2 Outside diameter.

\section{AMERICAIN PETROLEUM INSTITUTE, SPEC- IFICATIONS FOR STEEL AND IRON PIPE FOR OIL-COUNTRY TUBULAR GOODS, NO. 5-A, 1931}

\section{A. P. I. TUBULAR GAGES}

Each manufacturer of A. P. I. tubular threaded material shall provide himself with plug and ring reference gages which have been manufactured in accordance with the gage tables appended; such reference gages to be checked and certified by the Bureau of Standards, or by the National Physical Laboratory, England.

\section{SPECIFICATION FOR A. P. I. TOBOLAR GAGES}

\section{Plug}

The certified master plug shall be the control and shall be a physical reproduction of the thread on the pipe.

The lead may vary not more than \pm 0.0003 inch from basic for gages of $3 / 8$-inch taper per foot, and 0.0005 inch from basic for gages of $3 / 4$-inch taper per foot, to be measured over a length of axis beginning and ending not less than two full threads in from the ends of the threaded portion.

The included taper on diameter in the length $E$ shall lie between the limits (basic) and (basic +0.0005 inch) for gages of $3 / 8$-inch taper per foot, and (basic) and (basic 0.0010 inch) for gages of $3 / 4$-inch taper per foot, to be measured over a length of axis beginning and ending not less than two full threads in from the ends of the treaded portion.

The flanks of the tread may vary from the basic angle of $30^{\circ}$ to the normal by not more than \pm 10 minutes of arc.
The pitch diameter at the gage point may vary not more than \pm 0.0005 inch from basic for gages of $3 / 8$-inch taper per foot, and 0.0010 inch from basic for gages of $3 / 4$-inch taper per foot. The above tolerances are subject to the additional condition that the cumulative error must not exceed one-half turn for gages of $3 / 8$-inch taper per foot, and one-third turn for gages of $3 / 4$-inch taper per foot. This means that if the error in any element exceeds the specified unit tolerance, some other element or elements must be a sufficient amount inside of the specified tolerance to compensate for the excess error.

\section{Ring}

The ring is not standard and its definition of size is obtained by calibration from the certified master plug.

To be a reliable transfer medium it must be certified for lead, taper, and form of thread.

The lead may vary not more than \pm 0.0005 -inch from basic for gages of $3 / 8$-inch taper per foot and 0.0008 inch from basic for gages of $3 / 4$-inch taper per foot, to be measured over a length of axis beginning and ending not less than two full threads in from the ends of the threaded portion.

The included taper on diameter in the length "E" shall lie between the limits (basic -0.0002 inch) and (basic - 0.0012 inch) for all sizes, to be measured over a length of axis beginning and ending not less than two full threads in from the ends of the threaded portion.

The flanks of the thread may vary from the basic angle of $30^{\circ}$ to the normal by not more than 15 minutes of arc.

The above tolerances are subject to the additional condition that the cumulative error as calcu- 
lated from the unit errors must not exceed one-half turn for gages of 3/8-inch taper per foot and one-third turn for gages of $3 / 4$-inch taper per foot. This means that if the error in any element exceeds the specified unit tolerance, some other element or elements must be a sufficient amount inside of the specified tolerances to compensate for the excess error.
(General specifications are identical with those for tubular gages (line pipe), given above.)

\section{Casing Gages}

(Text and illustration are identical with Figure 665 in tubular gages (line pipe), given above. The latter illustration also carries the lettered dimensions referred to in the following table:)

\section{TABLE 1.-A.P. I. casing gages}

[All dimensions in inches at $68^{\circ} \mathrm{F}$.]

\begin{tabular}{|c|c|c|c|c|c|c|c|c|c|}
\hline Size & $\begin{array}{c}\text { Number } \\
\text { of threads } \\
\text { per inch, } \\
B\end{array}$ & $\begin{array}{c}\text { included } \\
\text { taper, } \\
\text { inches per } \\
\text { foot, } b\end{array}$ & $\begin{array}{c}\text { Pitch diam- } \\
\text { eter at gage } \\
\text { point, } C\end{array}$ & $\begin{array}{c}\text { Major di- } \\
\text { ameter at } \\
\text { gage point, } \\
D\end{array}$ & $\begin{array}{c}\text { Distance } \\
\text { from gage } \\
\text { point to } \\
\text { last } \\
\text { scratch, } g\end{array}$ & Width of notch, \\
$U$
\end{tabular}

1 The purchaser may specify the $S$ dimension to suit his requirements, ignoring the values given. In the absence of definite speciflcations for $S$ from the purchaser, the values given in above Table will be used. The calibrated value of $S$ in any case must be stamped on the ring gage. Fitting plates may be added to gages if desired.

2 External upset.

\section{Drill-Pipe Gages}

(Text and illustration are identical with Figure 665 in tubular gages (line pipe), given above. The latter illustration also carries the lettered dimensions referred to in the following table:)

\section{TABLE 2.-A.P. I. drill-pipe gages}

[All dimensions in inches at $68^{\circ} \mathrm{F}$.]

\begin{tabular}{|c|c|c|c|c|c|c|c|c|c|c|}
\hline Size & $\begin{array}{c}\text { Outside } \\
\text { diameter, } \\
O . D .\end{array}$ & $\begin{array}{c}\text { Number } \\
\text { of threads } \\
\text { per inch, } \\
\quad B\end{array}$ & $\begin{array}{c}\text { Included } \\
\text { taper, } \\
\text { inches per } \\
\text { foot, } b\end{array}$ & $\begin{array}{l}\text { Pitch diam- } \\
\text { eter at gage } \\
\text { point, } C\end{array}$ & $\begin{array}{c}\text { Major di- } \\
\text { ameter of } \\
\text { gage point, } \\
D\end{array}$ & $\begin{array}{c}\text { Distance } \\
\text { from gage } \\
\text { point to } \\
\text { last } \\
\text { scratch, } g\end{array}$ & Width of notch, & $\begin{array}{l}\text { Height of } \\
\text { thread } \\
\text { (trun- } \\
\text { cated), } h\end{array}$ & Pitch, $P$ & Stand-off, $2 S$ \\
\hline $\begin{array}{l}23 / 8 \\
23 / 8 \\
27 / 8 \\
31 / 2 \\
41 / 2\end{array}$ & $\begin{array}{l}2.375 \\
2.375 \\
2.875 \\
3.500 \\
4.500\end{array}$ & $\begin{array}{r}10 \\
8 \\
8 \\
8 \\
8\end{array}$ & $\begin{array}{l}3 / 8 \\
3 / 4 \\
3 / 4 \\
3 / 4 \\
3 / 4\end{array}$ & $\begin{array}{l}2.31640 \\
2.30075 \\
2.80075 \\
3.42575 \\
4.42575\end{array}$ & $\begin{array}{l}2.35200 \\
2.34900 \\
2.84900 \\
3.47400 \\
4.47400\end{array}$ & $\begin{array}{l}0.458 \\
.588 \\
.588 \\
.588 \\
.588\end{array}$ & $\begin{array}{l}0.20 \pm 0.100 \\
.25 \pm .125 \\
.25 \pm .125 \\
.25 \pm .125 \\
.25 \pm .125\end{array}$ & $\begin{array}{r}0.03560 \\
.04825 \\
.04825 \\
.04825 \\
.04825\end{array}$ & $\begin{array}{r}0.100 \\
.125 \\
.125 \\
.125 \\
.125\end{array}$ & $\begin{array}{r}0.208 \pm 0.100 \\
.338 \pm .100 \\
.338 \pm .100 \\
.338 \pm .100 \\
.338 \pm .100\end{array}$ \\
\hline $\begin{array}{r}5 \% 16 \\
65 \% \\
375 / 8 \\
385 \%\end{array}$ & $\begin{array}{l}5.563 \\
6.625 \\
7.625 \\
8.625\end{array}$ & $\begin{array}{l}8 \\
8 \\
8 \\
8\end{array}$ & $\begin{array}{l}3 / 4 \\
3 / 4 \\
3 / 4 \\
3 / 4\end{array}$ & $\begin{array}{l}5.43875 \\
6.55075 \\
7.55075 \\
8.55075\end{array}$ & $\begin{array}{l}5.53700 \\
6.59900 \\
7.59900 \\
8.59900\end{array}$ & $\begin{array}{l}.588 \\
.588 \\
.588 \\
.588\end{array}$ & $\begin{array}{l}.25 \pm .125 \\
.25 \pm .125 \\
.25 \pm .125 \\
.25 \pm .125\end{array}$ & $\begin{array}{l}.04825 \\
.04825 \\
.04825 \\
.04825\end{array}$ & $\begin{array}{l}.125 \\
.125 \\
.125 \\
.125\end{array}$ & $\begin{array}{l}.338 \pm .100 \\
.338 \pm .100 \\
.338 \pm .100 \\
.338 \pm .100\end{array}$ \\
\hline
\end{tabular}

1 Pitch diameter as determined by thread micrometers.

${ }^{2}$ The purchaser may specify the $S$ dimension to suit his requirements, ignoring the values given. In the absence of definite specifications for $S$ from the purchaser, the values given above will be used. The calibrated value of $S$ in any case must be stamped on the ring gage. Fitting plates may be added to gages if desired.

3 Data on the 75,8 and $85 \%$ inch sizes published for general information only. Due to the very limited demand for these sizes, manufacturers are not required to supply themselves with certified master gages unless they desire to do so. Manufacturers will furnish these sizes with threads made as closely as possible to dimensions given, without certified gages. The A. P. I. monogram can not be used on these sizes unless the manufacturer has the necessary master gages. One weight only listed; consult manufacturers if other weights are desired. 


\section{Tubing Gages}

(Text and illustration are identical with Figure 665 in tubular gages (line pipe), given above. The latter illustration also carries the lettered dimensions referred to in the following table:)

TABLE 3.-A. P. I. standard tubing gages

[All dimensions in inches at $68^{\circ} \mathrm{F}$.]

PLAIN TUBING GAGES

\begin{tabular}{|c|c|c|c|c|c|c|c|c|c|c|c|}
\hline $\begin{array}{c}\text { Nomi- } \\
\text { nal } \\
\text { size }\end{array}$ & $\begin{array}{c}\text { Outside } \\
\text { diam- } \\
\text { eter, } \\
O . D .\end{array}$ & $\begin{array}{c}\text { Outside } \\
\text { diameter } \\
\text { of exter- } \\
\text { nal upset, } \\
U . D .\end{array}$ & $\begin{array}{l}\text { Num- } \\
\text { ber of } \\
\text { threads } \\
\text { per } \\
\text { inch, } \\
B\end{array}$ & $\begin{array}{c}\text { In- } \\
\text { cluded } \\
\text { taper, } \\
\text { inches } \\
\text { per } \\
\text { foot, } b\end{array}$ & $\begin{array}{c}\text { Pitch } \\
\text { diameter } \\
\text { at gage } \\
\text { point, } C\end{array}$ & $\begin{array}{c}\text { Major } \\
\text { diameter } \\
\text { at gage } \\
\text { point, } D\end{array}$ & $\begin{array}{c}\text { Distance } \\
\text { from } \\
\text { gage } \\
\text { point } \\
\text { to last } \\
\text { scratch, } g\end{array}$ & $\begin{array}{l}\text { Width of } \\
\text { notch, } U\end{array}$ & $\begin{array}{l}\text { Height of } \\
\text { threads } \\
\text { (trun- } \\
\text { cated), } h\end{array}$ & Pitch, $P$ & Stand-off, $1 S$ \\
\hline $\begin{array}{l}11 / 2 \\
21 / 2 \\
3 \\
31 / 2 \\
4\end{array}$ & $\begin{array}{l}1.900 \\
2.875 \\
3.500 \\
4.000 \\
4.500\end{array}$ & $-1 .-1$ & $\begin{array}{l}111 / 2 \\
111 / 2 \\
111 / 2 \\
10 \\
10\end{array}$ & $\begin{array}{l}3 / 4 \\
3 / 4 \\
3 / 4 \\
3 / 4 \\
3 / 4\end{array}$ & $\begin{array}{l}1.83043 \\
2.80543 \\
3.43043 \\
3.92000 \\
4.42000\end{array}$ & $\begin{array}{l}1.88835 \\
2.86335 \\
3.48835 \\
3.98660 \\
4.48660\end{array}$ & $\begin{array}{r}0.4348 \\
.4348 \\
.4348 \\
.5000 \\
.5000\end{array}$ & $\begin{array}{r}0.261 \pm 0.087 \\
.261 \pm .087 \\
.261 \pm .087 \\
.300 \pm .087 \\
.300 \pm .087\end{array}$ & $\begin{array}{r}0.05791 \\
.05791 \\
.05791 \\
.06660 \\
.06660\end{array}$ & $\begin{array}{r}0.08696 \\
.08696 \\
.08696 \\
.10000 \\
.10000\end{array}$ & $\begin{array}{r}0.1848 \pm 0.100 \\
.1848 \pm .100 \\
.1848 \pm .100 \\
.2500 \pm .100 \\
.2500 \pm .100\end{array}$ \\
\hline
\end{tabular}

UPSET TUBING GAGES

\begin{tabular}{|l|l|l|l|r|r|r|r|r|r|r|r|r|}
\hline $11 / 4$ & 1.660 & 1.81250 & $111 / 2$ & $3 / 4$ & 1.74293 & 1.80085 & 0.4348 & $0.261 \pm 0.087$ & 0.05791 & 0.08696 & $0.1848 \pm 0.100$ \\
$11 / 2$ & 1.900 & 2.09375 & $111 / 2$ & $3 / 4$ & 2.02418 & 2.08210 & .4348 & $.261 \pm .087$ & .05791 & .05696 & $.1848 \pm .100$ \\
2 & 2.375 & 2.59375 & 10 & $3 / 4$ & 2.51375 & 2.58035 & .5000 & $.300 \pm .100$ & .06660 & .10000 & $.2500 \pm .100$ \\
$21 / 2$ & 2.875 & 3.09375 & 10 & $3 / 4$ & 3.01375 & 3.08035 & .5000 & $.300 \pm .100$ & .06660 & .10000 & $.2500 \pm .100$ \\
3 & 3.500 & 3.75000 & 10 & $3 / 4$ & 3.67000 & 3.73660 & .5000 & $.300 \pm .100$ & .06660 & .10000 & $.2500 \pm .100$ \\
$31 / 2$ & 4.000 & 4.25000 & 10 & $3 / 4$ & 4.17000 & 4.23660 & .5000 & $.300 \pm .100$ & .06660 & .10000 & $.2500 \pm .100$ \\
4 & 4.500 & 4.75000 & 10 & $3 / 4$ & 4.67000 & 4.73660 & .5000 & $.300 \pm .100$ & .06660 & .10000 & $.2500 \pm .100$ \\
\hline
\end{tabular}

1 The purchaser may specify the $S$ dimension to suit his rcquirements, ignoring the values given. In the absence of definite specifications for $S$ from the purchaser, the values given above will be uscd. The calibrated value of $S$ in any case must be stamped on the ring gage. Fitting plates may be added to gages if desired.

\section{AMERICAN PETROLEUM INSTITUTE, SPEC- IFICATIONS FOR ROTARY-DRILLING TAPER JOINTS, NO. 7-B, FEBRUARY, 1931}

\section{ROTARY-TOOI JOINT GAGES}

Anyone desiring to produce A. P. I. standard rotary-drilling taper joints shall provide himself with reference master gages which have been manufactured in accordance with the dimensions and tolerances given below, such reference gages to be checked and certified by any A. P. I. custodian or the Bureau of Standards.

\section{SPECIFICATION FOR MASTER GAGES}

All A. P. I. reference master gages (including grand and special reference masters) shall be hardened and ground steel gages, as specified in tables appended. They may be manufactured with a fitting plate or ring. The plug gage (or master pin gage) in all cases shall be the standard.

The thread form on these gages shall be the basic thread form specified for the rotary-drilling taper joints, except that the crests shall be truncated 0.04808 inch for four-thread and 0.03946 inch for five-thread in the 3 inch per foot taper, and 0.04820 inch for four-thread in the 2 inch per foot taper, while the roots may be undercut to prevent the gage from bearing on the crest of the thread on the product.

The first thread on both ends of the plug and ring gages shall be removed until the full form of the thread is obtained. (This provision is effective on new gages purchased subsequent to April 1, 1929.)

The lead and the taper on plug and ring gages shall be measured over a length of axis beginning and ending not less than one-half inch from the ends of the threaded portion.

The plug shall be the control.

The ring shall be controlled by its engagement with the mating plug, and the step value may vary between the limits 0.624 and 0.626 inch.

The pitch diameter at the gage point of the plug shall lie between the limits (basic $\pm T R / 10,000$ inches), but the limit for gages, the $T R$ length of which is less than 4 inches, shall be (basic $\pm 4 / 10,000$ inches); see Table 1.

The lead between any two threads within the lengths specified above may vary from the nominal by not more than the amounts shown in Table 1. (These provisions effective on new ring gages purchased subsequent to April 1, 1929.)

The included taper on diameter in the length $T R$ shall be within the limits shown in Table 1.

The flanks of the thread may vary from the basic angle of $30^{\circ}$ to the normal by not more than the fol$1^{\text {owing values: }}$

$$
\begin{aligned}
& \text { For plug gages } \pm 7 \text { minutes of arc. } \\
& \text { For ring gages } \pm 15 \text { minutes of arc. }
\end{aligned}
$$

The above tolerances are subject to the additional condition that plugs and rings must interchange with A. P. I. regional master ring and plug gages to step values within the limits of 0.621 and 0.629 inch.

\section{TABLE 1.-Tolerances on rotary-tool joint gages}

[All dimensions in inches at $68^{\circ} \mathrm{F}$.]

\begin{tabular}{|c|c|c|c|c|c|c|}
\hline \multirow{3}{*}{ Size } & \multirow{3}{*}{$\begin{array}{c}\text { Pitch } \\
\text { diameter } \\
\text { tolerance }\end{array}$} & \multicolumn{2}{|c|}{ Lead tolerances } & \multicolumn{3}{|c|}{$\begin{array}{l}\text { Taper (difference in diam } \\
\text { eter in } T R \text { length) }\end{array}$} \\
\hline & & \multirow{2}{*}{ Plug } & \multirow{2}{*}{ Ring } & \multirow{2}{*}{$\begin{array}{c}\text { Nomi- } \\
\text { nal }\end{array}$} & \multicolumn{2}{|c|}{ Tolerances } \\
\hline & & & & & Plug & Ring \\
\hline 1 & 2 & 3 & 4 & 5 & 6 & 7 \\
\hline 8. & \pm 0.0004 & \pm 0.0004 & \pm 0.0006 & 0.59375 & +0.0004 & \\
\hline $27 / 8-$ & \pm .0004 & \pm .0004 & \pm .0006 & .71875 & $\begin{array}{r}+.0004 \\
-.0000\end{array}$ & \\
\hline $31 / 2$ & \pm .0004 & \pm .0004 & \pm .0006 & .78125 & \pm & \\
\hline $41 / 2$ & \pm .0004 & \pm .0004 & \pm .0006 & .90625 &.+ & \\
\hline $5 \% 16$ & \pm .0004 & \pm .0004 & \pm .0006 & 1. 03125 & + & -. \\
\hline $65 / 8$ & \pm .0004 & \pm .0004 & \pm .0007 & .72917 & + & \\
\hline $75 / 8$ & \pm .0005 & \pm .0005 & \pm .0007 & 1. 15625 & +.0005 & -.00 \\
\hline $85 / 3$ & \pm .0005 & \pm .0005 & \pm .0007 & 1. 18750 & $\begin{array}{r}+.0005 \\
+.0000\end{array}$ & $\begin{array}{l}-.00 \\
-.00\end{array}$ \\
\hline
\end{tabular}

[Values apply to either right or left hand threads] 
Column 3. The tolerances on lead of plug gages whose $T R$ length is less than 4 inches are based upon a tolerance of \pm 0.0004 from basic, and for larger gages upon a tolerance of \pm 0.0001 per inch of $T R$ length.

Column 4. The tolerances on lead of ring gages whose $T R$ length is less than 4 inches are based upon \pm 0.0006 inch from basic, and for larger gages upon a tolerance of \pm 0.00015 inch per inch of $T R$ length. * Column 5. Above values show the difference in diameter over the $T R$ length. The $6 \% / 8$-inch size is based on a taper of 2 inches per foot; all others, 3 inches per foot.

Column 6. The tolerances on taper of plug gages whose $T R$ length is less than 4 inches are based on a tolerance of (basic) and (basic +0.0004 inch), and for large gages upon a tolerance of (basic) and (basic $+T R / 10,000$ inch).

Column 7. The tolerances on taper of ring gages are based upon the limits of (basic-0.0004 inch) and (basic-0.0012 inch).

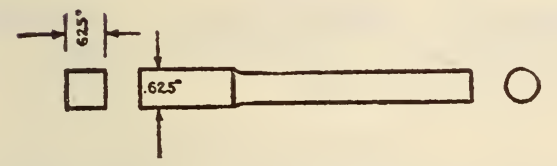

Figure 666. - Feeler gage

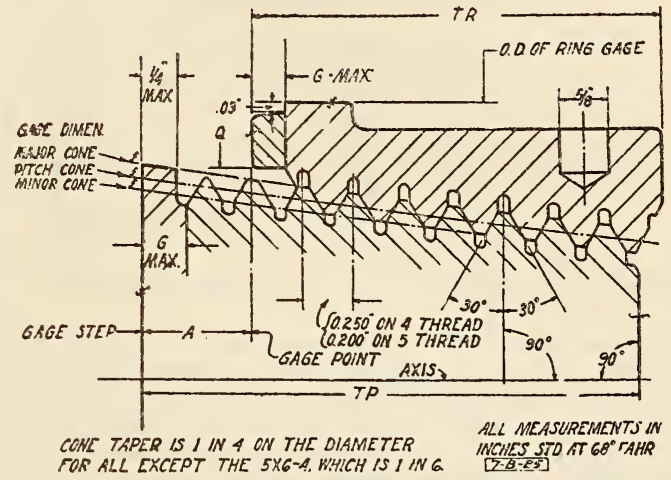

FIgURE 667.-Rotary drilling taper joint gages

Note. $-G$ (fitting plates), which is a maximum dimension, equals 0.437 inch for $65 \%, 75 \%$, and $81 / 2$ inch sizes, and equals 0.375 for all other
sizes. TABLE 2.-Gage dimensions for A. P. I. standard rotary-drilling taper joints

[See figs. 666, 667, and 668. All dimensions in inches at $68^{\circ} \mathrm{F}$. Taper joints shall be identified by the drill-pipe size]

\begin{tabular}{|c|c|c|c|c|c|c|c|c|c|c|c|}
\hline \multirow[t]{2}{*}{$\begin{array}{l}\text { Size } \\
\text { of } \\
\text { drill } \\
\text { pipe }\end{array}$} & \multirow[t]{2}{*}{ Nominal size of tapered joint } & \multirow[t]{2}{*}{ Pitch } & \multirow[t]{2}{*}{$\begin{array}{l}\text { Taper, } \\
\text { inches } \\
\text { per } \\
\text { foot }\end{array}$} & $\begin{array}{c}\text { Pitch diam- } \\
\text { eter at gage } \\
\text { point }\end{array}$ & $\begin{array}{c}\text { Major cone } \\
\text { diameter at } \\
\text { gage step }\end{array}$ & $\begin{array}{l}\text { Minor cone } \\
\text { diameter at } \\
\text { gage point }\end{array}$ & $\begin{array}{l}\text { Total } \\
\text { length } \\
\text { plug } \\
\text { gage } \\
T P\end{array}$ & $\begin{array}{c}\text { Total } \\
\text { length } \\
\text { ring } \\
\text { gage } \\
T R\end{array}$ & $\begin{array}{c}\text { Outside } \\
\text { diameter } \\
\text { of ring } \\
\text { gage } \\
O . D \text {. }\end{array}$ & $\begin{array}{c}\text { Diame- } \\
\text { ter of } \\
\text { recess } \\
Q\end{array}$ & $\begin{array}{l}\text { Step } \\
\text { value } \\
\qquad A\end{array}$ \\
\hline & & & & 1 & 2 & 3 & 4 & 5 & 6 & 7 & 8 \\
\hline $\begin{array}{r}23 / 8 \\
27 / 8 \\
31 / 2 \\
41 / 2 \\
59 \% 16 \\
65 / 8 \\
75 / 8 \\
85 \% 8\end{array}$ & $\begin{array}{l}17 / 8 \text { by } 25 / 8-5 \\
218 \text { by } 3-5 \\
2916 \text { by } 31 / 2-5 \\
396 \text { by } 45 / 8-5 \\
41 / 2 \text { by } 51 / 2-4 \\
5 \text { by } 6-4 \\
6 \text { by } 7-4 \\
7 \text { by } 8-4\end{array}$ & $\begin{array}{l}1 / 5 \\
1 / 5 \\
1 / 5 \\
1 / 5 \\
1 / 4 \\
1 / 4 \\
1 / 4 \\
1 / 4\end{array}$ & $\begin{array}{l}3 \\
3 \\
3 \\
3 \\
3 \\
2 \\
3 \\
3\end{array}$ & $\begin{array}{l}\text { 2. } 36537 \\
\text { 2. } 74037 \\
\text { 3. } 23987 \\
\text { 4. } 36487 \\
\text { 5. } 23402 \\
\text { 5. } 75780 \\
\text { 6. } 71453 \\
\text { 7. } 66658\end{array}$ & $\begin{array}{l}\text { 2. } 61500 \\
\text { 2. } 99000 \\
\text { 3. } 48950 \\
\text { 4. } 61450 \\
\text { 5. } 50950 \\
\text { 5. } 98157 \\
\text { 6. } 99000 \\
\text { 7. } 94206\end{array}$ & $\begin{array}{l}\text { 2. } 27199 \\
\text { 2. } 64699 \\
\text { 3. } 14649 \\
\text { 4. } 27149 \\
\text { 5. } 11479 \\
\text { 5. } 63820 \\
\text { 6. } 59531 \\
\text { 7. } 54735\end{array}$ & $\begin{array}{l}3 \\
31 / 2 \\
33 / 4 \\
41 / 4 \\
43 / 4 \\
5 \\
51 / 4 \\
53 / 8\end{array}$ & $\begin{array}{l}23 / 8 \\
27 / 8 \\
31 / 8 \\
358 \\
41 / 8 \\
43 / 8 \\
458 \\
43 / 4\end{array}$ & $\begin{array}{r}3.750 \\
4.250 \\
5.000 \\
6.250 \\
7.500 \\
8.250 \\
9.500 \\
10.750\end{array}$ & $\begin{array}{l}2.531 \\
\text { 2. } 906 \\
\text { 3. } 406 \\
4.531 \\
5.427 \\
5.949 \\
6.908 \\
7.858\end{array}$ & $\begin{array}{r}0.625 \\
.625 \\
.625 \\
.625 \\
.625 \\
.625 \\
.625 \\
.625\end{array}$ \\
\hline
\end{tabular}

The values contained in Table 2 apply to either right or left hand threads.

When ordering gages the purchaser should specify whether right-hand or left-hand threads are desired.

The gage point is to be 0.625 inch from the plug gage shoulder.

Tolerance:

Column $2= \pm 0.002$ inch; column $3= \pm 0.002$ inch; column $4= \pm 0.094$ inch. Column $5= \pm 0.094$ inch; column $6=$ diameter \pm 0.005 inch; column $7= \pm 0.015$ inch.

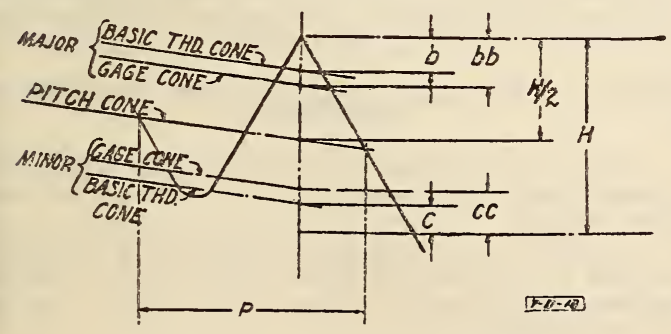

FIGURE 668. - Thread heights
TABLE 3.-Thread heights-Product and gages

[See fig. 668. All dimensions in inches at $65^{\circ} \mathrm{F}$.]

\begin{tabular}{|c|c|c|c|c|c|c|c|c|}
\hline \multirow{2}{*}{$\begin{array}{l}\text { Num- } \\
\text { ber of } \\
\text { threads } \\
\text { per } \\
\text { inch }\end{array}$} & \multirow{2}{*}{$\underset{P}{\text { Pitch }}$} & \multirow{2}{*}{$\begin{array}{c}\text { Taper } \\
\text { inches } \\
\text { per } \\
\text { foot }\end{array}$} & \multirow{2}{*}{$\begin{array}{l}\text { Height } \\
\text { before } \\
\text { trunca- } \\
\text { tion } H\end{array}$} & \multicolumn{2}{|c|}{$\begin{array}{c}\text { Crest trunca- } \\
\text { tion }\end{array}$} & \multicolumn{2}{|c|}{$\begin{array}{c}\text { Root trunca- } \\
\text { tion }\end{array}$} & \multirow{2}{*}{$\begin{array}{l}\text { Half } \\
\text { of } \\
\text { theo- } \\
\text { reti- } \\
\text { cal } \\
\text { thread } \\
\text { height } \\
\text { H/2 }\end{array}$} \\
\hline & & & & $\begin{array}{l}\text { On } \\
\text { prod- } \\
\text { uet } b\end{array}$ & $\begin{array}{l}\text { On } \\
\text { gage } \\
b h\end{array}$ & $\begin{array}{c}\text { On } \\
\text { prod- } \\
\text { uct } c\end{array}$ & $\underset{c c}{\operatorname{On}}$ & \\
\hline & $\begin{array}{r}0.200 \\
.250 \\
.250\end{array}$ & & $\begin{array}{r}0.172303 \\
.215379 \\
.216005\end{array}$ & $\begin{array}{r}0.03446 \\
.04308 \\
.04320\end{array}$ & $\begin{array}{r}0.03946 \\
.04808 \\
.04820\end{array}$ & $\begin{array}{r}0.03446 \\
.04308 \\
.04320\end{array}$ & $\begin{array}{r}0.03946 \\
.04808 \\
.04820\end{array}$ & $\begin{array}{r}0.08615 \\
.10769 \\
.10800\end{array}$ \\
\hline
\end{tabular}


AMERICAN PETROLEUM INSTITUTE, SPEC. IFICATIONS FOR OIL-WELI PUMPS (BARRELS, PLUNGERS, VALVES, ETC.), HO. 11-A, FEBRUARY, 1931

\section{WORKING-BARREL GAGES}

The inside diameters of cold-drawn working barrels and machined liners shall be of the dimensions

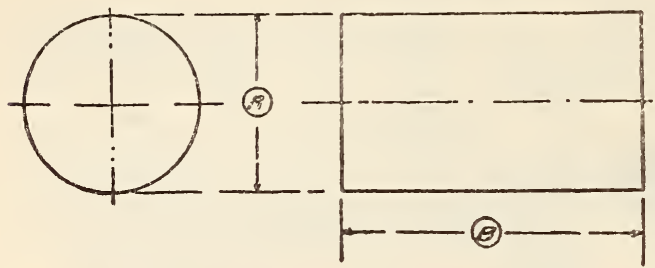

FIGURE 669. - Gage for cold-drawn working barrels

and within the tolerances stipulated. The use of the "go" plug gages specified by Figure 669 and Table 2 , and Figure 670 and Table 6 , is a necessary and sufficient check of straightness and minimum diameter. Use of an inside gage is necessary to insure that a barrel or liner is not oversize at any point. Gages other than those referred to may be used, but must be capable of measuring the diameter throughout the whole length and around the circumference. In any case it devolves upon the user

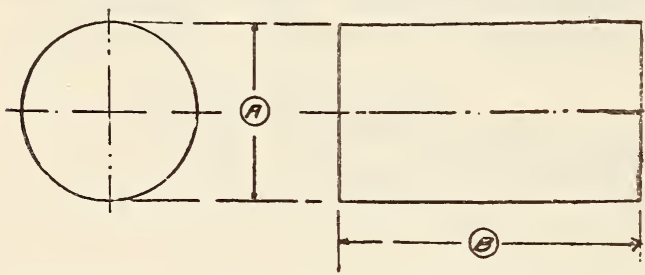

FIGURE 670.-Gage for machined working barrels

of the gage to demonstrate that the gage he chooses to use has an accuracy appropriate to the product being gaged.

TABLE 2.-Gages for cold-drawn barrels

[See fig. 669. All dimensions in inches at $68^{\circ} \mathrm{F}$.]

\begin{tabular}{|c|c|c|c|c|c|}
\hline \multirow{3}{*}{$\begin{array}{l}\text { Size of } \\
\text { work- } \\
\text { ing } \\
\text { barrel }\end{array}$} & \multicolumn{2}{|c|}{$\begin{array}{l}\text { Permissible limits } \\
\text { on inside diameter } \\
\text { of product }\end{array}$} & \multicolumn{3}{|c|}{ Permissible limits “go" gage } \\
\hline & \multirow{2}{*}{$\begin{array}{l}\text { Maxi- } \\
\text { mum }\end{array}$} & \multirow{2}{*}{$\begin{array}{l}\text { Mini- } \\
\text { mum }\end{array}$} & \multicolumn{2}{|c|}{ Diameter $A$} & \multirow{2}{*}{ 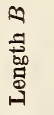 } \\
\hline & & & Nominal & Tolerance & \\
\hline 2. & 1.7892 & 1.7728 & 1.7728 & +0.0002 & 3 \\
\hline $21 / 2-$ & 2.2582 & 2.2418 & 2.2418 & $\begin{array}{r}-.0000 \\
+.0002\end{array}$ & 3 \\
\hline 3.- & 2.7582 & 2. 7418 & 2. 7418 & $\begin{array}{l}-.0000 \\
+.0002\end{array}$ & 3 \\
\hline 4.- & 3. 7602 & 3. 7398 & 3. 7398 & $\begin{array}{r}.0000 \\
+.0000\end{array}$ & 3 \\
\hline
\end{tabular}

TABLE 6.-Gages for machined liner working barrels [See fig. 670. All dimensions in inches at $68^{\circ} \mathrm{F}$.]

\begin{tabular}{|c|c|c|c|c|c|}
\hline \multirow{3}{*}{$\begin{array}{l}\text { Size of } \\
\text { work- } \\
\text { ing } \\
\text { barrel }\end{array}$} & \multicolumn{2}{|c|}{$\begin{array}{l}\text { Permissible limits } \\
\text { on inside diame- } \\
\text { ter of product }\end{array}$} & \multicolumn{3}{|c|}{ Permissible limits "go" gage } \\
\hline & \multirow{2}{*}{$\begin{array}{l}\text { Maxi- } \\
\text { mum }\end{array}$} & \multirow{2}{*}{$\begin{array}{l}\text { Mini- } \\
\text { mum }\end{array}$} & \multicolumn{2}{|c|}{ Diameter $A$} & \multirow{2}{*}{ 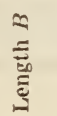 } \\
\hline & & & Nominal & Tolerance & \\
\hline 2 & 1. 7512 & 1. 7498 & 1. 7498 & +0.0002 & 12 \\
\hline $21 / 2$ & 2. 2512 & 2. 2498 & 2. 2498 & +.0002 & 12 \\
\hline 3 & 2. 7512 & 2. 7498 & 2. 7498 & $\begin{array}{l}-.0000 \\
+.0002\end{array}$ & 12 \\
\hline 4 & 3. 7512 & 3. 7498 & 3. 7498 & $\begin{array}{l}+.0002 \\
+.0000\end{array}$ & 12 \\
\hline
\end{tabular}

Nore.-The entire length of machined liners shall be checked with the "go" gage.

\section{AMERICAN PETROLEUM INSTITUTE,} SUCKER-ROD SPECIFICATIONS, NO. 11-B, JULY, 1931

\section{SUCKER-ROD GAGES}

Any manufacturer desiring to produce sucker-rod joints in accordance with A. P. I. standards shalı

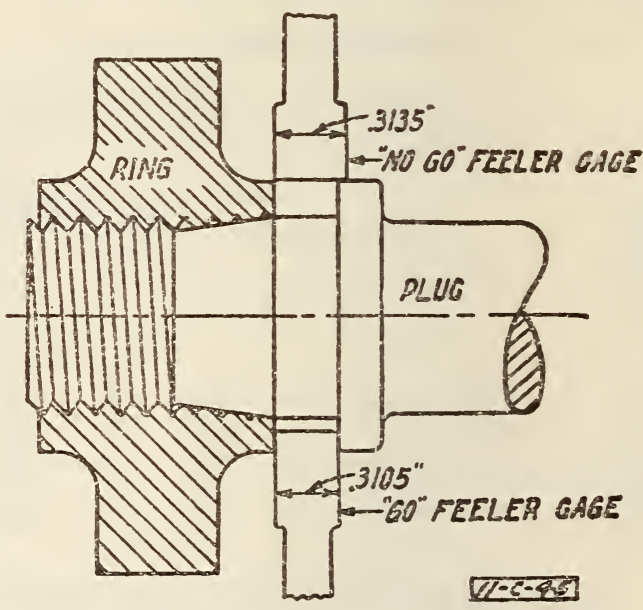

FIgURE 671.-Projection of mating gages

provide himself with a set of reference master gages, as specified below, such reference master gages to be checked and certified by the Bureau of Standards or by the National Physical Laboratory, England.

\section{SPECIFICATION FOR A. P. I. GRAND MASTER AND REFERENCE MASTER GAGES}

Grand master gages and manufacturer's reference master gages shall consist of the following for each size of joint:

A master "go" plug gage representing maximum thread and maximum cone of pin, and a mating reference ring for the master "go" plug. (See fig. 672 and Table 1.) 
A master "no go" plug gage representing the minimum pitch diameter of the pin, and a mating reference ring for the master "no go" plug gage. (See fig. 673 and Table 2.)

A master "go" ring gage representing the minimum thread and minimum cone of box, and a mating reference plug for the master "go" ring. (See fig. 674 and Table 3.)

A master "no go" ring gage representing the maximum pitch diameter of the box, and a mating reference plug for the master "no go" ring gage. (See fig. 675 and Table 4.)

"Go" and "no go" feeler gages to check cone dimensions.

A. P. I. grand master and manufacturer's reference master gages shall be hardened and ground steel gages and shall be manufactured in accordance with dimensions and tolerances given in tables and figures appended.

The form of the threads for the A. P. I. sucker-rod gages shall be the thread form specified for A. P. I. sucker-rod threads.

The mates of "go" master gages shall be a good gage fit on their respective masters, and shall assem-

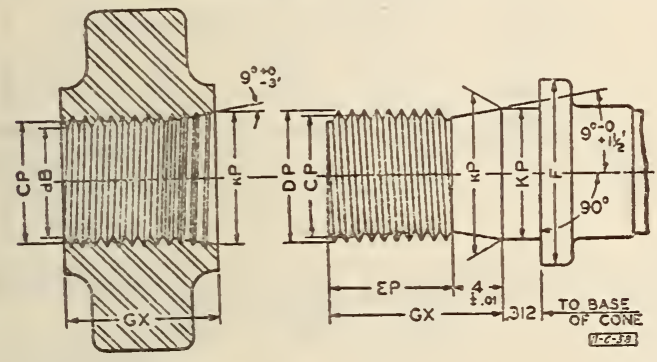

FIGURE 672.-Master "go" plug gage and mating

ble to cone contact by hand, without spinning, to a step value of $0.312 \mathrm{inch} \pm 0.0015 \mathrm{inch}$. (See fig.671.)

The mates of "no go" master gages shall be a good gage fit on their respective masters.

The ring gages may be of the adjustable type, if properly locked and sealed.

The permissible eccentricity of cone of "go" gages, with relation to axis of thread, shall not exceed 0.0002 inch for the plug gage or 0.0004 inch for the ring gage.

The allowable variation in lead between any two threads no farther apart than the length of engagement shall not exceed \pm 0.0002 inch. The flanks of the thread may vary from the basic angle of $30^{\circ}$ to the normal by not more than \pm 10 minutes of arc. A. P. I. gages shall be stamped with the certificate number, a suffix letter which indicates the name of the gage, the A.P. I. monogram, the nominal size of the rod, the words "go" or "no go," and the words "pin" or "box."

TABLE 1.-A. P. I. sucker-rod gages, master "go" plug gage and mating ring

[Maximum pin cone and threads]

[See fig. 672. All dimensions in inches at $68^{\circ} \mathrm{F}$.]

\begin{tabular}{|c|c|c|c|c|c|c|c|c|c|}
\hline 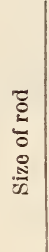 & 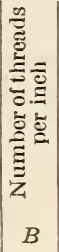 & 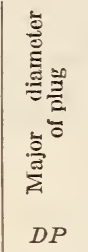 & 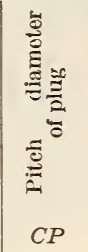 & 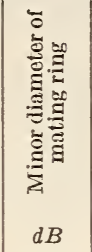 & 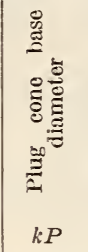 & 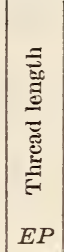 & 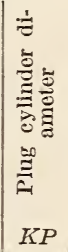 & 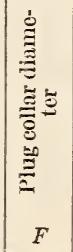 & 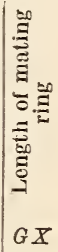 \\
\hline $5 / 8$ & 10 & $\begin{array}{r}0.93695 \\
+.0000 \\
-.0004\end{array}$ & $\begin{array}{r}0.87200 \\
+.0000 \\
-.0002\end{array}$ & $\begin{array}{r}0.82970 \\
+.0000 \\
-.0004\end{array}$ & $\begin{array}{r}0.94300 \\
+.0000 \\
-.0002\end{array}$ & $\begin{array}{c}0.63 \\
\pm .01\end{array}$ & $\begin{array}{r}0.943 \\
+.000 \\
-.003\end{array}$ & $\begin{array}{r}1.380 \\
+.000 \\
-.010\end{array}$ & \pm \\
\hline $3 / 4$ & 10 & $\begin{array}{r}1.06195 \\
+.0000 \\
-.0004\end{array}$ & $\begin{array}{r}.99700 \\
+.0000 \\
-.0002\end{array}$ & $\begin{array}{r}.95470 \\
+.0000 \\
-.0004\end{array}$ & $\begin{array}{r}1.068000 \\
+.0000 \\
-.0002\end{array}$ & $\begin{array}{r}.88 \\
\pm .01\end{array}$ & $\begin{array}{r}1.068 \\
+.000 \\
-.003\end{array}$ & $\begin{array}{r}1.505 \\
+.000 \\
-.010\end{array}$ & \\
\hline $7 / 8$ & 10 & $\begin{array}{r}1.18695 \\
+.0000 \\
-.0004\end{array}$ & $\begin{array}{r}1.12200 \\
+.0000 \\
-.0002\end{array}$ & $\begin{array}{r}1.07970 \\
+.0000 \\
-.0001\end{array}$ & $\begin{array}{r}1.19300 \\
+.0000 \\
-.0002\end{array}$ & $\begin{array}{r}.88 \\
\pm .01\end{array}$ & $\begin{array}{r}1.193 \\
+.000 \\
-.003\end{array}$ & $\begin{array}{r}1.692 \\
+.000 \\
-.010\end{array}$ & \\
\hline
\end{tabular}

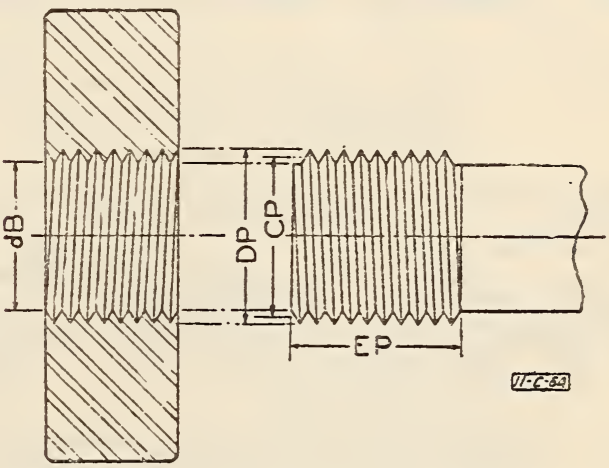

FIGURE 673.-Master "go" plug gage and
mating ring 
TABLE 2.-A. P. I. sucker-rod gages master "no go" plug gage and mating ring

[Minimum pin thread]

[See fig. 673. All dimensions in inches at $68^{\circ} \mathrm{F}$.]

\begin{tabular}{|c|c|c|c|c|c|}
\hline $\begin{array}{l}\text { Size of } \\
\text { rod }\end{array}$ & $\begin{array}{c}\text { Num- } \\
\text { ber of } \\
\text { threads } \\
\text { per } \\
\text { inch } \\
B\end{array}$ & $\begin{array}{c}\text { Major } \\
\text { diameter } \\
\text { of plug } \\
\\
D P\end{array}$ & $\begin{array}{c}\text { Pitch } \\
\text { diameter } \\
\text { of plug } \\
\\
C P\end{array}$ & $\begin{array}{c}\text { Minor } \\
\text { diameter } \\
\text { of mat- } \\
\text { ing ring } \\
\text { gage } \\
d B\end{array}$ & $\begin{array}{c}\text { Thread length } \\
E P\end{array}$ \\
\hline $58 \ldots$ & 10 & $\begin{array}{r}0.93695 \\
+.0000 \\
-.0004\end{array}$ & $\begin{array}{l}0.86900 \\
+.0002 \\
-.0000\end{array}$ & $\begin{array}{l}0.82970 \\
+.0004 \\
-.0000\end{array}$ & \\
\hline $3 / 4 \ldots$ & 10 & $\begin{array}{l}1.06195 \\
+.0000 \\
-.0004\end{array}$ & $\begin{array}{l}.99400 \\
+.0002 \\
-.0000\end{array}$ & $\begin{array}{l}.95470 \\
+.0004 \\
-.0000\end{array}$ & $\left\{\begin{array}{c}\text { Good commercial } \\
\text { practice (not less } \\
\text { than } 3 \text { perfect } \\
\text { threads) }\end{array}\right.$ \\
\hline $7 / 8$ & 10 & $\begin{array}{l}1.18695 \\
+.0000 \\
-.0004\end{array}$ & $\begin{array}{l}1.11900 \\
+.0002 \\
-.0000\end{array}$ & $\begin{array}{l}1.07970 \\
+.0004 \\
-.0000\end{array}$ & \\
\hline
\end{tabular}

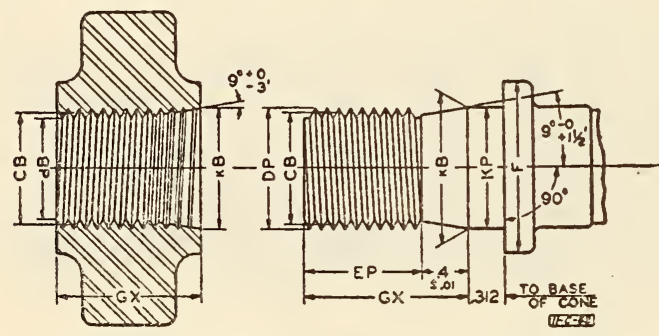

Figure 674.-Master "go" ring and mating plug

TABLE 3.-A. P. I. sucker-rod gages master "go" ring and mating plug

[Minimum box thread and cone]

[See fig. 674. All dimensions in inches at $68^{\circ} \mathrm{F}$. See Table 1 for dimension $F$ ]

\begin{tabular}{|c|c|c|c|c|c|c|c|c|}
\hline $\begin{array}{c}\text { Size } \\
\text { of } \\
\text { rod }\end{array}$ & $\begin{array}{c}\text { Num- } \\
\text { ber of } \\
\text { threads } \\
\text { per } \\
\text { inch } \\
B\end{array}$ & $\begin{array}{c}\text { Minor } \\
\text { diam- } \\
\text { eter of } \\
\text { ring } \\
d B\end{array}$ & $\begin{array}{c}\text { Pitch } \\
\text { diam- } \\
\text { eter of } \\
\text { mating } \\
\text { plug } \\
c B\end{array}$ & $\begin{array}{c}\text { Major } \\
\text { diam- } \\
\text { eter of } \\
\text { mating } \\
\text { plug } \\
D P\end{array}$ & $\begin{array}{l}\text { Plug } \\
\text { cyl- } \\
\text { inder } \\
\text { diam- } \\
\text { eter } \\
K P\end{array}$ & $\begin{array}{c}\text { Plug } \\
\text { cone } \\
\text { base } \\
\text { diam- } \\
\text { eter } \\
k B\end{array}$ & $\begin{array}{c}\text { Ring } \\
\text { length } \\
G X\end{array}$ & $\begin{array}{l}\text { Length } \\
\text { of } \\
\text { threads } \\
\text { on } \\
\text { mating } \\
\text { plug } \\
E P\end{array}$ \\
\hline $5 / 8 \ldots$ & 10 & $\begin{array}{r}0.82970 \\
+.0004 \\
-.0000\end{array}$ & $\begin{array}{r}0.87300 \\
+.0002 \\
-.0000\end{array}$ & $\begin{array}{r}0.93695 \\
+.0004 \\
-.0000\end{array}$ & $\begin{array}{r}0.943 \\
+.000 \\
-.003\end{array}$ & $\begin{array}{r}0.9430 \\
+.0000 \\
-.0002\end{array}$ & $\begin{array}{r}1.03 \\
\pm .01\end{array}$ & $\begin{array}{r}0.63 \\
\pm .01\end{array}$ \\
\hline $3 / 4 \ldots$ & 10 & $\begin{array}{r}.95470 \\
+.0004 \\
-.0000\end{array}$ & $\begin{array}{r}.99800 \\
+.0002 \\
-.0000\end{array}$ & $\begin{array}{r}1.06195 \\
+.0004 \\
-.0000\end{array}$ & $\begin{array}{r}1.068 \\
+.000 \\
-.003\end{array}$ & $\begin{array}{r}1.0680 \\
+.0000 \\
-.0002\end{array}$ & $\begin{array}{r}1.28 \\
\pm .01\end{array}$ & $\begin{array}{r}.88 \\
\pm .01\end{array}$ \\
\hline $7 / 8 \ldots$ & 10 & $\begin{array}{r}1.0797 \\
+.0004 \\
-.0000\end{array}$ & $\begin{array}{r}1.12300 \\
+.0002 \\
-.0000\end{array}$ & $\begin{array}{r}1.18695 \\
+.0004 \\
-.0000\end{array}$ & $\begin{array}{r}1.193 \\
+.000 \\
-.003\end{array}$ & $\begin{array}{r}1.1930 \\
+.0000 \\
-.0002\end{array}$ & $\begin{array}{r}1.28 \\
\pm .01\end{array}$ & $\begin{array}{r}.88 \\
\pm .01\end{array}$ \\
\hline
\end{tabular}

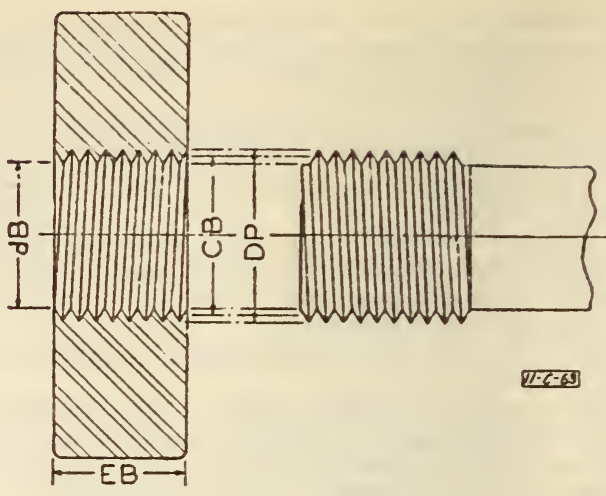

Figure 675. - Master "no go" ring gage and mating plug

TABLE 4.-A. P. I. sucker-rod gages master "no go" ring gage and mating plug

[Maximum box threads]

[See fig. 675. All dimensions in inches at $68^{\circ} \mathrm{F}$.]

\begin{tabular}{|c|c|c|c|c|c|c|}
\hline $\begin{array}{l}\text { Size of } \\
\text { rod }\end{array}$ & $\begin{array}{c}\text { Num- } \\
\text { ber of } \\
\text { threads } \\
\text { per } \\
\text { inch } \\
\\
B\end{array}$ & $\begin{array}{l}\text { Minor } \\
\text { diameter } \\
\text { of ring } \\
d B\end{array}$ & $\begin{array}{l}\text { Pitch } \\
\text { diameter } \\
\text { of mat- } \\
\text { ing plug } \\
c B\end{array}$ & $\begin{array}{c}\text { Major } \\
\text { diameter } \\
\text { of mat- } \\
\text { ing plug } \\
D P\end{array}$ & $\begin{array}{c}\text { Ring } \\
\text { length } \\
\text { EB }\end{array}$ & $\begin{array}{l}\text { Length } \\
\text { of } \\
\text { threads } \\
\text { on } \\
\text { mating } \\
\text { plug } \\
E B\end{array}$ \\
\hline $5 / 8 \ldots$ & 10 & $\begin{array}{l}0.82970 \\
+.0004 \\
-.0000\end{array}$ & $\begin{array}{r}0.87600 \\
+.0000 \\
-.0002\end{array}$ & $\begin{array}{r}0.93695 \\
+.0000 \\
-.0004\end{array}$ & \multicolumn{2}{|c|}{$\begin{array}{l}\text { Good commer- } \\
\text { ci a l prac- } \\
\text { tice (not less } \\
\text { than } 3 \text { perfect } \\
\text { threads) }\end{array}$} \\
\hline $3 / 4 \ldots$ & 10 & $\begin{array}{r}.95470 \\
+.0004 \\
-.0000\end{array}$ & $\begin{array}{r}1.00100 \\
+.0000 \\
-.0002\end{array}$ & $\begin{array}{r}1.06195 \\
+.0000 \\
-.0004\end{array}$ & & \\
\hline $7 / 8 \ldots \ldots$ & 10 & $\begin{array}{r}1.0797 \\
+.0004 \\
-.0000\end{array}$ & $\begin{array}{r}1.12600 \\
+.0000 \\
-.0002\end{array}$ & $\begin{array}{l}1.18695 \\
+.0000 \\
-.0004\end{array}$ & & \\
\hline
\end{tabular}

AMERICAN RAILWAY ASSOCIATION, MECHANICAL DIVISION ; GAGES AND TESTING DEVICES, RECOMMENDED PRACTICE, 1930

\section{IIMIT GAGES FOR ROUND IRON}

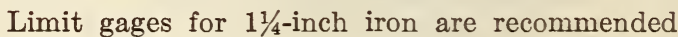
for use in procuring round iron to take the Sellers' standard screw threads; round iron used to be of such size as will enter the large or + end of the gage intended for that size, in any way, and also of such size as will not enter the small or - end in any way. 
The limiting diameters for certain nominal sizes of iron, together with the maximum variation allowable by such use of these gages, are given in the following table:

\section{Sizes of limit gages for round iron}

\begin{tabular}{|c|c|c|c|}
\hline $\begin{array}{l}\text { Nominal } \\
\text { diameter } \\
\text { of iron }\end{array}$ & $\begin{array}{c}\text { Large } \\
\text { size + } \\
\text { end }\end{array}$ & $\begin{array}{l}\text { Small } \\
\text { size - } \\
\text { end }\end{array}$ & $\begin{array}{c}\text { Total } \\
\text { variation }\end{array}$ \\
\hline $\begin{array}{c}\text { Inches } \\
1 / 4 \\
5 / 16 \\
3 / 8 \\
7 / 16 \\
3 / 2\end{array}$ & $\begin{array}{l}\text { Inches } \\
0.2550 \\
.3180 \\
.3810 \\
.4440 \\
.5070\end{array}$ & $\begin{array}{c}\text { Inches } \\
0.2450 \\
.3070 \\
.3690 \\
.4310 \\
.4930\end{array}$ & $\begin{array}{l}\text { Inch } \\
0.010 \\
.011 \\
.012 \\
.013 \\
.014\end{array}$ \\
\hline $\begin{array}{l}9 / 16 \\
5 / 8 \\
3 / 4 \\
7 / 8\end{array}$ & $\begin{array}{l}.5700 \\
.6330 \\
.7585 \\
.8840\end{array}$ & $\begin{array}{l}.5550 \\
.6170 \\
.7415 \\
.8660\end{array}$ & $\begin{array}{l}.015 \\
.016 \\
.017 \\
.018\end{array}$ \\
\hline $\begin{array}{l}1 \\
138 \\
11 / 4 \\
13 / 8\end{array}$ & $\begin{array}{l}1.0095 \\
1.1350 \\
1.2605 \\
1.3860\end{array}$ & $\begin{array}{r}.9905 \\
1.1150 \\
1.2395 \\
1.3640\end{array}$ & $\begin{array}{l}.019 \\
.020 \\
.021 \\
.022\end{array}$ \\
\hline $\begin{array}{l}13 / 2 \\
15 / 8 \\
13 / 4 \\
17 / 8\end{array}$ & $\begin{array}{l}1.5115 \\
1.6370 \\
1.7625 \\
1.8880\end{array}$ & $\begin{array}{l}1.4885 \\
1.6130 \\
1.7375 \\
1.8620\end{array}$ & $\begin{array}{l}.023 \\
.024 \\
.025 \\
.026\end{array}$ \\
\hline
\end{tabular}

Round iron 2 inches in diameter and over should be rolled to nominal diameter.
AMERICAN RAILWAY ASSOCIATION, MECHANICAL DIVISION ; GAGES AND TESTING DEVICES, STANDARD, 1895

\section{DECIMAL GAGE}

The micrometer caliper should be used for laboratory and tool-room work, and in the shop when specially desired.

The solid notch gage should be used for general shop purposes. The form of this gage shall be an ellipse whose major axis is 4 inches, the minor axis 2.5 inches, and the thickness 0.1 inch, with a central hole 0.75 inch in diameter.

The notches in this gage shall be as follows:

\begin{tabular}{|l|l|l|l|}
\hline Inch & Inch & Inch & Inch \\
0.002 & 0.022 & 0.065 & 0.135 \\
.004 & .025 & .070 & .150 \\
.006 & .028 & .075 & .165 \\
.008 & .032 & .080 & .180 \\
.010 & .036 & .085 & .200 \\
.012 & .040 & .090 & .220 \\
.014 & .045 & .095 & .240 \\
.016 & .050 & .100 & .250 \\
.018 & .055 & .110 &.. \\
.020 & .060 & .125 &...- \\
\hline
\end{tabular}

All notches to be marked as in above list.

The gage must be plainly stamped with the words "decimal gage" in capital letters 0.2 inch high, and below this the words "master mechanics."

In ordering material the term "gage" shall not be used, but the thickness ordered by writing the decimal as in above list. For sizes over one-fourth inch the ordinary common fractions may be used.

\section{AMERICAN RAILWAY ENGINEERING ASSOCIATION, TRACK GAGE, SEPTEMBER, 1929}

\section{TRACK GAGE}

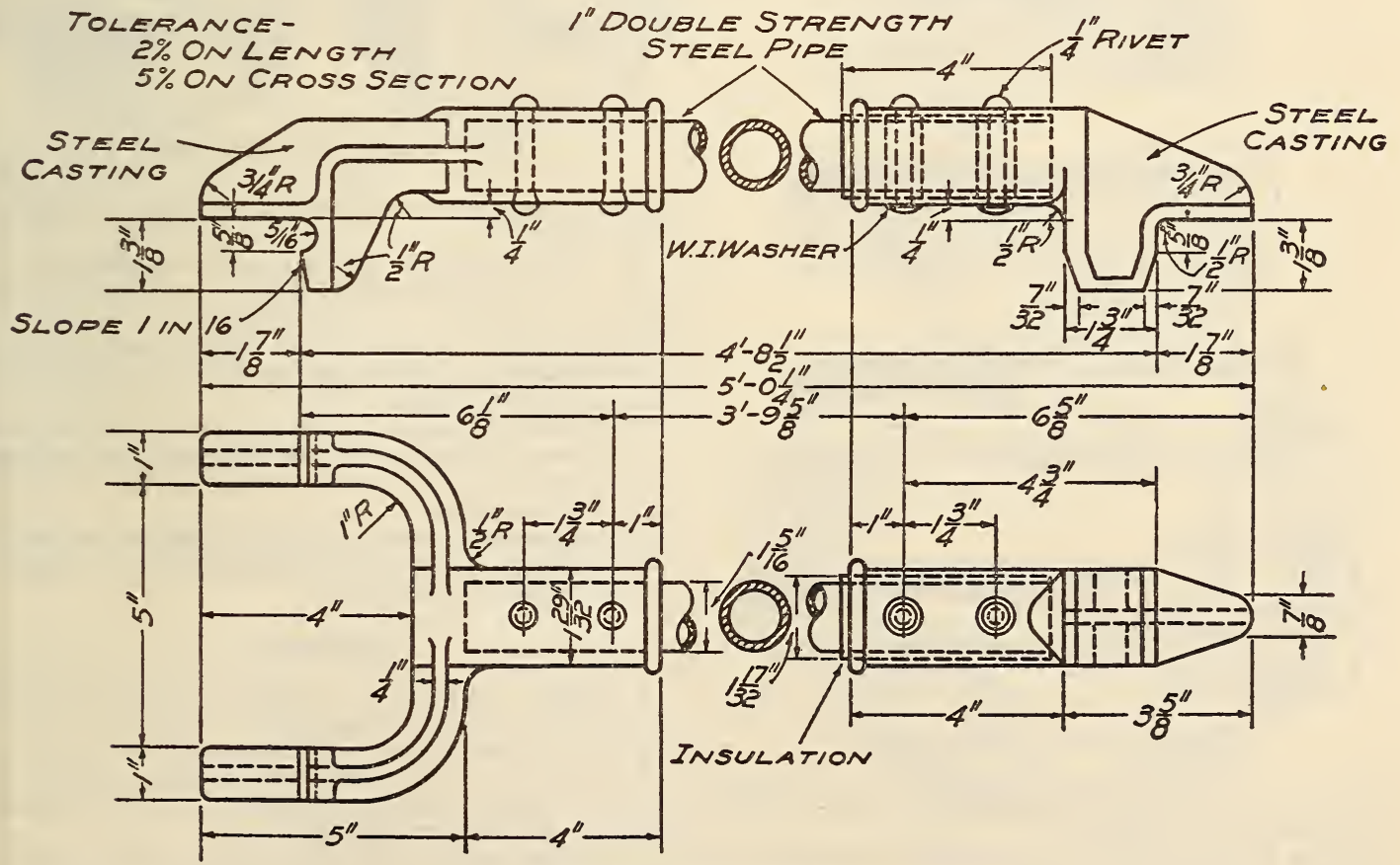

APPROX. WT. 17 LES.

FIGURE 676. - Track gage

Note.-Insulate by use of fiber bushings at both ends when required. Center line to be marked on gage when required. No tolerance allowed in gage distance. 
AMERICAN SOCIETY OF MECHANICAL ENGINEERS, TENTATIVE AMERICAN STANDARD TOLERANCES, ALLOWANCES, AND GAGES FOR METAL FITS. APPROVED BY THE AMERICAN STANDARDS ASSOCIATION, DECEMBER, 1925, B4a-1925

\section{TOLERANCES, ALIOWANCES, AND GAGES FOR INETAL FITS}

\section{FUNDAMENTALS}

1. Direction of tolerance on gages.-The extreme sizes for all plain limit gages shall not exceed the extreme limits of the part to be gaged. All varia-
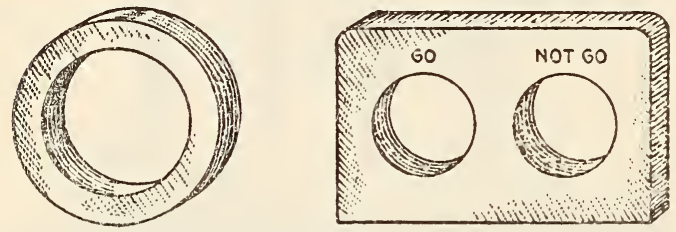

Figure 677.-Ring gages

tion in the gages, whatever their cause or purpose, shall bring these gages within these extreme limits. Thus a gage which represents a minimum limit may be larger, but never smaller, than the minimum size specified for the part to be gaged, likewise the gage which represents a maximum limit may be smaller,
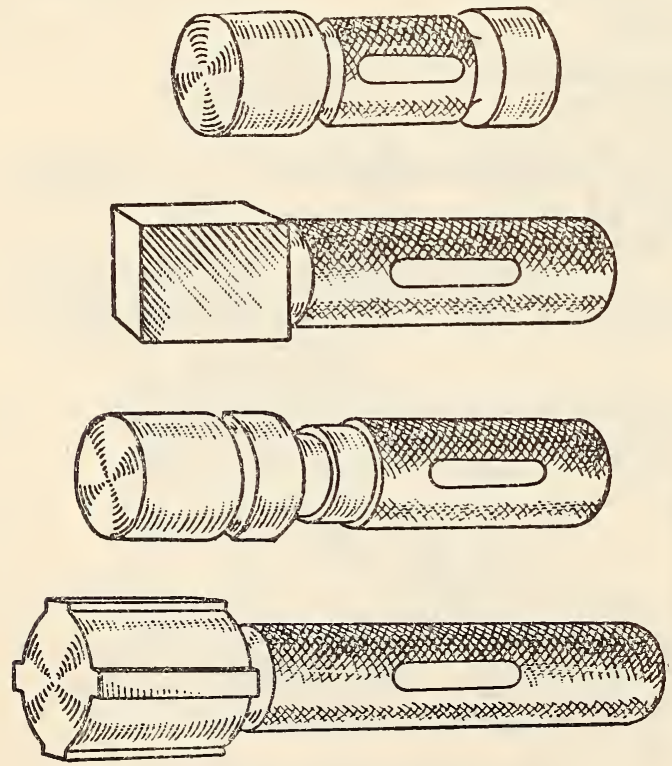

FigURe 678.-Plug gages

but never larger, than the maximum size specified for the part to be gaged.

2. The temperature of $68^{\circ} \mathrm{F}$. $\left(20^{\circ}\right.$ C. $)$ shall be the standard for plain limit gages.

3 . The final result sought by gaging is interchangeable manufacture in some degree. This means that the parts of a mechanism can be assembled without fitting one part to another and when assembled the parts will function properly.
4. Applied to manufactured material, the result sought is sufficient uniformity in size and contour to adapt the material without further fitting to the requirements of the industries. The fundamental principle involved in interchangeable manufacture requires that "a system of standardization and classification of fits shall establish a clearly defined line at which interference between mating parts begins." Hence,

(a) The standard or basic size, as physically represented by a correct standard master gage, represents the line at which this interference begins between mating parts.

(b) It is the minimum size of the external members of all mating parts of standardized practice, regardless of the kind of fit.

(c) It is the maximum size of internal members of all mating parts where interference begins or that fit metal to metal.

The condition of $(b)$ and $(c)$ is represented by a mating pair of correctly fitting standard gages.

(d) The limits of the component as physically represented by the limit master gages shall not be
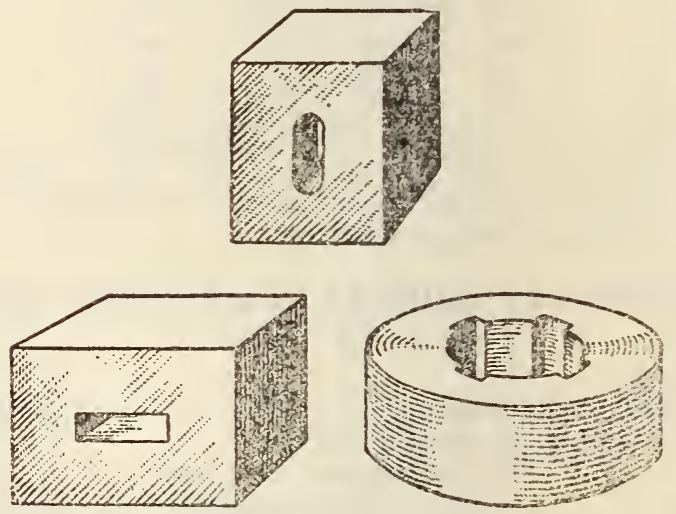

Figure 679.-Receiving gages

exceeded as a result either of tolerance or wear of the gages.

(e) "Go" gages, or the equivalent verification of all the factors involved in the fit, are necessary to prevent interference of mating parts.

In the case of force fits "go" gages are necessary to determine the maximum amount of interference between mating parts.

$(f)$ "Not go" gages, or the equivalent verification of the determining factor are necessary to prevent the maximum looseness of mating parts exceeding the limits specified.

In the case of force fits, "not go" gages are necessary to determine the minimum amount of interference between mating parts.

\section{DEFINITIONS}

5. Gaging.-A process of measuring manufactured materials to assure the specified uniformity of size and contour required by the industries.

6. Gage.-A device for determining whether or not one or more of the dimensions of a manufactured part are within specified limits.

(a) Ring gage.-(Fig 677.) One whose inside measuring surfaces are circular in form. The measuring surfaces may be cylindrical or conical.

(b) Plug gage.-(Fig. 678.) One whose outside measuring surfaces are arranged to verify the specified uniformity of holes. A plug gage may be straight or tapered and of any cross-sectional shape. 
(c) Receiving gage.-(Fig. 679.) One whose inside measuring surfaces are arranged to verify the specified uniformity of size and contour of manufactured material.

(d) Indicating gage.-(Fig. 680.) One that exhibits visually the variations in the uniformity of dimensions or contour, the amount of the variation being
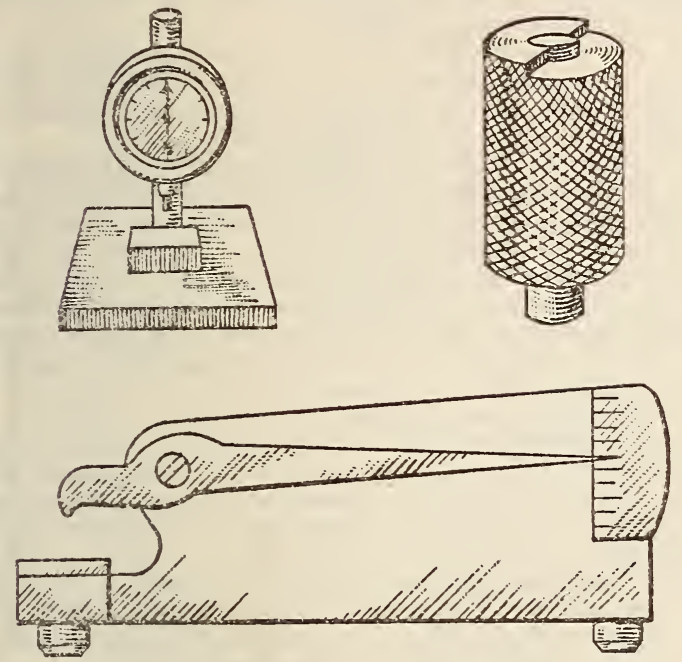

FIGURE 680.-Indicating gages

indicated by lever on graduated scale, dial, flush-pin, plunger gages, etc.

NotE.-For detailed information concerning the design and use of plain gages see "Methods of Gaging and Specifications for Plain Limit Gages" a standard developed by the committee on plain limit gages for general engineering work, and published by the American Society of Mechanical Engineers, 29 West Thirty-ninth Street, New York, N. Y.

(e) Snap gage.-(Fig. 681.) A fixed gage arranged with inside measuring surfaces for calipering diameters, lengths, thicknesses, etc.

wis $(f)$ Caliper gage. - (Fig. 682.) One which, for internal members, is similar to a snap gage, and for external members, is similar to a plug gage.

7. Standard.-A physical representation of a form, dimension or size established by law or by general usage and consent.

* Standard sizes.-A series of recognized or accepted sizes corresponding to various subdivisions of a recognized unit of length such as the yard or the meter. These are usually expressed in inches or in millimeters; sometimes by arbitrary numbers or letters.

Note.-The industries by common consent have chosen several different series important for interchangeable manufacture. For instance the common fractions of an inch are commonly called standard sizes. Such as: $1 / 8$ inch, $3 / 10$ inch, $1 / 4$ inch, $5 / 10$ inch, etc.

9. Nominal size.-A designation given to the subdivision of the unit of length having no specified limits of accuracy but indicating a close approximation to a standard size.

10. Basic size.-The exact theoretical size from which all limiting variations are made.

11. Allowance.-(Neutral zone.) An intentional difference in the dimensions of mating parts; or, the minimum clearance space which is intended between mating parts. It represents the condition of the tightest permissible fit, or the largest internal member mated with the smallest external member. It is to provide for different classes of fit.
EXAMPLE.-A shaft dimensioned 0.874 inch and a hole dimensioned 0.875 inch represents an allowance of 0.001 inch. The same hole with
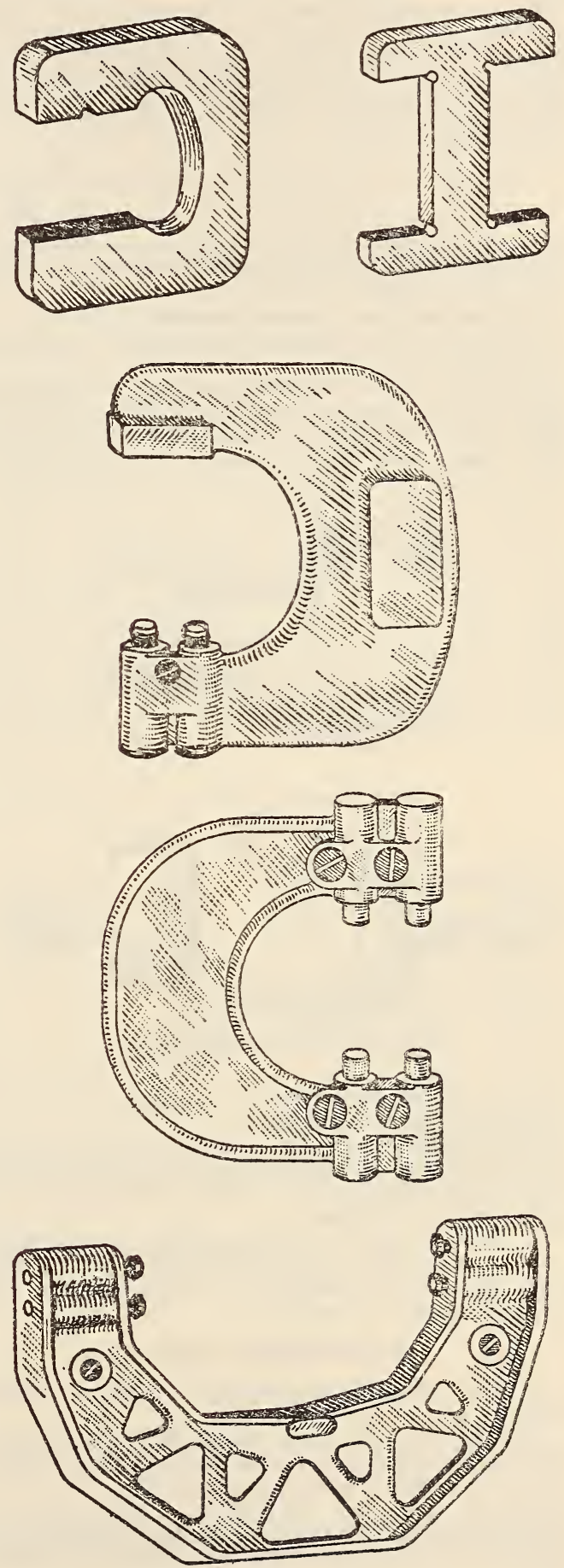

FIGURE 681.-Snap gages

a shaft dimensioned 0.876 inch represents an allowance of 0.001 inch also; but, as the shaft is larger than the hole, this allowance becomas a negative quantity. (See explanatory notes, below.) 
12. Tolerance. - The amount of variation permitted in the size of a part.

Nore. - In the example under allowance, the ideal condition and the tightest fit permissible have been given; but in manufacturing large numbers of pieces these sizes could not be produced exactly, so variations must be made that will not prevent their proper function. ing but will enable them to be produced. These variations must therefore tend toward greater looseness. Therefore, if a manufacturing tolerance of 0.001 inch is required on each member, they would be dimensioned as follows:

Inch

Shaft $0.874+0.000$

Hole.-. $-.001$ $-.000$

This defines a condition in which the greatest looseness is 0.003 inch and the greatest tightness gives a clearance of 0.001 inch. (See explanatory notes, below.)

13. Neutral zone.-(See allowance.)

14. Limits. - The extreme permissible dimensions of a part.

15. Master gage.-One whose gaging dimensions represent as exactly as possible the physical dimensions of the component. It is the gage to which all other gages and all dimensions of manufactured
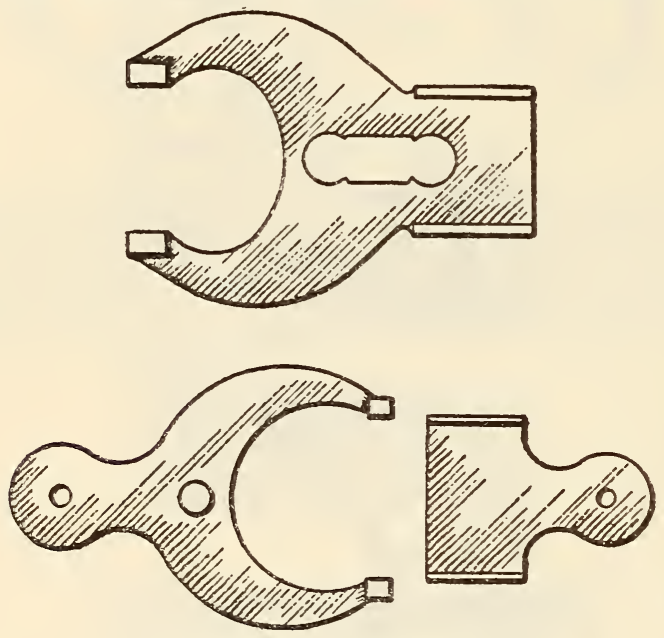

FIGURE 682.-Caliper gages

material are finally checked or compared, either by direct check or comparison.

16. Inspection gages. - Gages for the use of the manufacturer or purchaser in accepting the product. These gages must not accept any product which the master gages will reject.

17. Working gages. - Gages used by the manufacturer to check the work as it is produced. These gages should not accept any product which the inspection gages will reject.

\section{CLASSIFICATION OF FITS}

18. The following classification of fits is recommended:

Loose fit (class 1)-Large allowance.-This fit provides for considerable freedom and embraces certain fits where accuracy is not essential.

EXAMPLES.-Machine fits of agricultural and mining machinery: controlling apparatus for marine work; textile, rubber, candy, and bread machinery; general machinery of a similar grade; some ordnance material.

Free fit (class 2)-liberal allowance.-For running fits with speeds of 600 r. p. m. or over, and journal pressures of 600 pounds per square inch or over.

EXAMPLES.-Dynamos, engines, many machine-tool parts, and some automotive parts.

Medium fit (class 3)-medium allowance.-For running fits under 600 r. p. m. and with journal pressures less than 600 pounds per square inch; also for sliding fits; and the more accurate machine-tool and automotive parts.

Snug fit (class 4)-zero allowance.-This is the closest fit which can be assembled by hand and necessitates work of considerable precision. It should be used where no perceptible shake is permissible and where moving parts are not intended to move freely under a load.

Wringing fit (class 5)-Zero to negative allowance.This is also known as a "tunking fit" and it is practically metal to metal. Assembly is usually selective and not interchangeable.

Tight fit (class 6)-Slight negative allowance.Light pressure is required to assemble these fits, and the parts are more or less permanently assembled, such as the fixed ends of studs for gears, pulleys, rocker arms, etc. These fits are used for drive fits in thin sections or extremely long fits in other sections and also for shrink fits on very light sections. Used in automotive, ordnance, and general machine manufacturing.

Medium force fit (class 7)-Negative allowance.Considerable pressure is required to assemble these fits, and the parts are considered permanently assembled. These fits are used in fastening locomotive wheels, car wheels, armatures of dynamos and motors, and crank disks to their axles or shafts. They are also used for shrink fits on medium sections or long fits. These fits are the tightest which are recommended for cast-iron holes or external members as they stress cast iron to its elastic limit.

Heavy force and shrink fit (class 8)-Considerable negative allowance.-These fits are used for steel holes where the metal can be highly stressed without exceeding its elastic limit. These fits cause excessive stress for cast-iron holes. Shrink fits are used where heavy force fits are impractical, as on locomotive wheel tires, heavy crank disks of large engines, etc.

(Included in this tentative standard are tables showing the tolerances and allowances for establishing the limiting sizes of mating parts for interchangeable manufacture, and also tables showing the tolerances and allowances for establishing the limiting sizes for the manufacture of mating parts which are to be assembled by selection.)

\section{EXPIANATORY NOTES}

In the table reproduced below many of the terms in common use in the shops of the country are given with the equivalent term recommended by the committee on plain limit gages for general engineering work.

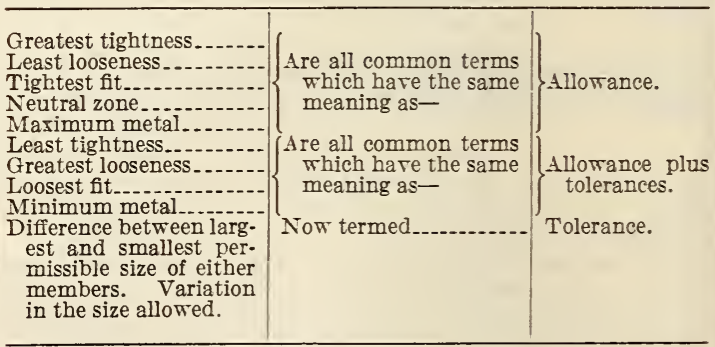

The basis for the foregoing recommended allowances and tolerances is a classification of fits quite commonly known to the manufacturing public. Interference between mating parts takes place at the exact basic value of the dimension; hence an accepted pair of mating standard gages become the "go" gages for mating parts which will prevent interference at the basic value of the dimension. 
On account of the lack of a uniform and acceptable method of measuring the fitting surfaces of the external members of a pair of mating parts, it is recommended that plugs, blocks, disks, and rods govern the checking of external members for the reason that usually the same measuring device will check for accuracy both the internal member and the gage for the external member, thus avoiding an interference when both members appear to have the same dimension.

To illustrate: If a 2-inch hole be measured with an inside micrometer and a shaft be measured with an outside micrometer, and both measure exactly 2 inches, there is no assurance they will go together; but, unless there were a definite understanding in a contract, the pieces might be ruled acceptable by law. If, however, the 2 -inch hole would receive a plug gage which measured exactly 2 inches by a measuring machine, it would also receive an accurately finished shaft of the same size when measured by the same machine.

It is well known that a correct pair of plug and ring gages 2 inches in diameter will each check parts that will go together. It is also well known that the character of fit depends on the rotundity, parallelism, size, and in some cases, contour of the mating parts, and that for cylindrical fits in mating parts, plug and ring gages are the only sure means of determining whether or not parts will go together. If either member of a pair of mating parts is three or five cornered, a snap gage or micrometer will not insure their going together.

In the foregoing allowances and tolerances, the best practice available was considered, and where differences occurred a compromise was attempted. The tolerances on holes apply only to reamed holes, or holes having a finish equivalent to reaming. From the standpoint of satisfactory performance, the tightest and loosest fits permissible are the allimportant considerations. From a manufacturing standpoint, tolerances are the important factors. Hence both the tightest and loosest fits permissible must combine the tolerance and allowance, and therefore both allowance and tolerance must be considered for all-around satisfactory conditions.

It is not deemed to be feasible to include in this report allowances and tolerances which will apply to all work. Many so-called standard fits are made in the industries which are not interchangeable, and an attempt to make them so would be prohibitive in cost. These fits, however, must be made on a production basis, and it is believed that the tables here given will be found to contain allowances and tolerances which are suitable for most work, even if some selection or individual fitting of parts is desired.

In choosing the class of fit for manufacture, the engineer should keep in mind that cost usually increases proportionately to the accuracy required, and no finer class of fit should be chosen than the functional requirements actually demand. It is axiomatic that the closer the fit the smaller the manufacturing tolerance, and usually the greater the cost. The length of engagement of the fit also plays an important part in the selection of the class of fit for a piece of work. It is obvious that a long engagement will tolerate more looseness than a short one, and due regard should be paid to this feature.

Formulas for recommended allowances and tolerances

\begin{tabular}{|c|c|c|c|c|c|}
\hline Class of fit & Method of assembly & Allowance & $\begin{array}{l}\text { Selected } \\
\text { average } \\
\text { interfer- } \\
\text { ence of } \\
\text { metal }\end{array}$ & $\begin{array}{l}\text { Hole toler- } \\
\text { ance }\end{array}$ & $\begin{array}{c}\text { Shaft toler } \\
\text { ance }\end{array}$ \\
\hline 1. Loose... & Strictly interchangeable... & $0.0025 \sqrt[3]{d^{2}}$ & & $0.0025 \sqrt[3]{d}$ & $0.0025 \sqrt[3]{d}$ \\
\hline 2. Free...... & . & $.0014 \sqrt[3]{d^{2}}$ & & $.0013 \sqrt[3]{d}$ & $.0013 \sqrt[3]{d}$ \\
\hline 3. Medium..... & [..... do do _. & $.0009 \sqrt[3]{d^{2}}$ & & $.0008 \sqrt[3]{\bar{d}}$ & $.0008 \sqrt[3]{a}$ \\
\hline 4. Snug & -.....do & .0000 & & $.0006 \sqrt[3]{d}$ & $.0004 \sqrt[3]{d}$ \\
\hline 5. Wringing. & Selective assembly. & & 0.0000 & $.0006 \sqrt[3]{d}$ & $.0004 \sqrt[3]{d}$ \\
\hline 6. Tight......... & - do do n & $\cdots$ & $.00025 d$ & $.0006 \sqrt[3]{d}$ & $.0006 \sqrt[3]{d}$ \\
\hline 7. Medium force........... & -.....do & $\ldots$ & $.0005 d$ & $.0006 \sqrt[3]{d}$ & $.0006 \sqrt[3]{\vec{d}}$ \\
\hline 9. Heavy force or shrink.... & -.... do _. . . & & $.001 d$ & $.0000 \sqrt[3]{d}$ & $.0006 \sqrt[3]{d}$ \\
\hline
\end{tabular}

$d=$ diameter of fit in inches.

The formulas for allowance values give the ideal condition of fit for classes 1 to 4 .

The formulas for selected average interference of metal give the ideal condition of fit for classes 5 to 8 . 


\section{GLASS CONTAINER ASSOCIATION OF AMERICA, GAGES FOR GLASS CON- TAINERS, AUGUST 1, 1929}

\section{GLASS-CONTAINER GAGES}

(This association has adopted as standard a number of different types of diameter and profile gages for glass bottles and jars. Details covering materials and dimensions of these gages are shomn on blue prints issued by the standardization committee.)

UNITED STATES GOVERNMENT, DEPARTMENT OF COMMERCE; PLAIN AND THREAD PLUG AND RING GAGE BLANKS, COMMERCIAL STANDARD CS8-30, OCTOBER 21, 1930

[Approved as American standard (B47-1932) by the American Standards Association, May, 1932]

\section{PLAIN AND THEEAD PLUG AND RING GAGE BLANKS}

\section{TERMINOLOGY}

The following glossary is intended to clarify the meaning of certain technical terms employed in this report. The definitions are not intended to be general; rather they are specific as to their application to the American gage design standards.

American gage design standard.-The caption "American gage design standard" has been adopted to designate gages made to the design specifications promulgated by the American gage design committee

A plain cylindrical plug gage is a complete unthreaded internal gage of single or double ended type for the size control of holes. It consists of handle and gaging member or members, with suitable locking means.

A progressive cylindrical plug gage is a complete unthreaded internal gage consisting of handle and gaging member in which the "go" and "not go" gaging sections are combined in a single unit secured to one end of the handle.

A thread plug gage is a complete internal thread gage of either single or double ended type, comprising handle and threaded gaging member or members, with suitable locking means.

The gaging member is that integral unit of a plug gage which is accurately finished to size and is employed for size control of the work. In taper lock gages, the gaging member consists of a shank and a gaging section.

The gaging section is that portion of the gage which comes into physical contact with the work. In the plug range above 1.510 to and including 4.510 inches, the gaging section is identical with the gaging member.

The shank (applied to taper lock gages only) is that portion of the gaging member which is employed for fixing the gaging member to the handle.

The term "taper lock" designates that construction in which the gaging member has a taper shank, which is forced into a taper hole in the handle. This design is standard for plug gages in the range above 0.059 inch to and including 1.510 inches, is optional for plain cylindrical and thread plug gages in the range above 1.510 inches to and including 2.510 inches, and is standard for pipe-thread plug gages up to and including 2-inch nominal pipe size.

A reversible plug gage is a plain cylindrical or thread plug gage, in which three wedge-shaped locking prongs on the handle are forced into corresponding grooves in the gaging member by means of a single through screw thus providing a self-centering support with a positive lock. This design is stand- ard for all plug gages in the ranges above 1.510 to and including 4.510 inches, with the exception of pipe thread plug gages, for which it is standard in the ranges above 2.510 to and including 4.510 inches.

Lightening holes are unfinished drilled holes provided in the heavier sizes of gaging members for the sole purpose of reducing the weight of the gage.

The handle is that portion of a plug gage which is employed as supporting means for the gaging member or members.

The drift hole or drift slot is a small hole or slot provided in the side of a taper lock gage handle near the "go" end through which a pin or drift may be inserted for the purpose of ejecting the gaging member from the handle.

A plain ring gage is an unthreaded external gage employed for the size control of external diameters. In the smaller sizes it consists of a gage body into which is pressed a bushing, the latter being accurately finished to size for gaging purposes.

A thread ring gage is an external thread gage employed for the size control of threaded work, means of adjustment being provided integral with the gage body.

The flange is that external portion of a large ring gage which is reduced in section for the purpose of lightening the gage.

The hub is the midsection of a flanged ring gage. It determines the length of the gaging section.

The thread ring gage locking derice is a means of expanding and contracting the thread ring gage during the manufacturing or resizing processes. It also provides an effectual lock. It comprises an adjusting screw, a locking screw, and a sleeve. For detailed description and illustration see below:

Adjusting slots are radial slots provided in thread ring gages in order to facilitate expansion and contraction of gage size by means of the adjusting device. An adjusting slot always terminates in an adjusting slot terminal hole.

The locking slot is that slot which passes entirely through the wall of a thread ring gage. In conjunction with the thread ring gage locking derice, it permits expansion and contraction of gage size.

\section{DETAILS OF CONSTRUCTION, AMERICAN GAGE DESIGN STANDARDS}

\section{Plain Cylindrical Plug Gage Blanks and Handles}

Two separate designs have been adopted for plain cylindrical plug gages-the taper lock design for the range from 0.059 to and including 1.510 inches, and the reversible design with reversible gaging members for the range from above 1.510 to and including 4.510 inches. For sizes above 0.240 inch to and including 2.510 inches, both straight and progressive gaging members are prorided. Use of the taper lock design is optional for the size range from above 1.510 to and including 2.510 inches.

(a) Taper lock design above 0.059 to and inciuding 1.510 inches. - It was felt that the taper lock design was particularly well suited for the smaller sizes of plain plug gages. This type of gage is simple and is economical of production and maintenance. The gaging member has a taper shank which is forced into a taper hole in the handle. When properly assembled, the taper lock gage possesses the rigidity of a solid gage and is entirely free of shake or "wink." Drift slots or drift holes are provided near one end of the handle, permitting gaging members to be removed when replacement is necessary. In the case of double-end gages, one end is remored by running a rod through the hollow handle. In the smaller size ranges above 0.059 inch to and including 0.240 inch, a groove is provided near one end of the handle to designate the "not go" end, as the length of the "go" member in this range is often insuffi- 
cient clearly to distinguish it from the "not go" member. The groove is omitted as unnecessary above 0.240 inch.

Complete dimensional tolerances have been established for the mating parts of gaging members and handles, insuring absolute interchangeability of gaging members and handles wherever manufac- grooves in the gaging member by a single through screw thus providing a self-centering support with a positive lock, and resulting in a degree of rigidity equivalent to that of a solid gage. The useful life of the plug is furthermore materially increased, as when one end is worn the plug can be reversed and is then, for most purposes, as good as new.

(a)

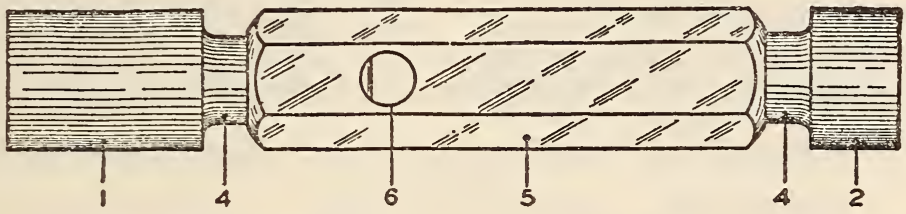

(a)

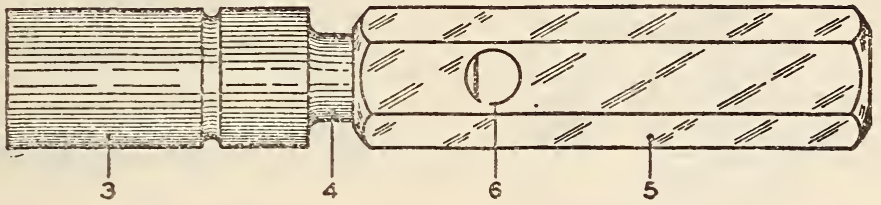

13

(b)

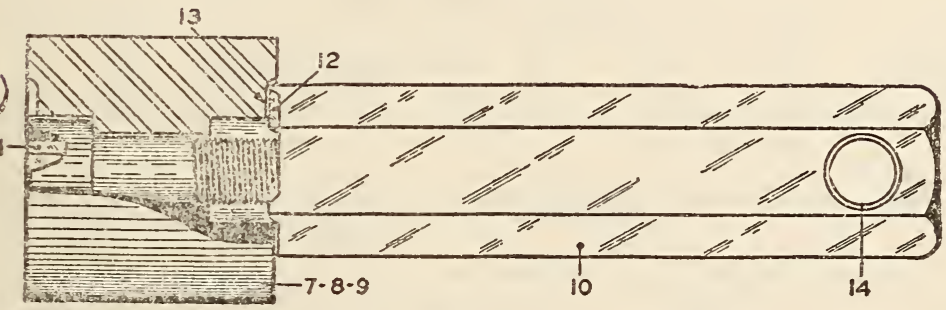

13

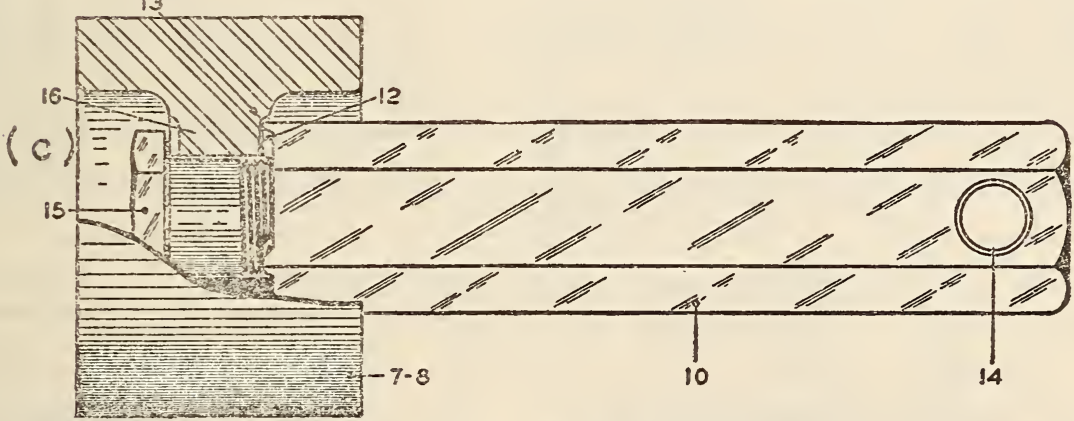

FIGURE 683.- American gage design standard plain cylindrical plug gages, details of construciion

(a) Range: Above 0.059 to and including 1.510 inches; optional above 1.510 to and including 2.510 inches.

(b) Range: Above 1.510 to and including 2.510 inches.

(c) Range: Above 2.510 to and including 4.510 inches.

$$
\begin{aligned}
& \text { 1, "go" gaging member. } \\
& \text { 2, "not go" gaging member. } \\
& \text { 9, progressive gaging member. } \\
& \text { 4, shank. } \\
& \text { 5, taper lock handle. } \\
& \text { 6, drift hole (or slot). } \\
& \text { 7, "go" gaging member. } \\
& \text { 8, "not go" gaging member. }
\end{aligned}
$$

tured. General details of construction will be apparent from Figure 683. See also Tables 1 to 4 .

(b) Reversible design, above 1.510 to and including 4.510 inches. - Considerations of rigidity of construction and long life have dictated the choice of the reversible design with reversible gaging members for the size range above 1.510 inches. With this construction there is no chance for shake or "wink" to interfere with the sensitive feel so necessary in gages of this type. Three wedge-shaped locking prongs on the handle are forced into corresponding
9, progressive gaging member.

10 , handle for reversible gage.

11 , socket head screw.

12 , locking prong.

13 , locking groove.

14 , cross-pin hole.

15 , hexagon head screw.

16 , web.

The construction is protected by carefully worked out dimensional limits, and interchangeability is insured between gaging members and handles wherever manufactured. Details of construction will be apparent from Figure 683 and Figure 688. See also Tables 5 and 6.

(c) Handles.-Handles for both taper lock and reversible gages are of the hexagonal type. However, the use of round medium-knurled handles, while not recommended is made optional in all sizes. 
Handles as designed for taper lock and reversible gages offer a feature of economy in that they may be disassembled from gaging members when the latter are worn out or discarded for any other reason, and may then be reassembled with new gaging members, thus giving them, with reasonable care, practically indefinite life.

\section{Thread Plug Gage Blanks and Handles}

The taper lock and reversible designs have been adopted for thread plug gage blanks and handles and follow the plain cylindrical plug gage designs described above with the exception that the length of thread-gaging members is slightly different in some instances and the use of taper lock blanks and handles for pie-thread plug gages is standard to and including 2 inches nominal pipe size. General details of construction will be apparent from Figure 689. Data sheets for thread plug gages are set forth below. A separate table specifying the taper lock handles and gaging members for pipe-thread plug gages is shown in Table 11 .

TABLE 1.-Plain cylindrical plug gage handles, taper lock design, range above 0.059 to and including $0.240 \mathrm{inch}$

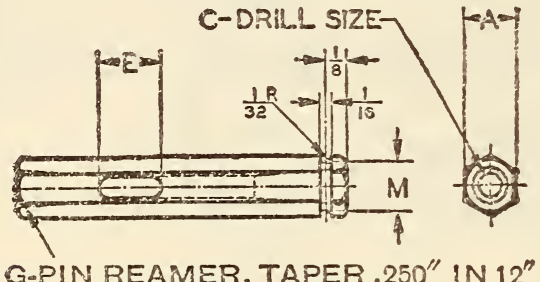

G-PIN REAMER, TAPER . $250^{\prime \prime}$ IN $12^{\prime \prime}$

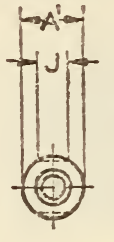

KPURL-

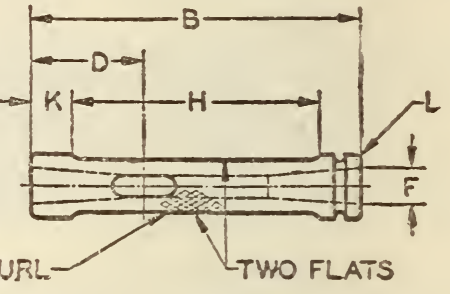

FIGURE 684

\begin{tabular}{|c|c|c|c|c|c|c|c|c|c|c|c|c|c|c|c|c|c|}
\hline \multirow{3}{*}{$\begin{array}{l}\text { Handle } \\
\text { size No. }\end{array}$} & \multicolumn{2}{|c|}{$\begin{array}{l}\text { Range, plain } \\
\text { plug diameters }\end{array}$} & \multicolumn{15}{|c|}{ General dimensions } \\
\hline & \multirow[b]{2}{*}{ Above- } & \multirow{2}{*}{$\begin{array}{l}\text { To and } \\
\text { includ- } \\
\text { ing- }\end{array}$} & \multirow[b]{2}{*}{$A$} & \multirow[b]{2}{*}{$A^{\prime}$} & \multirow[b]{2}{*}{$B$} & \multirow{2}{*}{$\begin{array}{c}C \\
\text { Drill } \\
\text { size }\end{array}$} & \multirow[b]{2}{*}{$D$} & \multirow[b]{2}{*}{$E$} & \multicolumn{2}{|c|}{$F$} & \multirow[b]{2}{*}{$G$} & \multirow[b]{2}{*}{$H$} & \multirow[b]{2}{*}{$J$} & \multirow[b]{2}{*}{$K$} & \multirow[b]{2}{*}{$L$} & \multicolumn{2}{|c|}{$M$} \\
\hline & & & & & & & & & $\begin{array}{l}\text { Mini- } \\
\text { mum }\end{array}$ & $\begin{array}{l}\text { Maxi- } \\
\text { mum }\end{array}$ & & & & & & $\begin{array}{l}\text { Mini- } \\
\text { mum }\end{array}$ & $\begin{array}{l}\text { Maxi- } \\
\text { mum }\end{array}$ \\
\hline & Inch & Inch & Inch & Inch & Inches & & Inch & Inch & Inch & Inch & No. & Inches & Inch & Inch & Inch & Inch & Inch \\
\hline $000 \ldots$ & 0.059 & 0.105 & $3 / 16$ & $1 / 4$ & 136 & $\begin{array}{l}\text { No. } 34 \\
(0.111)\end{array}$ & $9 / 16$ & $5 / 64$ by 14 & 0.125 & 0.126 & 000 & 1 & $5 / 32$ & 34 & $1 / 32$ & 0.172 & 0.177 \\
\hline $00 \ldots$ & .105 & .150 & $1 / 4$ & $5 / 16$ & $13 / 4$ & $\begin{array}{l}\text { No. } 29 \\
(0.136)\end{array}$ & $5 / 8$ & $3 / 32$ by $5 / 16$ & .155 & .156 & 0 & $11 / 4$ & $3 / 16$ & $3 / 4$ & $1 / 32$ & .235 & .240 \\
\hline $0 \ldots$ & .150 & .240 & $5 / 16$ & $3 / 8$ & 2 & $\begin{array}{l}\text { No. } 20 \\
(0.161)\end{array}$ & 1116 & $1 / 8$ by $3 / 8$ & .180 & .181 & 2 & 136 & $3 / 16$ & $1 / 4$ & $1 / 32$ & .297 & .302 \\
\hline
\end{tabular}

NoтE.-The purpose of the groove in the "not go" end of the handle is to distinguish the "not go" from the "go" end.

TABLE 2.-Plain cylindrical plug gaging members, taper lock design, range above 0.059 to and including 0.240 inch
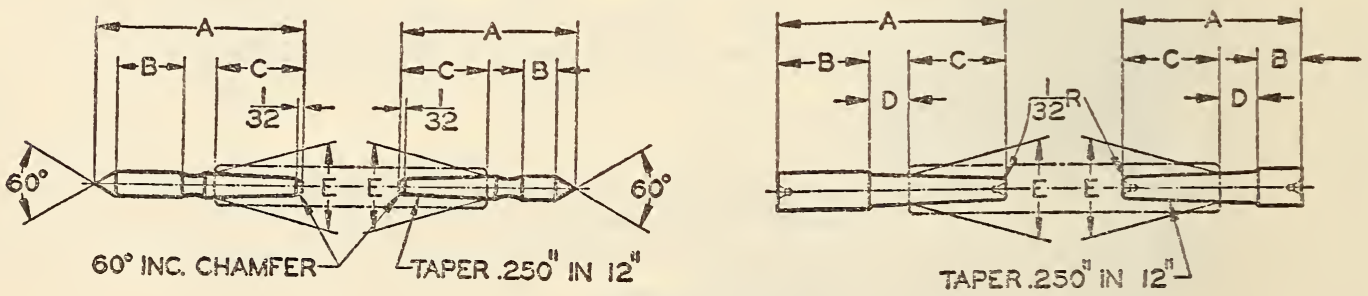

Figdre 685

\begin{tabular}{|c|c|c|c|c|c|c|c|c|c|c|c|c|c|c|}
\hline \multirow{4}{*}{ Handle size No. } & \multirow{2}{*}{\multicolumn{2}{|c|}{$\begin{array}{c}\text { Range in diam- } \\
\text { eters }\end{array}$}} & \multicolumn{12}{|c|}{ General dimensions } \\
\hline & & & \multicolumn{6}{|c|}{ "Go" } & \multicolumn{6}{|c|}{ "Not go" } \\
\hline & \multirow[b]{2}{*}{ Above- } & \multirow{2}{*}{$\begin{array}{l}\text { To and } \\
\text { includ- } \\
\text { ing- }\end{array}$} & \multirow[b]{2}{*}{$A$} & \multirow[b]{2}{*}{$B$} & \multirow[b]{2}{*}{$C$} & \multirow[b]{2}{*}{$D$} & \multicolumn{2}{|c|}{$E$} & \multirow[b]{2}{*}{$A$} & \multirow[b]{2}{*}{$B$} & \multirow[b]{2}{*}{$C$} & \multirow[b]{2}{*}{$D$} & \multicolumn{2}{|c|}{$E$} \\
\hline & & & & & & & $\begin{array}{l}\text { Mini- } \\
\text { mum }\end{array}$ & $\begin{array}{l}\text { Maxi- } \\
\text { mum }\end{array}$ & & & & & $\begin{array}{l}\text { Mini- } \\
\text { mum }\end{array}$ & $\begin{array}{l}\text { Maxi- } \\
\text { mum }\end{array}$ \\
\hline 000 & $\begin{array}{l}\text { Inch } \\
0.059 \\
.105 \\
.150\end{array}$ & $\begin{array}{l}\text { Inch } \\
0.105 \\
.150 \\
.240\end{array}$ & $\begin{array}{c}\text { Inches } \\
15 / 32 \\
111 / 32 \\
115 / 32\end{array}$ & $\begin{array}{c}\text { Inch } \\
3 / 8 \\
7 / 16 \\
19 / 32\end{array}$ & $\begin{array}{c}\text { Inch } \\
1 / 2 \\
9 / 16 \\
5 / 8\end{array}$ & \begin{tabular}{c} 
Inch \\
\hdashline $1 / 4$
\end{tabular} & $\begin{array}{l}\text { Inch } \\
0.125 \\
.155 \\
.180\end{array}$ & $\begin{array}{l}\text { Inch } \\
0.126 \\
.156 \\
.181\end{array}$ & $\begin{array}{c}\text { Inches } \\
31 / 32 \\
118 \\
15 / 32\end{array}$ & \begin{tabular}{r|} 
Inch \\
$3 / 16$ \\
$7 / 32$ \\
$9 / 32$
\end{tabular} & $\begin{array}{c}\text { Inch } \\
1 / 2 \\
9 / 16 \\
5 / 8\end{array}$ & $\begin{array}{c}\text { Inch } \\
1 / 4\end{array}$ & $\begin{array}{l}\text { Inch } \\
0.125 \\
.155 \\
.180\end{array}$ & $\begin{array}{l}\text { Inch } \\
0.126 \\
.155 \\
.181\end{array}$ \\
\hline
\end{tabular}


TABLE 3.-Plain cylindrical plug gage handles, taper lock design, range above 0.240 to and including 1.510 inches
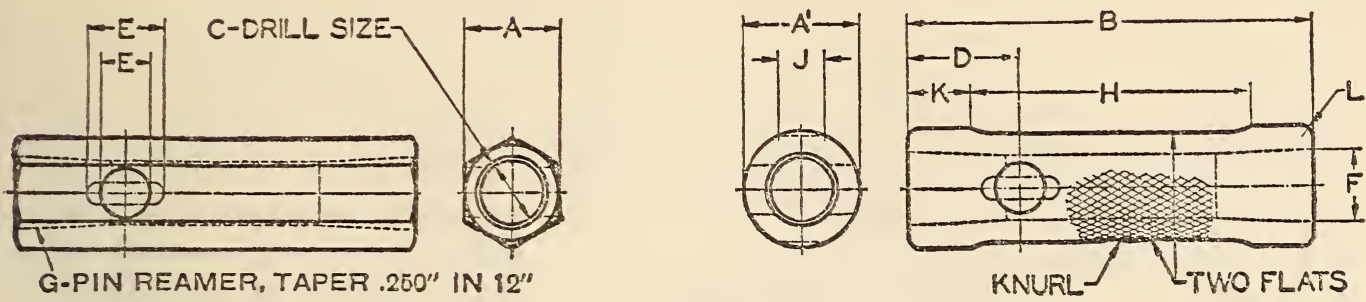

FIGURE 686

\begin{tabular}{|c|c|c|c|c|c|c|c|c|c|c|c|c|c|c|c|}
\hline \multirow{3}{*}{ Size } & \multicolumn{2}{|c|}{$\begin{array}{l}\text { Range, plain } \\
\text { plug diameters }\end{array}$} & \multicolumn{13}{|c|}{ General dimensions } \\
\hline & \multirow[b]{2}{*}{ Above- } & \multirow{2}{*}{$\begin{array}{c}\text { To and } \\
\text { includ- } \\
\text { ing- }\end{array}$} & \multirow[b]{2}{*}{$A$} & \multirow[b]{2}{*}{$A^{\prime}$} & \multirow[b]{2}{*}{$B$} & \multirow{2}{*}{$\begin{array}{c}C \\
\text { Drill } \\
\text { size }\end{array}$} & \multirow[b]{2}{*}{$D$} & \multirow[b]{2}{*}{$E$} & \multicolumn{2}{|c|}{$F$} & \multirow[b]{2}{*}{$G$} & \multirow[b]{2}{*}{$H$} & \multirow[b]{2}{*}{$J$} & \multirow[b]{2}{*}{$K$} & \multirow[b]{2}{*}{$L$} \\
\hline & & & & & & & & & $\begin{array}{l}\text { Mini- } \\
\text { mum }\end{array}$ & $\begin{array}{l}\text { Maxi- } \\
\text { mum }\end{array}$ & & & & & \\
\hline & $\begin{array}{r}\text { Inches } \\
0.240\end{array}$ & $\begin{array}{r}\text { Inches } \\
0.365\end{array}$ & $\begin{array}{c}\text { Inches } \\
3 / 8\end{array}$ & $\begin{array}{c}\text { Inches } \\
7 / 16\end{array}$ & $\begin{array}{r}\text { Inches } \\
23 / 4\end{array}$ & $7 / 32$ & $\begin{array}{r}\text { Inches } \\
25 / 32\end{array}$ & $\begin{array}{c}\text { Inch } \\
1 / 8 \text { by } 1 / 2\end{array}$ & $\begin{array}{l}\text { Inch } \\
0.239\end{array}$ & $\begin{array}{l}\text { Inch } \\
0.240\end{array}$ & $\mathrm{No}_{4}$ & $\begin{array}{r}\text { Inches } \\
13 / 4\end{array}$ & $\begin{array}{c}\text { Inch } \\
7 / 32\end{array}$ & $\begin{array}{r}\text { Inch } \\
1 / 2\end{array}$ & $\begin{array}{c}\text { Inch } \\
1 / 16\end{array}$ \\
\hline 5, optional, see note... & $\begin{array}{r}.365 \\
.510 \\
.825 \\
1.135 \\
1.510\end{array}$ & $\begin{array}{r}.510 \\
.825 \\
1.135 \\
1.510 \\
2.510\end{array}$ & $\begin{array}{l}1 / 2 \\
11 / 16 \\
7 / 8 \\
118 \\
118\end{array}$ & $\begin{array}{l}5 / 8 \\
13 / 16 \\
11 / 16 \\
15 / 16 \\
15 / 16\end{array}$ & $\begin{array}{l}3 \\
31 / 4 \\
35 / 8 \\
4 \\
4\end{array}$ & $\begin{array}{r}\mathrm{L}(0.290) \\
25 / 64 \\
37.64 \\
25 \% 32 \\
25 / 32\end{array}$ & $\begin{array}{c}25 / 32 \\
27 / 32 \\
63 / 64 \\
11 / 8 \\
11 / 8\end{array}$ & $\begin{array}{c}15 / 64 \\
11 / 32 \\
3 / 8 \\
7 / 16 \\
7 / 16\end{array}$ & $\begin{array}{l}.309 \\
.409 \\
.609 \\
.809 \\
.809\end{array}$ & $\begin{array}{l}.310 \\
.410 \\
.610 \\
.810 \\
.810\end{array}$ & $\begin{array}{r}6 \\
7 \\
10 \\
11 \\
11\end{array}$ & $\begin{array}{l}2 \\
21 / 4 \\
21 / 2 \\
23 / 4 \\
23 / 4\end{array}$ & $\begin{array}{l}3 / 8 \\
7 / 16 \\
7 / 16 \\
1 / 2 \\
1 / 2\end{array}$ & $\begin{array}{l}1 / 2 \\
1 / 2 \\
9 / 16 \\
5 / 8 \\
5 / 8\end{array}$ & $\begin{array}{l}1 / 16 \\
3 / 32 \\
3 / 32 \\
118 \\
1 / 8\end{array}$ \\
\hline
\end{tabular}

NOTE.-The use of taper lock plain cylindrical plug gaging members and handles is optional in the range above 1.510 to and including 2.510 inches, but the use of the reversible design is standard for all sizes above 1.510 inches.

TABLE 4.-Plain cylindrical plug gaging members, taper lock design, range above 0.240 to and including 1.510 inches

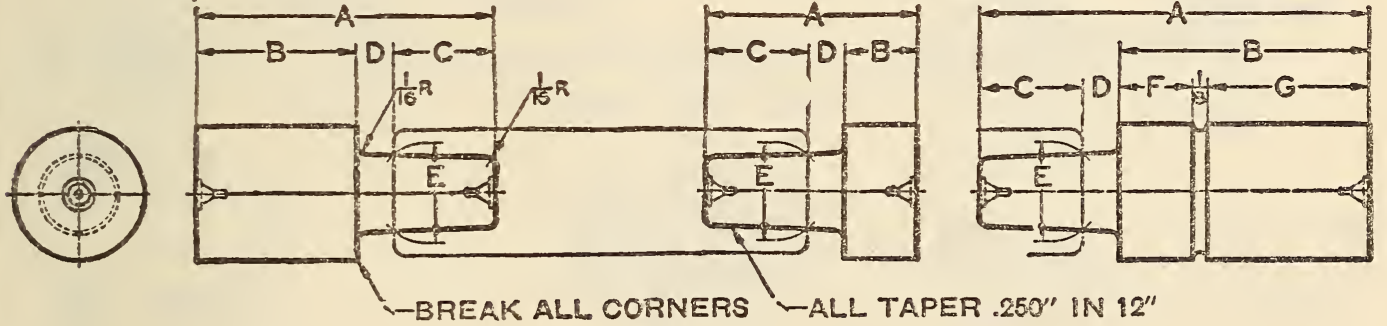

FIGURE 687

\begin{tabular}{|c|c|c|c|c|c|c|c|c|c|c|c|c|c|c|c|c|c|c|c|c|c|c|}
\hline \multirow{4}{*}{ Eandle size No. } & \multicolumn{2}{|c|}{$\begin{array}{l}\text { Range in } \\
\text { diameters }\end{array}$} & \multicolumn{20}{|c|}{ General dimensions } \\
\hline & \multirow{3}{*}{ Above- } & \multirow{3}{*}{$\begin{array}{l}\text { To } \\
\text { and } \\
\text { in- } \\
\text { clud- } \\
\text { ing- }\end{array}$} & \multicolumn{6}{|c|}{ "Go" } & \multicolumn{6}{|c|}{ "Not go" } & \multicolumn{8}{|c|}{ Progressive } \\
\hline & & & \multirow[b]{2}{*}{$A$} & \multirow[b]{2}{*}{$B$} & \multirow[b]{2}{*}{$C$} & \multirow[b]{2}{*}{$D$} & \multicolumn{2}{|c|}{$E$} & \multirow[b]{2}{*}{$A$} & \multirow[b]{2}{*}{$B$} & \multirow[b]{2}{*}{$C$} & \multirow[b]{2}{*}{$D$} & \multicolumn{2}{|c|}{$E$} & \multirow[b]{2}{*}{$A$} & \multirow[b]{2}{*}{$B$} & \multirow[b]{2}{*}{$C$} & \multirow[b]{2}{*}{$D$} & \multicolumn{2}{|c|}{$E$} & \multirow[b]{2}{*}{$F$} & \multirow[b]{2}{*}{$G$} \\
\hline & & & & & & & $\begin{array}{l}\text { Mini- } \\
\text { mum }\end{array}$ & $\begin{array}{l}\text { Maxi- } \\
\text { mum }\end{array}$ & & & & & $\begin{array}{l}\text { Mini- } \\
\text { mum }\end{array}$ & $\begin{array}{l}\text { Maxi- } \\
\text { mum }\end{array}$ & & & & & $\begin{array}{l}\text { Mini- } \\
\text { mum }\end{array}$ & $\begin{array}{l}\text { Maxi- } \\
\text { mum }\end{array}$ & & \\
\hline $\begin{array}{l}\text { optional, see note } \\
\text { Do.... }\end{array}$ & $\begin{array}{r}\text { In. } \\
0.240 \\
.365 \\
.510 \\
.825 \\
1.135 \\
1.510 \\
2.010\end{array}$ & $\begin{array}{l}\text { In. } \\
0.365 \\
.510 \\
.825 \\
1.135 \\
1.510 \\
2.010 \\
2.510\end{array}$ & $\begin{array}{l}\text { In. } \\
13 / 4 \\
2 \\
21 / 4 \\
2916 \\
278 \\
314 \\
33 / 8\end{array}$ & $\begin{array}{c}\text { In. } \\
3 / 4 \\
1 \\
11 / 4 \\
138 \\
11 / 2 \\
17 / 8 \\
2\end{array}$ & $\begin{array}{l}\text { In. } \\
3 / 4 \\
3 / 4 \\
3 / 4 \\
7 / 8 \\
1 \\
1 \\
1\end{array}$ & $\begin{array}{l}\text { In. } \\
1 / 4 \\
1 / 4 \\
1 / 4 \\
5 / 16 \\
3 / 8 \\
3 / 8 \\
3 / 8\end{array}$ & $\begin{array}{c}\text { In. } \\
0.239 \\
.309 \\
.408 \\
.608 \\
.808 \\
.808 \\
.808\end{array}$ & $\begin{array}{l}\text { In. } \\
0.240 \\
.310 \\
.410 \\
.610 \\
.810 \\
.810 \\
.810\end{array}$ & $\begin{array}{l}\text { In. } \\
15 / 16 \\
13 / 8 \\
11 / 2 \\
113 / 16 \\
21 / 8 \\
21 / 4 \\
21 / 4\end{array}$ & $\begin{array}{l}\text { In. } \\
5 / 16 \\
3 / 8 \\
1 / 2 \\
5 / 8 \\
3 / 4 \\
7 / 8 \\
7 / 8\end{array}$ & $\begin{array}{l}\text { In. } \\
3 / 4 \\
3 / 4 \\
3 / 4 \\
7 / 8 \\
1 \\
1 \\
1\end{array}$ & $\begin{array}{l}\text { In. } \\
1 / 4 \\
1 / 4 \\
1 / 4 \\
5 / 16 \\
3 / 8 \\
3 / 8 \\
3 / 8\end{array}$ & $\begin{array}{l}\text { In. } \\
0.239 \\
.309 \\
.408 \\
.608 \\
.808 \\
.808 \\
.808\end{array}$ & $\begin{array}{l}\text { In. } \\
0.2402 \\
.3102 \\
.4102 \\
.6103 \\
.8103 \\
.8104 \\
.8104\end{array}$ & $\begin{array}{l}\text { In. } \\
23 / 16 \\
21 / 2 \\
27 / 8 \\
35 / 16 \\
33 / 4 \\
41 / 4 \\
43 / 8\end{array}$ & $\begin{array}{l}\text { In. } \\
131 / 6 \\
112 \\
17 / 8 \\
218 \\
23 / 8 \\
27 / 8 \\
3\end{array}$ & \begin{tabular}{r|} 
In. \\
$3 / 4$ \\
$3 / 4$ \\
$3 / 4$ \\
$7 / 8$ \\
1 \\
1 \\
1
\end{tabular} & $\begin{array}{l}\text { In. } \\
1 / 4 \\
1 / 4 \\
1 / 4 \\
5 / 16 \\
3 / 8 \\
3 / 8 \\
3 / 8\end{array}$ & $\begin{array}{l}\text { In. } \\
0.239 \\
.309 \\
.408 \\
.608 \\
.808 \\
.808 \\
.808\end{array}$ & $\begin{array}{l}\text { In. } \\
0.240 \\
.310 \\
.410 \\
.610 \\
.810 \\
.810 \\
.810\end{array}$ & $\begin{array}{l}I n . \\
5 / 16 \\
3 / 8 \\
1 / 2 \\
5 / 8 \\
3 / 4 \\
7 / 8 \\
7 / 8\end{array}$ & $\begin{array}{l}\text { In } \\
3 / 4 \\
1 \\
114 \\
13 / 8 \\
11 / 2 \\
17 / 8 \\
2\end{array}$ \\
\hline
\end{tabular}

Nоте.-The use of taper lock plain cylindrical plug gaging members and handles is optional in the range above 1.510 to and including 2.510 inches, but the use of the reversible design is standard for all sizes above 1.510 inches. 


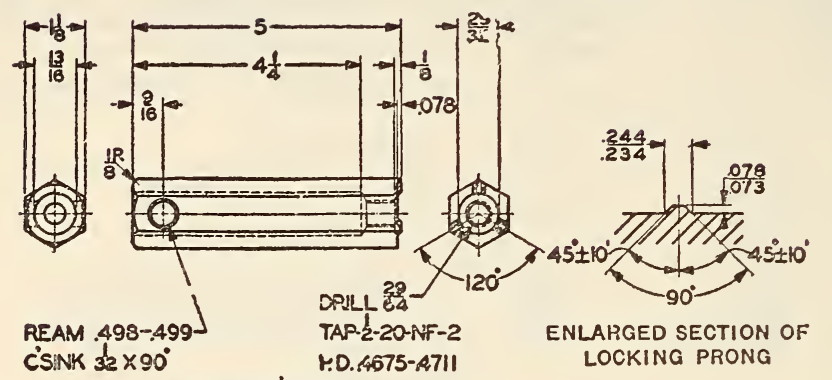
STRNDARD DESIGN

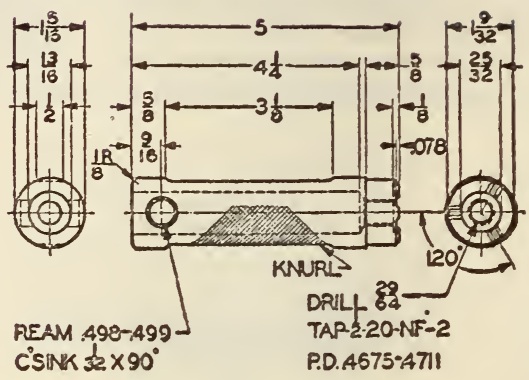

No. 6 handle. Range: Above 1.510 to and including 2.510 incless

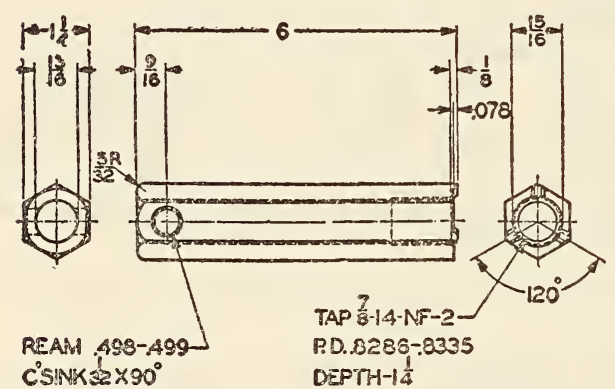

STANDARD DESIGN

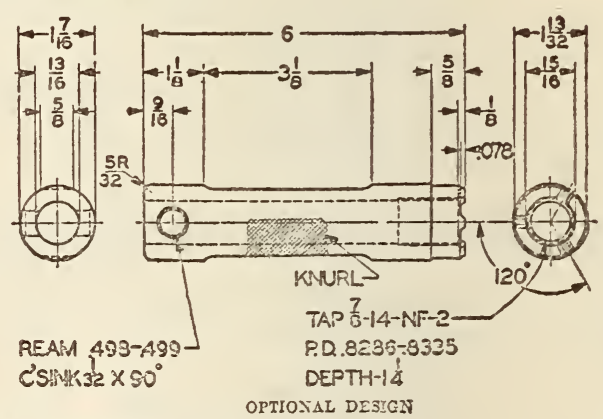

No. 7 handle. Range: Above 2.510 to and including 4.510 inches

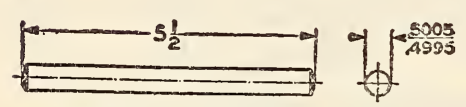

Cross bar, Nos. 6 and 7 handles (optional)

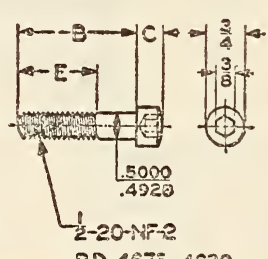

P.D. $4575-4639$

Sosiket head cap screr, No. 6 handls

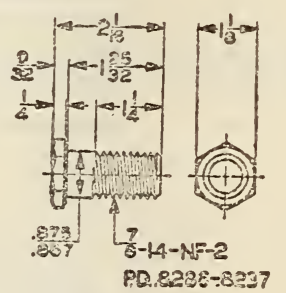

Hexвgon heed cap screw, Nio. 7 hand:3

FIGURE 688.-Plain cylindrical plug gage handles, reversible design, range above 1.510 to and including 4.510 inches

Dimensions of socket head cap screw, No. 6 handle

\begin{tabular}{|c|c|c|c|}
\hline Plug & B & $\mathrm{C}$ & $\mathrm{E}$ \\
\hline $\begin{array}{l}\text { Not go } \\
\text { Go } \\
\text { Progressive }-\end{array}$ & $\begin{array}{c}\text { Inches } \\
11 / 2 \\
21 / 4 \\
31 / 4\end{array}$ & $\begin{array}{c}\text { Inch } \\
3 / 8 \\
1 / 2 \\
1 / 2\end{array}$ & $\begin{array}{c}\text { Inches } \\
11 / 4 \\
11 / 2 \\
21 / 8\end{array}$ \\
\hline
\end{tabular}


TABLE 5.-Plain cylindrical plug gaging members, reversible design, range above 1.510 to and including 2.510 inches
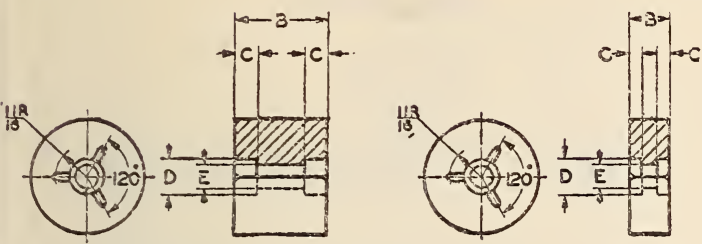

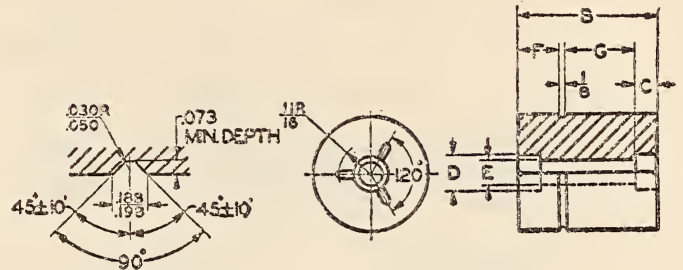

ENLARGEO SECTION OF LOCKING GROVE PEQUIRED ON BOTH ENOS CF ALL GAGES

FIgURE 689

\begin{tabular}{|c|c|c|c|c|c|c|c|c|c|c|c|c|c|c|c|c|c|c|}
\hline \multirow{3}{*}{ Handle size No. } & \multicolumn{4}{|c|}{ Plain plug diameters } & \multicolumn{4}{|c|}{ "Go" } & \multicolumn{4}{|c|}{ "Not go" } & \multicolumn{6}{|c|}{ Progressive } \\
\hline & \multicolumn{2}{|c|}{$\begin{array}{c}\text { Nominal range, } \\
\text { inclusive }\end{array}$} & \multicolumn{2}{|c|}{ Decimal range } & \multirow{2}{*}{$B$} & \multirow{2}{*}{ C } & \multirow{2}{*}{$D$} & \multirow{2}{*}{$E$} & \multirow{2}{*}{$B$} & \multirow{2}{*}{$C$} & \multirow{2}{*}{$D$} & \multirow{2}{*}{$E$} & \multirow{2}{*}{$B$} & \multirow{2}{*}{$C$} & \multirow{2}{*}{$D$} & \multirow{2}{*}{$E$} & \multirow{2}{*}{$F$} & \multirow{2}{*}{$G$} \\
\hline & From- & To- & Above- & $\begin{array}{l}\text { To and } \\
\text { includ- } \\
\text { ing- }\end{array}$ & & & & & & & & & & & & & & \\
\hline & $\begin{array}{r}\text { Inches } \\
11 / 2 \\
2\end{array}$ & $\begin{array}{r}\text { Inches } \\
2 \\
21 / 2\end{array}$ & $\begin{array}{l}\text { Inches } \\
1.510 \\
2.010\end{array}$ & $\begin{array}{l}\text { Inches } \\
2.010 \\
2.510\end{array}$ & $\begin{array}{c}\text { Inches } \\
17 / 8 \\
2\end{array}$ & $\begin{array}{r}\text { Inch } \\
1 / 2 \\
1 / 2\end{array}$ & \begin{tabular}{|c|} 
Inch \\
$25 / 32$ \\
$25 / 32$
\end{tabular} & $\begin{array}{c}\text { Inch } \\
17 / 32 \\
17 / 32\end{array}$ & $\begin{array}{r}\text { Inch } \\
7 / 8 \\
7 / 8\end{array}$ & $\mid \begin{array}{r}\text { Inch } \\
9 / 32 \\
9 / 32\end{array}$ & $\left|\begin{array}{r}\text { Inch } \\
25 / 32 \\
25 / 32\end{array}\right|$ & $\begin{array}{l}\text { Inch } \\
17 / 32 \\
17 / 32\end{array}$ & $\begin{array}{c}\text { Inches } \\
27,8 \\
3\end{array}$ & $\begin{array}{r}\text { Inch } \\
1 / 2 \\
1 / 2\end{array}$ & $\begin{array}{c}\text { Inch } \\
25 / 32 \\
25 / 32\end{array}$ & $\begin{array}{r}\text { Inch } \\
17 / 32 \\
17 / 32\end{array}$ & $\begin{array}{r}\text { Inch } \\
7 / 8 \\
7 / 8\end{array}$ & $\begin{array}{c}\text { Inches } \\
17 / 8 \\
2\end{array}$ \\
\hline
\end{tabular}

TABLE 6.-Plain cylindrical plug gaging members, reversible design, range above 2.510 to and including 4.510

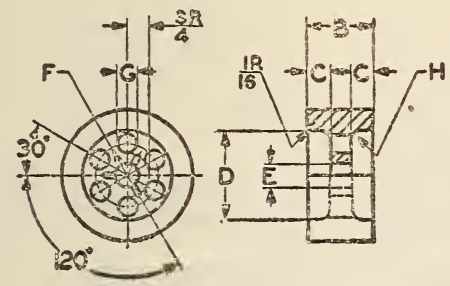

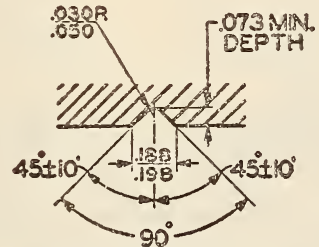

ENLARGED SECTION OF

LOCKING GROOVE

3 REQUIRED ON BOTH ENDS OF

ALL, GAGES

FigURe 690

\begin{tabular}{|c|c|c|c|c|c|c|c|c|c|c|c|c|c|c|c|c|c|c|}
\hline \multirow{3}{*}{$\begin{array}{l}\text { Handle } \\
\text { size No. }\end{array}$} & \multicolumn{4}{|c|}{ Plain plug diameters } & \multicolumn{7}{|c|}{ "Go" } & \multicolumn{7}{|c|}{ "Not go" } \\
\hline & \multicolumn{2}{|c|}{$\begin{array}{c}\text { Nominal range, } \\
\text { inclusive }\end{array}$} & \multicolumn{2}{|c|}{ Decimal range } & \multirow[b]{2}{*}{$B$} & \multirow[b]{2}{*}{$C$} & \multirow[b]{2}{*}{$D$} & \multirow[b]{2}{*}{$E$} & \multirow[b]{2}{*}{$F$} & \multirow[b]{2}{*}{$G$} & \multirow[b]{2}{*}{$H$} & \multirow[b]{2}{*}{$B$} & \multirow[b]{2}{*}{$C$} & \multirow[b]{2}{*}{$D$} & \multirow[b]{2}{*}{$E$} & \multirow[b]{2}{*}{$F$} & \multirow[b]{2}{*}{$G$} & \multirow[b]{2}{*}{$H$} \\
\hline & From- & To- & Above- & $\begin{array}{l}\text { To and } \\
\text { includ- } \\
\text { ing- }\end{array}$ & & & & & & & & & & & & & & \\
\hline & $\begin{array}{c}\text { Inches } \\
21 / 2 \\
3 \\
31 / 2 \\
4\end{array}$ & $\begin{array}{c}\text { Inches } \\
3 \\
31 / 2 \\
4 \\
41 / 2\end{array}$ & $\begin{array}{l}\text { Inches } \\
2.510 \\
3.010 \\
3.510 \\
4.010\end{array}$ & $\begin{array}{r}\text { Inches } \\
3.010 \\
3.510 \\
4.010 \\
4.510\end{array}$ & $\begin{array}{r}\text { Inches } \\
21 / 8 \\
21 / 4 \\
21 / 4 \\
21 / 4\end{array}$ & \begin{tabular}{|l} 
Inch \\
$11 / 16$ \\
$3 / 4$ \\
$3 / 4$ \\
$3 / 4$
\end{tabular} & \begin{tabular}{|c|} 
Inches \\
$17 / 8$ \\
$21 / 4$ \\
$25 / 8$ \\
3
\end{tabular} & $\begin{array}{c}\text { Inch } \\
29 / 32 \\
29 / 32 \\
29 / 32 \\
29 / 32\end{array}$ & $\begin{array}{l}\text { Inches } \\
11 / 16\end{array}$ & \begin{tabular}{|c|} 
Inch \\
6
\end{tabular} & $\begin{array}{r}\text { Inch } \\
3 / 16 \\
5 / 16 \\
5 / 16 \\
5 / 16\end{array}$ & \begin{tabular}{r|} 
Inch \\
1 \\
1 \\
1 \\
1
\end{tabular} & $\begin{array}{r}\text { Inch } \\
1 / 8 \\
1 / 8 \\
1 / 8 \\
1 / 8\end{array}$ & $\begin{array}{r}\text { Inches } \\
17 / 8 \\
21 / 4 \\
25 / 8 \\
3\end{array}$ & $\begin{array}{c}\text { Inch } \\
29 / 32 \\
29 / 32 \\
29 / 32 \\
29 / 32\end{array}$ & \begin{tabular}{|c|} 
Inches \\
1116 \\
\end{tabular} & $\begin{array}{l}\text { Inch } \\
6 \\
6 \\
3 / 4\end{array}$ & $\begin{array}{c}\text { Inch } \\
3 / 16 \\
3 / 16 \\
3 / 16 \\
3 / 16\end{array}$ \\
\hline
\end{tabular}


(a)

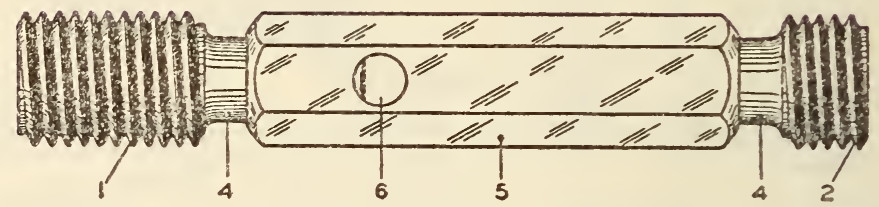

(b)
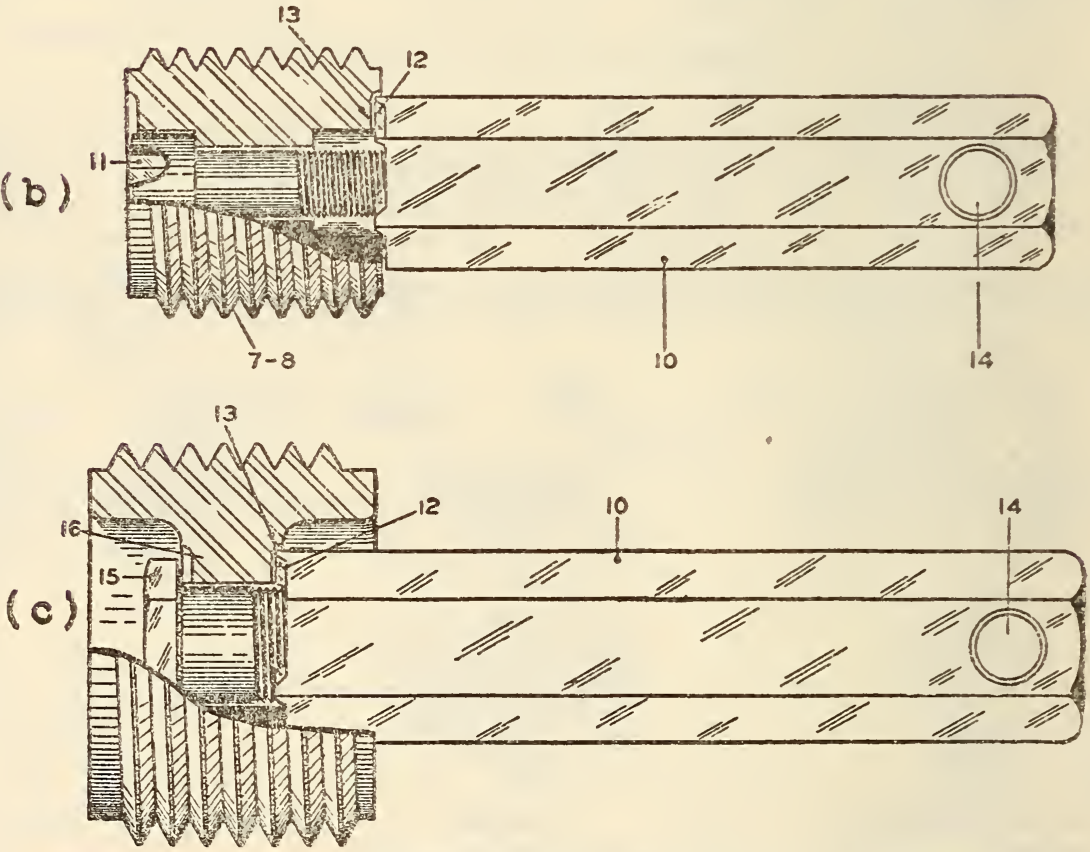

FIGURE 691.-American gage design standard thread plug gages, details of construction

(a) Range: No. 0 to and including 1.510 inches.

(b) Range: Above 1.510 to and including 2.510 inches.

(c) Range: Above 2.510 to and including 4.150 inches.

1 , "Go" gaging member.

1, "Not go" gaging member

10 , handle for reversible gage.

4 , shank.

5 , taper lock handle.

6 , drift hole (or slot).

7 " "Go" gaging member.

8 , "Not go" gaging member.

11, socket head screw.

12, locking prong.

13, locking roove.

14 , cross-pin hole.

15 , hexagon head screw

16 , web. 
TABLE 7.-Thread plug gage handles, taper lock design, range No. 0 to No. 12, inclusive

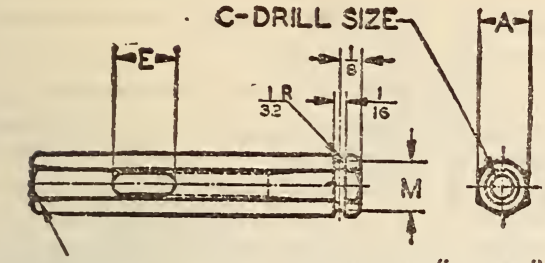

G.PIN REAMER, TAPER .250" IN $12^{\prime \prime}$

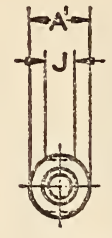

KNURL-

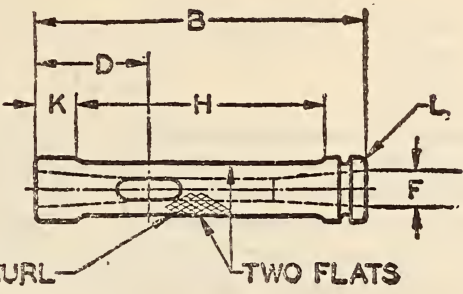

FiguRe 692

\begin{tabular}{|c|c|c|c|c|c|c|c|c|c|c|c|c|c|c|c|c|c|c|c|}
\hline \multirow{3}{*}{$\begin{array}{l}\text { Handle } \\
\text { size No. }\end{array}$} & \multicolumn{4}{|c|}{ Thread plug diameters } & \multicolumn{15}{|c|}{ General dimensions } \\
\hline & \multicolumn{2}{|c|}{$\begin{array}{c}\text { Nominal range, } \\
\text { inclusive }\end{array}$} & \multicolumn{2}{|c|}{ Decimal range } & \multirow{2}{*}{$A$} & \multirow{2}{*}{$A^{\prime}$} & \multirow{2}{*}{$B$} & \multirow{2}{*}{$\underset{\text { Dize }}{C}$} & \multirow{2}{*}{$D$} & \multirow{2}{*}{$E$} & \multicolumn{2}{|c|}{$F$} & \multirow{2}{*}{$G$} & \multirow{2}{*}{$H$} & \multirow{2}{*}{$J$} & \multirow{2}{*}{$K$} & \multirow{2}{*}{$L$} & \multicolumn{2}{|c|}{$M$} \\
\hline & From- & To- & Above- & $\begin{array}{l}\text { To and in- } \\
\text { cluding- }\end{array}$ & & & & & & & $\begin{array}{l}\text { Mini- } \\
\text { mum }\end{array}$ & $\begin{array}{l}\text { Max- } \\
\text { imum }\end{array}$ & & & & & & $\begin{array}{l}\text { Mini- } \\
\text { mum }\end{array}$ & $\begin{array}{l}\text { Max- } \\
\text { imum }\end{array}$ \\
\hline 000 & No. & No. & $\begin{array}{l}\text { Inch } \\
0.059\end{array}$ & $\begin{array}{l}\text { Inch } \\
0.105\end{array}$ & $\begin{array}{c}\text { In. } \\
3 / 16\end{array}$ & $\begin{array}{l}\text { In. } \\
14\end{array}$ & $\begin{array}{r}\text { Ins. } \\
11 / 2\end{array}$ & $\left\{\begin{array}{l}\text { No. } 34 \\
(0.111)\end{array}\right.$ & $\begin{array}{r}\text { Inch } \\
9 / 16\end{array}$ & $\begin{array}{c}\text { Inch } \\
5 \% 4 \text { by } 144\end{array}$ & $\begin{array}{l}\text { Inch } \\
0.125\end{array}$ & $\begin{array}{l}\text { Inch } \\
0.126\end{array}$ & $\begin{array}{r}\text { No. } \\
000\end{array}$ & Ins. & $\begin{array}{l}I n . \\
5 / 32\end{array}$ & $\begin{array}{r}\text { In. } \\
14\end{array}$ & $\begin{array}{l}\text { In. } \\
1 / 32\end{array}$ & $\begin{array}{l}\text { Inch } \\
0.172\end{array}$ & $\begin{array}{l}\text { Inch } \\
0.177\end{array}$ \\
\hline $00 \ldots$ & 4 & 6 & .105 & .150 & $1 / 4$ & $5 / 16$ & $13 / 4$ & $\left\{\begin{array}{l}\text { No. 29 } \\
(0.136)\end{array}\right.$ & $5 / 8$ & $3 / 32$ by $5 / 16$ & .155 & .156 & 0 & $11 / 4$ & $3 / 16$ & 14 & $1 / 32$ & .235 & .240 \\
\hline $0 \ldots$ & 8 & 12 & .150 & .240 & $5 / 16$ & $3 / 8$ & 2 & $\left\{\begin{array}{c}\text { No. } 20 \\
(0.161)\end{array}\right.$ & $11 / 16$ & $1 / 8$ by $3 / 8$ & .180 & .181 & 2 & $11 / 2$ & $3 / 16$ & $1 / 4$ & $1 / 32$ & .297 & .302 \\
\hline
\end{tabular}

NotE.-The purpose of the groore in the "not go" end of the handle is to distinguish the "not go" from the "go" end.

TABLE 8.-Thread plug gaging members, taper lock design, range No. 0 to No. 12 , inclusive
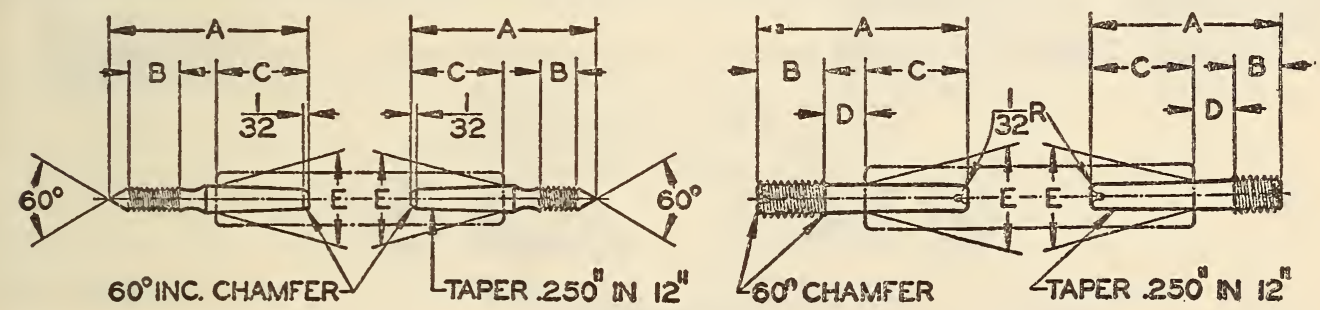

FIGURE 693

\begin{tabular}{|c|c|c|c|c|c|c|c|c|c|c|c|c|c|c|c|c|}
\hline \multirow{4}{*}{$\begin{array}{l}\text { Handle size } \\
\text { No. }\end{array}$} & \multicolumn{4}{|c|}{ Range, thread plug diameters } & \multicolumn{12}{|c|}{ General dimensions } \\
\hline & \multicolumn{2}{|c|}{$\begin{array}{l}\text { Nominal, in- } \\
\text { clusive }\end{array}$} & \multicolumn{2}{|c|}{ Decimal } & \multicolumn{6}{|c|}{ "Go" } & \multicolumn{6}{|c|}{ "Not go" } \\
\hline & \multirow[b]{2}{*}{ From- } & \multirow[b]{2}{*}{ To- } & \multirow[b]{2}{*}{ Above- } & \multirow{2}{*}{$\begin{array}{c}\text { To and } \\
\text { includ- } \\
\text { ing- }\end{array}$} & \multirow[b]{2}{*}{$A$} & \multirow[b]{2}{*}{$B$} & \multirow[b]{2}{*}{$C$} & \multirow[b]{2}{*}{$D$} & \multicolumn{2}{|c|}{$E$} & \multirow[b]{2}{*}{$A$} & \multirow[b]{2}{*}{$B$} & \multirow[b]{2}{*}{$C$} & \multirow[b]{2}{*}{$D$} & \multicolumn{2}{|c|}{$E$} \\
\hline & & & & & & & & & $\begin{array}{l}\text { Mini- } \\
\text { mum }\end{array}$ & $\begin{array}{l}\text { Maxi- } \\
\text { mum }\end{array}$ & & & & & $\begin{array}{l}\text { Mini- } \\
\text { mum }\end{array}$ & $\begin{array}{l}\text { Maxi- } \\
\text { mum }\end{array}$ \\
\hline $\begin{array}{l}000 . \\
00 .-\end{array}$ & $\begin{array}{r}\text { No. } \\
0 \\
4 \\
8\end{array}$ & $\begin{array}{r}\text { No. } \\
3 \\
6 \\
12\end{array}$ & $\begin{array}{c}\text { Inch } \\
0.059 \\
.105 \\
.150\end{array}$ & $\begin{array}{c}\text { Inch } \\
0.105 \\
.150 \\
.240\end{array}$ & $\begin{array}{r}\text { Inches } \\
1132 \\
17 / 32 \\
1 \% 32\end{array}$ & $\begin{array}{c}\text { Inch } \\
1 / 4 \\
5 / 16 \\
13 / 32\end{array}$ & $\begin{array}{r}\text { Inch } \\
1 / 2 \\
9 / 16 \\
5 / 8\end{array}$ & \begin{tabular}{c} 
Inch \\
\hdashline $1 / 4$
\end{tabular} & $\begin{array}{l}\text { Inch } \\
0.125 \\
.155 \\
.180\end{array}$ & $\begin{array}{c}\text { Inch } \\
0.126 \\
.156 \\
.181\end{array}$ & $\begin{array}{c}\text { Inches } \\
31 / 32 \\
11 / 8 \\
15 / 32\end{array}$ & $\begin{array}{r}\text { Inch } \\
3 / 16 \\
7 / 32 \\
9 / 32\end{array}$ & $\begin{array}{c}\text { Inch } \\
1 / 2 \\
9 / 16 \\
5 / 8\end{array}$ & $\mid \begin{array}{r}\text { Inch } \\
1 / 4 \\
\end{array}$ & $\begin{array}{l}\text { Inch } \\
0.125 \\
.155 \\
.180\end{array}$ & $\begin{array}{l}\text { Inch } \\
0.126 \\
.156 \\
.181\end{array}$ \\
\hline
\end{tabular}


TABLE 9.-Thread plug gage handles, taper lock design, range $1 / 4$ to $1 \frac{112}{2}$ inches, inclusive

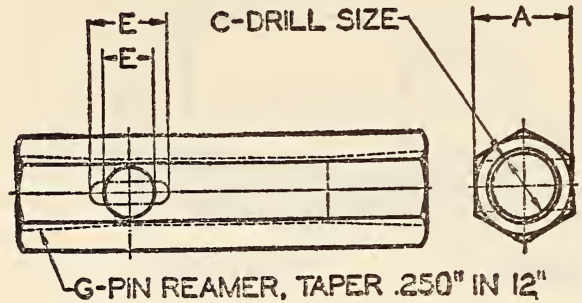

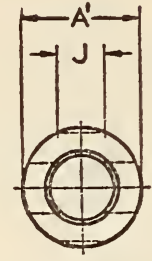

FIGURE 694
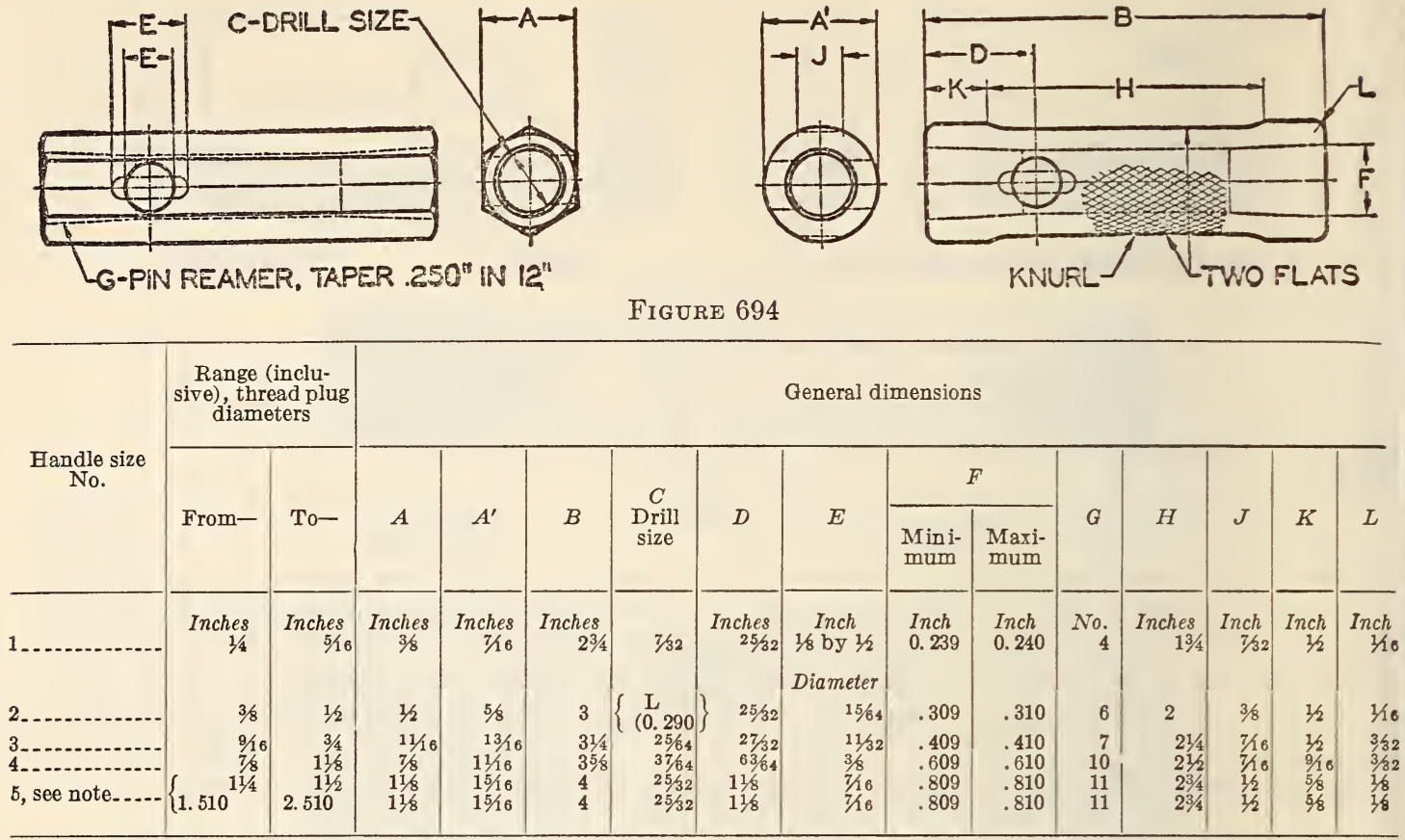

Note. - In the range above 1.510 to and including 2.510 inches the No. 5 handle is standard for taper pipe thread plug gages and optional or other thread plug gages. See footnote, Table 10.

TABLE 10.-Thread plug gaging members, taper lock design, range $1 / 4$ to $1 \frac{1}{2}$ inches, inclusive

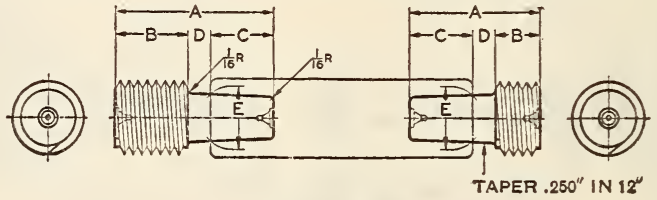

FIGURE 695

Not less than three full threads must remain on "not go" plug

\begin{tabular}{|c|c|c|c|c|c|c|c|c|c|c|c|c|c|c|c|c|c|}
\hline \multirow{4}{*}{$\begin{array}{c}\text { Handle } \\
\text { size No. }\end{array}$} & \multicolumn{5}{|c|}{ Range } & \multicolumn{12}{|c|}{ General dimensions } \\
\hline & \multicolumn{4}{|c|}{ Thread plug diameters } & \multirow{3}{*}{ Threads per inch } & \multirow{3}{*}{$A$} & \multirow{3}{*}{$B$} & \multicolumn{4}{|c|}{ "Go" } & \multicolumn{6}{|c|}{ "Not go" } \\
\hline & \multicolumn{2}{|c|}{$\begin{array}{l}\text { Nominal } \\
\text { range, in- } \\
\text { clusive }\end{array}$} & \multicolumn{2}{|c|}{ Decimal range } & & & & & & \multicolumn{2}{|c|}{$E$} & \multirow{2}{*}{$A$} & \multirow{2}{*}{$B$} & \multirow{2}{*}{$C$} & \multirow{2}{*}{$D$} & \multicolumn{2}{|c|}{$E$} \\
\hline & From- & To- & Above- & $\begin{array}{l}\text { To and } \\
\text { includ- } \\
\text { ing- }\end{array}$ & & & & & & $\begin{array}{l}\text { Mini- } \\
\text { mum }\end{array}$ & $\begin{array}{l}\text { Maxi- } \\
\text { mum }\end{array}$ & & & & & $\begin{array}{l}\text { Mini- } \\
\text { mum }\end{array}$ & $\begin{array}{l}\text { Maxi- } \\
\text { mum }\end{array}$ \\
\hline & \begin{tabular}{|c} 
Inches \\
$1 / 4$ \\
$3 / 8$ \\
$9 / 16$ \\
78 \\
$11 / 4$
\end{tabular} & $\begin{array}{c}\text { Inches } \\
5 / 16 \\
1 / 2 \\
3 / 4 \\
118 \\
11 / 2\end{array}$ & $\begin{array}{r}\text { Inches } \\
0.240 \\
.365 \\
.510 \\
.825 \\
1.135\end{array}$ & $\begin{array}{r}\text { Inches } \\
0.365 \\
.510 \\
.825 \\
1.135 \\
1.510\end{array}$ & Coarser than 12 & $\begin{array}{l}\text { In. } \\
11 / 2 \\
13 / 4 \\
17 / 8 \\
23 / 16 \\
25 / 8\end{array}$ & $\begin{array}{l}\text { In. } \\
1 / 2 \\
3 / 4 \\
1^{7 / 8} \\
11 / 4\end{array}$ & $\begin{array}{l}\text { In. } \\
3 / 4 \\
3 / 4 \\
3 / 4 \\
7 / 8 \\
1\end{array}$ & $\begin{array}{l}\text { In. } \\
114 \\
134 \\
1 / 4 \\
5 / 16 \\
3 / 8\end{array}$ & $\begin{array}{r}\text { Inch } \\
0.239 \\
.309 \\
.408 \\
.608 \\
.808\end{array}$ & $\begin{array}{r}\text { Inch } \\
0.240 \\
.310 \\
.410 \\
.610 \\
.810\end{array}$ & \begin{tabular}{|l|} 
In. \\
$15 / 16$ \\
$13 / 8$ \\
113 \\
$113 / 16$ \\
218
\end{tabular} & \begin{tabular}{|l|} 
In. \\
$5 / 16$ \\
$3 / 8$ \\
$1 / 2$ \\
$5 / 8$ \\
$3 / 4$
\end{tabular} & $\begin{array}{r}\text { In. } \\
3 / 4 \\
3 / 4 \\
3 / 4 \\
7 / 8 \\
1^{7 / 8}\end{array}$ & $\begin{array}{l}\text { In. } \\
14 \\
144 \\
1 / 4 \\
516 \\
3 / 8\end{array}$ & $\begin{array}{r}\text { Inch } \\
0.239 \\
.309 \\
.408 \\
.608 \\
.808\end{array}$ & $\begin{array}{l}\text { Inch } \\
0.240 \\
.310 \\
.410 \\
.610 \\
.810\end{array}$ \\
\hline 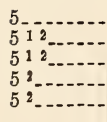 & $\begin{array}{l}11 / 4 \\
11 / 2 \\
11 / 2 \\
11 / 2 \\
11 / 2\end{array}$ & $\begin{array}{l}11 / 2 \\
2 \\
2 \\
2 \\
2\end{array}$ & $\begin{array}{l}1.135 \\
1.510 \\
1.510 \\
1.510 \\
1.510\end{array}$ & $\begin{array}{l}1.510 \\
2.010 \\
2.010 \\
2.010 \\
2.010\end{array}$ & $\begin{array}{l}12 \text { and finer } \\
\text { Coarser than } 12 . \\
12 \text { and finer } \\
7 \text { and coarser } \\
\text { Finer than } 7 \text {, coarser thar }\end{array}$ & $\begin{array}{l}23 / 8 \\
27 / 8 \\
23 / 8 \\
314 \\
25 / 8\end{array}$ & $\begin{array}{l}1 \\
11 / 2 \\
17 / 8 \\
17 / 4\end{array}$ & $\begin{array}{l}1 \\
1 \\
1 \\
1 \\
1\end{array}$ & $\begin{array}{l}3 / 8 \\
3 / 8 \\
3 / 8 \\
3 / 8 \\
3 / 8\end{array}$ & $\begin{array}{l}.808 \\
.808 \\
.808 \\
.808 \\
.808\end{array}$ & $\begin{array}{l}.810 \\
.810 \\
.810 \\
.810 \\
.810\end{array}$ & $\begin{array}{l}21 / 8 \\
21 / 8 \\
21 / 8 \\
21 / 4 \\
21 / 4\end{array}$ & \begin{tabular}{l|}
$3 / 4$ \\
$3 / 4$ \\
$3 / 4$ \\
$7 / 8$ \\
$7 / 8$
\end{tabular} & $\begin{array}{l}1 \\
1 \\
1 \\
1\end{array}$ & $\begin{array}{l}3 / 8 \\
3 / 8 \\
3 / 8 \\
3 / 8 \\
3 / 8\end{array}$ & $\begin{array}{l}.808 \\
.808 \\
.808 \\
.808 \\
.808\end{array}$ & $\begin{array}{l}.810 \\
.810 \\
.810 \\
.810 \\
.810\end{array}$ \\
\hline $\begin{array}{lll}5 & 2 & \\
5 & 2 & -\ldots \ldots \\
5 & 2 & -\ldots\end{array}$ & $\begin{array}{l}11 / 2 \\
2 \\
2 \\
2\end{array}$ & $\begin{array}{l}2 \\
2^{1 / 2} \\
2^{1 / 2} \\
2^{1 / 2}\end{array}$ & $\begin{array}{l}1.510 \\
2.010 \\
2.010 \\
2.010\end{array}$ & $\begin{array}{l}2.010 \\
2.510 \\
2.510 \\
2.510\end{array}$ & $\begin{array}{l}16 \text { and finer } \\
7 \text { and coarser } \\
\text { Finer than } 7, \text { coarser thar } \\
16 \text { and finer............ }\end{array}$ & $\begin{array}{l}214 \\
33 / 8 \\
234 \\
21 / 4 \\
13\end{array}$ & $\begin{array}{l}2^{7 / 8} \\
13 / 8 \\
7 / 8\end{array}$ & $\begin{array}{l}1 \\
1 \\
1 \\
1\end{array}$ & $\begin{array}{l}3 / 8 \\
3 / 8 \\
3 / 8 \\
3 / 8\end{array}$ & $\begin{array}{l}.808 \\
.808 \\
.808 \\
.808\end{array}$ & $\begin{array}{l}.810 \\
.810 \\
.810 \\
.810\end{array}$ & $\begin{array}{l}21 / 4 \\
21 / 4 \\
21 / 4 \\
21 / 4\end{array}$ & $\begin{array}{l}7 / 8 \\
7 / 8 \\
7 / 8 \\
7 / 8\end{array}$ & $\begin{array}{l}1 \\
1 \\
1 \\
1\end{array}$ & $\begin{array}{l}3 / 8 \\
3 / 8 \\
3 / 8 \\
3 / 8\end{array}$ & $\begin{array}{l}.808 \\
.808 \\
.808 \\
.808\end{array}$ & $\begin{array}{l}.810 \\
.810 \\
.810 \\
.810\end{array}$ \\
\hline
\end{tabular}

1 These blanks are intended for the American national coarse and fine thread series only.

2 The use of taper lock thread plug gaging members and handles is optional for straight thread plug gages in the ranges above 1.510 to and including 2.510 inches, but the reversible design is recommended as standard. For taper pipe thread plug gages, see Table 11. 
TABLE 11,-Pipe thread plug gage handles and gaging members, taper lock design, range $1 / 8$ to 2 inches, inclusive

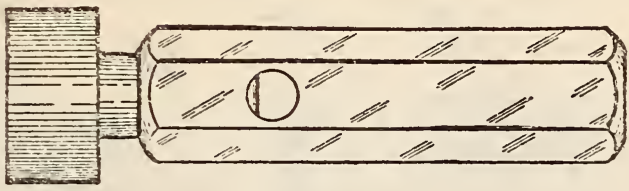

FIGURE 696

Taper lock gaging members and handles are standard for pipe thread plug gages to and including 2 inches nominal pipe size. The general dimensions of handles and gaging members which are referred to in this table are given in Tables 9 and 10.

\begin{tabular}{|c|c|c|c|c|c|}
\hline Nominal pipe size & $\begin{array}{l}\text { Handle } \\
\text { No. }\end{array}$ & $\begin{array}{l}\text { Gaging member, use "not } \\
\text { go" blank for range- }\end{array}$ & Nominal pipe size & $\begin{array}{c}\text { Handle } \\
\text { No. }\end{array}$ & $\begin{array}{l}\text { Gaging member, use "not } \\
\text { go" blank for range- }\end{array}$ \\
\hline $\begin{array}{l}18 \text { inch } \\
1 / 4 \text { inch } \\
36 \text { inch } \\
1 / 2 \text { inch } \\
3 / 4 \text { inch }\end{array}$ & $\begin{array}{l}2 \\
3 \\
3 \\
4 \\
4\end{array}$ & $\begin{array}{l}3 / 8 \text { to } 1 / 2 \text { inch. } \\
9 / 16 \text { to } 34 \text { inch. } \\
9 / 16 \text { to } 3 / 4 \text { inch. } \\
7 / 8 \text { to } 118 \text { inches. } \\
7 / 8 \text { to } 11 / 8 \text { inches. }\end{array}$ & $\begin{array}{l}1 \text { inch } \\
11 / 4 \text { inches. } \\
11 / 2 \text { inches.... } \\
2 \text { inches. }\end{array}$ & $\begin{array}{l}5 \\
5 \\
5 \\
5\end{array}$ & $\begin{array}{l}11 / 4 \text { to } 11 / 2 \text { inches. } \\
11 / 2 \text { to } 2 \text { inches. } \\
11 / 2 \text { to } 2 \text { inches. } \\
2 \text { to } 21 / 2 \text { inches. }\end{array}$ \\
\hline
\end{tabular}

TABLE 12.-Thread plug gaging members, reversible design, range above $1 \frac{112}{2}$ to and including $2 \frac{1}{2}$ inches

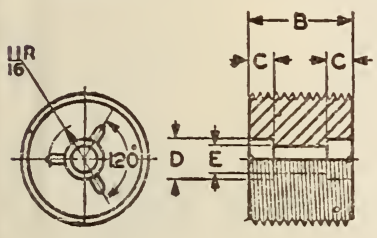

NOT LESS THAN THEEE

FULL THREADS MUST REMAN ON"NOT GÓPLUG.

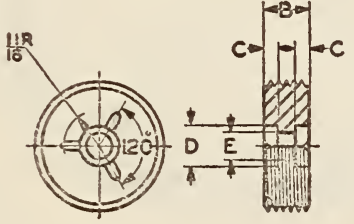

FigURE 697

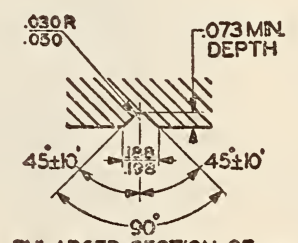

ENLARGEO SECTION OF NTEFWAL LOCKING MEMSER 3 REQUIRED ON BOTH ENDS OF ALL GAGES.

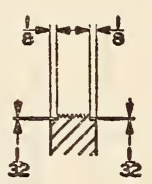

"NOT GO GAGES la Tí.I. \& FINER RELIFVED ON BOTH ENDS SE BELLW SHARP ROOT OF THRD AS SHOWN.

\begin{tabular}{|c|c|c|c|c|c|c|c|c|c|c|c|c|c|c|c|c|c|c|}
\hline \multirow{3}{*}{ Handle size No. } & \multicolumn{2}{|c|}{$\begin{array}{l}\text { Range, thread plug } \\
\text { diameters }\end{array}$} & \multicolumn{12}{|c|}{ "Go" } & \multicolumn{4}{|c|}{ "Not go" } \\
\hline & \multirow{2}{*}{ Above- } & \multirow{2}{*}{$\begin{array}{l}\text { To and } \\
\text { including- }\end{array}$} & \multicolumn{4}{|c|}{$\begin{array}{l}7 \text { threads per inch } \\
\text { and coarser }\end{array}$} & \multicolumn{4}{|c|}{$\begin{array}{c}\text { Finer than } 7 \text { threads } \\
\text { per inch and coarser } \\
\text { than } 16\end{array}$} & \multicolumn{4}{|c|}{$\begin{array}{l}16 \text { threads per inch } \\
\text { and finer }\end{array}$} & \multicolumn{4}{|c|}{ All pitches } \\
\hline & & & $B$ & $C$ & $D$ & $E$ & $B$ & $C$ & $D$ & $E$ & $B$ & $C$ & $D$ & $E$ & $B$ & C & $D$ & $E$ \\
\hline & $\begin{array}{l}\text { Inches } \\
1.510 \\
2.010\end{array}$ & $\begin{array}{l}\text { Inches } \\
2.010 \\
2.510\end{array}$ & $\begin{array}{c}\text { Inches } \\
17 / 8 \\
2\end{array}$ & $\begin{array}{r}\text { Inch } \\
1 / 2 \\
1 / 2\end{array}$ & $\begin{array}{l}\text { Inch } \\
25 / 32 \\
25 / 32\end{array}$ & $\begin{array}{l}\text { Inch } \\
17 / 32 \\
17 / 32\end{array}$ & $\begin{array}{r}\text { Inches } \\
11 / 4 \\
13 / 8\end{array}$ & $\begin{array}{r}\text { Inch } \\
3 / 8 \\
3 / 8\end{array}$ & $\begin{array}{l}\text { Inch } \\
25 / 32 \\
25 / 32\end{array}$ & $\begin{array}{l}\text { Inch } \\
17 / 32 \\
17 / 32\end{array}$ & $\begin{array}{r}\text { Inch } \\
7 / 8 \\
7 / 8\end{array}$ & $\begin{array}{r}\text { Inch } \\
9 / 32 \\
9 / 32\end{array}$ & $\begin{array}{l}\text { Inch } \\
25 / 32 \\
25 / 32\end{array}$ & $\begin{array}{l}\text { Inch } \\
17 / 32 \\
17 / 32\end{array}$ & $\begin{array}{r}\text { Inch } \\
7 / 8 \\
7 / 8\end{array}$ & $\begin{array}{r}\text { Inch } \\
9 / 32 \\
9 / 32\end{array}$ & $\begin{array}{l}\text { Inch } \\
25 / 32 \\
25 / 32\end{array}$ & $\begin{array}{l}\text { Inch } \\
17 / 32 \\
17 / 32\end{array}$ \\
\hline
\end{tabular}

(The illustration for thread plug gage handles, reversible design, range above 1.510 to and including 4.510 inches, is the same as for plain cylindrical plug gage handles shown above in Figure 688 . The dimensions of socket head cap screw, No. 6 handle, in this instance are as follows:)

\begin{tabular}{|c|c|c|c|}
\hline Plug & $B$ & $C$ & $E$ \\
\hline $\begin{array}{l}\text { "Not go," all pitches. Go, } 16 \text { threads per inch } \\
\text { and finer-a........... }\end{array}$ & $\begin{array}{r}\text { Inches } \\
11 / 2 \\
214\end{array}$ & $\begin{array}{r}\text { Inch } \\
3 / 8 \\
1 / 2\end{array}$ & $\begin{array}{r}\text { Inches } \\
11 / 4 \\
11 / 2\end{array}$ \\
\hline
\end{tabular}


TABLE 13.-Thread plug gaging members, reversible design, range above $21 / 2$ to and including $4 \frac{1}{2}$ inches
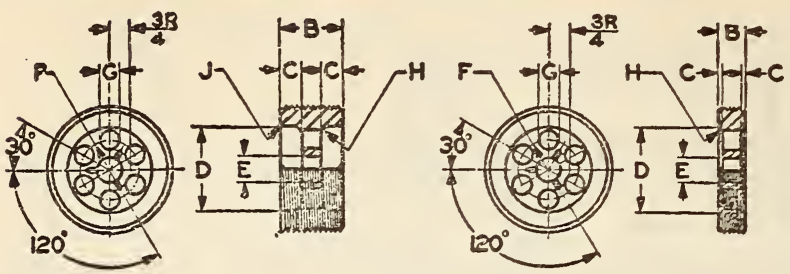

NOT LESS THAN THREE

FULL THREADS MUST

REMAIN ON"NOT GO" PLUG.

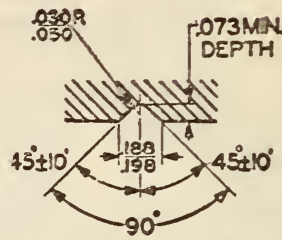

ENLARGED SECTION OF LOCKING GROOVE 3 REQUIRED ON BOTH ENDS OF ALL GAGES.

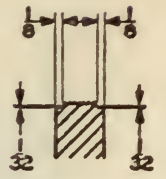

"NOT CO" CACES IST.P.1. SFTERR RELISVED ON BOTH ENDS 32 BEDOW SHARP ROOT OF THRD AS SHONN.

Figure 698

\begin{tabular}{|c|c|c|c|c|c|c|c|c|c|c|c|c|c|c|c|c|c|c|c|c|c|c|}
\hline \multirow{3}{*}{$\begin{array}{l}\text { Handle } \\
\text { size No. }\end{array}$} & \multicolumn{4}{|c|}{ Thread plug diameter } & \multicolumn{11}{|c|}{ "Go" } & \multicolumn{3}{|c|}{ "Not go" } & \multicolumn{4}{|c|}{ All } \\
\hline & \multicolumn{2}{|c|}{$\begin{array}{l}\text { Nominal } \\
\text { range, } \\
\text { inclusive }\end{array}$} & \multicolumn{2}{|c|}{ Decimal range } & \multicolumn{4}{|c|}{$\begin{array}{l}7 \text { threads per inch } \\
\text { and coarser }\end{array}$} & \multicolumn{4}{|c|}{$\begin{array}{c}\text { Finer than } 7 \text { threads } \\
\text { per inch and coarser } \\
\text { than } 16\end{array}$} & \multicolumn{3}{|c|}{$\begin{array}{l}16 \text { threads per } \\
\text { inch and finer }\end{array}$} & \multicolumn{3}{|c|}{ All pitches } & \multicolumn{4}{|c|}{ All pitches } \\
\hline & 竞 & $\bigsqcup_{E}^{1}$ & $\begin{array}{l}0 \\
0 \\
0 \\
0 \\
\frac{1}{4}\end{array}$ & 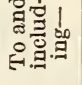 & $B$ & $C$ & $H$ & $J$ & $B$ & $C$ & $H$ & $J$ & $B$ & $C$ & $H$ & $B$ & $C$ & $H$ & $D$ & $E$ & $F$ & $G$ \\
\hline $\begin{array}{l}7 \\
7 \\
7 \\
7\end{array}$ & $\begin{array}{r}\text { Inches } \\
21 / 2 \\
3 \\
31 / 2 \\
4\end{array}$ & \begin{tabular}{|c} 
Inches \\
3 \\
$31 / 2$ \\
4 \\
$41 / 2$
\end{tabular} & $\begin{array}{r}\text { Inches } \\
2.510 \\
3.010 \\
3.510 \\
5.010\end{array}$ & $\begin{array}{r}\text { Inches } \\
3.010 \\
3.510 \\
4.010 \\
4.510\end{array}$ & \begin{tabular}{c|} 
In. \\
$21 / 8$ \\
214 \\
214 \\
214
\end{tabular} & $\begin{array}{r}\text { In. } \\
11 / 16 \\
3 / 4 \\
3 / 4 \\
3 / 4 \\
\text {. }\end{array}$ & $\begin{array}{c}\text { In. } \\
3 / 16 \\
5 / 16 \\
5 / 16 \\
5 / 16\end{array}$ & $\begin{array}{c}I n . \\
316 \\
116 \\
116 \\
1 / 16\end{array}$ & \begin{tabular}{r|}
$I n$. \\
116 \\
$11 / 2$ \\
$11 / 2$ \\
116
\end{tabular} & \begin{tabular}{r|} 
In. \\
$3 / 8$ \\
36 \\
$3 / 8$ \\
3,8
\end{tabular} & $\begin{array}{r}\text { In. } \\
3 / 16 \\
1 / 4 \\
1 / 4 \\
1 / 4 \\
\end{array}$ & $\begin{array}{l}\text { In. } \\
1 / 16 \\
1 / 16 \\
1 / 16 \\
1 / 16\end{array}$ & $\begin{array}{c}\text { In. } \\
1 \\
1 \\
1 \\
1\end{array}$ & $\begin{array}{r}\text { In. } \\
18 \\
38 \\
18 \\
18 \\
18\end{array}$ & $\begin{array}{c}\text { In. } \\
3 / 16 \\
3 / 16 \\
3 / 16 \\
3 / 16\end{array}$ & $\begin{array}{c}\text { In. } \\
1 \\
1 \\
1 \\
1\end{array}$ & $\begin{array}{r}I n . \\
18 \\
168 \\
38 \\
1 / 8\end{array}$ & $\begin{array}{c}\text { In. } \\
3 / 16 \\
3 / 16 \\
3 / 16 \\
3 / 16\end{array}$ & $\begin{array}{l}\text { In. } \\
17 / 8 \\
214 \\
25 \% 8 \\
3\end{array}$ & $\begin{array}{l}\text { In. } \\
29 / 32 \\
29 / 32 \\
29 / 32 \\
29 / 32\end{array}$ & $\begin{array}{l}\text { In. } \\
11 / 16\end{array}$ & \begin{tabular}{l} 
In. \\
\hdashline.. \\
$3 / 4$
\end{tabular} \\
\hline
\end{tabular}
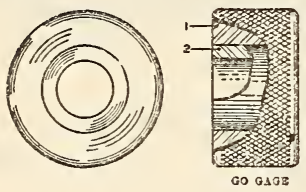

(a)
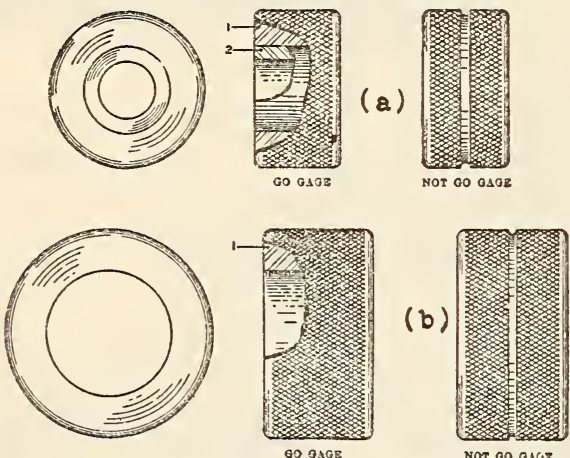

NoT 00 OAG3
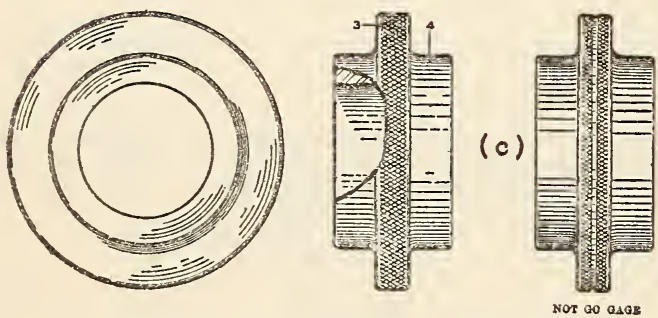

FIgURE 699.-American gage design standard plain ring gages, details of construction

(a) Range: Above 0.059 to and including 0.510 inch.

(b) Range: Above 0.510 to and including 1.510 inches.

(c) Range: A bove 1.510 to and including 4.510 inches.

1 , body.
2 , bushing. $\quad 3$, flange.

Plain Ring Gage Blanks

The use of the solid ring-gage design for external size control, being fairly well established, the committee's work on plain ring gages was con- cerned chiefly with matters of proportion. In the smaller sizes of plain ring gages it was felt desirable to employ a hardened bushing pressed into a soft gage body, in place of the 1-piece ring gage, and this design has been adopted in the range above 0.059 to and including 0.510 inch. The single-piece gage is employed in all cases above 0.510 inch, but gages in sizes above 1.510 inches are flanged, in order to eliminate unnecessary weight and facilitate handling.

No dimensional difference exists between "go" and "not go" blanks of identical size range, but an annular groove is provided in the periphery of "not go" blanks as a means of identification.

General details of construction are shown in Figure 699, and dimensions are given in Tables 14 and 15.

\section{Thread Ring Gage Blaniss}

The committee found universal accord as to the superiority of the adjustable thread ring gage over the solid type, with the result that all American gage design standard thread ring gage blanks are equipped with an effective device for adjusting and locking the gage in the manufacturing or resizing processes. Of the many locking devices considered the single-unit locking device was finally adopted as standard, as it permits a minimum diameter of blank for a given size range, and provides a simple adjustment and positive lock without introducing any mechanical stresses into the gage body which might tend to create distortion after setting. Referring to Figure 700 , the construction and operation of this device is as follows:

The adjusting screw, $\mathcal{S}$, is threaded externally and internally and split longitudinally. Turning this screw to the right exerts pressure on the sleeve, 2 , against the shoulder in the left-hand side of the gage here shown, thus spreading the ring. Once the ring has been properly adjusted by means of adjusting screw, 3 , the adjustment is locked by tightening locking screw, 1 . The tightening of locking screw, 1, 


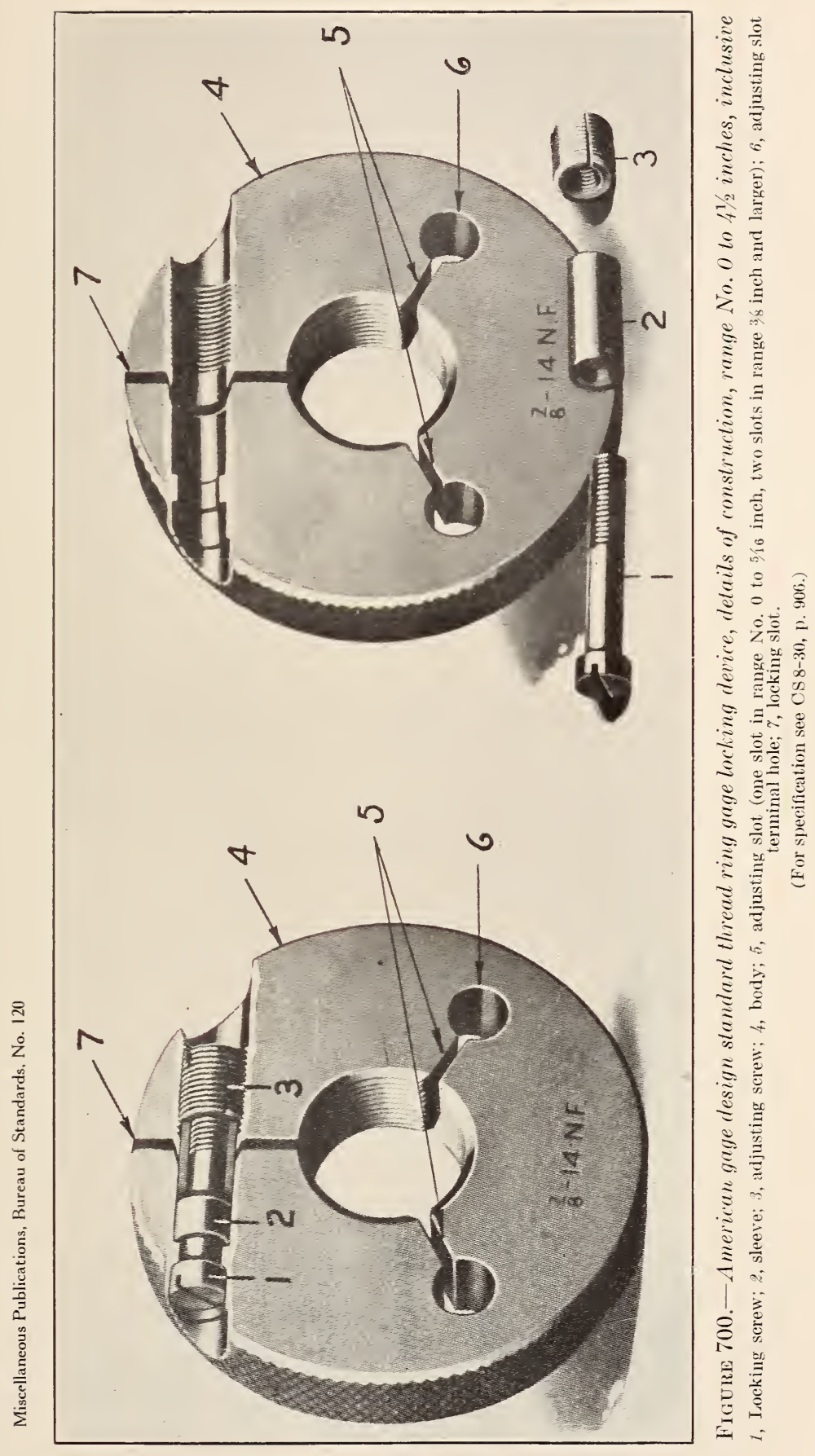



exerts a pull between the shoulder immediately under its head and the internal threads of the adjusting screw, 3 , which causes the adjusting screw to expand into the threads in the wall of the gage, the thrust of this action being taken up longitudinally by the sleeve, 2. Therefore, the clamping is accomplished by expansion of the adjusting screw equally in all directions and not by the application of
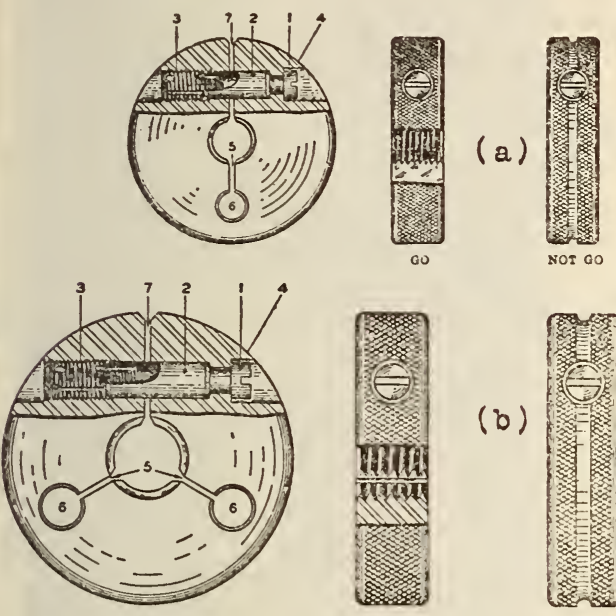

(b)
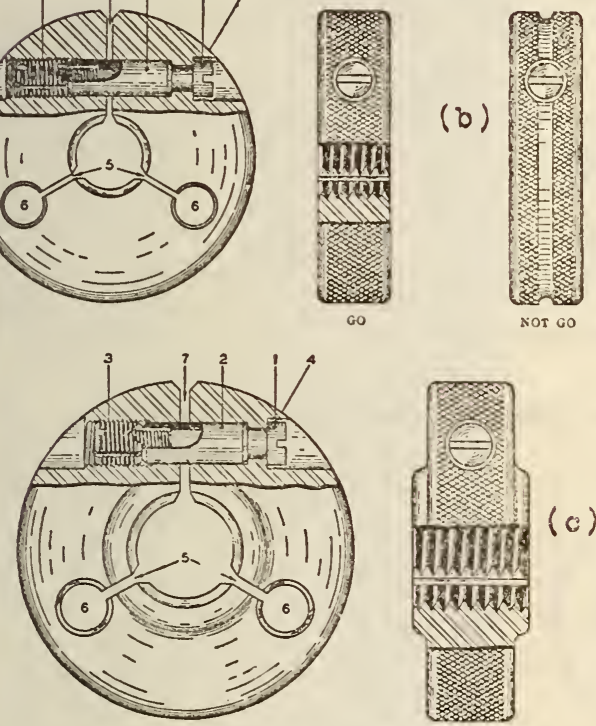

(c)

FIGURE 701.-American gage design standard

thread ring gages, details of construction, range

No. 0 to $4 \frac{1}{2}$ inches, inclusive

(a) Range: No.."0 to and including 0.365 inch, "go" and "not go"

(b) Range: 0.365 to and including 0.510 inch, "go" and "not go" gages, all pitches; 0.510 to and including 4.510 inches, "go" and "not go" gages, fine pitches; 0.510 to and including 4.510 inches "not go" gages only, coarse pitches.

(c) Range: 0.510 to and including 4.510 inches, "go" gages only,$$
1 \text {, locking screw. } \quad 5 \text {, adjusting slot. }
$$

2 , sleeve. $\quad 6$, adjusting slot terminal hole.

3 , adjusting screw. 7 , locking slot.

4 , body.

any eccentric forces that tend to distort the gage or upset the adjustment. The locking pressure, it is seen, is taken up centrally in the locking screw itself as the reacting support is directly under the head of the locking screw in the form of a shoulder in the gage. The sleeve, 2 , being accurately fitted, serves as a large dowel to maintain the alignment of the gage.

Three types of thread ring gage blanks have been provided as illustrated in Figure 701, namely:

1. A thin flat disk type with one adjusting slot for all sizes and pitches, both "go" and "not go," No. 0 to $5 / 16$ inch, inclusive.

2. A thin flat disk type with two adjusting slots for the following: (a) All sizes and pitches, "go" and "not go," above five-sixteenths to and including one-half inch; (b) fine pitches, ${ }^{91}$ "go" and "not go," above one-half to and including $4 \frac{1}{2}$ inches; (c) coarse pitches, "not go" only, above one-half to and including $4 \frac{1}{2}$ inches.

3. A thick flanged type with two adjusting slots for all "go" coarse pitch gages, above one-half to and including $41 / 2$ inches.

Dimensions for thread ring gage blanks in the range from No. 0 to $4 \frac{1}{2}$ inches, inclusive, and of parts for the thread ring gage locking device, are given in Tables 16, 17, 18, and 19 .

\section{Taper Plug and Ring Gages for Checking Taper Lock Handles and Gaging Members}

It has been deemed advisable to formulate specifications for a complete set of finished gages for inspecting the taper shanks and handles of gages of taper lock design.

A complete set consists of a taper plug and a taper ring for each size range. General details of construction will be apparent from drawings, and Tables 20 and 21.

In the range above 0.059 to and including 0.240 inch, the taper limits established by the American gage design committee for taper lock handles and shanks may be readily maintained by the use of gages shown in Table 20 , in which the taper plug gage is a double-end limit gage. Similarly, in the range above 0.240 to and including 1.510 inches, the taper limits established by the American gage design committee for taper lock handles and shanks may be readily maintained by the use of the gages shown in Table 21, in which the taper plug gage is of the single-end limit type, with a ground step representing the minimum size of hole. For either range equally satisfactory results may be secured by using a single-end taper plug gage, on which a scribed line represents the minimum size of hole and the shoulder of the gage represents the maximum size of hole. Both designs are sanctioned by the American gage design committee. Offlcial Monogram for Designating Products Made to American Gage
Design Standards

The optional use of the monogram shown in Figure 702, to identify gages made to American

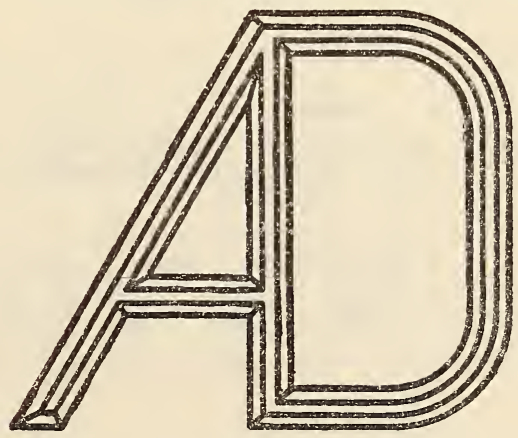

FIGURE 702.-Official monogram for designating products made to American gage design standards

gage design standards, is sanctioned by the committee. The monogram, it will be noted, consists of the initials "AD," the right-hand side of the "A" and the straight side of the "D" being common. The monogram, if used, should be placed adjacent to the maker's trade-mark.

01 Specific information as to the meaning of the terms "fine pitches" and "coarse pitches," as used above, is given in the footnote to Table 16. 
TABLE 14.-Plain ring gages, range above 0.059 to and including 1.510 inches
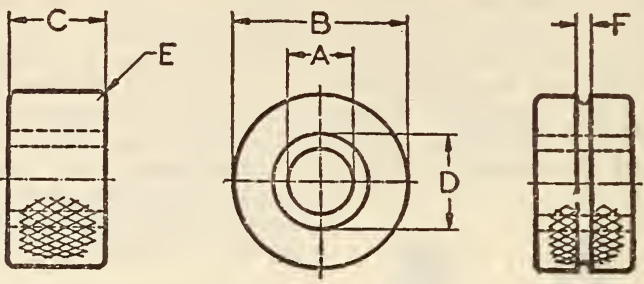

Figure 703

\begin{tabular}{|c|c|c|c|c|c|c|c|c|}
\hline \multirow[b]{2}{*}{ Ring size No. } & \multicolumn{2}{|c|}{$\stackrel{A}{\text { Range }}$} & \multicolumn{6}{|c|}{ General dimensions } \\
\hline & Above- & $\begin{array}{l}\text { To and in- } \\
\text { cluding- }\end{array}$ & $\underset{\text { Outside }}{B}$ & $\begin{array}{c}C \\
\text { Thick- } \\
\text { ness }\end{array}$ & $\begin{array}{c}D \\
\text { Bushing } \\
\text { diameter }\end{array}$ & $\underset{\text { Radius }}{E}$ & $\begin{array}{c}F \\
\text { "Not go" } \\
\text { groove } \\
\text { width }\end{array}$ & $\begin{array}{l}\text { Lengtho } \\
\text { bushing }\end{array}$ \\
\hline
\end{tabular}

1 Bushings are $1 / 16$ inch longer than ring thickness, but are ground flush after hole is finished.

2 Sizes 3,4 , and 5 are solid.

TABLE 15.-Plain ring gages, range above 1.510 to and including 4.510 inches
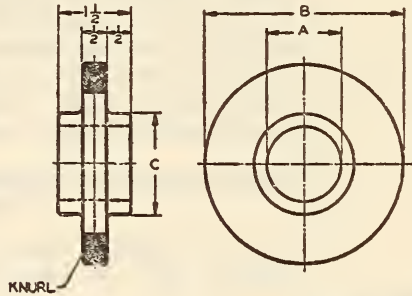

FIGURE 704

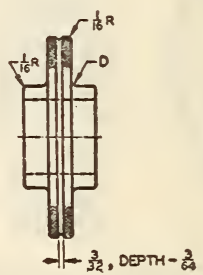

\begin{tabular}{|c|c|c|c|c|}
\hline \multicolumn{2}{|c|}{$\stackrel{A}{\text { Range }}$} & \multirow{2}{*}{ 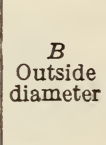 } & \multirow{2}{*}{$\begin{array}{l}C \\
\text { Hub di- } \\
\text { ameter }\end{array}$} & \multirow{2}{*}{$\begin{array}{c}D \\
\text { Radius }\end{array}$} \\
\hline Above- & $\begin{array}{l}\text { To and in- } \\
\text { cluding- }\end{array}$ & & & \\
\hline $\begin{array}{l}\text { Inches } \\
3.010 \\
3.510 \\
4.010\end{array}$ & $\begin{array}{l}\text { Inches } \\
3.510 \\
4.010 \\
4.510\end{array}$ & $\begin{array}{c}\text { Inches } \\
6 \\
67 / 8 \\
73 / 8\end{array}$ & $\begin{array}{c}\text { Inches } \\
A+1 \\
A+118 \\
A+138\end{array}$ & $\begin{array}{c}\text { Inch } \\
3 / 10 \\
1 / 4 \\
1 / 4\end{array}$ \\
\hline
\end{tabular}




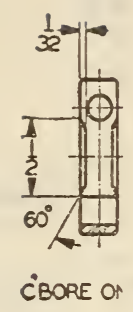

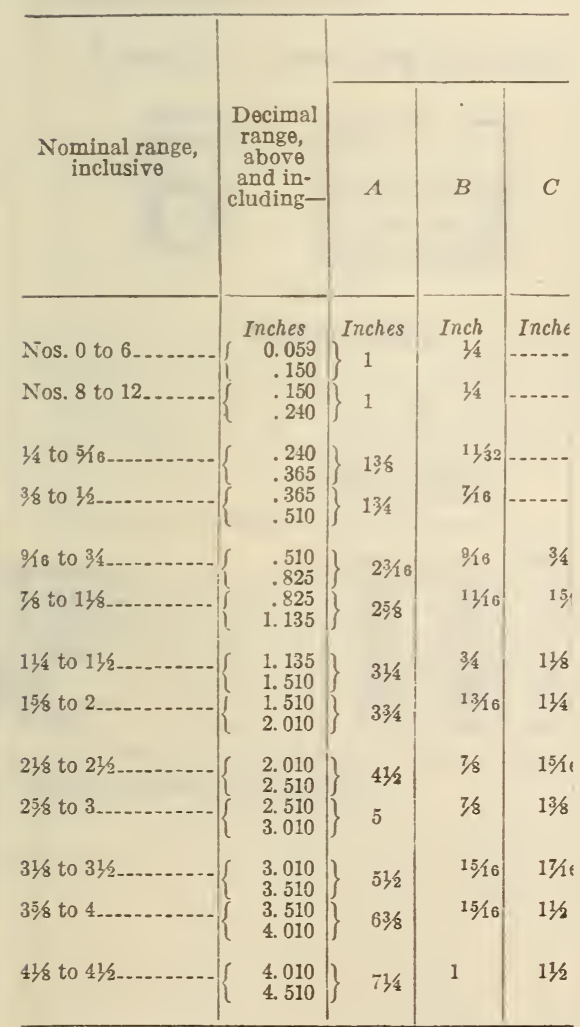

1 Approximate.

NOTE. - Thin gage blanks are to be used for all "I size. Thick blanks are to be used for all American n

It is recommended that standard thread ring gag

No. 0 to $1 / 2$ inch, inclusive, thin blanks for all pit Above $1 / 2$ inch to and including $11 / 8$ inches, thin $b$
Above $11 / 8$ inches, thin blanks for 10 threads per 1 


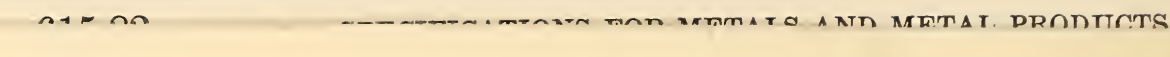




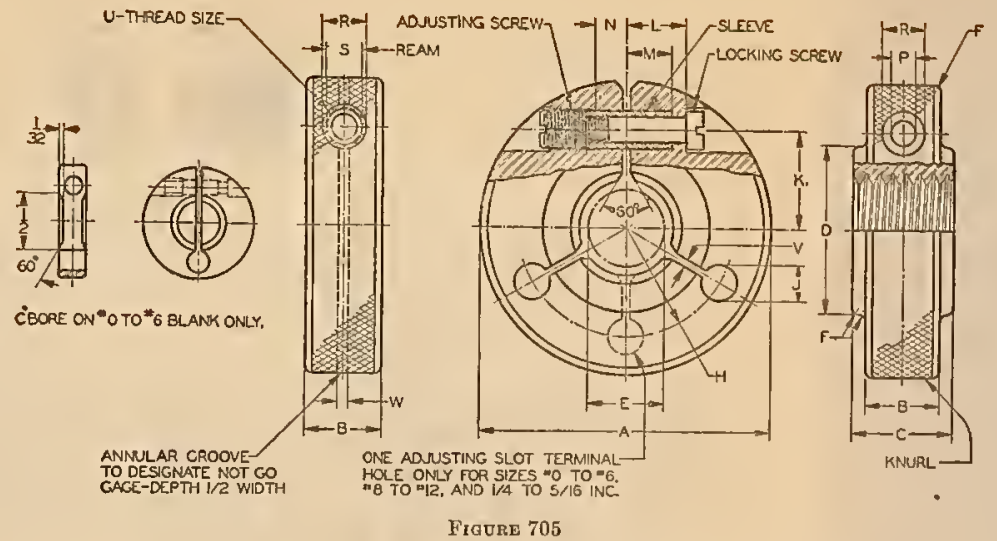

TABLE 16,-Thread ring gnges, range No. 0 to $4 \frac{1}{2}$ inches, inclusive

\begin{tabular}{|c|c|c|c|c|c|c|c|c|c|c|c|c|c|c|c|c|c|c|c|c|c|c|}
\hline \multirow{4}{*}{$\begin{array}{l}\text { Nominal range, } \\
\text { libclusive }\end{array}$} & \multirow{4}{*}{$\begin{array}{l}\text { Decimal } \\
\text { rango, } \\
\text { above } \\
\text { and in- } \\
\text { cluding- }\end{array}$} & \multicolumn{21}{|c|}{ General dimensions } \\
\hline & & \multirow{3}{*}{$A$} & \multirow{3}{*}{$B$} & \multirow{3}{*}{ C } & \multirow{3}{*}{$D$} & \multirow{3}{*}{$E$} & \multirow{3}{*}{$F$} & \multirow{3}{*}{ II } & \multirow{3}{*}{$J$} & \multirow{3}{*}{ IS } & \multirow{3}{*}{$L$} & \multirow{3}{*}{$M$} & \multirow{3}{*}{$N$} & \multirow{3}{*}{$\begin{array}{c}\text { Drill } \\
\text { siza }\end{array}$} & \multirow{3}{*}{$\underset{\substack{\text { Drim } \\
\text { size }}}{R}$} & \multicolumn{2}{|c|}{$\stackrel{s}{s}$} & \multicolumn{3}{|c|}{$u$} & \multirow{3}{*}{$V^{\prime}$} & \multirow{3}{*}{$w$} \\
\hline & & & & & & & & & & & & & & & & \multirow{2}{*}{$\begin{array}{l}\text { Mini- } \\
\text { muru }\end{array}$} & \multirow{2}{*}{$\begin{array}{l}\text { Mluri- } \\
\text { mum }\end{array}$} & \multirow[b]{2}{*}{ Slze } & \multicolumn{2}{|c|}{ Pltch disnieter } & & \\
\hline & & & & & & & & & & & & & & & & & & & $\begin{array}{l}\text { Minal- } \\
\text { zaun }\end{array}$ & $\begin{array}{l}\text { Mfaxi. } \\
\text { mumf }\end{array}$ & & \\
\hline Nos. 0 to $6 .$. & $\left.\begin{array}{c}\text { Inches } \\
0.050 \\
.150\end{array}\right)$ & $\left\{\begin{array}{l}\text { Inches } \\
1\end{array}\right.$ & $\underset{31}{\operatorname{Inch}}$ & Inches & Inches & Inches & Inch & $\begin{array}{r}\text { Inches } \\
\text { 1/10 }\end{array}$ & $\begin{array}{c}\text { Inch } \\
\text { \$S3 }\end{array}$ & $\begin{array}{r}\text { Ineses } \\
5,0\end{array}$ & $\underset{\text { Inda }}{\text { Inch }}$ & $\begin{array}{c}\text { Inch } \\
\text { \$5a }\end{array}$ & $\begin{array}{c}\text { Inch } \\
\text { Sis }\end{array}$ & $($ No. 41 & $(0.1749)$ & $\left|\begin{array}{c}\text { Inch } \\
0.370\end{array}\right|$ & $\begin{array}{c}\text { inch } \\
0.1373\end{array}$ & No. $8-3 \theta$ & $\begin{array}{c}1 \text { irch } \\
0.1 \mathrm{~N} S 0\end{array}$ & $\left|\begin{array}{c}\text { Inch } \\
0 . \text {. } 1 \text { tis }\end{array}\right|$ & $\left\{\begin{array}{l}\text { Inch } \\
0.010 \\
010\end{array}\right.$ & $\underbrace{\text { Inch }}_{j \mathrm{j}}$ \\
\hline Nos. 8 to 12 . . & $\left.\begin{array}{r}150 \\
.150 \\
.210\end{array}\right\}$ & 1 & 34 & & & & 3,82 & S10 & 4,53 & 510 & $7 / 3 a$ & 952 & & $\left\{\begin{array}{l}N=0.41 \\
(0.0 .90)\end{array}\right.$ & $\left|\begin{array}{l}0.116 \\
(0.17 i 9)\end{array}\right|$ & . $13: 0$ & .1373 & No. $8-36$ & .1400 & .1478 & 361 & 36 \\
\hline W to Fre... & $\begin{array}{l}.240 \\
.315\end{array}$ & 128 & ${ }_{1532}$ & & & 532 & 352 & T/10 & $33_{0}^{\circ}$ & 38 & ${ }^{13 / 32}$ & 4 & 18 & $\left\{\begin{array}{l}\text { No, } 31 \\
(0,1200)\end{array}\right.$ & $(0.21872)$ & b.1510 & .1813 & No. $12-23$ & .1023 & .1050 & $13:$ & Ho \\
\hline K to $34 .$. & $\begin{array}{l}.365 \\
.510\end{array}$ & $13 \%$ & 3io & & & $3 a$ & 364 & 13ร2 & 14 & 1958 & 13 & 36 & 316 & $\left\{\begin{array}{l}N o, 25 \\
0.1195)\end{array}\right.$ & $\begin{array}{c}1364 \\
(0.2606)\end{array}$ & .2180 & .2163 & $16-28$ & .2263 & $.22 \% 0$ & $1 s_{2}$ & 352 \\
\hline Ke to 3 . & $\begin{array}{l}.510 \\
.825\end{array}$ & $2 \pi, 16$ & S13 & $\$ 4$ & I3 10 & 13,12 & $3 / 6$ & 36 & Sio & $1 / 40$ & 1732 & 1932 & $7 / 38$ & $\left\{\begin{array}{l}\mathrm{No}_{0} 7 \\
(0.2010)\end{array}\right.$ & $\begin{array}{c}3164 \\
(0.3281)\end{array}$ & \}. .2720 & .2723 & $410-24$ & .2354 & .2378 & Mo. & ;/, \\
\hline hato 136. & $\begin{array}{r}.825 \\
1.135\end{array}$ & 248 & $13 i_{0}$ & $15 \mathbf{C}_{0}$ & $13 / 2$ & 310 & $K_{0}$ & 3152 & Yio & 76 & $173 / 2$ & $13 \% 2$ & 952 & $\left\{\begin{array}{l}150.1 \\
(0.2250)\end{array}\right.$ & (0. 35093 & \} .3360 & .3344 & $36-24$ & .3479 & .3503 & Bis & $3 / 30$ \\
\hline IS to $13 x$.... & $\left.\begin{array}{l}1,136 \\
1.510\end{array}\right\}$ & 344 & 3 & 1178 & 138 & $27,3=$ & $Y_{0}$ & $13 \%$ & 98 & 113 & 58 & 30 & 510 & $\left\{\begin{array}{l}1764 \\
(0.2650)\end{array}\right.$ & $\left(\begin{array}{c}2364 \\
(0.4531)\end{array}\right]$ & | .3500 & .3504 & $300-20$ & .4050 & .4070 & $\mathrm{HOO}$ & בנצ' \\
\hline 15y to 2 .. & $\left.\begin{array}{l}1.510 \\
2.010\end{array}\right\}$ & 396 & $13 \%$ & 134 & $2>8$ & $13 \%$ & 352 & $17 / 0$ & 38 & 198 & 98 & $3 i a$ & 510 & $\left\{\begin{array}{l}1780 \\
(0.2656)\end{array}\right.$ & $(0.4351)$ & \}$\cdot 340$ & .3894 & $30-20$ & .1050 & .6070 & 310 & sis \\
\hline 366 to $21 / 2 \ldots$ & $\left.\begin{array}{l}2.010 \\
2.510\end{array}\right\}$ & 43/2 & 78 & 1950 & $27 / 8$ & $1^{10,52}$ & 352 & 138 & 30 & $113 / 60$ & 1910 & 910 & 7/o & 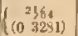 & $\left|\begin{array}{c}3366 \\
(0.3156)\end{array}\right|$ & .4510 & .4515 & $42-20$ & .4875 & .1701 & 932) & is \\
\hline 03 & $\left.\begin{array}{l}2.650 \\
3.010 \\
3.010\end{array}\right\}$ & 5 & $7 /$ & 138 & 334 & 2 & 352 & 2 & ko & 12516 & 13180 & Bío & 310 & $\left\{\begin{array}{l}2161 \\
(0,3221)\end{array}\right.$ & $\left(\begin{array}{c}3.36 \\
(0.5156)\end{array}\right)$ & $\{0.4510\}$ & .4515 & $34-20$ & .1676 & . $4: 01$ & 342 & 34 \\
\hline 315 to $31 / 2 . \ldots$ & $\left.\begin{array}{l}3.010 \\
3.510\end{array}\right\}$ & 533 & 1310 & $17 \%$ & $37 / 1$ & $27 / 0$ & 352 & 2753 & $3 \%$ & $2 K_{0}$ & 1390 & S10 & & $\left\{\begin{array}{l}{ }^{2} 3646 \\
(0,3231)\end{array}\right.$ & $\left.\begin{array}{c}3364 \\
(0.5156)\end{array}\right)$ & \}.1510 & . 4515 & $35-20$ & .4675 & - +701 & $3 / s_{3}$ & 36 \\
\hline & $\left.\begin{array}{l}3.610 \\
4.010\end{array}\right\}$ & 0.38 & 1510 & 132 & $4: 6$ & 21590 & 352 & 276 & $1 / 5$ & $2 \%$ & 1 & 31 & $\$ 8$ & $\left\{\begin{array}{l}1393 \\
10.4062\}\end{array}\right.$ & $\left.\begin{array}{l}(4 / 54 \\
(0,803)\end{array}\right\}$ & \} .5710 & .8715 & $3-18$ & .2880 & .8910 & $3 / 5=$ & 16 \\
\hline $1 / 610+1\} \ldots$ & $\left.\begin{array}{l}\text { 4. } 010 \\
\text { 4. } 510\end{array}\right\}$ & 736 & 1 & $13 / 3$ & 536 & 336 & 352 & 3152 & 35 & 3 & 1 & 34 & 58 & $\left\{\begin{array}{c}1.3 / 2 \\
(0.4062)\end{array}\right.$ & $\left|\begin{array}{c}4364 \\
(0.6106)\end{array}\right|$ & |. 5710 & .8715 & $44-18$ & . 3889 & .5010 & she & 18 \\
\hline
\end{tabular}

\footnotetext{
1 spproximate.
}

Nore - Thin page blanks are to he used for all "not go" thread ring gages, all American national finc-thread "go"

"ir, Thlek blanks are te he ured for ull American "

It is rerommonded that standord thread riag gage blants be used for speclal pitches as follotrs:

No.0 to 15 loch, laclusire, thin blanks for all pirches.

Abor 

TABLE 17.-Thread ring gage adjusting screws
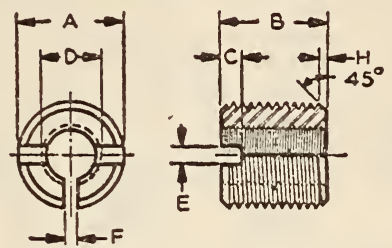

Figure 706

\begin{tabular}{|c|c|c|c|c|c|c|c|c|c|c|c|c|c|}
\hline \multirow{3}{*}{ Size } & \multicolumn{2}{|l|}{4} & \multicolumn{2}{|c|}{ Minor diameter } & \multirow{3}{*}{$B^{1}$} & \multirow{3}{*}{ C } & \multicolumn{4}{|c|}{$D$} & \multirow{3}{*}{$E$} & \multirow{3}{*}{$F$} & \multirow{3}{*}{$H$} \\
\hline & \multicolumn{2}{|c|}{ Pitch diameter } & \multirow{2}{*}{$\begin{array}{l}\text { Mini- } \\
\text { mum }\end{array}$} & \multirow{2}{*}{$\begin{array}{c}\text { Maxi- } \\
\text { mum }\end{array}$} & & & \multirow{2}{*}{ Size } & \multicolumn{2}{|c|}{ Pitch diameter } & \multirow{2}{*}{ Tap drill } & & & \\
\hline & $\begin{array}{l}\text { Mini- } \\
\text { mum }\end{array}$ & $\begin{array}{l}\text { Maxi- } \\
\text { mum }\end{array}$ & & & & & & $\begin{array}{l}\text { Mini- } \\
\text { mum }\end{array}$ & $\begin{array}{l}\text { Maxi- } \\
\text { mum }\end{array}$ & & & & \\
\hline $\begin{array}{r}\text { No. } 8-36 \\
\text { No. } 12-28 \\
1 / 4-28 \\
5 / 16-24\end{array}$ & $\begin{array}{c}\text { Inch } \\
0.1442 \\
.1906 \\
.2246 \\
.2830\end{array}$ & $\begin{array}{l}\text { Inch } \\
0.1460 \\
.1928 \\
.2268 \\
.2854\end{array}$ & $\begin{array}{l}\text { Inch } \\
0.1315 \\
.1744 \\
.2084 \\
.2641\end{array}$ & $\begin{array}{c}\text { Inch } \\
0.1333 \\
.1766 \\
.2106 \\
.2665\end{array}$ & $\begin{array}{c}\text { Inch } \\
3 / 16 \\
1 / 4 \\
5 / 16 \\
516\end{array}$ & $\begin{array}{r}\text { Inch } \\
3 / 64 \\
3 / 64 \\
1 / 16 \\
1 / 16\end{array}$ & $\begin{array}{l}\text { No. } 2-64-1- \\
\text { No. } 4-48- \\
\text { No. } 6-40 \\
\text { No. } 10-32\end{array}$ & $\begin{array}{c}\text { Inch } \\
0.0759 \\
.0985 \\
.1218 \\
.1697\end{array}$ & $\begin{array}{l}\text { Inch } \\
0.0773 \\
.1001 \\
.1235 \\
.1716\end{array}$ & $\begin{array}{ll} & \text { No. } \\
50 & (0.070) \\
42 & (.093) \\
32 & (.116) \\
20 & (.161)\end{array}$ & \begin{tabular}{r|} 
Inch \\
$1 / 32$ \\
132 \\
364 \\
$3 / 64$
\end{tabular} & \begin{tabular}{r|} 
Inch \\
$1 / 64$ \\
164 \\
$1 / 32$ \\
$1 / 32$
\end{tabular} & $\begin{array}{l}\text { Inch } \\
0.020 \\
.020 \\
.020 \\
1 / 32\end{array}$ \\
\hline 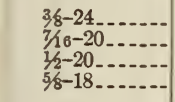 & $\begin{array}{l}.3455 \\
.4024 \\
.4649 \\
.5859\end{array}$ & $\begin{array}{l}.3479 \\
.4050 \\
.4675 \\
.5889\end{array}$ & $\begin{array}{l}.3266 \\
.3797 \\
.4422 \\
.5607\end{array}$ & $\begin{array}{l}.3290 \\
.3823 \\
.4448 \\
.5637\end{array}$ & $\begin{array}{l}3 / 8 \\
7 / 16 \\
1 / 2 \\
9 / 10\end{array}$ & $\begin{array}{l}5 / 64 \\
5 / 64 \\
3 / 32 \\
3 / 32\end{array}$ & $\begin{array}{r}\text { No. } 12-28 \ldots \\
1 / 4-28 \ldots \\
5 / 16-24 . \\
3 / 8-24 . .\end{array}$ & $\begin{array}{l}.1928 \\
.2268 \\
.2854 \\
.3479\end{array}$ & $\begin{array}{l}.1950 \\
.2290 \\
.2878 \\
.3503\end{array}$ & 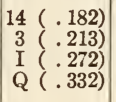 & $\begin{array}{l}1 / 16 \\
1 / 16 \\
5 / 64 \\
5 / 64\end{array}$ & $\begin{array}{l}3 / 64 \\
3 / 64 \\
1 / 16 \\
1 / 16\end{array}$ & $\begin{array}{l}1 / 32 \\
1 / 32 \\
3 / 64 \\
3 / 64\end{array}$ \\
\hline
\end{tabular}

1 Tolerance on length $B= \pm 364$ inch.

TABLE 18.-Thread ring gage sleeves
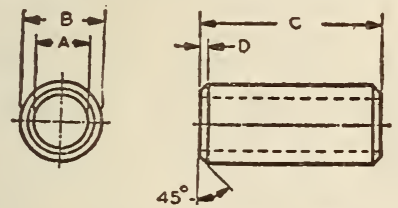

FigdRE 707

\begin{tabular}{|c|c|c|c|c|}
\hline \multirow{2}{*}{$A$} & \multicolumn{2}{|c|}{$B$} & \multirow{2}{*}{$C^{1}$} & \multirow{2}{*}{$D$} \\
\hline & $\begin{array}{l}\text { Mini- } \\
\text { mum }\end{array}$ & $\begin{array}{l}\text { Maxi- } \\
\text { mum }\end{array}$ & & \\
\hline $\begin{array}{l}\text { No. } 43(0.089) \\
\text { No. } 32(0.116) \\
\text { No. } 27(0.144) \\
\text { No. } 10(0.193) \\
\text { No. } 2(0.221) \\
\text { F }(0.257) \\
\text { P }(0.323) \\
2564(0.391)\end{array}$ & $\begin{array}{c}\text { Inch } \\
0.1368 \\
.1808 \\
.2148 \\
.2718 \\
.3337 \\
.3887 \\
.4507 \\
.5707\end{array}$ & $\begin{array}{c}\text { Inch } \\
0.1370 \\
.1810 \\
.2150 \\
.2720 \\
.3340 \\
.3890 \\
.4510 \\
.5710\end{array}$ & $\begin{array}{c}\text { Inches } \\
1 / 4 \\
7 / 16 \\
5 / 8 \\
13 / 16 \\
3 / 4 \\
13 / 16 \\
11 / 16 \\
11 / 2\end{array}$ & $\begin{array}{c}\text { Inch } \\
0.010 \\
.020 \\
.020 \\
1 / 32 \\
1 / 32 \\
1 / 32 \\
3 / 64 \\
3 / 64\end{array}$ \\
\hline
\end{tabular}

1 Tolerance on length $C= \pm 364$ inch.
TABLE 19.-Thread ring gage locking screws
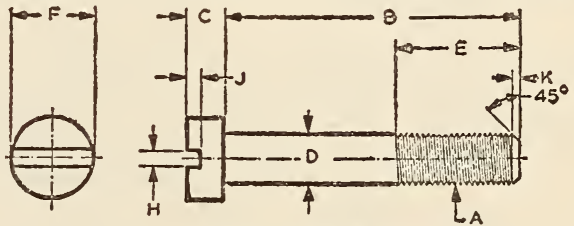

FIgURE 708

\begin{tabular}{|c|c|c|c|c|c|c|c|c|c|c|c|}
\hline \multicolumn{3}{|c|}{ A } & \multirow{3}{*}{$B^{1}$} & \multirow{3}{*}{$C$} & \multicolumn{2}{|c|}{$D$} & \multirow{3}{*}{$E$} & \multirow{3}{*}{$F$} & \multirow{3}{*}{$H$} & \multirow{3}{*}{$J$} & \multirow{3}{*}{ K } \\
\hline \multirow{2}{*}{ Size } & \multicolumn{2}{|c|}{$\begin{array}{c}\text { Pitch } \\
\text { diameter }\end{array}$} & & & \multirow{2}{*}{$\begin{array}{l}\text { Mini- } \\
\text { mum }\end{array}$} & \multirow{2}{*}{$\begin{array}{l}\text { Maxi- } \\
\text { mum }\end{array}$} & & & & & \\
\hline & $\begin{array}{l}\text { Mini- } \\
\text { mum }\end{array}$ & $\begin{array}{l}\text { Maxi- } \\
\text { mum }\end{array}$ & & & & & & & & & \\
\hline $\begin{array}{l}\text { No. } 2-64 \ldots \\
\text { No. } 4-48 \ldots \\
\text { No. } 6-40 . \\
\text { No. } 10-32 .-\end{array}$ & $\begin{array}{c}\text { Inch } \\
0.0745 \\
.0969 \\
.1201 \\
.1678\end{array}$ & \begin{tabular}{|c|} 
Inch \\
0.0759 \\
.0985 \\
.1218 \\
.1697
\end{tabular} & $\begin{array}{c}I n . \\
2964 \\
23 / 32 \\
11 \\
11 / 16\end{array}$ & $\begin{array}{l}\text { In. } \\
5 \% 64 \\
3 / 32 \\
1 / 8 \\
1 / 8\end{array}$ & $\begin{array}{c}\text { Inch } \\
0.0840 \\
.1100 \\
.1360 \\
.1880\end{array}$ & $\begin{array}{l}\text { Inch } \\
0.0860 \\
.1120 \\
.1380 \\
.1900\end{array}$ & $\begin{array}{l}\text { In. } \\
3 / 16 \\
5 / 16 \\
7 / 16 \\
7 / 16\end{array}$ & $\begin{array}{l}\text { In } \\
5 / 32 \\
3 / 16 \\
7 / 32 \\
9 / 32\end{array}$ & $\begin{array}{l}\text { In. } \\
1 / 32 \\
132 \\
3 / 64 \\
364\end{array}$ & $\mid \begin{array}{l}I n . \\
3 \% 64 \\
3 \% 64 \\
1 / 16 \\
1 / 16\end{array}$ & $\begin{array}{l}\text { Inch } \\
0.010 \\
.020 \\
.020 \\
1 / 32\end{array}$ \\
\hline $\begin{array}{l}\text { No. } 12-28 . \\
1 / 4-28 \\
5 / 16-24 \\
3 / 8-24\end{array}$ & $\begin{array}{l}.1906 \\
.2246 \\
.2830 \\
.3455\end{array}$ & $\begin{array}{l}.19281 \\
.2268 \\
.2854 \\
.3479\end{array}$ & $\begin{array}{l}13 / 16 \\
12364 \\
123 / 32 \\
23 / 16\end{array}$ & $\begin{array}{l}5 / 32 \\
3 / 16 \\
1 / 4 \\
5 / 16\end{array}$ & $\begin{array}{l}.2140 \\
.2480 \\
.3105 \\
.3730\end{array}$ & $\begin{array}{l}.2160 \\
.2500 \\
.3125 \\
.3750\end{array}$ & $\begin{array}{l}1 / 2 \\
9 / 16 \\
5 / 8 \\
3 / 4\end{array}$ & $\begin{array}{l}11 / 32 \\
13 / 32 \\
15 / 32 \\
19 / 32\end{array}$ & $\begin{array}{l}1 / 16 \\
1 / 16 \\
564 \\
5 \% 4\end{array}$ & $\begin{array}{l}1 / 16 \\
5 / 64 \\
3 / 32 \\
3 / 32\end{array}$ & $\begin{array}{l}1 / 32 \\
1 / 32 \\
3 / 64 \\
3 / 64\end{array}$ \\
\hline
\end{tabular}


TABLE 20.-Plug and ring gages for checking handles and gaging members of taper lock plug gages, range above 0.059 to and including 0.240 inch
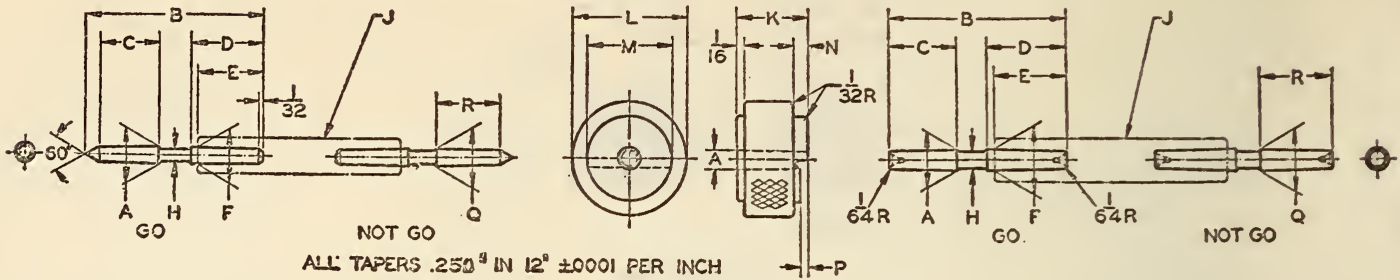

FIGURE 709

PLUG GAGES FOR CHECKING HANDLES

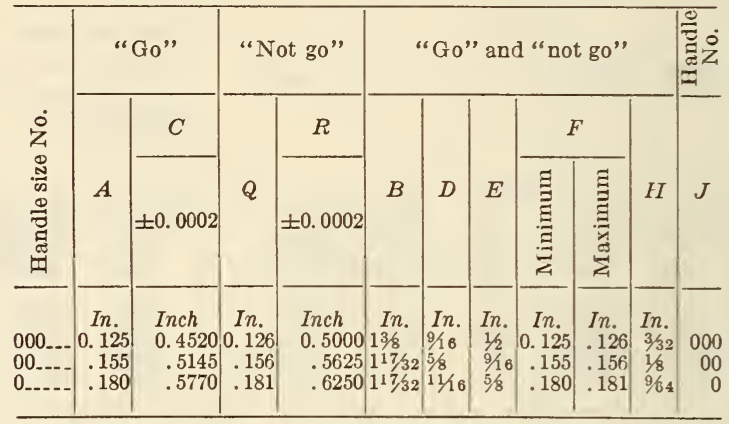

RING GAGES FOR CHECKING GAGING MEMBERS

\begin{tabular}{|c|c|c|c|c|c|c|}
\hline \multirow{2}{*}{ Size } & \multirow{2}{*}{$A$} & K & \multirow{2}{*}{$L$} & \multirow{2}{*}{$M$} & \multirow{2}{*}{$N$} & $P$ \\
\hline & & \pm 0.0002 & & & & \pm 0.0002 \\
\hline 000 & $\begin{array}{l}\text { Inch } \\
0.126 \\
.156 \\
.181\end{array}$ & $\begin{array}{c}\text { Inch } \\
0.5480 \\
.6105 \\
.6730\end{array}$ & \begin{tabular}{|c|} 
Inches \\
1 \\
1 \\
118
\end{tabular} & $\begin{array}{r}\text { Inch } \\
3 / 4 \\
3 / 4 \\
7 / 8\end{array}$ & $\begin{array}{r}\text { Inch } \\
1 / 8 \\
1 / 8 \\
1 / 8\end{array}$ & $\begin{array}{l}\text { Inch } \\
0.0480 \\
.0480 \\
.0480\end{array}$ \\
\hline
\end{tabular}

$\mathrm{T}_{\mathrm{ABLE}}$ 21.-Plug and ring gages for checking handles and gaging members of taper lock plug gages, range above 0.240 to and including 1.510 inches

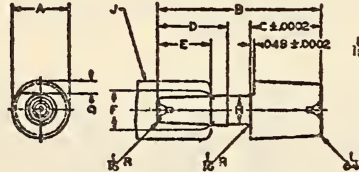

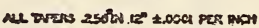

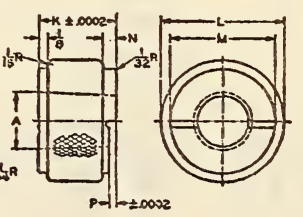

FIGURE 710

PLUG GAGES FOR CHECKING HANDLES

\begin{tabular}{|c|c|c|c|c|c|c|c|c|c|c|}
\hline \multirow{2}{*}{$\begin{array}{c}\text { Handle } \\
\text { size No. }\end{array}$} & \multirow{2}{*}{$A$} & \multirow{2}{*}{$B$} & \multirow{2}{*}{$C$} & \multirow{2}{*}{$D$} & \multirow{2}{*}{$E$} & \multicolumn{2}{|r|}{$F$} & \multirow{2}{*}{$H$} & $\begin{array}{l}\text { Han- } \\
\text { dle } \\
\text { No. }\end{array}$ & \multirow{2}{*}{$Q$} \\
\hline & & & & & & $\begin{array}{l}\text { Mini- } \\
\text { mum }\end{array}$ & - Maxi- & & $J$ & \\
\hline 4 & $\begin{array}{r}\text { Inch } \\
0.240 \\
.310 \\
.410 \\
.610 \\
.810\end{array}$ & \begin{tabular}{|l|} 
Ins. \\
2 \\
2 \\
2 \\
$23 / 16$ \\
$25 / 16$
\end{tabular} & $\begin{array}{r}\text { Inch } \\
0.7500 \\
.7500 \\
.7500 \\
.8750 \\
1.0000\end{array}$ & $\begin{array}{r}I n . \\
1 \\
1 \\
1 \\
1 \\
1\end{array}$ & $\begin{array}{r}I n . \\
3 / 4 \\
3 / 4 \\
3 / 4 \\
3 / 4 \\
3 / 4\end{array}$ & $\begin{aligned} & I n \\
& 0.239 \\
& .239 \\
& .309 \\
& .408 \\
& .408\end{aligned}$ & $\begin{array}{l}\text { In. } \\
.240 \\
.240 \\
.310 \\
.410 \\
.410\end{array}$ & $\begin{array}{c}\text { In. } \\
3 / 16 \\
1 / 4 \\
5 / 16 \\
13 / 32 \\
13 / 32\end{array}$ & $\begin{array}{l}1 \\
2 \\
3 \\
4 \\
5\end{array}$ & $\begin{array}{l}\text { In. } \\
0.025 \\
.025 \\
.050 \\
.100 \\
.200\end{array}$ \\
\hline
\end{tabular}

RING GAGES FOR CHECKING GAGING MEMBERS

\begin{tabular}{|c|c|c|c|c|c|c|}
\hline Size & $A$ & K & $L$ & $M$ & $N$ & $P$ \\
\hline 5 & $\begin{array}{c}\text { Inch } \\
.240 \\
.310 \\
.410 \\
.610 \\
.810\end{array}$ & $\begin{array}{r}\text { Inches } \\
0.7980 \\
.7980 \\
.8460 \\
.9710 \\
1.0960\end{array}$ & $\begin{array}{c}\text { Inches } \\
11 / 8 \\
11 / 4 \\
13 / 8 \\
19 / 16 \\
13 / 4\end{array}$ & $\begin{array}{c}\text { Inches } \\
7 / 8 \\
11 / 8 \\
118 \\
11 / 16 \\
11 / 2\end{array}$ & $\begin{array}{r}\text { Inch } \\
5 / 32 \\
5 / 32 \\
3 / 16 \\
316 \\
3 / 16\end{array}$ & $\begin{array}{c}\text { Inch } \\
0.0480 \\
.0480 \\
.0960 \\
.0960 \\
.0960\end{array}$ \\
\hline
\end{tabular}

UNITED STATES GOVERNMENT, DEPARTMENT OF COMMERCE, BUREAU OF STANDARDS; THE MEASUREMENT OF TAPER GAGES, LC22, AUGUST 15, 1919

\section{TAPER GAGES}

(A description of the various methods used by the gage section of the Bureau of Standards in measuring plug gages having a single taper, double taper, or triple taper; taper ring gages; and profile gages having tapered surfaces.)

\section{UNITED STATES GOVERNMENT, DEPART- MENT OF COMMERCE, BUREAU OF STANDARDS; MEASUREMENT OF PITCH DIAMETER OF SCREW-THREAD GAGES, IC23, AUGUST 25, 1919}

\section{SCREW-THREAD GAGES}

(A résumé of the methods used for the measurement of the pitch diameter of screw-thread gages. It is stated that, of the various methods tried, the 3 -wire method has been found to be the most accurate and satisfactory when properly carried out. This is the method used by the gage section of the Bureau of Standards. Appendixes to this publication contain in detail the complex mathematical relations involved in the measurement of pitch diameter by the 3-wire method.) 
UNITED STATES GOVERNMENT, DEPARTMENT OF COMMERCE, BUREAU OF STANDARDS; INTERFERENCE METHODS FOR STANDARDIZING AND TESTING PRECISION GAGE BLOCKS, NO. 436, MAY 2, 1922

\section{PRECISION GAGE BLOCKS}

Precision gages, which are blocks of metal (usually steel), having two opposite faces plane, parallel, and a specified distance apart, are used in the shop as reference end standards for checking micrometers and other measuring instruments, and also as distance pieces or size blocks for precise mechanical work. The extensive use of precision gages necessitated by the small tolerances allowed in the manufacture of interchangeable machine parts has required more accurately determined end standards and more rapid and precise methods for comparing gages with these standards than have been previously available.

Since comparisons of end standards with line standards by means of micrometer-microscopes and of precision gages $\pi$ ith end standards by means of contact instruments are subject to appreciable errors, methods which make use of the interference of light waves were used in making these measurements. With the interference methods described in this article the planeness and parallelism errors of precision surfaces can be measured, and the length of standard gages can be determined by direct comparison with the standard light waves with an uncertainty of not more than a few millionths of an inch. The errors of other gages can be determined by comparison with these calibrated standards with equal precision. This process makes the standard light waves, which have been determined to one part in four or five million relative to the international meter, the standards of length for this work.

The apparatus used for calibrating standards and comparing other gages with these standards is illustrated by line drawings and thoroughly explained.

UNITED STATES GOVERNMENT, NATIONAL SCREW THREAD COMMISSION, SPECIFICATIONS FOR GAGES. BUREAU OF
STANDARDS MISCELLANEOUS PUBLI-
CATION NO. 89,1928 GAGES

The following specifications are for the purpose of establishing definite limits for thread gages rather than for the purpose of specifying the gages required for the various inspection operations:

\section{Classification of Gages, and Gage Tolerances}

Screw-thread gages are classified according to accuracy into classes $\mathrm{X}, \mathrm{Y}$, and $\mathrm{Z}$, the class $\mathrm{X}$ being the most accurate. The tolerance limits on classes $\mathrm{Y}$ and Z "go" gages are placed inside of the extreme product limits to provide allowance for wear of the gages. The tolerances on all "not go" gages, however, are applied from the extreme product limit as the starting point, as no allowance for wear is necessary. The selection of gages from among these classes for use in the inspection of threaded product depends entirely upon the specifications for the product. For example, in the production of parts to class 4 , close fit specifications, class $\mathrm{X}$ gages may be required for all purposes. On the other hand, for parts made to class 1 , loose fit specifications, class Z gages may be sufficiently accurate for all purposes.

(a) Master gages. - No fixed tolerances are specified for master gages. These should be made to the basic size as accurately as possible and be within the tolerances specified for class $\mathrm{X}$ gages. The variations from basic size shall be plus. Each master gage shall be marked with an identification number or symbol, and be accompanied by a record of its measurement on major diameter, pitch diameter, lead, and angle. In case of question, the deviations of such gages from the exact standard shall be ascertained by the Bureau of Standards at Washington, D. C.

(b) Class $X$ gages.-Class $\mathrm{X}$ gages should be suitable for inspection and setting gages for all classes of fits. They may also be necessary for working gages for the class 4 fit. The tolerances on these gages are given in Table 18 . In all cases the tolerances shall be such that the gage does not fall outside of the component tolerances. For example, if a thread-plug gage is used as the "go" gage for checking a tapped hole, it can be larger, but not smaller than the minimum size specified. On the other hand, if a thread-plug gage is used as the "go" setting plug for thread-ring gages or for optical or other comparators, it can be smaller, but never larger, than the maximum size of the screw.

Class X tolerances, as given in Table 18, are specified for all " not go" gages.

(c) Class Y gages.-Class Y gages should be suitable for inspection gages for classes 1, 2, and 3 fits. They may also be desired as working gages for classes 2 and 3 fits. The tolerances on these gages are given in Table 19 .

(d) Class Z gages.-Class Z gages should be suitable for working gages for class 1 , loose fit. The tolerances on these gages are given in Table 20 .

(e) Wear on gages. "Go" gages may be permitted to wear to the extreme product limits. It is desirable, however, that working and inspection gages be so selected that the dimensions of the working gages are inside of the limiting dimensions represented by the inspection gages, in order that all parts passed by the working gage will be accepted by the inspection gage.

As to wear on "not go" gages, it is purely a question of economy as to when the "not go" gage should be discarded. Continued use reduces the available working tolerance on the product, and the resulting loss must be balanced against the cost of a new gage.

(f) Tolerances on lead.-The tolerances on lead given in Tables 18 to 20 , inclusive, are specified as an allowable variation between any two threads not farther apart than the length of engagement of the assembled threaded product. When this length of engagement is equal to the diameter, the permissible progressive lead errors per inch may be determined by dividing these lead tolerances by the corresponding diameters.

(g) Tolerances on angle of thread.-The tolerances on angle of thread, as specified in Tables 18 to 20, inclusive, for the various pitches, are tolerances on one-half of the included angle. This insures that the bisector of the included angle will be perpendicular to the axis of the thread within proper limits. The equivalent deviation from the true thread form caused by such irregularities as convex or concave sides of thread, rounded crests, or slight projections on the thread form, should not exceed the tolerances permitted on angle of thread.

Tolerances for Plain Gages

For plain plug gages, plain ring gages, and plain snap gages required for measuring diameters of screw-thread work, the gage tolerances specified in Table 18 should be used. Attention is directed to the fact that the tolerances on thread diameters vary in accordance with the number of threads per inch. In manufacturing a plain plug, ring, or snap gage, in the absence of information as to the number 
of threads per inch of the screw to be made, or for gage dimensions other than thread diameters, the tolerances for plain gages given in Table 21 may be used. This table contains recommended tolerances for classes $X, Y$, and $Z$ gages, which have been tentatively adopted by the A. S. A. sectional committee on the standardization of plain limit gages for general engineering work.

\section{Recommended Gage Practice}

There are given in Table 17 the recommended uses for the foregoing classes of gages. Tables 22, 23,24 , and 25 give limiting dimensions of gages of the several classifications for the American national coarse and American national fine thread series.

TABLE 17.-Recommended uses for classes $X, Y$, and $Z$ gages

\begin{tabular}{|c|c|c|c|}
\hline Class of fit & Setting gage & $\begin{array}{l}\text { Inspection } \\
\text { gage }\end{array}$ & $\begin{array}{c}\text { Working } \\
\text { gage }\end{array}$ \\
\hline 1 & 2 & 3 & 4 \\
\hline $\begin{array}{l}\text { Class 1, loose fit......... } \\
\text { Class 2, free fit.... } \\
\text { Class 3, medium fit..... } \\
\text { Class 4, close fit........ }\end{array}$ & 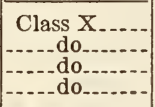 & 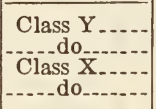 & $\begin{array}{l}\text { Class Z. } \\
\text { Class Y. } \\
\text { Do. } \\
\text { Class X. }\end{array}$ \\
\hline
\end{tabular}

TABLE 18.-Tolerances for class X "go" thread gages and all "not go" thread gages

\begin{tabular}{|c|c|c|c|c|c|c|c|}
\hline \multirow{2}{*}{ 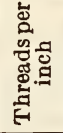 } & \multicolumn{2}{|c|}{$\begin{array}{c}\text { Tolerance on } \\
\text { pitch diameter }\end{array}$} & \multirow{2}{*}{$\begin{array}{l}\text { Toler- } \\
\text { ance in } \\
\text { lead }{ }^{2} \\
\pm\end{array}$} & \multirow{2}{*}{\multicolumn{2}{|c|}{$\begin{array}{l}\text { Toler- } \\
\text { ance } \\
\text { on half } \\
\text { angle of } \\
\text { thread } \pm\end{array}$}} & \multicolumn{2}{|c|}{$\begin{array}{l}\text { Tolerance on } \\
\text { major or minor } \\
\text { diameters }\end{array}$} \\
\hline & From- & To- & & & & From- & To- \\
\hline 1 & 2 & 3 & 4 & 5 & & 6 & 7 \\
\hline $\begin{array}{l}80 . \\
72 . \\
64 . \\
56 . \\
48 .\end{array}$ & $\begin{array}{c}\text { Inch } \\
0.0000 \\
.0000 \\
.0000 \\
.0000 \\
.0000\end{array}$ & $\begin{array}{c}\text { Inch } \\
0.0002 \\
.0002 \\
.0002 \\
.0002 \\
.0002\end{array}$ & $\begin{array}{c}\text { Inch } \\
0.0002 \\
.0002 \\
.0002 \\
.0002 \\
.0002\end{array}$ & $\begin{array}{ll}\circ & \\
0 & 3 \\
0 & 3 \\
0 & 3 \\
0 & 3 \\
0 & 3\end{array}$ & $\begin{array}{c} \\
30 \\
30 \\
30 \\
30 \\
30\end{array}$ & $\begin{array}{c}\text { Inch } \\
0.0000 \\
.0000 \\
.0000 \\
.0000 \\
.0000\end{array}$ & $\begin{array}{r}\text { Inch } \\
0.0003 \\
.0003 \\
.0004 \\
.0004 \\
.0004\end{array}$ \\
\hline $\begin{array}{l}44 . \\
40 . \\
36 . \\
32 . \\
28 .\end{array}$ & $\begin{array}{l}.0000 \\
.0000 \\
.0000 \\
.0000 \\
.0000\end{array}$ & $\begin{array}{l}.0002 \\
.0002 \\
.0002 \\
.0003 \\
.0003\end{array}$ & $\begin{array}{l}.0002 \\
.0002 \\
.0002 \\
.0003 \\
.0003\end{array}$ & $\begin{array}{ll}0 & 2 \\
0 & 2 \\
0 & 2 \\
0 & 1 \\
0 & 1\end{array}$ & $\begin{array}{l}20 \\
20 \\
20 \\
15 \\
15\end{array}$ & $\begin{array}{l}.0000 \\
.0000 \\
.0000 \\
.0000 \\
.0000\end{array}$ & $\begin{array}{l}.0004 \\
.0004 \\
.0004 \\
.0004 \\
.0005\end{array}$ \\
\hline $\begin{array}{l}24 \\
20 \\
18 \\
16 \\
14\end{array}$ & $\begin{array}{l}.0000 \\
.0000 \\
.0000 \\
.0000 \\
.0000\end{array}$ & $\begin{array}{l}.0003 \\
.0003 \\
.0003 \\
.0003 \\
.0003\end{array}$ & $\begin{array}{l}.0003 \\
.0003 \\
.0003 \\
.0003 \\
.0003\end{array}$ & $\begin{array}{ll}0 & 1 \\
0 & 1 \\
0 & 1 \\
0 & 1 \\
0 & 1\end{array}$ & $\begin{array}{l}15 \\
15 \\
10 \\
10 \\
10\end{array}$ & $\begin{array}{l}.0000 \\
.0000 \\
.0000 \\
.0000 \\
.0000\end{array}$ & $\begin{array}{l}.0005 \\
.0005 \\
.0005 \\
.0006 \\
.0006\end{array}$ \\
\hline $\begin{array}{l}13 \\
12 \\
11 \\
10\end{array}$ & $\begin{array}{l}.0000 \\
.0000 \\
.0000 \\
.0000\end{array}$ & $\begin{array}{l}.0003 \\
.0003 \\
.0003 \\
.0003\end{array}$ & $\begin{array}{l}.0003 \\
.0003 \\
.0003 \\
.0003\end{array}$ & $\begin{array}{ll}0 & 1 \\
0 & 1 \\
0 & 1 \\
0 & 1\end{array}$ & $\begin{array}{l}10 \\
10 \\
10 \\
10\end{array}$ & $\begin{array}{l}.0000 \\
.0000 \\
.0000 \\
.0000\end{array}$ & $\begin{array}{l}.0006 \\
.0006 \\
.0006 \\
.0006\end{array}$ \\
\hline $\begin{array}{l}9 . \\
8 . \\
7 . \\
6 .\end{array}$ & $\begin{array}{l}.0000 \\
.0000 \\
.0000 \\
.0000\end{array}$ & $\begin{array}{l}.0003 \\
.0004 \\
.0004 \\
.0004\end{array}$ & $\begin{array}{l}.0003 \\
.0004 \\
.0004 \\
.0004\end{array}$ & $\begin{array}{ll}0 & 1 \\
0 & \\
0 & \\
0 & \end{array}$ & $\begin{array}{r}10 \\
5 \\
5 \\
5\end{array}$ & $\begin{array}{l}.0000 \\
.0000 \\
.0000 \\
.0000\end{array}$ & $\begin{array}{l}.0007 \\
.0007 \\
.0007 \\
.0008\end{array}$ \\
\hline $\begin{array}{l}5 \\
41 \\
4 \\
31\end{array}$ & $\begin{array}{l}.0000 \\
.0000 \\
.0000 \\
.0000\end{array}$ & $\begin{array}{l}.0004 \\
.0004 \\
.0004 \\
.0004\end{array}$ & $\begin{array}{l}.0004 \\
.0004 \\
.0004 \\
.0004\end{array}$ & $\begin{array}{l}0 \\
0 \\
0 \\
0\end{array}$ & $\begin{array}{l}5 \\
5 \\
5 \\
5\end{array}$ & $\begin{array}{l}.0000 \\
.0000 \\
.0000 \\
.0000\end{array}$ & $\begin{array}{l}.0008 \\
.0008 \\
.0009 \\
.0009\end{array}$ \\
\hline
\end{tabular}

1 On "go" plugs the tolerance is plus, and on "go" rings the tolerance is minus. On "not go"' plugs the tolerance is minus, and on "not go" rings the tolerance is plus.

2 Allowable variation in lead between any 2 threads not farther apart than the standard length of engagement, which is equal to the basic major diameter.
It is suggested that, in case of question between the manufacturer and purchaser of threaded products in regard to their size, if the manufacturer produces limit gages which do not measure outside of the specified limits for the threaded components and which pass the parts in question, they be accepted as meeting the specifications for size. In case the dimensions of the gages are questioned, their sizes shall be determined by a disinterested third party, preferably the Bureau of Standards at Washington, D. C., which maintains a department for this service.

\section{Marking of Gages}

Each gage shall be plainly and permanently marked, for identification, with the diameter, pitch, thread series, and class of fit.

For example: A 1-inch 8-pitch gage of the American national coarse-thread series, class 2 , free fit, shall be marked $1^{\prime \prime}-8-\mathrm{NC}-2$.

A 1-inch 14-pitch gage of the American national fine-thread series, class 3 , medium fit, shall be marked $1^{\prime \prime}-14-\mathrm{NF}-3$.

\section{TABLE 19.-Tolerances for class $Y$ "go" thread gages}

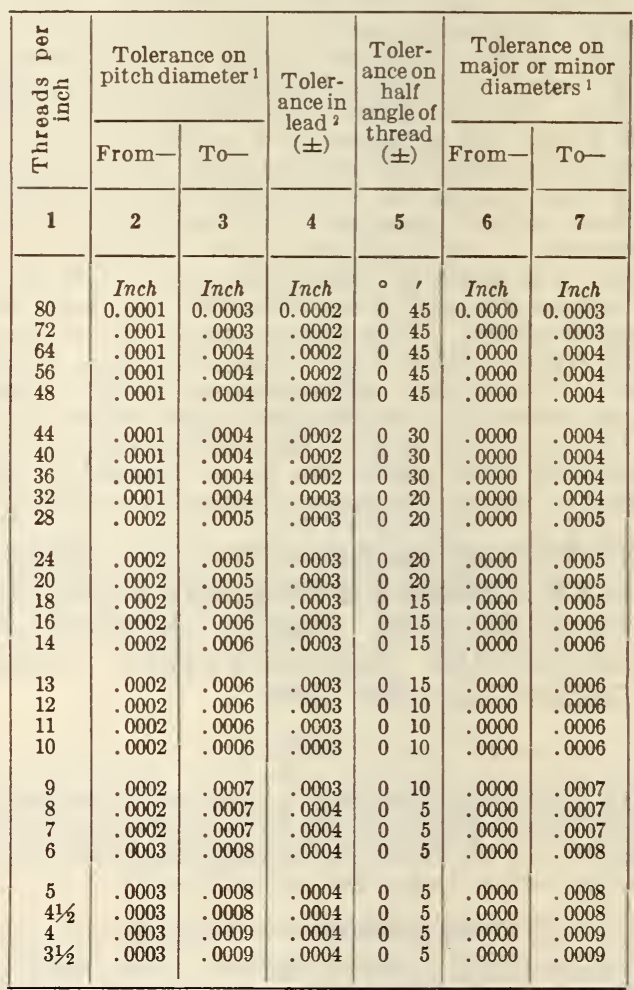

1 On "go" plugs the tolerance is plus and on "go" rings the tolerance is minus.

Allowable variation in lead between any 2 threads not farther apart than the standard length of engagement, which is equal to the basic major diameter. 
TABLE 20.-Tolerances for class Z "go" thread gages

\begin{tabular}{|c|c|c|c|c|c|c|}
\hline \multirow{2}{*}{ 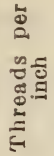 } & \multicolumn{2}{|c|}{$\begin{array}{c}\text { Tolerance on } \\
\text { pitch diameter }^{1}\end{array}$} & \multirow{2}{*}{$\begin{array}{l}\text { Toler- } \\
\text { ance in } \\
\text { lead }{ }^{2} \\
( \pm)\end{array}$} & \multirow{2}{*}{$\begin{array}{l}\text { Toler- } \\
\text { ance on } \\
\text { half } \\
\text { angle of } \\
\text { thread } \\
( \pm)\end{array}$} & \multicolumn{2}{|c|}{$\begin{array}{c}\text { Tolerance on } \\
\text { major or minor } \\
\text { diameters }^{1}\end{array}$} \\
\hline & From- & To- & & & From- & To- \\
\hline 1 & 2 & 3 & 4 & 5 & 6 & 7 \\
\hline $\begin{array}{l}80 \\
72 \\
64 \\
56 \\
48\end{array}$ & $\begin{array}{c}\text { Inch } \\
0.0002 \\
.0002 \\
.0002 \\
.0002 \\
.0002\end{array}$ & $\begin{array}{c}\text { Inch } \\
0.0006 \\
.0006 \\
.0006 \\
.0007 \\
.0007\end{array}$ & $\begin{array}{c}\text { Inch } \\
0.0002 \\
.0002 \\
.0002 \\
.0002 \\
.0002\end{array}$ & $\begin{array}{lc}0 & , \\
0 & 45 \\
0 & 45 \\
0 & 45 \\
0 & 45 \\
0 & 45\end{array}$ & $\begin{array}{c}\text { Inch } \\
0.0000 \\
.0000 \\
.0000 \\
.0000 \\
.0000\end{array}$ & $\begin{array}{c}\text { Inch } \\
0.0003 \\
.0003 \\
.0004 \\
.0004 \\
.0004\end{array}$ \\
\hline $\begin{array}{l}44 \\
40 \\
36 \\
32 \\
28\end{array}$ & $\begin{array}{l}.0002 \\
.0002 \\
.0003 \\
.0003 \\
.0003\end{array}$ & $\begin{array}{l}.0007 \\
.0007 \\
.0008 \\
.0008 \\
.0008\end{array}$ & $\begin{array}{l}.0002 \\
.0002 \\
.0002 \\
.0003 \\
.0003\end{array}$ & $\begin{array}{ll}0 & 30 \\
0 & 30 \\
0 & 30 \\
0 & 20 \\
0 & 20\end{array}$ & $\begin{array}{l}.0000 \\
.0000 \\
.0000 \\
.0000 \\
.0000\end{array}$ & $\begin{array}{l}.0004 \\
.0004 \\
.0004 \\
.0004 \\
.0005\end{array}$ \\
\hline $\begin{array}{l}24 \\
20 \\
18 \\
16 \\
14\end{array}$ & $\begin{array}{l}.0003 \\
.0003 \\
.0004 \\
.0004 \\
.0004\end{array}$ & $\begin{array}{l}.0009 \\
.0009 \\
.0010 \\
.0010 \\
.0010\end{array}$ & $\begin{array}{l}.0003 \\
.0003 \\
.0004 \\
.0004 \\
.0004\end{array}$ & $\begin{array}{ll}0 & 20 \\
0 & 20 \\
0 & 15 \\
0 & 15 \\
0 & 15\end{array}$ & $\begin{array}{l}.0000 \\
.0000 \\
.0000 \\
.0000 \\
.0000\end{array}$ & $\begin{array}{l}.0005 \\
.0005 \\
.0005 \\
.0006 \\
.0006\end{array}$ \\
\hline $\begin{array}{l}13 \\
12 \\
11 \\
10\end{array}$ & $\begin{array}{l}.0004 \\
.0004 \\
.0004 \\
.0005\end{array}$ & $\begin{array}{l}.0011 \\
.0011 \\
.0011 \\
.0012\end{array}$ & $\begin{array}{l}.0004 \\
.0004 \\
.0004 \\
.0004\end{array}$ & $\begin{array}{ll}0 & 15 \\
0 & 10 \\
0 & 10 \\
0 & 10\end{array}$ & $\begin{array}{l}.0000 \\
.0000 \\
.0000 \\
.0000\end{array}$ & $\begin{array}{l}.0006 \\
.0006 \\
.0006 \\
.0006\end{array}$ \\
\hline
\end{tabular}

1 On "go" plugs the tolerance is plus, and on "go" rings the tolerance is minus.

2 Allowable variation in lead between any 2 threads not farther apart than the standard length of engagement, which is equal to the basic major diameter.
TABLE 20.-Tolerances for class Z "go" thread gagesContinued

\begin{tabular}{|c|c|c|c|c|c|c|}
\hline \multirow{2}{*}{ 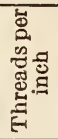 } & \multicolumn{2}{|c|}{$\begin{array}{l}\text { Tolerance on } \\
\text { pitch diameter }\end{array}$} & \multirow{2}{*}{$\begin{array}{l}\text { Toler- } \\
\text { ance in } \\
\text { lead } \\
( \pm)\end{array}$} & \multirow{2}{*}{$\begin{array}{l}\text { Toler- } \\
\text { ance on } \\
\text { half } \\
\text { angle of } \\
\text { thread } \\
( \pm)\end{array}$} & \multicolumn{2}{|c|}{$\begin{array}{l}\text { Tolerance on } \\
\text { major or minor } \\
\text { diameters }\end{array}$} \\
\hline & From- & To- & & & From- & To- \\
\hline 1 & 2 & 3 & 4 & 5 & 6 & 7 \\
\hline $\begin{array}{l}9 \\
8 \\
7 \\
6\end{array}$ & $\begin{array}{l}\text { Inch } \\
.0005 \\
.0006 \\
.0006 \\
.0006\end{array}$ & $\begin{array}{l}\text { Inch } \\
.0012 \\
.0013 \\
.0013 \\
.0014\end{array}$ & $\begin{array}{l}\text { Inch } \\
.0004 \\
.0005 \\
.0005 \\
.0005\end{array}$ & $\begin{array}{ll}\circ & , \\
0 & 10 \\
0 & \\
0 & \\
0 & \end{array}$ & $\begin{array}{l}\text { Inch } \\
.0000 \\
.0000 \\
.0000 \\
.0000\end{array}$ & $\begin{array}{l}\text { Inch } \\
.0007 \\
.0007 \\
.0007 \\
.0008\end{array}$ \\
\hline $\begin{array}{l}5 \\
41 / 2 \\
4 \\
31 / 2\end{array}$ & $\begin{array}{l}.0007 \\
.0007 \\
.0007 \\
.0007\end{array}$ & $\begin{array}{l}.0015 \\
.0015 \\
.0016 \\
.0016\end{array}$ & $\begin{array}{l}.0005 \\
.0005 \\
.0005 \\
.0005\end{array}$ & $\begin{array}{l}0 \\
0 \\
0 \\
0\end{array}$ & $\begin{array}{l}.0000 \\
.0000 \\
.0000 \\
.0000\end{array}$ & $\begin{array}{l}.0008 \\
.0008 \\
.0009 \\
.0009\end{array}$ \\
\hline
\end{tabular}

TABLE 21.-Tolerances for plain gages ${ }^{1}$

\begin{tabular}{|c|c|c|c|c|c|c|}
\hline \multirow{2}{*}{$\begin{array}{l}\text { Size of gage in } \\
\text { inches }\end{array}$} & \multicolumn{2}{|c|}{ Class $\mathrm{X}^{2}$} & \multicolumn{2}{|c|}{ Class Y } & \multicolumn{2}{|c|}{ Class Z } \\
\hline & From- & To- & From- & To- & From- & To- \\
\hline 1 & 2 & 3 & 4 & 5 & 6 & 7 \\
\hline $\begin{array}{l}0 \text { to } 1 \text {, inclusive. } \\
1 \text { to } 3 \text {, inclusive. }\end{array}$ & $\begin{array}{l}\text { Inch } \\
0.0000 \\
.0000\end{array}$ & $\begin{array}{r}\text { Inch } \\
0.0001 \\
.0002\end{array}$ & $\begin{array}{l}\text { Inch } \\
0.0001 \\
.0001\end{array}$ & $\begin{array}{r}\text { Inch } \\
0.0002 \\
.0003\end{array}$ & $\begin{array}{c}\text { Inch } \\
0.0002 \\
.0003\end{array}$ & $\begin{array}{r}\text { Inch } \\
0.0003 \\
.0005\end{array}$ \\
\hline
\end{tabular}

1 On "go" plugs the tolerance is plus, and on "go" rings the toler ance is minus. On "not go" plugs the tolerance is minus, and on not go" rings the tolerance is plus.

"All "not go" gages are made to class $\mathrm{X}$ tolerances.

T $\mathrm{TBLE}$ 22.-Limiting dimensions of setting plug and thread ring gages for screws of classes 1, 2, 3, and 4 fits, American national coarse-thread series

Machine screw number or nominal size

\begin{tabular}{l|l|l|l|l|l|l|l|l|l|l|l|l|l|l}
\hline 1 & 2 & 3 & 4 & 5 & 6 & 8 & 10 & 12 & $1 / 4$ & $5 / 6$ & $3 / 8$ & $7 / 16$ & $1 / 2$ \\
\hline
\end{tabular}

Threads per inch

\begin{tabular}{c|c|c|c|c|c|c|c|c|c|c|c|c|c}
\hline 64 & 56 & 48 & 40 & 40 & 32 & 32 & 24 & 24 & 20 & 18 & 16 & 14 & 13 \\
\hline 2 & $\mathbf{3}$ & 4 & $\mathbf{5}$ & $\mathbf{6}$ & $\mathbf{7}$ & $\mathbf{8}$ & $\mathbf{9}$ & $\mathbf{1 0}$ & $\mathbf{1 1}$ & $\mathbf{1 2}$ & $\mathbf{1 3}$ & $\mathbf{1 4}$ & $\mathbf{1 5}$ \\
\hline Inch & Inch & Inch & Inch & Inch & Inch & Inch & Inch & Inch & Inch & Inch & Inch & Inch & Inch
\end{tabular}

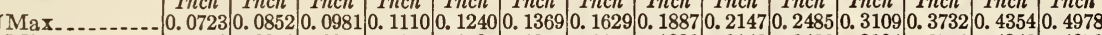

"GO" GAGES FOR SCREWS

Major diameter Class 1...-.--- Min

of setting plug. Classes $2,3,\{\operatorname{Max} . . . . . .$. and 4. Min

Max., class X. $\begin{array}{cl}\text { Class } 1, \text { loose } & \begin{array}{l}\text { Min., class X.- } \\ \text { Max., class Y.- } \\ \text { Min., class Y.- }\end{array} \\ \text { Min } & \end{array}$ fit. $\quad \begin{aligned} & \text { Min., class Y } \\ & \text { Max., class Z- }\end{aligned}$ Min., class Z Pitch diameter or ring gage.

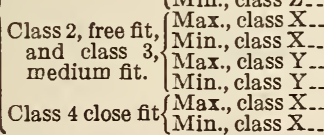

Minor diameter(Classes 1, 2, 3, $\{$ Max.1.......... of ring gage.

Min.......... "NOT GO" GAGES FOR SCREWS

(Min

Min

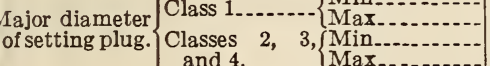
Class 1, loose $\{$ Min..........-

fit. 1 Max.-.-.---

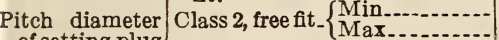
of setting plug
or ring gage. Class 3, me- Min Min

Class 4, close fit $\{$ Min Minor diameter Classes 1, 2, 3, Min. ${ }^{2}$ of ring gage. $\left\{\begin{array}{c}\text { and } 4 . \\ \text { anax. }\end{array}\right.$ \begin{tabular}{l|l|l|l|l|l|l|l|l|l|l|l|l|l|l|}
.0719 & .0848 & .0977 & .1106 & .1236 & .1365 & .1625 & .1882 & .2142 & .2480 & .3104 & .3726 & 0.4354 & 0.4978 \\
.04972
\end{tabular}

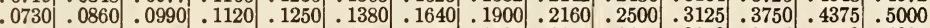

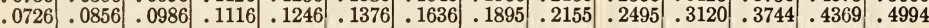

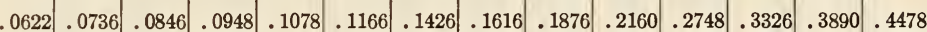
\begin{tabular}{l|l|l|l|l|l|l|l|l|l|l|l|l|l|l}
0620 & .0734 & .0844 & .0946 & .1076 & .1163 & .1423 & .1613 & .1873 & .2157 & .2745 & .3323 & .3887 & .4475
\end{tabular}

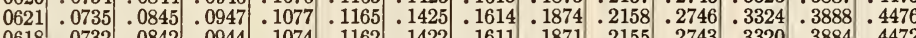
\begin{tabular}{ll|l|l|l|l|l|l|l|l|l|l|l|l|l}
0618 & .0732 & .0842 & .0944 & .1074 & .1162 & .1422 & .1611 & .1871 & .2155 & .2743 & .3320 & .3884 & .4472
\end{tabular}

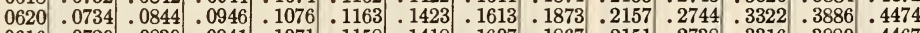

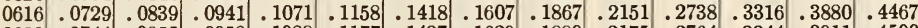

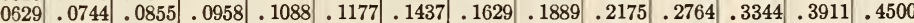

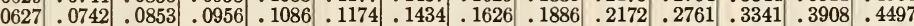

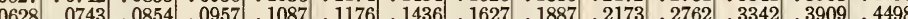

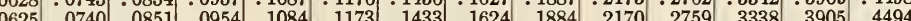
. $0740.081 \cdot 0954 \cdot 1084 \cdot 117 \cdot 1433 \cdot 1624 \cdot 1884 \cdot 217 \cdot 2759 \cdot 3338 \cdot 3905 \cdot 4494$

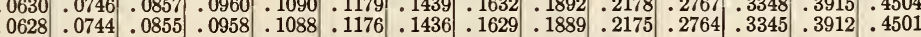

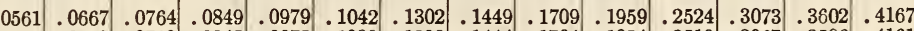

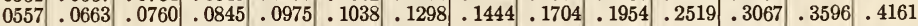

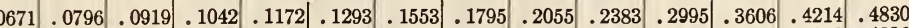

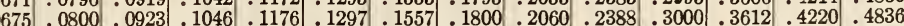

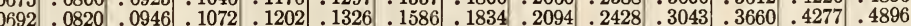

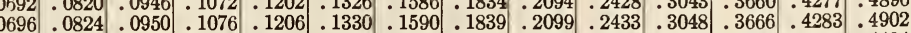
$0596.0708 .0815 .0914 .1044 .1128 .1388: 1570.1830 .2109 .2691 .3263 . .3820 .4404$ \begin{tabular}{ll|l|l|l|l|l|l|l|l|l|l|l|l|l|l|l|}
.0598 & .0710 & .0817 & .0916 & .1046 & .1131 & .1391 & .1573 & .1833 & .2112 & .2694 & .3266 & .3823 & .4407
\end{tabular} \begin{tabular}{ll|l|l|l|l|l|l|l|l|l|l|l|l|}
0610 & .0724 & .0833 & .0934 & .1064 & .1150 & .1410 & .1596 & .1856 & .2139 & .2723 & .3299 & .3862 & .4448
\end{tabular}

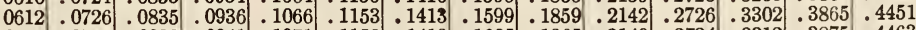

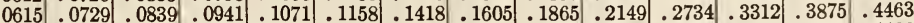

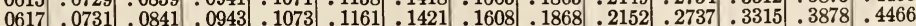

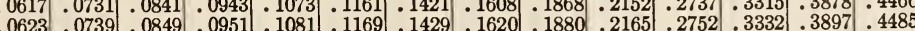

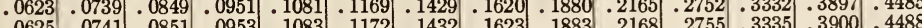

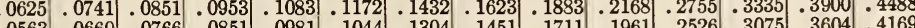
\begin{tabular}{l|l|l|l|l|l|l|l|l|l|l|l|l|l|}
.0563 & .0669 & .0766 & .0851 & .0981 & .1044 & .1304 & .1451 & .1711 & .1961 & .2526 & .3075 & .3604 & .4169
\end{tabular} \begin{tabular}{l|l|l|l|l|l|l|l|l|l|l|l|l|l|l|l|l|l|}
.0567 & .0673 & .0770 & .0855 & .0985 & .1048 & .1308 & .1456 & .1716 & .1966 & .2531 & .3081 & .3610 & .4175 \\
\hline
\end{tabular} 
TABLE 22.-Limiting dimensions of setting plug and thread ring gages for screws of classes 1, 2, 3, and 4 fits, American national coarse-thread series-Continued

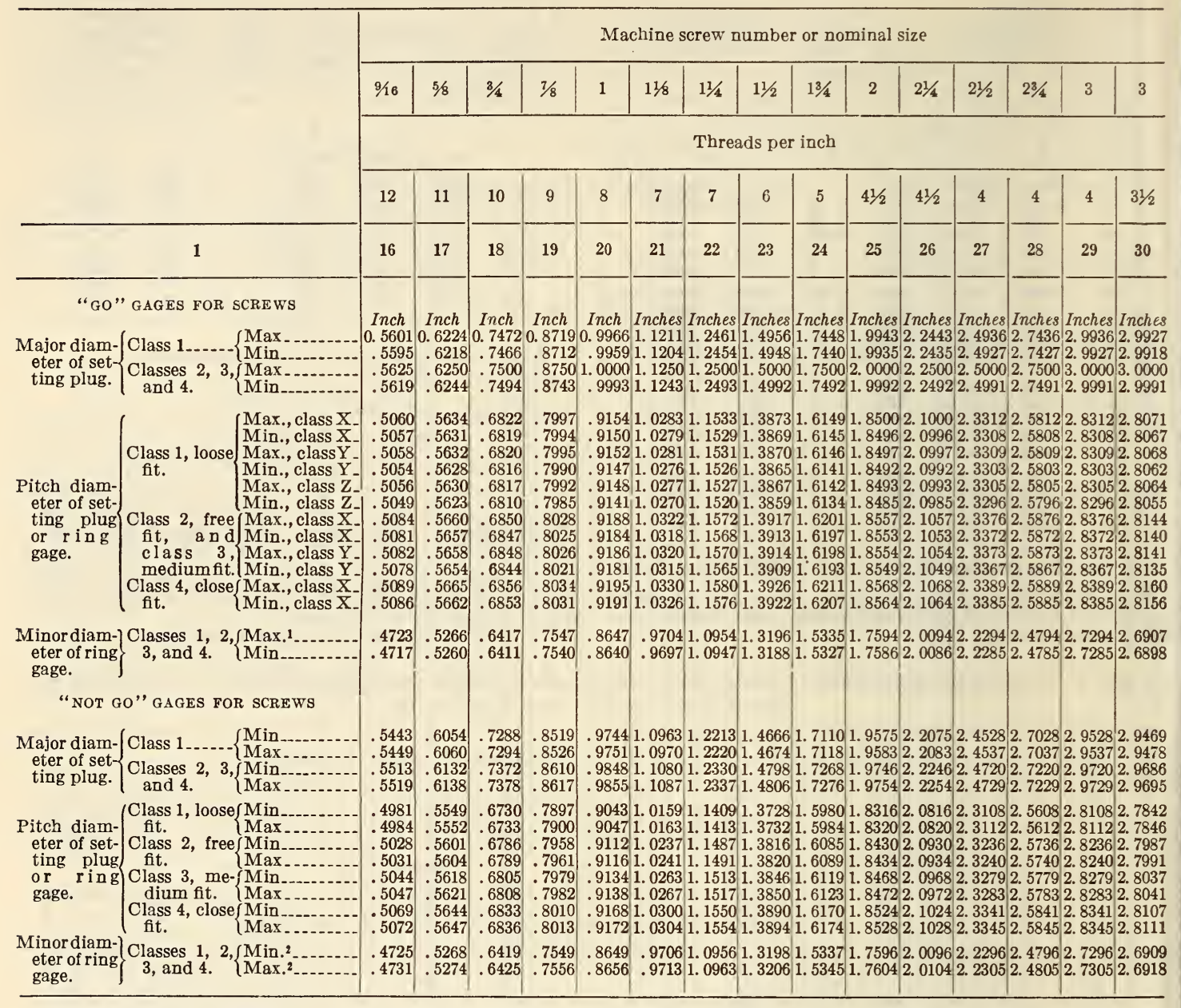

12 See footnotes to Table 23. 
TABLE 23.-Limiting dimensions of setting plug and thread ring gages for screws of classes 1, 2, 3, and 4 fits, American national fine-thread series

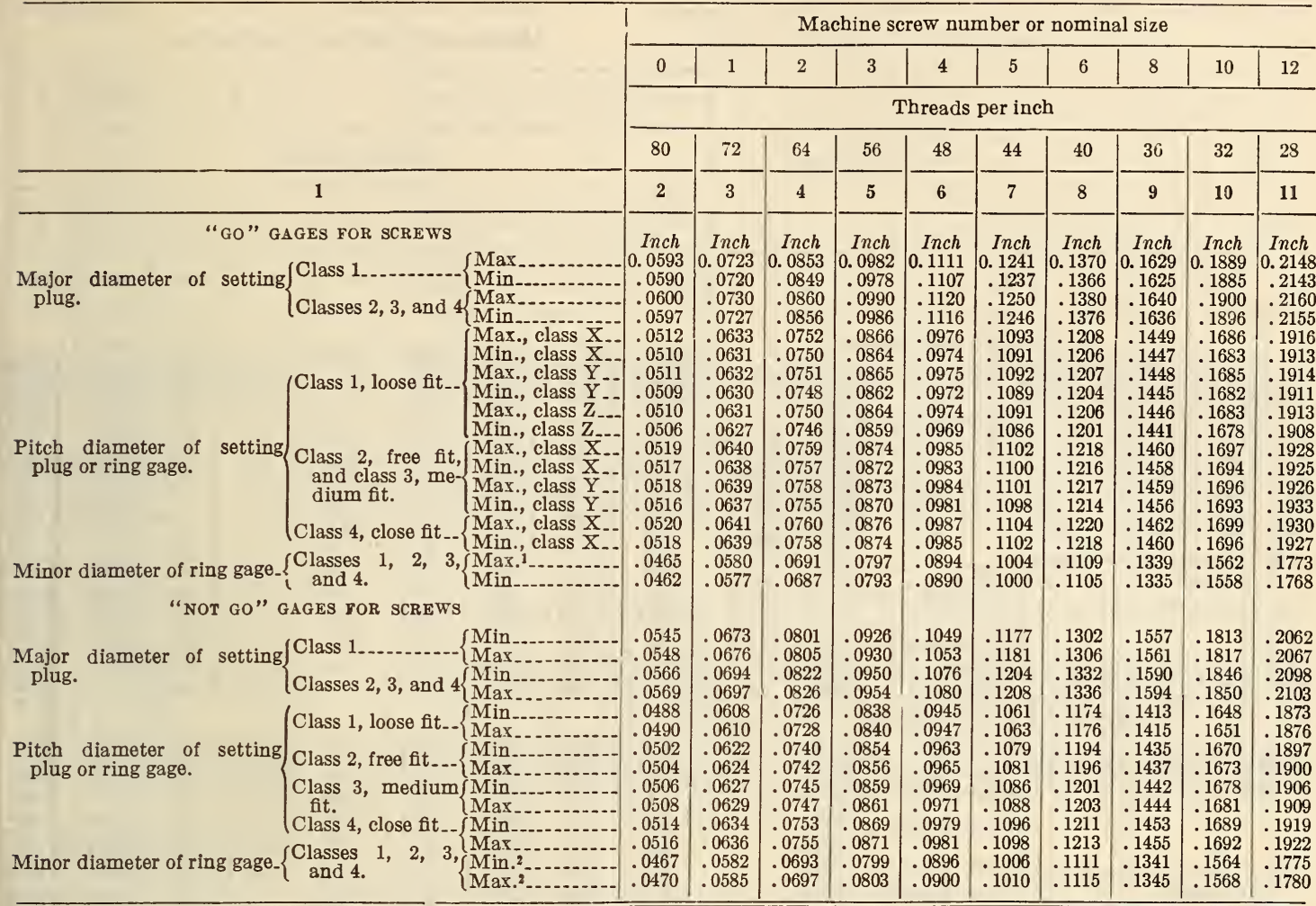

\begin{tabular}{|c|c|c|c|c|c|c|c|c|c|c|c|c|}
\hline \multirow{4}{*}{\multicolumn{3}{|c|}{ the }} & \multicolumn{10}{|c|}{ Machine screw number or nominal size } \\
\hline & & & $1 / 4$ & 516 & $3 / 8$ & $7 / 16$ & $1 / 2$ & $9 / 16$ & $5 / 8$ & $8 / 4$ & $7 / 8$ & 1 \\
\hline & & & \multicolumn{10}{|c|}{ Threads per inch } \\
\hline & & & 28 & 24 & 24 & 20 & 20 & 18 & 18 & 16 & 14 & 14 \\
\hline & 1 & & 12 & 13 & 14 & 15 & 16 & 17 & 18 & 19 & 20 & 21 \\
\hline Major diameter of setting plug- & $\begin{array}{l}\text { SOR SCRE WS } \\
\left\{\begin{array}{l}\text { Class } 1 \\
\text { Classes } 2,3, \text { and } \\
4 .\end{array}\right.\end{array}$ & $\begin{array}{l}\left\{\begin{array}{l}\text { Max } \\
\text { Min }\end{array}\right. \\
\left\{\begin{array}{l}\text { Max } \\
\text { Min }\end{array}\right.\end{array}$ & $\begin{array}{r}\text { Inch } \\
0.2488 \\
.2483 \\
.2500 \\
.2495\end{array}$ & $\begin{array}{r}\text { Inch } \\
0.3112 \\
.3107 \\
.3125 \\
.3120\end{array}$ & $\begin{array}{r}\text { Inch } \\
0.3737 \\
.3732 \\
.3750 \\
.3745\end{array}$ & $\begin{array}{c}\text { Inch } \\
0.4360 \\
.4355 \\
.4375 \\
.4370\end{array}$ & $\begin{array}{r}\text { Inch } \\
0.4985 \\
4980 \\
.5000 \\
.4995\end{array}$ & $\begin{array}{r}\text { Inch } \\
0.5609 \\
.5604 \\
.5625 \\
.5620\end{array}$ & $\begin{array}{r}\text { Inch } \\
0.6234 \\
.6229 \\
.6250 \\
.6245\end{array}$ & $\begin{array}{r}\text { Inch } \\
0.7482 \\
.7476 \\
.7500 \\
.7494\end{array}$ & $\begin{array}{c}\text { Inch } \\
0.8729 \\
.8723 \\
.8750 \\
.8744\end{array}$ & $\begin{array}{l}\text { Inch } \\
0.9979 \\
.9973 \\
1.0000 \\
.9994\end{array}$ \\
\hline $\begin{array}{l}\text { Pitch diameter of setting plug } \\
\text { or ring gage. }\end{array}$ & $\left\{\begin{array}{l}\text { Class } 1, \text { loose fit- } \\
\text { Class } 2 \text {, free fit, } \\
\text { and class } 3, \\
\text { medium fit. } \\
\text { Class } 4 \text {, close fit_- }\end{array}\right.$ & 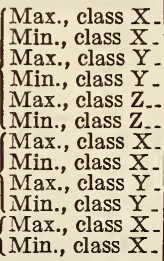 & $\begin{array}{l}.2256 \\
.2253 \\
.2254 \\
.2251 \\
.2253 \\
.2248 \\
.2268 \\
.2265 \\
.2266 \\
.2263 \\
.2270 \\
.2267\end{array}$ & $\begin{array}{l}.2841 \\
.2838 \\
.2839 \\
.2836 \\
.2838 \\
.2832 \\
.2854 \\
.2851 \\
.2852 \\
.2849 \\
.2857 \\
.2854\end{array}$ & $\begin{array}{l}.3466 \\
.3463 \\
.3464 \\
.3461 \\
.3463 \\
.3457 \\
.3479 \\
.3476 \\
.3477 \\
.3474 \\
.3482 \\
.3479\end{array}$ & $\begin{array}{l}.4035 \\
.4032 \\
.4033 \\
.4030 \\
.4032 \\
.4026 \\
.4050 \\
.4047 \\
.4048 \\
.4045 \\
.4053 \\
.4050\end{array}$ & $\begin{array}{l}.4660 \\
.4657 \\
.4658 \\
.4655 \\
.4657 \\
.4651 \\
.4675 \\
.4672 \\
.4673 \\
.4670 \\
.4678 \\
.4675\end{array}$ & $\begin{array}{l}.5248 \\
.5245 \\
.5246 \\
.5243 \\
.5244 \\
.5238 \\
.5264 \\
.5261 \\
.5262 \\
.5259 \\
.5267 \\
.5264\end{array}$ & $\begin{array}{l}.5873 \\
.5870 \\
.5871 \\
.5868 \\
.5869 \\
.5863 \\
.5889 \\
.5886 \\
.5887 \\
.5884 \\
.5892 \\
.5889\end{array}$ & $\begin{array}{l}.7076 \\
.7073 \\
.7074 \\
.7070 \\
.7072 \\
.7066 \\
.7094 \\
.7091 \\
.7092 \\
.7088 \\
.7098 \\
.7095\end{array}$ & $\begin{array}{l}.8265 \\
.8262 \\
.8263 \\
.8259 \\
.8261 \\
.8255 \\
.8286 \\
.8283 \\
.8284 \\
.8280 \\
.8290 \\
.8287\end{array}$ & $\begin{array}{l}.9515 \\
.9512 \\
.9513 \\
.9509 \\
.9511 \\
.9505 \\
.9536 \\
.9533 \\
.9534 \\
.9530 \\
.9540 \\
.9537\end{array}$ \\
\hline Minor diameter of ring gage.. & $\left\{\begin{array}{l}\text { Class } 1,2,3 \text {, and } \\
4 .\end{array}\right.$ & $\left\{\begin{array}{l}\operatorname{Max}{ }^{1} \ldots \ldots \\
\text { Min. }\end{array}\right.$ & .2113 & $\begin{array}{l}.2674 \\
.2669\end{array}$ & $\begin{array}{l}.3299 \\
.3294\end{array}$ & $\begin{array}{l}.3834 \\
.3829\end{array}$ & $\begin{array}{l}.4459 \\
.4454\end{array}$ & $\begin{array}{l}.5024 \\
.5019\end{array}$ & $\begin{array}{l}.5649 \\
.5644\end{array}$ & .6823 & $\begin{array}{l}.7977 \\
.7971\end{array}$ & $\begin{array}{l}.9227 \\
.9221\end{array}$ \\
\hline Major diamter of setting plug & $\begin{array}{l}\text { GES FOR SCRE WS } \\
\left\{\begin{array}{l}\text { Class } 1 \\
\text { Classes } 2,3, \text { and } \\
4 .\end{array}\right.\end{array}$ & $\begin{array}{l}\left\{\begin{array}{l}\text { Min } \\
\text { Max }\end{array}\right. \\
\left\{\begin{array}{l}\text { Min } \\
\text { Max }\end{array}\right.\end{array}$ & $\begin{array}{l}.2402 \\
.2407 \\
.2438 \\
.2443\end{array}$ & $\begin{array}{l}.3020 \\
.3025 \\
.3059 \\
.3064\end{array}$ & $\begin{array}{l}.3645 \\
.3650 \\
.3684 \\
.3689\end{array}$ & $\begin{array}{l}.4258 \\
.4263 \\
.4303 \\
.4308\end{array}$ & $\begin{array}{r}.4883 \\
.4888 \\
.4928 \\
.4933\end{array}$ & $\begin{array}{l}.5495 \\
.5500 \\
.5543 \\
.5548\end{array}$ & $\begin{array}{l}.6120 \\
.6125 \\
.6168 \\
.6173\end{array}$ & $\begin{array}{l}.7356 \\
.7362 \\
.7410 \\
.7416\end{array}$ & $\begin{array}{l}.8589 \\
.8995 \\
.8652 \\
.8658\end{array}$ & $\begin{array}{l}.9839 \\
.9845 \\
.9902 \\
.9908\end{array}$ \\
\hline $\begin{array}{l}\text { Pitch diamter of setting plug } \\
\text { or ring gage. }\end{array}$ & $\left\{\begin{array}{l}\text { Class } 1, \text { loose fit_- } \\
\text { Class } 2 \text {, free fit.- } \\
\text { Class } 3 \text {, medium } \\
\text { fit. } \\
\text { Class } 4 \text {, close fit_- }\end{array}\right.$ & $\begin{array}{l}\left\{\begin{array}{l}\text { Min } \\
\text { Max }\end{array}\right. \\
\left\{\begin{array}{l}\text { Min } \\
\text { Max }\end{array}\right. \\
\left\{\begin{array}{l}\text { Min } \\
\text { Max }\end{array}\right. \\
\left\{\begin{array}{l}\text { Min } \\
\text { Max }\end{array}\right.\end{array}$ & $\begin{array}{l}.2213 \\
.2216 \\
.2237 \\
.2240 \\
.2246 \\
.2249 \\
.2259 \\
.2262\end{array}$ & $\begin{array}{l}.2795 \\
.2798 \\
.2821 \\
.2824 \\
.2830 \\
.2833 \\
.2845 \\
.2848\end{array}$ & $\begin{array}{l}.3420 \\
.3423 \\
.3446 \\
.3449 \\
.3455 \\
.3458 \\
.3470 \\
.3473\end{array}$ & $\begin{array}{l}.3984 \\
.3987 \\
.4014 \\
.4017 \\
.4024 \\
.4027 \\
.4040 \\
.4043\end{array}$ & $\begin{array}{l}.4609 \\
.4612 \\
.4639 \\
.4642 \\
.4649 \\
.4652 \\
.4665 \\
.4668\end{array}$ & $\begin{array}{l}.5191 \\
.5194 \\
.5223 \\
.5226 \\
.5234 \\
.5237 \\
.5252 \\
.5255\end{array}$ & $\begin{array}{l}.5816 \\
.5819 \\
.5848 \\
.5851 \\
.5859 \\
.5862 \\
.5877 \\
.5880\end{array}$ & $\begin{array}{l}.7013 \\
.7016 \\
.7049 \\
.7052 \\
.7062 \\
.7065 \\
.7082 \\
.7085\end{array}$ & $\begin{array}{l}.8195 \\
.8198 \\
.8237 \\
.8240 \\
.8250 \\
.8253 \\
.8272 \\
.8275\end{array}$ & $\begin{array}{l}.9445 \\
.9448 \\
.9487 \\
.9490 \\
.9500 \\
.9503 \\
.9522 \\
.9525\end{array}$ \\
\hline Minor diameter of ring gage. & $\left\{\begin{array}{c}\text { Class } 1,2,3 \text {, and } \\
4 .\end{array}\right.$ & $\left\{\begin{array}{l}\operatorname{Min} .^{2} \ldots \\
\operatorname{Max} .^{2}\end{array}\right.$ & $\begin{array}{l}.2115 \\
.2120\end{array}$ & $\begin{array}{l}.2676 \\
.2681\end{array}$ & $\begin{array}{l}.3301 \\
.3306\end{array}$ & $\begin{array}{l}.3836 \\
.3841\end{array}$ & $\begin{array}{l}.4461 \\
.4466\end{array}$ & $\begin{array}{l}.5026 \\
.5031\end{array}$ & $\begin{array}{l}.5651 \\
.5656\end{array}$ & .6825 & .7979 & $\begin{array}{l}.9229 \\
.9235\end{array}$ \\
\hline
\end{tabular}

See footnotes at end of Table. 
TABLE 23.-Limiting dimensions of setting plug and thread ring gages for screws of classes 1, 2, 3, and 4 fits, American national fine-thread series-Continued

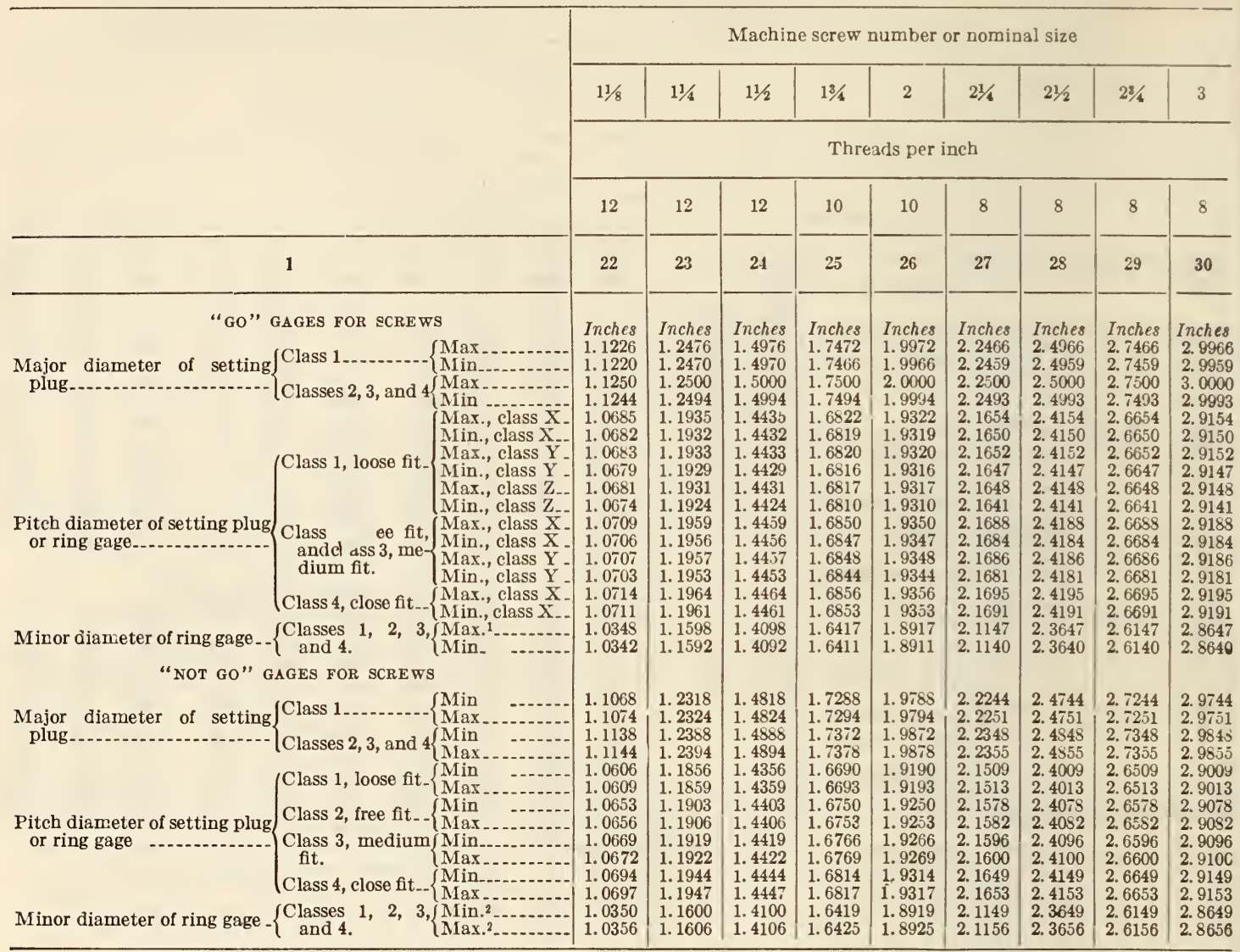

1 The maximum minor diameter of the "go" thread ring gage is the same as the minimum minor diameter of the tapped hole.

2 In order that the "not go" gage check pitch diameter only, it is necessary that the minor diameter of the "not go" ring gage be never less than that specified for the "go" ring gage. The limiting dimensions given in this table for the minor diameter of the "not go" ring gage represent this condition.

TABLE 24.-Limiting dimensions of thread plug gages for nuts of classes 1, 2, 3, and 4 fits, American national coarse-thread series

\begin{tabular}{|c|c|c|c|c|c|c|c|c|c|c|c|c|c|c|c|}
\hline & & \multicolumn{14}{|c|}{ Machine screw number or nominal size } \\
\hline & & 1 & 2 & 3 & 4 & 5 & 6 & 8 & 10 & 12 & $1 / 4$ & $5 / 10$ & $3 / 8$ & $7 / 16$ & $1 / 2$ \\
\hline & & \multicolumn{14}{|c|}{ Threads per inch } \\
\hline & & 64 & 56 & 48 & 40 & 40 & 32 & 32 & 24 & 24 & 20 & 18 & 16 & 14 & 13 \\
\hline & 1 & 2 & 3 & 4 & 5 & 6 & 7 & 8 & 9 & 10 & 11 & 12 & 13 & 14 & 15 \\
\hline \multicolumn{2}{|c|}{ "GO" GAGES FOR NUTS } & Inch & Inch & Inch & Inch & Inch & Inch & Inch & Inch & Inch & Inch & Inch & Inch & Inch & \\
\hline \multicolumn{2}{|c|}{$\begin{array}{l}\text { Major diameter of plug gage, }\left\{\begin{array}{l}\text { Min } \\
\text { classes } 1,2,3,\end{array} \text { and } 4 .\right.\end{array}$} & $\begin{array}{r}0.0730 \\
.0734\end{array}$ & $\begin{array}{r}0.0860 \\
.0864\end{array}$ & $\begin{array}{r}0.0990 \\
.0994\end{array}$ & 0.1120 & 0.1250 & $\begin{array}{l}0.1380 \\
.1384\end{array}$ & $\begin{array}{r}0.1640 \\
.1644\end{array}$ & $\begin{array}{l}0.1900 \\
.1905\end{array}$ & $\begin{array}{l}0.2160 \\
.2165\end{array}$ & $\begin{array}{r}0.2500 \\
.2505\end{array}$ & $\begin{array}{r}0.3125 \\
.3130\end{array}$ & $\begin{array}{l}0.37500 \\
.3756\end{array}$ & $\begin{array}{r}0.4375 \\
.4381\end{array}$ & $\begin{array}{l}0.5000 \\
.5006\end{array}$ \\
\hline $\begin{array}{l}\text { Pitch diameter } \\
\text { of plug gage. }\end{array}$ & 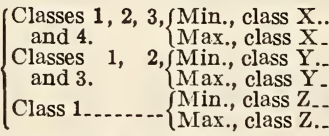 & $\begin{array}{l}.0629 \\
.0631 \\
.0630 \\
.0633 \\
.0631 \\
.0635\end{array}$ & $\begin{array}{l}.0744 \\
.0746 \\
.0745 \\
.0748 \\
.0746 \\
.0751\end{array}$ & $\begin{array}{l}.0855 \\
.0857 \\
.0856 \\
.0859 \\
.0857 \\
.0862\end{array}$ & $\begin{array}{l}.0958 \\
.0960 \\
.0959 \\
.0962 \\
.0960 \\
.0965\end{array}$ & $\begin{array}{l}.108 \overline{8} \\
.1090 \\
.1089 \\
.1092 \\
.1090 \\
.1095\end{array}$ & $\begin{array}{l}.1177 \\
.1180 \\
.1178 \\
.1181 \\
.1180 \\
.1185\end{array}$ & $\begin{array}{l}.1437 \\
.1440 \\
.1438 \\
.1441 \\
.1440 \\
.1445\end{array}$ & $\begin{array}{l}.1629 \\
.1632 \\
.1631 \\
.1634 \\
.1632 \\
.1638\end{array}$ & $\begin{array}{l}.1889 \\
.1892 \\
.1891 \\
.1894 \\
.1892 \\
.1898\end{array}$ & $\begin{array}{l}.2175 \\
.2178 \\
.2177 \\
.2180 \\
.2178 \\
.2184\end{array}$ & $\begin{array}{l}.2764 \\
.2767 \\
.2766 \\
.2769 \\
.2768 \\
.2774\end{array}$ & $\begin{array}{l}.3344 \\
.3347 \\
.3346 \\
.3350 \\
.3348 \\
.3354\end{array}$ & $\begin{array}{l}.3911 \\
.3914 \\
.3913 \\
.3917 \\
.3915 \\
.3921\end{array}$ & $\begin{array}{l}.4500 \\
.4503 \\
.4502 \\
.4506 \\
.4504 \\
.4511\end{array}$ \\
\hline \multicolumn{2}{|c|}{ "NOT GO" GAGES FOR NUTS } & & & & & & & & & & & & & & \\
\hline \multicolumn{2}{|c|}{$\begin{array}{l}\text { Major diameter of plug gage, }\left\{\begin{array}{l}\text { Max.1. } \\
\text { classes } 1,2,3, \text { and } 4 .\end{array}\right.\end{array}$} & $\begin{array}{l}.0728 \\
.0696\end{array}$ & $\begin{array}{l}.0858 \\
.0821\end{array}$ & $\begin{array}{l}.0988 \\
.0945\end{array}$ & .1118 & $\begin{array}{l}.1248 \\
.1196\end{array}$ & $\begin{array}{l}.1378 \\
.1312\end{array}$ & $\begin{array}{l}.1638 \\
.1572\end{array}$ & $\begin{array}{l}.1898 \\
.1810\end{array}$ & $\begin{array}{l}.2158 \\
.2070\end{array}$ & .2498 & $\begin{array}{l}.3123 \\
.3005\end{array}$ & $\begin{array}{l}.3748 \\
.3615\end{array}$ & $\begin{array}{l}.4373 \\
.4220\end{array}$ & $\begin{array}{l}.4998 \\
.4833\end{array}$ \\
\hline \multirow{4}{*}{$\begin{array}{l}\text { Pitch diameter } \\
\text { of plug gage. }\end{array}$} & $\left\{\begin{array}{c}\text { Class 1, loose }\left\{\begin{array}{l}\operatorname{Max} \\
\text { fit. }\end{array}\right. \\
\text { Class 2, free fit_\{ }\left\{\begin{array}{l}\text { Max } \\
\text { Min }\end{array}\right.\end{array}\right.$ & $\begin{array}{l}.0655 \\
.0653 \\
.0648 \\
.0646\end{array}$ & $\begin{array}{l}.0772 \\
.0770 \\
.0764 \\
.0762\end{array}$ & $\begin{array}{l}.0886 \\
.0884 \\
.0877 \\
.0875\end{array}$ & $\begin{array}{l}.0992 \\
.0990 \\
.0982 \\
.0980\end{array}$ & $\begin{array}{l}.1122 \\
.1120 \\
.1112 \\
.1110\end{array}$ & $\begin{array}{l}.1215 \\
.1212 \\
.1204 \\
.1201\end{array}$ & $\begin{array}{l}.1475 \\
.1472 \\
.1464 \\
.1461\end{array}$ & $\begin{array}{l}.1675 \\
.1672 \\
.1662 \\
.1659\end{array}$ & $\begin{array}{l}.1935 \\
.1932 \\
.1922 \\
.1919\end{array}$ & $\begin{array}{l}.2226 \\
.2223 \\
.2211 \\
.2208\end{array}$ & $\begin{array}{l}.2821 \\
.2818 \\
.2805 \\
.2802\end{array}$ & $\begin{array}{l}.3407 \\
.3404 \\
.3389 \\
.3386\end{array}$ & $\begin{array}{l}.3981 \\
.3978 \\
.3960 \\
.3957\end{array}$ & $\begin{array}{l}.4574 \\
.4571 \\
.4552 \\
.4549\end{array}$ \\
\hline & $\begin{array}{l}\text { Class 3, medi- }\left\{\begin{array}{l}\text { Max... } \\
\text { um fit. }\end{array}\right. \\
\text { Min.. }\end{array}$ & .0643 & $\begin{array}{l}.0759 \\
.0757\end{array}$ & $\begin{array}{l}.0871 \\
.0869\end{array}$ & \begin{tabular}{|l}
.0975 \\
.0973
\end{tabular} & $\begin{array}{l}.1105 \\
.1103\end{array}$ & $\begin{array}{l}.1196 \\
.1193\end{array}$ & $\begin{array}{l}.1456 \\
.1453\end{array}$ & $\begin{array}{l}.1653 \\
.1650\end{array}$ & $\begin{array}{l}.1913 \\
1910\end{array}$ & $\begin{array}{l}.2201 \\
.2198\end{array}$ & $\begin{array}{l}.2794 \\
.2791\end{array}$ & $\begin{array}{l}.3376 \\
.3373\end{array}$ & $\begin{array}{l}.3947 \\
.3944\end{array}$ & $\begin{array}{l}.4537 \\
.4534\end{array}$ \\
\hline & $\begin{array}{c}\text { Class } 4, \text { close } \\
\text { fit. } \\
N\end{array}$ & .0636 & .0751 & .0863 & .0967 & .1097 & .1187 & .1447 & .1641 & .1901 & 2188 & .2779 & .3360 & .3929 & 4519 \\
\hline & & .0007 & 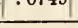 & & .0800 & 90 & 1184 & .1444 & .1038 & 1890 & 2180 & 2670 & 3357 & .3926 & 4516 \\
\hline
\end{tabular}


TABLE 24.-Limiting dimensions of thread plug gages for nuts of classes 1, 2, 3, and 4 fits, American national coarse-thread series-Continued

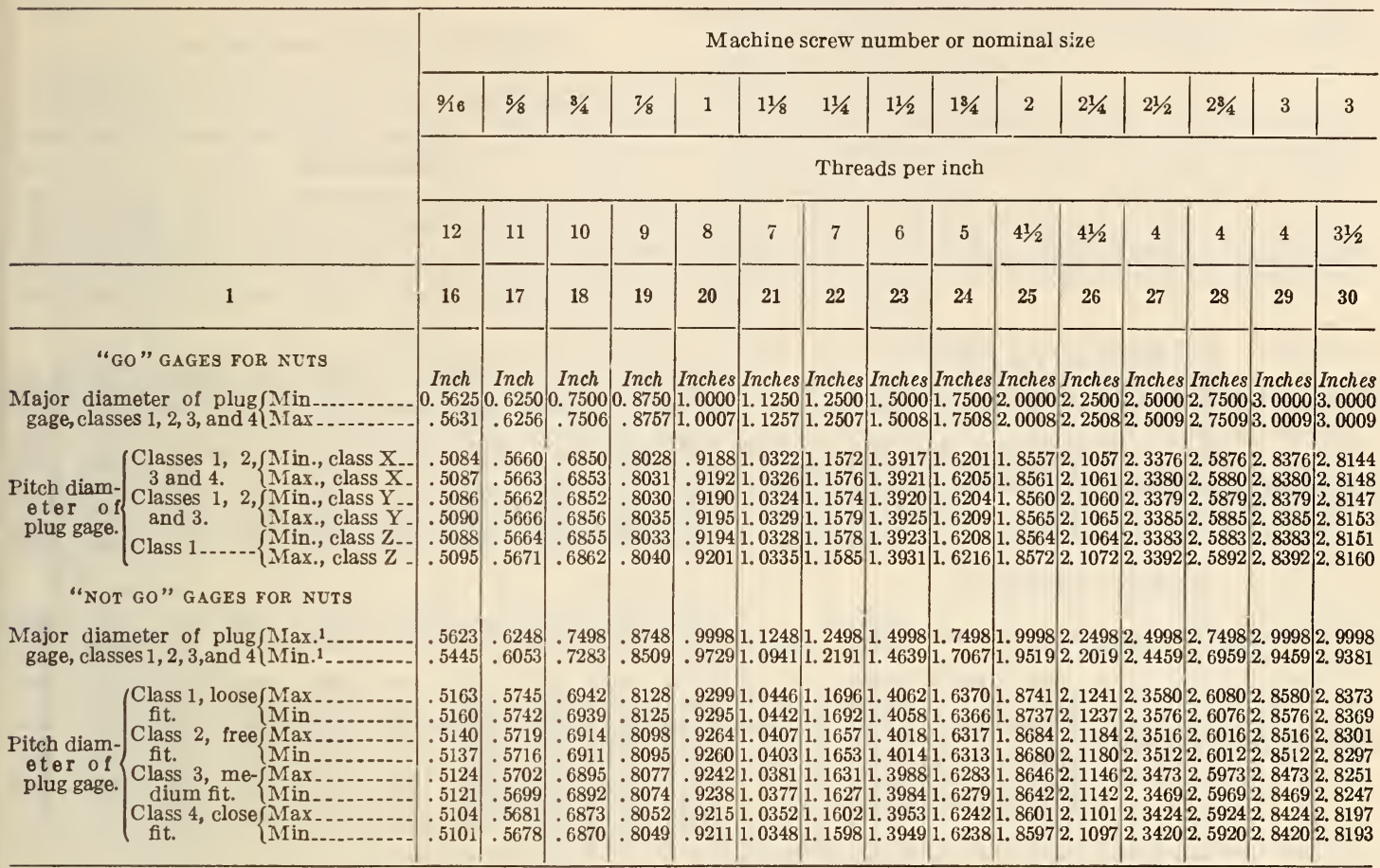

1 See footnote to Table 25.

TABLE 25.-Limiting dimensions of thread plug gages for nuts of classes 1, 2, 3, and 4 fits, American national fine-thread series

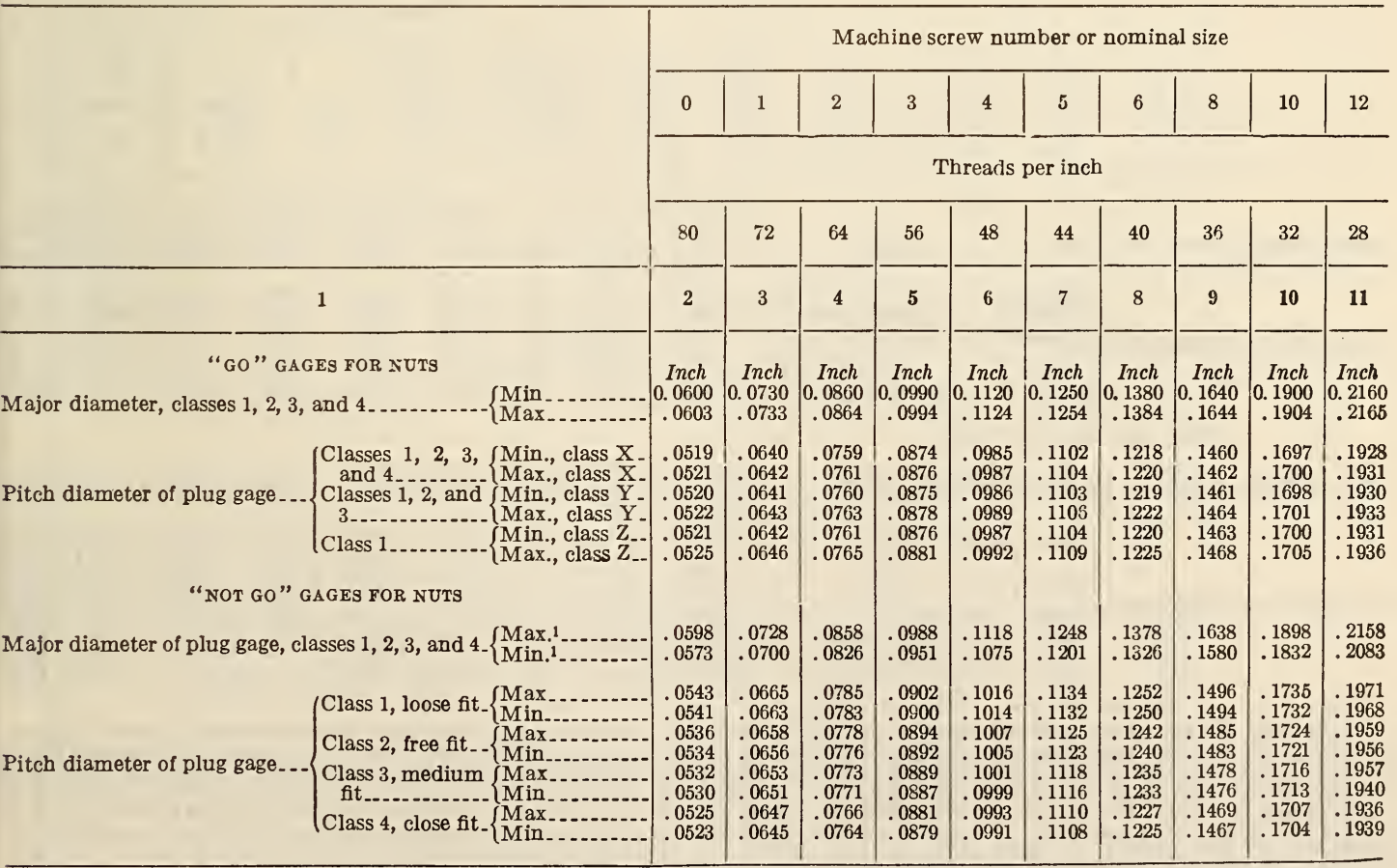

See footnote at end of Table. 
TABLE 25.-Limiting dimensions of thread plug gages for nuts of classes 1, 2, 3, and 4 fits, American national fine-thread series-Continued.

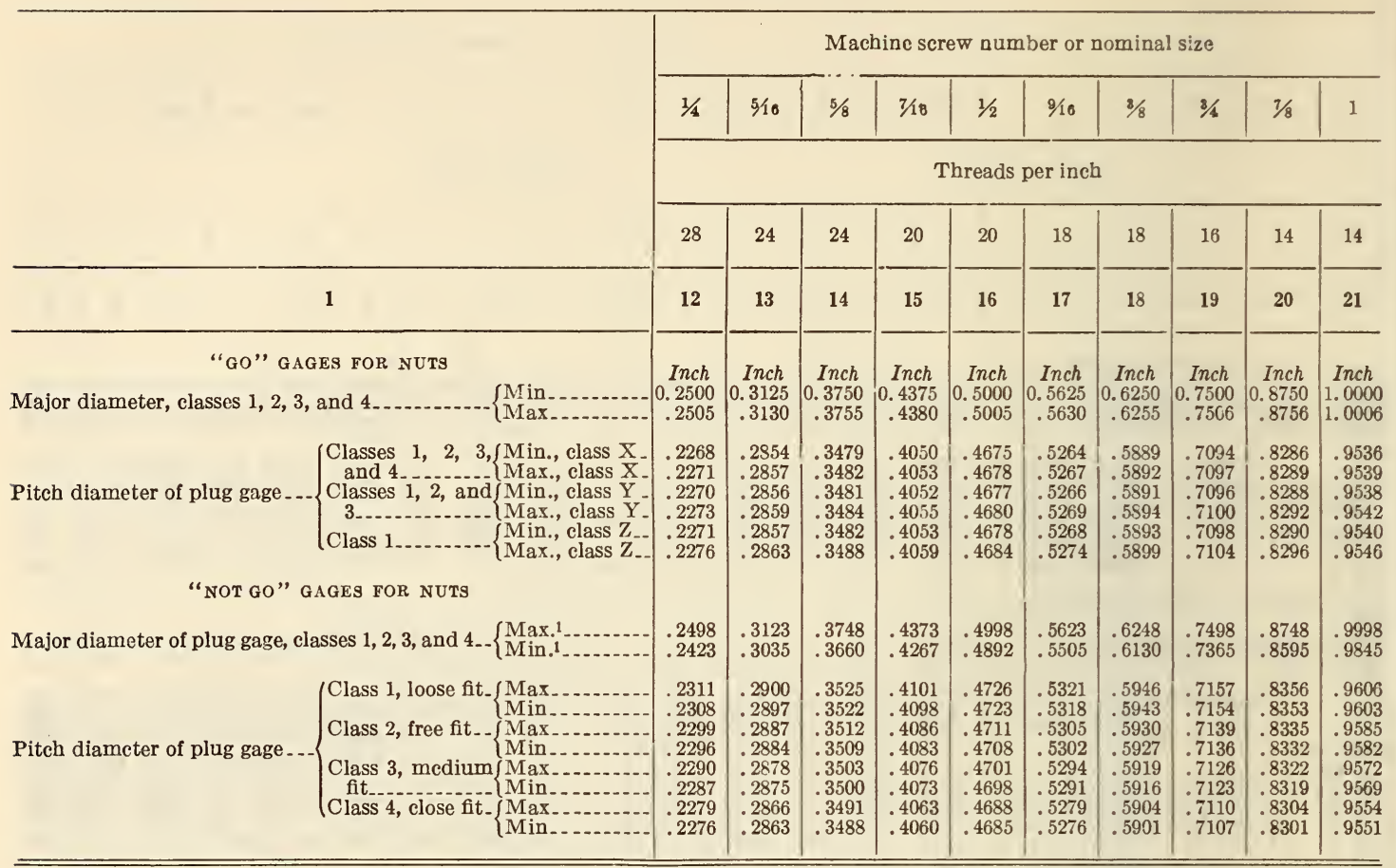

Machine screw number or nominal size

\begin{tabular}{l|l|l|l|l|l|l|l|l}
\hline $11 / 8$ & $11 / 4$ & $11 / 2$ & $13 / 4$ & 2 & $21 / 4$ & $21 / 2$ & $23 / 4$ & 3 \\
\hline
\end{tabular}

Threads per inch

\begin{tabular}{|c|c|c|c|c|c|c|c|c|}
\hline 12 & 12 & 12 & 10 & 10 & 8 & 8 & 8 & 8 \\
\hline 22 & 23 & 24 & 25 & 26 & 27 & 28 & 29 & 30 \\
\hline Inches & Inches & Inches & Inches & Inches & Inches & Inches & Inches & Inches \\
\hline 1. 1250 & 1.2500 & 1. 5000 & 1. 7500 & 2. 0000 & 2. 2500 & 2. 5000 & 2. 7500 & 3.0000 \\
\hline 1. 1256 & 1.2506 & 1.5006 & 1.7506 & 2.0006 & 2. 2507 & 2.5007 & 2. 7507 & 3. 0007 \\
\hline 1. 0709 & 1. 1959 & 1. 4459 & 1. 6850 & 1. 9350 & 2. 1688 & 2. 4188 & 2. 6688 & 2.9188 \\
\hline 1. 0712 & 1. 1962 & 1. 4462 & 1. 6853 & 1. 9353 & 2. 1692 & 2. 4192 & 2. 6692 & 2. 9192 \\
\hline 1.0711 & 1. 1961 & 1.4461 & 1.6852 & 1.9352 & 2. 1690 & 2. 4190 & 2. 6690 & 2. 9190 \\
\hline 1. 0715 & 1. 1965 & 1.4465 & 1. 6855 & 1. 9356 & 2. 1695 & 2. 4195 & 2. 6695 & 2. 9195 \\
\hline 1. 0713 & 1. 1963 & 1. 4463 & 1. 6855 & 1. 9355 & 2. 1694 & 2. 4194 & 2. 6694 & 2. 9194 \\
\hline 1.0720 & 1. 1970 & 1.4470 & 1. 6862 & 1. 9362 & 2. 1701 & 2. 4201 & 2. 6701 & 2. 9201 \\
\hline 1. 1248 & 1. 2498 & 1. 4998 & 1. 7498 & 1. 9998 & 2. 2498 & 2. 4998 & 2. 7498 & 2.9998 \\
\hline 1. 1070 & 1.2320 & 1.4820 & 1. 7283 & 1. 9783 & 2.2229 & 2.4729 & 2. 7229 & 2. 9729 \\
\hline 1.0788 & 1. 2038 & 1.4538 & 1. 6982 & 1. 9482 & 2. 1833 & 2. 4333 & 2. 6833 & 2. 9333 \\
\hline 1.0785 & 1.2035 & 1. 4535 & 1. 6979 & 1. 9479 & 2. 1829 & 2. 4329 & 2. 6829 & 2. 9329 \\
\hline 1.0765 & 1.2015 & 1.4515 & 1. 6950 & 1. 9450 & 2.1798 & 2. 4298 & 2. 6798 & 2. 9298 \\
\hline 1.0762 & 1. 2012 & 1.4512 & 1. 6947 & 1. 9447 & ๔. 1794 & 2. 4294 & 2. 6794 & 2. 9294 \\
\hline 1.0749 & 1.1999 & 1. 4499 & 1. 6934 & 1.9434 & 2. 1780 & 2.4280 & 2. 6780 & 2. 9280 \\
\hline 1.0746 & 1. 1996 & 1.4496 & 1. 6931 & 1.9431 & 2. 1776 & 2. 4276 & 2. 6776 & 2. 9276 \\
\hline 1. 0729 & 1. 1979 & 1. 4479 & 1. 6892 & 1. 9392 & 2. 1734 & 2. 4234 & 2. 6734 & 2. 9234 \\
\hline 1. 0726 & 1. 1976 & 1.4476 & 1. 6889 & 1. 9389 & 2. 1730 & 2.4230 & 2.6730 & 2. 9230 \\
\hline
\end{tabular}

1 The difference between these dimensions is not a manufacturing tolerance in the usual sense, but these dimensions represent the limits between which the dimensions may fall. In order that the "not go" gage check pitch diameter only, it is necessary that the crest of the thread be removed so that the major diameter of the "not go" plug gage be never greater than that specified for the "go" plug gage. The limiting dimensions given in this table for the major diameter of the "not go" plug gage represent this condition. On the other hand, it is desirable that the crest of the "not go" gage be truncated a considerable amount, in order to minimize the effect of angle error on the fit of the "not go" gage with the product. A truncation from basic dimensions corresponding to a width of flat equal to $14 \times p$ is recommended. and this condition is represented by the limiting dimensions given in this table as the minimum minor diameter of the plug gage. "Not go" and this condition is represented by the limiting dimensions given in this table as the minimum minor diameter of the plug gage. "Not go" these latter limits as starting points. 


\section{GAGES FOR SPECIAL THREADS}

Only one set of tolerances, namely, class $\mathrm{X}$, is specified for "not go" gages.

\section{Thread Form of Plug and Ring Thread Gages}

The minor diameter of the "go" thread ring gage is the same as the minimum minor diameter of the nut or tapped hole with a minus gage tolerance. The minor diameter of the "not go" ring gage is the minimum minor diameter of the nut plus at least 0.0002 inch, with a plus gage tolerance. Also the maximum major diameter of the "not go" thread plug gage is at least 0.0002 inch less than the basic major diameter, with a minus gage tolerance.

A relief is provided at the root of the "go" thread plug or ring gage, the width of which is not greater than one-eighth of the pitch. Also a relief is provided at the root of the "not go" plug or ring gage, the width of which is approximately one-fourth of the pitch.

Thus contact of the "not go" thread gage on the sides of the threads, rather than at the corners of the crest and root, is assured.

(Recommended uses for classes $\mathrm{X}, \mathrm{Y}$, and $\mathrm{Z}$ gages are the same as given above. Tolerances for like number of threads per inch are also the same as given above.)

\section{GAGES FOR AMERICAN NATIONAL FIRE-HOSE COUPLING THREADS}

It is recommended that American national firehose coupling threads be inspected in the field by means of gages made within the tolerances given in Table 41. Limiting dimensions for these gages are given in Tables 42 and 43.

It is further recommended that American national fire-hose coupling threads be given final inspection by the manufacturer by means of gages made within the limiting dimensions given in Tables 42 and 43 , by whatever amount may be desired, in order to avoid, as far as possible, disagreements which might otherwise arise as the result of slight differences in the sizes of gages.

\section{TABLE 41.-Tolerances on gages for American national fire-hose coupling threads}

\begin{tabular}{|c|c|c|c|}
\hline $\begin{array}{c}\text { Allowable variation in lead } \\
\text { between any 2 threads } \\
\text { not farther apart than } \\
\text { length of engagement }\end{array}$ & $\begin{array}{c}\text { Allowable } \\
\text { variation } \\
\text { in one-half } \\
\text { angle of } \\
\text { thread }\end{array}$ & $\begin{array}{c}\text { Tolerance } \\
\text { on diam- } \\
\text { eter of } \\
\text { minimum } \\
\text { thread } \\
\text { gage }\end{array}$ & $\begin{array}{c}\text { Tolerance } \\
\text { on diam- } \\
\text { eter of } \\
\text { maximum } \\
\text { thread } \\
\text { gage }\end{array}$ \\
\hline 1 & 2 & 3 & 4 \\
\hline Inch & $\pm 0^{\circ} 10^{\prime}$ & $\begin{array}{c}\text { Inch } \\
+0.000 \\
+.001\end{array}$ & \begin{tabular}{c} 
Inch \\
-0.000 \\
\hline 0.0005
\end{tabular} \\
\hline
\end{tabular}

TABLE 42.-Limiting dimensions of field inspection thread plug gages for couplings (internal threads) ${ }^{1}$

\begin{tabular}{|c|c|c|c|c|c|c|c|c|c|}
\hline \multirow{3}{*}{$\begin{array}{l}\text { Nominal } \\
\text { size }\end{array}$} & \multirow{3}{*}{$\begin{array}{l}\text { Threads } \\
\text { per inch }\end{array}$} & \multicolumn{4}{|c|}{ "Go" or minimum gage } & \multicolumn{4}{|c|}{ "Not go" or maximum gage } \\
\hline & & \multicolumn{2}{|c|}{ Major diameter } & \multicolumn{2}{|c|}{ Pitch diameter } & \multicolumn{2}{|c|}{ Major diameter } & \multicolumn{2}{|c|}{ Pitch diameter } \\
\hline & & $\begin{array}{l}\text { Maxi- } \\
\text { mum }\end{array}$ & $\begin{array}{l}\text { Mini- } \\
\text { mum }\end{array}$ & $\begin{array}{l}\text { Maxi- } \\
\text { mum }\end{array}$ & $\begin{array}{l}\text { Mini- } \\
\text { mum }\end{array}$ & $\begin{array}{l}\text { Maxi- } \\
\text { mum }\end{array}$ & $\begin{array}{l}\text { Mini- } \\
\text { mum }\end{array}$ & $\begin{array}{l}\text { Maxi- } \\
\text { mum }\end{array}$ & $\begin{array}{l}\text { Mini- } \\
\text { mum }\end{array}$ \\
\hline 1 & 2 & 3 & 4 & 5 & 6 & 7 & 8 & 9 & 10 \\
\hline $\begin{array}{l}2.500 \\
\text { 3. } 000 \\
3.500 \\
4.500\end{array}$ & $\begin{array}{l}7.5 \\
6.0 \\
6.0 \\
4.0\end{array}$ & $\begin{array}{l}\text { Inches } \\
\text { 3. } 0846 \\
\text { 3. } 6399 \\
\text { 4. } 2649 \\
\text { 5. } 7869\end{array}$ & $\begin{array}{l}\text { Inches } \\
\text { 3. } 0836 \\
\text { 3. } 6389 \\
\text { 4.2639 } \\
\text { 5. } 7859\end{array}$ & $\begin{array}{l}\text { Inches } \\
2.9980 \\
3.5316 \\
4.1566 \\
5.6245\end{array}$ & $\begin{array}{l}\text { Inches } \\
2.9970 \\
3.5306 \\
4.1556 \\
5.6235\end{array}$ & $\begin{array}{l}\text { Inches } \\
\text { 3. } 0836 \\
\text { 3. } 6389 \\
\text { 4.2639 } \\
\text { 5. } 7859\end{array}$ & $\begin{array}{l}\text { Inches } \\
\text { 3. } 0826 \\
\text { 3. } 6379 \\
\text { 4. } 2629 \\
\text { 5. } 7849\end{array}$ & $\begin{array}{l}\text { Inches } \\
\text { 3. } 0130 \\
3.5486 \\
\text { 4. } 1736 \\
5.6485\end{array}$ & $\begin{array}{l}\text { Inches } \\
\text { 3. } 0120 \\
\text { 3. } 5476 \\
\text { 4. } 1726 \\
\text { 5. } 6475\end{array}$ \\
\hline
\end{tabular}

1 See footnote to Table 43 .

$\mathrm{T}_{\mathrm{ABLE}}$ 43.-Limiting dimensions of field inspection thread ring gages for coupling nipples (external threads) ${ }^{1}$

\begin{tabular}{|c|c|c|c|c|c|c|c|c|c|}
\hline \multirow{3}{*}{$\begin{array}{l}\text { Nominal } \\
\text { size }\end{array}$} & \multirow{3}{*}{$\begin{array}{l}\text { Threads } \\
\text { per inch }\end{array}$} & \multicolumn{4}{|c|}{ "Go" or maximum gage } & \multicolumn{4}{|c|}{ "Not go" or minimum gage } \\
\hline & & \multicolumn{2}{|c|}{ Pitch diameter } & \multicolumn{2}{|c|}{ Minor diameter } & \multicolumn{2}{|c|}{ Pitch diameter } & \multicolumn{2}{|c|}{ Minor diameter } \\
\hline & & $\begin{array}{l}\text { Maxi- } \\
\text { mum }\end{array}$ & $\begin{array}{l}\text { Mini- } \\
\text { mum }\end{array}$ & $\begin{array}{l}\text { Maxi- } \\
\text { mum }\end{array}$ & $\begin{array}{l}\text { Mini- } \\
\text { mum }\end{array}$ & $\begin{array}{l}\text { Maxi- } \\
\text { mum }\end{array}$ & $\begin{array}{l}\text { Mini- } \\
\text { mum }\end{array}$ & $\begin{array}{l}\text { Maxi- } \\
\text { mum }\end{array}$ & $\begin{array}{l}\text { Mini- } \\
\text { mum }\end{array}$ \\
\hline 1 & 2 & 3 & 4 & 5 & 6 & 7 & 8 & 9 & 10 \\
\hline $\begin{array}{l}2.500 \\
\text { 3. } 000 \\
3.500 \\
4.500\end{array}$ & $\begin{array}{l}7.5 \\
6.0 \\
6.0 \\
4.0\end{array}$ & $\begin{array}{l}\text { Inches } \\
2.9820 \\
3.5156 \\
4.1356 \\
5.5985\end{array}$ & $\begin{array}{l}\text { Inches } \\
2.9810 \\
3.5146 \\
4.1346 \\
5.5975\end{array}$ & $\begin{array}{l}\text { Inches } \\
\text { 2. } 9104 \\
3.4223 \\
4.0473 \\
5.4611\end{array}$ & $\begin{array}{l}\text { Inches } \\
2.9094 \\
3.4213 \\
4.0463 \\
5.4601\end{array}$ & $\begin{array}{l}\text { Inches } \\
\text { 2. } 9670 \\
3.4986 \\
\text { 4. } 1186 \\
\text { 5. } 5745\end{array}$ & $\begin{array}{l}\text { Inches } \\
2.9660 \\
3.4976 \\
\text { 4. } 1176 \\
5.5735\end{array}$ & $\begin{array}{l}\text { Inches } \\
\text { 2. } 9114 \\
\text { 3. } 4233 \\
\text { 4. } 0483 \\
5.4621\end{array}$ & $\begin{array}{l}\text { Inches } \\
2.9104 \\
3.4223 \\
4.0473 \\
5.4611\end{array}$ \\
\hline
\end{tabular}

1 The minor diameters of plug gages and the major diameters of ring gages are undercut beyond the nominal diameters to give clearance for grinding or lapping. The allowable variation in lead between any 2 threads not farther apart than the length of engagement is \pm 50.000 inch. The allowable variation in one-half angle of thread is \pm 10 minutes. 


\section{GAGE FOR PIPE THREADS}

In order properly to maintain interchangeability of pipe threads, gages should consist of "master," "check" or "setting," "inspection," and "working" gages. The same fundamentals apply as those outlined in the section covering gages for fastening screws, with the single exception that, with taperthreaded gages, separate "go" and "not go" gages are not necessary.

\section{Classification of Gages}

1. Master gage.-The master gage is a taperthreaded plug gage. The roots of the threads are

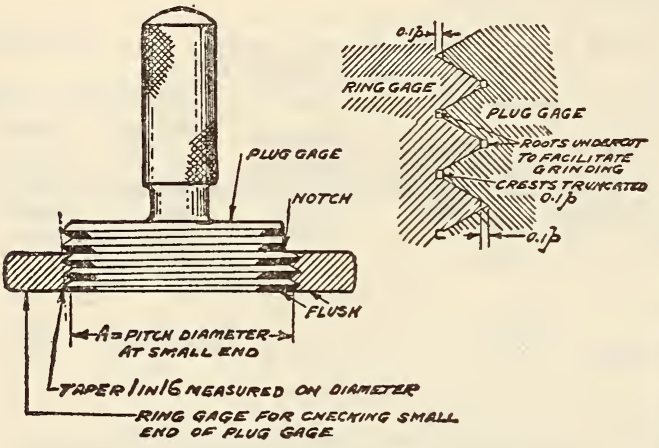

Figure 711.-Masier gages or checl gages for checking working gages

cut to a sharp $V$ or may be undercut to facilitate the making of the thread. The crests are truncated an amount equal to $0.1 \mathrm{p}$. (See fig. 711.) ${ }^{92}$ Basic dimensions of taper pipe thread gages are given in Table 55. This gage is provided with the gaging notch as illustrated in Figure 711. The master gage is the gage to which all other gages are ultimately referred, either by transfer of measurements or direct comparision by engagement. It is in-

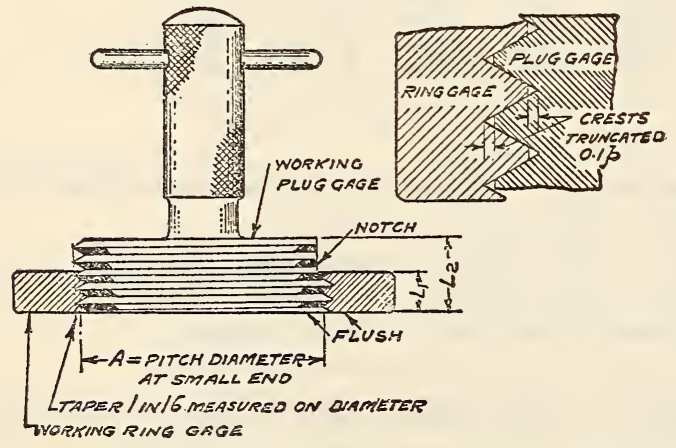

FIgURE 712.-Inspection or working gages for checking product

tended primarily for the use of gage and thread tool manufacturers.

2. Check (or reference) gages.-The check gages consist of a plug gage, similar in all respects to the master gage, and one ring gage. The ring gage has a thickness equal to dimension $L_{1}$, is the same diameter at the small end as the small end of the plug gage, and is flush with the plug gage at the small end and at the gaging notch when screwed on tight

${ }^{92}$ The object of truncating the crests on gages (truncation $0.1 p$ ) is to insure that, when gaging commercial threads cut with a slightly dull tool, the gage bears on the sides of the thread instead of on the roots. by hand. (See fig. 711.) The check plug gage is used to inspectinspection and working taper-threaded ring gages. The check ring gage is used to compare the check plug with the master plug, or the inspection and working plug gages with the check plug gage.

3. Inspection gages.-Inspection gages consist of one taper threaded plug gage and one taper threaded ring gage. The roots of the threads are cut to sharp $\mathrm{V}$ or may be undercut to facilitate making the thread. The crests are truncated an amount equal to $0.1 p$, but otherwise the gages are made to the dimensions given in Table 44.92 (See N. S. T. C. under 607.0, p.367.) The ring gage has a thickness equal to dimension $L_{1}$, and the same diameter at the small end as the small end of the plug gage. (See fig. 712.)

Inspection gages are for the use of the purchaser of pipe thread products. When used, the extreme tolerances on the work should be applied. This tolerance is one and one-half turns either way from the gaging notch in the case of internal threads inspected with the inspection plug gage, and when inspecting external threads the tolerance is one and one-half turns either way from the small end of the inspection ring. Inspection gages should be checked

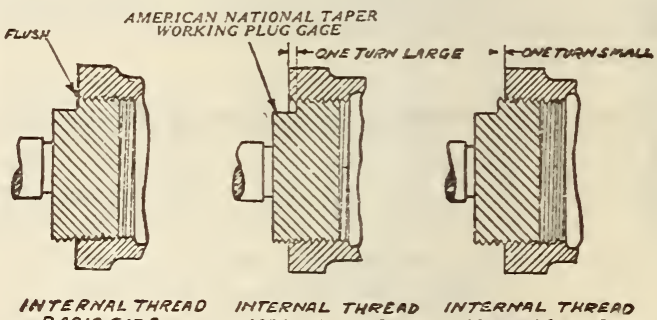

BASIC SIZE

Figure 713.-Gaging of internal American national taper pipe threads

frequently against the check gages, and in use their errors should be taken into account.

4. Working gages. - The working gages consist of one taper-threaded plug and one taper-threaded ring gage. These gages are similar in all respects to the inspection plug and ring gages. The working gages are used by the manufacturer to inspect his product. In using the working gages, the tolerance to be applied is one turn either way from the gaging notch in the case of internal threads inspected with the plug gage, and in the case of external threads the tolerance is one turn either way from the small end of the working ring gage.

\section{Gaging Practices}

1. Gaging internal threads.-The inspection and working plug gages, Figure 713 , should screw tight by hand into the fitting or coupling until the notch is flush with the face. When the thread is chamfered, the notch should be flush with the bottom of the chamfer. The fitting or coupling is within the working or net tolerance if the working gage notch is within one turn of the coupling or fitting face when screwed in tight by hand. In the same way the coupling or fitting is within the inspection or extreme tolerance if the inspection gage notch is within one and one-half turns of the coupling or fitting when screwed on tight by hand.

This method of gaging is used either for taper internal threads or for straight internally threaded couplings which screw together with taper external threads.

2. Gaging taper external threads.-The ring gage, Figure 714, should screw tight by hand on the pipe or external thread until the small end of the gage is 
flush with the end of the thread. The pipe or external thread is within the working or net tolerance if the working ring gage screws on until the end of pipe or external thread is within one turn of the small end of the gage. The pipe or external thread is within the inspection or extreme tolerance if the inspection ring screws on until the end of pipe is within one and one-half turns of the small end of the gage.

\section{Specifications for Gages}

1. Master gages.-Master gages shall be made within the closest possible limits of error. In no case errors for comparison with column 2. In Table 53 the results of errors in angle are expressed in terms of diameter. In Table 54 the results of errors in pitch are expressed in terms of diameter.

For example: A three-fourths inch, 14-thread, pipethread plug gage is reported as follows:

Pitch diameter, large end, 0.98881 inch.

Pitch diameter, small end, 0.96775 inch.

One-half included angle of thread, $29^{\circ} 58^{\prime}$.

Maximum error in lead, 0.00007 inch.

The correct pitch diameter at large end is 0.98886 inch. The error is 0.00005 inch. The correct pitch

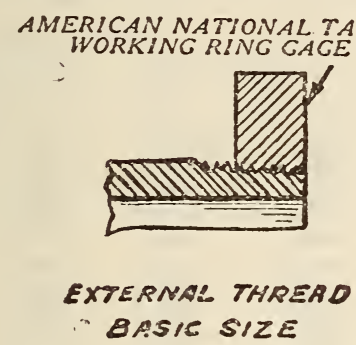

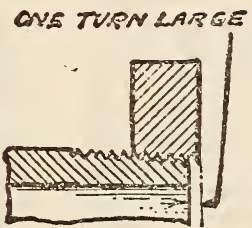

EXTERNAL THREAD MAXIMUM SIZE
ONE TURA SMALL

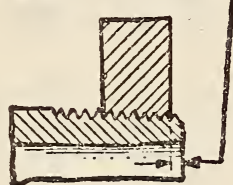

\section{EXTERAAL THREAD}

MINIMUMSIZE

FIGURE 714.-Gaging of external American national taper pipe threads

shall the accumulative error exceed the total accumulative tolerance on diameter given in Table 51 . Each master gage shall be accompanied by a report showing the error on each of the elements of thread and a statement of the accumulative error derived from the errors in the various elements. In case of question, the deviations of this gage from the basic size shall be ascertained by the Bureau of Standards at Washington, D. C.

2. Check (or reference) gages.-Column 2 of Table 51 gives the maximum allowable accumulation of

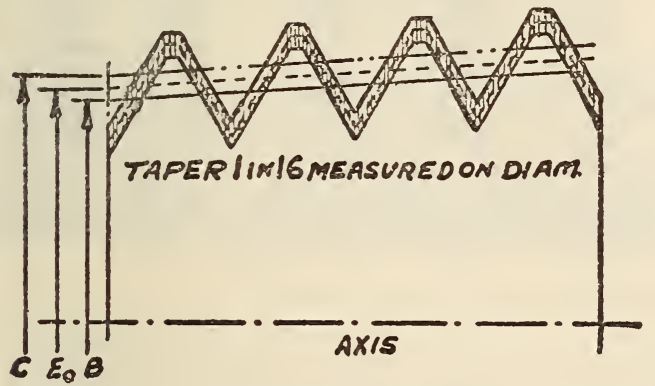

FIgURE 715.-Relation of tolerance to basic dimensions of a correct taper pipe thread gage

$E_{\circ}=$ basic pitch diameter at small end of gage.

$B=$ minimum pitch diameter at small end of gage.

$C=$ maximum pitch diameter at small end of gage

$B=E_{\text {o }}$, column 2 from Table 51 for check gages, or column 2 from Table 52 for new working gages.

$C=E_{0}+$ column 2 from Table 51 for check gages, or column 2 from $C=E_{\text {o }}+$ column 2 from Table
Table 52 for new working gages.

NOTE. - No point of the thread surface of the gage should be outside of the zone of tolerance indicated by the shaded portion of the illustration. The dotted line indicates the outline of a correct gage made exactly to the basic dimensions.

all errors in the thread surface of a check gage expressed in terms of diameter, as illustrated in Figure 715. No point on the thread surface of the gage should be outside the zone of tolerance indicated by the shaded portion of the illustration. This column is used when checking gages by measurement. If the errors in the gage are reported in terms of pitch, angle of thread, and diameter, Tables 53 and 54 may be used to determine the accumulation of these diameter at small end is 0.96768 inch. (See Table 44 , N. S. T. C. under 607.0, p. 367.)

Error of $2^{\prime}$ in angle is equivalent to 0.00005 inch; 0.00007 inch error in lead is equivalent to 0.00012 inch. (See Table 44.) The accumulative error at large end in terms of diameter $=0.00023$ inch. The accumulative error at small end equals 0.00025 inch.

The gage falls within the limits of the check gage (0.00028 inch as given in Table 51).

Column 3 of Table 51 gives the equivalent of column 2, expressed in terms of distance parallel to the axis, and represents the maximum distance which a check ring gage of correct thickness, or a check plug gage of correct length from small end to gaging notch, may vary from being flush at the gaging notch, or at the small end, when referred to basic dimensions. It is equal to sixteen times column 2 because of the basic taper of 1 in 16, measured on the diameter. This column is used when checking: check gages by comparison with a master gage. The necessary allowance must be made for the error in the master gage.

Column 4 of Table 51 gives the equivalent of column 3, expressed in terms of the decimal part of a turn. This column is also used when checking check gages by comparison with a master gage. The necessary allowance must be made for the error in the master gage.

A tolerance of plus or minus 0.0002 inch is allowed on the distance between the gaging notch and the small end of the check plug gage, or on the thickness of the check ring gage.

It is possible for check plug and ring gages, which come within all of the above tolerances, to vary from being flush with each other at the small end, or at the gaging notch, when screwed together tight by hand. The maximum variation which might occur, expressed in terms of distance, is given in column 5 of Table 51.

3. Inspection gages.-The tolerances on new inspection gages are the same as on working gages. (See Table 52.)

4. New working gages.-Column 2 of Table 52 gives the maximum allowable accumulation of all errors in the thread surface of new working gages, expressed in terms of diameter, as illustrated in Figure 715. No point in the thread surface of the gage should be outside of the zone of tolerance 
indicated by the shaded portion of the illustration. This column is used when checking gages by measurement.

Column 3 of Table 52 gives the equivalent of column 2, expressed in terms of distance parallel to the axis, and represents the maximum distance which a new working ring gage of correct thickness, or a new working plug gage of correct length from small end to gaging notch, may vary from being flush at the gaging notch, or at the small end, when referred to basic dimensions. It is equal to sixteen times column 2, because of the basic taper of 1 in 16, measured on the diameter. This column is used when checking working gages by comparison with a gage the error of which is known. The necessary allowance must be made for this error.

Column 4 of Table 52 gives the equivalent of column 3, expressed in terms of the decimal part of a turn. This column is also used when checking working gages by comparison with a gage the error of which is known. The necessary allowance must be made for this error.

A tolerance of plus or minus 0.0005 inch is allowed on the distance between the gaging notch and the small end of the working plug gage, or on the thickness of the working ring gage.

It is possible for working plug and ring gages which come within all of the above tolerances to vary from being flush with each other at the small end or at the gaging notch, when screwed together tight by hand. The maximum variation which might occur, expressed in terms of distance, is given in column 5 of Table 52 .

It is also possible for working plug and ring gages which come within all of the above tolerances to vary from being flush at the small end or at the gaging notch, when screwed tight by hand on a reference gage which comes within the tolerances specified for reference gages. The maximum variation which might occur, expressed in terms of distance, is given in column 6 of Table 52 .

5. Worn working gages.-The maximum wear on working gages must not be more than the equivalent of one-half turn from the basic dimensions.

In order that no work passed by the working gage shall be rejected by the inspection gage it will be necessary to discontinue the use of the working gage when it has worn one-half turn; that is, the working gage should always be kept within the tolerance equivalent of one turn from the basic dimensions.

TABLE 51.-Tolerances for check (or reference) gages, American national taper pipe threads

\begin{tabular}{|c|c|c|c|c|}
\hline $\begin{array}{l}\text { Nomi- } \\
\text { nal } \\
\text { sizes in } \\
\text { inches }\end{array}$ & $\begin{array}{c}\text { Total ac- } \\
\text { cumulative } \\
\text { tolerance } \\
\text { on diam- } \\
\text { eter. (See } \\
\text { Fig. 715) }\end{array}$ & $\begin{array}{l}\text { Equivalent } \\
\text { longitudi- } \\
\text { nal varia- } \\
\text { tion }(16 \times \\
\text { column } 2)\end{array}$ & $\begin{array}{c}\text { Equivalent } \\
\text { angular } \\
\text { variation } \\
\text { expressed } \\
\text { as decimal } \\
\text { part of } 1 \\
\text { turn }\end{array}$ & (1) \\
\hline 1 & 2 & 3 & 4 & 5 \\
\hline $\begin{array}{l}1 / 8 \ldots \\
1 / 4 \ldots \\
8 / 8 \ldots \\
1 / 2 \ldots \\
3 / 4 \ldots \\
\end{array}$ & $\begin{array}{l}\text { Inch } \\
0.00020 \\
.00022 \\
.00024 \\
.00026 \\
.00028\end{array}$ & $\begin{array}{l}\text { Inch } \\
0.0032 \\
.0035 \\
.0038 \\
.0042 \\
.0045\end{array}$ & $\begin{array}{l}0.086 \\
.063 \\
.068 \\
.059 \\
.063\end{array}$ & $\begin{array}{l}\text { Inch } \\
0.0068 \\
.0074 \\
.0080 \\
.0088 \\
.0094\end{array}$ \\
\hline $\begin{array}{l}1-\ldots \\
11 / 4 \ldots \\
11 / 2-\cdots \\
2-\ldots \\
21 / 2 \ldots\end{array}$ & $\begin{array}{l}.00030 \\
.00032 \\
.00034 \\
.00036 \\
.00035\end{array}$ & $\begin{array}{l}.0048 \\
.0051 \\
.0054 \\
.0058 \\
.0061\end{array}$ & $\begin{array}{l}.055 \\
.059 \\
.062 \\
.067 \\
.050\end{array}$ & $\begin{array}{l}.0100 \\
.0106 \\
.0112 \\
.0120 \\
.0126\end{array}$ \\
\hline $\begin{array}{l}3 \ldots \\
31 / 2 \ldots- \\
4 \ldots- \\
41 / 2 \ldots- \\
5 \ldots\end{array}$ & $\begin{array}{l}.00038 \\
.00041 \\
.00043 \\
.00045 \\
.00047\end{array}$ & $\begin{array}{l}.0061 \\
.0066 \\
.0069 \\
.0072 \\
.0075\end{array}$ & $\begin{array}{l}.050 \\
.053 \\
.055 \\
.058 \\
.060\end{array}$ & $\begin{array}{l}.0126 \\
.0136 \\
.0142 \\
.0148 \\
.0154\end{array}$ \\
\hline $\begin{array}{l}6 \ldots \\
7 \\
8 \\
9 \\
9 \\
10 \ldots . . .\end{array}$ & $\begin{array}{l}.00051 \\
.00055 \\
.00059 \\
.00063 \\
.00066\end{array}$ & $\begin{array}{l}.0082 \\
.0088 \\
.0094 \\
.0101 \\
.0106\end{array}$ & $\begin{array}{l}.065 \\
.070 \\
.075 \\
.080 \\
.085\end{array}$ & $\begin{array}{l}.0168 \\
.0180 \\
.0192 \\
.0206 \\
.0216\end{array}$ \\
\hline $\begin{array}{l}11 \ldots \\
12 \ldots . \\
14 \ldots- \\
15 \ldots- \\
16 \ldots-. .\end{array}$ & $\begin{array}{l}.00070 \\
.00074 \\
.00082 \\
.00086 \\
.00090\end{array}$ & $\begin{array}{l}.0112 \\
.0118 \\
.0131 \\
.0138 \\
.0144\end{array}$ & $\begin{array}{l}.090 \\
.095 \\
.105 \\
.110 \\
.115\end{array}$ & $\begin{array}{l}.0228 \\
.0240 \\
.0266 \\
.0279 \\
.0292\end{array}$ \\
\hline $\begin{array}{l}17 . . .- \\
18 \ldots- \\
20 .-- \\
22 \ldots \\
\end{array}$ & $\begin{array}{l}.00094 \\
.00093 \\
.00106 \\
.00113\end{array}$ & $\begin{array}{l}.0150 \\
.0157 \\
.0170 \\
.0181\end{array}$ & $\begin{array}{l}.120 \\
.125 \\
.135 \\
.145\end{array}$ & $\begin{array}{l}.0305 \\
.0318 \\
.0344 \\
.0366\end{array}$ \\
\hline $\begin{array}{l}24 \ldots . .- \\
26 \ldots-. .- \\
28 . . .- \\
30 \ldots-.-\end{array}$ & $\begin{array}{l}.00121 \\
.00129 \\
.00137 \\
.00144\end{array}$ & $\begin{array}{l}.0194 \\
.0206 \\
.0219 \\
.0230\end{array}$ & $\begin{array}{l}.155 \\
.165 \\
.175 \\
.185\end{array}$ & $\begin{array}{l}.0392 \\
.0416 \\
.0442 \\
.0464\end{array}$ \\
\hline
\end{tabular}

1 Maximum amount it is possible for plug and ring gages to vary from being flush at small end or at gaging notch when screwed together tight by hand ( 2 times column $3+0.0004$ inch). 
TABLE 52.-Tolerances for inspection and working gages, American national taper pipe threads

\begin{tabular}{|c|c|c|c|c|c|}
\hline $\begin{array}{l}\text { Nomi- } \\
\text { nal } \\
\text { sizes in } \\
\text { inches }\end{array}$ & $\begin{array}{l}\text { Total ac- } \\
\text { cumula- } \\
\text { tive toler- } \\
\text { ance on } \\
\text { diameter } \\
\text { (see fig. } \\
715 \text { ) }\end{array}$ & $\begin{array}{l}\text { Equiva- } \\
\text { lent lon- } \\
\text { gitudi- } \\
\text { nal vari- } \\
\text { ation } \\
(16 \times \\
\text { column } \\
2)\end{array}$ & $\begin{array}{c}\text { Equiv- } \\
\text { alent } \\
\text { angular } \\
\text { varia- } \\
\text { tion ex- } \\
\text { pressed } \\
\text { as a } \\
\text { decimal } \\
\text { part of } \\
1 \text { turn }\end{array}$ & (1) & (2) \\
\hline 1 & 2 & 3 & 4 & 5 & 6 \\
\hline $\begin{array}{l}1 / 8 \\
1 / 4 \\
3 / 8 \\
1 / 2 \\
3 / 4\end{array}$ & $\begin{array}{c}\text { Inch } \\
0.00040 \\
.00044 \\
.00048 \\
.00052 \\
.00056\end{array}$ & $\begin{array}{r}\text { Inch } \\
0.0064 \\
.0070 \\
.0077 \\
.0083 \\
.0090\end{array}$ & $\begin{array}{r}0.172 \\
.126 \\
.136 \\
.118 \\
.126\end{array}$ & $\begin{array}{r}\text { Inch } \\
0.0138 \\
.0150 \\
.0164 \\
.0176 \\
.0190\end{array}$ & $\begin{array}{r}\text { Inch } \\
0.0103 \\
.0112 \\
.0122 \\
.0132 \\
.0142\end{array}$ \\
\hline $\begin{array}{l}1 \\
11 / 4 \\
11 / 2 \\
2 \\
21 / 2\end{array}$ & $\begin{array}{l}.00060 \\
.00064 \\
.00068 \\
.00072 \\
.00076\end{array}$ & $\begin{array}{l}.0096 \\
.0102 \\
.0109 \\
.0115 \\
.0122\end{array}$ & $\begin{array}{l}.110 \\
.118 \\
.124 \\
.134 \\
.100\end{array}$ & $\begin{array}{l}.0202 \\
.0214 \\
.0228 \\
.0240 \\
.0254\end{array}$ & $\begin{array}{l}.0151 \\
.0160 \\
.0170 \\
.0180 \\
.0190\end{array}$ \\
\hline $\begin{array}{l}3 \\
31 / 2 \\
4 \\
41 / 2 \\
5\end{array}$ & $\begin{array}{l}.00076 \\
.00082 \\
.00086 \\
.00090 \\
.00094\end{array}$ & $\begin{array}{l}.0122 \\
.0131 \\
.0138 \\
.0144 \\
.0150\end{array}$ & $\begin{array}{l}.100 \\
.105 \\
.110 \\
.115 \\
.120\end{array}$ & $\begin{array}{l}.0254 \\
.0272 \\
.0286 \\
.0298 \\
.0310\end{array}$ & $\begin{array}{l}.0190 \\
.0204 \\
.0214 \\
.0223 \\
.0232\end{array}$ \\
\hline $\begin{array}{r}6 \\
7 \\
8 \\
9 \\
10\end{array}$ & $\begin{array}{l}.00102 \\
.00110 \\
.00118 \\
.00126 \\
.00132\end{array}$ & $\begin{array}{l}.0163 \\
.0176 \\
.0189 \\
.0202 \\
.0211\end{array}$ & $\begin{array}{l}.130 \\
.140 \\
.150 \\
.160 \\
.170\end{array}$ & $\begin{array}{l}.0336 \\
.0362 \\
.0388 \\
.0414 \\
.0432\end{array}$ & $\begin{array}{l}.0252 \\
.0271 \\
.0290 \\
.0310 \\
.0324\end{array}$ \\
\hline $\begin{array}{l}11 \\
12 \\
14 \\
15 \\
16\end{array}$ & $\begin{array}{l}.00140 \\
.00148 \\
.00164 \\
.00172 \\
.00180\end{array}$ & $\begin{array}{l}.0224 \\
.0237 \\
.0262 \\
.0275 \\
.0288\end{array}$ & $\begin{array}{l}.180 \\
.190 \\
.210 \\
.220 \\
.230\end{array}$ & $\begin{array}{l}.0458 \\
.0484 \\
.0534 \\
.0560 \\
.0586\end{array}$ & $\begin{array}{l}.0343 \\
.0362 \\
.0400 \\
.0420 \\
.0439\end{array}$ \\
\hline $\begin{array}{l}17 \\
18 \\
20 \\
22\end{array}$ & $\begin{array}{l}.00188 \\
.00196 \\
.00212 \\
.00226\end{array}$ & $\begin{array}{l}.0301 \\
.0314 \\
.0339 \\
.0362\end{array}$ & $\begin{array}{l}.240 \\
.250 \\
.270 \\
.290\end{array}$ & $\begin{array}{l}.0611 \\
.0638 \\
.0688 \\
.0734\end{array}$ & $\begin{array}{l}.0458 \\
.0478 \\
.0516 \\
.0550\end{array}$ \\
\hline $\begin{array}{l}24 \\
26 \\
28 \\
30\end{array}$ & $\begin{array}{l}.00242 \\
.00258 \\
.00274 \\
.00288\end{array}$ & $\begin{array}{l}.0387 \\
.0413 \\
.0438 \\
.0461\end{array}$ & $\begin{array}{l}.310 \\
.330 \\
.350 \\
.370\end{array}$ & $\begin{array}{l}.0784 \\
.0836 \\
.0886 \\
.0932\end{array}$ & $\begin{array}{l}.0588 \\
.0626 \\
.0664 \\
.0698\end{array}$ \\
\hline
\end{tabular}

I Maximum amount it is possible for new working plug and ring gages which come within the specified tolerances to vary from being flush at the small end or at the gaging notch when screwed together tight by hand ( 2 times column $3+0.0010$ inch).

2 Maximum amount it is possible for new working plug or ring gages which come within specified tolerances to vary from being flush at the small end or at the gaging notch when screwed on reference gage tight by hand. $\left\{\frac{\text { Column } 5 \text {, Table } 51+\text { column } 5 \text {, Table } 52}{2}\right\}$
TABLE 53.-Corrections in diameter for errors in half angle, American national taper pipe thread gages

\begin{tabular}{|c|c|c|c|c|c|}
\hline \multirow{2}{*}{$\begin{array}{l}\text { Error } \\
\text { in half } \\
\text { angle of } \\
\text { thread } \\
\text { in min- } \\
\text { utes, } a^{\prime}\end{array}$} & \multicolumn{5}{|c|}{ Correction in diameter, $E^{\prime \prime}$} \\
\hline & $\begin{array}{c}8 \\
\text { threads } \\
\text { per inch }\end{array}$ & $\begin{array}{c}11 \frac{1}{2} \\
\text { threads } \\
\text { per inch }\end{array}$ & $\begin{array}{c}14 \\
\text { threads } \\
\text { per inch }\end{array}$ & $\begin{array}{c}18 \\
\text { threads } \\
\text { per inch }\end{array}$ & $\begin{array}{c}27 \\
\text { threads } \\
\text { per inch }\end{array}$ \\
\hline 1 & 2 & 3 & 4 & 5 & 6 \\
\hline $\begin{array}{l}1 \\
2 \\
3 \\
4 \\
5\end{array}$ & $\begin{array}{c}\text { Inch } \\
0.00006 \\
.00011 \\
.00017 \\
.00022 \\
.00028\end{array}$ & $\begin{array}{c}\text { Inch } \\
0.00004 \\
.00008 \\
.00012 \\
.00016 \\
.00019\end{array}$ & $\begin{array}{c}\text { Inch } \\
0.00003 \\
.00006 \\
.00010 \\
.00013 \\
.00016\end{array}$ & $\begin{array}{c}\text { Inch } \\
0.00002 \\
.00005 \\
.00007 \\
.00010 \\
.00012\end{array}$ & $\begin{array}{c}\text { Inch } \\
0.00002 \\
.00003 \\
.00005 \\
.00007 \\
.00008\end{array}$ \\
\hline $\begin{array}{r}6 \\
7 \\
8 \\
9 \\
10\end{array}$ & $\begin{array}{r}.00034 \\
.00039 \\
.00045 \\
.00050 \\
.00056\end{array}$ & $\begin{array}{l}.00023 \\
.00027 \\
.00031 \\
.00035 \\
.00039\end{array}$ & $\begin{array}{l}.00019 \\
.00022 \\
.00026 \\
.00029 \\
.00032\end{array}$ & $\begin{array}{l}.00015 \\
.00017 \\
.00020 \\
.00022 \\
.00025\end{array}$ & $\begin{array}{l}.00010 \\
.00012 \\
.00013 \\
.00015 \\
.00017\end{array}$ \\
\hline $\begin{array}{l}11 \\
12 \\
13 \\
14 \\
15\end{array}$ & $\begin{array}{l}.00062 \\
.00067 \\
.00073 \\
.00078 \\
.00084\end{array}$ & $\begin{array}{l}.00043 \\
.00047 \\
.00051 \\
.00054 \\
.00058\end{array}$ & $\begin{array}{l}.00035 \\
.00038 \\
.00042 \\
.00045 \\
.00048\end{array}$ & $\begin{array}{l}.00027 \\
.00030 \\
.00032 \\
.00035 \\
.00037\end{array}$ & $\begin{array}{l}.00018 \\
.00020 \\
.00022 \\
.00023 \\
.00025\end{array}$ \\
\hline $\begin{array}{l}16 \\
17 \\
18 \\
19 \\
20\end{array}$ & $\begin{array}{l}.00089 \\
.00095 \\
.00101 \\
.00106 \\
.00112\end{array}$ & $\begin{array}{l}.00062 \\
.00066 \\
.00070 \\
.00074 \\
.00078\end{array}$ & $\begin{array}{l}.00051 \\
.00054 \\
.00058 \\
.00061 \\
.00064\end{array}$ & $\begin{array}{l}.00040 \\
.00042 \\
.00045 \\
.00047 \\
.00050\end{array}$ & $\begin{array}{l}.00027 \\
.00028 \\
.00030 \\
.00031 \\
.00033\end{array}$ \\
\hline $\begin{array}{l}21 \\
22 \\
23 \\
24 \\
25\end{array}$ & $\begin{array}{l}.00117 \\
.00123 \\
.00129 \\
.00134 \\
.00140\end{array}$ & $\begin{array}{l}.00082 \\
.00086 \\
.00089 \\
.00093 \\
.00097\end{array}$ & $\begin{array}{l}.00067 \\
.00070 \\
.00074 \\
.00077 \\
.00080\end{array}$ & $\begin{array}{l}.00052 \\
.00055 \\
.00057 \\
.00060 \\
.00062\end{array}$ & $\begin{array}{r}.00035 \\
.00036 \\
.00038 \\
.00040 \\
.00041\end{array}$ \\
\hline $\begin{array}{l}26 \\
27 \\
28 \\
29 \\
30\end{array}$ & $\begin{array}{l}.00145 \\
.00151 \\
.00157 \\
.00162 \\
.00168\end{array}$ & $\begin{array}{l}.00101 \\
.00105 \\
.00109 \\
.00113 \\
.00117\end{array}$ & $\begin{array}{l}.00083 \\
.00086 \\
.00089 \\
.00093 \\
.00096\end{array}$ & $\begin{array}{l}.00065 \\
.00067 \\
.00070 \\
.00072 \\
.00075\end{array}$ & $\begin{array}{l}.00043 \\
.00045 \\
.00046 \\
.00048 \\
.00050\end{array}$ \\
\hline $\begin{array}{l}45 \\
60\end{array}$ & $\begin{array}{l}.00252 \\
.00336\end{array}$ & $\begin{array}{l}.00175 \\
.00233\end{array}$ & $\begin{array}{l}.00144 \\
.00192\end{array}$ & $\begin{array}{l}.00112 \\
.00149\end{array}$ & $\begin{array}{l}.00075 \\
.00099\end{array}$ \\
\hline
\end{tabular}

$a^{\prime}=$ error in half included angle of thread. $E^{\prime \prime}=$ correction in diameter.

$E^{\prime \prime}=\frac{1.53812}{n} \times \tan a^{\prime}$

TABLE 54.-Corrections in diameter for errors in lead, $60^{\circ}$ threads

\begin{tabular}{|c|c|c|c|c|c|c|c|c|c|c|}
\hline \multirow{2}{*}{$\begin{array}{l}\text { Error in } \\
\text { lead in } \\
\text { inches, } p^{\prime}\end{array}$} & \multicolumn{10}{|c|}{ Correction in diameter, $E^{\prime}$} \\
\hline & 0.00000 & 0.00001 & 0.00002 & 0.00003 & 0.00004 & 0.00005 & 0.00006 & 0.00007 & 0.00008 & 0.00009 \\
\hline 1 & 2 & 3 & 4 & 5 & 6 & $\begin{array}{llllllll} & & & & & \end{array}$ & 8 & $\mathbf{9}$ & 10 & 11 \\
\hline $\begin{array}{c}\text { Inch } \\
0.00000 \\
.00010 \\
.00020 \\
.00030 \\
.00040 \\
.00050\end{array}$ & $\begin{array}{c}\text { Inch } \\
0.00000 \\
.00017 \\
.00035 \\
.00052 \\
.00069 \\
.00087\end{array}$ & $\begin{array}{c}\text { Inch } \\
0.00002 \\
.00019 \\
.00036 \\
.00054 \\
.00071 \\
.00088\end{array}$ & $\begin{array}{c}\text { Inch } \\
0.00003 \\
.00021 \\
.00038 \\
.00055 \\
.00073 \\
.00090\end{array}$ & $\begin{array}{c}\text { Inch } \\
0.00005 \\
.00023 \\
.00040 \\
.00057 \\
.00074 \\
.00092\end{array}$ & $\begin{array}{c}\text { Inch } \\
0.00007 \\
.00024 \\
.00042 \\
.00059 \\
.00076 \\
.00094\end{array}$ & $\begin{array}{c}\text { Inch } \\
0.00009 \\
.00026 \\
.00043 \\
.00061 \\
.00078 \\
.00095\end{array}$ & $\begin{array}{c}\text { Inch } \\
0.00010 \\
.00028 \\
.00045 \\
.00062 \\
.00080 \\
.00097\end{array}$ & $\begin{array}{c}\text { Inch } \\
0.00012 \\
.00029 \\
.00047 \\
.00064 \\
.00081 \\
.00099\end{array}$ & $\begin{array}{c}\text { Inch } \\
0.00014 \\
.00031 \\
.00048 \\
.00066 \\
.00083 \\
.00100\end{array}$ & $\begin{array}{c}\text { Inch } \\
0.00016 \\
.00033 \\
.00050 \\
.00068 \\
.00085 \\
.00102\end{array}$ \\
\hline $\begin{array}{l}.00080 \\
.00070\end{array}$ & $\begin{array}{l}.00104 \\
.00121 \\
.00139\end{array}$ & $\begin{array}{r}.00106 \\
.00123 \\
.00140\end{array}$ & & $\begin{array}{l}.00109 \\
.000126 \\
.00144\end{array}$ & $\begin{array}{l}.00111 \\
.00128 \\
.00145\end{array}$ & $\begin{array}{l}.00113 \\
.00130 \\
.00147\end{array}$ & & $\begin{array}{r}.00116 \\
.00133 \\
.00151\end{array}$ & & $\begin{array}{l}.00120 \\
.00137 \\
.00154\end{array}$ \\
\hline $\begin{array}{l}.00090 \\
.00100\end{array}$ & $\begin{array}{l}.00156 \\
00173\end{array}$ & $\begin{array}{r}.00158 \\
.00175\end{array}$ & $\begin{array}{r}.00159 \\
00177\end{array}$ & .00161 & $\begin{array}{r}.00163 \\
.00180\end{array}$ & $\begin{array}{r}.00165 \\
.0012\end{array}$ & $\begin{array}{l}.00166 \\
.00184\end{array}$ & $\begin{array}{l}.00168 \\
.00185\end{array}$ & .00170 & $\begin{array}{r}.00171 \\
00189\end{array}$ \\
\hline .0 & .001 & .00 & .00 & & .00 & .00 & & & & .00206 \\
\hline .0 & & & & & & & & & & \\
\hline .001 & .00 & .00 & & & & & & & & .00258 \\
\hline .00150 & . 00260 & .00262 & .00263 & .00265 & .0026 & .00268 & .002 & .00272 & .00274 & .00275 \\
\hline .00160 & .00277 & .0027 & .00281 & .00282 & .002 & .00286 & .00288 & .00289 & 00291 & .00293 \\
\hline .00 & .003 & .00 & .00 & .06 & .00 & .00 & & .00 & & .00327 \\
\hline .0000 & . & .00331 & - & 0 & & & & & & 00345 \\
\hline .00200 & . 000346. & .00348 & 年. & .00352 & .00353 & .00355 & .00357 & .00359 & .00360 & .00362 \\
\hline
\end{tabular}


TABLE 55.-Basic dimensions of threaded plug and ring gages for American national taper pipe threads

\begin{tabular}{|c|c|c|c|c|c|c|c|c|c|c|c|c|c|c|}
\hline \multirow{3}{*}{$\begin{array}{c}\text { Nomi- } \\
\text { nal size } \\
\text { of pipe } \\
\text { in } \\
\text { inches }\end{array}$} & \multirow{3}{*}{$\begin{array}{c}\text { Num- } \\
\text { ber of } \\
\text { threads } \\
\text { per } \\
\text { inch, } \\
n\end{array}$} & \multirow{3}{*}{$\begin{array}{l}\text { Pitch, } \\
\quad p\end{array}$} & \multicolumn{3}{|c|}{$\begin{array}{l}\text { Major diameters of plug } \\
\text { gages } 1\end{array}$} & \multicolumn{3}{|c|}{$\begin{array}{l}\text { Pitch diameters of plug } \\
\text { and ring gages }\end{array}$} & \multicolumn{3}{|c|}{$\begin{array}{c}\text { Minor diameter of ring } \\
\text { gages } 1\end{array}$} & \multirow{3}{*}{$\begin{array}{l}\text { Increase } \\
\text { in diam- } \\
\text { eter per } \\
\text { thread, } \\
0.0625 \\
n\end{array}$} & \multirow{3}{*}{$\begin{array}{l}\text { Thick- } \\
\text { ness of } \\
\text { thin } \\
\text { ring, } \\
L_{1}\end{array}$} & \multirow{3}{*}{$\begin{array}{l}\text { Thick- } \\
\text { ness of } \\
\text { full } \\
\text { ring, } \\
L_{2}\end{array}$} \\
\hline & & & $\begin{array}{c}\text { At small } \\
\text { end, } \\
E_{0}+\end{array}$ & $\begin{array}{l}\text { At gag- } \\
\text { ing } \\
\text { notch, }\end{array}$ & $\begin{array}{l}\text { At large } \\
\text { end, full } \\
\text { ring, }\end{array}$ & & $\begin{array}{c}\text { At gag- } \\
\text { ing }\end{array}$ & $\begin{array}{l}\text { At large } \\
\text { end, full }\end{array}$ & $\begin{array}{c}\text { At small } \\
\text { end, } \\
E_{0}-\end{array}$ & $\begin{array}{l}\text { At gag- } \\
\text { ing } \\
\text { notch, }\end{array}$ & $\begin{array}{c}\text { At large } \\
\text { end, full } \\
\text { ring, }\end{array}$ & & & \\
\hline & & & $\frac{660}{n}$ & $\frac{0.666025}{n}$ & $\frac{0.666025}{n}$ & & $\pi$ & III, & 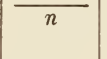 & $\frac{0.666025}{n}$ & $\frac{0.666025}{n}$ & & & \\
\hline 1 & 2 & 3 & 4 & 5 & 6 & 7 & 8 & 9 & 10 & 11 & 12 & 13 & 14 & 15 \\
\hline $\begin{array}{l}1 / 8 \\
1 / 4 \\
3 \\
1 / 2 \\
3 / 4\end{array}$ & $\begin{array}{l}27 \\
18 \\
18 \\
14 \\
14\end{array}$ & $\begin{array}{c}\text { Inch } \\
0.03704 \\
.05556 \\
.05556 \\
.07143 \\
.07143\end{array}$ & $\begin{array}{c}\text { Inches } \\
0.38818 \\
.51439 \\
.64902 \\
.80600 \\
1.01525\end{array}$ & $\begin{array}{c}\text { Inches } \\
0.39943 \\
.52689 \\
.66402 \\
.82600 \\
1.03644\end{array}$ & $\begin{array}{c}\text { Inches } \\
0.40467 \\
.53950 \\
.67450 \\
.83936 \\
1.04936\end{array}$ & $\begin{array}{c}\text { Inches } \\
0.36351 \\
.47739 \\
.61201 \\
.75843 \\
.96768\end{array}$ & $\begin{array}{l}\text { Inches } \\
0.37476 \\
.48989 \\
.62701 \\
.77843 \\
.98887\end{array}$ & $\begin{array}{c}\text { Inches } \\
0.38000 \\
.50250 \\
.63750 \\
.79179 \\
1.00179\end{array}$ & $\begin{array}{c}\text { 1nches } \\
0.33884 \\
.44039 \\
.57501 \\
.71086 \\
.92011\end{array}$ & $\begin{array}{c}\text { Inches } \\
0.35009 \\
.45289 \\
.59001 \\
.73086 \\
.94129\end{array}$ & $\begin{array}{c}\text { Inches } \\
0.35533 \\
.46550 \\
.60050 \\
.74421 \\
.95421\end{array}$ & $\begin{array}{c}\text { Inch } \\
0.00231 \\
.00347 \\
.00347 \\
.00446 \\
.00446\end{array}$ & $\begin{array}{r}\text { Inches } \\
0.180 \\
.200 \\
.240 \\
.320 \\
.339\end{array}$ & $\begin{array}{l}\text { Inches } \\
0.26385 \\
.40178 \\
.40778 \\
.53371 \\
.54571\end{array}$ \\
\hline $\begin{array}{l}1 \\
11 / 4 \\
11 / 2 \\
2 \\
21 / 2\end{array}$ & $\begin{array}{c}111 / 2 \\
111 / 2 \\
111 / 2 \\
111 / 2 \\
8\end{array}$ & $\begin{array}{l}.08696 \\
.08696 \\
.08696 \\
.08696 \\
.12500\end{array}$ & $\begin{array}{l}1.27155 \\
1.61505 \\
1.85400 \\
2.32694 \\
2.80278\end{array}$ & $\begin{array}{l}1.29655 \\
1.64130 \\
1.88025 \\
2.35419 \\
2.84541\end{array}$ & $\begin{array}{l}1.31422 \\
1.65922 \\
1.89922 \\
2.37422 \\
2.87388\end{array}$ & $\begin{array}{l}1.21363 \\
1.55713 \\
1.79609 \\
2.26902 \\
2.71953\end{array}$ & $\begin{array}{l}1.23863 \\
1.58338 \\
1.82234 \\
2.29627 \\
2.76216\end{array}$ & & & & & & $\begin{array}{l}.400 \\
.420 \\
.420 \\
.436 \\
.682\end{array}$ & .6827 \\
\hline $\begin{array}{l}3 \\
31 / 2 \\
4 \\
41 / 2 \\
5\end{array}$ & $\begin{array}{l}8 \\
8 \\
8 \\
8 \\
8 \\
8\end{array}$ & $\begin{array}{l}.12500 \\
.12500 \\
.12500 \\
.12500 \\
.12500\end{array}$ & $\begin{array}{l}\text { 3. } 42388 \\
3.92075 \\
4.41763 \\
4.91450 \\
5.47398\end{array}$ & $\begin{array}{l}\text { 3. } 47175 \\
\text { 3. } 97207 \\
4.47038 \\
\text { 4. } 96919 \\
5.53255\end{array}$ & $\begin{array}{l}\text { 3. } 49888 \\
\text { 3. } 99888 \\
4.49888 \\
\text { 4. } 99888 \\
\text { 5. } 56188\end{array}$ & $\begin{array}{l}\text { 3. } 34062 \\
\text { 3. } 33750 \\
\text { 4. } 33438 \\
\text { 4. } 83125 \\
\text { 5. } 39073\end{array}$ & $\begin{array}{l}\text { 3. } 38850 \\
\text { 3. } 88881 \\
4.38712 \\
4.88594 \\
5.44929\end{array}$ & $\begin{array}{l}3.41562 \\
3.91562 \\
4.41562 \\
4.91562 \\
5.47862\end{array}$ & $\begin{array}{l}3.25737 \\
3.75425 \\
4.25112 \\
4.74800 \\
5.30748\end{array}$ & $\begin{array}{l}\text { 3. } 30525 \\
3.80556 \\
4.30387 \\
4.80268 \\
5.36604\end{array}$ & & $\begin{array}{l}.00781 \\
.00781 \\
.00781 \\
.00781 \\
.00781\end{array}$ & $\begin{array}{l}.866 \\
.821 \\
.844 \\
.875 \\
.937\end{array}$ & \\
\hline $\begin{array}{r}6 \\
7 \\
8 \\
9 \\
10\end{array}$ & $\begin{array}{l}8 \\
8 \\
8 \\
8 \\
8\end{array}$ & $\begin{array}{l}.12500 \\
.12500 \\
.12500 \\
.12500 \\
.12500\end{array}$ & $\begin{array}{r}6.52935 \\
7.52310 \\
8.51685 \\
9.51060 \\
10.62857\end{array}$ & $\begin{array}{r}6.58922 \\
7.58560 \\
8.58328 \\
9.58122 \\
10.70419\end{array}$ & $\begin{array}{r}6.62388 \\
7.62388 \\
8.62388 \\
9.62388 \\
10.74888\end{array}$ & $\begin{array}{r}6.44609 \\
7.43984 \\
8.43359 \\
9.42734 \\
10.54531\end{array}$ & $\begin{array}{r}6.50597 \\
7.50234 \\
8.50003 \\
9.49797 \\
10.62094\end{array}$ & $\begin{array}{r}6.54062 \\
7.54062 \\
8.54062 \\
9.54062 \\
10.66562\end{array}$ & $\begin{array}{r}6.36284 \\
7.35659 \\
8.35034 \\
9.34409 \\
10.46206\end{array}$ & $\begin{array}{r}6.42272 \\
7.41909 \\
8.41678 \\
9.41472 \\
10.53768\end{array}$ & $\begin{array}{r}6.45737 \\
7.45737 \\
8.45737 \\
9.45737 \\
10.53237\end{array}$ & $\begin{array}{l}.00781 \\
.00781 \\
.00781 \\
.00781 \\
.00781\end{array}$ & $\begin{array}{l}.958 \\
1.000 \\
1.063 \\
1.130 \\
1.210\end{array}$ & 1.9250 \\
\hline $\begin{array}{l}11 \\
12 \\
214 \\
215 \\
216 \\
217 \\
218 \\
220 \\
222\end{array}$ & $\begin{array}{l}8 \\
8 \\
8 \\
8 \\
8 \\
8 \\
8 \\
8 \\
8 \\
8\end{array}$ & $\begin{array}{l}.12500 \\
.12500 \\
.12500 \\
.12500 \\
.12500 \\
.12500 \\
.12500 \\
.12500 \\
.12500\end{array}$ & \begin{tabular}{|l|}
11.62232 \\
12,61607 \\
13.85825 \\
14.85200 \\
15.84575 \\
16.83950 \\
17.83325 \\
19.82075 \\
21.80825
\end{tabular} & $\begin{array}{l}11.70263 \\
12,70107 \\
13.95588 \\
14.95744 \\
15.95900 \\
16.95825 \\
17.95825 \\
19.95357 \\
21.94888\end{array}$ & $\begin{array}{l}11.74888 \\
12,74888 \\
13.99888 \\
14.99888 \\
15.99888 \\
16.99888 \\
17.99888 \\
19.99888 \\
21.99888\end{array}$ & $\begin{array}{l}11.53906 \\
12,53281 \\
13.77500 \\
14.76875 \\
15.76250 \\
16.75625 \\
17.75000 \\
19.73750 \\
21.72500\end{array}$ & $\begin{array}{l}11.61938 \\
12,61781 \\
13.87262 \\
14.87419 \\
15.87575 \\
16.87500 \\
17.87500 \\
19.87031 \\
21.86562\end{array}$ & $\begin{array}{l}11.66562 \\
12,66562 \\
13.91562 \\
14.91562 \\
15.91562 \\
16.91562 \\
17.91562 \\
19.91562 \\
21.91562\end{array}$ & $\begin{array}{l}11.45531 \\
12,41956 \\
13.69175 \\
14.68550 \\
15.67925 \\
16.67300 \\
17.66675 \\
19.65425 \\
21.64175\end{array}$ & $\begin{array}{l}11.53612 \\
12,53456 \\
13.78937 \\
14.79093 \\
15.79250 \\
16.79175 \\
17.79175 \\
19.78706 \\
21.78237\end{array}$ & $\begin{array}{l}11.58237 \\
12,58237 \\
13.83237 \\
14.83237 \\
15.83237 \\
16.83237 \\
17.83237 \\
19.83237 \\
21.83237\end{array}$ & $\begin{array}{l}.00781 \\
.00781 \\
.00781 \\
.00781 \\
.00781 \\
.00781 \\
.00781 \\
.00781 \\
.00781\end{array}$ & $\begin{array}{l}1.285 \\
1.360 \\
1.562 \\
1.687 \\
1.812 \\
1.900 \\
2.000 \\
2.125 \\
2.250\end{array}$ & 3. 0500 \\
\hline $\begin{array}{l}324 \\
226 \\
228 \\
230\end{array}$ & $\begin{array}{l}8 \\
8 \\
8 \\
8\end{array}$ & $\begin{array}{r}.12500 \\
.12500 \\
.12500 \\
.12500\end{array}$ & $\begin{array}{l}23.79575 \\
25.78325 \\
27.77075 \\
29.75825\end{array}$ & $\begin{array}{l}23.94419 \\
25.93950 \\
27.93482 \\
29.93013\end{array}$ & $\begin{array}{l}23.99888 \\
25.99888 \\
27.99888 \\
29.99888\end{array}$ & $\begin{array}{l}23.71250 \\
25.70000 \\
27.68750 \\
29.67500\end{array}$ & $\begin{array}{l}23.86094 \\
25.85625 \\
27.85156 \\
29.84688\end{array}$ & $\begin{array}{l}23.91562 \\
25.91562 \\
27.91562 \\
29.91562\end{array}$ & $\begin{array}{l}23.62925 \\
25.61675 \\
27.60425 \\
29.59175\end{array}$ & $\begin{array}{l}23.77768 \\
25.77300 \\
27.76831 \\
29.76362\end{array}$ & $\begin{array}{l}23.83237 \\
25.83237 \\
27.83237 \\
29.83237\end{array}$ & $\begin{array}{l}.00781 \\
.00781 \\
.00781 \\
.00781\end{array}$ & $\begin{array}{l}2.375 \\
2.500 \\
2.625 \\
2.750\end{array}$ & 3. 85000 \\
\hline
\end{tabular}

1 These dimensions are based on a crest truncation of $0.1 p$ for pipe thread gages, which insures bearing of the gage on the sides of the thread, when cut with a slightly dull tool, instead of at the roots of the thread.

2 Outside diameter.

\section{GAGES FOR ACME THREADS}

\section{Limiting Dimensions}

Tables 82,83 , and 84 given herein are for the purpose of establishing definite limits for gages used in the inspection of Acme threads, rather than for the purpose of specifying the gages required for the various inspection operations. The dimensions as given are in accordance with the principles: (a) That the "go" gage should check simultaneously as many elements as possible and a "not go"gage can effectively check but one element; and (b), that permissible variations in the gages be within the extreme product limits.

\section{Tolerances on Lead}

The tolerances on lead given in Table 82 are specified as an allowable variation between any two threads not farther apart than 12 inches.

\section{Tolerances on Angles of Thread}

The tolerances on angle of thread, as specified in Table 82 for the various pitches, are tolerances on one-half of the included angle. This insures that the bisector of the included angle will be perpendicular to the axis of the thread within proper limits. The equivalent deviation from the true thread form caused by such irregularities as convex or concave sides of thread, or slight projections on the thread form, should not exceed the tolerances permitted on angle of thread.

\section{Fillets at Minor Diameter}

"Go" threaded plug gages for nuts have fillets at the minor diameter, the radii of which are not less than 0.010 inch for pitches of three threads per inch and finer, and not less than 0.020 inch for pitches coarser than three threads per inch. 
TABLE 82.-Tolerances for "go" and "not go" thread gages, American national acme threads

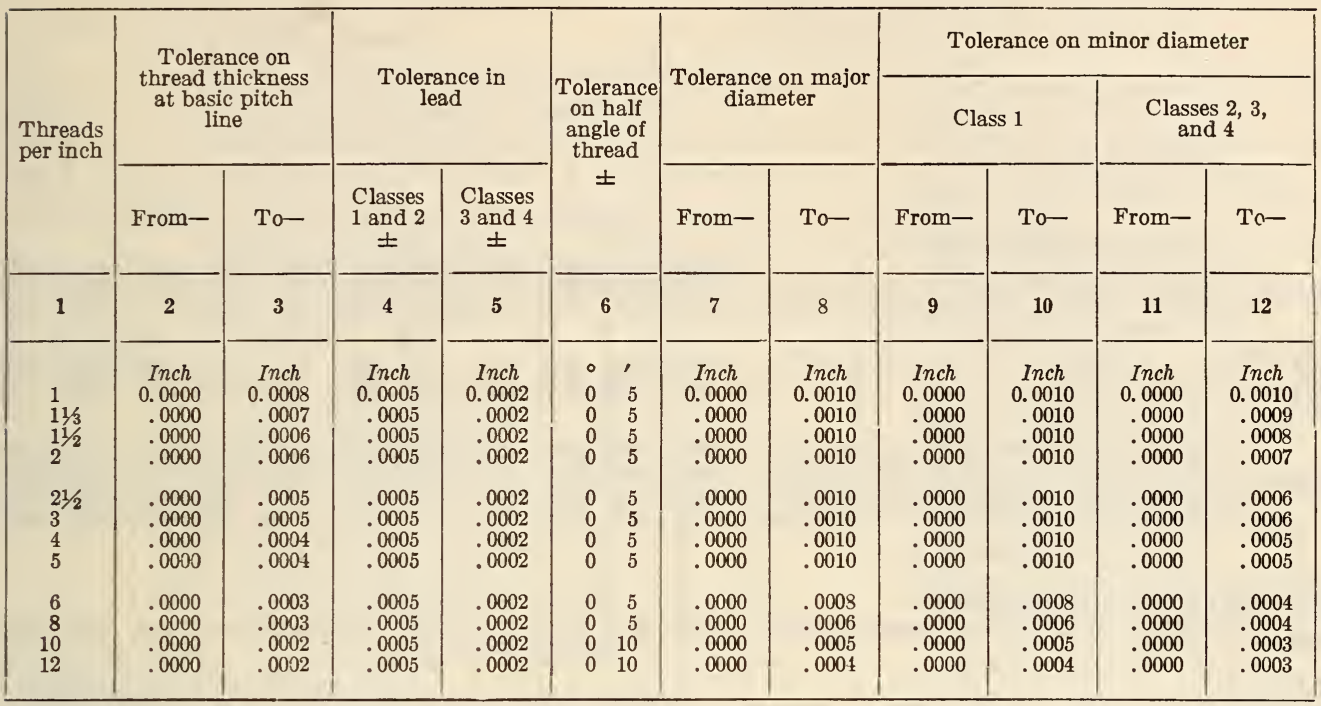

TABLE 83.-Limiting dimensions of gages for screws, classes 1, 2, 3, and 4 acme

Threads per inch

\begin{tabular}{c|c|c|c|c|c|c|c|c|c|c|c}
\hline 1 & $11 / 3$ & $11 / 2$ & 2 & $2 \frac{11 / 2}{3}$ & 3 & 4 & 5 & 6 & 8 & 10 & 12 \\
\hline 2 & 3 & 4 & 5 & 6 & 7 & 8 & 9 & 10 & 11 & 12 & 13 \\
\hline Inches & Inch & Inch & Inch & Inch & Inch & Inch & Inch & Inch & Inch & Inch & Inch
\end{tabular}

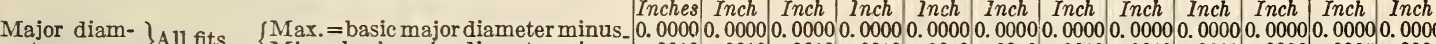

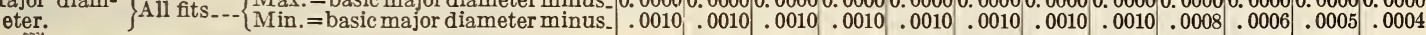

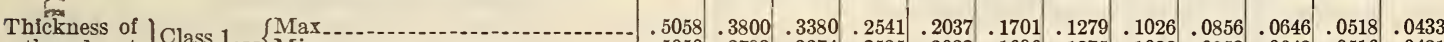
thread at Class 1.--\{ Min Min

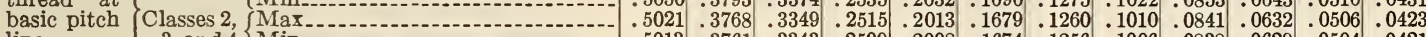

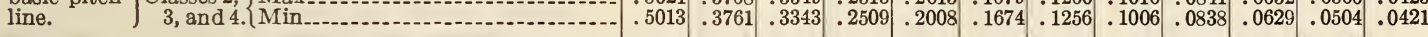

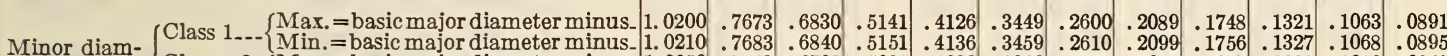

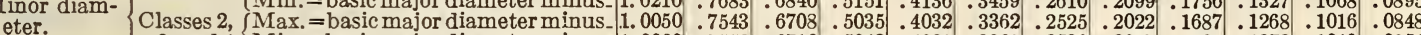

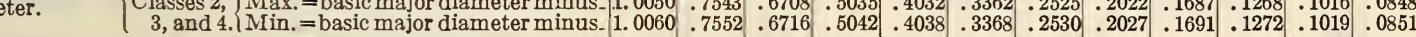
"NOT GO" GAGES FOR SCREWS

Threaded ring or snap gages for thread thickness only:

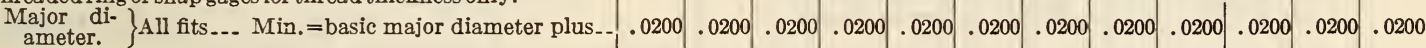

Thickness

$\left.\begin{array}{l}\text { of thread } \\ \text { at basic }\end{array}\right\}$ All fits..--\{Max.=pitch minus

pitch line.

Minimum thickness of thread of the screw at the basic pitch line

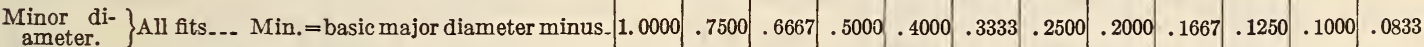
ameter.

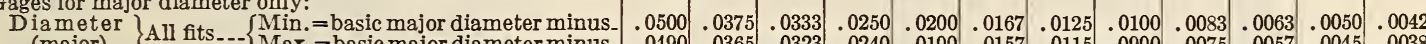

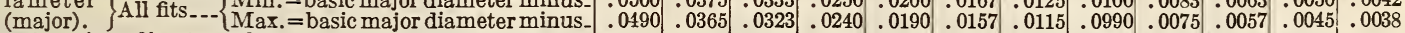

Gages for minor diamter only:

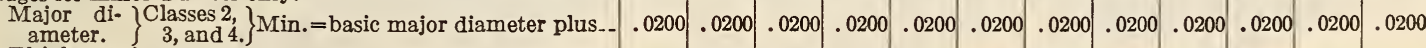

Thickness

of thread Classes 2, Max at basic $\{3$, and 4 .

pitch line.

$\begin{array}{lllllllll}.5000 .3750 .3333 .2500 .2000 .1667 .1250 & .1000 .0833 .0625 & .0500 & .0417\end{array}$

Minor di- $\{$ Classes 2, SMin.=basic major diameter minus 1.0150 .7630 .6790 .5106 .4095 .3420 .2575 . 2067.1728 .1303 .1048 .0877

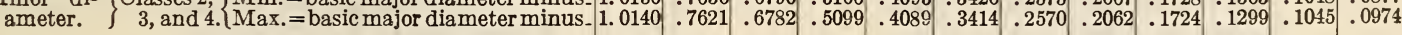


TABLE 84.-Limiting dimensions of gages for nuts, classes 1, 2, 3, and 4 acme

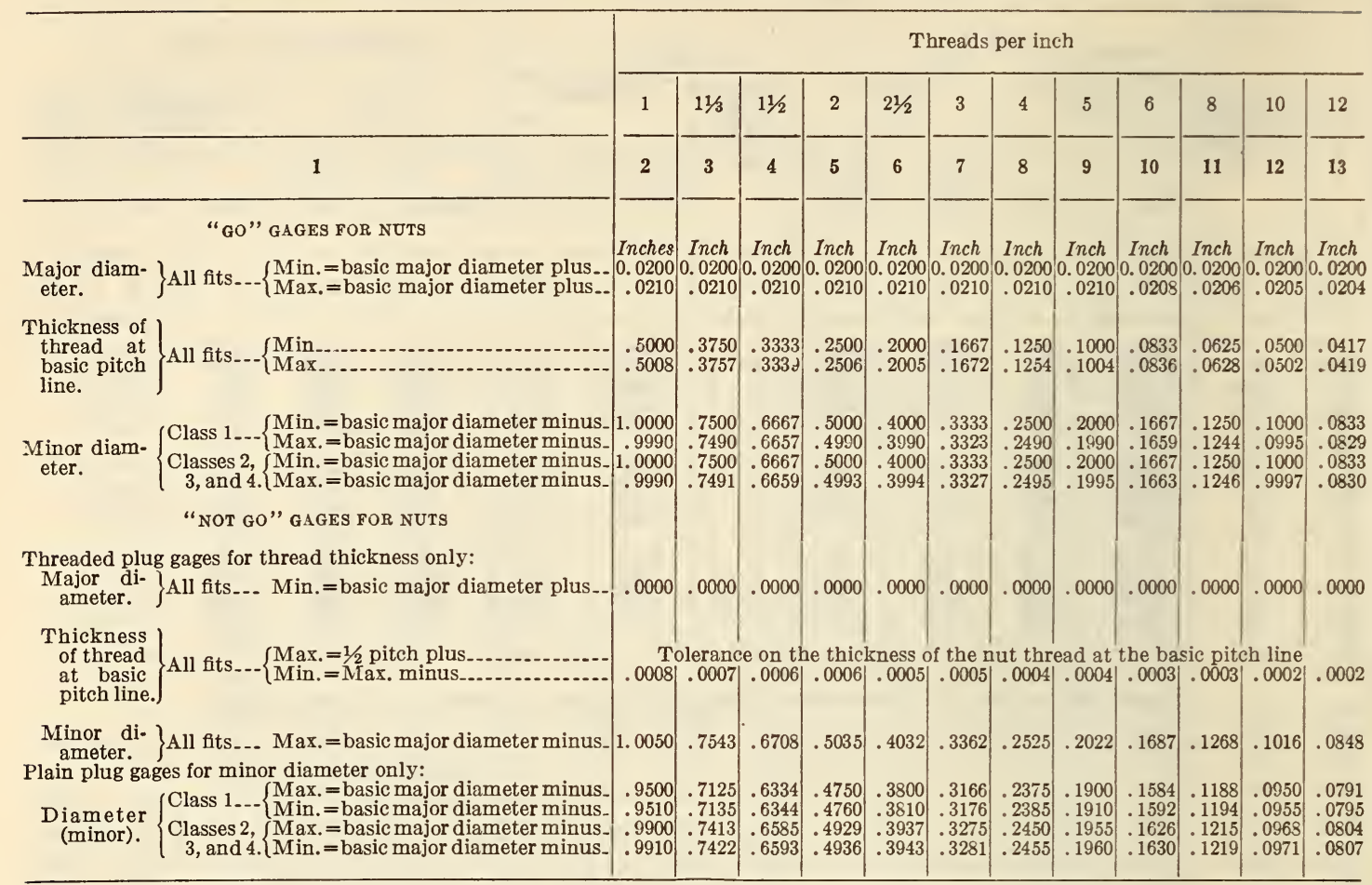

\section{GAGES FOR STANDARD HOSE CONNECTIONS FOR WELDING} AND CUTTING TORCHES

Dimensions and designs of gages for maintaining the hose connection standards for welding and cutting torches are recommended as follows:

NoTE.-In connection with screw-thread gages see also page 921 . Gage No.

1. "Go" and "not go" gage for depth of threaded recess and shank bore-

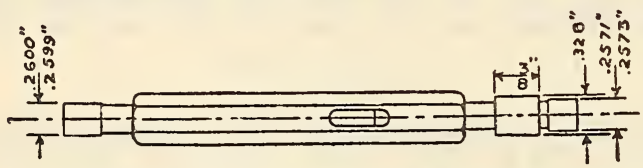

Figure 716._ "Go" and "not go" gage for depth of threaded recess, and shank bore, $A$ size

A size hose connection, as shown in Figure 716.

$\mathrm{B}$ size hose connection, as shown in Figure 721.

2. "Go" adjustable thread-ring gage for right-

hand nipple thread-

Minor diameter, maximum, 0.3299; minimum, 0.3294 inch.

Pitch diameter, maximum, 0.3477; minimum, 0.3474 inch.

$\mathrm{B}$ size, $9 / 16-18-\mathrm{NF}-3$

Minor diameter, maximum, 0.5024; minimum, 0.5019 inch.

Pitch diameter, maximum, 0.5262; minimum, 0.5259 inch.
3. "Go" adjustable thread-ring gage for left-hand nipple thread-

A size, $3 / 8-24$ L. H. $-\mathrm{NF}-3$

Minor diameter, maximum, 0.3299; minimum, 0.3294 inch.

Pitch diameter, maximum, 0.3477; minimum, 0.3474 inch.

B size, $9 / 10-18$ L. H. $-\mathrm{NF}-3$

Minor diameter, maximum, 0.5024; minimum, 0.5019 inch.

Pitch diameter, maximum, 0.5262; minimum, 0.5259 inch.

4. "Not go" adjustable thread-ring gage for righthand nipple thread-

A size, $3 / 8-24-N F-3$

Minor diameter, maximum, 0.3304; minimum, 0.3299 inch.

Pitch diameter, maximum, 0.3458; minimum, 0.3455 inch.

$\mathrm{B}$ size, $9 / 16-18-\mathrm{NF}-3$

Minor diameter, maximum, 0.5029; minimum, 0.5024 inch.

Pitch diameter, maximum, 0.5237; minimum, 0.5234 inch.

5. "Not go" adjustable thread-ring gage for lefthand nipple thread-

A size, $3 / 8-24$ L. H. $\mathrm{NF}-3$

Minor diameter, maximum, 0.3304; minimum, 0.3299 inch.

Pitch diameter, maximum, 0.3458; minimum, 0.3455 inch.

$\mathrm{B}$ size, $9116-18 \mathrm{~L}$. H. $-\mathrm{NF}-3$

Minor diameter, maximum, 0.5029; minimum, 0.5024 inch.

Pitch diameter, maximum, 0.5237; minimum, 0.5234 inch. 
6. "Go" and "not go" double-end threaded setting-plug gages for Nos. 2 and 4 -

A size, $3 / 8-24-\mathrm{NF}-3$

"Go" end-

Major diameter, maximum, 0.3750 ; minimum, 0.3740 inch.

Pitch diameter, maximum, 0.3477; minimum, 0.3474 inch.

"Not go" end-

Major diameter, maximum, 0.3689 ; minimum, 0.3684 inch.

Pitch diameter, maximum, 0.3458 ; minimum, 0.3455 inch.

B size, $9 / 16-18-\mathrm{NF}-3$

"Go" end-

Major diameter, maximum, 0.5625 ; minimum, 0.5620 inch.

Pitch diameter, maximum, 0.5262; minimum, 0.5259 inch.

"Not go" end-

Major diameter, maximum, 0.5548; minimum, 0.5543 inch.

Pitch diameter, maximum, 0.5237 ; minimum, 0.5234 inch.

7. "Go" and "not go" double-end threaded setting plug gage for Nos. 3 and 5

A size, $3 / 8-24$ L. H. $-\mathrm{NF}-3$

"Go" end-

Major diameter, maximum, 0.3750 ; minimum, 0.3745 inch.

Pitch diameter, maximum, 0.3477 ; minimum, 0.3474 inch.

"Not go" end-

Major diameter, maximum, 0.3689 ; minimum, 0.3684 inch.

Pitch diameter, maximum, 0.3458; minimum, 0.3455 inch.

B size, $9 / 16-18$ L. H. $-\mathrm{NF}-3$

"Go" end-

Major diameter, maximum, 0.5625; minimum, 0.5620 inch.

Pitch diameter, maximum, 0.5262; minimum, 0.5259 inch.

"Not go" end-

Major diameter, maximum, 0.5548 ; minimum, 0.5543 inch.

Pitch diameter, maximum, 0.5237; minimum. 0.5234 inch.

8. "Go" and "not go" double-end thread plug gage for right-hand nut thread-

A size, $3 / 8-24-\mathrm{NF}-3$

"Go" end-

Major diameter, maximum, 0.3755 ; minimum, 0.3750 inch.

Pitch diameter, maximum, 0.3484; minimum, 0.3481 inch.

Gaging notch, 0.125 from back.

"Not go" end-

Major diameter, maximum, 0.3665; minimum, 0.3660 inch.

Pitch diameter, maximum, 0.3503; minimum, 0.3500 inch.

$\mathrm{B}$ size, $9 / 16-18-\mathrm{NF}-3$

"Go" end-

Major diameter, maximum, 0.5630 ; minimum, 0.5625 inch.

Pitch diameter, maximum, 0.5269; minimum, 0.5266 inch.

Gaging notch, 0.125 from back.

"Not go" end-

Major diameter, maximum, 0.5510; minimum, 0.5505 inch.

Pitch diameter, maximum, 0.5294; minimum, 0.5291 inch.
9. "Go" and "not go" double-end thread plug gage for left-hand nut thread-

A size, $3 / 8-24$ L. H. $-\mathrm{NF}-3$

"Go" end-

Major diameter, maximum, 0.3755 ; minimum, 0.3750 inch.

Pitch diameter, maximum, 0.3784; minimum, 0.3481 inch.

Gaging notch, 0.125 from back.

"Not go" end-

Major diameter, maximum, 0.3665; minimum, 0.3660 inch.

Pitch diameter, maximum, 0.3503; minimum, 0.3500 inch.

B size. $9 / 16-18$ L. H. $-\mathrm{NF}-3$

"Go" end-

Major diameter, maximum, 0.5630; minimum, 0.5625 inch.

Pitch diameter, maximum, 0.5269; minimum, 0.5266 inch.

Gaging notch, 0.125 from back.

"Not go" end-

Major diameter, maximum, 0.5510; minimum, 0.5505 inch.

Pitch diameter, maximum, 0.5294; minimum, 0.5291 inch.

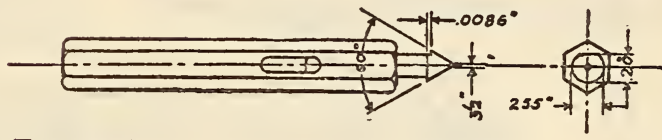

Figure 717.- Taper gage for nipple seat, $A$ size

10. Taper gage for nipple seat-

A size, as shown in Figure 717 .

$\mathrm{B}$ size, as shown in Figure 722 .

11. "Go" ring gage for diameter of shank shoulder and concentricity of serrated portion-

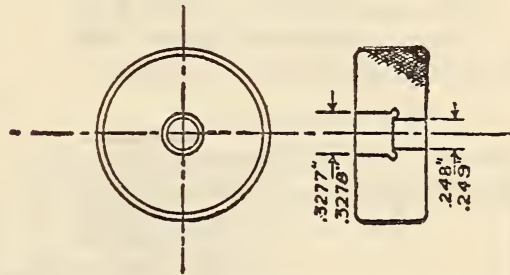

FIGURE 718.- "Go" ring gage for diameter of shank shoulder and concentricity of serrated portion, $A$ size

A size, as shown in Figure 718.

B size, as shown in Figure 723.

12. "Not go" snap gage for shank shoulder diameter-

A size, maximum, 0.3241; minimum, 0.3240 inch.

B size, maximum, 0.4961; minimum, 0.4960 inch.

13. "Go" and "not go" snap gage for diameter of $3 / 8$-inch shank-

B size-

"Go" end, maximum, 0.4298; minimum, 0.4297 inch.

"Not go" end, maximum, 0.4251; minimum, 0.4250 inch.

14. "Go" and "not go" snap gage for diameter of 5/16-inch shank, -

B size-

"Go" end, maximum, 0.3748; minimum 0.3747 inch.

"Not go" end, maximum, 0.3701; minimum, 0.3700 inch. 
15. "Go" and "not go" snap gage for diameter of 1'́-inch shank-

$\mathrm{B}$ size- $\mathrm{Go}$

"Go" end, maximum, 0.3118; minimum, 0.3117 inch.

"Not go" end, maximum, 0.3071; mimimum, 0.3070 inch.

16. "Go" and "not go" snap gage for diameter of $3 / 16$-inch shank-

A size-

"Go" end, maximum, 0.2478; minimum, 0.2477 inch.

"Not go" end, maximum, 0.2431; minimum, 0.2430 inch.

B size-Go" end, maximum, 0.2498; minimum, 0.2497 inch.

"Not go" end, maximum, 0.2451; minimum, 0.2450 inch.

17. "Go" and "not go" snap gage for diameter of $1 / 8$-inch shank-

$A$ and $B$ sizes-

"Go" end, maximum, 0.1868; minimum, 0.1867 inch.

"Not go" end, maximum, 0.1821; minimum, 0.1820 inch.

18. Master template for nose of shank-
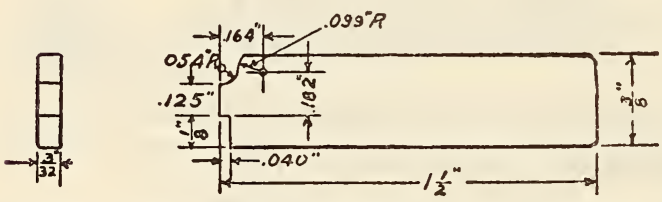

FigURE 719.-Master templaie for nose of shank, A size

A size, as shown in Figure 719 .

B size, as shown in Figure 724 .

19. Template gage for nose of shank-

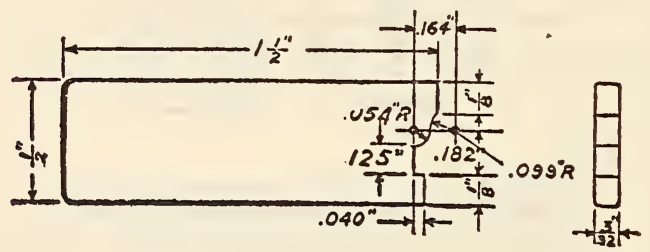

Figdre 720.-Template gage for nose of shank, A size

A size, as shown in Figure 720 .

$\mathrm{B}$ size, as shown in Figure 725 .

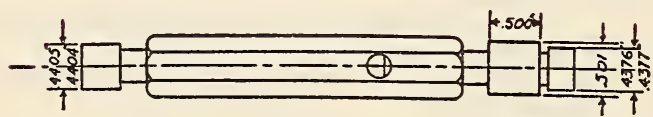

FIGURE 721.- "Go" and "not go" gage for depth of threaded recess, and shank bore, $B$ size

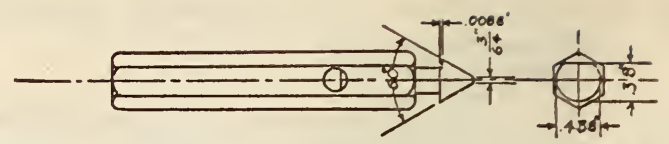

FiguRE 722.-Taper gage for nipple seat, $B$ size

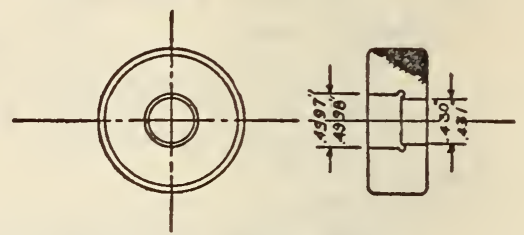

Figure 723.- "Go" ring gage for diameter of shank shoulder and concentricity of serrated portion, $B$ size.
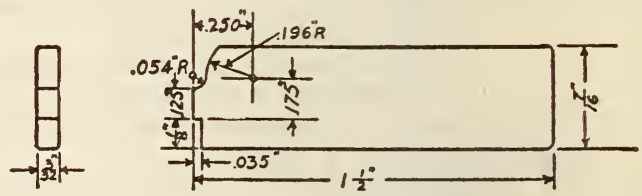

FIGURE 724.-Master template for nose of shank, $B$ size

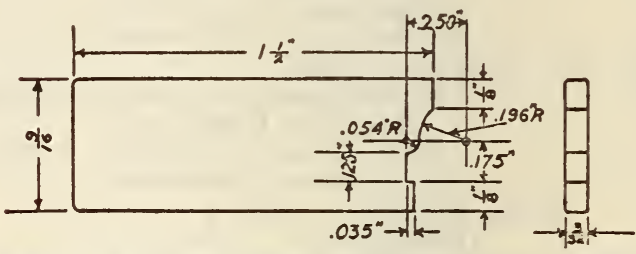

FigURE 725.-Template gage for nose of shank, $B$ size

\section{GAGES FOR 12-PITCH THREAD SERIES}

The specifications for gages given above are applicable. Tolerances on diameter, lead, and angle for classes $\mathrm{X}, \mathrm{Y}$, and $\mathrm{Z}$ gages are given in Table 89 .

TABLE 89.-Tolerances for thread gages, American national 12-pitch thread series

\begin{tabular}{|c|c|c|c|c|c|c|}
\hline \multirow[t]{2}{*}{ Class of gage } & \multicolumn{2}{|c|}{$\begin{array}{c}\text { Tolerance on } \\
\text { pitch } \\
\text { diameter }\end{array}$} & \multirow{2}{*}{$\begin{array}{l}\text { Toler- } \\
\text { ance on } \\
\text { lead } 2 \\
( \pm)\end{array}$} & \multirow{2}{*}{$\begin{array}{l}\text { Toler- } \\
\text { ance } \\
\text { on half } \\
\text { angle of } \\
\text { thread } \\
( \pm)\end{array}$} & \multicolumn{2}{|c|}{$\begin{array}{l}\text { Tolerance on } \\
\text { major or minor } \\
\text { diameters } 1\end{array}$} \\
\hline & From- & To- & & & From- & To- \\
\hline 1 & 2 & 3 & 4 & 5 & 6 & 7 \\
\hline $\begin{array}{l}\text { Class } X \text { and all } \\
\text { "not go" } \\
\text { Class Y } \\
\text { Class Z }\end{array}$ & $\begin{array}{l}\text { Inch } \\
0.0000 \\
.0002 \\
.0004\end{array}$ & $\begin{array}{l}\text { Inch } \\
0.0003 \\
.0006 \\
.0011\end{array}$ & $\begin{array}{l}\text { Inch } \\
0.0003 \\
.0003 \\
.0001\end{array}$ & $\begin{array}{ll}\circ & 1 \\
0 & 10 \\
0 & 10 \\
0 & 10\end{array}$ & $\begin{array}{l}\text { Inch } \\
0.0000 \\
.0000 \\
.0000\end{array}$ & $\begin{array}{l}\text { Inch } \\
0.0006 \\
.0006 \\
.0006\end{array}$ \\
\hline
\end{tabular}

1 On "go" plugs the tolerance is plus, and on "go" rings the tolerance is minus. On "not go" plugs the tolerance is minus, and on "not go" rings the tolerance is plus.

2 Allowable variation in lead between any 2 threads not farther apart than the length of engagement. 
615.9. MISCELLANEOUS METAL-WORKING TOOLS. COMPRESSED AIR SOCIETY, TRADE STANDARDS, PNEUMATIC TOOLS, 1930 PNEUMATIC TOOLS

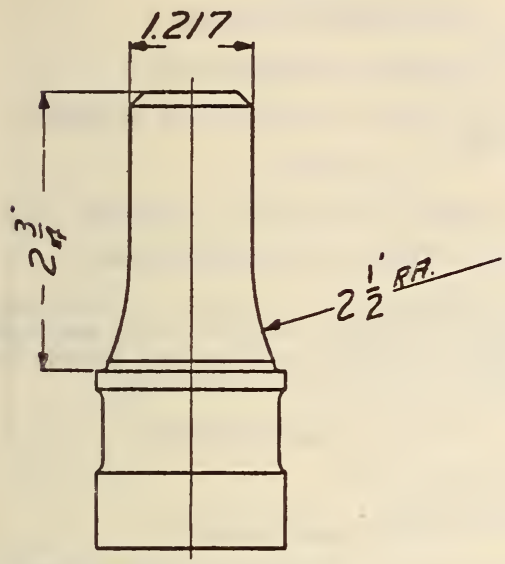

RIVETING HAMMER SPAPKER NOSE
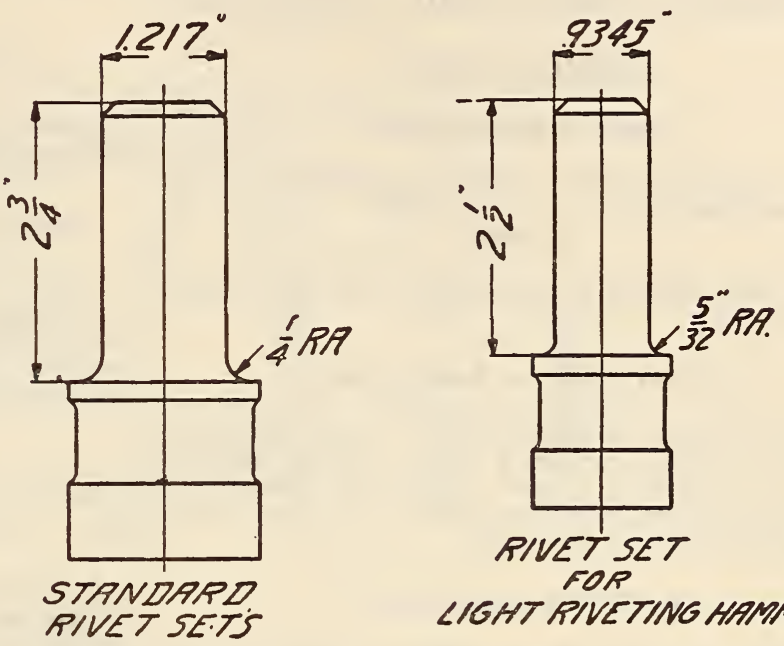

LIGHT RIVETING HAMMER

CHISELS FOP CHIPPING HAMMERS AND HEAVY SCALING HAMMEPS
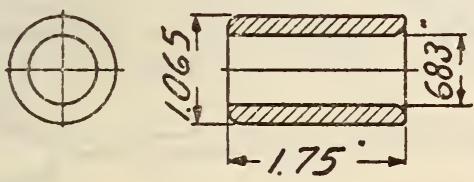

FOUND CHISELS
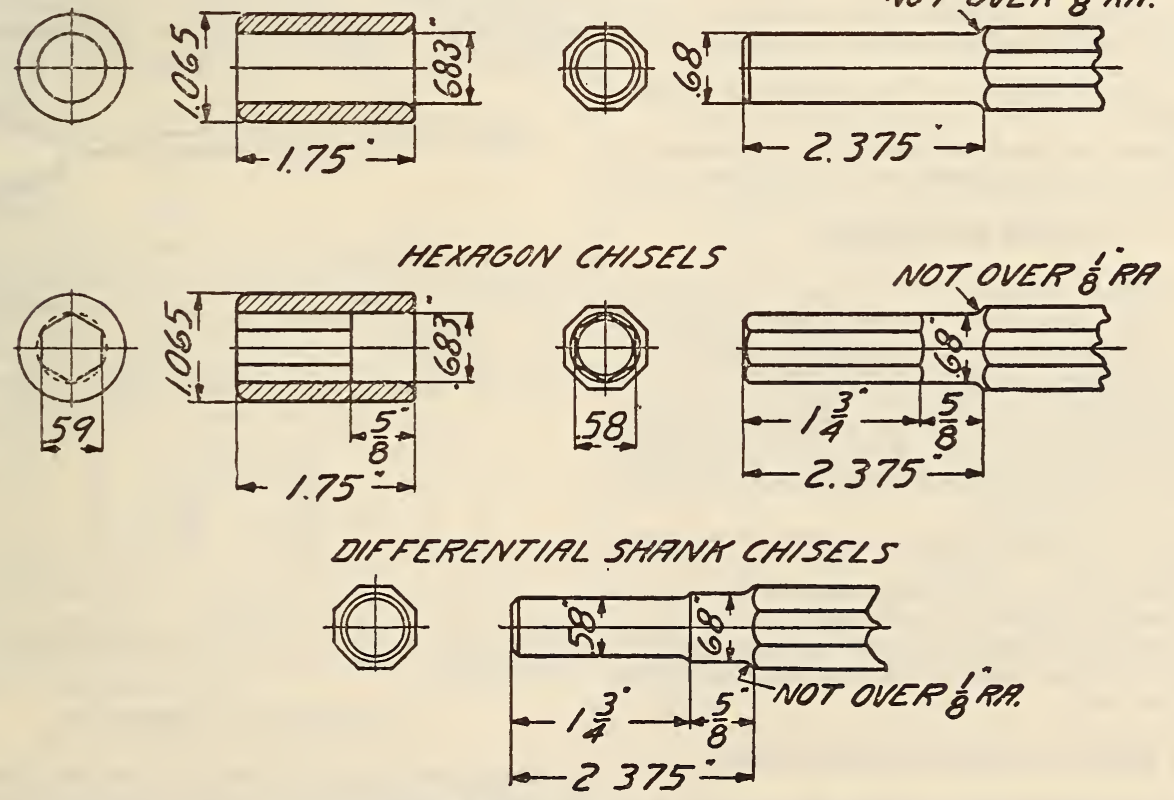

FigdRE 726.-Standard pneumatic tools

(This publication also contains sections covering nomenclature and terminology, definitions, test practice, compressor speeds, installation and care of air compressors, data and formulas, and uses of compressed air.) 


\section{UNITED STATES GOVERNMENT, FED- ERAL SPECIFICATIONS BOARD, FED- ERAL SPECIFICATION FOR SETS, HAND- SAW, NO. 440, SEPTEMBER 25, 1926 (CURRENT DESIGNATION : GGG-H-101).}

\section{HAND-SAW SETS}

\section{GENERAL SPECIFICATIONS}

There are no general specifications applicable to this specification.

\section{TYPE}

Saw sets shall be of the hinged lever type, operated by hand.

\section{MATERIAL AND WORKMANSHIP}

Saw sets shall be manufactured from high-grade material suitable for the purpose. They shall be made in accordance with the best commercial practice and free from any defects that may affect the serviceability of the tools.

\section{GENERAL REQUIREMENTS}

1. Saw sets shall be so designed that by the movement of the operating lever a plunger shall be caused to move forward and impinge upon an anvil located in the head of the tool. The levers shall be returned to their open position by means of a spiral spring inclosing the plunger, which causes the plunger to press against the short arm of the operating lever.

2. The exterior parts of all sets shall be finished and polished.

3. Each saw set shall be marked in a permanent manner with the manufacturer's name or trademark.

\section{DETAIL REQUIREMENTS}

1. The anvil located in the head of the tool shall be made from tool steel properly hardened and tempered. The anvil shall be rigidly secured to the head and shall be capable of adjustment.

2. The underside of the head shall be provided with an adjustment device for regulating the amount of set given the saw tooth. The upper side of the head shall be open so as to permit operator to witness the operation of the tool.

3 . The plunger shall be made of tool steel, properly hardened and tempered. The end of the plunger when in its normal position shall be approximately one-eighth inch from the anvil.

4. Saw sets shall have a capacity for setting saws, thickness on cutting edge 0.065 and less, teeth 4 to 16 points per inch.

\section{METHOD OF INSPECTION AND TESTS}

1. At least three representative samples shall be taken from each lot submitted for inspection. Each sample inspected shall conform to the requirements of this specification.

2. Result of inspection shall be considered as representative of the lot and acceptance or rejection will be based thereon.

\section{PACKING AND MARKING}

1. Saw sets shall be packed in accordance with the best commercial practice, unless otherwise specified.

\section{UNITED STATES GOVERNMENT, FEDERAI SPECIFICATIONS BOARD, FEDERAL SPEC- IFICATION FOR LADLES, PLUMBERS', GGG-I-51, MARCH 1, 1932}

\section{PLUMBERS' LADLES}

\section{GENERAL SPECIFICATIONS}

There are no general specifications applicable to this specification.

$$
\text { II. TYPE }
$$

Plumber's' ladles shall be of the double-lip type.

\section{MATERIAL AND WORKMANSHIP}

Plumbers' ladles shall be made of high-grade material and in accordance with the best commercial practice. They shall be free from any defects that may affect the serviceability of the ladles.

\section{GENERAL REQUIREMENTS}

See detail requirements.

\section{DETAIL REQUIREMENTS}

1. Each ladle shall have the maker's name or trade-mark stamped or otherwise marked on it in a permanent manner.

2. The ladles shall be made of wrought iron or mild steel and shall have a suitable handle attached to the bowl. The bowl shall be provided with two lips for pouring, one on each side, located at right angles to the center line of the handle. These ladles shall be similar to Figure 727 and shall conform to the requirements of table:

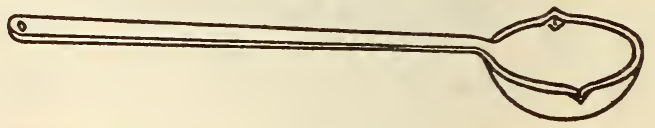

Figure 727.-Plumbers' ladle

\begin{tabular}{|c|c|c|c|c|}
\hline \multirow{2}{*}{$\begin{array}{c}\text { Diameter } \\
\text { of bowl } \\
\text { (approx- } \\
\text { imate) }\end{array}$} & \multirow{2}{*}{$\begin{array}{l}\text { Depth of } \\
\text { bowl } \\
\text { (approx- } \\
\text { imate) }\end{array}$} & \multirow{2}{*}{$\begin{array}{l}\text { Length of } \\
\text { handle } \\
\text { (approx- } \\
\text { imate) }\end{array}$} & \multicolumn{2}{|c|}{$\begin{array}{c}\text { Thickness of } \\
\text { bowl }\end{array}$} \\
\hline & & & $\begin{array}{l}\text { Min- } \\
\text { imum }\end{array}$ & $\begin{array}{l}\text { Max- } \\
\text { imum }\end{array}$ \\
\hline $\begin{array}{c}\text { Inches } \\
21 / 2 \\
3 \\
4 \\
5 \\
6 \\
7\end{array}$ & $\begin{array}{c}\text { Inches } \\
114 \\
116 \\
13 / 4 \\
23 / 16 \\
256 \\
27 / 8\end{array}$ & $\begin{array}{c}\text { Inches } \\
111 / 2 \\
11162 \\
131 / 2 \\
181 / 2 \\
181 / 2 \\
24\end{array}$ & $\begin{array}{r}\text { Inch } \\
3 / 32 \\
3 / 32 \\
7 / 64 \\
964 \\
9 / 64 \\
3 / 10\end{array}$ & $\begin{array}{c}\text { Inch } \\
7 / 64 \\
7 / 64 \\
16 \\
5 / 32 \\
5 / 32 \\
7 / 32\end{array}$ \\
\hline
\end{tabular}

NotE.-Where reference is made to approximate dimensions a plus or minus tolerance of 10 per cent from the specified dimensions will be allowed.

\section{METEOD OF INSPECTION}

Representative samples (not less than three) shall be taken from deliveries and inspected for compliance with the requirements of this specification.

\section{PACKING AND MARKING OF SHIPMENTS}

Plumbers' ladles shall be packed and marked in accordance with manufacturers' trade practice unless otherwise specified.

616. HAND TOOLS OTHER THAN METALWORKING TOOLS

\subsection{EDGE TOOLS.}




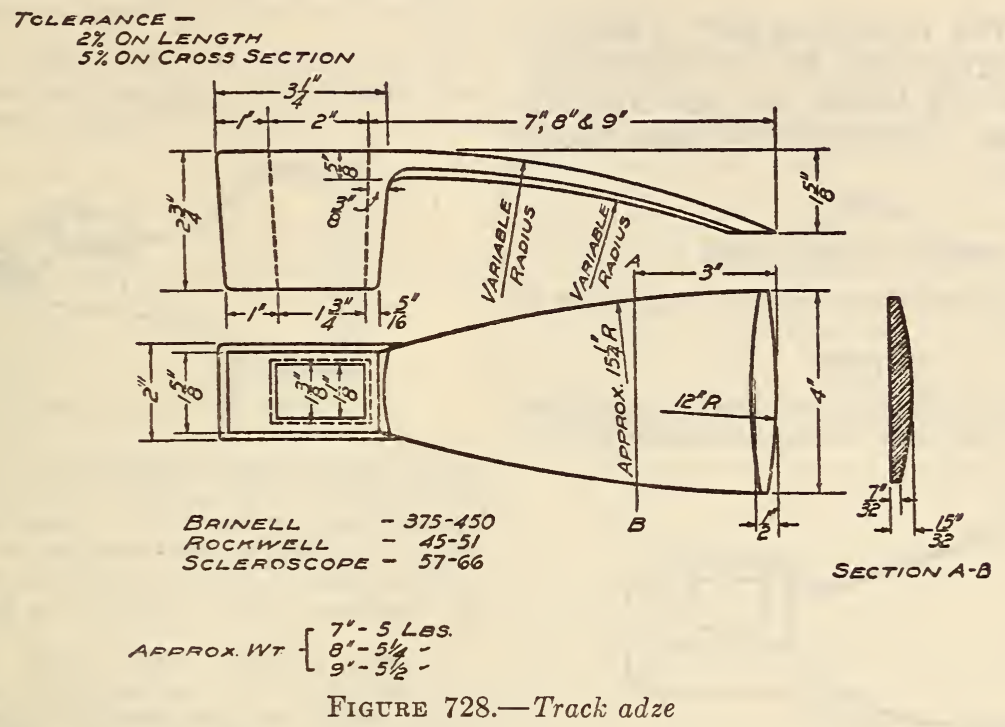

UNITED STATES GOVERNMENT, DEPARTMENT OF COMMERCE, BUREAU OF STANDARDS, SIMPLIFIED PRACTICE RECOMMENDATION FOR FORGED TOOLS, R17-31, JULY 1, 1931

\section{TRACK ADZES}

(See 616.60, p. 960.)

UNITED STATES GOVERNMENT, FEDERAL SPECIFICATIONS BOARD, FEDERAL SPECIFICATION FOR HATCHETS, NO. 433, SEPTEMBER 25, 1926. (CURRENT DESIGNATION : GGG-H-131)

\section{HATCHETS}

\section{GENERAL SPECIFICATIONS}

There are no general specifications applicable to this specification.

\section{TYPE}

Hatchets shall be of the type known commercially as shingling hatchets only.

\section{MATERIAL AND WORKMANSHIP}

All hatchets to be made from high-grade material of good workmanship, and free from all defects or blemishes which may affect their serviceability or appearance.

\section{GENERAL REQUIREMENTS}

Each hatchet shall be impress-stamped with the manufacturer's name.

\section{DETAIL REQUIREMENTS}

\section{Hatchets}

(a) Physical requirements.-They shall be made from high-grade forged tool steel.

Width of cutting edge, 4 inches toler- Per cent ance plus or minus _..... 10

Length from cutting edge to top, $6 \overline{1 / 2}$ inches tolerance plus or minus_...- 10

Weight, 1 pound 7 ounces tolerance plus or minus..... 20

(b) Treatment.-They shall be properly hardened, tempered, and ground.

(c) Shape and finish.-They shall be properly shaped, neatly finished, and coated with a suitable paint or enamel, except the top and that part within about $1 \frac{1}{2}$ inches of the cutting edge, which shall be polished. A slot shall be provided on the handle side of the blade for pulling nails.

\section{Hatchet Handles}

(a) All handles shall be furnished properly fitted and conforming to the requirements of the latest issue of United States Government master specifications for "hickory handles" in effect at opening of bids.

(b) Length of handle not less than 14 inches nor more than 15 inches.

\section{METHOD OF INSPECTION}

Representative samples shall be taken from each lot delivered and inspected for compliance with this specification, and shall be considered as representative of the lot and acceptance or rejection shall be based thereon. 
VII. PACKING AND MARKING

Hatchets shall be packed and marked in accordance with standard commercial practice, unless otherwise specified.

UNITED STATES GOVERNMENT, FEDERAL SPECIFICATIONS BOARD, FEDERALSPECIFICATION FOR ADZES, No. 449, NOVEMBER 22, 1926. (CURRENT DESIGNATION : GGG-A-151)

\section{ADZES}

\section{GENERAI SPECIFICATIONS}

There are no general specifications applicable to this specification.

\section{TYPES}

Adzes shall be of the following types, as specified: A, carpenters' adze, plain; B, ship carpenters' adze,

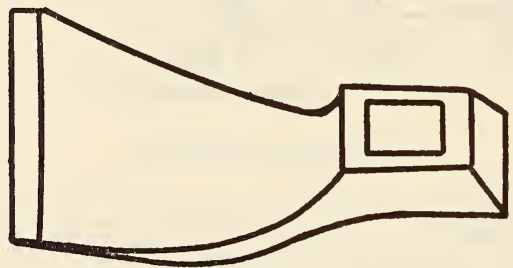

Frgore 729.-Type $A$ adze

plain; C, ship carpenters' adze, lipped; and D, railroad adze, plain.

\section{MATERIAL AND WORKMANSHIP}

1. All adzes shall be forged of high-grade tool steel and shall be furnished properly fitted with handles conforming to the requirements of United States Government master specification for "hickory handles" in effect at opening of bids.

2. All adzes shall be of good workmanship and made in accordance with the best commercial practice.

\section{GENERAL REQUIREMENTS}

1. Adzes shall be properly hardened, tempered, and ground. They shall be furnished with a durable black finish, except that the part of the blade within about $2 \frac{1}{2}$ inches of the cutting edge shall be polished.

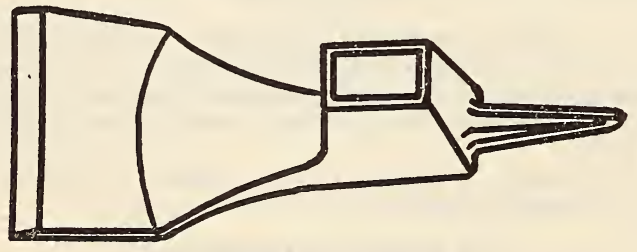

Figure 730.-Type $B$ adze

2. All adzes shall be stamped, or otherwise permanently marked, with the manufacturer's name or trade-mark.

\section{DETAIL REQUIREMENTS}

1. Carpenters' adze, plain, type A.-These shall consist of a full flat head; a rectangular eyehole; and a thin arching blade curving toward the handle and set at right angles to the handle. The blade shall have a beveled cutting edge. The bevel shall be on the side of the blade nearest the handle.

These adzes shall conform to the dimensions listed for this type in the table and be similar to Figure 729.

2. Ship carpenters' adze, plain, type B.-These shall consist of an octagonal spur head, which shall have a neat taper; a rectangular eyehole; and a thin

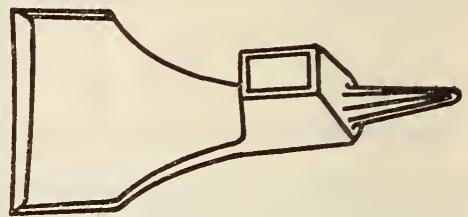

Figure 731.-Type $C$ adze

arching blade curving toward the handle and set at right angles to the handle. The blade shall have a beveled-cutting edge. The bevel shall be on the side of the blade nearest the handle.

These adzes shall conform to the dimensions listed for this type in the table and be similar to Figure 730.

3. Ship carpenters' adze, lipped, type C.-These shall consist of an octagonal spur head, which shall have a neat taper; a rectangular eyehole; and a thin arching blade curving toward the handle and set at right angles to the handle. The blade shall have a beveled cutting edge. The bevel shall be on the side of the blade nearest the handle. The extreme

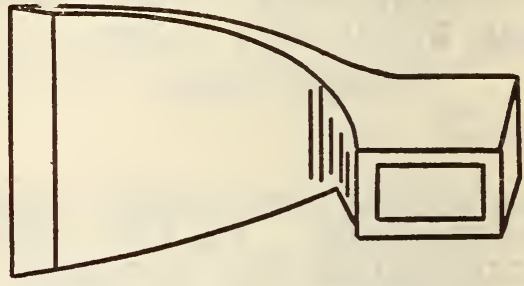

Figure 732.-Type $D$ adze

ends of the blades shall be turned up at right angles to the surface of the blade.

These adzes shall conform to the dimensions listed for this type in the table and be similar to Figure 731.

4. Railroad adze, plain, type D.-These shall consist of a full flat head; a rectangular eyehole; and a thin arching blade curving toward the handle and set at right angles to the handle. The blade shall have a beveled cutting edge. The bevel shall be on the side of the blade nearest the handle.

These adzes shall conform to the dimensions listed for this type in the table and be similar to Figure 732 .

\section{METHOD OF INSPECTION}

Samples shall be selected by the inspector from each lot of the same type submitted, and inspected for compliance with this specification.

\section{PACKING}

Adzes shall be packed in accordance with the best commercial practice unless otherwise specified. 
Table of adze dimensions

\begin{tabular}{|c|c|c|c|c|}
\hline & $\begin{array}{c}\text { Type A } \\
\text { (in inches) }\end{array}$ & $\begin{array}{c}\text { Type B (in } \\
\text { inches) }\end{array}$ & $\begin{array}{c}\text { Type C (in } \\
\text { inches) }\end{array}$ & $\begin{array}{c}\text { Type D } \\
\text { (in inches) }\end{array}$ \\
\hline $\begin{array}{l}\text { Length from cut- } \\
\text { ting edge to }\end{array}$ & $91 / 4$ to $93 / 4$.. & $\ldots$ & & $91 / 4-97 / 8$ \\
\hline $\begin{array}{l}\text { top. } \\
\text { Length from cut- }\end{array}$ & - & $103 / 4$ to $115 / 8$ & $103 / 4$ to $115 / 8$ & \\
\hline $\begin{array}{l}\text { Curve of the } \\
\text { blade in its } \\
\text { length. }\end{array}$ & $11 / 4$ to $13 / 4 \ldots$ & 114 to $15 / 8$ & $7 / 8$ to $11 / 2 \ldots$ & $\begin{array}{c}11 / 16 \\
15 / 8 .\end{array}$ \\
\hline $\begin{array}{l}\text { width of cutting } \\
\text { edge. }\end{array}$ & $31 / 2$ to $41 / 2$. & 414 to $43 / 4 \ldots$ & $41 / 2$ to $51 / 2 \ldots$ & 5 to 6 . \\
\hline $\begin{array}{l}\text { Length of eye- } \\
\text { hole. }\end{array}$ & & 214 to $23 / 4 \ldots$ & 214 to 234 & $21 / 4$ to $23 / 4$. \\
\hline $\begin{array}{l}\text { Inside of small } \\
\text { end eyehole. }\end{array}$ & $\begin{array}{c}15 / 8 \text { by } 1 \text { to } \\
13 / 4 \text { by }\end{array}$ & & & \\
\hline $\begin{array}{l}\text { Inside of large } \\
\text { end eyehole. }\end{array}$ & $\begin{array}{l}11 / 8 . \\
17 / 8 \text { by } 11 / 8 \\
\text { to } 21 / 4 \mathrm{by} \\
136 .\end{array}$ & \multicolumn{3}{|c|}{ Same as for type $A$. } \\
\hline Length of head.- & 1 to 134 & & 236 to 3 & 1 to 1144 . \\
\hline $\begin{array}{l}\text { Diam e ter of } \\
\text { spur, small }\end{array}$ & - & $5 / 16$ to $3 / 8 \ldots$ & $5 / 8$ to $3 \ldots$ & \\
\hline $\begin{array}{l}\text { Diameter of } \\
\text { spur, large } \\
\text { end. }\end{array}$ & $\ldots$ & $1 / 2$ to $9 / 16$ & 152 to $\% 16 \ldots$ & \\
\hline Height of lips.... & & & $3 / 16$ to $16 \ldots$ & \\
\hline $\begin{array}{l}\text { Length of han- } \\
\text { dle over all. }\end{array}$ & 34 to $36 \ldots$ & 34 to $36 \ldots$ & 34 to 36 & 34 to 36 . \\
\hline
\end{tabular}

Table of adze weights

\begin{tabular}{|c|c|c|c|c|}
\hline & Type A & Type B & Type C & Type D \\
\hline $\begin{array}{l}\text { Weight without } \\
\text { handle........ }\end{array}$ & $\begin{array}{r}\text { Pounds } \\
3-4\end{array}$ & $\begin{array}{l}\text { Pounds } \\
2152-3\end{array}$ & $\begin{array}{l}\text { Pounds } \\
21 / 2-3\end{array}$ & $\begin{array}{l}\text { Pounds } \\
41 / 2-7\end{array}$ \\
\hline
\end{tabular}

UNITED STATES GOVERNMENT, FEDERAL SPECIFICATIONS BOARD, FEDERAL SPECIFICATION FOR AXES, GGG-A-926, NOVEMBER 10, 1931

\section{AXES}

\section{A. APPLICABLE FEDERAL SPECIFICATIONS}

A-1. There are no other Federal specifications applicable to this specification.

\section{B. TYPE}

B-1. Axes shall be furnished in the following types as specified: A, chopping ax, 4 pounds; B, chopping ax, 5 pounds, and $\mathrm{C}$, fire ax with pick head, 6 pounds.

\section{MATERIAL AND WORKMANSHIP}

\section{C-1. Material.-}

C-1a. Body and bit.-High-grade steel as hereinafter specified, free from all imperfections that may affect serviceability.

C-1b. Handles.-Good weight, live growth, hickory, free from all defects that may affect serviceability. Handles shall have not over 22 rings of annual growth per inch of radius and be supplied in white, red, or mixed white and red wood. "NOTE.-Above specification covers handles graded as " $\mathrm{AW}$ " and States Department of Commerce.

C-2. Workmanship.-First class in every particular. Axes shall be smoothly finished throughout.

\section{GENERAL REQUIREMENTS}

D-1. Construction.-Axes shall be of bimetallic construction with bits of best-quality crucible tool steel overlaid or inserted by hammer welding to carbon-steel polls or heads, or they may be of onepiece construction, hammer forged from bestquality carbon or alloy tool steel. If of bimetallic construction, the welds shall be free from flaws, temper cracks, or other imperfections, and the crucible tool steel bit, which shall be properly hardened and tempered, shall be of not less than 1 inch depth, back from the cutting edge, to provide for maximum life of the ax. If of 1-piece construction the bit shall be properly hardened and tempered throughout for a distance not less than 1 inch back from the cutting edge.

D-2. Eye.-The difference in the thickness of the eye of any ax shall not exceed one thirty-second of an inch. A median line through the eye shall be in normal alignment parallel to the cutting edge of the ax. The eye section shall be of such size, shape, and strength as to prevent distortion.

D-3. Handles. - The handles shall be of oval cross section and of suitable size and shape throughout to provide a well-proportioned handle with a comfortable grip for the hands. Handles shall be properly fitted and secured to the head in such a manner as to preclude probability of the head working loose or flying off the handle under service conditions. When specifically required, the handles shall be dipped in hot linseed oil or some other equally effective coating to protect them from the powder-post beetle.

D-4. Marking and labeling.-Each ax shall be stamped or otherwise permanently marked in a legible manner on the side of the head with the manufacturer's name or known trade-mark and the weight. Each ax shall also be marked or labeled to show that the axes are the manufacturer's recognized first-quality brand. (Only the first-quality brand of a manufacturer will be acceptable.)

D-5. Weights.-The weights indicated herein shall be exclusive of the handles.

D-6. Tolerances. - A plus or minus tolerance of 5 per cent will be allowed in the weights herein specified. A plus or minus tolerace of 7 per cent will be allowed in the "approximate" dimensions herein specified.

\section{E. DETAIL REQUIREMENTS}

E-1. Chopping axes, 4 pounds, type A.-

$\mathrm{E}-2$. Style.-Axes of this type shall be of the style commonly known as the "Dayton pattern" and shall be suitable for general purposes.

$\mathrm{E}-3$. Hardness. - The bits of all axes of this type shall show a Rockwell hardness number of not less than $\mathrm{C}-52$ nor more than $\mathrm{C}-62$ at $3 / 4$ to 1 inch from the cutting edge. The eye and the head of axes of 1-piece construction shall show a Rockwell hardness number of not less than $\mathrm{C}-18$.

E-4. Finish. - The top face of the head of these axes shall be smoothly finished and polished. That part of axes of bimetallic construction beginning at the cutting edge and terminating at a minimum of one-half inch above the welded joint shall also be smoothly finished and polished. The bits of axes of 1-piece construction shall be smoothly finished and polished a distance of not less than one-half inch back from the cutting edge. The unpolished portion of the bit and head of all axes shall be coated with a suitable paint or enamel to provide a rustresisting finish. The cutting edge shall be ground and ready for service without further attention.

$\mathrm{E}-5$. Axes of this type shall be similar to illustration Figure 733 and shall conform with the dimensions and weight outlined in Table 1. 
TABLE 1.-Chopping ax, type A

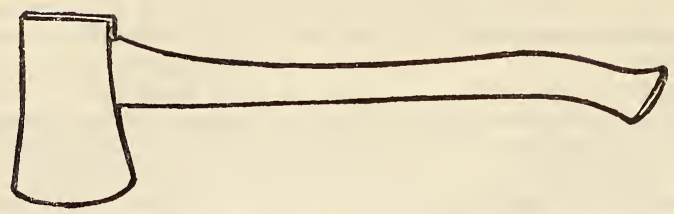

FIgURE 733

\begin{tabular}{|c|c|c|c|c|c|}
\hline Weight & $\begin{array}{c}\text { Width at } \\
\text { cutting } \\
\text { edge (ap- } \\
\text { proxi- } \\
\text { mate) }\end{array}$ & $\begin{array}{c}\text { Width at } \\
\text { top of } \\
\text { head (ap- } \\
\text { proxi- } \\
\text { mate) }\end{array}$ & $\begin{array}{c}\text { Thick- } \\
\text { ness at } \\
\text { top of } \\
\text { head } \\
\text { (approxi- } \\
\text { mate) }\end{array}$ & $\begin{array}{c}\text { Length } \\
\text { of head } \\
\text { (approxi- } \\
\text { mate) }\end{array}$ & $\begin{array}{c}\text { Length } \\
\text { of handle } \\
\text { over all } \\
\text { (approxi- } \\
\text { mate) }\end{array}$ \\
\cline { 1 - 6 } $\begin{array}{c}\text { Pounds } \\
4\end{array}$ & $\begin{array}{c}\text { Inches } \\
43 / 4\end{array}$ & $\begin{array}{c}\text { Inches } \\
31 / 4\end{array}$ & $\begin{array}{c}\text { Inches } \\
13 / 16\end{array}$ & $\begin{array}{c}\text { Inches } \\
71 / 2\end{array}$ & $\begin{array}{c}\text { Inches } \\
30\end{array}$ \\
\hline
\end{tabular}

NotE.-See paragraph D-6 for the tolerances in the above weight and dimensions.

E-6. Chopping axes, 5 pounds, type B.-

E-7. Axes of this type shall conform with the requirements outlined in sections $\mathrm{E}-2$ to $\mathrm{E}-5$, inclusive, for type A axes, except as to weight and dimensions. Axes of this type shall be similar to illustration Figure 733 and shall conform with the dimensions and weight outlined in Table 2.

TABLE 2

\begin{tabular}{|c|c|c|c|c|c|}
\hline Weight & $\begin{array}{c}\text { Width at } \\
\text { cutting } \\
\text { edge (ap- } \\
\text { proxi- } \\
\text { mate) }\end{array}$ & $\begin{array}{c}\text { Width at } \\
\text { top of } \\
\text { proxi- } \\
\text { mate) } \\
\text { mate }\end{array}$ & $\begin{array}{c}\text { Thick- } \\
\text { ness at } \\
\text { top of } \\
\text { head } \\
\text { (approxi- } \\
\text { mate) }\end{array}$ & $\begin{array}{c}\text { Length } \\
\text { of head } \\
\text { (approxi- } \\
\text { mate) }\end{array}$ & $\begin{array}{c}\text { Length } \\
\text { handle } \\
\text { over all } \\
\text { (approxi- } \\
\text { mate) }\end{array}$ \\
\cline { 1 - 3 } $\begin{array}{c}\text { Pounds } \\
5\end{array}$ & $\begin{array}{c}\text { Inches } \\
5\end{array}$ & $\begin{array}{c}\text { Inches } \\
31 / 2\end{array}$ & $\begin{array}{c}\text { Inches } \\
15 / 16\end{array}$ & $\begin{array}{c}\text { Inches } \\
8\end{array}$ & $\begin{array}{c}\text { Inches } \\
36\end{array}$ \\
\hline
\end{tabular}

Note.-See paragraph D-6 for tolerances in the above weight and dimensions.

E-8. Fire axes with pick heads, type C.-

E-9. Style.-Axes of this type shall be of the style commonly known as fire or firemen's axes with pick head. They shall be suitable for tearing up floors, battering down doors, hacking through all kinds of material, and all other conditions likely to be encountered under ordinary service conditions.

E-10. Hardness. - The bits of these axes shall show a Rockwell hardness number of not less than C-50 nor more than C-58 at $3 / 4$ to 1 inch from the cutting edge. The picks shall show a Rockwell hardness number of not less than C-42 nor more than C-58 at approximately one-half inch from the point.

$\mathrm{E}-11$. Finish.-The pick head and that portion of the bit about 4 inches back from the cutting edge shall be smoothly finished and polished. The remaining portion of the head and the entire surface of the handle shall be coated with good quality red paint or enamel.

$\mathrm{E}-12$. Fire axes shall be similar to illustration Figure 734 and shall conform with the dimensions outlined in Table 3.
TABLE 3.-Fire ax with pick head, Type C

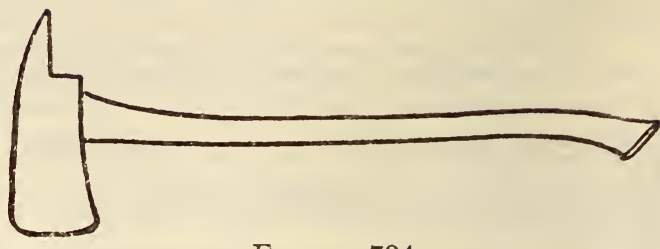

Figure 734

\begin{tabular}{|c|c|c|c|c|c|} 
Weight & $\begin{array}{c}\text { Width a t } \\
\text { cutting } \\
\text { edge } \\
\text { (approxi- } \\
\text { mate) }\end{array}$ & $\begin{array}{c}\text { Length } \\
\text { of head } \\
\text { (approxi- } \\
\text { mate) }\end{array}$ & $\begin{array}{c}\text { Length } \\
\text { from cen- } \\
\text { ter of } \\
\text { handle } \\
\text { to cut- } \\
\text { ting edge } \\
\text { (approxi- } \\
\text { mate) }\end{array}$ & $\begin{array}{c}\text { Length } \\
\text { from cen- } \\
\text { ter of } \\
\text { handle } \\
\text { to pick } \\
\text { end } \\
\text { (approxi- } \\
\text { mate) }\end{array}$ & $\begin{array}{c}\text { Length } \\
\text { of handle } \\
\text { over } \\
\text { all } \\
\text { (approxi- } \\
\text { mate) }\end{array}$ \\
\hline $\begin{array}{c}\text { Pounds } \\
6\end{array}$ & $\begin{array}{c}\text { Inches } \\
5\end{array}$ & $\begin{array}{c}\text { Inches } \\
12\end{array}$ & $\begin{array}{c}\text { Inches } \\
6\end{array}$ & $\begin{array}{c}\text { Inches } \\
6\end{array}$ & $\begin{array}{c}\text { Inches } \\
35\end{array}$ \\
\hline
\end{tabular}

Note.-See paragraph D-6 for tolerances in the above weight and dimensions.

\section{F. METHOD OF SAMPLING, INSPECTION, AND TESTS}

F-1. Axes shall be submitted to a careful surface inspection to determine whether they are in compliance with the requirements of this specification. Special attention should be given to the welded joint of axes of bimetallic construction to see that no apparent imperfections exist, also to the quality of the cutting edge of all axes and as to whether the handles are secured to the head in a proper manner. Representative samples, at least three of each size and type, shall be taken from those under inspection and in addition to the Rockwell hardness tests should be used in practical cutting tests of such duration as may be considered necessary (in cutting hardwood knots, etc.) to determine whether the axes are in compliance with the requirements of this specification.

\section{G. PACKAGING, PACKING, AND MARKING}

G-1. Packaging.- -Unless otherwise specified, commercial packages are acceptable, provided that not more than six axes (which shall be of the same type) shall be contained in any one box or package. Polished surfaces shall be coated with a rust preventive.

G-2. Packing.-Unless otherwise specified, the subject commodity shall be delivered in standard commercial containers so constructed as to insure acceptance by common or other carrier, for safe transportation, at the lowest rate, to the point of delivery.

G-3. Marking.-

G-3a. Unless otherwise specified, each box or package shall be marked with the name of the article, the size, the type, the quantity contained therein, and the name of the manufacturer.

G-3b. Unless otherwise specified, shipping containers shall be marked with the name of the article, the size, the type, and the quantity contained therein as defined by the contract or order under which shipment is made, the name of the contractor, and the number of the contract or order. 
I. NOTES

I-1. The illustrations are included for the convenience of those interested and are not intended to preclude the purchase of axes which are otherwise in accordance with this specification.

I-2. It is believed that this specification adequately describes the characteristics necessary to secure the desired material and that normally no samples will be necessary prior to award to determine compliance with this specification. If, for any particular purpose samples with bids are necessary, they should be specifically asked for in the invitation for bids, and the particular purpose to be served by the bid sample should be definitely stated, the specification to apply in all other respects.

\subsection{AUGERS AND CARPENTERS BRACES.}

UNITED STATES GOVERNMENT, FEDERAI SPECIFICATIONS BOARD, FEDERAL SPECIFICATION FOR BRACES, RATCHET, No. 416, JULY 9, 1926. (CURRENT DESIGNATION : GGG-B-671)

\section{BRACES}

\section{GENERAL SPECIFICATIONS}

There are no general specifications applicable to this specification.

\section{TYPES}

Ratchet braces shall be of one type only.

\section{MATERIAL AND WORKMANSHIP}

Ratchet braces shall be free from defects or blemishes which might affect their use. They shall be of good workmanship, and in accordance with the best commercial practice.

\section{GENERAL REQUIREMENTS}

Each ratchet brace shall be stamped with the brand or manufacturer's name.

\section{DETAIL REQUIREMENTS}

1. Ratchet braces shall consist of a malleable iron or steel chuck with two forged steel jaws engaging a steel spindle which is operated by means of a ratchet in a socket secured to a steel sweep provided with wood center and head.

2. The chuck sleeve shall be $23 / 4$ to $31 / 4$ inches long, about $1 \frac{1}{8}$ inches outside diameter at socket end, and be neatly knurled, and shall have a wellmade internal thread for screwing on to the spindle.

3 . The chuck jaw shall be forged, and of the alligator type or a type equally efficient to take round bit shanks from one-eighth to one-half inch, as well as taper shank bits. They shall be held together by means of a steel wire spring so as to permit of a parallel movement of the jaws and in the case of alligator jaws a proper articulation of the jaw tooth. They shall have a longitudinal V-shaped groove of such depth as to permit gripping one-eighth inch round shanks.

4. The chuck spindle shall be made of steel in one piece and provided with square or acme cut threads; it shall have a rectangular slot at one end, cut ratchet teeth at the socket end, and a turned square shoulder against which bears a hardened steel antifriction washer for reducing the thrust friction between the spindle and the ratchet socket. The spindle shall be held in the socket by means of a washer entering the end of the spindle and bearing against a square shoulder about one-eighth inch below the upper end of the socket. The ratchet tooth shall engage two steel pawls which are actuated by a spiral steel spring and are controlled by a neatly knurled slip ring. The ring shall be so designed as to permit the ratchet being operated in either direction or to lock same.

5. The sweep shall be solid and made of highgrade steel of such diameter as to resist bending under ordinary usage. The head of the sweep shall be made of cocobolo, rosewood, lignum-vitæ or other approved material, about $23 / 4$ inches in diameter and 1 inch thick, and be secured to the sweep by means of a revolving upper thrust bearing in such a manner as not to require the sweep passing through the center of the wooden head. The thrust pressure shall be transmitted by hardened steel balls running on hardened steel ball races which are integral parts of the bearings. The balls shall be suitably protected from exposure to dirt, etc. The center grip of the sweep shall be made of cocobolo, rosewood, lignum-vitæ or other approved material and be reinforced by brass rings inserted in each end so as to prevent splitting and be held in place by collars secured to the sweep at each end of the grip.

6. All exposed metal surfaces shall be heavily nickel plated. The head and center grip shall be smoothly finished and polished.

7. The size of the brace will be designated by the sweep; that is, the diameter of the circle traversed

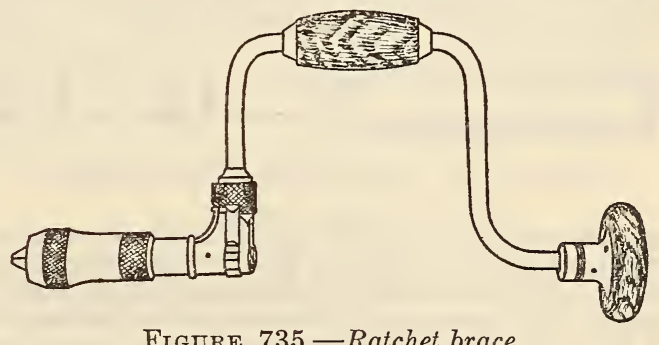

FIGURE 735.- Ratchet brace

by the center grip, and shall be 8,10 , or 12 inches, as may be specifically called for.

8. Ratchet braces shall be similar to Figure 735 .

\section{METHOD OF INSPECTION AND TESTS}

Ratchet braces shall be subjected to such inspection as will determine whether they comply with this specification.

\section{PACKING AND MARKING}

Commercial packing and marking unless otherwise specified.

\subsection{CHISELS,}

UNITED STATES GOVERNMENT, FEDERAI SPECIFICATIONS BOARD, FEDERAL SPEC. IFICATION FOR CHISELS, GOUGES AND SLICKS, WOODWORKERS', GGG-C-311, MARCH 1, 1932

\section{CHISELS, GOUGES, AND SLICKS}

\section{GENERAI SPECIFICATIONS}

There are no general specifications applicable to this specification.

$$
\text { II. TYPES }
$$

Woodworkers' chisels, gouges, and slicks shall be of the following types, as required: A, chisels, socket firmer; B, chisels, socket framing; C, chisels, 
tanged turning; D, gouges, socket firmer, outside or inside bevel (as specified); E, gouges, tanged turning; and F, slicks.

\section{MATERIAL AND WORKMANSHIP}

Chisels, gouges, and slicks shall be made of highgrade material and be free from all defects affecting their appearance or that may affect their serviceability. They shall be of high-grade workmanship and made in accordance with the best commercial practice.

\section{GENERAL REQUIREMENTS}

1. Each chisel, gouge, or slick shall have the manufacturer's name or trade-mark permanently marked on the blade or socket.

2 . All tools shall have the blades properly hardened to within 2 inches of socket or tang, tempered and ground. The hardened portion of all blades shall show a Rockwell hardness number of not less than 56 nor more than 66 on the $\mathrm{C}$ scale. They shall be smoothly finished and polished, with the cutting edge sharpened and honed ready for use.

3 . No welding of iron and steel shall be permitted on any tools that are less than 2 inches wide, except in the case of socket barrels and tangs, which may be of iron or steel. On tools that are more than 2 inches wide, the welding of an iron back on a steel face will be permitted.

4. Each tool shall be furnished with a handle as specified in description of details, Section V. The

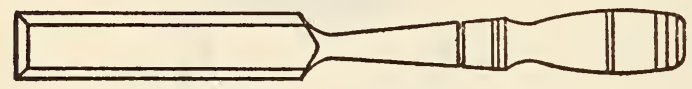

Figure 736.-Chisel, socket firmer, Type A

handles shall be made of high-grade,well-seasoned, straight-grained hardwood, free from knots, checks, holes or any other defects that may affect serviceability, shall be smoothly finished, and shall be given at least one coat of shellac, varnish or other protective material to prevent the absorption of moisture.

5. A plus or minus tolerance of 5 per cent will be allowed in the dimensions herein specified for the width of the blades. Dimensions referred to as "approximate" shall be interpreted as allowing a plus or minus tolerance of 15 per cent from the dimension specified.

\section{DETAIL REQUIREMENTS}

1. Chisels, socket firmer, type A.-These chisels shall be of the bevel-edge type and shall be furnished in the following widths of blade, as specified: $1 / 8,1 / 4,3 / 8,1 / 2,5 / 8,3 / 4,7 / 8,1,11 / 4,11 / 2,13 / 4$, and 2 inches.

2. They shall have a minimum length of blade of 6 inches, exclusive of the socket.

3. Each of these chisels shall be furnished with a leather-tipped handle of second-growth hickory, of suitable size, having at least three thicknesses of high-grade leather firmly secured to the top of the handle. The tipped end shall be properly shaped to prevent being easily distorted. The tapered end of the handle shall be a good fit in the socket. The largest section of the handle shall have a diameter of 1 to $1 \frac{3}{8}$ inches, and the length of the handle exclusive of the tapered portion shall be approximately $4 \frac{1}{2}$ inches.

4. These chisels shall be similar to Figure 736 .

5. Chisels, socket framing, type B.-These chisels shall be of the plain-edge type and shall be furnished in the following widths of blades, as specified: $1 / 4,3 / 8,1 / 2,5 / 8,3 / 4,7 / 8,1,1 \frac{1}{4}, 1 \frac{1}{2}, 13 / 4$, and 2 inches.

6 . They shall have a minimum length of blade of 8 inches, exclusive of the socket.
7. Each of these chisels shall be furnished with a handle of second-growth hickory, of suitable size, having a steel or malleable iron ring firmly secured to the top of the handle. The ring shall be of proper size to prevent the handle from splitting. Handles with improperly fitted rings will not be accepted. The tapered end of the handle shall be a good fit in the socket. The largest section of the handle shall have a diameter of 1 to $1 \frac{3}{8}$ inches, and the length of

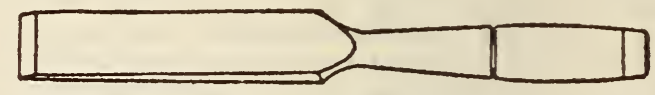

\section{FIGURE 737.-Chisel, socket framing, type B}

the handle exclusive of the tapered portion shall be approximately $4 \frac{1}{2}$ inches.

8. These chisels shall be similar to Figure 737 .

9. Chisels, tanged turning, type C.-These chisels shall be of the regular skew type and shall be suitable for either right or left hand turning. The cutting edge shall be bereled from both the flat sides.

10. These chisels shall have a minimum length of blade of $6 \frac{1}{2}$ inches, exclusive of the tang, and shall

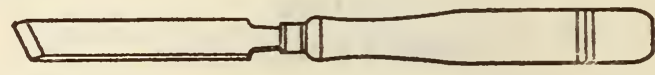

Figure 738.-Chisel, tanged turning, type C

be furnished in the following widths as specified: $1 / 4,3 / 8,1 / 2,5 / 8,3 / 4,7 / 8,1,1 \frac{1}{4}, 1 \frac{1}{2}, 13 / 4$, and 2 inches.

11. Each turning chisel shall be furnished with a properly shaped handle of ash, birch, maple, or second-growth hickory, having a minimum over-all length of approximately 9 inches and a diameter at the largest section of 1 to $13 / 8$ inches. The handle shall have a heavy brass ferrule firmly attached to it in such manner as to securely hold the tang of the

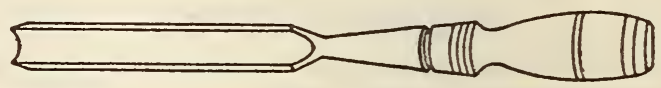

FIGURE 739.-Gouge, socket firmer, type $D$

chisel. Handles with loose or improperly fitted ferrules will not be accepted.

12. These chisels shall be similar to Figure 738 .

13. Gouges, socket firmer, inside or outside bevel, type D.-These gouges shall be furnished with inside bevel or outside bevel, as required, and shall be of the regular sweep type. They shall have a minimum length of blade of 6 inches, exclusive of

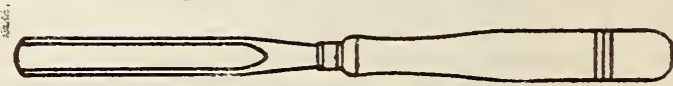

FIGURE 740.-Gouge, tanged turning, type $E$

the socket, and shall be furnished in the following widths, as specified: $1 / 4,3 / 8,1 / 2,5 / 8,3 / 4,7 / 8,1,1 \frac{1}{4}$, and $1 \frac{1}{2}$ inches.

14. Each of these gouges shall be furnished with a leather-tipped handle, as specified for socket firmer chisels, type A.

15. These gouges shall be similar to Figure 739 .

16. Gouges, tanged turning, type E.-These gouges shall be of the regular sweep type. They shall have a minimum length of blade of $61 / 2$ inches, exclusive of the tang, and shall be furnished in the following widths, as specified: $1 / 4,3 / 8,1 / 2,5 / 8,3 / 4,7 / 8,1,1 \frac{1}{4}, 1 \frac{1}{2}, 1 \frac{13}{4}$, and 2 inches.

17. Each of these gouges shall be furnished with a handle of about 9 inches length as specified for tanged turning chisels, type C.

18. These gouges shall be similar to Figure 740 . 
19. Slicks, type F.-These slicks shall be the oval back type carpenters' slick. They shall have a minimum length of blade of 10 inches, exclusive of the socket; the widths of blade shall be $2 \frac{1}{2}, 3,3 \frac{1}{2}$, and 4 inches. The over-all length of the blade and socket shall be approximately 15 inches.

20. Each slick shall be furnished with a handle having an over-all length of approximately 14 inches and a diameter of about $13 / 4$ inches at the thicker section. The handle shall be of the regular commercial type, tapered on one end to fit the socket of the slick, the other end being rounded to suit the palm of the hand.

21. These slicks shall be similar to Figure 741 .

\section{METHOD OF INSPECTION AND TESTS}

From each lot under inspection, representative samples (at least three) shall be taken at random and submitted to the respective test outlined below

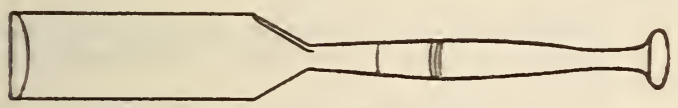

FIGTRE 741.-Slich, type F

with a view to determining whether they are in accordance with specifications.

Chisels, types $A$ and $B$, gouges, trpe D, and slicks type $\mathrm{F}$, shall be required to cut through at least three ordinary knots ( 1 inch or more in diameter) as found in seasoned black spruce or rellow pine, without showing undue wear, such as turning over, or breaking out of any part of the cutting edge, or damage to the handles.

Turning chisels, type $\mathrm{C}$, and turning gouges, type E, shall be used in turning hard oak or similar material under severe conditions for a period of at least 15 minutes, after which the cutting edges shall be carefully examined for undue wear, turning over, or breaking out of any part of the cutting edge.

The blade of each sample chisel, gouge, and slick shall also be subjected to a Rockwell hardness test to determine whether they are in compliance with the requirements prescribed in paragraph IV-5.

Chisels, gouges, or slicks failing to meet the above tests shall be considered as unsatisfactory and not in accordance with specifications.

\section{PACEING AND MAREING}

Chisels, gouges, and slicks shall be packed and marked in accordance with best commercial practice unless otherwise specified. Not more than six tools of the same size and type shall be contained in any one box or package.

\section{NOTES}

1. The illustrations are included for the convenience of those interested, and are not intended to prevent the purchase of tools which are otherwise in accordance with specifications.

2 . When so requested, bidders shall submit, free of expense to the Government, for examination and test, sample tools similar to those they propose to furnish. Samples shall remain the property of the exhibitors, but a sample may be accepted as a part of a delivery in the case of a successful bidder. However, the right is reserved to destroy the sample submitted, if such action is considered necessary to determine the quality of material and method of mar ufacture, in which case no expense to the Government shall accrue.
3. If award is made on samples submitted, it will be with the understanding that tools furnished shall be equal in all respects to samples.

\subsection{DRILIS.}

(No nationally recognized specifications available.) 616.15 KNIVES.

\section{UNITED STATES GOVERNMENT, FEDERAL SPECIFICATIONS BOARD, FEDERAL SPEC- IFICATION FOR KNIVES, SHOE, NO. 442, OCTOBER 28, 1926. (CURRENT DESIGNATION : GGG-K-491}

\section{SHOE KNIVES}

\section{GENERAL SPECIFICATIONS}

There are no general specifications applicable to this specification.

\section{TYPE}

These shall be the commercial type of shoe knives.

\section{MATERIAI AND WORKMANSHIP}

Knife blades shall be made from the best quality crucible tool steel, properly shaped and hardened, tempered, and ground. The blades shall be of such design as to withstand ordinary usage with a minimum breakage, and shall be free from all defects or blemishes which may affect their appearance or serviceability. The handles shall be properly designed to afford a comfortable grip for the user and shall be smoothly finished.

\section{GENERAL REQOIREMENTS}

All knives shall have the maker's name or trademark stamped or otherwise permanently marked on the blades.

\section{DETAII REQUIREMENTS}

1. Shoe knives shall consist of a steel blade, a shank, and a wooden handle. The blade shall be square pointed. The shank shall be of proper length and inserted in a round wooden handle in a perma-

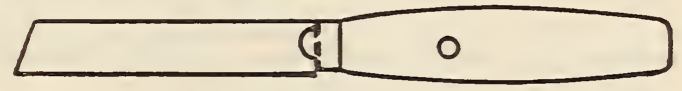

\section{Figdre 742.-Shoe knife}

nent manner. The handle shall be fitted with a heavy metal ferrule which shall be firmly secured.

2 . Shoe knives shall conform to the following approximate dimensions:

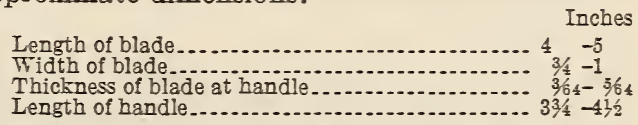

3. These knives shall be similar to Figure 742 .

\section{METHOD OF IISPECTION}

Representative samples shall be taken from each lot submitted and inspected for compliance with this specification.

\section{PACKING AND MARKING}

Packing and marking shall be in accordance with the manufacturer's trade practice, unless otherwise specified. 
UNITED STATES GOVERNMENT, FEDERAL SPECIFICATIONS BOARD, FEDERAL SPECIFICATION FOR KNIVES,

GGG-K-471, MARCH 1, 1932
DRAWING KNIVES

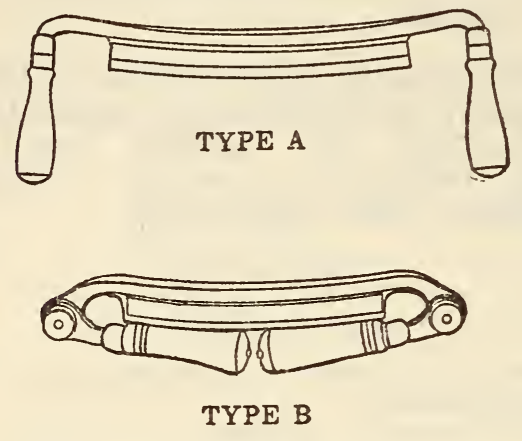

Type $A$ and type $B$ drawing knives
A-1. There are no other Federal specifications applicable to this specification.

\section{B. TYPE}

B-1. Drawing knives shall be furnished in two types, as specified.

A. Fixed handle.

B. Folding handle.

C. MATERIAL AND WORKMANSHIP, ETC.

\section{C-1. Material.}

C-1a. Blades.-Best quality tool steel.

C-1b. Handles. - Ash, birch, hard maple, hickory, or other wood of equal or better quality and suitable for the purpose.

C-1c. Ferrules and caps.-Brass or steel.

C-2. Workmanship. - Shall be first class.

\section{GENERAL REQUIREMENTS}

D-1. Markings.-The blade of each drawing knife shall be marked in a plain and permanent manner with the manufacturer's name or with a trade-mark or such known character that the source of manufacture may be readily determined.

\section{E. DETAIL REQUIREMENTS}

E-1. Fixed handle drawing knives, type A.-

E-1a. Sizes. - Unless otherwise specified, drawing knives shall be furnished in the 8,10 , and 14 inch sizes, size referring to the length of the cutting edge of the blades.

E-1b. Shanks.-The shanks or handle arms of drawing knives may be either an integral part of the blade or made from good quality mild steel securely welded to the blade. The shanks or handle arms shall be bent at an angle of approximately $90^{\circ}$ to the cutting edge of the blade and the ends shall be peened over caps or washers at end of the handles in such a manner as to prevent the handles from working off the arms when the knives are in use.

E-1c. Handles and ferrules. - The handles shall be so shaped as to afford a comfortable grip for the hand and shall be trimmed with substantial ferrules. The ferrules shall be made of brass or steel. If made of steel other than corrosion resisting they shall be heavily plated in accordance with the best commercial practice with nickel, cadmium, chromium, or other substance equally suitable for the purpose. The ferrules shall be smoothly finished and polished.

E-1d. Blades.-The blades of these knives shall have a polished finish. The cutting edges shall be suitably beveled and properly hardened, tempered, and ground. If welded form of knife is furnished, the tool steel portion shall have a minimum depth of $3 / 4$ inch back from the cutting edge, so as to permit any number of grinds that may be required during the life of the knife.

$\mathrm{E}-1 \mathrm{e}$. Drawing knives of this type shall be similar to illustration and shall also conform to the dimensions prescribed in Table 1.

TABLE 1

\begin{tabular}{|c|c|c|c|c|c|c|c|c|}
\hline \multirow{2}{*}{$\begin{array}{l}\text { Sizes, } \\
\text { inches }\end{array}$} & \multicolumn{2}{|c|}{$\begin{array}{l}\text { Full depth } \\
\text { cutting edge } \\
\text { to back of } \\
\text { blade }\end{array}$} & \multicolumn{2}{|c|}{$\begin{array}{l}\text { Length han. } \\
\text { dles }\end{array}$} & \multicolumn{4}{|c|}{$\begin{array}{c}\text { Diameter handles, ferruled } \\
\text { cap end }\end{array}$} \\
\hline & $\begin{array}{l}\text { Mini- } \\
\text { mum }\end{array}$ & $\begin{array}{l}\text { Maxi- } \\
\text { mum }\end{array}$ & $\begin{array}{l}\text { Mini- } \\
\text { mum }\end{array}$ & $\begin{array}{l}\text { Maxi- } \\
\text { mum }\end{array}$ & $\begin{array}{l}\text { Mini- } \\
\text { mum }\end{array}$ & $\begin{array}{l}\text { Maxi- } \\
\text { mum }\end{array}$ & $\begin{array}{l}\text { Mini- } \\
\text { mum }\end{array}$ & $\begin{array}{l}\text { Maxi. } \\
\text { mum }\end{array}$ \\
\hline $\begin{array}{l}8 . . \\
10 . \\
14 .\end{array}$ & $\begin{array}{c}\text { Inches } \\
1 \\
118 \\
138\end{array}$ & $\begin{array}{r}\text { Inches } \\
13 / 3 \\
11 \frac{1}{13} \\
13\end{array}$ & $\begin{array}{r}\text { Inches } \\
3^{3} 4 \\
3^{3} \\
3^{3}\end{array}$ & $\begin{array}{r}\text { Inches } \\
5 \\
5 \\
5\end{array}$ & $\begin{array}{r}\text { Inch } \\
3 / 4 \\
3 / 4 \\
3 / 4\end{array}$ & $\begin{array}{r}\text { Inch } \\
1 \\
1 \\
1\end{array}$ & $\begin{array}{r}\text { Inches } \\
11 / 4 \\
11 / 4 \\
11 / 4\end{array}$ & $\begin{array}{r}\text { Inches } \\
13 / 4 \\
13 / 4 \\
13 / 4\end{array}$ \\
\hline
\end{tabular}

E-2. Folding handle drawing knives, type B.-

E-2a. Drawing knives of this type shall conform to all the requirements above sutlined for type $A$ drawing knives except that the handles of the type $\mathrm{B}$ drawing knives shall be of the folding scale. The handles of this type of drawing knife shall be hinged near the outer ends of the shank so that the handles may be folded over in a manner to protect the cutting edge of the blade. The design shall be such that the handles are readily adjustable to the opened or closed position and securely fixed in these positions by turning wing or knurled thumb nuts mounted on the shank.

E-2b. Drawing knives of this type shall be similar to illustration.

\section{F. METHODS OF SAMPLING, INSPECTION, AND TESTS.}

F-1. Representative samples, at least three of each type, shall be taken at random from those under inspection and in addition to a careful surface examination shall be subjected to the following test.

The blade of each sample shall be tested for hardness and shall show about one-eighth inch back from the cutting edge a Rockwell hardness number of not less than 52 nor more than 62 on the $\mathrm{C}$ scale. As a further evidence that the knives are suitable for the purpose the samples selected for test shall also be subjected to practical cutting tests on hard woods such as knots found in seasoned black spruce or yellow pine. These tests shall be of sufficient duration to satisfy the inspector as to whether the knives under inspection are in compliance with the requirements of this specification. Under these tests the blade shall not show undue wear, such as turning over or the breaking out of any part of the cutting edge.

F-2. Results of the above tests shall be considered as representative of all the drawing knives under inspection and acceptance or rejection shall be based thereon.

\section{G. PACKAGING, PACKING, AND MARKING}

G-1. Packaging.-Unless otherwise specified, commercial packages are acceptable provided that not more than six drawing knives shall be contained in any one box or package. 
I. NOTES

I-1. The illustrations are included for the convenience of those interested and are not intended to preclude the purchase of drawing hnives which are otherwise in accordance with this specification.

UNITED STATES GOVERNMENT FEDERAL SPECIFICATIONS BOARD, FEDERAL SPECIFICATION FOR KNIVES, PUTTY AND SCRAPING, GGG-K-486, NOVEMBER 10,1931

\section{PUTTY KNIVES, SCRAPING KNIVES}

\section{A. APPLICABIE FEDERAL SPECIFICETIOKS}

A-1. There are no other Federal specifications applicable to this speeification.

\section{B. TYPES}

B-1. These knives shall be furnished in two types, as specified: Type A, putty, and type B, scraping.

\section{MATERIAL AND WORKMANSEIP}

\section{C-1. Material.}

C-1a. Blades.-Best quality, putty knife or cutlery steel.

C-1b. Handles.-Cocobolo.

C-1c. Bolsters.-Rust-resisting metal suitable for the purpose.

C-1d. Rivets,-Brass.

C-2. Workmanship.-First class in every respect.

\section{GENERAL REQUIREMENTS}

D-1. Putty and scraping knives shall be composed of a blade the tang of which shall extend entirely through the handle, a handle of suitable size and shape to afford a comfortable grip to the hand, a bolster at the front end of the handle and a sufficient quantity of rivets securely headed to praclude likelihood of the handle becoming loose in service. These knires shall be of the common commercial shape and designed so as to withstand with minimum breakage conditions likely to be encountered in service. They shall be free from all imperfections that may affect serviceability.

D-2. Marking. - The blades of all knives shall be marked in a plain and permanent manner with the manufacturer's name or with a trade-mark of such known character that the source of manufacture may be readily determined.

D-3. Finish.-All knives shall be smoothly fnished and polished throughout. The abrasive used in the final polish of the blades shall be not coarser than No. 150. The final polish shall be parallel with the long dimension of the blade, a distance not less than one-half its length, measured from the bolster to the extreme working edge of the blade.

\section{E. DETAIL REQUIREMENTS}

\section{E-1. Putty knives, type $A$.}

E-1a. Blades. - The blades of these knives shall be properly hardened and tempered during the process of manufacturing the blades. The blades shall be ground with a uniform taper from the end of the handle to the point of the blade and witn a slight bevel at the extreme working edge. The thicker portion of the blade shall be at the handle end. They shall show at any position on the blade a Rockwell hardness of not less than 48 nor more than 58 on the $\mathrm{C}$ scale. These blades shall also be flexible and suitable for glazing work and other operations of a similar nature. They shall show no permanent deformation or breakage when subjected to the bending test outlined under section F.

$\mathrm{E}-1 \mathrm{~b}$. Putty knives shall be similar to illustration Figure 743 and shall also conform with the dimensions outlined in Table 1.

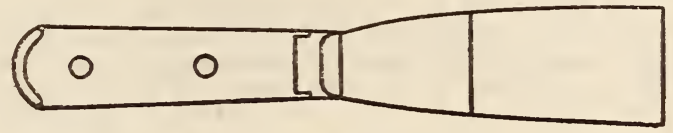

FigCRE 743

TABLE 1

\begin{tabular}{|c|c|c|c|c|c|}
\hline \multicolumn{2}{|c|}{ Orer-all length } & \multicolumn{2}{|c|}{ Width of blade } & \multicolumn{2}{|c|}{ Length of blade } \\
\hline $\begin{array}{l}\text { Mini- } \\
\text { mum }\end{array}$ & $\begin{array}{l}\text { Maxi- } \\
\text { mum }\end{array}$ & $\begin{array}{l}\text { Mini- } \\
\text { mum }\end{array}$ & $\begin{array}{l}\text { Maxi- } \\
\text { mum }\end{array}$ & $\begin{array}{l}\text { Mini- } \\
\text { mum }\end{array}$ & $\begin{array}{l}\text { Maxi- } \\
\text { mum }\end{array}$ \\
\hline $\begin{array}{c}\text { Inches } \\
7\end{array}$ & $\begin{array}{c}\text { Inches } \\
8\end{array}$ & $\begin{array}{c}\text { Inches } \\
11 / 4\end{array}$ & $\begin{array}{c}\text { Inches } \\
138\end{array}$ & $\begin{array}{c}\text { Inches } \\
31 / 2\end{array}$ & $\begin{array}{c}\text { Inches } \\
4\end{array}$ \\
\hline
\end{tabular}

E-2. Scraping knives, type $B$.

E-2a. Blades.-The blades of these knires shall be properly hardened and tempered during the process of manufacturing the blades. The blades shall be ground with a uniform taper from the end of the handle to the point of the blade and with a slight bevel at the extreme working edge. The thicker portion of the blade shall be at the handle end. They shall show at any position on the blade a Rockwell hardness of not less than 48 nor more than 58 on the $\mathrm{C}$ scale. The blades of these knives shall be stiff unless otherwise specified. When specifically required they shall be flexible or semiflexible. The blades of these knives shall be suitable for scraping operations. They shall show no permanent deformation or breakage when subjected to the bending test outlined under section $F$.

E-2b. Scraping knives shall be similar to illustration Figure 744 and shall also conform with the dimensions outlined in Table 2.

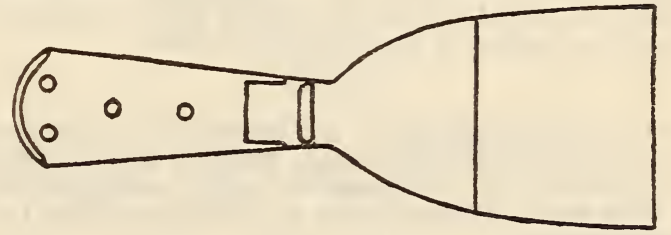

FIGURE 744

TABLE 2

\begin{tabular}{|c|c|c|c|c|c|}
\hline \multicolumn{2}{|c|}{ Orer-all length } & \multicolumn{2}{|c|}{ Width of blade } & \multicolumn{2}{|c|}{ Length of blade } \\
\hline $\begin{array}{l}\text { Mini- } \\
\text { mum }\end{array}$ & $\begin{array}{l}\text { Maxi- } \\
\text { mum }\end{array}$ & $\begin{array}{l}\text { Mini- } \\
\text { mum }\end{array}$ & $\begin{array}{l}\text { Maxi- } \\
\text { mum }\end{array}$ & $\begin{array}{l}\text { Mini- } \\
\text { mum }\end{array}$ & $\begin{array}{l}\text { Maxi- } \\
\text { mum }\end{array}$ \\
\hline $\begin{array}{c}\text { Inches } \\
712\end{array}$ & $\begin{array}{c}\text { Inches } \\
81 / 2\end{array}$ & $\begin{array}{c}\text { Inches } \\
2^{15} / 16\end{array}$ & $\begin{array}{l}\text { Inches } \\
31116\end{array}$ & $\begin{array}{c}\text { Inches } \\
4\end{array}$ & $\begin{array}{c}\text { Inches } \\
41 / 2\end{array}$ \\
\hline
\end{tabular}

\section{F. METHODS OF SAMPLING, INSPECTION AND TESTS}

F-1. All putty and scraping knives shall be inspected to determine whether they are in compliance with the requirements of this specification. In addition to a careful surface inspection at least three samples of each trpe of knife (in no instance less than 2 per cent of entire quantity of each type under inspection) shall be taken at random 
from those under inspection and subjected to the test outlined hereunder.

The Rockwell hardness of the blades shall be checked for compliance with the requirements outlined in section $\mathrm{E}$.

The front end of each blade shall be gripped in an ordinary bench vise with the front edge of the blade parallel with and set one-fourth inch below the top surface of the vise jaws. The outer end of the handle shall then be moved each side of the perpendicular through the arc given below for the respective style of blade.

\begin{tabular}{|c|c|}
\hline Style of blade & Arc \\
\hline $\begin{array}{l}\text { Flexible } \\
\text { Semiflexible } \\
\text { Stiff }\end{array}$ & $\begin{array}{l}45^{\circ} \text { from perpendicular }\left(90^{\circ} \text { in all }\right) \text {. } \\
30^{\circ} \text { from perpendicular }\left(60^{\circ} \text { in all }\right) \text {. } \\
15^{\circ} \text { from perpendicular }\left(30^{\circ} \text { in all }\right) \text {. }\end{array}$ \\
\hline
\end{tabular}

F-2. Knives failing to pass the above inspection test shal be rejected. Acceptance or rejection of entire quantity under inspection shall be based on the results of the above inspection test.

\section{G. PACKAGING, PACKING, AND MARKING}

G-1. Packaging.-Unless otherwise specified commercial packages are acceptable, provided that not more than six knives (which shall be of the same type) shall be contained in any one box or package.

G-2. Packing.- Unless otherwise specified the subject commodity shall be delivered in standard commercial containers so constructed as to insure acceptance by common or other carrier, for safe transportation, at the lowest rate, to the point of delivery.

G-3. Marking.

G-3a. Unless otherwise specified, each box or package shall be marked with the name of the article, the size, the type, the quantity contained therein, and the name of the manufacturer.

G-3b. Unless otherwise specified, shipping containers shall be marked with the name of the article, the size, the type, and the quantity contained therein as defined by the contract or order under which shipment is made, the name of the contractor. and the number of the contract or order.

\section{NOTES}

I-1. The illustrations are included for the convenience of those interested, and are not intended to preclude the purchase of putty and scraping knives which are otherwise in accordance with this specification.

I-2. It is believed that this specification adequately describes the characteristics necessary to secure the desired material, and that normally no samples will be necessary prior to award to determine compliance with this specification. If, for any particular purpose, samples with bids are necessary, they should be specifically asked for in the invitation for bids and the particular purpose to be served by the bid sample should be definitely stated, the specification to apply in all other respects.

616.16 PLANES AND SCRAPERS.

\section{UNITED STATES GOVERNMENT, FEDERAL SPECIFICATIONS BOARD; FEDERAL SPECIFICATION FOR SHAVES, SPOKE, GGG-S-271，MARCH 1, 1932}

\section{SPOKESHAVES}

\section{GENERAL SPECIFICATIONS}

There are no general specifications applicable to this specification.

\section{TYPE}

Spokeshaves shall be of the raised-handle type.

\section{MATERIAL AND WORKMATSHIP}

All spokeshaves shall be made of high-grade material and be free from all defects that may affect their serviceability. They shall be of the best workmanship and shall be made in accordance with the best commercial practice.

\section{GENERAL REQUIREMEYTS}

See Section V.

\section{DETAIL REQUIREMENTS}

1. Spokeshaves shall be essentially composed of a frame with two handles, a cutter, and a cap iron into which a thumbscrew shall be fitted. Each spokeshave shall have the maker's name or known trade-mark stamped or otherwise permanently marked thereon.

2 . The frame, handles, and cap iron shall be made of malleable or gray cast iron. The frame shall be finished on that portion where the cutter projects and which comes in direct contact with the work. The handles shall be japanned or enameled, of the raised type, and well proportioned to afford a good handgrip. The handles shall have a hole near the ends to enable the user to hang the tool up out of

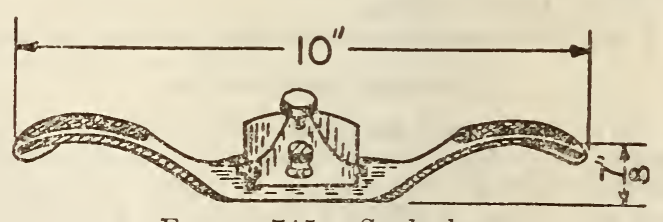

FIGURE 745. - Spokeshave

the way. The cutter shall ve made of high-grade tool steel, properly hardened and tempered a distance from the cutting edge to at least one-half the length of the cutter, and shall be ground and sharpened ready for use. The cutter shall show about one-eighth inch back from the cutting edge a Rockwell hardness number of not less than 58 nor more than 68 on the $\mathrm{C}$ scale. The cutter shall be held in position with a cap iron fastened by a thumbscrew in such manner as to exert an even pressure on the cutter edge and also to allow adjustment of the cutter without the use of a screw driver or other tool.

3. Spokeshaves shall be 10 inches long over all and, the width of cutter shall be $21 / 8$ inches.

4. A tolerance of plus or minus 5 per cent is permitted in the above dimensions.

5. Spokeshaves shall be similar to Figure 745 .

\section{METHOD OF INSPECTION AND TESTS}

Representative samples, at least three, shall be taken at random from those under inspection and, in addition to a careful surface, inspection and test of the cutters for hardness, shall be subjected to practical cutting tests of sufficient duration to satisfy the inspector whether the tools are of satisfactory quality and in compliance with this specification.

\section{PACKING AND MARKING}

Packaging.-Unless otherwise specified, commercial packages are acceptable, provided that not more than one spokeshave shall be contained in any one box or package. 
616.17 SAWS.

\section{UNITED STATES GOVERNMENT, FEDERAL SPECIFICATIONS BOARD; FEDERAL SPECIFICATION FOR SETS, HANDSAW, NO. 440, SEPTEMBER 25, 1926 (CURRENT DESIGNATION : GGG-H-101) \\ HANDSAW SETS}

(See 615.9, p. 940.)

\section{UNITED STATES GOVERNMENT, FEDERAL SPECIFICATIONS BOARD, FEDERAL SPECIFICATION FOR SAWS, No. 480, APRIL 25, 1927. (CURRENT DESIGNA- TION : GGG-S-61)}

\section{SAWS \\ II. TYPES}

Saws shall be of the following types, as specified: A, backsaw; B, miter-box saw; C, band saw, spring set, metal cutting; $\mathrm{D}$, band saw, spring set, wood cutting; E, band saw, swage set, wood cutting; $\mathrm{F}$, butcher saw; $\mathrm{G}$, circular saw, crosscut; $\mathrm{H}$, circular saw, rip; I, compass saw; J, crosscut saw, two-man; $\mathrm{K}$, hack saw, hand; $\mathrm{L}$, handsaw, crosscut; $\mathrm{M}$, handsaw, rip; $\mathrm{N}$, keyhole saw; and $\mathrm{O}$, web saw, felloe.

\section{MATERIAL AND WORKMANSHIP}

1. All saw blades, band saws and circular saws shall be made of the best grade crucible or electricfurnace steel, free from cracks, seams, imperfections, hammer marks, or other defects affecting their appearance or that may affect their serviceability. They shall be of good workmanship and equal in every respect to the best commercial product.

2. All handles furnished on saws covered by this specification shall be free from defects or blemishes and shall be made of thoroughly seasoned apple wood with the exception of those handles which are otherwise specified in Section V.

\section{GENERAL REQUIREMENTS}

1. All handles shall have polished and rounded edges with the exception of those for two-man crosscut saws, type J.

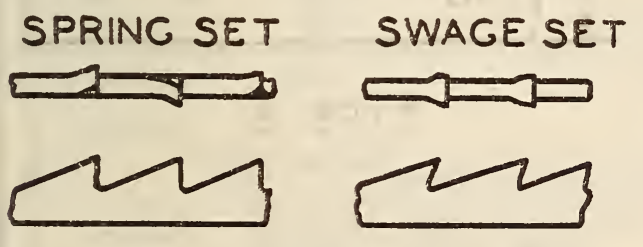

Figure 746

2. The nominal length of all saws shall be the length of the tooth edge, unless otherwise specified.

3. All saws furnished under this specification shall be etched, stamped, or otherwise permanently marked with the manufacturer's name, and shall also be coated with oil or other rust preventive.

4. Saws shall be without set, with spring set, or with swage set, as specifically called for under Section V. Swage-set saws shall have every tooth swaged to the same width of cutting edge and the same clearance on each side of the body of the blade. Spring-set saws shall have every tooth set an equal amount, alternate teeth being set to the same side of the saw.

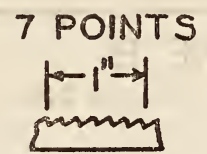

FIGURE 747

5. Points per inch shall be measured as shown in Figure 747.

\section{DETAIL REQUIREMENTS}

1. Backsaws, type A.-These saws shall be of uniform thickness throughout with the exception of a reinforcing steel back about seven-eighths inch wide and 0.109 inch thick. The teeth shall be spring set, bevel filed, and adapted for both crosscutting and ripping. The teeth shall be set to provide a proper clearance on each side of the blade.

The handle shall be secured to the blade by means of three brass screws and nuts. These saws shall be similar to Figure 748 and conform to the dimensions in the following table:

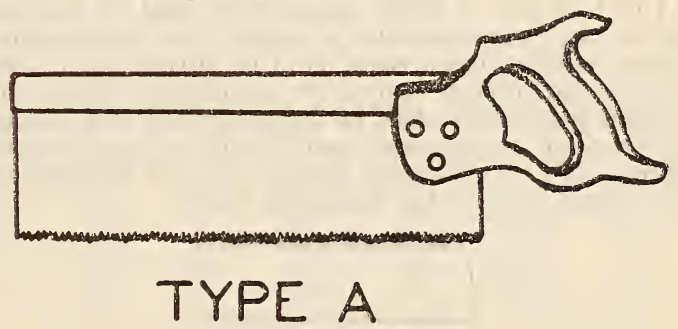

Figure 748

\begin{tabular}{|r|r|r|r|r|}
\hline $\begin{array}{r}\text { Length on } \\
\text { cutting } \\
\text { edge }\end{array}$ & $\begin{array}{c}\text { Width } \\
\text { under } \\
\text { back }\end{array}$ & $\begin{array}{c}\text { Points } \\
\text { per inch }\end{array}$ & \multicolumn{2}{|c|}{ Thickness of blade } \\
\cline { 4 - 5 } & Minimum & Maximum \\
\hline $\begin{array}{r}\text { Inches } \\
16\end{array}$ & $\begin{array}{r}\text { Inches } \\
31 / 2 \\
21 / 2\end{array}$ & 12 & $\begin{array}{r}\text { Inch } \\
0.032\end{array}$ & $\begin{array}{c}\text { Inch } \\
0.042 \\
.028\end{array}$ \\
\hline
\end{tabular}

2. Miter-box saws, type B.-These saws shall be of uniform thickness through out with the exception of a reinforcing steel back about 1 inch wide and about $0.109 \mathrm{inch}$ thick. The teeth shall be spring set, bevel filed, and adapted for both crosscutting and ripping. The teeth shall be set to provide proper clearance on each side of the blade. The tooth edge shall be 2 inches shorter than the length or size as specified in the table. 
The handles shall be secured to the blade by means of three brass screws and nuts. These saws shall be similar to Figure 749 and conform to the dimensions in the following table:

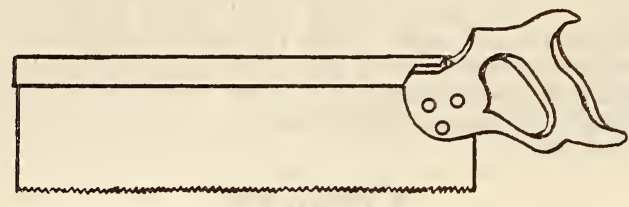

TYPE B

Figure 749

\begin{tabular}{|c|c|c|c|c|c|}
\hline \multirow{2}{*}{$\begin{array}{c}\text { Full } \\
\text { length of } \\
\text { blade }\end{array}$} & \multirow{2}{*}{$\begin{array}{l}\text { Width } \\
\text { under } \\
\text { back }\end{array}$} & \multicolumn{2}{|c|}{ Points per inch } & \multicolumn{2}{|c|}{ Thickness } \\
\hline & & $\begin{array}{l}\text { Mini- } \\
\text { mum }\end{array}$ & $\begin{array}{l}\text { Maxi- } \\
\text { mum }\end{array}$ & $\begin{array}{l}\text { Mini- } \\
\text { mum }\end{array}$ & $\begin{array}{l}\text { Maxi- } \\
\text { mum }\end{array}$ \\
\hline $\begin{array}{r}\text { Inches } \\
26\end{array}$ & $\begin{array}{r}\text { Inches } \\
4\end{array}$ & 11 & 12 & $\begin{array}{l}\text { Inch } \\
0.035\end{array}$ & $\begin{array}{l}\text { Inch } \\
0.049\end{array}$ \\
\hline
\end{tabular}

3. Band saw, spring set, metal cutting, type C.These saws shall be of uniform thickness throughout. The teeth shall be spring set, filed straight across, and shall provide a proper clearance on each side of the blade. These saws shall be tempered throughout or may be hardened and tempered on the tooth edge only; the temper shall be as high as possible consistent with allowing the teeth to be set. Every 14 feet or less of these band saws shall be marked with the manufacturer's name or trade-mark. Metal-cutting band saws shall be furnished in 200foot coils, unless otherwise specified. When required these saws shall be furnished cut and brazed to specified lengths and ready to fit on band wheel. They shall be similar to Figure 750 and conform to the dimensions in the following table:

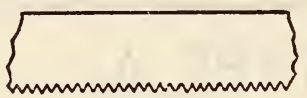

TYPE C

Figure 750

\begin{tabular}{|c|c|c|c|c|}
\hline \multirow{2}{*}{ Width } & \multicolumn{2}{|c|}{ Points per inch } & \multicolumn{2}{|c|}{ Thickness } \\
\hline & $\begin{array}{l}\text { Mini- } \\
\text { mum }\end{array}$ & $\begin{array}{l}\text { Maxi- } \\
\text { mum }\end{array}$ & $\begin{array}{l}\text { Mini- } \\
\text { mum }\end{array}$ & $\begin{array}{l}\text { Maxi- } \\
\text { mum }\end{array}$ \\
\hline $\begin{array}{c}\text { Inches } \\
3 / 8 \\
3 / 4 \\
3 / 4 \\
1 \\
1\end{array}$ & $\begin{array}{r}8 \\
8 \\
14 \\
14 \\
22\end{array}$ & $\begin{array}{l}10 \\
10 \\
16 \\
16 \\
24\end{array}$ & $\begin{array}{c}\text { Inch } \\
0.022 \\
.028 \\
.028 \\
.032 \\
.032\end{array}$ & $\begin{array}{c}\text { Inch } \\
0.028 \\
.035 \\
.035 \\
.042 \\
.042\end{array}$ \\
\hline
\end{tabular}

4. Band saw, spring set, wood cutting, type D.These saws shall be of uniform thickness throughout. The teeth shall be spring set, filed straight across, and adapted for either crosscutting or ripping. Every 14 feet or less of these band saws shall be marked with the manufacturer's name or trademark. The teeth shall be set to provide a proper clearance on each side. These saws shall be furnished in 200-foot coils unless otherwise specified. When required these saws shall be furnished cut and brazed to specified lengths and ready to fit on band wheel. They shall be similar to Figure 751 and conform to the dimensions in the following table:

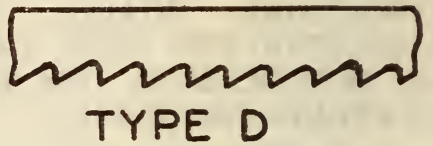

Figure 751

\begin{tabular}{|c|c|c|c|}
\hline & \multirow{2}{*}{ Width } & $\begin{array}{c}\text { Points per } \\
\text { inch }\end{array}$ & \multicolumn{2}{|c|}{ Thickness } \\
\cline { 3 - 4 } & & Minimum & Maximum \\
\cline { 3 - 4 } Inches & & Inch & Inch \\
$1 / 4$ & & 0.025 & 0.032 \\
$3 / 8$ & 6 & .028 & .035 \\
$1 / 2$ & $51 / 2$ & .028 & .035 \\
$1 / 4$ & 5 & .028 & .035 \\
$11 / 2$ & $31 / 2$ & .032 & .042 \\
2 & 3 & .035 & .049 \\
& 3 & .035 & .049 \\
\hline
\end{tabular}

5. Band saw, swage set, wood cutting, type E.These saws shall be of uniform thickness throughout and shall be crowned or uncrowned to suit the machine in which they will be used, as specified. The hook of the teeth shall be approximately fiveeighths of an inch for each inch of width of the saw.

The teeth shall be swaged to provide a proper clearance on each side of the blade. The length of the saws must be specified, also whether right or left hand; and the saws delivered shall be joined, set, and filed and ready to fit on the band wheels. These saws shall be similar to Figure 752 and conform to the dimensions in the following table:

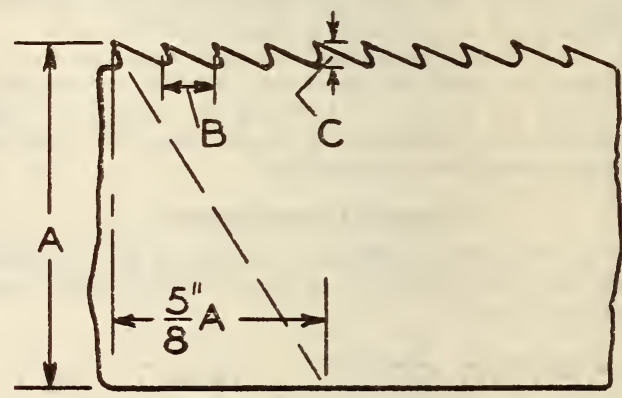

TYPE $E$

FIGURE 752

\begin{tabular}{|c|c|c|c|c|}
\hline \multirow{2}{*}{$\begin{array}{c}\text { Width } \\
\text { dimension } \\
A\end{array}$} & \multirow{2}{*}{$\begin{array}{l}\text { Point to } \\
\text { point di- } \\
\text { mension } \\
\qquad B\end{array}$} & \multirow{2}{*}{$\begin{array}{l}\text { Depth of } \\
\text { tooth di- } \\
\text { mension } \\
C\end{array}$} & \multicolumn{2}{|c|}{ Thickness } \\
\hline & & & Minimum & Maximum \\
\hline $\begin{array}{c}\text { Inches } \\
21 / 2 \\
3 \\
4 \\
6 \\
8 \\
10 \\
12\end{array}$ & $\begin{array}{r}\text { Inches } \\
11 / 4 \\
11 / 4 \\
11 / 4 \\
11 / 2 \\
13 / 4 \\
13 / 4 \\
13 / 4\end{array}$ & $\begin{array}{r}\text { Inch } \\
3 / 8 \\
3 / 8 \\
3 / 8 \\
1 / 2 \\
58 \\
3 / 4 \\
3 / 4\end{array}$ & $\begin{array}{l}\text { Inch } \\
0.035 \\
.035 \\
.035 \\
.042 \\
.058 \\
.065 \\
.072\end{array}$ & $\begin{array}{l}\text { Inch } \\
0.049 \\
.049 \\
.049 \\
.058 \\
.072 \\
.083 \\
.095\end{array}$ \\
\hline
\end{tabular}

6. Butcher saws, type F.-These saws shall include the frame and the blade. The frame shall be made of steel and bent to form. It shall have a flat 
back with square or round edges and shall have a cross section of about three-fourths by one-fourth inch and suitable for blades 24 inches in length. A suitable winged nut or equivalent device shall be provided for tightening the blade in place. The handle shall be made of apple or beech wood and secured to the frame by means of three nickelplated steel screws and nuts.

The blade shall be of uniform thickness throughout; the teeth shall be spring set and filed straight across. The teeth shall be set to provide a proper clearance on each side of the blade. All butcher saws, both frames and blades, shall be marked in accordance with "General requirements," Section IV. They shall be similar to Figure 753 and conform to the dimensions in the following table:

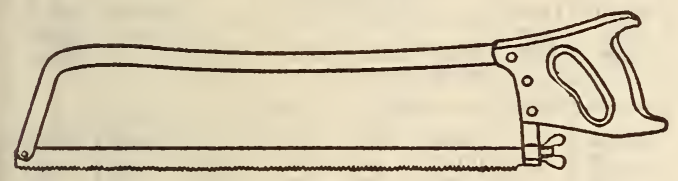

TYPE F

FigURE 753

\begin{tabular}{|c|c|c|c|c|}
\hline \multirow{2}{*}{$\begin{array}{l}\text { Length of } \\
\text { blade }\end{array}$} & \multirow{2}{*}{$\begin{array}{l}\text { Width of } \\
\text { blade }\end{array}$} & \multirow{2}{*}{$\begin{array}{l}\text { Points } \\
\text { per inch }\end{array}$} & \multicolumn{2}{|c|}{ Thickness of blade } \\
\hline & & & Minimum & Maximum \\
\hline $\begin{array}{r}\text { Inches } \\
24\end{array}$ & $\underset{3 / 4}{\text { Inch }}$ & 11 & $\begin{array}{l}\text { Inch } \\
0.022\end{array}$ & $\begin{array}{l}\text { Inch } \\
0.028\end{array}$ \\
\hline
\end{tabular}

7. Circular saws, crosscut, type G.-These saws shall be suitable for either right or left hand cutting and shall be of uniform thickness throughout. The teeth shall be spring set to provide a proper clearance on each side of the saw. The hook of the teeth shall be such that a line passing down the front or cutting edge shall go through the center of the saw. On the 44-inch saw there shall be a gullet extending into the front or cutting edge of the teeth. The depth of the teeth shall be approximately three-fourths of the point-to-point spacing.

These saws shall be tensioned for a rim speed of 10,000 feet per minute and shall be furnished with arbor holes as listed below. No undersize tolerance will be permitted on the arbor-hole diameter, but an oversize tolerance of 0.001 inch in diameter of hole will be allowed. Crosscut circular saws shall be similar to Figure 754 and conform to the dimensions in the following table:

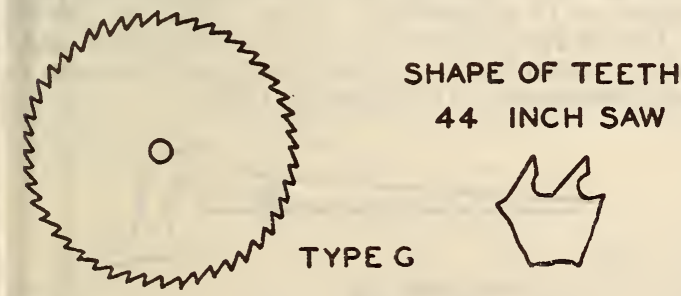

FIGURE 754

\begin{tabular}{|c|c|c|c|c|c|}
\hline \multirow[b]{2}{*}{ Diameter } & \multirow{2}{*}{$\begin{array}{l}\text { Number } \\
\text { of teeth }\end{array}$} & \multicolumn{2}{|c|}{ Thickness } & \multirow{2}{*}{$\begin{array}{l}\text { Revolu- } \\
\text { tions per } \\
\text { minute }\end{array}$} & \multirow{2}{*}{$\begin{array}{l}\text { Arbor- } \\
\text { hole dia- } \\
\text { ameter }\end{array}$} \\
\hline & & $\begin{array}{l}\text { Mini- } \\
\text { mum }\end{array}$ & $\begin{array}{l}\text { Maxi- } \\
\text { mum }\end{array}$ & & \\
\hline $\begin{array}{r}\text { Inches } \\
12 \\
14 \\
16 \\
18 \\
20 \\
24 \\
44\end{array}$ & $\begin{array}{l}90 \\
90 \\
80 \\
80 \\
80 \\
70 \\
90\end{array}$ & $\begin{array}{l}\text { Inch } \\
0.065 \\
.072 \\
.072 \\
.083 \\
.083 \\
.109 \\
.148\end{array}$ & $\begin{array}{l}\text { Inch } \\
0.083 \\
.095 \\
.095 \\
.109 \\
.109 \\
.134 \\
.180\end{array}$ & $\begin{array}{r}3,180 \\
2,730 \\
2,390 \\
2,120 \\
1,910 \\
1,590 \\
870\end{array}$ & $\begin{array}{r}\text { Inches } \\
11 / 4 \\
11 / 4 \\
11 / 4 \\
1114 \\
11 / 4 \\
136 \\
115 / 16\end{array}$ \\
\hline
\end{tabular}

8. Circular saws, rip, type H.-These saws shall be suitable for right or left hand cutting and shall be of uniform thickness throughout. The teeth shall be adapted for ripping and shall be spring or swage set. The teeth shall be set to provide a proper clearance on each side of the saw. The hook of the teeth where possible shall be such that a line passing down the front or cutting edge of the teeth shall, when extended, be tangent to a circle whose diameter is one-half that of the saw and whose center coincides with the center of the saw. The depth of the gullets shall be approximately equal to one-half the distance, point to point, of the teeth.

These saws shall be tensioned for a rim speed of 10,000 feet per minute and shall be furnished with arbor holes as listed. No undersize tolerance will be permitted on the arbor-hole diameter, but an oversize tolerance of 0.001 inch in diameter of hole will be allowed. Rip circular saws shall be similar to Figure 755 and conform to the dimensions in the following table:

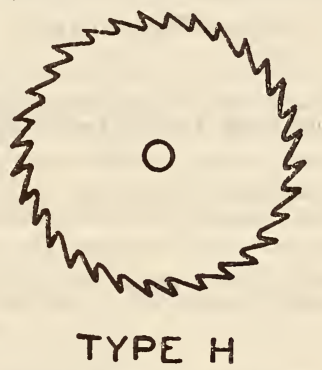

FIGURE 755

\begin{tabular}{|c|c|c|c|c|c|}
\hline \multirow[b]{2}{*}{ Diameter } & \multirow{2}{*}{$\begin{array}{l}\text { Number } \\
\text { of teeth }\end{array}$} & \multicolumn{2}{|c|}{ Thickness } & \multirow{2}{*}{$\begin{array}{l}\text { Revolu- } \\
\text { tions per } \\
\text { minute }\end{array}$} & \multirow{2}{*}{$\begin{array}{c}\text { Arbor- } \\
\text { hole } \\
\text { diameter }\end{array}$} \\
\hline & & $\begin{array}{l}\text { Mini- } \\
\text { mum }\end{array}$ & $\begin{array}{l}\text { Maxi- } \\
\text { mum }\end{array}$ & & \\
\hline $\begin{array}{r}\text { Inches } \\
12 \\
14 \\
16 \\
18 \\
20\end{array}$ & $\begin{array}{l}36 \\
36 \\
36 \\
36 \\
36\end{array}$ & $\begin{array}{l}\text { Inch } \\
0.065 \\
.072 \\
.072 \\
.083 \\
.083\end{array}$ & $\begin{array}{l}\text { Inch } \\
0.083 \\
.095 \\
.095 \\
.109 \\
.109\end{array}$ & $\begin{array}{l}3,180 \\
2,730 \\
2,390 \\
2,120 \\
1,910\end{array}$ & $\begin{array}{r}\text { Inches } \\
11 / 4 \\
11 / 4 \\
11 / 4 \\
11 / 4 \\
11 / 4\end{array}$ \\
\hline
\end{tabular}

9. Compass saws, type 1 .-These saws shall be of uniform thickness throughout the length of the cutting edge. The butt or heel on the back may be of the same thickness as the tooth or cutting edge, in which case there shall be a gradual taper from the heel to the point; or the back may be of uniform thickness throughout, in which case the back shall be tapered thin with reference to the cutting edge. The teeth shall be spring set, bevel filed, and adapted for both crosscutting and ripping. The teeth shall be set to provide proper clearance on each side of the blade.

The handle shall be secured to the blade by means of two brass screws and nuts. These saws shall be similar to Figure 756 and conform to the dimensions in the following table:

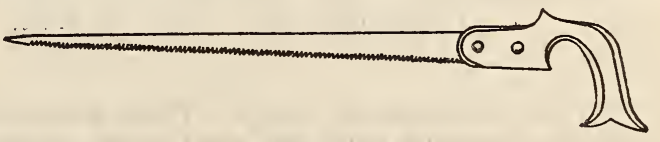

FigURe 756

\begin{tabular}{|c|c|c|c|c|c|c|}
\hline \multirow{2}{*}{$\begin{array}{l}\text { Length } \\
\text { on cut- } \\
\text { ting } \\
\text { edge }\end{array}$} & \multirow{2}{*}{$\begin{array}{l}\text { Points } \\
\text { per } \\
\text { inch }\end{array}$} & \multicolumn{2}{|c|}{$\begin{array}{l}\text { Thickness on } \\
\text { cutting edge }\end{array}$} & \multicolumn{2}{|c|}{$\begin{array}{l}\text { Thickness on } \\
\text { back at point }\end{array}$} & \multirow{2}{*}{$\begin{array}{l}\text { Width } \\
\text { at heel } \\
\text { at en- } \\
\text { trance } \\
\text { to } \\
\text { handle }\end{array}$} \\
\hline & & $\begin{array}{l}\text { Mini- } \\
\text { mum }\end{array}$ & $\begin{array}{l}\text { Maxi- } \\
\text { mum }\end{array}$ & $\begin{array}{l}\text { Mini- } \\
\text { mum }\end{array}$ & $\begin{array}{l}\text { Maxi- } \\
\text { mum }\end{array}$ & \\
\hline $\begin{array}{c}\text { Inches } \\
14 \\
12\end{array}$ & $\begin{array}{l}8 \\
8\end{array}$ & $\begin{array}{r}\text { Inch } \\
0.049 \\
.049\end{array}$ & $\begin{array}{r}\text { Inch } \\
0.065 \\
.065\end{array}$ & $\begin{array}{r}\text { Inch } \\
0.035 \\
.035\end{array}$ & $\begin{array}{r}\text { Inch } \\
0.048 \\
.048\end{array}$ & $\begin{array}{c}\text { Inches } \\
11 / 4 \\
11 / 8\end{array}$ \\
\hline
\end{tabular}


10. Crosscut saws, two-man, type J.-These saws shall be taper ground toward the back. The cutting edge shall be of uniform thickness throughout the length. These saws shall be furnished with handles of thoroughly seasoned hardwood, the handles to be of the loop type and secured to the saw by means of the loop screwing up into an internally threaded nut in the handle. The handle shall be from $91 / 2$ to $10^{1 / 2}$ inches long and $13 / 8$ inches largest diameter, the length to be exclusive of the loop.

These saws shall be furnished with a style of tooth as shown in Figure 757 and shall conform to the dimensions in the following table:

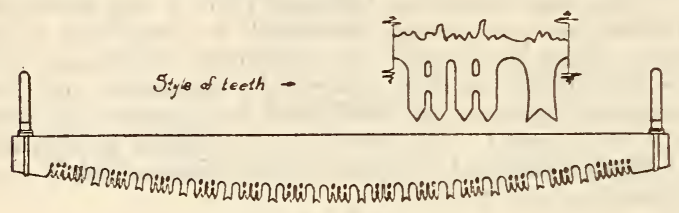

Figure 757.-Type $J$

\begin{tabular}{|c|c|c|c|c|c|c|c|c|}
\hline $\begin{array}{l}\frac{y}{0} \\
\text { oा } \\
0 \\
0\end{array}$ & $\begin{array}{l}\text { Thick } \\
\text { cuttin }\end{array}$ & $\begin{array}{l}\text { eess on } \\
\text { g edge }\end{array}$ & $\begin{array}{l}\text { Thick } \\
\text { back a }\end{array}$ & $\begin{array}{l}\text { ess on } \\
\text { center }\end{array}$ & $\begin{array}{l}\text { Thick } \\
\text { back }\end{array}$ & $\begin{array}{l}\text { eess on } \\
t \text { ends }\end{array}$ & $\begin{array}{r}\text { Wid } \\
\text { cen }\end{array}$ & $\begin{array}{l}\text { th at } \\
\text { nter }\end{array}$ \\
\hline 㷰 & $\begin{array}{l}\text { Mini- } \\
\text { mum }\end{array}$ & $\underset{\text { Maxi- }}{\text { Mam }}$ & $\begin{array}{l}\text { Mini- } \\
\text { mum }\end{array}$ & $\begin{array}{l}\text { Maxi- } \\
\text { mum }\end{array}$ & $\begin{array}{l}\text { Mini- } \\
\text { mum }\end{array}$ & $\begin{array}{l}\text { Maxi- } \\
\text { mum }\end{array}$ & $\begin{array}{l}\text { Mini- } \\
\text { mum }\end{array}$ & $\begin{array}{l}\text { Maxi- } \\
\text { mum }\end{array}$ \\
\hline $\begin{array}{c}\text { Feet } \\
5\end{array}$ & $\begin{array}{l}\text { Inch } \\
0.072\end{array}$ & $\begin{array}{l}\text { Inch } \\
0.095\end{array}$ & $\begin{array}{l}\text { Inch } \\
0.035\end{array}$ & $\begin{array}{l}\text { Inch } \\
0.049\end{array}$ & $\begin{array}{l}\text { Inch } \\
0.058\end{array}$ & $\begin{array}{l}\text { Inch } \\
0.072\end{array}$ & $\begin{array}{c}\text { Inches } \\
51 / 2\end{array}$ & $\begin{array}{c}\text { Inches } \\
61 / 2\end{array}$ \\
\hline
\end{tabular}

11. Hack saw, hand, type K.-These saws shall be of the straigth-back type and either taper ground or taper and hollow ground. They shall be of uniform thickness throughout the length of the cutting edge. The back of the saw may be of uniform gage throughout or may be tapered from the heel to the point. The teeth shall not be set but shall be milled straight across.

The handle shall be secured to the blade by means of four brass screws and nuts. These saws shall be similar to Figure 758 and conform to the dimensions in the following table:

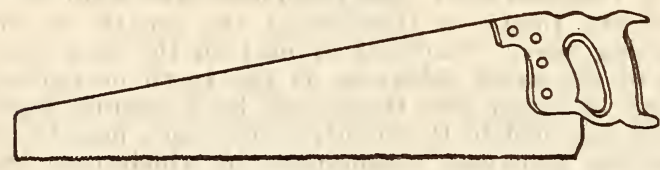

TYPE K

Figure 758

\begin{tabular}{|c|c|c|c|c|c|c|c|c|}
\hline \multirow{2}{*}{ 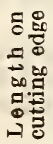 } & \multicolumn{2}{|c|}{$\begin{array}{l}\text { Points per } \\
\text { inch }\end{array}$} & \multicolumn{2}{|c|}{$\begin{array}{l}\text { Thickness on } \\
\text { cutting edge }\end{array}$} & \multicolumn{2}{|c|}{$\begin{array}{c}\text { Thickness on } \\
\text { back at } \\
\text { handle }\end{array}$} & \multicolumn{2}{|c|}{$\begin{array}{c}\text { Thickness on } \\
\text { back at } \\
\text { point }\end{array}$} \\
\hline & $\begin{array}{l}\text { Mini- } \\
\text { mum }\end{array}$ & $\begin{array}{l}\text { Maxi- } \\
\text { mum }\end{array}$ & $\begin{array}{l}\text { Mini- } \\
\text { mum }\end{array}$ & $\begin{array}{l}\text { Maxi- } \\
\text { mum }\end{array}$ & $\begin{array}{l}\text { Mini- } \\
\text { mum }\end{array}$ & $\begin{array}{l}\text { Mari- } \\
\text { mum }\end{array}$ & $\begin{array}{l}\text { Mini- } \\
\text { mum }\end{array}$ & $\begin{array}{l}\text { Maxi- } \\
\text { mum }\end{array}$ \\
\hline $\begin{array}{r}\text { Ins. } \\
18\end{array}$ & 12 & 15 & $\begin{array}{l}\text { Inch } \\
0.042\end{array}$ & $\begin{array}{l}\text { Inch } \\
0.049\end{array}$ & $\begin{array}{l}\text { Inch } \\
0.032\end{array}$ & $\begin{array}{l}\text { In } \\
0 .\end{array}$ & $\begin{array}{l}\text { Inch } \\
0.022\end{array}$ & $\begin{array}{l}\text { Inch } \\
0.025\end{array}$ \\
\hline
\end{tabular}

12. Handsaws, crosscut, type L.-These saws shall be of the skewback type and shall be of uniform thickness throughout the length of the cutting edge. The back shall have a gradual taper from the heel to the point. The teeth shall be spring set, bevel filed, and adapted for crosscutting. The teeth shall be set to provide proper clearance on each side of the blade.

The handle shall be secured to the blade by means of four brass screws and nuts in the case of the 20-inch saw and five brass screws and nuts in the case of a longer saw. These saws shall be similar to Figure 759 and conform to the dimensions in the following table:

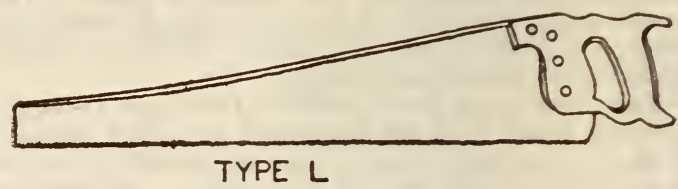

Figdre 759

\begin{tabular}{|c|c|c|c|c|c|c|c|}
\hline \multirow{2}{*}{$\begin{array}{c}\text { Length } \\
\text { on eut- } \\
\text { ting } \\
\text { edge }\end{array}$} & \multirow{2}{*}{$\begin{array}{c}\text { Points } \\
\text { per } \\
\text { inch }\end{array}$} & \multicolumn{2}{|c|}{$\begin{array}{l}\text { Thickness on } \\
\text { cutting edge }\end{array}$} & \multicolumn{2}{|c|}{$\begin{array}{l}\text { Thickness on } \\
\text { back at point }\end{array}$} & \multirow{2}{*}{$\begin{array}{l}\text { Width } \\
\text { butt }\end{array}$} & \multirow{2}{*}{$\begin{array}{l}\text { Width } \\
\text { point, } \\
\text { mini- } \\
\text { mum }\end{array}$} \\
\hline & & $\begin{array}{l}\text { Mini- } \\
\text { mum }\end{array}$ & $\begin{array}{l}\text { Maxi- } \\
\text { mum }\end{array}$ & $\begin{array}{l}\text { Mini- } \\
\text { mum }\end{array}$ & $\begin{array}{l}\text { Maxi- } \\
\text { mum }\end{array}$ & & \\
\hline $\begin{array}{r}\text { Inches } \\
20 \\
26\end{array}$ & $\begin{array}{r}10 \\
8\end{array}$ & $\begin{array}{l}\text { Inch } \\
0.032 \\
.032\end{array}$ & $\begin{array}{l}\text { Inch } \\
0.042 \\
.042\end{array}$ & $\begin{array}{l}\text { Inch } \\
0.022 \\
.022\end{array}$ & $\begin{array}{l}\text { Inch } \\
0.028 \\
.028\end{array}$ & $\begin{array}{r}\text { Inches } \\
51 / 2 \\
71 / 4\end{array}$ & $\begin{array}{r}\text { Inches } \\
216 \\
256\end{array}$ \\
\hline
\end{tabular}

13. Handsaws, rip, type $M$.-These saws shall conform to the requirements for handsaws, crosscut, type $\mathrm{L}$, except as to dimensions and that the teeth shall be filed straight across and adapted for ripping. These saws shall be similar to Figure 760 and conform to the dimensions in the following table:

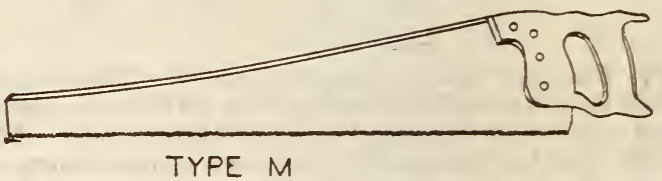

FigdRE 760

\begin{tabular}{|c|c|c|c|c|c|c|c|}
\hline \multirow{2}{*}{$\begin{array}{l}\text { Length } \\
\text { on cut- } \\
\text { ting } \\
\text { edge }\end{array}$} & \multirow{2}{*}{$\begin{array}{c}\text { Points } \\
\text { per } \\
\text { inch }\end{array}$} & \multicolumn{2}{|c|}{$\begin{array}{l}\text { Thickness on } \\
\text { cutting edge }\end{array}$} & \multicolumn{2}{|c|}{$\begin{array}{l}\text { Thickness on } \\
\text { back at point }\end{array}$} & \multirow{2}{*}{$\begin{array}{l}\text { Width } \\
\text { butt, } \\
\text { mini- } \\
\text { mum }\end{array}$} & \multirow{2}{*}{$\begin{array}{l}\text { Width } \\
\text { point }\end{array}$} \\
\hline & & $\begin{array}{l}\text { Mini- } \\
\text { mum }\end{array}$ & $\begin{array}{l}\text { Maxi- } \\
\text { mum }\end{array}$ & $\begin{array}{l}\text { Mini- } \\
\text { mum }\end{array}$ & $\begin{array}{l}\text { Maxi- } \\
\text { mum }\end{array}$ & & \\
\hline $\begin{array}{r}\text { Inches } \\
28 \\
26 \\
22 \\
20\end{array}$ & $\begin{array}{l}5 \\
6 \\
7 \\
7\end{array}$ & $\begin{array}{l}\text { Inch } \\
0.035 \\
.035 \\
.035 \\
.035\end{array}$ & $\begin{array}{l}\text { Inch } \\
0.049 \\
.048 \\
.048 \\
.048\end{array}$ & $\begin{array}{l}\text { Inch } \\
0.022 \\
.022 \\
.022 \\
.022\end{array}$ & $\begin{array}{l}\text { Inch } \\
0.028 \\
.028 \\
.028 \\
.028\end{array}$ & $\begin{array}{r}\text { Inches } \\
738 \\
71 / 4 \\
578 \\
51 / 2\end{array}$ & $\begin{array}{r}\text { Inches } \\
27 / 8 \\
25 / 8 \\
2 \\
2\end{array}$ \\
\hline
\end{tabular}

14. Keyhole saws, type $N$.-These saws shall conform to the requirements for compass saws, type I, except as to dimensions. They shall be similar to Figure 761-except the 6-inch saw, which shall have a metal handle or pad-and shall conform to the dimensions in the following table:

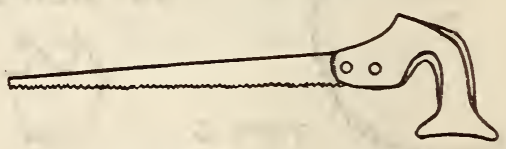

TYPE N

FigURE 761

\begin{tabular}{|c|c|c|c|c|c|c|}
\hline \multirow{2}{*}{$\begin{array}{l}\text { Length } \\
\text { on } \\
\text { cutting } \\
\text { edge }\end{array}$} & \multirow{2}{*}{$\begin{array}{l}\text { Points } \\
\text { per } \\
\text { inch }\end{array}$} & \multicolumn{2}{|c|}{$\begin{array}{l}\text { Thickness on } \\
\text { cutting edge }\end{array}$} & \multicolumn{2}{|c|}{$\begin{array}{l}\text { Thickness on } \\
\text { back at point }\end{array}$} & \multirow{2}{*}{$\begin{array}{l}\text { Width } \\
\text { at heel }\end{array}$} \\
\hline & & $\begin{array}{l}\text { Mini- } \\
\text { mum }\end{array}$ & $\begin{array}{l}\text { Maxi- } \\
\text { mum }\end{array}$ & $\begin{array}{l}\text { Mini- } \\
\text { mum }\end{array}$ & $\begin{array}{l}\text { Maxi- } \\
\text { mum }\end{array}$ & \\
\hline $\begin{array}{r}\text { Inches } \\
12 \\
6 \pm 162\end{array}$ & $\begin{array}{l}10 \\
10\end{array}$ & $\begin{array}{l}\text { Inch } \\
0.042 \\
.042\end{array}$ & $\begin{array}{l}\text { Inch } \\
0.058 \\
.058\end{array}$ & $\begin{array}{l}\text { Inch } \\
0.032 \\
.032\end{array}$ & $\begin{array}{l}\text { Inch } \\
0.042 \\
.042\end{array}$ & $\begin{array}{l}\text { Inch } \\
7 / 8 \\
12 \pm 18\end{array}$ \\
\hline
\end{tabular}


15. Web saws, felloe, type 0.-These saws shall be of uniform thickness throughout the length of the cutting edge, tapering to a uniform thickness on the back. The teeth shall be spring set, bevel filed, and adapted for both crosscutting and ripping. The teeth shall be set to provide proper clearance on each side of the blade. These saws shall have holes at each end for use in fastening the saws in machines. The length or size shall be the distance over all. These saw's shall be similar to Figure 762 and conform to the dimensions in the following table:

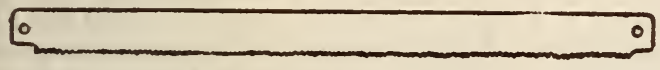

TYPE 0

FigURE 762

\begin{tabular}{|c|c|c|c|c|c|c|}
\hline \multirow{2}{*}{ Length } & \multirow{2}{*}{ Width } & \multirow{2}{*}{$\begin{array}{l}\text { Points } \\
\text { per } \\
\text { inch }\end{array}$} & \multicolumn{2}{|c|}{$\begin{array}{c}\text { Thickness on } \\
\text { back }\end{array}$} & \multicolumn{2}{|c|}{$\begin{array}{l}\text { Thickness on } \\
\text { cutting edge }\end{array}$} \\
\hline & & & $\begin{array}{l}\text { Mini- } \\
\text { mumm }\end{array}$ & $\begin{array}{l}\text { Maxi- } \\
\text { mum }\end{array}$ & $\begin{array}{l}\text { Mini- } \\
\text { mum }\end{array}$ & $\begin{array}{l}\text { Maxi- } \\
\text { mum }\end{array}$ \\
\hline $\begin{array}{r}\text { Inches } \\
14 \\
14 \\
14 \\
12 \\
12\end{array}$ & $\begin{array}{c}\text { Inch } \\
134 \\
3 / 8 \\
1 / 2 \\
1 / 4 \\
3 / 10\end{array}$ & $\begin{array}{l}8 \\
6312 \\
6 \\
9 \\
10\end{array}$ & $\begin{array}{c}\text { Inch } \\
0.028 \\
.028 \\
.032 \\
.022 \\
.022\end{array}$ & $\begin{array}{l}\text { Inch } \\
0.035 \\
.035 \\
.042 \\
.032 \\
.032\end{array}$ & $\begin{array}{l}\text { Inch } \\
0.042 \\
.042 \\
.049 \\
.032 \\
.032\end{array}$ & $\begin{array}{l}\text { Inch } \\
0.058 \\
.058 \\
.065 \\
.035 \\
.035\end{array}$ \\
\hline
\end{tabular}

VIII. NOTES

1. When requisitions are prepared for type $E$ band saws, all detailed information referred to in Section V should be furnished. If type C or D saws are required cut and brazed to length, the requisition should state the exact length desired.

\subsection{GLASS CUTTERS.}

UNITED STATES GOVERNMENT, FEDERAL SPECIFICATIONS BOARD; FEDERAL SPECIFICATION FOR CUTTERS, GLASS, (WHEEL TYPE), GGG-C-751, MARCH 1, 1932

\section{GLASS CUTTERS}

\section{B. TYPES.}

B-1. Glass cutters shall be of the following types, as specified: A. Single, fixed wheel. B. Single, renewable wheel. C. Turret head.

\section{MATERIAL AND WORKMANSHIP, ETC.}

C-1. Material.-Glass cutters shall be made of material of good quality throughout, which shall be suitable for the purpose.

G-2. Workmanship. - Shall be first class.

\section{GENERAL REQUIREMENTS.}

D-1. Markings.-Each glass cutter shall be marked in a plain and permanent manner with the manufacturer's name or with a trade-mark of such known character that the source of manufacture may be readily determined.

D-2. Cutting wheels.-The cutting wheels shall be of hardened tool steel, suitably designed with a cutting edge, ground and honed to the proper bevel for long life under service conditions. The cutting edge shall be continuous and free from perceptible dull spots or nicks.

D-3. Spindle.-The wheels shall be mounted on hard steel spindles, having a diameter of not less than 0.050 inch nor more than 0.060 inch.
D-4. Clearances.-A close working fit shall be provided between the hole in the wheel and the spindle on which it operates. The side fit or lateral clearance for the wheels shall not exceed 0.003 inch. All wheels shall operate freely.

\section{E. DETAIL REQUIREMENTS.}

E-1. Type A, single fixed wheel glass cutters.

E-1a. Component parts.-These cutters shall be composed essentially of a handle, and a shank with a cutting wheel mounted in a slot near the outer end of the shank.

E-1b. Handle.-Unless otherwise specified the handle shall be of metal and made integral with the shank. That portion of the handle adjacent to the shank shall be suitably shaped to provide a comfortable rest for the finger. The finger-rest portion of the handle shall be not less than seven-sixteenths inch nor more than nine-sixteenths inch in width. The upper portion of the handle which is held between the fingers under normal operating conditions shall have a diameter of not less than oneeighth inch nor more than three-sixteenths inch. The handle shall be smoothly finished and have a coating of baked enamel.

E-1c. Shank. - The shank shall be made of metal and provided with three glass grips or breakers. The depth of the grips or breakers shall be onefourth inch subject to a tolerance of plus or minus one thirty-second inch. The widths of the grips shall be one-eighth inch, five thirty-seconds inch, and three sixteenths-inch, respectively, with an allowable tolerance of plus or minus one sixty-fourth inch in all these dimensions. The corners and edges of the grips shall be clean and true. The edges and outer corners shall be beveled slightly to prevent abrasion to glass rules. When specifically required the head of the shank shall have a thickness of threesixteenth inch subject to a plus or minus tolerance of one sixty-fourth inch.

E-1d. Wheels.-The wheels of these cutters shall have a diameter of seven thirty-second inch subject to a plus or minus tolerance of one thirty-second inch.

E-1e. Glass cutters of this type shall be similar to illustration.

E-2. Type B, single, removable wheel glass cutters.

E-2a. Glass cutters of this type shall conform to the requirements outlined herein for type A glass cutters, with the following exceptions:

E-2a (1). Mounting. - The cutter wheel shall be mounted in a slot in the shank on a spindle of such quality that the useful life of the spindle will be equal to that of at least seven cutting wheels. The wheel spindle shall be so constructed that the worn wheel may be removed and replaced by a new wheel without damage to the spindle. Replacement of wheels shall be accomplished without the use of tools.

E-2a (2). Handle. - The outer end of the handle shall have a magazine or recess of ample size to contain six spare cutting wheels. The recess in the handle shall be threaded and capped with a metal screw to prevent loss of the spare wheels.

E-2a (3). Spare cutting wheels.-At least six spare cutting wheels shall be furnished with each of these glass cutters. The spare wheels shall be coated with a rust preventive and set in the recess in the handle.

E-2b. Glass cutters of this type shall be similar to illustration.

$\mathrm{E}-3$. Type $C$, turret head glass cutters.

E-3a. Component parts. - These cutters shall be composed essentially of a handle, a shank, and a turret with at least six cutting wheels mounted thereon.

E-3b. Handles. - The handles may be integral with and of the same material as that of the shank or they may be of wood or other suitable material and be 
separate from the shank. However, if other than integral construction is employed the handle shall be ferruled at the junction of the handle and shank and the handle shall be secured in a manner to prevent its working loose or coming off the shank in service. All handles shall be smoothly finished and have a coating of baked enamel.

E-3c. Shank. - The shank shall be made of metal and provided with two glass grips or breakers having a depth of one-fourth inch, subject to a plus or minus tolerance of one thirty-second inch. The width of these grips shall be five thirty-seconds inch and one-eighth inch, respectively, with an allowable tolerance of plus or minus one sixty-fourth inch. The corners and edges of the grips shall be clean and true. The edges and outer corners shall be beveled slightly to prevent abrasion to glass rules. When specifically required the wheel end of the cutter with wheels mounted on turret and attached to shank shall not exceed three-sixteenths inch in thickness, subject to a plus or minus tolerance of one sixtyfourth inch.

E-3d. Turret.-The turret shall be of hardened steel, circular in shape, and provided with at least six spindles, on each of which a cutting wheel shall be mounted. The turret shall be fastened to the shank of the cutter by a single, countersunk head, slotted, screw. When tightened the head of the screw shall not protrude above the face of the shank. Changing position of the cutter wheels shall be accomplished by loosening the retaining screw and revolving the turret. The turret shall be securely locked or clamped by tightening the retaining screw and when locked in position the side play between the wheel and the turret or the shank of the cutter shall not exceed the lateral clearance prescribed in paragraph $\mathrm{D}-4$. The turret shall be marked on the exposed face with Figures 1 to 6, inclusive. Each of these figures shall appear opposite a cutting wheel.

E-3e. Wheels. - The wheels of these cutters shall have a diameter of five thirty-seconds inch subject to a plus or minus tolerance of one sixty-fourth inch.

E3-f. Glass cutters of this type shall be similar to either of illustrations for type C.

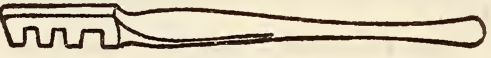

TYPE A
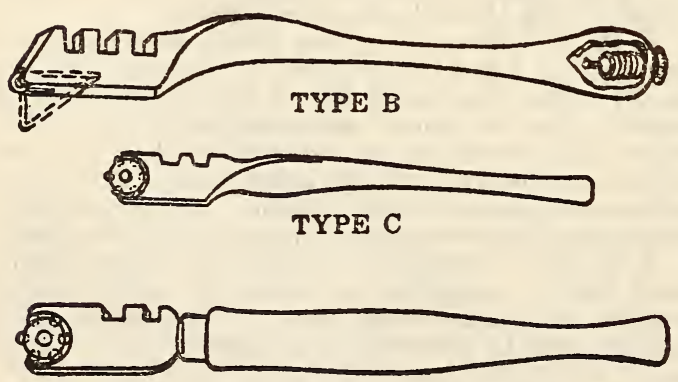

TYPE C

Glass cutter: Type A, single. fixed wheel; type $B$, single, renewable wheel; type $C$, turret head

\section{F. METEODS OF SAMPLIHG, INSPECTION, AND TESTS.}

F-1. Work value tests.-Tests as described herein will be conducted to determine the work values of glass cutters submitted by the various manufacturers. The results obtained in these tests will be entered on the list of work values maintained by the department concerned. The list will show the name of the manufacturer, the brand name, and the type of cutter.
F-2. Test procedure.-Work values will be determined by subjecting six sample glass cutting wheels to the following test:

Each sample cutter will be held, with the handle vertical, in a stationary position, and caused to bear, with pressure of 8 pounds, on a sheet of clear unpolished window glass, double strength, A quality, having a minimum thickness of 0.111 inch, a maximum thickness of 0.125 inch, and a weight of 24 to 25 ounces per square foot. (Type B clear window glass, Federal Specification DD-G-451.) The glass will be mounted on a reciprocating carriage having a 6 -inch stroke and operated at 30 strokes per minute. Each wheel will be tested in periods of 100 revolutions of the crank operating the reciprocating carriage, with the wheel bearing in one grooxe for each period. No lubricant will be used in testing wheels. At the end of each period the cutter will be removed from the testing machine to be determined by hand if failure of the wheel has occurred. Failure will be deemed to have occurred when the wheel does not make a clean continuous scratch on glass under a pressure of 10 pounds (with glass lying on platform scale) or when the clearance becomes so great that the wheel will not operate in a straight line. The work value of the wheel will be taken as the number of strokes or twice the number of crank revolutions of the testing machine required to bring the wheel to the point of failure. The work value of each style or make of cutter will be taken as the sum of the work values of six test wheels. Any make or style glass cutter showing a work factor of less than 5,000 in the above-prescribed test will not be approved.

$\mathrm{F}-3$. Inspection.-All glass cutters shall be inspected to determine whether they conform to the requirements of this specification. In addition to a careful surface inspection for workmanship and for general compliance with this specification, at least three samples (in no instance less than 2 per cent of the entire quantity of each type under inspection) shall be taken at random from those under inspection and subjected to the work value test prescribed herein. If any one of the test samples fails under inspection check test to equal 90 per cent of the original work value test, on which award was based, the entire quantity represented by the samples shall be rejected.

\section{G. PACKAGING, PACKING, AND MARKING.}

G-1. Packaging.-Unless otherwise specified, commercial packages are acceptable, provided that not more than twelve tools shall be contained in any one box or package and that suitable provision shall be made to prevent rust or corrosion of the cutting wheels and other finished parts of the tools.

\subsection{AWLS, PUNCHES, AND SCREW DRIVERS.}

616.21 AWLS.

(No nationally recognized specifications available.)

\subsection{PUNCHES.}

(For railway-track punches, see American Railway Engineering Association specifications for track tools. See 616.8 , p. 969.)

(For standard sizes of blacksmith punches, see United States Government, Department of Commerce, Bureau of Standards, simplified practice recommendation $\mathrm{R} 17-31$ for forged tools. See 616.60, p. 960.)

\subsection{SCREW DRIVERS.}

(No nationally recognized specifications available.) 
616.3 IEVELING AND MEASURING TOOIS.

\subsection{BEVELS AND MITER BOXES.}

able.)

\subsection{LEVELS AND PLUMBS.}

(No nationally recognized specifications available.)

\subsection{RULES.}

\section{UNITED STATES GOVERNMENT, FEDERAL SPECIFICATIONS BOARD; FEDERAL SPECIFICATION FOR RULES, NO. 419, JULY 18, 1928. (CURRENT DESIGNA- TION : GGG-R-791)}

\section{RULES}

\section{TYPES}

Rules shall be of the following types, as specified: $A$, caliper rules; B, carpenter's folding rules; $C$, multiple-folding rules; and $\mathrm{D}$, steel rules.

\section{IUATERIAL AND WORKMANSEIP}

All rules shall be of high-grade material and workmanship and free from defects and blemishes which may affect the appearance or serviceability of the rules.

\section{GENERAL REQUIREMENTS}

1. All rules shall be stamped or otherwise permanently marked with the manufacturer's name or trade-mark.

2. Inch graduations shall be plainly marked with the proper figures. All graduations and figures shall be black in order that they may be clear and distinct.

\section{DETAIL REQUIREMENTS}

1. Caliper rules, type A.-These shall be left-hand type and made from thoroughly seasoned boxwood, with ends and joints bound with brass. They shall be of the folding type which folds into two equal parts. The middle joint located at the 6-inch division on rule shall be of the square-joint type.

2 . The flanges or wings of the joints, of which there shall be two for each leg of the rule, shall cover the faces of the rule at the joint, and shall be held together by rivets passing through the flanges and the rule. One leg shall be slotted to receive the caliper slide.

3. The caliper slide shall be of brass, and machined to fit accurately the slot in the leg of the rule. It shall be graduated in inches, also sixteenths and thirty-seconds of an inch.

4. The body of the rule shall be graduated in inches, also eighths, tenths, twelfths, and sixteenths of an inch.

5. Caliper rules shall be $1 \frac{3 / 8}{8}$ inches wide, 1 foot length, double fold.

6. Folding rules, type B.-These shall be made from thoroughly seasoned sound boxwood with ends and joints bound with brass. Rules shall be of the folding type which fold into four equal parts. The middle joint located at the 12-inch division on rule, shall be of the type known as square joint, in which the flanges or wings of the joints, of which there shall be two for each leg of the rule, shall cover the face of the rule at the joint and shall be held together by rivets passing through the flanges and the rule. The joints located at the 6 and 18 inch divisions of the rule shall be of the type known as edge-plate joint, in which the flanges or wings of the joint shall be fastened to the outer edges of the rules and shall be secured to same by rivets passing through both wood and flanges, holding all parts securely together.

7. These rules shall be graduated in inches, also eighths, tenths, twelfths, and sixteenths of an inch.

8 . These rules shall be 1 inch wide, 2 -foot length, fourfold.

9. Multiple-folding rule, type C.-These shall be made from thoroughly seasoned sound hardwood. They shall be of the multiple-folding type and capable of being folded upon themselves by means of folding joints placed every 6 inches along the rules. The joints shall be of the inclosed spring type, so that the rules can be opened full length and hold themselves rigid in order to measure vertical or horizontal surfaces. The joints shall be made of brass-plated metal securely fastened to the wood, and provided with rivets passing through the wood and the metal joints. The ends of the rivets shall be peened over a suitable washer, which will prevent the rivet pulling through the wood.

10. The joints shall be so designed that they can not become loose, weak, or permit any movement of the wood where securad to the same. The ends of the rules shall be provided with brass plated tips so as to protect the ends of the rules from injury. These tips shall be secured in such a way as not to interfere with reading of end graduations.

11. These rules shall be graduated in inches and sixteenths of an inch, reading consecutively from the outside of the rule to the total length of rule.

12. Multiple-folding rules shall be five-eighths inch wide, 6-foot length, and yellow enameled.

13. Steel rules, type D.-These shall be made of tool steel-properly hardened, tempered, and ground - and accurately graduated into inches on both sides. One side shall be graduated into eighths and sixteenths, the other side shall be graduated into thirty-seconds and sixty-fourths of an inch.

14. The edges shall be ground parallel, the ends shall be similarly ground and at right angles to the edges.

15. Steel rules shall be furnished in accordance with the sizes and dimensions called for in the following table:

\begin{tabular}{|c|c|c|c|c|}
\hline \multirow{2}{*}{ Length } & \multicolumn{2}{|c|}{ Width } & \multicolumn{2}{|c|}{ Thickness } \\
\hline & $\begin{array}{l}\text { Mini- } \\
\text { mum }\end{array}$ & $\begin{array}{l}\text { Maxi- } \\
\text { mum }\end{array}$ & $\begin{array}{l}\text { Mini- } \\
\text { mum }\end{array}$ & $\begin{array}{l}\text { Maxi- } \\
\text { mum }\end{array}$ \\
\hline $\begin{array}{r}\text { Inches } \\
6 \\
12 \\
18\end{array}$ & $\begin{array}{l}\text { Inch } \\
11 / 16 \\
31 / 32 \\
1\end{array}$ & $\begin{array}{c}\text { Inches } \\
3 / 4 \\
1 \\
118\end{array}$ & $\begin{array}{r}\text { Inch } \\
3 / 64 \\
3,64 \\
3,64\end{array}$ & $\begin{array}{r}\text { Inch } \\
1 / 20 \\
1 / 16 \\
1 / 16\end{array}$ \\
\hline
\end{tabular}

\section{INSPECTION}

Representative samples shall be taken from each lot submitted for inspection. Each sample shall conform to the requirements of the specifications.

\subsection{SQUARES.} ble.)

(No nationally recognized specifications availa- 
616.35 TAPES.

AMERICAN ELECTRIC RAILWAY ENGINEERING ASSOCIATION, WHEEL CIRCUMFER -

ENCE MEASURING TAPE FOR CONTOURS A, B, C, AND D, RECOMMENDED DESIGN E115-26, 1926

WHEEL CONTOUR MEASURING TAPE

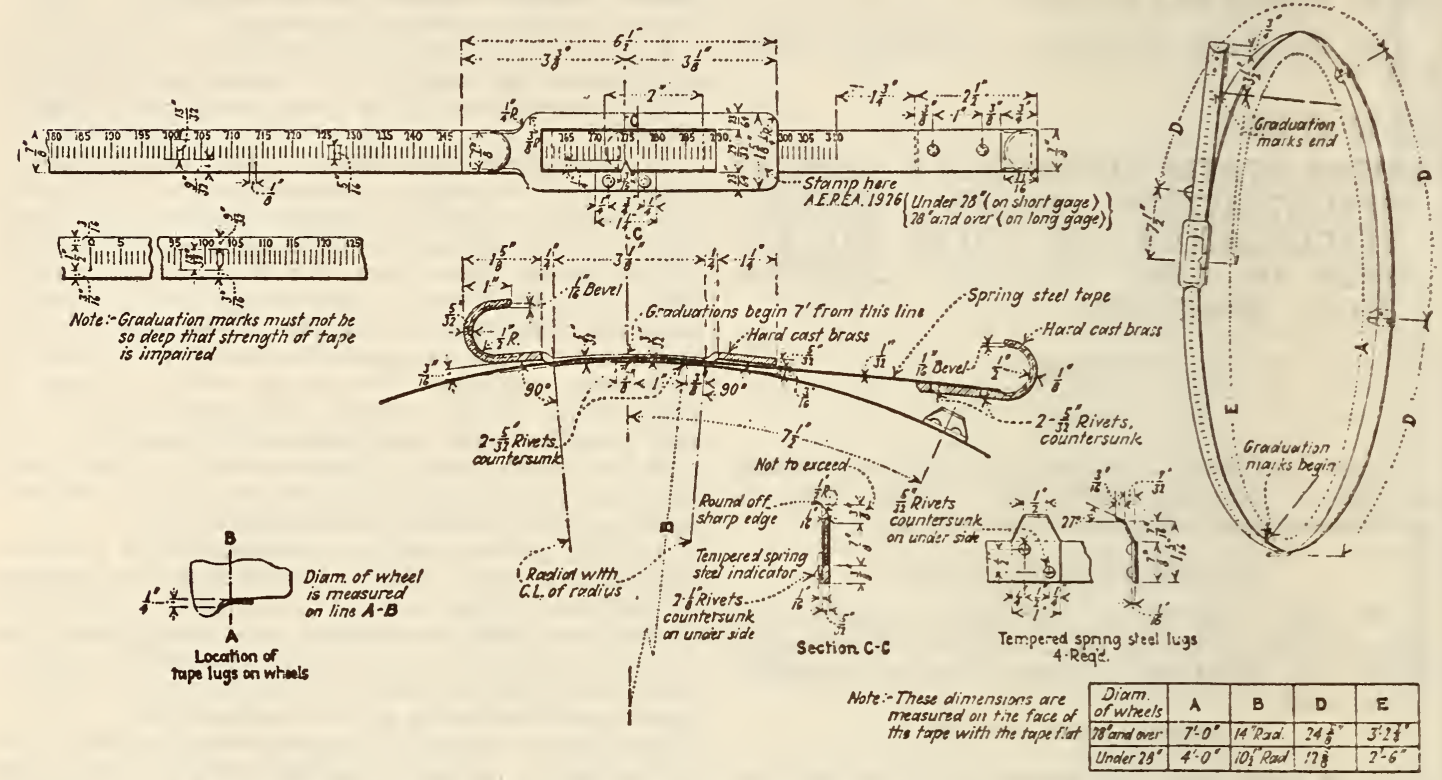

FigURE 763.- Wheel circumference measuring tape for contours $A, B, C$, and $D$ 
AMERICAN ELECTRIC RAILWAY ENGINEERING ASSOCIATION, WHEEL CIRCUMFERENCE MEASURING TAPE FOR CONTOURS E AND F, RECOMMENDED DESIGN E116-26, 1926

\section{WHEEL CONTOUR MEASURING TAPE}

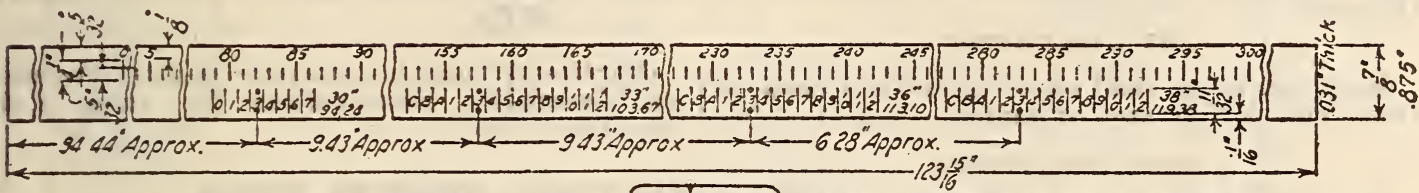

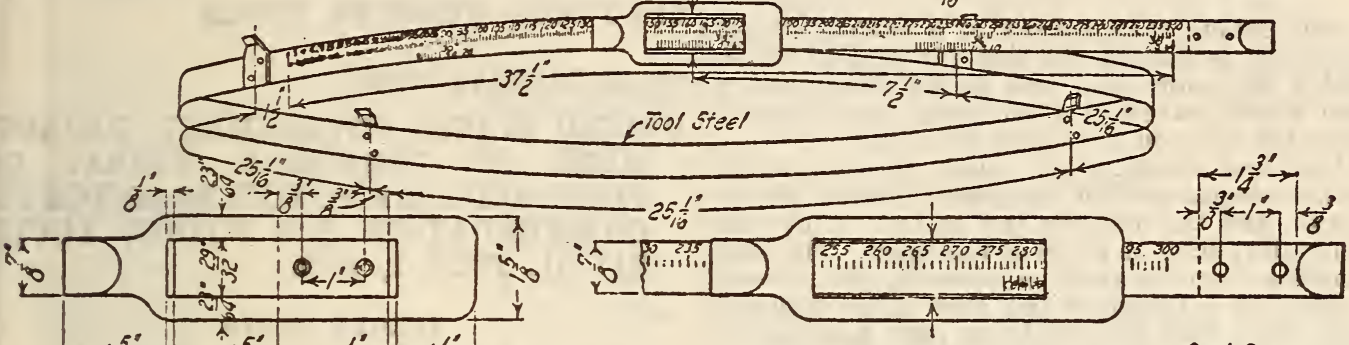

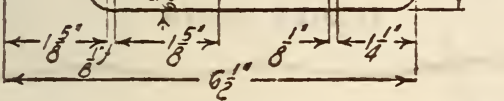

Limear Oimensions Shown Represent Measuremento of Actual Circumference of the Wheel and Not straight Length of the Tape. Graduations to be Spaced 1 "Apurt With Tape laid Flat, and Spaie Between lines 157 and 158 on the Upper side of Tape to Coincide With soace Representing Tape size Nla 3 for the $33^{\prime \prime}$ Diameter cost iron Wheels

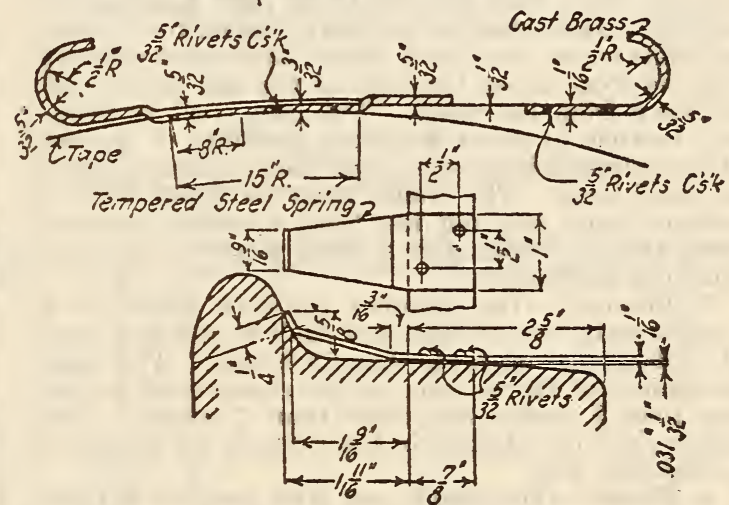

Figure 764.-Wheel circumference measuring tape for wheel contours $E$ and $F$

This tape is the same as that adopted by the American Railway Association in 1893 and modified in 1919.

616.4 HOOKS AND PULLERS.

616.41 HOOKS, FOR MANUAL HANDLING OF COMMODITIES.

UNITED STATES GOVERNMENT FEDERAL SPECIFICATIONS BOARD, FEDERAL SPECIFICATION FOR STANDARD MISCELLANEOUS CHAIN AND ATTACHMENTS, RR-C-271, DECEMBER 8, 1931

\section{HOOKS OF VARIOUS TYPES}

(See 603.57, p. 217.)

\subsection{NAIL PULLERS.}

UNITED STATES GOVERNMENT, FEDERAL SPECIFICATIONS BOARD: FEDERAI SPECIFICATION FOR PULLERS, NAIL, GGG-P-791, MARCH 1, 1932

\section{NAIL PULLERS}

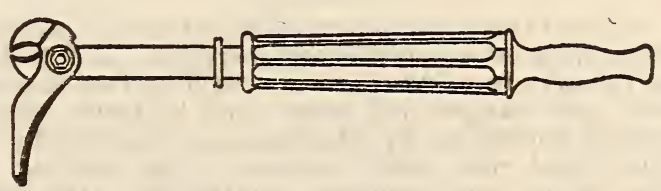

Nail puller

TYPES

Nail pullers shall be of the rammer type. 
MATERIAL AND WORKMANSHIP

Nail pullers shall be made of high-grade material and in accordance with the best commercial practice. The $y$ shall be free from all defects or blemishes that may affect the serviceability or appearance of the tools.

GENERAL REQUIREMENTS

Each nail puller shall have the maker's name or trade-mark legibly cast or impress stamped on it.

\section{DETAIL REQJIREMENTS}

1. Nail pullers shall consist of a shank, at one end of which is located the long jaw and to which is riveted the short jaw, and at the other end a rammer which travels over the shank, and thereby produces the blow on the end of the shank.

2. Jaws and shanks. - The shank, the jaw which forms an integral part of the shank (known as the long jaw), and the movable jaw or foot (known as the short jaw) shall be made of tool steel. The long jaw and the short jaw shall be joined by and pivoted on a steel pin. The short jaw shall be so designed that the jaws shall be held in an open position by gravity. Jaws shall be properly shaped for pulling out nails which have been driven into wood without slipping from under the head of the nail as the nail is drawn from the wood. The jaws shall be properly treated to stand abrasion, bending, or dulling at the gripping jaws when used under ordinary service conditions. The shank shall be fitted with a suitable hand grip not less than 4 inches long nor more than 5 inches and a hand guard to prevent pinching the hand.

3. Rammer. - The rammer shall be made of a good-qualtiy iron or steel and shall be not less than 11 inches long nor more than 14 inches. The axial movement of the rammer on the shank shall be not less than 5 inches nor more than 7 inches. The shank shall be retained in the rammer by means of a steel stop pin.

4. Finish.-The shank and jaws shall be natural steel finish or they may be polished or coated with japan or enamel. The rammer shall be japanned or black enameled.

5 . The length of the nail puller when closed shall be not less than 17 inches nor more than 20 inches. The weight shall be not less than $3 \frac{3 / 4}{\text { pounds }}$ nor more than 6 . The puller shall have a thrust of not less than 5 inches nor more than 7 .

6 . Nail pullers shall also be similar to the illustration.

\section{TESTS}

At least three nail pullers shall be taken at random from each lot submitted for inspection and tested as follows: In addition to a careful surface inspection, each sample nail puller shall be tested under service conditions by gripping and drawing 10 or more steel wire nails (common type) varying in size up to and including twelvepenny (148 mils diameter, $3 \frac{1}{4}$ inches long) which have been driven with heads flush into softwood.
Under the above test the sample nail pullers shall show no indications of weakness such as chipping, breaking, or bending of any of the parts.

\subsection{TOOLS FOR LINE-CONSTRUCTION MEN.}

(For timber tongs, see American Railway Engineering Association specifications for track tools. See 616.8, p. 969.$)$

\subsection{SOIL WORKING TOOLS.}

\subsection{GENERAL ITEMS.}

UNITED STATES GOVERNMENT, DEPARTMENT OF COMMERCE, BUREAU OF STANDARDS, SIMPLIFIED PRACTICE RECOMMENDATION FOR FORGED TOOLS, R17-31, JULY 1, 1931

\section{FORGED TOOLS}

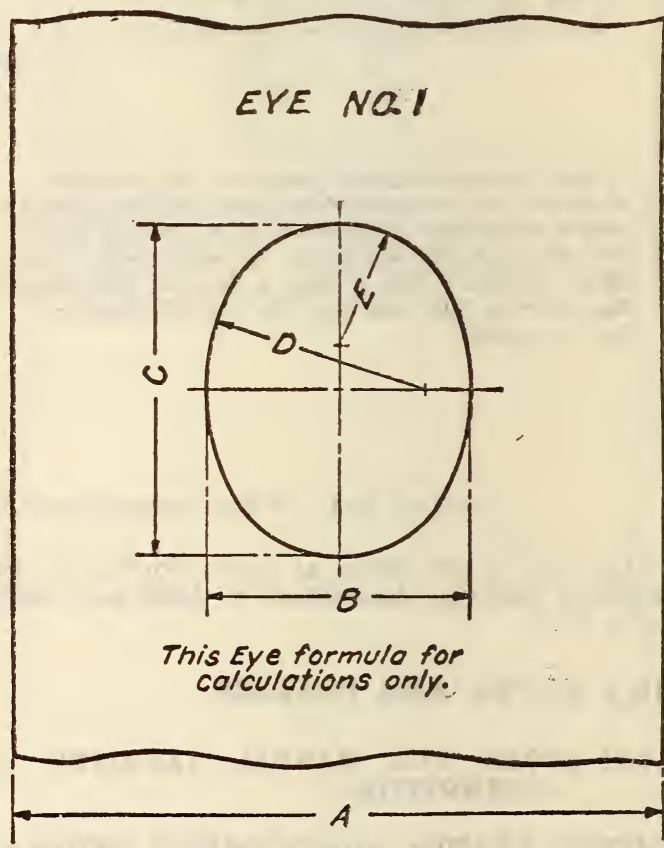

Figure 765

Eye formula:

$A=$ Size of stock

$B=40$ per cent of $A$

$C=1.25$ per cent of $B$ (approximate)

$D=5 / 6$ of $\mathrm{B}$

$E=$ To suit dimensions $\mathrm{B}, \mathrm{C}$, and D.

Note.-Manufacturers have a tolerance limit of $1 / 16$ inch above and $1 / 32$ inch under all eyes to allow for punch wear. 


\section{EYE NO.2}

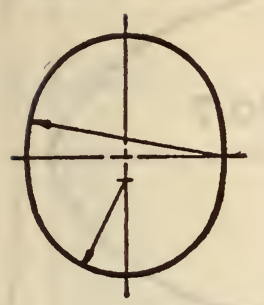

EYE NO.3

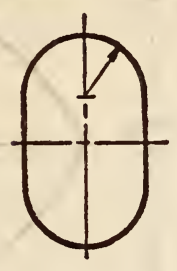

EVE NO. 5
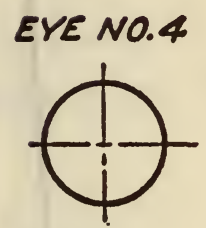

FigURE 766

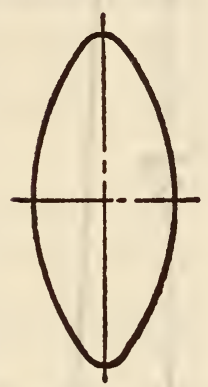

(Simplified practice recommended and accepted by industry establishing a limited list of standard sizes and types of forged tools.)

TaBle 1.-Picks

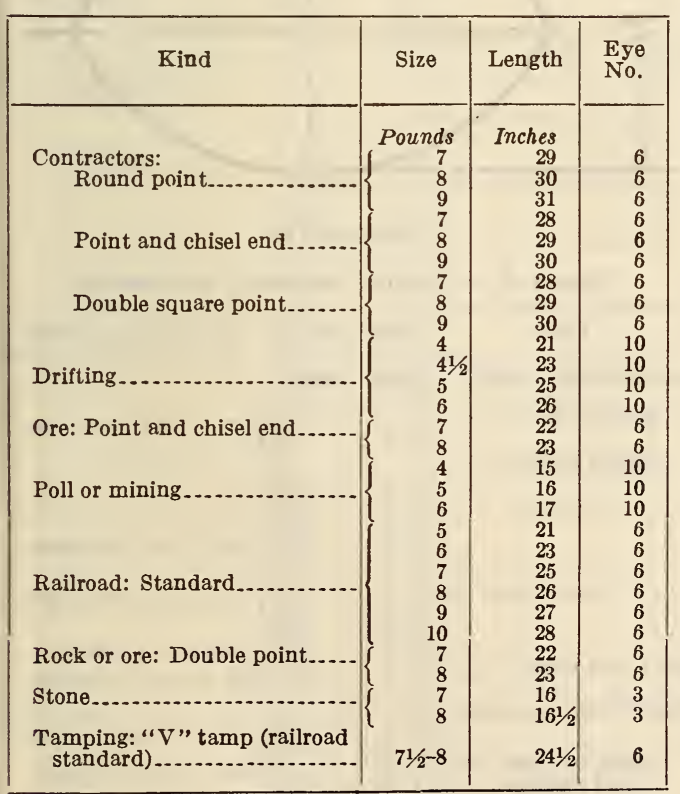

TABLE 2.-Mattocks

\begin{tabular}{|c|c|c|c|c|}
\hline Kind & Size & $\begin{array}{l}\text { Blade } \\
\text { width }\end{array}$ & $\begin{array}{l}\text { Over-all } \\
\text { length }\end{array}$ & $\begin{array}{l}\text { Eye } \\
\text { No. }\end{array}$ \\
\hline $\begin{array}{l}\text { Cutter. } \\
\text { Pick....... } \\
\text { Nursery } \\
\text { Brush } \\
\text { Asphalt: } \\
\quad \text { Single eye } \\
\text { Double eye }\end{array}$ & $\left\{\begin{array}{l}\text { Pounds } \\
3 \\
4 \\
5 \\
15 \\
15 \\
5 \\
6 \\
3 \\
6 \\
9 \\
10\end{array}\right.$ & $\begin{array}{c}\text { Inches } \\
3 \\
31 / 4 \\
3 \\
31 / 2 \\
4 \\
31 / 2 \\
4 \\
3 \\
31 / 2 \\
\\
23 / 4 \\
3\end{array}$ & $\begin{array}{l}\text { Inches } \\
13 \\
141 / 2 \\
15^{3} / 4 \\
15^{3} / 4 \\
16^{1} / 2 \\
19 \\
20 \\
15^{3} / 4 \\
181 / 2 \\
\\
20 \\
20\end{array}$ & \begin{tabular}{|c|}
7 \\
7 \\
6 \\
6 \\
6 \\
6 \\
6 \\
7 \\
6 \\
6 \\
Special.
\end{tabular} \\
\hline
\end{tabular}

1 Sizes in popular demand.
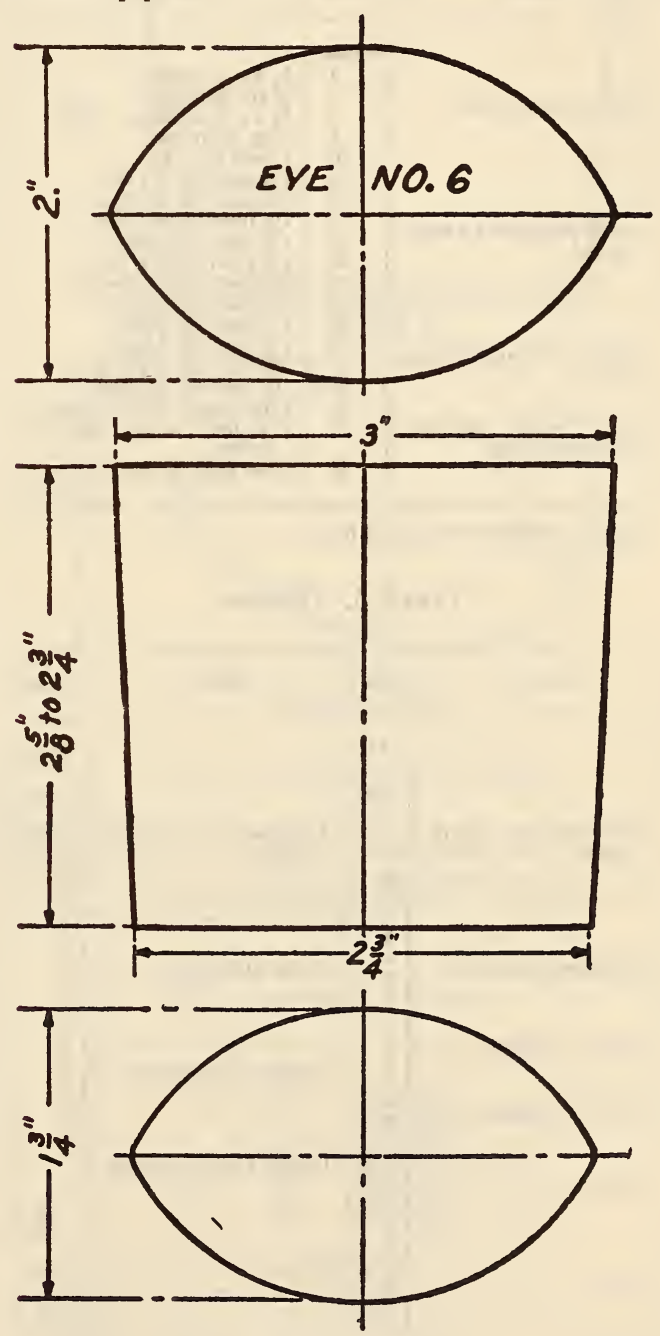

Figure 767 
TABLE 3.-Hoes, heavy eye type

\begin{tabular}{|c|c|c|c|c|}
\hline Kind & Size & $\begin{array}{l}\text { Blade } \\
\text { width }\end{array}$ & $\begin{array}{l}\text { Over-all } \\
\text { length }\end{array}$ & $\begin{array}{l}\text { Eye } \\
\text { No. }\end{array}$ \\
\hline $\begin{array}{l}\text { Grub..... } \\
\text { Hazel.... } \\
\text { Accomac.. }\end{array}$ & $\begin{array}{l}\text { Pounds } \\
\left\{\begin{array}{l}31 / 2 \\
4 \\
41 / 2 \\
3 \\
41 / 2 \\
5\end{array}\right.\end{array}$ & $\begin{array}{c}\text { Inches } \\
\quad 33 / 4 \\
4 \\
41 / 4 \\
6 \\
6 \\
61 / 2\end{array}$ & $\begin{array}{c}\text { Inches } \\
103 / 4 \\
111 / 4 \\
111 / 2 \\
91 / 2 \\
11 \\
111 / 2\end{array}$ & $\begin{array}{l}8 \\
8 \\
8 \\
9 \\
8 \\
8\end{array}$ \\
\hline
\end{tabular}

TABLE 4.- Bars $^{1}$

\begin{tabular}{|c|c|c|}
\hline Kind & Size & Dimensions \\
\hline Crou & $\begin{array}{l}\text { Pounds } \\
\left\{\begin{array}{c}3 \\
4 \\
6 \\
8 \\
10 \\
12 \\
14\end{array}\right.\end{array}$ & $\begin{array}{l}3 / 4 \text { by } 24 \text { inches. } \\
3 / 4 \text { by } 30 \text { inches. } \\
7 / 8 \text { by } 36 \text { inches. } \\
1 \text { by } 42 \text { inches. } \\
1 \text { inch by } 4 \text { feet. } \\
11 / 8 \text { inch by } 41 / 4 \text { feet. } \\
11 / 8 \text { inch by } 41 / 2 \text { feet. }\end{array}$ \\
\hline $\begin{array}{l}\text { Crow; pinch and wedge } \\
\text { point. } \\
\text { Lining; diamond shape } \\
\text { point. } \\
\text { Claw; with railroad } \\
\text { standard heel. }\end{array}$ & $\begin{array}{l}16 \\
18 \\
20 \\
24 \\
26 \\
30 \\
18 \\
20 \\
26 \\
20 \\
25\end{array}$ & $\begin{array}{l}11 / 4 \text { inch by } 48 / 4 \text { feet. } \\
11 / 4 \text { inch by } 5 \text { feet. } \\
11 / 4 \text { inch by } 51 / 4 \text { feet. } \\
13 / 8 \text { inch by } 51 / 2 \text { feet. } \\
11 / 2 \text { inch by } 51 / 2 \text { feet. } \\
11 / 2 \text { inch by } 6 \text { feet. } \\
11 / 4 \text { inch by } 5 \text { feet. } \\
11 / 4 \text { inch by } 51 / 4 \text { feet. } \\
11 / 2 \text { inch by } 51 / 2 \text { feet. } \\
4 \text { feet long; } 1 / 2 \text {-inch spike. } \\
41 / 2 \text { feet long; } 8 / 8 \text {-inch } \\
\text { spike. }\end{array}$ \\
\hline & 30 & 5 feet long; $5 / 8$-inch spike. \\
\hline
\end{tabular}

${ }^{1}$ See also railroad track tool list.

\section{TABLE 5.-Wedges}

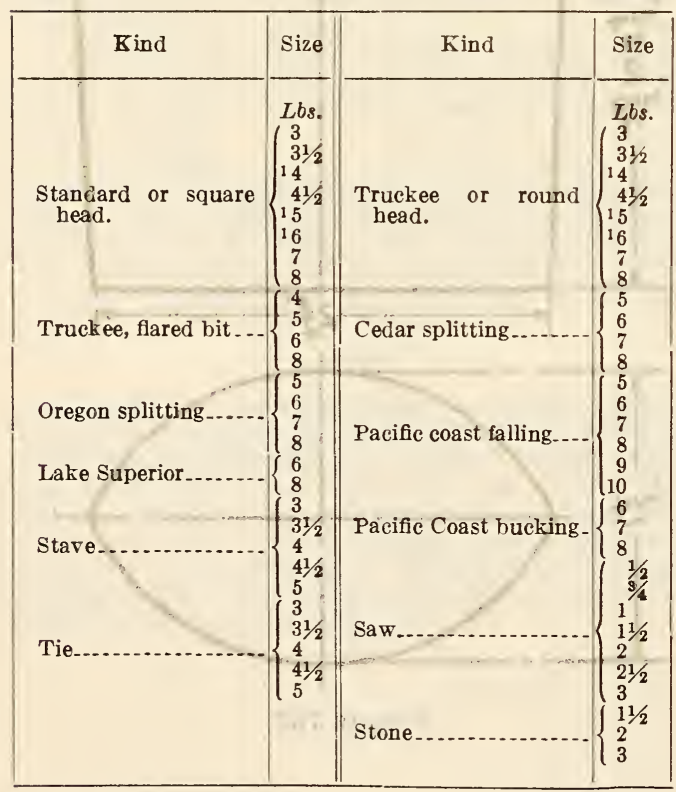

${ }^{1}$ Sizes in popular demand.
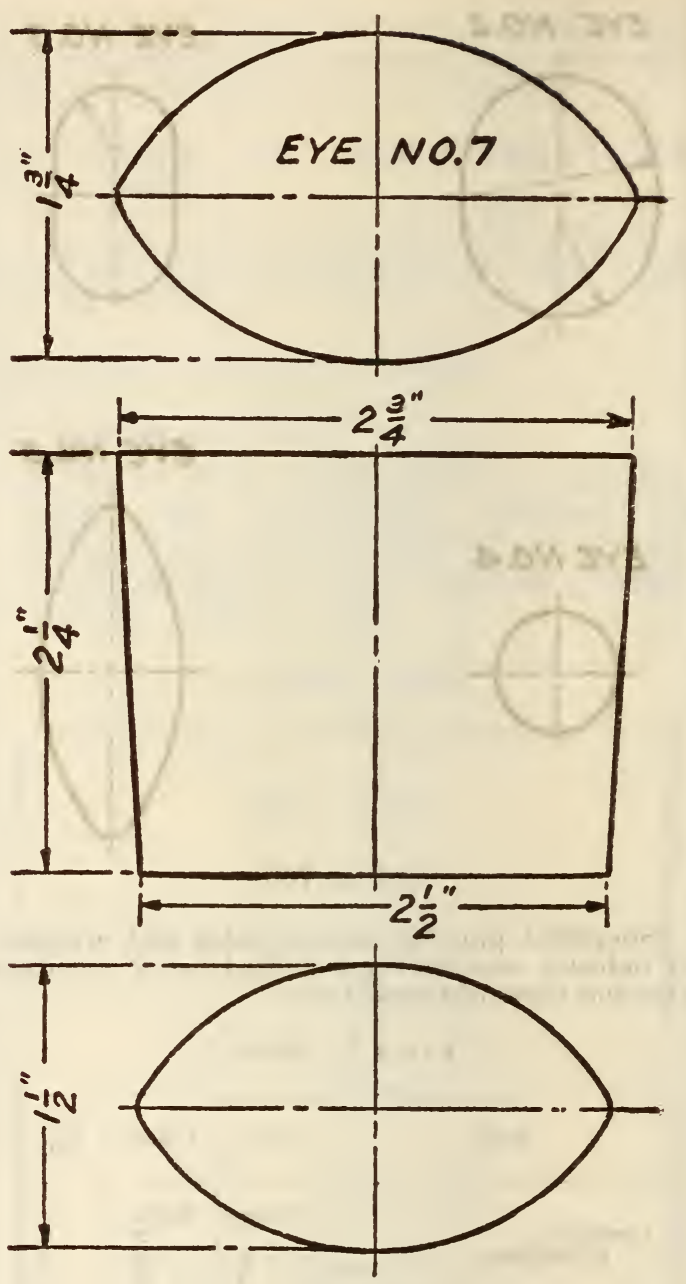

Figure 768

TABLE 6.-Sledges, hammers, and mauls

\begin{tabular}{|c|c|c|c|}
\hline Kind & Size & | Length & Eye (shape and size) \\
\hline $\begin{array}{l}\text { Woodehoppers' mauls: } \\
\text { Straight bit_....... }\end{array}$ & $\left\{\begin{array}{c}\text { Lbs. } \\
6 \\
7 \\
0\end{array}\right.$ & \begin{tabular}{|c|} 
Inches \\
8 \\
$81 / 4$
\end{tabular} & No. 2,1 by $1 \frac{1}{4}$ inches. \\
\hline Oregon pattern- & & & \\
\hline
\end{tabular}

Oregon pattern-

Oval eye.......

$\left\{\begin{array}{r}6 \\ 7 \\ 8 \\ 10 \\ 6 \\ 7 \\ 8 \\ 10 \\ 4 \\ 5 \\ 6\end{array}\right.$

Hand-drilling hammers:

Short, or New Eng. land pattern.

$\left.\begin{array}{l}81 / 2 \\ 87 / 8 \\ 9\end{array}\right\}$ Do.

$9 \frac{91 / 8}{9}$ No. 2,1 by $13 / 8$ inches.

Double-bit ax eye $81 / 2$ No. $5,7 / 8$ by 216 inches. 938

No. 2, 3/4 by 1 inch. $83 / 4$ No. 2, 1 by $11 / 4$ inches.

No. 4 , round, $3 / 4$-inch.

Long, or $\quad \begin{array}{ll}3 & 41 / 2 \\ 4 & 41 / 2\end{array}$ pattern. Colorado $\left\{\begin{array}{ll}3 & 51 / 4 \\ 31 / 2 & 51 / 2 \\ 4 & 53 / 4\end{array}\right\}$ No. 2, $3 / 4$ by 1 inch. 
TABLE 6.-Sledges, hammers, and mauls-Contd.

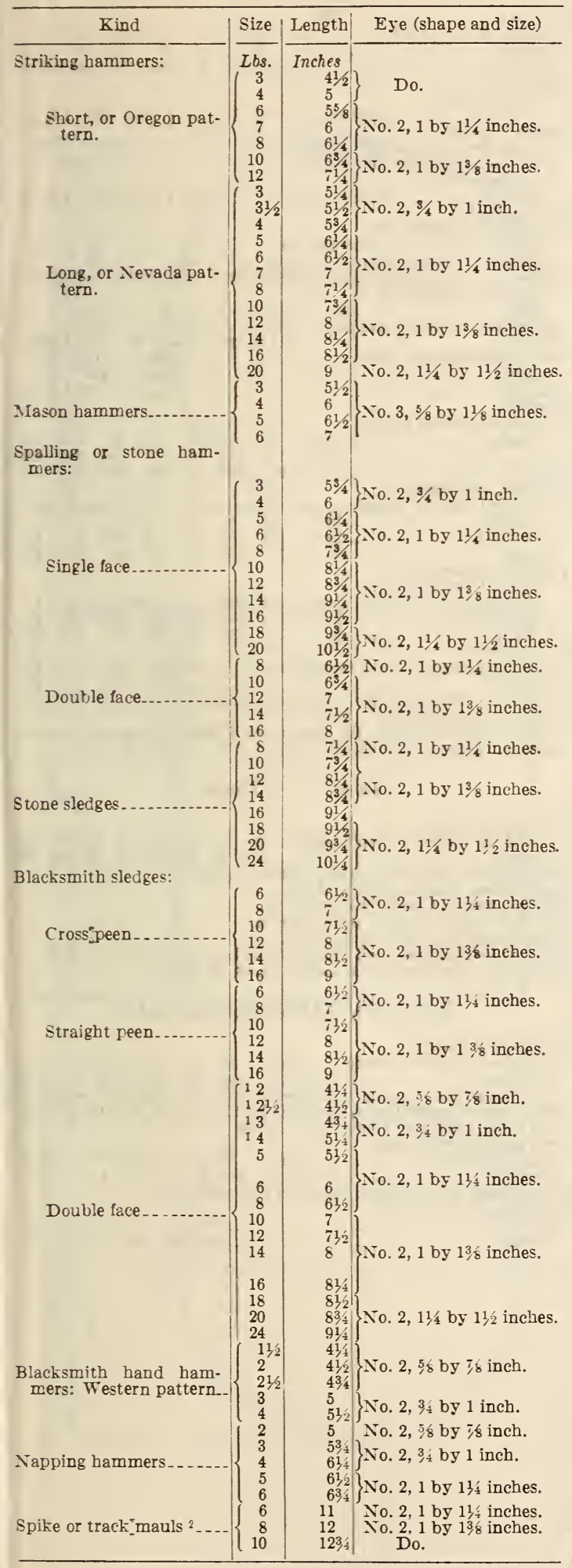

${ }^{1}$ Sizes $2,21,2,3$, and 4 pounds, known also as "engineers" and double face blacksmith hand hammers."

2 See track tool list for railroad's standard track mauls.

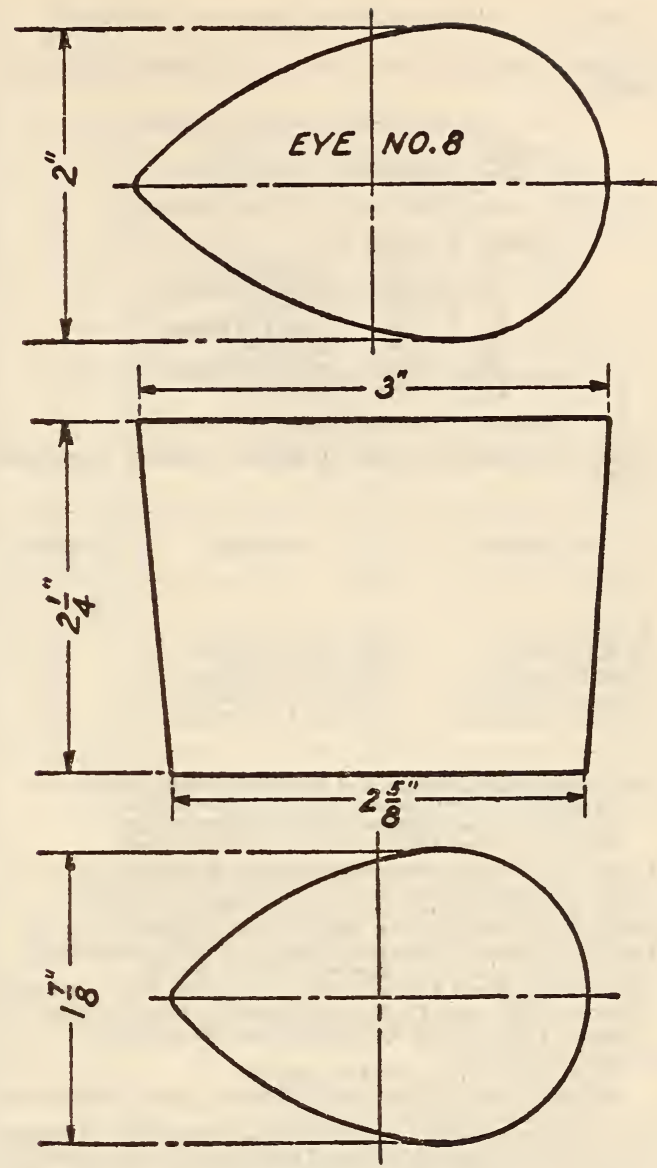

FigURE 769

TABLE 7.-Railroad track tools

Plan

1. Pick; clay, 7 pounds

3. Mauls, spike, pattern $\{$ No. 1,10 pounds $\}$ standard eye No. 2

Wrenche

4. Wrenches; single end (old style bolt):

\begin{tabular}{|c|c|c|}
\hline $\begin{array}{c}\text { Size of } \\
\text { bolt }\end{array}$ & Opening & \multicolumn{1}{|c|}{ Length } \\
\cline { 1 - 2 } Inches & Inches & \\
$3 / 4$ & $15 / 16$ & 30 inches. \\
$7 / 8$ & $11 / 2$ & 30 and 36 inches. \\
1 & $11 / 16$ & 36 inches. \\
$11 / 8$ & $17 / 8$ & 36 and 42 inches. \\
$11 / 4$ & $21 / 16$ & 42 inches. \\
$13 / 8$ & $21 / 4$ & 42 and 48 inches. \\
$11 / 2$ & $2 \frac{1}{2} / 16$ & 48 inches. \\
\hline
\end{tabular}

Wrenches; double end (old style bolt):

\begin{tabular}{|c|c|c|}
\hline Size of bolts & Openings & Length \\
\hline $\begin{array}{l}3 / 4 \text { and } 7 / 8 \text { inches.... } \\
7,8 \text { and } 1 \text { inch } \\
1 \text { and } 11 / 8 \text { inches.... } \\
11 / 8 \text { and } 11 / 4 \text { inches.... } \\
11 / 4 \text { and } 13 / 8 \text { inches..... }\end{array}$ & $\begin{array}{l}15,16 \text { and } 11 / 2 \text { inches.... } \\
11 / 2 \text { and } 1^{11 / 16} \text { inches.... } \\
111 / 16 \text { and } 1 / 8 \text { inches.... } \\
17 / 8 \text { and } 21 / 16 \text { inches.... } \\
21 / 16 \text { and } 21 / 1 \text { inches.... }\end{array}$ & $\begin{array}{r}\text { Inches } \\
36 \\
36 \\
42 \\
42 \\
48\end{array}$ \\
\hline
\end{tabular}

1 Plan numbers refer to illustrations shown in appendix of this report. 
TABLe 7.-Railroad track tools-Continued

Wrenches; single end (new American Standard Association nuts):

\begin{tabular}{|c|c|c|}
\hline $\begin{array}{c}\text { Size of } \\
\text { bolt }\end{array}$ & Opening & \multicolumn{1}{|c|}{ Length } \\
\cline { 1 - 2 } Inches & Inches & \\
$3 / 4$ & $13 / 16$ & 30 inches. \\
$7 / 8$ & 138 & 30 and 36 inches. \\
1 & 1916 & 36 inches. \\
$11 / 8$ & $13 / 4$ & 36 and 42 inches. \\
$11 / 4$ & 11516 & 42 inches. \\
$13 / 8$ & $21 / 8$ & 42 and 48 inches. \\
$11 / 2$ & 2516 & 48 inches. \\
\hline
\end{tabular}

W renches; double end (new American Standard Association nuts):

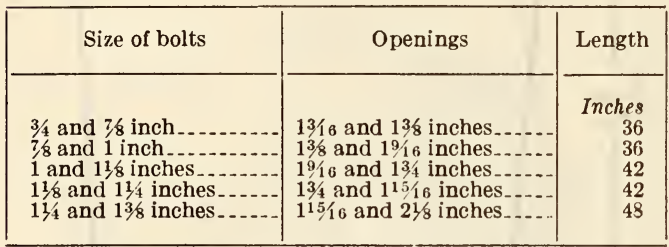

5. Bars, lining; pinch, wedge, and diamond shaped points: 18 pounds, 5 feet 0 inches long, 114 inches square.

20 pounds, 5 feet 3 inches long, 114 inches square.

26 pounds, 5 feet 6 inches long, $11 / 2$ inches square.

6. Tongs; rail, standard size, approximately 20 pounds.

7. Tongs; tie, standard size, approximately $71 / 2$ to 8 pounds

8. Tongs; timber, standard size, approximately 17 pounds.

9. Puller, spike, standard size, approximately 2 pounds.

10. Fork; rail, standard size, approximately 13 to 131 b́ pounds.

11. Bar; claw, standard size, approximately 30 pounds.

12. Adzes; track, blade 4 inches wide by 7 inches long, 5 pounds (standard eye No. 11) 4 inches wide by 8 inches long, 51 pounds; 4 inches wide by 9 inches long, $51 \frac{1}{2}$ pounds.

13. Sledges; double face:

Sizes: $6,8,10,12,14$, and 16 pounds.

Standard eye No. 2 for 6 and 8 pounds, 1 by 114 inches; for 10 to 16 pounds, 1 by $13 \%$ inches.

14. Bar; tamping, chisel end, standard size approximately 13 pounds 15. Bar; tamping, spear end, standard size approximately 15 pounds 16. Chisel; track, design No. 1, 512 and 612 pounds. (Standard eye No. $3,3 / 4$ by $13 / 8$ inches.)

17. Chisel; track, design No. 2, $51 \frac{1}{2}$ pounds. (Standard eye No. 3, $3 / 4$ by $13 / 8$ inches.)

18. Punch; tie plug, standard size approximately $31 / 2$ pounds. (Standard eye No. $3,3,4$ by 136 inches.)

19. Punch; track, round end, standard size approximately 5 pounds (Standard eye No. 3, 3/4 by 13,8 inches.)

20. Gage; track, standard design approximately 17 pounds.

\section{TABLE 8.-Blacksmith anvil tools}

\section{Chisels, cold:}

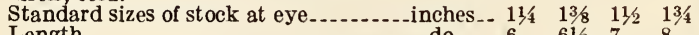
Length $\ldots \ldots \ldots$. Weight.....

(reights

Standard sizes of stock at eye..........inches_-_ $11 / 4 \quad 13 / 8 \quad 11 / 2 \quad 134$

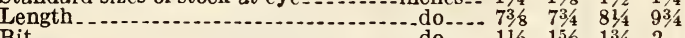
Bit

\section{TABLE 8.-Blacksmith anvil tools-Continued}

Flatters:

Standard sizes of face..............-inches_- $2 \quad 21 / 2 \quad 3 \quad 31 / 2 \quad 4$

$\begin{array}{lllllll}\text { Length } & \ldots \\ \text { Weight }\end{array}$

Fullers, top:

Standard sizes of groove -

(Shank for 1 -i

$23 / 8$ inches long anvil hole,

Standard sizes of

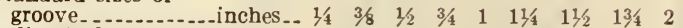
Heading tools:

Standard diameter of

hole
Length._...

Hength

Standard diameter of

hole

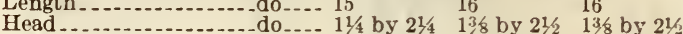

Standard diameter of hole............................

Standard diameter of hole.......... 11

Hengd

Hardies:

Standard sizes of square

$\begin{array}{llllllll}\text { shank.............inches._. } & 1 / 2 & 5 / 8 & 3 / 4 & 7 / 8 & 1 & 11 / 8 & 114\end{array}$

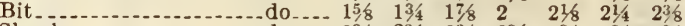
$\begin{array}{lllllll}\text { Shank.................do_... 23/8 } & 23 / 8 & 23 / 8 & 23 / 8 & 23 / 8 & 23 / 8 & 2^{3 / 6}\end{array}$

Punches, round end:

Standard sizes of round

$\begin{array}{llllllll}\text { punch........... } & 1 / 4 & 3 / 8 & 1 / 2 & 5 / 8 & 3 / 4 & 7 / 8 & 1\end{array}$

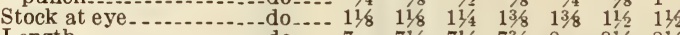

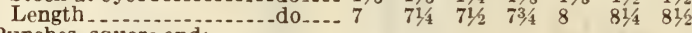
Punches, square end:

Standard sizes of square

$\begin{array}{llllllll}\text { punch................... } 1 / 4 & 3 / 8 & 1 / 2 & 5 / 8 & 3 / 4 & 7 / 8 & 1\end{array}$

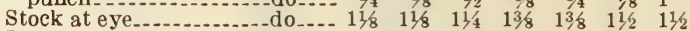

Length..............................

Set hammers:

Standard square sizes of stock at face........inches.. $114411 / 2 \quad 13 /$ Weight_................................. Swages, top: Standard sizes of groove, inches, $1 / 4,3,8,1 / 2,5 / 8,3 / 4,7 / 8,1$, $11 / 4,11 / 2,13 \frac{1}{4}, 2,21 / 2,3$.

Swages, bottom (shank for 1-inch anvil hole): Standard sizes of groove, inches, 1/4, 3/8, 1/2, 5/8, 3/4, 7/8, 1, 11/4, 11/2, 13/4, 2, 21/2, 3 .

\section{TABLE 9.-Tongs}

Bolt or curved lip:

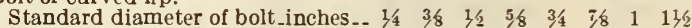

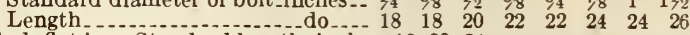
Gad, flat jaw: Standard length, inches, 18, 20, 24.

Horseshoers: Length, inches, 14, 16.

Pick-up, single: Length, inches, 20, 24.

Pick-up, double: Length, inches, 20, 24

Rivet: Length, inches, 18, 20, 22, 24 .

Riveters for structural and bridge work: Length, inches, 18, 24, 30

Straight lip: Standard sizes, inches, 16, 18, 20, 22, 24.

\section{TABLE 10.-Miscellaneous tools}

Backing out punches:

Standard diameter of face...inches_- $\begin{array}{llllll}3 / 8 & 1 / 2 & 5 / 8 & 3 / 4 & 7 / 8 & 1\end{array}$

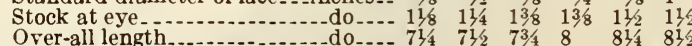
Bull points: Standard sizes, inches, $7 / 8$ by $12,7 / 8$ by 18,1 by 15,1 by 18 $11 / 8$ by $18,11 / 8$ by $24,11 / 4$ by 18,114 by 24 .

Drift pins (barrel shape)

Diameter of stock - . Rivet buster or side chisel: Standard size, pounds, 5 .

ivet header or rivet set

Size rivet. inches.- $\quad 1 / 2 \quad 5 / 8 \quad 3 / 4 \quad 7 / 8$ 

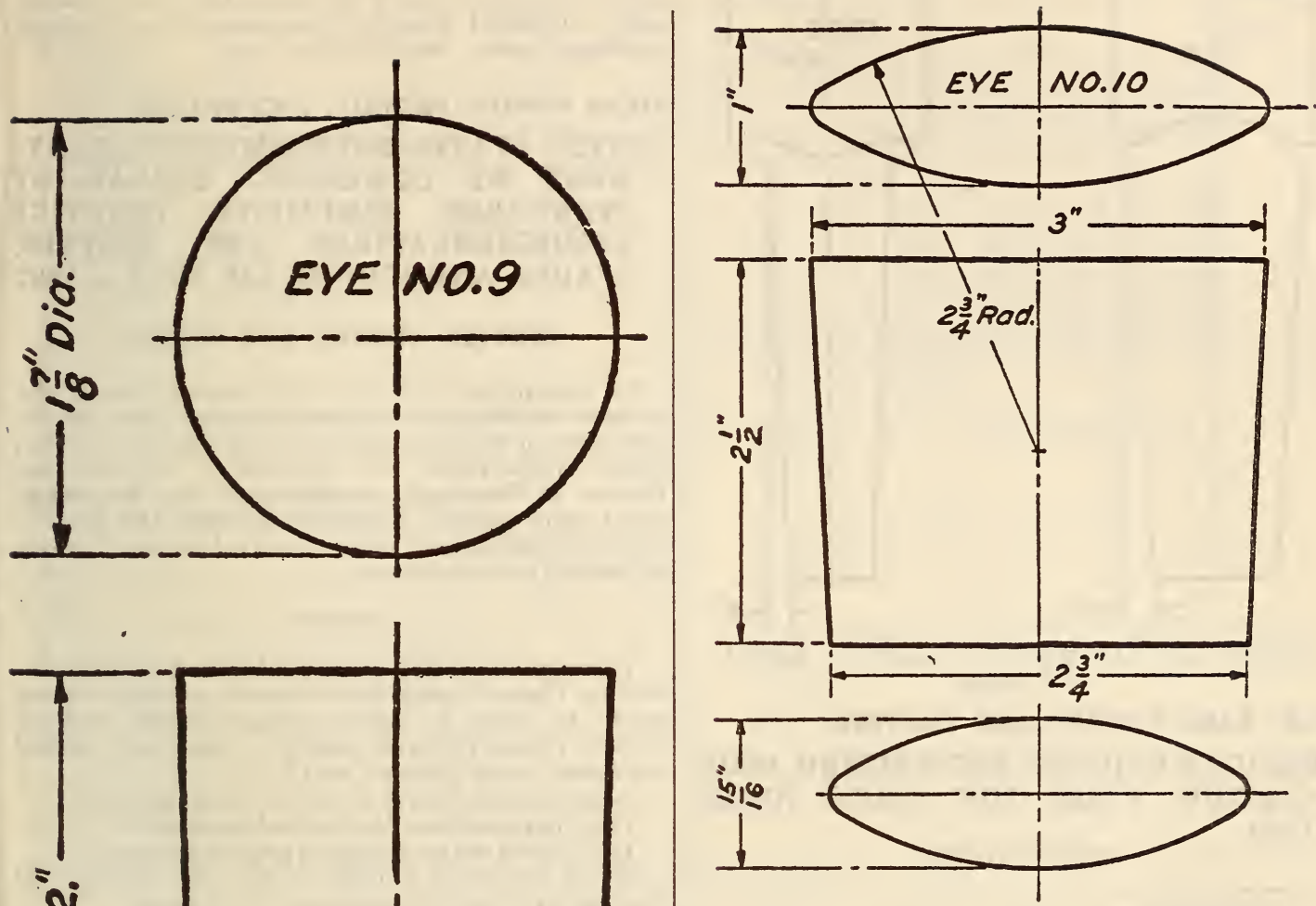

FIGURE 771
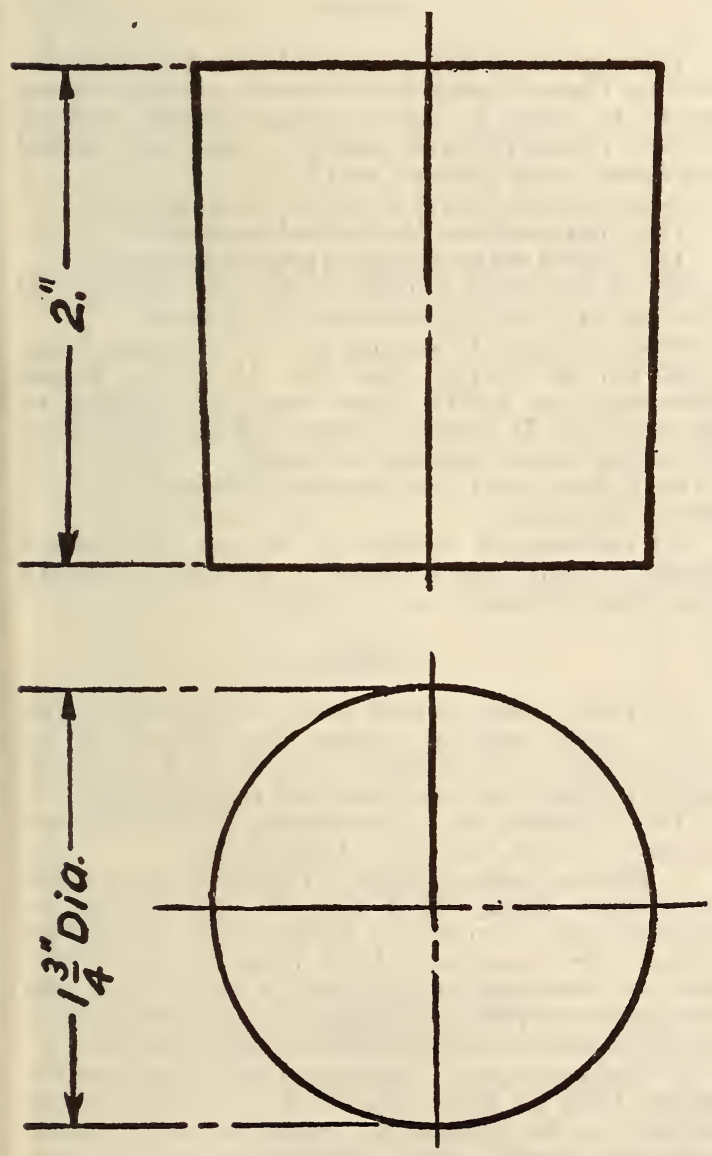

FiguRe 770

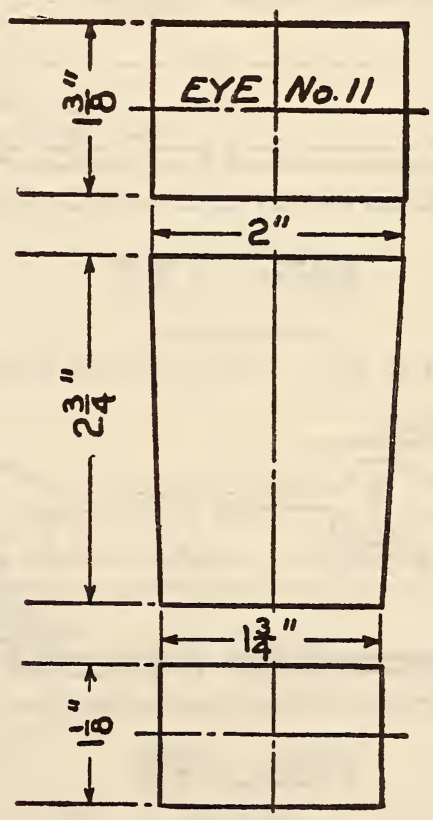

FIGURE 772 


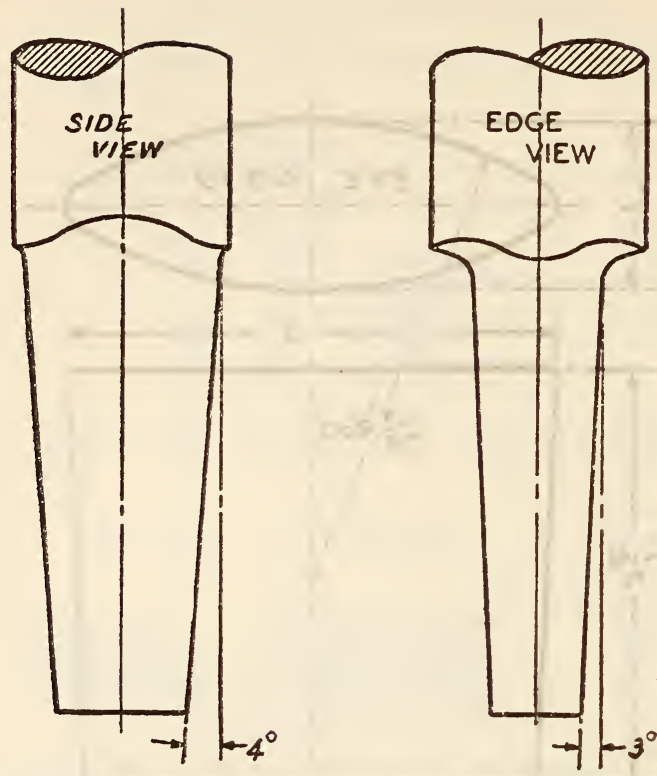

Figure 773. - Illustration of taper for typical tool handle

616.61 BARS, DIGGING AND TAMPING.

AMERICAN RAILWAY ENGINEERING ASSOCIATION, PLANS FOR TRACK TOOLS, 1930

TRACK TOOLS

TOLANANCE-

Sz ON Choss SECTION
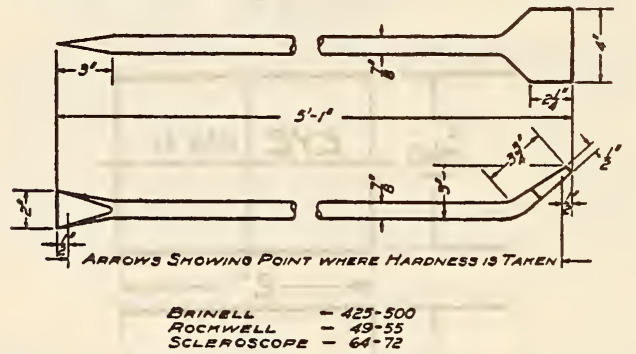

APPROX.WT. ISLES

Frgure 774.-Chisel and tamping bar

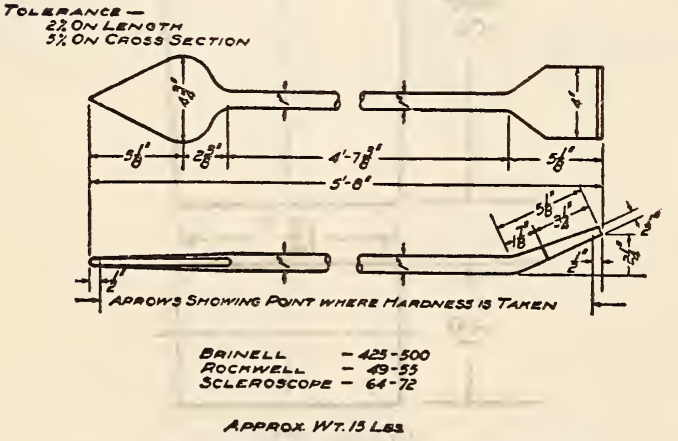

Figure 775. - Spear end tamping bar

(In addition to the standard chisel end tamping bar shown in Figure 774 this association has adopted a standard spear end tamping bar, Figure 775, with hardness requirements and dimension tolerances the same as for the standard chisel end tamping bar.)
616.62 HOES, RAKES, AND FORKS.

(For standard sizes and varieties see $\mathrm{U}$. S. Government, Department of Commerce, Bureau of Standards, simplified practice recommendation R17-31 for forged tools. See 616.60, p. 960.)

616.63 SCOOPS, SHOVELS, AND SPADES.

UNITED STATES GOVERNMENT, DEPART-
MENT OF COMMERCE, BUREAU OF
STANDARDS, SIMPLIFIED PRACTICE
RECOMMENDATIONS FOR SHOVELS,
SPADES, AND SCOOPS, R48, JULY 1, 1927

\section{SHOVELS, SPADES, AND SCOOPS}

In accordance with the unanimous action of the general conferences of manufacturers, distributors, and users of shovels, spades, and scoops, the United States Department of Commerce, through the Bureau of Standards, recommends that the recognized stock grades, multiples, finishes, and sizes of shovels, spades, and scoops carried as stock items be limited to the following:

\section{GRADES}

Three grades of shovels instead of four, to be classified as: Class A, high-grade shovel (replaces former grade A); class B, medium-grade shovel (replaces former grade B); and class C, low-grade shovel (replaces former grades $\mathrm{C}$ and $\mathrm{D}$ ).

No shovels of a lower grade to be manufactured.

The "narrow strap" to be used on class C.

The United States standard gage to be used.

No. 1 standard handle to be used in class $\mathrm{C}$ shovels, with waxing permitted if so desired.

Steel for class $\mathrm{C}$ shovels of the following types shall not be heavier than No. 15 United States standard gage, 0.0703: Plain back, hollow back, or riveted back, $D$ handle, square; long handle, square; $D$ handle, round; long handle, round.

Post, drain, and ditch spades to be made only in classes A and B.

All hollow-back sockets to be not less than 8 inches or more than 9 inches in the blank in length from head of blade to end of socket.

\section{FINISHES}

All plain back, riveted back, hollow back, and solid socket shovels, spades, and scoops of all grades shall be manufactured only in black or natural finish, full polished finish, and half polished finish.

The following is a description of finishes and manner in which they are to be produced:

1. Black or natural finish. - The blade to be left in its natural state, but it may be wire brushed to remove scale or rust, the blade not to be pickled before wire brushing. After brushing no further work or treatment of the blade is to be done, with the single exception of dipping the finished blade in shovel lacquer or lacquer with an asphaltum base.

2. Full polished.-The blade to be pickled, and the blades both on front and back, over their entire surface, to be finished on roughing and finishing polishing wheels. After polishing no further work is to be done on the blade, with the single exception of dipping the finished blade in the shovel lacquer or lacquer with an asphaltum base.

3. Half polished.-The lower half of the blade, both front and back, to be pickled and finished on roughing and finishing polishing wheels. The upper half, both front and back, to be left in the natural black condition or finished with a colored varnish, lacquer, or paint. 
Multipies and sizes

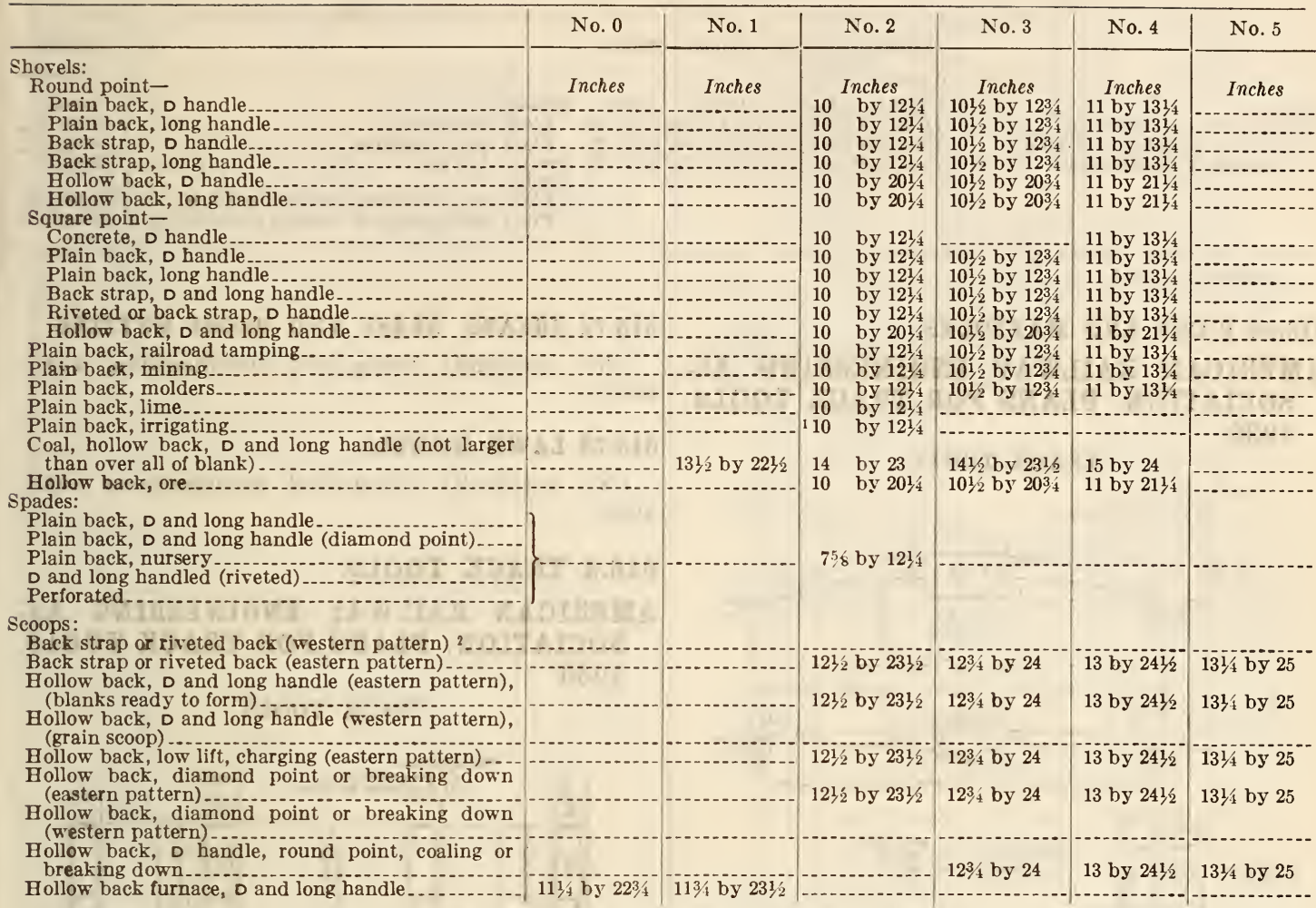

\section{Shovels:}

Round point-

Plain back, D handle

Plain back, long handle

Back strap, D handle

Back strap, long handle

Hollow back, handle

$11 \frac{1}{4}$ by $22^{3} / 4$ 113/4 by $231 / 2$

Square point-

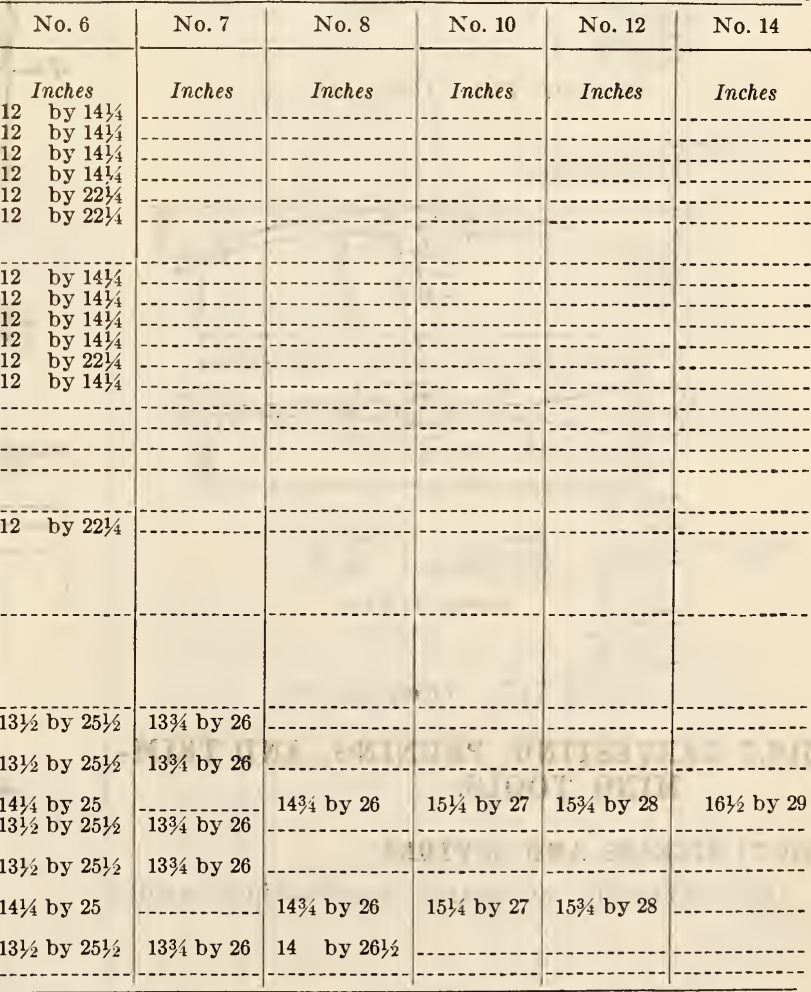

Concrete, $D$ handle.

Plain back, D handle

Plain back, long handle.

Back strap, D and long handle

Riveted or back strap, $D$ handle

Hollow back, D and long handle.

Plain back, railroad tamping.

Plain back, mining

Plain back, molders.

Plain back, lime

Plain back, irrigating.

Coal, hollow back, D and long handle (not larger than

over all of blank

Spades:

Plain back, D and long handle.

Plain back, D and long handle (diamond point)

Plain back, nursery

$D$ and long handled (riveted)

Perforated

Scoops:

Back strap or riveted back (western pattern) ${ }^{2}$

Back strap or riveted back (eastern pattern)

Hollow back, $D$ and long handle (eastern pattern), (blanks ready to form)

Hollow back, D and long handle (western pattern),

(grain scoop)

Hollow back, diamond point or breaking down (eastern

pattern)

pattern)

Hollow back, D handle, round point, coaling or breaking

Hollow back furnace, $\mathrm{D}$ and long handle. 
Multiples and sizes-Continued

\begin{tabular}{|c|c|c|c|c|c|c|}
\hline & 14-inch & 16-inch & 18-inch & 20-inch & & Regular \\
\hline $\begin{array}{l}\text { Spades: } \\
\text { Plain back post, } D \text { handle...... } \\
\text { Plain back ditching, } D \text { handle.. } \\
\text { Plain back drain, } D \text { handle.... }\end{array}$ & $\begin{array}{c}\text { Inches } \\
71 / 8 \text { by } 14 \\
718 \text { by } 14 \\
718 \text { by } 14\end{array}$ & \begin{tabular}{|c|} 
Inches \\
718 by 16 \\
718 by 16 \\
716 hy 16
\end{tabular} & $\begin{array}{c}\text { Inches } \\
71 / 8 \text { by } 18 \\
713 \text { by } 18 \\
713 \text { by } 18\end{array}$ & $\begin{array}{l}\text { Inches } \\
71 / 8 \text { by } 20 \\
71,8 \text { by } 20 \\
71,8 \text { by } 20\end{array}$ & 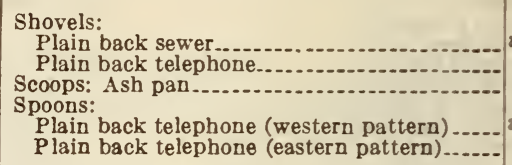 & $\begin{array}{l}\text { Inches } \\
10 \text { by } 121 / 4 \\
10 \text { by } 121 / 4 \\
12 \text { by } 31 \\
10 \text { by } 121 / 4 \\
10 \text { by } 121 / 4\end{array}$ \\
\hline
\end{tabular}

${ }^{3}$ Smaller if desired.

616.64 PICKS AND MATTOCKS.

AMERICAN RAILWAY ENGINEERING ASSOCIATION, PLANS FOR TRACK TOOLS, 1930

TRACK TOOLS

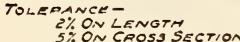
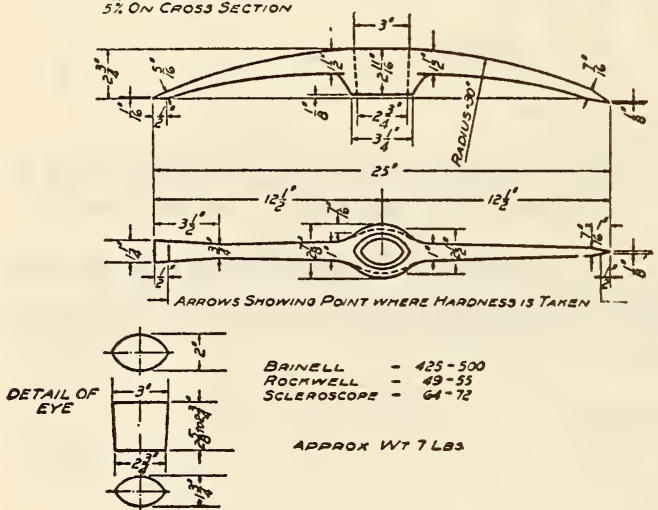

Figure 776.-Clay pick

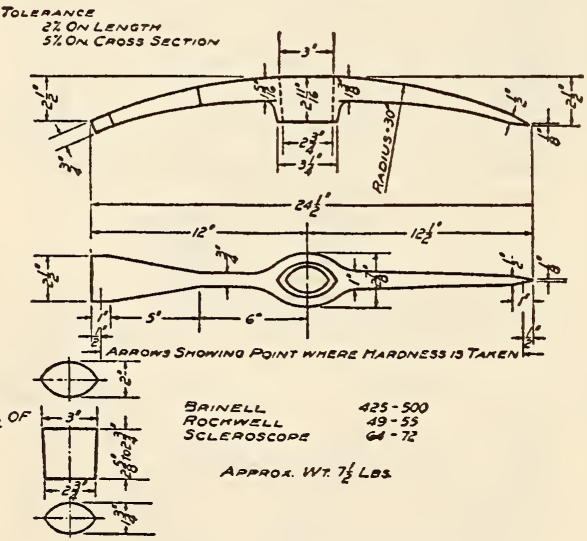

FIGURE 777.-Tamping pick

616.7 HARVESTING, PRUNING, AND TRIMMING TOOLS.

\subsection{SICKLES AND SCYTHES.}

(No nationally recognized specifications available.)
616.72 SHEARS, GRASS AND HEDGE COTTING.

(No nationally recognized specifications available.)

\subsection{LAWN MOWERS.}

(No nationally recognized specifications available.)

616.8 TRACK TOOLS.

AMERICAN RAILWAY ENGINEERING ASSOCIATION, PLANS FOR TRACK TOOLS, 1930

TRACK TOOLS
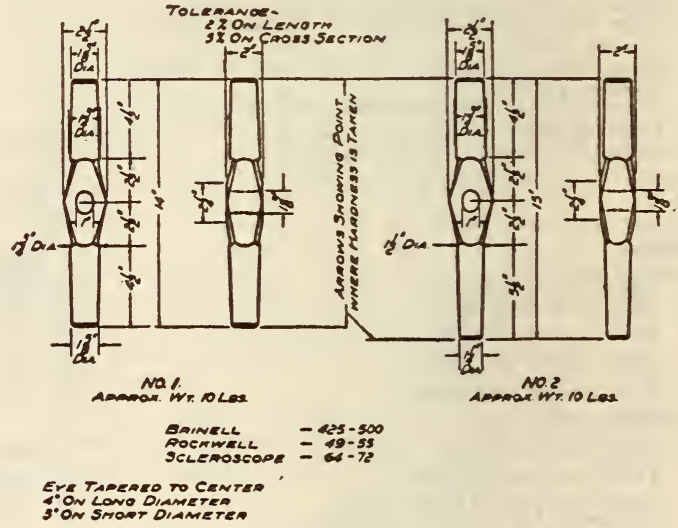

FIGURE 778. - Spike mauls
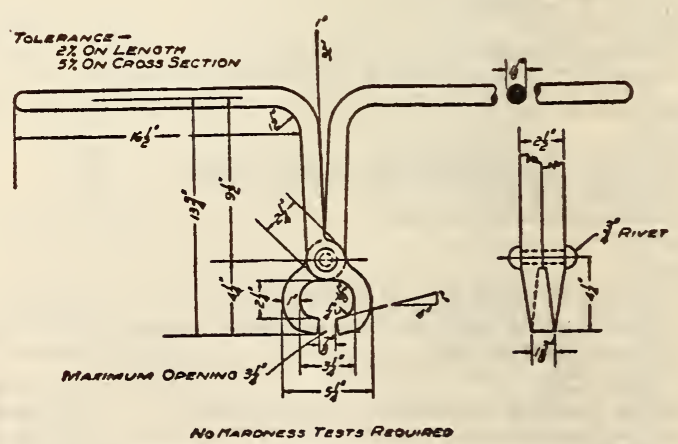

APpAOX un colos

FIGURE 779. - Rail tongs 

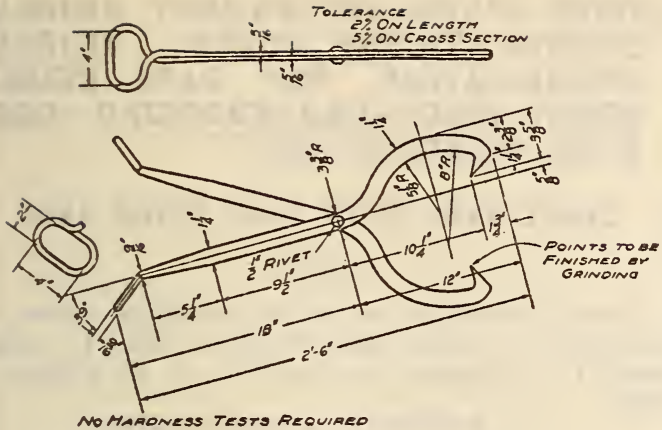

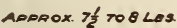

Figure 780.-Tie tongs

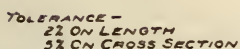

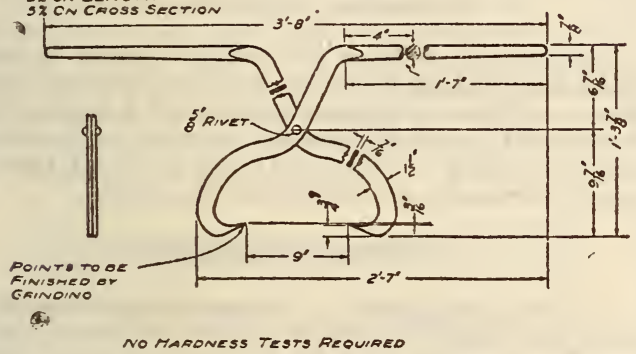

ASDOOX WT 17 LES

FIGURE 781. - Timber tongs

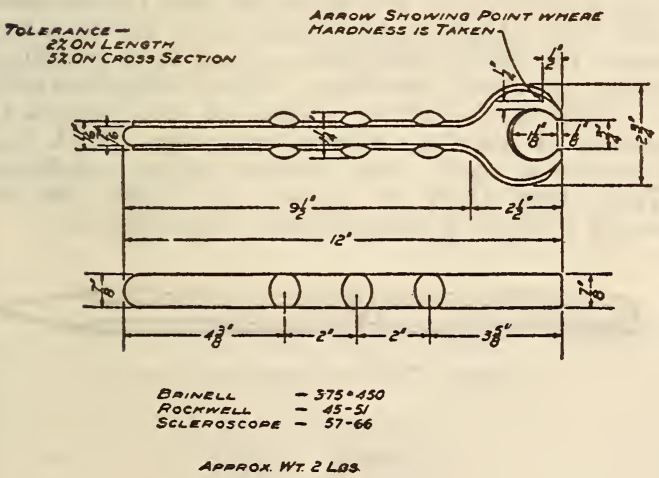

FIGURE 782.-Spike puller

Toueancer-

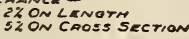

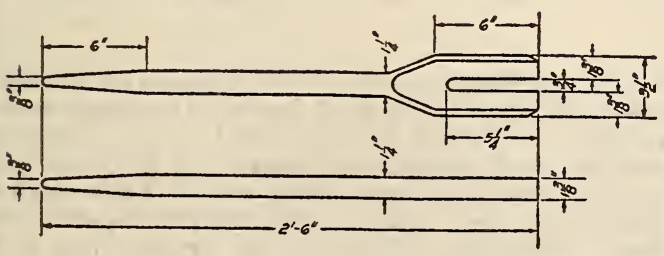

NOMARONESS TESTS REQUIRED

ADPROXWT:13TOISELCOS

FigURE 783. - Rail fork
ToLefance-

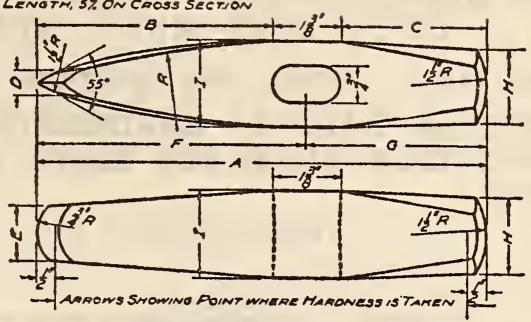

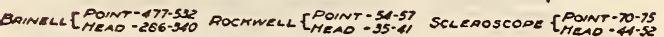

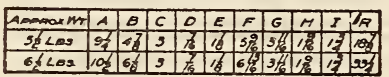

Eya tameaco to centen

Fon SMOat DIAMETEA

Figure 784. - Track chisel, design No. 1

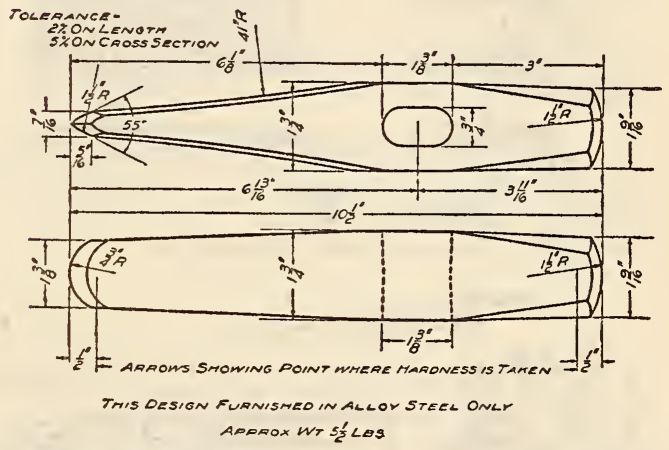

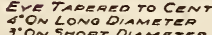

Figure 785. - Track chisel, design No. 2

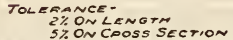

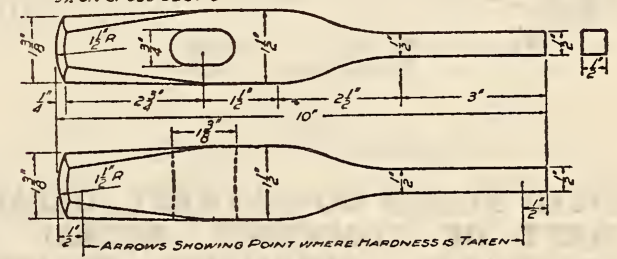

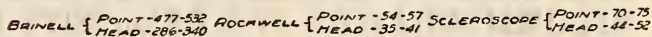
ADPROX WT 3 LES

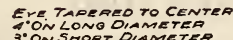

FigURE 786. - Tie plug punch

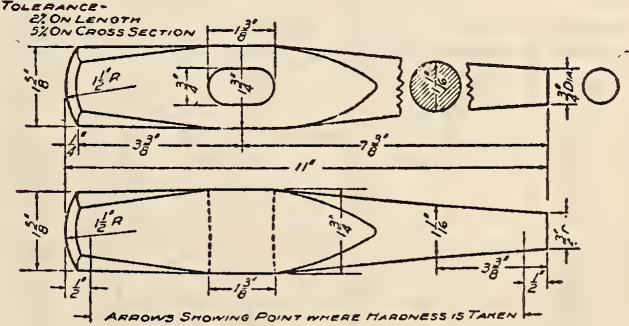

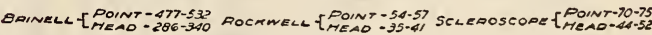
ADPROX WT 5 LES

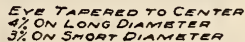

FIGURE 787.-Round track punch 


\subsection{MISCELLANEOUS OTHER THAN \\ HAND \\ TOOLS METAL WORKING.}

616.91 JACKS, LEVERS, AND LEVERAGE BARS.

AMERICAN RAILWAY ENGINEERING ASSOCIATION, PLANS FOR TRACK TOOLS, 1930

\section{TRACK TOOLS}

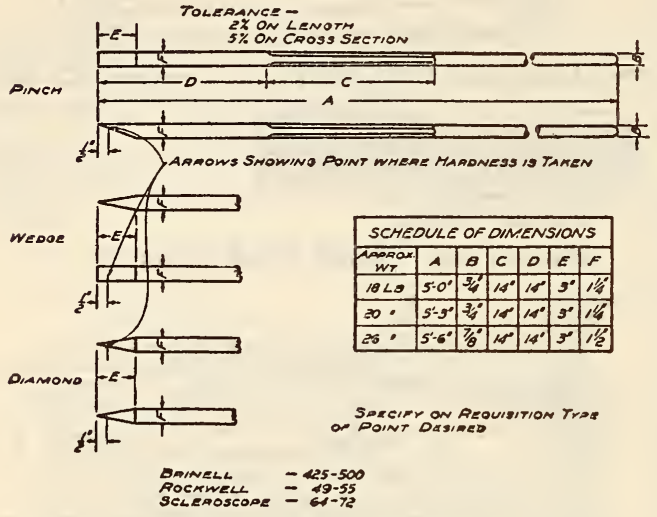

Figure 788.-Lining bar

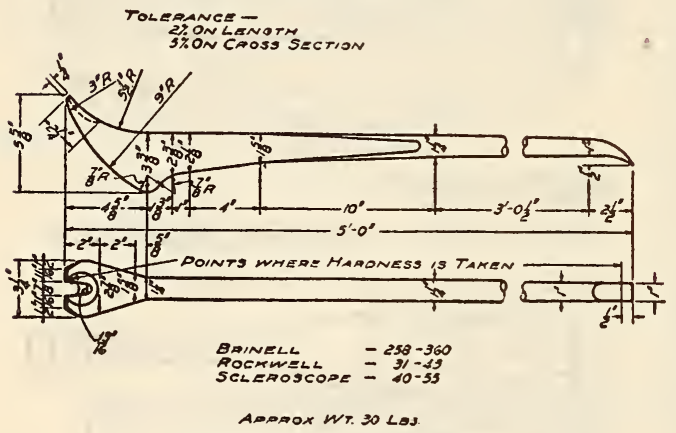

Figure 789. - Claw bar

UNITED STATES GOVERNMENT, DEPARTMENT OF COMMERCE, BUREAU OF STANDARDS; SIMPIIFIED PRACTICE RECOMMENDATION FOR BELL-BOTTOM SCREW JACKS, R97-30, OCTOBER 1, 1930

\section{BELL-BOTTOM SCREW JACKS}

(Simplified practice recommended and accepted by industry establishing a limited list of standard sizes.)

\begin{tabular}{|c|c|c|c|c|c|}
\hline $\begin{array}{l}\text { Diameter } \\
\text { of screw }\end{array}$ & $\begin{array}{l}\text { Height } \\
\text { of stand }\end{array}$ & $\begin{array}{l}\text { Height } \\
\text { over all, } \\
\text { closed }\end{array}$ & $\begin{array}{c}\text { Diameter } \\
\text { of screw }\end{array}$ & $\begin{array}{l}\text { Height } \\
\text { of stand }\end{array}$ & $\begin{array}{l}\text { Heignt } \\
\text { over all, } \\
\text { closed }\end{array}$ \\
\hline Inches & Inches & Inches & Inches & Inches & Inches \\
\hline 1 & 6 & 8 & 2 & 5 & $81 / 2$ \\
\hline $11 / 4$ & 4 & $61 / 4$ & 2 & 6 & $91 / 2$ \\
\hline $11 / 4$ & 6 & $81 / 2$ & 2 & 8 & $111 / 2$ \\
\hline $11 / 4$ & 8 & $10 \frac{1}{2}$ & 2 & 10 & $13^{1 / 2}$ \\
\hline $11 / 4$ & 10 & $121 / 2$ & 2 & 12 & $151 / 2$ \\
\hline $11 / 4$ & 12 & $141 / 2$ & 2 & 14 & $171 / 2$ \\
\hline & & & 2 & 16 & $191 / 2$ \\
\hline $11 / 2$ & 4 & $f^{3} 34$ & 2 & 18 & $211 / 2$ \\
\hline $11 / 2$ & 6 & $83 \frac{1}{4}$ & $21 / 2$ & 6 & 10 \\
\hline $11 / 2$ & 8 & $10 \frac{3}{4}$ & $21 / 2$ & 8 & 12 \\
\hline $11 / 2$ & 10 & $12 \frac{3}{4}$ & $21 / 2$ & 10 & 14 \\
\hline $11 / 2$ & 12 & $143 / 4$ & $21 / 2$ & 12 & 16 \\
\hline $11 / 2$ & 14 & $16^{3} / 4$ & $21 / 2$ & 14 & 18 \\
\hline $13 / 4$ & 6 & 9 & $\begin{array}{l}21 / 2 \\
21 \%\end{array}$ & $\begin{array}{l}16 \\
18\end{array}$ & $\begin{array}{l}20 \\
22\end{array}$ \\
\hline $13 \frac{1}{4}$ & 8 & 11 & $21 \%$ & 20 & $\begin{array}{l}22 \\
24\end{array}$ \\
\hline $13 \frac{3}{4}$ & 10 & 13 & $\overline{3}$ & 8 & 13 \\
\hline $13 / 4$ & 12 & 15 & 3 & 14 & 19 \\
\hline 13 & 14 & 17 & 3 & 18 & 23 \\
\hline & 16 & 19 & 3 & 24 & 29 \\
\hline
\end{tabular}

\section{UNITED STATES GOVERNMENT, FEDERAL SPECIFICATIONS BOARD; FEDERAL SPECIFICATION FOR BARS-CHISEL, CROW, PINCH AND WRECKING-GGG- B-101, MARCH 29, 1932}

\section{CHISEL BARS, CROW BARS, PINCH BARS}

II. TYPES

These bars shall be of the following types, as specified: $A$, chisel bars; $B$, crow bars; $\mathrm{C}$, pinch bars; D, wrecking, gooseneck; and E, wrecking, offset.

\section{MATERIAL AND WORKMANSHIP}

All bars shall be made of high-grade material and in accordance with the best commercial practice. They shall be free from scale, checks, cracks, or any other defects or blemishes which may affect their serviceability or appearance.

\section{GENERAL REQUIREMENTS}

The bars shall have the maker's name or trademark stamped or otherwise permanently marked on them. A plus or minus tolerance of 5 per cent will be allowed in all dimensions referred to herein except for width of edge of type (A) chisel bars for which tolerances are otherwise provided.

\section{DETAIL REQUIREMENTS}

1. Chisel bars, type A. - Unless otherwise specified, chisel bars shall be made of octagon tool steel having a carbon content of 0.80 to 0.90 per cent and a maximum sulphur and phosphorus content of 0.04 per cent or of a high-grade alloy tool steel. One end of the bar shall be forged, hardened, tempered, and ground to a chisel-shaped edge. The width of this edge shall be from one-sixteenth inch to one-fourth inch wider than the short diameter of the stock from which the bar is made, the amount to be in proportion to the size of the stock. The striking end of the bar shall be chamfered. This and shall possess the quality of resisting deformation when struck by a hammer or maul under normal service conditions. These bars shall be similar to Figure 790 and conform to the following table of dimensions:

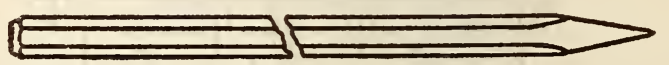

Figure 790.-Chisel bar, type $A$

\begin{tabular}{|c|c|}
\hline $\begin{array}{c}\text { Diameter } \\
\text { octagon steel }\end{array}$ & $\begin{array}{l}\text { Length } \\
\text { over all }\end{array}$ \\
\hline $\begin{array}{c}\text { Incines } \\
5 / 3 \\
3 / 4 \\
7 / 8 \\
1 \\
13\end{array}$ & $\begin{array}{c}\text { Inches } \\
18 \\
24 \\
30 \\
36\end{array}$ \\
\hline
\end{tabular}

2. Crow bars, type B. - Unless otherwise specified, crow bars shall be made of tool steel having a carbon content of 0.60 to 0.90 per cent and a maximum sulphur and phosphorus content of 0.05 per cent, or of good-quality alloy tool steel. They shall be the type known commercially as pinch-point crow bars. The formed end shall be square, with a flat chisel-shaped edge, the body shall be octagonal in shape, and the handle shall be round, with a slight taper toward the end. These bars shall be furnished in 60 -inch over-all length; the pointed end shall be $1 \frac{1}{4}$ inches wide, and shall be properly 
hardened and tempered. They shall be similar to Figure 791 and conform to the dimensions in the following table:

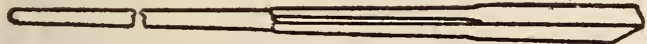

Figure 791. - Crow bar, type B

\begin{tabular}{|c|c|c|c|}
\hline \multirow{2}{*}{$\begin{array}{c}\text { Diameter } \\
\text { octagon } \\
\text { steel }\end{array}$} & \multicolumn{2}{|c|}{ Weight } & $\begin{array}{c}\text { Length } \\
\text { Over all }\end{array}$ \\
\cline { 2 - 3 } & $\begin{array}{c}\text { Mini- } \\
\text { mum }\end{array}$ & $\begin{array}{c}\text { Maxi- } \\
\text { mum }\end{array}$ & \\
\hline $\begin{array}{c}\text { Inches } \\
11 / 4\end{array}$ & $\begin{array}{c}\text { Pounds } \\
17\end{array}$ & $\begin{array}{c}\text { Pounds } \\
20\end{array}$ & $\begin{array}{c}\text { Inches } \\
60\end{array}$ \\
\hline
\end{tabular}

3. Pinch bars, type C. - Unless otherwise specified, pinch bars shall be made of octagon tool steel having a carbon content of 0.70 to 0.90 per cent with a maximum sulphur or phosphorus content of 0.04 per cent or of good-quality alloy tool steel. One end shall have a rounded offset and shall be properly hardened and tempered. The other end shall be round, smoothly finished, and tapered for bringing holes in steel plates into alignment. These bars shall be similar to Figure 792 and conform to the following table of diniensions:

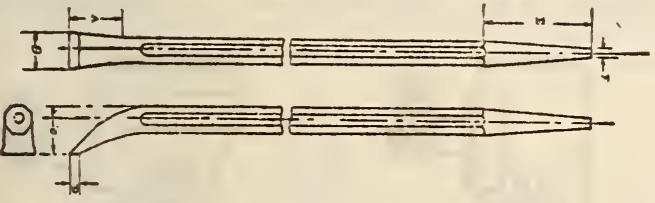

Figure 792.-Pinch bar, type $C$

Dimensions of type $C$, pinch bars

\begin{tabular}{|c|c|c|c|c|c|c|c|}
\hline $\begin{array}{c}\text { Diam- } \\
\text { eter } \\
\text { octagon } \\
\text { steel }\end{array}$ & $\begin{array}{c}\text { Length } \\
\text { over all }\end{array}$ & \multicolumn{6}{|c|}{ Dimensions (inches) } \\
\cline { 3 - 7 } & & $\mathrm{A}$ & $\mathrm{B}$ & $\mathrm{C}$ & $\mathrm{D}$ & $\mathrm{E}$ & $\mathrm{F}$ \\
\hline Inch & Inches & & & & & & \\
$3 / 4$ & 26 & $11 / 2$ & 1 & $11 / 4$ & $5 / 16$ & 3 & $5 / 16$ \\
$7 / 8$ & 30 & $13 / 4$ & $11 / 3$ & $11 / 2$ & $3 / 8$ & $31 / 2$ & $3 / 8$ \\
1 & 36 & 2 & $11 / 4$ & $13 / 4$ & $7 / 16$ & 4 & $7 / 16$ \\
\hline
\end{tabular}

4. Type D, wrecking bars (gooseneck, claw and pinch point). - These bars shall be made of $3 / 4$-inch hexagon tool steel having a carbon content of 0.70 to 0.90 per cent, with a maximum sulphur and phosphorus content of 0.04 per cent or of good quality alloy tool steel. One end of the bar shall be bent in the form of a gooseneck and shall be provided with a claw suitable for pulling nails. The opposite end of the har shall be wedge shaped and offset at an angle of approximately $30^{\circ}$. Both ends shall be properly heat-treated and tempered and the bars shall possess the quality of resisting bending or breaking under normal service conditions.

Bars of this type shall be furnished with over-all lengths of 30 or 36 inches as may be specified. They shall be similar to illustration Figure A-7921/2 and shall conform to the dimensions given therein except that the dimensions may vary within the tolerances provided by paragraph IV.

5. Type E, wrecking bars (offset claw and pinch point). These bars shall be made of hexagon tool steel of the same size and quality as that prescribed herein for type D wrecking bars. Both ends of type $\mathrm{E}$ wrecking bars shall be offset at an angle of approximately $30^{\circ}$, in opposite directions. One end shall be wedge-shaped and the other end shall be provided with a claw suitable for pulling nails. Both ends shall be properly heat-treated and tempered and the bars shall possess the quality of resisting bending or breaking under normal service conditions.

Bars of this type shall be furnished with over-all lengths of 30 or 36 inches as may be specified. They shall be similar to illustration Figure B-7921/2 and shall conform to the dimensions given therein except

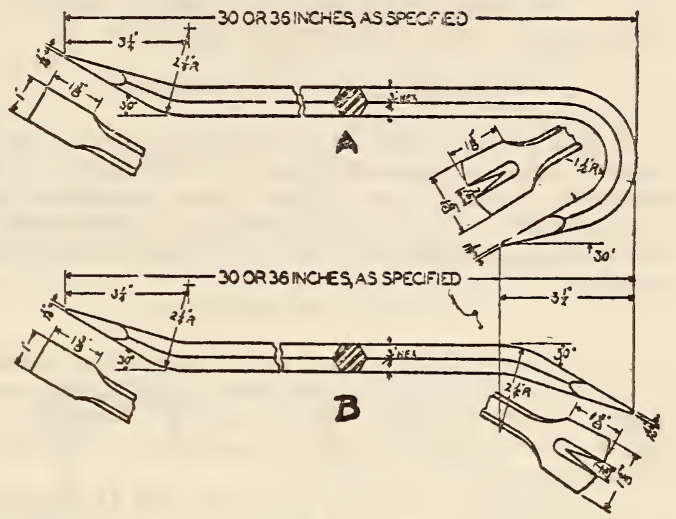

Figure 7921/2

A. Type D, wrecking bars, gooseneck claw and pinch point, B. Type E, wrecking bars, offset claw and pinch point.

that the dimensions may vary within the tolerances provided by paragraph IV.

\section{METHOD OF INSPECTION AND TESTS}

1. Representative samples totaling at least 5 per cent of each type and size shall be taken at random from each delivery and submitted to chemical (if of carbon tool steel) and physical tests as follows:

(a) Chemical test.-Two ounces of drillings shall be obtained from each sample bar of carbon tool steel for chemical analysis, which shall be in accordance with requirement specified under detail requirements.

(b) Physical tests.-Each sample chisel bar selected for test shall be subjected, at the option of the inspector, to either or both of the methods specified in paragraphs (1) and (2) below.

(1) Chisel bars shall be securely held in a vertical position by a vise or clamp with the striking end of chisel bar resting on a solid foundation. A medium steel bar at least three-quarters inch in diameter shall be held at right angles with the cutting edge and struck a sufficient number of blows with a heavy hammer to make a cut in the bar one-eighth inch deep without the cutting edge chipping or showing undue wear.

(2) Chisel bars shall show a Rockwell hardness of not less than 42 nor more than 58 on the $C$ scale (or equivalent Brinell of 398-587) about one-half inch back from the cutting edge.

F-1b (2). Crowbars and pinch bars shall show a Rockwell hardness of not less than 35 nor more than 48 on the C scale (or equivalent Brinell of 327-464) about 2 inches back from the chisel-shaped edge.

F-1c. Wrecking bars snall show a Rockwell hardness of not less than 35 nor more than 48 on the $\mathrm{C}$ scale (or equivalent Brinell of $327-464$ ) about three-fourths inch back from the wedge-shaped ends. 
UNITED STATES GOVERNMENT, FEDERAL SPECIFICATIONS BOARD ; FEDERAL SPECIFICATION FOR JACKS, No. 481, APRIL 25, $192 \%$. GGG-J-51) (CURRENT DESIGNATION :

\section{JACKS}

\section{TYPES}

Jacks shall be furnished in the following types as required: A, bevel-geared screw jacks; B, bevelgeared journal jacks; C, hydraulic jacks; D, lever jacks, automatic; E, planer or leveling jacks; $F$, screw jacks; and G, motor-truck jacks.

\section{MATERIAL AND WORYMANSEIP}

All jacks shall be made from best-quality materials, and be free from all defects affecting their appearance or that may affect their operation or serviceability. They shall be of good workmanship and made in accordance with the best commercial practice.

\section{GENERAL REQUIREMENTS}

1. All types of jacks shall be of safe construction for their rated capacity and shall have strong and rugged working parts. They shall be designed so that all parts will be readily accessible for repairs or lubrication.

2. Jacks, types A to D, inclusive, and $G$, shall be equipped with a warning device or stop to insure that the maximum lift is not exceeded.

3 . The capacities stated for the various types and sizes of jacks shall be based on the head lift. The claw or foot lift shall be based on one-half of the head lift.

4. Each jack shall be smoothly finished, carefully japanned or painted, and plainly and permanently marked with the name of the manufacturer.

5. The caps, tops, or rams shall be roughened on the top to prevent the slipping of the load, except that this requirement need not apply to type $E$ jacks.

\section{DETAIL REQUIREMENTS}

1. Bevel-geared screw jacks, type A.-These shall be of the type known as standard speed and shall be provided with a sleeve cast in one piece having a solid or removable top and equipped with a claw or ground lift. The base shall be of suitable size to prevent tilting over when load is placed on the claw. The following essential parts are included: A bevel gear and pinion; suitable shaft between the pinion and ratchet; sleeve, standard, and screw with suitable engaging threads in the standard lining; cap; ratchet; and lever. Handles shall also be provided for moving the jack from place to place.

The gear and pinion shall be made of steel and shall have machine-cut teeth. The pinion may be forged integrally with the part of the shaft between the pinion and ratchet or may be secured to the shaft in any other equally satisfactory manner. The design shall be such that the gear and pinion will be in proper mesh.

The ratchet shall be made of steel. The ratchet holder shall be made of malleable iron or steel. The pawl shall be of steel and shall engage the teeth of the ratchet gear. Means shall be provided to change the direction of operation of the jack.

The main bearing on which the screw rotates shall consist of two hardened steel plates, separated by balls or rollers made of hardened alloy steel. The bevel gear shall be securely fastened to the screw.

The sleeve shall be made of either steel or malleable iron. The standard and base shall be in one piece, and made of steel or malleable iron. A bushing or nut of alloy bronze, threaded internally to engage with the screw, shall be fitted inside the standard. The caps of these jacks shall be roughened on top. The claw may be plain or roughened in accordance with the manufacturer's practice.

The screw shall be made of steel, the threads shall be carefully cut and finished. The threaded portion of the screw shall engage with the threads in the lining or nut in the standard, the other end shall project through the bearing plates. The end of the screw which passes through the bearing plates shall rotate freely. All parts of the jack shall be easily accessible for inspection or repairs to the working parts.

For jacks larger than 50 tons' capacity, compound gearing may be used.

Each jack shall be furnished with a suitable operating lever.

These jacks shall be provided with a warning device or stop which will prevent the screw coming out of the nut or bushing when the limit of rise is reached.

Bevel-geared screw jacks shall be plainly and permanently marked to indicate their maximum lifting capacity and the jacks shall be capable of retaining any weight up to their rated capacity at any height within the range. Ther shall be capable of lifting one-half their rated capacity on the claw to any height within their range.

These jacks shall be similar to Figure 793 and conform to the requirements of the following table:
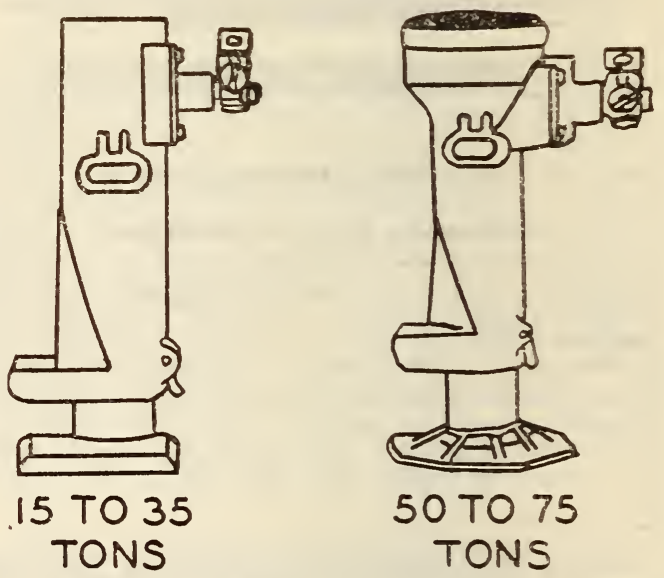

Figure 793.-Jack, type $A$

\begin{tabular}{|c|r|r|r|}
\hline Capacity & $\begin{array}{c}\text { iveight } \\
\text { (mini- } \\
\text { mum) }\end{array}$ & $\begin{array}{c}\text { Closed } \\
\text { height } \\
\text { (approxi- } \\
\text { mate) }\end{array}$ & $\begin{array}{c}\text { Rise (ap- } \\
\text { proxi- } \\
\text { mate) }\end{array}$ \\
\hline Tons & Pounds & Inches & Inches \\
$15 \ldots \ldots \ldots$ & 22 & 10 \\
$25 \ldots \ldots \ldots$ & 110 & 22 & 10 \\
$35 \ldots \ldots$ & 135 & 22 & 10 \\
$75 \ldots$ & 180 & 24 & 9 \\
\hline
\end{tabular}

2. Beveled-geared journal jacks, type B.-These shall be of the same type as the bevel-geared screw jacks. They shall consist essentially of the following parts: A bevel gear and pinion; suitable shaft between pinion and ratchet; casing, ram, and screw with suitable engaging threads in the ram; ratchet; and ratchet holder with lever.

The gear and pinion shall be made of steel and shall have machine-cut teeth. The pinion may be forged integrally with the part of the shaft between 
the pinion and ratchet or may be secured to the shaft in any other equally satisfactory manner. The design shall be such that the gear and pinion will be in proper mesh.

The ratchet shall be made of steel. The ratchet holder shall be made of malleable iron or steel. The pawl shall be of steel and shall engage the teeth of the ratchet gear. Means shall be provided to change the direction of operation of the jack.

The main bearing on which the screw rotates shall consist of two hardened steel plates separated by balls or rollers made of hardened alloy steel.

The casing of the jack shall be made of malleable iron or steel and equipped with a handle. The ram shall be made of malleable iron or steel, and fitted with a nut or bushing made of alloy bronze threaded to engage the threads of the screw.

The screw shall be made of steel; the threads shall be carefully cut and finished. One end of the screw shall screw into the ram, the other end shall be securely fastened to the bevel gear. These jacks shall be provided with a warning device or stop which will prevent the screw coming out of the nut or bushing when the limit of rise is reached.

Bevel geared journal jacks shall be plainly and permanently marked to indicate their maximum lifting capacity and the jacks shall be capable of retaining any weight up to their rated capacity at any height within the range.

Each jack shall be furnished with a suitable operating lever.

These jacks shall be similar to Figure 794 and shall conform to the requirements of the following table:

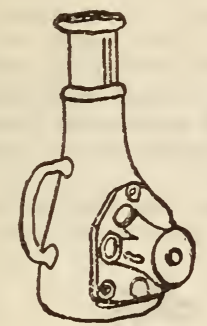

Figure 794.-

Jack, type B

\begin{tabular}{|c|c|c|c|}
\hline Capacity & $\begin{array}{c}\text { Weight } \\
\text { (mini- } \\
\text { mum) }\end{array}$ & $\begin{array}{c}\text { Closer } \\
\text { height } \\
\text { (approxi- } \\
\text { mate) }\end{array}$ & $\begin{array}{c}\text { Rise (ap- } \\
\text { proxi- } \\
\text { mate) }\end{array}$ \\
\hline Tons & $\begin{array}{c}\text { Pounds } \\
25\end{array}$ & $\begin{array}{c}\text { Inches } \\
93 / 4\end{array}$ & $\begin{array}{r}\text { Inches } \\
4\end{array}$ \\
\hline $25 \ldots \ldots$ & 50 & 11 & 5 \\
\hline
\end{tabular}

3. Hydraulic jacks, type C.-These shall be of the independent pump type and shall be capable of being operated either vertically or horizontally.

The jack shall consist of a cylinder and ram connected to a pump by means of a flexible copper pipe.

The cylinder and ram shall be made from highgrade steel and free from welds or seams. They shall be accurately machined so as to insure a proper fit between the ram and the bore of the cylinder.

The cylinder shall have two handles suitable for carrying the jack from place to place, also a suitable connection for the copper pipe.

The pump shall be the single-plunger type and suitable for the rated capacity of the jack. It shall be fitted with a suitable connection for the copper pipe. The reservoir shall be of ample capacity for the total displacement of the jack. The pump body shall be of bronze. The plunger shall be of tool steel properly hardened.

The connecting pipe shall be made of copper, annealed, and capable of withstanding a pressure of at least 10,000 pounds per square inch. It shall be approximately 8 feet in length and shall be furnished. with suitable fittings for connecting it to the pump and the jack. All joints in the jack, pump, and copper pipe shall be designed to prevent leakage of liquid under maximum working pressure.

The jacks shall be provided with a warning device or step which will prevent the ram coming out of the cylinder when the limit of rise is reached.

Hydraulic jacks shall be plainly and permanently marked to indicate their maximum lifting capacity, and the jacks shall be capable of retaining any weight up to their rated capacity at any height within the range. Each pump shall be furnished with a suitable operating lever.

These jacks shall be similar to Figure 795 and shall conform to the requirements of the following table:

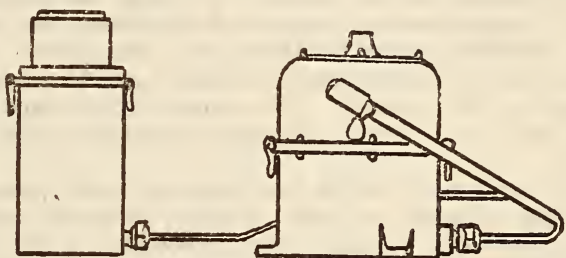

Figure 795.-Jack, tupe C

\begin{tabular}{|c|c|c|c|}
\hline Capacity & $\begin{array}{l}\text { Weight } \\
\text { of jack } \\
\text { (mini- } \\
\text { mum) }\end{array}$ & $\begin{array}{l}\text { Rise (ap- } \\
\text { proxi- } \\
\text { mate) }\end{array}$ & $\begin{array}{l}\text { Closed } \\
\text { height } \\
\text { (maxi- } \\
\text { mum) }\end{array}$ \\
\hline $\begin{array}{c}\text { Tons } \\
60 \\
100\end{array}$ & $\begin{array}{c}\text { Pounds } \\
200 \\
340\end{array}$ & $\begin{array}{c}\text { Inches } \\
8 \\
12\end{array}$ & $\begin{array}{c}\text { Inches } \\
16 \\
21\end{array}$ \\
\hline
\end{tabular}

4. Lever jacks, automatic, type D.-These shall be the single-acting automatic lever jacks which are operated on the downward stroke of the lever. They shall be essentially made up of the following: A base and frame, a rack bar, retaining and lifting pawls, trunnions, bearings, socket lever, and cap.

The base and frame shall be of one-piece construction and made of malleable iron or steel. The rack bar shall be solid and made of steel, properly hardened, and equipped with a claw or ground lift. The teeth shall be machine cut and smoothly finished.

The pawls shall be made of steel, properly heat treated, and so arranged in the jack mechanism that one or the other shall always be in mesh with the rack bar when the jack is under load.

The trunnions on which the pawls oscillate shall be made of steel and the bearings in which trunnions rotate shall be furnished with casehardened bushings.

The jacks shall be provided with a stop which will prevent the bar coming out of the frame when the limit of rise is reached.

These jacks shall be capable of retaining any weight up to their rated capacity at any height within the range.

Each jack shall be furnished with a suitable operating lever. 
These jacks shall be similar to Figure 796 and shall conform to the requirements of the following table:
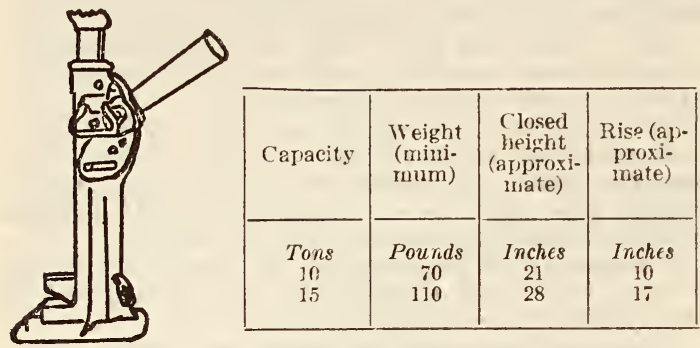

Figure 796 .-

Jack, type $D$

5. Planer or leveling jacks, type E.-These shall be essentially the same as screw jacks, type F, with the following exceptions:

The barrel shall be made of iron or steel and properly threaded for the screw. It shall be provided with a large bearing surface at the base, the latter to be finished square to the axis of the screw.

The screw shall be made of steel and threaded to engage in the barrel. The screw, ball, and head shall be in one piece, all parts revolving with the screw.

The head may either be furnished with holes for inserting a lever to turn or may be made with a hexagonal head suitable for a wrench. A cap shall be fitted on the top of the ball and shall swivel on the ball.

These jacks shall be similar to Figure 797 and shall conform to the requirements of the following table:

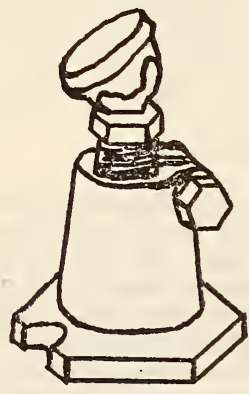

\begin{tabular}{|c|c|c|}
$\begin{array}{c}\text { Closed } \\
\text { height (ap- } \\
\text { proximate) }\end{array}$ & $\begin{array}{c}\text { Risa (ap- } \\
\text { proxi- } \\
\text { mate) }\end{array}$ & $\begin{array}{c}\text { Weight } \\
\text { (approxi- } \\
\text { mate) }\end{array}$ \\
\hline Inches & Inches & Pounds \\
3 & 1 & $11 / 2$ \\
4 & $11 / 2$ & 3 \\
$51 / 4$ & $21 / 4$ & 6 \\
$71 / 2$ & 4 & 12 \\
\hline
\end{tabular}

FIGURE 797 . Jack, type $E$

6. Screw jacks, type F.-These shall be of the ordinary screw-jack type and shall consist essentially of a barrel, screw, head, and cap. The head shall be of the four or six way type.

The barrel shall be made of cast or malleable iron and threaded internally to engage the threads on the screw. It shall be the form known as a bell base or the equivalent, the barrel widening out to give a large bearing surface at the base. The bottom of the barrel shall be finished square to the axis of the screw. The barrel shall also be equipped with a suitable handle.

The screw shall be made in one piece, of a good grade of steel, and shall be externally threaded to engage the threads in the barrel. The upper end of the screw shall extend up through the barrel, and at the top a cap shall be fitted.

The head shall be fixed to the screw below the cap, and holes made for inserting a lever to turn the head and screw and lift the load. The cap shall be made of steel and fastened to the screw so that there is no lateral motion. The cap, however, shall be free to rotate.

Each jack shall be furnished with a suitable operating lever.

These jacks shall be similar to Figure 798 and shall conform to the requirements of the following table:

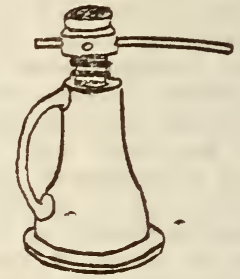

FIgURE 798 . Jack, type $F$

\begin{tabular}{|r|c|c|c|c|}
\hline Capacity & $\begin{array}{c}\text { Weight } \\
\text { (mini- } \\
\text { mum) }\end{array}$ & $\begin{array}{c}\text { Closed } \\
\text { height } \\
\text { (approxi- } \\
\text { mate) }\end{array}$ & $\begin{array}{c}\text { Safe rise } \\
\text { of screw } \\
\text { (approxi- } \\
\text { mate) }\end{array}$ & $\begin{array}{c}\text { Diameter } \\
\text { of screw } \\
\text { (min imum) }\end{array}$ \\
\hline Tons & Pounds & Inches & Inches & Inches \\
5 & 9 & 9 & 4 & $11 / 4$ \\
8 & 16 & $10 \frac{1}{2}$ & 5 & $11 / 2$ \\
12 & 25 & 14 & 8 & $13 / 4$ \\
15 & 35 & 16 & 9 & 2 \\
\hline
\end{tabular}

7. Motor-truck jacks, type G.-These shall be similar to automatic lever jacks, type D, except that they may be either single or double acting and especially designed for motor trucks. Consideration will be given to bids offering motor-truck jacks differing from the automatic-lever type, provided that a full description of the jacks offered is furnished with bid.

These jacks shall be similar to Figure 799 and shall conform to the requirements of the following table:

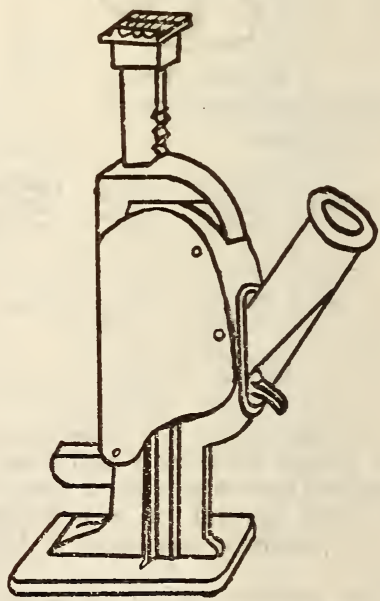

FigURE 799.-Jack, type G

\begin{tabular}{|c|c|c|c|}
\hline Capacity & $\begin{array}{l}\text { Weight } \\
\text { (mini- } \\
\text { mum) }\end{array}$ & $\begin{array}{l}\text { Closed } \\
\text { height } \\
\text { (maxi- } \\
\text { mum) }\end{array}$ & $\begin{array}{l}\text { Rise } \\
\text { (mini- } \\
\text { mum) }\end{array}$ \\
\hline $\begin{array}{r}\text { Tons } \\
1 \\
1 \\
3 \\
5\end{array}$ & $\begin{array}{c}\text { Pounds } \\
10 \\
11 \\
26 \\
32\end{array}$ & $\begin{array}{r}\text { Inches } \\
93 / 4 \\
113 / 4 \\
131 / 2 \\
131 / 2\end{array}$ & $\begin{array}{r}\text { Inches } \\
5 \\
6 \\
6 \\
6\end{array}$ \\
\hline
\end{tabular}




\section{METHOD OF INSPECTION AND TEST}

1. Jacks, types $\mathrm{E}$ and $\mathrm{F}$, will be carefully examined for defects in material and workmanship, and as to whether they comply with this specification.

2. Jacks, types A, B, C, D, and G, will be required to raise loads equivalent to those specified in the following table:

\begin{tabular}{|c|c|c|c|c|c|}
\hline \multirow{3}{*}{ Туре } & \multirow{3}{*}{ Kind of jack } & \multirow{3}{*}{$\begin{array}{c}\text { Rated } \\
\text { capac- } \\
\text { ity } \\
\text { of } \\
\text { jack }\end{array}$} & \multicolumn{3}{|c|}{$\begin{array}{l}\text { Test load jacks } \\
\text { shall lift }\end{array}$} \\
\hline & & & \multicolumn{2}{|c|}{ Head lift } & \multirow{2}{*}{$\begin{array}{c}\begin{array}{c}\text { Foot } \\
\text { lift }\end{array} \\
\\
\begin{array}{c}\text { To } \\
\text { maxi- } \\
\text { rise }\end{array}\end{array}$} \\
\hline & & & $\begin{array}{l}\text { To } 50 \\
\text { per } \\
\text { cent } \\
\text { of } \\
\text { maxi- } \\
\text { mum } \\
\text { rise }\end{array}$ & $\begin{array}{l}\text { To } \\
\text { maxi- } \\
\text { mume }\end{array}$ & \\
\hline $\begin{array}{l}\hat{\mathbf{A}} \\
\mathbf{A} \\
\hat{\mathbf{A}} \\
\hat{\mathbf{A}}\end{array}$ & $\begin{array}{l}\text { Berel-geared, screw } \\
\text { do do do }\end{array}$ & $\begin{array}{c}\text { Tons } \\
15 \\
25 \\
35 \\
50 \\
75\end{array}$ & $\begin{array}{c}\text { Tons } \\
15 \\
25 \\
35 \\
50 \\
75\end{array}$ & $\begin{array}{l}\text { Tons } \\
12 \\
20 \\
28 \\
40 \\
60\end{array}$ & $\begin{array}{l}\text { Tons } \\
7.5 \\
12.5 \\
17.5 \\
25 \\
37.5\end{array}$ \\
\hline $\begin{array}{l}\mathrm{B} \\
\mathrm{B} \\
\mathrm{C} \\
\mathrm{C}\end{array}$ & $\begin{array}{l}\text { Bevel-geared, journal } \\
\text { Hydraulic.. }\end{array}$ & $\begin{array}{r}15 \\
25 \\
60 \\
100\end{array}$ & $\begin{array}{r}18 \\
30 \\
72 \\
120\end{array}$ & $\begin{array}{r}15 \\
25 \\
60 \\
100\end{array}$ & $\begin{array}{ll}\cdots \\
\cdots \\
\cdots\end{array}$ \\
\hline $\begin{array}{l}D \\
D \\
G \\
G\end{array}$ & $\begin{array}{l}\text { Lever, automatic. } \\
\text { Motor-truck }\end{array}$ & $\begin{array}{r}10 \\
15 \\
3 \\
5\end{array}$ & & $\begin{array}{l}5 \\
7.5 \\
3 \\
5\end{array}$ & $\begin{array}{l}5 \\
7.5 \\
1.5 \\
2.5\end{array}$ \\
\hline
\end{tabular}

3. In addition to the above, jacks, types A, D, $\mathrm{F}$, and $\mathrm{G}$ shall withstand the compressive action of a load 20 per cent in excess of their rated capacity at 50 per cent of the maximum rise. They shall also sustain the compressive action of a load equal to their rated capacity at the maximum rise.

4. The jacks shall withstand all of the required tests without showing any signs of cracking, bending, or breaking, and shall also be free from mechanical defects at completion of tests.

616.92 MALLETS.

(No nationally recognized specifications available.) 616.93 TROWELS.

\section{UNITED STATES GOVERNMENT, FEDERAL SPECIFICATIONS BOARD; FEDERAL SPECIFICATION FOR TROWELS, PLAS- TERING, CEMENT, BRICK, AND POINTING, GGG-T-6\%1, MARCH 1, 1932 \\ TROWELS \\ II. TYPES}

Trowels shall be of the following types, as specified: A, plastering trowels; B, cement trowels; $\mathrm{C}$, brick trowels; and $\mathrm{D}$, pointing trowels.

\section{MATERIAL AND WORKMANSHIP}

1. Trowels shall be made of the best grade material, and of good workmanship and finish throughout. They shall be free from all defects and blemishes affecting their appearance or that may affect their serviceability.

2 . The blades of all trowels shall be made from high-grade tool steel, properly hardened and tempered.

\section{GENERAL REQUIREMENTS}

1. Each trowel shall be furnished with a handle properly shaped, smoothly finished, and of wellseasoned sound wood, suitable for the purpose.

2. The blade of each trowel shall be clearly and distinctly marked in a permanent manner with the manufacturer's name or trade-mark.

3. A plus or minus tolerance of 10 per cent will be allowed in all dimensions specified herein as approximate.

\section{DETAIL REQUIREMENTS}

1. Plastering trowels, type A.-(a) The blade shall be smoothly and evenly ground on the face and edges. The face shall be rolled to the side edges for clearance in use under service conditions.

(b) The mounting shall be made from malleable iron or steel and shall be secured to the blade by at least five flathead steel rivets, the heads of which shall be ground flush with the surface of the blade. The mounting shall be so shaped as properly to reinforce the blade.

(c) The tang of the mounting shall pass through the handle, which shall be bored in a manner suitable to receive the tang. The handle shall be secured in position by a nut which may be tightened upon a threaded tang over a suitable washer, or by riveting the end of the tang over a suitable washer. The end of tang shall be smoothly finished by either method.

(d) Plastering trowels shall be similar to Figure 800 and shall conform to the requirements of the table following:

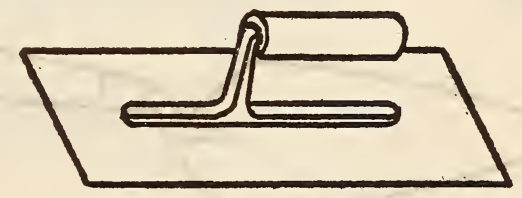

Figure 800. - Plastering trowels, type $A$

Dimensions of trowel, type $A$

\begin{tabular}{|c|c|c|c|c|}
\hline $\begin{array}{c}\text { Blade } \\
\text { length } \\
\text { plus or } \\
\text { minus } \\
\begin{array}{c}0.125 \\
\text { (toler- } \\
\text { ance) }\end{array}\end{array}$ & $\begin{array}{c}\text { Blade } \\
\text { width, } \\
\text { plus or } \\
\text { minus } \\
0.125 \\
\text { (toler- } \\
\text { ance) }\end{array}$ & $\begin{array}{c}\text { Blade } \\
\text { thickness, } \\
\text { plus or } \\
\text { minus } \\
0.003 \\
\text { (toler- } \\
\text { ance) }\end{array}$ & $\begin{array}{c}\text { Handie } \\
\text { length, } \\
\text { mate }\end{array}$ & $\begin{array}{c}\text { Largest } \\
\text { diameter } \\
\text { of handle, } \\
\text { approxi- } \\
\text { mate }\end{array}$ \\
\cline { 1 - 2 } $\begin{array}{c}\text { Inches } \\
11\end{array}$ & $\begin{array}{c}\text { Inches } \\
43 / 4\end{array}$ & $\begin{array}{c}\text { Inch } \\
0.032\end{array}$ & $\begin{array}{c}\text { Inches } \\
41 / 2\end{array}$ & $\begin{array}{c}\text { Inches } \\
11 / 2\end{array}$ \\
\hline
\end{tabular}

2. Cement trowels, type B.-(a) These trowels shall conform in general to the type A plastering trowels described in Section V, 1, except that the face of the blade shall be straight, and the mounting be secured to the blade by at least three flathead rivets. Cement trowels shall be similar to Figure 
801 and shall conform to the requirements of the table following:

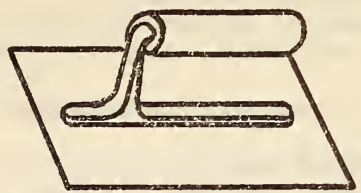

FI G URE 801 . - Cement trowels, type $B$

Dimensions of trowel, type $B$

\begin{tabular}{|c|c|c|c|c|}
\hline $\begin{array}{c}\text { Blade } \\
\text { length, } \\
\text { plus or } \\
\text { minus } \\
0.125 \\
\text { (toler- } \\
\text { ance) }\end{array}$ & $\begin{array}{c}\text { Blade } \\
\text { width, } \\
\text { plus or } \\
\text { minus } \\
0.125 \\
\text { (toler- } \\
\text { ance) }\end{array}$ & $\begin{array}{c}\text { Blade } \\
\text { thickness, } \\
\text { plus or } \\
\text { minus } \\
0.003 \\
\text { (toler- } \\
\text { ance) }\end{array}$ & $\begin{array}{c}\text { Handle } \\
\text { length, } \\
\text { mate }\end{array}$ & $\begin{array}{c}\text { Largest } \\
\text { diameter } \\
\text { of handle, } \\
\text { approxi- } \\
\text { mate }\end{array}$ \\
$\begin{aligned} \text { Inches } \\
51 / 2\end{aligned}$ & $\begin{array}{r}\text { Inches } \\
4\end{array}$ & $\begin{array}{c}\text { Inch } \\
0.032\end{array}$ & $\begin{array}{r}\text { Inches } \\
4\end{array}$ & $\begin{array}{r}\text { Inches } \\
11 / 2\end{array}$ \\
\hline
\end{tabular}

3. Brick trowels, type C.-(a) The blade of these trowels shall be smoothly and evenly ground and shall taper uniformly in thickness from heel to point.

(b) The tang shall be an integral part of the blade and shall be properly shaped to hold the handle securely.

(c) The handle shall be driven onto the tang and the front end where the tang enters shall be fitted with a substantial steel ferrule not less than $1 \frac{1}{2}$ inches long.

(d) Brick trowels shall be similar to Figure 802 and shall conform to the requirements of the table following:

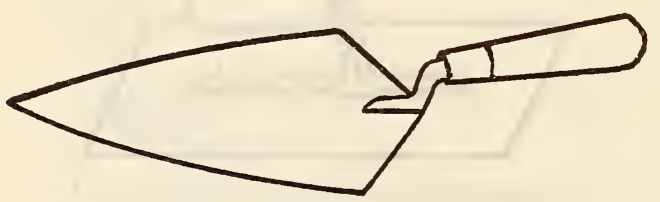

Figure 802.-Brick trowels, type $C$

Dimensions of trowel, type $C$

\begin{tabular}{|c|c|c|c|c|}
\hline $\begin{array}{l}\text { Blade } \\
\text { length, } \\
\text { point to } \\
\text { point, } \\
\text { plus or } \\
\text { minus } \\
0.125 \\
\text { (toler- } \\
\text { ance) }\end{array}$ & $\begin{array}{c}\text { Blade } \\
\text { width, } \\
\text { at widest } \\
\text { point, } \\
\text { plus or } \\
\text { minus } \\
0.250 \\
\text { (toler- } \\
\text { ance) }\end{array}$ & $\begin{array}{c}\text { Blade } \\
\text { thickness, } \\
\text { at heel, } \\
\text { plus or } \\
\text { minus } \\
0.010 \\
\text { (toler- } \\
\text { ance) }\end{array}$ & $\begin{array}{l}\text { Handle } \\
\text { length, } \\
\text { approxi- } \\
\text { mate }\end{array}$ & $\begin{array}{c}\text { Largest } \\
\text { diameter } \\
\text { of handle, } \\
\text { approxi- } \\
\text { mate }\end{array}$ \\
\hline $\begin{array}{r}\text { Inches } \\
10^{1 / 2}\end{array}$ & $\begin{array}{r}\text { Inches } \\
51 / 4\end{array}$ & $\begin{array}{l}\text { Inch } \\
0.074\end{array}$ & $\begin{array}{r}\text { Inches } \\
51 / 2\end{array}$ & $\begin{array}{r}\text { Inches } \\
11 / 4\end{array}$ \\
\hline
\end{tabular}

4. Pointing trowels, type D.-These trowels shall conform in general to the type $\mathrm{C}$ brick trowels described in Section V, 3, except that the handle shall be furnished with a substantial metal ferrule at leat five-eighths inch long. Pointing trowels shall be similar to Figure 803 and shall conform to the requirements of the table following:

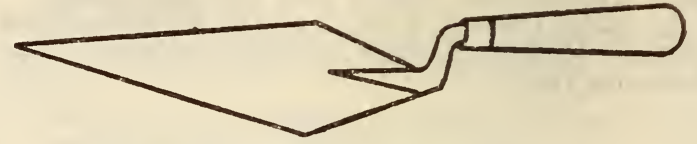

Figure 803.-Pointing trowels, type D

Dimensions of trowel, type $D$

\begin{tabular}{|c|c|c|c|c|}
\hline $\begin{array}{l}\text { Blade } \\
\text { length, } \\
\text { point to } \\
\text { point, } \\
\text { plus or } \\
\text { minus } \\
0.125 \\
\text { (toler- } \\
\text { ance) }\end{array}$ & $\begin{array}{l}\text { Blade } \\
\text { width, } \\
\text { at widest } \\
\text { point, } \\
\text { plus or } \\
\text { minus } \\
0.250 \\
\text { (toler- } \\
\text { ance) }\end{array}$ & $\begin{array}{c}\text { Blade } \\
\text { thickness, } \\
\text { at heel, } \\
\text { plus or } \\
\text { minus } \\
0.0 \mathrm{i} 0 \\
\text { (toler- } \\
\text { ance) }\end{array}$ & $\begin{array}{l}\text { Handle } \\
\text { length, } \\
\text { approxi- } \\
\text { mate }\end{array}$ & $\begin{array}{c}\text { Largest } \\
\text { diameter } \\
\text { of handle, } \\
\text { approxi- } \\
\text { mate }\end{array}$ \\
\hline $\begin{array}{r}\text { Inches } \\
6\end{array}$ & $\begin{array}{r}\text { Inches } \\
258\end{array}$ & $\begin{array}{l}\text { Inch } \\
0.067\end{array}$ & $\begin{array}{r}\text { Inches } \\
41 / 2\end{array}$ & $\begin{array}{r}\text { Inches } \\
1\end{array}$ \\
\hline
\end{tabular}

5. Trowels shall be packed in accordance with the manufacturer's trade practice, except that each package or box shall not contain more than six trowels, which shall be of one type and size.

\section{METHODS OF INSPECTION AND TESTS}

1. All trowels shall be inspected to determine whether they are in compliance with the requirements of this specification.

2. Sampling. - For purposes of inspection at least three sample trowels of each type shall be taken at random from those under inspection and submitted to the inspection test prescribed herein.

3. Inspection tests. - In addition to a careful surface inspection for workmanship, finish, marking, compliance with dimensional requirements, etc., the samples shall be subjected to the following tests:

\section{Types A and B Trowels}

One of the edges in alignment with the handle of types A and B trowels shall be gripped in an ordinary bench vise, with the edge of the trowel parallel with and set five-sixteenths inch below the top surface of the vise jaws. The opposing edge of the trowel shall then be moved each side of the perpendicular a distance of not less than 2 inches.

\section{Types C and D Trowels}

The pointed end of types $\mathrm{C}$ and $\mathrm{D}$ trowels shall be gripped in an ordinary bench vise, with the trowel in a vertical position and the point $1 \frac{1}{2}$ inches below the top surface of the vise jaws. The heel end of the blade shall then be moved each side of the perpendicular a distance of not less than 3 inches. Type $\mathrm{C}$ trowels shall also be tested in cutting not less than three face bricks in half.

4. Trowels showing permanent set, breakage, or turning of the edges under the above tests shall be regarded as unsatisfactory and not in complianca with the requirements of this specification. 
616.99 HAND TOOLS NOT ELSEWHERE CLASSIFIED AMERICAN RAILWAY ASSOCIATION, MECHANICAL DIVISION ; STANDARD PACKING TOOLS FOR JOURNAL BOXES, 1928

\section{PACKING TOOLS}

Two packing tools are required for packing journal boxes: (a) A packing iron, horn, or blade, and (b) a packing hook. uniformity in subject matter being due to differing requirements, such as a commercial standard failing to meet a specific Government need, or a Government need being too special to find place as a commercial standard.)

(Contained in CS22-30 are rules, practices, finishes, types, and sizes which are recommended for standard use. The finishes are identical with those given in FF-H-101. (See below.) Items of build-

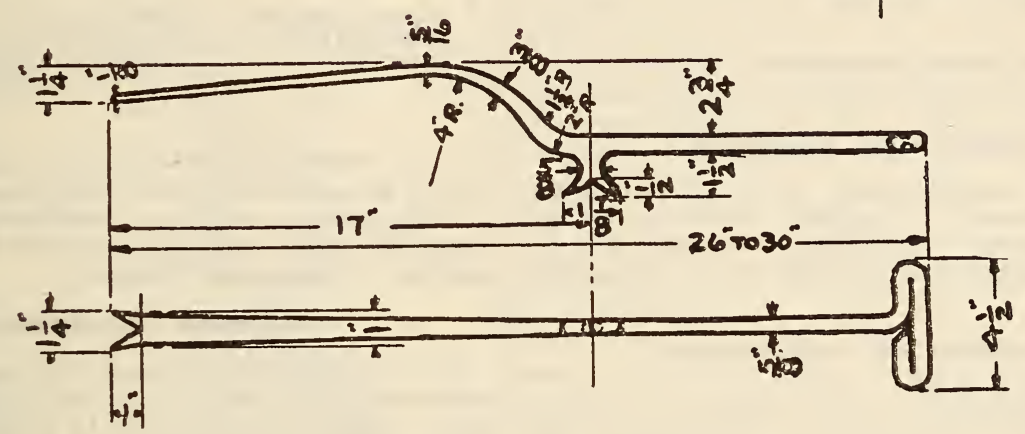

MATERIAL - SPRING STEEL

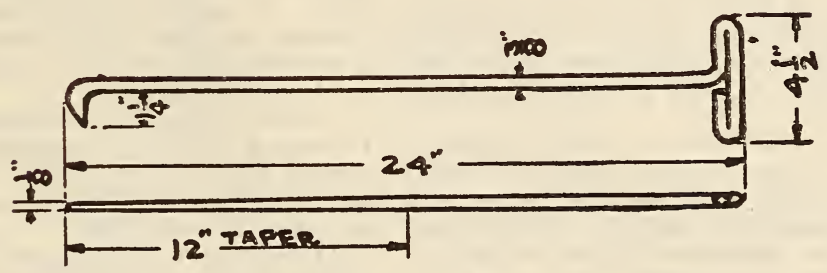

MATERIRL - SPRANG STEEL

Figure 804

The design of the packing iron and packing hook shall be in accordance with drawing.

\section{FURNITURE HARDWARE, BUILDERS' HARDWARE, AND PLUMBING}

\subsection{GENERAL ITEMS.}

UNITED STATES GOVERNMENT, DEPARTMENT OF COMMERCE; BUILDERS' HARDWARE (NONTEMPLATE), COMMERCIAL STANDARD CS22-30, DECEMBER 11,1930

\section{BUILDERS' HARDWARE (NONTEMPLATE)}

(This publication is similar to Federal Specification FF-H-101, given below, the latter being, as far as practicable, based upon commercial standard requirements. In respect to corresponding type numbers the two publications are identical. Each publication, though, contains type numbers that are not found in the other, the departures from

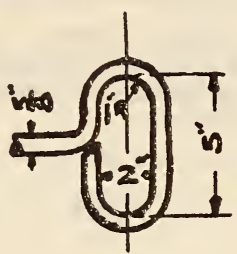

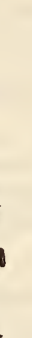




\section{UNITED STATES GOVERNMENT, FEDERAL SPECIFICATIONS BOARD, FEDERAL SPECIFICATION FOR BUILDERS' HARD. WARE (NONTEMPLATE), FF-H-101, AU- GUST 19, 1930}

\section{BUILDERS' HARDWARE (NONTEMPLATE)}

\section{A. APPLICABLE FEDERAL SPECIFICATIONS}

A-1. The following Federal specifications or parts thereof that are below indicated, of the issue in effect on date of invitation for bids, shall form a part of this specification:

\begin{tabular}{|c|c|c|c|}
\hline $\begin{array}{c}\text { Federal } \\
\text { specifica- } \\
\text { tion }\end{array}$ & $\begin{array}{c}\text { For- } \\
\text { mer } \\
\text { No. }\end{array}$ & Title & See \\
\hline RR-C-271 & 348 & Sash and transom chains_... & 603.56, p. 212 \\
\hline
\end{tabular}

\section{B. TYPES, GRADES, AND CLASSES}

B-1. These specifications cover such types, grades, and classes of builders' hardware as are enumerated under "Detail requirements" and do not include hardware for marine use or for hollow metal doors.

\section{MATERIAL AND WORKMANSHIP}

\section{C-1. Quality and fit.-}

C-1a. All hardware in each grade shall be free from all defects affecting the appearance and serviceability.

C-1b. All parts of hardware generally that are to be set flush when in place shall have accurately machined, true, parallel edges, except that edges of unfinished steel and iron and unpolished work need not be machine finished, but shall be smooth and reasonably true.

C-1c. Locks and knob latches, except types 4, $4 \mathrm{~A}, 59,80$, and steel cabinet locks, shall have heavy and substantial working and moving parts, and springs of suitable nonferrous metal. Working and moving parts shall be accurately fitted to smooth close bearings and free from rattle.

C-2. Finish.-

C-2a. Exposed finished parts shall have such of the standard finishes enumerated under paragraph $\mathrm{I}-4 \mathrm{a}$ and $\mathrm{I}-4 \mathrm{~b}$ as are specified.

C-2b. Bronze shall be polished with a bright finish, No. US9, when no other finish is specified.

C-2c. Plated work shall be heavily plated and buifed and of finish US9 for bronze and US14 for nickel when no other finishes are specified.

$\mathrm{C}-2 \mathrm{~d}$. When unfinished iron or steel is specified, galvanized, sherardized, japanned, coppered, or unfinished iron or steel may be furnished. Iron and steel shall be free from rust. Where wrought steel is specified, it is understood to include wrought iron.

C-3. Nickel bronze.-

C-3a. When nickei bronze (nickel silver or white metal) is specified or furnished, it shall be one of the following compositions according to whether cast nickel bronze or wrought nickel bronze is required. Nickel-bronze keys need not conform to either composition specified.
Cast nickel bronze:

\begin{tabular}{|c|c|}
\hline Desired composition & $\begin{array}{c}\text { Required minimum and marimum } \\
\text { by weight }\end{array}$ \\
\hline $\begin{array}{l}65 \text { per cent copper.... } \\
20 \text { per cent nickel.... } \\
4 \text { per cent tin } \\
5 \text { per cent lead } \\
6 \text { per cent zinc........ }\end{array}$ & $\begin{array}{l}63 \text { per cent minimum. } \\
19 \text { per cent minimum. } \\
6 \text { per cent maximum. } \\
10 \text { per cent maximum. } \\
1 \text { per cent manganese maximum. } \\
1 \text { per cent iron and other impurities } \\
\text { maximum. }\end{array}$ \\
\hline
\end{tabular}

Wrought nickel bronze:

\begin{tabular}{|l|l|}
\hline Desired composition & $\begin{array}{l}\text { Required minimum and maximum } \\
\text { by weight }\end{array}$ \\
\cline { 1 - 2 } 65 per cent copper....- & $\begin{array}{l}62 \text { per cent minimum. } \\
17 \text { per cent minimum. }\end{array}$ \\
17 per cezt nickel .... & $\begin{array}{l}18 \text { per cent maximum. } \\
0.5 \text { per cent manganese maximum. }\end{array}$ \\
\hline
\end{tabular}

C-3b. Solid nickel-bronze trimmings and fittings will be acceptable in lieu of nickel-plated brass.

C-4. Lacquer.-All natural color or plated finish hardware unless otherwise specified shall have a coating of transparent lacquer.

\section{GENERAL REQUIREMENTS}

D-1. Dimensions.-Unless otherwise specified dimensions given herein are to be considered approximate. Sizes of locks refer to dimensions of case where not otherwise stated. Minimum dimensions covering cross section and throw of latch bolts and dead bolts are given to the nearest sixty-fourth of an inch. In general, locks and panic bolts of larger dimensions than those specified will be acceptable if suitable and of equal quality.

D-2. Fastenings. -

D-2a. Hardware hereinafter specified shall be furnished with all necessary screws, bolts, etc., for proper application, which shall be of suitable size and type, and shall harmonize with the hardware as to material and finish.

D-2b. Screw sizes where given are the minimum acceptable and shall be considered standard. Longer lengths or other special fastenings may sometimes be required.

D-3. Lock fronts, strikes, and spindles.-

D-3a. All locks shall have a flat front unless otherwise specified. When beveled fronts are specified, the bevel shall be one-eighth inch in 2 inches. When rabbeted fronts are specified the depth of rabbet shall be one-half inch.

D-3b. Armored fronts will be accepted in lieu of solid fronts on locks.

D-3c. All strikes shall be plain unless otherwise specified. Lips of strikes shall project not more than one-eighth inch beyond amount necessary to protect trim.

D-3d. Triplex spindles will be accepted in lieu of threaded spindles.

D-4. Countersunk screw holes.-The countersunk part of screw holes in butts must form a good seat for the screw heads, with no sharp edges at back.

D-5. Japan coating.-Japan coating, where specified, shall cling tenaciously to the work and be dry 
and elastic. The surface shall be black and not subject to cracking, scratching, or flaking. The coating is considered to be dry when the pressure that can be exerted between the thumb and forefinger does not leave a mark which remains noticeable after the spot is lightly polished. The above test is to be made at room temperature (about $20^{\circ}$ C.) $\left(6 S^{\circ}\right.$ F.).

(For chromium plating, nickel plating, and zinc coatings; and methods of inspection and tests, see 600.3 , p. 70.)

\section{E. DETAIL REQUIREMENTS}

(E-1. For detail requirements see individual items of builders' hardware. In this compilation these items have been placed under their proper commodity heading as follows.)

617.11, p. 981:

Pole hangers.

Sash-pole hooks.

Sash-pull plates.

617.12, p. 994:

Hinges, blind.

Hinges, butt.

Hinges, checking floor.

Hinges, floor surface spring.

Hinges, garage.

Hinges, lavatory door spring.

Hinges, scuttle.

Hinges, spring.

Hinges, strap.

Hinges, $T$.

617.13 , p. 1004 :

Hinge hasps.

617.21, p. 1020:

Locks, cabinet.

Locks, cylindrical case, and latches.

Locks, door.

Locks, cylinder door.

Locks, tubular, and latches.

Locks, unit type, and latches.

617.22, p. 1034:

Latches, cylindrical case. (See 617.21, above.)

Latches, crlinder mortise night.

Latches, rim knob.

Latches, rim night.

Latches, secret gate.

Latches, thumb.

Latches, tubular. (See 617.21, above.)

Latches, unit type. (See 617.21, above.)

617.23, p. 1036 :

Cylinder collars.

Escutcheons.

Key plates.

Padlock eyes.

Roses.

617.31, p. 1037:

Door knobs, metal.
617.32, p. 1038:

Entrance door handles.

617.33, p. 1039:

Pulls, door.

Pulls, drawer.

Pulls, knob.

Pulls, on plate, door.

Pulls, sectional door.

617.34 , p. 1040 :

Bars, push.

Plates, push.

Plates, kick.

617.35 , p. 1041 :

Lifts, bar sash.

Lifts, flush sash.

Lifts, hook sash.

Lifts, screen.

617.41 , p. 1042 .

Door closers.

617.42 , p. 1043 :

Catches, elbow.

Catches, transom.

617.43, p. 1043 :

Fasteners, blind.

Fasteners, casement.

Fasteners, cellar window.

Fasteners, door, with chain.

Fasteners, sash.

Fasteners, scuttle.

Fasteners, window screen.

Turnbuckles for blinds.

617.44, p. 1045:

Door holders.

Door stops.

617.5 , p. 1049 :

Sash cord irons.

Sash operators.

Sash pulleys.

Sash weights.

Transom chains.

Transom eyes.

Transom lifters.

Transom operators.

Transom pivots.

Window-stop adjusters.

Casement adjusters.
617.61, p. 1056 :

Bolts, barrel.

Bolts, chain.

Bolts, cremone.

Bolts, espagnolette.

Bolts, exit.

Bolts, flush.

Bolts, foot.

Bolts, lavatory door.

Bolts, mortise.

Bolts, neck.

Bolts, square.

Bolts, surface.

Bolts, window spring.

\section{G. PACKAGING, PACKING, AND MARKING}

G-2. Packaging.-Unless otherwise specified, commercial packages are acceptable under this specification.

G-3. Packing.-Unless otherwise specified, the subject commodity shall be delivered in substantial commercial containers, so constructed as to insure acceptance by common or other carriers, for safe transportation, at the lowest rate, to the point of deliverv.

G-4. Marking.-Unless otherwise specified, shipping containers shall be marked with the name of the material, the size, type, and the quantity contained therein, as defined by the contract or order under which the shipment is made, the name of the contractor, and the number of the contract or order.

\section{NOTES}

I-1. Rules for hands of locks.-

I-1a. The hand of a lock including locks for French doors is determined from the outside of the door to which it is applied. The outside of a cupboard, bookcase, or closet door is the room side. For other doors the outside is usually the "push" or jamb side.

I-1b. If, standing outside of a door, the butts are on the right, it takes a right-hand lock; if on the left, it takes a left-hand lock.

I-1c. If, standing outside, the door opens from you, it takes a lock with a regular-bevel latch bolt; if opening toward you, it takes a lock with a reversebevel latch bolt.

I-2. Rules for hands of casement sash.-

I-2a. The hand of casement sash is taken from the room side. If the butts are on the right, it is a right-hand sash; if on the left, it is a left-hand sash. It is necessary to state whether sash opens in or out.

I-3. Dimensions.-Where more than one dimension is given for items of builders' hardware, the vertical dimension is listed first.

I-4. Finishes.-

I-4a. Where practicable, finishes should be confined to bronze, solid or plated; nickel plated; galvanized; or japanned.

I-4b. Where finishes other than those given above are required, selection should be confined to the following standards adopted by the manufacturers as listed in Commercial Standard for Builders' Hardware (Nontemplate) CS22-30: 
TABLE 1.-Standard hardware finishes

\begin{tabular}{|c|c|c|c|c|}
\hline U. S. No. & General description & Metal applied to- & $\begin{array}{l}\text { Samples selected as } \\
\text { standard }\end{array}$ & Restrictions \\
\hline USP. & Primed for painting. & & & \\
\hline US1B. & Bright japanned ..... & & & \\
\hline $\begin{array}{l}\text { US1D-- } \\
\text { US2C.. }\end{array}$ & $\begin{array}{l}\text { Dead black japanned..... } \\
\text { Cadmium plated......... }\end{array}$ & & & \\
\hline US2G. & Electro galvanized... & & & \\
\hline $\begin{array}{l}\text { US2H } \\
\text { US } 2 S_{--}\end{array}$ & $\begin{array}{l}\text { Hot galvanized } \\
\text { Sherardized }\end{array}$ & & $\ldots-.$. & \\
\hline US3.. & Bright brass.................... & Iron, steel, wrought brass, & Yale AZ10. & \\
\hline US4 & Dull brass.. & do & Corbin EA & \\
\hline US5. & $\begin{array}{l}\text { Dull brass, oxidized and re- } \\
\text { lieved. }\end{array}$ & - no... do & Russwin $9 \mathrm{C}_{-}$ & $\begin{array}{l}\text { Limited to ornamental designs. } \\
\text { Plain hardware to match to be } \\
\text { finish US4. }\end{array}$ \\
\hline US6 & $\begin{array}{l}\text { Sanded brass, oxidized and } \\
\text { relieved. }\end{array}$ & ........ & Sargent RD_.... & \\
\hline US7_- & $\begin{array}{l}\text { Sanded brass, oxidized and } \\
\text { relieved, raised ornamen- } \\
\text { tation polished. }\end{array}$ & {$\left[\ldots-d_{-\ldots}\right.$} & $\begin{array}{l}\text { Russwin 09B, edges not } \\
\text { polished. }\end{array}$ & $\begin{array}{l}\text { Limited to ornamental designs. } \\
\text { Polishing confined to raised or- } \\
\text { namentation, edges not pol- }\end{array}$ \\
\hline US8 & Antique copper..... & ... do & Chantrell 24 & show color only; mot- \\
\hline US9_. & Bright bronze......... & Iron, steel, wrought & Yale BZ10. & Wrought designs limited to two- \\
\hline US10 & Dull bronze & ... do do & Corbin DB & \\
\hline US11 & $\begin{array}{l}\text { Dull bronze, oxidized and } \\
\text { relieved. }\end{array}$ & ...... do & Sargent $06 \mathrm{P}$ & $\begin{array}{l}\text { Limited to ornamental designs. } \\
\text { Plain hardware to match to be } \\
\text { finish US10. }\end{array}$ \\
\hline US12.. & $\begin{array}{l}\text { Sanded bronze, oxidized and } \\
\text { relieved. }\end{array}$ & ............. & Penn SBZ .- & \\
\hline US13. & $\begin{array}{l}\text { Sanded bronze, oxidized and } \\
\text { relieved, raised ornamen- } \\
\text { tation polished. }\end{array}$ & ...... do_.. & Penn SBZ9. & $\begin{array}{l}\text { Limited to ornamental designs. } \\
\text { Polishing confined to raised or- } \\
\text { namentation, edges not pol- } \\
\text { ished. }\end{array}$ \\
\hline US14_. & Nickel plated.............. & $\begin{array}{l}\text { Iron, steel, wrought brass, } \\
\text { cast brass. }\end{array}$ & Russwin 4...... & \\
\hline US15 & Nickel plated, dull. . & ..... do & Lockwood 90 & $\begin{array}{l}\text { Also applicable to ornamental de- } \\
\text { signs as Lockwood } 91 \text { or Sar- } \\
\text { gent L5N. }\end{array}$ \\
\hline US16 & Nickel plated, sanded....... & $\begin{array}{l}\text { Iron, steel, wrought brass, } \\
\text { cast brass. }\end{array}$ & Yale NX10 & \\
\hline US17. & $\begin{array}{c}\text { Nickel plated, sanded, raised } \\
\text { ornamentation polished. } \\
\text { “Rustproof" black......... }\end{array}$ & Wrought steel, castiron ... & Penn SN9... & $\begin{array}{l}\text { Limited to ornamental designs. } \\
\text { Polishing confined to raised or- } \\
\text { namentation, edges not pol- } \\
\text { ished. } \\
\text { Wrought designs limited to two- }\end{array}$ \\
\hline US19... & Sanded, dull black.... & Iron, steel, wrought brass, & Yale $\mathrm{BX} 80$ & vel. \\
\hline US20 & Statuary bronze.... & $\begin{array}{l}\text { cast brass. } \\
\text { Wrought bronze, cast } \\
\text { bronze. }\end{array}$ & $\begin{array}{l}\text { Yale FX90 (steel) } \\
\text { Penn BBZ4....... }\end{array}$ & $\begin{array}{l}\text { Wrought designs limited to two- } \\
\text { round corner, and broad level. } \\
\text { Limited on iron and steel to } \\
\text { butts. } \\
\text { Do. }\end{array}$ \\
\hline $\begin{array}{l}\text { US21 } \\
\text { US22.. }\end{array}$ & $\begin{array}{l}\text { Statuary bronze, sanded } \\
\text { Verde antique........ }\end{array}$ & 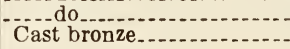 & $\begin{array}{l}\text { Penn SBZ4 } \\
\text { Yale BX67.......... }\end{array}$ & $\begin{array}{l}\text { Do. } \\
\text { Limited on iron and steel to butts }\end{array}$ \\
\hline US23. & Silver plated, dull, oxidized & Cast brass, cast bronze..... & Lockwood 72 & $\begin{array}{l}\text { plates, push plates, and butts. } \\
\text { Do. }\end{array}$ \\
\hline $\begin{array}{l}\text { US } 24 \ldots \\
\text { US25... }\end{array}$ & $\begin{array}{l}\text { Gold plated, dull } \\
\text { White bronze............. }\end{array}$ & $\begin{array}{l}\text { Cast nickel bronze, } \\
\text { wrought nickel bronze } \\
\text { in push plates, kick } \\
\text { plates, and butts. }\end{array}$ & $\begin{array}{l}\text { Corbin } \mathrm{DG}_{-.-} \\
\text {Sargent } \mathrm{EM} .\end{array}$ & 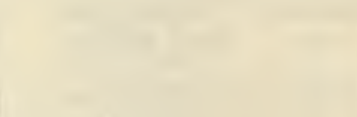 \\
\hline $\begin{array}{l}\text { US26- } \\
\text { US260- }\end{array}$ & $\begin{array}{l}\text { Bright chromium plated } \\
\text { Dull chromium plated.. }\end{array}$ & & & $\begin{array}{l}\text { Polished surfaces. } \\
\text { Do. }\end{array}$ \\
\hline
\end{tabular}

I-5. Type numbers.-Type numbers correspond, where the items are comparable, to those given in Commercial Standard CS22-3C, Builders' Hardware (Nontemplate).

I-6. Purchasers should exercise all options offered herein and should specify type, size, finish, handing, and master-keying as required.

I-7. It is believed that this specification adequately describes the characteristics necessary to secure the desired material, and that normally no samples will be necessary prior to award to determine compliance with this specification. If, for any particular purpose, samples with bids are necessary, they should be specifically asked for in the invitation for bids and the particular purpose to be served by the bid samples should be definitely stated, the specification to apply in all other respects.

617.1 HANGERS, HINGES, HASPS, AND STAPLES. 617.11 SASH POLE HANGERS, HOOKS, AND SASH PULL PLATES.

UNITED STATES GOVERNMENT, DEPARTMENT OF COMMERCE; BUILDERS' HARDWARE (NONTEMPLATE), COMMERCIAL STANDARD CS22-30, DECEMBER 11, 1930

POLE HANGERS, POLE HOOKS, PULL PLATES

(See 617.0, p. 977, also Federal specification FF$\mathrm{H}-101$ below.) 
UNITED STATES GOVERNMENT, FEDERAL SPECIFICATIONS BOARD; FEDERAL SPECIFICATION FOR BUILDERS' HARDWARE (NONTEMPLATE), FF-H-101, AUGUST 19,1930

(For types, grades, and classes; material and workmanship; general requirements; detail requirements; packaging, packing, and marking; notes; and table of standard finishes. See 617.0, p. 978 . For chromium plating, nickel plating, and zinc coatings; and methods of inspection and tests. See 600.3 , p. 70.)

\section{BUILDERS' HARDWARE (NONTEMPLATE)}

POLE HANGERS

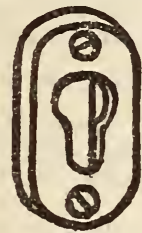

Frgure 805.

Pole hanger, type 1262

Type 1262.-Bronze. $23 / 8$ by $1 \frac{1}{8}$ inches.

Type 1262A.-Cast iron, plated. $23 / 8$ by $11 / 8$ inches: SASH POIE HOOKS

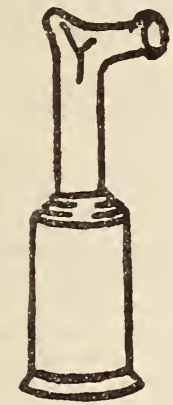

Frgure 806.-

Sash pole

hook, type

1264

Type 1264.-Cast bronze. Length, $4 \frac{1}{8}$ inches Diameter, $11 / 8$ inches.

Type 1264A.-Cast iron, plated. Length, 41/8 inches. Diameter, $11 / 8$ inches.

\section{SASH PULL PLATES}

Type 1266.-Sash pull plate minimum $1 \frac{1}{8}$ by 2 inches, cast bronze. Two screw holes.
Type 1266 A.-Same as type 1266, except cast iron. Type 126\%.- Sash pull plate minimum $11 / 4$ by 2 inches. Cast bronze. Four screw holes.

617.12 HINGES.

SOCIETY OF AUTOMOTIVE ENGINEERS BODY PARTS AND MATERIALS; DOOR HINGES, S. A. E. RECOMMENDED PRACTICE, FEBRUARY 1925

\section{DOOR HINGES}

CURVED AND SEMICURVED TYPE HINGES
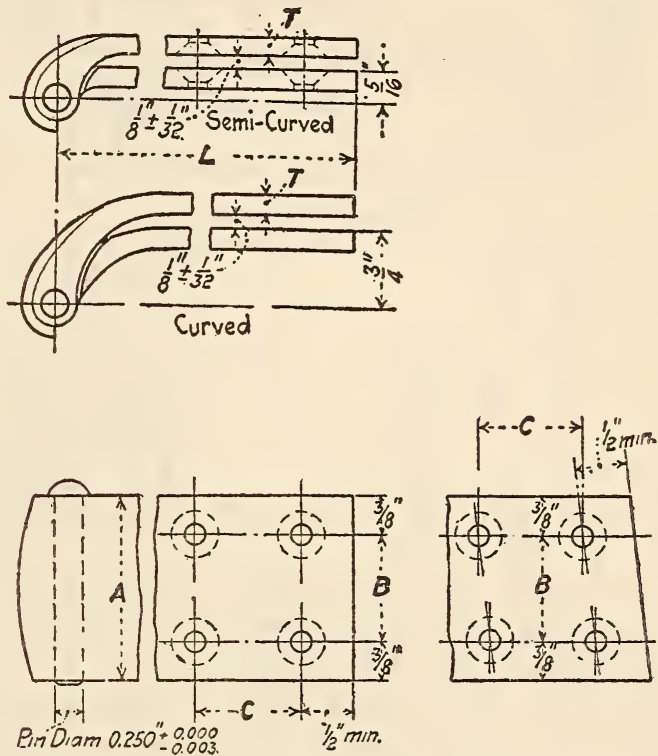

Figure 807.-Curved and semicurved type hinges

[Dimensions in inches]

\begin{tabular}{|c|r|c|c|c|}
\hline Width $A$ & $\begin{array}{r}\text { Number } \\
\text { of joints }\end{array}$ & $L$ & $B$ & $\begin{array}{c}C \text { (mini- } \\
\text { mum) }\end{array}$ \\
\hline $\mathbf{1}^{13 / 4}$ & $\begin{array}{r}3 \text { or } 5 \\
5\end{array}$ & $\begin{array}{r}3,4,5, \text { or } 51 / 2 \\
3,4,5, \text { or } 51 / 2\end{array}$ & $\begin{array}{l}1 \\
11 / 4\end{array}$ & \begin{tabular}{c}
1 \\
\hline
\end{tabular} \\
\hline
\end{tabular}

Screw holes shall be made to a minimum diameter of 0.438 in. and countersunk at $80^{\circ}$ for No. 12 wood screws.

Plate thickness (T).-For malleable and forged hinges, the thickness shall be one-fourth plus or minus one thirty-second of an inch; for pressedsteel hinges, three-sixteenths or one-fourth plus or minus 0.005 inch. 
STRAIGET TYPE DOOR HINGES

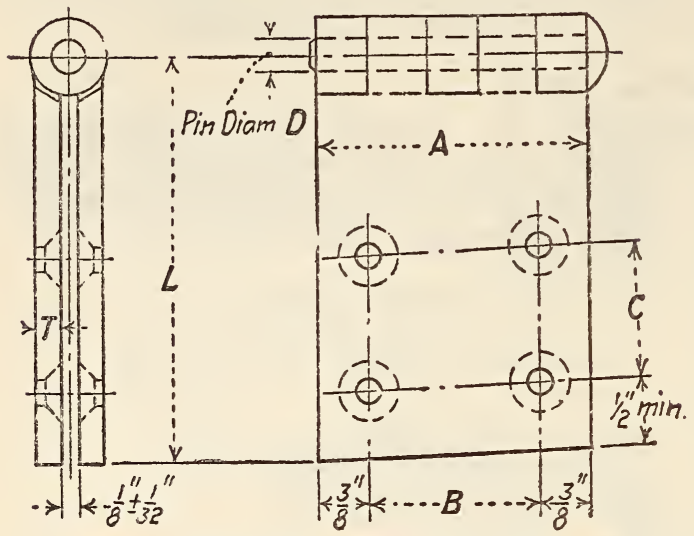

FIgURE 808. - Straight-type door hinges

[Dimensions in inches]

\begin{tabular}{|c|c|c|c|c|c|c|c|c|c|}
\hline \multirow[b]{2}{*}{ 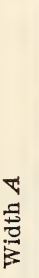 } & \multirow{2}{*}{ 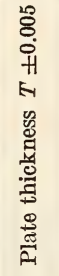 } & \multirow{2}{*}{ 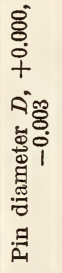 } & \multirow[b]{2}{*}{ 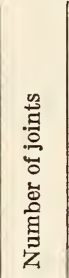 } & \multirow[b]{2}{*}{ 구 } & \multirow[b]{2}{*}{$\infty$} & \multirow[b]{2}{*}{ 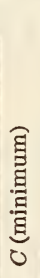 } & \multicolumn{3}{|c|}{$\begin{array}{c}\text { Number of } \\
\text { hinges }\end{array}$} \\
\hline & & & & & & & 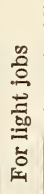 & 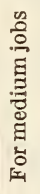 & 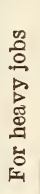 \\
\hline $13 / 4$ & 0.160 & 0.273 & 3 or 5 & $3,4,5$, or $51 / 2$ & 1 & 1 & 3 & 4 & \\
\hline $13 / 4$ & .187 & .273 & 3 & $3,4,5$, or $51 / 2$ & 1 & 1 & 3 & 4 & 4 \\
\hline 2 & .160 & .273 & 5 & $3,4,5$, or $51 / 2$ & $11 / 4$ & 1 & 3 & 3 & 4 \\
\hline 2 & .187 & .273 & 5 & $3,4,5$, or $51 / 2$ & $11 / 4$ & 1 & 3 & 3 & 3 \\
\hline
\end{tabular}

Screw holes shall be made to a minimum diameter of 0.438 inch and countersunk at $80^{\circ}$ for No. 12 wood screws.

\section{UNITED STATES GOVERNMENT, DEPART- MENT OF COMMERCE, BUILDERS' TEMPLATE HARDWARE, COMMERCIAL STANDARD CS9-29, SEPTEMBER 18, 1929}

(See also 617.21, p. 1020:)

\section{BUILDERS' TEMPIATE HARDWARE}

The following dimensions, clearances, tolerances, screw sizes, and varieties of template butt hinges are recommended as standard for application to hollow metal doors.

\section{TENFLATE BUTT HINGES}

1. Full mortise.-All full mortise template butt hinges shall be of the exact size as shown (both leaves), with straight edges and square corners. The width of mortise butt hinges is purposely omitted from these standards, and shall be such as to suit the architectural details.

2. Half surface, full surface, and half mortise.-All half surface, full surface, and half mortise template butt hinges shall be of the exact size as shown with square corners and straight edges on the mortise leaf. The surface leaves shall have beveled edges.

3. Tolerance on length.-A tolerance of $\left\{\begin{array}{l}+0.000 \\ -0.015\end{array}\right\}$ inch is allowed on the length of all butt hinges.

4. Tolerance on hole spacing.-A tolerance of \pm 0.005 inch is allowed on dimensions for hole spacing.

5. Tolerance on thickness.- (a) Cast butt hinges shall be of uniform thickness without taper, and within $\left\{\begin{array}{l}+0.005 \\ -0.010\end{array}\right\}$ inch of the specified thickness.

(b) A tolerance of \pm 0.005 inch is permitted on thickness of wrought butt hinges.

6. Paint clearance minimum.-The clearance between the inner edges of the leares and the barrel of the butt hinge shall be as follows:

(a) Butt hinges having gage of metal 0.090 or greater shall have a clearance of 0.090 plus or minus 0.008 inch.

(b) Butt hinges having gage of metal less than 0.090 shall be provided with a clearance of 0.050 plus or minus 0.008 inch.

(c) Cabinet butt hinges for painting shall be provided with a clearance of 0.40 plus or minus 0.005 inch between knuckles and edge of leaf.

7. Template identification symbol. - The first letter indicates thickness as $\mathrm{A}=$ regular thickness and $\mathrm{B}=$ extra heavy. The following numeral indicates template, as $1=$ regular template, and $2=$ narrow template. The next two digits indicate vertical height of butt hinge as $20=2$-inch, $25=2 \frac{1}{2} 2$-inch, $30=3$-inch, $35=31 / 2$-inch, etc. The last letter indicates type of butt hinge as $\mathrm{M}=$ full mortise, $\mathrm{H}=$ half surface, $\mathrm{S}=$ full surface, and $\mathrm{HM}=$ half mortise.

Thus A125M indicates a regular thickness, regular template, in $2 c f$ inch size, full mortise butt hinge. 
TABLE 1.-21/2 to 31/2 inch full-mortise template butt hinges

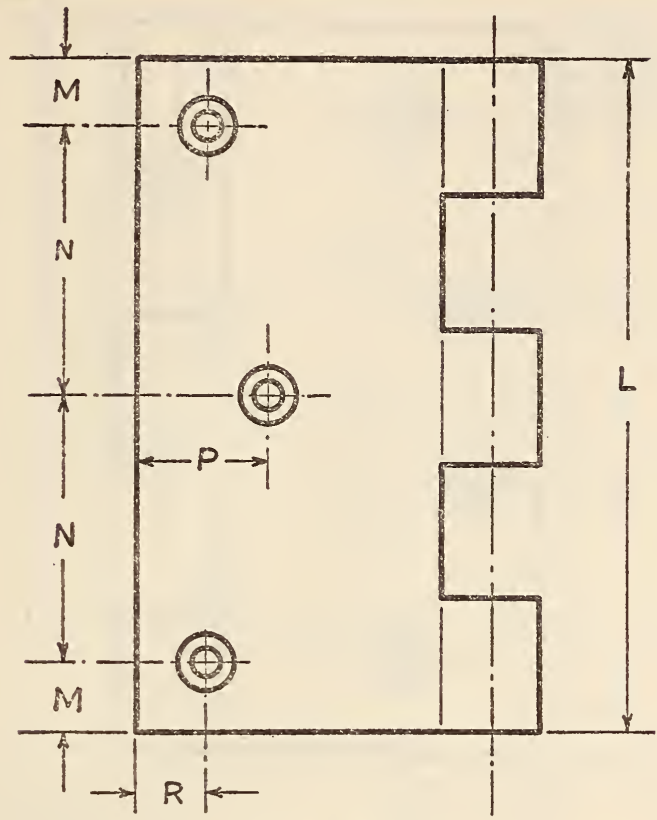

Figure 809

\begin{tabular}{|c|c|c|c|c|c|c|c|c|c|c|c|}
\hline \multirow{2}{*}{$\begin{array}{c}\text { Template } \\
\text { identification } \\
\text { symbol }\end{array}$} & \multicolumn{6}{|c|}{ Dimensions (inches) } & \multirow[b]{2}{*}{ Material of butt hinge } & \multicolumn{4}{|c|}{ Machine screws } \\
\hline & L & M & $\mathrm{N}$ & $\mathrm{P}$ & $\mathrm{R}$ & $\begin{array}{c}\text { Nomi- } \\
\text { nal } \\
\text { thick- } \\
\text { ness }\end{array}$ & & Туре & Length & $\begin{array}{l}\text { Diam- } \\
\text { eter }\end{array}$ & Threads \\
\hline $\begin{array}{l}\text { A125M } \\
\text { A130M } \\
\text { A130M } \\
\text { A135M.......... } \\
\text { A135M...... }\end{array}$ & $\begin{array}{l}21 / 2 \\
3 \\
3 \\
31 / 2 \\
31 / 2\end{array}$ & $\begin{array}{l}0.312 \\
.312 \\
.312 \\
.355 \\
.355\end{array}$ & $\begin{array}{l}0.938 \\
1.188 \\
1.188 \\
1.395 \\
1.395\end{array}$ & $\begin{array}{l}0.516 \\
.580 \\
.580 \\
.687 \\
.687\end{array}$ & $\begin{array}{l}0.320 \\
.312 \\
.312 \\
.360 \\
.360\end{array}$ & $\begin{array}{l}0.089 \\
.092 \\
.092 \\
.123 \\
.156\end{array}$ & $\begin{array}{l}\text { Wrought bronze or steel } \\
\text { Cast bronze or iron } \\
\text { Wrought bronze or steel } \\
\text { Cast bronze or iron }\end{array}$ & $\begin{array}{l}\mathrm{F} \cdot \mathrm{H} . \\
\mathrm{F} \cdot \mathrm{H} \\
\mathrm{F} \cdot \mathrm{H} \\
\mathrm{F} \cdot \mathrm{H} \\
\mathrm{F} . \mathrm{H} .\end{array}$ & $\begin{array}{l}1 / 2 \\
1 / 2 \\
1 / 2 \\
1 / 2 \\
1 / 2\end{array}$ & $\begin{array}{r}8 \\
10 \\
10 \\
10 \\
10\end{array}$ & $\begin{array}{l}32 \\
24 \\
24 \\
24 \\
24\end{array}$ \\
\hline
\end{tabular}


TABLE 2. -4 and $4 \frac{1}{2}$ inch full-mortise template butt hinges

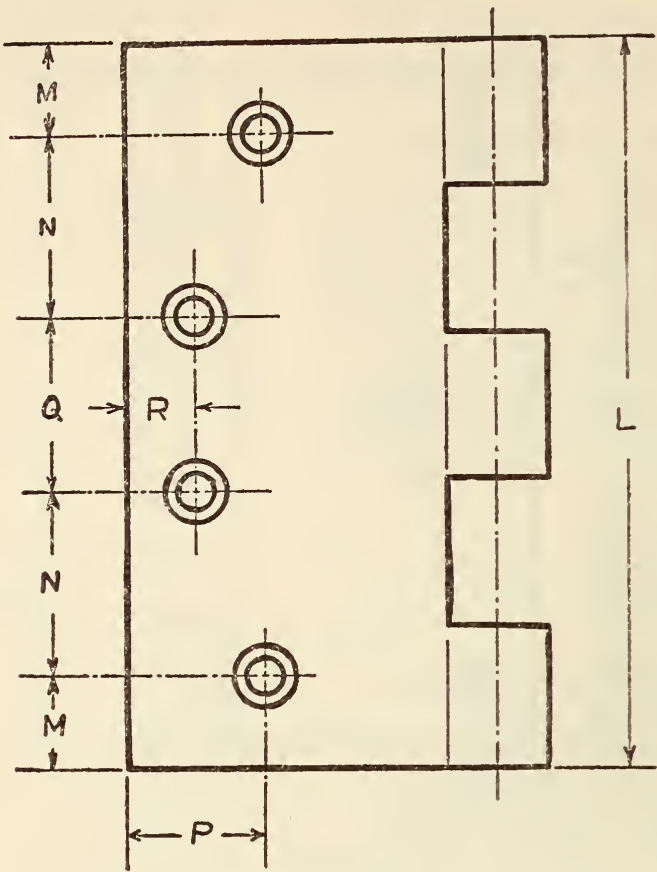

Figdre 810

\begin{tabular}{|c|c|c|c|c|c|c|c|c|c|c|c|c|}
\hline \multirow{2}{*}{$\begin{array}{c}\text { Template } \\
\text { identification } \\
\text { symbol }\end{array}$} & \multicolumn{7}{|c|}{ Dimensions (inches) } & \multirow[b]{2}{*}{ Material of butt hinge } & \multicolumn{4}{|c|}{ Machine screws } \\
\hline & $\mathrm{L}$ & M & $\mathrm{N}$ & $\mathrm{P}$ & Q & $\mathrm{R}$ & $\begin{array}{l}\text { Nomi- } \\
\text { nal } \\
\text { thick- } \\
\text { ness }\end{array}$ & & Tуре & Length & $\begin{array}{c}\text { Diam- } \\
\text { eter }\end{array}$ & Threads \\
\hline $\begin{array}{l}\text { A140M } \\
\text { A140M-..-- } \\
\text { B140M--.- } \\
\text { B140M--.- }\end{array}$ & $\begin{array}{l}4 \\
4 \\
4 \\
4\end{array}$ & $\begin{array}{r}0.512 \\
.512 \\
.512 \\
.512\end{array}$ & $\begin{array}{l}1.004 \\
1.004 \\
1.004 \\
1.004\end{array}$ & $\begin{array}{l}0.750 \\
.750 \\
.750 \\
.750\end{array}$ & $\begin{array}{r}0.968 \\
.968 \\
.968 \\
.968\end{array}$ & $\begin{array}{l}0.375 \\
.375 \\
.375 \\
.375\end{array}$ & $\begin{array}{r}0.130 \\
.172 \\
.170 \\
.250\end{array}$ & $\begin{array}{l}\text { Wrought bronze or steel. } \\
\text { Cast bronze or iron. } \\
\text { Wrought bronze or steel. } \\
\text { Cast bronze or iron...... }\end{array}$ & $\begin{array}{l}\text { F. } \frac{\mathrm{H}}{\mathrm{H}} \\
\mathrm{F} \cdot \mathrm{H} \\
\mathrm{F} \cdot \mathrm{H}\end{array}$ & $\begin{array}{l}1 / 2 \\
112 \\
1 / 2 \\
1 / 2\end{array}$ & $\begin{array}{l}12 \\
12 \\
12 \\
12\end{array}$ & $\begin{array}{l}24 \\
24 \\
24 \\
24\end{array}$ \\
\hline $\begin{array}{l}\text { A145M-...- } \\
\text { A145M-...- } \\
\text { B145M--.- } \\
\text { B145M-.-. }\end{array}$ & $\begin{array}{l}41 / 2 \\
41 / 2 \\
41 / 2 \\
41 / 2\end{array}$ & $\begin{array}{l}.508 \\
.508 \\
.508 \\
.508\end{array}$ & $\begin{array}{l}1.125 \\
1.125 \\
1.125 \\
1.125\end{array}$ & $\begin{array}{l}1.000 \\
1.000 \\
1.000 \\
1.000\end{array}$ & $\begin{array}{l}1.234 \\
1.234 \\
1.234 \\
1.234\end{array}$ & $\begin{array}{l}.375 \\
.375 \\
.375 \\
.375\end{array}$ & $\begin{array}{l}.134 \\
.187 \\
.180 \\
.250\end{array}$ & $\begin{array}{l}\text { Wrought bronze or steel. } \\
\text { Cast bronze or iron } \\
\text { Wrought bronze or steel. } \\
\text { Cast bronze or iron. }\end{array}$ & $\begin{array}{l}\text { F. H. } \\
\text { F. } \\
\text { F. } \\
\text { F. } \\
\text { H. }\end{array}$ & $\begin{array}{l}1 / 2 \\
1 / 2 \\
11 / 2 \\
1 / 2\end{array}$ & $\begin{array}{l}12 \\
12 \\
12 \\
12\end{array}$ & $\begin{array}{l}24 \\
24 \\
24 \\
24\end{array}$ \\
\hline 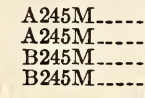 & $\begin{array}{l}41 / 2 \\
41 / 2 \\
41 / 2 \\
41 / 2\end{array}$ & $\begin{array}{l}.500 \\
.500 \\
.500 \\
.500\end{array}$ & $\begin{array}{l}1.166 \\
1.166 \\
1.166 \\
1.166\end{array}$ & $\begin{array}{l}.593 \\
.593 \\
.593 \\
.593\end{array}$ & $\begin{array}{l}1.166 \\
1.166 \\
1.166 \\
1.166\end{array}$ & $\begin{array}{l}.406 \\
.406 \\
.406 \\
.406\end{array}$ & $\begin{array}{l}.134 \\
.187 \\
.180 \\
.250\end{array}$ & $\begin{array}{l}\text { Wrought bronze or steel. } \\
\text { Cast bronze or iron } \\
\text { Wrought bronze or steel. } \\
\text { Cast bronze or iron. }\end{array}$ & $\begin{array}{l}\text { F. H. } \\
\text { F. } \frac{H}{H} \text {. } \\
\text { F. H. }\end{array}$ & $\begin{array}{l}1 / 2 \\
1 / 2 \\
1 / 2 \\
1 / 2\end{array}$ & $\begin{array}{l}12 \\
12 \\
12 \\
12\end{array}$ & $\begin{array}{l}24 \\
24 \\
24 \\
24\end{array}$ \\
\hline
\end{tabular}


$\mathrm{T}_{\triangle \mathrm{BLE}} 3 .-5$ and 6 inch full-mortise template butt hinges

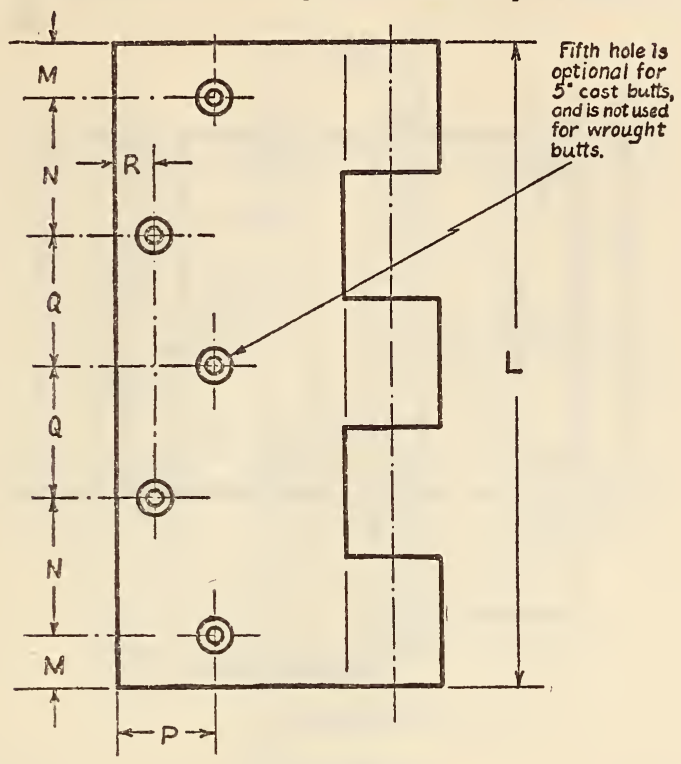

Figure 811

\begin{tabular}{|c|c|c|c|c|c|c|c|c|c|c|c|c|}
\hline \multirow{2}{*}{$\begin{array}{l}\text { Template } \\
\text { identification } \\
\text { symbol }\end{array}$} & \multicolumn{7}{|c|}{ Dimensions (inches) } & \multirow[b]{2}{*}{ Material of butt hinge } & \multicolumn{4}{|c|}{ Machine screws } \\
\hline & L & M & $\mathrm{N}$ & $\mathrm{P}$ & Q & $\mathrm{R}$ & $\begin{array}{c}\text { Nomi- } \\
\text { nal } \\
\text { thick- } \\
\text { ness }\end{array}$ & & Type & Length & $\begin{array}{c}\text { Diam- } \\
\text { eter }\end{array}$ & Threads \\
\hline $\begin{array}{l}\text { A150M } \\
\text { A150M-.... } \\
\text { B150M-...- } \\
\text { B150M-.... } \\
\text { A250M..... }\end{array}$ & $\begin{array}{l}5 \\
5 \\
5 \\
5 \\
5\end{array}$ & $\begin{array}{l}0.508 \\
.508 \\
.508 \\
.508 \\
.500\end{array}$ & $\begin{array}{l}1.250 \\
1.250 \\
1.250 \\
1.250 \\
1.250\end{array}$ & $\begin{array}{l}1.000 \\
1.000 \\
1.000 \\
1.000 \\
.687\end{array}$ & $\begin{array}{r}0.742 \\
.742 \\
.742 \\
.742 \\
.750\end{array}$ & $\begin{array}{l}0.375 \\
.375 \\
.375 \\
.375 \\
.312\end{array}$ & $\begin{array}{l}0.146 \\
.203 \\
.190 \\
.281 \\
.146\end{array}$ & $\begin{array}{l}\text { Wrought bronze or steel.-. } \\
\text { Cast bronze or iron.-... } \\
\text { Wrought bronze or steel.-. } \\
\text { Cast bronze or steel. } \\
\text { Wrought bronze or steel.-. }\end{array}$ & $\begin{array}{l}\text { F. H. } \\
\text { F. H. } \\
\text { F. H. } \\
\text { F. H. } \\
\text { F. H. }\end{array}$ & $\begin{array}{l}1 / 2 \\
1 / 2 \\
1 / 2 \\
3 / 4 \\
1 / 2\end{array}$ & $\begin{array}{l}12 \\
12 \\
12 \\
12 \\
12\end{array}$ & $\begin{array}{l}24 \\
24 \\
24 \\
24 \\
24\end{array}$ \\
\hline $\begin{array}{l}\text { A250M } \\
\text { B250M...... } \\
\text { B250M } \\
\text { B160M...... } \\
\text { B160M..... }\end{array}$ & $\begin{array}{l}5 \\
5 \\
5 \\
6 \\
6\end{array}$ & $\begin{array}{l}.500 \\
.500 \\
.500 \\
.500 \\
.500\end{array}$ & $\begin{array}{l}1.250 \\
1.250 \\
1.250 \\
1.281 \\
1.281\end{array}$ & $\begin{array}{l}.687 \\
.687 \\
.687 \\
.937 \\
.937\end{array}$ & $\begin{array}{r}.750 \\
.750 \\
.750 \\
1.219 \\
1.219\end{array}$ & $\begin{array}{l}.312 \\
.312 \\
.312 \\
.375 \\
.375\end{array}$ & $\begin{array}{l}.203 \\
.190 \\
.281 \\
.203 \\
.321\end{array}$ & $\begin{array}{l}\text { Cast bronze or iron } \\
\text { Wrought bronze or steel.-. } \\
\text { Cast bronze or iron } \\
\text { Wrought bronze or steel.- } \\
\text { Cast bronze or iron.....- }\end{array}$ & $\begin{array}{l}\text { F. } H . \\
\text { F. } H . \\
\text { F. }{ }_{\text {H. }} \\
\text { F. } \\
\text { F. } H .\end{array}$ & $\begin{array}{l}1 / 2 \\
1 / 2 \\
3 / 4 \\
112 \\
3 / 4\end{array}$ & $\begin{array}{l}12 \\
12 \\
12 \\
1 / 4 \\
1 / 4\end{array}$ & $\begin{array}{l}24 \\
24 \\
24 \\
20 \\
20\end{array}$ \\
\hline
\end{tabular}


TABLE 4.-3-inch half-surface template hinges

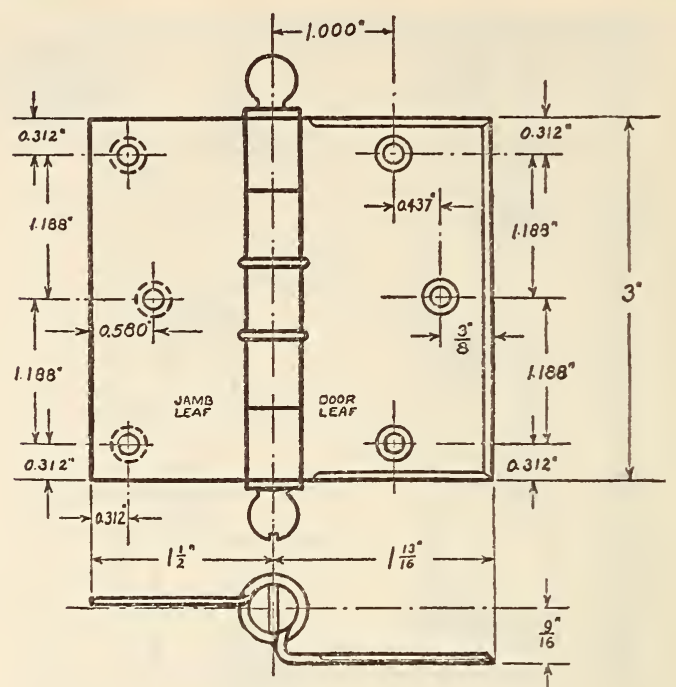

FIgURe 812

\begin{tabular}{|c|c|c|c|c|}
\hline $\begin{array}{c}\text { Template identifica- } \\
\text { tion symbol }\end{array}$ & $\begin{array}{c}\text { Nominal } \\
\text { thickness } \\
\text { (inch) }\end{array}$ & Material of butt hinge & Machine screws \\
\hline A130H...........- & 0.092 & Wrought steel and bronze.-Jamb leaf & $\begin{array}{c}\text { O. H. with grommet } \\
\text { nuts-Door leaf }\end{array}$ \\
\hline
\end{tabular}

TABLE 5.- $31 / 2-$ inch half-surface template hinges

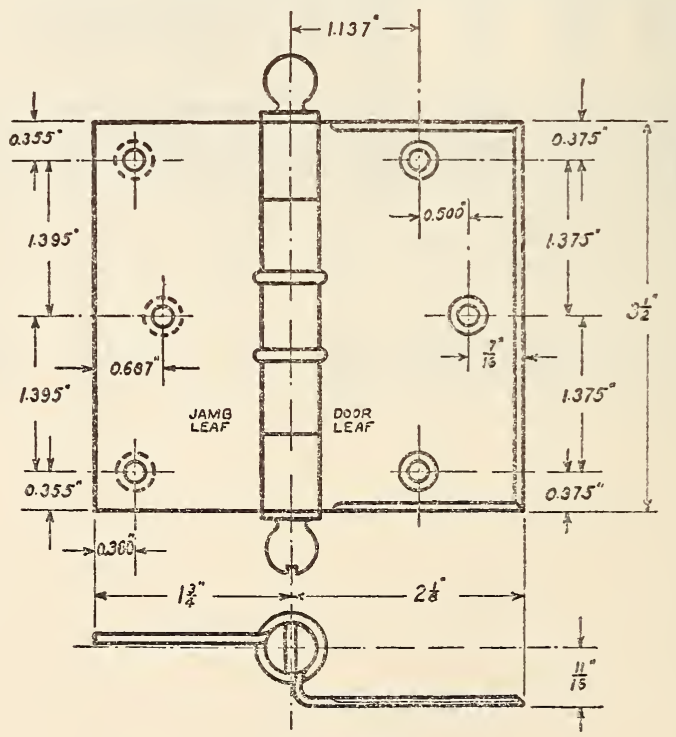

Figure 813

\begin{tabular}{|c|c|c|c|c|}
\hline $\begin{array}{c}\text { Template identifica- } \\
\text { tion symbol }\end{array}$ & $\begin{array}{c}\text { Nominal } \\
\text { thickness } \\
\text { (inch) }\end{array}$ & Material of butt hinge & Machine screws \\
\hline A135H. H._-Jamb leaf & $\begin{array}{c}\text { O. H. with grommet } \\
\text { nuts-Door leaf }\end{array}$ \\
\hline
\end{tabular}


TABLE 6.-4-inch half-surface template hinges

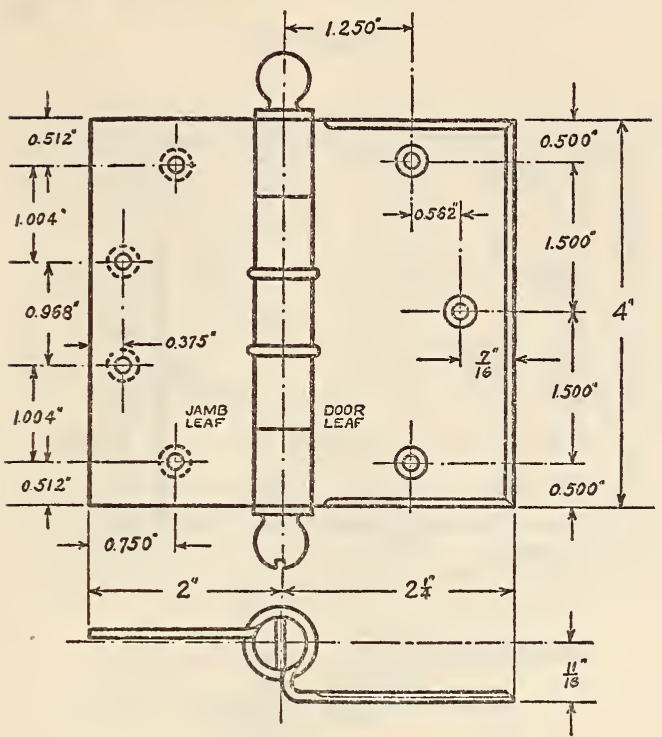

Figure 814

\begin{tabular}{|c|c|c|c|c|}
\hline \multirow{2}{*}{$\begin{array}{l}\text { Template identifica. } \\
\text { tion symbol }\end{array}$} & \multirow{2}{*}{$\begin{array}{l}\text { Nominal } \\
\text { thickness } \\
\text { (inch) }\end{array}$} & \multirow{2}{*}{ Material of butt hinge } & \multicolumn{2}{|c|}{ Machine screws } \\
\hline & & & F. H.-Jamb leaf & $\begin{array}{l}\text { O. H. with grommet } \\
\text { nuts-Door leaf }\end{array}$ \\
\hline A140H... & 0.130 & Wrought steel and bronze. & $1 / 2$ inch by $12-24$ & 2 inches by $1 / 4-20$. \\
\hline
\end{tabular}

TABLE 7.-4132-inch half-surface template hinges

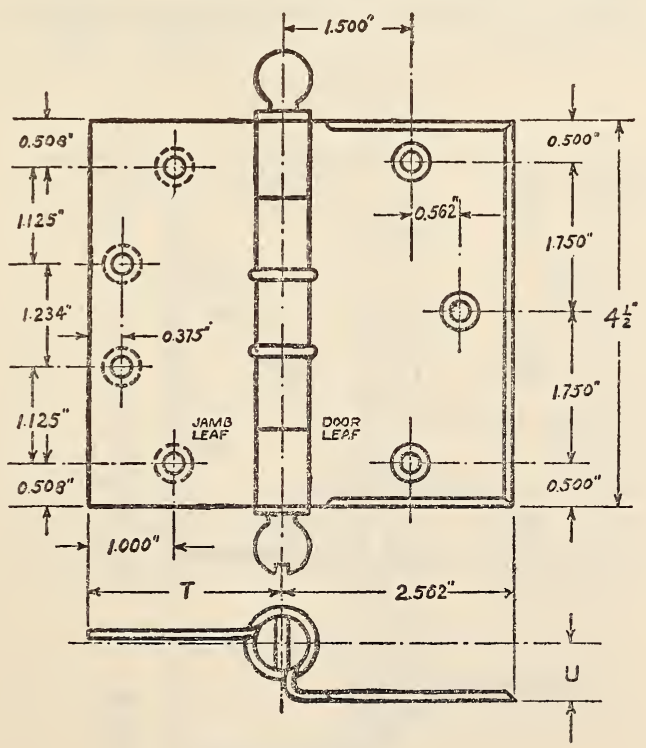

Figure 815

\begin{tabular}{|c|c|c|c|c|c|c|}
\hline \multirow{2}{*}{$\begin{array}{c}\text { Template } \\
\text { identification } \\
\text { symbol }\end{array}$} & \multicolumn{3}{|c|}{$\begin{array}{l}\text { Dimensions } \\
\text { (inches) }\end{array}$} & \multirow{2}{*}{ Material of butt hinge } & \multicolumn{2}{|c|}{ Machine screws } \\
\hline & $\mathrm{T}$ & U & $\left|\begin{array}{l}\text { Nominal } \\
\text { thickness }\end{array}\right|$ & & F. H.-Jamb leaf & $\begin{array}{l}\text { O. H. with grom- } \\
\text { met nuts-Door leaf }\end{array}$ \\
\hline A145H & $\begin{array}{l}21 / 4 \\
21 / 2\end{array}$ & $1^{3 / 4}$ & $\begin{array}{r}0.134 \\
.180\end{array}$ & Wrought steel and bronze & $\begin{array}{l}1 / 2 \text { inch by } 12-24 \\
1 / 2 \text { inch by } 12-24\end{array}$ & $\begin{array}{l}2 \text { inches by } 1 / 4-20 \\
21 / 4 \text { inches by } 1 / 4-20 .\end{array}$ \\
\hline
\end{tabular}


TABLE 8.-5-inch half-surface template hinges

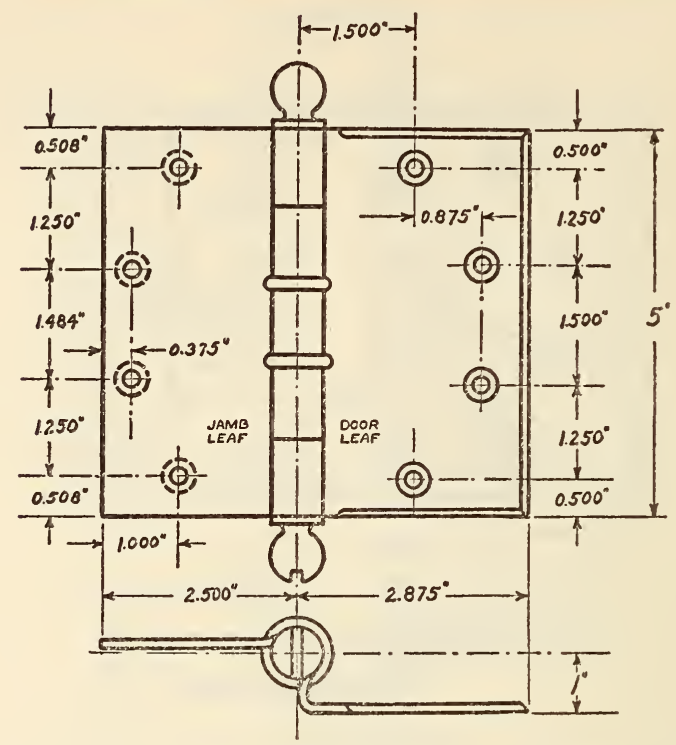

Figure 816

\begin{tabular}{|c|c|c|c|c|}
\hline \multirow{2}{*}{$\begin{array}{l}\text { Template identifica- } \\
\text { tion symbol }\end{array}$} & \multirow{2}{*}{$\begin{array}{l}\text { Nominal } \\
\text { thickness } \\
\text { (inch) }\end{array}$} & \multirow{2}{*}{ Material of butt hinge } & \multicolumn{2}{|c|}{ Machine screws } \\
\hline & & & F. H.-Jamb leaf & $\begin{array}{l}\text { O. H. with grommet } \\
\text { nuts-Door leaf }\end{array}$ \\
\hline B150H & $\begin{array}{r}0.146 \\
.190\end{array}$ & Wrought steel and bronze. & $\begin{array}{l}1 / 2 \text {-inch by } 12-24 \ldots \\
1 / 2 \text {-inch by } 12-24 \ldots\end{array}$ & $\begin{array}{l}2 \text { inches" by } 1 / 4-20 . \\
21 / 4 \text { inches by } 1 / 4-20 .\end{array}$ \\
\hline
\end{tabular}

TABLE 9.-6-inch half-surface template hinges

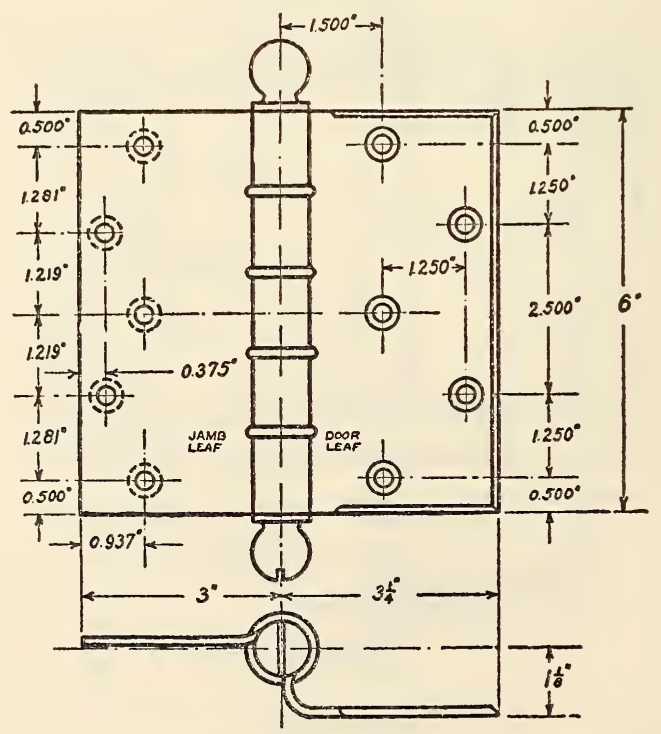

Figure 817

\begin{tabular}{|c|c|c|c|c|}
\hline \multirow{2}{*}{$\begin{array}{l}\text { Template identifica- } \\
\text { tion symbol }\end{array}$} & \multirow{2}{*}{$\begin{array}{l}\text { Nominal } \\
\text { thickness } \\
\text { (inch) }\end{array}$} & \multirow[b]{2}{*}{ Material of butt hinge } & \multicolumn{2}{|c|}{ Machine screws } \\
\hline & & & F. H.-Jamb leaf & O. H. with grommet \\
\hline B160H & 0.203 & Wrought steel and bronze.... & $1 / 2$-inch by $1 / 4-20 \ldots$ & $23 / 4$ inches by $1 / 4-20$. \\
\hline
\end{tabular}


$\mathrm{T}_{\mathrm{ABLE}}$ 10.-41\%2-inch full surface template hinges

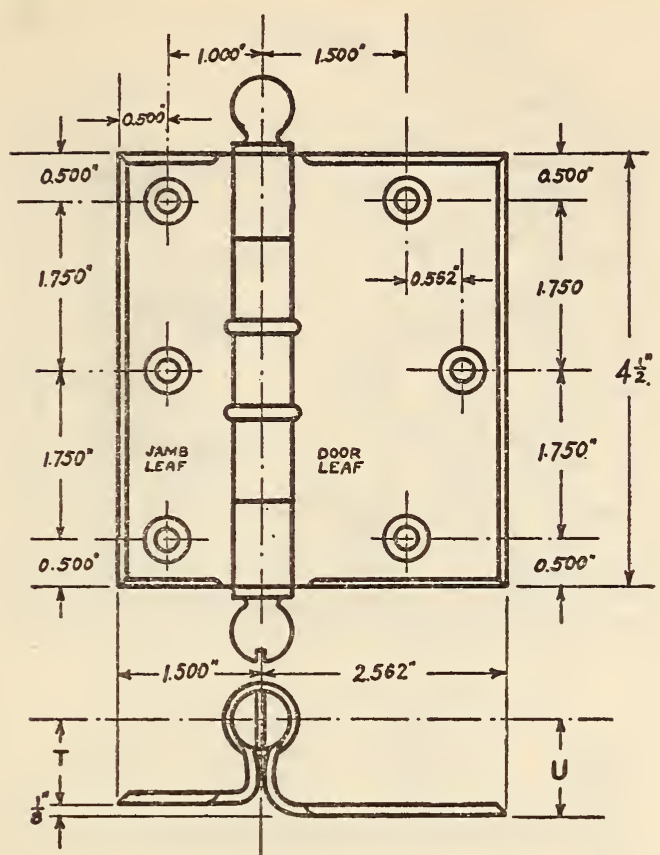

Figure 818

\begin{tabular}{|c|c|c|c|c|c|c|}
\hline \multirow{2}{*}{$\begin{array}{c}\text { Template } \\
\text { identification } \\
\text { symbol }\end{array}$} & \multicolumn{3}{|c|}{$\begin{array}{l}\text { Dimensions } \\
\text { (inches) }\end{array}$} & \multirow{2}{*}{ Material of butt hinge } & \multicolumn{2}{|c|}{ Machine screws } \\
\hline & $\mathrm{T}$ & U & $\begin{array}{l}\text { Nominal } \\
\text { thickness }\end{array}$ & & F. H.-Jamb leaf & $\begin{array}{l}\text { O. H. with grom- } \\
\text { met nuts-Door leaf }\end{array}$ \\
\hline A145S & $\begin{array}{r}13 / 16 \\
7 / 8\end{array}$ & $1^{15 / 16}$ & $\begin{array}{r}0.134 \\
.180\end{array}$ & Wrought steel and bronze & $\begin{array}{l}1 / 2 \text { inch by } 12-24 \ldots \\
1 / 2 \text { inch by } 12-24 \ldots\end{array}$ & $\begin{array}{l}2 \text { inches by } 1 / 4-20 \text {. } \\
21 / 4 \text { inches by } 1 / 4-20 \text {. }\end{array}$ \\
\hline
\end{tabular}


TABLE 11.-5-inch full surface template hinges

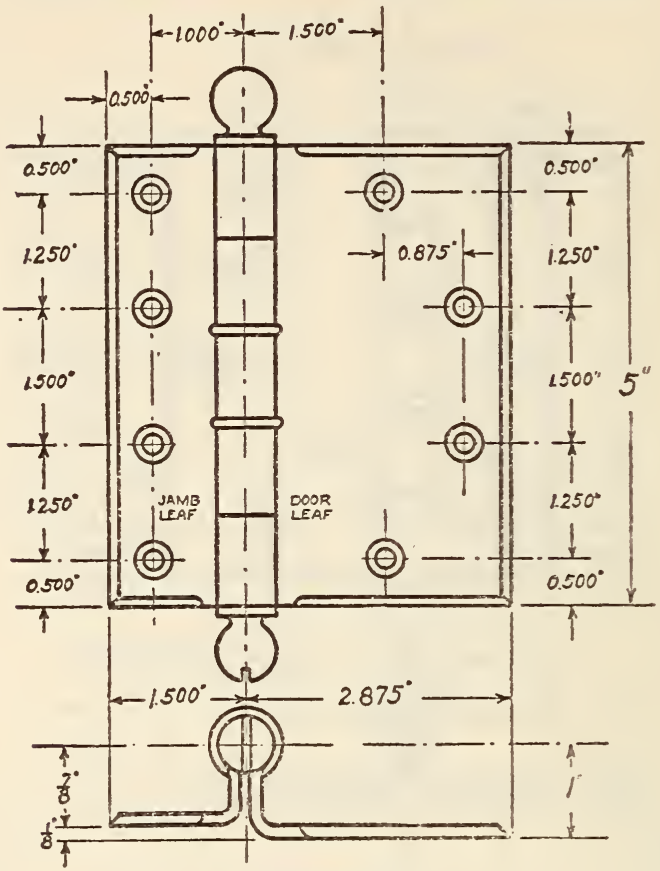

FIGURE 819

\begin{tabular}{|c|c|c|c|c|}
\hline \multirow{2}{*}{$\begin{array}{l}\text { Template identifica- } \\
\text { tion symbol }\end{array}$} & \multirow{2}{*}{$\begin{array}{l}\text { Nominal } \\
\text { thickness } \\
\text { (inch) }\end{array}$} & \multirow{2}{*}{ Material of butt hinge } & \multicolumn{2}{|c|}{ Machine screws } \\
\hline & & & F. H.-Jamb leaf & $\begin{array}{l}\text { O. H. with grommet } \\
\text { nuts-Door leaf }\end{array}$ \\
\hline A150S & $\begin{array}{r}0.146 \\
.190\end{array}$ & Wrought steel and bronze.. & $\begin{array}{l}1 / 2 \text { inch by } 12-24 \ldots \\
1 / 2 \text { inch by } 12-24 \ldots\end{array}$ & $\begin{array}{l}2 \text { inches by } 1 / 4-20 \\
21 / 4 \text { inches b } 1 / 4-20\end{array}$ \\
\hline
\end{tabular}


TABLE 12.-6-inch full-surface template hinges

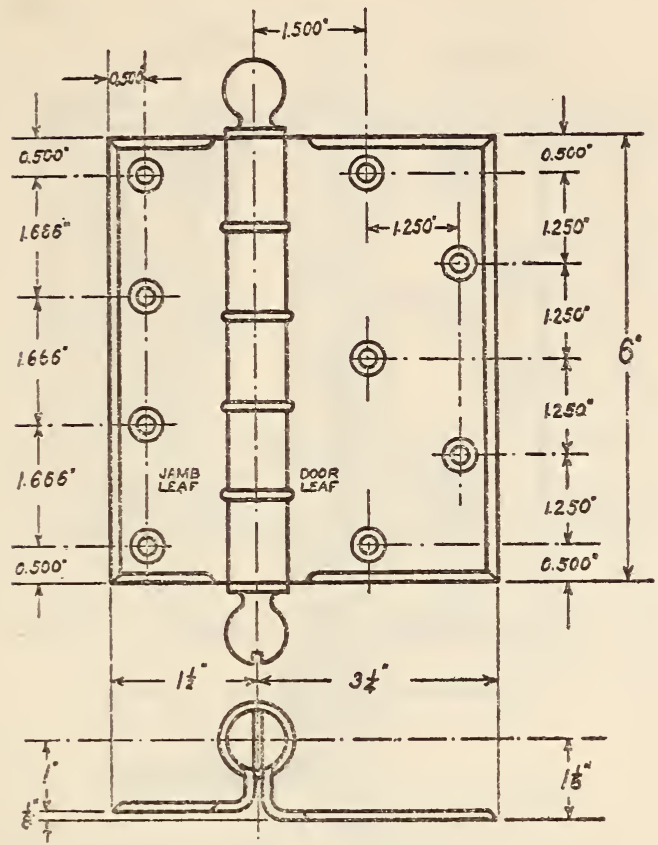

Figure 820

\begin{tabular}{|c|c|c|c|c|}
\hline \multirow{2}{*}{$\begin{array}{l}\text { Template identifica- } \\
\text { tion symbol }\end{array}$} & \multirow{2}{*}{$\begin{array}{l}\text { Nominal } \\
\text { thickness } \\
\text { (inch) }\end{array}$} & \multirow[b]{2}{*}{ Material of butt hinge } & \multicolumn{2}{|c|}{ Machine screws } \\
\hline & & & F.H.-Jamb leaf & $\begin{array}{l}\text { O. H. with grommet } \\
\text { nuts-Door leaf }\end{array}$ \\
\hline B160S... & 0.203 & Wrought steel and bronze. & $1 / 2$ inch by $1 / 4-20 \ldots$ & $23 / 4$ inches by $1 / 4-20$. \\
\hline
\end{tabular}


$\mathrm{T}_{\mathrm{ABLE}}$ 13.-412/2-inch half-mortise template hinges

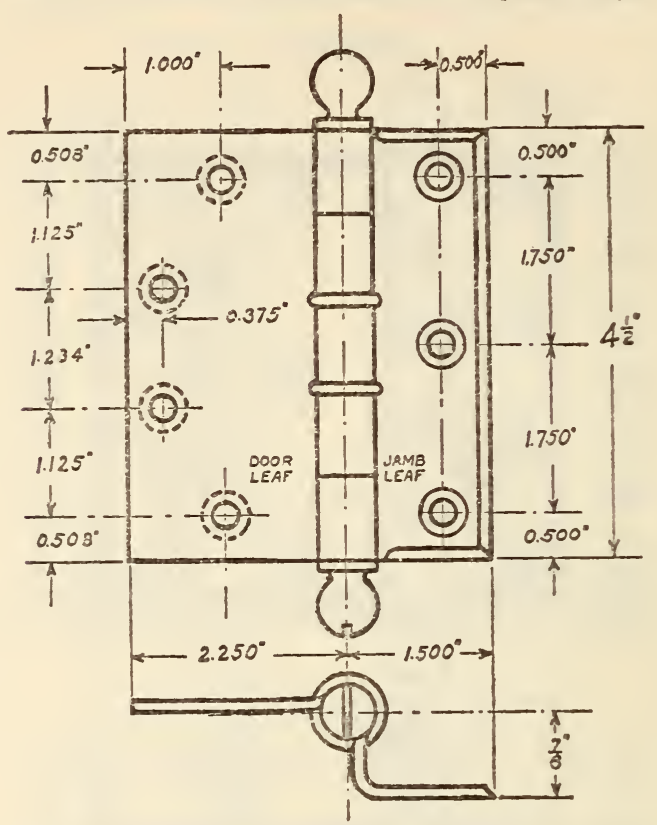

FigURE 821

\begin{tabular}{|c|c|c|c|c|}
\hline \multirow{2}{*}{$\begin{array}{l}\text { Template identifica- } \\
\text { tion symbol }\end{array}$} & \multirow{2}{*}{$\begin{array}{l}\text { Nominal } \\
\text { thickness } \\
\text { (inch) }\end{array}$} & \multirow{2}{*}{ Material of butt hinge } & \multicolumn{2}{|c|}{ Machine screws } \\
\hline & & & O. H. -Jamb leaf & F. H.-Door leaf \\
\hline B145HM_................ & 0.180 & Wrought steel and bronze.............. & $1 / 2$ inch by $12-24 \ldots \ldots \ldots$ & $1 / 2$ inch by $12-24$. \\
\hline
\end{tabular}

TABLE 14.-5-inch half-mortise template hinges

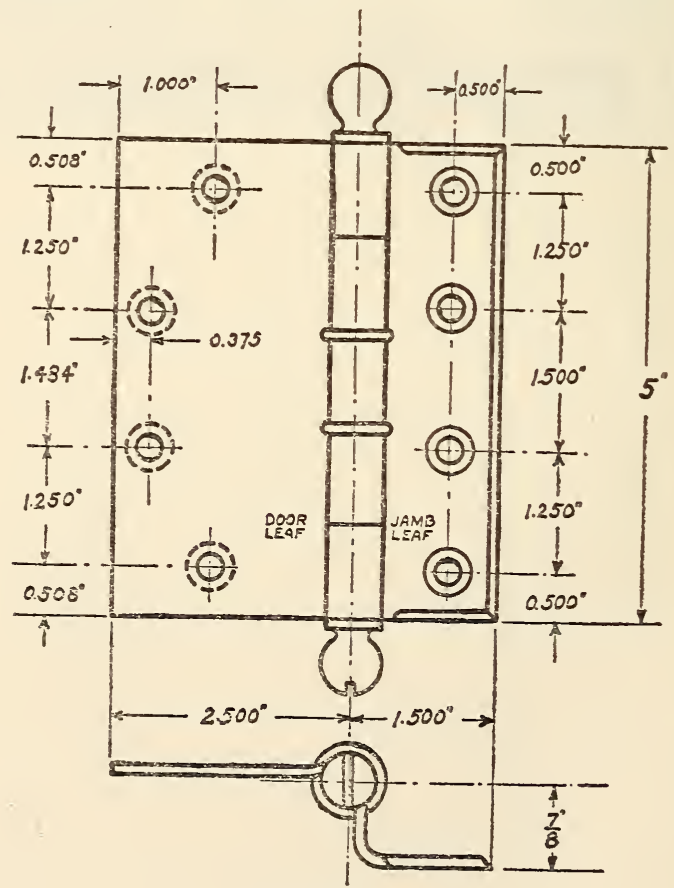

Figure 822

\begin{tabular}{|c|c|c|c|c|}
\hline \multirow{2}{*}{$\begin{array}{l}\text { Template identifica- } \\
\text { tion symbol }\end{array}$} & \multirow{2}{*}{$\begin{array}{l}\text { Nominal } \\
\text { thickness } \\
\text { (inch) }\end{array}$} & \multirow{2}{*}{ Material of butt hinge } & \multicolumn{2}{|c|}{ Machine screws } \\
\hline & & & O. H.-Jamb leaf & F. H.-Door leaf \\
\hline 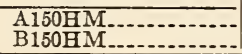 & $\begin{array}{r}0.146 \\
.190\end{array}$ & Wrought steel and bronze............ & 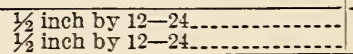 & $\begin{array}{l}1 / 2 \text { inch by } 12-24 . \\
1 / 2 \text { inch by } 12-24 .\end{array}$ \\
\hline
\end{tabular}


$T_{A B L E} 15 .-6$-inch half-mortise template hinges

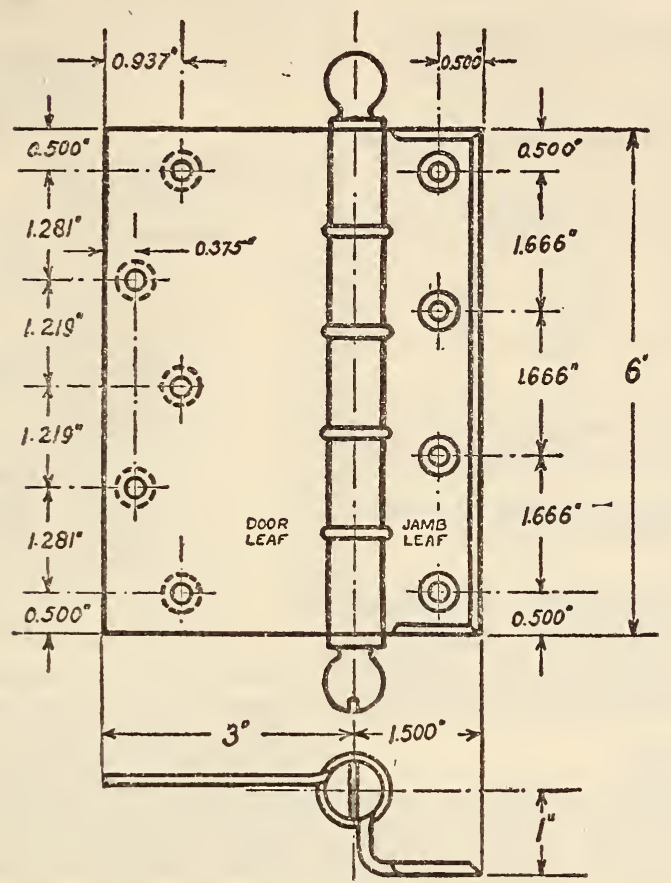

Figure 823

\begin{tabular}{|c|c|c|c|c|}
\hline \multirow{2}{*}{$\begin{array}{l}\text { Template identifica- } \\
\text { tion symbol }\end{array}$} & \multirow{2}{*}{$\begin{array}{l}\text { Nominal } \\
\text { thickness } \\
\text { (inch) }\end{array}$} & \multirow{2}{*}{ Material of butt hinge } & \multicolumn{2}{|c|}{ Machine screws } \\
\hline & & & O. H.-Jamb leaf & F.1H.-Door leaf \\
\hline B160HM...- & 0.203 & Wrought steel and bronze... & $1 / 2$ inch by $1 / 4-20 \ldots$ & $1 / 2$ inch by $1 / 4-20$. \\
\hline
\end{tabular}

UNITED STATES GOVERNMENT, DEPARTMENT OF COMMERCE, BUILDERS' HARDWARE (NONTEMPLATE), COMMERCIAL STANDARD CS22-30, DECEMBER 11, 1930

\section{HINGES}

(See 617.0 , p. 977, also Federal specification FF$\mathrm{H}-101$ below).

UNITED STATES GOVERNMENT, DEPARTMENT OF COMMERCE, BUREAU OF STANDARDS, COMMERCIAL STANDARD FOR STAPLE SEATS FOR WATER-CLOSET BOWLS, CS29-31, OCTOBER 1, 1931

\section{HINGES FOR WATER-CLOSET SEATS}

(A commercial standard selected and accepted by industry.)

The types of hinges shall be known as leaf hinge with bar, concealed hinge with bar, side-arm hinge, and arch hinge.

Hinges shall be made of nickel-plated polished brass. The brass mixture shall contain not less than 61 per cent copper and not more than 5 per cent lead.
The distance between hinge posts shall be 512 inches from center to center.

Hinge posts shall be provided with 4 rubber and 2 metal washers. Studs shall be not less than 2 inches in length below flange on post. Studs shall be threaded to within 1 inch of flange. Threads shall be U. S. standard-16 threads per inch for $3 / 8$-inchdiameter stud or 13 threads per inch for $1 / 2$-inchdiameter stud. Nuts for studs shall be not more than 1 inch in maximum diameter.

The bars for bar hinges used on varnished, sprayed, or sheet-covered seats shall be a brass tube at least seven-sixteenths inch in diameter. When not threaded it shall be at least No. 22 B. \& S. gage $(0.025$ inch) and when threaded it shall be at least No. 18 B. \& S. gage (0.040 inch).

The bars for bar hinges used on hard-rubber or molded-composition seats shall be a brass tube or bar at least five-eighths inch in diameter and shall be of same thicknesses as specified for hinges for varnished, sprayed, or sheet-covered seats.

The minimum weights of the various hinge types are specified in Table 1. The total weight of the bar hinges includes the weight of the finished bar, posts, nuts, and seat, and cover leaves. The total weight of the side-arm hinge includes the weight of 2 arms, 2 posts, studs, and nuts. 
TABLE 1.-Minimum weights of hinges

\begin{tabular}{|c|c|c|c|c|}
\hline \multirow{2}{*}{ Type of hinge } & \multicolumn{2}{|c|}{$\begin{array}{c}\text { Varnished, sprayed, } \\
\text { or sheet-covered } \\
\text { seats }\end{array}$} & \multicolumn{2}{|c|}{$\begin{array}{l}\text { Hard-rubber or } \\
\text { molded-composi- } \\
\text { tion seats }\end{array}$} \\
\hline & $\begin{array}{l}\text { With } \\
\text { cover }\end{array}$ & $\begin{array}{c}\text { Without } \\
\text { cover }\end{array}$ & $\begin{array}{l}\text { With } \\
\text { cover }\end{array}$ & $\begin{array}{c}\text { Without } \\
\text { cover }\end{array}$ \\
\hline $\begin{array}{l}\text { Leaf hinge with bar } \\
\text { Concealed hinge with bar. } \\
\text { Side-arm hinge } \\
\text { Arch hinge }\end{array}$ & $\begin{array}{r}\text { Ounces } \\
16 \\
14 \\
10 \\
40\end{array}$ & \begin{tabular}{r|} 
Ounces \\
12 \\
12 \\
7 \\
37
\end{tabular} & $\begin{array}{r}\text { Ounces } \\
\text { (1) } \\
18 \\
18 \\
50\end{array}$ & $\begin{array}{l}\text { Ounces } \\
\text { (1) } \\
\text { (1) } 15 \\
\end{array}$ \\
\hline
\end{tabular}

1 Not to be used.

All wood screws used in connection with hinges shall be made of brass and at least five-eighths inch in length.

White metal, chromium-plated, colored, or rubbercovered hinges shall be considered as specials.

\section{UNITED STATES GOVERNMENT, FEDERAL SPECIFICATIONS BOARD, FEDERAL SPECIFICATION FOR BUILDERS' HARDWARE (NONTEMPLATE), FF-H-101, AUGUST 19, 1930}

(For types, grades, and classes; material and workmanship; general requirements; detail requirements; packaging, packing, and marking; notes; and table of standard finishes see 617.0 , p. 978 . For chromium plating, nickel plating, and zinc coatings; and methods of inspection and tests see 600.3 , p. 70.)

\section{BUILDERS, HARDWARE (NONTEMPLATE)}

\section{BLIND HARDWARE}

\section{Hinges for Blinds}

Type 1801.-Wrought steel, end type, top and bottom patterns. Corrugated elbow. For frame construction. Hook mounted on hook plate drilled for wood screws. Hinge dimensions, 4 inches vertical by 5 inches horizontal. Width of hinge 1 inch, of hook plate $1 \frac{1}{8}$ inches. Offset 1 inch, throw 2 inches. Wood screws 1 inch by number 8 , minimum of three in hook plate, four in hinge. Galvanized unless plain steel is specified.

Type 1801 A. - Same as type 1801, except center pattern. Length 6 inches. Three screw holes.

Type 1802.- Same as type 1801, except for brick construction. Offset 2 inches, throw 4 inches.

Type 1802A.-Same as type 1801, except for brick construction. Center pattern. Offset 2 inches, throw 4 inches. Length 6 inches. Three screw holes.

Type 1803.-Cast iron or steel, galvanized, unless plain iron or steel is specified. Gravity type with screws, right and left. Shall hold open and closed. Furnish with catch. Self-locking.
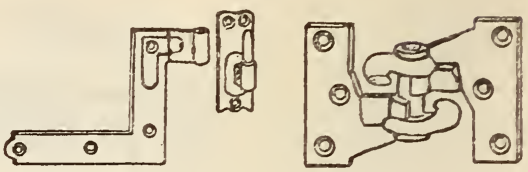

FigURE 824.-Hinges, for blinds, types 1801 and 1803

\begin{tabular}{|c|c|c|}
\hline No. & Size open & $\begin{array}{c}\text { Minimum } \\
\text { weight per } \\
\text { pair, includ- } \\
\text { ing screws } \\
\text { and catches }\end{array}$ \\
\hline & & Pounds \\
$11 / 2$ & Inches & $11 / 2$ \\
2 & 3 by $43 / 4$ & $11 / 4$ \\
$21 / 2$ & $23 / 4$ by $43 / 8$ & 1 \\
\hline
\end{tabular}

\section{BOTT HINGES}

E-12. All butt hinges shall bear the trade-mark or name of the manufacturer.

$\mathrm{E}-13$. Button tips shall be of the cylindrical type, having a square shoulder about one-eighth inch high and flush with the barrel of the butt hinge and having a flat or convex top. Where half surface, full surface, or other similar hinges (other than full mortise butt hinges) are necessary for the proper installation of metal or metal-covered doors in steel or metal frames, they shall be similar to and the equivalent of the types specified.

$\mathrm{E}-14$. Clearance between the leaves of butt hinges when open with leaves parallel shall be onesixteenth inch.

$\mathrm{E}-15$. Correct sizes of butt hinges for doors and transoms. -

$\mathrm{E}-15 \mathrm{a}$. The correct sizes of butt hinges for doors and transoms are as given in Table 3.

$\mathrm{E}-15 \mathrm{~b}$. Door sizes given are jamb opening dimensions.

E-15c. Butt-hinge sizes given refer to length of joint.

E-15d. Extra heavy butt hinges should be specified on doors where high-frequency service is expected. (See Table 4.) Extra heavy butt hinges are made in sizes 4 to 8 inches, inclusive. Whenever in Table 3, regular weight butt hinges are specified, but the door in question is of such a character as to come into the high-frequency classification, then extra heavy butt hinges of the same length and width should be substituted.

E-15e. Underwriters requirements.-Two butt hinges should be used for doors measuring 5 feet or less in height. Doors of a greater height shall require one butt hinge for each $2 \frac{1}{2}$ feet or fraction thereof in height. 
TABLE 3.-Rules for sizes of nontemplate butt hinges

[ Doors to and including 60 inches high take 2 butt hinges, over 60 to and including 90 inches high take 3 butt hinges, and over 90 to and including 120 inches high take 4 butt hinges. Transoms to and including 48 inches wide take 2 butt hinges, over 48 to and including 84 inches wide take 3 butt hinges.]

\begin{tabular}{|c|c|c|}
\hline Thickness in inches & $\begin{array}{l}\text { Width of doors or } \\
\text { height of tran- } \\
\text { soms in inches }\end{array}$ & $\begin{array}{l}\text { Height of } \\
\text { butt } \\
\text { hinges }\end{array}$ \\
\hline 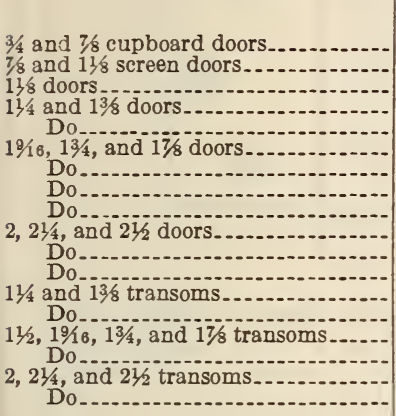 & 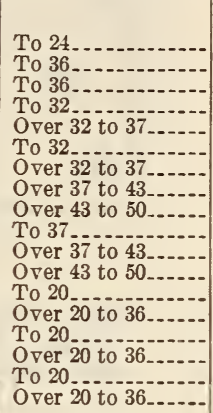 & $\begin{array}{l}\text { Inches } \\
21 / 2 \\
3 \\
31 / 2 \\
31 / 2 \\
4 \\
41 / 2 \\
5 \\
15 \\
16 \\
5 \\
15 \\
16 \\
21 / 2 \\
3 \\
3 \\
31 / 2 \\
31 / 2 \\
4\end{array}$ \\
\hline
\end{tabular}

\section{Extra heavy.}

TABLE 4.-Expected frequency of operation of doors [Number of operations of one leaf of door, opening and closing $=1$ cycle]

\begin{tabular}{|c|c|c|}
\hline \multirow{2}{*}{ Type of building and door } & \multicolumn{2}{|c|}{ Expected frequency } \\
\hline & Daily & Yearly \\
\hline 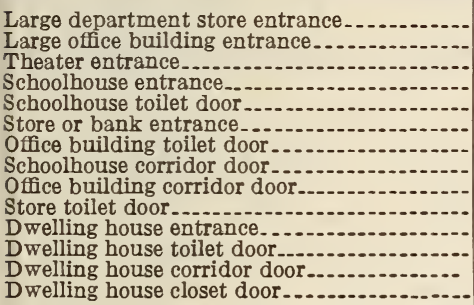 & $\begin{array}{r}5,000 \\
4,000 \\
2,000 \\
1,250 \\
1,250 \\
500 \\
400 \\
80 \\
75 \\
60 \\
40 \\
25 \\
10 \\
6\end{array}$ & $\begin{array}{r}11,500,000 \\
1,200,000 \\
1450,000 \\
1225,000 \\
1225,000 \\
1150,000 \\
1118,000 \\
15,000 \\
22,000 \\
18,000 \\
15,000 \\
9,000 \\
3,600 \\
2,200\end{array}$ \\
\hline
\end{tabular}

1 High frequency.

2 Per performance.

\section{Bronze Butt Hinges}

Type 2001.-Wrought bronze. Five knuckles. Ball bearings. Hardened steel ball races, hardened and polished steel balls. Ball bearings shall be attached to butts and shall be well packed with grease; retaining jackets when of visible type shall bear on one side only. Concealed ball bearings shall conform to tabulated requirements for size and number of balls. Inner edges of leaves shall be beveled. Nonrising, loose, steel pin with ball tips. Butt hinges for exterior doors opening out shall be so arranged that the pins can not be removed when the doors are closed.

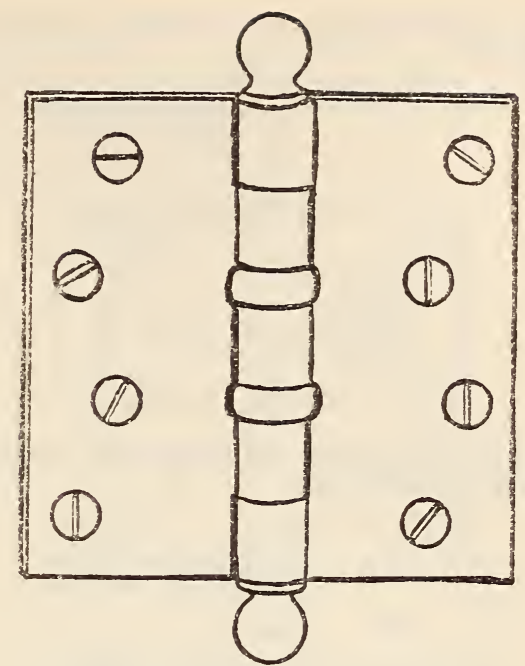

Figure 825.-Bronze butt hinge, 4 by 4 inch size, type 2001

\begin{tabular}{|c|c|c|c|}
\hline Size & $\begin{array}{c}\text { Required } \\
\text { thickness } \\
\text { of metal } \\
\pm 0.005 \text { inch }\end{array}$ & $\begin{array}{c}\text { Minimum } \\
\text { diameter } \\
\text { of balls in } \\
\text { concealed } \\
\text { bearings }\end{array}$ & $\begin{array}{c}\text { Minimum } \\
\text { number } \\
\text { of balls in } \\
\text { each con- } \\
\text { cealed bear- } \\
\text { ing }\end{array}$ \\
\hline $\begin{array}{c}\text { Inches } \\
\text { by } 4\end{array}$ & $\begin{array}{c}\text { Inch } \\
412 \text { by 41/2 }\end{array}$ & $\begin{array}{c}\text { Inch } \\
1 / 8\end{array}$ & 10 \\
5 by 5 & .134 & $1 / 8$ & 13 \\
6 by 6 & .160 & $1 / 8$ & 13 \\
\hline
\end{tabular}

Type 20011/2.- Same as type 2001, except with button tips.

Type 2002.-Same as type 2001, except extraheavy wrought bronze.

\begin{tabular}{|c|c|c|}
\hline Size & $\begin{array}{c}\text { Required } \\
\text { thickness } \\
\text { of metal } \\
\pm 0.005 \text { inch }\end{array}$ & $\begin{array}{c}\text { Number } \\
\text { of ball- } \\
\text { bearing } \\
\text { washers }\end{array}$ \\
\cline { 1 - 2 } $\begin{array}{c}\text { Inches } \\
41 / 2 \text { by } 41 / 2\end{array}$ & $\begin{array}{c}\text { Inch } \\
5 \text { by } 5\end{array}$ & \\
6 by 6 & .180 & 4 \\
8 by 6 & .203 & 4 \\
\hline
\end{tabular}

Type 20021/2.-Same as type 2001, except extraheavy wrought bronze with button tips.

\begin{tabular}{|c|c|c|}
\hline Size & $\begin{array}{c}\text { Required } \\
\text { thickness } \\
\text { of metal } \\
\pm 0.005 \text { inch }\end{array}$ & $\begin{array}{c}\text { Number } \\
\text { of ball- } \\
\text { bearing } \\
\text { washers }\end{array}$ \\
\hline Inches & Inch & \\
$41 / 2$ by $41 / 2$ & 0.180 & 4 \\
5 by 5 & .190 & 4 \\
6 by 6 & .203 & 4 \\
8 by 6 & .203 & 4 \\
\hline
\end{tabular}


Type 2003.-Same as type 2001, except cast bronze.

\begin{tabular}{|c|c|c|}
\hline & \multicolumn{1}{|c|}{$\begin{array}{c}\text { Minimum weight } \\
\text { per pair not in- } \\
\text { cluding screws }\end{array}$} \\
\cline { 2 - 3 } & Pounds & Ounces \\
\hline & & \\
4 Inches & & 14 \\
$41 / 2$ by 4112 & 2 & 8 \\
5 by 5 & 3 & 6 \\
6 by 6 & 5 & 2 \\
\hline
\end{tabular}

Type 20081/2.-Same as type 2001, except cast bronze with button tips.

\begin{tabular}{|c|c|c|}
\hline & \multicolumn{2}{|c|}{$\begin{array}{c}\text { Minimum weight per } \\
\text { pair not including } \\
\text { screws }\end{array}$} \\
\cline { 3 - 3 } Size & Pounds & Ounces \\
\hline & & \\
Inches & & 14 \\
$41 / 2$ by 41/2 & 1 & 8 \\
5 by 5 & 3 & 6 \\
6 by 6 & 5 & 2 \\
\hline
\end{tabular}

Type 2004.- Same as type 2001, except extraheavy cast bronze.

\begin{tabular}{|c|c|c|c|}
\hline \multirow{2}{*}{ Size } & \multicolumn{2}{|c|}{$\begin{array}{c}\text { Minimum weight } \\
\text { per pair not in- } \\
\text { cluding screws }\end{array}$} & $\begin{array}{c}\text { Number } \\
\text { of ball- } \\
\text { bearing } \\
\text { washers }\end{array}$ \\
\cline { 2 - 4 } & Pounds & Ounces & \\
\hline Inches & & & \\
5 5 by 5 & 4 & 2 & 4 \\
6 by 6 & 6 & 9 & 4 \\
8 by 6 & 8 & 8 & 4 \\
\hline
\end{tabular}

Type 20041/2.-Same as type 2001, except extraheavy cast bronze with button tips.

\begin{tabular}{|c|c|c|c|}
\hline Size & \multicolumn{2}{|c|}{$\begin{array}{c}\text { Minimum weight } \\
\text { per pair not in- } \\
\text { cluding screws }\end{array}$} & $\begin{array}{c}\text { Number } \\
\text { of ball- } \\
\text { bearing } \\
\text { washers }\end{array}$ \\
\cline { 2 - 4 } & Pounds & Ounces & \\
\hline Inches & & & \\
5 by 5 & 4 & 2 & 4 \\
6 by 6 & 6 & 9 & 4 \\
8 by 6 & 8 & 8 & 4 \\
\hline
\end{tabular}

Type 2005.-Wrought bronze. Steel bushed and self-lubricating. Five knuckles. Inner edges of leaves shall be beveled. Nonrising, loose, steel pin with ball tips. Butt hinges for exterior doors open- ing out shall be so arranged that the pins can not be removed when the doors are closed.

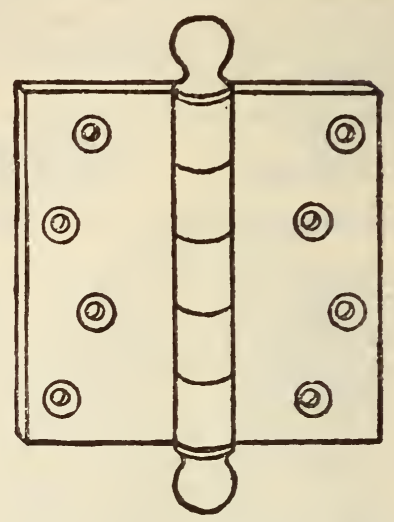

Figure 826.-Bronze butt hinge. 4 by 4 inch size, type 2005

\begin{tabular}{|c|c|}
\hline Size & $\begin{array}{c}\text { Required } \\
\text { thickness } \\
\text { of metal } \\
\pm 0.005 \\
\text { inch }\end{array}$ \\
\hline $\begin{array}{c}\text { Inches } \\
41 / 2 \text { by } 31 / 2\end{array}$ & $\begin{array}{c}\text { Inch } \\
4 \text { by } 4 \\
41 / 2 \text { by } 41 / 2\end{array}$ \\
.130 \\
.134 \\
\hline
\end{tabular}

Type 20051/2.-Same as type 2005, except with button tips.

Type 2006.-Same as type 2005, except cast bronze.

\begin{tabular}{|c|c|c|}
\hline \multirow{2}{*}{ Size } & \multicolumn{2}{|c|}{$\begin{array}{c}\text { Minimum weight } \\
\text { per pair not in- } \\
\text { cluding screws }\end{array}$} \\
\cline { 2 - 3 } & Pounds & Ounces \\
\hline Inches & & \\
$31 / 2$ by $31 / 2$ & 1 & 4 \\
4 by 4 & 1 & 14 \\
$41 / 2$ by $41 / 2$ & 2 & 6 \\
\hline
\end{tabular}

Type 200612.- Same as type 2005, except cast bronze with button tips.

\begin{tabular}{|c|c|c|}
\hline \multirow{2}{*}{ Size } & \multicolumn{2}{|c|}{$\begin{array}{l}\text { Minimum weight } \\
\text { per pair not in- } \\
\text { cluding screws }\end{array}$} \\
\hline & Pounds & Ounces \\
\hline $\begin{array}{c}\text { Inches } \\
31 / 2 \text { by } 31 / 2 \\
41 \text { by } 4 \\
41 / 2 \text { by } 41 / 2\end{array}$ & $\begin{array}{l}1 \\
1 \\
2\end{array}$ & $\begin{array}{r}4 \\
14 \\
6\end{array}$ \\
\hline
\end{tabular}


Type 2008.-Cast iron, polished and plated. Five knuckles. Inner edges of leaves shall be beveled. Nonrising, loose, steel pin with ball tips. Butt hinges for exterior doors opening out shall be so arranged that the pins can not be removed when the doors are closed.

\begin{tabular}{|c|c|c|}
\hline Size & \multicolumn{1}{|c|}{$\begin{array}{c}\text { Minimum weight } \\
\text { per pair not in- } \\
\text { cluding screws }\end{array}$} \\
\cline { 2 - 3 } & Pounds & Ounces \\
\hline $\begin{array}{c}\text { Inches } \\
\text { 31/2 by } 31 / 2\end{array}$ & 1 & 2 \\
$41 / 2$ by $41 / 2$ & 1 & 10 \\
& 2 & 2 \\
\hline
\end{tabular}

Type 20081/2.-Same as type 2008, except with button tips.

Type 2009.-Same as type 2008, except extra heavy.

\begin{tabular}{|c|c|c|}
\hline Size & \multicolumn{2}{|c|}{$\begin{array}{c}\text { Minimum weight } \\
\text { per pair not in- } \\
\text { cluding screws }\end{array}$} \\
\cline { 2 - 3 } & Pounds & Ounces \\
\hline Inches & & \\
5 by 5 & 3 & 7 \\
6 by 6 & 5 & 10 \\
\hline
\end{tabular}

Type 20091/2.- Same as type 2008, except extra heavy cast iron with button tips.

\begin{tabular}{|c|c|c|}
\hline & $\begin{array}{c}\text { Minimum weight } \\
\text { per pair not in } \\
\text { cluding screws }\end{array}$ \\
\cline { 2 - 3 } & Pounds & Ounces \\
\hline $\begin{array}{l}\text { Inches } \\
\text { by } 5\end{array}$ & 3 & 7 \\
6 by 6 & 5 & 10 \\
\hline
\end{tabular}

Wrought Steel But Hinges

Type 2010.-Wrought steel, plated. Five knuckles. Ball bearings. Hardened steel ball races, hardened and polished steel balls. Ball bearings shall be attached to butt and shall be well packed with grease; retaining jackets when of visible type shall bear on one side only. Concealed ball bearings shall conform to tabulated requirements for size and number of balls. Nonrising, loose, steel pin with ball tips. Butt hinges for exterior doors opening out shall be so arranged that the pins can not be removed when the doors are closed.

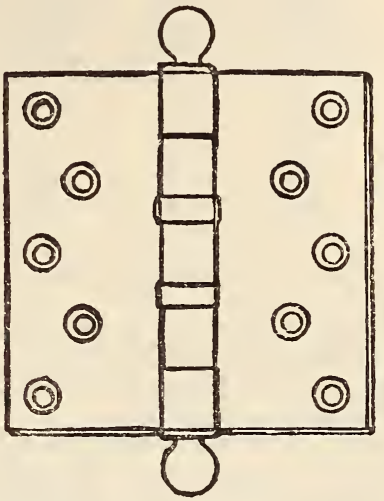

FIgURE 827.-Wrought steel butt hinge, 5 by 5 inch size, type 2010

\begin{tabular}{|c|c|c|c|}
\hline Size & $\begin{array}{c}\text { Required } \\
\text { thickness } \\
\text { of metal } \\
\pm 0.005 \text { inch }\end{array}$ & $\begin{array}{l}\text { Minimum } \\
\text { diameter of } \\
\text { balls in } \\
\text { concealed } \\
\text { bearings }\end{array}$ & $\begin{array}{c}\text { Minimum } \\
\text { number of } \\
\text { balls in each } \\
\text { concealed } \\
\text { bearing }\end{array}$ \\
\hline \begin{tabular}{l}
\multicolumn{1}{c}{ Inches } \\
4 by 4 \\
$41 / 2$ by $41 / 2$ \\
$5 \quad$ by 5 \\
$6 \quad$ by 6
\end{tabular} & $\begin{array}{l}\text { Inch } \\
0.130 \\
.134 \\
.146 \\
.160\end{array}$ & $\begin{array}{l}\text { Inch } \\
1 / 8 \\
1 / 8 \\
1 / 8 \\
1 / 8\end{array}$ & $\begin{array}{l}10 \\
13 \\
13 \\
13\end{array}$ \\
\hline
\end{tabular}

Type 20101/2.- Same as type 2010, except with button tips.

Type 2010B.- Same as type 2010, except wrought steel, polished and heavily plated with inner edges of leaves beveled.

Type 2010 $\frac{1}{2}$ B. - Same as type 2010, except wrought steel polished and heavily plated with inner edges of leaves beveled and with button tips.

Type 2010 $1 / 2 P$. - Same as type 2010, except for painting. Furnish with priming coat. Button tips. Clearance for paint between barrel and inner edges of leaves $0.090 \mathrm{inch} \pm 0.010 \mathrm{inch}$.

Type 2011.-Extra heavy wrought steel, polished and heavily plated, with inner edges of leaves beveled. Five knuckles. Ball bearings. Hardened steel ball races, hardened and polished steel balls. Ball bearings shall be attached to butts and shall be well packed with grease; retaining jackets when of visible type shall bear on one side only. Concealed ball bearings shall conform to tabulated requirements for size and number of balls. Nonrising loose, steel pin with ball tips. Butt hinges for exterior doors opening out shall be so arranged 
that the pins can not be removed when the doors are closed.

\begin{tabular}{|c|c|c|c|c|}
\hline Size & $\begin{array}{c}\text { Required } \\
\text { thickness } \\
\text { of metal } \\
\pm 0.005 \text { inch }\end{array}$ & $\begin{array}{c}\text { Number } \\
\text { of ball- } \\
\text { bearing } \\
\text { washers }\end{array}$ & $\begin{array}{l}\text { Minimum } \\
\text { diameter of } \\
\text { balls in } \\
\text { concealed } \\
\text { bearings }\end{array}$ & $\begin{array}{c}\text { Minimum } \\
\text { number of } \\
\text { balls in each } \\
\text { concealed } \\
\text { bearing }\end{array}$ \\
\hline 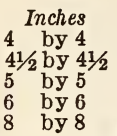 & $\begin{array}{r}\text { Inch } \\
0.170 \\
.180 \\
.190 \\
.203 \\
.203\end{array}$ & $\begin{array}{l}2 \\
4 \\
4 \\
4 \\
4\end{array}$ & $\begin{array}{c}\text { Inch } \\
118 \\
1 / 8 \\
1 / 8 \\
1 / 8 \\
1 / 8\end{array}$ & $\begin{array}{l}10 \\
13 \\
13 \\
13 \\
13\end{array}$ \\
\hline
\end{tabular}

Type 20111/2.-Same as type 2011, except with button tips.

Type $2011 \frac{1}{2}$ P. - Same as type 2011, except for painting. Furnish with priming coat. Button tips. Clearance for paint between barrel and inner edges of leaves $0.090 \mathrm{inch} \pm 0.010$ inch.

Type $20141 / 2$ P. - Wrought steel with priming coat for painting. Five knuckles. Nonrising loose steel pin, button tips. Clearance for paint between barrel and inner edges of leaves 0.050 inch \pm 0.010 inch, on $2 \frac{1}{2}$ inch size only. Other sizes clearance shall be $0.090 \mathrm{inch} \pm 0.010 \mathrm{inch}$.

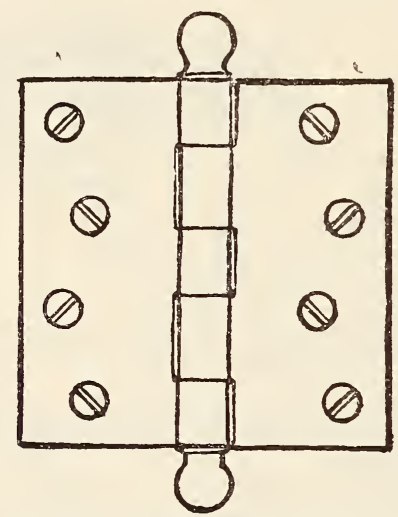

FIgURe 828.-Wrought steel butt hinge, 4 by 4 inch size, type 2014-1/2P

\begin{tabular}{|c|c|}
\hline Size & $\begin{array}{c}\text { Required } \\
\text { thickness } \\
\text { of metal } \\
\pm 0.005 \text { inch }\end{array}$ \\
\hline \begin{tabular}{l}
\multicolumn{1}{c}{ Inches } \\
$21 / 2$ by $21 / 2$ \\
3 by 3 \\
$31 / 2$ by $31 / 2$ \\
4 by 4 \\
$41 / 2$ by $41 / 2$ \\
5 by 5 \\
6 by 6
\end{tabular} & $\begin{array}{l}\text { Inch } \\
0.089 \\
.092 \\
.123 \\
.130 \\
.134 \\
.146 \\
.160\end{array}$ \\
\hline
\end{tabular}

Type 2015.-Wrought steel, plated. Five knuckles. Nonrising loose steel pin with ball tips.

\begin{tabular}{|c|c|}
\hline Sizo & $\begin{array}{c}\text { Required } \\
\text { thickness } \\
\text { of metal } \\
\pm 0.005 \text { inch }\end{array}$ \\
\hline $\begin{array}{l}\text { Inches } \\
21 / 2 \text { by } 21 / 2 \\
3 \text { by } 3 \\
31 / 2 \text { by } 31 / 2 \\
4 \text { by } 4 \\
41 / 2 \text { by } 41 / 2 \\
5 \text { by } 5 \\
6 \text { by } 6\end{array}$ & $\begin{array}{l}\text { Inch } \\
0.089 \\
.092 \\
.123 \\
.130 \\
.134 \\
.146 \\
.160\end{array}$ \\
\hline
\end{tabular}

Type 20151/2.-Same as type 2015, except wrought steel, plated with button tips.

Type 2015B.-Same as type 2015, except wrought steel, polished and heavily plated. Inner edges of leaves shall be beveled.

Type 20151/2B.-Same as type 2015, except wrought steel, polished and heavily plated. Inner edges of leaves shall be beveled. Button tips.

Light Loose Pin Butt Hinges îor Capboards

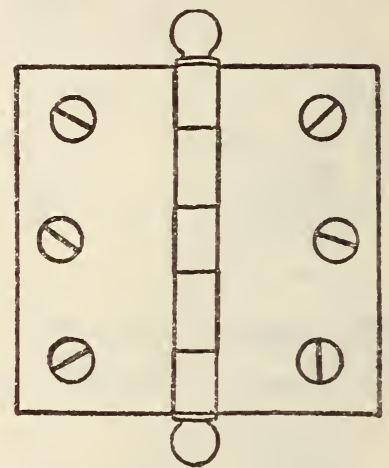

Figure 829. - Cast bronze light loose pin butt hinge for cupboards, 3 by 3 inch size, type 2017

Type 201\%.-Cast bronze. Five knuckles, steel pin, ball tips. Sizes, $2 \frac{1}{2}$ by $2 \frac{1}{2}$ and 3 by 3 inches. Minimum weight per pair not including screws, 7 and 12 ounces, respectively.

Type 20171/2. - Same as type 2017, except with button tips.

Type 2017 A.-Wrought bronze. Five knuckles, steel pin, ball tips. Sizes, $2 \frac{1}{2}$ by $2 \frac{1}{2}$ and 3 by 3 inches. Minimum thickness of metal required 0.062 inch \pm 0.005 inch and 0.071 inch \pm 0.005 inch, respectively.

Type 20171/2A. - Same as type $2017 \mathrm{~A}$, except with button tips.

Type 2018.-Wrought steel, plated. Five knuckles, steel pin, ball tips. Sizes, $2 \frac{1}{2}$ by $2,2 \frac{1}{2}$ by $2 \frac{1}{2}$ and 3 by 3 inches. Minimum thickness of metal required 0.062 inch \pm 0.005 inch, 0.062 inch \pm 0.005 inch and 0.071 inch \pm 0.005 inch, respectively.

Type 20181/2. - Same as type 2018, except with button tips.

Type 20181/2P.-Same as type 2018, except for painting. Furnish with priming coat. Button tips. Clearance for paint between barrel and inner edges of leaves 0.050 inch \pm 0.010 inch for $2 \frac{1}{2}$-inch sizes and 0.090 inch \pm 0.010 inch, for 3 -inch size.

\section{Light Narrow Butt Hinges}

Type 2021.-Wrought brass, three knuckles on $1 \frac{1}{2}$ and 2 inch sizes, five knuckles on $2 \frac{1}{2}$ and 3 inch sizes. Loose steel pin with ball tips.

\begin{tabular}{|c|c|}
\hline Size & $\begin{array}{l}\text { Required } \\
\text { thickness } \\
\text { of metal } \\
\pm 0.005 \\
\text { inch }\end{array}$ \\
\hline $\begin{array}{l}\text { Inches } \\
11 / 2 \text { by } 13 / 8 \\
2 \text { by } 1 \frac{3}{8} \\
21 / 2 \text { by } 13 / 4 \\
3 \text { by } 2\end{array}$ & $\begin{array}{l}\text { Inch } \\
0.047 \\
.062 \\
.062 \\
.071\end{array}$ \\
\hline
\end{tabular}


Type 20221/2P.-Same as type 2021, except wrought steel with priming coat for painting; clearance between barrel and inner edges of leaves, 0.050 inch \pm 0.010 inch. Button tips.

Transom Butt Hinges, Fast Pin

Type 2027.-Cast bronze. Five knuckles, steel pin, no tips. Inner edges of leaves shall be beveled.

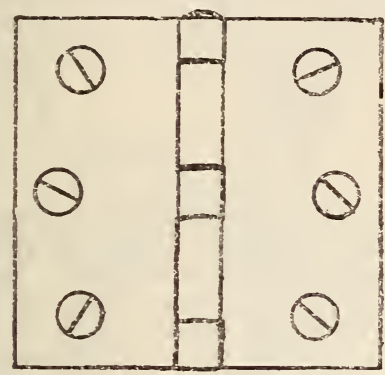

Figdre 830.-Cast bronze transom butt hinge, fast pin, type 2027

\begin{tabular}{|c|c|c|}
\hline \multirow{2}{*}{ Size } & \multicolumn{2}{|c|}{$\begin{array}{c}\text { Minimum weight } \\
\text { per pair not in- } \\
\text { cluding screws }\end{array}$} \\
\cline { 2 - 3 } & Pounds & Ounces \\
\hline $\begin{array}{c}\text { Inches } \\
21 / 2 \text { by } 21 / 2 \\
3 \text { by } 3 \\
31 / 2 \text { by } 31 / 2 \\
4 \text { by } 4\end{array}$ & & \\
\hline
\end{tabular}

Type 2028.-Wrought bronze. Five knuckles, steel pin, no tips. Inner edges of leaves shall be beveled.

\begin{tabular}{|c|c|}
\hline Size & $\begin{array}{c}\text { Required } \\
\text { thickness } \\
\text { of metal } \\
\pm 0.005 \\
\text { inch }\end{array}$ \\
\hline $\begin{array}{l}\text { Inches } \\
21 / 2 \text { by } 21 / 2 \\
3 \text { by } 3 \\
31 / 2 \text { by } 31 / 2 \\
4 \text { by } 4\end{array}$ & $\begin{array}{l}\text { Inch } \\
0.089 \\
.092 \\
.123 \\
.130\end{array}$ \\
\hline
\end{tabular}

Type 2029A.-Wrought steel, galvanized, five knuckles, steel pin, no tips.

\begin{tabular}{|c|c|}
\hline Size & $\begin{array}{l}\text { Required } \\
\text { thickness } \\
\text { of metal } \\
\pm 0.005 \\
\text { inch }\end{array}$ \\
\hline $\begin{array}{l}\text { Inches } \\
2 \text { by } 2 \\
21 / 2 \text { by } 21 / 2 \\
3 \text { by } 3 \\
31 / 2 \text { by } 31 / 2 \\
4 \\
4 \text { by } 4\end{array}$ & $\begin{array}{l}\text { Inch } \\
0.083 \\
.089 \\
.092 \\
.123 \\
.130\end{array}$ \\
\hline
\end{tabular}

Type 2029B.-Same as type 2029A, except plated. Type 2029P. - Same as type 2029A, except not galvanized and with priming coat for painting. Clearance for paint between barrel and inner edges of leaves 0.050 inch \pm 0.010 inch for $2 \frac{1}{2}$-inch size, other sizes clearance shall be 0.090 inch \pm 0.010 inch.

Type A2030.-Fast pin, full surface. Plain steel, without screw holes. Five knuckles.

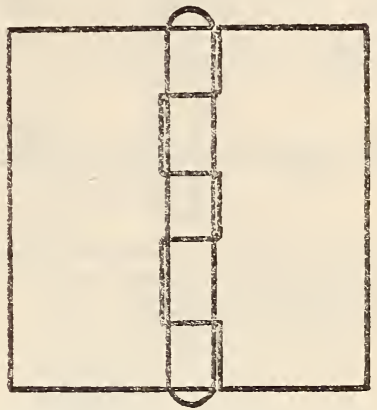

FigURE 831.-Plain steel transom butt hinge, fast pin, type A2030

\begin{tabular}{|c|c|}
\hline Size & $\begin{array}{l}\text { Required } \\
\text { thickness } \\
\text { of metal } \\
\pm 0.005 \\
\text { inch }\end{array}$ \\
\hline $\begin{array}{l}\text { Inches } \\
2 \text { by } 2 \\
21 / 2 \text { by } 21 / 2 \\
3 \text { by } 3 \\
31 / 2 \text { by } 31 / 2 \\
4 \text { by } 4\end{array}$ & $\begin{array}{l}\text { Inch } \\
0.083 \\
.089 \\
.092 \\
.123 \\
.130\end{array}$ \\
\hline
\end{tabular}

Type A2030A.-Same as type A2030, but with loose pin.

Type A2030B.-Same as type A2030, but full mortise.

Type A2030C.-Same as type A2030, but full mortise and with loose pin.

Type A2030D.-Same as type A2030, except galvanized and with brass pin.

Ioose Pin Reversible Butt Finges

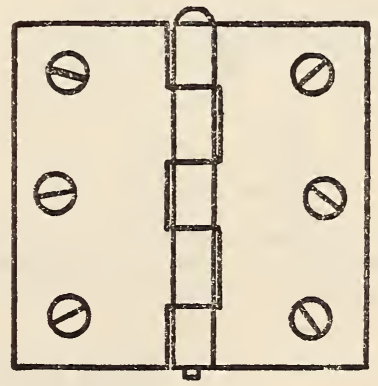

FIgURE 832.-Wrought steel loose pin reversible butt hinge, type 2031

Type 2031.-Wrought steel butt hinges with oval head loose pins. Plain steel. Sizes 2 by $2,21 / 2$ by 2 , $21 / 2$ by $21 / 2,3$ by $2 \frac{1}{2}, 3$ by $3,3 \frac{1}{2}$ by $3 \frac{1}{2}$, and 4 by 4 inches.

Butt Hinges for Wide Throw and for Folding Doors

Type 2040.-Wrought steel, plated. Five knuckles. Nonrising loose steel pin. Ball tips. 


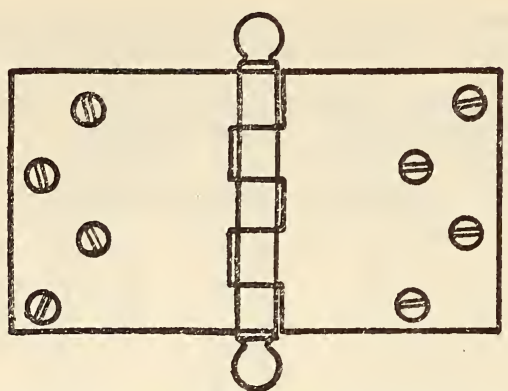

FIG URE 83 3.-Wrought steel butt hinge for wide throw and for folding doors, type 2040

\begin{tabular}{|c|c|}
\hline Size & $\begin{array}{c}\text { Required } \\
\text { thickness } \\
\text { of metal } \\
\pm 0.005\end{array}$ \\
\hline Inches & Inch \\
3 by 6 & 0.092 \\
$31 / 2$ by 5 & .123 \\
$31 / 2$ by 6 & .123 \\
4 by 6 & .130 \\
4 by 7 & .130 \\
5 by 7 & .146 \\
\hline
\end{tabular}

Type 2041.-Wrought steel, polished and heavily plated. Five knuckles. Ball bearings. Hardened steel ball races, hardened and polished steel balls. Ball bearings shall be attached to butts and shall be well packed with grease; retaining jackets when of visible type shall bear on one side only. Concealed ball bearings shall conform to tabulated requirements for size and number of balls. Nonrising loose steel pin with ball tips.

\begin{tabular}{|c|c|c|c|}
\hline Size & $\begin{array}{l}\text { Thickness } \\
\text { of metal } \\
\pm 0.005 \\
\text { inch }\end{array}$ & $\begin{array}{l}\text { Minimum } \\
\text { diameter } \\
\text { of balls in } \\
\text { concealed } \\
\text { bearings }\end{array}$ & $\begin{array}{l}\text { Minimum } \\
\text { number of } \\
\text { balls in } \\
\text { each } \\
\text { concealed } \\
\text { bearing }\end{array}$ \\
\hline $\begin{array}{r}\text { Inches } \\
3 \text { by } 6 \\
31 / 2 \text { by } 5 \\
31 / 2 \text { by } 6 \\
4 \text { by } 6 \\
4 \text { by } 7 \\
5 \text { by } 7\end{array}$ & $\begin{array}{r}\text { Inch } \\
0.092 \\
.123 \\
.123 \\
.130 \\
.130 \\
.146\end{array}$ & $\begin{array}{c}\text { Inch } \\
5 / 64 \\
5 / 64 \\
5 / 64 \\
1 / 8 \\
1 / 8 \\
1 / 8\end{array}$ & $\begin{array}{l}14 \\
14 \\
14 \\
10 \\
10 \\
13\end{array}$ \\
\hline
\end{tabular}

Gospical Type Ruit Firges

Type 2050.-Cast bronze, five knuckles, nonremovable bronze pin. Ends of barrel and pin shall be fully rounded to eliminate projecting edges.

\begin{tabular}{|c|c|c|}
\hline & \multicolumn{2}{|c|}{$\begin{array}{c}\text { Minimum weight } \\
\text { per pair, not in- } \\
\text { cluding screws }\end{array}$} \\
\cline { 2 - 3 } & Pounds & Ounces \\
\hline & & \\
Inches & 1 & 4 \\
4 by $31 / 2$ & 1 & 14 \\
$41 / 2$ by $411 / 2$ & 2 & 10 \\
5 by 5 & 3 & 4 \\
\hline
\end{tabular}

Type 2051.-Cast iron, plated. Nonremovable steel pin. Ends of barrel and pin shall be fully rounded to eliminate projecting edges.

\begin{tabular}{|c|c|c|}
\hline & $\begin{array}{c}\text { Minimum weight } \\
\text { per pair, not in- } \\
\text { cluding screws }\end{array}$ \\
\cline { 2 - 3 } & Pounds & Ounces \\
\hline Inches & & \\
$31 / 2$ by $31 / 2$ & 1 & 2 \\
4 by 4 & 1 & 12 \\
$41 / 2$ by $41 / 2$ & 2 & 6 \\
5 by 5 & 3 & 2 \\
\hline
\end{tabular}

Type 2060.-Wrought bronze. Reversible. Five knuckles. Ball bearings. Hardened steel ball races hardened and polished steel balls. Ball bearings shall be attached to butts and shall be well packed with grease; retaining jackets when of visible type shall bear on one side only. Concealed ball bearings shall conform to tabulated requirements for size and number of balls. Inner edges of leaves shall be beveled. Nonremovable pin. Ends of barrel and pin shall be fully rounded to eliminate projecting edges.

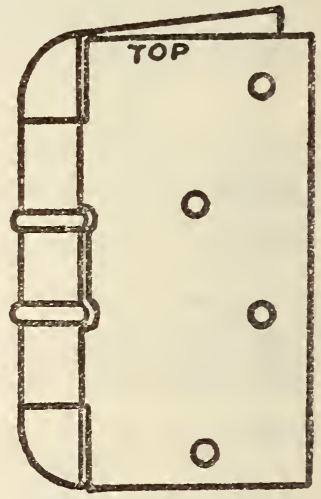

Figure 834.-Wrought bronze hospital type butt hinge, $41 / 2$ by $41 / 2$ inch size, type 2060

\begin{tabular}{|c|c|c|c|}
\hline Size & $\begin{array}{c}\text { Required } \\
\text { thickness } \\
\text { of metal } \\
\pm 0.005 \\
\text { inch }\end{array}$ & $\begin{array}{c}\text { Minimum } \\
\text { diameter } \\
\text { of balls in } \\
\text { concealed } \\
\text { bearings }\end{array}$ & $\begin{array}{c}\text { Minimum } \\
\text { number of } \\
\text { balls in } \\
\text { each } \\
\text { concealed } \\
\text { bearing }\end{array}$ \\
\hline Inches & Inch & Inch & \\
3 by 3 & 0.092 & $5 / 64$ & 14 \\
$31 / 2$ by $31 / 2$ & .123 & $5 / 64$ & 14 \\
4 by 4 & .130 & $1 / 8$ & 10 \\
$41 / 2$ by $41 / 2$ & .134 & 118 & 13 \\
5 by 5 & .146 & $1 / 8$ & 13 \\
6 by 6 & .160 & $1 / 8$ & 13 \\
\hline
\end{tabular}

Type 2061.-Same as type 2060, except wrought steel, polished and heavily plated.

Type 2061P.-Same as type 2060, except for painting. Wrought steel with priming coat. Clearance for paint between barrel and inner edges of leaves, 0.090 inch \pm 0.010 . 
Ialf Surface Butt Binges

Type 2080B.-Wrought steel, polished and heavily plated, reversible. Five knuckles. Ball bearings. Hardened steel ball races; hardened and polished steel balls. Ball bearings shall be attached to butts and shall be well packed with grease; retaining jackets when of visible type shall bear on one side only. Concealed ball bearings shall conform to tabulated requirements for size and number of balls. Inner edges of leaves shall be beveled. Nonrising loose steel pin with ball tips.

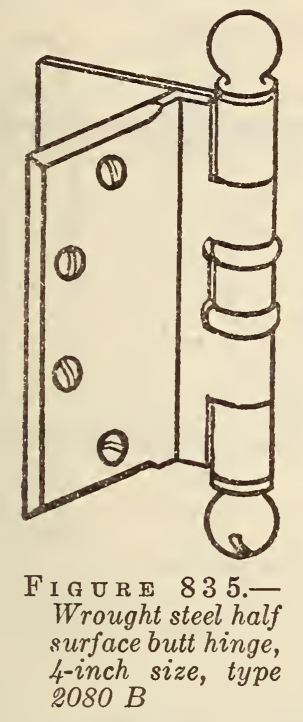

\begin{tabular}{|c|c|c|c|c|c|c|}
\hline $\begin{array}{l}\text { Butt } \\
\text { size }\end{array}$ & $\begin{array}{l}\text { Re- } \\
\text { quired } \\
\text { thick- } \\
\text { ness } \\
\text { of } \\
\text { metal } \\
\pm 0.005 \\
\text { inch }\end{array}$ & $\begin{array}{l}\text { Mini- } \\
\text { mum } \\
\text { diam- } \\
\text { eter of } \\
\text { balls in } \\
\text { con- } \\
\text { cealed } \\
\text { bearings }\end{array}$ & \begin{tabular}{|c|} 
Mini- \\
mum \\
number \\
of balls \\
in each \\
con- \\
cealed \\
bearing
\end{tabular} & $\begin{array}{l}\text { Offset } \\
\text { door } \\
\text { leaf }\end{array}$ & $\begin{array}{c}\text { Wood screws for } \\
\text { jamb leaf }\end{array}$ & $\begin{array}{l}\text { Oval head } \\
\text { machine } \\
\text { screws with } \\
\text { grommet } \\
\text { nuts for } \\
\text { door leaf }\end{array}$ \\
\hline $31 / 2$ & $\begin{array}{l}\text { Inch } \\
0.123\end{array}$ & $\begin{array}{l}\text { Inch } \\
5,64\end{array}$ & 14 & $\begin{array}{l}\text { Inch } \\
11 / 16\end{array}$ & \multirow{4}{*}{$\begin{array}{l}1 \text { inch by No. } \\
9 . \\
1 \text { inch by No. } \\
10 \text {. } \\
11 / 4 \text { inches by } \\
\text { No. } 10 \text {. } \\
11 / 4 \text { inches by } \\
\text { No. } 12 .\end{array}$} & \multirow{4}{*}{$\begin{array}{l}2 \text { inches by } \\
\text { No. 10-24. } \\
2 \text { inches by } \\
\text { No. 10-24. } \\
2 \text { inches by } \\
\text { No. 10-24. } \\
2 \text { inches by } \\
\text { No. 12-24. }\end{array}$} \\
\hline 4 & .130 & 18 & 10 & $11 / 16$ & & \\
\hline $41 / 2$ & .134 & 18 & 13 & $3 / 4$ & & \\
\hline 5 & 146 & $1 / 8$ & 13 & 1 & & \\
\hline
\end{tabular}

Type 20801/2P.-Same as type 2080B, except with priming coat for painting. Button tips. Clear- ance between barrel and inner edges of leaves shall be 0.090 inch \pm 0.010 inch.

Garage Hinges-Extra Eeavy Ball Bearing

Type 2140.-Wrought steel, galvanized. Reversed pad. Balls of hardened and polished tool steel. Hardened steel ball races. Fastened with wood screws, lag screws, or through bolts as ordered.

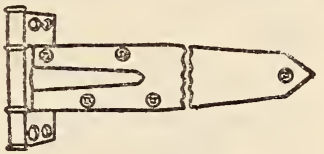

\section{FIGURE 836.-Wrought steel garage hinge, extra heavy ball bear- ing, type 2140}

\begin{tabular}{|c|c|c|c|}
\hline $\begin{array}{c}\text { Length } \\
\text { of strap }\end{array}$ & $\begin{array}{c}\text { Width of } \\
\text { strap at } \\
\text { joint }\end{array}$ & $\begin{array}{c}\text { Width of } \\
\text { jamb } \\
\text { leaf }\end{array}$ & $\begin{array}{c}\text { Length } \\
\text { of joint }\end{array}$ \\
\hline Inches & Inches & Inches & Inches \\
12 & 3 & $2^{13} / 16$ & 7 \\
18 & 3 & 21316 & 7 \\
24 & 3 & $213 / 16$ & 7 \\
36 & 4 & $35 / 16$ & 8 \\
\hline
\end{tabular}

Type 2140A.- Same as type 2140, except wrought steel, galvanized and japanned.

\section{Sirap Hinges}

Type 2203.-Wrought steel, galvanized; with brass pins.

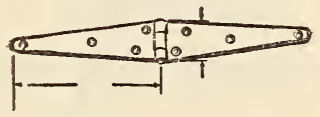

\section{FiguRe 837.-Wrought steel strap hinge, type 2203}

\begin{tabular}{|c|c|c|}
\hline $\begin{array}{c}\text { Length } \\
\text { closed }\end{array}$ & $\begin{array}{c}\text { Width of } \\
\text { strap at } \\
\text { at joint }\end{array}$ & $\begin{array}{c}\text { Minimum } \\
\text { thickness } \\
\text { of metal }\end{array}$ \\
\hline Inches & Inches & Inch \\
4 & 1916 & 0.080 \\
6 & $21 / 2$ & .118 \\
8 & 278 & .135 \\
10 & $33 / 8$ & .146 \\
12 & 4 & .158 \\
16 & $45 / 8$ & .150 \\
\hline
\end{tabular}




\section{T Hinges \\ Regular}

Type 2208.-Wrought steel, galvanized; with brass pins.

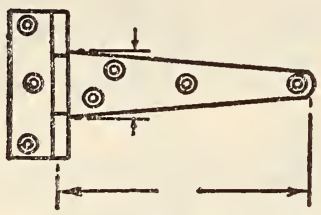

FIgURe 838.-Wrought steel $\mathrm{T}$ hinge, regular, type 2208

\begin{tabular}{|c|c|c|}
\hline $\begin{array}{c}\text { Length } \\
\text { closed }\end{array}$ & $\begin{array}{c}\text { Width of } \\
\text { strap at } \\
\text { joint }\end{array}$ & $\begin{array}{c}\text { Minimum } \\
\text { thickness } \\
\text { of metal }\end{array}$ \\
\hline $\begin{array}{c}\text { Inches } \\
4\end{array}$ & $\begin{array}{c}\text { Inches } \\
15 / 16 \\
1 \% 16\end{array}$ & $\begin{array}{c}\text { Inch } \\
0.072 \\
.087\end{array}$ \\
\hline
\end{tabular}

留 Type 2209.- Same as type 2208, except extra heavy.

\begin{tabular}{|c|c|c|}
\hline $\begin{array}{l}\text { Length } \\
\text { closed }\end{array}$ & $\begin{array}{l}\text { Width of } \\
\text { strap ar } \\
\text { joint }\end{array}$ & $\begin{array}{l}\text { Minimum } \\
\text { thickness } \\
\text { of metal }\end{array}$ \\
\hline $\begin{array}{c}\text { Inches } \\
8 \\
10 \\
12 \\
16\end{array}$ & $\begin{array}{c}\text { Inches } \\
25,8 \\
3 \\
4 \\
41 / 4\end{array}$ & $\begin{array}{r}\text { Inch } \\
0.135 \\
.140 \\
.160 \\
.160\end{array}$ \\
\hline
\end{tabular}

Reversed pad

Type 2212.-Wrought steel, galvanized; with brass pins.

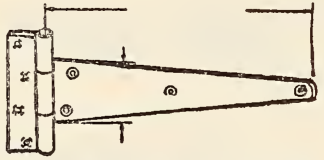

Figure 839.-Wrought steel $\mathrm{T}$ hinge, reversed pad, type 2212

\begin{tabular}{|c|c|c|}
\hline $\begin{array}{c}\text { Length } \\
\text { closed }\end{array}$ & $\begin{array}{c}\text { Width of } \\
\text { strap at } \\
\text { joint }\end{array}$ & $\begin{array}{c}\text { Minimum } \\
\text { thickness } \\
\text { of metal }\end{array}$ \\
\hline Inches & Inches & Inch \\
8 & 3 & 0.135 \\
10 & $31 / 2$ & .140 \\
12 & 378 & .160 \\
16 & 378 & .160 \\
\hline
\end{tabular}

Scuttle Kinges

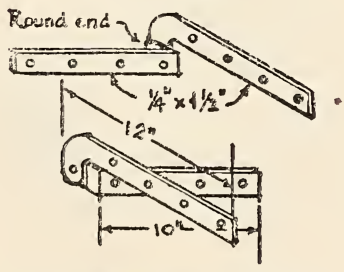

Figure 840.-Wrought iron scuttle hinge, type 2240
Type 2240.-Wrought iron, unfinished. Dimensions as shown. Right and left hand in pairs.

Type 2240 A. - Same as type 2240, except wrought iron or wrought steel, japanned.

Type 2240B.-Same as type 2240, except galvanized.

\section{Spring 푤es \\ Butt type}

Type 2330.-Wrought bronze. Double acting. For hanging strip. Covered, adjustable tension, coil springs. Antifriction washers, or ball bearings. Ball tips.

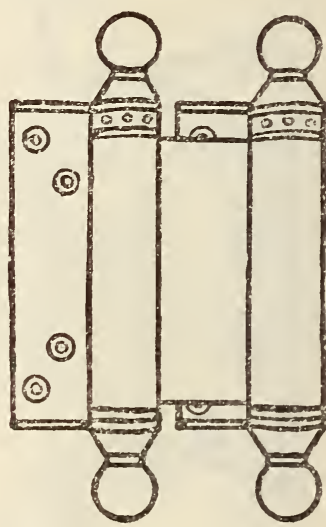

Frgure 841.-Wrought bronze spring hinge, butt type, type 2330

\begin{tabular}{|c|c|c|c|c|c|}
\hline \multirow{2}{*}{$\begin{array}{c}\text { Size } \\
\text { length } \\
\text { of flange }\end{array}$} & \multirow{2}{*}{$\begin{array}{c}\text { Minimum } \\
\text { thickness } \\
\text { of flange }\end{array}$} & \multirow{2}{*}{$\begin{array}{l}\text { Minimum } \\
\text { diameter } \\
\text { of barrel }\end{array}$} & \multirow{2}{*}{$\begin{array}{l}\text { Door, } \\
\text { thickness }\end{array}$} & \multicolumn{2}{|c|}{$\begin{array}{l}\text { Door, maxi- } \\
\text { mum width }\end{array}$} \\
\hline & & & & Feet & Inches \\
\hline $\begin{array}{c}\text { Inches } \\
3 \\
4 \\
5 \\
6 \\
7 \\
8 \\
10 \\
12\end{array}$ & $\begin{array}{r}\text { Inch } \\
0.078 \\
.081 \\
.091 \\
.102 \\
.114 \\
.128 \\
.144 \\
.162\end{array}$ & $\begin{array}{l}\text { Inches } \\
0.875 \\
.875 \\
1.00 \\
1.094 \\
1.219 \\
1.375 \\
1.50 \\
1.75\end{array}$ & $\begin{array}{l}\text { Inches } \\
3 / 4-1 \\
7 / 8-11 / 4 \\
11 / 8-11 / 2 \\
11 / 4-13 / 4 \\
13 / 8-2 \\
11 / 2-21 / 4 \\
13 / 4-21 / 2 \\
21 / 4-3\end{array}$ & $\begin{array}{l}2 \\
2 \\
2 \\
2 \\
2 \\
2 \\
3 \\
3\end{array}$ & $\begin{array}{r}2 \\
4 \\
6 \\
8 \\
9 \\
10 \\
0 \\
2\end{array}$ \\
\hline
\end{tabular}

Type 2330A.-Same as type 2330, except wrought steel, plated.

Type 2331.- Same as type 2330, except single acting. Wrought bronze.

Type 2331A.- Same as type 2330, except single acting, wrought steel, plated.

Floor Surface Spring Einges

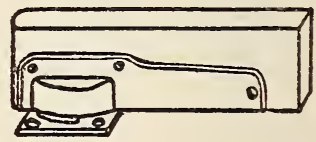

Figure 842.-Bronze mounted floor surface spring hinge, horizontal type, type 2334

Type 2334.-Double acting. Parts exposed to view, bronze. Hinge applied to bottom edge of door, with side plates to cover hinge mechanism. Socket and top pivot shall be provided. Weight of door carried on hardened steel balls and raceways. 
Parts subjected to thrust of spring to be casehardened steel, or malleable iron. Adjustable door alignment. Adjustable or nonadjustable spring tension acceptable. Automatic hold back when door is opened at or past $90^{\circ}$.

Size I. For doors $1 \frac{1}{8}$ inches to $13 / 4$ inches thick.

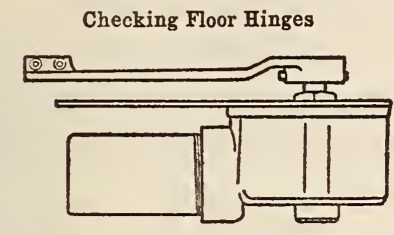

Figure 843.-Bronze mounted checking floor hinge, type 2350

Type 2350.-Liquid-controlled type, double acting, centrally pivoted under door. Mechanism set in floor. Exposed parts bronze. Where necessary, furnish separate outer case to be built into floor. Mechanism to operate in nonfreezing, lubricating, checking fluid. Closing speed controlled by accessible needle valve. Means for adjusting door alignment. Top pivot adjustable by screw. Automatic hold open unless nonhold open is specified:

Size I. For light, interior doors not over 2 feet 8 inches wide.

Size II. For medium weight interior doors not over 2 feet 10 inches wide.

Size III. For medium weight interior doors not over 3 feet 0 inches wide.

Type 2350A.- Liquid-controlled type, double acting, centrally pivoted under door. Mechanism set in floor. Where necessary, furnish separate outer case to be built into floor. Exposed parts bronze. Mechanism to operate in nonfreezing, lubricating, checking fluid. Ball-bearing pivot for carrying weight of door. Closing speed controlled by accessible needle valve. Top pivot adjustable by screw. Nonhold open type only.

Size I. For extra heavy or wide interior doors.

For ordinary entrance and vestibule doors not over 2 feet 10 inches wide.

Size II. For heavy entrance and vestibule doors over 2 feet 10 inches wide.

Type 2350B.- Same as type $2350 \mathrm{~A}$, but for singleacting doors. Nonhold open unless hold open is specified.

Size I. For light interior doors not over 3 feet 0 inches wide.

Size II. For medium and heavy interior doors not over 3 feet 6 inches wide.

For entrance and vestibule doors not over 2 feet 10 inches wide.

Size III. For heavy entrance and vestibule doors over 2 feet 10 inches wide.

Type 2352.-Liquid-controlled offset pivot type, single acting. Mechanism set in floor. Where necessary, furnish separate outer case to be built into floor. Exposed parts bronze. Mechanism to operate in nonfreezing, lubricating, checking fluid. Ball-bearing pivot for carrying weight of door. Closing speed controlled by accessible needle valve. Nonhold open unless hold open is specified.

Size I. For light interior doors not over 3 feet 0 inches wide.

Size II. For medium and heavy interior doors not over 3 feet 6 inches wide.

For entrance and vestibule doors not over 2 feet 10 inches wide.

Size III. For heavy entrance and vestibule doors over 2 feet 10 inches wide.
Lavatory Door Sprin \& Hinges

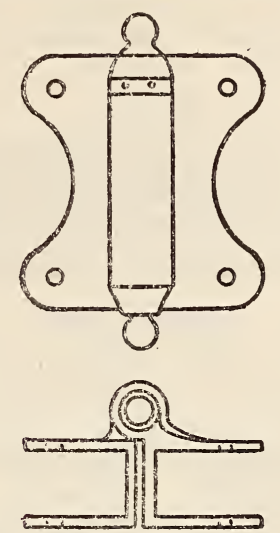

FI G U R E 844 . -

Bronze lavatory

door spring

hinge, type 2360

Type 2360.-Bronze, nickel plated. Single acting; self-opening, unless self-closing is specified. Covered, adjustable tension, coiled springs. Box flanges for $1 \frac{1}{4}$-inch stiles, adjustable for $1 \frac{1}{8}$ to $13 / 8$ inches. Size 4 inches (length of flange). Minimum thickness of metal at flanges, 0.090 inch. Fastenings, through bolts with rounded hexagonal heads and cap nuts.

Type $2360 A$.-Same as type 2360 , except nickel bronze.

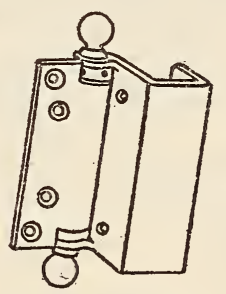

Figure 845 . -

Bronze single-

action clamp

flange spring

hinge, type 2362

Type 2362.-Four-inch single-action clamp flange spring hinge for partitions without hanging stile and with doors opening in. To hold door open (reverse acting) unless otherwise specified. Bronze, nickel plated. One, nonadjustable clamp flange. One butt flange. Barrel seven-eighth inch minimum outside diameter. Minimum thickness of metal at flanges 0.090 inch. Fastenings for clamp flanges, sleeve bolts with flat-head screws. For partition thickness $1,1 \frac{1}{4}, 1 \frac{1}{2}$, and $13 / 4$ inches.

Type 2362 A.-Same as type 2362 , except nickel bronze.

Type 2363.-Same as type 2362, except with two butt flanges for two doors opening in.

Type 2363A.- Same as type 2362 , except of nickel bronze with two butt flanges for two doors opening in.

Type 2364.- - Same as type 2362, except with one wall flange in place of clamp flange. Fastenings for wall flange, bolts or wood screws as required. 
Type 2364A.-Same as type 2362, except of nickel bronze with one wall flange in place of clamp flange. Fastenings for wall flange, bolts screws as required.

617.13 HASPS AND STAPLES.

UNITED STATES GOVERNMENT, DEPARTMENT OF COMMERCE; BUILDERS' HARD. WARE (NONTEMPLATE), COMMERCIAL STANDARD CS22-30, DECEMBER 11, 1930

\section{HINGE HASPS}

(See 617.0, p. 977 also Federal Specification FF-H-101 below.

UNITED STATES GOVERNMENT, FEDERAL SPECIFICATIONS BOARD; FEDERAL SPECIFICATION FOR BUILDERS' HARDWARE (NONTEMPLATE), FF-H-101, AUGUST 19, 1930

(For types, grades, and classes; material and workmanship; general requirements; detail requirements; packaging, packing, and marking; notes; and table of standard finishes, see 617.0 p. 978 . For chromium plating, nickel plating, and zinc coatings; and methods of inspection and tests, see 600.3, p. 70 .)

\section{BUILDERS' HARDWARE (NONTEMPLATE)} IINTGE HASPS

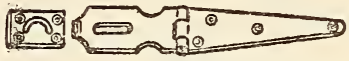

Frgure 846.-Unfinished steel hinge hasp, type 1401

Type 1401.-Open pattern. Unfinished steel.

\begin{tabular}{l|r|r}
\hline Length closed (inches) & Size of screws & $\begin{array}{r}\text { Minimum } \\
\text { thickness } \\
\text { of metal }\end{array}$ \\
\hline & $\begin{array}{r}\text { Inch } \\
0.069 \\
6 / 4 \text { inch by No. } 6\end{array}$ & .084 \\
\hline 8 & .096 \\
\hline
\end{tabular}

Type 1401A.- Same as type 1401, except galvanized steel.

Type 1420.- Safety pattern. Unfinished steel. Adjustable staple. Staple shall be rigidly secured to plate.

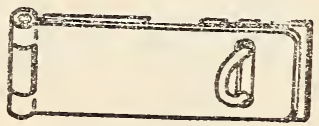

Figure 847.-Unfinished hinge hasp, type 1420

\begin{tabular}{c|r|r}
\hline Length closed (inches) & Size of screws & $\begin{array}{r}\text { Minimum } \\
\text { thickness } \\
\text { of metal }\end{array}$ \\
\hline & $\begin{array}{l}\text { Inch } \\
\text { 3/4 inch by No. } 7\end{array}$ \\
\hline 7 inch by No. 9 & .072 \\
\hline
\end{tabular}

Type 1420A.- Same as type 1420, except galvanized steel.
617.2 LOCKS, LATCHES, AND LOCK PARTS. 617.21 LOCKS.

AMERICAN MARINE STANDARDS COMMITTEE, LOCKS AND ACCESSORIES FOR SHIP DOORS, H NO. $61-1930$

\section{SHIP-DOOR LOCKS}

Section I. STAMDARD TYPES AND VARIATIONS

Type A. Rin Lock, Rezular Bevel, for Ordinary Wood Doors
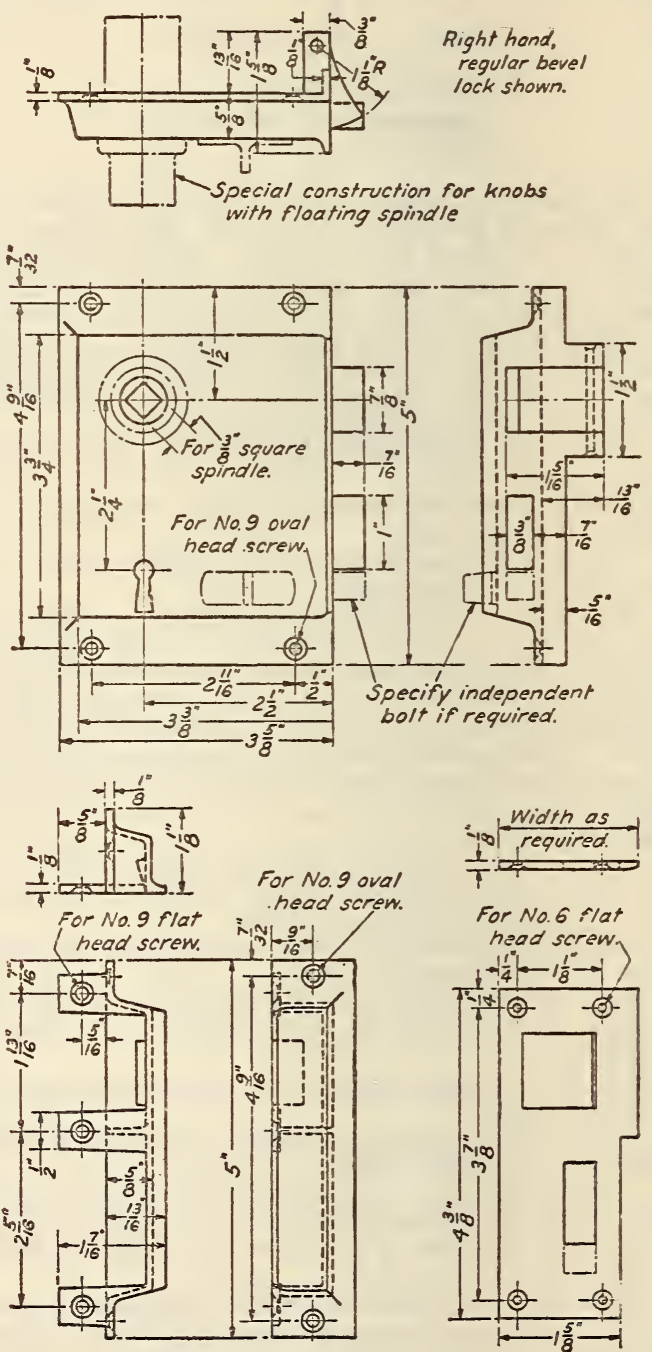

FIgURE 848.-Rim lock, type $A$, regular bevel

This lock has a nonreversible swinging latch and must be ordered for either right or left hand.

Application.-Interior doors which swing in to the space that they close.

Tumblers and key changes.-Either one tumbler capable of at least 40 changes to be operated by one master key, or three tumblers capable of at least 240 changes to be operated by one master key, as ordered. Keeper.-Box or plate type, as ordered.

Escutcheons.-One escutcheon plate, 2 by 6 inches, as per Diagrams 1 or 2 , or one rosette and key escutcheon, Diagrams 3 and 4, Figure 862, as ordered. 
Knobs.-One knob of each type, Diagrams 5 and 6 , Figure 863 .

Finish.-Lock and accessories shall be polished and lacquered on exposed surfaces.

Special features not included in standard lock set.(a) Special bolt. - This lock may be ordered with an independent bolt as indicated on Figure 848 .

(b) Optional type of accessories.-Ring handles, or a combination of one ring handle and one knob of appropriate types to be identified by Diagrams 5 to 10 on Figure 863, may be ordered in lieu of the standard knob set. This type of lock may also be ordered with knobs attached direct to the lock with floating spindle as per typical Diagram 11 on Figure 864 .

\section{Type B. Rim Lock, Reverse Bevel, for Ordinary Wood Dcors}

This lock has a nonreversible swinging latch and must be ordered either right or left hand.
Special features not included in standard lock set.(a) Special bolt. - This lock may be ordered with an independent bolt as indicated on Figure 849 .

(b) Optional type accessories.-Ring handles, or a combination of one ring handle and one knob of appropriate types to be identified by diagrams on Figure 863, may be ordered in lieu of the standard knob set. This type of lock may also be ordered with knobs attached direct to the lock with floating spindle as per typical diagram 11 on Figure 864.

Type C. Rim Lock, Reverse Bevel, for Heavy Wood Doors

This lock has a nonreversible swinging latch and must be ordered for either right or left hand.

Application.--Exterior doors which swing outward from the space that they close.

Tumblers and key changes.-One or more tumblers capable of at least 40 changes to be operated by one master key.

Keeper.-Angle type only.
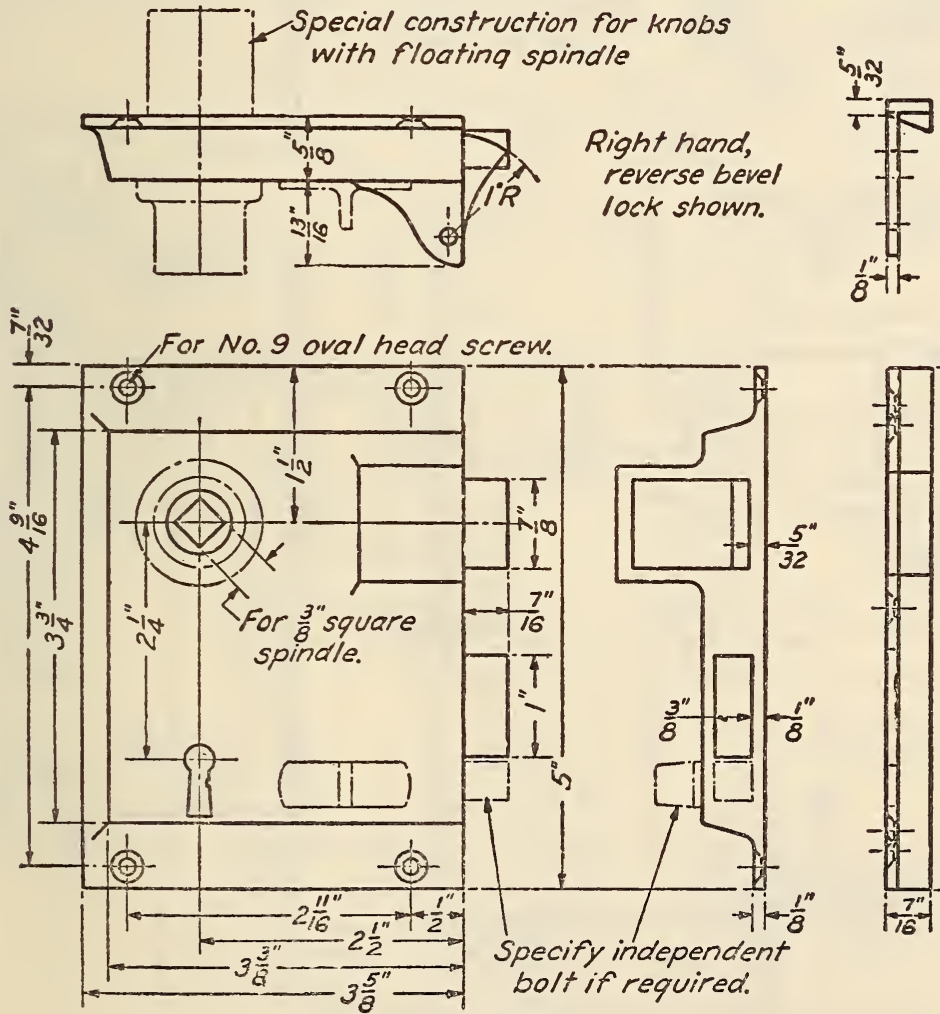

Figure 849.-Rim lock, Type $B$, reverse bevel

Application.-Interior doors which swing outward from the space that they close.

Tumblers and key changes.-Either one tumbler capable of at least 40 changes to be operated by one master key, or three tumblers capable of at least 240 ehanges to be operated by one master key, as ordered.

Keeper.-Angle type only.

Escutcheons.-One escutcheon plate, 2 by 6 inches, Diagrams 1 or 2 , or one rosette and key escutcheon, Diagrams 3 and 4 , Figure 862, as ordered.

$K$ nobs.-One knob of each type, Diagrams 5 and 6 , Figure 863.

Finish.-Lock and accessories shall be polished and lacquered on exposed surfaces.
Escutcheons.-One escutcheon plate, 2 by 6 inches, Diagrams 1 or 2, or one rosette and key escutcheon, Diagrams 3 and 4, Figure 862, as ordered.

Knobs.-One knob of each type, Diagrams 5 and 6 , Figure 863.

Finish.-Lock and accessories shall be polished and lacquered on exposed surfaces.

Special features not included in standard lock set.(a) Special bolt. - This lock may be ordered with an independent bolt as indicated on Figure 850 .

(b) Optional type of accessories.-Ring handles, or a combination of one ring handle and one knob of appropriate types to be identified by diagrams on Figure 863 , may be ordered in lieu of the standard knob set. This type of lock may also be ordered with knobs attached direct to the lock with floating spindle as per typical Diagram 11 on Figure 864. 
Type D. Rim Lock for Steel-Plate Doors, Unfinished

This lock has a reversible sliding spring latch only normally operated by knobs and locked by key.

Application.-Ordinary steel-plate doors where finished appearance is unimportant.

Tumblers and key changes.-One or more tumblers capable of at least 24 changes to be operated by one master key.

Keeper.-Box or plate type, as ordered.

Escutcheon.-None to be furnished unless specifically ordered. 851.

$K$ nobs.-Two special $\mathrm{T}$ knobs shown on Figure
Knobs.-For metal doors, two knobs, Diagram 5; for wood doors, one knob, Diagram 5 and one Diagram 6, Figure 863.

Finish.-Lock and accessories shall be polished and lacquered all over, except the bottom edge of the lock, which may be rough dipped only. Locks required for specific locations may be ordered with the side next to the door machined only for installation and unpolished.

Optional ring handles.-A pair of ring handles, or a combination of one ring handle and one knob of appropriate types to be identified by diagrams on Figure 863 , may be ordered instead of the standard knob set.

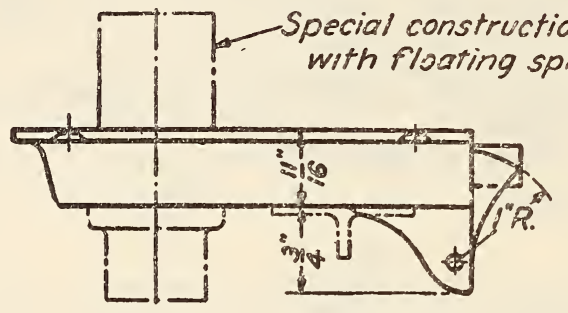

Right hand, reverse bevel lock shown.
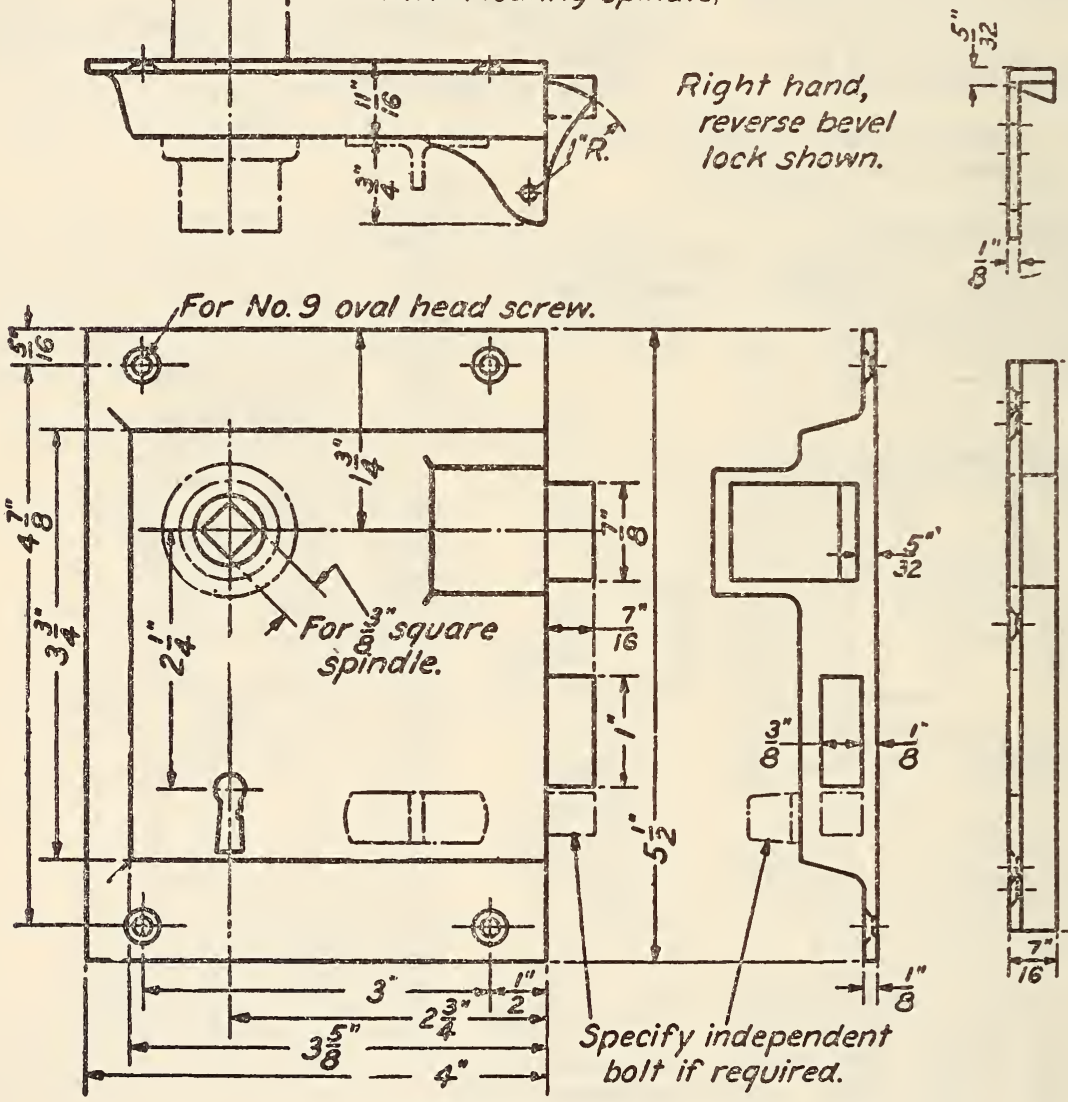

\section{for knoiss}

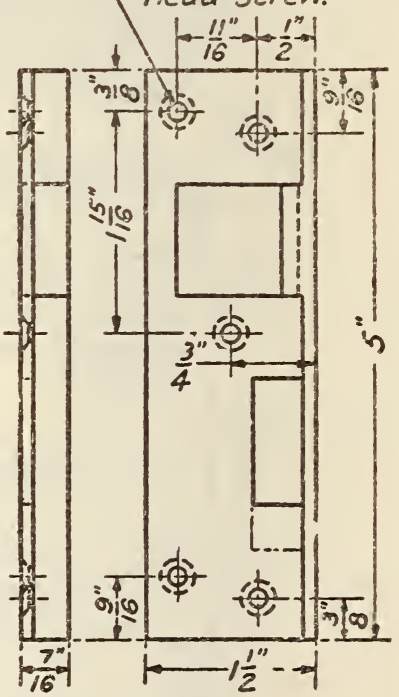

Figure 850.-Rim lock, type $C$, reverse bevel

Finish.-Lock and accessories shall be machined only where necessary for installation. Other surfaces shall be rough dipped.

Type ¿. Rim Lock, Reversıble, for Oräinary Metal Doors

Application.-Comparatively light steel-plate doors where finished appearance is required. May be used also on wood doors where finished appearance is unessential.

Tumblers and key changes.-One or more tumblers capable of at least 60 changes to be operated by one master key.

Keeper.-Box, angle, or plate type, as ordered.

Escutcheons.-None required for metal doors unless specifically ordered. Locks for wood doors require one escutcheon plate, 2 by 6 inches, Diagrams 1 or 2 , or one rosette and key escutcheon, Diagrams 3 and 4 , Figure 862 , as ordered.
Type F. Rim Lock, Reversible, for Yieavj Metal Doors

Application.-Heavy steel-plate doors where finished appearance is required. May be used also on wood doors where finished appearance is unessential.

Tumolers and key changes.-One or more tumblers capable of at least 60 changes to be operated by one master key.

Keeper.-Box, angle, or plate type, as ordered.

Escutcheons.-None required for metal doors unless specifically ordered. Locks for wood doors require one escutcheon plate, 2 by 6 inches, Diagrams 1 or 2 , or one rosette and key escutcheon, Diagrams 3 and 4, Figure 862, as ordered.

Knobs.-For metal doors, two knobs, Diagram 5; for wood doors, one knob, Diagram 5, and one, Diagram 6, Figure 863. 

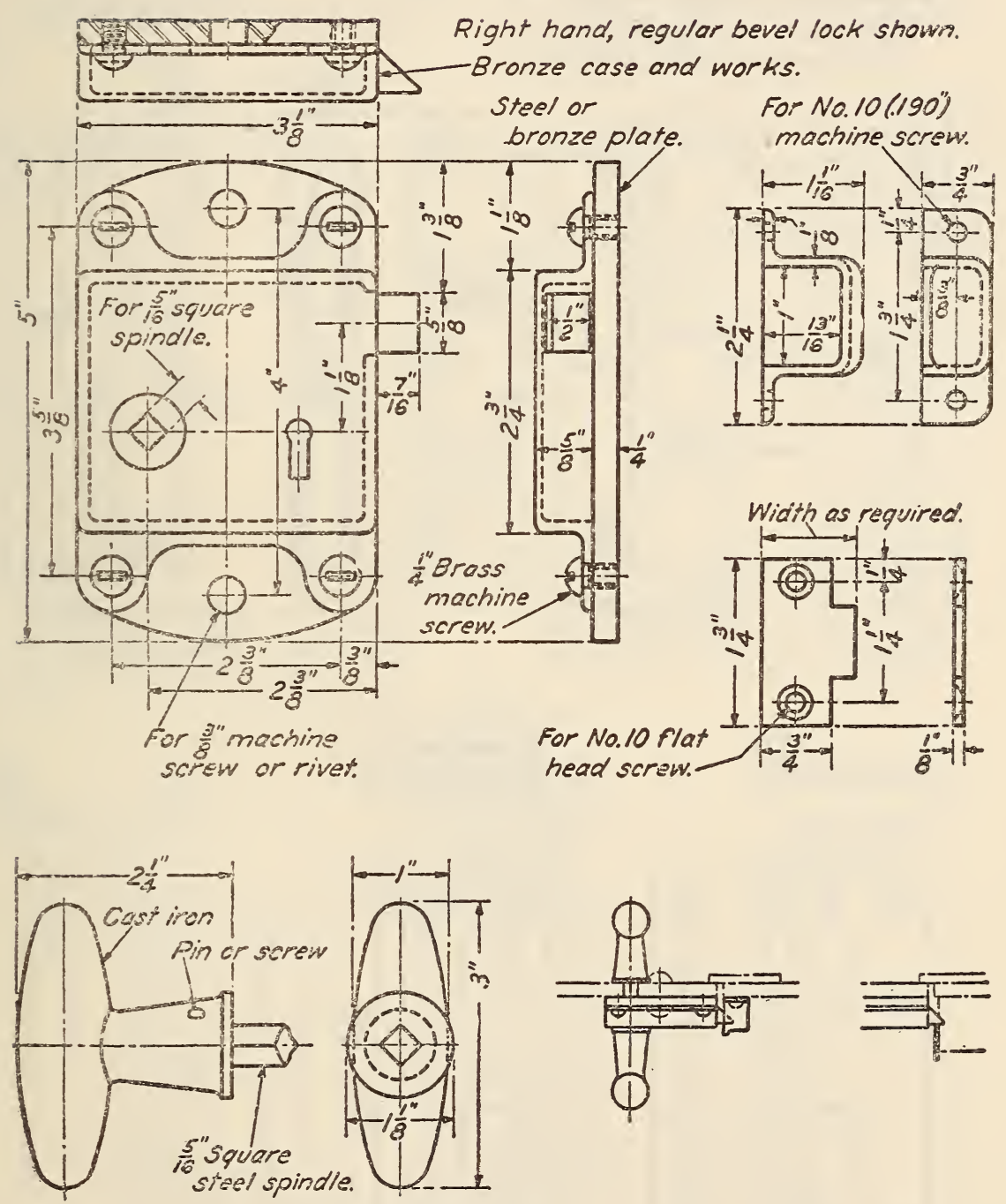

Figdre 851.-Rim lock, Type D, unfinished 

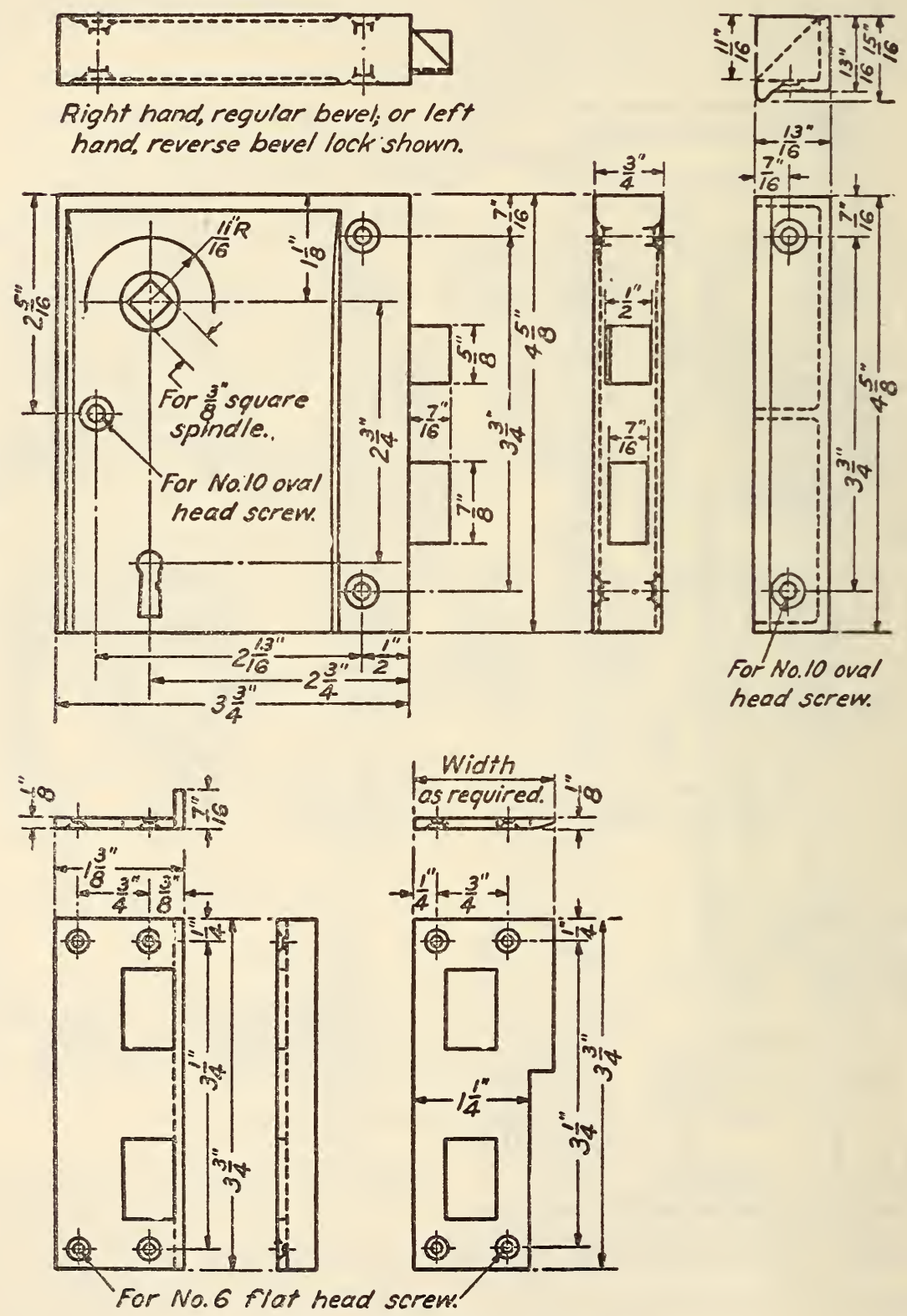

FIGURE 852.-Rim lock, type $E$ 

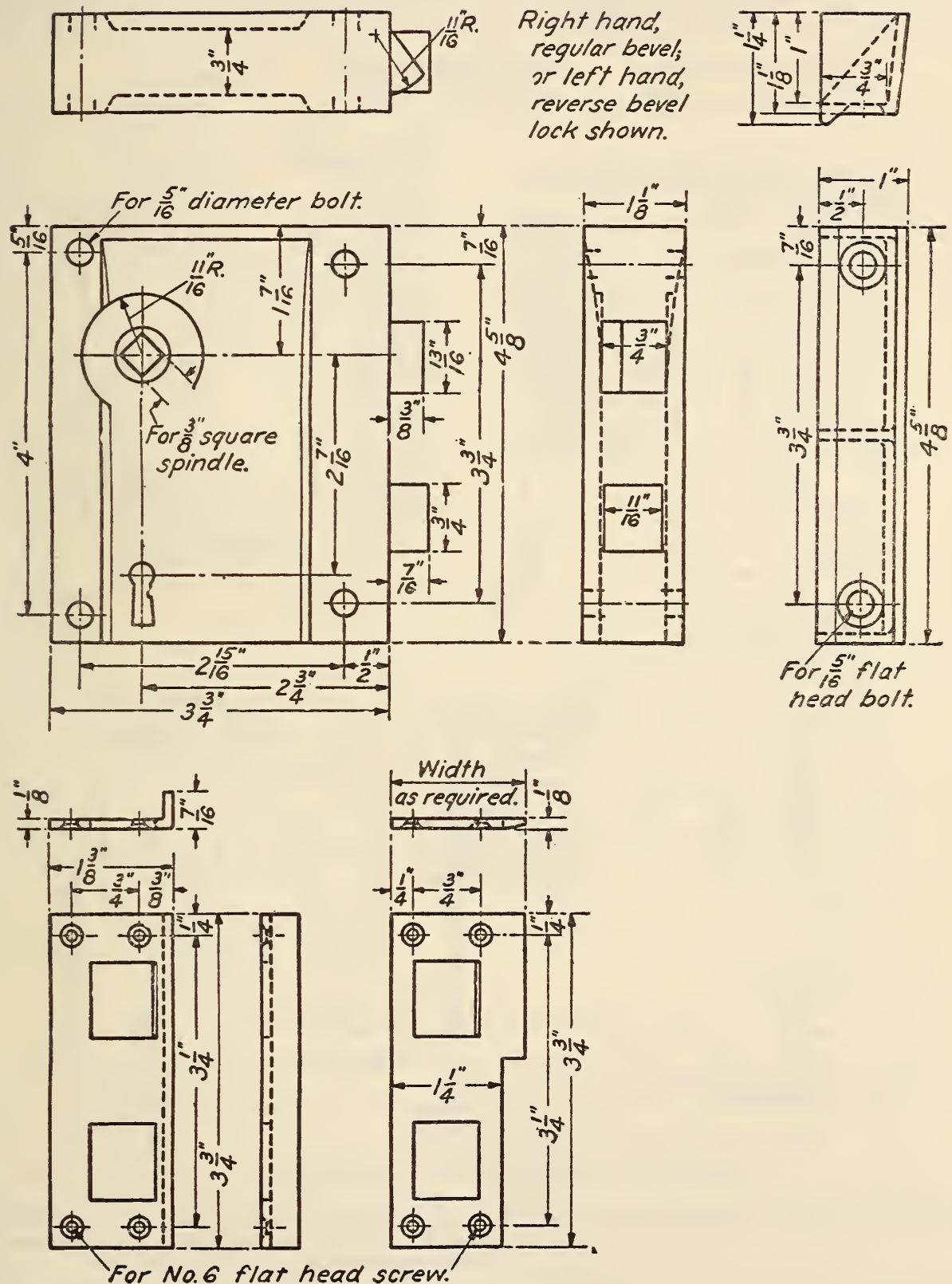

FIGURE 853.-Rim lock, type F 
Finish-Lock and accessories shall be polished and lacquered all over, except the bottom edge of the lock which may be rough dipped only. Locks required for specific locations may be ordered with the side next to the door machined only for installation and unpolished.

Optional ring handles.-A pair of ring handles, or a combination of one ring handle and one knob of appropriate types to be identified by diagrams on
Finish.-The lock shall be machine finished only where necessary for its proper installation. The exposed surfaces of escutcheon and knob shall be polished and lacquered.

Type R. Rim Lock, Rererse Zerel, for \$mail Locker and Cupboard ícot's

This lock has a nonreversible sliding spring latch only, normally operated by knob and locked by key. It must be ordered for either right or left hand.

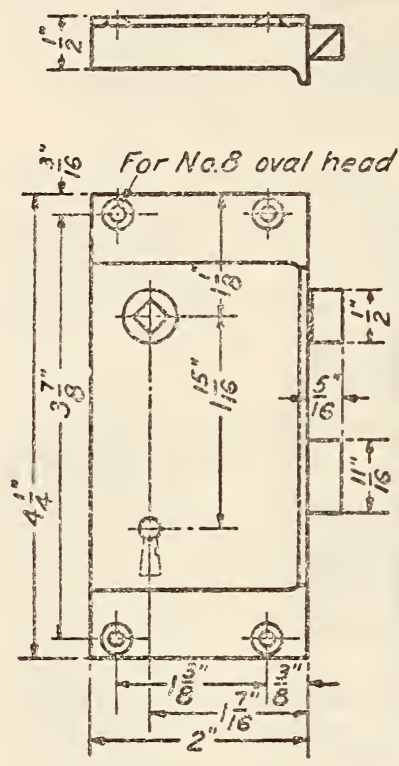

\section{Right hand, reverse bevel lock shown.}
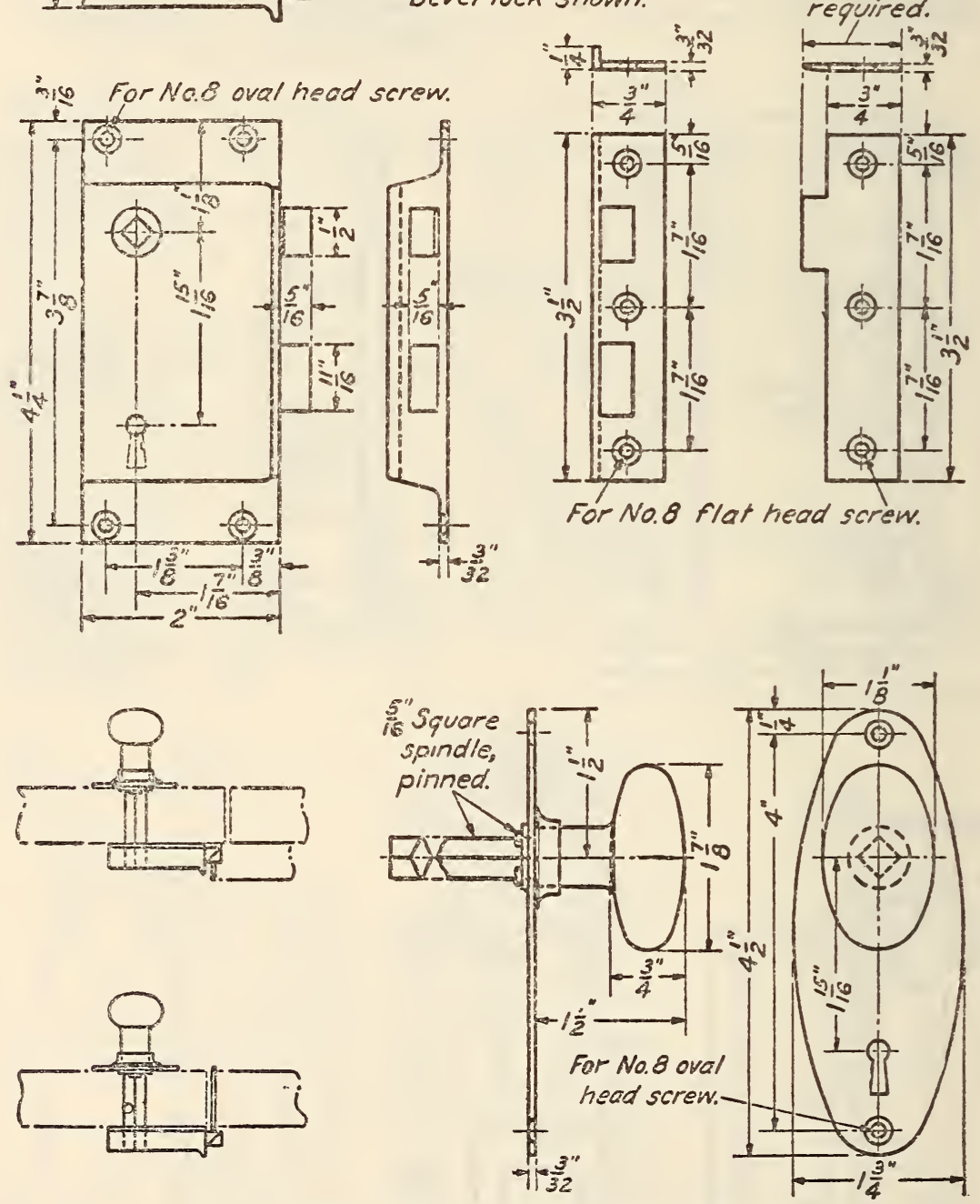

Figure 854.-Rim lock, type G, reverse bevel

Figure 863 , may be ordered instead of the standard knob set.

Type G. Rim Lock, Eeverse Bevol, for Wardrcbe Doors

This lock has a nonreversible sliding latch and must be ordered for either right or left hand.

Application.-Wood doors to wardrobes, lockers, or similar spaces which swing outward.

Tumblers and key changes.- Tumblers capable of at least 240 changes to be operated by one master key.

Keeper.-Angle or plate type, as ordered.

Escutcheon and knob. - Special escutcheon plate with knob attached as shown on Figure 854.
Application.-Wood doors on wardrobes of the smaller size and locker and cupboard doors which swing outward.

Tumblers and key changes.-Tumblers capable of at least 240 changes to be operated by one master key.

Keeper.-Angle or plate type, as ordered.

Escutcheon and knob.- Special escutcheon plate with knob attached as shown on Figure 855.

Finish.-The lock shall be machine finished only where necessary for its proper installation. The exposed surfaces of escutcheon and knob shall be polished and lacquered. 
Type I. Rim Latch, Reversible, for Light Wood Doors

Application.-Principally wood screen doors and lavatory-stall doors.

Keeper.-Box or plate type, as ordered. (Note alternative plate types.)

Rosette and knobs.-One rosette and two knobs of special type shown on Figure 856.

Finish.-All exposed surfaces of lock and accessories shall be polished and lacquered.
Optional ring handles.-A pair of ring handles, or a combination of one ring handle and one knob of appropriate types to be identified by diagrams on Figure 863 , may be ordered instead of the standard knob set.

Type $\mathrm{z}$. Mortise Iock, Reversible, for Heavy Wood Doors

Application.-Heavy interior wood doors of thicknesses over $1 \frac{1}{4}$ inches.

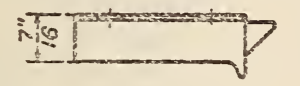

\section{Right hand, reverse bevel lock shown.}
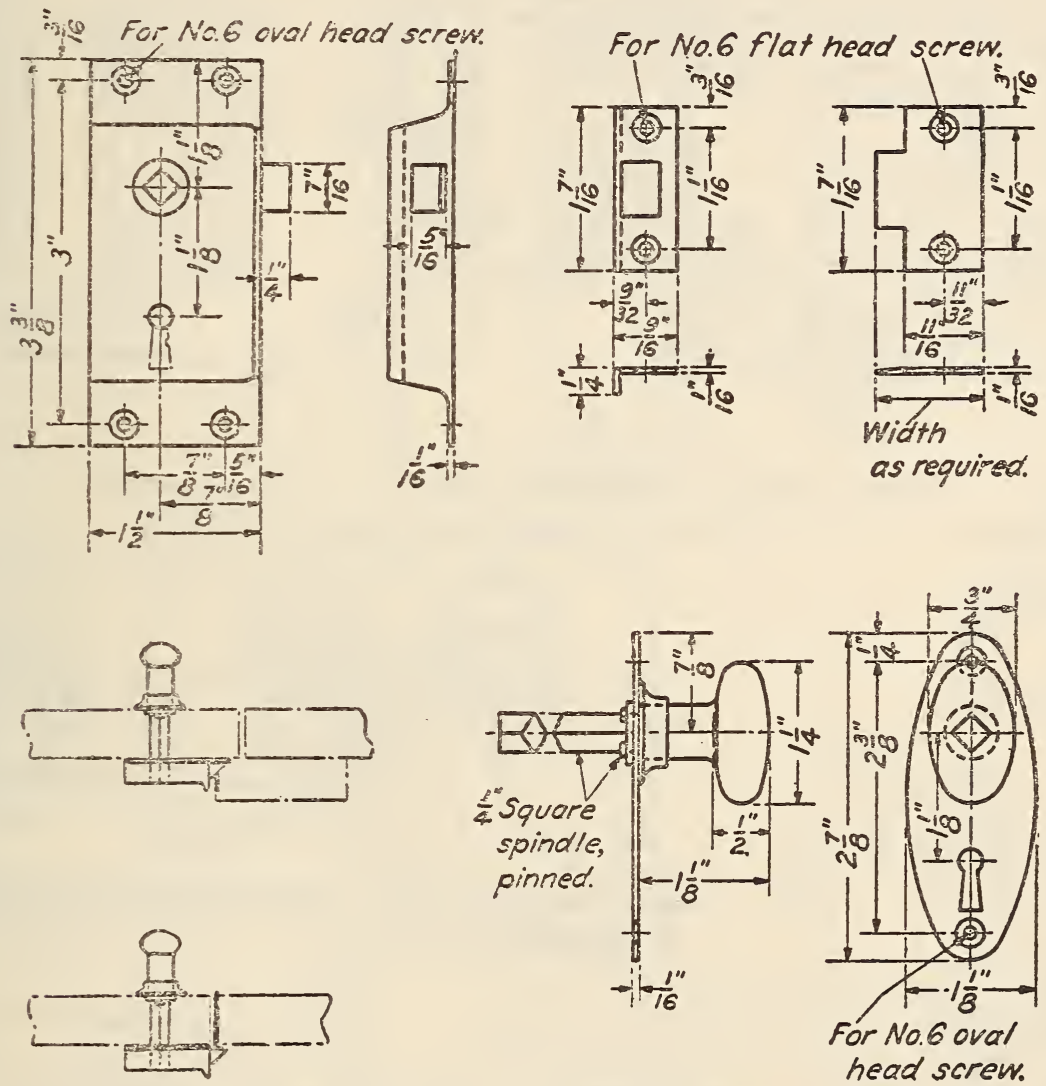

FIGURE 855.-Rim lock, type $H$, reverse bevel

Trpe I. Martise Lock, Reversikle, for Light Wood Doors

Application.-Interior wood doors of minimum thickness of $1 \frac{1}{8}$ inches.

Tumblers and key changes.-Either one tumbler capable of at least 24 changes to be operated by one master key, or three tumblers capable of 72 changes to be operated by one master key. (This type of lock can be made with tumblers capable of at least 200 changes for one master key in groups up to 72 locks.)

Keeper.-Reversible-plate type only.

Escutcheons.-Two escutcheon plates, 2 by 6 inches, both of which may be of type with low collar, Diagram 6, or one of each of types, Diagrams 5 and 6. Rosettes and key escutcheons may be ordered in lieu of escutcheon plates. All to be as shown on Figure 862.

Knobs.-Two knobs as per Diagram 6, Figure 863.

Finish.-The exposed surfaces of the lock and accessories shall be polished and lacquered.
Tumblers and key changes.-One or more tumblers capable of at least 40 changes to be operated by one master key.

Keeper.-Double-edged plate type only.

Escutcheons.-Two escutcheon plates, 2 by $65 / 8$ inches, both of which may be of type with low collar, Diagram 6, or one of each of types, Diagrams 5 and 6. Rosettes and key escutcheons may be ordered in lieu of escutcheon plates. All to be as shown on Figure 862.

Knobs.-Two knobs, Diagram 6, Figure 863.

Finish.-The exposed surfaces of the lock and accessories shall be polished and lacquered.

Special features not included in standard lock set.Ring handles or a combination of one ring handle and one knob of appropriate types to be identified by diagrams on Figure 863, may be ordered in lieu of the standard knob set. This type of lock may also be ordered with knobs attached direct to the lock with floating spindle as shown by Diagram 12 on Figure 864. 

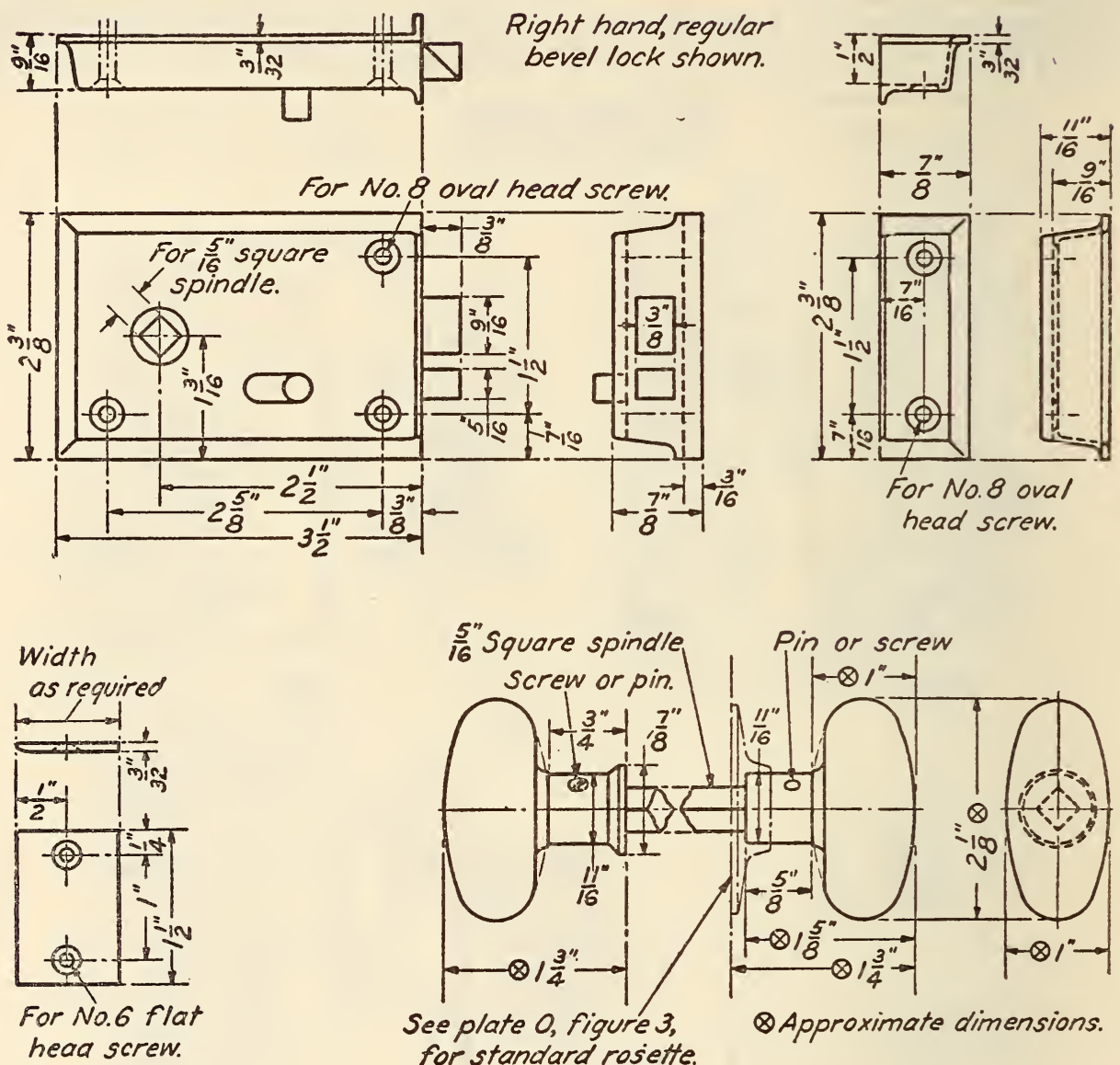

head screw.

See plate 0 , figure 3 ,

for standard rosette.

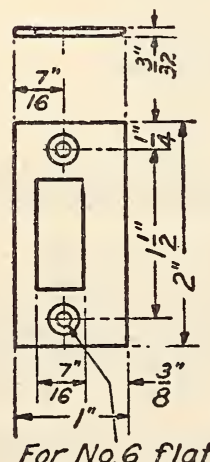

head screw.

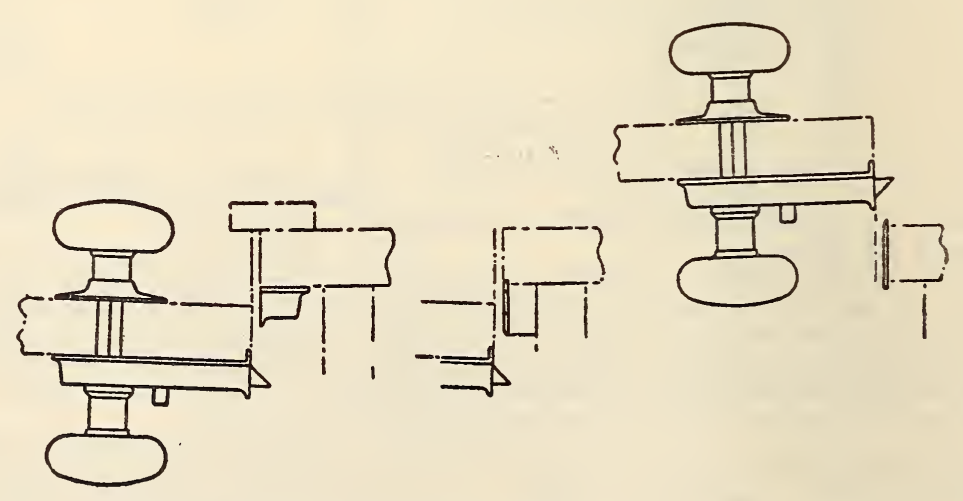

FIGURE 856.-Rim latch, type I 


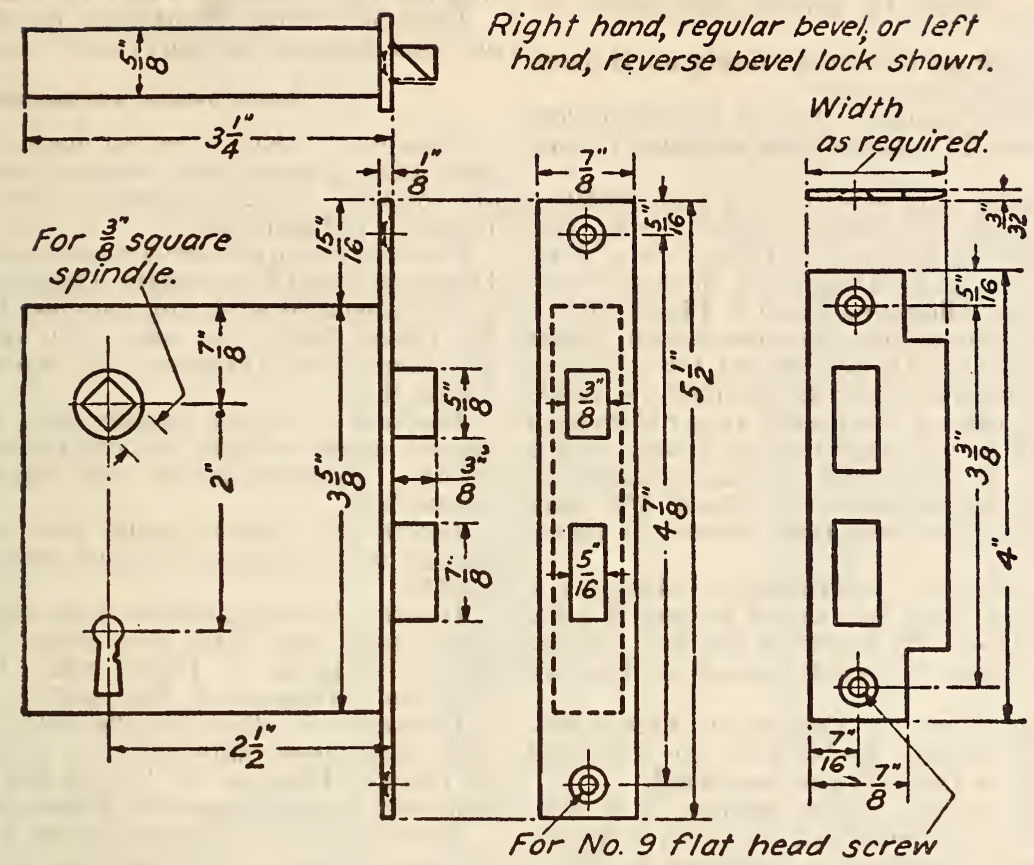

FIGURE 857.-Mortise lock, type J

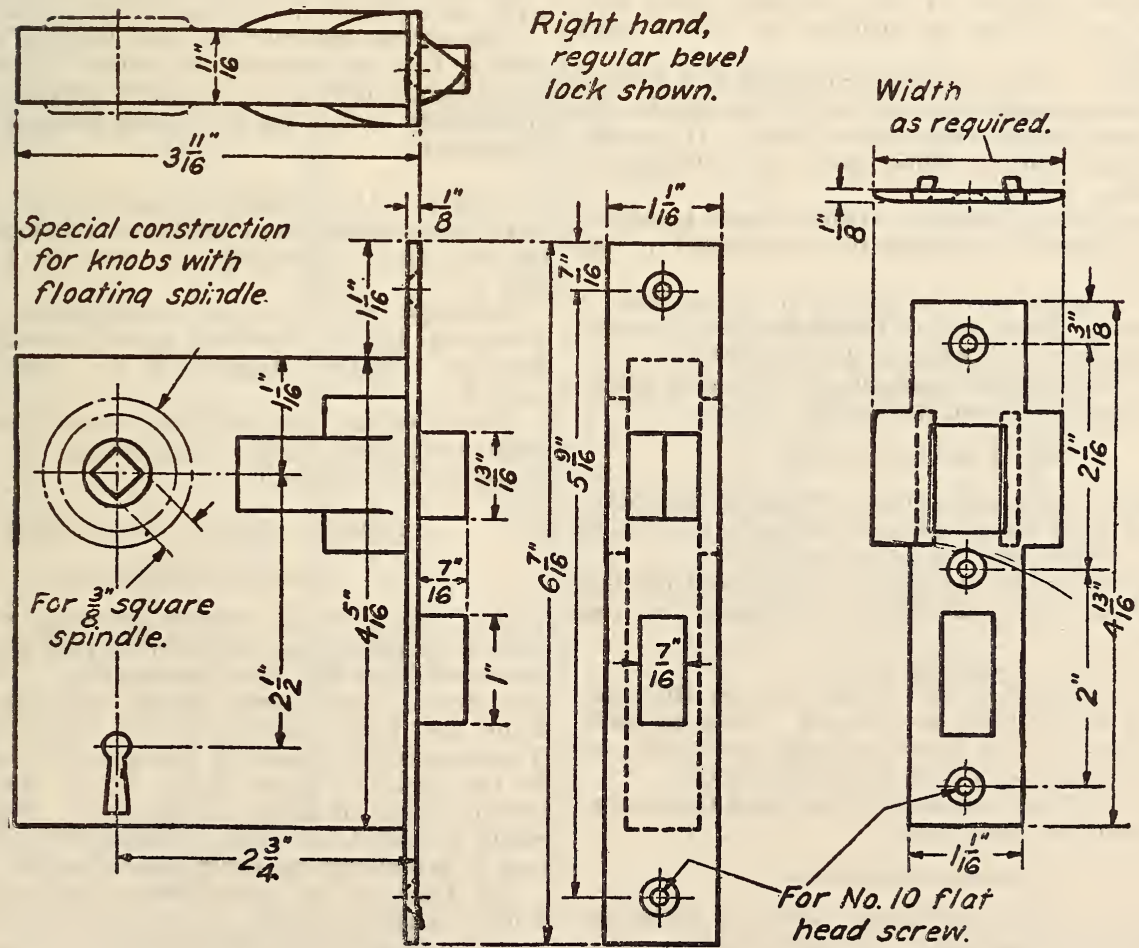

FIGURE 858.-Mortise lock, type $K$ 


\section{Type I. Mortise Lock for Sliding Doors} key.

Hook latch operated by spindle and locked by

Application.-Single or double sliding wood doors of thickness $13 / 8$; inches or over.

Tumblers and key changes.-One or more tumblers capable of at least 24 changes to be operated by one master key.

Keeper.-Special form as shown on Figure 859.

Escutcheons.-(a) For single sliding doors, two standard escutcheon plates 2 by $65 \%$ inches of type with low collar shown by Diagram 2 , or one of each of types shown by Diagrams 1 and 2, Figure 862 .

(b) For double doors, identical escutcheon plates for use with knobs may be ordered for both the active and the inactive door as specified for single doors. In such case, a dead plate as per sketch on Figure 859 shall be furnished for the spindle on the inactive door. If preferred, special escutcheon plates with pull recess, shown on Figure 859, may be ordered for the inactive door instead of plates with knobs.

(c) Rosettes and key escutcheons, Diagrams 3 and 4, Figure 862 , may be ordered instead of plate escutcheons for use with knobs or handles. These require a dead plate for knob spindle of inactive door.

Knobs.-Type shown by Diagram 6, Figure 863.

Finish.-The exposed surfaces of the lock and accessories shall be polished and lacquered.

Optional ring handles.-Ring handles, or a combination of one ring handle and one knob of appropriate types, to be identified by diagrams on Figure 863 , may be ordered in lieu of the standard knob set.

Type M. Hali-Mortise Lock, Nonreversıble, for Sliding Doors

Hook latch operated by lever in hand recess and locked by key. Must be ordered for either right or left hand.

Application.-This lock has no knobs or handles; its latch is operated by levers set in recesses in the lock case and special escutcheon plate. It is suitable for single sliding wood doors of thickness 1 inch or over.

Tumblers and key changes.-One or more tumblers capable of at least 24 changes to be operated by one master key.

Keeper.-Special form as shown on Figure 860

Escutcheon.-Plate with a recess for lever-handleengaging latch as indicated on Figure 860.

Finish.-All exposed surfaces of lock and accessories to be polished and lacquered.

\section{Type N. Mortise Dead Lock}

Application.-Double-acting swinging or other wood doors with a minimum thickness of $11 / 8$ inches on which a spring latch is not required.

Tumblers and key changes.-One or more tumblers capable of at least 12 changes operated by one master key.

Keeper.-Plate type only.

Escutcheons.-Either one or two key escutcheons, Diagram 4, Figure 862, as ordered. (One escutcheon for doors to lock from one side only; two escutcheons for doors locking from either side.)

Finish.-Exposed surfaces of lock and accessories to be polished and lacquered.

\section{Escutcheons and Rosette}

Diagram 1.-Plate escutcheon with low collar for use with knob, Diagram 6, or with ring handles, Diagrams 9 and 10, Figure 863.

Diagram 2.-Plate escutcheon with high collar for use with knob, Diagram 6, Figure 863, where screw or pin securing knob to spindle is not to be exposed.
Diagram 3.-Rosette for knob, Diagram 6, or ring handle, Diagrams 9 and 10, Figure 863.

Diagram 4.-Key escutcheon for use with rosette on wood doors, or without rosette on metal doors.

\section{Knobs, Handles, and Spindles}

Diagram 5.-Knob for use against metal surface (rim lock or metal door) without escutcheon plate or rosette. Interchangeable with ring handle, Diagram 7, Figure 863.

Diagram 6.-Knob for use with escutcheon plates, Diagrams 1 and 2, or rosette, Diagram 3, Figure 862. Interchangeable with ring handles, Diagrams 9 and 10 , Figure 863, when used with escutcheon plate with low collas, Diagram 1, or rosette, Diagram 3, Figure 862.

Diagram 7.-Ring handle with socket for use against metal surface, without escutcheon plate or rosette. Interchangeable with knob, Diagram 5 , Figure 863.

Diagram 8.-Ring handle with spindle for use against metal surface, without escutcheon plate or rosette.

Diagram 9.-Ring handle with socket for use with escutcheon plate with low collar, Diagram 1, or rosette, Diagram 3, Figure 862. Interchangeable with knob, Diagram 6, Figure 863.

Diagram 10.-Ring handle with spindle for use with escutcheon plate with low collar, Diagram 1, or rosette, Diagram 3, Figure 862. Interchangeable with knob, Diagram 6, Figure 863.

Spindles shall be of proper length to suit doors and types of locks. They shall, as far as practicable, be furnished securely attached to one knob or handle, with the other end drilled for pin or tapped for screw in several positions as necessary for adjustment. Bronze or brass washers of diameter matching the shank of the knob or handle shall be furnished with each spindle for intermediate adjustment.

Alternative construction with floating spindle (fig. 864). - This construction is extra to the standards hereinbefore defined and will be supplied only when specifically called for in asking for quotations and in ordering.

Diagram 11.-Typical construction of rim lock with knobs attaching directly to the lock and floating spindle. Applicable to locks of types A, B, and $\mathrm{C}$.

Diagram 12.-Typical construction of mortise lock with knobs attaching directly to the lock and floating spindle. Applicable to mortise lock of type $\mathrm{K}$.

Either of the constructions shown may be used with escutcheon plate and ferrule or with combined rose and ferrule.

\section{Section II.-GENERAI REQUIRENENTS}

\section{Application of Drawings}

(a) The above-mentioned drawings and the following specifications collectively define the marinestandard locks and their accessories. The drawings give general dimensions deemed necessary to determine the types and to obtain interchangeability. The accessories shown on Figures 862 and 863 apply to rim locks of types A, B, C, E, and F, and to mortise locks of types, J, K, and L. Special accessories required for locks of types D, G, H, I, L, M, and $\mathrm{N}$ are shown on their respective figures.

(b) The use of escutcheon plates or rosettes, knobs or ring handles, is optional; the one wanted must be identified by the diagram number in asking for quotations and in ordering.

(c) The styles of accessories shown on Figures 862 and 863 shall be furnished unless otherwise specified in asking for quotations and in ordering. Different 

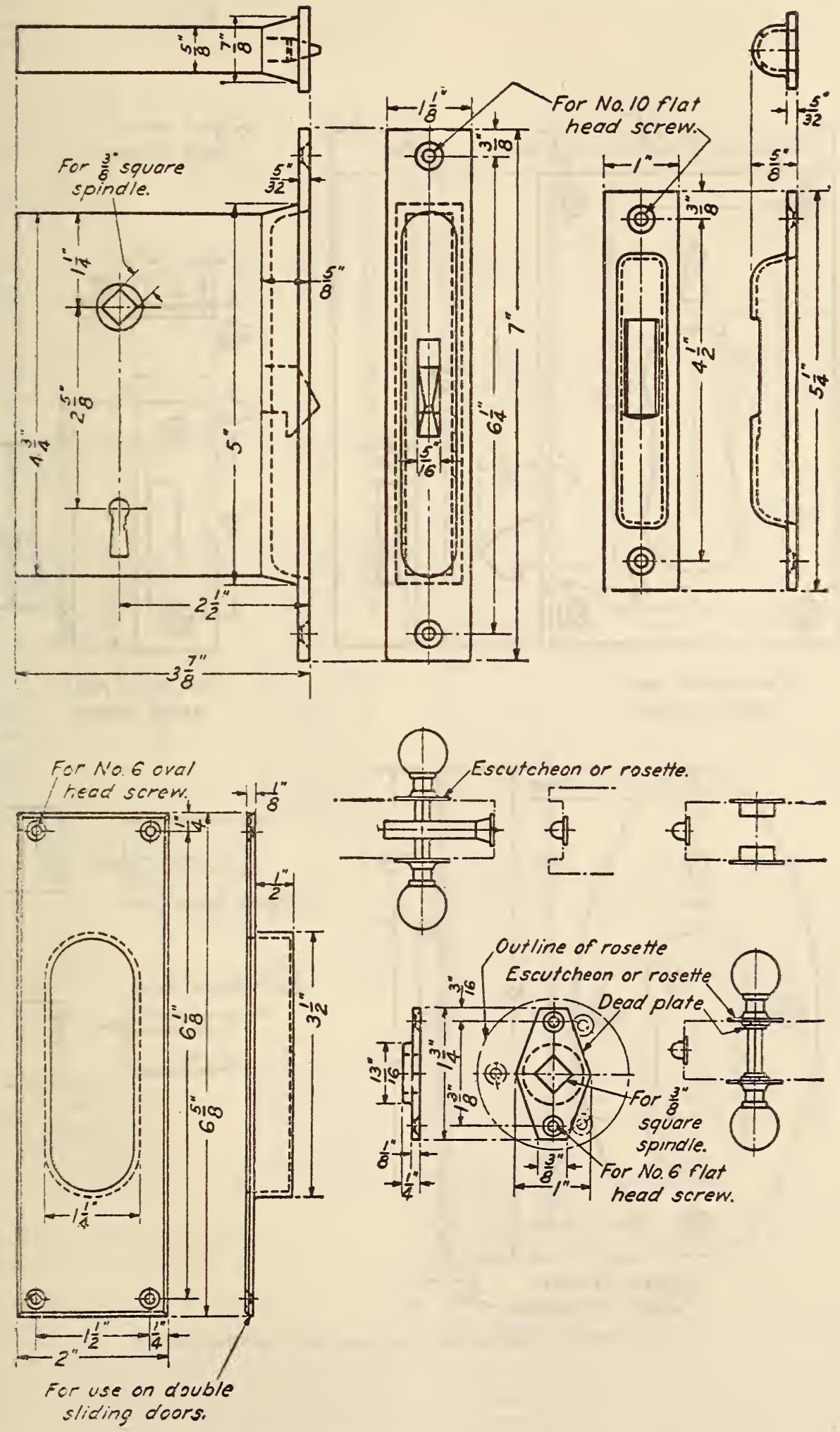

FIGURE 859.-Mortise lock, type L, for sliding doors 


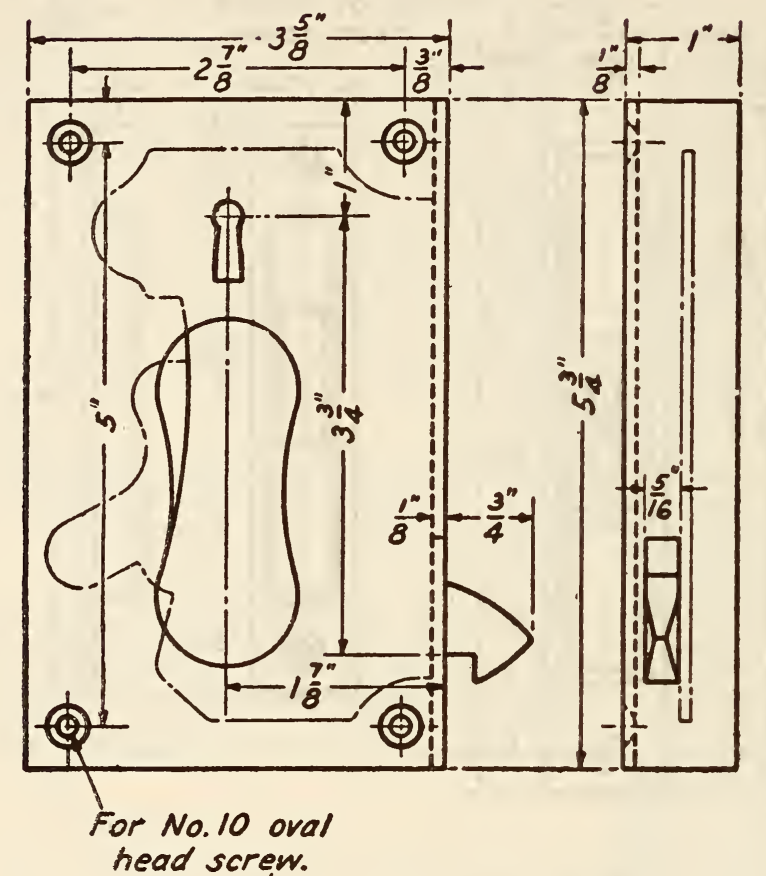

Right hand
lock shown.
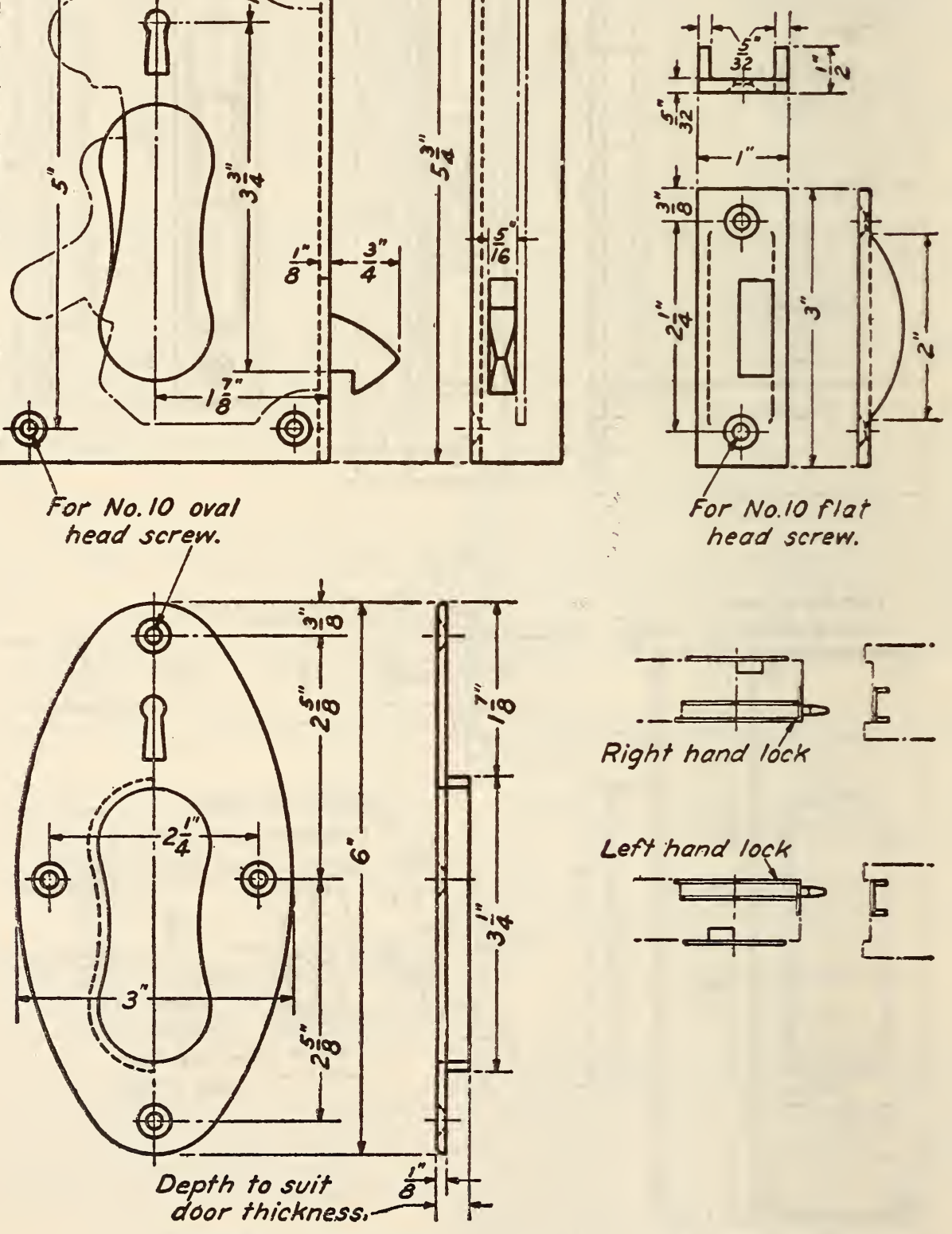

FIGURE 860.-Half-mortise lock, type $M$, for sliding doors 
styles of knobs or handles, such as horizontal lever handles, etc., or of escutcheon plates or rosettes may be ordered, but in such cases the neck of the knob or handle, and the recess in the escutcheon

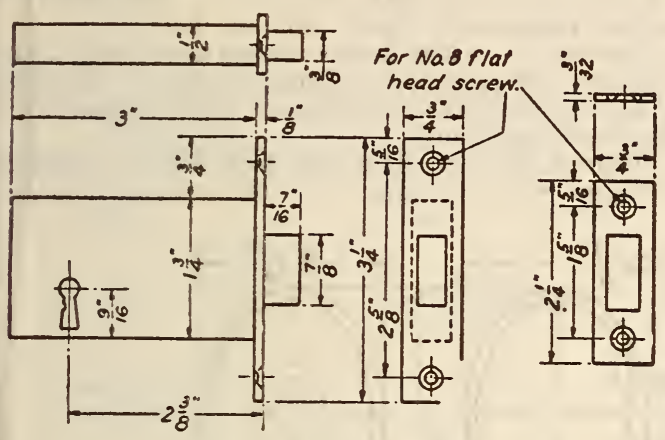

Figdre 861.-Mortise dead lock, type $N$

Jlate or rosette, should be made of the standard form and dimensions shown by the drawings.

\section{Materials}

(a) Unless otherwise specified on the drawings or in purchasing, all parts of locks and accessories, except springs and spindles, shall be of high-grade cast bronze which shall comprise at least 80 per cent
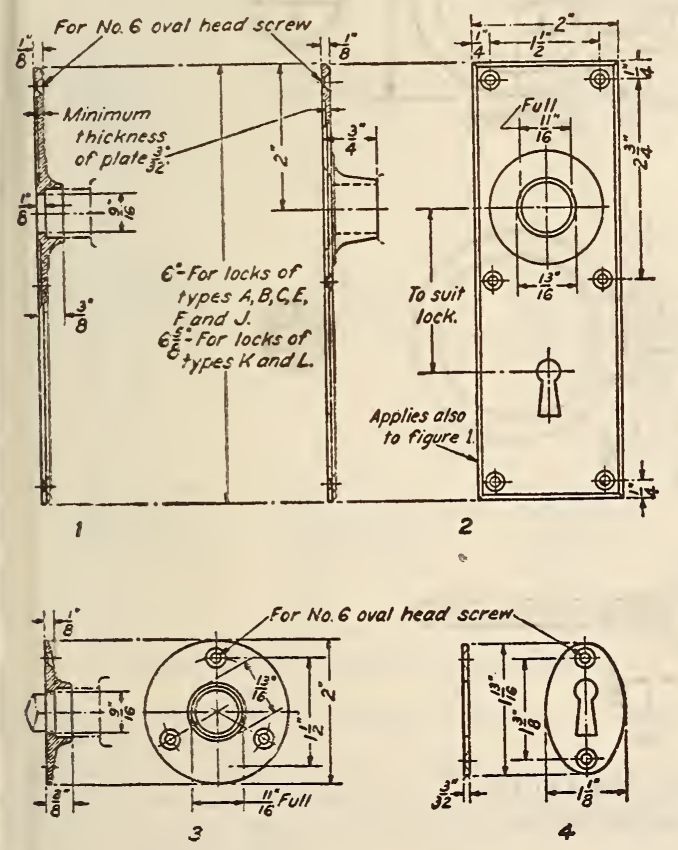

Figdre 862.-Diagrams 1 to 4

See Figure 865 for typical applications.

of copper, not more than 5 per cent of lead, and no scrap metal.

(b) White bronze (nickel silver or white metal) may be ordered in lieu of the above. When ordered it is recommended that one of the following compositions be used, according to whether the white bronze is to be cast or wrought. White-bronze keys need not, however, conform to either of these compositions.
White bronze (percentages of components)

\begin{tabular}{|c|c|c|c|c|}
\hline \multirow{2}{*}{ Components } & \multicolumn{2}{|c|}{$\begin{array}{l}\text { Desired com- } \\
\text { position }\end{array}$} & \multicolumn{2}{|c|}{$\begin{array}{l}\text { Minimum and maxi- } \\
\text { mum by weight }\end{array}$} \\
\hline & Cast & Wrought & Cast & Wrought \\
\hline $\begin{array}{l}\text { Copper } \\
\text { Nickel } \\
\text { Tin }\end{array}$ & $\begin{array}{r}65 \\
20 \\
4\end{array}$ & $\begin{array}{l}65 \\
18\end{array}$ & $\begin{array}{r}\text { Minimum } \\
63 \\
19 \\
\text { Optional. }\end{array}$ & $\begin{array}{r}\text { Minimum } \\
62 \\
17\end{array}$ \\
\hline Lead. & 5 & & $\underset{6}{\operatorname{Maximum}}$ & Maximum \\
\hline Zinc & 6 & 17 & $x_{-1}$ & 18 \\
\hline Iron and other impurities.- & & & $\frac{1}{1}$ & \\
\hline
\end{tabular}

(See appended notes relative to white-bronze castings, p. 109.)

(c) All springs shall be of phosphor bronze.

(d) Spindles shall be of wrought bronze or brass, except where otherwise shown on drawings.

(e) All parts shall be free from defects or blemishes affecting their appearance, operation, or serviceability.

\section{Construction}

The outlines and dimensions of the locks, the positions and dimensions of holes for screws or bolts, spindles, keys, and other features which are to govern cutting of doors or adjacent framework to fit and attach the locks and their accessories shall be in such close accordance with the drawings that any lock or accessory may be quickly replaced by any other lock or accessory of the same standard type, without requiring additional cutting or fitting. Reasonable variations in other features will be allowed which do not prevent interchangeability or impair good appearance or serviceability of the lock and accessories. Particulars of interior mechanism of the locks are onitted from these standards in order to leave all manufacturers free to apply their skill and manufacturing practice. All parts of such mechanism, however, must be substantial and serviceable.

\section{Screws}

Screws shall be furnished with the locks and accessories for their proper attachment. They shall be of material and finish to match the piece that they are to secure. They shall be of appropriate type and length for the purpose intended. Screws required for softwood should be as long as consistent with their purpose. Unless lengths are specifically listed in purchasing, they shall be furnished in accordance with the manufacturer's practice. They shall, as far as applicable, be of the countersunk ovalhead type. They shall conform to the standards approved by the National Screw Thread Commission. (Miscellaneous Publications, Bureau of Standards, No. 89.)

$$
\text { 5. Keys }
$$

Unless otherwise ordered, each lock shall be furnished with two bronze keys. Each key shall be provided with a hole for key ring. Duplicate master keys shall, when ordered, be furnished with each group of locks susceptible of operation by one such key.

$$
\text { 6. Workmanship and rinish }
$$

The workmanship on the locks and accessories shall be such as to produce good appearance and serviceability. It shall be so uniform in character as to insure interchangeability of all locks and accessories of like types. All parts shall conform to the standards herein defined to the satisfaction of the purchaser, and shall be guaranteed by the manufacturer or dealer for efficient operation and lasting service. The standard finish shall be as hereinbe- 
fore specified for each type of lock, and will be furnished unless other finish is specifically called for in asking for quotations and in purchasing.

\section{Marking and Packing}

(a) Each lock shall be legibly impress stamped with the name or trade-mark of the manufacturer, the letters AMS, and with marks to identify individual and master keys. Each individual or master key shall be legibly stamped for identification with the particular lock or the group of locks it applies to.
Diagram 13.-Knobs, Diagram 5, Figure 863, applied to metal door and rim lock.

Diagram 14.- Rim lock on wood door, with knob, Diagram 5, Figure 863, on lock; and knob, Diagram 6 , Figure 863 , with plate escutcheon, Diagram 1 , or rosette, Diagram 3, Figure 862, on door.

Diagram 15.-Rim lock on wood door, with knob, Diagram 5, Figure 863, on lock; and knob, Diagram 6 , Figure 863, with escutcheon plate, Diagram 2, Figure 862 , on door.

Diagram 16.-Mortise lock with knobs, Diagram 6 , Figure 863 , and both types of escutcheon plates,
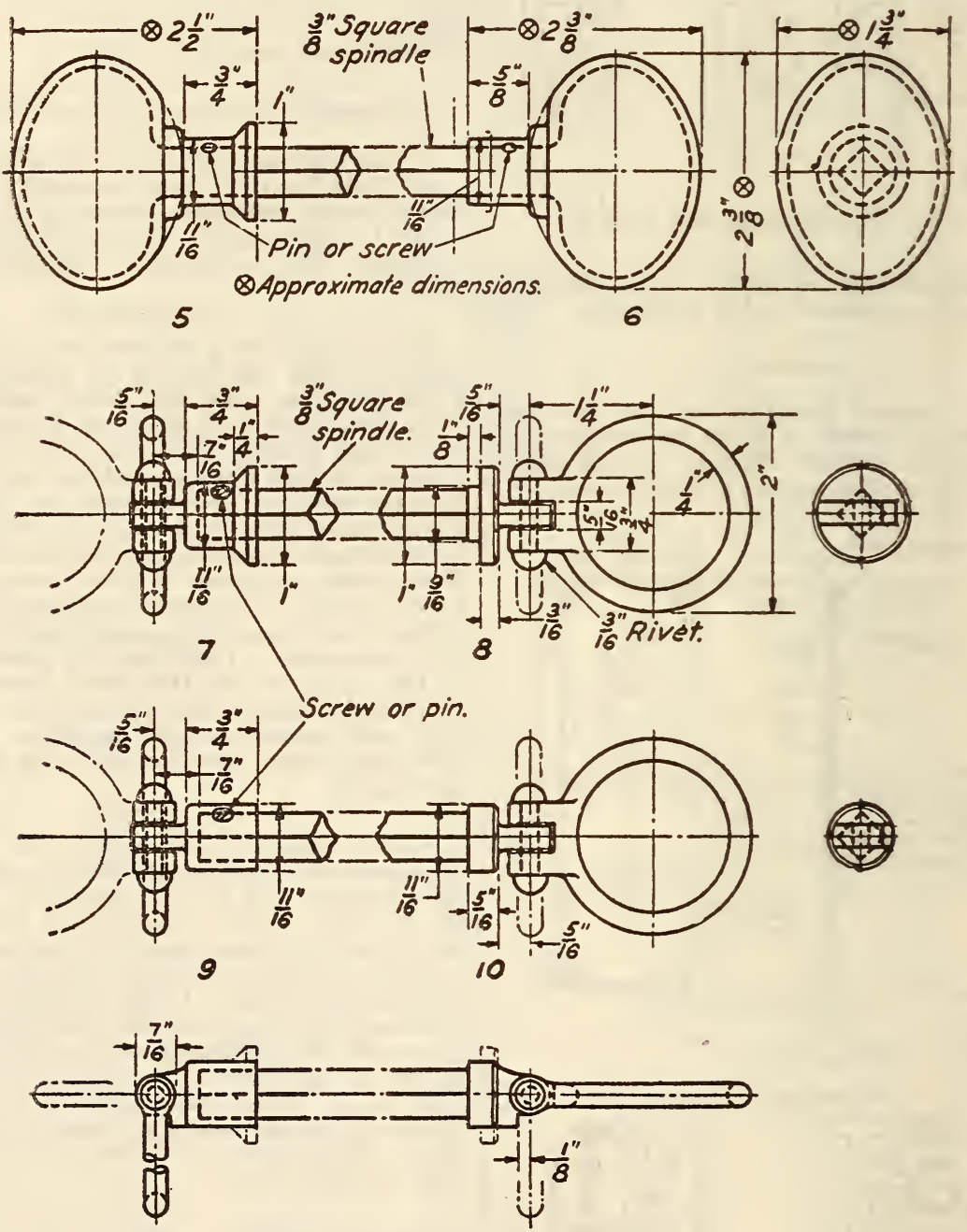

Figure 863.-Diagrams 5 to 10

See Figure 865 for typical applications.

(b) Locks, accessories, and screws shall, unless otherwise ordered, be packed one set in a carton, and the latter shall be properly labeled to show the type of the lock that it contains.

(c) Each container of a shipment shall be marked for identification of contents with the purchaser's order by number or date.

\section{Section III.-GENERAL INFORMATION}

1. Typical Applications of Standard Locks and Accessoriez

Figure 865 shows typical applications of the standard locks and accessories hereinbefore described as follows:
Diagrams 1 and 2, Figure 862. (Rosettes, Diagram 3 , Figure 862 , may be used instead of escutcheon plates.)

Diagram 1\%.-Ring handles, Diagrams 7 and 8 . Figure 863, applied to metal door and rim lock.

Diagram 18.- Ring handles, Diagrams 7 and 10 , Figure 863, applied to wood door and rim lock, using escutcheon plate, Diagram 1, or rosette, Diagram 3, Figure 862 , on door.

Diagram 19.-Ring handle, Diagram 10, Figure 863 , with escutcheon plate, Diagram 1 , or rosette Diagram 3, Figure 862, on door; and knob, Diagram 5 , Figure 863 , on rim lock. (Combination may be reversed by using knob, Diagram 6, with plate or 


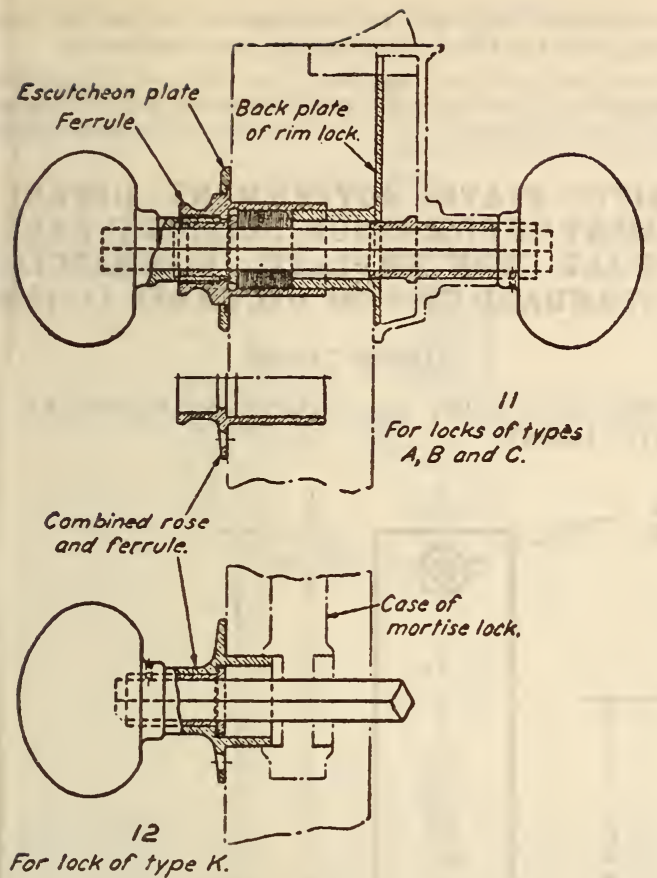

FIGURE 864.-Diagrams 11 and 12
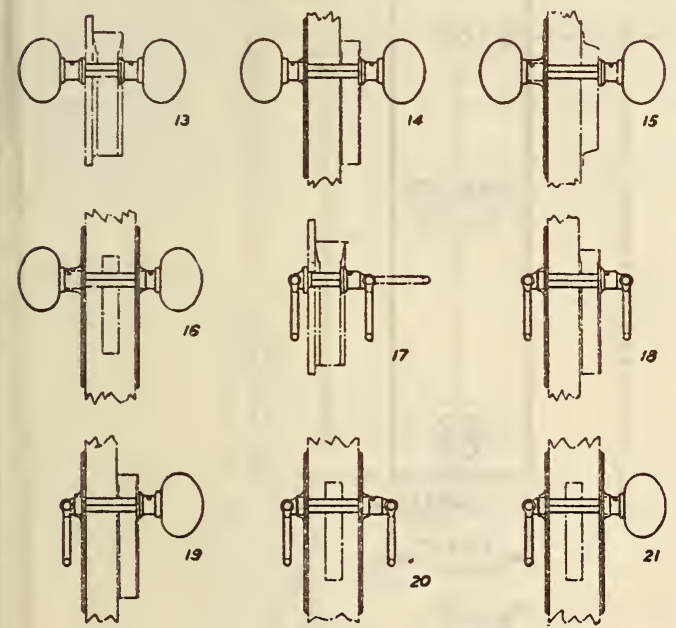

FIGURE 865.-Typical applications

rosette on door; and ring handle, Diagram 7, Figure 863 , on rim lock.)

Diagram 20.- Ring handles, Diagrams 9 and 10, Figure 863, applied to wood door with mortise lock; with either escutcheon plates, Diagram 1, or rosette, Diagram 3, Figure 862 .

Diagram 21. - Ring handle, Diagram 10, and knob, Diagram 6, Figure 863, applied to wood door with mortise lock, using escutcheon plates, Diagram 1, or rosettes, Diagram 3, Figure 862 .

\section{Hand and Bevel of Locks}

Diagrams 22 to 27, inclusive, Figure 866, show typically the hand and bevel of locks as defined in the hardware trade. These figures are included in these specifications for information and guidance in specifying correctly the hand and bevel in purchasing. Locks having a fixed bevel latch, being nonreversible, must be ordered specifically for either right or left hand. Those having reversible latches are adaptable to all conditions of door swing.

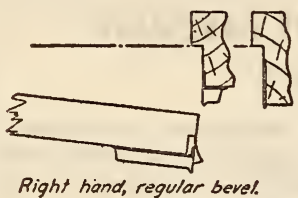

22

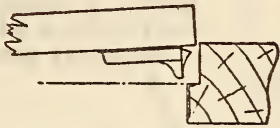

Right hand, reverse bayel.

24

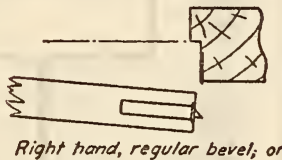

Right hand, regular bevel; or
left hand, reverse bevel.

26
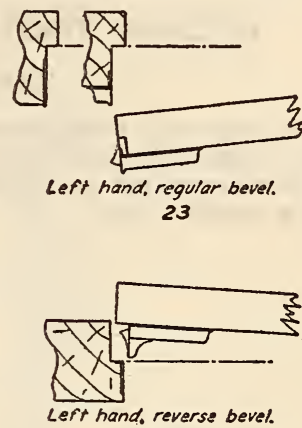

25

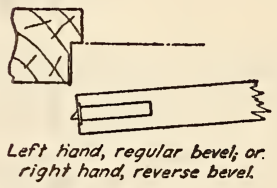

27
FIGURE 866.-Hand and bevel of locks, typical plan views

APPENDIX

Notes Relative to White-Bronze Castings

White-bronze fittings containing appreciably less than 20 per cent of nickel discolor and corrode badly even in locations on shore. Fittings of this class were recently observed on which it was practically impossible to remove surface corrosion which had accumulated in a few months. It was deemed expedient to remove these fixtures and to plate them with chromium to overcome the difficulty.

Experiments were made some years ago by the foundry section of the metallurgical division of the National Bureau of Standards to determine the causes of difficulties reported by manufacturers in producing sound castings of white bronze and in machining and polishing them.

The investigation was to determine the most suitable alloy from the standpoints of foundry practice, machining, polishing, and satisfactory color for hardware castings. Physical properties or ability of any of the compositions to withstand hydrostatic pressure were not determined. From the results and subsequent experience in manufacturing practice, the following conslusions have been reached:

1. Different compositions for cast and wrought white bronze are advisable.

2. Considering service requirements, the compositions specified under head of materials in the preceding specifications are the desirable alloys.

3. No difficulty should be experienced either in producing or machining castings of the composition specified, if proper methods are used and due care is exercised. 
UNITED STATES GOVERNMENT, DEPARTMENT OF COMMERCE, BUILDERS' TEMPLATE HARDWARE, COMMERCIAI STANDARD CS9-29, SEPTEMBER 18, 1929

(See also 617.12, p. 982.)

\section{BUILDERS' TEMPLATE HARDWARE GENERAI}

The following dimensions, clearances, tolerances, screw sizes, and varities of template cylinder locks are recommended as standard for application to hollow metal doors. are required they will be furnished as a part of the door jamb by the hollow-metal manufacturers.

Note.-Details of lock, lock front, and strike given herein are arranged to suit mortise details given in Simplified Practice Rccom. mendation R82-28, holiow metal single-acting swing doors, frames, and trim. (See 605.22, p. 280.)

UNITED STATES GOVERNMENT, DEPARTMENT OF COMMERCE ; BUILDERS' HARD. WARE (NONTEMPLATE), COMMERCIAL STANDARD CS22-30, DECEMBER 11, 1930

\section{DOOR LOCKS}

(See 617.0, p. 977 also Federal specification FF$\mathrm{H}-101$ below).

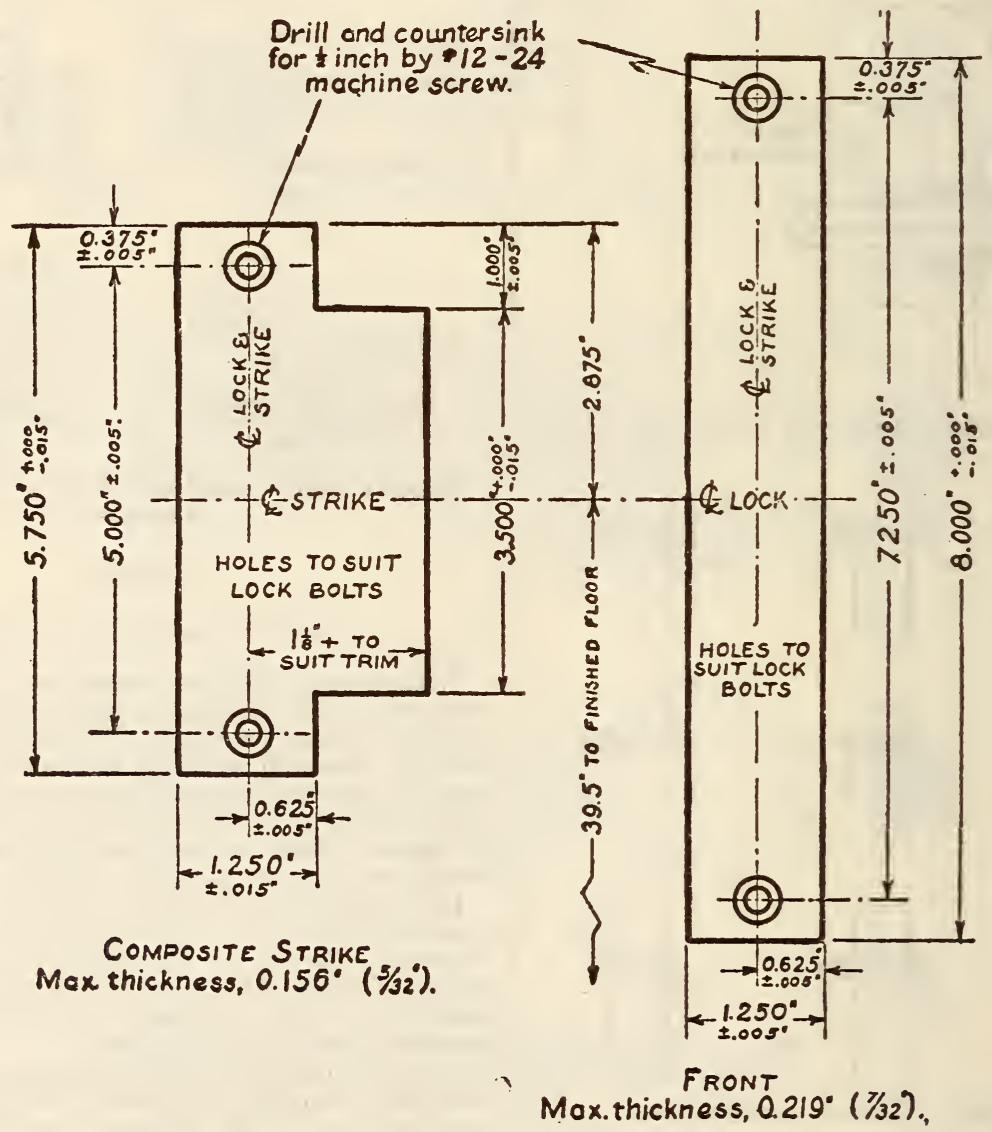

FIGURE 867.-Template front and flat strike for cylinder locks

TEMPLATE LOCKS

One and two bolt cylinder locks of following classification applicable to hollow metal doors shall be provided with standard fronts and strikes conforming to detail dimensions as shown below and in Figure 867.

Such locks are as follows:

Vertical dimension of case, $5 \frac{3}{4}$ inches maximum; 5 inches minimum.

Horizontal dimension of case, $4 \frac{1}{4}$ inches maximum; $3 \frac{3}{4}$ inches minimum.

Thickness of case, $7 / 8$ inch maximum; $3 / 4$ inch minimum.

Backset, $23 / 4$ inches.

Berel of front, $1 / 8$ inch in 2 inches.

Doors receiving such locks shall be reinforced to prevent more than one-eighth inch lateral movement of lock case at rear edge. When boxes for strikes
UNITED STATES GOVERNMENT, FEDERAL SPECIFICATIONS BOARD; FEDERAI SPECIFICATION FOR BUILDERS' HARD. WARE (NONTEMPLATE), FF-H-101, AUGUST 19, 1930

(For types, grades, and classes; material and workmanship; general requirements; detail requirements; packaging, packing, and marking; notes; and table of standard finishes, see 617.0, p. 978 . For chromium plating, nickel plating, and zinc coatings; and methods of inspection and tests, see 600.3, p. 70.) 
BUILDERS' HARDWARE (NONTEMPLATE) DOOR IOCKS

E-2. All locks shall bear the trade-mark or name of the manufacturer.

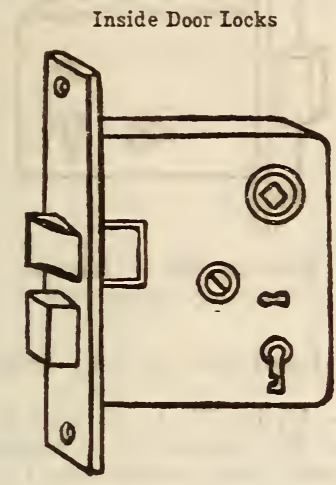

FIgURE 868.-Inside door lock, inexpensive grade, type 4

\section{Inexpensice grade}

Type $4 .-3 \frac{1}{2}$ to $3 \frac{3}{4}$ inches by $3 \frac{1}{4}$ to $3 \frac{1}{2}$ by $5 / 8$ inch japanned iron case. Backset $2 \frac{1}{2}$ inches. Front $5 \frac{1}{2}$ by 1 inch. Latch bolt, minimum $9 / 16$ by $7 / 16$ inch, minimum throw $3 / 8$ inch. Dead bolt, minimum $13 / 16$ by $3 / 8$ inch, minimum throw $7 / 16$ inch. Single extension easy spring; single compression spring, bar type; or double compression spring. Lock shall have one lever tumbler giving 24 changes. Cast bronze front and bolts. Bronze strike. One nickel-plated solid steel key.

Operation.-Latch bolt by knob from either side; dead bolt by key from either side. Reversible.

Medium grade

Type.4A.-Same as type 4, except three tumblers.

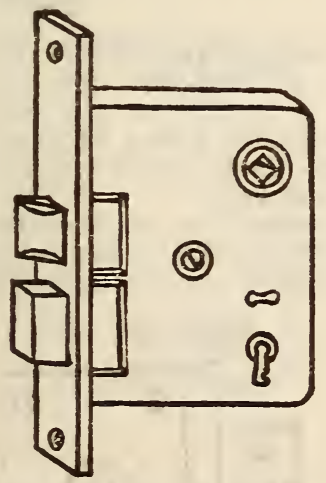

Figure 869.-Inside door lock, best grade, type 7

Best grade

Type 7. 4 to $4 \frac{1}{4}$ inches by $3 \frac{1}{2}$ by $5 / 8$ inch japanned iron case. Backset $2 \frac{3}{4}$ inches. Front $6 \frac{1}{8}$ by 1 inch. Latch bolt, minimum $5 / 8$ by $7 / 16$ inch, minimum throw $3 / 8$ inch. Dead bolt, minimum $7 / 8$ by $3 / 8$ inch, minimum throw $7 / 16$ inch. Double compression easy spring. Lock shall have three-lever tumblers giving 54 changes. Cast bronze front and bolts. Brass hub. Bronze strike. One nickel-plated solid steel kev.

Operation.-Latch bolt by knob from either side; dead bolt by key from either side. Reversible.

Erench Door Lock3

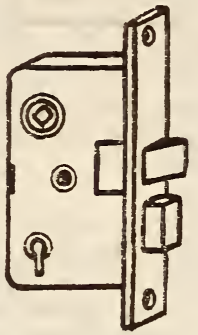

Figure 870.-French door lock, type $12 \mathrm{~A}$

Type $12 A .-3 \frac{1}{2}$ by $3 \frac{3}{4}$ by 2 by $5 / 8$ inch japanned iron case. Backset $1 \frac{1}{2}$ inches. Front 5 by $7 / 8$ (minimum 3/4) inch. Latch bolt, minimum $1 / 2$ by $5 / 16$ inch, minimum throw $5 / 16$ inch. Dead bolt, minimum $5 / 8$ by $5 / 16$ inch, minimum throw $5 / 16$ inch. Single extension or compression easy spring. Threelever tumblers iving 18 changes. Bronze front and bolts. Bronze strike. One nickel-plated solid steel key.

Operation.-Latch bolt by knob from eitner side, dead bolt by key from either side. Reversible.

Type 12B.-Same as type 12A, except with rabbeted front.

Bathroom Door Locks

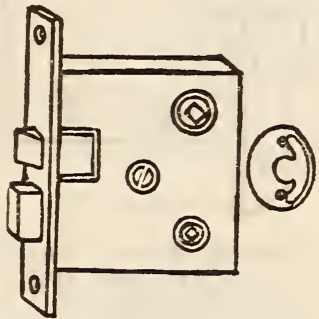

FIGURE 871.-Bathroom door lock, type $17 \mathrm{~A}$

Type 17 A. $-3 \frac{1}{2}$ to $3 \frac{3}{4}$ inches by $3 \frac{1}{2}$ by $5 / 8$ inch japanned iron case. Backset $2 \frac{1}{2}$ inches. Front $5 \frac{1}{2}$ by 1 inch. Latch bolt, minimum $\% / 16$ by $7 / 16$ inch, minimum throw $7 / 16$ inch. Dead bolt, minimum $5 / 8$ by $11 / 32$ inch, minimum throw $7 / 16$ inch. Doublecompression easy spring. One bronze turn knob. Bronze front, bolts, and strike. One emergency key.

Operation. - Latch bolt by knob from either side; dead bolt by turn knob from inside or emergency key from outside. Reversible. 
Communicating Door Locks

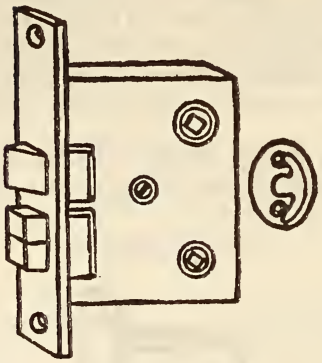

Figdre 872.-Communicating door lock, type 20

Type 20.-41/4 to $4 \frac{1}{2}$ inches by $3 \frac{1}{2}$ by $5 / 8$ inch japanned iron case. Backset $23 / 4$ inches. Front $61 / 8$ by 1 inch. Latch bolt, minimum $\% 16$ by $7 / 16$ inch, minimum throw $3 / 8$ inch. Dead bolt, minimum $7 / 8$ by $3 / 8$ inch, minimum throw $3 / 8$ inch. Double compression easy spring. Split dead bolt. Two bronze turn knobs. Cast bronze front and bolts. Bronze strike. Brass hub.

Operation.-Latch bolt by knob from either side; split dead bolt by turn knob from either side, each locking against the other. Reversible.

Front Door Locks

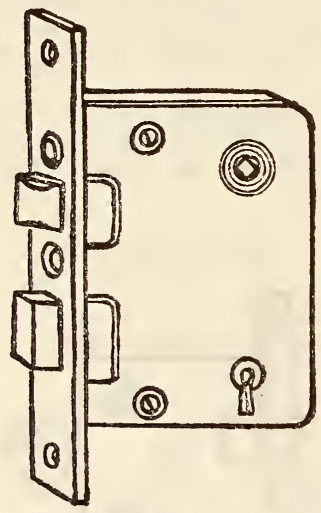

Figdre 873.-Front door lock, type $22 A$

Type 22 $A .-43 / 4$ to 5 inches by $3 \frac{5}{8}$ by $5 / 8$ inch japanned iron case. Backset $2 \frac{1}{2}$ or $23 / 4$ inches. Front 7 by 1 inch. Latch bolt, minimum $5 / 8$ by $7 / 16$ inch, minimum throw $3 / 8$ inch. Dead bolt, minimum $7 / 8$ by $3 / 8$ inch, minimum throw $1 / 2$ inch. Lock shall have three lever tumblers giving 24 changes. Bronze front, bolts, and strike. Twc nickel-plated solid steel keys.

Operation. - Latch bolt by knob from either side; outer knob set by stop in face; dead bolt by key from either side; latch bolt by key from outside when outer knob is stopped. Latch and dead bolts shall be operated by the same key through same keyhole. Reversible.

\section{Mortise Dead Locks}

Type $38 A .-2 \frac{1}{8}$ by $3 \frac{1}{2}$ by $5 / 8$ inch japanned iron case. Backset 2\% inches. Front 4 by 1 inch. Dead bolt, minimum $7 / 8$ by $7 / 10$ inch; minimum throw $7 / 16$ inch. Lock shall have three tumblers giving 54 changes. Cast bronze front and bolt. Bronze strike. One nickel-plated solid steel key.

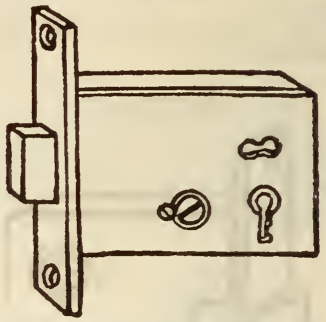

Figure 874.-Mortise dead lock, type $38 A$

Operation.-Dead bolt by key from!'either sides Reversible.

Type A $40 .-4 \frac{1}{2}$ by $3 \frac{1}{2}$ by $5 / 8$ inch japanned Hiron case. Backset $23 / 4$ inches. Cast bronze front 7 by $1 \frac{1}{8}$ inches. Dead bolt, minimum $11 / 16$ by $1 / 2$ inch, minimum throw $1 / 2$ inch. Lock shall have not less

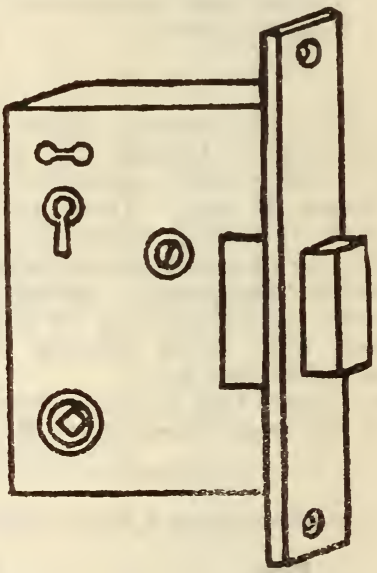

Figure 875.-Mortise dead lock, type $A_{4} 0$

than three tumblers giving 54 changes. Keyhole above knob. Cast bronze bolt. One nickelplated solid steel key.

Operation.-Dead bolt by knob from either side except when deadlocked by key from either side in locked or unlocked position. Reversible.

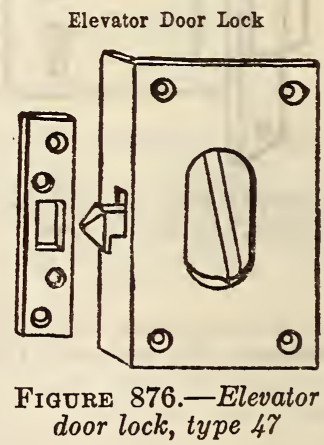

Type $47 .-5$ by $2 \%$ inches cast bronze case. Backset $1 \frac{1}{4}$ inches. Front $7 / 8$ inch wide. Latch bolt, minimum $1 / 2$ by $5 / 16$ inch, minimum lift of latch 
$7 / 32$ inch. Escutcheon for key $1 \frac{1}{4}$ by 1 inch. All cast bronze. One nickel-plated solid steel key.

Operation.- Latch bolt by finger pull from elevator side and by key only from outside. All locks in one project shall be operated by the same key unless otherwise specified. Reversible.

Opright Rim Knob Locks

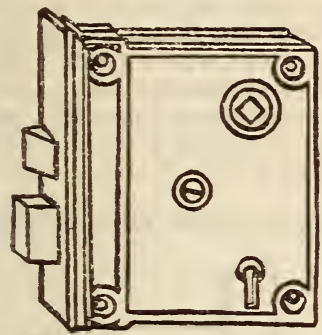

FigURe 877.-Upright rim knob lock, type 59

Type $59 .-4$ by $3 \frac{1 / 4}{4}$ by $3 / 4$ inch japanned iron case. Rim strike. Backset $2 \frac{1}{2}$ inches. Lock shall have one tumbler. Two iron bolts with stop. Latch bolt, minimum $1 / 2$ by $7 / 16$ inch with $3 / 8$ inch throw. Dead bolt, minimum $23 / 32$ by $9 / 32$ inch with $13 / 32$ inch throw. One nickel-plated solid steel key. Screws for lock and keeper shall be not less than $1 \frac{13}{4}$ inches long.

Operation. - Latch bolt by knob from either side; dead bolt by key from either side. Reversible.

\section{Cylinder Locks}

E-3. The key change number shall be stamped on shell or cam of all cylinder locks.

E-4. Except where otherwise specified bolts for cylinder locks shall have not less than 1/2-inch throw.

Cylinder Front Door Locks

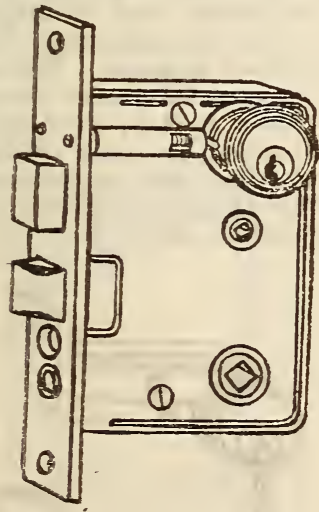

FIGURE 878.-Cylinder front door lock, type 88

7 ype $88 .-5$ to 6 inches by $33 / 4$ by $3 / 4$ inch japanned iron case. Backset $23 / 4$ inches. Solid front $8 \mathrm{by}$ $1 \frac{1}{4}$ inches. Latch bolt, minimum 1 by $5 / 8$ inch, minimum throw $1 / 2$ inch. Dead bolt, minimum $11 / 8$ by $1 / 2$ inch, minimum throw $1 / 2$ inch. Lock shall have one bronze cylinder, with or without removable and interchangeable core feature, with five or more pin tumblers, giving practically unlimited changes. Bronze front, bolts, and strike. Three nickelbronze keys.
Operation.-Latch bolt by knob from either side; outer knob set by stop in face; when outer knob is stopped, latch bolt by key from outside; dead bolt by key from outside and turn knob from inside. Reversible.

Type 88B.-Same as type 88, except beveled front. Not reversible.

Cylinder Vestibule or Office Docr Locks

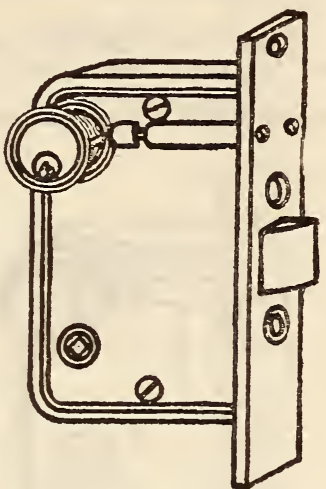

Figdre 879.-Cylinder vestibule or office door lock, type 91

Type 91 - 5 to 6 incnes by $3 \frac{3}{4}$ by $3 / 4$ inch japanned iron case. Backset $23 / 4$ inches. Solid front $71 / 4$ by $11 \frac{1}{8}$ inches. Latch bolt, minimum 1 by $5 / 8$ inch, minimum throw $1 / 2$ inch. Lock shall have one bronze cylinder, with or without removable and interchangeable core feature, with five or more pin tumblers, giving practically unlimited changes. Bronze front, latch bolt, and strike. Three nicklebronze keys.

Operation. - Latch bolt by knob from either side; outer knob set by stop in face; when outer knob is stopped latch bolt by key from outside. Reversible.

Type 91B.-Same as type 91 , except with two bronze cylinders and straight spindle. When knobs are stopped latch bolt shall be operated only by key from either side. Reversible.

Type 91C.- Same as type 91, except with no stop works. Outside knob always rigid. Reversible.

Cylinder Office Locke

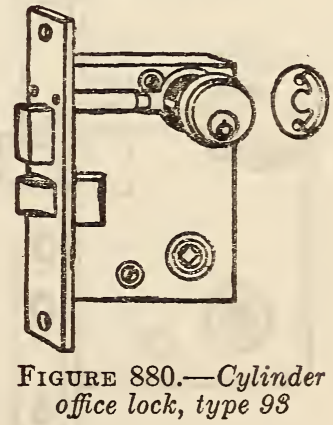

Type 93. -5 to 6 inches by $33 / 4$ by $3 / 4$ inch japanned iron case. Backset $23 / 4$ inches. Solid front 8 by $11 / 4$ inches. Latch bolt, minimum 1 by $5 / 8$ inch, minimum throw $1 / 2$ inch. Dead bolt, minimum $1 \frac{118}{8}$ by $1 / 2$ inch, minimum throw $1 / 2$ inch. Lock shall have one bronze cylinder, with or without removable and interchangeable core feature, with five or more pin 
tumblers giving practically unlimited changes. Bronze front, bolts, and strike. Three nickel-bronze keys.

Operation.-Latch bolt by knob from either side; dead bolt by key from outside and turn knob from inside. Reversible.

Type 93A.-Same as type 93, but with rabbeted front.

Type 94.- Same as type 93, but with two cylinders and no turn knob.

Type $94 A$.- Same as type 93 , except with two cylinders and no turn knob and with rabbeted front.

\section{Cylinder Office or Front Door Locks}

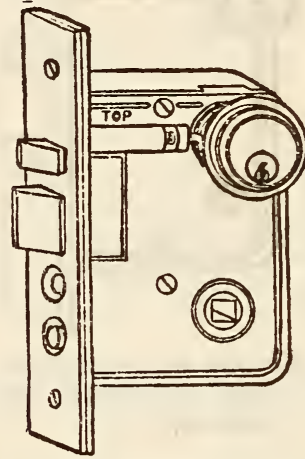

Figure 881.-Cylinder office or front door lock, type 97

Type $97 .-5$ to 6 inches by $37 / 8$ by $3 / 4$ inch, japanned iron case. Backset $23 / 4$ inches. Solid front $7 \frac{1}{4}$ by $1 \frac{1}{4}$ inches. Latch bolt, minimum 1 by $5 / 8$ inch, minimum throw $1 / 2$ inch. Lock shall have one bronze cylinder, with or without removable and interchangeable core feature, with five or more pin tumblers giving practically unlimited changes; auxiliary latch bolt and stop works. Bronze front, bolts, and strike. Three nickel-bronze keys.

Operation.- - Latch bolt by knob from either side by key from outside when outer knob is stopped. When door is closed auxiliary bolt shall deadlock the latch bolt. Not reversible.

Note: Either type of auxiliary bolt will be acceptable.

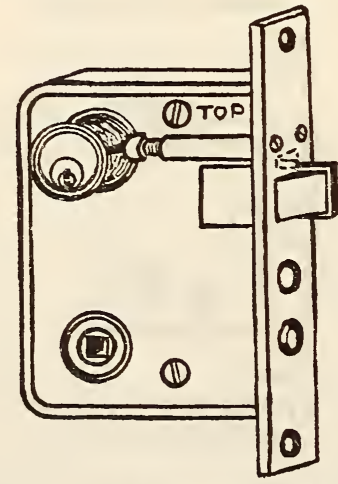

FIG U RE 882.-Cylinder office or front door lock, type 102

Type 102. $-5 \frac{1}{2}$ to 6 incnes by $3 \frac{7 / 8}{7}$ to $4 \frac{1}{2}$ by $3 / 4$ inch japanned iron case. Backset $23 / 4$ inches. Solid flat front minimum $73 / 4$ by $1 \frac{1}{4}$ inches. Latch bult, minimum 1 by $5 / 8$ inch, minimum throw $3 / 4$ inch.
Lock shall have one bronze cylinder with five or more pin tumblers giving practically unlimited changes; latch bolt and auxiliary bolt or slide, and stop works. Single or double compression spring. Front, bolts, and box strike bronze. Three nickel-bronze keys.

Operation. - Latch bolt by knob from either side; by key from outside only, when outer knob is stopped. Bolt and stop works shall be deadlocked by auxiliary bolt and deadlocking function shall be effective to the point where space between lock front and strike is five-sixteenths inch. Not reversible.

Type 102A.- Same as type 102, except with two cylinders. Inner cylinder shall operate stop work which are omitted from front of lock.

\section{Cylinder Locks for Public Toilo: Room Doo:s}

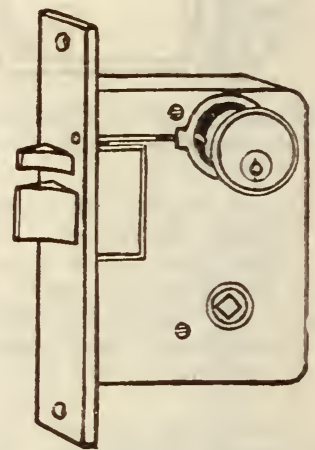

Figure 883.-Cylinder lock for public toilet room door, type 105

Type $105 .-5$ to 6 inches by $3 \frac{7}{8}$ by $3 / 4$ inch japanned iron case. Backset $23 / 4$ inches. Solid front $71 / 4$ by $1 \frac{1}{4}$ inches. Latch bolt, minimum 1 by $5 / 8$ inch, minimum throw $1 / 2$ inch. Lock shall have auxiliary latch bolt and two bronze cylinders with or without removable and interchangeable core feature, with five or more pin tumblers giving practically unlimited changes. Bronze front, bolts, and strike. Three nickel-bronze keys.

Operation. - Latch bolt by knob from either side; stop works controlled by inside cylinder; latch bolt by key from outside when outer knob is stopped; when door is closed auxiliary bolt shall automatically deadlock the latch bolt. Not required to be reversible.

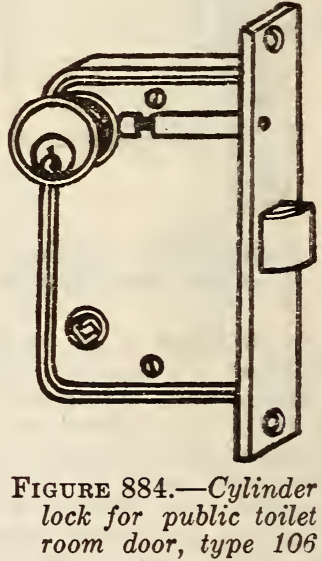

Type 106.-Same as type 105, except withoui auxiliary latch bolt. Reversible. 
Cylincer Mortise De adlocks

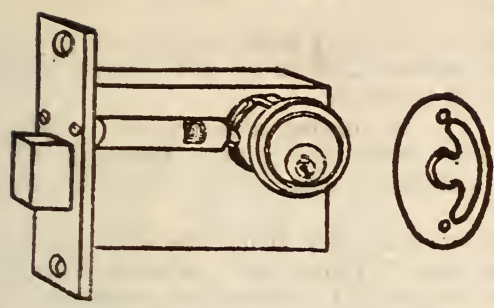

FiguRE 885.-Cylinder mortise dead lock, type 114

Type $114 .-2 \frac{1}{2}$ by $3 \frac{5}{8}$ by $3 / 4$ inch japanned iron case. Backset 23/4 inches. Solid front $41 / 8$ by $1 \frac{1}{8}$ inches. Dead bolt, minimum $15 / 16$ by $1 / 2$ inch, minimum throw $1 / 2$ inch. Lock shall have one bronze cylinder, with or without removable and interchangeable core feature, with five or more pin tumblers giving practically unlimited changes. Bronze front, bolt, strike and turn knob. Three nickel-bronze keys.

Operation.-Bolt by key from one side and turn knob from the other. Reversible.

Type 115.- Same as type 114, except with two bronze cylinders and no turn knob.

Type 116. $-4 \frac{1}{2}$ by $3 \frac{3}{4}$ inch japanned iron case. Backset, $23 / 4$ inches. Solid front $67 / 8$ by $1 \frac{1}{4}$ inches. Dead bolt, minimum $15 / 8$ by $1 / 2$ inch, minimum throw $\% / 16$ inch. Lock shall have one bronze cylinder with or without removable and interchangeable core feature, with five or more pin tumblers giving practically unlimited changes. Bronze front, bolt, strike, and turn knob. Three nickel-bronze keys.

Operation. - Bolt by key from one side and turn knob from the other.

Special backsets.-When so specified this lock shall be furnished in one of the following backsets $1 \frac{11}{2}, 2,2^{1 / 2}$, or $3^{1 / 2}$ inches. Reversible.

Type 11\%.-Same as type 116, except with two, bronze cylinders and no turn knob.

\section{Cylinčer Entrance Door Locks}

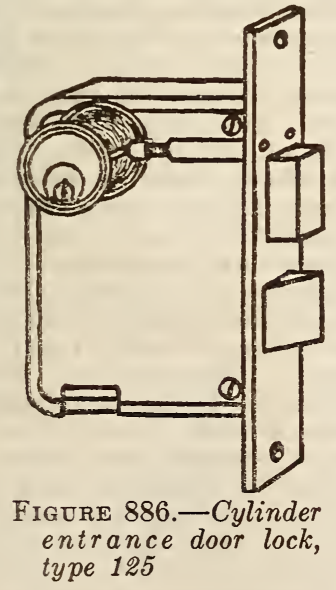

Type $125 .-5$ to 6 by $33 / 4$ by $13 / 16$ inch japanned iron case. Backset $23 / 4$ inches. Solid front $73 / 4$ by $1 \frac{1}{4}$ inches. Latch bolt, minimum 1 by $1 / 2$ inch, minimum throw $1 / 2$ inch. Dead bolt, minimum $1 \frac{1}{8}$ by $1 / 2$ inch, minimum throw $1 / 2$ inch. Lock shall have two bronze cylinders, with or without removable and interchangeable core feature, with five or more pin tumblers giving practically unlimited changes. Cast bronze front and bolts. Bronze strike. Three nickel-bronze keys.

Operation.- Latch bolt by thumb piece (handle) from either side at all times. Dead bolt by key from either side. Reversible.

Type 125 A.- Same as type 125, except rabbeted front.

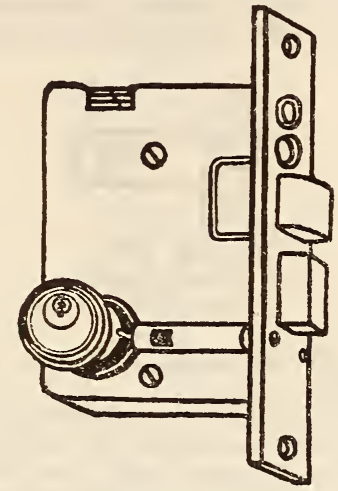

Figure 887.-Cylinder entrance door lock, type 126

Type $126 .-5$ by $3 \frac{3}{4}$ by $3 / 4$ inch japanned iron case. Backset $23 / 4$ inches. Solid front $7 \frac{1}{2}$ by $1 \frac{1}{4}$ inches. Latch bolt, minimum $7 / 8$ by $5 / 8$ inch, minimum throw $1 / 2$ inch. Dead bolt, minimum $1 \frac{1}{8}$ by $1 / 2$ inch, minimum throw $1 / 2$ inch. Lock shall have one bronze cylinder, with or without removable and interchangeable core feature, with five or more pin tumblers giving practically unlimited changes. Bronze front, bolts, and strike. Three nickel-bronze keys.

Operation. - Latch bolt by thumb piece (handle) from either side, except when outside thumb piece is set by stops in face of lock. Dead bolt by turn knob on inside; both dead and latch bolts by key from outside. Reversible.

Type 126A.-Same as type 126, except rabbeted front.

Type 126B.-Same as type 126, except with beveled front.

\section{Tubular Locks and Latches}

E-5. Tubular locks and latches are applied to door by inserting tubular case into one hole bored in edge of door stile. The knob, cylinder, or other operating device is attached to case through opening in face of door stile. Front and strike plates require a shallow mortise.

E-5a. Suitable combinations of separate latch and lock units may be applied to door to secure additional operating features not obtainable in a single unit.

E-6. These locks provide, in general, only $3 / 8$ inch throw of bolt, and naturally do not offer the same protection nor safeguard against shrinkage or forceful entry as locks with $1 / 2$ inch or more throw of bolt.

E-7. The following general requirements apply to all types of tubular locks and latches covered by this specification: Case shall contain bolt, spring, and operating hub and shall be tubular, $7 / 8$ to 1 inch outside diameter, by not more than $33 / 4$ inches long, and shall be of galvanized or cadmium plated steel, unless brass is specified, not less than 0.035 inch thick. Backset $2 \frac{1}{2}$ inches except where specification admits $23 / 8$ inches. Solid front $25 / 16$ by $1 \frac{1}{16}$ inches. Solid bolt not less than $9 / 16$ by $1 / 2$ inch, minimum throw $3 / 8$ inch except where otherwise specified. Compression spring. Key-controlled locks and. latches shall have one or more brass or bronze cylinders, as required, with or without removable 
and interchangeable core feature, each having five or more pin tumblers giving practically unlimited key changes. Brass or bronze bolt, front, and strike. Three nickel-bronze keys shall be supplied with all key-operated locks and latches. All units shall be reversible. (For knobs, spindles, escutcheons, etc., see types 205 to $330 \mathrm{~A}$, inclusive, under their respective commodity designations. For commodity designations, see under detail requirements in 617.0 p. 979.)

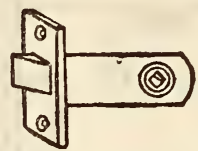

Figure 888.Knob latch, type 150

Type 150.-Knob Latch: No cylinders required. (See par. E-7.)

Operation. - By knob from either side at all times.

Type 150A.- Same as type 150, except that operation shall be by knob one side, turn knob one side.

Type 150B.- Same as type 150, except that operation shall be on one side only.

Operation.-By knob.

Type 151.-Knob latch with stop one side. Same as type 150, except with stop one side. Stop shall control outside knob from inside and prevent operation from outside. Emergency access shall. be provided.

Type 152.-Knob latch with stops both sides. Same as type 150 , except with stop both sides. Stop on either side shall control knob on side opposite and prevent operation from that side.

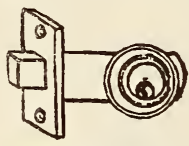

Figure 889 . Deadboltlock, type 153

Type 153.-Dead bolt lock. Cylinder one side. Turn knob one side. Backset $2 \frac{3}{8}$ or $2 \frac{1}{2}$ inches, minimum throw of bolt $1 / 2$ inch. (See par. E-7.)

Operation.-Bolt by key from one side, by turn knob from one side. When locked it shall not be possible to force bolt back except by key or turn knob. Cylinder shall permit key to be withdroiwn leaving bolt in unlocked position.

Type 153A.-Same as type 153, except that cylinder shall not permit key to be withdrawn leaving bolt in unlocked position.

Type 153B.- Same as type 153, except with cylinder on both sides.

Operation.-By key from both sides.

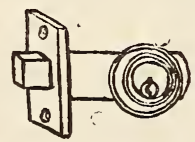

$$
\begin{aligned}
& \text { Figure } 890 .- \\
& \text { Deadbolt lock, } \\
& \text { type } 153 \mathrm{C} \text {, } \\
& \text { without turn } \\
& \text { knob }
\end{aligned}
$$

Type 153C.-Same as type 153, except without turn knob.

Operation.-By key from one side only.
Type 153D.- Same as type 153, except without turn knob.

Operation.-By key from one side only. Cylinder shall not permit key to be withdrawn leaving bolt in unlocked position.

Type 154.-Cylinder night latch. Cylinder one side. Turn knob one side. Backsets $2 \frac{3}{8}$ or $2 \frac{1}{2}$ inches, minimum throw of bolt $1 / 2$ inch. (See par. E-7.)

Operation.-By key from one side, by turn knob from one side. Turn knob or slide stop shall permit latch to be held in retracted position. Cylinder shall permit key to be withdrawn leaving bolt in retracted position.

Type 154A.- Same as type 154, except that cylinder shall not permit key to be withdrawn leaving latch in retracted position.

Type 154B.- Same as type 154, except with cylinder on both sides.

Operation.-By key both sides.

Type 154C.-Same as type 154, except with cylinder on both sides.

Operation.-By key both sides. Cylinder shall not permit key to be withdrawn leaving latch in retracted position.

\section{Cylindrical Case Locks and Iatche.}

E-8. Cylindrical case locks and latches are applied to door by inserting cylindrical case into one large hole bored through face of door stile, and the separable bolt into a smaller hole bored in edge of door stile. Case is secured in door by escutcheons or flanges attached to case, bolt is secured by attachment to edge of door and to case or operating mechanism. Front and strike plates require a shallow mortise.

$\mathrm{E}-8 \mathrm{a}$. These locks provide, in general, only three-eighths inch throw of bolt. and naturally do not offer the same protection nor safeguard against shrinkage or forceful entry as locks with one-half inch or more throw of bolt.

E-9. The following general requirements apply to all types of cylindrical case locks and latches covered by this specification: All cylindrical case locks and latches shall be furnished complete with knobs and escutcheons. Spindle or other members conveying knob or key action to retractor shall be an integral factory-assembled part of lock. Cylindrical case shall be firmly anchored in place by machine screws or through bolts. Solid front and bolt. Except where otherwise specified, bolt shall be not less than $3 / 4$ by $5 / 8$ inch, minimum throw $3 / 8$ inch. Compression springs. Key-controlled locks and latches shall have a cylinder in the center of one or both knobs, as required, each with not less than five pin tumblers giving practically unlimited key changes. Bronze bolt, front, and strike. Wrought-bronze escutcheons. Wrought-bronze knobs unless glass knobs are specified. Bronze knobs of spheroid type, 2 to $2 \frac{1}{4}$ inches diameter. Glass knobs of octagon type, clear glass unless milk-white glass is specified, diameter 2 inches. All steel working parts shall be heavily rustproofed. Two nickel-bronze keys shall be supplied with all key-operated locks and latches. Reversible to all hands and applicable to doors having either flat or beveled edges.

Type 160.-Knob latch. (See par. E-9.) Backset $2 \frac{3}{8}$ inches. Front not over 1 inch wide. Bolt shall be not less than $1 / 2$ by $3 / 8$ inch. Applicable to doors $15 / 16$ to $2 \frac{1}{4}$ inches thick.

Operation. - Latch bolt by knob from one side, by turn knob from one side. 
Type 161.-Knob latch. (See par. E-9.) Backset $2 \frac{3}{8}$ inches. Front 1 to $11 / 4$ inches wide. Applicable to doors $13 / 8$ to $21 / 4$ inches thick.

Operation.-Latch bolt by knob from either side at all times.

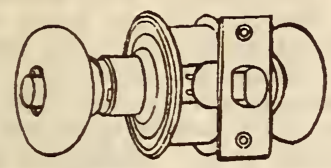

Figure 891.-Knoblatch, type 162

Type 162. - Knob latch with stop one side. Same as type 161, except with stop one side. Stop shall control outside knob or both knobs from inside and prevent operation from outside. Lock shall provide for emergency access.

Type 163.-Knob latch with stop both sides. Same as type 161, except with stop both sides. Stop on either side shall control both knobs or knob on side opposite and prevent operation from that side.

Type 164.-Cylinder vestibule or office door lock. Same as type 161, except with cylinder in outside knob. Strike shall be of protected type.

Operation.-By knob from either side except when stop is set from inside, then from outside by key only.

Type 165.-Cylinder front or office door lock. Same as type 161, except with cylinder in outside knob. Strike shall be of protected type.

Operation. - By knob from either side except when stop is set by key from outside or push button from inside. When stop is set, outside knob shall be inoperative and latch bolt shall be securely and positively deadlocked. Stops shall be released by key from outside or by push button from inside.

\section{Unit Type Locks and Latches}

E-10. Unit type locks and latches are applied complete without disassembling-case, bolts, escutcheons, and knobs being assembled at factory as a unit which is slipped into a cutaway made in the door stile. The escutcheons are then fastened to the door securing the entire mechanism in place.

E-11. All unit type locks and latches shall be assembled complete by manufacturer at factory with knobs and escutcheons attached to a heavy cast frame. Each lock shall be a complete unit ready to slip into cutaway to be made in the door stile. Types which are key controlled shall have a cylinder in the center of one or both knobs as required, each with not less than five pin tumblers giving practically unlimited key changes. Knobs shall be of cast bronze, spheroid type (unless ellipsoid type is specified), of $21 / 4$ inches minimum diameter. Escutcheons shall be vertical and shall be of cast bronze, not less than 8 by $2 \frac{1}{2}$ inches, with bracket-bearing sleeve or thimble. The two escutcheons shall be clamped to door by at least one through bolt from the inside in addition to screw fastenings. Locks shall be so constructed as to permit of adjustment for slight variation in thickness of doors, and shall be furnished for doors $13 / 8$ to 3 inches thick as ordered. Bolts shall be of cast bronze. Strikes shall be cast bronze of the box type for metal jambs and of the protected box type for wood jambs. All locks shall be beveled $1 / 8$ inch in 2 inches.

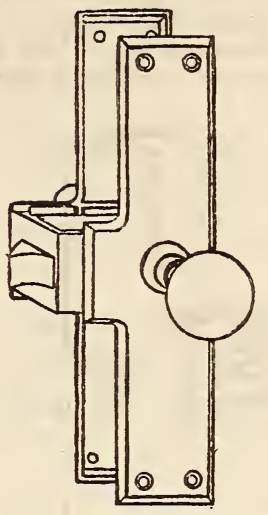

Figure 892.-Knob latch, type 170

Type 170.-Knob latch. (See par. E-11.) Case not less than 2 by $2 \frac{7}{8}$ inches; backset $23 / 4$ or 3 inches; heavy swinging latch bolt, minimum $5 / 8$ by $1 \frac{1}{4}$ inch, minimum throw $5 / 8$ inch. Both knobs without cylinders.

Operation.-By knob from either side at all times.

Type 171.-Bathroom door lock. Same as type 170 , except that inside escutcheon shall include a turn knob.

Operation. - Latch bolt by knob from either side. Turn knob on inside shall lock against outside by deadlocking the latch bolt.

Type 17\%.-Communicating door lock. Same as type 170, except that both escutcheons shall include a turn knob.

Operation. - Latch bolt by knobs from either side. Turn knob on each side shall lock against opposite side by deadlocking the latch bolt.

Type 173.-Cylinder front door lock. (See par. E-11.) Case not less than $23 / 8$ by $27 / 8$ inches; backset $23 / 4$ or 3 inches; heavy swinging latch bolt, minimum $5 / 8$ by $1 \frac{1}{4}$ inches, minimum throw $5 / 8$ inch; rectangular dead bolt, minimum $5 / 16$ by $7 / 8$ inch, minimum throw $1 / 2$ inch. Stop works in face of lock. The outside knob shall contain the cylinder. Three nickelbronze keys. Inside escutcheon shall include a turn knob.

Operation.-Latch bolt by knob from either side except when outer knob is set by stop in face. When outer knob is set by stop, latch bolt by key from outside; dead bolt by key from outside and turn knob from inside.

Type $174 .-$ Cylinder vestibule or office door lock. (See par. E-11.) Case not less than 2 by $27 / 8$ inches; backset $23 / 4$ or 3 inches; heavy swinging latch bolt, minimum $5 / 8$ by $11 / 4$ inches, minimum throw $5 / 8$ inch; stop works in face of lock. The outside knob shall contain the cylinder. Three nickel-bronze keys.

Operation.-Latch by knob from either side except when outside knob is set by stop in face, then by key from outside. 
Type 174A.- Same as type 174, except both knobs shall contain a cylinder. Three nickel-bronze keys.

Operation.-By knob from either side except when both knobs are stopped; latch bolt shall then be operated by key from either side.

Type 174B.- Same as type 174, except with no stop works; outside knob always rigid.

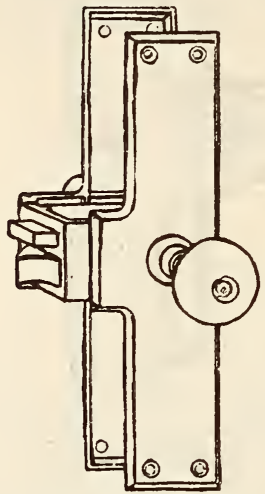

Figure 893.-Cylinder office lock, type 175

Type 175.-Cylinder office lock. (See par. E-11.) Case not less than $23 / 8$ by $2 \frac{7}{8}$ inches; backset $23 / 4$ or 3 inches; heavy swinging latch bolt, minimum $5 / 8$ by $1 \frac{1}{4}$ inches, minimum throw $1 / 2$ inch, rectangular dead bolt, minimum $5 / 16$ by $7 / 8$ inch, minimum throw $1 / 2$ inch. Three nickel-bronze keys. The outside knob shall contain the cylinder. The inside escutcheon shall include a turn knob.

Operation.- Latch bolt by knob from either side at all times; dead bolt by key from outside and by turn knob from inside.

Type $175 \mathrm{~A}$.- Same as type 175 , but with rabbeted strike for pairs of rabbeted doors.

Type 176.- Same as type 175, except without turn knob. Both knobs shall contain a cylinder. Three nickel-bronze keys.

Operation.- Latch bolt by knobs from either side at all times; dead bolt by key from either side.

Type 176 A.- Same as type 175, except without turn knob. Both knobs shall contain a cylinder. Three nickel-bronze keys. Rabbeted strike for pairs of rabbeted doors.

Operation.-Latch bolt by knobs from either side at all times; dead bolt by key from either side.

Type 17\%.-Cylinder office or front door locks. (See par. E-11.) Case not less than $2 \frac{3}{8}$ by $2 \frac{7}{8}$ inches; backset $23 / 4$ or 3 inches; heavy swinging latch bolt, minimum $5 / 8$ by $1 \frac{1 / 4}{\text { inches, minimum }}$ throw $5 / 8$ inch, and auxiliary latch bolt, stop works in face. The outside knob shall contain the cylinder. Three nickel-bronze keys.

Operation. - Latch by knob from either side except when outside knob is set by stop in face, then by key from outside. When door is closed auxiliary bolt shall automatically deadlock the latch bolt and stops.

Type 178.-Cylinder office or front door locks. (See par. E-11.) Case not less than $23 / 8$ by $2 \frac{7}{8}$ inches; backset $23 / 4$ or 3 inches; heavy antifriction or swinging latch bolt minimum $5 / 8$ by $1 \frac{1}{8}$ inch with $3 / 4$ inch minimum throw and auxiliary bolt. Stop works on inner knob or inner face. The outside knob shall contain the cylinder. Three nickelbronze keys.

Operation.-Latch bolt by knob from either side; by key from outside only when the outer knob is stopped. When door is closed the auxiliary bolt shall deadlock latch bolt. The deadlocking function shall be effective to the point where space between lock front and strike is $5 / 16$ inch.

Type 178A.-Same as type 178, except both knobs shall contain a cylinder. The cylinder in the inside knob shall operate concealed stop works to lock the outside knob. Retracing latch bolt by key from outside must not release the outside knob.

Type 179.-Cylinder locks for public toilet room doors. (See par. E-11.) Case not less than 2 by $27 / 8$ inches; backset $2 \frac{1}{4}$ or 3 inches; heavy swinging latch bolt, minimum $5 / 8$ by $1 \frac{1}{4}$ inches, minimum throw $5 / 8$ inch, and auxiliary bolt; concealed stop works. Each knob shall contain a cylinder. Three nickel-bronze keys.

Operatior. - Latch bolt by knobs from either side except when outside knob is set by key for inside cylinder; then by key from outside. Inside knob shall always be free. When door is closed auxiliary bolt shall automatically deadlock the latch bolt.

Type 179 A.-Same as type 179, except without auxiliary latch.

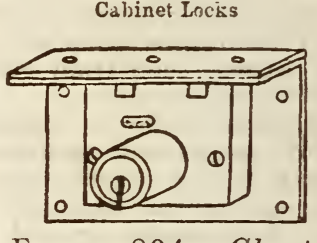

Figure 894.-Chest

lock, type 501

Chest locks

Type 501.-Brass. Square selvedge; solid square box, screwed; three secure levers; double link; cylinder with two flat steel keys. Size $15 / 16$ by 2 inches. Drop $7 / 8$ inch. Made for $3 / 4$-inch wood. Minimum thickness of plate 0.040 inch.

Type 501A.-Brass. Square selvedge; solid square box, screwed; four secure levers; double link; cylinder with two flat steel keys. Size $2 \frac{1}{4}$ by $3 \frac{1}{2}$ inches. Drop $1 \frac{1 / 4}{4}$ inches. Made for 1 -inch wood. Minimum thickness of plate 0.040 inch.

Type 501 A1. - Same as type 501A, except for $1 \frac{1}{4}$-inch wood.

Type 501 A2.-Same as type 501A, except for $11 / 2$-inch wood.

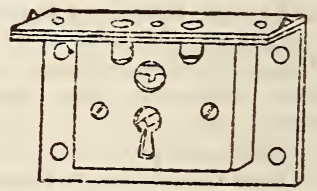

Figure 895.-Chest lock, type 520

Type 520.-Steel. Square selvedge; solid square box, screwed; double link; two secure levers; one barrel key. Size 11/8 by $11 / 2$ inches. Drop $11 / 16$ inch. Minimum thickness of plate 0.040 inch.

Type 520A.- Same as type 520, except size $1^{11 / 16}$ by $2 \frac{1}{2}$ inches, with $15 / 16$-inch drop. 
Cupboard locks

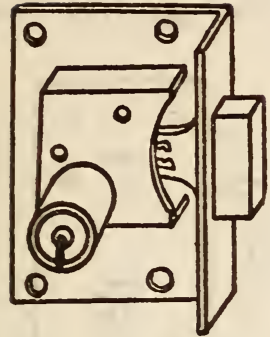

FIgURE 896.-Cupboard lock, type 540

Type 540.-Brass. Square selvedge; solid square box; broad, heavy bolt; three secure levers; cylinder with two flat steel keys; made for $7 / 8$ and $1 \frac{1}{4}$ inch wood. Size $2 \frac{1}{2}$ by $1 \frac{1}{2}$ inches. Backset $3 / 4$ inch.

See also draw-lock cut for cupboard, type 655, made for $7 / 8$-inch wood; size $13 / 4$ by $2 \frac{1 / 4}{4}$ inches, giving backset of $7 / 8$ inch.

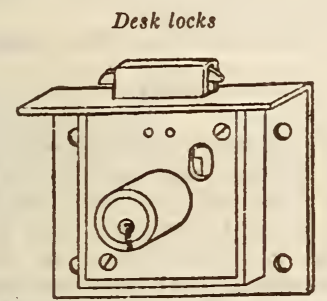

Figure 897.-Desk lock, type 601

Type 601.-All brass. Square selvedge; solid square box, screwed; three secure levers; doublehooked bolt; strike; cylinder with two flat steel keys. Size $1 \frac{5}{8}$ by 2 inches. Drop $7 / 8$ inch. Minimum thickness of plate 0.040 inch.

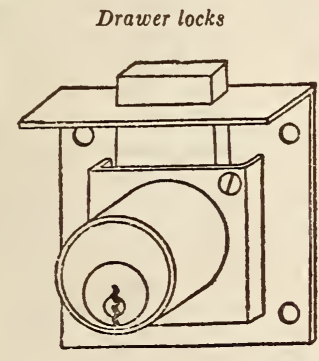

Figure 898.-Drawer lock, type 650

Type 650.-Brass. Pin tumbler; square selvedge; dead bolt; three keys. Drop 1 inch. Made for $7 / 8$-inch wood. Size $13 / 4$ by 2 inches. Cylinder $7 / 8$-inch diameter. Minimum thickness of plate 0.045 inch.

Type 650 A.-Same as type 650 , except size $1 \% 8$ by $21 / 4$ inches with $1 \frac{1}{8}$-inch diameter cylinder.
Type 650B.-Same as type 650A, except with spring bolt.

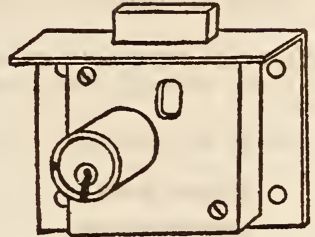

Figure 899.-Drawer lock, type 655

Type 655.-Brass. Cut for drawer or cupboard. Square selvedge; solid square box, screwed; broad heavy bolt; three secure levers; cylinder with two flat steel keys; made for $7 / 8$-inch wood. Size $13 / 4$ by $2 \frac{1}{4}$ inches. Drop $7 / 8$ inch. Minimum thickness of plate 0.040 inch.

Type 655 A.- Same as type 655, except steel.

Type 656.-Brass. Square selvedge; two secure levers; cylinder with two flat steel keys. Made for $7 / 8$-inch wood. Size $17 / 16$ by $13 / 4$ inches. Drop $3 / 4$ inch. Minimum thickness of plate 0.040 inch.

Type 656 A.-Same as type 656, except steel.

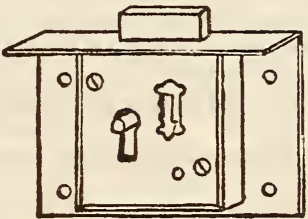

Figure 900.-Drawer lock, type 680

Type 680.-Steel, with brass bolt. Square selvedge; solid square box, screwed; broad heavy bolt; two secure levers; one barrel key. Size $1 \% 16$ by $2 \frac{1}{8}$ inches. Drop $5 / 8$ inch. Minimum thickness of plate 0.040 inch.

Type 680A.-Same as type 680, except steel. Size $13 / 4$ by $2 \frac{1}{2}$ inches. Drop $7 / 8$ inch. Minimum thickness of plate 0.040 inch.

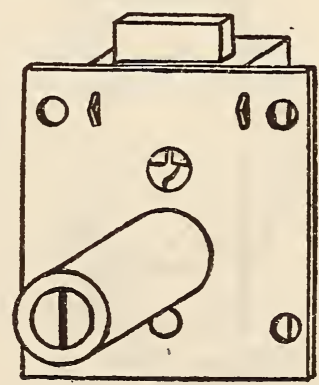

Figure 901.-Drawer lock, type 690

Type 690.-Brass. Surface; two secure levers; strike; cylinder with two flat steel keys. Size $17 / 8$ by $1 \frac{5}{8}$ inches. Drop $1 \frac{1}{8}$ inches. Minimum thickness of plate 0.040 inch. Made for $3 / 4$-inch wood, 
Type $690 A$.- Same as type 690 , except for $7 / 8$-inch wood.

Type 690A1.- Same as type 690 , except for $1 \frac{1}{8}-$ inch wood.

Type 690B.-Same as type 690, except steel.

Type 690B1.- Same as type 690, except steel for $7 / 8$-inch wood.

Type 690B2.- Same as type 690, except steel for $11 \frac{1}{8}$-inch wood.

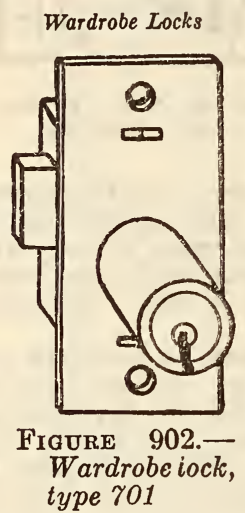

Type 701.-Brass. Solid square box; two secure levers; bolt shall shoot right and left; cylinder with two flat steel keys. Size $17 / 8$ by $7 / 8$ inches. Made for $7 / 8$-inch wood. Minimum thickness of plate, 0.040 inch.

Type 701 A.-Same as type 701, except steel with brass bolt.

Type 701B.-Same as type 701, except size 23/4 by $1 \frac{11}{4}$ inches. Made for $7 / 8$-inch wood.

Type 701B1.-Same as type 701B, except for 1 -inch wood.

Type 701B2.-Same as type 701B, except for $11 / 8$-inch wood.

Type 701C.- Same as type 701, except steel with brass bolt. Size $23 / 4$ by $1 \frac{1 / 4}{4}$ inches. Made for $7 / 8$-inch wood.

Type 701C1.-Same as type 701C, except for 1-inch wood.

Type 701C2.-Same as type 701C, except for $11 / 8$-inch wood.

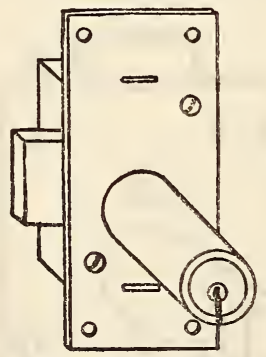

Figure 903.-

Wardrobe lock, type 702

Type 702.-Brass. Solid square box, screwed; two secure levers; bolt shall shoot right and left; cylinder with two flat steel keys. Size 3 by 15\% inches. Made for $7 / 8$-inch wood. Minimum thickness of plate 0.040 inch.

Type 702A.-Same as type 702, except for 1-inch wood.

Type 702 A1.-Same as type 702, except for $1 \frac{1}{8}$-inch wood.

Type 702B.-Same as type 702, except steel with brass bolt. Made for $7 / 8$-inch wood.
Type 702B1.-Same as type 702B, except for 1-inch wood.

Type 702B2.- Same as type 702B, except for $11 / 8$-inch wood.

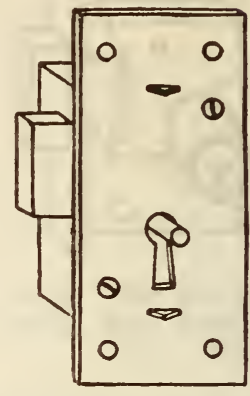

Figure 904.

Wardrobe lock, type 730

Type 730.-Brass. Solid square box, screwed: two secure levers; bolt shall shoot right and left; one barrel key. Size $23 / 4$ by $1 \frac{11}{4}$ inches. Minimum thickness of plate, 0.40 inch.

Type 780A.-Same as type 730, except steel with brass bolt.

\section{NOTES}

I-5a. In Table 2 below are listed for convenient reference the minimum size and throw required for latch and dead bolts for the locks, latches, transom catches, and cupboard turns covered by this specification. (Lock requirements are given above. Latch, transom-catch, and cupboard-turn requirements will be found under their respective commodity designations as listed in detail requirements in 617.0, p. 979.)

TABLE 2.-Minimum dimensions for lock and latch bolts

[Expressed to the nearest 1/64th inch]

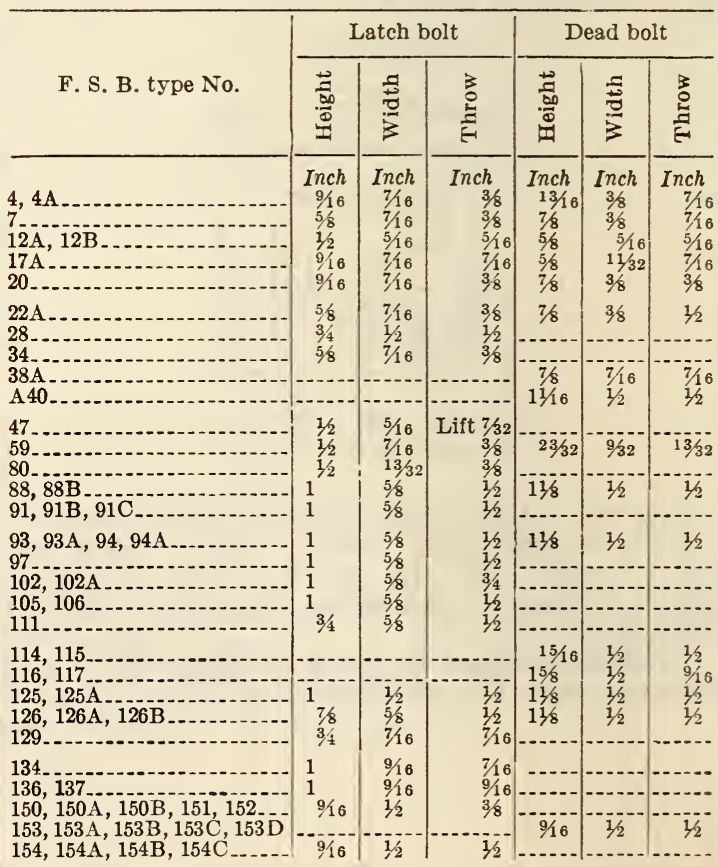


TABLE 2.-Minimum dimensions for lock and latch bolts-Continued

[Expressed to the nearest 1/64th inch]

\begin{tabular}{|c|c|c|c|c|c|c|}
\hline \multirow[b]{2}{*}{ F. S. B. type No. } & \multicolumn{3}{|c|}{ Latch bolt } & \multicolumn{3}{|c|}{ Dead bolt } \\
\hline & 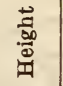 & $\frac{\pi}{3}$ & 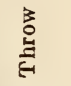 & 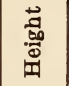 & $\underset{3}{\stackrel{0}{0}}$ & है \\
\hline $\begin{array}{l}160 \\
161,162,163,164,165 \\
170,171,172, \ldots \\
174,174 \mathrm{~A}, 174 \mathrm{~B}\end{array}$ & $\begin{array}{l}\text { Inch } \\
1 / 2 \\
3 / 4 \\
5 / 8 \\
58 \\
58\end{array}$ & $\begin{array}{l}\text { Inch } \\
3 / 8 \\
5 / 8 \\
11 / 4 \\
11 / 4 \\
11 / 4\end{array}$ & $\begin{array}{r}\text { Inch } \\
3 / 8 \\
3 / 8 \\
5 / 8 \\
5 / 8 \\
5 / 8\end{array}$ & $\left|\begin{array}{c|}\text { Inch } \\
\hdashline 5 / 16\end{array}\right|$ & $\begin{array}{l}\text { Inch } \\
7 / 8\end{array}$ & \begin{tabular}{|c|} 
Inch \\
\hdashline $1 / 2$
\end{tabular} \\
\hline $\begin{array}{l}175,175 \mathrm{~A}, 176,176 \mathrm{~A} \\
1778,178 \mathrm{~A} \\
179,179 \mathrm{~A}\end{array}$ & $\begin{array}{l}5 / 8 \\
58 \\
5 / 8 \\
5 / 8\end{array}$ & $\begin{array}{l}11 / 4 \\
11 / 4 \\
116 \\
11 / 4\end{array}$ & $\begin{array}{l}16 \\
5 / 8 \\
3 / 4 \\
5 / 8\end{array}$ & $5 / 16$ & 78 & \begin{tabular}{c}
$1 / 2$ \\
$-1-1$ \\
\hdashline-1
\end{tabular} \\
\hline $\begin{array}{l}1082,1082 \mathrm{~A} \\
1082 \mathrm{~B} \\
1082 \mathrm{C} 3,1083 \mathrm{~A}, 1083 \mathrm{~B}\end{array}$ & $\begin{array}{l}13 / 32 \\
3 / 8 \\
3 / 8 \\
1 / 2\end{array}$ & $\begin{array}{l}5 / 16 \\
11 / 32 \\
5 / 16 \\
3 / 8\end{array}$ & $\begin{array}{l}7 / 32 \\
7 / 32 \\
7 / 32 \\
5 / 16\end{array}$ & - & & 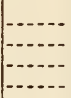 \\
\hline $\begin{array}{l}1083 \mathrm{C} \\
1097,1097 \mathrm{~A}-100 \\
1100 \mathrm{~A}\end{array}$ & $\begin{array}{l}36 \\
1 / 2 \\
2164 \\
1 / 2\end{array}$ & $\begin{array}{l}3,8 \\
3,8 \\
3 / 8 \\
3,8\end{array}$ & $\begin{array}{l}5 / 16 \\
5 / 16 \\
1 / 2 \\
7 / 16\end{array}$ & & & $\begin{array}{l}-- \\
-- \\
-\end{array}$ \\
\hline
\end{tabular}

UNITED STATES GOVERNMENT, FEDERAL SPECIFICATIONS BOARD, FEDERAL SPECIFICATION FOR PADLOCKS, FF-P-101, JUNE 24, 1930

\section{PADLOCKS}

\section{A. APPLICABLE FEDERAL SPECIFICATIONS}

A-1. There are no Federal specifications applicable to this specification.

\section{B. TYPES AND SIZES}

B-1. This specification covers the following general types and sizes of padlocks:

Type I.-Pin-tumbler mechanism, bronze or brass case. Sizes, $2,1^{3} \frac{1}{4}$, and $1 \frac{1}{2}$ inches.

Type II.-Pin-tumbler mechanism, push-up shackle, bronze or brass case. Size, $11 \frac{1}{8}$ inches.

Type III.-Lever-tumbler mechanism, bronze or brass case. Size, 21'4 inches.

Type IV.-Lever-tumbler mechanism, bronze or brass case. Size, $11^{\prime}$ inches.

Type V.-Lever-tumbler mechanism, brass-plated steel case. Size, 2 inches.

Type VI.-Lever-tumbler mechanism, bronze case. Steel shackle. Sizes, 11/2, 13/4, and 2 inches.

Type VII.- Warded mechanism secure type, laminated or solid steel case, cadmium plated. Sizes, $11^{\prime}, 13^{\prime}{ }^{\prime}$, and $17 / 8$ inches.

\section{MATERIAL AND WORKMANSHIP}

C-1. Parts specified to be of bronze or brass may be of either material. No particular composition is required for nickel-bronze keys.

C-2. All interior parts of locks, except as noted, shall be of suitable noncorrosive metals.

C-3. Padlocks shall be of substantial construction designed to withstand sever usage. Working parts shall be accurately fitted. Lock mechanism shall be so constructed that it can not be jarred open. All parts of padlocks shall be well finished.

C-4. Chains, where called for, shall be secured to padlocks with suitable clevises so attached as not to interfere with the operation of the lock, and may be attached to either case or shackle, except where otherwise indicated. Clevises for brass or bronze padlocks shall be of brass or bronze.

\section{GENERAL REQUIREMENTS}

D-1. Unless otherwise specified, all padlocks in any one order shall be operated only by their individual keys, within the limit of the number of key changes specified below.

D-2. The name or trade-mark of the manufacturer shall be legibly stamped or cast on each padlock. The change number of the lock shall be legibly stamped in a distinctive noncommercial code on all padlocks and keys of the pin-tumbler type. The code symbol shall indicate to the manufacturer that it is a Government padlock. Duplicate keys shall not be furnished except on recognized authority. Additional identification marks shall be provided when specified.

D-3. The principal dimensions and weights given are approximate minimum requirements, however, a tolerance of 5 per cent under will be permitted.

D-4. Plugs used to close openings in lock case, through which mechanism is inserted, shall be secured in such a manner as to prevent easy removal of same.

\section{E. DETAIL REQUIREMNETS}

Type I.-Pin-tumbler mechanism, 4 or more tumblers. With or without removable and interchangeable core feature. Case and shackle bronze or brass, polished finish. Self-locking spring shackle extension type; shackle shall be locked at toe or at both toe and heel. Lock shall be capable of providing at least 3,200 key changes absolutely safe from key interchanging. Master-keyed when so ordered. Two nickel-bronze milled keys. Size, 2, $13 \frac{1}{4}$, and $11 \% 2$ inches. Weight with 2 keys, 11,9 , and 5 ounces.
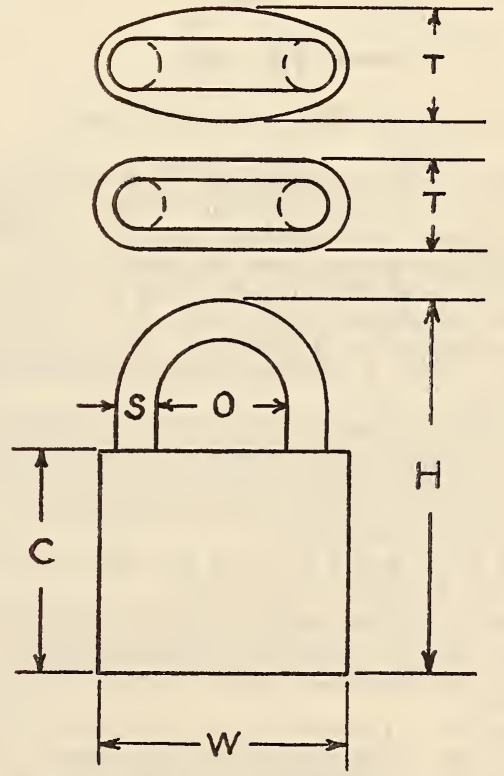

FIgure 905.-Padlock, Type $I$

\begin{tabular}{|c|c|c|c|c|c|c|}
\hline \multirow{2}{*}{$\begin{array}{c}\text { Size (inches) } \\
\text { W }\end{array}$} & \multicolumn{5}{|c|}{ Dimensions (inches) } & \multirow{2}{*}{$\begin{array}{l}\text { Weight } \\
\text { with } 2 \\
\text { keys }\end{array}$} \\
\hline & $\mathrm{C}$ & H & 0 & $\mathrm{~S}$ & $\mathbf{T}$ & \\
\hline $\begin{array}{l}2 \\
13 / 4 \\
11 / 2 \ldots\end{array}$ & $\begin{array}{l}15 / 8 \\
15 / 8 \\
11 / 4\end{array}$ & $\begin{array}{l}27 / 8 \\
25 / 8 \\
21 / 4\end{array}$ & $\begin{array}{r}15 / 16 \\
13 / 16 \\
5 / 8\end{array}$ & $\begin{array}{r}1132 \\
5 / 16 \\
1 / 4\end{array}$ & $\begin{array}{l}13 / 16 \\
13 / 16 \\
21 / 32\end{array}$ & $\begin{array}{r}\text { Ounces } \\
11 \\
9 \\
5\end{array}$ \\
\hline
\end{tabular}


Type Ia.-Same as Type I, but with bronze or brass chain 9 inches long, plus or minus one link, attached to body of lock. Chain shall be either flat lock link 0.072-inch wire (No. 4 triumph type), or No. 0 sash chain, 0.028-inch metal.

Type Ib.-Same as Type I, but with casehardened steel shackle, rustproofed.

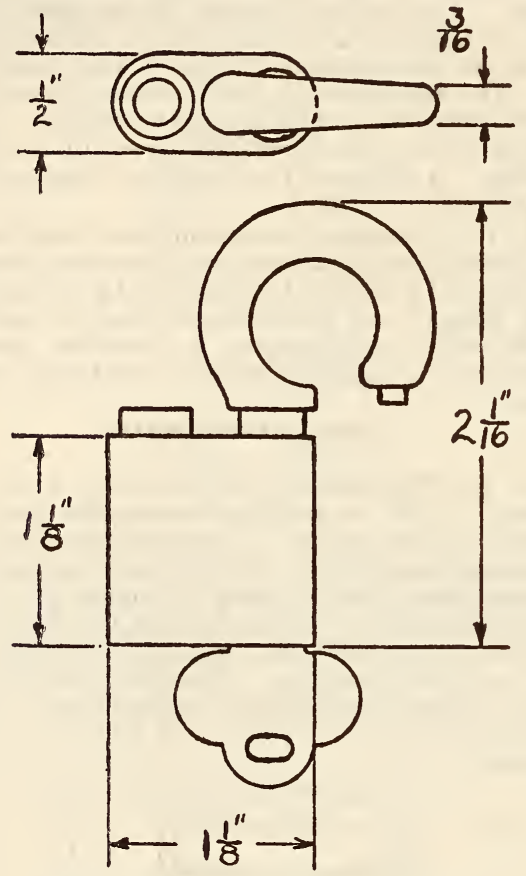

Figure 906.-Padlock, Type II

Type II.-Pin-tumbler mechanism, four or more tumblers. Case and shackle bronze or brass, polished finish. Self-locking push-up shackle type. Dutchman or key at base of shackle shall be steel. Lock shall be capable of providing at least 1,600 key changes absolutely safe from key interchanging. Two nickel-bronze milled keys. Shackle spring may be steel. Size, 11/8 inches. Weight with two keys, $3 \frac{3}{4}$ ounces.

Type II a.-Same as Type II, with bronze or brass chain 9 inches long, plus or minus one link, attached to body of lock. Chain shall be either flat lock link 0.072 -inch wire (No. 4 triumph type) or No. 0 sash chain, 0.028-inch metal.

Type IIb.-Same as Type II, but with casehardened steel shackle, rustproofed.

Type IIc.- Same as Type II, but with shackle not less than 0.220 inch in diameter and such that it will readily pass through a hole five-sixteenths inch in diameter.

Type III.-Lever-tumbler mechanism, three secure levers. Case and shackle cast bronze or brass. Wheel finish. Self-locking spring shackle, hinged type. One-hundred and twenty changes when not master-keyed. Master-keyed when so ordered.
Two nickel-plated flat steel keys. Size, $2 \frac{1}{4}$ inches. Weight, with two keys, $71 / 2$ ounces.

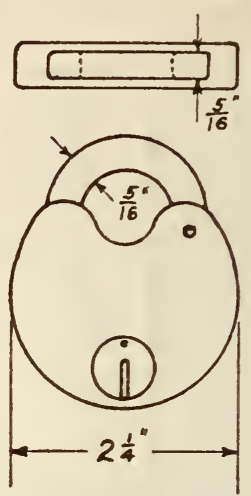

Figure 907.-Padlock, Type III

Type IIIa.-Same as Type III, but with bronze or brass chain 9 inches long, plus or minus one link. Chain shall be either flat lock link 0.072 -inch wire (No. 4 triumph type) or No. 0 sash chain, 0.028 -inch metal.
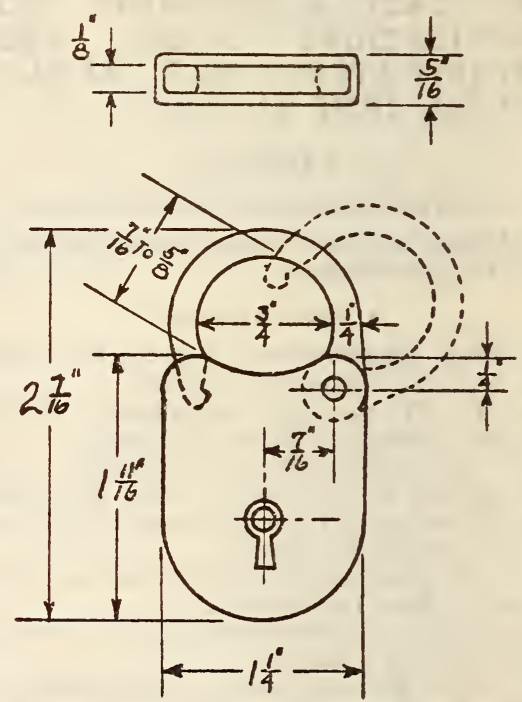

Figure 908.-Padlock, Type $I V^{\top}$

Type IV.-Lever-tumbler mechanism, three or more tumblers. Case and shackle wrought bronze or brass. Self-locking spring shackle, hinged type, shall be of flat section indicated, or round section of equivalent cross-sectional area. Not required to be master-keyed. Two brass or bronze barrel keys. Size, 11/4 inches. Weight with two keys, $21 / 4$ ounces. Type IVa.-Same as Type IV but with No. 0 size, 0.021 -inch bronze or brass safety chain 9 inches long, plus or minus one link. 
Type V.-Brass-plated heavy steel case. Brassplated malleable iron shackle; 6 lever tumblers; 12 changes. Two nickel-plated, double-bitted, drilled

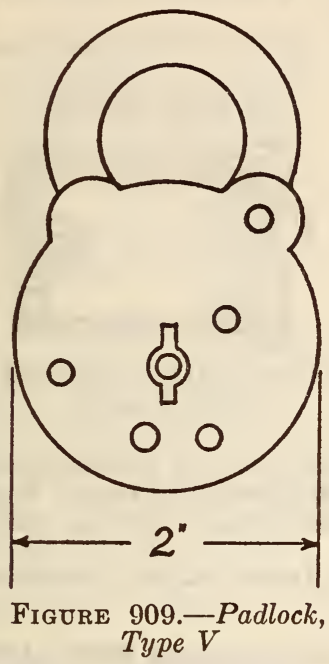

barrel keys. This lock is not required to pass the corrosion test. Size, 2 inches. Weight with two keys, $52 / 3$ ounces.

Type Va.-Same as Type V, but with chain 9 inches long plus or minus one iink. Chain shasl be either flat lock link, 0.072-inch wire (No. 4 triumph type) or No. 0 sash chain, 0.028 -inch metal.

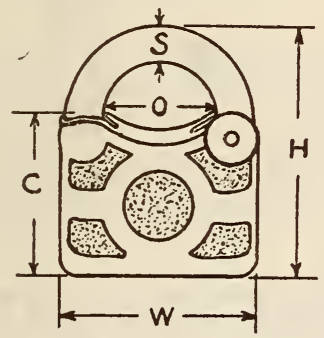

FigURE 910.-Padlock, Type VI

Type VI.-Lever-tumbler mechanism. Cast bronze case, wheel finish. Round or square casehardened steel shackle of self-locking hinged type. Not to be master-keyed. Two nickel-bronze or brass keys. This lock is not required to pass the corrosion test.

\begin{tabular}{|c|c|c|c|c|c|c|c|}
\hline \multirow{2}{*}{ Size (inches) } & \multirow{2}{*}{$\begin{array}{c}\text { Key } \\
\text { changes }\end{array}$} & \multirow{2}{*}{$\begin{array}{l}\text { Number } \\
\text { lever } \\
\text { tumblers }\end{array}$} & \multicolumn{4}{|c|}{ Dimensions (inches) } & \multirow{2}{*}{$\begin{array}{c}\text { Weight } \\
\text { with 2 } \\
\text { keys }\end{array}$} \\
\hline & & & $\mathrm{C}$ & H & $\mathrm{O}$ & $\mathrm{S}$ & \\
\hline $\begin{array}{l}1126 \\
13 / 4 \ldots \\
2\end{array}$ & $\begin{array}{r}48 \\
72 \\
144\end{array}$ & $\begin{array}{l}3 \\
4 \\
4\end{array}$ & $\begin{array}{l}11 / 4 \\
13 / 8 \\
11 / 2\end{array}$ & $\begin{array}{l}2 \\
21 / 4 \\
21 / 2\end{array}$ & $\begin{array}{l}13 / 16 \\
15 / 16 \\
11 / 16\end{array}$ & $\begin{array}{r}1 / 4 \\
5 / 16 \\
3 / 8\end{array}$ & $\begin{array}{c}\text { Ounces } \\
5 \\
61 / 3 \\
9^{1 / 2}\end{array}$ \\
\hline
\end{tabular}

Type VIa.-Same as Type VI, but with bronze or brass chain 9 inches long, plus or minus one link. Chain shall be either flat-lock link 0.072 -inch wire (No. 4 triumph type) or No. 0 sash chain 0.028-inch metal.
Type VII.-Warded mechanism-secure type with two or more springs locking shackle. Laminated or solid steel case, unground, cadmium plated. Selflocking spring shackle, extension type. Minimum

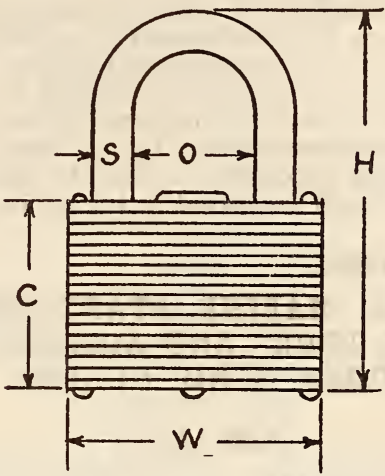

FI g U R 911 - Padlock, Type VII

number of key changes as specified in table. Master keyed when so ordered. Two nickel-plated steel keys.

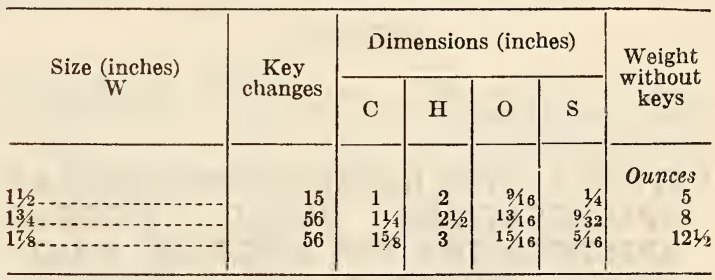

Type VIIa.-Same as Type VII except with cadmium-plated welded steel chain 9 inches long plus or minus one link. Twisted-wire type chain of 0.072 inch diameter wire.

\section{F. METHODS OF SAMPLING, INSPECTION, AND TESTS}

F-1. One lock per gross, or fraction thereof, shall be taken from any shipment and examined to determine whether they comply with this specification.

F-2. Samples of Types I, II, III, IV, and VII shall be subjected to the following corrosion test:

F-2a. Submerge padlock for 1 hour each day for one week in a 3.5 per cent (by weight) salt (sodium chloride) solution at room temperature (about $20^{\circ}$ C. or $68^{\circ} \mathrm{F}$.), and then hang to dry in the open air for 23 hours. At the end of the week the lock shall permit being opened by its own key without undue force, and shall permit of being relocked and opened without undue difficulty. Failure of samples to withstand this test will result in rejection of the entire delivery.

\section{G. PACKING AND MARKING}

G-1. Locks shall be packed separately with their keys in suitable containers.

G-2. Containers shall be labeled with the type, size, and number of locks contained.

G-3. Locks, when master keyed or when keyed alike, shall be packed in separate lots and labeled to indicate the method of keying.

G-4. Deliveries shall also be marked with the number of the contract or order under which delivery is made. 


\section{NOTES}

I-1. If more than two keys are required for each lock, the number of keys per lock should be specified in the contract or order.

I-2. Each contract or order should state whether all padlocks are to fit the same key, all different keys, or the number of different keys required.

I-3. When master-keyed locks are required, the manufacturer should be required to lay out additional changes than covered by the order to provide for such future expansion as may be contemplated, and which should be stated in the order.

617.22 LATCHES.

\section{AMERICAN MARINE STANDARDS COM- MITTEE, LOCKS AND ACCESSORIES FOR SHIP DOORS, H NO. 61-1930}

\section{RIM LATCH}

(See 617.21, p. 1012.)

UNITED STATES GOVERNMENT, DEPARTIMENT OF COMMERCE, BUILDERS' HARDWARE (NONTEMPLATE), COMMERCIAL STANDARD CS22-30, DECEMBER 11, 1930

\section{LATCHES}

(See 617.0, p. 977, also Federal specification FF-H-101 below.)

\section{UNITED STATES GOVERNMENT, FEDERAL SPECIFICATIONS BOARD, FEDERAL SPECIFICATION FOR BUILDERS' HARD- WARE (NONTEMPLATE), FF-H-101, AUGUST 19, 1930}

(For types, grades, and classes; material and workmanship; general requirements; detail requirements; packaging, packing, and marking; notes; and table of standard finishes, see 617.0, p.978. For chromium plating, nickel plating, and zinc coatings; and methods of inspection and tests, see 600.3, p. 70.)

\section{BUILDERS' HARDWARE (NONTEMPLATE)}

(Tubular locks and latches, cylindrical case locks and latches, and unit type locks and latches will be found under 617.21 , p. 1020.)

\section{MORTISE KNOB LATCEES}

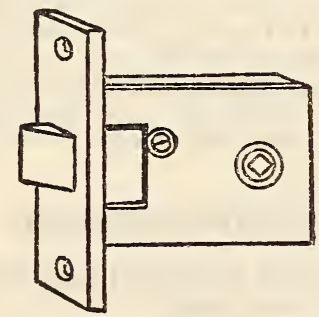

Figure 912.-Mortise knob latch, type 28

Type $28 .-2 \frac{1}{2}$ by $3 \frac{1}{2}$ by $5 / 8$ inch japanned iron case. Backset $23 / 4$ inches. Front $4 \frac{1}{2}$ by 1 inch. Latch bolt, minimum $3 / 4$ by $1 / 2$ inch, minimum throw onehalf inch. Double spring type. Cast bronze front and bolt. Brass hub. Bronze strike.

Operation. - Latch bolt by knob from either side.
Type $34 .-23 / 8$ by $2 \frac{1}{4}$ by $5 / 8$ inch japanned iron case. Backset $1 \frac{1}{2}$ inches. Front 4 by 1 (minimum $7 / 8$ ) inch. Latch bolt, minimum $5 / 8$ by $7 / 16$ inch, minimum throw $3 / 8$ inch. Bronze front and bolt. Brass hub. Bronze strike.

Operation.-Latch bolt by knob from either side. Reversible.

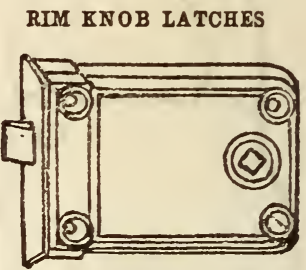

Figure 913.-Rim knob latch, type 80

Type $80 .-25 / 8$ by $33 / 4$ by $5 / 8$ inch japanned iron case. Rim strike. Backset $23 / 4$ inches. Single iron latch. Latch bolt, minimum $1 / 2$ by $13 / 32$ inch, with $8 / 8$-inch throw.

Operation.-Latch bolt by knob from either side. Reversible.

\section{CYIINDER MORTISE NIGHT LATCHES}

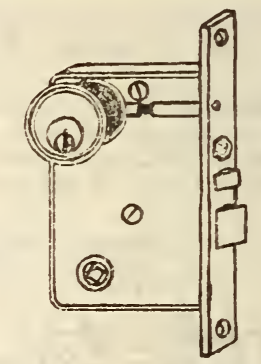

Figure 914.-Cylinder mortise night latch, type 111

Type 111. - $3 \frac{1}{2}$ by $3 \frac{5}{8}$ by $3 / 4$ inch japanned iron case. Backset $23 / 4$ inches. Solid front 5 by $1 \frac{1}{8}$ inches. Latch bolt, minimum $3 / 4$ by $5 / 8$ inch, minimum throw $1 / 2$ inch. Latch shall have one bronze cylinder with or without removable and interchangeable core feature, with five or more pin tumblers giving practically unlimited changes. Bronze front, bolts, hub, and strike. Three nickel-bronze keys.

Operation. - Latch bolt by knob from inside and key from outside. When door is closed, auxiliary bolt shall automatically deadlock the latch bolt. Bolt may be held back by stop in front. Not reversible.

\section{RIM NIGHT LATCHES}

Tubular

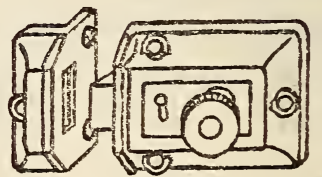

Figure 915.-Rim night latch, tubular, type 129

Type 129.- $-2 \frac{1}{4}$ by 3 inches, japanned iron case. Backset 2 inches. Latch bolt, minimum $3 / 4$ by $7 / 16$ inch, minimum throw $7 / 16$ inch. Latch shall have 12 changes. Bronze turn knob and slide stop. Bronze plated bolt and knob. Two polished steel flat keys. 
Operation.-Latch by key from outside and by turn knob from inside. Slide stop. Reversible.

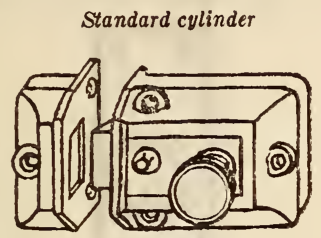

Figure 916.-Rim night latch, standard cylinder, type 134

Type $134 .-2 \frac{1}{2}$ by 3 inches, japanned iron case. Backset $15 / 8$ or 2 inches. Latch bolt, minimum 1 by $\% / 16$ inch, minimum throw $7 / 16$ inch. Latch shall have one standard bronze cylinder, with or without removable and interchangeable core feature, with five or more pin tumblers providing practically unlimited changes. Screws for latch and strike shall be at least $1 \frac{3 / 4}{4}$ inches long. Bronze bolt, knob, and slide stop. Three nickel-bronze keys.

Operation.-Latch by key from outside and by turn knob from inside. Slide stop. Reversible.

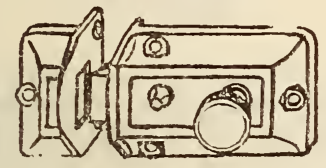

\section{FIGURE 917.-Rim night latch, standard cylinder type 136}

Fype $136 .-21 / 2$ by $3 \frac{1}{2}$ inches, japanned iron case. Backset $23 / 8$ inches. Latch bolt, minimum 1 by $\% / 10$ inch, minimum throw $\% 16$ inch. Latch shall have one standard bronze cylinder, with or without removable and interchangeable core feature, with five or more pin tumblers providing practically unlimited changes. Bronze bolt, knob, and slide stop. Three nickel-bronze keys.

Operation. - Latch by key from outside and by turn knob from inside. Slide stop. Reversible.

Type 137.- Same as type 136, except all bronze.

SECRET GATE LATCHES

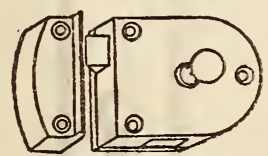

Figdre 918.-Secret gate latch, type A1187

Type A118\%.-Cast bronze. Single acting. Size 2 by $2 \frac{3}{8}$ inches.
THOMB LATCHES

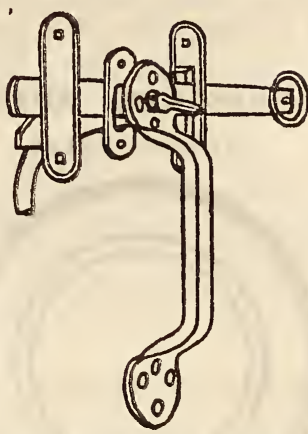

Figdre 919.-Thumb latch, type 1189

Type 1189.-Extra heavy. Wrought steel, galvanized. For doors $13 \% 4$ to $2 \frac{1}{2}$ inches thick. Handle 10 inches.

Type 1189 A.-Same as type 1189 , except wrought steel, japanned.

Type A1196.-Duplex type. Extra heavy wrought steel, galvanized or japanned. For doors $13 \%$ to $21^{\prime}$ inches thick. Handle plates $14 \frac{1}{2}$ inches, handles 10 inches long.

617.23 LOCK PARTS.

AMERICAN MARINE STANDARDS COMMITTEE, LOCKS AND ACCESSORIES FOR SHIP DOORS, H NO. 61-1930

ESCUTCHEONS AND ROSETTE

(See 617.21, p. 1014.)

UNITED STATES GOVERNMENT, DEPARTMENT OF COMMERCE, BUILDERS' HARDWARE (NONTEMPLATE), COMMERCIAL STANDARD CS22-30, DECEMBER 11, 1930

\section{PADIOCK EYES}

(See 617.0, p. 977, also Federal Specification FF-H-101 below.)

\section{UNITED STATES GOVERNMENT, FED. ERAL SPECIFICATIONS BOARD, FED. ERAL SPECIFICATION FOR BUILDERS' HARDWARE (NONTEMPLATE), FF-H- 101, AUGUST 19, 1930}

(For types, grades, and classes; material and workmanship; general requirements; detail requirements; packaging, packing, and marking; notes; 
and table of standard finishes, see 617.0, p. 978. For chromium plating, nickel plating, and zinc coatings; and methods of inspection and tests, see 600.3, p. 70.)

\section{BUILDERS' HARDWARE (NONTEMPLATE)} CYLINDER COLLAR

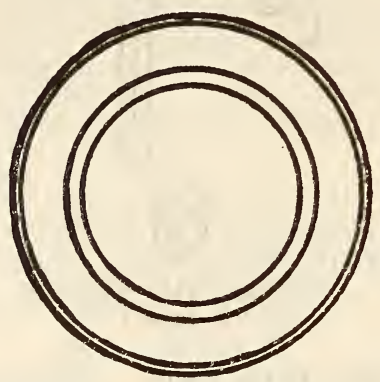

F1G URE $920 .-C y l i n d e r$ collar, type 320

Type 320.-Wrought or cast bronze, buffed. Diameter as required to fit cylinder.

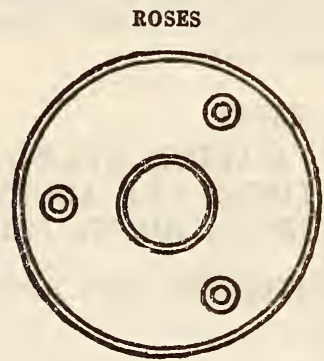

FI G U R 921 . - Rose, type 330

Type 330.- Wrought bronze, buffed, with wrought bracket bearing type thimble to suit knob shanks. Size, 2 inch diameter.

Type $330 A_{1}$ - Same as type 330, except cast bronze and thinbles with machine-finished bearings.

\section{KEY PLATES}

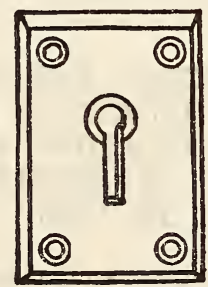

Figure 922.-

Key plate

type 350

Type 350.- Wrought bronze, buffed, with square corners, beveled edges, and two screw holes. Size $13 \frac{1}{4}$ by $1 \frac{118}{8}$ inches. Minimum thickness of plate 0.025 inch.

Type 350A.- Same as type 350, except cast bronze, and four screw holes.

Type 351. - Wrought bronze, buffed. Oval shape, 2 screw, plain surface. Size minimum $15 \%$ by $1 \frac{1}{8}$ nches. Minimum thickness of plate 0.022 inch.

Type 352.- Thread escutcheon. Cast brass 1 by $5 / 8$ inch.

\section{ESCOTCHEONS}

For mortise cylinder locks

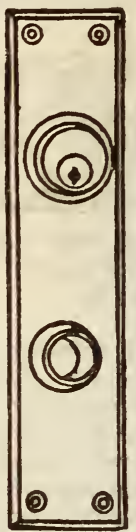

Figure 923. -

Escutcheon for mortise cylinder locks, type 300

Type 300.-Wrought bronze, with square corners and medium beveled edges; drilled to suit cylinder, and shall have bracket bearing type thimble to suit knob shanks. Thimbles shall be reinforced at back with washers to bear against door. Approximate sizes, 7 by $23^{\prime}$ and 10 by $2^{3}{ }_{4}^{\prime}$ inches. Minimum thickness of wrought plates, 0.028 and 0.035 inch, respectively.

Type 300 A.- Same as type 300 , except cast bronze and thimbles with machine-finished bearings.

For mortise bit key locks

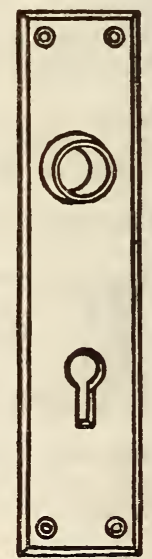

F1GURE 924.

Escutcheon for mortise bit key locks, type 301

Type 301.-Wrought bronze, with square corners and medium beveled edges; cut to suit key; shall have wrought bracket-bearing type thimble to suit knob shanks. Thimble shall be reinforced at back with washers to bear against door. Approximate sizes, 7 by $23^{\prime}, 8$ by $2 \frac{1}{\prime}$, and 10 by $2^{3}{ }^{\prime}$ inches. Minimum thickness of wrought plates, $0.028,0.028$, and 0.035 inch, respectively.

Type 301A.-Same as type 301 , except cast bronze, and thimbles with machine-finished bearings. 
Type 302.-Wrought bronze, with square corners and medium beveled edges; complete with bracketbearing thimble to suit knob shanks and $13 / 8$-inch turn knob. Thimbles shall be reinforced at back

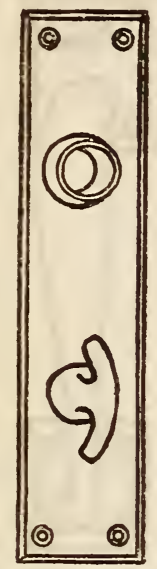

Figure 925.-

Escutcheon, type 302

with washers to bear against door. Approximate sizes, 7 by $2 \frac{1}{\prime}, 8$ by $2 \frac{1}{2}$, and 10 by $2^{3}{ }^{\prime}$ inches. Minimum thickness of wrought plates, $0.028,0.028$, and 0.035 inch, respectively.

Type 302A.- Same as type 302, except cast bronze, and thimbles with machine-finished bearings.

PADLOCK EYES

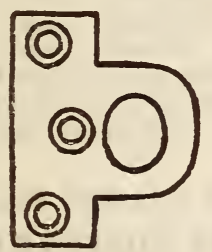

Figure 926.--

Padlock eye, type 1430

Type 1430.-Galvanized steel. Size of plate about $2 \frac{1}{8}$ by $17 / 8$ inches. Size of hole about $3 / 4$ by $5 / 8$ inch.

617.3 KNOBS, HANDLES, AND PULIS.

617.31 DOOR KNOBS.

AMERICAN MARINE STANDARDS COMMITTEE, LOCKS AND ACCESSORIES FOR SHIP DOORS, H. NO 61-1930

KNOBS

(See 617.21, p. 1014.)

UNITED STATES GOVERNMENT, DEPARTMENT OF COMMERCE, BUILDERS' HARDWARE (NONTEMPLATE), COMMERCIAL STANDARD CS22-30, DECEMBER 11, 1930

\section{DOOR KNOBS}

(See 617.0, p. 977 also Federal specification FF-H101, below.)
UNITED STATES GOVERNMENT, FEDERAL SPECIFICATIONS BOARD, FEDERAL SPECIFICATION FOR BUILDERS' HARDWARE (NONTEMPLATE), FF-H-101, AUGUST 19, 1930

(For types, grades, and classes; material and workmanship; general requirements; detail requirements; packaging, packing, and marking; notes; and table of standard finishes, see 617.0 , p. 978. For chromium plating, nickel plating, and zinc coatings; and methods of inspection and tests, see 600.3, p. 70.)

\section{BUILDERS' HARDWARE (NONTEMPLATE)}

DOOR KNOBS, METAL

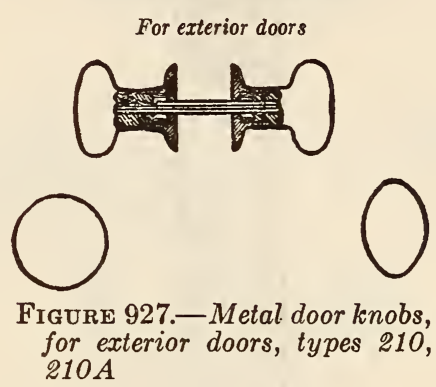

Type 210.-Seamless all cast bronze, buffed, for bracket-bearing type shank. Each pair of knobs complete with $5 / 16$-inch solid or swivel wrought steel spindle to fit and properly operate the lock for which required. Outside knob shall be secured to spindle with pin. Inside knob shall be secured with clamp or set screw. Shanks shall have machine-finished bearings. Spheroid type, size 21'4 inches diameter.

Type 210A.-Same as type 210, except ellipsoid type, $21^{\prime}$ inches minimum diameter on major axis.

Type 210B.-Same as type 210, except seamless wrought bronze, minimum thickness of metal 0.033 inch. Machine-finished bearings not required.

For interior doors

Type 211.- Same as type 210, except with threaded or triplex spindle.

Type 211 A.- Same as type 210, except ellipsoid type, $21^{\prime}$ inches minimum diameter on major axis, and with threaded or triplex spindle.

617.32 DOOR AND DRAWER HANDLES.

AMERICAN MARINE STANDARDS COMMITTEE, LOCKS AND ACCESSORIES FOR SHIP DOORS, H NO. 61-1930

\section{HANDLES}

(See 617.21, p. 1014.)

UNITED STATES GOVERNMENT, DEPARTMENT OF COMMERCE, BUIIDERS' HARDWARE (NONTEMPLATE), COMMERCIAL STANDARD CS22-30, DECEMBER 11, 1930

\section{HANDLES}

(See 617.0, p. 977, also Federal specifications FF-H-101 below.) 


\section{UNITED STATES GOVERNMENT, FEDERAL SPECIFICATIONS BOARD, FEDERAL SPECIFICATIONS FOR BUILDERS' HARD- WARE (NONTEMPLATE), FF-H-101, AU- GUST 19, 1930}

(For types, grades, and classes; material and workmanship; general requirements; detail requirements; packaging, packing, and marking; notes; and table of standard finishes, see 617.0, p. 978 . For chromium plating, nickel plating, and zinc coatings; and methods of inspection and tests, see 600.3 , p. 70.)

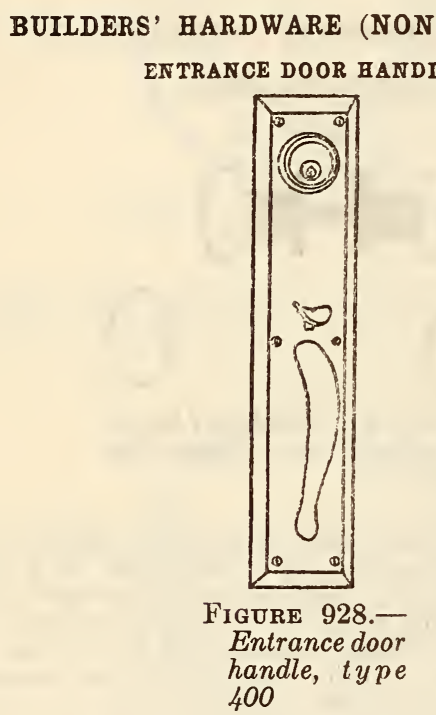

Type 400.-Wrought bronze plates with square corners and beveled edges, cut for cylinder or turn knob. Handles about 5-inch grip with thumb piece for operating latch bolt. Plates 14 by 3 inches. Minimum thickness of plate, 0.040 inch. Connections for handles shall have bosses on back to bear against door.

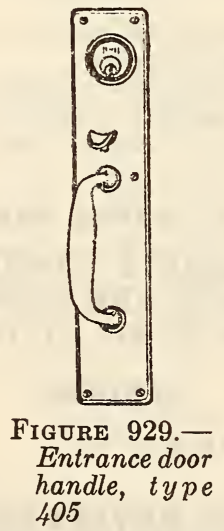

Type 405.-Cast iron, finish US18. Plate about 15 by 3 inches with round or square corners cut for cylinder or turn knob. Grip about $5 \frac{7 / 8}{8}$ inches on centers with thumb piece.

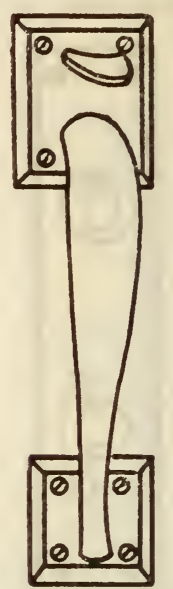

FrguRe 930.Entrance door handle, sectional trim, type 420

Type 420.-Cast bronze plates with square corners and beveled edges. Cylinder collar $21 / 4$ by $2 \frac{1}{4}$ inches. Grip about $5 \frac{3}{4}$ inches. Plates to suit.

\section{Dummy trim}

Type 430.-Dummy trim shall be furnished only when specified. Thimbles shall be required for knobs and turns. Dummy trim shall match the escutcheons specified for the lock for which dummy trim is required. Spindles shall extend through door and have plates to hold knob rigid.

617.33 DOOR AND DRAWER PULLS.

UNITED STATES GOVERNMENT, DEPART-

MENT OF COMMERCE, BUILDERS'

HARDWARE (NONTEMPLATE), COMMERCIAI STANDARD CS22-30, DECEMBER 11, 1930

\section{DOOR PULLS}

(See 617.0 , p. 977 , also Federal specification FF-H-101 below.)

\section{UNITED STATES GOVERNMENT, FEDERAI SPECIFICATIONS BOARD, FEDERAL SPECIFICATION FOR BUILDERS' HARD- WARE (NONTEMPLATE), FF-H-101, AUGUST 19, 1930}

(For types, grades, and classes; material and workmanship; general requirements; detail requirements; packaging, packing, and marking; note; and tables of standard finishes, see 617.0 , p. 978 . For chromium plating, nickel plating, and zinc coatings; and methods of inspection and tests, see 600.3 , p. 70.$)$ 
BUILDERS' HARDWARE (NONTEMPLATE)

DOOR PULLS

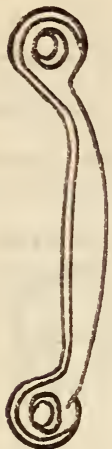

FIGURE 931.-

Door pull, type 1269

Type 1269.-Wrought bronze. Two screw holes; $31 / 2$ or $4 \frac{7}{8}$ inch handle.

Type 1269A.-Steel, plated. Two screw holes; $3 \frac{1}{2}$ or $47 / 8$ inch handle.

Type 1269B.-Steel, japanned. Two screw holes; $3 \frac{1}{2}$ or $4 \frac{7}{8}$ inch handle.

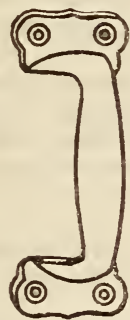

\section{FIgURE 932.- \\ Door pull, type 1274}

Type 1274.-Cast bronze. Four screw holes. 6 inches long.

Type 1274A.-Same as type 1274, except cast iron or wrought steel plated.

Type 1274B.-Same as type 1274, except cast iron or wrought steel, galvanized.

Type 1274C.-Same as type 1274, except cast iron or wrought steel, japanned.

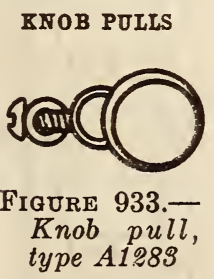

Type A1283.-Cast bronze. Not less than 1inch diameter. With machine screw.
DRAWER PULLS

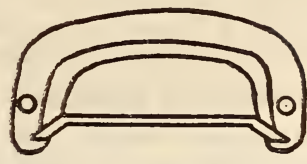

Figure 934.-Drawer pull, type 1296

Type 1296.-Cup pattern. Flat edge. Steel, plated. Size, $3 \frac{11}{4}$ inches.

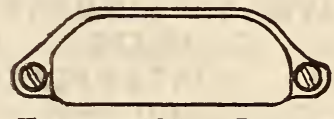

FigURE 935.-Drawer pull, type 1297

Type 1297.-Cup pattern. Narrow edge. Wrought bronze. Size, $3 \frac{1}{2}$ inches. Metal thickness not less than 0.035 inch.

Type 1298.-Cup pattern. Flat edge. Cast bronze. Size, $3 / 4$ inches.

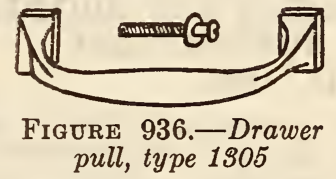

Type 1305.-Bar pattern. Rounded handle, rectangular ends. Cast bronze. With machine screws for fastening handles from inside. Size overall, 4 inches.

\section{DOOR PULLS ON PLATE}

Type 450.-Cast bronze with square corners and beveled edges. Size approximately 16 by $3 \frac{1}{2}$ inches. Pull handles shall be approximately 6 inches center to center, $3 / 4$-inch diameter at center of grip; and shall be secured with through bolts. Shall be drilled for cylinder outlets where same occur.

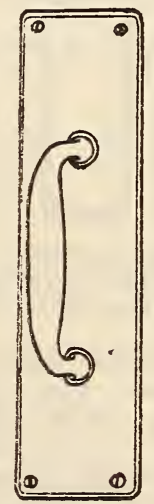

$$
\begin{aligned}
& \text { FigURe 937.- } \\
& \text { Door pull on } \\
& \text { plate, type } 451
\end{aligned}
$$

Type 451.-Wrought bronze with rounded corners and edges. Size approximately 10 by $2^{3 / 4}$ inches. 
Minimum thickness of plate 0.035 inch. Cast bronze pull handle secured with through bolts. Grip approximately 5 inches on centers. Shall be drilled for cylinder outlets where same occur.

DOOR PULLS, SECTIONAL

Type 452.-Plain cast bronze grip with through bolts. Grip approximately 6 inches on centers. Minimum diameter at center of grip three-fourths inch. Separate round cast bronze bases minimum diameter $1 \frac{3}{4}$ inches.

617.34 PUSH AND KICK PLATES AND BARS.

UNITED STATES GOVERNMENT, DEPARTMENT OF COMMERCE, BUILDERS' HARDWARE (NONTEMPLATE), COMMERCIAL STANDARD CS22-30, DECEMBER 11, 1930

\section{PUSH BARS, PUSH PLATES}

(See 617.0, p. 977, also Federal specification FF-H-101 below.)

\section{UNITED STATES GOVERNMENT, FEDERAL SPECIFICATIONS BOARD, FEDERAL SPECIFICATION FOR BUILDERS' HARD- WARE (NONTEMPLATE), FF-H-101, AUGUST 19, 1930}

(For types, grades, and classes; material and workmanship; general requirements; detail requirements; packaging, packing, and marking; notes; and table of standard finishes, see $617.0, \mathrm{p}$. 978. For chromium plating, nickel plating, and zinc coating; and methods of inspection and tests, see 600.3 , p. 70.)

\section{BUILDERS' HARDWARE (NONTEMPLATE)}

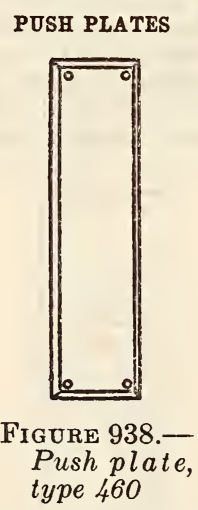

Type 460.-Cast bronze with square corners and medium beveled edges. Size approximately 16 by $3 \frac{1}{2}$ inches. Shall be drilled for cylinder outlets where same occur.

Type 461.- Cast bronze with square corners and medium beveled edges. Sizes 12 by $3 \frac{1}{2}$ inches and 16 by $3 \frac{1}{2}$ inches.

Type $461 \mathrm{~A}$.- Same as type 461 , except wrought bronze. Minimum thickness of plate 0.040 inch.

Type 462.-Wrought bronze with rounded corners and edges. Size 10 by $23 / 4$ inches. Minimum thickness of plate 0.035 inch.

Type 463.- Plate-glass push plates, polished, 12 by $3 \frac{1}{2}$ inches, with beveled edges, rubber washers, bronze rosettes and screws. Nickel bronze or nickel-plated bronze rosettes and screws shall be used in locations where nickel-bronze hardware is specified.
KICK PLATES

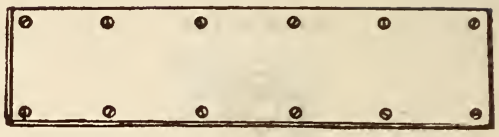

Figure 939.-Kick plate, type 1224

Type 1224.-Wrought bronze. Square corners, beveled edges. Plates 0.064 inch thick. Sizes as required.

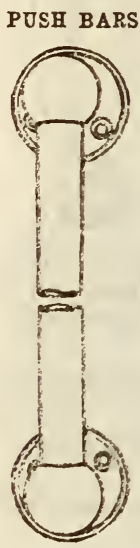

Figure 940.-Push bar, type 1009

Type 1009.-Cast bronze bases approximately 2 inches diameter. Wrought bronze tubing 1 inch diameter and $3 / 32$ inch thick, or tubing may be $1 / 32$ inch thick tightly shrunken over a steel core. Projection $23 \frac{1}{4}$ inches. Clearance $1 \frac{3}{8}$ inches. Lengths 24,30 , or 36 inches.

Type 1009A.-For double acting doors. Same as type 1009 except with cast bronze flat bracket for inner end.

Type A1009.-Cast bronze brackets three-fourths inch wide for surface of stiles. Solid wrought bronze bars $1 / 2$ to $5 / 8$ inch diameter, or bronze tubing $1 / 2$ to $5 / 8$ inch outside diameter with wall not less than 0.045 inch thick, 3 inches on centers; projection 1 inch, clearence $1 / 2$ inch. Length of bracket 6 inches with two bars. Length of bars as required.

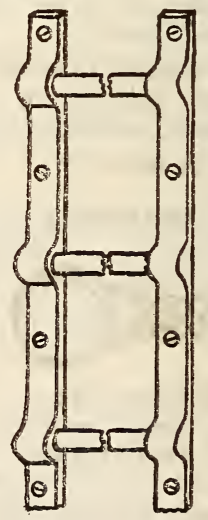

Figure 941Push bar, type 1009 A

Type A1009A.-Same as type A1009, except with length of bracket 9 inches and 3 bars. Length of bars as required. 
Type A1009B.-Same as type A1009, except with length of bracket 12 inches and 4 bars. Length of bars as required.

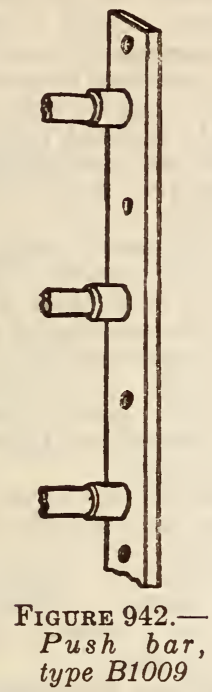

Type B1009.-Cast bronze brackets 1 inch wide for inside edges of stiles. Solid wrought bronze bars $1 / 2$-inch diameter, 3 inches on centers. Length of bracket 6 inches with two bars. Length of bars as required.

Type B1009A.-Same as type B1009, except with length of bracket 9 inches and 3 bars. Length of bars as required.

Type B1009B.- Same as type B1009, except with length of bracket 12 inches and 4 bars. Length of bars as required.

Type A1010.-Clothes-hanger bar, wrought steel telescoping tubing, plated; minimum wall thickness one thirty-second inch. Outer tube not less than 1 inch outside diameter. A metal wall plate shall be securely attached to each end of bar, and shall have at least two screw holes for attaching to hook strip in closet. Size 18 inches, adjustable from 18 to 30 inches; size 30 inches, adjustable from 30 to 48 inches; size 48 inches adjustable from 48 to 72 inches; and size 72 inches adjustable from 72 to 96 inches.

617.35 SASH LIFTS.

\section{AMERICAN MARINE STANDARDS COM- MITTEE, SASH HARDWOOD FOR SHIPS,} H NO. $63-1930$

\section{SASH HARDWARE FOR SHIPS}

(See 617.5, p. 1046.)

UNITED STATES GOVERNMENT, DEPARTMENT OF COMMERCE, BUILDERS' HARDWARE (NONTEMPLATE), COMMERCIAL STANDARD CS22-30, DECEMBER 11, 1930

\section{SASH LIFTS}

(See 617.0, p. 977; also Federal specification FF-H-101 below.)
UNITED STATES GOVERNMENT, FEDERAL SPECIFICATIONS BOARD, FEDERAL SPECIFICATION FOR BUILDERS' HARD. WARE (NONTEMPLATE), FF-H-101 AUGUST 19, 1930

(For types, grades, and classes; material and workmanship; general requirements; detail requirements; packaging, packing, and marking; notes; and table of standard finishes, see 617.0, p.978. For chromium plating, nickel plating, and zinc coatings; and methods of inspection and tests, see 600.3, p. 70.)

\section{BUILDERS' HARDWARE (NONTEMPLATE)}

HOOK SASH LIFTS

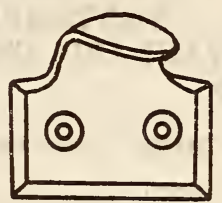

Figure 943.Hook sash lift, type 1201

Type 1201.-Wrought bronze. Beveled edge. Size 1 by $1 \frac{5}{8}$ inches. Minimum thickness of metal 0.057 inch.

Type 1201A.-Wrought steel plated. Beveled edge. Size 1 by $15 \% 8$ inches. Minimum thickness of metal 0.057 inch.

Type 1202. - Cast bronze. Beveled edge. Size $15 / 16$ by $1 \frac{1}{2}$ inches.

FLUSH SASH LIFTS

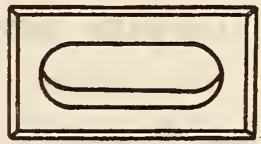

FrguRe 944.-Flush sash lift, type 1208

Type 1208.-Wrought bronze. Beveled edge. Square corners. Deep cup with side screw holes. Size $13 / 4$ by $3 \frac{1}{8}$ inches.

Type 1208A.- Same as type 1208, except wrought steel, plated.

Type 1212.-Cast bronze. Beveled edge. Square corners. Side screw holes. Size $13 / 4$ by 3 inches.

BAR SASH IIFTS

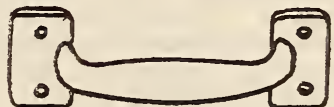

Figure 945.-Bar sash lift, type 1219

Type 1219.-Cast bronze. $13 / 8$ by $4 \frac{1}{2}$ to 5 inches. Round corners. Minimum weight 3 ounces each. 


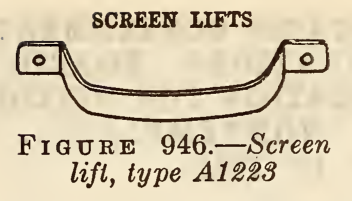

Type A1223. - Wrought brass. Length $3 \frac{1}{2}$ inches. Type A1223A. - Steel, japanned. Length $3 \frac{1}{2}$ inches.

617.4 CHECKS, CATCHES, AND FASTENERS.

617.41 DOOR CLOSERS AND CHECKS.

UNITED STATES GOVERNMENT, DEPARTMENT OF COMMERCE, BUILDERS' HARDWARE (NONTEMPLATE), COMMERCIAL STANDARD CS22-30, DECEMBER 11, 1930

\section{DOOR CLOSERS}

(See 617.0, p. 977; also Federal specification FF-H-101 below.

\section{UNITED STATES GOVERNMENT, FEDERAI SPECIFICATIONS BOARD, FEDERAL SPECIFICATION FOR BUILDERS' HARD. WARE (NONTEMPLATE), FF-H-101, AUG- UST 19,1930}

(For types, grades, and classes; material and workmanship; general requirements, detail requirements; packaging, packing, and marking; notes; and table of standard finishes, see 617.0, p.978. For chromium plating, nickel plating, and zinc coatings; and methods of inspection and tests, see 600.3 , p. 70.)

\section{BUILDERS' HARDWARE (NONTEMPLATE)}

\section{DOOR CLOSERS}

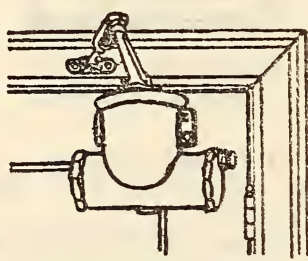

Figure 947 . - Door closer, type 1128

Type 1128.-Overhead type, single acting, combined spring and liquid check. Springs shall have adjustable tension. Closers having helical torsion spring shall have provision to prevent overwinding. Checking regulation accessible and close. Shall close the door positively and quietly. If a gylcerinalcohol mixture is used the acidity shall not exceed $0.30 \mathrm{mg} \mathrm{KOH}$ per gram of liquid. If other liquids are used, they shall conform to the following requirements: Viscosity, Saybolt Universal, 60 to 70 seconds at $100^{\circ} \mathrm{F}$.; pour point, minus $40^{\circ} \mathrm{F}$. or lower; acidity not over $0.10 \mathrm{mg} \mathrm{KOH}$ per gram of oil. Crankshaft or spindle shall be of 1-piece steel with 3 main bearings, one on either side of and close to the crank, cam, or pinion, and one near the operating lever, and 1 crank bearing if of crank type. When through shafts are used, they shall have 2 sets of packing, 2 main bearings, 1 crank bearing, if of crank type, and shall be provided with cap and gasket to completely cover lower end of shaft. Cams, arms, and brackets of forged steel or malleable iron. Arms and brackets finished to match case. Cases, cast bronze, unpolished. Closers shall be constructed so as to prevent the rise of liquid into the spring chamber and shall be equipped to prevent any leakage. Brackets shall be furnished when specified. Fastenings for sizes O, I, II, III, and IV shall be screws; for sizes V and VI, through bolts with washers and cap nuts. The following size designations are the equivalent of the various sizes designated by the manufacturers as suitable for the sizes and types of doors scheduled below:

O. For screen doors.

I. For screen, storm, pantry, closet, and very light interior doors not exceeding $1 \frac{1}{8}$ inches by 2 feet 6 inches by 6 feet 6 inches.

II. For light interior doors not over 3 feet wide and exterior doors not exceeding $1 \frac{3 / 4}{4}$ inches by 2 feet 8 inches by 7 feet.

III. For light exterior doors not over 2 feet 6 inches wide and ordinary interior doors.

IV. For very heavy interior doors and exterior doors not over 3 feet wide.

V. For heavy exterior doors 3 inches thick or less and doors operating against strong drafts.

VI. For extra-heavy exterior doors, tin-clad doors, and vault doors not exceeding 2,000 pounds in weight.

Type 1128A.-Same as type 1128, except with cast-iron case with smooth surface and prime coat suitable for painting.

Type 1128B.- Same as type 1128, except with cast-iron case commercial bronzed (sprayed).

\subsection{CATCHES.}

AMERICAN MARINE STANDARDS COMMITTEE, BOLTS AND CATCHES FOR SHIP DOORS, H No. 62-1930

\section{BOLTS AND CATCHES FOR SHIP DOORS}

(See 617.61, p. 1052.)

UNITED STATES GOVERNMENT, DEPARTMENT OF COMMERCE, BUILDERS' HARDWARE (NONTEMPLATE), COMMERCIAL STANDARD CS22-30, DECEMBER 11, 1930

\section{CATCHES}

(See 617.0, p. 977; also Federal specification FF$\mathrm{H}-101$ below.)

\section{UNITED STATES GOVERNMENT, FEDERAI SPECIFICATIONS BOARD, FEDERAL SPEC- IFICATION FOR BUILDERS' HARDWARE (NONTEMPLATE), FF-H-101, AUGUST 19, 1930}

(For types, grades, and classes; material and workmanship; general requirements; detail requirements; packaging, packing, and marking; notes; and table of standard finishes, see 617.0, p. 978 . For chromium plating, nickel plating, and zinc coatings; and methods of inspection and tests, see 600.3, p. 70.) 
BUILDERS' HARDWARE (NONTEMPLATE)

ELBOW CATCHES

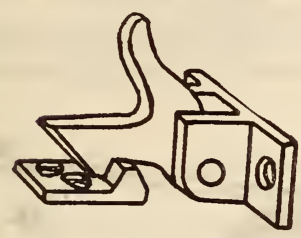

Frgure 948.-Elbow catch, type 1079

Type 1079.-Cast iron, plated. Size $5 / 8$ by $1 \frac{14}{4}$ inches.

Type 1080.-Unpolished brass. Size $5 / 8$ by $1 \frac{1}{4}$ inches.

\section{TRANSOM CATCHES}

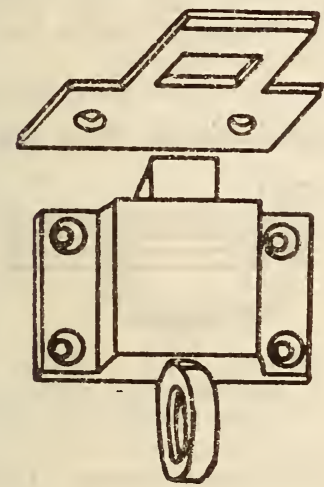

Figure 949.-Transom catch, type 1097

Type 109\%.-Rim type. Wrought bronze. Size $1 \frac{1}{2}$ by 2 inches. Latch bolt, minimum $1 / 2$ by $3 / 8$ inch, minimum throw $5 / 16$ inch. With flat strike unless otherwise specified. Minimum thickness of case 0.040 inch.

Type 1097 A.-Same as type 1097, except wrought steel, plated.

Type 1100.-Rim type. Cast bronze. Size 13/4 by $2 \frac{33}{4}$ inches. Latch bolt, minimum $21 / 64$ by $3 / 8$ inch, minimum throw $1 / 2$ inch. With flat strike unless otherwise specified.

Type $1100 A$.- Same as type 1100 , except iron, plated. Latch bolt, minimum $1 / 2$ by $3 / 8$ inch, minimum throw $7 / 16$ inch.

617.43 DOOR, CASEMENT, AND SASH FASTENERS. AMERICAN MARINE STANDARDS COMMITTEE, SASH HARDWARE FOR SHIPS, H NO. $63-1930$

\section{SASH HARDWARE FOR SHIPS}

(See 617.5, p. 1046.)

UNITED STATES GOVERNMENT, DEPARTMENT OF COMMERCE, BUILDERS' HARDWARE (NONTEMPLATE), COMMERCIAL STANDARD CS22-30, DECEMBER 11, 1930

\section{FASTENERS}

(See 617.0 , p. 977 also Federal specification FF-
UNITED STATES GOVERNMENT, FEDERAL SPECIFICATIONS BOARD, FEDERAI SPECIFICATION FOR BUILDERS' HARDWARE (NONTEMPLATE), FF-H-101, AUGUST 19, 1930

(For types, grades, and classes; material and workmanship; general requirements; detail requirements; packaging, packing, and marking; notes; and table of standard finishes, see 617.0, p.978. For chromium plating, nickel plating, and zinc coatings; and methods of inspection and tests, see 600.3, p. 70.)

\section{BUILDERS' HARDWARE (NONTEMPLATE)}

\section{CASEMENT FASTENERS}

Type 1131. -T handle. With rim strike. Cast bronze. Size $11 / 2$ by $1 \frac{1}{2}$ inches.

Type 1131 A.- Same as type 1131 , except cast iron, plated.

Type 1131B.-T handle. With surface strike. Cast bronze. Size $11 / 2$ by $1 \frac{1}{2}$ inches.

Type 1131C.--Same as type 1131B, except cast iron, plated.

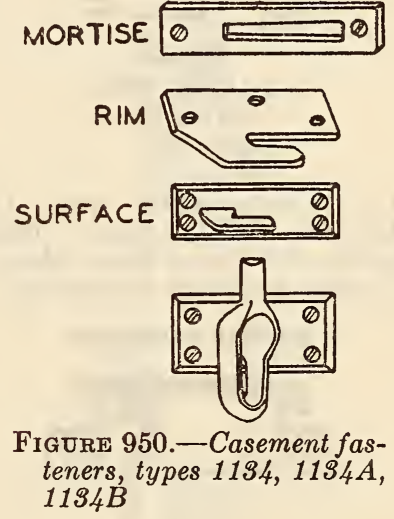

Type 1194.-D handle. With rim strike. Cast bronze. Size 3 by $13 / 8$ inches.

Type 1134A.- Same as type 1134, except with mortise strike.

Type 1134B.- Same as type 1134, except with surface strike.

CELLAR-WINDOW FASTENERS

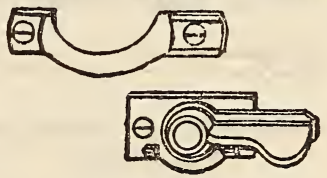

FIGURE 951.-Cellarwindow fastener, type A1137

Type A113\%.-Cast iron, galvanized. Size about $1 \frac{1}{8}$ by $21 / 2$ inches.

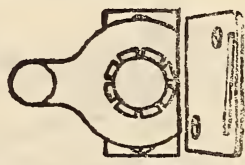

FIG URE 952 . Cellar - window fastener, $t$ y $p e$ B1137

Type B113\%.- Wrought steel, galvanized: Cam action and cam release with spring washer. Plates about $15 \% 8$ inches long. Universal strike. 
Type B1137A.-Same as type B1137, except japanned.

\section{SASH FASTENERS}

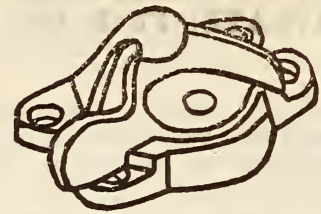

FIGURE $953 .-S a s h$ fastener, type 1139

Type 1139.-Crescent type. Cast bronze. Moving parts shall have cam action. For double-hung sash. Size of case about 1 by $23 / 4$ inches, approximately 40 pounds to the gross (boxed with screws).

Type 1199A.- Same as type 1139, except cast iron, plated.

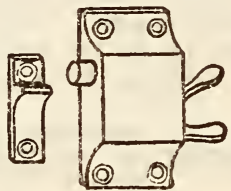

F I GURE 954 . Sash fastener, type 1146

Type 1146.-Car window type. Cast bronze approximately $2 \frac{1}{2}$ by $1 \frac{1}{4}$ inches. Right and left hand. Furnish with three stops, about $1 \frac{1}{2}$ by $7 / 16$ inch with $7 / 32$ inch minimum projection.

\section{SCUTTLE FASTENERS}

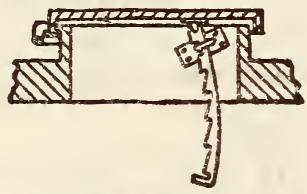

FigURE 955.-Scuttle fastener, type A1148

Type A1148.-Wrought iron, galvanized. Size $3 / 16$ by $1 \frac{1}{2}$ by 30 inches.

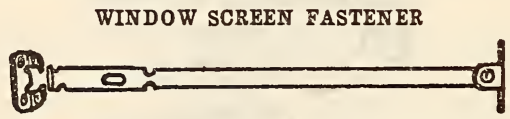

FIGURE 956.-Window screen fastener, type 1650

Type 1650.-Galvanized, 10-inch size. To hold screen open or closed.

\section{FASTENERS FOR BLINDS}
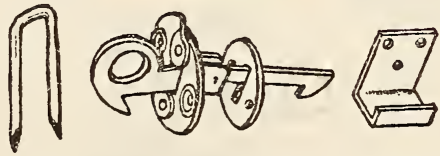

FigURE 957.-Fastener for blinds, type 1811

Type 1811.-Wrought steel, galvanized, unless plain steel is specified. Double acting gravity type with screws. When closed it shall not be possible to unlock blind from outside. Furnish with sill staple and back catch with screw holes for frame construction.

Type 1811A.-Same as type 1811, except with back catch shaped for driving into brick construction.

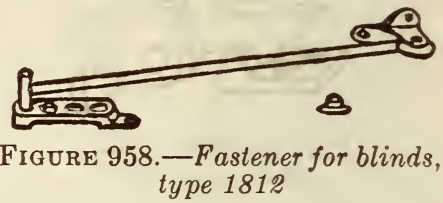

Type 1812.-Combined fastener and holder type. Cast-iron fittings with wrought steel holding rod. Shall hold open at four positions including wide open. When closed, rod shall engage hook on blind. Japanned, with screws. Sizes, 7, 9, 103/4, and 14 inches for $5 / 16$-inch rods; 16 and 18 inches for $3 / 8$-inch rods; 20 and 22 inches for $7 / 16$-inch rods; and 24 inches for $1 / 2$-inch rods.

Type 1812A.- Same as type 1812, except zinc coated.

DOOR FASTENERS, WITH CHANN

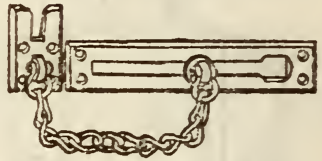

Figure $959 .-D o o r$ fastener, with chain, type 1116

Type 1116.- Wrought bronze. Length of plates about 6 inches.

Type 1116 A.-Wrought steel, plated. Length of plates about 6 inches.

Type 1118.-Cast bronze. Length of plates about 6 inches.

Type 1118A.-Cast iron, plated. Length of plates about 6 inches.

The above types (about 6 inches long) are suitable only for doors having stiles not less than 6 inches wide or having horizontal rails or muntins. When fasteners are specified for doors without horizontal rails or muntins or with stiles from 4 to less than 6 inches wide, their plates shall be 4 to $4 \frac{1}{2}$ inches long, as the case requires.

\section{TURNBUCKLES FOR BLINDS}

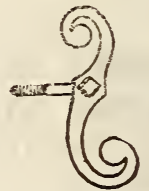

FiguRe 960--
Turnbuckle
for blinds,
type 1821

Type 1821.-Wrought steel $\mathrm{S}$ pattern right and left. Prime coat for painting. Minimum length, 6 inches. With lag screw.

Type 1821 A.- Same as type 1821, except with back plate. Four screw holes.

Type 1821 B.-Same as type 1821, except with drive pin for brick. 
617.44 DOOR HOLDERS AND STOPS.

UNITED STATES GOVERNMENT, DEPARTMENT OF COMMERCE, BUILDERS' HARDWARE (NONTEMPLATE), COMMERCIAL STANDARD CS22-30, DECEMBER 11,1930

\section{DOOR STOPS, DOOR HOLDERS}

(See 617.0 , p. 977 , also Federal specification FFH-101 below.)

\section{UNITED STATES GOVERNMENT, FEDERAL SPECIFICATIONS BOARD, FEDERAI SPECIFICATION FOR BUILDERS' HARD- WARE (NONTEMPLATE), FF-H-101, AUGUST 19, 1930}

\section{DOOR HOLDERS}

(For types, grades, and classes; material and workmanship; general requirements; detail requirements; packaging, packing, and marking; notes; and table of standard finishes, see 617.0, p. 978. For chromium plating, nickel plating, and zinc coatings; and methods of inspection and tests, see 600.3, p. 70.)

\section{BUILDERS' HARDWARE (NONTEMPLATE)}

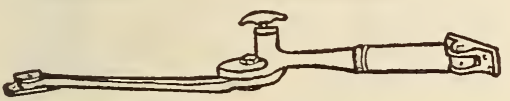

FIGURE 961.-Door holder, type A1149

Type A1149.-Overhead door holder, stop, and shock absorber. Folding disk jointed type. Locking handle adjustable for the neutral and locked positions. Cast bronze bearings and fittings, malleable iron arms, plated.

Size 1. For 24 to 28 inch doors. Minimum weight, 3 pounds 7 ounces.

Size 2. For 30 to 36 inch doors. Minimum weight, 3 pounds 10 ounces.

Size 3 . For doors 36 inches and over. Minimum weight, 3 pounds 14 ounces.

Type A1149A.- Same as type A1149, except cast phosphor bronze.

Size 1. For 24 to 28 inch doors. Minimum weight, 3 pounds 12 ounces.

Size 2. For 30 to 36 inch doors. Minimum weight, 4 pounds 2 ounces.

Size 3. For doors 36 inches and over. Minimum weight, 4 pounds 9 ounces.

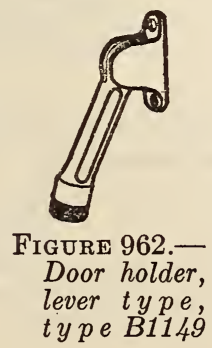

Type B1149.-Lever type. Cast bronze. Provided with circular replaceable rubber tip. Shall swing up and remain securely held in vertical position when out of action. Length 4 to $4 \frac{1}{2}$ inches for doors with 2 -inch or less floor clearance.
Type B1149A.-Same as type B1149, except with arm or lever 5 to $5 \frac{1}{2}$ inches long for doors with $3 \frac{1}{2}$-inch or less floor clearance.

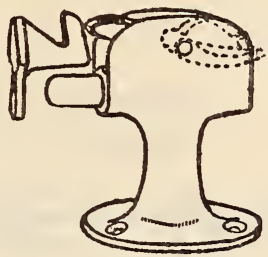

FIgURE 963.-Door holder, floor type, type C1149

Type C1149.-Floor type door holder, and bumper with an encased metal plunger and semiautomatic engagement of hook and tongue. Height $13 / 4$ inches minimum.

Type C1149A.-Same as type C1149, and equipped with $1 / 2$-inch steel stud and expansion shield.

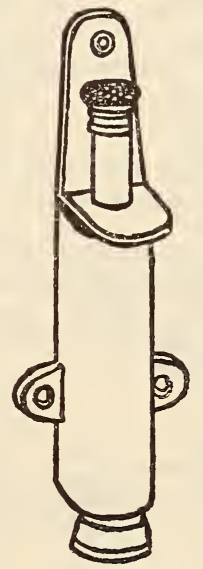

FIgURE 964Door holder, plunger type, type 1151

Type 1151.-Plunger type. Wrought bronze case. Rubber tip removable. Releasing lip shall be plainly evident. Size about 6 inches.

Type $1151 \mathrm{~A}$.- Same as type 1151, except wrought steel, plated.

Type 1153.-Plunger type. Cast bronze case. Rubber tip removable. Releasing lip shall be plainly evident. Size 6 inches.

Type 1153A.- Same as type 1153, except cast iron, plated.

Type 1154.--Plunger type. Cast bronze case. Rubber tip removable. Releasing lip shall be plainly evident. Size 8 inches.

Type 1154A.-Same as type 1154, except cast iron, plated.

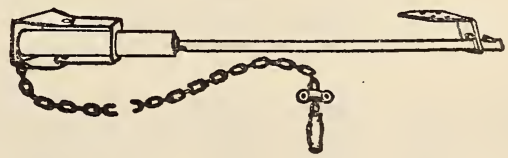

Figure 965.-Door holder, overhead garage type, type B1154

Type B1154.--Overhead garage type. Wrought steel japanned or galvanized. Bar not less than 30 
inches long. Catch on end of bar shall prevent pulling through bumper plate. Equipped with cushion spring. Release chain approximately 44 inches long.

Type B1154A.-Same as type B1154, except without cusion spring.

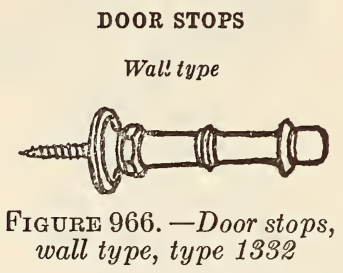

Type 1332.-Cast bronze, with rubber tip; 3 -inch projection. To screw. Diameter of base about 1 inch.

Type 1332A.-Same as type 1332, except cast ron, plated.

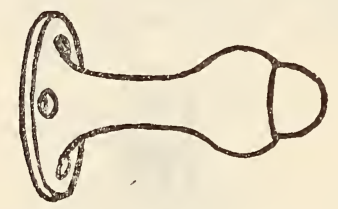

Figure 967. - Door stop, wall type, type 1334

Type 1334.- Cast bronze, with rubber tip; 31/2inch projection. Diameter of base about $2 \frac{1}{4}$ inches. Regular fastenings, three wood screws. When specified, furnish expansion sleeves for attaching to marble or stone work.

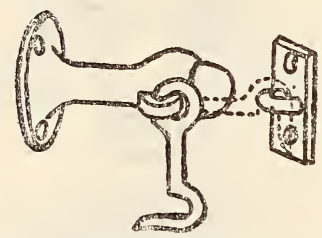

Figure 968. - Door stop, wall type, type 1336

Type 1336.- Same as type 1334, except with hook and keeper.

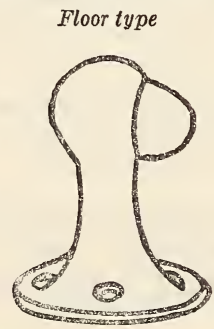

Figure 969. - Door stop, floor type, type 1340

Type 1340.-Cast bronze, with rubber tip. Height about 3 inches. Diameter of base about $2 \frac{1}{2}$ inches. Regular fastenings, three wood screws.
When specified, furnish expansion sleeves for attaching to marble or stone work.

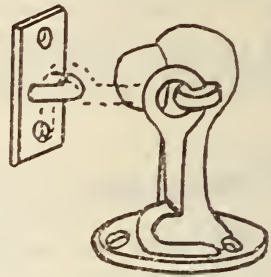

Figure 970.-Door stop, floor ty $p \boldsymbol{e}$, type 1341

Type 1341.- Same as type 1340, except with hook and keeper.

61\%.5 TRANSOM AND SASH DEVICES.

AMERICAN MARINE STANDARDS COMMITTEE, SASH HARDWARE FOR SHIPS, H NO. 63-1930

\section{SASH HARDWARE FOR SHIPS}

Meeting rail sash lock.-One size and hand only as shown by Figure 971.

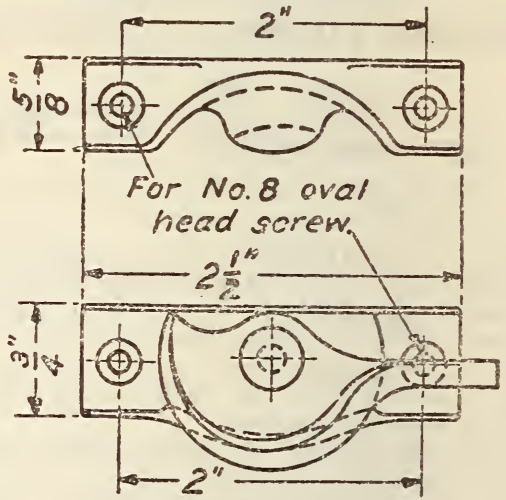

FIgURe 971

Spring bolt sash fastener.-One size only as shown by Figure 972(a). May be required of either right or left hand, with one or more individual ratchet stops or with multiples of the latter in form of strip, as indicated by Figure 972(b), or in lieu thereof, one or more individual plate keepers as shown by Figure $972(\mathrm{c})$. The hand of the fastener and full particulars of the stops or keepers must be given in asking for quotations and in purchasing.

Hook sash fastener.-One type and size only as shown by Figure 973.

Pawl sash catch.-One size only as shown by Figure 974(a). May be required of either right or left hand, with one or more individual ratchet stops or with multiples of the latter in form of strip as shown by Figure $974(\mathrm{~b})$. The hand of the catch and the particulars of the stop must be given in asking for quotations and in purchasing.

Antirattler, cam type.-One size only as shown by Figure 975. May be required for either left or right hand, the hand to be specified in asking for 


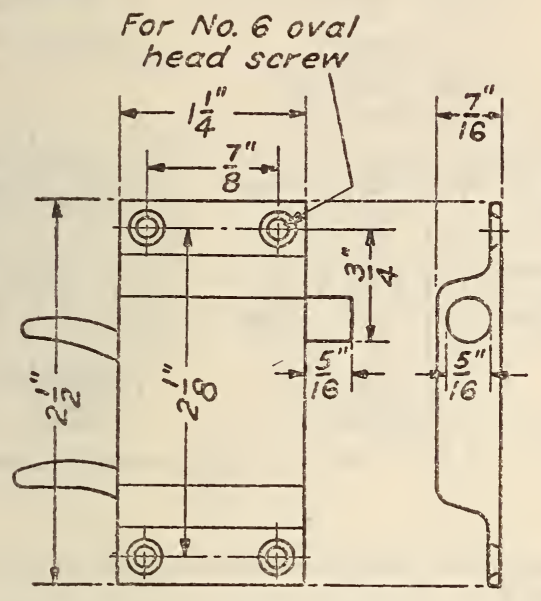

Right hand shown.
Stops may be individual or
in strips as ordered.

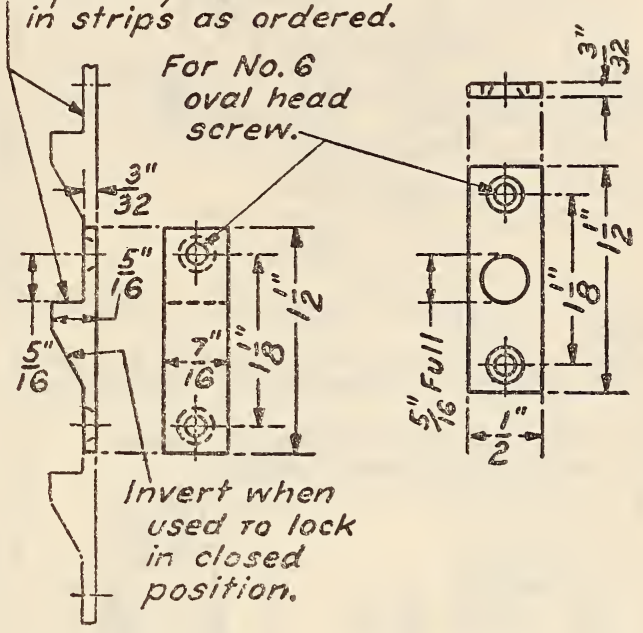

(c)

(b)

FigURE 972
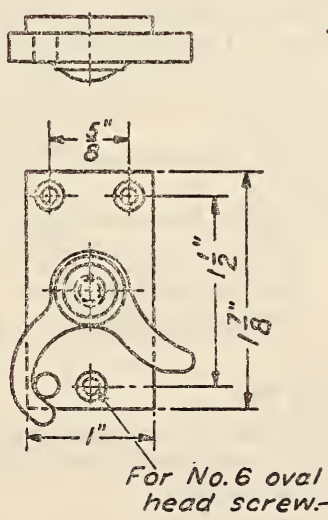

Right hand shown.
Stops may be

individual or in

strips as ordered.

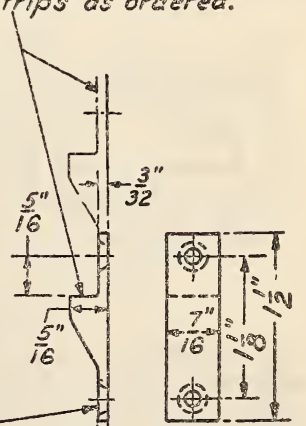
hecid screm

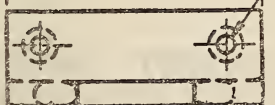

Figure 973

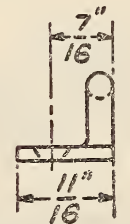

(a) FIGURE 974

(b) 
quotations and in purchasing. The face of the shoe, to be rough machined to a true plane.

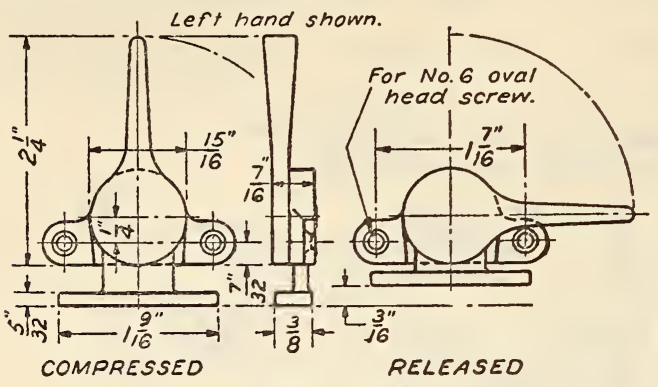

Figure 975

Antirattler, screw type.-One size only as shown by Figure 976.

Flush sash lifts.-Two optional types of like size, shown respectively by Figures 977 (a) and $977(\mathrm{~b})$.

Swivel cap with friction disc of rubber

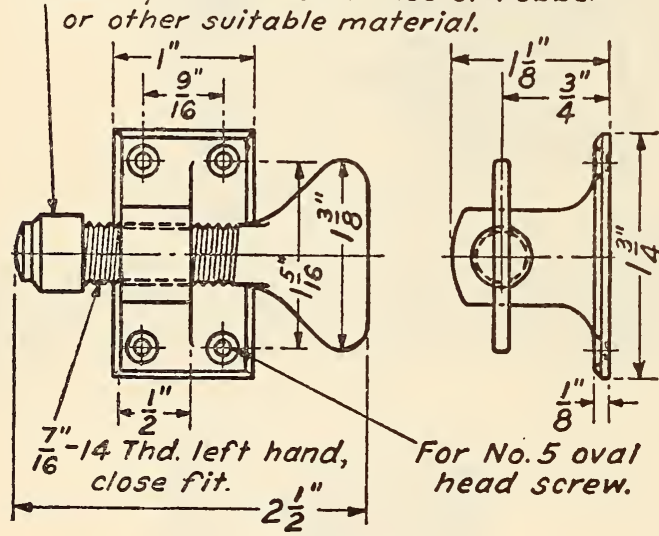

Figure 976

The type required must be specified in asking for quotations and in ordering. The type shown by

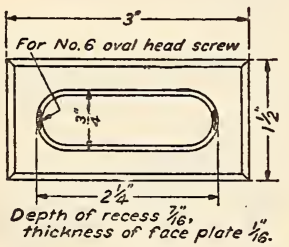

(a)

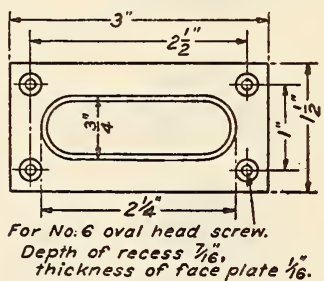

(b)
Figure 977

Figure $977(a)$ is secured by two screws, one at each end of the recess. The type shown by Figure 977(b) is secured by four screws on the face.

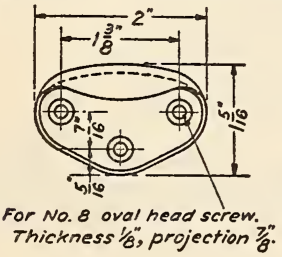

(a)

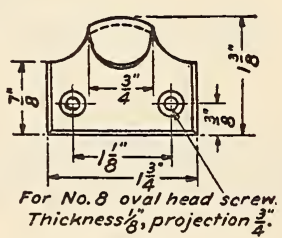

(b)
Figure 978

Hook sash lifts.-Two types each of single size. shown respectively by Figures 978 (a) and $978(\mathrm{~b})$,
The type required must be specified in asking for quotations and in ordering. The type shown by Figure $978(\mathrm{a})$ is cast. The type shown by Figure 978 (b) may be furnished either cast or wrought.
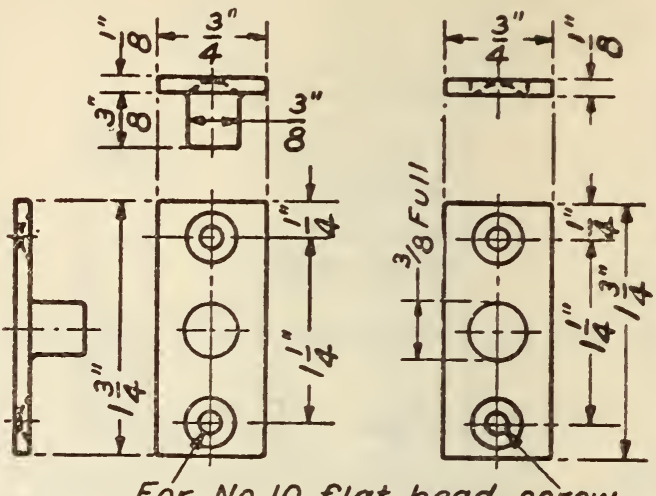

Figure 979

Sash pivot, straight.-One size only as shown by Figure 979.

Sash pivot, offset.-One size only as shown by Figure 980.

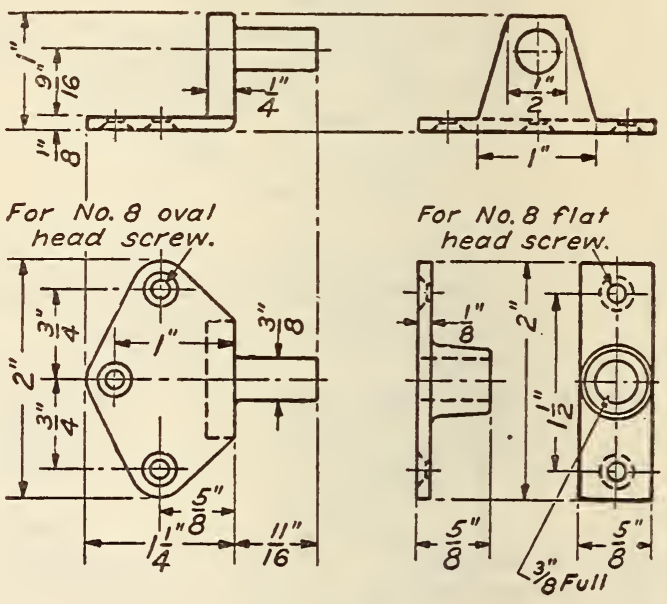

Figure 980

\section{GENERAL REQUIREMENTS}

(In respect to materials, including percentages of components of white bronze; screws, and marking and packing, this specification conforms to A. M. S. C. H No. 61-1930, specification for locks and accessories for ship doors. See 617.21, p. 1004. In respect to construction and workmanship and finish it comforms to A. M. S. C. H No. 62-1930, specification for bolts and catches for ship doors. See 617.61, p. 1052.)

UNITED STATES GOVERNMENT, DEPARTMENT OF COMMERCE, BUILDERS' HARDWARE (NONTEMPLATE), COMMERCIAL STANDARD CS22-30, DECEMBER 11, 1930

\section{ADJUSTERS, ROLLERS, SHEAVES, PIVOTS}

(See 617.0 , p. 977 , also Federal specification FF$\mathrm{H}-101$ below. 


\section{UNITED STATES GOVERNMENT, FEDERAL SPECIFICATIONS BOARD, FEDERAL SPECIFICATION RR-C-271 FOR STAND- ARD MISCELLANEOUS CHAIN AND AT- TACHMENTS, DECEMBER 8, 1931}

\section{SASH ATTACHMENTS}

(For general specifications, material and workmanship, general requirements, detail requirements, inspection and tests, packing and marking, see 603.50, p. 199.)

(See also 603.51 , p. $201 ; 603.52$, p. $204 ; 603.54$, p. $211 ; 603.56$, p. $212 ; 603.57$, p. 217 ; 631.9 , p. 1164 ; 642.9 , p. 1191 ; 645.9 , p. 1242 ; and 646.59 , p. 1258 .)

1. Sash attachments shall be as covered by the following sketches:

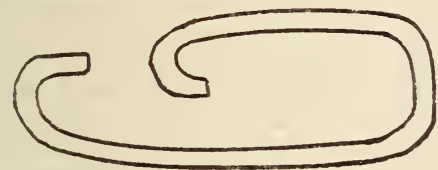

Figdre 991.-Attachment to weight

Dimensions, $33 / 4$ by $13 / 8$ by 0.135 inches. Steel wire.

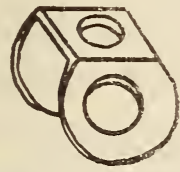

FIgURE 982. - Socket secured to sash

Dimensions, 1 by $3 / 4$ by 0.070 inches. Sheet steel.

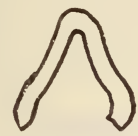

FIgURe 983. - Attachment to socket

Dimensions, 1 by 1 by 0.120 inches. Steel wire.

\section{UNITED |STATES GOVERNMENT, FEDERAL SPECIFICATIONS BOARD, FEDERAL SPECIFICATION 'FOR BUILDERS' HARD- WARE (NONTEMPLATE), FF - H - 101, AUGUST 19, 1930}

(For types, grades, and classes; material and workmanship; general requirements; detail requirements; packaging, packing, and marking; notes; and table of standard finishes, see 617.0, p. 978 . For chromium plating, nickel plating, and zinc coatings; and methods of inspection and tests, see 600.3 , p. 70.)
BUILDERS' HARDWARE (NONTEMPLATE)

SASH CORD IRONS

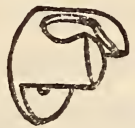

FIgURE 984. - Sash cord iron, type B1118

Type B1118.-Cast iron, about $5 / 8$-inch long with flat-head screw.

\section{SASH OPERATORS}

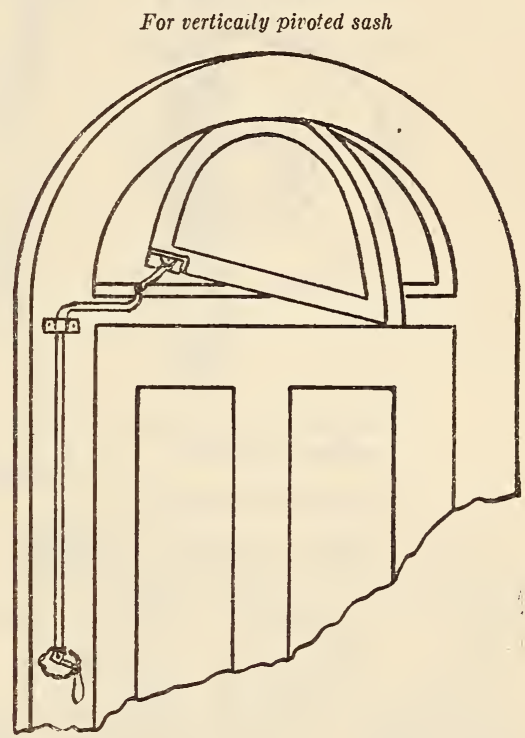

FIgURe 985.-Sash operator, type 1501

Type 1501.-Bronze.-Vertical rotating rod with arm and connection to sash. Jointed handle with locking mechanism. Rods $3 / 8$-inch diameter; lengths $3,4,5$, and 6 feet.

Type $1501 \mathrm{~A}$.- Same as type 1501, except steel, plated.

SASH WEIGHTS

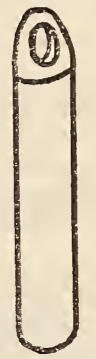

FIgURE 986. -Sash weight, type 1701

Type 1701.-Cast iron, round, reasonably sinooth, free from fins, with well-formed eyes. Weights as required. 
Type 1701 A.-Same as type 1701, except square.

Type 1701B.- Same as type 1701, except cast lead, round.

Type 1701C.-Same as type 1701, except cast lead, square.

\section{SASH PULLEYS}

Type 1248.-Polished iron or wrought steel wheels with round groove for cord. Plain iron or steel face for two wood screws. Wrought steel or cast-iron case, round

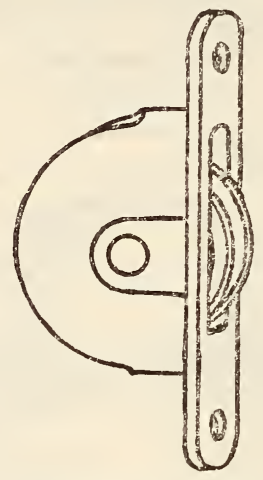

Figure 987. -Sash pulley, type 1248

[All dimensions in inches]

\begin{tabular}{|c|c|c|c|}
\hline $\begin{array}{c}\text { Size of } \\
\text { wheel }\end{array}$ & $\begin{array}{c}\text { Width of } \\
\text { front }\end{array}$ & $\begin{array}{c}\text { Length of } \\
\text { front }\end{array}$ & $\begin{array}{c}\text { Thickness } \\
\text { of front }\end{array}$ \\
\hline \multirow{2}{2}{$21 / 4$} & $11 / 8$ & 5 & $1 / 8$ \\
$21 / 2$ & $11 / 4$ & 5 & $1 / 8$ \\
\hline
\end{tabular}

Type 1249.-Turned iron wheel, with combination groove for cord or chain. Polished and plated iron face for two wood screws. Cast-iron case, with round ends. Three eighths inch plain steel axle.

[All dimensions in inches]

\begin{tabular}{|c|c|c|c|}
\hline $\begin{array}{c}\text { Size of } \\
\text { wheel }\end{array}$ & $\begin{array}{c}\text { Width of } \\
\text { front }\end{array}$ & $\begin{array}{c}\text { Length of } \\
\text { front }\end{array}$ & $\begin{array}{c}\text { Thickness } \\
\text { of iront }\end{array}$ \\
\cline { 1 - 1 } 2 & $11 / 8$ & 5 & $1 / 8$ \\
$21 / 4$ & $11 / 8$ & 5 & $1 / 8$ \\
$21 / 2$ & $11 / 4$ & $53 / 4$ & $5 / 32$ \\
\hline
\end{tabular}

Type 1249A.- Same as type 1249, except roller bearing with $1 / 4$-inch minimum diameter axle.

Type 1249C. - Same as type 1249, except with wrought steel wheel and case. Metal of wheel not less than 0.024 inch thick. Metal of case not less than 0.031 inch thick. Metal of face plate not less than 0.050 inch thick.

Type 1249D.-Same as type 1249 , except with wrought steel wheel and case, and with ball-bearing axle. Metal of wheel not less than 0.024 inch thick. Metal of case not less than 0.031 inch thick. Metal of face plate not less than 0.050 inch thick. Wheel sizes $2 \frac{1}{4}$ and $2 \frac{1}{2}$ inches.

Type 1250A.-Turned brass wheel, steel bushed with combination groove for cord and chain. Wrought bronze front. Cast-iron case with round ends. Roller bearing with 1/4-inch minimum diameter steel axle with rollers machined true.

[All dimensions in inches]

\begin{tabular}{|c|c|c|c|}
\hline $\begin{array}{c}\text { Size of } \\
\text { wheel }\end{array}$ & $\begin{array}{c}\text { Width of } \\
\text { front }\end{array}$ & $\begin{array}{c}\text { Length of } \\
\text { front }\end{array}$ & $\begin{array}{c}\text { Thickness } \\
\text { of front }\end{array}$ \\
& $11 / 8$ & 5 & $1 / 8$ \\
22 & $11 / 8$ & 5 & $1 / 8$ \\
$21 / 2$ & $11 / 4$ & $53 / 4$ & $5 / 32$ \\
\hline
\end{tabular}

Type 1250B.-Same as type $1250 \mathrm{~A}$, except ball bearing with $1 / 4$-inch minimum diameter axle.

Type 1250C.-Same as type $1250 \mathrm{~A}$, except with wrought steel case, wrought bronze face plate and wheel, with ball-bearing axle. Metal of wheel not less than 0.024 inch thick. Metal of case not less than 0.031 inch thick. Metal of face plate not less than 0.062 inch thick. Wheel sizes $2 \frac{1}{4}$ and $2 \frac{1}{2}$ inches.

Type 1250E.-Overhead type sash pulley. Turned face iron wheel. Wrought bronze front. Cast-iron case. Roller bearings, rollers machined true. Wheels fully housed to prevent chain from leaving wheel. Equipment shall include all necessary parts of suitable sizes for complete operation on doublehung counter-weighted sash in mullion windows without weights in the mullions.

\section{TRANSOM OPERATORS}

Type 1530.-For heavy sash, over $3 \frac{1}{2}$ feet wide, horizontally hinged or pivoted. Operator shall connect to each side of transom with brass screw and traveling nut to control movement. The transmission shall consist of solid rod and miter gears. The operation shall be by hand crank through inclosed pair of miter gears. The mechanism shall provide smooth and even operations of transom and be self-locking at all points. Steel rods onehalf inch diameter for transoms up to 5 by 4 feet not heavier than 100 pounds. Cast-iron brackets, plated.

Type 1530A.-Same as type 1530, except with $5 / 8$-inch diameter steel rods for larger or heavier transoms, but not heavier than 175 pounds.

\section{WINDOW STOP ADJUSTER}

Type 1343.-Flat, adjustable steel washer with 114-inch No. 8 round-head screw.

Type 1344.- Cup type adjustable bronze washer with 1-inch No. 8 round-head screw.

\section{TRANSOM CHAINS}

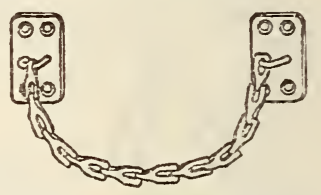

\section{FIgURE 988. - Transom chain, type 1120A}

Type 1120A.-Steel, plated. Four screws to each plate. Lengths 12 and 15 inches over all. Steel sash chain, 0.055-inch metal thickness as specified in Federal Specification RR-C-271.

Type 1120B.- Same as type 1120A, except galvanized.

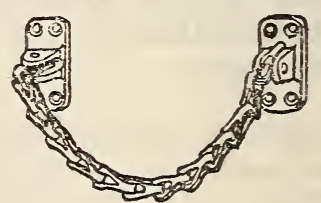

Figure 989. - Transom

Ars chain, type 1122

Type 1122.-Heavy type, bronze. Lugs and plate made in one casting. Four screws to each plate. Lengths 12 and 15 inches over all. Bronze sash chain, 0.055-inch metal thickness as specified in Federal Specification RR-C-271.

\section{TRAHSOM EYES}

Type 1268.-Cast bronze. Size 1 by $1 \frac{3}{8}$ inches or $13 / 4$ by $13 / 4$ inches. 
TRANSOM LIFTERS
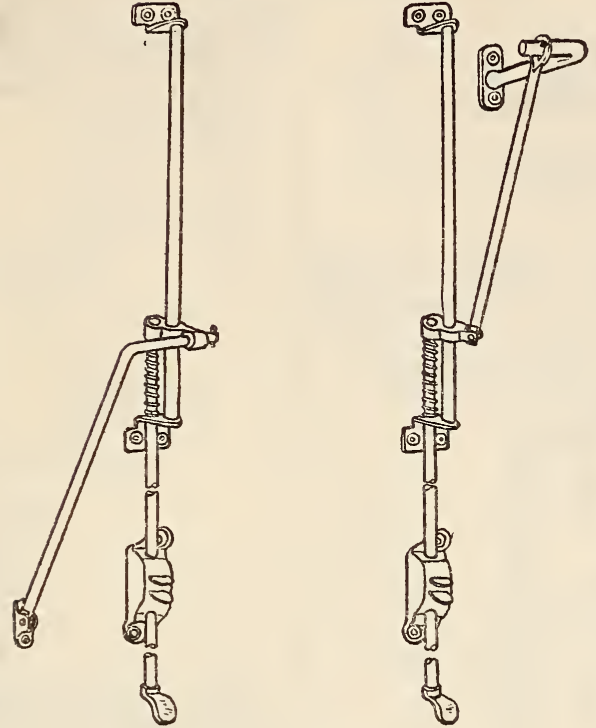

Figure 990.-Transom lifters

$A$ and $B$ lifters (spring not required on A).

$\mathrm{C}$ and $\mathrm{D}$ lifters (spring not required on $\mathrm{D}$ ).

All transom lifters shall have automatic grips.

\begin{tabular}{|c|c|c|c|c|c|c|}
\hline Type & Figure & For sash & Material & $\begin{array}{l}\text { Diam- } \\
\text { eter, } \\
\text { rod }\end{array}$ & $\begin{array}{l}\text { Lengths as re- } \\
\text { quired }\end{array}$ & $\begin{array}{l}\text { Safety cushion } \\
\text { spring }\end{array}$ \\
\hline 1198 & A & Top hung, opening out & Bronze & Inch & Feer & \multirow{16}{*}{$\begin{array}{l}\text { Not required. } \\
\text { Required. } \\
\text { Not required. } \\
\text { Required. } \\
\text { Not required. } \\
\text { Required. } \\
\text { Not required. } \\
\text { Required. } \\
\text { Not required. } \\
\text { Do. } \\
\text { Do. } \\
\text { Do. } \\
\text { Required. } \\
\text { Do. } \\
\text { Do. } \\
\text { Do. }\end{array}$} \\
\hline $1198 \mathrm{~A}$ & $\mathrm{~B}$ & Bottom hung, opening out & -_-_-_do_. & $5 / 16$ & 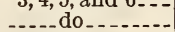 & \\
\hline $1198 \mathrm{~B}$ & $\overrightarrow{\mathrm{A}}$ & Top hung, opening out & Steel plated. & $5 / 16$ & -...- do & \\
\hline $1198 \mathrm{C}$ & $\mathrm{B}$ & Bottom hung, opening out & - do & $5 / 16$ & - do & \\
\hline 1199 & $\widetilde{A}$ & Top hung, opening out & Bronze... & 38 & $4,5,6$, and 8 & \\
\hline $1199 \mathrm{~A}$ & $\mathrm{~B}$ & Bottom hung, opening out & _.... do _. & $3 / 8$ & .... do . . & \\
\hline $1199 \mathrm{~B}$ & A & Top hung, opening out & Steel plated & $3 / 8$ & ......... & \\
\hline $1199 \mathrm{C}$ & $\mathrm{B}$ & Bottom hung, opening out & do & $3 / 8$ & do & \\
\hline $1199 \mathrm{D}$ & $\mathrm{D}$ & $\begin{array}{l}\text { Center hung sash opening in or out, top } \\
\text { hung sash opening in. }\end{array}$ & & $5 / 16$ & $3,4,5$, and 6 & \\
\hline 1199E & - & - & Steel plated & $5 / 16$ & ..... do & \\
\hline $1199 \mathrm{~F}$ & & 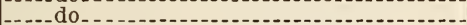 & Bronze... & $3 / 8$ & $4,5,6$, and 8 & \\
\hline $1199 \mathrm{G}$ & --- & 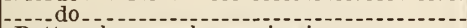 & Steel plated. & $3 / 8$ & -...-. do . & \\
\hline $1199 \mathrm{H}$ & $\mathrm{C}$ & Bottom hung sash, opening in & Bronze.-. & $5 \% 16$ & $3,4,5$, and 6 & \\
\hline $1199 \mathrm{~J}$ & & - & Steel plated & $5 / 16$ & ..... do ... & \\
\hline $1199 \mathrm{~K}$ & & $x^{-2}$ do & Bronze.... & $3 / 8$ & $4,5,6$, and & \\
\hline $1199 \mathrm{~L}$ & & (2) & Steel plated & $3 / 8$ & do... & \\
\hline
\end{tabular}

CASEMENT ADJUSTERS

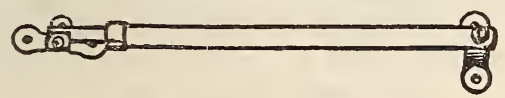

\section{Figure 991. - Casement adjuster, type}

Type 1000.-Friction type. Wrought-bronze sliding rod and tube, 8-inch extension. Friction adjustable. For out or in swinging casements. Lengths, 10 and 12 inches.

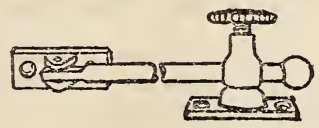

FIGURE 992.-Casement adjuster, type 1002

Type 1002.-For out-swinging casements. Bronze. Three-eighths-inch diameter round bar; swivel standard. Thumbserew grip with broad-knurled rim for finger hold. Lengths, 10 and 12 inches.

Type 1002A.- Same as type 1002 , except cast iron or steel, plated.
Type 1002B.-Same as type 1002, except cast iron or steel, galvanized.

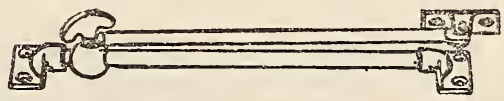

Figure 993. - Casement adjuster, type 1003

Type 1003.-Casement adjuster for outswinging sash, bronze, with solid stationary rod and solid movable rod, each five-sixteenths inch in diameter. Stationary rod shall have two cast base or sill plates, each about $3 / 4$ by $13 / 4$ inches. Movable rod shall have a cast sash plate about $5 / 8$ by $13 / 4$ inches and a thumb-set sliding connection on the stationary rod. Lengths over all approximately 9 and 13 inches, as specified.

Type 1003A.-Same as type 1003, except iron or steel, polished and plated.

Type 1006.-For in-swinging casements. Bronze. Three-eighths-inch diameter round bar. Thumbscrew grip with broad-knurled rim for finger hold. Lengths, 10 and 12 inches. 
Type 1006 A.-Same as type 1006, except cast iron or steel, plated.

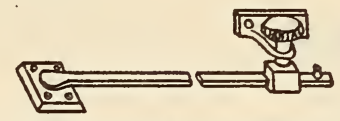

FIgURE 994.-Casement adjuster, type 1008

Type 1008.-For in-swinging casements. Cast bronze. Rectangular bar $5 / 16$ by $1 / 2$ inch. Thumbscrew grip with broad knurled rim for finger hold. Lengths, 12 and 15 inches.

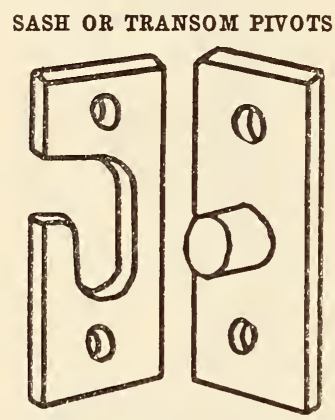

Figure 995. - Sash or transom pivot, type 1109

Type 1109.-Full mortise type. Cast iron, japanned. Plate $2 \frac{1}{4}$ by $7 / 8$ inch, with open slot; $3 / 8$-inch pin.
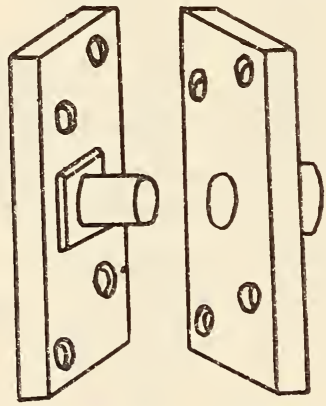

Figure 996. - Sash or transom pivot, type 1113

Type 1113.-Extra heavy full mortise type for vertical pivots. Cast iron, japanned. Plate 4 by $11 / 4$ inches about $3 / 16$ inch thick with round hole; 1/2-inch pin.

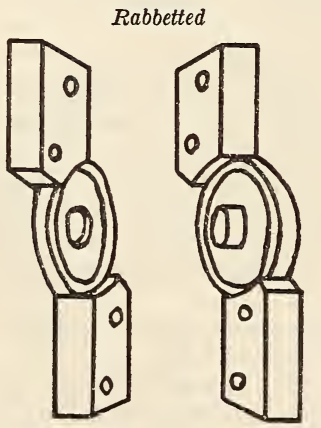

FIgURE 997.-Sash or transom pivot, rabetted, type 1114
Type 1114.- With interlocking weatherproof grooves. For $1 / 2$-inch rabbet. Cast bronze. Sizes, $37 / 8$ by $1 \frac{3}{8}, 37 / 8$ by $13 / 4,4$ or $4 \frac{1}{2}$ by $2 \frac{1}{4}$ inches. For sash thickness, $13 / 8,13 \% 4$ and $21 / 4$ inches.

Type 1114A.- Same as type 1114, except cast iron, plated.

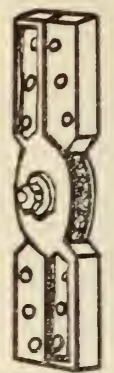

Figure 998. -

Sash or tran-

som pivot, rabetted, type

1115

Type 1115.-Friction type, with corrugated friction washers. For $1 / 2$-inch rabbet. Cast bronze, steel bushed or with steel balls and springs. Sizes $3 \frac{3}{4}$ by $13 \frac{3}{3}, 3 \frac{3}{4}$ by $1 \frac{3}{4}$, and $3 \frac{3}{4}$ by $2 \frac{1}{4}$ inches. For sash thickness, $13 / 8,13 / 4$, and $2 \frac{1}{4}$ inches.

Type 1115 A.- Same as type 1115 , except malleable iron, plated.

Type 1115B.-Same as type 1115A, except unfinished.

617.6 BOLT LOCKS, BRACKETS, AND HOOKS.

617.61 BOIT LOCKS.

AMERICAN MARINE STANDARDS COMMITTEE, BOLTS AND CATCHES FOR SHIP DOORS, H NO. 62-1930

\section{BOLTS AND CATCHES FOR SHIP DOORS}
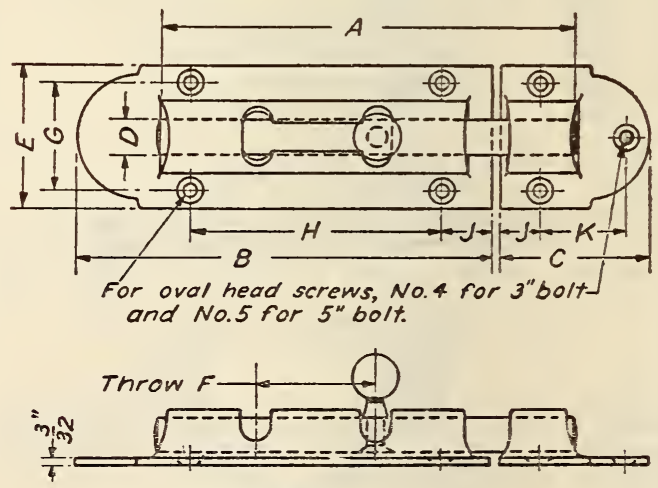

FiguRe 999.-Barrel bolt

Barrel bolts.-Two standard sizes as shown by Figure 999. These bolts are suitable for installation in horizontal position unly.

[Dimensions in inches]

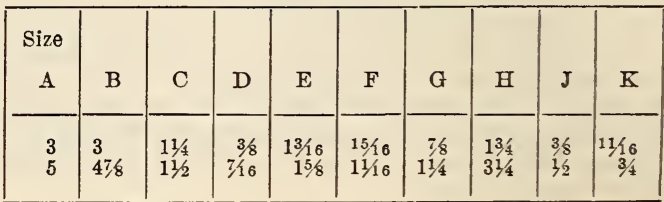


Light straight bolts.-Tnree standard sizes as shown by Figure 1000. These bolts are suitable for installation in any position on light and medium weight doors.
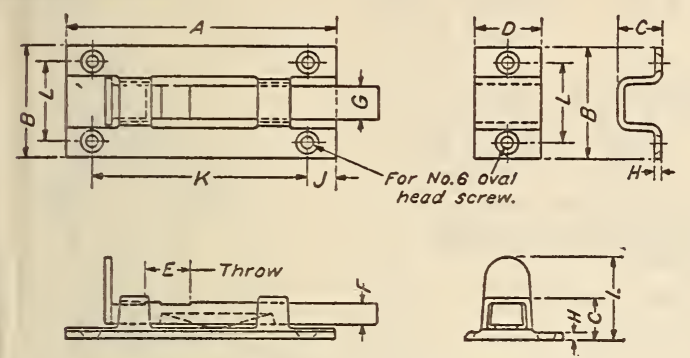

FIGURE 1000. - Light straight bolt

[Dimensions in inches]

\begin{tabular}{|c|c|c|c|c|c|c|c|c|c|c|}
\hline $\begin{array}{c}\text { Size } \\
\text { A }\end{array}$ & B & $\mathrm{C}$ & D & $\mathrm{E}$ & $F$ & G & H & $\mathrm{J}$ & K & L \\
\hline $\begin{array}{l}23 / 2 \\
3 \\
4\end{array}$ & $\begin{array}{l}111 \\
11,4 \\
138\end{array}$ & $\begin{array}{l}1 / 2 \\
1 / 2 \\
5 / 8\end{array}$ & $\begin{array}{r}3 / 4 \\
1^{3 / 4}\end{array}$ & $\begin{array}{l}1 / 2 \\
1 / 2 \\
3 / 2\end{array}$ & $\begin{array}{r}1 / 4 \\
1 / 4 \\
5 / 16\end{array}$ & $\begin{array}{l}3 / 8 \\
3 / 8 \\
1 / 2\end{array}$ & $\begin{array}{l}3 / 32 \\
3 / 32 \\
3 / 32\end{array}$ & $\begin{array}{l}5 / 16 \\
5 / 16 \\
7 / 16\end{array}$ & $\begin{array}{l}17 / 8 \\
23,8 \\
31 / 8\end{array}$ & $\begin{array}{l}7 / 8 \\
1^{7 / 8}\end{array}$ \\
\hline
\end{tabular}

Heavy straight bolts.-Two standard sizes as shown by Figure 1001. These bolts are suitable for installation in any position on the heavier class of doors.
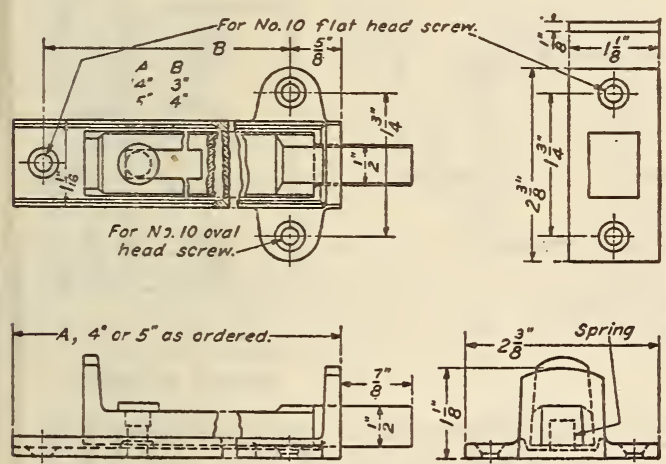

FIGURE 1001.-Heavy straight bolt

Light neck bolts.-Three standard sizes as shown by Figure 1002 . These bolts are suitable for installation in any position on light and medium weight doors.

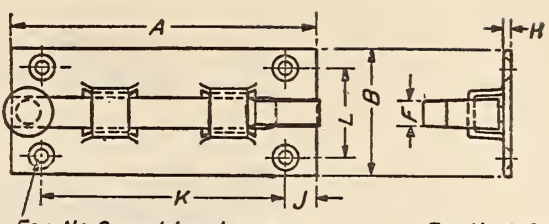

for No.6 oval head screw.

For No.6 flat
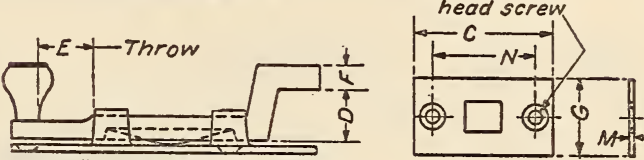

FIGURE 1002.-Light neck bolts

[Dimensions in inches]

\begin{tabular}{|c|c|c|c|c|c|c|c|c|c|c|c|c|}
\hline Size & & & & & & & & & & & & \\
$\mathrm{A}$ & $\mathrm{B}$ & $\mathrm{C}$ & $\mathrm{D}$ & $\mathrm{E}$ & $\mathrm{F}$ & $\mathrm{G}$ & $\mathrm{H}$ & $\mathrm{J}$ & $\mathrm{K}$ & $\mathrm{L}$ & $\mathrm{M}$ & $\mathrm{N}$ \\
\hline & & & & & & & & & & & & \\
\hline $21 / 2$ & $11 / 4$ & $13 / 8$ & $1 / 2$ & $1 / 2$ & $1 / 4$ & $3 / 1$ & $3 / 32$ & $5 / 16$ & $17 / 8$ & $7 / 8$ & $1 / 16$ & 1 \\
3 & $11 / 4$ & $13 / 8$ & $1 / 2$ & $1 / 2$ & $1 / 4$ & $3 / 4$ & $3 / 32$ & $5 / 16$ & $23 / 8$ & $7 / 8$ & $1 / 16$ & 1 \\
4 & $13 / 8$ & $13 / 4$ & $5 / 8$ & $3 / 4$ & $5 / 16$ & $3 / 4$ & $3 / 32$ & $7 / 16$ & $31 / 8$ & 1 & $13 / 8$ \\
\hline
\end{tabular}

Heavy neck bolts.-Two standard sizes as shown by Figure 1003. These bolts are suitable for installation in any position on the heavier class of doors.

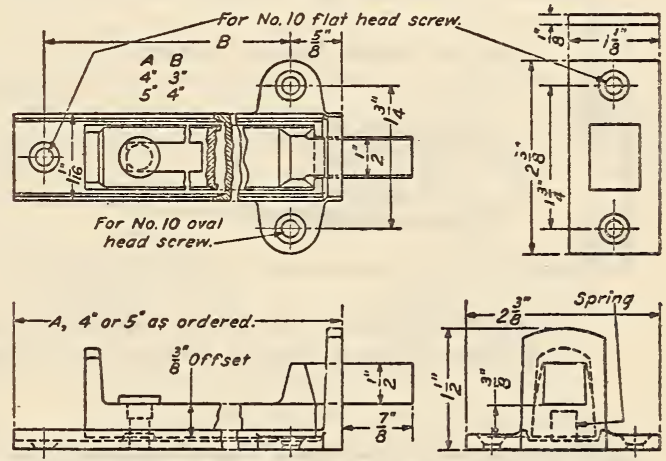

FIGURe 1003. - Heavy neck bolts

Light flush bolts.-Two standard sizes as shown by Figure 1004. These bolts are suitable for installation in any position on light and medium weight doors. 


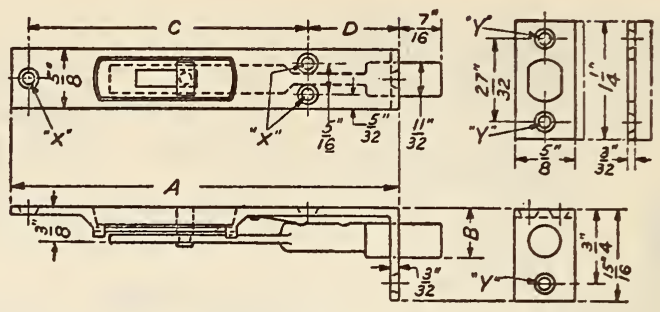

No. $4 \times 1$ "flat head screws at holes marked " $x$, No.4x 5 , oval hoad screws at holes marked " $Y$."

Figure 1004. - Light flush bolts

[Dimensions in inches]

\begin{tabular}{|c|c|c|c|}
\hline $\begin{array}{c}\text { Size } \\
\text { A }\end{array}$ & B & C & D \\
\hline $\begin{array}{l}3 \\
4\end{array}$ & $\begin{array}{l}15 / 32 \\
1 / 2\end{array}$ & $\begin{array}{l}21 / 8 \\
27 / 8\end{array}$ & $\begin{array}{l}11 / 16 \\
15 / 16\end{array}$ \\
\hline
\end{tabular}

Heavy flush bolts. - Two standard sizes as shown by Figure 1005. These bolts are suitable for installation in any position on the heavier class of doors.

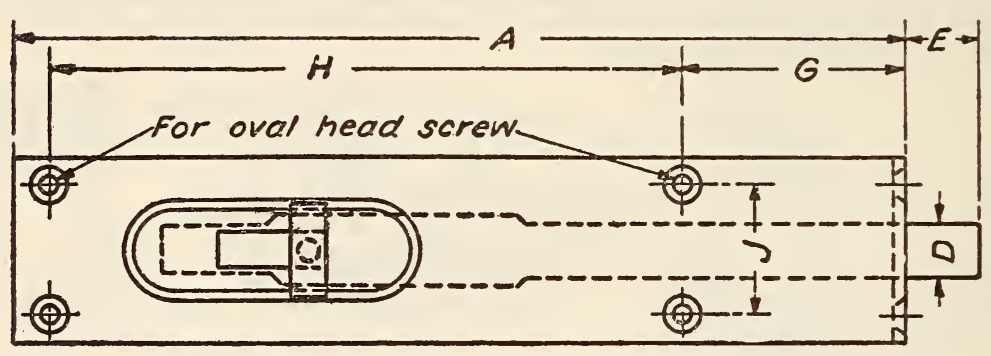

SCREW SIZES:- No. 6 for 6" bolt, No. 8 for

8 "bolt and both sizes of Keeper.
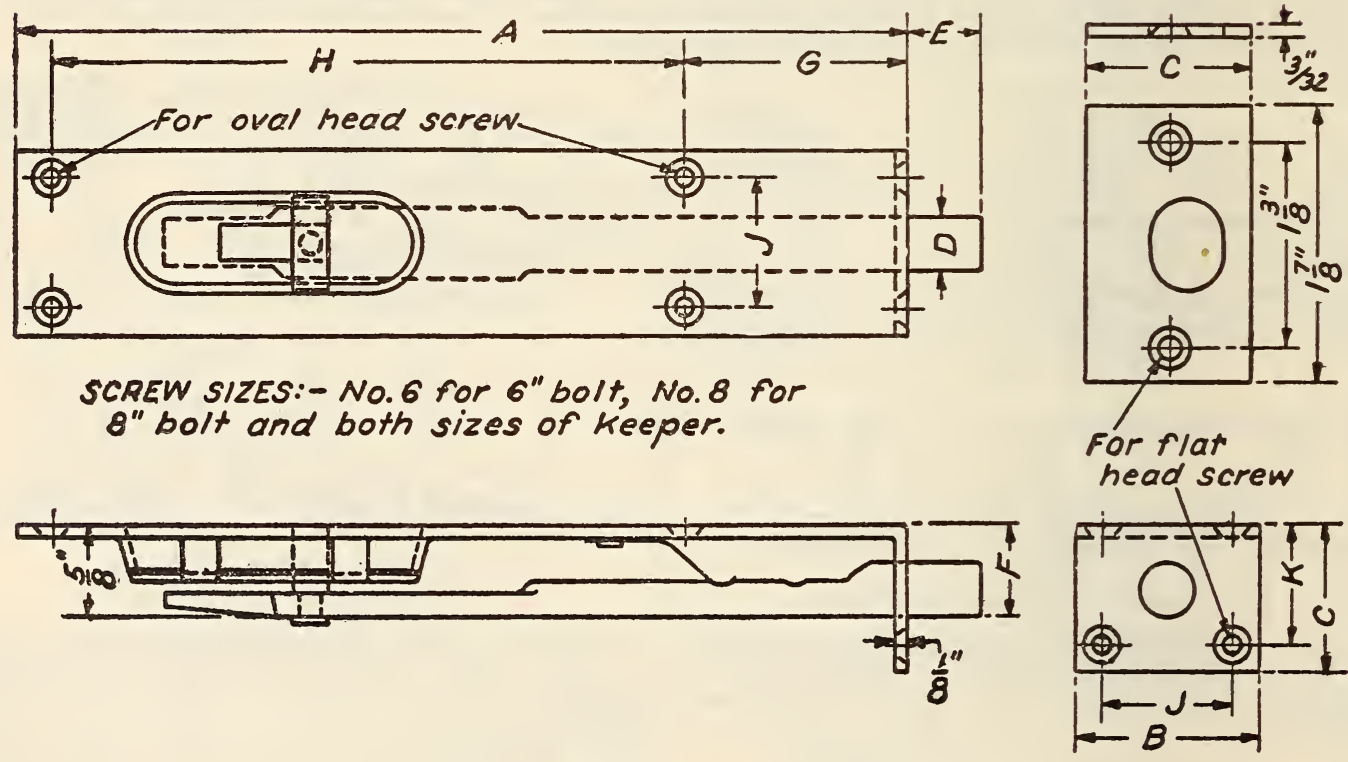

Figure 1005. - Heavy flush bolts

[Dimensions in inches]

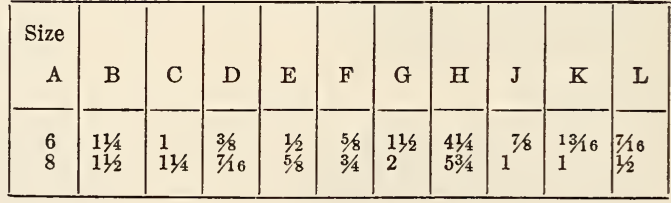




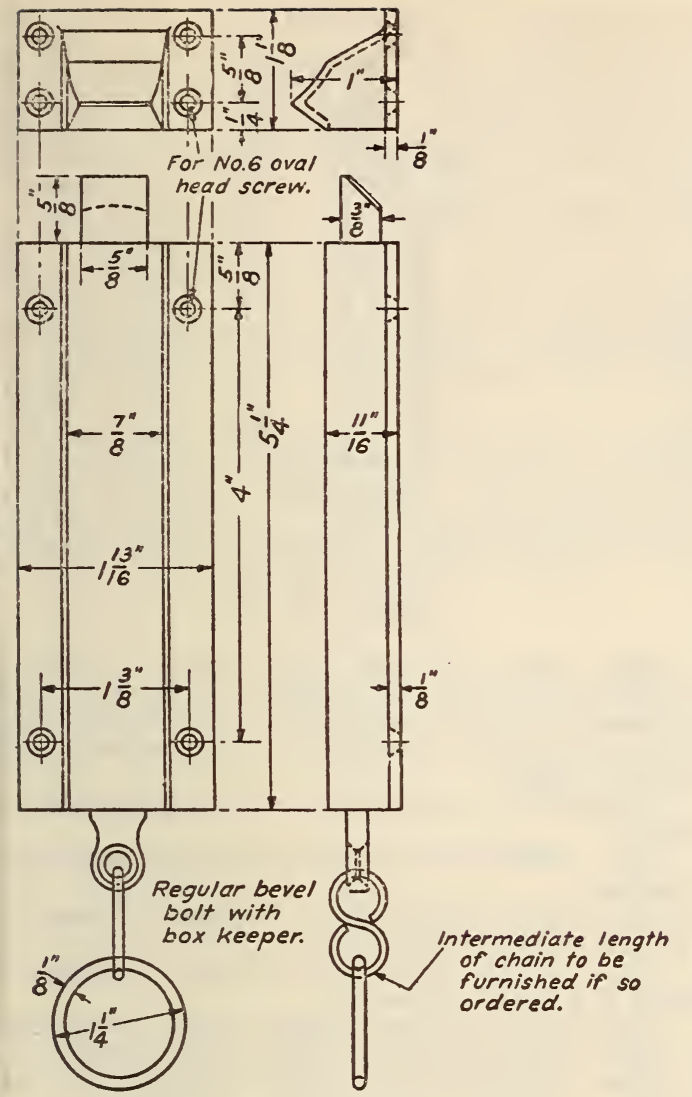

FIGURE 1006. - Spring bolt

Spring bolt (also known in the hardware trade as chain bolt). - One standard size as shown by Figure 1006. This bolt may be required of regular bevel with box keeper as per Figure 1006, or of reverse bevel with an angle keeper as per Figure 1007.

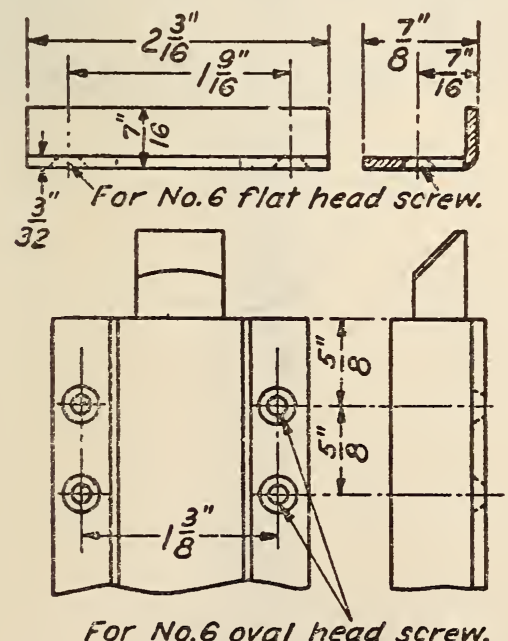

$$
\begin{aligned}
& \text { Reverse bevel bolt } \\
& \text { with angle keeper. }
\end{aligned}
$$

FIGURE 1007.-Spring bolt
The regular bevel bolt shall be attached by four screws; the reverse bevel bolt snall be attached by six screws. The standard includes a ring handle attached to the bolt by a short link as shown, but an intermediate length of chain shall be inserted in lieu of the short link when so ordered. This bolt is suitable for medium weight and heavy doors.

Turn catch for cupboard and locker doors.-One standard size as shown by Figure 1008.

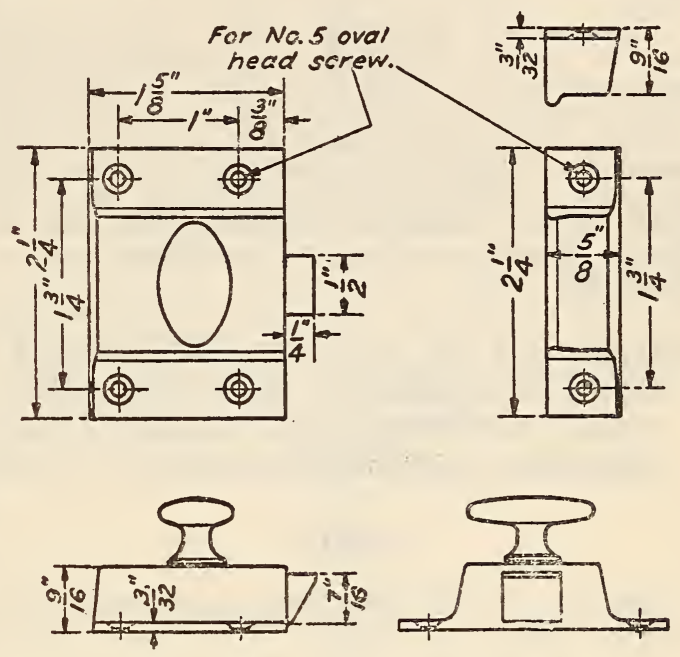

FIGURE 1008. - Turn catch for cupboard and locker doors

Elbow catch.-One standard size as shown by Figure 1009. This type of catch is applicable to the standing door of a pair of doors on cupboards and lockers.

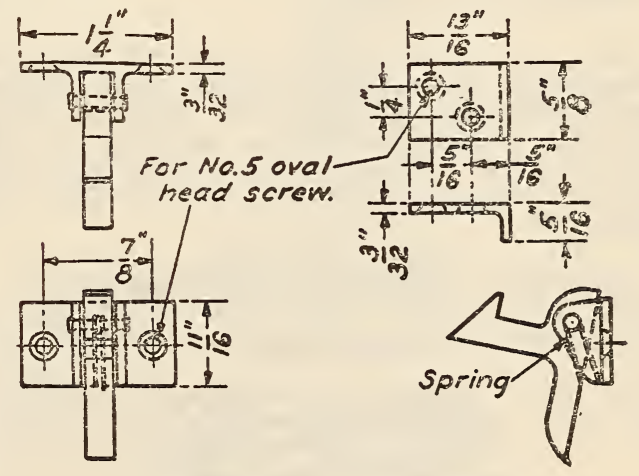

Figure 1009.-Elbow catch

\section{GENERAL REQUIREMENTS}

(In respect to materials, including percentages of components of white bronze, screws, and marking and packing, this specification conforms to A. M. S. C. H. No. $61-1930$, specification for locks and accessories for ship doors. (See 617.21, p. 1004.) Otherwise it provides as follows:)

Construction. - The fittings shall conform to the standards herein defined to the satisfaction of the purchaser and shall be guaranteed by the manufacturer or dealer for efficient operation and lasting service. All features, including the positions and dimensions of holes for screws, which are to govern cutting of doors or adjacent framework to fit and attach the bolts, catches, and their accessories, shall be in such close accordance with the drawings that 
any item may be quickly replaced by another one of the same standard type and size without requiring additional cutting or fitting. Reasonable variations in other features will be allowed which do not prevent interchangeability or impair good appearance or serviceability. Certain ones of the bolts may be required for metal doors and when ordered for such purpose, the underside of the base which is to fit on the metal door shall be machined to a true plane.

Workmanship and finish.-The workmanship shall be such as to produce good appearance and serviceability and shall be uniform in character. Exposed surfaces shall be polished and lacquered; other surfaces shall be rough dipped, except where machining is required for assembly, installation, and operation. This shall constitute the standard finish, and any variation desired therefrom must be clearly stated in asking for quotations and in purchasing.

\section{UNITED STATES GOVERNMENT, DEPART-} MENT OF COMMERCE, BUILDERS' HARDWARE (NONTEMPLATE), COMMERCIAL STANDARD CS22-30, DECEMBER 11, 1930

\section{BOITS}

(See 617.0 , p. 977, also Federal specification FF$\mathrm{H}-101$, below.)

\section{UNITED STATES GOVERNMENT, FEDERAL SPECIFICATIONS BOARD, FEDERAL SPECIFICATION FOR BUILDERS' HARD- WARE (NONTEMPLATE), FF-H-101, AUGUST 19, 1930.}

(For types, grades, and classes; material and workmanship; general requirements; detail requirements; packaging, packing, and marking; notes; and table of standard finishes, see 617.0, p. 978 . For chromium plating, nickel plating, and zinc coatings; and methods of inspection and tests, see 600.3 , p. 70. .)

\section{BUILDERS' HARDWARE (NONTEMPLATE)}

BARREL BOLTS

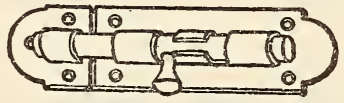

Figure 1010. - Barrel bolt, type 1019

Type 1019.- Heavy wrought steel, plated. Length of bolt, 3, 4, and 5 inches. Minimum thickness of plate, 0.051 inch.

Type 1019A.-Heavy wrought steel, galvanized. Length of bolt, 3, 4, 5, and 6 inches. Minimum thickness of plate, 0.051 inch.

Type 1019B.- Same as type 1019A, except japanned.

Type 1019C.-Same as type 1019, except wrought brass.

Type 1020.-Cast bronze polished. Length of bolt, 3,4 , and 5 inches.

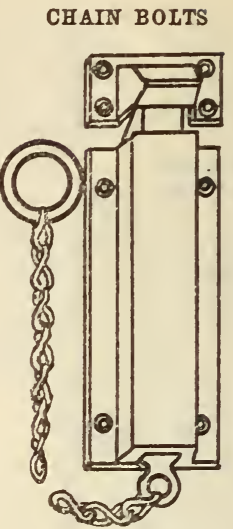

FIGURE 1011. Chain bolt, type 1022

Type 1022.-Wrought steel, plated. Round or square case acceptable. Length of bolt, 3,6 , and 8 inches. Minimum thickness of plate, $0.040,0.064$, and 0.064 inch, respectively. (For use with type 1049 or 1050 .)

Type 1022A.-Same as type 1022, except galvan ized.

Type 1022B.-Same as type 1022, except japanned.

Type 1025.-Cast bronze, polished. Length of bolt, 3,6 , and 8 inches.

Type 1025 A.-Cast iron, plated. Length of bolt, 3,6 , and 8 inches.

Type 1025 B.-Same as type 1025A, except galvanized. Length of bolt, 3, 6, and 8 inches.

Type 1025C.-Same as type 1025A, except japanned. Length of bolt, 3,6 , and 8 inches.

CREMONE BOLTS

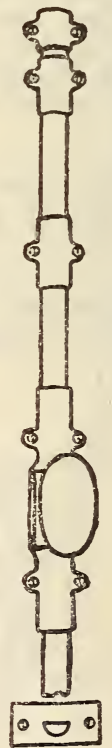

Figure 1012-Cremone bolt, type 1027

Type 1027.-All bronze with wrought bronze rod one-half inch wide. Half oval or half round rods, 
with guides for every 2 feet of rod. Constructed so as not to deface paint in operating. Oval knob or lever handle, right or left hand as specified. Quarter or half turn shall operate. Operation from inside only. Minimum size of knob $2 \frac{1}{4}$ by $1 \frac{3}{8}$ inches for French doors; and $13 / 4$ by $1 \frac{1}{8}$ inches for casement windows.

Type 1027 A.-Same as type 1027, except with steel rod, and iron or steel cases and guides, unfinished. Bronze knob or handle.

Type 1027B.-Same as type 1027, except with steel rod, and iron or steel cases and guides, plated.

Type 1028.- Same as type 1027, except with $5 / 8$-inch wrought bronze rod and bronze cases and guides.

Type A1028.-Extra heavy garage type, wrought steel, galvanized and japanned. Half oval rod $3 / 4$ inch wide, half oval bolt head $1 \frac{1}{2}$ inches wide. Handle 7 inches long. Throw of bolts $1 \frac{1}{2}$ inches. Standard for 8-foot doors with total adjustment of 6 inches. Furnished with extra floor keeper.

\section{ESPAGNOLETTE BOLTS}

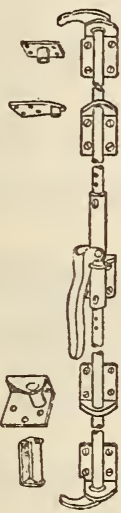

Figure 1013. -

Espagnolette bolt, type A1030

Type A1030.-Extra heavy garage type, wrought steel, galvanized and japanned. Diameter of rod 5/8 inch; 7-inch handle. Handle shall lock bolt when in closed position. Standard for 8-foot doors with total adjustment of $43 / 4$ inches.

\section{FLUSH BOLTS}

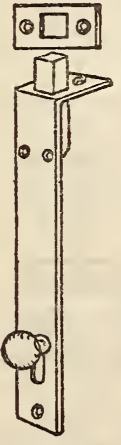

$$
\begin{gathered}
\text { Figure 1014.- } \\
\text { Flush bolt, } \\
\text { type } 1037 B
\end{gathered}
$$

Type 1087B.-Wrought steel, plated; 1/2-inch square bolt with knob for mounting on face of door stiles. Plates $1 \frac{1 / 4}{4}$ inches wide, minimum thickness 0.081 inch. Sizes $6,9,12$, and 18 inches.
EXTENSION FLUSH BOLTS

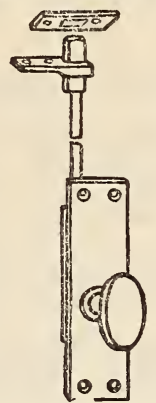

FIGURE 1015. - Extension flush bolt, type 1014

Type 1041.-Turn knob type for surface with oval turn knob. Cast bronze. Plate, $5 \frac{5}{8}$ by $11 / 2$ inches. Bolt head, $1 / 2$ inch. Length of rods, 9, 12, 18 , and 24 inches.

Type 1041 A.-Same as type 1041, except iron or steel, plated.

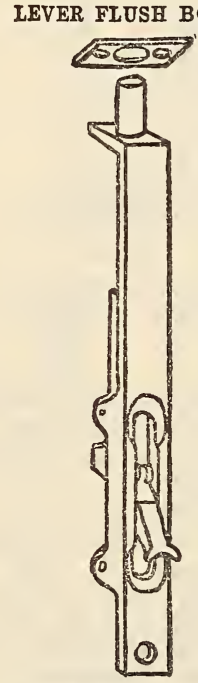

Figure 1016. - Lever flush bolt, type 1043

Type 1043.-Cast bronze. Plate, 6 by $5 / 8$ inch. Bolt head not less than $3 / 8$-inch diameter with $5 / 8$-inch throw.

Type A1043.-Cast bronze, plate 1 inch to $1 \frac{1 / 4}{4}$ inches wide by 9,12 , or 18 inches long. Bolt head not less than $3 / 8$ inch.

EXTENSION-LEVER FLUSH BOLTS

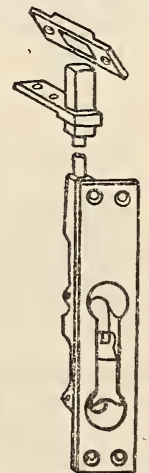

Figure 1017. - Extension lever flush bolt, type 1044 
Type 1044.-Cast bronze. Plate, 6 by $5 / 8$ inch. Bolt head, $3 / 8$ inch. Length of rods, 12,18 , and 24 inches.

Type A1044.- Same as type 1044, except cast iron, plated.

Type 1044 B.-Cast bronze. Plate $63 / 4$ by $1 \frac{1}{4}$ inches. Bolt head, $1 / 2$ inch. Length of rods, 9 12,18 , and 24 inches.

Type B1044B.- Same as type 1044B, except cast iron, plated.

Type 1044D.- Same as type 1044B, except wrought bronze. Minimum thickness of plate 0.092 inch.

Type 1044E.-Same as type 1044B, except wrought steel, plated. Minimum thickness of plate 0.092 inch.

\section{FOOT BOLTS}

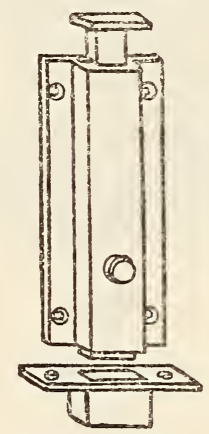

Figure 1018. Foot bolt, type $104 i$

Type 104\%.-Cast bronze, polished foot bolts with trip. Sizes 4,6 , and 8 inches.

Type 1047A.-Cast iron, plated. With trip. Sizes 4,6 , and 8 inches.

Type 1047B.-Same as type 1047A, except galvanized. Sizes 4,6 , and 8 inches.

Type 1047C.-Same as type 1047A, except japanned. Sizes 4,6 , and 8 inches.

Type 1049.-Wrought steel, plated. With trip. Round or square case acceptable. Size, 3, 6, and 8 inches. Minimum thickness of plate, 0.040, 0.064 , and 0.064 inch, respectively. (For use with type 1022.)

Type 1049 A.- Same as type 1049, except galvanized. Sizes 3,6 , and 8 inches.

Type 1049B.- Same as type 1049, except japanned. Sizes 3,6 , and 8 inches.

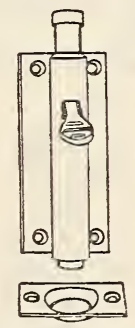

Figure 1019. -

Foot bolt, type 1050

Type 1050 .-Wrought steel positive action foot bolts. Plated. Sizes 6 and 8 inches. (For use with type 1022.)

Type 1050A.- Same as type 1050, except galvanized. Sizes 6 and 8 inches.
Type 1050B.- Same as type 1050, except japanned. Sizes 6 and 8 inches.

Type 1051.-Extra heavy cane bolt. Galvanized steel rod, minimum diameter $3 / 4$ inch. Cast-iron guides, japanned. Strikes to be furnished for wood or concrete. Suitable stops shall prevent bolt from slipping out of guides. Length of bolt 20 inches, minimum throw 12 inches.

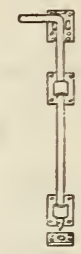

Figure 1020.-
Cane bolt, type
1051

Type 1051A.-Heavy wrought steel cane type. Galvanized steel rod, minimum diameter $5 / 8$ inch. Wrought steel guides japanned. Strikes to be furnished for wood or concrete. Suitable stops shall prevent bolt from slipping out of guides. Minimum throw 8 inches, length of bolt 18 and 24 inches.

\section{LAVATORY DOOR BOLTS}

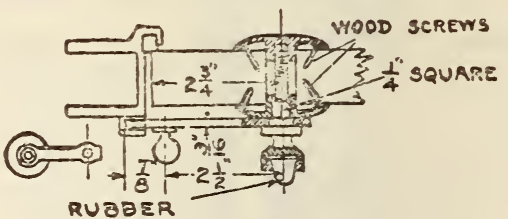

Figure 1021. - Lavatory door bolt, type A1051

Type A1051.-Bronze, nickel plated. Rubber stop on strike and rubber tip on knob. Adjustable for $1 \frac{1}{8}$ to $1 \frac{3}{8}$ inch door and stile. Strike secured with through bolts and cap nuts.

Type A1051A.- Same as type A1051, except nickel bronze.

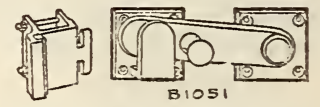

FIgure 1022.-Lavatory door bolt, type B1051

Type B1051.-Cast bronze, nickel plated. Reversible. Minimum length of bar 4 inches. Size of base about 2 by 2 inches.

Type B1051A.- Same as type B1051 except nickel bronze.
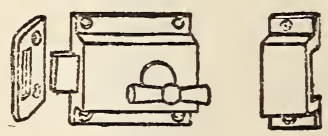

Figure 1023.-Lavatory door bolt, type C1051

Type C1051.-Cast bronze, nickel plated. $\mathrm{T}$ handle, without indicator. Rim or mortise strike. Size about $2 \frac{1}{2}$ by $2 \frac{5}{8}$ inches.

Type C1051A.- Same as type C1051, except nickel bronze. 
MORTISE BOLTS

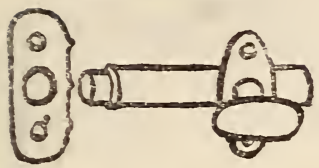

Figure 1024.-Mortise bolt, type $1053 \mathrm{~A}$

Type 1053A.- Wrought steel bolt. Approximate diameter of case $\% 16$ inch. Bronze turn, plate and strike. Back-set $15 \%$ inches.

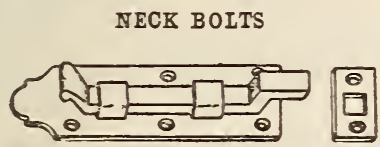

Figure 1025.-Neck bolt, type 1054

Type 1054.- Wrought brass plate and strike with wrought or cast brass bolt; size 3 inches, minimum weight 1.0 ounce each; size 4 inches, minimum weight 2.0 ounces each.

Type 1054A.- Same as type 1054, except cast brass. Size 3 inches, minimum weight 4.0 ounces each. Size 4 inches, minimum weight 5.6 ounces each.

Type 1056.- Wrought steel, japanned, plain square bolt, rust-proof spring. Minimum thickness of plate 0.033 inch. Minimum throw of bolt $1 / 2$ inch.

\begin{tabular}{|c|c|c|}
\hline Size & $\begin{array}{c}\text { Width of } \\
\text { plate }\end{array}$ & Offset \\
\cline { 1 - 2 } $\begin{array}{c}\text { Inches } \\
21 / 2 \\
4\end{array}$ & $\begin{array}{c}\text { Inches } \\
1\end{array}$ & $\begin{array}{r}\text { Inch } \\
1 / 3\end{array}$ \\
& $1 / 8$ \\
\hline
\end{tabular}

\section{SQOARE BOITS}

Type 1058.-Cast brass square bolt, size 4 inches. Type 1059.-Square bolt unfinished, 21/2, 3, and 4 inches with two strikes, flat and strap.

\section{SURFACE BOLTS}

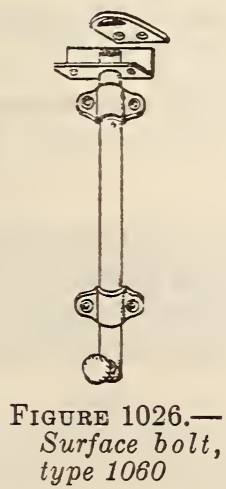

Type 1060.-Wrought bronze. Three-eighthsinch half round or oval rod. One-inch throw. Two guides which shall prevent bolt from touching woodwork. Bolts shall not rotate, and shall be secure in both extended and withdrawn positions. Furnish with angle and universal strikes. Lengths 4 and 6 inches.

\section{EXIT BOLT LOCKS}

Type 800.-For use with exit bolt type 831 . Rotary antifriction or roll-back latch bolt with auxiliary bolt. Japanned cast-iron case, $5 \frac{3}{8}$ by $3 \frac{3}{4}$ by $3 / 4$ inch. Solid front, 8 by $11^{\prime}$ inch. Backset, $23^{3} / 4$ inches. Lock shall have one or more bronze cylinders with five or more nin tumblers giving practically unlimited changes. Bronze front, bolts, and strike, not reversible. Three nickel-bronze keys. Master-keyed if so ordered.

Operation.-From inside by slight push or pull, or both, on bar at all times. From outside by key when locked and by handle when unlocked. When locked, latch bolt shall be deadlocked by auxiliary bolt upon engaging strike, and action of auxiliary bolt shall permit of space $3 / 16$ inch between lock front and strike without releasing deadlocking function. Latch bolt shall be capable of being locked in retracted position.

Type 801.- Same as type 800 , except operation from outside by knob when unlocked.

\section{EXIT BOLTS}

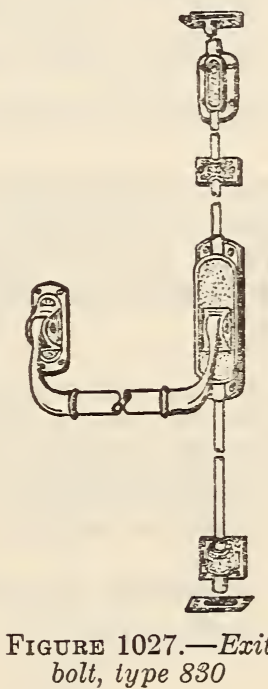

Type 830.- Surface type for single exit doors only. Top and bottom locking. Shall require no outside trim. All cast bronze except wrought-bronze cross bar and vertical rods. One-inch cross bar, $3 / 8$ to $1 / 2$ inch rods, $3 / 4$-inch (minimum 9/16) top and bottom bolts with not less than 1/2-inch throw. Not reversible. Bar capable of being locked in inactive position with bolts retracted.

Operation.-By slight push or pull, or both, on cross bar. Bolts shall remain retracted until door is closed.

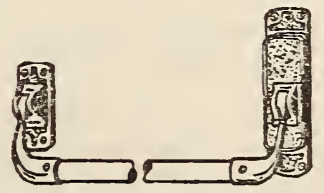
FIGURE 1028.-Exit bolt, side latching type, type 831

Type 831.-Side latching type, for single doors. All cast bronze except wrought-bronze cross bar, 1 -inch diameter. For $4 \frac{1}{2}$-inch stiles. Bar capable 
of being locked in inactive position with bolts retracted. To be used only with special lock, types 800 and 801.

Operation.-See type 800.

WINDOW SPRING BOLTS

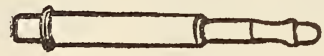

Figure 1029.-Window spring bolt, type $A 1060$

Type A1060.-Tinned or galvanized malleable iron or steel bolt. Tinned or galvanized wrought steel case. Brass spring. For sash $7 / 8$ to $17 / 8$ inches wide.

61\%.62 BRACKETS, HANDRAIL AND SHELF.

UNITED STATES GOVERNMENT, DEPARTMENT OF COMMERCE, BUILDERS' HARDWARE (NONTEMPLATE), COMMERCIAL STANDARD CS22-30, DECEMBER 11, 1930

\section{SHELF BRACKETS, SHELF RESTS}

(See 617.0 , p. 977, also Federal specification FFH-101 below.)

\section{UNITED STATES GOVERNMENT, FEDERAL SPECIFICATIONS BOARD; FEDERAI SPECIFICATION FOR BUILDERS' HARD- WARE (NONTEMPLATE), FF-H-101, AU- GUST 19, 1930}

(For types, grades, and classes; material and workmanship; general requirements; detail requirements; packaging, packing, and marking; notes; and table of standard finishes, see 617.0, p. 978. For chromium plating, nickel plating, and zinc coatings; and methods of inspection and tests, see 600.3 , p. 70.)

\section{BUILDERS' HARDWARE (NONTEMPLATE)} HANDRAIL BRACKETS

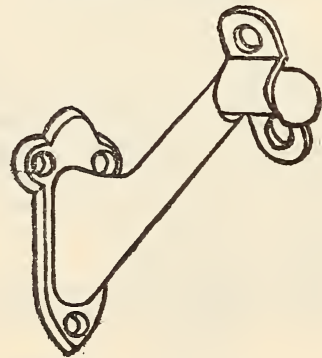

Figure 1030.-Handrail bracket, type 1064

Type 1064.-Cast bronze, polished. Adjustable. Projection $23 / 4$ inches from base to center of rail.

Type 1064A.- Same as type 1064, except cast iron, plated.
SHELF BRACKETS

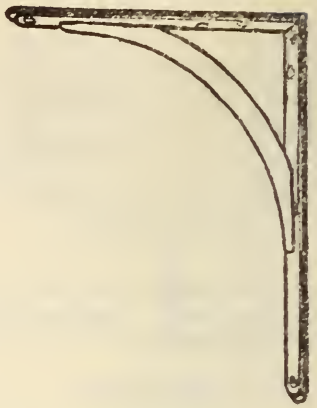

FIgURE 1031.-Shelf

bracket, type 1068

Type 1068.-Wrought steel, plated, with corrugated or tubular braces and embossed plates.

\begin{tabular}{|c|c|c|c|}
\hline Size & Shelf plate screws & Wall plate screws & $\begin{array}{c}\text { Minimum } \\
\text { thickness } \\
\text { of plate }\end{array}$ \\
\hline $\begin{array}{l}\text { Inches } \\
4 \text { by } 5 \\
6 \text { by } 8 \\
8 \text { by } 10 \\
10 \text { by } 12 \\
12 \text { by } 14 \\
16 \text { by } 18\end{array}$ & $\begin{array}{l}3 / 4 \text { inch by No. } 7 .- \\
3 / 4 \text { inch by No. } 7 . . \\
3 / 4 \text { inch by No. } 8 . \\
3 / 4 \text { inch by No. } 9 . . \\
3 / 4 \text { inch by No. } 10 . \\
1 \text { inch by No. } 10 . .\end{array}$ & $\begin{array}{l}11 / 4 \text { inches by No. } 7 \\
11 / 4 \text { inches by No. } 7 . . \\
11 / 4 \text { inches by No. } 8 . . \\
11 / 4 \text { inches by No. } 9 \\
11 / 4 \text { inches by No. } 10 . \\
11 / 2 \text { inches by No. } 10 .\end{array}$ & $\begin{array}{l}\text { Inch } \\
0.064 \\
.064 \\
.072 \\
.081 \\
.081 \\
.102\end{array}$ \\
\hline
\end{tabular}

Type 1068A.-Same as type 1068, except galvanized.

Type 1068B.-Same as type 1068, except japanned.

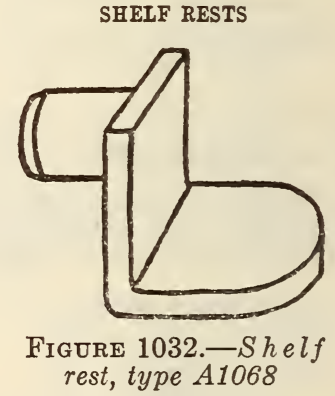

Type A1068.-Cast iron, coppered. Milled pin, 3/8-inch diameter.

61\%.63 HOOKS.

UNITED STATES GOVERNMENT, DEPARTMENT OF COMMERCE; BUILDERS'HARD-

WARE (NONTEMPLATE), COMMERCIAL

STANDARD CS22-30, DECEMBER 11, 1930

\section{HOOKS}

(See 617.0, p. 977, also Federal specification FFH-101 below:) 
UNITED STATES GOVERNMENT, FEDERAL SPECIFICATIONS BOARD; FEDERAL SPECIFICATION FOR PLUMBING FIXTURES (FOR IAND USE), INO. 448, NOVEMBER 22, 1926. (CURRENT DESIGNATION FF-P-451)

\section{COAT HOOKS}

(See 617.73, p. 1062.)

\section{UNITED STATES GOVERNMENT FEDERAL SPECIFICATIONS BOARD, FEDERAI SPECIFICATION FOR BUIIDERS' HARD- WARE (NONTEMPLATE), FF-H-101, AUG- UST 19,1930}

(For types, grades, and classes; material and workmanship; general requirements; detail requirements packaging, packing, and marking; notes; and table of standard finishes, see 617.0, p. 978. For chromium plating, nickel plating, and zinc coatings; and methods of inspection and tests, see 600.3 , p. 70.)

\section{BUILDERS' HARDWARE (NONTEMPLATE)}

CEILING HOOKS

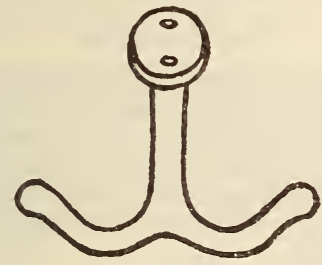

Figure 1033.-Ceiling hook, type $115 \%$

Type 115\%.-Cast bronze. Two screws. Projection $2 \frac{3}{8}$ inches.

Type $115 \%$ A.-Same as type 1157 , except cast iron, plated.

Type A115\%.-Steel wire with protective coating. Screw point. Projection $2 \frac{3}{8}$ inches.

\section{COAT AND HAT HOOKS}

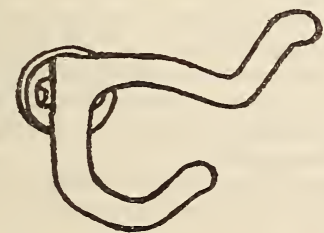

Figure 1034.-Coat and hat hook, type 1162

Type 1162.-Cast bronze. Upper prong bent. Two screws. Projection 3 inches.

Type 1162A.- Same as type 1162, except cast bronze, nickel plated.

Type 1162B. - Same as type 1162, except cast iron, plated.

Type A1162.-Steel wire with protective coating. Screw point. Projection 3 inches.
DOOR HOOKS

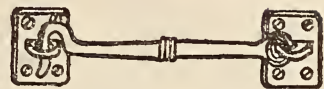

Figure 1035.-Door hook, type A1176

Type A1176.-Cast bronze. Length 4 inches.

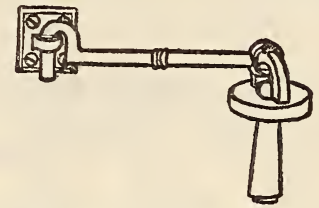

Figure 1036.-Door

hook, type A1176A

Type A1176A.-Cast bronze. With expansion bolt. Lengths 4 and 6 inches.

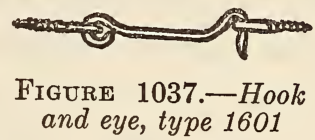

Type 1601.-Brass wire hooks with screw eyes. Lengths, 2, 3, 4, and 6 inches.

Type 1601A.- Same as type 1601, except steel wire, brass plated.

Type 1601B.-Same as type 1601, except steel wire, galvanized.

Type 1601C.- Same as type 1601, except unfinished steel wire.

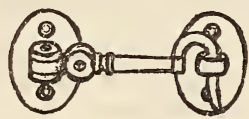

FI G URE 1038.Cabin door hook, type 1602

Type 1602.-Cabin door hook. Heavy jointed cast bronze hook and plates, sizes $2 \frac{1}{2}, 3 \frac{1}{2}, 5,6,8$, and 12 inches.

\section{1\%.7 PIUMBING FIXTURES.}

\subsection{VITREOUS AND PORCELAIN WARE.}

(For nationally recognized standards and specifications for vitreous and procelain ware, see Miscellaneous Publication No. 110 of the Bureau of Standards, entitled "Standards and Specifications for Nonmetallic Minerals and Their Products," published, April 1930.)

\section{1\%.72 SLATE, SOAPSTONE, MARBLE, AND GLASS PLUMBING.}

(For nationally recognized standards and specifications for slate, soapstone, marble, and glass plumbing, see Miscellaneous Publication No. 110 of the Bureau of Standards, entitled "Standards and Specifications for Nonmetallic Minerals and Their Products," published, April, 1930.) 
617.73 PIUMBING TRIMIMINGS AND FITTINGS.

UNITED STATES GOVERNMENT, DEPARTMENT OF COMMERCE, NATIONAL BUREAU OF STANDARDS, BRASS LAVATORY AND SINK TRAPS, SIMPLIFIED PRACTICE RECOMMENDATION NO. 21, 1925

\author{
BRASS LAVATORY AND SINK TRAPS
}

(See 645.4 , p. 1234.)

\section{UNITED STATES GOVERNMENT, FEDERAL SPECIFICATIONS BOARD, FEDERAL SPECIFICATION FOR PLUMBING FIX- TURES (FOR LAND USE), NO. 448, NOVEMBER 22, 1926. (CURRENT DES- IGNATION: FF-P-451)}

(See also 600.3, p. 70.)

\section{PLUMBING FIXTURES (FOR LAND USE)}

TYPES, GRADES, AND CLASSES

This specification covers such types, grades, and classes of plumbing fixtures as are enumerated under "Detail requirements," comprising vitreous ware and porcelain ware, enameled ironware, slate, soapstone, brass trimmings and fittings, supports, stops, cocks, valves, flushing mechanisms, pipe covering, etc.

(For vitreous ware, porcelain ware, slate, and soapstone, see "Standards and Specifications for Nonmetallic Minerals and Their Products," miscellaneous publication No. 110 of the Bureau of Standards.)

\section{GENERAL REQUIREMENTS}

Where United States Government specifications are referred to, the latest revision of such specifications shall apply in all cases.

\section{Enameled Ironware}

Quality of enameled ironware.-Enameled ironware shall be best quality cast iron of the necessary thickness to form fixtures of the best grade manufactured.

Ironware shall have a porcelain enamel coat thoroughly fused on required surfaces. Ename shall be smooth and of even thickness, white, without discoloration, chips, or other flaws, and free from craze. Exterior exposed surfaces not required to be enameled shall be treated with one coat of filler at factory.

Dimensions and variations.-The dimensions shown on the plates shall be followed where given, but a variation of not more than 3 per cent plus or minus will be allowed.

Labeling enamel ware.-All fixtures shall have the manufacturer's guarantee label indicating first quality. Omission of this label may be sufficient cause for rejection.

\section{Trimmings and Fittings}

Quality.-All trimmings and fittings in connection with the plumbing system shall be of best quality, of heavy and substantial pattern.

The brass mixture, except for red metal and where fittings for tanks are used on salt water, shall contain not less than 65 per cent copper and not more than 5 per cent lead.
Composition for fittings where salt water is used shall contain not less than 88 per cent copper and not less than 8 per cent tin.

Composition for cast trimmings and fittings where red metal is required shall contain not less than 85 per cent copper, 5 per cent tin, and not more than 7 per cent zinc, nor more than 4 per cent lead.

When white metal is desired in lieu of brass it shall be called for in the specification for the work and shall be of the following composition: Copper, 54 to 58 per cent; nickel, 14 to 20 per cent; lead, 12 per cent, maximum; tin, $2 \frac{1}{2}$ per cent, maximum; zine, 20 per cent, maximum; impurities, including iron and manganese, not more than 1 per cent.

Where red metal trimmings and fittings or saltwater fittings are desired, they will be called for in the specifications for the work.

Finish--All exposed brass, except red metal, shall be heavily nickel-plated in a manner to produce a finish of high polish and durability, except where otherwise specified. When chromium plating is desired, it shall be so stated in the specifications for the work.

Red metal shall be highly polished and finished and of uniform color for all castings, tubing, and fittings.

Stamping or marking trimmings and fittings.-All faucets, ball cocks, and stops shall have manufacturer's name or trade-mark cast or stamped on body of the piece.

(For nickel-plating of plumbing fixtures see 600.3, p. 70.)

$$
\text { Compression Stops }
$$

Shall be standard weight designed for 100 pounds working pressure, globe pattern, with full area opening having raised seat and composition disk; shall be finished brass, nickel-plated, and provided with stuffing boxes and cast brass milled-wheel tee or cross handles. Angle pattern stops may be used where practicable.

\section{Ball Cocks}

For flushing tanks shall consist of $1 / 2$-inch diameter brass, automatic, float-operated supply cock, guaranteed to close effectively against a water pressure of 100 pounds to the square inch, top supply pattern for high tanks, having brass refill and hush tube, and bottom supply with elevated ball cock and hush tube, or semielevated ball cock for low tanks. Float shall be of 16-ounce $(0.0216$ inch thickness) spun copper, vulcanized rubber, or of glass with brass stem connected to float and cock with screw threads; cock shall be constructed so it can readily be taken apart for repairs, pins forming bearings for levers shall be $1 / 8$-inch brass cotter pins. Supports for ball cocks and lever fulcrum on high tanks shall be secured with through bolts and nuts to lugs cast on tanks having lead washers between tank lugs and supports.

Supply pipes to ball cocks shall be $1 / 2$-inch diameter fitted with a compression stop.

\section{Flushing Tank Mechanism}

Flushing mechanism of high tanks shall consist of a cast brass gooseneck siphon with slotted guide sleeves and stable rubber washer. Siphons for urinal flush tanks shall be $11 / 8$ inches in diameter, and siphons for water-closet flush tanks shall be $1 \frac{1}{2}$ inches in diameter. Siphons shall be properly secured to the operating levers in such a manner that they will be properly balanced and guided to secure a straight lift and proper reseating. The lever post shall be provided with an adjustable stud bolt that will limit the pull on the lever and prevent the guide on the gooseneck siphon from being pulled out of its sleeve. 
Flushing mechanism shall be operated by means of a nickel-plated brass plumber's chain not thinner that No. 20 Brown \& Sharpe gage (0.032 inch), with a hardwood pull handle. Handle shall have a brass rod passing through same with nickel-plated brass ring nut and bottom nut sweated on rod. Chain shall be fastened to operating lever and ring nut by means of rings of $1 / 8$-inch diameter nickelplated brass with joints well soldered after chain is attached.

Flushing mechanism of low tanks shall be of rubber ball design, having a $23 / 4$-inch diameter, best quality, rubber ball, reinforced at top and center, with necessary brass stem, guide, and lever. Operation shall be quiet in action, shall be controlled by means of a nickel-plated brass or china lever handle, set at front of tank. A brass standpipe not less than 1-inch diameter, of thickness not less than No. 17 Brown \& Sharpe gage ( 0.045 inch), shall be properly installed for overflow.

Each discharge shall be succeeded by an afterflush or refill that will fill the fixture trap to its overflow weir.

\section{Flushing Valves}

Shall be of a true flushometer type and of a pattern approved by the bureau or office concerned, operated either by push button, lever, or oscillating handle. If of lever or oscillating handle type, the handle shall be all brass, nickel-plated or white metal (nickel brass) and, in addition to this, if of leverhandle type, the handle shall be integral with the lever.

Flushing valves shall be easily operated by hand, and so designed that they will cause absolutely no water hammer in the piping system and if a regulating device is used to adjust the amount of water and give the proper afterfill, the regulating device shall be accessible without disconnecting or taking the valve apart.

Flushing valves for water-closets shall provide successful flushing of fixture and refill the fixture trap at each operation with a discharge of not more than 4 gallons of water, under a pressure ranging from 15 to 100 pounds. Flushing valves for urinals shall discharge not less than 1 gallon nor more than 2 gallons of water under a pressure ranging from 15 to 100 pounds.

Each flushing valve shall be provided with a cutoff cock adjacent to flushing valve and connected thereto with a nickel-plated brass union or nipple. A lock shield gate valve may be used in lieu of the cut-off cock.

Flushing valves for urinals shall have not smaller than $1 / 2$-inch nor larger than $3 / 4$-inch diameter supply pipe connection; flushing valves for water-closets shall have not smaller than 1-inch nor larger than $11 / 4$-inch diameter supply pipe connection.

All exposed parts of flushing valves and cut-off cocks or gate valves shall be nickel-plated, finished brass, or white metal. All other parts of flushing valves shall be of very best noncorrodible metal that will withstand frequent operation of valve without wear or breakage.

\section{Roof Drains}

Shall be heavy pattern cast iron with integral cast-iron cup pattern fitting having an integral flange not less than 16 inches in diameter, and a satisfactory device for clamping or otherwise securing the roof covering so as to make a water-tight connection; or in lieu of the integral flange, shall have a 16 -ounce $(0.0216$ inch) soft-rolled copper or a 5-pound sheet lead flashing flange with not less than 4-inch lap on ail sides extending into the roofing.

For gravel or slag roofing the drain shall be provided with a suitable gravel stop.
Shall have a cast-iron beehive or dome shaped strainer, except on promenade roofs where strainer shall be flat. Openings in strainer shall have a combined area equal to twice the area of drain outlet.

Outlet shall be equipped with necessary parts to make proper connection to screw pipe or cast-iron pipe as required of same size as down spout.

When specified in specification for the work each roof drain shall be provided with a heavy pattern, cast brass or copper sleeve-expansion joint of thickness not lighter than No. 10 Stubbs gage (0.134 inch) constructed so as to form a water and air tight flexible joint. Gaskets and packing shall be of asbestos fiber, soft lead, or other suitable durable material which shall not be located in the flow line of the drainage. Means of adjustment shall be provided for tightening or replacing packing.

\section{Piping}

Cast-iron soil pipe, fittings, and connections.-Castiron soil pipe and fittings shall be in accordance with Federal specification No. 343. (Current designation WW-P-401.)

Wrought-iron pipe.-Shall be standard-weight genuine wrought-iron galvanized pipe in accordance with Federal specification WW-P-441.

Steel pipe.-Shall be standard weight galvanized steel pipe in accordance with Federal specification $\mathrm{WW}-\mathrm{P}-431$.

Fittings and connections for wrought-iron and steel pipe.-Fittings for soil and waste piping and for roof-drainage piping systems shall be cast iron, recessed and banded, screw-jointed drainage fittings, free from fins and burrs. Long-turn fittings shall be used where space and other conditions permit, and the consent of the Government representative shall be obtained before any short-radius fittings are installed. Fittings on soil, waste, and roof drainage piping may be galvanized or plain, uncoated.

Fittings for vent and water supply piping shall be standard banded cast or standard beaded or banded malleable iron. All fittings on water supply piping shall be galvanized. Cast fittings on vent piping may be galvanized or plain, uncoated. Malleable fittings on vent piping shall be galvanized.

Changes in pipe sizes shall be made with reducing fittings or fittings bushed in the sand.

Brass pipe, fittings, and connections.-Brass pipe shall be grade $\mathrm{B}$ or $\mathrm{C}$ in accordance with Federal specification $\mathrm{WW}-\mathrm{P}-351$.

When specified in specification for the work, brass pipe shall be nickel-plated.

Fittings and couplings on unfinished brass watersupply pipe shall be cast brass, beaded or banded malleable-iron pattern; couplings shall be brass.

Fittings on nickel-plated brass pipe shall be cast brass, malleable-iron pattern, finished and nickelplated.

Unions on pipes 2 inches and smaller in diameter shall be heavy pattern, all brass, ground joint unions with both screw ends hexagonal or octagonal; shall be finished and nickel-plated on nickel-plated pipes. Unions on pipes $2 \frac{1}{2}$ inches and larger in diameter shall be standard weight, galvanized, cast-iron flange unions with gasket of $1 / 16$-inch thick bestquality rubber.

Pipe threads. - Where iron-pipe size pipe threads are specified, they shall conform to Federal specification No. 238. (Current designation GGG-P-351.)

Hangers, Supports, Etc.

All hangers and collars shall be of a size proportionate to the weight of the pipe supported. Chain, perforated bar iron, or wire hangers will not be permitted. Pipe supports shall be installed in an approved manner. 


\section{Valves}

All gate valves shall be designed for a steam working pressure of 125 pounds per square inch, all globe and angle valves for a steam working pressure of 150 pounds per square inch, and shall have the name or trade-mark of the manufacturer and the guaranteed working pressure cast or stamped on the body.

Except as noted, valves 2 inches and smaller in diameter shall be all brass, screw ends with rough body and finished trimmings, except that those on nickel-plated brass pipe shall be finished and nickelplated. Valves $2 \frac{1}{2}$ inches and larger in diameter
The globe valves shall weigh not less than the following: $1 / 2$-inch, 1.25 pounds; $3 / 4$-inch, 2 pounds; 1 -inch, 2.75 pounds; $11 / 4$-inch, 4 pounds; $1 \frac{1}{2}$-inch, 5.5 pounds; 2-inch, 9 pounds. Unless otherwise specified, brass globe and angle valves of the union bonnet type with malleable-iron union ring and malleable-iron stuffing nut will be acceptable if the malleable-iron parts do not come in contact with water.

Check valves.-Shall be horizontal swing check valves with composition rubber, leather, or brass disks. Valves with brass disks shall be of the regrinding type.

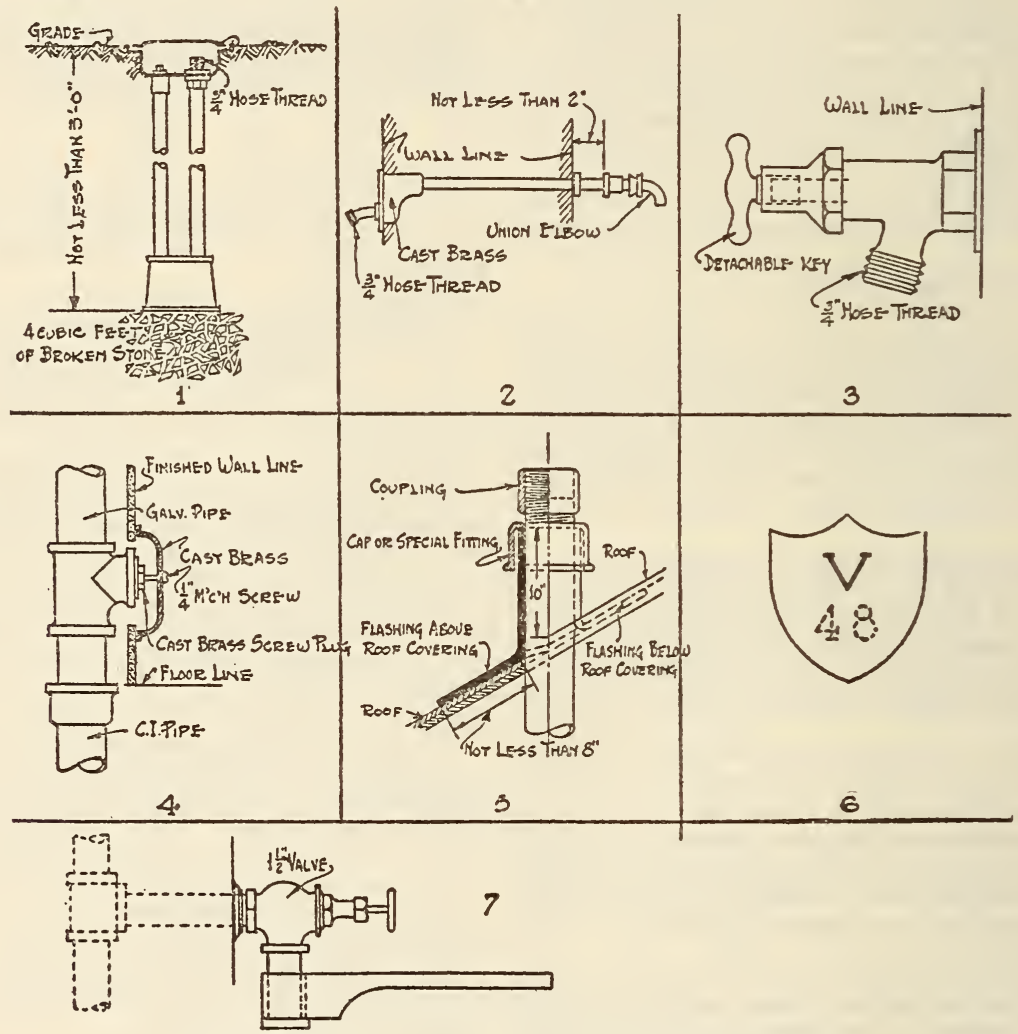

Figdre 1039.-Miscellaneous plumbing fixtures

shall have iron body, brass mounted, and shall have either screw or flange ends.

Brass for valves shall have a minimum copper content of 80 per cent and a maximum lead content of $51 / 2$ per cent.

All valves shall be gate valves unless otherwise specified.

Gate valves in connection with sinks and laundry tubs, and gate or other valves in connection with boilers and hot-water tanks are not considered as a part of the fixtures, but shall be installed on supply pipes between fixtures and supply mains at the most convenient point as may be shown or specified.

Gate valves.- Shall be of the solid wedge pattern, double-seat rising or nonrising stems with gland stuffing boxes, and iron wheels. Shall weigh not less than the following: $1 / 2$-inch, 0.93 pound; $3 / 4$-inch, 1.56 pounds; 1-inch, 2.18 pounds; 11/4-inch, 3.50 pounds; 11/2-inch, 5 pounds; 2-inch, 7.19 pounds; $2 \frac{1}{2}$-inch (screwed), 31 pounds, and (flanged), 41 pounds; 3 -inch (screwed), 42 pounds, and (flanged), 57 pounds; 4-inch (screwed), 65 pounds, and (flanged), 85 pounds.

Globe and angle valves.-Shall have renewable elastic disk, raised flat seats and gland stuffing boxes, iron wheel, ample lift and full-size openings.
Note

Trimmings and fittings for fixtures are generally specified to be nickel-plated brass. If white metal or red metal is desired, it should be so stated.

\section{Miscellaneous}

Detail No. 1-Street washers.-Shall be of type shown in Figure 1039; shall have an automatic non freezing drain, and shall be set above not less than 4 cubic feet of clean broken stone which will pass 2-inch mesh screen.

The valve shall be in a cast-iron box placed not less than 36 inches below the ground; from this two $3 / 4$-inch pipes shall extend to surface and be connected to a cast-iron box with hinged cover marked "water," having top set level with grade. One of the pipes shall be fitted at upper end with cast-brass $3 / 4$-inch hose connection with jam nut; the other shall contain a brass valve operating rod. Furnish with each washer a short T-handle key; all wrought-iron parts shall be galvanized.

Detail No. 2-Wall hydrant.-Shall have brass face and detachable $T$-handle and nozzle for $3 / 4$-inch hose connection; shall be of sufficient length to go through wall with shut-off valve on inside of build- 
ing wall and shall have heavy brass coupling and union elbow for $3 / 4$-inch pipe, as shown in Figure 1039. The outer casing pipe passing through wall shall be galvanized and the inside operating rod shall be brass.

Detail No. 3-Sill cock.-Shall be all brass compression type, with integral flange tapped for $3 / 4$-inch pipe, and a detachable T-handle key; hose connection shall be for $3 / 4$-inch diameter hose.

Detail No. 4-Clean-out plug. - Shall be heavy cast-brass screw-jointed clean-out plug screwed into cast-iron screw-jointed tee pattern, $90^{\circ} \mathrm{Y}$ fitting as shown in Figure 1039. On pipes 4 inches and smaller in diameter plug shall be same size as pipe; on pipes larger in diameter than 4 inches plug shall be 4 -inch diameter.

Drains, Traps, and Clean Outs

DetailNo. 1-Drain.-Shall be cast-brass shower or urinal drain as shown, with removable strainer; plug; shall be installed in manhole constructed for, and provided with cast-iron cover as shown. (Sketch 5, fig. 1040.) Cover shall have checkered top surface.

\section{Down-Spout Nozzle, Floor Flanges}

Down-spout nozzles.-Shall be unfinished cast brass of dimensions noted. Nozzle shall be screwed on pipe, screwed into drainage elbow at base of down spout, and extend beyond outside face of wall, as shown in Figure 1041.

Water-closet floor flanges.-Types A, B, and C shall be cast brass of dimensions shown. Type D shall be cast iron approximately of dimensions shown in Figure 1041.

Running Trap, Manholes

Detail No. 1-Running trap.-Where required shall be "extra heavy" cast iron, with fresh-air

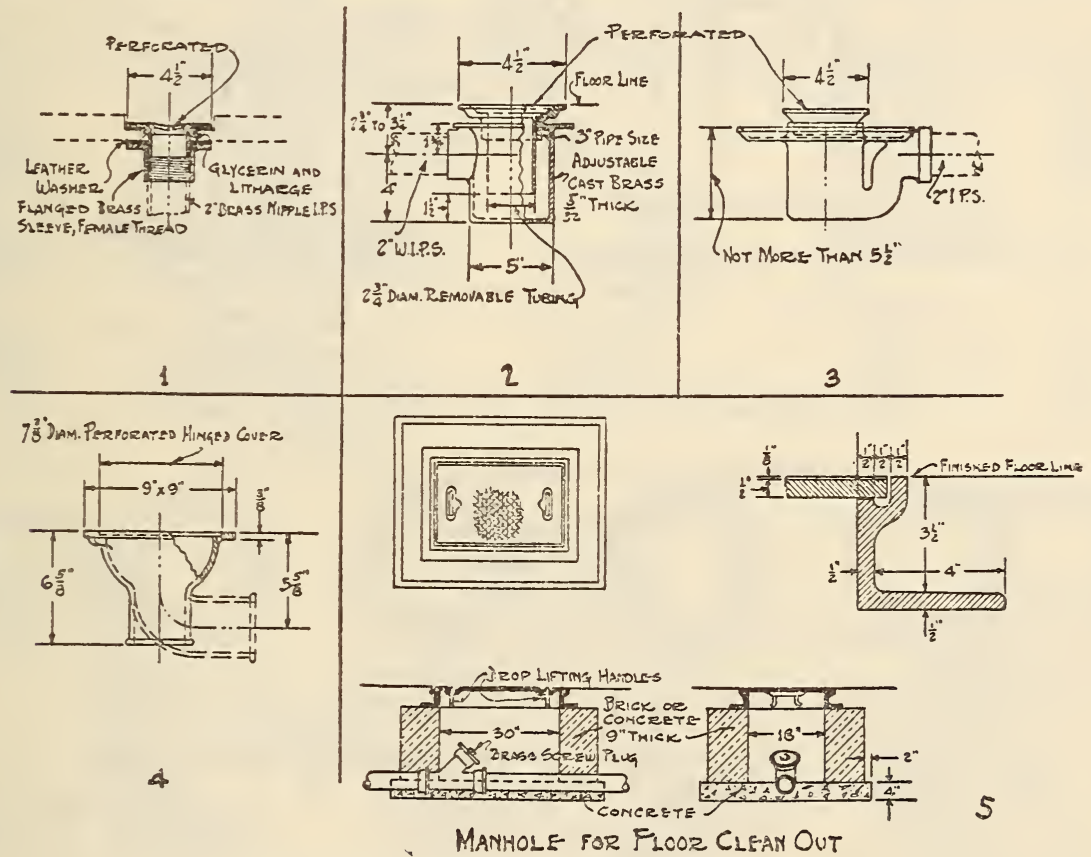

FIGURE 1040.-Drains, traps, and clean outs

nickel-plated or finished brass strainer and top flange. (Sketch 1, fig. 1040.)

Detail No. 2-Combined drain and trap.-Shall be cast-brass combined drain and trap as shown, with removable strainer; nickel-plated or finished brass strainer and top flange. Water seal shall be not less than 11/2 inches in depth. (Sketch 2, fig. 1040.)

Detail No. 3-Combined drain and trap.-Shall be heavy pattern cast iron combined drain and trap, as shown, with a cast-brass strainer. Strainer and top flange shall be nickel-plated or finished brass. Water seal shall be not less than $1 \frac{1}{2} 2$ inches in depth. (Sketch 3, fig. 1040.)

Detail No. 4-Area cesspool.-Shall be extra heavy cast iron approximately of dimensions shown with perforated hinged cast-iron cover plate and with 3-inch connection for cast-iron pipe; without bell trap. (Sketch 4, fig. 1040.)

Side outlet cesspool shall be used only where space does not permit use of a bottom outlet type.

Detail No. 5-Clean out on cast-iron pipe.-Fitting shall be extra heavy cast-iron $Y$ with heavy pattern brass ferrule and cast-brass screw jointed clean-out inlet on house side and with heavy pattern brass ferrule and cast-brass screw-jointed clean-out plug on sewer side. Fresh-air inlet shall be extended about 2 feet as indicated, and shall be provided with return bend at top and a 3/4-inch diameter hole shall be drilled into air-inlet pipe near bottom of manhole; 4, 5, and 6 inch traps shall have 4 -inch diameter fresh-air inlet and clean-out plug; 8-inch trap and larger shall have 6-inch diameter fresh-air inlet and clean-out plug. Shall be installed in a manhole constructed for and provided with cast-iron curb and perforated cover, as shown in Figure 1042.

Detail No. 2-Manhole for main sewer. - Shall be constructed for and provided with heavy-weight castiron curb and solid cover, as shown in Figure 1042.

Detail No. 3-Manhole for main sewer. - Shall be constructed for and provided with light-weight castiron curb and solid cover, as shown in Figure 1042.

\section{Shower Fixtures}

Outfit No. 48.- Shall consist of a shower fixture complete with curtain frame, and curtain for installation over a recessed or corner bathtub. 
Shower fixture shall consist of a combination compression valve fixture with $1 / 2$-inch supply to shower head, 1/2-inch union valves, elbows, and connections complete for connecting to concealed tees of bath faucet; cast-brass escutcheons at wall and support as shown in Figure 1043. All pipe and nipples shall be iron-pipe size and thickness brass pipe.

Shower head shall be cast-brass body and face. Face shall be threaded and removable, approximately 4 inches in diameter, with round perforations, not smaller than No. 58 drill (0.042-inch diameter) delivering a rain shower.

Curtain frame shall be straight for recessed tub and angle pattern for corner tub; shall be of suitable the building are not part of the fixture, and shall be as specified in the specification for the work.

(Shower head is the same as for No. 48.)

Drain No. 1, Figure 1040, shall be furnished except when specification for the work requires a combined floor drain and trap, in which case either drain No. 2 or No. 3, Figure 1040, shall be furnished.

Outfit No. 50.- Shall consist of a shower fixture complete with shower head, curtain, and curtain frame, and receptor as shown.

(Shower fixture is the same as for No. 49.)

(Shower head is the same as for No. 48.)

Curtain frame shall be of $3 / 4$-inch steel pipe with cast-iron or steel flanges at wall and $3 / 8$-inch steel

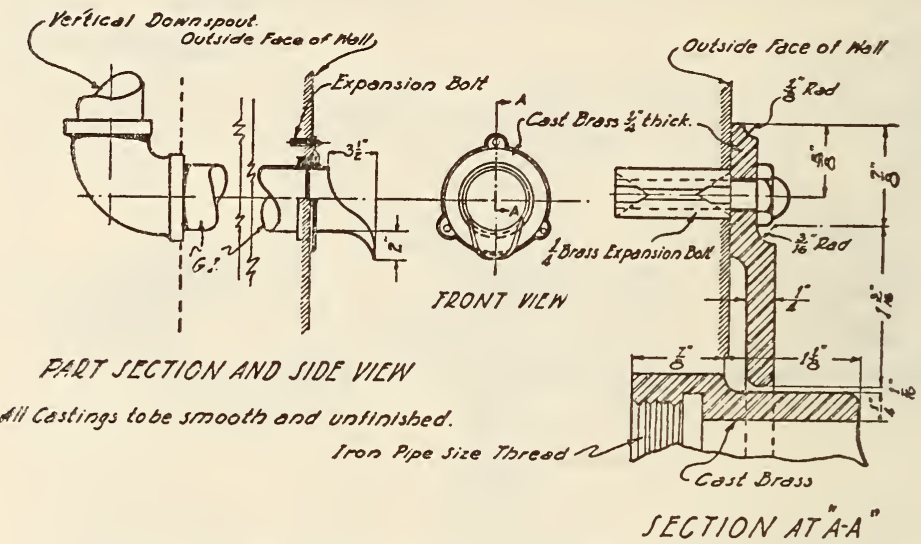

DETAIL OF DOWNSPOUTS DISCHARGING AT GRADE

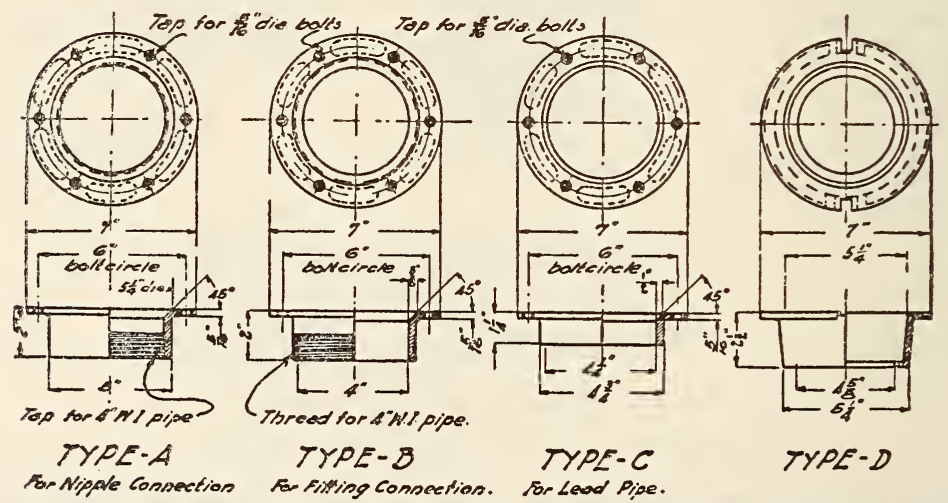

BRASS FLOOR FLANGES

CAST SRON FLOOR FLANGE.

FigURe 1041.-Down-spout nozzle, floor flanges

length for the recess and tub; shall be of not less than $7 / 8$-inch diameter brass tubing, of thickness not less than No. 17 Brown \& Sharpe gage $(0.045$ inch), with cast-brass flanges at wall; curtain hook for each grommet in curtain and holdback for curtain, with wood screws.

All metal parts shall be nickel-plated brass.

Curtain shall be not less than 10-ounce white duck, 6 feet long; shall be of width suitable for the outfit and shall have nickel-plated brass grommets spaced not over 6 inches apart. Duck shall conform to Federal specification CCC-D-751.

Outfit No. 49.- Shall consist of a shower fixture complete for installation in a shower bath inclosure Shower fixture shall consist of a combination compression valve fixture with $1 / 2$-inch union valve connections and $1 / 2$-inch supply to shower heads with supports as shown in Figure 1043. The supply piping from the union valves to the supply mains of pipe ceiling support with flange. All parts shall be galvanized, and shall have a nickel-plated brass curtain hook for each grommet in curtain.

Curtain shall be not less than 10-ounce white duck 6 feet 6 inches long; shall be in two pieces, each not less than 3 feet wide and shall have nickel-plated brass grommets spaced not over 6 inches apart. Duck shall conform to Federal Specifications Board specification CCC-D-751.

Receptor shall be 1-piece porcelain of dimensions shown, glazed over all exposed surfaces.

Drain No. 1, Figure 1040, shall be furnished except when specification for the work requires a combined floor drain and trap, in which case either drain No. 2 or No. 3, Figure 1040, shall be furnished.

Mixing valve.-When outfit number is followed by the letters $\mathrm{MV}$, as $48 \mathrm{MV}$, or $50 \mathrm{MV}$, the outfit shall be as specified for the respective numbers, except that the shower fixture shall have a mixing valve 
between the two compression valves, which shall be of lock shield type.

Mixing valve shall be of heavy construction, compression type, and if of the yoke type shall have renewable seats, china, or brass index, and shall have a capacity to deliver not less than 10 gallons of cold or mixed water per minute with an initial pressure at inlets of 45 pounds per square inch. Mixing valve shall be of a type that admits cold water first.

\section{Outfits}

No. 48. Shower fixture, with frame and curtain.

No. 49. Shower fixture, with floor drain.

No. 50. Shower fixture, with frame and curtain, receptor, and floor drain.

No. $48 \mathrm{MV}$. Shower fixture, with mixing valve, frame and curtain.

No. $49 \mathrm{MV}$. Shower fixture, with mixing valve, and floor drain. lavatory shall be supported on two concealed hangers of suitable design.

When two or more lavatories are set in battery there shall be a space of 4 inches between lavatories.

Waste, supplies, etc.-Unless otherwise specified, each lavatory shall be provided with waste complete with lavatory plug, $P$ or $S$ trap, as required, chain stay, hot and cold water supplies with compression faucets and stops, supplies and waste connections to wall or floor, as required.

For details of waste and supply connections, and chain stay, see Figures 1052 and 1053.

No. $24 \mathrm{CI}$.

Outfits

No. $21 \mathrm{RCIB}$.

No. 19 CCIB.

\section{Kitchen Sinks}

Kitchen sinks.-Shall be 1-piece cast iron, approximately 6 inches deep, with roll rim and integral

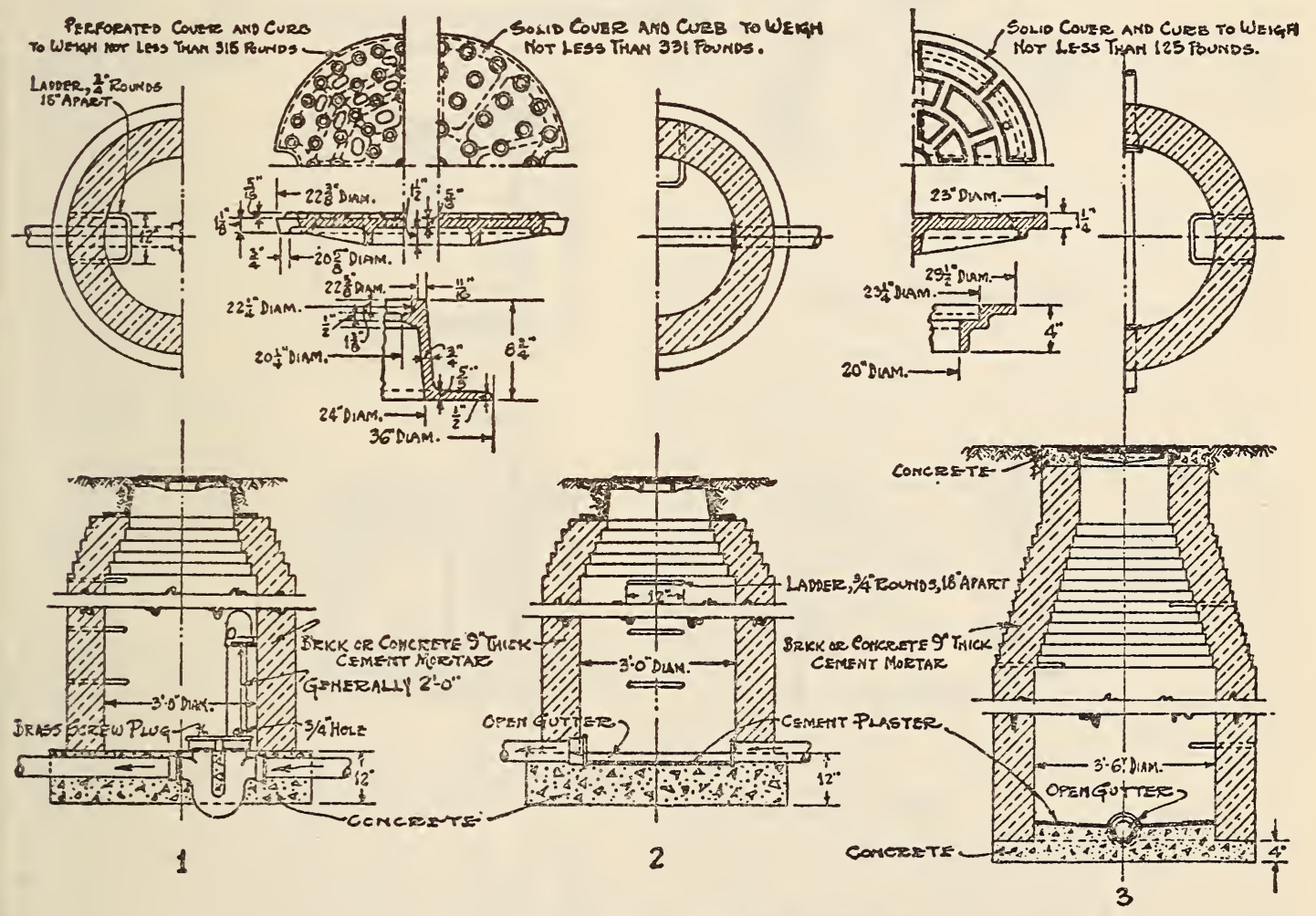

FigdRe 1042.-Running trap, manholes

No. 50MV. Shower fixture, with mixing valve, frame and curtain, receptor, and floor drain.

\section{Cast-Iron Lavatories, Cast-Iron Sinks}

Lavatories.-Shall be 1-piece cast iron with full apron, oval bowl, and integral overflow and roll rimenameled on inside, over rim, apron, and back.

Bowls shall be of the following approximate sizes: No. $24 \mathrm{CI}, 12$ by 15 inch bowl; No. $21 \mathrm{RCIB}, 10 \frac{1}{2}$ by 15 inches; No. 19 CCIB, $101 / 2$ by 15 inches.

Overflow shall have a cross-sectional area of not less than $1 \frac{1}{2}$ square inches at every point.

Supports. - No. 24 CI lavatory shall have a castiron pedestal base support, enameled on outside. No. 21RCIB lavatury shall be supported on a 1-piece concealed hanger of suitable design. No. $19 \mathrm{CCIB}$ back. Sink No. 52CI shall have integral drain board.

Sinks shall be enameled on inside, over rim, back, and drain board, if any.

Supports.-Sinks Nos. 30CI and 36CI shall be supported on a 1-piece cast-iron concealed hanger of suitable design. Sink No. 52CI shall have two painted cast-iron legs and two cast-iron concealed hangers of suitable design.

Waste, supplies, etc.-Unless otherwise specified, each sink shall be provided with sink plug and $P$ trap with connection to wall or $S$ trap with connection to floor as required and as detailed in Figure 1053 , also one hot and one cold water 1/2-inch bibb, detail No. 5, Figure 1052. Cold-water bibb shall have hose thread. 
The connections from the bibbs to the supply lines of the building siall be as called for in the specification for the work and are not parts of the fixtures.

No. $30 \mathrm{CI}$.

Outfits

No. $36 \mathrm{CI}$.

No. 52CIR with right-hand drain board, as shown.

No. 52CIL with left-hand drain board. have tapped inlet to receive the threaded shank of the strainer. Trap standard for wall connection shall be tappea for 3-inch pipe and shall be adjustable as to heignt; trap standard for floor connection to cast-iron hub and spigot pipe shall be provided with spigot end at outlet below floor, and loose flange at base for caulking into hub of cast-iron pipe; trap standard for floor connection to screw pipe shall be provided with horn at outlet and a neavy brass

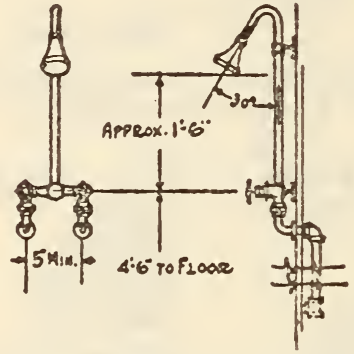

48

Fe USE OVER BATMTUB

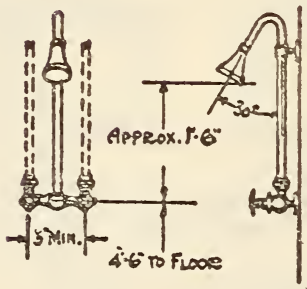

49

For USE IN SHOWER INELOSURE
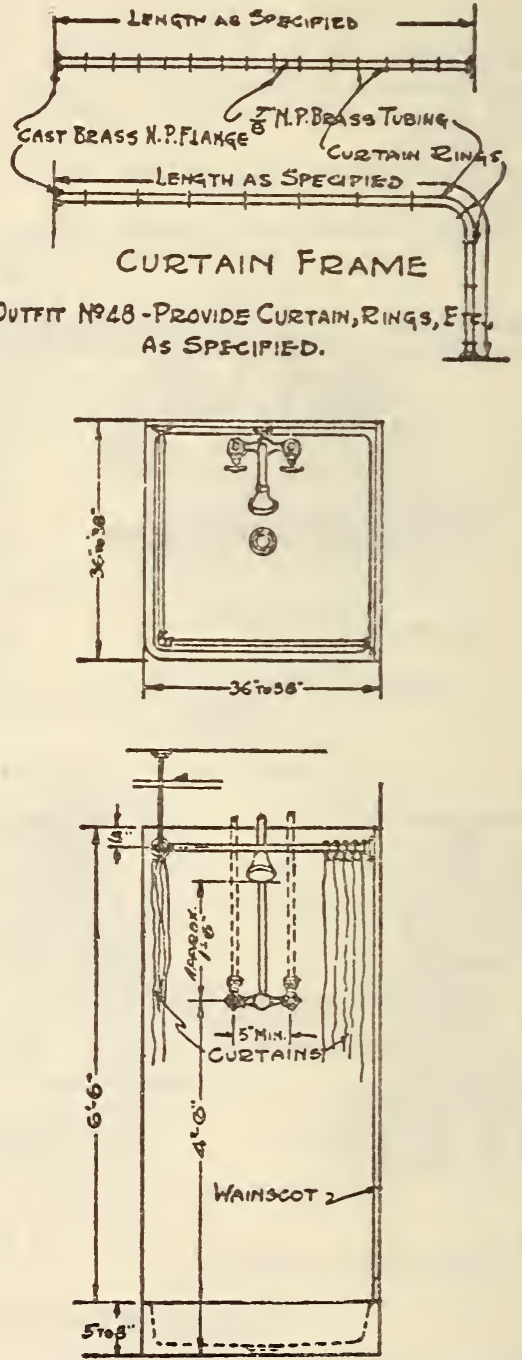

50

FIGURE 1043.-Shower fixtures

Slop Sink

Slop sink.-Shall be 1-piece cast iron, at lesst 12 inches deep, with roll rim and integral back enameled inside and over rim and back. Snall have a nickel-plated brass strainer with cast-brass threaded shank; shall be supported on and secured to a trap standard.

Trap standard.-Shall be cast iron, open pattern, enameled inside, 3-inch diameter, without vent, with brass screw clean-out plug, and with water seal not less than 2 inches in depth; shall be for floor or wall connection, as required for the work, and shall floor flange tapped for 3-inch pipe, or a cast-ir floor flange similar to type D, Figure 1041, shall be provided, with an asbestos-molded gasket and bolts for fastening fixture.

Supplies. - Each sink shall be provided with one hot and one cold water $3 / 4$-inen bibb, detail No. 5 , Figure 1052. Cold-water bibb shall have a hose thread.

Rim guard.- Shall be constructed of $3 / 8$-inch brass tubing of thickness not less than No. 14 Brown \& Sharpe gage (0.064 inch) in thickness with castbrass knobs on ends of rods and cast-brass spacing and holding bars. Holding bars shall have brass 
spring plates or thumbscrew attached for fastening guard to rim of sink. All parts of rim guard shall be nickel-plated.

\section{Outfits}

No. 24SCI. Sink without rim guard.

No. 24SCIG. Sink with rim guard.

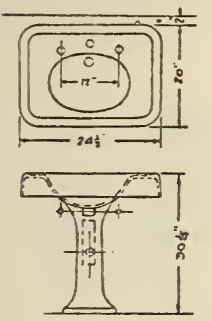

No. 24 C.I.
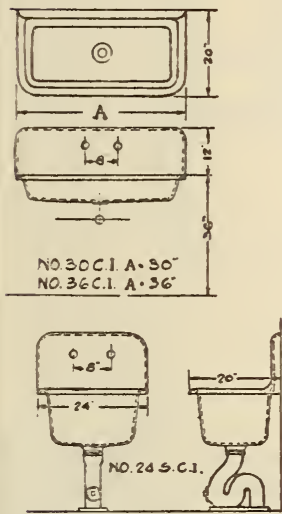

FIGURE 1044.-Cast-iron lavatories and sinks

Bathtubs

Recessed tubs. - Shall be 1-piece cast iron with flat rim at back and both ends, designed to be built into a recess and a full extended front, enameled on all exposed surfaces.

When 3-inch roll rim is required in lieu of the extended front it shall be so stated in the specification for the work. In such cases, tub shall be enameled on inside and over rim only.

Supplies shall consist of a concealed combination over rim nozzle supply fitting with $1 / 2$-inch valves connected to the nozzle by means of I. P. S. brass pipe and cast-brass fittings. Valves may be at a higher elevation than the nozzle or at the same elevation as shown. Hot and cold water supplies shill be fitted with $1 / 2$-inch compression stops and iron pipe size supplies furnished as a part of the fixture.

Valves shall be of the regular compression type with four arm china index handles and constructed so that the bonnet may be removed at the front after installation for repairs and renewals; shall have china escutcheons covering all metal parts except stem which snall be neld in place by adjustable threaded nuts; shall have ground joint unions for connections to $1 / 2$-inch diameter hot and cold water supplies, and heavy pattern nozzle of proper length to discharge over rim of tub and shall be fitted with a china escutcheon at wall.

Waste shall consist of a concealed connected waste and overflow fixture of $1 \frac{1}{2}$-inch brass tubing of thickness not less than No. 17 Brown \& Sharpe gage (0.045 inch) and cast-brass fittings; strainer on waste; strainer and chain stay on overflow. Attached to chain stay shall be a brass plumbers' chain of thickness not less than No. 20 Brown \& Sharpe gage ( 0.032 inch) with $1 \frac{1}{2}$-inch diameter rubber stopper. Chain shall be secured to chain stay and stopper with rings of $1 / 8$-inch thick brass with joints well soldered.

The waste tee shall have on the outside a $11 / 2$-inch standard male thread for a $1 \frac{1}{2}$-incb pipe fitting, and shall bave on inside a fine thread for $1 \frac{1}{2}$-inch tubing. The tee shall be reversible so that the outlet may be set parallel with the waste arm.

Supplies and waste shall be at right or left end as called for in specification for the work.

All exposed metal parts of supply and waste shall be nickel-plated.

The trap indicated on figure and supplies from the compression stops to the building mains shall be as called for in the specification for the work and are not parts of the fixture.

Corner tubs.- Tubs shall be 1-piece cast iron with flat rim at back and at one end designed to be built into the wall with full extended front and other end. Tub shall be enameled on all exposed surfaces. Concealed supplies and waste as specified for recessed tub shall be furnished.

If 3-inch roll rim in lieu of the full extended front and end is desired, it shall be so stated in the specification for the work. In such cases tub shall be enameled on inside and over rim only. Supplies shall consist of an exposed combination compressiontype faucet as shown on detail with 4-arm all brass, china index handles, and elbows with ground joint unions and connections with compression stops to floor or wall, as required. The outlet of faucet shall have a female thread to take the standard thread of a hose coupling. The minimum weight of faucet without elbows shall be 44 ounces.

Waste shall consist of an exposed connected waste and overflow fixture with chain stay, chain stopper, etc., as specified for recessed tubs, except that the waste tee is not required to be reversible. A castbrass escutcheon shall be provided at floor to cover the unfinished fitting.

All exposed metal parts of supplies and waste shall be nickel-plated.

Free standing tubs. - Shall be 1-piece cast-iron flat bottom pattern with 3 -inch roll rim, enameled on the inside and over rim. Tub shall set on four cast-iron feet or on separate 1-piece cast-iron base, as required by designation.

Supplies shall consist of a combination-type faucet as shown on detail with 4-arm all-brass china index handles and elbows with ground joint unions and connections with compression stops to floor or wall, as required. The outlet of faucet shall have a female thread to take the standard thread of a hose coupling. The minimum weight of faucet without elbows shall be 44 ounces.

Waste shall consist of a connected waste and overflow fixture of $1 \frac{1}{2}$-inch brass tubing of thickness not less than No. 17 Brown \& Sharpe gage (0.045 inch) and cast-brass fittings; strainer on waste; strainer and chain stay on overflow. Attached to chain stay shall be a brass plumber's chain of thickness not less than No. 20 Brown \& Sharpe gage ( 0.032 inch) with $1 \frac{1}{2}$-inch diameter rubber stopper. Chain shall be secured to chain stay and stopper with rings of $1 / 8$-inch thick brass with joints well soldered; shall have brass escutcheon at floor.

All metal parts of supply and waste shall be nickel-plated.

Connection from tailpiece of waste fitting shall be as called for in the specification for the work and is not part of the fixture. 
Outfits

No. 54R, recessed tub, nominal commercial length, 54 inches.

No. 60R, recessed tub, nominal commercial length, 60 inches.

No. 66R, recessed tub, nominal commercial length, 66 inches.
No. 60CL, corner tub for left-hand corner, nominal commercial length, 60 inches.

No. 66CL, corner tub for left-hand corner, nominal commercial length, 66 inches.

No. 54B, free-standing tub on base as shown, nominal commercial length, 54 inches.

No. 60B, free-standing tub on base as shown nominal commercial length, 60 inches.
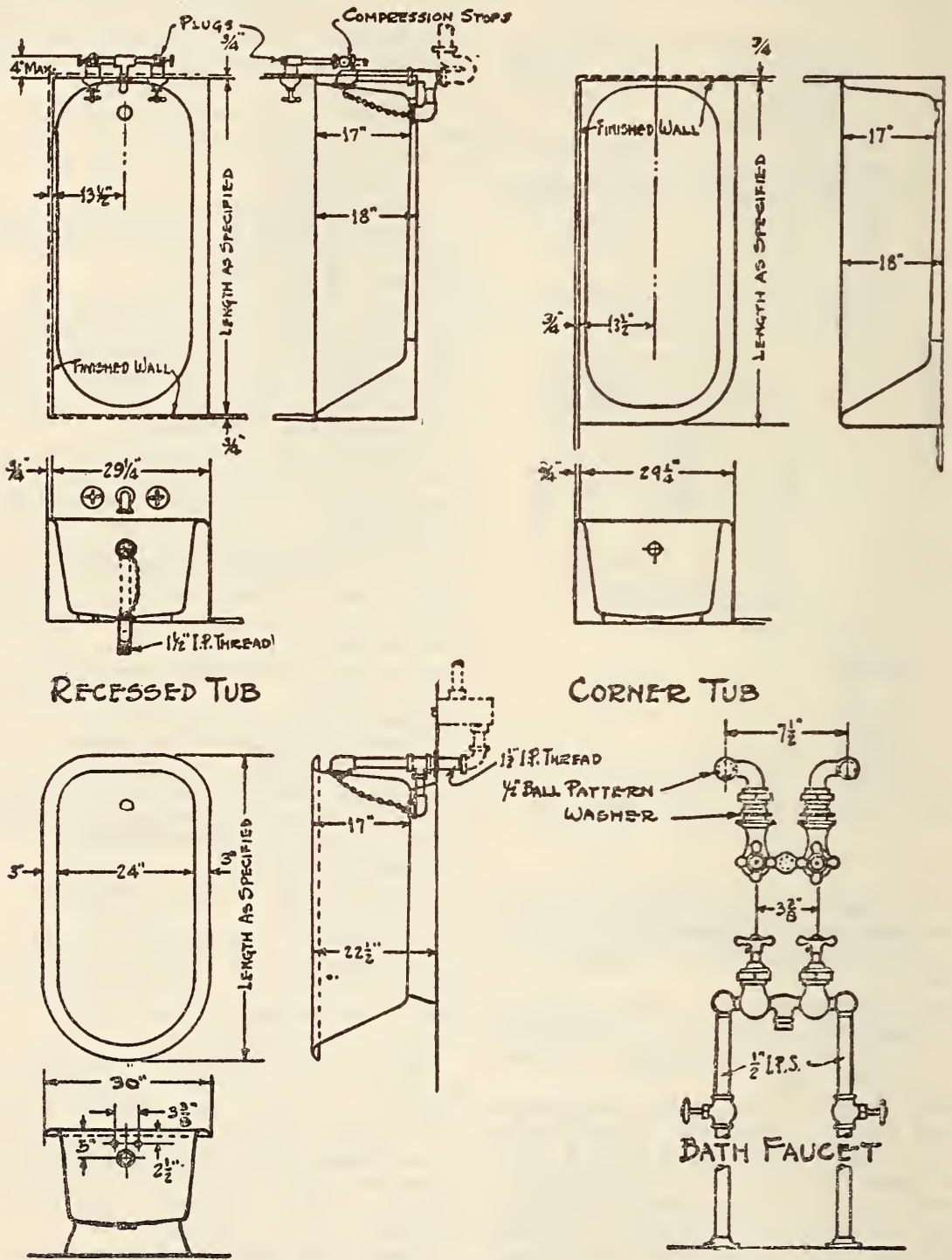

CORNER TUB

FREE STANDIRG TUB

Figure 1045.-Bath tubs

No. 54CR, corner tub for right-hand corner, as shown, nominal commercial length, 54 inches.

No. $60 \mathrm{CR}$, corner tub for right-hand corner, as shown, nominal commercial length, 60 inches.

No. 66CR, corner tub for right-hand corner, as shown, nominal commercial length, 66 inches.

No. 54CL, corner tub for left-hand corner, nominal commercial length, 54 inches.
No. 66B, free-standing tub on base as shown, nominal commercial length, 66 inches.

No. 54F, free-standing tub on feet, nominal commercial lentgh, 54 inches.

No. $60 \mathrm{~F}$, free-standing tub on feet, nominal commercial length, 60 inches.

No. $66 \mathrm{~F}$, free-standing tub on feet, nominal commercial length 66, inches. 
Steel Scullery Sink

Sink.-Shall be two compartments, with or without integral drain boards; integral back; roll rim at front and on back, leaving space behind back for supplies. (Fig. 1046.)

Sink shall be constructed of No. 12 United States standard gage (0.109 inch thick before galvanizing) galvanized steel, welded or riveted. Partition shall be welded or riveted to the bottom and both sides, gage (0.032 inch) brass, with $1 / 8$-inch thick ring at stopper and bibb with joints well soldered. Waste from sink plugs to building drain including grease trap or interceptor is not part of the fixture and shall be as called for in the specifications for the work.

\section{Outfits}

No. $60 \mathrm{DB}$, with drain boards, as shown.

No. 60 , without drain boards.
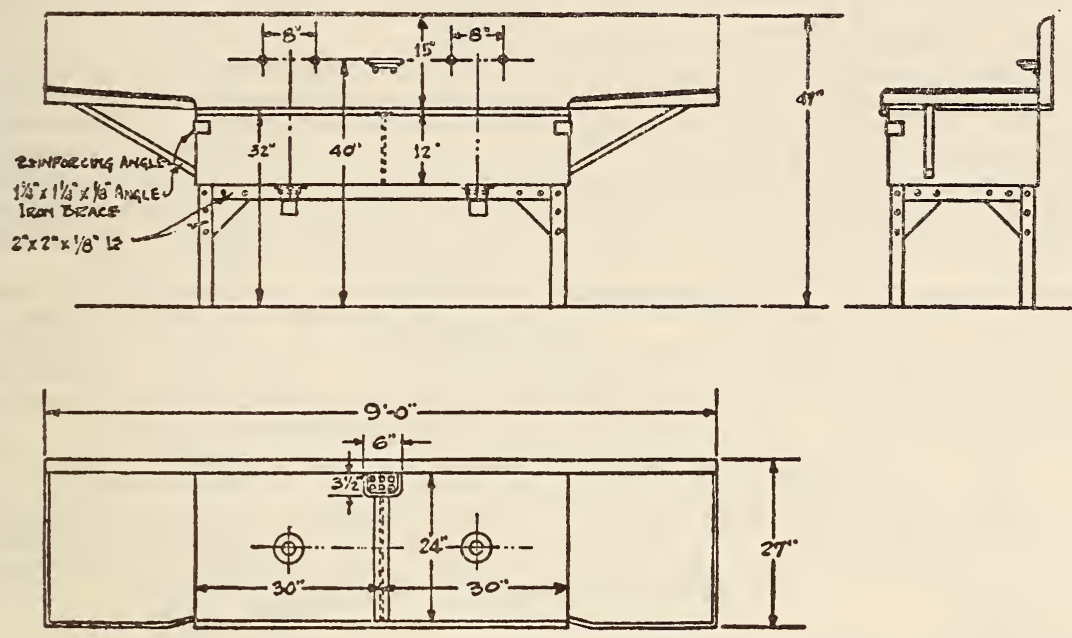

GODB

FIgURE 1046.-Steel scullery sink

and bordered on each side and at top with 1-inch galvanized band iron, riveted on.

Supports.- Sink shall be supported on galvanized steel angle frame, as shown, riveted to sink and bolted to floor.

Supplies.-Each compartment of sink shall be supplied with one cold and one hot water $3 / 4$-inch
Water Heater and Horizontal Storage Tank

Heaters. - Shall be circular in form and made of the best quality gray cast iron, free from imperfections. Inside surface of the fire section shall be corrugated and the fire pot not less than 14 inches deep. The design of the heater shall be such that

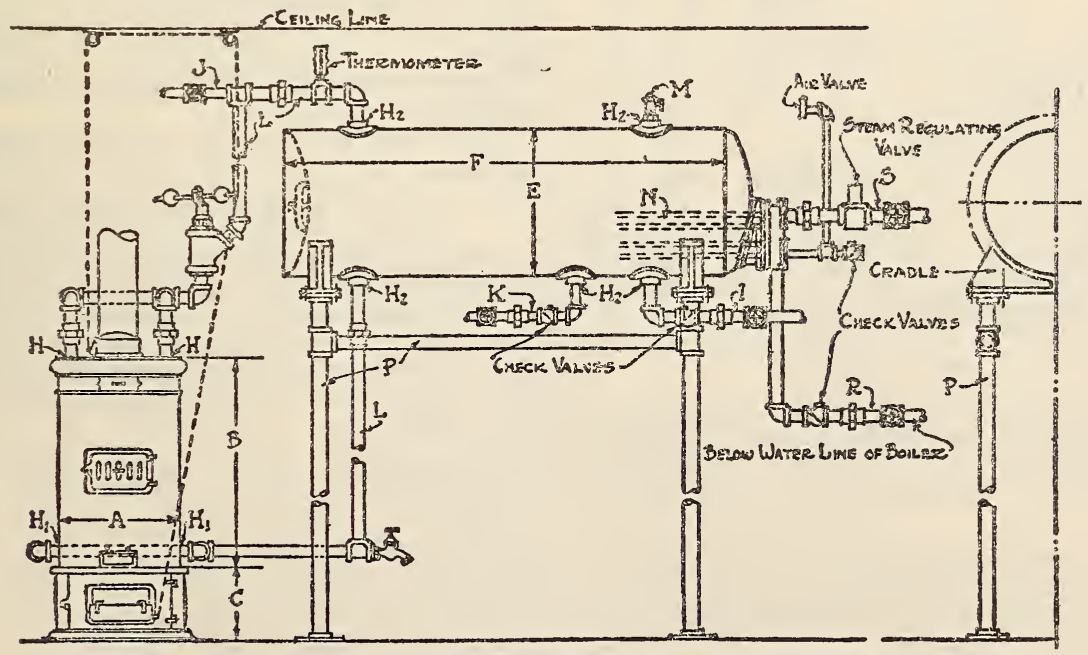

FIgURE 1047.-Water heater and horizontal storage tank

bibb, detail No. 5, Figure 1052. Cold-water bibb shall be provided with hose connections.

Waste. -Each compartment of sink shall have a 2 -inch sink plug with strainer bars and brass stopper. Stoppers shall be fastened to bibbs with plumber's chain of not lighter than No. 20 Brown \& Sharpe all surfaces may easily be cleaned of soot and dust. (Figure 1047.)

All heater sections shall be tested to 100 pounds hydrostatic pressure at the shop and also when assembled on the premises where same will be used. 
Grates shall be heavy cast iron, sectional, rocking and dumping, of sizes noted in figure, and easily removable without disturbing the heater. All doors and base shall be heavy cast iron.

Shaker lever and cleaning appliances shall be furnished.

Tanks.-Heads and shell shall be constructed of best quality homogeneous flange steel of a tensile strength between 55,000 and 65,000 pounds per square inch, and shall have all openings properly reinforced.

Tanks No. 20HP and 24HP shall have handhole in end; tank No. $36 \mathrm{HP}$ shall have a manhole in end. Handholes and manholes may be either in center of head or in head near bottom, and shall be provided with plate, yoke, and gasket.

Seams shall be riveted for not less than 100 pounds working pressure, and shall be tested at shop to a hydrostatic pressure of 150 pounds.

Marking. - The name of manufacturer and working pressure shall be marked on a brass plate secured to tank or be stenciled on the tank.

Supports.-Tank No. 20HP shall be supported from ceiling with strap hangers of $1 \frac{1 / 2}{\text { by }} 5 / 16$ inch strap iron or $3 / 4$-inch round iron around tank and secured to ceiling at both ends in an approved manner. Tanks No. 24HP and $36 \mathrm{HP}$ shall be provided with cast-iron cradles and supported from floor with a pipe frame as shown. Pipe frame shall be constructed of plain wrought iron or mild steel pipe, steam pattern fittings and floor flanges; floor flanges shall be fastened to floor and cradles with two bolts to each flange.

Trimmings. - Each tank shall be provided with a $3 / 4$-inch diameter spring loaded brass water-relief valve, set 25 pounds above high-water pressure, excepting fire pressure, and a $3 / 4$-inch angle pattern, hot-water mercury thermometer graduated from $40^{\circ}$ to $240^{\circ} \mathrm{F}$.

Heating section.-Each HP tank shall be provided with a removable heating section constructed of seamless drawn brass or seamless copper tubing either in the form of $U$ bends or straight tubes with a floating head. The heating surface of coils shall be not less than noted on the figure.

\begin{tabular}{|c|c|c|c|}
\hline Outfit number. & 100 & 200 & 300 \\
\hline Number of heater & 100 & 200 & 300 \\
\hline Diameter of grate & 12 & 15 & 18 \\
\hline Diameter of heater (approximately), $A_{-}$do & 18 & 20 & 23 \\
\hline Height of heater (minimum), $B$ & $271 / 2$ & 35 & 35 \\
\hline Height of base (minimum), $C$ & 12 & 12 & 13 \\
\hline Size of flow taps (2 per outfit), $H_{-}$ & $11 / 2$ & 2 & 2 \\
\hline $\begin{array}{l}\text { Size of return taps ( } 2 \text { per outfit), } H_{1} \\
\text { Number of tank }\end{array}$ & $11 / 2$ & $\stackrel{2}{24}$ & 2 \\
\hline Diameter of tank, $E$ & 20 & $\begin{array}{l}24 \\
24\end{array}$ & $\begin{array}{l}36 \\
36\end{array}$ \\
\hline Length of tank, $F$ & 60 & $\frac{24}{72}$ & \\
\hline Thickness of shell... & & & $5 / 16$ \\
\hline of heads.- & 3 & $x$ & $1 / 2$ \\
\hline Size of all taps, $\mathrm{H}_{2}$ & $11 / 2$ & $11 / 2$ & \\
\hline Size of cold-water supply, $I_{-}$ & & $11 / 4$ & $11 / 2$ \\
\hline $\begin{array}{l}\text { Size of hot-water supply to building, } J-\text { do } \\
\text { Size of hot-water circulation from building, } \bar{K}\end{array}$ & 1 & $11 / 4$ & $11 / 2$ \\
\hline Size of circulation between heater and tan & $3 / 4$ & 1 & $11 / 4$ \\
\hline Size of relief valve, $\lambda$ & 132 & $11 / 2$ & \\
\hline Square feet of surface in heating coil, $\bar{N}^{-}$ & 4.90 & 9. 75 & 18.2 \\
\hline Size of steam inlet, $S$ & 11 & 11 & \\
\hline Size of steam return, $R_{-}$ & 1 & $11 / 4$ & $1 \frac{1}{2}$ \\
\hline $\begin{array}{l}\text { Size of pipe stand, } P_{--} \\
\text {a As specified. }\end{array}$ & & & \\
\hline
\end{tabular}

When the letter $\mathrm{P}$ is omitted from designation of tank, the heating section and headpost shall be omitted, and all other requirements shall be the same as for the HP tanks.

Piping other than heating section is not part of the fixture, and shall be as called for in the specification for the work.

Regulator.-Each tank connected to a coalburning heater shall be provided with a thermostatic regulator with necessary chains and pulleys to operate the draft door and check draft on heater, adjusted so as to close draft door and open check draft when temperature of water is $140^{\circ} \mathrm{F}$. in tank.

In case gas burners are used in the heater, regulator shall be connected to operate the gas supply to heater.

Each tank containing a steam coil shall be provided with a thermostatic regulator with necessary connections and valve to control the steam supply to the coil, adjusted so as to close steam valve when temperature of water is $140^{\circ}$ F. in tank.

Regulator shall be inserted preferably in head of tank, and shall consist of a tube with necessary attachments containing a volatile liquid or an expanding tube containing a nonexpanding member, or both, the expansion and contraction of which shall operate valve direct or cause a metal diaphragm or metal bellows to expand and contract, thus controlling the steam supply.

Either type of regulator will be acceptable, viz, one that controls the steam supply to coil and operates the draft dampers or gas burners, as the case may be, or type that requires regulator for steam valve to coil with an independent regulator to control the dampers on heater.

Regulators that operate by water of the plumbing system will not be permitted.

Regulators shall have the name or trade-mark of manufacturer stamped or cast on body of valve, or shall have a separate plate with name of manufacturer bolted to valve.

\section{Outfits}

No. 100HP heater, No. 100CI, with No. 20HP tank having heating section.

No. $200 \mathrm{HP}$ heater, No. $200 \mathrm{CI}$, with No. $24 \mathrm{HP}$ tank having heating section.

No. $300 \mathrm{HP}$ heater, No. $300 \mathrm{CI}$, with No. $36 \mathrm{HP}$ tank having heating section.

No. $100 \mathrm{H}$ heater, No. $100 \mathrm{CI}$, with No. $20 \mathrm{H}$ tank without heating section.

No. $200 \mathrm{H}$ heater, No. $200 \mathrm{CI}$, with No. $24 \mathrm{H}$ tank without heating section.

No. $300 \mathrm{H}$ heater, No. $300 \mathrm{CI}$, with No. $36 \mathrm{H}$ tank without heating section.

No. 20HP tank having heating section, without heater.

No. $24 \mathrm{HP}$ tank having heating section, without heater.

No. 36HP tank having heating section, without heater.

\section{Water Heater and Vertical Storage Tank}

Heaters.- Shall be circular in form and made of the best quality gray cast iron, free from imperfections. Inside surface of the fire section shall be corrugated and the fire pot not less than 14 inches deep. The design of the heater shall be such that all surfaces may easily be cleaned of soot and dust. (Fig. 1048.)

Outfit number

Number of heater

Diameter of heater (approxi-

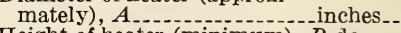

Height of heater (minimum), $B_{\text {ddo }}$ do..

Height of base (minimum), $C_{-- \text {do }}$

Diameter of grate

Size of flow openings ( 2 per out-

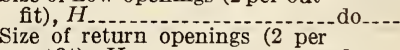

outfit), $H_{1}$

Number of tank

Size of tank, ExF-.---inches.-

Thickness of shell of tank -......-.do....

Thickness of concave head of

Thick

tank . Size of all pipe openings, $H_{2-. .-d o}$

$\begin{array}{rrr}100 & 200 & 300 \\ 100 & 200 & 300 \\ 18 & 20 & 23 \\ 271 / 2 & 35 & 35 \\ 12 & 12 & 12 \\ 12 & 15 & 18 \\ & & 2 \\ 11 / 2 & 2 & 2 \\ 11 / 2 & 2 & 2 \\ 36 \mathrm{~V} & 42 \mathrm{~V} & 48 \mathrm{~V} \\ \text { by } 60 & 42 \text { by } 60 & 48 \text { by } 60 \\ 1 / 4 & 1 / 4 & 1 / 4 \\ 5 / 16 & 5 / 16 & 5 / 16 \\ 3 / 8 & 3 / 8 & 7 / 6 \\ 11 / 2 & 2 & 2 \\ & & \end{array}$


All heater sections shall be tested to 100 pounds hydrostatic pressure at the shop and also when assembled on the premises where same will be used.

Grates shall be heavy cast iron, sectional, rocking and dumping, of sizes noted on plate and easily removable without disturbing the heater. All doors and base shall be heavy cast iron.

Shaker lever and cleaning appliances shall be furnished.

Tanks.- Shall be constructed of best quality homogeneous flange steel of tensile strength of between 55,000 and 65,000 pounds per square inch, and shall have all openings reinforced. Handhole shall be provided with cover plate, yoke, and gasket, and when tank is set on stand, handhole shall be located in an accessible position.

Seams shall be riveted for not less than 100 pounds working pressure, and shall be tested at shop to a hydrostatic pressure of 150 pounds. which shall operate the damper and check draft or regulator may be of the all-metal bulb type placed in pipe connection at $J$; within the bulb shall be an expansible metallic bellows surrounded by a volatile liquid, the expansion of which shall operate the damper and check draft.

\section{Outfits}

No. $100 \mathrm{~B}$, heater No. $100 \mathrm{CI}$, with tank No. $36 \mathrm{~V}$. No. 200B, heater No. 200CI, with tank No. $42 \mathrm{~V}$.

No. 300B, heater No. 300CI, with tank No. $48 \mathrm{~V}$.

\section{Trimmings for Water-Closet and Bath Inclosures}

Trimmings for slab work shall be of the design and dimensions shown; shall be cast brass, exposed surfaces finished and nickel-plated, with slots of proper size for the marble, slate, etc.; standards shall be $1 \frac{1}{1}$-inch brass tubing and all bracing shall

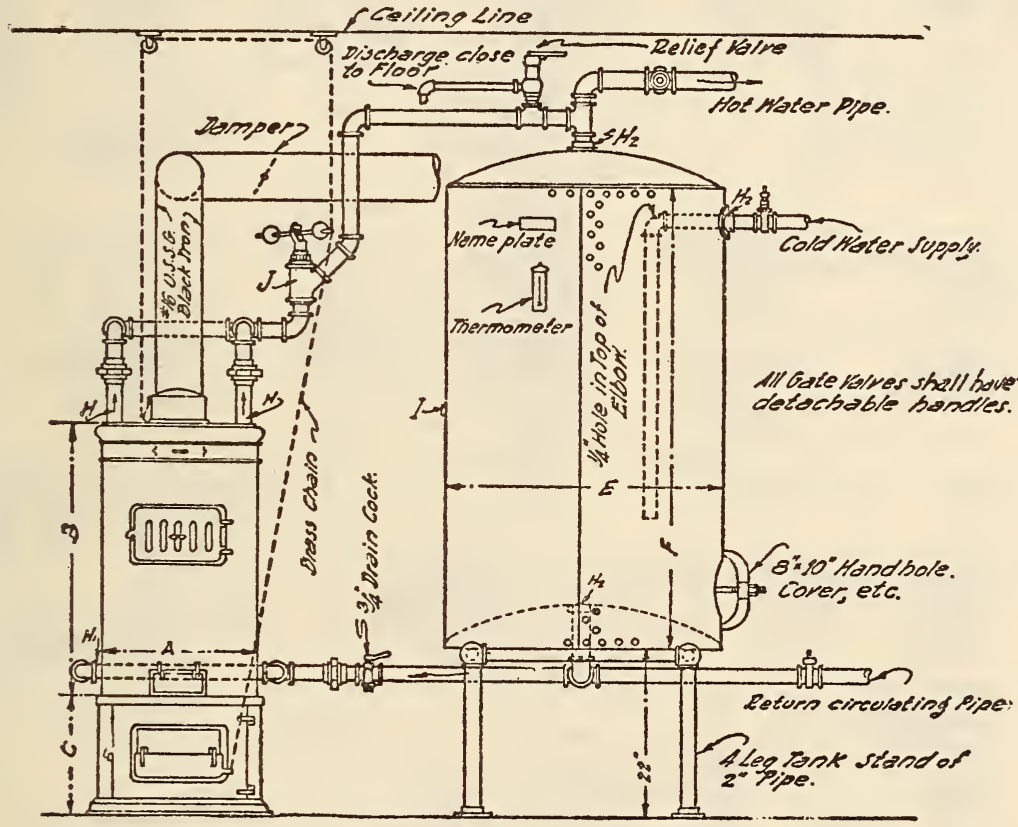

FIgURE 1048.-Water heater and vertical storage tank

Marking. - The name of manufacturer and working pressure shall be marked on a brass plate secured to tank or shall be stenciled on tank.

Supports.- Tanks shall be supported from floor with a pipe frame as shown, constructed of plain wrought iron or steel pipe, malleable railing fittings and floor flanges.

Trimmings.-Each tank shall be provided with a $3 / 4$-inch diameter spring loaded brass water relief valve set at 25 pounds above maximum watersupply pressure, excepting fire pressure, and a ${ }^{3}{ }_{4}^{\prime}$-inch hot-water mercury thermometer graduated from $40^{\circ}$ to $240^{\circ} \mathrm{F}$.

Piping.-Piping indicated is not part of the fixture and shall be as called for in the specification for the work.

Regulator.-Each tank shall be provided with a thermostatic regulator with necessary chains and pulleys to operate the draft door and check draft on heater, adjusted so as to close draft door and open check draft when temperature of water is $140^{\circ} \mathrm{F}$. in tank.

Regulator shall consist of either a tube inserted in tank at point marked $I$ containing a volatile liquid or containing an expanding tube with a nonexpanding member, the expansion or contraction of be 1-inch outside diameter, of thickness not less than No. 17 Brown \& Sharpe gage (0.045 inch) polished and nickel-plated. (Fig. 1049.)

Hinge $(J)$. - Shall be heavy pattern, cast or wrought brass, with clamp adjustable from $11 / 8$ to $13 / 8$ inches, not thinner than 0.09 inch, with inclosed adjustable spring.

\section{Accessories}

All metal shall be brass with exposed parts finished and nickel-plated, except brackets for wood seat, detail 8, which may be unfinished brass. (Fig. 1050.) (For nickel-plating or plumbing fixtures, see 600.3, p. 70 .)

\section{Lavatory Supplies}

All exposed parts shall be finished brass, nickelplated. (Fig. 1052.) (For nickel-plating of plumbing fixtures see 600.3 , p. 70.)

Detail No. 1-Lavatory supplies, with compression faucets. - Shall consist of a best quality cast-brass compression type faucet with 4 -arm brass handles with china index; shank, tailpiece, and compression stop with connections to wall or floor of brass, iron pipe size pipe with cast-brass wall or floor flange as required. Stop shall be straight way or angle 
pattern as required for the work. The minimum weight of faucets, including tailpiece, shall be $21 \frac{1}{2}$ ounces.

When self-closing faucets are called for in the specification for the work, the faucets shall be best quality cast-brass self-closing faucet with 4,5 , or 6 arm brass handle with china index shank and tailpiece. The mechanism of faucet shall be perfect in every respect; shall be closed against the water pressure by means of a phosphor bronze spring. The spring shall close faucet against 100 pounds the compression type with brass lever index handles, shanks, and tailpieces and compression stops with connections from tailpieces to wall or floor of brass, iron pipe size pipe with cast-brass wall or floor flange as required. Stops shall be straightway or angle pattern as required for the work. The minimum weight of faucet, including tailpieces, shall be 57 ounces. Connections from faucets to wall or floor and wall or floor flange are parts of the fixtures.

Detail No. 4-Sink bibb. - Shall be full $1 / 2$ or $3 / 4$ inch, as specified, brass compression type with 4 -arm

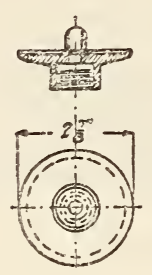

A

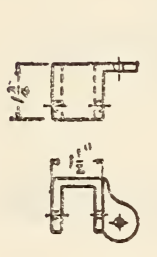

$\boldsymbol{F}$
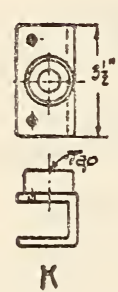

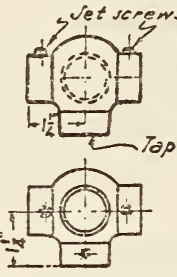

B
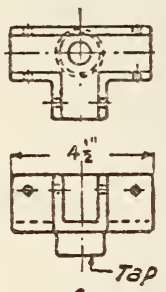

6

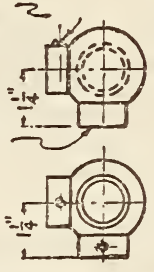

C
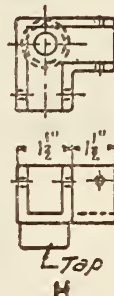

H

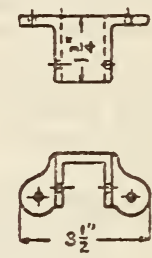

D

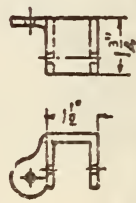

I
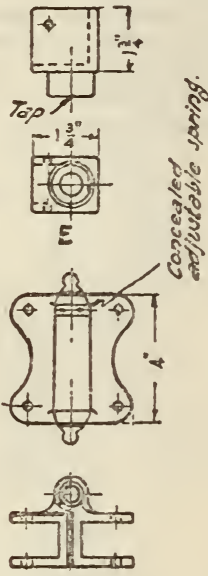

J
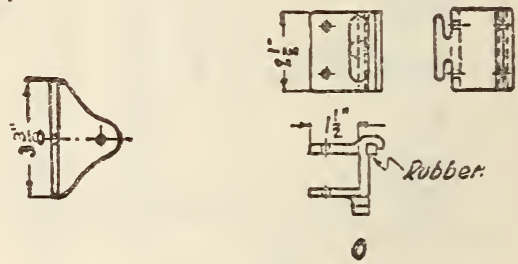

L

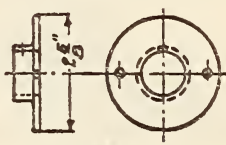

M

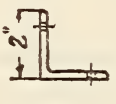

N

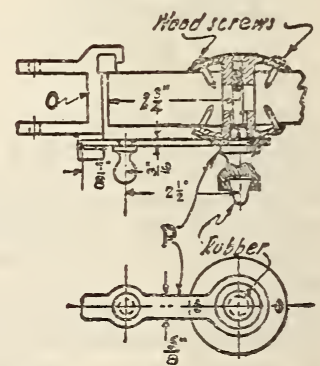

FIGURE 1049.-Trimmings for water-closet and bath inclosures

water pressure per square inch and the faucet shall be easily operated at any pressure up to 100 pounds. Faucet shall operate on ball or roller bearings, and check lugs or other suitable method shall be provided to prevent handle from making complete turns. The minimum weight of faucet including tailpiece shall be 26 ounces.

Connections from faucets to wall or floor, and wall or floor flange are parts of the fixtures.

Detail No. 2-Combination faucet.-Shall consist of a best quality cast-brass combination faucet of china index handle and standard male thread. When hose connection is specified bibb shall be provided with thread for $3 / 4$-inch diameter hose. Minimum weight of $1 / 2$-inch bibb shall be 14 ounces and of $3 / 4$-inch bibb shall be 18 ounces.

Detail No. 5-Sink bibb. - Shall be full $1 / 2$ or $3 / 4$ inch, as specified, brass compression type with 4-arm china index handle, shank as shown, cast-brass adjustable flange with set screw or flange threaded on shank; standard male thread. When hose con- 
nection is specified bibb shall be provided with thread for $3 \frac{1}{4}$-inch diameter hose.

The minimum weight of $1 / 2$-inch bibb, including flange, shall be 19 ounces, and the minimum weight of $3_{4}^{3}$-inch bibb, including flange, 20 ounces.

Detail No. 6-Laundry tray bibb. - Shall be brass compression type stub pattern with brass $T$ handle and integral female flange tapped for $1 / 2$ or $3 \frac{1}{4}$ inch pipe as specified; shall have thread for $3 / 4$-inch diameter hose.

The minimum weight of $1 / 2$-inch bibb shall be $12 \frac{1}{2}$ ounces, and of $3 / 4$-inch bibb shall be 13 ounces. Detail No. 7-Chain stay.-For lavatories shall be
Detail No. 1-Lavatory plug.-Shall be cast brass with rod strainer and with overflow openings for vitreous or cast-iron lavatory as required, jam nut, leather washer, opening for $1_{4}^{1}$-inch diameter rubber stopper; tailpiece of $1 \frac{1}{2}$-inch brass tubing of thickness not less than No. 17 Brown \& Sharpe gage (0.045 inch).

Detail No. 2-Sink plug.-Shall be cast brass with removable strainer; jam nut and leather washer; coupling and 11/2-inch tailpiece of thickness not less than No. 17 Brown \& Sharpe gage (0.045 inch).

Detail No. 3-Tray plug. - Shall be cast brass with

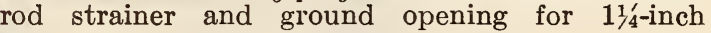
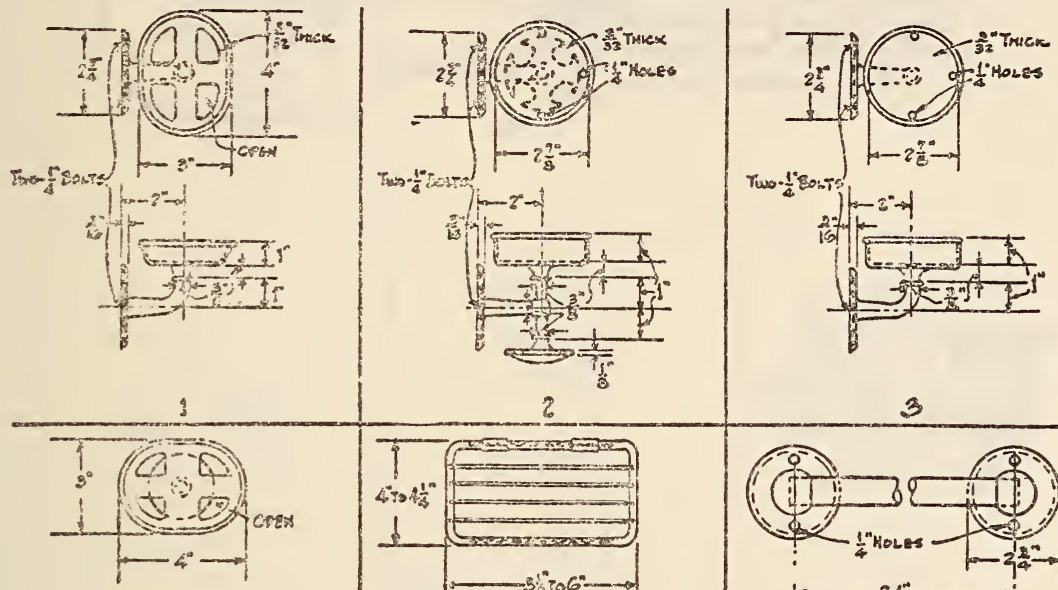

2

3
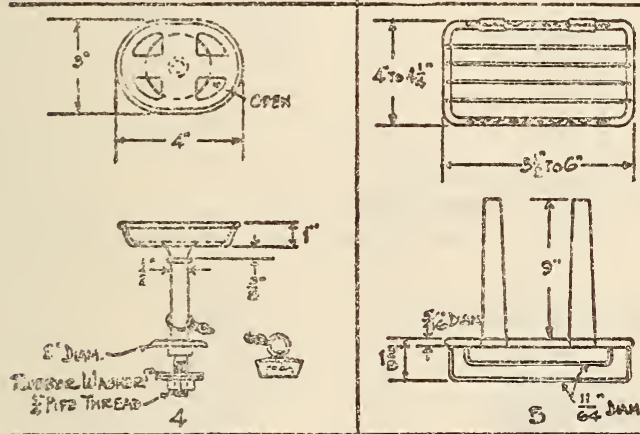

$-33^{\prime \prime} 706^{n}-$
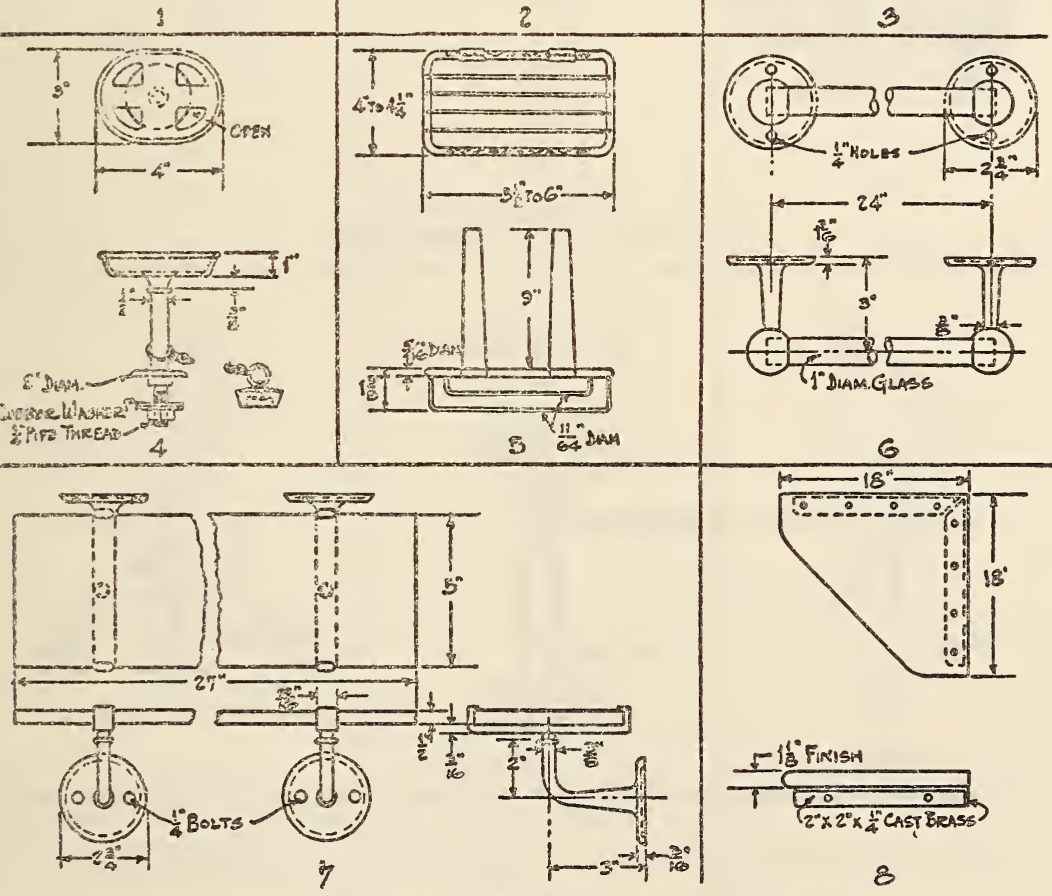

Figure 1050.-Accessories

cast-brass stay of dimensions shown. Attached to chain stay shall be a nickel-plated brass plumbers' chain not lighter than No. 20 Brown \& Sharpe gage (0.032 inch) with $11^{1} 4_{-}$-inch diameter rubber stopper, to fit the lavatory plug, detail No. 1, Figure 1053. Chain shall be attached to chain stay and stopper with rings of $1 / 8$-inch brass with joints well soldered.

\section{Lavatory Wastes}

All exposed parts shall be finished brass, nickelplated. (Fig. 1053.) (For nickel-plating of plumbing fixtures, see 600.3 , p. 70 .) diameter metal stopper; jam nut and leather washer; coupling and 11/2-inch tailpiece of thickness not less than No. 17 Brown \& Sharpe gage $(0.045$ inch).

Detail No. 4-P trap.- Shall consist of a 11/2-inch cast-brass $P$ trap with cast-brass clean-out plug; slip joint with cast-brass nut and rubber washer at inlet for connecting to the plugs, details Nos. 1, 2, or 3 ; band with a $1 \frac{1}{2}$-inch standard pipe tap at outlet.

Connection and wall flange indicated in the figure are part of the lavatory waste. 

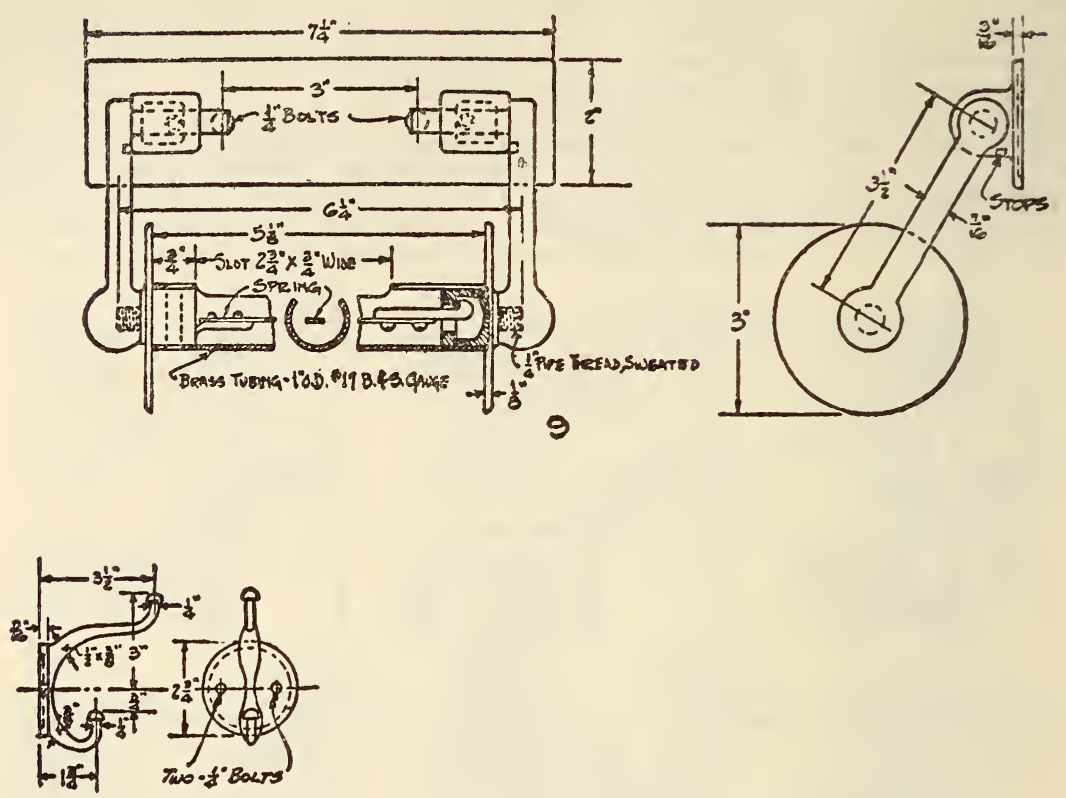

10

FIGURE 1051.-Accessories

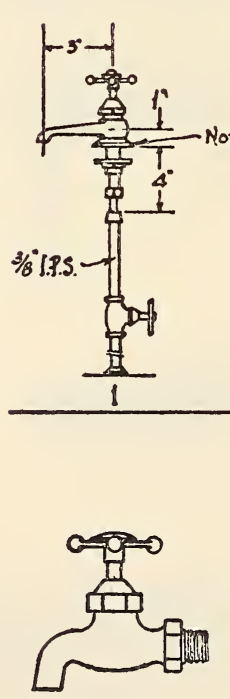

4

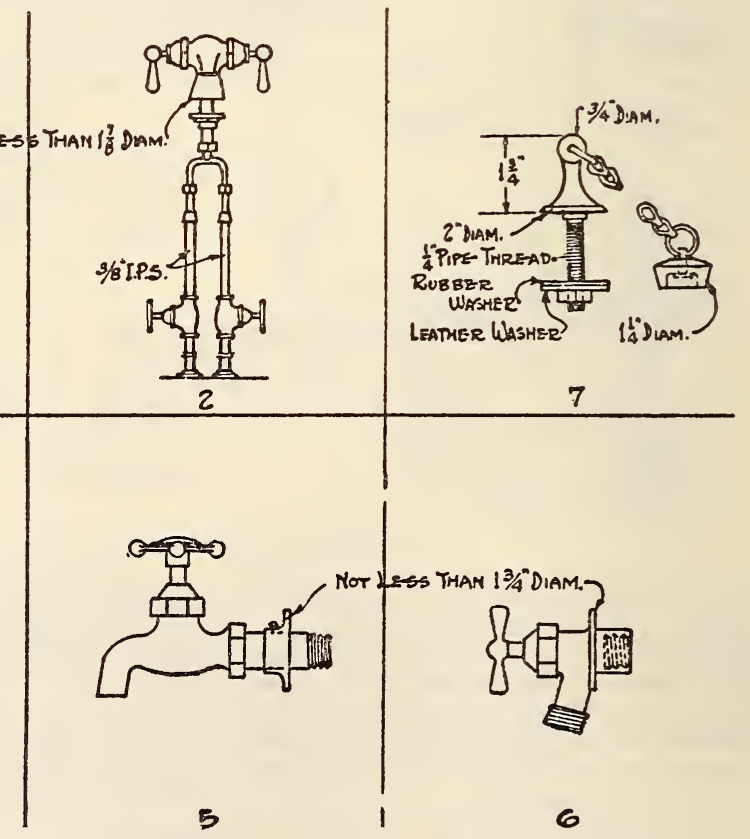

FIGURE 1052.-Lavatory supplies 
Detail No. 5-S trap.-Shall consist of a 11/2-inch cast-brass $S$ trap with cast-brass clean-out plug; slip joint with heavy cast-brass nut and rubber washer at inlet for connecting to the lavatory plugs, details Nos. 1, 2, or 3; ground joint union with a standard 11/2-inch pipe tap at outlet.

Connection and floor flange indicated on the plate are part of the lavatory waste.

Detail No. 6-Nonsiphoning trap.-Shall consist of a cast-brass nonsiphoning trap with a cast-brass necting to the lavatory plugs, details Nos. 1, 2, or 3; slip joint or union connection at outlet. Connection and wall flange indicated in figure are parts of the lavatory waste, and shall be of thickness not less than No. 17 Brown \& Sharpe gage (0.045 inch).

Detail No. 9.- Shall be as specified for trap in detail No. 8, except that tubing at outlet shall be for floor connection as shown.

Connection and floor flange indicated in figure are parts of the waste connection, and shall be of

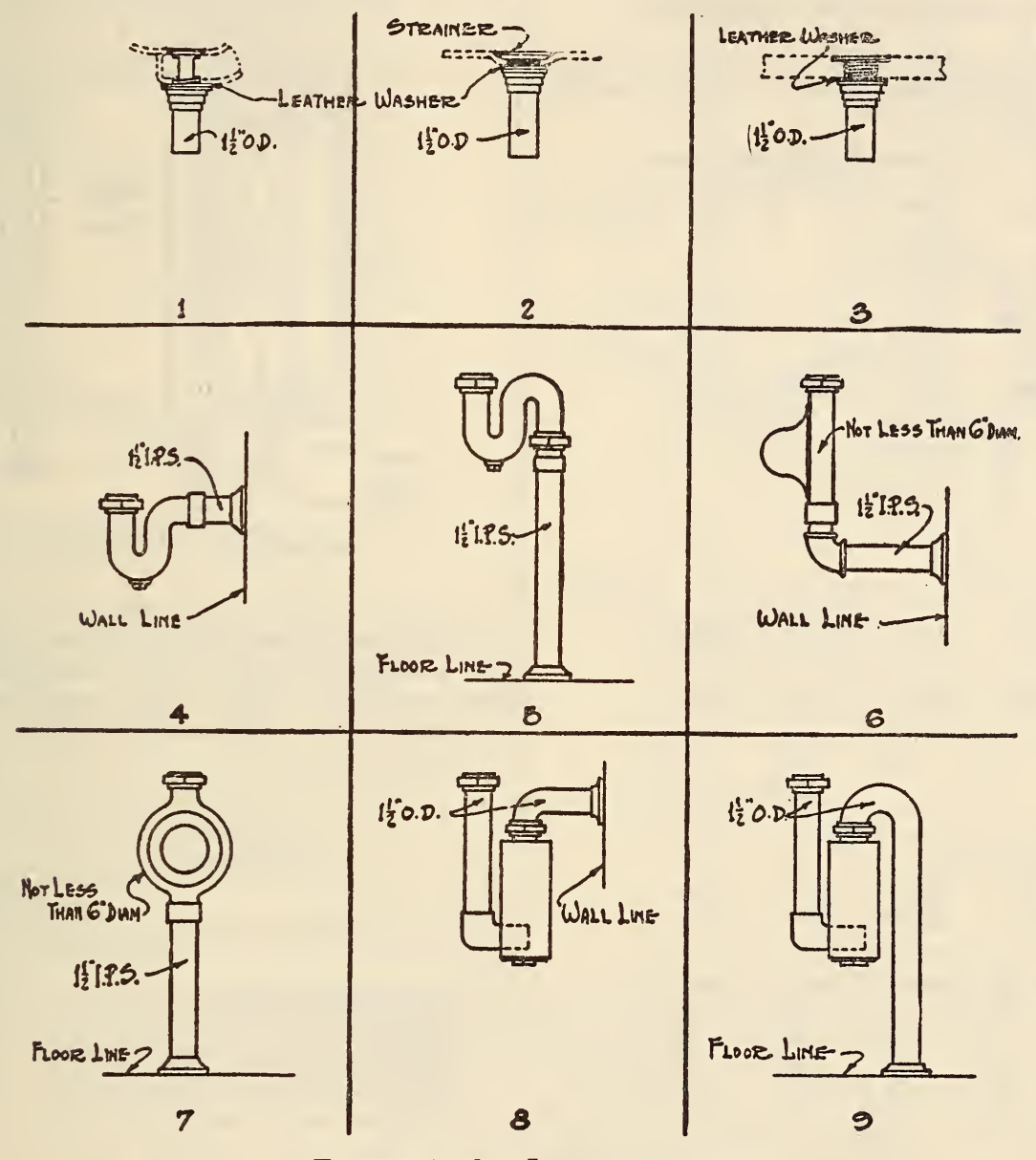

Figure 1053.-Lavatory wastes

interior partition, integral with body of the trap; slip joint with heavy cast-brass nut and rubber washer at inlet for connecting to the lavatory plugs, details Nos. 1,2 , or 3 ; band with a $1 \frac{11}{2}$-inch standard pipe tap at outlet.

Connection and wall flange indicated in the figure are part of the lavatory waste.

Detail No. 7-Nonsiphoning trap.-Shall be as specified for trap in detail No. 6 .

Connection and floor flange indicated in figure are part of the lavatory waste.

Detail No. 8-Clean-sweep trap.-Shall consist of a cast-brass body trap (commercially known as the clean sweep or centrifugal), slip joint with heavy cast-brass nut and rubber washer at inlet for con- thickness not less than No. 17 Brown \& Sharpe gage $(0.045$ inch).

\subsection{PLUMBING STOP COCKS AND VALVES.}

(See 607.6, p. 491.)

617.75 PIPE, PLUMBING.

(Plumbing practice and symbols, see 600.6, p. 113. Cast-iron pipe, see 607.11, p. 369 and 607.12, p. 374. Malleable-iron pipe, see 607.2 , p. 416 . Wrought-iron pipe, see 607.3, p. 430. Steel pipe, see 607.4, p. 435. Eaves troughs and conductor pipe, see 607.5, p. 491. Copper pipe, see 642.23, p. 1182. Brass pipe, see 645.23, p. 1216.) 
617.79 MISCELLANEOUS PLUMBING FIXTURES.

UNITED STATES GOVERNMENT, DEPARTMENT OF COMMERCE, BUREAU OF STANDARDS, SIMPLIFIED PRACTICE RECOMMENDATION FOR METAL PARTITIONS FOR TOILETS AND SHOWERS, R101-29, JULY 1, 1929

\section{TOILET AND SHOWER PARTITIONS}

(Simplified practice recommended and accepted by industry establishing a limited list of standard sizes and dimensions.)

\section{TOILET AND DRESSING ROOM INCLOSURES}

Center-to-center dimensions. $-30,32,34$, and 36 inches.

Stock sizes, partitions.-

\begin{tabular}{|c|c|c|c|}
\hline Depth & Height & Depth & Height \\
\hline $\begin{array}{r}\text { Inches } \\
36 \\
42 \\
48 \\
54 \\
60\end{array}$ & $\begin{array}{r}\text { Inches } \\
54 \\
54 \\
54 \\
54 \\
54\end{array}$ & $\begin{array}{r}\text { Inches } \\
48 \\
54\end{array}$ & $\begin{array}{r}\text { Inches } \\
60 \\
60\end{array}$ \\
\hline
\end{tabular}

Stock sizes, fronts.-

\begin{tabular}{|r|r||r|r|}
\hline Width & \multirow{2}{*}{ Height } & Width & Height \\
\cline { 1 - 1 } \cline { 3 - 4 } Inches & & & \\
\cline { 3 - 4 } 6 & Inches & Inches & Inches \\
8 & 54 & 6 & 60 \\
10 & 54 & 8 & 60 \\
12 & 54 & 10 & 60 \\
& & 60 \\
\hline
\end{tabular}

Stock sizes, doors.-

\begin{tabular}{|r|r||r|r|}
\hline Width & \multirow{2}{*}{ Height } & Width & Height \\
\cline { 1 - 1 } Inches & Inches & Inches & Inches \\
24 & 54 & 24 & 60 \\
26 & 54 & 26 & 60 \\
28 & 54 & 28 & 60 \\
30 & 54 & 30 & 60 \\
32 & 54 & 32 & 60 \\
34 & 54 & 34 & 60 \\
\hline
\end{tabular}

Stock sizes, posts.-All posts shall be 78 inches high (providing for head rail 12 inches above the tops of 54 -inch partitions and 6 inches above tops of 60 -inch partitions).
Hardware.-Standard hardware shall consist of the following: Self-closing hinge, latch, pull and bumper, and coat hook.

\section{TYPICAL INCLOSURES}

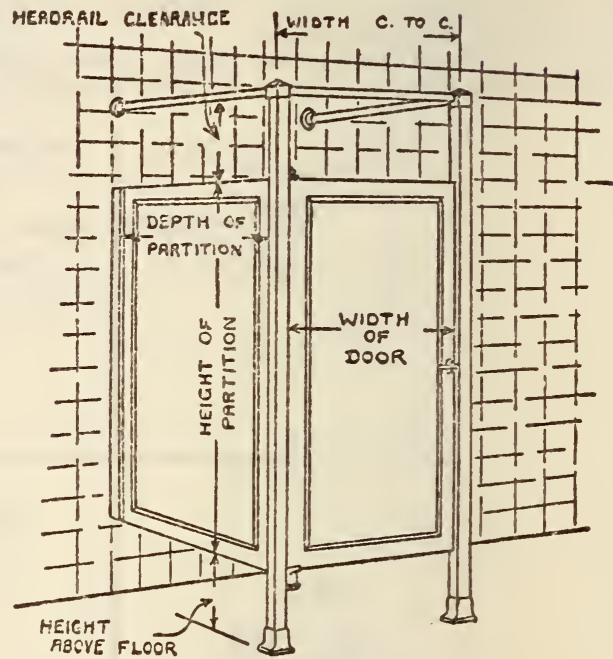

Figure 1054.-Metal toilet stall

Experience has shown that toilet inclosures of the following dimensions fulfill the requirements of the average installation, and these dimensions are recommended for use where special conditions do not make other dimensions preferable.

\begin{tabular}{|c|c|c|}
\hline \multirow[b]{2}{*}{ Arrangement } & \multicolumn{2}{|c|}{ Size of inclosures } \\
\hline & $\begin{array}{l}\text { Width } \\
\text { (center to } \\
\text { center) }\end{array}$ & Depth \\
\hline Inclosures without doors & Inches & Inches \\
\hline $\begin{array}{l}\text { Inclosures with full-width doors swing- } \\
\text { ing out }\end{array}$ & 32 & 48 \\
\hline $\begin{array}{l}\text { Inclosures with angle fronts and narrow } \\
\text { doors swinging in }\end{array}$ & 32 & 54 \\
\hline $\begin{array}{l}\text { Inclosures with full-width doors swing- } \\
\text { ing in }\end{array}$ & 32 & 60 \\
\hline
\end{tabular}

Door clearances.-Figure 1055 and the accompanying table are to be used in determining the conditions under which an inswinging door will 
clear the fixture, and the amount of such clearance, and to clarify terminology.

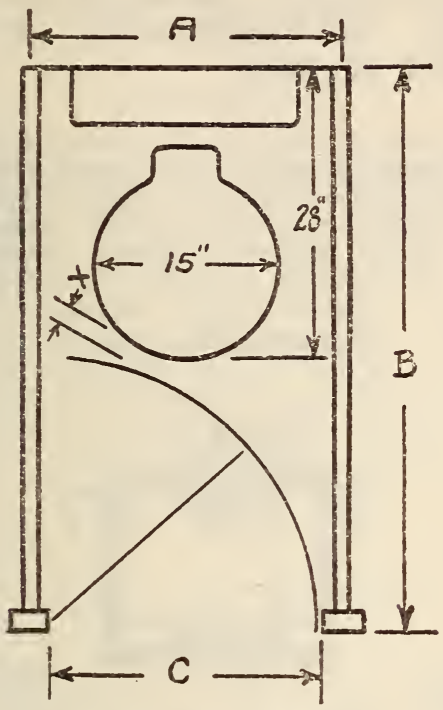

FigURE 1055

\begin{tabular}{|c|c|c|c|}
\hline A & B & C & $\mathrm{x}$ \\
\hline $\begin{array}{r}\text { Inches } \\
30 \\
32 \\
32 \\
36\end{array}$ & $\begin{array}{r}\text { Inches } \\
54 \\
54 \\
60 \\
60\end{array}$ & $\begin{array}{r}\text { Inches } \\
28 \\
30 \\
30 \\
34\end{array}$ & $\begin{array}{c}\text { Inches } \\
15 / 8 \\
1 / 16 \\
55 / 8 \\
23 / 8\end{array}$ \\
\hline
\end{tabular}

$A=$ Width of compartment.

$\mathrm{B}=$ Depth of compartment.

$\mathrm{C}=$ Door opening.

$\mathrm{X}=$ Clearance.

Note.-It is an exceptional fixture that projects more than 28 inches from wall to outside of seat.

Style of fronts.-Fronts (front partitions or front panels) of the style termed "corner fronts" or "angle fronts" are preferred to that style termed center fronts" or "T fronts."

Height off floor, partitions, and doors.-The standard clearance between the floor and bottom of partitions and doors shall be 12 inches.

Height off floor, backs.-Backs shall be terminated on curbs at least 4 inches above floor level.
SHOWER INCLOSURES

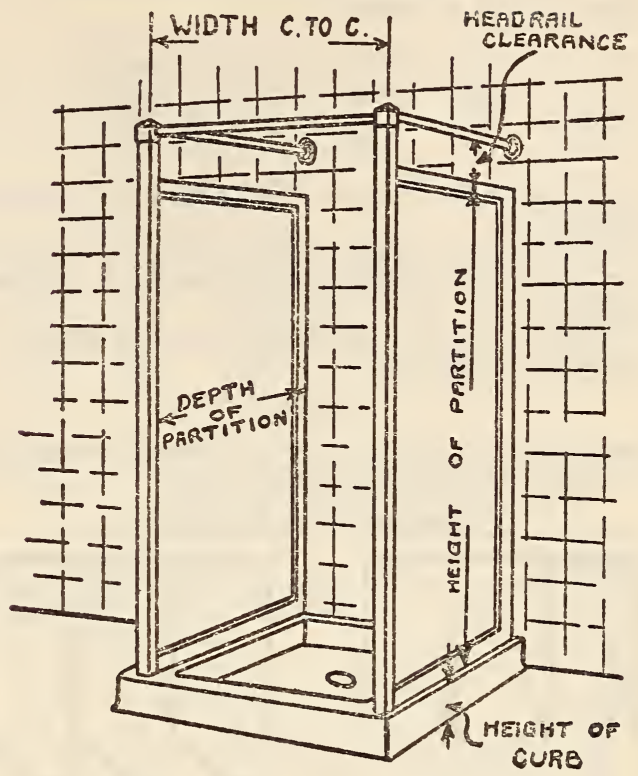

FIGURE 1056.-Metal shower stall

Center-to-center dimensions.-30,36, and 42 inches, Stock sizes, partitions.-

\begin{tabular}{|c|c|c|c|}
\hline Depth & Height & Depth & Height \\
\cline { 1 - 1 } \cline { 3 - 4 } Inches & Inches & Inches & Inches \\
36 & 66 & 36 & 72 \\
42 & 66 & 42 & 72 \\
\hline
\end{tabular}

Stock sizes, fronts.-

\begin{tabular}{|c|c|c|c|}
\hline Width & Height & Width & Height \\
\cline { 1 - 3 } Inches & Inches & Inches & Inches \\
12 & 66 & 12 & 72 \\
18 & 66 & 18 & 72 \\
\hline
\end{tabular}

Stock sizes, posts.-All posts shall be 78 inches high (providing for head rail 12 inches above tops of 66-inch partitions and 6 inches above tops of 72-inch partitions).

Style of fronts.-Fronts (front partitions or front panels), of the style termed "corner fronts" or "angle fronts" are preferred to that style termed "center fronts" or "T fronts."

Flush-type constructions.-Partitions and doors, panel-sheet steel shall be of 22 gage U. S. standard (0.0306 inch) or heavier. 
Panel-type constructions.-In partitions of the following sizes or larger:

\begin{tabular}{|c|c|c|c|}
\hline Depth & Height & Depth & Height \\
\hline Inches & Inches & Inches & Inches \\
54 & 54 & 54 & 60 \\
60 & 54 & 60 & 60 \\
\hline
\end{tabular}

panel-sheet steel shall be of 16 gage U. S. standard (0.0613 inch).

In partitions smaller than above mentioned, panel-sheet steel shall be of 18 gage U. S. standard (0.0490 inch).

Doors, panel-sheet steel shall be of 18 gage U. S. standard (0.0490 inch).

\section{FINISH}

All material shall be finished in a prime coat, further finish to be considered an extra.

TERMINOLOGY IN DRAWINGS
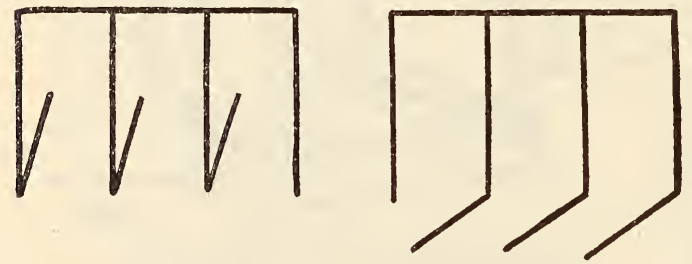

Figure 1057.-No fronts required, full width doors, inswinging and outswinging

The use of the following conventions is recommended in order to distinguish clearly between in-

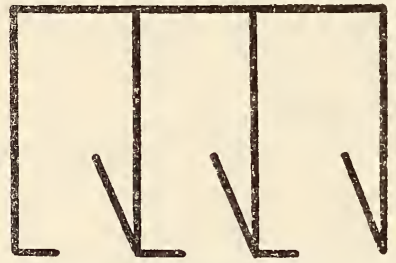

Figdre 1058.-Angle fronts required, narrow doors

closures which are to be constructed with wide doors and without fronts, and those with angle fronts and narrow doors.

UNITED STATES GOVERNMENT, FEDERAL SPECIFICATIONS BOARD, SPECIFICATION FOR PLUMBING FIXTURES (FOR LAND USE), NO. 448, NOVEMBER 22, 1926. (CURRENT DESIGNATION: FFP-451.)

\section{SHOWER FIXTURES}

(See 617.73, p. 1062.)

\subsection{MISCELLANEOUS BUILDERS AND FURNITURE HARDWARE.}

UNITED STATES GOVERNMENT, DEPARTMENT OF COMMERCE, BUILDERS' HARDWARE (NONTEMPLATE), COMMERCIAI STANDARD CS22-30, DECEMBER 11, 1930 BUILDERS' HARDWARE (NONTEMPLATE)

(See 617.0, p. 977, also Federal specification FF-H-101 below.)

\section{UNITED STATES GOVERNMENT, FEDERAL SPECIFICATIONS BOARD, FEDERAI SPECIFICATION FOR BUILDERS' HARD. WARE (NONTEMPLATE), FF-H-101, AUGUST 19, 1930}

(For types, grades, classes; material and workmanship; general requirements; detail requirements packaging, packing, and marking; notes; and table of standard finishes, see 617.0, p.978. For chromium plating, nickel plating, and zinc coatings; and methods of inspection and tests, see 600.3, p. 70 .)

\section{SCUTTLE OPERATORS}
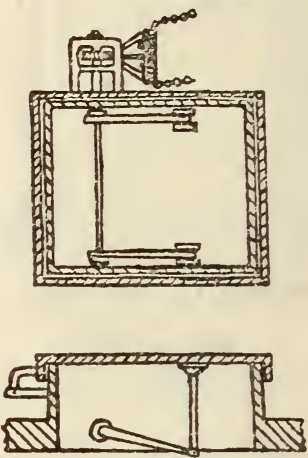

Figure 1059.-Scuttle operator, type 1510

Type 1510.-For heavy scuttles. Worm gear operated by sprocket wheel and endless chain. Rocker shaft 3/4-inch iron pipe. Arms and connections to scuttle of cast or wrought iron of lengths to open scuttle $60^{\circ}$. Gears and arms secured to shafts with set screws. Shaft bearings and brackets of cast iron. Length of shaft and chains as specified. Bearings, machine finished, and provided with oil holes. Worms and gears smoothly finished and close fitting. Fastening to scuttle, wood screws. Fastening to curb, through bolts. Finish, painted.

\section{SKYLIGHT OPERATORS}

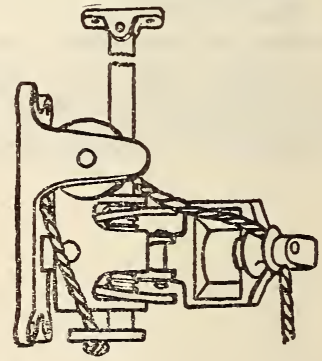

FigdRE 1060.-Skylight operator, type 1520

Type 1520.- - Lifting rod operated by single cord, self-lockin. Cord No. 10, braided cotton sash cord. Rods, steel, copper plated. If single rod, minimum diameter shall be one-half inch; if two rods, minimum diameter shall be three-eighths inch. Lift 18 inches. 
DOOR BUTTONS

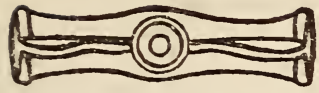

FIG URE 1061.-Door button, type 1069

Type 1069.-Cast iron or wrought steel, galvanized. Without plates. Lengths $1 \frac{1}{2}$ and 2 inches. With screws.

\section{CUPBOARD TORNS}

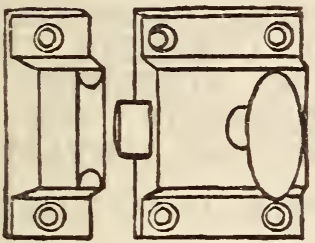

Figure 1062.-Cupboard turn, type 1082

Type 1082.-Cast bronze. Oval knob. Size, without strike, $17 / 8$ by $13 / 16$ inches. Latch bolt, minimum $11 / 32$ by $5 / 16$ inch, minimum throw $7 / 32$ inch.

Type 10824.-Same as type 1082, except nickel plated.

Type 1082B.-Same as type 1082, except wrought bronze. Minimum thickness of case 0.040 inch. Latch bolt, minimum $3 / 8$ by $11 / 32$ inch, with $7 / 32$-inch minimum throw.

Type 1082C.-Same as type 1082, except cast iron or wrought steel, plated. Minimum thickness of case 0.040 inch. Latch bolt, minimum $3 / 8$ by $5 / 10$ inch, with $7 / 32$-inch minimum throw.

Type 1083.-Cast bronze. Oval knob. Size, without strike, $2 \frac{1}{4}$ by $1 \frac{1}{2}$ inches. Latch bolt, minimum $1 / 2$ by $3 / 8$ inch, minimum throw five-sixteenths inch.

Type 1083A.-Same as type 1083, except nickel plated.

Type 1083B.-Same as type 1083, except wrought bronze. Minimum thickness of case 0.040 inch.

Type 1083C.- Same as type 1083, except cast iron or wrought steel, plated. Minimum thickness of case 0.040 inch. Latch bolt, minimum $3 / 8$ by $3 / 8$ inch, minimum throw $5 / 16$ inch.

\section{MISCELLANEOUS IRON AND STEEL MANUFACTURES}

\subsection{FIREARMS AND ORDNANCE.}

\section{UNITED STATES GOVERNMENT, DEPART- MENT OF COMMERCE, BUREAU OF STANDARDS, SIMPLIFIED PRACTICE RECOMMENDATION FOR LOADED PAPER SHOT SHELLS, R31-31, JANUARY 2, 1931}

\section{LOADED PAPER SHOT SHELLS}

(Simplified practice recommended and accepted by industry establishing a limited list of standard stock sizes and types.)
TABLE 1.-Black powder loads

[Now Club, New Chief, Nublack, Referee, and Romax shells]

\begin{tabular}{|c|c|c|c|c|c|}
\hline $\begin{array}{l}\text { Shell } \\
\text { gages }\end{array}$ & $\begin{array}{l}\text { Weight } \\
\text { of } \\
\text { powder }\end{array}$ & $\begin{array}{l}\text { Weight } \\
\text { of } \\
\text { shot }\end{array}$ & $\begin{array}{c}\text { Sizes of drop } \\
\text { shot }\end{array}$ & $\begin{array}{c}\text { Sizes of chilled } \\
\text { shot }\end{array}$ & $\begin{array}{l}\text { Length } \\
\text { of shells } \\
\text { un- } \\
\text { loaded }\end{array}$ \\
\hline $\begin{array}{l}16 \\
20\end{array}$ & $\left\{\begin{array}{r}\text { Drams } \\
4 \\
8 \\
3 \\
31 / 4 \\
6 \\
21 / 2 \\
21 / 2\end{array}\right.$ & \begin{tabular}{r} 
Ounces \\
$11 / 8$ \\
\hdashline 1 \\
$11 / 8$ \\
\hdashline $7 / 8$ \\
$7 / 8$
\end{tabular} & $\begin{array}{l}4,6 \\
\text { Blank } \\
2,4,5,6,7,8 \\
\text { BB, } 4,5,6 \\
\text { Blank } \\
4,5,6 \\
4,6\end{array}$ & ( & $\begin{array}{l}\text { Inches } \\
27 / 8 \\
27 / 8 \\
25 \% 8 \\
25 / 8 \\
25 \% 8 \\
2916 \\
21 \%\end{array}$ \\
\hline
\end{tabular}

TABLE 2.-Smokeless powder loads in high-cup shells

[Arrow, Ajax, Leader, Record, and Reliable shells]

\begin{tabular}{|c|c|c|c|c|c|}
\hline $\begin{array}{l}\text { Shell } \\
\text { gages }\end{array}$ & $\begin{array}{l}\text { Dram } \\
\text { equiv- } \\
\text { alent } \\
\text { powder }\end{array}$ & $\begin{array}{l}\text { Weight } \\
\text { of } \\
\text { shot }\end{array}$ & $\begin{array}{l}\text { Sizes of drop } \\
\text { shot }\end{array}$ & $\begin{array}{l}\text { Sizes of chilled } \\
\text { shot }\end{array}$ & $\begin{array}{l}\text { Length } \\
\text { of shells } \\
\text { un- } \\
\text { loaded }\end{array}$ \\
\hline $\begin{array}{l}16 \\
20\end{array}$ & $\begin{array}{l}41 / 4 \\
3 \\
31 / 4 \\
31 / 4 \\
31 / 2 \\
31 / 2 \\
23 / 4 \\
21 / 4 \\
21 / 2\end{array}$ & $\begin{array}{r}\text { Ounces } \\
11 / 4 \\
11 / 8 \\
118 \\
11 / 4 \\
118 \\
11 / 4 \\
1 \\
178 \\
1\end{array}$ & $\begin{array}{l}4,6 \\
4,5,6,7,8- \\
4,5,6,7,8 \\
\text { BB } \\
4,6,8 \\
4,6,8 \\
\end{array}$ & $\begin{array}{l}4,5,6 \\
4,5,6,71 / 2,8- \\
2,4,5,6,71 / 2- \\
4,5,6,71 / 2 \\
2,4,5,6,7,712- \\
2,4,5,6,7- \\
4,5,6,7112,8 \\
4,5,6,71 / 2,8 \\
4,5,6,71 / 2 . \\
\end{array}$ & $\begin{array}{l}\text { Inches } \\
27 / 8 \\
25 / 8 \\
258 \\
23 / 4 \\
23 / 4 \\
23 / 4 \\
2916 \\
21 / 2 \\
21 / 2\end{array}$ \\
\hline
\end{tabular}

12-GAGE TRAP LOADS

\begin{tabular}{|c|c|c|c|c|c|}
\hline 12 & $\begin{array}{l}3 \\
3\end{array}$ & $\begin{array}{l}11 / 8 \\
11 / 4\end{array}$ & - & $\begin{array}{l}71 / 2,8 \\
71 / 2,8\end{array}$ & $\begin{array}{l}23 / 4 \\
23 / 4\end{array}$ \\
\hline
\end{tabular}

1 See sec. (b).

TABLE 3.-Smokeless powder loads in medium-cup [Climax, Field, Nitro Club, Repeater, Target, and Favorite shells]

\begin{tabular}{|c|c|c|c|c|c|}
\hline 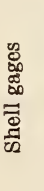 & 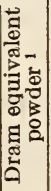 & 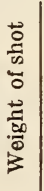 & Sizes of drop shot & $\begin{array}{l}\text { Sizes of chilled } \\
\text { shot }\end{array}$ & 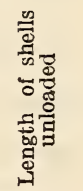 \\
\hline 10 & $41 / 4$ & $\begin{array}{c}O z . \\
11 / 4\end{array}$ & 4,6 & $4,5,6$ & $\begin{array}{r}\text { Inches } \\
27 / 8\end{array}$ \\
\hline 12 & $\left\{\begin{array}{l}23 / 4 \\
3 \\
3 \\
31 / 4 \\
31 / 4 \\
31 / 2 \\
31 / 2\end{array}\right.$ & \begin{tabular}{l|}
$11 / 8$ \\
1 \\
$11 / 8$ \\
$11 / 8$ \\
$11 / 4$ \\
118 \\
$11 / 4$
\end{tabular} & $\begin{array}{l}4,5,6,7,8,9 \\
4,5,6,7,71 \frac{1}{2}, 8,9,10 \\
4,5,6,7,71 / 2,8,9\end{array}$ & $\begin{array}{l}71 / 2,8 \\
4,5,6,7,71 / 2,8,9,10 \\
2,4,5,6,7,71 / 2,8,9 \\
4,5,6,71 / 2 \\
2,4,5,6,7,71 / 2 \\
2,4,5,6,7\end{array}$ & $\begin{array}{l}25 / 8 \\
25 / 8 \\
25 \% 8 \\
25 / 8 \\
23 / 4 \\
23 / 4 \\
23 / 4\end{array}$ \\
\hline 16 & $\left\{\begin{array}{l}21 / 2 \\
23 / 4 \\
3\end{array}\right.$ & $\begin{array}{l}1 \\
1 \\
1\end{array}$ & $\begin{array}{l}4,5,6,7,71 \frac{1}{2}, 8,9,10^{-} \\
4,5,6,7,7 \frac{1}{2}, 8,9,10\end{array}$ & $\begin{array}{l}4,5,6,71 / 2,8,9 \\
4,5,6,7,7132,8,9,10 \\
4,5,6,71 / 2 \ldots\end{array}$ & $\begin{array}{l}2 \% 16 \\
2 \% 16 \\
2 \% 16\end{array}$ \\
\hline 20 & $\left\{\begin{array}{l}21 / 4 \\
21 / 2\end{array}\right.$ & $1^{7 / 8}$ & $4,5,6,7,71 / 2,8,9,10$ & $\begin{array}{l}4,5,6,71 / 2,8,9,10 \ldots \\
4,5,6,71 / 2,8\end{array}$ & $\left\{\begin{array}{l}21 / 2 \\
21 / 2 \text { or } \\
23 / 4\end{array}\right.$ \\
\hline 28 & $\left\{\begin{array}{c}13 / 4 \\
2\end{array}\right.$ & $\begin{array}{l}5 / 8 \\
3 / 4\end{array}$ & & $\begin{array}{l}4,6,71 / 2, \ldots \\
4,5,6,7,8\end{array}$ & $\begin{array}{l}21 / 2 \\
27 / 8\end{array}$ \\
\hline 410 & & $3 / 8$ & & $4,5,6,71 / 2,8,9,10 \ldots$ & $21 / 2$ \\
\hline
\end{tabular}


TABLE 3.-Smokeless powder loads in medium-cup shells-Continued

12-GAGE TRAP LOADS

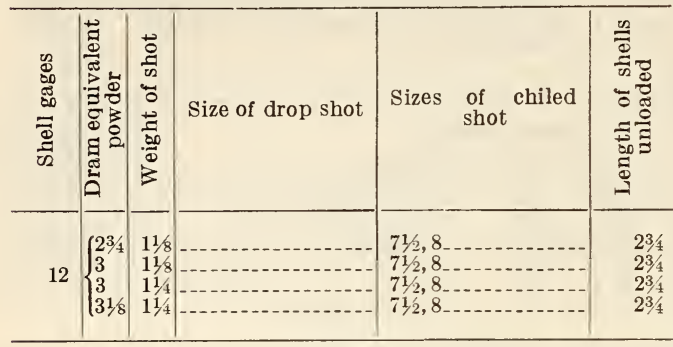

SHORT-RANGE LOADS

[Brush, Bush, Scatter, Spreader, and Thicket]

\begin{tabular}{|c|c|c|c|c|c|}
\hline 12 & 3 & $11 \frac{1}{1}$ & $6,71 / 2,8$ & $6,71 \frac{1}{2}, 8,9$ & $2^{3} 44$ \\
\hline 16 & $21 / 2$ & 1 & $8,9 \ldots$ & $6,71 / 2,8,9 \ldots$ & 2916 \\
\hline 20 & $21 / 4$ & $7 / 8$ & & $6,71 / 2,8,9$ & \\
\hline
\end{tabular}

SINGLE-BALL LOADS

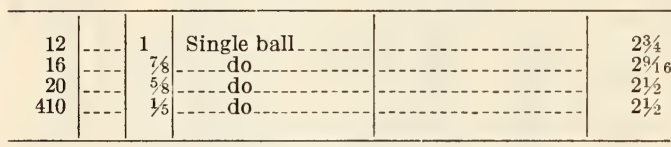

BUCKSHOT LOADS

\begin{tabular}{|c|c|c|c|c|c|c|}
\hline Shell gages & $\begin{array}{l}\text { Dram } \\
\text { equiv- } \\
\text { alent } \\
\text { pow- } \\
\text { der }{ }^{1}\end{array}$ & $\begin{array}{l}\text { Weight } \\
\text { of shot }\end{array}$ & $\begin{array}{l}\text { Buck- } \\
\text { shot } \\
\text { east- } \\
\text { ern } \\
\text { sizes }\end{array}$ & $\begin{array}{l}\text { Lay- } \\
\text { ers }\end{array}$ & $\begin{array}{l}\text { Pel- } \\
\text { lets }\end{array}$ & $\begin{array}{l}\text { Length of } \\
\text { shells } \\
\text { unloaded }\end{array}$ \\
\hline $10 \ldots$ & & Ounces & 0 & 4 & 16 & $\begin{array}{l}\text { Inches } \\
27,8\end{array}$ \\
\hline & & & $\begin{array}{r}00 \\
0\end{array}$ & $\begin{array}{l}3 \\
4\end{array}$ & $\begin{array}{r}9 \\
12\end{array}$ & $\begin{array}{l}23 / 4 \\
23 / 4\end{array}$ \\
\hline & & & 1 & 4 & 16 & $\begin{array}{l}23 \\
234 \\
2_{3}\end{array}$ \\
\hline $16 \ldots$ & & -...- & 1 & 4 & 12 & $\begin{array}{l}294 \\
2 \% 16\end{array}$ \\
\hline $20 \ldots$ & $\int-.-$ & - & $\begin{array}{l}3 \\
3\end{array}$ & $\begin{array}{l}5 \\
6\end{array}$ & $\begin{array}{l}20 \\
24\end{array}$ & $\begin{array}{l}21 / 6 \\
2^{3} 34\end{array}$ \\
\hline
\end{tabular}

1 See sec. $(b)$.

TABLE 4.-Smokeless powder loads in low-cup shells

[Defiance, Shur Shot, Xpert, Ranger, Victor, and Monark̀ shells]

\begin{tabular}{|c|c|c|c|c|c|}
\hline $\begin{array}{l}\text { Shell } \\
\text { gages }\end{array}$ & $\begin{array}{l}\text { Dram } \\
\text { equiv- } \\
\text { alent } \\
\text { pow- } \\
\text { der } 1\end{array}$ & $\begin{array}{l}\text { Weight } \\
\text { of shot }\end{array}$ & $\begin{array}{l}\text { Sizes of drop } \\
\text { shot }\end{array}$ & $\begin{array}{c}\text { Sizes of chilled } \\
\text { shot }\end{array}$ & $\begin{array}{c}\text { Length } \\
\text { of shels } \\
\text { un- } \\
\text { loaded }\end{array}$ \\
\hline $\begin{array}{l}16 \\
20\end{array}$ & $\begin{array}{l}23 / 4 \\
3 \\
3 \\
3 \\
3 \\
31 / 4 \\
31 / 2 \\
21 / 2 \\
23 / 4 \\
21 / 4\end{array}$ & $\begin{array}{c}\text { Ounces } \\
1 \\
1 \\
11 / 8 \\
118 \\
11 / 4 \\
11 / 8 \\
11 / 4 \\
1 \\
1 \\
7 / 8\end{array}$ & $\begin{array}{l}6,8,10 \\
4,5,6,7,8,9 \\
4,5,6,7,8,10 \\
\text { BB }, 2,4,5,6,7,8 \\
\text { BB } \\
4,5,6,7,8,10 \\
4,5,6,7,8,10\end{array}$ & $\begin{array}{l}4,5,6,71 / 2,9 \\
71 / 2 \\
71 / 2,8 \\
4,5,6,71 / 2 \\
2,4,5,6,7 \\
4,5,6,71 / 2,9 \\
4,5,6,7,71 / 2- \\
4,5,6,71 / 2,9\end{array}$ & 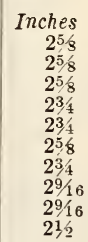 \\
\hline
\end{tabular}

1 6ee sec. (b).

(a) All loads in excess of any maximum load herein listed may be furnished at the option of the individual loading company, in progressive-burning powders only.

(b) Powder-brand markings are to be eliminated from all gages and all grades of loaded paper shot shells, with the exception of 12-gage trap loads in medium and high cup shells and progressive-burning powder loads in $10,12,16$, and 20 gage.

All specified loads in all gages in medium and high cup smokeless shells, with the exception of 12-gage trap loads and progressive-burning powder loads, are to be marked in drams with the words "equivalent" or "equivalent to"; for example, 3-dram equivalent, or equivalent to 3 drams.

(c) Loads not covered by the above are to be supplied as standard loads only until the stock on hand January 1, 1931, shall have become exhausted; thereafter they may be supplied only as special loads.

\section{UNITED STATES GOVERNMENT, DEPART- MENT OF COMMERCE, BUREAU OF STANDARDS, SIMPLIFIED PRACTICE RECOMMENDATION FOR METALLIC CARTRIDGES R62, JANUARY 1, 1927 \\ METAILIC CARTRIDGES}

In accordance with the unanimous action of representatives of manufacturers and distributors of metallic cartridges, the Department of Commerce, through the Bureau of Standards, recommends the establishment of the following simplified list of stock items:

\section{TABLE 1.-Rim-fire cartridges (ball)}

[The letter " $\mathrm{x}$ " indicates those cartridges recommended as standard]

\begin{tabular}{ll|l|l|l|l}
\hline & Name of cartridge & & & \\
& & & & & \\
\end{tabular}

TABLE 2.-Rim-fire cartridges (shot)

[The letter " $x$ " indicates those cartridges recommended as standard]

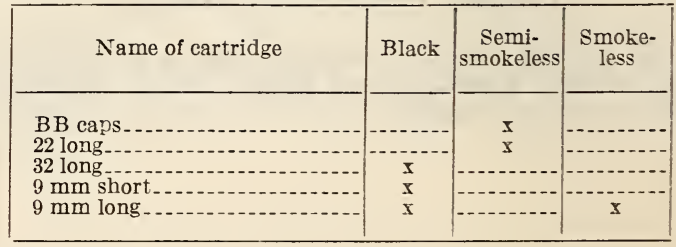


TABLE 3.-Rim-fire cartridges (blank)

["Semismokeless" and "smokeless" loads eliminated]

[The letter " $x$ " indicates those cartridges recommended as standard]

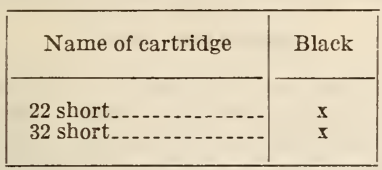

TABLE 4.-Center-fire pistol cartridges (ball)

[The letter " $x$ " indicates those cartridges recommended as standard]

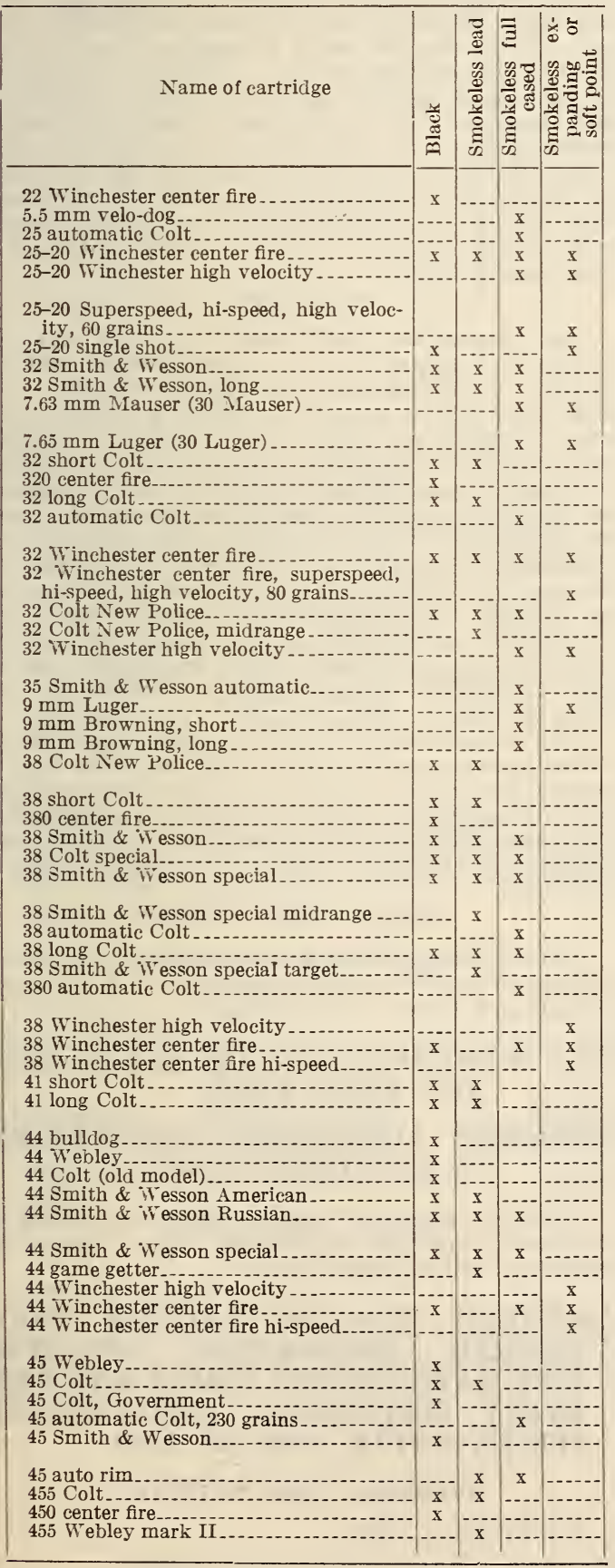

TABLE 5.-Center-fire pistol cartridges (shot)

[The letter " $x$ " indicates those cartridges recommended as standard]

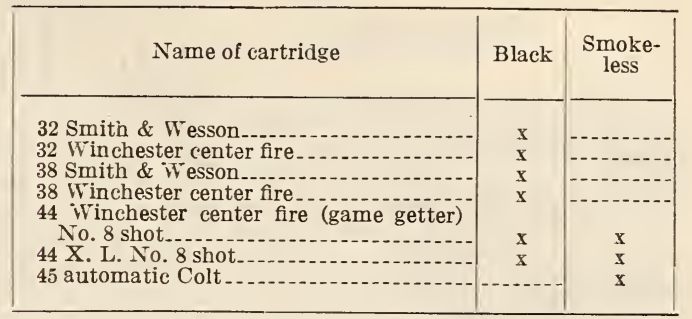

TABLE 6.-Center-fire pistol cartridges (blank)

[The letter " $x$ " indicates those cartridges recommended as standard]

\begin{tabular}{|c|c|}
\hline Name of cartridge & Black \\
\hline $\begin{array}{l}32 \text { Smith \& Wesson } \\
38 \text { Smith \& Wesson }\end{array}$ & $\begin{array}{l}x \\
x\end{array}$ \\
\hline
\end{tabular}

TABLE 7.-Center-fire rifle cartridges (ball)

["Smokeless lead" loads eliminated]

[The letter " $x$ " indicates those cartridges recommende $f$ as standard]

Name of cartridge $\mid$

$6 \mathrm{~mm} \mathrm{U} . \mathrm{S}$

$6.5 \mathrm{inm}$ Mannlicher

$7 \mathrm{~mm}$

22 Savage high power

25 Remington automatic

25 Remington superspeed

25-35 Winchester center fire

25-35 Winchester center fire superspeed

Varlin

250-3,000 Savage

$7.62 \mathrm{~mm}$ Russian

$7.65 \mathrm{~mm}$ Mauser

$8 \mathrm{~mm}$ Mauser

$8 \mathrm{~mm}$ Mannlicher schoenaue

$8 \mathrm{~mm}$ special

$9 \mathrm{~mm}$

270 Winchester center nire

275 Magnum

280 Ross pointed

30 Winehester center fire.

30 Winchester center fire superspeed

30 Remington automatic

30 Remington superspeed

300 Savage

300 Magnum

303 Savage

303 British

30 Army 220 grains

30 Army pointed, 150 grains

30 Army pointed, 180 grains

30 Army hi-speed

30 Government Model 1903

30 Government Model 1906, pointed, 150 grains 30 Government Model 1906, pointed, 180 grains 30 Government Model 1906, 220 grains ........-..

30 Government Model 1908, pointed, 145 grains 30 Government Model 1906, hi-speed.

32 Winchester special ...........................

32 Remington automatic

32 Remington superspeed

$32-40$

$32-40$ Winchester high velocity

33 Winchester center fire

35 Winchester center fire

35 Winchester self-loading

35 Remington automatic

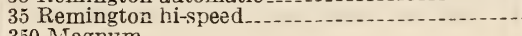

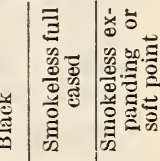


TABLE 7.-Center-fire rifle cartridges (ball)-Contd.

\begin{tabular}{l|c|c|c|c|}
\hline Name of cartridge & & & & \\
\end{tabular}

UNITED STATES GOVERNMENT, DEPARTMENT OF COMMERCE, STEAMBOAT INSPECTION SERVICE, GENERAL RULES AND REGULATIONS PRESCRIBED BY THE BOARD OF SUPERVISING INSPECTORS, OCEAN AND COASTWISE, 1929

\section{IINE-CARRYING GUNS}

All steam vessels of 150 gross tons and over navigating the ocean shall be equipped with at least three line-carrying projectiles and the means of propelling them, of a type and character which have been officially approved by the board of supervising inspectors.

\section{ALLOY OF IYLE-TYPE GONS}

The material entering into the construction of cast-bronze guns for use on board steam vessels as a means of propelling line-carrying projectiles shall have a tensile strength of not less than 52,000 pounds per square inch of section and a reduction of area of not less than 25 per cent.

\section{SAMPIE OF GON FOR TESTING}

At least one sample of the material shall be taken from the casting of each gun, and shall not be less than 7 inches in length, 2 inches in width, one-half inch in thickness, and have a section of 0.5 by 0.75 inch over a length of 2 inches.

(Specifications include provision for firing tests of both Lyle and Hunt guns.)

\section{STEEL GONS}

Before any steel gun of the cannon type shall be approved, the manufacturer shall furnish the supervising inspector of the district where the gun is tested a sample of the material of which it is constructed. Such sample is to be of the following dimensions: 7 inches in length and approximately $1 \frac{1}{2}$ inches wide and 1 inch thick, with a section $2 \frac{1}{2}$ inches in the center turned down to $1 / 2$-inch diameter, the sample to be accompanied by the same form of affidavit as required for bronze guns. That is, the samples for testing shall be furnished by the supervising inspector of the district where the gun is to be tested, and must be accompanied by an affidavit of the manufacturer of the gun that the samples actually and correctly represent the material of the gun. Samples must be distinctly marked with the same number appearing on guns which they represent, so as to be readily identified by the inspector.

\section{SHOOLDER GONS}

The gun known as the shoulder gun may be used on steamers of 300 tons and under as a means of propelling a line-carrying projectile. This gun is an ordinary breech-loading rifle with barrel shortened and bore smoothed, using a blank cartridge of 40-75 or 50-70, propelling a projectile about 8 inches long, weighing about 8 ounces, attached to a line not less than 400 feet in length.

The projectile must be thrown a distance of not less than 250 feet without fouling or breaking the line. The gun shall be of a type approved by the steamboat inspection service. The manufacturer of the gun shall furnish purchasers with an affidavit that it is of approved type, of safe material, and good workmanship. Each shoulder gun shall be provided with 3 shot lines, 10 projectiles, at least 25 cartridges, and the other necessary equipment. Each vessel using the shoulder gun shall carry auxiliary thereto, to be used for no other purpose, at least 500 feet of 3inch manila line.

\section{PROJECTILES}

The projectiles shall weigh not less than 18 pounds, be smoothly turned and finished, with a windage of not more than one sixty-fourth inch. No such projectile or means of propelling it shall hereafter be approved unless of such character that the projectile and propelling charge can be readily withdrawn from the gun when it becomes necessary or desirable to do so. Powder containers of centerfire type shall be of such construction that proper alignment of firing pin and percussion primer always obtains when propelling charge and projectile are set for use. When perforated metal powder containers are used, such perforations shall extend entirely around the base of such containers, and at least 25 efficient primers shall be carried for the firing thereof.

It is recommended that the charge of powder in actual service on shipboard shall be from 5 to 6 ounces, but may be exceeded under extraordinary circumstances, and in no case shall the charge exceed 8 ounces. The end of the line attached to the projectile should be thoroughly wet foi a length of about 1 fathom.

\section{REPORT OF TEST OF GON}

The supervising inspector shall furnish the manufacturer of any line-carrying gun a copy of the report on each gun tested and inspected.

\subsection{MANUFACTURES OF SHEET IRON AND SHEET STEEL.}

\section{AMERICAN AUTOMOBILE ASSOCIATION, CATALOGUE, 1929}

(Contains details of shapes, sizes, and colors of standard enameled sheet-iron signs conveying warnings, routes, speeds, etc.)

\section{AMERICAN ELECTRIC RAILWAY ENGI- NEERING ASSOCIATION, OVERHEAD LINE MATERIAL FOR DIRECT AND CAT- ENARY SUSPENSION, RECOMMENDED SPECIFICATION, D102-30, 1930}

\author{
OVERHEAD IINE MATERIAL
}

(See 611.29, p. 682.) 
AMERICAN SOCIETY FOR TESTING MATERIALS, STANDARD METHOD OF TEST FOR SIZE OF ANTHRACITE, DESIGNATION D310-31, 1931

\section{TEST SCREENS}

\section{SCOPE}

1. This method of test covers the screen tests of anthracite to determine the percentage of undersize or oversize in any given commercial size.

\section{APPARATUS}

2. The screens for testing the various sizes of anthracite shall consist of No. 16 U. S. gage metal plates with staggered round openings. Screens mounted in hardwood box frames 16 to 20 inches square are satisfactory for testing chestnut, pea, and buckwheat sizes of anthracite. For broken, egg, and stove sizes it is more convenient to use screens square or rectangular in shape having an area of 4 to 6 square feet. The screens may conveniently be mounted on a rack, so as to slide like a drawer, with a pan underneath to catch the undersize.

3. The screen openings for testing the different commercial sizes of anthracite ${ }^{92}$ shall be as follows:

\begin{tabular}{|c|c|}
\hline Size & $\begin{array}{l}\text { Size of round-hole openings in testing } \\
\text { screens }\end{array}$ \\
\hline Broken. & Passing a $47 / 16$-inch screen, retained on \\
\hline Egg- & Passing a 37/16-inch screen, retained on \\
\hline Stove & $\begin{array}{l}\text { a 2816-inch screen. } \\
\text { Passing a } 2816 \text {-inch screen, retained on }\end{array}$ \\
\hline Chestnut & $\begin{array}{l}\text { a } 19 / 16 \text {-inch screen. } \\
\text { Passing a } 19 / 16 \text {-inch screen, retained on }\end{array}$ \\
\hline & $\begin{array}{l}\text { a }^{11 / 16} \text {-inch screen. } \\
\text { Passing a } 12 / 16 \text {-inch screen, retained on }\end{array}$ \\
\hline No. 1 Buckwheat. & $\begin{array}{l}\text { a } 8 / 16 \text {-inch screen. } \\
\text { Passing a } \% 16 \text {-inch screen, retained on }\end{array}$ \\
\hline No. 2 Buckwheat & $\begin{array}{l}\text { a } 5 / 16 \text {-inch screen. } \\
\text { Passing a } 5 / 16 \text {-inch screen, retained on }\end{array}$ \\
\hline $\begin{array}{l}\text { No. } 3 \text { Buckwheat } \\
\text { (barley). }\end{array}$ & $\begin{array}{l}\text { Passing a } 3 / 16 \text {-inch screen, retained on } \\
\text { a } 3 / 32 \text {-inch screen. }\end{array}$ \\
\hline
\end{tabular}

AMERICAN SOCIETY FOR TESTING MATERIALS, TENTATIVE DEFINITION OF THE TERM SCREEN (SIEVE), DESIGNATION E13-28T, 1928

\section{DEFINITION OF SIEVE}

(See 645.31, p. 1226.)

Note.-Tentative specifications, E17-31T, entitled "Round-Hole Screens for Testing Purposes" was issued in 1932.

\section{NATIONAL ASSOCIATION OF SHEET METAL CONTRACTORS, STANDARD PRACTICE IN SHEET METAL WORK, 1929}

\section{SHEET METAL WORK}

(See 605.22, p. 276.)

92 The names of anthracite sizes and the size of screen openings for broken, egg, stove, chestnut, and pea sizes are in conformity with those recommended by the Anthracite Operators' Conference of Aug. 8, 1929.
619.3 SCREW POSTS, METAL FENCE POSTS. AMERICAN RAILWAY ENGINEERING ASSOCIATION, SPECIFICATIONS FOR METAL FENCE POSTS, 1928

\section{METAL FENCE POSTS}

Classes.-Metal fence posts shall be divided into two classes: (1) Posts which support the straightaway body of the fence shall be designated as line posts. (2) Such other special posts as are needed at the end or corner of the fence and at gates, shall be designated as end, corner, or gate posts.

Material.-Metal fence posts may be made of steel or wrought iron, or alloys of iron of any kind which will pass strength tests as described below.

Strength.- Material used for fence posts shall have a minimum tensile strength of 40,000 pounds per square inch; and with a finished post, clamped rigidly, a load of 300 pounds applied at 3 feet from the face of the clamp, the deflection shall not exceed 6 inches, and the permanent set shall not exceed $2 \frac{1}{2}$ inches. The load for this test shall be applied to the face of the post against which the fence material bears.

Ductility. - A finished post resting upon supports 5 feet apart, and with a load applied at the center, must withstand bending 9 inches from a straight line, with no signs of failure.

Tubular posts.-The cold rolling of tubular posts is sufficient test for ductility; therefore, tubular posts are exempt from the ductility test.

Workmanship.-All posts shall be smoothly rolled or formed and shall be straight throughout the length. Each finished post shall be free from burrs or other deformation caused by fabrication. They shall also be free from slivers, depressions, seams, crop ends, and evidence of being burnt. (The above does not refer to rough places caused by zinc coating when galvanized.)

Variations in length shall not exceed one-half inch over or under the prescribed length. Variations in other dimensions shall not be more than onesixteenth inch over or under the prescribed figures.

Coating-Zinc.-All galvanized posts shall be galvanized by the hot-dip process and shall possess a uniform coating of prime western spelter or better grade, wiih not less than 2 ounces per square foot of surface as determined by the spot test or by the Preece test with four dips.

Galvanized posts are to be desired. All posts not galvanized shall be painted or dipped in a protective coating of metallic paint.

Special fabrication for line posts. - Line posts shall be fabricated to the section agreed upon by the purchaser.

If the posts are not so designed as to make anchorage for alinement unnecessary, an anchorage device shall be rigidly fabricated to bottom portion of posts.

All posts shall be provided with some convenient means of fastening wire to same for at least 12 positions in a height of 5 feet, above the surface of the ground. All posts shall permit the refastening of the wire at least five times without damage to the connecting appliance, if an integral part of the post.

All posts shall be capable of being driven in ordinary earth without injury to the post.

All posts shall have sufficient length, so that when installed with the required height above the ground, one-third of the total length shall be underground, providing that the post shall extend into the ground not less than $2 \frac{1}{2}$ feet. 
Special fabrication for end, corner, and gate posts.All special posts shall be fabricated the same as line posts, except as follows:

All posts shall have sufficient length to permit installing 3 feet into the ground; and shall be equipped with some suitable form of bracing and anchorage which shall aid in keeping the post vertical.

Weight.-The main stem of all form sections, not including tubing, shall weigh at least 1.30 pounds per linear foot, and preferably should be 25 per cent heavier than that. Posts made of tubing shall be fabricated of at least 10-gage material and be not less than $13 / 4$ inches in diameter.

Inspection. - Inspection and approval of the posts shall be made by the engineer or other authorized representative of the purchaser. Such inspection shall be made at the plant of the manufacturer, who will allow the inspector access to all operations involved and shall facilitate as much as possible the work of inspection and provide necessary facilities for inspection. Two posts out of each 200, selected by the inspector at random, shall be inspected and tested and if they meet the requirements, the lot shall be accepted. If either fails to meet requirements, three other posts shall be selected in like manner by the inspector, and if either one of these shall fail, the lot shall be rejected.

\subsection{BUOYS OF IRON OR STEEL.}

(No nationally recognized specifications available.)

\subsection{WATER FILTERS.}

AMERICAN WATER WORKS ASSOCIATION, WATER WORKS PRACTICE MANUAL, 1925

\section{PRESSURE WATER FILTERS}

(Permissible rates of filtration dependent on use of water; requirements concerning dimensions and capacities for filters and pipe connections, and minimum wash water for various filtration rates. Required thickness of shell and head for various working pressures for vertical steel and cast-iron filters.)

\subsection{MANUFACTURES OF IRON OR STEEL NOT COVERED IN ABOVE CLAS- SIFICATION.}

\section{AMERICAN ELECTRIC RAILWAY ENGI-} NEERING ASSOCIATION, OVERHEAD LINE MATERIAL FOR DIRECT AND CATENARY SUSPENSION, RECOMMENDED SPECIFICATION, D102-30, 1930

\section{OVERHEAD IINE MATERIAL}

(D102-30, paragraphs 306, 307, 309, 310, etc., include requirements for various kinds of fittings. For specification, see 611.29 , p. 682.)

\section{AMERICAN MARINE STANDARDS COM- MITTEE, FIXED LIGHTS FOR SHIPS, H NO. 3-1925}

\section{TYPE A PRESSED STEEL FRAME}

The frames of types A and B are interchangeable; they are for use in places where the ship's structure holds the glass on one side and generally where no bright metal finish is required around the glass, although type B, if polished, is suitable for lights where bright metal finish is wanted on one side. Type C is designed for independent or shop assembly and is intended especially for places where bright metal finish is desired around the glass on both sides. (For types B and C, see 645.21, pp. 1207-1208.)

The glass used in these lights is separately covered by standard H No. 1-1925 as to dimensions and by

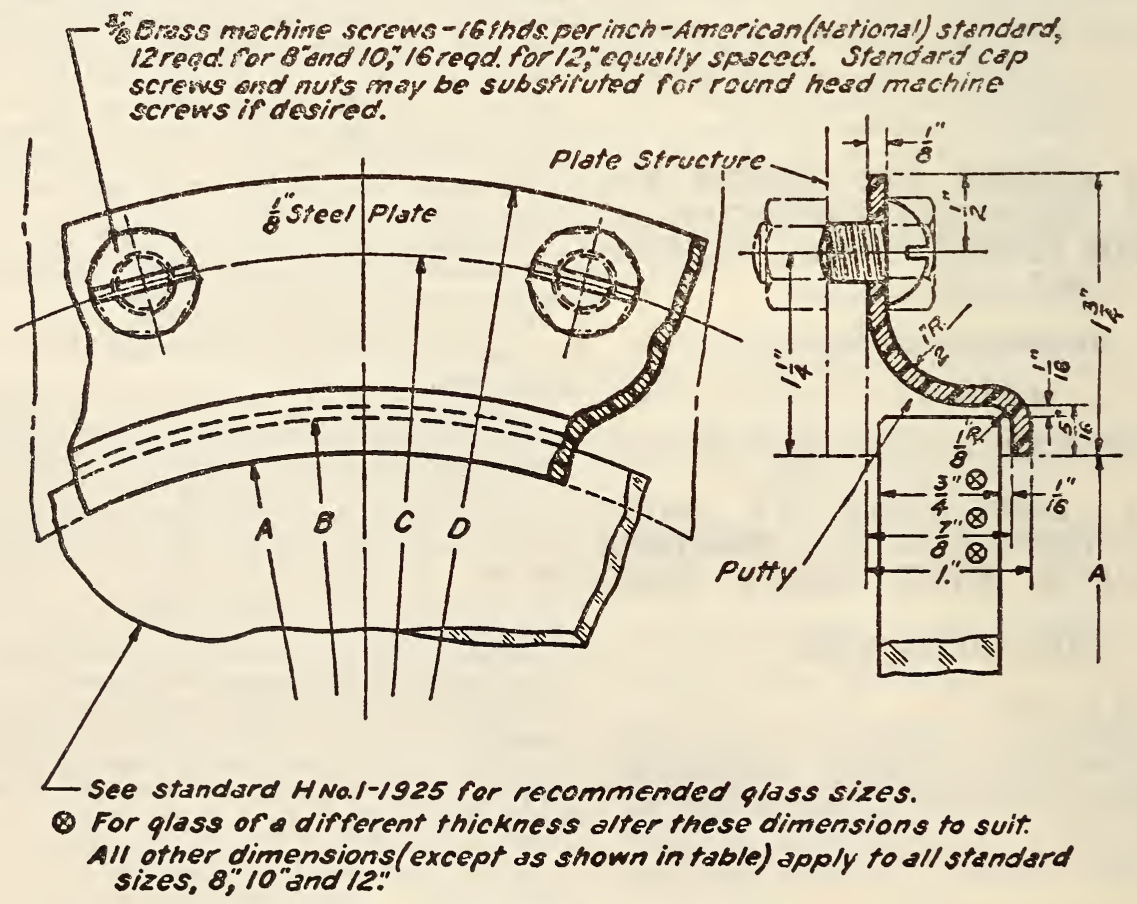

FIGURE 1063.-Fixed lights, type A, pressed steel frame 
Federal specification DD-G-451 issued by the Federal Specifications Board as to quality.

\section{[Diameters in inches]}

A-Sizes of lights

C-Pitch circle, frame screws

$\begin{array}{llll}-)- \text { Outside of frame } & \end{array}$

AMERICAN MARINE STANDARDS COMMITTEE, SHIP FITTINGS FOR DECKS AND RIGGING, H NO. 12-1926

THIMBLES FOR MANILA ROPE

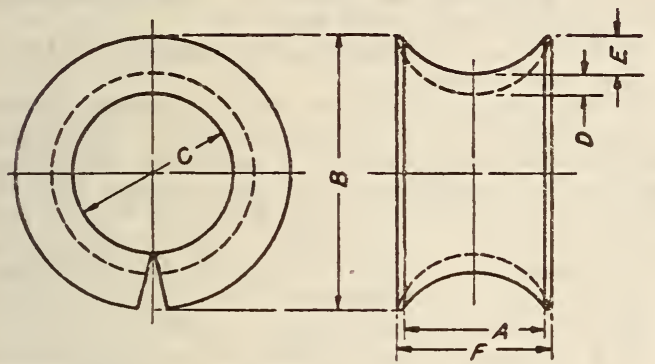

FIGURE 1064.-Thimbles for manila rope
Material.-Wrought steel, galvanized.

[All dimensions in inches]

\begin{tabular}{|c|c|c|c|c|c|c|c|}
\hline \multicolumn{2}{|c|}{ Size of rope } & \multirow{2}{*}{ Score } & \multirow[b]{2}{*}{ B } & \multirow[b]{2}{*}{ C } & \multirow[b]{2}{*}{$\mathrm{D}$} & \multirow[b]{2}{*}{$\mathbf{E}$} & \multirow[b]{2}{*}{$\mathrm{F}$} \\
\hline $\begin{array}{l}\text { Circum- } \\
\text { ference }\end{array}$ & $\begin{array}{c}\text { Approx- } \\
\text { imate } \\
\text { diam- } \\
\text { eter }\end{array}$ & & & & & & \\
\hline $\begin{array}{l}11 / 8 \\
11 / 4 \\
11 / 2 \\
13 / 4 \\
2\end{array}$ & $\begin{array}{l}3 / 8 \\
7 / 16 \\
1 / 2 \\
9 / 16 \\
5 / 8\end{array}$ & $\begin{array}{l}3 / 8 \\
7 / 16 \\
1 / 2 \\
9 / 16 \\
5 / 8\end{array}$ & $\begin{array}{l}3 / 4 \\
7 / 8 \\
1 \\
11 / 8 \\
11 / 4\end{array}$ & $\begin{array}{l}3 / 8 \\
7 / 16 \\
1 / 2 \\
17 / 32 \\
11 / 16\end{array}$ & $\begin{array}{l}5 / 64 \\
5 / 64 \\
3 / 32 \\
3 / 32 \\
7 / 64\end{array}$ & $\begin{array}{l}7 / 64 \\
1 / 8 \\
5 / 32 \\
11 / 64 \\
11 / 64\end{array}$ & $\begin{array}{l}7 / 16 \\
1 / 2 \\
9 / 16 \\
5 / 8 \\
11 / 16\end{array}$ \\
\hline $\begin{array}{l}21 / 4 \\
21 / 2 \\
23 / 4 \\
3 \\
31 / 4\end{array}$ & $\begin{array}{l}3 / 4 \\
13 / 16 \\
7 / 8 \\
1 \\
11 / 16\end{array}$ & $\begin{array}{l}11 / 16 \\
3 / 4 \\
1^{7 / 8} \\
13 / 16\end{array}$ & $\begin{array}{l}13 / 8 \\
11 / 2 \\
13 / 4 \\
2 \\
21 / 4\end{array}$ & $\begin{array}{l}3 / 4 \\
27 / 32 \\
1^{15 / 32} \\
15 / 16\end{array}$ & $\begin{array}{l}7 / 64 \\
1 / 8 \\
1 / 8 \\
9 / 64 \\
9 / 64\end{array}$ & $\begin{array}{l}13 / 64 \\
13 / 64 \\
1 / 4 \\
9 / 32 \\
21 / 64\end{array}$ & $\begin{array}{l}3 / 4 \\
7 / 8 \\
1 \\
11 / 8 \\
15 / 16\end{array}$ \\
\hline $\begin{array}{l}33 / 4 \\
41 / 4 \\
41 / 2 \\
5 \\
51 / 2\end{array}$ & $\begin{array}{l}11 / 4 \\
13 / 8 \\
11 / 2 \\
15 / 8 \\
13 / 4\end{array}$ & $\begin{array}{l}11 / 4 \\
17 / 16 \\
11 / 2 \\
15 / 8 \\
13 / 4\end{array}$ & $\begin{array}{l}21 / 2 \\
23 / 4 \\
3 \\
31 / 4 \\
31 / 2\end{array}$ & $\begin{array}{l}115 / 32 \\
15 / 8 \\
113 / 16 \\
131 / 32 \\
21 / 8\end{array}$ & $\begin{array}{l}5 / 32 \\
11 / 64 \\
11 / 64 \\
3 / 16 \\
3 / 16\end{array}$ & $\begin{array}{l}23 / 64 \\
25 / 64 \\
27 / 64 \\
29 / 64 \\
1 / 2\end{array}$ & $\begin{array}{l}13 / 8 \\
19 / 16 \\
15 / 8 \\
13 / 4 \\
115 / 16\end{array}$ \\
\hline $\begin{array}{l}6 \\
61 / 2 \\
7 \\
71 / 2 \\
8\end{array}$ & $\begin{array}{l}2 \\
21 / 16 \\
21 / 4 \\
21 / 2 \\
25 / 8\end{array}$ & $\begin{array}{l}17 / 8 \\
2 \\
25 / 16 \\
21 / 2 \\
23 / 4\end{array}$ & $\begin{array}{l}33 / 4 \\
4 \\
41 / 2 \\
5 \\
51 / 2\end{array}$ & $\begin{array}{l}29 / 32 \\
27 / 16 \\
23 / 4 \\
31 / 16 \\
336\end{array}$ & $\begin{array}{l}7 / 32 \\
7 / 32 \\
1 / 4 \\
17 / 64 \\
9 / 32\end{array}$ & $\begin{array}{l}33 / 64 \\
9 / 16 \\
5 / 8 \\
45 / 64 \\
25 / 32\end{array}$ & $\begin{array}{l}21 / 8 \\
21 / 4 \\
29 / 16 \\
23 / 4 \\
3\end{array}$ \\
\hline
\end{tabular}




\section{0-629 FERRO-ALLOYING ORES AND METALS, ALLOY STEELS, AND ALLOY-STEEL MANUFACTURES}

621. FERRO-ALLOYING ORES AND

621.1 FERRO-ALLOYING ORES.

621.11 CHROMIUM ORES.

AMERICAN SOCIETY FOR TESTING MA-
TERIALS, STANDARD METHODS OF
ULTIMATE CHEMICAL ANALYSIS OF
REFRACTORY MATERIALS, INCLUD-
ING CHROME ORES AND CHROME
BRICK, DESIGNATION C18-21, 1921

REFRACTORY MATERIALS, CHEMICAL ANALYSIS

(As implied by the title, this specification also includes the chemical analysis of refractory nonmetallic materials, such as lime and magnesia.)

Prefatory NOTE.-It will be understood that the making of a complete silicate analysis is a difficult procedure requiring a wide knowledge of the chemistry involved in the operations, and a thorough training in carrying out the work. A skilled analyst of good training is therefore required to do the work. The descriptions here given cover the vital points of procedure, but frequent reference in regard to the details of the various manipulations must be made to Bulletin No. 422, United States Geological Survey on Analysis of Silicate and Carbonate Rocks, by W. F. Hillebrand; also, to Treatis on the Ceramic Industries, vol. 1, 1913, by J. W. Mellor; and to sim lar publications.

\section{GENERAI REFRACTORIES}

General Considerations

1. The sample shall be crushed in a hardened toolsteel mortar, using a pestle of the same sort. Fine grinding shall be done in an agate mortar, either by hand, or by a mechanical sample grinder of the McKenna, Carling, or similar type, so constructed as to prevent the introduction of impurity.

2. Moisture shall be determined in the sample in its ordinary air-dried condition, and all other percentage composition shall be calculated to a moisture-free basis. Whenever a sample is weighed out for any determination, a moisture determination shall also be made. If preferred, the sample may be dried in a weighing bottle, from which the required samples shall be weighed out.

3. In all cases, check determinations shall be made, and the results shall be redetermined if satisfactory checks are not obtained. It shall be considered satisfactory if the differences between check determinations do not exceed the following limits:

For silica or other constituent amounting to 30 per cent or
over.
For alumina or other constituent amounting to $10-30$
per cent
For any other constituent amounting to under 10 per
cent

These figures are stated in terms of the whole sample as 100 per cent.

\section{Solutions Required}

4. The acids referred to as concentrated shall be of approximately the following specific gravities:

Hydrochloric Acid ( $\mathrm{HCl}$ )

Sulphuric Acid $\left(\mathrm{H}_{2} \mathrm{SO}_{4}\right)$ 1.19

Nitric Acid ( $\left.\mathrm{HNO}_{3}\right)$
5. Dissolve $1.5 \mathrm{~g}$ of ammonium carbonate in $50 \mathrm{c}$ c of cold water.

6. Dissolve $107 \mathrm{~g}$ of $\mathrm{NH}_{4} \mathrm{Cl}$ in $1,000 \mathrm{c}$ c of warm water.

7. Neutralize 20 c c of concentrated $\mathrm{HNO}_{3}$ with $\mathrm{NH}_{4} \mathrm{OH}$ and dilute to 1,000 c c. Test with litmus; the solution shall not be acid.

8. Dissolve $1 \mathrm{~g}$ of ammonium oxalate in $50 \mathrm{c} \mathrm{c}$ of water, heating gently.

9. The strength of the final standard titania solution shall be $1 \mathrm{c} c=0.0001 \mathrm{~g} \mathrm{TiO}_{2}$. To prepare the solution weigh out in a platinum crucible an amount of potassium titanium fluoride $\left(\mathrm{K}_{2} \mathrm{TiF}_{6}\right)$ sufficient to make from $1 / 2$ to 1 liter of "stock solution" in which 1 c c $=0.001$ g $\mathrm{TiO}_{2}$. Evaporate several times with $\mathrm{H}_{2} \mathrm{SO}_{4}$ without taking to dryness, thus driving out all fluorine. Take up the residue with water containing enough $\mathrm{H}_{2} \mathrm{SO}_{4}$ to make at least 5 per cent of the solution, when finally diluted to the standard solution strength. To standardize the stock solution take out two $50 \mathrm{c}$ c portions, dilute, boil, and precipitate with ammonia. Filter, wash with hot water until free from alkali, ignite, blast, and weigh. The duplicate should check very closely. From the weight of titania thus determined calculate the strength of the stock solution. The standard solution to be used is obtained from the stock solution by diluting until $1 \mathrm{c} \mathrm{c}=0.0001 \mathrm{~g}$ $\left(\mathrm{TiO}_{2}\right)$.

10. Dissolve about $0.4 \mathrm{~g}$ (a little less) of $\mathrm{KMnO}_{4}$ in 1 liter of water and check against an iron solution of known purity and strength. Dilute the solution until $1 \mathrm{c} \mathrm{c}=0.001 \mathrm{~g} \mathrm{Fe}_{2} \mathrm{O}_{3}$.

The ordinary chemical iron wire is not sufficiently pure for this use. If preferred the solution may be standardized against sodium oxalate, a pure form of which is prepared especially for such work by the Bureau of Standards, Washington, D. C.

\section{Methods}

11. Moisture.-To determine moisture, heat approximately $1 \mathrm{~g}$ of the sample at a temperature not under $105^{\circ}$ nor over $110^{\circ} \mathrm{C}$., to constant weight.

12. Loss on ignition.-To determine loss on ignition, heat about $1 \mathrm{~g}$ of the sample over a blast lamp or in an electric furnace at $900^{\circ}$ to $1,000^{\circ} \mathrm{C}$., to constant weight. Ignition loss may be determined in the sample from which the moisture has been removed. The percentage of ignition loss is calculated to a moisture-free basis.

13. Silica.-To determine silica, weigh out approximately $0.5 \mathrm{~g}$ of the sample and mix with $5 \mathrm{~g}$ of $\mathrm{Na}_{2} \mathrm{CO}_{3}$. Put a little $\mathrm{Na}_{2} \mathrm{CO}_{3}$ in the bottom of the crucible before introducing the mixture, and then cover the mixture with a little more $\mathrm{Na}_{2} \mathrm{CO}_{3}$. Fuse over a Meker burner or blast lamp until complete solution is obtained. Cool the fusion and as it solidifies, rotate the crucible to spread the mass up the side walls. Dissolve the fusion in about $100 \mathrm{c}$ c of water in a platinum or porcelain evaporating dish. Add about $20 \mathrm{c}$ c of concentrated $\mathrm{HCl}$, introducing it slowly by means of a pipette, keeping the dish covered with a watch glass. Evaporate to dryness and heat until the fumes of $\mathrm{HCl}$ are gone. Add about $5 \mathrm{c} \mathrm{c}$ of concentrated $\mathrm{HCl}$ and 30 to $40 \mathrm{c}$ c of water. Warm on a water bath for 10 to 15 minutes and break up the lumps. Decant the clear liquid onto a filter paper and collect the filtrate in a $400 \mathrm{c}$ c beaker. Add more $\mathrm{HCl}$ and 
water to the evaporating dish, warm again and decant. Repeat this a third time.

Finally transfer the contents of the dish to the filter paper. Wash with cold water until silver nitrate shows no chlorides to be left. Transfer the filtrate to the original evaporating dish, evaporate again to dryness, take up with a little $\mathrm{HCl}$ and water, transfer to a new filter paper and wash as before. Transfer both silica precipitates to a platinum crucible. Ignite carefully over a Bunsen flame until the filter paper is burned off, then blast for about 30 minutes; cool and weigh. Repeat blasting for 5 minutes, weigh again and repeat until constant weight is obtained.

To the residue in the crucible add about $5 \mathrm{c} c$ of water and 5 drops of $\mathrm{H}_{2} \mathrm{SO}_{4}$. Add $\mathrm{HF}$ drop by drop at first, and then slowly until the crucible is almost one-half filled. Warm on a hot plate until almost dry, add 2 or $3 \mathrm{c}$ c more of $\mathrm{HF}$ and evaporate to dryness. Heat the crucible to bright redness and then blast for 5 minutes. Cool and weigh and repeat blasting to constant weight. The loss in weight from the original silica residue represents the actual silica content $\left(\mathrm{SiO}_{2}\right)$, except for that part of silica which is later recovered from alumina, etc. The residue from the $\mathrm{HCl}$ evaporation is left in the crucible and the total precipitate of alumina, etc., is added to this same crucible in which it is ignited and weighed.

14. Alumina--Regarding the determination of alumina, reference should be made to Scientific Paper No. 286 of the Bureau of Standards by Doctor Blum on Determination of Alumina as Oxide.

To determine alumina, to the filtrate from the silica determination, add about $10 \mathrm{c}$ e of $\mathrm{NH}_{4} \mathrm{Cl}$ solution and heat to boiling. Then add $\mathrm{NH}_{4} \mathrm{OH}$ very slowly and with constant stirring until there is a slight excess. The $\mathrm{NH}_{4} \mathrm{OH}$ must be free from $\mathrm{CO}_{2}$. Filter hot and rapidly, and wash four times by decantation with hot solution of ammonium nitrate. Carefully wash the precipitate from the filter paper into a beaker, using hot water. Dissolve the precipitate in hot dilute $\mathrm{HCl}$. Repeat the precipitation with $\mathrm{NH}_{4} \mathrm{Cl}$ and $\mathrm{NH}_{4} \mathrm{OH}$. Decant four times as before, using hot ammonium nitrate. Transfer to the filter paper and wash with hot ammonium nitrate until the washings are free from chlorides when tested by silver nitrate. Evaporate the filtrate nearly to dryness, add a little $\mathrm{NH}_{4} \mathrm{OH}$ and continue the evaporation. Keep the solution alkaline to coagulate any iron and aluminum hydroxides. Transfer the precipitate to a filter paper and wash thoroughly. Transfer the moist filter paper to the platinum crucible containing the residue from the silica. Burn off the filter paper and blast the precipitate. Repeat blasting and weighing to a constant weight.

15. Iron oxide. - To determine iron oxide, fuse the ignited alumina precipitate with about six times its weight of potassium pyrosulfate or potassium bisulfate. Avoid sputtering. Heat carefully to redness and continue heating until the residue is all dissolved. Cool. Dissolve in warm water and add about $10 \mathrm{c}$ c of dilute $\mathrm{H}_{2} \mathrm{SO}_{4}(1: 5)$. Evaporate to a small volume. Then heat to a higher temperature until copious fumes of $\mathrm{H}_{2} \mathrm{SO}_{4}$ are evolved. Sufficient $\mathrm{H}_{2} \mathrm{SO}_{4}$ should be present to form a pasty mass when cooled. Take up with water, filter off, wash, ignite and weigh the silica as before and evaporate with $\mathrm{HF}$ as before. The loss in weight is extra silica to be added to the original silica determination and subtracted from the alumina. Fuse the residue with a little potassium pyrosulfate or potassium bisulfate, and add the solution of this cake to the main solution. Transfer the total filtrate to a 250-c c graduated flask and dilute to 250 c c, mixing thoroughly. Draw off $50 \mathrm{c}$ c of this solution and reserve for titania determination. Pass the remaining $200 \mathrm{c}$ c through a Jones reductor, or otherwise reduce the solution with pure zinc and $\mathrm{H}_{2} \mathrm{SO}_{4}$. To the solution add $3 \mathrm{c}$ c of 10 per cent $\mathrm{CuSO}_{4}$ solution and titrate with standard $\mathrm{KMnO}_{4}$ solution ( $1 \mathrm{c} \mathrm{c}$ equivalent to $0.001 \mathrm{~g} \mathrm{Fe}_{2} \mathrm{O}_{3}$ ), until a faint pink tinge is seen. If Jones reductor or similar apparatus is used, subtract the $\mathrm{KMnO}_{4}$ equivalent, which has previously been determined on blank samples run through the apparatus. From the result, calculate the iron as $\mathrm{Fe}_{2} \mathrm{O}_{3}$.

16. Titania.-To determine titania, place in a small Nessler tube the $50 \mathrm{c}$ c of filtrate previously set aside for titania determination, and fill the tube up to the graduation mark. Add $1 \mathrm{c} \mathrm{c}$ of $\mathrm{H}_{2} \mathrm{O}_{2}$ (free from fluorine) and shake well. The color of this solution is now compared in any standard color comparator with the color of a known standard solution, preferably of such strength that $1 \mathrm{c} c$ equals $0.0001 \mathrm{~g}$ of titanium dioxide $\left(\mathrm{TiO}_{2}\right)$. To make the comparison, put $10 \mathrm{c} c$ of this standard solution into a second small Nessler tube and dilute with water from a burette until the color is matched. Note the amount of water added and calculate the percentage of $\mathrm{TiO}_{2}$ in the sample.

17. Lime.-To determine lime, evaporate the filtrate from the alumina precipitation to about 250 c c. While still boiling add about $5 \mathrm{c} c$ of acetic acid. Meanwhile, dissolve about $1 \mathrm{~g}$ of oxalic acid in a little hot water and add to the solution. In about five minutes add a slight excess of $\mathrm{NH}_{4} \mathrm{OH}$ and boil until precipitation is complete. Cool thoroughly and filter. Wash three times by decantation with dilute $\mathrm{NH}_{4} \mathrm{OH}(1: 10)$ or a 1 per cent solution of ammonium oxalate. Dissolve the precipitate by adding about $50 \mathrm{c} \mathrm{c}$ of dilute $\mathrm{HNO}_{3}(1: 5)$. Again add a slight excess of $\mathrm{NH}_{4} \mathrm{OH}$ and a few drops of oxalic-acid solution. Boil well and let stand a couple of hours to cool. Filter and wash as before. Transfer the precipitate to a platinum crucible, carefully burn off the paper and ignite over a blast lamp for about 10 minutes. Cool and weigh quickly. Repeat the blasting to constant weight. The increase in weight of crucible is calcium oxide.

18. Magnesia. - To determine magnesia, add to the filtrate from lime precipitation about $2 \mathrm{~g}$ of sodium-ammonium-phosphate dissolved in $15 \mathrm{c} \mathrm{c}$ of water. Stir vigorously and while stirring add drop by drop about one-third of the volume of the solution of $\mathrm{NH}_{4} \mathrm{OH}$. Let stand 12 to 24 hours. Filter and wash the precipitate with dilute $\mathrm{NH}_{4} \mathrm{OH}(1: 10)$. Discard the filtrate. Wash the precipitate until the washings show no discoloration when tested with silver nitrate. Redissolve the precipitate in warm dilute $\mathrm{HNO}_{3}(1: 5)$, using about $50 \mathrm{c} \mathrm{c}$, and collect in a beaker. Precipitate and wash as before. Collect the precipitate on a Gooch asbestos crucible. Dry slowly and then heat over a Meker burner for about 10 minutes; cool and weigh. Re-ignite to constant weight.

The $\mathrm{Mg}_{2} \mathrm{P}_{2} \mathrm{O}_{7}$ is never pure, being contaminated by small amounts of calcium, barium, aluminum, iron, manganese, etc., but in the analysis of refractories this error is negligible, and correction for these impurities is not required unless previously agreed upon.

19. Alkalies.-To determine alkalies, the J. Lawrence Smith method shall be used. Weigh out about $0.5 \mathrm{~g}$ of the sample and mix well with $0.5 \mathrm{~g}$ of $\mathrm{NH}_{4} \mathrm{Cl}$ and $3 \mathrm{~g}$ of $\mathrm{CaCO}_{3}$. Transfer to a platinum crucible, placing about $0.5 \mathrm{~g}$ of $\mathrm{CaCO}_{3}$ in the bottom of the crucible and a similar amount over the top of the mixture. Heat gently over a low flame for about 15 minutes to volatilize $\mathrm{NH}_{4} \mathrm{Cl}$. Then raise the temperature until the lower threequarters of the crucible is dull red. Hold this temperature for about one hour. Cool, take up with about $50 \mathrm{c}$ c of water and heat over a water bath, 
adding water to replace that lost by evaporation. Break up any lumps with a small pestle. Decant the clear liquid through a filter paper and wash four times by decantation. Then transfer the residue to the filter. Wash until silver nitrate shows only a very faint turbidity. To the filtrate add $\mathrm{NH}_{4} \mathrm{OH}$ and ammonium carbonate and heat to boiling. Filter and again digest the precipitate with $\mathrm{NH}_{4} \mathrm{OH}$ and ammonium carbonate. Filter and allow the filtrate to collect with the previous filtrate, then evaporate to dryness in a platinum or porcelain dish. Remove ammonium salts by gentle ignition in a moving flame. Treat with water and remove the last trace of lime by adding ammonium oxalate to the boiling solution and let stand over night. Filter, evaporate to dryness, ignite gently and allow to cool. Then moisten the residue with $\mathrm{HCl}$, evaporate again to dryness, ignite gently and weigh. Dissolve in water. Ignite and weigh any insoluble residue, deducting this from the alkali chlorides.

Dilute the solution of the mixed chlorides and add hydrochloroplatinic acid in amount equal to $0.3 \mathrm{c} \mathrm{c}$ more than would be necessary if all chlorides were present as $\mathrm{NaCl}$. Evaporate to a sirupy consistency. Cool and treat with a few cubic centimeters of 80 per cent ethyl alcohol. Stir and decant through a weighed Gooch crucible. Treat again with 80 per cent alcohol and decant. Repeat washing and decantation until the alcohol leaving the Gooch crucible is colorless and the precipitate appears golden yellow, not orange. Transfer the precipitate to the Gooch crucible, wash with 80 per cent alcohol about six times and dry at $130^{\circ} \mathrm{C}$. to constant weight. This residue is potassium chloroplatinate.

The oxides are calculated as follows:

Weight of potassium chloroplatinate $\times 0.3068=\mathrm{KCl}$.

Weight of potassium chloroplatinate $\times 0.1941=\mathrm{K}_{2} \mathrm{O}$.

Total chlorides $-\mathrm{KCl}=\mathrm{NaCl}$

$\mathrm{NaCl} \times 0.5303=\mathrm{Na} 2 \mathrm{O}$

\section{CHROME ORES AND CHROME BRICK}

20. The sample shall be secured after the manner of any of the approved methods of sampling. If from ores or chrome-bearing refractories, it shall not be less than 10 pounds, in small nut-size particles when sent to the laboratory. If from chrome brick, the sample shall consist of a small portion from the interior of from 15 to 25 bricks. This 10 -pound sample shall be crushed to pass a No. 20 sieve ${ }^{93}$ and quartered until a sample of $100 \mathrm{~g}$ is secured, which shall be ground to pass a No. 100 sieve. ${ }^{93}$ From this portion, a smaller working sample shall be quartered and ground in an agate mortar to a very nearly gritless powder. In the case of hard burned brick or hard lump chrome ore the very fine grinding assists materially in solution of the sample.

21. Dry the fine, ground powder at $110^{\circ} \mathrm{C}$. for not less than one hour. Cool in desiccator. Fuse $0.5 \mathrm{~g}$ of the thoroughly dried sample in approximately $10 \mathrm{~g}$ of fused potassium bisulphate in a platinum crucible. The usual cautions of a bisulphate fusion should be observed; that is, the flame turned very low so that the bisulphate just melts, increasing the flame so that the bisulphate remains in a liquid condition, very gradually increasing until the crucible reaches a dull red heat. The object of the slow heating is to retain the sulphuric acid, which is the effective part of the flux. The most refractory ores should be thoroughly fused in one and one-half to two hours. Remove the crucible from the burner and allow to cool in the air. The bisulphate melt will shrink from the crucible and may be removed

${ }^{23}$ For detailed specifications for these sieves, see the standard pecifications for sieves for testing purposes, A. S. T. M. E11. (See 645.31 , p. 1226.) easily in one cake. Dissolve the fusion in 200 c c hot $\mathrm{HCl}(1: 1)$, boiling until the solution acquires a clear, bright green color. Transfer the solution to a porcelain evaporating dish and evaporate to dryness on a water bath. Bake at exactly $120^{\circ} \mathrm{C}$. until free from hydrochloric acid fumes. Moisten with hot $\mathrm{HCl}(1: 1)$, evaporate on water bath and bake a second time at $120^{\circ} \mathrm{C}$. Dissolve in $200 \mathrm{c} \mathrm{c}$ hot dilute $\mathrm{HCl}(1: 1)$, and filter out the silica. Ignite and weigh the silica in a platinum crucible. Add two drops concentrated $\mathrm{H}_{2} \mathrm{SO}_{4}$ and sufficient $\mathrm{HF}$ to remove all silica. Evaporate, ignite and weigh. The difference gives the amount of silica present in the sample. Should there be any appreciable residue left in the crucible it should be fused with a smail amount of fused $\mathrm{KHSO}_{4}$ and added to the filtrate from the silica.

Dilute the filtrate from the silica to about $500 \mathrm{c} \mathrm{c}$ and precipitate iron, aluminum, chromium, and titanium, if present, with $\mathrm{NH}_{4} \mathrm{OH}$, using care to have not more than two drops of $\mathrm{NH}_{4} \mathrm{OH}$ in excess in order to avoid solution of chromium and aluminum hydroxide in the alkaline solution. On account of the relatively large amount of magnesium that is usually present in chrome ores and chrome brick it is advisable to dissolve the precipitate in hot dilute $\mathrm{HCl}$ and precipitate a second time. Filter out the combined hydroxides and dissolve them on the filter paper with $\mathrm{HNO}_{3}$, catching the solution in a porcelain casserole. Add a few crystals of $\mathrm{KClO}_{3}$ and evaporate the nitric solution, adding a crystal of $\mathrm{KClO}_{3}$ from time to time until the chromium is completely oxidized, which is indicated by a change from a dull green to a brilliant orange color. Dilute this solution with hot water and again precipitate with $\mathrm{NH}_{4} \mathrm{OH}$, filtering as before. The filtrate contains a large percentage of the chromium, while the residue on the filter consists of iron and aluminum hydroxides and titanium, if present. Repeat the oxidation two or three times until the filtrate from the iron and aluminum hydroxide precipitation shows no further traces of chromium. Concentrate and combine the filtrates containing chromium to approximately $500 \mathrm{c}$ c. Add $\mathrm{H}_{2} \mathrm{SO}_{3}$ until the liquid changes from a bright orange to a brilliant green color and heat to boiling. Add $\mathrm{NH}_{4} \mathrm{OH}$ to precipitate chromium as hydroxide, having not more than two drops in excess, as statcd above. Filter, wash well with hot water, ignite, and weigh as $\mathrm{Cr}_{2} \mathrm{O}_{3}$. The iron and aluminum and titanium hydroxides are ignited and weighed in a platinum crucible. Fuse the combined oxides with a small amount of $\mathrm{KHSO}_{4}$, following the same precautions as mentioned above. Dissolve the fusion in dilute $\mathrm{H}_{2} \mathrm{SO}_{4}$, reduce with $\mathrm{H}_{2} \mathrm{~S}$ and titrate the iron with standard permanganate solution. Determine the titanium present by the colorimetric method, as described in section 16 . Subtract the combined amounts of $\mathrm{Fe}_{2} \mathrm{O}_{3}$ and $\mathrm{TiO}_{2}$ from the total weight of the three oxides and thus determine $\mathrm{Al}_{2} \mathrm{O}_{3}$. If titanium is absent, reduce the iron with metallic zinc and titrate with $\mathrm{K}_{2} \mathrm{MnO}_{4}$. which is a little quicker method than the $\mathrm{H}_{2} \mathrm{~S}$ reduction.

To the combined filtrates from the precipitations of iron, aluminum, chromium, and titanium hydroxides add bromine, allow to stand for a few minutes and then add sufficient $\mathrm{NH}_{4} \mathrm{OH}$ to precipitate the manganese hydroxide. Boil to concentrate the manganese hydroxide if any be present. Filter, ignite, and weigh as $\mathrm{Mn}_{3} \mathrm{O}_{4}$. In the filtrate from the manganese add $\mathrm{NH}_{4} \mathrm{OH}$ and $\left(\mathrm{NH}_{4}\right)_{2} \mathrm{C}_{2} \mathrm{O}_{4}$. Heat to boiling and when the calcium oxide has settled, filter with the usual precautions. The calcium oxide may be determined by ignition or by the volumetric method. (Refer to the determination of calcium oxide as described in sec. 17.) 
Evaporate the filtrate from the calcium oxalate to dryness with $\mathrm{HNO}_{3}$ to remove the excess of ammonium salts. Dissolve the residue in hot water, filter, allow to cool and determine $\mathrm{MgO}$ as described in section 18 .

Determine alkalies by the J. Lawrence Smith Method, as described in section 19.

RAPID ROUTINE METHOD FOR THE ANALYSIS OF CHROME BRICK AND CHROME ORE

22. The sample shall be secured after the manner of any of the approved methods of sampling. If from ores or chrome-bearing refractories it shall not be less than 10 pounds in small nut-sized particles when sent to the laboratory. If from chrome brick, the sample shall consist of a small portion from the interior of from 15 or 25 bricks. This 10-pound sample shall be crushed to pass a No. 20 sieve ${ }^{94}$ and quartered, until a 4-ounce sample is secured, which shall be ground to pass a No. 100 sieve. ${ }^{94}$ From this portion a smaller working sample shall be quartered and ground in an agate mortar to a very nearly gritless powder. In the case of hard burned brick or hard lump chrome ore the very fine grinding assists materially in solution of the sample. Dry the fine, ground powder at $110^{\circ} \mathrm{C}$. for not less than 1 hour. Cool in desiccator.

23. Chromium.-Fuse $0.5 \mathrm{~g}$ of the sample in 8 to $10 \mathrm{~g}$ of sodium peroxide in a nickel or porcelain crucible over a Bunsen burner, being careful not to heat too strongly. When fused, leach out with cold water, boil 15 minutes to decompose any sodium peroxide. Cool and add $100 \mathrm{c}$ c of $\mathrm{H}_{2} \mathrm{SO}_{4}$ (1:1). Cool to tap-water temperature and titrate with ferrous ammonium sulfate and potassium permanganate. Time 30 to 45 minutes.

24. Silica and iron.-Fuse $0.5 \mathrm{~g}$ of the sample in a nickel crucible over a Bunsen burner. Leach out with cold water, make acid with $\mathrm{HCl}$ and evaporate to dryness. Take up with $200 \mathrm{c}$ c water and add $10 \mathrm{c} \mathrm{c} \mathrm{HCl}$. Filter off silica and wash well with hot water and hot dilute $\mathrm{HCl}$. For high silica a second evaporation is necessary. The filtrate or combined filtrate is made alkaline with sodium hydroxide and about $5 \mathrm{c} \mathrm{c}$ of bromine added. The mass is allowed to digest for one-half hour on a steam bath. This oxidizes the iron and chromium, precipitates the iron, and dissolves the chromium and aluminum. After precipitating the iron twice it is filtered off on an asbestos plug and washed well with hot water. This precipitate of iron may be dissolved off the plug with hot $\mathrm{HCl}(1: 1)$ and determined by the stannous chloride reduction, and permanganate method.

A blank should be run in conjunction with this method and deductions made when necessary to determine the purity of the chemicals used.

\section{UNITED STATES GOVERNMENT, FEDERAL SPECIFICATIONS BOARD, FEDERAI SPECIFICATION FOR GROUND CHROME ORE, NO. 125, APRIL 1, 1924. (CUR- RENT DESIGNATION: TT-C-251)}

\section{CHROME ORE}

\section{GRADE}

1. This specification covers one grade of ground chrome ore.

${ }^{94}$ For detailed specifications for these sieves, see the standard specifications for sieves for testing purposes, A. S. T. M. E11. (See 645.31,
p. 1226.)
MATERIAI

2. The material shall be as required in the following paragraphs.

\section{GENERAL REQUIREMENTS}

3. Chrome ore furnished under these specifications shall be ground sufficiently fine to insure its suitability for use as a refractory for open-hearth furnaces.

\section{DETAIL REQUIREMENTS}

4. (a) Ground chrome ore shall contain not less than 45.0 per cent chromic oxide $\left(\mathrm{Cr}_{2} \mathrm{O}_{3}\right)$.

(b) An analysis of each shipment of ground chrome ore shall be furnished by the contractor showing the percentage of chromic oxide. The results of this analysis shall conform to the requirements specified in paragraph $4(a)$.

(c) Ground chrome ore not complying with all the requirements of this specification and of the contract or purchase order shall be rejected and the contractor notified.

\section{TESTS AND METHODS OF INSPECTION}

5. (a) Check analyses may be made by the inspector, or through him by any Government laboratory or other designated representative and without cost to the contractor. The chemical composition thus determined shall conform to the requirements specified in paragraph $4(a)$.

(b) Chrome ore shall be sampled at the contractor's plant, whenever practicable, otherwise at the point of delivery.

(c) Representative samples shall be obtained from at least 10 per cent of the containers in a lot or shipment, these individual samples crushed to $1 / 2$-inch size or smaller, thoroughly mixed, and the resultant mixture, quartered down to a 5-pound sample, shall be sent to the designated laboratory in an air-tight and moisture-proof container for analysis.

621.12 MANGANESE ORES.

UNITED STATES GOVERNMENT, DEPARTMENT OF COMMERCE, BUREAU OF STANDARDS, STANDARD SAMPLES, C25 AND SUPPLEMENT, 1927

\section{MANGANESE ORE, SAMPLE NO, 25b}

(This standard sample is prepared and sold by the Bureau of Standards for use by industrial organizations and others for checking the accuracy of analyses of manganese ores, etc. It is accompanied by a certificate giving its analysis as regards manganese and available oxygen.)

\section{UNITED STATES GOVERNMENT, DEPART- MENT OF COMMERCE, BUREAU OF STANDARDS, ANALYZED IRON AND MANGANESE ORES, METHODS OF ANALY- SIS, C26, 1921}

\section{METHODS OF ANALYSIS OF MANGANESE AND IRON ORES}

(Circular 26 contains methods of analysis which have been found satisfactory at the Bureau of Standards and which may be used in the analysis of the standard sample No. $25 \mathrm{~b}$ for manganese ore and standard samples No. 26 , No. 27 a, No. 28 , and No. 29, for crescent, Sibley, Norrie, and magnetite iron ores.) 


\section{UNITED STATES GOVERNMENT, FEDERAL SPECIFICATIONS BOARD; FEDERAL SPECIFICATION FOR MANGANESE ORE, QQ-M-71, MAY 3, 1932.}

\section{MANGANESE ORE}

\section{GENERAL REQUIREMENTS}

1. Federal specification QQ-M-151 (see 600.0, p. 3), general specifications for metals, forms a part of this specification.

2. Manganese ore furnished under these specifications shall consist of pyrolusite or psilomelane. It shall be supplied in lumps, crushed, or screened to size, as specified in the contract or purchase order.

\section{DETAIL REQUIREMENTS}

3. (a) Manganese ore shall contain not less than 40.0 per cent manganese.

(b) An analysis of each shipment of manganese ore shall be furnished by the contractor, showing the percentage of manganese. The results of this analysis shall conform to the requirements specified in paragraph $3(a)$.

(c) Manganese ore not complying with all the requirements of this specification and of the contract or purchase order shall be rejected, and the contractor notified.

\section{TESTS AND METHODS OF INSPECTION}

4. (a) Check analyses may be made by the inspector, or through him by any Government laboratory or other designated representative, and without cost to the contractor. The chemical composition thus determined shall conform to the requirements specified in paragraph $3(a)$.

(b) Manganese ore shall be sampled at the contractor's plant whenever possible, otherwise at the point of delivery.

(c) Representative samples shall be obtained from at least 10 per cent of the containers in a lot or shipment, consisting of at least 4 ounces for every 150 pounds, in the case of finely granulated or screened material. In the case of lump material, at least 5 pounds shall be taken for every 1,000 pounds or fraction thereof in the lot or shipment. These individual samples shall be crushed to onehalf inch or smaller, thoroughly mixed, and the resultant mixture quartered down to a 5-pound sample, which shall be sent to the designated laboratory in an air-tight and moisture-proof container for analysis.

\subsection{SILICON ORES.}

(No nationally recognized specifications available.)

\subsection{FERRO-ALLOYS.}

\subsection{GENERAL ITEMS.}

\section{AMERICAN SOCIETY FOR TESTING MATE- RIALS, STANDARD METHODS OF SAM- PLING FERRO-ALLOYS, DESIGNATION A103-27, $192 \%$}

\section{SAMPLING FERRO-ALLOYS}

The procedures recommended in these methods are considerably more elaborate than those in general use by the principal consumers of ferro-alloys in this country. However, the methods have been designed as standard procedure for use in important cases where large sums are often involved and they accordingly should represent the most approred modern practice.
FERROSILICON, HIGH-CARBON FERROMANGANESE, AND SMICOMANGANESE

These alloys are usually shipped in bulk in carloads and should be sampled while being loaded or unloaded. At these times the sampler can make the fairest estimate of the ratio of lump to fine material and should secure proportionate amounts of each. Frequently the percentage of fines is negligible.

While the shipment is being loaded or unloaded, samples aggregating 150 pounds or more shall be gathered from all parts of the car with due regard to the estimated ratio of lump to fine material. In breaking lumps or pigs, fragments representing the full cross section should be secured, a heary hammer or sledge being employed.

The gross sample thus obtained shall be crushed to pea size in a heavy crusher provided with smooth plates of manganese steel. The crushed product shall be repeatedly mixed and halved with a Jones divider until about 5 pounds remain. If a Jones divider is not available, the sampler may be reduced by quartering, but use of the divider is preferred. The sample shall be further reduced to approximately a size to pass a No. 10 sieve ${ }^{85}$ in laboratory rolls and again cut down by a Jones divider or by quartering to about $300 \mathrm{~g}$. This sample shall then be divided in the same way into four portions of approximately $75 \mathrm{~g}$ each and three of these shall be reserved. The fourth portion shall be pulverized until all has been passed through a No. 80 or No. 100 sieve. $^{95}$ A steel mortar with a close-fitting pestle, both of hardened allor steel, shall be used for this reduction. Rejection of any part of the sample because of failure to pass the screen is almost sure to produce error. The sample shall be thoroughly mixed, and dried for one hour at $105^{\circ}$ to $110^{\circ}$ C. It is then ready for analysis. If necessary, one-half rather than one-fourth of the 10-mesh sample shall be pulverized to pass a No. 80 or No. 100 sieve and the resulting sample divided for distribution to the interested parties.

\section{HIGH-CARBON FERROCHROMIOM, CHROMTOM METAL, LOW -} CARBON FERROMANGANESE, AND MANGANESE METAL

These alloys are usually shipped in stout kegs or barrels. Most of these products are relatively costly, and accuracy in sampling is extremely important eren when the tonnage involved is small.

If the shipment is believed to be fairly uniform in composition and contains only a small percentage of fines, a satisfactory sample can be obtained by removing the ends or cutting the sides of the packages and taking from each at least 0.5 per cent of the contents. Thus at least $2 \frac{1}{2}$ pounds would be taken from a package containing 500 pounds. If the product has been crushed to a small size, for example, $1 / 4$ to $3 / 4$ inch, the sample can be taken with a shove or scoop. If the material is in larger pieces, as is usual with high-carbon ferrochromium, the sample shall be taken by breaking fragments from a number of lumps until the requisite weight has been obtained as already described for the sampling of lumps of ferrosilicon. The gross sample shall be obtained by combining the samples taken from the individual packages and crushed and worked down in the manner described for the preceding group of alloys.

The relatively simple procedure of the preceding paragraph is justified only when experience has shown that the product being sampled contains no more than a small percentage of fines and is relatively uniform in composition. The fines usually differ somewhat in composition from the lumps and always work toward the bottom of the container

85 For detailed specifications for these sieres, see A. S. T. M. specification E 11 (645.31, p. 1226). 
while in transit. Products like tungsten powder or pulverized ferrosilicon (80 to 85 per cent $\mathrm{Si}$ ) are also liable to segregate while in transit and should be sampled in a manner to avoid error due to the accumulation of a heavy constituent in the bottom of the container. The term "relatively uniform in composition" applies to a shipment or a sublot of a shipment in which the composition of any package is approximately the same as that of any other. Frequently a shipment comprises two or more lots, each uniform in composition within itself, but differing in this respect from the other lots. In these cases, each lot shall be sampled separately. If the product is rather variable in composition or if it contains a considerable proportion of fines, all of the packages comprising the shipment or lot shall be emptied and from each shall be taken a sample amounting to at least 0.5 per cent of the contents. The percentage of fine and lump alloy in each barrel shall be estimated and the sampling conducted accordingly. In sampling a small lot which varies widely in composition within itself, it is good practice to empty all of the packages, combine the entire contents, and mix the lot thoroughly. The pile shall then be quartered repeatedly until a sample is obtained approximating 5 per cent of the weight of the entire lot. The gross sample obtained by combining samples from the individual barrels or by quartering the entire shipment shall be crushed and worked down in the manner described for the group containing ferrosilicon.

\section{LOW-CARBON FERROCHROMIUM}

Low-carbon ferrochromium is ordinarily shipped in stout kegs. The alloy usually contains about 70 per cent of chromium together with maximum contents of carbon ranging from 0.1 to 2 per cent. The combination of hardness and toughness characteristic of this product, particularly of the lower carbon grades, makes it the most difficult by far of any of the ferro-alloys to sample properly. In view of the great importance of the accurate determination of the carbon content-especially of the alloy containing a maximum of 0.1 per cent of carbon-the utmost care must be taken to avoid contamination of the sample with fragments of steel from the tools. Fortunately low-carbon ferrochromium is usually uniform in composition and free from fines.

If the alloy is known to be reasonably uniform in composition, 10 lumps shall be drawn from each package in the shipment or sublot. This can be accomplished by removing the head or cutting the side of the keg. If the composition is variable, each package of the shipment or sublot shall be emptied and about 20 lumps taken at random. The number of lumps should be varied according to the net weight of the package and the average size of the lumps found in it. One fragment about one-eighth inch in diameter shall be broken from each lump of the gross sample, using a heavy hammer and a plate of hardened alloy steel. As nearly as possible each fragment should offer a cross section of the lump from which it was taken. The resulting sample shall be crushed to approximately a size to pass a No. 10 sieve by means of a mortar and pestle of hardened alloy steel. This material shall be cut down with a Jones divider or quartered until 30 or $40 \mathrm{~g}$ remain. This quantity shall then be pulverized to pass a No. 40 sieve by means of the mortar and pestle. Especially in pulverizing the grades relatively low in carbon, an air hammer is almost indispensable. The material passing a No. 40 sieve shall be split into two or four parts according to the requirements, by means of a Jones divider or by quartering. The following procedure is recommended for those cases in which prolonged crushing has resulted in a powder which passes entirely through a No. 20 sieve but which passes a No. 40 sieve in part only. The entire sample shall be passed through a No. 20 sieve and then divided into fractions finer and coarser than No. 40. The fractions shall be weighed and preserved in separate packages upon which are recorded the percentages by weight of the two sizes. The fractions may be analyzed separately or a composite sample may be prepared for the determination of each element, by weighing and subsequently combining the proper proportions of the coarse and fine material.

\section{REQUIREMENTS FOR MORTAR AND PESTLE}

In the analysis of ferro-alloys, errors arising from containination of the sample by iron from the sampling appliances are both serious and common. A rapid and accurate method for the determination of unalloyed metallic iron in ferrochromium and in ferrosilicon is known, and by this means such contamination has been detected and measured. Contamination has often been due to iron from the mortar and pestle but can be avoided by the proper selection and treatment of the steel from which they are made. These tools are essential to the accurate sampling of ferro-alloys-especially lowcarbon ferrochromium-and when made in accordance with the following requirements are suitable for this purpose.

Chromium-nickel steel containing approximately 1.5 per cent $\mathrm{Cr}, 3$ per cent $\mathrm{Ni}$, and 0.35 per cent $\mathrm{C}$ is recommended for both tools. For the mortar a cylinder 3 by 3 in. shall be drilled axially to a diameter of $1 \frac{1}{2}$ inches and a depth of $2 \frac{1}{2}$ inches. The bottom of the hole shall be made perfectly flat and the entire inner surface brought to a smooth finish. The pestle shall be made from a rod originally $11 / 2$ inches in diameter by 5 inches long, and shall be smoothly faced and turned to a final diameter slightly less than $1 \frac{1}{2}$ inches. The mortar and pestle shall be heated for several hours in a laboratory muffle furnace at $750^{\circ}$ to $800^{\circ} \mathrm{C}$. and hardened by quenching in oil. The upper part of the pestle shall be slightly softer than the remainder in order to decrease the tendency to shatter. Both tools shall be polished with abrasive paper for the removal of scale. Such a mortar and pestle, tngether with an air hammer will pulverize alloys which will resist other equipment.

Other approved methods for the preparation of the pulverized sample from the gross sample may be substituted for the above provided they do not lead to contamination or loss of any portion of it. For example, the gross sample may be crushed entirely by rolls or in the absence of power machinery may be broken by hand to the required size. In the entire absence of power equipment the gross sample should be sent to a point where suitable facilities are availa ole.

\section{AMERICAN SOCIETY FOR TESTING MATE- RIALS, STANDARD METHODS OF CHEM- ICAL ANALYSIS OF FERRO-ALLOYS, DESIGNATION A104-2\%, 192\%}

\section{CHEMICAL ANALYSIS OF FERRO-ALLOYS \\ FERROSILICON}

Determination of Silicon by Fusion with Sodium Peroxide and Dehydration with Hydrochloric Acid

Transfer $0.5000 \mathrm{~g}$ (use $0.4000 \mathrm{~g}$ for 90 per cent silicon) of the finely powdered sample (passing a No. 150 sieve ${ }^{96}$ ) to a $50 \mathrm{c}$ c pure iron crucible made from No. 20 gage (0.32 inch thickness) ingot iron and add approximately $13 \mathrm{~g}$ of sodium peroxide. Mix thoroughly with a platinum or iron rod and carefully clean the rod of adhering particles by scraping with another rod. Cover the mixture with a layer of about $2 \mathrm{~g}$ of additional peroxide. Heat the crucible and contents on a hot plate for 5 to 10 minutes to expel any water in the peroxide which would cause spattering in the subsequent fusion.

96 For detailed specifications for this sieve see A. S. T. M. Specification E11 (645.31, p. 1226). 
Carefully fuse over a low flame by holding the crucible with a pair of tongs and slowly revolving it around the outer edge of the flame until the contents have melted down quietly. When the fusion is molten rotate the crucible carefully to stir up any unattacked particles on the bottom or sides, the crucible and contents being maintained at a low red heat. Just before completion of the fusion, which requires only three or four minutes, increase the temperature to bright redness for a minute. If the reaction has proceeded too violently, due to too rapid heating, to the use of insufficient sodium peroxide, or to the lack of thorough mixing, appreciable loss will occur and the work should be repeated.

Cover the crucible and allow it to cool until the fusion has solidified, and tap the crucible, while still warm, several times on an iron plate, which will loosen the fused mass in a solid cake. When the crucible and cake have completely cooled, transfer the cake to a large $(275 \mathrm{c} \mathrm{c})$ gold or preferably platinum dish. Cover the dish with a clock glass and cautiously add $50 \mathrm{c} \mathrm{c}$ of cold water. When the reaction ceases, wash the small amount of adhering matter in the crucible into the dish with a little water. Cool the solution and carefully add an excess of hydrochloric acid. Evaporate to dryness, preferably on a steam bath, but do not bake at a temperature above $110^{\circ} \mathrm{C}$. If platinum or gold dishes are not available for solution of the fused cake, the latter can be disintegrated with water in a pure nickel dish and the contents then transferred to a porcelain dish containing sufficient hydrochloric acid to provide an excess of acid. It is not desirable to dissolve the fusions directly in porcelain, because of the action of the alkaline solution.

When the residue is dry, allow the dish to cool. Add $10 \mathrm{c} \mathrm{c}$ of hydrochloric acid (specific gravity 1.13), cover the dish, and heat gently for a few minutes. Dilute with $100 \mathrm{c} \mathrm{c}$ of hot water, digest for a few minutes, and filter through an $11 \mathrm{~cm}$ paper. Wash about eight times with hot 5 per cent hydrochloric acid, three times with warm 5 per cent sulphuric acid, and a few times with hot water. Reserve this paper until the second evaporation has been completed.

Evaporate the filtrate to dryness and bake the covered casserole or dish at $110^{\circ} \mathrm{C}$. for one hour. Heating at a higher temperature is unnecessary and also disadvantageous. Cool, add $10 \mathrm{c}$ c of hydrochloric acid (1:1), digest on the steam bath for ten minutes, add $100 \mathrm{c}$ c of warm water, filter immediately, and wash about eight times with cold 1 per cent hydrochloric acid, twice with cold 5 per cent sulphuric acid, and a few times with cold water.

Place the paper and silica from the second dehydration in a large partially covered platinum crucible and char the paper without inflaming. Add the paper containing the silica from the first dehydration, char as before and then ignite carefully until the carbon has been burned off. Great care should be exercised in igniting the papers as the current of air produced by a burning filter is sufficient to carry finely divided silica out of the crucible. When the carbon of the filter paper has been completely burned, gradually heat the tightly covered crucible to the full heat of a blast lamp and continue the heating at $1,050^{\circ}$ to $1,100^{\circ} \mathrm{C}$. for 25 minutes. Cool in a desiccator, weigh, and check for constant weight by ten minutes' additional heating at the above temperature. Add sufficient sulphuric acid (1:1) to moisten the silica and then 15 to $20 \mathrm{c}$ c of pure hydrofiuoric acid (48 per cent). Evaporate to dryness, ignite, and weigh. The loss represents silica which contains 46.72 per cent of silicon. A blank, not omitting the same amount of hydrofluoric acid, should be carried through all stages of the operation.

Note.-The silica remaining in the filtrate after the second dehy. dration is practically negligible, averaging about 0.0008 to $0.0010 \mathrm{~g}$ in hydrochloric dehydration and 0.0010 to $0.0015 \mathrm{~g}$ in sulphuric dehydration.

\section{FERROMANGANESE}

Determination of Manganese by the Modified Bismuthate Method

\section{REAGENTS REQUIRED}

Dilute nitric acid (specific gravity 1.135).-Mix 300 c c of nitric acid (specific gravity 1.42) and $700 \mathrm{c}$ c of distilled water. Test for proper specific gravity.

Dilute nitric acid $(3: 97)$.- Mix $30 \mathrm{c}$ c of recently boiled nitric acid (specific gravity 1.42) and $970 \mathrm{c}$ c of distilled water. Add a little sodium bismuthate and shake well.

Asbestos.-Asbestos for this work should be digested in hot nitric acid (specific gravity 1.42) and then washed free from acid with distilled water.

Sodium bismuthate.-This reagent should be of 80 per cent grade and its oxidizing power tested as follows: Shake $0.5 \mathrm{~g}$ of the reagent with $4 \mathrm{~g}$ of potassium iodide and a little water in a stoppered flask. Add $15 \mathrm{c}$ c of hydrochloric acid and allow to stand in the dark, with occasional shaking until the bismuthate has entirely decomposed. Dilute to 300 c c and titrate with $0.1 N$ sodium thiosulphate, using starch as an indicator at the end. One cubic centimeter of $0.1 \quad N \quad \mathrm{Na}_{2} \mathrm{~S}_{2} \mathrm{O}_{3}$ equals $0.0140 \mathrm{~g}$ of $\mathrm{NaBiO}_{3}$.

Standard potassium permanganate solution.--See the determination of chromium in ferrochromium, given below. One cubic centimeter of $0.1 N^{\prime}$ $\mathrm{KMnO}_{4}$ equals $0.001099 \mathrm{~g}$ of manganese.

\section{METHOD}

(a) Solution of the sample

All samples should be crushed to approximately a size to pass a No. 100 sieve and dried at $105^{\circ}$ to $110^{\circ} \mathrm{C}$.

With high-carbon 80 per cent ferromanganese, dissolve $0.25 \mathrm{~g}$ of the prepared sample in $60 \mathrm{c} \mathrm{c}$ of nitric acid (specific gravity 1.42) in a 750 c c Erlenmeyer flask, provided with a clock-glass cover or trap to prevent loss by spraying.

Add 2 g of potassium nitrate and boil gently until the volume of the solution has been reduced to about $15 \mathrm{c}$ c. Cool, add $10 \mathrm{c} \mathrm{c}$ of $\mathrm{H}_{2} \mathrm{SO}_{4}(1: 1)$ and evaporate to strong fumes of sulphuric acid. Cool somewhat, dilute the solution with $200 \mathrm{c}$ c of $\mathrm{HNO}_{3}$ (specific gravity 1.135), heat to boiling, remove from the heat, and add sodium bismuthate a little at a time until a precipitate of manganese dioxide has formed. When this occurs, add sulphurous acid dropwise until the precipitate has dissolved and an excess of about $1 \mathrm{c} c$ has been provided to reduce any chromium to the trivalent form. Boil the solution about five minutes to expel oxides of nitrogen completely, dilute to $250 \mathrm{c}$ c with water, cool the flask and solution in crushed ice, and treat as described later. High-carbon ferromanganese containing 1 per cent or more of chromium may not dissolve completely in nitric acid. In this case, dilute the solution after treatment with nitric acid, filter, and fuse the washed residue with a little sodium carbonate. Dissolve the melt in a small volume of nitric acid (specific gravity 1.135), and add it to the main solution. Treat with potassium nitrate, etc., as previously described.

With low-carbon ferromanganese or manganese metal dissolve $0.2500 \mathrm{~g}$ of the sample in $250 \mathrm{c}$ c of 
nitric acid (specific gravity 1.135) in a covered 750 c c Erlenmever flask and boil the solution gently for a few minutes to expel oxides of nitrogen. Treat with bismuthate and sulphurous acid as described above.

With silico-manganese (60 to 70 per cent of manganese) and manganese-silicon (20 to 25 per cent of manganese) treat $0.3000 \mathrm{~g}$ of the former and $1.000 \mathrm{~g}$ of the latter with $5 \mathrm{c} \mathrm{c}$ of hydrofluoric acid in a large platinum dish equipped with a gold or platinum cover. (Some spiegeleisens are completely soluble in nitric acid, in which case the sample can be dissolved directly in $250 \mathrm{c}$ c of nitric acid (specific gravity 1.135). When the reaction moderates, add nitric acid (specific gravity 1.42) a few drops at a time until the sample has completely dissolved. Add $10 \mathrm{c} c$ of nitric acid (specific gravity 1.42) and evaporate to a sirupy consistency. Again add $10 \mathrm{c} \mathrm{c}$ of nitric acid (specific gravity 1.42) and evaporate as before to expel hydrofluoric acid. Dissolve the residue in nitric acid (specific gravity 1.135), transfer to a 750 c c Erlenmeyer flask, dilute with nitric acid (specific gravity 1.135 ) to $250 \mathrm{c}$ c, and treat as described before with sodium bismuthate, etc.

\section{(b) Oxidation of manganese}

If the preceding directions have been followed, the manganese will be present in a concentration of approximately $0.001 \mathrm{~g}$ per $1 \mathrm{c} \mathrm{c}$ of nitric acid (specific gravity 1.135). This concentration of manganese and nitric acid, together with a temperature of $10^{\circ}$ to $15^{\circ} \mathrm{C}$. are the conditions which insure maximum stability of the permanganic acid, formed as further described. To insure complete oxidation of the manganese to permanganic acid, it is essential that the sodium bismuthate $\left(80\right.$ per cent of $\mathrm{NaBiO}_{3}$ ) be used in the ratio of at least $26 \mathrm{~g}$ to every gram of manganese in solution. Add approximately $7.0 \mathrm{~g}$ of sodium bismuthate ( 80 per cent) to the flask, agitate briskly for one minute, dilute with $250 \mathrm{c} c$ of cold water, and filter immediately on a layer of acid-washed asbestos supported on a 2 -inch alundum or perforated porcelain plate resting in a large glass funnel. The filter can be washed free from manganese more readily if not allowed to run dry during the filtering and washing. Wash the filter and residue with cold 3 per cent nitric acid until the washings are entirely colorless and immediately treat the filtrate and washings as described in the following paragraph.

Weigh accurately $9.000 \mathrm{~g}$ of ferrous ammonium sulphate, taken from a bottle of the well-mixed salt, and add to the filtered solution of permanganic acid. Stir briskly and, as soon as the reduction is complete and the salt has dissolved, titrate the excess of ferrous salt with $0.1 \mathrm{~N}$ potassium permanganate. The manganese value of the ferrous salt is obtained in the following manner:

Two hundred and fifty cubic centimeters of nitric acid (specific gravity 1.135) contained in a $750 \mathrm{c} \mathrm{c}$ Erlenmeyer flask are treated with approximately $2.0 \mathrm{~g}$ of sodium bismuthate and the solution is vigorously agitated for one minute, diluted with $250 \mathrm{c}$ c of cold water, and immediately filtered on a layer of acid-washed asbestos as previously described. Five (5.000) grams of the ferrous ammonium sulphate are added to the filtrate and the resulting solution is immediately titrated with $0.1 \mathrm{~N} \mathrm{\textrm {KMnO } _ { 4 }}$ until a faint pink color appears. The excess of permanganate used to cause an end-point color must be determined by watching the color in another beaker containing the same amounts of acid and cold water.

Nores.-1. Chromium when less than 2 per cent does not cause any interference if the reactions are rapidly done in cold solutions. Larger amounts interfere to some extent and should be separated prior to the final oxidation with hismuthate.
2. The gravimetric determination of manganese by the pyrophos. phate method will give high values if the precipitate of manganese ammonium phosphate is allowed to stand more than 10 hours before ammonium

3. Volumetric methods such as Volhard's, Ford-Williams's, and Julian's do not permit the use of a theoretical titre. Each necessitates the use of an empirical titre obtained by titration of material of known manganese content which has been carried through all steps of the method.

\section{Determination of Carbon by the Direct-Combustion Method}

(For apparatus and reagents common to both the soda-asbestos and barium hydroxide methods of absorbing the carbon dioxide, also for factors influencing rapid combustion, as well as for the determination of carbon (carbon dioxide absorbed in soda-asbestos), and for diagram showing arrangement of apparatus, see A64 (601.20, p. 119). For the determination of carbon (carbon dioxide absorbed in barium hydroxide solution) and diagram showing apparatus for filtration, see A33 (600.1, p. 10).)

A furnace temperature of $1,050^{\circ}$ to $1,075^{\circ} \mathrm{C}$. suffices, and high-silicon material should be covered with 1 or $2 \mathrm{~g}$ of ingot iron.

Determination of Phosphorus by the Alkalimetric Method

\section{SOLUTIONS REQUIRED}

Dilute nitric acid (specific gravity 1.195).-Mix $300 \mathrm{c} \mathrm{c}$ of $\mathrm{HNO}_{3}$ (specific gravity 1.42) and $700 \mathrm{c} \mathrm{c}$ of distilled water.

Standard sodium hydroxide.-Dissolve $6.5 \mathrm{~g}$ of purified $\mathrm{NaOH}$ in $1,000 \mathrm{c}$ c of distilled water, add a slight excess of a 1 per cent solution of $\mathrm{Ba}(\mathrm{OH})_{2}$, let stand for 24 hours, decant the liquid, and standardize against Bureau of Standards standard benzoic acid as follows: Dissolve $0.6 \mathrm{~g}$ of benzoic acid in $15 \mathrm{c} c$ of alcohol in a small Erlenmever flask and titrate in the cold with phenolphthalein indicator, taking precautions to prevent access of carbon dioxide from the air during titration. The ratio of phosphorus to sodium hydroxide is taken as 1 to 23 in calculating the value of the sodium hydroxide. Protect the solution from carbon dioxide with a soda-lime tube.

Dilute ammonium hydroxiale (specific gravity $0.96)$.- Dilute $400 \mathrm{c}$ c of $\mathrm{NH}_{4} \mathrm{OH}$ (specific gravity 0.90 ) with $600 \mathrm{c}$ c of water. The strength of this solution should be established by titration or test with a hydrometer.

(Standard nitric acid and ammonium molybdate are identical with like solutions in the determination of phosphorus by the alkalimetric method in B28. (See 646.0, p. 1243.)

\section{METHOD}

Dissolve $2.000 \mathrm{~g}$ of the sample in $70 \mathrm{c} \mathrm{c}$ of nitric acid (specific gravity 1.42), evaporate to dryness, and bake for two or three hours at $120^{\circ} \mathrm{C}$. Highsilicon alloys may require the addition of a little hydrofluoric acid for complete decomposition. In this case, several evaporations with $15 \mathrm{c}$ c portions of nitric acid (specific gravity 1.42) should be made to remove hydrofluoric acid before the baking treatment. Moisten the residue with $25 \mathrm{c}$ c of dilute hydrochloric acid (1:1), digest a few minutes, ${ }^{97}$ add $75 \mathrm{c}$ c of water, heat until the salts are dissolved, and then filter to remove silica. Evaporate the filtrate to a sirupy condition, add $15 \mathrm{c}$ c of nitric acid (specific gravity 1.42), and again evaporate to a small volume. Again add $15 \mathrm{c} \mathrm{c}$ of nitric acid (specific gravity 1.42) and evaporate to a small volume. Add $100 \mathrm{c}$ c of nitric acid (specific gravity 1.135), heat to boiling, and add a 1.5 per cent solution of potassium permanganate until oxides of

97 Addition of a little sulphite or sulphurous acid facilitates solution after the baking treatment. 
manganese are precipitated. Dissolve the oxides of manganese by addition of sulphurous acid and boil to expel the oxides of nitrogen. To the cold solution add $40 \mathrm{c}$ c of $\mathrm{NH}_{4} \mathrm{OH}$ (specific gravity 0.96 ) and $50 \mathrm{c} \mathrm{c}$ of ammonium molybdate solution and shake for 5 minutes. Allow to settle for 10 minutes; for material containing less than 0.02 per cent of phosphorus, allow to stand 30 minutes. Filter and wash ten times with a 1 per cent solution of $\mathrm{KNO}_{3}$. Return the precipitate and paper to the precipitating vessel, add 1 to $3 \mathrm{c}$ c in excess of standard $\mathrm{NaOH}$ solution and $25 \mathrm{c}$ c of water, both free from carbon dioxide, and shake or stir until the precipitate is dissolved. Dilute to $100 \mathrm{c} \mathrm{c}$ with water free from carbon dioxide, add 3 drops of a 0.2 per cent solution of phenolphthalein, and discharge the pink color with standard acid. Finish the titration by adding standard alkali until the reappearance of the pink color. With very low phosphorus material this titration should be corrected by a blank carried through all the steps of the method.

Note.-If vanadium is present in amounts over 0.08 per cent it should be reduced by ferrous sulphate and sulphurous acid before precipitation with molybdate.

Determination of Sulphur by the Oxidation Method

Proceed as in the determination of sulphur by the oxidation method in plain carbon steel in A33. (See 600.1 p. 10.)

Note.-Routine determinations of sulphur can be made by the evolution method, provided concentrated hydrochlorir acid (specific gravity 1.19) is used, preferably in all-glass apparatus of the Pulsifer type.

Determination of Silicon by the Nitro-Sulphuric Acid Method SOLUTIONS REQUIRED

(Nitro-sulphuric acid is the same as in A64. See 601.20, p. 119.)

\section{METHOD}

Dissolve $0.9344 \mathrm{~g}$ of the sample passing a No. 100 sieve ${ }^{98}$ with $50 \mathrm{c} \mathrm{c}$ of the nitro-sulphuric acid in a $250 \mathrm{c}$ c porcelain casserole and evaporate until copious fumes of sulphuric acid are evolved. (Then proceed as in A64 (see 601.20, p. 119), until weighing, when) the difference in weights divided by 2 and multiplied by 100 represents the percentage of silicon in the alloy.

Note.-For silico-manganese and manganese-silicon fuse $0.4672 \mathrm{~g}$ of the former or $0.2336 \mathrm{~g}$ of the latter with $8 \mathrm{~g}$ of sodium peroxide in a pure iron crucible. Dissolve the fusion and evaporate to fumes of sulphuric acid. When acidifying the solution of the fusion with sulphuric acid it is necessary to add some sulphurous acid to dissolve the manganese dioxide which separates out.

\section{FERROCHROMIOM}

Determination of Chromium by Fusion with Sodium Peroxide

\section{SOLUTIONS REQUIRED}

Potassium permanganate, 0.1 N.-Dissolve $3.2 \mathrm{~g}$ of potassium permanganate in $1,000 \mathrm{c} \mathrm{c}$ of distilled water, allow to age for at least 10 days, and filter through purified asbestos. Standardize the permanganate solution against Bureau of Standards standard sodium oxalate as follows:

In a $400 \mathrm{c}$ c beaker dissolve 0.25 to $0.30 \mathrm{~g}$ of sodium oxalate in 200 to $250 \mathrm{c}$ c of hot water (80 to $90^{\circ}$ C.) and add $10 \mathrm{c}$ c of dilute $\mathrm{H}_{2} \mathrm{SO}_{4}(1: 1)$. Titrate at once with the permanganate solution, stirring the liquid vigorously and continuously. The permanganate must not be added more rapidly than 10 to $15 \mathrm{c} \mathrm{c}$ per minute, and the last 0.5 to to $1 \mathrm{c}$ c must be added drop wise, with particular care to allow each drop fully to decolorize before

${ }^{88}$ For detailed sperifications for this sieve see $\mathrm{E} 11$ (645.31, p. 1226). the next is introduced. The excess of permanganate used to cause an end-point color must be estimated by matching the color in another beaker containing the same bulk of acid and hot water. The temperature of the solution should not be below $60^{\circ}$ C. by the time the end point is reached. One cubic centimeter of $0.1 \mathrm{~N} \mathrm{KMnO}_{4}$ is equivalent to 0.001734 $\mathrm{g}$ of chromium.

Standard ferrous ammonium sulphate.-Dissolve $43 \mathrm{~g}$ of the salt in $250 \mathrm{c} \mathrm{c}$ of cold water, add $40 \mathrm{c} \mathrm{c}$ of sulphuric acid $(1: 3)$, dilute to one liter with distilled water, and mix thoroughly. Large amounts of the solution can be conveniently mixed by means of a stream of carbon dioxide from a cylinder. As the ferrous ammonium sulphate solution gradually weakens in reducing power it is necessary to standardize it daily or at the same time the ferrochromium is analyzed. The solution can be standardized against either potassium dichromate or sodium oxalate.

The first method is somewhat simpler and more satisfactory for routine work as the conditions prevailing in both the standardization and in the analysis are practically identical. No "blank" corrections are afterwards necessary where the amounts of dichromate, ferrous salt, and permanganate are approximately those prevailing in the analysis. The second method is employed chiefly for checking the accuracy of the results obtained with potassium dichromate and in cases where only occasional analyses are made and pure dichromate is not readily available.

To prepare pure potassium dichromate, recrystallize at least twice the purest grade of the salt obtainable, dry the crystals at $150^{\circ} \mathrm{C}$., grind to a fine powder, and again dry at $150^{\circ} \mathrm{C}$. to constant weight. Keep the prepared salt in a ground-glassstoppered bottle. Transfer $1.0000 \mathrm{~g}$ of the pure dichromate, which contains approximately the same amount of chromium as an $0.5000 \mathrm{~g}$ sample of 70 per cent ferrochromium, to an $800 \mathrm{c}$ c beaker. Dissolve in cold water, acidify with $40 \mathrm{c}$ c of sulphuric acid $(1: 3)$, and dilute with cold water to 500 c c. Add a slight excess of the ferrous sulphate solution, approximately $190 \mathrm{c}$ c, and titrate the excess ferrous salt with $0.1 N$ permanganate to the first faint permanent darkening of the clear green color.

Example.-The calculations inrolved are illustrated as follows: In a standardization, $190.0 \mathrm{c}$ c of the ferrous solution was used and $5.06 \mathrm{c} \mathrm{c}$ of $0.1 \mathrm{~N}$ permanganate was required to titrate the excess.

1 c c of $0.1 \mathrm{~N} \mathrm{~K}_{2} \mathrm{Cr}_{2} \mathrm{O}_{7}$ contains $0.004903 \mathrm{~g}$ of $\mathrm{K}_{2} \mathrm{Cr}_{2} \mathrm{O}_{7}$

$1 \mathrm{~g}$ of $\mathrm{K}_{2} \mathrm{Cr}_{2} \mathrm{O}_{7}$ equals $203.94 \mathrm{c}$ c of $0.1 \mathrm{~N} \mathrm{~K}_{2} \mathrm{Cr}_{2} \mathrm{O}_{7}$

then:

$203.94+5.06=209.0 \mathrm{c}$ c of equivalent $0.1 \mathrm{~N}$ solution reduced by the ferrous solution.

$$
\frac{209.0}{190}=1.100, \text { the } 0.1 \mathrm{~N} \text { normality factor }
$$

In standardizing through sodium oxalate, it is necessary to add the ferrous ammonium sulphate solution by means of an accurately calibrated pipette or burette instead of an automatic pipette. Transfer $100 \mathrm{c}$ c of the approximately $0.1 \mathrm{~N}$ ferrous solution to a $600 \mathrm{c}$ c beaker, dilute to $300 \mathrm{c} \mathrm{c}$ with cold 5 per cent sulphuric acid, add $2 \mathrm{c}$ c of phosphoric acid, and titrate immediately with $0.1 N \mathrm{KMnO}_{4}$ to a faint permanent pink color. Determine the "blank" on the same volume of water and acids and deduct. When this method is used it is necessary in the actual analysis to determine a "blank" to counteract the influence of the green color of the chromium sulphate upon the permanganate end point. This "blank" can be determined in the solution used in the analysis and is obtained as described later. 


\section{METHOD}

Transfer $0.5000 \mathrm{~g}$ of the sample of ferrochromium (dried at $105^{\circ}$ to $110^{\circ} \mathrm{C}$.) to a $30 \mathrm{c} \mathrm{c}$ heavy-walled porcelain crucible. Samples of low-carbon ferrochromium should be crushed to pass through a No. 40 screen, while high-carbon ferrochromium and chromium metal should be reduced to pass a No. 100 screen. Add approximately $8 \mathrm{~g}$ of sodium peroxide and thoroughly mix the contents of the crucible with a small rod, being careful to clean the rod, which can be done conveniently by scraping with another rod. Cover the mixture with 1 to $2 \mathrm{~g}$ of sodium peroxide. Carefully fuse the contents of the covered crucible, preferably in an electric muffle heated to $600^{\circ}$ to $700^{\circ} \mathrm{C}$. Fusion for five minutes at a low red heat after the mass has melted will insure complete decomposition. When the charge has melted, the crucible should be given a slight rotary motion to stir up any unattached particles.

Place the cooled crucible and cover in a $600 \mathrm{c} \mathrm{c}$ beaker and dissolve the contents in $200 \mathrm{c}$ c of water. When the melt has dissolved, remove the crucible and cover, add $1 \mathrm{~g}$ of sodium peroxide and boil the solution 10 minutes to decompose the excess of peroxide completely.

Allow the precipitate to settle and filter through a pad of ignited asbestos, preferably in a Büchner funnel. An asbestos rather than a paper filter should be used, for the alkaline solution extracts reducing matter from paper which would cause low results for chromium. Thoroughly wash the precipitate with hot water, and at no time allow the precipitate to run dry during the filtering and washing. If desired, the precipitate can be subsequently dissolved in dilute sulphuric acid and tested for chromium by adding a slight excess of sodium peroxide; the amount of chromium retained is negligible if the precipitate is filtered and washed as directed.

Cool the filtrate, acidify with $50 \mathrm{c}$ c of sulphuric acid (1:1), again cool to room temperature, transfer to an $800 \mathrm{c}$ c beaker, dilute with cold water to 500 c $c$ and add a measured excess of ferrous ammonium sulphate. The point at which reduction is complete can be told by changes in the deep green color which is developed. The ferrous salt can be added either as an approximately $0.12 \mathrm{~N}$ solution or in weighed portions from a bottle of the well-mixed salt. If the solution of ferrous salt is used, approximately $180 \mathrm{c}$ c will be required for a 70 per cent alloy; if the salt is added, $8 \mathrm{~g}$ will usually be sufficient. Titrate the excess of ferrous salt with standard $0.10 N$ potassium permanganate. The first faint permanent darkening of the clear green color is taken as the end point, which is quite sharp and well defined to the practiced eye. If the ferrous salt has been standardized against dichromate, subtract the required number of cubic centimeters of $0.1 \mathrm{~N}$ permanganate from the number of cubic centimeters of $0.1 N$ ferrous solution (obtained by multiplying the number of cubic centimeters of the approximately $0.1 \mathrm{~N}$ ferrous solution by the $0.1 \mathrm{~N}$ normality factor). The difference represents the number of cubic centimeters of $0.1 \mathrm{~N}$ ferrous solution which multiplied by $0.001733 \mathrm{~g}$ gives the number of grams of chromium present.

Where the ferrous solution has been standardized against sodium oxalate, it is necessary to subtract a "blank" from the permanganate titration. This "blank" is obtained as follows: When the end point is reached and the readings have been noted, add about $5 \mathrm{c} \mathrm{c}$ in excess of the ferrous solution and oxidize the excess of ferrous iron by adding $8 \mathrm{c} c$ of a 15 per cent solution of ammonium persulphate. Stir the liquid vigorously for one minute. Carefully add $0.1 N$ permanganate to the solution with constant stirring until the same end point is obtained as before. The volume of permanganate required, usually 0.2 to 0.4 c c, constitutes the "blank" to be deducted from the volume of the permanganate titration. For example, in the determination of chromium in an $0.5000 \mathrm{~g}$ sample, $190.0 \mathrm{c}$ c of a 0.11 $N$ ferrous solution and $11.00 \mathrm{c}$ c of an $0.1 N$ permanganate $(11.40-0.4 \mathrm{c}$ c blank) were employed.

The calculations are as follows:

$190.0 \times 1.100=209.0 \mathrm{c}$ c of $0.1 \mathrm{~N}$ ferrous solution $209.0-11.0=198.00 \mathrm{c} \mathrm{c}$

$198.0 \times 0.001734 \times 2 \times 100=68.65$ per cent of chromium.

The above calculations are given for exactly 0.1 $N$ permanganate; if the permanganate is not $0.1 \mathrm{~N}$, the volume used must be converted to the equivalent of $0.1 N$ by multiplying by the proper factor.

\section{Determination of Carbon by the Direct-Combustion Method}

(For apparatus and reagents common to both the soda-asbestos and barium hydroxide methods of absorbing the carbon dioxide, also for factors influencing rapid combustion, as well as for the determination of carbon (carbon dioxide absorbed in soda-asbestos), and for diagram showing arrangement of apparatus, see A64 (601.20, p. 119). For the determination of carbon (carbon dioxide absorbed in barium hydroxide solution) and diagram showing apparatus for filtration, see A33 (600.1, p. 10).)

1. Ferrochromium alloys require a furnace temperature of 1,125 to $1,150^{\circ} \mathrm{C}$. in order to yield all of the carbon in the material.

2. The determination is made as given in the reference except that the weighed sample of ferrochromium, $0.5000 \mathrm{~g}$ for 4 to 8 per cent carbon and 3 to $1 \mathrm{~g}$ for 0.10 to 2.00 per cent carbon, is mixed with $1 \mathrm{~g}$ of copper oxide and covered with $2 \mathrm{~g}$ of pure ingot-iron drillings in order to facilitate complete combustion.

3. On account of the small weight of sample used, the blanks are important and must be carefully determined.

\section{Determination of Silicon}

Low-carbon ferrochromium and chromium metal can be decomposed by means of dilute sulphuric acid, but the high-carbon alloy has to be fused with sodium peroxide. For low-carbon ferrochromium, transfer $0.9344 \mathrm{~g}$ (a double factor weight) to a 250 c c porcelain casserole, add $60 \mathrm{c}$ c of sulphuric acid $(1: 4)$ and warm the covered casserole gently until the reaction is complete. For high-carbon ferrochromium, transfer $0.9344 \mathrm{~g}$ of the sample passing a No. 100 sieve to a $30 \mathrm{c}$ c pure iron crucible made from No. 20 gage ( 0.032 inch thickness) ingot iron. Add approximately $8 \mathrm{~g}$ of sodium peroxide, mix thoroughly, and fuse as directed under the determination of chromium. When the fusion has solidified, tap the covered crucible, while still warm, on an iron plate to loosen its contents in a solid cake which is then transferred to a $250 \mathrm{c}$ c casserole. Rinse the crucible with a little water, transfer the solution to the casserole, cover, and carefully add $60 \mathrm{c} c$ of sulphuric acid (1:4) after which the cover glass is rinsed and removed.

Evaporate the solution obtained by either of the above procedures until fumes of sulphuric acid are given off. If chromium sulphate solutions are fumed at too high temperatures or for any great length of time or with too little free acid present, considerable difficulty will occur in dissolving the salts. The fuming should, therefore, be made at a temperatu :e not much higher than that required for evolving the acid. Allow the casserole and contents partly to cool (if permitted to become entirely cold 
difficulty may be experienced in getting chromium salts to dissolve), add $100 \mathrm{c}$ c of warm water while stirring to prevent the residue from caking on the bottom, and boil the solution gently for several minutes to dissolve all salts. Filter, washing the paper several times with cold dilute hydiochloric acid ( 2 per cent), and then with warm water. Transfer the filter and contents to a platinum crucible, char the paper, carefully ignite to remove carbon, and then heat the covered crucible over a blast lamp or in a muffle furnace at $1,050^{\circ}$ to $1,100^{\circ} \mathrm{C}$. for at least 15 minutes. Cool in a desiccator and weigh. Add sufficient dilute sulphuric acid $(1: 1)$ to moisten the silica and then a small amount of hydrofluoric acid. Evaporate to dryness, ignite, and weigh. The difference in weights represents silica which contains 46.72 per cent of silicon.

NoTE.--Crucibles made from pure ingot iron contain only traces of silicon and the amount of silica present in the sodium peroxide used is usually negligible, but "blanks" should be run on new lots of crucibles and of peroxide. All nickel crucibles, even the German "Rein" nickel, contains appreciable amounts of silicon.

\section{FERROVANADIOM}

Determination of Vanadium by the Ammonium Persulphate Method

\section{SOLUTIONS REQUIRED}

Ammonium persulphate (15 per cent).-Make up as needed by dissolving $15 \mathrm{~g}$ of the salt in $100 \mathrm{c} \mathrm{c}$ of distilled water. This salt should be 95 per cent pure and its purity tested by adding $30 \mathrm{c}$ c of a standard ferrous sulphate solution to $0.3 \mathrm{~g}$ of the persulphate, then $200 \mathrm{c}$ c of hot water, and titrating the excess ferrous salt with potassium permanganate. One cubic centimeter of $0.1 N$ ferrous solution equals $0.01141 \mathrm{~g}$ of $\left(\mathrm{NH}_{4}\right)_{2} \mathrm{~S}_{2} \mathrm{O}_{8}$.

Potassium ferricyanide indicator.-Dissolve $0.1 \mathrm{~g}$ of the salt in $100 \mathrm{c}$ c of water. This solution should be made up fresh daily.

Standard ferrous ammonium sulphate (approximately $0.1 \mathrm{~N}$ ). - See the determination of chromium in ferrochromium, given above.

Potassium Permanganate $(0.1 \mathrm{~N})$.- See the determination of chromium in ferrochromium given above. One cubic centimeter of $0.1 N \mathrm{KMnO}_{4}$ is equivalent to $0.005096 \mathrm{~g}$ of vanadium in the following method.

\section{METHOD}

Treat $0.5000 \mathrm{~g}$ of the allov, dried at $105^{\circ}$ to $110^{\circ}$ C., in a $250 \mathrm{c}$ c covered beaker with a mixture of $60 \mathrm{c}$ c of sulphuric acid $(1: 3)$ and $25 \mathrm{c}$ c of nitric acid $(1: 1)$. If the alloy fails to decompose readily because the silicon content is high, it may be necessary to add a small amount of hydrofluoric acid. In this event, a 200 c c platinum dish should be substituted for the beaker. When the reaction has abated, rinse the cover glass and evaporate the solution until copious fumes of sulphuric acid are evolved. Cool, add $100 \mathrm{c} \mathrm{c}$ of water, and heat until the salts are dissolved. Transfer the solution to an $800 \mathrm{c}$ c beaker, dilute to $400 \mathrm{c}$ c and to the cooled solution add an approximately $0.1 \mathrm{~N}$ solution of potassium permanganate until a strong pink color has developed which remains permanent for 30 seconds. Next reduce the vanadium by the use of an approximately $0.1 \mathrm{~N}$ solution of ferrous ammonium sulphate added until a drop of the solution withdrawn from the beaker and placed on a drop of potassium ferricyanide indicator shows ferrous iron present-about $35 \mathrm{c} \mathrm{c}$ of $0.1 \mathrm{~N}$ ferrous solution. Add $5 \mathrm{c}$ c of the ferrous ammonium sulphate in excess, stir the solution at least one minute, oxidize the excess of ferrous sulphate with $8 \mathrm{c} \mathrm{c}$ of 15 per cent ammonium persulphate and stir the solution vigorously for one minute. Titrate the solution with $0.1 N$ potassium permanganate solution, added with constant stirring until a faint rink color appears which remains permanent for 30 seconds. "Determine the "blank" by dissolving $0.4 \mathrm{~g}$ of "ingot iron" in $60 \mathrm{c} \mathrm{c}$ of $\mathrm{H}_{2} \mathrm{SO}_{4}(1: 3)$ and $5 \mathrm{c} \mathrm{c}$ of nitric acid (specific gravity 1.42), and putting the solution through all of the operations of the analysis. If the alloy contains an appreciable amount of chromium, a weight of pure $\mathrm{K}_{2} \mathrm{Cr}_{2} \mathrm{O}_{7}$ equivalent to the amount of chromium in the sample is dissolved in the acids with the ingot iron.

\section{Determination of Carbon}

(For apparatus and reagents common to both the soda-asbestos and barium hydroxide methods of absorbing the carbon dioxide, also for factors influencing rapid combustion, as well as for the determination of carbon (carbon dioxide absorbed in soda-asbestos) and for diagram showing arrangement of apparatus, see A64 (601.20, p. 119). For the determination of carbon (carbon dioxide absorbed in barium hydroxide solution) and for diagram showing apparatus for filtration, see A33 (600.1, p. 10).) A furnace temperature of $1,050^{\circ}$ to $1,100^{\circ} \mathrm{C}$. will suffice for ferrovanadium and complete combustion, except in very high silicon material, can be secured without the addition of ingot iron and copper oxide.

Determination of Phosphorus by the Molybdate Magnesia Method

\section{SOLUTIONS REQUIRED}

(Ammonium molybdate is the same as in the determination of phosphorus by the alkalimetric method in B28. See 646.0 , p. 1243.)

Magnesia mixture.-Dissolve $50 \mathrm{~g}$ of $\mathrm{MgCl}_{2} \cdot 6 \mathrm{H}_{2} \mathrm{O}$ and $200 \mathrm{~g}$ of $\mathrm{NH}_{4} \mathrm{Cl}$ in $1,000 \mathrm{c} \mathrm{c}$ of distilled water containing a few cubic centimeters of hydrochloric acid.

Transfer $4 \mathrm{~g}$ of the sample to a platinum dish of 150 to 200 c c capacity, add 70 c c of dilute $\mathrm{HNO}_{3}$ (5:6), and heat the covered dish until vigorous action ceases. Add 1 or 2 c c of hydrofluoric acid, stir the solution, evaporate to dryness, moisten with 10 c c of nitric acid (specific gravity 1.42), and again evaporate to drvness. Moisten the cooled residue with $10 \mathrm{c}$ c of $\mathrm{H}^{2} \mathrm{NO}_{3}$ (specific gravity 1.42 ), add $50 \mathrm{c}$ c of water, and wash the contents of the dish into a $400 \mathrm{c}$ c beaker, rubbing the dish well with a policeman. Remove the last traces of adhering salts with $5 \mathrm{c} \mathrm{c}$ of $\mathrm{HCl}$ (specific gravity 1.19) and hot water. Add $10 \mathrm{c}$ c of $\mathrm{HNO}_{3}$ (specific gravity 1.42) and evaporate to a sirupy consistency. Cool, add $25 \mathrm{c} \mathrm{c}$ of $\mathrm{HNO}_{3}$ (specific gravity 1.42), $60 \mathrm{c}$ c of water, $1 \mathrm{~g}$ of boric acid (to take care of any residual fluoride), and heat gently until solution occurs. Filter through a rapid paper into a 300 c c Erlenmeyer flask and wash with hot water. Heat the solution to boiling, add about $12 \mathrm{c} \mathrm{c}$ of potassium permanganate solution (1.5 per cent) and continue the boiling until manganese dioxide precipitates. Dissolve the precipitate by the addition of sufficient sulphurous acid added dropwise. About 1 c c usually suffices. Boil for a few minutes to expel completely oxides of nitrogen. Add to the cold solution $15 \mathrm{c}$ c of ammonium hydroxide (specific gravity 0.90 ) and cool the solution to $15^{\circ} \mathrm{C}$

Next reduce the vanadium in the cold $\left(15^{\circ} \mathrm{C}\right.$.) solution by ferrous sulphate added in approximately $1.5 \mathrm{~g}$ excess. The use of a 50 per cent solution of the salt furnishes a convenient way of adding the necessary amount. One gram of quinquevalent vanadium requires $5.5 \mathrm{~g}$ of $\mathrm{FeSO}_{4} \cdot 7 \mathrm{H}_{2} \mathrm{O}$ for reduction to the quadrivalent state. A $4 \mathrm{~g}$ sample of 30 per cent vanadium alloy, including 
an excess of $1.5 \mathrm{~g}$ would require $8.1 \mathrm{~g}$ of ferrous sulphate. Too large an excess of ferrous sulphate should be avoided. The addition of ferrous sulphate will change the color of the solution to a deep blue. Add 2 drops of a saturated solution of sulphurous acid, 100 c c of molybdate reagent, shake for 10 minutes, and allow to stand at $15^{\circ} \mathrm{C}$. for four to six hours, or preferably overnight. It is well to let the solution stand in a refrigerator. Filter on a paper of close texture, keeping as much of the precipitate in the flask as possible, and wash the flask and the precipitate with not more than $50 \mathrm{c} \mathrm{c}$ of cold, dilute nitric acid $(1: 99)$. Set the filtrate and washings aside after thorough mixing and see whether further separation of phosphomolybdate occurs. Dissolve the precipitate on the filter in $20 \mathrm{c}$ c of dilute ammonium hydroxide (1:1) to which have been added $2 \mathrm{~g}$ of citric acid, catch the solution in the original flask containing the bulk of the yellow precipitate, and finally wash the filter several times with dilute hydrochloric acid $(5: 95)$. If the ammoniacal solution of the phosphomolybdate is not clear at this point, heat it to boiling, filter through the same paper, and wash the paper with hot water. In this case, and whenever appreciable amounts of such elements as titanium or zirconium are known to be present in the original material, preserve the paper and ignite it in a small platinum crucible with the paper reserved below.

Render the ammoniacal solution acid with hydrochloric acid, add $20 \mathrm{c}$ c of magnesia mixture and then ammonium hydroxide (specific gravity 0.90) slowly until a crystalline precipitate appears, and then finally an excess of 3 to 5 per cent by volume. The volume of the solution at this point should not exceed 100 c c. Allow the solutions to stand in a cool place for four to six hours, or preferably over night.

Filter, keeping as much of the precipitate as possible in the flask, and wash the flask and the precipitate moderately with dilute ammonium hydroxide $(1: 20)$. Set the filtrate and washings aside after thorough mixing and see whether further precipitation occurs. Dissolve the precipitate on the filter in $25 \mathrm{c}$ c of dilute hydrochloric acid $(1: 1)$, catching the solution in the original flask containing the bulk of the precipitate. Wash the filter thoroughly with dilute hydrochloric acid (5:95). In case the original material contains appreciable amounts of the elements mentioned above (titanium or zirconium), add the paper to the one reserved above, ignite, fuse any residue with as little carbonate as possible (not over $0.5 \mathrm{~g}$ ), extract the cooled melt with hot water, filter, and add the water solution to the hydrochloric acid solution. Transfer the solution to a $200 \mathrm{c}$ c beaker. In case the amount of arsenic is known to be very small, add 0.5 to $1 \mathrm{~g}$ of ammonium bromide and boil the hydrochloric acid solution to a volume of 5 to $10 \mathrm{c} \mathrm{c}$. Where large. amounts of arsenic were originally present, treat with hydrogen sulphide, digest, filter, and wash with acidulated hydrogen sulphide water. Boil to expel hydrogen sulphide and to reduce the volume to 50 to $75 \mathrm{c} \mathrm{c}$. Add 0.1 to $0.2 \mathrm{~g}$. of citric acid, 2 to $3 \mathrm{c} \mathrm{c}$ of magnesia mixture, make ammoniacal as above, and allow to stand in a cool place for 4 to 24 hours.

Filter, wash with dilute ammonium hydroxide $(1: 20)$, and ignite the precipitate carefully and at as low a temperature as possible until the carbon has been destroyed and the residue is white. Finally, ignite at approximately $1,000^{\circ} \mathrm{C}$. to constant weight. Dissolve the ignited residue in $5 \mathrm{c} c$ of dilute nitric acid (1:1) and $20 \mathrm{c}$ c of water. Filter, wash the residue with hot water, ignite, and weigh. Treat with a few drops of hydrofluoric acid, evaporate to dryness, ignite, and reweigh. Subtract any loss of weight from the original weight of the pyrophosphate and calculate on the basis of this purified magnesium pyrophosphate.

\section{Determination of Sulphur by the Oxidation Method}

Transfer $4.57 \mathrm{~g}$ of the sample to a $600 \mathrm{c} \mathrm{c}$ beaker and dissolve it in $75 \mathrm{c}$ c of $\mathrm{HNO}_{3}$ (specific gravity 1.42). The reaction is apt to be very rapid and the acid should be added in small portions to the covered beaker, which is preferably kept cooled by immersion in ice water. Effect complete solution by gentle warming if necessary.

Add $30 \mathrm{c}$ c of $\mathrm{HCl}$ (specific gravity 1.19) and evaporate to dryness on the steam bath. Add $30 \mathrm{c} \mathrm{c}$ more of $\mathrm{HCl}$ (specific gravity 1.19), again evaporate to dryness, and bake for 30 minutes at $105^{\circ} \mathrm{C}$. Moisten the residue with $30 \mathrm{c}$ c of $\mathrm{HCl}$ (specific gravity 1.19) and evaporate on the steam bath to a volume of 5 c c. Add 5 c c of $\mathrm{HCl}$ (specific gravity 1.19), dilute to $150 \mathrm{c} \mathrm{c}$, and filter. Wash with warm water.

Adjust the volume of the solution to $250 \mathrm{c} c$, which should now contain about 3 to 4 per cent by volume of $\mathrm{HCl}$. Warm to $75^{\circ} \mathrm{C}$, add $30 \mathrm{c}$ c of $\mathrm{BaCl}_{2}(10$ per cent), and allow to stand for 24 to 40 hours.

Filter, with as little transfer of the precipitate as possible, through a paper of close texture. Wash the paper two or three times with cold 1 per cent $\mathrm{HCl}$ and seven times with warm water. Ignite the paper and contents in a platinum crucible. Treat the ignited residue with $5 \mathrm{c}$ c of $\mathrm{HCl}$ (specific gravity 1.19) and transfer the resulting solution to the original beaker containing most of the precipitate of $\mathrm{BaSO}_{4}$. Digest the combined portions of $\mathrm{BaSO}_{4}$ so as to dissolve any barium vanadate. Adjust to $150 \mathrm{c}$ c volume and 2 per cent acidity, add $8 \mathrm{c}$ c of 10 per cent $\mathrm{BaCl}_{2}$ solution, and allow to stand for 24 hours. Filter on a paper of close texture, wash three times with cold 1 per cent $\mathrm{HCl}$ and 10 times with warm water. Ignite the paper in platinum, cool in a desiccator, and weigh. A blank should be carried through all steps of the determination. The corrected weight of the ignited barium sulphate multiplied by 3 is equal to the percentage of sulphur.

Notes.-If desired, the washings can be kept separate and examined for $\mathrm{BaSO}_{4}$ as follows: Evaporate the washings to dryness. Dissolve the slight residue in $50 \mathrm{c}$ c of hot dilute $\mathrm{HCl}(2: 98)$, add $2 \mathrm{c} \mathrm{c}$ of $\mathrm{BaCl}_{2}$ solution (10 per cent), digest at $70^{\circ}$ to $80^{\circ} \mathrm{C}$. for two hours, avoiding solution (10 per cent), digest at $0^{\circ}$ to $80^{\circ}$. lor two hours, avoiding wash as above. The recovery of $\mathrm{BaSO}_{4}$ which is ordinarily obtained, wash as above. The recovery of $\mathrm{BaSO}_{4}$ which is ordinarily
represents approximately 0.001 to 0.002 per cent of sulphur.

represents approximately 0.001 to 0.002 per cent of sulphur.
In high-silicon alloys the residue from the initial acid digestion In high-silicon alloys the residue from the initial acid digestion
should be evaporated with hydrofluoric and nitric acids, fused with sodium carbonate, and the water extract of the melt added to the main solution.

Determination of Silicon by the Nitrosulphuric Method

\section{SOLUTIONS REQUIRED}

(Nitrosulphuric acid is the same as in the determination of silicon by the nitrosulphuric method in A64. See 601.20, p. 119.)

\section{METHOD}

Dissolve $0.9344 \mathrm{~g}$ of the sample passing a No. 100 sieve of low or medium silicon ferrovanadium in 40 c $\mathrm{c}$ of the nitrosulphuric acid in a $250 \mathrm{c}$ c porcelain or platinum dish and evaporate the solution until copious fumes of sulphuric acid are evolved.

Ferrovanadium containing 4 per cent or more of silicon is not completely soluble in nitric acid and sulphuric acid. In this case, fuse $0.9344 \mathrm{~g}$ of the alloy, thoroughly mixed with $8 \mathrm{~g}$ of sodium peroxide, in a $30 \mathrm{c}$ c crucible of pure iron. Five minutes heating at a dull red heat after the contents of the crucible have melted will insure complete decomposition of the sample. Allow the fusion to solidify, but before it has entirely cooled tap the covered crucible on an iron plate which will loosen the contents in a solid cake. Transfer the melt to a 250 c c porcelain casserole, rinse the crucible with warm water, and cautiously add the solution to the 
covered casserole. Next add $150 \mathrm{c}$ c of sulphuric acid ( 20 per cent by volume) and evaporate to copious fumes of sulphuric acid. Cool the sulphuric acid solution obtained in either procedure, add 125 c c of water, and heat for a few minutes with frequent stirring until all salts are dissolved. Immediately filter on a $9 \mathrm{~cm}$ paper. Wash the precipitate with cold dilute $\mathrm{HCl}(5: 95)$ and cold water alternately to complete the removal of iron salts, and finally with hot water until free from acid. Transfer the filter to a platinum crucible, char the paper without inflaming, and finally ignite the covered crucible over a blast lamp or in a muffle furnace at $1,050^{\circ}$ to $1,100^{\circ} \mathrm{C}$. for at least 15 minutes. Cool in a desiccator and weigh. Add sufficient dilute $\mathrm{H}_{2} \mathrm{SO}_{4}(1: 1)$ to moisten the silica and then a small amount of hydrofluoric acid. Evaporate to dryness, ignite, and weigh. The difference in weights divided by 2 and multiplied by 100 represents the percentage of silicon in the alloy.

Determination of Aluminum by the Ether-Hydrochloric Acid Method 9

Dissolve $2 \mathrm{~g}$ of the sample in 25 to $30 \mathrm{c} \mathrm{c}$ of dilute nitric acid (specific gravity 1.135). (Note 1.) When action ceases, add $5 \mathrm{c} c$ of hydrochloric acid and evaporate to dryness. Add $10 \mathrm{c}$ c of hydrochloric acid, evaporate to dryness, and bake the residue at a moderate temperature. Dissolve the residue in $10 \mathrm{c}$ c of hydrochloric acid, add $30 \mathrm{c} \mathrm{c}$ of water, boil, and filter. Wash the residue with hot dilute hydrochloric acid (1:9) followed by water. (Note 2.)

Evaporate the solution to dryness on a steam bath, add $20 \mathrm{c}$ c of hydrochloric acid, and warm gently until complete solution is obtained. Cool the solution in ice water and pass hydrochloric acid gas into it to saturation. Add $20 \mathrm{c} \mathrm{c}$ of ether and continue passing the gas into the cold solution until it is again saturated. Allow the mixture to stand for several hours, keeping it cold by immersing the container in ice water. If the solution becomes warm or if it is not allowed to stand, the separation of hydrated aluminum chloride is not quite complete.

Filter through asbestos (small Bückner-asbestos pad washed once with cold ether-hydrochloric acid mixture immediately before use), wash, by decantation, with a cold mixture of equal parts of strong hydrochloric acid and ether which has been saturated with hydrochloric acid gas. The aluminum chloride retains a little vanadium.

Dissolve the precipitated aluminum chloride in the least possible amount of dilute hydrochloric acid (1:9) back into the original beaker, from which all of the precipitate need not have been removed. Evaporate the solution to near dryness on a steam bath, add $10 \mathrm{c}$ c of strong hydrochloric acid and pass hydrochloric acid gas into the solution to saturation, add $15 \mathrm{c}$ c of ether and continue passing hydrochloric acid gas to saturation, keeping the solution cold by means of ice water. Let stand several hours, filter on asbestos, and wash with the ether-hydrochloric acid mixture as before.

Dissolve the aluminum chloride in dilute hydrochloric acid (1:9) into a $250 \mathrm{c}$ c beaker, add a few grams of ammonium chloride, make faintly alkaline with ammonia, boil, filter (using paper pulp), wash with hot water, dry, ignite, and weigh as $\mathrm{Al}_{2} \mathrm{O}_{3}$.

According to published data, this method gives a complete separation of aluminum from iron, beryllium, zinc, copper, mercury, and bismuth. A little vanadium is carried down in the first separation, but two chloride precipitations seem to give a practically complete separation. The proportion of vanadium retained in the final alumina is hardly appreciable when working on a 35 per cent vanadium alloy.

${ }_{99}$ Gooch-Havens, Am. J. Sci., vol. 4, pp. 11-416.
The effect of chromium is not known; presumably some of it would be included in the $\mathrm{Al}_{2} \mathrm{O}_{3}$. This could be corrected for by fusing the $\mathrm{Al}_{2} \mathrm{O}_{3}$ with sodium peroxide and estimating the $\mathrm{Cr}_{2} \mathrm{O}_{3}$ by color.

Notes.-1. Dilute nitric acid (specific gravity 1.135) is recommended for solution. While this requires rather long digestion at steam-bath temperature, solution seems to be quite complete even when the alloy is high in silicon. In some cases where silicon is very high it is necessary to use a little hydrofluoric acid with the nitric acid. In this case solution should be effected in a platinum dish, the solution evaporated to dryness, and the dry residue transferred to a beaker before treatment with hydrochloric acid. A little dilute hydrochioric acid may be used to loosen any oxides that adhere to the dish.

2. The siliceous residue may retain alumina; it should be ignited in platinum. Silica should be expelled by $\mathrm{HF}+\mathrm{H}_{2} \mathrm{SO}_{4}$ and the residue fused with $\mathrm{KHSO}_{4}$. After dissolving the fusion in water plus a little $\mathrm{HCl}$, the liquid is made faintly alkaline with ammonia and boiled. Any iron and alumina precipitate is removed by filtration, washed with water, dissolved in a little hot dilute $\mathrm{HCl}$ and added to the solution of the alumina obtained after the first etherhydrochloric acid separation.

\section{FERROTUNGSTEN AND TUNGSTEN METAL}

\section{Determination of Tungsten}

\section{SOLUTIONS REQUIRED}

(Cinchonine solution and cinchonine wash solution are the same as in the determination of tungsten in tungsten-steel by the acid digestion-cinchonine method in A55. (See 621.30, p 1107.)

Treat $1 \mathrm{~g}$ of the material passing a No. 100 sieve in a large $(60 \mathrm{c} \mathrm{c})$ platinum crucible with $5 \mathrm{c} \mathrm{c}$ of $\mathrm{HF}$; add $\mathrm{HNO}_{3}$ drop by drop until the metal dissolves. Add 3 to $4 \mathrm{c} \mathrm{c}$ of $\mathrm{H}_{2} \mathrm{SO}_{4}$ and evaporate on a steam bath until $\mathrm{HNO}_{3}+\mathrm{HF}$ is expelled. Shake gently over a small Bunsen flame, or heat slowly in a sheet iron "radiator" (described by Hillebrand) ${ }^{1}$ until $\mathrm{H}_{2} \mathrm{SO}_{4}$ fumes strongly. Cool, transfer to a $250 \mathrm{c}$ c beaker with water, finally wiping the crucible with a little filter paper. A little $\mathrm{WO}_{3}$ sticks to the crucible; it can not be removed by wiping. Reverse the crucible. Dilute the contents of the beaker to about $150 \mathrm{c}$ c with water, add $10 \mathrm{c}$ c of hydrochloric acid (specific gravity 1.19) and boil for a few minutes. Remove from the source of heat, add $10 \mathrm{c}$ c of cinchonine solution and digest the solution at $80^{\circ}$ to $90^{\circ} \mathrm{C}$. with occasional stirring, for 30 to 45 minutes.

Add some paper pulp to the solution, stir the solution and when the tungsten precipitate has settled filter on a $9 \mathrm{~cm}$ paper containing a little "ashless" filter pulp. Wash thoroughly with hot cinchonine solution and finally several times with warm 1 per cent hydrochloric acid. Gently ignite the filter and residue of crude $\mathrm{WO}_{3}$ in the crucible in which it was originally treated until the carbon is consumed. Add a few drops of nitric acid and dry in the radiator. Ignite the covered crucible for five minutes in the full heat of a Bunsen burner, cool, and weigh. Ignitions may be made at about $750^{\circ}$ C. in an electric muffle. As $\mathrm{WO}_{3}$ is slowly but steadily volatilized at temperatures above $750^{\circ} \mathrm{C}$., serious error may result from continued ignition of the tungsten trioxide at the temperature that is specified. Add about $5 \mathrm{~g}$ of $\mathrm{Na}_{2} \mathrm{CO}_{3}$ and fuse, running the fusion around the side of the crucible to remove all $\mathrm{WO}_{3}$. Dissolve the fusion in hot water, add alcohol, heat, filter, and wash with hot water. Place the filter in the crucible and ignite; add a little $\mathrm{Na}_{2} \mathrm{CO}_{3}$ and fuse again. Dissolve the fusion in water, filter, and wash very thoroughly with hot water to remove the last traces of $\mathrm{Na}_{2} \mathrm{CO}_{3}$; ignite in the same crucible as at first, cool, and weigh. The difference between the weight of crucible plus residue and the weight of crucible plus impure tungstic oxide is $\mathrm{WO}_{3} \times 0.7931=\mathrm{W}$.

Notes.-At the best, direct determination of tungsten in high grade metal is subject to inherent errors. Accuracy within 0.2 per cent is all that can be expected by any method. 


\section{Determination of Carbon}

(For apparatus and reagents common to both the soda-asbestos and barium hydroxide methods of absorbing the carbon dioxide, also for factors influencing rapid combustion, as well as for the determination of carbon (carbon dioxide absorbed in soda-asbestos) and for diagram showing arrangement of apparatus, see A64 (601.20, p. 119). For the determination of carbon (carbon dioxide absorbed in barium hydroxide solution) and diagram showing apparatus for filtration, see A33 (600.1, p. 10).) The alloy ground to pass a No. 100 sieve and mixed with $2 \mathrm{~g}$ of pure iron is burned in an electric furnace heated at $1,050^{\circ}$ to $1,100^{\circ} \mathrm{C}$. A "blank" on the ingot iron must be determined and deducted.

\section{Determination of Phosphorus}

Treat $1 \mathrm{~g}$ of the finely powdered sample in a platinum dish, fitted with a gold cover, with $15 \mathrm{c} c$ of $\mathrm{HNO}_{3}$ (specific gravity 1.42), add $3 \mathrm{c} c$ of $\mathrm{HF}$, and warm gentlv. When action subsides, add 3 c c more of $\mathrm{HF}$. After action subsides, boil, remove cover, and if decomposition is not complete add more $\mathrm{HF}$ and hoil again. When solution is complete, wash off the cover and evaporate at a low heat to a volume of about $10 \mathrm{c} c$, then add three or four drops of concentrated permanganate solution and continue evaporation until crusts of $\mathrm{WO}_{3}$ begin to form at the edges, that is to a volume of about $6 \mathrm{c} \mathrm{c}$. Add $5 \mathrm{c} \mathrm{c}$ of $\mathrm{H}_{2} \mathrm{SO}_{4}$ and evaporate on the stove at a low heat until $\mathrm{HF}$ and $\mathrm{HNO}_{3}$ are expelled and $\mathrm{H}_{2} \mathrm{SO}_{4}$ fumes are given off. (Strong heat causes spattering and also causes hard overbaked crusts to form on the bottom of the dish which resist subsequent treatment.) Cool, add $25 \mathrm{c}$ c of water, and boil (by agitating over a Bunsen flame) until all soluble salts are dissolved. Discharge pink color, due to excess permangnate, by adding sulphurous acid drop by drop. The pink color may not be very evident but the $\mathrm{SO}_{2}$ is added even so to reduce higher oxides of manganese. Boil for a minute or two after adding the $\mathrm{SO}_{2}$. Add $1.20 \mathrm{~g}$ of pure tartaric acid and, when this is dissolved and the solution is cooled to a temperature of about $50^{\circ} \mathrm{C}$., add $20 \mathrm{c}$ c of $\mathrm{NH}_{4} \mathrm{OH}$ (specific gravity 0.90 ) diluted with an equal volume of water. The precipated tungstic acid should dissolve completely, giving a clear solution. The solution becomes hot from the reaction between $\mathrm{H}_{2} \mathrm{SO}_{4}$ and $\mathrm{NH}_{4} \mathrm{OH}$. While it is still hot, add $10 \mathrm{c} \mathrm{c}$ of magnesia mixture and transfer it from the platinum dish to a 6-ounce glass-stoppered bottle. Set the bottle in ice water and when it is thoroughly cooled add four or five glass beads about $6 \mathrm{~mm}$ in diameter. Stopper it tightly and shake in an efficient shaking machine for at least 10 minutes. The agitation should be violent. The beads aid in starting the formation of the magnesium precipitate. After agitation add $15 \mathrm{c}$ c of strong $\mathrm{NH}_{4} \mathrm{OH}$ and return the bottle to the ice-water tank and put into a refrigerator to stand overnight. Phosphoros separates as magnesium ammonium phosphate free from tungsten but containing possibly basic magnesium compounds. After standing overnight filter the solution through a $9 \mathrm{~cm}$ paper containing a little paper pulp and wash the bottle and paper thoroughly by small additions of ammonia wash water ( 1 part $\mathrm{NH}_{4} \mathrm{OH}$ to 3 parts $\mathrm{H}_{2} \mathrm{O}$ ). Do not attempt to remove all the precipitate from the bottle, but remove the beads to the filter. The precipitate consists of magnesium ammonium phosphate and arsenate together with silica and other impurities. Tin, tungsten, molydenum, vanadium, and titanium are eliminated by precipitation with magnesia mixture in the presence of tartaric acid. Dissolve the magnesia precipitate in hot dilute $\mathrm{HCl}$ and evaporate to a small volume, say 7

1 U. S. Geological Survey Bull. No. 700, p. 33. or 8 c c. Add $10 \mathrm{c} \mathrm{c}$ of strong $\mathrm{HCl}$ and $0.5 \mathrm{~g}$ of $\mathrm{KBr}$ and evaporate to dryness. Take up with $5 \mathrm{c} \mathrm{c}$ of $\mathrm{HNO}_{3}$ and evaporate to dryness again. The $\mathrm{KBr}$ serves to reduce $\mathrm{As}^{\nabla}$ to $\mathrm{As}^{\prime \prime \prime}$. The addition of strong $\mathrm{HCl}$ and boiling followed by evaporation to dryness eliminates As. Dissolve residue in $60 \mathrm{c}$ c of $\mathrm{HNO}_{3}$ (specific gravity 1.135 ), filter into a $250 \mathrm{c}$ c shaking bottle; add $40 \mathrm{c}$ c of $\mathrm{NH}_{4} \mathrm{OH}$ (specific gravity 0.96 ), cool to $33^{\circ}$ C., add $30 \mathrm{c}$ c of molydate solution (Blair), and finish the determination by the alkalimetric method (Handy) described in Blair's Chemical Analysis of Iron.

Notes.-The usual proportions of $\mathrm{HNO}_{3}$ and $\mathrm{HF}$ are reversed in order to provide a constant excess of $\mathrm{HNO}_{3}$ to oxidize P. The procedure given takes a little longer than when the sample is treated with $\mathrm{H} \mathrm{F}$ first and $\mathrm{HNO} \mathrm{N}_{3}$ is added a little at a time, but the solution is finally complete. It is necessary to keep the platinum dish covred after action begins, as the reaction is somewhat violent.

The platinum dishes recommended are: $8 \mathrm{~cm}$ in diameter at top $7.8 \mathrm{~cm}$ in diameter at bottom, and $4 \mathrm{~cm}$ high. They have flat bot, toms and are wire rimmed at the top to give additional stiffness They weigh 58 to $60 \mathrm{~g}$ each and hold about $175 \mathrm{c} \mathrm{c}$. The covers are. made of pure gold (for economy), and dished like a crucible cover to fit the top of the dishes closely. The dishes have small lips to aid fit the top of the dishes closely. The dishes have small lips to aid pouring. The tongue of the cover overlaps the lip. Ordinary roundbottomed dishes may be used, but the manipulation is much more difficult; there is a greater tendency to spattering and danger of local baking or overheating in evaporating to fumes of $\mathrm{H}_{2} \mathrm{SO}_{4}$. If the

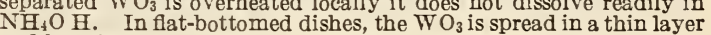
$\mathrm{H}_{4} \mathrm{OH}$. In flat-bottomed dishes, the $\mathrm{WO}_{3}$ is

A good shaking machine should be used. The magnesia precipitate may be started by shaking the bottles by hand, but it is a tiresome job. The solution must be cold, say $8^{\circ}$ or $10^{\circ} \mathrm{C}$., when shaking begins.

Permanganate solution is added to insure complete oxidation of phosphorous, as in steel analysis. The color of the permanganate gradually fades in the hydrofluoric nitric solution, but after evaporting to fumes and adding water the solution is usually slightly pink.

The amount of tartaric acid is limited to $1.20 \mathrm{~g}$ since ammonium tartrate retards the formation of the magnesia precipitate. Complete precipitation can only be obtained by brisk agitation and by keeping the solution very cold, followed by long standing in a cold place. By this method determinations started at $3 \mathrm{p}$. m. may be completed by noon the next day.

\section{Determination of Sulphur and Silicon}

\section{SOLUTIONS REQUIRED}

Cinchonine solution.-Thoroughly wash cinchonine reagent with hot water on a Büchner funnel, dry, and dissolve $125 \mathrm{~g}$ of the washed cinchonine in a mixture of $500 \mathrm{c}$ c of $\mathrm{HCl}$ (specific gravity 1.19) and $500 \mathrm{c}$ c of distilled water.

Cinchonine wash solution.-Dilute $30 \mathrm{c}$ c of the above solution to one liter.

Barium chloride solution.-One hundred grams of $\mathrm{BaCl}_{2} \cdot 2 \mathrm{H}_{2} \mathrm{O}$ to 1,000 c c.

\section{SULPHOR}

Fuse $2 \mathrm{~g}$ of the finely powdered metal, intimately mixed with $8 \mathrm{~g}$ of dry $\mathrm{Na}_{2} \mathrm{CO}_{3}$ and $2 \mathrm{~g}$ of powdered $\mathrm{KNO}_{3}$, in a large platinum crucible (40 c c). The fusion is best done over an alcohol blast flame or in an electric muffle to avoid contamination with sulphur contained in illuminating gas. Leach the fusion with $100 \mathrm{c}$ c of distilled water in a $250 \mathrm{c}$ c beaker. Filter into a $12 \mathrm{~cm}$ porcelain casserole and wash the residue several times with hot distilled water. The solution contains practically all of the sulphur and most of the tungsten and silica. The residue contains some of each, but not more than traces of sulphur.

Render the liquid acid with $20 \mathrm{c}$ c of $\mathrm{HCl}$ and evaporate to dryness on a steam bath, thus precipitating tungstic acid. After the residue is dry, heat it to $110^{\circ}$ to $120^{\circ} \mathrm{C}$. in an oven to dehydrate silica, take up with $5 \mathrm{c}$ c of $\mathrm{HCl}$ and $50 \mathrm{c} \mathrm{c}$ of water, add $3 \mathrm{c}$ c of a strong cinchonine solution, and boil, or heat just short of the boiling point, for 30 minutes. Let the solution stand for at least three hours and preferably overnight. $\mathrm{By}$ means of the cinchonine, all $\mathrm{WO}_{3}$ is rendered insoluble. Filter through an $11 \mathrm{~cm}$ filter paper (free from ash) which has been liberally treated with an emulsion of filter paper 
pulp and wash the residue with dilute cinchonine solution. Reserve the residue. The filtrate contains all the sulphur; heat it to boiling and add 10 c c of barium chloride solution, boil for 10 minutes, allow it to stand overnight, filter on a $9 \mathrm{~cm}$ filter paper (free from ash), wash repreatedly but not excessively with hot water, ignite in platinum, and weigh as $\mathrm{BaSO}_{4}$. If the work is properly carried out every trace of $\mathrm{WO}_{3}$ is removed from the solutions and there is no danger of including $\mathrm{BaWO}_{4}$ with the $\mathrm{BaSO}_{4}$. If one suspects that the $\mathrm{BaSO}_{4}$ is not quite pure, it should be fused with a little $\mathrm{Na}_{2} \mathrm{CO}_{3}$, leached with water and filtered; the filtrate should be acidulated with $\mathrm{HCl}$, a few drops of cinchonine solution added and the solution evaporated to dryness on a steam bath. The dry residue should be dissolved in a little water, a few drops of $\mathrm{HCl}$ added, and any residue filtered off. In the clear filtrate $\mathrm{BaSO}_{4}$ should be again precipitated as before. The weight of $\mathrm{BaSO}_{4} \times 0.1374=\mathrm{S}$.

"Blanks" on all reagents must be made parallel with the determination; this is important since all sodium carbonate obtainable contains sulphur and there is sometimes more or less of it in cinchonine. All evaporations should be made over steam and the operations conducted in a place free from sulphur gases.

Note.-Evolution of sulphur in a stream of hydrogen saturated with hydrochloric acid as in Johnson's method is optional.

\section{SILICON}

The residue of tungstic oxide from which the solution for the determination of sulphur was filtered contains most of the silica. The residue from leaching the original fusion contains the rest.

Wash the residue from $\mathrm{Na}_{2} \mathrm{CO}_{3}$ fusion from the filter paper with a fine jet of water into a small casserole, add an excess of $\mathrm{HCl}$, and evaporate to dryness on a steam bath; heat the drr residue at $110^{\circ}$ to $120^{\circ} \mathrm{C}$. to dehydrate the silica, drench with hydrochloric acid, dilute to $100 \mathrm{c} \mathrm{c}$ with hot water heat until salts are in solution, and filter on a small filter paper (free from ash). Wash thoroughly with hot dilute $\mathrm{HCl}(1: 100)$ and finally with hot water. Reserve the filter.

Wash the residue of tungstic oxide, silica, and filter paper pulp from the filter into a small casserole, add $5 \mathrm{c}$ c of $\mathrm{HCl}$ and heat for a few minutes, then filter again through the same paper and wash it thoroughly with hot water. This second washing is necessary to remove sodium salts completely. (The original washing sufficed to remove all of the small amount of sulphur present.) Transfer the well-washed filter and residue to a platinum crucible, add the small filter containing the little silica recovered from the $\mathrm{Na}_{2} \mathrm{CO}_{3}$ fusion residue, and ignite both to constant weight in a platinum crucible. On account of the presence of filter-paper pulp, the ignited residue is porous and friable; if paper pulp had not been used the $\mathrm{WO}_{3}$ would be dense and not easily susceptible to the subsequent treatment. Cool the ignited residue of $\mathrm{WO}_{3}$ and $\mathrm{SiO}_{2}$ and weigh it. Add 2 or 3 drops of $\mathrm{H}_{2} \mathrm{SO}_{4}$ and 5 or $6 \mathrm{c}$ c of pure HF. Digest at a gentle heat for some time, and then slowly evaporate off the HF. When $\mathrm{H}_{2} \mathrm{SO}_{4}$ fumes are evolved, cool and again add $\mathrm{HF}$, digest as before, evaporate off the HF, ignite gently to expel $\mathrm{H}_{2} \mathrm{SO}_{4}$ and heat strongly for 10 or 15 minutes. Cool and weigh. The loss in weight after expulsion is $\mathrm{SiO}_{2}$ which multiplied by 0.4672 equals silicon.

Note.-Tentative standard, A 104-32T, entitled “Tentative Methods of Chemical Analysis of Ferrotungsten and Ferromolybdenum "was issued in 1932 .
621.21 FERROCHROMIUM.

AMERICAN SOCIETY FOR TESTING MATERIALS, STANDARD SPECIFICATIONS FOR FERROCHROMIUM, DESIGNATION A10127, 1927

\section{FERROCHORMIUM}

\section{SCOPE}

1. These specifications cover ferrochromium in four grades, as follows: High carbon, grade A; low carbon, grades B, C, and D.

\section{BASIS OF PURCHASE}

2. (a) Ferrochromium, high carbon.-This material shall be furnished to size as specified.

(b) Ferrochromium, low carbon.-This material shall be crushed to the specified size and mixed before packing, so that the quality in each package is uniform with the lot.

\section{CHEMICAL REQUIREMENTS}

3. The material shall conform to the following requirements as to chemical composition:

\begin{tabular}{|c|c|c|c|c|}
\hline & High carbun & \multicolumn{3}{|c|}{ Low carbon } \\
\hline & Grade A & Grade B & Grade C & Grade D \\
\hline Chromium .. & $\begin{array}{c}\text { Per cent } \\
60.00 \text { to } 75.00\end{array}$ & $\begin{array}{c}\text { Per cent } \\
60.00 \text { to } 75.00\end{array}$ & $\begin{array}{c}\text { Per cent } \\
60.00 \text { to } 75.00\end{array}$ & $\begin{array}{c}\text { Per cent } \\
60.00 \text { to } 75.00\end{array}$ \\
\hline Carbon & $\begin{array}{c}4.00 \text { to } 8.00 \\
\text { as specified. }\end{array}$ & 1.50 to 2.00 & 1.00 to 1.50 & $\begin{array}{l}\text { Under } 1.00^{1} \\
\text { as specified. }\end{array}$ \\
\hline Silicon. & As specified & As specified. & As specificd. & As specified \\
\hline
\end{tabular}

1 Grade D material may be obtained with various maximum carbon contents down to 0.10 per cent of carbon.

\section{SAMPLING}

4. When agreed upon by the purchaser and seller, the sampling shall be carried out in accordance with the standard methods of sampling ferro-allors. (See A. S. T. M., A103-27 (621.20, p. 1092).)

\section{METHODS OF CHEMICAL ANALYSIS}

5. When agreed upon by the purchaser and seller, the chemical analysis shall be carried out in accrodance with the standard methods of chemical analysis of ferro-allors. (See A. S. T. M., A104-27 $(621.20$, p. 1093).)

\section{UNITED STATES GOVERNMENT, DEPART- MENT OF COMMERCE, BUREAU OF STANDARDS, STANDARD SAMPLES, C25 AND SUPPLEMENT, 1927}

\section{FERROCHROMIUM (HIGH CARBON), SAMPLE NO. 64}

(This standard sample is prepared and sold by the Bureau of Standards for use by industrial organizations and others for checking the accuracy of analysis of ferrochromium, etc. It is accompanied by a certificate giving its complete analysis.) 
UNITED STATES GOVERNMENT, FEDERAL SPECIFICATIONS BOARD, FEDERAL SPECIFICATION FOR FERROCHROMIUM, QQ-F-151 MAY 3, 1932

\section{FERROCHROMIUM}

\section{GRADES}

1. Ferrochromium shall be of the following grades: Grade $\mathrm{A}$, grade $\mathrm{B}$, grade $\mathrm{C}$, grade $\mathrm{D}$, grade $\mathrm{E}$, and grade $\mathrm{F}$.

\section{MATERIAL}

2. The material shall be as required in the following paragraphs.

\section{GENERAL REQUIREMENTS}

3. Ferrochromium shall be furnished in lumps, crushed, or screened to size, as specified in the contract or purchase order.

\section{DETAIL REQUIREMENTS}

4. (a) Ferrochromium shall conform to the requirements as to chemical composition specified in the following table for the grade ordered:

\begin{tabular}{|c|c|c|c|c|}
\hline Grades & $\begin{array}{c}\text { Chromium, } \\
\text { permissible } \\
\text { range }\end{array}$ & Iron & $\begin{array}{l}\text { Carbon, } \\
\text { maxi- } \\
\text { mum }\end{array}$ & $\begin{array}{l}\text { Alumi- } \\
\text { num, } \\
\text { maxi- } \\
\text { mum }\end{array}$ \\
\hline $\begin{array}{l}\text { A } \\
\text { B } \\
\text { C } \\
\text { E } \\
\mathrm{F}_{-}\end{array}$ & $\begin{array}{c}\text { Per cent } \\
196 \\
67-72 \\
67-72 \\
6 \bar{i}-72 \\
67-72 \\
67-72\end{array}$ & $\begin{array}{l}\text { Per cent } \\
\text { Remainder--- } \\
\text { Remainder--. } \\
\text { Remainder--- } \\
\text { Remainder--- } \\
\text { Remainder } \\
\text { Remainder---. }\end{array}$ & $\begin{array}{r}\text { Per cent } \\
0.10 \\
.20 \\
.50 \\
1.00 \\
(2)\end{array}$ & $\begin{array}{r}\text { Percent } \\
1.00 \\
1.00 \\
1.00 \\
1.00 \\
1.00\end{array}$ \\
\hline
\end{tabular}

1 Minimum.

${ }^{2}$ Greater than 1 as specified.

(b) An analysis of each shipment of ferrochromium shall be furnished by the contractor, showing the percentage of the elements specified.

\section{TESTS AND METHODS OF INSPECTION}

5. (a) Check analyses may be made by the inspector, or through him by any Government laboratory or other designated representative, and without cost to the contractor.

(b) Representative samples shall be obtained from at least 10 per cent of the containers in a lot or shipment, consisting of at least 4 ounces for every 150 pounds, in the case of finely granulated or screened material. In the case of lump material at least 5 pounds shall be taken for every 1,000 pounds, or fraction thereof, in the lot or shipment. The samples so selected shall be crushed to at least $1 / 2$-inch size, well mixed, and the resultant sample quartered down to 5 pounds, which shall be sent to the designated laboratory for analysis.

\section{NOTES}

Grade A ferrochromium covers what is known commercially as pure carbon-free chromium, or chromium metal, intended only for use in the manufacture of alloys where iron is considered an impurity.

Grades B, C, D, and E ferrochromium are suitable for use in the manufacture of special steels and alloys where high carbon content is objectionable. Grade B provides for ferrochromium of a quality known commercially as "carbon-free."
Grade $\mathrm{F}$ covers high-carbon ferrochromium suitable for use in the manufacture of alloy steels. The maximum carbon permitted should be stated in the proposal and contract or purchase order.

Federal Specification QQ-M-1j1, Metals; General Specifications for the inspection of, of the issue in effect on date of invitation for bids, forms a part of this specification. (See 600-0, p. 3.)

\subsection{FERROMANGANESE, SPIEGELEISEN.}

AMERICAN SOCIETY FOR TESTING MATERIALS, STANDARD SPECIFICATIONS FOR SPIEGELEISEN, DESIGNATION A98-27, 1927

\section{SPIEGEIEISEN}

SCOPE

1. These specifications cover spiegeleisen in two grades as follows: Grade A and grade B.

\section{BASIS OF PURCHASE}

2. The spiegeleisen shall be furnished in pigs, or lumps, and each shipment shall be uniform as to the grade specified.

\section{CHEMICAL REQUIREMENTS}

3. The material shall conform to the following requirements as to chemical composition:

\begin{tabular}{c|r|r|r|r|r} 
Grade & $\begin{array}{c}\text { Manga- } \\
\text { nese }\end{array}$ & $\begin{array}{c}\text { Carbon, } \\
\text { maximum }\end{array}$ & $\begin{array}{c}\text { Phos- } \\
\text { phorus, } \\
\text { maximum }\end{array}$ & $\begin{array}{c}\text { Sulphur, } \\
\text { maximum }\end{array}$ & $\begin{array}{c}\text { Silicon, } \\
\text { maximum }\end{array}$ \\
\hline & $\begin{array}{r}\text { Per cent } \\
19 \text { to } 21\end{array}$ & $\begin{array}{r}\text { Per cent } \\
6.50\end{array}$ & $\begin{aligned} \text { Per cent } \\
0.15\end{aligned}$ & $\begin{aligned} \text { Per cent } \\
0.04\end{aligned}$ & $\begin{array}{c}\text { Per cent } \\
\text { As specified. } \\
\text { Do. }\end{array}$ \\
\hline B...-- 19 & 6.50 & .25 & .05 & . \\
\hline
\end{tabular}

\section{SAMPLING AND CHEMICAL ANALYSIS}

4. (For sampling, see A. S. T. M., A103-27 (621.20, p. 1092). For methods of chemical analysis, see A. S. T. M., A104-27 (621.20, p. 1093).)

\section{AMERICAN SOCIETY FOR TESTING MA- TERIALS, STANDARD SPECIFICATION FOR FERROMANGANESE, DESIGNATION A99-27, $192 \%$}

\section{FERROMAGANESE SCOPE}

1. These specifications cover ferromanganese in one grade only.

\section{BASIS OF PURCHAS}

2. Ferromanganese shall be furnished in pigs, lumps, crushed, or screened to size, as specified.

\section{CHEMICAL REQUIREMENTS}

3. The material shall conform to the following requirements as to chemical composition:

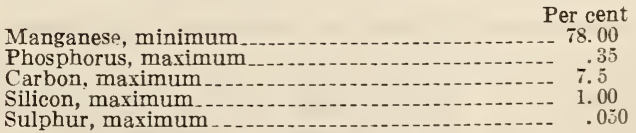

\section{SAMPLING AND CHEMICAL ANALYSIS}

4. (For sampling, see A. S. T. Mi., A103-27 (621.20, p. 1092). For methods of chemical analysis, see A. S. T. M., A104-27 (621.20, p. 1093) .) 
UNITED STATES GOVERNMENT, DEPARTMENT OF COMMERCE, BUREAU OF STANDARDS; STANDARD SAMPLES, C25 AND SUPPLEMENT, 1927

SPIEGELEISEN，SAMPLE NO. 66; MANGANESE METAL, SAMPLE NO. 67; FERROMANGANESE, SAMPLE NO. 68

(These standard samples are prepared and sold by the Bureau of Standards for use by industrial organizations and others for checking the accuracy of analysis of similar products. Each is accompanied by a certificate giving the complete analysis of the particular sample.)

UNITED STATES GOVERNMENT, FEDERAL SPECIFICATIONS BOARD, FEDERAL SPECIFICATION FOR FERROMANGANESE, QQ-F-161, MAY 26, 1931

\section{FERROMANGANESE}

\section{A. APPLICABLE FEDERAL SPECIFICATIONS}

A-1. Federal specification QQ-M-151 (formerly No. 339), general specifications for metals, of the issue in effect on date of invitation for bids, shall form a part of this specification. (See 600.0, p. 3.)

\section{B. GRADES}

B-1. Ferromanganese shall be of the following grades: Grade A and grade B.

\section{MATERIAL}

C-1. The material shall be as required in the following paragraphs.

\section{GENERAL REQUIREMENTS}

D-1. Ferromanganese shall be furnished in pigs, lumps, or crushed in screened sizes, as specified in the contract or purchase order.

\section{E. DETAIL REQUIREMENTS}

E-1. Ferromanganese shall conform to the requirements as to chemical composition specified in the following table for the grade ordered:

\begin{tabular}{|c|c|c|c|c|c|c|c|}
\hline Grade & $\begin{array}{l}\text { Manga- } \\
\text { nese, } \\
\text { mini- } \\
\text { mum }\end{array}$ & $\begin{array}{l}\text { Car- } \\
\text { bon, } \\
\text { maxi- } \\
\text { mum }\end{array}$ & $\begin{array}{c}\text { Phos- } \\
\text { phorus, } \\
\text { maxi- } \\
\text { mum }\end{array}$ & $\begin{array}{l}\text { Sul- } \\
\text { phur, } \\
\text { maxi- } \\
\text { mum }\end{array}$ & $\begin{array}{l}\text { Sili- } \\
\text { con, } \\
\text { maxi- } \\
\text { mum }\end{array}$ & $\begin{array}{l}\text { Alumi- } \\
\text { num, } \\
\text { maxi- } \\
\text { mum }\end{array}$ & $\begin{array}{l}\text { Iron, } \\
\text { maxi- } \\
\text { mum }\end{array}$ \\
\hline $\begin{array}{l}\mathrm{A} \\
\mathrm{B}\end{array}$ & $\begin{array}{c}\text { Per cent } \\
95.00 \\
78.00\end{array}$ & $\begin{array}{c}\text { Per cent } \\
0.25 \\
7.50\end{array}$ & $\begin{array}{c}\text { Per cent } \\
0.25 \\
.35\end{array}$ & $\begin{array}{c}\text { Per cent } \\
0.10 \\
.05\end{array}$ & $\begin{array}{c}\text { Per cent } \\
1.50 \\
1.00\end{array}$ & $\mid \begin{array}{c}\text { Per cent } \\
1.25 \\
.20\end{array}$ & $\begin{array}{l}\text { Per cent } \\
2.50 \\
\text { Remain- } \\
\text { der. }\end{array}$ \\
\hline
\end{tabular}

E-2. An analysis of each shipment of ferromanganese shall be made by the contractor showing the percentages of the elements specified.

\section{F METHODS OF SAMPLING, INSPECTION, AND TESTS}

F-1. Check analyses may be made by the inspector, or through him by any Government laboratory or other designated representative and without cost to the contractor.

F-2. Representative samples shall be obtained from at least 10 per cent of the containers in a lot or shipment, consisting of at least 4 ounces for every 150 pounds, in the case of finely granulated or screened material. In the case of lump material at least 5 pounds shall be taken for every 1,000 pounds, or fraction thereof, in the lot or shipment. The samples so selected shall be crushed to at least $1 / 2$-inch size, well mixed, and the resultant sample quartered down to 5 pounds, which shall be sent to the designated laboratory for analysis.

\section{NOTES}

I-1. Grade A ferromanganese covers what is known as pure carbon-free manganese metal, intended only for use in the manufacture of highgrade aluminum and nonferrous castings, and of crucible steel.

I-2. Grade B covers a good grade of commercial ferromanganese.

\section{UNITED STATES GOVERNMENT, FEDERAL} SPECIFICATIONS BOARD, FEDERAL SPECIFICATION FOR SPIEGELEISEN, NO. 273, JANUARY 22, 1925 (CURRENT DESIGNATION : QQ-S-611)

\section{SPIEGELEISEN}

In respect to material, general requirements, analysis of shipments, tests, sampling, packing, and marking this specification is identical with F. S. B. QQ-F-151. (See 621.21, p. 1103.) Like A. S. T. M., A98, given above, it covers two grades, $\mathrm{A}$ and $\mathrm{B}$, the chemical compositions of which, however, are as follows:

\begin{tabular}{|c|c|c|c|c|c|}
\hline Grade & $\begin{array}{l}\text { Manga- } \\
\text { nese }\end{array}$ & $\begin{array}{c}\text { Carbon, } \\
\text { maximum }\end{array}$ & $\begin{array}{c}\text { Phos- } \\
\text { phorus, } \\
\text { maximum }\end{array}$ & $\begin{array}{l}\text { Sulphur, } \\
\text { maximum }\end{array}$ & $\begin{array}{l}\text { Silicon, } \\
\text { maximum }\end{array}$ \\
\hline A.... & $\begin{array}{r}\text { Per cent } \\
19-22 \\
16-19\end{array}$ & $\begin{array}{r}\text { Per cent } \\
6.5 \\
6.0\end{array}$ & $\begin{array}{r}\text { Per cent } \\
0.10 \\
.25\end{array}$ & $\begin{array}{r}\text { Per cent } \\
0.04 \\
.05\end{array}$ & $\begin{array}{r}\text { Per cent } \\
1.50 \\
2.0\end{array}$ \\
\hline
\end{tabular}

\subsection{FERROMOLYBDENUM.}

UNITED STATES GOVERNMENT, FEDERAL SPECIFICATIONS BOARD, FEDERAL SPECIFICATION FOR FERROMOLYBDENUM, NO. 143, MAY 1, 1924 (CURRENT DESIGNATION : $Q Q-F-171$ )

\section{FERROMOLYBDENUM}

(In respect to material, general requirements, analysis of shipments, tests, sampling, packing, and marking this specification is identical with F.S. B. QQ-F-151.) (See 621.21, p.1103.) It covers one grade of ferromolybdenum, the chemical composition of which is as follows:)

\begin{tabular}{|c|c|c|c|}
\hline Molybdenum & $\begin{array}{c}\text { Carbon, } \\
\text { maximum }\end{array}$ & $\begin{array}{c}\text { Phos- } \\
\text { phorus, } \\
\text { maximum }\end{array}$ & $\begin{array}{c}\text { Sulphur, } \\
\text { maximum }\end{array}$ \\
\hline $\begin{array}{c}\text { Per cent } \\
50.0-60.0\end{array}$ & $\begin{array}{c}\text { Per cent } \\
2.00\end{array}$ & $\begin{array}{c}\text { Per cent } \\
0.10\end{array}$ & $\begin{array}{c}\text { Per cent } \\
0.25\end{array}$ \\
\hline
\end{tabular}

(The following additional requirements appear in this specification:)

1. If the results of the check analysis show a molybdenum content of more than 1 per cent below the minimum specified, such material may be accepted, in the discretion of the contracting officer, but the molybdenum content obtained by such analysis shall constitute the basis of payment. 
2. Except as provided for in the preceding paragraph, ferromolybdenum not complying with all the requirements of this specification and of the contract or purchase order shall be rejected.

3. If special packing be necessary to secure the acceptance of ferromolybdenum by common or other carriers for safe transportation at the lowest rate to the point of delivery, such packing shall be provided by the contractor without additional expense to the purchaser.

\section{ADDITIONAL INFORMATION}

4. The melting point of ferromolybdenum is about $2,700^{\circ} \mathrm{F}$.

621.24 FERROSILICON.

\section{AMERICAN SOCIETY FOR TESTING MATE- RIALS, STANDARD SPECIFICATIONS FOR FERROSILICON, DESIGNATION, A100-2\%,} 1927

\section{FERROSILICON}

SCOPE

1. These specifications cover ferrosilicon in three grades, as follows: Grade A, grade B, and grade $\mathrm{C}$.

\section{BASIS OF PURCHASE}

2. (a) The ferrosilicon shall be furnished in lumps, or crushed or screened to size, as specified.

(b) When furnished in lump or granulated form, the ferrosilicon shall be free from excessive disintegration.

\section{CHEMICAL REQUIREMENTS}

3. The material shall conform to the following requirements as to chemical composition:

\begin{tabular}{|c|c|c|c|}
\hline & Grade A & Grade B & Grade C \\
\hline Silicon..- & $\begin{array}{c}\text { Per cent } \\
47.00-53.00\end{array}$ & $\begin{array}{c}\text { Per cent } \\
72.00-78.00\end{array}$ & $\begin{array}{c}\text { Per cent } \\
85.00-95.00\end{array}$ \\
\hline
\end{tabular}

4. An analysis of each shipment of ferrosilicon shall be furnished the purchaser, showing the percentage of silicon.

\section{SAMPLING AND CHEMICAL ANALYSIS}

5. For sampling, see A. S. T. M., A103-27 (621.20, p. 1092). For methods of chemical analysis, see A. S. T. M., A104-27 (621.20, p. 1093).)

UNITED STATES GOVERNMENT, DEPARTMENT OF COMMERCE, BUREAU OF STANDARDS; STANDARD SAMPLES, C25 AND SUPPLEMENT, 1927

REFINED SILICON, SAMPLE NO. 57. FERROSILICON (75 PER CENT SILICON), SAMPLE NO. 58. FERROSILICON (50 PER CENT SILICON), SAMPLE NO. 59

(These standard samples are prepared and sold by the Bureau of Standards for use by industrial organizations and others for checking the accuracy of analysis of similar products, etc. Each is accompanied with a certificate giving the complete analysis of the particular sample.)
UNITED STATES GOVERNMENT, FEDERAL SPECIFICATIONS

SPECIFICATION FOR FERROSILICON, NO. 145, MAY 1, 1924. (CURRENT DES-

IGNATION : QQ-F-181)

\section{FERROSILICON}

(In respect to material, general requirements, analysis of shipments, tests, sampling, packing, and marking this specification is identical with F. S. B. QQ-F-151. (See 621.21, p. 1103.) It covers three grades of ferrosilicon, $\mathrm{A}, \mathrm{B}$, and $\mathrm{C}$, the chemical compositions of which are as follows:)

\begin{tabular}{|c|c|c|c|}
\hline & Grade A & Grade B & Grade C \\
\hline Silicon.....-- & $\begin{array}{c}\text { Per cent } \\
70.0-80.0\end{array}$ & $\begin{array}{c}\text { Per cent } \\
45.0-55.0\end{array}$ & $\begin{array}{c}\text { Per cent } \\
10.0-20.0\end{array}$ \\
\hline
\end{tabular}

(The following additional requirements appear in this specification):

1. If the results of the check analysis show a silicon content of more than 1 per cent below the minimum specified for grades $\mathrm{A}$ and $\mathrm{B}$, or more than 0.5 per cent below the minimum specified for grade $\mathrm{C}$, such material may be accepted, in the discretion of the contracting officer, but the silicon content obtained by such check analysis shall constitute the basis of payment.

2. Except as provided for in the preceding paragraph, ferrosilicon not complying with all the requirements of this specification and of the contract or purchase order shall be rejected.

3. If special packing be necessary to secure the acceptance of ferrosilicon by common or other carriers for safe transportation at the lowest rate to the point of delivery, such packing shall be provided by the contractor without additional expense to the purchaser.

\section{ADDITIONAL INFORMATION}

4. Grade A ferrosilicon is suitable for use in the manufacture of steels with a relatively high silicon content, such as steels for springs, and for use on certain electrical equipment.

5. Grade B ferrosilicon is suitable for use as a deoxidizer in the manufacture of steels.

6. Grade $\mathbf{C}$ ferrosilicon is also known as siliconeisen, high-silicon pig, or Bessemer ferrosilicon. It is used in the manufacture of high-silicon acid-resistant iron and in general cupola practice.

\subsection{FERROTUNGSTEN.}

\section{UNITED STATES GOVERNMENT, DEPART- MENT OF COMMERCE, BUREAU OF STANDARDS; STANDARD SAMPLES, C25 AND SUPPLEMENT, 1927}

\section{FERROTUNGSTEN SAMPLE NO. 75}

(This standard sample is prepared and sold by the Bureau of Standards for use by industrial organizations and others for checking the accuracy of analysis of ferrotungsten, etc. It is accompanied by a certificate giving its complete analysis.) 


\subsection{FERROTITANIUM, FERROURANIUM, FER- ROVANADIUM.}

AMERICAN SOCIETY FOR TESTING MATERIALS, STANDARD SPECIFICATIONS FOR FERROVANADIUM DESIGNATION A102-27, 1927

\section{FERROVANADIUI SCOPE}

1. These specifications cover ferrovanadium in four grades, as follows: Grade A, grade B, grade C, and grade $\mathrm{D}$.

\section{BASIS OF PURCHASE}

2. The material furnished under this specification shall be crushed to the specified size and mixed before packing, so that the quality in each package is uniform with the lot. The material shall be packed in sound containers sufficiently strong to prevent loss in transportation.

\section{CPIEMICAL REQUIREMENTS}

3. The material shall conform to the following requirements as to chemical composition:

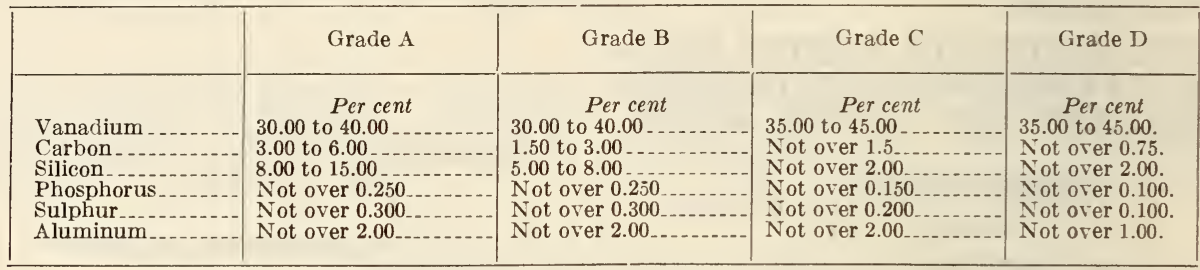

\section{SAMPLING AND CHEMICAL ANALYSIS}

4. (For sampling, see A. S. T. M., A103-27 (621.20 p. 1092). For methods of chemical analysis, see A. S. T. M., A104-27 (621.20, p. 1093).)

\section{UNITED STATES GOVERNMENT, DEPART- MENT OF COMMERCE, BUREAU OF STANDARDS, STANDARD SAMPLES, C25 AND SUPPLEMENT, $192 \%$}

\section{FERROVANADIUM, SAMPIE NO. 61}

(This standard sample of high-carbon ferrovanadium is prepared and sold by the Bureau of Standards for use by industrial organizations and others for checking the accuracy of analysis of ferrovanadium, etc. It is accompanied by a certificate giving its complete analysis.)

\section{UNITED STATES GOVERNMENT, FEDERAI SPECIFICATIONS BOARD; FEDERAL SPECIFICATION FOR FERROVANADIUM, QQ-F-201, MAY 31, 1932}

\section{FERROVANADIUM}

(In respect to material, general requirements, analysis of shipments, tests, packing, and marking this specification is identical with F. S. B. QQ-F-151. (See 621.21 p. 1103.) It covers two grades of ferrovanadium, the chemical compositions of which are as follows:)

\begin{tabular}{|c|c|c|c|c|c|c|c|}
\hline Grade & $\begin{array}{l}\text { Vana- } \\
\text { dium }\end{array}$ & $\begin{array}{l}\text { Alumi- } \\
\text { num, } \\
\text { maxi- } \\
\text { mum }\end{array}$ & $\begin{array}{l}\text { Sili- } \\
\text { con, } \\
\text { maxi- } \\
\text { mum }\end{array}$ & $\begin{array}{l}\text { Sul- } \\
\text { phur, } \\
\text { maxi- } \\
\text { mum }\end{array}$ & $\begin{array}{l}\text { Phos- } \\
\text { phorus, } \\
\text { maxi- } \\
\text { mum }\end{array}$ & $\begin{array}{l}\text { Car- } \\
\text { bon, } \\
\text { maxi- } \\
\text { mum }\end{array}$ & $\begin{array}{l}\text { Man- } \\
\text { ga- } \\
\text { nese, } \\
\text { maxi- } \\
\text { mum }\end{array}$ \\
\hline $\begin{array}{l}A_{-. .} \\
B_{-. .}\end{array}$ & $\begin{array}{c}\text { Per ct. } \\
\text { 30. 0-40. } 0 \\
30.0-40.0\end{array}$ & $\begin{array}{r}\text { Per ct. } \\
1.00 \\
2.00\end{array}$ & $\begin{array}{r}\text { Perct. } \\
1.00 \\
2.00\end{array}$ & $\begin{array}{c}\text { Perct. } \\
0.03 \\
.35\end{array}$ & $\begin{array}{r}\text { Per ct. } \\
0.04 \\
.08\end{array}$ & $\begin{array}{c}\text { Per ct. } \\
0.75 \\
2.0\end{array}$ & $\begin{array}{r}\text { Perct. } \\
0.75\end{array}$ \\
\hline
\end{tabular}

(Method of sampling is the same as in Federal Specification $\mathrm{QQ}-\mathrm{F}-191$ below for sampling lump ferrotitanium.)
1. Circular proposals and requests for bids on ferrovanadium should require that all bidders quote "per pound of contained vanadium." Payment should be made on the basis of vanadium content only.

\section{UNITED STATES GOVERNMENT, FEDERAL SPECIFICATIONS BOARD, FEDERAL SPECIFICATION FOR FERROTITANIUM, QQ-F-191, JANUARY 5, 1932}

\section{FERROTITANIUM}

\section{A. APPLICABLE FEDERAL SPECIFICATIONS}

A-1. Federal specification $Q Q-M-151$ (old No. 399a).-Metals; general specifications for-of the issue in effect on date of invitation for bids-forms a part of this specification. (See 600.0, p. 3.)

\section{B. GRADES}

B-1. Ferrotitanium shall be of the following grades: Grade $\mathrm{A}$ and grade $\mathrm{B}$.

\section{MATERIAL}

C-1. The material shall be as required in the following paragraphs.

\section{GENERAL REQUIREMENTS}

D-1. Ferrotitanium shall be furnished in lumps, crushed or screened to size, as specified in the contract or purchase order.

\section{E. DETAIL REQUIREMENTS}

E-1. Ferrotitanium shall conform to the requirements as to chemical composition specified in the following table for the grade ordered.

\begin{tabular}{|c|c|c|c|c|c|c|}
\hline Grade & $\begin{array}{l}\text { Tita- } \\
\text { nium }\end{array}$ & $\begin{array}{c}\text { Carbon, } \\
\text { maxi- } \\
\text { mum }\end{array}$ & $\begin{array}{l}\text { Silicon, } \\
\text { maxi- } \\
\text { mum }\end{array}$ & $\begin{array}{l}\text { Alumi- } \\
\text { num, } \\
\text { maxi- } \\
\text { mum }\end{array}$ & \begin{tabular}{|} 
Copper \\
phosphor- \\
us, sul- \\
fur, and \\
manga- \\
nese com- \\
bined, \\
maxi- \\
mum
\end{tabular} & Iron, per cent \\
\hline $\begin{array}{l}A_{1} \\
B_{-}\end{array}$ & $\begin{array}{c}\text { Per cent } \\
25-30 \\
15-18\end{array}$ & $\begin{array}{c}\text { Per cent } \\
0.25 \\
7.50\end{array}$ & $\begin{array}{c}\text { Per cent } \\
3.00 \\
3.00\end{array}$ & $\begin{array}{l}\text { Per cent } \\
6.00 \\
1.50\end{array}$ & $\begin{array}{r}\text { Per cent } \\
0.50 \\
.50\end{array}$ & $\begin{array}{c}\text { Remainder. } \\
\text { Do. }\end{array}$ \\
\hline
\end{tabular}


E-2. Analysis of each shipment of ferrotitanium shall be made by the contractor showing the percentages of the elements specified.

\section{F. METHODS OF SAMPIING, INSPECTION, AND TESTS}

F-1. Check analyses may be made by the inspector, or through him by any Government laboratory or other designated representative and without cost to the contractor.

F-2. Representative samples shall be obtained from at least 10 per cent of the containers in a lot or shipment, consisting of at least 4 ounces for every 150 pounds, in the case of finely granulated or screened material. In the case of lump material at least 5 pounds shall be taken for every 1,000 pounds, or fraction thereof, in the lot or shipment. The samples so selected shall be crushed to at least $1 / 2$-inch size, well mixed, and the resultant sarnple quartered down to 5 pounds, which shall be sent to the designated laboratory for analysis.

\section{NOTES}

I-1. Grade A ferrotitanium covers what is known as commercially carbon-free alloy, intended for use in the manufacture of products wherein this grade is specifically required.

I-2. Grade B covers a good grade of commercial ferrotitanium.

\subsection{FERROPHOSPHORUS.}

UNITED STATES GOVERNMENT, DEPARTMENT OF COMMERCE, BUREAU OF STANDARDS; STANDARD SAMPLES, C25 AND SUPPLEMENT, 1927

\section{FERROPHOSPHORUS, SAMPLE NO. 90}

(This standard sample is prepared and sold by the Bureau of Standards for use by industrial organizations and others for checking the accuracy of analysis of ferrophosphorus. It is accompanied by a certificate giving its analysis as regards phosphorus.)

\subsection{FINISHED AND SEMIFINISHED AL- LOY STEELS.}

621.30 GENERAL ITEMS.

\section{AMERICAN SOCIETY FOR TESTING MATE- RIAIS, STANDARD METHODS OF CHEMI- CAL ANALYSIS OF ALLOY STEELS, SERIAL DESIGNATION A55-24, 1924}

\section{CHEMICAL ANALYSIS OF ALLOY STEELS}

\section{SILICOMANGANESE STEELS}

All determinations are carried out as in the standard methods of chemical analysis of plain carbon steel as specified in A33. (See 600.1, p. 10.)

(In some respects the specifications in A33 are either identical with or substantially the same as the specifications in A64 and B28. Direct reference to all such identities will be found at the proper place in A33.)

\section{NICKEL STEEI}

\section{Determination of Carbon}

See the determination of carbon in plain carbon steel by the direct-combustion method in A33 600.1, p. 10) and also in sampling and chemical analysis of pig and cast iron in A64 (601.20, p. 119).
Determination of Manganese by the Bismuthate Method (Absence of Cobalt)

(Same as determination of manganese by the bismuthate method (absence of cohalt) in A33 (see 600.1, p. 10) except in the following particulars:)

\section{SOLUTIONS REQUIRED}

(Dilute nitric acid $(1: 3)$, dilute nitric acid (3:97), ferrous ammonium sulphate solution, standard potassium permanganate solution, and standard sodium arsenite solution, all of which are the same as like solutions in the determination of manganese by the bismuthate method in A64. See 601.20 , p. 119.)

\section{METHOD}

(Ferrous sulphate-permanganate titration (less than 0.05 per cent chromium) same as in A64. See 601.20 , p. 119.)

Determination of Manganese by the Persulphate Method (Routine, in the Preserce of Cobalt)

\section{SOLUTIONS REQUIRED}

(Conform to like solutions in the determination of manganese by the persulphate method of A64. See 601.20 , p. 119.)

\section{METHOD}

(Same as in the determination of manganese by the persulphate method in A33 (see 600.1, p. 10) until addition of $\mathrm{AgNO}_{3}$ solution ( 0.133 per cent), then proceed as indicated in A64 (see 601.20, p. 119).)

Determination of Phosphorus by the Molybdate-Magnesia Method

(Same as A33 (see 600.1, p. 10), except in respect to ammonium molybdate solution, which conforms to B28 (see 646.0, p. 1243).)

Determination of Phosphorus by the Alkalimetric Method (Routine)

(In respect to solutions required, same as molybdate-magnesia method in A33 (see 600.1, p. 10), except for ammonium molybdate, potassium nitrate (1 per cent), phenolphthalein indicator, standard sodium hydroxide, and standard nitric acid, all of which conform to B28 (see 646.0, p. 1243).)

\section{METHOD}

(Same as A33. See 600.1, p. 10.)

Notes.-(Same as A33. See 600.1, p. 10.)

$$
\text { Determination of Sulphur by the Oxidation Method }
$$

(Same as A33. See 600.1, p. 10.)

Determination of Sulphur by the Evolution-Titration Method (Routine)

(Same as A64 (see 601.20 , p. 119) in respect to diagram illustrating apparatus, solutions required, and method, except that here the chemical reactions in the potassium iodide solution are omitted, and for the starch solution in A64 is substituted the starch indicator specified in A33 (see 600.1, p. 10). The notes are identical with those given in A33, with the addition of the following:)

NOTE-The evolution-titration method should not be used with steels containing appreciable amounts of tungsten or molybdenum or more than 0.4 per cent of copper.

\section{Determination of Silicon by the Nitrosulphuric Method}

(In respect to solutions required, same as A33 (see 600.1 , p. 10), except for dilute hydrochloric acid (5:95) which conforms to the determination of silicon by the nitrosulphuric acid method in A64 (see 601.20, p. 119).) 


\section{METHOD}

(Identical with A64 (see 601.20, p. 119), except that $80 \mathrm{c} \mathrm{c}$ of the nitrosulphuric acid is added cautiously to $4.676 \mathrm{~g}$ of steel, and that the difference in weights in milligrams divided by 100 equals the percentage of silver, instead of being divided by 50 , as in $\mathrm{A} 64$.

Allowable error.-(Same as in A33: (See 600.1, p. 10).)

Note.-(Same as in A33 (see 600.1, p. 10).)

Determination of Silicon by the Sulphuric Acid Method (Optional)

(Same as A64 (see 601.20, p. 119), except that here $2.338 \mathrm{~g}$ of steel is substituted for the $2.336 \mathrm{~g}$ of iron specified in A64.)

Determination of Nickel by the Gravimetric Dimethylglyoxime Method

SOLUTIONS REQUIRED

Dilute nitric acid $(1: 3)$.- Mix 250 c c of $\mathrm{HNO}_{3}$ (specific gravity 1.42) with $750 \mathrm{c} \mathrm{c}$ of distilled water. Dilute hydrochloric acid $(1: 1)$. - Mix $500 \mathrm{c}$ c of $\mathrm{HCl}$ (specific gravity 1.19) and $500 \mathrm{c}$ c of distilled water.

Dimethylglyoxime (1 per cent).-(Same as A64. (See 601.20, p. 119.)

\section{METHOD}

For ordinary nickel steel take a $1 \mathrm{~g}$ sample; with less than 0.10 or more than 5 per cent nickel, larger or smaller samples may be taken. In the presence of less than 0.5 per cent chromium dissolve the sample in $50 \mathrm{c}$ c of hot dilute $\mathrm{HNO}_{3}(1: 3)$. When chromium is present in amounts greater than 0.5 per cent or under circumstances where chromium carbides are present, dissolve the sample in $60 \mathrm{c}$ c of dilute $\mathrm{HCl}(1: 1)$ with the aid of heat and then oxidize the iron and the carbides by adding $\mathrm{HNO}_{3}$ (specific gravity 1.42) drop by drop, until effervescence ceases. Boil to remove the products resulting from the decomposition of the $\mathrm{HNO}_{3}$ and then cool the solution. Add in succession, with stirring after each addition, $12 \mathrm{~g}$ of citric acid or equivalent solution, $20 \mathrm{c}$ c of $\mathrm{NH}_{4} \mathrm{OH}$ (specific gravity 0.90 ), sufficient solution of dimethylglyoxime to precipitate all nickel present, and finally enough more $\mathrm{NH}_{4} \mathrm{OH}$ to make the solution distinctly ammoniacal. The solution will usually have a volume of 200 to $250 \mathrm{c}$ c and precipitation is complete immediately if the amount of nickel is over 0.10 per cent. With less nickel or in umpire analyses, digestion for one hour is desirable. Stir the solution thoroughly and filter on an asbestos mat on a 2-inch perforated porcelain plate or a Büchner funnel, avoiding strong suction and taking care that the funnel always remains partly filled with liquid. Thoroughly wash the precipitate with water, discard the filtrate and washings, rinse the funnel stem and then with the mat still in place and with suction off, add $25 \mathrm{c}$ c of a mixture of $20 \mathrm{c} \mathrm{c}$ of $\mathrm{HCl}$ (specific gravity 1.19) and 5 c c of $\mathrm{HNO}_{3}$ (specific gravity 1.42). After one minute, apply suction till dry, repeat the treatment with $25 \mathrm{c}$ c of the mixture, drain, and wash thoroughly with water. Transfer the solution to a $400 \mathrm{c}$ c beaker and evaporate the solution to 100 c c. Filter if necessary and repeat the precipitation of the glyoxime by adding $5 \mathrm{~g}$ citric acid, nearly neutralizing the absolutely clear solution with $\mathrm{NH}_{4} \mathrm{OH}$ (specific gravity 0.90 ) and then adding dimethylglyoxime and $\mathrm{NH}_{4} \mathrm{OH}$ as before. Finally filter on a weighed Gooch crucible, thoroughly wash with hot water, dry at $110^{\circ}$ to $120^{\circ} \mathrm{C}$. and weigh. The precipitate contains 20.31 per cent of nickel.

Allowable error. - Percentages of nickel determined by the above method should be accurate to $\pm(0.005+(0.01 \times$ the percentage of nickel found)
Notes.-Direct weighing of the precipitate is desirable when small amounts of nickel are concerned. Large preripitates are more con. veniently ignited at red heat to $\mathrm{NiO}$. In this case, rare must be taken to prevent sublimation of the undecomposed salt. This may be accomplished by inclosing the wet paper and precipitate in or two wet ashless papers and slowly igniting so that the papers one or two wet ashless papers and slowly igniting so that the papers char before taking fire. In this way the compound is decomposed and ignition may proceed at full red heat.

It is difficult to precipitate nickel and to obtain it free from cobalt when cobalt is preponderant. Complete precipitation may be obtained by adding sufficient dimethylglyoxime to react with both cobalt and nickel and digesting for a longer period, or by oxidizing the cobalt to the trivalent condition by means of $\mathrm{NaClO}_{3}$ or $\left(\mathrm{NH}_{4}\right)_{2}$ $\mathrm{S}_{2} \mathrm{O}_{8}$ in $\mathrm{mm}$ (n) im in dissolved, and reprecipitated.

Determination of Nickel by the Cyanide-Titration Method (Routine)

\section{Preliminary Treatment}

The cyanide titration may be performed in a solution prepared as in $(a),(b)$, or $(c)$ below:

(a) Preliminary separation with dimethylglyoxime.-(Presence of chromium, copper, cobalt and small amounts of tungsten.)

Proceed as in the gravimetric method until the dimethylglyoxime precipitate has been dirsolved in aqua regia, and then evaporate the solution to 50 c c or until free from dimethylglyoxime and oxidizing gases. Cool the solution, with ice if desired, neutralize with $\mathrm{NH}_{4} \mathrm{OH}$ (specific gravity 0.90 ) (rosolic acid is a convenient indicator), add $5 \mathrm{c} \mathrm{c}$ in excess, dilute to $250 \mathrm{c} \mathrm{c}$ and proceed as in the final titration below.

(b) Preliminary ether extraction.-(Absence of cobalt and more than 1 per cent tungsten.)

\section{SOLUTIONS REQUIRED}

Dilute hydrochloric acid (3:2).-Mix 600 c c of $\mathrm{HCl}$ (specific gravity 1.19 ) and $400 \mathrm{c} \mathrm{c}$ of distilled water.

Acidulated hydrogen sulphide water.-Add 25 c c of $\mathrm{HCl}$ (specific gravity 1.19) to $500 \mathrm{c} \mathrm{c}$ of distilled water and saturate with $\mathrm{H}_{2} \mathrm{~S}$.

\section{METHOD}

In a $150 \mathrm{c}$ c beaker dissolve $1 \mathrm{~g}$ of the steel in $20 \mathrm{c} \mathrm{c}$ of the dilute $\mathrm{HCl}(3: 2)$, add about $2 \mathrm{c} \mathrm{c}$ of $\mathrm{HNO}_{3}$ (specific gravity 1.42) to oxidize the iron, and boil to expel the oxides of nitrogen. Cool and transfer the solution into an 8-ounce separatory funnel, rinsing the beaker with small portions of the dilute $\mathrm{HCl}(3: 2)$. Add $50 \mathrm{c} \mathrm{c}$ of ether, shake for five minutes, let settle for one minute, and then draw off the lower clear solution into another 8ounce separatory funnel. Add 10 c c of dilute $\mathrm{HCl}(3: 2)$ to the solution in the first separatory funnel, cool, shake thoroughly, allow to settle for one minute, and then draw off the lower clear solution into the second separatory funnel. To the combined solutions in the second separatory funnel add $50 \mathrm{c} \mathrm{c}$ of ether, shake for five minutes, let settle for 1 minute, and then draw off the clear layer into a $150 \mathrm{c}$ c beaker. Heat the aqueous solution gently to expel the ether, add $0.2 \mathrm{~g}$ of potassium chlorate, boil until the chlorate is decomposed, dilute to 100 c c with hot water, make faintly ammoniacal, and boil for five minutes. Filter and wash with hot water. To the filtrate add $10 \mathrm{c} \mathrm{c}$ of $\mathrm{HCl}$ (specific gravity 1.19) heat just short of boiling and precipitate the copper with hydrogen sulphide. Filter and wash with acidulated hydrogen sulphide water. Boil the filtrate to expel hydrogen sulphide, neutralize with ammunia as in $(a)$, add $5 \mathrm{c} c$ in excess, dilute to $250 \mathrm{c} \mathrm{c}$, and proceed as in the final titration below.

(c) Direct titration.-(Absence of copper, cobalt, and more than 1 per cent of tungsten). 


\section{SOLUTIONS REQUIRED}

Dilute nitric acid (specific gravity 1.20).-Mix 380 c c of $\mathrm{HNO}_{3}$ (specific gravity 1.42) and $620 \mathrm{c} \mathrm{c}$ of distilled water.

Ammonium persulphate (15 per cent).-Make up as needed by dissolving $15 \mathrm{~g}$ of the salt in $100 \mathrm{c} \mathrm{c}$ of distilled water.

Dilute ammonia (1:1).-Mix equal volumes of $\mathrm{NH}_{4} \mathrm{OH}$ (specific gravity 0.90 ) and distilled water. Sulphuric-citric acids.-Dissulve $200 \mathrm{~g}$ of citric acid in a cooled mixture of $100 \mathrm{c} \mathrm{c} \mathrm{H}_{2} \mathrm{SO}_{4}$ (specific gravity 1.84) and $900 \mathrm{c}$ c of distilled water.

\section{METHOD}

Transfer $1 \mathrm{~g}$ of the sample to a $400 \mathrm{c}$ c beaker, treat with $20 \mathrm{c}$ c of dilute $\mathrm{HNO}_{3}$ (specific gravity 1.20 ) and heat until the sample is dissolved. Add 8 to $10 \mathrm{c}$ c of ammonium persulphate solution (15 per cent) and continue the boiling for five minutes. Cool the solution, add $50 \mathrm{c}$ c of the sulphuric-citric acid mixture, and then dilute $\mathrm{NH}_{4} \mathrm{OH}$ (1:1) until the solution is just alkaline to litmus, when an excess of $5 \mathrm{c} c$ is added. In case a precipitate is formed, use more citric acid. The neutralization of the solution should be made as soon as possible after the addition of the citric acid, as otherwise this may reduce traces of iron. Cool to room temperature, dilute to $250 \mathrm{c}$ c and proceed as in the final titration below.

Note.-If chromium is present in amounts over 1 per cent, dissolve the steel in $20 \mathrm{c}$ c of dilute $\mathrm{HSO}_{4}(1: 4)$, oxidize with $5 \mathrm{c} \mathrm{c}$ of $\mathrm{HNO}_{3}$ (specific gravity 1.42), boil five minutes, cool, add $50 \mathrm{c}$ c of sulphuricitric acid mixture, and proceed as above.

\section{Final Titration}

\section{SOLUTIONS REQUIRED}

Potassium iodide (10 per cent).-Dissolve $10 \mathrm{~g}$ of $\mathrm{KI}$ in $100 \mathrm{c}$ c of distilled water.

Standard silver nitrate.-Dissolve $5.7903 \mathrm{~g}$ of $\mathrm{AgNO}_{3}$ in distilled water and dilute to exactly 1,000 c c.

Standard potassium cyanide.-Dissolve $4.5 \mathrm{~g}$ of $\mathrm{KCN}$ in $1,000 \mathrm{c} \mathrm{c}$ of distilled water containing $1 \mathrm{~g}$ of $\mathrm{KOH}$. Standardize this solution against the standard silver nitrate solution as follows: To a 400 c c beaker add 100 c c of water, 3 c c of $\mathrm{NH}_{4} \mathrm{OH}, 2$ c c of potassium iodide solution (10 per cent), and finally $30 \mathrm{c}$ c of silver nitrate solution from a burette. Titrate with the cyanide solution until the solution is perfectly clear, and then add more silver nitrate solution until a faint cloudiness is obtained which can be dispelled by two drops of the cyanide solution. Adjust the cyanide solution so that it is equivalent to the silver nitrate solution and check the theoretical value ( $0.001 \mathrm{~g}$ nickel) by standardizing, by the procedure chosen, against a steel of known nickel content as determined by the gravimetric dimethylglyoxime method.

\section{METHOD}

Add 2 c c of potassium iodide solution (10 per cent) to the solution prepared as in $(a),(b)$, or (c) and then from a burette add $3 \mathrm{c} \mathrm{c}$ of standard silver nitrate solution. Slowly add standard cyanide solution, with constant stirring, until the solution is perfectly clear. Continue the addition of the silver nitrate solution and titration with cyanide solution until the cloudiness of the solution, due to the addition of two drops of the standard silver nitrate, is just expelled by the final addition of two drops of the standard cyanide. Each cubic centimeter excess of standard cyanide solution required over standard silver nitrate solution used in the titration represents 0.1 per cent of nickel.
Nore.- If cobalt is present, an unsatisfactory end point will be obtained if the titration is carried out as directed after a preliminary separation by methods (b) or (c). Cobalt may, however, be titrated satisfactorily provided it is first oxidized to the trivalent condition by boiling the ammoniacal solution with $\mathrm{NaClO}_{3}$ prior to the titraby boiling the ammoniacal solution with $\mathrm{NaClO}_{3}$ prior to the titra-
tion with cyanide. In this case, the compound $\mathrm{K}_{2} \mathrm{Co}(\mathrm{CN})_{5}$ is formed and the titration represents the combined effect of nickel and cobalt. (See the determination of cobalt in tungsten steel by the cyanide titration method (routine), given below.)

\section{CHRONE-NICKEL STEEL}

Determination of Carbon

See the determination of carbon in plain carbon steel by the direct-combustion method in A33 (600.1, p. 10) and also in sampling and chemical analysis of pig and cast iron in A64 (601.20, p. 119).

Note.-Difficultly combustible high-chromium steel can usually be burned directly if the sample is heated for a few minutes before admitting oxygen. A high temperature $\left(1,100^{\circ} \mathrm{C}\right.$.) is desirable, and in extreme cases complete combustion can be had by mixing the the drilings with low-carbon iron or steel.

Determination of Manganese by the Hydrolysis-Bismuthate Method

(Absence of Cobalt)

\section{SOLUTIONS REQUIRED}

Dilute sulphuric acid (1:9).-Pour 100 c c of $\mathrm{H}_{2} \mathrm{SO}_{4}$ (specific gravity 1.84) slowly and with stirring into distilled water, cool, and dilute to $1,000 \mathrm{c} \mathrm{c}$.

Sodium bicarbonate ( 8 per cent).-Dissolve $80 \mathrm{~g}$ of $\mathrm{NaHCO}_{3}$ in $1,000 \mathrm{c}$ c of distilled water.

Dilute nitric acid $(3: 97)$.- Mix 30 c c of $\mathrm{HNO}_{3}$ (specific gravity 1.42) and $970 \mathrm{c}$ c of distilled water.

(Ferrous sulphate and standard potassium permanganate are the same as in the determination of manganese in sampling and chemical analysis of pig and cast iron by the bismuthate method in A64. (See 601.20, p. 119).)

METHOD

Dissolve $1.000 \mathrm{~g}$ of the sample contained in a $200 \mathrm{c} \mathrm{c}$ covered Erlenmeyer flask in $20 \mathrm{c} \mathrm{c}$ of dilute $\mathrm{H}_{2} \mathrm{SO}_{4}(1: 9)$. When action is complete, dilute to 100 $\mathrm{c} \mathrm{c}$ with boiling water. Add from a burette sodium bicarbonate solution ( 8 per cent) until a permanent precipitate is formed (approximately $36 \mathrm{c} \mathrm{c}$ ) and then $4 \mathrm{c}$ c more. Boil one minute, allow to settle, and filter on a rapid filter, washing four to five times with boiling water. If the precipitation has been properly performed there will be no more precipitate than can be conveniently handled on a $9 \mathrm{~cm}$ paper. The filtrate will become cloudy in the funnel stem and in the receiving vessel, owing to oxidation and hydrolysis. Heat the filtrate to boiling and oxidize with small portions of $\mathrm{HNO}_{3}$ (specific gravity 1.42), adding in all $12 \mathrm{c}$ c. Evaporate to about $25 \mathrm{c} \mathrm{c}$, cool to $15^{\circ} \mathrm{C}$., and add an excess of sodium bismuthate. Let stand for a few minutes, add $50 \mathrm{c} \mathrm{c}$ of dilute $\mathrm{HNO}_{3}(3: 97)$, and filter through asbestos. Wash with the same acid until the washings run through colorless. Add $3 \mathrm{c}$ c of $\mathrm{H}_{3} \mathrm{PO}_{4}$ (specific gravity 1.71) and titrate with ferrous sulphate and standard potassium permanganate, as in the determination of manganese in sampling and chemical analysis of pig and cast iron by the bismuthate method. (See A64 (601.20, p. 119).)

Allowable error.-Percentages of manganese determined by the above method should be accurate to $\pm(0.02+(0.02 \times$ the percentage of manganese found)).

Notes.-The bicarbonate precipitate does not ordinarily contain more than small amounts (about 0.005 per cent) of manganese. In very accurate work this should be recovered by transferring the precipitate to a beaker, dissolving it in aqua regia, fuming with sulprecipitate to a beaker, dissolving it in aqua regia, fuming with sulphuric acid, and then adding sodium hydroxide and sodium peroxide and boiling. Chromium and vanadium are thus separated from manganese and iron which should be filtered off, dissolved in $5 \mathrm{c} c$ of
$\mathrm{HNO}_{3}(1: 1)$ and added to the filtrate from the bicarbonate precipitation. A drop of sulphurous acid added to the nitric acid aids in the solution of the manganese dioxide. 
The determination of manganese by the Ford or Ford-Williams method (preliminary separation of manganese as dioxide by boiling with concentrated nitric acid and potassium chlorate) is satisfactory for steels containing over 0.1 per cent of manganese. This method should not be used, however, except in laboratories which have excellent ventilation facilities.

\section{Determination of Manganese by the Persulphate Method (Routine and in the Presence of Cobalt)}

\section{SOLUTIONS REQUIRED}

(Conform to like solutions in the determination of manganese by the persulphate method in A64. (See 601.20, p. 119).

\section{METHOD}

(Same as in A33 (see 600.1, p. 10) until addition of $\mathrm{AgNO}_{3}$ solution (0.133 per cent), then proceed as indicated, in A64 (see 601.20, p. 119).)

Note.-In making the titration, special care should be given to standardizing the end-point reading and the reading should be corrected by a blank, which varies with the amount of chromium present.

\section{Determination of Phosphorus}

(Same as the determination of phosphorus in plain carbon steel by the molybdate magnesia method in A33 (see 600.1, p. 10) except for ammonium molybdate solution, for which see B28 (646.0 p. 1243).j

Determination of Phosphorus by the Alkalimetric Method (Routine)

\section{SOLUTIONS REQUIRED}

(Dilute nitric acid (specific gravity 1.20), dilute nitric acid (2:100), potassium permanganate, and ammonium bisulphite-all are identical with like solutions in the molybdate magnesia method in A33. (See 600.1, p. 10.) Ammonium molybdate, potassium nitrate (1 per cent), phenolphthalein indicator, standard sodium hydroxide, and standard nitric acid-all are identical with like solutions in B28. See 646.0, p. 1243.)

\section{METHOD AND NOTES}

(Same as in A33. (See 600.1, p. 10).)

\section{DETERMINATION OF SULPHUR}

See the determination of sulphur in plain carbon steel by the oxidation method in $\mathrm{A} 33$ (600.1, p. 10).

(In the determination of sulphur by the evolution-titration method, this specification conforms, in respect to diagram illustrating apparatus, solutions required, and method, to A64 (see 601.20, p. 119), except that in A64 the potassium iodate solution includes the chemical reactions, and for the starch solution and notes in A64, there should be substituted the starch indicator and notes in A33 (see 600.1, p. 10).)

Notes.-The evolution-titration method should not be used with steels containing appreciable amounts of tungsten or molybdenum, or more than 0.4 per cent of copper.

The annealing of the steel drillings has been found by a number of investigators to increase the degree of refinement of the method.

\section{Determination of Silicon}

\section{(A) Low Chromidm}

\section{SOLUTIONS REQUIRED}

Nitrosulphuric acid.-(Same as in A33. See 600.1, p. 10.)

Dilute hydrochloric acid (5:95).-(Same as in A64. See 601.20, p. 119.)

\section{METHOD}

(Identical with A64 (see 601.20, p. 119), except that $80 \mathrm{c}$ c of the nitrosulphuric acid is added cau- tiously to $4.676 \mathrm{~g}$ of steel, and that the difference in weights in milligrams divided by 100 equals the percentage of silicon, instead of being divided by 50 as in A64.)

Allowable error.-(Same as in the determination of silicon in plain carbon steel by the nitrosulphuric method in A33. (See 600.1, p. 10.)

Note.-(Same as in the determination of silicon in plain carbon steel by the nitrosulphuric method in A33. See 600.1, p. 10.)

\section{(B) Нigh Сhromidm}

\section{SOLUTIONS REQUIRED}

Dilute sulphuric acid (1:9).-Pour 100 c c of $\mathrm{H}_{2} \mathrm{SO}_{4}$ (specific gravity 1.84 ) slowly and with stirring into $900 \mathrm{c}$ c of distilled water.

Dilute hydrochloric acid (5:95).-(Same as in A64. See 601.20, p. 119.)

\section{METHOD}

Dissolve $2.338 \mathrm{~g}$ of the sample in a covered porcelain dish in $30 \mathrm{c} \mathrm{c}$ of $\mathrm{HCl}$ (specific gravity 1.19) or $100 \mathrm{c}$ c of dilute sulphuric acid $(1: 9)$. If vanadium or molybdenum be present, add a few crystals of sodium chlorate when the action is complete, boil, and then evaporate to low bulk. If sulphuric acid has not been added, cautiously add $15 \mathrm{c} \mathrm{c}$ of $\mathrm{H}_{2} \mathrm{SO}_{4}$ (specific gravity 1.84) and continue the evaporation until fumes of $\mathrm{H}_{2} \mathrm{SO}_{4}$ begin to come off. Do not prolong this treatment or allow the solution to go to dryness lest insoluble chromium and nickel sulphates be formed. Cool, dilute with $125 \mathrm{c}$ c of distilled water, add $5 \mathrm{c} \mathrm{c}$ of $\mathrm{HCl}$ (specific gravity 1.19), and warm gently with frequent stirring until the soluble salts have dissolved. Immediately, filter through an 11-cm filter paper, wash with dilute $\mathrm{HCl}$ (5:95) until free from iron and then with hot water until free from acid. Transfer the filter to a platinum crucible, burn oft the paper carefully, and finally ignite, with the crucible covered, over a blast lamp or in a muffle furnace at $1,000^{\circ} \mathrm{C}$. for at least 10 minutes. Cool in a desiccator and weigh. Add sufficient dilute $\mathrm{H}_{2} \mathrm{SO}_{4}(1: 1)$ to moisten the silica, and then a small amount of HF. Evaporate to dryness, ignite, and weigh. The difference in weights represents the amount of silica.

Note.-(Same as in the determination of silicon in vlain carbon steel by the nitrosulphuric method in A33. See $600.1, \mathrm{p}, 10$.)

Determination of Chromium by the Persulphate-Oxidation Method

\section{SOLUTIONS REQUIRED}

Sulphuric-phosphoric acid mixture--Mix 320 c c of $\mathrm{H}_{2} \mathrm{SO}_{4}(1: 1), 80 \mathrm{c}$ c of $\mathrm{H}_{3} \mathrm{PO}_{4}$ (85 per cent), and $600 \mathrm{c} \mathrm{c}$ of distilled water.

Silver nitrate (2.5 per cent).-Dissolve $25 \mathrm{~g}$ of $\mathrm{AgNO}_{3}$ in $1,000 \mathrm{c} \mathrm{c}$ of distilled water.

Ammonium persulphate (15 per cent).-Make up as needed by dissolving $15 \mathrm{~g}$ of the salt in $100 \mathrm{c} \mathrm{c}$ of distilled water.

Sodium chloride (5 per cent).-Dissolve $50 \mathrm{~g}$ of $\mathrm{NaCl}$ in $1,000 \mathrm{c}$ c of distilled water.

Dilute nitric acid (specific gravity 1.20).-(Same as in determination of manganese by the persulphate method in A64. (See 601.20, p. 119.))

Standard ferrous sulphate.-(Same as in determination of manganese by the bismuthate method in A64. (See 601.20, p. 119.))

Standard permanganate solution.-(Same as in determination of manganese by the bismuthate method in A64. (See 601.20, p. 119.))

\section{METHOD}

Transfer $2 \mathrm{~g}$ of the sample to a $600 \mathrm{c}$ c beaker and add $60 \mathrm{c}$ c of sulphuric-phosphoric acid mixture. Heat until all action ceases, add $10 \mathrm{c}$ c of $\mathrm{HNO}_{3}$ 
(specific gravity 1.20), and boil until solution is complete and oxides of nitrogen are expelled. Add silver nitrate solution ( 2.5 per cent) in amount equal to $0.03 \mathrm{~g}$ of the salt for each 1.5 per cent of chromium present. Dilute to $300 \mathrm{c}$ c with boiling water, heat to boiling, and add $8 \mathrm{c} \mathrm{c}$ of ammonium persulphate solution (15 per cent). When the permanganate color has developed, add 5 c c of sodium chloride solution ( 5 per cent) and boil until the pink color disappears and the persulphate is decomposed, which requires about 10 minutes. Cool the solution, dilute to approximately $400 \mathrm{c} \mathrm{c}$, and titrate for chromium as follows: Add a measured excess of standard ferrous sulphate solution $(50 \mathrm{c}$ c for less than 1.5 per cent chromium, $100 \mathrm{c}$ c for 1.5 to 3 per cent chromium) and then titrate with the standard potassium permanganate solution until an end point is obtained which is permanent upon continued stirring for at least one minute. The titration must be corrected for dilution and color effect. If no chromium or nickel were present the dilution effect alone would be involved and satisfactorily eliminated by titrating the same volume of ferrous sulphate solution in a solution of like volume and acidity. In colored solutions the correction can be made (1) empirically, as judged by experience (2) by titration of the same volume of ferrous sulphate in a solution of like volume and acidity and containing the same amount of the coloring elements in their final valency; or (3) in the final titrated solution. The last is the most convenient and satisfactory and may be performed by destroying the slight excess of permanganate which is present in the titrated solution by a few drops of hydrogen peroxide, boiling until the excess of peroxide and the pervanadate are destroyed (10 minutes actual boiling), cooling to room temperature, and then titrating with the permanganate solution. The difference between the volumes of permanganate used is then a true oxidizing equivalent of the excess of ferrous sulphate employed and the percentage of chromium may be calculated in the usual manner. The final solution may be preserved for the determination of vanadium as described in the determination of vanadium in chrome-vanadium steel by reduction with ferrous sulphate and titration with permanganate method (routine), given below.

Allowable error.-Percentages of chromium determined by the above method should be accurate to $\pm(0.01+(0.01 \times$ the percentage of chromium found)).

\section{Determination of Nickel}

See the determination of nickel in nickel steel by the gravimetric dimethylglyoxime method, given above. (For dimethylglyoxime (1 per cent) solution, see A64 (601.20, p. 119).)

See the determination of nickel in nickel steel by the cyanide-titration method (routine), given above.

\section{VANADIOM STEEI}

All determinations carried out as in the methods of chemical analysis of chrome-vanadium steel, given below.

\section{CEROME-VANADIOM STEEL}

\section{Determination of Carbon}

See determination of carbon in plain carbon steel by the direct-combustion method in A33 (600.1, p. 10); and also in sampling and chemical analysis of pig and cast iron in A64 (601.20, p. 119).)

\section{Determination of Manganese}

(Conforms to the determination of manganese in chrome-nickel steel by the hydrolysis-bismuthate method, given above, except in solutions required, in which standard potassium permanganate and ferrous ammonium sulphate are the same as in the determination of manganese by the bismuthate method in A64 (see 601.20, p. 119).)

(Conforms to the determination of manganese by the persulphate method in A64 (see 601.20, p. 119) in respect to like solutions. In respect to method, it conforms to the determination of manganese by the persulphate method in A33 (see 600.1, p. 10) until addition of $\mathrm{AgNO}_{3}$ solution (0.133 per cent), then proceed as indicated, in A64 (see 601.20, p. 119). Note is the same as in the determination of manganese in chrome-nickel steel by the persulphate method, given above.)

Determination of Phosphorus by the Modified Molybdate Mrgnesia Method.

\section{SOLUTIONS REQUIRED}

(Same as in the determination of phosphorus by the molybdate magnesia method in A33 (see 600.1, p. 10) except ammonium molybdate, which is the same as in the determination of phosphorus by the alkalimetric method in B28 (see 646.0, p. 1243) and the following additional item:

Ferrous sulphate.-Dissolve $40 \mathrm{~g}$ of ferrous sulphate in $100 \mathrm{c}$ c of distilled water that has been acidified with $1 \mathrm{c}$ c of $\mathrm{H}_{2} \mathrm{SO}_{4}$ (specific gravity 1.84).

\section{METHOD AND NOTES}

(Same as in A33 (see 600.1, p. 10) except that after boiling to free from oxides of nitrogen, the solution is cooled to $15^{\circ}$ to $20^{\circ} \mathrm{C}$. (instead of to $80^{\circ} \mathrm{C}$. as in A33) and then there are added $5 \mathrm{c} c$ of the ferrous sulphate solution and two or three drops of concentrated sulphurous acid.)

Allowable error.-Percentages of phosphoris determined by the abore metbod should be accurate to $\pm(0.003+(0.02 \times$ the percentage of phosphorus found)).

Determination of Phosphorus by the Modified Alkalimetric Method (Routine)

\section{SOLUTIONS REQUIRED}

(Dilute nitric acid (specific gravity 1.20), dilute nitric acid for washing $(2: 100)$, potassium permanganate (2.5 per cent), ammonium bisulfite (3 per cent) - all are identical with the determination of phosphorus by the molybdate magnesia method in A33. (See 600.1, p. 10.) Ammonium molybdate, potassium nitrate (1 per cent), phenolphthalein indicator, standard sodium hydroxide, and standard nitric acid-all are identical $w$ ith the determination of phosphorus by the alkalimetric method in B28. (See 646.0 , p. 1243.) Ferrous sulphate solution is the same as given above in modified molybdate magnesia method.)

\section{METHOD AND NOTES}

(Same as in A33 (see 600.1, p. 10) except that after boiling to free from oxides of nitrogen, the solution is cooled to $15^{\circ}$ to $20^{\circ} \mathrm{C}$. (instead of to $80^{\circ} \mathrm{C}$. as in A33) and then there are added $5 \mathrm{c} c$ of the ferrous sulphate solution and two or three drops of concentrated sulphurous acid. Also omit here the notation reference to phosphomolybdate.)

Determination of Sulphur

See the determination of sulphur in plain carbon steel by the oxidation method in A33 (600.1, p. 10).

(In respect to the determination of sulphur by the evolution-titration method, this specification is identical to A64 (see 601.20, p. 119) as regards diagram of apparatus, solutions required, and method, except that the potassium iodate chemical reactions are not included here, and for the starch 
solution in A64 should be substituted the starch indicator in A33 (see 600.1, p. 10). Notes are the same as in A33.)

Notes.-(Same as for the determination of sulphur in chromenickel steel, given above.)

Determination of Silicon by the Nitrosulphuric Method

(In respect to solutions required, same as A33 (see 600.1, p. 10) except for dilute hydrochloric acid (5:95) which conforms to A64.) (See 601.20, p. 119.)

\section{METHOD}

(Identical with A64 (see 601.20, p. 119) except that $80 \mathrm{c} \mathrm{c}$ of the nitrosulphuric acid is added cautiously to $4.676 \mathrm{~g}$ of steel, and that the difference in weights in milligrams divided by 100 equals the percentage of silver, instead of being divided by 50 , as in A64.)

Allowable error.-(Same as in A33. See 600.1, p. 10.)

Note.-(Same as in A33. See 600.1 , p. 10.)

Determination of Silicon by the Sulphuric Acid Method (Optional)

(Same as A64 (see 601.20, p. 119) except that here $2.338 \mathrm{~g}$ of steel is substituted for the $2.336 \mathrm{~g}$ of iron specified in A64.)

\section{Determination of Chromium}

\section{SOLUTIONS REQUIRED}

(Sulphuric-phosphoric acid mixture, silver nitrate (2.5 per cent), ammonium persulphate (15 per cent), and sodium chloride ( 5 per cent) - all are identical with like solutions in the determination of chromium in chrome-nickel steel by the persulphate-oxidation method, given above. Dilute nitric acid (specific gravity 1.20) is identical with solution in determination of manganese by the persulphate method in A64. (See 601.20 , p. 119.) Standard ferrous sulphate and standard permanganate solution are identical with like solutions in determination of manganese by the bismuthate method in A64.) (See 601.20, p. 119.)

\section{METHOD}

(Same as in determination of chromium in chromenickel steel by the persulphate-oxidation method, given above.)

Note.-Reserve the titrated solution for the determination of vanadium by reduction with ferrous sulphate and titration with permanganate (routine) given below.

Determination of Vanadium by the Electrolytic-Separation Method

\section{SOLUTIONS REQUIRED}

Dilute sulphuric acid (1:9).-Carefully mix 100 c c of $\mathrm{H}_{2} \mathrm{SO}_{4}$ (specific gravity 1.84) and $900 \mathrm{c}$ c of distilled water.

Dilute sulphuric acid (1:1).-Add 500 c c of $\mathrm{H}_{2} \mathrm{SO}_{4}$ (specific gravity 1.84) slowly and with stirling to $500 \mathrm{c} \mathrm{c}$ of distilled water.

Sodium bicarbonate ( 8 per cent).-Dissolve $80 \mathrm{~g}$ of $\mathrm{NaHCO}_{3}$ in $1,000 \mathrm{c}$ c of distilled water.

Potassium ferricyanide.-Prepare a very dilute solution as needed, by dissolving a crystal the size of the head of a pin in $25 \mathrm{c}$ c of distilled water.

Standard potassium permanganate.-See the determination of manganese by the bismuthate method in $\mathrm{A} 64(601.20, \mathrm{p} .119$.

\section{METHOD}

Dissolve $2 \mathrm{~g}$ of the sample, contained in a $200 \mathrm{c} \mathrm{c}$ covered Erlenmeyer flask, in 30 c c of dilute $\mathrm{H}_{2} \mathrm{SO}_{4}$ (1:9). When action is complete, dilute to $100 \mathrm{c} \mathrm{c}$ with boiling water, heat to boiling, and add sodium bicarbonate solution ( 8 per cent) from a burette until a permanent precipitate appears (approximately $36 \mathrm{c} \mathrm{c}$ ) and then $4 \mathrm{c}$ c more. Let settle, filter on a rapid filter, and quickly wash the flask and precipitate with hot water. If the precipitation has been properly performed there will be no more precipitate than can be conveniently handled on a $9 \mathrm{~cm}$ paper. The filtrate will become cloudy in the funnel stem and in the receiving vessel, owing to oxidation and hydrolysis. (If the flask, paper, and precipitate have been well washed, the filtrate will contain all but traces of the managnese in the sample free from chromium and vanadium and therefore, it may be oxidized with nitric acid and the manganese determined by the bismuthate or persulphate methods.) Place the paper and precipitate in the original flask, add $20 \mathrm{c}$ c of $\mathrm{HNO}_{3}$ (specific gravity 1.42) shake until the paper is broken up, and then add $5 \mathrm{c}$ c of $\mathrm{H}_{2} \mathrm{SO}_{4}$ (specific gravity 1.84). Heat over a free flame until fumes of sulphuric acid are given off, cool, and then add more $\mathrm{HNO}_{3}$ and repeat the heating in case the organic matter has not been destroyed. Finally cool, wash down the sides of the flask, and evaporate again to the appearance of fumes of sulphuric acid and until all of the nitric acid has been expelled. Cool, dilute to $40 \mathrm{c} \mathrm{c}$, and nearly neutralize the solution with $\mathrm{NH}_{4} \mathrm{OH}$ (specific gravity 0.90 ). Transfer the weakly acid solution to the electrolyzing apparatus, rinsing the flask with small portions of water and keeping the total volume of the solution down to $70 \mathrm{c} \mathrm{c}$. The electrolysis may be performed with a mercury cathode in (1) a special modified separatory funnel, ${ }^{2}(2)$ in a small beaker with sealed-in platinum wire, ${ }^{3}$ or (3) in an ordinary beaker with the cathode wire enclosed in a glass tube extending into the mercury. In any case, the cathode wire should be embedded in about $200 \mathrm{~g}$ of mercury, and the solution electrolyzed (preferably with a stirring arrangement) using a current of 5 to 6 amperes at 6 to 7 volts. Continue the electrolysis (ordinarily 15 to 20 minutes if the solution is stirred) until iron is absent as indicated by a ferricyanide test on a small drop of the electrolyte. When iron is absent, remove the electrolyte and wash the mercury two or three times with 25 to $30 \mathrm{c}$ c of water while the current is continued. The solution must now give no test for iron. Add 2 to $3 \mathrm{c}$ c of dilute $\mathrm{H}_{2} \mathrm{SO}_{4}$ $(1: 1)$, heat to $70^{\circ}$ to $80^{\circ} \mathrm{C}$., and add permanganate solution to a strong pink color. Run sulphur dioxide into the boiling solution for a few minutes until the vanadium is reduced, and then a rapid stream of carbon dioxide until the escaping steam gives no test for $\mathrm{SO}_{2}$. Cool the solution to $70^{\circ}$ to $80^{\circ} \mathrm{C}$., and titrate with the standard permanganate solution. Repeat the reduction and titration until concordant results are obtained. The titration should be corrected by a blank determination on a solution of like volume and acidity.

Allowable errot.-Percentages of vanadium determined by the above method should be accurate to $\pm(0.02+(0.01 \times$ the percentage of vanadium found)).

Determination of Vanadium by Reduction with Ferrous Sulphate and Titration with Permanganate (Routine)

\section{SOLUTIONS REQUIRED}

(Sulphuric-phosphoric acid mixture, silver nitrate (2.5 per cent), ammonium persulphate (15 per cent) and sodium chloride ( 5 per cent) - all are identical with the determination of chromium in chromenickel steel by the persulphate-oxidation method, given above. Dilute nitric acid $(1: 1)$ is identical with the determination of manganese by the persulphate method in A64. (See 601.20, p. 119). Standard ferrous sulphate and standard potassium permanganate are identical with the determination

2 Reprint 161 from the Bulletin of the Bureau of Standards, Fol. 74, No. 3; Journal Industrial and Engineering Chemistry, vol. 3, p. $76,1911$.

Smith, Electroanalysis, 5th ed., P. Blakiston's Sons \& Co., p. 63. 
of manganese by the bismuthate method in A64. (See 601.20, p. 119).)

\section{Method}

See the determination of chromium in chromenickel steel by the persulphate-oxidation method given above.

Use the solution employed for the determination of chromium and add $10 \mathrm{c} c$ of ferrous sulphate solution if vanadium is under 0.80 per cent, and $20 \mathrm{c}$ c if the vanadium content is higher. Stir the solution thoroughly, add $8 \mathrm{c}$ c of ammonium persulphate solution (15 per cent) and stir for one minute. Titrate with the standard permanganate solution to a definite pink color which does not fade upon continued stirring for one minute. In this determination the ferrous sulphate reduces the vanadium, the persulphate oxidizes the excess of ferrous sulphate but does not oxidize the reduced vanadium and the reduced vanadium is oxidized by permanganate which does not react with the excess of persulphate. As in the determination of chromium, an excess of permanganate is used in order to overcome the dilution effect and the color interference, and the same blank correction should be subtracted as was there found.

\section{TONGSTEN STEEL}

\section{Determination of Carbon}

See the determination of carbon in plain carbon steel by the direct-combustion method in A33 (600.1, p. 10) and also in sampling and chemical analysis of pig and cast iron in A64 (601.20, p. 119).

Nore.-Difficulily combustible tungsten steel can often be burned directly if the sample is heated for a few minutes before admitting orggen. A high termperarure $\left(1,100^{\circ} \mathrm{C}\right.$.) is desirable, and in extreme cases complete combustion can be ha 1 by mixing the sample with lowearbon iron or steel.

Determination of Manganese by the Hydrolysis-Bismuthate Method (Absence of Cobalt)

(Same as the determination of manganese in chrome-nickel steel by the hydrolysis-bismuthate method, given above, except for standard potassium permanganate and ferrous sulphate solutions, for which see determination of manganese by the bismuthate method in A64 (601.20, p. 119), and the addition of the following:

Notes.-The retention of manganese by the bicarbonate precipitate is a little greater in steels containing tungsten than in steel containing no tungsten, but the retention is usually no more than from 0.01 to 0.02 per cent. In very accurate work this should be recovered as follow:

Place the paper and precipitate in the original flask, add $20 \mathrm{c} \mathrm{c}$ of $\mathrm{HNO}_{3}$ (specific gravity 1.42) shake until the paper is broken up, and then add $5 \mathrm{c} \mathrm{c}$ of $\mathrm{H}_{2} \mathrm{SO}_{4}$ (specific gravity 1.84). Heat over a free flame until fumes of sulphuric acid are given off, cool, and then add mor $\mathrm{HNO}_{3}$ and repeat the heating in case the organic matter has not been $\mathrm{HNO}_{3}$ and repeat the heating in case the organic matter has not been destroyed. Finally cool, wash down the sides of the flask, and evaporate again to the appearance of fumes of sulphuric acid and until al sodium hydroxide, then add sodium peroxide and boil. Chromium, vanadium, and tungsten are thus separated from manganese and iron, which should be filtered off and washed with hot water containing little sodium hydroxide. Dissolve the precipitate of iron and manganese in warm dilute 1:1 nitric acid with the aid of a fow drops of sulnese in warm dilute $1: 1$ nitric acid with the aid of a few drops of sul-
phurous acid if necessary, and add the solution to the original filtrate. phurous acid if necessary, and add the solution to the original filtrate. resent the persulphate method must be used with a fresh sample a directed below, or in the filtrate obtained after the bicarbonate precipitation. In the latter event, acidify the filtrate with nitric acid, add $10 \mathrm{c}$ c of dilute sulphuric acid $(1: 1)$ and evaporate to a volume of $20 \mathrm{c} c$. Add $10 \mathrm{c}$ c of $\mathrm{HNO}_{3}$ (specific gravity 1.20 ) and proceed as directed belcw. With material containing much cobalt use one fourth of the solution, which will represent $0.5 \mathrm{~g}$ of steel.

Allowable error.-Percentages of manganese determined by the above method should be accurate to $\pm(0.02+(0.02 \times$ the percentage of manganese found)).

Determination of Manganese by the Persulphate-Arsenite Method (Routine and in the Presence of Cobalt)

\section{SOLUTIONS REQUIRED}

Dilute sulphuric acid $(1: 1)$.-(Same as in determination of vanadium in chrome-vanadium steel by the electrolytic separation method, given above.)
Dilute nitric acid (specific gravity: 1.20).-(Same as in determination of phosphorus by the molybdatemagnesia method in A33. See 600.1, p. 10.)

Silver nitrate $(0.5$ per cent $)$.-Dissolve $5 \mathrm{~g}$ of $\mathrm{AgNO}_{3}$ in a mixture of $750 \mathrm{c} \mathrm{c}$ of distilled water with $250 \mathrm{c} \mathrm{c}$ of $\mathrm{H}_{2} \mathrm{SO}_{4}(1: 1)$.

Ammonium persulphate (15 per cent).-(Same as in determination of chromium in chrome-nickel steel by the persulphate-oxidation method, given above.) Sodium chloride $(0.5$ per cent).-Dissolve $5 \mathrm{~g}$ of $\mathrm{NaCl}$ in $1,000 \mathrm{c}$ c of distilled water.

Standard sodium arsenite solution.-Dissolve $2 \mathrm{~g}$ of sodium arsenite in $1,000 \mathrm{c}$ c of distilled water and standardize against a standard steel or by withdrawing $10 \mathrm{c}$ c of $0.03 \mathrm{~N}$ permanganate (or its equivalent) into a 250 c c beaker, redueing with a few drops of sulphurous acid, and then proceeding as described in the method below.

\section{METHOD}

Transfer $0.50 \mathrm{~g}$ of steel to a flask, add $25 \mathrm{c} \mathrm{c}$ of water, $5 \mathrm{c}$ c of dilute $\mathrm{H}_{2} \mathrm{SO}_{4}(1: 1)$, and $2 \mathrm{c} e$ of phosphoric acid ( 85 per cent), and heat until all effervescence ceases. Add $10 \mathrm{c}$ c of $\mathrm{HNO}_{3}$ (specific gravity 1.20), and boil to dissolve the tungstic acid. Add $50 \mathrm{c} \mathrm{c}$ of silver nitrate solution $(0.5$ per cent) (sufficient for 5 per cent chromium steel), boil, and add $15 \mathrm{c} \mathrm{c}$ of ammonium persulphate solution (15 per cent). Remove the flask from the heat, allow to stand in a warm place until the color has developed, and then rapidly cool. Transfer the solution to a $250 \mathrm{c}$ c beaker, dilute to a volume of $100 \mathrm{c} \mathrm{c}$ and add $30 \mathrm{c}$ c of sodium chloride solution $(0.5$ per cent) to precipitate the silver. Titrate with the standard sodium arsenite solution to the disappearance of the pink tint of permanganic acid. The addition of sodium chloride is unnecessary if the solution of permanganic acid is quite cold and the titration is conducted rapidly.

Determination of Phosphorus by the Molybdate Magnesia Method

\section{SOLUTIONS REQUIRED}

Dilute hydrochloric acid (1:1).-Dilute 500 c c of $\mathrm{HCl}$ (specific gravity 1.19) with $500 \mathrm{c} \mathrm{c}$ of distilled water.

Dilute hydrochloric acid (1:10).-Dilute $100 \mathrm{c}$ c of $\mathrm{HCl}$ (specific gravity 1.19) with $1,000 \mathrm{c} \mathrm{c}$ of distilled water.

Ferrous sulphate-Dissolve $40 \mathrm{~g}$ of ferrous sulphate in $100 \mathrm{c}$ c of water and acidify with $1 \mathrm{c} c$ of $\mathrm{H}_{2} \mathrm{SO}_{4}$ (specific gravity 1.84 ).

Ammonium molybdate.-(Same as in B28. See 646.0 , p. 1243.)

(Dilute nitric acid for washing $(2: 100)$, dilute ammonium hydroxide (1:2), and magnesia mixture-all are identical with like solutions in the determination of phosphorus in plain carbon steel by the molybdate-magnesia method in A33. See 600.1 , p. 10.)

\section{METHOD}

Treat $2 \mathrm{~g}$ of the sample, in a porcelain dish provided with a cover glass, with $20 \mathrm{c}$ c of $\mathrm{HNO}_{3}$ (specific gravity 1.42) and $60 \mathrm{c} c$ of $\mathrm{HCl}$ (specific gravity 1.19). Heat gently until the sample is completely decomposed and then evaporate to dryness. Remove the cover glass and bake until the acid is completely expelled. Cool, add $30 \mathrm{c} c$ of $\mathrm{HCl}$ (specific gravity 1.19), warm until the soluble salts are dissolved, and then evaporate to dryness on the sand bath. Add $30 \mathrm{c}$ c of dilute $\mathrm{HCl}(1: 1)$ and boil the solution until the soluble salts are dissolved. Dilute to $60 \mathrm{c} c$ with boiling water, filter from the silica and tungstic acid, and wash the residue with dilute $\mathrm{HCl}(1: 10)$ until free from iron. (See note.) Evaporate the filtrate to approx- 
imately $20 \mathrm{c}$ c, cool, and make ammoniacal. Dissolve the precipitated hydroxicles in the exact amount of $\mathrm{HNO}_{3}$ (specific gravity 1.42) necessary to effect complete solution and add $5 \mathrm{c} \mathrm{c}$ in excess. The further treatment depends on whether vanadium is absent or present. If vanadium is absent, proceed as in the determination of phosphorus in plain carbon steel by the molybdate-magnesia method in A33. (See 600.1, p. 10.) If vanadium is present, proceed as in the determination of phosphorus in chrome-vanadium steel by the modified molybdate-magnesia method, given above, in which will be found certain references to A33 and B28.

Allowatie error.-Percentages of phosphorus determined by the above method should be accurate to $\pm(0.004+(0.02 \times$ the percentage of phosphorus found)).

Note. - The residue of tungstic and silicic acids sometimes contains very small amounts of phosphorus and in accurate analyses it must be treated as follows: Ignite in a platinum crucible, fuse with little $\mathrm{Na}_{2} \mathrm{CO}_{3}$ (free from phosphorus), and dissolve the cooled melt in hot water. Acidify the solution slightly with $\mathrm{HCl}$, add a few crystal of $\mathrm{FeCl}_{3}$, and precipitate with $\mathrm{NH}_{4} \mathrm{OH}$ in slight excess. Filter, wash with a hot wash solution containing a little $\mathrm{NH}_{4} \mathrm{OH}$ and $\mathrm{NH} \mathrm{Cl}_{4}$, and discard the filtrate. Dissolve the precipitate of ferric hydroxide and ferric phosphate in a small amount of $\mathrm{HCl}$ and add to the main fil trate

Determination of Phosphorus by the Alkalimetric Method (Routine)

\section{SOLUTIONS REQUIRED}

Dilute nitric acid $(1: 3)$.-Dilute 250 c c of $\mathrm{HNO}_{3}$ (specific gravity 1.42) with $750 \mathrm{c} \mathrm{c}$ of distilled water. (Dilute hydrocholoric acid (1:1), dilute hydrochloric acid $(1: 10)$, and ferrous sulphate-all are identical with like solutions in the determination of phosphorus in tungsten steel by the molybdatemagnesia method, given above. Ammonium molybdate is the same as in the determination of phosphorus by the alkalimetric method in B28 (see 646.0 , p. 1243), as also are potassium nitrate (1 per cent), phenolphthalein indicator, standard sodium hydroxide, and standard nitric acid.)

\section{METHOD}

Treat $2 \mathrm{~g}$ of the sample, in a porcelain dish provided with a cover glass, with $50 \mathrm{c}$ c of dilute $\mathrm{HNO}_{3}$ (1:3), add $10 \mathrm{c}$ c of $\mathrm{HCl}$ (specific gravity 1.19), and evaporate to dryness. Dissolve the soluble salts in $30 \mathrm{c} \mathrm{c}$ of dilute $\mathrm{HCl}(1: 1)$ by boiling, dilute to $60 \mathrm{c} \mathrm{c}$ with water, filter, and wash the residue with dilute $\mathrm{HCl}(1: 10)$. Evaporate the filtrate to approximately $100 \mathrm{c} \mathrm{c}$, make the solution ammoniacal, add just enough $\mathrm{HNO}_{3}$ (specific gravity 1.42) to effect complete solution and then add $5 \mathrm{c}$ c in excess. The further treatment depends on whether vanadium is absent or present. If vanadium is absent, proceed as in the determination of phosphorus in plain carbon steel by the alkalimetric method (routine) in A33 (see 600.1, p 10). If vanadium is present, proceed as in the determination of phosphorus in chrome-vanadium steel by the modified alkalimetric method (routine), given above.

\section{Determination of Sulphur by the Gravimetric Method}

\section{SOLUTIONS REQUIRED}

(Dilute hydrochloric acid $(1: 10)$ and dilute hydrochloric acid $(1: 1)$ are the same as in the determination of phosphorus in tungsten steel by the molybdate-magnesia method, given above. Cinchonine and cinchonine wash solution are the same as in the determination of tungsten in tungsten steel by the acid digestion-cinchonine method, given below.)

\section{METHOD}

Treat $5 \mathrm{~g}$ of the sample, in a $600 \mathrm{c}$ c beaker or Erlenmever flask, with 75 c c of $\mathrm{HNO}_{3}$ (specific gravity 1.42) and heat gently. Certain steels react violently. In this event, do not heat but cool the vessel in cold water in order to prevent loss of incompletely oxidized sulphur. Some steels dissolve very slowly. In this case add $5 \mathrm{c} \mathrm{c}$ of $\mathrm{HCl}$ (specific gravity 1.19) in successive portions as the solution is digested on a steam bath. When the steel is dissolved, digest until the residue is bright yellow with no dark material, adding fresh portions of acid if necessary. Evaporate the solution to small volume, cool, add 30 c c of $\mathrm{HCl}$ (specific gravity 1.19), again evaporate to small volume and dilute with boiling water. Boil until soluble salts are in solution, filter, and wash the precipitate with dilute $\mathrm{HCl}(1: 10)$, catching the filtrate and washings in a 600 c c casserole. Evaporate the solution to dryness, add $40 \mathrm{c}$ c of $\mathrm{HCl}$ (specific gravity 1.19), and again evaporate to dryness and heat for 10 minutes at a temperature not to exceed $130^{\circ} \mathrm{C}$. Treat the residue with $60 \mathrm{c}$ c of dilute $\mathrm{HCl}(1: 1)$, warm until soluble salts are in solution, dilute with $50 \mathrm{c} \mathrm{c}$ of boiling water, and add $10 \mathrm{c} \mathrm{c}$ of cinchonine solution. After standing in a warm place for several hours, preferably overnight, filter, and wash with cinchonine wash solution. Evaporate the filtrate and washings until a slight film begins to form upon the surface of the solution and proceed as in the determination of sulphur in plain carbon steel by the oxidation method. The results should be corrected by blanks on reagents carried through all of the steps of the process, paring particular attention to sulphur derived from the cinchonine soluticn.

Allowabie error-Percenta es of sulpi.ur determined by the above method should be ac: urate $i( \pm(0.00)+(0 \%)$ Xthe percentage of ulthur found)

Determination of Silicon by Acid Attack and Baking at $120^{\circ} \mathrm{C}$.

\section{SOLUTIONS REQUIRED}

(Dilute hydrochloric acid $(1: 10)$ and dilute $h y-$ drochloric acid $(1: 1)$ are the same as in the determination of phosphorus in tungsten steel by the molybdate-magnesia method, given above. Dilute hydrochloric acid $(5: 95)$ same as in the determination of silicon by the nitro-sulphuric acid method in A64. See 601.20, p. 119.)

Dilute sulphuric acid $(1: 1)$. Cautiously pour, with constant stirring 500 c. c. of $\mathrm{H}_{2} \mathrm{SO}_{4}$ (specific gravity 1.84) into $500 \mathrm{c}$ c of distilled water.

Sodium hydroxide $(2 N)$. - Dissolve $80 \mathrm{~g}$ of $\mathrm{NaOH}$ in $1,000 \mathrm{c}$ c of distilled water.

\section{METHOD}

Treat $2 \mathbf{g}$ of the sample in a corered beaker with $50 \mathrm{c} \mathrm{c}$ of $\mathrm{HCl}$ (specific gravity 1.19) heat to incipient boiling and then when solution is complete add $\mathrm{HNO}_{3}$ (specific gravity 1.42) at intervals and a few drops at a time until the vigorous action which ensues ceases. Digest until the tungstic acid is bright yellow. Add $100 \mathrm{c}$ c of boiling water, boil for one minute, cool somewhat, and filter. Wash the residue thorougbly with dilute $\mathrm{HCl}(1: 10)$ and set the precipitate aside. Evaporate the filtrate to dryness and bake for one hour at a temperature of $120^{\circ} \mathrm{C}$. Take up in $15 \mathrm{c}$ c of $\mathrm{HCl}$ (specific gravity 1.19) and bake again. Take up in $10 \mathrm{c}$ c of dilute $\mathrm{HCl}$ (1:1) and $50 \mathrm{c} \mathrm{c}$ of hot water. Filter and wash with dilute $\mathrm{HCl}$ (5:95). Reserve the filtrate. Ignite the two residues and filter papers in an open deepform platinum crucible at a temperature high enough to dehydrate the silica and weigh. Treat the residue with a few drops of dilute $\mathrm{H}_{2} \mathrm{SO}_{4}(1: 1)$, a few cubic centimeters of hydrofluoric acid, and carefully evaporate to dryness. Ignite very cautiously (to avoid spattering) at a temperature not above 
S00 $0^{\circ}$ C. and weigh. The loss in weight is the weight of silica which multiplied by 0.4676 gives the weight of silicon.

Dissolve the ignited tuntstic oxide in a slight excess of $\mathrm{NaOH}$ solution $(2 N)$, boil, and filter off any residue. Wash the residue with hot water, dissolve it in a few cubic centimeters of hot dilute $\mathrm{HCl}(1: 1)$, add the solution to the reserved filtrate and preserve the combined solutions for the determination of cobalt.

Allowable error.-Percentages of silicon determined by the above method should be accurate to $\pm(0.02+(0.02 \times$ the percentage of s:licon found)

Determination of Tungsten by the Acid Digestion-Cinchonine Method

\section{SOLUTIONS REQUIRED}

Cinchonine.-Dissolve $125 \mathrm{~g}$ of cinchonine in a mixture of $500 \mathrm{c} \mathrm{c}$ of $\mathrm{HCl}$ (specific gravity 1.19) and $500 \mathrm{c} c$ of distilled water.

Cinchonine wash solution.-Dilute $30 \mathrm{c} \mathrm{c}$ of the above solution to 1 liter.

Ammonia-ammonium chloride.-Pour 200 c c of $\mathrm{NH}_{4} \mathrm{OH}$ (specific gravity 0.90 ) into $790 \mathrm{c}$ c of water and add $10 \mathrm{c}$ c of $\mathrm{HCl}$ (specific gravity 1.19).

(Dilute hydrochloric acid $(1: 1)$ is the same as in the determination of phosphorus in tungsten steel by the molybdate-magnesia method, given above.)

\section{METHOD}

Treat $2 \mathrm{~g}$ of the sample in a covered beaker with $50 \mathrm{c} \mathrm{c}$ of $\mathrm{HCl}$ (specific gravity 1.19), heat to incipient boiling and then when solution is complete, add $\mathrm{HNO}_{3}$ (specific gravity 1.42) at intervals and a few drops at a time until the vigorous action which ensues ceases. Digest until the tungstic acid is bright yellow. Dilute to $150 \mathrm{c} \mathrm{c}$, add $10 \mathrm{c}$ c of cinchorine solution, and digest at a temperature of about $90^{\circ}$ to $95^{\circ}$ C. for 30 minutes or longer, with occasional stirring. Decant the clear solution on a paper and wash the beaker and the precipitate by decantation four times with 30 to 40 cc furtions of the hot cinchonine wash solution, keeping as much of the precipitate as possible in the beaker. Place the beaker and precipitate under the fumnel and dissolve the precipitate on the paper on the least possible amount of the ammonia-ammonium chloride solution. Wash the paper several times with hot water, once with hot dilute $\mathrm{HCl}(1: 1)$, several more times with hot water, and finally with small portions of the ammonia-ammonium chloride wash. Reserve the paper. Warm the filtrate and add just enough $\mathrm{NH}_{4} \mathrm{OH}$ (specific gravity 0.90) to dissolve the tungstic acid. Evaporate the solution until most of the ammonia has been expelled and then filter through the reserved paper into a $400 \mathrm{c}$ c beaker. Wash the original beaker and the paper with small portions of hot water. Reserve the filtrate. The residue may contain a little tungsten, therefore treat it with successive small portions of hot dilute $\mathrm{HCl}(1: 1)$, hot water, and ammonia-ammonium chloride solution. Render this solution slightly alkaline, boil to expel most of the ammonia, filter through the oirginal paper and wash with a little hot water. Combine the ammoniacal filtrates. (The above treatments serve to remove any tantalum or columbium as well as iron and chromium and render unnecessary any purification of the final hydroflouric acid-treated tungstic oxide.) Dilute the faintly ammoniacal filtrates to $200 \mathrm{c}$ c, add 10 c c of $\mathrm{HCl}$ (specific gravity 1.19), and boil for 10 minutes. Add $10 \mathrm{c} c$ of cinchonine solution and digest in a warm place for 15 to 30 minutes with occasional stirring. Add a little paper pulp, filter, and wash the precipitate with hot cinchonine wash solution. Add $5 \mathrm{c}$ c of cinchonine to the filtrate and washings, set aside in a warm place for several hours, and, in case any tungstic acid settles out, filter it off, wash, and add to the main precipitate. Ignite the tungstic acid under oxidizing conditions in a deep-form platinum crucible until the carbon is destroyed and finally at $800^{\circ}$ to $900^{\circ} \mathrm{C}$. Cool the crucible and contents, moisten the oxide with hydrofluoric acid, carefully evaporate to dryness, and then cautiously (to avoid spattering) ignite as before and weigh as $\mathrm{WO}_{3}$. Multiply by 0.7931 to obtain the weight of tungsten.

Allowable error.-Percentages of tungsten determined by the above method should be accurate to $\pm(0.05+(0.01 \times$ the percentage of tung. sten found)).

Notes.-1. Tungstic acid is incompletely precipitated by acid digestion alone of solutions containing appreciable amounts of alkali or ammonium salts, phosphorus, or molybdenum, and only slowly by cincrionine in such solutions.

2. Tungstic acid carries down phosphorus pentoxide if it is present in appreciable amount. The small amount present in the solution of the steel is not carried down in sufficient amount to affect the determination of tungsten.

3. Molybdenum is carried down by tungstic acid and must be recovered from the final weighed oxide as specified under the determination of molybdenum in molybdenum steel by precipitation as sulphide and weighing as lead molybdate $((b)$ presence of tungsten) and derlucted as $\mathrm{MoO}_{3}$, given below.

Determination of Tungsten and Silicon by Digestion with Acids (Routine)

METHOD

(a) Determination of silicon.-Proceed exactly as in the determination of silicon above until the ignition of the tungstic and silicic acids. Then ignite in a weighed deep-form platinum crucible at a temperature of from $800^{\circ}$ to $900^{\circ} \mathrm{C}$. and under oxidizing conditions. Weigh, treat the residue with a few drops of dilute $\mathrm{H}_{2} \mathrm{SO}_{4}$ (1:1), and a few cubic centimeters of hydrofluoric acid, and carefully evaporate to dryness. Ignite very cautiously (to avoid spattering) at a temperature not above $800^{\circ}$ to $900^{\circ} \mathrm{C}$. and again weigh. The loss of weight represents silica which, multiplied by 0.4676 , gives silicon.

(b) Determination of tungsten. - The weight of the residue left after the above treatment represents impure tungstic oxide. To correct for the impurities, cover the residue with about its weight of pure dry sodium carbonate and heat until thoroughly fused. Extract the cooled melt with hot water, filter, and wash the residue thoroughly with hot water. Ignite the residue in the original crucible, weigh, subtract the weight from the weight of the crucible and impure tungstic oxide, and multiply the corrected weight of $\mathrm{WO}_{3}$ by 0.7931 to obtain tungsten.

Determination of Chromium by the Persulphate-Oxidation Method

See the determination of chromium in chromenickel steel by the persulphate-oxidation method, given above, in which will be found, under solutions required, certain references to $\mathbf{A 6 4}$.

Allowable error.--Percentages of chromium determined by the above method should be accurate to $\pm(0.01+(0.02 \times$ the percentage of chromium found)).

\section{Determination of Vanadium by the Electrolytic Separation Method}

See the determination of vanadium in chromevanadium steel by the electrolytic-separation method, given above, except for standard potassium permanganate, for which see the determination of manganese by the bismuthate method in A64. (See 601.20, p. 119.) Proceed exactly as in that method until the bicarbonate precipitate and paper have been dissolved in sulphuric and nitric acids and all of the nitric acid has been expelled. Cool the solution, dilute with three times as much water as there is sulphuric acid present, warm to dissolve the soluble salts, and filter through a small filter to 
remove the tungstic acid. Wash the paper and precipitate with small portions of dilute $\mathrm{H}_{2} \mathrm{SO}_{4}(1: 3)$. Nearly neutralize the filtrate with $\mathrm{NH}_{4} \mathrm{OH}$ (specific gravity 0.90 ), evaporate, if necessary, to a volume of approximately $70 \mathrm{c} c$ and proceed with the electrolysis of the weakly acid solution and the determination of vanadium as in the determination of vanadium in chrome-vanadium steel by the electrolytic separation method, given above.

Allowatle eiror.-Percentages of vanadium determined by the above method should be accurate to $\pm(0.03+(0.01 \times$ the percentage of vanadium found)). Determination of Vanadium by Reduction with Ferrous Sulphate and
Titration with Permanganate (Routine)

\section{SOLUTIONS REQUIRED}

Dilute hydrochloric acid (2:1).-Mix 2 parts of $\mathrm{HCl}$ (specific gravity 1.19) with 1 part of water.

Diluie hydrochloric acid (1:9).-Mix $100 \mathrm{c}$ c of $\mathrm{HCl}$ (specific gravity 1.19) and $900 \mathrm{c}$ c of distilled water.

(Dilute nitric acid (specific gravity 1.20 ) is the same as in the determination of phosphorus by the molybdate-magnesia method in A33. See 600.1, p. 10.)

\section{METHOD}

(a) (When cobalt does not exceed 1 per cent). Treat $2 \mathrm{~g}$ of the steel in a porcelain dish with $70 \mathrm{c} \mathrm{c}$ of dilute $\mathrm{HCl}(2: 1)$ and heat until all action ceases. Cautiously add $10 \mathrm{c}$ c of dilute $\mathrm{HNO}_{3}$ (specific gravity 1.20) and evaporate the solution to dryness or to low bulk. Treat the residue with $45 \mathrm{c} \mathrm{c}$ of dilute $\mathrm{HCl}(2: 1)$ and heat until the soluble portion dissolves. Add an equal volume of water, boil for three minutes, filter, and wash the residue thoroughly with dilute $\mathrm{HCl}(1: 9)$ until free from iron. Evaporate the solution to small volume and destroy hydrochloric acid by small additions of nitric acid (specific gravity 1.42) followed by boiling. If a residue appears, filter the solution. From this point proceed for chromium as in the determination of chromium in chrome-nickel steel by the persulphate-oxidation method, given above, in which will be found, under solutions required, certain references to A64 (see 601.20, p. 119); and for vanadium as in the determination of vanadium in chrome-vanadium steel by reduction with ferrous sulphate and titration with permanganate method (routine), given above, in which will be found certain references to A64.

(b) (When cobalt exceeds 1 per cent).- See the determination of vanadium in chrome-vanadium steel by the electrolytic-separation method, given above, except for standard potassium permanganate, for which see the determination of manganese by the bismuthate method in A64. (See 601.20, p. 119.)

Proceed as described in that method until the bicarbonate precipitate has been washed with hot water. Return the paper and precipitate to the flask and add $60 \mathrm{c} \mathrm{c}$ of dilute $\mathrm{HCl}(2: 1)$. Heat the solution, add $10 \mathrm{c}$ e of $\mathrm{HNO}_{3}$ (specific gravity 1.20), and evaporate the solution to dryness or to a small volume. Treat the residue with $45 \mathrm{c} \mathrm{c}$ of dilute $\mathrm{HCl}(2: 1)$ and proceed as described above.

Notes.-Tungsten interferes with the determination of ranadium by the above method and must be removed. While chromium can be determined in the presence of tungsten, it is conveniently deter $\mathrm{r}$ ined in the same saniple as is vanadium and the end point is easie to get when tungsten has been removed.

In case hydrochloric acid is present when silver nitrate is added, enough of the latter miust be added to take care of this before the regular adidition is made.

regular addition is made. If under 1 per cent, the color does not seriously affect the resuits, if over this, it should be remored.
Determination of Nickel by the Gravimetric Dimethylglyoxime Method

\section{SOLUTIONS REQUIRED}

(Dilute hydrochloric acid (1:10) and dilute hydrochloric acid $(1: 1)$ are the same as in the determination of phosphorus in tungsten steel by the molybdate-magnesia method, given above. Sodium hydroxide $(2 N)$ is the same as in the determination of silicon in tungsten steel by acid attack and baking at $120^{\circ} \mathrm{C}$, given above. Dimethylglyoxime ( 1 per cent) is the same as in the determination of nickel by the dimethylglyoxime method in A64. (See 601.20, p. 119.)

\section{METHOD}

Treat $2 \mathrm{~g}$ of the sample in a covered beaker with $50 \mathrm{c}$ c of $\mathrm{HCl}$ (specific gravity 1.19), heat to incipient boiling, and then when solution is complete add $\mathrm{HNO}_{3}$ (specific gravity 1.42) at intervals and a few drops at a time until the vigorous action which ensues ceases. Digest until the tungstic acid is bright yellow. Add $100 \mathrm{c}$ c of boiling water, boil for one minute, cool somewhat, and filter. Wash the residue thoroughly with dilute $\mathrm{HCl}(1: 10)$ and reserve the filtrate. Dissolve the tungstic acid in a slight excess of sodium hydroxide solution $(2 N)$, boil, and filter off any residue. Wash the residue with hot water, discard the filtrate, and dissolve the residue in a few cubic centimeters of hot dilute $\mathrm{HCl}(1: 1)$, add the solution to the main reserved filtrate and proceed with the determination of nickel as in the determination of nickel in nickel steel by the gravimetric dimethylglyoxime method, given above.

Allotuble error.-Percentages of nickel determined by the above method should be accurate to $\pm(0.02+(0.01 \times$ the perentaze of nickel iound)

Note.-It is difficult to precipitate nickel and to obtain it free from cobalt when cobalt is preponcierant. Complete precipitation may be obtained by adding sufficient dinethy lglyoxime to react with both cobalt and nickel and digesting for a longer pericd or by oxidizing the cobalt to the trivalent condition by means of $\mathrm{NaClO}$ or $\mathrm{NH}_{2} \mathrm{C}_{8}$ in ammoniacal solution before precipitating with dimethylgiyoxime. In either case, the first precipitate siould be filtered off, cissolved, and reprecipitated.

Direct weighing of nickel dimethylgloxime is desirsble when small amounts of nichel are concernerl. Larze precipitates are more conveniently ignited at red heat to $\mathrm{NiO}$. In this case, care must be taken to prevent sublimation of the undecomposed salt. This may be acompished br enclosing the wet pazer ond precipitote in one or two or two wet ashless papers and slowly igniting so that the papers
char before taking fire. In this way the coinpound is decomposed and ignition may proceed at full reu heat.

Determination of Nickel by the Cy\&nide Titration Method (Routine)

(The only solution required is dilute hydrochloric acid $(1: 10)$ which is the same as in the determination of phosphorus in tungsten steel by the molybdate-magnesia method, given above.)

\section{METHOD}

Treat $2 \mathrm{~g}$ of the sample in a covered beaker with $50 \mathrm{c}$ c of $\mathrm{HCl}$ (specific gravity 1.19), heat to incipient boiling, and then when solution is complete add $\mathrm{HNO}_{3}$ (specific gravity 1.42) at intervals and a few drops at a time until the vigorous action which ensues ceases. Digest until the tungstic acid is bright yellow. Add $100 \mathrm{c}$ c of boiling water, boil for one minute, cool somewhat and filter. Wash the residue thoroughly with dilute $\mathrm{HCl}$ (1:10) and determine nickel in the filtrate as in the determination of nickel in nickel steel by the cyanide titration method, given above.

NoTE.-In case cobalt is present, the preliminary separeticn $(\pi)$ as given in the cyanide titration method must be employed. 
Determination of Cobalt

\section{SOLUTIONS REQUIRED}

Sodium carbonate (10 per cent).-Dissolve $100 \mathrm{~g}$ of $\mathrm{Na}_{2} \mathrm{Co}_{3}$ in $1,000 \mathrm{c}$ c of distilled water. Ammonium acetate (5 per cent).-Dissolve $50 \mathrm{~g}$ of $\mathrm{NH}_{4} \mathrm{C}_{2} \mathrm{H}_{3} \mathrm{O}_{2}$ in $1,000 \mathrm{c} \mathrm{c}$ of distilled water.

Dilute hydrochloric acid $(1: 1)$.- (Same as in the determination of phosphorus in tungsten steel by the molybdate-magnesia method, given above.) Acidulated hydrogen sulphide water.-Mix $20 \mathrm{c}$ c of $\mathrm{H}_{2} \mathrm{SO}_{4}$ (specific gravity 1.84) and $1,000 \mathrm{c}$ c of distilled water, cool, and saturate with $\mathrm{H}_{2} \mathrm{~S}$.

Nitroso beta naphthol (7 per cent).-Prepare as needed by dissolving in the proportion of $7 \mathrm{~g}$ of the compound to $100 \mathrm{c}$ c of glacial acetic acid.

\section{METHOD}

See the determination of silicon in tungsten steel by the acid attack and baking at $120^{\circ} \mathrm{C}$. method, given above.

Employ the $\mathrm{HCl}$ solution reserved in this determination or prepare a similar solution by treatinent of a new $1 \mathrm{~g}$ sample in the sare fashion, with the difference, however, that the wet tungstic acid is not ignited but treated directly with $\mathrm{NaOH}$ solution (2N) for the recovery of its cobalt content.

Evaporate the $\mathrm{HCl}$ solution to a volume of approximately $100 \mathrm{c} \mathrm{c}$ and neutralize the acid solution by adding solid $\mathrm{Na}_{2} \mathrm{CO}_{3}$ until the acid is nearly destroyed and then continuing with care by adding sodium carbonate solution (10 per cent), drop by drop with vigorous stirring, until the solution has become amber colored and the precipitate dissolves with difficulty. Pour the feebly acid solution slowly and with constant stirring into $200 \mathrm{c} c$ of boiling ammonium acetate solution ( 5 per cent). Boil for two to three minutes, allow to settle, filter without washing on a large rapid filter, and reserve the filtrate which must be absolutely clear. Dissolve the precipitate in as little hot dilute $(\mathrm{HCl} 1: 1)$ as possible, catching the solution in the unwashed beaker and reprecipitate as before, taking the same precautions as to preliminary neutralization and the volumes employed. Again filter without washing, discard the precipitate, and combine the filtrate with that already reserved. The combined filtrates should now be free from iron, chromium, and vanadium but may contain such elements as molybdenum, copper, and small amounts of tungsten in addition to cobalt, nickel, and manganese. To eliminate molybdenum and tungsten and to concentrate the others, neutralize the combined solutions with $\mathrm{NH}_{4} \mathrm{OH}$ (specific gravity 0.90 ) add $30 \mathrm{c}$ c in excess, and saturate the solution with a rapid current of $\mathrm{H}_{2} \mathrm{~S}$. Allow the precipitate to settle, filter without washing, discard the clear filtrate, and transfer the paper and precipitate to a $250 \mathrm{c}$ c Erlenmeyer flask. Dissolve any precipitate adhering to the precipitation beaker in $20 \mathrm{c}$ c of $\mathrm{HNO}_{3}$ (specific gravity 1.42) and a little $\mathrm{HCl}$ (specific gravity 1.19), pour the solution on the paper and precipitate and shake until the paper has disintegrated. Add 5 c c of $\mathrm{H}_{2} \mathrm{SO}^{\dagger}$ (specific gravity 1.84) and heat over a free flame until fumes of $\mathrm{H}_{2} \mathrm{SO}_{4}$ are evolved. In case the organic matter has not been destroyed add a little more $\mathrm{HNO}_{3}$ and repeat the $\mathrm{HNO}_{3}$ treatment and the evaporation until fumes of $\mathrm{H}_{2} \mathrm{SO}_{4}$ appear. Cool, dilute to $100 \mathrm{c}$ c, add $2 \mathrm{cc}$ of $\mathrm{NH}_{4} \mathrm{OH}$ (specific gravity 0.90 ), and saturate the acid solution with a rapid current of $\mathrm{H}_{2} \mathrm{~S}$. Filter, wash with acidulated $\mathrm{H}_{2} \mathrm{~S}$ water, and discard the precipitate. Boil the filtrate untl $\mathrm{H}_{2} \mathrm{~S}$ has been expelled and the solution reduced to a volume of $100 \mathrm{c} \mathrm{c}$ and then proceed as in $(a)$ or (b) below. (a) Determination of cobalt by the electrolytic method.-Add $5 \mathrm{c}$ c of $\mathrm{H}^{2} \mathrm{SO}^{4}$ (specific gravity 1.84), neutralize with $\mathrm{NH}_{4} \mathrm{OH}$ (specific gravity 0.90 ), add $35 \mathrm{c} \mathrm{c}$ in excess and then $2 \mathrm{~g}$ of $\mathrm{NaHSO}_{3}$. Electrolyze in a volume of $150 \mathrm{c}$ c for six hours or overnight, using gauze electrodes and a current density of 0.2 to 0.3 ampere per $\mathrm{dm}^{2}$. Wash the cathode with cold water, dry at $100^{\circ} \mathrm{C}$., and weigh the deposit. The electrolyte usually contains from 0.1 to $1 \mathrm{mg}$ of nickel and cobalt (mainly cobalt) and should be tested as follows: Boil the electrolyte with an excess of ammonium persulphate, keeping the solution strongly ammoniacal, to precipitate manganese. Filter, wash, and discard the precipitate and saturate the clear ammoniacal filtrate with $\mathrm{H}_{2} \mathrm{~S}$. Digest at the side of the steam bath for one hour, filter if a precipitate has appeared and wash with water containing a little $\mathrm{NH}_{4} \mathrm{Cl}$ and $\left(\mathrm{NH}_{4}\right)_{2} \mathrm{~S}$. Ignite, weigh the combined oxides of nickel and cobalt, multiply by the empirical factor 0.75 , and add to the cathode weight. The weight of cobalt must then be found by deducting nickel as determined on a separate portion of the steel or by analysis of the deposit and the small recovery from the electrolyte. In either case, attention must be paid to the difficulty in precipitating small amounts of nickel as pure nickel dimethylglyoxime in the presence of large amounts of cobalt, as described under the determination of nickel in nickel steel by the gravimetric dimethylglyoxime method, given above.

NoTE.-Tungsten, molybdenum, and vanadium must be completely removed in order to obtain a satisfactory deposition of nickel and cobalt.

(b) Determination of cobalt by the nitroso-betanaphthol method.-Transfer the solution to a $600 \mathrm{c} \mathrm{c}$ beaker, add $\mathrm{NH}_{4} \mathrm{OH}$ (specific gravity 0.90) until the solution is slightly ammoniacal and then $\mathrm{HCl}$ (specific gravity 1.19) until in $5 \mathrm{c}$ c excess. Dilute to $350 \mathrm{c}$ c heat to boiling, and for each centigram of cobalt expected add, with constant stirring, $6 \mathrm{c}$ c of nitroso-beta-naphthol solution ( 7 per cent). Set aside until the precipitate has settled, filter on paper, and wash the precipitate twice, alternately with dilute $\mathrm{HCl}(1: 1)$ and water, and finally with hot water. Transfer to a porcelain crucible, heat gently until the carbonaceous matter has been destroyed, and then at a high temperature for 20 minutes. $\mathrm{Cool}$ in a desiccator and weigh as $\mathrm{Co}_{3} \mathrm{O}_{4}$.

Allowable error.-Percentages of cobalt determined by the above method should be accurate to $\pm(0.04+(0.01 \times$ the percentage of cobalt found)).

NOTES.-The ignited oxide does not have the exact formula $\mathrm{Co}_{3} \mathrm{O}_{4}$ and therefore in case the weight is appreciable it is safer to change the oxide to metallic cobalt by repeated ignition in hydrogen in a rose-form crucible until the weight is constant.

The precipitate is apt to be contaminated by nickel in case nickel is preponderant in the original solution.

\section{Determination of Cobalt by Cyanide Titration (Routine)}

\section{SOLUTIONS REQUIRED}

Dilute nitric acid (1:3).-Mix 200 c c of $\mathrm{HNO}_{3}$ (specific gravity 1.42) and $600 \mathrm{c}$ c of distilled water.

(Sulphuric-citric acid, potassium iodide (10 per cent), standard silver nitrate, and standard potassium cyanide, all identical with like solutions in determination of nickel in nickel steel by the cyanide titration method (routine), given above.)

\section{METHOD}

See the determination of silicon in tungsten steel by the acid attack and baking at $120^{\circ} \mathrm{C}$., given above. Then proceed as follows:

To the reserved $\mathrm{HCl}$ solution or one prepared in the same way from a new portion of steel, add $50 \mathrm{c} \mathrm{c}$ of the sulphuric-citric acid solution and then $\mathrm{NH}_{4} \mathrm{OH}$ (specific gravity 0.90 ) until in 10 to 15 c c excess.

$126987-33-72$ 
Bring the solution to boiling, add $1 \mathrm{~g}$ of $\mathrm{NaClO}_{3}$ and boil for five minutes. Cool, dilute to $200 \mathrm{c} \mathrm{c}$, add dilute $\mathrm{HNO}_{3}(1: 3)$, carefully, until the solution is just neutral and then add $5 \mathrm{c}$ c of $\mathrm{NH}_{4} \mathrm{OH}$ (specific gravity 0.90). Again cool if necessary, add $2 \mathrm{c} c$ of potassium iodide solution (10 per cent) and titrate as in the determination of nickel in nickel steel by the cyanide titration method, given above. The volume of standard cyanide solution which is actually consumed represents the sum of nickel and cobalt. The volume of standard cyanide solution consumed by the nickel may be found and deducted by a determination of nickel as in the determination of nickel in tungsten steel by the cyanide titration method (routine), given above, with preliminary separation $(a)$ or by calculation based on a determination of nickel as in the determination of nickel in tungsten steel by the gravimetric dimethyglyoxime method, given above. In either case, the volume of standard cyanide solution consumed by cobalt represents that required to convert cobalt to the compound $\mathrm{K}_{2} \mathrm{Co}(\mathrm{CN})_{5}$ and the cobalt titre of the cyanide solution is best found by titration of material of known cobalt content under the conditions that obtain in the method.

\section{Determination of Copper}

In steels containing little tungsten, proceed as in the determination of copper in A64 (see 601.20, p. 119), substituting $10 \mathrm{~g}$ of steel for $10 \mathrm{~g}$ of iron and dissolving the cooled melt in $\mathrm{HCl}$ instead of dilute $\mathrm{H}_{2} \mathrm{SO}_{4}$.

In steels containing considerable tungsten, proceed as in the determination of copper in A64 (making the substitutions noted above) with the single exception that the first sulphide precipitate and paper is digested with $\mathrm{HNO}_{3}$ and $\mathrm{H}_{2} \mathrm{SO}_{4}$ to complete destruction of organic matter instead of igniting the paper and residue. The $\mathrm{H}_{2} \mathrm{SO}_{4}$ solution is then cooled, diluted, boiled with an excess of $\mathrm{NaOH}$ solution $\left(2 N^{7}\right)$ and treated as in the ordinary procedure.

\section{MOLYBDENUM STEEL}

Determination of Carbon

See the determination of carbon in plain carbon steel by the direct-combustion method in A33 (600.1, p. 10) and also in sampling and chemical analysis of pig and cast iron in A64 (601.20, p. 119).

\section{Determination of Manganese}

(a) Determination of manganese (absence of chromium and cobalt).-(Same as determination of manganese by the bismuthate method (absence of cobalt) in A33 (see 600.1, p. 10), except in the following particulars:)

\section{SOLUTIONS REQUIRED}

(Dilute nitric acid (1:3), dilute nitric acid (3:97), ferrous ammonium sulphate solution, standard potassium permanganate solution, and standard sodium arsenite solution, all of which are the same as like solutions in the determination of manganese by the bismuthate method in A64. See 601.20, p. 119.)

\section{METHOD}

(Ferrous sulphate-permanganate titration (less than 0.05 per cent chromium) (Same as in A64 (see 601.20 , p. 119).)

Determination of manganese (presence of chromium and absence of cobalt). - (Same as determination of manganese by the hydrolysis-bismuthate method (absence of cobalt) in chrome-nickel steel, given above, except for ferrous ammonium sulphate and standard potassium permanganate solution, which conform to the determillation of manganese by the bismuthate method in A64. See 601.20, p. 119.)

Determination of manganese.-(Routine and in the presence of cobalt.)

\section{SOLUTIONS REQUIRED}

(Conforms to like solutions in the determination of manganese by the persulphate method in A64. See 601.20, p. 119.)

\section{METHOD}

(Conforms to the determination of manganese by the persulphate method (routine and in the presence of cobalt) in A33 (see 600.1, p. 10) wherein will be found certain references to A64.)

\section{Determination of $\mathrm{Phosphorus}$}

(a) Determination of phosphorus (absence of vanadium).- See the determination of phosphorus in plain carbon steel by the molybdate-magnesia method in A33 (600.1, p. 10), except ammoniummolybdate solution, for which see B28 (646.0, p. 1243).

(b) Determination of phosphorus (presence of vanadium).

\section{SOLUTIONS REQUIRED}

(Same as in the determination of phosphorus by the molybdate-niagnesia method in A33 see 600.i, p. 10) except ammonium molybdate, which is the same as in the determination of phosphorus by the alkalimetric method in B28 (see 646.0, p. 1243) and the following additional item:)

Ferrous sulphate.-Dissolve $40 \mathrm{~g}$ of ferrous sulphate in $100 \mathrm{c}$ c of distilled water that has been acidified with $1 \mathrm{c}$ c of $\mathrm{H}_{2} \mathrm{SO}_{4}$ (specific gravity 1.84 ).

\section{METHOD AND NOTES}

(Same as in A33 (see 600.1, p. 10), except that after boiling to free from oxides of nitrogen, the solution is cooled to $15^{\circ}$ to $20^{\circ} \mathrm{C}$. (instead of to $80^{\circ}$ C. as in A33) and then there are added $5 \mathrm{c} \mathrm{c}$ of the ferrous-sulphate solution and two or three drops of concentrated sulfurous acid.)

Allowable error.-(Same as in the determination of phosphorus in chrome-vanadium steel by the modified molybdate-magnesia method, given above.)

(c) Determination of phosphorus (routive and in presence of vanadium).

\section{SOLUTIONS REQUIRED}

(Dilute nitric acid (specific gravity 1.20), dilute nitric acid for washing $(2: 100)$, potassium permanganate (2.5 per cent), ammonium bisulphite (3 per cent), all are identical with the determination of phosphorus by the molybdate-magnesia method in A33. See 600.1, p. 10 . Ammonium molybdate, potassium nitrate (1 per cent), phenolphthalein indicator, standard sodium hydroxide, and standard nitric acid, all are identical with the determination of phosphorus by the alkalimetric method in B28. (See 646.0, p. 1243. Ferrous sulphate solution is the same as given above, in (b).)

\section{METHOD AND NOTES}

(Same as in A33 (see 600.1, p. 10), except that after boiling to free from oxides of nitrogen, the solution is cooled to $15^{\circ}$ to $20^{\circ} \mathrm{C}$. (instead of to $80^{\circ}$ C. as in A33) and then there are added $5 \mathrm{c} c$ of the ferrous sulphate solution and two or three drops of concentrated sulphurous acid. Also omit here the notation reference to phosphomolybdate.)

(d) Determination of phosphorus (routine in absence of vanadium). 


\section{SOLUTIONS REQUIRED}

(Dilute nitric acid (specific gravity 1.20), dilute nitric acid $(2: 100)$, potassium permanganate, and ammonium bisulphite, all are identical with the determination of phosphorus by the molybdatemagnesia method in A33. (See 600.1, p. 10.) Ammonium molybdate, potassium nitrate (1 per cent), phenolphthalein indicator, standard sodium hydroxide, and standard nitric acid all are identical with the determination of phosphorus by the alkalimetric method in B28. See 646.0, p. 1243.)

METHOD AND NOTES

(Same as in A33. See 600.1, p. 10.)

\section{Determination of Sulphur}

See the determination of sulphur in plain carbon steel by the oxidation method in A33 (600.1, p. 10).

See the determination of sulphur in plain carbon steel by the evolution-titration method (routine) in A33, in which will be found certain references to A64.

Notes.-The ordinary evolution method yields low values with molybdenum steel. Good results are obtained on annealed samples with $\mathrm{HCl}$ (specific gravity 1.19).

\section{Determination of Silicon}

Determination of silicon by the nitrosulphuric method.

\section{SOLUTIONS REQUIRED}

(Nitrosulphuric acid same as in A33. See 600.1' p. 10. Dilute hydrochloric acid (5:95) same as in A64. See 601.20 , p. 119.)

\section{METHOD}

(Identical with A64 (see 601.20, p. 119), except that $80 \mathrm{c} c$ of the nitrosulphuric acid is added cautiously to $4.676 \mathrm{~g}$ of steel, and that the difference in reights in milligrams divided by 100 equals the percentage of silicon, instead of being divided by 50 as in $\mathrm{A} 64$.)

(Allowable error and note are the same as in A33. See 600.1, p. 10.)

Determination of silicon by the sulphuric acid method (optional).-(Identical with A64 (see 601.20, p. 119) except that here $2.338 \mathrm{~g}$ of steel is substituted for the $2.336 \mathrm{~g}$ of iron specified in A64.)

Determination of Molybdenum by Precipitation as Sulphide and Weighing as Iead Molybdate

SOLUTIONS REQUIRED

Bromine water. - A saturated solution.

Ammonium persulphate (10 per cent).-Prepare as needed by dissolving $1 \mathrm{~g}$ of the persulphate in 10 c c of distilled water.

Acidulated hydrogen sulphide water.-Pour 10 c c of sulphuric acid (specific gravity 1.84) into $1,000 \mathrm{c} c$ of distilled water. Add $10 \mathrm{~g}$ of tartaric acid and saturate the solution with $\mathrm{H}_{2} \mathrm{~S}$.

Ammonium acetate (50 per cent).-Dissolve $100 \mathrm{~g}$ of ammonium acetate in $100 \mathrm{c} c$ of distilled water.

Lead acetate (1 per cent).-Dissolve $10 \mathrm{~g}$ of lead acetate in a mixture of $990 \mathrm{c}$ c of distilled water and $10 \mathrm{c}$ c of acetic acid. Filter if necessary.

Ammonium nitrate (2.5 per cent).-Dissolve 25 $\mathrm{g}$ of $\mathrm{NH}_{4} \mathrm{NO}_{3}$ in $1,000 \mathrm{c}$ c of distilled water, filter if necessary, and make sure that the solution is neutral.

(Dilute sulphuric acid $(1: 1)$ and sodium hydroxide $(2 N)$ are the same as in the determination of silicon in tungsten steel by acid attack and baking at $120^{\circ}$, given above. Dilute hydrochloric acid
$(1: 1)$ is the same as in the determination of phosphorus in tungsten steel by the molybdate-magnesia method, given above. Dilute hydrochloric acid $(1: 9)$ is the same as in the determination of vanadium in chrome-vanadium steel by reduction with ferrous sulphate and titration with permanganate, given above.)

\section{METHOD}

(a) Determination of molybdenum (absence of tungsten). - In an $800 \mathrm{c}$ c beaker, dissolve a sufficient amount of the sample to yield approximately $0.03 \mathrm{~g}$ of molybdenum in a mixture of $100 \mathrm{c} c$ of water and $50 \mathrm{c}$ c of $\mathrm{H}_{2} \mathrm{SO}_{4}(1: 1)$. When effervescence ceases, add $10 \mathrm{c}$ e of ammonium persulphate solution (10 per cent), boil to oxidize molybdenum and part of the iron and cool somewhat. (Note 1.) Add $5 \mathrm{~g}$ of tartaric acid (note 2) nearly neutralize with $\mathrm{NH}_{4}$ $\mathrm{OH}$ (specific gravity 0.90 ) heat nearly to boiling and pass in $\mathrm{H}_{2} \mathrm{~S}$ rapidly for 10 minutes or until the iron has been reduced and the molybdenum precipitated. Allow the solution to stand on the steam bath at $50^{\circ}$ to $60^{\circ} \mathrm{C}$. for three hours, filter and wash the sulphur and sulphides with acidulated hydrogen sulphide water. Treat the sulphides as below. Boil the filtrate to expel hydrogen sulphide, and until the volume is reduced to from 400 to $500 \mathrm{c} \mathrm{c}$. Add $10 \mathrm{c}$ c of ammonium persulphate solution (10 per cent), boil, and again precipitate with $\mathrm{H}_{2} \mathrm{~S}$ as above in order to recover any molybdenum which escaped the first precipitation. Wash the main precipitate and the recovered precipitate from the papers into a $400 \mathrm{c}$ c beaker and dissolve the part remaining on the papers in $20 \mathrm{c} c$ of hot dilute $\mathrm{HCl}(1: 1)$ and bromine water, the solution being received in the $400 \mathrm{c} c$ beaker. Finally wash the papers with a little water and ignite them with the paper reserved below. Dilute the solution to $100 \mathrm{c} \mathrm{c}$. add $20 \mathrm{c}$ c of $\mathrm{HCl}$ (specific gravity 1.19) and $3 \mathrm{~g}$ of sodium chlorate and boil until the molybdenum sulphide is dissolved. Filter, wash the paper and residue with water, and reserve the filtrate. Ignite the papers and residues at a very low temperature in a deep-form crucible (to avoid volatilization loss of any $\mathrm{MoO}_{3}$ ), treat any residue with warm $\mathrm{NaOH}$ solution $(2 N)$ and pour the solution into the main filtrate. Nearly neutralize the combined solutions with sodium hydroxide solution $(2 N)$ and pour the clear yellow solution slowly and with stirring into $100 \mathrm{c}$ c of boiling sodium hydroxide solution $(2 N)$ contained in a $400 \mathrm{c}$ c beaker. After the precipitate has settled, filter and wash thoroughly with hot water. Reserve the filtrate and dissolve the precipitate in hot dilute $\mathrm{HCl}(1: 1)$ and re-precipitate with sodium hydroxide solution $(2 N)$ as above. (Note 3.) Neutralize the combined filtrates with $\mathrm{HCl}$ (specific gravity 1.19), add $5 \mathrm{c} \mathrm{c}$ in excess and evaporate the solution to 250 c c. To the clear solution add $50 \mathrm{c} \mathrm{c}$ of ammonium acetate solution (50 per cent), heat to boiling and slowly add lead acetate solution (1 per cent) from a burette dropwise and with continual stirring, until in slight excess. This is usually shown by a clearing of the solution. At this point discontinue the addition of the acetate, boil the solution for two or three minutes while stirring, allow the precipitate to settle somewhat, and test the supernatant liquid with a few drops more of the acetate. When precipitation is complete, boil for 10 minutes, allow to settle and filter the supernatant liquid through an ashless filter. Wash three or four times by decantation with $75 \mathrm{c} \mathrm{c}$ portions of hot ammonium nitrate solution (2.5 per cent), and finally transfer and complete the washing of the precipitate on the paper. If the precipitate is large or much sulphate was present in the solution, it is advisable to dissolve the first precipitate in hot dilute $\mathrm{HCl}(1: 1)$ and re-precipitate it as above in order to avoid undue contamination by 
lead sulphate or sodium salts. The precipitate may be carefully ignited directly in a porcelain crucible under oxidizing conditions until the carbon is destroyed or else the paper and precipitate may be dried at $100^{\circ} \mathrm{C}$., the precipitate removed from the paper, and the paper carefully ignited separately before the ignition of the main precipitate. In either case the final ignition to $\mathrm{PbMoO}_{4}$ should be at a very dull red heat. (Note 4.)

(b) Determination of molybdenum (presence of tungsien).- Treat the sample, in a $400 \mathrm{c}$ c beaker, with $50 \mathrm{c}$ c of dilute $\mathrm{HCl}(1: 1)$, and when solution is complete add $10 \mathrm{c}$ c of $\mathrm{HNO}_{3}$ (specific gravity 1.42) to oxidize the iron and the tungsten. Digest until the tungstic acid is bright yellow, filter and wash with dilute $\mathrm{HCl}(1: 9)$. Reserve the precipitate. Add $30 \mathrm{c}$ c of dilute $\mathrm{H}_{2} \mathrm{SO}_{4}(1: 1)$ to the filtrate, evaporate until fumes of $\mathrm{H}_{2} \mathrm{SO}_{4}$ appear, cool, and add $40 \mathrm{c} \mathrm{c}$ of water. If a light residue of tungstic acid separates, filter it off on a small filter, wash with a little dilute sulphuric acid, and treat it together with the main tungstic acid precipitate. Add $3 \mathrm{~g}$ of tartaric acid to the clear solution, nearly neutralize with $\mathrm{NH}_{4} \mathrm{OH}$ (specific gravity 0.90 ), precipitate the molybdenum as sulphide in slightly acid solution and proceed as above. In order to recover the molybdenum which is always carried down by tungstic acid, dissolve the tungstic acid residues by pouring hot sodium hydroxide solution $(2 N)$ upon them and finally wash the papers with a little hot dilute sulphuric acid. To the filtrate add $5 \mathrm{~g}$ of tartaric acid, make slightly acid with sulphuric acid, and precipitate as above with hydrogen sulphide. Any molybdenum recovered here is apt to be contaminated by tungsten and therefore the precipitate must be dissolved in a little $\mathrm{HCl}$ and bromine water as above, treated with $2 \mathrm{c}$ c of $\mathrm{H}_{2} \mathrm{SO}_{4}$ (specific gravity 1.84) evaporated until fumes of $\mathrm{H}_{2} \mathrm{SO}_{4}$ appear, cooled and diluted with $6 \mathrm{c}$ c of water. After warm digestion, filter off the dissolved molybdenum, wash with water containing a little $\mathrm{H}_{2} \mathrm{SO}_{4}$, add the solution to the reserved major portion, and proceed as in method $(a)$.

Allowable error.-Percentages of molybdenum determined by the above method should be accurate to $\pm(0.02+(0.02 \times$ the percentage of molybdenum found)).

Notes.-1. Molybdenum is very slowly and incompletely pre cipitated by $\mathrm{H}_{2} \mathrm{~S}$ when in a reduced state.

2. Tartaric acid is necessary to prevent the partial precipitation of vanadium and tungsten by $\mathrm{H}_{2} \mathrm{~S}$. These are the main interfering elements in any method for the determination of molybdenum.

3 . The two sodium hydroxide precipitations are necessary to completely separate molybdenum from iron which would otherwise be precipitated upon boiling in dilute acetic acid solution.

4. $\mathrm{PbMOO}_{4}$ is somewhat soluble in ammonium acetate solution, but this reagent is necessary to prevent contamination by $\mathrm{PbSO}_{4}$. If the solution contained no sulphates a more desirable method would consist in precipitating the molybdate in a hot, very dilute $\mathrm{HNO}_{3}$ solution by means of $\mathrm{Pb}\left(\mathrm{NO}_{3}\right)_{2}$, and then, when the precipitate became granular, making the solution faintly alkaline with ammonia and faintly acid with acetic acid.

5. Molybdenum may also be satisfactorily determined by carefully igniting the sulphide to the oxide $\mathrm{MoO}_{3}$, weighing, and then correcting for iron and copper oxides or, by dissolving the sulphide in acid, freeing the solution from copper, arsenic, and iron, reducing in a Jones reductor containing ferric alum and phosphoric acid in the receiver, and titrating the reduced oxide $\left(\mathrm{Mo}_{2} \mathrm{O}_{3}\right)$ to $\mathrm{MoO}_{3}$ by means of a standard permanganate solution

Determination of Molybdenum by Sodium Hydroxide Separation, Lead Molybdate Precipitation (Routine)

\section{SOLUTIONS REQUIRED}

Dilute hydrochloric acid (1:4).-Pour $200 \mathrm{c} \mathrm{c}$ of $\mathrm{HCl}$ (specific gravity 1.19) into $800 \mathrm{c} \mathrm{c}$ of distilled water.

Dilute hydrochloric acid (5:100).-Pour $50 \mathrm{c}$ c of $\mathrm{HCl}$ (specific gravity 1.19) into $1,000 \mathrm{c} \mathrm{c}$ of distilled water.

(Sodium hydroxide $(2 N)$ same as in the determination of silicon in tungsten steel by acid attack and baking at $120^{\circ} \mathrm{C}$., given above. Ammionium acetate ( 50 per cent) and lead acetate (1 per cent) are the same as in the determination of molybdenum by precipitation as sulphide and weighing as lead molybdate, given above.)

\section{METHOD}

Determination of molybdenum by sodium hydroxide separation, lead molybdate precipitation (absence of tungsten and vanadium).-Dissolve $2 \mathrm{~g}$ of the sample in $20 \mathrm{c}$ c of $\mathrm{HCl}$ (specific gravity 1.19), oxidize with $5 \mathrm{c}$ c of $\mathrm{HNO}_{3}$ (specific gravity 1.42) and expel oxides of nitrogen. Add sodium hydroxide solution $(2 N)$ until the excess of free acid is neutralized, taking care not to produce a red solution (dissolved hydrate) or a precipitate (basic molybdate). Heat the solution to boiling, and run the solution of the steel in rapid drops from a tap funnel into $160 \mathrm{c} \mathrm{c}$ of sodium hydroxide solution $(2 N)$ with constant stirring of the mixture. Dilute the alkaline solution to exactly $500 \mathrm{c} \mathrm{c}$, filter through a dry filter, discard the first few cubic centimeters, and then take exactly $250 \mathrm{c}$ c of the filtrate. Neutralize the solution with $\mathrm{HCl}$ (specific gravity 1.19) add $5 \mathrm{c}$ c in excess, boil, add $20 \mathrm{c}$ c of ammonium acetate solution (50 per cent), and proceed as in the determination of molybdenum by precipitation as sulphide and weighing as lead molybdate, given above, omitting, however, a second precipitation of the lead molybdate.

Notr.s.-The above process is of fairly general application. Manganese, nickel, cobalt, aluminum, and the ordinary elements of steel are without influence in the method. Chromium may be partially oxidized to chromate in the alkali treatment but can be reduced to the harmless trivalent condition by adding a few drops of sulphurous acid to the acid solution, prior to the molybdate precipitation. Tungsten and vanadium interfere. Vanadium is precipitation. Tungsten and vanadium interfere. Vanadium is not easily removed but the interference of tungsten can be largely
overcome by the modification of the method indicated in overcome by
method $(b)$.

Determination of molybdenum by sodium hydroxide separation, lead molybdaie precipitation (presence of tungsten and absence of vanadium).-Dissolve $2 \mathrm{~g}$ of the sample in a covered beaker in $50 \mathrm{c} \mathrm{c}$ of $\mathrm{HCl}$ (specific gravity 1.19), heat to incipient boiling and digest on a hot plate at moderate heat. Add a few drops of $\mathrm{HNO}_{3}$ (specific gravity 1.42), wait until the vigorous action ceases, and continue the dropwise addition of the acid until the vigorous action ceases. If this is carefully done, no tungstic acid separates at this stage. Next evaporate the liquid as quickly as possible until tungstic acid begins to separate, and then more slowly until the solution is pasty. Now add $50 \mathrm{c}$ c of dilute $\mathrm{HCl}(1: 4)$, boil, and set aside for at least 15 minutes. Filter through paper pulp, wash with dilute $\mathrm{HCl}(5: 100)$ and treat the hydrochloric acid solution for molybdenum as above.

Determination of Molybdenum by the Colorimetric Method (O ptional Routine) (Absence of Tungsten and High Vanadium or Copper)

\section{SOLUTIONS REQUIRED}

Dilute sulphuric acid (1:7).-Carefully mix $100 \mathrm{c} \mathrm{c}$ of $\mathrm{H}_{2} \mathrm{SO}_{4}$ (specific gravity 1.84) and $700 \mathrm{c} c$ of distilled water.

Dilute nitric-sulphuric acid mixture.-Mix 833 c c of $\mathrm{HNO}_{3}$ (specific gravity 1.20) and $167 \mathrm{c}$ c of $\mathrm{H}_{2} \mathrm{SO}_{4}(1: 1)$.

Sulphuric-hydrochloric acid mixture.-Mix $450 \mathrm{c} \mathrm{c}$ of $\mathrm{H}_{2} \mathrm{SO}_{4}(1: 1), 100 \mathrm{c} \mathrm{c}$ of $\mathrm{HCl}(1: 1)$, and $450 \mathrm{c} \mathrm{c}$ of distilled water.

Ferric sulphate-Dissolve $70 \mathrm{~g}$ of ferric sulphate in a mixture of $120 \mathrm{c} \mathrm{c}$ of $\mathrm{HCl}(1: 1), 500 \mathrm{c} \mathrm{c}$ of $\mathrm{H}_{2} \mathrm{SO}_{4}(1: 1)$, and $380 \mathrm{c} \mathrm{c}$ oi distiliea water. Filter if necessary.

Chromic sulphate.-Dissolve $50 \mathrm{~g}$ of chromic potassium sulphate in $100 \mathrm{c}$ c of $\mathrm{H}_{2} \mathrm{SO}_{4}(1: 1)$, and 200 
c c of water and heat until the purple color changes to green. Dilute to $1,000 \mathrm{cc}$.

Stannous chloride.-Dissolve $250 \mathrm{~g}$ of stannous chloride in $200 \mathrm{c}$ c of $\mathrm{HCl}$ (specific gravity 1.19), and dilute to $1,000 \mathrm{c} \mathrm{c}$ with distilled water.

Standard molybdenum solution.-Weigh accurately such an amount of a standard ferro-molybdenum (preferably over 50 per cent molybdenum) as will represent exactly $0.5 \mathrm{~g}$ of molybdenum, and transfer the sample to a platinum dish. Add 20 c c of dilute $\mathrm{HNO}_{3}(1: 1)$, and a few cubic centimeters of hydrofluoric acid, warm until the alloy is dissolved and then add $20 \mathrm{c}$ c of $\mathrm{H}_{2} \mathrm{SO}_{4}(1: 1)$ and evaporate until fumes of $\mathrm{H}_{2} \mathrm{SO}_{4}$ appear. Cool, add $50 \mathrm{c} \mathrm{c}$ of water, boil until all salts are dissolved and then cool and transfer to a liter flask. Add $100 \mathrm{c} \mathrm{c}$ of $\mathrm{H}_{2} \mathrm{SO}_{4}$ $(1: 1)$ and dilute the solution to the mark.

Potassium thiocyanate ( 5 per cent).-Dissolve $50 \mathrm{~g}$ of $\mathrm{KCNS}$ in $1,000 \mathrm{c} \mathrm{c}$ of distilled water.

\section{METHOD}

Transfer $0.5 \mathrm{~g}$ of the steel to a $200 \mathrm{c}$ c casserole, dissolve in $10 \mathrm{c}$ c of the nitric-sulphuric mixture, and then evaporate until fumes of $\mathrm{H}_{2} \mathrm{SO}_{4}$ are evolved. Cool, add $30 \mathrm{c} \mathrm{c}$ of sulphuric-hydrochloric mixture and heat until the salts are in solution. Cool the solution, transfer to a comparison bath and place in a bath for further cooling. To another comparison tube add $25 \mathrm{c}$ c of the ferric sulphate solution $(0.5 \mathrm{~g}$ $\mathrm{Fe}), 1 \mathrm{c}$ c of the chromic sulphate solution for each per cent of chromium present, and then $1 \mathrm{c} c$ of the standard molybdenum solution for each 0.1 per cent of molybdenum expected. Cool both tubes to the same temperature and add to each from a burette $5 \mathrm{c}$ c of potassium thiocyanate solution ( 5 per cent). Mix the solution in each tube, add $10 \mathrm{c}$ c of the stannous chloride solution to each from a burette and well mix each solution for 10 seconds. With dilute $\mathrm{H}_{2} \mathrm{SO}_{4}(1: 7)$, dilute the standard to some multiple of its molybdenum content and dilute the test until the colors match. The amounts of molybdenum are then directly proportional to the heights of the columns of liquids.

Notes.-The above method is applicable to steels containing up to 0.5 per cent of molybdenum. Above this percentage the solution of the steel must be divided into aliquot portions and the iron and the hydrochloric acid in the standard varied accordingly.

If the analyst is dealing with steels of approximately the same composition, the iron, chromium (nickel) and molybdenum may be composition, the iron, chromium (nickel) and molybdenum may

The amount of hydrochloric acid must be regulated and be the same in the tests as excessive hydrochloric acid causes a fading of the color.

If the steel contains chromium in excess of 1 per cent, difficulty may be encountered in obtaining complete solution after the evaporation to the appearance of fumes of $\mathrm{H}_{2} \mathrm{SO}_{4}$. In this event, dissolve $0.5 \mathrm{~g}$ of the steel, contained in a $200 \mathrm{c}$ c flask, in $15 \mathrm{c}$ c of dilute $\mathrm{H}_{2} \mathrm{SO}_{4}$ (1:1), and $30 \mathrm{c} \mathrm{c}$ of distilled water. When solution is complete, add $15 \mathrm{c}$ c of $\left(\mathrm{NH}_{4}\right)_{2} \mathrm{~S}_{2} \mathrm{O}_{8}$ solution ( 15 per cent) and boil, add $3 \mathrm{c} \mathrm{c}$ of dilute HCl (1:1), boil 10 minutes to expel the excess of persulphate, and to reduce the volume to $30 \mathrm{c}$ c preliminary to comparison. If any residue remains, add paper pulp, filter, reserve the filtrate, and transfer the paper and pulp to a $150 \mathrm{c} \mathrm{c}$ breaker and add $10 \mathrm{c} \mathrm{c}$ of $\mathrm{HNO}_{3}$ (specific gravity 1.42), $5 \mathrm{~g}$ of sodium chlorate and $5 \mathrm{c} \mathrm{c}$ of $\mathrm{H}_{2} \mathrm{SO}_{4}$ (specific gravity 1.84). Evaporate to the appearance of copious fumes and repeat the treatment if the solution is not free from color due to organic matter. Cool and add to the reserved filtrate.

\section{Determination of Copper}

(Substantially the same as the determination of copper in A64. See 601.20, p. 119.)

\section{Determination of Nickel}

See determination of nickel in nickel steel by the gravimetric dimethylglyoxime method, given above, except in solutions required, where dimethylglyoxime is the same as in A64. (See 601.20, p. 119.)

See the determination of nickel in nickel steel by the cyanide titration method (routine), given above.
Determination of Chromium

\section{SOLUTIONS REQUIRED}

(Sulphuric-phosphoric acid mixture, silver nitrate (2.5 per cent), ammonium persulphate (15 per cent), sodium chloride ( 5 per cent), all are the same as in the determination of chromium in chrome-nickel steel by the persulphate oxidation method, given above. Dilute nitric acid (specific gravity 1.20) is the same as in the determination of manganese by the persulphate method in A64. See 601.20, p. 119. Standard ferrous sulphate and standard permanganate solution are the same as in the determination of manganese by the bismuthate method in A64. See 601.20, p. 119.)

\section{METHOD}

(Same as in the determination of chromium in chrome-nickel steel by the persulphate oxidation method, given above.)

\section{Determination of Vanadium}

See the determination of vanadium in chromevanadium steel by the electrolytic-separation method, given above, except for standard potassium permanganate in solutions required, for which see the determination of manganese by the bismuthate method in A64. (See 601.20, p. 119.)

Determination of Vanadium by Reduction with Ferrous Sulphate and Titration with Permanganate (Routine)

\section{SOLUTIONS REQUIRED}

(Sulphuric-phosphoric acid mixture, silver nitrate (2.5 per cent), ammonium persulphate (15 per cent), and sodium chloride ( 5 per cent), all are identical with the determination of chromium in chromenickel steel by the persulphate metbod, given above. Dilute nitric acid $(1: 1)$ is identical with the determination of manganese by the persulphate method in A64. See 601.20, p. 119. Standard ferrous sulphate and standard potassium permanganate are identical with the defermination of manganese by the bismuthate method in A64. See 601.20, p. 119.)

\section{METHOD}

See the determination of chromium in chromenickel steel by the persulphate oxidation method, given above. Then turn to the determination of vanadium by reduction with ferrous sulphate and titration with permanganate (routine) under chromevanadium steel, given above.

\section{Determination of Tungsten}

See the determination of tungsten in tungsten steel by the acid digestion-cinchonine method, given above, except for dilute hydrochloric acid (1:1), for which see the determination of phosphorus in tungsten steel by the molybdate-magnesia method, given above.

See the determination of tungsten in tungsten steel by the digestion with acids method (routine), given above.

\section{Determination of Cobalt}

\section{SOLUTIONS REQUIRED}

See the determination of cobalt in tungsten steel by the sulphide method, given above, except for dilute hydrochloric acid (1:1), for which see the determination of phosphorus in tungsten steel by the molybdate-magnesia method, given above. 


\section{METHOD}

See the determination of silicon in tungsten steel by the acid attack and baking at $120^{\circ} \mathrm{C}$. given above. Then turn to the determination of cobalt in tungsten steel by the sulphide method, given above.

Determination of Cobalt by Cyanide Titration (Routine)

\section{SOLUTIONS REQUIRED}

Dilute nitric acid $(1: 3)$ is identical with the determination of cobalt in tungsten steel by the cyanide titration method, given above. Sulphuriccitric acid, potassium iodide (10 per cent), standard silver nitrate, and standard potassium cyanide, all are identical with like solutions in the determination of nickel in nickel steel by the cyanide titration method (routine), given above.

\section{METHOD}

See the determination of silicon in tungsten steel by the acid attack and baking at $120^{\circ} \mathrm{C}$. given above. Then turn to the determination of cobalt in tungsten steel by the cyanide titration method, given above.

AMERICAN SOCIETY FOR TESTING MATERIALS, TENTATIVE METHODS OF TENSION TESTING OF METALIIC MATERIALS, DESIGNATION E8-27T, 1927

\section{TENSION TESTING OF METALLIC MATERIALS}

See 600.1 , p. 34 .

AMERICAN SOCIETY FOR TESTING MATERIALS, TENTATIVE METHODS OF COMPRESSION TESTING OF METALLIC MATERIALS, DESIGNATION E9-27T, 1927

\section{COMPRESSION TESTING OF METALLIC MIATE- RIALS}

(See 600.1, p. 38.)

UNITED STATES GOVERNMENT, DEPARTMENT OF COMMERCE, BUREAU OF STANDARDS, STANDARD SAMPLES, C25 AND SUPPLEMENT, 1927

CHROME-VANADIUM STEEL, SAMPLE NO. 30c CHROME-NICKEL STEEL, SAMPLE NO. 32b; NICKEL STEEL, SAMPLE NO. 33b; CHROME. TUNGSTEN VANADIUM STEEL, SAMPLE NO. 50a; CHROME-MOLYBDENUM STEEL, SAMPLE NO. 72; STAINLESS STEEL, SAMPLE NO. 73; MEDIUM MANGANESE STEEL, SAMPLE NO. 100; $18 \mathrm{Cr}$., $8 \mathrm{Ni}$. STEEL, SAMPLE NO. 101

(These standard samples are prepared and sold by the Bureau of Standards for use by industrial organizations and others for checking the accuracy of analysis of similar products. Each is accompanied by a certificate giving its analysis as regards the important constituents.)
621.31 ALLOY STEEL BILLETS, BARS, AND RODS. AMERICAN RAILWAY ASSOCIATION, MECHANICAI DIVISION, HIGH CHROME STEEL RIVET MATERIAL AND RIVETS FOR NITRIC ACID TANK CARS, RECOMMENDED PRACTICE, 1929

\section{HIGH CHROME STEEL RIVET MATERIAL}

(In respect to process, chemical properties, ladle and check analyses, and method of pickling, this specification is identical with the A. R. A. specification for high chrome steel tank plates for nitric acid tank cars (see 621.32, p. 1124). In respect to inspection, rejection, and rehearing it conforms to $\mathrm{A}$. S. T. M. standard specifications for steel (see 605.0, p. 247). Otherwise it provides for the following requirements:)

\section{PHYSICAL PROPERTIES AND TESTS}

1. Tension tests.-Bars to be used for manufacture of rivets shall conform to the following minimum requirements as to tensile properties:

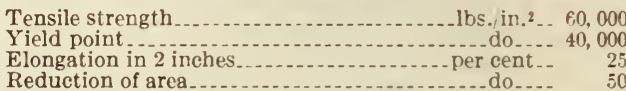

2. Bend tests.- (a) The test specimen shall stand being bent cold through $180^{\circ}$ flat on itself without cracking on the outside of the bent portion; subject to approval of the inspector.

(b) Oil quench bend tests.- The specimen shall be heated to $1,400^{\circ} \mathrm{F}$. and quenched in oil. These specimens shall stand bending $120^{\circ}$ around a pin equal to diameter of the bar without cracking on the outside of the bent portion. Oil quench bend tests may be omitted at the discretion of the inspector.

3 . Test specimens in section 2 shall be of the full diameter of the bars as rolled.

4. Test specimens.-Two tension, two cold bend, and two oil quench bend test specimens shall be selected from each annealing charge and stamped with an identification mark. Each test shall conform to the requirements specified in sections 1 and 2 .

5. If any specimen fails to meet the condition of the test, another specimen may be selected. If this also fails to meet the conditions of the test, the heat may be rejected at the discretion of the inspector.

\section{PERMISSIBLE VARIATIONS IN DIAMETER}

6. Permissible variations.-The diameter of the finished rivet bars shall not vary from that specified by more than the amount given in the following table:

\begin{tabular}{|c|c|c|c|}
\hline \multirow{2}{*}{ Diameter } & \multicolumn{2}{|c|}{$\begin{array}{l}\text { Variations in diam- } \\
\text { eter }\end{array}$} & \multirow{2}{*}{$\begin{array}{l}\text { Out of } \\
\text { round }\end{array}$} \\
\hline & Under & Orer & \\
\hline $\begin{array}{l}\text { Up to } 1 / 2 \text { inch, inclusive } \\
\text { Orer } 1 / 2 \text { to } 1 \text { inch, inclusive. }\end{array}$ & $\begin{array}{l}\text { Inch } \\
0.010 \\
.010\end{array}$ & $\begin{array}{c}\text { Inch } \\
0.010 \\
.015\end{array}$ & $\begin{array}{l}\text { Inch } \\
0.010 \\
.015\end{array}$ \\
\hline
\end{tabular}

FINISH

7. Finish.-The finished bars shall be free from seams, laps, slivers, etc., and shall have a work-man like finish. 


\section{MARKING}

8. Rivet bars shall, when loaded for shipment, be properly separated and marked with the name or brand of the manufacturer and heat numbers, as well as an identification mark corresponding with the test as specified in section 4 .

\section{REQUIREMENTS FOR RIVETS}

9. Rivets may be fabricated by either of the following methods unless one of them is specifically designated. No manufacturer may start the fabrication of rivets without first notifying the purchaser in sufficient time to enable his representative to be present.

(a) Hot fabrication. - The fabrication of rivets by this method shall be conducted in an indirect fired furnace whose temperature is uniform throughout, at a temperature not exceeding $1,400^{\circ} \mathrm{F}$. Furnaces for the fabrication of rivets shall be provided with a pyrometric apparatus which has recently been tested and is in good working condition, in order to ascertain that the temperature of $1,400^{\circ} \mathrm{F}$. is not being exceeded.

(b) Cold fabrication. - The fabrication of rivets by this method shall be conducted at a temperature not exceeding $250^{\circ} \mathrm{F}$.

10. The shape of the rivet head shall be in accordance with the purchaser's design.

11. All rivets shall carry a distinguishing mark or symbol on the head to indicate that the material of which they are made is chromium steel, in order to avoid the possibility of the accidental substitution of carbon-steel rivets in the operations of fabrication.

\section{PHYSICAL PROPERTIES AND TESTS}

12. Bend tests. - The rivet shank shall stand being bent cold through $180^{\circ}$ flat on itself without cracking on the outside of the bent portion, subject to the approval of the inspector.

13. Flattening test. - The rivet head shall flatten axially while hot (at the fabrication temperature specified above in section $9(a)$ ), to a diameter two and one-half times the diameter of the shank without cracking at the edges.

14. Number of tests. - Three bend and three flattening tests shall be made from each size in each lot of 500 rivets of less offered for inspection, each of which shall conform to the requirements specified.

\section{PERMISSIBLE VARIATIONS IN DIAMETER}

15. Permissible variations. - The diameter of finished rivets measured cold shall not vary from that specified more than the amounts given in the following table:

\begin{tabular}{c|c|c}
\hline Diameter & $\begin{array}{c}\text { Variations in } \\
\text { snap gage } \\
\text { measurement }\end{array}$ & $\begin{array}{c}\text { Diameter, ring } \\
\text { gage measure- } \\
\text { ment }\end{array}$ \\
\hline Up to 1 inch, inclusive_......... & $\begin{array}{c}0.0156 \text { inch un- } \\
\text { der. }\end{array}$ & $\begin{array}{c}0.0156 \text { inch } \\
\text { above. }\end{array}$ \\
\hline
\end{tabular}

Snap gage measurements shall be made at the point of minimum diameter, but it is not required that the rivet shall turn completely in the gage. Measurements of the maximum tolerance shall be made with the ring gage, all rivets to slip full to the head in the gage of the required size for the various diameters.
16. The dimensions of the rivet head shall not vary from that specified for standard boiler rivets more than the amount given in the following table:

\begin{tabular}{|c|c|c|}
\hline \multirow{2}{*}{$\begin{array}{l}\text { Diameter } \\
\text { of rivets } \\
\text { as or- } \\
\text { dered }\end{array}$} & \multicolumn{2}{|c|}{ Head tolerances } \\
\hline & Width & Height \\
\hline $\begin{array}{c}\text { Inch } \\
1 / 2 \\
5 / 8 \\
3 / 4 \\
7 / 8\end{array}$ & $\begin{aligned} & \text { Inch } \\
+1 / 16 & -1 / 32 \\
+1 / 16 & -1 / 32 \\
+116 & -1 / 32 \\
+1 / 16 & -1 / 32\end{aligned}$ & $\begin{array}{l}\text { Inch } \\
+1 / 32 \\
+1 / 32 \\
+1 / 32 \\
+1 / 32\end{array}$ \\
\hline
\end{tabular}

\section{WORKMANSHIP AND FINISH}

17. Finish. - All rivets shall be true to form and concentric, shall be made in a workmanlike manner and shall be free from injurious defects.

18. Bars and rivets, pickling.-All bars and rivets are to be thoroughly pickled by the method referred to above (see A. R. A., 621.32, p. 1124).

19. When required, the manufacturer shall furnish the purchaser with a statement as to the analysis of each lot of bars or rivets, including the number shipped, and that the bars or rivets on this lot have been tested and have met all the requirements of these specifications.

\section{AMERICAN SOCIETY FOR TESTING MATERIALS, STANDARD SPECIFICA- TIONS FOR STRUCTURAL NICKEL STEEL，DESIGNATION A8-29, 1929}

\author{
STRUCTURAL NICKEL STEEL
}

(See 621.33, p. 1126.)

AMERICAN SOCIETY FOR TESTING MATERIALS, STANDARD SPECIFICA. TIONS FOR CARBON-STEEL AND ALLOY-STEEL BLOOMS, BILLETS, AND SLABS FOR FORGINGS, DESIGNATION A17-29, 1929

STEEL BILLETS

(See 602.2, p. 131.)

\section{AMERICAN SOCIETY FOR TESTING MATERIALS, STANDARD SPECIFICA- TIONS FOR STRUCTURAL SILICON STEEL, DESIGNATION A94-29, 1929}

$$
\text { STRUCTURAL SILICON STEEL }
$$

(See 621.33, p. 1126.)

\section{ASSOCIATION OF AMERICAN STEEL MANUFACTURERS, STANDARD PER- MISSIBLE VARIATIONS IN GAGE WEIGHT, GAGE THICKNESS, SIZE, AND FLATNESS OF SHEETS AND LIGHT PLATES BLUE ANNEALED, 1929}

\section{PERMISSIBLE VARIATIONS IN SHEETS AND PLATES}

(See 600.7, p. 117.) 
SOCIETY OF AUTOMOTIVE ENGINEERS, IRON AND STEEL SPECIFICATIONS, CHEMICAL COMPOSITION, JUNE, 1929

(For prefatory remarks, see 600.0, p. 2; for specification details, see 600.1 , p. 49 ; for silicon range see footnote 1.)

NICKEL STEELS

\begin{tabular}{|c|c|c|c|c|c|}
\hline $\begin{array}{l}\text { S. A. E. } \\
\text { steel } \\
\text { No. }\end{array}$ & $\begin{array}{l}\text { Carbon } \\
\text { range }\end{array}$ & $\begin{array}{c}\text { Manganese } \\
\text { range }\end{array}$ & $\begin{array}{l}\text { Phos- } \\
\text { phorus, } \\
\text { maxi- } \\
\text { mum }\end{array}$ & $\begin{array}{c}\text { Sulphur, } \\
\text { maxi- } \\
\text { mum }\end{array}$ & $\begin{array}{l}\text { Nickel } \\
\text { range }\end{array}$ \\
\hline $\begin{array}{l}2015 \\
2115 \\
2315 \\
2320 \\
2330\end{array}$ & $\begin{array}{l}\text { Per cent } \\
0.10-0.20 \\
.10-.20 \\
.10-.20 \\
.15-.25 \\
.25-.35\end{array}$ & $\begin{array}{l}\text { Per cent } \\
0.30-0.60 \\
.30-.60 \\
.30-.60 \\
.30-.60 \\
.50-.80\end{array}$ & $\begin{array}{r}\text { Per cent } \\
0.04 \\
.04 \\
.04 \\
.04 \\
.04\end{array}$ & $\begin{array}{r}\text { Per cent } \\
0.05 \\
.05 \\
.05 \\
.05 \\
.05\end{array}$ & $\begin{array}{l}\text { Per cent } \\
0.40-0.60 \\
1.25-1.75 \\
3.25-3.75 \\
3.25-3.75 \\
3.25-3.75\end{array}$ \\
\hline $\begin{array}{l}2335 \\
2340 \\
2345 \\
2350 \\
2512\end{array}$ & $\begin{array}{r}.30-.40 \\
.35-.45 \\
.40-.50 \\
.45-.55 \\
\text { (max.) } .17\end{array}$ & $\begin{array}{l}.50-.80 \\
.50-.80 \\
.50-.80 \\
.50-.80 \\
.30-.80\end{array}$ & $\begin{array}{l}.04 \\
.04 \\
.04 \\
.04 \\
.04\end{array}$ & $\begin{array}{l}.05 \\
.05 \\
.05 \\
.05 \\
.05\end{array}$ & $\begin{array}{l}3.25-3.75 \\
3.25-3.75 \\
3.25-3.75 \\
3.25-3.75 \\
4.75-5.25\end{array}$ \\
\hline
\end{tabular}

NICKEL-CHROMIUM STEELS

\begin{tabular}{|c|c|c|c|c|c|c|}
\hline $\begin{array}{c}\text { S. A. E. } \\
\text { steel } \\
\text { No. }\end{array}$ & $\begin{array}{c}\text { Carbon } \\
\text { range }\end{array}$ & $\begin{array}{c}\text { Manga- } \\
\text { nese } \\
\text { range }\end{array}$ & $\begin{array}{l}\text { Phos- } \\
\text { phor- } \\
\text { us, } \\
\text { maxi- } \\
\text { mum }\end{array}$ & $\begin{array}{l}\text { Sul- } \\
\text { phur, } \\
\text { maxi- } \\
\text { mum }\end{array}$ & $\begin{array}{l}\text { Nickel } \\
\text { range }\end{array}$ & $\begin{array}{l}\text { Chro- } \\
\text { mium } \\
\text { range }\end{array}$ \\
\hline $\begin{array}{l}3115 \\
3120 \\
3125 \\
3130 \\
3135\end{array}$ & $\begin{array}{l}\text { Per cent } \\
0.10-0.20 \\
.15-.25 \\
.20-.30 \\
.25-.35 \\
.30-.40\end{array}$ & $\begin{array}{r}\text { Per cent } \\
0.30-0.60 \\
.30-.60 \\
.50-.80 \\
.50-.80 \\
.50-.80\end{array}$ & $\begin{array}{c}\text { Per } \\
\text { cent } \\
0.04 \\
.04 \\
.04 \\
.04 \\
.04\end{array}$ & $\begin{array}{c}\text { Per } \\
\text { cent } \\
0.05 \\
.05 \\
.05 \\
.05 \\
.05\end{array}$ & $\begin{array}{l}\text { Per cent } \\
1.00-1.50 \\
1.00-1.50 \\
1.00-1.50 \\
1.00-1.50 \\
1.00-1.50\end{array}$ & $\begin{array}{l}\text { Per cent } \\
0.45-0.75 \\
.45-.75 \\
.45-.75 \\
.45-.75 \\
.45-.75\end{array}$ \\
\hline $\begin{array}{l}3140 \\
3145 \\
3150 \\
3215\end{array}$ & $\begin{array}{l}.35-.45 \\
.40-.50 \\
.45-.55 \\
.10-. .20\end{array}$ & $\begin{array}{l}.50-.80 \\
.50-.80 \\
.50-.80 \\
.30-.60\end{array}$ & $\begin{array}{l}.04 \\
.04 \\
.04 \\
.04\end{array}$ & $\begin{array}{l}.05 \\
.05 \\
.05 \\
.045\end{array}$ & $\begin{array}{l}1.00-1.50 \\
1.00-1.50 \\
1.00-1.50 \\
1.50-2.00\end{array}$ & $\begin{array}{l}.45-.75 \\
.45-.75 \\
.45-.75 \\
.96-1.25\end{array}$ \\
\hline $\begin{array}{l}3220 \\
3230 \\
3240 \\
3245\end{array}$ & $\begin{array}{l}.15-.25 \\
.25-.35 \\
.35-.45 \\
.40-.50\end{array}$ & $\begin{array}{l}.30-.60 \\
.30-.60 \\
.30-.60 \\
.30-.60\end{array}$ & $\begin{array}{l}.04 \\
.04 \\
.04 \\
.04\end{array}$ & $\begin{array}{l}.045 \\
.045 \\
.045 \\
.045\end{array}$ & $\begin{array}{l}1.50-2.00 \\
1.50-2.00 \\
1.50-2.00 \\
1.50-2.00\end{array}$ & $\begin{array}{l}.90-1.25 \\
.90-1.25 \\
.90-1.25 \\
.90-1.25\end{array}$ \\
\hline $\begin{array}{l}3250 \\
3312 \\
3325 \\
3335\end{array}$ & $\begin{array}{c}.45-.55 \\
(\max .) \\
.20-.17 \\
.30-.40\end{array}$ & $\begin{array}{l}.30-.60 \\
.30-.60 \\
.30-.60 \\
.30-.60\end{array}$ & $\begin{array}{l}.04 \\
.04 \\
.04 \\
.04\end{array}$ & $\begin{array}{l}.045 \\
.045 \\
.045 \\
.045\end{array}$ & $\begin{array}{l}1.50-2.00 \\
3.25-3.75 \\
3.25-3.75 \\
3.25-3.75\end{array}$ & $\begin{array}{l}.90-1.25 \\
1.25-1.75 \\
1.25-1.75 \\
1.25-1.75\end{array}$ \\
\hline $\begin{array}{l}3340 \\
3415 \\
3435 \\
3450\end{array}$ & $\begin{array}{l}.35-.45 \\
.10-.20 \\
.30-.40 \\
.45-.55\end{array}$ & $\begin{array}{l}.30-.60 \\
.30-.60 \\
.30-.60 \\
.30-.60\end{array}$ & $\begin{array}{l}.04 \\
.04 \\
.04 \\
.04\end{array}$ & $\begin{array}{l}.045 \\
.045 \\
.045 \\
.045\end{array}$ & $\begin{array}{l}3.25-3.75 \\
2.75-3.25 \\
2.75-3.25 \\
2.75-3.25\end{array}$ & $\begin{array}{r}1.25-1.75 \\
.60-.95 \\
.60-.95 \\
.60-.95\end{array}$ \\
\hline
\end{tabular}

MOLYBDENUM STEEIS

\begin{tabular}{|c|c|c|c|c|c|c|c|}
\hline $\begin{array}{c}\text { S. A. } \\
\text { E. } \\
\text { steel } \\
\text { No. }^{\top} \text {. }\end{array}$ & $\begin{array}{l}\text { Carbon } \\
\text { range }\end{array}$ & $\begin{array}{l}\text { Man- } \\
\text { ganese } \\
\text { range }\end{array}$ & $\begin{array}{l}\text { Phos- } \\
\text { phor- } \\
\text { us, } \\
\text { maxi- } \\
\text { mum }\end{array}$ & $\begin{array}{l}\text { Sul- } \\
\text { phur, } \\
\text { maxi- } \\
\text { mum }\end{array}$ & $\begin{array}{l}\text { Chro- } \\
\text { mium } \\
\text { range }\end{array}$ & $\begin{array}{l}\text { Nickel } \\
\text { range }\end{array}$ & $\begin{array}{l}\text { Molyb- } \\
\text { denum } \\
\text { range }\end{array}$ \\
\hline $\begin{array}{l}4130 \\
4140 \\
4150 \\
4615\end{array}$ & $\begin{array}{c}\text { P.ct. } \\
0.25-0.35 \\
.35-.45 \\
.45-.55 \\
.10-. .20\end{array}$ & $\begin{array}{c}P . c t . \\
0.50-0.80 \\
.50-.80 \\
.50-.80 \\
.30-.60\end{array}$ & $\begin{array}{r}P . c t . \\
0.04 \\
.04 \\
.04 \\
.04\end{array}$ & $\begin{array}{r}P . c t . \\
0.05 \\
.05 \\
.05 \\
.05\end{array}$ & \begin{tabular}{l}
$P . c t$. \\
$0.50-0.80$ \\
$.80-1.10$ \\
$.80-1.10$ \\
\hdashline
\end{tabular} & $P . c t$. & $\begin{array}{c}P . c t . \\
0.15-0.25 \\
.15-.25 \\
.15-.25 \\
.20-.30\end{array}$ \\
\hline
\end{tabular}

CHROMIUM STEELS

\begin{tabular}{|c|c|c|c|c|c|}
\hline $\begin{array}{c}\text { S. A. E. } \\
\text { stecl } \\
\text { No. }\end{array}$ & $\begin{array}{l}\text { Carbon } \\
\text { range }\end{array}$ & $\begin{array}{c}\text { Manganese } \\
\text { range }\end{array}$ & $\begin{array}{l}\text { Phos- } \\
\text { phorus, } \\
\text { maxi- } \\
\text { mum }\end{array}$ & $\begin{array}{l}\text { Sulphur, } \\
\text { maxi- } \\
\text { mum }\end{array}$ & $\begin{array}{l}\text { Chromium } \\
\text { range }\end{array}$ \\
\hline $\begin{array}{r}5120 \\
5140 \\
5150 \\
52100\end{array}$ & $\begin{array}{l}\text { Per cent } \\
0.15-0.25 \\
.35-.45 \\
.45-.55 \\
.95-1.10\end{array}$ & $\begin{array}{l}\text { Per cent } \\
0.30-0.60 \\
.50-.80 \\
.50-.80 \\
.20-.50\end{array}$ & $\begin{array}{r}\text { Per cent } \\
0.04 \\
.04 \\
.04 \\
.03\end{array}$ & $\begin{array}{c}\text { Per cent } \\
0.05 \\
.05 \\
.05 \\
.035\end{array}$ & $\begin{array}{r}\text { Per cent } \\
0.60-0.90 \\
.80-1.10 \\
.80-1.10 \\
1.20-1.50\end{array}$ \\
\hline
\end{tabular}

CHROMIUM-VANADIUM STEELS

\begin{tabular}{|c|c|c|c|c|c|c|c|}
\hline \multirow{2}{*}{$\begin{array}{c}\text { S. A. } \\
\text { E. } \\
\text { steel } \\
\text { No. }\end{array}$} & \multirow{2}{*}{$\begin{array}{c}\text { Carbon } \\
\text { range }\end{array}$} & \multirow{2}{*}{$\begin{array}{c}\text { Manga- } \\
\text { nese } \\
\text { range }\end{array}$} & \multirow{2}{*}{$\begin{array}{l}\text { Phos- } \\
\text { phor- } \\
\text { us, } \\
\text { maxi- } \\
\text { mum }\end{array}$} & \multirow{2}{*}{$\begin{array}{l}\text { Sul- } \\
\text { phur, } \\
\text { maxi- } \\
\text { mum }\end{array}$} & \multirow{2}{*}{$\begin{array}{l}\text { Chro- } \\
\text { mium } \\
\text { range }\end{array}$} & \multicolumn{2}{|c|}{ Vanadium } \\
\hline & & & & & & $\begin{array}{l}\text { Mini- } \\
\text { mum }\end{array}$ & $\begin{array}{l}\text { De- } \\
\text { sirer }\end{array}$ \\
\hline $\begin{array}{l}6115 \\
6120 \\
6125 \\
6130 \\
6135 \\
6140 \\
6145 \\
6150 \\
6195\end{array}$ & $\begin{array}{c}\text { Per ct. } \\
0.10-0.20 \\
.15-.25 \\
.20-.30 \\
.25-.35 \\
.30-.40 \\
.35-.45 \\
.40-.50 \\
.45-.55 \\
.90-1.05\end{array}$ & $\begin{array}{c}\text { Per ct. } \\
0.30-0.60 \\
.30-.80 \\
.50-.80 \\
.50-.80 \\
.50-.80 \\
.50-.80 \\
.50-.80 \\
.50-.80 \\
.20-.85\end{array}$ & $\begin{array}{r}\text { Perct. } \\
0.04 \\
.04 \\
.04 \\
.04 \\
.04 \\
.04 \\
.04 \\
.04 \\
.03\end{array}$ & $\begin{array}{l}\text { Perct. } \\
0.045 \\
.045 \\
.045 \\
.045 \\
.045 \\
.045 \\
.045 \\
.045 \\
.035\end{array}$ & $\begin{array}{c}\text { Per ct. } \\
0.80-1.10 \\
.80-1.10 \\
.80-1.10 \\
.80-1.10 \\
.80-1.10 \\
.80-1.10 \\
.80-1.10 \\
.80-1.10 \\
.80-1.10\end{array}$ & $\begin{array}{r}\text { Perct. } \\
0.15 \\
.15 \\
.15 \\
.15 \\
.15 \\
.15 \\
.15 \\
.15 \\
.15\end{array}$ & $\begin{array}{l}\text { Per ct. } \\
0.18\end{array}$ \\
\hline
\end{tabular}

1 Silicon range of all S. A. E. hasic open-hearth allov steels shall be $0.15-0.30$ per cent. For the electric and acid open-hearth steels the silicon content shall be a minimum of 0.15 per cent.

UNITED STATES GOVERNMENT, FEDERAL SPECIFICATIONS BOARD, FEDERAL SPECIFICATION FOR STEEL, CARBON AND ALLOY; BARS, BILLETS, BLCOMS, AND SLABS (FOR) REFORGING, NO. 316, AUGUST 8, 1925. (CURKEIT DESIGNATION : QQ-S-675)

ALLOY-STEEL BLOOMS, BILLETS, SLABS, AND (See 602.2, p. 133.)

UNITED STATES GOVERNMENT, FEDERAL SPECIFICATIONS BOARD, SPECIFICATION NO. 372, FOR STRUCTURAL NICKEL STEEL, JANUARY 25, 1826. (CURRENT DESIGNATION : QQ-N-341)

STRUCTURAL NICKEL STEEL

(See 621.33, p. 1127.)

621.32 ALLOY-STEEL PLATES, SHEETS, AND STRIPS.

AMERICAN RAILWAY ASSOCIATION, MECHAIICAL DIVISION, HIGE CHROME STEEL TANK PLATES FOR NITRIC ACID TANK CARS, RECOMMENDED PRACTICE, 1929

\section{STEEL TANK PLATES}

\section{PROCESS}

1. The steel shall be made by the electric furnace process.

\section{CHEMICAL PROPERTIES AND TESTS}

2. The steel shail conform to the following requirements as to chemical composition:

Carbon, maximum Per cent

Manganese, maximum 0.12

Phosphorus, maximum .50

Sulphur, maximum

Nickel, maximum --

Silicon, maximum - 11.40

Chromium, minimum, unless otherwise specifed.... 16.0

Analysis of each heat shall be made by the manufacturer for the determination of the elements mentioned above. The analysis shall be made from a test ingot taken during the pouring of the heat. The chemical composition thus determined shall be 
reported to the purchaser or his representative, and shall conform to the requirements specified in section 2 .

\section{CHECK ANALYSES}

3. Check analyses may be made by the purchaser from finished material representing each heat. The chemical composition thus determined shall conform to the requirements specified in section 2 .

\section{PHYSICAL PROPERTIES AND TESTS}

4. Bend test.-Test specimens shall withstand bending cold around a pin, the diameter of which is equal to the thickness of the plate, $180^{\circ}$ for specimens taken longitudinally and $120^{\circ}$ for transverse specimens, without cracking on the outside of the bent portion.

5. Test specimens.-(a) Bend-test specimens for longitudinal bend tests, section 4 , shall be taken longitudinally (in direction of rolling) from the long edge of the rolled plate.

(b) Bend-test specimens for transverse bend test, section 4 , shall be taken transversely (normal to the direction of rolling) from the middle of the ends of the rolled plates.

(c) Bend-test specimens shall be of the full thickness of the material and shall be about 18 inches long by not less than 2 inches wide, and shall have machined edges.

6. Number of tests. - Three longitudinal and three transverse bend-test specimens shall be made for each annealing charge, one of which must be from each heat.

7. Retests. - If the results of the bend tests do not conform to the requirements specified in section 4 , a retest of one additional pair of bend-test specimens from the same lot may be made, at the discretion of the inspector.

8. As bend tests can not be made on a finished, flanged, and dished head because tests of the material made previous to heating and bending would give no indication of its condition after fabrication, the the only means to determine the condition of the metal in flanged and dished heads will be by comparison of the hardness of the finished head with the hardness of the satisfactory annealed plate, which has passed inspection. No manufacturer may flange and dish a head or do any hot operation upon a firished plate without first notifying the purchaser in sufficient time to have a representative present. Hardness will be measured by either Scleroscope or Brinell test, in as many places as required by the inspector, in which case the reading on the finished head should be within 10 per cent of either the Scleroscope or Brinell reading on the annealed plate.

\section{PERMISSIBLE VARIATIONS IN THICENESS}

9. Permissible variations. - The thickness of each plate shall not vary more than 0.01 inch under that ordered; for plates one-fourth-inch thick and under the thickness shall not be more than 0.02 inch over that ordered, and for plates over one-fourth-inch thick, not more than 0.03 inch over that ordered. The thickness is to be measured not over 3 inches from the edge along the long edge of the plate.

\section{FINISH}

10. Finish.-(a) The finished material shall be free from pits, seams, splits, slivers, and other injurious defects, and shall have a workmanlike finish. Unless otherwise specified, all pits, seams, and slivers, which can be removed by spot grinding to a depth such as will not go below the minimum thickness as specified under section 9 , shall not be classified as defects.

(b) If plates are to be finished by sand blasting, ail pits, seams, and slivers which show up after sand blasting, may be removed by spot grinding if in so doing the thickness of plate is not reduced below the minimum thickness as specified under section 9, above.

(c) All spot grinding to remove such defects shall be done in the presence of the inspector and acceptance or rejection shall be at his discretion.

11. Pickling. - All plates are to be thoroughly pickled by the method described below, or other approved method.

Method of pickling.-For the pickling of all high chrome material, made by any method of manufacture, in order to produce surfaces free from scale and as corrosive resistant as possible, the following procedure shall be carried out:

(a) The material shall first be immersed in a solution containing a mixture of not less than 10 per cent sulphuric acid, by weight, and not less than 10 per cent hydrochloric acid, by weight, and held there for approximately 30 minutes, depending on the condition of the material; the temperature of this solution should be approximately $110^{\circ} \mathrm{F}$.

(b) At the end of the above period of time the material shall be well rinsed in water and immersed in a 10 per cent solution of nitric acid at approximately $110^{\circ} \mathrm{F}$. for a sufficient period of time to thoroughly loosen all the remaining scale.

(c) The material shall then be thoroughly washed off with water, using a hose stream and a suitable brush.

\section{MARKING}

12. The name or brand of the manufacturer and the manufacturer's test identification number (including the heat number) shall be legibly stamped on each finished plate in two places not less than 12 inches from the edges. The manufacturer's test identification number shall be legibly stamped on each of the test specimens.

\section{INSPECTION AND REJECTION}

(Requirements covering inspection, rejection, and rehearing conform to those specified in A. S. T. M. standard specifications for steel. See 605.0, p. 247.)

\section{AMERICAN SOCIETY FOR TESTIYG MATE- RIALS, STANDARD SPECIFICATIONS FOR STRUCTURAL NICKEL STEEL, DESIGNA- TION A8-29, 1929}

STRUCTURAL NICKEL STEEL

(See 621.33, p. 1126.)

AMERICAN SOCIETY FOR TESTING MATERIALS, STANDARD SPECIFICATIONS FOR STRUCTURAL SILICON STEEL, DESIGNATION A94-28, 1929

STRUCTURAL SILICON STEEL

(See 621.33, p. 1126.)

ASSOCIATION OF AMERICAN STEEL MANUFACTURERS, STANDARD PERMISSIBLE VARIATIONS IN GAGE WEIGHT, GAGE THICKNESS, SIZE, AID FLATNESS OF SHEETS AND LIGET PLATES BLUE ANNEALED, 1929

\section{PERMISSIBIE VARIATIONS IN SHEETS AND} PIATES

(See 600.7, p. 117.) 
UNITED STATES GOVERNMENT, FEDERAL SPECIFICATIONS BOARD, FEDERAL SPECIFICATION FOR NICKEL STEEL, STRUCTURAL, NO. 372, JANUARY 25, 1926. (CURRENT DESIGNATION : QQ-N-341)

\section{STRUCTURAL NICKEL STEEL}

(See 621.33, p. 1127.)

621.33 STRUCTURAL SHAPES OF ALLOY STEELS. AMERICAN SOCIETY FOR TESTING MATERIALS, STANDARD SPECIFICATIONS FOR STRUCTURAL NICKEL STEEL, DESIGNATION A8-29, 1929

\section{STRUCTURAL NICKEL STEEL}

(With a few exceptions, this specification is identical with A. S. T. M. A7 (see 605.11, p. 249). The exceptions are:)
1. Discard.-A sufficient discard shall be made from each ingot intended for eyebars to secure freedom from injurious piping and undue segregation.

2. Chemical composition.-The steel shall conform to the following requirements as to chemical composition:

\begin{tabular}{|c|c|c|}
\hline & $\begin{array}{c}\text { Structural steel (per } \\
\text { cent) }\end{array}$ & $\begin{array}{l}\text { Rivet steel (per } \\
\text { cent) }\end{array}$ \\
\hline $\begin{array}{l}\text { Carbon } \\
\text { Manganese... } \\
\text { Phosphorus: } \\
\text { Acid } \\
\text { Basic } \\
\text { Sulphur } \\
\text { Nickel }\end{array}$ & $\begin{array}{l}\text { Not over } 0.45 \\
\text { Not over } 0.70 \\
\text { Not over } 0.05 \\
\text { Not over } 0.04 \\
\text { Not over } 0.05 \\
\text { Not under } 3.25\end{array}$ & $\begin{array}{l}\text { Not over } 0.30 \text {. } \\
\text { Not over } 0.60 \text {. } \\
\text { Not over } 0.04 \text {. } \\
\text { Not over } 0.03 \text {. } \\
\text { Not over } 0.045 \text {. } \\
\text { Not under } 3.25 \text {. }\end{array}$ \\
\hline
\end{tabular}

3. Check analyses.-Analyses may be made by the purchaser from finished material representing each melt. The chemical composition thus determined shall conform to the requirements specified in section 2.

4. Tension tests.-The material shall conform to the following requirements as to tensile properties:

\begin{tabular}{|c|c|c|c|c|}
\hline Properties considered & Rivet steel & $\begin{array}{l}\text { Plates, shapes, } \\
\text { and bars }\end{array}$ & $\begin{array}{l}\text { Eyebar flats and } \\
\text { rollers unannealeda }\end{array}$ & $\begin{array}{l}\text { Eyebar flats } b \\
\text { and pins }{ }^{\circ} \text { un- } \\
\text { annealed }\end{array}$ \\
\hline $\begin{array}{l}\text { Tensile strength } \\
\text { Yield point, minimum }\end{array}$ & $\begin{array}{r}70,000-80,000 \\
45,000\end{array}$ & $\begin{array}{r}85,000-100,000 \\
50,000\end{array}$ & $\begin{array}{r}95,000-110,000 \\
55,000\end{array}$ & $\begin{array}{r}90,000-105,000 \\
52,000\end{array}$ \\
\hline Elongation in 8 inches, minimum _...- per cent_- & $\frac{1,500,000}{\text { Tensile strength }}$ & $\frac{e 1,500.000}{\text { Tensile strength }}$ & $\frac{e 1,500,000}{\text { Tensile strength }}$ & \\
\hline $\begin{array}{l}\text { Elongation in } 2 \text { inches, minimum } \\
\text { Reduction of area, mirimum }\end{array}$ & 40 & $\begin{array}{l}18 \\
25\end{array}$ & $\begin{array}{l}16 \\
25\end{array}$ & $\begin{array}{l}20 \\
35\end{array}$ \\
\hline
\end{tabular}

a Elongation shall be measured in 2 inches.

- Tests of annealed specimens of eyebar flats shall be made for information only.

- See modification in elongation.

5. Modification in elongation.-For plates, shapes and unannealed bars over 1 inch in thickness, a deduction from the percentage of elongation in 8 inches specified in section 4 of 0.25 per cent shall be made for each increase of one thirty-second inch of the specified thickness above 1 inch to a minimum of 14 per cent.

6. Character of fracture.-All broken tension-test specimens shall show either a silky or a very fine granular fracture, of uniform color, and free from coarse crystals.

7. Bend tests.-Bend-test specimens shall stand being bent cold through $180^{\circ}$ without cracking on the outside of the bent portion as follows: For material three-fourths inch or under in thickness, around a pin the diameter of which is equal to the thickness of the specimen; and for material over three-fourths inch in thickness, around a pin the diameter of which is equal to twice the thickness of the specimen.

8. Drift tests.-Punched rivet holes pitched two diameters from a planed edge shall stand drifting until the diameter is enlarged 50 per cent, without cracking the metal.

9. Tests of eyebars.-Full-size tests of annealed eyebars shall conform to the following requirements as to tensile properties:

Tensile strength

Yield point, minimum

Elongation in 18 feet, minimum

Reduction of area, minimum

48,000

10
30

10. The yield point shall be determined by the halt of the gage of the testing machine.

(For general requirements relating to yield point, number of tests, permissible variations in weight and thickness of material, finish, marking, inspection, rejection, and rehearing, also test specimens Figures 128 and 129 , see A. S. T. M. specifications for steel, 605.0, p. 247.)
(For tables of permissible variations of rectangu lar plates ordered to weight, and permissible overweights of rectangular plates ordered to thickness, see A. A. S. M. Tables 1 and 2,605.0, p. 248.)

(Requirements relating to process, ladle analysis, bend test for rivet steel, and test specimens are identical with those in A. S. T. M. A7 (see 605.11, p. 249), par. $2,4,7(c)$, and $8(a),(b),(c),(d),(e)$, $(f),(g),(h),(i)$, and $(j)$.

\section{AMERICAN SOCIETY FOR TESTING MATE- RIALS, STANDARD SPECIFICATIONS FOR STRUCTURAI SILICON STEEL, DESIGNA- TION A94-29, 1929}

\section{STRUCTURAL SILICON STEEL}

1. Scope.-These specifications cover a special high-strength structural steel intended primarily for use as main stress-carrying material of structural members.

2. Process. - The steel shall be made by the openhearth process.

3. A sufficient discard shall be made from each ingot to secure freedom from injurious piping and undue segregation.

4. Chemical composition.-The steel shall conform to the following requirements as to chemical composition:

\begin{tabular}{|c|c|c|}
\hline & Ladle analysis (per cent) & $\begin{array}{l}\text { Check analysis } \\
\text { (per cent) }\end{array}$ \\
\hline Carbon..... & Not over 0.40 & Not over 0.44 \\
\hline $\begin{array}{l}\text { Phosphorus: } \\
\text { Acid }\end{array}$ & & Not orer 0.075 . \\
\hline Basic & Not over 0.04 . & Not over 0.05 . \\
\hline Sulphur_...... & Not over 0.05 & Not over 0.063 \\
\hline Silicon & Not under 0.20 & Not under 0.18 . \\
\hline
\end{tabular}


5. Ladle analysis.-An analysis of each melt shall be made by the manufacturer to determine the percentages of carbon, manganese, phosphorus, sulphur, and silicon. This analysis shall be made from a test ingot taken during the pouring of the melt. The chemical composition thus determined shall be reported to the purchaser or his representative, and the percentages of carbon, phosphorus, sulphur, and silicon shall conform to the requirements for ladle analysis specified in section 4 .

6. Check analysis.-(Same as A8, above.)

7. (a) Tension tests. - The material shall conform to the following requirements as to tensile properties:
Tensile strength .lbs./in.2.- 80, 000-95, 000
Yield point, minimum 45,000
Elongation in 8 inches, minimum_..per cent $a$ $1,500,000$
Reduction of area, minimum $\underset{b 30}{\text { Tensile strength }}$

(b) The yield point shall be determined by the drop of the beam of the testing machine when operated at a crosshead speed not greater than one-half inch per minute. The testing machine shall not be stopped to obtain the drop of the beam.

8. (a) Modifications in elongation.-For material over three-fourths inch in thickness, a deduction from the percentage of elongation specified in section $7(a)$ of 0.25 per cent shall be made for each increase of one-sixteenth inch of the specified thickness above three-fourths inch, to a minimum of 14 per cent.

9. Modifications of reduction in area.-For material over three-fourths inch in thickness, a deduction from the percentage of reduction of area specified in section $7(a)$ of 0.50 per cent shall be made for each increase of one-sixteenth inch of the specified thickness above three-fourths inch, to a minimum of 24 per cent.

10. Bend tests. - The test specimen shall stand being bent cold through $180^{\circ}$ without cracking on the outside of the bent portion around a pin the diameter of which shall have the following relation to the thickness of the specimen:
Specimen thickness

Up to $3 / 4$ inch, inclusire

Over $3 / 4$ inch to 1 inch, inclusire

Orer 1 inch to 1144 inches, inclusire

Orer 114 inches. .
Pin diameter

1 times the thickness. $11 / 2$ times the thickness. 2 times the thickness. $21 / 2$ times the thickness.
11. Test specimens.-(Paragraphs $(a),(b),(c)$, $(d)$, and $(g)$ are identical with paragraphs $8(a),(b)$, $(d),(e)$, and (j) of A.S. T. M. A7 (see 605.11, p. 249.) except that reference to evebar flats is omitted from paragraph $(d)$ in this specification. For test specimen diagram, see A. S. T. M. specifications (605.0, p. 247). The remaining paragraphs of this section are as follows:)

(e) Tension-test specimens for material over 1 inch in thickness or diameter may be machined to a thickness or diameter of at least three-fourths inch for a length of at least 9 inches.

(f) Bend-test specimens for material over $11 / 4$ inches in thickness or diameter may be machined to a thickness or diameter of at least three-fourths inch, or to 1 by $1 / 2$ inch in section.

(For general requirements relating to number of tests, permissible variations in weight and thickness of material, finish, marking, inspection, rejection, and rehearing, see A. S. T. M. specifications $(605.0$, p. 247), except that two tension and one bend test shall be made from each melt, and reference to 2-inch specimens is omitted from paragraph $12(c)$.)

(For tables of permissible variations of rectangular plates ordered to weight, and permissible over- weights of retangular plates ordered to thickness, see A. A. S. M. Tables 1 and 2, 605.0, p. 248.)

AMERICAN SOCIETY FOR TESTING MATERIALS, STANDARD SPECIFICATIONS FOR STRUCTURAL STEEL FOR LOCO. MOTIVES AND CARS, A. S. T. M. DESIGNATION A113-29, 1929

COPPER STEEL FOR LOCOMOTIVES AND CARS

(See 605.13, p. 266.)

SOCIETY OF AUTOMOTIVE ENGINEERS, IRON AND STEEL SPECIFICATIONS, CHEMICAL COMPOSITIONS, JUNE, 1929

\author{
NICKEL STEELS
}

(See 621.31, p. 1124.)

SOCIETY OF AUTOMOTIVE ENGINEERS, IRON AND STEEL SPECIFICATIONS, CHEMICAL COMPOSITIONS, JUNE, 1929

NICKEL-CHROMIUM STEELS

(See 621.31, p. 1124.)

UNITED STATES GOVERNMENT, FEDERAL SPECIFICATIONS BOARD, FEDERAL SPECIFICATION FOR NICKEL STEEL, STRUCTURAL, No. 372, JANUARY 25, 1926. (CURRENT DESIGNATION: QQ$\mathrm{N}-341)$

\section{STRUCTURAL NICKEL STEEL}

\section{GENERAL SPECIFICATIONS}

1. United States Government general specifications for metals, Federal Specifications Board specifications No. 339a (current designation: QQ-M-151), in effect on date of issuance of proposals, shall form part of this specification. (See 600.0, p. 3.)

\section{GRADES}

2. Structural nickel steel shall be of the following grades: A, plates, shapes, and bars; $\mathrm{B}$, rivet steel; and $\mathrm{C}$, eyebars and rollers (annealed and unannealed).

\section{NOMBER OF TESTS}

3. Two tension and two bend tests shall be made from each melt, unless the finished material from a melt is less than 25 short tons, when one tension and one bend test shall be sufficient. If, however, material from one melt differs three-eighths inch or more in thickness, the thickest and thinnest material rolled shall be represented by one tension and one bend test, regardless of the weight of the melt.

4. Plates three-sixteenths inch or under in thickness shall not be subject to tension tests.

5. On all bars the tension-test specimens shall be taken when size permits so that the axis shall be at a point one-half the distance between the center and the surface of the bar.

(In respect to process of manufacture, test specimens, and bend tests this specification conforms to A. S. T. M. A7-24 (see 605.11 , p. 249); in respect to workmanship, permissible variations, yield point, defective machining, percentage of elongation, packing and marking, it conforms to A. S. T. M. 
specifications for metals (see 605.0,p. 247); in respect to chemical composition, check analysis, tension requirements, modification of elongation, character of fracture, and drift tests it conforms to A. S. T. M. A8-24 (see 621.33, p. 1126); in respect to permissible variation of plates ordered to weight, permissible overweight of plates ordered to thickness, and allowable variation in size of rivet bars it conforms to the Association of American Steel Manufacturers' standard specifications for structural and boiler steel (see 605.0, p. 248).)

621.34 ALLOY STEEL FOR SPRINGS.

AMERICAN SOCIETY FOR TESTING MATERIALS, STANDARD SPECIFICATIONS FOR SILICO-MANGANESE-STEEL BARS FOR RAILWAY SPRINGS, DESIGNATION A59-2\%, 1927

\section{BARS FOR RAILWAY SPRINGS}

\section{SCOPE}

1. These specifications cover silicomanganese-steel bars to be used for the manufacture of railway springs.

\section{MANUFACTORE}

2. The steel shall be made by one or more of the following processes: Open-hearth, crucible, or electric furnace.

\section{CHEMICAL PROPERTIES AND TESTS}

3. The steel shall conform to the following requirements as to chemical composition:

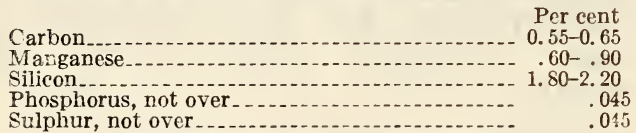

\section{LADLE AND CHECK ANALYSES}

(Same as in American Railway Association specification for steel-tank plates. See 621.32, p. 1124.)

PERMISSIBLE VARIATIONS IN DIMENSIONS

(Identical with A. S. T. M. A14. See 603.33, p. 152.)

(Workmanship and finish, marking, inspection and rejection, and rehearing conform to paragraphs $5,6,7,8$, and 9 in A. S. T. M. standard specifications for steel. See 605.0 , p. 247.)

\section{AIMERICAN SOCIETY FOR TESTING MATE- RIALS, STANDARD SPECIFICATIONS FOR CHROME-VANADIUM-STEEL BARS FOR RAILWAY SPRINGS, DESIGNATION A60-2\%, 1927}

\section{BARS FOR RAILWAY SPRINGS}

\section{SCOPE}

1. These specifications cover chrome-vanadiumsteel bars to be used for the inanufacture of railway springs.

\section{MANUFACTURE}

2. The steel shall be made by one or more of the following processes: Open-hearth, crucible, or electric-furnace.

\section{CHEMICAL PROPERTIES AND TESTS}

3. The steel shall conform to the following requirements as to chemical composition:

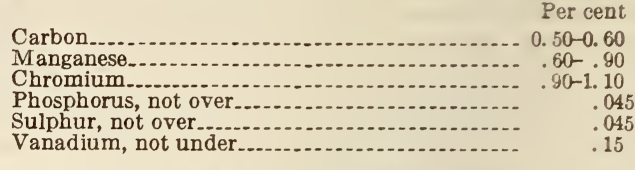

\section{LADLE AND CHECK ANALYSES}

(Same as in American Railway Association specification for steel tank plates. See 621.32, p. 1124.)

\section{PERMISSIBLE VARIATIONS IN DIMENSIONS}

(Identical with A. S. T. Mi. A14. See 603.33, p. 152.)

(Workmanship and finish, marking, inspection and rejection, and rehearing conform to paragraphs $5,6,7,8$, and 9 in A. S. T. M. standard specifications for steel. See 605.0, p. 247.)

\section{SOCIETY OF AUTOMOTIVE ENGINEERS,} IRON AND STEEL SPECIFICATIONS, CHEMICAL COMPOSITIONS, JUNE, 1929

\section{SILICOMANGANESE STEELS}

(For prefatory remarks, see 600.0, p. 2 ; for specification details, see 600.1 , p. 49.)

\section{CHEMICAL COMPOSITION}

[For silicon range see footnote 1]

\begin{tabular}{|c|c|c|c|c|c|}
\hline $\begin{array}{l}\text { S. A. E. } \\
\text { steel } \\
\text { No. }\end{array}$ & $\begin{array}{c}\text { Carbon } \\
\text { range }\end{array}$ & $\begin{array}{l}\text { Man- } \\
\text { ganese } \\
\text { range }\end{array}$ & $\begin{array}{l}\text { Phos- } \\
\text { phorus, } \\
\text { maxi- } \\
\text { mum }\end{array}$ & $\begin{array}{l}\text { Sul- } \\
\text { phur, } \\
\text { maxi- } \\
\text { mumn }\end{array}$ & $\begin{array}{l}\text { Silicon } \\
\text { range } 1\end{array}$ \\
\hline $\begin{array}{l}9250 \\
9260\end{array}$ & $\begin{array}{l}\text { Per cent } \\
0.45-0.55 \\
.55-.65\end{array}$ & $\begin{array}{l}\text { Per cent } \\
0.60-0.90 \\
.60-.90\end{array}$ & $\begin{array}{c}\text { Per cent } \\
0.045 \\
.045\end{array}$ & $\begin{array}{r}\text { Per cent } \\
0.05 \\
.05\end{array}$ & $\begin{array}{r}\text { Per cent } \\
1.80-2.20 \\
1.80-2.20\end{array}$ \\
\hline
\end{tabular}

1 Silicon range of all S. A. E. basic open-hearth alloy steels shall be $0.15-0.30$ per cent. For the electric and acid open-hearth steels the silicon content shall be a minimum of 0.15 per cent.

621.35 ALLOY STEEL FOR TOOLS.

AMERICAN RAILWAY ASSOCIATION, PURCHASES AND STORES DIVISION, STAND. ARDIZATION AND SIMPLIFICATION OF STORES STOCKS, D V1-238, 1930

\section{HIGH-SPEED TOOL STEEL}

(Recommended practice in stocking certain specified sizes of flat, square, and round high-speed tool steel.)

\section{AMERICAN SOCIETY FOR TESTING MATE- RIALS, STANDARD SPECIFICATIONS FOR HIGH-SPEED TOOL STEEL, DESIG- NATION A92-26, 1926}

\section{HIGH-SPEED TOOL STEEL}

(Identical with A. S. T. M. A71-26 (see 603.32, p. 151) except in the following particulars:) 


\section{MANUFACTORE}

The steei shall be furnished annealed unless otherwise specified. The microsctucture, Brinell hardness, and fracture tests shall be as agreed upon between the purchaser and the vendor.

\section{CHEMICAL COMPOSITION}

The composition of the tool steels shall be as agreed upon by the purchaser and the vendor with the following permissible variations in composition:

\begin{tabular}{|c|c|c|}
\hline & \multicolumn{2}{|c|}{$\begin{array}{c}\text { Permissible varia- } \\
\text { tions }\end{array}$} \\
\hline 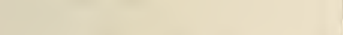 & Over & Under \\
\hline $\begin{array}{l}\text { Carbon } \\
\text { Manganese } \\
\text { Phosphorus. } \\
\text { Sulphur } \\
\text { Silicon } \\
\text { Chromium } \\
\text { Vanadium } \\
\text { Tungsten } \\
\text { Cobalt } \\
\text { Other elements as agreed. }\end{array}$ & $\begin{array}{c}\text { Per cent } \\
0.05 \\
.15 \\
.01 \\
.01 \\
.15 \\
.50 \\
.25 \\
.75 \\
.50\end{array}$ & $\begin{array}{c}\text { Per cent } \\
0.05 \\
.15 \\
0.15 \\
.50 \\
.25 \\
.75 \\
.50\end{array}$ \\
\hline
\end{tabular}

In case of dispute, the chemical analysis shall be made in accordance with the standard methods of chemical analysis of alloy steels. See A. S. T. M. A55 (621.30, p. 1107).

AMERICAN SOCIETY FOR TESTING MATERIAIS, STANDARD SPECIFICATIONS FOR ALIOY TOOL STEEL, DESIGNATION A115-28, 1928

\section{ALLOY TOOL STEEL}

(Identical with A. S. T. M. A71-26 (see 603.32, p. 151) except in the following particulars:)

\section{SCOPE}

These specifications cover alloy tool steels, the composition of which shall be as agreed upon by the purchaser and the vendor, with the permissible variations in chemical composition specified below. These steels are suitable for the manufacture of special purpose tools.

\section{MAN UFACTURE}

The steel shall be furnished annealed unless otherwise specified. The microstructure, Brinell harness, and fracture tests shall be as agreed upon by the purchaser and the vendor.

\section{CHEMICAL COMPOSITION}

The cornposition of the tool steels shall be as agreed upon by the purchaser and the vendor with the following permissible variations in composition:

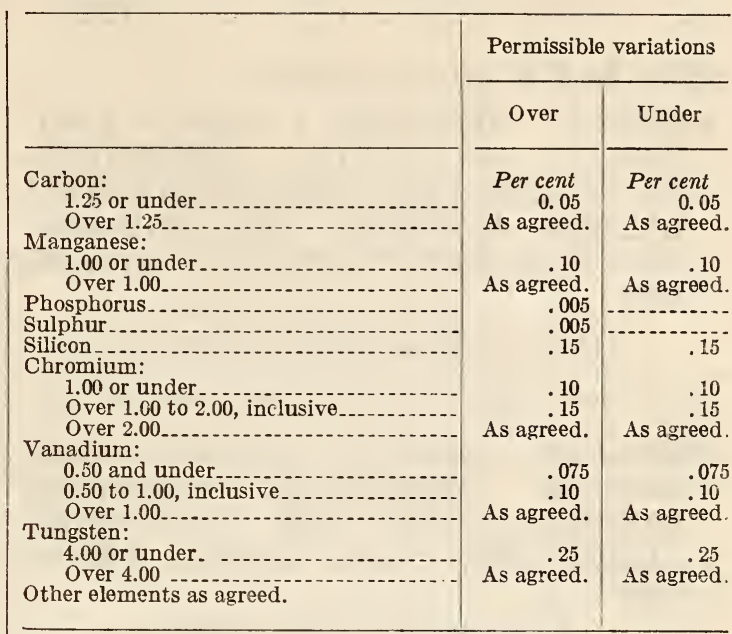

In case of dispute, the chemical analysis shall be made in accordance with the standard methods of chemical analysis of alloy steels. See A. S. T. M. A55 (621.30, p. 1107).

\section{SOCIETY OF AUTOMOTIVE ENGINEERS,} IRON AND STEEL SPECIFICATIONS, CHEMICAL COMPOSITIONS, JUNE, 1929

\section{CHROMIUM-VANADIUM STEELS}

(See 621.31, p. 1124.)

\section{SOCIETY OF AUTOMOTIVE ENGINEERS, IRON AND STEEL SPECIFICATIOIS, CHEMICAI COMPOSITIONS, JUNE, 1929}

\section{TUNGSTEN STEELS}

(For prefatory remarks, see 600.0, p. 2 ; for specification details, see 600.1 , p. 49 .)

\section{CHEMICAL COMPOSITION}

[For silicon range see footnote 1]

\begin{tabular}{|c|c|c|c|c|c|c|}
\hline $\begin{array}{c}\text { S. A. E. } \\
\text { steel } \\
\text { No. }\end{array}$ & $\begin{array}{l}\text { Carbon } \\
\text { range }\end{array}$ & \begin{tabular}{|} 
Man- \\
ganese, \\
maxi- \\
mum
\end{tabular} & $\begin{array}{l}\text { Phos- } \\
\text { phorus- } \\
\text { maxi- } \\
\text { mum }\end{array}$ & $\begin{array}{l}\text { Sulphur } \\
\text { maxi- } \\
\text { mum }\end{array}$ & $\begin{array}{l}\text { Chro- } \\
\text { mium } \\
\text { range }\end{array}$ & $\begin{array}{c}\text { Tungsten } \\
\text { range }\end{array}$ \\
\hline $\begin{array}{r}71360 \\
71660 \\
7260\end{array}$ & 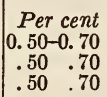 & \begin{tabular}{|c|} 
Per cent \\
0.30 \\
.30 \\
.30
\end{tabular} & $\begin{array}{r}\text { Per cent } \\
0.035 \\
.035 \\
.035\end{array}$ & $\begin{array}{c}\text { Per cent } \\
0.04 \\
.04 \\
.04\end{array}$ & $\begin{array}{l}\text { Per cent } \\
3.00-4.00 \\
3.00-4.00 \\
0.50-1.00\end{array}$ & $\begin{array}{c}\text { Per cent } \\
12.00-15.00 \\
15.00-18.00 \\
1.50-2.00\end{array}$ \\
\hline
\end{tabular}

1 Silicon range of all S. A. E. basic open-hearth alloy steels shall be $0.15-0.30$ per cent. For the electric and acid open-hearth steels the silicon content shall be a minimum of 0.15 per cent.

\subsection{ALLOY STEEL FOR MAGNETS.}

(For methods of test for magnetic properties of iron and steel see A. S. T. M. specification A34-28, $600.1, p .13$. No nationally recognized specifications for alloy magnet steels themselves are available.) 
622. MANUFACTURES OF ALLOY STEELS

622.1 BOLTS, ALLOY STEEL.

AMERICAN ELECTRIC RAILWAY ENGINEERING ASSOCIATION, RECOMMENDED SPECIFICATION FOR ALLOY-STEEL BOLTING MATERIAL FOR HIGH-TEMPERATURE SERVICE, SERIAL No. G101-25, 1925

\section{ALLOY-STEEL BOLTING MATERIAL}

(Identical with A. S. T. M. A96, given below.)

\begin{abstract}
AMERICAN ELECTRIC RAILWAY ENGINEERING ASSOCIATION, STANDARD SPECIFICATION FOR QUENCHED, ALLOY-STEEL TRACK BOLTS, W22-23, 1923
\end{abstract}

\section{ALLOY-STEEL TRACK BOLTS}

(See 606.4, p. 352.)

\section{AMERICAN ELECTRIC RAILWAY ENGI- NEERING ASSOCIATION, SPECIFICA- TION FOR MATERIALS FOR USE IN THE MANUFACTURE OF SPECIAL TRACK WORK, W104-28, 1928}

[Approved as American tentative standard (E10-1929) by the American Standards Association, January 23, 1929]

SPECIAL TRACK-WORK MATERIALS

(See 606.3, p. 342.)

AMERICAN RAILWAY ENGINEERING ASSOCIATION, SPECIFICATION FOR QUENCHED CARBON-STEEL AND ALLOYSTEEL TRACK BOLTS, 1924

ALLOY-STEEL TRACK BOLTS

(See 606.4, p. 352.)

AMERICAN SOCIETY OF MECHANICAL ENGINELRS, STEEL PIPE FLANGES AND FLANGE FITTINGS, B16e-1927

[Approved as American tentative standard by the American Standards Association, June, 1927]

ALIOY-STEEL BOLTING MATERIAL

(See 607.4, p. 465.)

AMERICAN SOCIETY OF MECHANICAI ENGINEERS, STEEL PIPE FLANGES AND FLANGED FITTINGS, B16e-1927

[Approved as American tentative standard by the American Standards Association, June, 1927]

STEEI PIPE FLANGES AND FLANGED FITTINGS (See 607.4, p. 465.)

AMERICAN SOCIETY FOR TESTING MATERIALS, STANDARD SPECIFICATIONS FOR QUENCHED ALLOY-STEEL TRACK BOLTS, DESIGNATION A51-21, 1921

\section{QUENCHED ALLOY-STEEL TRACK BOITS}

(See 606.4, p. 356.)
AMERICAN SOCIETY FOR TESTING MATERIALS, STANDARD SPECIFICATIONS FOR ALLOY-STEEL BOLTING MATERIAL FOR HIGH-TEMPERATURE SERVICE, DESIGNATION A96-31, 1931

\section{ALLOY-STEEL BOLTING MATERIAL}

1. (a) These specifications cover allov-steel bolting material for pressure vessels, valves, flanges, and fittings for high-temperature service (see explanatory note in A. S. T. M. A95, 611.41, p. 709). The term "bolting material" as used in these specifications cover rolled, forged or cold-drawn bars, and bolts, screws, and studs.

(b) Three classes of material are covered, A, B, and $\mathrm{C}$, classified in accordance with their physical properties as specified in section 10 , choice depending upon design and upon the stresses and service for which the material is to be used.

(c) Nickel, chrome-nickel, chrome-ranadium, chrome-manganese or any other types of alloy steel approved by the purchaser may be submitted under these specifications.

(d) Carbon-steel nuts and washers for use with this bolting material are covered in section 16 .

2 . When agreed upon between the manufacturer and the purchaser, Brinell hardness tests may be made to determine the acceptance of bolting material in lieu of the tension tests herein specified; but shall not be used as a basis for rejection without confirming tension tests being made.

3. Unless otherwise specified on the order, a certification that bolts, screws, and studs for valves and other fittings intended for stock and other purposes requiring assembly in the manufacturer's plant conform to the requirements of these specifications, shall be accepted in lieu of the tests herein specified.

\section{MANOFACTORE}

4. The steel shall be made by one or more of the following processes: Open-hearth, electric furnace,or crucible.

5. A sufficient discard shall be made from each ingot to secure freedom from injurious piping and undue segregation.

6. (a) Heat treatment shall consist of quenching and tempering.

(b) Immediately after rolling or forging, the bolting material shall be allowed to cool to a temperature below the critical range under suitable conditions to prevent injury by too rapid cooling. For quenching and tempering, the material shall then be uniformly reheated to the proper temperature to refine the grain (a group thus reheated being known as a quenching charge) and quenched in some medium under substantially uniform conditions for each quenching charge. The material shall then be uniformly reheated to the proper temperature for tempering or "drawing back" (a group thus reheated being known as a tempering charge) and allowed to cool uniformly.

\section{CHEMICAL PROPERTIES AND TESTS}

7. (a) The steel shall conform to the following requirements as to chemical composition:

Phosphorus, not over. Per cent

Sulphur, not over.

(b) The composition of the steel furnished under these specifications, other than phosphorus and sulphur, shall be agreed upon by the manufacturer and the purchaser.

NoтE.-It is recommended that the carbon content shall not be less than 0.20 nor more than 0.45 per cent, and that the carbon ranges shall be 0.10 per cent of carbon. 
8. An analysis of each melt of steel shall be made by the steel manufacturer to determine the percentages of the elements present. This analysis shall be made from a test ingot taken during the pouring of the melt. The chemical composition thus determined shall be reported to the purchaser or his representative, and shall conform to the requirements specified in section 7 .

9. Analyses may be made by the purchaser from samples representing the bolting material. The chemical composition thus determined shall conform to the requirements specified in section 7 .

\section{PHYSICAL PROPERTIES AND TESTS}

10. (a) The bolting material, after final heat treatment, shall conform to the following requirements as to minimum tensile properties:

\begin{tabular}{|c|c|c|c|c|}
\hline $\begin{array}{l}\text { Diameter } \\
\text { (inches) }\end{array}$ & & $\underset{\mathrm{A}}{\mathrm{Class}}$ & $\underset{\mathrm{B}}{\mathrm{Class}}$ & $\underset{\mathrm{C}}{\mathrm{Class}}$ \\
\hline $\begin{array}{l}21 / 2 \text { and } \\
\text { under }\end{array}$ & $\left\{\begin{array}{l}\text { Tensile strength } \\
\text { Yield point } \\
\text { Elongation in } 2 \text { inches }\end{array}\right.$ & $\begin{array}{l}95,000 \\
70,000\end{array}$ & $\begin{array}{r}105,000 \\
80,000\end{array}$ & $\begin{array}{l}125,000 \\
105,000\end{array}$ \\
\hline & $\begin{array}{l}\text { Reduction of area. } \\
\text { Tensile strength }\end{array}$ & $\begin{array}{r}20 \\
50 \\
90,000\end{array}$ & $\begin{array}{r}20 \\
50 \\
100,000\end{array}$ & $\begin{array}{r}16 \\
50 \\
115,000\end{array}$ \\
\hline $\begin{array}{l}\text { Over } 21 / 2 \\
\text { to } 4, \text { in- }\end{array}$ & $\left\{\begin{array}{l}\text { Yield point } \\
\text { Elongation in } 2 \text { inches }\end{array}\right.$ & 65,000 & 75,000 & 95,000 \\
\hline clusive & $\begin{array}{l}\text { Reduction of area } \\
\text { Tensile strength }\end{array}$ & $\begin{array}{r}20 \\
50 \\
90,000\end{array}$ & $\begin{array}{r}20 \\
50 \\
100,000\end{array}$ & $\begin{array}{r}16 \\
45 \\
110,000\end{array}$ \\
\hline $\begin{array}{l}\text { Over } 4 \text { to } \\
7 \text {, inclu- }\end{array}$ & $\left\{\begin{array}{l}Y \text { ield point } \\
\text { Elongation in } 2 \text { inches }\end{array}\right.$ & 65,000 & 75,000 & 85,000 \\
\hline sive & Reduction of area...-per cent.. & $\begin{array}{l}20 \\
50\end{array}$ & $\begin{array}{l}20 \\
50\end{array}$ & $\begin{array}{l}16 \\
45\end{array}$ \\
\hline
\end{tabular}

(b) The yield point shall be determined by the drop of the beam of the testing machine or by the use of dividers.

11. (a) When Brinell hardness tests are made, the bolting material, after final heat treatment, shall conform to the following requirements:

Class $\mathrm{A}$

Brinell hardness No $190-250$
$210-270$

Class $\mathrm{C}$ 260-320

(b) The Brinell hardness test shall be made in accordance with the standard methods of Brinell hardness testing of metallic materials in A. S. T. M.: E 10 (See 600.1, p. 40).

12. (a) The tension-test specimens taken from the bolting material shall conform to the dimensions shown in Figure 129 in A. S. T. M. standard specifications for steel (see 605.0, p. 247), except as specified in paragraph $(b)$. The ends shall be of a form to fit the holders of the testing machine in such a way that the load shall be axial.

(b) In the case of small sections which will not permit of taking the standard tension-test specimen specified in paragraph $(a)$, the tension-test specimen shall be as large as feasible and its dimensions shall be proportional to those shown in the standard-test specimen in Figure 129,605.0, p. 247. The gage length for measuring elongation shall be four times the diameter of the specimen.

(c) Specimens for tension tests shall be taken longitudinally. For sizes under $1 \frac{1}{2}$ inches in diameter the full section shall be turned to conform to requirements specified in paragraph $(a)$. For sizes $1 \frac{1}{2}$ inches and larger the specimens shall be taken halfway between the center and the surface.

13. (a) Except as specified in paragraph (b), one tension test shall be made for each lot of 100 pieces or less of bolting material from each tempering charge. If more than one quenching charge is rep- resented in a tempering charge, one teusion test shall be made from each lot of 100 pieces or less from each quenching charge. If more than one melt is represented in a quenching charge, one tension test shall be made from each lot of 100 pieces or less from each melt.

(b) Unless required on the order, tension tests shall not be made on an order of less than 100 bolts, screws, or studs; in which case acceptance shall be based upon the provisions in sections 2 or 3 .

(c) If any test specimen shows defective machining or develops flaws, it may be discarded and another specimen substituted.

(d) If the percentage of elongation of any test specimen is less than that specified in section $10(a)$ and any part of the fracture is outside of the middle three-fourths of the gage length, as indicated by scribe scratches marked on the specimen before testing, a retest shall be allowed.

14. If the results of the physical tests of any lot of bolting material do not conform to the requirments specified, the manufacturer may re-treat such lot one or more times and retests shall be made as specified in section $13(a)$.

\section{WORKMANSHIP AND FINISH}

15. The bolting material shall be free from injurious defects and shall have a workmanlike finish.

16. Bolts and studs shall be equipped with semifinished carbon-steel nuts of United States standard rough dimensions, chamfered and trimmed. If washers are used under nuts they shall be of forged or rolled steel. All nuts and washers shall be free from injurious defects and shall have a workmanlike finish. Nuts shall conform to the chemical requirements for phosphorus and sulphur as follows:

\begin{tabular}{|c|c|c|}
\hline & Class A & Class B \\
\hline Phosphorus, not over . ... & $\begin{array}{r}\text { Per cent } \\
0.045 \\
.05\end{array}$ & $\begin{array}{r}\text { Per cent } \\
0.05 \\
.15\end{array}$ \\
\hline Sulphur, not over....... & & \\
\hline
\end{tabular}

17. United States standard threads shall be used, except that no stud shall have less than eight threads per inch.

\section{MARKING}

18. Identification marks to be stamped on bolting material shall be agreed upon between the manufacturer and the purchaser.

\section{INSPECTION AND REJECTION}

(Conform to A. S. T. M. standard specifications for steel under 605.0, paragraphs 7 and 8, except that rejections based on tests shall be reported within 12 days instead of within 5 days, and that the following requirements are added:)

19. The purchaser may make the tests to govern the acceptance or rejection of the bolting material in his own laboratory or elsewhere. Such tests, however, shall be made at the expense of the purchaser.

20. Samples tested in accordance with section 19 which represent rejected bolting material, shall be preserved for two weeks from the date of the test report. In case of dissatisfaction with the results of the test, the manufacturer may make claim for a rehearing within that time.

\section{EXPLANATORY NOTE}

(Identical with explanatory note No. 1 in A. S. T. M. A95. See 611.41, p. 709.) 
SOCIETY OF AUTOMOTIVE ENGINEERS, CAP SCREWS, BOLTS, AND NUTS; CONNECTING-ROD BOLTS, S. A. E. STANDARD, AUGUST, 1930

CONNECTING-ROD BOLTS

(See 608.31, p. 601.)

SOCIETY OF AUTOMOTIVE ENGINEERS, PROPELLER - BLADE CLAMP RINGS, BOLTS, AND NUTS, AERONATUIC; S. A. E. RECOMMENDED PRACTICE, JUNE, 1929

PROPELIER-BLADE CLAMP RINGS, BOLTS, AND NUTS

(See 608.31, p. 603.)

622.2 RAILWAY RAILS, ALLOY STEEL.

AMERICAN ELECTRIC RAILWAY ENGINEERING ASSOCIATION, STANDARD DESIGN OF BOLTED MANGANESESTEEL CROSSINGS; STEAM RAILROAD OVER ELECTRIC RAILWAY, FOR ANGLES BELOW $45^{\circ}$ TO $30^{\circ}$, INCLUSIVE, W44-28, 1928

BOLTED MANGANESE-STEEL CROSSINGS

(See 606.3, p. 342.)

AMERICAN ELECTRIC RAILWAY ENGINEERING ASSOCAITION, SPECIFICATION FOR MIATERIALS FOR USE IN THE MIANUFACTURE OF SPECIAL TRACK WORK, W104-28, 1928

[A pproved as American tentative standard (E10-1929) by the American Standards Association, January 23, 1929]

SPECIAL TRACK-WORK MATERIALS

(See 606.3, p. 342.)

622.3 TUBULAR PRODUCTS AND FITTINGS, ALLOY STEEL.

AMERICAN RAILWAY ASSOCIATION, MECHANICAL DIVISION, SEAMIESS HIGH CHROME-STEEL TUBING FOR NITRICACID TANK CARS, RECOMIENDED PRACTICE, 1929

\section{HIGH CHROME-STEEL TUBING}

(In respect to process, chemical properties, ladle and check analyses, and method of pickling, this specification is identical with the A. R. A. specification for high chrome-steel tank plates for nitric-acid tank cars (see 621.32, p. 1124). The flaring tool and die block used in making flange tests is the same as that shown in A. S. T. M. B42-24 (see 642.23, p. 1182). In respect to inspection, rejection, and rehearing it conforms to A. S. T. M. standard specifi- cations for steel (see 605.0, p. 247). Otherwise it provides for the following requirements:)

\section{PHYSICAL PROPERTIES AND TESTS}

4. (a) For tubing to be used for heat exchanges and coolers, a test specimen not less than 4 inches in length shall have a flange turned over at right angles to the body of the tube without cracking or showing any flaws. This flange, measured from the outside of the tube, shall in no case be less than one-fourth inch nor more than one-half inch in width.

(b) For tubing which has been hot rolled, the inside may be reamed out, previous to testing, until the wall thickness is uniform.

(d) For all tubes, a test specimen 6 inches in length shall withstand flattening between parallel plates without cracking or showing any flaws until the distance between the plates is six times the wall thickness of the tube.

(e) For tubing 3 inches in diameter or under, a sufficient length of tubing shall stand being bent cold through $90^{\circ}$ around a cylindrical mandrel, the diameter of which is five times the nominal diameter of the tube, without developing cracks or seams in any portion.

5. Test specimens.-(a) Test specimens shall be from the finished material. They shall be smooth on the ends and free from surface defects.

(b) All tests specified under section 4 for the several specimens shall be made cold; that is, at temperatures not exceeding $250^{\circ} \mathrm{F}$.

6. Number of tests. - One of each of the physical tests specified in section 4 shall be made from each of two tubes in annealing charge, each lot of 250 tubes or less.

7. Retests. - If the results of the physical tests of only one tube from any lot do not conform to the requirements specified in section 6 , retest of two additional tubes from the same lot shall be made, each of which shall conform to the requirements specified.

\section{WORKMANSHIP AND FINISH}

8. (a) Finished tubes 4 inches or under in outside diameter shall be circular within 0.02 inch and the mean outside diameter shall not vary more than 0.02 inch from the size ordered. For tubes over 4 inches in outside diameter these variations shall not exceed 0.5 per cent of the outside diameter.

(b) The thickness of the wall of the tubes shall not be less than the gage thickness specified by the purchaser, nor greater than that specified by more than three gages.

(c) The length shall not be less, but may be oneeighth inch more than that specified.

9. Finish.-Finished tubes shall be free from injurious or surface defects and distortion, shall have smooth ends, and shall have a workmanlike finish.

ASSOCIATION OF AMERICAN STEEL MANUFACTURERS. STANDARD SPECIFICATIONS FOR SEAMLESS ALLOY STEEL STILL TUBES OF 4 TO 6 PER CENT CHROMIUM FOR GASOLINE CRACKING PLANTS, NO. 200, APRIL 1, 1932

\section{ALLOY STEEL STILL TUBES}

(This specification includes requirements on chemical composition, tension test, flattening test, flaring test, Brinell test, hydrostatic test, and permissible variations in dimensions, for oil carrying tubes subject to furnace temperatures.) 


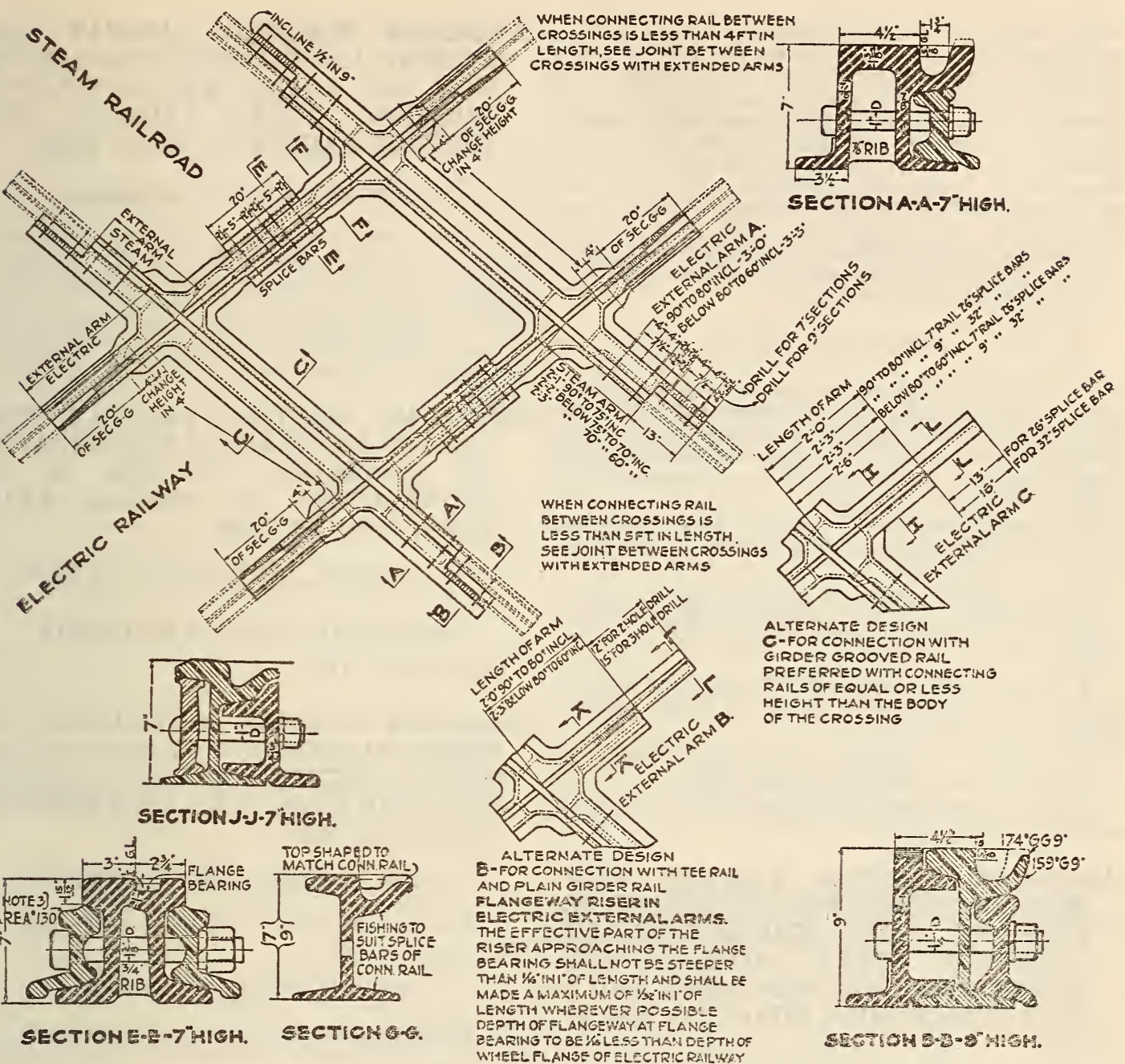

FIGURE 1065.-Solid manganese steel crossings, steam railroad over electric railway for 7 and 9 inch girder rails, angles $90^{\circ}$ to $60^{\circ}$, inclusive

(Identical with A. R. E. A. Plan No. 780.)

622.4 ALLOY STEEL WIRE AND IMANUFACTURES.

UNITED STATES GOVERNMENT, FEDERAL SPECIFICATIONS BOARD, FEDERAL SPECIFICATION FOR WELDING WIRE, IRON, AND STEEL, NO. 174, JUNE 30, 1924. (CURRENT DESIGNATION: QQ-W-351)

\section{WELDING WIRE}

(This specification includes requirements for a nickel-steel gas-welding wire designated grade $\mathrm{G}$. See 603.41, p. 157.)
622.5 ALLOY-STEEL CASTINGS.

AMERICAN ELECTRIC RAILWAY ENGINEERING ASSOCIATION, STANDARD DESIGN OF SOLID IIANGANESE-STEEL CROSSINGS; STEAM RAILROAD OVER ELECTRIC RAILWAY FOR \%-INCH AND 9-INCH GIRDER RAILS, SERIAL NO. W29-2\%, 1927

\section{MANGANESE-STEEL CROSSINGS}

(Specifications for the general character and quality of the materials and workmanship entering into manganese-steel rail crossings are contained in 
A. E. R. E. A. W32-30 (see 606.3, p. 335), ircluding references in W32 to A. E. R. E. A. W33. The drawing accompanying W29 is identical with A. R. E. A. plan No. 780. An excerpt from this drawing is shown below as Figure 1065. Similar drawings covering rail braces, switches, switch stands, frogs, guard rails, crossings, gages and flangeways, double-slip. switches, turnouts and crossovers, movable-point crossings, and track construction details will be found in a book of track-work plans published by the American Railway Engineering Association in collaboration with the Manganese Track Society.)

\section{NOTES TO PLAN NO. 780}

1. For external joints, bolts to be $1 \frac{3}{8} 8$ inches diameter. For external arms on steam railroad, bolts to be $1 \frac{1}{4}$ inches diameter for 9 -inch rail, 1 inch diameter for 7 -inch rail. For external arms on electric railway, bolts to be 1 inch diameter. All bolts to be high tensile steel.

2. Crossing may be furnished in four parts if so specified, with internal steam railroad joints as shown on plan No. 781 .

3. For internal joints on electric railway, planed bar steel plates may be substituted for the splice bars shown in sections $\mathrm{E}-\mathrm{E}$. Bars to be $1 \frac{1}{2}$ inches thick.

4. Mitered or lap joints may be used for internal joints on electric railway, similar to steam railroad joints on plan No. 781.

5. Portions of castings under intersections to be thoroughly and efficiently reinforced.

6. It is recommended that new rail be installed against all external arms of crossing.

\section{AMERICAN ELECTRIC RAILWAY ENGI- NEERING ASSOCIATION, STANDARD DE- SIGN OF SOLID MANGANESE-STEEL CROSSINGS; STEAM RAILROAD OVER ELECTRIC RAILWAY FOR 7-INCH AND 9- INCH GIRDER RAIIS, SERIAL NO. W30-2\%, 1927}

\section{MANGANESE-STEEL CROSSINGS}

(Observations identical with those given above for A. E. R. E. A. No. W29-27, apply in this case, except that the drawing accompanying W30 is identical with A. R. E. A. plan No. 781. This plan No. 781 provides for crossings at angles below $60^{\circ}$ to $40^{\circ}$.)

\section{AMERICAN ELECTRIC RAILWAY ENGI- NEERING ASSOCIATION, STANDARD DE- SIGN OF SOLID MANGANESE-STEEL CROSSINGS; STEAII RAILROAD OVER ELECTRIC RAILWAY, W37-28, 1928}

\section{MANGANESE-STEEL CROSSINGS}

(Observations identical with those given above for A. E. R. E. A. No. W29-27, apply in this case, except that the drawing accompanying W37 is identical with A. R. E. A. plan No. 776. This plan No. 776 provides for solid manganese-steel crossings at angles $90^{\circ}$ to $60^{\circ}$, inclusive. No mention is made of girder rails as in A. E. R. E. A. W29.)
AMERICAN ELECTRIC RAILWAY ENGINEERING ASSOCIATION, STANDARD DESIGN OF SOLID MANGANESE-STEEL CROSSINGS; STEAM RAILROAD OVER ELECTRIC RAILWAY, W38-28, 1928

\section{MANGANESE-STEEL CROSSINGS}

(Observations identical with those given above for A. E. R. E. A. No. W29-27, apply in this case, except that the drawing accompanying W38 is identical with A. R. E. A. plan No. 777. This plan No. 777 provides for solid manganese-steel crossings at angles below $60^{\circ}$ to $40^{\circ}$, inclusive. No mention is made of girder rails as in A. E. R. E. A. W29.)

\section{AMERICAN ELECTRIC RAILWAY ENGI- NEERING ASSOCIATION, SPECIFICATION FOR MATERIALS FOR USE IN THE MANUFACTURE OF SPECIAL TRACK WORK, W104-28, 1928}

[Approved as American tentative standard (E10-1929) by the American Standards Association, January 23, 1929]

SPECIAL TRACK-WORK MATERIALS

(See 606.3, p. 342.)

AMERICAN RAILWAY ASSOCIATION, MECHANICAL DIVISION, HIGH CHROMESTEEL CASTINGS FOR VALVES FOR NITRIC-ACID TANK CARS, RECOMMENDED PRACTICE, 1929

\section{HIGH CHROME-STEEL CASTINGS}

1. Process. - The steel shall be made by the electric furnace process.

\section{CHEMICAL PROPERTIES AND TESTS}

2. Chemical properties.-(a) The steel shall conform to the following requirements as to chemical composition:

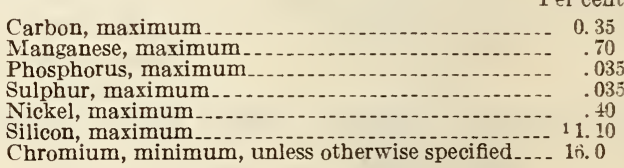

(b) Analysis of each heat shall be made by the manufacturer to determine the percentage of the elements specified in section $2(a)$. This analysis shall be made from drillings taken at least onequarter inch beneath the surface of a test ingot obtained during the pouring of the heat. The chemical composition thus determined shall be reported to the purchaser or his representative, and shall conform to the requirements specified in section $2(a)$.

3. Check analyses.-Check analysis may be made by the purchaser from broken test specimens or from a casting representing each heat. The chemical composition thus determined shall conform to the requirements specified in section 2 . Drillings for analysis shall be taken not less than one-quarter inch beneath the surface, except in the case of cast-

1 For chromium 16 to 19 per cent. 
ings which are too thin and shall be taken in such manner as not to impair the usefulness of the casting.

\section{HEAT TREATMENT}

4. Heat treatment.-(a) All castings shall receive a heat treatment prior to shipment from the manufacturer's works. This heat treatment shall consist of a normalizing and drawing treatment according to the following:

(b) The procedure for normalizing shall consist in allowing the castings to cool, after pouring, to a temperature below the critical range. They shall then be heated to a proper temperature to refine the grain and allowed to cool freely in air.

(c) After normalizing, the castings shall receive a drawing treatment by heating to $1,450^{\circ}$ to $1,500^{\circ} \mathrm{F}$. and holding for a sufficient time for a thorough soaking, then cooling slowly in the furnace.

\section{WORKMANSHIP AND FINISH}

5. Workmanship.-(a) All castings shall be of good workmanship and of such dimensions as will comply with dimensions shown on blue prints after machining is finished.

(b) Finish.-The castings shall be free from injurious defects such as blowholes, scabs, and porosity. Castings which have been calked will be rejected. All castings sliall be freed from scale by sand-blasting and acid pickle as required, and followed by nitric-acid dip.

6. Porosity test.-Unles:s otherwise specified on the drawings, all castingis shall be subjected to a porosity test and each shall show no leakage or sweating under 150 pounds hydrostatic pressure.

\section{INSPECTION AND REJECTION}

(Inspection, rejection, and rehearing conform to A. S. T. M. standard specifications for steel (see 605.0 , p. 247) with the following additional provision:)

7. When required, the manufacturer shall furnish the purchaser with a statement as to the analysis of each lot of castings, including the number shipped, and that the castings on this lot have been tested and have met all the requirements of these specifications.

\section{AMERICAN RAILWAY ENGINEERING AS- SOCIATION AND MANGANESE TRACK SOCIETY, SPECIFICATIONS FOR SWITCHES, FROGS, CROSSINGS, AND GUARD RAILS, TRACK-WORK PLANS, 1929}

\section{TRACK WORK}

(See A. R. E. A., 606.3, p. 349.)

\section{AMERICAN SOCIETY FOR TESTING MATE- RIALS, TENTATIVE SPECIFICATIONS FOR AUSTENITIC MANGANESE-STEEL CAST- INGS, DESIGNATION A128-30T, 1930}

\section{AUSTENITIC MANGANESE-STEEL CASTINGS}

1. These specifications cover austenitic manganese-steel castings; this material is also often referred to as Hadfield's manganese steel.

\section{MANUFACTURE}

2. The steel shall be made by one or more of the following processes: Open-hearth, electric furnace, converter, or crucible.
3. The castings shall receive a suitable heat treatment to render them tough and ductile. This heat treatment shall consist of uniformly heating the castings to a temperature of at least $1,000^{\circ} \mathrm{C}$. $\left(1,832^{\circ} \mathrm{F}.\right)$, holding them at the maximum temperature until they are uniformly heated throughout, and quenching them in water.

\section{CHEMICAL PROPERTIES AND TESTS}

4. The steel shall conform to the following requirements as to chemical composition:

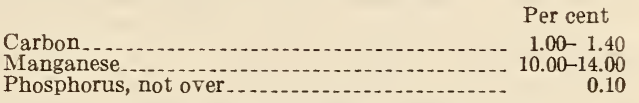

5. An analysis of each melt of steel shall be made by the manufacturer to determine the percentages of the elements specified in section 4 . This analysis shall be made from drillings taken at least one-quarter inch beneath the surface of a test ingot obtained during the pouring of the melt. The chemical composition thus determined shall be reported to the purchaser or his representative, and shall conform to the requirements specified in section 4 .

6. Analyses may be made by the purchaser on samples obtained from a casting. The samples for analyses shall be taken not less than one-quarter inch below the surface in the case of rough castings. In the case of castings finished by grinding or machining, the samples for analysis may be taken at the finished surface, provided at least one-quarter inch of metal has been removed from the casting at the point selected for sampling. The phosphorus content thus determined shall not exceed 0.11 per cent. The carbon content thus determined shall not be less than 0.90 per cent nor more than 1.50 per cent.

NotE.-Contamination of the drillings by drill chips must be care fully guarded against. Flat drills of the best high-speed steels, or drills of some of the newer tool materials, will generally be satisfactory for drilling manganese steel. Manganese steel may be drilled best after it has been annealed for several hours at from $500^{\circ}$ to $600^{\circ} \mathrm{C}$.

\section{PHYSICAL PROPERTIES AND TESTS}

7. (a) Bend tests shall be required orly when specified in the purchase order.

(b) When bend tests are specified, the test specimens shall stand being bent cold without breaking, around a pin 1 inch in diameter, to an angle of $150^{\circ}$. The bending may be by any method preferred by the manufacturer.

8. In the case of castings weighing not over 50 pounds, and if satisfactory to the manufacturer and inspector, a test to destruction may be made as an alternative to bend tests when the latter are specified. For this test the castings shall be grouped in lots, each lot containing castings from the same melt and from the same heat-treatment charge. From each such lot the inspector shall select one casting to represent the lot. The representative casting shall be tested to destruction by pressure or by blows. This test shall show the material to be tough and ductile, and suitable for the purpose intended. The cost of all such test castings shall be provided for in the purchase order.

9. (a) Bend-test specimens, when specified, shall not be cast attached to the castings, but shall be poured in separate molds, from the same heat or melt of steel as the castings they represent. They shall be $1 / 2$ by $3 / 4$ inch in cross section and 12 inches in length, and shall be heat treated and tested without being machined or ground except when necessary to remove irregularities of surface, or to separate them from the runner or sprue through which they are poured.

(b) Bend-test specimens, when specified, shall be heat treated with the castings they represent. When 
bend-test specimens are heat treated with castings of comparatively heavy section, the test bars should be protected from oxidation by inclosing them in pipes or containers containing a small amount of carbonaceous material, and suitably sealed or luted to prevent the furnace gases from coming in contact with the test bars. To permit the expansion of the gases within these containers, tney should not be completely sealed.

10. (a) When bend tests are specified, one such test shall be made from eacn melt or heat of steel in each heat-treatment charge. When so specified by the purchaser, one bend test shall be made from tne steel used for pouring each casting weighing 500 pounds or over.

(b) If any test specimen develops fiaws and does not conform to the requirements specified, it shall be discarded, in which case another specimen from the same lot shall be substituted.

11. If the results of the bend tests for any lot do not conform to the requirements specified, such lot may be re-heat-treated, but not more than twice. Retests shall be made as specified in section $7(b)$. In the case of re-heat-treatment and retest, when bend tests are specified in the order, two bend tests from a lot, or from each casting weighing 500 pounds or over, instead of one as specified in section $10(a)$, shall be required, both of which shall conform to the requirements specified in section $7(b)$.

\section{WORKMANSHIP AND FINISH}

12. The castings shall conform to the shapes and sizes indicated by the patterns and drawings submitted by the purchaser, with such tolerances as are customarily allowed in the case of parts not finished to exact dimensions by grinding or machining.

13. (a) The casting snall be free from injuricus defects.

(b) Defects which do not impair the strength of twe castings may be welded by an approved process. The defects shall be cleaned out to solid metal, before welding, and when so required by the inspector, shall be submitted to him in this condition for his approval. No welding shall be done except such as is approved by the inspector.

\section{INSPECTION AND REJECTION}

(Inspection, rejection, and rehearing conform to the requirements in A. S. 'T. M. standara specifications for steel, paragraphs 7,8 , and 9 . See 605.0, p. 247.)

\subsection{ALLOY-STEEL FORGINGS.}

\section{AMERICAN RAILWAY ASSOCIATION, ME- CHANICAL DIVISION, AXLES, SHAFTS, AND OTHER FORGINGS, ALIOY STEEL, QUENCHED AND TEMPERED, 1925}

\section{ALLOY-STEEL FORGINGS}

\section{SCOPE}

These specifications cover the various classes of chrome-nickel and chrome-vanadium alloy steel forgings now commonly used in locomotive construction.

The purpose for which these classes are frequently used are as follows:

Class A.-Forgings for main and side rods, straps, and piston rods, and all other forgings which are to be machined with milling cutters or complicated forming tools, or where there is an abrupt change in section.

Class B.-Forgings for driving and trailer axles, crank pins, plain piston rods, crosshead pins, and other forgings not requiring the use of milling cutters or complicated forming tools.

\section{MANUFACTURE}

(Requirements covering manufacture are practically the same as for carbon-steel and alloy-steel forgings in A. S. T. M. A18-30 (see 611.51, p. 754), including the heat-treatment specified in paragraph $6(b)$.

\section{CHEMICAL PROPERTIES AND TESTS}

Chemical composition.-The steel shall conform to the following requirements as to chemical composition:

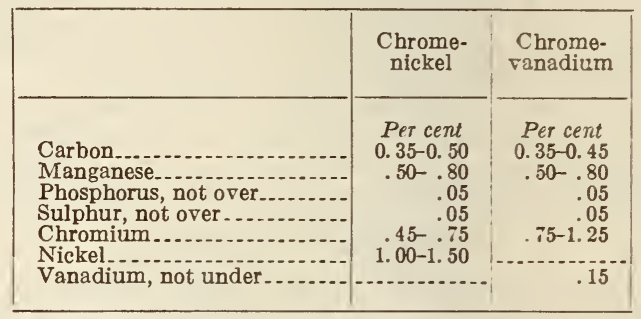

(Ladle and check analyses are the same as in A. S. T. M. A18-30, with the addition of the following paragraph:)

In addition to the complete analysis a phosphorus determination may be made by the purchaser from each tension-test specimen, and this determination shall conform to the requirements for phosphorus specified in the table given above.

\section{PHYSICAL PROPERTIES AND TESTS}

Tension tests. - The forgings shall conform to the requirements as to tensile properties in Table 1.

TABLE 1.-For forgings whose maximum diameter or thickness is not over 10 inches when solid

CLASS A

\begin{tabular}{|c|c|c|c|c|}
\hline & $\begin{array}{l}\text { Tensile } \\
\text { strength }\end{array}$ & $\begin{array}{l}\text { Elastic } \\
\text { limit, } \\
\text { mini- } \\
\text { mum }\end{array}$ & $\begin{array}{l}\text { Elon- } \\
\text { gation } \\
\text { in } 2 \\
\text { inches, } \\
\text { mini- } \\
\text { mum }\end{array}$ & $\begin{array}{l}\text { Reduc- } \\
\text { tion of } \\
\text { area } \\
\text { mini- } \\
\text { mum }\end{array}$ \\
\hline $\begin{array}{l}\text { Main and side rods, straps, } \\
\text { piston rods }\end{array}$ & $\begin{array}{l}\text { Lbs./in. }{ }^{2} \\
90,000 \text { to } \\
110,000\end{array}$ & $\begin{array}{c}\text { Lhs.iin. } \\
65,000\end{array}$ & $\begin{array}{c}\text { Per cent } \\
20\end{array}$ & Per cent \\
\hline
\end{tabular}

CLASS B

\begin{tabular}{|c|c|c|c|c|}
\hline $\begin{array}{l}\text { Up to } 7 \text { inches diameter or } \\
\text { thickness when solid, or } 31 / 2 \text { - } \\
\text { inch maximum wall....... }\end{array}$ & $\begin{array}{r}100,000 \text { to } \\
120,000\end{array}$ & 75,000 & 20 & 50 \\
\hline $\begin{array}{l}7 \text { to } 10 \text {-inch diameter or thick- } \\
\text { ness when solid, or } 5 \text {-inch } \\
\text { maximum wall. }\end{array}$ & $\begin{array}{r}100,000 \text { to } \\
120,000\end{array}$ & 75,000 & 18 & 45 \\
\hline
\end{tabular}

(Requirements covering classification of forgings by size, determination of elastic limit by means of the extensometer, time of testing forgings, tension and bend test specimens (including illustration), are practically the same as for carbon-steel and alloy steel forgings in A. S. T. M. A18-30. See 611.51, p. 754.)

Bend tests.-If specified by the purchaser, bend tests shall be made as follows:

(a) For forgings up to 7 inches in diameter or thickness when solid, $180^{\circ}$ on a 1 -inch flat mandrel having a rounded edge of $1 / 2$-inch radius without cracking on the outside of the bent portion.

(b) For forgings 7 to 10 inches in diameter or thickness when solid or, 5-inch maximum wall, the 
test specimen shall bend cold through $180^{\circ}$ around a $1 \frac{1}{2}$-inch flat mandrel having a rounded edge of $3 /$-inch radius without cracking on the outside of the bent portion.

Proof test.-Unless otherwise specified by the purchaser, ail forgings shall be subjected to an impact proof test. The details of this test shall be agreed upon by the manufacturer and the purchaser.

Bend-test specimens shall be one-half inch square in section with corners rounded to a radius not over one-sixteenth inch and need not exceed 6 inches in length.

Number of tests.-(a) One tension, and if specified by the purchaser, one bend test shall be made from each tempering charge. If more than one quenching charge is represented in a tempering charge, one tension, and, if specified, one bend test shall be made from each quenching charge. If more than one melt is represented in a quenching charge, one tension and, if specified, one bend test shall be made from each melt.

(b) If more than one class of forgings by size is represented in any lot, one tension and, if specified, one bend test from a forging of each class by size shall be made as specified.

(c) If any test specimen shows defective machining or develops flaws, it may be discarded and another specimen substituted.

(d) If the percentage of elongation of any tension test specimen is less than that specified above and any part of the fracture is more than three-fourths inch from the center of the gage length, as indicated by scribe scratches marked on the specimen before testing, a retest shall be allowed.

Retests.-(a) If the fracture of any tension-test specimen shows over 15 per cent crystalline, a second test shall be made. If the fracture of the second specimen shows over 15 per cent crystalline, the forgings represented by such specimen shall be retempered or requenched and retempered.

(b) If the results of the physical tests of any lot do not conform to the requirements specified, the manufacturer may retemper or requench and retemper such lot, but not more than three additional times, unless authorized by the purchaser, and retests shall be made as specified above.

(Requirements covering workmanship and finish, marking, inspection and rejection, and rehearing conform with those specified in A. S. T. M. standard specifications for steel, paragraphs $5,6,7,8$, and 9 . See 605.0, p. 247).

Freight charges.-All rejected forgings will be returned to the manufacturer, who shall pay freight charge both ways.

\section{AMERICAN SOCIETY FOR TESTING MA- TERIALS, STANDARD SPECIFICATIONS FOR CARBON-STEEL AND ALLOY-STEEL FORGINGS, DESIGNATION A18-30, 1930}

CARBON-STEEL AND ALLOY STEEL FORGINGS

(See 611.51, p. 754.)

AMERICAN SOCIETY FOR TESTING MATERIALS, STANDARD SPECIFICATIONS FOR QUENCHED - AND - TEMPERED ALLOY - STEEL AXLES, SHAFTS, AND OTHER FORGINGS FOR LOCOMOTIVES AND CARS, DESIGNATION A63-27, 1927

\section{ALLOY-STEEL FORGINGS}

(Practically the same as the specification for alloy-steel forgings, quenched and tempered, of the American Railway Association, given above, in- cluding references therein to A. S. T. M. A18-30, and standard specifications for steel in 605.0 , pars. 5 , $6,7,8$, and 9 , except that the percentage of phosphorus in basic steel should be not over 0.04. Also the requirements as to boring are the same as in A. S. T. M. A19-27. (See 611.52, p. 771.)

AMERICAN SOCIETY FOR TESTING MATERIALS, TENTATIVE SPECIFICATIONS FOR NORMAIIZED AND TEMPERED ALLOY-STEEL FORGINGS FOR LOCOMOTIVES, DESIGNATION A133-31T, 1931

\section{ALLOY-STEEL FORGINGS FOR LOCOMOTIVES}

\section{SCOPE}

These specifications cover two classes of normalized and tempered alloy-steel forgings for use in locomotive construction. The characteristics of the two classes are as follows:

Class A.-Medium tensile strength with high yield point and high ductility.

Class B.-High tensile strength with considerable ductility.

\section{MANUFACTURE}

(Requirements covering process, discard, prolongation for tests, and boring are the same as for carbon-steel and alloy-steel forgings in A. S. T. M. A18-30. See 611.51, p. 754.)

\section{HEAT TREATMENT}

Cooling.-After forging, the objects shall be allowed to cool to a temperature below the critical range, under suitable conditions to prevent injury by too rapid cooling.

Normalizing.-For normalizing, the forgings shall be uniformly reheated to a proper temperature to refine the grain, and after being held a sufficient length of time at this temperature, shall be withdrawn from the furnace and allowed to cool uniformly in still air, protected from rain or snow, to a temperature below the critical range. A group of forgings thus heated shall be known as a normalizing charge.

Tempering.-For tempering, after normalizing as described in the preceding paragraph, the forgings shall be uniformly reheated to a temperature below the critical range, and after being held a proper time at this temperature shall be allowed to cool uniformly either in the furnace or in the atmosphere protected from rain or snow. A group of forgings thus treated shall be known as a tempering charge.

\section{CHEMICAL PROPERTIES AND TESTS}

The steel shall conform to the following requirements as to chemical composition:

Phosphorus, maximum
Sulphur, maximum

The composition other than phosphorus and sulphur shall be agreed upon by the manufacturer and the purchaser.

Note.-Compositions which have been used successfully to give the physical properties specified below are as follows:

\begin{tabular}{|c|c|c|}
\hline & Jlass A & Class B \\
\hline $\begin{array}{l}\text { Carbon } \\
\text { Manganese } \\
\text { Silicon } \\
\text { Nickel } \\
\text { Chromium } \\
\text { Vanadium }\end{array}$ & $\begin{array}{l}\quad \text { Per cent } \\
0.20 \text { to } 0.30 \\
0.80 \text { to } 1.00 \\
\text { Not less than } 0.15 \\
2.50 \text { to } 3.00 \\
\text { Not over } 0.40 \\
\text { Not over } 0.05\end{array}$ & $\begin{array}{l}\quad \text { Per cent } \\
0.42 \text { to } 0.57 \text {. } \\
0.70 \text { to } 0.95 \text {. } \\
\text { Not less than } 0.15 \text {. } \\
\text { Not over } 0.25 \text {. } \\
\text { Not over } 0.40 \text {. } \\
\text { Not less than } 0.15 \text {. }\end{array}$ \\
\hline
\end{tabular}


(Ladle and check analyses are the same as in A. S. T. M. A 18-30, referred to above.)

\section{PHYSICAL PROPERTIES AND TESTS}

The forgings shall conform to the following minimum requirements as to tensile properties:

\begin{tabular}{|c|c|c|}
\hline & $\begin{array}{c}\text { Class A, } \\
\text { not over } \\
14 \text { inches in } \\
\text { diameter or } \\
\text { thickness, } \\
\text { 7-inch } \\
\text { maximum } \\
\text { wall }\end{array}$ & $\begin{array}{c}\text { Class B, } \\
\text { not over } \\
14 \text { inches in } \\
\text { diameter or } \\
\text { thickness, } \\
\text { 7-inch } \\
\text { maximum } \\
\text { wall }\end{array}$ \\
\hline 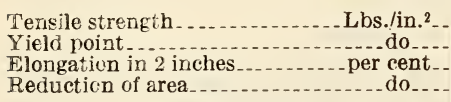 & $\begin{array}{r}80,000 \\
10.67 \\
28 \\
56\end{array}$ & $\begin{array}{r}90,000 \\
10.62 \\
22 \\
42\end{array}$ \\
\hline
\end{tabular}

1 Of tensile strength.

The yield point shall be determined by the drop of the beam or halt in the gage of the testing machine. The speed of the testing machine shall be such that when running light the crosshead speed shall be not less than one-sixteenth inch nor more than one-eighth inch per minute.

Tests of forgings shall be made only after final treatment. If specified by the purchaser, bend tests shall be made as follows: The test specimen shall stand being bent cold through $180^{\circ}$ around a pin one-half inch in diameter without cracking on the outside of the bent portion.

(Requirements covering test specimens, including illustration, are the same as in A. S. T. M. A18, referred to above. Requirements covering bend test specimens, number of tests, and retests are the same as in the American Railway Association specification for axles, shafts, and other forgings, alloy steel, quenched and tempered, given above, except that in this specification normalizing is substituted for quenching and tempering in the A. R. A. specification.) (Requirements covering workmanship and finish, marking, inspection and rejection, and rehearing conform to those specified in A. S. T. M. standard specifications for steel. See 605.0, p. 247.)

\subsection{ALLOY STEEL SPRINGS.}

(Alloy steel stock for springs, see 621.34, p. 1128.)

\subsection{MISCELLANEOUS MANUFACTURES OF ALLOY STEEL.}

AMERICAN ELECTRIC RAILWAY ENGI-
NEERING ASSOCIATION, STANDARD
DESIGN OF BOLTED MANGANESE-STEEL
CROSSINGS; STEAM RAILROAD OVER
ELECTRIC RAILWAY, FOR ANGLES BE-
LOW $45^{\circ}$ TO $30^{\circ}$, INCLUSIVE, W44-28,
1928

\section{BOLTED MANGANESE-STEEL CROSSINGS}

(See 606.3, p. 342.)
AMERICAN RAILWAY ASSOCIATION, MECHANICAL DIVISION, HIGH CHROME STEEL RIVET MATERIAL AND RIVETS FOR NITRIC ACID TANK CARS, RECOMMENDED PRACTICE, 1929

\author{
HIGH CHROME STEEL RIVETS
}

(See 621.31, p. 1122.)

UNITED STATES GOVERNMENT, DEPARTMENT OF COMMERCE, BUREAU OF STANDARDS, COMMERCIAL STANDARD FOR STEEL BONE PLATES AND SCREWS, CS37-31, NOVEMBER 16, 1931

\section{STEEL BONE PLATES}

(This commercial standard has been selected and accepted by industry and is promulgated by the Department of Commerce through the Bureau of Standards.)

\section{SCOPE}

1. This commercial standard covers the phrsical and metallurgical requirements, design, dimensions, workmanship, and finish of steel bone plates and screws available for use in the reduction of bone fractures, together with the packaging, identification, and certification of quality to buyers.

\section{TYPE AND GRADE}

2. Steel bone plates and screws shall be of the form known as the Sherman type. They shall be made in one grade only.

\section{GENERAL REQUIREMENTS}

3. Workmanship and finish.-Steel bone plates and screws shall be of good workmanship without apparent flaws or defects. They shall be thoroughly cleaned and polished smooth after tempering and then given a blued steel finish.

4. Metallurgical requrrements. ${ }^{4}-(a)$ The steel for both plates and screws shall be chromium-ranadium steel conforming to the following requirements as to chemical composition:

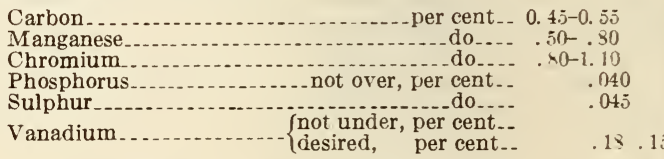

(b) The plates and screws, after forming, shall be heat treated to a hardness of not less than 43 or more than 53 , as determined by the Rockwell hardness tester using the $\mathrm{C}$ scale and the $150 \mathrm{~kg}$ load on the diamond cone penetrator.

\section{DETAIL REQUIREMENTS}

(a) Bone Plates

5. Design.-Bone plates shall conform to the Sherman design as shown in Figures 1066 and 1067,

4 These requirements are the same as for Society of Automotive Engineers steel 6150, according to 1931 S. A. E. handbook. 


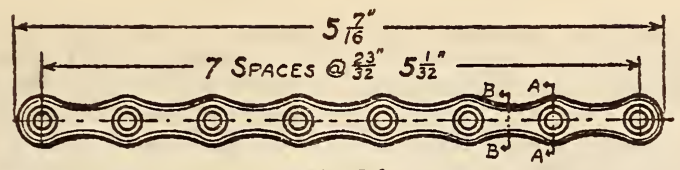

No. 00
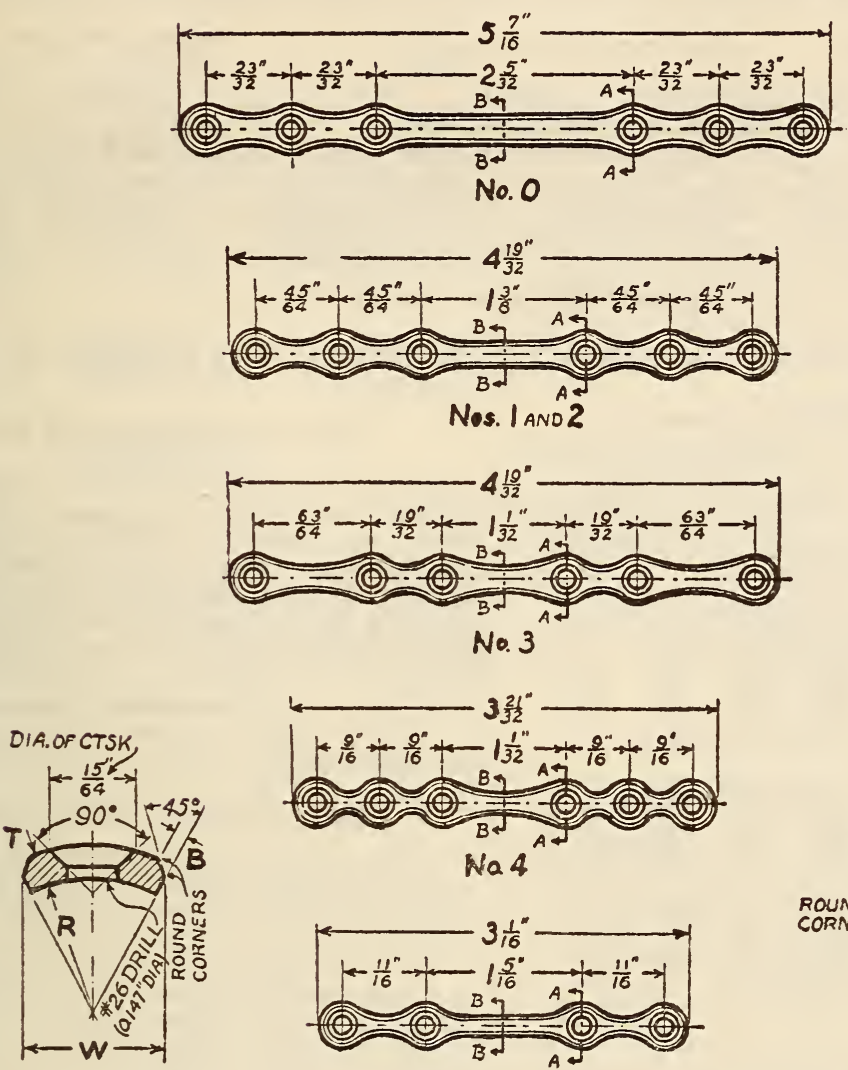

SECTIONAA (ENLARGED)
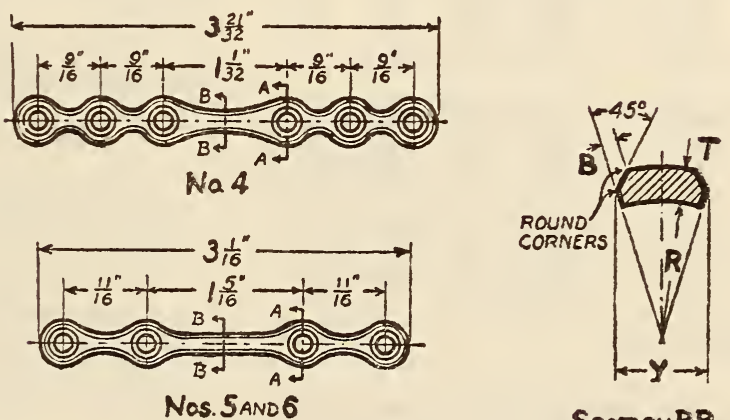

Section BB

FIgURe 1066.-Curved bone plates
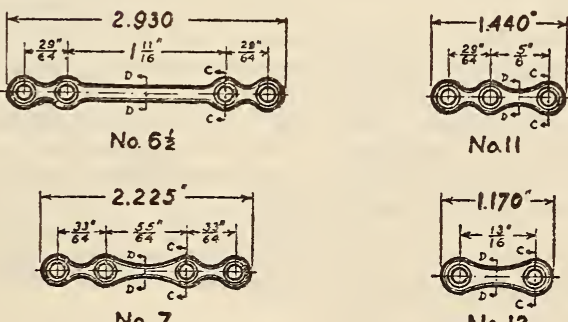

No. 7

No. 12
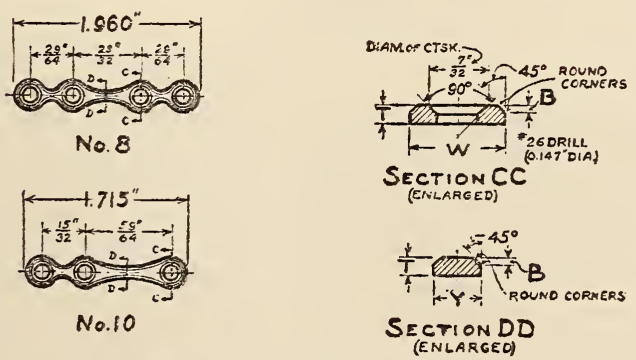

Figure 1067.-Flat bone plates 
and shall be of good workmanship, provided with envelope must be broken to remove the plate. accurately drilled and countersunk screw holes. The envelope shall bear a mark showing the size Screw holes in curved plates shall be countersunk after plate is curved.

6. Sizes and dimensions.-Bone plates shall be made in 14 sizes with dimensions and contour to conform with Table 1 and Figures 1066 and 1067.

number of the plate contained therein.

11. Guarantee label.-Each envelope containing a plate shall bear a label worded as follows: "The ........ Co. certifies this steel bone plate to conform to all requirements of Commercial Stand-

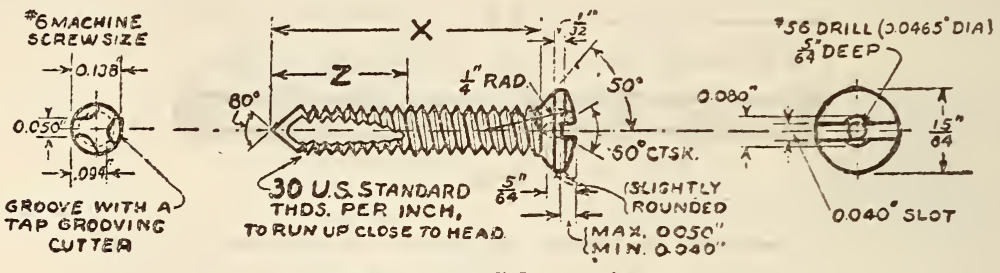

Figure 1068.-Self-tapping screw

7. Tolerances.-Tolerances are as given in Table 1.

TABLE 1.-Plates, sizes, and dimensions

\begin{tabular}{|c|c|c|c|c|c|c|c|}
\hline \multirow{2}{*}{ No. } & \multirow{2}{*}{$\begin{array}{c}\text { Total } \\
\text { length } \\
\pm 1 / 16\end{array}$} & \multirow{2}{*}{$\begin{array}{l}\text { Thick- } \\
\text { ness } \\
(T)^{1} \\
\pm 0.015\end{array}$} & \multirow{2}{*}{$\begin{array}{l}\text { Num } \\
\text { ber of } \\
\text { holes }\end{array}$} & \multicolumn{2}{|c|}{ Width } & \multirow{2}{*}{$\begin{array}{l}\text { Bevel } \\
(B) 1\end{array}$} & \multirow{2}{*}{$\begin{array}{l}\text { Radius } \\
\text { of cur- } \\
\text { vature } \\
(R)^{1}\end{array}$} \\
\hline & & & & $\begin{array}{c}W^{1} \\
\pm 0.015\end{array}$ & $\begin{array}{c}Y^{1} \\
\pm 0.015\end{array}$ & & \\
\hline & Inches & Inch & & Inch & Inch & Inch & \\
\hline 00 & $57 / 16$ & 0.095 & 8 & 0.406 & 0.260 & 3,64 & $3 / 8$ \\
\hline 0 & $57 / 16$ & .095 & 6 & .406 & .260 & 364 & $3 / 8$ \\
\hline 1 & $4^{19} / 32$ & .095 & 6 & 406 & .215 & $3 / 64$ & $3 / 8$ \\
\hline 2 & $419 / 32$ & .083 & 6 & 406 & .215 & .041 & $3 \% 8$ \\
\hline 3 & $419 / 32$ & .083 & 6 & .406 & .228 & .041 & $3 / 8$ \\
\hline 4 & $3^{21} / 32$ & .065 & 6 & .375 & .146 & .025 & $3 / 8$ \\
\hline 5 & $31 / 16$ & .083 & 4 & .375 & .156 & .041 & $3 / 8$ \\
\hline 6 & $31 / 16$ & .065 & 4 & .375 & .156 & .025 & $3 / 8$ \\
\hline $61 / 2$ & 2. 930 & .065 & 4 & .336 & .165 & .025 & Flat. \\
\hline 7 & 2.227 & .065 & 4 & .336 & .125 & .025 & Flat. \\
\hline 8 & 1.960 & .065 & 4 & .336 & .125 & .025 & Flat. \\
\hline 10 & 1.715 & .065 & 3 & 325 & .112 & .025 & Flat. \\
\hline 11 & 1. 440 & .065 & 3 & .360 & .186 & .025 & Flat. \\
\hline 12 & 1. 170 & .065 & 2 & .360 & .186 & .025 & Flat. \\
\hline
\end{tabular}

1 See Figs. 1066 and 1067.

8. Inspeciion and tests.-Each plate shall be tested for hardness before sale or offering for sale as conforming to this specification. Hardness impressions shall be made as near the ends of the plate as practicable, and not in the mid section of any plate which is to be put into service

9. Stamping. - Each bone plate shall be stamped near an end with the name or trade-mark of the manufacturer and with the designation CS37-31, indicating conformity to this specification. No stamping shall be allowed on the mid section. Stamping shall not be so deep as to appreciably weaken the plate, and shall be done before hardening.

10. Packaging.-Each individual plate shall be sealed in a transparent envelope so that the seal or ard CS37-31 issued by the U. S. Department of Commerce.'

(b) Screws for Attaching Steel Bone Plates

12. Material.-The steel for screws shall be as specified in paragraph $4(a)$, and shall be heat treated as specified in paragraph $4(b)$.

13. Design.-Screws for attaching steel bone plates shall be of the Sherman self-tapping design, provided with accurately cut self-tapping flutes and center hole for use with special self-centering screw driver.

14. Sizes and dimensions.-Screws shall be made in 11 sizes with dimensions as shown in Table 2 and Figure 1068.

TABLE 2.-Screws, sizes, and dimensions

\begin{tabular}{|c|c|c|c|c|c|}
\hline Size & $\begin{array}{l}\text { Dimen- } \\
\text { sion } \\
X\end{array}$ & $\begin{array}{l}\text { Dimen- } \\
\text { sion } \\
Z\end{array}$ & Size & $\begin{array}{l}\text { Dimen- } \\
\text { sion } \\
X\end{array}$ & $\begin{array}{l}\text { Dimen- } \\
\text { sion } \\
\quad Z\end{array}$ \\
\hline $\begin{array}{l}A \\
B \\
C \\
D \\
E \\
F \\
F\end{array}$ & $\begin{array}{l}\text { Inch } \\
3 / 8 \\
1 / 2 \\
5,8 \\
3 / 4 \\
7 / 3 \\
1^{7 / 3}\end{array}$ & $\begin{array}{r}\text { Inch } \\
3 / 16 \\
9 / 32 \\
11 / 32 \\
38 \\
3 / 8 \\
3 / 8\end{array}$ & $\begin{array}{c}G \\
H \\
I \\
J \\
K \\
K\end{array}$ & $\begin{array}{c}\text { Inches } \\
118 \\
11 / 4 \\
13 / 8 \\
11 / 2 \\
15 / 8\end{array}$ & $\begin{array}{c}\text { Inch } \\
3 / 8 \\
3 / 8 \\
3 / 8 \\
3 / 8 \\
3 / 8\end{array}$ \\
\hline
\end{tabular}

15.-Packaging.- Screws shall be packaged in lots of 3 or 6 of one size, in transparent envelopes so sealed that the seal or envelope must be broken to remove screws. Each envelope containing screws shall bear a label stating the size and number of screws contained therein.

16. Guarantee label.-Each envelope containing screws shall bear a guarantee label worded as follows: "The -....... Co. certifies these screws to conform to all requirements of Commercial Standard CS37-31 issued by the U. S. Department of Commerce." 


\section{0-639 ALUMINUM, ANTIMONY, BISMUTH, CADMIUM, AND COBALT}

630.0 GENERAL ITEMS.

\section{AMERICAN SOCIETY FOR TESTING MATE- RIALS, STANDARD METHODS OF METAL- LOGRAPHIC TESTING OF NONFERROUS METALS AND ALLOYS, DESIGNATION E5-27, 1927}

\section{METALIOGRAPHIC TESTING OF NONFERROUS METALS}

(See 600.1, p. 28.)

631.

\section{ALUMINUM}

631.0 GENERAL ITEMS.

AMERICAN SOCIETY FOR TESTING MATERIALS, TENTATIVE METHODS OF CHEMICAL ANALYSIS OF ALUMINUM AND LIGHT ALUMINUM ALLOYS, DESIGNATION B40-28T, 1928

\section{CHEMICAI ANAIYSIS OF AIUMINUM AND LIGHT ALUMINUM ALIOYS}

The methods of analysis listed under "analysis of aluminum" contemplate the analysis of aluminum containing more than 98 per cent aluminum and containing only silicon, titanium, iron, copper, carbon, and traces of manganese as impurities.

Unless it is positively known that such elements as tin, antimony, zinc, lead, nickel, magnesium, sodium, nitrogen or oxygen (as aluminum oxide) are absent, the methods listed under the Analysis of Light Aluminum Alloys should be employed.

\section{ANALYSIS OF ALUMINUM}

(Not less than 98 per cent aluminum)

\section{Determination of Silicon and Titanium}

Determination of Total Silicon

SOLUTIONS REQUIRED

Acid mixture.-1,200 c c of $\mathrm{H}_{2} \mathrm{SO}_{4}$ (25 per cent by volume), 600 c c $\mathrm{HCl}$ (specific gravity 1.20) and 200 c c $\mathrm{HNO}_{3}$ (specific gravity 1.42).

25 per cent sulphuric acid.-Pour 250 c c of $\mathrm{H}_{2} \mathrm{SO}_{4}$ (specific gravity 1.84) into distilled water, and dilute to $1,000 \mathrm{c}$ c.

\section{METHOD}

Dissolve $1 \mathrm{~g}$ of the well-mixed sample in $35 \mathrm{c} \mathrm{c}$ of the acid mixture in a covered flat-bottomed porcelain dish or casserole. When the drillings are completely dissolved, boil the solution to complete expulsion of $\mathrm{HNO}_{3}$ and $\mathrm{HCl}$ and heat until copious fumes of $\mathrm{H}_{2} \mathrm{SO}_{4}$ have been given off for five minutes. Cool and take up the residue with $10 \mathrm{c}$ c of 25 per cent $\mathrm{H}_{2} \mathrm{SO}_{4}$ and $100 \mathrm{c}$ c of water. Warm to complete solution of the sulphates and filter on an ashless filter paper. Wash the residue with hot water until free from sulphates and ignite in a platinum crucible. Fuse the residue with eight to ten times its weight of $\mathrm{Na}_{2} \mathrm{CO}_{3}$ and treat the fused mass in a casserole or beaker with 20 c c of $\mathrm{H}_{2} \mathrm{SO}_{4}(1: 3)$, washing out the crucible. Evap- orate the solution until it fumes strongly to separate the $\mathrm{SiO}_{2}$. Cool, dilute to about $100 \mathrm{c}$ c, boil till the salts are dissolved, filter, wash well, ignite in a platinum crucible and weigh. Treat the ash with $\mathrm{HF}$ and a few drops of $\mathrm{H}_{2} \mathrm{SO}_{4}(1: 3)$, carefully evaporate till dry, ignite and again weigh.

From the difference between the two weights deduct a blank obtained from the reagents and filter. The rest of the loss in weight represents $\mathrm{SiO}_{2}$ corresponding to the Si in the metal.

$$
\mathrm{SiO}_{2} \times 0.4672=\mathrm{Si}
$$

Notes.-Blanks should be carried along with all reagents and the proper corrections made.

In routine analysis, fusion of the first $\mathrm{H}_{2} \mathrm{SO}_{4}$ residue with sodium carbonate and subsequent treatment to the point of treating the ash with HF may be omitted. The first residue may be weighed and treated with $\mathrm{HF}$, etc., as directed for the second $\mathrm{H}_{2} \mathrm{SO}_{4}$ residue. This, however, should not be done if the material contains much graphitic silicon or if the residue after ignition is dark colored.

Since there may be a slight loss of silicon in the filtrates from the silica filtrations, this amount should be recovered by repeated evapo-
. rations of the filtrates, for accurate work.

Determination of Titanium

APPARATUS AND SOLUTIONS REQUIRED

Nessler tubes or some form of colorimeter.

Hydrogen peroxide U.S. P.-This reagent must be free from $\mathrm{HF}$.

Standard titanium solution.-This solution is best prepared and standardized as follows: ${ }^{5}$

"Potassium titanium fluoride, $\mathrm{K}_{2} \mathrm{TiF}$, best serves as the starting point for the preparation of the standard solution. A quantity of this is recrystallized from boiling water one or more times, dried, and preserved in a glass-stoppered bottle Enough of it to make 0.5 to 1 liter of the standard sulphate solution is put into a platinum dish and evaporated several times with strong sulphuric acid without bringing to dryness, till the fluorine is completely expelled. The residue is then taken up with water containing enough sulphuric acid to make at least 5 per cent of the latter when fully diluted. Two 50 to 100 c c portions of the prepared solution are then further diluted, boiled, and precipitated with ammonia. The precipitates are collected on paper, washed with hot water till free from alkali, ignited moist in the filter, blasted, and weighed. Duplicates should agree almost exactly. From the weights found, the strength of the standard is calculated and the result affixed to the bottle containing it. The stopper of the bottle should be coated with vaseline and the needed quantities of solution should be withdrawn by a pipette, never poured. In a solution so prepared there is not enough alkali sulphate to weaken the color when peroxidized.'

\section{METHOD}

See determination of total silicon.

Fuse the nonvolatile residue remaining after the silicon determination in a small amount of $\mathrm{K}_{2} \mathrm{~S}_{2} \mathrm{O}_{7}$, take up in a little 5 per cent $\mathrm{H}_{2} \mathrm{SO}_{4}$ and add to the filtrates and washings from the silicon determination. Evaporate the solution to approximately $100 \mathrm{c}$ c, and treat with $5 \mathrm{c} \mathrm{c} \mathrm{H}_{2} \mathrm{SO}_{4}$ (specific gravity 1.84 ) and $3 \mathrm{~g}$ of iron-free zinc. Heat until the zinc

5 W. F. Hillebrand, The Analysis of Silicate and Carbonate Rocks, Bulletin No. 700, U. S. Geological Survey, p. 157. 
is nearly dissolved and the reduction of copper is complete. Decant the solution into another beaker. wash the zinc and copper with hot water and continue the evaporation of the solution to approximately 75 c c. Cool and transfer to a 100 c c Nessler's comparison tube, add $5 \mathrm{c}$ c of 3 per cent $\mathrm{H}_{2} \mathrm{O}_{2}$ and dilute to the $10.0 \mathrm{c} \mathrm{c}$ mark. In another tube, place 88 c c $\mathrm{H}_{2} \mathrm{O}, 5 \mathrm{c}$ c of $\mathrm{H}_{2} \mathrm{SO}_{4}$ (specific gravity 1.84) and cool. Add 5 c c of 3 per cent $\mathrm{H}_{2} \mathrm{O}_{2}$ and then from a burette add the standard titanium solution until the colors match. The volume of the standard solution required multiplied by one hundred times its titer represents the percentage of titanium in the sample.

Notes.-The filtrate and washings from the silicon determination should ordinarily be colorless. If light yellow owing to iron, the standard comparison solution should be brought to the same tint standard comparison solution should be brought to the same tint

In case the amount of titanium is high the solution may be diluted to a definite volume and aliquot portions taken for the colorimetric test.

\section{Determination of Iron}

SOLUTIONS REQUIRED

Acid mixture.-1,200 c c of $\mathrm{H}_{2} \mathrm{SO}_{4}$ (25 per cent by volume), $600 \mathrm{c} \mathrm{c}$ of $\mathrm{HCl}$ (specific gravity 1.20) and $200 \mathrm{c}$ c of $\mathrm{HNO}_{3}$ (specific gravity 1.42).

Tartaric acid solution.-Dissolve $20 \mathrm{~g}$ of tartaric acid crystals in $100 \mathrm{c} \mathrm{c}$ of distilled water.

Dilute ammonium sulphide solution.-Add $15 \mathrm{c} \mathrm{c}$ of $\mathrm{NH}_{4} \mathrm{OH}$ (specific gravity 0.90 ) to $15 \mathrm{c}$ c of distilled water and saturate with $\mathrm{H}_{2} \mathrm{~S}$; then dilute to $200 \mathrm{c}$ c with distilled water. Use freshly made.

Dilute sulphuric acid solution for reductor (5 per cent).-Mix $50 \mathrm{c}$ c of $\mathrm{H}_{2} \mathrm{SO}_{4}$ (specific gravity 1.84) and $1,000 \mathrm{c}$ c of distilled water.

Standard potassium permanganate solution.-Dissolve $1 \mathrm{~g} \mathrm{KMnO}_{4}$ in $1,000 \mathrm{c} \mathrm{c}$ of $\mathrm{H}_{2} \mathrm{O}$, and allow to stand for several days in a glass-stoppered bottle in a dark closet. Filter the solution through prepared asbestos and standardize against $0.1000 \mathrm{~g}$ portions of pure sodium oxalate. Each cubic centimeter is equivalent to approximately $0.00177 \mathrm{~g}$ of iron.

\section{METHOD}

Dissolve $1 \mathrm{~g}$ of the well-mixed sample in $35 \mathrm{c} \mathrm{c}$ of the acid mixture and proceed as in determination of total silicon, until the first insoluble residue is ignited. Treat this with a little concentrated $\mathrm{HNO}_{3}$ and $\mathrm{HF}$ to remove the silicon, then evaporate to aryness. Fuse the unvolatilized part with $\mathrm{K}_{2} \mathrm{~S}_{2} \mathrm{O}_{7}$, dissolve in a little $\mathrm{H}_{2} \mathrm{SO}_{4}$ (5 per cent) and add to the main solution. Saturate this solution with $\mathrm{H}_{2} \mathrm{~S}$ and filter. To the filtrate add $25 \mathrm{c} c$ of the tartaric acid solution, and then with constant stirring, add $\mathrm{NH}_{4} \mathrm{OH}$ (specific gravity 0.90), finally drop by drop, until it is in slight excess. Pass in $\mathrm{H}_{2} \mathrm{~S}$ for a few moments, warm somewhat and allow the solution to stand. Filter and wash the sulphide precipitate thoroughly with a freshly made dilute ammonium sulphide solution, until all tartaric acid is washed out.

Dissolve the sulphide precipitate through the filter paper with warm dilute $\mathrm{H}_{2} \mathrm{SO}_{4}(1: 10)$, washing the paper thoroughly, alternately with hot distilled water and with 5 per cent $\mathrm{H}_{2} \mathrm{SO}_{4}$, using in all about 100 c c. Boil until $\mathrm{H}_{2} \mathrm{~S}$ is expelled and add, while hot, a few drops of concentrated solution of $\mathrm{KMnO}_{4}$ until a distinct pink color persists. Boil again, cool and pass the solution through the reductor. Wash the beaker and reductor with $150 \mathrm{c}$ c of dilute $\mathrm{H}_{2} \mathrm{SO}_{4}$ for reductor and then with $100 \mathrm{c} \mathrm{c}$ of distilled water. Titrate with standard $\mathrm{KMnO}_{4}$ solution.

Notes.-Results of titration should be checked with a blank determination made on corresponding amounts of solutions used.

A small quantity of liquid should always be left in the reductor funnel and air should never be allowed to enter the body of the reductor.
For further details of the use of reductor see The Chemical Analysis of Iron, by A. S. Blair, or Quantitive Chemical Analysis, by H. P. Talbot.

The effect of tartaric acid is to form complexes with aluminum, vanadium, chromium, titanium, etc., preventing their precipitation as hydroxides.

Where the iron exists in very small quantities, a gravimetric method is preferable. In this case, double precipitation of iron sul. phide is necessary to eliminate all aluminum. Dissolve the second sulphide precipitate in warm dilute $\mathrm{H}_{2} \mathrm{SO}_{4}(1: 10)$, boil, and oxidize the iron with $\mathrm{H}_{2} \mathrm{O}_{2}$. Add 3 to $4 \mathrm{~g}$ of $\mathrm{NH}_{4} \mathrm{Cl}$ and then $\mathrm{NH}_{6} \mathrm{OH}$ (specific gravity 0.90) in considerable excess, with stirring. Filter quickly, dry and ignite the precipitate, weighing as ferric oxide. If an accurate determination of iron is desired, it is necessary to correct for the small accumulation of silica by treatment of the ignited precipitate with $\mathrm{H}_{2} \mathrm{SO}_{4}(-) \mathrm{H}$.

In adding the few drops of concentrated solution of $\mathrm{KMnO}_{4}$ for oxidation, only a very slight excess is used. There may be precipitated a very slight amount of manganese oxide but this can be disregarded in passing the solution through the reductor.

\section{Determination of Copper by Electrolytic Method}

\section{APPARATUS FOR ELECTROLYSIS}

See the determination of copper by the electrolytic method in B27 (647.20, p. 1261).

\section{SOLUTIONS REUUIRED}

Sodium hydroxide solution.-Dissolve $250 \mathrm{~g}$ of $\mathrm{NaOH}$ in distilled water and dilute to $1,000 \mathrm{c}$ c.

Nitric acid $(1: 1)$.-Pour 500 c c of $\mathrm{HNO}_{3}$ (specific gravity 1.42) into distilled water and dilute to $1,000 \mathrm{c} \mathrm{c}$.

\section{METHOD}

Dissolve $10 \mathrm{~g}$ of the sample in a large casserole or beaker with $150 \mathrm{c}$ c of the $\mathrm{NaOH}$ solution.

This should be added in small portions as the action is vigorous for a time. After the action slackens, complete solution mav be hastened by warming. When no evidence of further reaction can be seen, dilute to about $700 \mathrm{c} c$ with water at the boiling temperature. Filter through a strong paper and wash.

Dissolve the residue from the paper with $25 \mathrm{c} c$ of hot $\mathrm{HNO}_{3}(1: 1)$ into a beaker for electrolyzing, and wash the filter thoroughly with hot water. Boil to expel oxides of nitrogen, add 6 to $8 \mathrm{c} \mathrm{c}$ of $\mathrm{H}_{2} \mathrm{SO}_{4}(1: 1)$, dilute to about $150 \mathrm{c}$ c and electrolyze. Use a weighed sand-blasted cathode and a current of from 3 to 5 amperes at approximately 10 volts. When the solution is colorless and $1 \mathrm{c} c$ of the solution gives no copper test with $\mathrm{H}_{2} \mathrm{~S}$ water on a porcelain plate, remove the solution from the electrodes quickly without interrupting the current. Quickly rinse the cathode in distilled water and then dip it in two successive baths of alcohol. Shake off the excess alcohol and ignite the remainder by bringing it to the flame of an alcohol lamp. Keep the cathode moving continually while the alcohol burns. Weigh as metallic copper.

Notes.-The electrolytic method is preferred for the determination of copper no matter what the percentage.

It is not anticipated that the aluminum will contain tin. In case tin has been found it will be preferable to precipitate with hydrogen sulphide. The sulphides are then to be dissolved, digested in $\mathrm{HNO}_{8}$ $(1: 1)$, the metastannic acid filtered off and the electrolysis then carried out.

A staining of the anode indicates the presence of lead or manganese. Copper, when present in small amounts, may also be determined by potassium cyanide titration. See standard methods of chemical analysis of alloys of lead, tin, antimony and copper B18(692.0, p. 1317)

If the electrolytically deposited copper is not bright in appearance, it should be dissolved from the cathode and redeposited from a sulphuric-nitric acid solution, the operation being carried through in the same way as in the first electrolysis.

\section{Determination of Manganese}

(a) Persulphate method.-(For samples containing under 1.5 per cent of manganese.) 
SOLUTIONS REQUIRED

Acid mixture.-Pour $400 \mathrm{c}$ c of $\mathrm{H}_{2} \mathrm{SO}_{4}$ (specific gravity 1.84) into distilled water, cool and add $400 \mathrm{c}$ c of $\mathrm{HNO}_{3}$ (specific gravity 1.42). Then dilute the mixture with distilled water to a total volume of $2,000 \mathrm{c}$ c.

Silver nitrate.-Dissolve $1.33 \mathrm{~g}$ of $\mathrm{AgNO}_{3}$ in 1,000 c $\mathrm{c}$ of distilled water.

Stock sodium arsenite solution.-To $15 \mathrm{~g}$ of arsenious oxide $\left(\mathrm{As}_{2} \mathrm{O}_{3}\right)$ in $300 \mathrm{c}$ c Erlenmeyer flask, add $45 \mathrm{~g}$ of $\mathrm{Na}_{2} \mathrm{CO}_{3}$ and $150 \mathrm{c} \mathrm{c}$ of distilled water. Heat the flask and contents on the steam bath until the $\mathrm{As}_{2} \mathrm{O}_{3}$ is dissolved. Cool the solution, filter and make up to $1,000 \mathrm{c}$ c with distilled water.

Standard sodium arsenite solution.-Mix $200 \mathrm{c}$ c of the stock sodium arsenite solution with 2,500 c c of distilled water, and standardize against a measured volume of a standard $\mathrm{KMnO}_{4}$ solution that has been reduced and treated as in the method, or against a suitable standard metal or alloy of the United States Bureau of Standards. One cubic centimeter of this solution should be equivalent to approximately $0.00035 \mathrm{~g}$ of manganese.

Ammonium persulphate.-Dissolve $6 \mathbf{g}$ of ammonium persulphate in $100 \mathrm{c}$ c of distilled water. (This solution should be made up as needed as it deteriorates rapidly.)

\section{METHOD}

Use $1 \mathrm{~g}$ of the sample if the manganese is not over 0.2 per cent. For a higher manganese content adjust the size of sample so that it will not contain more than $0.002 \mathrm{~g}$ of manganese. Place it in a 250 c c Erlenmeyer flask with 30 c c of the acid mixture and heat until solution is complete. Boil until oxides of nitrogen are expelled. Dilute to 100 c c with boiling-hot water, add 20 c c of $\mathrm{AgNO}_{3}$ solution and $30 \mathrm{c} c$ of the persulphate solution, then remove to a moderately warm place to stand for a few minutes for complete development of the permanganic acid. Cool to below $25^{\circ} \mathrm{C}$. and titrate with the standard sodium arsenite solution to the disappearance of the pink color.

Notes.-Large amounts of permanganic acid are unstable; on that account samples which contain large amiounts of manganese should have correspondingly small samples weighed out.

The ammonium persulphate must be tested for its strength. A good grade should contain about 95 per cent of $\left(\mathrm{NH}_{4}\right)_{2} \mathrm{~S}_{2} \mathrm{O}_{8}$; some lots contain no more than 25 per cent of the salt.

If it is desired, larger amounts of the sample can be used and correspondingly greater amounts of $\mathrm{AgNO}_{3}$ and $\left(\mathrm{NH}_{4}\right)_{2} \mathrm{~S}_{2} \mathrm{O}_{3}$ (preferably in the form of stronger solutions).

(b) Bismuthate method.-- For samples containing less than 1.50 per cent manganese.) (Optional.)

\section{SOLUTIONS REQUIRED}

Acid mixture.-(Same as in (a), above.)

Nitric acid (1:3).-Pour $250 \mathrm{c}$ c of $\mathrm{HNO}_{3}$ (specific gravity 1.42) into distilled water and dilute to 1,000 c c.

Dilute nitric acid (3 per cent).-Pour $30 \mathrm{c}$ c of $\mathrm{HNO}_{3}$ (specific gravity 1.42) into distilled water and dilute to 1,000 c c.

Standard permanganate solution.-Dissolve $1 \mathrm{~g}$ of $\mathrm{KMnO}_{4}$ in 1,000 c c of distilled water. Allow it to stand for at least one week and then filter through purified asbestos. Standardize against $0.1 \mathrm{~g}$ portions of pure sodium oxalate. One cubic centimeter of this solution should be equal to approximately $0.00035 \mathrm{~g}$ of manganese.

Standard ferrous ammonium sulphate solution.Dissolve $12.4 \mathrm{~g}$ of ferrous ammonium sulphate crystals in $950 \mathrm{c}$ c distilled water, and add $50 \mathrm{c} \mathrm{c}$ of $\mathrm{H}_{2} \mathrm{SO}_{4}$ (specific gravity 1.84).
METHOD

In a $250 \mathrm{c}$ c Erlenmeyer flask, dissolve $1 \mathrm{~g}$ of the sample in $30 \mathrm{c} c$ of acid mixture by warming on a water bath until solution is complete.

When solution is complete heat to expel all oxides of nitrogen and cool. Dilute with $50 \mathrm{c}$ c of $\mathrm{HNO}_{3}$ $(1: 3)$ and add $0.5 \mathrm{~g}$ sodium bismuthate. Heat for a few minutes until the purple color has disappeared with or without the precipitation of manganese dioxide. Add a little ferrous ammonium sulphate solution until the solution becomes clear and boil until the oxides of nitrogen are expelled. Cool, add an excess of sodium bismuthate and agitate for a few minutes. Add $50 \mathrm{c}$ c of 3 per cent $\mathrm{HNO}_{3}$ and filter through an alundum crucible or an asbestos pad. Wash with $50 \mathrm{c}$ c of 3 per cent $\mathrm{HNO}_{3}$. Add from a pipette or a burette 10 to $50 \mathrm{c} \mathrm{c}$ (depending on the amount of permanganic acid) of ferrous ammonium sulphate solution and titrate with the $\mathrm{KMnO}_{4}$ solution.

In exactly the same manner carry through a blank determination using the same amounts of acid mixture, $\mathrm{HNO}_{3}(1: 3)$, and sodium bismuthate as was done with the regular sample. Finally, add the exact volume of ferrous ammonium sulphate solution employed and titrate with the $\mathrm{KMnO}_{4}$ solution.

The difference between the volumes required in the two titrations represents the manganese in the sample, and the percentage is found by multiplying this volume by two hundred times the manganese titre of the permanganate solution.

NoTES. - Large amounts (above $0.01 \mathrm{~g}$ of manganese per $100 \mathrm{c} \mathrm{c}$ of solution) of permanganic acid are unstable; the method can be used with satisfaction for alloys containing high percentages of manganese by decreasing the size of the sample.

The filtrate from the bismuthate must be perfectly clear, as the least particle of bismuthate carried into the filtrate will vitiate the results.

The solution must be cold at time of filtration and titration.

Instead of employing the method of reducing permanganic acid by means of standardized ferrous ammonium sulphate solution and titrating the excess of the reagent, it is possible to reduce the permanganic acid by standard sodium-arsenite solution. See determination of manganese by the persulphate method.

The sodium bismuthate reagent should be tested for manganese and used only if its absence is shown.

\section{ANALYSIS OF LIGHT ALUNINUM ALLOYS}

\section{(a) Alloys Containing 1 Per Cent or Less of SilicoN}

Determination of Total Silicon

Proceed as described under determination of total silicon under analysis of aluminum.

Nores.-In case $\mathrm{PbSO}_{4}$ is present (as evidenced by a white crystallin precipitate quickly settling after stirring) the filtered residue must be washed free from the sulphate with hot dilute HCl $(1: 3)$. must final washing must be made with hot water.

If the alloy contains much tin an insoluble compound is apt to form on redissolving the sulphates after the first fuming for $\mathrm{SiO}_{2}$ To avoid this, thoroughly wet all the salts with $\mathrm{H}_{2} \mathrm{SO}_{4}$ (specific gravity 1.84) or $\mathrm{HCl}$ instead of dilute acid, before adding water.

$$
\text { Determination of Iron }
$$

Proceed as described under determination of iron under analysis of aluminum.

\section{Determination of Copper by Electrolytic Method}

\section{APPARATUS AND SOLUTIONS REQUIRED}

See the determination of copper by electrolytic method under analysis of aluminum.

\section{METHOD}

For copper contents up to 1 per cent proceed as described under determination of copper by electrolytic method under analysis of aluminum. 
If the copper content is more than 1 per cent, dissolve a $1 \mathrm{~g}$ sample in a medium size beaker with 20 c c of the $\mathrm{NaOH}$ solution, warming after the reaction slakens. When no further action can be seen, dilute to $200 \mathrm{c}$ c with hot water, filter and wash with hot water.

Dissolve the metallic residue from the paper into an electrolytic beaker with $10 \mathrm{c}$ c of hot $\mathrm{HNO}_{3}(1: 1)$ followed by thorough washing. Add dilute $\mathrm{H}_{2} \mathrm{SO}_{4}$ equivalent to $2 \mathrm{c}$ c of concentrated acid. Dilute to 150 c c. Electrolyze and finish as described under determination of copper by electrolytic method under analysis of aluminum.

\section{Determination of Manganese}

Proceed as described under determination of manganese under analysis of aluminum.

Note.-If the manganese content is over 2 per cent there is danger of precipitation at the peroxidizing stage. In that case a suitable aliquot of a new solution should be taken for developing the permanganate form.

\section{Determination of Calcium and Magnesium}

\section{SOTUTIONS REGUIRED}

Sodium hydroxide solution.-Same as in the determination of copper by electrolytic method under analysis of aluminum.

Sodium carbonate wash.-Dissolve $1 \mathrm{~g}$ of $\mathrm{Na}_{2} \mathrm{CO}_{3}$ in $100 \mathrm{c}$ e of distilled water.

Hydrochloric acid (1:1).-Mix equal volumes of $\mathrm{HCl}$ (specific gravity 1.19) and distilled water.

Ammonium sulphide wash.-Take $10 \mathrm{c}$ c of $\mathrm{NH}_{4}$ $\mathrm{OH}$ (specific gravity 0.90 ) dilute to $500 \mathrm{c} \mathrm{c}$, add $10 \mathrm{~g}$ of $\mathrm{NH}_{4} \mathrm{Cl}$ and saturate with $\mathrm{H}_{2} \mathrm{~S}$.

Bromine water.-Place 20 to $30 \mathrm{c}$ c of liquid bromine in a $500 \mathrm{c}$ c bottle, fill with cold distilled water, and shake thoroughly. Be very careful to avoid contact of the bromine with the skin.

Ammonium oxalate solution.-Make a saturated solution by adding $\left(\mathrm{NH}_{4}\right)_{2} \mathrm{C}_{2} \mathrm{O}_{4}$ in excess to distilled water.

Sulphuric acid (25 per cent).- - See the determination of total silicon in analysis of aluminum.

Standard potassium permanganate.-See the determination of iron in analysis of aluminum.

Microcosmic salt solution.-Prepare a clear saturated solution as required.

Ammonium nitrate wash.-Mix 80 c c of $\mathrm{HNO}_{3}$ (1:1) with $100 \mathrm{c} \mathrm{c}$ of $\mathrm{NH}_{4} \mathrm{OH}$ and dilute to 1 liter.

\section{METHOD}

Dissolve $2 \mathrm{~g}$ of the sample in $35 \mathrm{c}$ c of the $\mathrm{NaOH}$ solution, add about $0.5 \mathrm{~g}$ of $\mathrm{Na}_{2} \mathrm{CO}_{3}$. When dissolved, dilute to $250 \mathrm{c} \mathrm{c}$ with hot water filter, and wash five times with hot soaium carbonate wash. Dissolve the residue from the filter with $40 \mathrm{c} \mathrm{c}$ of hot $\mathrm{HCl}(1: 1)$ and a few drops of $\mathrm{HNO}_{3}$, receiving it in a beaker, and wash the filter. Neutralize this solution with $\mathrm{NH}_{4} \mathrm{OH}$ and add from 2 to $5 \mathrm{c}$ c in excess. Pass in $\mathrm{H}_{2} \mathrm{~S}$ for two or three minutes. Filter, and wash a few times with an ammonium sulphide wash.

Boil the filtrate vigorously to drive off $\left(\mathrm{NH}_{4}\right)_{2} \mathrm{~S}$. When sulphur precipitates add a little bromine water and continue boiling until clear and the odor and color of bromine are gone. Add a drop or two of methyl red as indicator to the solution, then $\mathrm{NH}_{4} \mathrm{OH}$ carefully till the yellow color appears. Boil for a minute, filter and wash. Add a few drops of $\mathrm{NH}_{4} \mathrm{OH}, 10 \mathrm{c} \mathrm{c}$ of $\left(\mathrm{NH}_{4}\right)_{2} \mathrm{C}_{2} \mathrm{O}_{4}$ solution and heat near boiling for 30 minutes, keeping ammoniacal. If a precipitate forms, filter through a small, close-textured paper, and wash eight times with small portions of hot water, reserving the filtrate for determining magnesium.

Return the filter to the precipitation beaker, add $130 \mathrm{c}$ c of hot water and $20 \mathrm{c}$ c of $\mathrm{H}_{2} \mathrm{SO}_{4}(25$ per cent), then titrate with standard $\mathrm{KMnO}_{4}$ solution. Deduct a determined blank.

The $\mathrm{Na}_{2} \mathrm{C}_{2} \mathrm{O}_{4}$ value of the $\mathrm{KMnO}_{4}$ multiplied by 0.2990 equals the calcium value.

The filtrate from the calcium determination, or the unfiltered solution if clear, is made just acid with $\mathrm{HCl}$, and 20 to $30 \mathrm{c}$ c of microcosmic salt solution added. Cool, and add $\mathrm{NH}_{4} \mathrm{OH}$ drop by drop, stirring vigorously until a crystallin piecipitate begins to form. Continue the very slow addition of $\mathrm{NH}_{4} \mathrm{OH}$ with stirring until the precipitate no longer forms, and then add one-tenth the volume of $\mathrm{NH}_{4} \mathrm{OH}$ (specific gravity 0.90 ) and let stand for at least three hours. Filter, and wash with a cold ammonium nitrate wash solution. Place in a weighed porcelain or vitreosil crucible and ignite at about $1,000^{\circ} \mathrm{C}$. until completely white Weigh as $\mathrm{Mg}_{2} \mathrm{P}_{2} \mathrm{O}_{7}$ and deduct a blank.

\section{$\mathrm{Mg}_{2} \mathrm{P}_{2} \mathrm{O}_{7} \times 0.2184=\mathrm{Mg}$}

Notes.-If much Mg is present (over 1 per cent) some of it may be carried down with the sulphides. In that case, dissolve the precipitate with $10 \mathrm{c} \mathrm{c}$ of $\mathrm{HCl}(1: 1)$, neutralize and add $2 \mathrm{c} \mathrm{c}$ in excess of $\mathrm{NH}_{4} \mathrm{OH}$, precipitate, and filter off the sulphides as before, then combine the filtrates.

If the alloy contains manganese the ignited phosphate should be dissolved and tested for manganese by the $(a)$ persulphate method as described in the determination of manganese in the analysis of aluminum, deduction being made for pyrophosphate corresponding to the manganese found.

Determination of Zinc

(a) Zinc oxide method.-(For samples containing 1 per cent of zinc or less.)

\section{SOLUTIONS REQUIRED}

Hydrochloric acid $(1: 1)$.- Mix equal volumes of $\mathrm{HCl}$ (specific gravity 1.19) and distilled water.

Acid wash water.-Slowly stir 10 c c of $\mathrm{H}_{2} \mathrm{SO}_{4}$ (specific gravity 1.84) into $1,000 \mathrm{c}$ c of distilled water, and saturate with $\mathrm{H}_{2} \mathrm{~S}$.

Formic acid mixture.-Treat 200 c c of formic acid with $30 \mathrm{c} \mathrm{c}$ of $\mathrm{NH}_{4} \mathrm{OH}$ (specific gravity 0.90) and dilute to $1,000 \mathrm{c}$ c.

Formic acid wash water.-Mix 25 c c of formic acid mixture and $1,000 \mathrm{c} \mathrm{c}$ of distilled water and saturate with $\mathrm{H}_{2} \mathrm{~S}$.

Hydrochloric acid $(1: 10)$.- Mix $100 \mathrm{c}$ c of concentrated $\mathrm{HCl}$ and $1,000 \mathrm{c} \mathrm{c}$ of distilled water.

\section{METHOD}

Dissolve $2 \mathrm{~g}$ of the sample in a covered $400 \mathrm{c}$ c beaker in $45 \mathrm{c}$ c of $\mathrm{HCl}(1: 1)$ added cautiously, followed by 1 c c of $\mathrm{HNO}_{3}$ (specific gravity 1.42) toward the end of the reaction. Boil to complete the solution and to expel oxides of nitrogen. Dilute to 200 c c, precipitate with $\mathrm{H}_{2} \mathrm{~S}$, filter, and wash with acid wash water. Boil thoroughly to expel $\mathrm{H}_{2} \mathrm{~S}$.

Cool somewhat, add $10 \mathrm{~g}$ of citric acid, dilute to $300 \mathrm{c} \mathrm{c}$, neutralize with $\mathrm{NH}_{4} \mathrm{OH}$ using methyl red as indicator, then add $25 \mathrm{c}$ c of the formic acid mixture. Heat nearly to boiling, and pass in a rapid stream of $\mathrm{H}_{2} \mathrm{~S}$ for 15 minutes. Filter through a close-textured paper set with a little of the liquid to be filtered. Refilter the first part if cloudy. Wash five times with the formic acid wash water. Dissolve the $\mathrm{ZnS}$ from the filter into the original beaker with bot $\mathrm{HCl}(1: 10)$, washing thoroughly with hot water. Boil the solution to expel $\mathrm{H}_{2} \mathrm{~S}$. Cool, add $1 \mathrm{~g}$ of citric acid and make just alkaline to methyl red with $\mathrm{NH}_{4} \mathrm{OH}$, dilute to $100 \mathrm{c} \mathrm{c}$, heat, and pass in $\mathrm{H}_{2} \mathrm{~S}$ for three minutes. Add $10 \mathrm{c} \mathrm{c}$ of the formic acid mixture and continue the $\mathrm{H}_{2} \mathrm{~S}$ for five minutes more. Filter through a close-textured. ashless paper set with a little of the liquid to be filtered, and wash five times with the formic acic wash water. To avoid creeping of the precipitate 
do not fill the paper too near to its top, and do not wet the funnel above the paper.

Ignite the paper and precipitate in a weighed porcelain or vitreosil crucible, starting at a dull red heat and finishing at a moderate red. Cool, and weigh as $\mathrm{ZnO}$. Deduct a determined blank.

\section{$\mathrm{ZnO} \times 0.8034=\mathrm{Zn}$}

Fote-By starting the second sulphide precipitation in the alkaline condition adherence of sulphide to the container is a voided.

(b) Ferrocyanide method.-(For samples containing over 1 per cent of zinc.)

\section{SOLUTIONS REQUIRED}

Ferrous sulphate indicator.-Dissolve $0.2 \mathrm{~g}$ of $\mathrm{FeSO}_{4} \cdot\left(\mathrm{NH}_{4}\right)_{2} \mathrm{SO}_{4} \cdot 6 \mathrm{H}_{2} \mathrm{O}$ in $50 \mathrm{c}$ c of distilled water. Prepare fresh, as required.

Standard potassium ferrocyanide solution.-Dissolve approximately $22 \mathrm{~g}$ of $\mathrm{K}_{4} \mathrm{~F}_{e}(\mathrm{CN})_{6} \cdot 3 \mathrm{H}_{2} \mathrm{O}$ per liter of distilled water. It is necessary for this solution to contain a small amount of ferricyanide for indicator purposes. If the solution can be allowed to stand for several weeks enough ferricyanide will be formed by oxidation. For immediate use add $0.15 \mathrm{~g}$ of $\mathrm{K}_{3} \mathrm{FE}(\mathrm{CN})_{6}$ per liter. The ferrocyanide known solution is standardized by titrating solutions containing known amounts of pure zinc, in accordance with the directions for titration given in this method.

\section{METHOD}

If the zinc content is between 1 and 3 per cent dissolve $2 \mathrm{~g}$ of the sample in $45 \mathrm{c}$ c of the $\mathrm{HCl}$. (1:1). If over 3 per cent dissolve $1 \mathrm{~g}$ of the sample in $25 \mathrm{c} \mathrm{c}$ of the HCl. Proceed as in (a) zinc oxide method until the first $\mathrm{ZnS}$ precipitate has been washed. Return this precipitate on its paper to the original beaker and add $20 \mathrm{c}$ c of $\mathrm{HCl}(1: 1)$. When the sulphide has dissolved, filter, and wash with hot water. Boil the solution containing the zinc to expel $\mathrm{H}_{2} \mathrm{~S}$, cool, and wash down. Add two drops of methyl red solution ( 0.1 per cent), then neutralize with $\mathrm{NH}_{4} \mathrm{OH}$. Make the solution just acid again with $\mathrm{HCl}$ and add $3 \mathrm{c} \mathrm{c}$ of concentrated $\mathrm{HCl}$ in excess. Dilute to $200 \mathrm{c} \mathrm{c}$ and heat almost to boiling for titration.

Add five drops of the ferrous sulphate indicator then titrate with the standard potassium ferrocyanide solution. Shortly after starting, a blue color appears and obscures the methyl red. When the ferrocyanide has acted on all the zinc, the red tint reappears since the red is stronger than the light green which would otherwise show. Toward the end the standard solution should be added slowly, stirring vigorously after each addition. The end point is reached when an additional drop causes no visible change from blue to red.

Determination of Nickel by the Dimethylglyoxime Method

\section{SOLUTIONS REQUIRED}

Sulphuric acid (1:1).- Slowly stir 500 c c of $\mathrm{H}_{2} \mathrm{SO}_{4}$ (specific gravity 1.84 ) into distilled water, cool, and dilute to $1,000 \mathrm{c} \mathrm{c}$.

Hydrogen sulphide wash water.-Slowly stir $10 \mathrm{c} \mathrm{c}$ of $\mathrm{H}_{2} \mathrm{SO}_{4}$ (specific gravity 1.84) into $1,000 \mathrm{c}$ c of distilled water and saturate with $\mathrm{H}_{2} \mathrm{~S}$.

Tartaric acid solution (20 per cent).-Dissolve 20 $\mathrm{g}$ of tartaric acid in distilled water, dilute to $100 \mathrm{c} \mathrm{c}$ and filter if necessary.

Dimethylglyoxime solution (1 per cent).-Dissolve $10 \mathrm{~g}$ of dimethylglyoxime in $1,000 \mathrm{c}$ c of alcohol (95 per cent).

\section{METHOD}

Dissolve $1 \mathrm{~g}$ of the sample in $25 \mathrm{c}$ c of $\mathrm{H}_{2} \mathrm{SO}_{4}(1: 1)$ by warming and adding a drop or two of $\mathrm{HNO}_{3}$ (specific gravity 1.42) occasionally until solution is complete. After oxides of nitrogen have been expelled by boiling, add $14 \mathrm{c}$ c of $\mathrm{NH}_{4} \mathrm{OH}$ (specific gravity 0.90 ) in order to bring down the acidity to approximately $5 \mathrm{c}$ c of $\mathrm{H}_{2} \mathrm{SO}_{4}$ (specific gravity 1.84) per $100 \mathrm{c}$ c of solution. Treat with $\mathrm{H}_{2} \mathrm{~S}$. Filter off the sulphides, wash with hydrogen sulphide wash water and boil the filtrate until $\mathrm{H}_{2} \mathrm{~S}$ is expelled. Add a few crystals of $\left(\mathrm{NH}_{4}\right)_{2} \mathrm{~S}_{2} \mathrm{O}_{8}$ and boil thoroughly to destroy sulphur and oxidize iron. Add $20 \mathrm{c} c$ of tartaric acid solution and make slightly ammoniacal. If no precipitate appears add $\mathrm{HCl}$ (specific gravity 1.19) to slight acidity. If a precipitate appears, dissolve in $\mathrm{HCl}$, add $10 \mathrm{c}$ c of tartaric acid and again make ammoniacal, repeating the operation, if necessary, to get a clear solution. To the weakly acid solution add dimethylglyoxime solution in such an amount that the ratio of the reagent to nickel is at least four to one. Heat the solution to boiling and make slightly ammoniacal. Allow to digest, while cooling, for two hours. Collect on a weighed Gooch crucible and wash thoroughly with hot water. Dry for 45 minutes at from $110^{\circ}$ to $120^{\circ} \mathrm{C}$. and weigh. The nickel dimethylglyoxime contains 20.32 per cent of nickel.

(b) Alloys Containing Over 1 Per Cent of SILICON

\section{Determination of Silicon}

SOLUTIONS REQUIRED

See the determination of total silicon under the analysis of aluminum.

\section{METHOD}

If the silicon content is under 10 per cent use a $1 \mathrm{~g}$ sample and if a content of higher percentage is expected use an $0.5 \mathrm{~g}$ sample. Place the sample in a 350-c c casserole. Keeping covered as much as possible, cautiously add $35 \mathrm{c}$ c of the acid mixture. When no further action can be seen evaporate to dryness, taking care to prevent spattering. Heat to strong fuming of $\mathrm{SO}_{3}$. Cool, moisten with $10 \mathrm{c} \mathrm{c}$ of $\mathrm{H}_{2} \mathrm{SO}_{4}$ (25 per cent), add $100 \mathrm{c}$ c of hot water, and boil to dissolve the salts. Stir in some ashless paper pulp, then filter through a close-textured paper. Wasn three or four times with hot water. Evaporate the filtrate to fumes, cool, dissolve with water, add paper pulp, filter, and wash as before.

Ignite the residues in a large platinum crucible. After cooling, add from 5 to $8 \mathrm{~g}$ (depending on the amount of residue) of $\mathrm{Na}_{2} \mathrm{CO}_{3}$. Stir well and cover with a layer of $\mathrm{Na}_{2} \mathrm{CO}_{3}$. Fuse cautiously till nearly quiet, then finish with a full Meker flame or its equivalent. Run the melt up the sides of the crucible, cool, and place in a casserole with 60 to $80 \mathrm{c} \mathrm{c}$ of $\mathrm{H}_{2} \mathrm{SO}_{4}$ (25 per cent). When the melt has dissolved remove the crucible, washing it into the casserole. Evaporate, and finally heat till heavy fumes are given off, then remove from the heat. When still moderatel $y$ warm add a little cold water followed by $100 \mathrm{c} \mathrm{c}$ of hot water. Heat to complete solution of soluble salts, but avoid too long a treatment as the $\mathrm{SiO}_{2}$ tends to redissolve. Filter the liquid, preferably after stirring in some paper pulp, and wash thoroughly with hot water.

Evaporate the filtrate and heat to fuming again to separate any silica which may have escaped the 
first treatment. This is collected in the same manner as the main portion.

Dry the filters with contents, then ignite in a platinum crucible, cool, and weigh. Moisten with a few drops of $\mathrm{H}_{2} \mathrm{SO}_{4}(1: 3)$ and add several cubic centimeters of HF. Evaporate until dry, ignite, cool, and weigh again. The loss in weight represents $\mathrm{SiO}_{2}$. After correcting for a determined blank multiply by 0.4672 to find the amount of $\mathrm{Si}$ in the sample taken.

Note.-In diluting the solution after the fuming which succeeds the fusion, adding a little cold water to the warm solution throws out the silica in a more granular form which is less soluble and more convenient to filter.

\section{Determination of Iron}

\section{METHOD}

Dissolve $1 \mathrm{~g}$ of the sample in the acid mixture and proceed as under determination of total silicon under analysis of aluminum till the first insoluble residue is ignited. Treat this with a little concentrated $\mathrm{HNO}_{3}$ and $\mathrm{HF}$ to remove the silicon, then evaporate to dryness. Fuse the unvolatilized part with $\mathrm{K}_{2} \mathrm{~S}_{2} \mathrm{O}_{7}$, dissolve, and add to the main solution. From this point continue by the sulphide separation method as described under determination of iron under analysis of aluminum.

\section{Determination of Copper}

\section{METHOD}

Dissolve $5 \mathrm{~g}$ of the sample in a large casserole or beaker by adding, in small portions, $150 \mathrm{c}$ c of the acid mixture used for starting the silicon determination. When the aluminum is dissolved, evaporate to dryness and heat until copious fumes of $\mathrm{H}_{2} \mathrm{SO}_{4}$ are given off, cool, dissolve by heating with $300 \mathrm{c} \mathrm{c}$ of water, filter, and wash a few times.

Ignite the dark residue in a platinum dish, add $10 \mathrm{c}$ c of $\mathrm{HNO}_{3}$ (specific gravity 1.42), 2 c c of $\mathrm{H}_{2} \mathrm{SO}_{4}(1: 1)$, and then add $\mathrm{HF}$ drop by drop until the silicon has dissolved. Evaporate to fumes to remove the $\mathrm{HF}$. Take up with water and add to the main filtrate.

Pass $\mathrm{H}_{2} \mathrm{~S}$ into this liquid for several minutes, and filter off the precipitated CuS, washing with 1 per cent $\mathrm{H}_{2} \mathrm{SO}_{4}$ saturated with $\mathrm{H}_{2} \mathrm{~S}$. Ignite the precipitate in a porcelain crucible, cool, dissolve by heating with some $\mathrm{HNO}_{3}(1: 1)$, transfer to a suitable beaker and finish electrolytically as under determination of copper by electrolytic method under the analysis of aluminum.

\section{Determination of Manganese}

\section{METHOD}

Proceed as under determination of manganese, (a) persulphate method, under the analysis of aluminum, except that a few drops of HF should be added to remove the silicon when dissolving the sample. After the solution is complete boil a short time to remove most of the $\mathrm{HF}$, then proceed as usual.

\section{AMERICAN SOCIETY FOR TESTING MA- TERIALS, TENTATIVE METHODS OF TENSION TESTING OF METALIIC MA- TERIALS, DESIGNATION E 8-27T, 1927}

\section{TENSION TESTING OF METALIIC MATERIAIS}

(See 600.1, p. 34.)
AMERICAN SOCIETY FOR TESTING MATERIALS, TENTATIVE METHODS OF COMPRESSION TESTING OF METALIIC MATERIALS, DESIGNATION E 9-27T, 1927

\section{COMPRESSION TESTING OF METALLIC MATE- RIALS}

(See 600.1, p. 38.)

UNITED STATES GOVERNMENT, DEPARTMENT OF COMMERCE, BUREAU OF STANDARDS, STANDARD SAMPLES, CIRCULAR NO. 25 AND SUPPLEMENT, 1927

\section{ALUMINUM SAMPLE}

(Sample No. 44b is a standard sample of aluminum metal prepared and sold by the Bureau of Standards for use by industrial organizations and others as a standard for calibrating pyrometers, etc. Each sample is accompanied by a certificate giving the melting point of that particular sample.)

(Circular No. 66 of the Bureau of Standards gives information on the preparation of these samples and the method of use.)

\subsection{ALUMINUM AND ALUMINUM ALLOY INGOTS AND BARS.}

\subsection{ALLOYS, ALUMINUM.}

SOCIETY OF AUTOMOTIVE ENGINEERS, NONFERROUS METAL SPECIFICATIONS : ALLOYS NOS. 26, 27, 28, AND 29; SPECIFICATIONS NOS. 30, 31, 31A, 32, 33, 34, $35,36,37,38$, AND 39, ALUMINUM ALLOYS, JULY, 1930

\section{ALUMINUM ALLOYS}

ALIOY NO. 28

1. Composition in percentagePer cen

Copper Magnesium . . Manganese........ 4 1.0 Aluminum, not less than......................... 92.0

2. General information.-This alloy is the alloy commonly designated "duralumin," "dural," or "17S." It is used in the wrought condition either rolled, drawn-forged, or extruded. In the form of sheet this alloy is supplied to the following mechanical property requirements:

3. Duralumin or $17 \mathrm{~S}$ sheet, heat-treated

\begin{tabular}{|c|r|r|r|}
\hline Thickness & $\begin{array}{c}\text { Minimum } \\
\text { tensile } \\
\text { strength }\end{array}$ & $\begin{array}{c}\text { Minimum } \\
\text { vield } \\
\text { point }\end{array}$ & $\begin{array}{r}\text { Minimum } \\
\text { elongation } \\
\text { in 2 inches }\end{array}$ \\
\hline Inches & Lbs./in.2 & Lbs./in.2 & Per cent \\
$0.010-0.020$ & 55,000 & 30,000 & 15 \\
$.021-.128$ & 55,000 & 30,000 & 118 \\
$.129-.258$ & 55,000 & 30,000 & 15 \\
$.259-.500$ & 55,000 & 30,000 & 12 \\
$.501-1.75$ & 55,000 & 30,000 & 9 \\
\hline
\end{tabular}

1 Sheets 30 inches or more in width in thickness of 0.021 to 0.04 inch may show a minimum elongation of 17 per cent. 
4. In the annealed temper, the tensile strength shall not exceed 35,000 pounds per square inch.

5. The following thickness tolerances are commercial for this product:

Thickness tolerances for duralumin or $17 \mathrm{~S}$ sheet

\begin{tabular}{|c|c|c|c|}
\hline \multirow[b]{2}{*}{$\begin{array}{l}\text { Thickness } \\
\text { (inch) }\end{array}$} & \multicolumn{3}{|c|}{ Plus or minus tolerances, widths } \\
\hline & $\begin{array}{l}18 \text { inches } \\
\text { and less }\end{array}$ & $\begin{array}{l}\text { Over } 18 \\
\text { inches to } \\
36 \text { inches }\end{array}$ & $\begin{array}{l}\text { Over } 36 \\
\text { inches }\end{array}$ \\
\hline $\begin{array}{l}0.013-0.036 \\
0.037-0.050 \\
0.051-0.070 \\
0.071-0.090 \\
0.092 \text { and heavier. }\end{array}$ & $\begin{array}{l}\text { Inch } \\
0.0015 \\
.002 \\
.0025 \\
.003 \\
5 \% T\end{array}$ & $\begin{array}{l}\text { Inch } \\
0.002 \\
.0025 \\
.003 \\
.003 \\
5 \% T\end{array}$ & $\begin{array}{l}\text { Inch } \\
0.0025 \\
.003 \\
.004 \\
.004 \\
\end{array}$ \\
\hline
\end{tabular}

( $T=$ nominal thickness of sheet.)

6 . In the form of tubing and extruded shapes, the same tensile strength and yield point are obtained, the elongation varying with the cross-sectional area of the test section. Rod and bar will also show these same properties, except in certain of the larger sizes, where manufacturing limitations may result in slightly lower values.

7. This alloy is extensively used in the construction of motor coaches and trucks, and aircraft (both heavier and lighter than air). It is available in all the forms in which metals are commonly used-sheet, plate, strip, tubing (including streamline and other special shapes), bar, rod, wire, rivets, bolts, nuts, screws and other screw-machine products, structural shapes, and extruded sections specially designed for aircraft construction.

\section{ALLOY NO. 27}

\section{Composition in percentage.-}

Copper

Per cent

Manganese 3. $9-5.0$

Silicon-..................

. $5-1.1$

$5-1.1$

2. General information.-This alloy is known as $25 \mathrm{~S}$ and, like duralumin, is used in the wrought condition. In the fully heat-treated temper, the tensile strength is from 55,000 to 63,000 pounds per square inch. The elongation, except for very thin or very heavy sections, is between 16 and 22 per cent in 2 inches. If the alloy be given the solution heat treatment, but not the precipitation heat treatment, it is in a more workable condition and has the following properties:

Tensile strength Yield-point _. Elongation in 2 inches..............

3. In the annealed temper the tensile strength varies from 25,000 to 35,000 pounds per square inch.

4. This alloy is commonly available in a variety of forms, including sheet, plate, bar, and structural shapes. It is especially adapted for the manufacture of forgings, because of its excellent hotworking properties. Forgings of this alloy in common use include connecting rods for automobile engines, propellers for aircraft, automobile hardware, and fittings of various types.

\section{ALIOY NO. 28}

\section{Composition in percentage.-}

Magnesium

Per cent

Sluminum, not less than.$0.45-0.9$

$6-1.2$
96.3
2. General information.-This alloy is designated in the trade as 51S. It is used in the wrought form and is one of the alloys susceptible to heat treatment. In the fully heat-treated temper (designated as $T$ ), the tensile strength is from 45,000 to 50,000 pounds per square inch; the elongation varies from 10 to 18 per cent in 2 inches, except in very thin or very heavy sections. The yieldpoint is from 30,000 to 40,000 pounds per square inch, substantially the same as duralumin or $25 \mathrm{~S}$, although the ultimate strength is about 10,000 pounds lower than that of these alloys. If the alloy is not subjected to the precipitation heat treatment after it has been heated and quenched, it has a tensile strength in the range 30,000 to 40,000 pounds per square inch, and an elongation of 20 to 30 per cent in 2 inches. In this temper (designated $W$ ) the alloy can be formed with considerable ease. In the annealed temper the tensile strength varies from 14,000 to 19,000 pounds per square inch, and the elongation from 22 to 32 per cent in 2 inches.

3. This alloy is available in practically all the wrought forms in which aluminum and other metals are produced; these include sheet, plate, tubing, rod, bar, wire, shapes, moldings, both rolled and extruded, forgings, etc.

4. Because of the readiness with which this alloy can be worked hot, it is used for the manufacture of complicated forgings, such as radial aircraft-engine crank cases. It is also used for less highly stressed parts, such as automobile hardware.

ALLOY NO. 29

\section{Composition in percentage.-}

Manganese

Per cent

Copper, not over $1.0-1.5$

2. General information.-This alloy is used to replace pure aluminum where somewhat higher tensile strength and hardness are desired. It is not quite so easily formed as is commercial aluminum in the corresponding tempers. This alloy shows substantially the same resistance to atmospheric corrosion and to mist forms of chemical attack as does aluminum of commercial purity.

3. As is the case with commercial aluminum, the various tempers are obtained b; varying the amount of cold work done on the alloy after its final annealing. It is regularly manufactured in the form of sheet, plate, tubing, rod, bar, and wire, extruded shapes, moldings, etc.

4. The alloy in the various standard tempers has the tensile strengths shown in the following table. The elongation values for flat sheet are also shown. In the case of strip sheet in the half-hard temper, the elongation may be slightly lower than the values shown for flat sheet.

\begin{tabular}{|c|c|c|c|c|c|c|c|c|}
\hline \multirow{3}{*}{ Temper } & \multirow{3}{*}{$\begin{array}{c}\text { Tensile } \\
\text { strength, } \\
\text { mini- } \\
\text { mum }\end{array}$} & \multicolumn{7}{|c|}{ Thickness (inch) } \\
\hline & & $\left|\begin{array}{c}0.013 \\
t_{0} \\
0.019\end{array}\right|$ & $\begin{array}{l}0.020 \\
\text { to } \\
0.031\end{array}$ & $\mid \begin{array}{c}0.032 \\
\text { to } \\
0.050\end{array}$ & $\left|\begin{array}{c}0.051 \\
\text { to } \\
0.113\end{array}\right|$ & $\mid \begin{array}{c}0.114 \\
\text { to } \\
0.162\end{array}$ & $\begin{array}{l}0.163 \\
\text { to } \\
0.258\end{array}$ & $\begin{array}{l}3.259 \\
\text { to } \\
0.375\end{array}$ \\
\hline & & \multicolumn{7}{|c|}{ Elongation in 2 inches } \\
\hline Soft & Lbs./in. ${ }^{2}$ & P.ct. & P.ct. & P.ct. & P.ct. & P.ct. & P.ct. & P. ct. \\
\hline One-half hard........ & 19,500 & $\begin{array}{r}20 \\
3\end{array}$ & $\begin{array}{r}20 \\
4\end{array}$ & $\begin{array}{r}20 \\
5\end{array}$ & $\begin{array}{r}20 \\
6\end{array}$ & 7 & 8 & 8 \\
\hline Three-fourths hard. & 24,000 & 1 & 2 & 3 & 4 &.---- & $-\ldots$ & $-\ldots$ \\
\hline Hard & 27,000 & 1 & 2 & 3 & 4 & $-\ldots$ & $-\ldots$ & $-\ldots$ \\
\hline
\end{tabular}


(Thickness tolerances for flat sheet and for strip sheet are identical with those tabulated in S. A. E. specification No. 78. See 631.23, p. 1156.)

\section{SPECIFICATION NO. 30}

\section{Composition in percentage.-}

Copper

Zinc, maximum.

Silicon, iron,

Silicon, iron, zinc, manganese, and tin, maximum

Other impurities

Aluminum
Per cent

7. $00-8.50$

.20

1. 70
None.

Remainder.
2. General information.-The tensile strength of test specimens about one-half inch in diameter of this alloy cast in sand and tested without machining off the skin should be ab sut 18,000 to 20,000 pounds per square inch, and the elongation 1 to 2 per cent in 2 inches.

3. This is a light alloy, having a specific gravity of about 2.83, and is used more extensively in the automotive industry than all other light castirg alloys ccmbined. A shrinkage of $0.156(5 / 32)$ inch per foot should be allowed in pattern designs. Thi alloy is used for crank cases, oil pans, steering-wheel spiders, differential carriers, transmission cases, cam-shaft housings, hub caps, and similar parts.

4. For more difficult castings, alloy No. 36, which has similar mechanical properties and superior casting characteristics, is now commonly specified.

\section{SPECIFICATION NO. S1}

\section{Composition in percentage.-}

\section{Copper}

Silicon, iron, manganese, and tin, maximum.

Other impurities

Aluminum

Per cent

2. $25-3.25$

12. $50-14.50$

1. 70

Remainder.

2. General information.-The tensile strength of test specimens about one-half inch in diameter of this alloy cast in sand and tested without machining off the skin should be about 25,000 to 30,000 pounds per square inch, with an elongation of more than 1 per cent in 2 inches.

3 . The specific gravity is about 3.0 , and a shrinkage of $0.156(5 / 32)$ inch per foot should be allowed in pattern designs.

4. This alloy is used extensively in England for such parts as crank cases, oil pans, steering-wheel spiders, and transmission cases.

\section{SPECIFICATION NO. S1A}

\section{Composition in percentage.-}

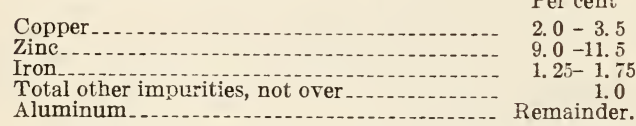

2. General information.-Test specimens cast ir sand and tested without machining give tensile strengths in the range 25,000 to 36,000 pounds per square inch and elongations from 3 to 7 per cent in 2 inches.

3. The specific gravity is about 3.0. A shrinkage of $0.156(5 / 32)$ inch per foot should be allowed in pattern designs.

4. This alloy is used where somewhat higher me chanical properties are desired than are obtained with alloys Nos. 30,33 , and 36 . In common with other alloys containing considerable percentages of zinc, this alloy loses its strength more rapidly as the temperature is raised than do some of the other alloys, hence it is not recommended for use at elevated temperatures.
SPECIFICATION NO. 32

\section{Composition in percentage.-}

Copper

Per cent

11. $00-13.50$

Zinc, maximum

Silicon, iron, zinc, manganese, and tin, maximum. .20

Other impurities

1. 70

Remainder.

2. General information. - The tensile strength of test specimens about one half inch in diameter of this alloy cast in sand and tested without machining off the skin should be about 19,000 to 23,000 pounds per square inch, and the elongation will be practically nothing.

\section{SPECIFICATION NO. 33}

\section{Composition in percentage.-}

Copper

Zine, not over

silicon, not over

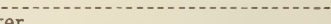

Total other impurities, not orer

Aluminum

2. General information.-This is a light alloy, having a specific eravity of 2.83 to 2.86 , and has been the most extensively used alloy in the automotive industry. It is similar to specifications Nos. 30 and 36 , and is used for crank cases, oil pans, steeringwheel spiders, differential carriers, transmission cases, cam-shaft housings, hub caps, and similar parts. A shrinkage of $0.156(5 / 32)$ inch per foot should be allowed in pattern designs.

3. Test specimens cast in sand and tested without machining show tensile strengths in the range 19,000 to 24,000 pounds per square inch and elongation from 1.0 to 2.5 per cent in 2 inches. It is customary to omit elongation requirements from specifications for this alloy.

\section{SPECIFICATION NO 0.34}

\section{Composition in percentage.-}

Copper

Iron

Ill other elements, not orer-a-s-a

Aluminum

2. General information.-Test-bars cast in a chill mold show a tensile strength of 24,000 to 30,000 pounds per square inch, and the elongation in 2 inches is usually less than 1 per cent. The specific gravity should not exceed 2.95. The Brinell hardness number, using a 500 or $1,000 \mathrm{~kg}$ load with a ball $10 \mathrm{~mm}$ in diameter, should not be less than 85 , and should average about 105 .

3. This alloy cast in permanent molds is used principally for pistons, cam-shaft bearings, valve tappet guides, and other parts where high hardness and good bearing qualities are essential.

\section{SPECIFICATION NO. 35}

\section{Composition in percentage.-}

\section{Silicon.}

Copper, not over

Iron, not over

Zinc, not over

Manganese, not over

Iagnesium, not over

therinumes, not

2. General information.-The minimum tensile strength of test specimens about one-half inch in diameter of this alloy cast in sand and tested without machining off the skin should be about 16,000 pounds per square inch, with a minimum elongation of 3.5 per cent in 2 inches. 
3. This alloy is intended for automobile body parts and other parts that must be cast in thin sections. The alloy withstands salt-water corrosion very well and is therefore suitable for aircraftengine parts or other parts that may be subjected to severe corroding influences. The alloy has a relatively low yield point and therefore can not be used where great strength or stiffness is required.

\section{SPECIFICATION NO. 36}

1. Composition in percentage.-

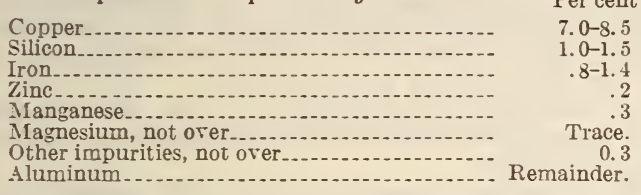

2. General information.-Standard tensile test specimens cast in sand and tested without machining give tensile strengths in the range 19,000 to 24,000 pounds per square inch. The elongation is usually from 1 to 3 per cent in 2 inches, but because of the difficulty of measuring elongations in this range with accuracy it is customary to omit elongation requirements from specifications for this alloy.

3. This alloy was developed from alloy No. 30 , and differs from it only in that iron and silicon are added in carefully controlled amounts instead of being allowed to vary at random in the range permitted by the limit on impurities. In this alloy iron and silicon are definite alloying constituents, and are added to improve the casting characteristics of the old aluminum-copper casting alloy. With this alloy the tendency for cracks and shrinks is very greatly decreased. It has largely superseded No. 30 , particularly for more difficult castings that require pouring temperatures higher than are usually used.

4. This is a light alloy, having a specific gravity of about 2.83, and is commonly used where alloy No. 30 has previously been specified. A shrinkage of $0.156(5 / 32)$ inch per foot should be allowed in pattern design. This alloy and No. 33 are most commonly used for casting crank cases, oil pans, differential carriers, transmission cases, and other such castings.

SPECIFICATION NO. 37

\section{Composition in percentage.-}

Silicon.

Iron, not over

(n)

Zinc not over

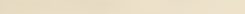

Manganese, not over

Magnesium, not over

Total other impurities, not over.

Aluminum.

Per cent 12. $0-13.0$

\section{.8
.3}

5

Trace. Remainder.

2. General information.-Standard test bars cast to size in sand show tensile strengths from 24,000 to 31,000 pounds per square inch, and elongations from 5.0 to 15.0 per cent. These properties are obtained only if the molten alloy is subjected to a special process, known as "modification," immediately before it is poured. The specific gravity is 2.68 .

3. Like alloy No. 35 , this alloy is especially resistant to salt-water corrosion. Because of its good foundry characteristics it can be used for complicated castings consisting of both thin and heavy sections. The ratio of yield point to ultimate tensile strength is somewhat lower than is the case with some of the other aluminum casting alloys, which fact should be considered in connection with the properties shown in the preceeding paragraph.

\section{SPECIFICATION NO. 38 (בEAT-TREATED CASTINGS)}

\section{Composition in percentage-}

Copper-

Per cent

Silicon, not over

Iron, not over - -

Zinc, not over

Total of all constituents except aluminum and copper, not over.

2. General information.-Castings from this alloy can be heat treated to produce mechanical properties distinctly higher than those of the ordinary aluminum casting alloys. The heat treatment may be varied, depending upon the service requirements.

3 . Where maximum toughness and resistance to shock are desired, a solution heat-treatment alone is used which produces in sand-cast test specimens tensile strengths from 28,000 to 38,000 pounds per square inch, and elongations from 6 to 12 per cent in 2 inches. On standing at room temperatures there is an aging effect which is practically complete in a few months. The tensile strength increases by a few thousand pounds per square inch, and the elongation decreases by a few per cent in 2 inches. The greatest change occurs in the yield point, which increases to a value usually well in excess of 20,000 pounds per square inch.

4. If somewhat greater initial strength, hardness, and yield point are desired, the solution heat treatment may be followed by a precipitation heat treatment in which tensile strength from 30,000 to 40,000 pounds per square inch, and elongations from 3 to 8 per cent in 2 inches are developed.

5. If still greater strength and hardness are desired, the heat treatment may be varied to produce tensile strengths from 36,000 to 50,000 pounds per square inch, and elongations from 0 to 5 per cent in 2 inches.

6. Since the introduction of this alloy its use has developed into very substantial tonnages. It is used in windshield frames, fire engine, motor coach engine, and aircraft engine crank cases, and for a variety of parts in both motor vehicle and aircraft assemblies, where a high-strength light-weight casting is desired. It is very much more resistant to corrosion than the ordinary alloys containing copper, and is extensively used in the manufacture of outboard motors and for castings to be used on board ships.

SPECIFICATION NO. 39

\section{Composition in percentage-}

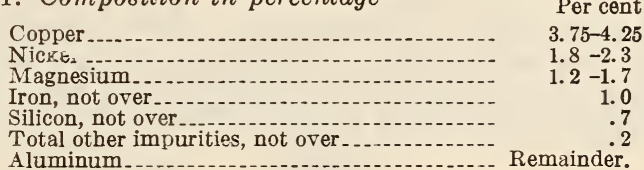

Aluminum Remainder.

2. General information.--Standard test specimens cast to size in sand, after proper heat treatment, show tensile strengths from 30,000 to 42,000 pounds per square inch, and elongations from 0 to 2 per cent. If the alloy is not heat treated, the tensile strength is about one-third less than these values. Because of its property of retaining its strength better at elevated temperatures than do many of the other aluminum alloys, it is used for pistons, and cylinder-heads of aircraft engines and for other parts where this property is an advatage. 
631.12 INGOTS, ALUMINUM.

\section{AMERICAN SOCIETY FOR TESTING MATE- RIALS, STANDARD SPECIFICATIONS FOR ALUMINUM INGOTS FOR REMELTING, DESIGNATION B24-29, 1929}

\section{ALUMINUM INGOTS}

\section{SCOPE}

1. These specifications cover three grades of virgin aluminum, as follows:

\begin{tabular}{|c|c|}
\hline Grade & $\begin{array}{c}\text { Aluminum } \\
\text { minimum }\end{array}$ \\
\cline { 2 - 2 } & Per cent \\
\hline & 99.5 \\
99.5 & 99.0 \\
98.5 & 98.5 \\
\hline
\end{tabular}

2. The presence in any of the above grades of more than a trace of manganese, magnesium, zinc, calcium, or similar metals commonly used in the light alloys, is not admissible, and such ingot can not be classed as virgin aluminum under these specifications. Copper is an allowable impurity up to a maximum of 0.1 per cent in the 99.5 grade; 0.25 per cent in the 99.0 grade; and 0.45 per cent in the 98.5 grade. Iron and silicon are also allowable impurities. The sum total of the impurities, however, shall not exceed 0.5 per cent in the 99.5 grade, 1.0 per cent in the 99.0 grade, nor 1.5 per cent in the 98.5 grade.

\section{MANUFACTURE}

3. No scrap shall be used other than that resulting in the manufacturer's own plant from the production of material of composition similar to that specified.

\section{PHYSICAL DEFECTS}

4. Aluminum ingots shall have a clean surface and shall be free from dirt, slag, and foreign material.

\section{SAMPLING}

5. If the aluminum is shipped in carload lots, then random samples shall be taken, but not less than five ingots for each car. If the shipment is in less than carload lots, one ingot shall be taken for each 10,000 pounds $(4,536 \mathrm{~kg})$ or fractional part thereof. When it is deemed necessary, a sample may be taken from each heat of 500 pounds (226.8 $\mathrm{kg}$ ) or more of metal.

6. The ingots selected shall be drilled without lubricant completely through, or halfway through from top and bottom. The drillings shall be fine, thin chips. They shall be thoroughly mixed and the sample for analysis shall not be less than $60 \mathrm{~g}$.

\section{ANAIYSES}

7. The chemical analysis shall be made in accordance with the tentative methods of chemical analysis of aluminum and light aluminum alloys in A. S. T. M. B40. (See 631.0, p. 1141.)

\section{MARKING}

8. The maker's brand and the grade of aluminum shall be cast on each ingot. When this is not practicable, the grade shall be indicated by spotting each large ingot, or one ignot in each bundle of small ingots, with a designating color of painting-white indicating the 99.5 grade; blue, the 99.0 grade; and red the 98.5 grade.

\section{CLAIMS}

(Same as B30. See 644.11, p. 1196.)
AMERICAN SOCIETY FOR TESTING MATERIAIS, STANDARD SPECIFICATIONS FOR ALUMINUM FOR USE IN THE MANUFACTURE OF IRON AND STEEL, DESIGNATION B37-26, 1926

\section{ALUMINUM FOR STEEL MANUFACTURE}

\section{SCOPE}

1. These specifications cover two grades of virgin and four grades of secondary aluminum, as follows:

(a) Virgin aluminum.-(1) 98.0 grade, containing not less than 98 per cent aluminum.

(2) 94.0 grade, containing not less than 94 per cent aluminum. The remainder may be chiefly iron and silicon.

(b) Secondary aluminum.-(1) 98.0 grade, containing not less than 98 per cent aluminum. This grade is generally made from sheet clippings. The remainder may be chiefly copper, iron, and silicon.

(2) 94.0 grade, containing not less than 94 per cent aluminum. A maximum of 0.5 per cent of zinc will be allowed in this grade. The remainder may be chiefly copper.

(3) 90.0-A grade, containing not less than 90 per cent aluminum. A maximum of 0.5 per cent of zinc will be allowed in this grade. The remainder may be chiefly copper.

(4) 90.0-B grade, containing not less than 90 per cent aluminum. A maximum of 4 per cent of zinc will be allowed in this grade. The remainder may be chiefly copper.

\section{MANUFACTURE}

2. The manufacturer shall use care to have each lot of metal as uniform as possible.

3. The aluminum may be in the form of ingots, rods, or shot.

Ingots should be of convenient size for use. Ingots with a minimum of five notches, weighing approximately 3 pounds, will meet the requirements of these specifications.

Rods may be cither rolled or cast and threeeighth to one-half inch in diameter.

Shot should be of convenient size. That produced by running the molten metal through oneeighth inch diameter holes is satisfactory.

\section{SAMPLING}

(Requirements covering sampling are the same as in A. S. T. M. B24, given above, except for the omission from B37 of taking samples from each heat of 500 pounds or more of metal, and the addition of the following two items:)

5. Rods shall be sampled in a similar manner, except that the drilling shall be radial.

An equivalent amount of shot shall be taken as a sample and reduced by quartering to a sample of convenient size.

\section{ANALYSES}

(Same as in A. S. T. M. B40-28T. See 631.0, p. 1141.)

\section{MARKING}

7. The maker's brand and the grade of aluminum shall be cast or rolled on each ingot or rod and marked plainly on each container of shot metal. When marking the grade by casting or rolling is not practicable, the grade shall be indicated by spotting each large ingot or one ingot or rod from each bundle with a designating color of paint, red indicating the 98.0 virgin grade, yellow the 94.0 virgin grade, red and white the secondary 98.0 grade, red and yellow the secondary 94.0 grade, 
red and blue the secondary $90.0-\mathrm{A}$ grade, and red and black the secondary $90.0-\mathrm{B}$ grade.

\section{CLAIMS}

(Same as in A. S. T. M. B51-27. See 682, p. 1307.)

\section{AMERICAN SOCIETY FOR TESTING MATE- RIALS, SPECIFICATIONS FOR ALU- MINUM-BASE SAND-CASTING ALLOYS IN INGOT FORM, DESIGNATION B58- 31,1931}

\section{ALUMINUM-ALLOY INGOTS}

1. These specifications cover commercial aluminum-base sand-casting alloys in ingot form for remelting. The specific gravity of these alloys does not exceed 3 . Ten alloys are specified and are designated alloys $\mathrm{A}$ to $\mathrm{H}$, inclusive, and $\mathrm{J}$ and K. (See explanatory note.)

\section{MANUFACTURE}

2. The alloys may be made by any approved process.

3. The material covered by these specifications shall be of uniform quality, and shall be free from dross, slag, and other harmful contamination.

\section{CHEMICAL COMPOSITION AND TESTS}

4. (The alloys shall conform to the requirements as to chemical composition specified in Table 1 in A. S. T. M. B26-31 (see 631.41, p. 1160) except for percentage of iron, which is 0.2 per cent lower in B58.)

(Sampling requirements are identical with those in A. S. T. M. B10. See 643.4, p. 1192. Inspection and rejection requirements are identical with those in A. S. T. M. B59. See 647.15, p. 1259.)

Note. - Explanatory notes pertaining to these alloys when used in the form of sand castings are given in connection with the tentative specifications for aluminum-base alloy sand castings in A. S. T. M. B26-31. (See 631.41, p. 1160.)

UNITED STATES GOVERNMENT, FEDERAL SPECIFICATIONS BOARD, FEDERAL SPECIFICATION FOR ALUMINUM INGOTS, NO. 134, MAY 1, 1924. (CURRENT DESIGNATION : QQ-A-451)

\section{ALUMINUM INGOTS}

(This specification is the same as A. S. T. M. serial designation B24-29, given above, with the exception that only two grades of aluminum ingots are specified herein while B24-29 covers three grades. The chemical composition of like grades in both specifications is practically identical. In this speci- fication grade A corresponds to the 99.0 grade, and grade B to the 98.0 grade in B24-29.)

631.13 BARS, ALUMINUM.

SOCIETY OF AUTOMOTIVE ENGINEERS, NONFERROUS METAL SPECIFICATIONS, ALUMINUM ALLOYS, ALLOYS NOS. 26, 27, 28, AND 29, JULY, 1930

\section{ALUMINUM-ALLOY BARS}

(See 631.11, p. 1146.)

631.2 ALUMINUM AND ALUMINUMALLOY PLATES, SHEETS, SHAPES, AND STRIPS.

631.21 PLATES, ALUMINUM.

SOCIETY OF AUTOMOTIVE ENGINEERS, NONFERROUS METAL SPECIFICATIONS, ALUMINUM ALLOYS, ALLOYS NOS. 26, 27, 28, AND 29, JULY, 1930

\section{ALUMINUM-ALLOY PLATES}

(See 631.11, p. 1146.)

UNITED STATES GOVERNMENT, DEPARTMENT OF COMMERCE, BUREAU OF STANDARDS, STANDARD THICKNESSES, WEIGHTS, AND TOLERANCES OF SHEET METAL (CUSTOIMARY PRACTICE) CIRCULAR NO. 391, MARCH 24, 1931

AMERICAN WIRE GAGE-ALUMINUM PLATES

(See 640.1, p. 1165.)

631.22 SHAPES, ALUMINUM.

SOCIETY OF AUTOMOTIVE ENGINEERS, NONFERROUS METAI SPECIFICATIONS, ALLOYS, ALUMINUM ALLOYS NOS. 26, 27, 28, AND 29, JULY, 1930

\section{ALUMINUM-ALLOY SHAPES}

(See 631.11, p. 1146.)

SOCIETY OF AUTOMOTIVE ENGINEERS, BODY PARTS AND MATERIALS; RECOMMENDED PRACTICE, ALUMINUM BELT MOLDING AND DRIP MOLDING, FEBRUARY, 1928

ALUMINUM BELT MOLDING AND DRIP MOLDING

(See 631.9, p. 1163.) 


\section{SOCIETY OF AUTOMOTIVE ENGINEERS, AERONAUTIC; EXTRUDED STRUCTURAL SHAPES, ALUMINUM ALLOY S. A. E. RECOMMENDED PRACTICE, JANUARY, 1931}

\section{EXTRUDED STRUCTURAL SHAPES}

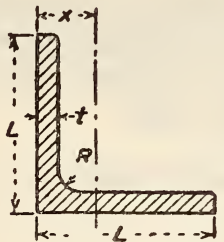

Seg TABLE 1

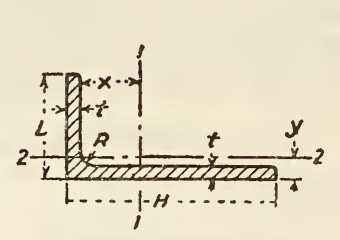

See Table 2

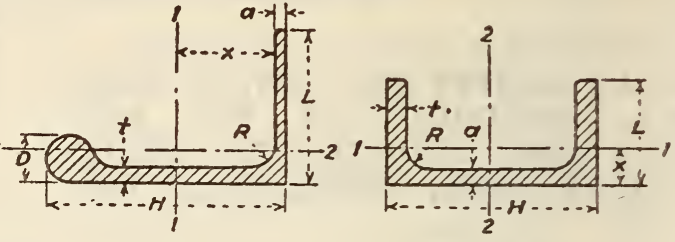

SEE TABLE 3

SEE TABLE 4

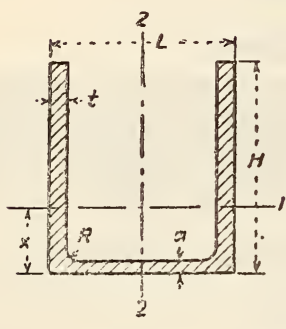

SED TABLE 5

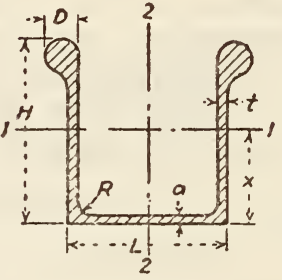

See Table 6

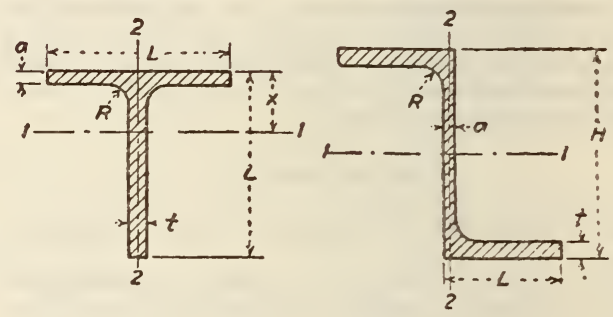

See Table 7

SEE TABLE 3

FIGURE 1069.-Exiruded structural shapes

TABLE 1.-Extruded equal angles

\begin{tabular}{|c|c|c|c|c|c|c|c|c|c|c|}
\hline Size & $t$ & $L$ & $R$ & $X$ & Area & $\begin{array}{l}\text { Moment } \\
\text { of izertia }\end{array}$ & $\begin{array}{l}\text { Section } \\
\text { modulus }\end{array}$ & $\begin{array}{l}\text { Weight } \\
\text { per foot }\end{array}$ & $\begin{array}{l}\text { Radius of } \\
\text { gyration }\end{array}$ & $\begin{array}{l}\text { Least ra- } \\
\text { dius of } \\
\text { gyration }\end{array}$ \\
\hline $\begin{array}{l}5 / 8 \\
3 / 4 \\
7 / 8 \\
1 \\
11 / 4 \\
11 / 2 \\
2\end{array}$ & $\begin{array}{l}0.063 \\
.075 \\
.088 \\
.100 \\
.125 \\
.150 \\
.200\end{array}$ & $\begin{array}{l}5 / 8 \\
3 / 4 \\
7 / 8 \\
1 \\
11 / 4 \\
11 / 2 \\
2\end{array}$ & $\begin{array}{r}0.063 \\
.075 \\
.085 \\
.100 \\
.125 \\
.150 \\
.200\end{array}$ & $\begin{array}{l}0.181 \\
.215 \\
.253 \\
.257 \\
.359 \\
.431 \\
.574\end{array}$ & $\begin{array}{r}0.0763 \\
.1031 \\
.1468 \\
.1922 \\
.3003 \\
.4321 \\
.7688\end{array}$ & $\begin{array}{l}0.002 \$ 43 \\
.005711 \\
.01053 \\
.01805 \\
.04404 \\
.09138 \\
.2588\end{array}$ & $\begin{array}{l}0.008325 \\
.01058 \\
.01723 \\
.0253 \\
.04941 \\
.0 \times 539 \\
.2024\end{array}$ & $\begin{array}{l}0.092 \\
.130 \\
.178 \\
.231 \\
.360 \\
.520 \\
.922\end{array}$ & $\begin{array}{l}0.194 \\
.231 \\
.272 \\
.305 \\
.386 \\
.453 \\
.6 ! 7\end{array}$ & $\begin{array}{l}0.123 \\
.147 \\
.172 \\
.193 \\
.244 \\
.293 \\
.391\end{array}$ \\
\hline
\end{tabular}

TABLE 2.-Extruded unequal angles

\begin{tabular}{|c|c|c|c|c|c|c|c|c|c|c|c|c|c|c|}
\hline Size & $L$ & $H$ & $t$ & $R$ & $x$ & $y$ & Area & $I_{1-1}$ & $S_{1-1}$ & $K_{1-1}$ & $I_{2-2}$ & $S_{2-2}$ & $K_{2-2}$ & $\begin{array}{l}\text { Weight } \\
\text { per } \\
\text { foot }\end{array}$ \\
\hline $\begin{array}{l}1 / 2 \text { by } 1 \\
5 / 8 \text { by } 11 / 4 \ldots \\
3 / 4 \text { by } 11 / 2 \\
7 / 8 \text { by } 13 / 4 \\
1 \text { by } 2 \\
11 / 4 \text { by } 21 / 2\end{array}$ & $\begin{array}{l}1 / 2 \\
5 / 8 \\
3 / 1 \\
7 / 8 \\
1 \\
11 / 4\end{array}$ & $\begin{array}{l}1 \\
11 / 4 \\
11 / 2 \\
13 / 4 \\
2 \\
21 / 2\end{array}$ & $\begin{array}{r}0.063 \\
.078 \\
.094 \\
.109 \\
.125 \\
.156\end{array}$ & $\begin{array}{r}0.063 \\
.078 \\
.094 \\
.109 \\
.125 \\
.156\end{array}$ & $\begin{array}{r}0.357 \\
.442 \\
.533 \\
.618 \\
.709 \\
.884\end{array}$ & $\begin{array}{r}0.108 \\
.133 \\
.161 \\
.186 \\
.214 \\
.267\end{array}$ & $\begin{array}{r}0.09216 \\
.14127 \\
.20517 \\
.27588 \\
.36281 \\
.56508\end{array}$ & $\begin{array}{r}0.009766 \\
.022943 \\
.048415 \\
.087529 \\
.151297 \\
.367230\end{array}$ & $\begin{array}{r}0.015007 \\
.028484 \\
.049861 \\
.077739 \\
.117239 \\
.227898\end{array}$ & $\begin{array}{r}0.326 \\
.403 \\
.486 \\
.564 \\
.646 \\
.806\end{array}$ & $\begin{array}{r}0.001700 \\
.003994 \\
.008429 \\
.015238 \\
.026340 \\
.063932\end{array}$ & $\begin{array}{r}0.004290 \\
.008142 \\
.014253 \\
.022222 \\
.033513 \\
.065146\end{array}$ & $\begin{array}{l}0.136 \\
.168 \\
.203 \\
.235 \\
.270 \\
.337\end{array}$ & $\begin{array}{r}0.111 \\
.170 \\
.246 \\
.331 \\
.435 \\
.678\end{array}$ \\
\hline
\end{tabular}

$I=$ Moment of inertia. $\quad K=$ Radius of gyration. $\quad S=$ Section modulus.

TABLE 3.-Extruded bulb angles

\begin{tabular}{|c|c|c|c|c|c|c|c|c|c|c|c|c|c|}
\hline Size & $H$ & $L$ & $t$ & $a$ & $D$ & $R$ & Area & $I_{1-1}$ & $S_{1-1}$ & $K_{1-1}$ & $I_{2-2}$ & $X$ & $\begin{array}{l}\text { Weight } \\
\text { per } \\
\text { foot }\end{array}$ \\
\hline $\begin{array}{l}15 / 16 \\
11 / 8 \\
15 / 16 \\
11 / 2 \\
17 / 8 \\
21 / 4 \\
3\end{array}$ & $\begin{array}{l}15 / 16 \\
11 / 8 \\
15 / 16 \\
11 / 2 \\
17 / 8 \\
21 / 4 \\
3\end{array}$ & $\begin{array}{l}5 / 8 \\
3 / 4 \\
7 / 8 \\
1^{71 / 4} \\
11 / 2 \\
2\end{array}$ & $\begin{array}{r}0.063 \\
.075 \\
.088 \\
.100 \\
.125 \\
.150 \\
.200\end{array}$ & $\begin{array}{r}0.063 \\
.063 \\
.063 \\
.070 \\
.088 \\
.105 \\
.140\end{array}$ & $\begin{array}{l}0.188 \\
.225 \\
.264 \\
.300 \\
.375 \\
.450 \\
.600\end{array}$ & $\begin{array}{l}0.094 \\
.112 \\
.132 \\
.150 \\
.188 \\
.225 \\
.300\end{array}$ & $\begin{array}{c}0.12518 \\
.17111 \\
.19700 \\
.2544 \\
.3975 \\
.5724 \\
1.0176\end{array}$ & $\begin{array}{r}0.01289 \\
.02475 \\
.04333 \\
.07222 \\
.17621 \\
.56560 \\
1.15549\end{array}$ & $\begin{array}{r}0.02231 \\
.03671 \\
.06273 \\
.09211 \\
.17989 \\
.31087 \\
.73688\end{array}$ & $\begin{array}{r}0.321 \\
.381 \\
.469 \\
.533 \\
.666 \\
.799 \\
1.066\end{array}$ & $\begin{array}{l}0.005196 \\
.00866 \\
.02113 \\
.04384 \\
.13856\end{array}$ & $\begin{array}{r}0.367 \\
.451 \\
.630 \\
.715 \\
.895 \\
1.074 \\
1.432\end{array}$ & $\begin{array}{r}0.150 \\
.205 \\
.236 \\
.305 \\
.47 \\
.687 \\
1.220\end{array}$ \\
\hline
\end{tabular}

= Moment of inertia. $\quad K=$ Radius of gyration. $S=$ Section modulus . 
TABLE 4.-Extruded channels (shallow,

\begin{tabular}{|c|c|c|c|c|c|c|c|c|c|c|c|c|c|c|}
\hline Size & $L$ & $H$ & $t$ & $a$ & $R$ & $x$ & Area & $I_{1-1}$ & $S_{1-1}$ & $K_{1-1}$ & $I_{2-2}$ & $S_{2-2}$ & $K_{2-2}$ & $\begin{array}{l}\text { Weight } \\
\text { per foot }\end{array}$ \\
\hline $\begin{array}{l}3.4 \\
78 \\
7 / 8 \\
1 / 8 \\
114 \\
132\end{array}$ & $\begin{array}{l}3 / 8 \\
3 / 16 \\
3 / 16 \\
1 / 2 \\
5,8 \\
3 / 4\end{array}$ & $\begin{array}{l}3 / 4 \\
3 / 8 \\
7 / 8 \\
1^{7 / 8} \\
11,4 \\
11,2\end{array}$ & $\begin{array}{r}0.063 \\
.063 \\
.088 \\
.100 \\
.125 \\
.150\end{array}$ & $\begin{array}{r}0.063 \\
.063 \\
.063 \\
.070 \\
.088 \\
.105\end{array}$ & $\begin{array}{r}0.063 \\
.063 \\
.088 \\
.100 \\
.125 \\
.150\end{array}$ & $\begin{array}{r}0.117 \\
.132 \\
.150 \\
.171 \\
.214 \\
.257\end{array}$ & $\begin{array}{r}0.08902 \\
.10490 \\
.12567 \\
.16030 \\
.25047 \\
.36067\end{array}$ & $\begin{array}{r}0.001119 \\
.001836 \\
.002277 \\
.003775 \\
.009211 \\
.028322\end{array}$ & $\begin{array}{r}0.004308 \\
.005950 \\
.007920 \\
.011470 \\
.022401 \\
.038711\end{array}$ & $\begin{array}{r}0.112 \\
.132 \\
.135 \\
.153 \\
.192 \\
.230\end{array}$ & $\begin{array}{c}0.007195 \\
.11823 \\
.014443 \\
.023909 \\
.058338 \\
.121039\end{array}$ & $\begin{array}{c}0.019035 \\
.026880 \\
.033013 \\
.04782 \\
.09339 \\
.16139\end{array}$ & $\begin{array}{r}0.029 \\
.336 \\
.339 \\
.386 \\
.482 \\
.579\end{array}$ & $\begin{array}{r}0.107 \\
.126 \\
.151 \\
.192 \\
.301 \\
.433\end{array}$ \\
\hline
\end{tabular}

$I=$ Noment of inertia. $\quad K=$ Radius of gyration. $S=$ Section modulus.

TABLE 5.-Extruded channels (deep)

\begin{tabular}{|c|c|c|c|c|c|c|c|c|c|c|c|c|c|c|}
\hline Size & $L$ & H & $a$ & $t$ & $R$ & $x$ & Area & $I_{1-1}$ & $S_{1-1}$ & $K_{1-1}$ & $I_{2-2}$ & $S_{2-2}$ & $K_{2-2}$ & $\begin{array}{l}\text { Weight } \\
\text { per } \\
\text { foot }\end{array}$ \\
\hline $\begin{array}{l}1 \\
11,4 \\
11,2 \\
13 / 4 \\
2\end{array}$ & $\begin{array}{c}7 / 8 \\
13: 32 \\
15116 \\
117,32 \\
13 / 4\end{array}$ & $\begin{array}{l}1 \\
11 / 4 \\
11 / 2 \\
13,4 \\
2\end{array}$ & $\begin{array}{r}0.063 \\
.078 \\
.094 \\
.109 \\
.125\end{array}$ & $\begin{array}{r}0.077 \\
.094 \\
.113 \\
.131 \\
.150\end{array}$ & $\begin{array}{r}0.063 \\
.078 \\
.094 \\
.109 \\
.125\end{array}$ & $\begin{array}{r}0.317 \\
.392 \\
.473 \\
.548 \\
.629\end{array}$ & $\begin{array}{r}0.20016 \\
.30682 \\
.44560 \\
.59916 \\
.78797\end{array}$ & $\begin{array}{r}0.02208 \\
.05186 \\
.10944 \\
.19785 \\
.34199\end{array}$ & $\begin{array}{r}0.03194 \\
.06063 \\
.10613 \\
.16546 \\
.24953\end{array}$ & $\begin{array}{r}0.332 \\
.411 \\
.495 \\
.574 \\
.659\end{array}$ & $\begin{array}{r}0.02382 \\
.05597 \\
.11811 \\
.21352 \\
.36908\end{array}$ & $\begin{array}{r}0.05402 \\
.10253 \\
.17948 \\
.27984 \\
.42202\end{array}$ & $\begin{array}{r}0.345 \\
.427 \\
.515 \\
.597 \\
.685\end{array}$ & $\begin{array}{r}0.240 \\
.368 \\
.535 \\
.719 \\
.946\end{array}$ \\
\hline
\end{tabular}

$I=$ Moment of inertia. $\quad K=$ Radius of gyration. $\quad S=$ Section modulus.

TABLE 6.-Extruded bulb channels

\begin{tabular}{|c|c|c|c|c|c|c|c|c|c|c|c|c|c|c|c|}
\hline Size & $L$ & $D$ & $t$ & $h$ & $a$ & $R$ & $x$ & Area & $\mathrm{I}_{1-1}$ & $S_{1-1}$ & $K_{1-1}$ & $I_{2-2}$ & $S_{2-2}$ & $K_{2-2}$ & $\begin{array}{l}\text { Weight } \\
\text { per foot }\end{array}$ \\
\hline $\begin{array}{l}1 \\
114 \\
11 / 2 \\
13 / 4 \\
2\end{array}$ & $\begin{array}{c}7 \% \\
13,82 \\
13,16 \\
117,32 \\
13.4\end{array}$ & $\begin{array}{r}316 \\
1564 \\
932 \\
21,64 \\
3,8\end{array}$ & $\begin{array}{l}1 / 16 \\
564 \\
3 / 32 \\
7 / 64 \\
1 / 8\end{array}$ & $\begin{array}{l}1 \\
11 / 4 \\
11 / 2 \\
13 / 4 \\
2\end{array}$ & $\begin{array}{r}1 / 16 \\
5,64 \\
0.070 \\
.082 \\
3 / 32\end{array}$ & $\begin{array}{l}1 / 16 \\
5 / 64 \\
3 / 32 \\
7 / 64 \\
1 / 8\end{array}$ & $\begin{array}{r}0.464 \\
.574 \\
.728 \\
.844 \\
.968\end{array}$ & $\begin{array}{r}0.21671 \\
.33219 \\
.45594 \\
.61306 \\
.80625\end{array}$ & $\begin{array}{l}0.028629 \\
.067255 \\
.13117 \\
.23715 \\
.40992\end{array}$ & $\begin{array}{r}0.05049 \\
.09584 \\
.15505 \\
.24174 \\
.36457\end{array}$ & $\begin{array}{l}0.363 \\
.450 \\
.537 \\
.622 \\
.714\end{array}$ & $\begin{array}{c}0.033059 \\
.077663 \\
.16108 \\
.29121 \\
.50337\end{array}$ & $\begin{array}{l}0.060735 \\
.115288 \\
.20755 \\
.32359 \\
.48802\end{array}$ & $\begin{array}{l}0.390 \\
.484 \\
.594 \\
.689 \\
.790\end{array}$ & $\begin{array}{l}0.260 \\
.399 \\
.547 \\
.736 \\
.968\end{array}$ \\
\hline
\end{tabular}

$I=$ Moment of inertia. $\quad K=$ Radius of gyration. $S=$ Section modulus.

TABLe 7.-Extruded tees

\begin{tabular}{|c|c|c|c|c|c|c|c|c|c|c|c|c|c|}
\hline Size & $t$ & $L$ & $a$ & $R$ & $X$ & Area & $I_{1-1}$ & $S_{1-1}$ & $K_{1-1}$ & $I_{2-2}$ & $S_{2-2}$ & $K_{2-2}$ & $\begin{array}{l}\text { Weight } \\
\text { per foot }\end{array}$ \\
\hline $\begin{array}{l}1^{3 / 4} \\
11 / 4 \\
11 / 2 \\
13 / 4 \\
2\end{array}$ & $\begin{array}{l}0.075 \\
.100 \\
.125 \\
.150 \\
.175 \\
.200\end{array}$ & $\begin{array}{l}1^{3 / 4} \\
11 / 4 \\
11 / 2 \\
13 / 4 \\
2\end{array}$ & $\begin{array}{r}0.063 \\
.070 \\
.083 \\
.105 \\
.123 \\
.140\end{array}$ & $\begin{array}{l}0.075 \\
.100 \\
.125 \\
.150 \\
.175 \\
.200\end{array}$ & $\begin{array}{r}0.224 \\
.314 \\
.393 \\
.472 \\
.550 \\
.629\end{array}$ & $\begin{array}{r}0.1012 \\
.1673 \\
.2614 \\
.3764 \\
.5124 \\
.6692\end{array}$ & $\begin{array}{c}0.005565 \\
.01694 \\
.04133 \\
.05576 \\
.15888 \\
.27104\end{array}$ & $\begin{array}{r}0.01057 \\
.02471 \\
.04826 \\
.08340 \\
.13242 \\
.19768\end{array}$ & $\begin{array}{r}0.235 \\
.318 \\
.398 \\
.477 \\
.557 \\
.636\end{array}$ & $\begin{array}{c}0.002246 \\
.006935 \\
.01692 \\
.03511 \\
.06504 \\
.11096\end{array}$ & $\begin{array}{r}0.00599 \\
.01387 \\
.02709 \\
.04681 \\
.07433 \\
.11096\end{array}$ & $\begin{array}{r}0.149 \\
.204 \\
.254 \\
.305 \\
.356 \\
.407\end{array}$ & $\begin{array}{r}0.121 \\
.201 \\
.314 \\
.452 \\
.615 \\
.803\end{array}$ \\
\hline
\end{tabular}

$I=$ Moment of irertia. $\quad L=$ Radius of gyration. $S=$ Section modulus.

TABLE 8.-Extruaed zees

\begin{tabular}{|c|c|c|c|c|c|c|c|c|c|c|c|c|c|}
\hline Size & $L$ & $H$ & $a$ & $t$ & $R$ & Area & $I_{1-1}$ & $S_{1-1}$ & $K_{1-1}$ & $I_{2-2}$ & $S_{2-2}$ & $K_{2-2}$ & $\begin{array}{l}\text { Weight } \\
\text { per foot }\end{array}$ \\
\hline $\begin{array}{l}i \xi \delta \\
11^{1 / 4} \\
11 / 2\end{array}$ & $\begin{array}{r}1 / 2 \\
5 / 6 \\
11 / 16 \\
0.825\end{array}$ & $\begin{array}{l}1^{7 / 8} \\
11 / 4 \\
11 / 2\end{array}$ & $\begin{array}{r}0.063 \\
.063 \\
.063 \\
.075\end{array}$ & $\begin{array}{r}0.063 \\
.080 \\
.100 \\
.120\end{array}$ & $\begin{array}{r}3 / 32 \\
0.125 \\
.125 \\
.150\end{array}$ & $\begin{array}{r}0.1150 \\
.1510 \\
.2132 \\
.3022\end{array}$ & $\begin{array}{r}0.01340 \\
.02375 \\
.05500 \\
.11049\end{array}$ & $\begin{array}{r}0.03038 \\
.04713 \\
.08730 \\
.14736\end{array}$ & $\begin{array}{r}0.341 \\
.397 \\
.508 \\
.604\end{array}$ & $\begin{array}{r}0.00446 \\
.00824 \\
.01947 \\
.03911\end{array}$ & $\begin{array}{r}0.01012 \\
.01538 \\
.02943 \\
.04968\end{array}$ & $\begin{array}{r}0.197 \\
.234 \\
.302 \\
.360\end{array}$ & $\begin{array}{r}0.138 \\
.181 \\
.256 \\
.363\end{array}$ \\
\hline $\begin{array}{l}13 / 4 \\
2 \\
21,2 \\
3\end{array}$ & $\begin{array}{r}.963 \\
1.10 \\
13 / 5 \\
1.65\end{array}$ & $\begin{array}{l}13 / 4 \\
2 \\
21 / 2 \\
3\end{array}$ & $\begin{array}{l}.088 \\
.100 \\
.125 \\
.150\end{array}$ & $\begin{array}{l}.140 \\
.160 \\
.200 \\
.240\end{array}$ & $\begin{array}{l}.175 \\
.200 \\
.250 \\
.300\end{array}$ & $\begin{array}{r}.4160 \\
.5372 \\
.8394 \\
1.2087\end{array}$ & $\begin{array}{r}.20952 \\
.34920 \\
.85206 \\
1.76785\end{array}$ & $\begin{array}{r}.23750 \\
.34920 \\
.68199 \\
1.17855\end{array}$ & $\begin{array}{r}.709 \\
.806 \\
1.007 \\
1.209\end{array}$ & $\begin{array}{l}.07416 \\
.12360 \\
.30160 \\
.62575\end{array}$ & $\begin{array}{r}.08017 \\
.11772 \\
.22991 \\
.39730\end{array}$ & $\begin{array}{l}.422 \\
.480 \\
.600 \\
.720\end{array}$ & $\begin{array}{r}.499 \\
.645 \\
1.007 \\
1.450\end{array}$ \\
\hline
\end{tabular}

$I=$ Moment of inertia. $\quad K=$ Radius of gyration. $\quad S=$ Section modulus. 
631.23 SHEETS, ALUMINUM.

\section{AMERICAN SOCIETY FOR TESTING MATE- RIALS, STANDARD SPECIFICATIONS FOR ALUMINUM SHEET, DESIGNATION B25- 29, 1929}

\section{ALUMINUM SHEET}

\section{SCOPE}

1. These specifications cover flat aluminum sheet. Strip or coil sheet may show somewhat lower elongations than those specified but is required to conform to the requirements of the same bend test as the flat sheet.

\section{MAN UFACTURE}

2. No scrap shall be used in the manufacture of aluminum sheet except such as shall accumulate at the manufacturer's own plants from material of similar composition and of his own manufacture.

\section{CHEMICAL PROPERTIES AND TESTS}

3. The sheet shall contain a minimum of 99 per cent aluminum as determined by chemical analysis.

4. Samples for analysis shall be obtained from a random sheet, representing each 500 pounds $(226.8 \mathrm{~kg})$ of aluminum or any lot weighing less than 500 pounds $(226.8 \mathrm{~kg})$, as agreed upon between the seller and the purchaser. Samples so prepared shall be divided into three equal parts, each of which shall be placed in a sealed package, one for each party and one for an umpire if necessary. The sample for analysis may be prepared by shearing or by drilling or machining without tne use of any lubricant in such a manner as to give a sample representative of the entire cross section of the sheet.

\section{PHYSICAL PROPERTIES AND TESTS}

5. (a) The sheet shall be supplied in the temper specified in the contract or purchase order and shall conform to the following minimum requirements as to tensile properties for the specified temper:

\begin{tabular}{|c|c|c|c|c|c|c|c|}
\hline \multicolumn{2}{|c|}{ Thickness } & \multicolumn{2}{|c|}{$\begin{array}{l}\text { Temper } \\
\text { No. } 1 \text {, soft }\end{array}$} & \multicolumn{2}{|c|}{$\begin{array}{c}\text { Temper } \\
\text { No. 2, } \\
\text { half hard }\end{array}$} & \multicolumn{2}{|c|}{$\begin{array}{c}\text { Temper } \\
\text { No. 3, hard }\end{array}$} \\
\hline B. \& S. gage & Inches & 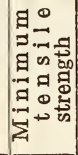 & 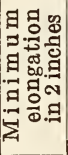 & 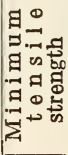 & 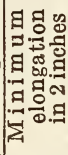 & 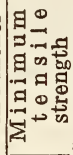 & 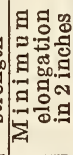 \\
\hline $\begin{array}{l}2 \text { too } 16 \\
\text { Thinner than } 10\end{array}$ & $0.258-0.051$ & $\begin{array}{c}\text { Lbs./ } \\
\text { in. }{ }^{2} \\
12,000\end{array}$ & 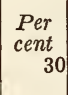 & $\begin{array}{l}L b s . / \\
\text { in.2 } \\
16,000\end{array}$ & $\begin{array}{l}\text { Per } \\
\text { cent } \\
\quad 7\end{array}$ & $\begin{array}{c}\text { Lbs./ } \\
\text { in.2 }\end{array}$ & $\begin{array}{l}\text { Per } \\
\text { cent }\end{array}$ \\
\hline $\operatorname{than} 16$ & & & & & & & \\
\hline $\begin{array}{l}\text { to } 20 \\
\text { Thinner than } 20\end{array}$ & $.050-.032$ & 212,000 & & 16,000 & & 24,000 & \\
\hline & $.031-.020$ & 012,000 & & 16,000 & & 426,000 & \\
\hline $\begin{array}{l}\text { Thinner than } 24 \\
\text { to } 28\end{array}$ & $.019-.013$ & 312,000 & & & & 330,000 & \\
\hline
\end{tabular}

(b) Tension-test specimens for sheet in temper No. 2, half hard, shall be cut from the sheet parallel to the direction of final rolling. From sheet in temper No. 1, soft, or temper No. 3, hard, the tension-test specimens may be cut in any direction.

6. Bend-test specimens cut in any direction from sheet in temper No. 1, soft, or No. 2, half hard, shall stand being bent cold through $180^{\circ}$ flat upon themselves without cracking.
7. One tension and one bend test specimen shall be cut from a random sheet representing each 500 pounds $(226.8 \mathrm{~kg})$ or fraction thereof of aluminum, as mutually agreed upon between the manufacturer and purchaser.

\section{PERMISSIBLE VARIATIONS IN DIMENSIONS}

8. The thickness of sheets shall not vary from that specified by more than the following tolerances:

\begin{tabular}{|c|c|c|c|c|}
\hline \multicolumn{2}{|l|}{ Thickness } & \multicolumn{3}{|c|}{ Tolerance in thickness } \\
\hline B. \& S. gage & Inches & $\begin{array}{l}20 \text { inches } \\
\text { in width } \\
\text { and } \\
\text { under }\end{array}$ & $\begin{array}{l}\text { Over } 20 \\
\text { to } 36 \\
\text { inches, } \\
\text { inclusive, } \\
\text { in width }\end{array}$ & $\begin{array}{l}\text { Over } 36 \\
\text { to } 60 \\
\text { inches, } \\
\text { inclu- } \\
\text { sive, in } \\
\text { width }\end{array}$ \\
\hline $\begin{array}{l}2 \text { to } 3 \\
\text { Thinner than } 3 \text { to } 9 \\
\text { Thinner than } 9 \text { to } 13 \\
\text { Thinner than } 13 \text { to } 21 .- \\
\text { Thinner than } 21 \text { to } 24 \\
\text { Thinner than } 24 \text { to } 27 \\
\text { Thinner than } 27\end{array}$ & $\begin{array}{c}0.258-0.220 \\
.219-.115 \\
.114-.073 \\
.072-.030 \\
.029-.019 \\
.018-.014 \\
.013 \text { and } \\
\text { less. }\end{array}$ & $\begin{array}{l}\text { Inch } \\
\pm 0.007 \\
\pm .005 \\
\pm .003 \\
\pm .0025 \\
\pm .002 \\
\pm .002 \\
\pm .0015\end{array}$ & $\begin{array}{l}\text { Inch } \\
\pm 0.008 \\
\pm .006 \\
\pm .0035 \\
\pm .0025 \\
\pm .002 \\
\pm .002 \\
\pm .0015\end{array}$ & $\begin{array}{l}\text { Inch } \\
\pm 0.009 \\
\pm .007 \\
\pm .004 \\
\pm .003 \\
\pm .003 \\
\pm .002 \\
\pm .0015\end{array}$ \\
\hline
\end{tabular}

\section{WOREMANSHIP AND FINISH}

9. All sheets shall be commercially flat and free from buckles; they shall be free from injurious surface defects and shall have a workmanlike finish.

\section{INSPECTION AND REJECTION}

10. The manufacturer shall afford the inspector representing the purchaser, without charge, all reasonable facilities to satisfy him that the material is being furnished in accordance with these specifications.

11. Materials which fail to conform to these specifications will be rejected and the manufacturer shall be notified.

NotEs. - In the case of sheet in the half-hard temper, there is an appreciable difference in the elongation depending upon the direction in which the test specimen is taken from the sheet. This measurement made parallel to the direction of final rolling is distinctly higher than if the specimen were cut perpendicular to this direction. This difference is not observed in hard or soft sheet. The inspection of half-hard sheet calls for the use of specimens parallel to the direction of final rolling because of the greater contrast with the values obtained from hard sheet. The fact that the bend-test specimens may be taken without regard to the direction of rolling is evidence that the lower elongation across the grain does not indicate a lack of workability.

The use of grips which insure a uniform axial loading of the test specimen is essential, if satisfactory results are to be obtained.

AMERICAN SOCIETY FOR TESTING MATERIAIS, STANDARD SPECIFICATIONS FOR ALUMINUM-ALIOY (DURALUMIN) SHEET, DESIGNATION B\%8-31, 1931

\section{DURALUMIN}

(In respect to manufacture, chemical analysis, workmanship and finish, inspection and rejection, this specification conforms with A. S. T. M. B25, given above.)

\section{CHEMICAL COMPOSITION}

2. The sheet shall conform to the following requirements as to chemical composition:

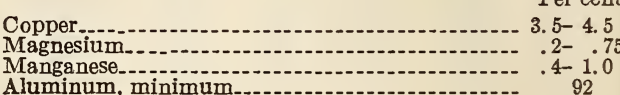

Mangesiumese

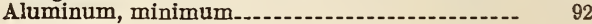


PHYSICAL PROPERTIES AND TESTS

4. (a) The sheet shall be supplied in the temper specified in the contract or purchase order and shall conform to the following requirements as to tensile properties for the specified temper:

\begin{tabular}{|c|c|c|c|c|c|c|}
\hline \multicolumn{2}{|c|}{ Thickness } & \multicolumn{3}{|c|}{$\begin{array}{l}\text { Temper No. 1, } \\
\text { quenched and aged }\end{array}$} & \multicolumn{2}{|c|}{$\begin{array}{l}\text { Temper No. 2, } \\
\text { annealed }\end{array}$} \\
\hline $\begin{array}{c}\text { B. \& S. } \\
\text { gage }\end{array}$ & Inch & $\begin{array}{c}\text { Ten- } \\
\text { sile } \\
\text { strength, } \\
\text { mini- } \\
\text { num }\end{array}$ & $\begin{array}{l}\text { Yield } \\
\text { point, } \\
\text { mini- } \\
\text { mum }\end{array}$ & $\begin{array}{l}\text { Elon- } \\
\text { ga- } \\
\text { tion } \\
\text { in } 2 \\
\text { inch- } \\
\text { es, } \\
\text { mini- } \\
\text { mum }\end{array}$ & \begin{tabular}{|} 
Ten- \\
sile \\
strength, \\
maxi- \\
num
\end{tabular} & $\begin{array}{l}\text { Elon- } \\
\text { ga- } \\
\text { tion } \\
\text { in } 2 \\
\text { inch- } \\
\text { es, } \\
\text { mini- } \\
\text { mum }\end{array}$ \\
\hline 2 to 10 & $0.258-0.102$ & $\begin{array}{r}\text { Lbs./in. } \\
55,000\end{array}$ & $\begin{array}{c}\text { Lbs./in. }{ }^{2} \\
30,000\end{array}$ & $\begin{array}{r}\text { Per } \\
\text { cent } \\
15\end{array}$ & $\begin{array}{c}\text { Lbs./in. }{ }^{2} \\
35,000\end{array}$ & $\begin{array}{l}\text { Per } \\
\text { cent } \\
12\end{array}$ \\
\hline $\begin{array}{l}\text { Thinner than } \\
10 \text { to } 16 \\
\text { Thinner than }\end{array}$ & $.101-.051$ & 55,000 & 30,000 & 18 & 35,000 & 12 \\
\hline $\begin{array}{l}16 \text { to } 20 \\
\text { Thinner than }\end{array}$ & $.050-.032$ & 55,000 & 30,000 & 18 & 35,000 & 12 \\
\hline 20 to 24 & $.031-.020$ & 55,000 & 30,000 & 15 & 35,000 & 10 \\
\hline $\begin{array}{l}\text { Thinner than } \\
24 \text { to } 28\end{array}$ & $.020-.013$ & 55,000 & 30,000 & 15 & 35,000 & 8 \\
\hline
\end{tabular}

(b) Tension-test specimens for sheet in either of the tempers may be cut in any direction, irrespective of the direction of rolling.

5. Bend-test specimens cut in any direction from aluminum-alloy (duralumin) sheet shall stand being bent cold through an angle of $180^{\circ}$, around a pin the diameter of which is equal to $n$ times the thickness of the sheet without cracking; the values of $n$ for tempers Nos. 1 and 2 being as follows:

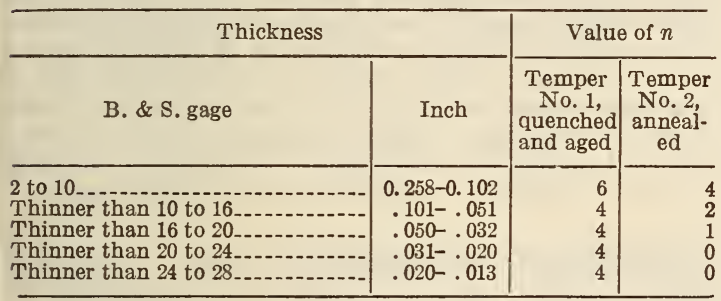

6. One tension and one bend-test specimen shall be cut from a random sheet representing each 500 pounds $(226.8 \mathrm{~kg}$ ) or fraction thereof of aluminum alloy (duralumin), as mutually agreed upon between the manufacturer and purchaser.
PERMISSIBLE VARIATIONS IN DIMENSIONS

7. The thickness of sheets shall not vary from that specified by more than the following tolerances:

\begin{tabular}{|c|c|c|c|c|}
\hline \multicolumn{2}{|c|}{ Thickness } & \multicolumn{3}{|c|}{ Tolerances in thickness } \\
\hline B. \& S. gage & Inch & $\begin{array}{c}18 \text { inches } \\
\text { in width } \\
\text { and } \\
\text { wider }\end{array}$ & $\begin{array}{l}\text { Over } 18 \\
\text { to } 36 \\
\text { inches } \\
\text { inclu- } \\
\text { sive, in } \\
\text { width }\end{array}$ & $\mid \begin{array}{c}\text { Over } 36 \\
\text { inches } \\
\text { in } \\
\text { width }\end{array}$ \\
\hline $\begin{array}{l}\text { Thicker than } 11 \\
11 \text { to } 13 \\
14 \text { to } 15 \\
16 \text { to } 18 \\
19 \text { to } 28\end{array}$ & $\begin{array}{r}0.092 \text { and thicker. } \\
0.091-0.072 \\
.064-.057 \\
.051-.040 \\
.036-.013\end{array}$ & $\begin{array}{c}\text { Inch } \\
(1) \\
\pm 0.003 \\
\pm .0025 \\
\pm .002 \\
\pm .0015\end{array}$ & 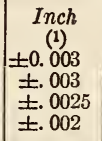 & $\begin{array}{c}\begin{array}{l}\text { Inch } \\
(1)\end{array} \\
\pm 0.004 \\
\pm .004 \\
\pm .003 \\
\pm .0025\end{array}$ \\
\hline \multicolumn{5}{|c|}{$1 \pm 5$ per cent of sheet thickness. } \\
\hline \multicolumn{5}{|c|}{$\begin{array}{l}\text { AMERICAN SOCIETY FOR TESTING MATE- } \\
\text { RIALS, STANDARD SPECIFICATIONS FOR } \\
\text { ALUMINUM-NANGANESE ALIOY SHEET, } \\
\text { DESIGNATION B79-31, } 1931\end{array}$} \\
\hline
\end{tabular}

\section{ALUMINUM-MANGANESE ALLOY SHEET}

(In respect to manufacture, chemical analysis, workmanship and finish, inspection and rejection, and explanatory notes, this specification conforms with A. S. T. M. B25, given above.)

\section{SCOPE}

1. These specifications cover flat aluminum-alloy (duralumin) sheet. Strip or coil sheet may show somewhat lower elongations that those specified, but it is required to conform to the requirements of the same bend test as the flat sheet.

\section{CHEMTCAL COMPOSITION}

3. The sheet shall conform to the following requirements as to chemical composition:

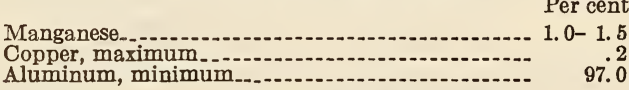

\section{PHYSICAL PROPERTIES AND TESTS}

5. (a) The sheet shall be supplied in the temper specified in the contract or purchase order and shall conform to the following requirements as to tensile properties for the specified temper:

\begin{tabular}{|c|c|c|c|c|c|c|c|}
\hline \multicolumn{2}{|l|}{ Thickness } & \multicolumn{2}{|c|}{ Temper No. 1, soft } & \multicolumn{2}{|c|}{$\begin{array}{c}\text { Temper No. } 2, \text { half } \\
\text { hard }\end{array}$} & \multicolumn{2}{|c|}{ Temper No. 3 , hard } \\
\hline B. \& S. gage & Inch & $\begin{array}{c}\text { Tensile } \\
\text { strength, } \\
\text { minimum }\end{array}$ & $\begin{array}{c}\text { Elonga- } \\
\text { tion in } \\
2 \text { inches } \\
\text { minimum }\end{array}$ & $\begin{array}{c}\text { Tensile } \\
\text { strength, } \\
\text { minimum }\end{array}$ & $\begin{array}{c}\text { Elonga- } \\
\text { tion in } \\
2 \text { inches, } \\
\text { minimum }\end{array}$ & $\begin{array}{c}\text { Tensi'e } \\
\text { strength, } \\
\text { minimum }\end{array}$ & $\begin{array}{l}\text { Elonga- } \\
\text { tion in } \\
2 \text { inches, } \\
\text { minimum }\end{array}$ \\
\hline $\begin{array}{l}2 \text { to } 16 \\
\text { Thinner than } 10 \text { to } 16 \\
\text { Thinner than } 16 \text { to } 20 \\
\text { Thinner than } 20 \text { to } 24 \\
\text { Thinner than } 24 \text { to } 28\end{array}$ & $\begin{array}{r}0.258-0.051 \\
.102-.051 \\
.050-.032 \\
.031-.020 \\
.019-.013\end{array}$ & $\begin{array}{l}\text { Lbs./in.2 } \\
14,509 \\
14,500 \\
14,500 \\
14,500\end{array}$ & $\begin{array}{l}\text { Per cent } \\
25 \\
-\overline{23} \\
20 \\
20\end{array}$ & $\begin{array}{l}\text { Lbs./in.2 } \\
19,500 \\
19,500 \\
19,500 \\
19,500\end{array}$ & $\begin{array}{c}\text { Per cent } \\
6 \\
-\overline{5} \\
4 \\
3\end{array}$ & $\begin{array}{l}\text { Lbs./in. }{ }^{2} \\
27,000 \\
27,000 \\
20,000 \\
33,000\end{array}$ & $\begin{array}{c}\text { Per cent } \\
-- \\
4 \\
3 \\
2 \\
1\end{array}$ \\
\hline
\end{tabular}


(b) Tension-test specimens for sheet in Temper No. 2, half hard, shall be cut from the sheet parallel to the direction of final rolling. From sheet in temper No. 1, soft, or temper No. 3, hard, the tension-test specimens may be cut in any direction.

6. (a) Bend-test specimens cut in any direction from sheet in temper No. 1, soft, shall stand being bent cold through $180^{\circ}$ flat upon themselves without cracking.

(b) Bend-test specimens cut in any direction from sheet in temper No. 2, half hard, shall stand being bent cold through $180^{\circ}$ around a pin the diameter of which is equal to twice the thickness of the sheet.

(c) The bend test shall not be required on sheet in temper No. 3, hard.

7. One tension and one bend-test specimen shall be cut from a random sheet representing each 500 pounds $(226.8 \mathrm{~kg})$ or fraction thereof of aluminummanganese alloy as mutually agreed upon between the manufacturer and purchaser.

\section{PERMISSIBLE VARIATIONS IN DIMENSIONS}

8. The thickness of sheets shall not vary from that specified by more than the following tolerances:

\begin{tabular}{|c|c|c|c|c|}
\hline \multicolumn{2}{|c|}{ Thickness } & \multicolumn{3}{|c|}{ Tolerances in thickness } \\
\hline B. \& S. gage & Inch & $\begin{array}{l}20 \text { inches } \\
\text { in width } \\
\text { and } \\
\text { under }\end{array}$ & $\begin{array}{l}\text { Over } 20 \\
\text { to } 36 \\
\text { inches, } \\
\text { inclusive, } \\
\text { in width }\end{array}$ & $\begin{array}{l}\text { Over } 36 \\
\text { to } 60 \\
\text { inches, } \\
\text { inclu- } \\
\text { sive, in } \\
\text { width }\end{array}$ \\
\hline $\begin{array}{l}2 \text { to } 3 \\
\text { Thinner than } 3 \text { to } 9 \\
\text { Thinner than } 9 \text { to } 13 \\
\text { Thinner than } 13 \text { to } 21 \\
\text { Thinner than } 21 \text { to } 24 \\
\text { Thinner than } 24 \text { to } 27 \\
\text { Thinner than } 27\end{array}$ & $\begin{array}{l}0.258-0.229 \\
.228-.114 \\
.113-.072 \\
.071-.028 \\
.027-.020 \\
.019-.014 \\
.013 \text { and less. }\end{array}$ & $\begin{array}{l}\text { Inch } \\
\pm 0.007 \\
\pm .005 \\
\pm .003 \\
\pm .0025 \\
\pm .002 \\
\pm .002 \\
\pm .0015\end{array}$ & $\begin{array}{l}\text { Inch } \\
\pm 0.003 \\
\pm .006 \\
\pm .0035 \\
\pm .0025 \\
\pm .002 \\
\pm .002 \\
\pm .0015\end{array}$ & $\begin{array}{l}\text { Inch } \\
\pm 0.009 \\
\pm .007 \\
\pm .004 \\
\pm .003 \\
\pm .003 \\
\pm .002 \\
\pm .0015\end{array}$ \\
\hline
\end{tabular}

SOCIETY OF AUTOMOTIVE ENGINEERS, NONFERROUS METAL SPECIFICATIONS, ALUMINUN ALLOYS, ALLOYS NOS. 26, 27, 28, AND 29, JULY, 1930

\section{ALUMINUM-ALLOY SHEETS}

(See 631.11, p. 1146.)

\section{SOCIETY OF AUTOMOTIVE ENGINEERS NONFERROUS METAL SPECIFICATIONS, SPECIFICATION NO. \%8, ALUMINUMI SHERT AND STRIP, JULY, 1930}

\section{AIUMINUM SHEET AND STRIP}

\section{Composition in percentage.-}

Aluminum, minimum.

Per cent

2. Physıcal properties.-Aluminum sheet or strip is furnished in several tempers or degrees of hardness. These tempers or degrees of hardness are indicated by the reduction in thickness produced by cold-rolling after annealing and are expressed in terms of the number of American wire (Brown \& Sharpe) gage sizes included between the final annealing thickness and the finish thickness. Thus temper No.4indicates that the sheet was given its final annealing at a thickness four gage numbers thicker than the finish gage, to which it was subse quently reduced by cold-rolling. Temper No. 0 indicates that the sheet or strip is in the soft annealed state. Hard temper indicates that the sheet or strip has been reduced in thickness after its final annealing by 12 or more gage numbers by coldrolling. The mechanical properties of aluminum sheet or strip of tempers 0,4 , and 6 and hard temper are given in the accompanying table.
3. Physical properties of aluminum flat sheet.-

\begin{tabular}{|c|c|c|c|c|c|c|}
\hline \multirow{3}{*}{ Temper } & \multicolumn{4}{|c|}{ Thickness } & \multirow{3}{*}{$\begin{array}{l}\text { Mini- } \\
\text { mum } \\
\text { tensile } \\
\text { strength }\end{array}$} & \multirow{3}{*}{$\begin{array}{l}\text { Mini- } \\
\text { mum } \\
\text { elonga- } \\
\text { tion in } \\
2 \text { inches }\end{array}$} \\
\hline & \multicolumn{2}{|c|}{ A. w. g. } & \multicolumn{2}{|c|}{ Inch } & & \\
\hline & From- & $\mid \begin{array}{l}\mathrm{Tc}-(\mathrm{in}- \\
\text { cluding) }\end{array}$ & From- & $\begin{array}{l}\text { To-(in- } \\
\text { cluding) }\end{array}$ & & \\
\hline 0 (soft).. & $\begin{array}{r}2 \\
9 \\
16 \\
20 \\
24\end{array}$ & $\begin{array}{r}9 \\
16 \\
20 \\
24 \\
28\end{array}$ & $\begin{array}{l}0.258 \\
.114 \\
.051 \\
.032 \\
.020\end{array}$ & $\begin{array}{l}0.114 \\
.051 \\
.032 \\
.020 \\
.013\end{array}$ & $\begin{array}{r}\text { Lbs. in. }{ }^{2} \\
12,000 \\
12,000 \\
12,000 \\
12,000 \\
12,000\end{array}$ & $\begin{array}{r}\text { Per cent } \\
30 \\
30 \\
25 \\
20 \\
15\end{array}$ \\
\hline & $\begin{array}{r}6 \\
9 \\
16 \\
20 \\
24\end{array}$ & $\begin{array}{r}9 \\
16 \\
20 \\
24 \\
28\end{array}$ & $\begin{array}{l}.162 \\
.114 \\
.051 \\
.032 \\
.020\end{array}$ & $\begin{array}{l}.114 \\
.051 \\
.032 \\
.020 \\
.013\end{array}$ & $\begin{array}{l}16,000 \\
16,000 \\
16,000 \\
16,000 \\
16,000\end{array}$ & $\begin{array}{l}7 \\
7 \\
5 \\
4 \\
3\end{array}$ \\
\hline & $\begin{array}{l}10 \\
16 \\
20 \\
24\end{array}$ & $\begin{array}{l}16 \\
20 \\
24 \\
23\end{array}$ & $\begin{array}{l}.102 \\
.051 \\
.032 \\
.020\end{array}$ & $\begin{array}{l}.051 \\
.032 \\
.020 \\
.013\end{array}$ & $\begin{array}{l}18,000 \\
18,000 \\
18,000 \\
18,000\end{array}$ & 2 \\
\hline Hard. & $\begin{array}{l}10 \\
16 \\
20 \\
24\end{array}$ & $\begin{array}{l}16 \\
20 \\
24 \\
28\end{array}$ & $\begin{array}{l}.102 \\
.051 \\
.032 \\
.020\end{array}$ & $\begin{array}{l}.051 \\
.032 \\
.020 \\
.013\end{array}$ & $\begin{array}{l}22,000 \\
24,000 \\
26,000 \\
30,050\end{array}$ & 1 \\
\hline
\end{tabular}

4. Aluminum strip sheet shows the same physical properties as flat sheet, with the exception that in tempers Nos. 4 and 6 the elongations may be slightly lower than those shown for flat sheet.

5. The tensile-test specimen shall be cut from the sheet or strip parallel to the direction of rolling. The bend-test specimen may be cut without regard to the direction of rolling. Sheet or strip of tempers Nos. 0 and 4 shall withstand being bent cold flat upon itself, without cracking. Although tensile tests of sheet strip of temper No. 6 incicate an elongation only slightly greater than that of the hard-temper sheet, it is considerably more ductile, as is evidenced by the fact that it will withstand cold bending through an angle of $180^{\circ}$ around a diameter equal to twice the thickness of the sheet, without cracking. Hard aluminum sheet or strip will not endure any considerable amount of bending without cracking, especially in the thicker gages.

6. Thickness tolerances for flat sheet.-

\begin{tabular}{|c|c|c|c|c|}
\hline \multirow[b]{2}{*}{ B. \& S. gage } & \multirow[b]{2}{*}{ Thickness 1} & \multicolumn{3}{|c|}{ Width } \\
\hline & & $\begin{array}{c}20 \text { inches } \\
\text { and } \\
\text { under }\end{array}$ & $\begin{array}{l}\text { Orer } 20 \\
\text { inches to } \\
30 \text { inches, } \\
\text { inclusire }\end{array}$ & $\begin{array}{l}\text { Over } 36 \\
\text { inches to } \\
60 \text { inches, } \\
\text { inclusire }\end{array}$ \\
\hline $\begin{array}{l}2 \text { and } 3-- \\
4 \text { to } 9 \\
10 \text { to } 13- \\
14 \text { to } 21- \\
22 \text { to } 24- \\
25 \text { to } 27- \\
28 \text { to } 36-\end{array}$ & $\begin{array}{c}\text { Inch } \\
0.250-0.220 \\
.219-.115 \\
.111-.073 \\
.0 .2-.030 \\
.029-.019 \\
.018-.014 \\
.013-.005\end{array}$ & $\begin{array}{l}\text { Inch } \\
\pm 0.00 \% \\
\pm .005 \\
\pm .003 \\
\pm .0025 \\
\pm .002 \\
\pm .002 \\
\pm .0015\end{array}$ & $\begin{array}{l}\text { Inch } \\
\pm 0.009 \\
\pm .006 \\
\pm .0035 \\
\pm .0025 \\
\pm .002 \\
\pm .002 \\
\pm .0015\end{array}$ & $\begin{array}{l}\text { Inch } \\
\pm 0.009 \\
\pm .007 \\
\pm .004 \\
\pm .003 \\
\pm .003 \\
\pm .002 \\
\pm .0015\end{array}$ \\
\hline
\end{tabular}

1 Material having thickness greater than 0.250 inch is consideroi plate and is covered by plate tcierances.

7. Thickness tolerances for strip sheet.-

\begin{tabular}{|c|c|c|c|}
\hline \multirow[b]{2}{*}{ B. \&. S. gage } & \multirow[b]{2}{*}{ Thickness } & \multicolumn{2}{|c|}{ Width } \\
\hline & & $\begin{array}{l}14 \text { inch to } \\
12 \text { inches } \\
\text { inclusive }\end{array}$ & $\begin{array}{l}\text { Crer } 12 \\
\text { inches to } \\
24 \text { inches, } \\
\text { inclusire }\end{array}$ \\
\hline $\begin{array}{l}10 \text { to } 18 \\
19 \text { and } 20 \\
21 \text { to } 25 \\
26 \text { to } 29 \\
30 \text { to } 36 \ldots\end{array}$ & $\begin{array}{l}\text { Inci } \\
0.102-0.041 \\
.040-.030 \\
.029-.017 \\
.016-.011 \\
.010-.005\end{array}$ & $\begin{array}{l}\text { Inch } \\
\pm 0.003 \\
\pm .002 \\
\pm .002 \\
\pm .0015 \\
\pm .001\end{array}$ & $\begin{array}{l}\text { Inch } \\
\pm 0.003 \\
\pm .0025 \\
\pm .0025 \\
\end{array}$ \\
\hline
\end{tabular}


§. Aluminum body sheets.-

S. A. E. recommended practice

\begin{tabular}{|c|c|}
\hline No. 14 gage & No. 16 gage \\
\hline $\begin{array}{l}\text { Inches } \\
24 \text { by } 72 \\
30 \text { by } 96 \\
36 \text { by } 120 \\
36 \text { by } 144\end{array}$ & $\begin{array}{c}\text { Inches } \\
24 \text { by }-2 \\
30 \text { by } 96 \\
36 \text { by } 120 \\
36 \text { by } 144\end{array}$ \\
\hline $\begin{array}{l}40 \text { by } 144 \\
48 \text { by } 144 \\
60 \text { by } 120 \\
60 \text { by } 144\end{array}$ & 45 by 144 \\
\hline
\end{tabular}

9. Aluminum sheet of No. 14 A. w. g. (B. \& S.) should be obtained in tempers Nos. 4 and 6 for body panels. Sheets of No. 16 A. w. g. should be obtained in temper No. 6 for body panels.

UNITED STATES GOVERNMENT, DEPARTMENT OF COKMERCE, BUREAU OF STANDARDS; STANDARD THICKNESSES, WEIGHTS, AND TOLERANCES OF SHEET METAL (CUSTOMARY PRACTICE), CIRCULAR NO. 391, MARCH 24, 1931

AMERICAN WIRE GAGE-ALUMINUM SHEETS (See 640.1, p. 1165.)

631.24 STRIPS, ALUMINUM.

SOCIETY OF AUTOMOTIVE ENGINEERS, NONFERROUS METAL SPECIFICATIONS, ALUMINUM ALLOYS, ALLOYS NOS. 26, 27, 28, AND 29, JULY, 1930

\section{ALUMINUM-ALLOY STRIPS}

(See 631.11, p. 1146.)

SOCIETY OF AUTOMOTIVE ENGINEERS, NONFERROUS METAL SPECIFICATIONS, SPECIFICATION HO. 78, ALUMINUM SHEET AND STRIP, JULY, 1930

\section{ALUMTNUM SHEET AND STRIP}

(See 631.23, p. 1156.)

UNITED STATES GOVERNIIENT, DEPARTMENT OF COMMERCE, BUREAU OF STANDARDS; STANDARD THICKNESSES, WEIGETS, AND TOLERANCES OF SHEET METAI (CUSTOMARI PRACTICE), CIRCULAR NO. 391, MARCH 24, 1931

AMERICAN WIRE GAGE-ALUMINUM STRIPS

(See 640.1, p. 1165.)
631.3 ALUMINUM AND ALUMINUM-ALLOY RODS, WIRES, AND RIVETS.

631.31 RODS, ALUMINUM.

AMERICAN RAILWAY ASSOCIATION, MECHANICAL DIVISION; SPECIFICATIONS FOR WELDING WIRE AND RODS, 1924

\section{WEIDING WIRE AND RODS}

1. Aluminum welding rods for gas welding all parts made of aluminum.

2. Chemical composition.-Welding wire and rods shall conform to the following chemical composition:

\begin{tabular}{l|r|r}
\hline & Class E & Class F \\
\hline Aluminum & $\begin{array}{r}\text { Per cent } \\
99.00\end{array}$ & $\begin{array}{r}\text { Per cent } \\
92.00 \\
8.00\end{array}$ \\
Copper \\
Tron \\
mum other elements and impurities, maxi-
\end{tabular}

(For specification details see 603.41, p. 156.)

SOCIETY OF AUTOMOTIVE ENGINEERS, NONFERROUS METAL SPECIFICATIONS, ALUMINUM ALLOYS, ALLOYS NOS. 26, 27, 28, AND 29, JULY, 1930

\section{ALUMINUM-ALLOY RODS}

(See 631.11, p. 1146.)

UNITED STATES GOVERNMENT, FEDERAL SPECIFICATIONS BOARD; FEDERAL SPECIFICATION FOR RODS, WELDING, NONFERROUS, FOR GAS WELDING, QQ-R - $5 \%$, MAY 3, 1932

\section{ALUMINUM AND ALUMINUM-ALIOY RODS}

(For general specifications, types, material, general requirements, detail requirements, methods of inspection, tests, and chemical analysis, packing and marking of shipments, and notes see F. S. B. QQ$\mathrm{R}-571,600.0$, p. 3.)

1. Aluminum rods may be used for general welding of aluminum.

2. Aluminum-alloy rods may be used for general welding of aluminum-copper alloys.

3. Welding rods shall conform to the following chemical requirements:

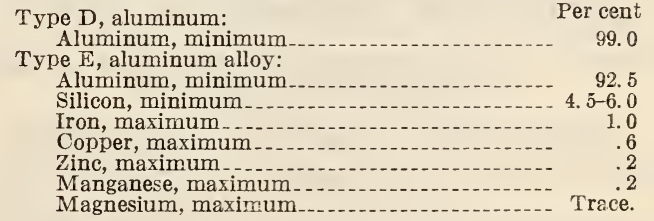

4. Standard sizes for both aluminum and aluminum-alloy rods for gas welding are:

Diameter (inch) $1 / 16,1 / 8,3 / 16,1 / 4,5 / 16$, and $3 / 8$ 
631.32. WIRES, ALUMINUM.

\section{AMERICAN ELECTRIC RAILWAY ENGINEERING ASSOCIATION, MISCELLANEOUS METHODS AND PRACTICES; HARD-DRAWN ALUMINUM WIRE, SERIAL NO. D201-10, 1910}

\section{HARD-DRAWN ALUMINUM WIRE}

Hard-drawn aluminum wire at $20^{\circ} \mathrm{C} .\left(\right.$ or $68^{\circ} \mathrm{F}$.) American wire gage (B. \& S.) English units

\begin{tabular}{|c|c|c|c|c|c|c|c|}
\hline \multirow{2}{*}{$\begin{array}{l}\text { Gage } \\
\text { No. }\end{array}$} & \multirow{2}{*}{$\begin{array}{c}\text { Diameter } \\
\text { in mils }\end{array}$} & \multicolumn{2}{|c|}{ Cross section } & \multirow{2}{*}{$\underset{\text { feet }}{\text { Ohms per } 1,000}$} & \multirow{2}{*}{$\begin{array}{l}\text { Pounds per } \\
1,000 \text { feet }\end{array}$} & \multirow{2}{*}{ Pounds per ohm } & \multirow{2}{*}{ Feet per ohm } \\
\hline & & Circular mils & Square inches & & & & \\
\hline $\begin{array}{r}0000 \\
000 \\
00 \\
0 \\
1\end{array}$ & $\begin{array}{l}460.0 \\
410.0 \\
365.0 \\
325.0 \\
289.0\end{array}$ & $\begin{array}{r}212,000.0 \\
168,000.0 \\
133,000.0 \\
106,000.0 \\
83,700.0\end{array}$ & $\begin{array}{l}0.166 \\
.132 \\
.105 \\
.0829 \\
.0657\end{array}$ & $\begin{array}{l}0.0804 \\
.101 \\
.128 \\
.161 \\
.203\end{array}$ & $\begin{array}{r}195.0 \\
154.0 \\
122.0 \\
97.0 \\
76.9\end{array}$ & $\begin{array}{r}2,420.0 \\
\mathbf{1}, 520.0 \\
957.0 \\
602.0 \\
379.0\end{array}$ & $\begin{array}{r}12,400.0 \\
9,860.0 \\
7,820.0 \\
6,200.0 \\
4,920.0\end{array}$ \\
\hline $\begin{array}{l}2 \\
3 \\
4 \\
5 \\
6\end{array}$ & $\begin{array}{l}258.0 \\
229.0 \\
204.0 \\
182.0 \\
162.0\end{array}$ & $\begin{array}{l}66,400.0 \\
52,600.0 \\
41,700.0 \\
33,100.0 \\
26,300.0\end{array}$ & $\begin{array}{l}.0521 \\
.0413 \\
.0328 \\
.0260 \\
.0206\end{array}$ & $\begin{array}{l}.256 \\
.323 \\
.408 \\
.514 \\
.648\end{array}$ & $\begin{array}{l}61.0 \\
48.4 \\
38.4 \\
30.4 \\
24.1\end{array}$ & $\begin{array}{r}238.0 \\
150.0 \\
94.2 \\
59.2 \\
37.2\end{array}$ & $\begin{array}{l}3,900.0 \\
3,090.0 \\
2,450.0 \\
1,950.0 \\
1,540.0\end{array}$ \\
\hline $\begin{array}{r}7 \\
8 \\
9 \\
10 \\
11\end{array}$ & $\begin{array}{r}144.0 \\
128.0 \\
114.0 \\
102.0 \\
91.0\end{array}$ & $\begin{array}{r}20,800.0 \\
16,500.0 \\
13,100.0 \\
10,400.0 \\
8,230.0\end{array}$ & $\begin{array}{l}.0164 \\
.0130 \\
.0103 \\
.00815 \\
.00647\end{array}$ & $\begin{array}{l}.817 \\
1.03 \\
1.30 \\
1.64 \\
2.07\end{array}$ & $\begin{array}{c}19.1 \\
15.2 \\
12.0 \\
9.55 \\
7.57\end{array}$ & $\begin{array}{l}23.4 \\
14.7 \\
9.26 \\
5.83 \\
3.66\end{array}$ & $\begin{array}{r}1,220.0 \\
970.0 \\
770.0 \\
610.0 \\
484.0\end{array}$ \\
\hline $\begin{array}{l}12 \\
13 \\
14 \\
15 \\
16\end{array}$ & $\begin{array}{l}81.0 \\
72.0 \\
64.0 \\
57.0 \\
51.0\end{array}$ & $\begin{array}{l}6,530.0 \\
5,180.0 \\
4,110.0 \\
3,260.0 \\
2,580.0\end{array}$ & $\begin{array}{l}.00513 \\
.00407 \\
.00323 \\
.00256 \\
.00203\end{array}$ & $\begin{array}{l}2.61 \\
3.29 \\
4.14 \\
5.22 \\
6.59\end{array}$ & $\begin{array}{l}6.00 \\
4.76 \\
3.78 \\
2.99 \\
2.37\end{array}$ & $\begin{array}{l}2.30 \\
1.45 \\
0.911 \\
.573 \\
.360\end{array}$ & $\begin{array}{l}384.0 \\
304.0 \\
241.0 \\
191.0 \\
152.0\end{array}$ \\
\hline $\begin{array}{l}17 \\
18 \\
19 \\
20 \\
21\end{array}$ & $\begin{array}{l}45.0 \\
40.0 \\
36.0 \\
32.0 \\
28.5\end{array}$ & $\begin{array}{r}2,050.0 \\
1,620.0 \\
1,290.0 \\
1,020.0 \\
810.0\end{array}$ & $\begin{array}{l}.00161 \\
.00128 \\
.00101 \\
.000802 \\
.000636\end{array}$ & $\begin{array}{l}8.31 \\
10.5 \\
13.2 \\
16.7 \\
21.0\end{array}$ & $\begin{array}{l}1.88 \\
1.49 \\
1.18 \\
.939 \\
.745\end{array}$ & $\begin{array}{l}.227 \\
.143 \\
.0897 \\
.0564 \\
.0355\end{array}$ & $\begin{array}{r}120.0 \\
95.5 \\
75.7 \\
60.0 \\
47.6\end{array}$ \\
\hline $\begin{array}{l}22 \\
23 \\
24 \\
25 \\
26\end{array}$ & $\begin{array}{l}25.3 \\
22.6 \\
20.1 \\
17.9 \\
15.9\end{array}$ & $\begin{array}{l}642.0 \\
509.0 \\
404.0 \\
320.0 \\
254.0\end{array}$ & $\begin{array}{l}.000505 \\
.000400 \\
.000317 \\
.000252 \\
.000200\end{array}$ & $\begin{array}{l}26.5 \\
33.4 \\
42.1 \\
53.1 \\
67.0\end{array}$ & $\begin{array}{l}.591 \\
.468 \\
.371 \\
.295 \\
.234\end{array}$ & $\begin{array}{l}.0223 \\
.0140 \\
.00882 \\
.00555 \\
.00349\end{array}$ & $\begin{array}{l}37.8 \\
29.9 \\
23.7 \\
18.8 \\
14.9\end{array}$ \\
\hline $\begin{array}{l}27 \\
28 \\
29 \\
30 \\
31\end{array}$ & $\begin{array}{r}14.2 \\
12.6 \\
11.3 \\
10.0 \\
8.9\end{array}$ & $\begin{array}{r}202.0 \\
160.0 \\
127.0 \\
101.0 \\
79.7\end{array}$ & $\begin{array}{l}.000158 \\
.000126 \\
.0000995 \\
.0000789 \\
.0000626\end{array}$ & $\begin{array}{r}84.4 \\
106.0 \\
134.0 \\
169.0 \\
213.0\end{array}$ & $\begin{array}{l}.185 \\
.147 \\
.117 \\
.0924 \\
.0733\end{array}$ & $\begin{array}{l}.00219 \\
.00138 \\
.000868 \\
.000546 \\
.000343\end{array}$ & $\begin{array}{c}11.8 \\
9.39 \\
7.45 \\
5.91 \\
4.68\end{array}$ \\
\hline $\begin{array}{l}32 \\
63 \\
34 \\
35 \\
36\end{array}$ & $\begin{array}{l}8.0 \\
7.1 \\
6.3 \\
5.6 \\
5.0\end{array}$ & $\begin{array}{l}63.2 \\
50.1 \\
39.8 \\
31.5 \\
25.0\end{array}$ & $\begin{array}{l}.0000496 \\
.0000394 \\
.0000312 \\
.0000248 \\
.0000196\end{array}$ & $\begin{array}{l}269.0 \\
339.0 \\
428.0 \\
540.0 \\
681.0\end{array}$ & $\begin{array}{l}.0581 \\
.0461 \\
.0365 \\
.0290 \\
.0230\end{array}$ & $\begin{array}{l}.000216 \\
.000136 \\
.0000854 \\
.0000537 \\
.0000338\end{array}$ & $\begin{array}{l}3.72 \\
2.95 \\
2.34 \\
1.85 \\
1.47\end{array}$ \\
\hline $\begin{array}{l}37 \\
38 \\
39 \\
40\end{array}$ & $\begin{array}{l}4.5 \\
4.0 \\
3.5 \\
3.1\end{array}$ & $\begin{array}{r}19.8 \\
15.7 \\
12.5 \\
9.9\end{array}$ & $\begin{array}{l}.0000156 \\
.0000123 \\
.00000979 \\
.000007 \\
77\end{array}$ & $\begin{array}{r}858.0 \\
1,080.0 \\
1,360.0 \\
1,720.0\end{array}$ & $\begin{array}{l}.0182 \\
.0145 \\
.0115 \\
.0091\end{array}$ & $\begin{array}{lll}.000 & 021 & 2 \\
.000 & 013 & 4 \\
.000 & 008 & 40 \\
.000 & 005 & 28\end{array}$ & $\begin{array}{l}1.17 \\
.924 \\
.733 \\
.581\end{array}$ \\
\hline
\end{tabular}

Note. - This table is the same as Table XIV, p. 54, Circular 31, 2d ed., of the Bureau of Standards and is based on data furnished by the Aluminum Co. of America as representing the mean conductivity of its output for all sizes and wire for five years.

\section{AMERICAN INSTITUTE OF ELECTRICAL ENGINEERS, HARD-DRAWN ALUMINUM CONDUCTORS, DECEMBER 10, 1926}

Approved as American Standard (C11-1927) by the American Standards Association, May 2, 1927]

\section{HARD-DRAWN ALUMINUM CONDUCTORS SCOPE}

The standards of this section cover the electrical and related properties of standard hard-drawn aluminum conductors. The following shall be taken as normal values for standard hard-drawn aluminum conductors:

Resistivity.-At a temperature of $20^{\circ} \mathrm{C}$. the electrical resistivity is 2.828 microhm-cm. (See note 1.)

Resistance-temperature coefficient. - The $20^{\circ}$ C. resistance-temperature coefficient is 0.00403 per degree centigrade. (See note 2.)

Density.-At a temperature of $20^{\circ} \mathrm{C}$. the density

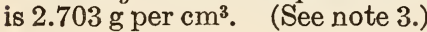

Length-temperature coefficient.-The $20^{\circ} \mathrm{C}$. lengthtemperature coefficient is 0.000023 per degree centigrade. (See note 4.)

Change in resistivity with change in temperature.The change in resistivity with change in temperature is 0.0115 microhm-cm per degree centigrade. (See note 5.)

Temperature.-In determining the resistivity of commercial aluminum conductors, measurements shall be made at a temperature $(t)$ not less than $10^{\circ} \mathrm{C}$. nor more than $30^{\circ} \mathrm{C}$., and the values obtained corrected to $20^{\circ} \mathrm{C}$. (See note 6.)

\section{EXPLANATORY NOTES}

1. The values given above are consistent with resistivities at $20^{\circ} \mathrm{C}$. of $17.01 \mathrm{ohm}$ circular mil per foot and $0.07644 \mathrm{ohm}$ gram $/ \mathrm{meter}^{2}$, and a conductivity at $20^{\circ}$ C., as compared with the annealed copper standard at $20^{\circ} \mathrm{C}$. of 60.97 per cent.

2. The value taken for the resistance-temperature coefficient is the amount by which the resistance of a conductor of standard hard-drawn aluminum 
changes when the temperature is changed, divided by the change in temperature expressed in degrees centigrade, and divided by the resistance of the same conductor when at a temperature of $20^{\circ} \mathrm{C}$.

3. The value taken for the density may be considered to be the same as the ratio of the mass of a quantity of standard hard-drawn aluminum to the mass of a quantity of pure water when the volume of the aluminum at a temperature of $20^{\circ} \mathrm{C}$. is equal to the volume of the water at a temperature of $4^{\circ} \mathrm{C}$.; or the specific gravity at $20^{\circ} \mathrm{C}$. as compared with pure water at $4^{\circ} \mathrm{C}$.

4. The value taken for the length-temperature coefficient (linear expansion coefficient) is the amount by which the length of a condutor of standard hard-drawn aluminum changes when the temperature is changed, divided by the change in temperature expressed in degrees centigrade, and divided by the length when at a temperature of $20^{\circ} \mathrm{C}$.

5. The value taken for the change in resistivity with change in temperature, as given above, is the sum of the resistance-temperature coefficient and the length-temperature coefficient, multiplied by the resistivity at $20^{\circ}$ C., as given above.

6. A temperature correction of $0.0115(t-20)$ microhm-cm corresponds to $0.069(t-20)$ ohm circular mil per foot and $0.000305(t-20) \mathrm{ohm}$ gram/meter ${ }^{2}$.

\section{AMERICAN RAILWAY ASSOCIATION, SIG- NAL SECTION, AERIAI ALUMINUM CABLE, STEEL REINFORCED, SPECIFI- CATION NO. 5416, 1916}

\section{ALUMINUM CABLE}

\section{GEIERAL}

(a) The intent of this specification is to provide for the furnishing of aerial aluminum cable, steel reinforced.

(b) The cable shall be composed of one extra high strength galvanized steel wire for a core and six aluminum wires concentrically laid around the steel core.

(c) The wires composing the cable shall be cvlindrical in form, free from scales, flaws, irregularities, splits, and all imperfections. Each length shall contain no weld, joint, or splice.

(d) The galvanizing for the steel core shall consist of a continuous coating of pure zinc of uniform thickness so applied that it adheres firmly to the wire and presents a smooth surface.

\section{PROPERTIES}

(a) The mechanical properties of the separate wires and of the finished cable shall conform to the following requirements:

\section{COMPLETE CABLE}

\begin{tabular}{|r|c|c|c|c|c|}
\hline $\begin{array}{c}\text { Nomi- } \\
\text { nal size }\end{array}$ & $\begin{array}{c}\text { Diam- } \\
\text { eter }\end{array}$ & $\begin{array}{c}\text { Cir- } \\
\text { cular } \\
\text { mils }\end{array}$ & $\begin{array}{c}\text { Elastic } \\
\text { limit }\end{array}$ & $\begin{array}{c}\text { Break- } \\
\text { ing } \\
\text { strength }\end{array}$ & $\begin{array}{c}\text { Approxi- } \\
\text { mate } \\
\text { weight } \\
\text { per 1,000 } \\
\text { feet }\end{array}$ \\
\hline A. vo. g. & Inch & & Pounds & Pounds & Pounds \\
0000 & 0.564 & 247,275 & 5,950 & 8,452 & 290 \\
000 & .502 & 195,766 & 4,692 & 6,666 & 231 \\
00 & .447 & 155,423 & 3,740 & 5,315 & 184 \\
0 & .398 & 123,118 & 2,964 & 4,215 & 145 \\
1 & .355 & 97,580 & 2,354 & 3,344 & 116 \\
2 & .316 & 77,432 & 1,864 & 2,646 & 91.9 \\
3 & .281 & 61,402 & 1,482 & 2,104 & 72.6 \\
4 & .250 & 48,697 & 1,158 & 1,646 & 57.5 \\
6 & .198 & 30,625 & 732 & 1,040 & 35.1 \\
\hline
\end{tabular}

STEEL WIR

\begin{tabular}{|r|c|r|r|r|r|}
\hline $\begin{array}{c}\text { Gage of } \\
\text { com- } \\
\text { plete } \\
\text { cable }\end{array}$ & $\begin{array}{c}\text { Diam- } \\
\text { eter }\end{array}$ & $\begin{array}{c}\text { Cir- } \\
\text { cuiar } \\
\text { mils }\end{array}$ & $\begin{array}{c}\text { Mini- } \\
\text { mum } \\
\text { elonga- } \\
\text { tion } \\
\text { in 18 } \\
\text { inches }\end{array}$ & $\begin{array}{c}\text { Elastic } \\
\text { limit }\end{array}$ & $\begin{array}{l}\text { Breaking } \\
\text { strength }\end{array}$ \\
\hline & & & & \\
\hline 0000 & 0.1880 & 35,325 & 4 & 3,600 & 4,450 \\
000 & .1670 & 27,966 & 4 & 2,850 & 3,500 \\
00 & .1491 & 22,203 & 4 & 2,280 & 2,800 \\
0 & .1327 & 17,588 & 4 & 1,800 & 2,240 \\
1 & .1182 & 13,940 & 4 & 1,440 & 1,760 \\
2 & .1052 & 11,062 & 4 & 1,140 & 1,380 \\
3 & .0933 & 8,772 & 4 & 895 & 1,110 \\
4 & .0834 & 6,957 & 4 & 720 & 830 \\
6 & .0661 & 4,375 & 4 & 450 & 555 \\
\hline
\end{tabular}

\section{ALUMTNUM WIRE}

(Gage of complete cable, diameter in inches, and circular mils are the same for aluminum wire as for steel wire, given above. The minimum elongation in 18 inches, per cent, is 1 . The elastic limit and breaking strength are as follows:)

\begin{tabular}{|c|c|c|c|c|c|c|c|c|c|}
\hline & \multicolumn{9}{|c|}{ Gage } \\
\hline & 0000 & 000 & 00 & 0 & 1 & 2 & 3 & 4 & 6 \\
\hline $\begin{array}{l}\text { Elastic limit_-_..-_pounds. } \\
\text { Breaking strength... do }\end{array}$ & $\begin{array}{l}391 \\
667\end{array}$ & $\begin{array}{l}307 \\
527 \\
\end{array}$ & $\begin{array}{l}243 \\
419 \\
\end{array}$ & $\begin{array}{l}194 \\
329 \\
\end{array}$ & $\begin{array}{l}152 \\
264 \\
\end{array}$ & $\begin{array}{l}121 \\
211\end{array}$ & $\begin{array}{r}98 \\
165 \\
\end{array}$ & $\begin{array}{r}73 \\
127\end{array}$ & $\begin{array}{l}47 \\
81\end{array}$ \\
\hline
\end{tabular}

(b) The aluminum wire shall be smooth and true and shall not vary in diameter more than 1 per cent from the specified diameter.

(c) The conductivity of aluminum wire shall not be less than 60 per cent of Matthiessen's annealed copper standard at zero degrees centigrade.

\section{GALVANIZING TEST}

(a) Galvanizing shall be in accordance with A. R. A. specification No. 2912. (See 600.3, p. 61.)

\section{INSPECTION AND TESTS}

(a) The purchaser is to have the right to make such inspection and tests of the completed products as are necessary to assure him that the requirements of this specification have been met, except that samples for test if taken from the finished cable shall not exceed 4 feet in length, and from not more than 5 per cent of the reels.

(b) The manufacturer must provide, at the mill all apparatus and labor for making the required tests under the supervision of the purchaser.

(c) Tests shall be made at the mill or on samples submitted by the manufacturer, and may also be made on the cable upon its arrival at destination.

(d) If, upon arrival at destination, the cable does not meet the requirements of this specification, it will be rejected and returned to the manufacturer. who shall pay all freight charges.

\section{PACEING FOR SHIPMENT}

(a) The cable shall be furnished in coils or on reels as specified, and of not less than the following lengths unless otherwise specified:

\begin{tabular}{|c|c|c|}
\hline $\begin{array}{c}\text { Gage of } \\
\text { cable }\end{array}$ & Preferred arrangement & $\begin{array}{c}\text { Minimum } \\
\text { length }\end{array}$ \\
\hline $\begin{array}{r}0000 \\
000 \\
00 \\
0 \\
1\end{array}$ & $\begin{array}{l}\text { Reel } \\
\text { Coil }\end{array}$ & $\begin{array}{l}\text { Feet } \\
3,500 \\
4,400 \\
5,600 \\
3,550 \\
4,500\end{array}$ \\
\hline $\begin{array}{l}2 \\
3 \\
4 \\
6\end{array}$ & 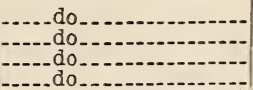 & $\begin{array}{l}5,600 \\
3,550 \\
4,500 \\
4,100\end{array}$ \\
\hline
\end{tabular}


(b) When furnished on reels, each reel shall have the weight, length of cable, with arrow indicating direction of unreeling, the name of the manufacturer, and purchaser's order and inspection number plainly and indelibly marked on a strong tag securely fastened to the cable and also stenciled on the outside of the reel.

(c) When furnished in coils, each coil shall be thoroughly wrapped with burlap and shall have the weight, length, and size of cable, the name of the maker, the purchaser's order, and inspection number, and the proper shipping address plainly and indelibly marked on a strong tag, securely fastened to the coil.

\section{SOCIETY OF AUTOMOTIVE ENGINEERS NONFERROUS METAL SPECIFICATIONS, ALUMINUM ALLOYS, ALLOYS NOS. 26 , 27, 28, AND 29, JULY, 1930}

\section{ALUMINUM ALLOY WIRE}

(See 631.11, p. 1146.)
631.33 RIVETS, ALUMINUM.

SOCIETY OF AUTOMOTIVE ENGINEERS, AERONAUTIC; RIVETS, ALUMINUM TYPE, S. A. E. STANDARD, JANUARY, 1931

\section{ALUMINUM TYPE RIVETS}
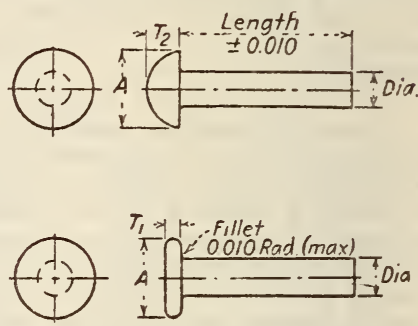

Figure 1070.-Aluminum type rivets

TABLE 1.-Aluminum rivet dimensions

\begin{tabular}{|c|c|c|c|c|c|c|c|c|}
\hline & \multicolumn{8}{|c|}{ Body diameter (inch) } \\
\hline & $\begin{array}{r}1 / 16 \\
+0.003 \\
-.001\end{array}$ & $\begin{array}{r}3 / 52 \\
+0.003 \\
-.001\end{array}$ & $\begin{array}{r}1 / 8 \\
+0.0035 \\
-.001\end{array}$ & $\begin{array}{r}5 / 32 \\
+0.004 \\
-.001\end{array}$ & $\begin{array}{l}3 / 16 \\
+0.004 \\
-.001\end{array}$ & $\begin{array}{r}1.1 \\
+0.004 \\
-.001\end{array}$ & $\begin{aligned} & 516 \\
+ & 0.004 \\
- & .001\end{aligned}$ & $\begin{array}{r}36 \\
+0.004 \\
-.001\end{array}$ \\
\hline $\begin{array}{l}\mathrm{A} \ldots \\
\mathrm{T}_{1 \ldots} \ldots \\
\mathrm{T}_{2 \ldots} \ldots\end{array}$ & $\begin{array}{r}1 / 8 \\
\pm 0.006 \\
.025 \\
\pm .005 \\
.047 \\
\pm .005\end{array}$ & $\begin{array}{r}3 / 16 \\
\pm 0.009 \\
.038 \\
\pm .005 \\
.070 \\
\pm .005\end{array}$ & $\begin{array}{r}1 / 4 \\
\pm 0.012 \\
.050 \\
\pm .005 \\
.094 \\
\pm .005\end{array}$ & $\begin{array}{r}5 / 16 \\
\pm 0.016 \\
.062 \\
\pm .005 \\
.117 \\
\pm .005\end{array}$ & $\begin{array}{r}3 / 8 \\
\pm 0.019 \\
.075 \\
\pm .005 \\
.141 \\
\pm .007\end{array}$ & $\begin{array}{r}1 / 2 \\
\pm 0.025 \\
.100 \\
\pm .005 \\
.188 \\
\pm .009\end{array}$ & $\begin{aligned} & 58 \\
& \pm 0.031 \\
& .125 \\
& \pm .005 \\
& .234 \\
& \pm .012\end{aligned}$ & $\begin{array}{r}3 / 4 \\
\pm 0.037 \\
.150 \\
\pm .007 \\
.281 \\
\pm .104\end{array}$ \\
\hline
\end{tabular}

TABLE 2.-Aluminum rivet lengths

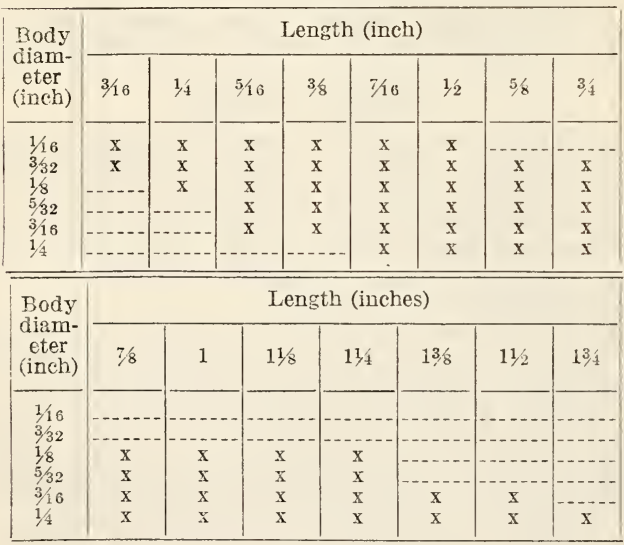

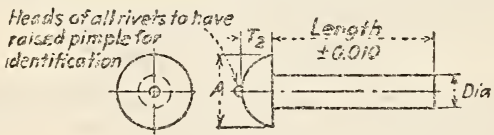

Heads of all rivets to have

reised pimple for

identification
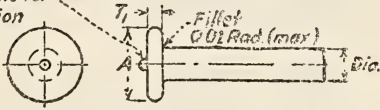

FIGURE 1071.-Aluminum alloy rivets

(Aluminum alloy rivets have the same dimensions as those given above for aluminum type rivets. The lengths of aluminum-alloy rivets are as follows:)
TABLE 3.-Aluminum-alloy rivet lengths

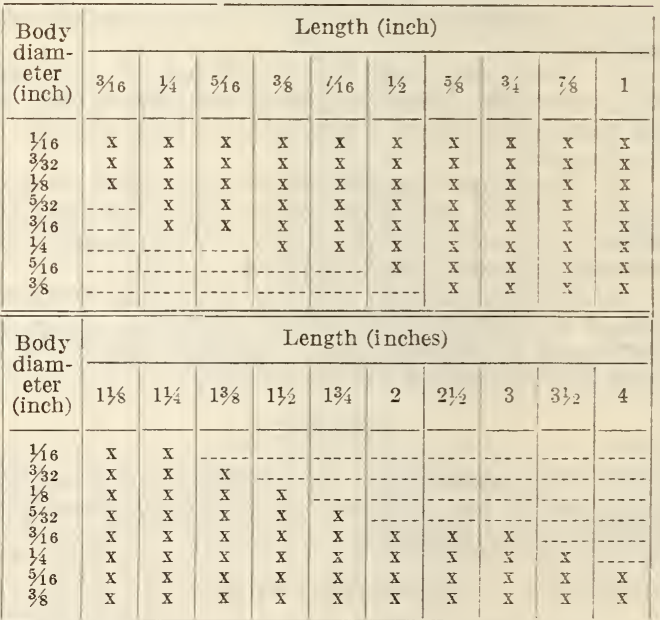

631.4 ALUMINUM AND AIUMINUN ALIOY CASTINGS AND TUBES.

631.41 CASTINGS, ALUMINUM.

AMERICAN SOCIETY FOR TESTING HATERIALS, STANDARD SPECIFICATIONS FOR ALUMINUM-BASE ALIOY SAND CASTINGS, DESIGNATION B26-31, 1931

\section{ALUMINUM ALIOY CASTINGS}

1. These specifications cover commercial aluminum-base alloy sand-castings having a specific grav- 
ity of 3 and less. Ten alloys are specified and are designated alloys $\mathrm{A}$ to $\mathrm{H}$, inclusive, and $\mathrm{J}$ and $\mathrm{K}$. (See explanatory notes.)

\section{MANUFACTURE}

2. The alloy may be made by any approved method.

\section{CHEMICAL COMPOSITION AND TESTS}

3. The alloys shall conform to the requirements as to chemical composition specified in Table 1. (See explanatory notes.)

(Sampling requirements are identical with those in A. S. T. M. B10 (see 643.4 , p. 1192). Inspection and rejection requirements are identical with those in A. S. T. M. B59 (see 647.15, p. 1259).)

\section{PHYSICAL PROPERTIES AND TESTS}

5. The alloys shall conform to the minimum requirements as to tensile properties specified in Table 2.

6 . The test bars shall be separately cast in green sand and shall be "cast to size" according to the dimensions shown in Figure 1072. If the castings are heat treated, the test bars representing such castings shall be similarly heat treated. They shall not be machined prior to test except to adapt the grip

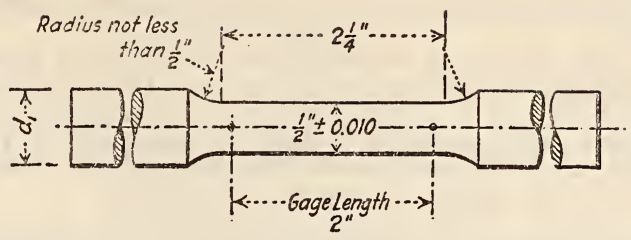

MOTE :

The Gage Length, Parallel Section and Fillers shall be as shown, but thie Ends maybe of any Shape to fit the Holders of the Testing Machine in such a way, that the Load shall be Axial.

In Orider to promote the Breaking of the Specimen in the Center it is permissible to uss a Taper in the feduced Section from the Fillets to the center of not to excesd $0.010 \mathrm{in}$.

It is recommended, that Dianieter $d$, be from s"tol."

\section{FIgURE 1072.-Tension test specimen}

to the holders of the testing machine in such a manner as to insure an axial load.

7. (a) The tests shall so far as possible be made by heats or melts, but unless otherwise agreed upon two tension tests shall be made for each unit of 500 pounds or fraction thereof.

TABLE 1.-Chemical compositions of aluminum-base alloy sand-castings

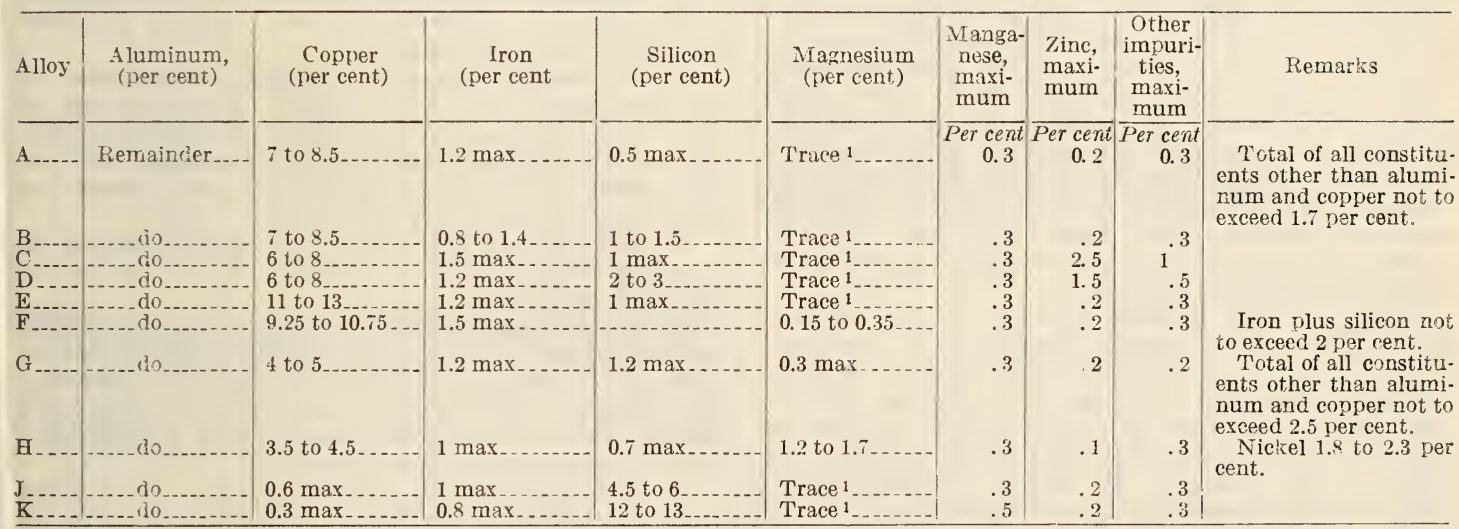

1 For the purpose of these specinications "trace" shall be considered as not over 0.05 per cent.

TABLE 2.-Physcial properties of aluminum-base alloy castings

\begin{tabular}{|c|c|c|}
\hline Alloy & $\begin{array}{l}\text { Tensile } \\
\text { strength, } \\
\text { minimum }\end{array}$ & $\begin{array}{l}\text { Elongation } \\
\text { in } 2 \text { inches, } \\
\text { minimum }\end{array}$ \\
\hline & Lbs./in. ${ }^{2}$ & Per cent \\
\hline & 19,000 & -..- \\
\hline 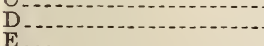 & 18,000 & $\cdots$ \\
\hline & $\begin{array}{l}19,000 \\
19,000\end{array}$ & \\
\hline $\int_{\text {heat treatment No. }}^{\text {heat }}$ & 28,000 & \\
\hline $\begin{array}{l}\text { heat treatment No. } 3 \\
\text { H. Heat treated } b \ldots \ldots\end{array}$ & $\begin{array}{l}50,000 \\
32,000\end{array}$ & \\
\hline $\mathbf{K}$ & $\begin{array}{l}16,000 \\
24,000\end{array}$ & $\begin{array}{l}3 \\
3 \\
5\end{array}$ \\
\hline
\end{tabular}

a See explanatory notes, alloy $\mathrm{G}$.

$\checkmark$ See explanatory notes, alloy $\mathrm{H}$.

(b) If any test specimen shows defective machining or develops flaws, it may be discarded; in which case the manufacturer and the purchaser or his representative shall agree upon the selection of another specimen in its stead.

\section{EXPLANATORY NOTES}

Alloy A.-Alloy A has a specific gravity of about 2.83. In pattern design a shrinkage of about $0.156(5 / 32)$ inch per foot should be allowed. This alloy was for years the standard and is still somewhat used, but is being superseded by alloy $\mathrm{B}$.

Alloy B.-This alloy has a specific gravity of about 2.83. The pattern shrinkage is about 0.156 $(5 / 32)$ inch per foot. It is superseding alloy $\mathrm{A}$ as it possesses better casting characteristics, especially in the more difficult work where higher pouring temperatures than those usually employed are found necessary. The tendency toward cracks and shrinks is considerably overcome by the careful control of the iron and silicon contents. This alloy is used for general casting purposes.

Alloy C.-The specific gravity of alloy $\mathrm{C}$ is about 2.83 to 2.86 . The pattern shrinkage is about 0.156 $(5 / 32)$ inch per foot. It has somewhat better machining qualities than alloys $\mathrm{A}$ and $\mathrm{B}$. It is used for about the same purposes as alloy B, particularly for automotive work, such as crank and transmission cases and similar parts.

Alloy D. - This alloy has a specific gravity of about 2.83. Due to its higher silicon content it has greater fluidity and is less subject to cracking. 
It is used for the more intricate general casting work.

Alloy E.-Alloy E has a specific gravity of about 2.95 and the pattern shrinkage is $0.156(5 / 32)$ inch per foot. It is used for pumps, manifolds, and general castings which should be leak proof. The high copper content makes the alloy more brittle and lowers its shock resistance.

Alloy $F$.-The specific gravity of this alloy is about 2.90 and the pattern shrinkage about 0.156 $(5 / 32)$ inch per foot. The alloy is used for pistons, cylinder heads, valve guides, and bearings or other parts where hardness and good bearing qualities are essential. Together with alloy $\mathrm{H}$ it possesses the property of retaining at higher temperatures a greater percentage of its room-temperature strength than any other alloy in this group. This alloy may be heat-treated, in which case substantially higher strength and hardness or other desirable characteristics are obtained.

Alloy G.-This alloy has a specific gravity of about 2.78. It is for general casting purposes where a combination of lightness, higher strength, and ductility is desired. The various strengths are obtained through different heat treatments. Heat treatment No. 1 gives maximum elongation and resistance to shock. It is a solution heat treatment and may be specified where the most rugged service is required. An aging effect takes place on standing which increases the strength and slightly decreases the elongation. Heat treatment No. 2 is a solution and precipitation heat treatment, and should be specified where a higher initial strength, yield point, and hardness are desired. Heat treatment No. 3 should be specified where the highest strength and hardness are desired, and lower ductility is not objectionable. This alloy with the various heat treatments is used in general for high strength parts in street cars and buses, parts for internal combustion engines, outboard motor parts, and other marine castings.

Alloy $H$.- The specific gravity of this alloy is about 2.80. It is usually employed in the heattreated condition. The alloy is used for pistons, cylinder heads, valve guides, and bearings or other parts where hardness or good bearing qualities are essential. Together with alloy $\mathrm{F}$ it possesses the property of retaining at higher temperatures a greater percentage of its room-temperature strength than any other alloy in this group.

Alloy J.-This alloy is somewhat lighter than aluminum, having a specific gravity of about 2.69 . It withstands corrosion better than the copper alloys. The free-flowing property of the alloy when molten makes it easier to pour castings of thin sections or intricate design. Its leak-proof properties are valuable where pressure tightness is essential. It has a lower strength and yield point than the copper alloys and poorer machining qualities. It is especially suitable for automobile body parts, cooking utensils, architectural and marine castings.
Alloy $K$.-The specific gravity of this alloy is about 2.68. Immediately before pouring, the alloy is treated by a process called "modification." Its corrosion resistance is good, especially to salt water. It has good foundry characteristics and can be used for castings having both thin and heavy sections. It is not so readily machined and has a lower yield point in proportion to tensile strength than most of the copper alloys. It is used for marine castings, bus, street car, and engine parts, and for thin castings such as radiators, small motor housings, and meter cases.

\section{AMERICAN SOCIETY FOR TESTING MATE- RIALS, TENTATIVE SPECIFICATIONS FOR ALUMINUM-BASE ALIOY DIE CAST- INGS, DESIGNATION B85-31T, 1931}

\section{ALUMINUM-BASE ALLOY DIE CASTINGS}

\section{SCOPE}

1. These specifications cover die castings die cast from aluminum-base alloys. Seven alloy co-positions are specified, designated alloys Nos. IV to IX, inclusive, and XII.

\section{CHEMICAL COMPOSITION AND TESTS}

2. The alloys shall conform to the requirements as to chemical composition given in Table 1 within the limits specified therein.

3. (a) It is understood that iron contributes to the hardness in all these alloys. While the use of steel or iron containers for the commercial manufacture of die castings unavoidably introduces some of this element into the alloy, it shall not exceed the percentages specified in Table 1 .

(b) Manganese and nickel are not regarded as harmful elements in these alloys up to the limits specified in Table 1.

4. Samples for chemical analysis may be taken either by sawing, drilling, or milling a representative group of castings, and shall represent the average cross section of the material. The castings and cutter shall be thoroughly cleaned and no lubricant shall be used during the operations.

5. Chemical analysis shall be made in accordance with the tentative methods of chemical analysis of aluminum and light aluminum alloys in A. S. T. M. B40-28T. (See 631.0, p. 1141.)

\section{SOUNDINESS}

6. No casting shall be acceptable which weighs less than a minimum agreed upon by the manufacturer and purchaser for the individual order.

\section{WORKMANSHIP AND FINISH}

7. Castings shall be produced with smooth surfaces, free from cracks and disfiguring blemishes.

TABLE 1.-Chemical compositions of aluminum-base die-casting alloys

\begin{tabular}{|c|c|c|c|c|c|c|c|c|c|c|c|c|c|c|}
\hline \multirow{2}{*}{$\begin{array}{l}\mathrm{Al}- \\
\text { loy }{ }^{1} \\
\text { No. }\end{array}$} & \multicolumn{4}{|c|}{ Desired composition } & \multicolumn{4}{|c|}{ Permissible variations in alloying elements, per cent } & \multicolumn{6}{|c|}{ Other elements, maximum } \\
\hline & $\begin{array}{l}\text { Cop- } \\
\text { per }\end{array}$ & $\begin{array}{l}\text { Sili- } \\
\text { con }\end{array}$ & $\begin{array}{c}\text { Nick- } \\
\text { el }\end{array}$ & $\begin{array}{c}\text { Alu- } \\
\text { mi- } \\
\text { num }\end{array}$ & Copper & Silicon & Nickel & Aluminum & Iron & Zinc & $\begin{array}{l}\text { Man- } \\
\text { ganese }\end{array}$ & $\begin{array}{c}\text { Mag } \\
\text { ne- } \\
\text { sium }\end{array}$ & Tin & $\begin{array}{l}\text { Total, } \\
\text { other } \\
\text { impu- } \\
\text { rities }\end{array}$ \\
\hline IV & P.ct. & $\underset{5}{P . c t .}$ & P.ct. & ${ }_{95}^{P . c t .}$ & 0.6 maximum & 4.5 to 6.0 & 0.5 maximum & Bal & P. ct. & P. ct. & P.ct. & P.ct. & $P$.ct. & P.ct. \\
\hline V & 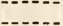 & & (-..- & & 0.6 maximum & 11 to 13 & 0.5 maximum & $\begin{array}{c}-.-\mathrm{d} 0_{2} . . . . \\
\end{array}$ & 2. & .75 & $\begin{array}{r}0.0 \\
.3\end{array}$ & 0.1 & 0.1 & 0.2 \\
\hline VI & 2 & & & & 1.5 to $2.5 \ldots$ & 2.5 to $3.5 \ldots$ & 0.5 maximum. & 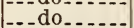 & 2.5 & 75 & .3 & .1 & .1 & 2 \\
\hline VII & $\tilde{4}$ & & & & 3.5 to 4.5 & 4.5 to 5.5 & 0.5 maximum & do & 2.25 & 3 & 3 & 1 & 1 & 02 \\
\hline VIII & 1. 5 & & 2.25 & 95.25 & 1 to 2 & 0.75 to $2 \ldots$ & 1.75 to 2.5 & - do do.n. & 2.5 & 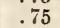 &. & 1 & 1 & .2 \\
\hline IX & 4 & 1. 75 & 4 & 90.25 & 3.5 to 4.5 & 1.0 to 2.5 & 3.5 to 4.5 & ... do & 2.5 & .7 & .3 & .1 & 1 & \\
\hline $\mathrm{XII}$ & 8 & 1.5 & -- & 90.50 & 7.0 to 9.0 & 1.0 to 2.0 & 0.5 maximum & ...do_ & 2.5 & 1. 0 & .3 & 1 & 2 & .2 \\
\hline
\end{tabular}

1 The alloy designations correspond to numbers used in the investigation on aluminum-base die-casting alloys carried out under the jurisdiction of committee B-6 on die-cast metals and alloys (formerly subcommittee XV on die-cast metals and alloys of committee B-2 on nonferrous metals and alloys), the alloy numbers omitted correspond to alloy compositions which were investigated by the committee, but which were not considered desirable of inclusion in these specifications. See Proc. A. S. T. M., rol. 29, p. 192, 1929. 
8. Dimensional tolerances shall be within the limits specified on the drawings describing the casting or castings on order or shall be within the limits specified in the order.

\section{REJECTION}

9. Castings which show injurious defects subsequent to acceptance may be rejected. The manufacturer's liability shall be confined to replacing rejected castings without charge to the purchaser.

\section{APPENDIX}

The data in the following table do not constitute a part of these specifications. They will indicate to the purchaser the physical properties that may be expected of tension and impact test specimens when made in a die, the test specimens being cast in regular production routine and conforming to the chemical composition specified. It should be thoroughly understood that the following figures represent die-cast test specimens and not specimens cut from commercial die-cast parts. Rockwell hardness values have not been shown because they are considered too unreliable on die castings.

\begin{tabular}{|c|c|c|c|}
\hline \multirow{2}{*}{$\begin{array}{l}\text { Alloy } \\
\text { No. }\end{array}$} & \multicolumn{2}{|c|}{$\begin{array}{l}\text { Tension tests on round } \\
\text { specimens }\end{array}$} & \multirow{2}{*}{$\begin{array}{l}\text { Charpy } \\
\text { impact on } \\
\text { square } \\
\text { specimens }\end{array}$} \\
\hline & $\begin{array}{c}\text { Tensile } \\
\text { (strength) }\end{array}$ & $\begin{array}{l}\text { Elonga- } \\
\text { tion in } 2 \\
\text { inches }\end{array}$ & \\
\hline $\begin{array}{l}\text { IV } \\
\text { V } \\
\text { VI } \\
\text { VII } \\
\text { VIII } \\
\text { IX } \\
\text { XII }\end{array}$ & $\begin{array}{c}\text { Lbs./in. }{ }^{2} \\
29,000 \\
33,000 \\
30,000 \\
32,000 \\
29,000 \\
31,000 \\
33,000\end{array}$ & $\begin{array}{c}\text { Per cent } \\
3.5 \\
1.5 \\
3.5 \\
2.0 \\
4.0 \\
1.5 \\
1.0\end{array}$ & $\begin{array}{c}F t .-l b s . \\
4.5 \\
2.0 \\
5.0 \\
2.5 \\
4.5 \\
2.0 \\
1.5\end{array}$ \\
\hline
\end{tabular}

SOCIETY OF AUTOMOTIVE ENGINEERS, NONFERROUS-METAL SPECIFICATIONS, SPECIFICATIONS NOS. $30,31,31 \mathrm{~A}, 32$, $33,34,35,36,37,38$, AND 39, ALUMINUM ALLOYS, JULY, 1930

\section{ALUMINUM-ALIOY CASTINGS}

(See 631.11, p. 1146.)

UNITED STATES GOVERNMENT, DEPARTMENT OF COMMERCE, BUREAU OF STANDARDS, STANDARD SAMPLES, CIRCULAR NO. 25 AND SUPPLEMENT, 1927

\section{ALUMINUM-BASE CASTING ALLOY SAMPLE}

(Sample No. 86 is a standard sample of aluminumbase casting alloy prepared and sold by the Bureau of Standards for use by industrial organizations and others as a comparison standard for checking the accuracy of analysis of similar material. Each sample is accompanied by a certificate giving the complete analysis of that particular sample.)

631.42 TUBES, ALUMINUM.

SOCIETY OF AUTOMOTIVE ENGINEERS, NONFERROUS-METAL SPECIFICATIONS, ALUMINUM ALLOYS, ALLOYS NOS. 26, 27, 28, AND 29, JULY, 1930

\section{ALUMINUM-ALLOY TUBES}

(See 631.11, p. 1146.)
631.5 ALUMINUM AND ALUMINUM-ALLOY HOLLOW WARE.

(No nationally recognized specifications available.)

\subsection{MISCELLANEOUS ALUMINUM AR- TICLES.}

SOCIETY OF AUTOMOTIVE ENGINEERS, BODY PARTS AND MATERIALS, RECOMMENDED PRACTICE, ALUMINUM BELT MOLDING AND DRIP MOLDING, FEBRU. ARY, 1928

\section{ALUMINUM BELT MOLDING}
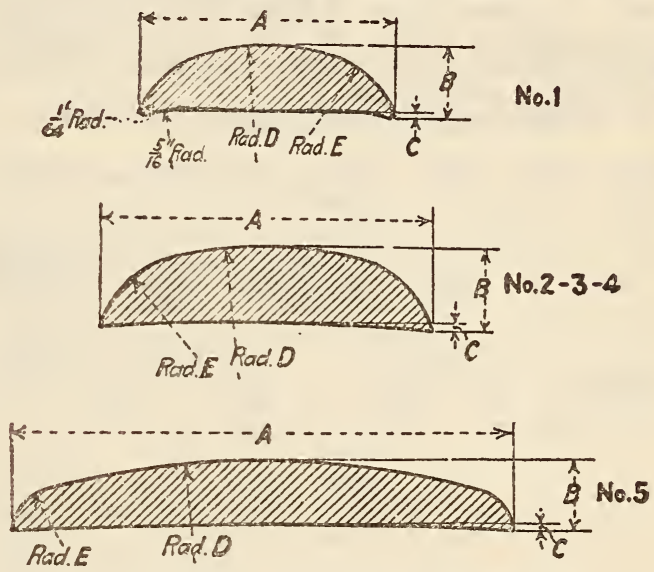

FIgURE 1073.-Aluminum belt molding

\begin{tabular}{|c|c|c|c|c|c|c|}
\hline No. & A. & B & C & D & $\mathrm{E}$ & $\begin{array}{l}\text { Estimated } \\
\text { weight per } \\
\text { foot }\end{array}$ \\
\hline $\begin{array}{l}1 \\
2 \\
3 \\
4 \\
5\end{array}$ & $\begin{array}{l}0.770 \\
7 / 8 \\
1 \\
11 / 4 \\
11 / 2\end{array}$ & $\begin{array}{c}0.223 \\
1 / 4 \\
1 / 4 \\
1 / 4 \\
7 / 32\end{array}$ & $\begin{array}{r}0.016 \\
.025 \\
.025 \\
.025 \\
.016\end{array}$ & $\begin{array}{l}0.625 \\
.875 \\
1.094 \\
11 / 4 \\
23 / 8\end{array}$ & $\begin{array}{l}0.313 \\
.281 \\
.281 \\
.344 \\
964\end{array}$ & $\begin{array}{r}\text { Pound } \\
0.143 \\
.186 \\
.228 \\
.286 \\
.331\end{array}$ \\
\hline
\end{tabular}

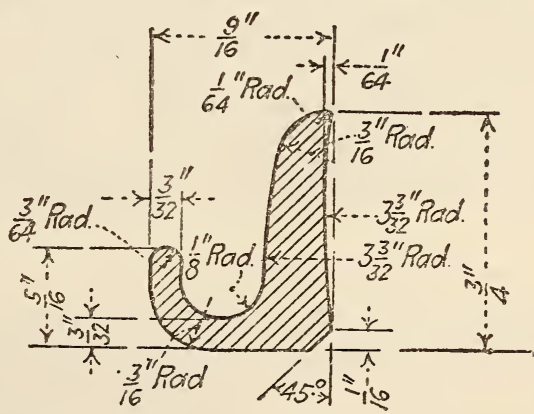

Figure 1074.-Aluminum drip molding

SOCIETY OF AUTOMOTIVE ENGINEERS, NONFERROUS METAL SPECIFICATIONS, ALUMINUN ALLOYS, ALLOYS NOS. 26, 27, 28, AND 29, JULY, 1930

ALUMINUM ALLOY RIVETS, BOLTS, NUTS, SCREWS

(See 631.11, p. 1146.) 


\section{UNITED STATES GOVERNMENT, FEDERAL SPECIFICATIONS BOARD, FEDERAI
SPECIFICATION FOR STANDARD MIS- CELLANEOUS CHAIN AND ATTACH- MENTS RR-C-271, DECEMBER 8, 1931}

SAFETY CHAIN, WELDLESS

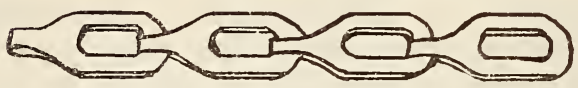

FIGURE 1075.-Safety chain, weldless

(For general specifications, material and workmanship, detail requirements, and methods of inspection and tests, see F. S. B. RR-C-271 (603.50, p. 199). For other kinds of chain and chain attachments specified in F. S. B. RR-C-271, see 603.51, $603.53,603.54,603.56,603.57$, and 617.5. See also $642.9,645.9$, and 646.59 .)

1. Weldless chain will be paid for by the foot. TABLE 50.- Safety $\begin{aligned} & \text { chain (dimensions, strength, } \\ & \text { weights) }\end{aligned}$

\begin{tabular}{|c|c|c|c|c|}
\hline Material & $\begin{array}{c}\text { Metal } \\
\text { gage }\end{array}$ & \begin{tabular}{|c|} 
Approxi- \\
mate num- \\
ber of links \\
per foot
\end{tabular} & $\begin{array}{l}\text { Minimum } \\
\text { tensile } \\
\text { strength }\end{array}$ & $\begin{array}{c}\text { Approxi- } \\
\text { mateweight } \\
\text { per } 100 \\
\text { feet }\end{array}$ \\
\hline $\begin{array}{l}\text { Aluminur } \\
\text { Do }\end{array}$ & $\begin{array}{l}\text { Inch } \\
0.018 \\
.028\end{array}$ & $\begin{array}{l}22 \\
27\end{array}$ & $\begin{array}{r}\text { Pounds } \\
50 \\
30\end{array}$ & $\begin{array}{r}\text { Pounds } \\
1.5 \\
.75\end{array}$ \\
\hline
\end{tabular}

632.

\section{ANTIMONY}

632.1 GENERAL SPECIFICATIONS FOR ANTIMONY.

(No nationally recognized specifications available.)

\subsection{ANTIMONY INGOT METAL.}

(No nationally recognized specifications available.)

\subsection{ANTIMONY ALLOYS.}

(For antimony-lead alloys, see classification 651.9 , p. 1279,692 , p. $1317,693.1$, p. 1330 .)

633.

\section{BISMUTH}

(No nationally recognized specifications available.) 634.

\section{CADMIUM}

(No nationally recognized specifications available.)

635.

\section{COBALT}

(No nationally recognized specifications avail- 
640.

\author{
GENERAL ITEMS
}

AMERICAN SOCIETY FOR TESTING MATERIALS, STANDARD RULES GOVERIING THE PREPARATION OF MICROGRAPHS OF METALS AND ALLOYS, DESIGNATION E2-30, 1930

\section{MICROGRAPHS}

(See 600.1, p. 20.)

AMERICAN SOCIETY FOR TESTING MATERIALS, STANDARD METHODS OF METALLOGRAPHIC TESTING OF NONEERROUS METALS AND ALLOYS, DESIGNATION E5-27, 1927

\section{METALIOGRAPHIC TESTING OF NONFERROUS METALS}

(See 600.1, p. 28.)

AMERICAN SOCIETY FOR TESTIYG MATERIALS, TENTATIVE METHODS OF TENSION TESTING OF METALIIC MATERIALS, DESIGNATION E8-27T, 1927

TENSION TESTING OF METALIIC MATERIALS

(See 600.1, p. 34.)

AMERICAN SOCIETY FOR TESTING MATERIALS, TENTATIVE IHETHODS OF CONPRESSION TESTING OF METALLIC MATERIALS, DESIGNATION E9-27T, $192 \%$

COMPRESSION TESTING OF METALLIC MATERIALS

(See 600.1, p. 38.)

640.1 THICKNESSES, WEIGHTS, AND TOLERANCES OF NONEERROUS SHEETS AND PLATES.

SOCIETY OF AUTOMOTIVE ENGINEERS, WROUGHT NONFERROUS METAL ALLOYS, PERIIISSIBLE VARIATIONS IN THICKNESS OF SHEETS AND STRIPS, JULY, 1924

\section{PERMISSIBLE VARIATIONS}

(Same as given in tables in F. S. B. specification QQ-C-501 under classification 641.21, p. 1177; and in tables in F. S. B. specification No. 468 (current designation QQ-N-321) under classification 655.2, p. 1287.)
UNITED STATES GOVERNMENT, DEPARTMENT OF COMMERCE, BUREAU OF STANDARDS; STANDARD THICKNESSES, WEIGHTS, AND TOLERAMCES OF SHEET METAL (CUSTOIMARY PRACTICE), CIRCULAR NO. 391, MARCH 24, 1931

(See also 604.0, p. 224.)

\section{NONFERROUS PLATE, SHEET, AND STRIP}

1. AMERICAN WIRE GAGE (FOR COPPER, ALUMINUM AND NONFERROUS ALIOYS)

The American wire gage, also commonly known as the Brown \& Sharpe gage, is extensively used in the United States for nearly all nonferrous sheets, particularly copper, aluminum, and nonferrous alloy sheets, as well as for wire of the same materials. It was devised by J. R. Brown and Lucian Sharpe, founders of the Brown \& Sharpe Manufacturing Co., in 1856, and was adopted by the Association of Brass Manufacturers in February, 1857, eight of the leading brass manufacturers signing the resolutions. Its gage numbers, like those of the U. S. standard gage and many other gages, are retrogressive, a larger number denoting a smaller size. The gage is based on a simple mathematical law of geometrical progression, which may be expressed in either of three following manners: (a) The ratio of any size to the next smaller is a constant number, namely

$\sqrt[39]{\frac{0.460}{0.005}}$, which is $1.1229322 ;$ (b) the difference

between any two successive sizes is a constant percentage of the smaller of the two sizes, namely, 0.1229322 ; and $(c)$ the difference between any two successive sizes is a constant ratio times the next smaller difference between two successive sizes, namely, 1.1229322 .

When the gage was developed, the size No. 0000 was defined as 0.4600 and No. 36 as $0.005 \mathrm{inch}$, and it was specified that there should be 38 sizes between the two which should advance by geometrical progression. The sixth power of the ratio 1.229322 is 2.0050 , so that the thickness and, consequently, the weight per unit area of a sheet six sizes heavier than a given size, is approximately twice as great as for the given size.

\section{(a) Approximate Weights per Square Foot}

In Table 13 the gage numbers and thicknesses of the American wire gage are given, together with the approximate weights per square foot of rolled copper and aluminum sheets.

Copper.-The weights of copper sheets given in Table 13 are based on the density of 8.89 grams per cubic centimeter, which is the value adopted by the International Electrotechnical Commission and the American Institute of Electrical Engineers in their "definition of the annealed copper standard"; and is used in some specifications of the American Society for Testing Materials. This value for density is equivalent to 0.321 pounds per cubic inch, or 46.25 
pounds per square foot per inch thick. The weights given in the table are, therefore, for cold-rolled and annealed copper sheets. Hot-rolled copper plates having a thickness of five-sixteenths inch and over are about one-half per cent heavier, the density being 8.94 grams per cubic centimeter, 0.323 pound per cubic inch, or 46.51 pounds per square foot per inch thick, according to A.S.T. M. standard specifications for copper plates for locomotive fireboxes, B11-18 (see 641.21, p. 1176). The International Critical Tables give 8.94 grams per cubic centimeter as also the value for pure copper.

Aluminum. - The weights of aluminum sheets, given in Table 13 , are based on the density 2.71 grams per cubic centimeter, 169.2 pounds per cubic foot, or 14.10 pounds per square foot per inch thick, this value being commonly accepted as the average density of commercially pure (99.0 to 99.4 per cent) aluminum (2S) rolled commercial sheets. The National Metals Handbook gives the value 2.706 grams per cubic centimeter for 99.4 per cent aluminum.

Copper alloys.- The weights of brass and some other copper-alloy sheets and plates rolled to the American wire gage are given in Table 14. These are alloys covered by certain United States Government or United States Army specifications. The weights of gilding metal and cupro-nickel are based on determinations of density of samples of sheet made to such specifications, whereas those for commercial brass, naval brass, and nickel silver are those generally accepted as representative of rolled sheets of these materials. The densities are as follows:

\begin{tabular}{|c|c|c|c|}
\hline Alloy & $\begin{array}{l}\text { Grams } \\
\text { per cu- } \\
\text { bic cen- } \\
\text { timeter }\end{array}$ & $\begin{array}{l}\text { Pounds } \\
\text { per cu- } \\
\text { bic inch }\end{array}$ & $\begin{array}{l}\text { Pounds } \\
\text { per } \\
\text { square } \\
\text { foot per } \\
\text { inch } \\
\text { thick }\end{array}$ \\
\hline $\begin{array}{l}\text { Commercial brass } \\
\text { Naval brass. } \\
\text { Gilding metal } \\
\text { Cupro-nickel (Benedict nickel) } \\
\text { Nickel silver._............ }\end{array}$ & $\begin{array}{l}8.47 \\
8.41 \\
8.79 \\
8.92 \\
8.75\end{array}$ & $\begin{array}{l}0.306 \\
.304 \\
.318 \\
.322 \\
.316\end{array}$ & $\begin{array}{l}44.06 \\
43.75 \\
45.73 \\
46.41 \\
45.52\end{array}$ \\
\hline
\end{tabular}

Tests of samples showed that, in general, the density of nonferrous sheets is somewhat higher than that of bars. Also a variation averaging about onehalf per cent among different thicknesses of sheet samples of brass of the same nominal composition was noted, but a sufficient number of samples was not tested and chemically analyzed to determine a consistent relationship.

Aluminum alloys. - The weights of certain aluminum-alloy sheets and plates rolled to the American wire gage are given in Table 15 . These weights are based on densities obtained from Aluminum Co. of America data sheet No. 389, November 15, 1929, as follows:

\begin{tabular}{|c|c|c|c|}
\hline Alloy & $\begin{array}{l}\text { Grams } \\
\text { per cu- } \\
\text { bic cen- } \\
\text { timeter }\end{array}$ & $\begin{array}{l}\text { Pounds } \\
\text { per cu- } \\
\text { bic inch }\end{array}$ & $\begin{array}{l}\text { Pounds } \\
\text { per } \\
\text { square } \\
\text { foot per } \\
\text { inch } \\
\text { thick }\end{array}$ \\
\hline $\begin{array}{l}\text { Aluminum manganese alloy }(3 \mathrm{~S}) \\
\text { Aluminum alloy } 17 \mathrm{~S} \text { and duralumin. } \\
\text { Aluminum alloy } 25 \mathrm{~S} \\
\text { Aluminum alloy } 51 \mathrm{~S}\end{array}$ & $\begin{array}{l}2.74 \\
2.79 \\
2.79 \\
2.68\end{array}$ & $\begin{array}{r}0.099 \\
.101 \\
.101 \\
.097\end{array}$ & $\begin{array}{l}14.25 \\
14.51 \\
14.51 \\
13.94\end{array}$ \\
\hline
\end{tabular}

TABLE 13.-American wire gage, weights of copper and aluminum sheets and plates

\begin{tabular}{|c|c|c|c|c|c|c|c|c|c|}
\hline \multirow{3}{*}{$\begin{array}{c}\text { Gage } \\
\text { No. } \\
1\end{array}$} & \multirow{2}{*}{\multicolumn{2}{|c|}{ Thickness }} & \multicolumn{2}{|c|}{$\begin{array}{l}\text { Approxi- } \\
\text { mate weight } \\
\text { per square } \\
\text { foot } 1\end{array}$} & \multirow{3}{*}{$\begin{array}{l}\text { Gage } \\
\text { No. } \\
1\end{array}$} & \multirow{2}{*}{\multicolumn{2}{|c|}{ Thickness }} & \multicolumn{2}{|c|}{$\begin{array}{l}\text { A pproxi- } \\
\text { mate weight } \\
\text { per square } \\
\text { foot } 1\end{array}$} \\
\hline & & & \multirow{2}{*}{$\begin{array}{c}\begin{array}{c}\text { Cop- } \\
\text { per }\end{array} \\
4\end{array}$} & \multirow{2}{*}{$\begin{array}{c}\begin{array}{c}\text { Alu- } \\
\text { mi- } \\
\text { num }\end{array} \\
5\end{array}$} & & & & $\begin{array}{l}\text { CoD- } \\
\text { per }\end{array}$ & $\begin{array}{c}\text { Alu- } \\
\text { mi- } \\
\text { num }\end{array}$ \\
\hline & 2 & 3 & & & & 2 & 3 & 1 & 5 \\
\hline $\begin{array}{l}0000 \\
000\end{array}$ & $\begin{array}{l}\text { Inch } \\
0.4600 \\
.4096 \\
.3648 \\
.3249 \\
.2893\end{array}$ & \begin{tabular}{r|}
$m m$ \\
11.68 \\
10.40 \\
9.266 \\
8.252 \\
7.348
\end{tabular} & $\begin{array}{l}\text { Lb.s. } \\
21.27 \\
18.94 \\
16.87 \\
15.03 \\
13.38\end{array}$ & $\begin{array}{l}\text { Lbs. } \\
6.49 \\
5.78 \\
5.14 \\
458 \\
4.08\end{array}$ & $\begin{array}{l}19 \\
20 \\
21 \\
22 \\
23\end{array}$ & $\begin{array}{c}\text { Inch } \\
0.0359 \\
.0320 \\
.0285 \\
.0253 \\
.0225\end{array}$ & \begin{tabular}{|c|}
$m m$ \\
0.9116 \\
.8118 \\
.7230 \\
.6438 \\
.5733
\end{tabular} & $\begin{array}{r}L b 8 . \\
1.660 \\
1.480 \\
1.318 \\
1.170 \\
1.045\end{array}$ & $\begin{array}{c}\text { Lb8. } \\
0.506 \\
.451 \\
.402 \\
.3567 \\
.3186\end{array}$ \\
\hline & $\begin{array}{l}.2576 \\
.2294 \\
.2043 \\
.1819 \\
.1620\end{array}$ & $\begin{array}{l}6.544 \\
5.827 \\
5.189 \\
4.621 \\
4.115\end{array}$ & \begin{tabular}{|r|}
1.91 \\
10.61 \\
9.45 \\
8.41 \\
7.49
\end{tabular} & $\begin{array}{l}3.632 \\
3.234 \\
2.880 \\
2.565 \\
2.284\end{array}$ & $\begin{array}{l}24 .- \\
25 .- \\
26- \\
27- \\
28 .\end{array}$ & $\begin{array}{l}.0201 \\
.0179 \\
.0159 \\
.0142 \\
.0126\end{array}$ & $\begin{array}{l}.5106 \\
.4547 \\
.4049 \\
.3606 \\
.3211\end{array}$ & $\begin{array}{l}.930 \\
.828 \\
.735 \\
.657 \\
.583\end{array}$ & $\begin{array}{l}.2834 \\
.2524 \\
.2242 \\
.2002 \\
.1776\end{array}$ \\
\hline & $\begin{array}{l}.1413 \\
.1285 \\
.1144 \\
.1019\end{array}$ & $\begin{array}{l}3.665 \\
3.264 \\
2.906 \\
2.588\end{array}$ & $\begin{array}{l}\text { 6. } 67 \\
5.94 \\
5.29 \\
4.713\end{array}$ & $\begin{array}{l}2.034 \\
1.812 \\
1.613 \\
1.137\end{array}$ & $\begin{array}{l}29 \ldots \\
30 \ldots \\
31 \ldots \\
32 \ldots\end{array}$ & $\begin{array}{l}.0113 \\
.0100 \\
.00893 \\
.00795\end{array}$ & $\begin{array}{l}.2859 \\
.2546 \\
.2268 \\
.2019\end{array}$ & $\begin{array}{l}.523 \\
.4625 \\
.4130 \\
.3677\end{array}$ & $\begin{array}{l}.1593 \\
.1410 \\
.1259 \\
.1121\end{array}$ \\
\hline $\begin{array}{l}11 . . . . \\
12 . . . \\
13 . . . \\
14 \ldots \\
\end{array}$ & $\begin{array}{l}.0907 \\
.0808 \\
.0720 \\
.0641\end{array}$ & $\begin{array}{l}2.305 \\
2.053 \\
1.828 \\
1.628\end{array}$ & $\begin{array}{l}4.195 \\
3.737 \\
3.330 \\
2.965\end{array}$ & $\begin{array}{r}1.279 \\
1.139 \\
1.015 \\
.904\end{array}$ & $\begin{array}{l}33 . . \\
34 .- \\
35 .- \\
36 . .\end{array}$ & $\begin{array}{l}.00708 \\
.00630 \\
.00561 \\
.00500\end{array}$ & $\begin{array}{l}.1798 \\
.1601 \\
.1426 \\
.1270\end{array}$ & $\begin{array}{l}.3274 \\
.2914 \\
.2595 \\
.2312\end{array}$ & $\begin{array}{l}.0998 \\
.0888 \\
.0791 \\
.0705\end{array}$ \\
\hline $\begin{array}{l}15 . \\
16 . \\
17- \\
18 .\end{array}$ & $\begin{array}{l}.0571 \\
.0508 \\
.0153 \\
.0403\end{array}$ & $\begin{array}{l}1.450 \\
1.291 \\
1.150 \\
1.024\end{array}$ & $\begin{array}{l}\text { 2. } 641 \\
\text { 2. } 349 \\
\text { 1. } 095 \\
\text { 1. } 864\end{array}$ & $\begin{array}{l}.805 \\
.716 \\
.639 \\
.568\end{array}$ & $\begin{array}{l}37 \\
38 \\
39 \\
40\end{array}$ & $\begin{array}{l}.00445 \\
.00397 \\
.00353 \\
.00314\end{array}$ & $\begin{array}{l}.1131 \\
.1007 \\
.0897 \\
.0799\end{array}$ & $\begin{array}{l}.2058 \\
.1836 \\
.1633 \\
.1452\end{array}$ & $\begin{array}{l}.0627 \\
.0560 \\
.0498 \\
.0443\end{array}$ \\
\hline
\end{tabular}

1 The equivalent weights correspond to the gage thicknesses as rounded off in column 2 , and are carried out further than they should be used practically.

TABLE 14.-American wire gage, weights of copperalloy sheets and plates

\begin{tabular}{|c|c|c|c|c|c|c|c|}
\hline \multirow{3}{*}{$\begin{array}{c}\text { Gage } \\
\text { No. } \\
1\end{array}$} & \multirow{2}{*}{\multicolumn{2}{|c|}{ Thickness }} & \multicolumn{5}{|c|}{ A pproximate weight per square foot 1} \\
\hline & & & $\begin{array}{l}\text { Com- } \\
\text { mercial }\end{array}$ & Naval & $\begin{array}{l}\text { Red } \\
\text { brass }\end{array}$ & Cupro- & Nickel \\
\hline & 2 & 3 & 4 & 5 & 6 & 7 & 8 \\
\hline $\begin{array}{l}0000 \\
000 \\
00 \\
0\end{array}$ & $\begin{array}{c}\text { Inch } \\
0.4600 \\
.4096 \\
.3648 \\
.3249 \\
.2893\end{array}$ & $\begin{array}{c}m m \\
11.68 \\
10.40 \\
9.266 \\
8.252 \\
7.348\end{array}$ & $\begin{array}{l}\text { Pounds } \\
20.27 \\
18.05 \\
16.07 \\
14.32 \\
12.75\end{array}$ & \begin{tabular}{|l} 
Pounds \\
20.13 \\
17.92 \\
15.96 \\
14.21 \\
12.66
\end{tabular} & $\begin{array}{l}\text { Pounds } \\
21.04 \\
18.73 \\
16.68 \\
14.86 \\
13.23\end{array}$ & \begin{tabular}{|c|} 
Pounds \\
21.35 \\
19.01 \\
16.93 \\
15.08 \\
13.42
\end{tabular} & $\begin{array}{c}\text { Pounds } \\
20.94 \\
18.64 \\
16.61 \\
14.79 \\
13.17\end{array}$ \\
\hline $\begin{array}{l}2 \\
3 \\
3 \\
4 \\
5 \\
6 \\
6\end{array}$ & $\begin{array}{l}.2576 \\
.2294 \\
.2043 \\
.1819 \\
.1620\end{array}$ & $\begin{array}{l}6.544 \\
5.827 \\
5.189 \\
4.621 \\
4.115\end{array}$ & $\begin{array}{r}11.35 \\
10.11 \\
9.00 \\
8.01 \\
7.14\end{array}$ & $\begin{array}{r}11.27 \\
10.04 \\
8.94 \\
7.96 \\
7.09\end{array}$ & $\begin{array}{r}11.78 \\
10.49 \\
9.34 \\
8.32 \\
7.41\end{array}$ & $\begin{array}{r}11.95 \\
10.65 \\
9.48 \\
8.44 \\
7.52\end{array}$ & $\begin{array}{r}11.73 \\
10.44 \\
9.30 \\
8.28 \\
7.37\end{array}$ \\
\hline $\begin{array}{l}7 . . . \\
8 \\
9 \\
9 \\
10 \\
11 . . . \\
\end{array}$ & $\begin{array}{l}.1443 \\
.1285 \\
.1144 \\
.1019 \\
.0907\end{array}$ & $\begin{array}{l}3.665 \\
3.264 \\
2.906 \\
2.588 \\
2.305\end{array}$ & $\begin{array}{l}6.36 \\
5.66 \\
5.04 \\
4.490 \\
3.996\end{array}$ & $\begin{array}{l}6.31 \\
5.62 \\
5.01 \\
4.458 \\
3.968\end{array}$ & $\begin{array}{l}6.60 \\
\text { 5. } 88 \\
5.23 \\
4.660 \\
4.148\end{array}$ & $\begin{array}{l}\text { 6. } 70 \\
5.96 \\
5.31 \\
4.729 \\
4.209\end{array}$ & $\begin{array}{l}6.57 \\
5.85 \\
5.21 \\
4.638 \\
4.129\end{array}$ \\
\hline $\begin{array}{l}12 \\
13-. . \\
14-. . \\
15 \\
16-. . \\
16\end{array}$ & $\begin{array}{l}.0808 \\
.0720 \\
.0641 \\
.0571 \\
.0508\end{array}$ & $\begin{array}{l}2.053 \\
1.828 \\
1.628 \\
1.450 \\
1.291\end{array}$ & \begin{tabular}{|l}
3.560 \\
3.172 \\
2.824 \\
2.516 \\
2.238
\end{tabular} & 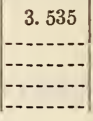 & $\begin{array}{l}3.695 \\
3.292 \\
2.931 \\
2.611 \\
2.323\end{array}$ & $\begin{array}{l}3.750 \\
3.341 \\
2.975 \\
2.650 \\
2.357\end{array}$ & $\begin{array}{l}3.678 \\
\text { 3. } 277 \\
2.918 \\
2.599 \\
2.312\end{array}$ \\
\hline
\end{tabular}


TABLE 14.-American wire gage, weights of copperalloy sheets and plates-Continued

\begin{tabular}{|c|c|c|c|c|c|c|c|}
\hline \multirow[b]{2}{*}{$\begin{array}{l}\text { Gage } \\
\text { No. }\end{array}$} & \multirow{2}{*}{\multicolumn{2}{|c|}{ Thickness }} & \multicolumn{5}{|c|}{ Approximate weight per square foot } \\
\hline & & & $\begin{array}{c}\text { Com- } \\
\text { mercial } \\
\text { (high) } \\
\text { brass }\end{array}$ & $\begin{array}{l}\text { Navas } \\
\text { brass }\end{array}$ & $\begin{array}{c}\text { Red } \\
\text { brass } \\
\text { or } \\
\text { gilding } \\
\text { metal }\end{array}$ & $\begin{array}{l}\text { Cupro- } \\
\text { nickel }\end{array}$ & $\begin{array}{l}\text { Nickel } \\
\text { silver }\end{array}$ \\
\hline 1 & 2 & 3 & 4 & 5 & 6 & 7 & 8 \\
\hline$\therefore$ & $\begin{array}{l}\text { Inch } \\
0.0453 \\
.0403 \\
.0359 \\
.0320 \\
.0285\end{array}$ & $\begin{array}{l}m m \\
1.150 \\
1.024 \\
.9116 \\
.8118 \\
.7230\end{array}$ & \begin{tabular}{|l|} 
Pounds \\
1.996 \\
1.776 \\
1.582 \\
1.410 \\
1.256
\end{tabular} & \begin{tabular}{|c|} 
Pounds \\
\\
-
\end{tabular} & $\begin{array}{l}\text { Pounds } \\
2.072 \\
1.843 \\
1.642 \\
1.463 \\
1.303\end{array}$ & \begin{tabular}{|c|} 
Pounds \\
2.102 \\
1.870 \\
1.666 \\
1.485 \\
1.323
\end{tabular} & $\begin{array}{l}\text { Pounds } \\
2.062 \\
1.834 \\
1.634 \\
1.457 \\
1.297\end{array}$ \\
\hline & $\begin{array}{l}.0253 \\
.0226 \\
.0201 \\
.0179 \\
.0159\end{array}$ & $\begin{array}{r}.6438 \\
.5733 \\
.5106 \\
.4547 \\
.4049\end{array}$ & $\begin{array}{r}1.115 \\
.996 \\
.886 \\
.789 \\
.701\end{array}$ & & $\begin{array}{r}1.157 \\
1.033 \\
.919 \\
.819 \\
.727\end{array}$ & $\begin{array}{r}1.174 \\
1.049 \\
.933 \\
.831 \\
.738\end{array}$ & $\begin{array}{r}1.152 \\
1.029 \\
.915 \\
.815 \\
.724\end{array}$ \\
\hline 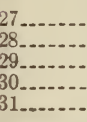 & $\begin{array}{l}.0142 \\
.0126 \\
.0113 \\
.0100 \\
.00893\end{array}$ & $\begin{array}{r}.3606 \\
.3211 \\
.2859 \\
.2546 \\
.2268\end{array}$ & $\begin{array}{l}.626 \\
.555 \\
.498 \\
.4406 \\
.3935\end{array}$ & & $\begin{array}{l}.649 \\
.576 \\
.517 \\
.4573 \\
.4084\end{array}$ & $\begin{array}{l}.659 \\
.555 \\
.524 \\
.4641 \\
.4144\end{array}$ & $\begin{array}{l}.646 \\
.574 \\
.514 \\
.4552 \\
.4065\end{array}$ \\
\hline S & $\begin{array}{l}.00795 \\
.00703 \\
.00630 \\
.00561 \\
.00500\end{array}$ & $\begin{array}{r}.2019 \\
.1795 \\
.1601 \\
.1426 \\
.1270\end{array}$ & $\begin{array}{l}.3503 \\
.3119 \\
.2776 \\
.2472 \\
.2203\end{array}$ & & $\begin{array}{l}.3035 \\
.3238 \\
.2881 \\
.2565 \\
.2286\end{array}$ & $\begin{array}{l}.3659 \\
.3286 \\
.2924 \\
.2604 \\
.2320\end{array}$ & $\begin{array}{r}.3619 \\
.3223 \\
.2868 \\
.2554 \\
.2276\end{array}$ \\
\hline & $\begin{array}{l}.00445 \\
.00397 \\
.00353 \\
.00314\end{array}$ & $\begin{array}{l}.1131 \\
.1007 \\
.0897 \\
.0799\end{array}$ & $\begin{array}{l}1961 \\
.1749 \\
.1555 \\
.1383\end{array}$ & & $\begin{array}{l}.2035 \\
.1815 \\
.1614 \\
.1436\end{array}$ & $\begin{array}{l}.2065 \\
.1842 \\
.1635 \\
.1457\end{array}$ & $\begin{array}{l}.2026 \\
.1807 \\
.1607 \\
.1429\end{array}$ \\
\hline
\end{tabular}

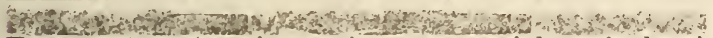
TABLE 15.-American wire gage, weights of aluminum-alloy sheets and plates

\begin{tabular}{|c|c|c|c|c|c|}
\hline \multirow[b]{2}{*}{ Gage No. } & \multirow{2}{*}{\multicolumn{2}{|c|}{ Thickness }} & \multicolumn{3}{|c|}{$\begin{array}{l}\text { Approximate weight per } \\
\text { square foot } 1\end{array}$} \\
\hline & & & $\begin{array}{l}\text { Alumi- } \\
\text { num } \\
\text { manga- } \\
\text { nese } \\
\text { alloy } 3 S\end{array}$ & $\begin{array}{l}\text { Alumi- } \\
\text { num } \\
\text { alloys } \\
17 \mathrm{~S}, 25 \mathrm{~S} \text {, } \\
\text { and du- } \\
\text { ralumin }\end{array}$ & $\begin{array}{l}\text { Alumi- } \\
\text { num } \\
\text { alloy } \\
51 \mathrm{~S}\end{array}$ \\
\hline 1 & 2 & 3 & 4 & 5 & 6 \\
\hline $\begin{array}{l}0000 \ldots \\
000 \ldots \\
00 \\
0 . . .- \\
1 . . . . .\end{array}$ & $\begin{array}{l}\text { Inch } \\
0.4600 \\
.4096 \\
.3648 \\
.3249 \\
.2893\end{array}$ & $\begin{array}{l}m m \\
11.68 \\
10.40 \\
9.266 \\
8.252 \\
7.348\end{array}$ & $\begin{array}{c}\text { Pounds } \\
6.56 \\
5.84 \\
5.20 \\
4.63 \\
4.12\end{array}$ & $\begin{array}{c}\text { Pounds } \\
6.68 \\
5.95 \\
5.29 \\
4.72 \\
4.20\end{array}$ & $\begin{array}{r}\text { Pounds } \\
6.41 \\
5.71 \\
5.09 \\
4.53 \\
4.03\end{array}$ \\
\hline $\begin{array}{l}2 \ldots \ldots . \\
3 \ldots \ldots . \\
4 \ldots \ldots \\
5 \ldots . . . \\
6\end{array}$ & $\begin{array}{l}.2576 \\
.2294 \\
.2043 \\
.1819 \\
.1620\end{array}$ & $\begin{array}{l}\text { 6. } 544 \\
5.827 \\
5.189 \\
4.621 \\
4.115\end{array}$ & $\begin{array}{l}\text { 3. } 671 \\
\text { 3. } 269 \\
\text { 2. } 911 \\
\text { 2. } 592 \\
2.308\end{array}$ & $\begin{array}{l}\text { 3. } 738 \\
3.329 \\
2.964 \\
2.640 \\
2.351\end{array}$ & $\begin{array}{l}\text { 3. } 591 \\
\text { 3. } 198 \\
2.848 \\
2.536 \\
2.258\end{array}$ \\
\hline
\end{tabular}

1 The equivalent weights correspond to the gage thickness as rounded off in column 2, and are carried out further than they should be used practically.
TABLE 15.-American wire gage, weights of aluminum-alloy sheets and plates-Continued

\begin{tabular}{|c|c|c|c|c|c|}
\hline \multirow[b]{2}{*}{ Gage No. } & \multirow{2}{*}{\multicolumn{2}{|c|}{ Thickness }} & \multicolumn{3}{|c|}{$\begin{array}{l}\text { Approximate weight per } \\
\text { square foot }\end{array}$} \\
\hline & & & $\begin{array}{l}\text { Alumi- } \\
\text { num } \\
\text { manga- } \\
\text { nese } \\
\text { alloy } 3 \mathrm{~S}\end{array}$ & $\begin{array}{l}\text { Alumi- } \\
\text { num } \\
\text { alloys } \\
17 \mathrm{~S}, 25 \mathrm{~S}, \\
\text { and du- } \\
\text { ralumin }\end{array}$ & $\begin{array}{l}\text { Alumi- } \\
\text { num } \\
\text { alloy } \\
51 \mathrm{~S}\end{array}$ \\
\hline 1 & 2 & 3 & 4 & 5 & 6 \\
\hline $\begin{array}{l}7 \\
8 \\
9 \\
10\end{array}$ & $\begin{array}{l}\text { Inch } \\
0.1443 \\
.1285 \\
.1144 \\
.1019 \\
.0907\end{array}$ & $\begin{array}{l}m m \\
3.665 \\
3.204 \\
2.906 \\
2.588 \\
2.305\end{array}$ & $\begin{array}{l}\text { Pounds } \\
2.056 \\
1.831 \\
1.630 \\
1.452 \\
1.292\end{array}$ & $\begin{array}{c}\text { Pounds } \\
2.094 \\
1.865 \\
1.660 \\
1.479 \\
1.316\end{array}$ & $\begin{array}{r}\text { Pounds } \\
2.012 \\
1.792 \\
1.595 \\
1.420 \\
1.264\end{array}$ \\
\hline 12 & $\begin{array}{l}.0508 \\
.0720 \\
.0641 \\
.0571 \\
.0508\end{array}$ & $\begin{array}{l}2.053 \\
1.828 \\
1.628 \\
1.450 \\
1.291\end{array}$ & $\begin{array}{r}1.151 \\
1.026 \\
.913 \\
.814 \\
.724\end{array}$ & $\begin{array}{r}1.172 \\
1.045 \\
.930 \\
.829 \\
.737\end{array}$ & $\begin{array}{r}1.126 \\
1.004 \\
.894 \\
.796 \\
.708\end{array}$ \\
\hline 17 & $\begin{array}{l}.0453 \\
.0403 \\
.0359 \\
.0320 \\
.0285\end{array}$ & $\begin{array}{l}1.150 \\
1.024 \\
.9116 \\
.8118 \\
.7230\end{array}$ & $\begin{array}{l}.645 \\
.574 \\
.512 \\
.456 \\
.406\end{array}$ & $\begin{array}{l}.657 \\
.585 \\
.521 \\
.464 \\
.414\end{array}$ & $\begin{array}{l}.631 \\
.562 \\
.501 \\
.446 \\
.397\end{array}$ \\
\hline $\begin{array}{l}22 \\
23 \\
24 \\
25 \\
26\end{array}$ & $\begin{array}{l}.0253 \\
.0226 \\
.0201 \\
.0179 \\
.0159\end{array}$ & $\begin{array}{l}.6438 \\
.5733 \\
.5106 \\
.4547 \\
.4049\end{array}$ & $\begin{array}{l}.3605 \\
.3221 \\
.2864 \\
.2551 \\
.2266\end{array}$ & $\begin{array}{l}.3671 \\
.3280 \\
.2917 \\
.2597 \\
.2307\end{array}$ & $\begin{array}{l}.3527 \\
.3150 \\
.2802 \\
.2495 \\
.2216\end{array}$ \\
\hline $\begin{array}{l}27 \\
28 \\
29 \\
30 \\
31\end{array}$ & $\begin{array}{l}.0142 \\
.0126 \\
.0113 \\
.0100 \\
.00893\end{array}$ & $\begin{array}{l}.3606 \\
.3211 \\
.2859 \\
.2546 \\
.2263\end{array}$ & $\begin{array}{l}.2024 \\
.1796 \\
.1610 \\
.1425 \\
.1273\end{array}$ & $\begin{array}{l}.2060 \\
.1828 \\
.1640 \\
.1451 \\
.1296\end{array}$ & $\begin{array}{l}.1980 \\
.1756 \\
.1575 \\
.1394 \\
.1245\end{array}$ \\
\hline $\begin{array}{l}32 \\
33 \\
34 \\
35 \\
36\end{array}$ & $\begin{array}{l}.00795 \\
.00708 \\
.00630 \\
.00561 \\
.00500\end{array}$ & $\begin{array}{l}.2019 \\
.1798 \\
.1601 \\
.1426 \\
.1270\end{array}$ & $\begin{array}{l}.1133 \\
.1009 \\
.0898 \\
.0800 \\
.0713\end{array}$ & $\begin{array}{l}.1154 \\
.1027 \\
.0914 \\
.0814 \\
.0726\end{array}$ & $\begin{array}{l}.1108 \\
.0987 \\
.0878 \\
.0782 \\
.0697\end{array}$ \\
\hline $\begin{array}{l}37 \\
38 \\
39 \\
40\end{array}$ & $\begin{array}{l}.00445 \\
.00397 \\
.00353 \\
.00314\end{array}$ & $\begin{array}{l}.1131 \\
.1007 \\
.0897 \\
.0799\end{array}$ & $\begin{array}{l}.0634 \\
.0566 \\
.0503 \\
.0447\end{array}$ & $\begin{array}{l}.0646 \\
.0576 \\
.0512 \\
.0456\end{array}$ & $\begin{array}{l}.0620 \\
.0553 \\
.0492 \\
.0438\end{array}$ \\
\hline
\end{tabular}

(b) Permissible Variations in Thickness and Weight

(The available data as to tolerances applied to thicknesses of copper, aluminum, brass, and other nonferrous-alloy plates, sheets, and strips are contained in tabulations in this circular. One tabulation gives the permissible variations in thickness of wrought nonferrous sheets and strips of naval brass, wrought aluminum bronze, phosphor bronze, cupronickel, nickel silver, and manganese bronze. This is identical with the table of permissible variations in thickness of German silver sheets and strips in F.S. B. specification QQ-N-321. (See 655.2, p.1287.) The other tabulation gives the permissible variations in thickness of wrought nonferrous sheets and strips of copper, low and rich low brass, commercial brass, and gilding metal. This is identical with the table of permissible variations in thickness of copper sheets and strips in F. S. B. specification QQ-C-501. (See 641.21, p. 1177.)) 
TABLE 18.--Permissible variations in thickness of flat aluminum sheets, plates, and strips (commercially pure aluminum (2S) and aluminum manganese (1.25 per cent) alloy (3S))

\begin{tabular}{|c|c|c|c|c|c|c|c|c|}
\hline \multicolumn{2}{|c|}{ Ameican wire gage No. } & \multicolumn{2}{|c|}{ Thickness } & \multicolumn{3}{|c|}{ Sheets only 1} & \multicolumn{2}{|c|}{$\begin{array}{l}\text { Sheets, plates, and } \\
\text { strips } 2\end{array}$} \\
\hline From- & $\begin{array}{l}\text { To and } \\
\text { includ- } \\
\text { ing- }\end{array}$ & From- & $\begin{array}{l}\text { To and } \\
\text { includ- } \\
\text { ing- }\end{array}$ & $\begin{array}{l}20 \text { inches } \\
\text { and un- } \\
\text { der }\end{array}$ & $\begin{array}{l}\text { Over } 20 \\
\text { to } 36 \\
\text { inches, } \\
\text { inclusive }\end{array}$ & $\begin{array}{c}\text { Over } 36 \\
\text { to } 60 \\
\text { inches, } \\
\text { inclusive }\end{array}$ & $\begin{array}{l}30 \text { inches } \\
\text { and un- } \\
\text { dar }\end{array}$ & $\begin{array}{l}\text { Over } 30 \\
\text { inclies }\end{array}$ \\
\hline 1 & 2 & 3 & 4 & 5 & 6 & 7 & 8 & 9 \\
\hline $\begin{array}{l}2 \\
5 \\
10 \\
14\end{array}$ & \begin{tabular}{r}
3 \\
\hdashline 9 \\
13 \\
17
\end{tabular} & $\begin{array}{r}\text { Inch } \\
0.2576 \\
.2043 \\
.1819 \\
.1019 \\
.0641\end{array}$ & $\begin{array}{c}\text { Inch } \\
0.2294 \\
.1144 \\
.0720 \\
.0453\end{array}$ & $\begin{array}{r}\text { Inch } \\
\pm 0.0070 \\
\pm .0050 \\
\pm .0050 \\
\pm .0030 \\
\pm .0025\end{array}$ & $\begin{array}{r}\text { Inch } \\
\pm 0.0080 \\
\pm .0060 \\
\pm .0060 \\
\pm .0035 \\
\pm .0025\end{array}$ & $\begin{aligned} & \text { Inch } \\
& \pm 0.0090 \\
& \pm .0070 \\
& \pm .0070 \\
& \pm .0040 \\
& \pm .0030\end{aligned}$ & $\begin{array}{r}\text { Inch } \\
\pm 0.0100 \\
\pm .0100 \\
\pm .0060 \\
\pm .0030 \\
\pm .0030\end{array}$ & $\begin{aligned} & \text { Inch } \\
& \pm 0.0100 \\
& \pm .0100 \\
& \pm .0065 \\
& \pm .003 .5 \\
& \pm .0035\end{aligned}$ \\
\hline $\begin{array}{l}18 \ldots \\
22 \\
25 \\
27 \\
28\end{array}$ & $\begin{array}{l}21 \\
24 \\
26 \\
-36\end{array}$ & $\begin{array}{l}.0403 \\
.0253 \\
.0179 \\
.0142 \\
.0126\end{array}$ & $\begin{array}{l}.0285 \\
.0201 \\
.0159 \\
.0050\end{array}$ & $\begin{array}{l} \pm .0025 \\
\pm .0020 \\
\pm .0020 \\
\pm .0020 \\
\pm .0015\end{array}$ & $\begin{array}{l} \pm .0025 \\
\pm .0020 \\
\pm .0020 \\
\pm .0020 \\
\pm .0015\end{array}$ & $\begin{array}{l} \pm .0030 \\
\pm .0030 \\
\pm .0020 \\
\pm .0020 \\
\pm .0015\end{array}$ & $\begin{array}{l} \pm .0025 \\
\pm .0020 \\
\pm .0020 \\
\pm .0015 \\
\pm .0015\end{array}$ & $\begin{array}{l} \pm .0030 \\
\pm .0025 \\
\pm .0025 \\
\pm .0020 \\
\pm .0020\end{array}$ \\
\hline
\end{tabular}

Presented in American Society for Testing Materials standard specification B25-29 (see 631.23, p. 1154), and Aluminum Co. of A merican data sheet No. 669.742, June 1, 1929. Material having thickness greater than 0.250 inch is considered plate, and is corered by plate tolerances. 2 Presented in-

1. United States Army metal specifications No. 57-151, May 9, 1923. The tolerances given in column 8 also apply to aluminum-alloy plates, sheets, and strips, United States Arma metal specifications No. 57-152, May 25, 1923.

2. Society of Automotive Engineers specification No. 78, July, 1930. (See 631.23, p. 1156.)

TABLE 19.-Permissible variations in thickness of coiled aluminum sheet, commercial practice

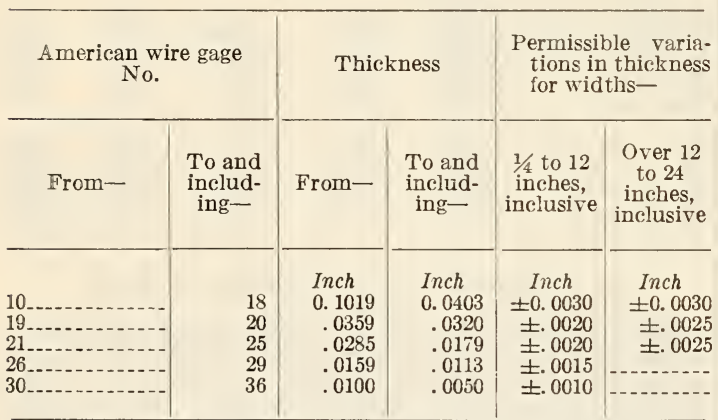

Note.-The above tolerances are taken from Aluminum Co. of America data sheet No. 669.742, June 1, 1929.

\section{OTHER GAGES FOR COPPER SHEETS}

Copper sheets are frequently made in definite weights per square foot. This practice is customary in the heavier flat sheets. Table 20 shows the corresponding approximate thicknesses, which are based on a density of 8.89 grams per cubic centimeter, 555 pounds per cubic foot, or 46.25 pounds per square foot per inch thick.

Copper sheets can also be obtained in fractionalinch thicknesses varying by sixteenths of an inch from one-sixteenth to 2 inches. Also the Birmingham wire gage has been used in designating thicknesses of copper sheets. Permissible variations in weight and thickness of certain fractional-inch sizes will be found in A. S. T. M., B11-18 (see 641.21, p. 1176).
TABLE 20.-Ccpper sheets furnished in weights per square foot

\begin{tabular}{|c|c|c|c|c|c|}
\hline $\begin{array}{l}\text { Weight } \\
\text { per } \\
\text { square } \\
\text { foot } \\
\text { (ounces) }\end{array}$ & $\begin{array}{l}\text { Approxi- } \\
\text { mate } \\
\text { thick- } \\
\text { ness }\end{array}$ & $\begin{array}{l}\text { Weight } \\
\text { per } \\
\text { square } \\
\text { foot } \\
\text { (ounces) }\end{array}$ & $\begin{array}{l}\text { Approxi- } \\
\text { mate } \\
\text { thick- } \\
\text { ness }\end{array}$ & $\begin{array}{c}\text { Weight } \\
\text { per } \\
\text { square } \\
\text { foot } \\
\text { (pounds) }\end{array}$ & $\begin{array}{l}\text { Approxi- } \\
\text { mate } \\
\text { thick- } \\
\text { ness }\end{array}$ \\
\hline $\begin{array}{l}2 \\
4 \\
6 \ldots \ldots \\
7 \\
8\end{array}$ & $\begin{array}{l}\text { Inch } \\
0.0027 \\
.0054 \\
.0081 \\
.0095 \\
.0105\end{array}$ & $\begin{array}{l}24 \\
26 \ldots \\
28 \\
32 \\
36 \ldots\end{array}$ & $\begin{array}{l}\text { Inch } \\
0.0324 \\
.0351 \\
.0378 \\
.0432 \\
.0486\end{array}$ & $\begin{array}{l}5 \ldots \\
51 / 2 \ldots \\
6 \ldots \\
61 / 2 \ldots \\
7 \ldots\end{array}$ & $\begin{array}{l}\text { Inch } \\
0.1081 \\
.1189 \\
.1297 \\
.1405 \\
.1514\end{array}$ \\
\hline $\begin{array}{l}9 \\
10 \\
11 \\
12 \\
13\end{array}$ & $\begin{array}{l}.0122 \\
.0135 \\
.0149 \\
.0162 \\
.0176\end{array}$ & $\begin{array}{l}40 \\
44 \\
46 \\
48 \\
52\end{array}$ & $\begin{array}{l}.0511 \\
.0595 \\
.0622 \\
.0649 \\
.0703\end{array}$ & $\begin{array}{l}71 / 2 \\
8- \\
81 / 2- \\
9- \\
91 / 2-\end{array}$ & $\begin{array}{l}.1622 \\
.1730 \\
.1838 \\
.1946 \\
.2054\end{array}$ \\
\hline $\begin{array}{l}14 \\
15 \\
16 \\
18 \\
20\end{array}$ & $\begin{array}{l}.0189 \\
.0203 \\
.0216 \\
.0243 \\
.0270\end{array}$ & $\begin{array}{l}56 \ldots \\
64 \ldots \\
72 \ldots \\
76 \ldots\end{array}$ & $\begin{array}{l}.0757 \\
.0865 \\
.0973 \\
.1027\end{array}$ & $\begin{array}{l}10 \\
11 \\
12 \\
13 \\
14 \\
15 \\
16\end{array}$ & $\begin{array}{l}.2162 \\
.2378 \\
.2595 \\
.2811 \\
.3027 \\
\\
.3243 \\
.3460\end{array}$ \\
\hline
\end{tabular}

\section{MONEL METAL (NICEEL-COPPER ALLOY)}

Monel-metal sheets (approximately 67 per cent nickel, 28 per cent copper, and $j$ per cent of other elements) are rolled in thicknesses corresponding to the wrought-iron thicknesses of the U. S. standard gage for sheet and plate iron and steel. The corresponding weights per unit area are given in Table 22. The weights per square foot are based on a density of 8.850 grams per cubic centimeter, 552.5 pounds per 
cubic foot, or 46.04 pounds per square foot per inch thick.

Inasmuch as the U. S. standard gage is strictly a weight gage, this practice with regard to thicknesses of Monel-metal sheets represents a deviation from the standard practice. Monel-metal sheets are usually used to replace sheet steel, or steel sheets coated with zinc, both of which come in U. S. standard gage weights. If Monel-metal sheets were rolled to the same weight per unit area as steel sheets, according to the U. S. standard gage, the resulting thicknesses would be quite different from those of standard steel sheets of corresponding gage number, because of the large difference in density of the two metals.

The tolerances on thickness of Monel metal, as given in Table 22, are the practice of the International Nickel Co. When rolled to weight, the tolerances correspond to sheet-steel practice. Permissible variations in thickness given in F. S. B. No. QQ-C-541, will be found in 654.52 , p. 1285 .

TABLE 22.-Monel-metal sheets

\begin{tabular}{l|c|c|c|c|c}
\hline & \multicolumn{3}{|c|}{ Thickness } & \multicolumn{2}{c}{ Weight per square } \\
foot
\end{tabular}

1 To the nearest $1 / 4$ ounce.

\section{AMERICAN ZINC GAGE}

The American zinc gage, commonly used by manufacturers of zinc sheet in the United States, is given in Table 24. The weights per square foot for the thickness given are based on a density of 0.2596 pounds per cubic inch, 37.38 pounds per square foot per inch thick, or 7.186 grams per cubic centimeter, the value given in the specifications cited below.

Permissible viarations in thicknesses of sheet zinc are presented in United States Government, Federal specification QQ-Z-301, and in the American Society for Testing Materials standard specifications for rolled zinc, B69-29 (see 683.2, p. 1311.), wherein rolled zinc is classified as follows:

Type $A$, ribbon zinc and sheets or strips cut from ribbon zinc.-Type A rolled zinc shall be rolled from a single bar in one continuous direction. The permissible variations in thickness of Type A rolled zinc shall be as given in B69-29. Thicknesses of metal falling between the gages shown shall take the tolerances of the nearest gage.

Type B, sheet zinc or strips cut from sheet zinc.-Type B rolled zinc shall be rolled by the pack-rolling method. The permissible variations in thickness of type B rolled zinc shall be as given in B 69-29, wherein such tolerances are expressed as percentages, plus or minus, of the equivalent weights as given in Table 24, and apply only to units or lots of 500 pounds or more.

No individual sheet shall weigh more than the theoretical weight of a sheet of the gage next above. nor less than a sheet of the next gage below.

Type $C$, boiler plate, name plates, tags, etc.-Type $\mathrm{C}$ rolled zinc may be rolled either from a single bar or by the pack-rolling method. The permissible variations in thickness of type $\mathrm{C}$ rolled zinc shall be the same as those for either type A or type B rolled zinc, depending upon whether the material is cut from a ribbon or a sheet.

\section{TABLE 24.-American zinc gage}

\begin{tabular}{|c|c|c|c|c|c|c|c|c|}
\hline $\begin{array}{l}\text { Ameri- } \\
\text { can zinc } \\
\text { gage No. }\end{array}$ & 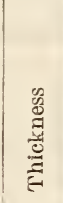 & 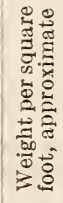 & 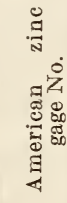 & 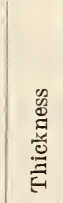 & 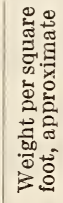 & 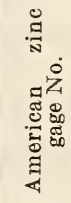 & $\begin{array}{l}\text { Dै } \\
\text { है } \\
\text { है } \\
\text { Eี }\end{array}$ & 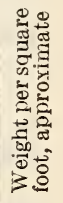 \\
\hline & $\begin{array}{r}\text { Inch } \\
0.002 \\
.004 \\
.006 \\
.008 \\
.010\end{array}$ & $\begin{array}{c}\text { Lbs. } \\
0.075 \\
.150 \\
.224 \\
.299 \\
.374\end{array}$ & $\begin{array}{l}11 \\
12 \\
13 \\
14 \\
15\end{array}$ & $\begin{array}{r}\text { Inch } \\
0.024 \\
.028 \\
.032 \\
.036 \\
.040\end{array}$ & $\begin{array}{r}\text { Lbs. } \\
0.897 \\
1.05 \\
1.20 \\
1.35 \\
1.50\end{array}$ & $\begin{array}{l}21 \\
22 \\
23 \\
24 \\
25\end{array}$ & $\begin{array}{r}\text { Inch } \\
0.080 \\
.090 \\
.100 \\
.125 \\
.250\end{array}$ & $\begin{array}{r}\text { Lbs. } \\
2.99 \\
3.36 \\
3.74 \\
4.67 \\
9.35\end{array}$ \\
\hline $\begin{array}{l}6 .- \\
7 .- \\
8 .- \\
9 \ldots \\
10 .\end{array}$ & $\begin{array}{r}.012 \\
.014 \\
.016 \\
.018 \\
.020\end{array}$ & $\begin{array}{r}.449 \\
.523 \\
.598 \\
.673 \\
.748\end{array}$ & $\begin{array}{l}16- \\
17- \\
18 \\
19 \\
20\end{array}$ & $\begin{array}{l}.045 \\
.050 \\
.055 \\
.060 \\
.070\end{array}$ & $\begin{array}{l}1.68 \\
1.87 \\
2.06 \\
2.24 \\
2.62\end{array}$ & $\begin{array}{l}26 . \\
27 \\
28\end{array}$ & $\begin{array}{r}.375 \\
.500 \\
1.000\end{array}$ & $\begin{array}{l}14.02 \\
18.69 \\
37.38\end{array}$ \\
\hline
\end{tabular}

Note.-Manufacturers' catalogues usually omit Nos. 1 and 2.

\section{SHEET LEAD}

Sheet lead is commonly ordered on the basis of weight per square foot in units as given in column 1 of Table 27. The average weight per cubic foot of sheet lead is about 707.6 pounds, which corresponds to 59.0 pounds per square foot per inch thick. (The value 59.5 is commonly used.) The decimal thicknesses given in column 2, Table 27, are computed on the basis 59.0. The fractional thicknesses in column 3 are those commonly listed in catalogues and handbooks.

United States Government, Federal specification QQ-I-201 specifies the permissible variation in weight of any sheet as \pm 5 per cent (see 651.5, p. 1277). Grade A lead sheet contains no reclaimed lead, and the thickness of any sheet of grade A sheet lead, corresponding to the weight per square-foot specified, may vary within \pm 0.003 inch. 
TABLE 27.-Lead sheets furnished by weight

\begin{tabular}{|c|c|c|c|c|c|c|c|c|}
\hline \multirow{2}{*}{$\begin{array}{l}\text { Weight } \\
\text { per } \\
\text { square } \\
\text { foot (in } \\
\text { pounds) }\end{array}$} & \multicolumn{2}{|c|}{$\begin{array}{l}\text { Approxi- } \\
\text { mate } \\
\text { thick- } \\
\text { ness }\end{array}$} & \multirow{2}{*}{$\begin{array}{l}\text { Weight } \\
\text { per } \\
\text { square } \\
\text { foot (in } \\
\text { pounds) }\end{array}$} & \multicolumn{2}{|c|}{$\begin{array}{l}\text { Approxi- } \\
\text { mate } \\
\text { thick- } \\
\text { ness }\end{array}$} & \multirow{2}{*}{$\begin{array}{l}\text { Weight } \\
\text { per } \\
\text { square } \\
\text { foot (in } \\
\text { pounds) }\end{array}$} & \multicolumn{2}{|c|}{$\begin{array}{l}\text { Approxi- } \\
\text { mate } \\
\text { thick- } \\
\text { ness }\end{array}$} \\
\hline & $\begin{array}{l}\text { Deci- } \\
\text { mal }\end{array}$ & \begin{tabular}{|} 
Bi- \\
nary \\
frac- \\
tion 1
\end{tabular} & & $\begin{array}{c}\text { Deci- } \\
\text { mal }\end{array}$ & $\begin{array}{c}\text { Bi- } \\
\text { nary } \\
\text { frac- } \\
\text { tion t }\end{array}$ & & $\begin{array}{l}\text { Deci- } \\
\text { mal }\end{array}$ & $\begin{array}{l}\text { Bi- } \\
\text { nary } \\
\text { frac- } \\
\text { tion }\end{array}$ \\
\hline 1 & 2 & 3 & 1 & 2 & 3 & 1 & 2 & 3 \\
\hline $\begin{array}{l}11 / 2 \ldots \\
21 / 2 \ldots\end{array}$ & \begin{tabular}{|c|} 
Inch \\
0.0169 \\
.0254 \\
.0339 \\
.0424
\end{tabular} & $\begin{array}{c}\text { Inch } \\
1 / 64 \\
3 / 128 \\
1 / 32 \\
5 / 128\end{array}$ & $\begin{array}{l}5 . \\
6 .- \\
8 . \\
10 \\
\end{array}$ & $\begin{array}{l}\text { Inch } \\
0.0847 \\
.1017 \\
.1356 \\
.1695\end{array}$ & \begin{tabular}{c|} 
Inch \\
$5 / 64$ \\
$3 / 32$ \\
118 \\
$5 / 32$
\end{tabular} & $\begin{array}{l}16 \ldots \ldots \\
20 \ldots \ldots \\
24 \ldots \ldots \\
30 \ldots \ldots\end{array}$ & $\begin{array}{l}\text { Inches } \\
0.2712 \\
.3390 \\
.4068 \\
.5085\end{array}$ & $\begin{array}{l}\text { Inch } \\
9 / 32 \\
11 / 32 \\
13 / 32 \\
1 / 2\end{array}$ \\
\hline $\begin{array}{l}3 \\
31 / 2 \ldots \ldots \\
4\end{array}$ & $\begin{array}{l}.0508 \\
.0593 \\
.0678\end{array}$ & \begin{tabular}{l|}
$3 / 64$ \\
$7 / 128$ \\
$1 / 16$
\end{tabular} & $12 \ldots$ & $\begin{array}{l}.2034 \\
.2373 \\
.2542\end{array}$ & $\begin{array}{l}3 / 16 \\
7 / 32 \\
1 / 4\end{array}$ & $40 \ldots$ & $\begin{array}{r}.6790 \\
1.0169\end{array}$ & $1^{11 / 16}$ \\
\hline
\end{tabular}

1 As commonly listed in catalogues.

641. COPPER

\subsection{GENERAI ITEMS.}

\section{AMERICAN SOCIETY FOR TESTING MATE- RIAIS, STANDARD METHODS OF BAT- TERY ASSAY OF COPPER, SERIAI DESIG- NATION B34-20, 1920}

[A pproved as American tentative standard by the American Standards Association, June 15, 1921]

\section{BATTERY ASSAY OF COPPER}

[Approved by the supervisory committee on standard methods of analysis, American Chemical Society]

The methods that follow are those originally proposed by Heath, ${ }^{6}$ whose work has been checked and elaborated by Keller, Ferguson, and others.

The methods are those generally accepted in the United States for standard analysis in all the larger laboratories of both producers and consumers of copper and copper products.

\section{GENERAL INSTRUCTIONS}

Sampling ingots.-Select 6 ingots at random from the lot. Considering 3 ingots as a rectangular unit, drill 1 hole in each ingot, 1 at the center and 1 at each end of a diagonal of the rectangle. Drill each hole entirely through the ingot, starting from the bottom. Start the drill on the surface sufficiently to remove the oxide before commencing to collect the drillings for the sample. Run the drill so as to prevent oxidation of the chips. Discard all drillings carrying oxide from the "set," or burned by the drill. Use no lubricant on the drill, and if the sample shows oil or grease, remove this with ether. Sift all drillings on a screen with 250 meshes per square centimeter in order to remove material which is ground between the drill and sides of the hole. Extract with a strong magnet any iron which may have come from the drill. Keep the drillings in air-tight bottles.

Weights.-Weights shall be calibrated and frequently rechecked. It is recommended 7 to use a special $5 \mathrm{~g}$ brass weight in weighing the samples and to have the weight of the sample always within $5 \mathrm{mg}$ of $5 \mathrm{~g}$. When the cathode with its deposit is finally weighed the identical weights are to be used with which the cylinder was tared, plus the special $5 \mathrm{~g}$ brass weight. This brings all the change

6 Heath, Transactions, Am. Inst. Min. Engrs., vol. 27, pp. 390$400,1897$.

Greenwood, U.S. Metals Refining Co., Personal Communication. or deficiency in the final weight on a small fractional weight and the beam and rider.

\section{ASSAY OF ELECTROLYTIC AND LOW-RESISTARCE LAKB}

The method described below is applicable to such grades of electrolytic and low-resistance lake copper as are covered by the standard specifications for lake copper wire bars, cakes, slabs, billets, ingots and ingot bars, serial designation: B 4, (see 641.11, p. 1173.) and for electrolytic copper wire bars, cakes, slabs, billets, ingots and ingot bars, serial designation: B 5, (see 641.11, p. 1174.) of the American Society for Testing Materials. The silver is deposited with the copper and is included as such; when it is desired to correct for the silver this is determined in a larger separate sample and the amount found is deducted from the deposit of copper plus silver.

\section{APPARATOS FOR ELECTROIYSIS}

(Specifications for electrodes and cathodes are identical with those in B27. (See 647.20, p. 1261.)

Anodes.-Spiral platinum anodes should be used, made from $1.02 \mathrm{~mm}$ or $1.14 \mathrm{~mm}$ wire formed into a spiral of seven turns having a height of approximately $51 \mathrm{~mm}$ and a diameter of $16 \mathrm{~mm}$, the length over all being approximately $145 \mathrm{~mm}$.

\section{SOLUTION REQULRED}

"Assay solution" for dissolving.-Mix 300 c c of $\mathrm{H}_{2} \mathrm{SO}_{4}$ (specific gravity 1.84), 210 c c of $\mathrm{HNO}_{3}$ (specific gravity 1.42) and $750 \mathrm{c}$ c of distilled water.

\section{METHOD}

Place $5 \mathrm{~g}$ of the drillings in a tall lipless beaker which is provided with a well-fitting cover glass, and add $42 \mathrm{c}$ c of "assay solution." The electrolysis beakers are from 100 to $125 \mathrm{~mm}$ tall and $55 \mathrm{~mm}$ in diameter across the bottom, with a total capacity of 225 to 300 c c. After the assays have stood for a few minutes and the action has nearly ceased, place them on a steam bath having a temperature of $80^{\circ}$ to $90^{\circ} \mathrm{C}$. and allow them to remain there until solution is completed and the red fumes have disappeared. Wash the cover and the sides of the beaker and dilute the solution to $150 \mathrm{c} c$ and electrolyze, keeping the top of the cathode 5 or $6 \mathrm{~mm}$ below the surface of the solution.

During electrolysis, cover the beakers with two pairs of split watch glasses placed at right angles to each other. For each solution use a current of 1 ampere at approximately 10 volts, or else use a current of 4 amperes at approximately 10 volts continued for about 4 hours. The latter case requires the use of gauze cathodes. In the former case, it is convenient to start the assays at night; after about 15 hours wash the cover glasses, electrodes and beakers and reduce the current to 0.6 ampere per solution. As soon as the cathodes begin to erolve gas reduce the current to 0.4 ampere per solution. When this occurs take out about $1 \mathrm{c}$ c of liquid, place it in a depression in a porcelain test plate and add two or three drops of fresh $\mathrm{H}_{2} \mathrm{~S}$ water. If the slightest discoloration occurs continue the electrolysis, repeating the test until there is no discoloration whatever. ${ }^{8}$ If possible the assay should finish without decided evolution of gas.

8 It has been shown by Heath, J. Ind. Eng. Chem., vol. 4, p. 404, 1912, that a few thousandths of a per cent of copper may remain in 1912, that a few thousandths of a per cent of copper may remain in The solution without being detected by the test on the spot plate. This amount of copper is negligible in many instances, but when necessary to make exact determinations, as in the complete analysis neutralize with $\mathrm{HCl}$ (specific gravity 1.19), adding 3 c c excess, erap. orate to $50 \mathrm{c} \mathrm{c}$ and precipitate with $\mathrm{H}_{2} \mathrm{~S}$. Filter off the copper sulphide, treat with $\mathrm{HNO}_{3}$ and $\mathrm{H}_{2} \mathrm{SO}_{4}$, deposit the small amount of copper from the solution by electrolysis and add it to that obtained in the original determination. 
Without interrupting the current, siphon off the acid solution, at the same time filling the beakers with distilled water. Remove the cathodes quickly and rinse them in distilled water and two successive baths of alcohol. An experienced operator can quickly remove a cathode and immerse it in water without loss. Throw off the excess alcohol by a quick motion of the hand and ignite the remainder by bringing the cathode quickly to the flame of an alcohol lamp; then keep the cathode moving continually while the alcohol burns. Weigh as metallic copper.

Accuracy.-All assays shall be made in duplicate and shall check within an outside limit of 0.015 per cent of copper.

Notes.-Use of the "Assay Solution" prevents a violent reaction and a consequent loss of copper with the vapors. The solution should not be boiled, since this likewise may cause a loss of copper. By the use of rotating anodes, gauze or perforated electrodes or the Frary solenoid, it is possible to use a high current density and to reduce the time required for depositing the copper to about four hours. Good results can be obtained by experienced workers, but there is much more chance for error. The slow or overnight method, which is more certain and which requires less of the operator's time is therefore recommended.

The deposition of the silver with the copper is said to work well up to the limit of 0.3 per cent silver, that is, 100 ounces per ton.

The method of burning off the alcohol in the flame of an alcohol lamp dries the cathode without causing oxidation, whereas drying in an oven to $100^{\circ} \mathrm{C}$. generally causes increase in weight.

\section{ASSAY OF LOW-GRADE OR CASTING COPPER}

For all grades of copper which are not included in the specifications of the American Society for Testing Materials, previously referred to, as electrolytic and low-resistance lake copper, the procedures given below are recommended. These brands may contain a considerable amount of arsenic and some antimony, tellurium, bismuth, and nickel. (See notes.)

\section{Solution Required}

"Assay solution" for dissolving.-Mix 300 c c of $\mathrm{H}_{2} \mathrm{SO}_{4}$ (specific gravity 1.84), 210 c c of $\mathrm{HNO}_{3}$ (specific gravity 1.42) and $750 \mathrm{c}$ c of distilled water.

\section{METHOD I}

Carry out the assay exactly as given for electrolytic and low-resistance lake copper. Place the cathode in a clean beaker, cover with a watch glass perforated with a small hole just large enough to accommodate the stem of the electrode, add $42 \mathrm{c}$ c of "assay solution" and water enough to cover the sheet. Let it stand upon the steam bath until the coating is dissolved. Reelectrolyze in the regular manner.

\section{METHOD II 9}

Dissolve a $5 \mathrm{~g}$ sample in $42 \mathrm{c}$ c of the "assay solution" and evaporate until all the $\mathrm{HNO}_{3}$ is expelled. Redissolve in $70 \mathrm{c} c$ of water and add $3 \mathrm{c} \mathrm{c}$ of ferric nitrate solution ( $1 \mathrm{c} \mathrm{c}=0.01 \mathrm{~g}$ of iron). Transfer the solution to a lipped beaker and place the original beaker under a funnel fitted with a small filter paper. Precipitate the iron from the hot liquid by ammonia, filter and wash out salts. Place the solution on a hot plate to concentrate, reprecipitate the hydroxide from dilute $\mathrm{H}_{2} \mathrm{SO}_{4}$ in a very small volume of solution and add the filtrate to the main solution. Reprecipitate the hydroxide once more from dilute $\mathrm{H}_{2} \mathrm{SO}_{4}$, filter wash thoroughly and add the filtrate to the main solution. Make acid with dilute $\mathrm{H}_{2} \mathrm{SO}_{4}$ and add $2 \mathrm{c} \mathrm{c}$ of $\mathrm{HNO}_{3}$ (specific gravity 1.42). Deposit the copper as in the method for electrolytic and low-resistance lake copper.

Heath (Senter modified), J., Am. Chem. Soc. vol. 26, p. 1123, 1904.

\section{METHOD III 10}

[For copper containing only traces of antimony or bismuth but sufficient selenium or tellurium to interfere]

Dissolve the $5 \mathrm{~g}$ sample in $42 \mathrm{c} \mathrm{c}$ of "assay solution" and evaporate until fumes appear, or until the residue is white. Redissolve in $60 \mathrm{c} \mathrm{c}$ of water and saturate the boiling hot solution for 10 minutes with $\mathrm{SO}_{2}$ gas, removing the solution from the lamp when the gas is started. The gas may be generated by dropping cold saturated sodium sulphite solution into strong $\mathrm{H}_{2} \mathrm{SO}_{4}$. It is also conveniently obtainable in cylinders which are fitted with a valve. Let the precipitate settle for a few hours, filter and wash with a little hot water. Boil the filtrate gently to remove most of the $\mathrm{SO}_{2}$ gas. Ignite the filter in a porcelain crucible to volatilize the selenium and tellurium, redissolve the oxidized residue in 1.5 to 2 $\mathrm{c} \mathrm{c}$ of $\mathrm{HNO}_{3}$ and add to the main solution. Deposit the copper as in the method for electrolytic and lowresistance lake copper.

Note.-The selenium is said to be carried down by $\mathrm{SO}_{2}$ as a selenide of silver, but the roasting sufficiently removes the elements selenium and tellurium. When the silver is to be removed, the selenium, etc., are thrown down with the silver by the $\mathrm{SO}_{2}$ gas and the precipitation of silver is then completed by the addition of chlorides or $\mathrm{HCl}$

METHOD IV 11

[For copper high in arsenic only]

Modification A.-Dissolve the sample in $42 \mathrm{c} \mathrm{c}$ of the "assay solution" in the regular way and add $5 \mathrm{~g}$ of ammonium nitrate. Electrolyze as usual.

Modification B.-Dissolve the sample in $60 \mathrm{c} c$ of the "assay solution" instead of $42 \mathrm{c}$ c. Electrolyze as usual.

Accuracy.-All assays shall be made in duplicate and shall check with an outside limit of 0.015 per cent of copper.

Notes.-It has been recommended 12 to use with solutions of the low-grade or casting copper about $2 \mathrm{c}$ c of an addition agent prepared by boiling "lard oil" with strong HNO $\mathrm{H}_{3}$, cooling and removing the grease. This, it is said, will permit the use of high current densities and yet allow the copper to be deposited pure and bright. Moreover it is said that arsenic and antimony, when present, do not contaminate the copper. It seems probable, 13 however, that copper deposited from such solutions is not quite pure and that it is more accurate purify the cathode deposit (Method I) or to give a preliminary treatpurify the cathode deposit (Method

Method II is recommended in preference to Method I, which should be used only in the presence of small amounts of impurities. In Method IV for copper high in arsenic, Modification B is to be preferred.

\section{UNITED STATES GOVERNMENT, DEPART.} MENT OF COMMERCE, BUREAU OF STANDARDS, STANDARD SAMPIES, CIRCULAR NO. 25 AND SUPPLEMENT, $192 \%$

\section{COPPER SAMPLE}

(Sample No. 45a is a standard sample of copper metal prepared and sold by the Bureau of Standards for use by industrial organizations and others as a temperature standard for calibrating pyrometers, etc. Each sample is accompanied by a certificate giving the melting point of that particular sample.)

(Circular No. 66 of the Bureau of Standards gives information on the preparation of these samples and the method of use.)

10 Heath. Ibid. vol, 26, p. 1123, 1904; Keller, Trans. Am. Inst.

Min. Engrs., p. 2098, 1913, Bulletin No. 80. 11 Modification A.-Heath, J., Am. Chem. Soc., vol. 26, p. 1124,
1904; Modification B.-Heath, J. Ind. Eng. Chem., vol. 3, p. 75, 1911. ${ }_{12}$ Guess, Transactions, Am. Inst. Min. Engrs., vol. 36, p. 605, 1905 13 Keller, Ibid., p. 2099, 1913, Bulletin No. 80. Heath, J. Ind. Eng. Chem., vol. 3, p. 75, 1911. 


\section{UNITED STATES GOVERNMENT, FEDERAL SPECIFICATIONS BOARD, FEDERAI SPECIFICATION FOR COPPER RODS, BARS, SHAPES, PLATES, SHEETS, AND STRIPS, QQ-C-501, FEBRUARY 2, 1932}

\section{COPPER RODS, BARS, SHAPES, PLATES, SHEETS, STRIPS}

\section{GENERAL SPECIFICATIONS}

1. United States Government General Specification for Metals, Federal Specifications Board specification No. 339a (current designation: QQ-M-151) in effect on date of issuance of proposals, shall form part of this specification. (See 600.0, p. 3.)

\section{GRADE, FORMS, AND TEMPERS}

2. This specification covers one grade of copper in the following forms and tempers:

Rods, soft and hard. (See also 642.11, p. 1180.)

Bars, soft and hard. (See also 642.11, p. 1180.)

Shapes, soft and hard. (See also 641.22, p. 1179 .)

Plates, soft and hard. (See also 641.21, p. 1177. .

Sheets, soft and hard. (See also 641.21, p. 1177.)

Strips, soft and hard. (See also 641.21, p. 1177.)

\section{IIATERIAL AND WORKMANSHIP}

3. Material shall be made from only the best grades of virgin metals, or from virgin metals and scrap of known and approved composition.

4. The material shall be uniform in quality and temper, clean, sound, smooth, commercially straight or flat, and free from foreign material, pipes, slivers, laps, cracks, twists, seams, scale, damaged ends or edges, buckles, and other injurious defects.

\section{DETAIL REQUIREMENTS}

5. The material shall contain not less than 99.90 per cent copper, silver being counted as copper.

\section{TENSILE STRENGTH}

6. The requirements as to tensile strength are as shown in Table 1.

\section{TABLE 1.-Physical properties}

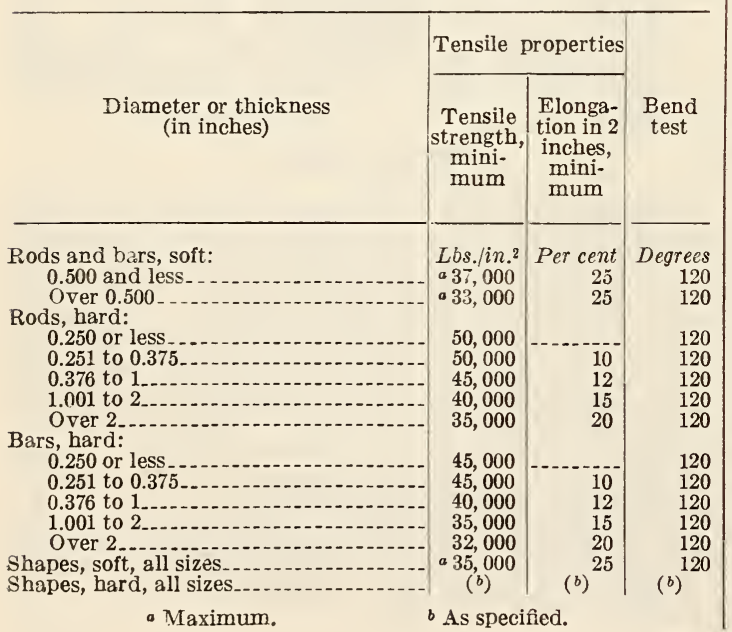

TABLE 1.-Physical properties-Continued

\begin{tabular}{|c|c|c|c|}
\hline \multirow[b]{2}{*}{$\begin{array}{l}\text { Diameter or thickness } \\
\text { (in inches) }\end{array}$} & \multicolumn{2}{|c|}{ Tensile properties } & \multirow[b]{2}{*}{$\begin{array}{l}\text { Bend } \\
\text { test }\end{array}$} \\
\hline & $\begin{array}{c}\text { Tensile } \\
\text { strength, } \\
\text { mini- } \\
\text { mum }\end{array}$ & $\begin{array}{l}\text { Elonga- } \\
\text { tion in } 2 \\
\text { inches, } \\
\text { mini- } \\
\text { mum }\end{array}$ & \\
\hline $\begin{array}{l}\text { Plates, sheets, and strpis, soft: } \\
0.005 \text { to } 0.010 \\
0.011 \text { to } 0.021 \\
0.022 \text { to } 0.031 \\
0.032 \text { to } 0.375 \\
\text { Over } 0.375 \\
\text { Plates and sheets, hard: } \\
0.0 \text { to } 0.071 \\
0.072 \text { to } 0.3755 \\
\text { Over } 0.375 \\
\text { Strips, hard, maximum width } 8 \text { inches: } \\
0.071 \text { and less. } \\
0.072 \text { to } 0.375 \\
\text { Over } 0.375\end{array}$ & $\begin{array}{l}\text { Lbs./in } 2 \\
\text { c None. } \\
\text { eNone. } \\
\text { cNone. } \\
\text { c None. } \\
\text { c None. } \\
35,000 \\
35,000 \\
35,000 \\
40,000 \\
40,000 \\
35,000\end{array}$ & $\begin{array}{r}\text { Per cent } \\
15 \\
20 \\
20 \\
25 \\
25\end{array}$ & $\begin{array}{r}\text { Degrees } \\
\text { d } 180 \\
\text { d } 180 \\
d 180 \\
d 180 \\
d 180 \\
120 \\
120 \\
120 \\
120 \\
120 \\
120\end{array}$ \\
\hline
\end{tabular}

$c$ Tensile strength to be recorded, but not to he a hasis for acceptance.

d Flat.

7. Special tempers not covered by Table 1 may be agreed upon between the contractor and purchaser, in which case they will be specifically defined and designated in the contract or purchase order.

Material shall withstand bending cold, without fracture on the outside of the bent portion, through the angle specified in Table 1 , on a radius equal to the diameter or thickness of the material, except where the angle is noted as " $180^{\circ}$ flat" the material shall be bent flat back upon itself.

\section{TESTS AND METHODS OF INSPECTION}

8. When check analyses are required, the samples shall be obtained from each lot of 1,000 pounds or fraction thereof, of each size in the shipment. In the case of thin sheets and strips, at least 10 represenative pieces shall be taken. In all other cases at least four representative pieces shall be taken. From the pieces so selected equal amounts of clean chips shall be obtained and the chips combined and thoroughly mixed. The inspector shall forward for analysis not less than 2 ounces of the resultant mixture.

9. Unless otherwise specified in the contract or purchase order, 1 tension, 1 bend, and 1 hammer test shall be made of every lot of 5,000 pounds or fraction thereof of each size in the shipment. The tension test specimen and the bend test specimen to represent a given lot shall not be selected from the same piece.

\section{TENSION TESTS}

10. All rods, bars, and shapes may be pulled in full size when practicable. When a machined specimen becomes necessary, enough metal may be removed from the gage section to meet the limitations of the testing machine, or the specimen may be machined to the form and dimensions of trpe 1 , general specifications for metals. (See 600.0 , p. 3.)

11. For rods, bars, and shapes up to $1 \frac{1}{2}$ inches in diameter or thickness, the axis of the test specimen shall coincide with the central axis of the piece. Over $1 \frac{1}{2}$ inches, the axis shall be located midway between the center and surface of the piece.

12. The test specimen for plates, sheets, and strips shall be machined to the form and dimensions 
of type 2 , general specification for metals.

(See, 600.0 p. 3.)

13. The axis of tension test specimens for rods, bars, and shapes shall be parallel to the direction of rolling or drawing.

\section{BENDING TESTS}

14. Bending test specimens from rods, bars, and shapes may be the full size of the section or may be machined to the form indicated in paragraph 27 of general specification for metals. (See 600.0, p. 3.) When polygonal sections are bent in full size the edges may be rounded to 116 -inch radius.

15. Bending test specimens from plates, sheets, and strips may consist of a strip from 1 to 2 inches wide by 6 to 12 inches long, with edges rounded to $1 / 16$-inch radius, or may be machined to the form indicated in paragraph 19 of general specification for metals. (See 600.0, p. 3.) Material less than 1 inch wide shall be bent in full section.

16. The axis of all bending test specimens shall be parallel to the direction of rolling or drawing.

17. Specimens shall be bent cold, by either pressure or blows.

\section{PACKING AND MAREING OF SHIPMENTS}

18. All material shall be properly separated by size when loaded for shipment.

19. Each bundle, box, or crate shall be legibly and indelibly marked with the dimensions, weight, and temper of contents; name, brand, or trade-mark of contractor, and the contract or purchase order number.

Notes.-Round, square, hexagonal, etc., sections are classed as rods. Rectangular sections with the ratio of the two dimensions of the section relatively small, are classed as bars. Rectangular sections with the ratio of the two dimensions relatively large, are classed as plates, sheets, or strips. All other sections are classed as shapes.

The material covered herein is suitable for the manufacture of copper pipes, tubing, shapes, receptacles, and for general coppersmith work. For purposes of calculation the weight per cubic inch of soft or hard copper may be taken as 0.321 pound.

\subsection{COPPER INGOTS AND BARS.}

641.11 INGOTS AND CAST BARS, COPPER.

AMERICAN SOCIETY FOR TESTING MATERIAIS, STANDARD SPECIFICATIONS FOR LAKE COPPER WIRE BARS, CAKES, SLABS, BILLETS, INGOTS, AND INGOT BARS, SERIAL DESIGNATION B4-2\%, $192 \%$

[Approved as American Standard, No. H 17.1, 1932, by the American Standards Association]

\section{LAKE COPPER WIRE BARS, CAKES, SLABS, BILLETS, INGOTS AND INGOT BARS}

1. In order to be classed as lake, copper must originate on the northern peninsula of Michigan, U.S.A.

2. All wire bars, cakes, slabs, and billets shall be stamped with the maker's brand and furnace charge mark. Ingots and ingot bars shall have a brand stamped or cast in, but need have no furnace charge mark.

3. The refiner shall arrange carloads or lots so that as far as possible each shall contain pieces from but one furnace charge, in order to facilitate testing by the user.

4. (a) Low resistance lake.-Lake copper offered for electrical purposes, whether fire or electrolytically refined, shall be known as "low resistance lake."
Low resistance lake wire bars shall have a resistivity not to exceed 0.15436 international ohms per meter-gram at $20^{\circ} \mathrm{C}$. (annealed). All ingots and ingot bars shall have a resistivity not to exceed 0.15694 international ohms per meter-gram at $20^{\circ}$ C. (annealed).

Cakes, slabs, and billets shall come under the ingot classification, except when specified for electrical use at time of purchase; in which case wire-bar classification shall apply.

(b) High resistance lake.-Lake copper having a resistivity greater than 0.15694 international ohms per meter-gram at $20^{\circ} \mathrm{C}$. shall be known as "high resistance lake."

5. (a) Low resistance lake copper shall have a purity of at least 99.900 per cent as determined by electrolytic assay, silver being counted as copper.

(b) High resistance lake copper shall have a purity of at least 99.900 per cent, copper, silver, and arsenic being counted together. The arsenic content of high resistance lake copper, when required for special purposes, shall be the subject of agreement at time of purchase.

6. Wire bars, cakes, slabs, and billets shall be substantially free from shrink holes, cold sets, pits,

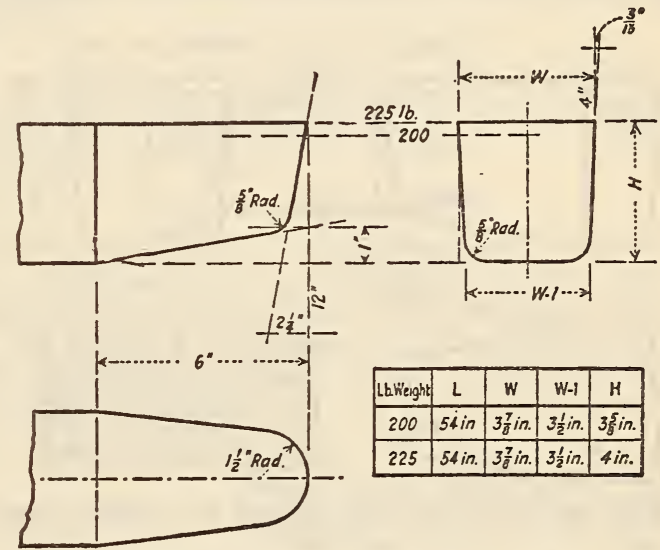

FIgURE 1076.-Mold for casting 200 to 230 pound wire bars

sloppy edges, concave tops and similar defects in set or casting. This clause shall not apply to ingots or ingot bars, in which case physical defects are of no consequence.

7. (a) One mold shall be used for casting 200 to 230 pound wire bars, the bottom width of this bar to be $3 \frac{1}{2}$ inches, the listed weights being 200 and 225 pounds. (See fig. 1076.)

(b) One mold shall be used for casting 240 to 300 pound wire bars, the bottom width of this bar to be 4 inches, the listed weights being 250, 275, and 300 pounds. (See fig. 1077.)

(c) The length dimension of all bars shall be 54 inches. The side draft or taper shall be $3 / 8$ inch in 4 inches ( $3 / 16$ inch in 4 inches on each side of the bar). The radius of the corners at the bottom of the bars shall be $5 / 8$ inch. The end taper at the bottom shall be 6 inches long over all and approximately 2 inches per foot.

The end taper of the side shall be approximately $2 \frac{3}{8}$ inches per foot and the end of the bar shall be approximately $3 \frac{3}{8}$ inches deep at the point.

8. Five per cent variation in weight or $1 / 4$ inch variation in any dimension from the refiner's published list or purchaser's specified size shall be considered good delivery; provided, however, that wire bars may vary in length 1 per cent from the listed 
or specified length, and cakes 3 per cent from the listed or specified size in any dimension greater than 8 inches. The weight of ingot and ingot-bar copper shall not exceed that specified by more than 10 per cent, but otherwise its variation is not important.

9. Claims shall be made in writing within 30 days of receipt of copper at the customer's mill, and the results of the customer's test shall accompany such claims. The refiner shall be given one week from date of receipt of complaint to investigate his records, and shall then either agree to replace the defective copper or send a representative to the mill. No claims will be considered unless made as above stated, and if the copper in question, unused, can not be shown to the refiner's representative.

Claims against quality will be considered as follows:

(a) Resistivity by furnace charges, ingot lots, or ingot-bar lots.

(b) Metal contents by furnace charges, ingot lots, or ingot-bar lots.

(c) Physical defects by individual pieces.

(d) Variation in weights or dimensions by individual pieces.

10. (a) The refiners representative shall inspect all pieces where physical defects or variation in

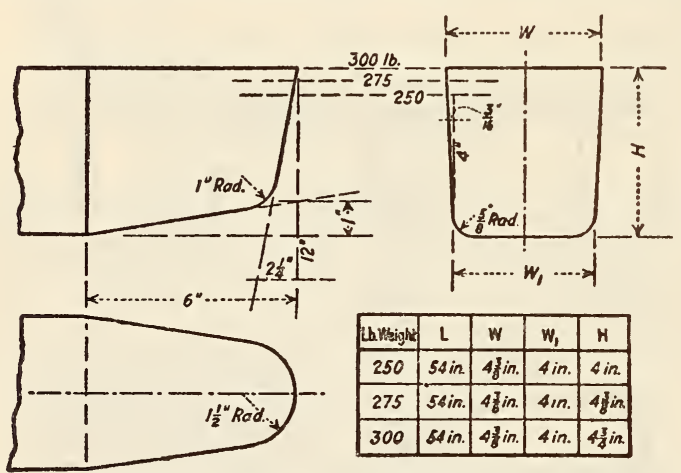

FIgURE 1077.-Mold for casting 240 to 300 pound wire bars

weignt or dimension are claimed. If agreement is not reached, the question of fact shall be submitted to a mutually agreeable umpire, whose decision shall be final.

(b) In a question of metal contents, each party shall select a sample of three pieces from the consignment or lot to be investigated. These shall be sampled in the presence of botn parties by drilling five holes, approximately one-half inch in diameter, at points equally spaced between the ends of the pieces. With bars, billets, ingots, and ingot bars, these holes should be along a middle line and with slabs and cakes on a diagonal line projected between opposite corners. The drilling shall be from top to bottom and completely through each piece. Scale from set and any surface dirt shall be rejected. No lubricant shall be used and drilling shall not be forced sufficiently to cause oxidation of the chips. In the case of sections having a depth greater tnan 5 inches, drillings may be made from both top and bottom for a depth of not less than 2 inches in each direction instead of completely through, but the drilling shall be otherwise conducted as before described.

The resulting sample shall be cut up, mixed and separated into three equal portions, each of which shall be placed in a sealed package, one for each party and the third for the umpire, if necessary.
Each party shall make an analysis in accordance with the standard methods of battery assay of copper (see A. S. T. M. B34, 641.0, p. 1170), and if the results do not establish or dismiss the claim to the satisfaction of both parties concerned, the third sample shall be submitted to a mutually agreeable umpire, who shall determine the question of fact and whose determination shall be final.

(c) In a question of resistivity each party shall select two samples, and in the presence of both parties these shall be rolled hot and drawn cold into wire of 0.080 inch in diameter, approximately, which shall be annealed at approximately $500^{\circ}$ C. Three samples shall be cut from each coil and the same procedure followed as described inthe previous paragraph.

11. The expenses of the shipper's representative and of the umpire shall be paid by the loser, or divided in proportion to the concession made in case of compromise. In case of rejection being establisned, the damage shall be limited to payment of freight both ways by the refiner for substitution of an equivalent weight of copper meeting these specifications.

AMERICAN SOCIETY FOR TESTING MATERIALS, STANDARD SPECIFICATIONS FOR ELECTROLYTIC COPPER WIRE BARS, CAKES, SLABS, BILLETS, INGOTS, AND INGOT BARS, SERIAL DESIGNATION B5-27, 1927

[Approved as American Standard, No. H 17.2, 1932, by the American Standar ds Association]

ELECTROLYTIC-COPPER WIRE BARS, CAKES,

(This specification is identical with A. S. T. M., $\mathrm{B} 4$, given above, with the exception that the copper does not have to originate on the northern peninsula of Michigan; that definitions and character of lowresistance and high-resistance lake copper are omitted; and that the following two paragraphs are included:)

1. Metal content.-The copper in all shapes shall have a purity of at least 99.900 per cent, as determined in accordance with the standard methods of battery assay of copper (see A. S. T. M., B34, 641.0 , p. 1170), silver being counted as copper.

2. Resistivity. - All wire bars shall have a resistivity not to exceed 0.15436 international ohms per meter-gram at $20^{\circ} \mathrm{C}$. (annealed;) all ingots and ingot bars shall have a resistivity not to exceed 0.15694 international ohms per meter-gram at $20^{\circ} \mathrm{C}$. (annealed).

\section{AMERICAN SOCIETY FOR TESTING MA- TERIALS, TENTATIVE SPECIFICATIONS FOR FIRE - REFINED COPPER OTHER THAN LAKE, SERIAL DESIGNATION B72- 28T, 1928}

\section{FIRE-REFINED COPPER OTHER THAN LAKE}

1. (a) These specifications cover fire-refined copper, other than lake, and not usually electrolytically refined.

(b) Fire-refined copper, other than lake, is intended for use in rolling into sheets and shapes for mechanical purposes, and for certain grades of wrought-copper alloys, and is not intended for electrical purposes. 


\section{CHEMICAL COMPOSITION}

2. The copper in all shapes shall conform to the following requirements as to chemical composition:

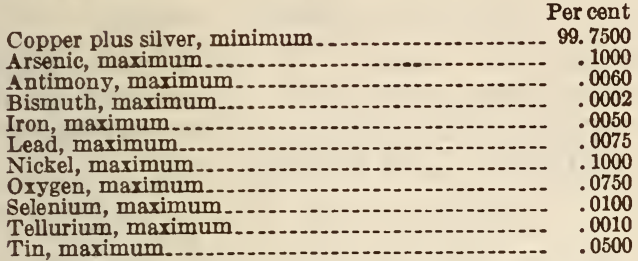

(In respect to physical defects, permissible variations in weight and dimensions, marking, lots, claims, and chemical analysis, this specification is identical with A. S. T. M., B4, given above.)

NATIONAL METAL EXCHANGE (INC.) AND NATIONAL METAL CLEARING ASSOCIATION (INC.), BY-LAWS AND RULES, 1931

\section{BY-LAWS CONCERNING COPPER}

TENDERABLE GRADES OF COPPER FOR OLD STANDARD CONTRACT

Section 40-A.-The following grades of copper may be delivered against the National Metal Exchange (Inc.), old standard contract:

Contract grades-

Prime electrolytic copper.

Prime lake copper, assaying 99.90 per cent.

In ingots and/or ingot bars and/or wire bars of standard weights and sizes.

Discount grades-

Best selected copper, assaying 99.80 per cent.

Casting copper, assaying 99.50 per cent.

Casting copper, assaying 99.50 per cent.

In ingots and/or ingot bars of the usual weights and sizes. Discount grades-

(Covers 5 grades of rough or blister copper, in 1 per cent assay steps, for copper assaying 98.00 to 94.00 per cent.)

In slabs and/or pigs weighing not more than 500 pounds.

Prime electrolytic copper to be deliverable as herein provided shall conform to the specifications and conditions given in American Society for Testing Materials specification B5-27.

(These by-laws also include discounts allowed from the contract price when any of the various discount grades above are delivered by the seller.)

\section{TENDERABLE GRADES OF COPPER FOR "A" CONTRACT}

Section 40-B.-The contract (basis) grade for the "A" contract shall be rough or blister copper assaying 99 per cent or better, deliverable at the contract price. (This by-law also includes the premiums and discounts applicable on any of 11 other grades which may be tendered against the "A" contract at the option of the seller.)

No copper assaying less than 94 per cent shall be a good delivery.

\section{TENDERABLE GRADES OF COPPER FOR NEW STANDARD}

Section 40-C.-The contract (basis) grade for the new standard contract shall be rough or blister copper assaying 99 per cent or better, deliverable at the contract price. (This by-law also includes 6 premium grades and 32 discount grades, with the premiums and discounts from the contract price specified, which may be tendered agianst the new standard contract.

\section{OFFICIAI IIST OF BRANDS OF COPPER}

Section 42-A.-There shall be on file in the office of the secretary of the exchange descriptions and/or replicas of the brands of copper which may be delivered against National Metal Exchange copper contracts, which shall be official when approved by the board of governors of the exchange.

\section{UNITED STATES GOVERNMENT, FEDERAL SPECIFICATIONS BOARD; FEDERAI SPECIFICATION FOR INGOT COPPER, QQ-C-521, MAY 13, 1932}

\section{INGOT COPPER}

(This specification is basically similar to A. S. T. M., B4, given above. It provides for two grades of copper ingots, A and B, as shown below. Grade A corresponds to low-resistance lake in B4.)

\section{GRADES}

1. Ingot copper shall be furnished in two grades as required: Grade $\mathrm{A}$, and grade $\mathrm{B}$.

\section{MATERIAL}

2. Electrolytic copper or lake copper shall be furnished except as modified in paragraph 4 (b) below:

\section{GENERAL REQUIREMENTS}

3. Ingot copper shall be furnished in standard commercial-shaped ingots, between 9 and 12 inches in length, unless otherwise specified.

\section{DETAIL REQOIREMENTS}

4. (a) Grade A ingot copper shall be that known in the trade as "prime electrolytic" or "lake copper" and shall conform to the following table:

(b) Grade B ingot copper may be refined from ore or reclaimed from scrap and shall conform to the following table:

\begin{tabular}{|c|c|c|c|c|c|c|}
\hline \multirow[t]{2}{*}{ Grade } & \multirow{2}{*}{$\begin{array}{c}\text { Copper, } \\
\text { min- } \\
\text { mum 1 }\end{array}$} & $\begin{array}{l}\text { Bis- } \\
\text { muth, } \\
\text { maxi- } \\
\text { mum }\end{array}$ & \multicolumn{2}{|c|}{$\begin{array}{l}\text { Anti- } \\
\text { mony, } \\
\text { maxi- } \\
\text { mum }\end{array}$} & $\begin{array}{l}\text { Arsenic, } \\
\text { maxi- } \\
\text { mum }\end{array}$ & $\begin{array}{l}\text { Sul- } \\
\text { phur, } \\
\text { maxi- } \\
\text { mum }\end{array}$ \\
\hline & & $\begin{array}{c}\text { Per cent } \\
\quad(2) \\
0.01\end{array}$ & $\begin{array}{r}P e r \\
0.00 \\
.05\end{array}$ & 25 & $\begin{array}{c}\text { Per cent } \\
0.0025 \\
.0300\end{array}$ & $\begin{array}{c}\text { Per cent } \\
0.0035 \\
.006\end{array}$ \\
\hline Grade & $\begin{array}{l}\text { Iron, } \\
\text { maxi- } \\
\text { mum }\end{array}$ & $\begin{array}{l}\text { Lea } \\
\text { max } \\
\text { mu }\end{array}$ & & & $\begin{array}{l}\text { ickel, } \\
\text { naxi- } \\
\text { num }\end{array}$ & $\begin{array}{l}\text { Tin, } \\
\text { maxi- } \\
\text { mum }\end{array}$ \\
\hline A & $\begin{array}{r}\text { Per cent } \\
0.004 \\
.10\end{array}$ & $\begin{array}{r}\text { Per } \\
0 . \\
.\end{array}$ & $\begin{array}{l}\text { cent } \\
005 \\
05\end{array}$ & $\mathrm{Pe}$ & er cent & Per cent \\
\hline
\end{tabular}

1 Silver to be computed as copper.

2 None as determined from a $50 \mathrm{~g}$ sample.

\section{TESTS AND METHOD OF INSPECTION}

5. (a) Samples shall be taken as follows: One representative ingot shall be taken from each lot of 8,000 pounds or fraction thereof of an order. Two one-half inch holes shall be drilled from the top of ingot as cast to one-fourth inch from the bottom of each ingot selected for test. No lubricant shall be used and drilling shall not be forced sufficiently to cause oxidation of the chips. The drillings from the first one-eighth inch shall be discarded, and the inspector shall forward for analysis not less than 5 ounces of the remaining drillings, from each sample ingot, in a separate package for analysis. Drillings from all the samples of approximately equal weight for each full lot and not less 
than 5 ounces from an order shall be thoroughly mixed and a portion taken therefrom for analysis unless a question of homogeneity of the metal arises, in which case separate analysis shall be made as may be deemed expedient. Copper shall be determined by electrolytic assay. Silver shall be counted as copper.

(b) Check analyses may be made by the inspector, or through him by any Government laboratory or other designated representative, and without cost to the contractor. The chemical composition thus determined shall conform to the requirements specified.

\subsection{ROLLED BARS, COPPER.}

AMERICAN SOCIETY FOR TESTING IMATERIALS, STANDARD SPECIFICATIONS FOR COPPER BARS FOR LOCOMOTIVE STAY BOLTS, SERIAL DESIGNATION B12-21, 1921

\section{COPPER BARS FOR LOCOMOTIVE STAY BOLTS} SCOPE

1. These specifications cover two grades of copper for locomotive stay bolts; namely, arsenical and nonarsenical.

\section{MANUFACTURE}

2. The copper shall be fire-refined or electrolytic, and shall be finished to dimensions by hot-rolling from suitable bars.

\section{CHEMICAL PROPERTIES AND TESTS}

(Identical with A. S. T. M., B11 (see 641.21, p. 1176.), except that:)

3. An analysis may be made from one sample for each 5,000 pounds or fraction thereof of finished bars.

\section{PHYSICAL PROPERTIES AND TESTS}

(In respect to tensile strength, elongation, and retests, this specification is identical with B11, referred to above. In the matter of tests it varies from B11 in the following particulars:)

4. The test specimen shall bend cold through $180^{\circ}$ flat on itself without cracking on the outside of the bent portion.

5. Tension and bend test specimens shall be of the full-size section of the bars as rolled.

6 . One tension and one bend test shall be made from each 5,000 pounds or fraction thereof.

\section{PERMISSIBLE VARIATIONS IN DIAMETER}

7. The bars shall be truly round within 0.01 inch, and shall not vary more than 0.01 inch over nor more than 0.005 inch under the specified size.

(In respect to workmanship and finish, and marking, this specification is identical with A. S. T. M., B11, referred to above. In respect to inspection and rejection, and rehearing, it conforms to $\mathrm{A}$. S T. M. standard specifications for steel. (See 605.0 , p. 247).

\section{UNITED STATES GOVERNMENT, FEDERAL SPECIFICATIONS BOARD; FEDERAI SPECIFICATION FOR COPPER RODS, BARS, SHAPES, PLATES, SHEETS, AND STRIPS, QQ-C-501, February 2, 1932}

\section{COPPER BARS}

(See 642.11, p. 1180.)
641.2 COPPER PLATES, SHEETS, SHAPES, AND STRIPS.

641.21. PLATES AND SHEETS. COPPER.

AMERICAN BUREAU OR SHIPPING, RULES FOR BUILDING AND CLASSING STEEL VESSELS; MATERIALS FOR MACHINERY, BOILERS, AND PIPING, 1930

\section{COPPER PLATES AND SHAPES}

(For general information and requirements see 600.0 , p. 1. For test requirements see 600.1 , p. 7.)

The material must be clean, smooth, of uniform color and quality, and free from defects. At least one tension test and one cold and one hot bend test should be made from each heat of the finished material. The material is to have a tensile strength of not less than 28,000 pounds per square inch, with an elongation of at least 25 per cent on a gage length of 8 inches. A test specimen must stand being bent cold through $180^{\circ}$ flat on itself without fracture, and this specimen is to be subsequently drawn out cold by hammering to a fine point; a test specimen when heated to a dull red must stand being bent through $180^{\circ}$ flat on itself in both directions without fracture. Sheets less than 0.072 inches in thickness will be subjected to bend tests only.

\section{AMERICAN SOCIETY FOR TESTING MATE-} RIALS, STANDARD SPECIFICATIONS FOR COPPER PLATES FOR IOCOMOTIVE FIRE BOXES, SERIAL DESIGNATION B11-18, 1918

\section{COPPER PLATES FOR LOCOMOTIVE FIRE BOXES} SCOPE

1. These specifications cover two grades of copper for locomotive fire boxes, namely, arsenical and nonarsenical.

\section{MANUFACTURE}

2. The copper shall be fire-refined or electrolytic, and hot-rolled from suitable cakes.

\section{CHEMICAL PROPERTIES AND TESTS}

3. The copper shall conform to the following requirements as to chemical composition:

(a) Arsenical copper.-Arsenical copper shall contain 0.25 to 0.50 per cent of arsenic, and shall not contain more than 0.120 per cent of impurities, exclusive of arsenic and silver.

(b) Nonarsenical copper-Nonarsenical copper shall have a purity of at least 99.880 per cent as determined by electrolytic assay, silver being counted as copper. The total of impurities other than silver shall not exceed 0.120 per cent.

4. An analysis may be made by the purchaser from a broken tension-test specimen representing each plate as rolled. The chemical composition thus determined shall conform to the requirements specified in section 3 .

\section{PHYSICAL PROPERTIES AND TESTS}

5. The material shall conform to the following minimum requirements as to tensile properties:

\begin{tabular}{l|r|r}
\hline & $\begin{array}{c}\text { Arseni- } \\
\text { cal }\end{array}$ & $\begin{array}{r}\text { Nonar- } \\
\text { senical }\end{array}$ \\
\hline $\begin{array}{l}\text { Tensile strength_. } \\
\text { Elongation in 8 inches._._....... }\end{array}$ & $\begin{array}{r}31,000 \\
35\end{array}$ & $\begin{array}{r}30,000 \\
30\end{array}$ \\
\hline
\end{tabular}


6. The specimen shall bend both hot $\left(500^{\circ}\right.$ to $700^{\circ}$ C.) and cold through $180^{\circ}$ without cracking on the outside of the bent portion as follows: For material 1 inch or under in thickness, flat on itself; and for material over 1 inch in thickness, around a pin, the diameter of which is equal to the thickness of the specimen.

7. Tension and bend test specimens shall be taken from the finished rolled material. They shall be of the full thickness of material as rolled, and shall be machined to the form and dimensions shown in Figure 128 in A. S. T. M. standard specifications for steel (see 605.0 , p. 247.) except that bend-test specimens may be machined with both edges parallel.

8. (a) One tension, one cold-bend, and one hotbend test shall be made from each plate as rolled.

(b) If any test specimen develops flaws it may be discarded and two other specimens substituted, each of which shall be free from flaws. If flaws appear in either or both of the substituted specimens, the lot shall be rejected.

(c) If the percentage of elongation of any tensiontest specimen is less than that specified in section 5, and any part of the fracture is outside the middle third of the gage length, as indicated by scribe scratches marked on the specimen before testing, a retest shall be allowed.

(d) If any test specimen shows defective machining it may be discarded and another specimen substituted.

\section{PERMISSIBLE VARIATIONS IN WEIGHT AND THICKNESS}

9. The thickness of each plate shall not vary more than 0.04 inch under that ordered. An excess over the nominal weight corresponding to the dimensions on the order shall be allowed for each plate if not more than that shown in Table 1 . One cubic inch of rolled copper is assumed to weigh 0.323 pound.

\section{FINISH}

10. The finished plates shall be free from injurious defects and shall have a workmanlike finish.

\section{MARKING}

11. The name or brand of the manufacturer, plate number, and grade shall be stamped on each plate. The plate number shall be legibly stamped on each test specimen.

TABLE 1.-Permissible overweights of plates ordered to thickness

\begin{tabular}{|c|c|c|c|c|c|}
\hline \multirow{2}{*}{$\begin{array}{l}\text { Ordered thick- } \\
\text { ness (in inches) }\end{array}$} & \multirow{2}{*}{$\begin{array}{l}\text { Weight } \\
\text { per } \\
\text { square } \\
\text { foot }\end{array}$} & \multicolumn{4}{|c|}{$\begin{array}{l}\text { Permissible excess in average weights per } \\
\text { square foot of plates for widths given } \\
\text { (expressed in percentages of nominal } \\
\text { weights) }\end{array}$} \\
\hline & & $\begin{array}{c}\text { Under } 75 \\
\text { inches }\end{array}$ & $\begin{array}{l}75 \text { to } 100 \\
\text { inches } \\
\text { exclusive }\end{array}$ & $\begin{array}{l}100 \text { to } 115 \\
\text { inches } \\
\text { exclusive }\end{array}$ & $\begin{array}{l}115 \\
\text { inches } \\
\text { or over }\end{array}$ \\
\hline $\begin{array}{l}5 / 16 \\
3 / 8 \\
7 / 16 \\
1 / 2 \\
9 / 16 \\
\text { Over } 5 / 8\end{array}$ & $\begin{array}{r}\text { Pounds } \\
14.53 \\
17.44 \\
20.34 \\
23.25 \\
26.16 \\
29.06\end{array}$ & $\begin{array}{r}\text { Per cent } \\
8 \\
7 \\
6 \\
5 \\
5 \\
5 \\
5\end{array}$ & $\begin{array}{c}\text { Per cent } \\
12 \\
10 \\
8 \\
7 \\
6.5 \\
6 \\
5\end{array}$ & $\begin{array}{c}\text { Per cent } \\
16 \\
13 \\
10 \\
9 \\
8.5 \\
8 \\
6.5\end{array}$ & $\begin{array}{r}\text { Per cent } \\
17 \\
13 \\
12 \\
11 \\
10 \\
9\end{array}$ \\
\hline
\end{tabular}

\section{INSPECTION AND REJECTION}

(Inspection, rejection, and rehearing conform to the requirements specified in A. S. T. M. standard specifications for steel. See 605.0, p. 247.)
SOCIETY OF AUTOMOTIVE ENGINEERS, NONFERROUS METAL SPECIFICATIONS, SPECIFICATION NO. \%1, COPPER SHEET, FEBRUARY, 1929

\section{COPPER SHEET}

1. Composition in percentage:

Per cent

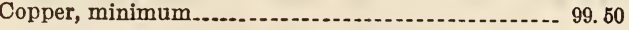

2. Tensile strength and elongation.-

\begin{tabular}{|c|c|c|c|}
\hline Copper & Thickness & $\left|\begin{array}{l}\text { Tensile strength } \\
\text { per square inch }\end{array}\right|$ & $\begin{array}{l}\text { Elonga- } \\
\text { tion in } \\
2 \text { inches } \\
\text { (mini- } \\
\text { mum) }\end{array}$ \\
\hline Soft_.... & $\left\{\begin{array}{c}\text { Inch } \\
0.005-0.031 \\
.032-.375\end{array}\right.$ & $\begin{array}{c}\text { Pounds } \\
\text { (Max.) } 37,000 \\
\text { (Max.) } 36,000\end{array}$ & $\begin{array}{r}\text { Per cent } \\
20.0 \\
25.0\end{array}$ \\
\hline Hard $1 .$. & $\left\{\begin{array}{l}.072-.375 \\
\text { Over } .375\end{array}\right.$ & 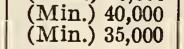 & $\begin{array}{r}8.0 \\
15.0\end{array}$ \\
\hline
\end{tabular}

1 Hard copper less than 0.072 inch thick should be made as hard as possible and still stand cold bending through an angle of $180^{\circ}$ flat back without fracture on the outside of the bent portion.

3. The thickness tolerances of sheet or strip made of this alloy shall be within the tolerances specified in S. A. E. Table 2. (See Table 5, F. S. B. QQ-C501 , given below.)

4. These should be considered as general specifications. Since copper is used for many purposes where the requirements of the operations used are too particular to be specified by any of the ordinary physical tests, it is frequently advisable to submit samples or drawings to the manufacturer and secure an adjustment of anneal or temper to suit the actual operations to which the material is to be submitted.

UNITED STATES GOVERNMENT, DEPARTMENT OF COMMERCE, BUREAU OF STANDARDS; STANDARD THICKNESSES, WEIGHTS, AND TOLERANCES OF SHEET METAL (CUSTOMARY PRACTICE), CIRCULAR NO. 391, MARCH 24, 1931

\section{AMERICAN WIRE GAGE COPPER PLATES AND SHEETS}

(See 640.1, p. 1165.)

\section{UNITED STATES GOVERNMENT, FEDERAL SPECIFICATIONS BOARD; FEDERAL SPECIFICATION FOR COPPER RODS, BARS, SHAPES, PLATES, SHEETS, AND STRIPS, QQ-C-501, FEBRUARY 2, 1932}

\section{COPPER PLATES, SHEETS, STRIPS}

(For general specifications; grade, forms, and tempers; material and workmanship; detail requirements; physical properties; tests and methods of inspection; packing and marking; and notes, see 641.0, p. 1172.)

1. Plates, sheets, and strips, soft, shall be furnished hot-rolled, or cold-rolled and annealed, unless the particular process is specified in the contract or purchase order.

2. Sheets and strips, when ordered for spinning or drawing, shall be of such quality that after spin- 
ning or drawing the material may be finished and present a good smooth surface which will not be spotted or open grained.

3. When specified in the contract or purchase order, plates, sheets, and strips shall be supplied with finished edges, as detailed hereinafter.

\section{PERMISSIBLE VARIATIONS; PLATES, SHEETS, AND STRIPS}

4. The permissible variations in thickness for sheets and strips 0.460 inch or less thick and 20 inches or less wide shall be as shown in Table 5 .

TABLE 5.-Permissible variations in thickness of sheets and strips (plus or minus)

\begin{tabular}{|c|c|c|c|c|}
\hline $\begin{array}{l}\text { Thick- } \\
\text { ness }\end{array}$ & $\begin{array}{l}\text { Up to } 6 \\
\text { inches } \\
\text { wide, } \\
\text { inclusive }\end{array}$ & $\begin{array}{c}\text { Over } 6 \text { to } \\
9 \text { inches } \\
\text { wide, } \\
\text { inclusive }\end{array}$ & $\begin{array}{l}\text { Over } 9 \text { to } \\
14 \text { inches } \\
\text { wide, } \\
\text { inclusive }\end{array}$ & $\begin{array}{l}\text { Over } 14 \text { to } \\
20 \text { inches } \\
\text { wide, } \\
\text { inclusive }\end{array}$ \\
\hline $\begin{array}{c}\text { Inch } \\
0.460 \\
.4096 \\
.3648 \\
.3249 \\
.2893\end{array}$ & $\begin{array}{c}\text { Inch } \\
0.0043 \\
.0042 \\
.0041 \\
.0040 \\
.0039\end{array}$ & $\begin{array}{c}\text { Inch } \\
0.0045 \\
.0044 \\
.0043 \\
.0042 \\
.0041\end{array}$ & $\begin{array}{c}\text { Inch } \\
0.0047 \\
.0046 \\
.0045 \\
.0044 \\
.0043\end{array}$ & $\begin{array}{c}\text { Inch } \\
0.0049 \\
.0048 \\
.0047 \\
.0046 \\
.0045\end{array}$ \\
\hline $\begin{array}{l}.2576 \\
.2294 \\
.2043 \\
.1819 \\
.1620\end{array}$ & $\begin{array}{l}.0038 \\
.0037 \\
.0036 \\
.0035 \\
.0034\end{array}$ & $\begin{array}{l}.0040 \\
.0039 \\
.0038 \\
.0037 \\
.0036\end{array}$ & $\begin{array}{l}.0042 \\
.0041 \\
.0040 \\
.0039 \\
.0038\end{array}$ & $\begin{array}{l}.0044 \\
.0043 \\
.0042 \\
.0041 \\
.0040\end{array}$ \\
\hline $\begin{array}{l}.1443 \\
.1285 \\
.1144 \\
.1019 \\
.0907\end{array}$ & $\begin{array}{l}.0033 \\
.0032 \\
.0031 \\
.0030 \\
.0029\end{array}$ & $\begin{array}{l}.0035 \\
.0034 \\
.0033 \\
.0032 \\
.0031\end{array}$ & $\begin{array}{l}.0037 \\
.0036 \\
.0035 \\
.0034 \\
.0033\end{array}$ & $\begin{array}{l}.0039 \\
.0038 \\
.0037 \\
.0036 \\
.0035\end{array}$ \\
\hline $\begin{array}{l}.0808 \\
.0719 \\
.0640 \\
.0570 \\
.0508\end{array}$ & $\begin{array}{l}.0028 \\
.0027 \\
.0026 \\
.0025 \\
.0024\end{array}$ & $\begin{array}{l}.0030 \\
.0029 \\
.0028 \\
.0027 \\
.0026\end{array}$ & $\begin{array}{l}.0032 \\
.0031 \\
.0030 \\
.0029 \\
.0028\end{array}$ & $\begin{array}{l}.0034 \\
.0033 \\
.0032 \\
.0031 \\
.0030\end{array}$ \\
\hline $\begin{array}{l}.0452 \\
.0403 \\
.0359 \\
.0320 \\
.0284\end{array}$ & $\begin{array}{l}.0023 \\
.0022 \\
.0021 \\
.0020 \\
.0019\end{array}$ & $\begin{array}{l}.0025 \\
.0024 \\
.0023 \\
.0021 \\
.0020\end{array}$ & $\begin{array}{l}.0027 \\
.0026 \\
.0025 \\
.0023 \\
.0021\end{array}$ & $\begin{array}{l}.0029 \\
.0028 \\
.0026 \\
.0024 \\
.0022\end{array}$ \\
\hline $\begin{array}{l}.0253 \\
.0225 \\
.0201 \\
.0179 \\
.0159\end{array}$ & $\begin{array}{l}.0018 \\
.0017 \\
.0016 \\
.0015 \\
.0014\end{array}$ & $\begin{array}{l}.0019 \\
.0018 \\
.0017 \\
.0016 \\
.0015\end{array}$ & $\begin{array}{l}.0020 \\
.0019 \\
.0018 \\
.0017 \\
.0016\end{array}$ & $\begin{array}{l}.0021 \\
.0020 \\
.0019 \\
.0018 \\
.0017\end{array}$ \\
\hline $\begin{array}{l}.0142 \\
.0126 \\
.0112 \\
.0100 \\
.0089\end{array}$ & $\begin{array}{l}.0013 \\
.0012 \\
.0011 \\
.0011 \\
.0010\end{array}$ & $\begin{array}{l}.0014 \\
.0013 \\
.0012 \\
.0012 \\
.0011\end{array}$ & $\begin{array}{l}.0015 \\
.0014 \\
.0013 \\
.0013 \\
.0012\end{array}$ & $\begin{array}{l}.0016 \\
.0015 \\
.0014 \\
.0014 \\
.0013\end{array}$ \\
\hline $\begin{array}{l}.0079 \\
.0070 \\
.0063 \\
.0056 \\
.0050\end{array}$ & $\begin{array}{l}.0010 \\
.0009 \\
.0009 \\
.0008 \\
.0008\end{array}$ & $\begin{array}{l}.0010 \\
.0010 \\
.0010 \\
.0009 \\
.0009\end{array}$ & $\begin{array}{l}.0012 \\
.0011 \\
.0011 \\
.0010 \\
.0010\end{array}$ & $\begin{array}{l}.0013 \\
.0012 \\
.0012 \\
.0011 \\
.0011\end{array}$ \\
\hline
\end{tabular}

5. The permissible variations in thickness for plates, sheets, and strips of all thicknesses and widths not covered in Table 5 , shall be as shown in Table 6, except in no case shall the tolerance be less than that permitted by Table 5 for the same thickness.

\section{TABLE 6.-Permissible variations in thickness of plates, sheets, and strips}

Width (in inches)

Up to 48 , inclusive

Over 48 to 60 , inclusive

Over 60 .

\begin{tabular}{r|l}
\multicolumn{2}{c}{$\begin{array}{c}\text { Permissible vari- } \\
\text { ations }\end{array}$} \\
\hline \multicolumn{1}{c|}{ Plus } & Minus \\
\hline Per cent & Per cent \\
5 & 5 \\
5 & 7 \\
5 & 8
\end{tabular}

6. The permissible variations in width for plates, sheets, and strips shall be as shown in Table 7:

TABLE 7.-Permissible variations in width of plates, sheets, and strips

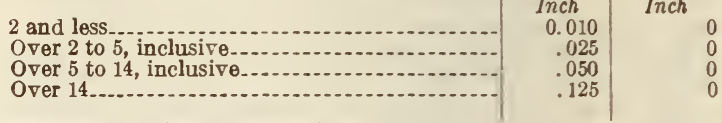

7. Plates, sheets, and strips of all sizes may be ordered to exact lengths, in which case a variation of plus one-eighth inch on length shall be permitted.

8. When plates, sheets, and strips up to 14 inches wide are ordered in stock lengths, the pieces shipped shall be cut to the nominal or stock length as a maximum. The shortest acceptable lengths; the maximum permissible percentage by weight of short lengths; and the required percentage by weight of stock lengths in any one shipment shall be as shown in Table 8.

TABLE 8.-Permissible variations in length of plates, sheets, and strips up to 12 inches wide

\begin{tabular}{|c|c|c|c|c|c|c|}
\hline \multirow{2}{*}{$\begin{array}{l}\text { Nominal or } \\
\text { stock length } \\
\text { (in feet) }\end{array}$} & \multirow{2}{*}{$\begin{array}{c}\text { Required } \\
\text { per cent } \\
\text { by weight } \\
\text { of stock } \\
\text { lengths }\end{array}$} & \multicolumn{5}{|c|}{$\begin{array}{l}\text { Maximum permissible percentage by } \\
\text { weight of short lengths }\end{array}$} \\
\hline & & $\begin{array}{c}8 \text { to } 10 \\
\text { feet }\end{array}$ & $\begin{array}{c}6 \text { to } 8 \\
\text { feet }\end{array}$ & $\begin{array}{c}4 \text { to } 6 \\
\text { feet }\end{array}$ & $\begin{array}{c}2 \text { to } 4 \\
\text { feet }\end{array}$ & $\begin{array}{c}\text { Cnder } 2 \\
\text { feet }\end{array}$ \\
\hline $\begin{array}{l}10 . \\
8 \ldots \\
6 \ldots\end{array}$ & $\begin{array}{l}60 \\
70 \\
80\end{array}$ & $\begin{array}{r}40 \\
- \\
-\end{array}$ & $\begin{array}{r}30 \\
30 \\
- \\
-\end{array}$ & $\begin{array}{l}20 \\
20 \\
20\end{array}$ & $\begin{array}{l}10 \\
10 \\
10\end{array}$ & $\begin{array}{l}0 \\
0 \\
0\end{array}$ \\
\hline
\end{tabular}

For copper strips and sheets, tinned one side, ordered under this specification in weights per square foot as listed below, the permissible variations in thickness shall be as shown in Table 6 and 9 .

TABLE 9

\begin{tabular}{|c|c|c|c|c|c|}
\hline \multirow{2}{*}{$\begin{array}{l}\text { Weight } \\
\text { per } \\
\text { square } \\
\text { foot }\end{array}$} & \multirow{2}{*}{$\begin{array}{c}\text { Thick- } \\
\text { ness }\end{array}$} & \multicolumn{4}{|c|}{$\begin{array}{l}\text { Permissible variations (plus or minus) } \\
\text { in thickness for widths }\end{array}$} \\
\hline & & $\begin{array}{l}\text { Up to } 6 \\
\text { inches, } \\
\text { inclusive }\end{array}$ & $\begin{array}{l}\text { Over } 6 \text { to } \\
9 \text { inches, } \\
\text { inclusive }\end{array}$ & $\begin{array}{l}\text { Over } 9 \text { to } \\
14 \text { inches, } \\
\text { inclusive }\end{array}$ & $\begin{array}{l}\text { O ver } 14 \text { to } \\
20 \text { inches, } \\
\text { inclusive }\end{array}$ \\
\hline $\begin{array}{c}\text { Ounces } \\
12 \\
14 \\
16 \\
18 \\
20\end{array}$ & $\begin{array}{c}\text { Inch } \\
0.0162 \\
.0189 \\
.0216 \\
.0243 \\
.0270\end{array}$ & $\begin{array}{c}\text { Inch } \\
0.0014 \\
.0015 \\
.0016 \\
.0017 \\
.0018\end{array}$ & $\begin{array}{c}\text { Inch } \\
0.0015 \\
.0016 \\
.0017 \\
.0018 \\
.0019\end{array}$ & $\begin{array}{c}\text { Inch } \\
0.0016 \\
.0017 \\
.0018 \\
.0019 \\
.0020\end{array}$ & $\begin{array}{c}\text { Inch } \\
0.0017 \\
.0018 \\
.0019 \\
.0020 \\
.0021\end{array}$ \\
\hline
\end{tabular}

When specified, sheets and strips shall be suitable for either shallow cupping or deep cupping as required without the formation of symmetrically located ears resulting from directional properties in the strip or sheet.

By "shallow cupping" is meant the forming of any cup where the inside depth is less than the inside diameter.

By "deep cupping" is meant the forming of any cup where the inside depth is equal to or greater than the inside diameter.

9. When finished edges of plates and sheets are specified in the contract or purchase order, the following descriptions shall apply: 
10. "Square edge" material shall be supplied with finishing edge, with sharp, square corners, without bevel or rounding of any sort.

11. "Round edge" material shall be supplied with finished edge, semicircular in form, the diameter of the circle forming the edge being equal to the thickness of the material.

12. "Round corners" material shall be supplied with square-finished edge, and corners rounded to $a$ definite radius which, unless otherwise specified in the contract or purchase order, may be any radius less than one-half the thickness of the material.

13. When no description of any required form of edge is given, it shall be understood that edges such as would result from slitting, sawing, or shearing will be acceptable.

14. Plates, sheets, and strips shall be packed in boxes or crates sufficiently strong to protect them from injury and loss during shipment.

15. When material is ordered in the form of plates, sheets, or strips, it will be understood that these terms refer merely to the general form and dimensions of the material and do not have any technical significance as to the method of manufacture.

16. Contracts or purchase orders for sheets and strips should state the purpose for which the material is to be used, as, "for cupping," "for deep cupping," "for spinning," "for edgewise winding," "for bending at sharp right angles," etc.

17. The thickness of plates, sheets, and strips should be stated in decimals of an inch.

18. Plates, sheets, and strips should be ordered in as narrow widths as can be used.

641.22 SHAPES, COPPER.

AMERICAN BUREAU OF SHIPPING, RULES FOR BUILDING AND CLASSING STEEL VESSELS; MATERIALS FOR MACHINERY, BOILERS, AND PIPING, 1930

\section{COPPER SHAPES}

(See 641.21, p. 1176.)

UNITED STATES GOVERNMENT, FEDERAL SPECIFICATIONS BOARD; FEDERAL SPECIFICATION FOR COPPER RODS, BARS, SHAPES, PLATES, SHEETS, AND STRIPS, QQ-C-501, FEBRUARY 2, 1932

\section{COPPER SHAPES}

(This specification is identical with that for copper rods and bars (see 642.11, p. 1180) except in the following particulars:

1. For shapes, the permissible variation shall be as specified in the contract or purchase order.

2. Since the physical properties obtainable in shapes, hard temper, are dependent on the size and intricacy of the shape, the requirements for each particular order should be agreed upon between the contractor and purchaser.

641.23 STRIPS, COPPER.

UNITED STATES GOVERNMENT, DEPARTMENT OF COMMERCE, BUREAU OF STANDARDS ; STANDARD THICKNESSES, WEIGHTS, AND TOLERANCES OF SHEET METAI (CUSTOMARY PRACTICE), CIRCULAR NO. 391, MARCH 24, 1931

\section{AMERICAN WIRE GAGE COPPER STRIPS}

(See 640.1, p. 1165.)
UNITED STATES GOVERNMENT, FEDERAI SPECIFICATIONS BOARD; FEDERAI SPECIFICATION FOR COPPER RODS, BARS, SHAPES, PLATES, SHEETS, AND STRIPS, QQ-C-501, FEBRUARY 2, 1932

COPPER STRIPS

(See 641.21, p. 1177.)

\section{MANUFACTURES OF COPPER}

642.1 COPPER RODS, WIRES, AND SLEEVES.

642.11 RODS, COPPER.

AMERICAN SOCIETY FOR TESTING MATERIALS, STANDARD SPECIFICATIONS FOR HOT-ROLLED COPPER RODS FOR WIRE DRAWING, DESIGNATION B49-26, 1926

\section{COPPER RODS FOR WIRE DRAWING}

SCOPE

1. These specifications cover black hot-rolled copper rods for wire-drawing purposes.

\section{MAN UFACTURE}

2. The copper shall conform in quality and purity to the requirements of either A. S. T. M. B4 or B5. (See 641.11, p. 1173.)

3. (a) The rod shall be hot-rolled from standard wire bars.

(b) The rods shall be furnished in one continuous length without joints.

\section{PHYSICAI PROPERTIES AND TESTS}

4. (a) The rods shall conform to the following requirements as to tensile properties:

The elongation in 10 inches shall not be less than 25 per cent. The elongation shall be measured between bench marks placed upon the wire originally 10 inches apart and the fracture shall be between the bench marks and not closer than 1 inch to either bench mark. If, upon testing a sample from any coil or reel, the results do not conform to the specified requirements, two additional samples shall be tested and the average of the three tests shall determine acceptance or rejection of the coil or reel.

(b) Two tests shall be made from each carload of 50,000 pounds or less.

5. Electric resistivity shall be determined upon fair samples by resistance measurements at a temperature of $20^{\circ}$ C. $\left(68^{\circ}\right.$ F.) and shall not exceed 891.58 pounds per mile-ohm.

\section{DIMENSIONS AND PERMISSIBLE VARIATIONS}

6. (a) The size shall be expressed as the diameter of the rod in decimal fractions of an inch.

(b) The rods shall be hot rolled to nominal size within the following permissible variations:

\begin{tabular}{|c|c|c|c|}
\hline Diameter (inch) & $\begin{array}{l}\text { Permissi- } \\
\text { ble vari- } \\
\text { ations }\end{array}$ & Diameter (inches) & $\begin{array}{l}\text { Permissi- } \\
\text { ble vari- } \\
\text { ations }\end{array}$ \\
\hline $\begin{array}{l}0.250 \\
.3125 \\
.375 \\
.500 \\
.625 \\
.750\end{array}$ & $\begin{array}{l}\text { Inch } \\
+0.020 \\
\pm .010 \\
\pm .010 \\
\pm .015 \\
\pm .015 \\
\pm .015\end{array}$ & $\begin{array}{l}0.875 \\
1.00 \\
1.125 \\
1.250 \\
1.375\end{array}$ & $\begin{array}{l}\text { Inch } \\
\pm 0.020 \\
\pm .020 \\
\pm .020 \\
\pm .020 \\
\pm .030\end{array}$ \\
\hline
\end{tabular}


Sizes between those listed shall take the variation for the next larger size in the table.

7. For the purpose of calculating weights, cross sections, etc., the density of copper shall be taken as $8.89 \mathrm{~g}$ per $\mathrm{cu} \mathrm{cm}$ at $20^{\circ} \mathrm{C}$. $\left(68^{\circ} \mathrm{F}\right.$.).

\section{FINISH}

8. The rod shall be free from all imperfections not consistent with the best commercial practice.

\section{PACKING}

9. Unless otherwise agreed upon, the rods shall be shipped in coils approximately 24 inches in inside diameter and each coil shall be bound tightly together with copper wire at two points approximately opposite to each other.

\section{INSPECTION}

10. The tests and inspection shall be made at the place of manufacture. The manufacturer shall afford the inspector representing the purchaser all reasonable facilities to assure him that the material conforms to all the requirements of the specifications.

\section{UNITED STATES GOVERNMENT, FEDERAL SPECIFICATIONS BOARD; FEDERAL SPECIFICATION FOR RODS, WELDING, NONFERROUS, FOR GAS WELDING, QQ- $\mathrm{R}-571$, MAY 3, 1932}

\section{COPPER RODS}

(For general specifications; types; material; general requirements; detail requirements; methods of inspection, tests, and chemical analysis; packing and marking of shipments; and notes see 600.0, p. 3.)

1. Copper rods may be used for general welding of copper sheets and rods.

2. Welding rods shall conform to the following chemical requirements:

\section{TYPE C}

Per cent

Copper, minimum

Balance shall contain at least:

Phosphorus

Or silicon

3. Standard sizes of copper rods for gas welding are:

Diameter (inch) $-16,3 / 16,1 / 4,5 / 16$, and $3 / 8$

UNITED STATES GOVERNMENT, FEDERAL SPECIFICATIONS BOARD; FEDERAL SPECIFICATION FOR COPPER RODS, BARS, SHAPES, PLATES, SHEETS, AND STRIPS, QQ-C-501, FEBRUARY 2, 1932

\section{COPPER RODS, BARS, SHAPES}

(For general specifications; grade, forms, and tempers; material and workmanship; detail requirements; physical properties; tests and methods of inspection; packing and marking; and notes, see $641.0, \mathrm{p} .1172$.)

\section{MANUFACTORE}

1. Rods, bars, and shapes may be manufactured by hot-rolling or extrusion, finished by such coldrolling or cold-drawing as may be required, and straightened.

2. All rods and bars shall withstand being hammered hot to a fine point.

\section{Permissible Variations; Rods, Bars, and Shapes}

3. Rods and bars, except forging stock, measured on their diameters, or between parallel faces, shall not vary from the specified dimensions by more than the amounts shown in Table 2.

TABLE 2.-Permissible variations in diameter or thickness, rods and bars, except forging stock

\begin{tabular}{|c|c|c|}
\hline \multirow[b]{2}{*}{ Diameter or thickness (in inches) } & \multicolumn{2}{|c|}{ Permissible variations } \\
\hline & Rounds & $\begin{array}{l}\text { Rectangu- } \\
\text { lar, square, } \\
\text { heragonsl, } \\
\text { etc. }\end{array}$ \\
\hline $\begin{array}{l}0.50 \text { and less. } \\
\text { Over } 0.50 \text { to } 1 \text {, inclusive } \\
\text { Over } 1 \text { to } 2.5 \text {, inclusive } \\
\text { Over } 2.5\end{array}$ & $\begin{array}{l}\text { Inch } \\
\pm 0.0015 \\
\pm .0020 \\
\pm .0025 \\
\pm .0030\end{array}$ & $\begin{array}{l}\text { Inch } \\
\pm 0.0030 \\
\pm .0040 \\
\pm .0050 \\
\pm .0060\end{array}$ \\
\hline
\end{tabular}

4. Rods and bars for forging, measured on their diameters or between parallel faces, shall not vary from the specified dimensions by more than the amounts shown in Table 3.

TABLE 3.-Permissible variations in diameter or thickness, rods and bars for forging

\begin{tabular}{l|c}
\hline Diameter or thickness (in inches) & $\begin{array}{c}\text { Permissible } \\
\text { variations } \\
\text { for all } \\
\text { sections }\end{array}$ \\
\hline Inch \\
\pm 0.005 \\
\pm 0 and less 008 \\
Over 0.50 to 1 , inclusive \\
Over 1 to 2.5, inclusive 010 \\
Over 2.5.... \\
\hline
\end{tabular}

5. Unless the contract or purchase order expressly stipulates that rods and bars are intended for forging, it shall be understood that the permissible variations specified in Table 2 shall apply.

6 . For shapes, the permissible variation shall be as specified in the contract or purchase order.

7. Round rods, except forging stock, shall not be out of round more than one-half the permissible variation in diameter stated in Table 2 .

8. Square, rectangular, and hexagonal rods, bars, and shapes shall have practically exact angles and shall have sharp corners unless otherwise specified.

9. Rods, bars, and shapes of all sizes may be ordered to exact lengths, in which case a variation of plus one-eighth inch on length shall be permitted.

10. When rods and bars one inch or less in diameter or thickness are ordered in stock lengths, the pieces shipped shall be cut to the nominal or stock length as a maximum. The shortest acceptable lengths and the maximum permissible percentage by weight of short lengths in any one shipment shall be as shown in Table 4 .

TABLE 4.-Permissible variations in length of rods and bars

\begin{tabular}{|c|c|c|}
\hline $\begin{array}{c}\text { Nominal or } \\
\text { stock length }\end{array}$ & $\begin{array}{c}\text { Shortest } \\
\text { acceptable } \\
\text { length }\end{array}$ & $\begin{array}{c}\text { Maximum } \\
\text { permissible } \\
\text { percentage, } \\
\text { by weight, of } \\
\text { short lengths }\end{array}$ \\
\hline Feet & Feet & 80 \\
20 & 12 & 80 \\
18 & 10 & 80 \\
16 & 10 & 80 \\
14 & 8 & 40 \\
12 & 8 & 40 \\
10 & 6 & 40 \\
8 & 6 & 40 \\
6 & 4 & \\
\hline
\end{tabular}


11. Unless exact lengths are specified, rods and bars over 1 to and including 2 inches in diameter or thickness will be acceptable in random lengths of 4 to 10 feet, but in no case shall more than 40 per cent of lengths shorter than 10 feet be accepted.

12. Rods and bars over 2 inches in diameter or thickness will be ordered in special lengths.

13. Rods, bars, and shapes may be bundled for shipment.

\subsection{WIRES, COPPER.}

(Specifications and standards for electrical supplies are not included in this publication since, in the general classification of commodities in the "Encyclopedia series," electrical supplies are being placed with the classification division "machinery and vehicles." It is anticipated that standards and specifications for machinery and vehicles, including electrical supplies, will be the subject of a separate volume in the Encyclopedia series to be issued at a later date.

However, a list of the current nationally recognized standards and specifications for electrical copper wire, insulated electrical cord, and electrical cables, is given herein along with the names of the organizations sponsoring them.

These standards include standard sizes; construction of cables; dimensions of wire and cable; thicknesses of insulating coverings and metallic sheaths; requirements on materials entering into conductor insulating covering, and sheaths; dielectric and insulation resistance requircments; flexibility requirements, etc.; and methods of test.)

American Bureau of Shipping, rules for building and classing steel vessels, 1930. Conductors and cables.

American Electric Railway Engineering Association; standard copper-wire tables, D1-16; round and grooved hard-drawn copper trolley wire, D2-30 high-voltage three-conductor paper-insulated leadcovered cable, D3-14; single-conductor paperinsulated lead-covered cable for 1,200 volts, D4-12; rubber-insulated wire and cable for power-distribution purposes, D10-30; motor leads, E216-27.

American Institute of Electrical Engineers; wires and cables, standard No. 30; recommended practice for electrical installations on shipboard, standard No. 45, includes cables and wires; tinned soft or annealed copper wire, standard No. 60; soft or annealed copper wire, standard No. 61; cottoncovered round copper magnet wire, standard No. 69 ; silk-covered round copper magnet wire, standard No. 70; enameled round copper magnet wire, standard No. 71.

American Railway Association, Electrical Section; electric wires and cables, XIX-a-21.

American Railway Association, Signal Section; enameled copper magnet wire, specification No. 4113; double-braided, weatherproof hard-drawn copper line wire, No. 7218; aerial braided cable for 660 volts or less, No. 8930; armored submarine cable for 660 volts or less and for 2,300 volts or less; lead-covered cable for 660 volts or less and for 2,300 volts or less, No. 9131; underground braided cable for 660 volts or less, No. 9420; copper bond wire, No. 10820; mineral-matter rubber-compound insulated signal wire, No. 11129; parkway cable, No. 14529.

American Railway Association, Telegraph and Telephone Section; paper-insulated lead-covered telegraph and telephone cable with high insulation, specification 1-A-9; hard-drawn bare copper wire, specification 1-A-13; annealed copper tie wires, specification 1-A-15; lead sheath office cable, specification 2-G-1; braided office cable, specification $2-\mathrm{G}-2$; single conductor cord, specification $2-\mathrm{G}-11$; twisted pair inside wire, specification 2-G-15; single-conductor inside wire, 2-G-16; lead-sheath office cable quadded, 2-G-19; braided office cable of quadded rubber insulated No. 16 A. W. G. conductors, 2-G-22; double-conductor patching cord, 2-G-28; double-conductor switchboard cord, $2-\mathrm{G}-29$; reverse double-conductor cord, 2-G-32; single-conductor cord, 2-G-33.

American Society for Testing Materials; harddrawn copper wire, B1-27; medium-drawn copper wire, B2-27; bare concentric-lay copper cablehard, medium hard, or soft-B8-27; tinned soft or annealed copper wire for rubber insulation, B33-21; round and grooved hard-drawn copper trolley wire, B47-32; soft rectangular copper wire, B48-27; insulated wire and cable, D-27-31T.

Association of Electragists, International; electragist standards for wiring installations.

Association of Railway Electrical Engineers; tinned soft or annealed copper wire for rubber insulation, G-I-1; soft or annealed copper wire, G-I-2; 30 per cent rubber insulation for wire and cable for general purposes, G-I-3; cotton-covered round copper magnet wire, G-I-4; silk-covered round copper magnet wire, G-I-5; enameled round copper magnet wire, G-I-6.

Insulated Power Cable Engineers Association; impregnated paper-insulated lead-covered cable; recommended walls of insulation and of lead sheaths for paper-lead power cables; varnished cambric insulated cables.

International Association of Municipal Electricians; underground and aerial cable for fire-alarm, police, telegraph, traffic-signal, and street-lighting service.

National Board of Fire Underwriters; national electrical code, includes requirements for conductors.

National Electrical Manufacturers Association: handbook of supply standards, includes standards for intercommunicating telephone cable; handbook of radio standards, includes standards for connection cords.

Radio Manufacturers Association (Inc.); power cable, M4-505 and M4-506.

Society of Automotive Engineers; 1931 handbook, includes specifications for insulated cables, lighting and starting motor cable, magnet wire, soft or annealed copper wire.

Underwriters' Laboratories; armored cable and armored cord; flexible cord; heat-resisting fixture wire and flexible cord; heater cord; motion-picture cable and stove wire; nonmetallic sheathed cables; rubber-covered fixture wires; rubber-covered wire; rubber-sheathed cords; slow-burning and slowburning weatherproof wires; varnished cloth wires and cables.

United States Government, Department of Commerce, Bureau of Mines; schedule $2 \mathrm{C}$, explosionproof mine equipment, includes requirements for trailing cables.

United States Government, Federal Specifications Board; rubber-covered wire and cables, specification No. 65.

642.13 SIEEVES, COPPER.

(No nationally recognized specifications available.)

642.2 COPPER CASTINGS, FERR ULES, PIPES, AND TUBES.

\subsection{CASTINGS, COPPER.}

(No nationally recognized specifications available.) 
642.22 FERRULES, COPPER.

(No nationally recognized specifications available.)

642.23 PIPES, COPPER.

AMERICAN BUREAU OF SHIPPING, RULES FOR BUILDING AND CLASSING STEEL VESSELS; MATERIALS FOR MACHINERY, BOILERS, AND PIPING, 1930

\section{COPPER PIPES AND TUBES}

(For general information and requirements see 600.0, p. 1 . For test requirements see 600.1, p. 7 .

1. Seamless-drawn copper pipes and tubes subject to a working pressure of over 150 pounds are to be tested and inspected by the surveyor in accordance with the following requirements: The material used must be of the best quality, and the tubes must be true to form, of uniform thickness throughout, clean and smooth, and free from defects.

2. The test specimens are to be selected by the surveyor, and at least one set of tests is to be made for each lot of 2,000 pounds or less. The test specimens are to be annealed.

\section{TENSILE TEST}

3. A standard test piece must show the material to have a minimum tensile strength of 28,000 pounds per square inch, and a minimum elongation of 25 per cent.

\section{FLATTENING TEST}

4. A pipe 4 inches long must stand being flattened until the sides meet without showing signs of cracks or flaws. For brazed pipes the bending tests for plates may be applied. (See 641.21, p. 1176.)

\section{FLANGING TEST}

5. The end of the pipe must stand flanging cold without signs of cracks or flaws, the flange to be at an angle of $90^{\circ}$ with the center of the pipe, and the width of the flange to be not less than one-fourth of the inside diameter of the pipe.

\section{HYDROSTATIC TEST}

6. All pipes are to be subjected to a hydrostatic test of not less than twice the working pressure for working pressures up to 1,000 pounds per square inch. For working pressures above 1,000 pounds per square inch the test pressure is to be equal to the working pressure plus 1,000 pounds per square inch.

\section{GAGING AND MARKING}

7. The minimum thickness shall not be less than specified by the purchaser and required by the rules.

8. Upon satisfactory compliance with the requirements, all pipes are to be legibly stenciled with the identification mark of the manufacturer, the test pressure, and A.B., showing that they have been satisfactorily tested and inspected by a surveyor to the bureau. Pipes developing defects previous to or during their installation shall be rejected, notwithstanding any previous acceptance.

\section{AMERICAN BUREAU OF SHIPPING, RULES FOR BUILDING AND CLASSING STEEL VESSELS; PUMPS AND PIPING SYSTEMS, 1930}

\section{COPPER AND BRASS PIPES}

(For general requirements see 607.4, p. 435.)

1. Copper pipes for pressures over 100 pounds per square inch should be seamless drawn. Copper pipes should not be used to carry superheated steam. Copper pipes for steam, feed, and blow-off must be properly annealed before installation.

2. The minimum thickness of pipes shall be determined by the following formulas:

$$
\begin{aligned}
& \text { Brazed copper and brass pipe } t=\frac{D W}{6000}+C \\
& \text { Seamless copper and brass pipe } t=\frac{D W}{7000}+C
\end{aligned}
$$

3. For special seamless brass pipe made of composition having a minimum tensile strength of 55,000 pounds per square inch and elongation of at least 30 per cent

Where

$$
t=\frac{D W}{8000}+C
$$

$t=$ Thickness in inches.

$D=$ Internal diameter in inches.

$W=$ Working pressure in pounds per square inch. For feed pipes this should be 1.2 times the boiler pressure.

$C=0.06$ inch for steam and water; 0.03 inch for air.

4. The reduction in thickness at the outer radius of bent pipes is to be considered in determining the required thickness.

AMERICAN SOCIETY FOR TESTING MATERIALS, STANDARD SPECIFICATIONS FOR COPPER PIPE, STANDARD SIZES, DESIGNATION B42-24, 1924

\section{COPPER PIPE}

\section{SCOPE}

1. These specifications cover seamless copper tubes and seamless copper pipe suitable for use in plumbing, boiler-feed lines, etc.

\section{MANUFACTURE}

2. (a) The pipe shall be cold-drawn to size.

(b) When pipe is required for bending, the purchaser shall so specify, in which case the pipe shall be furnished with a proper bending temper.

\section{CHEMICAL PROPERTIES AND TESTS}

3. The copper shall have a purity of at least 99.880 per cent as determined by electrolytic assay, silver being counted as copper.

4. The sample for chemical analysis shall consist of drillings, millings, or clippings, taken from the test specimens specified in section 9, equal quantities being taken from each specimen and thoroughly mixed. Samples so prepared shall be divided into three equal parts, each of which shall be placed in $\mathbf{a}$ sealed package-one for the seller, one for the purchaser, and one for an umpire if necessary.

\section{PHYSICAL PROPERTIES AND TESTS}

\begin{tabular}{|c|c|c|}
\hline & $\begin{array}{l}\text { Tensile } \\
\text { strength }\end{array}$ & $\begin{array}{l}\text { Elonga- } \\
\text { tion in } 4 \\
\text { inches }\end{array}$ \\
\hline Pipe less than 2 inches outside diameter & $\begin{array}{r}\text { Lbs./in. }{ }^{2} \\
40,000\end{array}$ & $\begin{array}{l}\text { Per cent } \\
10\end{array}$ \\
\hline $\begin{array}{l}\text { Pipe } 2 \text { inches or over outside diameter (after } \\
\text { annealing) }\end{array}$ & 30,000 & 25 \\
\hline
\end{tabular}

5. The material shall conform to the following minimum requirements as to tensile properties: 
6. (a) The test specimen, after being annealed, shall withstand being bent cold through $180^{\circ}$ without cracking on the outside of the bent portion as follows: For material less than one-half inch in thickness, flat on itself; and for material one-half inch or over in thickness, around a pin the diameter of which is twice the thickness of the specimen. (Note that in F. S. B. No. WW-P-378 (see 642.24, p. 1187) this test says "around a diameter equal to its thickness.")

(b) In the case of pipe for bending, annealed full sections of the pipe shall withstand being bent cold through $180^{\circ}$ without cracking on the outside of the bent portion, around a pin the diameter of which is one and one-half times the inside diameter of the pipe. This test shall be in addition to the test specified in paragraph $(a)$.

7. (a) An annealed test specimen not more than 4 inches in length shall have a flange turned over at right angles to the body of the pipe without showing cracks or flaws. The width of this flange, as measured from the outside of the pipe, shall be onefourth of the inside diameter of the pipe.

(b) In making the flange test it is recommended that the flaring tool and die block shown in Figure 1078 be used.

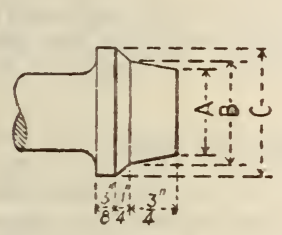

$A=$ Ouis. Diam. of Tute less $\frac{5}{8}^{\prime \prime}$ $B=$ Outs. Diam. of Tube less $\frac{3}{8}$ $C=$ Ouis. Diom of Tube flus $\frac{3}{16}$

$$
\text { Flaring Tool }
$$

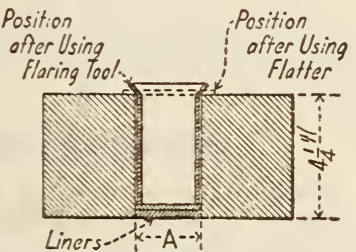

$A=$ Outs. Diam. of Tube plus $\frac{1}{32}$

Die Block

Figure 1078.-Flaring tool and die block

8. Each pipe shall withstand, without showing weakness or defects, an internal hydrostatic pressure sufficient to subject the material to a fiber stress of 6,000 pounds per square inch, determined by the formula for thin hollow cylinders under tension:

$$
P=\frac{2 t S}{D}
$$

in which $P$ equals the pressure in pounds per square inch, $t$ equals the thickness of wall in inches, $D$ equals the outside diameter of the pipe in inches, and $S$ equals the allowable unit stress of the material or 6,000 pounds per square inch. No pipe shall be tested beyond a pressure of 1,000 pounds per square inch unless so specified.

9. (a) Tension-test specimens for pipe less than 2 inches in outside diameter shall consist of a full section of the unannealed pipe; the ends shall be plugged with metal plugs which shall not extend within the gage marks.

(b) In the case of pipe 2 inches or over in outside diameter and not exceeding 6 inches in outside diameter, the test specimen shall be cut longitudinally. In the case of pipe exceeding 6 inches in outside diameter, the test specimen shall be cut circumferentially. (Note that, in F. S. B. No. WW-P-378 (see 642.24 , p. 1187), the corresponding specification calls for "six inches inside diameter.")

(c) The test specimen taken as specified in paragraph (b) shall be heated to a cherry red and straightened while hot. It shall then be machined to conform to Figure 1079. The width of the specimen shown in Figure 1079 shall be governed by the thickness of the material. For material one-fourth inch or less in thickness, the width of the narrow portion of the test specimen shall be approximately one inch; for material over one-fourth inch in thickness, the width shall be such as to give a cross section of approximately one-fourth square inch in area, but in no case shall the width be less than the thickness of the material. The drawn surfaces shall not be machined, but be left in their original condition.

(d) The bend-test specimen shall consist of a strip, not over $1 \frac{1}{2}$ inches in width, cut longitudinally from the pipe.

10. One bend test shall be made for each 2,000 pounds or less of the finished product. For the remaining tests a sufficient number of pipe shall be taken at random to constitute 0.5 per cent of the shipment.

\section{DIMENSIONS AND PERMISSIBLE VARIATIONS}

11. The standard weights and dimensions for pipe of various outside diameters are given in Table 1.

12. (a) No single piece of pipe that exceeds the calculated weight by more than 5 per cent will be accepted. One cubic inch of the material is assumed to weigh 0.323 pound.

(b) The weight of the pipe shall vary not more than the following percentages from the weight specified in section 11 ; pipe 6 inches or less in diameter, 5 per cent; pipe from 6 to 8 inches in diameter, 7 per cent; pipe over 8 inches in diameter, 8 per cent.

TABLE 1.-Standard weights and dimensions for

\begin{tabular}{|c|c|c|c|c|c|}
\hline \multirow[b]{2}{*}{$\begin{array}{l}\text { Size of } \\
\text { pipe }\end{array}$} & \multirow[b]{2}{*}{$\begin{array}{l}\text { Outside } \\
\text { diameter }\end{array}$} & \multicolumn{2}{|c|}{ Standard pipe } & \multicolumn{2}{|c|}{ Extra-strong pipe } \\
\hline & & $\begin{array}{c}\text { Thick- } \\
\text { ness }\end{array}$ & $\begin{array}{l}\text { Nominal } \\
\text { weight } \\
\text { per foot } \\
\text { of length }\end{array}$ & $\begin{array}{c}\text { Thick- } \\
\text { ness }\end{array}$ & $\begin{array}{l}\text { Nominal } \\
\text { weight } \\
\text { per foot } \\
\text { of length }\end{array}$ \\
\hline $\begin{array}{c}\text { Inches } \\
1 / 8 \\
1 / 4 \\
3 / 8 \\
1 / 2 \\
3 / 4\end{array}$ & $\begin{array}{r}\text { Inches } \\
0.405 \\
.540 \\
.675 \\
.840 \\
1.050\end{array}$ & $\begin{array}{c}\text { Inch } \\
0.0620 \\
.0825 \\
.0905 \\
.1075 \\
.1140\end{array}$ & $\begin{array}{c}\text { Pounds } \\
0.259 \\
.460 \\
.643 \\
.957 \\
1.30\end{array}$ & $\begin{array}{r}\text { Inch } \\
0.100 \\
.123 \\
.127 \\
.149 \\
.157\end{array}$ & $\begin{array}{c}\text { Pounds } \\
0.371 \\
.624 \\
.847 \\
1.25 \\
1.71\end{array}$ \\
\hline $\begin{array}{l}1 \\
11 / 4 \\
11 / 2 \\
2 \\
21 / 2\end{array}$ & $\begin{array}{l}1.315 \\
1.660 \\
1.900 \\
2.375 \\
2.875\end{array}$ & $\begin{array}{l}.1265 \\
.1460 \\
.1500 \\
.1565 \\
.1875\end{array}$ & $\begin{array}{l}1.83 \\
2.69 \\
3.20 \\
4.23 \\
6.14\end{array}$ & $\begin{array}{l}.182 \\
.194 \\
.203 \\
.221 \\
.280\end{array}$ & $\begin{array}{l}2.51 \\
3.46 \\
4.19 \\
5.79 \\
8.84\end{array}$ \\
\hline $\begin{array}{l}3 \\
31 / 2 \\
4 \\
41 / 2 \\
5\end{array}$ & $\begin{array}{l}3.500 \\
4.000 \\
4.500 \\
5.000 \\
5.563\end{array}$ & $\begin{array}{l}.2190 \\
.2500 \\
.2500 \\
.2500 \\
.2505\end{array}$ & $\begin{array}{r}8.75 \\
11.41 \\
12.94 \\
14.46 \\
16.21\end{array}$ & $\begin{array}{l}.304 \\
.321 \\
.341 \\
.375 \\
.375\end{array}$ & $\begin{array}{l}11.82 \\
14.37 \\
17.25 \\
21.10 \\
23.67\end{array}$ \\
\hline $\begin{array}{r}6 \\
7 \\
8 \\
9 \\
10\end{array}$ & $\begin{array}{r}6.625 \\
7.625 \\
8.625 \\
9.625 \\
10.750\end{array}$ & $\begin{array}{l}.2500 \\
.2815 \\
.3125 \\
.3440 \\
.3655\end{array}$ & $\begin{array}{l}19.41 \\
25.17 \\
31.63 \\
38.83 \\
46.22\end{array}$ & $\begin{array}{l}.437 \\
.500 \\
.500 \\
.500 \\
.500\end{array}$ & $\begin{array}{l}32.93 \\
43.34 \\
49.42 \\
55.56 \\
62.40\end{array}$ \\
\hline
\end{tabular}
copper pipe

\section{WORKMANSHIP AND FINISH}

13. (a) The pipe shall be round and of uniform thickness throughout. It shall be free from cracks, slivers, seams, scale, and other surface defects.

(b) The thickness at any point shall not be less than that specified in section 11 by more than the following percentages: Pipe 6 inches or less in diameter, 5 per cent; pipe from 6 to 8 inches in diameter, 7 per cent; pipe over 8 inches in diameter, 8 per cent.

\section{INSPECTION AND REJECTION}

14. When the pipe is finished and ready for shipment the inspector shall make the tests to govern 
the acceptance of the pipe. If any specimen fails to meet the requirements of sections 5 to 8 , inclusive, two additional specimens may be taken, each of which shall conform to the requirements specified.

(The inspection requirements are identical with those for seamless admiralty condenser tubes and ferrule stock in B44. (See 645.24, p. 1220.))

UNITED STATES GOVERNMENT, DEPARTMENT OF COMMERCE, STEAMBOAT INSPECTION SERVICE; GENERAL RULES AND REGULATIONS, MAY 4, 1929

\section{COPPER PIPE}

No copper pipe constructed after June 30,1905 , shall have any bend whose radius is less than one and one-half times the diameter of the pipe, and such pipe shall be so led and flanges so placed that they may be readily taken down if required. Such pipes shall be protected by iron casings when run through coal bunkers, and shall be clear of the coal chutes. The thickness of material, according to the working pressure, shall be determined by the following formula:

Where

$$
T=\frac{P \times D}{8000}+0.0625
$$

$T=$ thickness in inches.

$P=$ working pressure.

$D=$ inside diameter of pipe in inches.

Example.-Required, the thickness of material of a 5-inch copper pipe for a working pressure of 175 pounds per squale inch.

Substituting values, we have

$$
T=\frac{175 \times 5}{8000}+0.0625=0.171 \text { inch }
$$

Provided, however, that all copper pipe subject to pressure and installed for use on steam vessels after July 1, 1911, shall have a thickness of material according to the working pressure, to be determined by the following formula:

$$
T=\frac{P \times D}{6000}+0.0625
$$

Where

$$
T=\text { thickness in inches. }
$$

$P=$ working pressure.

$D=$ inside diameter of pipe in inches.

Example.-Required, the thickness of material of a 5-inch copper pipe for a working pressure of 175 pounds per square inch.

Substituting and solving, we have

$$
T=\frac{175 \times 5}{6000}+0.0625=0.208 \text {. }
$$

UNITED STATES GOVERNMENT, FEDERAL SPECIFICATIONS BOARD; FEDERAI SPECIFICATION FOR PIPE AND TUBING ; COPPER, SEAMIESS : PIPE (STAND. ARD IRON-PIPE SIZE); TUBING (FOR PRESSURES UP TO 450 POUNDS PER SQUARE INCH), WW-P-378, MAY $2 \%$, 1930

\section{SEAMLESS COPPER PIPE}

(See 642.24, p. 1187.)
642.24 TUBES, COPPER.

AMERICAN BUREAU OF SHIPPING, RULES FOR BUILDING AND CLASSING STEEL VESSELS; MATERIALS FOR MACHINERY, BOILERS, AND PIPING, 1930

\author{
COPPER PIPES AND TUBES
}

(See 642.23, p. 1182.)

AMERICAN SOCIETY FOR TESTING MATERIALS, STANDARD SPECIFICATIONS FOR SEAMLESS COPPER BOILER TUBES, DESIGNATION B13-18, 1918

\section{SEAMLESS COPPER BOILER TUBES}

\section{SCOPE}

1. These specifications cover two grades of copper tubes for locomotive boilers; namely, arsenical and nonarsenical.

\section{MANUFACTURE}

2. The copper shall be fire refined or electrolytic and shall be made into tubes either by the hotrolling or cold-drawing process. It shall be finished by cold drawing.

\section{CHEMICAL PROPERTIES AND TESTS}

(Requirements covering both arsenical and nonarsenical copper are identical with those for copper plates for locomotive fireboxes in A. S. T. M. B11 (see 641.21, p. 1176) except that in this case the arsenical copper shall contain from 0.15 to 0.35 per cent of arsenic.)

3. Analyses of two tubes in each lot of 250 or less may be made by the purchaser. The chemical composition thus determined shall conform to the requirements specified above. Drillings for analyses shall be taken from several points around each tube, or turnings may be taken from the end of the tube.

4. If the analysis of any one tube does not conform to the requirements specified, analyses of two additional tubes from the same lot shall be made, each of which shall conform to the requirements specified.

\section{PHYSICAL PROPERTIES AND TESTS}

5. Tubes will be furnished hard or annealed, as specified. Test specimens taken from hard tubes shall be annealed before testing.

6. A test specimen not more than 4 inches in length shall have a flange turned over at right angles to the body of the tube, without showing cracks or flaws. This flange, as measured from the outside of the tube, shall be three-eighths inch wide for tubes $2 \frac{1}{2}$ inches or under in outside diameter, and one-half inch wide for tubes over $2 \frac{1}{2}$ inches in outside diameter.

(For illustration of flaring tool and die block used in making the flange test, see A. S. T. M. B42 (642.23, p. 1182).)

7. A test specimen $2 \frac{1}{2}$ inches in length shall withstand crushing longitudinally until the outside folds are in contact, without showing cracks or flaws.

8. All tubes shall withstand an internal hydrostatic pressure of 750 pounds per square inch. The hydrostatic-pressure test shall be applied to the finished tubes before annealing.

9. (a) Test specimens shall consist of sections cut from tubes selected by the inspector representing 
the purchaser from the lot offered for shipment. They shall be smooth on the ends and free from burrs.

(b) All specimens shall be tested cold.

10. One flange and one crush test shall be made from each of two tubes in each lot of 250 or less. Each tube shall be subjected to the hydrostatic test.

11. If the results of the physical tests of only one tube from any lot do not conform to the requirements specified, retests of two additional tubes from the same lot shall be made, each of which shall conform to the requirements specified.

\section{STANDARD WEIGHTS}

12. The standard weights for tubes of various outside diameters and thicknesses are as follows:

\begin{tabular}{|c|c|c|c|c|c|c|}
\hline \multicolumn{2}{|c|}{ Thickness } & \multicolumn{5}{|c|}{ Outside diameter (inches) } \\
\hline \multirow{2}{*}{ B. w. g. } & \multirow{2}{*}{ Inch } & $11 / 2$ & $13 / 4$ & 2 & $21 / 4$ & $21 / 2$ \\
\hline & & \multicolumn{5}{|c|}{ Weight per foot } \\
\hline $\begin{array}{r}13 \\
12 \\
11 \\
10 \\
9\end{array}$ & $\begin{array}{r}0.095 \\
.109 \\
.120 \\
.134 \\
.148\end{array}$ & $\begin{array}{c}\text { Pounds } \\
1.624 \\
1.844 \\
2.015 \\
2.227 \\
2.434\end{array}$ & $\begin{array}{c}\text { Pounds } \\
1.913 \\
2.176 \\
2.380 \\
2.634 \\
2.884\end{array}$ & $\begin{array}{c}\text { Pounds } \\
2.202 \\
2.507 \\
2.744 \\
3.042 \\
3.334\end{array}$ & $\begin{array}{c}\text { Pounds } \\
2.491 \\
2.839 \\
3.109 \\
3.449 \\
3.785\end{array}$ & $\begin{array}{c}\text { Pounds } \\
2.779 \\
3.170 \\
3.474 \\
3.857 \\
4.235\end{array}$ \\
\hline
\end{tabular}

13. The weight of the tubes shall not vary more than 5 per cent from that specified in section 12 .

\section{WORKMANSHIP AND FINISH}

14. (a) The finished tubes shall be circular within $0.02 \mathrm{inch}$, and the mean outside diameter shall not vary more than 0.015 inch from the size ordered.

(b) The thickness at any point shall not vary more than 10 per cent from that specified.

(c) The length shall not be less, but may be 0.125 inch more than that ordered.

15. The finished tubes shall be free from injurious defects and distortion and shall have a workmanlike finish.

\section{MARKING}

16. The name or brand of the manufacturer and "tested at 750 pounds" shall be legibly stenciled on each tube.

\section{INSPECTION AND REJECTION}

(Requirements covering inspection and rejection are identical witn those for copper plates for locomotive fire boxes in A. S. T. M. B11 (see 641.21, p. 1176), except that tubes are substituted for plates, and the addition of the following paragraph:)

17. Tubes when inserted in the boiler shall withstand expanding and bending without showing cracks or flaws. Tubes which fail in this manner will be rejected, and the manufacturer shall be notified.

\section{AMERICAN SOCIETY FOR TESTING MATE- RIALS, TENTATIVE SPECIFICATIONS FOR BRIGHT ANNEALED SEAMLESS COPPER TUBING, B68-30T, 1930}

\section{ANNEALED SEAMLESS COPPER TUBING}

1. These specifications cover annealed copper tubing suitable for use in refrigerators, oil lines, gasoline lines, etc., where tubing absolutely free from scale and dirt is required.

\section{MANUFACTURE}

2. The tubing shall be made from copper that has been deoxidized by means of phosphorus.

3. The tubing shall be bright annealed after the last drawing operation or after the coiling, if coiling is required.

4. The tubing shall be thoroughly recrystallized and have an average grain size not exceeding 0.040 $\mathrm{mm}$ in diameter.

\section{CHEMICAL PROPERTIES AND TESTS}

5. The material shall conform to the following requirements as to chemical composition:

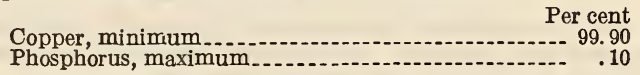

6. (Sampling is the same as for copper pipe in A. S. T. M. B42 (see 642.23 , p. 1182), except that the sample shall be taken from each lot of 5,000 pounds or fraction thereof.)

\section{PHYSICAL PROPERTIES AND TESTS}

7. (a) The material shall conform to the following minimum requirements as to tensile properties:

Tensile strength. lbs./in.2.-- 30,000

(b) Five test specimens shall be selected for phy ical test from each lot of 5,000 pounds or fraction thereof.

\section{PERIISSIBLE VARIATIONS IN DIMENSIONS}

8. The mean inside and outside diameters and the wall thickness of the tubing shall not vary more than the following amounts from that specified:

\begin{tabular}{l|c}
\hline Mean inside and outside diameters (in inches) & $\begin{array}{c}\text { Permis- } \\
\text { sible vari- } \\
\text { ations }\end{array}$ \\
\hline Up to 1, inclusive & $\begin{array}{c}\text { Inch } \\
\pm 0.0025 \\
\pm .003\end{array}$ \\
\hline Over 1 to 2, inclusive...
\end{tabular}

Wall thickness

\begin{tabular}{|c|c|c|}
\hline Stubbs gage No. & Inches & $\begin{array}{l}\text { Permis- } \\
\text { sible vari- } \\
\text { ations }\end{array}$ \\
\hline $\begin{array}{l}\text { Under } 14 \text { to } 17 \text {, inclusive....- } \\
\text { Under } 17 \text { to } 20 \text {, inclusive...-. } \\
\text { Under } 20 \text { to } 23 \text {, inclusive.... } \\
\text { Under } 23 . .\end{array}$ & $\begin{array}{l}0.082 \text { to } 0.058 \text {, inclusive. } \\
0.057 \text { to } 0.035 \text {, inclusive... } \\
0.034 \text { to } 0.025 \text {, inclusive... } \\
\text { Under } 0.025\end{array}$ & $\begin{array}{l}\text { Inch } \\
\pm 0.004 \\
\pm .003 \\
\pm .0025 \\
\pm .0025\end{array}$ \\
\hline
\end{tabular}

No combination of variations on the same tube shall make the thickness of the wall vary from the nominal thickness by more than these amounts.

9. The lengths of the tubing, when measured at a temperature of $68^{\circ} \mathrm{F}$. $\left(20^{\circ} \mathrm{C}\right.$.) shall not vary from that specified on the purchase order by more than the following amounts:

\section{For straight lengths}

\begin{tabular}{|c|c|c|}
\hline \multirow{2}{*}{ Ordered length (in feet) } & \multicolumn{2}{|c|}{$\begin{array}{l}\text { Permissible varia- } \\
\text { tions }\end{array}$} \\
\hline & Minus & Plus \\
\hline $\begin{array}{l}\text { Up to } 8 \text {, inclusive } \\
\text { Over } 8 \text { to } 15 \text {, inclusive } \\
\text { Over } 15 \text { to } 20 \text {, inclusive }\end{array}$ & $\begin{array}{r}\text { Inch } \\
0 \\
0 \\
0\end{array}$ & $\begin{array}{c}\text { Inch } \\
110 \\
18 \\
310 \\
310\end{array}$ \\
\hline
\end{tabular}


For coils

\begin{tabular}{|c|c|c|}
\hline \multirow{2}{*}{ Ordered length (in feet) } & \multicolumn{2}{|c|}{$\begin{array}{c}\text { Permissible varia- } \\
\text { tions }\end{array}$} \\
\hline & Minus & Plus \\
\hline $\begin{array}{l}\text { Up to } 10 \\
\text { Over } 10 \text { to } 20 \text {, inclusive } \\
\text { Over } 20 \text { to } 40 \text {, inclusive } \\
\text { Over } 40 \text { to } 80 \text {, inclusive } \\
\text { Over } 80\end{array}$ & $\begin{array}{r}\text { Inch } \\
0 \\
0 \\
0 \\
0 \\
0\end{array}$ & $\begin{array}{r}\text { Inches } \\
2 \\
3 \\
6 \\
12 \\
24\end{array}$ \\
\hline
\end{tabular}

\section{WORKMANSHIP AND FINISH}

10. The finished tubing shall be smooth, free from interior and exterior mechanical imperfections, and shall have a clean, bright appearance.

\section{PACKING AND MARKING}

11. The tubing shall be packed in such a manner as to prevent damage in ordinary handling and transportation.

12. All boxes, bundles, or barrels shall bear the following information: Purchase order number, manufacturer's name, size and kind of material, and part number, if any.

\section{REJECTION}

13. Material failing to comply with the above specifications may be rejected and returned for replacement or full credit at the option of the purchaser.

\section{AMERICAN SOCIETY FOR TESTING MA- TERIALS, SPECIFICATIONS FOR SEAM- LESS COPPER TUBES, DESIGNATION B75-30, 1930}

\section{SEAMLESS COPPER TUBES}

SCOPE

1. These specifications cover seamless copper tubes suitable for general engineering uses.

\section{MANUFACTURE}

2. The tubes shall be cold drawn to size.

3. (a) Unless otherwise specified, the tubes shall be furnished hard drawn.

(b) When tubes are ordered "hard," test specimens shall be annealed before testing.

\section{CHEMICAL PROPERTIES AND TESTS}

(Requirements covering chemical properties and tests are the same as those for copper pipe in A. S. T. M. B42 (see 642.23, p. 1182), except that in this case the copper shall have a purity of at least 99.90 per cent.)

\section{PHYSICAL PROPERTIES AND TESTS}

4. The tubes selected for test shall be capable of standing the following test without showing cracks or flaws: Pieces 4 inches in length cut from the ends of the test tubes shall be hammered flat so that a gage set at three times the thickness of the metal shall pass over the tube freely throughout the flattened part.

5. Pieces 3 inches in length cut from each tube selected for test shall, when split, stand opening out flat without showing cracks or flaws.

6. (a) A strip not over $1 \frac{1}{2}$ inches in width shall be taken from each test tube which, after annealing, shall stand being bent flat on itself without showing signs of fracture on the outside bent surface, and the ends of the bend-test specimen shall stand hammering down hot or cold to a knife-edge without showing signs of cracks.

(b) When tubes 2 inches in diameter or under are ordered fully annealed, full sections of the tubes shall stand being bent $180^{\circ}$ around a diameter one and one-half times the inside diameter of the tube without cracking on the outside bent surface.

(Requirements covering flange test, including flaring tool and die block, and hydrostatic test are identical with those for copper pipe in A. S. T. M. B42 (see 642.23, p. 1182), substituting the word tube for pipe.)

7. A number of tubes equivalent to 0.5 per cent of the shipment shall be selected at random for purpose of tests. One hammering, one opening, one bend, and one flange test shall be made on each tube so selected.

\section{DIMENSIONS AND PERMISSIBLE VARIATIONS}

8. The standard weight of any tube shall be based upon the assumption that one cubic inch of the material weighs 0.323 pound.

9. (a) The mean inside or outside diameters of the tubing shall not vary from that specified by more than the following amounts:

\begin{tabular}{|c|c|}
\hline Mean diameter (in inches) & $\begin{array}{c}\text { Permissible } \\
\text { variation }\end{array}$ \\
\hline $\begin{array}{l}\text { Up to } 1 \text {, inclusive } \\
\text { Over } 1 \text { to } 2 \text {, inclusive } \\
\text { Over } 2 \text { to } 3 \text {, inclusive } \\
\text { Over } 3 \text { to } 4 \text {, inclusive }\end{array}$ & $\begin{array}{l}\text { Inch } \\
\pm 0.0025 \\
\pm .003 \\
\pm .004 \\
\pm .005\end{array}$ \\
\hline $\begin{array}{l}\text { Over } 4 \text { to } 5 \text {, inclusive } \\
\text { Over } 5 \text { to } 6 \text {, inclusive } \\
\text { Over } 6 \text { to } 8 \text {, inclusive } \\
\text { Over } 8 \text { to } 10 \text {, inclusive. }\end{array}$ & $\begin{array}{l}\text { 土. } 006 \\
\pm .007 \\
\pm .008 \\
\pm .010\end{array}$ \\
\hline
\end{tabular}

(b) No combination of variations on the same tube shall make the thickness of the wall vary from the nominal thickness by more than the amount specified in Table 1.

TABLE 1.-Permissible variations from the standard tube thickness

\begin{tabular}{|c|c|c|c|c|c|}
\hline \multicolumn{2}{|c|}{ Standard tube thickness } & \multicolumn{4}{|c|}{ Diameter (inches) } \\
\hline Stubbs gage No. & Inch & $\begin{array}{l}\text { Up to } 2, \\
\text { inclusive }\end{array}$ & $\begin{array}{l}\text { Over } 2 \text { to } \\
4 \text {, inclu- } \\
\text { sive }\end{array}$ & $\begin{array}{l}\text { Over } 4 \text { to } \\
7, \text { inclu- } \\
\text { sive }\end{array}$ & $\begin{array}{l}\text { Over } 7 \text { to } \\
10 \text {, inclu- } \\
\text { sive }\end{array}$ \\
\hline $\begin{array}{l}\text { Over } 2-0 \\
\text { Under } 2-0 \text { to } 2 \text {, inclusive } \\
\text { Under } 2 \text { to } 5 \text {, inclusive } \\
\text { Under } 5 \text { to } 8 \text {, inclusive } \\
\text { Under } 8 \text { to } 11 \text {, inclusive } \\
\text { Under } 11 \text { to } 14 \text {, inclusive } \\
\text { Under } 14 \text { to } 17 \text {, inclusive } \\
\text { Under } 17 \text { to } 20 \text {, inclusive } \\
\text { Under } 20 \text { to } 23 \text {, inclusive } \\
\text { Under } 23 .\end{array}$ & $\begin{array}{l}\text { Over } 0.380 \\
0.379 \text { to } 0.284 \text {, inclusive } \\
0.283 \text { to } 0.220 \text {, inclusive } \\
0.219 \text { to } 0.165 \text {, inclusive } \\
0.164 \text { to } 0.120 \text {, inclusive } \\
0.119 \text { to } 0.083 \text {, inclusive } \\
0.082 \text { to } 0.058 \text {, inclusive } \\
0.057 \text { to } 0.035 \text {, inclusive } \\
0.034 \text { to } 0.025 \text {, inclusive } \\
\text { Under } 0.025\end{array}$ & $\begin{array}{l}\text { Per cent } \\
\pm 5 \\
\text { Inch } \\
\pm 0.012 \\
\pm .010 \\
\pm .008 \\
\pm .006 \\
\pm .005 \\
\pm .004 \\
\pm .003 \\
\pm .0025 \\
\pm .0025\end{array}$ & $\begin{array}{l}\text { Per cent } \\
\pm 5 \\
\text { Inch } \\
\pm 0.014 \\
\pm .012 \\
\pm .010 \\
\pm .008 \\
\pm .007 \\
\pm .006 \\
\pm .005 \\
\pm .004\end{array}$ & $\begin{array}{l}\text { Per cent } \\
\pm 6 \\
\text { Inch } \\
\pm 0.016 \\
\pm .014 \\
\pm .012 \\
\pm .010 \\
\pm .009 \\
\pm .008 \\
\pm .007\end{array}$ & $\begin{array}{l}\text { Per cent } \\
\pm 6 \\
\text { Inch } \\
\pm 0.018 \\
\pm .016 \\
\pm .014 \\
\pm .012 \\
\pm .011 \\
\pm .010\end{array}$ \\
\hline
\end{tabular}


(In workmanship and finish, and in rejection, this specification conforms to A. S. T. M. B42 (see 642.23, p. 1182), while inspection is identical with B44 (see 645.24, p. 1220).)

\section{SOCIETY OF AUTOMOTIVE ENGINEERS, NONFERROUS METAL SPECIFICATIONS ; SPECIFICATION NO. 75 , COPPER TUB- ING, MARCH, 1922}

\section{COPPER TUBING}

Composition in percentage.

Per cent

Copper, minimum

99.50

1. Hard-drawn tubes; bend test.-One test specimen cut longitudinally and one cut transversely from the tube shall be tested. Test specimens must withstand cold-bending through an angle of $180^{\circ}$ on a radius equal to the thickness of the wall of the tube without cracking.

2. Annealed tubes; bend test.-One test specimen cut longitudinally and one cut transversely from the tube shall be tested. Test specimens must withstand bending through an angle of $180^{\circ}$; that is, flat on itself, without cracking. The metal must not crack when hammered to a fine edge.

(In respect to dimensional tolerances, expanding test, appearance, special limits, and footnotes, this specification is identical with S. A. E. specification No. 74 , see 645.24 , p. 1221.)

UNITED STATES GOVERNMENT, FEDERAL SPECIFICATIONS BOARD, FEDERAI SPECIFICATION FOR PIPE AND TUBING; COPPER, SEAMLESS; PIPE (STANDARD IRON-PIPE SIZE); TUBING (FOR PRESSURES UP TO 450 POUNDS PER SQUARE INCH) WW-P-377 AND WW-P-378, MAY $2 \%, 1930$

\section{SEAMLESS COPPER TUBING AND PIPE}

\section{APPLICABLE FEDERAL SPECIFICATIONS}

The following Federal specifications, of the issue in effect on date of invitation for bids, shall form a part of this specification:

\begin{tabular}{|c|c|c|c|}
\hline $\begin{array}{l}\text { Federal } \\
\text { specifi- } \\
\text { cation }\end{array}$ & $\begin{array}{l}\text { Former } \\
\text { No. }\end{array}$ & Title & $\mathrm{SeO}-$ \\
\hline $\begin{array}{r}\text { GGG-P-351 } \\
\text { QQ-M-151 }\end{array}$ & $\begin{array}{l}238 \\
339\end{array}$ & $\begin{array}{l}\text { Pipe threads } \\
\text { General specifications for metals. }\end{array}$ & $\begin{array}{l}607.0, \text { p. } 365 . \\
600.0, \text { p. } 3\end{array}$ \\
\hline
\end{tabular}

\section{B. GRADE AND TYPES}

B-1. Seamless copper tubing and pipe shall each be of but one grade.

B-2. Seamless copper tubing shall be furnished in the following types:

\begin{tabular}{|c|r|}
\hline Type & $\begin{array}{c}\text { Maximum } \\
\text { nominal } \\
\text { working } \\
\text { pressure }\end{array}$ \\
\hline & Lbs./in.2 \\
A & 100 \\
B & 200 \\
C & 300 \\
D & 450 \\
\hline
\end{tabular}

B-2a. These types are selected to give a stress not greater than 4,000 pounds per square inch in the formula $t=\frac{P \times D}{2 S}$ under hydrostatic pressures as indicated opposite each type.

$t=$ thickness in inches.

$P=$ pressure, pounds per square inch.

$D=$ inside diameter in inches.

$S=$ stress in pounds per square inch.

B-3. For test pressures, see Table 3 .

\section{MATERIAL AND WORKMANSHIP}

C-1. The pipe or tubing shall be made of copper, tubing having a purity of at least 99.88 per cent and pipe having a purity of 99.90 per cent, as determined by electrolytic assay, silver being counted as copper.

C-2. The pipe or tubing shall be clean, smooth, and free from grooving and indentations, cracks, flaws, or other defects; round, straight, of the proper dimensions, and with ends cut square.

\section{GEN LRAL REQUIREMENTS}

(Requirements for assumed weight of a cubic inch of the material, and tolerances for underweight and gage are the same as those for copper pipe in A. S. T. M. B42.) (See 642.23, p. 1182.)

D-2. The ends of the pipe or tubing shall not be threaded unless so ordered. Pipe threads shall be in accordance with the dimensions given in the table of standard taper pipe threads as contained in the Federal specification GGG-P-351 for standard pipe threads for pipe and pipe fittings (F. S. B. No. 238), in effect on date of invitation for bids. (See 607.0, p. 365.) Tubing-thread details shall be furnished by the purchaser.

D-3. When tubes or pipes are ordered to exact length, a variation of plus one-eighth inch on the length shall be permitted. No minus tolerance shall be permitted.

D-4. The permissible variations in average outside diameter of copper pipe and tubing shall be as given in Table 1 and Table $1 \mathrm{~A}$.

TABLE 1.-Permissible variations in average outside diameter of seamless tubes

\begin{tabular}{|c|c|}
\hline Outside diameter & $\begin{array}{l}\text { Permissible } \\
\text { variation } \\
\text { in average } \\
\text { outside di- } \\
\text { ameter }\end{array}$ \\
\hline $\begin{array}{l}0.50 \text { and less } \\
\text { Over } 0.50 \text { to } 0.75 \text {, inclusive } \\
\text { Over } 0.75 \text { to } 1.00 \text {, inclusive } \\
\text { Over } 1.00 \text { to } 1.25 \text {, inclusive } \\
\text { Over } 1.25 \text { to } 1.50 \text {, inclusive } \\
\text { Over } 1.50 \text { to } 1.75 \text {, inclusive } \\
\text { Over } 1.75 \text { to } 2.00 \text {, inclusive } \\
\text { Over } 2.00\end{array}$ & $\begin{array}{l}\text { Inch } \\
\pm 0.0020 \\
\pm .0025 \\
\pm .0030 \\
\pm .0035 \\
\pm .0040 \\
\pm .0004 \\
\pm .0045 \\
\text { 土. } 0050 \\
\text { (1) }\end{array}$ \\
\hline
\end{tabular}

$1 \pm 0.25$ per cent of outside diameter.

TABLE 1A.-Permissible variations in outside diameter of seamless copper pipe

\begin{tabular}{|c|c|c|c|}
\hline $\begin{array}{c}\text { Iron pipe } \\
\text { size }\end{array}$ & $\begin{array}{c}\text { Permissible } \\
\text { variations } \\
\text { in average } \\
\text { outside } \\
\text { diameter }\end{array}$ & $\underset{\text { size }}{\text { Iron pipe }}$ & $\begin{array}{c}\text { Permissible } \\
\text { variations } \\
\text { in average } \\
\text { outside } \\
\text { diameter }\end{array}$ \\
\hline $\begin{array}{r}\text { Inches } \\
1 / 8 \\
1 / 4 \\
3 / 8 \\
1 / 2 \\
3 / 4 \\
1 \\
11 / 4 \\
11 / 2 \\
2\end{array}$ & $\begin{array}{l}\text { Inch } \\
\pm 0.0020 \\
\pm .0025 \\
\pm .0025 \\
\pm .0030 \\
\pm .0035 \\
\pm .0040 \\
\pm .0045 \\
\pm .0050 \\
\pm .0060\end{array}$ & $\begin{array}{c}\text { Inches } \\
21 / 2 \\
3 \\
31 / 2 \\
4 \\
5 \\
6 \\
8 \\
10\end{array}$ & $\begin{array}{l}\text { Inch } \\
\pm 0.0070 \\
\pm .0085 \\
\pm .0100 \\
\pm .0115 \\
\pm .0140 \\
\pm .0165 \\
\pm .0215 \\
\pm .0270\end{array}$ \\
\hline
\end{tabular}




\section{E. DETAIL REQUIREMENTS}

(In respect to pipe size, outside diameter, thickness, and weight per foot in pounds, this specification is identical with A. S. T. M. B42 (see 642.23 p. 1182), except that B42 includes data on $4 \frac{1}{2}, 7$, and 9 inch pipes. Additional data on copper pipe, iron pipe size, are as follows:)

TABLE 2.-Additional data on copper pipe, iron-pipe size

\begin{tabular}{|c|c|c|}
\hline $\begin{array}{c}\text { Iron-pipe } \\
\text { size }\end{array}$ & $\begin{array}{l}\text { Diameter, } \\
\text { inside }\end{array}$ & $\begin{array}{l}\text { Minimum } \\
\text { test pres- } \\
\text { sure }\end{array}$ \\
\hline $\begin{array}{r}\text { Inches } \\
1 / 8 \\
1 / 4 \\
3 / 8 \\
1 / 2 \\
3 / 4\end{array}$ & $\begin{array}{c}\text { Inches } \\
0.281 \\
.375 \\
.494 \\
.625 \\
.822\end{array}$ & $\begin{array}{r}\text { Lbs./in. }{ }^{2} \\
1,000 \\
1,000 \\
1,000 \\
1,000 \\
1,000\end{array}$ \\
\hline $\begin{array}{l}1 \\
11 / 4 \\
11 / 2 \\
2\end{array}$ & $\begin{array}{l}1.062 \\
1.368 \\
1.600 \\
2.062\end{array}$ & $\begin{array}{r}1,000 \\
1,000 \\
1,000 \\
900\end{array}$ \\
\hline $\begin{array}{l}21 / 2 \\
3 \\
31 / 2 \\
4\end{array}$ & $\begin{array}{l}2.500 \\
3.062 \\
3.500 \\
4.000\end{array}$ & $\begin{array}{l}900 \\
900 \\
900 \\
800\end{array}$ \\
\hline $\begin{array}{r}5 \\
6 \\
8 \\
10\end{array}$ & $\begin{array}{r}5.062 \\
6.125 \\
8.000 \\
10.019\end{array}$ & $\begin{array}{l}600 \\
500 \\
500 \\
400\end{array}$ \\
\hline
\end{tabular}

E-2. Copper tubing.-The dimensions and weights of the various sizes of copper tubing shall be as given in Table 3 . Other diameters and thicknesses may be specified when required.

TABLE 3.-Standard weights and dimensions of seamless copper tubes ${ }^{1}$

TYPE A

\begin{tabular}{|c|c|c|c|c|}
\hline $\begin{array}{c}\text { Outside } \\
\text { diameter }\end{array}$ & $\begin{array}{l}\text { Thick- } \\
\text { ness }\end{array}$ & $\begin{array}{c}\text { Inside } \\
\text { diameter }\end{array}$ & $\begin{array}{l}\text { Weight } \\
\text { per foot } \\
\text { of length }\end{array}$ & $\begin{array}{c}\text { Mini- } \\
\text { mum test } \\
\text { pressure }\end{array}$ \\
\hline $\begin{array}{r}\text { Inches } \\
2.875 \\
3.500 \\
4.000\end{array}$ & $\begin{array}{l}\text { Inch } \\
0.065 \\
.065 \\
.065\end{array}$ & $\begin{array}{r}\text { Inches } \\
2.745 \\
3.370 \\
3.870\end{array}$ & $\begin{array}{c}\text { Pounds } \\
2.224 \\
2.719 \\
3.115\end{array}$ & $\begin{array}{c}\text { Lbs./in. }{ }^{2} \\
300 \\
250 \\
200\end{array}$ \\
\hline $\begin{array}{r}4.500 \\
5.563 \\
6.625 \\
8.625 \\
10.750\end{array}$ & $\begin{array}{l}.065 \\
.068 \\
.081 \\
.105 \\
.131\end{array}$ & $\begin{array}{r}4.370 \\
5.427 \\
6.463 \\
8.415 \\
10.488\end{array}$ & $\begin{array}{r}3.510 \\
4.550 \\
6.455 \\
10.893 \\
16.939\end{array}$ & $\begin{array}{l}200 \\
150 \\
150 \\
150 \\
150\end{array}$ \\
\hline \multicolumn{5}{|c|}{ TYPE B } \\
\hline $\begin{array}{l}1.900 \\
2.375 \\
2.875 \\
3.500 \\
4.000\end{array}$ & $\begin{array}{l}0.065 \\
.065 \\
.068 \\
.083 \\
.095\end{array}$ & $\begin{array}{l}1.770 \\
2.245 \\
2.739 \\
3.334 \\
3.810\end{array}$ & $\begin{array}{l}1.452 \\
1.828 \\
2.324 \\
3.453 \\
4.517\end{array}$ & $\begin{array}{l}400 \\
300 \\
300 \\
300 \\
300\end{array}$ \\
\hline $\begin{array}{r}4.500 \\
5.563 \\
6.625 \\
8.625 \\
10.750\end{array}$ & $\begin{array}{l}.107 \\
.132 \\
.158 \\
.205 \\
.256\end{array}$ & $\begin{array}{r}4.286 \\
5.299 \\
6.309 \\
8.215 \\
10.238\end{array}$ & $\begin{array}{r}5.724 \\
8.730 \\
12.442 \\
21.018 \\
32.713\end{array}$ & $\begin{array}{l}300 \\
300 \\
300 \\
300 \\
300\end{array}$ \\
\hline \multicolumn{5}{|c|}{ TYPE C } \\
\hline $\begin{array}{l}1.315 \\
1.660 \\
1.900 \\
2.375\end{array}$ & $\begin{array}{r}0.065 \\
.065 \\
.066 \\
.083\end{array}$ & $\begin{array}{l}1.185 \\
1.530 \\
\text { 1. } 768 \\
2.209\end{array}$ & $\begin{array}{l}0.989 \\
1.262 \\
1.474 \\
2.316\end{array}$ & $\begin{array}{l}700 \\
500 \\
450 \\
450\end{array}$ \\
\hline $\begin{array}{l}2.875 \\
3.500 \\
4.000 \\
4.500\end{array}$ & $\begin{array}{l}.100 \\
.122 \\
.140 \\
.157\end{array}$ & $\begin{array}{l}2.675 \\
\text { 3. } 256 \\
\text { 3. } 720 \\
\text { 4. } 186\end{array}$ & $\begin{array}{l}\text { 3. } 379 \\
5.018 \\
6.580 \\
8.303\end{array}$ & $\begin{array}{l}450 \\
450 \\
450 \\
450\end{array}$ \\
\hline $\begin{array}{r}5.563 \\
6.625 \\
8.625 \\
10.750\end{array}$ & $\begin{array}{l}.194 \\
.231 \\
.301 \\
.375\end{array}$ & $\begin{array}{r}5.175 \\
6.163 \\
8.023 \\
10.000\end{array}$ & $\begin{array}{l}12.683 \\
17.985 \\
30.509 \\
47.375\end{array}$ & $\begin{array}{l}450 \\
450 \\
450 \\
450\end{array}$ \\
\hline
\end{tabular}

1 It will be noted that actual outside diameters correspond to ironpipesizes; however, all tubing should be designated by actual outside diameter and wall thickness.
TABLE 3.-Standard weights and dimensions of seamless copper tubes-Continued

TYPE D

\begin{tabular}{|c|c|c|c|c|}
\hline $\begin{array}{l}\text { Outside } \\
\text { diameter }\end{array}$ & $\begin{array}{l}\text { Thick- } \\
\text { ness }\end{array}$ & $\begin{array}{l}\text { Inside } \\
\text { diameter }\end{array}$ & $\begin{array}{l}\text { Weight } \\
\text { per foot } \\
\text { of length }\end{array}$ & $\begin{array}{c}\text { Mini- } \\
\text { mum test } \\
\text { pressure }\end{array}$ \\
\hline $\begin{array}{r}\text { Inches } \\
0.405 \\
.540 \\
.675 \\
.840 \\
1.050\end{array}$ & $\begin{array}{l}\text { Inch } \\
0.065 \\
.065 \\
.065 \\
.065 \\
.065\end{array}$ & $\begin{array}{r}\text { Inches } \\
0.275 \\
.410 \\
.545 \\
.710 \\
.920\end{array}$ & $\begin{array}{c}\text { Pounds } \\
0.269 \\
.376 \\
.483 \\
.613 \\
.780\end{array}$ & $\begin{array}{c}\text { Lbs./in.2 } \\
1,000 \\
1,000 \\
1,000 \\
1,000 \\
800\end{array}$ \\
\hline $\begin{array}{l}1.314 \\
1.660 \\
1.900 \\
2.375\end{array}$ & $\begin{array}{l}.066 \\
.084 \\
.096 \\
.120\end{array}$ & $\begin{array}{l}\text { 1. } 183 \\
\text { 1. } 492 \\
1.709 \\
\text { 2. } 135\end{array}$ & $\begin{array}{l}\text { 1. } 004 \\
1.612 \\
\text { 2. } 109 \\
\text { 3. } 295\end{array}$ & $\begin{array}{l}650 \\
650 \\
650 \\
650\end{array}$ \\
\hline $\begin{array}{l}2.875 \\
3.500 \\
4.000 \\
4.500\end{array}$ & $\begin{array}{l}.145 \\
.177 \\
.202 \\
.228\end{array}$ & $\begin{array}{l}\text { 2. } 585 \\
\text { 3. } 146 \\
\text { 3. } 596 \\
\text { 4. } 044\end{array}$ & $\begin{array}{r}4.820 \\
7.162 \\
9.342 \\
11.861\end{array}$ & $\begin{array}{l}650 \\
650 \\
650 \\
650\end{array}$ \\
\hline $\begin{array}{r}5.563 \\
6.625 \\
8.625 \\
10.750\end{array}$ & $\begin{array}{l}.281 \\
.335 \\
.436 \\
.544\end{array}$ & $\begin{array}{l}5.001 \\
5.955 \\
7.753 \\
9.662\end{array}$ & $\begin{array}{l}18.073 \\
25.658 \\
43.476 \\
67.607\end{array}$ & $\begin{array}{l}650 \\
650 \\
650 \\
650\end{array}$ \\
\hline
\end{tabular}

E-3. When copper pipe (I. P. S.) is ordered in random stock lengths, it shall be furnished in any lengths from 12 to 16 feet, inclusive.

$\mathrm{E}-4$. When tubes are ordered in nominal stock lengths, the pieces shipped shall be cut to the nominal length as a maximum. The shortest acceptable lengths and the maximum permissible percentage by weight of short lengths shall be the same as for copper rods and bars in F. S. B. QQ-C-501. (See 642.11, p. 1180 .)

\section{F. METHODS OF SAMPIING, INSPECTION, AND TESTS}

F-1. Every pipe or tube shall withstand an internal hydrostatic pressure in accordance with Tables 2 and 3 and shall be perfectly tight under pressure and show no signs of bulging, cracks, flaws, porous places, or other defects.

F-2. All pipe shall be surface inspected for defects by rolling on a set of wooden horses or other suitable supports so that the entire surface of each pipe may be properly examined. In the event of failure of 10 per cent of any lot presented for inspection to pass the surface inspection and gaging tests, that lot shall be rejected.

F-3. A strip not over $1 \frac{1}{2}$ inches wide shall be taken from each lot of 2,000 pounds or less of pipe or tubing and shall stand the following tests:

F-3a. If less than half an inch thick, it shall stand after annealing bending flat on itself without signs of fracture on the outer surface.

F-3b. If one-half inch thick or over, it shall stand after annealing, a $180^{\circ}$ bend around a diameter equal to its thickness without showing signs of fracture on the outer surface.

F-3c. The ends of all specimens used for the bending tests shall stand hammering down hot and cold to a knife edge without showing signs of cracks.

F-4. Each lot of pipe or tubing of the same diameter, $23 / 8$ inches outside diameter or less, shall be subjected to a hot bend test. One piece of full section in sufficient length shall be selected from each lot of 1,000 pounds or less, to represent the lot. The sample shall withstand bending while hot (low red heat, about $1,300^{\circ}$ to $1,400^{\circ} \mathrm{F}$.) $180^{\circ}$ around a mandrel of one and one-half times the inside diameter of the tubing or pipe without sign of cracks or draws on the outside of the bent portion.

F-5. Additional test specimens from pipes or tubes from which tension, bend, and hammer test specimens have been taken shall stand flanging.cold 
after annealing; the width of the flange measured from the outside of the pipe or tube shall be onefourth of the inside diameter of the pipe or tube.

F-6. Each lot of pipe or tubing of the same diameter, 2 inches inside diameter and over, shall be subjected to tension tests. One piece from each lot of 1,000 pounds or less shall be selected to represent the lot. If from 2 to 6 inches inside diameter for tubes and 2 to 5 inches I. P. S. for pipe, the test specimens shall be cut longitudinally; if over 6 inches inside diameter for tubes and 5 inches I. P. S. for pipe, they shall be cut circumferentially. All the test specimens shall be heated to a cherry red and straightened when hot, then annealed and machined to the shape of the standard type 8-inch test specimen. (See Figure No. 1079.)

F-7. For thicknesses up to and including one-half inch the width of the narrow part of the test specimen shall be about $1 \frac{1}{2}$ inches. For the thicker pieces the width shall be such as to give a cross section of about half a square inch, but the breadth shall not in any case be less than the thickness.

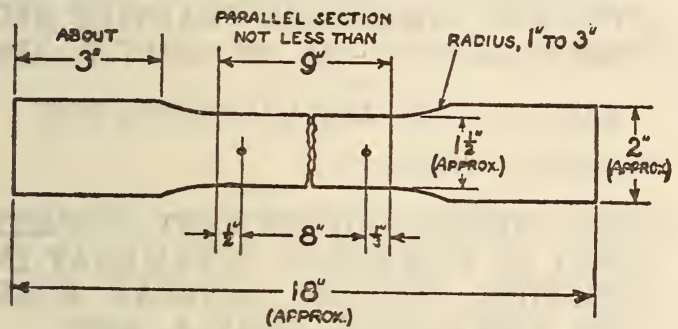

FIgURE 1079.-Standard type 8-inch test specimen

The surfaces shall not be machined but shall be left in their original condition.

F-8. Each test specimen shall show an ultimate tensile strength (after being annealed) of at least 30,000 pounds per square inch and an elongation of at least 25 per cent in 8 inches.

F-9. One piece of pipe selected from the completed lot (ready for shipment) shall stand threading in a satisfactory manner with the standard pipe threads for pipe and pipe fittings for that size of pipe.

F-10. Drillings, millings, or clippings for chemical analysis shall be taken from several points around each piece selected for physical tests and the chips from each lot combined and thoroughly mixed. The resultant sample shall be free from dirt, grit, and other foreign matter and shall consist of at least 2 ounces.

\section{G. PACKAGING, PACKING, AND MARKING OF SHIPMENTS}

G-2. Packaging.-Unless otherwise specified, commercial packages are acceptable under this specification.

G-3. Packing.-For domestic shipment, current commercial packing which will insure delivery in good order; for export, respective specifications of individual departments shall govern.

G-4. Marking.-Unless otherwise specified, shipments of the purchases of departments and establishments of the United States Government shall be marked with the name of the material, the size, type, and the quantity contained therein, as defined by the contract or order under which the shipment is made, the name of the contractor, and the number of the contract or order.

\section{NOTES}

I-1. Pipe is regularly designated by iron-pipe size.

I-2. Tubing is regularly designated by outside diameter and thickness.
I-3. When tubes or pipes are required to exact length it should be so stated on the requisition.

I-4. Tubing shall be furnished annealed or dead soft annealed when so specified by the order.

I-5. It is believed that this specification is adequate and that normally no samples will be necessary to determine compliance with the requirements, prior to award. If, for a particular purpose, samples with bids are necessary, they should be specifically asked for in the invitation for bids.

\subsection{GRAVITY BATTERY COPPERS.}

AMERICAN RAILWAY ASSOCIATION, SIGNAL SECTION, SPECIFICATION FOR GRAVITY BATTERY COPPERS, NO. 3328, JULY, 1928

\section{GRAVITY BATTERY COPPERS \\ PURPOSE}

The purpose of this specification is to provide coppers to be used as the cathodes of gravity battery cells.

\section{DRAWINGS}

Figure 1080 forms an essential part hereof.

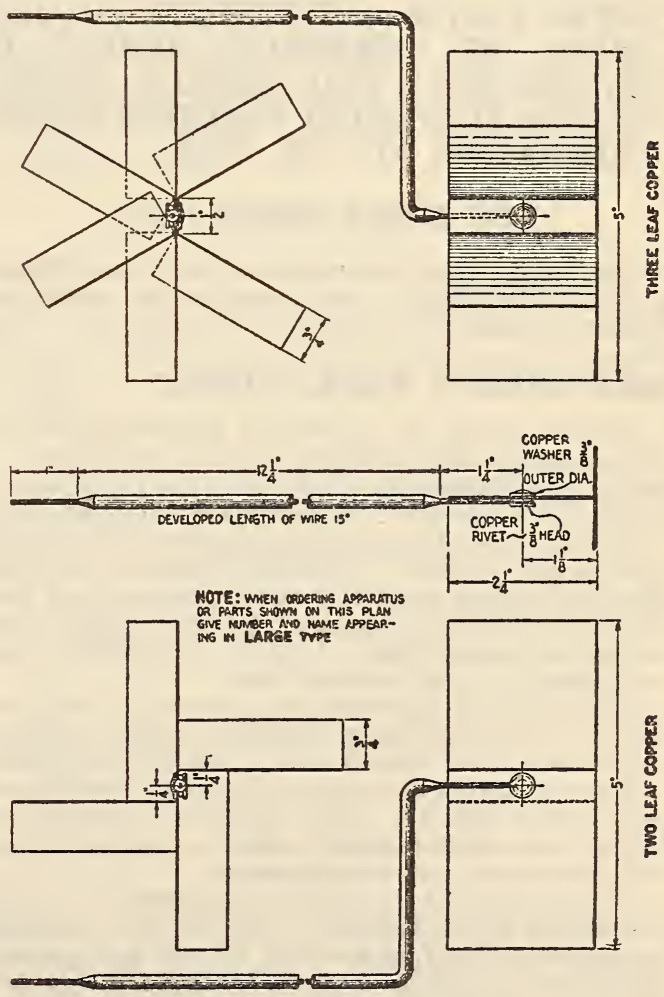

FIGURE 1080.-Coppers

\section{MATERIAL AND WORKMANSHIP}

Material and workmanship shall be first class in every respect.

Coppers shall be of 2-leaf. As an alternate 3-leaf may be specified. Leaves shall be of No. $30 \mathrm{~A}$. W. G. hard-rolled bright copper not less than 98 per cent pure. The lead wire shall be No. 14 A. W. G. solid soft-drawn copper, insulated throughout the entire length, except 1 inch at each end. The insulation shall consist of $3 / 64$-inch wall of rubber, of a character 
suitable to withstand the action of the battery solution, and must adhere tightly to the wire.

Rivets and washers shall be of copper not less than 98 per cent pure. Insulation of the wire shall be trimmed either tapered or square at the ends and in the operation the wire must not be scored. The end of the wire attached to the copper must be thoroughly cleaned and tightly riveted to the copper. Leaves, rivets, and wire shall be properly assembled.

\section{PACKING}

Coppers shall be carefully and securely packed in lots of 50. As an alternate, packing in lots of 100 may be specified.

642.4 COPPER WIRE CLOTH.

AMERICAN SOCIETY FOR TESTING MATERIALS, STANDARD SPECIFICATIONS FOR NONFERROUS INSECT SCREEN CLOTH, DESIGNATION B50-29, 1929

\section{INSECT SCREEN CLOTH}

(See 645.39, p. 1228.)

UNITED STATES GOVERNMENT, DEPARTMENT OF COMMERCE, BUREAU OF STANDARDS, WIRE INSECT SCREEN CLOTH, SIMPLIFIED PRACTICE RECOMMENDATION NO. 122, 1931

\section{WIRE INSECT SCREEN CLOTH}

(For steel wire insect screen cloth, see 603.43, p. 195. For copper wire insect screen cloth, see 646.52, p. 1256 .)

\subsection{HOLLOW WARE, COPPER.}

(Specifications for containers frequently include requirements applicable to copper containers. A list of specifications in which there are requirements applicable to copper containers is given herewith:

Underwriters' Laboratories, standards for electric lighting plants, 1921. Includes fuel tanks for installation at engine, made of copper, brass, or cast iron. Requirements on capacity, general design, wall thickness, construction of joints, connections, and vent openings, and pressure test.

Underwriters' Laboratories, standards for gasoline stoves for cooking and heating, 1928. Includes requirements for lift-out tanks and pressure tanks.

Underwriters' Laboratories, standards for internalcombustion engines, 1920. Includes requirements for fuel tanks installed at the engine, made of copper, cast iron, or protected sheet steel.

Underwriters' Laboratories, standards for kerosene stoves for cooking and heating, 1927. Includes requirements for fuel tanks of lift-out and pressure types, made of glass, copper, brass, or steel sheets.

United States Government, Federal Specifications Board, Federal specification for sterilizers, dressing containers, etc., No. 68a, 1925. (Current designation GG-S-751.) Includes requirements on construction, dimensions, thickness of metal, burner or electric heater requirements, for 6 sizes of sheet copper sterilizers for instruments and for sheet copper sterilizer for utensils, with requirements for pressure test.)

\subsection{BRUSH COPPER AND MATS.}

642.61 BRUSH COPPER.

able.)

642.62 MATS, COPPER.

(No nationally recognized specifications available.)

642.9 MISCELLANEOUS OF COPPER.

MANUFACTURES

UNITED STATES GOVERNMENT, DEPARTMENT OF COMMERCE, BUREAU OF STANDARDS, EAVES TROUGH, CONDUCTOR PIPE, CONDUCTOR ELBOWS, AND FITTINGS, SIMPLIFIED PRACTICE RECOMMENDATION NO. 29, JULY 1, 1925

\section{EAVES TROUGH AND CONDUCTOR PIPE}

(See 607.5, p. 491.)

UNITED STATES GOVERNMENT, DEPARTMENT OF COMMERCE, STEAMBOAT INSPECTION SERVICE, GENERAL RULES AND REGULATIONS, MAY 4, 1929

\section{SLIP JOINTS}

The wearing surface of the male pipe in all slip joints made after June 30,1908, for use in steam pipes shall be made of copper or composition, and shall be of sufficient length and so adjusted as to prevent accidental withdrawal from the packing box. When made of composition, same shall be of not less than 95 per cent copper, tin, and zinc, possessing a tensile strength of not less than 30,000 pounds to the square inch. It may be used for sizes up to and including 12 inches in diameter, and for all pressures not exceeding 300 pounds per square inch.

The minimum thickness of such fittings shall be determined by the following formula:

$$
T=\frac{P \times D}{4,000}+0.22
$$

Where

$P=$ working pressure in pounds.

$D=$ diameter in inches.

$T=$ thickness in inches.

UNITED STATES GOVERNMENT, FEDERAL SPECIFICATIONS BOARD, FEDERAL SPECIFICATION FOR NAILS, SPIKES, TACKS, AND STAPLES, FF-N-101, MAY 3, 1932

NAIIS, SPIKES, TACKS, AND STAPLES

(See 608.11, p. 560.) 
UNITED STATES GOVERNMENT, FEDERAL SPECIFICATIONS BOARD, FEDERAL SPECIFICATION FOR STANDARD MISCELLANEOUS CHAIN AND ATTACHMENTS, RR-C-271, DECEMBER 8, 1931

\section{PLOMBER'S CHAIN, WELDLESS}

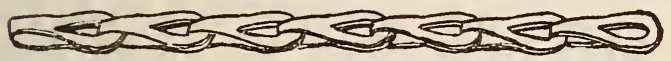

Figure 1081.-Plumbers' chain, weldless

(For eneral specifications, material and workmanship, detail requirements, and methods of inspection and tests, see F. S. B. RR-C-271, (603.50, p. 199.) For other kinds of chain and chain attachments, specified in F. S. B. RR-C-271 see 603.51, $603.52603 .54,603.56,603.57$, and 617.5. See also $631.9,645.9$, and 646.59.

1. Weldless chain shall be automatic machinemade chain. It will be paid for by the foot.

TABLE 51.-Plumber's chain (dimensions, strength, weights)

\begin{tabular}{|c|c|c|c|c|}
\hline Material & $\begin{array}{c}\text { Metal } \\
\text { gage }\end{array}$ & $\begin{array}{c}\text { Approxi- } \\
\text { mate } \\
\text { number } \\
\text { of links } \\
\text { per foot }\end{array}$ & $\begin{array}{c}\text { Mini- } \\
\text { mum } \\
\text { tensile } \\
\text { strength }\end{array}$ & $\begin{array}{c}\text { Approxi- } \\
\text { mate } \\
\text { weight } \\
\text { per 100 } \\
\text { feet }\end{array}$ \\
\hline Copper.....-. & $\begin{array}{c}\text { Inch } \\
0.032\end{array}$ & 21 & $\begin{array}{c}\text { Pounds } \\
150\end{array}$ & $\begin{array}{c}\text { Pounds } \\
31 / 2\end{array}$ \\
\hline
\end{tabular}

643.

\section{COPPER ALLOYS}

\subsection{MANGANESE COPPER.}

(No nationally recognized specifications available.)

\subsection{PHOSPHOR COPPER.}

AMERICAN SOCIETY FOR TESTING MATERIALS, STANDARD SPECIFICATIONS FOR PHOSPHOR COPPER, SERIAL DESIGNATION B52-27, 1927

\section{PHOSPHOR COPPER}

(In respect to quality of material, sampling marking, claims, and umpire analysis, these specifications are identical with A. S. T. M. B51. See 682 , p. 1307.)

\section{SCOPE}

1. These specifications cover phosphor copper in two grades, as follows: Grade A and grade $\mathrm{B}$.

\section{MANUFACTURE}

2. The manufacturer shall use new metal, or new metal and scrap resulting from the manufacture of ingots of similar composition, in the manufacture of this material.

3. The manufacturer or vendor shall furnish phosphor copper as ingots in the form of notched slabs weighing from 15 to 20 pounds, unless otherwise specified in the contract.

\section{CHEMICAL PROPERTIES}

4. The material shall conform to the following requirements as to chemical composition:

\begin{tabular}{|c|c|c|c|}
\hline Grade & $\begin{array}{c}\text { Phosphor- } \\
\text { us, mini- } \\
\text { mum }\end{array}$ & $\begin{array}{c}\text { Phosphor- } \\
\text { us plus } \\
\text { copper, } \\
\text { minimum }\end{array}$ & $\begin{array}{c}\text { Iron, maxi- } \\
\text { mum }\end{array}$ \\
\hline & $\begin{array}{c}\text { Per cent } \\
\text { A }\end{array}$ & $\begin{array}{c}\text { Per cent } \\
99.75\end{array}$ & $\begin{array}{c}\text { Per cent } \\
0.15 \\
\text { B }\end{array}$ \\
10.0 & 99.75 & .15 \\
\hline
\end{tabular}

UNITED STATES GOVERNMENT, FEDERAL SPECIFICATIONS BOARD, FEDERAL SPECIFICATION FOR PHOSPHOR COP. PER, QQ-C-571, MAY 31, 1932

\section{PHOSPHOR COPPER}

(Except for the omission of claims and umpire chemical analysis, this specification is similar to A. S. T. M. B52-27, given above. In respect to sampling and marking it is similar to A. S. T. M. B51-27. (See 682, p. 1307.))

\subsection{SILICON COPPER.}

AMERICAN SOCIETY FOR TESTING MATERIALS, STANDARD SPECIFICATIONS FOR SILICON COPPER, DESIGNATION B53-27, 1927

\section{SILICON COPPER}

(In respect to manufacture, these specifications are identical with A. S. T. M. B52. (See 643.2, p. 1191.) In respect to quality of material, sampling, marking, claims, and umpire analysis, these specifications are identical with A. S. T. M. B51. See 682, p. 1307.)

\section{SCOPE}

1. These specifications cover silicon copper in one grade.

\section{CHEMICAL PROPERTIES}

2. The material shall conform to the following requirements as to chemical composition:

\begin{tabular}{lr} 
Silicon & $\begin{array}{r}\text { Per cent } \\
\text { Tin, maximum }\end{array}$ \\
Zinc, maximum & .25 \\
Iron, maximum & .25 \\
Aluminum, maximum & .75 \\
Copper. & 25 \\
\hline
\end{tabular}

3. The sum of the copper, silicon, and iron content shall be not less than 99.4 per cent.

\section{UNITED STATES GOVERNMENT, FEDERAL SPECIFICATIONS BOARD, FEDERAI SPECIFICATION FOR SILICON COPPER, QQ-C-581, MAY 31, 1932}

\section{SILICON COPPER}

(Except for the omission of claims and umpire chemical analysis, this specification is similar to A. S. T. M. B53-27, given above. In respect to sampling and marking it is similar to A. S. T. M. B51-27. See 682, p. 1307.) 
643.4 COPPER-TIN-ZINC ALLOY.

AMERICAN SOCIETY FOR TESTING MATERIALS, STANDARD SPECIFICATIONS FOR THE ALLOY: COPPER, 88 PER CENT; TIN, 10 PER CENT; ZINC, 2 PER CENTDESIGNATION B10-18, 1918

\section{ALLOY 88-10-2}

\section{SCOPE}

1. (a) These specifications cover the alloy known commercially as government bronze, admiralty gun metal, gun metal, or 88-10-2 mixture, when used in castings.

(b) It is recommended that this alloy shall not be used where castings are subjected to a temperature exceeding $260^{\circ} \mathrm{C}$. $\left(500^{\circ} \mathrm{F}\right.$.).

\section{MANOFACTURE}

2. The alloy may be made by any approved method.

\section{CHEMICAL PROPERTIES AND TESTS}

3. The alloy shall conform to the following requirements as to chemical composition:

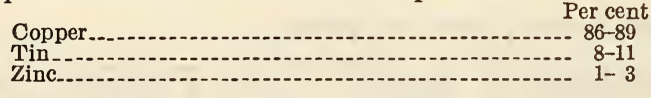

\section{CHEMICAL ANALYSIS}

4. An analysis of each melt shall be made by the manufacturer. The chemical composition thus determined shall be reported to the purchaser or his representative, and shall conform to the requirements specified in section 3.

\section{SAMPIING}

5. (a) The sample for chemical analysis may be taken either by sawing, drilling, or milling the casting or tension-test specimen, and shall represent the average cross section of the piece.

(b) The saw, drill, cutter, or other tool used shall be thoroughly cleaned. No lubricant shall be used in the operation, and the sawings or metal chips shall be carefully treated with a magnet to remove any particles of iron derived from the tools.

\section{PHYSICAL PROPERTIES AND TESTS}

6. The alloy shall conform to the following minimum requirements as to tensile properties:

Tensile strength
Elongation in 2 inches......

\section{TEST SPECIMENS}

7. (a) Two test bars (suitable for turning down to standard tension test specimens), shall be an integral part of large castings, or cast separately in the case of small castings, to represent a lot or melt, and shall be molded in a manner similar to the castings which they represent. If the castings are heat treated, the test bars representing such castings shall be similarly heat treated.

(b) The manufacturer and purchaser shall agree whether test bars can be attached to castings, on the location of bars on the castings, on the castings to which bars are to be attached, and on the method of casting unattached bars. Unless otherwise agreed upon, the minimum lot shall be considered as 500 pounds.

(c) (Tension-test specimen requirements conform with Figure 129 in A. S. T. M. standard specifications for steel. See 605.0, p. 247.)
NOMBER OF TESTS

8. (a) Two tension tests shall be made from each lot or melt.

(Requirements with respect to defective test specimens, and inspection and rejection are identical with those in B59. See 647.15, p. 1259.)

AMERICAN SOCIETY FOR TESTING MATERIALS, STANDARD SPECIFICATIONS FOR SAND CASTINGS OF THE ALLOY: COP. PER, 88 PER CENT; TIN, 8 PER CENT; ZINC, 4 PER CENT-DESIGNATION B6028, 1928

\section{ALLOY 88-8-4}

SCOPE

1. (a) These specifications cover alloy castings, the alloy being a composition of copper, tin, and zinc, known commercially as 88-8-4.

(b) The castings are intended for use where an excellent steam metal (withstanding in the cast state hydrostatic pressures satisfactorily) is required, such as expansion joints, pipe fittings, valves, pump pistons-in general where good strength and resistance to salt water are required.

\section{MANUFACTURE}

2. (a) The alloy may be made by any approved method.

(b) The castings shall be of uniform quality.

\section{CHEMICAL PROPERTIES AND TESTS}

3. (a) The alloy shall conform to the following requirements as to chemical composition:

\begin{tabular}{|c|c|c|c|}
\hline & Desired & Minimum & Maximum \\
\hline $\begin{array}{l}\text { Copper } \\
\text { Tin } \\
\text { Lead } \\
\text { Zinc } \\
\text { Iron } \\
\text { Nickel }\end{array}$ & $\begin{array}{r}\text { Per cent } \\
88.00 \\
8.00 \\
\text { None. } \\
4.00 \\
\text { None. }\end{array}$ & $\begin{array}{r}\text { Per cent } \\
86.00 \\
7.50 \\
1.50\end{array}$ & $\begin{array}{r}\text { Per cent } \\
89.00 \\
11.00 \\
.30 \\
4.50 \\
.10 \\
.50\end{array}$ \\
\hline $\begin{array}{l}\text { Phosphorus.... } \\
\text { Aluminum } \\
\text { Sulphur.... } \\
\text { Antimony. } \\
\text { Total other impurities...... }\end{array}$ & $\begin{array}{l}\text { None. } \\
\text { None. } \\
\text { None. } \\
\text { None. }\end{array}$ & 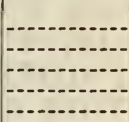 & $\begin{array}{r}.05 \\
\text { None. } \\
.05 \\
.25 \\
.15\end{array}$ \\
\hline
\end{tabular}

(b) Where "none" is specified it shall be construed to refer to none as determined on a $10 \mathrm{~g}$ sample.

\section{PHYSICAL PROPERTIES AND TESTS}

4. (a) The alloy shall conform to the following minimum requirements as to tensile properties:

Tensile strength $\ldots$.

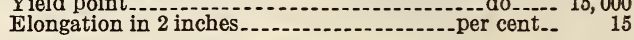

(b) Castings made from this alloy shall conform to such requirements as to pressure tests as may be mutually agreed upon between the purchaser and the manufacturer, and as specified in the contract or purchase order.

5. The coupon attached to the casting shall be in accordance with the dimensions shown in Figure 1082. The fin gate along the side shall not be less than five-sixteenths inch in thickness at any point along its length.

(Specifications covering tension-test specimen requirements are identical with those in paragraph $11(c), A 18$. See 611.51, p. 754 . In respect to chemi- 
cal analyses, determination of yield point, workmanship and finish, marking, inspection and rejection, this specification is identical with B59. See 647.15, p. 1259. In respect to number of tests, paragraphs 7 $(a),(b),(c)$, and $(d)$ in this specification are identical

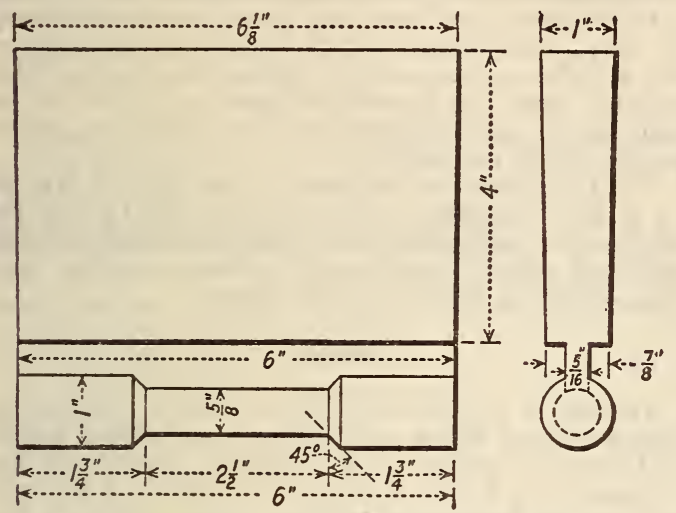

FigURE 1082.-Test specimen coupon

with Paragraphs VI 2, 3, 4, and 13 in F. S. B. 172a. See 646.41, p. 1252.)

(For other copper-tin-zinc alloys see 646.41.)

AMERICAN SOCIETY FOR TESTING MATERIALS, STANDARD RULES GOVERNING THE PREPARATION OF MICROGRAPHS OF METALS AND ALLOYS, DESIGNATION E2-30, 1930

\section{MICROGRAPHS}

(See 600.1 , p. 20.)

\subsection{COPPER-NICKEL ALLOY.}

(Monel metal and nickel-copper alloys, see items under the general class 654, p. 1282.)

\subsection{COPPER-NICKEL-ZINC ALLOY.}

(Nickel silver, German silver, nickel-copper-zinc alloys, see items under the general class $655, \mathrm{p} .1286$. .)

\subsection{COPPER-BEARING METALS.}

(Bearing metals other than Babbitt metal, see 692.2, p. 1324.)

\subsection{MISCELLANEOUS COPPER ALLOYS.}

(Silver solder, see 693.3, p. 1335.)

644.

BRASS

644.0 GENERAL ITEMS.

AMERICAN SOCIETY FOR TESTING MATERIALS, STANDARD METHODS OF CHEMICAL ANALYSIS OF BRASS INGOTS AND SAND CASTINGS, DESIGNATION B45-2\%, 1927

\section{CHEMICAL ANALYSIS OF BRASS INGOTS AND SAND CASTINGS}

1. In the alloys containing the higher amounts of zinc this element is taken by difference. For determination of smaller amounts of zinc, see B28 (646.0, p. 1243).
DETERMINATION OF COPPER BY THE ELECTROLYTIC METHOD

(Same as B27 (see 647.20,p. 1261) except that height of anode is $130 \mathrm{~mm}$ instead of $137 \mathrm{~mm}$ and notes omit reference to zinc determination.)

\section{DETERMINATION OF LEAD BY THE ELECTROLYTIC METHOD}

(Same as B27 (see 647.20, p. 1261) except that notes omit reference to manganese.)

\section{DETERMINATION OF LEAD AS SULPHATE}

(Same as B27 (see 647.20, p. 1261) except in the following particulars:)

\section{Method}

In a $150 \mathrm{c}$ c beaker, dissolve $1 \mathrm{~g}$ of brass in $10 \mathrm{c} \mathrm{c}$ of $\mathrm{HNO}_{3}$ (specific gravity 1.42). Allow the solution to bake dry on the edge of a hot plate, or by standing over night on the steam bath. Add $10 \mathrm{c} \mathrm{c}$ of $\mathrm{HNO}_{3}$ (specific gravity 1.42), and after digesting for a short time, add $50 \mathrm{c}$ c of hot water and heat to boiling for a few minutes. Allow the solution to stand for several hours at a temperature just below the boiling point. Filter off the precipitated $\mathrm{H}_{2} \mathrm{SnO}_{3}$ on double closely woven filter papers, being careful to keep the solution hot throughout the process of filtration. Wash with boiling hot water. To the combined filtrate and washings, add $40 \mathrm{c}$ c of lead acid, and evaporate until copious fumes of $\mathrm{H}_{2} \mathrm{SO}_{4}$ are evolved. Cool, add $35 \mathrm{c}$ c of distilled water to dissolve (continue as in B 27 (see 647.20 , p. 1261) from the point where 105 c $\mathrm{c}$ of distilled water is added).

NotES.-Copper and lead may be determined on the same samples if so desired. In such a case, after filtering off the lead sulphate and washing with lead-acid, and before washing with dilute alcohol, remove the filtrate, add $3 \mathrm{c} c$ of $\mathrm{HNO}_{3}$ (specific gravity 1.42), dilute to $120 \mathrm{c}$ c and determine copper by electrolysis. See the determina. tion of copper by the electrolytic method B27 (647.20, p. 1261).

\section{DETERMINATION OF COPPER AND IEAD SIMULTANEOUSLY}

(Same as B27. See 647.20, p. 1261.)

\section{DETERMLNATION OF TIN}

(Same as B27 (see 647.20 , p. 1261) except that in solutions required the $\mathrm{HCl}$ has a specific gravity of 1.19 instead of 1.20 , and in method $1 \mathrm{~g}$ of brass is substituted for $2 \mathrm{~g}$ of bronze.)

NOTE.-In alloys containing an appreciable amount of antimony and in presence of a large excess of tin, the antimony, as determined by titration according to the method given below, should be calculated to $\mathrm{Sb}^{2} \mathrm{O}^{4}$, and a corresponding amount deducted from the weight of the ignited $\mathrm{SnO}^{2}$ before calculating to tin.

\section{DETERMINATION OF IRON}

(Same as B27. See 647.20 , p. 1261.)

\section{DETERMINATION OF ANTIMONY BY THE VOLOMETRIC} METHOD

Solutions Required

Potassium permanganate.-Dissolve $0.3 \mathrm{~g}$ of potassium permanganate in $1,000 \mathrm{c}$ c of distilled water. Standardize by dissolving $25 \mathrm{mg}$ of pure antimony in $15 \mathrm{c} \mathrm{c}$ of boiling sulphuric acid and proceed according to the method from this point.

\section{Method}

In a $250 \mathrm{c}$ c beaker dissolve $5 \mathrm{~g}$ of brass in $25 \mathrm{c} \mathrm{c}$ of $\mathrm{HNO}_{3}$ (specific gravity 1.42). When the action has ceased, boil until the oxides of nitrogen are expelled, add $125 \mathrm{c} \mathrm{c}$ of boiling water and allow to stand and settle for one hour or longer, keeping the temperature just below the boiling point. Filter on double $9 \mathrm{~cm}$ closely woven papers, keeping the solution hot. Wash with boiling water. Discard the filtrate.

Transfer the papers containing the tin and antimony to a $350 \mathrm{c} \mathrm{c}$ Erlenmeyer flask and add 25 
c c of $\mathrm{HNO}_{3}$ (specific gravity 1.42), $5 \mathrm{~g}$ of ammonium persulphate, and $15 \mathrm{c}$ c of $\mathrm{H}_{2} \mathrm{SO}_{4}$ (specific gravity 1.84). Boil to strong fumes of $\mathrm{SO}_{3}$. If the solution is brown, cool and add 5 c c of $\mathrm{HNO}_{3}$ (specific gravity 1.42) and a little more (1 g or less) persulphate, if necessary, and boil again to strong fumes of $\mathrm{SO}_{3}$. When the solution is colorless, cool, add $20 \mathrm{c}$ c of distilled water $20 \mathrm{c} \mathrm{c}$ of $\mathrm{HCl}$ (specific gravity 1.19) and $1 \mathrm{~g}$ of sodium sulphite. Boil gently for 10 minutes to expel $\mathrm{SO}_{2}$ completely. Dilute with $200 \mathrm{c}$ c of distilled water and cool to $10^{\circ}$ to $12^{\circ} \mathrm{C}$. in ice or under running water.

Titrate with standard permanganate solution until the appearance of a decided pink color.

Notes.-1. In alloys containing a large excess of tin, the antimony is precipitated quantitatively with the meta-stannic acid.

2. Ammonium persulphate and nitric acid are used to destroy the filter paper, and subsequent boiling with sulphuric acid effects the solution of the tin and antimony. A mixture of fuming nitric and sulphuric acids might be used to accomplish the same results, but the persulphate mixture is much more efficient.

3. If the solution becomes cool during the first filtration some of the meta-stannic acid may dissolve.

4. If arsenic is present, correction should be made for it.

\section{DETERMINATION OF SULPHOR}

\section{Method}

Weigh out four $5 \mathrm{~g}$ samples of brass into $250 \mathrm{c} \mathrm{c}$ beakers. Dissolve each in $25 \mathrm{c}$ c of $\mathrm{HNO}_{3}$ (specific gravity 1.42) and boil until the disappearance of red fumes. Add $125 \mathrm{c}$ c of boiling water and settle for one hour or longer, keeping the temperature just below the boiling point. Filter on double $12.5 \mathrm{~cm}$ closely woven papers, keeping the solutions hot. Wash with boiling water. Discard the meta-stannic acid precipitates, combine the four filtrates in a tall $700 \mathrm{c}$ c beaker, and add $0.5 \mathrm{~g}$ of $\mathrm{Na}_{2} \mathrm{CO}_{3}$, and evaporate the solution until sirupy. Cool somewhat, then dilute to the lip with warm water. There should be present a layer about one-quarter inch $(6 \mathrm{~mm})$ in thickness, composed of basic salts of copper. Electrolyze, using stationary electrodes, at a current of 4 amperes, or using the Frary solenoid at a current of about 10 amperes. The lead must be removed at the anode as the copper is taken out at the cathode. When copper and lead are out, evaporate the electrolyte to small volume, cover the beaker, add $75 \mathrm{c}$ c of $\mathrm{HCl}$ (specific gravity 1.19), and boil down to small volume. Add $75 \mathrm{c}$ c more $\mathrm{HCl}$ and evaporate to dryness to dehydrate any silica present. Moisten with about $0.5 \mathrm{c} \mathrm{c}$ of $\mathrm{HC}$ and take up with $20 \mathrm{c}$ c of hot water. Filter into a small beaker. Heat the filtrate to boiling and add 10 c c of 5 per cent $\mathrm{BaCl}_{2}$ solution, drop by drop, with stirring. Allow to settle at least five hours, filter on a small ashless paper, and wash with hot water. Ignite cautiously in a small porcelain or platinum crucible, and then heat to bright redness for 20 minutes. Cool and weigh the $\mathrm{BaSO}_{4}$ and calculate to sulphur.

Notes.-1. Throughout the entire analysis, the utmost precautions must be taken to prevent contamination by sulphur in any form, $\mathrm{H}_{2} \mathrm{SO}_{4}$ fumes, $\mathrm{H}_{2} \mathrm{~S}$, and $\mathrm{SO}_{2}$ gases especially. All heating with gas burners must be avoided. Run a blank with each analysis, adding all the reagents and subjecting it to the same operations as the beakers containing the drillings. Subtract the amount of $\mathrm{BaSO}_{4}$ found in the blank from that in the determinations. If this blank amounts to 0.002 $\mathrm{g}$, it is a sign of poor work or impure reagents, and the analysis should be repeated. Wash out all beakers, funnels, etc., with distilled water before using.

2. It is necessary to keep the solution containing the meta-stannic acid hot during filtration. If the solution becomes cool, some of the meta-stannic acid may dissolve.

Determination of Aluminum in Small Quantities

(a) General method for accurate work-

Solutions Required

Electrolytic solution.-Mix 300 c c of $\mathrm{HNO}_{3}$ (specific gravity 1.42), 1,700 c c of distilled water, and $500 \mathrm{c}$ c of $\mathrm{H}_{2} \mathrm{SO}_{4}$ (specific gravity 1.84).
Acidified $\mathrm{H}_{2} \mathrm{~S}$ water for washing.-Dilute $10 \mathrm{c}$ c of $\mathrm{HCl}$ (specific gravity 1.19) with $1,000 \mathrm{c} \mathrm{c}$ of distilled water and saturate with $\mathrm{H}_{2} \mathrm{~S}$.

Phenylhydrazine solution for washing.-Add saturated $\mathrm{SO}_{2}$ water to a few cubic centimeters of phenylhydrazine until the crystallin sulphite first formed is redissolved, and then add phenylhydrazine, drop by drop, with vigorous agitation, until the odor of $\mathrm{SO}_{2}$ is no longer perceptible; dilute 5 to $10 \mathrm{c} \mathrm{c}$ of this solution with $100 \mathrm{c}$ c of hot water and allow to remain at the boiling point for a few minutes so as to get rid of the excess $\mathrm{SO}_{2}$.

$\mathrm{NH}_{4} \mathrm{Cl}$ solution for washing (2 per cert).-Mix 30 c c of $\mathrm{HCl}$ (specific gravity 1.19) with $200 \mathrm{c} \mathrm{c}$ of distilled water, add methyl red, neutralize with $\mathrm{NH}_{4} \mathrm{OH}$ until the solution changes to a distinct yellow, and then dilute to $1,000 \mathrm{c} \mathrm{c}$ with water.

\section{Method}

Dissolve $1 \mathrm{~g}$ of brass or bronze in $35 \mathrm{c}$ c of electrolytic solution, dilute with $100 \mathrm{c}$ c of distilled water, and remove copper by electrolysis. Evaporate the electrolyte to fumes of $\mathrm{SO}_{3}$. (If much tin is present, boil the sulphuric acid solution vigorously for a few minutes.) Cool, and dilute with $100 \mathrm{c}$ c of water. Filter off $\mathrm{PbSO}_{4}$ and any tin which may be precipitated at this point, washing with hot water. Dilute the filtrate to at least $200 \mathrm{c} \mathrm{c}$.

Pass $\mathrm{H}_{2} \mathrm{~S}$ into the filtrate for 30 minutes. This will precipitate any copper, lead, tin, arsenic, or antimony which may be present. Allow the sulphides to settle, and filter, washing with acidified $\mathrm{H}_{2} \mathrm{~S}$ water. Boil the filtrate to one-half volume to expel $\mathrm{H}_{2} \mathrm{~S}$, add $3 \mathrm{c} \mathrm{c} \mathrm{HNO}_{3}$ (specific gravity 1.42) and boil for a few minutes to oxidize iron.

Precipitate iron and aluminum according to the following ${ }^{14}$ : Add $10 \mathrm{c}$ c of $\mathrm{HCl}$ (specific gravity 1.19) and just neutralize with $\mathrm{NH}_{4} \mathrm{OH}$, using methyl red as an indicator. Boil for two minutes and filter, washing with hot $\mathrm{NH}_{4} \mathrm{Cl}$ (2 per cent). Dissolve the precipitate from the paper with boiling hot $\mathrm{HCl}(1: 1)$ and wash alternately with hot water and $\mathrm{HCl}(1: 1)$ into the beaker in which the precipitation was made. Reprecipitate the iron and aluminum, following the same procedure as before.

Dissolve the precipitate again with hot $\mathrm{HCl}(1: 1)$, dilute to $100 \mathrm{c} \mathrm{c}$ with water, make just ammoniacal, then only acid enough to hold iron in solution. Add sufficient saturated solution of ammonium bisulphite to reduce the iron (5 to 20 drops). Add a few drops of methyl orange, quickly bring to neutrality with $\mathrm{NH}_{4} \mathrm{OH}$, and then add 6 to 7 drops of $\mathrm{HCl}(1: 1)$ in excess. Finally, add from 1 to 3 c c of phenylhydrazine and stir until the precipitate becomes flaky. The supernatant liquid should be plainly acid to litmus and the precipitate may be colored owing to organic matter. Filter and wash with phenylhydrazine wash solution. Ignite in a weighed platinum crucible, finishing with the blast for five minutes. Weigh as $\mathrm{Al}_{2} \mathrm{O}_{3}$. The weighing should be made rapidly and with the crucible covered, as $\mathrm{Al}_{2} \mathrm{O}_{3}$ is somewhat hygroscopic.

Notes.-1. For small amounts of aluminum, a $5 \mathrm{~g}$ sample should be used. In this case dissolve in $60 \mathrm{c}$ c of "assay solution" of the following proportions:

7 volumes $\mathrm{HNO}_{3}$ (specific gravity 1.42).

10 volumes $\mathrm{H}_{2} \mathrm{SO}_{4}$ (specific gravity 1.84 ).

25 volumes $\mathrm{H}_{2} \mathrm{O}$.

2. When phosphorus is present the final precipitate will be $\mathrm{Al}_{2} \mathrm{O}_{3}+$ $\mathrm{P}_{2} \mathrm{O}_{5}$. In this case it is necessary to fuse the residue with $\mathrm{Na}_{2} \mathrm{CO}$, dissolve with hot water, acidify with $\mathrm{HNO}_{3}$, and determine phosphorus in accordance with the determination of phosphorus by the alkalimetric method of the standard methods of chemical analysis of bronze bearing metal: B46 (see 646.0, p. 1246). The phosphorus is calculated to $\mathrm{P}_{2} \mathrm{O}_{5}$ and subtracted.

14 Blum: B. S. Sci. Paper, No. 286, 1916; J. Am. Chem. Soc., rol. 38 , p. $1282,1916$. 
3. For accurate work the $\mathrm{Al}_{2} \mathrm{O}_{3}$ residue is treated with $\mathrm{HF}$ and $\mathrm{H}_{2} \mathrm{SO}_{4}$ for the removal of possible $\mathrm{SiO}_{2}$.

4. Ammonium bisulphite solution may be prepared by saturating cold $\mathrm{NH}_{1} \mathrm{OH}(1: 1)$ with $\mathrm{SO}_{2}$ until yellow.

5. This method is especially satisfactory for small amounts of aluminum in brass or bronze since no reagents are used which may contaminate the precipitate with aluminum, iron, or silica. A precipitate with phenylhydrazine gives positive indication of the presence of aluminum.

(b) Rapid method for control work-

\section{Solutions Required}

Sodium hydroxide solution (2.5 per cent).-Dissolve $25 \mathrm{~g}$ of $\mathrm{NaOH}$ (free from aluminum) in water and dilute to 1,000 c c.

Sodium sulphide solution.-Dissolve $150 \mathrm{~g}$ of $\mathrm{NaOH}$ (free from aluminum) in 1,000 c c of water, saturate $500 \mathrm{c} \mathrm{c}$ of this solution with hydrogen sulphide, and mix with the remaining $500 \mathrm{c}$ c of solution. Dilute hydrochloric acid $(1: 3)$. - Mix $200 \mathrm{c} \mathrm{c}$ of $\mathrm{HCl}$ (specific gravity 1.19) with $600 \mathrm{c}$ c of water. Acidified hydrogen sulphide water.-(Same as in (a) above.)

Ammonium chloride solution (2 per cent).--(Same as in (a) above.)

Method

Dissolve $2 \mathrm{~g}$ of the sample in $20 \mathrm{c}$ c of $\mathrm{HCl}$ (specific gravity 1.19) and $5 \mathrm{c}$ c of $\mathrm{HNO}_{3}$ (specific gravity 1.42). Boil the solution to expel chlorine and dilute with $50 \mathrm{c}$ c of water. Nearly neutralize the cold solution with $\mathrm{NaOH}$ solution (2.5 per cent, or stronger if much free acid is present), and pour it slowly and with constant shaking into a 500 c c volumetric flask containing $100 \mathrm{c}$ c of sodium sulphide solution. Dilute to the mark with $\mathrm{NaOH}$ solution (2.5 per cent) and mix thoroughly. Filter on a large, dry, No. 42 Whatman filter paper (or its equivalent) and keep the paper well filled with the solution lest iron be oxidized and dissolved. Reject the first 20 to 25 c c and catch exactly 250 c c which will represent $1 \mathrm{~g}$ of sample. In routine analyses of material containing not more than 1 per cent of aluminum, the aliquot portion can be gathered in a 250 c c graduate.

Transfer the aliquot portion to a $600 \mathrm{c} \mathrm{c}$ beaker, neutralize the solution with dilute $\mathrm{HCl}(1: 3)$, and finally add $25 \mathrm{c}$ c in excess. Digest at $40^{\circ}$ to $60^{\circ} \mathrm{C}$. for one hour, filter, and wash the paper and precipitate with acidified $\mathrm{H}_{2} \mathrm{~S}$ water.

Boil the filtrate and washings to expel $\mathrm{H}_{2} \mathrm{~S}$, add a few drops of methyl red indicator, and then dilute ammonia $(1: 2)$ until the solution is just distinctly yellow. Boil for one to two minutes and filter at once through a small filter. Wash the beaker, paper, and precipitate two or three times with hot $\mathrm{NH}_{4} \mathrm{Cl}$ solution (2 per cent) and discard the filtrate. Dissolve the precipitate in $200 \mathrm{c}$ c of hot dilute $\mathrm{HCl}$ $(1: 3)$, wash the filter thoroughly with small portions of hot water, and reserve it for the second filtration. Dilute the filtrate to $50 \mathrm{c}$ c, add methyl red, and precipitate with dilute ammonia (1:2) as before. Filter, wash with hot $\mathrm{NH}_{4} \mathrm{Cl}$ solution (2 per cent), ignite, and weigh.

The ignited residue is prone to carry silica and must be purified before weighing as follows: Add 1 or 2 drops of water, 1 drop of diluted $\mathrm{H}_{2} \mathrm{SO}_{4}$, and 1 to $5 \mathrm{c}$ c of HF. Evaporate to dryness, increase the heat slowly, and finally heat with a blast lamp or its equivalent. Weigh as $\mathrm{Al}_{2} \mathrm{O}_{3}$. The weight of $\mathrm{Al}_{2} \mathrm{O}_{3}$ corrected for the blank and multiplied by 52.94 gives the percentage of aluminum.

Notes. - The first aluminum hydroxide precipitate will carry down some $\mathrm{NaCl}$ and much of any silica that was dissolved in the $\mathrm{NaOH}$ solution; hence, it is not safe to omit the second precipitation and the HF treatment. There is no attack on the glassware during the short contact with the weak, cool, alkaline solution. It is, of course, preferable that the $\mathrm{NaOH}$ be free from aluminum. The most satisfactory test for it lies in running an analysis with nonferrous alloys containing no aluminum. Direct test, by acidification followed by precipitation with ammonia, is sufficient if no precipitate is obtained, but is of doubtful value in case one appears, for it may contain other elements, such as iron, which do not affect the results.
If phosphorus is known to be present, the method should be followed through the first addition of ammonia. If aluminum is indicated, reacidify the solution with $\mathrm{HCl}$, add macerated paper, two drops of methyl orange, and $10 \mathrm{c} c$ of a solution of diammonium phosphate (10 per cent). Render the solution just ammoniacal, then just restore the pink color with dilute $\mathrm{HCl}(1: 3)$, heat to boiling, and add $30 \mathrm{c}$ c of a solution of ammonium acetate (25 per cent). Boil for five minutes, filter on an $11 \mathrm{~cm}$ No. 42 Whatman or similar filter paper, and wash with hot $\mathrm{NH}_{4} \mathrm{NO}_{3}$ solution (5 per cent) until $5 \mathrm{c} \mathrm{c}$ of the washings no longer give a test for chlorides with acidified AgNO. Ignite in platinum or porcelain, heat at approximately $1,100^{\circ} \mathrm{C}$. for 10 minutes, and weigh as AlP . This method is not strictly accurate 10 minutes, and wictire sufficiently accurate for all but the most painstaking analyses.

\section{UNITED STATES GOVERNMENT, FEDERAL SPECIFICATIONS BOARD, FEDERAL SPECIFICATION FOR BRASS RODS, BARS, SHAPES, PLATES, SHEETS, AND STRIPS, COMMERCIAL, QQ-B-611, MARCH 1, 1932}

\section{BRASS RODS, BARS, SHAPES, PLATES, SHEETS, STRIPS}

(In respect to general specifications, material and workmanship, tests and methods of inspection, packing and marking, this specification is substantially the same as F. S. B. QQ-C-501 and notes (see $641.0, p .1172)$. It differs from the latter in requiring check analyses samples to be obtained from each lot of 5,000 pounds or fraction thereof and that resultant samples be not less than 5 ounces, the replacing of a hammer test by a flattening test, and in the following additional particulars:)

\section{TYPES AND GRADES}

1. This specification covers three grades of commercial brass in the following forms and tempers:

Grade A, rods for forging. See also 645.11, p. 1205.

Grade B, rods, soft and half hard, free cutting. See also 645.11. Grade A, bars for forging. See also 645.11.

Grade B, bars, soft and half hard, free cutting. See also 645.11.

Grade A, shapes for forging. See also 645.11.

Grade B, shapes, soft and half hard, free cutting. See also 645.11 Grade C, shapes, various tempers. See also 645.11.

Grade C, plates, various tempers. See also 644.21, p. 1202

Grade C, sheets, various tempers. See also 644.21, p. 1202

Grade C, strips, various tempers. See also 644.21 , p. 1202.

2. Grades B and C brass may be ordered as type 1 or type 2 .

3. The chemical requirements are as shown in Table 1 .

TABLE 1.-Chemical composition

\begin{tabular}{|c|c|c|c|c|c|c|}
\hline Grade & Composition & Copper & $\begin{array}{l}\text { Zinc (per } \\
\text { cent) }\end{array}$ & Lead & $\begin{array}{l}\text { Iron, } \\
\text { maxi- } \\
\text { mum }\end{array}$ & $\begin{array}{l}\text { Other } \\
\text { im- } \\
\text { pur- } \\
\text { ities, } \\
\text { maxi- } \\
\text { mum }\end{array}$ \\
\hline $\begin{array}{l}\text { A. } \\
\text { B. } \\
\text { C. }\end{array}$ & $\begin{array}{l}\left\{\begin{array}{l}\text { Desired } \\
\text { Permissible }\end{array}\right. \\
\left\{\begin{array}{l}\text { Desired } \\
\text { Permissible }\end{array}\right. \\
\left\{\begin{array}{l}\text { Desired } \\
\text { Permissible.. }\end{array}\right.\end{array}$ & $\begin{array}{r}\text { Per cent } \\
60.0 \\
58.5-61.5 \\
61.5 \\
60.0-63.0 \\
65.0 \\
64.5-67.5\end{array}$ & $\begin{array}{l}\text { Remainder. } \\
\\
\\
\end{array}$ & $\begin{array}{r}\text { Per cent } \\
2.00 \\
1.50-2.50 \\
3.00 \\
-2.25-3.75 \\
0.00 \\
0-0.30\end{array}$ & $\begin{array}{c}\text { Per } \\
\text { cent } \\
0.00 \\
.15 \\
.00 \\
.15 \\
.00 \\
.05\end{array}$ & $\begin{array}{c}\text { Per } \\
\text { cent } \\
0.00 \\
.35 \\
.00 \\
.50 \\
.00 \\
.10\end{array}$ \\
\hline
\end{tabular}

When grades $\mathrm{B}$ and $\mathrm{C}$ are ordered as type 2, they shall be in accordance with the following chemical composition: Copper, 59 to 68 per cent; tin, maximum, 1.5 per cent; zinc, remainder; lead, maximum, 3.5 per cent; iron, maximum, 0.2 per cent.

4. Grade A brass shall be malleable, as determined by the flattening test defined hereinafter.

5. Commercial brass shall conform to the physical requirements as shown in Table 2 . Special tempers not covered herein may be agreed upon between the contractor and purchaser, in which case they will be specifically defined and designated in the contract or purchase order. 
TABLE 2.-Physical properties

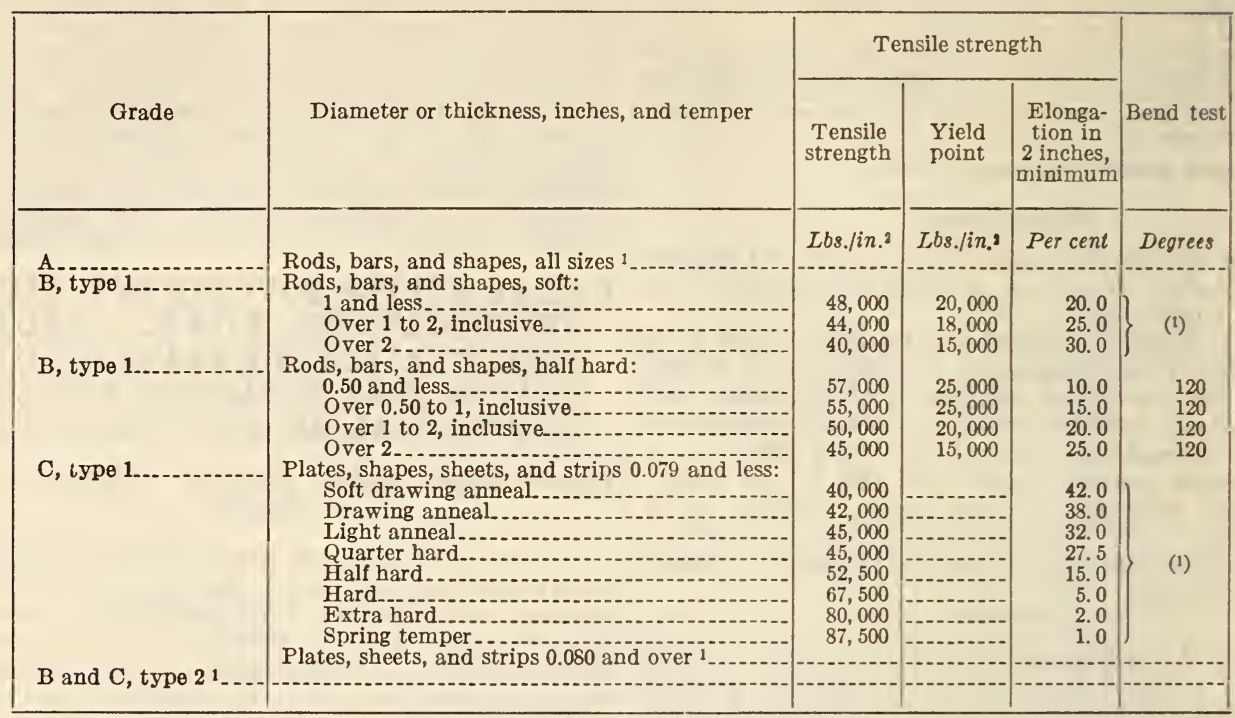

1 No requirements.

6. Grade B, type 1, brass rods, bars, and snapes, half hard, shall withstand bending cold, withcut fracture on the outside of the bent portion, through the angle specified in Table 2 , on a radius equal to the diameter or thickness of the material. At the option of the purchaser, any or all of the physical tests may be waived.

7. Grade C, brass plates, sheets, and strips 0.080 inch and over in thickness shall conform to the Brinell hardness requirements shown in Table 3:

TABLE 3.-Brinell hardness, grade C plates, sheets, and strips, 0.080 inch and over

\begin{tabular}{|c|c|}
\hline Temper & $\begin{array}{l}\text { Brinell } \\
\text { hardness }\end{array}$ \\
\hline Soft drawing anneal & $47-55$ \\
\hline Drawing anneal & $55-65$ \\
\hline Light anneal & $65-75$ \\
\hline Quarter hard & $75-95$ \\
\hline Half hard & $95-115$ \\
\hline Hard & $130-150$ \\
\hline Extra hard & $150-170$ \\
\hline Spring & $160-180$ \\
\hline
\end{tabular}

\section{TESTS}

(Rockwell hardness requirements are also included in the full text of this specification.)

8. For grade A brass (forging brass), specimens cut from the rods or bars, and having a length equal to the diameter or thickness of the specimen, shall stand flattening in an axial direction while hot until reduced to 20 per cent of their original length without cracking.

9. For sheets and strips 0.080 inch or over in thickness, at least 10 pieces shall be selected from every 5,000 pounds of metal, or fraction thereof, of each size, and subjected to the Brinell test, using a $10 \mathrm{~mm}$ ball, $500 \mathrm{~kg}$ pressure held for 30 seconds. The average hardness of the 10 sheets shall conform to the requirements specified.

\section{NOTES}

10. Round, square, hexagonal, etc., sections are classed as rods. Rectangular sections having greater width than thickness are classed as bars, plates, sheets, or strips. All other sections are classed as shapes.

11. These alloys are susceptible to season cracking, and are intended for structural uses in locations not exposed to weather or corrosive influences. When exposure to corrosive influences is unavoidable, naval brass should be used in the form of rods, bars, or shapes, or low brass in the form of plates, sheets, and strips.

\subsection{BRASS INGOTS AND BARS.}

\subsection{INGOTS, BRASS.}

\section{AMERICAN SOCIETY FOR TESTING MATE- RIALS, TENTATIVE SPECIFICATIONS FOR COPPER-BASE ALLOYS IN INGOT FORM FOR SAND CASTINGS, DESIGNA- TION B30-31T, 1931}

\section{COPPER-BASE ALLOYS IN INGOT FORM}

1. These specifications cover copper-base alloys In ingot form for sand castings in 20 different compositions, regularly sold by the trade and arbitrarily herein given numbers 1 to 20, inclusive, to differentiate them from one another. These numbers have no other significance.

\section{MANUFACTURE}

2. The manufacturer shall use care to have each lot of ingot metal as uniform in quality as possible. This metal may be manufactured by any refining process which will yield a satisfactory quality of product.

\section{CHEMICAL PROPERTIES AND TESTS}

3. The alloys shall conform to the requirements as to chemical composition given in Table 1, within the limits specified therein.

NoTr. - The limits specified in Table 1 indicate the maximum and minimum percentages within which variations of different elements shall be permitted, with the understanding that every precaution shall be permitted, with the understanding that every precaution of manufacture to insure that the resulting ingot metal will come within these specified limits. 


\section{SAMPLES FOR CHEMICAL ANALYSIS}

4. (a) Ten ingots shall be selected by the inspector to represent each 40,000 pounds, and five ingots shall be selected for less than carload lots.

(b) The samples for chemical analysis may be taken by drilling the ingots, and shall represent the average cross section of all the ingots selected for sampling.

(c) The drill used shall be thoroughly cleaned. No lubricant shall be used in the operation, and the drillings shall be carefully treated with a magnet to remove any particles of steel introduced in taking the sample.

\section{METHODS OF CHEMICAL ANALYSIS}

5. The chemical analysis shall be made in accordance with the standard methods of chemical analysis of brass ingots and sand castings in A. S. T. M. B45. (See 644.0 , p. 1193 , including references in B45 to A. S. T. M. B27.)

\section{MAREING}

6. The designating mark of the manufacturer, the proper lot number, and the numerical designation of the alloy shall be marked on each ingot for identification.

\section{INSPECTION AND REJECTION}

(Identical with B59 (see 647.15, p. 1259) with the addition of the following item:)

7. If the test ingots selected to represent a lot fail to conform to the requirements specified in section 3 , all ingots in such lot shall be rejected.

\section{CLAMS}

8. Claims, to be considered, shall be made in writing within 30 days of receipt of material at the purchaser's plant, and the results of the purchaser's tests shall be given. The manufacturer shall, within one week of receipt of such claim, either agree to satisfy the claim, or send a representative to the purchaser's plant to resample the shipment in accordance with section 4 . Samples so taken shall be sealed and submitted to a mutually agreeable umpire, whose determinations shall be final.

9. The expense of umpire analysis shall be paid by the loser, or divided in proportion to the concession made in case of a compromise. In case of rejection being established, the damages shall be limited to the payment of freight both ways by the manufacturer for the substitution of an equivalent weight of ingot metal conforming to these specifications.

TABLE 1.-Chemical compositions of copper-base alloys

BRONZE ALLOYS

\begin{tabular}{|c|c|c|c|c|c|c|c|c|c|c|c|c|c|c|c|c|c|c|c|c|}
\hline \multirow[b]{2}{*}{ Alloy grade } & \multicolumn{2}{|c|}{ Copper } & \multicolumn{3}{|c|}{ Tin } & \multicolumn{3}{|c|}{ Lead } & \multicolumn{3}{|c|}{ Zinc } & & \multicolumn{6}{|c|}{ Other elements, maximum } & \multicolumn{2}{|c|}{$\begin{array}{l}\text { Total con- } \\
\text { stituents }\end{array}$} \\
\hline & 亲 & 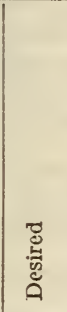 & 音 & 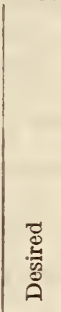 & 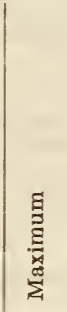 & 昜 & 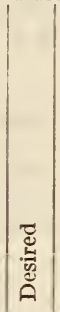 & 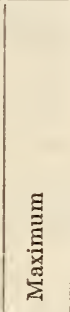 & 員 & 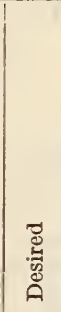 & 范 & 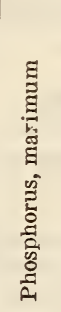 & 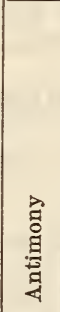 & 总 & 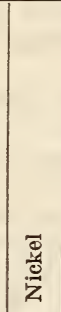 & 壱 & 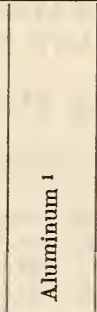 & 蒫 & 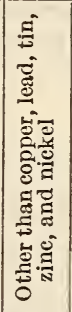 & 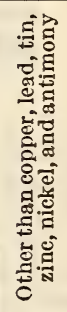 \\
\hline $\begin{array}{l}\text { No. } 1 \\
\text { No. } 2 \\
\text { No. } 3\end{array}$ & $\begin{array}{l}P e r \\
\text { cent } \\
87 \\
84 \\
84\end{array}$ & \begin{tabular}{|l} 
Per \\
cent \\
89 \\
85 \\
85
\end{tabular} & \begin{tabular}{|l} 
Per \\
cent \\
5.5 \\
9 \\
7.25
\end{tabular} & \begin{tabular}{|c} 
Per \\
cent \\
6 \\
10 \\
8
\end{tabular} & $\begin{array}{c}\text { Per } \\
\text { cent } \\
6.5 \\
11 \\
8.75\end{array}$ & $\begin{array}{l}\text { Per } \\
\text { cent } \\
1.5 \\
1.5 \\
1.5\end{array}$ & $\begin{array}{c}\text { Per } \\
\text { cent } \\
2 \\
2 \\
2\end{array}$ & $\begin{array}{l}\text { Per } \\
\text { cent } \\
2.5 \\
2.5 \\
2.5\end{array}$ & $\begin{array}{l}\text { Per } \\
\text { cent } \\
2 \\
1.5 \\
3.5\end{array}$ & $\begin{array}{l}\text { Per } \\
\text { cent } \\
3 \\
2 \\
4 \\
4\end{array}$ & $\begin{array}{l}\text { Per } \\
\text { cent } \\
4 \\
2.5 \\
4.5\end{array}$ & $\begin{array}{c}\text { Per } \\
\text { cent } \\
0.03 \\
.03 \\
.03\end{array}$ & $\begin{array}{c}\text { Per } \\
\text { cent } \\
0.25 \\
.25 \\
.25\end{array}$ & $\begin{array}{c}\text { Per } \\
\text { cent } \\
0.25 \\
.25 \\
.25\end{array}$ & $\begin{array}{c}P e r \\
\text { cent } \\
0.50 \\
.50 \\
.50\end{array}$ & \begin{tabular}{|c|} 
Per \\
cent \\
0.08 \\
.08 \\
.08
\end{tabular} & $\begin{array}{c}\text { Per } \\
\text { cent } \\
\text { None.-- } \\
\text { None-- } \\
\text { None.- }\end{array}$ & $\begin{array}{l}\text { Per } \\
\text { cent } \\
0.05 \\
.05 \\
.05\end{array}$ & $\begin{array}{c}P e r \\
c c n t \\
0.50 \\
.50 \\
.50\end{array}$ & $\begin{array}{l}\text { Per } \\
\text { cent }\end{array}$ \\
\hline
\end{tabular}

RED BRASS ALLOYS

\begin{tabular}{|c|c|c|c|c|c|c|c|c|c|c|c|c|c|c|c|c|c|c|c|c|}
\hline $\begin{array}{l}\text { No. } 4 \\
\text { No. } 5 \\
\text { No. } 6\end{array}$ & $\begin{array}{l}84 \\
83 \\
82\end{array}$ & $\begin{array}{l}85 \\
85 \\
83\end{array}$ & $\begin{array}{l}4.5 \\
4.25 \\
6.25\end{array}$ & $\begin{array}{l}5 \\
5 \\
7\end{array}$ & $\begin{array}{l}5.5 \\
5.75 \\
7.75\end{array}$ & $\begin{array}{l}4.5 \\
4.25 \\
6.25\end{array}$ & $\begin{array}{l}5 \\
5 \\
7\end{array}$ & $\begin{array}{l}5.5 \\
5.75 \\
7.75\end{array}$ & $\begin{array}{l}4.5 \\
4.25 \\
2\end{array}$ & $\begin{array}{l}5 \\
5 \\
3\end{array}$ & $\mid \begin{array}{l}5.5 \\
5.75 \\
-\cdots\end{array}$ & $\begin{array}{r}0.03 \\
.03 \\
.03\end{array}$ & $\begin{array}{r}0.25 \\
.25 \\
.25\end{array}$ & $\begin{array}{r}0.25 \\
.25 \\
.25\end{array}$ & $\begin{array}{r}0.50 \\
.50 \\
.50\end{array}$ & $\begin{array}{r}0.08 \\
.08 \\
.08\end{array}$ & $\begin{array}{l}\text { None.- } \\
\text { None.- } \\
\text { None.- }\end{array}$ & $\begin{array}{r}0.05 \\
.05 \\
.05\end{array}$ & $\begin{array}{r}0.50 \\
.50 \\
.50\end{array}$ & \\
\hline
\end{tabular}

SEMIRED BRASS ALLOYS

\begin{tabular}{|c|c|c|c|c|c|c|c|c|c|c|c|c|c|c|c|c|c|c|c|c|}
\hline $\begin{array}{l}\text { No. } 8 \\
\text { No. } 9 \\
\text { No. } 10\end{array}$ & $\begin{array}{l}83 \\
82 \\
79 \\
77\end{array}$ & $\begin{array}{l}84 \\
83 \\
80 \\
78\end{array}$ & $\begin{array}{l}2.5 \\
3.25 \\
2.5 \\
2.5\end{array}$ & $\begin{array}{l}4 \\
3\end{array}$ & $\begin{array}{l}3.5 \\
4.75 \\
3.5 \\
3.5\end{array}$ & $\begin{array}{l}2.5 \\
5 \\
6.25 \\
4.25\end{array}$ & $\begin{array}{l}3 \\
6 \\
7 \\
5\end{array}$ & $\begin{array}{l}3.5 \\
7 \\
7.75 \\
5.75\end{array}$ & $\begin{array}{c}9 \\
5 \\
7.5 \\
11\end{array}$ & $\begin{array}{r}10 \\
7 \\
9.5 \\
13.5\end{array}$ & 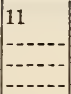 & $\begin{array}{r}0.03 \\
.03 \\
.03 \\
.03\end{array}$ & $\begin{array}{r}0.25 \\
.25 \\
.25 \\
.25\end{array}$ & $\begin{array}{r}0.35 \\
.35 \\
.35 \\
.35\end{array}$ & $\begin{array}{r}0.50 \\
.50 \\
.50 \\
.50\end{array}$ & $\begin{array}{r}0.08 \\
.08 \\
.08 \\
.08\end{array}$ & $\begin{array}{l}\text { None.- } \\
\text { None.-- } \\
\text { None.- } \\
\text { None.- }\end{array}$ & $\begin{array}{l}0.05 \\
.05 \\
.05 \\
.05\end{array}$ & $\begin{array}{l}0.50 \\
.50 \\
.50 \\
.50\end{array}$ & \\
\hline
\end{tabular}

YEILOW BRASS ALLOYS

\begin{tabular}{|c|c|c|c|c|c|c|c|c|c|c|c|c|c|c|c|c|c|c|c|c|}
\hline $\begin{array}{l}\text { No. } 11 \\
\text { No. } 12 . . . . \\
\text { No. } 13 . \ldots . \\
\text { No. } 14 . . .\end{array}$ & $\begin{array}{l}70 \\
67 \\
64 \\
60\end{array}$ & $\begin{array}{l}71 \\
69 \\
66 \\
62\end{array}$ & $\begin{array}{l}1 \\
0 \\
0 \\
0\end{array}$ & $\begin{array}{l}1.5 \\
0 \\
0 \\
0\end{array}$ & $\begin{array}{l}2 \\
1.5 \\
1 \\
1\end{array}$ & $\begin{array}{l}2 \\
2 \\
1 \\
1\end{array}$ & $\begin{array}{l}3 \\
3 \\
2 \\
2\end{array}$ & $\begin{array}{l}4 \\
4 \\
3 \\
3\end{array}$ & $\begin{array}{l}20 \\
25 \\
30 \\
30\end{array}$ & $\begin{array}{l}24 \\
27 \\
31 \\
35\end{array}$ & $\begin{array}{l}--- \\
-- \\
-- \\
--\end{array}$ & $\begin{array}{r}0.01 \\
.01 \\
.01 \\
.01\end{array}$ & $\begin{array}{r}0.10 \\
.10 \\
.10 \\
.10\end{array}$ & $\begin{array}{r}0.30 \\
.30 \\
.30 \\
.30\end{array}$ & $\begin{array}{r}0.50 \\
.50 \\
.50 \\
.50\end{array}$ & $\begin{array}{l}0.05 \\
.05 \\
.05 \\
.05\end{array}$ & $\begin{array}{c}\text { None.-- } \\
\text { None.-- } \\
\text { None.- } \\
0.50\end{array}$ & $\begin{array}{r}0.10 \\
.10 \\
.10 \\
.10\end{array}$ & $\begin{array}{r}0.50 \\
.50 \\
.50 \\
1.00\end{array}$ & \\
\hline
\end{tabular}

HIGH LEAD ALLOYS

\begin{tabular}{|c|c|c|c|c|c|c|c|c|c|c|c|c|c|c|c|c|c|c|c|c|}
\hline $\begin{array}{l}\text { No. } 15 \\
\text { No. } 16 \\
\text { No. } 17 \\
\text { No. } 18 \\
\text { No. } 19 \\
\text { No. } 20\end{array}$ & $\begin{array}{l}84 \\
79 \\
76 \\
72 \\
71 \\
70\end{array}$ & $\begin{array}{l}85 \\
80 \\
77 \\
73 \\
71.5 \\
70.5\end{array}$ & $\begin{array}{l}4.25 \\
9 \\
9 \\
7 \\
3.5 \\
5.25\end{array}$ & $\begin{array}{l}5 \\
10 \\
10 \\
8 \\
4.5 \\
6\end{array}$ & $\begin{array}{l}5.75 \\
11 \\
11 \\
9 \\
5.5 \\
6.75\end{array}$ & $\begin{array}{l}8 \\
9 \\
9 \\
13.5 \\
14 \\
18\end{array}$ & $\begin{array}{r}9 \\
10 \\
10 \\
15 \\
17 \\
20\end{array}$ & $\begin{array}{l}10 \\
11 \\
11 \\
16.5 \\
20 \\
22\end{array}$ & \begin{tabular}{l}
0.5 \\
\hdashline 1 \\
.5
\end{tabular} & \begin{tabular}{l}
1 \\
\hdashline .75 \\
1.5 \\
1
\end{tabular} & $\begin{array}{l}2 \\
i^{.25} \\
2 \\
6 \\
1.5\end{array}$ & $\begin{array}{l}0.01 \\
2.05 \\
.01 \\
.05 \\
.05 \\
.05\end{array}$ & $\begin{array}{r}0.25 \\
.50 \\
.50 \\
1.00 \\
1.00 \\
1.00\end{array}$ & $\begin{array}{r}0.25 \\
.25 \\
.25 \\
.25 \\
.25 \\
.25\end{array}$ & $\begin{array}{r}0.50 \\
.25 \\
.50 \\
.50 \\
.50 \\
.50\end{array}$ & $\begin{array}{l}0.08 \\
.08 \\
.08 \\
.08 \\
.08 \\
.08\end{array}$ & $\begin{array}{l}\text { None.-- } \\
\text { None_-- } \\
\text { None.-- } \\
\text { None.- } \\
\text { None_-- } \\
\text { None_- }\end{array}$ & $\begin{array}{l}0.03 \\
.03 \\
.03 \\
.03 \\
.03 \\
.03\end{array}$ & 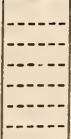 & $\begin{array}{r}0.50 \\
.50 \\
.50 \\
.50 \\
.50 \\
.50\end{array}$ \\
\hline
\end{tabular}

1 The term "none" as applied to aluminum allowance is defined as a maximum of 0.005 per cent when determined in accordance with section 5 .

${ }^{2}$ Minimum. 
(An appendix to this specification gives information on the tensile strength, uses, machining qualities, and other properties of these alloys.)

\section{AMERICAN SOCIETY FOR TESTING MATE- RIALS, STANDARD METHODS OF CHEM- ICAL ANALYSIS OF BRASS INGOTS AND SAND CASTINGS, DESIGNATION B45-27, 1927}

(See 644.0, p. 1193.)

644.12 BARS, BRASS.

FEDERAL SPECIFICATIONS BOARD, SPECIFICATION, FOR BRASS RODS, BARS, SHAPES, PLATES, SHEETS, AND STRIPS, COMMERCIAL, QQ-B-611, MARCH 1, 1932

\section{BRASS BARS}

(See 645.11, p. 1205.)

644.2 BRASS PLATES, SHEETS, SHAPES, AND STRIPS.

644.21 PLATES AND SHEETS, BRASS.

AMERICAN BUREAU OF SHIPPING, RULES FOR BUILDING AND CLASSING STEEL VESSELS; MATERIALS FOR MACHINERY, BOILERS, AND PIPING, 1930

\section{BRASS PLATES AND SHAPES}

(For general information and requirements see 600.0 , p. 1.)

(For test requirements see 600.1, p. 7.)

1. The material is to have a tensile strength of not less than 40,000 pounds per square inch, with an elongation of at least 15 per cent on a gage length of 8 inches. Otherwise the test and requirements are to be as specified for copper plates and shapes (See 641.21, p. 1176.)

\section{AMERICAN SOCIETY FOR TESTING MATE- RIALS, STANDARD SPECIFICATIONS FOR CARTRIDGE BRASS, DESIGNATION B19- 29, 1929}

\section{CARTRIDGE BRASS}

\section{SCOPE}

1. These specifications cover sheet brass for making rifle and other small arms cartridge cases.

\section{MANUFACTURE}

2. The brass shall be made from lake or electrolytic copper, meeting the requirements of the standard specifications for lake copper wire bars, cakes, slabs, billets, ingots, and ingot bars in A. S. T. M. B4 (see 641.11 , p. 1173), or the standard specifications for electrolytic copper wire bars, cakes, slabs, billets, ingots, and ingot bars in A. S. T. M. B5 (see 641.11, p. 1174), and zinc of grades Nos. 1 or 2 of the standard specifications for slab zinc (spelter) in A. S. T. M. B6 (see 683.1, p. 1310).

3. No scrap shall be used except that arising from the manufacture of brass or cases made from materials complying with the requirements of section 2 .

\section{CHEMICAL PROPERTIES AND TESTS}

4. The brass shall conform to the following requirements as to chemical composition:

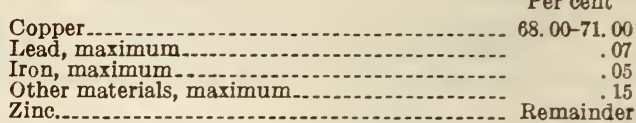

5. Analyses may be made on each iot of 50,000 pounds or fraction thereof. Drillings or millings shall be made from at least 10 separate pieces, equal quantities being taken from each and thoroughly mixed. Samples so prepared shall be divided into three equal parts, each of which shall be placed in a sealed package, one for the manufacturer, one for the purchaser, and one for an umpire, if necessary.

6. In case of dissatisfaction, resampling shall be done in the presence of representatives of both parties. The thoroughly mixed sample shall be divided into three equal parts, each of which shall be placed in a sealed package, one for the manufacturer, one for the purchaser, and one for an umpire, if necessary.

\section{PIYSICAL PROPERTIES AND TESTS}

7. The brass shall be so annealed that the average of 10 Brinell hardness readings from a lot will be within the limits of 49 to 65 , using a $10 \mathrm{~mm}$ ball and a pressure of $500 \mathrm{~kg}$. (See notes 1 and 2.)

\section{PERMISSIBLE VARIATIONS IN DIMENSIONS}

8. A permissible variation of \pm 0.002 inch from the specified thickness when measured one-half inch from the edge of the strip will be allowed. The width of the strip may vary \pm 0.010 inch.

9. Cartridge metal may be furnished in coils or flat strips as required. When furnished in flat strips, no strip shall be of less length than 60 per cent of that specified. No lot shall contain more than 40 per cent of such short lengths.

\section{WORKMANSHIP AND FINISH}

10. The metal shall be free from injurious external and internal defects of a nature which will interfere with the purpose for which it is intended. It shall be well pickled and free from dirt. Strips shall be substantially straight and flat. (See note 3. )

\section{INSPECTION AND REJECTION}

11. The manufacturer shall afford the inspector representing the purchaser, without charge, all reasonable facilities to satisfy him that the material is being furnished in accordance with these specifications.

12. Material which fails to conform to these specifications will be rejected and the manufacturer shall be notified.

\section{EXPLANATORY NOTES}

Note 1.-A comparative study of Brinell hardness and grain size indicates the following relations:

\begin{tabular}{|c|c||c|c|}
\hline $\begin{array}{c}\text { Grain size } \\
(\mathrm{mm})\end{array}$ & $\begin{array}{c}\text { Equiva- } \\
\text { lent } \\
\text { Brinell } \\
\text { hardness } \\
\text { number }\end{array}$ & $\begin{array}{c}\text { Grain size } \\
(\mathrm{mm})\end{array}$ & $\begin{array}{c}\text { Equiva- } \\
\text { lent } \\
\text { Brinell } \\
\text { hardness } \\
\text { number }\end{array}$ \\
\hline 0.150 & 47 & 0.058 & 55 \\
.111 & 49 & .050 & $571 / 2$ \\
.100 & 49.7 & .044 & 60 \\
.098 & 50 & .040 & $621 / 2$ \\
.087 & 51 & .034 & 65 \\
.075 & $521 / 4$ & .030 & 68 \\
.060 & 5434 & .029 & 69 \\
\hline
\end{tabular}


As it is easier and quicker to take the Brinell hardness than to polish and etch samples for study with the microscope, the Brinell hardness is much to be preferred. Furthermore, through straightening or flattening operations, cartridge metal may be hardened to an appreciable extent without affecting the grain size or producing any noticeable distortion.

Many operations in making rapid determinations of grain size slight the polishing and etch in strong acids, so that it is frequently impossible to distinguish between twinned crystals and separate crystals and a false grain count is obtained.

crystals and a false grain count is obtained.
Note 2.-These specifications are intended to cover the requireNote 2.-These specifications are intended to cover the require-
ments of commercial practice which do not in all cases agree with those of the Ordnance Department, U.S. Army. These departures involve only the degree of final annealing.

Note 3.-The differentiation between immaterial and injurious defects is a matter which requires a considerable degree of knowledge as to the causes of the various kinds of imperfections which are encountered in material of this character, as well as a thorough understanding of the effects thereof upon its further fabrication and use. In general, two classes may be readily distinguished, namely, those which are imposed and those which are inherent. In the former class are dents and scratches caused by blows or abrasion, and foreign matter which, adhering to the surface of the material, may have been rolled into it. Dents and scratches may be of considerable magnitude and still disappear completely in subsequent operations of fabrication. Similarly the presence of imposed foreign matter may be relatively unimportant. The point to be observed in this connection is that the presence of this class of defects is indicative of nothing beyond their presence.

Inherent defects are those which occur as a result of the presence either of gas or foreign matter included in the material during the process of casting. Metal is seldom entirely free from such defects and visual evidence of these argues the presence of others not visible. Experience is needed to determine whether any such encountered are of a magnitude and character sufficient to establish a departure from correct casting practice sufficient to disqualify the material.

A third character of defect, and one of a grave nature, is caused by failure to cumpletely remove any piping which may have occurred in casting. The indications of this defect are, however, well pronounced and characteristic.

\section{AMERICAN SOCIETY FOR TESTING MATE- RIAIS, STANDARD SPECIFICATIONS FOR CARTRIDGE BRASS DISKS, DESIGNA- TION B20-29, 1929}

\section{CARTRIDGE BRASS DISKS}

SCOPE

1. These specifications cover brass disks for making artillery cartridge cases.

2. The disks shall be made of cartridge brass conforming to the requirements of the A. S. T. M. standard specifications for cartridge brass, B19-29 (see above), except as to dimensions and permissible variations thereof, which shall conform to the requirements specified in section 4 .

3. The brass shall be so annealed that the average of 10 Brinell hardness readings from a lot will be within the limits of 49 to 65 , using a $10-\mathrm{mm}$ ball and a pressure of $500 \mathrm{~kg}$.

Note.-These specifications are intended to cover the requirements of commercial practice which do not in all cases agree with those of the Ordnance Department, U.S. Army. These departures involve only the degree of final annealing.

4. (a) Variations in dimensions shall conform to the following limits:

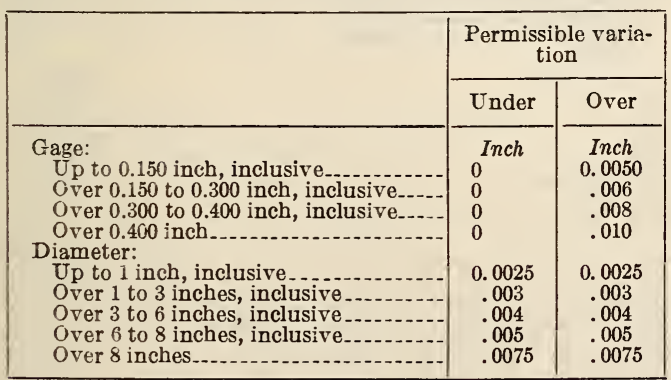

(b) The thickness of disks shall be measured not less than one-half inch from the edge and on the diameter at right angles to the direction in which the bar was rolled.

\section{AMERICAN SOCIETY FOR TESTING MATE- RIALS, STANDARD SPECIFICATIONS FOR SHEET HIGH BRASS, DESIGNATION B36- 27, 1927}

\section{SHEET HIGH BRASS \\ SCOPE}

1. These specifications cover commercial sheet brass commonly used for drawing, forming, stamping, and bending. It should be understood that they are general. Since high brass is used for many purposes where the requirements of the operations used are too particular to be specified by any of the ordinary physical tests, it is frequently advisable to submit samples or drawings to the manufacturer and secure an adjustment of anneal or temper to suit the actual operations to which the material is to be submitted.

\section{MAN UFACTORE}

2. (a) (Same as A. S. T. M. specification for cartridge brass (see above), except that for sheet high brass grade 3 zinc is used instead of grade 1 or 2.)

(b) The brass shall be manufactured by the coldrolling and annealing process and may be furnished in either the cold-rolled or annealed condition, as required.

\section{CHEMICAL PROPERTIES}

3. The brass shall conform to the following requirements as to chemical composition:

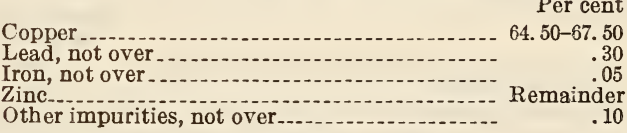

4. Analysis shall be made on each lot of 10,000 pounds or less. Drilling, millings, or clippings shall be taken from at least 10 separate coils or pieces. Drillings or millings shall be taken on materia thicker than 0.040 inch. Clippings may be taken with shears from metal thinner than 0.040 inch Equal quantities shall be taken from each piece and thoroughly mixed together. Samples so prepared shall be divided into three equal parts, each of which shall be placed in a sealed package, one for each party and one for an umpire, if necessary.

5. (a) Analyses of separate pieces may be made by the purchaser. The copper, lead, and iron determined shall be within the following limits:

Per cent

Copper

Lead, not over. 35

Iron, not over --.-. 07

(b) In case of dissatisfaction the metal snall be resampled in the presense of representatives of both parties. The thoroughly mixed sample shall be divided into three equal parts, each one of which shall be placed in a sealed package, one for each party and one for an umpire, if necessary.

\section{TEMPER AND ANNEAL}

6. The temper of sheet brass shall be designated as follows:

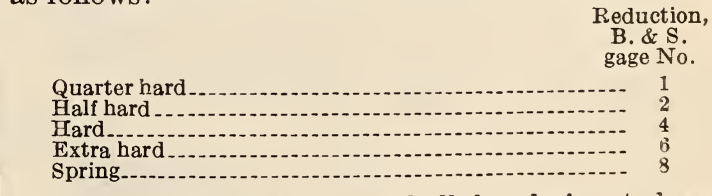

7. Annealed sheet brass shall be designated as follows:

Light anneal.

Drawing anneal.

Soft drawing anneal. 


\section{PHYSICAL PROPERTIES AND TESTS}

(a) Hard brass

8. The average Brinell hardness of 10 samples of cold-rolled brass 0.080 inch or over in thickness shall be within the following limits:

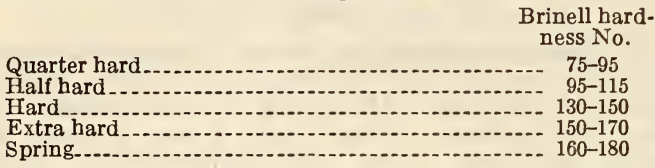

9. The average of tension tests of two samples of cold-rolled brass thinner than 0.080 inch shall conform to the following minimum requirements:

\begin{tabular}{|c|c|c|}
\hline & $\begin{array}{l}\text { Tensile } \\
\text { strength }\end{array}$ & $\begin{array}{l}\text { Elongation } \\
\text { in } 2 \text { inches }\end{array}$ \\
\hline $\begin{array}{l}\text { Quarter hard } \\
\text { Half hard } \\
\text { Hard } \\
\text { Extra hard. } \\
\text { Spring }\end{array}$ & $\begin{array}{l}\text { Lbs./in.2 } \\
45,000 \\
52.500 \\
67,500 \\
80,000 \\
87,500\end{array}$ & $\begin{array}{l}\text { Per cent } \\
27.5 \\
15.0 \\
5.0 \\
2.0 \\
1.0\end{array}$ \\
\hline
\end{tabular}

(b) Soft brass

10. The average Brinell hardness of 10 samples of annealed brass 0.080 inch or over in thickness shall be as follows:

Light anneal.

Brinell hardness No.

Drawing anneal - $65-75$

Soft drawing anneal

11. The average of tension tests of two samples of annealed brass thinner than 0.080 inch shall conform to the following minimum requirements:

\begin{tabular}{|l|c|c|}
\hline & $\begin{array}{c}\text { Tensile } \\
\text { strength }\end{array}$ & $\begin{array}{c}\text { Elongation } \\
\text { in 2 inches }\end{array}$ \\
\hline & Lbs./in.2 & Per cent \\
Light anneal _.......... & 45,000 & 32 \\
Drawing anneal ........ & 42,000 & 38 \\
Soft drawing anneal_....- & 40,000 & 42 \\
\hline
\end{tabular}

\section{PERMISSIBLE VARIATION IN DIMENSIONS}

12. The width of sheared metal shall not vary more than 0.010 inch.

13. The standard method of specifying thickness shall be in terms of Brown \& Sharpe gage. When the thickness is specified in either common or decimal fractions of an inch, the tolerances shall be those of the corresponding group of Brown \& Sharpe sizes in the following table:
TABLE 1.-Permissible variations in thickness, plus or minus

\begin{tabular}{|c|c|c|c|c|c|}
\hline \multicolumn{2}{|c|}{ Thickness } & \multicolumn{4}{|c|}{ Width } \\
\hline $\begin{array}{c}\text { B. \& S. } \\
\text { gage } \\
\text { No. }\end{array}$ & Inch & $\begin{array}{l}\text { Up to } 6 \\
\text { inches, } \\
\text { inclusive }\end{array}$ & $\begin{array}{c}\text { Over } 6 \\
\text { to } 9 \\
\text { inches, } \\
\text { inclusive }\end{array}$ & $\begin{array}{c}\text { Over } 9 \\
\text { to } 14 \\
\text { inches, } \\
\text { inclusive }\end{array}$ & $\begin{array}{l}\text { Over } 14 \\
\text { to } 20 \\
\text { inches, } \\
\text { inclusive }\end{array}$ \\
\hline $\begin{array}{r}0000 \\
000 \\
00 \\
0 \\
1\end{array}$ & $\begin{array}{l}0.460 \\
.4096 \\
.3648 \\
.3249 \\
.2893\end{array}$ & $\begin{array}{c}\text { Inch } \\
0.0043 \\
.0042 \\
.0041 \\
.0040 \\
.0039\end{array}$ & $\begin{array}{c}\text { Inch } \\
0.0045 \\
.0044 \\
.0043 \\
.0042 \\
.0041\end{array}$ & $\begin{array}{l}\text { Inch } \\
0.0047 \\
.0046 \\
.0045 \\
.0044 \\
.0043\end{array}$ & $\begin{array}{c}\text { Inch } \\
0.0049 \\
.0048 \\
.0047 \\
.0046 \\
.0045\end{array}$ \\
\hline $\begin{array}{l}2 \\
3 \\
4 \\
5 \\
6\end{array}$ & $\begin{array}{l}.2576 \\
.2294 \\
.2043 \\
.1819 \\
.1620\end{array}$ & $\begin{array}{l}.0038 \\
.0037 \\
.0036 \\
.0035 \\
.0034\end{array}$ & $\begin{array}{l}.0040 \\
.0039 \\
.0038 \\
.0037 \\
.0036\end{array}$ & $\begin{array}{l}.0042 \\
.0041 \\
.0040 \\
.0039 \\
.0038\end{array}$ & $\begin{array}{l}.0044 \\
.0043 \\
.0042 \\
.0041 \\
.0040\end{array}$ \\
\hline $\begin{array}{r}7 \\
8 \\
9 \\
10 \\
11\end{array}$ & $\begin{array}{l}.1443 \\
.1285 \\
.1144 \\
.1019 \\
.0907\end{array}$ & $\begin{array}{l}.0033 \\
.0032 \\
.0031 \\
.0030 \\
.0029\end{array}$ & $\begin{array}{l}.0035 \\
.0034 \\
.0033 \\
.0032 \\
.0031\end{array}$ & $\begin{array}{l}.0037 \\
.0036 \\
.0035 \\
.0034 \\
.0033\end{array}$ & $\begin{array}{l}.0039 \\
.0038 \\
.0037 \\
.0036 \\
.0035\end{array}$ \\
\hline $\begin{array}{l}12 \\
13 \\
14 \\
15 \\
16\end{array}$ & $\begin{array}{l}.0808 \\
.0719 \\
.0640 \\
.0570 \\
.0508\end{array}$ & $\begin{array}{l}.0028 \\
.0027 \\
.0026 \\
.0025 \\
.0024\end{array}$ & $\begin{array}{l}.0030 \\
.0029 \\
.0028 \\
.0027 \\
.0026\end{array}$ & $\begin{array}{l}.0032 \\
.0031 \\
.0030 \\
.0029 \\
.0028\end{array}$ & $\begin{array}{l}.0034 \\
.0033 \\
.0032 \\
.0031 \\
.0030\end{array}$ \\
\hline $\begin{array}{l}17 \\
18 \\
19 \\
20 \\
21\end{array}$ & $\begin{array}{l}.0452 \\
.0403 \\
.0359 \\
.0320 \\
.0284\end{array}$ & $\begin{array}{l}.0023 \\
.0022 \\
.0021 \\
.0020 \\
.0019\end{array}$ & $\begin{array}{l}.0025 \\
.0024 \\
.0023 \\
.0021 \\
.0020\end{array}$ & $\begin{array}{l}.0027 \\
.0026 \\
.0025 \\
.0023 \\
.0021\end{array}$ & $\begin{array}{l}.0029 \\
.0028 \\
.0026 \\
.0024 \\
.0022\end{array}$ \\
\hline $\begin{array}{l}22 \\
23 \\
24 \\
25 \\
26\end{array}$ & $\begin{array}{l}.0253 \\
.0225 \\
.0201 \\
.0179 \\
.0159\end{array}$ & $\begin{array}{l}.0018 \\
.0017 \\
.0016 \\
.0015 \\
.0014\end{array}$ & $\begin{array}{l}.0019 \\
.0018 \\
.0017 \\
.0016 \\
.0015\end{array}$ & $\begin{array}{l}.0020 \\
.0019 \\
.0018 \\
.0017 \\
.0016\end{array}$ & $\begin{array}{l}.0021 \\
.0020 \\
.0019 \\
.0018 \\
.0017\end{array}$ \\
\hline $\begin{array}{l}27 \\
28 \\
29 \\
30 \\
31\end{array}$ & $\begin{array}{l}.0142 \\
.0126 \\
.0112 \\
.0100 \\
.0089\end{array}$ & $\begin{array}{l}.0013 \\
.0012 \\
.0011 \\
.0011 \\
.0010\end{array}$ & $\begin{array}{l}.0014 \\
.0013 \\
.0012 \\
.0012 \\
.0011\end{array}$ & $\begin{array}{l}.0015 \\
.0014 \\
.0013 \\
.0013 \\
.0012\end{array}$ & $\begin{array}{l}.0016 \\
.0015 \\
.0014 \\
.0014 \\
.0013\end{array}$ \\
\hline $\begin{array}{l}32 \\
33 \\
34 \\
35 \\
36\end{array}$ & $\begin{array}{l}.0079 \\
.0070 \\
.0063 \\
.0056 \\
.0050\end{array}$ & $\begin{array}{l}.0010 \\
.0009 \\
.0009 \\
.0008 \\
.0008\end{array}$ & $\begin{array}{l}.0011 \\
.0010 \\
.0010 \\
.0009 \\
.0009\end{array}$ & $\begin{array}{l}.0012 \\
.0011 \\
.0011 \\
.0010 \\
.0010\end{array}$ & $\begin{array}{l}.0013 \\
.0012 \\
.0012 \\
.0011 \\
.0011\end{array}$ \\
\hline
\end{tabular}

14. Metal may be furnished in coils or flat strips as required. Coils shall consist of not more than three lengths, no one of which shall be less than 10 feet long. When furnished in flat strips a variation of one-sixteenth inch over the specified length will be permitted on full-length sheets.

15. (a) When ordered in lengths over 10 to 12 feet:

40 per cent may be 10 feet and over.

30 per cent may be 8 to 10 feet.

20 per cent may be 6 to 8 feet.

10 per cent may be 4 to 6 feet. 
Not more than 40 per cent of any one shipment may be less than the length ordered.

(b) When ordered in 10-foot lengths:

40 per cent may be 8 feet and over.

30 per cent may be 6 to 8 feet.

20 per cent may be 4 to 6 feet.

10 per cent may be 2 to 4 feet.

Not more than 40 per cent of any one shipment may be less than 10 feet.

(c) When ordered in 8-foot lengths:

30 per cent may be 6 feet and over.

20 per cent may be 4 to 6 feet.

10 per cent may be 2 to 4 feet.

Not more than 30 per cent of any one shipment may be less than 8 feet.

(d) When ordered in 6-foot lengths:

20 per cent may be 4 feet and over.

10 per cent may be 2 to 4 feet.

Not more than 20 per cent of any one shipment may be less than 6 feet.

(e) When special lengths are required, they shall be specified in the order.

\section{WORKMANSHIP AND FINISH}

16. The brass shall be free from injurious external and internal defects of a nature which would interfere with the purpose for which it is intended. It shall be well cleaned and free drom dirt.

\section{INSPECTION AND REJECTION}

(Same as A. S. T. M. B19-29, given above.)

Note 1.-In the making of brass the best results are obtained if a minimum of one-third of previously alloyed material is used. It is also frequently desirable to employ previously alloyed materia composed of copper and zinc in proportions other than provided for in these specifications, sufficient copper and zinc being added as may be required to properly correct the composition.

\section{AMERICAN SOCIETY FOR TESTING MATE- RIALS, STANDARD SPECIFICATIONS FOR MUNTZ METAL CONDENSER TUBE PLATES, DESIGNATION B57-27, 1927}

\section{MUNTZ METAL CONDENSER TUBE PLATES}

\section{SCOPE}

1. These specifications cover rolled Muntz metal plates for surface condenser tube sheets.

\section{MANOFACTURE}

2. The plates shall be made by hot rolling from castings of suitable dimensions.

\section{CHEMTCAL PROPERTIES AND TESTS}

3. The plates shall conform to the following requirements as to chemical composition:

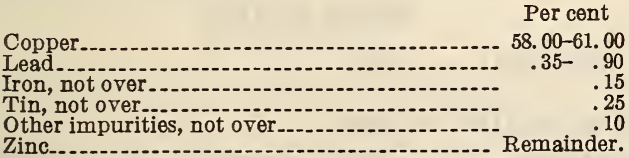

4. Analysis may be made on each lot of 5,000 pounds or less.

5. The sample shall be taken by drilling or milling representative plates in such a way that a sample of the entire thickness shall be obtained. Equal quantities from each plate shall be thoroughly mixed. Samples so prepared shall be divided into three equal parts, one for the seller, one for the purchaser, and one for an umpire, if necessary.

\section{PHYSICAL PROPERTIES AND TESTS}

6. The material shall conform to the following minimum requirements:

Tensile strength
Yield point

Elongation in 2 inches...

7. The bend-test specimen shall stand being bent cold through $120^{\circ}$ around a pin, the diameter of which is equal to twice the thickness of the specimen, without cracking on the outside of the bent portion.

8. Tension-test specimens may be 1 inch in width and of the full thickness of the plate as rolled, or may be machined to conform to the dimensions shown in Figure 129 in A. S. T. M. standard specifications for steel (see 605.0, p. 247), in which case the ends shall be of a form to fit the holders of the testing machine in such a way that the load shall be axial.

9 . Bend test specimens shall be 0.5 by 1 inch in section with corners rounded to a radius not over one-sixteenth inch. In the case of thin plates, the specimen may have the same thickness as the plate, with corners rounded to a radius equal to one-fourth of the thickness of the plate.

\section{PERMISSIBLE VARIATIONS IN DIMENSIONS}

10. The thickness shall not vary from that specified by more than plus 3 per cent or minus 2 per cent.

\section{WORKMANSHIP AND FINISH}

11. The plates shall be free from injurious defects and shall have a smooth clean surface. The fracture shall be uniform in color and grain throughout.

INSPECTION AND REJECTION

(Same as A. S. T. M. B19-29, given above.)

SOCIETY OF AUTOMOTIVE ENGINEERS, NONFERROUS METAL SPECIFICATIONS, SPECIFICATION NO. \%O, COMMERCIAL BRASS SHEET, FEBRUARY, 1929

\section{COMMERCIAL BRASS SHEET}

\section{Composition in percentage-}

Per cent

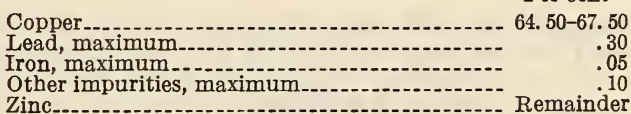

Other impurities, maximum

\section{MECHANICAL REQUIREMENTS}

2. Temper and anneal.--The temper of sheet brass shall be designated as follows:

Quarter hard.......
Half hard.
Hard
Extra hard \& S.
gage No.
Spring

3. The annealed sheet shall be designated as follows:

Light anneal

Drawing anneal

Soft drawing anneal 
4. The average Brinell hardness of 10 samples of sheet 0.080 inch or over in thickness shall be within the following limits:

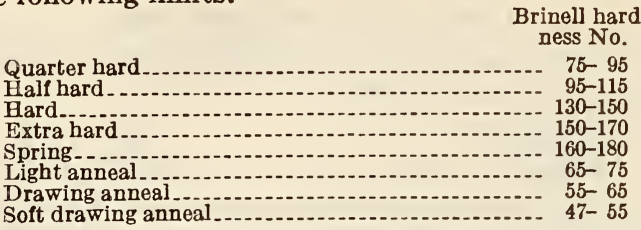

5. The average of tension tests of two samples of sheet thinner than 0.080 inch shall conform to the following minimum requirements:

\begin{tabular}{|c|c|c|}
\hline Temper & $\begin{array}{l}\text { Minimum } \\
\text { ultimate } \\
\text { strength }\end{array}$ & $\begin{array}{l}\text { Minimum } \\
\text { elongation } \\
\text { in } 2 \text { inches }\end{array}$ \\
\hline $\begin{array}{l}\text { Quarter hard } \\
\text { Half hard. } \\
\text { Hard. } \\
\text { Extra hard }\end{array}$ & $\begin{array}{c}\text { Lbs./in. }{ }^{2} \\
45,000 \\
52,500 \\
67,500 \\
80,000\end{array}$ & $\begin{array}{c}\text { Per cent } \\
27.5 \\
15.0 \\
5.0 \\
2.0\end{array}$ \\
\hline $\begin{array}{l}\text { Spring } \\
\text { Light anneal } \\
\text { Drawing anneal drawing anneal }\end{array}$ & $\begin{array}{l}87,500 \\
45,000 \\
42,000 \\
40,000\end{array}$ & $\begin{array}{r}1.0 \\
32.0 \\
38.0 \\
42.0\end{array}$ \\
\hline
\end{tabular}

In very thin strips, on account of the difficulties in testing, the elongation may be considerably less than the figures given above.

6. These should be considered as general specifications. Since high brass is used for many purposes where the requirements of the operations used are too particular to be specified by any of the ordinary physical tests, it is frequently advisable to submit samples or drawings to the manufacturer and secure an adjustment of anneal or temper to suit the actual operations to which the material is to be submitted.

7. The thickness tolerances of sheet or strip made of this alloy shall be within the tolerances specified in Table 5 under United States Government, F. S. B. QQ-C-501. (See 641.21, p. 1177.)

UNITED STATES GOVERNMENT, DEPARTMENT OF COMMERCE, BUREAU OF STANDARDS, STANDARD SAMPLES, CIRCULAR NO. 25 AND SUPPLEMENT, $192 \%$

\section{SHEET BRASS SAMPLE}

(Sample No. 37b is a standard sample of sheet brass prepared and sold by the Bureau of Standards for use by industrial organizations and others as a comparison standard for checking the accuracy of analysis of sheet brass. Each sample is accompanied by a certificate giving the complete analysis of that particular sample.)

UNITED STATES GOVERNMENT, DEPARTMENT OF COMMERCE, BUREAU OF STANDARDS, STANDARD THICKNESSES, WEIGHTS, AND TOLERANCES OF SHEET METAL (CUSTOMARY PRACTICE), CIRCULAR NO. 391, IMARCH 24, 1931

\section{AMERICAN WIRE GAGE BRASS PLATES AND SHEETS}

(See 640.1, p. 1165.)
UNITED STATES GOVERNMENT, FEDERAL SPECIFICATIONS BOARD, FEDERAI SPECIFICATION, FOR BRASS RODS, BARS, SHAPES, PLATES, SHEETS, AND STRIPS, C OMMERCIAL, QQ-B-611, MARCH 1, 1932

\section{BRASS PLATES, SHEETS, STRIPS}

(For general specifications, types and grades, material and workmanship, detail requirements, physical properties, tests and methods of inspection packing and marking, and notes, see 644.0, p. 1195. Otherwise the specifications for brass plates, sheets, and strips are identical with those for copper plates, sheets, and strips in F. S. B. QQ-C-501 (see 641.21, p. 1177) with the following exceptions:)

1. The permissible variations in thickness of sheets and strips (grade C, type 1) 0.460 inch or less thick and 20 inches or less wide shall be the same as for copper sheets and strips in Table 5. (See 641.21, p. 1178 .)

2. The permissible variations in thickness of plates, sheets, and strips (grade C, type 1) of all thicknesses and widths not covered in Table 5 (see 641.21, p. 1178) and of plates, sheets, and strips (grade C, type 2) of all thicknesses and widths shall be as shown in Table 6 (see 641.21, p. 1178), except that in no case shall the tolerances be less than that permitted for the same thickness given in Table 5 .

3. Sheets and strips in long lengths shall not exhibit sidewise bend or curvature in excess of 0.25 inch per foot.

4. Grade C, type 1, brass (plates, sheets, and strips) is a common high brass. It is malleable and suitable for a variety of drawing, forming, stamping, and bending operations. Grade C, type 2, is an ordinary sheet brass suitable for name plates, brass tags, and similar applications.

644.22 SHAPES, BRASS.

AMERICAN BUREAU OF SHIPPING, RULES FOR BUILDING AND CLASSING STEEL VESSELS; MATERIALS FOR MACHINERY, BOILERS, AND PIPING, 1930

BRASS PLATES AND SHAPES

(See 644.21, p. 1198.)

UNITED STATES GOVERNMENT, FEDERAL SPECIFICATIONS BOARD, FEDERAL SPECIFICATION, FOR BRASS RODS, BARS, SHAPES, PLATES, SHEETS, AND STRIPS, COMMERCIAL, QQ-B-611, MARCH 1, 1932

\section{BRASS SHAPES}

(See 645.11, p. 1205.)

644.23 STRIPS, BRASS.

SOCIETY OF AUTOMOTIVE ENGINEERS, NONFERROUS METAL SPECIFICATIONS, SPECIFICATION NO, 70, COMMERCIAL BRASS SHEET, FEBRUARY, 1929

\section{COMMERCIAI BRASS SHEET}

(See 644.21, p. 1201.) 
ONITED STATES GOVERNMENT, DEPARTMENT OF COMMERCE, BUREAU OF STANDARDS, STANDARD THICKNESSES, WEIGHTS, AND TOLERANCES, OF SHEET METAL (CUSTOMARY PRACTICE), CIRCULAR NO. 391, MARCH 24, 1931

\section{AMERICAN WIRE GAGE BRASS STRIPS}

(See 640.1, p. 1165.)

UNITED STATES GOVERNMENT, FEDERAL SPECIFICATIONS BOARD, FEDERAL SPECIFICATION, FOR BRASS RODS, BARS, SHAPES, PLATES, SHEETS, AND STRIPS, COMMERCIAL, QQ-B-611, MARCH 1, 1932

\section{BRASS STRIPS}

(See 644.21, p. 1202.)

\section{MANUFACTURES OF BRASS}

\subsection{BRASS RODS AND WIRES.}

645.11 RODS, BRASS.

AMERICAN RAILWAY ASSOCIATION, MECHANICAL DIVISION, SPECIFICATIONS FOR WELDING WIRE AND RODS, 1924

\section{WELDING WIRE AND RODS}

1. Brass welding rods for gas welding of miscellaneous brass and grey cast-iron parts.

2. Chemical composition.-Welding wire and rods shall conform to the following chemical composition:

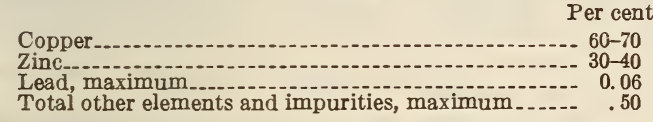

(For specification details, see 603.41 , p. 156.)

AMERICAN SOCIETY FOR TESTING MATERIALS, STANDARD SPECIFICATIONS FOR BRASS FORGING ROD, DESIGNATION B15-18, 1918

[Approved as American tentative standard (H7-1925) by the American Standards Association, June 8, 1925]

\section{BRASS FORGING ROD} SCOPE

1. These specifications cover brass rods of any specified cross section capable of being readily forged hot, and easily machined.

\section{MANUFACTURE}

2. The rods may be manufactured either by hot rolling or extrusion, and may be finished by cold drawing if necessary to meet the requirement for size.

\section{CHEMICAL ANALYSES}

3. Analyses may be made on each lot of 5,000 pounds or less. Drillings or millings shall be made from at least four separate rods, equal quantities being taken from each rod and thoroughly mixed. Samples so prepared shall be divided into three equal parts, each of which shall be placed in a sealed package, one for each party and one for an umpire, if necessary.
CHECK ANALYSES

4. (a) Analyses of separate rods may be made by the purchaser. The copper and lead contents thus determined shall be within the following limits:

Copper Per cont 57. 9-62.1

(b) In case of dissatisfaction the rod or rods shall be sampled in the presence of representatives of both parties. The thoroughly mixed sample shall be divided into three equal parts, each of which shall be placed in a sealed package, one for each party and one for an umpire, if necessary.

\section{PHYSICAL PROPERTIES AND TESTS}

5. (a) One flattening test shall be made from each lot of 2,000 pounds or fraction thereof.

(b) In case of failure of any test specimen representing a lot, two additional specimens shall be chosen each of which shall conform to the requirements as specified in paragraph 8 of F. S. B. QQ-B-611. (See 644.0, p. 1195.)

(With respect to chemical composition and flattening tests, this specification is identical with F. S. B. QQ-B-611. (See 644.0, p. 1195.) Permissible variations in dimensions and requirements as to stock lengths from 12 to 6 feet are the same as for copper rods, bars, and shapes in F. S. B. QQ-C-501. (See 642.11, p. 1180.) Workmanship and finish, inspection and rejection are identical with B57.' (See 644.21, p. 1201.))

NotE.-The material intended to be covered by these specifications exhibits a very considerable variation in properties with slight changes in composition. Its strength, stiffness, and ability to stand forging operations are in inverse proportion to the copper content; so also is the amount of lead which the alloy can contain without producing hot shortness. Toughness, on the other hand, is directly proportional to the copper content.

AMERICAN SOCIETY FOR TESTING MATERIALS, STANDARD SPECIFICATIONS FOR FREE-CUTTING BRASS ROD FOR USE IN SCREW MACHINES, DESIGNATION B1629, 1929

\section{FREE-CUTTING BRASS ROD} SCOPE

1. These specifications cover free-cutting brass rods of any specified cross section, suitable for highspeed screw machine work. (See note 1.)

\section{MANUFACTURE}

2. The rods shall be finished by cold drawing and straightening, unless otherwise specified.

\section{CHEMICAL PROPERTIES AND TESTS}

3. The brass shall conform to the following requirements as to chemical composition. (See note 2.)

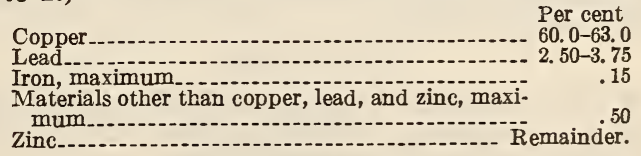

CHECK ANALYSES

4. In case of dissatisfaction, the rod or rods shall be sampled in the presence of representatives of both parties. The thoroughly mixed sample shall be divided into three equal parts, each of which shall be placed in a sealed package, one for the manufacturer, one for the purchaser, and one for an umpire, if necessary. 


\section{PHYSICAL PROPERTIES AND TESTS}

5. Rods or bars shall stand being bent cold through an angle of $120^{\circ}$ around a pin, the diameter of which is equal to twice the diameter of the round rods or four times the thickness of other shapes, without fracturing. (See note 3.)

6. (a) One bend test shall be made from each lot of 5,000 pounds or fraction thereof.

(b) In case of failure of any test specimen representing a lot, two additional specimens shall be chosen, each of which shall conform to the requirements specified in section 5 .

(With respect to chemical analyses, this specification is identical with B15, given above. Permissible variations and requirements as to stock lengths from 12 to 6 feet are the same as in F. S. B. QQ-C-501 (see 642.11, p. 1180) except that in this case for diameters over $2 \frac{1}{2}$ inches the permissible variation in diameter for rounds is \pm 0.15 , and for other shapes \pm 0.30 per cent. Workmanship and finish, inspection and rejection are identical with requirements in B57. (See 644.21, p. 1201.))

Notes.-1. The material intended to be covered by these specifcations is suitable for ordinary manufacturing purposes. In order to machine freely it must contain lead, and possess a considerable degree of temper which is obtained by cold working. The former requirement reduces strength and ductility while the latter adds firmness and stiffness.

2. There are four major constituents encountered in all brass alloys, the control and determination of which present very perplexing problems. They are copper, zinc, lead, and iron. The high volatility of zinc, the variations in temperature which occur in the alloying operation and the variation in the proportion in which it is added to the crucible charge in unalloyed form, causes its proportion in the casting to occasionally fluctuate over wide limits. Except when certain expensive grades of zinc are employed, substantially free from lead, the latter element will also show very considerable variation. Iron is subject to accidental introduction.

On account of the fact that the ordinary crucible charge is only about 200 pounds, it is not commercially practicable to determine the four main elements in each cast. On the other hand, the determination by chemical analysis of the constitutents of an individual sample from a lot of a given size tells little about the mean values of the proportions, or the extreme variations which exist in the lot. It is proposed, though not as a very satisfactory solution of this problem, to require that a sample composed of four equal portions from four individual pieces be taken to represent the mean com. position of a lot, and that individual samples be permitted to rary between wider limits. It is appleciated that this is not a satisfactory between wider limits. It is appleciated that this is not a sat

There are many uses for which alloys outside of the specified limits are perfectly suitable. The limits given, however, represent standard practice. On the other hand, there are special requirements which can not be most advantageously satisfied by alloys meeting these specifications.

3. It is desirable that a limit be imposed on the hardness due to cold drawing. It is felt that the bend test proposed accomplishes the purpose as satisfactorily as any other test.

\section{AMERICAN SOCIETY FOR TESTING IMATE- RIALS, STANDARD SPECIFICATIONS FOR NAVAL BRASS RODS FOR STRUCTURAL PURPOSES, DESIGNATION B21-29, 1929}

\section{NAVAL BRASS RODS}

SCOPE

1. These specifications cover naval brass rods of any uniform cross section, such as round, hexagonal, square, etc. (Note 1.)

\section{MANUFACTURE}

2. The rods shall be manufactured either by hot rolling or extrusion, finished by such cold drawing as may be required, and straightened.

\section{CHEMICAL PROPERTIES AND TESTS}

3. The brass shall conform to the following requirements as to chemical composition:

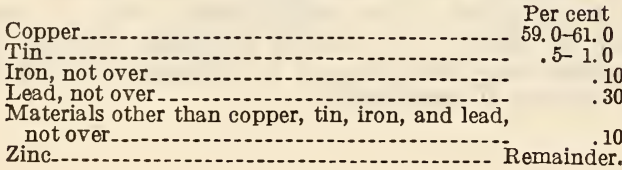

PHYSICAL PROPERTIES AND TESTS

4. (a) The rods shall conform to the following minimum requirements as to tensile properties:

\begin{tabular}{|c|c|c|c|}
\hline $\begin{array}{c}\text { Diameter, or distance between } \\
\text { parallel faces (in inches) }\end{array}$ & $\begin{array}{l}\text { Yield } \\
\text { point }\end{array}$ & $\begin{array}{l}\text { Tensile } \\
\text { strength }\end{array}$ & $\begin{array}{l}\text { Elonga- } \\
\text { tion in } 2 \\
\text { inches }\end{array}$ \\
\hline $\begin{array}{l}\text { Up to } 1 \text {, inclusive } \\
\text { Over } 1 \text { to } 21 / 2 \text {, inclusive } \\
\text { Over } 21 / 2 \text { to } 31 / 2 \text {, inclusive } \\
\text { Over } 31 / 2\end{array}$ & $\begin{array}{r}\text { Lbs./in. }{ }^{2} \\
31,000 \\
30,000 \\
25,000 \\
22,000\end{array}$ & $\begin{array}{r}\text { Lbs./in.2 } \\
62,000 \\
60,000 \\
56,000 \\
54,000\end{array}$ & $\begin{array}{r}\text { Per cent } \\
25.0 \\
30.0 \\
35.0 \\
40.0\end{array}$ \\
\hline
\end{tabular}

(b) Yield point in tension shall be determined as the stress producing an elongation under load of 0.5 per cent; that is, 0.01 inch in a gage length of 2 inches.

5. The test specimen shall stand being bent cold through $120^{\circ}$ to a radius equal to its diameter, without fracturing.

6. A 9-inch length of the test specimen shall stand without cracking an immersion for 15 minutes in an aqueous mercurous-nitrate solution containing $100 \mathrm{~g}$ of mercurous nitrate and $13 \mathrm{cc}$ of $\mathrm{HNO}_{3}$ (specific gravity 1.42) per liter of solution. (Note 2.)

7. (a) Tension test specimens shall be as nearly as possible of the same diameter, or distance between parallel faces, as the rods.

(b) Bend test specimens may be a piece of the full size rod, or a piece 1 inch in width by one-half inch in thickness. In the case of bend test specimens of rectangular section, the edges may be rounded off to a radius equal to one-fourth of the thickness.

(c) Strain test specimens shall be of the full size of the rod, at least 12 inches in length, and without bending, springing, polishing, or any other preparation.

8. (a) Rods shall be grouped into lots of not more than 5,000 pounds.

(b) One tension, one bend, and one strain test specimen shall be cut from one rod for each lot or fraction thereof.

(c) If any tension test specimen breaks outside the middle third of the gage length, a retest shall be allowed.

(With respect to chemical analyses, this specification is identical with B15, given above. Permissible variations and requirements as to stock lengths from 12 to 6 feet are the same as in F. S. B. QQ-C-501 (see 642.11, p. 1180) except that in this case for diameters over $2 \frac{1}{2}$ inches the permissible variation in diameter for rounds is \pm 0.15 , and for other shapes \pm 0.30 per cent. Workmanship and finish, inspection and rejection are identical with requirements in B57 (see 644.21, p. 1201).)

Notes.-1. Material intended to be covered by these specifications is suitable for structural purposes, and may be used as rods, bolts, etc. It is capable of being forg $=d$ hot, but is not free cutting.

2. Bars which have been properly stiaightened or sprung will have internal stresses so broken up as not to be in danger of splitting or cracking. The mercurous-nitrate test is designed to determin whether the internal stresses have been properly broken up and rendered safe.

\section{SOCIETY OF AUTOMOTIVE ENGINEERS, NONFERROUS METAL SPECIFICATIONS, SPECIFICATION NO. 88, BRASS ROD, JULY 1924}

\section{BRASS ROD}

(In respect to scope, manufacture, and analysis of separate rods this specification is identical with A. S. T. M. B15, given above. The flattening test is identical with paragraph 8 , and the chemical 
composition with grade A, in F. S. B. QQ-B-611 (see 644.0, p. 1195).

Dimensions and tolerances are identical with Table 3 in F. S. B. QQ-C-501 (see 642.11, p. 1180).)

\section{SOCIETY OF AUTOMOTIVE ENGINEERS, NONFERROUS METAL SPECIFICATIONS, SPECIFICATION NO. 72, FREE-CUTTING BRASS ROD, FEBRUARY 1929}

\section{FREE-CUTTING BRASS ROD}

\section{Composition in percentage.-}

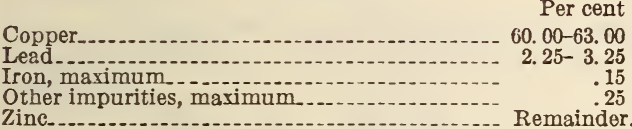

2. When several bars or rods are analyzed they should meet this specification. If only one rod or bar is sampled, the following limits will be acceptable:

Copper-10r cent
Lead

\section{MECHANICAL REQUIREMENTS}

3. Bend test.-Rods or bars shall bend cold without fracture through an angle of $120^{\circ}$ around a pin the radius of which is equal to the diameter or thickness of the rod or bar.

(Dimensions and tolerances are identical with those given in Table 2, F. S. B. QQ-C-501. See 642.11, p. 1180.)

SOCIETY OF AUTOMOTIVE ENGINEERS, NONFERROUS METAL SPECIFICATIONS, SPECIFICATION NO. 73, NAVAL BRASS (TOBIN BRONZE) ROD, JULY 1924

\section{NAVAL BRASS RODS}

1. Composition in percentage.-

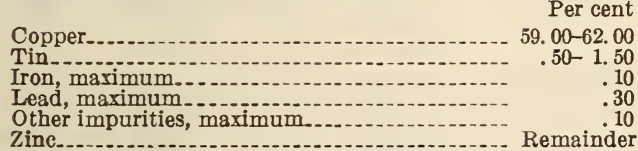

MECHANICAL REQUIREMENTS

2. Tensile test data.-

\begin{tabular}{|r|r|r|r|r|}
\hline \multicolumn{2}{|c|}{$\begin{array}{c}\text { Diameter or } \\
\text { thickness }\end{array}$} & $\begin{array}{c}\text { Minimum } \\
\text { tensile } \\
\text { strength }\end{array}$ & $\begin{array}{c}\text { Minimum } \\
\text { yield } \\
\text { point }\end{array}$ & $\begin{array}{c}\text { Minimum } \\
\text { elongation } \\
\text { in 2 inches }\end{array}$ \\
\cline { 1 - 2 } Over & $\begin{array}{c}\text { To and } \\
\text { including }\end{array}$ & & & \\
\cline { 1 - 2 } Inches & Inches & Lbs./in.2 & Lbs./in.2 & Per cent \\
0 & 1 & 62,000 & 31,000 & 25.0 \\
1 & $21 / 2$ & 60,000 & 30,000 & 30.0 \\
$21 / 2$ & $31 / 2$ & 56,000 & 25,000 & 35.0 \\
$31 / 2$ & $-\ldots 4,000$ & 22,000 & 40.0 \\
\hline
\end{tabular}

3. General information. - This material is intended for use wherever brass rod that is stronger, tougher, and less corrodible than commercial brass rod is required.

(Strain test requirements are the same as those for admiralty condenser tubes and ferrule stock in A. B. S. (See 645.24, p. 1219.) Bend test requirements are identical with those given above in specification No. 72. Dimensions and tolerances are identical with those given in Table 2, F. S. B. CQ-C-501. See 642.11, p. 1180.)
UNITED STATES GOVERNMENT, FEDERAL SPECIFICATIONS BOARD, FEDERAL SPECIFICATION FOR RODS, WELDING, NONFERROUS, FOR GAS WELDING QQ$\mathrm{R}-571$, MAY 3, 1932

\section{NAVAL BRASS RODS}

(For general specifications, types, material, general requirements, detail requirements, methods of inspection, tests, and chemical analysis, packing and marking of shipments, and notes, see 600.0 , p. 3.)

1. Naval brass rods may be used for general welding of brass.

2. Welding rods shall conform to the following chemical requirements:

\section{Type $B$}

\begin{tabular}{|c|c|}
\hline Copper....... & $59.0-62.0$ \\
\hline 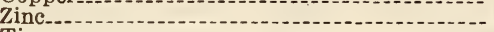 & Remainder. \\
\hline - & $0.5-1.5$ \\
\hline naximum & 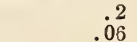 \\
\hline um, maximum & \\
\hline
\end{tabular}

3. Standard sizes of naval brass rods for gas welding are:

Diameter (inch)

UNITED STATES GOVERNMENT, FEDERAL SPECIFICATIONS BOARD, FEDERAL SPECIFICATION FOR BRASS RODS, BARS, SHAPES, PLATES, SHEETS, AND STRIPS, COMMERCIAL, QQ-B-611, MARCH 1, 1932

\section{BRASS RODS, BARS, SHAPES}

(For general specifications, types and grades, material and workmanship, detail requirements, physical properties, tests and methods of inspection, packing and marking, and notes, see 644.0, p. 1195. Otherwise the specifications for brass rods, bars, and shapes are identical with those for copper rods, bars, and shapes in F. S. B. QQ-C-501 (see 642.11, p. 1180) with the following exceptions:)

1. Free-cutting brass rods and bars (grade B, type 1), measured on their diameters or between parallel faces, shall not vary from the specified dimensions by more than the amounts shown for copper rods and bars in Table 2. (See 642.11, p. 1180.) Shapes (grade C, type 1) shall be measured on any cross-sectional dimension.

2. Forging brass rods and bars (grade A), freecutting brass rods and bars (grade $\mathrm{B}$, type 2), and shapes (grade C, type 2), measured on their diameters or between parallel faces, shall not vary from the specified dimensions by more than the amounts shown for copper rods and bars for forging in Table 3. (See 642.11, p. 1180.)

3. Grade A brass (rods, bars, and shapes) is easily forged hot, and machines well.

4. Grade B brass (rods, bars, and shapes) is suitable for use as trim and in machined parts where strength and incorrodibility are not required. It takes a good finish, especially the half hard, which is to be preferred where accuracy is essential. Type 1 should be ordered where physical requirements are important or close tolerances desired, but type 2 will be satisfactory for less exacting needs. Grade $\mathrm{B}$, type 1 , half hard, is also known as drill rod. 
645.12 WIRES, BRASS.

SOCIETY OF AUTOMOTIVE ENGINEERS, NONFERROUS METAL SPECIFICATIONS, SPECIFICATION NO. 80, BRASS WIRE, AUGUST, 1922

\section{BRASS WIRE}

1. Composition in percentage.-

\begin{tabular}{|c|c|c|}
\hline & Grade A & Grade B \\
\hline Cooper. & $70.00-74.00$ & $64.00-68.00$ \\
\hline Lead, maximum & .10 & .10 \\
\hline Iron, maximum & .06 & .07 \\
\hline Zinc. & Remainder. & Remainder. \\
\hline
\end{tabular}

2. Physical properties.-This wire shall have a tensile strength of at least 100,000 pounds per square inch, but should be capable of being bent through an angle of $180^{\circ}$ around a wire of the same diameter without breaking.

3. Appearance.-The wire shall be uniform in quality and temper, cylindrical in shape and smooth and free from injurious defects.

4. Dimensional tolerances.-The wire shall not vary from the specified diameter by more than plus or minus 1 per cent for sizes larger than 0.050 inch. From 0.050 to 0.025 inch the variation in size shall not be more than plus or minus 0.0005 inch. For sizes smaller than 0.025 inch the variation shall not be more than plus or minus 0.00025 inch.

5. General information.-Brass wire made to this specification is used for springs. Grade A is for use where the requirements are especially severe. Grade B is for use under ordinary conditions, and will be furnished unless otherwise specified.

\section{SOCIETY OF AUTOMOTIVE ENGINEERS, NONFERROUS METAL SPECIFICATIONS, SPECIFICATION NO. 82, BRASS WIRE, JUIY, 1924}

BRASS WIRE

(See 693.2, p. 1334.)

\section{UNITED STATES GOVERNMENT, FEDERAL SPECIFICATIONS BOARD, FEDERAL SPECIFICATION FOR WIRE, BRASS, QQ- W-321, JUNE 24, 1930}

\section{BRASS WIRE}

\section{A. APPLICABLE FEDERAL SPECIFICATIONS}

A-1. The following Federal specifications, or parts thereof that are below indicated, of the issue in effect on date of invitation for bids, shall form a part of this specification. Federal specification QQ-M-151. (F. S. B. No. 339a); general specifications for metals. (See 600.0, p. 3.)

\section{B. GRADES}

B-1. Brass wire shall be furnished in the following three grades, as specified: Grade A, annealed, soft; grade B, as drawn; and grade C, spring temper.

\section{MATERIAL AND WORKMANSHIP}

C-1. Brass wire shall be made from virgin metals, except scrap as may accumulate in the manufac- turer's plant from materials of the same composition, and of the manufacturer's own make, may be used.

\section{GENERAL REQUIREMENTS}

D-1. The wire shall be uniform in composition and quality, free from all injurious internal and surface defects, cracks, seams, laps, slivers, kinks, and scale. It shall be new, bright, smooth, and of uniform size.

D-2. Wire shall not vary from the specified diameter by more than the amounts shown in Table 1.

TABLE 1

\begin{tabular}{|c|c|}
\hline Diameter ofwire & $\begin{array}{c}\text { Permissible } \\
\text { variation }\end{array}$ \\
\hline $\begin{array}{c}\text { Inch } \\
0.0250-0.0375 \\
.0376-.050 \\
.0510-.080 \\
.0810-.10 \\
.0110-.150 \\
.1510-.18750 \\
.1880-.250\end{array}$ & $\begin{array}{l}\text { Inch } \\
\pm 0.00040 \\
\pm .00050 \\
\pm .00080 \\
\pm .0010 \\
\pm .0015 \\
\pm .0020 \\
\pm .0025\end{array}$ \\
\hline
\end{tabular}

\section{E. DETALL REQUIREMENTS}

E-1. The chemical composition of the wire shall be as covered by Table 2 .

TABLE 2

\begin{tabular}{|c|c|c|c|c|}
\hline Grades & Copper & \begin{tabular}{|c|} 
Lead, max- \\
imum
\end{tabular} & $\begin{array}{c}\text { Iron, max- } \\
\text { imum }\end{array}$ & Zinc, per cen \\
\hline $\mathrm{A}$ and $\mathrm{B}$ & $\begin{array}{r}\text { Per cent } \\
64-69 \\
69-74\end{array}$ & $\begin{array}{r}\text { Per cent } \\
0.150 \\
.075\end{array}$ & $\begin{array}{r}\text { Per cent } \\
0.06 \\
.06\end{array}$ & $\begin{array}{c}\text { Remainder. } \\
\text { Do. }\end{array}$ \\
\hline
\end{tabular}

E-2. The wire shall meet the physical requirements of Table 3.

TABLE 3

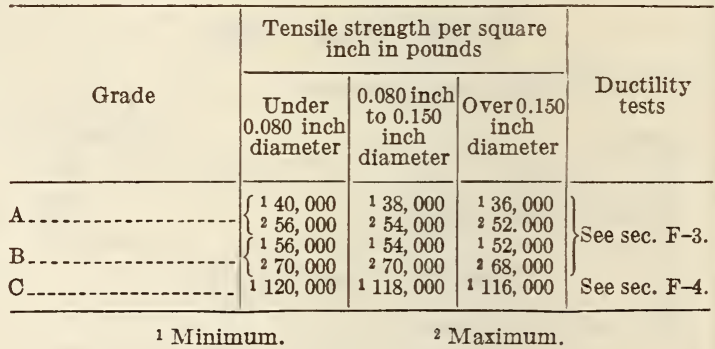

\section{F. METHODS OF SAMPLING, INSPECTION, AND TESTS}

F-1. One tension and one ductility test specimen shall be selected from each 500 pounds or fraction thereof.

F-2. One chemical analysis sample shall be taken to represent each 1,000 pounds or fraction thereof.

F-3. When required by the contract, the following test shall be included: Grades $A$ and $B$ wire shall be capable of being wrapped eight times around a rod having a diameter not more than five times the diameter of the wire. Upon release of the free end, the spring shall not be greater than that given in the following table:

\begin{tabular}{l|r|r}
\hline \multirow{2}{*}{ Diameter (inch) } & \multicolumn{2}{|c}{$\begin{array}{l}\text { Degree unwind of } \\
\text { free end }\end{array}$} \\
\cline { 2 - 4 } & Grade A & Grade B \\
\hline Wnder 0.080 & 30 & 35 \\
\hline Wire over 0.080 & 25 & 40 \\
\hline
\end{tabular}


F-4. Grades A, B, and C wire shall withstand being bent cold $360^{\circ}$ around a wire of the same diameter without showing cracks or fracture.

\section{G. PACKAGING, PACKING, AND MARKING}

G-1. Packaging.-Unless otherwise specified, the outside and inside diameters of the coils shall be optional with the manufacturer, and the brass wire shall be furnished in 1-pound spools, 5-pound coils, or 10-pound coils, as called for.

\section{NOTES}

I-1. The brass wire covered by this specification is intended for use only where commercial brass wire is applicable.
AMERICAN BUREAU OF SHIPPING, RULES FOR BUIIDING AND CLASSING STEEL VESSELS; PUMPS AND PIPING SYSTEMS, 1930

\section{BRASS CASTINGS}

(See 646.41, p. 1250.)

AMERICAN MARINE STANDARDS COMMITTEE, FIXED LIGHTS FOR SHIPS, H No. 4-1925

\section{TYPE B. CAST BRASS FRAME}

The frames of types $\mathrm{A}$ and $\mathrm{B}$ are interchangeable (for type A, see 619.9, p. 1086); they are for use in

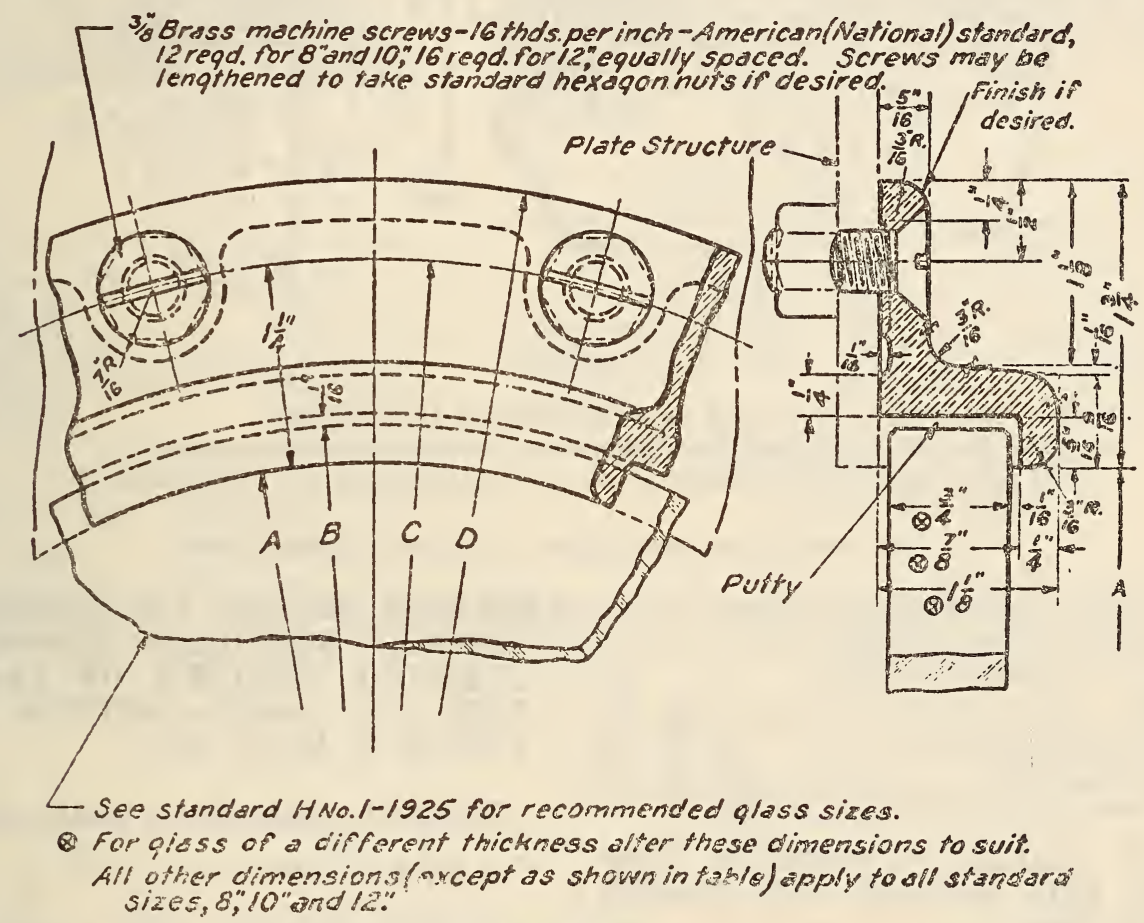

FIGURE 1083.-Fixed lights, type B, cast-brass frame

I-2. Grade A brass wire is used for beading, seizing, etc.

I-3. Grade B brass wire is used for hinge-pins, rivets, lamp guards, etc.

I-4. Grade C brass wire is used for springs, locking rings, etc.

645.2 BRASS CASTINGS, FERRULES, PIPES, AND TUBES.

645.21 CASTINGS, BRASS.

AMERICAN BUREAU OF SHIPPING, RULES FOR BUILDING AND CLASSING STEEL VESSELS; MATERIALS FOR MACHINERY, BOILERS, AND PIPING, 1930

\section{BRASS CASTINGS}

(See 646.41, p. 1250.) places where the ship's structure holds the glass on one side and generally where no bright metal finish is required around the glass, although type B, if polished, is suitable for lights where bright metal finish is wanted on one side. Type $\mathrm{C}$ is designed for independent or shop assembly and is intended especially for places where bright metal finish is desired around the glass on both sides. (For type C, see below.) The glass used in these lights is separately covered by standard $H$ No. $1-1925$ as to dimensions, and by United States specification DD-G-451 issued by the Federal Specifications Board as to quality.

\section{[Diameters in inches]}

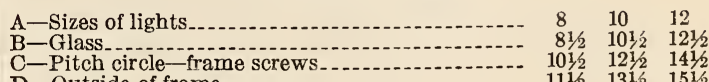


AMERICAN MARINE STANDARDS COMMITTEE, FIXED LIGHTS FOR SHIPS, H NO. 5,1925

TYPE C. CAST BRASS FINISHED FRAME

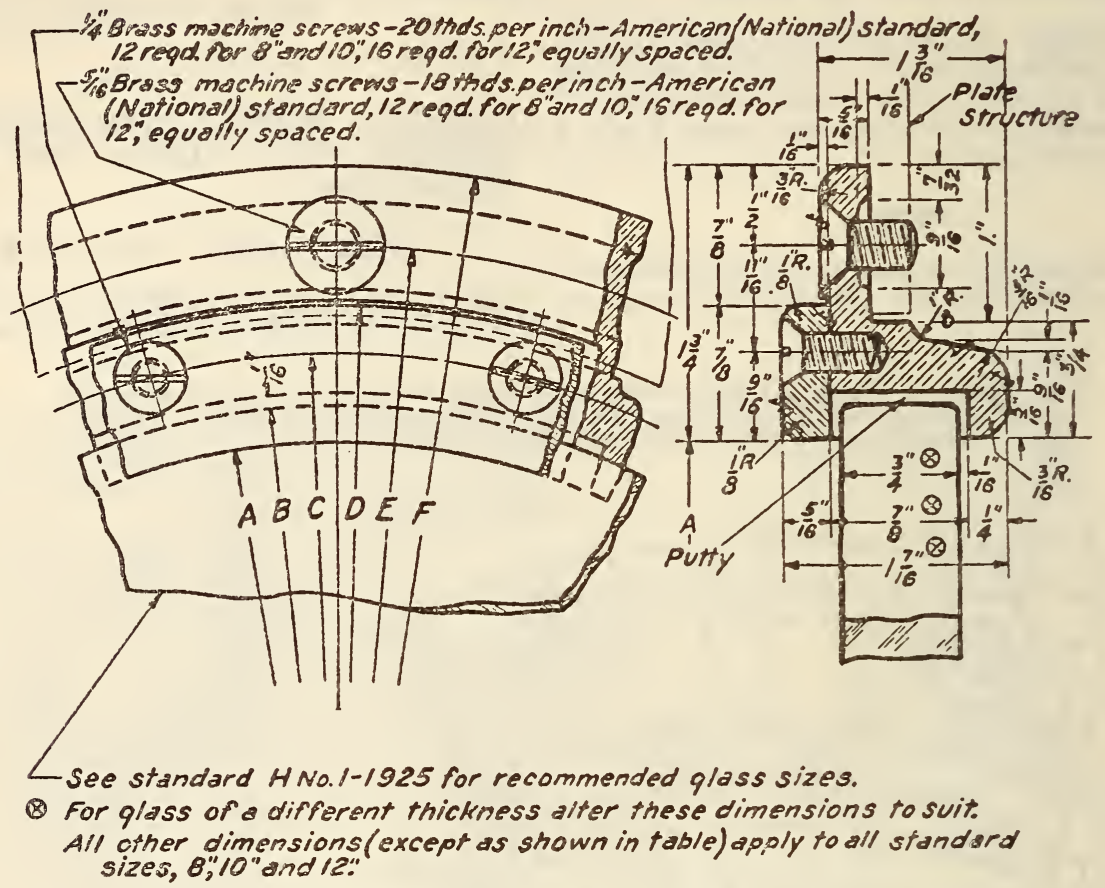

FIGURE 1084.-Fixed lighls, type $C$, cast-brass finished frame

(Requirements covering frame and glass are the same as given above for type B lights.)

[Diameters in inches]

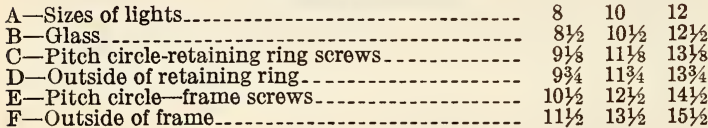

AMERICAN MARINE STANDARDS COMMITTEE, SHIP SCUPPER AND DRAINS, H NO. 38-1928

\section{DECK DRAIN STRAINER MATERIAL}

Brass.- No finished surfaces except as required for fitting. (See fig. 1085.)

\section{AMERICAN MARINE STANDARDS COM- MITTEE, SHIP SCUPPER AND DRAINS, H NO. 39-1928}

\section{DECK DRAIN STRAINER MATERIAL}

Brass.-Finish surfaces as required for fitting, also top when ordered. (See fig. 1086.)
AMERICAN SOCIETY FOR TESTING MATERIALS, STANDARD METHODS OF CHEMICAL ANALYSIS OF BRASS INGOTS AND SAND CASTINGS, DESIGNATION B $45-27,1927$

\section{CHEMICAL ANALYSIS OF BRASS INGOTS}

(See 644.0, p. 1193.)

AMERICAN SOCIETY FOR TESTING MATERIALS, STANDARD SPECIFICATIONS FOR COMPOSITION BRASS OR OUNCEMETAL SAND CASTINGS, DESIGNATION B62-28, 1928

\section{OUNCE METAI}

\section{SCOPE}

1. These specifications cover alloy castings, the alloy being a composition of copper, tin, lead, and zinc, known commercially as composition metal, 85-5-5-5, or ounce metal. Castings of this alloy are commonly used in hydrostatic pressures up to 350 pounds per square inch. 

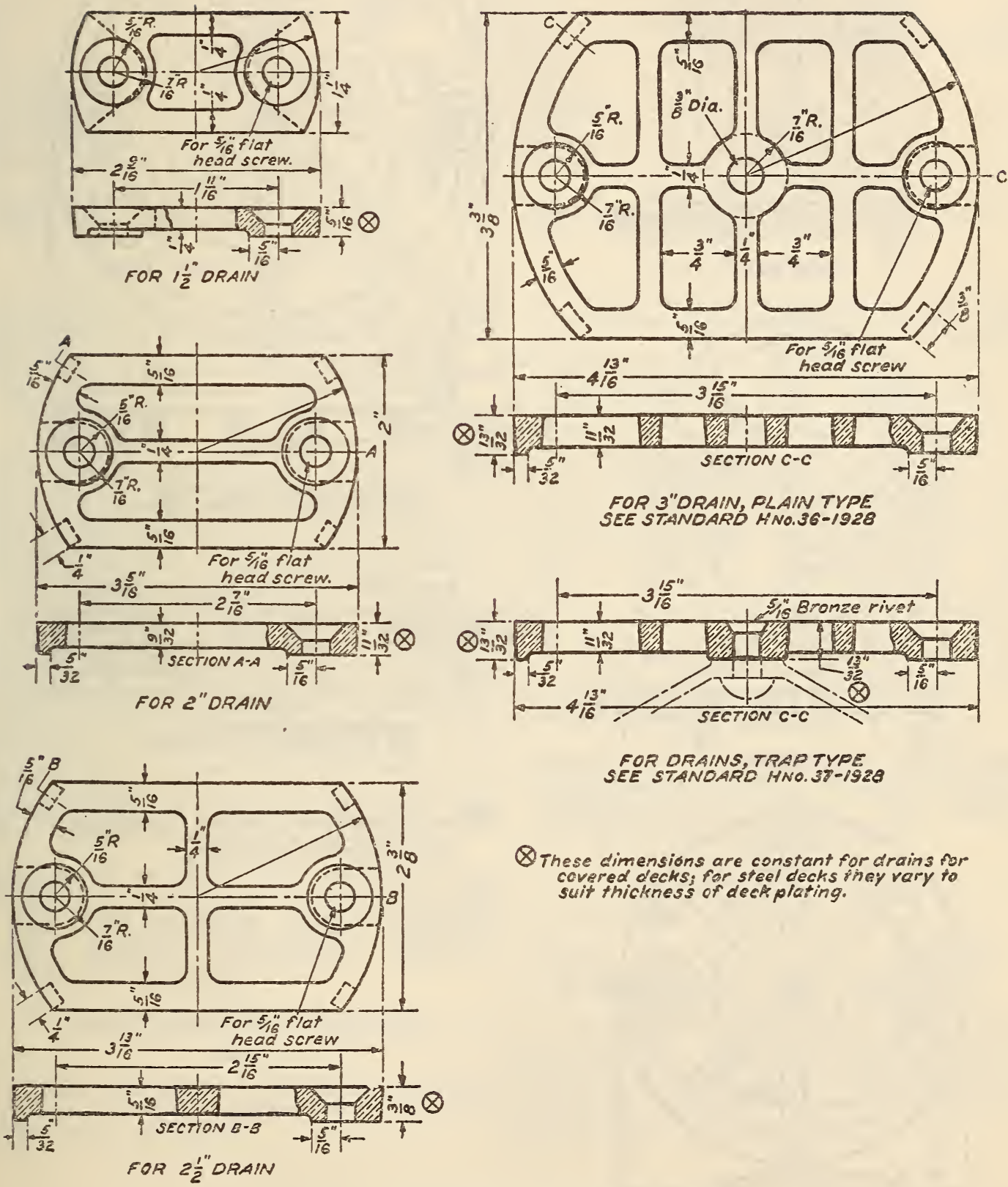

QThese dimensions are constant for drains por covered decks; for steel decks riney vary to suit thickness of deck plating.

FIGURE 1085.-Deck drain strainers, type $A$ 

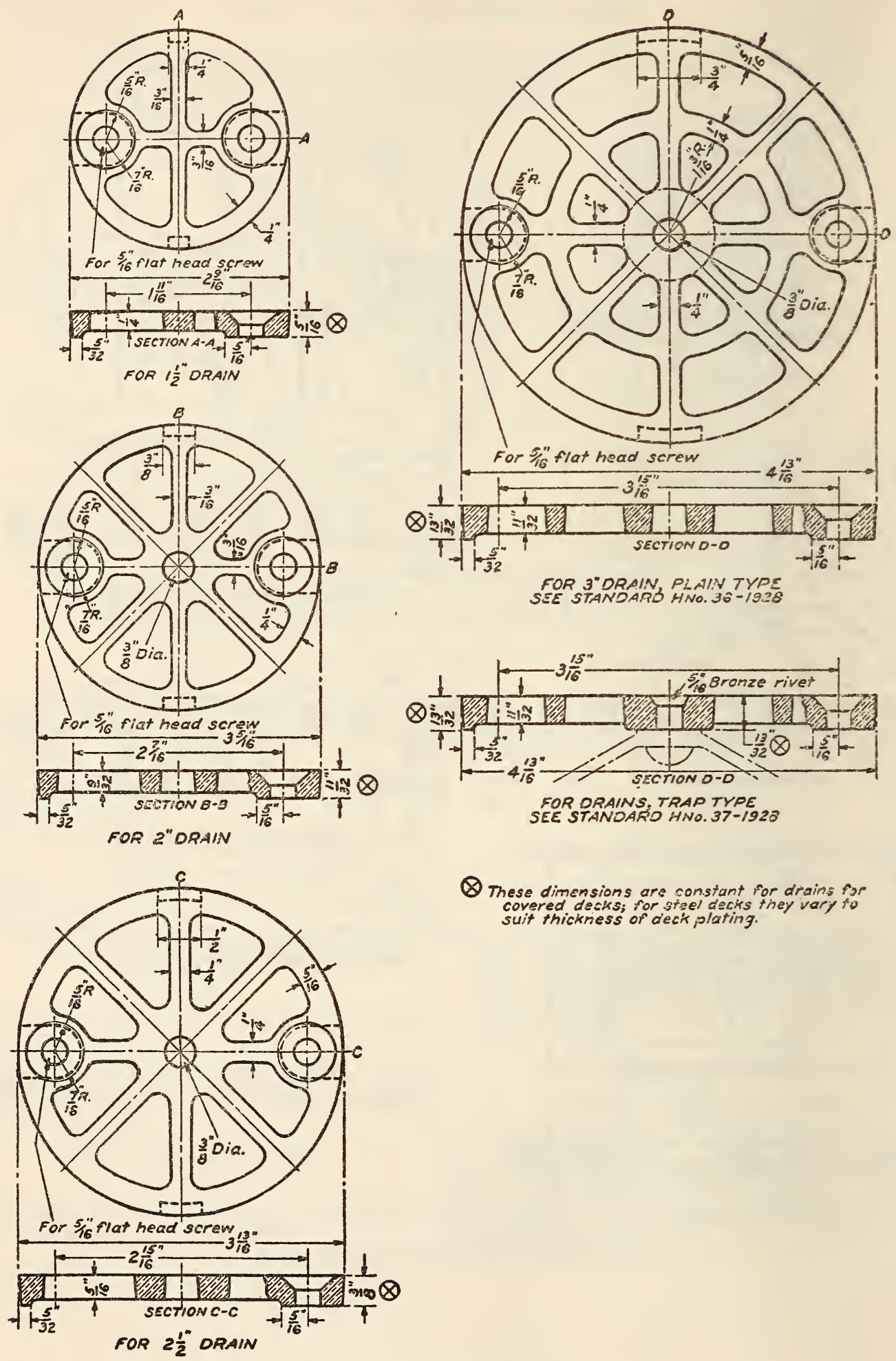

Q These dimensions are constant for drains for covered decks; for stiel decks they vary to suit thickness of deck plating.

FIgURE 1086.-Deck drain strainers, type $B$ 
CHEMICAL PROPERTIES AND TESTS

2. The alloy shall conform to the following requirements as to chemical composition:

\begin{tabular}{|c|c|c|c|}
\hline & Desired & Minimum & Maximum \\
\hline $\begin{array}{l}\text { Copper } \\
\text { Tin } \\
\text { Lead } \\
\text { Zinc } \\
\text { Iron } \\
\text { Nickel }\end{array}$ & $\begin{array}{r}\text { Per cent } \\
85.00 \\
5.00 \\
5.00 \\
5.00 \\
\text { None. }\end{array}$ & $\begin{array}{r}\text { Per cent } \\
84.00 \\
4.00 \\
4.00 \\
4.00 \\
\end{array}$ & $\begin{array}{r}\text { Per cent } \\
8600 \\
6.00 \\
6.00 \\
600 \\
25 \\
75\end{array}$ \\
\hline $\begin{array}{l}\text { Phosphorus } \\
\text { Aluminum } \\
\text { Sulphur } \\
\text { Antimony } \\
\text { Total other impurities..... }\end{array}$ & None. & - & $\begin{array}{r}.05 \\
\text { None. } \\
0.5 \\
25 \\
15\end{array}$ \\
\hline
\end{tabular}

Where "none" is specified it shall be construed to refer to none as determined on a $10 \mathrm{~g}$ sample.

\section{PEYSICAI PROPERTIES AND TESTS}

3. (a) Where desired by the purchaser and so specified in the contract or purchase order, the alloy shall conform to the following minimum requirements as to tensile properties:

Tensilestrength

Yield point

lbs./in.2-- 26, 000

Elongation in 2 inches

per cent- 12,000

(b) Where physical requirements are not specified in the contract or purchase order, a fracture of the test specimens shall be made and the fractured surface shall indicate the soundness and uniformity of the metal upon examination with the naked eye. If the test bars do not indicate a uniform soundness and quality of metal, the castings may be rejected.

(Specifications covering tension-test-specimen requirements are identical with those in paragraph 11 (c), A18. (See 611.51, p. 754.) In respect to chemical analyses, determination of yield point, workmanship and finish, marking, and inspection and rejection, this specification is identical with B59. (See 647.15, p.1259.) In respect to manufacture, pressure-test requirements, and dimensions of test-specimen coupon, it is identical with $\mathrm{B} 60$. (See 643.4, p. 1192.) Paragraphs $7(a),(b),(c)$, and $(d)$ in this specification, covering number of tests, are identical with Paragraphs VI $2,3,4$, and 13 in F.S. B. QQ-B-691. See 646.41, p. 1252.)

\section{AMERICAN SOCIETY FOR TESTING MA- TERIALS, STANDARD SPECIFICATIONS FOR YELLOW-BRASS SAND CASTINGS FOR GENERAL PURPOSES, DESIGNA- TION B65-28, 1928}

\section{YELLOW-BRASS SAND CASTINGS SCOPE}

1. These specifications cover castings made from one of the many yellow brasses commonly used for a wide variety of castings.

\section{CHEMICAL PROPERTIES AND TESTS}

2. The alloy shall conform to the following requirements as to chemical composition:

\begin{tabular}{|c|c|c|c|}
\hline & Desired & Minimum & Maximum \\
\hline $\begin{array}{l}\text { Copper } \\
\text { Tin } \\
\text { Lead } \\
\text { Zinc } \\
\text { Iron } \\
\text { Nickel }\end{array}$ & $\begin{array}{r}\text { Per cent } \\
63.5 \\
\text { None. } \\
2.5 \\
34.0 \\
\text { None. } \\
\text { None. }\end{array}$ & $\begin{array}{r}\text { Per cent } \\
62.0 \\
\text { None. } \\
1.5 \\
\text { Remainder. }\end{array}$ & $\begin{array}{r}\text { Per cent } \\
67.0 \\
1.0 \\
3.5 \\
\text { Remainder. } \\
0.75 \\
.25\end{array}$ \\
\hline $\begin{array}{l}\text { Phosphorus... } \\
\text { Aluminum } \\
\text { Sulphur. } \\
\text { Antimony. } \\
\text { Total other impurities. }\end{array}$ & $\begin{array}{l}\text { None. } \\
\text { None. } \\
\text { None. } \\
\text { None. } \\
\text { None. }\end{array}$ & 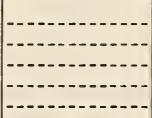 & $\begin{array}{l}.03 \\
.3 \\
.05 \\
.15 \\
.15\end{array}$ \\
\hline
\end{tabular}

Where "none" is specified it shall be construed to refer to none as determined on a $10 \mathrm{~g}$ sample.

\section{PHYSICAI PROPERTIES AND TESTS}

3. Where desired by the purchaser and so specified in the contract or purchase order, the alloy shall conform to the following minimum requirements as to tensile properties:

Tensile strength
Elongation in 2 inches.......... lbs./in. ${ }^{2}$-. 20, 000

(Specifications covering tension-test-specimen requirements are identical with those in paragraph 11 (c), A18. (See 611.51, p. 754.) In respect to chemical analyses, workmanship and finish, marking, and inspection and rejection, this specification is identical with B59. (See 647.15, p. 1259.) In respect to manufacture and dimensions of testspecimen coupon, it is identical with B60. (See 643.4, p. 1192.) In respect to fracture of test specimens when physical requirements are not specified it is identical with B62. (See 645.21, p. 1208.) Paragraphs $7(a),(b),(c)$, and $(d)$ in this specification, covering number of tests, are identical with Paragraphs VI 2, 3, 4, and 13 in F. S. B. QQ-B-691. (See 646.41, p. 1252.))

\section{SOCIETY OF AUTOMOTIVE ENGINEERS, NONFERROUS METAL SPECIFICATIONS, SPECIFICATION NO. 44, CAST BRASS TO BE BRAZED, MARCH, 1922}

\section{CAST BRASS TO BE BRAZED}

\section{Composition in percentage.-}

Copper Per cent 83. 00-86. 00 Zinc Lead, maximum

2. General information.-This brass starts to melt at approximately $1,830^{\circ} \mathrm{F}$., and is entirely melted at approximately $1,870^{\circ} \mathrm{F}$. As a brazing material on this brass to be brazed, either silver solder or a brazing brass melting at a temperature lower than than the brass to be brazed should be used. 
5. An analysis of each melt of castings shall be furnished by the contractor, showing the percentages of the elements designated.

6. The physical requirements for naval brass castings are:

\begin{tabular}{|c|c|}
$\begin{array}{c}\text { Tensile } \\
\text { strength } \\
\text { minimum }\end{array}$ & $\begin{array}{c}\text { Elongation } \\
\text { in 2 inches } \\
\text { minimum }\end{array}$ \\
\cline { 1 - 2 } $\begin{array}{c}\text { Lbs./in.2 } \\
30,000\end{array}$ & $\begin{array}{c}\text { Per cent } \\
15.0\end{array}$ \\
\hline
\end{tabular}

7. No physical requirements are specified for commercial brass castings, and no test coupons need be provided.

\section{TESTS AND METHODS OF INSPECTION}

8. Check chemical analyses may be made by the inspector, or through him by any Government laboratory or other designed representative, and without cost to the contractor.

9. Samples for check analyses shall be taken from tensile-test specimens or from a representative casting or gate selected by the inspector from each melt or lot. The drillings shall be taken from sound metal below the surface. The inspector shall forward for analysis not less than 5 ounces of drillings.

10. (The Brinell tests called for in QQ-B-691 are omitted from this specification.)

\section{PACKING AND MARKING}

Notes.-Naval brass may be used for corrosion-resisting castings, where strength or bearing requirements are unimportant, such as valves, handwheels, handrail fittings, chest hinges, etc.

Commercial brass may be used for castings that are not to be subected to water or air pressure, and where iron or steel is unsuitable because of corrodibility, such as name and number plates, instrument cases, oil cups, distribution boxes, trimmings, etc. Commercial brass will machine with greater facility than naval brass because of its higher lead content, but its physical properties are much lower.

UNITED STATES GOVERNMENT, FEDERAT SPECIFICATIONS BOARD; FEDERAL SPECIFICATION FOR BRASS CASTINGS TO BE BRAZED, QQ-B-601, MAY 31, 1932.

\section{BRASS CASTINGS TO BE BRAZED}

(In respect to general requirements, and packing and marking of shipments, this specification is identical with F. S. B. QQ-B-691. (See 646.41, p. 1252.) Otherwise its provisions are as follows:)

\section{GRADES}

1. Brass castings to be brazed shall be furnished in two grades: Grade A and grade B.

\section{MATERIAL}

2. The castings shall be made only from the best grades of virgin metals, or from virgin metals and scrap of known and approved composition.

\section{DETAIL REQUIREMENTS}

3. Castings shall conform to the chemical requirements shown below:

\begin{tabular}{|c|c|c|c|c|c|}
\hline Composition & Copper & Zinc & $\begin{array}{l}\text { Lead } \\
\text { (maxi- } \\
\text { mum) }\end{array}$ & $\begin{array}{l}\text { Iron } \\
\text { (maxi- } \\
\text { mum) }\end{array}$ & $\begin{array}{l}\text { Other } \\
\text { impuri- } \\
\text { ties } \\
\text { (maxi- } \\
\text { mum) }\end{array}$ \\
\hline $\begin{array}{l}\text { Desired } \\
\text { Permissible: }\end{array}$ & $\begin{array}{r}\text { Per cent } \\
85.0\end{array}$ & $\begin{array}{r}\text { Per cent } \\
15.0\end{array}$ & $\left|\begin{array}{c}\text { Per cent } \\
0\end{array}\right|$ & $\begin{array}{c}\text { Per cent } \\
0\end{array}$ & $\begin{array}{c}\text { Per cent } \\
0\end{array}$ \\
\hline $\begin{array}{l}\text { Grade A } \\
\text { Grade } \mathrm{B} .\end{array}$ & $\begin{array}{l}83.0-86.0 \\
83.0-86.0\end{array}$ & $\begin{array}{l}\text { Remainder. } \\
\text { Remainder. }\end{array}$ & $\begin{array}{r}0.3 \\
.5\end{array}$ & $\begin{array}{r}0.06 \\
.15\end{array}$ & $\begin{array}{r}0.04 \\
.10\end{array}$ \\
\hline
\end{tabular}

4. An analysis of each melt of castings shall be furnished by the contractor, showing the percentages of the elements designated.

5. Castings shall conform to such physical requirements as may be specified in the contract or purchase order.

6. Fracture tests may be prescribed by the department or bureau concerned.

\section{METHODS OF INSPECTION AND TESTS}

7. Check chemical analysis may be made by the inspector, or through him by any Government laboratory or other designated representative, and without cost to the contractor.

8. Samples for check analyses shall be taken from a representative casting selected by the inspector from each melt or lot. The drillings shall be taken from sound metal below the surface. The inspector shall forward for analysis not less than 5 ounces of drillings.

9. Where castings are of such form and dimensions as to make calipering of their walls impracticable, and the inspector so requests, one-quarter inch holes shall be drilled through at doubtful places to establish the exact thickness. Before loading for shipment, these holes shall be tapped out and filled by screwing in suitable brass plugs, or by welding, wherever such method is approved by the inspector. Such plugs shall carry the inspector's stamp.

Notes.-This brass starts to melt at approximately $1,830^{\circ} \mathrm{F}$. and is entirely melted at $1,870^{\circ} \mathrm{F}$.

As brazing material on this brass, either silver solder or a lower melting higher-zinc brass should be used.

645.22 FERRULES, BRASS.

\section{AMERICAN MARINE STANDARDS COM- MITTEE, E NO. 1-1931}

\section{CONDENSER-TUBE FERRULES}

\section{GENERAL NOTES}

1. Scope.-The ferrules and tube sheet stuffing boxes shown by this standard apply especially to condensers in which soft fibrous packing is used. They are usable with hard fiber and metallic packings also, but as these packings require but little compression in setting them and none after they are set, no reserve thread is, therefore, needed, and the length of ferrules for permanent use with such packings may be shortened by one-eighth inch in the thread, or from three-fourths inch to five-eighths inch over all. All of the other features of the shortened ferrules and the tube lengths would be in accordance with the standard. The length of thread in the stuffing box would be correspondingly shortened. Hard fiber or metallic packings should be ordered to suit the stuffing box and the length of ferrule to be used.

2. Types.-The standard comprises three types of ferrules, the details of which are shown in Figure 1087.

Type 1 is for application especially at the inlet end of tubes packed at both ends. It may also be used in lieu of type 2 at the outlet end of such tubes, but it is not advocated for that purpose except in an emergency.

Type 2 is companion to type 1 and is for application exclusively at the outlet end of the same tubes.

Type 3 is for application at the outlet end of tubes which are rigidly secured to the tube sheet at the inlet end by expansion or rolling.

Typical applications of all three types with soft packings appear herewith, which shows the approximate positions of the ferrules when the tubes are newly packed and when the packing is compressed to the limit and the ferrules are fully engaged. 
3. Materials.-High character of material for condenser-tube ferrules is of the utmost importance for reliable service and economical results. The American Marine Standards Committee contemplates the issuance of standard specifications for condenser tubes and ferrule stock, in which present
Tubes; condenser and ferrule admiralty /stock metal specification No. 374 (WW-T-781), issued by the Federal Specifications Board. (See 645.24, p. 1222.)

4. Packings.-The packings for condenser tubes must be of such character as to be unaffected by any
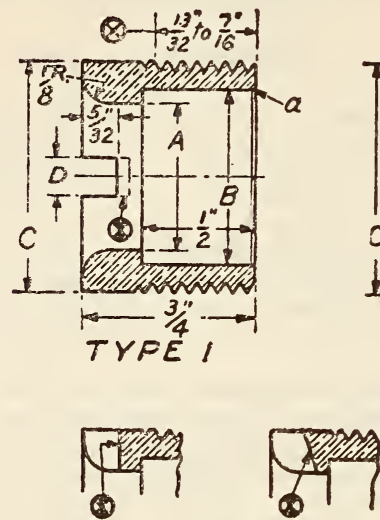

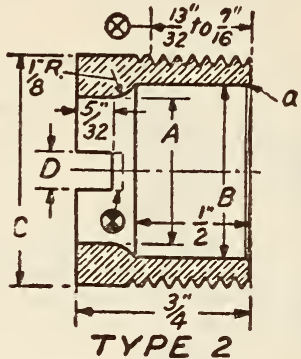

a-slight chamfer.

- Slots may be cut with a flat or concave bottom provided that the latter. leaves a thickness of at least $1 / 32$ between the slot and the shouider of types 1 and 2.

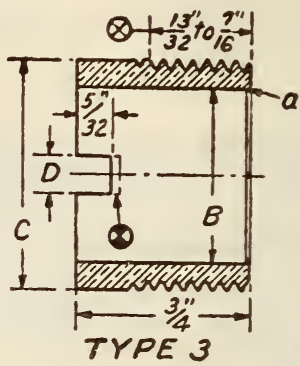

FERRULE DETAILS

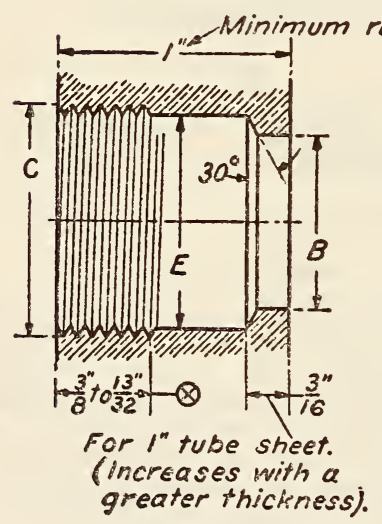

\section{$T$}

\section{TUBE SHEET}
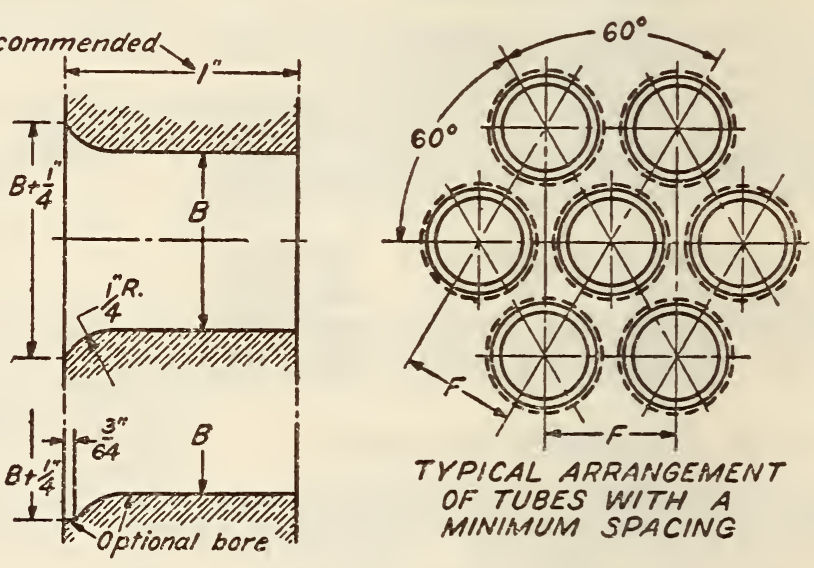

TYPICAL ARRANGEMENT

OF TUBES WITH A MINIAUM SPACING

FIGURE 1087

divergent requirements are to be reconciled. Pending issuance of such specifications, the use of either of the following is recommended as basis for purchasing these materials:

Seamless admiralty condenser tubes and ferrule stock, specifications B 44, promulgated by the American Society for Testing Materials. (See 645.22 , given below.) extremes or changes of temperature in the condenser. Soft packing may be of any suitable fibrous material, such as corset lacing, susceptible to moderate compression. Hard fiber packing may be of any appropriate composition, molded or cut to the requisite form and size. For metallic packing and its application, see American Marine Standard E No. 201928, published in A. M. S. C. 41. 

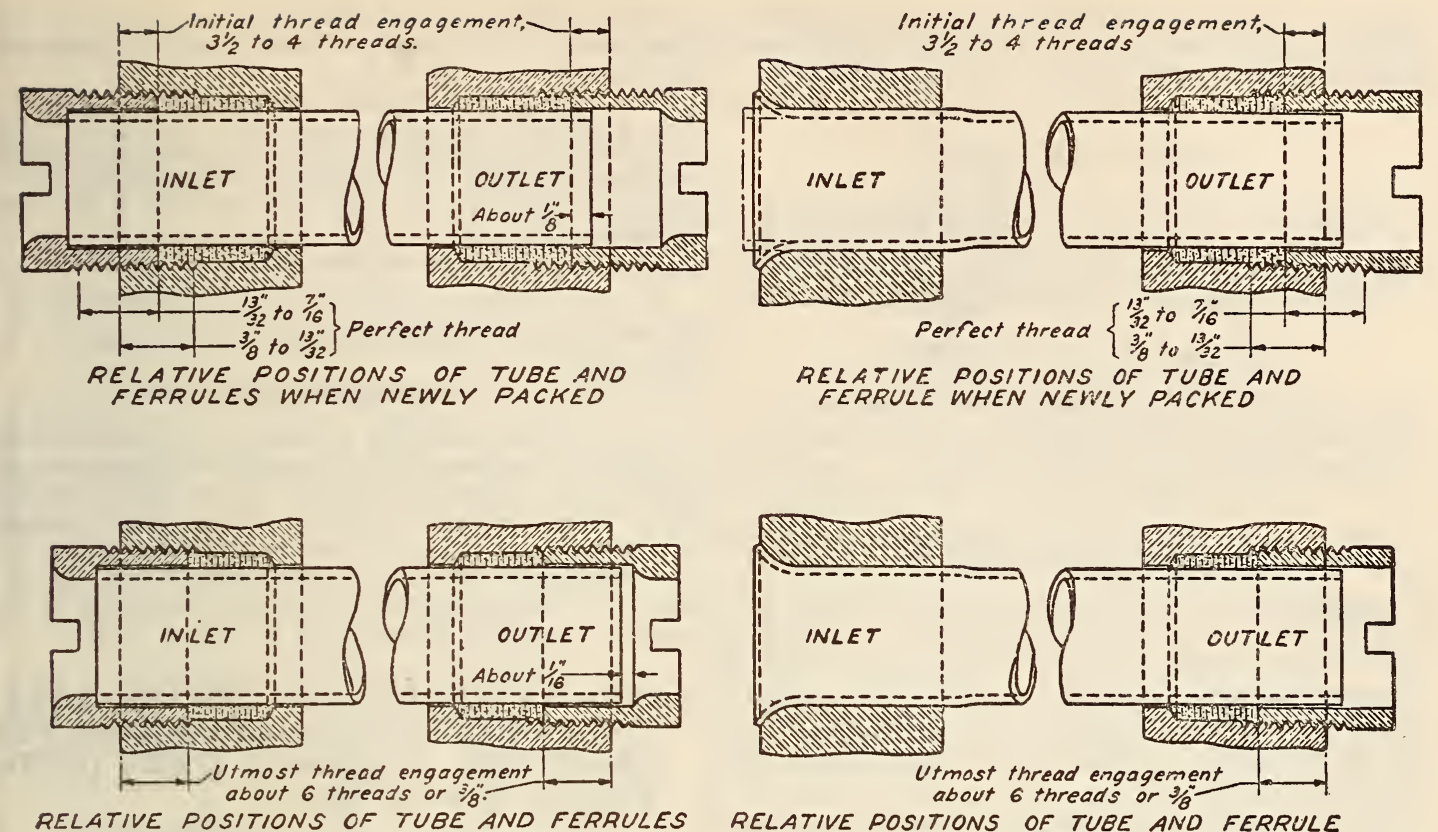

RELATIVE POSITIONS OF TUBE AND FERRULES

RELATIVE POSITIONS OF TUBE AND FERRULE
AT MAXIMUM COMPRESSION OF PACKING

FIGURE 1088.-Types 1 and 2, tube packed at both ends; type 3, tube expanded at one end. Typical applications of ferrules

[Dimensions given in inches]

\begin{tabular}{|c|c|c|c|c|c|c|c|c|c|c|c|c|c|}
\hline & $\begin{array}{l}\text { Size, out- } \\
\text { side } \\
\text { diameter }\end{array}$ & \multicolumn{3}{|c|}{$5 / 8$} & \multicolumn{3}{|c|}{$3 / 4$} & \multicolumn{3}{|c|}{78} & \multicolumn{3}{|c|}{1} \\
\hline & $\begin{array}{l}\text { Thickness, } \\
\text { B. w. g. }\end{array}$ & $\begin{array}{l}\text { No. } 18 \\
0.049\end{array}$ & $\begin{array}{l}\text { No. } 17 \\
0.058\end{array}$ & $\begin{array}{l}\text { No. } 16 \\
0.065\end{array}$ & $\begin{array}{l}\text { No. } 18 \\
0.049\end{array}$ & $\begin{array}{l}\text { No. } 17 \\
0.058\end{array}$ & $\begin{array}{l}\text { No. } 16 \\
0.065\end{array}$ & $\begin{array}{l}\text { No. } 18 \\
0.049\end{array}$ & $\begin{array}{l}\text { No. } 17 \\
0.058\end{array}$ & $\begin{array}{l}\text { No. } 16 \\
0.065\end{array}$ & $\begin{array}{l}\text { No. } 18 \\
0.049\end{array}$ & $\begin{array}{l}\text { No. } 17 \\
0.058\end{array}$ & $\begin{array}{l}\text { No. } 16 \\
0.065\end{array}$ \\
\hline & $\begin{array}{l}\text { Inside } \\
\text { diameter }\end{array}$ & 0.527 & 0.509 & 0.495 & 0.652 & 0.634 & 0.620 & 0.777 & 0.759 & 0.745 & 0.902 & 0.884 & 0.870 \\
\hline $\begin{array}{l}\text { Ferrules and tube } \\
\text { sheets }\end{array}$ & $\begin{array}{l}\mathrm{A} \\
\mathrm{B} \\
\mathrm{C} \\
\mathrm{D} \\
\mathrm{E} \\
\mathrm{E}\end{array}$ & & $\begin{array}{r}0 . \\
0.875\end{array}$ & $\begin{array}{r}509 \\
.635 \\
(7 / 8) \\
5 / 32 \\
.796 \\
1\end{array}$ & & & $\begin{array}{l}634 \\
762 \\
000 \\
5 / 32 \\
921 \\
11 / 8\end{array}$ & & $\begin{array}{r}0 . \\
1.125(1 \\
1 .\end{array}$ & $\begin{array}{l}759 \\
889 \\
118) \\
3 / 16 \\
046 \\
5 / 16\end{array}$ & & 1. 250 & $\begin{array}{r}884 \\
.016 \\
(11 / 4) \\
3 / 16 \\
171 \\
17 / 16\end{array}$ \\
\hline
\end{tabular}

Pitch diameter of thread:

Ferrules shall be gaged at a distance $3 / 8$-inch from end.

Tolerance: Undersize 0.002 inch; no oversize.

Tube sheet shall be gaged at the face.

Tolerance: Oversize 0.002 inch; no undersize.

Subject to these requirements, the threads may taper up to 0.004 inch per inch.

Q Length of perfect thread: 16 per inch, American (national) standard form.

Diameters A, B, C, and E may be plus 0.005 inch, no undersize; eccentricity shall not exceed 0.002 inch.

Tube length for all types equals distance from face to face of tube sheet plus $3 / 16$ inch, subject only to a tolerance of $1 / 32$ inch, plus or minus.

(An appendix to this specification covers general requirements for effective condenser-tube packing, and the details of an experiment conducted by the United States Shipping Board with an installation of condenser tubes without ferrules on the steamship Bakersfield. The results of this experiment will be found in Vol. XLII, No. 4, November, 1930, of the American Society of Naval Engineers.)

\section{SOCIETY OF AUTOMOTIVE ENGINEERS, ELECTRICAL EQUIPMENT, FLEXIBLE- CONDUIT FERRULES, S. A. E. STAND- ARD, AUGUST, 1928}

\section{FLEXIBLE-CONDUIT FERRULES}

1. Material.-Soft sheet brass, S. A. E. specification No. 70 (see 644.21 , p. 1201) or S. A. E. No. 1010 , dead-soft sheet steel. 
2. All steel ferrules must be zinc or cadmium plated to withstand salt-spray test for 24 hours without showing rust.
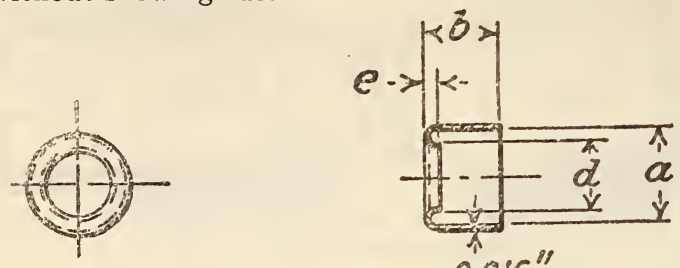

$0.016^{\prime \prime}$

Figdre 1089.-Flexible conduit ferrules

\begin{tabular}{|c|c|c|c|c|c|c|c|c|}
\hline & Conduit diameter..- & $3 / 16$ & \multicolumn{2}{|c|}{$1 / 4$} & $5 / 16$ & $3 / 8$ & $7 / 16$ & $1 / 2$ \\
\hline & $\begin{array}{l}\text { Inside diameter ..... } \\
\text { Length }\end{array}$ & $\underset{1 / 4}{0.285}$ & \multicolumn{2}{|c|}{$\begin{array}{c}0.355 \\
1 / 4\end{array}$} & $\begin{array}{c}0.425 \\
144\end{array}$ & $\begin{array}{c}0.485 \\
3 / 8\end{array}$ & $\begin{array}{l}0.555 \\
3 / 8\end{array}$ & $\begin{array}{c}0.630 \\
3 / 8\end{array}$ \\
\hline$d$ & $\begin{array}{l}\text { Shoulder diameter.-- } \\
\text { Shoulder........ }\end{array}$ & $\begin{array}{c}0.188 \\
1 / 16\end{array}$ & \multicolumn{2}{|c|}{$\begin{array}{l}0.250 \\
1 / 16\end{array}$} & $\begin{array}{c}0.313 \\
1 / 16\end{array}$ & $\begin{array}{c}0.375 \\
1 / 16\end{array}$ & $\begin{array}{c}0.438 \\
116\end{array}$ & $\begin{array}{l}0.500 \\
1 / 16\end{array}$ \\
\hline & Conduit diameter. & \multicolumn{2}{|c|}{$5 / 8$} & \multicolumn{2}{|c|}{$3 / 4$} & $7 / 8$ & 1 & 1144 \\
\hline 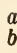 & $\begin{array}{l}\text { Inside diameter.-. } \\
\text { Length }\end{array}$ & \multicolumn{2}{|c|}{$0_{1 / 2}^{0.760}$} & \multicolumn{2}{|c|}{$\begin{array}{c}0.930 \\
1 / 2\end{array}$} & $\begin{array}{l}1.040 \\
1 / 2\end{array}$ & 1.175 & $\begin{array}{l}1.435 \\
5 / 8\end{array}$ \\
\hline $\begin{array}{l}d \\
e\end{array}$ & $\begin{array}{l}\text { Shoulder diameter } \\
\text { Shoulder }\end{array}$ & \multicolumn{2}{|c|}{$\begin{array}{c}0.625 \\
3 / 32\end{array}$} & \multicolumn{2}{|c|}{$\begin{array}{l}0.750 \\
3 / 32\end{array}$} & $\begin{array}{l}0.875 \\
3 / 32\end{array}$ & $\begin{array}{c}1.000 \\
3 / 8\end{array}$ & ${ }_{1.8}^{1.250}$ \\
\hline
\end{tabular}

645.23 PIPES, BRASS.

AMERICAN BUREAU OF SHIPPING, RULES FOR BUILDING AND CLASSING STEEL VESSELS; PUMPS AND PIPING SYSTEMS, 1930

\section{BRASS PIPES}

(See 642.23, p. 1182.)

\section{AMERICAN BUREAU OF SHIPPING, RULES FOR BUILDING AND CLASSING STEEL VESSELS; MATERIALS FOR MACHIN- ERY, BOILERS, AND PIPING, 1930}

\section{BRASS PIPES AND TUBES}

(For general information and requirements see 600.0 , p. 1. For test requirements see 600.1, p. 7.)

1. Seamless-drawn brass pipes and tubes subject to a working pressure of over 150 pounds are to be tested and inspected by the surveyor in accordance with the following requirements: The material used must be of the best quality; the tubes must be true to form, of uniform thickness throughout, clean and smooth, and free from defects.

2. Test specimens are to be selected by the surveyor, and at least one set of tests is to be made for each lot of 2,000 pounds or less. The test specimens are to be annealed. All tubes are to be at least semiannealed if full annealing is not required by the purchaser.

\section{TENSILE TEST}

3. A standard tension-test piece must show the material to have a minimum tensile strength of 40,000 pounds per square inch, and a minimum elongation of 15 per cent.

\section{FLATTENING TEST}

4. A section of pipe 4 inches long must stand flattening until the sides are brought parallel to a distance between the parallel sides of twice the thickness of the metal without showing cracks or flaws.

\section{BENDING TEST}

5. For fully annealed pipes a test tube shall stand bending through $180^{\circ}$ around a diameter of one and one-half times the inside diameter of the pipe or tube.

\section{EXPANDING TEST}

6. A test specimen 6 inches long must stand an expansion of three-eighths inch without cracking or splitting.

\section{HYDROSTATIC TEST}

7. All tubes are to be subjected to a hrdrostatic pressure of not less than twice the working pressure for working pressures up to 1,000 pounds per square inch. For working pressures above 1,000 pounds per square inch the test pressure is to be equal to the working pressure plus 1,000 pounds per square inch. (The gaging and marking are the same as for copper pipes and tubes. See 642.23, p. 1182.)

\section{AMERICAN SOCIETY FOR TESTING MATE- RIALS, STANDARD SPECIFICATIONS FOR BRASS PIPE, STANDARD SIZES, DESIG- NATION B43-24, 1924}

\section{BRASS PIPE, STANDARD SIZES SCOPE}

1. These specifications cover seamless brass pipe suitable for use in plumbing, boiler-feed lines, etc.

\section{MANUFACTURE}

2. The pipe shall be cold-drawn to size.

3. (a) The pipe shall be annealed sufficiently to enable it to pass the physical tests herein specified.

(b) All pipe shall be annealed sufficiently to prevent cracking. Semiannealed pipe, which is suitable for ordinary uses, shall be furnished unless otherwise specified.

(c) When pipe is required for bending, the purchaser shall so specify, in which case the pipe shall be furnished fully annealed.

\section{CHEMICAL PROPERTIES AND TESTS}

4. The brass shall conform to the following requirements as to chemical composition:

\begin{tabular}{|c|c|c|}
\hline & $\begin{array}{l}\text { Hot pierced } \\
\text { process }\end{array}$ & Cold process \\
\hline $\begin{array}{l}\text { Lopper maximum } \\
\text { Iron, maximum }\end{array}$ & $\begin{array}{r}\text { Per cent } \\
59.00-63.00 \\
0.50 \\
.07 \\
\text { Remainder. }\end{array}$ & $\begin{array}{r}\text { Per cent } \\
65.00-68.00 \\
0.80 \\
.07 \\
\text { Remainder. }\end{array}$ \\
\hline
\end{tabular}

(Sampling for chemical analysis is identical with that for copper pipe in A. S. T. M., B42. See 642.23, p. 1182.)

\section{PHYSICAL PROPERTIES AND TESTS}

(The hammering test is the same as that for condenser tubes and ferrule stock in A. B. S. (see 645.24 , p. 1219); also the strain test in an aqueous mercurous nitrate solution, except that in this specification the strain-test specimen is only 3 inches long.)

5. A test specimen 3 inches in length, when split, shall withstand opening out flat without showing cracks or flaws. 
6. The pipe shall withstand threading in a satisfactory manner.

7. A sufficient number of pipes shall be taken at random to constitute 0.5 per cent of the shipment. One flattening, one strain, and one threading test shall be made on each pipe so selected.

(Requirements covering bend test and hydrostatic test are the same as those for copper pipe in A. S. T. M., B 42 (see 642.23, p. 1182), except that in this case the fiber stress is 7,000 pounds per square inch instead of 6,000 as in B42.

\section{DIMENSIONS AND PERMISSIBLE VARIATIONS}

(The dimensions for brass pipe are the same as for copper pipe, as are the variations in both weight and thickness, as given in B42, referred to above. The standard weights for brass pipe of various outside diameters are shown in the accompanying table:)

\section{TABLE 1.-Standard weights for brass pipe}

\begin{tabular}{|c|c|c|}
\hline Size of pipe & $\begin{array}{l}\text { Standard } \\
\text { pipe, nomi- } \\
\text { nal weight } \\
\text { per foot of } \\
\text { length }\end{array}$ & $\begin{array}{l}\text { Extra-strong } \\
\text { pipe, nomi- } \\
\text { nal weight } \\
\text { per foot of } \\
\text { length }\end{array}$ \\
\hline $\begin{array}{c}\text { Inches } \\
1 / 8 \\
1 / 4 \\
3 / 8 \\
1 / 2 \\
3 / 4\end{array}$ & $\begin{array}{c}\text { Pounds } \\
0.246 \\
.437 \\
.612 \\
.911 \\
1.24\end{array}$ & $\begin{array}{c}\text { Pounds } \\
0.353 \\
.593 \\
.805 \\
1.19 \\
1.62\end{array}$ \\
\hline $\begin{array}{l}1 \\
11 / 4 \\
11 / 2 \\
2 \\
21 / 2\end{array}$ & $\begin{array}{l}1.74 \\
2.56 \\
3.04 \\
4.02 \\
5.83\end{array}$ & $\begin{array}{l}\text { 2. } 39 \\
3.30 \\
3.99 \\
5.51 \\
8.41\end{array}$ \\
\hline $\begin{array}{l}3 \\
31 / 2 \\
4 \\
41 / 2 \\
5\end{array}$ & $\begin{array}{r}8.31 \\
10.85 \\
12.29 \\
13.74 \\
15.40\end{array}$ & $\begin{array}{l}11.24 \\
13.67 \\
16.41 \\
20.07 \\
22.52\end{array}$ \\
\hline $\begin{array}{r}6 \\
7 \\
8 \\
9 \\
10\end{array}$ & $\begin{array}{l}18.44 \\
23.92 \\
30.05 \\
36.94 \\
43.91\end{array}$ & $\begin{array}{l}31.32 \\
41.23 \\
47.02 \\
52.81 \\
59.32\end{array}$ \\
\hline
\end{tabular}

8. No single piece of pipe that exceeds the calculated weight by more than 5 per cent will be accepted. One cubic inch of the material is assumed to weight 0.307 pound.

9. All pipes shall be acid cleaned after the final annealing.

(Requirements covering workmanship and inspection and rejection are identical with those for copper pipe in B42, with the exception that:)

10. Pipes that show nonuniform annealing under handling shall be rejected from the lot.

\section{UNITED STATES GOVERNMENT, FEDERAI SPECIFICATIONS BOARD, FEDERAI SPECIFICATION FOR PIPE; BRASS, SEAMLESS, IRON-PIPE SIZE, STANDARD AND EXTRA STRONG, WW-P-351, JULY 22, 1930 \\ BRASS PIPE}

\section{A. APPLICABLE FEDERAL SPECIFICATIONS}

A-1. The following Federal specifications, of the issue in effect on date of invitation for bids, shall form a part of this specification:
Federal specification GGG-P-351, pipe threads. (See 607.0, p. 365.)

Federal specification QQ-M-151. General specification for metals. (See 600.0 p. 3.)

\section{B. GRADES AND WEIGHTS}

B-1. Seamless brass pipe shall be furnished in three grades, A, B, C, and as required.

B-2. Each grade shall be furnished in two weights, standard and extra strong, as required.

\section{MATERIAL AND WORKMANSHIP}

C-1. The pipe shall be clean, smooth, round, straight, of the proper dimensions, and with ends cut square. It shall be free from grooving, indentations, cracks, flaws, or other defects.

\section{GENERAL REQUIREMENTS}

$\mathrm{D}-2$. One cubic inch of grade A material is assumed to weigh 0.316 pound. One cubic inch of grades B or C material is assumed to weigh 0.307 pound. No single piece that exceeds the calculated weight by more than 5 per cent shall be accepted. The tolerance for underweight and gage shall be 5 per cent for pipe up to and including 6 inches; 7 per cent from 6 inches to and including 8 inches; and 8 per cent above 8 inches, respectively.

D-3. The ends of the pipe shall not be threaded unless so ordered. Pipe threads when ordered shall be standard taper pipe threads conforming to Federal specification GGG-P-351, as referred to in paragraph $\mathrm{A}-1$.

D-4. Random stock lengths of from 12 feet to 16 feet, inclusive, will be accepted, except when exact lengths are ordered.

D-4a. When exact lengths are ordered, a variation of plus one-eighth inch in the length will be permitted.

D-5. All pipe shall be furnished semi-annealed, unless otherwise specified.

D-6. All fully annealed pipe shall be acid cleaned.

D-7. The name or trade-mark of the manufacturer and the grade letter shall be permanently marked on each pipe.

\section{E. DETAIL REQUIREMENTS}

E-1. Seamless brass pipe shall be made of material conforming in composition to the requirements in Table 1.

TABLE 1.-Chemical composition of brass pipe

\begin{tabular}{|c|c|c|c|c|}
\hline Grade & Copper & Zinc & Lead & Iron \\
\hline 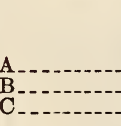 & $\begin{array}{l}\text { Per cent } \\
83 \text { to } 68 \\
65 \text { to } 68 \\
59 \text { to } 68\end{array}$ & $\mid \begin{array}{l}\text { Remainder....... } \\
\end{array}$ & $\begin{array}{r}\text { Per cent } \\
\text { maximum } \\
0.06 \\
.80 \\
.80\end{array}$ & $\begin{array}{r}\text { Per cent } \\
\text { maximum } \\
0.05 \\
.07 \\
.07\end{array}$ \\
\hline
\end{tabular}

E-2. The dimensions, weights, and number of threads per inch of the various sizes of pipe when ordered as standard iron-pipe size, regular, shall be as shown in Table 2 . 
TABLE 2.-Standard weights and dimensions of brass pipe, iron-pipe size

\begin{tabular}{|c|c|c|c|c|}
\hline \multirow[b]{2}{*}{$\begin{array}{l}\text { Iron- } \\
\text { pipe } \\
\text { size }\end{array}$} & \multicolumn{2}{|c|}{ Weight per foot } & \multirow{2}{*}{$\begin{array}{l}\text { Mini- } \\
\text { mum } \\
\text { internal } \\
\text { hydro- } \\
\text { static } \\
\text { test } \\
\text { pressure } \\
\text { per } \\
\text { square } \\
\text { inch }\end{array}$} & \multirow[b]{2}{*}{$\begin{array}{l}\text { Threads } \\
\text { per inch }\end{array}$} \\
\hline & Grade A & $\begin{array}{l}\text { Grades } \\
\mathrm{B} \text { and } \mathrm{C}\end{array}$ & & \\
\hline $\begin{array}{c}\text { Inches } \\
1 / 8 \\
1 / 4 \\
3 / 8 \\
1 / 2 \\
3 / 4\end{array}$ & $\begin{array}{c}\text { Pounds } \\
0.253 \\
.450 \\
.630 \\
.938 \\
1.27\end{array}$ & $\begin{array}{c}\text { Pounds } \\
0.246 \\
.437 \\
.612 \\
.911 \\
1.24\end{array}$ & $\begin{array}{c}\text { Pounds } \\
1,000 \\
1,000 \\
1,000 \\
1,000 \\
1,000\end{array}$ & $\begin{array}{l}27 \\
18 \\
18 \\
14 \\
14\end{array}$ \\
\hline $\begin{array}{l}1 \\
11 / 4 \\
11 / 2 \\
2\end{array}$ & $\begin{array}{l}1.79 \\
2.63 \\
3.13 \\
4.14\end{array}$ & $\begin{array}{l}1.74 \\
2.56 \\
3.04 \\
4.02\end{array}$ & $\begin{array}{l}1,000 \\
1,000 \\
1,000 \\
1,000\end{array}$ & $\begin{array}{l}111 / 2 \\
111 / 2 \\
111 / 2 \\
111 / 2\end{array}$ \\
\hline $\begin{array}{l}21 / 2 \\
3 \\
31 / 2 \\
4\end{array}$ & $\begin{array}{r}6.00 \\
8.56 \\
11.17 \\
12.66\end{array}$ & $\begin{array}{r}5.83 \\
8.31 \\
10.85 \\
12.29\end{array}$ & $\begin{array}{r}1,000 \\
1,000 \\
1,000 \\
900\end{array}$ & $\begin{array}{l}8 \\
8 \\
8 \\
8\end{array}$ \\
\hline $\begin{array}{r}5 \\
6 \\
8 \\
10\end{array}$ & $\begin{array}{l}15.85 \\
18.99 \\
30.95 \\
45.20\end{array}$ & $\begin{array}{l}15.40 \\
18.44 \\
30.05 \\
43.91\end{array}$ & $\begin{array}{l}700 \\
600 \\
500 \\
500\end{array}$ & $\begin{array}{l}8 \\
8 \\
8 \\
8\end{array}$ \\
\hline
\end{tabular}

(Outside diameter and wall thickness for the various sizes are the same as for standard-wieght copper pipe given in A. S. T. M. specification B42. (See 642.23, p. 1182.)

E-3. The dimensions, weights, and number of threads per inch of the various sizes of pipe when ordered as extra strong shall be as shown in Table 3.

\section{TABLE 3.-Weights and dimensions of brass pipe, iron-pipe size, extra strong}

\begin{tabular}{|c|c|c|c|c|}
\hline \multirow[b]{2}{*}{$\begin{array}{l}\text { Iron- } \\
\text { pipe } \\
\text { size }\end{array}$} & \multicolumn{2}{|c|}{ Weight per foot } & \multirow{2}{*}{$\begin{array}{l}\text { Mini- } \\
\text { mum in- } \\
\text { ternal hy- } \\
\text { drostatic } \\
\text { test pres- } \\
\text { sure per } \\
\text { square } \\
\text { inch }\end{array}$} & \multirow[b]{2}{*}{$\begin{array}{l}\text { Number } \\
\text { of threads } \\
\text { per inch }\end{array}$} \\
\hline & $\begin{array}{c}\text { Grade A } \\
\text { (plain } \\
\text { ends) }\end{array}$ & $\begin{array}{l}\text { Grades } \\
\text { B and C } \\
\text { (plain } \\
\text { ends) }\end{array}$ & & \\
\hline $\begin{array}{c}\text { Inches } \\
1 / 8 \\
1 / 4 \\
3 / 8 \\
1 / 2 \\
3 / 4\end{array}$ & $\begin{array}{c}\text { Pounds } \\
0.363 \\
.611 \\
.829 \\
1.23 \\
1.67\end{array}$ & $\begin{array}{c}\text { Pounds } \\
0.353 \\
.593 \\
.805 \\
1.19 \\
1.62\end{array}$ & $\begin{array}{c}\text { Pounds } \\
1,000 \\
1,000 \\
1,000 \\
1,000 \\
1,000\end{array}$ & $\begin{array}{l}27 \\
18 \\
18 \\
14 \\
14\end{array}$ \\
\hline $\begin{array}{l}1 \\
11 / 4 \\
11 / 2 \\
2\end{array}$ & $\begin{array}{l}2.46 \\
3.39 \\
4.10 \\
5.67\end{array}$ & $\begin{array}{l}2.39 \\
3.30 \\
3.99 \\
5.51\end{array}$ & $\begin{array}{l}1,000 \\
1,000 \\
1,000 \\
1,000\end{array}$ & $\begin{array}{l}111 / 2 \\
111 / 2 \\
111 / 2 \\
111 / 2\end{array}$ \\
\hline $\begin{array}{l}21 / 2 \\
3 \\
31 / 2 \\
4\end{array}$ & $\begin{array}{r}8.66 \\
11.57 \\
14.07 \\
16.89\end{array}$ & $\begin{array}{r}8.41 \\
11.24 \\
13.67 \\
16.41\end{array}$ & $\begin{array}{l}1,000 \\
1,000 \\
1,000 \\
1,000\end{array}$ & $\begin{array}{l}8 \\
8 \\
8 \\
8\end{array}$ \\
\hline $\begin{array}{r}5 \\
6 \\
8 \\
10\end{array}$ & $\begin{array}{l}23.18 \\
32.21 \\
48.39 \\
61.05\end{array}$ & $\begin{array}{l}22.52 \\
31.32 \\
47.02 \\
59.32\end{array}$ & $\begin{array}{r}1,000 \\
1,000 \\
900 \\
700\end{array}$ & $\begin{array}{l}8 \\
8 \\
8 \\
8\end{array}$ \\
\hline
\end{tabular}

(Outside diameter and wall thickness for the various sizes are the same as for extra-strong copper pipe given in A. S. T. M. specification B42. See 642.23, p. 1182.)

\section{F. METHOD OF SAMPLING INSPECTION AND TESTS}

F-1. Each pipe shall be subjected to a minimum internal hydrostatic pressure in accordance with the fourth column of Tables 2 and 3, without showing signs of bulging, cracks, flaws, porous places, or other defects.

F-2. All pipe shall be surface inspected for defects by rolling on a set of wooden horses or other suitable supports, so that the entire surface of each pipe may be properly examined. In the event of failure of 10 per cent of any lot presented for inspection to pass the surface inspection and gaging tests, that lot shall be rejected.

F-3. For purposes of inspection, 1,000 pounds or fraction thereof of the same size of pipe shall be considered a "lot."

$\mathrm{F}-3 \mathrm{a}$. The inspector shall select and subject 1 per cent of the lengths of each lot of pipe of the same size submitted for inspection to the following tests:

F-3b. Each test pipe shall have a piece 3 inches long cut from it, which piece when slit on one side shall stand bending out flat without showing cracks or flaws.

F-3c. A full-section test specimen 6 inches in length shall withstand total immersion for $15 \mathrm{~min}$ utes without cracking in an aqueous mercurous nitrate solution containing $100 \mathrm{~g}$ of mercurous nitrate and $13 \mathrm{c}$ c of nitric acid (specific gravity 1.42) per liter of solution.

F-3d. For pipe under 2 inches in diameter, the length of test pipe remaining after tests $\mathrm{F}-3 \mathrm{~b}$, and F-3c shall be flattened on different elements throughout its length so that a gage set at three times the thickness of the metal shall pass over the pipe freely throughout the flattened part, except at such points where changes in the elements of flattening take place. The pipe shall be rotated approximately $90^{\circ}$ between each successive flattened element. For pipe 2 inches and over in diameter a section 4 inches long shall be flattened so that a gage set at three times the wall thickness shall pass over the pipe freely throughout the flattened part. Pipe shall develop no cracks or flaws as a result of these tests.

F-4. One piece of pipe selected by the inspector from each size submitted for inspection shall stand threading satisfactorily with a hand die, with the standard pipe thread for pipe and pipe fittings for that size of pipe.

$\mathrm{F}-5$. If any specimen fails to meet the requirements of subparagraphs $\mathrm{F}-3 \mathrm{~b}, \mathrm{~F}-3 \mathrm{~d}, \mathrm{~F}-4$, two additional specimens may be taken, each of which shall conform to the requirements specified.

F-6. When 2-inch pipe and under is ordered fully annealed, full sections of pipe shall withstand bending $180^{\circ}$ around a diameter of one and one-half times the inside diameter of the pipe without cracking on the outside bent portion. Flattening of pipe due to bending is permitted.

F-7. Chemical analysis may be made by the inspector or through him by any Government laboratory or other designated representative without cost to the contractor.

F-7a. Drillings, millings, or clippings for chemical analysis shall be taken from several points around each piece selected for physical tests and the chips from each lot combined and thoroughly mixed. The resultant sample shall be free from dirt, grit, and other foreign matter and shall consist of at least 2 ounces.

\section{NOTES}

I-1. Pipe is regularly designated by iron-pipe size, grades $\mathrm{A}, \mathrm{B}$, or $\mathrm{C}$, standard or extra strong.

$\mathrm{I}-2$. When pipe is required for severe bending, the requisition or purchase order should specifically require "fully annealed pipe."

I-3. When pipes are required to exact length, it shall be so stated on the requisition.

$\mathrm{I}-4$. As regards corrosion resistance, it is considered that the grades may be rated in the following order: $\mathrm{A}, \mathrm{B}$, and $\mathrm{C}$, grade $\mathrm{A}$ being the most resistant 
645.24 TUBES, BRASS.

AMERICAN BUREAU OF SHIPPING, RULES FOR BUILDING AND CLASSING STEEL VESSELS; MATERIALS FOR MACHINERY, BOILERS, AND PIPING, 1930

\section{BRASS PIPE \& AND TUBES}

(See 645.23, p. 1216.)

AMERICAN BUREAU OF SHIPPING, RULES FOR BUILDING AND CLASSING STEEL VESSELS; MATERIALS FOR MACHINERY, BOILERS, AND PIPING, 1930

\section{CONDENSER TUBES AND FERRULE STOCK}

(For general information and requirements, see 600.0 , p. 1. For test requirements, see 600.1, p. 7 .)

1. The tube shall be cold-drawn to a size, of a standard alloy in which scrap shall not be used except such as may result from the manufacture of articles of the same composition.

2. Condenser tubes shall be annealed sufficiently to enable the tubes to pass the physical and metallographic tests specified. Tubes for ferrule stock shall be sufficiently annealed to prevent cracking, but need not be held within the close limits specified for condenser tubes.

3. The material shall conform to the following requirements as to chemical composition:

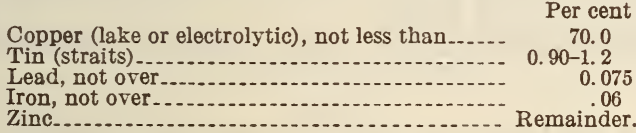

4. The sample for chemical analysis shall consist of drillings, millings, or clippings taken from the last three separate tubes from each lot of 600 tubes or less.

5. A microscopic examination of the tubes shall show sufficient annealing for complete recrystallization with a uniform grain size not in excess of 0.045 $\mathrm{mm}$ in average diameter, the grain size approaching as closely as possible a mean diameter of not over $0.035 \mathrm{~mm}$. The determination of the grain size shall be made by comparison of micrographs of the samples to those of standard specimens for which the grain size has been accurately determined, the standard magnification to be used being 75 diameters.

\section{HAMMERING TEST}

6. The tubes shall be capable of withstanding the following test without showing cracks or flaws: Pieces cut from the ends of the tubes shall be hammered flat on different elements throughout their length, so that a gage set at three times the thickness of the metal shall pass freely over the tube throughout the flattened part, except at such points where changes in the elements of flattening take place.

\section{EXPANDING TEST}

7. The tubes shall be capable of withstanding the following test without showing signs of cracking or rupture: A pin having a taper of $1 \frac{1}{2}$ inches per foot shall be driven into the tube until the diameter has been increased 20 per cent.

\section{STRAIN TEST}

8. A test specimen 6 inches long shall withstand an immersion in an aqueous mercurous nitrate solution containing $100 \mathrm{~g}$ of mercurous nitrate and 13 c c of nitric acid (specific gravity 1.42) per liter of solution for 15 minutes without cracking.

\section{NOMBER OF TESTS}

9. The foregoing physical tests should be made on two tubes from each lot of 600 tubes or less.

\section{HYDROSTATIC TEST}

10. All tubes shall withstand an internal hydrostatic pressure of 1,000 pounds per square inch without leakage, and shall show no sign of strain or cracking.

\section{TOLERANCES}

11. No tube at its thinnest point shall be less than the thickness specified. The lengths shall not be less but may be one-sixteenth inch more than that specified when measured at a temperature of $68^{\circ} \mathrm{F}$. $\left(20^{\circ} \mathrm{C}\right)$. No single tube shall exceed the calculated weight by more than 5 per cent. One cubic inch of the material is assumed to weigh 0.308 pound.

\section{WORKMANSHIP AND FINISH}

12. The tubes shall be round, straight, and of uniform thickness throughout. They shall be free from cracks, seams, slivers, scale, and other surface defects, both inside and outside. All tubes shall be acid cleaned after the final annealing.

13. If any test specimen representing a lot fails to meet the requirements, two additional specimens, at the option of the manufacturer, may be taken as before and submitted for check analysis or subjected to any tests in which the original failed, but each of these specimens shall conform to the requirements specified.

\section{AMERICAN INSTITUTE OF ELECTRICAL ENGINEERS, RECOMNENDED PRAC- TICE FOR ELECTRICAL INSTALLA- TIONS ON SHIPBOARD, NO. 45, OCTOBER, 1930}

\section{CONDUIT TERMINAL AND BULKHEAD STUFFING TUBES}

1. Conduit terminal tubes should consist of bronze, brass, or galvanized malleable iron. They should be machined with a standard pipe thread, and be designed so as to receive a flanged nut or gland nut of composition or brass, which when screwed into the body, should force a loose collar or gland ring against a soft rubber gasket or other suitable water-tight packing, thereby obtaining a water-tight joint.

2. The bulkhead or deck stuffing tubes should conform to the recommendations listed above, and in addition the body or tubes should have a nipple of sufficient length to pass through the bulkhead or deck with room for a gasket and lock nut on each side, thereby securing a water-tight joint through the deck or bulkhead. Deck stuffing tubes should be provided with a nut on each side.

AMERICAN SOCIETY FOR TESTING MATERIALS, STANDARD SPECIFICATIONS FOR SEAMLESS BRASS BOILER TUBES, DESIGNATION B14-18, 1918

\section{SEAMLESS BRASS BOILER TUBES}

\section{SCOPE}

1. These specifications cover brass tubes for locomotive boilers.

\section{MANUFACTURE}

2. The tubes shall be cold-drawn to size and semiannealed. They shall be fully annealed at both ends, if so ordered. 


\section{CHEMICAL PROPERTIES AND TESTS}

3. The material shall conform to the following requirements as to chemical composition:

70-30 alloy

Copper, not under.

Lead, not over.

Iron, not over

than copper and zinc, not over

Zinc... Remainder.

4. Drillings or turnings for analyses may be taken from the end of the tube.

\section{PHYSICAL PROPERTIES AND TESTS}

5. Test specimens taken from semiannealed tubes shall be fully annealed before testing.

6. A test specimen not more than 4 inches in length shall have a flange turned over at right angles to the body of the tube without showing cracks or flaws. The outside diameter of this flange shall measure not less than one and one-fourth times the original outside diameter of the tube.

7. The tubes shall be capable of standing the following test, when cold, without showing cracks or flaws: A piece of the tube shall be flattened down until the interior surfaces of the tube meet, and then doubled over on itself; that is, bent through an angle of 180, the bend being at right angles to the length of the tube.

8. One flange and one flattening test shall be made from each of two tubes in each lot of 250 or less. Each tube shall be subjected to the hydrostatic test.

\section{STANDARD WEIGHTS}

9. The standard weight for tubes of various outside diameters and thicknesses are as follows:

Weight in pounds, per foot of length

\begin{tabular}{|c|c|c|c|c|c|c|}
\hline \multicolumn{2}{|c|}{ Thickness } & \multicolumn{5}{|c|}{ Outside diameter (in inches) } \\
\hline B.w.g. & Inch & $11 / 2$ & $13 / 4$ & 2 & $21 / 4$ & $21 / 2$ \\
\hline $\begin{array}{r}13 \\
12 \\
11 \\
10 \\
7\end{array}$ & $\begin{array}{r}0.095 \\
.109 \\
.120 \\
.134 \\
.148\end{array}$ & $\begin{array}{c}\text { Pounds } \\
1.551 \\
1.761 \\
1.924 \\
2.127 \\
2.325\end{array}$ & $\begin{array}{c}\text { Pounds } \\
1.827 \\
2.078 \\
2.272 \\
2.516 \\
2.755\end{array}$ & $\begin{array}{c}\text { Pounds } \\
2.102 \\
2.395 \\
2.621 \\
2.905 \\
3.184\end{array}$ & $\begin{array}{c}\text { Pounds } \\
2.378 \\
2.711 \\
2.969 \\
3.294 \\
3.614\end{array}$ & $\begin{array}{c}\text { Pounds } \\
2.654 \\
3.028 \\
3.318 \\
3.683 \\
4.04\end{array}$ \\
\hline
\end{tabular}

(In respect to other physical properties and tests, permissible variations in weights, workmanship, and finish, marking, and inspection and rejection, this specification is identical with A. S. T. M., B13-18. See 642.24 , p. 1184.)

\section{AMERICAN SOCIETY FOR TESTING MATE- RIALS, STANDARD SPECIFICATIONS FOR SEAMLESS ADMIRALTY CONDENSER TUBES AND FERRULE STOCK, DESIGNA- TION B44-29, 1929}

\section{SEAMLESS ADMIRALTY CONDENSER TUBES AND FERRULE STOCK}

(These specifications are identical with those of the American Bureau of Shipping, given above, except that, instead of specifying the use of a standard alloy, B44 states specifically the use of lake or electrolytic copper conforming to the requirements of either the'standard specifications for lake-copper wire bars, cakes, slabs, billets, ingots, and ingot bars in A. S. T. M., B4 (see 641.11, p. 1173), or the standard specifications for electrolytic-copper wire bars, cakes, slabs, billets, ingots, and ingot bars in A. S. T. M., B5 (see 641.11 , p. 1174); zinc of grade No. 1 in the standard specifications for slab zinc (spelter) in A. S. T. M., B6 (see 683.1, p. 1310); and straits tin or its equivalent. The following additional matter is contained in these specifications).

\section{PHYSICAL PROPERTIES AND TESTS}

1. The standard micrographs shall be taken from specimens the grain size of which has been determined by Jeffries's method for grain-size measurements as described in the standard rules governing the preparation of micrographs of metals and alloys in A. S. T. M., E2 (see 600.1 , p. 20), at a magnification of 250 diameters. Reproductions of micrographs so prepared will be found under that commodity designation.

\section{PERMISSIBLE VARIATIONS IN DIMENSIONS}

2. The outside diameter of the tubes shall not vary from that specified by more than the following amounts, as measured by "go" and "no go" ring gages:

\begin{tabular}{|c|c|}
\hline Nominal diameter (in inches) & $\begin{array}{c}\text { Permissible } \\
\text { variation }\end{array}$ \\
\hline $\begin{array}{l}\text { Up to } 0.500 \text {, inclusive } \\
\text { Over } 0.500 \text { to } 0.740 \text {, inclusive } \\
\text { Over } 0.740 \text { to } 1.000 \text {, inclusive } \\
\text { Over } 1.000 \text { to } 1.250 \text {, inclusive } \\
\text { Over } 1.250 \text { to } 1.500 \text {, inclusive }\end{array}$ & $\begin{array}{l}\text { Inch } \\
\text { 土0.002 } \\
\pm .0025 \\
\pm .003 \\
\text { 土.0035 } \\
\pm .004\end{array}$ \\
\hline
\end{tabular}

3. No tube at its thinnest point shall be less than the thickness specified.

4. The length shall not be less but may be onesixteenth inch more than that ordered when measured at a temperature of $68^{\circ} \mathrm{F}$. $\left(20^{\circ} \mathrm{C}\right.$. $)$.

5. No single tube that exceeds the calculated weight by more than 5 per cent will be accepted. One cubic inch of the material is assumed to weigh 0.308 pound.

\section{INSPECTION}

(Conforms with standard specifications for steel, A. S. T. M. (See 605.0, p. 247.)

\section{EXPLANATORY NOTES}

Note 1.-The ideal composition for admiralty metal is 70 per cent of copper, 29 per cent of zinc, and 1 per cent of tin. Better tubes will be obtained by adhering as closely as possible to this composition. particularly as to tin. It does not, therefore, seem wise to make 1 per cent of tin the minimum, but rather in order to obtain as nearly 1 per cent as possible to allow some variation under 1 per cent and make the minimum limit for tin 0.90 per cent. Owing to the fact that a minimum of 1 per cent tin has heretofore existed, cases will occur, until the present specifications become universal, where the tin may exceed the 1.20 per cent maximum. It is not considered that this would be harmful or should be made a cause for rejection.

Note 2-The so-called compression test requiring that tubes of varying sizes and thicknesses should be subjected to certain arbitrary pressures without more than 0.005 inch permanent deformaton. has not been included because the physical properties of the tube under compression are functions of the temperature at which it receives its final heat treatment, and the metallographic examination sufficiently indicates the temper of the tube to decide whether it may be properly packed in the condenser head.

Until concrete information is available relative to the effect of grain sizes on corrosion, the committee points out that section $6(a)$ (see par. 5 in A. B. S. above) has been prepared solely for the purpose of getting a measurement of the compressibility and freedom from stress. Note 3.-Since the specifications require that the tubes shall be annealed sufficiently to establish crystalline equilibrium and show a grain size within certain definite limits, it would seem that a test for internal strains might be unnecessar 5 . The mercurous nitrate test has been included, however, to guard against the hardening of tubes by light drawing operations which would not deform the crystals to an extent recognizable by microscopic examination.

Note 4.-Provision is made for the testing of each tube separately to 1,000 pounds per square inch hydrostatic pressure in order 
to develop and make evident fractures or punctures which may have occurred in the process of manufacture. This is a 100 per cent test and it is not necessary to test all the tubes again by subjecting them to 80 pounds per square inch pneumatic pressure while immersed in water. Both of these tests are made to detect the same kind of faults, and either, if carried out to the intent of the specifications, should be sufficient. The 1,000-pound hydrostatic pressure test is the more searching and has been specified. The multiplication of tests to ness in applying one or another should not be encouraged.

AMERICAN SOCIETY FOR TESTING MATERIALS, STANDARD SPECIFICATIONS FOR SEAMLESS $\% 0-30$ BRASS CONDENSER TUBES AND FERRULE STOCK, DESIGNATION B55-25, 1925

\section{SEAMLESS 70-30 BRASS CONDENSER TUBES AND FERROLE STOCK \\ SCOPE}

1. These specifications cover seamless tubes and ferrule stock made from 70-30 brass for condenser use.

(In all other respects these specifications are identical with B44-29, given above, except of course that B55 contains no reference to tin as a component.)

\section{AMERICAN SOCIETY FOR TESTING MATE- RIAIS, STANDARD SPECIFICATIONS FOR SEAMLESS MUNTZ METAL CONDENSER TUBES AND FERRULE STOCK, DESIGNA- TION B56-25, 1925}

\section{SEAMLESS MUNTZ METAL CONDENSER TUBES AND FERRULE STOCK SCOPE}

1. These specifications cover seamless tubes and ferrule stock made from Muntz metal for condenser use.

\section{CHEMICAL PROPERTIES}

2. The material shall conform to the following requirements as to chemical composition:

Copper.5er
Irod, not over

(Otherwise these specifications are identical with B44-29, given above, except that the tubes shall be made by any approved process and finished by colddrawing; zinc of grades 1,2 , or 3 may be used; no reference is made to tin as a component; scrap of equivalent grades may be used; and the tubes and ferrule stock shall be suitable for packing in tube plates. The microscopic examination is similar, but there is no reference to determining the grain size by Jeffries's method, nor reproductions of micrographs, with accompanying notes. In the expanding test the diameter of the tube is increased only 15 per cent, instead of 20 per cent as in B44; and 1 cubic inch of the material is assumed to weigh 0.306 pound instead of 0.308 pound as in B44.)

SOCIETY OF AUTOMOTIVE ENGINEERS, NONFERROUS METAL SPECIFICATIONS, SPECIFICATION NO. 74, ANNEALED SEAMLESS BRASS TUBING, MARCH, 1922

\section{ANNEALED SEAMLESS BRASS TUBING}

Composition in percentage.-

Copper, minimum

Per cent

Lead, maximum

60.00

Iron, maximum

1.00

作, 10

\section{MECHANICAI REQOIREMENTS}

1. Tensile test.-Ultimate strength, minimum pounds per square inch, 40,000. Elongation in 2 inches, or proportionate gage length, minimum, per cent, 25.

2. Expanding test.-A pin having a taper of 1 to 8 shall be driven into one end of a tube until the diameter is increased 15 per cent. The tube shall withstand this test without showing cracks, splits, or other defects.

3. Appearance.-These tubes shall be clean, smooth, and free from injurious defects, both inside and outside.

Dimensional tolerances

\begin{tabular}{|c|c|c|c|c|c|}
\hline \multicolumn{2}{|c|}{$\begin{array}{l}\text { Outside diam- } \\
\text { eter }\end{array}$} & \multirow{2}{*}{$\begin{array}{l}\text { Tolerances, } 1 \\
\text { plus or } \\
\text { minus }\end{array}$} & \multicolumn{2}{|c|}{ Wall thickness } & \multirow{2}{*}{$\begin{array}{l}\text { Toler- } \\
\text { ances, } \\
\text { plus or } \\
\text { minus }\end{array}$} \\
\hline Over- & To- & & Over- & To- & \\
\hline $\begin{array}{c}\text { Inches } \\
0 \\
1 / 2 \\
3 / 4 \\
1 \\
11 / 4 \\
11 / 2 \\
13 / 4 \\
2\end{array}$ & $\begin{array}{c}\text { Inches } \\
1 / 2 \\
3 / 4 \\
1 \\
11 / 4 \\
11 / 2 \\
13 / 4 \\
2\end{array}$ & $\begin{array}{c}\text { Inch } \\
0.002 \\
.0025 \\
.003 \\
.0035 \\
.004 \\
.0045 \\
.005 \\
.0025 \times \\
\text { Diameter. }\end{array}$ & $\begin{array}{l}\text { Inch } \\
0 \\
1 / 64 \\
1 / 32 \\
1 / 16 \\
1 / 3 \\
1 / 4 \\
5 / 16\end{array}$ & $\begin{array}{l}\text { Inch } \\
1 / 64 \\
1 / 32 \\
1 / 16 \\
1 / 8 \\
1 / 4 \\
5 / 16 \\
3 / 8\end{array}$ & $\begin{array}{c}\text { Inch } \\
0.001 \\
.002 \\
.003 \\
.005 \\
.008 \\
.0125 \\
.015\end{array}$ \\
\hline
\end{tabular}

1 Either inside or outside diameter.

No combinations of variations on the same tube shall make the thickness of the wall vary from the nominal by more than these amounts.

4. Special limits.-On all stock where the above commercial variations are not permissible, the limits shall be specified in the order.

\section{SOCIETY OF AUTOMOTIVE ENGINEERS,} NONFERROUS METAL SPECIFICATIONS, SPECIFICATION NO. 76, NAVAL BRASS (TOBIN BRONZE) TUBING, AUGUST, 1922

\section{NAVAL BRASS TUBING}

Composition in percentage.-

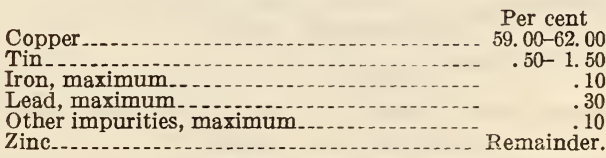

MECHANICAL REQUIREMENTS

Tensile-test data

\begin{tabular}{|c|c|c|c|c|}
\hline \multicolumn{2}{|c|}{ Thickness of wall } & \multirow{2}{*}{$\begin{array}{c}\text { Minimum } \\
\text { tensile } \\
\text { strength }\end{array}$} & \multirow{2}{*}{$\begin{array}{l}\text { Minimum } \\
\text { yield } \\
\text { point }\end{array}$} & \multirow{2}{*}{$\begin{array}{l}\text { Minimum } \\
\text { elongation } \\
\text { in } 2 \text { inches }\end{array}$} \\
\hline Over- & $\begin{array}{l}\text { To and } \\
\text { includ- } \\
\text { ing- }\end{array}$ & & & \\
\hline $\begin{array}{l}\text { Inch } \\
0 \\
1 / 8 \\
1 / 4\end{array}$ & $\begin{array}{c}\text { Inch } \\
1 / 8 \\
1 / 4\end{array}$ & $\begin{array}{r}\text { Lbs./in. }{ }^{2} \\
60,000 \\
55,000 \\
50,000\end{array}$ & $\begin{array}{r}\text { Lbs./in. }{ }^{2} \\
24,000 \\
22,000 \\
20,000\end{array}$ & $\begin{array}{c}\text { Per cent } \\
28 \\
32 \\
35\end{array}$ \\
\hline
\end{tabular}

(In respect to dimensional tolerances, expanding test, appearance, special limits, and footnotes, this specification is identical with S. A. E. specification No. 74 , given above.) 
UNITED STATES GOVERNMENT, FEDERAI SPECIFICATIONS BOARD; FEDERAL SPECIFICATION FOR CONDENSER TUBES AND TUBES FOR FERRULE STOCK (ADMIRALTY METAL), NO.374, JANUARY $25, \quad 1926$ WW-T-781)

\section{CONDENSER TUBES AND TUBES FOR FERRULE}

TYPES

1. Tubes in accordance with this specification shall be furnished in two types as required: Type $A$, condenser tubes, suitable for packing or expanding into tube sheets; type B, tubes for ferrule stock. (In respect to material, general requirements, and detail requirements, this specification is substantially the same as A. S. T. M., B44-29, given above, (including references in B44 to other specifications) with the following exceptions:)

2. Each lot of tubes shall, in the presence of the inspector, be immediately boxed for shipment unless waived by the purchaser.

3 . A tolerance of 0.0025 inch over the specified outside diameter of condenser tubes, and a minus tolerance of 0.0025 inch will be permitted.

4. For ferrule stock the stock length specified in the purchase order shall be the maximum. The

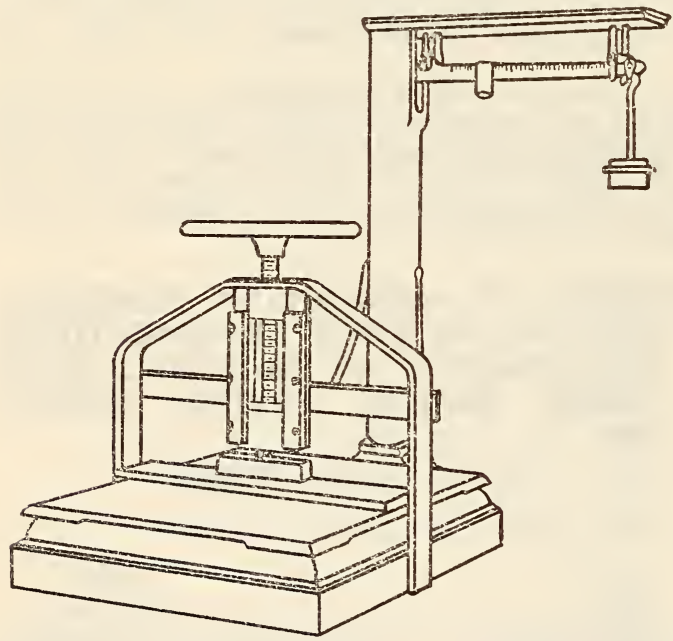

FIGURE 1090.- Sketch of approved testing machine

shortest acceptable lengths and the maximum permissible percentage by weight of short lengths shall be the same as for copper rods, bars, and shapes in Federal Specification QQ-C-501. (See 642.11, p. 1180.)

\section{METHOD OF INSPECTION AND TESTS}

5. All condenser tubes shall be inspected and tested as follows:

6 . The tubes shall be divided into lots of 606 tubes. Six tubes for test to destruction shall be selected by the inspector to represent each lot of 600 or less tubes. These test tubes, and additional tubes if required, shall be exclusive of the number required on the order and furnished at the contractor's expense.

7. All tubes shall be subjected to a hydrostatic pressure of 1,000 pounds per square inch. All tubes found defective shall be rejected.
8. The tubes of each lot shall be assembled for inspection in a well-lighted place and so arranged as to simplify the inspection as much as practicable. The tubes that do not show uniform annealing under handling shall be rejected from the lot.

9. All tubes shall be surface inspected and shall be of workmanlike finish; straight, true to form, and of an equal thickness throughout; free from cracks, seams, and other defects. All burrs resulting from cutting tubes to length shall be removed from the ends.

10. Each tube showing slivers or "spilly" metal either during the process of manufacture or upon final inspection shall be rejected. If 10 per cent of the tubes on final inspection of any lot show slivers on the inside, the lot shall be rejected.

11. To insure that the tubes conform to weight requirements, each lot of tubes shall be weighed, or the weight of any lot may at the discertion of the inspector be determined by weighing the test tubes representing that lot.

12. Samples for chemical analysis shall be taken by a milling machine or such other method as will insure clean chips the size of a medium pinhead, and they shall be from the entire cross section of each of the six test tubes representing a test lot. The whole amount taken from the six tubes shall be not less than 8 ounces.

13. These samples thus obtained shall be thoroughly blended one half given to the manufacturer and the other half retained by the inspector, the amount in each case being enough for three analyses.

14. In case the material is rejected on account of failure of the first check analysis to meet the requirements, a second check analysis by umpire methods may be made if requested by the contractors.

15. The inspector shall select two specimens 1 inch in length from each test tube. These specimens shall be subjected to a compression test in a testing machine approved by the purchaser. Each specimen shall be held between two parallel blocks, giving contact on opposite sides of the specimen over its entire length. The pressure shall be applied gradually until a total pressure of 300 pounds is reached. The pressure shall then be released, and the resulting deformation between the points of contact along the specimen shall not exceed 0.005 inch. An approved type of testing machine is shown in Figure 1090.

16. A specimen cut from each end of each test tube shall be subjected to an expanding test. This shall be performed by driving a pin having a taper in its diameter of $1 \frac{1}{2}$ inches per foot, into the tube until the internal diameter has been increased 20 per cent.

17. One 6-inch test specimen shall be taken from each test tube and submitted to the aqueous mercurous nitrate strain-test specified in the A. B. S. specification above. The failure of one or more specimens to stand this test shall cause the rejection of the lot represented.

18. One specimen at least one-half inch long for metallographic examination shall be taken from each of the six test tubes, and the six specimens thus obtained shall be marked with the lot number. The location from which each specimen is taken shall be selected by the inspector. The specimens shall be split longitudinally. One half of each specimen shall be prepared by the manufacturer for metallographic examination by the inspector. The other half of each specimen properly marked with the lot number for easy identification shall be sent to the laboratory designated by the purchaser, for use in case of dispute arising from the action of the inspector. 
19. In preparing the specimens for examination, the longitudinal surfaces of the two walls shall be polished and etched. For etching, any reagent may be used that will develop the structure so as to show clearly the grain boundaries and surface details. In case any specimen does not show complete recrystallization the lot shall be rejected.

20. All test tubes shall be flattened without cracks on different elements throughout the lengths remaining after specimens for the expanding, compression, and metallographic tests have been taken. Each element shall be slowly flattened by one stroke of a press. The term "flattened" shall be interpreted as follows: A micrometer caliper set at three times the wall thickness shall pass over the tube freely throughout the flattened part except at the points where the change in element of flattening takes place.

21. The failure of more than one test tube to stand all the tests required in paragraphs 15,16 , and 20 , or the same tube failing in more than one of these tests, shall cause the rejection of the lot represented. In the case of the failure of one test tube under a single one of these tests, six additional tubes shall be selected and subjected to all the required tests except check analysis. The failure of any one of the additional tubes to stand any one of the tests shall cause the rejection of the lot represented.

\section{TOBES FOR FERROLE STOCK}

22. The tubes shall be divided into lots of 600 lengths or less. (In respect to hydrostatic tests, assembly for inspection, finish and freedom from defects, weighing of tubes, samples for chemical analysis, and strain test by aqueous mercurous nitrate solution, the specifications for tubes for ferrule stock conform to those for condenser tubes given above. The following additional items cover both kinds of tubes:)

\section{PACKING AND MAREING}

23. Unless otherwise ordered, all tubes shall be packed in substantial boxes. The net weight of contents of each box shall not exceed 600 pounds.

24. Each box shall be marked with contract or order number; the lot number; the type, number, and dimensions of tubes contained; name of contractor; and manufacturer.

\section{NOTES}

25. The condenser tubes supplied in accordance with this specification are suitable for installation by packing or expanding and do not require further annealing at the place of delivery.

26. The properties of the material covered by this specification are based on the assumption that all 5\%-inch diameter condenser tubes will be ordered 0.065 inch thick; all $3_{4}^{\prime}$-inch diameter condenser tubes will be ordered 0.072 inch thick; all $7 / 8$ and 1 inch diameter condenser tubes will be ordered 0.083 inch thick.

27. It is assumed that the lateral compression strength of condenser tubes varies direcrly as the square of the wall thickness and inversely as the tube diameter. In event that it becomes necessary to purchase condenser tubes with dimensions other than given, the compression-test pressure should be calculated on the above assumption, using the dimensions of the $5 / 8$-inch tube as a basis of calculation.
28. Ferrule stock should be ordered in stock lengths, giving the maximum length desired. The order should also state the outside and inside diameter desired.

\section{UNITED STATES GOVERNMENT, FEDERAI SPECIFICATIONS BOARD, FEDERAI SPECIFICATION FOR TUBING, BRASS, SEAMLESS, WW-T-791, JULY 21, 1931}

\section{BRASS TUBING}

\section{A. APPLICABLE FEDERAL SPECIFICATIONS}

A-1. The following Federal specifications, or parts thereof that are below indicated, of the issue in effect on date of invitation for bids, shall form a part of this specification:

Federal specification QQ-M-151 (F. S. No. 339), general specifications for metals. (See 600.0, p. 3.)

\section{B. GRADES AND TYPES}

B-1. Seamless brass tubing shall be furnished in three grades-1, 2, and 3-according to chemical composition as specified in Table 4.

B-2. Seamless brass tubing shall be furnished in four types, as follows:

Type A, maximum nominal working pressure, 100 pounds per square inch.

Type B, maximum nominal working pressure, 200 pounds per square inch.

Type C, maximum nominal $\pi$ rrking pressure, 300 pounds per square inch.

Type D, maximum nominal working pressure, 450 pounds per square inch.

B-2a. The thicknesses for the various types are designed to give a stress not greater than 4,000 pounds per square inch in the formula

$t=\frac{P \times D}{2 S}$ under hydrostatic pressures as indicated opposite each type.

$t=$ thickness, in inches.

$P=$ pressure, pounds per square inch.

$D=$ inside diameter, in inches.

$S=$ stress in pounds per square inch.

B-3. For test pressures, see Table 5 .

\section{MATERIAL AND WORKMANSHIP}

C-1. The tubing shall be clean, smooth, round, straight, of the proper dimensions and with ends cut square. It shall be free from grooving, indentations, cracks, flaws, or other defects.

\section{GENERAL REQUIREMENTS}

D-1. The ends of the tubing shall not be threaded unless so ordered. (See par. I-4.)

D-1a. Tubes may be ordered with special threads, the details of which shall be furnished by the purchaser.

$\mathrm{D}-2$. One cubic inch of grade 1 material is assumed to weigh 0.316 pound. One cubic inch of grades 2 and 3 material is assumed to weigh 0.307 pound. The weight of any lot of tubes shall be not less than the calculated weight of the size specified.

D-2a. The maximum tolerance permitted for overweight of any lot shall be as given in Table 1 . 
TABLE 1.-Overweight tolerances for seamless brass tubing

\begin{tabular}{|c|c|c|c|c|c|c|c|c|}
\hline \multirow{2}{*}{ Wall thickness } & \multicolumn{2}{|c|}{$\begin{array}{l}\text { Up to } 2 \\
\text { inches } \\
\text { diame- } \\
\text { ter }\end{array}$} & \multicolumn{2}{|c|}{$\begin{array}{l}\text { Over } 2 \\
\text { inches } \\
\text { to } 4 \\
\text { inches } \\
\text { diame- } \\
\text { ter }\end{array}$} & \multicolumn{2}{|c|}{$\begin{array}{l}\text { Over } 4 \\
\text { inches } \\
\text { to } 7 \\
\text { inches } \\
\text { diame- } \\
\text { ter }\end{array}$} & \multicolumn{2}{|c|}{$\begin{array}{c}\text { Over } 7 \\
\text { inches } \\
\text { to } 10 \\
\text { inches } \\
\text { diame- } \\
\text { ter }\end{array}$} \\
\hline & 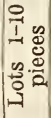 & 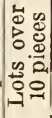 & $\mid \begin{array}{ll}0 \\
-1 \\
1 & 0 \\
-1 & 0 \\
0 & 0 \\
0 & 0 \\
0 & 0 \\
11\end{array}$ & 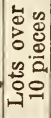 & $\mid \begin{array}{ll}0 \\
0 \\
1 & 0 \\
-1 & 0 \\
n & 0 \\
0 & 0 \\
0 & 0 \\
1 & 0\end{array}$ & $\mid \begin{array}{ll}1 & 0 \\
0 & 0 \\
0 & 0 \\
0 & 0 \\
0 & 0 \\
0 & 0 \\
0 & 0 \\
9 & 0\end{array}$ & $\mid \begin{array}{ll}0 \\
1 & 0 \\
1 & 0 \\
0 & 0 \\
0 & 0 \\
0 & 0\end{array}$ & $\begin{array}{ll}4 & 0 \\
0 & 0 \\
0 & 0 \\
0 & 0 \\
0 & 0 \\
0 & 0\end{array}$ \\
\hline & $\begin{array}{c}\text { Per } \\
\text { cent }\end{array}$ & $\begin{array}{l}\text { Per } \\
\text { cent }\end{array}$ & $\begin{array}{l}\text { Per } \\
\text { cent }\end{array}$ & $\begin{array}{l}\text { Per } \\
\text { cent }\end{array}$ & $\begin{array}{l}\text { Per } \\
\text { cent }\end{array}$ & $\begin{array}{l}\text { Per } \\
\text { cent }\end{array}$ & $\begin{array}{l}\text { Per } \\
\text { cent }\end{array}$ & $\begin{array}{l}\text { Per } \\
\text { cent }\end{array}$ \\
\hline Over 0.380 inch & (1) & (1) & $(1)$ & (1) & (1) & (1) & 8 & \\
\hline 0.379 inch to 0.284 inch, inclusive & (1) & (1) & (1) & (1) & 8 & 4 & 10 & \\
\hline 0.283 inch to 0.220 inch, inclusive & (1) & (1) & 7 & 4 & 10 & 5 & 12 & \\
\hline 0.219 inch to 0.165 inch, inclusive & 7 & 4 & 8 & 4 & 12 & 5 & 14 & 6 \\
\hline 0.164 inch to 0.120 inch, inclusive & 8 & 4 & 10 & 5 & 14 & 6 & 16 & \\
\hline 0.119 inch to 0.083 inch, inclusive & 10 & 5 & 12 & 5 & 16 & 6 & 18 & \\
\hline 0.082 inch to 0.058 inch, inclusive & 12 & 5 & 14 & 6 & 18 & 7 & & \\
\hline
\end{tabular}

1 These sizes are not covered by this specification. When required, the proper tolerances should be specified.

D-3. The walls of the tubes at any point shall be not less than the thickness specified.

D-4. The permissible variations in average outside diameter of brass tubing shall be as given in Table 2.

\section{TABLE 2.-Permissible variations in average outisde diameter}

(Tolerances on outside diameter are the same as given in Table 1 of F. S. B. specification WW-P-378 for copper tubing. See 642.24, p. 1187.)

D-5. When tubes are ordered to exact length, a variation of plus 0.125 inch on the length shall be permitted. No minus tolerance shall be permitted.

D-6. When tubes are ordered in nominal stock lengths the pieces shipped shall be cut to the nominal length as a maximum. The shortest acceptable lengths and the maximum permissible percentage by weight of short lengths shall be as given in Table 3.

TABLE 3.-Permissible variations in length

\begin{tabular}{|c|c|c|}
\hline $\begin{array}{c}\text { Nominal } \\
\text { stock length } \\
\text { (feet) }\end{array}$ & $\begin{array}{c}\text { Shortest } \\
\text { acceptable } \\
\text { length (feet) }\end{array}$ & $\begin{array}{c}\text { Maximum } \\
\text { permissible } \\
\text { percentage, } \\
\text { by weight } \\
\text { of lengths } \\
\text { shorter than } \\
\text { nominal }\end{array}$ \\
\hline & & 80 \\
20 & 12 & 80 \\
18 & 10 & 80 \\
16 & 10 & 40 \\
14 & 8 & 40 \\
10 & 8 & 40 \\
8 & 6 & 40 \\
6 & 4 & \\
\hline
\end{tabular}

D-7. All tubing shall be furnished semiannealed, unless otherwise specified.

D-7a. Fully annealed tubing shall be acid cleaned.

D-8. The name or trade-mark of the manufacturer and the type number shall be permanently marked on each tube.

\section{E. DETAIL REQUIREMENTS}

$\mathrm{E}-1$. Seamless brass tubing shall be made of material conforming in composition to the requirements in Table 4.
TABLE 4.-Chemical composition of brass tubing

\begin{tabular}{|c|c|c|c|c|}
\hline Grade & $\begin{array}{c}\text { Copper } \\
\text { (per cent) }\end{array}$ & Zinc (per cent) & $\begin{array}{l}\text { Lead (per } \\
\text { cent, maxi- } \\
\text { mum) }\end{array}$ & $\begin{array}{l}\text { Iron (per } \\
\text { cent, maxi- } \\
\text { mum) }\end{array}$ \\
\hline $\begin{array}{l}1 \\
2 \\
3\end{array}$ & $\begin{array}{l}83-86 \\
65-68 \\
59-68\end{array}$ & Remainder.... & $\begin{array}{r}0.06 \\
.80 \\
.80\end{array}$ & $\begin{array}{l}0.05 \\
.07 \\
.07\end{array}$ \\
\hline
\end{tabular}

E-2. The dimensions and weights of the various sizes of brass tubing shall be as given in Table 5 . Other diameters and thicknesses may be specified when required.

E-3. All tubing shall pass the tests prescribed in section $\mathrm{F}$ for the material ordered.

TABLE 5.-Standard weights and dimensions of seamless brass tubing

\begin{tabular}{|c|c|c|c|c|c|}
\hline \multirow{3}{*}{$\begin{array}{l}\text { Outside } \\
\text { diameter }\end{array}$} & \multicolumn{2}{|c|}{ TYPE A } & \multicolumn{3}{|c|}{ TYPE C } \\
\hline & \multicolumn{2}{|c|}{$\begin{array}{l}\text { Nominal weight per } \\
\text { foot of length }\end{array}$} & \multicolumn{2}{|c|}{$\begin{array}{l}\text { Nominal weight per } \\
\text { foot of length }\end{array}$} & \multirow{2}{*}{$\begin{array}{l}\text { Mini- } \\
\text { mum } \\
\text { test } \\
\text { pressure }\end{array}$} \\
\hline & Type 1 & $\begin{array}{l}\text { Types } 2 \\
\text { and } 3\end{array}$ & Туре 1 & $\begin{array}{c}\text { Types } 2 \\
\text { and } 3\end{array}$ & \\
\hline Inches & Pounds & Pounds & Pounds & Pounds & Lbs./in. ${ }^{2}$ \\
\hline $\begin{array}{l}1.315 \\
1.660 \\
1.900 \\
2.375\end{array}$ & 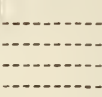 & - & $\begin{array}{l}0.968 \\
1.235 \\
1.442 \\
2.266\end{array}$ & $\begin{array}{l}0.940 \\
1.200 \\
1.401 \\
2.202\end{array}$ & $\begin{array}{l}700 \\
500 \\
500 \\
500\end{array}$ \\
\hline $\begin{array}{l}2.875 \\
3.500 \\
4.000 \\
4.500\end{array}$ & $\begin{array}{l}2.176 \\
2.660 \\
3.047 \\
3.434\end{array}$ & $\begin{array}{l}2.114 \\
2.584 \\
2.960 \\
3.336\end{array}$ & $\begin{array}{l}3.306 \\
4.910 \\
6.436 \\
8.123\end{array}$ & $\begin{array}{l}3.212 \\
4.770 \\
6.254 \\
7.891\end{array}$ & $\begin{array}{l}500 \\
500 \\
500 \\
500\end{array}$ \\
\hline $\begin{array}{r}5.563 \\
6.625 \\
8.625 \\
10.750\end{array}$ & $\begin{array}{r}4.451 \\
6.315 \\
10.657 \\
16.573\end{array}$ & $\begin{array}{r}4.325 \\
6.135 \\
10.354 \\
16.101\end{array}$ & $\begin{array}{l}12.408 \\
17.596 \\
29.848 \\
46.349\end{array}$ & $\begin{array}{l}12.055 \\
17.094 \\
28.998 \\
45.030\end{array}$ & $\begin{array}{l}500 \\
500 \\
500 \\
300\end{array}$ \\
\hline 10.750 & \multicolumn{2}{|c|}{ TYPE $\mathrm{B}$} & \multicolumn{3}{|c|}{ TYPE $D$} \\
\hline $\begin{array}{r}0.405 \\
.540 \\
.675 \\
.840 \\
1.050\end{array}$ & & & $\begin{array}{l}0.253 \\
.368 \\
.472 \\
.600 \\
.763\end{array}$ & $\begin{array}{l}0.246 \\
.357 \\
.459 \\
.583 \\
.741\end{array}$ & $\begin{array}{r}1,000 \\
1,000 \\
1,000 \\
1,000 \\
800\end{array}$ \\
\hline $\begin{array}{l}1.315 \\
1.660 \\
1.900 \\
2.375\end{array}$ & $\begin{array}{l}1.421 \\
1.789\end{array}$ & $\begin{array}{l}1.380 \\
1.738\end{array}$ & $\begin{array}{l}.982 \\
1.577 \\
2.053 \\
3.224\end{array}$ & $\begin{array}{l}.954 \\
1.532 \\
1.995 \\
3.132\end{array}$ & $\begin{array}{l}700 \\
800 \\
800 \\
700\end{array}$ \\
\hline $\begin{array}{l}2.875 \\
3.500 \\
4.000 \\
4.500\end{array}$ & $\begin{array}{l}2.274 \\
3.378 \\
4.420 \\
5.600\end{array}$ & $\begin{array}{l}\text { 2. } 209 \\
\text { 3. } 282 \\
4.294 \\
5.440\end{array}$ & $\begin{array}{r}4.716 \\
7.006 \\
9.139 \\
11.603\end{array}$ & $\begin{array}{r}4.581 \\
6.807 \\
8.879 \\
11.273\end{array}$ & $\begin{array}{l}700 \\
700 \\
700 \\
700\end{array}$ \\
\hline $\begin{array}{r}5.563 \\
6.625 \\
8.625 \\
10.750\end{array}$ & $\begin{array}{r}8.540 \\
12.172 \\
20.563 \\
32.004\end{array}$ & $\begin{array}{r}8.297 \\
11.826 \\
19.977 \\
31.093\end{array}$ & $\begin{array}{l}17.681 \\
25.102 \\
42.534 \\
66.142\end{array}$ & $\begin{array}{l}17.178 \\
24.387 \\
41.323 \\
64.259\end{array}$ & $\begin{array}{l}700 \\
700 \\
700 \\
700\end{array}$ \\
\hline
\end{tabular}

It will be noted that actual outside diameters correspond to ironpipe sizes; however, all tubing should be designated by actual outside diameter and wall thickness.

(Dimensions of the tubing for all four types are the same as for copper tubing given in Table 3 of F. S. B. specification WW-P-378. Test pressures for type A and type B tubing are the same as given in the above table. See 642.24, p. 1187.)

\section{F. METHODS OF SAMPLING INSPECTION AND TESTS}

F-2. Hydrostatic test.-Each tube shall withstand an internal, hydrostatic pressure which will subject 
the metal to a fiber stress of 7,000 pounds per square inch, the test pressure being calculated by the following formula for thin, hollow cylinders, but in no case will a test pressure of over 1,000 pounds per square inch be required.

in which

$$
P=\frac{2 t S}{D}
$$

$P=$ safe internal pressure.

$D=$ inside diameter, in inches.

$S=$ allowable unit stress of the material, 7,000 pounds per square inch.

$t=$ wall thickness, in inches.

Every tube shall be perfectly tight under pressure and show no signs of bulging, cracks, flaws, porous places, or other defects. The test pressures for specified sizes of tubing are listed in Table V.

F-3. Surface inspection.-All tubing shall be surface inspected for defects by rolling on a set of wooden horses or other suitable supports, so that the entire surface of each tube may be properly inspected. In the event of failure of 10 per cent of any lot presented for inspection to pass the surface inspection and gaging tests, that lot shall be rejected.

F-4. Lot.-For the purpose of inspection, 1,000 pounds or fraction thereof of the same size of tubes shall be considered a "lot."

F-5. The inspector shall select and subject 1 per cent by weight of each lot of tubes of the same size submitted for inspection to the following tests.

F-6. Mercurous nitrate test. - A full section test specimen 6 inches in length shall be taken from each tube selected for test and shall withstand total immersion, for 15 minutes without cracking, in an aqueous mercurous nitrate solution containing $100 \mathrm{~g}$ of mercurous nitrate and $13 \mathrm{ml}$ of nitric acid (specific gravity 1.42) per liter of solution.

F-7. Flattening test.-For all tubing under 2 inches in outside diameter of grades 1,2 , and 3 the length of tube selected for test remaining after test F-6 shall be flattened on different elements throughout its length so that a gage set at three times the thickness of the metal shall pass over the tube freely throughout the flattened part, except at such points where changes in the elements of flattening take place. The tube shall be rotated approximately $90^{\circ}$ between each successive flattening element. For tubing 2 inches and over in outside diameter, a section 4 inches long shall be flattened so that a gage set at three times the wall thickness shall pass over the tube freely throughout the flattened part. Tubing shall develop no cracks or flaws as a result of the tests.

F-8. Bending test.-When tubing 2 inches and under in diameter, of grades 1,2 , and 3 , is ordered fully annealed, full sections of tubing shall withstand bending $180^{\circ}$ around a diameter of one and one-half times the inside diameter of the tubing without cracking on the outside bent portion. Flattening of pipe due to bending is permitted.

F-8a. Bending test. - A strip not over $1 \frac{1}{2}$ inches wide shall be taken from each tube selected for test of grade 1 tubing, and after annealing shall stand bending flat on itself without showing signs of fracture on the outer surface. The ends of the specimens used in this test shall stand hammering down hot and cold to a knife-edge without showing signs of cracks.

F-9. Flanging test.-A specimen shall be taken from each tube selected for test of grade 1 tubing and shall stand flanging cold, after annealing. The width of the flange, measured from the outside of the tube, shall be one-fourth of the inside diameter of the tube.
F-10. Tension test.-Each lot of grade 1 tubing 2 inches inside diameter and over shall be subjected to tension tests. One piece from each tube selected for test shall be tested to represent the lot. If from 2 to 6 inches inside diameter, the test specimens shall be cut longitudinally; if over 6 inches inside diameter, they shall be cut circumferentially. All the test specimens shall be heated to a cherry red and straightened when hot, then annealed and machined to the dimensions of Type II standardtest specimen with 8-inch gage length, as required in Specification QQ-M-151 referred to in paragraph $A-1$. For thicknesses up to and including one-fourth inch, the width of the narrow part of the test specimen shall be about $1 \frac{1}{2}$ inches. For the thicker pieces the width shall be such as to give a cross section of about half a square inch, but the breadth shall not in any case be less than the thickness. The surfaces shall not be machined but shall be left in their original condition.

F-11. The test specimens of grade 1 tubing, as described in section $\mathrm{F}-10$, shall show an ultimate tensile strength of at least 35,000 pounds per square inch and an elongation of at least 30 per cent in 8 inches.

F-12. Chemical analysis.-Chemical analysis may be made by the inspector or through him by any Government laboratory or other designated representative without cost to the contractor.

F-12a. Drillings, millings, or clippings for chemical analysis shall be taken from several points around each piece selected for physical tests and the chips from each lot combined and thoroughly mixed. The resultant sample shall be free from dirt, grit, and other foreign matter and shall consist of at least 2 ounces.

\section{NOTES}

I-1. It is suggested that tubing be purchased on a footage basis (per foot of length) in preference to weight.

I-2. Tubing is regularly designated by actual outside diameter and thickness and should be so specified on all orders.

I-3. When tubes are required to be exact lengths it should be so stated in the requisition. When tubing is ordered in stock lengths the nominal stock lengths desired should be stated in the invitation for bids. (See Table 3.)

1-4. Tubing covered by Table 5 is suitable for the respective pressures given in that table only when straight and not threaded. If bending or threading is required, tubing with adequate wall thickness should be specified.

I-5. When tubing is required for severe bending, the requisition or purchase order should specifically require "fully annealed tubing."

I-6. Grade 1 is intended for use where a material with special resistance to corrosion by salt water, salt air, or gases is required.

I-7. Grade 2 is intended for fresh-water service where corrosive conditions are more severe than ordinary.

I-8. Grade 3 is intended for ordinary fresh-water service.

I-9. When tubing is required for instrument making and similar uses and the dimensions and/or tolerances in this specification are not suitable, the dimensions and/or tolerance desired should be stated in the invitation for bids.

\subsection{PIPE FITTINGS, BRASS.}

(See 645.4, p. 1230.) 
645.3 BRASS-WIRE MANUFACTURES.

645.31 SIEVES, BRASS.

\section{AMERICAN SOCIETY FOR TESTING MATE- RIALS, TENTATIVE DEFINITION OF THE TERM “SCREEN" (SIEVE), DESIGNATION E13-28T, 1928}

\section{SCREEN (SIEVE)}

A plate or sheet or a woven cloth, or other device, with regularly spaced apertures of uniform size, mounted in a suitable frame or holder, for use in separating material according to size.

Note 1.-The shape and spacing of apertures, size of wires or threads, thickness of plate or sheet, allowable variations and similar properties should be taken care of in specifications.

NOTE 2.-In mechanical analysis testing work, when not otherwise specified, the term "sieve" shall apply to an apparatus in which the apertures are rectangular, and the term "screen" shall apply to an apparatus in which the apertures are circular.

\section{AMERICAN SOCIETY FOR TESTING MATE- RIALS, STANDARD SPECIFICATIONS FOR SIEVES FOR TESTING PURPOSES, DESIG- NATION E11-26, 1926}

\section{SIEVES FOR TESTING}

1. These specifications cover sieves for use in the usual cases of precision testing for the fineness of materials (classification of materials according to size). The sieves have been designated according to the size of openings in terms of microns, the corresponding United States standard sieve series designation in arbitrary numbers being given for the convenience of those familiar with the latter scheme of designation.

\section{PHYSICAL REQUIREMENTS}

2. Wire cloth for standard sieves shall be woven (not twilled, except that the cloth of 44,53 , and 62 micron sieves may be twilled until further notice) from brass, bronze, or other suitable wire and mounted on the frames without distortion. To prevent the material being sieved from catching in the joint between the cloth and the frame, the joint shall be smoothly filled with solder, or so made that the material will not catch. The sieve frames should be circular, about $20 \mathrm{~cm}$ ( 8 inches) in diameter and about $5 \mathrm{~cm}$ ( 2 inches) or $2.5 \mathrm{~cm}$ ( 1 inch) between the top of the frame and the cloth

NoTE.-The size specified above is for the regular standard testing sieve, but for special purposes other sizes may be used, as, for example, he 3-inch sieve, which is designed especially for use on laboratory balances as in the testing of pigments.
Requirements for sieve openings and wire diameter with permissible variations

\begin{tabular}{|c|c|c|c|c|c|c|c|c|c|}
\hline \multirow{3}{*}{$\begin{array}{c}\text { Designa- } \\
\text { tion (in } \\
\text { microns) }\end{array}$} & \multirow{3}{*}{ 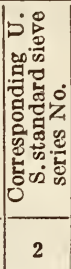 } & \multirow{2}{*}{\multicolumn{2}{|c|}{$\begin{array}{l}\text { Sieve open- } \\
\text { ing }\end{array}$}} & \multirow{2}{*}{\multicolumn{2}{|c|}{ Wire diameter }} & \multirow{3}{*}{ 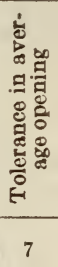 } & \multicolumn{2}{|c|}{$\begin{array}{l}\text { Toler- } \\
\text { ance on } \\
\text { wire di- } \\
\text { ameter }\end{array}$} & \multirow{2}{*}{ 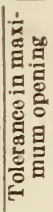 } \\
\hline & & & & & & & 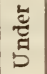 & 岂 & \\
\hline & & 3 & 4 & 5 & 6 & & 8 & 9 & 10 \\
\hline $\begin{array}{l}4,700 \\
4,000 \\
3,360 \\
2,830 \\
2,380\end{array}$ & $\begin{array}{l}4 \\
5 \\
6 \\
7 \\
8\end{array}$ & $\begin{array}{l}m m \\
4.76 \\
4.00 \\
3.36 \\
2.83 \\
2.38\end{array}$ & $\begin{array}{l}\text { Inch } \\
0.187 \\
.157 \\
.132 \\
.111 \\
.0937\end{array}$ & $\begin{array}{r}m m \\
1.27 \\
1.12 \\
1.02 \\
.92 \\
.84\end{array}$ & $\begin{array}{l}\text { Inch } \\
0.050 \\
.044 \\
.040 \\
.036 \\
.0331\end{array}$ & $\begin{array}{c}\text { Per } \\
\text { cent } \\
\pm 3 \\
\pm 3 \\
\pm 3 \\
\pm 3 \\
\pm 3\end{array}$ & $\begin{array}{c}\text { Per } \\
\text { cent } \\
15 \\
15 \\
15 \\
15 \\
15\end{array}$ & $\begin{array}{c}\text { Per } \\
\text { cent } \\
30 \\
30 \\
30 \\
30 \\
30\end{array}$ & $\begin{array}{r}\text { Per } \\
\text { cent } \\
10 \\
10 \\
10 \\
10 \\
10\end{array}$ \\
\hline - & $\begin{array}{l}10 \\
12 \\
14 \\
16 \\
18\end{array}$ & $\begin{array}{l}2.00 \\
1.68 \\
1.41 \\
1.19 \\
1.00\end{array}$ & $\begin{array}{l}.0787 \\
.0661 \\
.0555 \\
.0469 \\
.0394\end{array}$ & $\begin{array}{l}.76 \\
.69 \\
.61 \\
.54 \\
.48\end{array}$ & $\begin{array}{l}.0299 \\
.0272 \\
.0240 \\
.0213 \\
.0189\end{array}$ & $\begin{array}{l} \pm 3 \\
\pm 3 \\
\pm 3 \\
\pm 3 \\
\pm 3\end{array}$ & $\begin{array}{l}15 \\
15 \\
15 \\
15 \\
15\end{array}$ & $\begin{array}{l}30 \\
30 \\
30 \\
30 \\
30\end{array}$ & $\begin{array}{l}10 \\
10 \\
10 \\
10 \\
10\end{array}$ \\
\hline $\begin{array}{l}840 \\
710 \\
590\end{array}$ & $\begin{array}{l}20 \\
25 \\
30 \\
35 \\
40\end{array}$ & $\begin{array}{l}.84 \\
.71 \\
.59 \\
.50 \\
.42\end{array}$ & $\begin{array}{l}.0331 \\
.0280 \\
.0232 \\
.0197 \\
.0165\end{array}$ & $\begin{array}{l}.42 \\
.37 \\
.33 \\
.29 \\
.25\end{array}$ & $\begin{array}{l}.0165 \\
.0146 \\
.0130 \\
.0114 \\
.0098\end{array}$ & $\begin{array}{l} \pm 5 \\
\pm 3 \\
\pm 5 \\
\pm 5 \\
\pm 5\end{array}$ & $\begin{array}{l}5 \\
15 \\
15 \\
15 \\
15\end{array}$ & $\begin{array}{l}30 \\
30 \\
30 \\
30 \\
30\end{array}$ & $\begin{array}{l}25 \\
25 \\
25 \\
25 \\
25\end{array}$ \\
\hline $\begin{array}{l}350 . \\
297 \\
250 \\
210\end{array}$ & $\begin{array}{l}45 \\
50 \\
60 \\
70 \\
80\end{array}$ & $\begin{array}{l}.35 \\
.297 \\
.250 \\
.210 \\
.177\end{array}$ & $\begin{array}{l}.0138 \\
.0117 \\
.0098 \\
.0083 \\
.0070\end{array}$ & $\begin{array}{l}.22 \\
.188 \\
.162 \\
.140 \\
.119\end{array}$ & $\begin{array}{l}.0087 \\
.0074 \\
.0064 \\
.0055 \\
.0047\end{array}$ & $\begin{array}{l} \pm 5 \\
\pm 6 \\
\pm 6 \\
\pm 6 \\
\pm 6\end{array}$ & $\begin{array}{l}15 \\
15 \\
15 \\
15 \\
15\end{array}$ & $\begin{array}{l}30 \\
35 \\
35 \\
35 \\
35\end{array}$ & $\begin{array}{l}25 \\
40 \\
40 \\
40 \\
40\end{array}$ \\
\hline $\begin{array}{l}149 \\
125 \\
105 \\
88\end{array}$ & $\begin{array}{l}100 \\
120 \\
140 \\
170\end{array}$ & $\begin{array}{l}.149 \\
.125 \\
.105 \\
.088\end{array}$ & $\begin{array}{l}.0059 \\
.0049 \\
.0041 \\
.0035\end{array}$ & $\begin{array}{l}.102 \\
.086 \\
.074 \\
.063\end{array}$ & $\begin{array}{l}.0010 \\
.0031 \\
.0029 \\
.0025\end{array}$ & $\begin{array}{l} \pm 6 \\
\pm 6 \\
\pm 8 \\
\pm 8\end{array}$ & $\begin{array}{l}15 \\
15 \\
15 \\
15\end{array}$ & $\begin{array}{l}35 \\
35 \\
35 \\
35\end{array}$ & $\begin{array}{l}40 \\
40 \\
60 \\
60\end{array}$ \\
\hline $\begin{array}{l}74 \\
62 \\
53 \\
44 \ldots-\end{array}$ & $\begin{array}{l}200 \\
230 \\
270 \\
325\end{array}$ & $\begin{array}{l}.074 \\
.062 \\
.053 \\
.044\end{array}$ & $\begin{array}{l}.0029 \\
.0024 \\
.0021 \\
.0017\end{array}$ & $\begin{array}{l}.053 \\
.046 \\
.041 \\
.036\end{array}$ & $\begin{array}{l}.0021 \\
.0018 \\
.0016 \\
.0014\end{array}$ & $\begin{array}{l} \pm 8 \\
\pm 8 \\
\pm 8 \\
\pm 8\end{array}$ & $\begin{array}{l}15 \\
15 \\
15 \\
15\end{array}$ & $\begin{array}{l}35 \\
35 \\
35 \\
35\end{array}$ & $\begin{array}{l}60 \\
90 \\
90 \\
90\end{array}$ \\
\hline
\end{tabular}

NoTE.-Screens having larger openings than those included in the table may be specified, but in specifying screens of larger openings the actual size of the opening (in millimeters or in inches) shall be stated. A tolerance of \pm 3 per cent on average openings and of 10 per cent in maximum openlngs shall be permitted.

3. The average opening between the adjacent warp and the adjacent shoot wires, taken separately, shall be that given in column 3 (or 4) of the table, within the "tolerance in average opening" given in column 7. The average diameter of the warp and of the shoot wires, taken separately, of the cloth of any given sieve shall be that given in column 5 (or 6) of the table, within the "tolerance on wire diameter" given in columns 8 and 9 . The maximum opening between adjacent parallel wires shall not exceed the nominal width of opening for that sieve by more than the "tolerance in maximum opening" given in column 10 of the table. 


\section{WORKMANSHIP}

4. The sieves shall be free from imperfections in the sieve cloth or its mounting, as, for example, punctured, loose or wavy cloth, imperfections in soldering, etc.

\section{MARKING}

5. The sieves shall bear a label upon which the manufacturer's nominal opening in metric units (preferably in microns) with the equivalent in inches, together with the size of wire should be given.

Nore.-It is recommended that, until the new micron designation shall have been thoroughly established, in addition the corresponding United States standard sieve series number be given.

\section{METHODS OF TESTING SIEVES}

6. (a) To determine whether the cloth conforms to the requirements of these specifications, the wire diameter shall be measured in each direction by means of a micrometer-microscope or projection apparatus or other suitable apparatus of requisite precision, care being taken in all cases to avoid diffraction effects in measuring to the edge of a wire. Micrometer calipers and steel scales should not be used for this purpose. The mesh shall be determined by counting the number of openings for a distance of at least 6 inches in both directions of the sieve, using a scale, either glass or steel, and a microscope. The use of scales with special ruling for obtaining mesh by counting "interference bands" is recommended in cases where large numbers of any of the sieves with the smaller openings are to be measured. From the wire diameter and the mesh the average opening shall be computed.

Note.-No general rule can be given for the number of observations to be taken, except that they be sufficient to cause no change in the result if, say, three or four additional measurements are made.

(b) Measurements of large openings shall be made by means of the micrometer-microscope or the projection lantern or other suitable apparatus in the same way as wire diameters are measured.

Note.-In order to insure the securing of standard sieves it is possible to obtain from the manufacturer or dealer sieves which have been certified by the Bureau of Standards.

UNITED STATES GOVERNMENT, DEPARTMENT OF COMMERCE, BUREAU OF STANDARDS, 3-INCH SIEVES, LETTER CIRCULAR, LC58, MARCH 1, 1925

\section{3-INCH SIEVES (FOR PAINT PIGMENTS)}

Wire cloth for 3 -inch sieves shall conform to the specifications for cloth of sieves of the United States standard sieve series. (See A. S. T. M. specification E11-26 above.) The cloth shall be woven (not twilled, except that the cloth of No. 230, No. 270 , and the No. 325 sieve, may be twilled until further notice), from brass, bronze, or other suitable wire and mounted on the frames without distortion.

To prevent the material being sieved from catching in the joint between the cloth and the frame, the joint shall be smoothly filled with solder, or so made that the material will not catch. The sieve frames shall be circular, about $7.6 \mathrm{~cm}$ (3 inches) inside diameter and shall not vary from this by more than $0.4 \mathrm{~cm}(0.16 \mathrm{inch})$. The depth of the sieve from the top of the frame to the cloth shall not be less than $1.9 \mathrm{~cm}(0.75 \mathrm{inch})$. It is recommended that the frames be constructed of first-quality sheet brass in such a manner as to be permanently rigid. The weight of the completed sieve must not exceed 49 grams.

The Bureau of Standards also reserves the right to reject sieves for obvious imperfections in the sieve cloth or its mounting, as for example, punc- tured, loose, or wavy cloth, imperfections in soldering, etc.

(The Bureau of Standards issues reports on each 3-inch sieve submitted to it for test and stamps those which conform to this specification with the letters "B. S." and year of test.)

UNITED STATES GOVERNMENT, DEPARTMENT OF COMMERCE, BUREAU OF STANDARDS, SIEVE-TESTING APPARATUS, LETTER CIRCULAR, LC72, JULY 26 , 1922

\section{SIEVE TESTING APPARATUS}

(This circular describes apparatus developed at the Bureau of Standards for measuring openings and wire diameters in sieves by projecting an enlarged image of the sieve on a ground-glass screen, measuring the image of the wires and measuring the magnification.)

\section{UNITED STATES GOVERNMENT, DEPART- MENT OF COMMERCE, BUREAU OF STANDARDS, SPECIFICATIONS FOR SIEVES, UNITED STATES STANDARD SIEVE SERIES, LC74, 1926, OCTOBER 1, 1926}

\section{SIEVES}

(These specifications are identical in respect to nominal dimensions and tolerances with A. S. T. M. E11-26, given above. Section 3 of E11 gives specific requirements as to the sieve label. The Bureau of Standards at present has no requirements as to sieve label, except that it shall adequately describe the sieve and not be deceptive.)

\section{UNITED STATES GOVERNMENT, DEPART-} MENT OF COMMERCE, BUREAU OF STANDARDS, STANDARDIZATION OF NO. 200 CEMENT SIEVES, TECHNOLOGIC PAPER NO. 42, JULY 30, 1914

\section{NO. 200 SIEVES}

(This paper describes the investigation and discusses the results of a number of observations made to determine methods of standardizing the 200-mesh sieve of the United States standard sieve series. Specifications for these sieves will be found in A. S. T. M. specification E11-26 above. This paper also treats of the establishment of a standard for the No. 200 sieve and the keeping of primary standards at the Bureau of Standards.)

\section{ADOPTION AND MAINTENANCE OF A STANDARD VALUE OF}

In order to insure a greater uniformity in fineness determinations, and to enable other investigators to make comparative tests, the Bureau of Standards will henceforth maintain a standard of fineness. The real test of the preservation of fineness standards will consist in retests from time to time of a number of sieves which are in substantial agreement with the adopted standard and are set aside as fundamental standards. Three of the standard sieves now owned by the Bureau of Standards have been set aside as temporary fundamental standards.

In the future the bureau will be prepared to furnish standard samples of cement at a nominal price, for tests of sieves in other laboratories. The fineness of these samples will be guaranteed to within 0.2 per cent on the fundamental standard $\varepsilon$ 
The sieving values of all new sieves with one sample of cement will in the future be given with the certificates of the standardized sieves, but while these values will, in general, be correct within 0.2 or 0.3 per cent they will probably be guaranteed within 0.5 per cent. This standardization test will ordinarily be made with a cement having a fineness between 75 and 80 per cent passing the No. 200 sieve, and will furnish a correction factor which will only be directly applicable to cements of similar fineness. (This paper also includes methods for the construction of simple calibration curves which may be used for determining the proper correction to sieves.)

(The Bureau of Standards, as referred to in above paragraph, issues certificates with sieves submitted to it for test.)

645.32 RINGS AND HOOKS, BRASS AND BRONZE. UNITED STATES GOVERNMENT, FEDERAL SPECIFICATIONS BOARD, FEDERAL SPECIFICATION FOR BUILDERS' HARDWARE (NONTEMPLATE), FF-H-101, AUGUST 19, 1930

\section{HOOKS AND EYES}

(See 617.63, p. 1061.)

\subsection{MISCELLANEOUS BRASS-WIRE MANUFAC- TURES.}

AMERICAN SOCIETY FOR TESTING MATERIALS, STANDARD SPECIFICATIONS FOR NONFERROUS INSECT SCREEN CLOTH DESIGNATION B50-29, 1929

\section{INSECT SCREEN CLOTH SCOPE}

1. These specifications cover insect screen cloth woven from nonferrous wire and intended to be used as a protection against insects or small animals.

\section{MANUFACTURE}

2. There shall be two classes of standard insect screen cloth depending upon the material used as follows: Class A, comprising cloth made from copper wire. Class B, comprising cloth made from a 90 copper-10 zinc alloy wire.

3 . Insect screen cloth shall be manufactured in five weights or sizes of mesh as follows:
14-mesh regular
16-mesh regular.
18-mesh regular.
22-mesh heavy.

16-mesh heavy.

4. The same quality and size of wire shall be used for both the warp and filling.

\section{CHEMICAI PROPERTIES}

5. The composition of the wire used for class A insect screen cloth is the same as specified under manufacture in A. S. T. M. B49; that is, it conforms to the requirements of either A. S. T. M. B4 or B5. (See 641.11 , p. 1173.)

6 . The wire for class B insect screen cloth shall conform to the following chemical requirements:

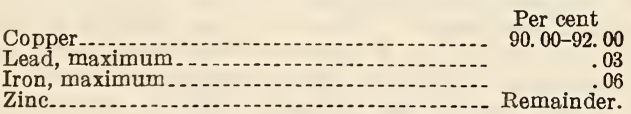

\section{PHYSICAL PROPERTIES OF WIRE}

7. The physical properties for all classes of screen wire shall be subject to agreement between the producer of the wire and the purchaser.
Note.-Wire for weaving insect screen cloth is usually furnisher with a drawn temper. The needs of different weavers of wire cloth apparently call for difference in drawn temper and it is consequentI be satisfactory to the entire trade. It has, therefore, seemed better to leave this matter to specific agreement between the producers of wire and the manufacturers of wire cloth.

\section{STANDARD SLES, WEIGHTS, DIMENSIONS, ETC.}

8. (a) The nominal diameter of wires to be used for the various weights of standard insect screen cloth shall be in accordance with the following:

\begin{tabular}{|c|c|c|c|}
\hline Mesh & Diameter & Mesh & Diameter \\
\hline $\begin{array}{l}14 \text { regular..... } \\
16 \text { regular...... } \\
16 \text { heavy...... }\end{array}$ & $\begin{array}{c}\text { Inch } \\
0.0119 \\
.0113 \\
.0150\end{array}$ & $\begin{array}{l}18 \text { regular } \\
22 \text { heavy }\end{array}$ & $\begin{array}{l}\text { Inch } \\
0.0100 \\
.0150\end{array}$ \\
\hline
\end{tabular}

(b) The permissible variation from the nominal diameter of any of the wires specified above shall be \pm 0.00025 inch.

9. The mesh as determined by counting the openings per linear inch for a length of one foot on any samples selected at random shall not vary from the nominal value in the warp, but an average variation of not more than one-half mesh per linear inch shall be permissible in the filling.

10. Insect screen cloth shall be furnished in rolls of approximately 100 linear feet. At least 87.5 per cent of the rolls ordered shall be in one continuous length; the remaining rolls may be in two lengths.

11. The widths of standard insect screen cloth shall be from 18 to 48 inches in multiples of 2 inches. The permissible variation in over all width as measured from outside wire to outside wire shall be $\pm 1 / 8$ inch.

\section{WORKMANSHIP AND FINISH}

12. (a) The insect screen cloth shall be free from all imperfections and blemishes not consistent with best commercial practice.

(b) Well-made splices in individual wires are permitted at any point in the screen cloth.

\section{INSPECTION}

13. (a) All testing and inspection, both of individual wires and completed cloth, shall be made at the place of manufacture. Tests on individual wires shall be made on samples taken before weaving and not on wires removed from finished cloth.

(b) The manufacturer shall afford the inspector representing the purchaser all reasonable facilities to satisfy him that the material conforms to these specifications.

\section{APPENDIX}

The following table giving the weights of various mesh of insect screen cloth is not a part of these specifications, but is appended as a source of information for the purchaser:

\begin{tabular}{|c|c|}
\hline Mesh & $\begin{array}{c}\text { Approximate } \\
\text { weight per } 100 \\
\text { square feet of } \\
\text { insect screen } \\
\text { cloth wrapped } \\
\text { and labeled } \\
\text { ready for ship- } \\
\text { ment }\end{array}$ \\
\hline $\begin{array}{l}14 \text { regular } \\
16 \text { regular } \\
16 \text { heavy } \\
18 \text { regular } \\
22 \text { heavy }\end{array}$ & $\begin{array}{l}\text { Pounds } \\
141 / 2 \\
15 \\
273 / 4 \\
14 \\
36\end{array}$ \\
\hline
\end{tabular}


SOCIETY OF AUTOMOTIVE ENGINEERS, PARTS AND FITTINGS, WIRE CIOTH, S. A. E. STANDARD, JULY, 1924

WIRE CLOTH

(See 646.52, p. 1256.)

UNITED STATES GOVERNMENT, DEPARTMENT OF COMMERCE, BUREAU OF STANDARDS, COMMERCIAI STANDARD FOR FOURDRINIER WIRE CLOTH, CS3631, NOVEMBER 1, 1931

\section{FOURDRINIER WIRE CLOTH}

(This commercial standard has been selected and accepted by industry and is promulgated by the Bureau of Standards.)

\section{GENERAI}

It is the desire of manufacturers of Fourdrinier wire cloth for paper-making machines that their product shall be of high-grade workmanship and material and thereby provide maximum service and satisfaction in use. Adherence to this policy requires but one high-grade product described below that shall be known as the commercial standard grade. Other grades anc meshes are to be considered as special.

\section{PURPOSE}

This commercial standard is a basis for clearer understanding between the manufacturers and users of Fourdrinier wire cloth. Its adoption and general use should result in a closer understanding between manufacturer and user through the establishment of standard mesh classifications, nomenclature, wire sizes, methods of inspection, labeling, handling, storage, and installation.

\section{SCOPE}

The standard applies to one grade only of Fourdrinier wire cloth - a flexible endless woven wire cloth used on paper-making machines. It establishes the number of wires in both directions for the several mesh classifications and the thickness of the wire. It further provides for inspection, labeling, and method of handling. An appendix includes recommendations of the manufacturers on the installation and use of the product.

\section{GENERAL REQUIREMENTS}

Commercial standard Fourdrinier wire cloth shall contain no imperfections that will shorten its period of usefulness or cause a mark or defect in the finished paper.

\section{DETAIL REQUIREMENTS}

1. Kinds of wire.-Wire used in the manufacture of Fourdrinier wire cloth may be of any metal or alloy with the necessary tensile strength, toughness, and elasticity to stand the physical strain and of sufficient resistance to the action of chemicals used in the preparation of pulp. Brass, bronze, phosphor bronze, Monel, nickel, and stainless steel are in common usage. One or more kinds of material may be used for the warp and shoot wires.

2. Mesh of wire cloth and size of wire.-The number of warp wires per inch (number of dents per inch in the reed) and the number of shoot wires (number of beats per inch) determines the mesh of the cloth.
The diameter of the single wire is varied according to the mesh of the wire cloth. The mesh, the corresponding number of warp and shoot wires per inch, and the corresponding diameter of wire listed in Table 1 are standard.

3. Seam.-The seam, which is one of the most important features of this commodity, shall suit the machine on which it is to be used and shall be flat and straight.

TABLE 1.-Details of standard mesh Fourdrinier wire cloth

\begin{tabular}{|c|c|c|c|c|c|c|c|c|}
\hline \multirow{3}{*}{$\begin{array}{l}\text { Stand } \\
\text { ard } \\
\text { mesh } \\
\text { desig- } \\
\text { nation }\end{array}$} & \multicolumn{2}{|c|}{$\begin{array}{l}\text { Warp wires } \\
\text { per inch }\end{array}$} & \multicolumn{2}{|c|}{$\begin{array}{l}\text { Shoot wires } \\
\text { per inch }\end{array}$} & \multicolumn{4}{|c|}{$\begin{array}{c}\text { Diameter of wire bofore } \\
\text { weaving }\end{array}$} \\
\hline & \multirow{2}{*}{ Dents } & \multirow{2}{*}{$\begin{array}{l}\text { Toler- } \\
\text { ance }\end{array}$} & \multirow{2}{*}{ Beats } & \multirow{2}{*}{$\begin{array}{l}\text { Toler- } \\
\text { ance }\end{array}$} & \multicolumn{2}{|c|}{ Warp wire } & \multicolumn{2}{|c|}{ Shoot wire } \\
\hline & & & & & $\begin{array}{l}\text { Mini- } \\
\text { mum }\end{array}$ & $\begin{array}{l}\text { Maxi- } \\
\text { mum }\end{array}$ & $\begin{array}{l}\text { Mini- } \\
\text { mum }\end{array}$ & $\begin{array}{l}\text { Maxi- } \\
\text { mum }\end{array}$ \\
\hline & Num- & Num- & Num- & Num- & & & & \\
\hline 40 & $\begin{array}{l}\text { ber } \\
40\end{array}$ & $\begin{array}{l}\text { ber } \\
-2\end{array}$ & $\begin{array}{c}\text { ber } \\
34\end{array}$ & $\begin{array}{l}\text { ber } \\
\quad \pm 2\end{array}$ & $\begin{array}{c}\text { Inch } \\
0.0105\end{array}$ & $\begin{array}{r}\text { Inch } \\
0.0115\end{array}$ & $\begin{array}{c}\text { Inch } \\
0.0105\end{array}$ & $\begin{array}{r}\text { Inch } \\
0.0115\end{array}$ \\
\hline 50 & 50 & -2 & 36 & \pm 2 & .0095 & .0105 & .0095 & $\begin{array}{r}.0105 \\
\end{array}$ \\
\hline 60 & 60 & -2 & 40 & \pm 2 & .0090 & .0095 & .0090 & .0105 \\
\hline 65 & 65 & $( \pm 1)$ & 46 & \pm 2 & .0080 & .0085 & .0080 & .0090 \\
\hline 70 & 70 & & 52 & \pm 2 & .0075 & .0080 & .0075 & .0085 \\
\hline 75 & 75 & $( \pm 1)$ & 56 & \pm 2 & .0070 & .0075 & .0070 & .0080 \\
\hline 80 & 80 & -2 & 60 & \pm 2 & .0065 & .0070 & .0065 & .0075 \\
\hline 85 & 85 & $( \pm 1)$ & 64 & \pm 2 & .0057 & .0063 & .0057 & .0065 \\
\hline 90 & 90 & -2 & 74 & \pm 2 & .0055 & .0060 & .0055 & .0060 \\
\hline 100 & 100 & -2 & 86 & \pm 4 & .0045 & .0050 & .0045 & .0055 \\
\hline
\end{tabular}

\section{METHOD OF INSPECTION}

The wire cloth shall be given a very rigid inspection while on the stretching table. It shall run true on the rolls. Particular attention shall be given to the seam. The entire area shall be examined for defects.

In measuring the diameter of a strand of wire a micrometer caliper, or $V$ gage, shall be used and the diameters recorded to the nearest ten-thousandth of an inch.

\section{PACKING}

The wire cloth shall be wound on three spars, each not less than $2 \frac{1}{2}$ inches in diameter, two at the center of the roll and the third inside the loose end. In order to prevent damage to the wire cloth all three spars shall be firmly bound in position at the ends. The wire cloth shall be securely wrapped in heavy moisture-resistant paper, tied, and carfully supported in the packing case by jamming both ends of the spars with pieces of wood securely fastened to them. Each Fourdrinier wire shall be packed in a case made for that partucular wire and every precaution shall be taken to guard against the possibility of damage in transit.

\section{LABELING}

The wrapper on all commercial standard Fourdrinier wire cloth shall be labeled according to mesh classification, kind of wire (both warp and shoot), and width and length of the cloth, and, in addition, shall contain the following statement:

"The - (Name of company)

wire cloth conforms to the requirements of the U.S. Commercial Standard CS36-31 as issued by the United States Department of Commerce."

The shipping case shall also be stenciled on side and end according to mesh classification, kind, size, and date of shipment. 
STORAGE, UNPACKING, AND INSTALLATION

1. Storage.-The Fourdrinier wires shall be stored in a clean, dry place in their original packing cases until required, and shall be used in the order received at the paper mill. No complaints will be recognized by wire-cloth manufacturers unless made within six months of delivery date.

2. Unpacking. - The packing case shall be opened with care. The covering of the wire cloth shall be carefully removed with the fingers only, never with a knife or other sharp instrument. The wire cloth shall then be placed on a clean, flat surface covered with heavy wrapping paper, the first turn unwound and the spar under the loose end slowly and evenly withdrawn, carefully followed by insertion of the porter bar.
3. Installation.-(The full text of this standard includes requirements on the installation of the wire cloth at the paper machine and recommendations on the proper care of wire cloth.)

\section{UNITED STATES GOVERNMENT, FEDERAL SPECIFICATIONS BOARD, FEDERAL SPECIFICATION FOR STANDARD MIS- CELLANEOUS CHAIN AND ATTACH- MENTS, RR-C-271, DECEMBER 8, 1931}

\section{LADDER CHAIN-REGISTER CHAIN}

(See 645.9, p. 1243.)

\subsection{BRASS FITTINGS AND PIUMBING FIXTURES.}

\section{AMERICAN BOTTLERS OF CARBONATED BEVERAGES, STANDARDS FOR BEVERAGE MANUFACTURING EQUIPIENT AND RECOMMENDED PRACTICES, EDUCATIONAL BULLETIN NO. 4, 1930}

STANDARD CARBONATOR-FILLER CARBONATED WATER CONNECTIONS
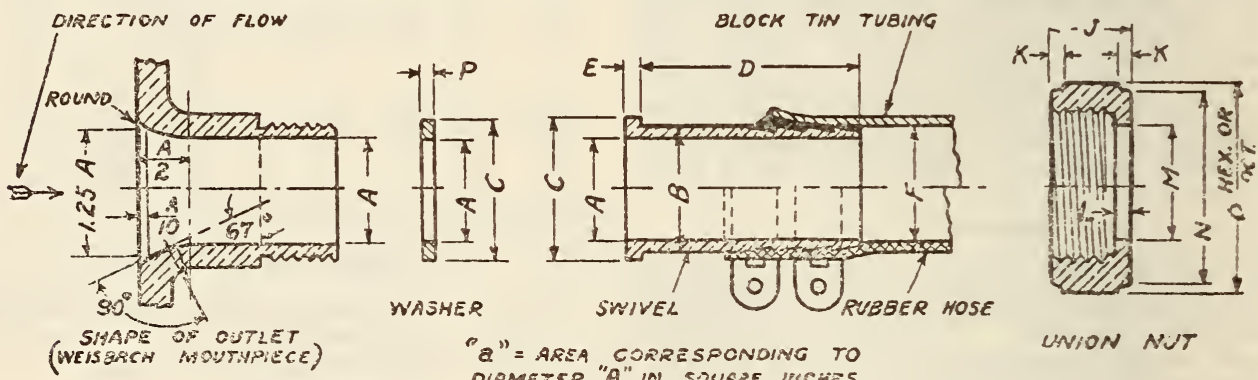

"Q" = AREA "CORRESPCNOING to

UNION AUT

Figure 1091.-Carbonated water connections

[Dimensions in inches]

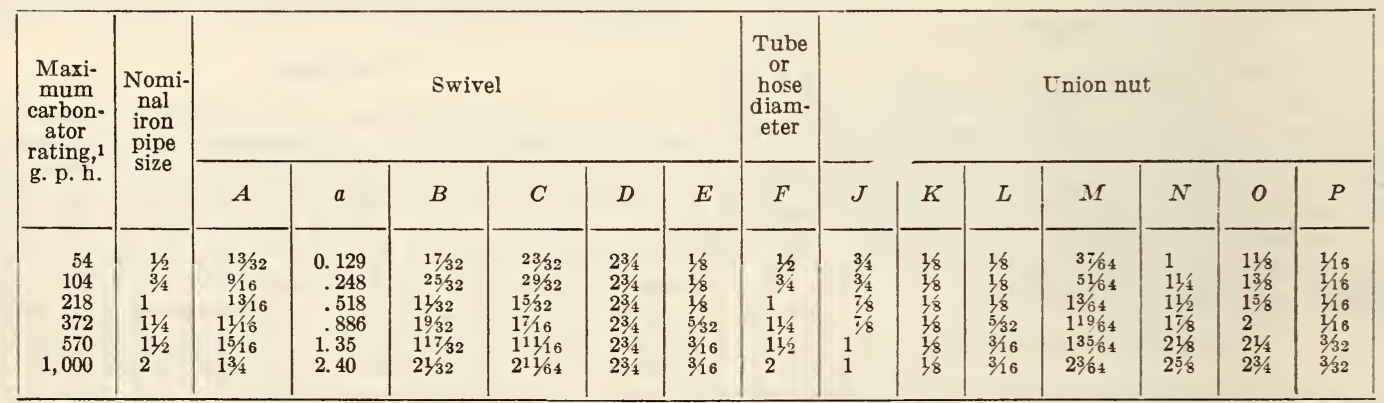

1 "Maximum carbonator rating" in United States gallons per hour based on a maximum allowable carbonated water velocity at the outlet of 2.25 feet per second. Example: To find the maximum capacity of a carbonator having an outlet of $A$ equals $15 / 16$ inches clear opening or $a$ equals 1.353 square inches outlet area. Answer: Formula: Rating equals $186.9 a V$, equals 186.9 by 1.353 by 2.25 , equals 570 United States gallons per hour.

Threads.-American standard straight pipe threads.

(See U. S. Government National Screw Thread Commission, 607.0, p. 365.) 

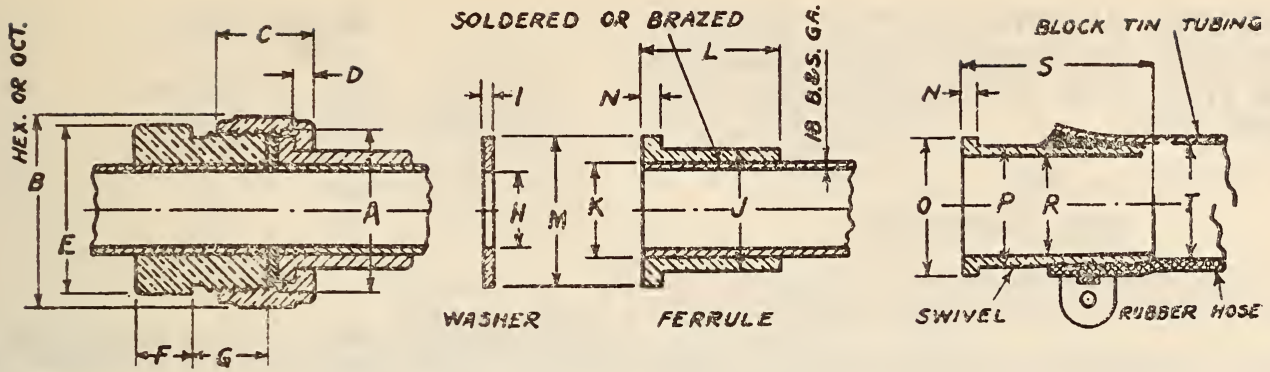

LINE COUPLING

FigURE 1091.-Sirup line connections

[Dimensions in inches]

\begin{tabular}{|c|c|c|c|c|c|c|c|c|c|c|c|c|c|c|c|c|c|c|c|}
\hline 䙔 & 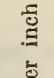 & \multicolumn{6}{|c|}{ Line coupling } & \multicolumn{2}{|c|}{ Washer } & \multicolumn{5}{|c|}{ Ferrule } & \multicolumn{5}{|c|}{ Swivel } \\
\hline$A$ & $\stackrel{\breve{J}}{\mathrm{E}}$ & $B$ & $C$ & $D$ & $E$ & $F$ & $G$ & $H$ & & $J$ & $K$ & $L$ & $M$ & $N$ & 0 & $P$ & $R$ & $S$ & $T$ \\
\hline $\begin{array}{l}1^{3 / 4} \\
11 / 4 \\
11 / 2 \\
2\end{array}$ & $\begin{array}{l}14 \\
111 / 2 \\
111 / 2 \\
111 / 2 \\
111 / 2\end{array}$ & $\begin{array}{l}11 / 8 \\
13 / 8 \\
13 / 4 \\
2 \\
21 / 2\end{array}$ & $\begin{array}{l}11 / 16 \\
3 / 4 \\
1^{7 / 8} \\
1\end{array}$ & $\begin{array}{r}1 / 8 \\
1 / 8 \\
5 / 32 \\
3 / 16 \\
3 / 16\end{array}$ & $\begin{array}{l}11 / 8 \\
13 / 8 \\
13 / 4 \\
2 \\
23 / 8\end{array}$ & $\begin{array}{l}5 / 16 \\
3 / 8 \\
7 / 16 \\
1 / 2 \\
5 / 8\end{array}$ & $\begin{array}{r}1 / 2 \\
9 / 16 \\
5 / 8 \\
11 / 16 \\
11 / 16\end{array}$ & $\begin{array}{r}5 / 8 \\
7 / 8 \\
11 / 8 \\
15 / 16 \\
111 / 16\end{array}$ & $\begin{array}{l}1 / 16 \\
1116 \\
3 / 32 \\
3 / 32 \\
3 / 32\end{array}$ & $\begin{array}{r}25 / 32 \\
11 / 32 \\
1932 \\
11732 \\
21 / 32\end{array}$ & $\begin{array}{r}5 / 8 \\
7 / 8 \\
118 \\
138 \\
134\end{array}$ & $\begin{array}{l}3 / 4 \\
7 / 8 \\
1 \\
118 \\
11 / 4\end{array}$ & $\begin{array}{r}59 / 64 \\
11164 \\
12964 \\
14564 \\
2^{116464}\end{array}$ & $\begin{array}{r}1 / 8 \\
1 / 8 \\
5 / 32 \\
3 / 16 \\
3 / 16\end{array}$ & $\begin{array}{r}59 / 64 \\
111 / 64 \\
12964 \\
145 / 64 \\
211 / 16\end{array}$ & $\begin{array}{c}25 / 32 \\
11 / 32 \\
19 / 32 \\
117 / 32 \\
21 / 32\end{array}$ & $\begin{array}{l}5 / 8 \\
7 / 8 \\
13 / 32 \\
15 / 16 \\
13 / 4\end{array}$ & $\begin{array}{l}15 / 8 \\
13 / 4 \\
17 / 8 \\
2 \\
21 / 4\end{array}$ & $\begin{array}{l}1^{3 / 4} \\
11 / 4 \\
11 / 2 \\
2\end{array}$ \\
\hline
\end{tabular}

Threads.-American standard straight pipe threads. (See U. S. Government National Screw Thread Commission, 607.0, p. 365 .)

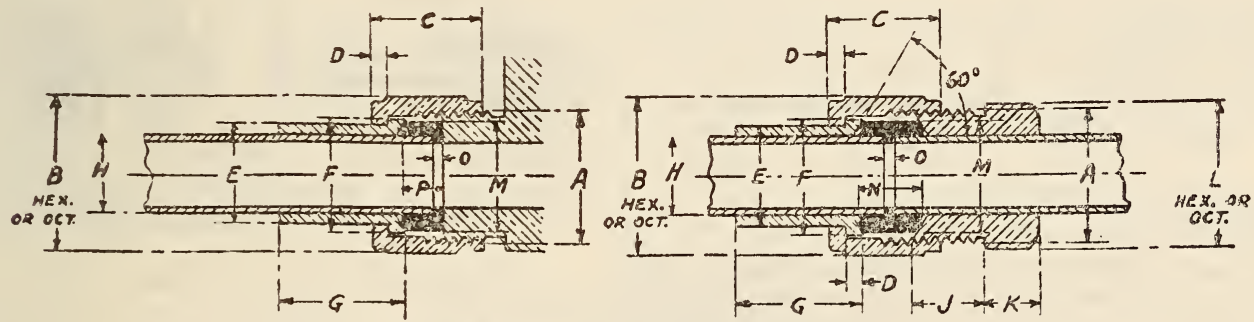

FIGURE 1092.-Compression coupling

[Dimensions in inches]

\begin{tabular}{|c|c|c|c|c|c|c|c|c|c|c|c|c|c|c|c|}
\hline \multirow{2}{*}{$\begin{array}{c}\text { Nomi- } \\
\text { nal } \\
\text { pipe } \\
\text { size }\end{array}$} & \multirow{2}{*}{$\begin{array}{l}\text { Threads } \\
\text { per } \\
\text { inch }\end{array}$} & \multicolumn{3}{|c|}{ Union nut } & \multicolumn{3}{|c|}{ Follower } & \multirow{2}{*}{ 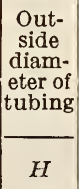 } & \multicolumn{3}{|c|}{ Coupling body } & \multicolumn{3}{|c|}{$\begin{array}{l}\text { Full-coupling } \\
\text { rubber washer }\end{array}$} & \multirow{2}{*}{$\begin{array}{c}\text { Half- } \\
\text { coup- } \\
\text { ling } \\
\text { rubber } \\
\text { washer }\end{array}$} \\
\hline & & $B$ & $C$ & $D$ & $E$ & $F$ & $G$ & & $J$ & $K$ & $L$ & $M$ & $N$ & 0 & \\
\hline $\begin{array}{l}1^{3 / 4} \\
114 \\
11 / 2 \\
2\end{array}$ & $\begin{array}{l}14 \\
111 / 2 \\
111 / 2 \\
111 / 2 \\
111 / 2\end{array}$ & $\begin{array}{l}11 / 8 \\
138 \\
13 / 4 \\
2 \\
21 / 2\end{array}$ & $\begin{array}{l}15 / 16 \\
13 / 16 \\
13 / 8 \\
13 / 2\end{array}$ & $\begin{array}{r}1 / 8 \\
1 / 8 \\
5 / 32 \\
3 / 16 \\
3 / 16\end{array}$ & $\begin{array}{l}25 / 32 \\
11 / 32 \\
19 / 32 \\
117 / 32 \\
21 / 32\end{array}$ & $\begin{array}{r}59 / 64 \\
111 / 64 \\
12964 \\
145 / 64 \\
2^{11} 1 / 64\end{array}$ & $\begin{array}{l}1^{7 / 8} \\
11 / 8 \\
11 / 4 \\
13 / 8\end{array}$ & $\begin{array}{r}5 / 8 \\
7 / 8 \\
11 / 8 \\
13 / 8 \\
13 \%\end{array}$ & $\begin{array}{l}3 / 8 \\
3 / 8 \\
7 / 16 \\
1 / 2 \\
9 / 16\end{array}$ & $\begin{array}{r}5 / 16 \\
3 / 8 \\
7 / 16 \\
1 / 2 \\
9 / 16\end{array}$ & $\begin{array}{l}11 / 8 \\
13 / 8 \\
13 / 4 \\
2 \\
23 / 8\end{array}$ & $\begin{array}{l}29 / 32 \\
11 / 8 \\
17 / 16 \\
1^{21 / 32} \\
21 / 8\end{array}$ & $\begin{array}{l}1 / 2 \\
9 / 16 \\
5 / 8 \\
11 / 16 \\
3 / 4\end{array}$ & $\begin{array}{l}3 / 32 \\
3 / 32 \\
3 / 32 \\
1 / 8 \\
1 / 8\end{array}$ & $\begin{array}{l}5 / 16 \\
11 / 32 \\
3 / 8 \\
13 / 32 \\
7 / 16\end{array}$ \\
\hline
\end{tabular}

The bottling equipment section of the B. A. I. C. |necessary to thread the tubing, the tubing being cut also recommends the use of a compression coupling off square and the joint made by compressing a adapted to the use of light-gage metal tubing lllus- rubber ring around it. It will be very easy to take trated above. This coupling is so constructed that the possibility of bacterial contamination in the
joint itself is reduced to a minimum. It is not por cleansing. The method of joint
pipe line to tanks or valves is also shown. 


\section{ASSOCIATED FACTORY MUTUAL FIRE IN- SURANCE COMPANIES, SPECIFICATIONS FOR FIRE HOSE, COUPLINGS, AND PLAY PIPES, 1930}

(See also 607.0, p. 363.)

\section{HOSE COUPLINGS}

Workmanship.-Couplings must be made, finished, and fitted in a workmanlike manner throughout.

Pattern.-Couplings must be of the expansionring pattern, of ample strength, and of the best form to resist the strain of expanding the binding ring in the coupling, and must have a tail part sufficiently long to extend it one-eighth inch beyond the end of the expansion ring. The female portion of the coupling shall have a swivel either of the tongueand-groove type, the wire-and-groove type, or a still thread.

Expansion rings.-Expansion rings must be made of well-annealed brass or other noncorrodible alloy of equally suitable properties. For $2 \frac{1}{2}$-inch couplings the ring must be at least $11 / 4$ inches long and

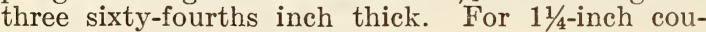
plings the ring must be at least three-fourths inch long and one thirty-second inch thick.

Washer.-Each coupling must be fitted with a rubber washer at least three-sixteenths inch thick for $2 \frac{1}{2}$-inch couplings, and at least one-eighth inch thick for $1 \frac{1}{2}$-inch couplings.

Composition.-Couplings must be made of an alloy of copper, tin, and zinc (and lead if desired) of the following proportions:

$\begin{array}{lr}\text { Copper, not less than } & \text { Per cent } \\ \text { Tin, not less than } & 53 \\ \text { Zinc, not more than } & \\ \text { Lead, not more than } & \end{array}$

Weight.-Couplings, including both male and female expansion rings, must weigh not less than:

21/2-inch for cotton rubber-lined hose Pound

$21 / 2$-inch for linen hose..................

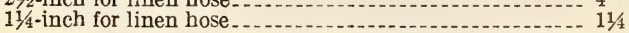

Marking.-Both male and female couplings must be stamped with the name or registered trade-mark of the maker, the date of manufacture, and the $\widehat{F M}\rangle$ identification mark, the marking to consist of letters and figures not less than one-eighth inch high.

Gasket.-Couplings for cotton rubber-lined hose must be provided with rubber gaskets of best quality, accurately fitted and not extending into the waterway.

Thread.-(See 607.0, p. 633.)

SOCIETY OF AUTOMOTIVE ENGINEERS, PARTS AND FITTINGS, DRAIN COCKS, S. A. E. RECOMMENDED PRACTICE, FEBRUARY, 1921
DRAIN COCKS

\begin{tabular}{|c|c|}
\hline Thread size 1 & $\begin{array}{c}\text { Equivalent } \\
\text { passage } \\
\text { diameter, } \\
\text { minimum 2 }\end{array}$ \\
\hline $1 / 8$ & $5 / 32$ \\
$1 / 4$ & $1 / 4$ \\
$3 / 8$ & $11 / 32$ \\
$1 / 2$ & $7 / 16$ \\
\hline
\end{tabular}

1 Taper pipe thread.

2 The cross-sectional area of the passages throughout their lengths shall not be less than those obtained with the minimum diameters given.

SOCIETY OF AUTOMOTIVE ENGINEERS, PARTS AND FITTINGS; FUEL AND LU. BRICATION TUBE FITTINGS, S. A. E. STANDARD, JULY, 1925

\section{FUEL AND LUBRICATION FITTINGS} SOLDERED TYPE
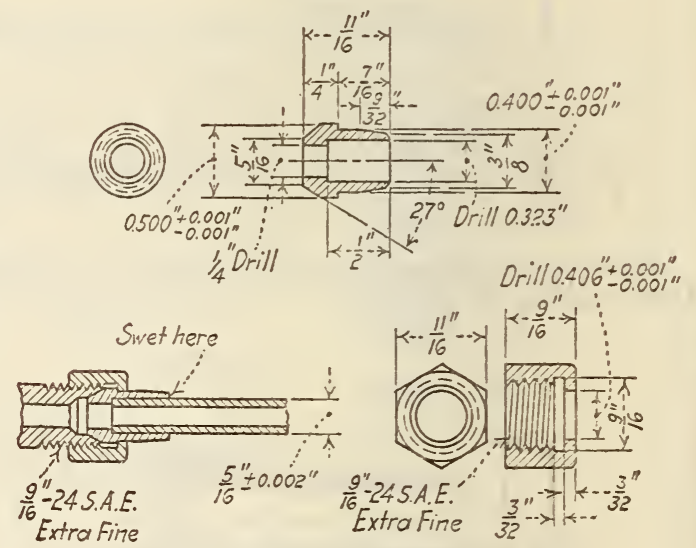

FIGURE 1094. - Soldered type

Thread tolerances for fittings and nuts

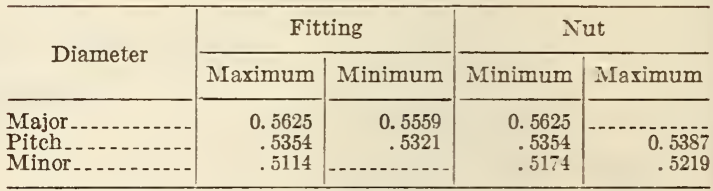

These tolerances are the same as are specified for 24 threads per inch for the S. A. E. fine-thread free (class 2) fit tolerances.

The corners at both ends of the nut shall be chamfered $45^{\circ}$ to the flats.

The sleeve shall be made of brass and the nut of steel, nickel-plated over a coating of copper. 
FLARED TYPE
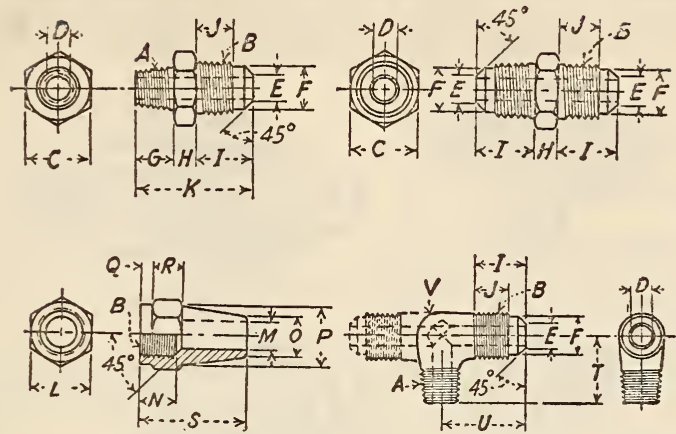

FIGURE 1095.-Flared type

[All dimensions in inches]

\begin{tabular}{|c|c|c|c|c|c|c|c|c|c|c|c|c|}
\hline $\begin{array}{l}\text { Tubing } \\
\text { diame- } \\
\text { ter } \\
\pm 0.002\end{array}$ & $A^{1}$ & $B^{2}$ & $C$ & $D$ & $E$ & $F$ & $G$ & $H$ & $I$ & $J$ & $K$ & $L$ \\
\hline $\begin{array}{l}1 / 8 \\
3116 \\
114 \\
5 / 16 \\
3 / 8 \\
7 / 16 \\
1 / 2\end{array}$ & $\begin{array}{l}1 / 8 \\
1 / 8 \\
1 / 8 \\
1 / 8 \\
1 / 4 \\
114 \\
3 / 8\end{array}$ & $\begin{array}{l}5 / 16-24 \\
3 / 8-24 \\
7 / 16-20 \\
1 / 2-20 \\
5 / 8-18 \\
11 / 16-16 \\
3 / 4-16\end{array}$ & $\begin{array}{l}7 / 16 \\
7 / 16 \\
7 / 16 \\
1 / 2 \\
5 / 8 \\
11 / 16 \\
3 / 4\end{array}$ & $\begin{array}{l}5 / 64 \\
1 / 8 \\
3 / 16 \\
7 / 32 \\
9 / 32 \\
5 / 16 \\
13 / 32\end{array}$ & $\begin{array}{l}7 / 64 \\
5 / 32 \\
7 / 32 \\
1 / 4 \\
5 / 16 \\
11 / 32 \\
7 / 16\end{array}$ & $\begin{array}{l}15 / 64 \\
19 / 64 \\
11 / 32 \\
13 / 32 \\
17 / 32 \\
37 / 64 \\
41 / 64\end{array}$ & $\begin{array}{l}3 / 8 \\
3 / 8 \\
3 / 8 \\
3 / 8 \\
7 / 16 \\
7 / 16 \\
1 / 2\end{array}$ & $\begin{array}{l}5 / 32 \\
3 / 16 \\
3 / 16 \\
7 / 32 \\
1 / 4 \\
9 / 32 \\
5 / 16\end{array}$ & $\begin{array}{l}5 / 16 \\
3 / 8 \\
1 / 2 \\
9 / 16 \\
5 / 8 \\
11 / 16 \\
3 / 4\end{array}$ & $\begin{array}{l}1 / 4 \\
9 / 32 \\
11 / 32 \\
3 / 8 \\
13 / 32 \\
7 / 16 \\
1 / 2\end{array}$ & $\begin{array}{l}27 / 32 \\
15 / 16 \\
11 / 16 \\
15 / 32 \\
15 / 16 \\
113 / 32 \\
19 / 16\end{array}$ & $\begin{array}{l}3 / 8 \\
7 / 16 \\
9 / 16 \\
5 / 8 \\
3 / 4 \\
13 / 16 \\
7 / 8\end{array}$ \\
\hline \multirow{2}{*}{$\begin{array}{l}\text { Tubing } \\
\text { diame- } \\
\text { ter }\end{array}$} & \multicolumn{2}{|c|}{$M$} & \multirow[b]{2}{*}{$N$} & \multirow[b]{2}{*}{0} & \multirow[b]{2}{*}{$P$} & \multirow[b]{2}{*}{$Q$} & \multirow[b]{2}{*}{$R$} & \multicolumn{2}{|c|}{$S$} & \multirow[b]{2}{*}{$T$} & \multirow[b]{2}{*}{$U$} & \multirow[b]{2}{*}{$V$} \\
\hline & $\begin{array}{l}\text { Mini- } \\
\text { mum }\end{array}$ & $\begin{array}{l}\text { Maxi- } \\
\text { mum }\end{array}$ & & & & & & Long & Short & & & \\
\hline $\begin{array}{l}1 / 8 \\
3 / 16 \\
1 / 4 \\
5 / 16 \\
3 / 8 \\
7 / 16 \\
1 / 2\end{array}$ & $\begin{array}{r}0.130 \\
.192 \\
.255 \\
.317 \\
.380 \\
.442 \\
.505\end{array}$ & $\begin{array}{l}0.135 \\
.197 \\
.260 \\
.322 \\
.385 \\
.447 \\
.510\end{array}$ & $\begin{array}{l}7 / 32 \\
9 / 32 \\
11 / 32 \\
3 / 8 \\
7 / 16 \\
1 / 2 \\
9 / 16\end{array}$ & $\begin{array}{l}1 / 4 \\
51 / 6 \\
3 / 8 \\
7 / 16 \\
1 / 2 \\
9 / 16 \\
5 / 8\end{array}$ & $\begin{array}{l}3 / 8 \\
7 / 16 \\
9 / 16 \\
5 / 8 \\
3 / 4 \\
13 / 16 \\
7 / 8\end{array}$ & $\begin{array}{l}3 / 32 \\
3 / 32 \\
1 / 8 \\
1 / 8 \\
1 / 8 \\
1 / 8 \\
1 / 8\end{array}$ & $\begin{array}{l}1 / 8 \\
3 / 16 \\
1 / 4 \\
5 / 16 \\
3 / 8 \\
7 / 16 \\
1 / 2\end{array}$ & $\begin{array}{l}3 / 4 \\
13 / 16 \\
15 / 16 \\
11 / 8 \\
15 / 16 \\
11 / 2 \\
15 / 8\end{array}$ & $\begin{array}{l}1 / 2 \\
5 / 8 \\
3 / 4 \\
7 / 8 \\
1 \\
11 / 16 \\
11 / 8\end{array}$ & $\begin{array}{l}11 / 16 \\
3 / 4 \\
3 / 4 \\
3 / 4 \\
7 / 8 \\
15 / 16 \\
1\end{array}$ & $\begin{array}{l}5 / 8 \\
3 / 4 \\
7 / 8 \\
15 / 16 \\
1 \\
11 / 8 \\
13 / 16\end{array}$ & $\begin{array}{l}1 / 16 \\
3 / 32 \\
1 / 8 \\
1 / 4 \\
9 / 32 \\
5 / 16 \\
11 / 32\end{array}$ \\
\hline
\end{tabular}

1 A merican standard taper pipe threads.

2 All threads shall be in accordance with the fine-thread series, free (class 2) fit.
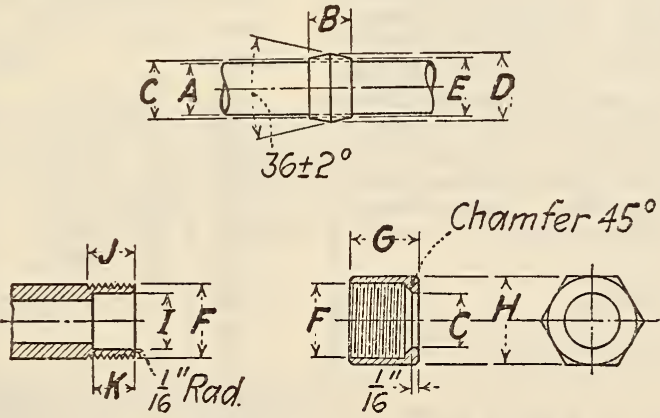

FIGURE 1096.-Compression type

Dimensions for sleeves, nuts, and threaded ends

[All dimensions in inches]

\begin{tabular}{|c|c|c|c|c|c|c|c|c|c|c|}
\hline $\begin{array}{c}\text { Tubing } \\
\text { diame- } \\
\text { ter } A \\
\pm 0.002\end{array}$ & $B$ & $\begin{array}{c}\text { Sleeve } \\
\text { bore } C \\
\pm 0.002\end{array}$ & $\begin{array}{c}\text { Stock } \\
\text { diame- } \\
\text { ter }{ }^{1} D\end{array}$ & $E^{2}$ & $F$ & $G$ & $H$ & $\begin{array}{c}I \\
\pm 0.002\end{array}$ & $\begin{array}{c}J, \\
\text { mini- } \\
\text { mum }\end{array}$ & $K$ \\
\hline $1 / 8$ & $3 / 16$ & 0.130 & $3 / 16$ & 0.140 & $5 / 16-24$ & $3 / 8$ & $3 / 8$ & 0.136 & $1 / 4$ & $3 / 16$ \\
$3 / 16$ & $7 / 32$ & .193 & $17 / 64$ & .205 & $3 / 8-24$ & $13 / 32$ & $7 / 16$ & .196 & $9 / 32$ & $7 / 32$ \\
$1 / 4$ & $1 / 4$ & .257 & $11 / 32$ & .269 & $7 / 16-24$ & $7 / 16$ & $1 / 2$ & .261 & $5 / 16$ & $1 / 4$ \\
$5 / 16$ & 114 & .323 & $13 / 32$ & .335 & $112-24$ & $7 / 16$ & $9 / 16$ & .328 & $11 / 32$ & $9 / 32$ \\
$3 / 8$ & $1 / 4$ & .386 & $15 / 32$ & .398 & $9516-24$ & $15 / 32$ & $5 / 8$ & .391 & $3 / 8$ & $5 / 16$ \\
$7 / 16$ & $5 / 16$ & .444 & $17 / 32$ & .455 & $5 / 8-24$ & $1 / 2$ & $11 / 16$ & .449 & $13 / 32$ & $11 / 32$ \\
$1 / 2$ & $3 / 8$ & .515 & $5 / 8$ & .527 & $11 / 16-20$ & $5 / 8$ & $13 / 16$ & .531 & $7 / 16$ & $3 / 8$ \\
\hline
\end{tabular}

A snort flat will appear on the sleeves for the $1 / 4,5 / 16$, and $3 / 8$ inch sizes as a result of the stock diameters selected. Small diameter of tapered sleeve. 
The adoption of this standard by the society does / patent that may exist and does not constitute a not insure users of constructions incorporating this recommendation of any patented or proprietary apstandard against liability for infringement of any plication that may be involved.

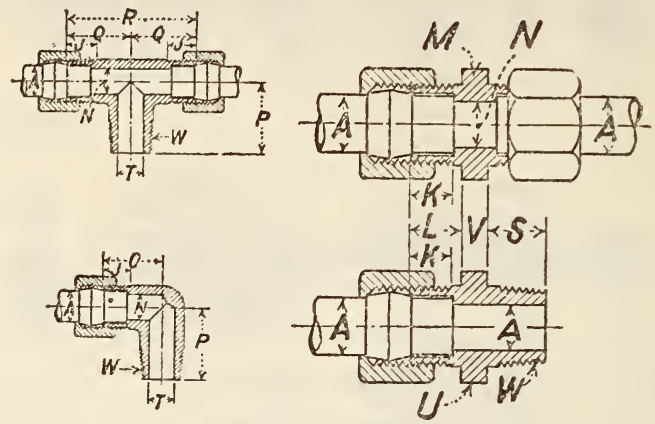

FIGURE 1097.-Compression type

Dimensions for elbows, tees, and unions

[All dimensions in inches]

\begin{tabular}{|c|c|c|c|c|c|c|c|c|c|c|c|c|}
\hline $\begin{array}{l}\text { Tubing } \\
\text { diame- } \\
\text { ter } A\end{array}$ & $L$ & $\begin{array}{l}\text { Hex- } \\
\text { agonal } \\
\text { diame- } \\
\text { ter } M\end{array}$ & $N$ & 0 & $P$ & $Q$ & $R$ & $S$ & $T$ & $\begin{array}{l}\text { Hex- } \\
\text { agonal } \\
\text { diame- } \\
\text { ter } U\end{array}$ & $V$ & $W^{1}$ \\
\hline $\begin{array}{l}18 \\
3 / 16 \\
1 / 4 \\
5 / 16 \\
3 / 8 \\
7 / 16 \\
1 / 2\end{array}$ & $\begin{array}{l}1 / 4 \\
9 / 32 \\
5 / 16 \\
11 / 32 \\
3 / 8 \\
13 / 32 \\
7 / 16\end{array}$ & $\begin{array}{l}5 / 16 \\
3 / 8 \\
7 / 16 \\
1 / 2 \\
9 / 16 \\
5 / 8 \\
11 / 16\end{array}$ & $\begin{array}{l}3 / 32 \\
1 / 8 \\
3 / 16 \\
1 / 4 \\
5 / 16 \\
11 / 32 \\
13 / 32\end{array}$ & $\begin{array}{l}5 / 8 \\
5 / 8 \\
5 / 8 \\
5 / 8 \\
3 / 4 \\
27 / 32 \\
15 / 16\end{array}$ & $\begin{array}{l}11 / 16 \\
11 / 16 \\
3 / 4 \\
3 / 4 \\
7 / 8 \\
1 \\
11 / 8\end{array}$ & $\begin{array}{l}11 / 16 \\
11 / 16 \\
11 / 16 \\
11 / 16 \\
13 / 16 \\
29 / 32 \\
1\end{array}$ & $\begin{array}{l}13 / 8 \\
13 / 8 \\
13 / 8 \\
13 / 8 \\
15 / 8 \\
1^{13 / 16} \\
2^{3 / 3}\end{array}$ & $\begin{array}{l}3 / 8 \\
3 / 8 \\
3 / 8 \\
3 / 8 \\
7 / 16 \\
7 / 16 \\
1 / 2\end{array}$ & $\begin{array}{l}3 / 16 \\
3 / 16 \\
3 / 16 \\
15 / 64 \\
5 / 16 \\
5 / 16 \\
13 / 32\end{array}$ & $\begin{array}{l}7 / 16 \\
7 / 16 \\
7 / 16 \\
1 / 2 \\
9 / 16 \\
5 / 8 \\
11 / 16\end{array}$ & $\begin{array}{l}5 / 32 \\
3 / 16 \\
3 / 16 \\
3 / 16 \\
7 / 32 \\
7 / 32 \\
7 / 32\end{array}$ & $\begin{array}{l}1 / 8 \\
1 / 8 \\
1 / 8 \\
1 / 8 \\
1 / 4 \\
1 / 4 \\
3 / 8\end{array}$ \\
\hline
\end{tabular}

1 American standard pipe thread.

Both ends of the unions shall be chamfered $45^{\circ}$ to the flats.

UNITED STATES GOVERNMENT, DEPARTMENT OF COMIMERCE, BRASS PIPE NIPPLES, COMMERCIAI STANDARDS CS1029, JULY 1, 1929

\section{BRASS PIPE NIPPLES}

(In respect to dimensions, this specification is identical with Federal Specifications Board specification WW-N-351, given below. In respect to material, this specification is identical with Federal Specifications Board specification WW-P-351 (see 645.23, p. 1217), except for slight tolerance differences in the copper and lead components of grade A (red brass).)

(Taper pipe-thread dimensions are identical with those of the National Screw Thread Commission under commodity designation 607.0, p. 365. Stock sizes of standard weight contained in carton are as follows:)

\begin{tabular}{|c|c|c|c|c|c|c|c|c|c|c|c|c|}
\hline \multirow{2}{*}{$\begin{array}{l}\text { Iron } \\
\text { pipe } \\
\text { size }\end{array}$} & \multirow{2}{*}{ Close } & \multicolumn{10}{|c|}{ Long (in inches) } & \multirow{2}{*}{ Total } \\
\hline & & $11 / 2$ & 2 & $21 / 2$ & 3 & $31 / 2$ & 4 & $41 / 2$ & 5 & $51 / 2$ & 6 & \\
\hline $\begin{array}{c}\text { Inch } \\
3 / 8 \\
1 / 2 \\
1^{3 / 4}\end{array}$ & $\begin{array}{l}20 \\
20 \\
20 \\
15\end{array}$ & $\begin{array}{c}10 \\
10 \\
10 \\
-. .-\end{array}$ & $\begin{array}{l}15 \\
15 \\
15 \\
12\end{array}$ & $\begin{array}{r}10 \\
10 \\
10 \\
5\end{array}$ & $\begin{array}{l}10 \\
10 \\
10 \\
12\end{array}$ & $\begin{array}{l}\mathbf{5} \\
\mathbf{5} \\
\mathbf{5} \\
\mathbf{3}\end{array}$ & $\begin{array}{l}10 \\
10 \\
10 \\
12\end{array}$ & $\begin{array}{l}5 \\
5 \\
5 \\
3\end{array}$ & $\begin{array}{l}5 \\
5 \\
5 \\
5\end{array}$ & $\begin{array}{l}5 \\
5 \\
5 \\
3\end{array}$ & $\begin{array}{l}5 \\
5 \\
5 \\
5\end{array}$ & $\begin{array}{r}100 \\
100 \\
100 \\
75\end{array}$ \\
\hline $\begin{array}{r}3 / 8 \\
1 / 2 \\
3 / 1 \\
1^{3 / 4}\end{array}$ & $\begin{array}{l}12 \\
12 \\
12 \\
10\end{array}$ & $\begin{array}{r}5 \\
5 \\
5 \\
-\end{array}$ & $\begin{array}{r}5 \\
5 \\
5 \\
10\end{array}$ & $\begin{array}{l}5 \\
5 \\
5 \\
5\end{array}$ & $\begin{array}{l}5 \\
5 \\
5 \\
5\end{array}$ & $\begin{array}{l}\mathbf{5} \\
\mathbf{5} \\
5 \\
\mathbf{3}\end{array}$ & $\begin{array}{l}\mathbf{5} \\
\mathbf{5} \\
\mathbf{5} \\
\mathbf{5}\end{array}$ & $\begin{array}{l}2 \\
2 \\
2 \\
2\end{array}$ & $\begin{array}{l}2 \\
2 \\
2 \\
4\end{array}$ & $\begin{array}{l}2 \\
2 \\
2 \\
2\end{array}$ & $\begin{array}{l}2 \\
2 \\
2 \\
4\end{array}$ & $\begin{array}{l}\mathbf{5 0} \\
\mathbf{5 0} \\
\mathbf{5 0} \\
\mathbf{5 0}\end{array}$ \\
\hline
\end{tabular}

UNITED STATES GOVERNMENT, DEPARTMENT OF COMMERCE, BUREAU OF STANDARDS, BRASS LAVATORY AND SINK TRAPS, SIMPLIFIED PRACTICE RECOMMENDATION R21, 1925

\section{BRASS LAVATORY AND SINK TRAPS}

In accordance with the unanimous action of the general conference of representatives of manufac- turers, distributors, and users of brass lavatory and sink traps, the United States Department of Commerce, through the Bureau of Standards, recommends that the recognized sizes, styles, and gages of brass lavatory and sink traps be reduced to the following list:

WROUGHT TRAPS

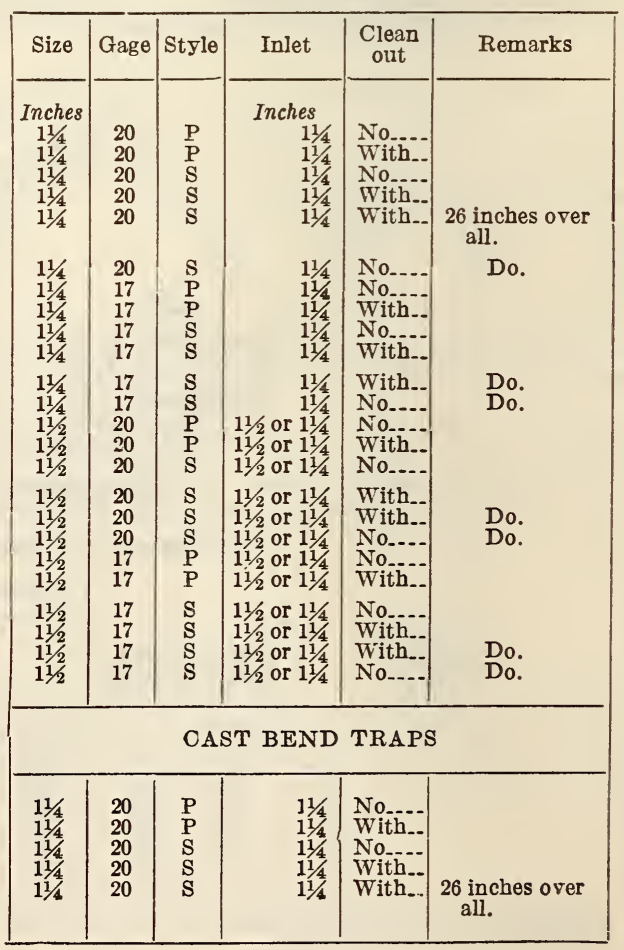


NEW YORK AND BOSTON REGULATION TRAPS

\begin{tabular}{|c|c|c|c|}
\hline Size & Gage & Style & Inlet \\
\hline $\begin{array}{r}\text { Inches } \\
11 / 4 \\
11 / 2 \\
2 \\
11 / 2 \\
11 \\
11 \\
11 \\
11 \\
112\end{array}$ & $\begin{array}{c}\text { Bag offset... } \\
\\
\\
\end{array}$ & \begin{tabular}{l}
$\mathrm{P}$ \\
$\mathrm{P}$ \\
$\mathrm{P}$ \\
$\mathrm{P}$ \\
\hdashline- \\
\hdashline- \\
\hdashline-1 \\
-1.
\end{tabular} & $\begin{array}{l}11 / 4 \text { inches external. } 1 \\
11 / 2 \text { or } 11 / 4 \text { inches external. } 1 \\
2,11 / 2 \text {, or } 11 / 4 \text { inches external } 1 \\
11 / 2 \text { or } 11 / 4 \text { inches internal. } \\
11 / 4 \text { inches tubing outlet. } \\
11 / 2 \text { or } 11 / 4 \text { inches tubing outlet. } \\
11 / 4 \text { inches I P outlet. } \\
11 / 2 \text { or } 11 / 4 \text { inches I } \mathrm{P} \text { outlet. }\end{array}$ \\
\hline
\end{tabular}

${ }^{1}$ Antisyphon traps (S) to be furnished with either straight or offset outlet tube as required.

It is further recommended that-

1. All traps be listed and supplied less connections.

2. All traps be stamped with the name of the manufacturer and with gage.

3. Elimination of all gages except No. 20 B. \& S. and No. 17 B. \& S. gage. (Every effort to be made to bring practice to use No. 17 B. \& S. gage minimum, thus reducing the present variety and adopting one standard gage for all types of traps.)

4. All crown-vented traps to be eliminated.

5. All traps made in accordance with special city codes, such as Kansas City, Los Angeles, and Cleveland, to be eliminated. (The eliminations under items 4 and 5 were intended as a step forward, as sufficient combinations and types were included in the recognized varieties to meet all local conditions. Local health authorities and engineers are urged to revise codes and specifications in order to keep abreast of the advance in practice as recommended by the industry.)

These recommendations became effective January 1,1925 , subject to regular annual revision by a similar conference of the industry.

UNITED STATES GOVERNMENT, FEDERAI SPECIFICATIONS BOARD; FEDERAI SPECIFICATION FOR PLUMBING FIXTURES (FOR LAND USE), NO. 448, NOVEMBER 22, 1926. (CURRENT DESIGNATION : FF-P-451)

\section{PLOMBING FIXTURES (FOR LAND USE)}

(See 617.73, p. 1062.)

UNITED STATES GOVERNMENT, FEDERAI
SPECIFICATIONS BOARD; FEDERAI SPECIFICATION FOR NIPPLES, PIPEBRASS, STEEL, AND WROUGHT IRON, WW-N-351, JUNE 24, 1930

\section{BRASS NIPPLES}

(See 607.4, p. 485.)

\section{UNITED STATES GOVERNMENT, FEDERAL SPECIFICATIONS BOARD; FEDERAL SPECIFICATION FOR PIPE FITTINGS- BRASS OR BRONZE (THREADED), 125 POUND, WW-P-448, MAY 26, 1931}

\section{BRASS OR BRONZE PIPE FITTINGS}

\section{A. APPLICABLE FEDERAI SPECIFICATIONS}

$A-1$. The following Federal specifications, of the issue in effect on date of irvitation for bids, shall form a part of this specification:

\begin{tabular}{|c|c|c|c|}
\hline $\begin{array}{l}\text { Federal Speci- } \\
\text { fication }\end{array}$ & $\begin{array}{c}\text { Former } \\
\text { No. }\end{array}$ & Title & See \\
\hline $\begin{array}{l}\text { GGG-P-351.. } \\
Q Q-M-151 \ldots\end{array}$ & $\begin{array}{l}238 \\
339\end{array}$ & $\begin{array}{l}\text { Pipe threads. } \\
\text { General specifications for } \\
\text { metals. }\end{array}$ & $\begin{array}{l}607.0, \text { p. } 365 . \\
600.0, \text { p. } 3\end{array}$ \\
\hline
\end{tabular}

\section{B. CLASS AND COMPOSITION}

B-1. Fittings shall be threaded and of the 125pound class.

B-2. Fittings shall be of the following chemical compositions as specified in paragraph $\mathrm{E}-1$ : Composition $\mathrm{A}$ and composition $\mathrm{B}$.

\section{MATERIAL AND WORKMANSHIP}

C-1. All cast fittings shall be sound, smoothly cored, true to form, uniform in texture and free from cold shuts, porosity, or any other defect which might affect their serviceability. They shall be thoroughly cleaned both inside and outside, and all fins and roughness shall be removed.

C-2. All wrought fittings shall be free from defects and shall be machined to dimensions required.

\section{GENERAL REQUIREMENTS}

D-1. The name or trade-mark of the manufacturer or his initial shall be cast upon the outside of the body of each rough cast fitting or stamped upon the outside of each wrought fitting.

$\mathrm{D}-2$. All fittings shall be threaded in accordance with Federal specification GGG-P-351, pipe threads, referred to in paragraph $\mathrm{A}-1$.

D-2a. The threads of all fittings shall be sharp and clean and shall not cut the walls of the fittings. Threading shall be concentric with the axis of the fitting.

D-2b. All fittings with external threads shall be chamfered at the end at an angle of about $45^{\circ}$ with the axis of the thread.

D-2c. All fittings with internal threads shall be countersunk at the end a distrance of not less than one-half the pitch of one thread and at an angle of about $45^{\circ}$ with the axis of the thread.

D-3. In measuring the length of threads specified in all tables of dimensions, the countersink or chamfer shall be included.

$\mathrm{D}-4$. The band on all fittings shall be rectangular in section with or without rounded corners.

D-5. A bevel on the face of the fittings not exceeding $5^{\circ}$ will be permitted.

D-6. Fitting dimensions shall be in accordance with the sketches and tables incorporated herein with the option permitted in paragraph $\mathrm{D}-5$ as to bevel.

D-7. The following tolerances shall be permitted in center to end dimensions for all fittings:

\begin{tabular}{|c|c||c|c|}
\hline $\begin{array}{c}\text { Iron pipe } \\
\text { size }\end{array}$ & $\begin{array}{c}\text { Tolerance } \\
\text { (plus or } \\
\text { minus) }\end{array}$ & $\begin{array}{c}\text { Iron pipe } \\
\text { size }\end{array}$ & $\begin{array}{c}\text { Tolerance } \\
\text { (plus or } \\
\text { minus) }\end{array}$ \\
\hline Inches & Inch & Inches & Inch \\
$1 / 8$ & 0.03 & $11 / 2$ & 0.08 \\
$1 / 4$ & .04 & 2 & .08 \\
$3 / 8$ & .05 & $21 / 2$ & .10 \\
$1 / 2$ & .06 & 3 & .10 \\
$3 / 4$ & .06 & $31 / 2$ & .10 \\
11 & .07 & 4 & .12 \\
$11 / 4$ & .07 & & \\
\hline
\end{tabular}

\section{E. DETAIL REQUIREMENTS}

E-1. The chemical composition of 125 -pound castbronze fittings shall be as follows:

\begin{tabular}{|c|c|c|c|c|c|c|}
\hline Composition & $\begin{array}{l}\text { Cop- } \\
\text { per }\end{array}$ & Tin & Zinc & Lead & $\begin{array}{l}\text { Iron } \\
\text { (maxi- } \\
\text { mum) }\end{array}$ & $\begin{array}{l}\text { Other } \\
\text { elements } \\
\text { (maxi- } \\
\text { mum) }\end{array}$ \\
\hline A & $\begin{array}{c}\text { Pet cent } \\
78-82 \\
84-86\end{array}$ & $\begin{array}{c}\text { Per cent } \\
4-6 \\
4-6\end{array}$ & $\begin{array}{r}\text { Per cent } \\
10-16 \\
4-6\end{array}$ & $\begin{array}{c}\text { Per cent } \\
2-3 \\
4-6\end{array}$ & $\begin{array}{c}\text { Per cent } \\
0.35 \\
.25\end{array}$ & $\begin{array}{r}\text { Per cent } \\
1.00 \\
1.00\end{array}$ \\
\hline
\end{tabular}


E-2. The chemical composition of 125-pound wrought-bronze fittings shall be as follows (see notes, Tables 4, 7, and 10):

Copper

Lead, maximum

Per cent

$83-86$

Iron, maximum .06

Zinc.... Remainder.

E-3. Fittings shall conform to dimensions specified in Tables 1 to 11 , inclusive.

E-4. Wall thickness of reducing fittings shall be that required for the iron pipe sizes involved.

E-5. All fittings shall pass the tests prescribed in section $\mathrm{F}$.

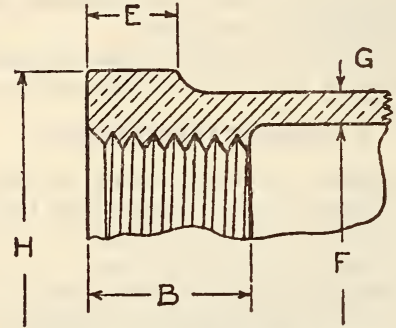

FIGURE 1098. - Detail dimensions, end of fittings

TABLE 1.-Detail dimensions

\begin{tabular}{|c|c|c|c|c|c|c|}
\hline \multirow{3}{*}{$\begin{array}{l}\text { Iron pipe size } \\
\text { (in inches) }\end{array}$} & $B$ & $E$ & & & $G$ & $H$ \\
\hline & \multirow{2}{*}{$\begin{array}{l}\text { Length } \\
\text { of } \\
\text { thread, } \\
\text { mini- } \\
\text { mum }\end{array}$} & \multirow{2}{*}{$\begin{array}{l}\text { Width } \\
\text { of band } \\
\text { mini- } \\
\text { mum }\end{array}$} & \multicolumn{2}{|c|}{$\begin{array}{l}\text { Inside diameter } \\
\text { of fittings }\end{array}$} & \multirow{2}{*}{$\begin{array}{l}\text { Metal } \\
\text { thick- } \\
\text { ness, } \\
\text { mini- } \\
\text { mum }\end{array}$} & \multirow{2}{*}{$\begin{array}{c}\text { Band } \\
\text { diame- } \\
\text { ter, } \\
\text { mini- } \\
\text { mum }\end{array}$} \\
\hline & & & $\begin{array}{l}\text { Mini- } \\
\text { mum }\end{array}$ & $\begin{array}{l}\text { Maxi- } \\
\text { mum }\end{array}$ & & \\
\hline & $\begin{array}{r}\text { Inches } \\
0.25 \\
.32 \\
.36 \\
.43 \\
.50\end{array}$ & $\begin{array}{c}\text { Inch } \\
0.14 \\
.16 \\
.17 \\
.19 \\
.23\end{array}$ & $\begin{array}{r}\text { Inches } \\
0.41 \\
.54 \\
.68 \\
.84 \\
1.05\end{array}$ & $\begin{array}{r}\text { Inches } \\
0.44 \\
.58 \\
.72 \\
.90 \\
1.11\end{array}$ & $\begin{array}{c}\text { Inch } \\
0.08 \\
.08 \\
.09 \\
.09 \\
.10\end{array}$ & $\begin{array}{r}\text { Inches } \\
0.67 \\
1.81 \\
1.00 \\
1.17 \\
1.42\end{array}$ \\
\hline & $\begin{array}{l}.58 \\
.67 \\
.70 \\
.75\end{array}$ & $\begin{array}{l}.27 \\
.31 \\
.34 \\
.41\end{array}$ & $\begin{array}{l}1.32 \\
1.66 \\
1.90 \\
2.38\end{array}$ & $\begin{array}{l}1.39 \\
1.73 \\
1.97 \\
2.45\end{array}$ & $\begin{array}{l}11 \\
.12 \\
.13 \\
.15\end{array}$ & $\begin{array}{l}1.72 \\
2.10 \\
2.38 \\
2.92\end{array}$ \\
\hline & $\begin{array}{r}.92 \\
.98 \\
1.03 \\
1.08\end{array}$ & $\begin{array}{l}.48 \\
.55 \\
.60 \\
.66\end{array}$ & $\begin{array}{l}2.88 \\
3.50 \\
4.00 \\
4.50\end{array}$ & $\begin{array}{l}2.98 \\
3.60 \\
4.10 \\
4.60\end{array}$ & $\begin{array}{l}.17 \\
.19 \\
.20 \\
.22\end{array}$ & $\begin{array}{l}3.49 \\
4.20 \\
4.75 \\
5.31\end{array}$ \\
\hline
\end{tabular}

TABLE 2.-Center to end dimensions of $90^{\circ}$ elbows, tees, crosses, and $45^{\circ}$ elbows, straight

[Detail dimensions shall be as given in Table 1]

\begin{tabular}{|c|c|c|}
\hline \multirow[b]{2}{*}{ Iron pipe size (in inches) } & $A$ & $C$ \\
\hline & $\begin{array}{l}\text { Center to } \\
\text { end } 90^{\circ} \\
\text { elbows, } \\
\text { tees, } \\
\text { crosses }\end{array}$ & $\begin{array}{l}\text { Center to } \\
\text { end } 45^{\circ} \\
\text { elbows }\end{array}$ \\
\hline & $\begin{array}{r}\text { Inches } \\
0.54 \\
.71 \\
.82 \\
1.01 \\
1.18\end{array}$ & $\begin{array}{r}\text { Inches } \\
0.42 \\
.56 \\
.63 \\
.78 \\
.89\end{array}$ \\
\hline $1 \frac{1}{1} 1 / 42$ & $\begin{array}{l}1.43 \\
1.69 \\
1.84 \\
2.12\end{array}$ & $\begin{array}{l}1.06 \\
1.22 \\
1.30 \\
1.45\end{array}$ \\
\hline $\begin{array}{l}21 / 2 \\
31 / 2 \\
4\end{array}$ & $\begin{array}{l}2.70 \\
3.08 \\
3.42 \\
3.79\end{array}$ & $\begin{array}{l}1.95 \\
2.17 \\
2.39 \\
2.61\end{array}$ \\
\hline
\end{tabular}

Note.-Sizes $2 \frac{1}{2}$ to 4 inches, inclusive, have the same center to end dimensions " $A$ " and " $C$ " as adopted for malleable iron screwed fittings conforming to specification B16c of A. S. M. E. (See 607.2, p. 422.)
(For illustrations of above fittings, see Figure 258 under American Society of Mechanical Engineers specification B16c for malleable-iron screwed fittings 607.2 , p. 422).)

$\mathrm{T}_{\mathrm{ABLE}}$ 3.-Dimensions of street tees and $90^{\circ}$ and $45^{\circ}$ elbows, straight

[Detail dimensions other than those given shall be as in Table 1]

\begin{tabular}{|c|c|c|c|c|c|c|c|}
\hline \multirow[b]{2}{*}{$\begin{array}{l}\text { Iron pipe size } \\
\text { (in inches) }\end{array}$} & $A$ & $C$ & $G$ & $J$ & $K$ & $L$ & $\mathrm{~N}$ \\
\hline & $\begin{array}{l}\text { Centel } \\
\text { to end }\end{array}$ & $\begin{array}{l}\text { Center } \\
\text { to end } \\
45^{\circ} \text { ell }\end{array}$ & $\begin{array}{l}\text { Metal } \\
\text { thick- } \\
\text { ness, } \\
\text { mini- } \\
\text { mum }\end{array}$ & $\begin{array}{l}\text { Center } \\
\text { to end }\end{array}$ & $\begin{array}{l}\text { Center } \\
\text { to end } \\
45^{\circ} \text { ell }\end{array}$ & $\begin{array}{l}\text { Length } \\
\text { of exter- } \\
\text { nal } \\
\text { thread } \\
\text { mini- } \\
\text { mum }\end{array}$ & $\begin{array}{l}\text { Port } \\
\text { diam- } \\
\text { eter, } \\
\text { maxi- } \\
\text { mum }\end{array}$ \\
\hline & $\begin{array}{c}\text { Inches } \\
0.54 \\
.71 \\
.82 \\
1.01 \\
1.18\end{array}$ & $\begin{array}{c}\text { Inches } \\
0.42 \\
.56 \\
.63 \\
.78 \\
.89\end{array}$ & $\begin{array}{r}\text { Inch } \\
0.08 \\
.08 \\
.09 \\
.09 \\
.10\end{array}$ & $\begin{array}{c}\text { Inches } \\
0.92 \\
1.11 \\
1.24 \\
1.48 \\
1.65\end{array}$ & $\begin{array}{c}\text { Inches } \\
0.78 \\
.88 \\
.92 \\
1.06 \\
1.23\end{array}$ & $\begin{array}{c}\text { Inch } \\
0.27 \\
.41 \\
.41 \\
.54 \\
.55\end{array}$ & $\begin{array}{r}\text { Inches } \\
0.18 \\
.28 \\
.40 \\
.53 \\
.72\end{array}$ \\
\hline 2 & $\begin{array}{l}1.43 \\
1.69 \\
1.84 \\
2.12\end{array}$ & $\begin{array}{l}1.06 \\
1.22 \\
1.30 \\
1.45\end{array}$ & $\begin{array}{l}.11 \\
.12 \\
.13 \\
.15\end{array}$ & $\begin{array}{l}1.98 \\
2.24 \\
2.46 \\
2.88\end{array}$ & $\begin{array}{l}1.40 \\
1.64 \\
1.80 \\
2.14\end{array}$ & $\begin{array}{l}.69 \\
.71 \\
.73 \\
.76\end{array}$ & $\begin{array}{r}.93 \\
1.25 \\
1.47 \\
1.91\end{array}$ \\
\hline
\end{tabular}

Note.-Both thread designs shown in Figure 262 are acceptable.

(For illustrations of above fittings, see Figure 262 under American Society of Mechanical Engineers, specification B16c for malleable-iron screwed fittings (607.2, p. 422)
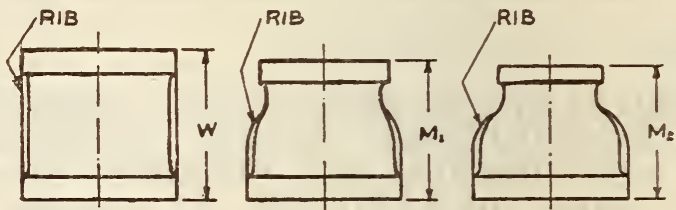

FIgURE 1099. - Straight coupling

Reducer (reducing one size)

Reducer (reducing two sizes).

TABLE 4.-End to end dimensions of cast couplings and reducers

[Detail dimensions shall be as given in Table 1]

\begin{tabular}{|c|c|c|c|}
\hline \multirow{2}{*}{ Iron pipe size (in inches) } & $W$ & $M_{1}$ & $M_{2}$ \\
\hline & $\begin{array}{c}\text { End to } \\
\text { end } \\
\text { (straight) }\end{array}$ & $\begin{array}{l}\text { End to } \\
\text { end (re- } \\
\text { ducing } \\
\text { one size) }\end{array}$ & $\begin{array}{l}\text { End to } \\
\text { end (re- } \\
\text { ducing } \\
\text { two sizes) }\end{array}$ \\
\hline & $\begin{array}{r}\text { Inches } \\
0.80 \\
.97 \\
1.05 \\
1.29 \\
1.43\end{array}$ & $\begin{array}{l}\text { Inches } \\
0.88 \\
1.01 \\
1.17 \\
1.36\end{array}$ & $\begin{array}{l}\text { Inches } \\
0.92 \\
1.13 \\
1.24\end{array}$ \\
\hline $11 / 42-1$ & $\begin{array}{l}1.68 \\
1.86 \\
1.92 \\
2.20\end{array}$ & $\begin{array}{l}1.56 \\
1.77 \\
1.89 \\
2.06\end{array}$ & $\begin{array}{l}1.49 \\
1.65 \\
1.80 \\
2.03\end{array}$ \\
\hline $\begin{array}{l}21 / 2 \\
31 / 2 \\
4\end{array}$ & $\begin{array}{l}2.88 \\
3.18 \\
3.43 \\
3.69\end{array}$ & $\begin{array}{l}3.25 \\
3.69 \\
4.00 \\
4.38\end{array}$ & $\begin{array}{l}3.25 \\
3.69 \\
4.00 \\
4.38\end{array}$ \\
\hline
\end{tabular}

NOTE.-Couplings may be cast or wrought in sizes $1 / 8$ to $3 / 4$ inch, inclusive. Dimensions of wrought couplings are given in Table 10. 2. Right-hand cast couplings have 2 ribs. Right and left cast couplings have 4 ribs on sizes smaller than 1 inch and 6 ribs on sizes 1 inch and larger.

3. End to end dimensions on sizes $2 \frac{1}{2}$ inches and larger are same as adopted for malleable-iron screwed fittings conforming to specification B16c of A. S. M. Ev (See 607.2, p. 422.) 
TABLE 5.-Center to end dimensions of $45^{\circ} \mathrm{Y}$ branch, straight

[Detail dimensions shall be as given in Table 1]

\begin{tabular}{|c|c|c|c|}
\hline \multirow[b]{2}{*}{ Iron pipe size (in inches) } & $T$ & $U$ & $\boldsymbol{V}$ \\
\hline & $\begin{array}{l}\text { Center } \\
\text { to end, } \\
\text { inlet }\end{array}$ & $\begin{array}{l}\text { Center } \\
\text { to end, } \\
\text { outlet }\end{array}$ & $\begin{array}{l}\text { End to } \\
\text { end }\end{array}$ \\
\hline $\begin{array}{l}1 / 8 \\
1 / 4 \\
3 / 8 \\
1 / 2 \\
3 / 4\end{array}$ & $\begin{array}{r}\text { Inches } \\
0.34 \\
.43 \\
.50 \\
.61 \\
.72\end{array}$ & $\begin{array}{r}\text { Inches } \\
0.81 \\
1.07 \\
1.28 \\
1.58 \\
190\end{array}$ & $\begin{array}{r}\text { Inches } \\
1.15 \\
1.50 \\
1.78 \\
2.19 \\
2.62\end{array}$ \\
\hline $11 / 21 / 2-10$ & $\begin{array}{l}.85 \\
1.02 \\
1.10 \\
1.24\end{array}$ & $\begin{array}{l}2.33 \\
2.83 \\
3.14 \\
3.76\end{array}$ & $\begin{array}{l}3.18 \\
3.85 \\
4.24 \\
5.00\end{array}$ \\
\hline $31 / 2$ & $\begin{array}{l}1.52 \\
1.71 \\
1.85 \\
2.01\end{array}$ & $\begin{array}{l}4.73 \\
5.55 \\
6.25 \\
6.97\end{array}$ & $\begin{array}{l}6.25 \\
7.26 \\
8.10 \\
8.98\end{array}$ \\
\hline
\end{tabular}

NOTE.-End to end dimensions on sizes $2 \frac{1}{2}$ inches and larger are same is adopted for malleable-iron screwed fittings conforming to specification B16c of A. S. M. E. (See 607.2, p. 422.)

(For illustration of above fittings, see Figure 261 under American Society of Mechanical Engineers, specification B16c for malleable-iron screwed fittings (607.2, p. 422).)

TABLE 6.-Center to center dimensions of close, medium, and open pattern return bends

[Detail dimensions sha]l be as given in Table 1]

\begin{tabular}{|c|c|c|c|}
\hline & $R_{\mathrm{l}}$ & $R_{2}$ & $R_{3}$ \\
\hline Iron pipe"size (in inches) & $\begin{array}{c}\text { Center } \\
\text { to } \\
\text { center, } \\
\text { close } \\
\text { pattern }\end{array}$ & $\begin{array}{c}\text { Center } \\
\text { to } \\
\text { center, } \\
\text { medium } \\
\text { pattern }\end{array}$ & $\begin{array}{c}\text { Center } \\
\text { to } \\
\text { center, } \\
\text { open } \\
\text { pattern }\end{array}$ \\
\hline $1 / 1 / 20$ & $\begin{array}{r}\text { Inches } \\
1.00 \\
1.25 \\
1.50 \\
1.75\end{array}$ & $\begin{array}{r}\text { Inches } \\
1.25 \\
1.50 \\
1.88 \\
2.25\end{array}$ & $\begin{array}{r}\text { Inches } \\
1.50 \\
2.00 \\
2.50 \\
3.00\end{array}$ \\
\hline $11 / 2 \ldots$ & $\begin{array}{l}\text { 2. } 19 \\
2.63 \\
3.25 \\
4.00\end{array}$ & $\begin{array}{l}\text { 2. } 50 \\
\text { 3. } 00 \\
\text { 3. } 75 \\
\text { 4. } 50\end{array}$ & $\begin{array}{l}3.50 \\
4.00 \\
450 \\
5.00\end{array}$ \\
\hline
\end{tabular}

Note.-Center to center dimension $R_{1}, R_{3}$, and $R_{3}$ are the same as adopted for malleable-iron screwed fittings conforming to specification B16c of A. S. M. E. (See 607.2, p. 422.)

(For illustrations of above fittings, see Figure 265 under American Society of Mechanical Engineers, specification B16c for malleable-iron screwed fittings (607.2, p. 422).)

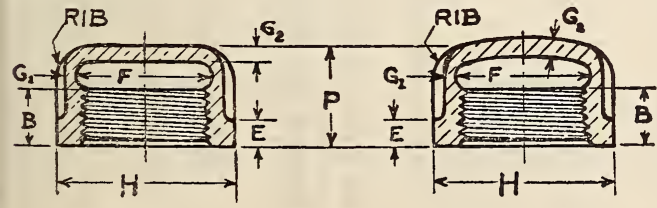

Figure 1100.-Flat top cap; crowned top cap
TABLE 7.-Detail dimensions of cast caps

\begin{tabular}{|c|c|c|c|c|c|c|c|c|c|}
\hline \multirow{3}{*}{$\begin{array}{l}\text { Iron pipe } \\
\text { size } \\
\text { (in inches) }\end{array}$} & $B$ & $E$ & \multicolumn{2}{|c|}{$F$} & $G_{1}$ & $G_{2}$ & $H$ & $P$ & $g$ \\
\hline & $\begin{array}{l}\dot{a} \\
\dot{a} \\
\tilde{\Xi} \\
\tilde{\Phi}\end{array}$ & 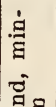 & \multicolumn{2}{|c|}{$\begin{array}{l}\text { Inside diam- } \\
\text { eter of fit- } \\
\text { tings }\end{array}$} & 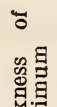 & 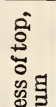 & 寻 & & $\begin{array}{l}\text { 昌 } \\
\text { 竞 } \\
0\end{array}$ \\
\hline & 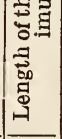 & 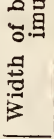 & 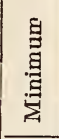 & 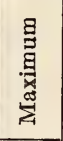 & 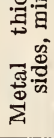 & 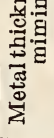 & 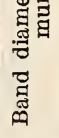 & 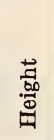 & 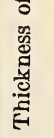 \\
\hline & $\begin{array}{l}\text { Ins. } \\
0.25 \\
.32 \\
.36 \\
.43 \\
.50\end{array}$ & $\begin{array}{c}I n . \\
0.14 \\
.16 \\
.17 \\
.19 \\
.23\end{array}$ & $\begin{array}{l}\text { Ins. } \\
0.41 \\
.54 \\
.68 \\
.84 \\
1.05\end{array}$ & $\begin{array}{r}\text { Ins. } \\
0.44 \\
.58 \\
.72 \\
.90 \\
1.11\end{array}$ & $\begin{array}{c}I n . \\
0.08 \\
.08 \\
.09 \\
.09 \\
.10\end{array}$ & $\begin{array}{c}\text { In. } \\
0.09 \\
.10 \\
.11 \\
.12 \\
.13\end{array}$ & $\begin{array}{l}\text { Ins. } \\
0.67 \\
.81 \\
1.00 \\
1.17 \\
1.42\end{array}$ & $\begin{array}{l}\text { Ins. } \\
0.49 \\
.59 \\
.64 \\
.76 \\
.84\end{array}$ & $\begin{array}{c}\text { In. } \\
0.08 \\
.08 \\
.09 \\
.09 \\
.10\end{array}$ \\
\hline & $\begin{array}{l}.58 \\
.67 \\
.70 \\
.75\end{array}$ & $\begin{array}{l}.27 \\
.31 \\
.34 \\
.41\end{array}$ & $\begin{array}{l}1.32 \\
1.66 \\
1.90 \\
2.38\end{array}$ & $\begin{array}{l}1.39 \\
1.73 \\
1.97 \\
2.45\end{array}$ & $\begin{array}{l}.11 \\
.12 \\
.13 \\
.15\end{array}$ & $\begin{array}{l}.15 \\
.17 \\
.19 \\
.22\end{array}$ & $\begin{array}{l}1.72 \\
2.10 \\
2.38 \\
2.92\end{array}$ & $\begin{array}{l}.99 \\
1.10 \\
1.15 \\
1.32\end{array}$ & $\begin{array}{l}.11 \\
.12 \\
.13 \\
.15\end{array}$ \\
\hline & $\begin{array}{r}.92 \\
.98 \\
1.03 \\
1.08\end{array}$ & $\begin{array}{l}.48 \\
.55 \\
.60 \\
.66\end{array}$ & $\begin{array}{l}2.88 \\
3.50 \\
4.00 \\
4.50\end{array}$ & $\begin{array}{l}2.98 \\
3.60 \\
4.10 \\
4.60\end{array}$ & $\begin{array}{l}.17 \\
.19 \\
.20 \\
.22\end{array}$ & $\begin{array}{r}.25 \\
.29 \\
.32 \\
.36\end{array}$ & $\begin{array}{l}3.49 \\
4.20 \\
4.75 \\
5.31\end{array}$ & $\begin{array}{l}1.70 \\
1.80 \\
1.90 \\
2.08\end{array}$ & $\begin{array}{r}.17 \\
.19 \\
.20 \\
.22\end{array}$ \\
\hline
\end{tabular}

Note 1.-Size $1 / 8$ to $3 / 4$ inch, inclusive, may be cast or wrought. Dimensions of wrought caps made from rod are given in Table 10.

2. Both flat-top and crowned-top designs are acceptable.

3. Height dimensions of sizes $1 / 8$ to 2 inches, inclusive, do not include allowance for draft or crown of top.

4. Height dimensions of size $2 \frac{1}{2}$ inches and larger, are same as adopted for malleable-iron screwed fittings, conforming to specification B16c of A. S. M. E. (See 607.2, p. 422.)

TABLE 8.-Center to end dimensions of elbows and crosses, reducing

[Detailed dimensions shall be as given in Table 1]

\begin{tabular}{|c|c|c|c|c|c|}
\hline \multicolumn{3}{|c|}{ Elbows } & \multicolumn{3}{|c|}{ Crosses } \\
\hline \multirow{2}{*}{$\begin{array}{l}\text { Iron pipe size } \\
\text { (in inches) }\end{array}$} & \multicolumn{2}{|c|}{ Center to end } & \multirow{2}{*}{$\begin{array}{l}\text { Iron pipe size } \\
\text { (in inches) }\end{array}$} & \multicolumn{2}{|c|}{ Center to end } \\
\hline & $x$ & $z$ & & $X$ & $z$ \\
\hline $\begin{array}{l}1 / 4 \text { by } 1 / 8 \\
3 / 8 \text { by } 1 / 4, \ldots \\
1 / 2 \text { by } 3 / 8 \\
3 / 4 \text { by } 1 / 2 \ldots \\
\end{array}$ & $\begin{array}{r}\text { Inches } \\
0.65 \\
.75 \\
.93 \\
1.08\end{array}$ & $\begin{array}{r}\text { Inches } \\
0.60 \\
.78 \\
.90 \\
1.11\end{array}$ & $\begin{array}{l}1 / 4 \text { by } 1 / 8 \\
3 / 8 \text { by } 1 / 4 \\
1 / 2 \text { by } 3 / 8 \\
3 / 4 \text { by } 1 / 2\end{array}$ & $\begin{array}{r}\text { Inches } \\
0.65 \\
.75 \\
.93 \\
1.08\end{array}$ & $\begin{array}{r}\text { Inches } \\
0.60 \\
.78 \\
.90 \\
1.11\end{array}$ \\
\hline $\begin{array}{l}3 / 4 \text { by } 8 / 8 \\
1 \text { by } 3 / 4- \\
1 \text { by } 1 / 2 \\
11 / 4 \text { by } 1\end{array}$ & $\begin{array}{l}1.00 \\
1.30 \\
1.20 \\
1.52\end{array}$ & $\begin{array}{l}1.00 \\
1.31 \\
1.24 \\
1.60\end{array}$ & $\begin{array}{l}3 / 4 \text { by } 3 / 8 \\
1 \text { by } 3 / 4- \\
1 \text { by } 1 / 2 \ldots \\
11 / 4 \text { by } 1 . .\end{array}$ & $\begin{array}{l}1.00 \\
1.30 \\
1.20 \\
1.52\end{array}$ & $\begin{array}{l}1.00 \\
1.31 \\
1.24 \\
1.60\end{array}$ \\
\hline $\begin{array}{l}11 / 2 \text { by } 11 / 4 \\
2 \text { by } 11 / 2 \\
2 \text { by } 11 / 2 \\
2 \text { by } 1\end{array}$ & $\begin{array}{l}1.72 \\
1.89 \\
1.77 \\
1.59\end{array}$ & $\begin{array}{l}1.81 \\
2.07 \\
2.04 \\
1.95\end{array}$ & $\begin{array}{l}11 / 4 \text { by } 1 / 2 \\
11 / 2 \text { by } 11 / 4 \\
11 / 2 \text { by } 3 / 4 \\
2 \text { by } 11 / 2 .\end{array}$ & $\begin{array}{l}1.29 \\
1.72 \\
1.42 \\
1.89\end{array}$ & $\begin{array}{l}1.41 \\
1.81 \\
1.60 \\
2.07\end{array}$ \\
\hline $\begin{array}{l}21 / 2 \text { by } 2 \\
3 \text { by } 21 / 2- \\
4 \text { by } 3\end{array}$ & $\begin{array}{l}2.39 \\
2.83 \\
3.30\end{array}$ & $\begin{array}{l}2.60 \\
2.99 \\
3.60\end{array}$ & $\begin{array}{l}2 \text { by } 3 / 4-\ldots \\
21 / 2 \text { by } 2 \\
3 \text { by } 21 / 2 \ldots \\
4 \text { by } 3 \ldots\end{array}$ & $\begin{array}{l}1.47 \\
2.39 \\
2.83 \\
3.30\end{array}$ & $\begin{array}{l}1.84 \\
2.60 \\
2.99 \\
3.60\end{array}$ \\
\hline
\end{tabular}

Nore.-Fittings having one or more ports $2 \frac{1}{2}$-inch size or larger have same center to end dimensions as adopted for malleable-iron screwed fittings conforming to specification B16c of A. S. M. $\mathbf{E}$. (See 607.2, p. 422.)

(For illustrations of above fittings, see Figure 25. under American Society of Mechanical Engineers, specification $\mathrm{B} 16 \mathrm{c}$ for malleable-iron serewed fittings. $(607.2$, p. 422). 
TABLE 9.-Center to end dimensions of tees, reducing

[Detail dimensions shall be as given in Table]

\begin{tabular}{|c|c|c|c|}
\hline \multirow{2}{*}{ Iron pipe size (in inches) } & \multicolumn{3}{|c|}{ Center to end } \\
\hline & $X$ & $Y$ & $Z$ \\
\hline $\begin{array}{l}1 / 8 \text { by } 1 / 8 \text { by } 1 / 4 \\
1 / 4 \text { by } 1 / 4 \text { by } 3 / 8 . \\
1 / 4 \text { by } 1 / 4 \text { by } 1 / 8 \\
1 / 4 \text { by } 1 / 8 \text { by } 1 / 4 \\
1 / 4 \text { by } 1 / 8 \text { by } 1 / 8\end{array}$ & $\begin{array}{c}\text { Inches } \\
0.60 \\
.78 \\
.65 \\
.71 \\
.65\end{array}$ & $\begin{array}{c}\text { Inches } \\
0.60 \\
.78 \\
.65 \\
.60 \\
.54\end{array}$ & $\begin{array}{c}\text { Inches } \\
0.65 \\
.75 \\
.60 \\
.71 \\
.60\end{array}$ \\
\hline $\begin{array}{l}3 / 8 \text { by } 3 / 8 \text { by } 1 / 2-- \\
3 / 8 \text { by } 3 / 8 \text { by } 1 / 4- \\
3 / 8 \text { by } 3 / 8 \text { by } 1 / 8- \\
3 / 8 \text { by } 1 / 4 \text { by } 3 / 8- \\
3 / 8 \text { by } 1 / 4 \text { by } 1 / 4--\end{array}$ & $\begin{array}{l}.90 \\
.75 \\
.69 \\
.82 \\
.75\end{array}$ & $\begin{array}{l}.90 \\
.75 \\
.69 \\
.78 \\
.71\end{array}$ & $\begin{array}{l}.93 \\
.78 \\
.67 \\
.82 \\
.78\end{array}$ \\
\hline $\begin{array}{l}1 / 2 \text { by } 1 / 2 \text { by } 3 / 4- \\
1 / 2 \text { by } 1 / 2 \text { by } 3 / 8- \\
1 / 2 \text { by } 3 / 8 \text { by } 1 / 2 \\
1 / 2 \text { by } 3 / 8 \text { by } 3 / 8 \\
3 / 4 \text { by } 3 / 4 \text { by } 1 .\end{array}$ & $\begin{array}{l}1.11 \\
.93 \\
1.01 \\
.93 \\
1.31\end{array}$ & $\begin{array}{r}1.11 \\
.93 \\
.90 \\
.82 \\
1.31\end{array}$ & $\begin{array}{l}1.08 \\
.90 \\
1.01 \\
.90 \\
1.30\end{array}$ \\
\hline $\begin{array}{l}8 / 4 \text { by } 3 / 4 \text { by } 1 / 2-- \\
3 / 4 \text { by } 3 / 4 \text { by } 3 / 8-- \\
3 / 4 \text { by } 3 / 4 \text { by } 1 / 4- \\
3 / 4 \text { by } 112 \text { by } 3 / 4- \\
8 / 4 \text { by } 1 / 2 \text { by } 1 / 2-\end{array}$ & $\begin{array}{l}1.08 \\
1.00 \\
.93 \\
1.18 \\
1.08\end{array}$ & $\begin{array}{l}1.08 \\
1.00 \\
.93 \\
1.11 \\
1.01\end{array}$ & $\begin{array}{l}1.11 \\
1.00 \\
.96 \\
1.18 \\
1.11\end{array}$ \\
\hline $\begin{array}{l}1 \text { by } 1 \text { by } 11 / 4 \ldots \\
1 \text { by } 1 \text { by } 3 / 4 \\
1 \text { by } 1 \text { by } 1 / 2 \ldots \\
1 \text { by } 3 / 4 \text { by } 1 \ldots \\
1 \text { by } 3 / 4 \text { by } 3 / 4-\end{array}$ & $\begin{array}{l}1.60 \\
1.30 \\
1.20 \\
1.43 \\
1.30\end{array}$ & $\begin{array}{l}1.60 \\
1.30 \\
1.20 \\
1.31 \\
1.18\end{array}$ & $\begin{array}{l}1.52 \\
1.31 \\
1.24 \\
1.43 \\
1.31\end{array}$ \\
\hline $\begin{array}{l}1 \text { by } 3 / 4 \text { by } 1 / 2-. \\
1 \text { by } 1 / 2 \text { by } 1 \\
1 \text { by } 1 / 2 \text { by } 3 / 4- \\
11 / 4 \text { by } 11 / 4 \text { by } 11 \\
11 / 4 \text { by } 11 / 4 \text { by } 1 .\end{array}$ & $\begin{array}{l}1.20 \\
1.43 \\
1.30 \\
1.81 \\
1.52\end{array}$ & $\begin{array}{l}1.08 \\
1.24 \\
1.11 \\
1.81 \\
1.52\end{array}$ & $\begin{array}{l}1.24 \\
1.43 \\
1.31 \\
1.72 \\
1.60\end{array}$ \\
\hline $\begin{array}{l}11 / 4 \text { by } 11 / 4 \text { by } 3 / 4 \\
11 / 4 \text { by } 11 / 4 \text { by } 1 / 2 \\
11 / 4 \text { by } 1 \text { by } 11 / 4- \\
11 / 4 \text { by } 1 \text { by } 1 .- \\
11 / 4 \text { by } 1 \text { by } 8 / 4-\end{array}$ & $\begin{array}{l}1.39 \\
1.29 \\
1.69 \\
1.52 \\
1.39\end{array}$ & $\begin{array}{l}1.39 \\
1.29 \\
1.60 \\
1.43 \\
1.30\end{array}$ & $\begin{array}{l}1.48 \\
1.41 \\
1.69 \\
1.60 \\
1.48\end{array}$ \\
\hline $\begin{array}{l}11 / 4 \text { by } 3 / 4 \text { by } 11 / 4 \\
11 / 2 \text { by } 1 / 2 \text { by } 2 \\
11 / 2 \text { by } 11 / 2 \text { by } 11 / 4 \\
11 / 2 \text { by } 11 / 2 \text { by } 1 \\
11 / 2 \text { by } 11 / 2 \text { by } 3 / 4 \\
11 / 2 \text { by } 11 / 2 \text { by } 112 \\
11 / 2 \text { by } 11 / 4 \text { by } 11 / 2 \\
11 / 2 \text { by } 11 / 4 \text { by } 11 / 4 \\
11 / 2 \text { by } 11 / 4 \text { by } 11 \\
11 / 2 \text { by } 3 / 4 \text { by } 11 / 2\end{array}$ & $\begin{array}{l}1.69 \\
2.07 \\
1.72 \\
1.55 \\
1.42 \\
1.32 \\
1.84 \\
1.72 \\
1.55 \\
1.84\end{array}$ & $\begin{array}{l}1.48 \\
2.07 \\
1.72 \\
1.55 \\
1.42 \\
1.32 \\
1.81 \\
1.69 \\
1.52 \\
1.60\end{array}$ & $\begin{array}{l}1.69 \\
1.89 \\
1.81 \\
1.72 \\
1.60 \\
1.53 \\
1.84 \\
1.81 \\
1.72 \\
1.84\end{array}$ \\
\hline $\begin{array}{l}2 \text { by } 2 \text { by } 21 / 2- \\
2 \text { by } 2 \text { by } 1112- \\
2 \text { by } 2 \text { by } 114- \\
2 \text { by } 2 \text { by } 1 \\
2 \text { by } 2 \text { by } 3 / 4-\end{array}$ & $\begin{array}{l}2.60 \\
1.89 \\
1.77 \\
1.59 \\
1.47\end{array}$ & $\begin{array}{l}2.60 \\
1.89 \\
1.77 \\
1.59 \\
1.47\end{array}$ & $\begin{array}{l}2.39 \\
2.07 \\
2.04 \\
1.95 \\
1.84\end{array}$ \\
\hline $\begin{array}{l}2 \text { by } 11 / 2 \text { by } 2 \\
2 \text { by } 11 / 2 \text { by } 11 / 2 \\
2 \text { by } 11 / 2 \text { by } 11 / 4 \\
2 \text { by } 3 / 4 \text { by } 2 \\
21 / 2 \text { by } 21 / 2 \text { by } 3\end{array}$ & $\begin{array}{l}2.12 \\
1.89 \\
1.77 \\
2.12 \\
2.99\end{array}$ & $\begin{array}{l}2.07 \\
1.84 \\
1.72 \\
1.84 \\
2.99\end{array}$ & $\begin{array}{l}2.12 \\
2.07 \\
2.04 \\
1.12 \\
2.83\end{array}$ \\
\hline $\begin{array}{l}21 / 2 \text { by } 21 / 2 \text { by } 2 \\
21 / 2 \text { by } 21 / 2 \text { by } 1 \\
21 / 2 \text { by } 2 \text { by } 21 / 2 \\
21 / 2 \text { by } 2 \text { by } 2 \\
3 \text { by } 3 \text { by } 21 / 2 \\
.\end{array}$ & $\begin{array}{l}2.39 \\
1.87 \\
2.70 \\
2.39 \\
2.83\end{array}$ & $\begin{array}{l}2.39 \\
1.87 \\
2.60 \\
2.25 \\
2.83\end{array}$ & $\begin{array}{l}2.60 \\
2.37 \\
2.70 \\
2.60 \\
2.99\end{array}$ \\
\hline $\begin{array}{l}3 \text { by } 3 \text { by } 2 \\
3 \text { by } 3 \text { by } 11 / 2 \\
3 \text { by } 3 \text { by } 11 / 4 \\
3 \text { by } 21 / 2 \text { by } 3 \\
3 \text { by } 21 / 2 \text { by } 21 / 2 \\
\end{array}$ & $\begin{array}{l}2.52 \\
2.29 \\
2.17 \\
3.08 \\
2.83\end{array}$ & $\begin{array}{l}2.52 \\
2.29 \\
2.17 \\
2.99 \\
2.70\end{array}$ & $\begin{array}{l}2.89 \\
2.80 \\
2.74 \\
3.08 \\
2.99\end{array}$ \\
\hline $\begin{array}{l}4 \text { by } 4 \text { by } 3 \\
4 \text { by } 4 \text { by } 112-2 \\
4 \text { by } 3 \text { by } 4 \\
4 \text { by } 3 \text { by } 3\end{array}$ & $\begin{array}{l}3.30 \\
2.51 \\
3.79 \\
3.30\end{array}$ & $\begin{array}{l}3.30 \\
2.51 \\
3.60 \\
3.08\end{array}$ & $\begin{array}{l}3.60 \\
3.32 \\
3.79 \\
3.60\end{array}$ \\
\hline
\end{tabular}

Note.-Fittings having one or more ports $2 \frac{1}{2}$-inch size or larger, have same center to end dimensions as adopted for malleable-iron
screwed fittings conforming to specification B16c of A.S. M. E. (See 607.2, p. 422.)
(For illustration of above fitting, see Figure 260 under American Society of Mechanical Engineers, specification B16c for malleable-iron screwed fittings $(607.2$, p. 422).)
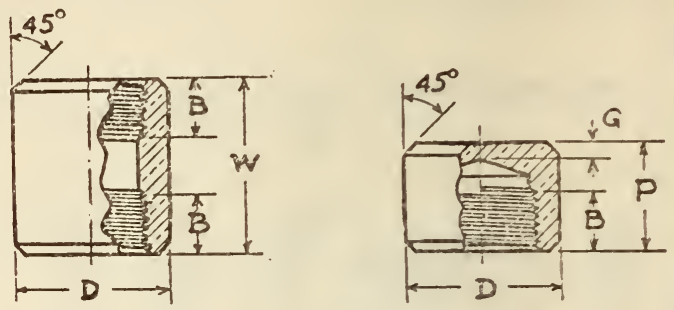

FIGURE 1101.-Wrought coupling; wrought cap

$\mathrm{T}_{\mathrm{ABLE}}$ 10.-Dimensions of wrought couplings and caps

\begin{tabular}{|c|c|c|c|c|c|}
\hline \multirow[b]{2}{*}{$\begin{array}{l}\text { Iron pipe size } \\
\text { (inch) }\end{array}$} & $B$ & $D$ & $G$ & $P$ & $W$ \\
\hline & $\begin{array}{l}\text { Length } \\
\text { perfect } \\
\text { thread, } \\
\text { minimum }\end{array}$ & $\begin{array}{l}\text { Outside } \\
\text { diameter, } \\
\text { minimum }\end{array}$ & $\begin{array}{l}\text { Metal } \\
\text { thickness, } \\
\text { minimum }\end{array}$ & $\begin{array}{c}\text { Height of } \\
\text { cap }\end{array}$ & $\begin{array}{l}\text { Length of } \\
\text { coupling }\end{array}$ \\
\hline & $\begin{array}{c}\text { Inch } \\
0.25 \\
.32 \\
.36 \\
.43 \\
.50\end{array}$ & $\begin{array}{r}\text { Inches } \\
0.56 \\
.69 \\
.84 \\
1.05 \\
1.31\end{array}$ & $\begin{array}{c}\text { Inch } \\
0.11 \\
.13 \\
.13 \\
.14 \\
.15\end{array}$ & $\begin{array}{c}\text { Inch } \\
0.49 \\
.59 \\
.68 \\
.84 \\
.94\end{array}$ & $\begin{array}{l}\text { Inches } \\
0.83 \\
1.03 \\
1.11 \\
1.36 \\
1.50\end{array}$ \\
\hline
\end{tabular}

NorEs. - These fittings will be accepted in lieu of cast fittings, Figures 1099 and 1100, when purchased on a per piece basis.

For dimensions of cast couplings, see Table 4.

For dimensions of cast caps, see Table 7.
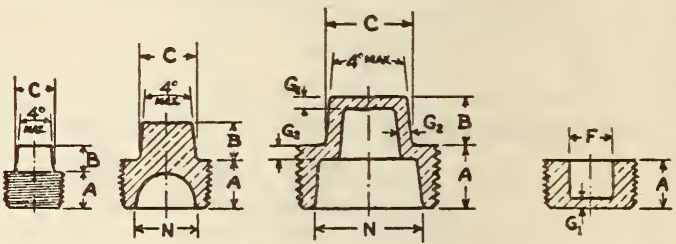

Figdre 1102.-Pipe plugs, square head, and countersunk patterns

TABLE 11.-Dimensions of pipe plugs

\begin{tabular}{|c|c|c|c|c|c|c|c|}
\hline \multirow[b]{2}{*}{$\begin{array}{l}\text { Iron pipe size (in } \\
\text { inches) }\end{array}$} & $A$ & $B$ & $C^{1}$ & $F^{\prime}$ & $G_{1}$ & $G_{2}$ & $1 / 2$ \\
\hline & 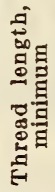 & 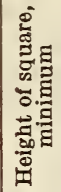 & 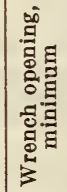 & 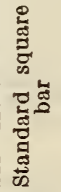 & 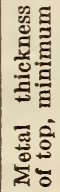 & 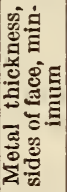 & 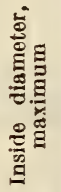 \\
\hline \multirow{9}{*}{$1 / 8-18$} & $\begin{array}{l}n s . \\
0.27\end{array}$ & $\begin{array}{l}\text { Inch } \\
0.24\end{array}$ & $\begin{array}{c}\text { Ins. } \\
9 / 22 \\
\text {. }\end{array}$ & Ins. & Inch & $\overline{\text { Inch }}$ & Ins. \\
\hline & .41 & $\begin{array}{l}.28 \\
\end{array}$ & $\begin{array}{l}.377 \\
.347\end{array}$ & & [-... & -o- & \\
\hline & $\begin{array}{l}.54 \\
.54\end{array}$ & $\begin{array}{r}.38 \\
\end{array}$ & $\begin{array}{l}0766 \\
5 \\
5606\end{array}$ & -8 & 0.09 & 0.12 & 0.53 \\
\hline & $\begin{array}{l}.55 \\
.69\end{array}$ & $\begin{array}{l}.44 \\
.50\end{array}$ & $\begin{array}{l}.629 \\
.818\end{array}$ & $1 / 2$ & $\begin{array}{l}1.11 \\
11\end{array}$ & $\begin{array}{l}.13 \\
.14\end{array}$ & $\begin{array}{l}.72 \\
.93\end{array}$ \\
\hline &.${ }_{73}^{71}$ & $\begin{array}{l}.56 \\
.62\end{array}$ & $\begin{array}{r}943 \\
132\end{array}$ & $\begin{array}{l}3 / 4 \\
3\end{array}$ & $\begin{array}{l}.12 \\
13\end{array}$ & $\begin{array}{r}15 \\
16\end{array}$ & $\begin{array}{l}1.25 \\
1.47\end{array}$ \\
\hline & .76 & .68 & 1.320 & $7 / 8$ & $\begin{array}{l}.15 \\
.15\end{array}$ & .17 & 1. 91 \\
\hline & 1. 07 & $\begin{array}{l}74 \\
80\end{array}$ & . 5099 & $11 / 8$ & $\begin{array}{l}.17 \\
19\end{array}$ & $\begin{array}{l}.18 \\
.19\end{array}$ & $\begin{array}{l}2.32 \\
2.92\end{array}$ \\
\hline & 1. 18 & .86 & 885 & $11 / 2$ & 20 & .20 & 3. 36 \\
\hline & & & & & & & \\
\hline
\end{tabular}

1 Square head of plug shall fit these minimum wrench openings. Maximum total taper of opposite sides head shall be $4^{\circ}$.

' Square socket of countersunk patterns shall fit standard square bars of these sizes. 
F-1. One or more fittings shall be selected by the inspector from each lot of the same size for chemical analysis to determine conformity with the composition required.

F-2. Representative samples shall be selected by the inspector from every lot of 20 or less of the same kind or size submitted for inspection and shall be subjected to an air pressure of not less than 60 pounds per square inch while under water. As an alternative, the test may be made under hydrostatic pressure of 250 pounds per square inch for one minute. Under the hydrostatic pressure the fittings shall not leak water or sweat at any part of the surface. Under the air pressure the fittings shall not leak air at any part of the surface.

F-3. Representative samples shall be surface inspected and checked for conformity with the dimensions as given in the tables.

\section{G. PACKAGING, PACKING, AND MARKING OF SHIPMENTS}

G-1. Packaging.-Ten fittings of the same kind and size up to and including 1 inch shall be securely tied together, or a like number may be packed in a commercial container.

G-1a. Five fittings of the same kind and size above 1 inch shall be securely tied together, or a like number may be packed in a commercial container.

\section{NOTES}

I-1. This specification contemplates the purchase of fittings by the piece, and it is recommended that bids be requested on that basis.

I-2. In ordering reducing fittings, the kind of fitting and the sizes of connecting ends shall be specified as shown in the following paragrapbs.

$I-3$. In specifying tees $(T)$ and wyes $(Y)$, the run should be given first, then the outlet, thus:

(The order in which opening sizes are stated is the same as given and illustrated in specification B16c of A. S. M. E. See 607.2 , p. 422 .)

I-4. Pipe nipples are covered by Federal specification No. WW-N-351. (See 607.4, p. 485.)

I-5. Compositions A or B may be furnished at the option of the bidder, unless one of these compositions is specified in the invitation for bids.

I-6. Rough fittings will regularly be furnished under this specification unless otherwise specified. When polished or plated fittings are required, it should be so specified in the invitation for bids.

I-7. It is the standard practice in some Government departments to use 250-pound fittings with standard weight pipe.

I-8. It is believed that this specification is adequate and that normally no samples will be necessary prior to award to determine compliance with this specification. If for any particular purpose, samples with bids are necessary, they should be specifically asked for in the invitation for bids and the particular purpose to be served by the bid sample should be definitely stated, the specification to apply in all other respects.

\subsection{BRASS OILERS.}

(No nationally recognized specifications available.)
645.6 BUILDERS' HARDWARE, BRASS.

UNITED STATES GOVERNMENT, DEPARTMENT OF COMMERCE, BUILDERS' HARDWARE (NONTEMPLATE), COMMERCIAL STANDARD CS22-30, DECEMBER 11, 1930

\author{
BUILDERS' HARDWARE (NONTEMPLATE)
}

(See 617.0, p. 977.)

\section{UNITED STATES GOVERNMENT, FEDERAL SPECIFICATIONS BOARD, FEDERAL SPECIFICATIONS FOR BUILDERS' HARDWARE (NONTEMPLATE), FF-H-101, AUGUST 19, 1930}

\section{BUILDERS' HARDWARE (NONTEMPLATE)}

(For applicable Federal specifications; types, grades, and classes; material and workmanship; general requirements; detail requirements; packaging, packing, and marking; notes; and table of standard finishes, see 617.0, p. 978. For chromium plating, nickel plating, and zinc coatings, and methods of inspection and tests, see 600.3, p. 70.)

(Specific commodities, such as locks, latches, hinges, bolts, etc., will be found under their respective commodity designations. For a list of these commodities, see under detail requirements in 617.0, p. 979.)

\subsection{MISCELLANEOUS MANUFACTURES OF BRASS.}

\section{AMERICAN GAS ASSOCIATION, STAND- ARD GAS FIXTURES SPECIFICATIONS, 1920}

\section{BRASS PIPE OR TUBING}

Brass pipe or tubing should be either seamless drawn or brazed; in either case of best qulity to allow bending and threading without splitting.

The thickness of walls of brass pipe or tubing should not be less than No. 18 B. \& S. gage $(0.04030$ inch), except when it is used as casing or covering for iron or steel pipe, in which case it should be not less than No. 22 B. \& S. gage $(0.02534$ inch). Both of these gages are subject to the usual gage tolerance of plus or minus 0.002 inch. Particular attention should be paid to having tubing which runs the full gage.

(While No. 18 gage is very generally in use, it is believed that it is very much better to use No. 17 gage. This is not because the 18-gage tubing is not strong or rigid enough but because, when threads are cut on it, there is not sufficient metal to be sure that a good joint is made. If the 18 gage always ran full size, or a little oversize, there would be no objection to it being used, but as it is necessary to allow some variation in the gaging of tubing, it is thought that the No. 17 gage should be specified.)

All threads on brass tubing should be straight threads to conform to the standard specification of the American Society of Mechanical Engineers for such threads.

When brass tube, steel or iron are connected, the thread should be gas-fixture size brass-tubing thread on both the brass and steel or iron; but is recom- 
mended that where possible, instead of connecting the parts in this manner, a brass bushing be used, threaded on the outside with standard I. P. S. thread to screw into the iron body, the bushing being tapped on the inside with a straight gasfixture size brass-tubing tap, for the connection of the brass tube.

(This specification also provides for assembling requirements, for gas-fixture fittings, including fixture cocks, chain pulls, and for sizes of pipe or tubing used in fixture stems and arms. These sizes are as follows:)

\section{SIZES OF PIPE OR TUBING USED IN FIXTURE STEMS}

[All dimensions in inches]

\begin{tabular}{|c|c|c|c|c|c|c|c|}
\hline & \multirow{2}{*}{ 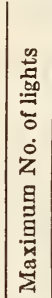 } & \multicolumn{2}{|c|}{$\begin{array}{l}\text { When } \\
\text { made of } \\
\text { iron or } \\
\text { steel } \\
\text { pipe }\end{array}$} & \multirow{2}{*}{ 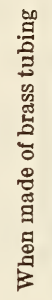 } & \multicolumn{2}{|c|}{$\begin{array}{l}\text { When } \\
\text { made of } \\
\text { brass } \\
\text { chain }\end{array}$} & \multirow{2}{*}{ 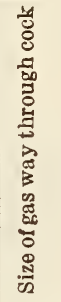 } \\
\hline & & $\begin{array}{c}\tilde{D} \\
\mathbb{Z} \\
\tilde{Z} \\
\tilde{E}\end{array}$ & 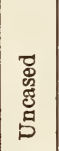 & & 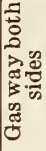 & 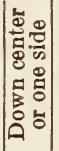 & \\
\hline ider.-......... & 1 & $1 / 8$ & $1 / 4$ & $\frac{8}{3 / 8}$ & $\begin{array}{l}1 / 4 \\
1 / 4\end{array}$ & $\frac{1 / 4}{3 / 8}$ & \\
\hline ches and under 42 & & & & & & & \\
\hline Over 42 inches and under 72 & 2 & $1 / 4$ & $3 / 8$ & $1 / 2$ & $3 / 8$ & 8 & \\
\hline $\begin{array}{l}\text { inches } \\
\text { Any length to } 42 \text { inches. }\end{array}$ & $\begin{array}{l}2 \\
4\end{array}$ & $\frac{3}{3 / 8}$ & $\begin{array}{l}3 / 8 \\
3 / 8\end{array}$ & $\begin{array}{l}1 / 2 \\
1 / 2\end{array}$ & $\begin{array}{l}3 / 8 \\
3 / 8\end{array}$ & $3 / 8$ & $\frac{3}{3}$ \\
\hline $\begin{array}{l}\text { Any length, } 42-72 \text { inches } \\
\text { Do } \\
\text { Do } \\
\text { Do }\end{array}$ & $\begin{array}{r}4 \\
7 \\
12 \\
20\end{array}$ & $\begin{array}{l}8 / 8 \\
3 / 8 \\
1 / 2 \\
8 / 4\end{array}$ & $\begin{array}{c}3 / 8 \\
1 / 2 \\
3 / 4 \\
1^{3 / 4}\end{array}$ & $\begin{array}{l}1 / 2 \\
3 / 4 \\
3 / 4 \\
1\end{array}$ & $\begin{array}{l}\frac{8}{3} 8 \\
3 / 4 \\
3 / 8 \\
1 / 2\end{array}$ & $\begin{array}{l}3 / 8 \\
1 / 2 \\
1 / 2 \\
5 / 8\end{array}$ & 5 \\
\hline
\end{tabular}

Size of pipe or tubing to be used in 1-piece or harp pendants:

[All dimensions in inches]

\begin{tabular}{|c|c|c|c|c|}
\hline \multirow{2}{*}{$\begin{array}{l}\text { Number of lights } \\
\text { not exceeding }\end{array}$} & \multicolumn{2}{|c|}{$\underset{\text { size } 18 \text { by } 12 \text { inches) }}{\text { Harp (maximum }}$} & \multicolumn{2}{|c|}{$\begin{array}{l}\text { Stem (maximum } \\
\text { length } 36 \text { inches) }\end{array}$} \\
\hline & $\begin{array}{l}\text { Steel or } \\
\text { iron pipe }\end{array}$ & $\begin{array}{l}\text { Brass } \\
\text { tubing }\end{array}$ & $\begin{array}{l}\text { Iron or } \\
\text { steel pipe }\end{array}$ & $\begin{array}{c}\text { Brass } \\
\text { tubing }\end{array}$ \\
\hline $1 \ldots$ & $\begin{array}{l}1 / 8 \\
11 / 4 \\
3 / 8\end{array}$ & $\begin{array}{l}3 / 8 \\
11 / 2 \\
1 / 2\end{array}$ & $\begin{array}{l}1 / 4 \\
3 / 8 \\
3 / 8\end{array}$ & $\begin{array}{l}1 / 2 \\
1 / 2 \\
8 / 4\end{array}$ \\
\hline
\end{tabular}

\section{SIZES OF PIPING OR TUBING FOR ARMS}

Arms of pendants, or of wall brackets; that is those parts which carry gas for only one burner, should be made not smaller than the following sizes:

[All dimensions in inches]

\begin{tabular}{|c|c|c|c|}
\hline \multirow{2}{*}{ Length of arm } & \multicolumn{2}{|c|}{$\begin{array}{l}\text { When made of iron } \\
\text { or steel pipe }\end{array}$} & \multirow{2}{*}{$\begin{array}{l}\text { When } \\
\text { made of } \\
\text { brass } \\
\text { pipe }\end{array}$} \\
\hline & Cased & Uncased & \\
\hline $\begin{array}{l}\text { s and under } \\
\text { inches to and inclu }\end{array}$ & $1 / 8$ & & $5 / 10$ \\
\hline $\begin{array}{l}\text { inches } \\
\text { Over } 18 \text { inches to and including } 24 \\
\text { inches }\end{array}$ & $8 / 8$ & $\begin{array}{l}1 / 4 \\
8 / 8\end{array}$ & \\
\hline Over 24 inch & 88 & $3 / 8$ & \\
\hline
\end{tabular}

NOTE.-When over 24 inches in length, the arms should be properly supported by some means other than their attachment to the wall outlet in case of a bracket, or the body in case of an arm.
AMERICAN RAILWAY ASSOCIATION, PURCHASES AND STORES DIVISION; STANDARDIZATION AND SIMPLIFICATION OF STORES STOCKS, D. VI-238, MAY 20,1930

\section{SCREWS}

(Recommended practice in stocking certain specified sizes and types of screws-wood and machine screws of brass with flat, oval, and round heads; wood and machine screws of iron with flat and round heads; and machine screws of iron with fillister heads.)

\section{AMERICAN SOCIETY OF MECHANICAI ENGINEERS, SLOTTED-HEAD PROPOR- TIONS, MACHINE SCREWS, CAP SCREWS, AND WOOD SCREWS, B18c-1930}

[Approved as an American standard by American Standards Association, April, 1930]

\section{BRASS WOOD SCREWS}

(See 608.2, p. 575.)

AMERICAN SOCIETY FOR TESTING MATERIALS, STANDARD SPECIFICATIONS FOR CARTRIDGE BRASS DISKS, DESIGNATION B20-29, 1929

\section{CARTRIDGE BRASS DISKS}

(See 644.21, p. 1199.)

SOCIETY OF AUTOMOTIVE ENGINEERS, PARTS AND FITTINGS, SPLIT AND TUBULAR RIVETS, S. A. E. RECOMMENDED PRACTICE, JUNE, 1929

\section{SPLIT AND TOBOLAR RIVETS}

\section{TUBOLAR RIVETS}
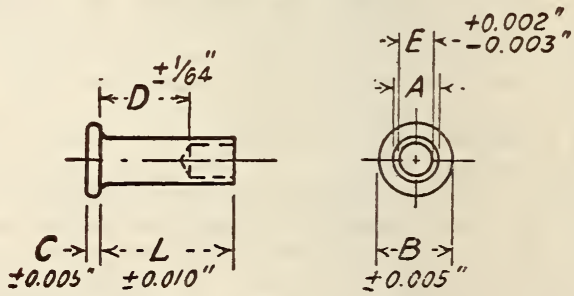

FigURE 1103. - Flat head tubular rivet Flat head

\begin{tabular}{|c|c|c|c|c|c|c|}
\hline $\begin{array}{l}\text { Nominal } \\
\text { diameter }\end{array}$ & $A$ & $B$ & $C$ & $D$ & $E$ & $\mathcal{L}$ \\
\hline $\begin{array}{r}16 \ldots \\
964 \ldots \ldots \\
3 / 16 \ldots \ldots .\end{array}$ & $\begin{array}{l}0.123 \pm 0.003 \\
.145 \pm .003 \\
.186 \pm .005\end{array}$ & $\begin{array}{r}1 / 4 \\
6 / 16 \\
7 / 16\end{array}$ & $\begin{array}{r}0.020 \\
364 \\
1 / 16\end{array}$ & $\begin{array}{l}\mathrm{L}-\mathrm{H} / \mathrm{i} \\
\mathrm{L}-5 / 32 \\
\mathrm{~L}-3 / 16\end{array}$ & $\begin{array}{r}0.086 \\
.098 \\
.133\end{array}$ & $\begin{array}{l}3 / 32 \text { to } 3 / 8 \text { by } 1 / 32 . \\
3 / 10 \text { to } 5 / 8 \text { by } 1 / 32 . \\
1 / 4 \text { to } 5 / 8 \text { by } 1 / 32 .\end{array}$ \\
\hline
\end{tabular}




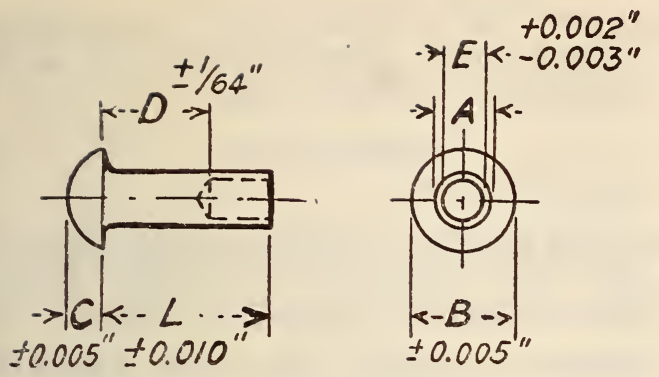

FIGURE 1104. - Oval head tubular rivet Oval head

\begin{tabular}{|c|c|c|c|c|c|c|}
\hline $\begin{array}{l}\text { Nominal } \\
\text { diameter }\end{array}$ & $A$ & $B$ & $C$ & $D$ & $E$ & $L$ \\
\hline $\begin{array}{l}16 \ldots \ldots \ldots \\
964 \ldots \ldots \ldots \\
964 \ldots \ldots \\
964 \ldots \ldots \ldots \\
3 / 16 \ldots \ldots \ldots \\
316 \ldots \ldots\end{array}$ & $\begin{array}{l}0.123 \pm 0.003 \\
.145 \pm .003 \\
.145 \pm .003 \\
.145 \pm .003 \\
.186 \pm .005 \\
.186 \pm .005\end{array}$ & $\begin{array}{l}7 / 32 \\
5 / 10 \\
5 / 16 \\
5 / 16 \\
3 / 8 \\
3 / 8\end{array}$ & $\begin{array}{l}1 / 32 \\
3 / 64 \\
3 / 64 \\
3 / 64 \\
1 / 16 \\
1 / 16\end{array}$ & $\begin{array}{l}\mathrm{L}-1 / 16 \\
\mathrm{~L}-18 \\
\mathrm{~L}-3 / 16 \\
\mathrm{~L}-1 / 4 \\
\mathrm{~L}-1 / 8 \\
\mathrm{~L}-3 / 16\end{array}$ & $\begin{array}{l}0.086 \\
.098 \\
.098 \\
.098 \\
.133 \\
.133\end{array}$ & $\begin{array}{l}3 / 32 \text { to } 5 / 8 \text { by } 1 / 32 . \\
3 / 16 \text { to } 5 / 8 \text { by } 1 / 32 \\
14 \text { to } 5 / 8 \text { by } 1 / 32 . \\
5 / 16 \text { to } 5 / 8 \text { by } 1 / 32 . \\
3 / 16 \text { to } 3 / 4 \text { by } 1 / 32 \\
1 / 4 \text { to } 3 / 4 \text { by } 1 / 32 \text {. }\end{array}$ \\
\hline
\end{tabular}
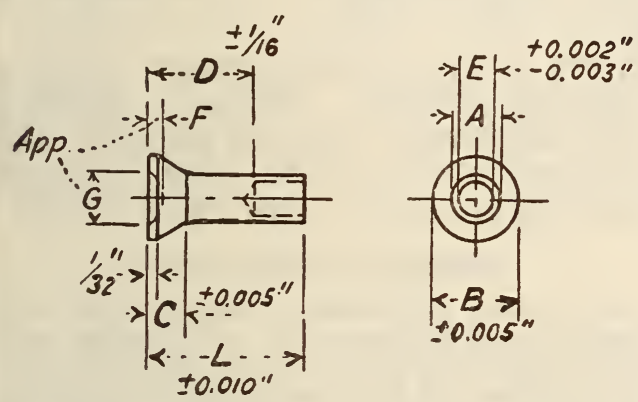

FIGURE 1105.-Cupped countersunk head tubular rivet

Cupped countersunk head

\begin{tabular}{|c|c|c|c|c|c|c|c|c|}
\hline $\begin{array}{l}\text { Nominal } \\
\text { diameter }\end{array}$ & $A$ & $B$ & $C$ & $D$ & $E$ & $F$ & $G$ & $L$ \\
\hline & 0.14 & $\begin{array}{l}10 \\
36\end{array}$ & & & $\begin{array}{l}0.098 \\
.13\end{array}$ & $3 / 64$ & $\begin{array}{r}1 / 4 \\
19 \% 4\end{array}$ & $\begin{array}{l}9 / 32 \text { to } \\
9 / 32 \text { to }\end{array}$ \\
\hline
\end{tabular}

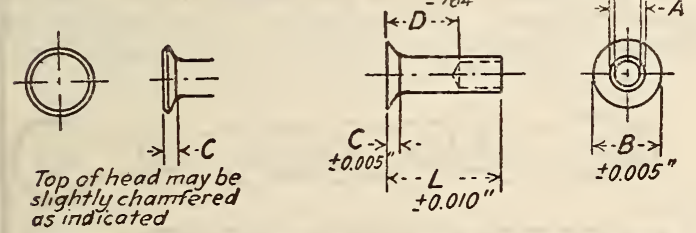

FIGURE 1106. - Flat countersunk head tubular rivet

Flat countersunk head

\begin{tabular}{|c|c|c|c|c|c|c|}
\hline $\begin{array}{l}\text { Nominal } \\
\text { diameter }\end{array}$ & $A$ & $B$ & $C$ & $D$ & $E$ & $L$ \\
\hline $\begin{array}{l}1 / 8 \ldots \ldots \\
9 \% 4 \ldots \ldots \\
9 / 64 \ldots \\
964 \ldots \\
3 / 16 \ldots \ldots \\
3 / 16 \ldots \ldots \\
316 \ldots \ldots\end{array}$ & $\begin{array}{l}0.123 \pm 0.003 \\
.145 \pm .003 \\
.145 \pm .003 \\
.145 \pm .003 \\
.186 \pm .005 \\
.186 \pm .005 \\
.186 \pm .005\end{array}$ & $\begin{array}{r}1 / 4 \\
5 / 16 \\
5 / 16 \\
5 / 16 \\
3 / 8 \\
3 / 8 \\
3 / 8\end{array}$ & $\begin{array}{l}1 / 32 \\
3 / 64 \\
3 / 64 \\
3 / 64 \\
3 / 64 \\
3 / 64 \\
3 / 64\end{array}$ & $\begin{array}{l}\mathrm{L}-5 / 32 \\
\mathrm{~L}-1 / 8 \\
\mathrm{~L}-3 / 16 \\
(1) \\
\mathrm{L}-1 / 8 \\
\mathrm{~L}-3 / 16 \\
(1)\end{array}$ & $\begin{array}{c}0.086 \\
.098 \\
.098 \\
.098 \\
.133 \\
.133 \\
.133\end{array}$ & $\begin{array}{l}1 / 4 \text { to } 1 / 2 \text { by } 1 / 32 . \\
1 / 4 \text { to } 58 \text { by } 1 / 32 . \\
1 / 4 \text { to } 58 \text { by } 1 / 32 . \\
5 / 16 \text { to } 1 / 2 \text { by } 1 / 32 \text {. } \\
1 / 4 \text { to } 3 / 4 \text { by } 1 / 32 \text {. } \\
1 / 4 \text { to } 3 / 4 \text { by } 1 / 32 \\
5 / 16 \text { to } 1 / 2 \text { by } 1 / 32 \text {. }\end{array}$ \\
\hline
\end{tabular}

1 Drilled to head.

The above specifications are for steel and brass rivets for a roll clinch; where star clinch or wrought-iron rivet is used, the drill size should be 0.006 in. larger.
SPIIT RIVETS

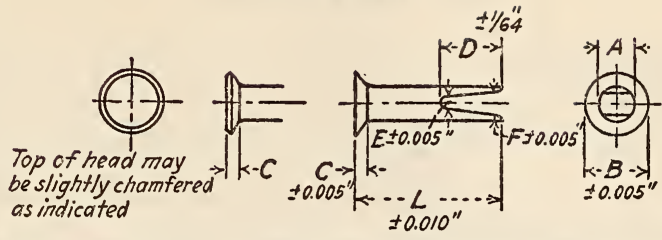

FIGURE 1107. - Flat countersunk-head split rivet

Flat countersunk head

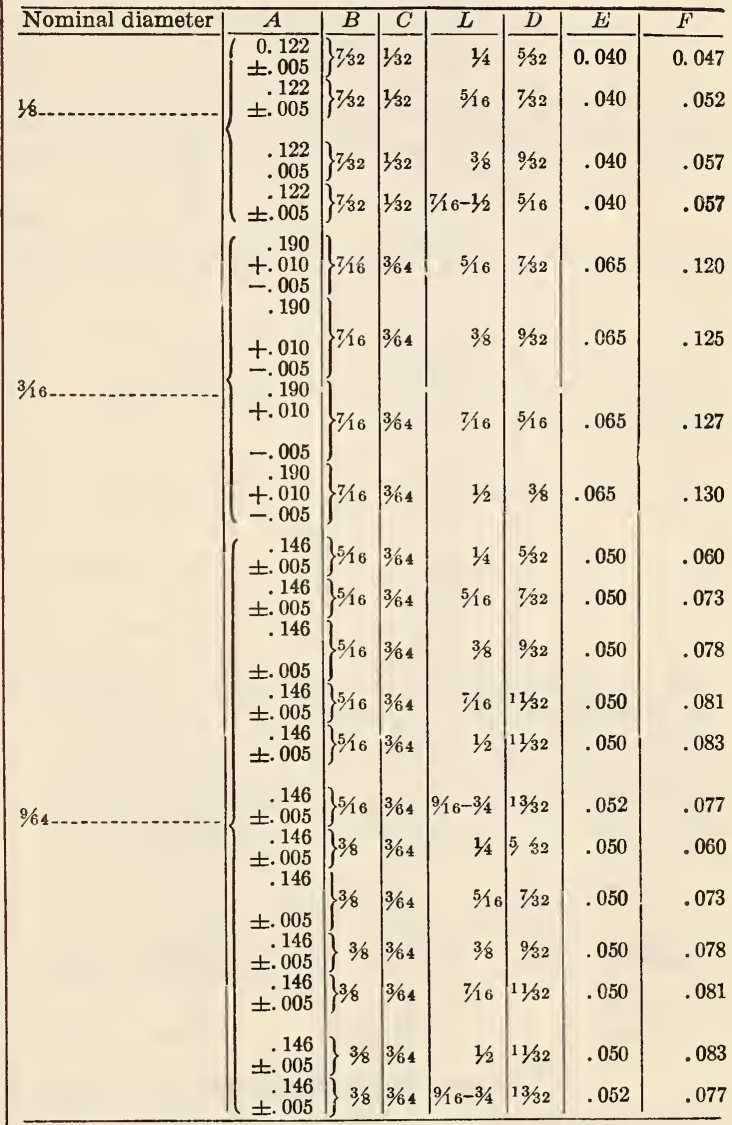

Lengths increase in $1 / 16$-inch steps.

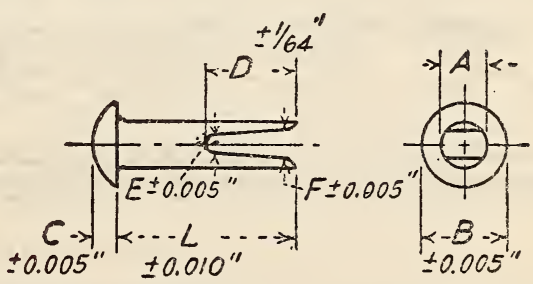

Figure 1108. - Oval-head split rivet

Oval head

\begin{tabular}{|c|c|c|c|c|c|c|c|}
\hline Nominal diameter & $\bar{A}$ & $\bar{B}$ & $C$ & $\bar{L}$ & $D$ & $E$ & $F$ \\
\hline \multirow{4}{*}{$3 / 32$} & $\begin{array}{r}0.086 \\
+.005\end{array}$ & 964 & 0.025 & $3 / 16$ & $5 / 32$ & 0.030 & 0.037 \\
\hline & $\begin{array}{r}.086 \\
+.005\end{array}$ & 964 & .025 & $1 / 4$ & $7 / 32$ & .030 & .039 \\
\hline & .086 & $\% 4$ & .025 & $5 / 26$ & $9 / 32$ & .030 & .039 \\
\hline & $\begin{array}{r}.086 \\
\pm .005\end{array}$ & 964 & .025 & $3 / 8-1 / 2$ & $5 / 16$ & .030 & .039 \\
\hline
\end{tabular}


Oval head-Continued

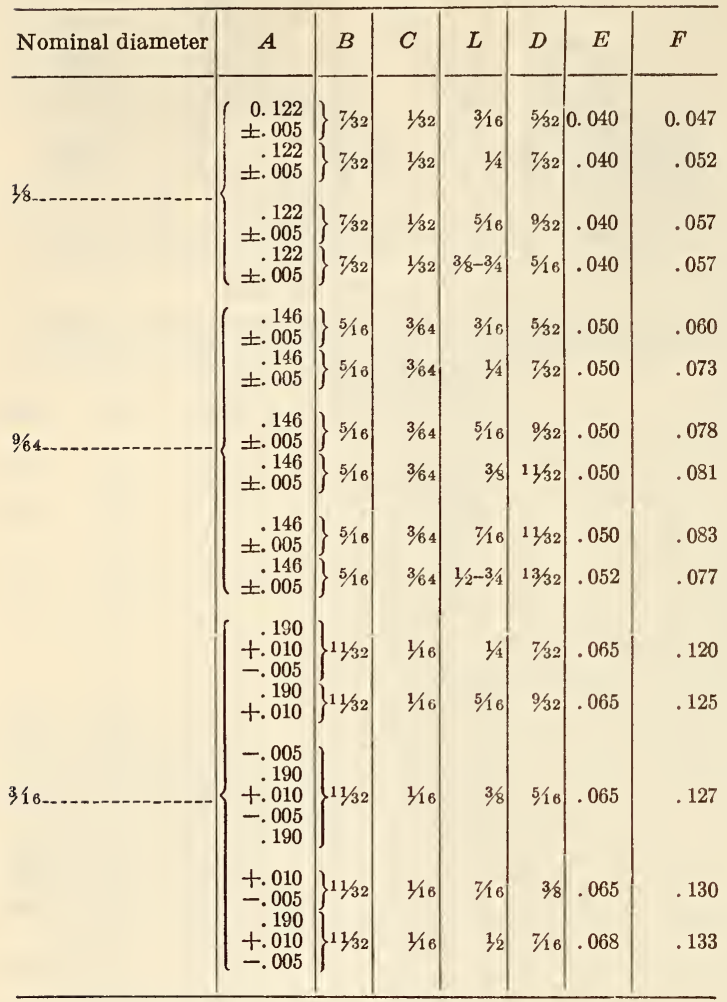

NOTE: Lengths increase in 1/16-inch steps.
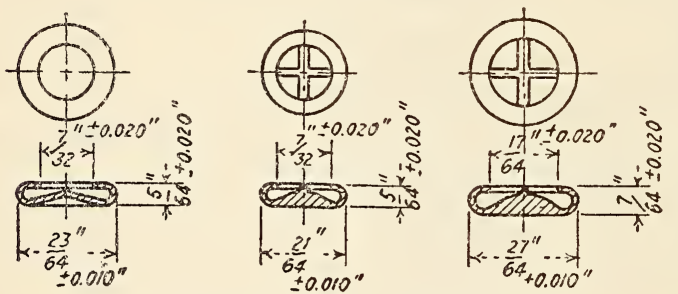

Figure 1109.-Rivet caps

For use with split rivets and wrought

For use with steel and iron tubular rivets.
SOCIETY OF AUTONOTIVE ENGINEERS, AERONAUTIC; TURNBUCKLES, S. A. E. STANDARD, AUGUST, 1928

\section{TURNBUCKLES}

(See 608.7, p. 630.)

UNDERWRITERS' IABORATORIES, STAND. ARD FOR WINDOW CLEANERS' BELTS AND ANCHORS, MARCH 30, 1930

WINDOW CLEANERS' BELTS AND ANCHORS

(See 654.59, p. 1286.)

UNITED STATES GOVERNMENT, FEDERAL SPECIFICATIONS BOARD, FEDERAL SPECIFICATION FOR SCREWS, WOOD, FF-S-111, APRIL 28, 1931

\section{BRASS WOOD SCREWS}

(Specifications and dimensions identical with those of the National Screw Thread Commission. See 608.2, Table 63, p. 586.)

\section{UNITED STATES GOVERNMENT, FEDERAL SPECIFICATIONS BOARD, FEDERAL SPECIFICATION FOR STANDARD MIS- CELLANEOUS CHAIN AND ATTACH- MENTS, RR-C-271, DECEMBER 8, 1931 \\ WELDLESS BRASS CHAIN}

(For general specifications, material and workmanship, detail requirements, and methods of inspection and tests, see 603.50 , p. 199 . For other kinds of chain and chain attachments specified in RR-C-271, see 603.51, 603.52, 603.54, 603.56, 603.57, and 617.5. See also 631.9,642.9, and 646.59.)

\section{BRASS CHAIN FOR MECHANICAL COMMUNICATION}

1. Brass chain for mechanical communication is required for use in pulleys having a $5 / 16$-inch groove used on a line of signal transmission of mechanical telegraphs.

2. Each end of the completed chain shall be fitted with a large brass link for the attachment of transmission wires.

3. The total length of the finished chain shall be about 30 inches. Weldless chain will be paid for by the foot. It shall be automatic-machine-made chain.
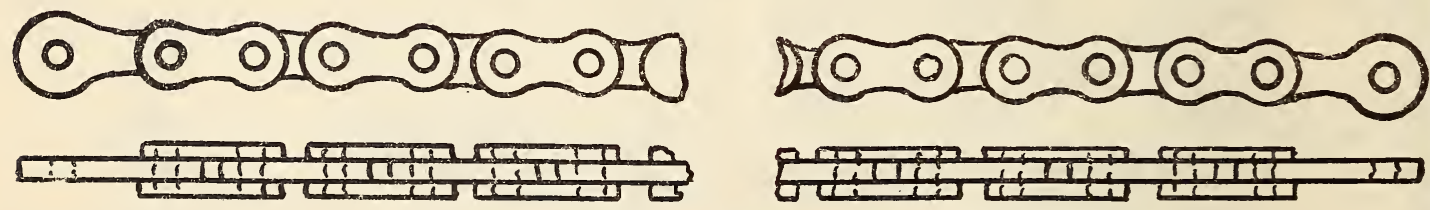

Figure 1110.-Weldless brass chain

TABLE 58.-Brass chain for mechanical communication (dimensions and strength)

\begin{tabular}{|c|c|c|c|c|c|}
\hline \multicolumn{2}{|c|}{ Thickness } & \multirow{2}{*}{$\begin{array}{l}\text { Width, } \\
\text { maxi- } \\
\text { mum }\end{array}$} & \multirow{2}{*}{$\begin{array}{l}\text { Pitch be- } \\
\text { tween } \\
\text { centers } \\
\text { of pins }\end{array}$} & \multirow{2}{*}{$\begin{array}{l}\text { Approxi- } \\
\text { mate } \\
\text { number } \\
\text { of links, } \\
\text { per foot }\end{array}$} & \multirow{2}{*}{$\begin{array}{c}\text { Mini- } \\
\text { mum } \\
\text { tensile } \\
\text { strength }\end{array}$} \\
\hline $\begin{array}{l}\text { Outside } \\
\text { links }\end{array}$ & $\begin{array}{l}\text { Inside } \\
\text { links }\end{array}$ & & & & \\
\hline $\begin{array}{l}\text { Inch } \\
1 / 16\end{array}$ & $\begin{array}{c}\text { Inch } \\
1 / 8\end{array}$ & $\begin{array}{c}\text { Inch } \\
1 / 4\end{array}$ & Inch & 15 & $\begin{array}{c}\text { Pounds } \\
800\end{array}$ \\
\hline
\end{tabular}


DOUBLE LOOP PATTERN CHAIN

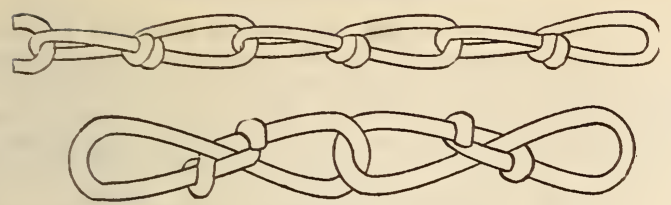

FIGURE 1111.-Double loop pattern chain

(For double loop pattern chain of brass, see 603.56, p. 212.)

SAFETY CHAIN

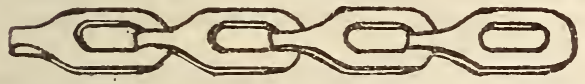

FIGURE 1112.-Safety chain

TABLE 53.-Safety chain (dimensions, strength, weights)

\begin{tabular}{c|r|r|r|r}
\hline Materia] & $\begin{array}{c}\text { Metal } \\
\text { gage }\end{array}$ & $\begin{array}{c}\text { Approxi- } \\
\text { mate } \\
\text { number of } \\
\text { links per } \\
\text { foot }\end{array}$ & $\begin{array}{c}\text { Minimum } \\
\text { tensile } \\
\text { strength }\end{array}$ & $\begin{array}{c}\text { Approxi- } \\
\text { mate } \\
\text { weight per } \\
100 \text { feet }\end{array}$ \\
\hline Brass_... & & & Pounds & Pounds \\
Inch & 27 & 150 & $11 / 4$ \\
0.018 & 25 & 175 & $13 / 4$ \\
.022 & 21 & 235 & $31 / 4$ \\
.027 & 21 & 300 & $31 / 2$ \\
.032 & 16 & 350 & $41 / 4$ \\
.037 & & &
\end{tabular}

SINGLE JACK CHAIN

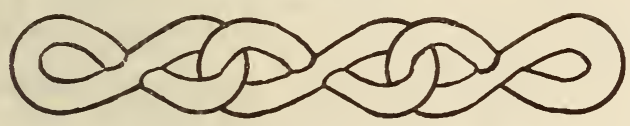

FiguRe 1113.-Single jack chain

TABLE 54.-Single jack chain (dimensions, strength, weights)

(Dimensions, strength, and weights for brass single jack chain are the same as for steel single jack chain. See 603.56 , p. 213.)

\section{DOUBLE JACK CHAIN}

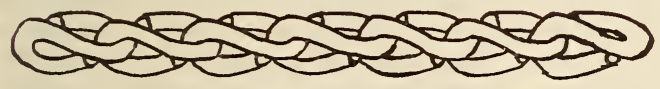

Figure 1114.-Double jack chain

TABLE 55.-Double jack chain (dimensions, strength weights)

(Dimensions, strength, and weights for brass double jack chain are the same as for steel double jack chain. See 603.56 , p. 213.)

\section{IADDER CHAIN}

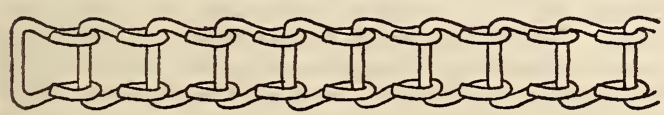

FiguRe 1115.-Ladder chain
TABLE 56.-Ladder chain (dimensions, strength, weights)

\begin{tabular}{c|r|r|r|r}
\hline Material & $\begin{array}{c}\text { Wire } \\
\text { gage }\end{array}$ & $\begin{array}{c}\text { Approxi- } \\
\text { mate } \\
\text { number of } \\
\text { links per } \\
\text { foot }\end{array}$ & $\begin{array}{c}\text { Minimum } \\
\text { tensile } \\
\text { strength }\end{array}$ & $\begin{array}{c}\text { Approxi- } \\
\text { mate } \\
\text { weight per } \\
100 \text { feet }\end{array}$ \\
\hline Brass... & & & Pounds & Pounds \\
0.036 & 64 & 40 & 0.8 \\
.048 & 42 & 60 & 2.5 \\
.055 & 34 & 75 & 3.5 \\
.080 & 25 & 120 & 6 \\
\hline
\end{tabular}

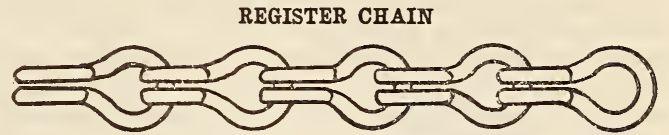

Frgure 1116.-Register chain

TABLE 57.-Register chain (dimensions, strength, weights)

\begin{tabular}{|c|c|c|c|c|}
\hline Material & $\begin{array}{l}\text { Wire } \\
\text { gage }\end{array}$ & $\begin{array}{l}\text { Approxi- } \\
\text { mate } \\
\text { number of } \\
\text { links per } \\
\text { foot }\end{array}$ & $\begin{array}{l}\text { Minimum } \\
\text { tensile } \\
\text { strength }\end{array}$ & $\begin{array}{l}\text { Approxi } \\
\text { mate } \\
\text { weight per } \\
100 \text { feet }\end{array}$ \\
\hline Brass.. & $\begin{array}{r}\text { Inch } \\
0.036 \\
.062 \\
.091 \\
.148\end{array}$ & $\begin{array}{l}35 \\
251 / 4 \\
143 / 4 \\
11\end{array}$ & $\begin{array}{r}\text { Pounds } \\
20 \\
90 \\
165 \\
450\end{array}$ & $\begin{array}{r}\text { Pounds } \\
1.6 \\
41 / 2 \\
101 / 4 \\
2614\end{array}$ \\
\hline
\end{tabular}

UNITED STATES GOVERNMENT, NATIONAL SCREW THREAD COMMISSION, AMERICAN NATIONAL WOOD SCREW SIZES, BUREAU OF STANDARDS MISCELLANEOUS PUBLICATION NO. 89, JUNE 22, 1928

\section{BRASS WOOD SCREWS}

(See 608.2, p. 584.)

646.

BRONZE

646.0 GENERAL ITEMS.

AMERICAN SOCIETY FOR TESTING MATERIALS, STANDARD METHODS OF CHEMICAL ANALYSIS OF GUN METAL, DESIGNATION B28-19, 1919

[Approved as American tentative standard (K1-1921) by the American Standards Association, December 28, 1921]

\section{CHEMICAI ANALYSIS OF GUN METAL GENERAL CONSIDERATIONS}

Phosphorus should not be present in this alloy except in very small amounts. A rapid qualitative test for it is given, whereby it is easily seen whether or not it is necessary to make a correction for its presence in the determination of tin.

A special method is also given for the determination of tin in alloys such as phosphor bronze which contain considerable phosphorus. 
DETERMIN ATION OF COPPER BY THE ELECTROLYTIC METHOD

APPARATUS FOR ELECTROLYSIS

(Electrodes, cathodes, and anodes are identical with those in B27. See 647.20, p. 1261.)

\section{METHOD}

In a $150 \mathrm{c} \mathrm{c}$ beaker dissolve $1 \mathrm{~g}$ of gun metal in 10 c c of $\mathrm{HNO}_{3}$, specific gravity 1.42 . When the action has ceased, boil until the oxides of nitrogen are expelled, add $50 \mathrm{c}$ c of hot distilled water, and allow to stand and settle for about one hour, keeping the temperature just below the boiling point. Filter on double $7 \mathrm{~cm}$ ashless papers, being careful to keep the solution hot throughout the process of filtration, and receiving the filtrate in a $200 \mathrm{c}$ c beaker of the tall type used in electrolysis. Wash with boiling not water. Retain the papers containing the precipitate for subsequent use in the tin determination.

Add to the filtrate $5 \mathrm{c} \mathrm{c}$ of $\mathrm{H}_{2} \mathrm{SO}_{4}$, specific gravity 1.84 , and determine copper by electrolysis, retaining the electrolyte, after the removal of the copper, for the subsequent determination of zinc. See the determination of copper by the electrolytic method, standard methods of chemical analysis of manganese bronze in B27. (See 647.20, p. 1261.)

Accuracy.-Duplicate determinations should check within 0.10 per cent of copper.

Nores.-Copper, zinc, and tin are conveniently determined on the same sample, tin being first removed, and any zinc tested for after copper has been deposited by electrolysis.

If the solution becomes cool during the first filtration some of the meta-stannic acid $\left(\mathrm{H}_{2} \mathrm{SnO}_{3}\right)$ may dissolve.

\section{DETERMINATION OF TIN}

[For samples free from phosphorus]

\section{METHOD}

See the determination of copper by the electrolytic method, given above.

Use the papers containing the $\mathrm{H}_{2} \mathrm{SnO}_{3}$ which was filtered off in the determination of copper by the electrolytic method. Ignite the papers with the precipitate, while moist, in a porcelain or platinum crucible, slowly at first, and finally at the full heat of the burner. If the precipitate weighs more than $20 \mathrm{mg}$ heat to constant weight, using a blast lamp. Weigh as $\mathrm{SnO}_{2}$, which contains 78.81 per cent of tin. Accuracy.-Duplicate determinations should check within 0.04 per cent of tin.

Notes.-This method is not accurate for alloys which contain arsenic, antimony, or phosphorus, or which contain over 0.25 per cent of iron. The $\mathrm{SnO}_{2}$ may be contaminated with a slight amount of copper and purification is required in very accurate work, but is generally neglected in routine analysis.

generally neglected in routine analysis.
The ignited tin oxide contains any phosphorus which may be present in the alloy. The following test is here made use of to detect phosphorus, and to estimate its amount if any is found.

\section{Qualitative Test for Phosphorus}

\section{SOLUTIONS REQUIRED}

Ferric chloride.-Dissolve $25 \mathrm{~g}$ of ferric chloride in $100 \mathrm{c}$ c of distilled water, and add $25 \mathrm{c} \mathrm{c}$ of $\mathrm{HCl}$, specific gravity 1.20 .

\section{METHOD}

Dip a small piece of the alloy into a few cubic centimeters of ferric chloride for about 10 seconds, and then rinse it in running water. Alloys containing phosphorus are darkened noticeably where they have been dipped; alloys containing over 0.25 per cent of phosphorus are rendered almost black by this test. Arsenic and antimony act in a manner similar to phosphorus, but should not be present in this alloy.
Correction for Phosphorus

In alloys containing up to 0.20 per cent of phosphorus a correction for its presence in the ignited tin oxide may be made according to the following empirical method: From the phosphorus content of the alloy, which must be determined on a separate sample, compute the weight of $\mathrm{P}_{2} \mathrm{O}_{5}$ and subtract two-thirds of this weight from the weight of the ignited tin oxide containing phosphorus. The remainder is considered as pure $\mathrm{SnO}_{2}$, which contains 78.81 per cent of tin. The factor for converting phosphorus to $2 / 3 \times \mathrm{P}_{2} \mathrm{O}_{5}$ is 152.6 per cent, or approximately one and one-half times the phosphorus content of the sample.

If the alloy contains over 0.20 per cent of phosphorus a special method for the determination of tin should be used.

Arsenic and antimony should not be present in weighable amounts in the tin oxide resulting from a sample of gun metal.

Iron may be present in the $\mathrm{H}_{2} \mathrm{SnO}_{3}$ in very small amounts, but its weight is negligible in this determination.

In alloys which contain over 0.25 per cent of iron, use the method for the determination of tin in manganese bronze, standard methods of chemical analysis of manganese bronze, B27. (See 647.20, p. 1261.)

\section{DETERMINATION OF TIN}

[For samples containing phosphorus]

\section{SOLUTIONS REQUIRED}

Yellow ammonium sulphide.-Saturate $150 \mathrm{c} \mathrm{c}$ of $\mathrm{NH}_{4} \mathrm{OH}$, specific gravity 0.90 , with $\mathrm{H}_{2} \mathrm{~S}$ gas and then add $50 \mathrm{c}$ c more $\mathrm{NH}_{4} \mathrm{OH}$. Dissolve in this solution 3 or $4 \mathrm{~g}$ of sulphur flour and about $1 \mathrm{~g}$ of $\mathrm{NH}_{4} \mathrm{Cl}$. Make up this solution freshly and filter immediately before using.

Dilute yellow ammonium sulphide for washing.Mix $20 \mathrm{c}$ c of yellow ammonium sulphide, prepared as above, and $400 \mathrm{c} c$ of distilled water.

Ammonium acetate for washing.-Dissolve $10 \mathrm{~g}$ of ammonium acetate in $300 \mathrm{c}$ c of distilled water, made slightly acid with acetic acid, and saturate with $\mathrm{H}_{2} \mathrm{~S}$ gas.

\section{METHOD}

See the determination of copper by the electrolytic method, given above.

Use the papers containing the freshly filtered $\mathrm{H}_{2} \mathrm{SnO}_{3}$ which was filtered off in the determination of copper by the electrolytic method. Transfer the papers with the precipitate to a $150 \mathrm{c}$ c beaker, and cover them with 40 to $50 \mathrm{c}$ c of yellow ammonium sulphide. Warm for about 15 minutes, or until the $\mathrm{H}_{2} \mathrm{SnO}_{3}$ has dissolved. Filter and wash thoroughly with dilute yellow ammonium sulphide. Acidify the combined filtrate and washings cautiously with 50 per cent acetic acid. Warm, and allow the precipitated tin sulphide and sulphur to settle. Filter on double papers and wash with ammonium acetate solution. Dry the precipitate and place it with the papers in a porcelain crucible which projects part way through a hole in a piece of asbestos board. Heat slowly until the free sulphur has been driven off, but without allowing the sulphur to burn. When the sulphur has been expelled, place the crucible on a triangle and ignite slowly at first, and finally at the full heat of the burner. If the precipitate weighs more than $20 \mathrm{mg}$, heat to constant weight, using a blast lamp. Weigh as $\mathrm{SnO}_{2}$, which contains 78.81 per cent of tin. 
Accuracy.-Duplicate determinations should check within 0.06 per cent of tin.

Notes.-If during the first filtration the solution becomes cool, some of the $\mathrm{H}_{2} \mathrm{SnO}_{3}$ may dissolve.

During ignition, if the sulphur is allowed to burn, some tin sulphate may be formed, causing high results. On the other hand, low result may be caused by too high heat, which causes spattering, and volatilizes some tin sulphide.

\section{DETERMINATION OF IEAD}

(Specifications covering the determination of lead, both by the electrolytic method and as a sulphate, for samples containing up to 2 per cent of lead, are identical with those specified in B27. See 647.20, p. 1261.)

\section{DETERMINATION OF ZINC}

SOLUTIONS REQUIRED

Dilute hydrochloric acid.-Mix 500 c c of $\mathrm{HCl}$, specific gravity 1.20 , and $500 \mathrm{c}$ c of distilled water.

\section{METHOD}

See the determination of copper by the electrolytic method, given above.

After the copper has been completely deposited by electrolysis, quickly remove the cathode and wash it thoroughly in distilled water, catching the washings in the beaker containing the electrolyte. Heat the solution and saturate with $\mathrm{H}_{2} \mathrm{~S}$ gas. Filter off any precipitate and boil the solution to expel $\mathrm{H}_{2} \mathrm{~S}$. Make the solution barely alkaline with $\mathrm{NH}_{4} \mathrm{OH}$, and add $25 \mathrm{c}$ c of 85 per cent formic acid. Dilute the solution with distilled water to $300 \mathrm{c} \mathrm{c}$, heat to boiling, and saturate with $\mathrm{H}_{2} \mathrm{~S}$ gas. Filter and wash with hot water. Dissolve the precipitate with hot dilute $\mathrm{HCl}$, and transfer the solution to a weighed platinum or porcelain dish or crucible. Add a few drops of $\mathrm{H}_{2} \mathrm{SO}_{4}$, specific gravity 1.84 , and evaporate the solution until copious fumes escape. If the solution is not clear and colorless, cool, add a few cubic centimeters of $\mathrm{HNO}_{3}$, specific gravity 1.42 , and again evaporate the solution until fumes of $\mathrm{H}_{2} \mathrm{SO}_{4}$ come off freely. Repeat the treatment with $\mathrm{HNO}_{3}$, if necessary, until the organic matter is destroyed, and the solution is colorless. Remove the excess of $\mathrm{H}_{2} \mathrm{SO}_{4}$ by heating the aish cautiously, and finally heat to dull redness. Weigh as zinc sulphate, which contains 40.49 per cent of zinc.

Accuracy.-Duplicate determinations should check within 0.05 per cent of zinc.

\section{DETERMINATION OF PHOSPHORUS BY THE ALKALIMETRIC} METHOD

\section{SOLUTIONS REFUIRED}

Nitric acid for dissolving.-Mix 1,000 c c of $\mathrm{HNO}_{3}$, specific gravity 1.42 , and 1,200 c c of distilled water.

Nitric acid for washing.-Mix $20 \mathrm{c}$ c of $\mathrm{HNO}_{3}$, specific gravity 1.42 , and $1,000 \mathrm{c}$ c of distilled water. Ammonium molybdate-Solution No. 1.-Place in a beaker $100 \mathrm{~g}$ of 85 per cent molybdic acid, mix it thoroughly with $240 \mathrm{c}$ c of distilled water, add 140 c c of $\mathrm{NH}_{4} \mathrm{OH}$, specific gravity 0.90 , filter, and add 60 c c of $\mathrm{HNO}_{3}$, specific gravity 1.42 .

Solulion No. 2.- Mix 400 c c of $\mathrm{HNO}_{3}$, specific gravity 1.42 , and $960 \mathrm{c}$ c of distilled water.

When the solutions are cold, add solution No. 1 to solution No. 2, stirring constantly; then add $0.1 \mathrm{~g}$ of ammonium phosphate dissolved in $10 \mathrm{c}$ c of distilled water and let stand at least 24 hours before using.

Potassium nitrate, 1 per cent.-Dissolve $10 \mathrm{~g}$ of $\mathrm{KNO}_{3}$ in $1,000 \mathrm{c} \mathrm{c}$ of distilled water.
Phenolphthalein indicator.-Dissolve $0.2 \mathrm{~g}$ of phenolphthalein in $50 \mathrm{c} \mathrm{c}$ of 95 per cent ethyl alcohol and $50 \mathrm{c}$ c of distilled water.

Standard sodium hydroxide.-Dissolve $6.5 \mathrm{~g}$ of purified $\mathrm{NaOH}$ in $1,000 \mathrm{c}$ c of distilled water, add a slight excess of 1 per cent solution of barium hydroxide, let stand for 24 hours, decant the liquid, and standardize it against a steel of known phosphorus content as determined by the molybdate-magnesia method, so that $1 \mathrm{c} \mathrm{c}$ will be equivalent to $0.01 \mathrm{per}$ cent of phosphorus on the basis of a $2 \mathrm{~g}$ sample (see notes). Protect the solution from carbon dioxide with a soda-lime tube.

Ferric chloride.-Dissolve $100 \mathrm{~g}$ of ferric chloride (phosphorus free), in $100 \mathrm{c} \mathrm{c}$ of distilled water.

Standard nitric acid.-Mix 10 c c of $\mathrm{HNO}_{3}$, specific gravity 1.42 , and $1,000 \mathrm{c} \mathrm{c}$ of distilled water. Titrate the solution against standardized $\mathrm{NaOH}$ using phenolphthalein as indicator, and make it equivalent to the $\mathrm{NaOH}$ by adding distilled water.

\section{METHOD}

In a $400 \mathrm{c}$ c casserole dissolve $1 \mathrm{~g}$ of gun metal in $10 \mathrm{c}$ c of $\mathrm{HNO}_{3}$ specific gravity 1.42 . Add $20 \mathrm{c} \mathrm{c}$ of $\mathrm{HCl}$, specific gravity 1.20 , and evaporate to dryness. Moisten with $\mathrm{HCl}$, evaporate to dryness again, and bake to dull redness. Moisten with $\mathrm{HCl}$ again, add $3 \mathrm{c}$ c of ferric chloride solution, and dilute to about $200 \mathrm{c}$ c with distilled water. Add $\mathrm{NH}_{4} \mathrm{OH}$, specific gravity 0.90 , until the basic salts of copper have dissolved and the solution has become a deep blue. Boil, allow to settle, and filter on a loosely woven filter paper. Wash with dilute ammonia and with hot water. Dissolve the precipitate on the filter with hot dilute $\mathrm{HCl}$, dilute the solution to about 200 c c, add $\mathrm{NH}_{4} \mathrm{OH}$, specific gravity 0.90 , until the precipitate which forms at first dissolves rather slowly, and saturate with $\mathrm{H}_{2} \mathrm{~S}$ gas. Filter off and reject the precipitate. Boil the filtrate to expel $\mathrm{H}_{2} \mathrm{~S}$ and add $\mathrm{HNO}_{3}$, specific gravity 1.42 , until the iron is oxidized. Add $\mathrm{NH}_{4} \mathrm{OH}$, specific gravity 0.90 , until the solution is alkaline. Boil and filter on a loosely woven filter paper. Wash with dilute ammonia and with hot water. Dissolve the precipitate on the filter with $\mathrm{HNO}_{3}$ for dissolving, receiving the solution in a $350 \mathrm{c}$ c Erlenmeyer flask. Add $\mathrm{NH}_{4} \mathrm{OH}$, specific gravity 0.90 , until the iron is entirely precipitated, and then add $\mathrm{HNO}_{3}$, specific gravity 1.42 cautiously until the solution just becomes clear. Bring the solution to a temperature of about $80^{\circ}$ C., and add $40 \mathrm{c}$ c of ammonium molybdate at room temperature. Allow to stand for one minute, shake or agitate for three minutes, and filter on a $9 \mathrm{~cm}$ paper. Wash the precipitate three times with the 2 per cent $\mathrm{HNO}_{3}$ solution to free it from iron, and continue the washing with the 1 per cent $\mathrm{KNO}_{3}$ solution until the precipitate and flask are free from acid.

Transfer the paper and precipitate to a solution flask, add $20 \mathrm{c}$ c of distilled water, five drops of phenolphthalein solution as indicator, and an excess of standard $\mathrm{NaOH}$ solution. Insert a rubber stopper and shake vigorously until solution of the precipitate is complete. Wash off the stopper with distilled water and determine the excess of $\mathrm{NaOH}$ solution by titrating with standard $\mathrm{HNO}_{3}$ solution. Each cubic centimeter of standard $\mathrm{NaOH}$ solution represents 0.02 per cent of phosphorus،

Accuracy.-Duplicate determinations should check within 0.01 per cent of phosphorus.

Notes.-The ammonium molybdate solution should be kept in a cool place and should always be filtered before using.

All distilled water used in titration should be freed from carbon dioxide by boiling or otherwise.

Bureau of Standards standard steel No. $19(a)$ is recommended as a suitable steel for standardization of the $\mathrm{NaOH}$ solution. 
DETERMINATION OF PHOSPHORUS BY THE FERRIC ALUM METHOD

[Optional]

\section{SOLUTIONS REQUIRED}

Ferric chloride.-Dissolve $100 \mathrm{~g}$ of ferric chloride (phosphorus free) in $100 \mathrm{c}$ c of distilled water.

Dilute ammonia.-Mix $100 \mathrm{c}$ c of $\mathrm{NH}_{4} \mathrm{OH}$, specific gravity 0.90 , and $900 \mathrm{c}$ c of distilled water.

Dilute hydrochloric acid.-Mix 500 c c of $\mathrm{HCl}$, specific gravity 1.20 , and $500 \mathrm{c}$ c of distilled water.

Dilute sulphuric acid for dissolving.-Mix $200 \mathrm{c} \mathrm{c}$ of $\mathrm{H}_{2} \mathrm{SO}_{4}$, specific gravity 1.84 , and $800 \mathrm{c} \mathrm{c}$ of distilled water.

Dilute sulphuric acid for reductor.-Mix $50 \mathrm{c} \mathrm{c}$ of $\mathrm{H}_{2} \mathrm{SO}_{4}$, specific gravity 1.84 , and $1,000 \mathrm{c}$ c of distilled water. This solution is used boiling hot.

Ammonium molybdate.-(Same as in alkalimetric method, given above.)

Acid ammonium sulphate.-Mix 25 c c of $\mathrm{H}_{2} \mathrm{SO}_{4}$ specific gravity 1.84 , and $1,000 \mathrm{c}$ c of distilled water, and then add $15 \mathrm{c} \mathrm{c}$ of $\mathrm{NH}_{4} \mathrm{OH}$, specific gravity 0.90 .

Ferric alum.-Dissolve $200 \mathrm{~g}$ of ferric ammonium sulphate crystals in $1,950 \mathrm{c}$ c of distilled water. Add $50 \mathrm{c} \mathrm{c}$ of $\mathrm{H}_{2} \mathrm{SO}_{4}$, specific gravity 1.84 , and 80 c c of phosphoric acid, 85 per cent.

Potassium permanganate.-Dissolve from 3.0 to 3.2 $\mathrm{g}$ of $\mathrm{KMnO}_{4}$ in $1,000 \mathrm{c} \mathrm{c}$ of distilled water. Allow the solution to stand for about one week, and then filter it through an asbestos filter. Standardize by using about $0.200 \mathrm{~g}$ portions of pure sodium oxalate.

\section{METHOD}

(Method is identical with the alkalimetric method given above until after boiling and filtering on a loosely woven filter paper, and washing with dilute ammonia and with hot water. Then proceed as follows:)

Dissolve the precipitate on the filter with hot dilute $\mathrm{H}_{2} \mathrm{SO}_{4}$, receiving the solution in a $350 \mathrm{c}$ c Erlenmeyer flask. Add $\mathrm{NH}_{4} \mathrm{OH}$, specific gravity 0.90 , until the iron is entirely precipitated, and then add $\mathrm{HNO}_{3}$, specific gravity 1.42 , cautiously until the solution just becomes clear. Bring the solution to a temperature of about $80^{\circ} \mathrm{C}$., and add $40 \mathrm{c}$ c of ammonium molybdate at room temperature. Allow to stand for one minute, shake or agitate for three minutes, filter on a $9 \mathrm{~cm}$ paper, and wash very thoroughly (about 25 times) with acid ammonium sulphate. Dissolve the precipitate on the paper using $50 \mathrm{c} \mathrm{c}$ of dilute ammonia. Add sufficient $\mathrm{H}_{2} \mathrm{SO}_{4}$ to adjust the acidity to 2.5 per cent by volume, and immediately pass the solution through a Jones reductor, which has the reductor tube prolonged and reaching nearly to the bottom of the flask, dipping into $50 \mathrm{c} \mathrm{c}$ of ferric alum solution. Wash through the reductor with $150 \mathrm{c} \mathrm{c}$ of the $\mathrm{H}_{2} \mathrm{SO}_{4}$ for reductor and follow with $100 \mathrm{c} c$ of distilled water.

Titrate with the standard $\mathrm{KMnO}_{4}$ solution.

By this method the molybdenum which in passing through the reductor has been reduced entirely to the form corresponding to the oxide $\mathrm{Mo}_{2} \mathrm{O}_{3}$, is partially oxidized by the ferric alum, an equivalent amount of iron being reduced to the ferrous condition. The resultant solution is not rapidly oxidized through contact with air during the titration, and the calculations can therefore be made on the $\mathrm{Mo}_{2} \mathrm{O}_{3}-2 \mathrm{MoO}_{3}$ basis.

Accuracy.-Duplicate determinations

check within 0.01 per cent of phosphorus.

Notes. - The ammonium molybdate solution should be kept in a cool place and siould always be filtered before using.

A blank determination should be made on corresponding amounts of acid and water, passing through the reductor in to the usual amount of ferric alum solution in the flask.

A small quantity of liquid should always be left in the reductor funnel, and air should never be allowed to enter the reductor.

For description and further details of the use of reductor see "Chem ical Analysis of Iron" by A. A. Blair, or "Quantitative Chemical Analysis" by H. P. Talbot.
AMERICAN SOCIETY FOR TESTING MATERIALS, STANDARD METHODS OF CHEMICAL ANALYSIS OF BRONZE BEARING METAL, DESIGNATION, B46-27, 1927

\section{CHEMICAI ANALYSIS OF BRONZE BEARING} METAL

DETER MIN ATION OF COPPER BY THE ELECTROLYTIC METHOD

APPARATUS FOR ELECTROLYSIS

(Identical with B27. See 647.20, p. 1261.)

\section{METHOD}

In a $150 \mathrm{c}$ c beaker, dissolve $1 \mathrm{~g}$ of the bronze in $10 \mathrm{c} \mathrm{c}$ of $\mathrm{HNO}_{3}$ (specific gravity 1.42). When the action has ceased, boil until the oxides of nitrogen are expelled; add $50 \mathrm{c}$ c of hot distilled water; and allow to stand and settle for about one hour, keeping the temperature just below the boiling point. Filter on double $7 \mathrm{~cm}$ ashless papers, being careful to keep the solution hot throughout the process of filtration, and receive the filtrate in a $200 \mathrm{c} c$ beaker of the tall type used in electrolysis. Wash with boiling hot water. Retain the papers containing the precipitate for subsequent use in the tin determination.

Add to the filtrate $5 \mathrm{c} \mathrm{c}$ of $\mathrm{H}_{2} \mathrm{SO}_{4}$ (specific gravity 1.84), and determine copper by electrolysis, retaining the electrolyte, after the removal of the copper, for the subsequent determination of zinc. See the determination of copper by the electrolytic method, B27 (647.20, p. 1261).

Notes.-Copper, zinc, and tin are conveniently determined on the same sample, tin being first removed, and any zinc tested for after copper has been deposited by electrolysis.

If the solution becomes cool during the first filtration, some of the meta-stannic acid $\left(\mathrm{H}_{2} \mathrm{SnO}_{3}\right)$ may dissolve.

\section{DETERMINATION OF TIN}

[For samples free from phosphorus]

(Same as B28, above.)

Qualitative Test for Phosphorus

(Same as B28, above, except that the specific gravity of the $\mathrm{HCl}$ is 1.19 instead of 1.20 and no reference is made to arsenic and antimony.)

\section{DETERMINATION OF TIN}

[For samples containing phosphorus]

(Same as B28, above, except for note 1, which says: "The ignited tin oxide will contain any arsenlc or antimony that was present in the sample.")

\section{DETERMINATION OF LEAD BY THE ELECTROLYTIC METHOD}

APPARATUS

Electrodes and other apparatus used in electrolysis; see the determination of copper by the electrolytic method, B27 (647.20, p. 1261).

\section{METHOD}

In a $150 \mathrm{c}$ c beaker, dissolve $1 \mathrm{~g}$ of the bronze in $10 \mathrm{c}$ c of $\mathrm{HNO}_{3}$ (specific gravity 1.42). When the action has ceased, boil until the oxides of nitrogen are expelled, add $50 \mathrm{c} c$ of boiling hot distilled water, and allow to stand and settle for about one hour, keeping the temperature just below the boiling point. Filter on double $7 \mathrm{~cm}$ ashless papers, being careful to keep the solution hot throughout the process of filtration. Wash with boiling hot water. Add to the filtrate $9 \mathrm{c}$ c of $\mathrm{HNO}_{3}$ (specific gravity 1.42) and dilute to about $150 \mathrm{c} \mathrm{c}$ with distilled 
water. Insert a sand-blasted platinum gauze electrode in the anode terminal; this electrode may be one of the type used as cathode in the determination of copper by the electrolytic method, or it may be one of the sand-blasted gauze anodes therein described. Insert in the cathode terminal one of the electrodes used as anode in the determination of copper by the electrolytic method. (See B27, 647.20, p. 1261.) Cover with a pair of split watch glasses and electrolyze. For each solution use a current of 2 amperes at 10 volts continued for about two hours. Wash down the cover glasses, electrodes, and sides of the beakers, raising the level of the liquid 5 or 6 $\mathrm{mm}$, and continue the electrolysis for 15 minutes. If no darkening of the newly exposed surface of the platinum can be detected, the lead has been entirely deposited. If the newly exposed surface of the platinum is darkened, continue the electrolysis until no such darkening can be detected when the current has been continued for 15 minutes after the level of the liquid has been raised.

When the lead has been entirely deposited, remove the anode quickly and rinse it in distilled water and then in alcohol, and dry in an oven at $210^{\circ} \mathrm{C}$. for 30 minutes. Weigh as $\mathrm{PbO}_{2}$ using the factor for lead 0.8643 instead of the theoretical value.

Notes. - 1 . It is impossible to remove the tin quantitativcly as $\mathrm{H}_{2} \mathrm{SnO}_{3}$ in the case of alloys containing over 0.25 per cent of iron, and traces of tin are sometimes found in the $\mathrm{PbO}_{2}$ deposit.

2. In this and in the following method, a slight amount of lead may be retained by the $\mathrm{H}_{2} \mathrm{SnO}_{3}$. This should be recovered in very accurate work, but is neglected in a routine analysis.

3. Samples for analysis. - When lead is less than 0.10 per cent, a 5 or $10 \mathrm{~g}$ sample is taken. The $5 \mathrm{~g}$ sample should be dissolved in $25 \mathrm{c} \mathrm{c}$ of $\mathrm{HNO}_{3}$. The $10 \mathrm{~g}$ sample should be dissolved in $45 \mathrm{c} \mathrm{c}$ of $\mathrm{HNO}_{3}$ When the lead content is greater than 15 per cent, dissolve the sample in $\mathrm{HNO}_{3}$ as described under "Method," transfer the solution to a in $\mathrm{HNO}_{3}$ as described under "Method," transfer the solution to a to exceed the above-mentioned limit of lead to be deposited on the anode as $\mathrm{PbO}_{2}$. The lead peroxide will tend to flake off the electrode when the amount deposited becomes exccssive.

\section{DETERMINATION OF LEAD AS SULPHATE}

Solutions Required

(Same as B27. See 647.20, p. 1261.)

\section{METHOD}

In a $150 \mathrm{c} c$ beaker, dissolve $1 \mathrm{~g}$ of the bronze in $10 \mathrm{c}$ c of $\mathrm{HNO}_{3}$ (specific gravity 1.42). When the action has ceased, boil until the oxides of nitrogen are expelled, add $50 \mathrm{c} \mathrm{c}$ of boiling hot distilled water, and allow to stand and settle for about one hour, keeping the temperature just below the boiling point. Filter on double $7 \mathrm{~cm}$ ashless papers, being careful to keep the solution hot throughout the process of filtration. Wash with boiling hot water. Add 40 c c of lead acid and evaporate until copious fumes of $\mathrm{H}_{2} \mathrm{SO}_{4}$ are evolved. Cool; add $35 \mathrm{c} \mathrm{c}$ of distilled water to dissolve the salts. Heat to boiling and allow to cool and settle for five hours, or overnight if convenient. Filter on a weighed Gooch crucible and wash with lead acid. Wash out the lead acid with dilute alcohol, set the Gooch crucible inside a porcelain crucible, ignite for five minutes at the full heat of a Tirrell burner, cool, and weigh as $\mathrm{PbSO}_{4}$, and calculate to $\mathrm{Pb}$.

Notes.-1. Copper and lead may be determined on the same samples if so desired. In such a casc, after filtering off the lead sulphate and washing with lead acid, and before washing with dilute alcohol, remove the filtrate, add $3 \mathrm{e} \mathrm{c}$ of $\mathrm{NHO}_{3}$ (specific gravity 1.42 ), dilute to $120 \mathrm{c} c$ and determine copper by electrolysis. See the determination of copper by the electrolytic method.

2. Copper and lead may also be determined simultaneously by the electrolytic method. See the standard methods of chemical analysis of manganese bronze B27 (647.20, p. 1261).

\section{DETERMIN ATION OF IRON}

\section{SOLUTIONS REQUIRED}

Yellow ammonium sulphide.-Saturate $150 \mathrm{c}$ c of $\mathrm{NH}_{4} \mathrm{OH}$ (specific gravity 0.90) with hydrogen sulphide gas, and then add 50 c c more of $\mathrm{NH}_{4} \mathrm{OH}$ Dissolve in this solution 3 or $4 \mathrm{~g}$ of sulphur flour, and about $1 \mathrm{~g}$ of $\mathrm{NH}_{4} \mathrm{Cl}$. Make up this solution fresh, and filter it immediately before use.

Dilute yellow ammonium sulphide for washing.Mix $20 \mathrm{c}$ c of yellow ammonium sulphide, prepared as above, and $400 \mathrm{c}$ c of distilled water.

Other solutions. - See the determination of iron in the standard methods of chemical analysis of brass ingots and sand castings B45 (644.0, p. 1193).

\section{METHOD}

In a $150 \mathrm{c}$ c beaker, dissolve $1 \mathrm{~g}$ of bronze in 10 c c of $\mathrm{HNO}_{3}$ (specific gravity 1.42). When the action has ceased, boil until the oxides of nitrogen are expelled, add $50 \mathrm{c} \mathrm{c}$ of boiling water, and allow to stand and settle for about one hour, keeping the temperature just below the boiling point. Filter on double $7 \mathrm{~cm}$ papers, keeping the solution hot, and wash with boiling hot water. Retain both the filtrate and the precipitate. Transfer the papers with the precipitate to a $150 \mathrm{c}$ c beaker, and cover them with 40 to $50 \mathrm{c}$ c of yellow ammonium sulphide. Warm about 15 minutes, or until the meta-stannic acid is dissolved. Filter and wash thoroughly with hot, dilute, yellow ammonium sulphide.

Dissolve the small black residue on the filter with hot dilute $\mathrm{HCl}$, receiving the solution in a small beaker. Add $5 \mathrm{c}$ c of $\mathrm{HNO}_{3}$ (specific gravity 1.42) and boil to oxidize sulphur and iron. Add this small solution to the original filtrate from the metastannic acid. Add about $5 \mathrm{~g}$ of $\mathrm{NH}_{4} \mathrm{Cl}$, then $\mathrm{NH}_{4} \mathrm{OH}$, (specific gravity 0.90 ) and proceed as in the determination of iron in the standard methods of chemical analysis of manganese bronze in B27. (See 647.20, p. 1261.)

\section{DETERMINATION OF NICKEL BY THE DIMETHYLGLYOXIME} METHOD

\section{SOLUTIONS REQUIRED}

Dimethylglyoxime solution.-Dissolve $5 \mathrm{~g}$ of dimethylglyoxime in $500 \mathrm{c}$ c of grain alcohol, and filter before using.

Sodium potassium tartrate solution.-Dissolve $200 \mathrm{~g}$ of the salt in 1 liter of distilled water.

Dilute ammonia for washing.-Solution containing $10 \mathrm{c} \mathrm{c}$ of $\mathrm{NH}_{4} \mathrm{OH}$ (specific gravity 0.90 ) per liter of water.

\section{METHOD}

In a 200 c c electrolytic beaker, dissolve a $1 \mathrm{~g}$ sample exactly as in the determination of copper by the electrolytic method, B27 (see 647.20, p. 1261), and remove the copper by electrolysis. Evaporate the solution to about $100 \mathrm{c}$ c, filter, add about 1 $\mathrm{g}$ of $\mathrm{NH}_{4} \mathrm{Cl}$, then $10 \mathrm{c}$ c of sodium potassium tartrate solution, and nearly neutralize with $\mathrm{NH}_{4} \mathrm{OH}$ (specific gravity 0.90). Heat nearly to boiling, add 5 c c of dimethylglyoxime solution for each $0.01 \mathrm{~g}$ or fraction of nickel present and then $\mathrm{NH}_{4} \mathrm{OH}$ in slight excess. Let stand at least 30 minutes on the st eam bath, filter, and wash with dilute ammonia solution. For accurate work, the precipitate should be dissolved in hot $\mathrm{HCl}(1: 1)$ and reprecipitated as before. Filter on a weighed Gooch crucible, wash with dilute ammonia solution and then with hot water, dry at $110^{\circ}$ to $120^{\circ} \mathrm{C}$. for about two hours, and weigh as the glyoxime salt which contains 20.31 per cent of nickel. If it is preferred instead of by weighing the glyoxime salt, the nickel can be determined electrolytically as follows: Dissolve the red precipitate of nickel glyoxime in hot $\mathrm{HCl}(1: 1)$, add $10 \mathrm{c}$ c of $\mathrm{H}_{2} \mathrm{SO}_{4}(1: 1)$ and evaporate to strong fumes. Dilute, neutralize with $\mathrm{NH}_{4} \mathrm{OH}$ (specific gravity 0.90 ), and add $20 \mathrm{c}$ c in excess. The final volume should be about $150 \mathrm{c} \mathrm{c}$ 
Electrolyze with a current of 0.3 ampere per solution. Test the solution for completion of deposition with fresh $\mathrm{H}_{2} \mathrm{~S}$ water as is done in the determination of copper by the electrolytic method. Remove the cathode, wash with water, and dry with alcohol as in the determination of copper by the electrolytic method. Weigh the deposit as metallic nickel.

\section{DETERMIN ATION OF ZINC}

(Same as B28, above, except that "solutions required" item is omitted and the hot $\mathrm{HCl}$ is proportioned $1: 1$.)

\section{DETERMINATION OF PHOSPHORUS PY THE ALKALIMETRIO}

SOLUTIONS REQUIRED

(Same as B28 above.)

\section{METHOD}

In a $400 \mathrm{c}$ c beaker, dissolve a $1 \mathrm{~g}$ sample in 10 c c of $\mathrm{HNO}_{3}$ (specific gravity 1.42) and heat until brown fumes are driven off; then add $15 \mathrm{c} c$ of $\mathrm{HCl}$ (specific gravity 1.19) and evaporate to dryness. Add $10 \mathrm{e}$ c of $\mathrm{HCl}$ (specific gravity 1.19) and again evaporate to dryness, but do not bake.

Add $15 \mathrm{c}$ c of $\mathrm{HCl}$ (specific gravity 1.19) and heat to solution, dilute with $50 \mathrm{c} \mathrm{c}$ of distilled water, and bring to a boil. Take off the hot plate and wash down the cover glass and sides of the beaker; add 20 -mesh c. p. zinc, 2 or $3 \mathrm{~g}$ at a time, until the solution becomes colorless, that is until all the copper, tin, and lead are precipitated. Filter, receiving the filtrate in a $400 \mathrm{c} \mathrm{c}$ beaker and wash well with hot water.

Add $3 \mathrm{c} \mathrm{c}$ of the ferric chloride solution to this filtrate and make faintly ammoniacal; bring to a boil, let settle and filter. Dissolve this precipitate in hot dilute $\mathrm{HCl}$ and again make ammoniacal; heat to boiling, let settle, and filter, washing well with hot water.

Dissolve this precipitate in as small a quantity of hot dilute $\mathrm{HNO}_{3}$ as possible, receiving the solution in a 300 c c Erlenmeyer flask, wash filter free of iron with hot dilute $\mathrm{HNO}_{3}$ and hot distilled water. Evaporate to about $15 \mathrm{c} \mathrm{c}$, cool, make ammoniacal, then neutralize with $\mathrm{HNO}_{3}$ (specific gravity 1.42 ) and add $5 \mathrm{c}$ c in excess. Cool to $80^{\circ}$ C. and add $60 \mathrm{c} \mathrm{c}$ of the ammonium molybdate solution. Let stand one minute, then shake three minutes and let settle. Filter on an $11 \mathrm{~cm}$ filter (using a small quantity of paper pulp on the filter) and wash free of iron with 2 per cent nitric acid wash, then free of acid with the 1 per cent potassium nitrate solution.

Place the paper in the thoroughly washed acid-free flask in which the original precipitation was made, add $25 \mathrm{c}$ c of distilled water and an excess of the standard sodium hydroxide solution. By using a long glass rod, the paper can be pulped and the yellow precipitate dissolved in a very few seconds. Wash off the rod and the sides of the flask and add a few drops of the phenolphthalein solution and titrate the excess of standard $\mathrm{NaOH}$ with the standard $\mathrm{HNO}_{3}$. The strength of the standard $\mathrm{NaOH}$ and the standard $\mathrm{HNO}_{3}$ in terms of phosphorus can be determined by using a standard steel or preferably a standard phosphor bronze in which the phosphorus has been determined by the molybdate-magnesia method.

Accuracy.-Duplicate determinations should check within 0.01 per cent of phosphorus.

Notes. -1 . The ammonium molybdate solution should be kept in a cool place and should always be filtered before using.

2. All distilled water useo in titration should be freed from carbon dioxide by boiling or otherwise.
3. Bureau of Standards standard steel No. 19 (a) or a bronze of known phosphorus content are recommended as suitable materials for standardization of the sodium hdyroxide solution.

4. (Gravimetric method, ortional.) It is considered by some that more accurate results can be obtained by a gravimetric determina tion of phosphorus as $\mathrm{M}_{2} \mathrm{P}_{2} \mathrm{O}_{7}$. This can be carried out by dissolv. ing the yellow molybdate precipitate in $20 \mathrm{c}$ c of $\mathrm{NH}_{4} \mathrm{OH}(1: 1)$ to which has been added $2 \mathrm{~g}$ of citric acid, precipitating with magnesis mixture, and proceeding as usual, finally weighing as $\mathrm{Mg}_{2} \mathrm{P}_{2} \mathrm{O}_{7}$. 5. Determination of phosphorus by the ferric alum method is optional. See the standard methods of chemicul analysis of gun metal B2S abore.

\section{DETERMINATION OF ANTIMONY BY THE VOLUMETRIC METHOD}

(Same as B45. See 644.0, p. 1193.)

IETERMINATION OF SULPHUR

(Same as B45. See 644.0, p. 1193.)

DETERMIN ATION OF ARSENIC

(Same as B45. See 644.0, p. 1193.)

DETERMINATIOIN OF ALUMINUM IN SMALL QUANTITLES

(Same as B45. See 644.0, p. 1193.)

646.1 BRONZE INGOTS AND BARS.

646.11 INGOTS, BRONZE.

AMERICAN SOCIETY FOR TESTING IMA. TERIALS, TENTATIVE SPECIFICATIONS FOR COPPER-BASE ALIOYS IN INGOT FORM FOR SAND CASTINGS, DESIGNATION B30-31T, 1932

COPPER-BASE ALLOYS-INGOT FORM

(See 644.11, p. 1196.)

UNITED STATES GOVERNMENT, FEDERAL SPECIFICATIONS BOARD; FEDERAI SPECIFICATION FOR BRONZE INGOTS (FOR REMELTING), QQ-B-701, MAY 31, 1932

\section{BRONZE INGOTS}

\section{GENERAL SPECIFICATIONS}

Federal specification for metals, Federal Specifications Board specification No. 339 (Current designation: QQ-M-151.), in effect on date of invitation for bids, shall form part of this specification.

\section{GRADES}

This specification covers 10 grades of bronze in the form of ingots for remelting, as shown in Table 1.

\section{MATERIAL}

Bronze ingots shall be made of the best grades of virgin metals or from scrap of known and approved composition.

\section{GENERAL REQUIREMENTS}

Ingots for remelting shall be furnished in commercial standard forms or shapes with brand name cast in unless otherwise specified in the contract or purchase order, and shall be of uniform quality, clean, and free from burned metal or foreign materials.

\section{DETAII REQUIREMENTS}

Bronze ingots shall conform to the chemical requirements shown in Table 1. 


\section{METHODS OF INSPECTION AND TESTS}

1. An analysis of each melt of ingots shall be furnished by the contractor, showing the percentage of the elements designated.

2. Check analysis of the ingots may be made by the inspector, or through him by any Government laboratory or other designated representative and without cost to the contractor.

3. The drillings for check analysis of ingots shall be taken from one ingot selected by the inspector from each melt. The drillings shall be taken from metal below the surface of the ingot, and shall consist of not less than 5 ounces.

\section{PACKING AND MARKING OF SHIPMENTS}

Each ingot shall bear its proper heat or melt number, either stamped or cast in, and shall be packed in such manner as to facilitate identification and handling at the point of delivery.

\section{NOTE}

The various grades of ingots mentioned herein are suitable for stock for castings of the corresponding grade prescribed in Federal specification for bronze castings, Federal Specifications Board, specification QQ-B-691.

TABLE 1.-Chemical composition

\begin{tabular}{|c|c|c|c|c|c|c|c|c|c|c|c|}
\hline Grade & Copper & Tin & Zinc & Lead & $\begin{array}{l}\text { Iron, } \\
\text { maxi- } \\
\text { mum }\end{array}$ & $\begin{array}{l}\text { Nickel, } \\
\text { maxi- } \\
\text { mum }\end{array}$ & $\begin{array}{l}\text { Phos- } \\
\text { phorus, } \\
\text { maximum }\end{array}$ & $\begin{array}{l}\text { Sulphur, } \\
\text { maxi- } \\
\text { mum }\end{array}$ & $\begin{array}{l}\text { Anti- } \\
\text { mony, } \\
\text { maxi- } \\
\text { mum }\end{array}$ & $\begin{array}{l}\text { Other } \\
\text { elements, } \\
\text { maxi- } \\
\text { mum }\end{array}$ & \\
\hline $\begin{array}{l}1 \\
2\end{array}$ & $\begin{array}{l}\text { Per cent } \\
\left\{\begin{array}{c}88.0 \\
86-91 \\
85.0 \\
84-86\end{array}\right.\end{array}$ & $\begin{array}{r}\text { Per cent } \\
6.5 \\
6.0-7.0 \\
5.0 \\
4.0-6.0\end{array}$ & $\begin{array}{r}\text { Per cent } \\
4.0 \\
1.5-5.0 \\
5.0 \\
4.0-6.0\end{array}$ & $\begin{array}{r}\text { Per cent } \\
1.5 \\
1.0-2.0 \\
5.0 \\
4.0-6.0\end{array}$ & $\begin{array}{l}\text { Per cent } \\
0 \\
0.15 \\
0 \\
0.25\end{array}$ & $\begin{array}{c}\text { Per cent } \\
0.075 \\
.75\end{array}$ & $\begin{array}{c}\text { Per cent } \\
0 \\
0.05 \\
0 \\
0.05\end{array}$ & $\begin{array}{c}\text { Per cent } \\
0 \\
0.05 \\
0 \\
0.05\end{array}$ & $\begin{array}{c}\text { Per cent } \\
0 \\
0.25 \\
0 \\
0.25\end{array}$ & \begin{tabular}{|c|} 
Per cent \\
0 \\
0.15 \\
0 \\
0.15
\end{tabular} & $\begin{array}{l}\text { Desired. } \\
\text { Permissible. } \\
\text { Desired. } \\
\text { Permissible. }\end{array}$ \\
\hline $\begin{array}{l}3 \\
4\end{array}$ & $\begin{array}{l}80.0 \\
78-82 \\
81.5 \\
80-85\end{array}$ & $\begin{array}{r}4.5 \\
4.0-6.0 \\
2.5 \\
2.0-3.0\end{array}$ & $\begin{array}{r}13.0 \\
10.0-16.0 \\
10.0 \\
8.0-12.0\end{array}$ & $\begin{array}{r}2.5 \\
2.0-3.0 \\
6.0 \\
5.0-7.0\end{array}$ & $\begin{array}{l}0 \\
0.35 \\
0 \\
0.35\end{array}$ & $\begin{array}{c}.75 \\
.75\end{array}$ & $\begin{array}{l}0 \\
0.05 \\
0 \\
0.05\end{array}$ & $\begin{array}{l}0 \\
0.05 \\
0 \\
0.05\end{array}$ & $\begin{array}{l}0 \\
0.25 \\
0 \\
0.25\end{array}$ & $\begin{array}{l}0 \\
0.75 \\
0 \\
1.00\end{array}$ & $\begin{array}{l}\text { Desired. } \\
\text { Permissible. } \\
\text { Desired. } \\
\text { Permissible. }\end{array}$ \\
\hline $\begin{array}{l}5 \\
6\end{array}$ & $\begin{array}{l}\left\{\begin{array}{l}88.0 \\
86-89 \\
88.0 \\
85-89\end{array}\right.\end{array}$ & $\begin{array}{r}8.0 \\
7.5-11.0 \\
8.0 \\
7.5-11.0\end{array}$ & $\begin{array}{r}4.0 \\
1.5-4.5 \\
4.0 \\
1.5-4.5\end{array}$ & 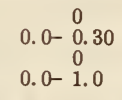 & $\begin{array}{l}0 \\
0.10 \\
0 \\
0.25\end{array}$ & $\begin{array}{c}.75 \\
.75\end{array}$ & $\begin{array}{l}0 \\
0.05 \\
0 \\
0.50\end{array}$ & $\begin{array}{l}0 \\
0.05 \\
0 \\
0.05\end{array}$ & $\begin{array}{l}0 \\
0.25 \\
0 \\
0.25\end{array}$ & $\begin{array}{l}0 \\
0.15 \\
0 \\
0.35\end{array}$ & $\begin{array}{l}\text { Desired. } \\
\text { Permissible } \\
\text { Desired. } \\
\text { Permissible. }\end{array}$ \\
\hline $\begin{array}{l}7 \\
8\end{array}$ & $\begin{array}{l}76.0 \\
73-80 \\
83.5 \\
82-85\end{array}$ & $\begin{array}{r}6.0 \\
5.0-7.0 \\
8.0 \\
7.0-9.0\end{array}$ & 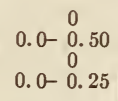 & $\begin{array}{r}18.0 \\
15.0-20.0 \\
8.0 \\
7.0-9.0\end{array}$ & $\begin{array}{l}0 \\
0.25 \\
0 \\
0.15\end{array}$ & 1.00 & $\begin{array}{r}0 \\
0.05 \\
0.50 \\
0.30-0.60\end{array}$ & $\begin{array}{l}0 \\
0.25 \\
0 \\
0.15\end{array}$ & $\begin{array}{l}0 \\
0.25 \\
0 \\
0.15\end{array}$ & $\begin{array}{l}0 \\
0.35 \\
0 \\
0.25\end{array}$ & $\begin{array}{l}\text { Desired. } \\
\text { Permissible. } \\
\text { Desired. } \\
\text { Permissible. }\end{array}$ \\
\hline $\begin{array}{c}9 \\
10\end{array}$ & $\begin{array}{l}85.5 \\
84-86 \\
81.0 \\
80-82\end{array}$ & $\begin{array}{r}13.5 \\
13.0-15.0 \\
18.5 \\
18.0-20.0\end{array}$ & $\begin{aligned} 0.0- & 1.0 \\
& 1.50 \\
0- & 0.25\end{aligned}$ & $\begin{aligned} & 0 \\
0- & 0.20 \\
& 0 \\
0- & 0.50\end{aligned}$ & $\begin{array}{l}0 \\
0.10 \\
0 \\
0.10\end{array}$ & $\begin{array}{c}.75 \\
.75\end{array}$ & $\begin{aligned} & 0 \\
& 0.05 \\
& 0.50 \\
& 0.40-0.60\end{aligned}$ & $\begin{array}{l}0 \\
0.05 \\
0 \\
0.05\end{array}$ & $\begin{array}{l}0 \\
0.15 \\
0 \\
0.15\end{array}$ & $\begin{array}{l}0 \\
0.25 \\
0 \\
0.15\end{array}$ & $\begin{array}{l}\text { Desired. } \\
\text { Permissible. } \\
\text { Desired. } \\
\text { Permissible. }\end{array}$ \\
\hline
\end{tabular}

646.12 BARS, BRONZE.

(Aluminum bronze bars, see 647.12 , p. 1258.)

(Manganese bronze bars, see 647.22, p. 1266.)

646.2 BRONZE PLATES, SHEETS, SHAPES, AND STRIPS.

646.21 PLATES AND SHEETS, BRONZE.

(Aluminum bronze plates and sheets, see 647.12, p. 1258.)

(Manganese bronze plates and sheets, see 647.24. p. 1266.$)$

UNITED STATES GOVERNMENT, DEPARTMENT OF COMMERCE, BUREAD OF STANDARDS; STANDARD THICKNESSES, WEIGHTS, AND TOLERANCES OF SHEET METAL (CUSTOMARY PRACTICE), CIRCULAR NO. 391, MARCH 24, 1931

\section{AMERICAN WIRE GAGE-BRONZE PLATES AND SHEETS}

(See 640.1 , p. 1165.)

\subsection{SHAPES, BRONZE.}

(Aluminum bronze shapes, see 647.12 , p. 1258.) (Manganese bronze shapes, see 647.23, p. 1266.)

\subsection{STRIPS, BRONZE.}

(Aluminum bronze strips, see 647.12 , p. 1258.)

(Phosphor bronze strips, see 647.36, p. 1269.)

\subsection{BRONZE RODS AND WIRES.}

\subsection{RODS, BRONZE.}

(Tobin bronze (naval brass) rods, see 645.11, p. 1205.)

(Aluminum bronze rods, see 647.12 , p. 1258).

(Manganese bronze rods, see 647.23 , p. 1266.)

\subsection{WIRES, BRONZE.}

(Phosphor bronze wire, see 647.38, p. 1270.)

(Specifications for bronze trolley wire are not included in this publication. See reference to electrical materials and supplies under 642.12, p. 1181. Specifications for bronze trolley wire have been prepared jointly by the American Electric Railway Engineering Association and the American Society for Testing Materials. These specifications cover round and grooved trolley wire of two strength grades with copper according to A. S. T. M. specifications, and include requirements on tensile strength, elongation, resistivity and dimensions, twist test for round wire, and standard sections for grooved wire. These specifications are designated as follows: A. E. R. E. A. bronze trolley wire, designation D12-30: A. S. T. M. bronze trolley wire, designation $\mathrm{B} 9-30$.) 
646.4 BRONZE CASTINGS AND TUBES. 646.41 CASTINGS, BRONZE.

AMERICAN BUREAU OF SHIPPING, RULES FOR BUILDING AND CLASSING STEEL VESSELS ; MATERIALS FOR MACHINERY, BOILERS, AND PIPING, 1930

\section{BRONZE CASTINGS}

(For general information and requirements see 600.0, p. 1. For test requirements see 600.1, p. 7.)

1. Brass and bronze castings subject to test and inspection shall be free from blowholes, porous spots, cracks, and other defects. The material must satisfactorily comply with the following physical characteristics:

\begin{tabular}{l|r|r|c|c}
\hline Grade & $\begin{array}{r}\text { Mini- } \\
\text { mum } \\
\text { tensile } \\
\text { strength }\end{array}$ & $\begin{array}{r}\text { Mini- } \\
\text { mum } \\
\text { elonga- } \\
\text { tion in } \\
2 \text { inches }\end{array}$ & Purpose & Stamp \\
\hline & $\begin{array}{r}\text { Lbs./in.2 } \\
30,000\end{array}$ & Per cent \\
15 & For general purposes.......- \\
60,000 & 20 & $\begin{array}{c}\text { For propeller blades and simi- } \\
\text { lar high-grade castings. }\end{array}$ & A. B. \\
\hline
\end{tabular}

2. The number of tests for brass and bronze castings is to be in accordance with the requirements for steel castings (see 611.41, p. 706, par. 2), but no bend tests are required.

AMERICAN BUREAU OF SHIPPING, RULES FOR BUILDING AND CLASSING STEEL VESSELS ; PUMPS AND PIPING SYSTEMS, 1930

\section{MATERIAL OF VALVES AND FITTINGS}

(For general requirements see 607.4 , p. 435.)

1. Composition having the physical characteristics for grade 1 material (bronze castings) specified above under materials for machinery, boilers, and piping may be used in the construction of valves and fittings for temperatures up to $470^{\circ} \mathrm{F}$. For temperatures greater than $470^{\circ} \mathrm{F}$. the chemical and physical characteristics of the composition are to be specially submitted for approval. The minimum thickness in inches for material of composition grade 1 quality shall be determined by the formula:

$$
t=\frac{\text { Working pressure } \times \text { internal diameter }}{5,000}+0.125
$$

AMERICAN ELECTRIC RAILWAY ENGINEERING ASSOCIATION, OVERHEAD IINE MATERIAL FOR DIRECT AND CATENARY SUSPENSION, RECOMMENDED SPECIFICATION, D102--30, 1930

\section{OVERHEAD IINE MATERIAL}

(D102-30, paragraphs 330, 332, 333, etc., include requirements for bronze castings. For specification see 611.29 , p. 682.)

AMERICAN MARINE STANDARDS COMMITTEE, SPECIFICATION FOR BUILT-UP PROPELLERS, E NO. 23-1928

\section{BUILT-UP PROPELLERS}

(See 611.19, p. 661.)
AMERICAN MARINE STANDARDS COMMITTEE, SPECIFICATION FOR PROPELLERS CAST IN ONE PIECE, E NO. 25-1928

CAST PROPELIERS

(See 611.19, p. 663.)

AMERICAN MARINE STANDARDS COMMITTEE, LOCKS AND ACCESSORIES FOR SHIP DOORS, H NO. 61-1930

BRONZE CASTINGS

(See 617.21, p. 1004.)

AMERICAN MARINE STANDARDS COMMITTEE, BOLTS AND CATCHES FOR SHIP DOORS, H NO. 62-1930

\section{BRONZE CASTINGS}

(See 617.61, p. 1052.)

AMERICAN RAILWAY ASSOCIATION, MECHANICAL DIVISION; SPECIFICATIONS FOR BEARINGS, BRONZE, FOR LOCOMOTIVES, 1928

BRONZE BEARINGS FOR LOCOMOTIVES (See 692.2, p. 1324.)

AMERICAN RAILWAY ENGINEERING ASSOCIATION, SPECIFICATIONS FOR RAILWAY BUILDINGS, MISCELLANEOUS METAL WORK, 1929

\section{BRONZE CASTINGS}

Bronze castings shall be made from electroplated metal patterus in molds with special facings and shall be hand filed, chased, and carefully finished.

AMERICAN SOCIETY FOR TESTING MATERIALS, STANDARD SPECIFICATIONS FOR THE ALIOY; COPPER, 88 PER CENT; TIN, 10 PER CENT; ZINC, 2 PER CENTDESIGNATION B10-18, 1918

$$
\text { AIIOY 88-10-2 }
$$

(See 643.4, p. 1192.)

AMERICAN SOCIETY FOR TESTING MATERIALS, STANDARD SPECIFICATIONS FOR SAND CASTINGS OF THE ALLOY; COPPER, 88 PER CENT; TIN, 8 PER CENT; ZINC, 4 PER CENT-DESIGNATION B6028,1928

$$
\text { ALIOY 88-8-4 }
$$

(See 643.4, p. 1192.)

AMERICAN SOCIETY FOR TESTING MATERIAIS, STANDARD SPECIFICATIONS FOR STEAM OR VALVE BRONZE SAND CASTINGS, DESIGNATION B61-28, 1928

\section{BRONZE SAND CASTINGS} SCOPE

1. These specifications cover alloy castings, the alloy being a composition of copper, tin, lead, and 
zinc, commonly used as a high-grade steam metal or valve bronze.

\section{CHEMICAL PROPERTIES AND TESTS}

2. The alloy shall conform to the following requirements as to chemical composition:

\begin{tabular}{|c|c|c|c|}
\hline & Desired & Minimum & Maximum \\
\hline $\begin{array}{l}\text { Copper. } \\
\text { Tin } \\
\text { Lead } \\
\text { Zinc. } \\
\text { Iron } \\
\text { Nickel. }\end{array}$ & $\begin{array}{r}\text { Per cent } \\
88.00 \\
6.50 \\
1.50 \\
4.00 \\
-\end{array}$ & $\begin{array}{r}\text { Per cent } \\
86.00 \\
6.00 \\
1.00 \\
\text { Remainder. }\end{array}$ & $\begin{array}{r}\text { Per cent } \\
91.00 \\
7.00 \\
2.00 \\
\text { Remainder. } \\
.15 \\
.50\end{array}$ \\
\hline $\begin{array}{l}\text { Phosphorus } \\
\text { Aluminum } \\
\text { Antimony } \\
\text { Sulphur } \\
\text { Total other impurities in addi- } \\
\text { tion to those specified above }\end{array}$ & None. & 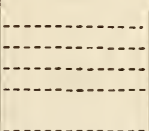 & $\begin{array}{r}.05 \\
\text { None. } \\
.25 \\
.05\end{array}$ \\
\hline
\end{tabular}

Where "none" is specified it shall be construed to refer to none as determined on a 10-gram sample.

\section{PHYSICAL PROPERTIES AND TESTS}

The alloy shall conform to the following minimum requirements as to tensile properties:

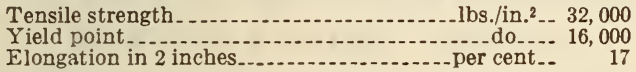

(Specifications covering tension-test specimen test requirements are identical with those in paragraph 11 (c), A18. See 611.51, p. 754. In respect to chemical analyses, determination of yield point, workmanship and finish, marking, and inspection and rejection, this specification is identical with B59. See 647.15 , p. 1259 . In respect to manufacture, pressure-test requirements, and dimensions of testspecimen coupon, it is identical with B60. See 643.4 , p. 1192 . In respect to number of tests, paragraphs $7(a),(b),(c)$, and $(d)$ in this specification are identical with Pars. VI 2, 3, 4, and 13 in F. S. B. QQ-B-691. See 646.41, p. 1252.)

\section{AMERICAN SOCIETY FOR TESTING MA- TERIALS, STANDARD SPECIFICATIONS FOR BRONZE CASTINGS IN THE ROUGH FOR LOCOMOTIVE WEARING PARTS, DESIGNATION B66-28, 1928}

\section{BRONZE CASTINGS}

\section{SCOPE}

1. These specifications cover bronze castings in the rough for locomotive wearing parts.

\section{BASIS OF PURCHASE}

2. Unless otherwise specified on the purchase order or in the contractural arrangement, the manufacturer shall furnish hard bronze for connecting-rod bearings, valve-motion bushings, crosshead gibs, and miscellaneous bearings subject to hard wear. Hard bronze shall also be furnished for pedestal wedges and eccentric straps should these appear on the purchase order or contract.

Medium bronze shall be furnished for driving-box brasses, engine-truck brasses, hub liners, and bearings which require lining metal for facing or lining.

Phosphor bronze and soft bronze will be furnished only when specified.
CHEMICAL PROPERTIES AND TESTS

3. (a) The material shall conform to the following requirements as to chemical composition:

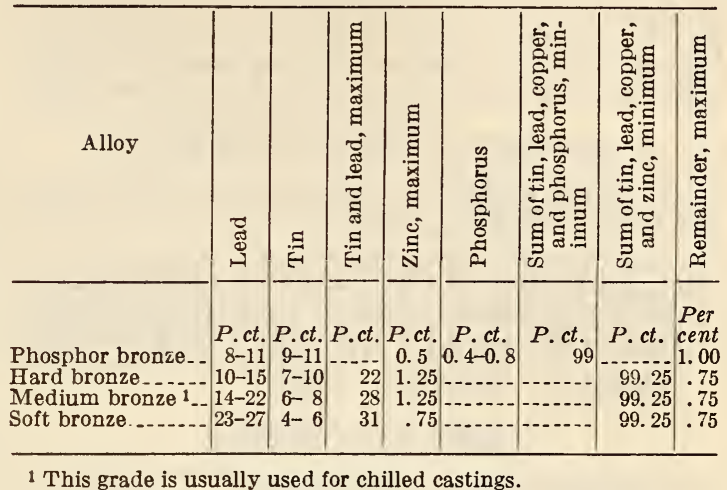

(b) Where no figures are given for impurity maximum, these elements may be present up to any amount to come within the total impurity specified provided the material otherwise meets the chemical and physical tests. Where maximum percentages are specified, the elements referred to are considered as impurities and shall not be intentionally added.

\section{CHEMICAL ANALYSIS}

4. An analysis shall be made by the purchaser or his representative from one casting representing each lot of 100 castings or fraction thereof. The chemical composition thus determined shall conform to the requirements as specified in section $3(a)$ for the particular alloy.

\section{SAMPLE FOR CHEMICAL ANALYSIS}

5. (a) The sample for chemical analysis shall be taken from the casting at three points along the fractured surface, as described in section $6(a)$. Drillings through the skin shall be rejected. Equal quantity of drillings from each hole thoroughly mixed shall constitute the sample for chemical analysis.

(b) All samples shall be kept free of oil and shall be carefully treated with a magnet to remove any iron introduced in taking the sample.

\section{PHYSICAL PROPERTIES AND TESTS}

6. (a) The casting representing a lot for acceptance, shall be broken through the center and parallel with the longitudinal axis of the bearing in order to ascertain the uniformity of the grain of the metal When this fracture shows separation or imperfect mixing of the component parts, or dross or dirt spots, the lot shall be rejected.

(b) One casting may be taken for fracture test from each lot of 100 castings or fraction thereof.

\section{PERMISSIBLE VARIATIONS IN DIMENSIONS}

7. The purchaser will furnish drawings showing the dimensions of the castings ordered and permissible variations therefrom, and all castings shall conform to the gages and dimensions shown on the drawings.

\section{WORKMANSHIP AND FINISH}

8. The castings shall be sound, free from blowholes, flaws, and shrinkage cracks, and shall show good foundry practice and workmanship. 


\section{MAREING}

9. Each lot of 100 castings or fraction thereof shall bear a serial number, commencing with the numeral one at the beginning of the year and continuing consecutively until the end of the year. All castings shall also have cast on them the manufacturer's initials or trade-mark, the pattern number, and such other marks as are shown on the drawings.

INSPECTION, REJECTION, AND REHEARING

(Conform to A. S. T. M. standard specifications for steel. See 605.0 , p. 247.)

SOCIETY OF AUTOIMOTIVE ENGINEERS, RONFERROUS METAL SPECIFICATIONS, SPECIFICATION NO. 62, HARD CAST BRONZE, MARCE, 1922

\section{HARD CAST BRONZE}

1. Composition in percentage.-

Topper.

Per cent

Lead, maximum

Iron, maximum 86. $00-89.00$

Zinc.. 9. $00-11.00$

$1.00-3.00$

2. General information.-Good castings made of this bronze should give the following minima in physical characteristics:

Ultimate strength

Yield point lbs./in. ${ }^{2}-30,000$

Elongation in 2 inches or proportionate gage length per cent.

14

3. This alloy is suitable wherever a strong general utility bronze is required. It may be used for severe working conditions where heavy pressures obtain, as in gears and bearings.

4. Bearings or gears made of bronze alloys should be used only against hardened steel.

SOCIETY OF AUTOMOTIVE ENGINEERS, NONFERROUS METAL SPECIFICATIONS, SPECIFICATION NO. 63, LEADED GUN METAL, MARCH, 1922

\section{LEADED GUN METAL}

1. Composition in percentage.-

Copper

Tin...

Phosphorus, maximum

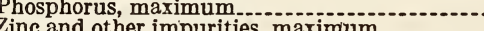

Zinc and other impurities, maximum $86.00-89.00$ $9.00-11.00$

2. General information.-Good castings made of this alloy should give the following minima in physical characteristics:

Ultimate strength............................lbs./in.2.. 30,000 Yield point

Elongation in 2 inches, or proportionate gage length per cent.10

3. Combining strength with fair machining qualities, this general-utility bronze is especially good for bushings subjected to heavy loads and severe working conditions.

SOCIETY OF AUTOMOTIVE ENGINEERS, NONFERROUS METAI SPECIFICATIONS, SPECIFICATION NO. 66, BRONZE BACKING FOR LINED BEARINGS, MARCH, 1922

BRONZE BACKING FOR IINED BEARINGS

(See 692.2, p. 1326.)
SOCIETY OF AUTOMOTIVE ENGINEERS, NONFERROUS METAL SPECIFICATIONS, SPECIFICATION NO. 67, SEMIPLASTIC BRONZE, MARCH, 1922

\section{SEMIPLASTIC BRONZE}

(See 692.2, p. 1326.)

(For other copper-tin-zinc alloys, see 643.4.)

UNITED STATES GOVERNMENT, DEPARTMENT OF COMIIERCE, BUREAU OF STANDARDS, STANDARD SAMPLES, CIRCULAR NO. 25 AND SUPPLEMENT, 1927

\section{CAST BRONZE SAMPLES}

(Sample No. 52 is a standard sample of cast bronze prepared and sold by the Bureau of Standards for use by industrial organizations and others as a comparison standard for checking the sccuracy of analysis of cast bronze. Each sample is accompanied by a certificate giving a complete analysis of that particular sample.)

UNITED STATES GOVERNIENT, DEPARTMENT OF COMMERCE, BUILDERS' TEMPLATE HARDWARE, COMMERCIAI STANDARD CS9-29, SEPTEMBER 18, 1929

\section{BUILDERS' TEMPLATE HARDWARE}

(For template butt hinges, see 617.12 , p. 982. For template cylinder locks, see 617.21 , p. 1020.)

UNITED STATES GOVERNMENT, DEPARTMENT OF COMMERCE, BUILDERS' HARDWARE (NONTEMPLATE), COMMERCIAL STANDARD CS22-30, DECEMBER 11, 1930 BUILDERS' HARDWARE (NONTEMPLATE.)

(See 617.0, p. 977.)

UNITED STATES GOVERNMENT, FEDERAL SPECIFICATIONS BOARD, FEDERAL SPECIFICATION FOR BUILDERS' HARD. WARE (NONTEMPLATE), FF-H-101, AUGUST 19, 1930

\section{BUILDERS' HARDWARE (NONTEMPLATE.)}

(For applicable Federal specifications; types, grades, and classes; material and workmanship; general requirements; detail requirements; packaging, packing, and marking; notes; and table of standard finishes, see 617.0 , p. 978. For chromium plating, nickel plating, and zinc coatings; and methods of inspection and tests, see 600.3 , p. 70.)

(Specific commodities, such as locks, latches, hinges, bolts, etc., will be found under their respective commodity designations. For a list of these commodities, see under detail requirements in 617.0 , p. 979 .)

\section{UNITED STATES GOVERNMENT, FEDERAI SPECIFICATIONS BOARD, FEDERAL SPECIFICATION FOR BRONZE CASTINGS, QQ-B-691, MAY 31, 1932}

\section{BRONZE CASTINGS}

(In respect to general specifications, this specification conforms to F. S. B. 339 under commodity des- 
ignation 600.0, p. 3 (current designation: QQ-M-151). In other respects it is as follows:)

\section{GRADES}

This specification covers 10 grades of bronze in the form of castings.

\section{MATERIAI}

The castings shall be made only from ingots of the corresponding grade of Federal Specifications Board specification QQ-B-701 of latest revision, from the best grades of virgin metals, or from the best grades of virgin metals and scrap of known and approved composition and cleanliness, with the exception of grades 4 and $6 \mathrm{X}$, which may be made from scrap only, if desired.

\section{GENERAL REQUIREMENTS}

1. The castings shall be of uniform quality, free from blowholes, porosity, hard spots, shrinkage defects, cracks, or other injurious defects, and shall be smooth and well cleaned before inspection by sandblasting, tumbling, chipping, or other process approved by the inspector.

2. Castings shall not be repaired, plugged, or welded without permis ${ }^{\circ}$ on from the inspector. Such permission will be given only when the defects are small and do not adversely affect the strength, use, or machineability of the castings.

3. Each repair shall be encircled with a ring of white paint prior to shipment.

4. The responoibility for furnishing castings that can be laid out and machined to the finished dimensions, within the specified tolerances, as shown on the blueprints or drawings, and that will conform to such gages as may be specified in individual cases, shall rest with the contractor. Sufficient stock shall be allowed for shrinkage, and, where required, for finishing, but castings or excessive size or weight shall not be furnished.

\section{DETAII REQUIREMENTS}

1. Castings shall conform to the chemical requirements shown in Table 1 , except that grade 4 shall show upon analysis a copper-tin-lead-zinc alloy of the minimum copper content shown in Table 1.

TABLE 1.-Chemical composition

\begin{tabular}{|c|c|c|c|c|c|c|c|c|c|c|c|}
\hline Grade & Copper & Tin & Zinc & Lead & $\begin{array}{l}\text { Iron, } \\
\text { maxi- } \\
\text { mum }\end{array}$ & $\mid \begin{array}{c}\text { Nickel, } \\
\text { maxi- } \\
\text { mum }\end{array}$ & $\begin{array}{l}\text { Phos- } \\
\text { phorus, } \\
\text { maxi- } \\
\text { mum }\end{array}$ & $\begin{array}{l}\text { Sulphur, } \\
\text { maxi- } \\
\text { mum }\end{array}$ & $\begin{array}{l}\text { Anti- } \\
\text { mony, } \\
\text { maxi- } \\
\text { mum }\end{array}$ & $\begin{array}{l}\text { Other } \\
\text { elements, } \\
\text { maxi- } \\
\text { mum }\end{array}$ & \\
\hline $\begin{array}{l}1 \\
2\end{array}$ & $\left\{\begin{array}{l}\text { Per cent } \\
88.0 \\
86-91 \\
85.0 \\
84-86\end{array}\right.$ & $\begin{array}{r}\text { Per cent } \\
6.5 \\
6.0-7.0 \\
5.0 \\
4.0-6.0\end{array}$ & $\begin{array}{r}\text { Per cent } \\
4.0 \\
1.5-5.0 \\
5.0 \\
4.0-6.0\end{array}$ & $\begin{array}{r}\text { Per cent } \\
1.5 \\
1.0-2.0 \\
5.0 \\
4.0-6.0\end{array}$ & $\begin{array}{c}\text { Per cent } \\
0 \\
0.15 \\
0 \\
0.25\end{array}$ & $\begin{array}{c}\text { Per cent } \\
0.75 \\
.75\end{array}$ & $\begin{array}{l}\text { Per cent } \\
0 \\
0.05 \\
0 \\
0.05\end{array}$ & $\begin{array}{c}\text { Per cent } \\
0 \\
0.05 \\
0 \\
0.05\end{array}$ & $\begin{array}{c}\text { Per cent } \\
0 \\
0.25 \\
0 \\
0.25\end{array}$ & $\begin{array}{c}\text { Per cent } \\
0 \\
0.15 \\
0 \\
0.15\end{array}$ & $\begin{array}{l}\text { Desired. } \\
\text { Permissible. } \\
\text { Desired. } \\
\text { Permissible. }\end{array}$ \\
\hline $\begin{array}{l}3 \\
4\end{array}$ & $\left\{\begin{array}{r}80.0 \\
78-82 \\
81.5 \\
180\end{array}\right.$ & $\begin{array}{r}4.5 \\
4.0-6.0 \\
2.5\end{array}$ & $\begin{array}{r}13.0 \\
10.0-16.0 \\
10.0\end{array}$ & $\begin{array}{r}2.5 \\
2.0-3.0 \\
6.0\end{array}$ & $\begin{array}{l}0 \\
0.35 \\
0\end{array}$ & .75 & $\begin{array}{l}0 \\
0.05 \\
0\end{array}$ & $\begin{array}{l}0 \\
0.05 \\
0\end{array}$ & $\begin{array}{l}0 \\
0.25 \\
0\end{array}$ & $\begin{array}{l}0 \\
0.75 \\
0\end{array}$ & $\begin{array}{l}\text { Desired. } \\
\text { Permissible. } \\
\text { Desired. } \\
\text { Permissible. }\end{array}$ \\
\hline $\begin{array}{l}5^{2} \\
6^{2}\end{array}$ & $\left\{\begin{array}{r}88.0 \\
86-89 \\
88.0 \\
85-89\end{array}\right.$ & $\begin{array}{r}8.0 \\
7.5-11.0 \\
8.0 \\
7.5-11.0\end{array}$ & $\begin{array}{r}4.0 \\
1.5-4.5 \\
4.0 \\
1.5-4.5\end{array}$ & $\begin{array}{c}0 \\
0.0-30 \\
0 \\
0.0-1.00\end{array}$ & $\begin{array}{l}0 \\
0.10 \\
0 \\
0.25\end{array}$ & $\begin{array}{c}.75 \\
-.75\end{array}$ & $\begin{array}{l}0 \\
0.05 \\
0 \\
0.50\end{array}$ & $\begin{array}{l}0 \\
0.05 \\
0 \\
0.05\end{array}$ & $\begin{array}{l}0 \\
0.25 \\
0 \\
0.25\end{array}$ & $\begin{array}{l}0 \\
0.15 \\
0 \\
0.35\end{array}$ & $\begin{array}{l}\text { Desired. } \\
\text { Permissible. } \\
\text { Desired. } \\
\text { Permissible. }\end{array}$ \\
\hline 8 & $\left\{\begin{array}{r}76.0 \\
73-80 \\
83.5 \\
82-85\end{array}\right.$ & $\begin{array}{r}6.0 \\
5.0-7.0 \\
8.0 \\
7.0-9.0\end{array}$ & $\begin{array}{c}0 \\
0-0.50 \\
0 \\
0-0.25\end{array}$ & $\begin{array}{r}18.0 \\
15.0-20.0 \\
8.0 \\
7.0-9.0\end{array}$ & $\begin{array}{l}0 \\
0.25 \\
0.15\end{array}$ & $\begin{array}{l}1.00 \\
1.00\end{array}$ & $\begin{array}{r}0 \\
0.05 \\
0.50 \\
{ }^{3} 0.30-0.60\end{array}$ & $\begin{array}{l}0.25 \\
0 \\
0.15\end{array}$ & $\begin{array}{l}0 \\
0.25 \\
0 \\
0.15\end{array}$ & $\begin{array}{l}0 \\
0.35 \\
0 \\
0.25\end{array}$ & $\begin{array}{l}\text { Desired. } \\
\text { Permissible. } \\
\text { Desired. } \\
\text { Permissible. }\end{array}$ \\
\hline $\begin{array}{l}9 \\
10\end{array}$ & $\left\{\begin{array}{r}85.5 \\
84-86 \\
81.0 \\
80-82\end{array}\right.$ & $\begin{array}{r}13.5 \\
13.0-15.0 \\
18.5 \\
18.0-20.0\end{array}$ & $\begin{array}{c}1.0 \\
0-1.50 \\
0 \\
0-0.25\end{array}$ & $\begin{array}{l}0 \\
0-0.20 \\
0 \\
0-0.50\end{array}$ & $\begin{array}{l}0 \\
0.10 \\
0 \\
0.10\end{array}$ & $\begin{array}{c}.75 \\
.75\end{array}$ & $\begin{array}{c}0 \\
0.05 \\
0 \\
0.40-0.60\end{array}$ & $\begin{array}{l}0 \\
0.05 \\
0 \\
0.05\end{array}$ & $\begin{array}{l}0 \\
0.15 \\
0 \\
0.15\end{array}$ & $\begin{array}{l}0 \\
0.25 \\
0 \\
0.15\end{array}$ & $\begin{array}{l}\text { Desired. } \\
\text { Permissible. } \\
\text { Desired. } \\
\text { Permissible. }\end{array}$ \\
\hline
\end{tabular}

1 Minimum.

2 See Sec. VIII for details of application.

3 Minimum and maximum limits.

Grade $6 \mathrm{X}$, which may be made of scrap only, has the same chemical composition requirements as grade 6 given in the above Table 1 .

2. An analysis of each melt of castings shall be furnished by the contractor, showing the percentage of the elements designated.
3. Castings shall conform to the physical requirements shown in Table 2.

4. The physical properties of castings of bronze No. 3 need not be determined unless specified in the contract or purchase order. 
TABLe 2.-Physical properties

\begin{tabular}{|c|c|c|c|}
\hline Grade & $\begin{array}{c}\text { Tensile } \\
\text { strength, } \\
\text { minimum }\end{array}$ & $\begin{array}{l}\text { Elonga- } \\
\text { tion in } 2 \\
\text { inches, } \\
\text { minimum }\end{array}$ & $\begin{array}{l}\text { Brinell } \\
\text { hardness } \\
\text { No., min- } \\
\text { imum }\end{array}$ \\
\hline $\begin{array}{l}1 \\
2 \\
4 \\
5\end{array}$ & $\begin{array}{r}\text { Lbs./in. }{ }^{2} \\
32,000 \\
27,000 \\
28,000 \\
25,000 \\
40,000\end{array}$ & $\begin{array}{r}\text { Per cent } \\
17.0 \\
15.0 \\
15.0 \\
12.0 \\
20.0\end{array}$ & 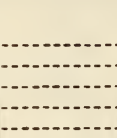 \\
\hline $6^{b}-$ & 35,000 & 18.0 & \\
\hline 9 & $\begin{array}{r}25,000 \\
30,000 \\
e 45,000\end{array}$ & $\begin{array}{r}8.0 \\
1.0\end{array}$ & 160 \\
\hline
\end{tabular}

a See Sec. V, 4.

$b$ See Sec. VIII for details of application.

$c$ Not required, but may be obtained in sound castings.

Grade $6 X$ shall have a tensile strength of 30,000 pounds per square inch and an elongation of 12 per cent.

5. Castings made of bronzes Nos. 1,2 , or 3 shall conform to such requirements as to pressure as may be specified in the contract or purchase order.

\section{METHODS OF INSPECTION AND TESTS}

1. Samples for check analyses shall be taken from tensile-test specimens or from a representative

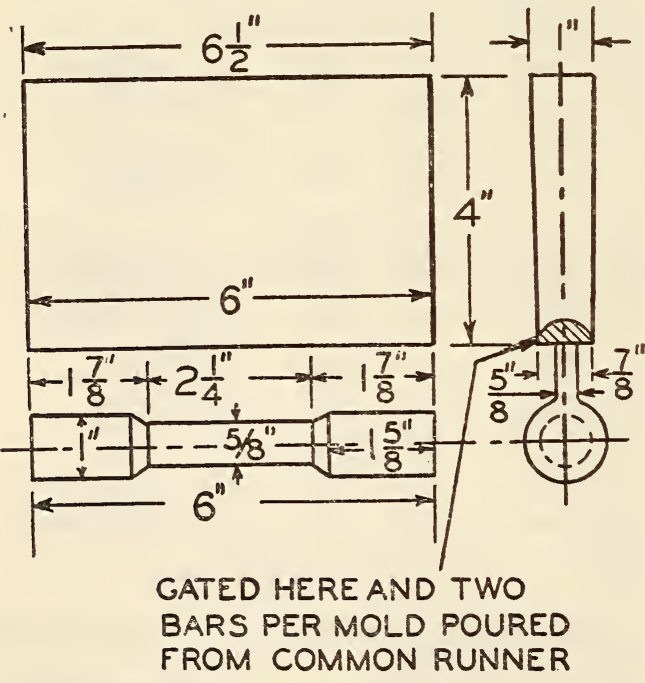

FIGURE 1117

casting selected by the inspector from each melt. The drillings shall be taken from sound metal below the surface. The inspector shall forward for analysis not less than five ounces of drillings.

2. Each casting weighing 250 pounds or more shall have at least one test coupon attached thereto. The responsibility of furnishing sufficient test specimens shall rest with the contractor.

3 . In the case of castings weighing less than 250 pounds each, at least one test coupon shall be attached to one or more castings from each melt or heat, or from such groups of melts or heats as the purchaser may specify, but in no case shall a lot consist of more than 1,000 pounds of castings.

4. In the case of castings where, in the opinion of the inspector, it is impracticable to attach a test coupon to the casting, bars shall be cast separately in sand, at least one test bar being poured from each melt or heat of metal, or such groups of melts or heats as the purchaser may specify, but in no case shall a lot consist of more than 1,000 pounds of castings. The bars shall be cast from the first metal poured from the heat, and the inspector shall witness the pouring of the test mold.

5. Test specimens may be of two types, namely, machined or cast to size.

6. Unless otherwise specified in the contract or purchase order, machined test specimens will be

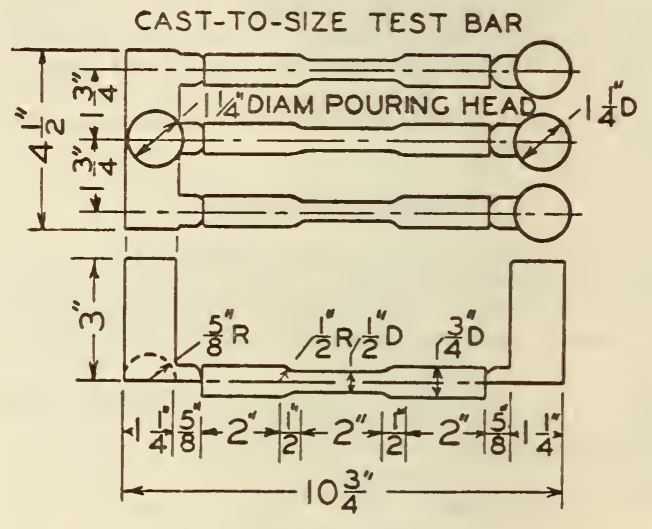

FigURE 1118

used. In this case the test coupon or separately cast bar shall be of the dimensions shown in Figure 1117, and the test specimen machined therefrom shall conform to the dimensions shown in Figure 129 under 605.0, p. 247. The fin connecting the test coupon with the casting or sink head shall be not less than five-eighths inch thick.

7. When required by the contract or purchase order, test bars cast in green sand in accordance with the method and to the dimensions show $\mathrm{n}$ in Figure 1118 shall be furnished by the contractor. These bars shall not be machined prior to testing, except to adapt the grips to the holders of the testing machine in such manner as to insure an axial load.

8. Separately cast test coupons shall be drawn from the sand in the presence of the inspector, who will mark the coupons or bars for future identification. Test coupons shall not be removed from castings except in the presence of the inspector, or until he has stamped them for future identification.

9. When the manufacturer so desires, extra castings may be made in order to provide for test specimens in lieu of the test bars or coupons attached.

10. The chilling of test coupons or bars shall cause the rejection of the material represented, unless otherwise specified in the contract or purchase order.

11. Brinell hardness determinations shall be made one-sixteenth inch below the surface with a $10-\mathrm{mm}$ ball and a pressure of $3,000 \mathrm{~kg}(6,615$ pounds) sustained for 30 seconds.

12. Where castings are of such form and dimensions as to make calipering of their walls impracticable, and the inspector so requests, $1 / 4$-inch holes shall be drilled through at doubtful places to establish the exact thickness. Before loading for shipment, these holes shall be tapped out and filled by screwing in suitable bronze plugs, or by welding, wherever such method is approved by the inspector. Such plugs shall carry the inspector's stamp.

13. If any test specimens contain flaws which result in the failure of the specimens, at the discretion of the inspector, additional specimens from the same melt or heat may be selected to replace them. If additional test coupons are not arailable, the 
replacement specimens, at the discretion of the inspector, may be taken from the body of a casting selected by the inspector.

14. Castings in which injurious defects are revealed by manufacturing operations subsequent to delivery shall be rejected.

\section{MARKING AND PACKING OF SHIPMENTS}

1. Castings shall be marked with the pattern or mark number, and when practicable with the melt or lot number, in a position on the casting where they will not be machined off in manufacturing to finished dimensions.

2. Castings which are impracticable to so mark shall be properly separated by melts or lots when presented for inspection, and shall be so marked that their identity will be clear at the point of delivery.

3. A lot shall consist of castings from the same melt unless otherwise specified in the contract or purchase order or mutually agreed upon by the contractor and the inspector.

4. Large castings having projections that may be damaged in handling shall be crated. Small, rough castings may be packed rigidly in boxes or crates, or may be bundled. Small polished castings shall be well wrapped in paper and rigidly packed in boxes.

5. If special packing be necessary to secure the acceptance of castings by common or other carriers for safe transportation at the lowest rate to the point of delivery, such packing shall be provided by the contractor without additional expense to the purchaser.

VIII. NOTES

1. The material covered by this specification is not intended for use in the manufacture of mail locks for the Post Office Department and this specification is not mandatory when purchasing castings therefor.

2. Grade No. 1 bronze is a high grade steam or valve bronze.

3. Grade No. 2 bronze is a standard composition for general service, also known as ounce metal, red brass, and hydraulic bronze.

4. Grade No. 3 bronze is a medium grade bronze which may be used for standard and extra heavy water, air, or gas fittings. Ordinarily it may be purchased without physical test.

5. Grade No. 4 bronze is a cheap bronze which may be used for ornamental or electrical fittings, instruction and marker plates, handwheels, brackets, and in general where the characteristics of a bronze are essential, but composition and physical properties are relatively unimportant.

6. Grade No. 5 bronze is an excellent steam metal and structural bronze which may be used for expansion joints, special pipe fittings, gears, bolts and nuts, valves, pump pistons and casings, bushings, bearings, and similar applications where strength requirements combined with the necessity for casting properties and corrosion resistance of tin-bronze and weight limitations make this high tensile bronze necessary.

7. Grade No. 6 bronze is a leaded phosphor bronze or leaded gun metal which serves in the same way as grade No. 5 as a steam metal and general structural bronze. It machines more easily than grade No. 5 bronze, but may, in general, be used for expansion joints, special pipe fittings, gears, valves, pump pistons and casings, bushings, bearings, and similar applications requiring good strength and resistance to sea-water corrosion.

Grade No. 6X is a leaded phosphor bronze or leaded gun metal which may be made from scrap or like composition, and is intended for use where the stresses are low or where structural strength is not required.

8. Grade No. 7 bronze is a good bearing bronze for machinery carrying heavy loads and running at very low speeds.
9. Grade No. 8 bronze is a good bearing bronze for general service.

10. Grade No. 9 bronze is a hard bearing bronze, also known as hard gear bronze.

11. Grade No, 10 bronze is an extra hard bearing bronze or gear bronze, intended only for special applications.

646.42 TUBES, BRONZE.

AMERICAN INSTITUTE OF ELECTRICAL ENGINEERS, RECOMMENDED PRACTICE FOR ELECTRICAL INSTALLATIONS ON SHIPBOARD, NO. 45, CONDUIT TERMINAL AND BULKHEAD STUFFING TUBES, OCTOBER, 1930

\section{STUFFING TUBES}

(See A. I. E. E. 645.24 , p. 1219.)

646.5 MANUFACTURES OF BRONZE.

646.51 FITTINGS, BRONZE.

AMERICAN MARINE STANDARDS COMMITTEE, BOLTS AND CATCHES FOR SHIP DOORS, II NO. 62-1930

BRONZE FITTINGS

(See 617.61, p. 1052.)

AMERICAN MARINE STANDARDS COMMITTEE, LOCKS AND ACCESSORIES FOR SHIP DOORS, H NO. 61-1930

\section{BRONZE FITTINGS}

(See 617.21, p. 1004.)

\section{INTERNATIONAL ASSOCIATION OF MILK DEALERS, STANDARD SANITARY FIT- TING, 1923}

\section{SANITARY FITTING}

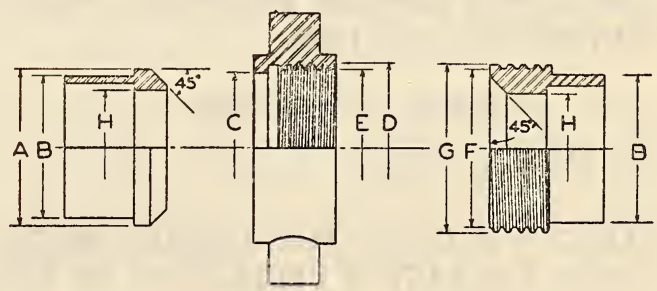

Figure 1119.-Sanitary fiting

Dimensions of standard sanitary fitting

[Inches]

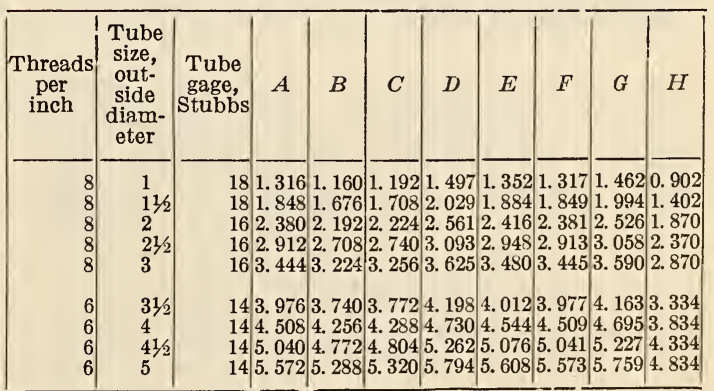

Threads are Acme standard thread. 


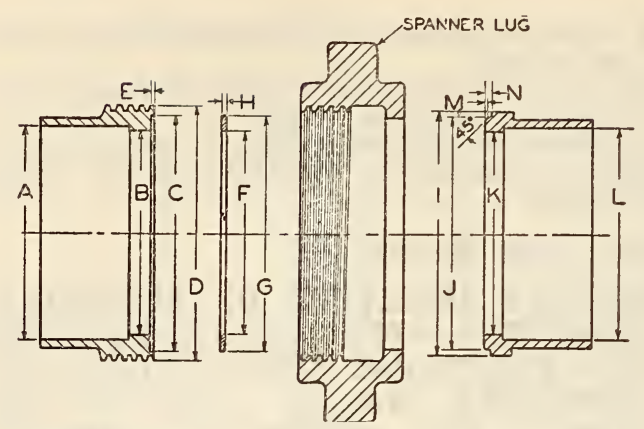

FIGURE 1120.-Sanitary pipe union with paper gasket

Dimensions of sanitary pipe union with paper gasket [Inches]

\begin{tabular}{|c|c|c|c|c|c|c|c|}
\hline $\begin{array}{c}\text { Size } \\
\text { diam- } \\
\text { eter }\end{array}$ & $A$ & $B$ & $C^{1}$ & $D$ & $E$ & $F$ & $G$ \\
\hline $\begin{array}{l}11 / 2 \\
2 \\
21 / 2 \\
3\end{array}$ & $\begin{array}{l}1.505 \\
2.005 \\
2.505 \\
3.005\end{array}$ & $\begin{array}{l}1.402 \\
1.870 \\
2.370 \\
2.870\end{array}$ & $\begin{array}{l}1.750 \\
2.250 \\
2.750 \\
3.312\end{array}$ & $\begin{array}{l}\text { 1. } 994 \\
\text { 2. } 526 \\
3.058 \\
3.590\end{array}$ & $\begin{array}{l}1 / 16 \\
1116 \\
1 / 16 \\
1 / 16\end{array}$ & $\begin{array}{l}1.402 \\
1.870 \\
2.370 \\
2.870\end{array}$ & \begin{tabular}{|l}
1.750 \\
2.250 \\
2.750 \\
3.312
\end{tabular} \\
\hline $\begin{array}{c}\text { Size } \\
\text { diam- } \\
\text { eter }\end{array}$ & $H$ & $I$ & $J$ & $K$ & $L$ & $M$ & $N$ \\
\hline $\begin{array}{l}11 / 2 \\
2 \\
21 / 2\end{array}$ & $\begin{array}{r}0.040 \\
.040 \\
.040 \\
.040\end{array}$ & $\begin{array}{r}1.848 \\
2.380 \\
\hdashline-344\end{array}$ & $\begin{array}{l}1.718 \\
2.218 \\
2.718 \\
3.281\end{array}$ & \begin{tabular}{|l|}
1.402 \\
1.870 \\
2.370 \\
2.870
\end{tabular} & $\begin{array}{l}1.505 \\
2.005 \\
2.505 \\
3\end{array}$ & $\begin{array}{l}1 / 32 \\
1332 \\
1332\end{array}$ & $\begin{array}{l}3 / 32 \\
3 / 32 \\
3 / 32 \\
3,32\end{array}$ \\
\hline
\end{tabular}

1 Tolerances on dimension $C$ are, $+0.001,-0.000$ inches for all sizes.

646.52 BRONZE-WIRE MANUFACTURES.

AMERICAN SOCIETY FOR TESTING MATERIAIS, STANDARD SPECIFICATIONS FOR SIEVES FOR TESTING PURPOSES, DESIG NATION E11-26, 1926

\section{SIEVES FOR TESTING}

(See 645.31, p. 1226.)

\section{AMERICAN SOCIETY FOR TESTING MATE- RIALS, TENTATIVE DEFINITION OF THE TERM SCREEN, DESIGNATION E13-28T, 1928 \\ DEFINITION OF SCREEN}

(See 645.31, p. 1226.)
SOCIETY OF AUTOMOTIVE ENGINEERS,

PARTS AND FITTINGS, WIRE CLOTH,

S. A. E. STANDARD, JULY, 1924

\section{WIRE CLOTH}

1. Mesh denotes the number of openings per inch.

2. The weave shall be what is known as "plain," except that in meshes 80 and finer, "twilled" weave may be used.

3. Double-crimped wire shall be used and woven so as to give square openings.

4.

Wire cloth dimensions

\begin{tabular}{|c|c|c|c|c|c|c|c|}
\hline Mesh & $\begin{array}{l}\text { Diam- } \\
\text { eter of } \\
\text { wire }\end{array}$ & $\begin{array}{c}\text { Size of } \\
\text { opening }\end{array}$ & $\begin{array}{l}\text { Propor- } \\
\text { tion of } \\
\text { open- } \\
\text { ings } \\
\text { to total } \\
\text { area } 1\end{array}$ & Mesh & $\begin{array}{l}\text { Diam- } \\
\text { eter of } \\
\text { wire }\end{array}$ & $\begin{array}{l}\text { Size of } \\
\text { opening }\end{array}$ & $\begin{array}{l}\text { Propor- } \\
\text { tion of } \\
\text { open- } \\
\text { ings } \\
\text { to total } \\
\text { area } 1\end{array}$ \\
\hline $\begin{array}{l}8 . \\
10 \\
12 . \\
14 . \\
16 .\end{array}$ & $\begin{array}{l}\text { Inch } \\
0.028 \\
.025 \\
.023 \\
.020 \\
.018\end{array}$ & $\begin{array}{l}\text { Inch } \\
0.097 \\
.075 \\
.060 \\
.051 \\
.044\end{array}$ & $\begin{array}{c}\text { Per cent } \\
60 \\
56 \\
52 \\
51 \\
51\end{array}$ & $\begin{array}{l}45 \\
50\end{array}$ & $\begin{array}{l}\text { Inch } \\
0.0095 \\
.009 \\
.008 \\
.007 \\
.006\end{array}$ & $\begin{array}{c}\text { Inch } \\
0.0130 \\
.0110 \\
.0090 \\
.0073 \\
.0068\end{array}$ & $\begin{array}{r}\text { Per cent } \\
34 \\
30 \\
29 \\
26 \\
29\end{array}$ \\
\hline $\begin{array}{l}20 . \\
24 . \\
30 . \\
35 \\
40\end{array}$ & $\begin{array}{l}.016 \\
.015 \\
.013 \\
.011 \\
.010\end{array}$ & $\begin{array}{l}.034 \\
.027 \\
.021 \\
.018 \\
.015\end{array}$ & $\begin{array}{l}46 \\
42 \\
40 \\
40 \\
36 \\
\end{array}$ & $\begin{array}{r}90 \\
100 \\
120 \\
150 \\
200\end{array}$ & $\begin{array}{l}.005 \\
.0045 \\
.004 \\
.003 \\
.0021\end{array}$ & $\begin{array}{l}.0059 \\
.0055 \\
.0043 \\
.0037 \\
.0029\end{array}$ & $\begin{array}{l}28 \\
30 \\
26 \\
31 \\
33 \\
\end{array}$ \\
\hline
\end{tabular}

1 This column is given for general information only. The areas are based on the nominal wire sizes.

5. The accuracy of spacing wires shall be such as to give openings within 10 per cent, plus or minus, of the openings specified.

6 . The variation in the diameter of the wire shall not exceed the following plus and minus tolerances:

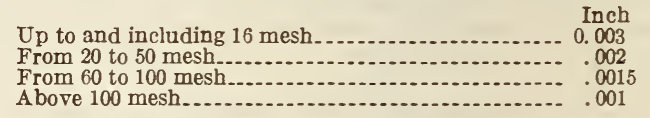

7. The material shall be specified by the purchaser, taking account of the fact that at present manufacturers of wirecloth stock these meshes in steel and brass up to about 80 mesh; above this it is common practice to supply phosphor-bronze and monel metal.

\section{UNITED STATES GOVERNMENT, DEPART-} MENT OF COMMERCE, BUREAU OF STANDARDS, WIRE INSECT SCREEN CLOTH, SIMPLIFIED PRACTICE RECOMMENDATION R122-31, 1931

\section{WIRE INSECT SCREEN CLOTH}

(For steel wire insect screen cloth, see 603.43, p. 195.)

In accordance with the unanimous action of a general conference of the industry held on June 18, 1931, the United States Department of Commerce submits for the acceptance of the industry the following recommendation for the stock varieties of wire insect screen cloth: 
TABLE 2.-Copper and commercial bronze wire insect screen cloth

[Hard-drawn copper and commercial bronze, selvaged both edges]

\begin{tabular}{|c|c|c|c|c|c|c|c|}
\hline \multirow{2}{*}{ Description } & \multirow{2}{*}{ Finish } & \multirow{2}{*}{$\begin{array}{c}\text { Mesh } \\
\text { open- } \\
\text { ings per } \\
\text { linear } \\
\text { inch } 1\end{array}$} & \multicolumn{2}{|c|}{$\begin{array}{l}\text { Wire size befo } \\
\text { weaving ? }\end{array}$} & \multirow{2}{*}{ Width of roll (inches) ${ }^{3}$} & \multirow{2}{*}{$\begin{array}{l}\text { Length of } \\
\text { roll (ap- } \\
\text { proxi- } \\
\text { mate) }\end{array}$} & \multirow{2}{*}{ Packing } \\
\hline & & & Warp & Filling & & & \\
\hline $\begin{array}{l}\text { Commercial bronze } 90 \text { to } 92 \text { per } \\
\text { cent copper; balance zinc. }\end{array}$ & Bright. & $\begin{array}{l}14 \\
16 \\
18\end{array}$ & $\left\{\begin{array}{c}\text { Inch } \\
0.0113\end{array}\right.$ & $\begin{array}{c}\text { Inch } \\
0.0113\end{array}$ & $\left\{\begin{array}{c}18,20,22,24,26,28,30,32, \\
34,36,38,40,42,48 .\end{array}\right.$ & $\begin{array}{l}\text { Feet } \\
\quad 100\end{array}$ & Heavy wrapping paper. \\
\hline $\begin{array}{l}\text { Hard-drawn copper (at least } 99.8 \\
\text { per cent copper). }\end{array}$ & & $\begin{array}{l}14 \\
16 \\
18\end{array}$ & 0113 & .0113 & $\left\{\begin{array}{c}18,20,22,24,26,28,30,32, \\
34,36,38,40,42,48 .\end{array}\right.$ & 100 & Do. \\
\hline
\end{tabular}

1 Commercially exact for warp wires; one-half mesh variation plus or minus in filler wires in any 1 inch.

2 Permissible variation from nominal diameter plus or minus 0.00025 inch.

2 Permissible variation in width plus or minus 18 inch.

4 The process of manufacture requires the inclusion of an occasional 2-piece roll; all 2-piece rolls have an extra footage of 1 to 2 linear feet.

Nore.-The approximate weight of 100 square feet of copper and commercial bronze screen cloth, exclusive of wrapping, is as follows. 14 mesh, 13 to 1334 pounds; 16 mesh, 1434 to 15 pounds; 18 mesh, 1632 to 1634 pounds.

This recommendation, if accepted by the industry, is effective 1 month after the date of the department's general letter announcing the necessary degree of support has been recorded through the medium of signed acceptance.

\subsection{MISCELLANEOUS MANUFACTURES OF BRONZE.}

AMERICAN ELECTRIC RAILWAY ENGINEERING ASSOCIATION, RECOMMENDED SPECIFICATION FOR TROLLEY POLES, WHEEIS, HARPS, AND BASES; MANUAL SECTION E124-29, 1929

\section{TROLLEY WHEEL}

Wheels shall be made of highest grade electrolytic ingot copper, Strait's tin, and highest grade zinc in the following proportions: Copper, 92 per cent; tin, 8 per cent; zinc, approximately 0.5 per cent. Brinell hardness of finished wheels shall be between 60 and 71 .

Finished wheel shall be made accurately to the dimensions shown in Figure 1121. The outer surface of the wheel flange shall be turned accurately to dimensions. The wheel shall be perfectly balanced.

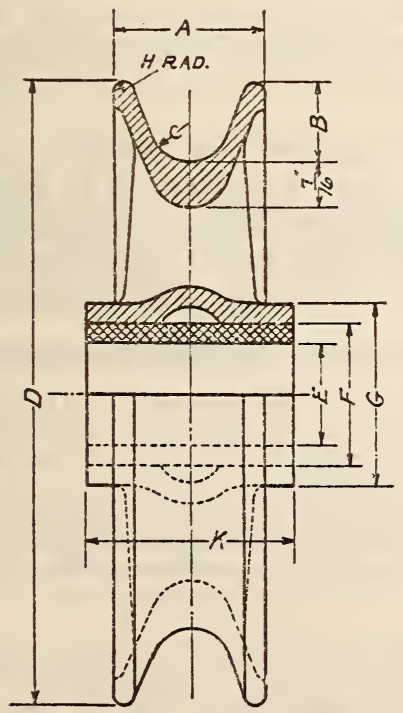

FiguRE 1121.-Recommended designs of trolley wheels of 41/4, 5 , and 6 inch diameter
Table of principal dimensions

[All dimensions in inches]

\begin{tabular}{|c|c|c|c|c|c|c|c|c|}
\hline$A$ & $B$ & $C$ & $D$ & $E$ & $F$ & $G$ & $H$ & $K$ \\
\hline $17 / 16$ & $3 / 4$ & $3 / 8$ & $41 / 4$ & $5 / 8$ & $11 / 8$ & $11 / 2$ & $3 / 32$ & $11 / 2$ \\
$17 / 16$ & $3 / 4$ & $3 / 8$ & 5 & $3 / 4$ & 138 & $11 / 2$ & $3 / 32$ & $11 / 2$ \\
$17 / 16$ & $3 / 4$ & $3 / 8$ & 6 & 1 & $13 / 8$ & $13 / 4$ & $3 / 32$ & 112,2 \\
\hline
\end{tabular}

AMERICAN ELECTRIC RAILWAY ENGINEERING ASSOCIATION, TROLLEY WHEEL PRACTICE, MANUAL SECTION E209-24, 1924

\section{TROLLEY WHEELS}

(This is a compilation of representative practice of trolley wheels now in use, including nine representative compositions for the bronze, representative diameters for different types of service, types of groove, sizes of bushings, types of lubrication, and recommendations on daily inspection.)

\section{AMERICAN MARINE STANDARDS COM- MITTEE, SPECIFICATION E NO. 11 , PROPELLER HUB STUDS, NUTS, AND LOCK SCREWS, A. M. S. C. 30, SEPTEM- BER, 20, 1927}

(See also 608.2 , p. $574 ; 608.31$, p. 586 ; and 608.32 , p. 605).

\section{LOCK SCREWS}

(Contains specifications, dimensions, and illustrations of propeller hub studs, nuts, and lock screws.)

\section{UNITED STATES GOVERNMENT, DE- PARTMENT OF COMMERCE, BUILD- ERS' TEMPLATE HARDWARE, COM- MERCIAL STANDARD CS9-29, SEP- TEMBER 18, 1929}

\section{BUILDERS' TEMPLATE HARDWARE}

(For template butt hinges see 617.12, p. 982. For template cylinder locks, see 617.21, p. 1020.) 
UNITED STATES GOVERNMENT, DEPARTMENT OF COMMERCE, BUILDERS' HARDWARE (NONTEMPLATE), COMMERCIAL STANDARD CS22-30, DECEMBER 11, 1930

\section{BUILDERS' HARDWARE (NONTEMPLATE)}

(See 617.0, p. 977.)

UNITED STATES GOVERNMENT, FEDERAL SPECIFICATIONS BOARD, FEDERAL SPECIFICATION FOR STANDARD MISCELLANEOUS CHAIN AND ATTACHMENTS, RR-C-271, DECEMBER 8, 1931

SASH CHAIN, WELDLESS
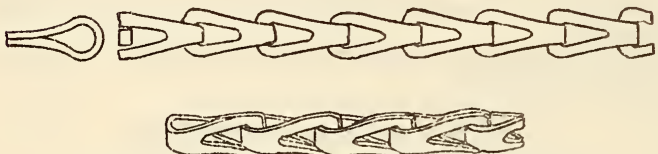

Figure 1122.-Sash chain, weldless

(For general specifications, material and workmanship, detail requirements, and methods of inspection and tests, see F. S. B. RR-C-271 (603.50, p. 199). For other kinds of chain and chain attachments specified in F. S. B. RR-C-271, see 603.51, $603.52,603.54,603.56,603.57$, and 617.5. See also $631.9,642.9$, and 645.9.

1. Weldless chain shall be automatic machinemade chain. It will be paid for by the foot.

TABLE 58.-Sash chain (dimensions, strength, weights)

\begin{tabular}{|c|c|c|c|c|}
\hline Material & Metal gage & $\begin{array}{l}\text { Approx- } \\
\text { imate } \\
\text { number } \\
\text { of links } \\
\text { per foot }\end{array}$ & $\begin{array}{l}\text { Mini- } \\
\text { mum } \\
\text { tensile } \\
\text { strength }\end{array}$ & $\begin{array}{l}\text { Approx- } \\
\text { imate } \\
\text { weight } \\
\text { per } 100 \\
\text { feet }\end{array}$ \\
\hline $\begin{array}{l}\text { Bronze } \\
\text { Do } \\
\text { Do } \\
\text { Do } \\
\text { Do } 0.2 .\end{array}$ & $\begin{array}{r}\text { Inch } \\
0.035 \\
0.042-0.045 \\
.049 \\
.054-.062 \\
.062\end{array}$ & $\begin{array}{l}21 \\
22 \\
22 \\
191 / 2 \\
17\end{array}$ & $\begin{array}{r}\text { Pounds } \\
425 \\
550 \\
650 \\
800 \\
800\end{array}$ & $\begin{array}{r}\text { Pounds } \\
53 / 4 \\
71 / 2 \\
81 / 2 \\
111 / 2 \\
12\end{array}$ \\
\hline
\end{tabular}

64\%. SPECIAL BRONZES（BRONZE ALIOYS)

\subsection{ALUMINUM BRONZE.}

647.11 INGOTS, ALUMINUM BRONZE.

UNITED STATES GOVERNMENT, FEDERAL SPECIFICATIONS BOARD, FEDERAL SPECIFICATION FOR ALUMINUM BRONZE INGOTS (FOR REMELTING), NO. 173A, OCTOBER 28, 1926. (CURRENT DESIGNATION: QQ-B-681)

(In respect to general specifications, material and workmanship, general requirements, methods of inspection and tests, and packing and marking, this specification conforms to F. S. B. No. 290a (current designation: QQ-B-701) (see 646.11, p. 1248). Otherwise its requirements are as follows:)
GRADE

This specification covers one grade of aluminum bronze in the form of ingots for remelting.

\section{DETAIL REQUIREMENTS}

The chemical requirements for aluminum bronze ingots are as follows:

\begin{tabular}{|c|c|c|c|c|}
\hline Copper & $\begin{array}{c}\text { Alumi- } \\
\text { num }\end{array}$ & Iron & $\begin{array}{c}\text { Tin (max- } \\
\text { imum) }\end{array}$ & $\begin{array}{r}\text { Total other } \\
\text { impurities } \\
\text { (maximum) }\end{array}$ \\
\hline $\begin{array}{r}\text { Per cent } \\
85-89\end{array}$ & $\begin{array}{r}\text { Per cent } \\
7.0-9.0\end{array}$ & $\begin{array}{r}\text { Per cent } \\
2.5-4.5\end{array}$ & $\begin{array}{r}\text { Per cent } \\
0.5\end{array}$ & $\begin{array}{r}\text { Per cent } \\
0.25\end{array}$ \\
\hline
\end{tabular}

Aluminum bronze differing in composition from that specified may be supplied, provided such differences are clearly noted in the bids, and are acceptable to the purchaser.

The physical properties of the material shall be as follows:

\begin{tabular}{|c|c|c|}
\hline $\begin{array}{c}\text { Tensile } \\
\text { strength, } \\
\text { (minimum) }\end{array}$ & $\begin{array}{r}\text { Yield point, } \\
\text { (minimum) }\end{array}$ & $\begin{array}{r}\text { Elongation } \\
\text { in 2 inches } \\
\text { (minimum) }\end{array}$ \\
\hline $\begin{array}{c}\text { Lbs./in.2 } \\
75,000 . \ldots\end{array}$ & $\begin{array}{r}\text { Lbs./in.2 } \\
30,000\end{array}$ & $\begin{array}{r}\text { Per cent } \\
30\end{array}$ \\
\hline
\end{tabular}

The yield point is obtained by the drop of the beam of the testing machine.

\section{METHOD OF INSPECTION AND TESTS}

One test ingot shall be selected by the inspector from each melt. The tension-test specimen shall be cut from one corner near the bottom of the ingot, and shall be machined to the standard form and dimensions shown in the general specification for metals. (See 600.0, p. 3.)

\section{PACKING AND MARKING}

All shipments shall be marked with the contract or purchase order number, name of manufacturer, and contents.

NotE.-Attention of the Federal Specifications Board has been called to the fact that the formula covered by this specification is alleged to be covered by letters patent.

647.12 BARS, ALUMINUM BRONZE.

SOCIETY OF AUTOMOTIVE ENGINEERS,

NONFERROUS METAL SPECIFICATIONS,

SPECIFICATION NO. 69, WROUGHT ALUMINUM BRONZE, FEBRUARY, 1929

\section{WROUGHT ALUMINUM BRONZE}

1. Composition in percentage-(Identical with specification No. 68 for cast aluminum bronze, except that copper component is 2 per cent larger. (See 647.15, p. 1260.)

2 . The thickness tolerances of sheet or strip made of this alloy shall be within the tolerances specified in Table 4 in Federal specification No. 468 (QQ-N321) for nickel silver sheets and strips. (See 655.2, p. 1287.)

3. General information.-Wrought shapes, rods, and bars of this alloy, the composition of which is dentical with specification No. 68 (see 647.15, p. 1260) combine unusual strength with good bearing and anticorrosive qualities. 
4. Wrought bars made of this alloy, when annealed or hot-rolled, should give the following minima in physical characteristics:

\begin{tabular}{|c|c|c|c|c|}
\hline \multicolumn{2}{|c|}{$\begin{array}{l}\text { Diameter or thick- } \\
\text { ness }\end{array}$} & \multirow{2}{*}{$\begin{array}{l}\text { Ultimate } \\
\text { strength }\end{array}$} & \multirow{2}{*}{$\begin{array}{l}\text { Yield } \\
\text { point }\end{array}$} & \multirow{2}{*}{$\begin{array}{c}\text { Elongation } \\
\text { in } 2 \text { inches } \\
\text { or propor- } \\
\text { tionate gage } \\
\text { length }\end{array}$} \\
\hline Orer & $\begin{array}{l}\text { To and in- } \\
\text { cluding }\end{array}$ & & & \\
\hline $\begin{array}{c}\text { Inch } \\
0 \\
1 / 2 \\
1\end{array}$ & $\begin{array}{c}\text { Inch } \\
1 / 2 \\
1\end{array}$ & $\begin{array}{c}\text { Lbs./in. }{ }^{2} \\
80,000 \\
75,000 \\
72,000\end{array}$ & $\begin{array}{c}\text { Lbs./in. }{ }^{2} \\
40,000 \\
37,500 \\
35,000\end{array}$ & $\begin{array}{c}\text { Per cent } \\
20 \\
25 \\
30\end{array}$ \\
\hline
\end{tabular}

647.13 RODS, ALUMINUM BRONZE.

SOCIETY OF AUTOMOTIVE ENGINEERS, NONFERROUS METAL SPECIFICATIONS, SPECIFICATION NO. 69, WROUGHT ALUMINUM BRONZE, FEBRUARY, 1929

\section{ALUMINUM BRONZE RODS}

(See 647.12, p. 1258.)

647.14 PLATES AND SHEETS, ALUMINUM BRONZE. SOCIETY OF AUTOMOTIVE ENGINEERS, NONFERROUS METAI SPECIFICATIONS, SPECIFICATION NO. 69, WROUGHT AL. UMINUM BRONZE, FEBRUARY, 1929

\section{ALUMINUM BRONZE SHEETS}

(See 647.12, p. 1258.)

UNITED STATES GOVERNMENT, DEPARTMENT OF COMMERCE, BUREAU OF STANDARDS, STANDARD THICKNESSES, WEIGHTS, AND TOLERANCES OF SHEET METAL (CUSTOMARY PRACTICE), CIRCULAR NO. 391, MARCH 24, 1931

\section{AMERICAN WIRE GAGE-ALUMINUM BRONZE PLATES AND SHEETS}

(See 640.1, p. 1165.)

647.15 CASTINGS, ALUMINUM BRONZE.

AMERICAN SOCIETY FOR TESTING MATERIALS, STANDARD SPECIFICATIONS FOR ALUMINUM BRONZE CASTINGS, DESIGNATION B59-28, 1928

\section{ALUMINUM BRONZE CASTINGS}

SCOPE

1. These specifications cover alloy castings, the alloy being a composition known commercially as aluminum bronze. Two grades are specified: Namely, grade A, not responding to heat treatment, and grade B, capable of heat treatment.

\section{MANUFACTURE}

2. (a) The bronze may be made by any approved method.

(b) No scrap shall be used in the manufacture of the bronze, excepting that of known composition and origin. (c) The copper used in these castings shall conform to the requirements of the standard specifications for lake copper wire bars, cakes, slabs, billets, ingots, and ingot bars in B4 (see 641.11, p. 1173) or of the standard specifications for electrolytic copper wire bars, cakes, slabs, billets, ingots, and ingot bars in B5 (see 641.11 , p. 1174), or copper of equal purity.

(d) The aluminum shall be that commercially known as grade No. 1 , or that carrying less than 0.30 per cent of silicon. The iron shall be a pure grade of iron free from dirt or other detrimental foreign material.

\section{CHEMICAL PROPERTIES AND TESTS}

3. The bronze shall conform to the following requirements as to chemical composition:

\begin{tabular}{|c|c|c|}
\hline & Grade A & Grade B \\
\hline Copper & $\begin{array}{r}\text { Per cent } \\
87-89\end{array}$ & $\begin{array}{l}\text { Per cent } \\
89.5-90.5\end{array}$ \\
\hline Aluminum & $7-9.6$ & $9.5-10.5$ \\
\hline Iron & 2. 5-4 & 11 . \\
\hline $\begin{array}{l}\text { Tin, maximum } \\
\text { Total other impurities, maximum }\end{array}$ & i. 5 & \\
\hline
\end{tabular}

1 Maximum

\section{CHEMICAL ANALYSES}

4. (a) An analysis of each melt may be made at the option of the purchaser and at his own expense.

(b) The sample for chemical analysis shall be taken from cuttings from the test bars.

\section{PHYSICAL PROPERTIES AND TESTS}

5. (a) The bronze shall conform to the following minimum requirements as to physical properties:

\begin{tabular}{|c|c|c|}
\hline & Grade A & Grade B \\
\hline Tensile strength (as cast) _...........lbs./in. ${ }^{2}$ & 65,000 & 65,000 \\
\hline $\begin{array}{l}\text { Tensile strength (as heat treated, quenched, } \\
\text { and drawn)... }\end{array}$ & & 80,0 \\
\hline Yield point (as cast) & 25,000 & 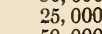 \\
\hline Yield point (as heat treated) & $-\cdots$ & 50 \\
\hline Elongation in 2 inches (as cast) ...... per cent & 20 & \\
\hline Elongation in 2 inches (as heat treated) & & \\
\hline
\end{tabular}

(b) The yield point in tension shall be determined as the stress producing an elongation under load of 0.5 per cent; that is, 0.01 inch in a gage length of 2 inches.

6. (a) The tension-test specimen shall be machined from coupons to the dimensions shown in Figure 550, A.S. T. M. A. 18 . (See 611.51, p. 754.) The ends shall be of a form to fit the holders of the testing machine in such a way that the load shall be axial.

(b) The coupon attached to the casting, or cast separately in the case of small castings, shall conform to the form and dimensions shown in Figure 1123. In the case of coupons cast separately, they shall be molded in a manner similar to the castings which they represent. If the castings are heat treated, the coupons representing such castings shall be similarly heat treated.

7. (a) Two coupons or test bars shall be cast as an integral part of large castings, or cast separately in the case of small castings, to represent each lot or melt of 500 pounds or less. Additional coupons may be provided at the option of the manufacturer.

(b) The manufacturer and purchaser shall agree whether coupons can be attached to the castings, on the location of coupons on the castings, on the castings to which the coupons are to be attached and on the method of casting unattached bars 
Unless otherwise agreed upon, the minimum lot shall be considered as 500 pounds.

(c) If any test specimen shows defective machining or develops flaws, it may be discarded; in which case the manufacturer and purchaser, or his representative, shall agree upon the selection of another specimen in its stead.

\section{WORKMANSHIP AND FINISH}

8. (a) The castings shall be free from blowholes, porosity, hard spots, shrinkage defects or cracks, or other injurious defects; and shall be smooth and well cleaned before inspection by sand blasting, tumbling, chipping, or other process approved by the inspector.

(b) The castings shall not be repaired, plugged, welded, or "burned in" unless permission from the inspector has been previously secured. This permission will be given only when the defects are such that after the approved repair the usefulness and strength of the casting has not been impaired. Each such repair shall be encircled by a ring of white paint at the time of shipment.

(c) All castings shall be true to pattern, free from swells, etc. Wall thickness shall be uniform throughout the lot of castings, and all cores shall be accurately set.

(d) Where thick and thin sections join, the manufacturer shall be permitted to add (where not previously provided) fillets of proper size to avoid cracking upon cooling, subject to the approval of the inspector.

\section{MARKING}

9. The castings shall be marked with pattern or mark number, and when practicable with the melt or lot number, at a position on the castings where they will not be machined off in manufacture to finished dimensions.

\section{INSPECTION AND REJECTION}

10. (a) Inspection may be made at the manufacturer's works, or at the point of delivery. Inspection shall be made at the option of the purchaser.

(b) If the purchaser elects to have the inspection made at the manufacturer's works, the inspector representing the purchaser shall have free entry, at all times while work on the contract is being performed, to all parts of the manufacturer's works which concern the manufacture of the material ordered. The manufacturer shall afford the inspector, without charge, all reasonable facilities to satisfy him that the material is being furnished in accordance with these specifications. All tests and inspection shall be so conducted as not to interfere unnecessarily with the operation of the works.

11. Castings which show injurious defects revealed by machining operations subsequent to acceptance may be rejected; and if rejected, shall be replaced by the manufacturer without charge to the purchaser. The full weight of the original material rejected shall be returned to the manufacturer.

\section{SOCIETY OF AUTOMOTIVE ENGINEERS, NONFERROUS METAL SPECIFICATIONS, SPECIFICATION NO. 68, CAST ALUMI- NUM BRONZE, MARCH, 1922}

\section{CAST ALUMINOM BRONZE}

\section{Composition in percentage.-}

Copper.

Per cent

Aluminum

85. 00-87.00

Tin (none desired), maximum

Th (nono desired), maxum.

25
2. General information.-Good castings made of this alloy should give the following minima in mechanical properties:

Ultimate strength Ibs./in. 2. 65,000

longation in 2 inches, or proportionate gage length

3. This is a noncorrodible alloy of great strength, with a hardness equal to that of manganese bronze, and good bearing qualities. It is suitable for use in worm wheels, gears, and similar parts.

4. Bearings or gears made of bronze alloys should be used only against hardened steel.

UNITED STATES GOVERNMENT, FEDERAL SPECIFICATIONS BOARD, FEDERAI SPECIFICATION FOR ALUMIN UM BRONZE CASTINGS, NO. 369a, OCTO. BER 28, 1926. (CURRENT DESIGNA. TION : QQ-B-671)

\section{ALUMINUM BRONZE CASTINGS}

(In respect to general specifications, this specification conforms to Federal specification 339a (current designation QQ-M-151) (see 600.0, p. 3); in respect to general requirements and methods of inspection and tests it is substantially the same as Federal specification QQ-B-691. See 646.41, p. 1252. It differs from the latter, however, in that it does not include requirements relative to samples for check analysis, Brinell tests, calipering or drilling tests, encircling repairs with a ring of white paint, and in the following additional particulars:)

GRADE

1. This specification covers one grade of aluminum bronze in the form of castings.

\section{MATERIAL}

2. Aluminum bronze castings shall be made only from the best grade of virgin metals, or from virgin metals and scrap of known and approved composition.

\section{DETAIL REQUIREMENTS}

3. The chemical requirements are as shown in the table below:

\begin{tabular}{|c|c|c|c|c|c|}
\hline & \multicolumn{5}{|c|}{ Composition } \\
\hline 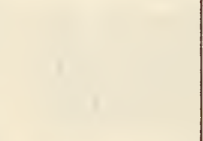 & Copper & $\begin{array}{l}\text { Alumi- } \\
\text { num }\end{array}$ & Iron & Tin & $\begin{array}{l}\text { Total } \\
\text { other } \\
\text { ele- } \\
\text { ments } \\
\text { maxi- } \\
\text { mum }\end{array}$ \\
\hline $\begin{array}{l}\text { Desired } \\
\text { Permissible....... }\end{array}$ & $\begin{array}{r}\text { Per cent } \\
88.0 \\
85.0-89.0\end{array}$ & $\begin{array}{r}\text { Per cent } \\
9.0 \\
7.0-9.0\end{array}$ & $\begin{array}{r}\text { Per cent } \\
3.0 \\
2.5-4.5\end{array}$ & $\begin{array}{c}\text { Per cent } \\
0 \\
.5\end{array} \mid$ & $\begin{array}{r}\text { Per cent } \\
0 \\
.40\end{array}$ \\
\hline
\end{tabular}

4. Aluminum bronze differing in composition from that specified above may be supplied, provided that such differences are clearly noted in the bids, and are acceptable to the purchaser.

5. The physical requirements are as shown below:

\begin{tabular}{|c|c|}
\hline $\begin{array}{c}\text { Tensile } \\
\text { strength, } \\
\text { mini- } \\
\text { mum }\end{array}$ & $\begin{array}{c}\text { Elong8- } \\
\text { tion in 2 } \\
\text { inches, } \\
\text { mini- } \\
\text { mum }\end{array}$ \\
\hline $\begin{array}{r}\text { Lbs./in.2 } \\
70,000\end{array}$ & $\begin{array}{r}\text { Per cent } \\
25.0\end{array}$ \\
\hline
\end{tabular}


METHODS OF INSPECTION AND TESTS

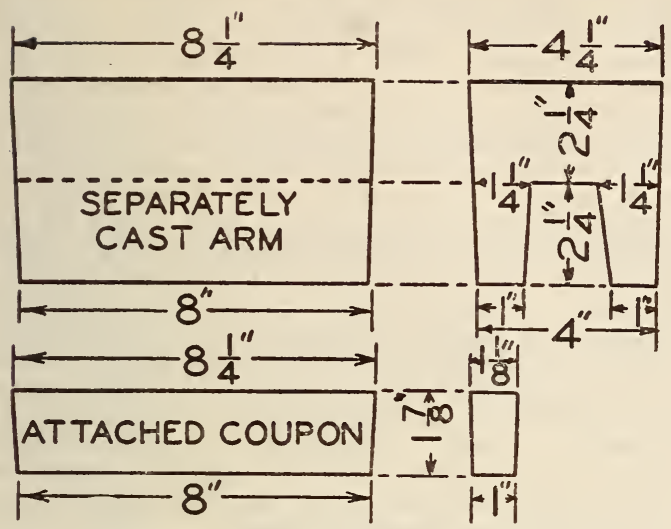

Figdre 1123.-Test coupon

6. One tension test is required for each lot of 1,000 pounds of castings.

7. The test coupons or separately cast bars shall conform to the dimensions of Figure 1123, and the test specimen machined therefrom shall conform to the dimensions of Figure 129, under 605.0, page 247 .

\section{PACKING AND MARKING}

8. Large castings shall be stamped with the melt or lot number, and when practicable, with the pattern or mark number.

9. Small castings shall be properly separated by melts or lots when presented for inspection, and when shipped shall be so marked that their identity will be clear at the point of delivery.

10. All shipments shall be marked with the contract or order number, name of manufacturer, and contents.

Notes.-Aluminum bronze is an alloy of great strength, with a hardness equal to that of manganese bronze, and greater resistance to corrosion, shock, and fatigue. It may be used in gun slides, gears, and similar parts.

647.16 SHAPES, ALUMINUM BRONZE.

SOCIETY OF AUTOMOTIVE ENGINEERS, NONFERROUS METAL SPECIFICATIONS, SPECIFICATION NO. 69, WROUGHT ALU. MINUM BRONZE, FEBRUARY, 1929

\section{ALUMINOM BRONZE SHAPES}

(See 647.12, p. 1258.)

647.17 STRIPS, ALUMINUM BRONZE.

SOCIETY OF AUTOMOTIVE ENGINEERS, NONFERROUS METAL SPECIFICATIONS, SPECIFICATION NO. 69, WROUGHT ALUMINUM BRONZE, FEBRUARY, 1929

\section{ALUMINOM BRONZE STRIPS}

(See 647.12, p. 1258.)
647.2 MANGANESE BRONZE.

647.20 GENERAL ITEMS.

AMERICAN SOCIETY FOR TESTING MATERIALS, STANDARD METHODS OF CHEMICAL ANALYSIS OF MANGANESE BRONZE, DESIGNATION B27-19, 1919

[Approved as American tentative standard (K3-1921) by the Ameri can Standards Association, December 28, 1921]

\section{CHEMICAL ANALYSIS OF MANGANESE BRONZE} GENERAL CONSIDERATIONS

Methods are given for the determination of lead, using a large sample when lead is present up to about 0.2 per cent. A method is also given for the electrolytic determination of lead and copper in the same small sample when lead is present in amounts over 0.2 per cent.

In this alloy zinc is taken by difference. For a method of determining small amounts of zinc, see standard methods of chemical analysis of gun metal, in B28 (646.0, p. 1243).

DETERMINATION OF COPPER BY THE ELECTROLYTIC METHOD

\section{APPARATUS FOR ELECTROLYSIS}

Electrodes.-The electrodes recommended are of the stationary and not of the rotating type, as the stationary require less operator's time and there is less chance for erroneous results to be obtained than with the other kind. Rapid and accurate results are obtained with stationary electrodes made from platinum gauze.

Cathodes.-Platinum cathodes may be formed either from plain or perforated sheets, or from wire gauze, and may be either open or closed cylinders. They should give a depositing surface, counting both sides, of approximately 100 square centimeters. Gauze cathodes are recommended and are best made from gauze containing approximately 400 meshes per square centimeter (50 meshes per linear inch). The cathode should be stiffened by doubling the gauze for about $3 \mathrm{~mm}$ at the top and at the bottom of the cylinder. The cylinder should be approximately $30 \mathrm{~mm}$ in diameter and $30 \mathrm{~mm}$ in height. The stem should be made from $1.14 \mathrm{~mm}$ or $1.29 \mathrm{~mm}$ wire, flattened and welded the entire width of the gauze; the height over all should be approximately $130 \mathrm{~mm}$, and the gauze should be sand blasted.

Anodes.-Platinum anodes may be of the spiral type when used in the determination of copper by electrolysis, or in the electrolytic determination of lead when it is present in amounts not over 0.2 per cent. When used in electrolytic determination of copper and lead together in samples containing 0.2 per cent lead or over, the anodes should be of wire gauze. Spiral anodes should be made from 1.02 $\mathrm{mm}$ or $1.14 \mathrm{~mm}$ wire formed into a spiral of seven turns having a height of approximately $51 \mathrm{~mm}$ and a diameter of $16 \mathrm{~mm}$, the length over all being approximately $145 \mathrm{~mm}$. Platinum gauze used in making anodes should contain approximately 400 meshes per square centimeter (50 meshes per linear inch). The gauze should be formed into closed cylinders approximately $12 \mathrm{~mm}$ in diameter and 30 
$\mathrm{mm}$ in height. The cylinders should be stiffened by doubling the gauze for about $3 \mathrm{~mm}$ at the top and at the bottom. The stem should be made from $1.02 \mathrm{~mm}$ or $1.29 \mathrm{~mm}$ wire, flattened and welded the entire width of the gauze; the height over all should be approximately $137 \mathrm{~mm}$, and the gauze should be sand blasted.

\section{METHOD}

In a $150 \mathrm{c}$ c beaker dissolve $1 \mathrm{~g}$ of bronze in $10 \mathrm{c} \mathrm{c}$ of $\mathrm{HNO}_{3}$, specific gravity 1.42 . When the action has ceased, evaporate the solution to dryness, and bake thoroughly on the edge of a hot plate. Moisten thoroughly with $\mathrm{HNO}_{3}$, specific gravity 1.42, and after digesting for a short time dilute to $50 \mathrm{c} \mathrm{c}$ with hot distilled water, heat to boiling, and allow to stand and settle for about one hour, keeping the temperature just below the boiling point. Filter on double $7 \mathrm{~cm}$ papers, keeping the solution hot, and receive the filtrate in a $200 \mathrm{c}$ c electrolysis beaker. Wash with boiling hot water. Add to the filtrate $5 \mathrm{c} \mathrm{c}$ of $\mathrm{H}_{2} \mathrm{SO}_{4}$, specific gravity 1.84 , and evaporate until copious fumes of $\mathrm{H}_{2} \mathrm{SO}_{4}$ are evolved. Dilute to about $100 \mathrm{c} \mathrm{c}$ with distilled water, add $1.5 \mathrm{c} \mathrm{c}$ of $\mathrm{HNO}_{3}$, specific gravity 1.42, insert electrodes, cover with a pair of split watch glasses and electrolyze. For each solution use a current overnight of 0.5 ampere at approximately 10 volts, or else use a current of 4 amperes at approximately 10 volts continued for about $2 \frac{1}{2}$ hours. The latter case requires the use of gauze cathodes. When the solution is colorless, wash down cover glasses, electrodes, and sides of beaker, raising the level of the liquid slightly and continue the electrolysis about 15 minutes, noting whether or not copper is deposited on the newly exposed surface of the platinum. If no copper appears, transfer about $1 \mathrm{c} \mathrm{c}$ of the colorless solution to the cavity of a porcelain test plate, and add a few drops of fresh $\mathrm{H}_{2} \mathrm{~S}$ water. If the slightest discoloration occurs continue the electrolysis, repeating the test. Remove the cathode quickly, rinse it in distilled water, and then dip it in two successive baths of alcohol. Shake off the excess alcohol and ignite the remainder by bringing it to the flame of an alcohol lamp. Keep the cathode moving continually as the alcohol burns. Weigh as metallic copper.

Accuracy.-Duplicate determinations should check within 0.10 per cent of copper.

Notes.-In alloys of the type of manganese bronze, which carry considerable iron, it is impossible to remove the tin quantitatively as metastannic acid $\left(\mathrm{H}_{2} \mathrm{SnO}_{3}\right)$, notwithstanding the preliminary baking. Upon testing (by the addition of $\mathrm{H}_{2} \mathrm{~S}$ water), the liquid in the pits of the test plate may be colored yellow, due to the tin present, but there should be no darkening due to the presence of copper. If the cathode appears dark, as though tin had been deposited on it, dissolve the coating in $10 \mathrm{c} \mathrm{c}$ of $\mathrm{HNO}_{3}$, specific gravity 1.42 , filter off the tin as before, add to the filtrate 5 c c of $\mathrm{H}_{2} \mathrm{SO}_{4}$, specific gravity 1.84 , and redeposit copper by electrolysis. It is rarely necessary thus to purify the deposited copper.

If zinc is to be determined in the alloy be careful to keep the solution quantitative when the electrodes are removed, catching all washings in the beaker containing the electrolyte.

A slight amount of copper is retained by the metastannic acid $\left(\mathrm{H}_{2} \mathrm{SnO}_{3}\right)$, which should be recorered in very accurate work but is generally neglected in routine analysis.

\section{DETERMINATION OF IEAD BY THE ELECTROIYTIC METHOD}

(For samples containing up to $\mathbf{0 . 2}$ per cent of lead)

\section{METHOD}

In a $250 \mathrm{c} \mathrm{c}$ beaker dissolve $5 \mathrm{~g}$ of bronze in $25 \mathrm{c} \mathrm{c}$ of $\mathrm{HNO}_{3}$, specific gravity 1.42 . Allow the solution to bake dry on the edge of a hot plate or by standing over night on the steam bath. Add $17 \mathrm{c} \mathrm{c}$ of $\mathrm{HNO}_{3}$, specific gravity 1.42 , and after digesting for a short time, add $85 \mathrm{c} \mathrm{c}$ of hot water and heat to boiling for a few minutes. Allow the solution to stand for several hours at a temperature just below the boiling point. Filter off the precipitated $\mathrm{H}_{2} \mathrm{SnO}_{3}$ on double closely woven filter papers, being careful to keep the solution hot throughout the process of filtration Wash with boiling hot water. Receive the combined filtrate and washings in a $250 \mathrm{c}$ c beaker. Dilute to about $150 \mathrm{c} c$ with distilled water and insert electrodes. In the positive terminal use a sand-blasted platinum gauze electrode such as is used for the cathode in the determination of copper; in the negative terminal insert an electrode such as is used for the anode in the determination of copper. Cover with a pair of split watch glasses and electrolyze. For each solution use a current of 1.25 to 1.5 amperes at 10 volts continued for about one hour. Wash down the cover glasses, electrodes, and sides of beaker, raising the level of the liquid slightly, and continue the electrolysis for 15 minutes. If no darkening of the newly exposed surface of the platinum can be detected the lead has been entirely deposited. Continue the electrolysis until no darkening of the platinum can be detected when the current has been passed for 15 minutes after the level of the liquid has been raised.

When the lead has been entirely deposited, remove the anode quickly, rinse it in distilled water and then in alcohol, and dry it at $210^{\circ} \mathrm{C}$. for one-half hour. Weigh as $\mathrm{PbO}_{2}$, using the factor for lead 0.8643 instead of the theoretical value.

Accuracy. - Duplicate determinations should chech within 0.01 per cent of lead. Results obtained by this method are likely to be about 0.01 per cent too high. For exceptionally accurate or investigation work redissolve the coating from the electrode and determine the lead as sulphate. See the determination of lead as sulphate.

Notes. - It is impossible to remore the tin quantitatively as $\mathrm{H}_{2} \mathrm{SnO}_{3}$ in the case of alloys containing over 0.25 per cent of iron, and traces of tin are sometimes found in the $\mathrm{PbO}_{2}$ deposit. Small amounts of manganese also are occasionally deposited with the $\mathrm{PbO}_{2}$. Tin and manganese are rarely present in the $\mathrm{PbO}_{1}$ in amounts which necessitate a purification of the deposit.

In this and the following method a slight amount of lead may be retained by the $\mathrm{H}_{2} \mathrm{SnO}_{3}$. This should be recovered in very accurate work, but is neglected in routine analysis.

\section{DETERMINATION OF LEAD AS SULPHATE}

(For samples containing up to 0.2 per cent of lead, optional)

\section{SOLUTIONS REQUIRED}

Lead acid.-Mix $300 \mathrm{c} \mathrm{c}$ of $\mathrm{H}_{2} \mathrm{SO}_{4}$, specific gravity 1.84 , and $1,800 \mathrm{c}$ c of distilled water. Dissolve $1 \mathrm{~g}$ of lead acetate, c. p., in $300 \mathrm{c} c$ of distilled water and add this to the hot solution, stirring meanwhile. Let stand at least 24 hours and syphon through a thick asbestos filter.

Dilute alcohol for washing.-Mix equal parts of denatured alcohol and distilled water.

\section{METHOD}

In a $250 \mathrm{c}$ c beaker dissolve $5 \mathrm{~g}$ of bronze in $25 \mathrm{c} \mathrm{c}$ of $\mathrm{HNO}_{3}$, specific gravity 1.42 . Allow the solution to bake dry on the edge of a hot plate, or by standing over night on the steam bath. Add $17 \mathrm{c} c$ of $\mathrm{HNO}_{3}$, specific gravity 1.42 , and after digesting for a short time, add $85 \mathrm{cc}$ of hot water and heat to boiling for a few minutes. Allow the solution to stand for several hours at a temperature just below the boiling point. Filter off the precipitated $\mathrm{H}_{2} \mathrm{SNO}_{3}$ on double closely woven filter papers, being careful to keep the solution hot throughout the process of filtration. Wash with boiling hot water. To the combined filtrate and washings add $120 \mathrm{c} \mathrm{c}$ of lead acid, and evaporate until copious fumes of $\mathrm{H}_{2} \mathrm{SO}_{4}$ are evolved. Cool, add $105 \mathrm{c} \mathrm{c}$ of distilled water to dissolve the salts and to make the acid concentration the same as in lead acid, heat to boiling, and allow to cool and settle for five hours, or over night if convenient. Filter on a weighed porcelain Gooch crucible and wash with lead acid. 
Wash out the lead acid with dilute alcohol, set the Gooch crucible inside a porcelain crucible, dry and ignite for five minutes at the full heat of a Tirrill burner, cool and weigh as $\mathrm{PbSO}_{4}$ which contains 68.29 per cent lead.

Accuracy.-Duplicate determinations should check within 0.01 per cent of lead. Results obtained by this method are likely to be about 0.01 per cent too low.

Note.-It is impossible to remove the tin quantitatively as $\mathrm{H}_{2} \mathrm{SnO}_{3}$ in the case of alloys containing over 0.25 per cent of iron, but by the preliminary baking and long standing, most of the $\mathrm{H}_{2} \mathrm{SnO}_{3}$ is precipitated.

\section{DETERMINATION OF COPPER AND LEAD SIMUITANEOUSLY BY THE ELECTROLYTIC METHOD}

(For samples containing over 0.2 per cent of lead)

\section{MFTHOD}

In a $150 \mathrm{c}$ c beaker dissolve $1 \mathrm{~g}$ of bronze in 10 c c of $\mathrm{HNO}_{3}$, specific gravity 1.42. When the action has ceased, evaporate the solution to dryness, and bake thoroughly on the edge of a hot plate. Add $10 \mathrm{c} \mathrm{c}$ of $\mathrm{HNO}_{3}$, specific gravity 1.42 , and after digesting for a short time add $50 \mathrm{c}$ of hot distilled water, heat to boiling, and allow to stand and settle for about one hour, keeping the temperature just below the boiling point. Filter on double $7 \mathrm{~cm}$ filter papers, keeping the solution hot, and receive the filtrate in a $200 \mathrm{c}$ c electrolysis beaker. Wash with boiling hot water, dilute to about $100 \mathrm{c} \mathrm{c}$ and insert electrodes. In the positive terminal insert one of the platinum gauze anodes previously described, and in the negative terminal insert a platinum gauze cathode. Cover with a pair of split watch glasses and electrolyze, using a current of from 3 to 5 amperes at approximately 10 volts for each solution. After about 45 minutes the lead will have been entirely deposited on the anode as $\mathrm{PbO}_{2}$; without interrupting the current add to the electrolyte 3 to $4 \mathrm{c}$ c of $\mathrm{H}_{2} \mathrm{SO}_{4}$, specific gravity 1.84, and continue the electrolysis. When the solution is colorless, wash down cover glasses, electrodes, and sides of beaker, raising the level of the liquid slightly, and continue the passage of the current about 15 minutes, noting whether or not copper is deposited on the newly exposed surface of the platinum. If no copper appears, transfer about $1 \mathrm{c} \mathrm{c}$ of the colorless solution to the cavity of a porcelain test plate, and add a few drops of fresh $\mathrm{H}_{2} \mathrm{~S}$ water. If the slightest discoloration occurs continue the electrolysis until there is no discoloration whatever upon repeating the test. Remove the solution from the electrodes quickly without interrupting the current. Rinse the electrodes in distilled water and then dip them in two successive baths of alcohol. Dry the anode at $210^{\circ} \mathrm{C}$. for one-half hour. Weigh as $\mathrm{PbO}_{2}$ using the factor for lead 0.8643 instead of the theoretical value. Shake off the excess alcohol from the cathode and ignite the remainder by bringing it to the flame of an alcohol lamp. Keep the cathode moving continually while the alcohol burns. Weigh as metallic copper.

Accuracy.-Duplicate copper determinations should check within 0.10 per cent of copper. Duplicate lead determinations should check within 0.02 per cent of lead. Lead results obtained by this method are likely to be about 0.01 per cent too high. See notes under the determination of lead by the electrolytic method.

\section{DETERMINATION OF TIN}

SOLUTIONS REQUIRED

Dilute hydrochloric acid.-Mix 500 c c of $\mathrm{HCl}$, specific gravity 1.20 , and $500 \mathrm{c}$ c of distilled water.
Dilute sulphuric acid.-Mix 300 c c of $\mathrm{H}_{2} \mathrm{SO}_{4}$, specific gravity, 1.84 , and $600 \mathrm{c}$ c of distilled water. Dilute ammonia.-Mix $100 \mathrm{c}$ c of $\mathrm{NH}_{4} \mathrm{OH}$, specific gravity 0.90 , and $900 \mathrm{c}$ c of distilled water.

Ammonium-acetate solution for washing.-Dissolve $10 \mathrm{~g}$ of ammonium acetate in $300 \mathrm{c} \mathrm{c}$ of distilled water. Make slightly acid with acetic acid and saturate with $\mathrm{H}_{2} \mathrm{~S}$ gas.

\section{METHOD}

In a $150 \mathrm{c}$ c beaker dissolve $2 \mathrm{~g}$ of bronze in a mixture of 10 e c of $\mathrm{HCl}$, specific gravity 1.20 , and $5 \mathrm{c}$ c of $\mathrm{HNO}_{3}$, specific gravity 1.42. Dilute to about $75 \mathrm{c}$ c with distilled water. Add $\mathrm{NH}_{4} \mathrm{OH}$, specific gravity 0.90 , until the basic salts of copper dissolve and the solution has become a deep blue. Boil and allow to settle, and filter on a closely woven filter paper. Wash with dilute ammonia and with hot water. Dissolve the precipitate on the filter with hot dilute $\mathrm{HCl}$. Dilute the solution to about 100 c c. Add $\mathrm{NH}_{4} \mathrm{OH}$, specific gravity 0.90 , until a permanent precipitate forms. Heat the solution to boiling, allow to settle, filter and wash as before. Dissolve the precipitate on the filter with boiling hot dilute $\mathrm{H}_{2} \mathrm{SO}_{4}$, washing the paper very thoroughly with this acid. Add $\mathrm{NH}_{4} \mathrm{OH}$, specific gravity 0.90 , cautiously until the precipitate which forms at first dissolves rather slowly. Allow the solution to stand for some hours and if any lead sulphate forms filter it off. Dilute the solution to about $200 \mathrm{c} \mathrm{c}$ and saturate it with $\mathrm{H}_{2} \mathrm{~S}$ gas. Filter the precipitated tin sulphide off on double papers and wash with ammonium-acetate solution, retaining the filtrate and washings for the iron determination. Dry the precipitate and place it with the papers in a porcelain crucible which projects part way through a hole in a piece of asbestos board. Heat slowly until any free sulphur has been driven off, but without allowing the sulphur to burn. When the sulphur has been expelled, place the crucible on a triangle and ignite slowly at first and finally at the full heat of the burner. If the precipitate weighs more than $20 \mathrm{mg}$ heat to constant weight, using a blast lamp. Weigh as $\mathrm{SnO}_{2}$, which contains 78.81 per cent of tin.

Accuracy.-Duplicate determinations should check within 0.06 per cent of tin.

Notes.-If, during ignition, the sulphur is allowed to burn, some tin sulphate may be formed, causing high results. On the other hand, low results may be caused by too high heat, which causes spattering and voltailizes some tin sulphide.

Tin and iron are best determined on the same sample, retaining the filtrate from the tin sulphide precipitate to use for the iron determination.

\section{DETERMINATION OF IRON}

\section{SOLUTIONS REQUIRED}

See determination of tin, given above, and the following:

Dilute sulphuric acid for reductor.-Mix $50 \mathrm{c} \mathrm{c}$ of $\mathrm{H}_{2} \mathrm{SO}_{4}$ specific gravity 1.84 , and $1,000 \mathrm{c} \mathrm{c}$ of distilled water. This solution is used boiling hot.

Potassium permanganate.-Dissolve $0.2 \mathrm{~g}$ of $\mathrm{KMnO}_{4}$ in water, filter through asbestos, and dilute to $1,000 \mathrm{c}$ c with distilled water. Standardize against $0.020^{\prime} \mathrm{g}$ portions of pure sodium oxalate. Each cubic centimeter is equivalent to approximately $0.00033 \mathrm{~g}$ of iron.

\section{METHOD}

Proceed exactly as in the determination of tin through the filtration and washing of the precipitated tin sulphide. Combine the filtrate and washings from the tin sulphide precipitate and boil until all $\mathrm{H}_{2} \mathrm{~S}$ is expelled. Add $\mathrm{HNO}_{3}$, specific gravity 1.42 , until the iron is oxidized, which is shown by the solution becoming clear and of $a$ 
yellowish color. Add about $5 \mathrm{~g}$ of $\mathrm{NH}_{4} \mathrm{Cl}$, then $\mathrm{NH}_{4} \mathrm{OH}$, specific gravity 0.90 , until a permanent precipitate forms. Boil, allow to settle, filter on a loosely woven filter paper and wash with dilute ammonia and with hot water. Dissolve the precipitate on the paper with hot dilute $\mathrm{HCl}$ and add $\mathrm{NH}_{4} \mathrm{OH}$, specific gravity 0.90 , to the solution again until a permanent precipitate forms. Boil, allow to settle, filter on a loosely woven filter paper, and wash with dilute ammonia and with hot water until all $\mathrm{NH}_{4} \mathrm{Cl}$ is removed. Dissolve the precipitate and wash the filter paper with $100 \mathrm{c}$ c of dilute $\mathrm{H}_{2} \mathrm{SO}_{4}$ for reductor. Pass the solution through a Jones reductor, wash first with $150 \mathrm{c}$ c of the $\mathrm{H}_{2} \mathrm{SO}_{4}$ for reductor and then with $100 \mathrm{c} \mathrm{c}$ of distilled water. Titrate with the standard $\mathrm{KMnO}_{4}$ solution.

Accuracy.-Duplicate determinations check within 0.03 per cent of iron.

Notes.-A blank determination should be made on corresponding amounts of acid and water passed through the reductor and the results should be corrected accordingly. About $0.5 \mathrm{c} \mathrm{c}$ of the permanganate will be required to give a permanent coloration to the manganate

A small quantity of liquid should always be left in the reductor funnel, and air should never be allowed to enter the body of the reductor.

For description and further details of use of reductor see The Chemical Analysis of Iron, by A. A. Blair, or Quantitative Chemical Analysis, by H. P. Talbot.

\section{DETERMINATION OF MANGANESE BY THE PERSULPHATE}

SOLUTIONS REQUIRED

Solution for dissolving.-Mix 500 c c of $\mathrm{H}_{2} \mathrm{SO}_{4}$ specific gravity $1.84,200 \mathrm{c}$ c of $\mathrm{HNO}_{3}$ specific gravity 1.42 , and $1,700 \mathrm{c} \mathrm{c}$ of distilled water.

Silver nitrate.-Dissolve $1.33 \mathrm{~g}$ of $\mathrm{AgNO}_{3}$ in 1,000 c $\mathrm{c}$ of distilled water.

Stock sodium arsenite.-To $15 \mathrm{~g}$ of arsenious oxide $\left(\mathrm{As}_{2} \mathrm{O}_{3}\right)$ in a $300 \mathrm{c}$ c Erlenmeyer flask, add $45 \mathrm{~g}$ of $\mathrm{Na}_{2} \mathrm{CO}_{3}$ and $150 \mathrm{c}$ c of distilled water. Heat the flask and contents on the steam bath until the $\mathrm{As}_{2} \mathrm{O}_{3}$ is dissolved. Cool the solution, filter and make up to $1,000 \mathrm{c} \mathrm{c}$ with distilled water.

Standard sodium arsenite.-Mix 200 c c of stock sodium-arsenite solution with $2,500 \mathrm{c}$ c of distilled water, and standardize against a steel or iron of known manganese content as determined by the bismuthate method, or standardize against one of the Bureau of Standards' standard irons or steels. One c c of this solution should be equivalent to approximately $0.00050 \mathrm{~g}$ of manganese.

Ammonium persulphate.-Dissolve $60 \mathrm{~g}$ of ammonium persulphate in $1,000 \mathrm{c} \mathrm{c}$ of distilled water.

\section{METHODS}

Adjust the size of sample to the expected percentage so that the portion taken does not contain more than $0.002 \mathrm{~g}$ manganese. Place it in a $250 \mathrm{c}$ c Erlenmeyer flask with 24 c c of the "solution for dissolving" and heat until solution is complete. Boil until oxides of nitrogen are expelled. Dilute to $100 \mathrm{c}$ c with boiling-hot water, add $20 \mathrm{c}$ c of $\mathrm{AgNO}_{3}$ solution and $30 \mathrm{c} \mathrm{c}$ of the persuphate solution, then remove to a moderately warm place to stand for a few minutes for complete development of the permanganic acid. Cool to below $25^{\circ} \mathrm{C}$., and titrate with the standard arsenite solution to the disappearance of the pink color.

(The method given above differs from that in the 1919 specification; it will be found in the A. S. T. M. tentative standards for 1930.)

Accuracy.-Duplicate determinations check within 0.02 per cent of manganese.

should

Notes.-Large amounts of permanganic acid are unstable; on that account, samples which contain large amounts of manganese should have correspondingly small samples weighed out. For instance, with metal containing 1.5 per cent of manganese use a $0.10 \mathrm{~g}$ sample, while if metal contains 0.75 per cent manganese use a $0.20 \mathrm{~g}$ sample.

The solution must be cold when it is titrated, for high temperatures cause low results to be obtained.

\section{DETERMINATION OF MANGANESE BY THE BISMUTHATE METHOD}

(Optional)

\section{SOLUTIONS REQUIRED}

Nitric acid (1:3).- Mix 250 c c of $\mathrm{HNO}_{3}$ specific gravity 1.42 , and $750 \mathrm{c} \mathrm{c}$ of distilled water.

Nitric acid for washing.-Mix 30 c c of $\mathrm{HNO}_{3}$ specific gravity 1.42 , and $970 \mathrm{c} \mathrm{c}$ of distilled water. Ferrous ammonium sulphate.-Dissolve $12.4 \mathrm{~g}$ of ferrous ammonium sulphate crystals in $950 \mathrm{c}$ c of distilled water, and add $50 \mathrm{c}$ c of $\mathrm{H}_{2} \mathrm{SO}_{4}$ specific gravity 1.84 .

Potassium permanganate.-Dissolve $1 \mathrm{~g}$ of $\mathrm{KMnO}_{4}$ in $1,000 \mathrm{c}$ c of distilled water. Allow it to stand for about one week and then filter it through asbestos. Standardize against $0.10 \mathrm{~g}$ portions of pure sodium oxalate.

\section{METHOD}

In a $250 \mathrm{c}$ c Erlenmeyer flask dissolve $1 \mathrm{~g}$ of bronze in $50 \mathrm{c} \mathrm{c}$ of $\mathrm{HNO}_{3}(1: 3)$. Without filtering off the $\mathrm{H}_{2} \mathrm{SnO}_{3}$, cool and add $0.5 \mathrm{~g}$ of sodium bismuthate. Heat for a few minutes until the purple color has disappeared, with or without the precipitation of manganese dioxide. Add a little ferrous ammonium sulphate solution until the solution becomes clear, and boil until the oxides of nitrogen are expelled. Cool, add an excess of sodium bismuthate and agitate for a few minutes. Add $50 \mathrm{c} \mathrm{c}$ of $\mathrm{HNO}_{3}$ for washing and filter through an alundum crucible or an asbestos pad. Wash with $50 \mathrm{c} \mathrm{c}$ of the $\mathrm{HNO}_{3}$ for washing. Add from a pipette or a burette $10 \mathrm{c} c$ of ferrous ammonium sulphate solution and titrate with $\mathrm{KMnO}_{4}$. Owing to the presence of considerable copper, the end point is somewhat different from the normal pink color.

In exactly the same manner carry through a blank determination, using the same amounts of $\mathrm{HNO}_{3}$ and sodium bismuthate as was done with the regular sample. Finally add exactly $10 \mathrm{c} c$ of ferrous ammonium sulphate solution and titrate with $\mathrm{KMnO}_{4}$. The difference between the two titrations is due to the manganese. Since one manganese as permanganate oxidized five irons, the iron value of the permanganate multiplied by the factor $\frac{M n}{5 F e}$ (or 0.1967 ) gives the value in terms of manganese.

Accuracy.-Duplicate determinations should check within 0.02 per cent of manganese.

Notes.-Instead of employing the method of reducing the permanganic acid by means of standardized ferrous ammonium sulphate solution and titrating the excess of this reagent, it is possible to reduce the permanganic acid by standard sodium-arsenite solution. See determination of manganese by the persulphate method.

The filtrate from the bismuthate must be perfectly clear, as the least particle of bismuthate carried into the filtrate $w$-ill vitiate the results.

\section{UNITED STATES GOVERNMENT, DEPART- MENT OF COMMERCE, BUREAU OF STANDARDS, STANDARD SAMPLES, CIR- CULAR NO. 25 AND SUPPLEMENT, 1927}

\section{MANGANESE BRONZE SAMPLE}

(Sample No. 62 is a standard sample of manganese bronze prepared and sold by the Bureau of Standards for use by industrial organizations and others as a comparison standard for checking the accuracy of analysis of manganese bronze. Each sample is accompanied by a certificate giving a complete analysis of that particular sample.) 
UNITED STATES GOVERNMENT, FEDERAL SPECIFICATIONS BOARD, FEDERAL SPECIFICATION FOR BRONZE, MANGANESE, RODS, SHAPES, AND PLATES, NO. 552, APRIL 30, 1928. (CURRENT DESIGNATION : QQ-B-721)

\section{MANGANESE BRONZE RODS, BARS, SHAPES,} PLATES

(In respect to general specifications, material and workmanship, tests and methods of inspection, packing and marking, this specification is substantially the same as F. S. B. QQ-C-501. (See 641.0, p. 1172.) It differs from the latter in requiring the material to be free from "season cracking"; check analyses samples are to be taken from 5,000-pound lots, or fraction there of ; reference is made to a hammer test; the bending in full section of material less ss than 1 inch wide is omitted; and in the following additional respects:)

\section{GRADES, FORMS, AND TEMPERS}

1. This specification covers one grade of manganese bronze in the following forms and tempers:

\begin{abstract}
Rods, soit, half-hard, and hard. (See also 647.23.)
Bars, soft, half-hard, and hard. (See also 617.22.)

Shapes, soft. (See also 647.25.)

Plates, soft, half-hard. (See also 647.24.)
\end{abstract}

\section{DETAIL REQUIREMENTS}

2. The chemical requirements are as shown in Table 1.

TABLE 1.-Chemical composition

\begin{tabular}{|c|c|c|c|c|c|c|c|}
\hline Copper & Zinc & Tin & Iron & $\begin{array}{l}\text { Man- } \\
\text { gan- } \\
\text { ese, } \\
\text { maxi- } \\
\text { mum }\end{array}$ & $\begin{array}{l}\text { Alu- } \\
\text { mi- } \\
\text { num, } \\
\text { maxi- } \\
\text { mum }\end{array}$ & $\begin{array}{l}\text { Lead, } \\
\text { maxi- } \\
\text { mum }\end{array}$ & $\begin{array}{l}\text { Other } \\
\text { im- } \\
\text { puri- } \\
\text { ties, } \\
\text { maxi- } \\
\text { mum }\end{array}$ \\
\hline $\begin{array}{c}\text { Per cent } \\
57-60\end{array}$ & $\begin{array}{c}\text { Per cent } \\
\text { Remainder. }\end{array}$ & $\begin{array}{c}\text { Per } \\
\text { cent } \\
0.5-1\end{array}$ & $\begin{array}{c}\text { Per } \\
\text { cent } \\
0.8-2\end{array}$ & $\begin{array}{l}\text { Per } \\
\text { cent } \\
0.50\end{array}$ & $\begin{array}{l}\text { Per } \\
\text { cent } \\
0.25\end{array}$ & $\begin{array}{l}\text { Per } \\
\text { cent } \\
0.20\end{array}$ & $\begin{array}{l}\text { Per } \\
\text { cent } \\
0.10\end{array}$ \\
\hline
\end{tabular}

TENSILE STRENGTH

3. The requirements as to tensile strength are as shown in Table 2.

4. Special tempers not covered in Table 2 may be agreed upon between the contractor and purchaser, in which case they will be specifically defined and designated in the contract or purchase order.

TABLE 2.-Physical properties

\begin{tabular}{|c|c|c|c|c|}
\hline $\begin{array}{l}\text { Diameter or thickness } \\
\text { (in inches) }\end{array}$ & $\begin{array}{c}\text { Tensile } \\
\text { strength, } \\
\text { mini- } \\
\text { mum }\end{array}$ & $\begin{array}{l}\text { Yield } \\
\text { point, } \\
\text { mini- } \\
\text { mum }\end{array}$ & $\left|\begin{array}{c}\text { Elonga- } \\
\text { tion in } \\
2 \text { inches, } \\
\text { mini- } \\
\text { mum }\end{array}\right|$ & $\begin{array}{l}\text { Bend } \\
\text { test }\end{array}$ \\
\hline Rods and bars, soft all sizes & Lbs./in..$^{2}$ & Lbs./in. ${ }^{2}$ & Per cent & $\begin{array}{r}\text { Degrees } \\
180\end{array}$ \\
\hline Rods and bars, half hard: & & & & \\
\hline 1 and less & 72,000 & 36,000 & 20 & 120 \\
\hline $\begin{array}{l}1.001 \text { to } 2.50 \\
2.501 \text { and over. }\end{array}$ & 70,000 & 35,000 & 25 & 120 \\
\hline $\begin{array}{l}2.501 \text { and over } \\
\text { Rods and bars, hard: }\end{array}$ & 65,000 & 32,000 & 30 & 120 \\
\hline 1 and less & 85,000 & 60,000 & 8 & 90 \\
\hline $\begin{array}{l}1,001 \text { to } 2.50 \\
2.501 \text { and over... }\end{array}$ & 83,000 & 57,000 & 13 & 90 \\
\hline $\begin{array}{l}2.501 \text { and over } \\
\text { Shapes, soft, all sizes. }\end{array}$ & 80,000 & 54,000 & 18 & 90 \\
\hline $\begin{array}{l}\text { Shapes, soft, all sizes } \ldots \ldots \\
\text { Plates, soft: }\end{array}$ & 55,000 & 22,000 & 25 & 180 \\
\hline 0.50 and less, up to 30 inches & & & & \\
\hline 0.50 and less, over 30 inches & 57,000 & 22,000 & 20 & 180 \\
\hline wide.... & 56,000 & 22,000 & 25 & 180 \\
\hline $\begin{array}{l}0.501 \text { and over, all widths.-- } \\
\text { Plates, half-hard: }\end{array}$ & 56,000 & 22,000 & 25 & 180 \\
\hline 0.50 and less, up to 30 inches & & & & \\
\hline $\begin{array}{l}\text { wide } \\
0.50 \text { and less, over } 30 \text { inches }\end{array}$ & 60,000 & 24,000 & 18 & 120 \\
\hline wide & 58,000 & 23,000 & 22 & 120 \\
\hline 0.501 and over, all widt & 58,000 & 23.000 & 22 & 120 \\
\hline
\end{tabular}

NOTE.-Soft rods and bars are intended for reforging.
5. Material shall withstand bending cold, without check or fracture on the outside of the bent portion, through the angle specified in Table 2 , on a radius equal to the diameter or thickness of the material.

6. Specimens of material shall withstand immersion for 15 minutes without cracking in an aqueous solution containing $100 \mathrm{~g}$ of mercurous nitrate and $13 \mathrm{c}$ c of nitric acid (specific gravity 1.42) per liter.

\section{STRAIN TESTS}

7. Strain test specimens shall be the full cross section size of the material. At least 6 inches of the specimen shall be immersed in the solution.

8. The specimen shall receive no annealing, bending, springing, polishing, or other preparation preliminary to this test.

Notes.-Round, square, hexagonal, etc., sections are classed as rods. Rectangular sections having greater width than thickness are classed as bars. All other sections are classed as shapes.

The material covered hereby is suitable for purposes requiring great strength and incorrodibility, such as valve stems, propellerblade bolts, air pump and condenser bolts, etc., and may be used in lieu of Tobin bronze and naval brass where higher strength is required.

The grade of manganese bronze covered herein is not commercially available in sheet and strip form, nor in any thickness less than one-eighth inch. Plates are generally furnished hot rolled. For purposes of calculation the weight per cubic inch may be taken as 0.304 pound.

647.21 INGOTS, MANGANESE BRONZE.

AMERICAN SOCIETY FOR TESTING MATERIALS, STANDARD SPECIFICATIONS FOR MANGANESE-BRONZE INGOTS FOR SAND CASTING, DESIGNATION B $7-2 \%$, 1927

\section{MANGANESE-BRONZE INGOTS FOR SAND CASTING}

\section{SCOPE}

1. These specifications cover the copper-zinc alloy known commercially as manganese bronze.

\section{MANUFACTURE}

2. The bronze shall be cast in the form of ingots having notched flat bottoms, approximately 3 by 2.75 inches wide by 12 inches long, properly tapered to strip easily from an iron mold.

\section{CHEMICAL PROPERTIES}

3. The bronze shall conform to the following requirements as to chemical composition:

\begin{tabular}{|c|c|c|}
\hline & Maximum & Minimum \\
\hline $\begin{array}{l}\text { Copper } \\
\text { Zinc } \\
\text { Tin } \\
\text { Manganese } \\
\text { Aluminum } \\
\text { Iron } \\
\text { Lead }\end{array}$ & $\begin{array}{c}\text { Per cent } \\
60 \\
42 \\
1.5 \\
3.5 \\
1.5 \\
2 \\
.2\end{array}$ & $\begin{array}{r}\text { Per cent } \\
55 \\
38 \\
0 \\
0 \\
0 \\
0 \\
0\end{array}$ \\
\hline
\end{tabular}

\section{PHYSICAL PROPERTIES AND TESTS}

4. The bronze shall conform to the following minimum requirements as to tensile properties:

Tensile strength. lbs./in.2-- 70,000

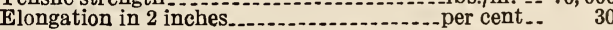

5. (a) The tension-test specimen shall be cut from one corner near the bottom of the test ingot.

(b) (Tension-test specimen requirements conform to fig. 129 in A. S. T. M. standard specifications for steel. See 605.0, p. 247.) 
6. (a) One test ingot shall be selected by the inspector to represent 10,000 pounds of ingots or fraction thereof.

(b) If any test specimen shows defective machining or develops flaws, it may be discarded, in which case two additional test specimens may be selected by the inspector from the same lot, and tested to represent the lot.

7. Each ingot shall be stamped with its proper heat or charge number.

\section{INSPECTION AND REJECTION}

(Requirements covering inspection and rejection are identical with those for brass ingot metal for sand castings in A. S. T. M. B30-31T. See 644.11, p. 1196.)

\section{SOCIETY OF AUTOMOTIVE ENGINEERS,} SPECIFICATION NO. 43, MANGANESE BRONZE, MARCH, 1922

\section{MANGANESE BRONZE}

(This specification is substantially the same as A. S. T. M. B7-27, given above.)

UNITED STATES GOVERNMENT, FEDERAL SPECIFICATION BOARD, FEDERAL SPECIFICATION FOR MANGANESE BRONZE INGOTS (FOR REMELTING), QQ-B-731, MAY 31, 1932

\section{MANGANESE BRONZE INGOTS}

(This specification conforms with A. S. T. M: B7-27, given above, except as to iron content. The minimum iron content here is 0.40 per cent as against zero content in B7.)

647.22 BARS, MANGANESE BRONZE.

UNITED STATES GOVERNMENT, FEDERAL SPECIFICATIONS BOARD, FEDERAL SPECIFICATION FOR BRONZE, MANGANESE, RODS, BARS, SHAPES, AND PLATES, NO. 552, APRIL 30, 1928. (CURRENT DESIGNATION : QQ-B-721)

\section{MANGANESE BRONZE BARS}

(This specification is identical with that given for manganese bronze rods (see 647.23, p. 1266) with the following exception:)

1. Round bars, except forging stock, shall not be out of round more than one-half the permissible variation in diameter shown in the accompanying table.

TABLE 3.-Permissible variations in diameter or thickness of rods and bars, except forging stock

\begin{tabular}{|c|c|c|}
\hline \multirow[b]{2}{*}{ Diameter or thickness (in inches) } & \multicolumn{2}{|c|}{$\begin{array}{c}\text { Permissible varia- } \\
\text { tions }\end{array}$} \\
\hline & Rounds & $\begin{array}{l}\text { Rectan- } \\
\text { gular, } \\
\text { square, } \\
\text { hexago- } \\
\text { nal, etc. }\end{array}$ \\
\hline $\begin{array}{l}0.50 \text { and less } \\
\text { Over } 0.50 \text { to } 1 \text {, inclusive } \\
\text { Over } 1 \text { to } 2.5 \text {, inclusive } \\
\text { Over } 2.5\end{array}$ & $\begin{array}{l}\text { Inch } \\
\pm 0.0015 \\
\pm .0020 \\
\pm .0025 \\
\pm .0030\end{array}$ & $\begin{array}{l}\text { Inch } \\
\pm 0.0030 \\
\pm .0040 \\
\pm .0050 \\
\pm .0060\end{array}$ \\
\hline
\end{tabular}

647.23 RODS, MANGANESE BRONZE.

UNITED STATES GOVERNMENT, FEDERAL SPECIFICATIONS BOARD, FEDERAL SPECIFICATION FOR RODS, WELDING, NONFERROUS, FOR GAS WELDING, QQR-571, MAY 3, 1932

\section{MANGANESE BRONZE RODS}

(For general specifications, types, material, general requirements, detail requirements, methods of inspection, tests, and chemical analysis, packing, and marking of shipments, and notes see 600.0, p. 3.)

1. Manganese bronze rods may be used for general welding of brass and bronze sheets, plates, shapes, and castings.

2. Welding rods shall conform to the following chemical requirements:

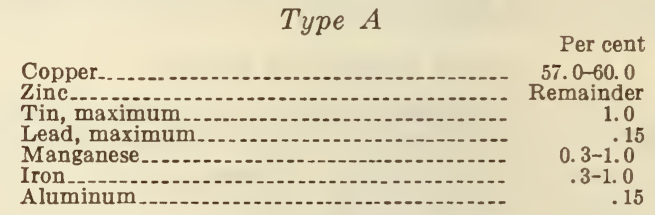

3. Standard sizes of manganese bronze rods for gas welding are:

Diameter (inch) ................... 18, 3/16, 34, 516, and 38

UNITED STATES GOVERNMENT, FEDERAL SPECIFICATIONS BOARD, FEDERAL SPECIFICATION FOR BRONZE, MANGANESE, RODS, BARS, SHAPES, AND PLATES, NO. 552, APRIL 30, 1928. (CURRENT DESIGNATION : QQ-B-721)

\section{MANGANESE BRONZE RODS, BARS, SHAPES}

(For general specifications; grades, forms, and tempers; materialand workmanship; detail requirements; physical properties; tests and methods of inspection; packing and marking; and notes, see 647.20 , p. 1265. Otherwise the specification for manganese bronze rods, bars, and shapes is identical with that for copper rods, bars, and shapes in F.S. B. QQ-C-501 (see 642.11, p. 1180), with the following exception:)

$\mathrm{T}_{\mathrm{ABLE}}$ 4-Permissible variations in diameter or thickness of rods and bars for forging stock

\begin{tabular}{|c|c|}
\hline Diameter or thickness (in inches) & \begin{tabular}{|l|} 
Permis- \\
sible va- \\
riations \\
for all sec- \\
tions
\end{tabular} \\
\hline $\begin{array}{l}0.50 \text { and less. } \\
\text { Over } 0.50 \text { to } 1 \text {, inclusive } \\
\text { Over } 1 \text { to } 2.5 \text {, inclusive } \\
\text { Over } 2.5\end{array}$ & $\begin{array}{c}\text { Inch } \\
\pm 0.007 \\
\pm .010 \\
\pm .015 \\
\pm .022\end{array}$ \\
\hline
\end{tabular}

647.24 PLATES AND SHEETS, MANGANESE BRONZE.

UNITED STATES GOVERNMENT, DEPARTMENT OF COMMERCE, B U R E A U OF STANDARDS, STANDARD THICKNESSES, WEIGHTS, AND TOLERANCES OF SHEET METAL (CUSTOMARY PRATICE), CIRCULAR NO. 391, MARCH 24, 1931

AMERICAN WIRE GAGE-MANGANESE BRONZE PLATES AND SHEETS

(See 640.1, p. 1165.) 
UNITED STATES GOVERNMENT, FEDERAL SPECIFICATIONS BOARD, FEDERAL SPECIFICATION FOR BRONZE, MANGANESE, RODS, BARS, SHAPES, AND PLATES, No. 552, APRIL 30, 1928. (CURRENT DESIGNATION : QQ-B-721)

\section{MANGANESE BRONZE PIATES}

(For general specifications; grades, forms, and tempers; material and workmanship; detail requirements; physical properties; tests and methods of inspection; packing and marking; and notes. see 647.20 , p. 1266. Otherwise the specification for manganese bronze plates is identical with the specification for copper plates in F. S. B. QQ-C-501 (see 641.21 , p. 1177), with the following exceptions:)

TABLE 8.-Permissible variations in width of plates

\begin{tabular}{|c|c|c|}
\hline \multirow{2}{*}{ Width (in inches) } & \multicolumn{2}{|c|}{$\begin{array}{c}\text { Permissible varia- } \\
\text { tion }\end{array}$} \\
\cline { 2 - 3 } & Plus & Minus \\
\hline & & \\
\hline & Inch & Inch \\
14 and less_......... & 0.050 & 0 \\
Over 14_......... & 0 \\
\hline
\end{tabular}

1. Plates of all sizes may be ordered to exact lengths, in which case a variation of one-half inch in length shall be permitted.

(In this specification the table of permissible variations in thickness of sheets and strips shows the B. \& S. gage number of each thickness; but the range of thickness is only from 0.460 inch downward to 0.0808 inch, while in F. S. B. QQ-C-501 the range is from 0.460 inch downward to 0.0050 inch.)

647.25 SHAPES, MANGANESE BRONZE.

UNITED STATES GOVERNMENT, FEDERAL SPECIFICATIONS BOARD, FEDERAL SPECIFICATION FOR BRONZE, MAN GANESE, RODS, BARS, SHAPES, AND PLATES, NO. 552, APRIL 30, 1928. (CURRENT DESIGNATION : QQ-B-721)

\section{MANGANESE BRONZE SHAPES}

(See 647.23, p. 1266.)

647.26 CASTINGS, MANGANESE BRONZE.

AMERICAN SOCIETY FOR TESTING MATERIALS, STANDARD SPECIFICATIONS FOR MANGANESE BRONZE SAND CASTINGS, DESIGNATION B54-2\%, 1927

\section{MANGANESE BRONZE SAND CASTINGS \\ SCOPE}

1. These specifications cover castings made from the copper-zinc alloy known commercially as manganese bronze.

\section{MANUFACTURE}

2. Material used in these castings shall conform to the requirements of the standard specifications for manganese bronze ingots for sand castings as given in A. S. T. M. B7 (see 647.21, p. 1265). At least 35 per cent of the mixture shall consist of ingots manufactured in accordance with the standard specifications for manganese bronze ingots and the remainder shall consist of risers, sprues, and defective castings, accruing from the manufacture of castings in accordance with these specifications. Zinc additions shall be of zinc conforming to the requirements of Grade 2 or better of the standard specifications for spelter as given in A. S. T. M. B6 (see 683.1, p. 1310).

\section{CHEMICAL COMPOSITION}

(Same as A. S. T. M. B7 (see 647.21, p. 1265) except that the maximum percentage of lead in B54 is 0.4 instead of 0.2 .)

\section{PHYSICAL PROPERTIES AND TESTS}

4. The bronze should conform to the following minimum requirements as to tensile properties:

Tensile strength..........................lbs./in. ${ }^{2}$-. 65, 000

Elongation in 2 inches....................................

5. (a) The tension-test specimen shall be machined from coupons to the dimensions as shown in Figure 129 in A. S. T. M. standard specifications for steel (see 605.0, p. 247).

(b) The coupon attached to the castings shall be in accordance with Figure 1123 in F. S. B. QQ-B-671 (see 647.15 , p. 1261), unless special authority to use coupons of other forms and dimensions is obtained from the purchaser.

(c) Each casting weighing 250 pounds or more as delivered from the foundry shall have at least two test coupons cast attached.

(d) When the castings constituting a heat weigh less than 250 pounds each, one coupon shall be cast attached to the first and one to the last casting poured in each heat.

(e) Castings which are too small to permit the casting of a coupon attached shall, when possible, be represented by coupons cast in the same flask and gated to the castings or runners. One coupon shall be cast gated to the first and one to the last casting poured in the heat.

(f) Only in cases when it is impossible to cast coupons in the same flask with small castings shall such coupons be cast separately. In case it is necessary to cast coupons separately, one coupon shall be cast to the first of the heats and one to the last.

(g) At least two ounces of drillings from each coupon tested shall be forwarded for chemical analysis.

6. Each casting shall be clearly stamped with the heat number, and the heat number shall also be stamped on the test coupon representing that heat.

\section{INSPECTION AND REJECTION}

(Inspection conforms to A. S. T. M. B59 (see 647.15 , p. 1259). Rejection is as follows:)

8. (a) Any casting may be rejected upon the failure of the test coupon to meet the physical requirements specified or for the noncompliance with the chemical composition. If the physical or chemical tests indicate that the heat of castings represented by its test coupon does not conform to the physical or chemical requirements, or both, the entire heat shall be rejected.

(b) In case tests on the first test piece do not conform to these specifications, a second test piece shall be tested. If such a test on the second piece is thoroughly satisfactory the castings or heat shall be accepted; if unsatisfactory, the castings or heat shall be rejected. 
UNITED STATES GOVERNMENT, FEDLERA SPECIFICATIONS BOARD, FEDERAL SPECIFICATION FOR MANGANESE BRONZE CASTINGS, QQ-B-726, MAY 31, 1932

\section{BRONZE CASTINGS}

(This specification is identical with Federal specification 369a (current designation: QQ-B-671) (see 647.15, p. 1260), except in the following particulars:)

GRADE

1. This specification covers one grade of manganese bronze in the form of castings.

\section{MATERIAI}

2. Manganese bronze castings shall be made only from the best grades of virgin metals, or from virgin metals and scrap of known and approved composition.

\section{DETAIL REQUIREMENTS}

3. The chemical requirements are as shown in the table below:

\begin{tabular}{|c|c|c|c|c|c|c|c|}
\hline Copper & Zinc & $\begin{array}{l}\text { Lead, } \\
\text { maxi- } \\
\text { mum }\end{array}$ & $\begin{array}{l}\text { Alumi- } \\
\text { num, } \\
\text { maxi- } \\
\text { mum }\end{array}$ & $\begin{array}{l}\text { Man- } \\
\text { ganese } \\
\text { maxi- } \\
\text { mum }\end{array}$ & Iron & Tin & $\begin{array}{l}\text { Other } \\
\text { ele- } \\
\text { ments, } \\
\text { maxi- } \\
\text { mum }\end{array}$ \\
\hline $\begin{array}{c}\text { Per cent } \\
55-60\end{array}$ & $\begin{array}{l}\text { Per } \\
\text { cent } \\
38-42\end{array}$ & $\begin{array}{c}\text { Per } \\
\text { cent } \\
0.20\end{array}$ & $\begin{array}{c}\text { Per } \\
\text { cent } \\
1.50\end{array}$ & $\begin{array}{l}\text { Per } \\
\text { cent } \\
3.50\end{array}$ & $\begin{array}{l}\text { Per cent } \\
0.40-2.0\end{array}$ & $\begin{array}{l}\text { Per } \\
\text { cent } \\
1.50\end{array}$ & $\begin{array}{l}\text { Per } \\
\text { cent } \\
0.20\end{array}$ \\
\hline
\end{tabular}

4. The physical requirements are as shown below:

\begin{tabular}{|c|c|}
\hline $\begin{array}{c}\text { Tensile } \\
\text { strength, } \\
\text { mini- } \\
\text { mum }\end{array}$ & $\begin{array}{c}\text { Elonga- } \\
\text { tion in 2 } \\
\text { inches, } \\
\text { minimum }\end{array}$ \\
\hline $\begin{array}{r}\text { Lbs./in.2 } \\
65,000\end{array}$ & $\begin{array}{r}\text { Per cent } \\
20.0\end{array}$ \\
\hline
\end{tabular}

Note.-Manganese bronze may be used for castings such as propeller hubs, propeller blades, engine framing, gears, worm wheels, torpedo spoons, torpedo-tube castings, gun-mount castings, etc., where strength, toughness, or resistance to corrosion of sea water are required.

\subsection{WIRES, MANGANESE BRONZE.}

(No nationally recognized specifications available.)

\subsection{PHOSPHOR BRONZE.}

\subsection{INGOTS AND BEARING METALS, PHOS- PHOR BRONZE.}

AMERICAN RAILWAY ENGINEERING ASSOCIATION, SPECIFICATIONS FOR MOVA. BLE BRIDGES, BRONZES, 1922

(See also 603.32, p. 151; 611.41, p. 707; 611.51, p. 753; 692.1, p. 1320.)

\section{BRONZES}

710. Phosphor bronze shall be a homogeneous alloy, of crystalline structure. It shall be made from new metals, except that scrap of known composition produced by the foundry at which the bronze is cast may be used. It shall not contain sulphur. The phosphorus shall be introduced in the form of phosphor-tin or phosphor-copper. Castings shall be sound, clean, and free from blowholes, porous places, cracks, and other defects.

711. The hardness of the finished castings shall be tested by the Brinell ball method and a record of the test furnished. The ball shall be of hardened steel $10 \mathrm{~mm}$ in diameter. The load shall be $500 \mathrm{~kg}$ and shall be applied for 30 seconds to a finished plane surface. No fewer than two hardness tests shall be made on each heat. A test shall be made on each trunnion bearing and on each disk.

712. The alloy shall be cast into ingots and allowed to cool, and the castings shall be poured from the remelted ingots. Care shall be exercised that the metal is not overheated and that the temperature at pouring and the conditions of cooling are such as will be most likely to secure dense castings.

713. There shall be four grades.

Grade A is to be used for contact with hardened steel disks under pressures exceeding 1,500 pounds per square inch, such as are used in turntables and center-bearing swing bridges.

Grade B is to be used for contact with soft steel at low speeds under pressures not exceeding 1,500 pounds per square inch, such as trunnions and journals of bascule and lift bridges.

Grade $\mathrm{C}$ is to be used for ordinary machinery bearings.

Grade D is to be used for gears, worm wheels, nuts, and similar parts which are subjected to other than compressive stresses.

714. The chemical and physical properties shall conform to the requirements in the following table:

\begin{tabular}{|c|c|c|c|c|}
\hline \multirow{3}{*}{ Alloy of- } & \multicolumn{4}{|c|}{ Grade } \\
\hline & $\mathbf{A}$ & B & C & $\mathrm{D}$ \\
\hline & $\begin{array}{l}\text { Cop- } \\
\text { per } \\
\text { and } \\
\text { tin }\end{array}$ & $\begin{array}{l}\text { Cop- } \\
\text { per } \\
\text { and } \\
\text { tin }\end{array}$ & $\begin{array}{l}\text { Cop- } \\
\text { per, } \\
\text { tin, } \\
\text { and } \\
\text { lead }\end{array}$ & $\begin{array}{l}\text { Cop- } \\
\text { per, } \\
\text { tin, } \\
\text { and } \\
\text { zinc }\end{array}$ \\
\hline Copper & a 20 & 17 & $\begin{array}{l}a \\
\cdot 82 \\
\cdot 11\end{array}$ & $\begin{array}{l}.89 \\
-11\end{array}$ \\
\hline 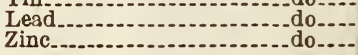 & (2) & 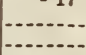 & 11 & 2.25 \\
\hline Phosphorus............... do & a 1.0 & a 1.0 & a 1.0 & $\cdot 0.25$ \\
\hline $\begin{array}{l}\text { Other elements } \\
\text { Elastic limit in compress ion }\end{array}$ & a 0.5 & a 0.5 & 0.5 & - 0.5 \\
\hline Permanent set in inches under & $\left\{\begin{array}{l}24,000 \\
60.06 \\
00.10\end{array}\right.$ & $\begin{array}{r}\text { b } 18,000 \\
c 0.10 \\
\text { a } 0.20\end{array}$ & & \\
\hline $\begin{array}{l}\text { Permanent set in inches under } \\
50,000 \\
\text { Yield point in tension..... }\end{array}$ & (c) & (c) & (c) & $\begin{array}{l}\text { (c) } \\
\text { (c) }\end{array}$ \\
\hline Ultimate strength in tension & & & & b 33,0 \\
\hline Elongation in 2 inches -per cent.- & & & & b 14 \\
\hline
\end{tabular}

- Maximum.

b Minimum.

- To be recorded.

Cracks and other evidences of excessive brittleness in compression-test specimens may be cause for rejection.

The chemical analysis of each heat shall be furnished.

715. Test specimens shall be made from coupons which are a part of the castings and which have been fed and cooled under the same conditions as the casting.

Compression-test specimens shall be cylinders 1 inch high and of 1 square inch area. The elastic limit in compression shall be the load which gives a permanent set of 0.001 inch.

Tension-test specimens shall be turned from a coupon not less than 1 inch in diameter to the form 
shown in A. S. T. M. standard specifications for steel. (See 605.0, p. 247.) The diameter of the turned specimen shall be one-half inch.

716. One or more compression tests shall be made from each melt for grades A, B, and C; and one compression and one tension test for grade $D$. For castings weighing over 100 pounds finished, the prescribed tests shall be made for each casting.

647.32 BARS, PHOSPHOR BRONZE.

(No nationally recognized specifications available.)

647.33 PLATES AND SHEETS, PHOSPHOR BRONZE. UNITED STATES GOVERNMENT, DEPARTMENT OF COMMERCE, BUREAU OF STANDARDS, STANDARD THICKNESSES, WEIGHTS, AND TOLERANCES OF SHEET METAL (CUSTOMARY PRACTICE), CIRCULAR NO. 391, MARCH 24, 1931

(See 640.1, p. 1165.)

647.34 CASTINGS, PHOSPHOR BRONZE.

SOCIETY OF AUTOMOTIVE ENGINEERS, NONFERROUS METAL SPECIFICATIONS, SPECIFICATION NO. 64, PHOSPHOR BRONZE, MARCH, 1922

\section{PHOSPHOR BRONZE}

1. Composition in percentage.-

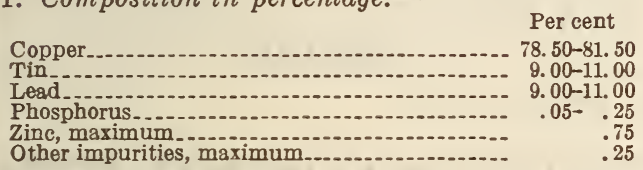

2. General information.-Good castings made of this alloy should give the following minima in physical characteristics:

Ultimate strength_.

Yield point

8

3. This metal is an excellent composition for use where antifriction qualities are desired, standing up exceedingly well under heavy loads and severe usage.

4. Bearings or gears made of bronze alloys should be used only against hardened steel.

SOCIETY OF AUTOMOTIVE ENGINEERS, NONFERROUS METAL SPECIFICATIONS, SPECIFICATION NO. 65, PHOSPHOR GEAR BRONZE, MARCH, 1922

\section{PHOSPHOR GEAR BRONZE}

1. Composition in percentage.-

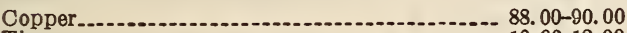
Tin Phosphorus Lead, zinc, and other impurities, maximum.... $\quad .50$

2. General information.-Good castings made of this alloy should give the following minima in physical characteristics:

Ultimate strength lbs./in. ${ }^{2}--35,000$

Yield point 20,000

longation in 2 inches, or proportionate gage length
3. This is a very hard bronze and may be used for gears and worm wheels where the requirements are severe.

4. Bearings or gears made of bronze alloys should be used only against hardened steel.

UNITED STATES GOV ERNMENT, FEDERAL SPECIFICATIONS BOARD, FEDERAL SPECIFICATION FOR BRONZE CASTINGS, QQ-B-691 MAY 31, 1932

\section{BRONZE CASTINGS}

(See 646.41, p. 1252.)

\subsection{SHAPES, PHOSPHOR BRONZE.}

(No nationally recognized specifications available.)

647.36 STRIPS, PHOSPHOR BRONZE.

SOCIETY OF AUTOMOTIVE ENGINEERS, NONFERROUS METAL SPECIFICATIONS SPECIFICATION NO. 7\%, PHOSPHORBRONZE STRIP, AUGUST, 1922

\section{PHOSPHOR-BRONZE STRIP}

1. This specification covers bronze strips up to 0.080 -inch gage, and includes a variety of tempers in two alloys, from which a suitable quality can be chosen for any ordinary purpose.

2. Composition in percentage.-

\begin{tabular}{|c|c|c|}
\hline & Grade A & Grade B \\
\hline & $\begin{array}{l}\text { Per cent } \\
4.00-6.00\end{array}$ & $\begin{array}{l}\text { Per cent } \\
7.00-9.00\end{array}$ \\
\hline Phosphorus........ & $.03-.40$ & $.03-.20$ \\
\hline Zinc, maximum & .20 & .20 \\
\hline $\begin{array}{l}\text { Iron, maximum } \\
\text { Lead, maximum }\end{array}$ & .10 & $\begin{array}{l}10 \\
10\end{array}$ \\
\hline Copper & Remainder. & Remainder. \\
\hline
\end{tabular}

3. Temper designation.-The temper of strip phosphor bronze shall be designated as follows:

\begin{tabular}{|c|c|}
\hline Temper & $\begin{array}{r}\text { Reduction } \\
\text { B.\& S.No. }\end{array}$ \\
\hline Half hard. & 2 \\
Hard. & 4 \\
Extra hard. & 6 \\
Spring & 8 \\
\hline
\end{tabular}

4. Minimum physical properties.-

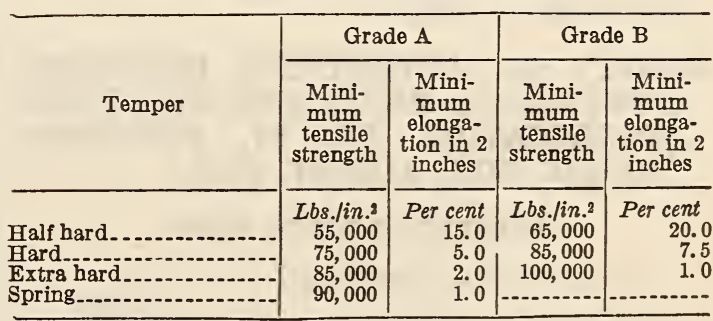

5. Physical properties.-The average tension test of two samples of sheet thinner than 0.080 inch should conform to the minimum requirements specified in the accompanying table. 
In very thin strips, on account of difficulties in testing, the elongation may be considerably less than the values given.

6. Dimensional tolerances.-The width of the strip shall not vary more than 0.01 inch from the size specified in the order.

The thickness tolerances of sheet or strip made of this alloy shall be within the tolerances specified in Table 4. (See 655.2, p. 1287.)

7. General information.-These should be considered as general specifications. Phosphor-bronze strip is used for various kinds of springs where the manufacturing requirements and the uses to which the springs are put are too particular to be specified by ordinary physical tests. It is advisable to submit samples or drawings to the manufacturer and secure an adjustment of temper to suit the manufacturing and service requirements of the article.

8. Flat springs formed with easy bands across the grain are usually made of the grade A alloy, "spring" temper.

9. Flat springs with easy bends either across or with the grain are usually made of the grade B alloy, "extra hard" temper.

10. Clips or contact springs with most difficult bends are usually made of the grade B alloy, "hard" temper.

\subsection{TUBING, PHOSPHOR BRONZE.} able.)

647.38 WIRES AND RODS, PHOSPHOR BRONZE.

AMERICAN MARINE STANDARDS COMMITTEE, STANDARD WIRE ROPES FOR MARINE USES, 0 NO. 25-1930

WIRE ROPES FOR MARINE USES

(See 603.42, p. 163.)

AMERICAN MARINE STANDARDS COMMITTEE, IOCKS AND ACCESSORIES FOR SHIP DOORS, H NO. 61-1930

PHOSPHOR-BRONZE SPRINGS

(See 617.21, p. 1004.)

AMERICAN MARINE STANDARDS COMMITTEE, BOLTS AND CATCHES FOR SHIP DOORS, H NO. 62-1930

PHOSPHOR-BRONZE SPRINGS

(See 617.61, p. 1052.)

SOCIETY OF AUTOMOTIVE ENGINEERS, NONFERROUS METAL SPECIFICATIONS, SPECIFICATION NO. 81, PHOSPHORBRONZE WIRE, AUGUST, 1922

\section{PHOSPHOR-BRONZE WIRE}

1. Composition in percentage.-

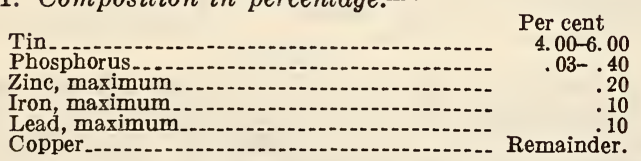

2. Bend test.-The wire shall be capable of being bent through an angle of $180^{\circ}$ flat back on itself without fracture on the outside of the bent portion.
3. Appearance.-The wire shall be uniform in quality and temper, cylindrical in shape, and smooth and free from all injurious defects.

4. Dimensional tolerances.-The wire shall not vary from the specified diameter by more than plus or minus 1 per cent for larger sizes than 0.050 inch.

\section{Mechanical requirements.-}

\begin{tabular}{|c|c|c|}
\hline \multicolumn{2}{|c|}{ Wire diameter } & $\begin{array}{c}\text { Minimum } \\
\text { tensile } \\
\text { strength }\end{array}$ \\
\hline Over & $\begin{array}{c}\text { To and } \\
\text { including }\end{array}$ & \\
\hline Inch & Inch & Lb8./in.2 \\
0.0000 & 0.0625 & 130,000 \\
.0625 & .1250 & 120,000 \\
.1250 & .2500 & 110,000 \\
.2500 & .3750 & 100,000 \\
\hline
\end{tabular}

From 0.050 to 0.025 inch the variation in size shall not be more than plus or minus 0.0005 inch.

For sizes smaller than 0.025 inch the variation shall not be more than plus or minus 0.00025 inch.

6. General information.-Phosphor-bronze wire made to this specification is intended primarily for use in springs.

\section{UNITED STATES GOVERNMENT, FEDERAI} SPECIFICATIONS BOARD, FEDERAI SPECIFICATION FOR ROPE, WIRE, NO. 297, 1925. (CURRENT DESIGNATION : $\mathrm{RR}-\mathrm{R}-\mathbf{5 7 1}$ )

\section{WIRE ROPE}

(See 603.42, p. 183.)

UNITED STATES GOVERNMENT, FEDERAL SPECIFICATIONS BOARD; FEDERAI SPECIFICATION FOR PHOSPHOR-BRONZE SPRING WIRE, QQ-W-401, MAY 31, 1932

\section{PHOSPHOR-BRONZE SPRING WIRE}

\section{GENERAL SPECIFICATIONS}

United States Government general specification for metals, Federal Specification Board No. 339 (current designation: QQ-M-151) in effect on date of invitation for bids, shall form part of this specification.

$$
\text { II. GRADE }
$$

This specification covers one grade of phosphorbronze spring wire as shown in Table 1 .

\section{MATERIAL}

1. Phosphor-bronze spring wire furnished under this specification may be either hard drawn or hard rolled and shall be in accordance with the requirements of Section V.

2. Scrap shall not be used in the manufacture, except as it may accumulate in the manufacturer's plant from material of the same composition, and of the manufacturer's own make.

\section{GENERAL REQUIREMENTS}

The material shall be free from all injurious internal and surface defects, clean, smooth, and of uniform color, quality and size. It shall be capable of being formed into helical springs without surface cracking. 


\section{DETAIL REQUIREMENTS}

1. The wire shall be truly circular in cross section within 0.0005 inch; permissible variation from nominal diameter shall be: For wire 0.100 inch in diameter and larger, 1 per cent over or under. For wire less than 0.100 inch diameter, 0.001 inch over or under.

2. The chemical composition shall conform to the requirements shown in Table 1.

TABLE 1

\begin{tabular}{|c|c|c|c|c|c|}
\hline $\begin{array}{c}\text { Copper, } \\
\text { mini- } \\
\text { mum }\end{array}$ & $\begin{array}{c}\text { Tin, } \\
\text { mini- } \\
\text { mum }\end{array}$ & $\begin{array}{c}\text { Zinc, } \\
\text { maxi- } \\
\text { mum }\end{array}$ & $\begin{array}{c}\text { Iron, } \\
\text { maxi- } \\
\text { mum }\end{array}$ & $\begin{array}{c}\text { Lead, } \\
\text { maxi- } \\
\text { mum }\end{array}$ & Phosphorus \\
\hline $\begin{array}{c}\text { Per cent } \\
94\end{array}$ & $\begin{array}{c}\text { Per cent } \\
3.50\end{array}$ & $\begin{array}{c}\text { Per cent } \\
0.02\end{array}$ & $\begin{array}{c}\text { Per cent } \\
0.10\end{array}$ & $\begin{array}{c}\text { Per cent } \\
0.20\end{array}$ & $\begin{array}{c}\text { Per cent } \\
0.05-0.50\end{array}$ \\
\hline
\end{tabular}

3. The physical properties shall be in accordance with the requirements shown in Table 2.

TABLE 2

\begin{tabular}{|c|c|c|}
\hline Diameter or thickness of material (inch) & $\begin{array}{c}\text { Tensile } \\
\text { strength, } \\
\text { minimum }\end{array}$ & $\begin{array}{l}\text { Elonga- } \\
\text { tion in } 2 \\
\text { inches, } \\
\text { minimum }\end{array}$ \\
\hline $\begin{array}{l}\text { Up to and including } 0.025 \\
\text { A bove } 0.025 \text { and including } 0.0625 \\
\text { A bove } 0.0625 \text { and including } 0.125 \\
\text { A bove } 0.125 \text { and including } 0.250 \\
\text { Above } 0.250 \text { and including } 0.375 \\
\text { Above } 0.375 \text { and including } 0.500\end{array}$ & $\begin{array}{c}\text { Lbs./in. }{ }^{2} \\
150,000 \\
135,000 \\
130,000 \\
125,000 \\
120,000 \\
105,000\end{array}$ & $\begin{array}{r}\text { Per cent } \\
1.5 \\
2.0 \\
3.5 \\
5.0 \\
9.0\end{array}$ \\
\hline
\end{tabular}

4. Test pieces shall withstand being bent cold through an angle of $120^{\circ}$ to a radius equal to the diameter of thickness of the material.

5 . The color of the fractured section of test pieces and the grain of the material shall be uniform throughout.

\section{METHODS OF INSPECTION AND TESTS}

1. Each coil or when furnished in straight lengths, each length, shall be gaged at three places, one near each end, and one approximately at the middle to determine compliance with the detail requirements given above.

2. When furnished in coils, one tension and one bend test specimen shall be taken from each alternate coil. When furnished in straight lengths, one tension and one bend test specimen shall be taken to represent each 500 pounds or less.

3. Tension tests shall be made on the full cross section of the wire.

4. One chemical analysis sample shall be taken to represent each four coils or each 1,000 pounds of straight lengths. If machined from the tension test specimen, equal parts of each of the two specimens shall constitute the sample; otherwise equal amounts shall be obtained from each of the coils or lengths. Sample shall consist of not less than 5 ounces of millings or drillings.

\section{PACKING AND MARKING OF SHIPMENTS}

1. Packages shall be legibly and indelibly marked with the name and size of material; the quantity contained; name, brand, or trade-mark of manufacturer or contractor; and the contract or purchase order number.

2. When furnishing in coils, each coil shall weigh not more than 200 pounds.

\section{NOTES}

1. The material covered by this specification is intended for use in the manufacture of phosphorbronze helical springs. It is not intended for use in the manufacture of mail-bag locks. 
650. GENERAL ITEMS

AMERICAN SOCIETY FOR TESTING MATERIALS, STANDARD METHODS OF METALLOGRAPHIC TESTING OF NONFERROUS METALS AND ALLOYS, DESIGNATION E5-27, 1927

METALLOGRAPHIC TESTING OF NONFERROUS

(See 600.1, p. 28.)

AMERICAN SOCIETY FOR TESTING MATERIALS, TENTATIVE METHODS OF TENSION TESTING OF METALLIC MATERIALS, DESIGNATION E8-27T, 1927

TENSION TESTING OF METALLIC MATERIALS

(See 600.1, p. 34.)

AMERICAN SOCIETY FOR TESTING MATERIALS, TENTATIVE METHODS OF COMPRESSION TESTING OF METALLIC MATERIALS, DESIGNATION E9-27T, 1927

COMPRESSION TESTING OF METALLIC MATERIALS

(See 600.1, p. 38.)

651. IEAD

\subsection{GENERAL ITEMS.}

AMERICAN SOCIETY FOR TESTING MATERIALS, STANDARD METHODS OF CHEMICAL ANALYSIS OF PIG LEAD, SERIAL DESIGNATION B35-24, 1924

\section{CHEMICAL ANALYSIS OF PIG LEAD}

\section{A. DETERMIIUATION OF SILVER}

Weigh $100 \mathrm{~g}$ of the sample into a 3-inch scorifier and heat in a muffle furnace until the assay "covers." Pour into an iron mold and allow to cool. Free the resulting lead button from $\mathrm{PbO}$, scorify again, and pour as before. The button last obtained should not weigh over $20 \mathrm{~g}$ and can be cupelled directly. Weigh the silver button obtained upon an assay balance.

Note.-If the amount of silver is large, the button should be examined for gold, in the usual manner.

\section{B. DETERMINATION OF BISMUTH}

\section{SOLUTIONS REQUIRED}

Sodium carbonate.-Dissolve $100 \mathrm{~g} \mathrm{Na}_{2} \mathrm{CO}_{3}$ in a liter of distilled water.

Ammonium carbonate.-Make a half-saturated solution.

Alkaline sulphide wash solution.-Dissolve $200 \mathrm{~g}$ of $\mathrm{KOH}$ in a liter of distilled water and mix one part of this solution with 4 parts of $\mathrm{H}_{2} \mathrm{~S}$ water.

\section{METHOD}

(a) Determination for ordinary amounts of bismuth.-Dissolve $20 \mathrm{~g}$ of the sample in a $400 \mathrm{c}$ c beaker with $100 \mathrm{c}$ c of $\mathrm{HNO}_{3}(1: 4)$, with the aid of heat. When solution is complete, add dilute ammonia (1:2), with constant stirring, drop by drop from a burette, until a faint opalescence appears. If an actual precipitate is formed, redissolve by the addition of a small amount of $\mathrm{HNO}_{3}$ $(1: 4)$ and repeat the addition of ammonia. Now add $5 \mathrm{c}$ c of $\mathrm{HCl}(1: 9)$, fill the beaker with hot water, bring to boiling, and allow to stand on a steam bath for two hours. The assay, while standing, must not reach the boiling temperature. Filter through a $7 \mathrm{~cm}$ paper. Transfer the precipitate completely to the paper by means of a "policeman" and wash twice with hot water. Carefully examine the filtrate, washings, and any decanted liquid and reject if clear. Dissolve the precipitate by dropping around the edges, from a $5 \mathrm{c}$ c pipette, $5 \mathrm{c} \mathrm{c}$ of boiling $\mathrm{HCl}(1: 9)$. receiving the solution in the original beaker. Wash the paper thoroughly with hot water, fill the beaker with water, bring to boiling, and allow to stand as before. Filter the bismuth oxychloride upon a weighed Gooch crucible, wash thoroughly with water, once with alcohol, once with ether, and dry upon the hot plate. Cool and weigh.

Note.-If time permits it is convenient to allow the assay to stand overnight. In that case, the precipitate of bismuth oxychloride generally settles so completely that the clear supernatant solution can be decanted.

(b) Determination for amounts of bismuth smaller than can be determined by (a).-Dissolve $100 \mathrm{~g}$ of the sample in $500 \mathrm{c}$ c of dilute $\mathrm{HNO}_{3}(1: 4)$. When solution is complete, allow to cool and add $\mathrm{Na}_{2} \mathrm{CO}_{2}$ solution little by little until a slight permanent precipitate has formed. Then add $50 \mathrm{c} c$ of the $\mathrm{Na}_{2} \mathrm{CO}_{3}$ solution, bring to boiling, allow to stand warm until the supernatant liquor is clear again, filter, and reject the filtrate. Dissolve the precipitate without washing by slowly pouring hot $\mathrm{HNO}_{3}$ $(1: 4)$ around the edges of the filter paper, using no more acid than necessary. Wash the paper once with hot water and determine bismuth in the filtrate as described in $(a)$.

Nore. - When the sample contains a small amount of bismuth, it is often difficult to ascertain when the correct amount of ammonia has been added to the nitrate solution. In this case, place a small piece of litmus paper in the solution, and add the ammonia very gradually until the litmus paper indicates a neutral reaction.

(c) Determination of bismuth in samples containing appreciable amounts of tin and antimony.-Use a piece of litmus paper as in note under $(b)$, and after the first precipitation of bismuth oxychloride has been filtered off and washed (see $(a)$ ) proceed as follows: Dissolve the bismuth by dropping around the edges of the paper $10 \mathrm{c} c$ of boiling $\mathrm{HCl}(1: 2)$, receiving the solution in the original beaker. Discard the paper after washing. Dilute the solution to about $200 \mathrm{c} \mathrm{c}$ with fresh $\mathrm{H}_{2} \mathrm{~S}$ water and then pass $\mathrm{H}_{2} \mathrm{~S}$ gas through the hot solution for 15 minutes. Filter and wash with hot water. Remove any tin or antimony present by washing three times with alkaline sulphide wash solution. Wash the precipitate again with hot water, place it, together with the filter paper, in a $100 \mathrm{c}$ c beaker, add $20 \mathrm{c} \mathrm{c}$ of $\mathrm{HNO}_{3}(1: 4)$, boil until sulphides are completely 
dissolved and the paper well pulped. Filter the solution, receiving the filtrate in the original beaker, and wash well. Determine bismuth in the filtrate as in $(a)$.

Note. - When the sample contains comparatively large amounts of tin or antimony, the residue left after the solution of the lead in the nitric acid obscures the opalescence found upon the addition of ammonia.

If the original sample contains more than 0.25 per cent of bismuth It is preferable to use only a $10 \mathrm{~g}$ charge.

\section{DETERMINATION OF ARSENIC}

\section{METHOD}

Dissolve $111.11 \mathrm{~g}$ of the sample in $550 \mathrm{c} \mathrm{c}$ of $\mathrm{HNO}_{3}(1: 4)$. When solution is complete wash into a graduated liter flask, add $75 \mathrm{c} \mathrm{c}$ of $\mathrm{H}_{2} \mathrm{SO}_{4}(1: 1)$, cool, and make up to the mark with water. Transfer to a large beaker, rinsing out the flask with $25 \mathrm{c} c$ of water. Mix thoroughly, allow to settle and filter off $900 \mathrm{c}$ c, equivalent to a $100 \mathrm{~g}$ charge. Evaporate in a large porcelain dish until only enough $\mathrm{H}_{2} \mathrm{SO}_{4}$ is left to moisten the residue. When cool, wash into a small distilling flask with $60 \mathrm{c}$ c of $\mathrm{HCl}$ (specific gravity 1.19) and $20 \mathrm{c}$ c of water, cleaning the dish carefully. Add $10 \mathrm{~g}$ of ferrous sulphate and distill, boiling to as small a volume as possible. When cool add $50 \mathrm{c} \mathrm{c}$ more of $\mathrm{HCl}$ (specific gravity 1.19) and redistill. Pass $\mathrm{H}_{2} \mathrm{~S}$ gas through the cold distillate for 45 minutes. Filter, and weigh the $\mathrm{As}_{2} \mathrm{~S}_{3}$ on a Gooch crucible, washing with cold water, alcohol, and $\mathrm{CS}_{2}$. After drying and weighing, redissolve with $\left(\mathrm{NH}_{4}\right)_{2} \mathrm{CO}_{3}$ solution and reweigh the Gooch crucible, calculating the loss in weight to arsenic.

Note.-The $25 \mathrm{c}$ c of water added is equivalent to the volume of precipitated lead sulphate.

\section{DETERMINATION OF REMAINING METALS}

\section{SOLUTIONS REQUIRED}

Tartaric acid.-Dissolve $50 \mathrm{~g}$ of tartaric acid in $250 \mathrm{c} \mathrm{c}$ of distilled water to which has been added 250 c c $\mathrm{HCl}$ (specific gravity 1.20 ).

\section{METHOD}

Dissolve $222.23 \mathrm{~g}$ of the sample in $1,100 \mathrm{c} \mathrm{c}$ of $\mathrm{HNO}_{3}(1: 4)$, using a 1,300 c c beaker. When solution is complete examine for color and turbidity. If clear, wash the solution at once into a $2,000 \mathrm{c} \mathrm{c}$ graduated flask. In case of a residue (note 1), however, dilute to about $1,100 \mathrm{c}$ c and allow to stand until the supernatant liquor is clear. Decant as much as possible into a $2,000 \mathrm{c}$ c flask, filter the remainder, and receive the filtrate in the same flask. Wash the precipitate well and then place it, together with the filter paper, in a $100 \mathrm{c}$ c beaker and add 20 c c of the tartaric acid mixture. Heat to boiling and when the paper is well pulped allow to digest warm for 30 minutes. Now add $50 \mathrm{c}$ c of hot water, filter, and wash. (Note 2.) Carefully dry the residue and ignite. If any appreciable residue remains, brush it into a small silver dish containing $1 \mathrm{~g}$ of molten KOH. Fuse for five minutes, and after cooling dissolve in as little hot water as possible and add to the above-mentioned tartaric acid filtrate. Render this solution just alkaline with ammonia and then just acid with $\mathrm{HCl}$, and saturate hot with $\mathrm{H}_{2} \mathrm{~S}$ gas. After digesting for 30 minutes on the steam bath, pass $\mathrm{H}_{2} \mathrm{~S}$ through the solution again for 15 minutes. Filter and wash with slightly acidified $\mathrm{H}_{2} \mathrm{~S}$ water. Reject the filtrate. Wash the sulphides from the paper into the original beaker and add $5 \mathrm{c} \mathrm{c}$ of $\mathrm{KOH}$ solution $(1: 5)$ for every 25 c c volume present. Digest hot for five minutes and filter through the original paper into a small flask graduated to $110 \mathrm{c} \mathrm{c}$. After washing with $\mathrm{H}_{2} \mathrm{~S}$ water containing a little of the $\mathrm{KOH}$ solution, cool the filtrate and make up to the mark. Mix and reserve $100 \mathrm{c} \mathrm{c}$ as alkaline sulphide solution No. 1. The precipitate may be discarded.

Add slowly to the main solution in the $2,000 \mathrm{c} \mathrm{c}$ flask $150 \mathrm{c}$ c of $\mathrm{H}_{2} \mathrm{SO}_{4}(1: 1)$. After cooling and filling up to the mark, pour into a clean 3-liter flask provided with a rubber stopper. Rinse the flask out with $50 \mathrm{c}$ c of water, which is equivalent to the volume of lead sulphate present, and is added to the portion. After mixing thoroughly by shaking, allow the precipitate to settle and filter off $1,800 \mathrm{c} \mathrm{c}$ of the liquid. This is equivalent to a $200 \mathrm{~g}$ charge. Place this in a No. 9 porcelain evaporating dish and evaporate, first over a free flame and later on the hot plate, until only enough $\mathrm{H}_{2} \mathrm{SO}_{4}$ is left to moisten the residue remaining. Add $50 \mathrm{c}$ c of water and, after digesting warm for a short time, wash the solution into a 250 c c beaker, cleaning the dish carefully. Allow the solution to digest on the steam bath for four or five hours. (Note 3.) Then filter, wash, and evaporate the filtrate to 200 to $250 \mathrm{c} \mathrm{c}$. Place any residue (note 4 ), together with the filter paper, in a $100 \mathrm{c} \mathrm{c}$ beaker and treat with $20 \mathrm{c} \mathrm{c}$ of the tartaric acid mixture. Boil for five minutes, dilute with $50 \mathrm{c}$ c of hot water, and filter. Make the filtrate alkaline with ammonia and just acid with $\mathrm{HCl}$, and obtain the tin, antimony, and arsenic as previously described, reserving the whole of the alkaline sulphide solution as solution No. 2. Reject the sulphide residue.

To filtrate from the lead sulphate, add ammonia until the neutral point is reached, and then for every $50 \mathrm{c} \mathrm{c}$ of the solution present add $2 \mathrm{c} \mathrm{c}$ of $\mathrm{HCl}$ (specific gravity 1.19). Pass $\mathrm{H}_{2} \mathrm{~S}$ gas into the hot solution until saturated, digest for 30 minutes on the steam bath, and again pass $\mathrm{H}_{2} \mathrm{~S}$ into the solution. Filter and wash with $\mathrm{H}_{2} \mathrm{~S}$ water slightly acidified. (Note 5.) Separate tin, antimony, and arsenic in the precipitate with $\mathrm{KOH}$ solution as usual, obtaining an alkaline sulphide solution No. 3. (Note 6.)

To sum up, three alkaline sulphide solutions have been obtained containing tin, antimony, and arsenic; a precipitate of metallic sulphides containing copper, lead, etc.; and a solution containing iron, zinc, nickel, etc.

Notes.-1. A residue indicates the presence of antimony, tin, possibly arsenic, or sulphur as lead sulphate.

2. It has been found that even this treatment occasionally fails to dissolve stannic acid completely.

3. If it is preferred, allow the solution to stand overnight to insure he complete solution of all soluble salts.

4. Any residue of lead sulphate may contain some tin, antimony, or possibly arseric.

5. The filtrate will contain any iron, zinc, nickel, cobalt, and manganese; while in the precipitate will be found any copper, cadmium, lead, silver, bismuth, tin, antimony, and arsenic.

6. When separating the sulphides of arsenic, antimony, and tin from sulphides of copper, lead, etc., it is necessary to wash all th sulphides back into the beaker in which they were precipitated. These sulphides sometimes cling so tenaciously to the paper that in dislodging them more water than the $25 \mathrm{c} \mathrm{c}$ specified is required. In this case allow the sulphides to settle and then decant the clear supernatant liquor through the filter until the volume is reduced to $25 \mathrm{c} c$ Before rejecting the decanted fluid always test with $\mathrm{H}_{2} \mathrm{~S}$ water.

In washing sulphide precipitates with water, much trouble is experienced from the tendency of the precipitate to pass through the filter in the colloidal form. This is particularly true in washing sulphides that have been digested with KOH. Time and trouble will be saved by washing all the sulphides precipitated from mineralacid solutions with $\mathrm{H}_{2} \mathrm{~S}$ water containing a little of the acid in which they were precipitated. The same is true of sulphides precipitated in or filtered from an alkaline solution.

When working with alkaline solutions in which tin is to be determined, avoid the use of Jena or other glass that contains zinc. The zinc content of the glass may influence the result.

\section{DETERMINATION OF ANTIMONY AND TIN}

\section{SOLUTIONS REQUIRED}

Potassium iodide.-Dissolve $100 \mathrm{~g}$ in a liter of distilled water.

Standard sodium thiosulphate.-Dissolve $24.8 \mathrm{~g}$ of $\mathrm{Na}_{2} \mathrm{~S}_{2} \mathrm{O}_{3} \cdot 5 \mathrm{H}_{2} \mathrm{O}$ in $1,000 \mathrm{c} \mathrm{c}$ of distilled water and allow to stand for 24 hours. Standardize against antimony metal chemically pure, using the same 
quantity of reagents and same procedure as under method. Each cubic centimeter is equivalent to approximately $0.006 \mathrm{~g}$ of antimony.

\section{METHOD}

Wash the alkaline sulphide solutions Nos. 1, 2, and 3 into a 600 c c beaker, and acidify with 5 c c of $\mathrm{HNO}_{3}$ (specific gravity 1.42) and 20 c c of $\mathrm{HCl}$ (specific gravity 1.19). Evaporate the solution to dryness on the steam bath. Dissolve the residue in $200 \mathrm{c} c$ of water, add $10 \mathrm{~g}$ of oxalic acid and $10 \mathrm{~g}$ of ammonium oxalate, and heat the solution until it is clear. Then pass $\mathrm{H}_{2} \mathrm{~S}$ gas through the boiling solution for 45 minutes. Filter off the precipitate, consisting of arsenic and antimony sulphides, and wash with hot water. Determine tin electrolytically in the filtrate, continuing the electrolysis until all the oxalic acid is decomposed and the solution becomes alkaline. Dissolve the deposit on the cathode with a small amount of $\mathrm{HCl}$ and examine qualitatively for tin.

Dissolve the sulphides of arsenic and antimony in $\mathrm{KOH}$ as usual, collecting the filtrate in a $500 \mathrm{c} \mathrm{c}$ Erlenmeyer flask. Add $50 \mathrm{c}$ c of $\mathrm{HCl}$ (specific gravity 1.19) and boil the solution until about $30 \mathrm{c} \mathrm{c}$ are left. Expel the arsenic as chloride. Now oxidize the solution with a pinch of $\mathrm{KClO}_{3}$ and boil until no more chlorine remains. Cool and add $5 \mathrm{c} \mathrm{c}$ of potassium iodide solution. Titrate the liberated iodine with $0.1 \mathrm{~N}$ sodium thiosulphate solution, using carbon disulphide as an indicator.

\section{DETERMINATION OF COPPER AND CADMIOM}

\section{SOLUTIONS REQUIRED}

Sodium chloride (solution No. 1).-Dissolve $1 \mathrm{~g}$ of $\mathrm{NaCl}$ in $100 \mathrm{~g}$ of distilled water.

Sodium chloride (solution No. 2).-Dissolve $10 \mathrm{~g}$ of $\mathrm{NaCl}$ in $100 \mathrm{~g}$ of distilled water.

Standard potassium cyanide.-Dissolve $2 \mathrm{~g}$ of $\mathrm{KCN}$ in a liter of distilled water and standardize against a known amount of copper as treated in the analysis.

Sodium carbonate (solution No. 1).-Dissolve $50 \mathrm{~g}$ of $\mathrm{Na}_{2} \mathrm{CO}_{3}$ in 1 liter of distilled water.

Sodium carbonate (solution No. 2).-Dissolve $\mathrm{Na}_{2} \mathrm{CO}_{3}$ in distilled water to saturation.

\section{METHOD}

(a) Determination where copper exceeds 0.0025 per cent.-Place the filter containing the sulphides in a $100 \mathrm{c}$ c beaker and add $20 \mathrm{c} c$ of $\mathrm{HNO}_{3}(1: 4)$. Heat with occasional stirring until the paper is thoroughly pulped and the sulphides are completely dissolved Filter into a 250 c c beaker. Dry the residue, which generally contains a small amount of copper, ignite in a porcelain crucible, boil with $5 \mathrm{c}$ c of $\mathrm{HNO}_{3}(1: 1)$, and wash into the main portion, keeping the volume below 100 c c. Render it strongly alkaline with ammonia, and add $5 \mathrm{~g}$ of potassium cyanide, then saturate it in the cold with $\mathrm{H}_{2} \mathrm{~S}$ gas. (Note 1.) Filter the solution and evaporate the filtrate to a volume of 20 to $30 \mathrm{c} \mathrm{c}$ in a 4 -inch porcelain casserole. Boil until solution is complete. Add $20 \mathrm{c}$ c of $\mathrm{H}_{2} \mathrm{SO}_{4}(1: 1)$ and evaporate the solution under a hood until dense fumes of $\mathrm{H}_{2} \mathrm{SO}_{4}$ escape. Cool, dilute, and warm until copper sulphate is all dissolved. Now filter, if necessary, into a $200 \mathrm{c}$ c beaker, render just alkaline with ammonia, make acid by the addition of $3 \mathrm{c}$ c of $\mathrm{HNO}_{3}$ per $100 \mathrm{c} \mathrm{c}$ solution, and electrolyze for copper.

Dissolve the precipitate of sulphides in the usual manner with $20 \mathrm{c} \mathrm{c}$ of $\mathrm{HNO}_{3}(1: 4)$. Add $1 \mathrm{c} \mathrm{c} \mathrm{NaCl}$ solution No. 1 to the solution, still containing the pulped filter, and digest for one-half hour. Filter off the $\mathrm{AgCl}$, wash, and reject. Make the filtrate, not exceeding $100 \mathrm{c} \mathrm{c}$, alkaline with a slight excess of $\mathrm{Na}_{2} \mathrm{CO}_{3}$ and add $5 \mathrm{~g}$ of $\mathrm{KCN}$. Digest for one hour. Filter and wash with $\mathrm{Na}_{2} \mathrm{CO}_{3}$ solution No. 1. Reject the precipitate of bismuth. Now add a few cubic centimeters of ammonium sulphide solution to the filtrate to precipitate any cadmium as yellow cadmium sulphide. Filter upon a weighed Gooch crucible and weigh as cadmium sulphide. (Note 2.)

Notes.-1. Copper remains in the solution while lead, silver, bismuth, and cadmium are precipitated.

2. If an appreciable amount of cadmium sulphide is found, it should be converted to and weighed as cadmium sulphate, according to method for cadmium under standard methods of chemical analysis of spelter, in B38 (see 683.0 , p. 1308).

(b) Determination where copper is less than 0.0025 per cent.-Place the paper containing the sulphides in a porcelain crucible, dry carefully, and ignite. When the carbon has been all burned off, cool and dissolve the residue in 5 to 10 of $\mathrm{HNO}_{3}(1: 1)$. After evaporating to a volume of 1 to $2 \mathrm{c} \mathrm{c}$, add $1 \mathrm{c} \mathrm{c}$ of $\mathrm{H}_{2} \mathrm{SO}_{4}(1: 1)$. Then evaporate the solution until fumes appear, cool, dilute, add a few drops of $\mathrm{NaCl}$ solution No. 2, and filter off the lead sulphate and silver chloride. Again evaporate the filtrate until fumes of $\mathrm{H}_{2} \mathrm{SO}_{4}$ appear, and when cold dilute and neutralize with $\mathrm{Na}_{2} \mathrm{CO}_{3}$ solution No. 2. Then add about 6 drops of concentrated ammonia and titrate the solution with standard $\mathrm{KCN}$ solution until the blue color is discharged.

The cadmium can be obtained by making this solution, titrated for copper, strongly alkaline, diluting a little, and adding $5 \mathrm{~g}$ of $\mathrm{KCN}$. Saturate the solution cold with $\mathrm{H}_{2} \mathrm{~S}$ gas, filter, discard the filtrate, and treat the precipitate for cadmium as described in method $(a)$.

\section{DETERMINATION OF IRON}

\section{METHOD}

Evaporate the filtrate containing iron, zinc, etc., to 100 c c and oxidize with a few drops of $\mathrm{HNO}_{3}$. Separate the iron with ammonia as usual, making two separations, and receive the filtrate in a $500 \mathrm{c}$ c Erlenmeyer flask. Redissolve the iron hydroxide with hot $\mathrm{HCl}(1: 1)$ or dilute $\mathrm{H}_{2} \mathrm{SO}_{4}$ and determine the iron volumetrically by any of the standard methods.

\section{DETERMINATION OF ZINC}

\section{SOLUTIONS REQUIRED}

Nitric acid (1 : 4).-Mix 200 c c of $\mathrm{HNO}_{3}$ (specific gravity 1.42) with $800 \mathrm{c}$ c of distilled water.

Sulphuric acid $(1: 1)$.-Carefully pour, with stirring, 500 c c of $\mathrm{H}_{2} \mathrm{SO}_{4}$ (specific gravity 1.84) into $500 \mathrm{c}$ c of distilled water.

Acidulated hydrogen sulphide water.-Add 20 c c of $\mathrm{HCl}$ (specific gravity 1.19) to $1,000 \mathrm{c} \mathrm{c}$ of distilled water and saturate with hydrogen sulphide.

Ammonium thiocyanate solution ( 2 per cent).Dissolve $20 \mathrm{~g}$ of $\mathrm{NH}_{4}$ CNS in $1,000 \mathrm{c} \mathrm{c}$ of distilled water.

Hydrochloric acid (1:3).-Mix 100 c c of $\mathrm{HCl}$ (specific gravity 1.19) and $300 \mathrm{c} \mathrm{c}$ of distilled water.

Standard zinc solution (0.1 $\mathrm{mg}$ of zinc per $c \mathrm{c}$ ).Dissolve exactly $0.1 \mathrm{~g}$ of Bureau of Standards pure zinc in $5 \mathrm{c} \mathrm{c}$ of $\mathrm{HCl}$ (specific gravity 1.19) and dilute to exactly $1,000 \mathrm{c} \mathrm{c}$ with distilled water.

Potassium ferrocyanide solution.-Dissolve $34.8 \mathrm{~g}$ of $\mathrm{K}_{4} \mathrm{Fe}(\mathrm{Cn})_{6} \cdot 3 \mathrm{H}_{2} \mathrm{O}$ in $1,000 \mathrm{c} \mathrm{c}$ of distilled water.

\section{METHOD}

Dissolve $222.23 \mathrm{~g}$ of the sample in $1,100 \mathrm{cc}$ of $\mathrm{HNO}_{3}$ (1 : 4), using a $1,300 \mathrm{c}$ c beaker. When the lead is dissolved, transfer the solution to a 2,000 c c graduated flask and add slowly $150 \mathrm{c}$ c of $\mathrm{HS}_{2} \mathrm{O}_{4}$ $(1: 1)$. Cool, fill the flask to the mark, and then pour the solution into a clean 3,000 c c flask pro- 
vided with a rubber stopper. Rinse the measuring flask with exactly $50 \mathrm{c}$ c of water, which is equivalent to the volume of lead sulphate which is present. Mix the solution thoroughly by shaking, allow the precipitate to settle and filter through a dry filter until 1,800 c c of filtrate has been obtained.

Place exactly $1,800 \mathrm{c}$ c of filtrate (equivalent to a $200 \mathrm{~g}$ charge) in a No. 9 porcelain evaporating dish and evaporate the solution to approximately 100 c c. Transfer the solution to a $600 \mathrm{c}$ c beaker, neutralize with ammonia, and then add $5 \mathrm{c} \mathrm{c}$ of $\mathrm{HCl}$ (specific gravity 1.19) for every $100 \mathrm{c}$ c of solution. Warm the solution and pass in a rapid current of hydrogen sulphide until it is saturated. Digest for 30 minutes on the steam bath, add an equal volume of water, and again saturate with hydrogen sulphide. Filter and wash with acidulated $\mathrm{H}_{2} \mathrm{~S}$ water.

Discard the precipitate and evaporate the filtrate in glassware containing no zinc (such as Pyrex) until the volume of the solution is approximately $100 \mathrm{c} \mathrm{c}$.

Neutralize the solution with ammonium hydroxide, add $5 \mathrm{~g}$ of citric acid, and warm until the acid is dissolved. Add small portions of calcium carbonate to the hot citric acid solution until about $1 \mathrm{~g}$ of calcium citrate has separated and then pass in a rapid current of $\mathrm{H}_{2} \mathrm{~S}$ as the solution is allowed to cool. Allow the solution to stand for from two to four hours, part of the time on a water bath, until the supernatant liquid is clear.

Collect the precipitate on a filter, wash with a 2 per cent solution of ammonium thiocyanate, and then dissolve the precipitate in hot dilute hydrochloric acid $(1: 3)$. If the solution has a reddish color (due to iron) the zinc must be reprecipitated as above. If the solution is clear, evaporate it to dryness on the steam bath, take up the residue in $3 \mathrm{c} \mathrm{c}$ of $\mathrm{HCl}$ (specific gravity 1.19), add $20 \mathrm{c} \mathrm{c}$ of water, and filter if not perfectly clear.

Transfer the solution (note 2) to a $50 \mathrm{c}$ c Nessler jar and dilute to $45 \mathrm{c} \mathrm{c}$. Prepare other Nessler jars containing $3 \mathrm{c}$ c of $\mathrm{HCl}$ (specific gravity 1.19), definite volumes of standard zinc solution, and diluted to $45 \mathrm{c}$ c. Add $5 \mathrm{c}$ c of potassium ferrocyanide solution to each jar, mix quickly, and compare the turbidities by viewing longitudinally as the jars are held over a sheet of fine print. Add more of the standard zine solution from a burette to the jar which approximates the turbidity of the unknown most closely, until the turbidities match each other, and calculate the percentage of zinc on the basis of a $200 \mathrm{~g}$ sample or the aliquot portion taken.

Nores.-1. All glassware that contains zinc must be avoided and in umpire work a blank test should be carried along with the test.

2. The whole solution can be used if the lead contains no more than 0.002 per cent of zinc. If more zinc is present it is best to take such an aliquot portion of the solution as will give approximately $4 \mathrm{mg}$ of zinc and then to add enough $\mathrm{HCl}$ to provide $3 \mathrm{c} \mathrm{c}$.

3. For further details concerning the turbidmetric test, consult the "Determination of Small Quantities of Zinc" by Mr. Bodansky, in J. Ind. Eng. Chem., vol. 13, pp. 696-697, 1921.

4. The addition of calcium carbonate with the formation of a precipitate of calcium citrate serves the purpose of giving a clear filtrate, and prevents the loss of colloidal sulphide.

\section{DETERMINATION OF NICKEL AND COBALT}

\section{SOLUTIONS REQUIRED}

Hydrogen sulphide wash water.-To each $100 \mathrm{c} \mathrm{c}$ of hydrogen sulphide water add $20 \mathrm{c}$ c neutral ammonium acetate.

\section{METHOD}

Render the filtrate just alkaline with ammonia and saturate with $\mathrm{H}_{2} \mathrm{~S}$. Heat to boiling and then make just acid with acetic acid, add $20 \mathrm{c}$ c of neutral ammonium acetate solution, and boil until the sulphides of nickel and cobalt separate out. Filter and wash with warm $\mathrm{H}_{2} \mathrm{~S}$ wash water. Dry the precipitate and paper in a porcelain crucible and carefully ignite. If there is an appreciable amount of residue after ignition, dissolve by boiling with 10 c c of aqua regia, wash into a $250 \mathrm{c}$ c beaker, add 10 c c of $\mathrm{H}_{2} \mathrm{SO}_{4}(1: 1)$, evaporate until fumes appear, cool, dilute to $200 \mathrm{c}$ c, make alkaline with ammonia, and add $15 \mathrm{c}$ c of concentrated ammonia. Then electrolyze the solution and weigh the nickel and cobalt as such.

Notes.-If the amount of nickel and cobalt is small it can be weighed as oxide.

If the filtrate from the nickel and cobalt sulphides shows a brown color, it indicates that the precipitation has not been complete. In this case render the solution ammoniacal and repeat the above process.

\section{UNITED STATES GOVERNMENT, DEPART- MENT OF COMMERCE, BUREAU OF STANDARDS, STANDARD SAMPLES, CIR- CULAR NO. 25 AND SUPPLEMENT, 1927}

\section{LEAD SAMPLE}

(Sample No. 49 is a standard sample of lead prepared and sold by the Bureau of Standards for use by industrial organizations and others as a standard for calibrating pyrometers and temperature-measuring instruments. Each sample is accompanied with a certificate giving the melting point of that particular sample.)

\subsection{Lead Ingots.}

\section{AMERICAN SOCIETY FOR TESTING MA. TERIALS, STANDARD SPECIFICATIONS FOR PIG LEAD, DESIGNATION B29-23, 1923}

\section{PIG LEAD}

$S C O P E$

1. These specifications are intended to cover lead in pig form, made from ore or similar raw materials, by processes of reduction and refining, and not produced from reworked material.

2. Under these specifications pig lead is considered in three grades, as follows: Grade I, corroding lead; Grade II, chemical lead; and Grade III, common lead.

\section{MANUFACTURE}

3. The maker shall use care to have each carload of as uniform quality as possible.

\section{CHEMICAL PROPERTIES AND TESTS}

\section{(A) Chemical Composition}

4. Corroding lead (Grade I) shall conform to the following requirements as to chemical composition:

\begin{tabular}{ll} 
Silver, not over & Per cent \\
Copper, not over & 0015 \\
Copper and silver together, not over & 0015 \\
Arsenic, not over & .0025 \\
Antimony and tin together, not over & 0015 \\
Zinc, not over & Iron, not over \\
Bismuth, not over & 0095 \\
\hline
\end{tabular}

Bismuth, not over.... 05

NoтE.-The maximum limits for bismuth, copper, and silver have been given, but it is not expected that any delivery will contain the maximum of all three.

5. Chemical lead (Grade II) is a designation that has been used for many years in the trade to describe the undesilverized lead produced from southeastern Missouri ores. This lead contains from 0.04 to 0.08 per cent of copper, from 0.005 to 0.015 per cent ( $11 / 2$ to $4 \frac{1}{2}$ ounces per ton) of silver and carries less than 0.005 per cent of bismuth. 
6. Common lead (Grade III) shall conform to the following requirements as to chemical composition:

(a) Desilverized lead.-

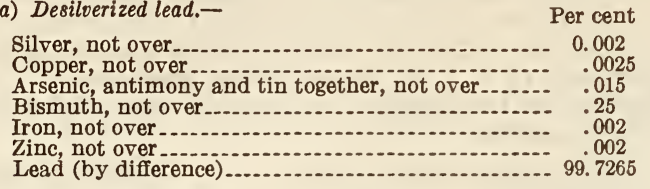

(b) Soft Missouri lead.-

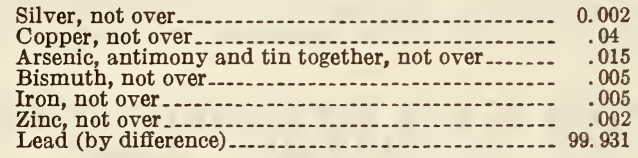

(B) Sampling and Analysis

7. (a) In sampling, a carload or less shall be considered a lot or unit. One pig shall be taken to represent each ton of lead in the lot. The pigs
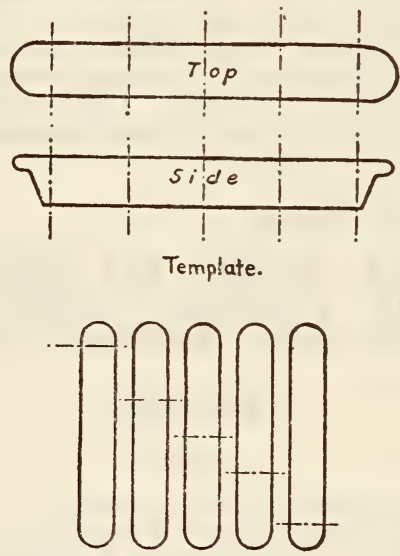

The Bars are Sampied in Sets of Five According to Template as Shc.rn above.

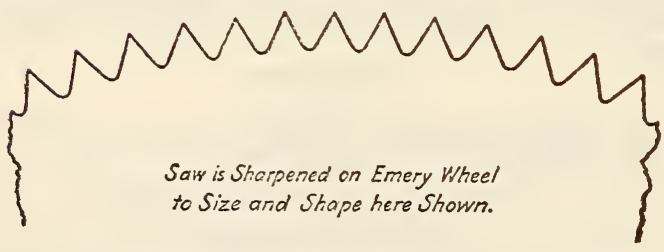

FIguRE 1124.-Saw method of sampling lead

thus taken shall be sampled by one of the following methods:

(1) By sawing completely through, as illustrated in Figure 1124.

(2) By punching completely through (if equipment permits) or punching halfway through from two opposite sides, as illustrated in Figures 1125 and 1126.

(3) By drilling at least halfway through.

(b) Whenever the surface of the pigs to be sampled is dirty, care shall be taken to see that none of the dirt or foreign material gets into the sample.
8. If the sample is taken by sawing, the sawings from all the pigs shall be thoroughly mixed and quartered and the sample for analysis drawn from the mixed material. Care shall be taken that the sawings are free from all extraneous material.

9. If the sample is taken by punching or drilling, the holes shall be spaced along a diagonal line from one corner of the pig to the other. Sampling in this
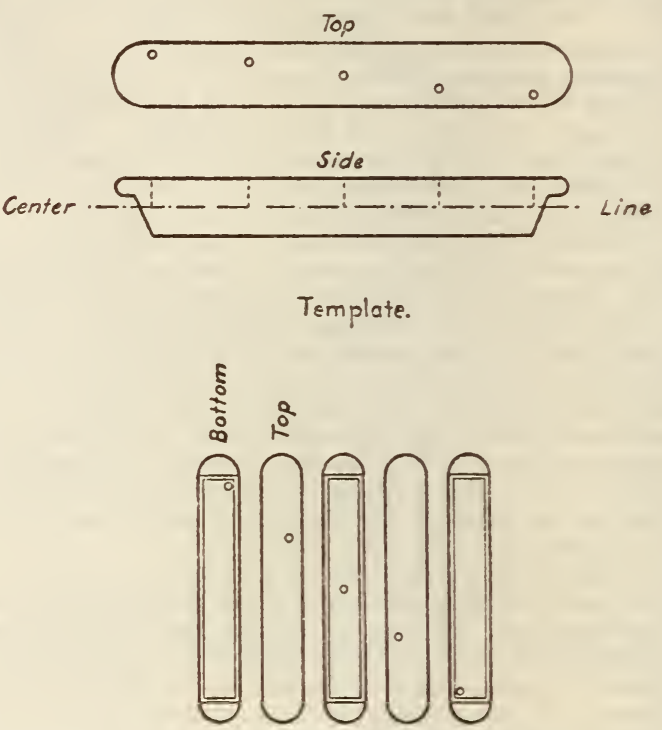

Figure 1125.-Punch method of sampling lead

The bars selected as samples are placed in a line with every other bar, bottom side up. The sampling is done according to tamplate in sets of five bars each, as indicated above. The punch must be driven halfway through the bar. In case a larger sample is desired, the bars are turned over and sampled on the other diagonal.

manner may be so arranged that one or more holes are made in each of several pigs of a group in such positions that they represent consecutive positions on the diagonal of a single pig. (See illustration in fig. 1125.)

(a) If punchings are taken, they may be carefully melted in a clean graphite crucible, and either granulated by carefully pouring into distilled water and thoroughly drying, or casting into thin slabs which shall be sawed completely through in several places and the sawdust treated as described in section 8.

(b) If drillings are taken, they may be clipped and mixed, and the sample for analysis drawn from the mixed material; or they may be melted as in the case of punchings.

10. The chemical analysis shall be made in accordance with the standard methods of chemical analysis of pig lead in A. S. T. M. B35 (see 651.0, p. 1272).

\section{MARKING}

11. A brand shall be cast or otherwise plainly marked upon each pig by which the maker and grade may be identified.

\section{CLAIMS}

(Conform to A.S. T. M. B6-18. See 683.1, p. 1310.) 


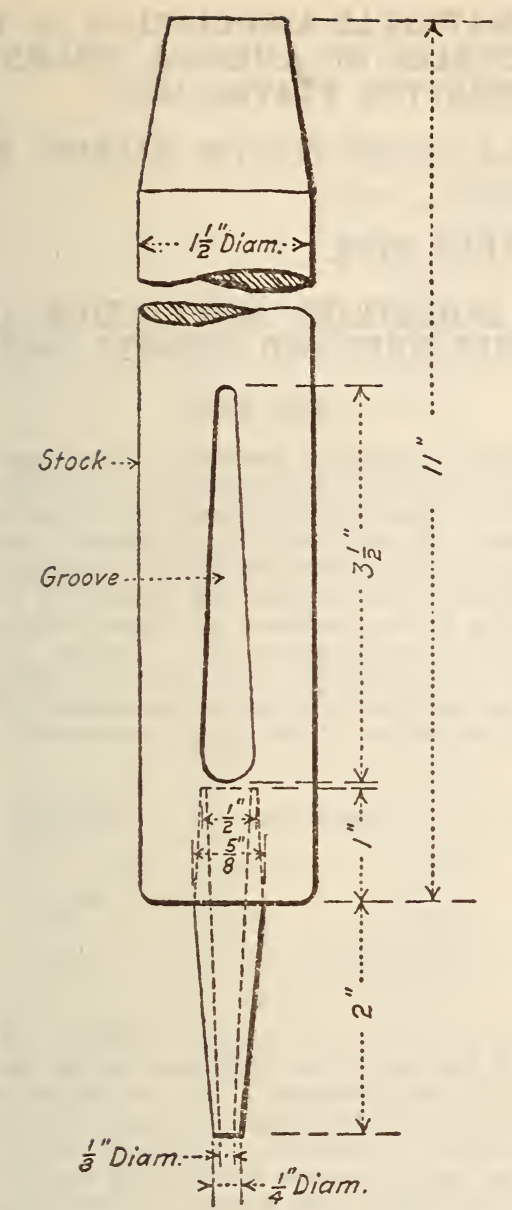

Figure 1126.-Sample punch for lead

UNITED STATES GOVERNMENT, FEDERAL SPECIFICATIONS BOARD, FEDERAL SPECIFICATION FOR LEAD; PIG, QQI-171, JUNE 23, 1931

\section{PIG IEAD}

A. APPIICABLE FEDERAL SPECIFICATIONS

A-1. Federal specification QQ-M-151, general specifications for metals, of the issue in effect on date of invitation for bids, shall form a part of this specification. (See 600.0, p. 3.)

\section{B. GRADES}

B-1. Pig lead shall be of the following grades, as specified: Grade A and grade B.

\section{MATERIAL AND WORKMANSHIP}

C-1. The material shall be as required in sections $D$ and $E$.

\section{GENERAL REQUIREMENTS}

D-1. Pig lead shall be clean and reasonably free from adhering foreign matter.

D-2. Pigs shall weigh from 80 to 110 pounds each, unless otherwise specified.

D-3. Each pig shall be legibly and indelibly marked with a brand, by which the maker may be identified.

\section{E. DETAIL REQUIREMENTS}

E-1. The chemical composition of the pig lead shall be respectively as follows:

Lead (minimum)
Grade A B

NoTE.-An analysis of each heat in a delivery shall be furnished by the contractor, showing the percentage of lead present.

\section{F. METHODS OF SAMPLING, INSPECTION, AND TESTS}

F-1. Check analyses shall be made by the inspector, or through him by any Government laboratory or other designated representative, without cost to the contractor.

F-2. From each 2 tons of grade $A$ and from each 10 tons of grade B pig lead presented for inspection, one pig shall be selected by the inspector, and an equal amount of clean, fine drillings or sawdust shall be taken from each pig representing a particular grade. The samples obtained shall be thoroughly mixed. The saw or drill shall penetrate the entire cross section, only the surface material being discarded. The sample representing a delivery shall weigh not less than 15 ounces.

\section{NOTES}

I-1. Grade A pig lead is intended for foundry use.

I-2. Grade B pig lead is intended for weights, ballasts, etc.

\subsection{BAR LEAD.}

(See lead ingots above.)

651.3 CASTINGS, LEAD.

UNITED STATES GOVERHMENT, FEDERAI SPECIFICATIONS BOARD, FEDERAL SPECIFICATION FOR BUILDERS' HARDWARE (NONTEMPLATE), FF-H-101, AUGUST 19, 1930

\section{BUILDERS' HARDWARE (NONTEMPLATE) SASH WEIGHTS}

(See 617.0 , p. 978, for general requirements; for detail requirements see 617.5, p. 1049.)

651.4 STRIPS AND SLEEVES, LEAD.

(No nationally recognized specifications available.)

651.5 SHEET LEAD.

UNITED STATES GOVERIMENT, DEPARTMENT OF COMMERCE, BUREAU OF STANDARDS, STANDARD THICKNESSES, WEIGHTS, AND TOLERANCES OF SHEET METAL (CUSTOMARY PRACTICE), CIRCULAR NO. 391, MARCH 24, 1931

\section{AMERICAN WIRE GAGE-SHEET LEAD}

(See 640.1, p. 1165.)

UNITED STATES GOVERNMENT, FEDERAI SPECIFICATIONS BOARD, FEDERAL SPECIFICATION FOR SHEET LEAD, NO. 308, JULY 6, 1925. (CURRENT DESIGNATION : QQ-L-201)

\section{GRADES}

There shall be two grades of sheet lead, as follows: Grade A and grade B. 


\section{MATERIAL AND WORKMANSHIP}

All sheet lead shall be flat and free from pits, dents, buckles, waves, scratches, grit, foreign matter and porosity. No reclaimed lead shall be used in the manufacture of grade A sheet lead.

\section{GENERAI REQUIREMENTS}

1. The tolerance for uniformity of thickness of any sheet of grade A sheet lead shall be not greater than 0.003 inch over or under the thickness corresponding to the weight per square foot specified in the contract or order.

2. No sheets will be accepted which show a variation of more than 5 per cent over or under the estimated weight, assuming 1 square foot of lead 1 inch thick to weight 59.5 pounds.

3. The tolerance for widths and lengths shall be one-eighth inch over or under that specified in contract or order.

\section{DETAIL REQUIREMENTS}

Chemical properties shall be as shown in the accompanying table.

\begin{tabular}{|c|c|c|c|c|c|c|c|c|}
\hline & $\begin{array}{l}\text { Lead, } \\
\text { mini- } \\
\text { mum }\end{array}$ & $\begin{array}{l}\text { Anti- } \\
\text { mony, } \\
\text { tin, } \\
\text { and } \\
\text { arsenic, } \\
\text { maxi- } \\
\text { mum }\end{array}$ & $\begin{array}{l}\text { Iron, } \\
\text { maxi- } \\
\text { mum }\end{array}$ & $\begin{array}{l}\text { Nickel } \\
\text { and } \\
\text { cobalt, } \\
\text { maxi- } \\
\text { mum }\end{array}$ & $\begin{array}{l}\text { Bis- } \\
\text { muth, } \\
\text { maxi- } \\
\text { mum }\end{array}$ & $\begin{array}{l}\text { Zinc, } \\
\text { maxi- } \\
\text { mum }\end{array}$ & $\begin{array}{l}\text { Cop- } \\
\text { per, } \\
\text { maxi- } \\
\text { mum }\end{array}$ & $\begin{array}{l}\text { Sil- } \\
\text { ver, } \\
\text { maxi- } \\
\text { mum }\end{array}$ \\
\hline $\begin{array}{l}\text { Grade A } \\
\text { Grade B... }\end{array}$ & $\begin{array}{c}\text { Per } \\
\text { cent } \\
99.90 \\
99.50\end{array}$ & $\begin{array}{c}\begin{array}{c}\text { Per } \\
\text { cent } \\
0.015\end{array} \\
-\end{array}$ & $\begin{array}{c}\text { Per } \\
\text { cent } \\
0.005\end{array}$ & $\begin{array}{c}\text { Per } \\
\text { cent } \\
0.008\end{array}$ & $\begin{array}{c}\text { Per } \\
\text { cent } \\
0.005\end{array}$ & $\begin{array}{c}\text { Per } \\
\text { cent } \\
0.002 \\
-\end{array}$ & $\begin{array}{c}\text { Per } \\
\text { cent } \\
0.060 \\
-\cdots\end{array}$ & \begin{tabular}{l} 
Per \\
cent \\
0.005 \\
\hdashline$\ldots .$.
\end{tabular} \\
\hline
\end{tabular}

\section{METHOD OF INSPECTION AND TESTS}

From each shipment of 500 pounds or less one chemical analysis consisting of at least 2 ounces shall be taken. The chemical results obtained from this sample shall be in accordance with the grade ordered under Section IV.

\section{PACKING AND MARKING}

1. Sheet lead shall be shipped in rolls properly slotted or boxed.

2. Shipments shall be marked with the name of the material, grade, name of contractor or manufacturer, quantity, and contract or order number under which delivery is made.

\section{ADDITIONAL INFORMATION}

Sheet lead will ordinarily be ordered on the weight-per-square-foot basis.

\subsection{IEAD ALIOYS.}

(Babbitt metal, see 692.1, p. 1319.)

(Solder, see 693.1, p. 1330.)

\section{AMERICAN SOCIETY FOR TESTING MA- TERIALS, STANDARD RULES GOVERN- ING THE PREPARATION OF MICRO- GRAPHS OF METALS AND ALLOYS, DESIGNATION E2-30, 1930}

\section{MICROGRAPH WORK}

(See 600.1, p. 20.)

\section{INTERNATIONAL ASSOCIATION OF ELEC- TROTYPERS OF AMERICA, STANDARDS IN PRINTING PLATES, 1930}

\section{FORMULA OF ELECTROTYPE BACKING METAL}

(See 651.9, p. 1279.)

\subsection{LEAD PIPE.}

\section{LEAD INDUSTRIES ASSOCIATION, LEAD PIPE SIZES AND WEIGHTS, 1932}

\section{IEAD PIPE}

The Lead Industries Association has approved a new standard of lead pipe sizes which is being adopted by the principal manufacturers as an aid to consumers. In addition to eliminating numerous variations in pipe sizes now found among lead pipe manufacturers, an outstanding feature of the new standard is that all sizes of lead pipe in the A, AA, and AAA classifications (or "strong," "extra strong," and "double extra strong") will safely withstand constant cold-water pressures of 50,75 , and 100 pounds per square inch, respectively.

\begin{tabular}{|c|c|}
\hline Classification & $\begin{array}{l}\text { Safe pres- } \\
\text { sures }\end{array}$ \\
\hline $\begin{array}{l}\text { A or } \mathrm{S} \\
\mathrm{AA} \text { or } \mathrm{X} S \mathrm{~S} \\
\mathrm{AAA} \text { or } \mathrm{X} \overline{\mathrm{X}} \mathrm{S}\end{array}$ & $\begin{array}{c}\text { Lbs./in. } \\
50 \\
75 \\
100\end{array}$ \\
\hline
\end{tabular}

Heretofore the safe working pressure of these classes of lead pipe has decreased as the diameter of the pipe has increased. The principal changes occur in the wall thicknesses of the sizes from $1 \frac{1}{4}$ to 2 inches. Alterations made in sizes below $1 \frac{1}{4}$ inches are of minor importance and small in number but have been made to introduce a uniformity which does not now exist among the lead pipe sizes of various manufacturers. The new standard is as follows:

\section{Lead Industries Association standard}

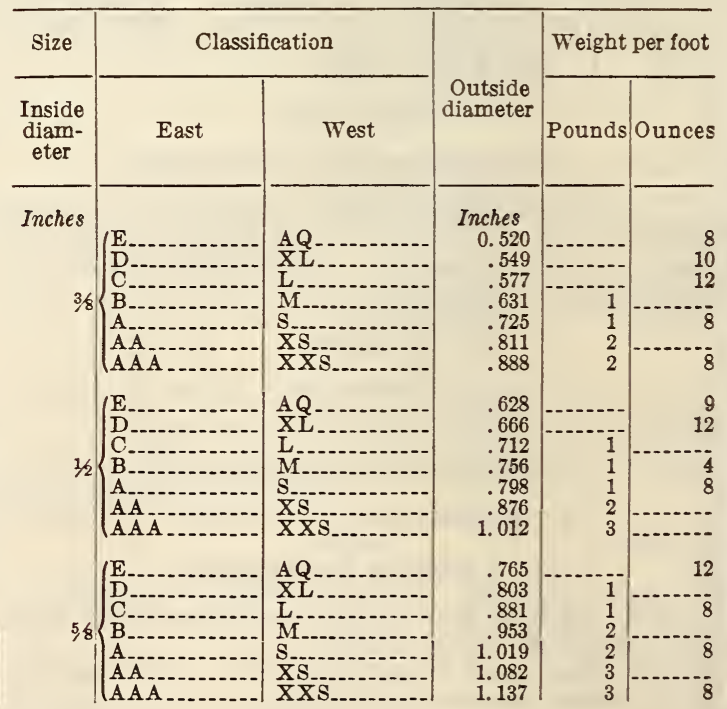


Lead Industries Association standard-Continued

\begin{tabular}{|c|c|c|c|c|c|}
\hline \multirow{2}{*}{$\begin{array}{c}\text { Size } \\
\begin{array}{l}\text { Inside } \\
\text {;iam- } \\
\text { eter }\end{array}\end{array}$} & \multicolumn{2}{|c|}{ Classification } & \multirow{2}{*}{$\begin{array}{l}\text { Outside } \\
\text { diameter }\end{array}$} & \multicolumn{2}{|c|}{ Weight per foot } \\
\hline & East & West & & Pounds & Ounces \\
\hline $\begin{array}{r}\text { Inches } \\
\\
3 / 4\end{array}$ & $\left\{\begin{array}{l}\mathrm{E} \\
\mathrm{D} \\
\mathrm{C} \\
\mathrm{B} \\
\mathrm{A} \\
\mathrm{AA} \\
\mathrm{AAA}\end{array}\right.$ & $\begin{array}{l}\text { AQ } \\
\text { XL. } \\
\text { L } \\
\text { M } \\
\text { S } \\
\text { XS } \\
\text { XXS... }\end{array}$ & $\begin{array}{c}\text { Inches } \\
0.906 \\
.940 \\
1.006 \\
1.068 \\
1.156 \\
1.212 \\
1.336\end{array}$ & $\begin{array}{l}1 \\
1 \\
1 \\
2 \\
3 \\
3 \\
4\end{array}$ & $\begin{array}{r}-5 \\
4 \\
12 \\
4 \\
8 \\
12\end{array}$ \\
\hline 1 & 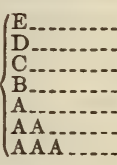 & 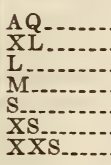 & $\begin{array}{l}1.192 \\
1.232 \\
1.284 \\
1.356 \\
1.428 \\
1.492 \\
1.596\end{array}$ & $\begin{array}{l}1 \\
2 \\
2 \\
3 \\
4 \\
4 \\
6\end{array}$ & $\begin{array}{r}10 \\
-8 \\
4 \\
12 \\
-.\end{array}$ \\
\hline $11 / 4$ & $\left\{\begin{array}{l}\mathrm{E} \\
\mathrm{D} \\
\mathrm{C} \\
\mathrm{B} \\
\mathrm{B} \\
\mathrm{A} \\
\mathrm{AA} \\
\mathrm{AAA}\end{array}\right.$ & $\begin{array}{l}\text { AQ } \\
\text { XL } \\
\text { L } \\
\text { M } \\
\text { S } \\
\text { XS } \\
\text { XX } \\
\end{array}$ & $\begin{array}{l}1.442 \\
1.486 \\
1.528 \\
1.592 \\
1.670 \\
1.765 \\
1.889\end{array}$ & $\begin{array}{l}2 \\
2 \\
3 \\
3 \\
4 \\
6 \\
7\end{array}$ & $\begin{array}{r}-8 \\
-12 \\
12 \\
-12\end{array}$ \\
\hline $11 / 2$ & $\left(\begin{array}{l}\mathrm{E} \\
\mathrm{D} \\
\mathrm{C} \\
\mathrm{B} \\
\mathrm{A} \\
\mathrm{AA} \\
\mathrm{AAA}\end{array}\right.$ & 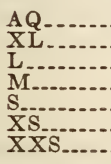 & $\begin{array}{l}1.740 \\
1.776 \\
1.830 \\
1.882 \\
1.984 \\
2.076 \\
2.272\end{array}$ & $\begin{array}{r}3 \\
3 \\
4 \\
5 \\
6 \\
8 \\
11\end{array}$ & $\begin{array}{r}-8 \\
4 \\
-8 \\
-4\end{array}$ \\
\hline $13 / 4$ & $\left\{\begin{array}{l}\text { D } \\
\text { C } \\
\mathrm{B} \\
\mathrm{A} \\
\mathrm{AA} \\
\mathrm{AAA}\end{array}\right.$ & $\begin{array}{l}\mathrm{XL} \\
\mathrm{L} \\
\mathrm{M} \\
\mathrm{M} \\
\mathrm{S} \\
\mathrm{S} \\
\mathrm{XS} \\
\mathrm{X} \mathrm{XS}\end{array}$ & $\begin{array}{l}2.024 \\
2.086 \\
2.146 \\
2.193 \\
2.404 \\
2.624\end{array}$ & $\begin{array}{r}4 \\
5 \\
6 \\
6 \\
10 \\
14\end{array}$ & $\begin{array}{r}-. . \\
12 \\
8 \\
12\end{array}$ \\
\hline 2 & $\left\{\begin{array}{l}\mathrm{E} \\
\mathrm{D} \\
\mathrm{C} \\
\mathrm{B} \\
\mathrm{A} \\
\mathrm{AA} \\
\mathrm{AAA}\end{array}\right.$ & $\begin{array}{l}\mathrm{A} Q \\
\mathrm{X} L \\
\mathrm{~L} \\
\mathrm{M} \\
\mathrm{S} \\
\mathrm{X}\end{array}$ & $\begin{array}{l}2.185 \\
2.284 \\
2.354 \\
2.410 \\
2.503 \\
2.751 \\
3.008\end{array}$ & \begin{tabular}{r|}
3 \\
4 \\
6 \\
7 \\
8 \\
13 \\
19
\end{tabular} & $\begin{array}{r}12 \\
12 \\
12 \\
8\end{array}$ \\
\hline $21 / 2$ & & & $\begin{array}{l}2.75 \\
3.00\end{array}$ & $\begin{array}{r}5 \\
10\end{array}$ & 10 \\
\hline 3 & & & $\begin{array}{l}3.25 \\
\text { 3. } 50\end{array}$ & $\begin{array}{r}6 \\
12\end{array}$ & 8 \\
\hline 4 & & & $\begin{array}{l}4.25 \\
4.50\end{array}$ & $\begin{array}{r}7 \\
16\end{array}$ & $\begin{array}{r}14 \\
6\end{array}$ \\
\hline 5 & & & $\begin{array}{l}5.25 \\
5.50\end{array}$ & $\begin{array}{r}9 \\
20\end{array}$ & $\begin{array}{r}14 \\
4\end{array}$ \\
\hline 6 & & & $\begin{array}{l}6.25 \\
6.50\end{array}$ & $\begin{array}{l}11 \\
24\end{array}$ & $\begin{array}{r}13 \\
2\end{array}$ \\
\hline
\end{tabular}

Lead pipe in sizes and weights per running foot other than indicated made to specification.

\subsection{MISCELLANEOUS MANUFACTURES OF LEAD.}

\section{INTERNATIONAL ASSOCIATION OF ELEC- TROTYPERS OF AMERICA, STANDARDS IN PRINTING PLATES, 1930}

\section{PRINTING PLATES}

\section{THICKNESS OF UNMOUNTED ELECTROTYPES}

(a) When made for use as "ad plates," 0.105 inches in thickness. Tolerance, plus or minus, 0.002 inch.

(b) When made for "patent bases," and recognizing as a standard base height 0.759 of an inch, plates are to be 0.154 inch thick, with 0.002 plus or minus tolerance.
For regular book printing this will allow an underlay of 0.005 of an inch, and in case the pressman does not wish to place underlays between plate and base (many do insist on this procedure), he can slip a sheet of 0.005 -inch tympan paper under the entire form.

Because there are "patent bases" in use that vary quite materially from the suggested standard, plates of special thickness must of necessity be manufactured, but it is the thought to build for the future in the manner suggested.

\section{THICKNESS OF CURVED ELECTROTYPES}

Naturally, with rotary presses of many differing cylinder diameters in use, the electrotyper must continue manufacturing curved plates to the specifications of his customers, but it seemed desirable to set up a standard of thickness to enable the press designers to intelligently cooperate with the electrotypers in the future.

Variations in cylinder diameters there will always be, of necessity, but the suggested standard of plate thickness is 0.187 of an inch, with a plus or minus tolerance of 0.002 of an inch, for cylinders of usual diameter; and a thickness of 0.250 of an inch for very large cylinders-also carrying the same tolerance.

\section{BEVEL OF EDGES OF "PATENT BASE" PLATES}

The makers of patent bases have apparently pretty well standardized the "hook" angles, so patent base plates should be so beveled that the "hook" will engage exactly with the edge of the plate.

A bevel of $20^{\circ}$ (which is $70^{\circ}$ from the horizontal) is recommended as standard.

\section{FORMULA OF ELECTROTYPE BACEING METAL}

Having in mind that deterioration through "drossing" begins as soon as backing metal is melted, and that what is termed as standard metal shall have certain well-defined characteristics, the recommendation for backing metal is 4 per cent tin, $3 \frac{1}{2}$ per cent antimony, $92 \frac{1}{2}$ per cent lead.

652.

\section{MERCURY}

(No nationally recognized specifications available.)

653.

\section{NICKEI}

653.0 GENERAL ITEMS.

\section{AMERICAN SOCIETY FOR TESTING MATERIALS, STANDARD METHODS OF CHEMICAL ANALYSIS OF NICKEL, DESIG- NATION B41-23, 1923}

\section{CHEMICAL ANALYSIS OF NICKEL}

\section{DETERMINATION OF IRON}

(a) By the cuprous chloride method.-

\section{SOLUTIONS REQUIRED}

Cuprous chloride solution.-Place into a 2-liter heavy glass bottle $400 \mathrm{~g} \mathrm{NaCl}, 100 \mathrm{c} \mathrm{c} \mathrm{HCl}$ and $20 \mathrm{~g}$ $\mathrm{Cu}_{2} \mathrm{Cl}_{2}$. Add 2 liters of warm water; shake well; place into the solution a few copper rods and let stand overnight in a cool place. After shaking, the solution is ready for use.

One cubic centimeter of $\mathrm{Cu}_{2} \mathrm{Cl}_{2}$ should reduce 0.003 to $0.005 \mathrm{~g}$ of iron. 


\section{METHOD}

Dissolve $10 \mathrm{~g}$ of nickel drillings in $100 \mathrm{c}$ c dilute $\mathrm{HNO}_{3}(1: 2)$ in a $600 \mathrm{c}$ c covered beaker, add 1 or 2 g $\mathrm{KClO}_{3}$, boil for 30 minutes, add about $100 \mathrm{c}$ c cold water, make alkaline with $\mathrm{NH}_{4} \mathrm{OH}$, and add about $50 \mathrm{c}$ c in excess. Boil a few minutes, filter, wash the precipitate with hot water two or three times.

Redissolve the precipitate in hot $\mathrm{HCl}$ (1:3). After the precipitate is dissolved make the solution alkaline with $\mathrm{NH}_{3} \mathrm{OH}$, boil a few minutes, filter, and wash with hot water. Dissolve the $\mathrm{Fe}(\mathrm{OH})_{3}$ in 30 c c hot $\mathrm{HCl}(1: 3)$ in a 24 -ounce flask, cool, add about $300 \mathrm{c}$ c cold water, and titrate with a standard $\mathrm{Cu}_{2} \mathrm{Cl}_{2}$ solution using two drops of a saturated solution of KCNS as an inside inclicator. Titrate to faint opalescence.

NotES.-The $\mathrm{Cu}_{2} \mathrm{Cl}_{2}$ solution should be standardized each time, before using, with $10 \mathrm{c} \mathrm{c}$ of standard ferric chloride, to which $5 \mathrm{c} \mathrm{c}$ o $\mathrm{HCl}$ is added, diluted to $300 \mathrm{c} \mathrm{c}$ with cold water, and titrated to faint $\mathrm{HCl}$ is added, diluted to $300 \mathrm{c}$ c with cold water, and titrated to faint
opalescence. The ferric chloride is made by dissolving $5 \mathrm{~g}$ of standard iron wire in $150 \mathrm{c} \mathrm{c}$ of $\mathrm{HCl}(1: 1)$; then $5 \mathrm{~g} \mathrm{KClO}_{3}$ is added to oxidize the iron. Add 1 liter of water and boil until all the free chlorine is driven off. Boil about eight hours, keeping the volume of the solution about 1 liter, then cool and make the solution up to 2 liters.

The above method is used for determining iron in material with less than 5 per cent iron, using a $1 \mathrm{~g}$ sample, and when less than 0.5 per cent using a $10 \mathrm{~g}$ sample.

The solution gains in $\mathrm{Cu}_{2} \mathrm{Cl}_{2}$, and should now and then be diluted with a solution containing 20 per cent of $\mathrm{NaCl}$ and 5 per cent of $\mathrm{HCl}$. If the solution should fall off in $\mathrm{Cu}_{2} \mathrm{Cl}_{2}$, add about $5 \mathrm{~g} \mathrm{Cu}_{2} \mathrm{Cl}_{2}$.

\section{(b) By potassium permanganate method.-}

\section{SOLUTIONS REQUIRED}

Standard potassium permanganate solution.Standardized by means of sodium oxalate (as furnished by the Bureau of Standards).

\section{METHOD}

Dissolve the $\mathrm{Fe}(\mathrm{OH})_{3}$ in dilute $\mathrm{H}_{2} \mathrm{SO}_{4}$ and reduce the iron with $\mathrm{Zn}$, and then titrate with $\mathrm{KMnO}_{4}$.

NotE.-This method is just as accurate as the cuprous chloride method providing $5 \mathrm{~g}$ of the drillings are taken.

\section{DETERMINATION OF CARBON BY THE DIRECT-COMBOSTION METHOD}

\section{APPARATUS}

Purifying train.-The purifying train should consist of a 5 -inch $\cup$ tube filled with glass beads and moistened with $\mathrm{H}_{2} \mathrm{CrO}_{4}$ solution, with a loose plug of glass wool in the forward end. This serves to oxidize any $\mathrm{SO}_{2}$ and to absorb the $\mathrm{SO}_{3}$ formed from the sulphur in the nickel drillings during combustion. This tube should be washed out daily and fresh glass wool inserted.

Material for lining boats. - The boat may be filled with ignited alumina. Alundum, " $R R$ Alundum, alkali-free, specially prepared for carbon determination," is also recommended.

Catalyzers.-The forward end of the platinum combustion tube should contain a plug of loosely wound platinum gauze, $5 \frac{1}{2}$ inches long, completely filling the bore of the tube and a similar roll 2 inches long, fitted with a loop, pushed in after the boat.

Combustion apparatus. - The apparatus may be heated by gas or electricity and combustion tubes may be of platinum, quartz, or porcelain glazed on one or both sides.

Boats or other containers of samples being burned.These may be of alundum, but porcelain, quartz, clay, and platinum are also recommended.

Absorption train. - It is recommended that the absorption train consist of a Geissler potash bulb with a calcium chloride tube attached.

\section{REAGENTS}

Oxygen.-Oxygen of not less than 97 per cent purity is recommended. Endeavor should be made to obtain oxygen which gives no blank since the correction for or elimination of this is troublesome and uncertain. For the most accurate work the blank should be completely eliminated by the use of a catalyzer before the furnace with a $\mathrm{CO}_{2}$ absnrbent interposed between the furnace and the catalyzer.

Potassium hydroxide solution.-A KOH solution (specific gravity 1.27) should be used as the absorbent for $\mathrm{CO}_{2}$ but the use of "Ascarite" is also recommended.

\section{FACTORS INFLUENCING RAPID COMBUSTION}

Size of particles of sample.-The finer the chips the better, except with samples which burn too vigorously. Particles too coarse to pass a 20 -mesh sieve are not recommended, nor long, curly drillings which will not pack closely. Oil, dust, and other foreign matter should be carefully excluded.

Manner of distributing sample in boat.-This is of considerable importance. With all samples, close packing in a small space is conducive to rapid combustion. In the case of samples which burn too vigorously, a satisfactory regulation may sometimes be attained by spreading the sample loosely over the lining in the boat.

Rate of admitting oxygen.-The valve controlling the supply of oxygen must be regulated so as to keep a slow current of gas passing through the absorption apparatus at all times and not to exceed three bubbles in a second.

\section{METHOD}

After having properly set up and tested the apparatus, fill the alundum boat with ignited alumina and make a V-shaped depression in the middle with a spatula, pressing the material against the sides of the boat. Ignite the boat and its contents strongly to expel any $\mathrm{CO}_{2}$ present and burn off any carbonaceous matter that the alumina may contain. Weigh out $3 \mathrm{~g}$ of nickel drillings which have been broken down so as to pass through a No. 20 sieve and transfer it to the boat. Introduce the boat into the combustion apparatus. Burn the carbon by starting a slow current of oxygen and lighting one of the burners at the forward end of the tube, and as soon as the tube is red hot increase the heat slowly by lighting the other burners. Nickel is burned at a temperature of $1,200^{\circ} \mathrm{C}$. for 20 minutes.

\section{DETERMINATION OF SULPHOR}

\section{SOLUTIONS REQUIRED}

Barium chloride.-A 20 per cent solution should be used.

\section{METHOD}

Weigh duplicate $10 \mathrm{~g}$ samples of drillings, place each into an $800 \mathrm{c}$ c casserole, and treat as follows: Add $3 \mathrm{~g}$ of $\mathrm{KClO}_{3}$, cover, and then add $200 \mathrm{c} \mathrm{c}$ strong $\mathrm{HNO}_{3}$. Place on steam bath until action has ceased and then evaporate to dryness on the hot plate. Cool and then add $50 \mathrm{c} \mathrm{c} \mathrm{HCl}$, and again evaporate to dryness. Repeat this operation. Take up with $150 \mathrm{c} \mathrm{c}$ water, add $8 \mathrm{c} \mathrm{c} \mathrm{HCl}$, heat to boiling, filter, and wash, taking care that the filtrate and washings do not exceed $300 \mathrm{c} c$ in volume. Heat the filtrate to boiling, add $25 \mathrm{c}$ c of 20 per cent $\mathrm{BaCl}_{2}$ solution and allow it to stand in a warm place overnight. Filter through a small double ashless filter paper and wash with rery dilute $\mathrm{HCl}$, and finally with hot water. Ignite in a small platinum crucible and then heat to bright redness for 20 minutes. Cool and weigh the $\mathrm{BaSO}_{4}$.

Notes. $-\mathrm{BaSO}_{4} \times 0.1373=$ sulphur.

Run a blank analysis, that is, take an $800 \mathrm{c} c$ casserole, add to it all the reagents and subject the contents to the same operations as the samples of drillings at the same time. Deduct any $\mathrm{BaSO}_{4} \mathrm{ob}$ tained from the result of the actual assay. If this blank amounts to $0.0040 \mathrm{~g}$, the reagents are unfit for the above analysis and the determinations should be repeated with other reagents. 
DETERMINATION OF NICKEL AND COBAIT

(a) By electrolysis.-

\section{APPARATUS}

Electrodes.-It is recommended that the cathode consist of a platinum cylinder 50 by $38 \mathrm{~mm}$ in diameter; with stem diameter $1 \frac{11}{2} \mathrm{~mm}$ and height $127 \mathrm{~mm}$.

The anode should consist of a spiral of iron wire $11 / 2 \mathrm{~mm}$ in diameter.

\section{METHOD}

Dissolve $2 \mathrm{~g}$ of nickel drillings in $30 \mathrm{c}$ c of $\mathrm{HNO}_{3}$ (1:1) in a $400 \mathrm{c}$ c covered beaker, add $30 \mathrm{c} \mathrm{c} \mathrm{HS}_{2} \mathrm{O}_{4}$ $(1: 1)$, and evaporate until fumes of $\mathrm{H}_{2} \mathrm{SO}_{4}$ are given off copiously. Dilute with $250 \mathrm{c}$ c of water. Boil, cool, and precipitate the copper with $\mathrm{H}_{2} \mathrm{~S}$. Filter into a $600 \mathrm{c}$ c plain beaker, wash the precipitate with hot water, boil the filtrate gently until about onethird of the volume is evaporated to remove the $\mathrm{H}_{2} \mathrm{~S}$. Add 10 e c $\mathrm{H}_{2} \mathrm{O}_{2}$ to oxidize the iron, cool, add $100 \mathrm{c} \mathrm{c} \mathrm{NH_{4 }} \mathrm{OH}$ water, then plate out the nickel and cobalt on a weighed platinum cylinder using a current of 0.6 ampere. It takes about 14 hours to plate out the nickel.

Make a replate on solution to recover the small amount of nickel which is left.

To make the replate, remove the beaker, add $\mathrm{H}_{2} \mathrm{SO}_{4}$ to the solution until acid, then add about 5 c c in excess, cool, add $60 \mathrm{c} \mathrm{c} \mathrm{NH}_{4} \mathrm{OH}$ water, then plate out nickel with a current of 1 ampere for two hours. Wash the cathode with water and alcohol, heat over an alcohol lamp, and when cool weigh. Increased weight equals nickel and cobalt.

The cathode on which the nickel and cobalt have been plated is placed in a 125 c c plain beaker. Dissolve the nickel and cobalt in dilute $\mathrm{HNO}_{3}$ and remove the cathode. Evaporate the solution to a syrupy consistency, cool, add 25 c c water, boil, neutralize with $\mathrm{K}_{2} \mathrm{CO}_{3}$ solution, make slightly acid with 50 per cent acetic acid, then add $10 \mathrm{c} \mathrm{c}$ of 50 per cent acetic acid and make the volume up to 50 c c. In another beaker, dissolve $25 \mathrm{~g} \mathrm{KNO}_{2}$ in 50 c c water and make acid with acetic acid. Add the nitrite solution to the solution containing the nickel and cobalt, while boiling. Let stand in a warm place overnight. Filter and wash the precipitate with a 5 per cent $\mathrm{KNO}_{2}$ solution made acid with acetic acid. The precipitate is transferred to a 300 c c beaker by washing with hot water and dilute $\mathrm{H}_{2} \mathrm{SO}_{4}$, add 20 c c $\mathrm{H}_{2} \mathrm{SO}_{4}(1: 1)$ and evaporate until fumes of sulphuric acid are given off copiously. Dilute with $150 \mathrm{c}$ c water. Boil, cool, and add 100 c c $\mathrm{NH}_{4} \mathrm{OH}$ water. Plate overnight, using a current of 0.5 ampere. Weigh as cobalt.

Subtract the percentage of cobalt found from the percentage of cobalt and nickel. The difference is nickel.

Note.- When manganese is present, a solution containing $30 \mathrm{c} \mathrm{c}$ of water and $2 \mathrm{~g}$ of $\mathrm{Na}_{2} \mathrm{O}_{2}$ is added to the alkaline solution just before plating out the nickel and cobalt and also just before making th replate on the solution.

653.1 NICKEL METAL, INGOTS, AND SHOT. AMERICAN SOCIETY FOR TESTING MATERIALS, STANDARD SPECIFICATIONS FOR NICKEL, DESIGNATION B39-22, 1922

\section{NICKEI}

SCOPE

1. (a) These specifications cover four grades of virgin nickel; that is, nickel made from ore or matte or similar raw material by refining processes, and not produced from melted metal, as follows: Electrolytic; X shot; A shot; ingot. (b) The uses for which these four grades of virgin nickel ar intended to be especially suitable are as follows:

Electrolytic, suitable for the manufacture of the highest grades of malleable alloys.

$X$ shot, suitable for the manufacture of nonferrous alloys and nickel steel.

$A$ shot, suitable for the manufacture of anodes.

Ingot, suitable for the manufacture of open-hearth and electric-furnace nickel steel.

\section{MANUFACTURE}

2. The maker shall use care to have each carload of as uniform quality as possible.

\section{CHEMICAL REQUIREMENTS AND TESTS}

3. The nickel shall conform to the following chemical requirements:

(a) Electrolytic. -

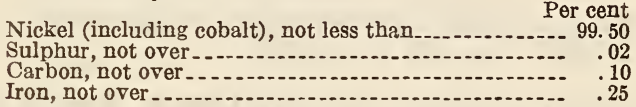

(b) $X$ shot.-

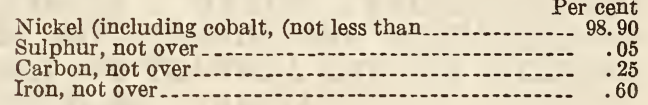

(c) A shot.-

Nickel (including cobalt), not less than Per cent

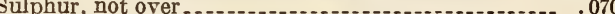

Carbon, not over---

Iron, not over

(d) Ingot.-

Nickel (including cobalt), not less than
It shall be reasonably free from surface corrosion and adhering foreign matter.

4. (a) Electrolytic. ${ }^{15}$ - In all shipments cathodes shall be taken representing 5 per cent of the total weight shipped, but in no case shall the number of cathodes taken as samples be less than three.

The cathodes taken as samples shall be drilled by templet. In the case of a shipment represented by a sufficient number of cathodes, one line of holes on the templet shall be used for each cathode; that is, cathode No. 1 shall be drilled at the base; cathode No. 2 shall be drilled with one line of hose farther up, etc., the resultant sample being a representation of the total sample of one whole cathode. In cases where the cathodes in the sample are less than the number of lines of holes in the templet, the sample shall be drilled, using sufficient, lines and fractions thereof to insure at all times a sample representative of one whole cathode. In all cases the drilling shall be done through the cathodes. The drillings obtained in the above manner shall be thoroughly mixed and divided by split sampler until the sample is about $100 \mathrm{~g}$.

(b) $X$ shot.-In receiving shipments of shot, the following method of sampling shall govern: All barrels from the same melt shall be opened and a sample taken from each barrel-top, center, and bottom. These barrel samples shall be mixed quartered down to 8 ounces, and broken into small pieces. This broken sample shall then be quartered down to $100 \mathrm{~g}$, only material passing through a No. 20 sieve being used.

(c) $A$ shot.-The sample shall be taken as described in paragraph (b).

15 The requirement for carbon in electrolytic nickel covers both contained and mechanically attached carbon from the starting sheet and it should be recognized that while lower values can be obtained by separating the cathode sheets from the starting sheet and pickling before drilling, this method of taking the sample is ouiside of these specifications. 
(d) Ingot.-Ingot nickel shall be sampled according to carloads whenever possible. From each carload 50 ingots shall be taken at random. In case of less than carload shipments, 5 per cent of the ingots shall be taken as a sample, but in no case shall the number of ingots taken as a sample be less than 10. (The drilling of the ingots is identical with that for electrolytic cathodes, specified above.)

\section{METHODS OF ANALYSIS}

5. The chemical analysis shall be made in accordance with the standard methods of chemical analysis of nickel. (See A. S. T. M. B41 (653.0, p. 1279).)

\section{PACKAGES}

6. All packages and barrels shall be marked with a brand by which the maker and grade can be identified.

\section{CLAIMS}

(Conform with specifications for spelter in A. S. T. M. B6. See 683.1, p. 1310.)

\section{UNITED STATES GOVERNMENT, FEDERAL SPECIFICATIONS BOARD, FEDERAI SPECIFICATION FOR NICKEL FOR RE- MELTING, NO. 371, JANUARY 25, 1926. (CURRENT DESIGNATION: QQ-N-301)}

\section{NICKEL FOR REMELTING}

\section{GENERAL SPEDIFICATIONS}

1. General specifications for metals, Federal Specifications Board specification No. 339a, (current designation: $Q Q-M-151$ ), in effect on date of proposal, shall form part of this specifications. (See 600.0, p. 3.)

\section{GRADES}

2. This specification covers three grades of nickel as follows: Grade A, grade B, and grade C.

\section{MATERIAL}

3. The material shall be made by refining processes, or may be produced from remelted metal.

\section{GENERAL REQUIREMENTS}

4. Nickel shall be furnished in cathodes, plates, pigs, ingots, cubes, or shot form, as specified in the contract or purchase order, except grade A, which shall be furnished in cathodes only.

5. Nickel shall be reasonably free from surface oxidation and adhering foreign matter.

\section{DETAIL REQUIREMEITS}

(The chemical requirements in respect to percentages of nickel, iron, carbon, and sulphur for grades A, B, C, and are the same as for electrolytic, $\mathrm{X}$ shot, and A shot, respectively, in A. S. T. M. B39, except for slight difference in content of sulphur in $\mathrm{B}$ and iron in $\mathrm{A}$ given above. This specification also provides for the following additional components:)

\begin{tabular}{|c|c|c|c|}
\hline Grade & $\begin{array}{l}\text { Copper, } \\
\text { maximum }\end{array}$ & $\begin{array}{l}\text { Silicon, } \\
\text { maximum }\end{array}$ & $\begin{array}{l}\text { Cobalt, } \\
\text { maximum }\end{array}$ \\
\hline $\begin{array}{l}\stackrel{\mathrm{A}}{\mathrm{B}} \\
\mathrm{C}\end{array}$ & $\begin{array}{c}\text { Per cent } \\
0.10 \\
.25 \\
.25\end{array}$ & $\begin{array}{c}\text { Per cent } \\
0.00 \\
.15 \\
.25\end{array}$ & $\begin{array}{c}\text { Per cent } \\
0.60 \\
1.00 \\
1.00\end{array}$ \\
\hline
\end{tabular}

\section{METHOD OF INSPECTION AND TEST}

6. An analysis of each shipment of nickel shall be furnished by the contractor, showing the percentages of the elements specified.

7. In the case of shot or cubes, representative samples shall be taken from at least 10 per cent of the containers in a lot or shipment, consisting of at least 4 ounces for every 150 pounds. In the case of cathodes, plates, pigs, or ingots, a sample shall be obtained from one piece for every 5,000 pounds or fraction thereof of metal, although not more than 10 pieces need be taken from a carload.

\section{PACKING AND MARKING}

8. Nickel cathodes, plates, pigs, or ingots may be shipped in bulk, in sealed box cars unless otherwise specified.

9. Nickel cathodes, plates, pigs, or ingots shall be legibly and indelibly marked with the maker's trade-mark or brand, and the grade number.

10. Nickel shot and cubes when loaded for shipment shall be packed in bags, boxes, barrels, or other suitable containers unless otherwise specified.

11. Containers shall be legibly and indelibly marked with the manufacturer's name, trademark, or brand; the weight, grade number, and contract or purchase order number.

Notes.-Grade A nickel is furnished in the form of the original cathode, and consists of thin plates with a peculiar warty surface on one side.

Grades B and C, while usually furnished in the form of the original cathode, may be procured in the form of cubes or ingots.

\subsection{NICKEL BARS.}

(No nationally recognized specifications available.)

\subsection{NICKEL MANUFACTURES.}

\subsection{NICKEL SHEETS.}

(No nationally recognized specifications available.) 653.32 NICKEL WIRE.

(No nationally recognized specifications available.)

654. NICKEL-COPPER ALLOYS (MONEL METAL)

654.0 GENERAL ITEMS.

AMERICAN SOCIETY FOR TESTING MATERIALS, STANDARD RULES GOVERNING THE PREPARATION OF MICROGRAPHS OF METALS AND ALLOYS, DESIGNATION E2-30, 1930

\section{MICROGRAPHS}

(See 600.1, p. 20.)

UNITED STATES GOVERNMENT, FEDERAL SPECIFICATIONS BOARD, FEDERAL SPECIFICATION FOR COPPER-NICKEL ALLOY RODS, BARS, SHAPES, PLATES, SHEETS, AND STRIPS, No. 585, DECEMBER 8, 1928. (CURRENT DESIGNATION: QQ-C-541)

\section{COPPER-NICKEL ALLOY RODS, BARS, SHAPES,} PLATES, SHEETS, AND STRIPS

\section{GENERAL SPECIFICATIONS}

1. United States Government general specification for metals, Federal Specifications Board speci- 
fication No. 339a (current designation: QQ-M-151), in effect on date of issuance of proposals, shall form part of this specification. (See 600.1 p. 3.)

\section{GRADE}

2. This specification covers one grade of coppernickel alloy in the form of:

Rods. (See also 654.53.)

Bars. (See also 654.53.)

Shapes. (See also 654.53.)

Plates. (See also 654.52.)

Sheets. (See also 654.52.)

Strips. (See also 654.52.)

\section{MATERIAL}

3. The material shall be copper-nickel alloy containing approximately 65 per cent nickel and 28 per cent copper, and shall be manufactured from raw material of good quality. Scrap of known and approved composition may be used.

4. The material shall be uniform in quality, clean sound, smooth, commercially straight or flat, and free from pipes, slivers, laps, cracks, twists, seams, scale, damaged ends or edges, buckles, or other injurious defects.

5. The material may be furnished in the hot finished or cold finished annealed condition, unless otherwise specified.

\section{DETAIL REQUIREMENTS}

6. The chemical properties shall be as specified in Table 1.

\section{TABLE 1.-Chemical properties}

\begin{tabular}{|c|c|c|c|c|c|c|}
\hline $\begin{array}{c}\text { Copper, } \\
\text { mini- } \\
\text { mum }\end{array}$ & $\begin{array}{c}\text { Nickel, } \\
\text { mini- } \\
\text { mum }\end{array}$ & $\begin{array}{c}\text { Iron, } \\
\text { maxi- } \\
\text { mum }\end{array}$ & $\begin{array}{c}\text { Alumi- } \\
\text { num, } \\
\text { maxi- } \\
\text { mum }\end{array}$ & $\begin{array}{c}\text { Man- } \\
\text { ganese, } \\
\text { maxi- } \\
\text { mum }\end{array}$ & $\begin{array}{c}\text { Carbon, } \\
\text { maxi- } \\
\text { mum }\end{array}$ & $\begin{array}{c}\text { Silicon, } \\
\text { maxi- } \\
\text { mum }\end{array}$ \\
\hline $\begin{array}{c}\text { Per cent } \\
23.0\end{array}$ & $\begin{array}{c}\text { Per cent } \\
60.0\end{array}$ & $\begin{array}{c}\text { Per cent } \\
3.5\end{array}$ & $\begin{array}{c}\text { Per cent } \\
0.5\end{array}$ & $\begin{array}{c}\text { Per cent } \\
3.5\end{array}$ & $\begin{array}{c}\text { Per cent } \\
0.3\end{array}$ & $\begin{array}{c}\text { Per cent } \\
0.5\end{array}$ \\
\hline
\end{tabular}

7. The physical properties shall be as specified in Table 2.

TABLE 2.-Physical properties

\begin{tabular}{|c|c|c|c|c|}
\hline $\begin{array}{r}\text { Diamet } \\
\text { (i. }\end{array}$ & $\begin{array}{l}\text { Tensile } \\
\text { strength, } \\
\text { mini- } \\
\text { mum }\end{array}$ & $\begin{array}{l}\text { Yield } \\
\text { point, } \\
\text { mini- } \\
\text { mum }\end{array}$ & $\begin{array}{l}\text { Elonga- } \\
\text { tion } \\
\text { in } 2 \\
\text { inches, } \\
\text { mini- } \\
\text { mum }\end{array}$ & $\begin{array}{c}\text { Cold } \\
\text { bend } a\end{array}$ \\
\hline 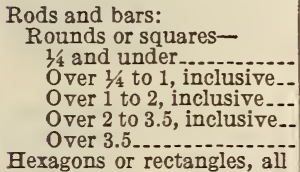 & $\begin{array}{r}\text { Lbs./in. }{ }^{2} \\
75,000 \\
80,000 \\
85,000 \\
80,000 \\
80,000\end{array}$ & $\begin{array}{r}\text { Lbs./in. }{ }^{2} \\
30,000 \\
40,000 \\
48,000 \\
35,000 \\
40,000\end{array}$ & $\begin{array}{r}\text { Per cent } \\
30.0 \\
30.0 \\
30.0 \\
35.0 \\
32.0\end{array}$ & $\begin{array}{r}\text { Degrees } \\
180 \\
180 \\
180 \\
180 \\
180\end{array}$ \\
\hline Hexagons or rectangles, all & 80,000 & 40,000 & 30.0 & 180 \\
\hline $\begin{array}{l}\text { Shapes, plates, sheets, and } \\
\text { strips: } \\
\text { Under } 0.25 \\
0.25 \text { and over }\end{array}$ & $\begin{array}{l}(b) \\
60,000\end{array}$ & $\begin{array}{l}(b) \\
25,000\end{array}$ & $\begin{array}{l}(b) \\
25.0\end{array}$ & ${ }^{(b)} 120$ \\
\hline
\end{tabular}

a Radius equal to diameter or thickness of test bar.

$b$ No requirements.

8. Material in which injurious defects are revealed by manufacturing operations subsequent to acceptance shall be rejected. It shall be replaced by the contractor without cost to the purchaser.

\section{METHODS OF INSPECTION AND TESTS}

9. An analysis of each melt or lot of material shall be furnished by the contractor, showing the percentages of the elements designated.
10. Check analysis may be made by the inspector, or through him by any Government laboratory or other designated representative, and without cost to the contractor.

11. Samples for check analyses shall be obtained from each lot of 5,000 pounds or fraction thereof of each size in the shipment. In the case of thin sheets and strips, at least 10 representative pieces shall be taken. In all other cases at least four representative pieces shall be taken. From the pieces so taken, equal amounts of clean chips shall be

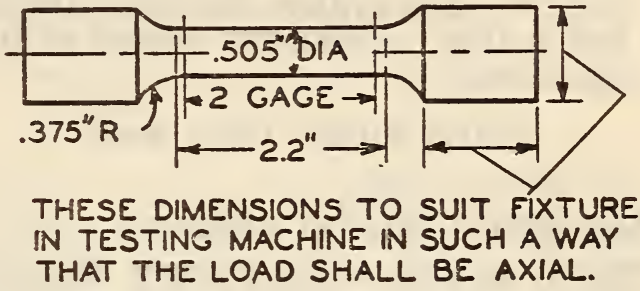

FIGURE 1127.-Standard tension test specimen

obtained. These chips shall be combined and thoroughly mixed. The inspector shall forward for analysis not less than 2 ounces of the resultant mixture.

12. Unless otherwise specified in the contract or purchase order, one tension and one hammer test shall be made for every lot of 5,000 pounds or fraction thereof of each size in the shipment.

13. All rods and bars shall withstand being hammered hot to a fine point. Such test shall be made when deemed advisable by the inspector.

14. All rods, bars, and shapes may be pulled in full size when practicable. When a machined specimen becomes necessary, enough metal may be removed from the pull section to meet the limitations of the testing machine, or the specimen may be machined to the form and dimensions of Figure 1127.

\section{FULL THICKNESS} OF MATERIAL $=T$

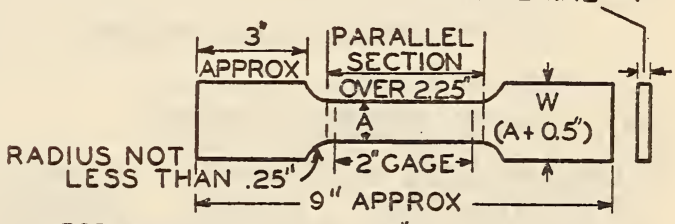

FOR MATERIAL UP TO $0.5^{\prime \prime}$ THICK, $A=0.5$ FOR MATERIAL OVER $0.5^{n}$ THICK, A $=T$

FIGURE 1128.- -Standard flat tension test specimen

15. For rods, bars, and shapes, up to $1 \frac{11}{2}$ inches in diameter or thickness, the axis of the test specimen shall coincide with the central axis of the piece; over $1 \frac{1}{2}$ inches, the axis shall be located midway between the center and surface of the piece.

16. The test specimen for plates, sheets, and strips shall be machined to the form and dimensions of Figure 1128, and may be taken either with or across the grain.

(Packing and marking of shipments are identical with that for copper rods, bars, shapes, plates, sheets, and strips given under 641.0, p. 1172.)

Nores.-Round, square, hexagonal, etc., sections are classed as rods. Rectangular sections having greater width than thickness are classed as bars. All other sections are classed as shapes.

Copper-nickel alloy, rolled or shaped, is suitable for parts requiring great strength or incorrodibility, such as propeller blade bolts, air pump and condenser bolts, pump rods, and sheathing for airplane propellers. For purposes of calculation, the weight per cubic inch of this material may be taken as 0.323 pound. 
654.1 INGOTS AND SHOT, NICKEL-COPPER METAL.

(No nationally recognized specifications available.)

654.2 BARS, NICKEL-COPPER.

UNITED STATES GOVERNMENT, FEDERAL SPECIFICATIONS BOARD, FEDERAL SPECIFICATION FOR COPPER-NICKEL ALLOY RODS, BARS, SHAPES, PLATES, SHEETS, AND STRIPS, NO. 585, DECEMBER 8, 1928. (CURRENT DESIGNATION: QQ-C-541)

\section{COPPER-NICKEL ALLOY BARS}

(See 654.53, p. 1285.)

654.3 STRIPS, NICKEL-COPPER.

UNITED STATES GOVERNMENT, DEPARTMIENT OF COMMERCE, NATIONAL BUREAU OF STANDARDS, STANDARD THICKNESSES, WEIGHTS, AND TOLERANCES OF SHEET METAL (CUSTOMARY PRACTICE), CIRCULAR NO. 391, MARCH 24, 1931

AMERICAN WIRE GAGE-COPPER-NICKEL STRIPS (See 640.1, p. 1165.)

UNITED STATES GOVERNMENT, FEDERAL SPECIFICATIONS BOARD, FEDERAL SPECIFICATIONS FOR COPPER-NICKEL ALLOY RODS, BARS, SHAPES, PLATES, SHEETS, AND STRIPS, NO. 585, DECEMBER 8, 1928. (CURRENT DESIGNATION: QQ-C-541)

\section{COPPER-NICKEL ALLOY STRIPS}

(See 654.52, p. 1285.)

654.4 SHAPES, NICKEL-COPPER.

UNITED STATES GOVERNMENT, FEDERAL SPECIFICATIONS BOARD, FEDERAL SPECIFICATION FOR COPPER-NICKEL ALIOY RODS, BARS, SHAPES, PLATES, SHEETS, AND STRIPS, NO. 585, DECEMBER 8, 1928. CURRENT DESIGNATION: QQ-C-541)

\section{COPPER-NICKEL ALLOY SHAPES}

(See 654.53, p. 1285.)

654.5 MANUFACTURES OF NICKEL-COPPER.

654.51 CASTINGS AND FORGINGS, NICKELCOPPER.

SOCIETY OF AUTOMOTIVE ENGINEERS, NONFERROUS METAL SPECIFICATIONS, SPECIFICATION NO. 42, WHITE NICKEL BRASS, MARCH, 1922

\section{WHITE NICKEL BRASS}

(See 655.9, p. 1288.)
UNITED STATES GOVERNMENT, FEDERAL SPECIFICATIONS BOARD, FEDERAL SPECIFICATION FOR COPPER-NICKEL ALLOY CASTINGS NO. 578, JUNE 28, 1928. (CURRENT DESIGNATION : QQ-C551)

\section{COPPER-NICKEL ALLOY CASTINGS}

(In respect to general specifications, general requirements, methods of inspection and tests, and special packing provisions, this specification is substantially the same as F. S. B. QQ-B-691. (See 646.41, p. 1252.) It differs from $Q Q-B-691$ in making no reference to encircling each repair with a ring of white paint, and in the following additional particulars:)

GRADE

1. This specification covers one grade of coppernickel alloy in the form of castings.

\section{GENERAL REQTIREMENTS}

2. The castings shall be a copper-nickel alloy, containing approximately 65 per cent nickel and 28 per cent copper.

\section{DETAIL REQUIREMENTS}

3. The chemical properties shall be as follows:

\begin{tabular}{|c|c|c|c|c|c|}
\hline $\begin{array}{c}\text { Copper, } \\
\text { mini- } \\
\text { mum }\end{array}$ & $\begin{array}{c}\text { Nickel, } \\
\text { mini- } \\
\text { mum }\end{array}$ & $\begin{array}{c}\text { Iron, } \\
\text { maxi- } \\
\text { mum }\end{array}$ & $\begin{array}{c}\text { Alum- } \\
\text { inum, } \\
\text { maxi- } \\
\text { mum }\end{array}$ & $\begin{array}{c}\text { Man- } \\
\text { maxi- } \\
\text { mum }\end{array}$ & $\begin{array}{c}\text { Carbon } \\
\text { plus } \\
\text { silicon, } \\
\text { maxi- } \\
\text { mum }\end{array}$ \\
\hline $\begin{array}{c}\text { Per cent } \\
23.0\end{array}$ & $\begin{array}{c}\text { Per cent } \\
60.0\end{array}$ & $\begin{array}{c}\text { Per cent } \\
3.5\end{array}$ & $\begin{array}{c}\text { Per cent } \\
0.5\end{array}$ & $\begin{array}{c}\text { Per cent } \\
3.5\end{array}$ & $\begin{array}{c}\text { Per cent } \\
2.0\end{array}$ \\
\hline
\end{tabular}

4. The physical requirements are as follows:

\begin{tabular}{|c|c|c|}
\hline $\begin{array}{c}\text { Tensile } \\
\text { strength, } \\
\text { minimum }\end{array}$ & $\begin{array}{c}\text { Yield point, } \\
\text { minimum }\end{array}$ & $\begin{array}{c}\text { Elongation } \\
\text { in 2 inches, } \\
\text { minimum }\end{array}$ \\
\hline $\begin{array}{c}\text { Lbs./in.2} \\
65,000\end{array}$ & $\begin{array}{c}\text { Lbs./in. }{ }^{2} \\
32,500\end{array}$ & $\begin{array}{c}\text { Per cent } \\
25.0\end{array}$ \\
\hline
\end{tabular}

METHODS OF INSPECTION AND TESTS

5. Check analyses of the castings may be made by the inspector, or through him by any Government laboratory or other designated representative, without cost to the contractor.

6 . The drillings for check analyses of castings shall be taken from the test coupons or from representative castings selected by the inspector. The drillings shall be taken from sound metal below the surface.

7. The test coupon or separately cast bar shall be of the dimensions shown in Figure 1123. The test specimen machined therefrom shall conform to the dimensions shown in Figure 129 (p. 247) except that the length of the shank shall be 2.25 inches instead of 2.2 inches.

\section{PACKING AND MARKING}

8. All patterns furnished by the purchaser shall be properly cared for, and when being returned to the purchaser shall be suitably boxed or crated by the contractor to prevent damage or breakage. Before a shipment from the foundry, the patterns shall be inspected by a representative of the purchaser, and report shall be made by him if they are found to be in an unsatisfactory condition. 
654.52 SHEETS AND PLATES, NICKEL-COPPER. ONITED STATES GOVERNMENT, DEPARTMENT OF COMMERCE, NATIONAL BUREAU OF STANDARDS, STANDARD THICKNESSES, WEIGHTS, AND TOLERANCES OF SHEET METAL (CUSTOMARY PRACTICE), CIRCULAR NO. 391, MARCH 24, 1931

\section{AMERICAN WIRE GAGE-COPPER-NICKEL SHEETS AND PLATES}

(See 640.1, p. 1165.)

UNITED STATES GOVERNMENT, FEDERAL SPECIFICATIONS BOARD, FEDERAL SPECIFICATION FOR COPPERNICKEL ALLOY RODS, BARS, SHAPES, PLATES, SHEETS, AND STRIPS, NO. 585, DECEMBER 8, 1928 . (CURRENT DESIGNATION: QQ-C-541)

\section{COPPER-NICKEL ALLOY PLATES, SHEETS, STRIPS}

(For general specifications, grade, material, detail requirements, physical properties, methods of inspection and tests, packing and marking, and notes, see 654.0 , p. 1282. Otherwise the specifications for copper-nickel alloy plates, sheets, and strips are identical with those for copper plates, sheets, and strips in F. S. B. QQ-C-501 (see 641.21, p. 1177), with the following exceptions:

1. Sheets and strips in long length shall not exhibit sidewise bend or curvature in excess of 0.25 inch per foot.

2. The permissible variations in thickness for plates, sheets, and strips shall be as shown in Table 1.

TABLE 1.-Permissible variations in thickness of plates, sheets, and strips

\begin{tabular}{|c|c|c|}
\hline Thickness (in inches) & Widths & $\begin{array}{l}\text { Permissi- } \\
\text { ble varia- } \\
\text { tions }\end{array}$ \\
\hline $\begin{array}{l}0.02 \text { and less } \\
\text { Over } 0.02 \text { to } 0.04 \text {, inclusive } \\
\text { Over } 0.04 \text { to } 0.065 \text {, inclusive. } \\
\text { Over } 0.065 \text { to } 0.08 \text {, inclusive } \\
\text { Over } 0.08 \text { to } 0.1 \text {, inclusive } \\
\text { Over } 0.1 \text { to } 0.12 \text {, inclusive } \\
\text { Over } 0.12 \text { to } 0.25 \text {, inclusive }\end{array}$ & 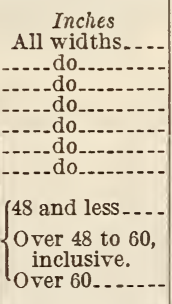 & $\begin{array}{l}\text { Inch } \\
\text { 土0. } 002 \\
\pm .003 \\
\pm .004 \\
\pm .005 \\
\pm .006 \\
\pm .007 \\
\pm .008 \\
\text { Per cent } \\
\pm 5 \\
+5 \\
\pm 7 \\
+5 \\
-8\end{array}$ \\
\hline
\end{tabular}

654.53 RODS, NICKEL-COPPER.

UNITED STATES GOVERNMENT, FEDERAL SPECIFICATIONS BOARD, FEDERALSPECIFICATION NO. 269 A, FOR RODS, WELDING, NONFERROUS, FOR GAS WELDING, QQ-R-5\%1, MAY 3, 1932

\section{COPPER-NICKEL ALLOY RODS}

(For general specifications, types, material, general requirements, detail requirements, methods of inspection, tests, and chemical analysis; packing and marking of shipinents; and notes see 600.0, p. 3.)
1. Copper-nickel alloy rods may be used for general welding of copper-nickel alloy castings and gray-iron castings.

2. Welding rods shall conform to the following chemical requirements:

\section{Type $F$}

Per cent

Copper, minimum

Nickel, minimum

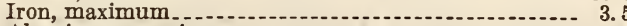

Aluminum, maximum.

Manganese, maximum ................................... 35

Carbon, maximum

Silicon, maximum -.-.--

3. Standard sizes of copper-nickel alloy rods for gas welding are:

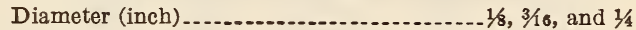

UNITED STATES GOVERNMENT, FEDERAI SPECIFICATIONS BOARD, FEDERAI SPECIFICATION FOR COPPER-NICKELALIOY RODS, BARS, SHAPES, PLATES, SHEETS, AND STRIPS, NO. 585, DECEMBEP 8, 1928. (CURRENT DESIGNATION : QQ-C-541)

\section{COPPER-NICKEL ALLOY RODS, BARS, SHAPES}

(For general specifications, grade, material, detail requirements, physical properties, methods of inspection and tests, packing and marking, and notes, see 654.0, p. 1282. Otherwise the specifications for copper-nickel-alloy rods, bars, and shapes are identical with those for copper rods, bars, and shapes in F. S. B. QQ-C-501 (see 642.11, p. 1180) with the following exceptions:)

PERMISSIBLE VARIATIONS IN DIAMETER OR THICKNESS OF RODS AND BARS

1. Unless otherwise specified, rods and bars, measured on their diameters or between parallel faces, shall not vary from the specified dimensions by more than the amount shown in Table 1.

TABLE 1.-Permissible variations in diameter or thickness of rods and bars

\begin{tabular}{|c|c|c|}
\hline \multirow{2}{*}{ Diameter or thickness (in inches) } & \multicolumn{2}{|c|}{$\begin{array}{l}\text { Permissible varia- } \\
\text { tions }\end{array}$} \\
\hline & Plus & Minus \\
\hline Over 1 to 2 , inclusive.-. & $\begin{aligned} 0.010 \\
.031\end{aligned}$ & $\begin{array}{r}0.010 \\
.016\end{array}$ \\
\hline Over 2 to 4 , inclusive.... & .047 & .031 \\
\hline $\begin{array}{l}\text { Over } 4 \\
\text { Fiexagonsand squares, hot-rolled: }\end{array}$ & . 125 & \\
\hline $\begin{array}{l}1.2 \text { and less } \\
\text { Over } 1.2\end{array}$ & .016 & .016 \\
\hline $\begin{array}{l}\text { Hexagons and squares, forged: } \\
1.5 \text { and less }\end{array}$ & .031 & 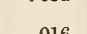 \\
\hline 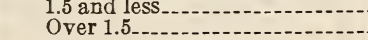 & .031 & .016 \\
\hline
\end{tabular}

2. Unless exact lengths are specified, rods and bars over 1 inch in diameter or thickness shall be furnished in stock lengths as follows (but in no case shall more than 80 per cent of lengths shorter than the maximum specified below be accepted):

Over 1 to 2 inches, inclusive, diameter or thickness, 8 to 20 feet.

Over 2 to 3 inches, inclusive, diameter or thickness, 6 to 15 feet.

Over 3 inches diameter or thickness, as specified in the contract or purchase order. 
3. For shapes, the permissible variation shall be as specified in the contract or purchase order.

\subsection{WIRES AND CABLES, NICKEL-COPPER.}

(No nationally recognized specifications available.)

654.59 MISCELLANEOUS MANUFACTURES OF NICKEL-COPPER.

\section{UNDERWRITERS' LABORATORIES, STAND. ARD FOR WINDOW CLEANERS' BELTS AND ANCHORS, MARCH 30, 1930}

\section{DESIGN AND CONSTRECTION}

BELTS

Window cleaners' belts shall be so designed and constructed that they may be readily put on by the wearer and will allow the wearer the requisite degree of freedom in use.

Belt hardware or fittings shall be forgings made of brass, Monel metal, or a copper-nickel alloy equivalent to Monel metal in physical properties. Belt terminal fittings shall be provided with a locking device to prevent accidental removal from anchors in service.

NoтE.-Brass forgings shall contain not less than 58 per cent nor more than 62 per cent copper with not less than 36 per cent zinc. Material used for such forgings shall be extruded brass.

Copper-nickel alloys shall show the following chemical analysis:

Copper, not less than
Nickel, not less than
Iron, not more than
Aluminum, not more than
Manganese, not more than
Carbon, not more than
Silicon, not more than

Ropes or straps secured to eyes or rings shall be provided with metal thimbles to prevent wear. Rope, if used, shall be not smaller than 1/2-inch yacht manila or its equivalent.

Belts shall be made with a sufficient degree of uniformity so that parts will be interchangeable. Each belt shall be marked so as to identify the manufacturer and the model or type.

\section{ANCEORS}

Anchors shall be forgings made of brass, Monel metal, or copper-nickel alloy as specified in "NoTE" above, under belts.

Anchors shall be of the twin or double-head type designed for attachment to window frames or mullions. Anchors for wood frames or mullions shall be designed for attachment by bolts not less than three-eighths inch diameter made of the same material as the anchor and provided with washers and lock nuts or equivalent. Anchors for metal frames shall be designed for attachment by at least two machine screws not less than five-sixteenths inch in diameter and of the same material as the anchor. Each anchor shall be marked so as to identify the manufacturer and his type or model. The identification marking shall be visible after installation.

\section{PERFORMANCE TESTS}

The waist band, ropes of the belt, and all interconnecting parts shall be separately subjected to tension tests and shall show a strength of at least 1,000 pounds.

Anchors shall withstand a load of at least 1,500 pounds applied by a special test terminal loaded in a direction $30^{\circ}$ from the face and $30^{\circ}$ to the side of the vertical center line of the anchor.
(The full text of this standard includes requirements on the installation of anchors on metal window frames and on wooden window frames.)

\section{NICKEL - COPPER - ZINC ALLOYS (NICKEL SILVER AND GERMAN SILVER)}

\subsection{GENERAL ITEMS.}

UNITED STATES GOVERNMENT, FEDERAL SPECIFICATIONS BOARD, FEDERAL SPECIFICATION FOR SILVER, NICKEL, RODS, BARS, SHAPES, PLATES, SHEETS, AND STRIPS (GERMAN SILVER), NO. 468, JANUARY 25, 1927. (CURRENT DESIGNATION : $Q Q-N-321)$

\section{GERMAN SILVER RODS, BARS, SHAPES, PLATES, SHEETS AND STRIPS}

(Requirements covering general specifications conform to F. S. B. QQ-M-151 (see 600.0, p. 3). Material and workmanship, packing and marking, and notes conform to F. S. B. QQ-C-501 (see $641.0, p .1172$ ). It differs from the latter in that no tests are required-only the usual optional check analyses-and in the following respects:)

\section{GRADE}

1. This specification covers two grades of nickelsilver, grades $\mathrm{A}$ and $\mathrm{B}$, in the following forms:

Rods. (See also 655.5.)

Bars. (See also 655.5.)

Shapes. (See also 655.5.)

Plates. (See also 655.2.)

Sheets. (See also 655.2.)

Strips. (See also 655.2.)

\section{DETAII REQUIREMENTS}

2. The chemical requirements are as shown in Table 1.)

\section{TABLE 1.-Chemical composition}

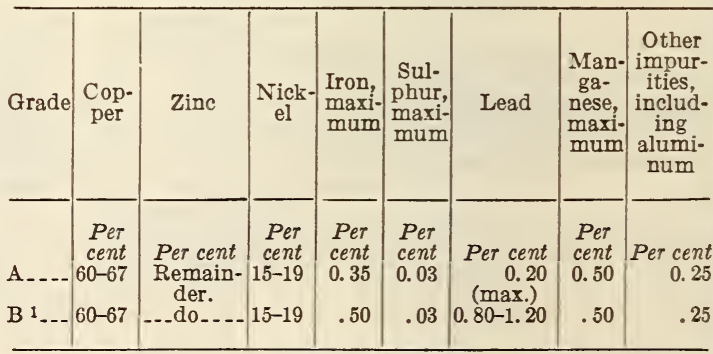

1 Grade B shall contain from 0.80 to 1.20 per cent lead.

\section{PHYSICAL PROPERTIES}

3. Nickel silver shall conform to such requirements as to physical properties as may be specified in the contract or purchase order.

Notes.-Nickel silver, or German silver, is suitable for trimmings and other parts requiring a metallic white finish and resistance to atmospheric corrosion. It is very ductile, can be rolled, hammered. stamped, or drawn, and at the same time is hard, tough, and not easily corroded. For purposes of calculation, the weight per cubic inch may be taken as 0.316 pound.

Grade A nickel silver machines readily with a fine finish, but will yield curly chips, as does naval brass. Grade B nickel silver is freecutting, similar to commercial or leaded brass, and is particularly suited to screw-machine work.

The material covered by this specification is not intended for electrical resistance work. 
AMERICAN SOCIETY FOR TESTING MATERIALS, STANDARD RULES GOVERNING THE PREPARATION OF MICROGRAPHS OF METALS AND ALLOYS, DESIGNATION E2-30, 1930

\section{MICROGRAPHS}

(See 600.1, p. 20.)

655.1 BARS, NICKEL-COPPER-ZINC ALLOY. UNITED STATES GOVERNMENT, FEDERAL SPECIFICATIONS BOARD, FEDERAL SPECIFICATION FOR SILVER, NICKEL, RODS, BARS, SHAPES, PLATES, SHEETS, AND STRIPS (GERMAN SILVER), NO. 468, JANUARY 25, 192\%. (CURRENT DESIGNATION : $Q Q-N-321)$

\section{GERMAN SILVER BARS}

(See 655.5 , p. 1288 , including references therein to various Federal specifications.)

655.2 SHEETS AND PLATES, NICKEL-COPPERZINC ALLOY.

UNITED STATES GOVERNMENT, DEPARTMENT OF COMMERCE, NATIONAL BUREAU OF STANDARDS, STANDARD THICKNESSES, WEIGHTS, AND TOLERANCES OF SHEET METAL (CUSTOMARY PRACTICE), CIRCULAR NO. 391, MARCH 24, 1931

AMERICAN WIRE GAGE-NICKEL-SILVER SHEETS AND PLATES

(See 640.1, p. 1165.)

UNITED STATES GOVERNMENT, FEDERAL SPECIFICATIONS BOARD, FEDERAL SPECIFICATION FOR SILVER, NICKEL, RODS, BARS, SHAPES, PLATES, SHEETS, AND STRIPS (GERMAN SILVER), No. 468, JANUARY 25, 192\%. (CURRENT DESIGNATION : QQ-N-321)

GERMAN SILVER PLATES, SHEETS, AND STRIPS

(Requirements covering general specifications conform to F. S. B. QQ-M-151. See 600.0, p 3. For grade, chemical composition, physical properties, and notes on German silver see F. S. B. QQ-N-321 under 655.0, p. 1286 . Material and workmanship, packing and marking, and notes conform to F. S. B. QQ-C-501, except that no tests are required-only the usual optional check analyses-and there is no reference to manufacture, hammering hot to a fine point, and forging stock.)
TABLE 4.-Permissible variations in thickness of sheets and strips (plus or minus)

\begin{tabular}{|c|c|c|c|c|}
\hline $\begin{array}{l}\text { Thick- } \\
\text { ness }\end{array}$ & $\begin{array}{l}\text { Up to } 6 \\
\text { inches } \\
\text { wide, in- } \\
\text { clusive }\end{array}$ & $\begin{array}{l}\text { Over } 6 \text { to } \\
9 \text { inches } \\
\text { wide, in- } \\
\text { clusive }\end{array}$ & $\begin{array}{l}\text { Over } 9 \text { to } \\
14 \text { inches } \\
\text { wide, in- } \\
\text { clusive }\end{array}$ & $\begin{array}{l}\text { Over } 14 \text { to } \\
20 \text { inches } \\
\text { wide, in- } \\
\text { clusive }\end{array}$ \\
\hline $\begin{array}{c}\text { Inch } \\
0.460 \\
.4096 \\
.3648 \\
.3249 \\
.2893\end{array}$ & $\begin{array}{c}\text { Inch } \\
0.0054 \\
.0053 \\
.0051 \\
.0050 \\
.0049\end{array}$ & $\begin{array}{c}\text { Inch } \\
0.0056 \\
.0055 \\
.0054 \\
.0053 \\
.0051\end{array}$ & $\begin{array}{c}\text { Inch } \\
0.0059 \\
.0058 \\
.0056 \\
.0055 \\
.0054\end{array}$ & $\begin{array}{c}\text { Inch } \\
0.0061 \\
.0060 \\
.0059 \\
.0058 \\
.0056\end{array}$ \\
\hline $\begin{array}{l}.2576 \\
.2294 \\
.2043 \\
.1819 \\
.1620\end{array}$ & $\begin{array}{l}.0048 \\
.0046 \\
.0045 \\
.0044 \\
.0043\end{array}$ & $\begin{array}{l}.0050 \\
.0049 \\
.0048 \\
.0046 \\
.0045\end{array}$ & $\begin{array}{l}.0053 \\
.0051 \\
.0050 \\
.0049 \\
.0048\end{array}$ & $\begin{array}{l}.0055 \\
.0054 \\
.0053 \\
.0051 \\
.0050\end{array}$ \\
\hline $\begin{array}{l}.1443 \\
.1285 \\
.1144 \\
.1019 \\
.0907\end{array}$ & $\begin{array}{l}.0041 \\
.0040 \\
.0039 \\
.0038 \\
.0036\end{array}$ & $\begin{array}{l}.0044 \\
.0043 \\
.0041 \\
.0040 \\
.0039\end{array}$ & $\begin{array}{l}.0046 \\
.0045 \\
.0044 \\
.0043 \\
.0041\end{array}$ & $\begin{array}{l}.0049 \\
.0048 \\
.0046 \\
.0045 \\
.0044\end{array}$ \\
\hline $\begin{array}{l}.0808 \\
.0719 \\
.0640 \\
.0570 \\
.0508\end{array}$ & $\begin{array}{l}.0035 \\
.0034 \\
.0033 \\
.0031 \\
.0030\end{array}$ & $\begin{array}{l}.0038 \\
.0036 \\
.0035 \\
.0034 \\
.0033\end{array}$ & $\begin{array}{l}.0040 \\
.0039 \\
.0038 \\
.0036 \\
.0035\end{array}$ & $\begin{array}{l}.0043 \\
.0041 \\
.0040 \\
.0039 \\
.0038\end{array}$ \\
\hline $\begin{array}{l}.0452 \\
.0403 \\
.0359 \\
.0320 \\
.0284\end{array}$ & $\begin{array}{l}.0029 \\
.0028 \\
.0026 \\
.0025 \\
.0024\end{array}$ & $\begin{array}{l}.0031 \\
.0030 \\
.0029 \\
.0026 \\
.0025\end{array}$ & $\begin{array}{l}.0034 \\
.0033 \\
.0031 \\
.0029 \\
.0026\end{array}$ & $\begin{array}{l}.0036 \\
.0035 \\
.0033 \\
.0030 \\
.0028\end{array}$ \\
\hline $\begin{array}{l}.0253 \\
.0225 \\
.0201 \\
.0179 \\
.0159\end{array}$ & $\begin{array}{l}.0023 \\
.0021 \\
.0020 \\
.0019 \\
.0018\end{array}$ & $\begin{array}{l}.0024 \\
.0023 \\
.0021 \\
.0020 \\
.0019\end{array}$ & $\begin{array}{l}.0025 \\
.0024 \\
.0023 \\
.0021 \\
.0020\end{array}$ & $\begin{array}{l}.0026 \\
.0025 \\
.0024 \\
.0023 \\
.0021\end{array}$ \\
\hline $\begin{array}{l}.0142 \\
.0126 \\
.0112 \\
.0100 \\
.0089\end{array}$ & $\begin{array}{l}.0016 \\
.0015 \\
.0014 \\
.0014 \\
.0013\end{array}$ & $\begin{array}{l}.0018 \\
.0016 \\
.0015 \\
.0015 \\
.0014\end{array}$ & $\begin{array}{l}.0019 \\
.0018 \\
.0016 \\
.0016 \\
.0015\end{array}$ & $\begin{array}{l}.0020 \\
.0019 \\
.0018 \\
.0018 \\
.0016\end{array}$ \\
\hline $\begin{array}{l}.0079 \\
.0070 \\
.0063 \\
.0056 \\
.0050\end{array}$ & $\begin{array}{l}.0013 \\
.0011 \\
.0011 \\
.0010 \\
.0010\end{array}$ & $\begin{array}{l}.0014 \\
.0013 \\
.0013 \\
.0011 \\
.0011\end{array}$ & $\begin{array}{l}.0015 \\
.0014 \\
.0014 \\
.0013 \\
.0013\end{array}$ & $\begin{array}{l}.0016 \\
.0015 \\
.0015 \\
.0014 \\
.0014\end{array}$ \\
\hline
\end{tabular}

1. The permissible variations in thickness for plates, sheets, and strips of all thicknesses and widths not covered in Table 4 shall be those of the nearest smaller thickness of the corresponding width.

\subsection{SHAPES AND CASTING, NICKEL-} COPPER-ZINC ALLOY.

AMERICAN MARINE STANDARDS COMMITTEE, LOCKS AND ACCESSORIES FOR SHIP DOORS, H No. 61-1930

\section{WHITE BRONZE}

(See 617.21, p. 1004.) 
AMERICAN MARINE STANDARDS COMMITTEE, BOLTS AND CATCHES FOR SHIP DOORS, H NO. 62-1930

\section{WHITE BRONZE}

(See 617.61, p. 1052.)

UNITED STATES GOVERNMENT, FEDERAL SPECIFICATIONS BOARD, FEDERAL SPECIFICATION FOR BUILDERS' HARDWARE (NONTEMPLATE), FF-H-101, AUGUST 19,1930

\section{BUILDERS' HARDWARE (NONTEMPLATE)}

(For applicable Federal specifications; types, grades, and classes; material and workmanship; general requirements; detail requirements; packaging, packing, and marking; notes; and table of standard finishes. See 617.0,p.978. For chromium plating nickel plating, and zinc coatings; and methods of inspection and tests. See 600.3, p. 70.)

(Specific commodities, such as locks, latches, hinges, bolts, etc., will be found under their respective commodity designations. For a list of these commodities see under detail requirements in 617.0, p. 979.)

UNITED STATES GOVERNMENT, FEDERAL SPECIFICATIONS BOARD, FEDERAL SPECIFICATION FOR SILVER, NICKEI, RODS, BARS, SHAPES, PLATES, SHEETS, AND STRIPS (GERMAN SILVER), NO. 468, JANUARY 25, 192\%. (CURRENT DESIGNATION : QQ-N-321

\section{GERMAN SILVER SHAPES}

(See 655.5 , p. 1288, including references therein to various Federal specifications.)

655.4 STRIPS, NICKEL-COPPER-ZINC ALIOY.

UNITED STATES GOVERNMENT, FEDERAL SPECIFICATIONS BOARD, FEDERAI SPECIFICATION FOR SILVER, NICKEL, RODS, BARS, SHAPES, PLATES, SHEETS, AND STRIPS (GERMAN SILVER), NO. 468, JANUARY 25, 192\%. (CURRENT DESIGNATION : QQ-N-321)

\section{GERMAN SILVER STRIPS}

(See 655.2, p. 1287, including references therein to various Federal specifications.)

655.5 WIRES AND RODS, NICKEL-COPPERZINC ALLOY.

UNITED STATES GOVERNMENT, FEDERAL SPECIFICATIONS BOARD, FEDERAI SPECIFICATION FOR SILVER, NICKEL, RODS, BARS, SHAPES, PLATES, SHEETS, AND STRIPS (GERMAN SILVER), NO. 468, JANUARY 25, 192\%. (CURRENT DESIGNATION : $Q Q-N-321)$

\section{GERMAN SILVER RODS, BARS, SHAPES}

(Requirements covering general specifications conform to F. S. B. QQ-M-151. See 600.0, p. 3 .
For grade, chemical composition, physical properties, and notes on German silver, see F. S. B. QQ-N-321 under 655.0, p. 1286 . Material and wormanship, packing and marking, and notes conform to F. S. B. QQ-C-501 (see 641.0 , p. 1172), except that in QQ-N321 there is no reference to manufacture, hammering hot to a fine point, and forging stock.)

\subsection{HOLLOW AND STAMPED WARE, NICKEL-COPPER-ZINC ALLOY.}

AMERICAN RAILWAY ASSOCIATION, TELEGRAPH AND TELEPHONE SECTION, SPECIFICATIONS FOR FUSE BLOCK ARA1-A AND FUSE BLOCK ARA-2-A, JANUARY, 1924

\section{FUSE BLOCKS}

(Fuse blocks are classifiable under electrical supplies and as such are not included in this publication. See remarks under 642.12, p. 1181. However the metal requirements specified in the A. R. A. specification are as given herewith.)

\section{CLIPS}

The clips shall be made of No. 21 A. W. G. spring German silver which shall show a hardness of from 70 to 82 points when measured by the Shore scleroscope (magnifier hammer). They shall be made exactly according to the dimensions shown on the drawing with the grain of the metal running in the direction of the longest dimension. An analysis of the composition of the German silver used shall show the following.

\begin{tabular}{|c|c|c|}
\hline & Minimum & Maximum \\
\hline $\begin{array}{l}\text { Copper... } \\
\text { Nickel } \\
\text { Zinc.... } \\
\text { Iron_... }\end{array}$ & $\begin{array}{c}\text { Per cent } \\
53.50 \\
16.50 \\
25.50\end{array}$ & $\begin{array}{c}\text { Per cent } \\
56.50 \\
19.50 \\
28.50 \\
.35\end{array}$ \\
\hline
\end{tabular}

655.9 MISCELIANEOUS SPECIFICATIONS FOR NICKEL-COPPER-ZINC ALIOY.

SOCIETY OF AUTOMOTIVE ENGINEERS, NONFERROUS METAL SPECIFICATIONS, SPECIFICATION NO. 42, WHITE NICKEL BRASS, MARCH, 1922

\section{WHITE NICKEL BRASS}

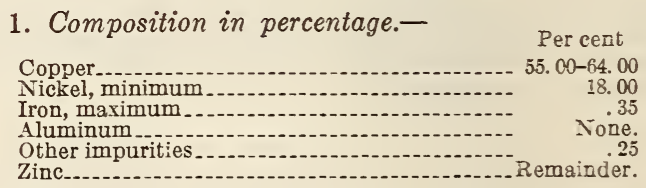

2. General information.-Good castings made of this alloy should give the following minima in physical characteristics:

Ultimate strength

Elongation in 2 inches, or proportionate gage

3. This brass is intended for use for trimmings or other parts requiring a metallic-white finish. The higher the nickel content, the more permanent will be the color. 
UNITED STATES GOVERNMENT, FEDERAL SPECIFICATIONS BOARD, SPECIFICATION FOR PLUMBING FIXTURES (FOR LAND USE), NO. 448, NOVEMBER 22, 1926. (CURRENT DESIGNATION: FFP-451.)

(See 617.73, p. 1062.)

PIUMBING FIXTURES (FOR LAND USE)

656. NICKEL-CHROMIUM ALLOY

656.0 GENERAL ITEMS.

AMERICAN SOCIETY FOR TESTING MATERIALS, TENTATIVE METHODS OF CHEMICAL ANALYSIS OF METALLIC MATERIALS FOR ELECTRIC HEATING, DESIGNATION B71-29T, 1929

\section{CHEMICAL ANALYSIS OF METALIIC MATERIALS FOR ELECTRIC HEATING}

DETERMINATION OF NICKEL

\section{SOLUTIONS REQUIRED}

Concentrated sulphuric acid (specific gravity 1.84).Concentrated nitric acid (specific gravity 1.42).Concentrated hydrochloric acid (specific gravity 1.19).-

Dilute sulphuric acid (1:1).- - Slowly stir $100 \mathrm{c}$ c of $\mathrm{H}_{2} \mathrm{SO}_{4}$ (specific gravity 1.84) into $100 \mathrm{c} \mathrm{c}$ of water. Dilute sulphuric acid (1:99). - Slowly stir $10 \mathrm{c} \mathrm{c}$ of $\mathrm{H}_{2} \mathrm{SO}_{4}$ (specific gravity 1.84) into $990 \mathrm{c}$ c of water.

Tartaric acid (20 per cent).-

Ammonium hydroxide (specific gravity 0.90).-

Dilute acetic acid $(1: 3)$. Mix $1 \mathrm{c}$ c of acetic acid (specific gravity 1.04) and $3 \mathrm{c} \mathrm{c}$ of water.

Nitrohydrochloric acid.-Mix $10 \mathrm{c}$ c of concentrated $\mathrm{HNO}_{3}$ (specific gravity 1.42), $30 \mathrm{c} \mathrm{c}$ of concentrated $\mathrm{HCl}$ (specific gravity 1.19), and $40 \mathrm{c} \mathrm{c}$ of water.

Ammonium bisulphite.-Dissolve $1 \mathrm{~g}$ of anmonium bisulphite in $5 \mathrm{c} \mathrm{c}$ of hot water.

Sodium dimethylglyoxime.-Dissolve $3 \mathrm{~g}$ of dimethylglyoxime in $100 \mathrm{c} c$ of $\mathrm{NaOH}$ (3 per cent), made fresh each time used.

\section{METHOD}

Dissolve exactly $1 \mathrm{~g}$ of the alloy sample in $20 \mathrm{c} \mathrm{c}$ of nitrohydrochloric acid in a covered $600 \mathrm{c}$ beaker. Add $20 \mathrm{c}$ c of dilute $\mathrm{H}_{2} \mathrm{SO}_{4}$ (1:1) and evaporate the liquid carefully to fumes of sulphur trioxide. When the beaker and its contents have cooled, add $100 \mathrm{c} \mathrm{c}$ of water and allow the solution to digest at a low heat until all salts have dissolved. Filter the solution on a $9 \mathrm{~cm}$ paper to remove any silica, and wash the paper and residue thoroughly with hot water or, if an insoluble residue is present, wash with dilute $\mathrm{H}_{2} \mathrm{SO}_{4}(1: 99)$. Ignite the precipitate at a low heat, and treat with $2 \mathrm{c} \mathrm{c}$ of $\mathrm{HF}$, and several drops of concentrated $\mathrm{H}_{2} \mathrm{SO}_{4}$ in a platinum crucible, and evaporate to dryness. Fuse the residue with a little sodium carbonate, dissolve in $\mathrm{HCl}$ and add to the main nickel solution.

Transfer the nickel solution to a $250 \mathrm{c}$ c graduated flask and ailute to the mark with cold water. Mix and draw off exactly 50 c c by means of an accurately calibrated pipette, and transfer this aliquot portion to a $600 \mathrm{c}$ c beaker. This aliquot portion represents $0.2 \mathrm{~g}$ of the original sample. Add 25 c c of a 20 per cent solution of tartaric acid (enough to hold all the iron and chromium in solution), make the solution alkaline with $\mathrm{NH}_{4} \mathrm{OH}$, and then make it acid with an excess of $15 \mathrm{c} \mathrm{c}$ of concen- trated $\mathrm{HCl}$ (specific gravity 1.19). Add 35 c c of a freshly prepared solution of sodium dimethylglyoxime, and make the solution ammoniacal, and then make it just acid with dilute acetic acid $(1: 3)$. Stir the solution vigorously and allow it to stand at a temperature of from $75^{\circ}$ to $90^{\circ} \mathrm{C}$., with occasional stirring, for one hour. If an alcoholic solution of dimethylgloxime was used to precipitate the nickel, the contents of the beaker should be digested at a temperature just short of the boiling point until the odor of alcohol can no longer be detected.

Filter the scarlet precipitate of nickel dimethylglyoxime on a tared Gooch crucible. Wash thoroughly with hot water and dry at $110^{\circ} \mathrm{C}$. to constant weight. Cool in a desiccator and weigh. The weight of nickel dimethylglyoxime multiplied by 20.32 and again by 5 gives the percentage of nickel in the sample. This method yields results that are correct within \pm 0.35 per cent.

If greater accuracy is desired, use a larger aliquot portion with proportionally larger amounts of reagents, and filcer the nickel cimethylglyoxime precipitate on an $11 \mathrm{~cm}$ paper and wash thoroughly 18 or 20 times with hot water. Return the paper and precipitate to the $600 \mathrm{c}$ c beaker, treat with $5 \mathrm{c} c$ of concentrated $\mathrm{H}_{2} \mathrm{SO}_{4}$ (specific gravity 1.84) and $25 \mathrm{c}$ c of concentrated $\mathrm{HNO}_{3}$ (specific gravity 1.42) and boil the liquid gently until strong fumes of sulphur trioxide are evolved.

Having allowed the beaker and its contents to cool somewhat, add $10 \mathrm{c}$ c of concentrated $\mathrm{HNO}_{3}$ (specific gravity 1.42 ) and repeat the evaporation. Rinse the cover and sides of the beaker with a fine jet of water and fume the solution again to insure the expulsion of every trace of the $\mathrm{HNO}_{3}$. When cool add approximately $50 \mathrm{c}$ c of cold water and boil the contents of the beaker for several minutes, which should result in a perfectly clear solution.

Neutralize with $\mathrm{NH}_{4} \mathrm{OH}$ and add an excess of $20 \mathrm{c} \mathrm{c} \mathrm{NH}_{4} \mathrm{OH}$ (specific gravity 0.90 ). Add $1 \mathrm{~g}$ of ammonium bisulphide dissolved in $5 \mathrm{c}$ c of hot water. New electrolize the nickel sulphate solution with a current of from 1 to 2 amperes, using a platinumgauze weighed cathode and a spiral platinum anode. Continue the electrolysis until the solution has become colorless, and then continue for at least 15 minutes longer. Now test the solution by adding one or two drops of it to a solution of potassium sulphocarbonate. Pink color indicates the presence of nickel. The amount of the solution withdrawn for this test will not affect the results and may be neglected. This test will show whether or not the electrolysis has been completed. Wash the metal deposited on the cathode with water. Remove the cathode and dip it first into a beaker of distilled water and then into absolute alcohol, dry for a few minutes at a temperature of $80^{\circ}$ to $100^{\circ}$ C., and weigh. The increase in weioht of the cathode multiplied by 100 and divided by the weight of the sample taken gives the percentage of nickel in the sample.

Accuracy. - In the case of a nickel content of 60 to 80 per cent, duplicate determinations should check within \pm 0.35 per cent nickel by the gravimetric method and \pm 0.15 per cent nickel by the electrolytic method.

\section{DETERMINATION OF CHROMHUM BY THE PERSUIPHATE} METHOD

\section{SOLUTIONS REQUIRED}

Silver nitrate solution ( 0.5 per cent).-Dissolve $5 \mathrm{~g}$ of $\mathrm{AgNO}_{3}$ in 1,000 c c of water.

Ammonium persulphate (15 per cent).-Dissolve $15 \mathrm{~g}$ of the salt in $100 \mathrm{c} \mathrm{c}$ of water. (The solution should be kept in a cool place and should not be used if over three days old. A fresh solution is preferable). 
Sodium chloride (10 per cent).-Dissolve $100 \mathrm{~g}$ of $\mathrm{NaCl}$ in $1,000 \mathrm{c}$ c of water.

Nitro-hydrochloric acid.-Mix 30 c c of concentrated $\mathrm{HCl}$ (specific gravity 1.19), $10 \mathrm{c}$ e of concentrated $\mathrm{HNO}_{3}$ (specific gravity 1.42), and $40 \mathrm{c} \mathrm{c}$ of water.

Sulphuric acid (1:1).-Wlowly stir 500 c c of concentrated $\mathrm{H}_{2} \mathrm{SO}_{4}$ (specific gravity 1.84) into $500 \mathrm{c}$ c of water.

Standardized ferrous ammonium sulphate solution.Dissolve $39.25 \mathrm{~g}$ of ferrous ammonium sulphate in distilled water, add $20 \mathrm{c} c$ of dilute $\mathrm{H}_{2} \mathrm{SO}_{4}(1: 1)$, dilute to 1 liter, mix thoroughly, and standardize against $0.1 \mathrm{~N}$ potassium permanganate, which has in turn been standardized against the Bureau of Standards sodium oxalate. This solution should be standardized just before using, as its value may change from day to day. One cubic centimeter of $0.1 \mathrm{~N}$ solution equals $0.001733 \mathrm{~g}$ of chromium.

Standard potassium permanganate solution (0.1 $N)$.-Dissolve approximately $3.18 \mathrm{~g}$ of pure potassium permanganate in $300 \mathrm{c} \mathrm{c}$ of hot distilled water, boil for 10 to 15 minutes, cool to room temperature, and filter on asbestos. Transfer the solution to a a glass-stoppered bottle and allow it to stand for two weeks, at the end of which time filter again on asbestos and transfer to a liter flask and dilute to the mark. This solution should be kept in a dark bottle. After a preliminary standardization against the Bureau of Standards sodium oxalate, dilute so as to be exactly $0.1 N$, and verify this strength by a second comparison with sodium oxalate.

\section{METHOD}

Treat approximately $0.2000 \mathrm{~g}$ of the sample in a $500 \mathrm{c}$ c flask with $20 \mathrm{c}$ c of nitro-hydrochloric acid and $0.5 \mathrm{c} \mathrm{c}$ of HF (48 per cent), warm at a moderate temperature (about $60^{\circ}$ C.) until the reaction is complete. Add $20 \mathrm{c}$ c of dilute $\mathrm{H}_{2} \mathrm{SO}_{4}(1: 1), 50 \mathrm{c} \mathrm{c}$ of water, and evaporate just to fumes of sulphuric acid. If any salts separate out, redissolve by adding $250 \mathrm{c}$ c of water and digest on a hot plate until an entirely clear solution results. Remove from the plate and add $1 \mathrm{c}$ c of $\mathrm{AgNO}_{3}$ solution (0.5 per cent) and sufficient ammonium persulphate (approximately $15 \mathrm{c}$ c for 10 to 12 per cent chromium alloys and $25 \mathrm{c}$ c for 20 per cent chromium alloy) thoroughly to oxidize the manganese, and boil for three minutes. Add $20 \mathrm{c} \mathrm{c}$ of $\mathrm{NaCl}$ solution (10 per cent), heat on a plate until the manganese dioxide has dissolved completely, and boil for five minutes. Cool to room temperature, and add from a burette standardized ferrous ammonium sulphate until the solution turns green, and then add $5 \mathrm{c} c$ in excess. Titrate this excess back with $0.1 \mathrm{~N}$ potassium permanganate.

Calculate the percentage of chromium from the following formula where

$$
\text { Percentage of chromium }=\frac{[(A \times B)-C] \times 0.001733}{0.2 \text {, or weight of sample }} \times 100
$$

$A=$ volume in cubic centimeters of ferrous ammonium sulphate used.

$B=$ normality factor of ferrous ammonium sulphate, determined daily.

$C=$ volume in cubic centimeters of $0.1 \mathrm{~N}$ potassium permanganate used.

Accuracy. - In the case of a chromium content of 10 to 20 per cent, duplicate determinations should check within \pm 0.15 per cent chromium.

\section{DETERMINATION OF IRON BY THE CUPFERRON METHOD}

\section{SOLUTIONS REQUIRED}

Dilute hydrochloric acid $(1: 1)$.- - Mix equal parts of concentrated $\mathrm{HCl}$ (specific gravity 1.19) and water.
Dilute hydrochloric acid (1:19).-Mix 5 e c of concentrated $\mathrm{HCl}$ (specific gravity 1.19 ) and $95 \mathrm{c} \mathrm{c}$ of water.

Dilute ammonium hydroxide $(1: 19)$.- Mix $1 \mathrm{c} \mathrm{c}$ of $\mathrm{NH}_{4} \mathrm{OH}$ (specific gravity 0.90 ) and $19 \mathrm{e}$ e of water.

Dilute sulphuric acid $(1: 4)$. - Slowly stir $1 \mathrm{c} \mathrm{c}$ of concentrated $\mathrm{H}_{2} \mathrm{SO}_{4}$ (specific gravity 1.84 ) into $4 \mathrm{c} \mathrm{c}$ of water.

Dilute sulphuric acid $(1: 9)$. - Slowly stir $1 \mathrm{c}$ c of concentrated $\mathrm{H}_{2} \mathrm{SO}_{4}$ (specific gravity 1.84 ) into $9 \mathrm{c} \mathrm{c}$ of water.

Stannous chloride solution.-Dissolve $50 \mathrm{~g}$ of stannous chloride in $100 \mathrm{c}$ c of concentrated $\mathrm{HCl}$ (specific gravity 1.19 ) and dilute to $1,000 \mathrm{c}$ c.

Cupferron (6 per cent).-Freshly prepared filtered cupferron $\left(\mathrm{C}_{6} \mathrm{H}_{5}(\mathrm{NO}) \mathrm{ONH}_{4}\right)$.

\section{METHOD}

For alloys containing 10 per cent or more of iron, use a 1 -g sample, and for alloys containing 2 per cent or less of iron use a 5-g sample. Dissolve the sample in a 400-c c covered beaker in 25 to 75 c c of concentrated $\mathrm{HCl}$ (specific gravity 1.19) at a temperature of about $65^{\circ} \mathrm{C}$. Add $2 \mathrm{c} \mathrm{c}$ of concentrated $\mathrm{HNO}_{3}$ (specific gravity 1.42 ) and from 20 to $25 \mathrm{c} \mathrm{c}$ of concentrated $\mathrm{H}_{2} \mathrm{SO}_{4}$ (specific gravity 1.84), and evaporate the solution until fumes of sulphur trioxide are given off. After having allowed the beaker and its contents partly to cool, add $50 \mathrm{c}$ c of warm water and boil the solution for several minutes to dissolve all salts. Filter the liquid on an 11-cm paper containing some paper pulp, and wash the residue and filter thoroughly with warm water. Reserve the filtrate. Ignite any residue in a platinum crucible until the carbon of the filter paper has burned off. After allowing the crucible to cool, add one or two drops of concentrated $\mathrm{H}_{2} \mathrm{SO}_{4}$ (specific gravity 1.84) and several cubic centimeters of $\mathrm{HF}$ and evaporate the solution to dryness. Any remaining residue is fused with a few grams of sodium carbonate. Dissolve the fusion in dilute $\mathrm{HCl}(1: 1)$ and boil to expel carbon dioxide. Add a slight excess of dilute $\mathrm{NH}_{4} \mathrm{OH}(1: 19)$ and boil the solution for several minutes. Filter, and wash well with hot water. Dissolve the precipitate off of the filter in $20 \mathrm{c} \mathrm{c}$ of hot dilute $\mathrm{H}_{2} \mathrm{SO}_{4} \quad(1: 4)$ and add it to the main filtrate which should have a total volume of not more than $150 \mathrm{c} \mathrm{c}$. Cool the solution to about $15^{\circ}$ C., treat with a slight excess of cold, freshly prepared solution of cupferron ( 6 per cent), which should cause the complete precipitation of the iron while all the nickel and chromium should remain in solution. A brownish-red, partly amorphous, partly crystalline precipitate should separate out.

As soon as a drop of the reagent causes the formation of a transient snow-white crystalline precipitate, all of the iron is down. Add some ashless paper pulp, and filter the contents of the beaker on an 11-cm paper, wash the precipitate eighteen to twenty times with cold dilute $\mathrm{H}_{2} \mathrm{SO}_{4}(1: 9)$, and finally wash five or six times with cold dilute $\mathrm{NH}_{4} \mathrm{OH}(1: 19)$. Discard the filtrate. This ammonia wash dissolves out the excess of cupferron, and will also dissolve any vanadium that might be present.

Ignite the cupferron precipitate, first at a low-red heat and finally at $900^{\circ}$ to $1,000^{\circ} \mathrm{C}$. Transfer the iron oxide to a $150-\mathrm{c}$ c covered beaker and wash the crucible with $20 \mathrm{c} \mathrm{c}$ of hot $\mathrm{HCl}(1: 1)$. Add $10 \mathrm{ce}$ of concentrated $\mathrm{HCl}$ (specific gravity 1.19) and digest the solution at a temperature somewhat short of boiling. Add stannous chloride solution from time to time in amounts sufficient to discharge the yellow color of the ferric chloride, and to provide a few drops in excess. Finish the determination by the Reinhardt-Zimmerman method. 
Accuracy.-In the case of an iron content of 0.5 per cent, duplicate determinations should check within \pm 0.05 per cent iron. In the case of an iron content of 25 per cent, duplicate determinations should check within \pm 0.2 per cent iron.

\section{DETERMINATION OF MANGANESE}

SOLUTIONS REQUIRED

Nitrohydrochloric acid.-Mix $10 \mathrm{c}$ c of concentrated $\mathrm{HNO}_{3}$ (specific gravity 1.42), 30 c c concentrated $\mathrm{HCl}$ (specific gravity 1.19) and $40 \mathrm{c}$ c of water.

Concentrated nitric acid (specific gravity 1.42).-

Dilute nitric acid (1:200). - Mix 1 c c of concentrated $\mathrm{HNO}_{3}$ (specific gravity 1.42) and $200 \mathrm{c}$ c of water.

Dilute sulphuric acid (1:3).-Slowly stir $1 \mathrm{c}$ c of concentrated $\mathrm{H}_{2} \mathrm{SO}_{4}$ (specific gravity 1.84) into 3 c c of water.

Standardized ferrous ammonium sulphate solution.-See the determination of chromium by the persulphate method, given above.

NotE.-One cubic centimeter of exactly $0.1 \mathrm{~N}$ solution equals $0.002769 \mathrm{~g}$ of manganese.

Standard potassium permanganate solution (0.1 $N)$.- See the determination of chromium by the persulphate method, given above.

\section{METHOD}

Dissolve exactly $2.000 \mathrm{~g}$ of drillings in a $400 \mathrm{c} \mathrm{c}$ beaker with $40 \mathrm{c}$ c of nitrohydrochloric acid, and when in solution evaporate to a sirupy consistency and take up with $50 \mathrm{c}$ c of concentrated $\mathrm{HNO}_{3}$ (specific gravity 1.42). Evaporate this again and add $100 \mathrm{c} \mathrm{c}$ of concentrated $\mathrm{HNO}_{3}$ and boil down to $80 \mathrm{c}$ c. Add slowly, $0.5 \mathrm{~g}$ at a time, about $3 \mathrm{~g}$ of potassium chlorate. Boil for at least five minutes after the last addition of potassium chlorate to insure complete oxidation of the chromium. Add $100 \mathrm{c} \mathrm{c}$ of water and filter through a thin asbestos pad with suction, washing the beaker and pad thoroughly with dilute $\mathrm{HNO}_{3}(1: 200)$, leaving no trace of oxidized chromium. Transfer the pad and the precipitate into the original beaker, being sure to wash out all precipitate adhering to the sides of the funnel with cold water.

Now add 100 c c of distilled water, 50 c c of dilute $\mathrm{H}_{2} \mathrm{SO}_{4}(1: 3)$, and $5 \mathrm{c} \mathrm{c}$ in excess of enough ferrous ammonium sulphate to dissolve all of the manganese dioxide. (About 20 c c should be required for the weight of alloy taken). The normality factor of the ferrous ammonium sulphate must be determined just before using. Stir until all the manganese dioxide is dissolved, and titrate the excess of ferrous ammonium sulphate with $0.1 \mathrm{~N}$ potassium permanganate. Occluded oxidized chromium is usually the cause for high manganese results.

Calculate the percentage of manganese from the following formula:

$$
\text { Percentage of manganese }=\frac{[(A \times B)-C] \times 0.002769}{2, \text { or weight of sample }} \times 100
$$

where

$A=$ volume in cubic centimeters of ferrous ammonium sulphate used

$B=$ normality factor of ferrous ammonium sulphate, determined daily

$C=$ volume in cubic centimeters of exactly $0.1 \mathrm{~N}$ potassium permanganate used.

Accuracy. - In the case of a manganese content of 2 to 3 per cent, duplicate determinations should check within \pm 0.1 per cent manganese.

\section{DETERMINATION OF CARBON BY TEE VOLUMETRIC}

This method for the determination of carbon is the method for determining carbon by absorption of the carbon dioxide in barium hydroxide solution. Reference should be made to the determination of carbon by the direct-combustion method as described in the standard methods of chemical analysis of plain carbon steel in A33. (See 600.1, p. 10.)

In a laboratory where accurate results are being obtained by the gravimetric method, or where a large number of samples are to be analyzed, and good weighing methods are in use, the gravimetric method may be used as described in method $(a)$ of the determination of carbon by the direct-combustion method described in A64. (See 601.20, p. 119.) Where only occasional samples are to be run, it is probable that the volumetric method will give more satisfactory results.

\section{SAMPLE}

The sample should weigh at least $4 \mathrm{~g}$ and should be rather finely divided in order that such a large sample may be placed in the combustion boat. Ordinary turnings are satisfactory, except that they are bulky. Usual precautions should be used in obtaining a perfectly clean sample.

\section{APPARATUS}

Boats and lining material.-Zirkite boats $100 \mathrm{~mm}$ long, $13 \mathrm{~mm}$ wide, and $8 \mathrm{~mm}$ deep (inside dimensions) are most satisfactory, although alundum or porcelain boats may be used. The boats should be ignited in a muffle furnace before using. The bottom of the boat should be lined with a trench of 40 mesh high-grade chrome ore, ignited at $1,000^{\circ} \mathrm{C}$. and preserved in a glass-stoppered bottle. The sample should be covered with a very thin layer of alundum. Some alundum powder should be sprinkled inside the combustion tube to prevent the boat from sticking.

Flux.-Exactly $2 \mathrm{~g}$ of fine drillings of ingot iron of known carbon content should be sprinkled over the sample before the alundum is placed over the top of the whole charge. The purpose of the ingot iron is not to flux the sample, but is a source of additional heat due to its rapid combustion when the oxygen is turned on. The results must be corrected for the amount of carbon added through the use of ingot iron.

Furnace and combustion tube-The standard electric combustion furnace in common use may be used, but as the temperatures are considerably higher the life of the heating element will be shorter than usual. A pyrometer should always be used. A fused-silica tube 24 inches in length and 1 inch in diameter gives the best results. The ends of the tubes should be encased in water-cooled jackets, or strips of blotting paper partly immersed in water should be placed over the ends of the tubes. At least 8 inches of the tube next to the absorption apparatus is filled with asbestos fiber which has been saturated with a solution of ferric nitrate and heated overnight at $1,000^{\circ} \mathrm{C}$. Ferric oxide will serve to oxidize any carbon monoxide formed, and will convert any sulphur dioxide to ferric sulphate. In case a combustion tube 30 inches in length is used, the dead space in the end next to the absorption apparatus should be filled with a glass plunger, which will decrease the amount of gas that has to be flushed out at each determination. The glass plunger may consist of a glass cylinder with the ends closed.

Detailed description of apparatus.-Figure 1129 illustrates the details of the apparatus. Oxygen from the 100 cubic feet cylinder $A$, passes through the reducing valve $B$, to the lower 8-liter-capacity bottle $C$, from which it forces distilled water to the upper bottle $D$, which is 2.5 feet higher than $C$ When the lower bottle is nearly full of oxygen, the valve $B$ is closed and the gas, now under reduced pressure, can be supplied to the apparatus at the desired rate by means of the petcock $H$. One of the 
essential parts of the apparatus is the mercury pressure gage $E$, which has a 6 -inch scale with $1 / 8$ inch subdivisions, for indicating the pressure, and, when calibrated, the rate of flow of the oxygen.

To calibrate the gage, the Meyer tube $O$, is filled to the usual point and the oxygen is regulated until the mercury stands at the desired mark, say $11 / 8$ inches. The volume of oxygen passing through the apparatus per minute is then determined by collecting it over water in a graduated cylinder, the connection being made to the exit end of the Meyer tube by means of a rubber tube. The rate of flow at 1-inch pressure should be about 100 c c per minute, although, of course, this varies with the resistance offered to the flow of the oxygen by the reagents and materials in the train.

The capillary tube $N$, in the stopper at the exit of the combustion tube $J$, is an essential part of the rubber stoppers and with water jackets, $K$ and $L$, at the ends. The clear solution of barium hydroxide (20 g per liter) in the bottle $S$, is arranged so that it can be conveniently siphoned into the 8-bulb Meyer tube, $O$. The barium carbonate is filtered on the Büchner funnel $X$, shown fitted to the filter flask $P$, and the precipitate is washed with carbondioxide-free distilled water (prepared by bubbling through the water compressed air previously freed from carbon dioxide by passage through the sodalime bottle $T$, delivered by gravity from the bottle $U)$. The standard $0.1 N$ solutions of $\mathrm{NaOH}$ and $\mathrm{HCl}$ are delivered by gravity to the burettes, $R$, from the bottles $V$ and $W$. The small hot plate $Q$ is used for warming the flask containing the barium carbonate and the measured amount of $0.1 \mathrm{~N}$ $\mathrm{HCl}$, in order to cause rapid and complete solution of the precipitate.

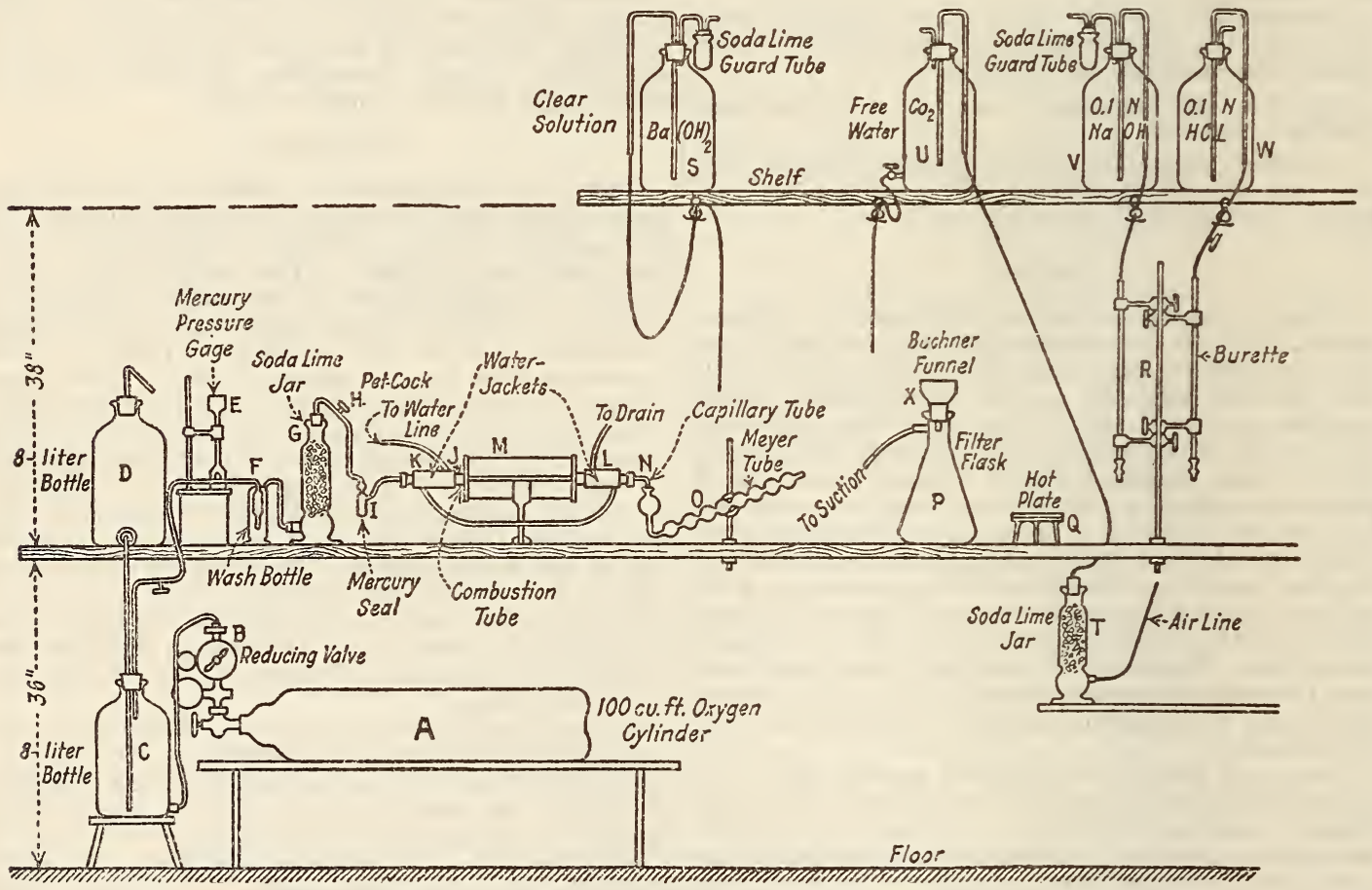

Figure 1129.- Apparatus for the determination of carbon by the volumetric method

apparatus. The size of the capillary opening is adjusted by heating in a flame so that when the mercury gage indicates a certain definite pressure (about 1 inch), the rate of flow through the capillary-the Meyer tube, $O$, being filled and connected-closely approximates but does not exceed $100 \mathrm{c} \mathrm{c}$ per minute. If the rate of flow much exceeds 100 c c per minute some carbon dioxide will be swept through the barium hydroxide without being absorbed, while if it is much less than $100 \mathrm{c} c$ all of the carbon dioxide will not be flushed out of the combustion tube in the specified time. Removal of any carbon dioxide in the oxygen is effected by passage through $\mathrm{NaOH}$ solution (specific gravity 1.27) contained in the wash bottle $F$, and the 12mesh soda lime in the jar $G$, although the principal function of the liquid in $F$ is to serve as an indicator of the rate of flow of the gas. The mercury seal $I$ prevents any possibility of back flow of the gases in the combustion tube. After passing through the mercury seal the oxygen enters the combustion tube $J$, which is provided with tightly fitting 1-hole

\section{METHOD}

The furnace having been previously heated to approximately $1,100^{\circ}$ C., all air having been displaced from the apparatus by passage of a stream of pure oxygen, and the Meyer tube having been filled with sufficient barium hydroxide solution (20 g per liter) to fill seven of the eight bulbs when the oxygen is passing at the maximum rate, the boat is quickly inserted, by means of a stout copper rod, into the hottest part of the furnace and the stopper immediately replaced. Allow the boat and its contents to heat for about one minute with no oxygen passing, this will result in some oxygen being absorbed by the iron, and the barium hydroxide being drawn back into the large bulb as a consequence of the partial vacuum resulting therefrom.

At the expiration of about one minute, admit oxygen to the tube at a rate of approximately 340 c c per minute (as indicated by the gage, which should read about $1 \frac{1}{2}$ inches). Complete combustion of the ingot iron, and the alloy sample, 
with vivid incandescence, should result in $1 \frac{1}{2}$ to 2 minutes. Preliminary heating followed by the rapid admission of oxygen are absolutely essential to securing complete combustion in the short time allowed. Only in case the oxides are so thoroughly fused as to show no trace of the shape of the original drillings when the boat is removed from the furnace should the combustion be considered to have been successful. Upon the appearance of the faintest cloudiness in the first bulb of the Meyer tube, decrease the rate of flow immediately to $100 \mathrm{c}$ per minute (about 1 inch on the gage), and maintain it so to the end of the operation. If the rate of flow after the first noticeable formation of barium carbonate is much less or much greater than $100 \mathrm{c} \mathrm{c}$ per minute, low carbon results will be obtained, due either to some carbon dioxide not having been swept out of the apparatus in the time specified, or to some having escaped absorption in the barium hydroxide. Passage of oxygen for a total of five minutes is sufficient to sweep all caroon dioxide from the combustion tube.

At whe conclusion of the above-described operations (six minutes after the boat was inserted into the furnace) remove the boat, and filter the contents of the Meyer tube on two superimposed, opengrain, $11 \mathrm{~cm}$ filter papers supported on a Büchner funnel fitted to a suction flask. Wash the Meyer tube three times with water free from carbon dioxide (prepared by passing compressed air through soda lime and then bubbling it through the distilled water), taking care to reach all points of the tube; then give the precipitate and paper four additional washings. Delivery of the water from an overhead bottle rather than from a wash flask avoids danger of contamination from the carbon dioxide of the breath, besides being more convenient and sanitary. Transfer the paper and precipitate to a $500 \mathrm{c}$ c Erlenmeyer flask ana add from a burette enough standard $0.1 \mathrm{~N} \mathrm{HCl}$ solution to dissolve the barium carbonate and add $5 \mathrm{c} \mathrm{c}$ in excess, a portion of the acid being used to rinse the Meyer tube, which is then further rinsed with water. Place the flask containing the precipitate on a hot plate until the carbonate has dissolved; long heating should be avoided as it apparently causes some action on the filter paper involving a slight error in the determination. Introduce five or six drops methyl orange into the solution and determine the excess of $\mathrm{HCl}$ by titration with a standard $0.1 \mathrm{~N}$ solution of $\mathrm{NaOH}$, the end point being marked by the color change from red to yellow. One cubic centimeter of standard $0.1 N \mathrm{HCl}$ equals $0.000600 \mathrm{~g}$ of carbon.

A blank should be run on the ingot iron, boat, filter paper, etc., and any carbon found should be deducted from that obtained in the analysis. A $2 \mathrm{~g}$ sample of ingot iron should be used, and the determination made by the method as just described.

Accuracy. - In the case of a carbon content of 0.05 per cent, duplicate determinations should check within \pm 0.01 per cent carbon.

\section{DETERMINATION OF INSOLUBIE RESIDUE}

\section{METHOD}

The sample should weigh at least $5 \mathrm{~g}$ and if the alloy is known to be low in insoluble residue a $10 \mathrm{~g}$ sample should be used. Treat the sample with from 200 to $400 \mathrm{c}$ c of $\mathrm{HCl}$ (specific gravity 1.19) at a temperature of from $60^{\circ}$ to $70^{\circ} \mathrm{C}$. until the reaction appears to be complete, then add 1 to 2 c c of $\mathrm{HNO}_{3}$ (specific gravity 1.42). Boil the solution for about two minutes, dilute with an equal volume of hot water, filter, and wash the filter thoroughly with hot dilute $\mathrm{HCl}(5: 95)$.

Ignite the residue at a low heat in a platinum crucible, treat with $\mathrm{HF}$ and several drops of dilute
$\mathrm{H}_{2} \mathrm{SO}_{4}(1: 1)$, and evaporate the solution just to fumes of sulphur trioxide. If any residue remains, add $10 \mathrm{c} c$ of water to the crucible, filter on a $9 \mathrm{~cm}$ paper and wash the residue with hot water. Ignite any residue remaining on the paper and weigh. The increase in weight multiplied by 100 and divided by the weight of sample taken gives the percentage of insoluble residue.

Accuracy. - In the case of an insoluble residue of 0.05 per cent, duplicate determinations should check within \pm 0.02 per cent insoluble residue.

\section{DETERMINATION OF SILICON}

\section{METHOD}

Treat a $2 \mathrm{~g}$ factor-weight sample $(0.9344 \mathrm{~g}) 3$ to $\mathrm{a}$ $5 \mathrm{~g}$ factor-weight sample $(2.3360 \mathrm{~g})$ in a $300 \mathrm{c} \mathrm{c}$ porcelain casserole with 30 to $50 \mathrm{c} \mathrm{c}$ of $\mathrm{HCl}$ (specific gravity 1.19), the casserole being covered with a watch glass, and warm until the reaction is complete. Add 30 to $50 \mathrm{c}$ c of dilute $\mathrm{H}_{2} \mathrm{SO}_{4}(1: 1)$, and evaporate just to fumes of sulphur trioxide. Allow the casserole partly te cool, add $200 \mathrm{c}$ c of warm water, and boil briskly for several minutes to dissolve all salts. Filter the silica oil an $11 \mathrm{~cm}$ paper containing some ashless paper pulp, wash five or six times with dilute $\mathrm{HCl}(1: 100)$, and finally with hot water until free from acid.

Ignite the paper and the precipitate in a small platinum crucible, first at a dull-red heat until the carbon of the filter paper has been oxidized, and finally at $1,050^{\circ}$ to $1,100^{\circ} \mathrm{C}$. Allow the crucible to cool in a desiccator and weigh. Add one or two drops of $\mathrm{H}_{2} \mathrm{SO}_{4}$ (specific gravity 1.84), several cubic centimeters of $\mathrm{HF}$, and evaporate the solution until all the $\mathrm{H}_{2} \mathrm{SO}_{4}$ has been expelled. Ignite the crucible again, conl, and weigh. The difference between the first and the second weights, multiplied by 100 and divided by the weight of sample take.l, gives the percentage of silicon in the sample. In case the silica precipitate contains any undissolved material, ignite the paper and the precipitate at a dull-red heat until the carbon of the filter paper has been oxidized, transfer to a 30 c c pure-iron crucible, and fuse with approximately $5 \mathrm{~g}$ of sodium peroxide. When cool, transfer the melt to a 300 c c porcelain cassarole and dissolve in $50 \mathrm{c}$ c of water. Add $50 \mathrm{c} \mathrm{c}$ of dilute $\mathrm{H}_{2} \mathrm{SO}_{4}(1: 1)$, and evaporate the solution just to fumes of sulphur trioxide. From this point proceed as described above.

Accuracy.- In the case of a silicon content of 0.2 per cent, duplicate determinations should check within \pm 0.03 per cent silicon.

\section{DETERMINATION OF SULPHUR BY THE EVOLUTION-
TITRATION METHOD}

This method is based on (1) the evolution of practically all the sulphur in the alloy as hydrogen sulphide when the alloy is dissolved in $\mathrm{HCl}$ (specific gravity 1.19) (see note); (2) absorption of the evolved gas by means of an ammoniacal solution of cadmium chloride; and (3) determination of the sulphur by titration with a standard solution of potassium iodate $(0.0312 \mathrm{~N})$.

\section{SOLUTIONS REQUIRED}

Potassium permanganate solution (2.5 per cent).Dissolve $2.5 \mathrm{~g}$ of $\mathrm{KMnO}_{4}$ in $100 \mathrm{c} \mathrm{c}$ of water.

Sodium hydroxide (30 per cent).-Dissolve $30 \mathrm{~g}$ of $\mathrm{NaOH}$ in $100 \mathrm{c}$ c of water.

Cadmium chloride solution.-Dissolve $10 \mathrm{~g}$ of $\mathrm{CdCl}_{2} \cdot 2 \mathrm{H}_{2} \mathrm{O}$ in $400 \mathrm{c} \mathrm{c}$ of water and $600 \mathrm{c} \mathrm{c}$ of $\mathrm{NH}_{4} \mathrm{OH}$ (specific gravity 0.90 ).

Starch solution.-Dissolve $1.2 \mathrm{~g}$ of soluble starch in $100 \mathrm{c}$ c of boiling water, cool to room temperature, and add a solution of $0.5 \mathrm{~g}$ of KI dissolved in $10 \mathrm{c} \mathrm{c}$ of 
cold, freshly distilled water. The starch solution shall not be used if it is over one day old.

Standard potassium permanganate $(0.03 \mathrm{~N})$.-Dissolve $0.99 \mathrm{~g}$ of pure $\mathrm{KMnO}_{4}$ in $1,000 \mathrm{c} \mathrm{c}$ of hot distilled water, boil for $\$ 10$ minutes, cool to room temperature, and filter through asbestos. Standardize against the Bureau of Standards standard sodium oxalate as follows: In a $200 \mathrm{c}$ c beaker, dissolve $0.1 \mathrm{~g}$ of sodium oxalate in 75 to $100 \mathrm{c} c$ of hot water $\left(80^{\circ}\right.$ to $90^{\circ}$ C.) and add $4 \mathrm{c} \mathrm{c}$ of $\mathrm{H}_{2} \mathrm{SO}_{4}(1: 1)$. Titrate at once with the permanganate solution, stirring the liquid vigorously and continuously. The permanganate must not be added more rapidly than 10 to $15 \mathrm{c}$ c per minute, and the last 0.5 to $1 \mathrm{c} \mathrm{c}$ must be added dropwise, with particular care to allow each drop to be fully decolorized before the next is introduced. The excess of permanganate used to cause an end-point color must be estimated by matching distilled water. To $250 \mathrm{c}$ c of cold distilled water in a $500 \mathrm{c}$ c flask, add $10 \mathrm{c}$ c of $\mathrm{HCl}(1: 1)$ and then as the flask is gently swirled, add $35 \mathrm{c} \mathrm{c}$ of the iodate solution from a burette. Titrate the liberated iodine with the standard sodium thiosulphate solution $(0.03 N)$ to a light straw color, add several cubic centimeters of the freshly prepared starcb solution and continue titration to the disappearance of the blue color. Calculate the normality of the iodate solution and adjust the solution so that $1 \mathrm{c} \mathrm{c}$ is equivalent to $0.0005 \mathrm{~g}$ of sulphur.

\section{APPARATUS}

Figure 1130 illustrates the details of the apparatus employed. Hydrogen supplied from the Kipp generator, $A$, is freed from any sulphur by passage through a solution of potassium permanganate $(2.5$
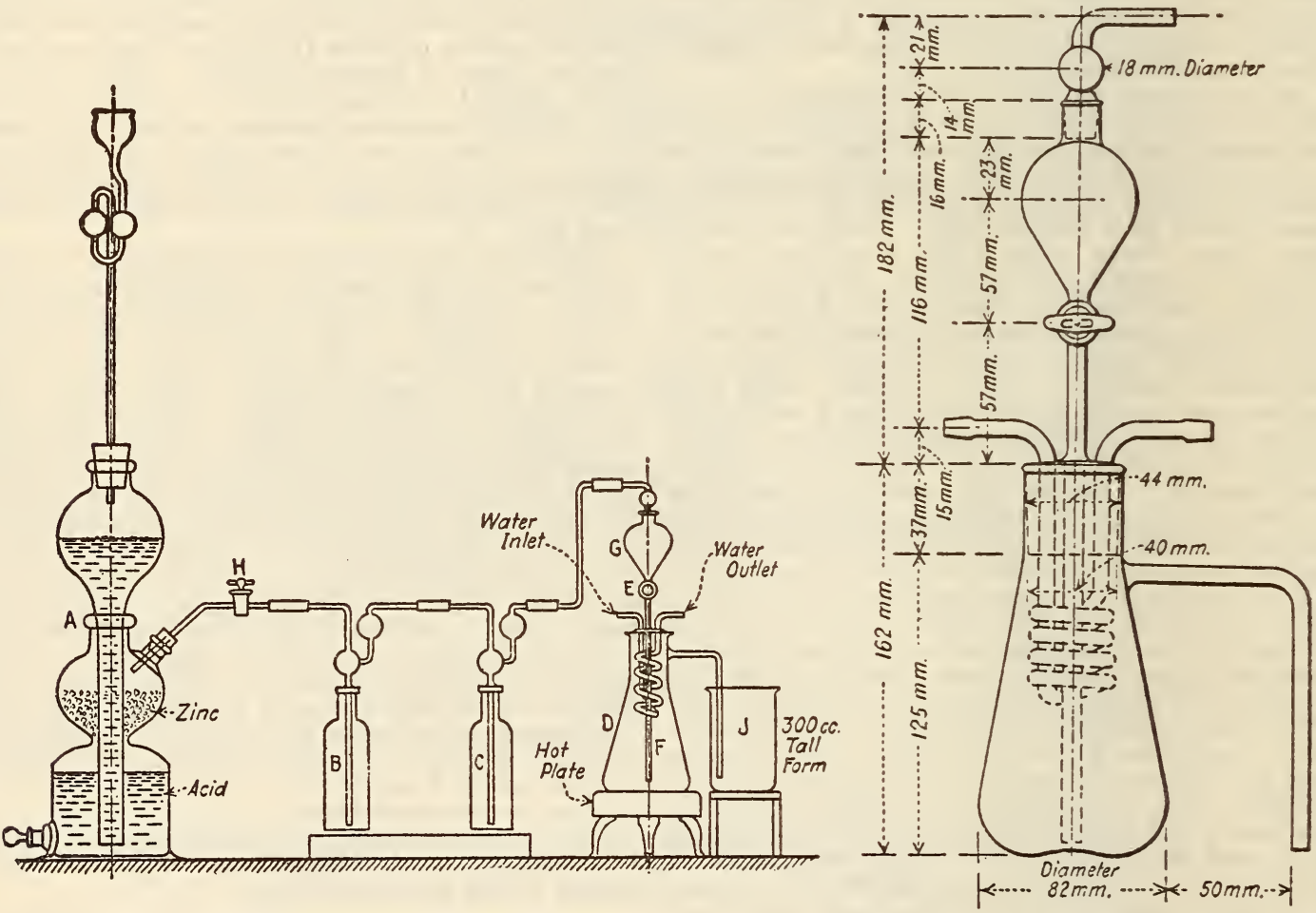

Decomposition Flask, Pulsifer Design.,

FIgURE 1130.-Apparatus for determination of sulphur by the evolution-titration method

the color in another beaker containing the same bulk of acid and hot water. The temperature of the solution should not be below $60^{\circ} \mathrm{C}$. by the time the end point is reached. Calculate the exact normality of potassium permanganate from the sodium oxalate.

Standard sodium thiosulphate $(0.3 \mathrm{~N})$.- Dissolve $7.5 \mathrm{~g}$ of $\mathrm{Na}_{2} \mathrm{~S}_{2} \mathrm{O}_{3} \cdot 5 \mathrm{H}_{2} \mathrm{O}$ in $1,000 \mathrm{c} \mathrm{c}$ of freshly boiled distilled water at room temperature. To $250 \mathrm{c} \mathrm{c}$ of distilled water in a $500 \mathrm{c} \mathrm{c}$ flask add $1 \mathrm{~g}$ of $\mathrm{KI}$ and $10 \mathrm{c} \mathrm{c}$ of $\mathrm{HCl}(1: 1)$. Cool and add $35 \mathrm{c} \mathrm{c}$ of the standard potassium permanganate solution $(0.03 \mathrm{~N})$ from a burette. Swirl gently and titrate the liberated iodine with the thiosulphate solution to a light straw color. Add several cubic centimeters of the freshly prepared starch solution and continue the titration to the disappearance of the blue color. Calculate the normality of the thiosulphate solution from the standard permanganate solution.

Standard potassium iodate $(0.0312 N)$.-Dissolve $1.12 \mathrm{~g}$ of pure $\mathrm{KIO}_{3}$, and $12 \mathrm{~g}$ of $\mathrm{KI}$ in $1,000 \mathrm{c} \mathrm{c}$ of per cent) contained in a Drechsel bottle, $B$, and sodium hydroxide (30 per cent) held in the Drechsel bottle, $C$. Hydrogen is admitted through a glass stopcock, $E$, to the decomposition flask, $D$. The decomposition flask (Pulsifer's design) is provided with a ground-glass stopper, $E$, a funnel, $G$, and a cooling coil, $F$, with inlet and outlet tubes. An ammoniacal solution of cadmium chloride or some other suitable absorbent is held in a $300 \mathrm{c}$ c beaker, $J$. The absorption should not be carried on in direct sunlight.

NotE.-It is advisable to use an all-glass apparatus because the length of time ( 30 minutes) required for the solution of the alloy gives opportunity for fumes of hydrochloric acid to attack rubber stoppers and rubber hose connections, which always contain sulphur, and thus cause erroneous results.

\section{METHOD}

Transfer to the decomposition flask $5.00 \mathrm{~g}$ of the alloy ribbon which has been cut into very small pieces or of the wire sample which has been flat- 
tened by means of a hammer and cut into small pieces. Pass a stream of hydrogen from the Kipp generator through the apparatus to displace the air. Pour $25 \mathrm{c}$ c of cadmium chloride solution into the beaker, $J$, and add sufficient water to give a depth of 3 inches of the solution. Stop the hydrogen by closing the control stopcock, $H$, on the Kipp generator. Close the stopcock $E$. Remove the groundglass tube leading into the funnel, $G$, and introduce $100 \mathrm{c} \mathrm{c}$ of $\mathrm{HCl}$ (specific gravity 1.19) (see note) into the decomposition flask through the funnel. As soon as the acid has been added, replace the ground-glass inlet tube, open the stopcock, $E$, and pass a steady stream of hydrogen through the apparatus during the remainder of the determination. Apply sufficient heat to the decomposition flask to maintain a steady decomposition of the sample. Passage of a stream of cold water through the cooling coil, $F$, of the decomposition flask serves to cool the stopper and to aid in maintaining the strength of the acid in the flask. Thirty minutes of this treatment is ordinarily sufficient to cause complete decomposition. When there is no further action, boil the solution in the decomposition flask for several minutes and continue the stream of hydrogen for five minutes longer.

At the end of this time remove the absorption beaker, $J$, close stopcock, $E$, and the control stopcock, $H$, and discontinue heating the decomposition flask. Filter the cadmium chloride solution through an $11 \mathrm{~cm}$ filter paper and wash the precipitate several times with water. Return the paper and precipitate to a $600 \mathrm{c} \mathrm{c}$ beaker, add $300 \mathrm{c} \mathrm{c}$ of cold water and $40 \mathrm{c} \mathrm{c}$ of dilute $\mathrm{HCl}(1: 1)$ and titrate the liberated hydrogen sulphide with the standard solution of potassium iodate, starch solution being used as an indicator.

Carry a blank through all steps of the process and deduct any sulphur that is found.

If the potassium iodate solution has been adjusted to $0.0312 N$ and $5 \mathrm{~g}$ of the alloy has been dissolved, then the percentage of sulphur is 0.01 times the number of cubic centimeters of the iodate solution used.

Note.-There is a question whether concentrated $\mathrm{HCl}$ or dilute $\mathrm{HCl}$ should be used. This method calls for concentrated $\mathrm{HCl}$. See article by Pulsifer, A Standard Apparatus for the Determination of Sulphur in Iron and Steel by the Evolution Method in Ind. Eng. Chem., p. 545,1918 .

\section{OPTIONAL METHOD}

For an optional method see the determination of sulphur by the evolution-titration method (routine) In the standard methods of chemical analysis of plain carbon steel A33 (see 600.1, p. 10). This optional method may be used for nickel-chromium and for nickel-iron-chromium alloys with the following precautions:

1. Due to the length of time (30 minutes) required for solution of the alloy, closer observation is necessary than in the analysis of steel.

2. During the solution of the sample care must be be exercised in order that the absorbing solution is not drawn back into the flask.

3. There is danger of contamination from rubber hose connections and rubber stoppers.

4. The use of a gas flame is a possible source of sulphur contamination and an electric heater is recommen ded.

Accuracy.-In the case of a sulphur content of 0.05 per cent, duplicate determinations should check within \pm 0.003 per cent sulphur.

\section{DETERMTNATION OF COPPER}

A. Nickel-Copper Alloys Containing 10 to 90 per cent Copper SOLUTIONS REQUIRED

Dilute nitric acid $(1: 3)$.-Slowly stir $50 \mathrm{c}$ c of $\mathrm{HNO}_{3}$ (specific gravity 1.42) into $150 \mathrm{c} \mathrm{c}$ of distilled water.
Dilute sulphuric acid (1:1).-Slowly stir 10 c c of concentrated $\mathrm{H}_{2} \mathrm{SO}_{4}$ (specific gravity 1.84 ) into $10 \mathrm{c}$ c of water.

\section{METHOD}

Dissolve exactly $2.000 \mathrm{~g}$ of the alloy in $25 \mathrm{c} \mathrm{c}$ of the dilute $\mathrm{HNO}_{3}$ (specific gravity 1.135) and, when solution is complete, boil to expel nitrous fumes and dilute with about $175 \mathrm{c} \mathrm{c}$ of cold water. Add $5 \mathrm{c} \mathrm{c}$ of the dilute sulphuric acid (1:1).

Determine the copper by electrolysis. Use a current of 0.5 ampere unless the solution is agitated, in which case a larger current up to 4 amperes may be used.

Accuracy.-Duplicate determinations should check within \pm 0.10 per cent of copper.

Note.-A little copper may be retained by the iron precipitate. The presence of molybdenum or silver will cause high results for copper.

B. Copper up to 5 per cent in Allogs of Chromium with Iron or Nickel or Both

SOLUTIONS REQUIRED

Ferric sulphate.-Dissolve $0.8 \mathrm{~g}$ of ferric sulphate $\left(\mathrm{Fe}_{2}\left(\mathrm{SO}_{4}\right)_{3} \cdot 9 \mathrm{H}_{2} \mathrm{O}\right)$ in $100 \mathrm{c} \mathrm{c}$ of distilled water.

Dilute sulphuric acid $(1: 4)$.- Slowly stir $50 \mathrm{c} c$ of $\mathrm{H}_{2} \mathrm{SO}_{4}$ (specific gravity 1.84 ) into $200 \mathrm{c}$ c of distilled water.

Dilute sulphuric acid $(1: 1)$.-Slowly stir 100 c c of $\mathrm{H}_{2} \mathrm{SO}_{4}$ (specific gravity 1.84) into $100 \mathrm{c}$ c of distilled water.

Acidified hydrogen sulphide water.-Mix $10 \mathrm{c}$ c of $\mathrm{HCl}$ (specific gravity 1.19) and $1,000 \mathrm{c}$ c of distilled water and saturate with $\mathrm{H}_{2} \mathrm{~S}$.

Ammonium hydroxide (specific gravity 0.90).-

\section{METHOD}

Dissolve exactly $5.000 \mathrm{~g}$ of the alloy in a $400 \mathrm{c} \mathrm{c}$ covered beaker in $100 \mathrm{c}$ c of $\mathrm{HCl}$ (specific gravity 1.19) at about $65^{\circ} \mathrm{C}$. When the reaction is complete, dilute to approximately $300 \mathrm{c}$ c with warm water. Introduce ammonium hydroxide (specific gravity 0.90 ) until the solution is practically neutral and then add sufficient $\mathrm{HCl}$ (specific gravity 1.19) to give an acidity of $3 \mathrm{c} \mathrm{c}$ of $\mathrm{HCl}$ (specific gravity 1.19) per $100 \mathrm{c}$ c of solution. Treat the solution with a brisk stream of hydrogen sulphide for at least 30 minutes.

Filter the precipitate of sulphide on a $9 \mathrm{~cm}$ paper containing a small amount of paper pulp and wash thoroughly with the acidified hydrogen sulphide water. Discard the filtrate.

Return the paper and the precipitate to a 250 c c covered beaker and treat with $3 \mathrm{c} \mathrm{c}$ of $\mathrm{H}_{2} \mathrm{SO}_{4}$ (specific gravity 1.84) and $25 \mathrm{c}$ c of $\mathrm{HNO}_{3}$ (specific gravity 1.42) and evaporate slowly to fumes of sulphur trioxide. Add sufficient $\mathrm{HNO}_{3}$ (specific gravity 1.42 ) in small quantities to oxidize completely the filter paper and subsequently heat the solution until strong fumes of sulphur trioxide are evolved and all $\mathrm{HNO}_{3}$ has been expelled.

Add $25 \mathrm{c}$ c of the ferric sulphate solution to the cooled sulphuric acid solution and add ammonium hydroxide (specific gravity 0.90) in small excess. Heat the solution to boiling and after the precipitated ferric hydroxide has settled, filter on a $9 \mathrm{~cm}$ paper and wash with hot water.

Dissolve the precipitate in a small amount of hot dilute $\mathrm{H}_{2} \mathrm{SO}_{4}(1: 4)$ and wash the filter with water. Repeat the ammonia precipitation and the filtration and combine the filtrates. Acidify with an excess of $5 \mathrm{c} c$ of $\mathrm{H}_{2} \mathrm{SO}_{4}(1: 1)$, add $1 \mathrm{c}$ c of $\mathrm{HNO}_{3}$ (specific gravity 1.42 ) and evaporate to approximately $100 \mathrm{c}$ c.

Determine the percentage of copper by electrolysis, using a perforated platinum cathode and a spiral anode. 
In case a trace of copper is to be determined, start with four $5 \mathrm{~g}$ portions of sample and combine them after the hydrogen sulphide precipitates have been filtered.

Accuracy.-Duplicate determinations should check within \pm 0.02 per cent copper.

Nоте.-A little copper may be retained by the jron precipitate. The presence of molybdenum or silver will cause high results for copper.

\subsection{NICKEL-CHROMIUM HEATING ELE- MENTS.}

AMERICAN SOCIETY FOR TESTING MA TERIALS, TENTATIVE SPECIFICATIONS FOR DRAWN OR ROLLED ALLOY, 80 PER CENT NICKEL, 20 PER CENT CHROMIUM, FOR ELECTRICAL HEATING ELEMENTS, DESIGNATION B82-31T, 1931

\section{NICKEL-CHROMIUM HEATING ELEMENTS}

1. These specifications cover annealed, drawn, or rolled shapes for electrical heating element purposes having a nominal chemical composition of 80 per cent nickel and 20 per cent chromium.

2. Wire and ribbon shall be thoroughly and uniformly annealed.

\section{CHEMICAL PROPERTIES AND TESTS}

3. The material shall conform to the following requirements as to chemical composition:

Nickel ${ }^{16}$
Chromium $\begin{array}{r}\text { Per cent } \\ \text { Iron, maximum } \\ \text { Manganese, maximum } \\ \text { Carbon, maximum } \\ \text { Silicon, maximum } \\ \text { Sulphur, maximum.... }\end{array}$

4. Samples for chemical analysis shall consist of clean drillings, millings, or clippings taken from the wire or ribbon selected for purposes of test. The sample shall be taken from a spool or coil representative of the lot in question. The sample so prepared shall be divided into three equal parts, each of which shall be placed in a sealed package, one for the manufacturer, one for the purchaser, and one for an umpire, if necessary.

5. Chemical analyses shall be determined in accordance with the tentative methods of chemical analysis of metallic materials for electrical heating in B71. (See 656.0, p. 1289.)

\section{PHYSICAL PROPERTIES AND TESTS}

6. Wire of No. 39 A. w. g. (0.0035 inch) and larger shall have a minimum elongation of 20 per cent in 10 inches, and from Nos. 40 to 44 A. w. g. $(0.0031$ to 0.002 inch), a minimum elongation of 10 per cent.

7. (a) The nominal specific resistance of the round wire shall be taken as 650 ohms per circular mil foot (108 microhms per centimeter cube) at $77^{\circ}$ F. $\left(25^{\circ}\right.$ C. $)$.

(b) The nominal specific resistance of the ribbon shall be taken as 510 ohms per square mil foot (108 microhms per centimeter cube) at $77^{\circ} \mathrm{F}$. $\left(25^{\circ} \mathrm{C}\right.$. $)$.

8. The specific resistance shall be determined in accordance with the standard method of test for resistivity of metallic materials for resistors in A. S. T. M. B63.

9. (a) Round wire-The resistance per foot of round wire shall be calculated from the nominal

16 Small quantities of cobalt included shall be reported as nickel. specific resistance and the cross section of the material. The limits on resistance per foot shall be as follows:

No. $30 \mathrm{~A}$. w. g. (0.0100 inch) and larger

Per cent

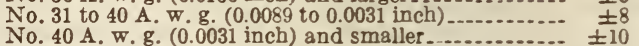

(b) Strip material.-The resistance per foot of strip material shall be calculated from the nomina] specific resistance and the cross section of the material. In order to compensate for error in the calculated cross section on ribbon with rounded edges, the calculated values arrived at by multiplying the nominal width by nominal thickness shall be corrected by multiplying by the factor indicated in the following table:

\begin{tabular}{|c|r|}
\hline Ratio of width to thickness & \multirow{2}{*}{ Factor } \\
\cline { 1 - 2 } 15 and under & \\
\hline Over 15 & 0.94 \\
& \\
\hline
\end{tabular}

10. Temperature coefficient of resistance shall, after the wire has been slowly cooled from an annealing temperature, be measured in accordance with the standard method of test for change of resistance with temperature of metallic materials for electrical heating in A. S. T. M. B70. The temperature coefficient of resistance shall be such as will bring the resistance within \pm 20 per cent of the following:

\begin{tabular}{|c|c|}
\hline Temperature & Resistance \\
\hline 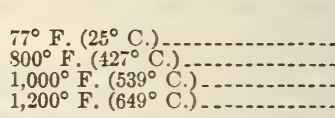 & $\begin{array}{c}\text { Per cent } \\
100.0 \\
103.7 \\
104.0 \\
103.3\end{array}$ \\
\hline 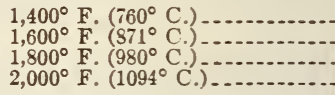 & $\begin{array}{l}103.2 \\
103.6 \\
104.1 \\
104.8\end{array}$ \\
\hline
\end{tabular}

11. (a) When it is desired to determine the durability of the material at high temperatures, life: tests shall be made in accordance with the tentative accelerated life test for metallic materials for electrical heating in A. S. T. M. B76. Test results shall be reported as a percentage of a standard of comparison.

(b) The life or durability value shall be the average of at least three simultaneous determinations each, on the wire in question and on the standard. If any test result varies more than 10 per cent from the average of the three determinations, that group shall be discarded.

\section{FINISE}

12. The material shall be as uniform and free from surface defects such as splits, kinks, laminations, scale, and other irregularities as the best commercial practice will permit. Finish shall be either bright annealed or oxidized, as specified.

\section{PACKING AND MARKING}

13. (a) Round wire or rod.-Round wire or rod in sizes No. $16 \mathrm{~A}$. w. g. or larger shall be supplied in coils consisting of not more than two pieces per 40pound coil. Round wire sizes smaller than No. 16 gage shall be supplied in spools containing not 
more than two pieces and shall be spooled in accordance with the following requirements:

\begin{tabular}{|c|c|c|c|c|}
\hline \multirow{2}{*}{$\begin{array}{l}\text { Weight } \\
\text { of wire }\end{array}$} & \multicolumn{2}{|c|}{ Diameter } & \multicolumn{2}{c|}{ Spool dimensions } \\
\cline { 2 - 3 } & Flange & Traverse & $\begin{array}{c}\text { Diameter } \\
\text { of hole }\end{array}$ & $\begin{array}{c}\text { Diameter } \\
\text { or barrel }\end{array}$ \\
\cline { 5 - 6 } Pounds & Inches & Inches & Inches & Inches \\
1 & 2 & 3 & $5 / 8$ & $11 / 4$ \\
2 & $23 / 4$ & 3 & $5 / 8$ & $11 / 2$ \\
5 & 4 & 3 & $5 / 8$ & $11 / 2$ \\
10 & 6 & 3 & 13 & $13 / 4$ \\
25 & 9 & 4 & 1 & $3 \frac{3}{4}$ \\
\hline
\end{tabular}

(b) Ribbon.-Ribbon over one-fourth inch in width shall be supplied in coils containing not more than one piece, and shall be securely wrapped so as to avoid injuring the edges of the strips. Ribbon of one-fourth inch in width or narrower, shall be supplied on suitable spools containing not more than two pieces per spool.

\section{MARKING}

14. Coils or spools shall be marked with the size and resistance per foot and a yellow tag or label shall be used to indicate this allor.

\section{AMERICAN SOCIETY FOR TESTING MA- TERIALS, TENTATIVE SPECIFICATIONS FOR DRAWN OR ROLLED ALLOY, 60 PER CENT NICKEL, 15 PER CENT CHROMIUM, AND BALANCE IRON FOR ELECTRICAL HEATING ELEMENTS, DESIGNATION B83-31T, 1931}

\section{NICKEL-CHROMIUM HEATING ELEMENTS}

(This specification is identical with A. S. T. M. B82-31T, given above, except that the ratio of nickel to chromium is 60 to 15 per cent instead of 80 to 20 per cent, with the following differences in respect to chemical composition, physical properties, and temperature coefficient of resistance:)

\section{CHEMICAL COMPOSITION}

3. The material shall conform to the following requirements as to chemical composition:

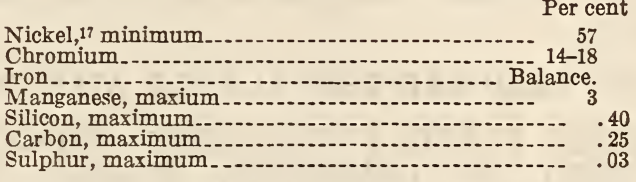

\section{PHYSICAL PROPERTIES}

6. Wire of No. 39 A. w. g. (0.0035 inch) and larger shall have a minimum elongation of 20 per cent in 10 inches and from Nos. 40 to $44 \mathrm{~A}$. w. g. (0.0031 to 0.002 inch) a minimum elongation of 10 per cent.

7. (a) The nominal specific resistance of round wire shall be taken as 675 ohms per circular mil foot (112.22 microhms per centimeter cube) at $77^{\circ}$ F. $\left(25^{\circ}\right.$ C. $)$.

(b) The nominal specific resistance of the ribbon shall be taken as 530 ohms per square mil foot (112.22 microhms per centimeter cube) at $77^{\circ} \mathrm{F}$. $\left(25^{\circ}\right.$ C. $)$.

Resistance increase with temperature

\begin{tabular}{|c|c|}
\hline Temperature & Resistance \\
\hline $\begin{array}{l}77^{\circ} \mathrm{F} .\left(25^{\circ} \mathrm{C}^{\circ}\right) \\
500^{\circ} \mathrm{F} \cdot\left(260^{\circ}{ }^{\circ} \mathrm{C} .\right) \\
600^{\circ} \mathrm{F} \cdot\left(315^{\circ} \text { C. }\right) \\
800^{\circ} \mathrm{F} .\left(427^{\circ} \text { C. }\right)\end{array}$ & $\begin{array}{c}\text { Per cent } \\
100 \\
104.0 \\
104.8 \\
106.3\end{array}$ \\
\hline $\begin{array}{l}1,000^{\circ} \mathrm{F} \cdot\left(537^{\circ} \mathrm{C} .\right) \\
1,200^{\circ} \mathrm{F} \cdot\left(649^{\circ}\right. \\
1,400^{\circ} \mathrm{C} \cdot \mathrm{C} \cdot\left(760^{\circ} \mathrm{C}\right) \\
1,600^{\circ} \mathrm{F} \cdot\left(871^{\circ} \text { C. }\right)\end{array}$ & $\begin{array}{l}107.4 \\
108.0 \\
108.6 \\
109.7\end{array}$ \\
\hline
\end{tabular}

${ }_{17}$ Small quantities of cobalt included shall be reported as nickel. 


\section{PLATINUM AND PLATINUM JEWELRY}

(No nationally recognized specifications available.)

\section{GOLD AND DENTAL GOLD ALLOYS}

\section{UNITED STATES GOVERNMENT, DEPART- MENT OF COMMERCE, BUREAU OF STANDARDS, C43, 1921}

\section{GOLD CARATS}

The carat weights in use previous to July 1, 1913, in different countries had differed greatly, scarcely any two of the important countries having the same standard. Even within the United States there was not agreement in the standard used, the various makers of weights using slightly different standards. This led to considerable confusion in the weighing of gems, and was the more serious because of the great value of the article.

Beginning July 1,1913 , the international metric carat of $200 \mathrm{mg}$ as the unit of weight for diamonds and other precious stones was put into commercial use in the United States by practically all the dealers in gems and precious stones through the efforts of a committee representing all the principal firms handling gems. On the same date the Treasury Department of the United States Government also began the use of this unit in the customs service for the levying of import duties on precious stones, and the Bureau of Standards recognized this unit for purposes of certification of all carat weights submitted to the bureau for test.

The movement for the adoption of a uniform, decimally divided standard was a decided step forward and therefore has met with success. The new metric carat of $200 \mathrm{mg}$ is universally used in the United States and has been officially adopted by Belgium, Bulgaria, Denmark, England, France, Germany, Holland, Italy, Japan, Norway, Portugal, Rumania, Spain, Sweden, and Switzerland. The Bureau of Standards in 1913 prepared tables for converting "old" carats to new metric carats, and vice versa, and published them as Circular No. 43. These tables were of great aid at the time to the transition from the old unit of about $205.3 \mathrm{mg}$ and binary fractions to the new unit and decimal fractions.

The inconvenience and inefficient use of the present system of pennyweights and grains as opposed to the benefits derived from the use of the new metric decimally divided carat has become so pronounced that the bureau was invited to prepare a practical working outline of the metric system that would suit the needs of the jewelry trade and allied industries. The purpose of this is to make it easily possible for jewelers and silversmiths to substitute the gram for the dual unit of pennyweights and grains ${ }^{18}$ and also to use the metric system in all of their work.

\section{UNITED STATES GOVERNMENT, DEPART. MENT OF COMMERCE, BUREAU OF STANDARDS, ANALYSIS OF DENTAL GOLD ALLOYS, S532, 1926}

\section{DENTAL GOLD ALLOYS}

Dental gold alloys are, in general, composed chiefly of gold, silver, copper, and platinum. The porportionate amounts of these metals are usually of the order of 60 per cent of gold, 10 to 15 per cent of silver, 10 to 15 per cent of copper, and 10 per cent of platinum. Palladium is often substituted for part or all of the platinum. Many alloys contain small amounts of iridium and occasionally one is found to contain rhodium. Of the base metals besides copper, small amounts of nickel, manganese, zinc, and tin are found in many of the alloys and occasionally iron and magnesium occur. As many as nine of the above-named metals have been found in a single alloy. A number of these were undoubt. edly present as impurities in the major constituents.

(The various steps for determining the composition of dental gold alloys are discussed in the order in which they would generally be carried out in making a complete analysis. The steps include sampling, assembling reagents, and a qualitative examination for the detection of minor constituents, such as palladium, iridium, rhodium, tin, nickel, manganese, zinc, iron, and magnesium. Gold, silver, platinum, and copper are the major constitents. The accompanying table shows analyses of typical dental gold alloys:)

\begin{tabular}{|c|c|c|c|c|c|c|c|c|c|c|c|c|c|}
\hline $\begin{array}{l}\text { Alloy } \\
\text { No. }\end{array}$ & Sample & $\begin{array}{l}\text { Argen- } \\
\text { tum }\end{array}$ & $\begin{array}{l}\text { Irid- } \\
\text { ium }\end{array}$ & $\begin{array}{l}\text { Stan- } \\
\text { num }\end{array}$ & Aurum & $\begin{array}{c}\text { Pallad- } \\
\text { ium }\end{array}$ & Copper & $\begin{array}{l}\text { Plati- } \\
\text { num }\end{array}$ & \begin{tabular}{|} 
Rhodi- \\
um
\end{tabular} & Zinc & Nickel & $\begin{array}{l}\text { Fer- } \\
\text { rum }\end{array}$ & Total \\
\hline & & Per cent & Per cent & Per & Per cent & Per cent & Per cent & Per cent & Per cent & Per cent & ген сели & Per & er cent \\
\hline 1 & $\left\{\begin{array}{l}\text { A-- } \\
\text { B.-. }\end{array}\right.$ & $\begin{array}{l}7.14 \\
7.12\end{array}$ & $\begin{array}{l}0.15 \\
.13\end{array}$ & $\begin{array}{l}0.82 \\
.81\end{array}$ & $\begin{array}{l}71.31 \\
71.25\end{array}$ & 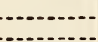 & $\begin{array}{l}9.86 \\
9.90\end{array}$ & $\begin{array}{l}10.65 \\
10.59\end{array}$ & {$[-\ldots . .$.} & $\begin{array}{l}0.08 \\
.07\end{array}$ & & & 99.87 \\
\hline 2 & $\left\{\begin{array}{l}A- \\
B\end{array}\right.$ & . & 1.23 & & 75.60 & 16.07 & & 6.95 & & .06 & & & 99.91 \\
\hline & A & .09 & 1.21 & & $\begin{array}{l}75.55 \\
90.60\end{array}$ & 16.04 & 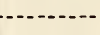 & 6. 97 & & .07 & & & 99.84 \\
\hline 3 & $\left\{\begin{array}{l}x_{-} \\
\mathrm{B}\end{array}\right.$ & .08 & ........ & & 90.54 & . & . & $\begin{array}{l}9.31 \\
9.33\end{array}$ & & .02 & 0.01 & & $\begin{array}{r}100.03 \\
99.98\end{array}$ \\
\hline 4 & $\left\{\begin{array}{l}A \\
B\end{array}\right.$ & 9.31 & .38 & & 62. 23 & .45 & 4.52 & 23.00 & & .09 & .02 & 0.15 & 100.15 \\
\hline & A. & $\begin{array}{l}9.35 \\
4.97\end{array}$ & $\begin{array}{l}41 \\
.15\end{array}$ & - & 62. 37 &. .53 & 4. 49 & 22. 64 & & .08 & .02 & .15 & 100. 04 \\
\hline 5 & B. & 4.94 & .15 & & 63. 91 & 10.22 & 7.12 & $\begin{array}{l}13.49 \\
13.43\end{array}$ & & 18 & & & $\begin{array}{l}100.02 \\
99.96\end{array}$ \\
\hline 6 & $\left\{\begin{array}{l}A- \\
B-\end{array}\right.$ & 10.98 & .23 & .13 & 60.41 & 5.89 & 8.83 & 12. 90 & $\cdots$ & .72 & - & -- & 100.09 \\
\hline & & $\begin{array}{r}11.00 \\
5.98\end{array}$ & .24 & .12 & $\begin{array}{l}60.43 \\
71.42\end{array}$ & 5.86 & $\begin{array}{r}8.80 \\
15.59\end{array}$ & $\begin{array}{r}12.99 \\
2.73\end{array}$ & & $\begin{array}{r}.71 \\
2.36\end{array}$ & 199 & & 100.15 \\
\hline 7 & B. & 6.02 & $\cdots$ & & 71.37 & & 15. 53 & 2.83 & & 2. 39 & $\begin{array}{l}1.97 \\
\text { 1. }\end{array}$ & & 100.11 \\
\hline 8 & $\left\{\begin{array}{l}\mathrm{A} \\
\mathrm{B}\end{array}\right.$ & $\begin{array}{l}5.88 \\
5.95\end{array}$ & .03 & $-\cdot-$ & 65.76 & 5. 73 & 10.02 & 10. 60 & 0.61 & 1. 35 & ... & $\cdots$ & 99.88 \\
\hline 9 & A. & 8.31 & .05 & .18 & $\begin{array}{l}65.89 \\
83.31\end{array}$ & 5.70 & $\begin{array}{r}10.16 \\
8.33\end{array}$ & & .50 & 1.33 & & & $\begin{array}{l}100.13 \\
100.13\end{array}$ \\
\hline y & B. & $\begin{array}{l}8.39 \\
9.67\end{array}$ & $\ldots$ & & 83.29 & $-\ldots$ & 8.29 & & & & & & \\
\hline 10 & & 9.65 & .07 & & $\begin{array}{l}66.34 \\
66.34\end{array}$ & $\begin{array}{l}7.79 \\
7.76\end{array}$ & $\begin{array}{l}\text { 12. } 94 \\
\text { 12. } 94\end{array}$ & $\begin{array}{l}2.04 \\
2.08\end{array}$ & & $\begin{array}{l}1.18 \\
1.19\end{array}$ & & ......... & 100.03 \\
\hline 11 & & 11. 67 & & & 67.77 & & 10.55 & 10.13 & $\ldots$ & & & & 100.12 \\
\hline & & 11.65 & & & & $\ldots$ & & 10. & & & & & 100 \\
\hline 12 & & & & & & $\ldots$ & 10. & 10.10 & & $\begin{array}{l}1.04 \\
\text { 1. }\end{array}$ & 2. 74 & & 100.11 \\
\hline 13 & A. & .22 & .46 & & 66. 23 & 19. 41 & .23 & & & .10 & & & 99.96 \\
\hline & & & & & & & & 13.32 & & & & & 100.09 \\
\hline
\end{tabular}

${ }_{18}$ It is desirable to note in this connection that all medical prescriptions of the U. S. Army must be expressed in metric units, not in grains. 
663. SILVER

\subsection{GENERAL ITEMS.}

UNITED STATES GOVERNMENT, CONGRESS, 34 STATUTES AT IARGE, 260, JUNE 13, 1906

\section{NATIONAL STAMPING ACT}

An act forbidding the importation, exportation or carriage in interstate commerce of falsely or spuriously stamped articles of merchandise made of gold or silver or their alloys, and for other purposes.

(For gold or part gold articles this act requires that the actual fineness of such gold or alloy shall not be less by more than one-half of 1 carat than the fineness indicated by the mark or word stamped or marked thereon; except for watchcases and flat ware the actual fineness shall be not less by more than 0.003 parts than the fineness indicated.)

(For silver or part silver articles the act requires that the actual fineness of the silver or alloy shall not be less by more than 0.004 parts than the actual fineness indicated by any mark or word, other than the word "sterling" or "coin"; no article shall be marked with the word "sterling" unless such article contains 0.925 parts pure silver; and no article shall be marked with the word "coin" or "coin silver" unless such article contains 0.900 parts pure silver: Provided, that in the case of all such articles, whose fineness is indicated by the word "sterling" or the word "coin" there shall be allowed a divergence in the fineness of 0.004 parts from the foregoing standards.)

\subsection{SILVER METAL AND ALIOYS.}

\section{AMERICAN DENTAL ASSOCIATION, TEN- TATIVE SPECIFICATION FOR DENTAI AMALGAM ALLOYS, OCTOBER 10, 1929}

\section{DENTAL AMALGAM ALLOYS}

(In regard to types, material, general requirements, and detail requirements, this specification is identical with specification U-A-451 of the U. S. Government Federal Specifications Board, given below. Methods of sampling and tests, and packing requirements are as given herewith.)

\section{METHOD OF SAMPLING AND TESTS}

The manufacturer shall specify the conditions under which test specimens are to be made, including alloy to mercury ratio, method and time of trituration and method of packing. These directions must fall within the limits of his published directions for the use of the product. The following details relative to test methods will be observed:

Ultimate compressive strength.-Specimens shall be cylinders $6 \mathrm{~mm}$ in diameter and $12 \mathrm{~mm}$ long. These shall be prepared by condensing the amalgam into a cavity of these dimensions in a rigid block, using a reasonable technic (that given in the sheet of instructions accompanying the alloy.)

The ends of the cylinder shall be surfaced plane at right angles to the axis. Strength tests shall be made five days after condensing, and the actual time consumed in applying the load, starting from the instant that the specimen is subjected to a load of 100 pounds, shall not be over three minutes nor under one minute. Tests shall be made at temperatures between $20^{\circ}$ and $25^{\circ} \mathrm{C}$.

(Test methods for flow test and setting changes are the same as given in Federal specification U-A-451 below.)

\section{PACKING}

Alloy shall be packed in moisture-resisting containers and in quantities of 1,2 , or 5 troy ounces. Accurate and adequate instructions for proportioning and manipulation shall accompany each package.

Each package of alloy shall be marked with a serial number or a combination of letters and numbers which shall refer to the manufacturer's records for the particular lot or batch or alloy. The date of manufacture (year and month) shall be indicated on the package as a separate item or as a part of the serial number.

Containers shall not be made, in whole or in part, of materials which will amalgamate readily with mercury.

\section{NATIONAL METAL EXCHANGE (INC.) AND NATIONAI METAL CLEARING ASSOCI- ATION (INC.), BY-LAWS AND RULES,} 1931

\section{BY-LAWS CONCERNING SILVER}

\section{TENDERABLE SILVER}

Section 40-D.- - In fulfillment of every contract of silver, the seller must deliver 25,000 ounces ( 2 per cent more or less) of bar silver in usual commercial large bars only, assaying not less than 99.9 fineness and made up of one or more of the officially listed brands or markings as provided in section 42-B of these by-laws, current at the date of delivery of such silver.

\section{OFFICIAL LIST OF BRANDS OR MARKINGS OF SILVER BARS}

Section 42-B.-There shall be on file in the office of the secretary of the exchange descriptions and/or replicas of the brands or markings of silver bars which may be delivered against National Metal Exchange silver contract, and said official list shall at all times be available to members for inspection.

\section{UNITED STATES GOVERNMENT, FEDERAL SPECIFICATIONS BOARD, FEDERAI SPECIFICATION FOR ALLOY; DENTAL, AMALGAM, U-A-451, MARCH 31,1931}

\section{ALLOY : DENTAL, AMALGAM}

\section{A. APPLICABIE FEDERAL SPECIFICATIONS}

A-1. There are no other Federal specifications applicable to this specification.

\section{B. TYPES}

B-1. Dental amalgam alloy shall be of the following types as specified: Type A, shavings, and type B, filings.

\section{MATERIAL AND WORKMANSHIP}

C-1. Material.-The comminuted alloy shall be free of foreign materials and shall be uniform.

\section{GENERAL REQUIREMENTS}

D-1. Ainalgams shall possess the following features, known as satisfactory working qualities:

D-1a. Thorough amalgamation in three minutes.

D-1b. Absence of granular or sandy consistency when amalgamated.

D-1c. Susceptibility to carving for at least 15 minutes after amalgamation.

D-1d. Susceptibility to receiving and retaining a polish 24 hours after amalgamation. 


\section{E. DETAIL REQUIREMENTS}

E-1. Chemical composition.-The chemical composition shall be within the following limits:

Per cent

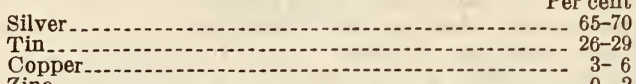

Copper........ $3-6$

E-2. Ultimate compressive strength.-The average ultimate compressive strength values of three or more tests shall not fall below $2,500 \mathrm{~kg}$ per square centimeter (approximately 35,500 pounds per square inch).

E-3. Flow.-Specimens subjected to a constant pressure of $250 \mathrm{~kg}$ per square centimeter (approximately 3,550 pounds per square inch) shall not show more than 5 per cent flow (that is, more than 5 per cent shortening in length of specimen) in a period of 24 hours.

E-4. Setting changes.-Twenty-four hours after amalgamation the length shall have increased between $1 \mu$ and $10 \mu$ per centimeter. If at any time during the test a contraction of more than $4 \mu$ per centimeter is detected, the alloy shall be rejected.

\section{F. METHODS OF SAMPLING, INSPECTION, AND TESTS}

F-1. Sampling.-At least 1 ounce shall be taken at random from every 500 ounces or fraction thereof for test at time of delivery.

$\mathrm{F}-2$. Inspection and tests.-

F-2a. Ultimate compressive strength.-Specimens shall be cylinders $6 \mathrm{~mm}$ in diameter and $12 \mathrm{~mm}$ long. These shall be prepared by condensing the amalgam into a cavity of these dimensions in a rigid block, using a reasonable technique (approximately that given in the sheet of instructions accompanying the alloy). The ends of the cylinder shall be surfaced plane at right angles to the axis. Strength tests shall be made five days after condensing and the actual time consumed in applying the load shall not be over five minutes nor under two minutes. Tests shall be made at temperatures between $20^{\circ}$ and $25^{\circ} \mathrm{C}$.

F-2b. Flow.-Specimens shall be cylinders $4 \mathrm{~mm}$ in diameter and $8 \mathrm{~mm}$ long and shall be prepared in the same manner as those for crushing. Three hours after condensing the specimen shall be subjected to a constant pressure of $250 \mathrm{~kg}$ per square centimeter (approximately 3,550 pounds per square inch). During this test the specimen shall be maintained at a temperature between $20^{\circ}$ and $25^{\circ} \mathrm{C}$.

F-2c. Setting changes.-Specimens shall be prepared by condensing into a cavity or matrix. These shall be removed as soon as condensation is completed and shall not be subject to restraint during the test. Measurements shall begin 15 minutes after amalgamation. (Time spent in mulling and condensing to be included as a part of the 15 minutes.)

\section{G. PACKAGING, PACKING, AND MARXING}

G-1. Packaging.-Dental amalgam alloy shall be furnished in moisture-resisting containers having either 1 or 5 ounce capacity, as specified.

(Packing and marking conform to the requirements specified in WW-N-351. See 607.4, p.485.)

\section{NOTES}

I-1. It is believed that this specification adequately describes the characteristics necessary to secure the desired material, and that normally no samples will be necessary prior to award to determine compliance with this specification. If, for any particular purpose, samples with bids are necessary, they should be specifically asked for in the invitation for bids, and the particular purpose to be served should be definitely stated, the specification to apply in all other respects.
663.2 SILVERWARE.

\section{AMERICAN MARINE STANDARDS COM- MITTEE, O NO. 9-1926}

\section{SHIP SILVERWARE}

This is a maximum list of kinds and sizes, from which can be selected articles required for any ship service. It is to be supplemented by standard specifications to define the characteristics of articles listed, the whole to constitute a simplified but adequate basis for manufacture and distribution, as well as a convenient and reliable one for purchasing, with resultant economy to all concerned.

(Under hollow ware are included casters, shakers, chafing dishes, dishes, covers, and accessories, pots, trays and waiters, tureens, and miscellaneous items. Under flatware will be found spoons, forks, knives, and miscellaneous items. Eighteen per cent alloy metal unplated (known to the trade as Lasherware) is specified for the crew and steerage.)

\section{UNITED STATES GOVERNMENT, DEPART-} MENT OF COMMERCE, BUREAU OF STANDARDS, STERLING SILVER FLATWARE, SIMPLIFIED PRACTICE RECOMMENDATION R54, 1926

\section{STERLING SILVER FLATWARE}

In accordance with the unanimous action, on March 2, 1926, of the general conference of representatives of manufacturers, distributors, and users of sterling silver flatware, the United States Department of Commerce, through the Bureau of Standards, recommends that the following schedule constitute the accepted list of stock items in sterling silver flatware patterns:

\section{STAPLES}

Item

Description

1. Teaspoons, trade (to be stamped with letter "T"), 8 ounces (tolerance, $1 / 2$ ounce over or under).

2. Teaspoons, regular (to be stamped with letter "R"), 10 ounces (tolerance, $1 / 2$ ounce over or under).

3. Teaspoons, heavy (to be stamped with letter "H"), 12 ounces (tolerance, $1 / 2$ ounce over or under).

4. Desert spoons. ${ }^{19}$

5. Tablespoons. ${ }^{19}$

6. Soup spoons.

7. Desert forks. ${ }^{19}$

8. Desert knives (hollow handle).

9. Dinner forks. ${ }^{10}$

10. Dinner knives (hollow handle).

\section{FANCY DOZENS}

11. Bouillon spoons.

12. Butter spreaders.

13. Coffee spoons.

14. Fish forks (individual).

15. Fruit knives (hollow handle) (one style of blade only).

16. Ice cream forks.

17. Iced tea spoons.

18. Orange or grapefruit spoons (one size only).

19. Oyster forks.

20. Salad forks (individual).

10 This item will be made in one or two wieghts at the manufacturer's option. If made and listed in one weight only, it shall in no case carry any designating mark referring to weight. 


\section{CARVING SETS}

21. Meat carving knife.

22. Meat carving fork.

23. Meat carving steel.

24. Game carving knife.

25. Game carving fork.

26. Steak carving knife.

27. Steak carving fork.

28. Baby fork.

BABY SET

29. Baby knife (hollow handle).

30. Baby spoon, short.

31. Baby spoon, bent.

\section{CHILD'S SET}

32. Child's knife (hollow handle; may be listed also as "tea knife").

33. Child's fork.

Child's spoon (same as item No. 1).

\section{FANCY SINGLE PIECES}

34. Bonbon or almond spoon.

35. Butter knife.

36. Butter pick.

37. Cake server (hollow handle).

38. Cheese server (hollow handle, one size only).

39. Cream ladle.

40. Cucumber server.

41. Duck, "poultry or game" shears (hollow handle)

42. Egg server (hollow handle).

43. Gravy ladle.

44. Jelly server.

45. Lemon fork.

46. Mayonnaise or sauce ladle.

47. Olive fork.

48. Olive spoon.

49. Pie server (hollow handle).

50. Salad fork, serving.

51. Cold meat fork.

52. Salad or berry spoon.

53. Preserve of salad spoon.

54. Serving spoon.

55. Sugar spoon.

56. Sugar tongs, one size only.

57. Tomato server.

\section{WEIGHTS}

Teaspoons have not infrequently been listed in four weights, and then under no definitely established terminology indicating those weights. The recommended standard list eliminates one of these weights entirely and establishes standard terminology for the three weights retained.

Dessert spoons, tablespoons, dessert forks, and dinner forks have not infrequently been made in three weights. The recommended standard list eliminates one of these weights for each of the four items, and it is believed will tend more and more to the manufacturing of but one weight in the items noted.

Standardization of the terminology applied to weights will of itself be recognized as a forward step. The recommendations here made are equivalent to dropping out of the list at least five items.

UNITED STATES GOVERNMENT, FEDERAL SPECIFICATIONS BOARD, FEDERAI SPECIFICATION FOR TABLEWARE; SILVER-PLATED, RR-T-51, JULY 21, 1931

\section{SILVER-PLATED TABLEWARE}

\section{A. FEDERAL SPECIFICATIONS}

A-1. There are no other Federal specifications applicable to this specification.

\section{B. GRADE AND CLASSES}

B-1. Silver-plated tableware covered by this specification shall be of a grade equal to that required for high-class hotel and restaurant service.

B-2. Silverware of this grade shall be divided into the following classes: Class Ia, flatware for frequent use; class Ib, flatware for occasional use; class IIa, hollow ware for frequent use and class IIb, hollow ware for occasional use.

\section{MATERIAL AND WORKMANSHIP}

\section{C-1. Base metal composition.}

C-1a. Unless white metal is specified, the base metal of bodies, spouts, and handles shall consist of "18 per cent" nickel brass (also known as nickel silver or German silver), except that the base metal of articles manufactured by spinning shall be of " 15 per cent" nickel brass. Tips and other small parts shall consist of " 12 per cent" nickel brass. For each of these alloys a minus tolerance of 1 per cent of nickel will be permitted.

C-1b. When "White metal" (Britannia) is specified, it shall be a tin-base alloy with not less than 80 per cent of tin by weight.

C-1c. Unless silver-plated blades are specified, the blades of the knives shall be of high-grade corrosion resisting cutlery steel properly heat treated and shall conform to the following chemical composition, and shall meet the corrosion test specified in paragraph $\mathrm{F}-4$.

\begin{tabular}{|c|c|}
\hline & Per cent \\
\hline Carbon & $0.30-0.4$ \\
\hline maximum & \\
\hline 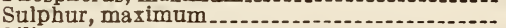 & \\
\hline 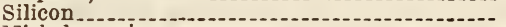 & $.10-$ \\
\hline & \\
\hline 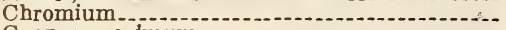 & \\
\hline ax & \\
\hline
\end{tabular}

C-1d. On hollow-handle knives the handles shall be of 18 per cent nickel brass. On solid-handle knives, the handle shall be of steel.

C-1e. All soldering on hollow ware shall be with hard or silver solder, except when in contact with "white metal" or insulators, in which case soft solder may be used.

C-1f. Shells of hollow-handle cutlery shall be silver soldered. Blades and tines may be soldered into the handles with soft solder.

C-1g. Where nonconducting handles are specified, they shall consist of or be provided with insulators consisting of wood, fiber, or other approved composition.

C-2. Base metal thickness.-

C-2a. Flatware: The blanks for flatware shall weigh not less than the following amounts:

Avor. 0z./doz.

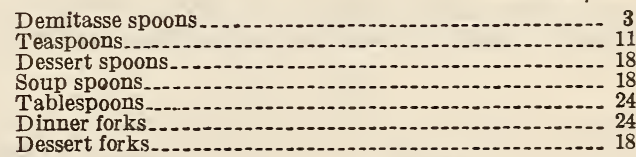

Dessert forks

Bidders shall state in their bids the weight of blanks for other pieces of flatware.

C-2b. Hollow ware: Unless specifically stated in drawings, the thickness of the metal used for each piece of hollow ware shall be such as will provide the strength and durability required for the service for which the particular piece is intended.

C-3. Finish.-

C-3a. Flatware--Unless otherwise specified, flatware shall have the "Butler" (dull) finish except for the bowls, tines, and plated blades, which shall be burnished, and left bright.

C-3b. Hollow ware.-Unless otherwise specified, hollow ware shall have the "Butler" (dull) finish. 


\section{GENERAL REQUIREMENTS}

D-1. The size, shape, design, and insignia of each article will be shown by appropriate drawings or samples, to be furnished by the purchasing officer.

D-2. The amount of silver to be applied to each article will be specified by weight. In general, this weight will represent approximately for each class, the average thickness corresponding to Table 1 . Except for those articles listed in paragraph E-2 of this specification, the purchaser will indicate on each drawing the required weight of silver.

TABLE 1.-Approximate average thickness and weight of silver

\begin{tabular}{l|c|c}
\hline Class & $\begin{array}{c}\text { Average } \\
\text { thick- } \\
\text { ness }\end{array}$ & $\begin{array}{c}\text { Weight of } \\
\text { silver }\end{array}$ \\
\hline Ia and IIa (flatware and hollow ware) & $\begin{array}{c}\text { Inch } \\
\text { Ib and IIb (flatware and hollow ware) }\end{array}$ & $\begin{array}{r}\text { Dot. } \\
0.0008\end{array}$ \\
\hline
\end{tabular}

D-3. The back of the bowls of spoons and tines of forks in Class Ia shall have on the middle line of the bearing surface a coating of silver not less than 0.0012 inch thick at the thickest point.

D-4. On flatware and hollow ware, a minus tolerance of 15 per cent of the specified weight of silver shall be permitted on individual samples, and of 5 per cent on the average of three or more samples of the same lot of shipment.

\section{E. DETAIL REQUIREMENTS}

E-1. The four classes of tableware included in this specification cover the following articles:

Class Ia-Flatware for frequent use-

Forks, dessert, dinner.

Spoons, dessert, soup, tea, table.

Knives, tea, dessert, dinner.

Class Ib-Flatware for occasional use-

All articles of flatware not listed in Class Ia.

Class IIa-Hollow ware for frequent use-

Boats, gravy.

Bowls, ice, salad, sugar.

Dishes and covers, chafing, butter, fish, fruit, meat, vegetable.

Pitchers, cream, ice, water.

Pots, chocolate, coffee, tea.

Scrapers, crumb.

Shakers, salt, pepper.

Trays.

Tureens, soup.

Waiters.

Class IIb-Hollow ware for occasional use-

All articles of hollow ware not listed in Class IIa.

E-2. The actual weight of silver required for flatware of Class Ia, shall be as shown in Table 2 .

TABLE 2

\begin{tabular}{|c|c|c|}
\hline Article & $\begin{array}{l}\text { Approxi- } \\
\text { mate area }\end{array}$ & $\begin{array}{c}\text { Required } \\
\text { weight of } \\
\text { silver }\end{array}$ \\
\hline $\begin{array}{l}\text { Forks: } \\
\text { Dessert } \\
\text { Dinner. } \\
\text { Knives: } \\
\text { Tea hollow handle } \\
\text { Dessert hollow handle } \\
\text { Dinner hollow handle } \\
\text { Dinner hollow handle (handle oniy) } \\
\text { Dinner solid handle } \\
\text { Dinner solid handle (handle only) } \\
\text { Spoons: } \\
\text { Dessert } \\
\text { Soup. } \\
\text { Tea } \\
\text { Table }\end{array}$ & $\begin{array}{r}\text { Square } \\
\text { inches } \\
9 \\
11 \\
10 \\
15 \\
19 \\
9 \\
17 \\
7 \\
13 \\
14 \\
9 \\
17\end{array}$ & $\begin{array}{r}\text { Tr.02.loross } \\
8 \\
10 \\
6 \\
10 \\
12 \\
6 \\
10\end{array}$ \\
\hline
\end{tabular}

\section{F. METHOD OF SAMPLING. INSPECTION, $\triangle N D$ TESTS}

F-1. Sampling for weight of silver plating.-

F-1a. On flatware or hollow ware, one piece shall be tested for each gross or less of that article or class purchased. If this sample fails to have within 15 per cent of the specified weight of silver, two more samples shall be selected at random. Unless the average of the three samples tested shall have within 5 per cent of the specified weight of silver the shipment will be rejected.

F-1b. The purchaser will pay for all samples tested upon accepted deliveries, but not for any samples tested on rejected deliveries. All rejected samples of hollow ware shall be returned to the contractor.

F-2. Determination of the weight of silver.-

F-2a. On nickel brass. - The articles are thoroughly cleaned from grease by washing with alcohol or an alkaline solution, and are then dried and weighed. They are then introduced into a suitable vessel containing a mixture of 19 parts by volume of C. P. concentrated sulphuric acid (specific gravity 1.84) and 1 part by volume of C. P. concentrated nitric acid (specific gravity 1.42) which mixture has been heated (for example on a sand bath) to $80^{\circ} \mathrm{C}$. $\left(176^{\circ}\right.$ F.). (The stripping bath should be kept covered when not in use, to prevent absorption of water.) The articles are kept in the solution until all the silver is dissolved as indicated by the production of a dark color over the entire surface. They are then thoroughly rinsed, dried, and reweighed, and the loss in weight is calculated as silver.

$$
\begin{aligned}
& \text { grams } \times 0.0322=\text { troy } 0 z . \\
& \text { grams } \times 0.6440=\text { dwt. }
\end{aligned}
$$

F-2b. On steel and white metal.-The articles are cleaned, dried, and weighed as before, and are then hung as anodes in a solution containing $30 \mathrm{~g} / \mathrm{L}(4 \mathrm{oz} . /$ gal.) of sodium cyanide, in which an iron or silver cathode is suspended. A potential of 3 to 4 volts is applied and the articles are shaken or the solution is agitated until all the silver has dissolved. The articles are then rinsed, dried, and weighed, and the loss in weight is considered as silver.

F-2c. Alternate method.-The purchaser may require the contractor to give notice as to when the material is to be plated so that the purchaser may send an inspector to the factory at that time. The inspector shall then weigh one or more pieces from each gross or one hundred pieces before plating and after plating and finishing, to determine whether the specified amount of silver is present.

F-2d. Umpire method. - In case of disagreement upon the results by the methods in $(a),(b)$, or $(c)$, three additional samples shall be tested by the following method. The article is treated with nitric acid (specific gravity 1.2 , prepared by mixing equal volumes of water and concentrated nitric acid) until all the silver is dissolved. The solution is evaporated to dryness on a stream bath, treated with a few drops of nitric acid, taken up in water, filtered if necessary, and diluted to a definite volume. An aliquot portion containing about 0.5 gram of silver is then precipitated with hydrochloric acid and the silver chloride is filtered on a Gooch crucible, washed with dilute nitric acid, dried, and weighed. Weight of silver chloride $\times 0.753=$ silver.

F-3. Determination of the thickness of silver on spoons and forks.-One piece representing each gross or less shall be subjected to the following test. On the back of the bowls of spoons and tines of forks of Class Ia, the maximum thickness of silver will be determined by microscopic examination of the cross section. This is conducted by first plating that portion of the spoon or fork with copper from an acid copper sulphate solution, to a thickness of at least 0.01 inch. The bowl of the spoon,
or the body of the fork is then cut with a fine saw 
along the center axis; and the surface is carefully polished on a plane perpendicular to the surface of the spoon or fork at the point of contact until all saw marks and polishing scratches are obliterated. The thickness of the silver coating on the back of the spoon or fork, at the point where it would normally rest on a plane surface, is then measured with a suitable microscope and scale. The thickness of silver on that part at the thickest point shall be not less than 0.0012 inch.

F-4. Corrosion test of steel knife blades.-From each gross or less of knives submitted for inspection, the inspector shall select not less than one knife for the following test to determine the resistance to corrosion. Upon the clean surface of the blade three drops of copper sulphate solution shall be placed at intervals. After remaining on the blade for six minutes, the solution shall be washed off with fresh water. If the blade is stained by the solution, the material represented by that particular lot shall be considered unsatisfactory and shall be rejected. The copper sulphate solution shall be made up as follows:

Copper sulphate $\left(\mathrm{CuSO}_{1}-5 \mathrm{H}_{2} \mathrm{O}\right)$
Sulphuric acid $\left(\mathrm{H}_{2} \mathrm{SO}_{4}\right) \ldots \ldots \ldots \ldots$
Water $\left(\mathrm{H}_{2} \mathrm{O}\right) \ldots \ldots \ldots$

\section{G. PACKING AND MARKING}

G-1. Packing.-Unless otherwise specified, the subject commodity shall be delivered in standard commercial containers, so constructed as to insure acceptance by common or other carriers, for safe transportation, at the lowest rate, to the point of delivery. All articles of flatware in Class Ia shall be securely wrapped in packages of 12 each.

G-2. Marking. - All articles shall bear the manufacturers' name or trade-mark. Unless otherwise specified, shipping containers shall be marked with the name of the material, the size, type, and quantity contained therein as defined by the contract or order under which the shipment is made, the name of the contractor and the number of the contract or order.

\section{NOTES}

I-1. Method of measuring areas and specifying weights of silver.-

I-1a. For all articles for which the required amount of silver has not been previously specified, the purchaser will estimate the total area of the particular piece, and compute the amount of silver to be applied to produce the average thickness indicated in Table 1 , and state on the drawing the required weight of silver.

I-1b. Measurement of areas: It is not possible by any simple methods to measure the area of irregularly shaped articles with high precision. By making a few measurements and the following arbitrary assumptions, however, the area can usually be estimated with a probable accuracy within 10 per cent.

I-1b (1). Flat or nearly flat surfaces such as the handles of spoons and forks, blades of knives, etc., may be considered as rectangles, with a length equal to the maximum length (measured with a flexible scale) and a width equal to the average of the maxi- mum and minimum widths. The area on the two sides is therefore equal to the length multiplied by the sum of the minimum and maximum widths. Thus if the length of the handle of a teaspoon is 4 inches, the maximum width 0.75 inch and the minimum width 0.20 inch, the total area of the handle may be considered as $4(0.75+0.20)=3.80$ square inches. (In this calculation it is assumed that the error involved in omitting the thickness of the handle usually about balances the error involved by the assumption that the average width is the mean of the minimum and maximum widths.

I-1b (2). For surfaces which approach a cylinder or cone (e. g. a knife handle or the body of a pitcher), the area may be estimated by multiplying the length by the average circumference, i. e., the mean of the minimum and maximum circumferences, as measured with a string or flexible scale. Thus if a knife handle is 4.5 inches long, the maximum circumference 2.75 inches and the minimum 1.55 inches,

the area is assumed to be $4.5\left(\frac{1.55+2.75}{2}\right)=9.7$ square inches.

I-1b (3). For ovoid or elliptical surfaces, such as the bowls of spoons, dishes, etc., it has been found by a series of measurements and approximations, that the area on each side is approximately equal to that of an ellipse, the two axes of which are equal respectively to the extreme length and width of the bowl. (These measurements may be made on the outside of the bowl with a flexible scale.) Thus if the extreme length of the bowl of a teaspoon is equal to 2.20 inches, and the extreme width 1.44 inches, the area on each side is equal to 3.14 (that is $\pi$ ) $\times$

$\left(\frac{2.20 \times 1.44}{4}\right)=2.49$ inches. A simple formula for

the area on the two sides of the bowl is therefore

$$
a=\frac{\pi l w}{2}
$$

where

$a=$ total area of bowl.

$\pi=3.14$

$l=$ extreme length.

$w=$ extreme width.

The total area of the teaspoon considered in this paragraph is 4.98 (bowl) +3.80 handle) $=8.8$ square inches or for practical purposes 9 square inches.

I-1c. Relation between the area and the weight and average thickness of the deposit.-For such computations the following factors may be employed:

1. Specific gravity of silver $=10.5$.

2. A silver coating 0.001 inch in thickness, weighs 0.80 troy oz./ft. ${ }^{2}$, or $16 \mathrm{dwt} . / \mathrm{ft}^{2}$, or $0.11 \mathrm{dwt} . / \mathrm{in.} .^{2}$.

3. Dwt./doz. $\times 0.6=$ troy oz./gross.

4. Average thickness in inches $\times 800=$ troy oz. $/ \mathrm{ft}^{2}$

5. Average thickness in inches $\times 16000=\mathrm{dwt} . / \mathrm{ft} .^{2}$

6. Area of a piece in square inches=number of square feet per gross.

(An extended table contains the stock numbers, plan numbers, classes of silverware, and minimum deposit in pennyweights for each item of silverware for naval use. The items range from admiral's smoking sets to officers' mess finger bowl trays.) 
671.

\section{CLOCKS}

(No nationally recognized specifications available.) 672.

\section{WATCHES}

NATIONAL WATCH CASE MANUFACTURERS ASSOCIATION, RULING OF THE FEDERAL TRADE COMMISSION INDORSED BY THE N. W. C. M. A. AND BY MANUFACTURERS OF GOLD-FILLED AND GOLD-PLATED WATCHCASES, JULY 25, 1931

\section{WATCHCASES}

Rule A.-The industry recommends that in every instance the manufacturer and the dealer place the maker's trade-mark conspicuously and indelibly on the inner surface of the lid or cap of any watches disposed of, and in the case of wrist watches, on the inner surface of the back.

Rule B.-The industry recommends that the line of time guarantee cases be definitely restricted in number to three: (a) 25-year cases, (b) 20-year cases, and (c) 10-year cases.

Rule C.-The industry recommends that the official standard for cases entitled to bear a 25-year guarantee stamp be as follows:

The sheet of gold or of its alloy affixed to the outer surface of the backs, center, open-faced bezel, pendant, crown and bow, shall not be less than four one-thousandths of 1 inch in thickness; the sheet of gold or its alloy affixed to the inner surfaces of the backs, to the inner and outer surfaces of the caps, and to the outer surface of the hunting bezel, shall not be less than one one-thousandth of an inch in thickness.

That whenever the thickness of the sheets of gold or its alloy in gold-filled watchcases is indicated, the mark indicating such thickness shall only refer to the thickness of the sheets of gold or its alloy so affixed to the outer surfaces of the backs, center, open face, bezel, pendant, crown and bow, the mark accurately indicating such thickness which shall be expressed in decimals indicating thousandths of an inch, in tests to ascertain the thickness, measurements being taken at a point where no gold has been added or taken away from decoration or ornament.

Rule D.-The industry recommends that the official standard for cases entitled to bear a 20-year guarantee stamp be as follows:
(This rule is similar to rule $\mathrm{C}$ for 25 -year cases except that the thickness of plate for outer surfaces shall be not less than three one-thousandths of an inch and for inner surfaces not less than one onethousandth of an inch.)

Rule E.-The industry recommends that the offcial standard for cases entitled to bear a 10-year guarantee stamp be that such cases shall have to contain as a minimum, a layer of gold of a thickness of one and one-half thousandths of an inch on the outer surface. The recognized marking for cases of this standard shall be "(blank) carat rolled gold plate, guaranteed (or warranted) 10 years."

(Rule $\mathrm{F}$ forbids the use of the word gold where the gold content is less than prescribed in above rules. Rule $\mathrm{G}$ limits the use of the time guarantee clause to the description of the wearing quality of the precious metal content of the watchcase.)

Rule H.-The industry recommends that all nickel or base metal cases be stamped with the word "nickel" or with the words "base metal" and condemns the manufacture and sale of such cases unless so stamped.

\section{UNITED STATES GOVERNMENT, DEPART-} MENT OF COMMERCE, BUREAU OF STANDARDS, JEWELERS' AND SILVERSMITHS' WEIGHTS AND MEASURES, CIRCULAR NO. 43, JANUARY 24, 1921

\section{SIZES OF WATCHES}

Watch sizes are based upon the diameter of the pillar plate. Watch movements made on the continent of Europe have their diameters expressed either in millimeters or in lignes, the former method being somewhat uncommon. A watch movement made in the United States has its diameter expressed in terms of a certain "Size No." The diameter of the 0 -size watch is thirty-five thirtieths of an inch; the size number increases for each thirtieth of an inch. The diameter of a 12-size watch movement is therefore forty-seven thirtieths of an inch (1.567 inches, or 39.79 millimeters).

From the third column of Table 1 it is seen that an 18-ligne watch equals almost exactly a 13-size and that a 15-ligne equals very closely a 5-size. In connection with the most common sizes it is well to note that the diameter of a 16 -size watch is nearest to 19 lignes, 12 -size to 18 lignes, and 0 -size to 13 lignes. 
TABLE 1.-Watch sizes

[Based upon the diameter of pillar plate. 1 ligne $=2.2559$ millimeters; 1 inch $=25.40005$ millimeters, Size No. $=$ Number of thirtieths (30th's) of an inch in excess of 35 thirtieths $(35 / 30)$ of an inch]

\begin{tabular}{|c|c|c|c|c|c|c|c|c|c|}
\hline \multirow{2}{*}{ 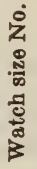 } & \multicolumn{4}{|c|}{ Pillar plate diameter } & \multirow{2}{*}{ 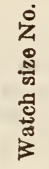 } & \multicolumn{4}{|c|}{ Pillar plate diameter } \\
\hline & 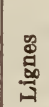 & $\mathrm{mm}$ & Inches & $\begin{array}{l}\text { Thir- } \\
\text { tieths } \\
\text { of an } \\
\text { inch }\end{array}$ & & 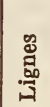 & $\mathrm{mm}$ & Inches & $\begin{array}{l}\text { Thir- } \\
\text { tieths } \\
\text { of an } \\
\text { inch }\end{array}$ \\
\hline 32 & 25 & 56.73 & 2. 233 & 67 & 14 & & 41.49 & 1.633 & 49 \\
\hline 31 & & 55.88 & 2. 200 & 66 & & 18 & $\begin{array}{l}40.64 \\
40.61\end{array}$ & $\begin{array}{l}1.600 \\
1.599\end{array}$ & 48 \\
\hline $\begin{array}{l}30 \\
29\end{array}$ & & $\begin{array}{l}55.03 \\
54.19\end{array}$ & $\begin{array}{l}2.167 \\
2.133\end{array}$ & $\begin{array}{l}65 \\
64\end{array}$ & $\begin{array}{l}12 \\
11\end{array}$ & 12 & $\begin{array}{l}39.79 \\
38.95\end{array}$ & $\begin{array}{l}1.567 \\
1.533\end{array}$ & $\begin{array}{l}47 \\
46\end{array}$ \\
\hline & 24 & 54.14 & 2. 132 & & & 17 & 38.35 & 1.510 & \\
\hline 28 & & 53. 34 & 2.100 & 63 & 10 & & 38. 10 & 1.500 & 45 \\
\hline 27 & 23 & $\begin{array}{l}52.49 \\
51.89\end{array}$ & $\begin{array}{l}2.067 \\
2.043\end{array}$ & 62 & $\begin{array}{l}9 \\
8\end{array}$ & $\cdots$ & $\begin{array}{l}37.25 \\
36.41\end{array}$ & $\begin{array}{l}1.467 \\
1.433\end{array}$ & $\begin{array}{l}44 \\
43\end{array}$ \\
\hline 26 & & 51.65 & 2.033 & 61 & & & & & \\
\hline 25 & $\cdots$ & $\begin{array}{l}50.80 \\
49.95\end{array}$ & 2. 000 & $\begin{array}{r}60 \\
59\end{array}$ & 7 & $\ldots$ & 35.56 & 1.400 & 42 \\
\hline & 22 & $\begin{array}{l}49.63 \\
49.63\end{array}$ & 1. 954 & & $\begin{array}{l}6 \\
5\end{array}$ & $\cdots$ & $\begin{array}{l}34.71 \\
33.87\end{array}$ & $\begin{array}{l}1.367 \\
1.333\end{array}$ & $\begin{array}{l}41 \\
40\end{array}$ \\
\hline $\begin{array}{l}23 \\
22\end{array}$ & & $\begin{array}{l}49.11 \\
48.26\end{array}$ & $\begin{array}{l}\text { 1. } 933 \\
1.900\end{array}$ & $\begin{array}{l}58 \\
57\end{array}$ & 4 & 15 & $\begin{array}{l}33.84 \\
33.02\end{array}$ & $\begin{array}{l}1.332 \\
\text { 1. } 332 \\
1.300\end{array}$ & 39 \\
\hline 21 & & 47. 41 & 1.867 & 56 & 3 & & 32.17 & 1. 267 & 38 \\
\hline 20 & & $\begin{array}{l}47.37 \\
46.57\end{array}$ & $\begin{array}{l}1.865 \\
1.833\end{array}$ & 55 & 2 & 14 & $\begin{array}{l}31.58 \\
31.33\end{array}$ & $\begin{array}{l}1.243 \\
1.233\end{array}$ & 37 \\
\hline 19 & & 45. 72 & 1.800 & 54 & & & & & 36 \\
\hline & & 45.12 & 1.77 & & 1 & $-\cdots$ & $\begin{array}{l}30.48 \\
29.63\end{array}$ & $\begin{array}{l}1.200 \\
1.167\end{array}$ & \\
\hline 18 & $\ldots$. & 44.87 & 1.767 & 53 & & 13 & 29.33 & 1.155 & \\
\hline 17 & & 44. 03 & 1. 733 & 52 & $2 / 0$ & 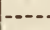 & 28.79 & 1. 133 & 34 \\
\hline 16 & & 43. 18 & 1.700 & 51 & $3 / 0$ & $\cdots$ & 27. 94 & 1.100 & 33 \\
\hline & 19 & 42.86 & 1.68 & & $4 / 0$ & & 27.09 & 1.0 & 32 \\
\hline 15 & & 42.33 & 1.667 & & 50 & 12 & 27.07 & 1.066 & \\
\hline
\end{tabular}

TABLE 1.-Watch sizes-Continued

\begin{tabular}{|c|c|c|c|c|c|c|c|c|c|}
\hline \multirow{2}{*}{ 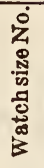 } & \multicolumn{4}{|c|}{ Pillar plate diameter } & \multirow{2}{*}{ 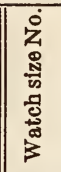 } & \multicolumn{4}{|c|}{ Pillar plate diameter } \\
\hline & $\begin{array}{l}\text { : } \\
\stackrel{\infty}{0}\end{array}$ & $\mathrm{~mm}$ & Inches & $\begin{array}{l}\text { Thir- } \\
\text { tieths } \\
\text { of an } \\
\text { inch }\end{array}$ & & $\begin{array}{l}\mathscr{8} \\
\stackrel{80}{3}\end{array}$ & $\mathrm{~mm}$ & Inches & $\begin{array}{l}\text { Thir- } \\
\text { tieths } \\
\text { of an } \\
\text { inch }\end{array}$ \\
\hline $\begin{array}{l}5 / 0 \\
6 / 0\end{array}$ & & $\begin{array}{l}26.25 \\
25.40\end{array}$ & $\begin{array}{l}1.033 \\
1.000\end{array}$ & $\begin{array}{l}31 \\
30\end{array}$ & $10 / 0$ & $93 / 2$ & $\begin{array}{l}22.01 \\
21.43\end{array}$ & $\begin{array}{r}0.867 \\
.844\end{array}$ & 26 \\
\hline $7 / 0$ & 11 & $\begin{array}{r}24.81 \\
24.55\end{array}$ & $\begin{array}{r}.977 \\
.967\end{array}$ & 29 & & $\begin{array}{l}9 \\
81 / 2\end{array}$ & $\begin{array}{l}20.30 \\
18.18\end{array}$ & $\begin{array}{r}.799 \\
.755\end{array}$ & \\
\hline $8 / 0$ & & 23 & .933 & 28 & -.... & 8 & 18.05 & .711 & \\
\hline $9 / 0$ & $101 / 2$ & $\begin{array}{l}23 . \\
22 .\end{array}$ & .933 & 27 & - & $73 / 2$ & & .666 & 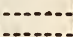 \\
\hline & 10 & 22.56 & .888 & & & 6 & 13.54 & .533 & \\
\hline
\end{tabular}

UNITED STATES GOVERNMENT, DEPARTMENT OF COMMERCE, BUREAU OF STANDARDS, TESTING OF TIMEPIECES, CIRCULAR NO. 392, JUNE 18, 1931

\section{TESTING OF TIMEPIECES}

(This circular describes in detail six standard tests for watches and chronometers adopted by the Bureau of Standards. These tests consist of four tests for pocket watches-namely, "class A," "class B," "railroad precision," and "business precision" - a short test for stop watches, and a short test for chronometers. Directions for the submittal of timepieces for test and other items of general information are also given.)

\section{3.}

\section{DIAIS} able.)

(No nationally recognized specifications avail- 
680.

GENERAL ITEMS

AMERICAN SOCIETY FOR TESTING MATERIALS, TENTATIVE METHODS OF TENSION TESTING OF METALLIC MATERIALS, DESIGNATION E8-27T, 1927

TENSION TESTING OF METALLIC MATERIALS

(See 600.1, p. 34.)

AMERICAN SOCIETY FOR TESTING MATERIALS, TENTATIVE METHODS OF COMPRESSION TESTING OF METALLIC MATERIALS, DESIGNATION E9-27T, 1927

\section{COMPRESSION TESTING OF METALLIC MATERIALS}

(See 600.1, p. 38.)

AMERICAN SOCIETY FOR TESTING MATERIALS, STANDARD METHODS OF METALLOGRAPHIC TESTING OF NONFERROUS METALS AND ALLOYS, DESIGNATION E5-27, 1927

\section{METALLOGRAPHIC TESTING OF NONFERROUS METALS}

(See 600.1, p. 28.)

681.

TIN

681.0 GENERAL ITEMS.

UNITED STATES GOVERNMENT, DEPARTMENT OF COMMERCE, BUREAU OF STANDARDS, STANDARD SAMPLES, CIRCULAR NO. 25 AND SUPPLEMENT, 1927

\section{TIN SAMPLE}

(Sample No. $42 \mathrm{~b}$ is a standard sample of tin prepared and sold by the Bureau of Standrads for use by industrial organizations and others as a standard for calibrating pyrometers, etc. Each sample is accompanied with a certificate giving the melting point of that particular sample.)

(Circular No. 66 of the Bureau of Standards entitled "Standard Samples for Thermometric Fixed Points" gives a description of the preparation of pure samples of tin and other metals, their chemical analyses, determination of freezing points, and methods of using these standard-temperature samples.)

\subsection{INGOTS AND PIG METAL, TIN.}

NATIONAL METAL EXCHANGE (INC.) AND NATIONAL METAL CLEARING ASSOCIATION (INC.), BY-LAWS AND RULES, 1931

\section{BY-IAWS CONCERNING TIN}

TENDERABLE GRADES OF TIN

Section 40.-Tin bearing the following brands and/or descriptions may be delivered against the $\mathrm{Na-}$ tional Metal Exchange (Inc.), standard tin contract.
Class A.-Banka tin, Straits tin ( 4 brands listed in full text of this by-law), English refined tin (4 brands listed), German refined tin (3 brands listed), Belgian refined (1 brand listed), Australian refined (3 brands listed).

Class B.-English common (4 brands listed), German common (3 brands listed), Chinese (1 brand listed).

(This by-law specifies the discount applicable to contract price when any one of the brands in class $\mathrm{B}$ is tendered.)

\section{UNMERCHANTABLE TIN}

Section 43.-If a majority of the board of governors shall at any time determine that any one brand tenderable against exchange contracts has depreciated in its metallurgical assay to a percentage below that represented by the producers of said tin and/or as generally recognized by the trade, thereupon the board of governors may exclude said brand from tenderability on exchange contracts unless deliveries of said brand are accompanied by certificates of analysis of one of the official assayers of the exchange showing a minimum tin content as follows:

traits, Banka, Australian, and German Australian, and/or English, Belgian

English, German, or Chinese common brands

UNITED STATES GOVERNMENT, FEDERAI SPECIFICATIONS BOARD, FEDERAI SPECIFICATION FOR PIG TIN, QQ-T-371. MAY 31, 1932

\section{PIG TIN \\ GRADES}

1. There shall be two grades of pig tin conform. ing to the requirements as stated below.

\section{MATERIAL}

2. (a) Grade A tin shall be new metal, free from scrap or remelted metal.

(b) Grade B tin may be Chinese tin, or may contain remelted metal.

\section{GENERAL REQUIREMENTS}

3. The general requirements shall be as stated in the following paragraph.

\section{DETAIL REQUIREMENTS}

4. The chemical properties of pig tin shall be as follows:

[Per cent]

\begin{tabular}{|c|c|c|c|c|c|c|c|c|c|c|}
\hline 芯 & 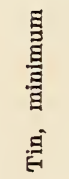 & 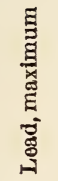 & 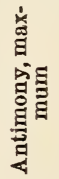 & 茪 & 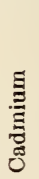 & 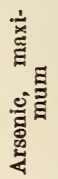 & 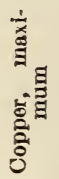 & 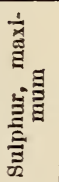 & 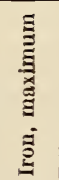 & 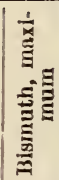 \\
\hline$\underset{B}{A}$ & $\begin{array}{l}99.75 \\
98.00\end{array}$ & $\begin{array}{l}0.10 \\
1.50\end{array}$ & $\begin{array}{l}0.10 \\
1.50\end{array}$ & $\begin{array}{l}x \\
x\end{array}$ & $\begin{array}{l}x \\
x\end{array}$ & $\begin{array}{r}0.10 \\
.10\end{array}$ & $\begin{array}{r}0.10 \\
.10\end{array}$ & $\begin{array}{r}0.01 \\
.01\end{array}$ & $\begin{array}{r}0.01 \\
.10\end{array}$ & $\begin{array}{l}0.01 \\
.10\end{array}$ \\
\hline
\end{tabular}

$\mathbf{x}=$ none as determined from a 20-gram sample.

The material shall be clean and of uniform quality. 
TEST AND INSPECTION

5 (a) Sample shall be taken as follows: Four representative pigs shall be taken from each lot of 5,000 pounds or fraction thereof of an order. A half-inch hole shall be drilled from the top (as cast) of each pig, to one-quarter inch from the bottom, the drillings from the first one-eighth of an inch being discarded in each instance. No lubricant shall be used and drilling shall be under uniform pressure and not forced sufficiently to cause oxidation of the chips.

(b) Approximately equal weights of drillings from each pig shall be thoroughly mixed unless the question of homogeneity of the metal arises (when drillings shall remain unmixed and analyzed separately). The composite sample thus formed shall weigh not less than 15 ounces, and analysis shall be made of this mixture.

(c) Check analyses may be made by the inspector, or through him by any Government laboratory or other designated representative, and without cost to the contractor. The chemical composition thus determined shall conform to the requirements specified.

\section{MARKING}

6. All ingots both when presented for inspection and when loaded for shipment shall be legibly and indelibly marked with the brand name.

\subsection{SHEETS, TIN.}

(Roofing tin, terneplate. See 604.31, p. 241.)

681.3 CASTINGS, TIN.

(No nationally recognized specifications available.)

\subsection{MANUFACTURES OF TIN.}

681.41 HOLLOW WARE, TIN.

AMERICAN RAILWAY ASSOCIATION, MECHANICAL DIVISION, TINWARE, STANDARDIZATION OF, RECOMMENDED PRACTICE, 1915

\section{TINWARE}

(See 612.29, p. 819.)

681.42 COATINGS, TIN.

(Test of tin coatings; see 600.3 , p. 64.)

(Roofing tin, terneplate; see 604.31, p. 241.)

681.43 ALLOYS, TIN.

(Bronzes. See 643., p. 1191; 646., p. 1243; 647., p. $1258 ; 692.3$, p. 1327.)

(Phosphor-Tin. See 682., p. 1307.)

(Babbitt Metal. See 692.1, p. 1319.)

(Solder. See 693.2, p. 1333.)

681.49 MISCELIANEOUS MANUFACTURES OF TIN.

AMERICAN BOTTLERS OF CARBONATED BEVERAGES, STANDARDS FOR BEVERAGE MANUFACTURING EQUIPMENT AND RECOMMENDED PRACTICES, EDUCATIONAL BULLETIN NO. 4, 1930

\section{RUBBER HOSE AND BLOCK TIN TUBING RECOMMENDED PRACTICE}

While all commercial piping and fittings threaded according to the National or American Briggs standard pipe thread system may be used with the A. B. C. B. connection standards, it will be particularly necessary that the inside diameters of rubber hose and block tin tubing as well as the outside diameters of light gage sirup line (metal) be selected to conform to those thread standards if their use is to be completely successful.

The following inside diameters for rubber hose and block tin tubing are recommended as satisfactory and every effort should be made to promote such sizes as standard: $3^{\prime}, 1,11_{4}^{\prime}, 11 / 2$, and 2 inch sizes.

The following outside diameters for light-gage metal sirup line tubing are also recommended as most satisfactory and as standard: $5 / 8,7 / 8,11 / 8,13 / 8$, and $13 \%$ inch sizes.

\section{AMERICAN BOTTLERS OF CARBONATED BEVERAGES, STANDARDS FOR BEVER- AGE MANUFACTURING EQUIPMENT AND RECOMMENDED PRACTICES, EDUCA- TIONAL BULLETIN NO. 4, 1930}

\section{FITTINGS}

(For fittings for tin and other metal tubing see 645.4, p. 1230.)

682.

PHOSPHOR TIN

AMERICAN SOCIETY FOR TESTING MATERIALS, STANDARD SPECIFICATIONS FOR PHOSPHOR TIN, SERIAL DESIGNATION B51-27, 1927

\section{PHOSPHOR TIN \\ $\mathrm{SCOPE}$}

1. These specifications cover phosphor tin in one grade.

\section{MANUFACTORE}

2. The manufacturer shall use new tin of uniform quality throughout in the manufacture of phosphor tin.

3. The manufacturer or vendor shall furnish phosphor tin ingots in the form of notched slabs of a size that can be handled conveniently.

4. Phosphor tin shall be clean, free from defects, and uniform in quality.

\section{CHEMICAL PROPERTIES AND TESTS}

5. The material shall conform to the following requirements as to chemical composition:

Phosphorus, minimum

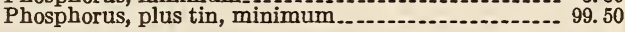

6. One ingot from each melt or portion of melt shall be selected, and not less than $140 \mathrm{~g}$ of clean fine drillings or sawdust shall be taken from the ingot and mixed thoroughly. The ingots selected shall be drilled completely through, or one-half through, from top to bottom, the surface material being rejected.

\section{MARKING}

7. A brand and melt number shall be cast or otherwise plainly marked upon each slab or ingot by which the manufacturer and heat may be identified. Marking may be done by either stamping or painting.

\section{CLATMS}

(Claims are the same as in A. S. T. M. B30-31T (see 644.11 , p. 1196) except that they must be made in writing within 10 days instead of the 30 days specified in $\mathrm{B} 30-31 \mathrm{~T}$.) 


\section{UNITED STATES GOVERNMENT, FEDERAL SPECIFICATIONS BOARD, FEDERAL SPECIFICATION FOR PHOSPHOR TIN, QQ-T-351 MAY 3, 1932}

\section{PHOSPHOR TIN}

(Except for the omission of claims and umpire chemical analysis, this specification is identical with A. S. T. M. B51-27, given above except that a sample of $150 \mathrm{~g}$ is required.)

683.

ZINC

\subsection{GENERAL ITEMS.}

\section{AMERICAN SOCIETY FOR TESTING MATE- RIALS, STANDARD METHODS OF CHEM- ICAL ANALYSIS OF SPELTER, SERIAI DESIGNATION B38-31, 1921}

\section{CHEMICAL ANALYSIS OF SPELTER}

\section{DETERMINATION OF LEAD BY THE ELECTROLYTIC METHOD}

\section{APPARATUS}

Platinum cylindrical gauze electrodes containing approximately 400 meshes per square centimeter (50 meshes per linear inch) are recommended for anode and cathode. They should be stiffened by doubling the gauze for about $3 \mathrm{~mm}$ at the top and at the bottom of the cylinders. The anode should be approximately $30 \mathrm{~mm}$ in diameter and $30 \mathrm{~mm}$ in height. The stem should be made from $1.14 \mathrm{~mm}$ or $1.29 \mathrm{~mm}$ wire, flattened and electrically welded the entire width of the gauze; the height over all should be approximately $130 \mathrm{~mm}$, and the gauze should be sand-blasted. The cathode should be approximately $12 \mathrm{~mm}$ in diameter by $30 \mathrm{~mm}$ in height, the stem being of the same dimensions and welded in the same way as the anode.

\section{METHOD}

Place $8.643 \mathrm{~g}$ (note 1 ) of the sample in a $400 \mathrm{c}$ c beaker, add sufficient water to cover, and then add 30 c c $\mathrm{HNO}_{3}$ (specific gravity 1.42) gradually. When action is complete, boil the solution for 10 minutes, wash off the watch glass, and transfer the solution to a $200 \mathrm{c}$ c electrolytic beaker, washing out the original $400 \mathrm{c}$ c beaker. Dilute to $125 \mathrm{c} \mathrm{c}$ and electrolyze (note 2) with a current of 5 amperes for 45 minutes. (Note 3.) Test the solution for complete precipitation of lead by washing the watch glasses and sides of the beaker, increasing the depth of the solution about $12 \mathrm{~mm}$. Continue the current for 15 minutes and, if the newly exposed surface is still bright, wash the anode (note 4) three or four times with distilled water, once with alcohol, and then dry in an oven (note 5) at $210^{\circ} \mathrm{C}$. for 30 minutes, cool in a desiccator, and weigh.

The weight of the deposited $\mathrm{PbO}_{2}$, in milligrams, divided by 100 , gives the percentage of lead. (Note 6.)

Notes.-1. The empirical factor weight, $8.643 \mathrm{~g}$, is used instead of the theoretical one $(8.660 \mathrm{~g})$ as dried dioxide contains some adherent and included water, not entirely expelled.

2. Before electrolyzing, the beaker should be covered with split watch glasses to prevent loss by spraying.

3. The time required for complete deposition is usually 30 to 45 minutes. For small amounts of lead 30 minutes will be found sufficient.

4. The washing should be done immediately, as the deposit of $\mathrm{PbO}_{2}$ tends to dissolve.

5. The anode may also be dried on a hot plate, although a drying oven is to be preferred.

6. The $\mathrm{PbO}_{2}$ deposit can be readily removed by immersing the anode in hot dilute solution of $\mathrm{HNO}_{3}$ to which oxalic acid has been added, or by covering with dilute $\mathrm{HNO}_{2}$ and inserting a rod of copper.

\section{DETERMIN ATION OF LEAD BY THE LEAD ACID METBOD}

\section{BOLUTIONS REQUIRED}

Lead acid.-Pour 300 c c of $\mathrm{H}_{2} \mathrm{SO}_{4}$ (specific gravity 1.84) into $1,800 \mathrm{c} \mathrm{c}$ of distilled water; dissolve $1 \mathrm{~g}$ of lead acetate in $300 \mathrm{c}$ c of distilled water, and add to the acid solution with stirring. Allow to settle for several days and siphon off the clear solu tion through a thick asbestos pad. (Note 1.)

Nitric acid.-Mix 1,000 c c of $\mathrm{HNO}_{3}$ (specific gravity 1.42) with $1,000 \mathrm{c}$ c of distilled water.

Alcohol.-Mix 1,000 c c of ethyl alcohol (95 per cent) with $1,000 \mathrm{c} \mathrm{c}$ of distilled water.

\section{METHOD}

Place sample (note 2) of $25,15,10$, or $5 \mathrm{~g}$ in 8 $350 \mathrm{c}$ c beaker and add $300,180,120$, or 60 c. c, respectively, of lead acid. After all but about $1 \mathrm{~g}$ is dissolved, filter on a close filter and wash out the beaker twice with lead acid from a wash bottle. Wash the undissolved matter from the filter into the original beaker with water and dissolve with $10 \mathrm{c}$ c of $\mathrm{HNO}_{3}$ Add $40 \mathrm{c}$ c of lead acid and evaporate until copious fumes of $\mathrm{H}_{2} \mathrm{SO}_{4}$ are evolved. When cool, add $35 \mathrm{c}$ c (Note 3 ) of water, heat to boiling and add the first filtrate (note 4), stir well and allow to stand overnight (note 5). Filter on a Gooch crucible, wash with lead acid, then with alcohol-water mixture, and finally with alcohol alone. Set the Gooch crucible inside of a larger porcelain crucible (note 6) and heat for five minutes at the full heat of a Tirrill burner. Cool and weigh as $\mathrm{PbSO}_{4}$.

Notes. - 1 . When lead acid is used, it is unnecessary to consider the solubility of $\mathrm{PbSO}_{4}$. since the solution is always brought back to the same volume as the lead acid used originally, consequently it will retain no $\mathrm{PbSO}_{4}$ from the sample.

2. The amount of sample taken is governed by the grade of spelter: Grade No. 1, $25 \mathrm{~g}$. $\quad$ Grade No. $3,10 \mathrm{~g}$.

Grade No, $5,5 \mathrm{G}$.

3. The $35 \mathrm{c} \mathrm{c}$ of water added is the quantity of water evaporated from the lead acid.

4. The first filtrate, which contains the greater part of the zinc, may also contain a small amount of $\mathrm{PbSO}_{4}$.

5. It is preferable to allow the solution and precipitate of $\mathrm{PbSO}_{4}$ to stand overnight, although this time may be shortened to not less than five hours.

6. The Gooch crucible is heated inside of a porcelain crucible to avoid reduction of the $\mathrm{PbSO}_{4}$ by flame gases and mechanical disntegration of the asbestos mat.

\section{DETERMIN ATION OF IRON}

\section{SOLUTIONS REQUIRED}

Dilute ammonia water.-Mix $100 \mathrm{c}$ c of $\mathrm{NH}_{4} \mathrm{OH}$ (specific gravity 0.90 ) with $1,000 \mathrm{c}$ c of distilled water.

Sulphuric acid (1:4).-Mix $250 \mathrm{c} \mathrm{c}$ of $\mathrm{H}_{2} \mathrm{SO}_{4}$ (specific gravity 1.84) with $1,000 \mathrm{c}$ c of distilled water.

Sulphuric acid (1:1).-Mix 500 c c of $\mathrm{H}_{2} \mathrm{SO}_{4}$ (specific gravity 1.84) with $500 \mathrm{c}$ c of distilled water.

Dilute sulphuric acid for reductor.-Mix $50 \mathrm{c} \mathrm{c}$ of $\mathrm{H}_{2} \mathrm{SO}_{4}$ (specific gravity 1.84) with $1,000 \mathrm{c}$ c of distilled water. This solution is used boiling hot.

Potassium permanganate.-Dissolve $0.2 \mathrm{~g}$ of $\mathrm{KMnO}_{4}$, chemically pure, in $1,000 \mathrm{c}$ c of distilled water. Allow to stand for several days in a glassstoppered bottle in a dark closet, and then filter through prepared asbestos. Standardize against $0.020 \mathrm{~g}$ portions of pure sodium oxalate, dissolved in $200 \mathrm{c}$ c of the dilute $\mathrm{H}_{2} \mathrm{SO}_{4}$ for reductor. Each cubic centimeter of $\mathrm{KMnO}_{4}$ is equivalent to approximately $0.00033 \mathrm{~g}$ of iron.

\section{METHOD}

Place $25 \mathrm{~g}$ of the sample in a tall $700 \mathrm{c}$ c beaker and dissolve cautiously with $125 \mathrm{c} \mathrm{c}$ of $\mathrm{HNO}_{3}$ (spe- 
cific gravity 1.42). Boil, dilute to about $300 \mathrm{c} \mathrm{c}$, add $10 \mathrm{~g}$ of $\mathrm{NH}_{4} \mathrm{Cl}$ and then ammonia water (specific gravity 0.90$)$ until the precipitated $\mathrm{Zn}(\mathrm{OH})_{2}$ has redissolved. Boil, let settle, and filter on an $11 \mathrm{~cm}$ filter paper. (Note 1.) Wash with dilute ammonia water and then with hot water until free from chlorides. Dissolve precipitate from the paper with hot $\mathrm{H}_{2} \mathrm{SO}_{4}(1: 4)$, add $40 \mathrm{c} \mathrm{c}$ of $\mathrm{H}_{2} \mathrm{SO}_{4}(1: 1)$, cool, and pass through a Jones reductor. (Note 2.) Wash out the reductor first with $150 \mathrm{c} c$ of dilute $\mathrm{H}_{2} \mathrm{SO}_{4}$ for reductor and then with $100 \mathrm{c}$ c of distilled water and titrate with standard $\mathrm{KMnO}_{4}$ solution. (Note 3.)

Nores.-1. A filter paper which will retain $\mathrm{Fe}(\mathrm{OH})_{3}$ and filter rapidly should be used. Whenever the amount of iron is at all large or when great accuracy is demanded, a second precipitation of $\mathrm{Fe}(\mathrm{OH})$ should be made by dissolving the precipitate on the paper in hot dilute $\mathrm{HCl}$, adding ammonia water and filtering, in order in hot dilute $\mathrm{HCl}$, adding ammo

2 . The reductor should be clean, preferably washed out with 150 c c of dilute $\mathrm{H}_{2} \mathrm{SO}_{4}$ and $100 \mathrm{c}$ c of water first. If, before passing through the reductor, a large amount of $\mathrm{PbSO}_{4}$ is present, it is well to filter it off so as to prevent it from clogging the reductor. A small quantity of liquid should always be left in the reductor funnel, and air should never be allowed to enter the body of the reductor. For description and further details of use of reductor, see "The Chemical Analysis of Iron," by A. A. Blair, or "Quantitative Chemical Analysis," by Iron," by A.

3. A blank determination should be made on corresponding amounts of acid and water passed through the reductor and the results should be corrected accordingly. About $0.5 \mathrm{c} \mathrm{c}$ of the $\mathrm{KMnO}_{4}$ solution will be required to give a permanent coloration to the solution.

\section{DETERMINATION OF CADMIOM GRAVIMETRICALLY}

\section{SOLUTIONS REQUIRED}

Sulphuric acid (1:1).-Mix 500 c c of $\mathrm{H}_{2} \mathrm{SO}_{4}$ (specific gravity 1.84) with $500 \mathrm{c}$ c of distilled water. Sulphuric acid $(1: 5)$.- Mix 200 c c of $\mathrm{H}_{2} \mathrm{SO}_{4}$ (specific gravity 1.84 ) with $1,000 \mathrm{c}$ c of distilled water.

Hydrochloric acid (1:3).- Mix 250 c c of $\mathrm{HCl}$ (specific gravity 1.20) with $750 \mathrm{c}$ c of distilled water.

\section{METHOD}

Place $25 \mathrm{~g}$ of drillings in a tall $500 \mathrm{c}$ c beaker; add $250 \mathrm{c} c$ of water and $55 \mathrm{c} \mathrm{c}$ of $\mathrm{HCl}$ (specific gravity 1.20 ) and stir. When the action has almost ceased add more acid with stirring, using about $2 \mathrm{c} c$ at a time and allowing to stand after each addition, until finally all but about $2 \mathrm{~g}$ of the zinc has been dissolved. (Note 1.) Filter, first transferring one of the undissolved pieces of zinc to the filter, and wash twice with water. Discard the filtrate. Wash the undissolved matter on the filter paper into the $\mathbf{5 0 0}$ c c beaker, cover, and dissolve in $10 \mathrm{c} c$ of water and $10 \mathrm{c}$ c of $\mathrm{HNO}_{3}$ (specific gravity 1.42). Transfer to a casserole, add $20 \mathrm{c}$ c of $\mathrm{H}_{2} \mathrm{SO}_{4}(1: 1)$, and evaporate until fumes appear. Take up with $100 \mathrm{c} \mathrm{c}$ of water, boil, cool, and let settle over night. (Note 2.) Filter off the $\mathrm{PbSO}_{4}$ on paper, wash twice with water, retain the filtrate but discard the paper and precipitate. Dilute the filtrate to $400 \mathrm{c}$ c, add about $10 \mathrm{~g}$ of $\mathrm{NH}_{4} \mathrm{Cl}$, and pass $\mathrm{H}_{2} \mathrm{~S}$ gas through for one hour. (Note 3.) Allow to stand until the precipitate has settled, and filter off the impure CdS (note 4) on a loose-bottom Gooch crucible. Remove the precipitate by punching out the bottom into a tall 200 c c beaker, wiping off the sides of the crucible with a little asbestos pulp. Add 60 c c of $\mathrm{H}_{2} \mathrm{SO}_{4}$ $(1: 5)$ and boil for 30 minutes. (Note 5.) Filter, wash, and dilute to $300 \mathrm{c} \mathrm{c}$, add about $5 \mathrm{~g}$ of $\mathrm{NH}_{4} \mathrm{Cl}$; and precipitate with $\mathrm{H}_{2} \mathrm{~S}$ gas as before. (Note 6.) Repeat, making a third precipitation if the amount of cadmium present is large. (Note 7.) After the final precipitation, let settle, filter, and transfer to a weighed platinum dish, cover, and dissolve in $\mathrm{HCl}$ (1:3). Dissolve the precipitate remaining on the paper in hot $\mathrm{HCl}(1: 3)$ and add also to the solution in the platinum dish. (Note 8.) Add $10 \mathrm{c} \mathrm{c}$ of $\mathrm{H}_{2} \mathrm{SO}_{4}$ $(1: 1)$ and evaporate the solution until copious fumes are evolved. (Note 9.) Remove the excess of $\mathrm{H}_{2} \mathrm{SO}_{4}$ by heating the dish cautiously, and finally heat to between $500^{\circ}$ and $600^{\circ} \mathrm{C}$. Cool and weigh as $\mathrm{CdSO}_{4}$.

Notes. - 1. The addition of $\mathrm{HCl}$ will dissolve the zinc and a small amount of the cadmium. By adding the acid in small quantities, that is, keeping the acidity low, and also keeping the solution cold, the amount of cadmium dissolved will be small. Since it requires some time to reprecipitate completely any cadmium dissolved, it is desirable for the solution to stand overnight following the addition of the $55 \mathrm{c}$ c of $\mathrm{HCl}$ at the start. For the same reason the solution should be allowed to stand after the last addition of acid, and care be taken that the stated amount of spelter remain in the undissolved residue. If the solution is heated, or if time is not allowed for the action of the acid to cease completely, small amounts of cadmium may pass into the solution and be lost.

Equally satisfactory or, in some cases, more satisfactory results are obtained by using $\mathrm{H}_{2} \mathrm{SO}_{4}$ to dissolve the major portion of the zinc. The rate of solution is slower and the tendency for cadmium to pass into solution less. The quantity of acid required can be calculated, measured out at the beginning and small quantities added from time to time as the action becomes slow.

2. It is desirable to allow it to settle overnight to precipitate all the $\mathrm{PbSO}_{4}$ from the solution and to give good conditions for filtering.

3. It is occasionally necessary to add a drop or two of ammonia water to the solution to start the precipitation of $\mathrm{CdS}$

4. All the cadmium will be precipitated as sulfide together with some $\mathrm{ZnS}$. The major portion of the zinc will remain in solution.

5. In case of high cadmium content, more acid may be required.

6. The second precipitation should give practically pure CdS.

7. A third precipitation will remove any uncertainty as to the completeness of the separation of cadmium from zinc.

8. A crucible may also be used if the volumes are kept small or evaporated before transferring to the crucible.

9. On evaporating to fumes, darkening of the solution may occur from filter paper shreds. This may be destroyed by diluting slightly with water and adding 5 c c $\mathrm{HNO}_{3}$ specific gravity 1.42) and again evaporating to fumes.

\section{DETERMINATION OF CADMIOM BY THE ELECTROLYTIC METHOD}

\section{SOLUTIONS REQUIRED}

Potassium hydroxide (note 1).-Dissolve $20 \mathrm{~g}$ of $\mathrm{KOH}$, chemically pure, in $200 \mathrm{c} \mathrm{c}$ of distilled water. Potassium cyanide (note 2).-Dissolve $20 \mathrm{~g}$ of $\mathrm{KCN}$, chemically pure, in $200 \mathrm{c}$ c of distilled water.

\section{APPARATUS}

See determination of lead by the electrolytic method, given above. The anode, as used in the lead determination, is here used as the cathode, and vice versa.

See determination of cadmium gravimetrically, given above.

Dissolve the final precipitate of $\mathrm{CdS}$ in hot $\mathrm{HCl}$ ( $1: 3)$, boil to remove $\mathrm{H}_{2} \mathrm{~S}$, adding a few drops of $\mathrm{HNO}_{3}$ (specific gravity 1.42) near the end, and filter off the separated sulphur. Transfer the solution to a 200 c c electrolytic beaker, add a drop or two of phenolphthalein and then $\mathrm{KOH}$ solution until a permanent red color is obtained. Add $\mathrm{KCN}$ solution with constant stirring until the precipitate 
of $\mathrm{Cd}(\mathrm{OH})_{2}$ is completely dissolved. (Note 3.) Dilute to $150 \mathrm{c}$ c and electrolize with a current of 5 amperes for two hours. (Note 4.) Test for completeness of deposition by washing the watch glasses and sides of the beaker to increase the depth of the solution. Continue electrolysis for 20 minutes and if the newly exposed surface of the cathode is still bright, remove the beaker with electrolyte and wash the electrodes three times with distilled water and twice with ethyl alcohol (95 per cent). Dry the cathode at $100^{\circ} \mathrm{C}$., cool, and weigh. The increase is metallic cadmium.

Notes.-1. Pure $\mathrm{NaOH}$ may be used in place of $\mathrm{KOH}$ 2. Pure NaCN may be used in place of KCN.

2. Pure NaCN may be used in place

4. The test for completeness of deposition may be made after one hour when the quantity present is not large.

\section{UNITED STATES GOVERNMENT, DEPART-} MENT OF COMMERCE, BUREAU OF STANDARDS, STANDARD SAMPLES, CIRCULAR NO. 25 AND SUPPLEMENT, 1927

\section{ZINC ORE AND ZINC SAMPLES}

(Sample No. 2 is a standard sample of zinc ore D prepared and sold by the Bureau of Standards for use by industrial organizations and others as a comparison standard for checking the accuracy of analysis of zinc ores. Each sample is accompanied by a certificate giving the content of zinc in the particular sample.)

(Sample No. $43 \mathrm{~b}$ is a standard sample of the metal zinc for use in calibrating pyrometers, etc. Each sample is accompanied with a certificate giving the melting point of that particular sample.)

(Circular No. 66 of the Bureau of Standards entitled "Standard Samples for Thermometric Fixed Points" gives information on the preparation of these standard samples, their chemical analyses, determination of freezing points, and methods of use.)

683.1 INGOT METALS, ZINC OR SPELTER. AMERICAN SOCIETY FOR TESTING MATERIALS, STANDARD SPECIFICATIONS FOR SPELTER, SERIAL DESIGNATION B6-18, 1918

\section{SPELTER}

SCOPE

1. Under these specifications virgin spelter; that is, spelter made from ore or similar raw material by a process of reduction and distillation, or by electrolysis, and not produced from reworked metal, is considered in five grades, as follows: 1 , high grade; 2 , intermediate; 3 , brass special; 4 , selected; and 5 , prime western.

2. A brand shall be cast in each slab by which the maker and grade can be identified.

\section{QUALITY}

3. The maker shall use care to have each carload of as uniform quality as possible.

\section{COMPOSITION}

4. (1) High grade. ${ }^{20}$ - The spelter shall not contain over-

Lead

Per cent

Iron 0.07

(1) 03

20 For slush castings and certain zinc-aluminum alloys, a special grade of spelter may be required, as noted in "Spelter, Its Grades and Uses," published as Appendix 1 to the report of Committee B-2 on nonferrous metals and alloys, Proc., Am. Soc. Testing Mats., on nonferrous metals and
It shall be free from aluminum.

The sum of lead, iron, and carmium shall not exceed 0.10 per cent.

(2) Intermediate.-The spelter shall not contain over-

Lead.

Per cent

Iron 0.20

. . . .

Cadmium

It shall be free from aluminum.

The sum total of lead, iron, and"cadmium shall not exceed 0.50 per cent.

(3) Brass special.- The spelter shall not contain over-

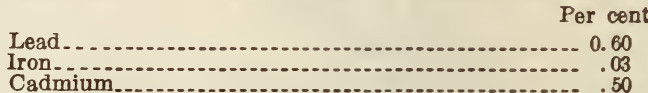

It shall be free from aluminum.

The sum total of lead, iron, and cadmium shall not exceed 1.0 per cent.

(4) Selected. - The spelter shall not contain overPer cent

Lead . . . .

Iron

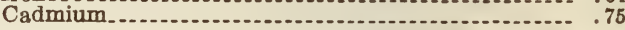

It shall be free from aluminum.

The sum total of lead, iron, and cadmium shall not exceed 1.25 per cent.

(5) Prime western.-The spelter shall not contain over-

Per cent

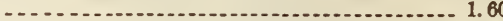

\section{PHYSICAL}

5. The slabs shall be reasonably free from surface corrosion or adhering foreign matter.

\section{SAMPLING}

6. Take 10 slabs ${ }^{21}$ at random from a carload and saw each slab completely across from the middle of one long side to the middle of the other, and use the sawdust as the sample; or, drill three 9-mm holes along one diagonal of each slab, boring completely through and taking care to make fine drillings; the holes should be drilled as nearly as possible at the middle and halfway between either end and the middle of such diagonals. Go over the drillings or sawings with a powerful magnet to take out any iron which may have come from the drill or saw, and mix the sample thoroughly. The drill or saw shall be thoroughly cleaned before use, and no lubricant shall be used in either drilling or sawing.

\section{CLAMMS}

Claims to be considered shall he in writing within 30 days of receipt of material at customer's plant, and the results of customer's test shall be given The shipper shall be given one week from date of receipt of such claim to investigate his records, and then shall either agree to satisfy the claim or send a representative to the plant.

(a) Analysis by car lots.-No claims shall be considered unless the minimum samples as specified for the grade in question can be shown to such representative.

(b) Physical defects of individual pieces.-No claims shall be considered unless the spelter in question, unused, can be shown to such representative.

11 Sec. 6 on sampling is based on the report of the subcommittee on metho methods of analysis of nonferrous alloys of the Division of Industrial ety, approved by the supervisory committee on standard methods ety, approved by the supervisory committee on standard methods
of analysis of that society: J. Ind. Eng. Chem., vol. 7, p. 547, 1915. 


\section{PLANT TREATMENT}

8. Where the spelter satisfies the chemical and physical requirements of these specifications, it shall not be condemned for defects of alloys in which it is used, or for defects in the coating of galvanized products.

\section{INVESTIGATION OF CLAIMS}

9. The maker's representative shall inspect all pieces where physical defects are claimed. If agreement is not reached the question of fact shall be submitted to a mutually agreeable umpire, whose decision shall be final.

On a question of metal contents a sample shall be drawn by representatives of both parties as described under "Sampling," section 6. The properly mixed and quartered sample shall be separated into three parts, each of which shall be placed in a sealed package, one for each party and one for the umpire if necessary. Each party shall make an analysis, and if the results do not establish or dismiss the claim to the satisfaction of both parties, the third sample shall be submitted to a mutually agreeable umpire, who shall determine the question of quality, and whose determination shall be final.

\section{SETTLEMENT OF CLAIMS}

10. The expenses of the seller's representative and of the umpire shall be paid by the loser, or divided in proportion to concession made in case of compromise.

In case of rejection being established, damages shall be limited to the payment of freight both ways by the seller for the substitution of an equivalent weight of spelter meeting these specifications.

UNITED STATES GOVERNMENT, FEDERAL SPECIFICATIONS BOARD, FEDERAL SPECIFICATION FOR ZINC SLAB (SPELTER) QQ-Z-351, MAY 31, 1932

\section{ZINC SLAB}

(In common with other Government specifications for metals, this specification includes $F$. B. S. No. 339a (QQ-M-151). (See 600.1, p. 3.) In respect of grades, material, general requirements, chemical composition (except that tin is specifically disallowed), and methods of inspection and tests, it is identical with A. S. T. M. B6, given above. It differs from B6 in that, instead of extended specifications on claims and settlement thereof, it states briefly:)

1 . Sample slabs for analysis shall be selected, one for each 4,000 pounds or fraction thereof, so as to represent as nearly as possible the average quality of the metal. The sample from each slab shall weigh not less than 5 ounces and shall be kept separate.

2. Check analysis may be made by the inspector, or through him by any Government laboratory or other designated representative and without cost to the contractor. The chemical composition thus determined shall conform to the requirements specified.

683.2 SHEETS AND PLATES, ZINC.

\section{AMERICAN SOCIETY FOR TESTING MATERIALS, STANDARD SPECIFICA- TIONS FOR ROLLED ZINC, DESIGNATION B-69-29, 1929}

\section{ROLIED ZINC SCOPE}

1. These specificatons cover three types of commercial rolled zinc, as described in section 2 . It should be understood that the specifications are general. Any closer limitations on permissible variations shall be a matter of agreement between the buyer and seller at the time of purchase.

\section{SPECIFICATIONS}

2. The rolled zinc shall be divided into the following types: Type $A$, ribbon zinc and sheets or strips cut from ribbon zinc; type $B$, sheet zinc or strips cut from sheet zinc; and type $C$, boiler plate, name plates, tags, etc.

\section{Manufacture}

3. (a) Type A rolled zinc shall be rolled from a single bar in one continuous direction.

(b) Type B rolled zinc shall be rolled by the pack rolling method.

(c) Type $\mathrm{C}$ rolled zinc may be rolled either from a single bar or by the pack rolling method.

\section{CLASSIFICATION ACCORDING TO TEMPER}

4. Rolled zinc of the three types shall be classified according to temper as follows:

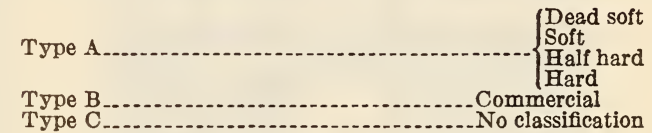

5. The classification of rolled zinc according to temper shall be determined by means of the dynamic ductility test and the temper test described in sections 18 to 23 , inclusive. The requirements for the several types shall be as specified in sections 6 and 7 .

6. Type A.-The requirements for temper for the several grades of type A rolled zinc shall be as given in Table 1.

TABLE 1.-Requirements for temper, type A rolled zinc

\begin{tabular}{|c|c|c|c|c|c|c|c|c|c|}
\hline \multirow[b]{2}{*}{$\begin{array}{l}\text { Amer- } \\
\text { ican } \\
\text { zinc } \\
\text { gage }\end{array}$} & \multirow[b]{2}{*}{$\begin{array}{c}\text { Thick- } \\
\text { ness }\end{array}$} & \multicolumn{2}{|c|}{ Dead soft } & \multicolumn{2}{|c|}{ Soft } & \multicolumn{2}{|c|}{ Half hard } & \multicolumn{2}{|c|}{ Hard } \\
\hline & & 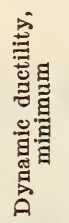 & 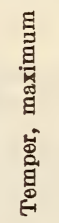 & 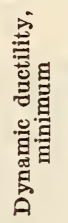 & 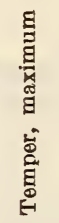 & 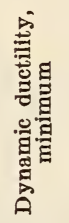 & 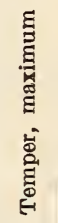 & 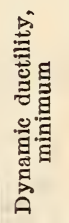 & 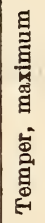 \\
\hline $\begin{array}{l}3 \\
4 \\
5 \\
6 \\
7\end{array}$ & $\begin{array}{r}\text { Inch } \\
0.006 \\
.008 \\
.010 \\
.012 \\
.014\end{array}$ & $\begin{array}{r}\text { Inch } \\
0.275 \\
.280 \\
.285 \\
.290 \\
.295\end{array}$ & $\begin{array}{l}\text { Per } \\
\text { cent } \\
70 \\
65 \\
60 \\
551 / 2 \\
52\end{array}$ & $\begin{array}{r}\text { Inch } \\
0.255 \\
.260 \\
.265 \\
.270 \\
.275\end{array}$ & $\begin{array}{l}\text { Per } \\
\text { cent } \\
76 \\
72 \\
66 \\
62 \\
58\end{array}$ & $\begin{array}{c}\text { Inch } \\
0.215 \\
.220 \\
.225 \\
.230 \\
.235\end{array}$ & $\begin{array}{l}\text { Per } \\
\text { cent } \\
80 \\
75 \\
70 \\
65 \\
61\end{array}$ & $\begin{array}{r}\text { Inch } \\
0.165 \\
.170 \\
.175 \\
.180 \\
.185\end{array}$ & $\begin{array}{l}\text { cent } \\
86 \\
83 \\
80 \\
77 \\
74\end{array}$ \\
\hline $\begin{array}{r}8 \\
9 \\
10 \\
11\end{array}$ & $\begin{array}{l}.016 \\
.018 \\
.020 \\
.022 \\
.024\end{array}$ & $\begin{array}{l}.300 \\
.305 \\
.310 \\
.315 \\
.320\end{array}$ & $\begin{array}{l}481 / 2 \\
46 \\
431 / 2 \\
411 / 2 \\
40\end{array}$ & $\begin{array}{r}.280 \\
.285 \\
.290 \\
.295 \\
.300\end{array}$ & $\begin{array}{l}54 \\
51 \\
48 \\
45 \\
43\end{array}$ & $\begin{array}{r}.240 \\
.245 \\
.250 \\
.255 \\
.260\end{array}$ & $\begin{array}{l}58 \\
54 \\
51 \\
48 \\
45\end{array}$ & $\begin{array}{r}.190 \\
.195 \\
.200 \\
.205 \\
.210\end{array}$ & $\begin{array}{l}69 \\
68 \\
65 \\
63\end{array}$ \\
\hline $\begin{array}{l}12 \\
13\end{array}$ & $\begin{array}{l}.026 \\
.028 \\
.030 \\
.032 \\
.034\end{array}$ & $\begin{array}{r}.325 \\
.330 \\
.335 \\
.340 \\
.345\end{array}$ & $\begin{array}{l}39 \\
371 / 2 \\
37 \\
361 / 2 \\
36\end{array}$ & $\begin{array}{l}.305 \\
.310 \\
.315 \\
.320 \\
.324\end{array}$ & $\begin{array}{l}40 \\
37 \\
37 \\
361 / 2 \\
36\end{array}$ & $\begin{array}{r}.265 \\
.270 \\
.275 \\
.280 \\
.285\end{array}$ & $\begin{array}{l}431 / 2 \\
421 / 2 \\
42 \\
41 \\
401 / 2\end{array}$ & $\begin{array}{r}.215 \\
.220 \\
.225 \\
.230 \\
.235\end{array}$ & $\begin{array}{l}61 \\
59 \\
58 \\
57 \\
56\end{array}$ \\
\hline $\begin{array}{l}14 \\
15\end{array}$ & $\begin{array}{l}.036 \\
.038 \\
.040 \\
.042\end{array}$ & $\begin{array}{r}.347 \\
.349 \\
.350 \\
.352\end{array}$ & & $\begin{array}{r}.326 \\
.328 \\
.329 \\
.330\end{array}$ & & $\begin{array}{r}.290 \\
.295 \\
.300 \\
.300\end{array}$ & & $\begin{array}{l}.240 \\
.245 \\
.250 \\
.250\end{array}$ & 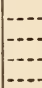 \\
\hline 17 & $\begin{array}{l}.044 \\
.046 \\
.048 \\
.050\end{array}$ & $\begin{array}{r}.353 \\
.354 \\
.355 \\
.356\end{array}$ & & $\begin{array}{r}.331 \\
.332 \\
.333 \\
.334\end{array}$ & & $\begin{array}{r}.305 \\
.305 \\
.310 \\
.310\end{array}$ & & $\begin{array}{r}.255 \\
.255 \\
.260 \\
.260\end{array}$ & \\
\hline
\end{tabular}

7. Type B.-The requirements for temper for type $B$ rolled zinc shall be as given in Table 2 . 


\section{PERMISSIBLE VARIATIONS IN DIMENSIONS}

(a) THICKNESS

8. The thickness of rolled-zinc plates, sheets, and ribbons of types $\mathrm{A}, \mathrm{B}$ and $\mathrm{C}$, shall be stated in decimals of an inch.

9. Type A.-The permissible variations in thickness of type A rolled zinc shall be as given in Table 3. Thicknesses of metals falling between the gages shown shall take the tolerances of the nearest gage.

TABLE 2.-Requirements for temper, type $B$ rolled zinc

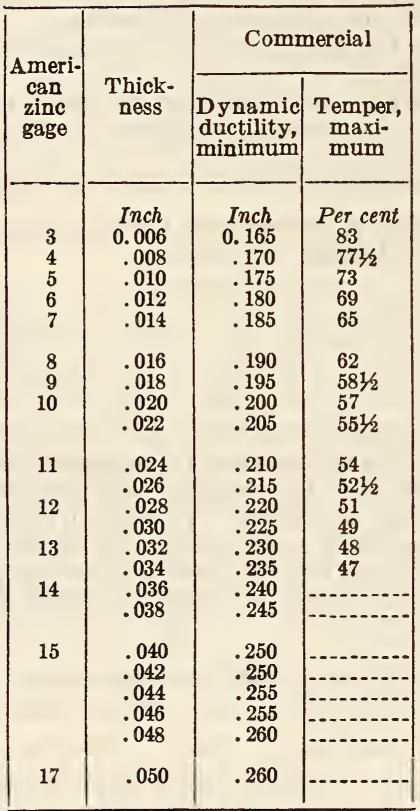

TABLE 3.-Permissible variations in thickness, inches, plus or minus, of type $A$ rolled zinc

\begin{tabular}{|c|c|c|c|c|c|}
\hline \multirow[b]{2}{*}{$\begin{array}{c}\text { Ameri- } \\
\text { can } \\
\text { zinc } \\
\text { gage }\end{array}$} & \multirow[b]{2}{*}{$\begin{array}{c}\text { Thick- } \\
\text { ness }\end{array}$} & \multicolumn{4}{|c|}{ Width } \\
\hline & & $\begin{array}{c}0 \text { to } 6 \\
\text { inches, } \\
\text { inclusive }\end{array}$ & $\begin{array}{c}\text { Over } 6 \\
\text { to } 9 \\
\text { inches, } \\
\text { inclusive }\end{array}$ & $\begin{array}{c}\text { Over } 9 \\
\text { to } 14 \\
\text { inches, } \\
\text { inclusive }\end{array}$ & $\begin{array}{c}\text { Over } 14 \\
\text { to } 20 \\
\text { inches, } \\
\text { inclusive }\end{array}$ \\
\hline $\begin{array}{l}3 \\
4 \\
5 \\
6 \\
7\end{array}$ & $\begin{array}{l}\text { Inch } \\
0.006 \\
.008 \\
.010 \\
.012 \\
.014\end{array}$ & $\begin{array}{r}0.0009 \\
.0010 \\
.0011 \\
.0011 \\
.0013\end{array}$ & $\begin{array}{r}0.0010 \\
.0011 \\
.0012 \\
.0012 \\
.0014\end{array}$ & $\begin{array}{r}0.0011 \\
.0012 \\
.0013 \\
.0013 \\
.0015\end{array}$ & $\begin{array}{r}0.0012 \\
.0013 \\
.0014 \\
.0014 \\
.0016\end{array}$ \\
\hline $\begin{array}{r}8 \\
9 \\
10 \\
11 \\
12\end{array}$ & $\begin{array}{l}.016 \\
.018 \\
.020 \\
.024 \\
.028\end{array}$ & $\begin{array}{l}.0014 \\
.0015 \\
.0016 \\
.0018 \\
.0019\end{array}$ & $\begin{array}{l}.0015 \\
.0016 \\
.0017 \\
.0019 \\
.0020\end{array}$ & $\begin{array}{l}.0016 \\
.0017 \\
.0018 \\
.0020 \\
.0021\end{array}$ & $\begin{array}{l}.0017 \\
.0018 \\
.0019 \\
.0021 \\
.0022\end{array}$ \\
\hline $\begin{array}{l}13 \\
14 \\
15 \\
16\end{array}$ & $\begin{array}{l}.032 \\
.036 \\
.040 \\
.045\end{array}$ & $\begin{array}{l}.0020 \\
.0021 \\
.0022 \\
.0023\end{array}$ & $\begin{array}{l}.0021 \\
.0023 \\
.0024 \\
.0025\end{array}$ & $\begin{array}{l}.0023 \\
.0025 \\
.0026 \\
.0027\end{array}$ & $\begin{array}{l}.0024 \\
.0026 \\
.0028 \\
.0029\end{array}$ \\
\hline $\begin{array}{l}17 \\
18 \\
19 \\
20\end{array}$ & $\begin{array}{l}.050 \\
.055 \\
.060 \\
.070\end{array}$ & $\begin{array}{l}.0024 \\
.0025 \\
.0026 \\
.0027\end{array}$ & $\begin{array}{l}.0026 \\
.0027 \\
.0028 \\
.0029\end{array}$ & $\begin{array}{l}.0028 \\
.0029 \\
.0030 \\
.0031\end{array}$ & $\begin{array}{l}.0030 \\
.0031 \\
.0032 \\
.0033\end{array}$ \\
\hline $\begin{array}{l}21 \\
22 \\
23 \\
24\end{array}$ & $\begin{array}{l}.080 \\
.090 \\
.100 \\
.125\end{array}$ & $\begin{array}{r}.0028 \\
0029 \\
.0030 \\
.0032\end{array}$ & $\begin{array}{l}.0030 \\
.0031 \\
.0032 \\
.0033\end{array}$ & $\begin{array}{l}.0032 \\
.0033 \\
.0034 \\
.0035\end{array}$ & $\begin{array}{l}.0034 \\
.0035 \\
.0036 \\
.0037\end{array}$ \\
\hline
\end{tabular}

10. Type B.-The permissible variations in thickness of type B rolled zinc shall be as given in Table 4. The gage tolerances on sheet zinc is the percentage plus or minus by weight based on the theoretical weight of any gage or size of sheet for each

TABLE 4.-Permissible variations in thickness of type $B$, rolled zinc

\begin{tabular}{|c|c|c|c|c|c|}
\hline $\begin{array}{l}\text { Ameri- } \\
\text { can } \\
\text { zinc } \\
\text { gage }\end{array}$ & $\begin{array}{c}\text { Thick- } \\
\text { ness }\end{array}$ & $\begin{array}{l}\text { Varia- } \\
\text { tion, per } \\
\text { cent by } \\
\text { weight }\end{array}$ & $\begin{array}{c}\text { Ameri- } \\
\text { can } \\
\text { zinc } \\
\text { gage }\end{array}$ & $\begin{array}{c}\text { Thick- } \\
\text { ness }\end{array}$ & $\begin{array}{l}\text { Varia- } \\
\text { tion, per } \\
\text { cent by } \\
\text { weight }\end{array}$ \\
\hline & Inch & & & Inch & \\
\hline 3 & 0.006 & \pm 16 & 14 & 0.036 & \pm 6 \\
\hline 4 & .008 & \pm 13 & 15 & .040 & \pm 6 \\
\hline 5 & .010 & \pm 10 & 16 & .045 & \pm 6 \\
\hline 6 & .012 & \pm 9 & 17 & .050 & \pm 6 \\
\hline 7 & .014 & \pm 8 & 18 & .055 & \pm 6 \\
\hline 8 & .016 & \pm 6 & 19 & .060 & \pm 6 \\
\hline 9 & .018 & \pm 6 & 20 & .070 & \pm 6 \\
\hline 10 & .020 & \pm 6 & 21 & .080 & \pm 6 \\
\hline 11 & .024 & \pm 6 & 22 & .090 & \pm 6 \\
\hline 12 & .028 & \pm 6 & 23 & .100 & \pm 6 \\
\hline 13 & .032 & \pm 6 & & & \\
\hline
\end{tabular}

unit of 500 pounds. One cubic inch of the material is assumed to weigh 0.2596 pound. No individual sheet shall weigh more than the theoretical weight of a sheet of the gage next above, nor less than a sheet of the gage next below.

11. Type C.-The permissible variations in thickness of type $\mathrm{C}$ rolled zinc shall be the same as those for either type A or type B rolled zinc, depending upon whether the material is cut from a ribbon or a sheet.

(b) WIDTH

12. (a) The permissible variations in width for all types of rolled zinc up to 0.100 inch in thickness shall be as follows:

\begin{tabular}{|c|c|c|}
\hline \multirow{2}{*}{ Width (in inches) } & \multicolumn{2}{|c|}{$\begin{array}{c}\text { Permissible } \\
\text { variation }\end{array}$} \\
\hline & Plus & Minus \\
\hline $\begin{array}{l}2 \text { and under } \\
\text { Over } 2 \text { to } 5 \text {, inclusive } \\
\text { Over } 5 \text { to } 14 \text {, inclusive } \\
\text { Over } 14\end{array}$ & $\begin{array}{l}\text { Inch } \\
0.010 \\
.025 \\
.050 \\
.125\end{array}$ & Inch $\begin{array}{r}0 \\
0 \\
0 \\
0\end{array}$ \\
\hline
\end{tabular}

(b) The permissible variations in width for all types of rolled zinc from 0.100 inch to 0.250 inch in thickness shall be plus or minus one-sixteenth inch, and from 0.250 inch to 0.500 inch in thickness plus or minus one-eighth inch, and for material over 0.500 inch in thickness plus one-fourth inch.

\section{(c) LENGTH}

13. (a) Sheets, strips, and plates may be ordered to exact length up to 12 feet, in which case a variation in length of plus one-fourth inch shall be permitted.

(b) When sheets, strips, and plates are ordered in stock lengths the pieces shall be cut to the nominal or stock length as a maximum. The shortest acceptable lengths, the maximum permissible percentage by weight of short lengths, and the required percentage by weight of stock lengths in any one shipment shall be as follows: 


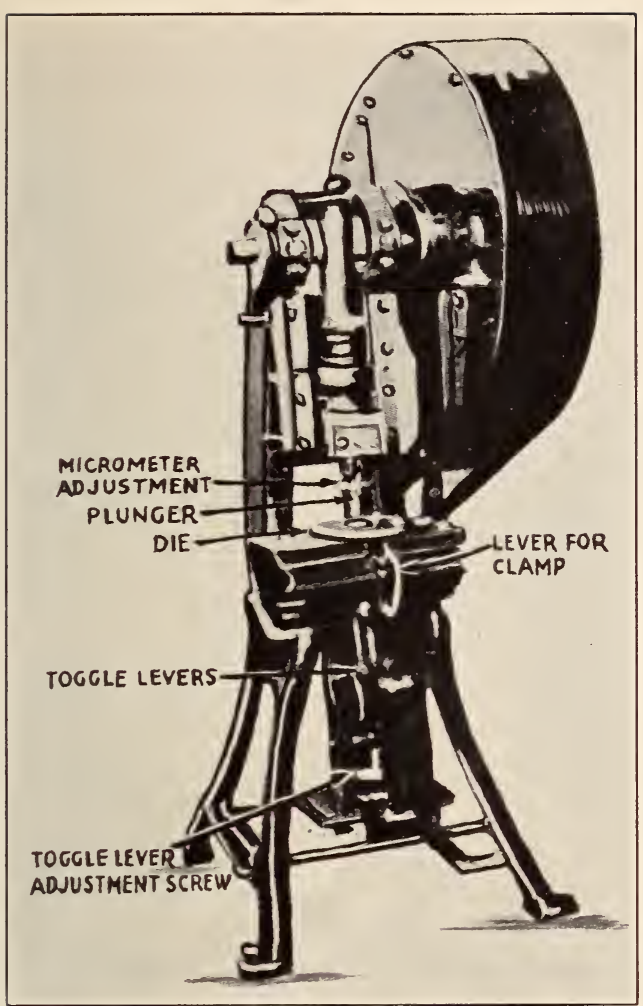

Figure 1131.-Dynamic ductility test machine (For specification see p. 1313.)

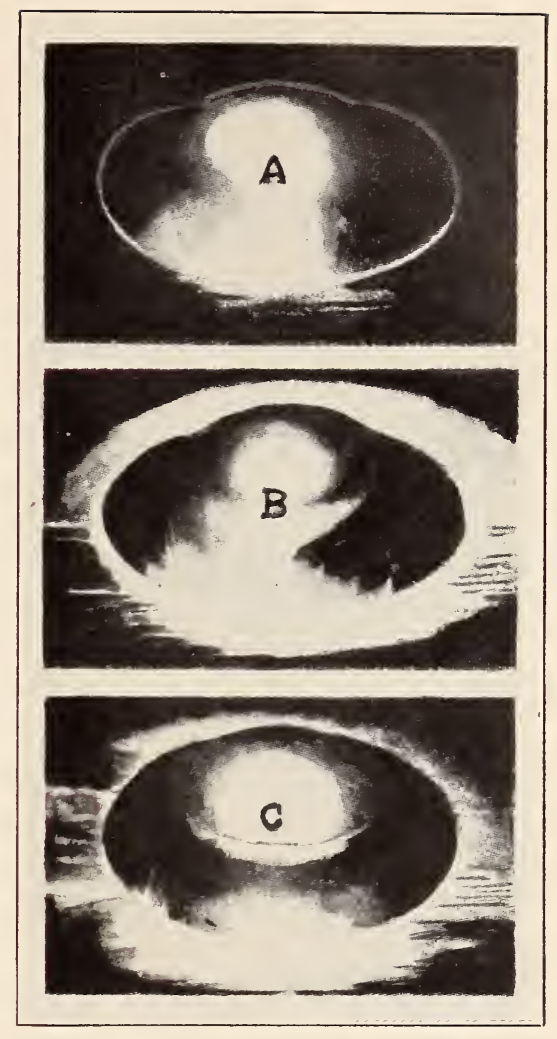

Figure 1132.- Showing typical cups formed in ductility test

$A$, Before rupture; $B$, at rupture; $C$, past rupture. (For specification see p. 1313.)

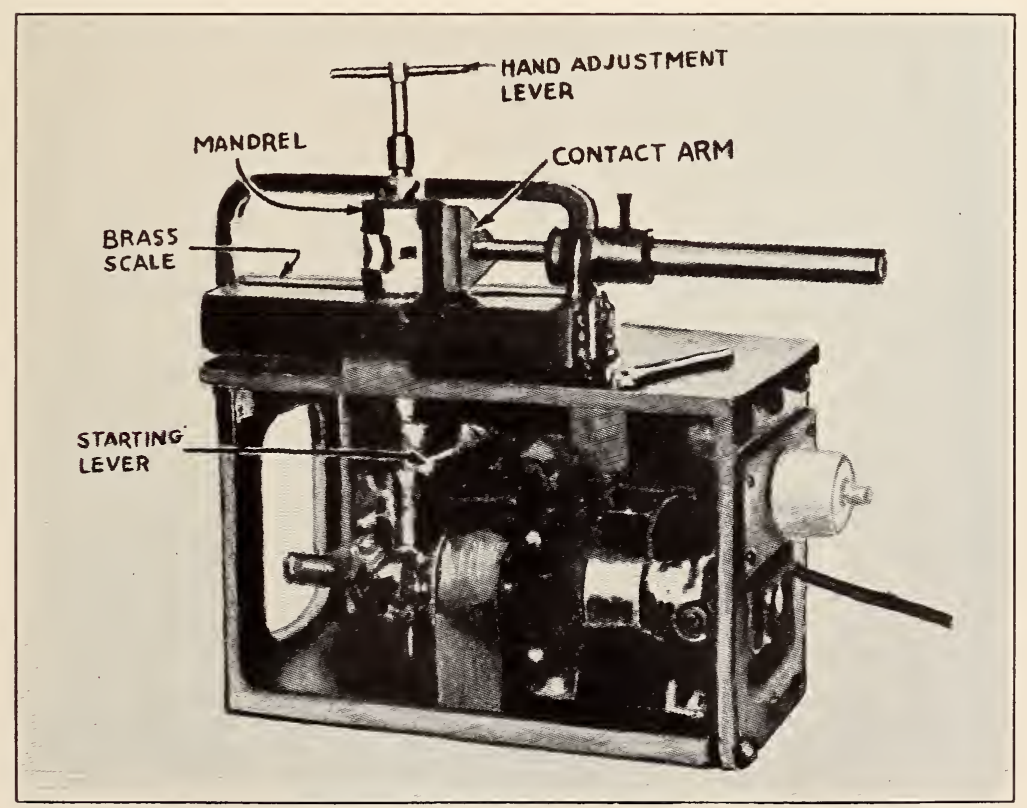

FIgURE 1133.-Temper test machine

(For specification see p. 1313.) 

Permissible variations in length of plates, sheets, and strips up to 20 inches in width

\begin{tabular}{|c|c|c|c|c|c|c|}
\hline \multirow{2}{*}{$\begin{array}{l}\text { Nom- } \\
\text { inal or } \\
\text { stock } \\
\text { length, } \\
\text { (feet) }\end{array}$} & \multirow{2}{*}{$\begin{array}{l}\text { Re- } \\
\text { quired } \\
\text { percent- } \\
\text { age by } \\
\text { weight } \\
\text { of stock, } \\
\text { lengths }\end{array}$} & \multicolumn{5}{|c|}{$\begin{array}{l}\text { Maximum permissible percentage by } \\
\text { weight of short lengths }\end{array}$} \\
\hline & & $\begin{array}{l}8 \text { to } 10 \\
\text { feet }\end{array}$ & $\begin{array}{l}6 \text { to } 8 \\
\text { feet }\end{array}$ & $\begin{array}{l}4 \text { to } 6 \\
\text { feet }\end{array}$ & $\begin{array}{l}2 \text { to } 4 \\
\text { feet }\end{array}$ & $\begin{array}{l}\text { Under } \\
2 \text { feet }\end{array}$ \\
\hline $\begin{array}{r}10 \\
8 \\
6\end{array}$ & $\begin{array}{l}60 \\
70 \\
80\end{array}$ & $\begin{array}{c}40 \\
0\end{array}$ & $\begin{array}{l}30 \\
30 \\
-\end{array}$ & $\begin{array}{l}20 \\
20 \\
20\end{array}$ & $\begin{array}{l}10 \\
10 \\
10\end{array}$ & $\begin{array}{l}0 \\
0 \\
0\end{array}$ \\
\hline
\end{tabular}

14. When type A rolled zinc is supplied in coils it shall be consistent with good commercial practice and in no case shall a coil consist of more than 4 pieces. No piece shall be less than 10 feet long.

15. Type A rolled zinc in lengths over 10 feet shall not exhibit sidewise bend or curvature in excess of 1 inch in any length of 10 feet.

\section{Packing and Marking}

16. (a) All material shall be properly separated according to size when loaded for shipment. In the case of shipments of plates, sheets, and ribbons packed in boxes or crates, the containers shall be sufficiently strong to protect them from injury and loss during shipment.

(b) Bulk shipments shall be properly braced in the car.

17. Each bundle, box or crate shall be legibly marked with the type letter, dimensions, and gross, tare, and net weight.

\section{METHODS OF TEST}

Sampling

18. For purposes of test in shipments of type A or B rolled zinc, 10 samples shall be taken for every 5,000 pounds. These samples shall be taken from representative coils or sheets throughout the shipment, and not more than one sample shall be taken from the same coil or sheet. Test specimens shall be cut from the samples as described in the various test methods.

\section{Dynamic Ductility Test}

19. The dynamic ductility machine for conducting the dynamic ductility test is a modified inclinable power press fitted with an adjustable die as shown in Figure 1131. The speed of the machine shall be 85 to 90 oscillations of the plunger per minute.

20. (a) The test specimen shall consist of a strip cut across the full width of a ribbon or sheet, the width of the strip being approximately $3 \frac{1}{2}$ inches. When this results in a specimen of less than 12 inches in length, as from narrow unslit ribbon zinc, two specimens $3 \frac{1}{2}$ inches in width shall be cut.

(b) Test specimens from narrow strips such as ribbon or sheet zinc after slitting shall be cut to 20 -inch lengths.

NoTE.-The construction of the machine will not permit test specimens of greater thickness than 0.060 inch.

21. (a) The temperature of the test specimens, testing machine and room throughout the test shall be kept within $70^{\circ}$ and $90^{\circ} \mathrm{F}$. The specimen shall be inserted in the machine and firmly clamped. The testing shall be started at one end and the strip moved with each successive cup. The series of cups shall be along the middle line of a $3 \frac{1}{2}$-inch strip so that clamping will be uniform around the cup as it is formed. With the motor running the clutch shall be thrown in, lowering the plunger, which, with the die in the clamp base, presses the cup in the test strip. If the cup does not show rupture, the plunger is advanced by increments of 0.005 inch and the cupping repeated with each increased depth until rupture occurs. Having determined the rupturing point additional cups shall be formed as a check. The rupturing point shall not be considered to be that point where an open crack is formed, but where the drawing of the bottom of the cup has just caused "necking" of the surface prior to rupture (see fig. 1132).

NotE.-An experienced operator should catch the rupture point after two or three trial cups.

(b) The depth of the cup at which rupture just occurs is read from the micrometer attachment on the plunger and is reported as the dynamic ductility of the specimen. The average ductility of the test specimens shall be considered the dynamic ductility of the shipment.

Note.-The micrometer attachment on the plunger mechanism measures the distance that the plunger extends beyond the zero point. The zero point is where no impression would be made on the test strip. The clamp base is adjusted for the varying thicknesses. The micrometer attachment also serves to advance and withdraw the plunger.

\section{Temper Test}

22. The apparatus consists of a temper-test machine as shown in Figure 1133.

23. Duplicate test specimens shall be cut from each sample and shall be rectangular in shape and cut accurately to $1 \frac{1 / 2}{2}$ inches in width and $4^{31 / 32}$ inches in length, with the length taken in the direction of rolling. With ribbon zinc the duplicate test specimens shall be cut from adjacent places across the width of a sample.

Note.-The construction of the machine does not permit test specimens of greater thickness than 0.035 inch.

24. (a) The temperature of test specimens, testing machine, and room throughout the test shall be kept within $70^{\circ}$ and $90^{\circ} \mathrm{F}$. The test specimens shall be inserted to the full depth of the vise of the mandrel and securely held by tightening the vise. The diamater of the mandrel is $2 \frac{1}{2}$ inches. Inserting the test specimen as specified to the full depth of the vise leaves extended a length exactly one-half the circumference of the mandrel. In the rotation of the mandrel the test specimen is pressed by the contact arm against the mandrel. When the outer or free end passes the contact arm it springs out according to its temper. The speed of rotation of the mandrel shall be between 50 and 55 r. p. m. With the motor running, the clutch shall be thrown in, rotating the mandrel counterclockwise. When the outer end of the specimen has passed the contact arm the rotation of the mandrel shall be stopped by throwing out the clutch. The mandrel shall be turned to the 100 per cent position which is indicated by a mark on the mandrel and the fixed pointer, and the percentage of temper shall be read from the position of the outer end of the specimen on the underlying graduated scale, consisting of a half circle graduated counterclockwise from 0 to 100 per cent.

(b) The dulpicate specimen shall be similarly tested and, in order to eliminate the effect of the curvature of the specimens due to coiling, shall be inserted in the vise in the reverse position to that of the first specimen.

That is, if one specimen is inserted and pressed with its curvature toward the mandrel, the duplicate specimen should be inserted with its curvature away from the mandrel.

(c) The average of the readings on two test specimens shall be reported as the temper of the material.

\section{Test for sidewise bend or curvature}

25. The coil shall be supported on a reel at one end of the table and the ribbon drawn along a 10- 
foot straight edge, keeping both ends of the straight edge touching the edge of the ribbon. No point on the straight edge shall then be more than 1 inch from the edge of the ribbon.

\section{Gaging}

26. Type A rolled zinc shall be gaged with a micrometer caliper which has previously been checked with precision blocks. No two measurements along the length of the coil shall be made within 12 inches of each other, nor shall measurements in any one line across the width of the coil be used as a basis of rejection.

UNITED STATES GOVERNMENT, DEPARTMENT OF COMMERCE, BUREAU OF STANDARDS, STANDARD THICKNESSES, WEIGHTS, AND TOLERANCES OF SHEET METAL (CUSTOMARY PRACTICE), CIRCULAR NO. 391, MARCH 24, 1931

\section{AMERICAN WIRE GAGE-ZINC SHEETS}

(See 640.1, p. 1165.)

UNITED STATES GOVERNMENT, FEDERAL SPECIFICATIONS BOARD, FED. ERAI SPECIFICATION, FOR ZINC PLATES, SHEETS, AND STRIPS, NO. 531, NOVEMBER 23, 192\%. (CURRENT DESIGNATION : QQ-Z-301)

\section{ZINC PLATES, SHEETS, AND STRIPS}

(In respect to grade and types; material and workmanship; permissible variations in thickness, width, and length; maximum permissible percentage by weight of short lengths; and method of inspection and tests, this specification is in conformity with A. S. T. M. B69-29, given above. In respect to quality of material, bending tests, general specifications, and packing and marking of shipments, it is in conformity with F. S. B. QQ-C-501. (See 641.0, p. 1172. Otherwise it provides that:)

The chemical requirements are as shown in Table 1 .

TABLE 1.-Chemical composition

\begin{tabular}{|c|c|c|c|c|c|}
\hline $\begin{array}{l}\text { Lead, } \\
\text { maxi- } \\
\text { mum }\end{array}$ & $\begin{array}{l}\text { Iron, } \\
\text { maxi- } \\
\text { mum }\end{array}$ & $\begin{array}{l}\text { Cad- } \\
\text { mium } \\
\text { maxi- } \\
\text { mum }\end{array}$ & $\begin{array}{l}\text { Alumi- } \\
\text { num } \\
\text { maxi- } \\
\text { mum }\end{array}$ & $\begin{array}{c}\text { Total, } \\
\text { of lead, } \\
\text { iron, } \\
\text { and } \\
\text { cad- } \\
\text { mium, } \\
\text { maxi- } \\
\text { mum }\end{array}$ & Zinc \\
\hline $\begin{array}{c}\text { Per cent } \\
0.80\end{array}$ & $\begin{array}{c}\text { Per cent } \\
0.04\end{array}$ & $\begin{array}{c}\text { Per cent } \\
0.75\end{array}$ & $\begin{array}{l}\text { Per cent } \\
\text { None }{ }^{1}\end{array}$ & $\mid \begin{array}{c}\text { Per cent } \\
1.25\end{array}$ & $\begin{array}{c}\text { Percent } \\
\text { Remainder. }\end{array}$ \\
\hline
\end{tabular}

1 As determined on a $25 \mathrm{~g}$. sample.

Material shall withstand transverse bending at a temperature of $65^{\circ}$ to $100^{\circ} \mathrm{F}$. through the angle noted in Table 2 without fracture on the outside of the bent portion, on a radius equal to the multiple of the thickness of the material indicated in Table 2.
TABLE 2.-Bending test requirements

\begin{tabular}{|c|c|c|c|}
\hline \multicolumn{2}{|c|}{ Thickness } & \multicolumn{2}{|c|}{ Bending test } \\
\hline From- & To- & Degree & $\begin{array}{l}\text { Radius of } \\
\text { pin equal } \\
\text { to- }\end{array}$ \\
\hline $\begin{array}{l}\text { Inch } \\
0.002^{\text {In }} .040 \\
.100 \text { and over }\end{array}$ & $\begin{array}{l}\text { Inch } \\
.0 .036 \\
.090 \\
\end{array}$ & $\begin{array}{r}180 \\
120 \\
45\end{array}$ & $\begin{array}{r}12 \mathrm{~T} \\
13 \mathrm{~T} \\
0.5 \mathrm{inch}\end{array}$ \\
\hline
\end{tabular}

$1 \mathrm{~T}=$ Thickness of material.

Unless otherwise specified in the contract or purchase order, two bend tests shall be made for every lot of 5,000 pounds or fraction thereof, of each size in the shipment.

NotES.-Contracts or purchase orders for sheets and strips should state the purpose for which the material is to be used; as "for cup, ping," "for deep cupping," "for spinning," for edgewise winding," "for bending at sharp right angles," etc.

Zinc plates, sheets, and strips are commercially obtainable in the following thicknesses:

\begin{tabular}{|r|r|r|r|}
\hline Inch & Inch & Inch & Inch \\
\cline { 1 - 1 } 0.006 & 0.020 & 0.050 & 0.125 \\
.008 & .024 & .055 & .250 \\
.010 & .028 & .060 & .375 \\
.012 & .032 & .070 & .500 \\
.014 & .036 & .080 & 1.000 \\
.016 & .040 & .090 & \\
.018 & .045 & .100 & \\
\hline
\end{tabular}

Plates, sheets, and strips should be ordered in as narrow widths as can be used.

For purposes of calculations, the weight of rolled zinc per cubic inch may be taken as 0.2596 pound.

When finished edges of plates, sheets, and strips are specified in the contract or purchase order, the following descriptions should apply: (a) "Square edge" material shall be supplied with finished edge with sharp, square corners, without bevel or rounding of any sort.

(b) "Round edge" material shall be supplied with finished edge semicircular in form, the diameter of the circle forming the edge being equal to the thickness of the material.

(c) "Round corners" material shall be supplied with square finished edges, and the corners rounded to a definite radius which, unless otherwise specified in the contract or purchase order, may be any radius less than one-half the thickness of the material.

(d) When no description of a required form of edge is given, it shall be understood that edges such as would be left after slitting, sawing, or shearing will be acceptable.

683.3 STRIPS, ZINC.

AMERICAN SOCIETY FOR TESTING MATERIALS, STANDARD SPECIFICATIONS FOR ROLLED ZINC, DESIGNATION B69-29 1929

$$
\text { ROLLED ZINC }
$$

(See 683.2, p. 1311.)

UNITED STATES GOVERNMENT, FEDERAL SPECIFICATIONS BOARD, FEDERAI SPECIFICATION FOR ZINC PLATES, SHEETS, AND STRIPS, NO. 531, NOVEMBER 23, 192\%. (CURRENT DESIGNATION : QQ-Z-301)

\section{ZINC STRIPS}

(See 683.2, p. 1314.) 
UNITED STATES GOVERNMENT, DEPARTMENT OF COMMERCE, BUREAU OF STANDARDS, STANDARD THICKNESSES, WEIGHTS, AND TOLERANCES OF SHEET METAL (CUSTOMARY PRACTICE), CIRCULAR NO. 391, MARCH 24, 1931

\section{AMERICAN WIRE GAGE-ZINC STRIPS}

(See 640.1, p. 1165.)

683.4 MANUFACTURES OF ZINC.

683.41 DIE CASTINGS, ZINC-BASE.

AMERICAN SOCIETY FOR TESTING MATERIALS, TENTATIVE SPECIFICATIONS FOR ZINC-BASE ALLOY DIE CASTINGS, DESIGNATION B86-31T, 1931

\section{ZINC-BASE ALIOY DIE CASTINGS}

SCOPE

1. These specifications cover commercial zinc-base alloy die castings.

\section{MANOFACTORE}

2. (a) The alloy may be made by any approved method.

(b) The casting shall be of uniform quality and no scrap shall be used other than the sprues, gates, rejected castings, etc., of this alloy which are produced in the manufacturer's own plant. The rejected castings used shall be free from finishes, solder, or inserts which may contaminate the alloy.

3 . The base metal for all alloys shall be slab zinc conforming to the requirements of the standard specifications for slab zinc (spelter) A. S. T. M., B6 (see 683.1, p.1310), except that the total impurities including lead, iron, tin, and cadmium shall not exceed 0.02 per cent.

\section{CHEMICAL COMPOSITION AND TESTS}

4. The alloy shall conform to the following requirements as to chemical composition:

Copper....

Per cent

$2.5-3.5$

Aluminum

Magnesium.

Iron, maximum.

.010

Lead, maximum................................

Cadmium, maximum

.010

Tin, maximum

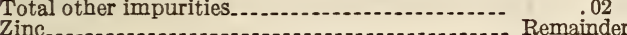

5. (a) The sample for chemical analysis may be taken either by sawing, drilling, or milling the casting or tension-test specimen, and shall represent the average cross section of the piece.

(b) The saw, drill, cutter, or other tool used shall be thoroughly cleaned. No lubricant shall be used in the operation, and the sawings or metal chips shall be carefully treated with a magnet to remove any particles of iron introduced in taking the sample.

\section{PHYSICAL PROPERTIES AND TESTS}

6. The average expansion of any five or more castings when aged as described in section 8 shall not exceed 0.0025 inch per inch. An expansion of 0.0025 inch will be allowable in any dimension less than 1 inch.

7. (a) Unless specified in the order, acceptance of castings under these specifications shall not depend upon the physical properties as determined by tension or impact test specimens. (b) When specified, the alloy shall conform to the following requirements as to physical properties:

\begin{tabular}{|c|c|c|}
\hline 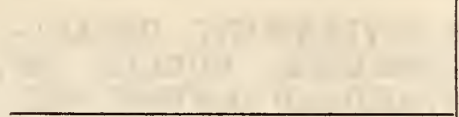 & $\begin{array}{l}\text { Minimum } \\
\text { average } \\
\text { of } 5 \\
\text { specimens }\end{array}$ & $\begin{array}{l}\text { Minimum } \\
\text { of individual } \\
\text { specimens }\end{array}$ \\
\hline 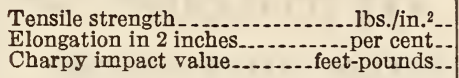 & $\begin{array}{r}44,000 \\
2.0 \\
6.0\end{array}$ & $\begin{array}{r}35,000 \\
0.5 \\
4.0\end{array}$ \\
\hline
\end{tabular}

8. (a) The alloy shall conform to the following requirements as to physical properties after being exposed to water vapor at $95^{\circ} \mathrm{C}$. for 10 days.

A verage of 5 specimens

Tensile strength, minimum 5 specimen

Elongation in 2 in., minimum............... per cent.- 0.5

Impact Charpy, minimum . ...........-. feet-pounds.- $\quad 0.75$

Expansion per inch, maximum

(b) The humidity cabinet shall contain no wood or organic matter and the specimens shall be supported only by glass, procelain, zinc, or zinc-coated metal.

9. (a) The test specimens shall be die cast according to the dimensions shown in Figures 1134

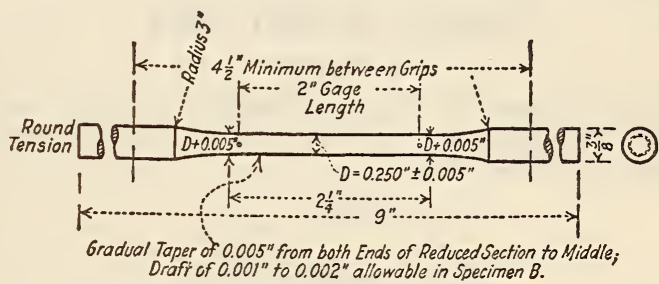

FIgURE 1134.-Tension test specimen

and 1135 and shall be tested without machining. No ejector pins shall be used on reduced sections. Specimens shall be removed from dies as soon as cast and cooled in still air with gates attached.

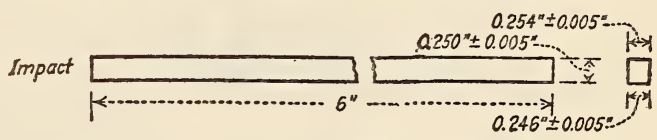

Figdre 1135.-Charpy impact test specimen

(b) Impact-test specimens shall be made by cutting the 6 -inch bar into two pieces 3 inches in length. The anvil of the testing machine shall have a 40 $\mathrm{mm}$ span. The parting line of the test specimen shall be placed against the anvil.

(c) In case any of the test specimens contains a flaw or flaws which results in failure of the alloy to meet the specifications, the manufacturer and purchaser shall agree upon the selection of specimens for retests, or the defective bars shall be disregarded.

10. Five of each type of test specimen shall be tested.

11. Heavy section die castings are subject to spongy centers. The extent of this sponginess if objectionable shall be mutually agreed upon by the manufacturer and purchser for each individual casting.

\section{WORKMANSHIP AND FINISH}

12. The die casting shall be produced free from cracks and other disfiguring blemishes.

\section{REJECTION}

13. Die castings which show injurious defects revealed by machining operations subsequent to 
acceptance may be rejected, and if rejected the manufacturer's responsibility shall be limited to replacing rejected parts free of charge to the purchaser.

\section{UNITED STATES GOVERNMENT, DEPART-} MENT OF COMMERCE, BUREAU OF STANDARDS, STANDARD SAMPLES, CIRCULAR NO. 25 AND SUPPLEMENT, 1927

\section{ZINC-BASE DIE-CASTING ALLOY SAMPLES}

(Samples No. 94, No. 95, and No. 96 are standard samples of zinc-base die-casting alloy prepared and sold by the Bureau of Standards for use by industrial organizations and others as comparison standards for checking the accuracy of analysis of similar alloys. Each sample is accompanied by a certificate giving a complete analysis of that particular sample.)

683.42 GRAVITY-BATTERY ZINCS.

AMERICAN RAILWAY ASSOCIATION, SIGNAL SECTION, SPECIFICATION FOR GRAVITY-BATTERY ZINCS, NO. 3428, JULY, 1928

\section{GRAVITY-BATTERY ZINCS}

1. PURPOSE

The purpose of this specification is to provide gravity-battery zincs to be used as the anodes of gravity-battery cells.

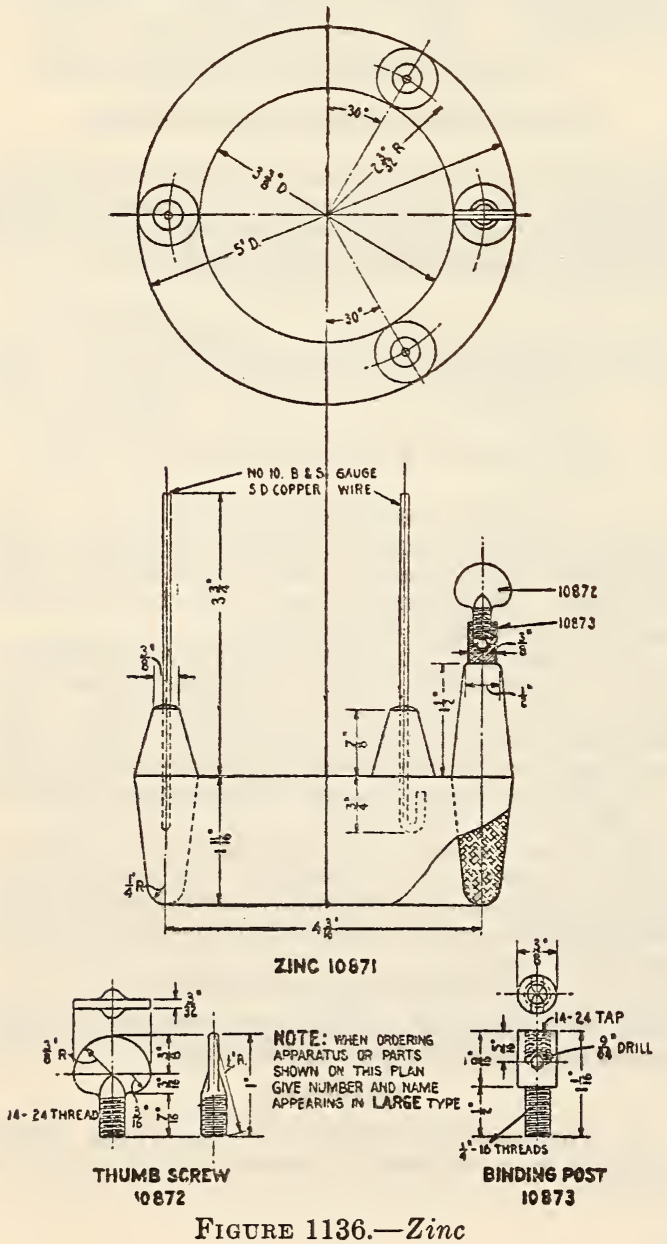

\section{DRA WINGS}

Figure 1136 forms an essential part hereof.

\section{MATERIAL AND WOREMANSHIP}

Material and workmanship shall be first class in every respect.

Zincs shall weigh 4 pounds each and shall be made of virgin spelter cast at a low temperature and thoroughly amalgamated with mercury. The chemical composition of the finished zincs shall be as follows:

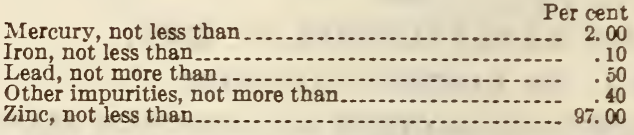

Binding post and screw shall be made of brass.

Zincs shall be uniform in size and weight, free from flaws and mechanical defects, and shall have a smooth outer surface and a firm close grain.

Binding post must be firmly connected both mechanically and electrically to the zinc. The binding screw must be perfectly threaded and fit closely in the binding post.

Manufacturer's name shall be cast on the upper flat surface of the zinc in letters raised to not less than three-thirty-seconds inch and as large as the surface will permit. In addition the manufacturer's name or trade-mark shall be stamped on some other part in such a position as not to be effaced by the action of the electrolyte or by the process of cleaning.

683.49 MISCELIANEOUS MANUFACTURES OF
ZINC.

UNITED STATES GOVERNMENT, FEDERAL SPECIFICATIONS BOARD, FEDERAL SPECIFICATION FOR NAILS, SPIKES, TACKS, AND STAPLES, FF-N-101, MAY 3, 1932

NAILS, SPIKES, TACKS, AND STAPLES

(See 608.11, p. 560.)

\subsection{ZINC ALLOYS.}

(Zinc-base die-casting alloys. See 683.41, p. 1315.) (Copper-tin-zinc alloys. See 643, p. 1191; 646, p. $1243 ; 647$, p. 1258 .)

(Brass. See 644 , p. $1193 ; 645$, p. 1203.)

(Nickel-copper-zinc alloys. See 655, p. 1286.)

(Brazing solder, brass. See 693.2, p. 1333.)

\section{ZINC COATINGS (GALVANIZING)}

(Zinc coatings. See 600.3, p. 60.)

(Zinc-coated sheets. See 604.32, p. 243.)

AMERICAN SOCIETY FOR TESTING MATERIALS, STANDARD METHODS OF DETERMINING WEIGHT OF COATING ON ZINC-COATED ARTICLES, DESIGNATION A90-30, 1930

\section{WEIGHT OF ZINC COATING}

(See 600.3, p. 62.)

SOCIETY OF AUTOMOTIVE ENGINEERS, ELECTRICAL EQUIPMENT, FLEXIBLE CONDUIT FERRULES, S. A. E. STANDARD, AUGUST, 1928

\section{FLEXIBLE CONDUIT FERRULES}

(See 645.22, p. 1215-.) 


\section{0-699 MISCELLANEOUS ORES, METALS, ALLOYS, AND METAL}

MANUFACTURERS

691.

SILVER

(For silver and silverware, see 663.)
692. BABBITT AND OTHER BEARING
METALS

692.0 GENERAL ITEMS.

AMERICAN SOCIETY FOR TESTING MATERIALS, TENTATIVE METHODS OF TENSION TESTING OF METALLIC MATERIAIS, DESIGNATION E8-27T, 1927

TENSION TESTING OF METALLIC MATERIALS

(See 600.1, p. 34.)

AMERICAN SOCIETY FOR TESTING MATERIALS, TENTATIVE METHODS OF COMPRESSION TESTING OF METALLIC MATERIALS, DESIGNATION E9-27T, 1927

\section{COMPRESSION TESTING OF METALLIC MATERIALS}

(See 600.1, p. 34.)

AMERICAN SOCIETY FOR TESTING MATERIALS, STANDARD METHODS OF CHEMICAL ANALYSIS OF ALLOYS OF LEAD, TIN, ANTIMONY, AND COPPER, SERIAL DESIGNATION B18-21, 1921

(Approved as American tentative standard ( $\mathrm{K} 5-1922)$ by the American Standards Association, Norember 13, 1922)

\section{CHEMICAL ANALYSIS OF ALLOYS OF LEAD, TIN,} ANTIMONY, AND COPPER

These methods apply particularly to white-metal bearing alloys (known commercially as Babbitt metal) and to similar lead and tin base alloys.

Two sets of methods are here given. The first method is somewhat slow but is recommended as giving the more accurate results where the analysis of these alloys is of comparatively infrequent occurrence. The second method is rapid and is suitable for control work, giving good results after the analyst has become familiar with it.

\section{GENERAL METHOD 22}

B. Determination of Lead, Copper, Antimony, and Tin. DETERMINATION OF LEAD

SOLTTIONS REQUIRED

Mixed acid.-Dissolve $20 \mathrm{~g}$ of $\mathrm{KCl}$ in $500 \mathrm{c} \mathrm{c}$ of water, add $400 \mathrm{c} \mathrm{c}$ of $\mathrm{HCl}$ (specific gravity 1.20), mix and add $100 \mathrm{c}$ c of $\mathrm{HNO}_{3}$ (specific gravity 1.42).

Alcohol-hydrochloric acid mixture.-Mix $400 \mathrm{c} \mathrm{c}$ of 95 per cent ethyl alcohol and $100 \mathrm{c}$ c of $\mathrm{HCl}$ (specific gravity 1.20 ).

Acid ammonium acetate. - To $500 \mathrm{c}$ c of $\mathrm{NH}_{4} \mathrm{OH}$ (specific gravity 0.90), add $500 \mathrm{c}$ c of water and then acetic acid ( 80 per cent), until slightly acid to litmus.

2. G. W. Thompson's method. See J., Soc. Chem. Ind. vol. 15,
METHOD

Dissolve $1 \mathrm{~g}$ of the finely divided alloy by boiling in 70 to $100 \mathrm{c}$ c of "mixed acid" solution in a covered beaker. Add more mixed acid if a complete solution of the alloy is difficult to obtain, and continue boiling until evaporated to about $50 \mathrm{c}$ c. Add $5 \mathrm{c}$ c of $\mathrm{HCl}$ (specific gravity 1.20), cool in ice water until the bulk of $\mathrm{PbCl}_{2}$ has crystallized out, then add slowly, with constant stirring, $50 \mathrm{c}$ c of alcohol (95 per cent), continue stirring for a few minutes, and cool in ice water for 10 minutes. Add $50 \mathrm{c} \mathrm{c}$ more alcohol from a pipette, allow to stand in ice water for 20 minutes, and filter through $9 \mathrm{~cm}$ paper into an 800 c c beaker. Wash by decantation three times with alcohol-hydrochloric acid mixture and finally wash the paper twice with the same mixture. Wash the $\mathrm{PbCl}_{2}$ from the paper back into the beaker; wash paper several times with hot water, catching washings in beaker with the bulk of the chloride. Finally, wash paper with hot acid ammonium acetate solution. Heat until all $\mathrm{PbCl}_{2}$ is dissolved. Add $15 \mathrm{c} c$ of saturated solution of $\mathrm{K}_{2} \mathrm{Cr}_{2} \mathrm{O}_{7}$; heat until precipitate is of good orange color; filter on weighed Gooch crucible; wash with water, alcohol, and ether; dry at $110^{\circ} \mathrm{C}$; ; and weigh. Calculate percentage of lead by the empirical factor 63.75 .

Notes-1. With proper manipulation, the $\mathrm{PbCl}_{2}$ precipitate should contain consistently all but $0.003 \mathrm{~g}$ of lead.

2. During the heating of the acid ammonium acetate to dissolve the $\mathrm{PbCl}_{2}$, the solution should remain perfectly clear, any turbidity indicating the presence of tin or antimony, as even $1 \mathrm{mg}$ of tin or antimony will cause a slight but distinct turbidity.

\section{DETERMINATION OF COPPER AND ANTIMONY}

SOLUTIONS REQUTRED

Potassium hydroxide.-Dissolve $100 \mathrm{~g}$ of $\mathrm{KOH}$ in $500 \mathrm{c}$ c of water.

Potassium cyanide.-Dissolve $3.5 \mathrm{~g}$ of $\mathrm{KCN}$ in $1,800 \mathrm{c} c$ of water and standardize against copper of known purity.

Sodium thiosulphate.-Dissolve $24.8 \mathrm{~g}$ of $\mathrm{Na}_{2} \mathrm{~S}_{2} \mathrm{O}_{3}$. $5 \mathrm{H}_{2} \mathrm{O}$ in $1,000 \mathrm{c}$ c of water, and allow to stand for 24 hours. Standardize against antimony metal chemically pure, using same quantity of reagents and same procedure as under (c) determination of antimony, given below.

METHOD

See determination of lead, given above.

Evaporate the filtrate from $\mathrm{PbCl}_{2}$ filtration by boiling in the loosely covered $800 \mathrm{c}$ c beaker, and finally take to dryness on a water bath. Add $10 \mathrm{c} \mathrm{c}$ of $\mathrm{KOH}$ solution and after a few minutes add 20 c c of 3 per cent $\mathrm{H}_{2} \mathrm{O}_{2}$. Add more $\mathrm{KOH}$, if solution is acid, until an alkaline reaction is shown by litmus paper. Heat on the water bath for 20 minutes, add $10 \mathrm{~g}$ of ammonium oxalate, $10 \mathrm{~g}$ of oxalic acid, and $200 \mathrm{c}$ c of water, and heat to boiling. Pass in $\mathrm{H}_{2} \mathrm{~S}$ for 45 minutes with solution near boiling, filter at once, and wash precipitate with hot dilute solution of oxalic acid saturated with $\mathrm{H}_{2} \mathrm{~S}$, catching washings with filtrate.

Wash the precipitate of copper and antimony sulphides from the filter paper back into the beaker with the least amount of water possible, treat with $10 \mathrm{c}$ c of $\mathrm{KOH}$ solution, heat on water bath until the undissolved matter is distinctly black, and filter through same paper into a 300 c c Erlenmeyer flask. Wash the precipitate with hot water containing a small amount of $K_{2} \mathrm{~S}$. 
(a) Determination of copper by potassium cyanide titration.--Dry and ignite the precipitate with paper in a small casserole, dissolve in nitric acid (specific gravity 1.42), boil to expel nitrous fumes, neutralize with $\mathrm{Na}_{2} \mathrm{CO}_{3}$, add a few drops of $\mathrm{NH}_{4} \mathrm{OH}$, and titrate with standard $\mathrm{KCN}$ solution.

(b) Determination of copper by electrolytic method.See determination of copper by potassium cyanide titration, given above.

Dry and ignite the sulphide precipitate in a small casserole, dissolve in $10 \mathrm{c}$ c of $\mathrm{HNO}_{3}$ (specific gravity 1.42), boil until oxides of nitrogen are expelled and add $50 \mathrm{c}$ c of distilled water. Transfer to a tall 200 c c beaker, wash out casserole, add 5 c c of $\mathrm{H}_{2} \mathrm{SO}_{4}$ (specific gravity 1.84) and determine copper by electrolysis. See the determination of copper by the electrolytic method in B27. (See 647.20, p. 1261).

Nores. -1 . On filter after heating with $\mathrm{KOH}$ solution, the copper remains as sulphide with a small amount of lead sulphide which failed to be precipitated as chloride. If it is desired to determine this lead, it can be done by separation from the copper by ordinary lead, it

2 . If the amount of antimony and copper is small (less than $10 \mathrm{mg}$ ) the lead which failed to be precipitated as chloride may also fail to come down as sulphide on passing $\mathbf{H}_{2} \mathrm{~S}$ through the oxalic solution It will remain in the filtrate from the sulphides and be deposited electrolytically with the tin on the cathode. This can be prevented by adding an oxalic acid solution of a pure antimony salt containing about $100 \mathrm{mg}$ of antimony just before passing $\mathrm{H}_{2} \mathrm{~S}$. In this case antimony must be determined on a separate portion.

3 . If the amount of copper present is from 8 to $10 \mathrm{mg}$ or more, it should be determined by the electrolytic method. In this case, the small amount of lead precipitated as sulphide with the copper is deposited on the anode as $\mathrm{PbO}_{2}$ and may be weighed, calculated to lead by factor 86.43 , and added to that obtained under determination of lead.

(c) Determination of antimony.-See determination of copper by potassium cyanide titration, given above.

Add $50 \mathrm{c}$ c of $\mathrm{HCl}$ (specific gravity 1.20) to the $\mathrm{KOH}$ solution of antimony sulphide, and boil down to small volume until all arsenic has been expelled as arsenious chloride.

Add $25 \mathrm{c}$ c of $\mathrm{HCl}$ (specific gravity 1.20) and $1 \mathrm{~g}$ of $\mathrm{KClO}_{3}$ and boil until colorless. Filter into a 300 c c Erlenmeyer flask, through glass wool if sulphur has separated, wash out original flask with $\mathrm{HCl}$ (specific gravity 1.20), cool, add $1 \mathrm{~g}$ of $\mathrm{KI}, 1 \mathrm{cc}$ of $\mathrm{CS}_{2}$, and titrate with standard thiosulphate solution.

\section{DETERMINATION OF TIN}

APPARATUS FOR ELECTROLYSIS

Electrodes.-Cylindrical cathodes of platinum wire gauze -2 by $13 \%$ inches in diameter.

Platinum-wire spiral anodes.

\section{METHOD}

See determination of copper and antimony, given above.

Concentrate the filtrate and washings from the precipitated sulphides, if necessary, to a volume of $200 \mathrm{c} c$ and add $5 \mathrm{~g}$ more of oxalic acid in case the amount of tin is over $0.5 \mathrm{~g}$. Electrolyze until the solution reacts alkaline, using a current of about 0.5 ampere. Remove the cylinder; wash twice with distilled water and once with 95 per cent ethyl alcohol; dry at $110^{\circ} \mathrm{C}$., and weigh.

NoTE.-If the electrolysis is carried out over night it will usually be found by morning that the solution has become alkaline and it may be taken for granted that the tin has all been precipitated on the cathode. The best results are obtained by regulating the current, or the time, so as to render the solution alleline only a very short the very short the cathode is to be removed. The cathode should be placed close to the bottom of the beaker to insure proper agitation

\section{B. Determination of Arsenic}

\section{SOLUTIONS REQUIRED}

Ferric chloride.-Solution of specific gravity 1.43 . Ammonium carbonate.-Dissolve $75 \mathrm{~g}$ in $500 \mathrm{c}$ c of water.
METHOD

Weigh $1 \mathrm{~g}$ of sample into a 250 c c distillation flask and add $10 \mathrm{c} \mathrm{c}$ of $\mathrm{FeCl}_{3}$ solution, $60 \mathrm{c} \mathrm{c}$ of $\mathrm{HCl}$ (specific gravity 1.20) $20 \mathrm{c}$ c of water and $5 \mathrm{~g}$ of $\mathrm{KCl}$. Connect the flask with a condenser, heat slowly until solution is complete and boil down to as small a volume as possible, catching distillate in a tall 400 c c beaker. Cool flask, add $50 \mathrm{c} \mathrm{c} \mathrm{HCl}$ (specific gravity 1.20) and redistill as before, catching distillate in same beaker. Cool distiliate and pass $\mathrm{H}_{2} \mathrm{~S}$ through for one hour, allow to settle, and filter the arsenious sulphide on a Gooch crucible. Wash twice with $\mathrm{HCl}(1: 4)$, four times with cold water, three times with alcohol, and four times with $\mathrm{CS}_{2}$ Dry at $110^{\circ} \mathrm{C}$. for 10 minutes and weigh after cooling. Dissolve the arsenious sulphide with $\left(\mathrm{NH}_{4}\right)_{2} \mathrm{CO}_{3}$ solution wash thoroughly with water, dry for 30 minutes at $110^{\circ}$ C., and reweigh after cooling. The difference in weights multiplied by 0.60911 gives the percentage of arsenic.

\section{RAPID METHOD FOR CONTROL WORK 22}

\section{A. Determination of Lead and Copper}

SOLUTIONS REQUIRED

Lead acid.-Mix $300 \mathrm{c}$ c of $\mathrm{H}_{2} \mathrm{SO}_{4}$ (specifie gravity 1.84) and $1,800 \mathrm{c} c$ of distilled water. Dissolve $1 \mathrm{~g}$ of lead acetate chemically pure in $300 \mathrm{c}$ c of distilled water and add this to the hot solution, stirring meanwhile. Let stand at least 24 hours and siphon through a thick asbestos filter.

Dilute alcohol for washing.-Mix equal parts of denatured alcohol and distilled water.

\section{METHOD}

(a) Determination of lead.-In a covered $300 \mathrm{c} \mathrm{c}$ Erlenmeyer flask dissolve $1 \mathrm{~g}$ of the alloy in $20 \mathrm{c} \mathrm{c}$ of $\mathrm{H}_{2} \mathrm{SO}_{4}$ (specific gravity 1.84 ); heat the solution nearly to boiling until the metal is completely decomposed and the $\mathrm{PbSO}_{4}$ is white (this may take 30 minutes or more), and finally boil for several minutes. Allow to cool, but not below $60^{\circ} \mathrm{C}$., and then add slowly 50 c c of water while the solution is agitated. Heat to boiling for several minutes in order to insure complete solution of antimony sulphate. Allow the $\mathrm{PbSO}_{4}$ to settle out until the solution is clear, not letting the temperature fall below $60^{\circ} \mathrm{C}$. If the liquid does not clear quickly it must be heated longer. When clear, pour the solution through a weighed porcelain Gooch crucible with asbestos mat, decanting the solution as completely as possible without allowing more than a very small amount of $\mathrm{PbSO}_{4}$ to go over into the crucible. Now add $10 \mathrm{c}$ c more of $\mathrm{H}_{2} \mathrm{SO}_{4}$ (specific gravity 1.84 ) to the $\mathrm{PbSO}_{4}$ in the original flask, and boil for several minutes. Cool, add slowly $30 \mathrm{cc}$ of water, and again heat to boiling for a few minutes; allow the solution to cool to about $60^{\circ} \mathrm{C}$. and completely transfer the $\mathrm{PbSO}_{4}$ to the Gooch crucible. Wash with lead acid, retaining the filtrate and these washings for the copper determination. Remove the beaker containing these solutions and wash out the lead acid with dilute alcohol; set the Gooch crucible inside a porcelain crucible; dry and ignite for five minutes at the full heat of a Tirrill burner; cool and weigh as $\mathrm{PbSO}_{4}$, which contains 68.29 per cent lead.

(b) Determination of copper. - To the filtrate from the $\mathrm{PbSO}_{4}$, which contains the copper and which has been transferred to an Erlenmeyer flask, add $\mathrm{NH}_{4} \mathrm{OH}$ (specific gravity 0.90) until the solution is slightly alkaline; then add $2 \mathrm{c}$ c of $\mathrm{H}_{2} \mathrm{SO}_{4}$ (specific gravity 1.84); heat nearly to boiling; add $2 \mathrm{~g}$ of

23 See The Analysis of Alloys of Lead, Tin, Antimony, and Copper, by D. J. Demorest, J. Ind. Eng. Chem., vol. 5, p. 842; Rapid Analysis of Alloys for Tin, Antimony, and Arsenic, by F. A. Stief, J. Ind Eng. Chem., vol. 7, p. 211; and Technical Analysis of Brass, 1917. Price and Meade, p. 182 . 
$\mathrm{Na}_{2} \mathrm{SO}_{3}$ and when this is entirely dissolved add $1 \mathrm{~g}$ of KCNS dissolved in $10 \mathrm{c}$ c of water. Shake well and allow the precipitated CuCNS to settle for one hour while the solution is kept hot. Filter on a close filter paper, wash with cold water and ignite paper and precipitate in a porcelain crucible. Proceed by one of the two following methods:

(1). Dissolve in $\mathrm{HNO}_{3}$ (specific gravity 1.42), add 5 c c $\mathrm{H}_{2} \mathrm{SO}_{4}$ (specific gravity 1.84); evaporate until fumes of $\mathrm{H}_{2} \mathrm{SO}_{4}$ are evolved. Dilute to about $100 \mathrm{c} \mathrm{c}$ with distilled water. Add $1.5 \mathrm{c} \mathrm{c}$ of $\mathrm{HNO}_{3}$ (specific gravity 1.42) and determine copper by electrolysis. See the determination of copper by the electrolytic method in B27 (647.20, p. 1261).

(2). Dissolving the ignited precipitate in $\mathrm{HNO}_{3}$ (specific gravity 1.42), as in (1); boil to expel nitrous fumes, neutralize with $\mathrm{Na}_{2} \mathrm{CO}_{3}$ and determine volumetrically with cyanide as in A of general method.

NotE.-If the amount of copper is very small it may escape detection by this method, in which case it should be determined as in A of "general method."

\section{B. DETERMINATION OF ARSENIC, ANTIMONY, AND TIN}

APPARATUS

Arsenic still.-A condenser is made from glass tubing in the form of a letter $\mathrm{S}$, about 18 inches long and one-half inch in inside diameter, tapering to about one-fourth inch at the upper end and to about one-eighth inch at the lower end. One curve is nearly filled with water and is submerged in cold water in a $500 \mathrm{c}$ c beaker. The lower end dips into about $75 \mathrm{c}$ c of water in a $300 \mathrm{c}$ c beaker and the upper end is connected by a delivery tube with a 300 c c Florence flask, closed with a rubber stopper which is fitted with a delivery tube and with a thermometer reaching to about 1 inch above the surface of the liquid in the flask.

\section{SOLUTIONS REQUIRED}

Iodine.-Dissolve $10.7 \mathrm{~g}$ of iodine in $50 \mathrm{c} \mathrm{c}$ of distilled water which contains $20 \mathrm{~g}$ of $\mathrm{KI}$ in solution and dilute to $1,000 \mathrm{c} c$ with distilled water. Standardize against pure tin having exactly the same conditions for titration as are specified for the analysis of the alloy. Each cubic centimeter is equivalent to approximately $0.00500 \mathrm{~g}$ of $\mathrm{tin}$ or $0.00315 \mathrm{~g}$ of arsenic.

Potassium permanganate.-Dissolve $2.7 \mathrm{~g}$ of $\mathrm{KMnO}_{4}$ in distilled water, filter through asbestos, and dilute to $1,000 \mathrm{c} \mathrm{c}$ with distilled water. Standardize against pure antimony having exactly the same conditions for titration as are specified for the analysis of the alloy. Each cubic centimeter is equivalent to approximately $0.00500 \mathrm{~g}$ of antimony or $0.00465 \mathrm{~g}$ of iron.

Ferrous ammonium sulphate.-Dissolve $12.4 \mathrm{~g}$ of ferrous ammonium sulphate crystals in $950 \mathrm{c} \mathrm{c}$ of distilled water and add $50 \mathrm{c}$ c of $\mathrm{H}_{2} \mathrm{SO}_{4}$ (specific gravity 1.84).

Starch.-To 1,000 e c of boiling water, add a cold suspension of $6 \mathrm{~g}$ of starch in $100 \mathrm{c} \mathrm{c}$ of distilled water; cool, add a few drops of chloroform, and mix thoroughly.

\section{METHOD}

(a) Determination of arsenic.-In a 300 c c Florence flask, dissolve $0.5 \mathrm{~g}$ of the alloy in exactly $8 \mathrm{c} \mathrm{c}$ of $\mathrm{H}_{2} \mathrm{SO}_{4}$ (specific gravity 1.84). The metal must be finely divided, preferably in the form of thin foillike shavings, although sawings or very fine drillings may be used. Heat the solution to boiling; cool, add about $5 \mathrm{c}$ c of water and a bulk of about $0.5 \mathrm{c}$ c of clean granulated pumice stone, and boil the covered solution very gently for about five minutes, or until the strong odor of $\mathrm{SO}_{2}$ can no longer be detected. Finally, being careful to have 5 c c of water present, cool the solution to about $18^{\circ} \mathrm{C}$. and cautiously add $20 \mathrm{c}$ c of $\mathrm{HCl}$ (specific gravity 1.20). Insert in the flask a rubber stopper fitted with a thermometer and delivery tube and connect the delivery tube with the $S$ condenser. Heat the solution to gentle boiling for from 10 to 15 minutes, keeping the vapor temperature at $107^{\circ}$ C. for at least 5 minutes. Wash out the condenser into a $300 \mathrm{c}$ c beaker and add an excess of about $2 \mathrm{~g}$ of $\mathrm{NaHCO}_{3}$. Bring the volume of the solution to about $200 \mathrm{c} \mathrm{c}$; warm to about $27^{\circ} \mathrm{C}$. and titrate with standard iodine solution and starch to the appearance of a deep blue color.

Note.-A blank determination should be made on corresponding amounts of reagents treated as above, and the results should be corrected accordingly.

(b) Determination of antimony.-Cool the solution which remains in the flask after the arsenic distillation; add about $130 \mathrm{c}$ c of cold, recently boiled distilled water to which has been added about $3 \mathrm{c} \mathrm{c}$ of $\mathrm{HCl}$ (specific gravity 1.20), and titrate with standard $\mathrm{KMnO}_{4}$ solution to the appearance of a decided permanent pink color. From a burette add an excess of standard ferrous ammonium sulfate solution until the pink color is discharged and then titrate with standard $\mathrm{KMnO}_{4}$ solution to the reappearance of a pink color.

(c) Determination of tin. - If the sample does not contain 14 per cent (or $0.07 \mathrm{~g}$ ) of antimony, add enough dissolved $\mathrm{SbCl}_{3}$ to the solution to bring the contents of antimony up to about $0.07 \mathrm{~g}$ for the purpose of insuring perfect reduction of the tin and uniform conditions for the titration. Then add exactly 6 c c of $\mathrm{H}_{2} \mathrm{SO}_{4}$ (specific gravity 1.84) and $60 \mathrm{c} \mathrm{c}$ of $\mathrm{HCl}$ (specific gravity 1.20) and add about 6 inches of clean, soft No. 14 pure iron wire cut into 2-inch lengths and cleaned with dilute $\mathrm{HCl}$ just before using. Boil gently for 30 minutes, add about 6 inches more wire, and boil 30 minutes longer. Remove from heat, close flask loosely with a rubber stopper, and allow about two minutes for all air to be expelled by hydrogen and acid vapors. Close flask tightly and quickly place it in cold water cooling the solution to about $20^{\circ} \mathrm{C}$. Transfer the solution quickly to a $500 \mathrm{c}$ c beaker (leaving the iron wires in the flask) and rinse flask and contents with $150 \mathrm{c}$ c of cold, recently boiled distilled water. Add rinsings to main solution in the beaker, bring final volume to about 300 to $350 \mathrm{c}$ c and titrate quickly with standard iodine solution and starch to the appearance of a strong blue color.

NoTES.-A blank determination should be made on corresponding amounts of reagents treated as above, and the results should be corrected accordingly.

If the copper content of the alloy is as high as about 3 per cent or over, it prevents a good end point in the tin titration when the tin percentage is low. Under such conditions, just before titrating, add about $1 \mathrm{~g}$. of KI crystals, stir until nearly dissolved, and titrate immediately. If allowed to stand too long at this point, trouble may be encountered due to the precipitation of some of the other constituents.

Other forms of pure iron may be employed, but the wire as above specified has been found convenient.

For accurate work it is desirable to reduce and titrate the solution of tin in an atmosphere of $\mathrm{CO}_{2}$.

692.1 BABBITT METALS.

\section{AMERICAN ELECTRIC RAILWAY ENGI- NEERING ASSOCIATION, ARMATURE AND AXLE IINERS, E212-25, 1925}

\section{ARMATURE AND AXLE LINERS}

(See 692.2, p. 1324.) 
AMERICAN RAILWAY ASSOCIATION, MECHANICAI DIVISION; BEARINGS, JOURNAL, IINED, RECOMMENDED PRACTICE, 1926

JOURNAL BEARINGS, LINED

(See 692.3, p. 1328.)

\section{AMERICAN RAILWAY ENGINEERING AS- SOCIATION, SPECIFICATIONS FOR MOV. ABLE RAILWAY BRIDGES, 1929.}

(See also 603.32 , p. $151 ; 611.41$, p. $707 ; 611.51$, p. 753; 647.31, p. 1268.)

\section{BABBITT METAL}

Babbitt metal shall be approximately of the following composition:

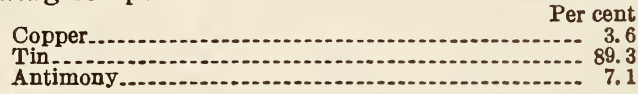

Babbitt metal shall be poured in the bearings in such a way that the thickness of the lining after boring will be uniform.

\section{AMERICAN SOCIETY FOR TESTING MATE- RIALS, STANDARD SPECIFICATIONS FOR WHITE METAL BEARING ALIOYS (BAB- BITT METAL), DESIGNATION B23-26, 1926}

\section{BABBITT METAL}

SCOPE

1. These specifications cover white metal bearing alloys, known commercially as "babbitt metal." Twelve typical babbitt metals are specified, cover ing the range of alloys commercially used and are designated as grades Nos. 1 to 12 , in accordance with their decreasing tin content as specified in section 4 .

\section{MANUFACTURE}

2. The manufacturer shall use care to have each lot of babbitt metal as uniform in quality as possible.

3. The standard bar, unless otherwise specified, shall have the following approximate dimensions:

Top face. 134 inches wide, $81 / 2$ inches long.

Thickness. Not over $11 / 4$ inches.

\section{CHEMICAL PROPERTIES AND TESTS}

4. The alloys shall conform to the following re quirements as to chemical composition, within the limits specified in section 5 :

\begin{tabular}{|c|c|c|c|c|c|c|c|c|}
\hline $\begin{array}{l}\text { Alloy } \\
\text { grade } \\
\text { No. }\end{array}$ & Tin & $\begin{array}{l}\text { Anti- } \\
\text { mony }\end{array}$ & Lead & $\begin{array}{l}\text { Cop- } \\
\text { per }\end{array}$ & $\begin{array}{l}\text { Iron, } \\
\text { maxi- } \\
\text { mum }\end{array}$ & $\begin{array}{l}\text { Arse- } \\
\text { nic, } \\
\text { maxi- } \\
\text { mum }\end{array}$ & Zinc & $\underset{\text { num }}{\text { Alumi- }}$ \\
\hline $\begin{array}{l}1 \\
2 \\
3 \\
4\end{array}$ & $\begin{array}{l}\text { Per } \\
\text { cent } \\
91 \\
89 \\
831 / 3 \\
75\end{array}$ & $\begin{array}{l}\text { Per } \\
\text { cent } \\
41 / 2 \\
71 / 2 \\
81 / 3 \\
12\end{array}$ & $\begin{array}{c}\text { Per } \\
\text { cent } \\
10.35 \\
1.35 \\
1.35 \\
10\end{array}$ & $\begin{array}{l}\text { Per } \\
\text { cent } \\
41 / 2 \\
31 / 2 \\
81 / 3 \\
3\end{array}$ & $\begin{array}{l}\text { Per } \\
\text { cent } \\
0.08 \\
.08 \\
.08 \\
.08\end{array}$ & $\begin{array}{l}\text { Per } \\
\text { cent } \\
0.10 \\
.10 \\
.10 \\
.15\end{array}$ & $\begin{array}{c}\text { Per } \\
\text { cent } \\
\text { None. } \\
\text { None. } \\
\text { None. } \\
\text { None. }\end{array}$ & $\begin{array}{c}\text { Per } \\
\text { cent } \\
\text { None. } \\
\text { None. } \\
\text { None. } \\
\text { None. }\end{array}$ \\
\hline $\begin{array}{l}5 \\
6 \\
7 \\
8\end{array}$ & $\begin{array}{r}65 \\
20 \\
10 \\
5\end{array}$ & $\begin{array}{l}15 \\
15 \\
15 \\
15\end{array}$ & $\begin{array}{l}18 \\
6316 \\
75 \\
80\end{array}$ & $\begin{array}{r}2 \\
111 / 2 \\
10.50 \\
1.50\end{array}$ & $\begin{array}{r}.08 \\
.08\end{array}$ & $\begin{array}{l}.15 \\
.15 \\
.20 \\
.20\end{array}$ & $\begin{array}{l}\text { None. } \\
\text { None. } \\
\text { None. } \\
\text { None. }\end{array}$ & $\begin{array}{l}\text { None. } \\
\text { None. } \\
\text { None. } \\
\text { None. }\end{array}$ \\
\hline $\begin{array}{r}9 \\
10 \\
11 \\
12\end{array}$ & \begin{tabular}{c}
5 \\
2 \\
\hdashline- \\
-2
\end{tabular} & $\begin{array}{l}10 \\
15 \\
15 \\
10\end{array}$ & $\begin{array}{l}85 \\
83 \\
85 \\
90\end{array}$ & $\begin{array}{l}1.50 \\
1.50 \\
1.50 \\
1.50\end{array}$ & & $\begin{array}{l}.20 \\
.20 \\
.25 \\
.25\end{array}$ & $\begin{array}{l}\text { None. } \\
\text { None. } \\
\text { None. } \\
\text { None. }\end{array}$ & $\begin{array}{l}\text { None. } \\
\text { None. } \\
\text { None } \\
\text { None. }\end{array}$ \\
\hline
\end{tabular}

1 Maximum.
5. The following permissible variations in the percentages of the desired elements specified in section 4 will be allowed, but shall not apply to the maximum percentages of impurities specified:

\begin{tabular}{|c|c|}
\hline Percentage of element specified & $\begin{array}{l}\text { Permissible } \\
\text { variations } \\
\text { over or } \\
\text { under the } \\
\text { specifled } \\
\text { value, units } \\
\text { of per cent }\end{array}$ \\
\hline 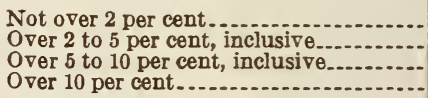 & $\begin{array}{r}0.25 \\
.50 \\
.75 \\
1.00\end{array}$ \\
\hline
\end{tabular}

6. (a) Three bars shall be selected to represent a shipment of less than 1,000 pounds, five bars to represent a shipment of over 1,000 pounds to 10,000 pounds, inclusive, and 10 bars to represent a shipment of over 10,000 pounds to one carload.
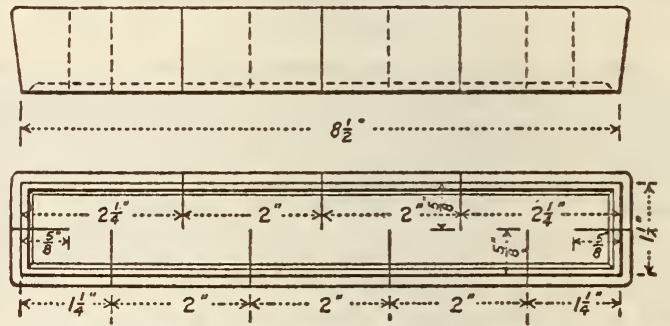

FIGURE 1137.-Method of sampling

(b) Saw cuts shall be made at points in the bars indicated in Figure 1137. No lubricants shall be used for sawing. The sawings shall be carefully treated with a magnet to remove any particles of steel introduced in taking the sample.

(c) Sawings thoroughly mixed shall constitute the sample for chemical analysis.

7. The chemical analysis shall be made in accordance with the A. S. T. M. standard methods of chemical analysis of alloys of lead, tin, antimony and copper (see 692.0 , p. 1317).

\section{MARKING}
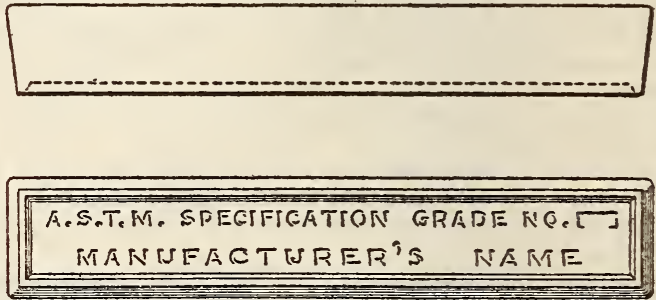

Figure 1138.-Preferred arrangement of marking

8. The name of the manufacturer shall be cast on each bar. The numerical designation of the grade supplied shall be stamped or cast on each bar for identification.

Note.-A preferred arrangement of marking is shown in Figure 1138.

\section{CLAIMS}

(Conforms to A. S. T. M. B30-31T. See 644.11, p. 1196.)

\section{APPENDIX}

The data in the following table do not constitute a part of these specifications. They are given 
merely to indicate to the purchaser the physical metal is to be used. The alloys were prepared at properties of the various alloys specified which can be expected of carefully manufactured alloys of the formulas indicated, and to constitute a guide to the purchaser in selecting the grade best suited for meeting the service condition for which the babbitt

the Bureau of Standards from pure Banka tin, high grade "Star" antimony and a commercially pure lead (99.94 per cent $\mathrm{Pb})$, and the values given are the results of tests made in the bureau laboratories. Composition and physical properties ${ }^{1}$ of white metal bearing alloys

\begin{tabular}{|c|c|c|c|c|c|c|c|c|c|c|c|c|}
\hline \multirow{2}{*}{ Alloy No. } & \multicolumn{4}{|c|}{ Specified composition of alloys } & \multirow{2}{*}{$\begin{array}{l}\text { Specific } \\
\text { gravity }\end{array}$} & \multicolumn{5}{|c|}{ Compositions of alloys tested } & \multicolumn{2}{|c|}{ Yield point 2} \\
\hline & Copper & Tin & $\begin{array}{l}\text { Anti- } \\
\text { mony }\end{array}$ & Lead & & Copp & & & $\begin{array}{l}\text { Anti- } \\
\text { mony }\end{array}$ & Lead & $20^{\circ} \mathrm{C}$. & $100^{\circ} \mathrm{C}$. \\
\hline 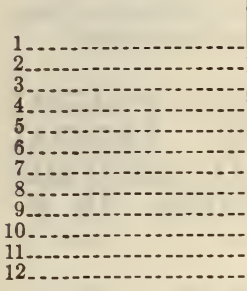 & \begin{tabular}{c} 
Per cent \\
4.5 \\
3.5 \\
$81 / 3$ \\
3.0 \\
2.0 \\
1.5 \\
\hdashline \\
\hdashline
\end{tabular} & $\begin{array}{c}\text { Per cent } \\
91.0 \\
89.0 \\
8813 \\
75.0 \\
65.0 \\
20.0 \\
10.0 \\
5.0 \\
5.0 \\
2.0\end{array}$ & \begin{tabular}{|r} 
Per cent \\
4.5 \\
7.5 \\
813 \\
12.0 \\
15.0 \\
15.0 \\
15.0 \\
15.0 \\
10.0 \\
15.0 \\
15.0 \\
-10.0
\end{tabular} & $\begin{array}{r}\text { Per cent } \\
10.0 \\
18 . \\
63 . \\
75 . \\
80 . \\
85 . \\
83 . \\
85.0 \\
90.0\end{array}$ & $\begin{array}{r}7 . \\
7 . \\
7 . \\
7 . \\
7 . \\
9 . \\
9 . \\
10 . \\
10 . \\
10 . \\
10 . \\
10 .\end{array}$ & \begin{tabular}{c|c} 
& Per ce \\
34 & 4. \\
39 & 3. \\
6 & 8. \\
5 & 3. \\
5 & 2. \\
3 & 1. \\
3 & 0. \\
4 & 0. \\
4 & 0. \\
7 & 0. \\
7 & 0. \\
7 & 0.
\end{tabular} & & & $\begin{array}{c}\text { Per cent } \\
4.52 \\
7.4 \\
8.2 \\
11.6 \\
14.1 \\
14.6 \\
14.5 \\
14.9 \\
9.9 \\
15.7 \\
14.8 \\
9.9\end{array}$ & $\begin{array}{c}\text { Per cent } \\
\text { None. } \\
0.03 \\
0.03 \\
10.2 \\
18.2 \\
63.7 \\
75.0 \\
79.4 \\
84.6 \\
82.0 \\
84.7 \\
89.4\end{array}$ & $\begin{array}{r}\text { Lbs./in.2 } \\
4,400 \\
6,100 \\
6,600 \\
5,550 \\
5,050 \\
3,800 \\
3,550 \\
3,400 \\
3,400 \\
3,350 \\
3,050 \\
2,800\end{array}$ & $\begin{array}{r}\text { Lbs./in. } \\
2,650 \\
3,000 \\
3,150 \\
2,150 \\
2,150 \\
2,050 \\
1,600 \\
1,750 \\
1,550 \\
1,850 \\
1,400 \\
1,250\end{array}$ \\
\hline \multirow[t]{2}{*}{ Alloy No. } & \multicolumn{2}{|c|}{$\begin{array}{l}\text { Johnson's appar- } \\
\text { ent elastic limit }\end{array}$} & \multicolumn{2}{|c|}{ Ultimate strength 4} & \multicolumn{2}{|c|}{ Brinell hardness 5} & \multicolumn{2}{|c|}{ Melting point } & \multicolumn{2}{|c|}{$\begin{array}{l}\text { Temperature of } \\
\text { complete liquida- } \\
\text { tion }\end{array}$} & \multicolumn{2}{|c|}{$\begin{array}{l}\text { Proper pouring } \\
\text { temperature }\end{array}$} \\
\hline & $20^{\circ} \mathrm{C}$. & $100^{\circ} \mathrm{C}$. & $20^{\circ} \mathrm{C}$. & $100^{\circ} \mathrm{C}$. & $20^{\circ} \mathrm{C}$ & $100^{\circ} \mathrm{C}$. & ${ }^{\circ} \mathrm{F}$. & ${ }^{\circ} \mathrm{C}$. & $\circ \mathrm{F}$. & ${ }^{\circ} \mathrm{C}$. & $\circ \mathrm{F}$. & ${ }^{\circ} \mathrm{C}$. \\
\hline $\begin{array}{r}3 \\
4 \\
5 \\
6 \\
7 \\
8 \\
9 \\
10 \\
11 \\
12\end{array}$ & $\begin{array}{r}\text { Lbs./in. }{ }^{2} \\
2,450 \\
3,350 \\
5,350 \\
3,200 \\
3,750 \\
3,550 \\
2,500 \\
2,650 \\
2,400 \\
2,250 \\
2,750 \\
2,250\end{array}$ & $\begin{array}{r}\text { Lbs./in. }{ }^{2} \\
1,050 \\
1,100 \\
1,300 \\
1,550 \\
1,500 \\
1,800 \\
1,350 \\
1,200 \\
950 \\
1,200 \\
1,100 \\
950\end{array}$ & $\begin{array}{r}\text { Lbs./in. }{ }^{2} \\
12,850 \\
14,900 \\
17,600 \\
16,150 \\
15,050 \\
14,550 \\
15,650 \\
15,600 \\
14,700 \\
15,450 \\
12,800 \\
12,900\end{array}$ & \begin{tabular}{|c|} 
Lbs./in. ${ }^{2}$ \\
6,950 \\
8,700 \\
9,900 \\
6,900 \\
6,750 \\
8,050 \\
6,150 \\
6,150 \\
5,850 \\
5,750 \\
5,100 \\
5,100
\end{tabular} & $\begin{array}{l}17.0 \\
24.5 \\
27.0 \\
24.5 \\
22.5 \\
21.0 \\
22.5 \\
20.0 \\
19.0 \\
17.5 \\
15.0 \\
14.5\end{array}$ & $\begin{array}{r}8.0 \\
12.0 \\
14.5 \\
12.0 \\
10.0 \\
10.5 \\
10.5 \\
9.5 \\
8.5 \\
9.0 \\
7.0 \\
6.5\end{array}$ & $\begin{array}{l}464 \\
459 \\
459 \\
468 \\
471 \\
473\end{array}$ & $\begin{array}{l}223 \\
241 \\
240 \\
184 \\
181 \\
181 \\
240 \\
237 \\
237 \\
242 \\
244 \\
245\end{array}$ & $\begin{array}{l}669 \\
792 \\
583 \\
565 \\
531 \\
514 \\
522 \\
493 \\
507 \\
504 \\
498\end{array}$ & $\begin{array}{l}354 \\
422 \\
306 \\
296 \\
277 \\
268 \\
272 \\
256 \\
264 \\
262 \\
259\end{array}$ & $\begin{array}{l}825 \\
795 \\
915 \\
710 \\
690 \\
655 \\
640 \\
645 \\
620 \\
630 \\
630 \\
625\end{array}$ & $\begin{array}{l}441 \\
424 \\
491 \\
377 \\
366 \\
346 \\
338 \\
341 \\
327 \\
332 \\
332 \\
329\end{array}$ \\
\hline
\end{tabular}

1 The compression test specimens were cylinders $11 / 2$ inches in length and $1 / 2$ inch diameter, machined from chill castings 2 inches in length and $3 / 4$ inch in diameter. The Brinell tests were made on the bottom face of parallel machined speciments cast in a 2-inch diameter by $5 / 8$-inch deep steel mold at room temperature.

The values for yield point were taken from stress-strain curves at a deformation of 0.125 per cent reduction of gage length.

3 Johnson's apparent elastic limit is taken as the unit stress at the point where the slope of the tangent to the curve is one and one-half times its slope at the origin.

1 The ultimate strength values were taken as the unit load necessary to produce a deformation of 25 per cent of the length of the specimen.

5 These values are the average Brinell number of 3 impressions on each alloy using a $10 \mathrm{~mm}$ ball and a $500 \mathrm{~kg}$ load applied for 30 seconds.

AMERICAN SOCIETY FOR TESTING MATERIAIS, STANDARD SPECIFICATIONS FOR CAR AND TENDER JOURNAL BEARINGS, IINED, DESIGNATION B67-28, 1928

JOURNAL BEARINGS, LINED

(See 692.3, p. 1329.)

\section{SOCIETY OF AUTOMOTIVE ENGINEERS, NONFERROUS METAL SPECIFICATIONS, WHITE BEARING METALS, SPECIFICA- TIONS NOS. 10,11,12,13,14, BABBITT, AUGUST, 1922}

\section{WHITE BEARING METALS}

The limits for the chemical compositions specified for metal in ingot form are closer than the limits specified for cast products, as allowances have been made for variations in the chemical content due to casting.
SPECIFICATION NO. 10, BABBITT

1. Composition in percentage.-

\begin{tabular}{|c|c|c|}
\hline & $\begin{array}{c}\text { Cast } \\
\text { products }\end{array}$ & Ingots \\
\hline \begin{tabular}{|l} 
Tin, minimum \\
Copper \\
Antimony \\
Lead, maximum
\end{tabular} & $\begin{array}{c}\text { Per cent } \\
90 \\
4-5 \\
4-5 \\
\quad .35\end{array}$ & $\begin{array}{r}\text { Per cent } \\
90.75 \\
\text { 4. } 25-4.75 \\
\text { 4. } 25-4.75 \\
.35\end{array}$ \\
\hline $\begin{array}{l}\text { Iron, maximum } \\
\text { Arsenic, maximum } \\
\text { Bismuth, maximum } \\
\text { Zine and aluminum }-. .- \\
\end{array}$ & $\begin{array}{r}.08 \\
.10 \\
.08 \\
\text { None. }\end{array}$ & $\begin{array}{r}.08 \\
.10 \\
.08 \\
\text { None. }\end{array}$ \\
\hline
\end{tabular}

2. When finished bronze-backed bearings are purchased a maximum of 0.6 per cent lead is permissible in scraped samples, provided a lead-tin solder has been used in bonding the bronze and the Babbitt.

3. General information.-This Babbit is very fluid and may be used for bronze-backed bearings, particularly for thin linings such as are used in aircraft engines. It is also suitable for die castings. 
SPECIFICATION NO. 11, BABBITT

1. Composition in percentage.-

\begin{tabular}{|c|c|c|}
\hline & $\begin{array}{c}\text { Cast } \\
\text { products }\end{array}$ & Ingots \\
\hline $\begin{array}{l}\text { Tin, minimum } \\
\text { Copper_... } \\
\text { Antimony } \\
\text { Lead, maximum }\end{array}$ & $\begin{array}{r}\text { Per cent } \\
86.00 \\
5.00-6.50 \\
6.00-7.50 \\
.35\end{array}$ & $\begin{array}{r}\text { Per cent } \\
\quad 87.25 \\
5.50-6.00 \\
6.50-7.00 \\
.35\end{array}$ \\
\hline $\begin{array}{l}\text { Iron, maximum } \\
\text { Arsenic, maximum } \\
\text { Bismuth, maximum } \\
\text { Zinc and aluminum }\end{array}$ & $\begin{array}{r}.08 \\
.10 \\
.08 \\
\text { None. }\end{array}$ & $\begin{array}{r}.08 \\
.10 \\
.08 \\
\text { None. }\end{array}$ \\
\hline
\end{tabular}

2. (Same as par. 2 in specification No. 10, given above.)

3. General information. - This is a rather hard Babbitt which may be used for lining connectingrod and shaft bearings which are subjected to heavy pressures. Its "wiping" tendency is very slight. It is also suitable for die castings.

SPECIFICATION NO. 12, BABBITT

1. Composition in percentage.-

\begin{tabular}{|c|c|c|}
\hline & $\begin{array}{c}\text { Cast } \\
\text { products }\end{array}$ & Ingots \\
\hline $\begin{array}{l}\text { Tin, minimum } \\
\text { Lead, maximum } \\
\text { Antimony } \\
\text { Copper } \\
\text { Iron, maximum } \\
\text { Bismuth, maximum } \\
\text { Zinc and aluminum }\end{array}$ & $\begin{array}{r}\text { Per cent } \\
59.50 \\
26.00 \\
9.50-11.50 \\
2.25-3.75 \\
.08 \\
.08 \\
\text { None. }\end{array}$ & $\begin{array}{r}\text { Per cent } \\
60.00 \\
25.25 \\
10.25-10.75 \\
2.75-3.25 \\
.08 \\
.08 \\
\text { None. }\end{array}$ \\
\hline
\end{tabular}

2. General information.-This is a relatively cheap Babbitt and is intended for bearings subjected to moderate pressures. It is also suitable for die castings.

SPECIFICATION NO. 13, BABBITT

1. Composition in percentage.-

\begin{tabular}{|c|c|c|}
\hline & $\begin{array}{c}\text { Cast } \\
\text { products }\end{array}$ & Ingots \\
\hline $\begin{array}{l}\text { Lead, maximum } \\
\text { Antimony } \\
\text { Tin } \\
\text { Copper, maximum } \\
\text { Arsenic, maximum and aluminum }\end{array}$ & $\begin{array}{r}\text { Per cent } \\
86.00 \\
9.25-10.75 \\
4.50-5.50 \\
.50 \\
.20 \\
\text { None. }\end{array}$ & $\begin{array}{r}\text { Per cent } \\
85.50 \\
9.75-10.25 \\
4.75-5.25 \\
.50 \\
.20 \\
\text { None. }\end{array}$ \\
\hline
\end{tabular}

2. General information.-This is a cheap Babbitt and serves successfully where the bearings are large and the service light. It should not be used as a substitute for a Babbitt with a high tin content. It is also suitable for die castings.

SPECIFICATION NO. 14, BABBITT

\section{Composition in percentage.-}

\begin{tabular}{|c|c|c|}
\hline & $\begin{array}{c}\text { Cast } \\
\text { products }\end{array}$ & Ingots \\
\hline Lead, maximum... & $\begin{array}{r}\text { Per cent } \\
76.00\end{array}$ & $\begin{array}{c}\text { Per cent } \\
75.25\end{array}$ \\
\hline Antimony......... & $14.00-16.00$ & 14. $75-15.2$ \\
\hline Tin & $9.25-10.75$ & $9.75-10.2$ \\
\hline Copper & .50 & $\begin{array}{r}.50 \\
20\end{array}$ \\
\hline $\begin{array}{l}\text { Arsenic, maximum } \\
\text { Zinc and aluminum }\end{array}$ & None. & None. \\
\hline & & \\
\hline
\end{tabular}

2. (Same as par. 2 in specification No. 13, given above.)
UNITED STATES GOVERNMENT, DEPARTMENT OF COMMERCE, BUREAU OF STANDARDS, STANDARD SAMPLES, CIRCULAR NO. 25 AND SUPPLEMENT, 1927

\section{LEAD-BASE AND TIN-BASE BEARING METAL SAMPLES}

(Standard sample No. 53 for lead-base bearing metal and standard sample No. 54a for tin-base bearing metal are prepared and sold by the Bureau of Standards for use by industrial organizations and others as comparison standards for checking the accuracy of analysis of similar bearing metals. Each sample is accompanied by a certificate giving the complete analysis of that particular sample.)

\section{UNITED STATES GOVERNMENT, FEDERAL SPECIFICATIONS BOARD, FEDERAL SPECIFICATION FOR METAL, ANTIFRIC- TION, INGOTS AND CASTINGS, QQ-M- 161, FEBRUARY 2, 1932}

\section{ANTI-FRICTION METAL}

\section{GENERAL SPECIFICATIONS}

United States Government general specification for metals, Federal Specifications Board specification No. 339 (current designation, QQ-M-151) in effect on date of invitation for bids, shall form part of this specification. (See 600.0, p. 3.)

\section{GRADE}

This specification covers seven grades of antifriction metal in the form of ingots for remelting or castings as shown in Table 1.

\section{MATERIAL}

The ingots or castings shall be made only from the best grades of virgin metals, or from virgin metals and scrap of known and approved composition.

\section{GENERAL REQUIREMENTS}

The metal, on both surface and fracture, shall be free from abnormal amounts of dross, thoroughly homogeneous, and white in color.

\section{DETAIL REQUIREMENTS}

1. The chemical composition shall conform to the requirements shown in Table 1.

2 . Unless otherwise specified in the contract or purchase order, antifriction metal ingots shall be provided with a central $V$ notch and shall have a maximum cross section of $2 \frac{1}{2}$ inches and a maximum weight of 10 pounds.

3. Ingots shall be made in a manner consistent with the best practice, particularly in respect to the temperature of the metal and that of the mold parts at time of pouring. They shall be of uniform quality, clean, and free from excessive dross and foreign material.

4. Castings shall be made in a manner consistent with the best practice, particularly in respect to the temperature of the metal and that of the mold parts at time of pouring, and shall be sound, clean, free from segregation, excessive grain size, blowholes. porous places, cracks, or any other defects which may materially affect their strength or appearance or which indicate an inferior quality of metal.

Castings shall be in accordance with the form and dimensions shown on plans accompanying the contract or purchase order. 
5. Each ingot shall have cast or stamped thereon, the nanie, brand, or trade-mark of the manufacturer and the grade number.

6. Large castings shall be marked with the pattern or mark number, and when practicable, with the lot number, in a position on the casting where they will not be machined off in manufacturing to finished dimensions.

7. Small castings shall be properly separated by melts or lots and shall be so marked that their identity will be clear at the point of delivery.

\section{METHODS OF INSPECTION AND TEST}

1. A lot shall consist of ingots or castings from the same melt, unless otherwise specified in the contract or purchase order or mutually agreed upon by the contractor and the inspector.

2. Chemical analysis of the ingots or castings may be made by the inspector or through him by any Government laboratory or other designated representative and without cost to the contractor.

3. The sample for analysis of ingots shall be obtained by selecting 3 ingots to represent a ship-
6. Samples for fracture test of castings shall be taken from 10 representative castings selected by the inspector from each lot of 1,000 pounds or fraction thereof. The inspector shall designate the points in the castings where fracture tests are to be made and may use heads or gates if satisfied that they are fully representative.

\section{PACKING AND MARKING OF SHIPMENTS}

1. Ingots may be shipped in bulk in sealed cars, or in suitable containers.

2. Castings shall be so boxed or crated as to insure safe transportation to the point of delivery.

3. Each container shall be legibly and indelibly marked with the grade number; weight of contents; melt or lot number (in the case of castings); name, brand, or trade-mark of contractor; and the contract or purchase order number.

\section{NOTES}

1. Contracts and purchase orders should state the grade of metal required.

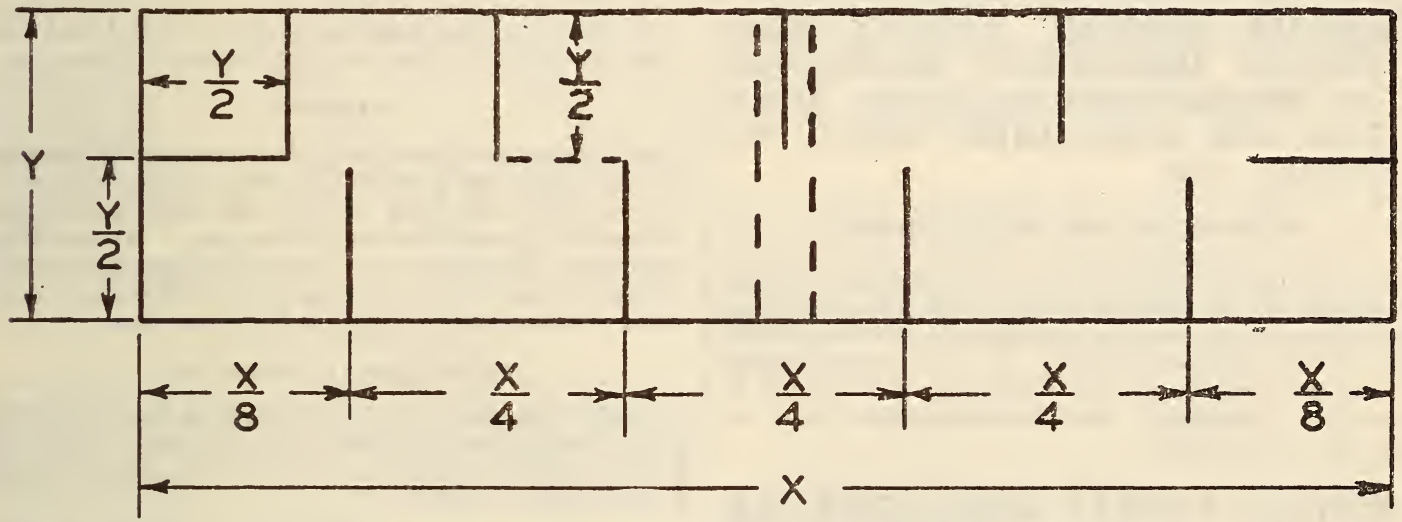

FIGURE 1139.-Locations in ingots from which samples shall be taken

ment of less than 1,000 pounds, 5 ingots to represent a shipment of from 1,000 to 10,000 pounds, and 10 ingots to represent a shipment of from 10,000 pounds to one carload. The samples for analysis shall be taken by saw cuts made in the ingots at the locations and of the depth shown in Figure 1139. No lubricant shall be used for sawing. The sawings shall be carefully treated with a magnet to remove any particles of steel introduced in taking the sample. The sawings thus obtained, thoroughly mixed, shall constitute the sample for analysis.

4. The samples for analysis of castings shall be taken from two representative castings selected by the inspector from each melt or lot of 250 pounds or fraction thereof. If a melt or lot consists of more than 250 pounds, two castings shall be selected from each 500 pounds or fraction thereof. Saw cuts shall be made at such points in the castings or heads or gates as the inspector may designate, the sawings cleaned with a magnet and thoroughly mixed before analysis. Where sawing is inadvisable, the sample may be obtained by scraping or other approved method.

5. To determine compliance with the fracture-test requirements of Section IV, 6 ingots shall be selected to represent a shipment of less than 1,000 pounds, 10 ingots to represent a shipment of from 1,000 to 10,000 pounds, and 20 ingots to represent a shipment of from 10,000 pounds to one carload.
2. Grade No. 1 antifriction metal is a medium hard Babbitt metal and may be used for aircraft engine bearings.

3. Grade No. 2 antifriction metal is geniune Babbitt metal and may be used for all bearing surfaces requiring a hard white metal alloy and where conditions are moderately severe such as general automotive engine use.

4. Grade No. 3 antifriction metal is a rather hard Babbitt metal which may be used for bearings subject to moderately heavy pressures.

5. Grade No. 4 antifriction metal is a hard bearing metal which may be used for service involving very heavy pressure and high speed.

6. Grade No. 5 antifriction metal is one of intermediate cost and may be used for electric motor and other bearings subject to rather low pressures at high speed.

7. Grade No. 6 antifriction metal is a clean Babbitt and may be used where the bearings are large and the service light.

8. Grade No. 7 antifriction metal is somewhat similar to grade No. 6, but may be used for slightly more severe service than No. 6 .

9. Alternate bids based on furnishing antifriction metal with a range of composition differing from the above will be considered, provided the range of composition of the metal that the bidder proposes to furnish is clearly noted and is approved by the department concerned. 
TABLE 1.-Percentage composition

\begin{tabular}{|c|c|c|c|c|c|c|}
\hline Grade & \multicolumn{2}{|c|}{ Tin } & \multicolumn{2}{|c|}{ Antimony } & Lead 1 & Copper \\
\hline $\begin{array}{l}1 \\
2 \\
3 \\
5 \\
6\end{array}$ & $\begin{array}{l}90 \\
87.5 \\
83 \\
80.5 \\
61 \\
4.5\end{array}$ & $\begin{array}{l}-93 \\
-89.5 \\
-85 \\
-82.5 \\
-63 \\
-5.5 \\
-10\end{array}$ & $\begin{array}{r}3 . \\
7 \\
7 . \\
12 \\
9 . \\
14 \\
14\end{array}$ & $\begin{array}{l}5-5 \\
-8 \\
5-8.5 \\
-14 \\
5-10.5 \\
-16 \\
-16\end{array}$ & $\begin{array}{r}0.50 \\
.35 \\
.35 \\
.25 \\
24-26 \\
79-81 \\
74-76\end{array}$ & $\begin{array}{l}3.5-5 \\
3.5-4.5 \\
7.5-8.5 \\
5 \quad-6 \\
2.5-3.5 \\
2.50 \\
2.50\end{array}$ \\
\hline Grade & Iron ${ }^{1}$ & Arse & nic 1 & Zinc? & $\begin{array}{c}\text { Alum- } \\
\text { num ? }\end{array}$ & $\begin{array}{c}\text { Other } \\
\text { impuri- } \\
\text { ties } 1\end{array}$ \\
\hline $\begin{array}{l}1 \\
2 \\
3 \\
4 \\
5 \\
6 \\
7\end{array}$ & $\begin{array}{r}0.08 \\
.08 \\
.08 \\
.08 \\
.08 \\
\\
\end{array}$ & & $\begin{array}{l}10 \\
10 \\
10 \\
15 \\
20 \\
20\end{array}$ & $\begin{array}{l}\text { None } \\
\text { None } \\
\text { None } \\
\text { None } \\
\text { None } \\
\text { None }\end{array}$ & $\begin{array}{l}\text { None. } \\
\text { None. } \\
\text { None. } \\
\text { None. } \\
\text { None. } \\
\text { None. }\end{array}$ & $\begin{array}{r}0.10 \\
.10 \\
.10 \\
.10 \\
.30 \\
.60 \\
.60\end{array}$ \\
\hline
\end{tabular}

1 Maximum.

I On a 20-gram sample.

692.2 BEARING METALS, OTHER THAN BABBITT.

\section{AMERICAN ELECTRIC RAILWAY ENGI- NEERING ASSOCIATION, MISCELLANE- OUS METHODS AND PRACTICES, ARMA- TURE AND AXLE LINERS, SERIAL NO. E212-25, 1925}

\section{ARMATURE AND AXLE LINERS}

(Contains information concerning present-day methods of treating armature and axle bearings, including bronze bearing shells, both hard and soft, with thin Babbitt linings. It also covers clearances; percentages of tin, antimony, and copper in "genuine Babbitt"; and recommendations for babbitting bearings.)

AMERICAN RAILWAY ASSOCIATION, MECHANICAL DIVISION, SPECIFICATIONS FOR BEARINGS, BRONZE, FOR LOCOMOTIVES, 1928

\section{BRONZE BEARINGS FOR LOCOMOTIVES SCOPE}

1. These specifications cover rough bronze castings for locomotive wearing parts.

\section{CHEMICAL PROPERTIES AND TESTS}

2. Chemical composition.-The material shall conform to the following requirements as to chemical composition:

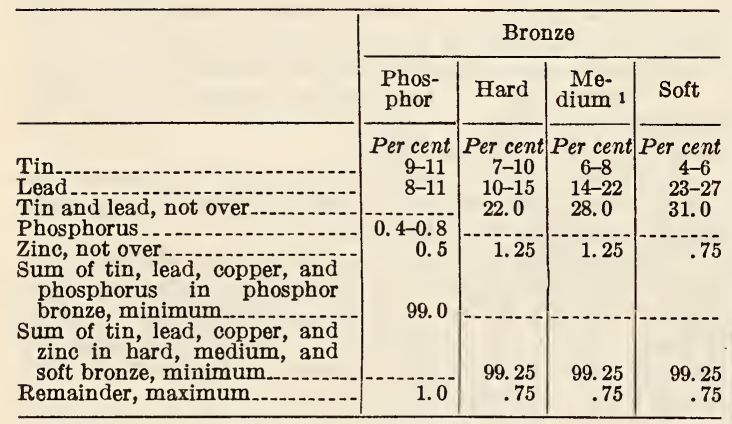

1 This grade is frequently used for chilled castings.
3. Analysis. - The sample for chemical analysis shall be taken from the bearing at three points along the fractured surface, as described in section 4, either by drilling or by using cuttings thus obtained, well mixed.

\section{PHYSICAL PROPERTIES AND TESTS}

4. Tests.-The casting representing a lot for acceptance shall be broken through the center and parallel with the longitudinal axis of the bearing in order to ascertain the uniformity of the grain of the metal. When this fracture shows separation or imperfect mixing of component parts, or dross or dirt spots, the lot shall be rejected.

5. Number of tests.-One sample may be taken for chemical analyses from each lot of 5,000 pounds or less. One casting may be taken for fracture test from each lot of 100 castings or less.

\section{PERMISSIBLE VARIATIONS}

6. Gaging.-All bearings shall conform to gages and dimensions shown on drawings.

FINISH

7. Finish.-The castings shall be sound and free from blowholes, dross, or mechanical defects.

\section{MARKING}

8. Marking.-Each lot of 100 castings or fraction thereof shall bear a serial number, commencing with one at the beginning of the year and continuing consecutively until the end of the year. All bearings shall also have cast on them the manufacturer's initials or trade-mark, the pattern number, and such other marks as are shown on the drawings.

\section{INSPECTION AND REJECTION}

(Requirements covering inspection and rejection, and rehearing conform with those specified in $\mathrm{A}$. S. T. M. standard specifications for steel, pars. 7, 8, and 9 (see 605.0 p. 247).)

\section{AMERICAN RAILWAY ENGINEERING ASSOCIATION, MOVABLE RAILWAY BRIDGES, MANUAL, 1929}

\section{BRONZES}

(See also 647.31, p. 1268, and 692.1, p. 1320.)

AMERICAN SOCIETY FOR TESTING MATERIALS, STANDARD SPECIFICATIONS FOR BRONZE BEARING METALS FOR TURNTABLES AND MOVABLE RAILROAD BRIDGES, SERIAL DESIGNATION B22-21, 1921

\section{BRONZE BEARING METALS}

SCOPE

1. (a) These specifications cover four classes of bronze bearing metals for turntables and movable railroad bridges.

(b) The purposes for which these classes are frequently used are as follows:

Class A, for contact with hardened steel disks under pressures over 1,500 pounds per square inch; for example, bearing metals used in turntables and center-bearing swing bridges.

Class B, for contact with soft steel at low speeds under pressures not over 1,500 pounds per square inch; for example, trunnions and journals of bascule and lift bridges. 
Class C, for ordinary machine bearings.

Class D, for gears, worm wheels, nuts, and similar parts which are subjected to other than compressive stresses.

\section{MANUFACTORE}

2. (a) The bronze shall be a homogeneous alloy of copper and tin. The bronze shall be made from new metal, except that scrap of known composition produced by the foundry at which the bronze is cast may be used.

(b) Care shall be exercised that the metal is not overheated and that the temperature at pouring and the conditions of cooling are such as will be most likely to secure dense castings.

\section{CHEMICAL PROPERTIES AND TESTS}

3. The bronze shall conform to the following requirements as to chemical composition:

\begin{tabular}{|c|c|c|c|c|}
\hline \multirow{2}{*}{ Elements considered } & \multicolumn{4}{|c|}{ Class } \\
\hline & A & B & C & $\mathrm{D}$ \\
\hline $\begin{array}{l}\text { Copper } \\
\text { Tin, maximum } \\
\text { Lead, maximum }\end{array}$ & $\begin{array}{c}\text { Per cent } \\
\text { (1) } \\
20\end{array}$ & $\begin{array}{c}\text { Per cent } \\
(1) \\
17\end{array}$ & $\begin{array}{c}\text { Per cent } \\
282 \\
11 \\
11\end{array}$ & $\begin{array}{c}\text { Per cent } \\
889 \\
11\end{array}$ \\
\hline $\begin{array}{l}\text { Zinc, maximum } \\
\text { Iron, maximum }\end{array}$ & (n) & (n) & (n) & 2.25 \\
\hline Phosphorus.................. & 21.0 & 1.0 & $0.7-1.0$ & 20.25 \\
\hline $\begin{array}{l}\text { Other elements, maxi- } \\
\text { mum. }\end{array}$ & 0.5 & 0.5 & 0.5 & 0.5 \\
\hline
\end{tabular}

1 Remainder.

2 Not over.

\section{CHEMICAL ANALYSES}

4. (a) An analysis of each melt may be made at the option of the purchaser and at the purchaser's expense. The chemical composition thus determined shall be reported to the purchaser or his representative, and shall conform to the requirements specified in section $3 .^{24}$

(b) The compression-test coupon shall be used for analysis.

\section{PHYSICAL PROPERTIES AND TESTS}

5. (a) The bronze shall conform to the following requirements as to compressive and tensile properties:

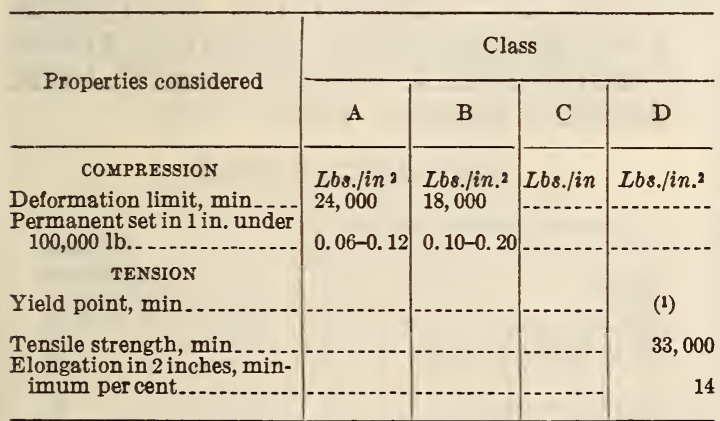

1 To be recorded.

(b) The deformation limit in compression shall be determined as that load which produces a permanent set of 0.001 inch in the compression-test specimen described in section $6(b)$.

24 Material with a range of composition differing from that specified in section 3, or containing elements not specified, may be used, provided the manufacturer shall submit in writing previous to the execution of the contract the range of composition of the material he proposes to use, and provided this range of composition shall be accepted by the purchaser.

\section{TEST SPECIMENS}

6. (a) A test bar of the form and dimensions shown in Figure 129 in A. S. T. M. standard specifications for steel. (See 605.0, p. 247), to be used for the tension-test specimen, and a suitable test bar for the compression-test specimen, shall be an integral part of the casting, and shall be fed and cooled under the same conditions as the castings.

(b) Compression-test specimens shall be cylinders 1 square inch in cross-sectional area and 1 inch high.

7. (a) One compression test shall be made from each melt for class $A$ and class $B$ castings; and one tension test from each melt for class $D$ castings. For castings of any grade weighing over 100 pounds finished, the specified tests shall be made for each casting. (Tension-test specimen requirements conform with Figure 129, (page 247). With respect to copper used in manufacture, yield point in tension, finish, and inspection and rejection, requirements are identical with those in B59 (see 647.15, p. 1259).)

\section{AMERICAN SOCIETY FOR TESTING MATE. RIALS, STANDARD SPECIFICATIONS FOR BRONZE BEARING METAL IN INGOT FORM, SERIAL DESIGNATION B31-21, 1921}

\section{BRONZE BEARING METAL SCOPE}

1. These specifications cover copper-tin-lead alloys in ingot form, known commercially as "bronze bearing metal," made wholly or partly from scrap materials. Six typical alloys are specified and are designated as grades Nos. 1 to 6 , in accordance with their decreasing copper content as specified in section 2 .

\section{CHEMICAL PROPERTIES AND TESTS}

2. The alloy shall conform to the following requirements as to chemical composition, within the limits specified in section 3 :

\begin{tabular}{|c|c|c|c|c|c|c|c|c|c|c|}
\hline $\begin{array}{l}\text { Alloy, } \\
\text { grade } \\
\text { No. }\end{array}$ & 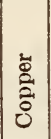 & $\stackrel{\Xi}{E}$ & 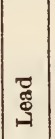 & $\frac{\mathfrak{g}}{\mathrm{N}}$ & $\frac{\text { है }}{\frac{0}{0}}$ & 泀 & 혼 & 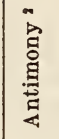 & $\underset{\Xi}{\Xi}$ & 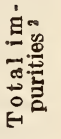 \\
\hline & $\begin{array}{c}\text { Per } \\
\text { cent } \\
85 \\
80 \\
80 \\
77 \\
73 \\
70\end{array}$ & \begin{tabular}{|c|} 
Per \\
cent \\
10 \\
10 \\
10 \\
8 \\
7 \\
5
\end{tabular} & $\begin{array}{c}P e r \\
\text { cent } \\
5 \\
10 \\
10 \\
15 \\
20 \\
25\end{array}$ & $\begin{array}{l}\text { Per } \\
\text { cent } \\
0.25 \\
.50 \\
2.00 \\
.50 \\
.50 \\
.50\end{array}$ & $\begin{array}{c}\text { Per } \\
\text { cent } \\
10.70 \\
1.70 \\
2.05 \\
3.25 \\
2.05 \\
\text { None. }\end{array}$ & $\begin{array}{l}\text { Per } \\
\text { cent } \\
0.05 \\
.05 \\
.05 \\
.05 \\
.05 \\
.05\end{array}$ & $\begin{array}{l}\text { Per } \\
\text { cent } \\
0.25 \\
.25 \\
.25 \\
.25 \\
.25 \\
.25\end{array}$ & $\begin{array}{l}\text { Per } \\
\text { cent } \\
0.50 \\
.50 \\
.50 \\
.50 \\
.50 \\
.50\end{array}$ & $\begin{array}{c}\text { Per } \\
\text { cent } \\
\text { None. } \\
\text { None. } \\
\text { None. } \\
\text { None. } \\
\text { None. } \\
\text { None. }\end{array}$ & $\begin{array}{c}\text { Per } \\
\text { cent } \\
0.50 \\
.75 \\
2.50 \\
.75 \\
1.00 \\
1.00\end{array}$ \\
\hline
\end{tabular}

\section{PERMIISSIBLE VARIATIONS}

3. The following permissible variations in the percentages of the desired elements specified in section 2 will be allowed, but shall not apply to the maximum percentages of impurities specified:

\begin{tabular}{|c|c|}
\hline Percentage of element specified & $\begin{array}{c}\text { Permissible } \\
\text { variations } \\
\text { over and } \\
\text { under the } \\
\text { specified } \\
\text { value }\end{array}$ \\
\hline $\begin{array}{l}\text { Not over } 5 \text { per cent. } \\
\text { Over } 5 \text { to } 10 \text { per cent, inclusive. } \\
\text { Over } 10 \text { per cent. }\end{array}$ & $\begin{array}{c}\text { Units of per } \\
\text { cent } \\
0.50 \\
.75 \\
2.00\end{array}$ \\
\hline
\end{tabular}




\section{SAMPLES FOR CHEMICAL ANALYSIS}

4. Three ingots shall be selected by the inspector to represent 10,000 pounds of ingot or fraction thereof.

(Requirements with respect to sampling are identical with those in B10. See 643.4, p. 1192. With respect to manufacture, marking, rejection, and claims, they are identical with those in B30. See 644.11, p. 1196. With respect to inspection, they are identical with those in B59. See 647.15, p. 1259.)

AMERICAN SOCIETY FOR TESTING MATERIALS, STANDARD METHODS OF CHEMICAL ANAIYSIS OF BRONZE BEARING METAI, DESIGNATION B46-27, 1927

\section{CHEMICAI ANALYSIS OF BRONZE BEARING METAL}

(See 646.0, p. 1246.)

AMERICAN SOCIETY FOR TESTING MATERIAIS, STANDARD SPECIFICATIONS FOR BRONZE CASTINGS IN THE ROUGH FOR LOCOMOTIVE WEARING PARTS, DESIG NATION B66-28, 1928

\section{BRONZE CASTINGS}

(See 646.41, p. 1251.)

AMERICAN SOCIETY FOR TESTING MATERIALS, TENTATIVE SPECIFICATIONS FOR SAND CASTINGS OF THE ALLOY: COPPER, 80 PER CENT; TIN, 10 PER CENT; LEAD, 10 PER CENT, DESIGNATION B74-28T, 1928

\section{ALLOY 80-10-10}

\section{SCOPE}

1. (a) These specifications cover alloy castings, the alloy being a composition of copper, tin, and lead, known commercially as 80-10-10, deoxidized with phosphorus.

(b) The castings are intended for use for bearings and bushings in the cast state. This alloy is frequently used also for castings which are called upon to resist some of the mild acids such as are found in mine waters.

\section{CHEMICAL PROPERTIES AND TESTS}

2. The alloy shall conform to the following requirements as to chemical composition:

\begin{tabular}{|c|c|c|c|}
\hline & Desired & Minimum & Maximum \\
\hline 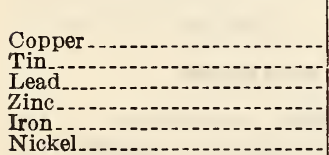 & $\begin{array}{r}\text { Per cent } \\
80 \\
10 \\
10 \\
\text { None. } \\
\text { None. }\end{array}$ & $\begin{array}{r}\text { Per cent } \\
79 \\
9 \\
9 \\
9 \\
\text { None. }\end{array}$ & $\begin{array}{r}\text { Per cent } \\
81 \\
11 \\
11 \\
0.25 \\
.10 \\
.50\end{array}$ \\
\hline impurities. & $\begin{array}{l}\text { Trace. } \\
\text { None. } \\
\text { None. } \\
\text { None. } \\
\text { None. }\end{array}$ & $\begin{array}{l}\text { Trace. } \\
\text { None. }\end{array}$ & $\begin{array}{r}\text { None. } \\
.05 \\
.25 \\
.15\end{array}$ \\
\hline
\end{tabular}

Where "none" is specified it shall be construed to refer to none determined on a $10 \mathrm{~g}$ sample.

\section{PHYSICAL PROPERTIES AND TESTS}

3. The alloy shall conform to the following minimum requirements as to tensile properties:

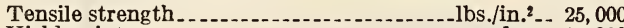
Yield point

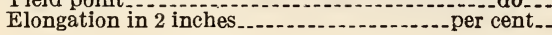

(Specifications covering tension-test specimen requirements are identical with those in paragraph 11 (c), A18. (See 611.51, p. 754.) In respect to chemical analyses, determination of yield point. workmanship and finish, marking, inspection, and rejection, this specification is identical with B59. (See 647.15, p. 1259.) In respect to manufacture, pressure-test requirements, and dimensions of test-specimen coupon, it is identical with B60. (See $643.4, p .1192$.) In respect to number of tests, paragraphs $7(a),(b),(c)$, and $(d)$ in this specification are identical with paragraphs VI $2,3,4$ and 13 in F. S. B. 172a (QQ-B-691). (See 646.41, p. 1252.)

SOCIETY OF AUTOMOTIVE ENGINEERS, NONFERROUS METAL SPECIFICATIONS, SPECIFICATION NO. 64, PHOSPHOR BRONZE, MARCH, 1922

\section{PHOSPHOR BRONZE}

(See 647.34, p. 1269.)

SOCIETY OF AUTOMOTIVE ENGINEERS, NONFERROUS METAL SPECIFICATIONS, SPECIFICATION NO. 66, BRONZE BACKING FOR LINED BEARINGS, MARCH, 1922

\section{BRONZE BACKING FOR LINED BEARINGS}

\section{Composition in percentage.-}

\begin{tabular}{lr} 
Copper & $\begin{array}{r}\text { Per cent } \\
\text { Tin }\end{array}$ \\
Lead & \\
Zinc, maximum & \\
Impurities, maximum & $4.00-86.00$ \\
\hline
\end{tabular}

2. General information.-Good castings made of this alloy should give the following minima in physical characteristics:

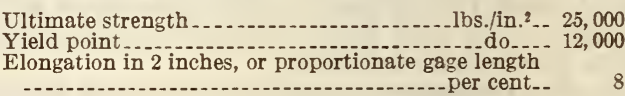

This composition is recommended as an inexpensive but suitable alloy for bronze-backed bearings.

4. Bearings or gears made of bronze alloys should be used only against hardened steel.

SOCIETY OF AUTOMOTIVE ENGINEERS, NONFERROUS METAL SPECIFICATIONS, SPECIFICATION NO. 67, SEMIPLASTIC BRONZE, MARCH, 1922

\section{SEMIPLASTIC BRONZE}

1. Composition in percentage.-

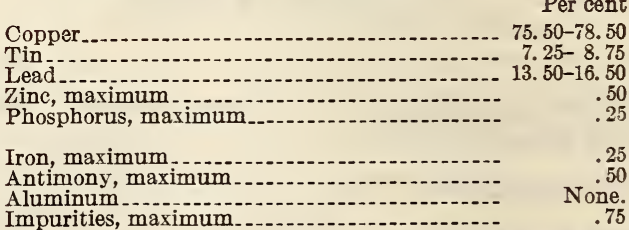

2. General information.-Good castings made of this alloy should give the following minima in physical characteristics:

Ultimate strength

Elongation in 2 inches, or proportionate gage length -.per cent.- $\quad 10$

3. This metal is intended for use where a soft bronze with good antifriction qualities is desired. 
UNITED STATES GOVERNMENT, FEDERAL SPECIFICATIONS BOARD, FEDERAL SPECIFICATION FOR BRONZE CASTINGS, QQ-B-691, MAY 31, 1932

\section{BRONZE CASTINGS}

(See 646.41, p. 1252.)

UNITED STATES GOVERNMENT, DEPARTMENT OF COMMERCE, BUREAU OF STANDARDS, STANDARD SAMPLES, CIRCULAR NO. 25 AND SUPPLEMENT, 1927

\section{PHOSPHOR BRONZE BEARING METAL SAMPLE}

(Sample No. 63 is a standard sample of phosphor bronze bearing metal prepared and sold by the Bureau of Standards for use by industrial organizations and others as a comparison standard for checking the accuracy of analysis of similar material. Each sample is accompanied by a certificate giving the complete analysis of that particular sample.)

692.3 METAL BEARINGS.

AMERICAN ELECTRIC RAILWAY ENGINEERING ASSOCIATION, STANDARD JOURNAL BOXES AND CONTAINED PARTS, SERIAL NO. E2-27, 1927 JOURNAL BOXES

(See 611.22, p. 667.)

AMERICAN ELECTRIC RAILWAY ENGINEERING ASSOCIATION, STANDARD DESIGN GAGES FOR JOURNAL BEARINGS AND WEDGES, SERIAL NO. E12-31, 1931 GAGES FOR JOURNAL BEARINGS AND WEDGES
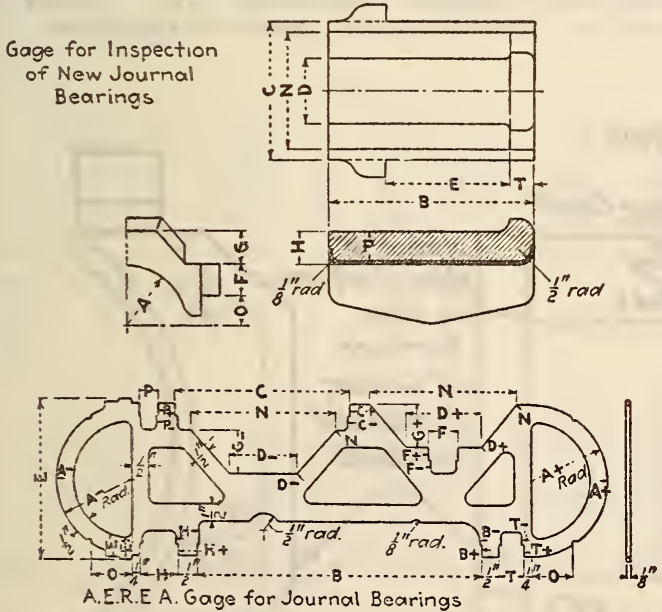

FIGURE 1140.-Dimension drawing of gage for journal bearing

Detail dimensions for each size of journal bearing gage are given in Table 1.
TABLE 1.-Dimensions of gages for journal bearings [Dimensions in inches]

\begin{tabular}{|c|c|c|c|c|c|c|c|c|}
\hline \multirow{2}{*}{ Size of journal } & \multicolumn{2}{|c|}{ A } & \multicolumn{2}{|c|}{ B } & \multicolumn{2}{|c|}{$\mathrm{C}$} & \multicolumn{2}{|c|}{$\mathrm{D}$} \\
\hline & + & - & + & - & + & - & + & - \\
\hline $\begin{array}{l}3 \text { by } 6 \\
31 / 4 \text { by } 6 \\
33 / 4 \text { by } 7 \\
41 / 4 \text { by } 8 \\
5 \text { by } 9 \\
51 / 2 \text { by } 10\end{array}$ & $\begin{array}{r}19 / 16 \\
111 / 16 \\
115 / 16 \\
23 / 16 \\
29 / 16 \\
213 / 16\end{array}$ & $\begin{array}{r}133 / 64 \\
14164 \\
157 / 64 \\
2946 \\
2^{33} 364 \\
2^{49} \% 4\end{array}$ & $\begin{array}{l}5^{29} / 32 \\
529 / 32 \\
629 / 32 \\
725 / 32 \\
825 / 32 \\
9^{25} / 32\end{array}$ & $\begin{array}{l}5^{27 / 32} \\
5^{27 / 32} \\
6^{27 / 32} \\
7^{23} / 32 \\
8^{23} / 32 \\
9^{23} / 32\end{array}$ & $\begin{array}{r}3^{27 / 32} \\
3^{27 / 32} \\
4^{17 / 32} \\
4^{29 / 32} \\
5^{21 / 32} \\
65 / 32\end{array}$ & $\begin{array}{r}313 / 16 \\
3^{13} / 16 \\
41 / 2 \\
47 / 8 \\
558 \\
61 / 8\end{array}$ & $\begin{array}{r}125 / 32 \\
125 / 32 \\
21 / 32 \\
211 / 32 \\
35 / 32 \\
35 / 32\end{array}$ & $\begin{array}{r}123 / 32 \\
123 / 32 \\
131 / 32 \\
29 / 32 \\
33 / 32 \\
33 / 32\end{array}$ \\
\hline \multirow{2}{*}{ Size of journal } & \multicolumn{2}{|c|}{$\mathrm{E}$} & \multicolumn{2}{|c|}{$\mathrm{F}$} & \multicolumn{2}{|c|}{$\mathrm{G}$} & \multicolumn{2}{|c|}{$\mathrm{H}$} \\
\hline & + & 一 & + & 一 & + & - & + & - \\
\hline $\begin{array}{l}3 \text { by } 6 \\
31 / 4 \text { by } 6 \\
33 / 4 \text { by } 7 \\
41 / 4 \text { by } 8 \\
5 \text { by } 9 \\
51 / 2 \text { by } 10\end{array}$ & $\begin{array}{r}35 / 8 \\
3,58 \\
41 / 8 \\
49116 \\
51 / 4 \\
53 / 4\end{array}$ & $\begin{array}{r}3^{17} / 32 \\
3^{17} / 32 \\
41 / 32 \\
4^{15 / 32} \\
55 / 32 \\
5^{21 / 32}\end{array}$ & $\begin{array}{l}25 / 32 \\
29 / 32 \\
29 / 32 \\
27 / 32 \\
13 / 32 \\
13 / 32\end{array}$ & $\begin{array}{l}45 / 64 \\
53 / 64 \\
53 / 64 \\
49 / 14 \\
11 / 64 \\
11 / 64\end{array}$ & $\begin{array}{r}13 / 64 \\
13 / 64 \\
111 / 64 \\
115 / 64 \\
115 / 64 \\
123 / 64\end{array}$ & \begin{tabular}{c|}
1 \\
1 \\
$11 / 8$ \\
$13 / 16$ \\
$13 / 16$ \\
$15 / 16$
\end{tabular} & $\begin{array}{l}11 / 16 \\
11116 \\
11 / 16 \\
11116 \\
13 / 16 \\
13 / 16\end{array}$ & $\begin{array}{l}31 / 32 \\
31 / 32 \\
31 / 32 \\
31 / 32 \\
13 / 32 \\
13 / 32\end{array}$ \\
\hline \multirow{2}{*}{ Size of journal } & \multirow{2}{*}{\multicolumn{2}{|c|}{$\mathrm{N}$}} & \multirow{2}{*}{$\mathrm{O}$} & \multicolumn{2}{|c|}{$\mathbf{P}$} & \\
\hline & & & & + & - & & + & - \\
\hline $\begin{array}{l}3 \text { by } 6 \\
31 / 4 \text { by } 6 \\
33 \text { by } 7 \ldots \ldots \\
41 / 4 \text { by } 8 \\
5 \text { by } 9 \\
51 / 2 \text { by } 10\end{array}$ & & $\begin{array}{l}31 / 4 \\
31 / 4 \\
33 / 4 \\
41 / 8 \\
43 / 4 \\
53 / 8\end{array}$ & $\begin{array}{r}25 / 32 \\
25 / 32 \\
29 / 32 \\
111 / 32 \\
113 / 32 \\
117 / 32\end{array}$ & $\begin{array}{l}15 / 16 \\
15 / 16 \\
15 / 16 \\
15 / 16 \\
11 / 16 \\
11 / 16\end{array}$ & & $\begin{array}{l}7 / 8 \\
7 / 8 \\
7 / 8 \\
7 / 8\end{array}$ & $\begin{array}{l}21 / 32 \\
21 / 32 \\
21 / 32 \\
21 / 32 \\
21 / 32 \\
21 / 32\end{array}$ & $\begin{array}{l}9 / 16 \\
9 / 16 \\
9 / 16 \\
9 / 16 \\
9 / 16 \\
9 / 16\end{array}$ \\
\hline
\end{tabular}

Gage for Inspection of New Journal Bearing Wedges
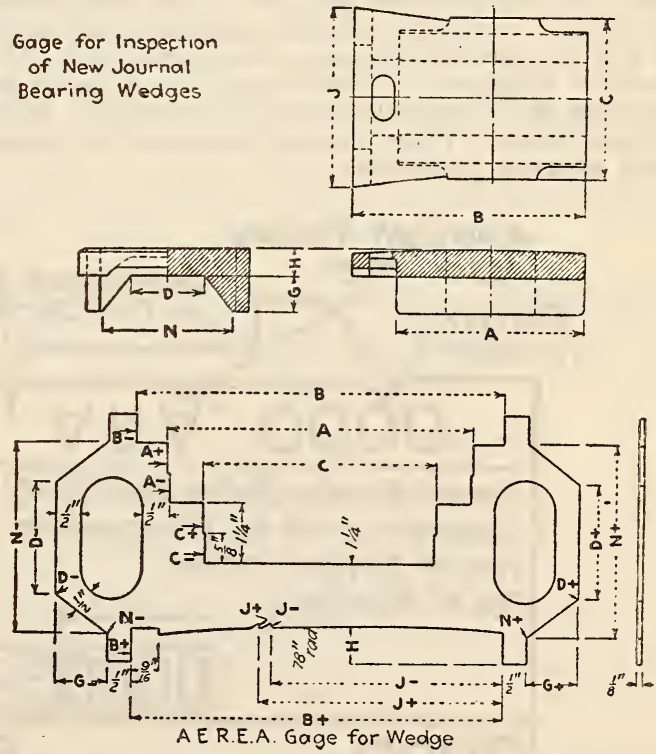

Figure 1141.-Dimension drawing of gage journal bearing wedges

Detail dimensions for each size of journal bearing wedge gage are given in Table 2 . 
TABLE 2.-Dimensions of gages for journal bearing wedges

[Dimensions in inches]

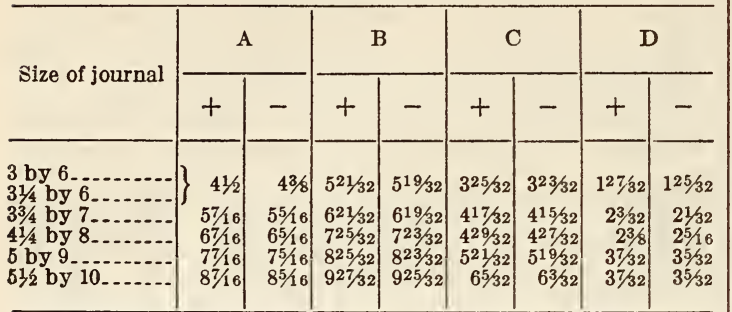

\begin{tabular}{|c|c|c|c|c|c|c|c|c|}
\hline \multirow{2}{*}{ Size of journal } & \multicolumn{2}{|c|}{ G } & \multicolumn{2}{|c|}{ H } & \multicolumn{2}{|c|}{$\mathrm{J}$} & \multicolumn{2}{|c|}{$\mathrm{N}$} \\
\hline & + & - & + & - & + & - & + & - \\
\hline $\begin{array}{l}3 \text { by } 6 \\
31 / 4 \text { by }\end{array}$ & $7 / 8$ & $53 / 64$ & $25 / 32$ & $11 / 16$ & $45 / 8$ & $43 / 8$ & $35 / 32$ & \\
\hline $33 / 4$ by 7 & 1. & $61 / 64$ & $25 / 32$ & $11 / 10$ & 5138 & 478 & $3^{21} 1 / 32$ & \\
\hline & 1116 & $11 / 64$ & $29 / 32$ & $13 / 10$ & $51 / 8$ & 478 & $43 / 32$ & $41 / 16$ \\
\hline s.s by 10 & $\begin{array}{l}1316 \\
13 / 16\end{array}$ & $\begin{array}{l}1164 \\
1964\end{array}$ & $\begin{array}{l}1132 \\
11 / 32\end{array}$ & $\begin{array}{l}1516 \\
15 / 16\end{array}$ & $\begin{array}{l}078 \\
67 / 8\end{array}$ & $\begin{array}{l}0 \% 8 \\
658\end{array}$ & $\begin{array}{r}40 / 32 \\
51 / 4\end{array}$ & $\begin{array}{r}4 \% / 4 \\
57 / 32\end{array}$ \\
\hline
\end{tabular}

AMERICAN RAILWAY ASSOCIATION, MECHANICAL DIVISION, BEARINGS, JOURNAL, LINED, RECOMMENDED PRACTICE, 1926

\section{JOURNAL BEARINGS, LINED}

1. Scope.-These specifications cover journal bearings for use on locomotive tenders and passenger and freight equipment cars.

2. Manufacture.-Before lining, the brass backs shall be bored and thoroughly tinned in accordance with the best standard practice. The thickness of linings which is desired shall be specified in the order. If it is not specified, linings one-quarter inch thick shall be furnished. After lining, the ends of the bearings shall be made smooth by scraping, filing, or machining. They must not be ground or rubbed with abrasive materials.

\section{CHEMICAL PROPERTIES AND TESTS}

3. Composition of back.-

\begin{tabular}{lr} 
Lead. & Per cent \\
Tin \\
Total of tin, lead, and copper minimum................. \\
Remainder, including zinc, maximum & $5-27$ \\
Remainder, excluding zinc, maximum.................. & 97 \\
\hline
\end{tabular}

Within the ranges permitted, the tin and lead shall vary, if at all, in opposite directions, the tin being increased for the lower percentages of lead.

\section{Composition of lining.-}

Per cent

Tin

Tin

Antimony, not less than

Tin and antimony

Arsenic, maximum

Sum of tin, antimony, lead, and arsenic, minimum... 99. 25

Remainder, maximum ............................. 75

5. Analysis. - The sample for chemical analysis of the back shall consist of a thorough mixture of equal quantities of fine drillings taken at three points on the bearing, surface metal being discarded. The sample for analysis of the lining shall be taken by scraping the lining, after removing surface metal.

\section{PHYSICAL PROPERTIES AND TESTS}

6. Tests.-The finished casting representing a lot for acceptance shall be broken, either longitudinally or transversely, or both, in order to ascertain the uniformity of the grain of the metal. When this fracture shows separation or imperfect mixing of component parts or dross or dirt spots, the lot shall be rejected.

7. Number of tests.-Bearings shall be divided into lots of 300 or less, and one bearing shall be taken for test and chemical analysis from each lot.

\section{PERMISSIBLE VARIATIONS}

8. Gaging.-All bearings shall conform to the latest standards of the mechanical section of the American Railway Association, with respect to form and dimensions and variations therefrom.

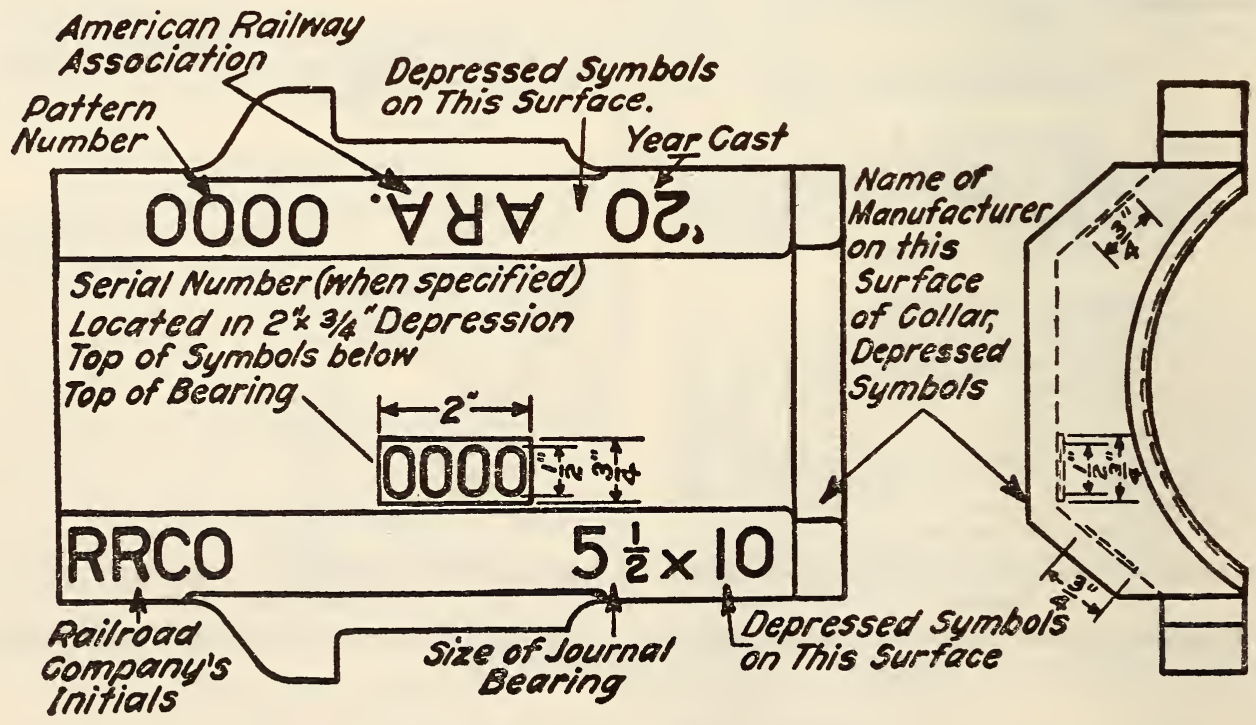

Figdre 1142.-Journal bearings 


\section{FINISH}

9. Finish.-The castings shall be sound and free from blowholes, dross, and mechanical defects. All bearing surfaces, including ends, before and after lining, shall be smooth finished and free from sand.

\section{MARKING}

10. Marking. - The bearings shall be cast with the marks as shown on Figure 1142. When a serial number is specified it shall be located as shown on Figure 1142. Each lot of 300 or less shall bear the same serial number, which shall commence with 1 with the opening of the year and continue consecutively until the end of the year, at each manufacturer's plant.

(Requirements covering inspection, rejection, and rehearing conform to those specified for A. R. A. M. galvanized sheets (see 604.32, p. 243).)

\section{AMERICAN RAILWAY ASSOCIATION, ME-} CHANICAL DIVISION, JOURNAL BEARINGS, STANDARD, 1920

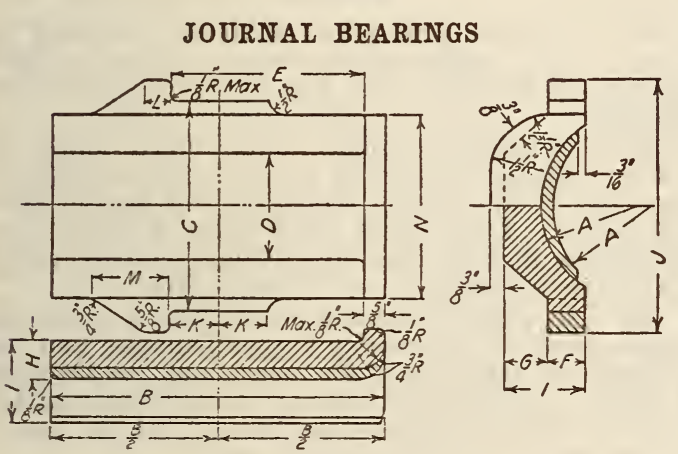

Figure 1143.-Journal bearings [Dimension in inches]

\begin{tabular}{|c|c|c|c|c|c|c|c|c|}
\hline $\begin{array}{l}\text { Classi } \\
\text { fica- } \\
\text { tion of } \\
\text { bear- } \\
\text { ing }\end{array}$ & $\begin{array}{l}\text { Size of } \\
\text { journal }\end{array}$ & $A$ & $B$ & $C$ & $D$ & $E$ & $F$ & $G$ \\
\hline $\begin{array}{l}\mathrm{A} \ldots . . \\
\mathrm{B} \ldots \\
\mathrm{C} \ldots \ldots \\
\mathrm{D} \ldots \ldots \\
\mathrm{E} \\
\mathrm{F} \ldots \ldots\end{array}$ & $\begin{array}{l}33 / 4 \text { by } 7 \\
41 / 4 \text { by } 8 . \\
5 \text { by } 9 \\
51 / 2 \text { by } 10 \\
6 \text { by } 11 \\
61 / 2 \text { by } 12 \\
\end{array}$ & $\begin{array}{l}129 / 32 \\
2^{5} / 32 \\
2^{17} / 32 \\
225 / 32 \\
31 / 32 \\
39 / 32\end{array}$ & $\begin{array}{r}63 / 4 \\
73 / 4 \\
83 / 4 \\
93 / 4 \\
103 / 4 \\
113 / 4\end{array}$ & $\begin{array}{l}41 / 2 \\
47 / 8 \\
55 / 8 \\
618 \\
71 / 8 \\
75 / 8\end{array}$ & \begin{tabular}{l|}
2 \\
$25 / 16$ \\
$31 / 8$ \\
$31 / 8$ \\
$31 / 2$ \\
4
\end{tabular} & $\begin{array}{l}4 \\
41 / 2 \\
53 / 16 \\
511 / 16 \\
65 / 8 \\
71 / 8\end{array}$ & $\begin{array}{l}7 / 8 \\
27 / 32 \\
11 / 32 \\
11 / 16 \\
11 / 4 \\
17 / 16\end{array}$ & $\begin{array}{l}118 \\
19 / 32 \\
17 / 32 \\
15 / 16 \\
11 / 2 \\
19 / 16\end{array}$ \\
\hline $\begin{array}{l}\text { Classi- } \\
\text { fica- } \\
\text { tion of } \\
\text { bear- } \\
\text { ing }\end{array}$ & $\begin{array}{c}\text { Size of } \\
\text { journal }\end{array}$ & $H$ & $I$ & $J$ & $K$ & $L$ & $M$ & $N$ \\
\hline $\begin{array}{l}\mathrm{A} \ldots \\
\mathrm{B} \ldots \\
\mathrm{C} \ldots \\
\mathrm{D} \ldots \\
\mathrm{E} \\
\mathrm{F}\end{array}$ & $\begin{array}{l}33 / 4 \text { by } 7 \\
41 / 4 \text { by } 8 . \\
5 \text { by } 9 \\
51 / 2 \text { by } 10 . \\
6 \text { by } 11 \\
61 / 2 \text { by } 12\end{array}$ & $\begin{array}{l}1 \\
1 \\
11 / 8 \\
11 / 8 \\
11 / 4 \\
13 / 8\end{array}$ & $\begin{array}{l}2 \\
21 / 8 \\
21 / 4 \\
23 / 8 \\
23 / 4 \\
3\end{array}$ & $\begin{array}{l}51 / 2 \\
57 / 8 \\
67 / 8 \\
73 / 8 \\
83 / 8 \\
87 / 8\end{array}$ & $\begin{array}{l}11 / 4 \\
11 / 4 \\
17 / 16 \\
17 / 16 \\
17 / 8 \\
17 / 8\end{array}$ & $\begin{array}{l}1 / 2 \\
5 / 8 \\
11 / 16 \\
3 / 4 \\
13 / 16 \\
7 / 8\end{array}$ & $\begin{array}{l}15 / 8 \\
13 / 4 \\
21 / 16 \\
21 / 4 \\
27 / 16 \\
25 / 8\end{array}$ & $\begin{array}{l}33 / 4 \\
43 / 8 \\
47 / 8 \\
53 / 8 \\
61 / 4 \\
61 / 2\end{array}$ \\
\hline
\end{tabular}

Note.-Journal bearings must bear markings as called for in specifications for lined journal bearings.
Journal bearings, minimum thickness for backs of, recommended practice, 1921

\begin{tabular}{|c|c|c|c|c|c|c|}
\hline & \multicolumn{6}{|c|}{ Class of bearing } \\
\hline & $A$ & $B$ & $C$ & $D$ & $E$ & $F$ \\
\hline $\begin{array}{l}\text { Size of journal- } \\
\text { Thickness of } \\
\text { back, mini- } \\
\text { mum. }\end{array}$ & $\begin{array}{r}\text { Inches } \\
33 / 4 \text { by } 7 \\
1 / 2\end{array}$ & $\begin{array}{r}\text { Inches } \\
41 / 4 \text { by } 8 \\
916\end{array}$ & $\begin{array}{r}\text { Inches } \\
5 \text { by } 9 \\
5 / 8\end{array}$ & $\left|\begin{array}{r}\text { Inches } \\
51 / 2 \text { by } 10 \\
11 / 10\end{array}\right|$ & $\begin{array}{l}\text { Inches } \\
6 \text { by } 11\end{array}$ & $\begin{array}{r}\text { Inches } \\
61 / 2 \text { by } 12 \\
13 / 16\end{array}$ \\
\hline
\end{tabular}

AMERICAN RAILWAY ASSOCIATION, MECHANICAL DIVISION, JOURNAL BEARING WEDGES, STANDARD, 1931

\section{JOURNAL BEARING WEDGES}

(See 611.22, p. 681.)

\section{AMERICAN SOCIETY FOR TESTING MA- TERIALS, STANDARD SPECIFICATIONS FOR CAR AND TENDER JOURNAL BEAR- INGS, IINED, DESIGNATION B67-28, 1928}

\section{JOURNAL BEARINGS, LINED}

(Identical with specification of American Railway Association, given above, with the following additions:)

All samples shall be kept free of oil and shall be carefully treated with a magnet to remove any iron introduced in taking the sample.

A second test shall be made after application of the lining, as follows:

The suspended brass when tapped with a hammer shall give a distinct ring.

The lining when chipped with a cold chisel shall tear from the soldered surface as distinguished from clean shearing.

\section{SOCIETY OF AUTOMOTIVE ENGINEERS, BALL AND ROLLER BEARINGS, S. A. E. STANDARD, JANUARY, 1930}

\section{BALL AND ROLLER BEARINGS}

(In these specifications essential dimensions of the several kinds of ball bearings and of roller bearings are contained in 28 tables, as indicated in the accompanying summary of tabular contents. As shown in the fifth column, in some cases the unit of measurement is in inches, in others it is in millimeters, and in still others it is in both inches and millimeters. The fourth column gives the range in the size of bore for each type of bearing.)

\begin{tabular}{|c|c|c|c|c|}
\hline Kind of bearing & Type & Series & Range in bore size & Unit of measure \\
\hline $\begin{array}{l}\text { Annular ball } \\
\text { Annular ball and roller- } \\
\text { Angular contact ball... } \\
\text { Thrust ball }\end{array}$ & $\left\{\begin{array}{l}\text { Single row } \\
\text { Wide } \\
\text { Separable (open) } \\
\text { (Steering knuckle } \\
\text { Single direction flat face } \\
\text { Single direction self-aligning } \\
\text { Double direction fiat face } \\
\text { Double direction self-aligning }\end{array}\right.$ & $\begin{array}{l}\text { Light, medium, heavy. } \\
\text { Light, medium, heavy.. } \\
\text { Light, medium medium, heavy. } \\
\text { Light, medium } \\
\text { Light, medium, heavy }\end{array}$ & 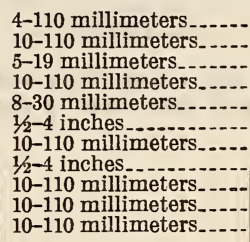 & $\begin{array}{l}\text { Inch and millimeter. } \\
\text { Do. } \\
\text { Do. } \\
\text { Do. } \\
\text { Do. } \\
\text { Inch. } \\
\text { Millimeter. } \\
\text { Inch. } \\
\text { Millimeter. } \\
\text { Do. } \\
\text { Do. }\end{array}$ \\
\hline
\end{tabular}


(S. A. E. standard roller bearings, arranged according to bore, are dimensioned in both inches and millimeters in tabular form, the diameter of the bore ranging from $5 / 8$ inch to 8 inches.)

693. SOLDERS

693.1 SOLDER, TIN-LEAD BASE.

AMERICAN RAILWAY ASSOCIATION, TELE-
GRAPH AND TELEPHONE SECTION,
SPECIFICATION $2-$ G-20, ROSIN CORE
SOLDER, 1926

\section{ROSIN CORE SOLDER}

1. The purpose of this specification is to guide the seller/contractor in the manufacture of rosin core solder.

GENERAL

2. The rosin core solder shall consist of a seamless tube of solder in the form of a cylindrical wire, filled with a core of rosin flux.

3. The quality of the material used and the methods of manufacture shall be such as to insure for the completed solder the properties called for in this specification.

4. The seller/contractor must make sure that all material and work is in accordance with the specification before the solder is offered.

\section{MATERIAI}

5. The solder employed in the wire shall consist of an alloy of lead and tin containing by weight approximately 38 per cent of tin and 62 per cent of lead.

6 . The proportion of tin contained in the alloy shall in no case be less than 37.75 per cent of the total weight. The remainder of the alloy shall consist of lead, except that antimony, bismuth, copper, and iron, in quantities not greater than hereinafter permitted, may also be present.

7. The proportion of copper or iron present shall not be more than 0.05 per cent by weight for each metal.

8. The proportion by weight of bismuth present shall not be greater than 0.10 per cent, and the total proportion by weight of antimony, bismuth, copper, and iron present in the solder shall not be greater than 0.5 per cent by weight.

9. Other metallic impurities, if present, shall amount to not more than a trace of each metal, the maximum limit for a trace being 0.005 per cent by weight.

10. The solder shall be uniform in composition throughout.

11. The rosin core shall consist of a good grade of commercial rosin, equivalent to grade $\mathrm{F}$ or better.

12. The per cent by weight of rosin in the finished solder shall be nominal, 3 per cent; minimum, $2 \frac{1}{2}$ per cent; maximum, 4 per cent.

\section{MANUFACTORE}

13. The diameter of the finished rosin core solder measured between the outside surfaces, shall not be greater than 0.102 inch nor less than 0.086 isch.

14. The core of rosin shall be well centered in the finished solder.

\section{INSPECTION}

15. The purchaser shall have the right to make such inspection and tests as he may desire of the rosin core solder at any stage of its manufacture. Such inspection is not to include the process of manufacture. The inspector of the purchaser shall have the right to reject any material or finished solder which is defective in any respect. Inspection, however, shall not relieve the seller/contractor, from obligation of furnishing first-class material and sound, reliable work.

16. Any improper material or unfaithful work that may be discovered before the final acceptance of the completed solder shall be corrected immediately, upon the requirement of the purchaser, notwithstanding that it may have been overlooked by the inspector. If the requirements of this specification are not fulfilled when the solder is offered for final acceptance, the purchaser shall have the right to reject the solder.

17. The seller/contractor shall provide at his factory apparatus and other facilities needed for making the required tests, and shall notify the purchaser when the solder is ready for test.

\section{PACKING}

18. The rosin core solder shall be furnished on spools. The weight of the finished solder on each spool shall be either 1 or 5 pounds, as specified on the order.

17. The length of spools containing 5 pounds of solder shall not be greater than $4 \frac{3}{4}$ inches, and the diameter of the ends of the spools not greater than $47 / 8$ inches. Each spool shall have a hole ninesixteenths inch in diameter, extending throughout its length symmetrical to its longitudinal axis.

18. The length of spools containing 1 pound of solder shall not be greater than $4 \frac{3}{4}$ inches, and the diameter of the ends of the spools not greater than 2 inches. Each spool shall have a hole three-eighths inch in diameter, extending throughout its length symmetrical to its longitudinal axis.

19. Each spool shall bear on one end an inscription in bold type specifying the name of material, its size, weight, and name of seller/contractor.

20. The spools of solder shall be packed in strong packing cases to prevent their being damaged in transit.

\section{MARKING}

21. The number of spools of solder, total net weight, purchaser's order and requisition numbers, name of consignor, name and address of consignee, and such other information as may be specified on the order shall be plainly marked on the outside of each case.

22. Detailed list of containers and their contents shall be furnished for each shipment.

\section{WARRANTY}

23. The seller/contractor hereby expressly warrants that the material furnished under this specification shall be free from defects in design, material, and workmanship. The seller's/contractor's obligation under this warranty shall not apply to any material which has been subject to misuse, negligence, or accident after acceptance.

24. The seller/contractor hereby convenants and agrees to save harmless and indemnify the purchaser against all claims, suits, actions, or proceedings, damages, costs, fees, and expenses by reason of alleged infringement of patents, or for patent royalties involved, in consequence of the purchase or the use of the material covered hereby. 
AMERICAN SOCIETY FOR TESTING MATERIALS, STANDARD METHODS OF CHEMICAL ANALYSIS OF ALLOYS OF LEAD, TIN, ANTIMONY, AND COPPER, DESIGNATION B18-21, 1921

\section{DETERMINATION OF LEAD, COPPER, ANTIMONY, AND TIN}

(See 692.0, p. 1317.)

\section{AMERICAN SOCIETY FOR TESTING MA- TERIALS, STANDARD SPECIFICATIONS FOR SOLDER METAL, DESIGNATION B32-21, 1921}

\section{SOIDER METAL}

\section{SCOPE}

1. (a) These specifications cover lead-tin alloys used for solder metal and commonly known as soft solder.

(b) Two classes, A and B, are given, in each of which several compositions are specified covering the range of alloys commercially used and designated as grades Nos. 0 to 5 , in accordance with their decreasing tin content as specified in section 4 .

The choice of the class and grade of solder for any specified purpose depends on the material in connection with which it is to be used and the method of applying. For galvanized iron and zinc, only class A should be used. Recommended practice is given in an appendix to these specifications, together with a table showing melting points, to constitute a guide to the purchaser in selecting the grade best suited for meeting the conditions required.

\section{MANUFACTURE}

2. The manufacturer shall use care to have each lot of solder metal as uniform in quanity as possible. Each bar, ingot, or other form in which the solder is sold shall be uniform in composition with the entire lot. All grades in class A shall be made from new or virgin metals. All grades in class B shall be made from at least one-half new or virgin metals, the balance being recovered or secondary metals.

3. When specified in bar or ingot form, the sizes or shapes, unless otherwise specified, shall be approximately as follows:

\begin{tabular}{|c|c|c|c|}
\hline & Length & Width & $\begin{array}{c}\text { Thick- } \\
\text { ness }\end{array}$ \\
\hline $\begin{array}{l}\text { A. Bar: } \\
\text { Top } \\
\text { Bottom }\end{array}$ & $\begin{array}{c}\text { Inches } \\
131 / 2 \\
131 / 2\end{array}$ & $\begin{array}{c}\text { Inches } \\
3 / 4 \\
5 / 8\end{array}$ & $\begin{array}{r}\text { Inches } \\
3 / 8\end{array}$ \\
\hline $\begin{array}{l}\text { B. Ingot: } \\
\text { Top-1-........ } \\
\text { Bottom }\end{array}$ & $\begin{array}{l}51 / 2 \\
41 / 2\end{array}$ & $\begin{array}{l}21 / 2 \\
11 / 2\end{array}$ & $11 / 2$ \\
\hline
\end{tabular}

CHEMICAL PROPERTIES AND TESTS

4. The alloys shall conform to the following requirements as to chemical composition, within the limits specified in section 5:
CLASS A

\begin{tabular}{|c|c|c|c|c|c|c|}
\hline $\begin{array}{c}\text { Grade } \\
\text { No. }\end{array}$ & Tin & $\begin{array}{l}\text { Lead, } \\
\text { approxi- } \\
\text { mate }\end{array}$ & $\begin{array}{l}\text { Anti- } \\
\text { mony, } \\
\text { maxi- } \\
\text { mum }\end{array}$ & $\begin{array}{c}\text { Copper, } \\
\text { maxi- } \\
\text { mum }\end{array}$ & $\begin{array}{l}\text { Zinc and } \\
\text { alumi- } \\
\text { num }\end{array}$ & $\begin{array}{l}\text { Other } \\
\text { impuri- } \\
\text { ties, } \\
\text { maxi- } \\
\text { mum 1 }\end{array}$ \\
\hline $\begin{array}{l}0 \\
1 \\
2 \\
3 \\
4 \\
5\end{array}$ & $\begin{array}{c}\text { Per cent } \\
63.00 \\
50.00 \\
45.00 \\
40.00 \\
37.50 \\
33.00\end{array}$ & $\begin{array}{c}\text { Per cent } \\
37.00 \\
50.00 \\
55.00 \\
60.00 \\
62.50 \\
67.00\end{array}$ & $\begin{array}{c}\text { Per cent } \\
0.12 \\
.12 \\
.12 \\
.12 \\
.12 \\
.12\end{array}$ & $\begin{array}{c}\text { Per cent } \\
0.08 \\
.08 \\
.08 \\
.08 \\
.08 \\
.08\end{array}$ & $\begin{array}{l}\text { Per cent } \\
\text { None. } \\
\text { None. } \\
\text { None. } \\
\text { None. } \\
\text { None. } \\
\text { None. }\end{array}$ & $\begin{array}{c}\text { Per cent } \\
0.10 \\
.10 \\
.10 \\
.10 \\
.10 \\
.10\end{array}$ \\
\hline
\end{tabular}

CLASS B

\begin{tabular}{|l|r|r|r|r|r|r|}
\hline 1 & 49.25 & 50.00 & 0.75 & 0.15 & None. & 0.10 \\
2 & 43.50 & 55.00 & 1.50 & .15 & None. & .10 \\
3 & 38.00 & 60.00 & 2.00 & .15 & None. & .10 \\
4 & 35.50 & 62.50 & 2.00 & .15 & None. & .10 \\
5 & 31.00 & 67.00 & 2.00 & .15 & Nohe. & .10 \\
\hline
\end{tabular}

1 Exclusive of bismuth.

\section{PERMISSIBLE VARIATIONS}

5. The permissible variation plus or minus in the percentage of tin shall not be over 1 per cent of the tin contents specified in section 4.

\section{SAMPLE FOR CHEMICAL ANALYSIS}

6. (a) Three bars or ingots shall be selected to represent a shipment of less than 1,000 pounds; 5 bars or ingots to represent a shipment of over 1,000 to 10,000 pounds, inclusive; and 10 bars or ingots to represent a shipment of over 10,000 pounds to one carload. In case the shipment is in other form, such as wire, wire segments, drops, pigs, or slabs, an equivalent amount of metal representative of the lot shall be taken, but in no case shall less than one whole piece (except wire) represent a shipment.

(b) The preferable method of preparing the sample for chemical analysis is to cut exactly in half the bars selected, marking each half and retaining one of each of the half bars to preserve their identity and for check analysis. The remaining half of each bar shall then be remelted in a perfectly clean utensil at a temperature a little above the complete liquation point of the alloy, mixed thoroughly, and poured into a cold mold, forming a thin bar approximately one-fourth inch thick. Saw cuts shall then be made entirely across this bar not more than 1 inch apart and evenly distributed throughout its length.

If it is impracticable to remelt the sample as above, saw cuts shall be made entirely across each piece at several points not more than 1 inch apart and evenly distributed throughout their length. No lubricants shall be used for sawing.

(c) Sawings thoroughly mixed shall constitute the sample for chemical analysis.

\section{METHODS OF CHEMICAL ANALYSIS}

7. The chemical analysis shall be made in accordance with the standard methods of chemical analysis of alloys of lead, tin, antimony, and copper of the American Society for Testing Materials. See A. S. T. M. B18-21 (692.0, p. 1317).

\section{MARKING}

8. The name of the manufacturer may be cast, at the option of the purchaser, on each bar or ingot. The numerical designation of the grade supplied 
shall be stamped or cast on each bar or ingot for identification.

(Requirements covering inspection and rejection and claims are indentical with those for brass ingot metal for sand castings in A. S. T. M. B30-31T. See 644.11, p. 1196).

\section{APPENDIX}

9. It is recommended that the grade of solder metal be selected which contains the least amount of tin required to give suitable flowing and adhesive qualities for the work in hand.

10. A table is given showing the melting points of the different grades of solder metal and of tin and lead for comparison. It is to be noted that the alloys are completely solid below the lower point given, designated "melting point," and completely liquid only above the higher point given, designated "complete liquation point." In the range of temperature between these two points the alloys are partly solid and partly liquid. In grade No. 1 the amount of solid portion is so small in the range that it is practically unnoticeable. In grade No. 4 the proportion of solid and fluid metal in the range given makes this alloy suitable for use as wiping solder.

Table showing melting points of solder metal

\begin{tabular}{|c|c|c|c|c|c|c|c|}
\hline \multirow{2}{*}{ Grade } & \multicolumn{3}{|c|}{ Formula } & \multicolumn{2}{|c|}{ Melting point } & \multicolumn{2}{|c|}{$\begin{array}{l}\text { Complete } \\
\text { liquidation } \\
\text { point }\end{array}$} \\
\hline & Tin & Lead & $\begin{array}{l}\text { Anti- } \\
\text { mony }\end{array}$ & ${ }^{\circ} \mathrm{C}$. & ${ }^{\circ} \mathrm{F}$. & ${ }^{\circ} \mathrm{C}$. & ${ }^{\circ} \mathrm{F}$. \\
\hline $\begin{array}{l}\text { Tin } \\
0 \mathrm{~A} \\
1 \mathrm{~A} \\
\mathbf{1 B}\end{array}$ & $\begin{array}{c}\text { Per cent } \\
100.00 \\
63.00 \\
50.00 \\
49.25 \\
45.00\end{array}$ & \begin{tabular}{r} 
Per cent \\
\hdashline 37.00 \\
50.00 \\
50.00 \\
55.00
\end{tabular} & $\mid \begin{array}{r}\text { Per cent } \\
0.12 \\
.12 \\
.75 \\
.12\end{array}$ & $\begin{array}{l}232 \\
181 \\
181 \\
185 \\
181\end{array}$ & $\begin{array}{l}449.6 \\
357.8 \\
357.8 \\
365.0 \\
357.8\end{array}$ & $\begin{array}{l}232 \\
181 \\
213 \\
208 \\
225\end{array}$ & $\begin{array}{l}449.6 \\
357.8 \\
415.4 \\
397.4 \\
437.0\end{array}$ \\
\hline $\begin{array}{l}2 \mathrm{~B} \\
3 \mathrm{~A} \\
3 \mathrm{~B} \\
4 \mathrm{~A}\end{array}$ & $\begin{array}{l}43.50 \\
40.00 \\
38.00 \\
37.50\end{array}$ & $\begin{array}{l}55.00 \\
60.00 \\
60.00 \\
62.50\end{array}$ & $\begin{array}{r}1.50 \\
.12 \\
2.00 \\
.12\end{array}$ & $\begin{array}{l}188 \\
181 \\
188 \\
181\end{array}$ & $\begin{array}{l}370.4 \\
357.8 \\
370.4 \\
357.8\end{array}$ & $\begin{array}{l}220 \\
237 \\
228 \\
241\end{array}$ & $\begin{array}{l}428.0 \\
458.6 \\
442.4 \\
467.6\end{array}$ \\
\hline $\begin{array}{l}4 \mathrm{~B} \\
5 \mathrm{~A} \\
5 \mathrm{~B} \\
\text { Lead..... }\end{array}$ & $\begin{array}{r}35.50 \\
33.00 \\
31.00\end{array}$ & $\begin{array}{r}62.50 \\
67.00 \\
67.00 \\
100.00\end{array}$ & $\begin{array}{r}2.00 \\
.12 \\
2.00\end{array}$ & $\begin{array}{l}188 \\
181 \\
188 \\
327\end{array}$ & $\begin{array}{l}370.4 \\
357.8 \\
370.4 \\
620.6\end{array}$ & $\begin{array}{l}231 \\
252 \\
235 \\
327\end{array}$ & $\begin{array}{l}411.8 \\
485.6 \\
455.0 \\
620.6\end{array}$ \\
\hline
\end{tabular}

11. The data in this appendix do not constitute a part of these specifications. They are given merely to indicate to the purchaser the physical properties of the various alloys specified which can be expected of carefully manufactured alloys of the formulas indicated, and to constitute a guide to the purchaser in selecting the grade best suited for meeting the service condition for which the solder metal is to be used.

\section{SOCIETY OF AUTOMOTIVE ENGINEERS, NONFERROUS METAL SPECIFICATIONS, SPECIFICATIONS NOS. 1, 2, AND 3, SOLDER, MARCH, 1922}

\section{SOLDER}

(These three specifications practically conform with A. S. T. M. B32-21, given above. The conformings are as follows:

\begin{tabular}{|c|c|}
\hline S. A. E. Handbook & A. S. T. M. B32-21 \\
\hline $\begin{array}{l}\text { Specification No. 1: } \\
\text { Class A } \\
\text { Class B B } \\
\text { Specification No. } 2 \text { : } \\
\text { Class A } \\
\text { Class B } \\
\text { Specification No. } 3 \text { : } \\
\text { Class A } \\
\text { Class B }\end{array}$ & $\begin{array}{l}\text { Grade No. } 1 \text {, Class A. } \\
\text { Grade No. } 1 \text {, Class B. } \\
\text { Grade No. 2, Class A. } \\
\text { Grade No. 2, Class B. } \\
\text { Grade No. 3, Class A. } \\
\text { Grade No. 3, Class B. }\end{array}$ \\
\hline
\end{tabular}

There are, however, in the S. A. E. specifications no classes that correspond to the other grades specified in B32, given above.)

SOCIETY OF AUTOMOTIVE ENGINEERS, NONFERROUS METAL SPECIFICATIONS, SPECIFICATION NO. 4, FOR SOLDER, AUGUST, 1922

\section{SOLDER}

\section{Composition in percentage.-}

\begin{tabular}{|c|c|c|}
\hline 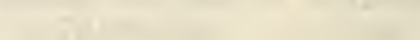 & Class A & Class B \\
\hline $\begin{array}{l}\text { Tin } \\
\text { Lead, approximate } \\
\text { Copper, maximumum } \\
\text { Zinc and aluminum } \\
\text { Other impurities, maximum }\end{array}$ & $\begin{array}{r}\text { Per cent } \\
24.50-25.50 \\
75.00 \\
.12 \\
.08 \\
\text { None. } \\
.10\end{array}$ & $\begin{array}{r}\text { Per cent } \\
22.50-23.50 \\
75.00 \\
2.00 \\
.15 \\
\text { None. } \\
.10\end{array}$ \\
\hline
\end{tabular}

2. General information.-This solder is recommended for use on work that is to be coated with enamel and then baked, as it will withstand higher baking temperatures than solders containing larger amounts of tin.

3. The choice of the class and grade of solder for any specified purpose depends on the material in connection with which it is to be used and the method of applying. For galvanized iron and zinc, only Class A should be used. Class A solder should be furnished under this specification unless otherwise specified.

4. It is recommended that the grade of solder metal be selected that contains the least amount of tin required to give suitable flowing and adhesive qualities for the work in hand.

\begin{tabular}{|c|c|c|c|c|}
\hline Class & \multicolumn{2}{|c|}{ Melting point } & \multicolumn{2}{|c|}{$\begin{array}{l}\text { Complete liquida- } \\
\text { tion point }\end{array}$} \\
\hline$\underset{\mathbf{B}}{\mathbf{A}}$ & $\begin{array}{l}{ }^{\circ} \mathrm{C} . \\
181 \\
188\end{array}$ & $\begin{array}{l}\text { ․ F. } \\
357.8 \\
370.4\end{array}$ & $\begin{array}{l}{ }^{\circ} \mathrm{C} . \\
268 \\
258\end{array}$ & $\begin{array}{l}{ }^{\circ} \mathrm{F} . \\
514.5 \\
496.5\end{array}$ \\
\hline
\end{tabular}

\section{UNITED STATES GOVERNMENT, FEDERAL SPECIFICATIONS BOARD, FEDERAL SPECIFICATION FOR TIN-LEAD SOLDER, QQ-S-571, MAY 31, 1932}

\section{TIN-LEAD SOLDER}

(In scope, manufacture, inspection and tests, marking, and melting points, this specification practically conforms with A. S. T. M. B32-31, given above. Otherwise it provides for the following:)

\section{GENERAL SPECIFICATIONS}

1. General specifications for metals, Federal Specifications Board specification No. 339a, (QQ-M151 ) in effect on date of issuance of proposals, shall form part of this specification. (See 600.0, p. 3.)

\section{GRADES}

2. This specification covers five grades of tin-lead solder, as follows: Grade A, grade B, grade C, grade $\mathrm{D}$, and grade $\mathrm{E}$.

Grades A, B, C, and F solder shall be manufactured from new metals. Grades $\mathrm{D}$ and $\mathrm{E}$ solder may contain reworked metals. 


\section{DETAIL REQUIREMENTS}

3. The chemical requirements are as given in the table below:

\begin{tabular}{|c|c|c|c|c|c|}
\hline Grade & $\begin{array}{l}\text { Tin plus } \\
\text { lead, } \\
\text { minimum }\end{array}$ & $\begin{array}{l}\text { Tin, } \\
\text { mini- } \\
\text { mum }\end{array}$ & $\begin{array}{l}\text { Antimony, } \\
\text { maximum }\end{array}$ & $\begin{array}{l}\text { Cop- } \\
\text { per, } \\
\text { maxi- } \\
\text { mum }\end{array}$ & $\begin{array}{l}\text { Sum of } \\
\text { zinc, alu- } \\
\text { minum, } \\
\text { and cad- } \\
\text { mium, } \\
\text { maxi- } \\
\text { mum }\end{array}$ \\
\hline $\begin{array}{l}\mathrm{A} \\
\mathbf{B} \\
\mathrm{C} \\
\mathrm{D} \\
\mathbf{E} \\
\mathbf{F}\end{array}$ & $\begin{array}{c}\text { Per cent } \\
99.65 \\
99.65 \\
99.65 \\
97.50 \\
97.50 \\
99.65\end{array}$ & $\begin{array}{c}\text { Per cent } \\
49.0 \\
44.0 \\
38.0 \\
35.0 \\
30.0 \\
70.0\end{array}$ & $\begin{array}{l}\text { Per cent } \\
0.12 \\
.12 \\
.12 \\
1.25-1.50 \\
.50-.75 \\
.12\end{array}$ & $\begin{array}{c}\text { Per cent } \\
0.08 \\
.08 \\
.08 \\
.15 \\
.15 \\
.08\end{array}$ & $\begin{array}{r}\text { Per cent } \\
1 \text { None. } \\
1 \text { None. } \\
1 \text { None. } \\
40.50 \\
2.50 \\
1 \text { None. }\end{array}$ \\
\hline
\end{tabular}

1 As determined on a $5 \mathrm{~g}$ sample.

Not more than 0.30 per cent of zinc, aluminum, or cadmium individually will be permitted.

4. The inspector shall forward for analysis not less than 5 ounces of mixed sawings.

\section{ADDITIONAL INFORMATION}

5. Grade A solder is suitable for use with galvanized iron and zinc, and in general for all purposes for which "half-and-half" solder is customarily employed. It starts to melt at about $360^{\circ} \mathrm{F}$, and is completely liquid at about $415^{\circ} \mathrm{F}$.

6. Grade B solder is suitable for use with galvanized iron and zinc, but is not so adaptable for sweating as grade $\mathrm{A}$. It starts to melt at about $360^{\circ} \mathrm{F}$., but is not completely liquid until about $430^{\circ}$ to $450^{\circ} \mathrm{F}$.

7. Grade $\mathrm{C}$ solder is suitable for use with galvanized iron and zinc and, in general, for all purposes for which 40-60 solder is customarily employed. It starts to melt at about $360^{\circ} \mathrm{F}$., but is not completely liquid until about $460^{\circ}$ to $470^{\circ} \mathrm{F}$.

8. Grade D solder is suitable for ordinary plumbing and wiping purposes where fine finish and strength of joint are not required. It starts to melt at about $370^{\circ} \mathrm{F}$., but is not completely liquid until about $415^{\circ}$ to $440^{\circ} \mathrm{F}$.

9. Grade E solder is suitable for such work as dip work on auto radiators. It starts to melt at about $370^{\circ} \mathrm{F}$., but is not completely liquid until about $460^{\circ} \mathrm{F}$.

10. Grade F solder is intended for making and sealing liners for small-arms ammunition packing cases and soldering zinc. It starts to melt at about $330^{\circ}$ F. but is not completely liquid until about $355^{\circ} \mathrm{F}$.

11. Only Grades A, B, and C, should be used for galvanized iron or zinc. It is recommended that the grade of solder be selected which contains the least amount of tin required to give suitable flowing and adhesive qualities for the work in hand.

\subsection{SOLDERS, COPPER-ZINC BASE.}

\section{AMERICAN SOCIETY FOR TESTING MATE- RIALS, STANDARD SPECIFICATIONS FOR BRAZING SOLDER, DESIGNATION B64-28, 1928}

\section{BRAZING SOLDER} SCOPE

1. These specifications cover two grades of granular brazing solder, in three classifications: Round grain, long grain, and lump, as described in sections 6,7 , and 8 , respectively.

\section{MANUFACTURE}

2. These solders shall be produced by grinding or other process of granulation.

\section{CHEMICAL PROPERTIES AND TESTS}

3. The material shall conform to the following requirements as to chemical composition:

\begin{tabular}{|c|c|c|}
\hline & $50-50$ grade & $52-48$ grade \\
\hline $\begin{array}{l}\text { Copper } \\
\text { Lead, maximum } \\
\text { Zron, maximum }\end{array}$ & $\begin{array}{r}\text { Per cent } \\
50.00-53.00 \\
.50 \\
.10 \\
\text { Remainder. }\end{array}$ & $\begin{array}{r}\text { Per cent } \\
\text { 52.00-55. } 00 \\
.50 \\
.10 \\
\text { Remainder. }\end{array}$ \\
\hline
\end{tabular}

4. A composite sample of not less than 2 ounces shall be taken from each 2,000 pounds or fraction thereof in the shipment by taking, as nearly as possible, equal parts from each package or container. Samples shall consist of the grains as prepared for round grain and long grain solder or, in the case of lump solder, of material obtained by drilling, milling, or sawing the lumps. The samples shall be clean and free from oil, dirt, oxide, or other foreign material.

5. In the case of dissatisfaction the solder shall be sampled in the presence of representatives of both the seller and the purchaser. The throughly mixed sample shall be divided into three equal parts, each of which shall be placed in a sealed package-one for the seller, one for the purchaser, and one for an umpire, if necessary.

\section{CLASSIFICATION AND SIZES}

Round Grain Solder

6. (a) Round grain solder shall be furnished according to the following classification and sizes:

\section{Fine grain}

Retained on No. 8 standard screen ( $2.38 \mathrm{~mm}$ opening), none.

Retained on No. 40 standard screen $(0.42 \mathrm{~mm}$ opening), not more than 5 per cent.

Retained on No. 100 standard screen ( $0.149 \mathrm{~mm}$ opening), not less than 90 per cent.

Passing No. 100 standard screen (0.149 mm opening), not more than 5 per cent.

\section{Coarse grain}

Retained on No. 8 standard screen (2.38 mm opening), not more than 8 per cent.

Retained on No. 40 standard screen ( $0.42 \mathrm{~mm}$ opening), not less than 90 per cent.

Passing No. 40 standard screen (0.42 mm opening), not more than 2 per cent.

(b) Mixed round grain solder shall be a mixture of all grains from fine to coarse.

\section{Long Grain Solder}

7. Long grain solder shall be furnished in the following sizes:

(a) Fine long grain solder shall consist of fairly uniform grains about one-half inch in length and about one-sixteenth inch in maximum diameter.

(b) Coarse long grain solder shall consist of irregular aggregates of long grains about one-quarter inch to 1 inch in length and about one-quarter inch in maximum diameter.

(c) Mixed long grain solder shall be a mixture of all grains from fine to coarse.

\section{Lump Solder}

8. Lump solder shall consist of irregular lumps of long grain solder, about 2 to 4 inches in dimensions. 


\section{PACKING AND MARKING}

9. Round and long grain solder shall be packed in strong wooden boxes, not to exceed 200 pounds per box. The fine grain solders shall be packed in paper-lined wooden boxes. Lots weighing less than 100 pounds shall be packed in tin containers inclosed in wooden boxes unless otherwise specified.

10. Lump solder shall be packed in strong barrels, not to exceed 500 pounds per barrel.

11. The coarse grain or mixed solders may be packed in strong barrels when so specified.

12. Each package shall be legibly marked with the grade and size, the weight of the material, brand or trade-mark of the manufacturer, and the contract or purchase order number.

\section{IMPURITIES}

13. The brazing solder shall be free from oxide, dirt, or any foreign material which will impair its working qualities.

\section{INSPECTION AND REJECTION}

14. The manufacturer shall afford the inspector representing the purchaser, without charge, all reasonable facilities to satisfy himself that the solder is being furnished in accordance with these specifications. All tests and inspections shall be so conducted as not to interfere unnecessarily with the operation of the works.

15. Solder which fails to conform to these specifications will be rejected and the manufacturer shall be notified.

\section{SOCIETY OF AUTOMOTIVE ENGINEERS, NONFERROUS METAL SPECIFICATIONS, SPECIFICATION NO. 82, BRASS WIRE, JULY, 1924.}

\section{BRASS WIRE}

\section{Composition in percentage.-}

Copper.

Lead, maximum

Iron, maximum

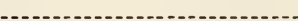

Aluminum _. None.

The surface should be clean and free from scale or other foreign materials.

3. Wire in accordance with this specification is suitable for brazing and torch welding.

\section{SOCIETY OF AUTOMOTIVE ENGINEERS, SPECIFICATION NO. 45, BRAZING SOL- DER, MARCH, 1922}

\section{BRAZING SOLDER}

\section{Composition in percentage.-}

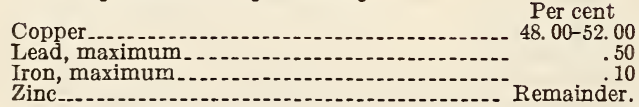

2. General information.-This solder starts to melt at approximately $1,560^{\circ} \mathrm{F}$., and is entirely melted at $1,600^{\circ} \mathrm{F}$. It may be used by melting it in a crucible under a flux of borax, with or without the addition of boric acid, and dipping the material to be brazed in the melted brazing solder; or this brazing solder, in powdered form, may be mixed with the flux applied to the material to be brazed and melted either in a furnace or by the use of a brazing torch.
UNITED STATES GOVERNMENT, FEDERAL SPECIFICATIONS BOARD; FEDERAL SPECIFICATION FOR SPELTER SOLDER (FOR BRAZING), NO. 306, JULY 6, 1925. CURRENT DESIGNATION: QQ-S-566)

\section{SPELTER SOLDER}

General specifications for metals, Federal specifications board specification No. 339a (QQ-M-151) (see 600.0, p. 3), in effect on date of issuance of proposals, shall form part of this specification.

\section{GRADES}

I. This specification covers four grades of spelter solder, as follows: Grade A, grade B, grade C, and grade $\mathrm{D}$.

\section{MATERIAL AND WORKMANSHIP}

II. Spelter solder furnished under this specification shall be of the best commercial quality.

III. Grades A and B spelter solder shall be furnished in granulated form. Grades C and D spelter solder shall be furnished in the form of ingots, bars, or wire, as may be specified.

IV. Chemical requirements.-The chemical requirements are as given in the following table:

\begin{tabular}{|c|c|c|c|c|c|c|}
\hline Grade & Copper & Tin & $\begin{array}{l}\text { Lead, } \\
\text { maxi- } \\
\text { mum }\end{array}$ & $\begin{array}{l}\text { Iron, } \\
\text { maxi- } \\
\text { mum }\end{array}$ & $\begin{array}{l}\text { Alumi- } \\
\text { num, } \\
\text { maxi- } \\
\text { mum }\end{array}$ & $\begin{array}{l}\text { Zinc (per } \\
\text { cent) }\end{array}$ \\
\hline $\begin{array}{l}\mathrm{A}-. \\
\mathrm{B}- \\
\mathrm{C}- \\
\mathrm{D}_{-}\end{array}$ & $\begin{array}{c}\text { Per cent } \\
49.0-52.0 \\
49.0-52.0 \\
68.0-72.0 \\
78.0-82.0\end{array}$ & $\begin{array}{l}\text { Per cent } \\
\text { None. } \\
3.0-4.0 \\
1 \text { None. } \\
1 \text { None. }\end{array}$ & $\begin{array}{c}\text { Per cent } \\
0.50 \\
.50 \\
.30 \\
.20\end{array}$ & $\begin{array}{r}\text { Per cent } \\
0.10 \\
.10 \\
.10 \\
.10\end{array}$ & $\begin{array}{c}\text { Per cent } \\
0.10 \\
0\end{array}$ & $\begin{array}{c}\text { Remainder. } \\
\text { Do. } \\
\text { Do. } \\
\text { Do. }\end{array}$ \\
\hline
\end{tabular}

1 As determined on a $1 \mathrm{~g}$ sample.

\section{METHOD OF INSPECTION AND TESTS}

V. Samples for check analysis, when made, shall be taken from at least five pieces or packages in each lot of 500 pounds, or fraction thereof, and shall be free from oil, dirt, or foreign material. The individual samples shall be thoroughly mixed and not less than 5 ounces forwarded for analysis.

\section{PACKING AND MARKING}

VI. 1. Grade A spelter solder shall be packed in substantial wooden boxes, each containing 100 pounds net weight.

2. Grade B spelter solder shall be packed in 1pound packages. These packages shall be shipped in suitable containers.

3. Grades $\mathrm{C}$ and $\mathrm{D}$ spelter solder may be packed in accordance with the contractor's usual practice.

4. Containers shall be legibly and indelibly marked with the grade of material, weight of contents, name, brand, or trade-mark of contractor, and the contract or purchase order number.

\section{ADDITIONAL INFORMATION}

VII. 1. The grain size desired should be specified by the department concerned. Long-grain solder is suitable for brazing steel; fine-grain for brazing nonferrous material.

2. The melting point of grades $\mathrm{A}$ and $\mathrm{B}$ is about $1,600^{\circ} \mathrm{F}$. Grades C and D are suitable for brazing steel parts that are to be subjected to heat treatment after brazing. The melting point of grade $\mathrm{C}$ varies 
from $1,650^{\circ} \mathrm{F}$. to $1,760^{\circ} \mathrm{F}$., and that of grade $\mathrm{D}$ from $1,725^{\circ} \mathrm{F}$. to $1,825^{\circ} \mathrm{F}$. On account of its higher melting point, grade $\mathrm{D}$ is more difficult of application than grade $\mathrm{C}$, but it is preferable with chrome-vanadium and chrome-molybdenum steel.

\subsection{SILVER SOLDER.}

AMERICAN SOCIETY FOR TESTING MATERIALS, STANDARD SPECIFICATIONS FOR SILVER SOLDERS, DESIGNATION B73-29, 1929

\section{SILVER SOLDERS}

SCOPE

1. (a) These specifications cover eight grades of silver-copper-zinc alloys in wire, strip, sheet, or granular form, used for brazing purposes and commercially known as silver solders.

(b) The eight grades of silver solders covered by these specifications shall be designated as grades Nos. 1 to 8 in accordance with their chemical composition as specified in section 6 .

(c) The choice of the grade of solder for any specified purpose depends on the material in connection with which it is to be used. Recommendations regarding applications and data regarding colors and melting ranges are given in the Appendix to these specifications.

\section{MANUFACTORE}

2. The alloys shall be made from virgin metals or such clean scrap as may result from the manufacture of articles of the same or similar composition.

3. Strip or sheet solder shall be cold-rolled to size and, unless otherwise specified, shall be furnished in the hard condition.

4. Wire shall be cold-drawn to size and, unless otherwise specified, shall be annealed after colddrawing and pickled bright.

5. Granular solder shall be produced by filing, grinding, or other process of granulation. The material shall be supplied in sizes specified in accordance with the requirements for the size of sieve opening of the standard specifications for sieves for testing purposes, in A. S. T. M., E11-26. (See 645.31, p. 1226.) The material shall contain not more than 10 per cent over the specified size.

\section{CHEMICAL PROPERTIES AND TESTS}

6. The alloys shall conform to the following requirements as to chemical composition, within the permissible variations specified in section 7:

\begin{tabular}{|c|c|c|c|c|c|}
\hline $\begin{array}{c}\text { Grade } \\
\text { No. }\end{array}$ & Silver & Copper & Zinc & $\begin{array}{l}\text { Cad- } \\
\text { mium }\end{array}$ & $\begin{array}{l}\text { Impur- } \\
\text { ities, } \\
\text { maxi- } \\
\text { mum }\end{array}$ \\
\hline & Per cent & Per cent & Per cent & Per cent & Per cent \\
\hline 1 & 10 & 52 & 38 & (1) & 0.15 \\
\hline 2 & 20 & 45 & 35 & (1) & .15 \\
\hline 3 & 20 & 45 & 30 & 5 & 15 \\
\hline 4 & 45 & 30 & 25 & nil. & .15 \\
\hline 5 & 50 & 34 & 16 & nil. & .15 \\
\hline 6 & 65 & 20 & 15 & nil. & 15 \\
\hline 7 & 70 & 20 & 10 & nil. & .15 \\
\hline 8 & 80 & 16 & 4 & nil. & .15 \\
\hline
\end{tabular}

1 The addition not to exceed 0.50 per cent of cadmium to assist in fabricating grades Nos. 1 and 2 shall not be considered as a harmful impurity.
7. The permissible variation from the percentages, specified in section 6, of silver, copper, and zinc, for all grades, shall be plus or minus 1 per cent in the case of silver and copper, and 2 per cent in the case of zinc. The permissible variation in the percentage of cadmium for grade No. 3 shall be plus or minus 1 per cent.

8. A composite sample of not less than 1 ounce shall be taken from each lot of 50 pounds or fraction thereof of grades Nos. 1, 2, and 3; and from each lot of 20 pounds or fraction thereof of grades Nos. $4,5,6,7$, and 8 . Samples of granulated solder shall consist of the grains as prepared. Samples from wire, sheet, or strip shall be taken by shearing or clipping pieces from the entire cross section of the same, remelting in a clean container at a temperature slightly above the melting range, mixing thoroughly and pouring into a cold mold of convenient size for milling, drilling, or sawing. The samples so prepared shall be milled, drilled, or sawed in such a manner as to represent the entire cross section. The saw, drill, cutter, or other tool used shall be thoroughly cleaned. No lubricant shall be used in the operation, and the sawings of metal chips shall be carefully treated with a magnet to remove any particles of steel introduced in taking the sample.

9. In the case of disagreement, the solder shall be re-sampled in the presence of the manufacturer and purchaser. The sample shall consist of not less than 3 ounces of solder prepared as described in section 8 . The thoroughly mixed sample shall be divided into three equal parts, each of which shall be placed in a sealed package-one for the manufacturer; one for the purchaser; and one for an umpire, if necessary.

\section{PACKING AND MARKING}

10. The silver solder shall be packed in such a manner as to prevent injury during shipment.

11. Each package shall be legibly marked with the grade and size, the weight of the material, brand or trade-mark of the manufacturer, and the contract or purchase order number.

\section{WORKMANSHIP AND FINISH}

12. All wire, sheet, and strip shall be reasonably smooth and bright, and substantially free from slivers, splitting, or other injurious or objectionable defects.

13. Granular solder shall be substantially free from oxide, dirt, or any foreign material which will impair its working qualities.

\section{REJECTION}

14. Solder which fails to conform to these specifications will be rejected, and the manufacturer shall be notified.

\section{APPENDIX}

15. The approximate melting points, flow points, and colors of the grades are as follows:

\begin{tabular}{|c|c|c|c|c|c|}
\hline $\begin{array}{c}\text { Grade } \\
\text { No. }\end{array}$ & \multicolumn{2}{|c|}{ Melting point } & \multicolumn{2}{|c|}{ Flow point } & Color \\
\hline $\begin{array}{l}1 \\
2 \\
3 \\
4\end{array}$ & $\begin{array}{l}{ }^{\circ} F \text {. } \\
1,510 \\
1,430 \\
1,430 \\
1,250\end{array}$ & $\begin{array}{l}{ }^{\circ} C . \\
820 \\
775 \\
775 \\
675\end{array}$ & $\begin{array}{l}{ }^{\circ} F . \\
1,600 \\
1,500 \\
1,500 \\
1,370\end{array}$ & $\begin{array}{l}{ }^{\circ} C . \\
870 \\
815 \\
815 \\
745\end{array}$ & $\begin{array}{l}\text { Yellow. } \\
\text { Do. } \\
\text { Do. } \\
\text { Nearly white. }\end{array}$ \\
\hline $\begin{array}{l}5 \\
6 \\
7 \\
8\end{array}$ & $\begin{array}{l}1,280 \\
1,280 \\
1,335 \\
1,360\end{array}$ & $\begin{array}{l}695 \\
695 \\
725 \\
740\end{array}$ & $\begin{array}{l}1,425 \\
1,325 \\
1,390 \\
1,460\end{array}$ & $\begin{array}{l}775 \\
720 \\
755 \\
795\end{array}$ & $\begin{array}{l}\text { Do. } \\
\text { White. } \\
\text { Do. } \\
\text { Do. }\end{array}$ \\
\hline
\end{tabular}




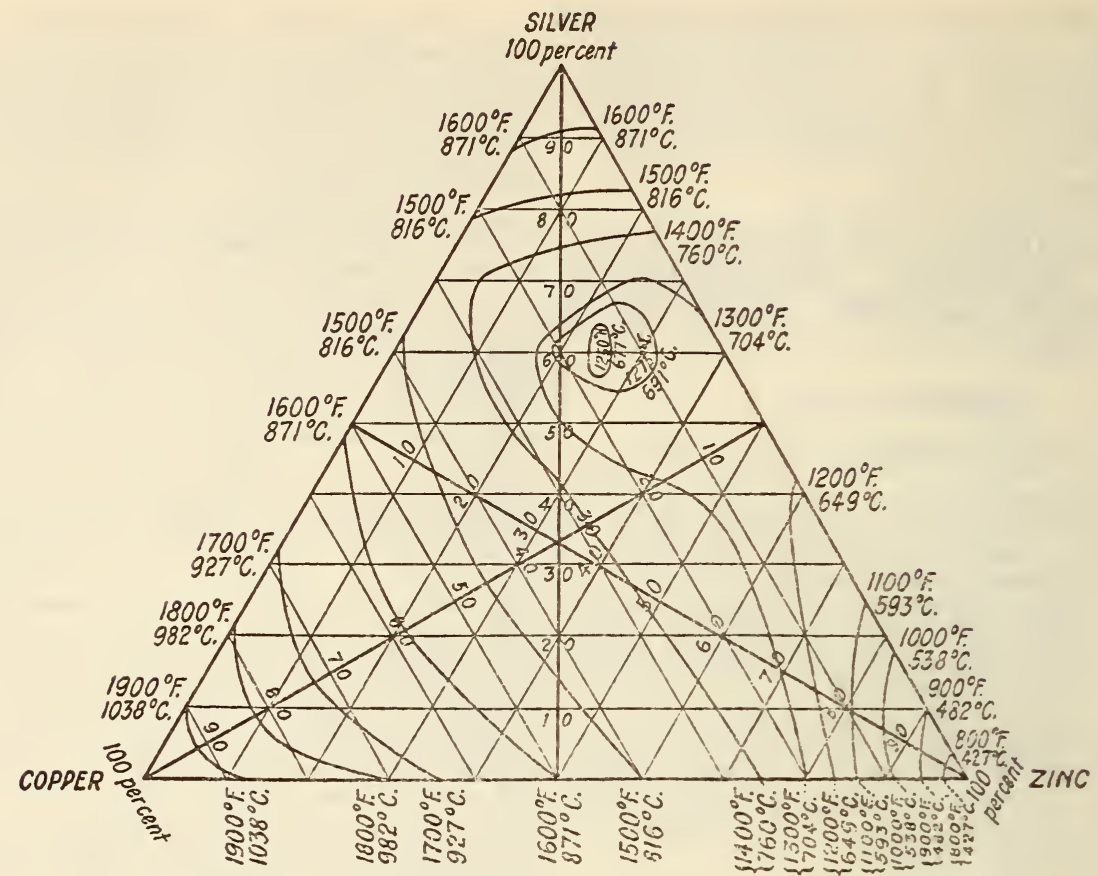

FIgURE 1144.-Liquidus diagram of silver-copper-zinc alloys

16. Solders of grades Nos. 1, 2, and 3 are suitable for brazing purposes which require a solder that flows more readily than the ordinary copper-zinc brazing solders. Grades Nos. 3 and 4 flow freely at a still lower temperature and are recommended for use where strong joints are required, and in such cases where heating to a sufficiently high temperature to flow grades Nos. 1,2 , or 3 , would be injurious to the article being soldered. Grades Nos. 6, 7 , and 8 , are higher-grade silver solders and are recommended for special cases where a high degree of malleability and ductility is required.

Nоте.-The liquidus diagram of silver-copper-zinc alloys shown in Figure 1144 is based upon a large number of cooling curres made in the research laboratory of Handy \& Harman, Bridgeport, Conn.

\section{AMERICAN SOCIETY FOR TESTING MATE- RIALS, TENTATIVE METHODS OF CHEM- ICAL ANALYSIS OF SILVER SOLDERS, DESIGNATION B81-31T, 1931}

\section{CHEMICAL ANALYSIS OF SILVER SOLDERS}

\section{General Considerations}

In the analysis of silver solder the usual determinations are silver, copper, cadmium, and zinc. In routine analysis, however, it is customary to take zinc by difference.

Methods are provided for the determination of lead, tin, iron, and nickel as impurities, starting with a separate $5 \mathrm{~g}$ sample. The methods for determination of impurities are based on the consideration that any of these will not exceed a few tenths of a per cent in amount; in case it is necessary to determine larger quantities, the size of the sample may be reduced accordingly.

In the scheme as outlined below, the procedure for the determination of main constituents is kept separate from the determination of impurities, in the belief that such an arrangement will provide continuous procedures convenient for general use.

\section{DETERMIN ATION OF SIIVER}

\section{METHOD}

Weigh $1 \mathrm{~g}$ of the sample into a $250 \mathrm{c} \mathrm{c}$ beaker, add $20 \mathrm{c}$ c of $\mathrm{HNO}_{3}(1: 4)$, cover, gently warm until solution is complete, and boil to expel $\mathrm{NO}_{2}$ fumes; dilute to a volume of $175 \mathrm{c}$ c with distilled water, and heat to boiling. Add, drop by drop, from a pipette, a dilute solution of $\mathrm{HCl}(3: 100)$ ( $1 \mathrm{c} \mathrm{c}$ of this solution should precipitate about $0.038 \mathrm{~g}$ of silver) until precipitation is complete. It is important to avoid a large excess of the precipitant. When the solution has become cold and the precipitate has settled, test the clear solution with a few drops of the dilute $\mathrm{HCl}$ to determine whether or not precipitation is complete. Filter on a weighed porcelain Gooch crucible, washing the precipitate several times with a cold dilute solution of $\mathrm{HNO}_{3}(1: 200)$ and once with cold distilled water. Save the filtrate for the determination of copper. Place the Gooch crucible inside a porcelain crucible, dry and heat the latter to dull red in the oxidizing flame of a Tirrill burner, cool, and weigh as $\mathrm{AgCl}$. Calculate to $\mathrm{Ag}$.

Notes.-1. If tin is present, as shown by a precipitate of metastannic acid when dissolving in nitric acid, it should be filtered off as directed under determination of tin before proceeding with the determination of silver. It is particularly important to observe the precautions regarding contamination by chlorides, noted under determination of tin.

2. $\mathrm{AgCl}$ melts at $451^{\circ} \mathrm{C}$. and, if heated too long at too high a tem. perature, low results are obtained. The final ignition of this precipitate should not be carried beyond the point of incipient fusion.

\section{DETERMINATION OF SILVER BY THE COMBINATION ASSAY}

\section{SOLUTIONS REQUIRED}

Sodium chloride solution.-Dissolve $107 \mathrm{~g}$ of $\mathrm{NaCl}$ in 2,000 c c of distilled water. One cubic centimeter of this solution will precipitate $100 \mathrm{mg}$ of $\mathrm{Ag}$.

\section{METHOD}

Weigh out an amount of sample which will be equivalent to 300 to $500 \mathrm{mg}$ of silver. Dissolve in 
dilute $\mathrm{HNO}_{3}(1: 4)$, allowing $20 \mathrm{c} \mathrm{c}$ of dilute acid for each gram of sample, in a $400 \mathrm{c}$ c beaker. Cover and then boil to remove $\mathrm{NO}_{2}$ fumes and dilute to 350 c e with hot $\mathrm{H}_{2} \mathrm{O}$. Precipitate $\mathrm{AgCl}$ in the hot solution with $\mathrm{NaCl}$ solution. Avoid an excess. As long as some $\mathrm{Ag}$ remains unprecipitated, the solution will clear rapidly on stirring; towards the end the cloudiness will persist for some time. Allow to settle (overnight for best results).

Test with a drop of sodium chloride solution. If clear, filter through a double $11 \mathrm{~cm}$ closely woven paper. Wash well with hot water to aid in removing copper. Add $5 \mathrm{~g}$ of test lead to precipitate in the paper. Place in a $2 \frac{1}{2}$-inch scorifier which has been lined with a sheet of lead meighing approximately $10 \mathrm{~g}$. Incinerate in an oven or muffle. Cover ash with $20 \mathrm{~g}$ of test lead, add $1 \mathrm{~g}$ of a mixture composed of equal parts of silica and borax glass. Scorify to about a 14 to $16 \mathrm{~g}$ button, pour in mold, clean off slag, hammer into a cube, and cupel in a moderately hot muffle, no attempt being made to feather. When cupellation is finished, set a hot cupel inverter over the one holding the head and withdraw both cupels from the furnace. Clean and weigh button as Ag. Corrections are made as determined by running proofs of approximately the same composition as the solder being analyzed.

NoтE.-All lead should be bismuth and silver free.

DETERMIN ATION OF COPPER BY THE ELECTROLYTIC METHOD

\section{APPARATUS FOR ELECTROLYSIS}

See the determination of copper by electrolytic method in B27 (647.20, p. 1261).

\section{METHOD}

Transfer the filtrate from $\mathrm{AgCl}$ (determination of silver) to a $400 \mathrm{c} \mathrm{c}$ beaker, add $5 \mathrm{c}$ c of $\mathrm{H}_{2} \mathrm{SO}_{4}$ (specific gravity 1.84) and evaporate to fumes, cool, dilute to a volume of $50 \mathrm{c}$. c with distilled water, and boil. Add $2 \mathrm{c}$ c of $\mathrm{HNO}_{3}$ (specific gravity 1.42), transfer to a $200 \mathrm{c}$ c lipless beaker, dilute to a volume of $100 \mathrm{c} \mathrm{c}$ with cold distilled water and electrolyze for copper, using stationary electrodes and a current of 1.0 ampere. When the solution is colorless, wash down cover glasses, electrodes, and sides of beaker, raising the level of the liquid slightly, and continue the electrolysis about 15 minutes, noting whether or not copper is deposited on the newly exposed surface of the platinum. If no copper appears, remove the cathode quickly, washing it with distilled water, and catching all washings in the beaker containing the electrolyte. Save the electrolyte for the determination of cadmium and zinc. Dip the cathode in two successive baths of alcohol, shake off the excess alcohol, and ignite the remainder by bringing it to the flame of an alcohol lamp. Keep the cathode moving continually as the alcohol burns. Weigh metallic copper.

NoTE.-If denatured alcohol is used for drying cathode with copper deposits, it should not show any weighable residue on evaporation to dryness. Alcohol denatured only by the addition of methyl alcohol is suitable for this purpose.

\section{DETERMINATION OF CADMIOM BY TEE ELECTROLYTIC METHOD}

\section{SOLUTIONS REQUIRED}

Potassium hydroxide (note 1).-Dissolve $20 \mathrm{~g}$ of $\mathrm{KOH}$, c. p., in $200 \mathrm{c}$ c of distilled water.

Potassium cyanide (note 2).-Dissolve $20 \mathrm{~g}$ of $\mathrm{KCN}$, c. p., in $200 \mathrm{c}$ c of distilled water.

\section{METHOD}

Transfer the electrolyte from the determination of copper to a $600 \mathrm{c}$ c beaker and boil to expel dis- solved gases. Make slightly alkaline with $\mathrm{NH}_{4} \mathrm{OH}$ $(1: 1)$, and then just neutral with $\mathrm{H}_{2} \mathrm{SO}_{4}(1: 4)$; finally add $25 \mathrm{c}$ c of $\mathrm{H}_{2} \mathrm{SO}_{4}(1: 4)$ in excess, dilute to a volume of $300 \mathrm{c}$ c with distilled water, bring to a boil, and pass $\mathrm{H}_{2} \mathrm{~S}$ for one hour. (Note 3.) Allow to stand until the precipitate has settled and filter off impure CdS (note 4) on a loose-bottom Gooch crucible. Remove the precipitate by punching out the bottom into a tall $200 \mathrm{c} \mathrm{c}$ beaker, washing and wiping off the sides of the crucible with a little asbestos pulp. Add $60 \mathrm{c}$ c of $\mathrm{H}_{2} \mathrm{SO}_{4}(1: 5)$ and boil for 30 minutes. (Note 5.) Filter, wash, and dilute to $300 \mathrm{c} c$ add about $5 \mathrm{~g}$ of $\mathrm{NH}_{4} \mathrm{Cl}$, and precipitate with $\mathrm{H}_{2} \mathrm{~S}$ gas as before. (Note 6.) Repeat, making a third precipitation if the amount of cadmium present is large. (Note 5.) Save combined filtrates from CdS for the determination of zinc. Dissolve the final precipitate of CdS in $25 \mathrm{c}$ c hot $\mathrm{HCl}(1: 3)$, boil to remove $\mathrm{H}_{2} \mathrm{~S}$, add a few drops of $\mathrm{HNO}_{3}$ (specific gravity 1.42) near the end, and filter off the separated sulphur. Transfer the solution to a $200 \mathrm{c} \mathrm{c}$ tall form lipless beaker, add a drop or two of phenolphthalein, and then $\mathrm{KOH}$ solution until a permanent red color is obtained. Add $\mathrm{KCN}$ solution with constant stirring until the precipitate $\mathrm{Cd}(\mathrm{OH})_{2}$ is completely dissolved. (Note 8.) Dilute to 150 c c and electrolyze with a current of 5 amperes for two hours. (Note 9.) Test for completeness of deposition by washing the watch glasses and sides of the beaker to increase the depth of the solution. Continue the electrolysis for 20 minutes and, if the newly exposed portion of the cathode is still bright, remove the beaker with the electrolyte and wash the electrode three times with distilled water and twice with ethyl alcohol (95 per cent). Dry the cathode at $100^{\circ} \mathrm{C}$., cool, and weigh. The increase is metallic cadmium.

\section{Notes. - 1. Pure NaOH may be used in place of $\mathrm{KOH}$.}

2. Pure NaCN may be used in place of KCN

3. It is occasionally necessary to add a drop or two of ammonia water to the solution to start the precipitation of CdS.

4. All the cadmium will be precipitated as sulphide together with some $\mathrm{ZnS}$. The major portion of the zinc will remain in solution.

5. In case of high cadmium content, more acid may be required. The final acidity should not exceed $10 \mathrm{c} \mathrm{c}$ of $\mathrm{H}_{2} \mathrm{SO}_{4}$ (specific gravity 1.81) in a volume of $300 \mathrm{c} \mathrm{c}$.

6. The second precipitation should give practically pure CdS.

7. A third precipitation will remove any uncertainty as to the completeness of the separation of cadmium from zinc.

8. Aroid using an excess of KCN.

9. The test for completeness of deposition may be made after one hour when the quantity present is not large.

\section{DETERMINATION OF CADMIUM BY THE GRAVIMETRIC}

[See determination of cadmium by the electrolytic method]

\section{METHOD}

Dissolve the final precipitate of CdS with $25 \mathrm{c} \mathrm{c}$ of hot $\mathrm{HCl}(1: 3)$ into a weighed platinum dish (note 1), add $10 \mathrm{c}$ c of $\mathrm{H}_{2} \mathrm{SO}_{4}(1: 1)$, and evaporate the solution until copious fumes are evolved. (Note 2.) Remove the excess of $\mathrm{H}_{2} \mathrm{SO}_{4}$ by heating the dish cautiously, and finally heat to between $500^{\circ}$ and $600^{\circ} \mathrm{C}$. Cool and weigh as $\mathrm{CdSO}_{4}$.

Notes.-1. A crucible may also be used if the volumes are kept small or evaporated before transferring to the crucible.

2. On evaporating to fumes, darkening of the solution may occur from filter-paper shreds. This may be destroyed by diluting slightly with water and adding $5 \mathrm{c}$ c of $\mathrm{HNO}_{3}$ (specific gravity 1.42) and again evaporating to fumes. It is not advisable to add $\mathrm{H}_{\mathrm{NO}}$ unless it is certain that all $\mathrm{HCl}$ has been expelled.

\section{DETERIINATION OF ZINC BY THE VOLORETRIC METHOD}

\section{SOLUTIONS REQUIRED}

Potassium ferrocyanide.-Dissolve $34.8 \mathrm{~g}$ of $\mathrm{K}_{4} \mathrm{Fe}(\mathrm{CN})_{6}$ in $1,000 \mathrm{c}$ c of distilled water and age this solution for at least 90 days. If necessary, add $0.3 \mathrm{~g}$ of $\mathrm{K}_{3} \mathrm{Fe}(\mathrm{CN})_{6}$ to the solution. (Note 1.) Standardize against c. p. zinc as follows: 
Weigh 0.3 to $0.35 \mathrm{~g}$ of c. p. zinc into a tall $400 \mathrm{c} \mathrm{c}$ beaker, add about $75 \mathrm{c} c$ of water and $13 \mathrm{c} \mathrm{c}$ of $\mathrm{HCl}$ (specific gravity 1.20). Cover and when in complete solution boil for about five minutes. Cool, add 13 c c of $\mathrm{NH}_{4} \mathrm{OH}$ (specific gravity, 0.90), neutralize with $\mathrm{HCl}$ (specific gravity 1.20), and add an excess of 2.5 c c. Dilute to $250 \mathrm{c}$ c, heat to boiling, and titrate immediately with the potassium ferrocyanide solution as described under the method below. Calculate the factor of the $\mathrm{K}_{4} \mathrm{Fe}(\mathrm{CN})_{6}$ solution as follows:

$$
\text { Factor of } \mathrm{K}_{4} \mathrm{Fe}(\mathrm{CN})_{6} \text { solution }=\frac{B}{A}
$$

where

$A=$ the cubic centimeters of $\mathrm{K}_{4} \mathrm{Fe}(\mathrm{CN})_{6}$ solution for the c. p. zinc; and

$B=$ the weight of the c. p. zinc

Ferrous sulphate.-Weigh $1.25 \mathrm{~g}$ of $\mathrm{FeSO}_{4} \cdot 7 \mathrm{H}_{2} \mathrm{O}$ into a tall $150 \mathrm{c}$ c beaker and add about $3 \mathrm{~g}$ of granular aluminum. Fill the beaker about half full with water and add about $0.15 \mathrm{c} \mathrm{c}$ of $\mathrm{HCl}$ (specific gravity 1.20). Heat to boiling and boil until the solution clears. Cool, transfer to a $250 \mathrm{c}$ c measuring flask, and dilute to the mark. Mix well before using.

Sulphuric acid (1:1).-Mix cautiously 100 c c of $\mathrm{H}_{2} \mathrm{SO}_{4}$ (specific gravity 1.84) with $100 \mathrm{c}$ c of cold distilled water.

\section{METHOD}

\section{(Refer to determination of cadmium)}

Add $10 \mathrm{c} c$ of $\mathrm{H}_{2} \mathrm{SO}_{4}(1: 1)$ to the combined filtrates from the cadmium determination and evaporate to dryness. Take up with $100 \mathrm{c} c$ of water and $10 \mathrm{c}$ c of $\mathrm{HCl}$ (specific gravity 1.20) and heat until all is in solution. Transfer the solution to a $400 \mathrm{c} \mathrm{c}$ beaker, neutralize (note 2) with $\mathrm{NH}_{4} \mathrm{OH}$ (specific gravity 0.90), and add exactly $2.5 \mathrm{c}$ c of $\mathrm{HCl}$ (specific gravity 1.20). Dilute to $250 \mathrm{c}$ c, heat to boiling, and titrate immediately with the potassium ferrocyanide solution as follows:

Transfer about one-half of the solution to be titrated into a $150 \mathrm{c}$ c beaker. Add $0.5 \mathrm{c} \mathrm{c}$ of the ferrous sulphate solution to the portion remaining in the original beaker and run the $\mathrm{K}_{4} \mathrm{Fe}(\mathrm{CN})_{6}$ solution into this portion with constant stirring until the blue color changes to pea green, then add an excess of $3 \mathrm{c} c$ of $\mathrm{K}_{4} \mathrm{Fe}(\mathrm{CN})_{\text {B. }}$ (Note 3.) Pour the solution from the $150 \mathrm{c} \mathrm{c}$ beaker into the solution in the $400 \mathrm{c} \mathrm{c}$ beaker, retaining from 2 to $3 \mathrm{c} \mathrm{c}$, and titrate until the end point is passed by two to three drops. Wash the remaining solution from the side beaker into the main portion. Add $0.05 \mathrm{c} \mathrm{c}$ of $\mathrm{K}_{4} \mathrm{Fe}(\mathrm{CN})_{6}$ solution at a time until the end point is reached. The end point is a change from blue to pea green. (Note 4.)

Calculate the percentage of zinc as follows:

where

$$
\text { Zinc, per cent }=\frac{A \times C \times 100}{\text { Weight of sample }}
$$

$A=$ the cubic centimeters of $\mathrm{K}_{4} \mathrm{Fe}(\mathrm{CN})_{6}$ solution required for the sample; and

$C=$ the factor of the $\mathrm{K}_{4} \mathrm{Fe}(\mathrm{CN})_{6}$ solution (grams of zinc per cubic centimeter).

Notes. - 1. The purpose of aging is to remove any oxidizing agents by the reducing action of the $\mathrm{K}_{4} \mathrm{Fe}(\mathrm{CN})_{6}$. A small amount of $\mathrm{K}_{3} \mathrm{Fe}$ $(\mathrm{CN})_{6}$ is formed. If this is sufficient to give the proper blue color with the ferrous iron in the standardization, no more need be added.

2. Use litmus paper as the indicator.

3 . The titration is carried out in this manner in order to insure proper composition of the precipitate.

4. The end point is best observed by looking down through the solution. The blue color in the solution is due to Turnbull's blue formed by the interaction of ferrous iron with $\mathrm{K}_{3} \mathrm{Fe}(\mathrm{CN})_{6}$. This ferrous ferricyanide is stable until the $\mathrm{K}_{4} \mathrm{Fe}(\mathrm{CN})_{6}$ is present in ercess when it forms the ferrous ferrocyanide which is colorless.

\section{DETERMINATION OF ZINC BY TAE ZINC OXIDE MRTEOD}

\section{METHOD}

Evaporate the combined filtrates from $\mathrm{CdS}$ to a volume of about $100 \mathrm{c} \mathrm{c}$. Make the solution just alkaline with $\mathrm{NH}_{4} \mathrm{OH}$ and add $30 \mathrm{c} \mathrm{c}$ of formic acid (specific gravity 1.2) for every $200 \mathrm{c} c$ of final volume. Heat nearly to boiling, and pass in a rapid stream of $\mathrm{H}_{2} \mathrm{~S}$ for 15 minutes. Filter and wash with hot 2 per cent formic acid solution saturated with $\mathrm{H}_{2} \mathrm{~S}$. Dissolve the $\mathrm{ZnS}$ from the filter into the original beaker with $50 \mathrm{c}$ c of hot $\mathrm{HCl}(1: 10)$ washing thoroughly with hot water. Boil to expel $\mathrm{H}_{2} \mathrm{~S}$. Make just alkaline with ammonia, then add $20 \mathrm{c}$ c of formic acid (specific gravity 1.2) and dilute to $200 \mathrm{c} \mathrm{c}$ with distilled water. Heat to boiling and pass $\mathrm{H}_{2} \mathrm{~S}$ for 15 minutes. Filter and wash with formic acid wash water. To avoid creeping of the precipitate do not fill the paper too near to its top and do not wet the funnel above the paper. Ignite the paper and precipitate under good oxidizing conditions in a weighed porcelain or vitreosil crucible, raising the temperature gradually to a dull red heat and finishing with a moderate red. $\mathrm{Cool}$ and weigh as $\mathrm{ZnO}$.

\section{DETERMINATION OF TIN}

\section{METHOD}

Dissolve a separate $5 \mathrm{~g}$ sample with $100 \mathrm{c}$ c of $\mathrm{HNO}_{3}(1: 4)$ in a $400 \mathrm{c} \mathrm{c}$ beaker. Boil to expel $\mathrm{NO}_{2}$ fumes, and dilute to a volume of $250 \mathrm{c} \mathrm{c}$ with boiling distilled water. Allow to stand and settle for about one hour, keeping the temperature just below the boiling point. Filter on double $7 \mathrm{~cm}$ ashless papers, being careful to keep the solution hot throughout the process of filtration, washing with boiling hot water. Save the filtrate for the determination of lead. Ignite the paper and precipitate under good oxidizing conditions in a weighed porcelain crucible, and weigh as $\mathrm{SnO}_{2}$.

NoтE--Every precaution must be taken to avoid contamination by chlorides from any source, in order to prerent the precipitation of $\mathrm{AgCl}$ during the determination of tin. All distilled water must be free from chlorides, beakers and apparatus must be carefully rinsed before using, and all chloride fumes must be avoided.

\section{DETERMINATION OF LEAD}

\section{SOLUTIONS REQUIRED}

Lead acid.-Mix 300 c c of $\mathrm{H}_{2} \mathrm{SO}_{4}$ (specific gravity 1.84) and $1,800 \mathrm{c} c$ of distilled water. Dissolve $1 \mathrm{~g}$ of lead acetate, c. p., in $300 \mathrm{c}$ c of distilled water and add this to the hot solution, stirring meanwhile. Let stand for at least 24 hours and siphon through a thick asbestos filter.

Dilute alcohol for washing.-Mix equal parts of denatured alcohol and distilled water.

\section{METHOD}

Heat the filtrate from the meta-stannic acid to boiling, and precipitate silver with dilute $\mathrm{HCl}$ as in the determination of silver, avoiding a large excess of the precipitant. When the solution has become cold and the precipitate has settled, test the clear solution with a few drops of the dilute $\mathrm{HCl}$ to determine whether or not precipitation is complete. Filter on a closely woven paper, washing with cold dilute $\mathrm{HNO}_{3}(1: 200)$. Add 80 c c of "lead acid" and evaporate to fumes. To insure complete removal of chlorides, cool, add a few cubic centimeters of $\mathrm{H}_{2} \mathrm{O}$ and fume again. Cool, add $70 \mathrm{cc}$ of distilled water to dissolve the salts and to make the acid concentration the same as in "lead acid." Heat to boiling, and allow to cool and settle for 5 
hours, or overnight if convenient. Filter on a weighed porcelain Gooch crucible and wash with "lead acid." Remove the filtrate and save for the determination of iron. Continue washing the precipitate with dilute alcohol, set the Gooch crucible inside a porcelain crucible, dry and ignite for five minutes at the full heat of a Tirrill burner, cool and weigh as $\mathrm{PbSO}_{4}$.

Note.-In order to avoid "spitting" during evaporation with "lead acid" it is suggested to heat over a burner, holding the beaker with a pair of tongs and twirling as the evaporation is carried on.

\section{DETERMINATION OF IRON}

\section{SOLUTIONS REQUIRED}

Dilute sulphuric acid solution for reductor.-Mix 50 c c of $\mathrm{H}_{2} \mathrm{SO}_{4}$ (specific gravity 1.84) and $1,000 \mathrm{c}$ c of distilled water.

Standard potassium permanganate solution.-Dissolve $1 \mathrm{~g}$ of $\mathrm{KMnO}_{4}$ in $1,000 \mathrm{c}$ c of $\mathrm{H}_{2} \mathrm{O}$ and allow to stand for several days in a glass-stoppered bottle in a dark closet. Filter the solution through prepared asbestos and standardize against $0.1000 \mathrm{~g}$ portions of pure sodium oxalate. Each cubic centimeter is equivalent to approximately $0.00177 \mathrm{~g}$ of iron.

\section{METHOD}

To the filtrate from the $\mathrm{PbSO}_{4}$ add $5 \mathrm{c} \mathrm{c}$ of $\mathrm{HNO}_{3}$ (specific gravity 1.42) and remove copper by electrolysis. To the electrolyte add $5 \mathrm{~g}$ of $\mathrm{NH}_{4} \mathrm{Cl}$, and 2 to $3 \mathrm{c}$ c of $\mathrm{H}_{2} \mathrm{O}_{2}$ or $\mathrm{HNO}_{3}$ (specific gravity 1.42 ), and boil to oxidize iron. Make ammoniacal, adding sufficient $\mathrm{NH}_{4} \mathrm{OH}$ to dissolve any basic salts, and boil. Filter and wash with hot water. Dissolve the precipitate through the filter paper with hot dilute $\mathrm{HCl}$ and reprecipitate iron with ammonia. Filter and wash with hot water. Combine and save filtrates for the determination of nickel. Dissolve the precipitate through the filter with $100 \mathrm{c}$ c of hot dilute $\mathrm{H}_{2} \mathrm{SO}_{4}$, for reductor. Add a few drops of concentrated solution of $\mathrm{KMnO}_{4}$ until a distinct pink color persists, boil, cool, and pass the solution through the reductor. Wash the beaker and reductor with $150 \mathrm{c}$ c of dilute $\mathrm{H}_{2} \mathrm{SO}_{4}$ solution for reductor and then with $100 \mathrm{c}$ c of distilled water. Titrate with standard $\mathrm{KMnO}_{4}$ solution.

Notes.-1. Results of titration should be checked with a blank determination made on corresponding amounts of solutions used.

2. A small quantity of liquid should always be left in the reductor funnel and air should never be allowed to enter the reductor.

3. For further details of the use of reductor see "Chemical Analysis of Iron" by A. A. Blair, "Quantitative Chemical Analysis" by H. P Talbot, or "Applied Inorganic Analysis" by Hillebrand and Lundell.

\section{DETERMINATION OF NICEEL}

SOLUTIONS REQUIRED

Dimethylglyoxime solution.-Dissolve $5 \mathrm{~g}$ ot dimethylglyoxime in $500 \mathrm{c}$ c of ethyl alcohol and filter before using.

\section{METHOD}

Evaporate the combined filtrates from the precipitation of iron to a convenient volume and make barely acid with $\mathrm{HCl}$ (specific gravity 1.20). Heat nearly to boiling and add $5 \mathrm{c} \mathrm{c}$ of dimethylglyoxime solution for each $0.1 \mathrm{~g}$ or fraction of nickel present and then $\mathrm{HN}_{4} \mathrm{OH}$ in slight excess. Let stand at least 30 minutes on the steam bath. Filter and wash with dilute ammonia solution $(1: 100)$. For accurate work, the precipitate should be dissolved in hot $\mathrm{HCl}(1: 1)$ and reprecipitated as before. Filter on a weighed Gooch crucible, wash with hot water, dry at $110^{\circ}$ to $120^{\circ} \mathrm{C}$. for about two hours and weigh as the glyoxime salt which contains 20.31 per cent nickel. If it is preferred, instead of weighing the glyoxime salt, the nickel can be determined electrolytically as follows: Dissolve the red precipitate of nickel glyoxime in hot $\mathrm{HCl}(1: 1)$, add $10 \mathrm{c} \mathrm{c}$ of $\mathrm{H}_{2} \mathrm{SO}_{4}(1: 1)$ and evaporate to strong fumes. Dilute, neutralize with $\mathrm{NH}_{4} \mathrm{OH}$ (specific gravity 0.90 ), and add $20 \mathrm{c}$ c in excess. The final volume should be about 150 c c. Electrolyze with a current density overnight of 0.3 amperes per square decimeter $(2.7$ amperes per square foot.) Test the solution for completeness of deposition by removing $1 \mathrm{c} \mathrm{c}$ to a porcelain test plate and adding fresh $\mathrm{H}_{2} \mathrm{~S}$ water. Remove the cathode, wash with water, and dry with alcohol as in the determination of copper by the electrolytic method. Weigh the deposit as metallic nickel.

\section{UNITED STATES GOVERNMENT, FEDERAL SPECIFICATIONS BOARD; FEDERAL SPECIFICATION FOR SILVER SOLDERS, JULY 6, 1925, NO. 30\%. (CURRENT DESIGNATION : QQ-S-561)}

\section{SILVER SOLDERS}

\section{GENERAL SPECIFICATIONS}

General specifications for metals, Federal Specifications Board No. 339a, (QQ-M-151) shall form part of this specification. (See 600.0, p. 3.)

I. This specification covers three grades of silver solder, as follows: Grade 0, grade 1, and grade 2.

\section{MATERIAL AND WORKMANSHIP}

II. Silver solder shall be made only from the best grades of virgin metals or from scrap of known and approved composition.

\section{GENERAI REQUIREMENTS}

III. 1. Silver solder shall be supplied in strips in form $\mathrm{X}, \mathrm{A}$, or $\mathrm{B}$, or wire, as specified.

2. Form X solder shall be not over 0.02 inch thick and not over 3 inches wide.

3. Form A solder shall be not over 0.003 inch thick and not over three-quarters inch wide.

4. Form B solder shall be not over 0.03 inch thick and not over one-quarter inch wide, and not less than 12 inches long.

5. Silver solder shall be uniform in color, quality, and size; clean, bright, sound, and smooth; and free from ragged edges, damaged ends, or other injurious defects.

\section{DETAIL REQUIREMENTS}

IV. Chemical requirements.-The chemical requirements are as given in the accompanying table.

\section{Chemical composition}

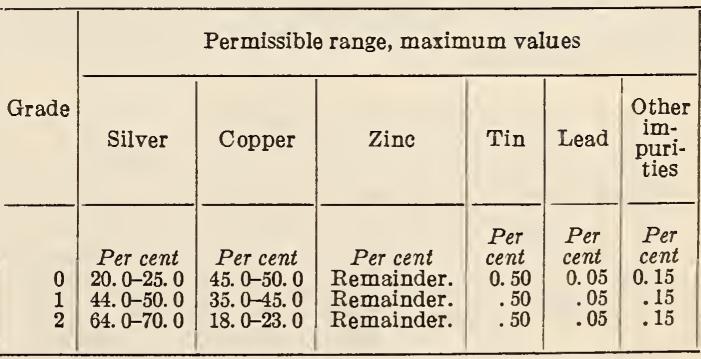

METHODS OF INSPECTION AND TESTS

V. Samples for check analysis, when made, shall be taken from each lot of 50 pounds, or fraction thereof, of grade 0 solder, and from each lot of 20 pounds, or fraction thereof, of grades 1 and 2 solder, and shall be free from oil, dirt, or foreign material. 


\section{PACKING AND MARKING}

VI. Solder ordered in form A shall be packed in metal containers containing not more than 2 ounces each, unless otherwise specified.

\section{ADDITIONAL INFORMATION}

VII. 1. Grade 0 solder is suitable for ordinary brazing purposes where a solder is required that possesses higher physical properties than provided by spelter solders, and where the service or appearance does not require a high silver content.

2 . Grade 1 solder is a high-grade silver solder suitable for the general range of silver-soldering requirements. It may be used for soldering turbine blades.

3. Grade 2 solder provides a very high silver content and should be used only where the application requires high strength, resistance to corrosion, and appearance.

4. Form A should be specified on grades 1 and 2 solder for very light work, such as the soldering of parts of delicate instruments, etc. Form B should be specified on Grades 1 and 2 when intended for soldering light and medium weight parts.

5. This specification does not include silver solder for dental purposes.

\subsection{SOLDERS FOR ALUMINUM.} ble.)

(No nationally recognized specifications availa-

\section{MAGNESIUM AND MAGNESIUM AILOYS}

\section{AMERICAN SOCIETY FOR TESTING MATE- RIALS, TENTATIVE SPECIFICATIONS FOR MAGNESIUM-BASE ALLOY CASTINGS, DESIGNATION B80-31T, 1931}

\section{MAGNESIUM-BASE ALLOY CASTINGS $S C O P E$}

1. These specifications cover commercial magnesium-base alloy sand castings having a specific gravity of 1.9 or less. Three typical alloys are specified and are designated alloys Nos. 1, 2, and 3.

\section{MANUFACTURE}

2. The alloy may be made from virgin metal or from purified scrap metal of known composition.

3. Castings from alloy No. 2 may be subjected to such heat treatment as the manufacturer desires to produce material that will conform to the requirements specified. Heat treatment shall be performed on the whole of a casting, never on a part only, and shall be applied in a manner that will produce the utmost uniformity.

\section{CHEMICAL COMPOSITION}

4. The alloys shall conform to the following requirements as to chemical composition:

\begin{tabular}{|c|c|c|c|c|c|c|}
\hline $\begin{array}{l}\text { Alloy } \\
\text { No. }\end{array}$ & $\begin{array}{c}\text { Magnesium } \\
\text { (per cent) }\end{array}$ & $\begin{array}{l}\text { Alumi- } \\
\text { num }\end{array}$ & $\begin{array}{l}\text { Manga- } \\
\text { nese, } \\
\text { mini- } \\
\text { mum }\end{array}$ & Copper & $\begin{array}{l}\text { Cadmi- } \\
\text { um }\end{array}$ & $\begin{array}{l}\text { Other } \\
\text { impuri- } \\
\text { ties } \\
\text { maxi- } \\
\text { mum }\end{array}$ \\
\hline & $\begin{array}{l}\text { Remainder.- } \\
\text { do }\end{array}$ & $\begin{array}{l}\text { Per cent } \\
5.5-7.0 \\
6.5-8.5 \\
1.7-2.3\end{array}$ & $\begin{array}{c}\text { Per cent } \\
0.2 \\
.2 \\
.2\end{array}$ & $\begin{array}{r}\text { Per cent } \\
10.1 \\
1.1 \\
3.5-4.5\end{array}$ & $\begin{array}{l}\text { Per cent } \\
1.6-2.4\end{array}$ & $\begin{array}{r}\text { Per cent } \\
0.3 \\
.3 \\
.3\end{array}$ \\
\hline
\end{tabular}

1 Maximum.

\section{PHYSICAL PROPERTIES AND TESTS}

5. The castings shall conform to the following requirements as to tensile properties:

\begin{tabular}{|c|c|c|c|}
\hline $\begin{array}{l}\text { Alloy } \\
\text { No. }\end{array}$ & Condition & $\begin{array}{l}\text { Tensile } \\
\text { strength, } \\
\text { mini- } \\
\text { mum }\end{array}$ & $\begin{array}{l}\text { Elonga- } \\
\text { tion in } 2 \\
\text { inches, } \\
\text { minimum }\end{array}$ \\
\hline $\begin{array}{l}1 .-- \\
2 .-- \\
3 .-.\end{array}$ & 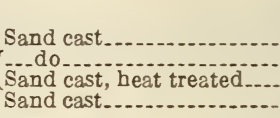 & $\begin{array}{c}\text { Lbs./in. }^{2} \\
24,000 \\
24,000 \\
29,000 \\
21,000\end{array}$ & $\begin{array}{c}\text { Per cent } \\
4 \\
4 \\
6 \\
2\end{array}$ \\
\hline
\end{tabular}

(Sampling requirements are identical with those in A. S. T. M. B10. See 643.4, p. 1192 . Test specimens and number of tests are identical with those in A. S. T. M. B26-31. See 631.41, p. 1160 . Inspection and rejection requirements are identical with those in A. S. T. M. B59. See 647.15, p. 1259.)

\section{WORRMANSHIP AND FINISH}

6. The castings shall be of uniform quality and condition, free from cracks or other injurious defects, and shall be well cleaned before inspection by sand-blasting, tumbling, chipping, pickling in sulphuric acid, or other approved process.

Note.-Alloy No.1.-Alloy No. 1 has a specific gravity of about 1.78. It is used only in the as-cast condition. The copper content should be kept as low as possible in order to minimize corrosion.

Alloy No. 2.-Alloy No. 2 has a specific gravity of about 1.80. It is susceptible to heat treatment and is used both in the as-cast and heat-treated conditions. The most beneficial effects of heat-treatment are reflected in the increase in tensile strength percentage of elongation, and notched-bar impact toughness. The copper content should be kept as low as possible in order to minimize corrosion.

Alloy No. 3. - Alloy No. 3 has a specific gravity of about 1.82 . It thermal conductirity is 50 to 75 per cent greater than that of alloys 5 Nos. 1 and 2, and is recommended where this property is of great importance.

\section{ORES, METALS, AND ALLOYS NOT} ELSEWHERE CLASSIFIED

\section{AMERICAN SOCIETY FOR TESTING NATE- RIAIS, STANDARD SPECIFICATIONS FOR TUNGSTEN POWDER, SERIAL DESIGNA- TION A97-27, 1927}

\section{TUNGSTEN POWDER}

1. These specifications cover tungsten powder in one grade only.

\section{BASIS OF PURCHASE}

2. The tungsten powder shall be thoroughly mixed and packed in sound containers, preferably metallic or metallic lined, and sufficiently strong to prevent loss in transportation.

\section{CHEMICAL REGUIREMENTS}

3. The material shall conform to the following requirements as to chemical composition:

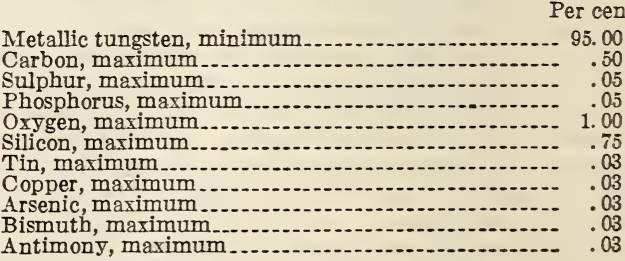

\section{SAMPIING AND CEEMICAL ANAIYSIS}

4. (For sampling, see A. S. T. M. A103 (621.20, p. 1092.) For methods of chemical analysis, see A. S. T. M. A104 (621.20, p. 1093).) 


\section{TECHNICAL SOCIETIES, TRADE ASSOCIATIONS, AND OTHER ORGANIZA- TIONS ISSUING STANDARDS AND SPECIFICATIONS DEALING WITH METALS AND METAL PRODUCTS}

There are given below the names and addresses of organizations the standards and specifications of which are reproduced herein either in full or in substance by means of abstracts and cross references. Under the procedure of many of these organizations provision is made for periodic revision of standards ois an annual, biannual, or similar basis. Persons interested in obtaining information concerning the status or revisions of standards should communicate with the organizations at the offices as listed below:

American Association of State Highway Officials. W. C. Markham, secretary, National Press Building, Washington, D. C.

American Automobile Association, Ernest N. Smith, executive vice president, Pennsylvania Avenue at Seventeenth Street NW., Washington, D. C.

American Bottlers of Carbonated Beverages, Junior Owens, secretary, 726 Bond Building, Washington, D. C.

American Bureau of Shipping, J. W. Cantillion, secretary, Stevenson Taylor Memorial Building, 24 Old Slip, New York, N. Y.

American Electric Railway Association, G. C. Hecker, general secretary, 292 Madison Avenue, New York, N. Y. (Name changed to American Transit Association.)

American Gas Association (Inc.), Alexander Forward, managing director, 420 Lexington Avenue, New York, N. Y.

American Gear Manufacturers Association, J. C. McQuiston, secretary, First National Bank Building, Wilkinsburg, $\mathrm{Pa}$.

American Institute of Architects, F. Leo Smith, technical secretary, structural service department, 1741 New York Avenue NW., Washington, D. C.

American Institute of Electrical Engineers, H. $H$. Henline, acting national secretary, 33 West Thirty-ninth Street, New York, N. Y.

American Institute of Steel Construction (Inc.), Charles F. Abbott, executive director, 200 Madison Avenue, New York, N. Y.

American Marine Standards Committee, A. V. Bouillon, secretary, U. S. Department of Commerce Building, Washington, D. C.

American Mining Congress, J. F. Callbreath, secretary, Munsey Building, Washington, D. C.

American Oil Chemists' Society, J. O. P. Helm, secretary, 705 Tchoupitoulas Street, New Orleans, La.

American Petroleum Institute, E. R. Boyd, jr., executive vice president, 250 Park Avenue, New York, N. Y.

American Railway Association, Electrical Section, E. H. Fritch, secretary, 59 East Van Buren Street, Chicago, IIl.

American Railway Association, Mechanical Division, V. R. Hawthorne, secretary, 59 East Van Buren Street, Chicago, Ill.

American Railway Association, Purchases and Stores Division, W. J. Farrell, secretary, 30 Vesey Street, New York, N. Y.

American Railway Association, Signal Section, R. H. C. Balliet, secretary, 30 Vesey Street, New York, N. Y.
American Railway Association, Telegraph and Telephone Section, W. A. Fairbanks, secretary, 30 Vesey Street, New York, N. Y.

American Railway Engineering Association, E. H. Fritch, secretary, 59 East Van Buren Street, Chicago, Ill.

American Society of Civil Engineers, George $\mathrm{T}$ Seabury, secretary, 33 West Thirty-ninth Street, New York, N. Y.

American Society of Heating and Ventilating Engineers, A. V. Hutchinson, secretary, 51 Madison Avenue, New York, N. Y.

American Society of Mechanical Engineers, Calvin W. Rice, secretary, 29 West Thirty-ninth Street, New York, N. Y.

American Society of Municipal Engineers, C. W. S. Sammelman, executive director, 4359 Lindell Boulevard, St. Louis, Mo.

American Society for Steel Treating, W. H. Eisenman, secretary, 7016 Euclid Avenue, Cleveland, Ohio.

American Society for Testing Materials, C. L. Warwick, secretary-treasurer, 1315 Spruce Street, Philadelphia, Pa.

American Standards Association, P. G. Agnew, secretary, 29 West Thirty-ninth Street, New York, N. Y.

American Transit Association, G. C. Hecker, general secretary, 292 Madison Avenue, New York, N. Y. (Formerly known as the American Electric Railway Association.)

American Water Works Association, Malcolm Pirnie, chairman, committee on water works practice, 25 West Forty-third Street, New York, N. $Y$.

Asphalt Shingle and Roofing Institute, J. S. Bryant, manager, 2 West Forty-fifth Street, New York, N. Y.

Associated Cooperage Industries of America, Louis F. Horn, secretary, 411 Olive Street, St. Louis, Mo.

Associated Factory Mutual Fire Insurance Companies, C. W. Mowry, manager, Inspection Department, 184 High Street, Boston, Mass.

Associated Manufacturers of Water Purifying Equipment, Arthur M. Crane, secretary, P. O. Box 307, Gary, Ind.

Association of American Steel Manufacturers, J. O. Leech, secretary, 616 Investment Building, Pittsburgh, $\mathrm{Pa}$.

Association of Edison Illuminating Companies, Preston S. Millar, secretary, Eightieth Street and East End Avenue, New York, N. Y.

Association of Iron and Steel Electrical Engineers, John F. Kelly, managing director, 1010 Empire Building, Pittsburgh, $\mathrm{Pa}$.

Association of Manufacturers of Chilled Car Wheels, G. E. Doke, president and secretary, McCormick Building, Chicago, Ill.

Association of Railway Electrical Engineers, J. A. Andreucetti, secretary, Chicago \& Northwestern Terminal Station, Chicago, Ill.

Chlorine Institute, Robert T. Baldwin, secretary, 30 East Forty-second Street, New York, N. Y. 
Compressed Air Society, C. H. Rohrbach, secretary, 90 West Street, New York, N. Y.

Concrete Reinforcing Steel Institute, R. W. Johnson, engineer, 333 North Michigan Blvd., Chicago, Ill.

Contracting Plasterers' International Association, Edward McDonnell, secretary-treasurer, 4755 Commonwealth Avenue, Detroit, Mich.

Glass Container Association of America, Victor L. Hall, secretary of the standardization committee, 19 West Forty-fourth Street, New York, N. Y.

Heating and Piping Contractors National Association, Joseph C. Fitts, secretary, 50 Union Square, East, New York, N. Y.

Institute of Boiler and Radiator Manufacturers, F. W. Herendeen, secretary, Geneva, N. Y.

International Association of Electrotypers, Neal Gross, field secretary, 949 Leader Building, Cleveland, Ohio.

International Association of Milk Dealers, R. E. Little, executive secretary, 228 North La Salle Street, Chicago, Ill.

Malleable Iron Research Institute, Robert E. Belt, secretary, Union Trust Building, Cleveland, Ohio.

Manganese Track Society, W. Homer Hartz, chairman standardization committee, care of Morden Frog and Crossing Works, Chicago, Ill.

Manufacturers Standardization Society of the Valve and Fittings Industry, John J. Harman, general secretary, 103 Park Ávenue, New York, N. Y.

National Association of Master Plumbers of the United States, Jere L. Murphy, chairman, standardization committee, 340 East Forty-fourth Street, New York, N. Y.

National Association of Sheet Metal Contractors of the United States, W. C. Markle, secretary, 429 Fourth Avenue, Pittsburgh, $\mathrm{Pa}$.

National Association of Waste Material Dealers, Charles M. Haskins, secretary, Times Building, New York, N. Y.

National Board of Fire Underwriters, W. E. Mallalieu, general manager, 85 John Street, New York, N. Y.

National District Heating Association, D. L. Gaskill, executive secretary, 603 Broadway, Greenville, Ohio.

National Electrical Manufacturers' Association, A. W. Berresford, managing director, 570 Lexington Avenue, New York, N. Y.
National Engineering Inspection Association, Burton H. Witherspoon, secretary, Pittsburgh Testing Laboratory, Pittsburgh, $\mathrm{Pa}$.

National Fire Protection Association, Franklin H. Wentworth, managing director, 60 Batterymarch Street, Boston, Mass.

National Machine Tool Builders' Association, Frida F. Selbert, secretary, 617 Vine Street, Cincinnati, Ohio.

National Metal Exchange (Inc.), J. J. Murphy, secretary, 27 William Street, New York, N. Y.

National Safety Council, W. H. Cameron, managing director, 20 North Wacker Drive, Chicago, Ill.

National Warm Air Heating Association, Allen W. Williams, managing director, 3440 A. I. U. Building, Columbus, Ohio.

National Watch Case Manufacturers Association, Alexander Vincent, secretary, 20 West Fortyseventh Street, New York, N. Y.

Power Piping Society, Stuart J. Swenson, secretary, 1714 Clark Building, Pittsburgh, $\mathrm{Pa}$.

Railway Fire Protection Association, R. R. Hackett, secretary, Baltimore and Ohio Building, Baltimore, Md.

Refrigerating Machinery Association, Fred Nolde, secretary, 23 South Fifty-second Street, Philadelphia, $\mathrm{Pa}$.

Society of Automotive Engineers (Inc.), John A. C. Warner, secretary and general manager, 29 West Thirty-ninth Street, New York, N. Y.

Steel Joist Institute, Frank Burton, Dime Bank Building, Detroit, Mich.

Underwriters' Laboratories, Dana Pierce, president, 207 East Ohio Street, Chicago, Ill.

United States Government, Washington, D. C.: Congress-

Statutes.

Executive Departments-

Department of Agriculture-

Bureau of Public Roads.

Department of Commerce-

Bureau of Standards-

Division of building and housing.

Division of simplified practice.

Division of trade standards (commercial standards).

Steamboat Inspection Service.

Independent establishments-

Federal Specifications Board.

Federal Trade Commission.

Interstate Commerce Commission.

National Screw Thread Commission. 


\section{N D E X}

A

Acetylene equipment for lighting, heating, and cooking...

Acme screw thread gages

Acme screw threads

Adjusters, casement window stop.

Admiralty metal castings. (See Castings, bronze.)

Adzes. track.

Air-brake release valves.

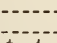

Air-brake valves, method of test

Bars, tool, alloy steel.)

Alloys, aluminum. (See Aluminum alloys.)

yeat treatment of

amalgam, dental

gold, chental gold, dental
silver, dental

Alloys and metals, nonferrous, metallographic testing of

Alpha brass, definition of

Aluminum, chemical analysis of methods of test of, metallographic.

scrap, classification of

Aluminum alloys, chemical analysis of heat treatment of

Aluminum bronze bars, castings, etc. (See Bars, aluminum bronze; castings, aluminum bronze; etc.)

Aluminum metal, standard sample

Aluminum plates, bars, etc. (See Plates, aluminum; bars, aluminum; etc.)

Aluminum manganese alloy sheet

Amalgam alloys, dental

A malgam alloys, dental

Ammunition, shot-gun

Ammunition, shot-gun

Analysis, chemical. (See Chemical analysis.)

Anchor chain.

Anchor rods.--

Anchor shackles.

Anchors, cast-steel

$$
\text { window cleaners. }
$$

Angle valres

Angle valres

ngles. (See Shapes.)

structiral. (See Shapes, structural.)
nnealing of steels. (See Heat treatme

Annealing of steels. (See Heat treatment of steel.)

Antimony.

copper, lead, and tin alloys, chemical analysis of

Antirattler, sash

Anvils.

046,1048
$--\quad 884$

Apple corers

Appliances, gas, methods of test... 858

Arbitration test bar, cast-iron

Arch bars for railway car trucks

Arms, trolley bracket...

Ash-can covers . .

Ash cans

Assay of copper, battery.

Attachments (wire rope). (See Fittings, rope, wire.)

Augers - ${ }_{\text {Automobile springs. (See Springs, automobile.) }}$

Automotive steel bars
Automotive steels, inspection and sampling for chemical

analysis steels, inspection and sampling for chemical Awls

Axes.

Axles, drop-test machine for

electric motor

railway car alloy steel forgings for ......... 1136 electric, forgings for forgings for $7 \overline{2}, \overline{757}, \overline{759}, \overline{765}, 7 \overline{71}, 1136$ railway electric motor

$\mathbf{B}$

Babbitt, scrap, classification of -

Babbitt metel (1320, 1321, 1322, 1328 chemical analysis of standard sample

Bale hooks

Ball bearings

219

Ball cocks for fiushing tanks

Bands, automotive, steel

$$
\text { pole. }
$$

Bar, arbitration test, cast-iron -----

Barbed nails
Barge spikes

Page

Barrel hooks

Barrel hoops, steel

Barrel nails

570
219

219

Barrels, working, oil-well pump--

Bars, alloy steel aluminum alloy arch, railway car truck

automotive, steel

bearing metal (Babbitt)

bolt stock, alloy steel .................................. 1130

brass. (See also Bars, solder, copper-zinc.)

brass .-. 1195, 1205

bronze. (See also Bars, aluminum bronze; Bars, man-

ganese bronze; Bars, phosphor bronze.)

chromium steel

chromium-vanadium steel

law

or railway springs.

commercial quality, steel

copper

chemical analysis of
chere

copper-nickel alloy

- 962,970

engine bolt, iron

forging. (See also Blooms and billets.)

forging-quality, steel

German silver. (See Bars, nickel silver.)

ingot, copper........

1173,1174

iron (method of designating class, kind, or grade, by paint-

ing the ends) -

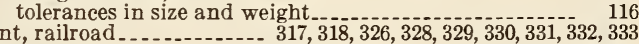
lead, ralload.......... lining . . magnet, alloy steel mannet, ane bronze manganese bronze

Monel metal. (See Bars, copper-nickel.)

nickel.

1282

nickel silver

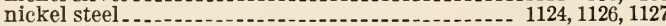

nickel-chromium steel _._._._._._._.

phosphor bronze ... .

pinch

push, door

reinforcement, steel 1122 screw stock, steel $144,233,249,250,251,252,254,266,267,269,270,273$ (14teel slicon steel steel

ilicomanganese steel,

nanese steel, for springs

$\begin{array}{lll}\text { silver } & \text { solder, brazing }\end{array}$ copper zinc....... 1334

soft

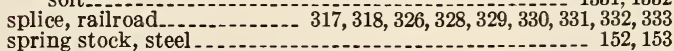
stay bolt, copper

iron

el. (See also Bars, reinfor cement, steel.)

steel. $150,151,152,153,233,249,250,251,252,254,266,267,269,270,346$
. for forgings.

galvanizing of (method of designating class, kind or grade, by paint-

ing the ends)

sampling for chemical analysis

(stock sizes)

structural, nickel steel silicon steel._._. $249,250,251,252,254,266,267,269,270,346,1126$
steel._._.

tamping

tool steel

alloy steel.

heat treatment of. (See Tool steel.)

tungsten steel, suitable for tools

wrecking

Bases, metal. (See Lath, metal.)

559,562 
Basket tacks (sizes)

Baskets, sheet metal

Bath fixtures, shower.

Bath inclosures, trimmings for

Baths, foot, metal.

shower, curtains for inclosures for

Bathtubs. (See also Baths.)

$$
\text { cast-iron. }
$$

colors for

Battery anodes (gravity), zinc.

Battery assay of copper

Battery copper, gravity battery

Battery zincs, gravity battery

Beams, brake. (See Brake beams.)

Bearing metal, Babbitt. chemical analysis of standard sample

bronze................. chemical analysis of

phosphor bronze.--
standard sample

Bearing wedges, journal

Bearings, ball.

bronze

bronze backing for (railway)

bronze castings for

bronze ingots for

journal, railway

roller

Bed casters

$$
\text { gages for }
$$

Bed shoes, wooden

Bed springs.

Beds. (See also Berths.)

hospital

metal.

surgical

wooden (sizes)

Bell cords, wire

Bellows, blacksmith

Belt rivets, steel

Belts, window cleaners

Bench plates and stakes

Bends, return, pipe. (See Fittings, pipe.)

Berths, ship
Beta brass, definition of
Bevels

Biggins, coffee

Bill posters' tacks

Billets, alloy steel.

copper

chemical analysis of

steel...

Bismuth---

Blacksmith tools (sizes)

Blades, hack saw

propeller, marine, bronze

razor, packaging of

shear, heat treatment of.

Blanks, gage, plain screw thread.

Blinds, fasteners for hinges for

turnbuckles for

Blocks, die. (See Die blocks.)

gage, precision

Blocks and sheaves, hoisting

Blooms, alloy-steel. iron

Blow torches

Boards, wash

Boat nails

Boat spikes

Boats, gravy. (See also Silverware.)

Boiler plate, zinc

Boiler steel. (See Plates, boiler, steel.)

Boiler tubes, brass. copper

Boilers, coffee
gas, house heating

gas, house heating

house heating.

coal burning

range

oil burning

Bollards, ship
Bolsters, truck, railway car

Bolt locks gages for cremone, for doors espagnolette, for doors

exit, for doors.

flush, for doors

$$
\text { ship door. }
$$

foot, for doors.
Page

Bolt locks, lavatory door

Bolts........... $586,589,590,594,595,596,601,602,603,604,683,684,690,810,1130$ aeronautic

cap. (See Cap screws.)

carriage

\section{packing of}

$590,596,683$

connecting-rod, automobile

engine, iron bars for

602,683

lag

packing of

machine.

packi
plow
pole line.

propeller, aeronautic

round unslotted-head

screw threads for -

pring center, for automobile springs

stay, copper bars for

tlexible (sizes) - 588

iron bars for

steel bars for

steel tubing for

step

Stove

Stud_-1

tap slot

track

Bonding wires, galvanizing of . ..................... 60

Bone plates, alloy steel._._._._._. 1138

oom fittings, cargo........ 441

Booms, carro, tubular, steel

Bottling equipment, carbonated water, fittings for

Bowls. (See also Silverware.)
metal

Box hooks.

Box nails

818

flour, metal

journal

dust guards for

gages for

lids for

packing tools for

lunch, metal._._._.

sugar, metal.

Braces, carpenter's.

cross arm

Bracket arms, trolley

Brackets, handrail

Brads. (See also Nails.)

common.

flooring-

molding nails

Brake beams, railway

Brake-shoe gages, railway.....

Brake shoes, railway ...... $720,726,727$

Brass, alpha, definition of ......... 34

beta, definition of

nickel. (See also Copper-nickel alloy.)

nickel plating on, methods of test.... specification

scrap, classification of

tack and nail

Brass rods, bars, etc. (See Rods, brass; Bars, brass; ete.)

Brass wire nails . . chemical analysis of (silver so!der) -

Bread boxes, metal_._._._._._. 818

Bread plates, metal 817

Breast drills

Bridges, highway, steel._._.

railway -..-.....movable.

structural steel for

Bronze, aluminum (bars, castings, etc.) (See Bars, aluminum bronze; Castings, aluminum bronze; etc.)

chemical analysis. (See ulso Manganese bronze, chemical analysis of.)

chemical analysis of 


\section{INDEX}

Page

Bronze, manganese, chemical analysis of ................. 1261 standard sample

methods of test of, metallographic.-

nickel (castings)

nickel plating on (specification)

phosphor (bars, castings, etc.). (See Bars, phosphor

bronze; castings, phosphor bronze; etc.)

scrap, classification of

white. (See also Nickel silver.)

Bronze bars, castings, etc. (See Bars, bronze; Castings, bronze; etc.)

ingots, etc. (See Bars, bronze; Ingots, bronze, etc.)

Bronze bearing metal, chemical analysis of

Bronze castings, Government. (See Castings, bronze.)

Broom nails

Brush copper

Bucket wheols turbine--.-.

Buclets, chals, tur

covered.

dinner-

Casement adjusters

Casement fasteners

Comb, sheet-metal

file, steel-

transfer. (See Cases, file.)

Casing, metal. (See Trim, door.)

oil well, screw-thread gages for

450

neils threads for

Cast iron. (See Castings, iron, gray, and Castings, iron,

malleable.)

Casters, bed

Castings, admiralty metal. (See Castings, bronze.)

alloy-steel-_.......- treatment of

aluminum alloy

standard sample

aluminum bronze

Babbitt metal

bearing metal, bronze

brass.

chemical analysis of

trolley suspension.

industrial

sheet-metal work for

structural steel for

bronze. (See also Castings, aluminum-bronze; Castings,

manganese-bronze; Castings, phosphor-bronze.)

bronze bearing -..-.-. $1324,1325,1326$ standard sample............................. 1252

chromium-steel, for valves for acid-tank cars.........-.- 1134

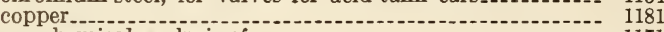
chemical analysis of

copper-nickel

die, aluminum-alloy

dies for, heat treatment of

zinc-base

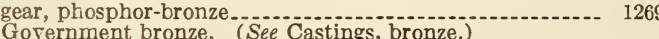

Government bronze. (See Castings, bronze.)

gun-metal. (See Castings, bronze.)

iron. (See also Pig iron.)

chemical analysis of

gray

method of test

malleable

ornamental, for build

magnesium-base alloy.

manganese-bronze

manganese-steel, austeniti

metal, radiographic testing of

astings, copper-nickel.

nickel-silver

ounce-metal. (See Castings, brass.)

phosphor-bronze

radiographic testing of

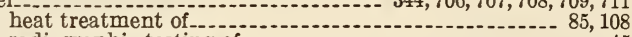
radiographic testing of

valve and high-temperature fitting ...............-. $\quad 709$

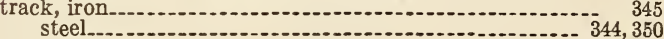

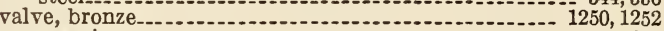

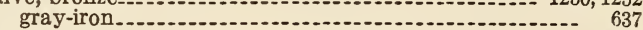

zinc
nuts

Castle nuts

Catches, door -...........-...-1043, 1055

elbow

sash

turn, for cupboard and locker doors.

Cathodes, copper (battery)

nickel - $-1-128$

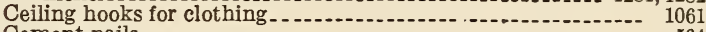

Cement nails

Center plates for railway cars-1--_-

Cesspool, plumbing - . .

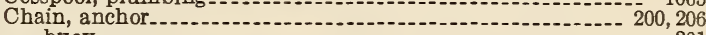

buoy

commercial grade-stel

commercial grade-1.

crane

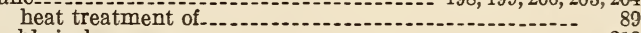

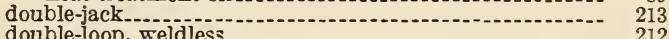

dredge (sizes)

flat-link. (See Chain, weldless.)

harness (sizes)

ladder

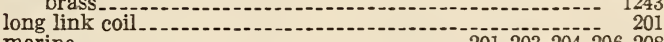

marine

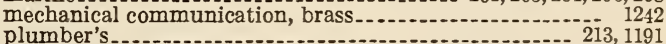

plumber's

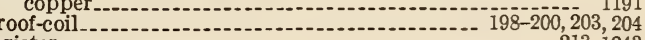

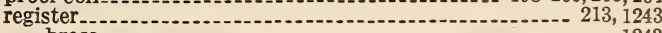

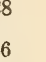


Page

Chain, safety, "aluminum.......................... 1164 ress.brass... sash sash attachments for ship. (See Chain, marine.)

silent

single-loop, weldless

sling

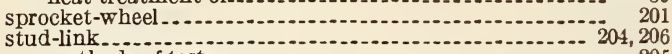

methods of test

submarine-net.

testing of

transom link coil

welded (sizes and varieties)

weldless.

brass - double-jack

double-loop pattern

flat-link

single-loop pattern

wire-link. (See Chain, weldless.)

Chain dogs.

Chain fittings...-

Chain plates, cast steel

Chain rings

Chain shackles --

method of test

Chain shackles and shackle pins

Chain swivels

Chain toggles

Channel nails (sizes)

Channels, structural. (See Shapes, structiral.)

Chasers, die-head (sizes) .

Check. valves

rubber facings for

Chemical analysis of alloy steels

Chemical analysis of aluminum and aluminum alloys.-

Chemical analysis of bearing metal (Babbitt)

Chemical analysis of brass castings

Chemical analysis of bronze

Chemical analysis of bronze bearing metal

Chemical analysis of chromium brick

Chemical analysis of chromium-nickel steel

Chemical analysis of chromium ores

Chemical analysis of chromium-vanadium steel

Chemical analysis of copper castings

Chemical analysis of copper nickel alloys

Chemical analysis of copper wire bars, billets, slabs, ingots, and ingot bars.

Chemical analysis of ferro-ailoys.

Chenical analysis of ferrochromium

Chemical analysis of ferromenganese

Chemical analysis of ferrosilicon -

Chemical analysis of ferrotungsten-

Chemical analysis of gun metal

Chemical analysis of iron (pig and cast iron)

Chemical anaiysis of lead in pig form

Chemical analysis of manganese bronze

Chemical analysis of metallic materials for electric heating

Chemical analysis of molybdenum steel

Chemical analysis of nickel

Chemical analysis of nickel-chromium alloy.-.

Chemical analysis of nickel steel..

Chemical analysis of refractory materials.

Chemical analysis of silicomanganese steels

Chemical analysis of silver solders.

Chemical analysis of solder (tin-lead)

Chemical analysis of spelter

Chemical analysis of steel, carbon

Chemical analysis of steel products, rolled and forged.

Chemical analysis of tungsten metal

Chemical anaiysis of tungsten steel

Chemical analysis of vanadium steel

Chemical analysis of zinc

Chest locks.

Chisel bar

Chisels:

backsmiths, (sizes)

heat treatment of --

track

woodworkers

Chlorine valves

Chrome. (See Chromium.)

Chromium metal, methods of sanipling.

Chromium ores

chemical analysis of

Chromium plating, testing of

Chromium piating on nonferrous metals (specification)

Chromium steel bars

sheets, plates.-.-

\section{C}

列

Chromium steel tank plates

Chromium steel tubing for acid tank cars

Chromium-molybdenum steel, standard sample standard sample

Chromium-tungsten-vanadium steel, standard sample

Chromium-vanadium steel, chemical analysis of ............... standard sample............................

Chromium-vanadium steel bars, sheets, plates

Chromium-vanadium steel bars for railway springs.

Circular saws

messenger dead end

trolley feed wire

$$
\text { vise... }
$$

Classification of materials and supplies for electric 1 ailways.

Classification of metals

Classification of railway metallic supplies

Classification of scrap metals (nonferrous)

Claw bars.

Clinch nails.

Clips, cable (electrical)

conduit (electrical)

fuse

spring rebound, automobile

Clocks tubing (electrical)

$$
\text { testing of.-. }
$$

Closers, door-

Cloth, Fourdrinier wire

wire, insect screen

Clout nails

Coal ho

Coat and hat hooks

Coating of metals

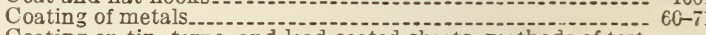

ing.............................

Coatings, zinc. (See Galvanizing.)

Cobalt

Cobbler's tacks (sizes)

Cocks, bali, for flushing tanks drain, automobile.

Code, safety, gas.

Coffee biggins

Coffee boilers

Coffee pots

Coin silver -

Collars, cylinder, door lock

stovepipe

Colors for sanitary ware

Comb cases, sheet metal-1._.
Compass saws

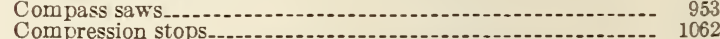

Condenser tube ferrules, brass...-

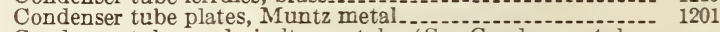

Condenser tubes, admiralty metal. (See Condenser tubes, brass.)

Mrass

Conductors, electrical. (See Wire.)

Conduit, flexible, ferrules for

steel, galvanizing of

Conduit clips (electrical)

Conduit terminal tubes

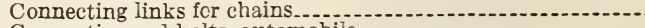

Connecting-rod bolts, automobile..............................

Containers, glass, gages for

Coolers, water, sheet metal.

Coopers' rivets, steel

Copper, battery, gravits

lead, tin and antimony alioys, chemical analysis of

manganese - . - of metaliographic

methods of test of, metaliographic-.-c-

phosphor

silicon.

tack and nail

Copper bars, plates, etc. (See Bars, copper; Plates, copper etc.

Copper bearing steel, for tacks and nails

Copper metal, standard sample.-.

Copper slating nails

Copper tacks (sizes)

Copper wire nails

Copper-clad nails, common

Copper-nickel alloys, chemical analysis of -....................

Copper-nickel-zinc rods, bars, etc. (See Rods, nickel silver; Bars, nickel silver; etc.)

Copper-tin-zine castings. (See Castings, bronze.)

Coppers, soldering

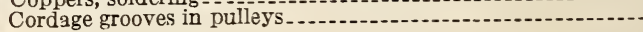


Page

Cords, bell, wire

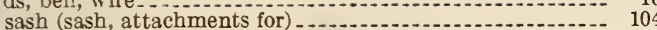
wire, sash and bell.

Corers, apple

Cot, hospita

$$
\text { metal - }
$$

Cotter-pin holes in bolt ends

Cotter pins.

Coupler gages, railway (type $\mathrm{D}$ coupler)

Coupler knuckle pivot pins, railway

Coupler yokes for railway cars

Couplers, drop-test machine for

$$
\begin{aligned}
& \text { railway (electric railway) } \\
& \text { type } \mathrm{D}- \\
& \text { type } \mathrm{E}
\end{aligned}
$$

Coupling, fire hose, screw thread gages for

Couplings, hose (fire hose)

$$
\text { screw threads for. }
$$

pipe. (See Fittings, pipe.)

Covers, ash can garbage can.manhole,

$$
\text { heat treatment of }
$$

167
1049

Crank pins, locomotive, steel forgings for

Cranks, oil well rig

$\begin{array}{lrr}\text { Cremone bolt locks for doors } & \\ \text { Cross arm braces } & & 656 \\ \end{array}$

Crosscut saws - (See Fittings, pipe.)

Crossings, railroad. manganese-steel

$$
\text { trolley }
$$

Crow bars

Cubes, nickel
Culvert pipe, cast-iron method of test of

Culverts, metal, corrugated

method of test of corrugated metal culverts

$336,339,341,348,349,1133$

Cupboard locks

Cupboard turn

Cups, metal

Curtain shower bath

Cuspidors. (See also Spittoons.) sheet metal

Cutlery, kitchen

Cut nails, copper.

iron, commoting -

finishing

flooring

shingle

Muntz metal

slating

steel, common

finishing.

flooring

shingle

slating -

Cutters, glass

keyway, Woodruff

milling, heat treatment of

pastry

pipe

Cylinder collar, door lock

\section{D}

Dating nails

Decimal gage, solid notch

Deck drain strainer (brass)

Deck drains, brass

cast-iron.

$\begin{array}{r}370 \\ -\quad 369 \\ \hline\end{array}$

488,490

Deck scupper------

Definitions of terms relating to magnetic testing

Definitions of terms relating to metallographic testing

Definitions of terms relating to methods of testing

Definitions of terms relating to specific gravity

Definitions of terms relating to wrought iron

Dental amalgam alloy

Dental amalgam alloys.

Dental gold alloys

Dental silver alloys

Derricks, oil-

Desk locks

Dials

Die blocks, plain carbon and alloy-steel, heat treatment of

Die castings, aluminum alloy

dies for, heat treatment of

$$
\text { zinc-base. }
$$

Die-head chasers (sizes)

Dies, blanking, heat treatment of

Dies for die castings, heat treatment of

Dinner buckets

Dishes. (See also Silverware.)
Disks, cartridge, brass $\quad$ Page

Dividers (tool)

Dogs, chain ...

ring

Door catches.

Door closers _._.

Door fasteners, with chain

Door frames, metal (sizes)

Door handles, entrance._._.

Door holders

Door hooks

Door knobs, metal

Door latehes . .

Door locks bathroom................ 1021

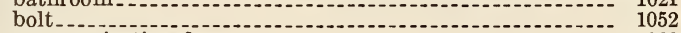

communicating door.

cylinder collar for

$\begin{array}{ll}\text { elevator } & \\ \text { entrance, cylinder } & \end{array}$

escutcheons and rosette for

escutcheons for

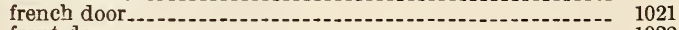

front door .

cylinder-1._- 1023

inside
key plates for -1036

knobs, handles, and spindles, for
office, cylinder...

roses for -1036

ship__._- 1004

vestibule, cylinder.

Door pulls

Door shock absorber...

Door stops

$\begin{array}{ll}\text { catches for } & \\ \text { cremone bolt locks for } & \end{array}$

espagnolette bolt locks for

exit bolt locks for.

flush bolt locks for

flush bolt locks for bolt locks for

$\begin{array}{lr}\text { foot bolt locks for } & 1058 \\ \text { Kalamein (sizes) } & \end{array}$

kick plates for

metal

metal-clad.-.

push bars for

push plates for

$\begin{array}{ll} & 1052\end{array}$

catches for
flush bolt locks for

pring bolt locks for _._._.

tin-clad. (See Doors, metal-clad.)

213

Double loop weldless chain._.

Dowel pins, metal._...

Down spout nozzles for roof drains

$\begin{array}{lll}\text { Drain and trap combined, plumbing } & 1065 \\ \text { Drain cocks, automobile } & \end{array}$

Drain pipe, cast-iron.

(See also Soil pipe, cast-iron.)

Drain plugs, crank case.

Drain strainer, deck (brass) 483

Drain traps . . . .

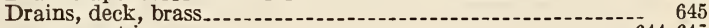

cast-iron

plumbing

roof down spout nozzles for

Draw knives .

Drawer locks.

Drawer pulls

Drift pins (sizes)

Drill pipe, oil well

threads for

$\begin{array}{lll}\text { Drill pipe thread gages } & 895 \\ \text { Drill sizes for various tap sizes. }\end{array}$

Drill sizes for various tap sizes._.

Drill tool screw thread gages

$\begin{array}{lll}\text { Drill tools (oil well), thread form for }-\ldots . . . & 362 \\ \text { Drills, breast }\end{array}$ hand..-.

Drivers, screw

Drop-test machine for railroad materials

Drop-test mache for railroad materials

Dry pipe valves, rubber facings for
Duralumin sheets, plates, etc. (See Sheets, aluminum alloy;

Duralumin sheets, plates, etc.
plates, aluminum alloy; etc.)
Dust guards for journal boxes.-

Dust pans

\section{$\mathbf{E}$}

$\begin{array}{ll}\text { Ears, trolley } & \\ \text { Eaves trough and conductor pipe (sizes) } & \end{array}$

Eaves trough and conductor pipe (sizes)
Egg poacher $\ldots$ catches
Elbow 1043,1055 


\section{SPECIFICATIONS FOR METALS AND METAL PRODUCTS}

Elbows. (See Fittings, pipe.)

Page

Electric heating, chemical analysis of materials for............. 1289 Electrician's staples

Electrodes, welding, iron steel...

Electrolytic nckel. (See Cathodes, nickel.)

Electrotypes chemical analysis of nickel chromium $155,156,157$ 1279 1296,1297 1296,1297

Elevator door lock

Enameled ironware

Enamelling ovens

Escutcheon pins

Escutcheons, doorlock

Espagnolette bolt locks for doors

Exit bolt locks for doors

Expansion tanks

Eyebolts

aeronautic

wire rope

Eyes, pad, ship

padlock

Fabric, fence. (See Fencing.)

Facings, rubber, for dry pipe valres and check ralves.......- 498

Fasteners, blind.

casement

door, with chain

sash.

scuttle.

window .....-.

window screen

Fence. (See also Fencing.)

Fence fabric, woren wire. (See Fencing, woven wire.)

Fence nails

Fence posts, metal

Fence staples, wire.

Fencing, barbed wire

chain-link

field.

hog and cattle (types and sizes)

poultry

railwa

wolf-proof, (types and sizes) woven wire

Ferro-alloys, chemical analysis of methods of sampling

Ferrochromium

chemical analysis of

methods of sampling

standard sample

Ferromanganese

chemical analysis of

methods of sampling

stanciard sample

Ferromolybdenum

Ferrophosphorus, standard sample

Ferrosilicon

chemical analysis of

methods of samplin

standard sample

Ferrotitanium

methods of sampling

Ferrotungsten, chemical analysis of standard sample

Ferrovanadium

chemical analysis of

standard sample

Ferrule stock, brass

Ferrules, condenser-tube, brass copper

flexible-conduit wire, aeronautic

Fetter drive screw

File cases, steel

Filters, water, pressur

Finishing nails

Finishing nails
Fire doors. (See Doors, fire.)

Fire hose coupling screw thread gages

Firebox steel. (See Plates, firebox, steel.)

Fireproofing building

carbonated water bottling equipment

chain.

fuel tubing, automobile

lubrication, automobile

pipe. (See also Nipples, pipe.)

(See also Unions, pipe.)

ammonia

brass.-

$435,1234,123$

$435,1235,1255$

bronze castings for

cast-iron

415,435 $191,192,193,195,196$ 1093 $0.102,1092$
$-1092,1093,1103$ $0.102,1092$
$-1092,1093,1103$

929
275
Fittings, pipe, gas, cast-iron high temperature, steel. iron. (See also Fittings, pipe, cast iron.
(See also Fittings, pipe, malleable i

malleable iron

malleable iron

sanitary

sewer,
sizes.

soil, cast-iron-

steel

steel castings for

union, combination

water, cast-iron

welding neck

plumbing

rope, wire............... 158, 161, 162, 191, 183

sprinkler pipe, cast-iron

- 407

brass and copper

Firtures, gas, tubing for

shower bath

Flanges, floor, water-closet-

pipe, automobile.

cast-iron_.................... iron.

steel

high temperature

$414,465,4=8$

welding neck

Flatters, blacksmiths, sizes

Floor flanges, water-closet

Floor hinges, checking

Flooring brads

Flooring nails

Flour boxes, metal

Four sieves

Flushing tank mechanism -

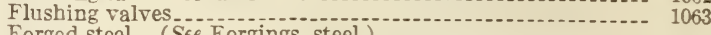

Forged steel. (See Forgings, steel.)

Forged steel products, methods of sampling
methods of sampling and chemical analysis...-............

Forges . blooms, billets, slabs, and bars for axle, allor-steel drum, steel iron locomotive, alloy steel. heat treatment of.

shaft, alloy steel

steel.

$346,750,752,753,754,75 ;, 759,765,771$

annealing of

bars, for

blooms, billets, and slabs for

proof testing of

Forks. (See Tableware.)

964,969

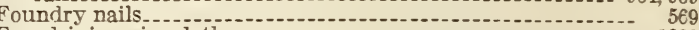

Fourdrinier wire cloth

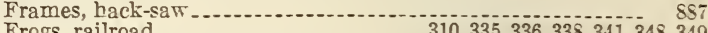

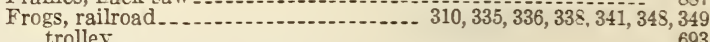

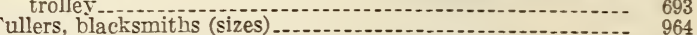

Fullers, blacksmiths (sizes)

Furnaces, hot air. (See Furnaces, warm air.)

house heating. (See also Boilers, house heating.)

warm air, for house heating

gas, for house heating

$\$ 61,866$

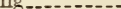

\section{G}

Gase, thickness, aluminum alloy sheet and plate

aluminum sheet and plate

brass sheet and plate

copper sheet and plate

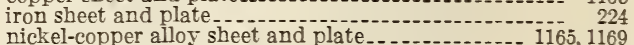

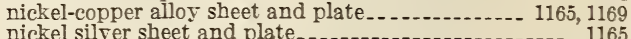

nickel silver sheet and plate

nonferrous plate, sheet, and

steel sheet and plate rire-

Gage blanks, plain.

screw thread.

Gage blocks, precision

Gages, barrel, oil well pum

brake beam, railway car

brake shoe, railway car

coupler, railway car

decimal, solid notch

glass container -

iron rod

journal bearing and wedge

journal box

journal length

\section{4} .

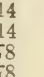
(2) . 
Gages, metal fit.................................... pipe thread

plain

round iron

screw thread

Acme-

coupling, fire hose

drill tool

drill tool

hose,

nut

oil well casing

oil well drill pipe

oil well tubing

pipe-pitch thread series

sucker-rod, oil well

taper, measurement of

tire, railway car wheel

truck bolster, railway car

truck side. railway car

wheel, railway car $652,773,774,775,777,779,780,785,786,787,791,792$

Galvanized tack

$$
652,7
$$

Galvanizing. (See also Coating.)

Galvanizing for rigid steel conduit

Galvanizing of bonding wire

Galvanizing on iron and steel
Galvanizing, specifications for testing of .

Garage heaters, gas

extra heavy ball bearing 1001

Garages, rail motor car

Garbage can

Garbage cans

Gas boilers for house heating

Gas fixture tubes.

Gas garage heaters

Gas heater.

Gas pipe, cast-iron

Gas piping

920,921

934

896

Gas, piping for

Gas ranges...

Gas shut-off valves

Gas stoves. hot plate.

Gas stores and appliances, method of test

Gas warm air furnaces for house heating.

Gas water heaters

Gas-fired steam and hot water radiator.

Gasoline cans.

Gasoline heaters, portable.

Gasoline hose ralves.

Gasoline stores.

Gate latches, secret

Gate valves.

Gears, alloy steel, oil-hardening, heat treatment of. carburizing and heat treatment of

electric rail and he

phosphor bronze castings for

spur, tooth form for.

German silver bars, rods, etc. (See bars, nickel-silver; rods,

nickel-silver; etc.).

Gimp tacks

Glass container gages

Glass cutters

Globe valves

Gouges, woodworkers

Government bronze castings. (See Castings, bronze.)

Grab hooks

Grading screens .

Graters, household.

Grooves in pulleys, manila rope.

Grooves in sheaves, wire rope.

Guards, dust, for journal boxes. hub (pole)

Gun cartridges, metallic (sizes and types)

Gun metal. (See Bronze.)

Gun metal castings. (See Castings, bronze.)

Gun shells, loaded

226,1227

, for ships

Gutter spikes

Guy clamps.

Guy hooks

Guy strand.

Guy wire rope

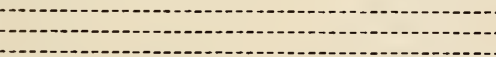

H

Hack-saw blades

Hack-saw frames

Hack saws

Hammers, blacksmiths (sizes)

pneumatic

(sizes)

sledge -

Page

Hand-saw sets.........

Hand saws .

Handhole frames and covers, cast-iron

entrance

Handrail brackets.

Hanger, messenger.

Hangers, pole, sash

trolley.- (See Heat treatment of steel.)

Hardies, blacksmiths (sizes)

Hardness testing, Rockwell

Shore.

Hardness testing of metallic materials (Brinell)

Hardware, builders' (nontemplate) (template)

Harness hooks

Hasps, hinge

Hat and coat hooks

Hatchets, shingling

Hawsers, wire rope fo

Header, rivet (sizes)

Heading tools, blacksmiths (sizes)

eat treatment, aluminum alloys of high strength

automotive carbon steels.

blanking dies and punches.

camshafts - objects

chain, sling and crane

chisels

definitions of terms

die blocks

dies for die castings

gears

(oil-hardening, alloy steel)

locomotive forgings

metals

milling cutter

piston pins.

rivet sets.

spline shrfts

spring steel, (carbon and alloy steel)

spring steel, (carbon and alloy steel)

steel castings, alloy steel
carbon steel

steel products, (annealing of roll

(Steel objects)_ 106

tool steel, (alloy steel)

tool steel (finishing steel)

nonshrinking, nondeforming, oil hardening tool steels).

(plain carbon)

tungsten high-speed steel.

tool steels

Heaters, gas

gasoline, portable

incubator, oil

kerosene, portable

oil -

oil, portable

water, coal burnin

gas

Heating elements, electrical

chemical analysis of

nickel-chromium.

906

955

1064

1298

945

Heel nails

rich speed steel,

High-speed tool steel bars

Highway bridges, steel.

Hinge hasps..... 1004

Hinge nalls

blind

bronze

cast-iron

cupboard

folding door

half-surface

hospital type

narrow

steel.

transom

cupboard.....-

floor, checking

garage, extra heavy ball bearing

alf mortise.

half surface.

scuttle

spring, butt type

floor surface.

lavatory door

strap

water-closet seat

$982,994,995,997,998,999,1000,1001$

water-closet seat.

Hods, coal

toy, coal 


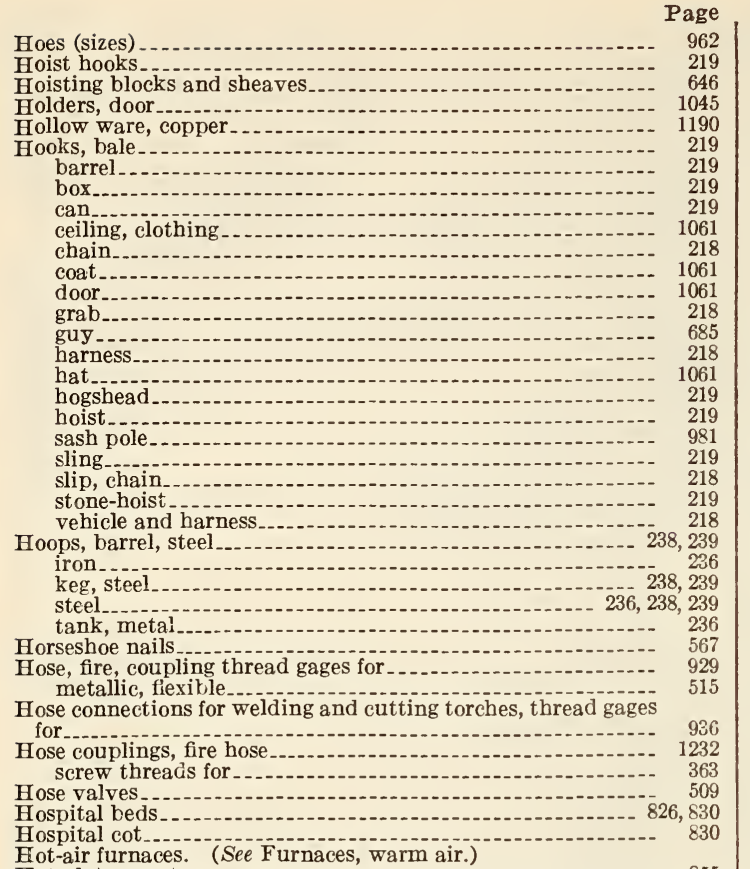

Eot-air furnaces. (See Furnaces, warm air.)

Hot-plate gas stove

Hot-water tanks
Hub guards (pole)

Hungarian nails. (See also Nails, hob.)

Hungarian nails (sizes)

Hyodrants

$853,866,1072,1073$

685

$497,505,1064$

\section{I}

Inclosure, bath, trimmings for

Inclosures, dressing room shower bath

toilet and dressing room

water-closet, trimmings for

Incubators, oil heaters for

Indicator post for concealed valves

Industrial buildings

Ingots, aluminum.

for steel manufacture

aluminum alloy ...

aluminum

antimony .....

bearing metal, Babbitt

bronze

brass. (See also Ingots, solder, copper-zinc.)

brass

chemical analysis of -
bronze. (See also Ingots, aluminum bronze; Ingots, manganese bronze.)

bronze bearing.

$1196,1248,1325,1326$

copper -

chemical analysis of

copper-nickel

lead

manganese-bronze.

nickel

phosphor copper

phosphor tin.

silicon copper.

solder, brazing

copper zinc.

soft.

spelter

zinc.-

Insect screen wire cloth

Insulator pins

messenger

Iron, cast. (See Castings, iron.)

engine bolt. (See Bars, engine, bolt, iron.)

galvanizing or sherardizing

magnetic testing of

metallographic testing of -

pig, charcoal. (See Iron, pig, foundry.)

chemical analysis of -

coke. (See Iron, pig, foundry.)

foundry

crap, classification of

sherardizing of

wrought, definitions of terms relating to
Iron castings. (See Castings, iron, gray, and Castings, iron,

malleable.)

Iron forgings. (See Forgings, iron.)

Iron ores, standard samples.

Iron products test specimens for

Iron wire. (See Wire, iron.)

Irons, rig, oil well .........

sash cord

soldering

1049

Ironware, enameled.......................

Jacks.

970,972

screw (sizes) -

Jam nuts

Japanned ware (sizes)

Joint bars, railro

Joint plates, railroa

Joints, slip, brass (pipe) copper (pipe)

Joists, open-web, steel

Journal bearing wedges

Journal bearings, railway

Journal bearings and wedges, cages for

Journal box gages

Journal box lids

Journal boxes

dust guards for

locomotive

packing tools for

Journal length gage

$317,318,326,328,329,330,331,332,333$ $317,318,326,328,329,330,331,332,333$

\section{K}

Kalamein doors (sizes)

Karats. (See Carats.)

Keg hoops, steel

Kerosene heaters, portable

Kerosene stoves

Kettles, milk

wash -

Key plates, door lock

Key slots. (See Key ways.)

Key way cutters, Woodruff

ey ways, shaft

Woodruff

Keyhole saws

Keys, propeller, marine

shaft, iWoodruff.

Woodruff----

Kick plates, door - Kitchen sinks, cast-iron
Kate

Knives. (See also Tableware.)

Knives, draw

kitchen

putty

scraping

shoe

table

Knob pulls

Knobs, door, metal

Laboratory oven, constant temperature - . - -

Lace tacks (sizes)

Ladder chain, brass

Ladles, plumbers'

sheet metal.

Lag bolts, packing of ..

Lamp frames, kitchen, metal.-.... 818

Latches, door

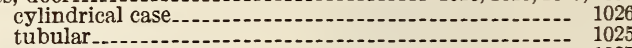
unit type

gate, secret

thumb

(1) 1035

Laundry stoves

Laundry tables

Laundry tray plug

Lavatories, cast-iron

colors for

Lavatory door spring hinges

Lavatory plug

Lavatory supplies

Lavatory traps, brass

968

Lead, methods of test of, metallographic scrap, classification of

standard sample

tin, antimony, and copper alloys, chemical analysis of .... 1317

Lead bars, sheets, etc. (See Bars, lead; Sheets, lead; etc.)

Lead sheets, pipe, etc. (See Sheets, lead; Pipe, lead; etc.)

8

(6)

.

,


Lead-coated sheets, method of testing coating................... 64

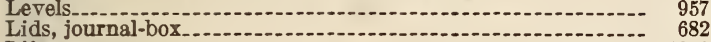

Lifters, transom

Lifts, sash

window screen

Lights, fixed, for ships

Line shafts. (')rass) Shafts, line.)

$\overline{1041}, 1048$

1048

1086

Line-carrying guns for ships.

Lining bars

1207,1208

Lining tacks, solid head, sizes

Links, chain (connecting) repair or end lap

Links and pad eyes, ship.

Lock washers

Lor

steel portable, for use in conjunction with sterilizers -.-.-8 840

Locks, bolt-.-..-10 espagnolette, door -...-..-...-. 1057 exit, door

flush, door-..

$$
\text { ship door. }
$$

foot, door

lavatory door

ship doo

spring, ship door

cabinet

chest................. foors

cupboard.

desk--1See also Fasteners, door.)

door.-.-. 1004, 1020, 1021, 1022, 1023, 1025, 1026, 1027, 1052, 1056

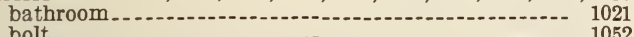
bolt.

communicating

cylinder collar for

cylindrical case.-.

elevator

entrance, cylinder

escutcheons and rosette for

escutcheons for

French

ront cylinder

inside

key plates for-----.-.-.-.- 1036

knobs, handles, and spindles for ........................ 1018

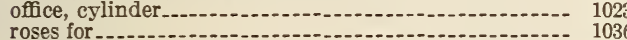

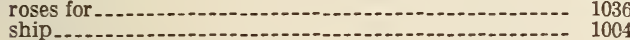

tubular

unit type...

vestibule, cylinder

drawer

padlock ...

sash. (See Fasteners, sash.)

scuttle. (See Fasteners, scuttle.)

transom. (See Fasteners, transom.)

wardrobe.

Locomotive wheels. (See Car wheels, railway.)

Loops, wire, aeronautic

Lunch boxes, metal. -

M

Machine bolts. (See Bolts.)

Machine screw threads

Machine screws

Machinery, structural steel for

Magnesium-base alloy castings

Magnetic testing (definitions of terms)

Magnetic testing of iron and steel

Magnets, alloy steel bars for.

Malleable iron. (See Castings, iron, malleable.)

Mallets

Manganese bronze, chemical analysis of standard sample

Manganese bronze bars, rods, etc. (See Rods, manganese

bronze; Bars, manganese bronze; etc.)

Manganese copper-- methods of sampling. standard sample

Manganese ore

$$
\text { standard sample }
$$

Manganese-steel castings, austenitic

Manganese-steel railway crossings

Manganese steel, standard sample

Manhole covers, sewer

Manhole frames and covers, cast-iron

Manholes, semer

Marking pots

Match safes, meta

Mates, railroad.

Mats, copper

Mattocks (sizes)

Mattresses (sizes)

Mauls

1191

\section{.}

Measures, dry, metal.

liquid.

metal

Mercury

Messenger dead end clamp

Messenger hanger

Messenger wire rope

Messenger wire rope-..-..-

Metallographic testing, definition of terms

iron and steel, methods of ........ micrographs..

nonferrous metals and alloys

Metals, classification of

coating of

general specification for

heat treatment of

methods of test of -

Micrographs of metals and

Milk kettles

Milk strainers

Milling cutters, heat treatment of

Miter boxes

Mixing valves for shower baths

Molding nails

Moldings, aluminum

aluminum alloy

Molds, cooking, metal

Molybdenum steel, chemical analysis of

Molybdenum steel bars, sheets, plates

Monel metal bars, rods, etc. (See Bars, copper-nickel; Rods, copper-nickel; etc.)

Mooring bitts, cast-iron cast steel.

Mortar and pestle for analysis of ferro-alloys

Motor axles, electric railway

Mounting of railway car wheels.

Mowers, lawn

Muntz metal for tacks and nails

Muntz metal plates, sheets, etc. (See Plates, brass; Sheets, brass; etc.)

$\mathbf{N}$

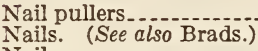

Nails

barbed.

barrel-1.

boat__-

brass $-1-2-564,565$

brass wire

broom

car

casing

cement

channel (sizes)

clinch

cobbler's

common

copper.

slating

storm (rivets)

wire

copper-clad, common

slating --.

iron, common

flooring

tooring

shingling.

Muntz metal

slating

small (sizes)

steel, common

finishing

flooring

shingle

slating.

zinc - slating

dating

-

double-head wire

fence.

finishing

flooring-

foundry -100

hinge

hob.

hob

Hungarian. (See also Nails, hob.)

lath sizes
819

820
(279

247

$-51$


Nails, molding

Page

plaster-board

purlin

roofing

saddle

saddlery.

sheathing

shingle

shoe (cobbiers)

siding.

slating.

stem

storm, copper (rivets)

testing of -

trunk (sizes)

upholsterer's

zinc

Name plates, zinc

Net, submarine, chain for

Netting, fence. (See Fencing, poultry.)

Nickel, chemical analysis of

Nickel bars, ingots, etc. (See Bars, nickel; Ingots, nickel; etc.)

Nickel brass. (See also Copper-nickel alloy.)

Nickel bronze castings.

Nickel chromium alloy, chemical analysis of

Nickel chromium shapes, ribbon, etc. (See Shapes, nickel chromium; Ribbon, nickel chromium; etc.)

Nickel plating

Shapes, nickel

Nickel silver castings

Nickel silver rods, bars, etc. (See Rods, nickel silver; bars, nickel silver; etc.)

Nickel steel. (See also Chromium-nickel steel.)

Nickel steel, chemical standard sample.

Nickel steel bars, sheets plates.

Nickel chromium steel, bars, sheets, plates...................- 1124

Nickel copper bars, rods, etc. (See Bars, copper nickel; Rods, copper nickel; etc.)

Nickel copper-zinc rods, bars, etc. (See Rods, nickel silver;

Bars, nickel silver; etc.)

Nipples, pipe, brass....

$$
\text { iron. }
$$

Nonferrous scrap, classification of

Normalizing steels. (See Heat treatment of steel.)

Nozzles, down spout, roof drain.

Nut slots, castle, gaging of

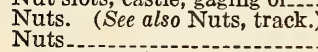
aeronautic castle.

high.

jam.

propeller, aeronautic.

screw-thread gages for

screw threads for

tube fitting

$522,586,588,603,606-609,611,683$

Oil burners, domestic

Oil-burning furnaces, house heating

Oil cans

hand.-

$588,607,608$

Oil heaters, incubator

portable

Oil pumps, Baltimore

Oil stoves

Oil-well casing thread gages

Oil-well drill tool thread and

Oil-well pipe. (See Pipe, drill; pipe, oil.)

Oil-well pumps. (See Pumps, oil-well.)

Oil-well sucker-rod gages

Oil-well tubing. (See Tubing, oil-well.)

Oilers, brass

Operators, sash

scuttle.

skylight

Ores, chromium

chemical analysis of

iron, standard samples.

manganese.

$$
\text { standard sample. }
$$

$$
\text { zinc, standard sample }
$$

Ornamental iron castings for building

Ounce metal castings, etc. (See Castings, brass, etc.)

Outdoor substations, electrical

Ovens, constant temperature, laboratory enamelling and japanning

Over-support rods, trolley.-.

\section{$\mathbf{P}$}

Packing tools for journal boxes..

Pad eyes and links, ship

Padlock eyes

Padlocks. (See Buckets.)
Pans, cooking and kitchen, metal

817,818

dust dust -

refrigerator

Pastry cutters

Pedestals, railway car truck

Phosphor bronze bars, castings, etc. (See Bars, phosphor

bronze; Castings, phosphor bronze; etc.)

Phosphor bronze bearing metal, standard sample

Phosphor bronze wire rope. (See Rope, wire, phosphor bronze.)

Phosphor copper

Phosphor tin ingots. (See Ingots, phosphor tin.)

Picks.

Pie plates, metal

Pig iron, charcoal. (See Pig iron, foundry.)

chemical analysis of
coke. (See Pig iron, foundry.)
foundry

foundry

Pig lead

Pig nickel

Pig tin.....

Pincers

Pinch bars .

Pinions, electric railway_.........

Pins, cotter

holes for, in bolt ends coupler knuckle pivot, railway dowel, metal drift (sizes)

escutcheon.

flat-head, aeronautic

insulator

piston, carburizing and heat treatment of

shackle, chain. (See also Shackles and shackle pins.)

taper

Pipe, brass.

cast-iron

$70,371,182,1216,1217,1238$

copper

testing of

culvert, cast-iron testing of metal, corrugated
rain, cast-iron. (See also Pipe, soil, cast-iron.)

drill, oil well

screw thread gages for.

eave conductor (sizes)

gas, cast-iron

high temperature, steel.

iron. (See also Pipe, cast-iron.)

(See also Pipe, culvert, metal, corrugated.)

(See also Pipe, sheet iron and steel.)

Pipe, iron ............ 365, 431, 433, 444, 457, 483

oil country.............

(sizes) ......

lead testing of

lead, iron

steel

ewer, cast-iron.-

sheet iron and steel.

signal, iron

steel.

soil, cast-iron

(See also Pipe, sheet iron and steel)

Pipe, steel._...... 365, 436, 444, 457, 463, 476, 479, 482, 483, 487 oil country..... 444,457 (sizes)

testing of.

water, cast-iron

Pipe fittings. (See also Fittings, pipe.) 882

Pipe flanges, steel, high temperature

Pipe flanges and flanged fittings, cast-iron.- $385,395,401,402,413,414$ iron steel

Pipe markings._.

Pipe nipples, brass iron steel

Pipe slip joints, brass._.

Pipe thread gage blanks.

Pipe thread gages...........

Pipe threads. (See Threads, screw, pipe.)

Pipe unions .

Pipe unions and combination union fittings.
Pipe valves. (See Valves, pipe.)

identification of 361,364

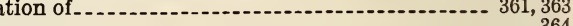
power

Piston pins, carburizing and heat treatment of 
Page

Piston rods, locomotive, steel forgings for Pitchers. (See also Silverware.)

Pivots, sash

Plaster-board nails

Plates, alloy steel

aluminum, thickness gage for

weights of

thickness gage for

weights of

bench.

boiler, steel

$$
\text { zinc. }
$$

bone, alloy steel

screws for

rass

thickness gage for

weights of

bread, metal

bronze. (See also Plates, manganese Bronze; plates, non-

ferrous.

center, railway car

chain, cast steel

chromium steel

chromium-vanadium steel

condenser tube, Muntz metal

copper

method of test

thickness gage for

weights of

copper-nickel alloy

firebox, copper steel.

flange quality, iron

(forge welding), steel

German silver. (See Plates, nickel silver.)

hot, gas stove.

iron.

thickness gage for

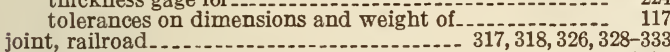

key, door lock

kick, door

manganese bronze

molybdenum stee

Monel metal. (See Plates, copper-nickel.)

name, zinc

nicke

ckel silver

thickness gage for

weights

nickel steel

nickel-chromium steel nickel-copper alloy, thickness gage for
weights of

nonferrous, thickness gage for

weights of

pie, metal

printing-

push, doo

sash pull silicon steel

98
steel_ $227,229-233,235,239,249-252,254,266-270,272,1124,1126,1127$

galvanizing of

tolerances on dimensions and weight of

$\overline{11} \overline{1} \overline{2}, \overline{1} \overline{17} 6,1177$

1172

116

1282,1285

$227,230,233$

$229,230,233,254$

$229,230,272$ tolerances on dimensions and weight of

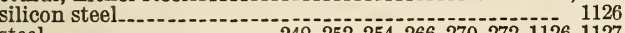

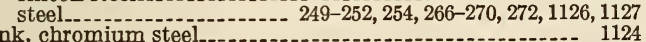

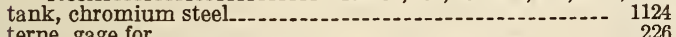

terne, gage for method of testing coating

$$
\text { (sizes) }
$$

tie, railroad

zinc

thickness gage for -

$328-330,333,334$

Plating, chromium, on nonferrous metals (specification) -..... 1169

nickel, for plumbing fixtures

on brass, methods of test of

on nonferrous metals (specification)

zinc. (See Galvanizing.)

Plating on metals. (See also Coating on metals.)

Pliers and plier cutters

Plugs, drain, crank-case

laundry tray

lavatory

pipe. (See Fittings, pipe.)

lumb bobs

Plumber's chain

$$
\text { copper }
$$

1165

1166

1124

166,1169

1165

817

1279

1040

1126

copper-----

Plumbing, recommended minimum requirements for.-....... 113

Plumbing cesspool

Plumbing drain and trap combined.
Page

Plumbing drains...........................

Plumbing fixtures, nickel plating of

Plumbing symbols ................................. 113-115

Plumbing trimmings and fittings................................. 1062

Pneumatic tools

Poacher, egg--

Pocketknives.

Pole bands...-..-

Pole hooks, sash

Pole steps

Posts, fence, metal

indicator, for concealed valves

Pots, coffee

marking

soldering

tea....

watering

Powder cans

Pressure regulators for gas cylinders

Printing plates

1279

Proof coil chain

Proof coil chain

Propeller keys, marine

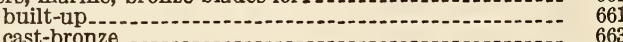
cast-bronze...........-. 663

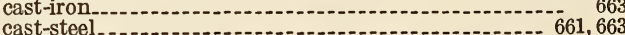

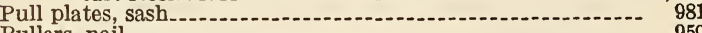

Pullers, nail

spike-
Pulleys. (See also sheaves.)

manila rope grooves in.

railway car lighting generator-

Pullover, trolley

Pulls, door

drawer

knob.

Pumps, oil, Baltimore

oil well, gages for barrel of

working barrels for

Punches, backing out, (sizes)

blacksmiths, (sizes)

blanking die, heat treatment of

tie plug

track

Purlin nails

Push bars, door

Push plates, door

Putty knives.

\section{$\mathbf{R}$}

Radiator requirements for buildings

Radiator requirements for houses

Radiator valves

Radiators, house heating

steam and hot water, gas-fired

Radiographic testing of metal castings

Rail forks

Rail splice bars. (See Bars, splice, railroad.)

Railroad tie plates. (See Tie plates, railroad.)

Railroad track tools.

Rails, girder, railroad

railroad.

Railway axles. (See Axles, railway.)

Railway car wheels. (See Wheels, railway car.)

Railway cars, structural steel for $-. .-\ldots-1 .-. .$.
Railway crossings. (See Crossings, railway.)

Railway metallic supplies, classification of

Railway springs. (See Springs, railway.)

Railway track material

Range boilers

Ranges, gas -

Rasps (sizes) - .

Razor blades, packaging of

Reamers.

Refractory materials, chemical analysis of

Refrigerator pans

Refrigerators, sheet metal

Register chain $\ldots+\ldots \ldots \ldots$
brass

Regulators, pressure, gas cylinder

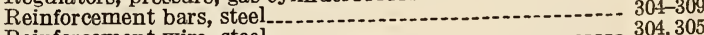

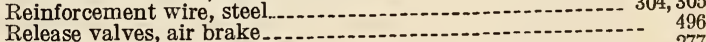

Release valves, air brake

Rests, shelf

1060

Return bends, pipe. (See Fittings, pipe.)

1296,1297

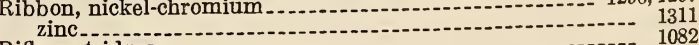

Rifle cartridges.-.-. 
Rig irons, oil well

Rigging screws for ships

Ring dogs.

Rings, chain

Rivet bars, chromium steel

steel. (See Bars, rivet, steel.)

Rivet header (sizes) -

Rivet sets, heat treatment of... riveting hammer. (sizes)

Rivet steel. (See Bars, rivet.)

Riveted joints, bibliography on

Riveters, pneumatic

Rivets, aluminum alloy

aluminum type.

belt, steel.

boiler

chromium steel, for nitric acid tank cars.

coopers', steel.

(copper storm nails)

iron

railway car, steel.

ship construction.

small, steel

split

steel bars for

(1)

steel bars for

tap

tinners, steel

tubular

Rods, aluminum. (See aıso Rods, welding, aluminum.)

aluminum alloy. (See also Rods, welding, aluminum alloy.)

aluminum alloy

aluminum bronze

anchor

brass. (See also Rods, welding, brass.)

bross... (See also Rods, aluminum bronze; rods, mangan-

cast iron. (See also Rods, welding, iron.)

method of test

copper-nickel ailoy

(Serman silver. (See Rods, nickel silver.)

$$
\text { gages for }
$$

locomotive, steel forgings for-ar

manganese bronze. (See also Rods, welding, manganese

bronze.

manganese bronze

Monel metal. (See Rods, copper-nickel.)

naval brass. (See Rods, brass.)

nickel silver

oversupport, trolley

piston, locomotive, steel forgings for

reinforcement. (See Bars, reinforcement.)

steel. (See also Bars, steel.)

(See also Rods, welding, steel.)

sucker, oil well, gages for.

tie, track

trolley oversupport

welding. (See also Wire, welding.)

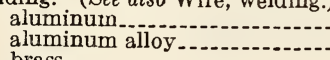

brass -

copper-nickel

gas, iron-

steel

iron-..--.--.-.-.

steel.

Roller bearings

$1172,1179,1180$

Page

769
626

218

222

96

98
939

246

939
1160

1160

614

1123

614

612

141

$\begin{array}{r}612 \\ 1240 \\ \hline\end{array}$

44,249

522,615

614
1240

1150

1146

6

\section{Sast}

Sash cord irons

Sash fasteners

Sash lifts.

Sash lock.

Sash operators

Sash pole hangers

Sash pole hooks

Sash pull plates

Sash pulleys

Saw blades, hack

Saw frames, hack

(1) 885,887

Saw sets, hand saw

Saws

band.-- 952

butcher

circular

crosscut.-.

hack

hack

hand keyhole_-a-

web

Scissors, office
Scoops

grocers, spice and thumb._. 817

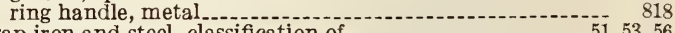

Scrap iron and steel, classification of

Scrap metals, nonferrous, classification of

Scraping knives.-10

Screen lifts, window screen

Screen wire cloth

Screens, grading

testing, brass

Screw jacks_._.

Screw stock, steel bars for

Screw thread gage blanks....... 906

- 915

Screw thread gages.... 920,921

acme

hose couplings (fire hose)

pipe

12-pitch thread series

Screw threads. (See also Threads, screw.)

acme

machine screw.

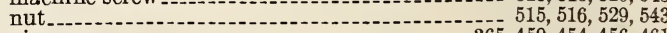

pipe

gages for

Screw drivers.-1eel, high temperature._.

bone plate, alloy steel._.

cap

Fetter drive-ar

lag packing of

Rope grooves in sheaves (wire rope)

Rope socket. (See Sockets, ropes) . 049

57

S

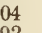
. 119 092 816 (1)

.


INDEX

Page

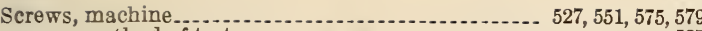
method of test screw threads for. rigging, ship set. wood

brass

Scribers

Scullery sink, steel

Scupper, deck............

Scuttle íasteners

Scuttle hinges....

Scythes

Secret gate latches

Servers. (See also Tableware.)

Set screws

ets, rivet, heat treatment of

saw, hand saw.

Sewer manholes and covers

Sewer pipe, cast-iron

Shackles, aeronautic

anchor.

chain

method of test

wire rope

Shades, electric light, metal

Shaft keys. (See Keys, shaft; Woodruff keys; pins; etc.)

Shafts, alloy steel forgings for................................

cam,

oil-well rig

$761,771,772$

spline, heat treatment of

steel bars for

steel forgings for

transmission. (See Shafts, line.)

Shapes, aluminum alloy

bronze. (See also Shapes, aluminum bronze; Shapes,

manganese bronze; Shapes, phosphor bronze.)

copper

method of test

$752,759,765,771,1136$

method of test

German silver. (See Shapes, nickel silver.)

iron.

manganese bronze

Monel metal. (See Shapes, copper-nickel.)

nickel chromium

nickel silver

nickel steel

open-web joists, stee

phosphor bronze.

ship, iron

silicon steel

steel. galvanizing of

structural, aluminum alloy copper nickel steel silicon steel

haves, spoke

Shear blades, heat treatment of

Shears, grass and hedge cutting

$$
\text { office. }
$$

Sheathing nails

Sheares. (See also Pulleys.)

Sheaves and hoisting blocks

Sheares, wire rope grooves in

Sheet iron and steel pipe

1146,1152

Sheets.

$$
\text { aluminum }
$$

$$
\text { thickness gage for }
$$
weights of -

aluminum alloy... thickness gage for

aluminum bronze

aluminum-manganese alloy

$249-252,254,266-270,346,1126,1127$

1179,1180

1296,1297

1286,1288

253

1269
234

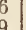

standard sample

thickness gage for weights of

bronze. (See also Sheets, aluminum bronze.)

chromium steel

chromium-vanadium steel

copper

method of test.

thickness gage for

weights of

copper-nickel alloy

duralumin. (See Sheets, aluminum alloy.)

galvanized, gage for

roofing (sizes)

steel

testing of.
Sheets, German silver. (See Sheets, nickel silver.)

iron.

gage for

testing of

tolerances on dimensions and weight of

lead

thickness gage for

weights of .....

molybdenum steel

Monel metal. (See sheets, copper-nickel.)

nickel.

nickel-chromium steel

nickel-copper alloy, thickness gage for

$$
\text { weights of. }
$$

nickel silver

thickness gage for

weights of.

nickel steel

nonferrous, thickness gage for

roofing. (See also Sheets, galvanized.)

iron (sizes)

roofing tin....

roofing tin...
solder, silver

steel.

gage for

sizes

testing of

tolerances on dimensions and weight

terneplate

method of test for

tin coated

method of test for

tin plate, gage for

zinc

thickness gage for

zinc-coated. (See Sheets, galvanized.)

Shelf brackets

Shelf rests

Shelf supports

Shells, shot, paper, loaded

shotgun

Sherardizing. (See Galvanizing.)

Ship chain. (See Chain, marine.)

Ships, structural steel for

Shock absorber, door

Shoe knives

Shoe nails (cobblers)

Shoe tacks

Shoes, bed, wooden

brake, railway

Shops, repair, rail motor cars

for steel manufacture

copper-nickel alloy.

nickel

Shot shells, paper, loaded.-

Shotgun shell

Shovels

Shower bath curtains

Shower bath fixtures.

Shower bath inclosures

Shower baths, mixing valves for

Shutters, fire

metal clad

Sickles

Siding nails

Sieve testing apparatus

Sieves, brass.

champion

flour-

41, 243, 244

224

242, 244

1277

1169

1170

1124

$\overline{11} \overline{6}, 1169$

1166,1169

1286,1287

1165

1166

1124

1165

246

246

$236,239,241,243,244,246$

236, 246

242,244

117

241

242

64,242

226
, 1314

1169

1060

1060

1060

108

1081

568

testing

Signals, railway, steel pipe fittings for

steel pipe for
Silent chains

Silicomanganese steel bars for springs

Silicomanganese steels, chemical analysis of

methods of sampling

Silicon, refined, standard sample...

Silicon copper steel bars, shapes, plates.....

Silicon steel bars, shapes, plates.--manganese. (See Silicomanganese.)

Sill, center, railway car.- 261

Silver, coin 261

German (bars, rods, etc.) (See Bars, nickel-silver; Rods, nickel-silver; etc.)

nickel (castings)

sterling

Silver alloys, dental.

Silver solders

chemical analysis of

Silverware analysis of.-_...

ship.

Single jack chain

Single loop chain

Sink plug traps, brass. 


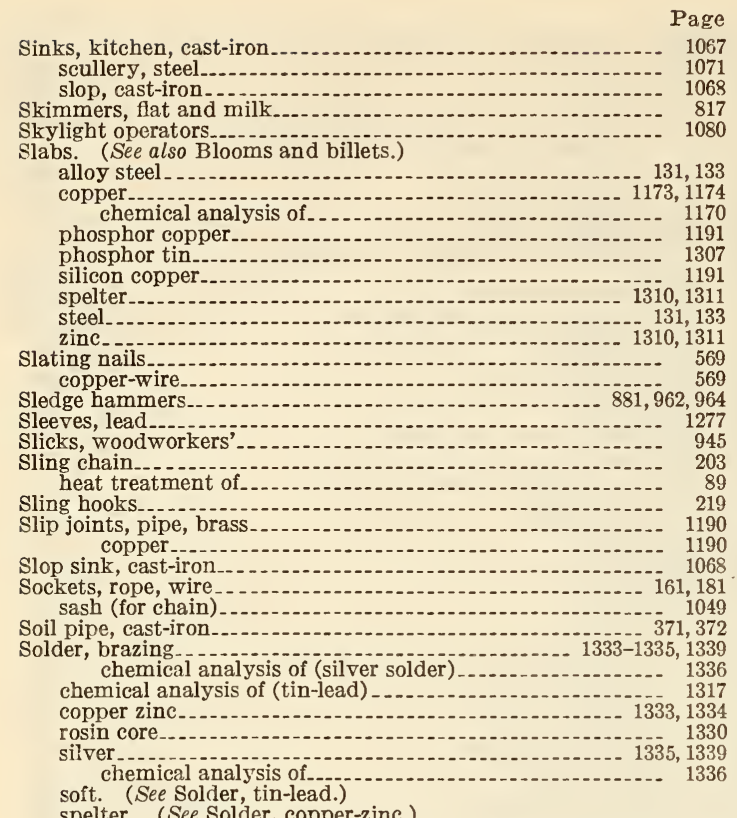

soft. (See Solder, tin-lead.)
spelter. (See Solder, copper-zinc.)

chemical analysis of wire

Solder for aluminum

Soldering coppers

Soldering irons

Soldering pots

Spacer links, trolley

Spades.

Specific gravity, definitions of terms relating to

Spelter, chemical analysis of

Spelter solder. (See Solder, copper-zinc.)

Spiegeleisen

standard sample

Spike maul, track

Spike pullers

Spikes, barge

cut iron or steel, common

gutter

gutter

wire, round

Spindles, door-lock

Spittoons. (See also Cuspidors.)

Spittoons, sheet metal

Splice bars, railroad

Spline shafts, heat treatment of

Spoke shaves

Spoons. (See Tableware.)

Spout nozzles for roof drains

Spouting, eave (sizes)

Spring caps, railway-car

Spring center bolts for automobile springs.

Spring hinges, butt type

Floor surface.

Spring rebound clips, space-.-...-..-...

biles_......... 810

Spring steel, alloy and carbon, heat treatment of

Springs, automobile.

bed.

general purpose, steel bars for

$826,828-831,833$

helical, for railroad frogs and switches.-1

phosphor-bronze wire for

railway.

carbon-steel bars for

chromium-vanadium steel bars for

$347,351,794,801,803,805$

silicomesteel bars for

silicomanganese steel bars for

track

vehicle, steel bars for

Sprinkler pipe fittings, cast-iron

Sprocket wheel chain.

Squares

Stainless steel, standard sample

Stakes, bench

Stamping act, national

Stands, toilet, sheet metal

Staples

electricians

metal-lath

$560,570,571,573$
Staples, poultry-netting

wire-fence...........

Stay bolts, copper bars for

flexible (sizes) . . -

iron bars for .............. 136, 137, 139

sizes

steel bars for

steel tubing for

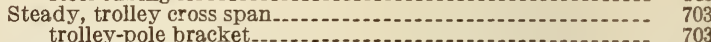

Steamers, sheet metal (tinware)

Steel, alloy, chemical analysis of .............................. 1107 tool, heat treatment of

automotive, carburizing and heat treatment of.-.......-.- 83,109

inspection and sampling............................... 49

bar steel. (See Bars, steel.)

boiler. (See Plates, boiler, steel.)

carbon, chemical analysis of.

$\mathrm{SAE}$ series, heat treatment of

chromium plating on

chromium-molybdenum, standard sample

chromium-nickel, chemical analysis of

chromium-nickel, standard sample

chromium-tungsten-vanadium, standard sample.-.-.-.-.-. 1122

chromium-vanadium, chemical analysis of .................-. 1111

chromium-vanadium, standard sample................. 1122

copper bearing, tack and nail

finishing, heat treatment of

firebox. (See Plates, firebox, steel.)

magnetic testing of -

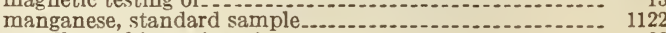

metallographic testing of

molybdenum, chemical analysis of

standard sample

nickel plating on (specification)

rivet. (See Bars, rivet, steel.)

sampling of

scrap, classification of

screw stock. (See Bars, screw stock, steel.)

sheradizing of

silicomanganese, chemical analysis of

spring. (See also Bars, spring, steel.)

heat treatment of.

stainless, standard sample

standard samples of . .

stay bolt. (See Bars, stay bolt.)

structural, bridge............................... 249-251, 273 building -

galvanizing of-

machinery

railway car

(1) 250, 268-270

thermal analysis of

tool. (See Bars, tool, steel.)

tool, finishing, heat treatment of

heat treatment of
high-speed, heat treatment of

nonshrinking, nondeforming, oil hardening, heat treatment of

plain carbon, heat treatment of -

tungsten, chemical analysis of

high-speed, heat treatment of

ranadium, chemical analysis of -...-......... 111

Steel blooms, billets, and slabs, etc. (See Blooms, steel;

Billets, steel; Slabs, steel; etc.)

Steel castings. (See Castings, steel.)

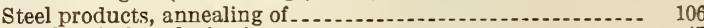

chemical analysis of

sampling of $-1-----1$

test specimens for

Steel scrap, classification of

Steel wire. (See Wire, steel.)

Stem nails

Step bolt

Steps, pole-...-.

Sterling silver

Stone-hoist hoo

Stools, metal-_-ars

tops, compression $\quad$ door

Storage cabinets. (See Cabinets.)

Storm nails, copper (rivets)

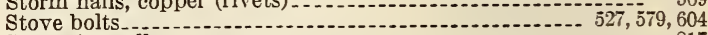

Stove bolts collars.

Stoves, gas

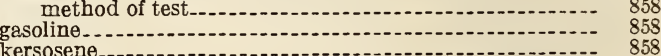

kersosene-10.- 858

laundry

Strainers, deck drain (brass)

gravy

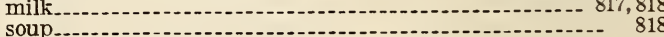

tea

Strand. (See Rope, wire.)

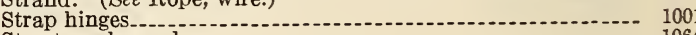

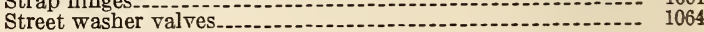


Strips. (See also Sheets.)

aluminum.

aluminum alloy.

aluminum bronze

automotive, steel

brass.

bronze. (See also

phor bronze.)

cartridge, brass

copper

method of test

copper-nickel alloy

German silver. (See Strips, nickel silver.)

lead.

Monel metal. (See Strips, copper-nickel.)

nickel-silver -.-.-.-.-.---.-.-.

nonferrous, thick

weights of -.-

phosphor bronze.

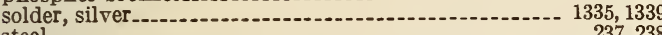

steel

1311,1314

zinc

thickness gage for

1169

weights of -

$\overline{11} \overline{2} 6,1127$

Structural nickel steel bars, shapes, plates

Structural silicon steel bars, shares, plates

Shapes, structural; etc.)

Shapes, structural; etc.)
Structural steel for bridges.

Structural steel for bridges.---
Structural steel for buildings.

$249,250,251,273$

Structural steel for machinery

$250,252,254$

Structural steel for railway cars and locomotives....- 250, 254, 266, 267

Structural steel for ships.

Structural steel shapes, plates, and bars, galvanizing of

Stud bolts

552,554

Stuffing tubes, bulkhead...

Submarine net chain

Substations, electrical, outdoor
Substructures, steel, for tanks. (See Towers, tank.)

Sucker-rod gages, oil well.

Sugar boxes, metal

Supports, shelf.

Surgical beds.

Suspensions, trolley

(sizes) - 964

Switches, railroad

Swivels, chain .

Symbols, engineering

magnetic testing

plumbing

\section{$\mathbf{T}$}

T Hinges slots, their bolts, nuts, tongues, and cutters

Tables, dispatchers' laundry

Tableware, silver silver-plated

Tacks, basket (sizes)

bill posters

brass

cobblers' (sizes)

copper

cut (sizes)

double-pointed

galvanized

gimp.

hide (sizes)

lace (sizes)

saddle.

shoe.-

testing of

thumb

trimmers' (sizes)

trunk (sizes)

upholsterer's

Tags, zinc.

Tamping bars.

Tank heating equipments

Tank hoops, metal (See also Plates, iron; Plates, steel.)

chromium stee

flammable liquid. (See Tanks, oil.)

flushing, flushing mechanism for.

gasoline. (See Tanks, oil.)

hot water

iron.

oil, iron

oil, iron
steel
pressure, steel

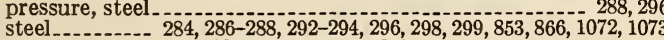

substructures for. (See Towers, tank.

(See also Tanks, hot water.)

Tap bolts.

sizes

Tap drill sizes

522,615

Tape, railway car wheel

Taps, screw thread.

Tea pots.-

Tea strainers

Tea trays, sheet metal

Tees, pipe. (See Fittings, pipe.)

(tructural.)

Tempering of steels. (See Heat treatment of steel.)

Tension test specimen,

Terms used in specifications. (See Definitions of terms.)

Terneplates, gage for

method of test

roofing

Ternes. (See Terneplate.)

Test, method of (air brake valves)

(boiler material)

(castings, gray-iron)

(chain)

iron)

(chain shackles)

(chromium plating)

(compression and tension)

(copper rods, bars, shapes, plates, sheets, strips)

(galvanizing)

(galvanizing of nails)

(galvanizing of sheets)

(galvanizing of wire rope)

(hardness)

(lead coating on sheets and plates)

(magnetic properties of iron and steel)

(micrographs, preparation of).

(nails)

(nickel plating on brass)

(pipe, cast-iron)

(pipe, copper)

(pipe, culvert, cast-iron)

(pipe, culvert, corrugated-metal)

(pipe, iron)

(radiographic testing of metal castings)

(screws)

(sherardizing of iron and steel)

(ship materials)

(stoves and appliances, gas)

(tacks)

(tension and compression)

terneplates and tin-coated sheets)

(test specimens)

(testing of metals)

(testing machines)

(thermal analysis of steel)

(tin coating on sheets and plates)

(valves, air brake).

(wheels, railway car, cast-iron)

(wire rope)

ar, arbitration, cast-iron

Test specimen, iron and steel

tension, cast-iron

$636,637,640$

640
209
205

205

215
5

5
172

$60,61,66,69,71$ $67,68,571$

244
168

49,50

64

sting, definitions of terms relating to magnetic testing _._. 13,18 definitions of terms relating to methods of testing metallic materials (Brinell hardness testing) (compression testing) (tension testing)

metallographic, (definitions of terms) iron and steel-1-1 and alloys

Testing apparatus, sieve... 1227

Testing machine, coupler and axle...

drop, railroad materials

Testing machines, method of verification of
Testing screens.-. brass

Testing sieves.

Thermal analysis of steel.

Thimbles, cables, aeronautic... 183 manila rope...

rope, wire

Thread gage blanks.

Thread gages...

A cme

fre-hose coupling

hose, welding and cutting torch._. 936

nut._-_

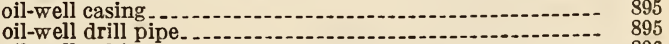

oil-well drill pipe.

pipe

12-pitch-thread series.

Threads, casing, oil-well

machine screw. (See Threads, screw, machine screw.)

pipe. (See Threads, screw, pipe.)

screw, Acme

drill-tool

hose coupling 
Page

Threads, screw, machine screw screw, nut pipe.-. gages for

Thumb latches

Thumb tacks

Tie plug punch.

Tie-rods, track.

Timber tongs

Timber tongs.... , antimony, copper, and lead alloys, chemical analysis of.- 1317

roofing - - standard sample.

Tin ingots, tubes, etc. (See Ingots, tin; Tubes, tin; etc.)

Tin-clad doors. (See Doors, metal clad.)

Tin-coated sheets, (See Sheets, tin-coated.)

Tinners' rivets, steel

Tinners shears.

Tin-plate gage

Tires, railway car wheel, steel. 817 gages for.-

Toggles, chain

Toilet and dressing room inclosures

Toilet stands, sheet metal.

Tolerances on dimensions and weights of iron and steel bars

Tolerances on dimensions and weights of iron and steel sheets

Tolerances on dimensions and weights of sheets, plates, bars,

etc. (See also Sheets, steel; Plates, steel; etc.) Tolerances on dimensions and weights of steel plates, shapes,

Tongs, hand tool (sizes)
tie.

timber

Tool steel, heat treatment of

Tool steel bars. (See Bars, tool, steel.)

Tools, blacksmith (sizes) drill (oil well), thread form for.

packing, journal box

pneumatic. track.

Tooth form, spur-gear.

$\begin{array}{ll}\text { welding and cutting, thread gages for hose connections } & 891 \\ \end{array}$

Towers, tank, steel .

Toy coal hod.

Track adzes.

Track bolts

Track bolts

Track castings, iron. (See Castings, track, iron.)

steel. (See Castings, track, steel.)

Track chisels.

Track forgings. (See Forgings, track.)

Track maul-

Track punches

Track punches

Track tie rods

Track work, special

bolted

cast steel hard center construction.

iron-bound hard center.

materials for.

rail-bound insert type

Transfer cases. (See Cases, file.)

$7 \overline{2}, \overline{7}, 75,76$

Transmission shafts. (See Shafts, line.)

Transom butt hing

Transom chains

Transom eyes.

Transom lifters

Transom operator

Transoms, metal (sizes)

Traps, drain

plumbing

Tray plug, laundry tray

Trays, tea, sheet metal

Trim, door, metal (sizes and types)

Trimmers' tacks (sizes)

Trimmings, plumbing

water-closet and bath inclosur

Triple-valve for air brakes, test of

Trolley bracket arms.

Trolley cross span steady......

Trolley crossings.

Trolley ears.

Trolley feed wire clamp

Trolley frogs.

Trolley hangers.

Trolley oversupport rods

Trolley pole bracket steady

Trolley pullover

Trolley spacer links $310,901,964$
$350,352,353,356,357$
$-9-964,969$ 964,969
$-360,570$
Trolley suspensions

Trolley wheels.

Trolley wire splicers

Trowels.

Truck bolsters, railway car

gages for

Truck side frames, railway car

Trucks, railway car, arch bars for

(gages for truck sides)

pedestals for

Trunk nails (sizes)

Trunk tacks (sizes)

Tubes, alloy steel

boiler, brass......

boiler, copper.-184

brass - _._._._- 1216, 1219-1223, 1239

chromium steel, for acid tank cars

condenser, admiralty metal. (See Tubes, condenser, brass.)

ferrules for (brass)

1219-1222

Muntz metal. (See Tubes, condenser, brass.)

conduit terminal.

copper

method of test

gas fixture

-1239
444

naval brass. (See Tubes, brass.)

phosphor bronze.

steel.

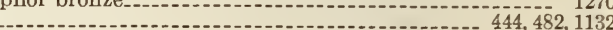

Tubing. (See also Tubes.)

screw-thread gages for

$\begin{aligned} & \text { stay bolt, steel } \\ & \text { ubing clips (electrical) }\end{aligned}$

Tubing clips (electrical)
Tubs, bath. (See also Baths.)

cast-iron

colors for

toy, wash

Tungsten metal, chemical analysis of

Tungsten powder

Tungsten steel, chemical analysis of ..... 1111

Tungsten steel bars. (See Bars, tungsten steel.)

Turbine bucket wheels

Turn, cupboard

locker

aeronautic_._.

Turnbuckles for blinds

Turnbuckles (sizes)

$\begin{array}{ll}\text { Turners, cake } & \\ \text { Twist link chain } & \end{array}$

Umbrella stands, sheet metal

Union pipe fittings

Unions, pipe

Upholsterer's tacks
Urinals, flushing mechanism for

Vacuum oven, constant temperature..._- 867

Valves. (See also Cocks.)

acid tank car, chromium steel castings for............. 1134

air brake, method of test

angle. (See Valves, pipe.)

bronze castings for

carbonated water and sirup. 491

check

rubber facings for

chlorine container-10 507

compression stop

flammable liquid._- 509

flushing -

flushing tank cylinder

gas shut-ofi

gasoline hose

gate. (See Valves, pipe.)

globe. (See Valves, pipe.)

gray-iron castings for

casting 502,509

mixing, shower bath

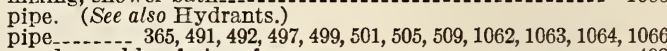

dry, rubber facings for

$\begin{array}{ll}\text { dry, rubber facings for } & \\ \text { (sizes) } & \end{array}$

radiator

release air brake

safety, boiler

locomotive.... 492

(sizes) 
Valves, steel castings for street washer sirup (carbonated beverages)

triple, air brake, test of

(See chemical analysis of

Variations in dimensions, weight, etc. (See also Tolerances on dimensions, weight, etc.)

Vehicle and harness hooks

Vessels, unfired pressure. (See Tanks, pressure.)

Vise clamps

Vises

Wardrobe locks.

W

Warm-air furnaces, gas, house heating house heating -

Warm-air heating systems for house heating

Warps, wire ropes for

boards

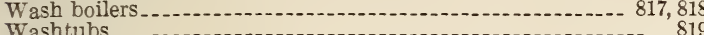

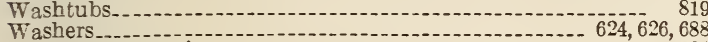

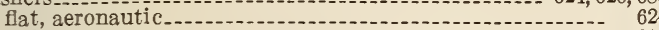
lock plain

Watchcases -... 1304

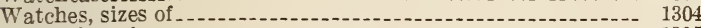
testing of

Water, carbonated, pipe fittings for

Water-closet floor flanges

Water-closet inclosures, trimmings for

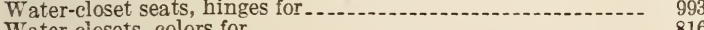

Water closets, colors for -....-..- 816 flushing mechanism for

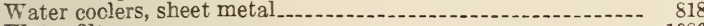

Water filters, pressure

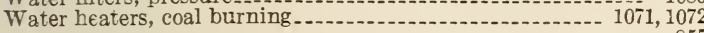

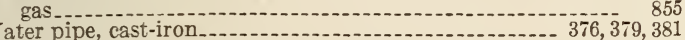

Water pipe flanges, automobile

Water pots $-1-\ldots$

Water tanks, hot water

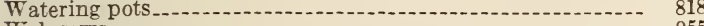

Web saws

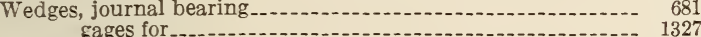
(sizes)

Weights, carat_.-...-. 1298 sash

Welding electrodes, iron

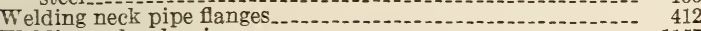

Welding rods, aluminum aluminum alloy aluminum alloy -

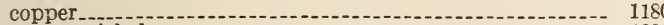

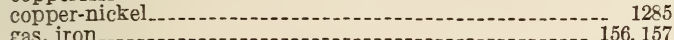
gas, iron $-1 .-156,157$ manganese bronze........- 1266 steel

Welding torches, screw thread gages for hose connections...-.- 936

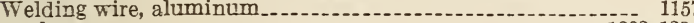
brass $-1-1.1-1203,1334$ iron and steel-ar

Well drilling equipment. (See Oil well.)

Wheel contour measuring tape

Wheels, crane track, steel--ive. (See Wheels, railway car.)
locomotive.

railway car, cast-iron.................... 649,653, 654, 656, 661, 775 gage for cast steel

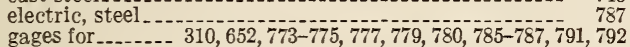
gages for --1-_-- 310, 652, 773-775, 777, 779, $780,785-787,791,792$ steel steel tire for trolley trolley
Window cleaners belts and anchors

Window fasteners

Window lifts ............

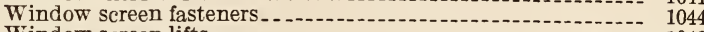

Window screen lifts $-1 \ldots$

Window screen wire cloth

Window stop adjuster

Windows. (See also Casements.)

Windows. (See also Sash.)

Windows, steel

Wire, aluminum. (See also Wire, welding, aluminum)

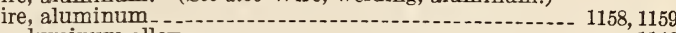

aluminum alloy -

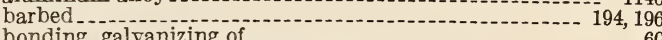

brass. (See also Wire, solder, copper zinc.)

(See also Wire, welding, brass.)

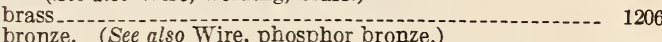

bronze. (See also Wire, phosphor bronze.)

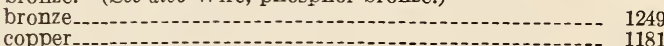

(rods for drawing into wire)

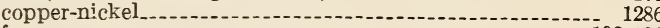

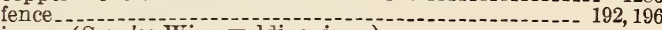

iron. (See also Wire, welding, iron.)

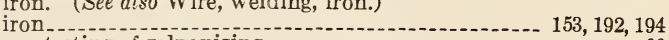
testing of galvanizing manganese bronze messenger. (See Rope, wire, messenger.)

nickel-ackel-chromium ${ }^{-}$

phosphor-bronze, for springs

reinforcement, steel

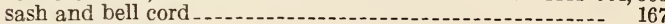

solder, brazing

solder, copper-zinc-silver-....... 1335, 1339 tin-lead -...-...-...- 1330

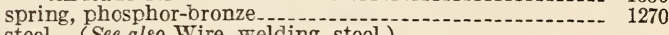
steel. (See also Wire, welding, steel.)

steel tding. (See also Rods, welding.)

aluminum -.--1.-- 1157

brass

(electric), iron and steel -

(gas). (See also Rods, welding, (gas).)

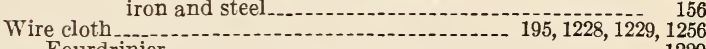

Fourdrinier -

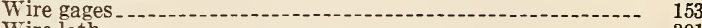

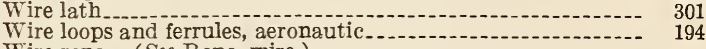

Wire rope. (See Rope, wire.)

Wood screws

brass

Whoodruff key way cutters.

Woodruff key ways for shafts

Woodruff keys ays for sha

Wood workers' chisels, gouges, and slicks

Wrecking bar -.--

Wrench openings

Wrought-iron, definitions of terms relating to

Wrought-iron sheets, plates, etc. (See Sheets, iron; Plates, iron; etc.)

$\mathbf{Y}$

Y branch, pipe. (See Fittings, pipe.)

Yokes, coupler, railway car

Ys, pipe. (See Fittings, pipe.)

\section{$\mathbf{Z}$}

Zine, battery, gravity

chemical analysis of

standard sample

Zinc coatings. (See Galvanizing.)

Zinc ore, standard sample

Zinc plates, strips, etc. (See Plates, zinc; Strips, zinc; etc.)

Zinc plating. (See Galvanizing.)

Zinc-coated (galvanized) steel sheets. (See Sheets, galvanized.) 


